eCommons@AKU

April 2006

\title{
The diagnostic yield of various tests for Helicobacter pylori infection in patients on acid reducing drugs
}

javed Yakoob

Aga Khan University, javed.yakoob@aku.edu

Wasim Jafri

Aga Khan University, wasim.jafri@aku.edu

ZAbbas

Aga Khan University, zaigham.abbas@aku.edu

Shahab Abid

Aga Khan University, shahab.abid@aku.edu

Muhammad Islam

Aga Khan University, muhammad.islam@aku.edu

See next page for additional authors

Follow this and additional works at: http://ecommons.aku.edu/

pakistan_fhs_mc_med_gastroenterol

Part of the Gastroenterology Commons

\section{Recommended Citation}

Yakoob, j., Jafri, W., Abbas, Z., Abid, S., Islam, M., Hamid, S., Shah, S. (2006). The diagnostic yield of various tests for Helicobacter pylori infection in patients on acid reducing drugs. Gastroenetrology, 130(4), A-570-A-570.

Available at: http://ecommons.aku.edu/pakistan_fhs_mc_med_gastroenterol/129 
Authors

javed Yakoob, Wasim Jafri, Z Abbas, Shahab Abid, Muhammad Islam, Saeed Hamid, and Syed H Shah 
Endoscopic Diagnosis of Early Gastrointestinal Graft-Versus-Host Disease William Ross, Alexander A. Dekovich, Karen R. Cleary, Shubra Ghosh, Jeffrey H. Lee, Daniel R. Couriel

BACKGROUND: Gastrointestinal (GI) graft-versus-host disease (GVHD) is a major cause of morbidity and mortality following allogenic hematopoeitic stem cell transplantation (HSCT). Diagnostic yield of endoscopic biopsy of various GI tract segments as well as the correlation between endoscopic findings and histology are currently debated. METHODS: All allogenic HSCT patients from July 2002 to Dec. 2004 who underwent same day esophagogastroduodenoscopy (EGD) and flexible sigmoidoscopy (FS) in the first 100 days post-transplant were studied. All patients were on prophylaxis for GVHD. There were 72 patients with same day biopsies from the stomach, duodenum and rectosigmoid. Endoscopic findings were recorded. Pathological specimens were reviewed by a single pathologist for possible GVHD. RESULTS: Fifty-four patients had histologic evidence for GHVD and 18 did not. Rectosigmoid biopsies were the most sensitive and had the highest negative predictive value (NPV) at $92.5 \%$ and $81.8 \%$ respectively. Gastric biopsies were the least sensitive with the lowest NPV at 57.4\% and $43.9 \%$ respectively. Duodenal biopsies were intermediate at $66.7 \%$ and $50 \%$ respectively. Agreement between gastric and duodenal biopsy findings was good with a kappa score of .665. However, agreement between UGI sites and rectosigmoid biopsies was only fair with kappa score of .402 for stomach and .471 for duodenum. Correlation between endoscopic findings and histology were poor in the stomach and rectum, p values of 404 and 267 respectively. However, the degree of duodenal injury was correlated with histologic evidence for GVHD with $\mathrm{p}=.008$. However, 12 of 35 patients with duodenal GVHD had an endoscopically normal duodenum. Symptoms were not help in selecting those patients most likely to have GHVD. Of the 49 patients with nausea, vomiting and diarrhea $77.5 \%$ had GI GVHD. Of the 14 with diarrhea only, $78.6 \%$ had GVHD. Of the nine with vomiting only, $62.5 \%$ had GVHD. Presenting symptoms did not alter the enhanced sensitivity of the rectosigmoid biopsies. CONCLUSIONS: Rectosigmoid biopsies was the most sensitive and had the highest negative predictive value for the diagnosis of GVHD in the first 100 days post-HSCT compared to upper GI sites. Presenting symptoms were not helpful in determining which portion of GI tract was most likely to yield a diagnosis. The degree of endoscopic injury was poorly correlated with presence of GVHD except in the duodenum.

Impact of Double-Balloon Endoscopy On Clinical Management of Obscure Gastrointestinal Bleeding

Keigo Mitsui, Shu Tanaka, Akihito Ehara, Tsuyoshi Kobayashi, Yoshihisa Sekita, Tsuguhiko Seo, Masaoki Yonezawa, Kazuhiro Nagata, Yoshiaki Shibata, Atsushi Tatsuguchi, Shunji Fujimori, Teruyuki Kishida, Katya Gudis, Choitsu Sakamoto

Background: Obscure gastrointestinal bleeding (OGIB) has several difficulties in clinical management. Recently, capsule endoscopy (CE) is exertive tool in the diagnosis of OGIB. However, its diagnostic yields are often equivocal, and the reliability of a definitive diagnosis depends a lot on further diagnostic procedures, including push or intraoperative enteroscopy and laparotomy. Double-balloon endoscopy (DBE) enables us to observe the entire small intestine without laparotomy. DBE is a promising tool for diagnoses and treatments of OGIB and may complement a diagnosis of CE. However, there have been a few reports concerning its clinical benefits and safety in OGIB. In addition, a favorable management of the enteroscopically negative OGIB (EN-OGIB) patients is still unknown. Aims: To determine the value of DBE in diagnosis and therapy of OGIB and analyze the management of EN-OGIB. Patients \& Methods: 59 consecutive patients of OGIB underwent DBEs between June 2003 and November 2005 at Nippon Medical School Hospital, Tokyo, Japan. We used EN-450 P5 and T5 double-balloon endoscopes (Fujinon, Japan). We evaluated patient characteristics, clinical manifestation, a positive finding rate, diagnostic yields, therapeutic procedures, complications, outcomes, and outcomes of EN-OGIB in 1 year follow-up. Results: Patients were 24 females and 35 males. Mean age was 58.6 (19-82) years old. Mean follow-up term was 371 (21-862) days. Hematochezia, melena, and iron deficiency anemia were in 22, 22, 15 cases, respectively. Positive findings were observed in $39(66.1 \%)$ of 59 cases. In positive finding rate, ongoing bleedings (100\%; all of 10 cases) were higher than discontinuation of bleedings ( $55.1 \%$; 27 of 49 cases). Diagnostic yields were tumor, ulcer, angiodysplasia, stenosis, and miscellaneous in $14,13,6,3$, and 3 cases. 7 (58.3\%) of 12 cases of tumor were confirmed pathologically diagnoses before surgical treatments. One case underwent polypectomy. All the 6 patients (100\%) of angiodysplasia were performed the cauterization. DBE was a well-tolerated and no complication was encountered. 37 (94.9\%) of 39 patients had no recurrence of bleeding for one year. In 2 cases of malignancies, their bleedings was continued. In EN-OGIB cases for one year follow-up, only one patient underwent a push enteroscopy at another hospital a month later, and a bleeding angiodysplasia was detected. The other patients of EN-OGIB have been still in hemostasis. Conclusions: DBE enables scrutiny of the entire small bowel, tissue sampling, and therapeutic intervention in OGIB patients. Diagnoses using DBE lead patients to desirable outcomes. A rebleeding risk of ENOGIB patients is low.

Extensive Gut Mucosal Involvement in Diarrhea-Prone IBS Patients Disclosed By Jejunal Mast Cell Infiltration

Mar Guilarte, Javier Santos, Ines de Torres, Carmen Alonso, Laura Ramos, Maria Vicario, Francesc Casellas, Cristina Martinez, Esteve Saperas, Juan Ramon Malagelada

Background: Stress-induced mast cell activation has been associated with both symptom onset and severity in irritable bowel syndrome (IBS). Furthermore, IBS patients show increased mast cells numbers in distal ileum/colonic mucosa. Upper gut symptoms are common in IBS. However, mast cells have not been thoroughly evaluated in the proximal gut of these patients. Methods: Jejunal biopsies by Watson's capsule, aspiration of intestinal fluid and a blood sample were obtained in 20 diarrhea-prone-IBS (D-IBS), 12 of them suffering also from dyspepsia (Rome II), and 14 healthy volunteers (H). Psychological stress (Holmes-Rahe
Scale) and depression (Beck's questionnaire) were evaluated at baseline in all participants. Biopsies were processed for $\mathrm{H} \& \mathrm{E}$ staining and scored for mucosal inflammation (1: normalto- 3: criptitis \& PMNs). In addition, immunohistochemistry with CD117 (c-kit) was used to evaluate mast cells in the lamina propria. Mast cells were blindly counted in 8 noncontiguous high power fields (HPF)/sample and tryptase levels measured in both intestinal fluid and serum. Food and respiratory allergy was excluded in all participants. Results: D IBS patients showed higher psychological stress (D-IBS:205 \pm 113 vs. H:112 $\pm 99 ; \mathrm{p}=0.007$ ) and depression levels (D-IBS:8.3+7.4 vs. H:2.8 $\pm 3.7 ; \mathrm{p}=0.007$ ) at the time of biopsies, compared to healthy. Jejunal mucosa of both $\mathrm{H}$ and D-IBS appeared normal on H\&E staining with no additional differences on histological inflammatory score (D-IBS: $1.3 \pm 0.47$ vs. H:1.2 \pm 0.43 ). In contrast, D-IBS showed a marked increase in jejunal mast cell numbers (D-IBS: $34 \pm 9.3$ mast cells/HPF; H:14.3 4.4 mast cells/HPF; $<<0.0001$ ), a finding paralleled by highe tryptase levels in jejunal fluid (D-IBS: $0.41 \pm 0.38 \mu \mathrm{g} / \mathrm{L} ; \mathrm{H}: 0.09 \pm 0.11 \mu \mathrm{g} / \mathrm{L} ; \mathrm{p}=0.014$ ) although not in serum (D-IBS: $5.91 \pm 1.31 \mu \mathrm{g} / \mathrm{L} ; \mathrm{H}: 5.49 \pm 2.32 \mu \mathrm{g} / \mathrm{L} ; \mathrm{p}=\mathrm{ns})$. Tryptase release correlated with depression scores $(r=0.51 ; p=0.025)$ and mast cell numbers with stress $(r=0.35 ; p=0.05)$. However, upper gut symptoms were not associated to gender, mast cell numbers, tryptase release, stress or depression. Conclusion: Psychologically-related mast cell hyperplasia and activation, a heretofore unravelled profile in the upper gut mucosa of IBS, may be a helpful diagnostic tool for D-IBS patients.

\section{0}

Effect of Nitazoxanide in Treating Rotavirus Gastroenteritis in Hospitalized Pediatric Patients

Jean-Francois Rossignol, Mona Abu Zekry

Rotavirus is a leading cause of severe, dehydrating gastroenteritis among children worldwide It is estimated to be responsible for 500,000 deaths each year accompanied by enormous morbidity, medical and social costs. There is no effective treatment for rotavirus infection Renewed efforts to develop a safe and effective rotavirus vaccine are ongoing. Nitazoxanide is a gastro-intestinal anti-infective approved in the United States for treating diarrhea caused by Cryptosporidium parvum and Giardia lamblia in patients down to 12 months of age Nitazoxanide is active in vitro against a broad range of viruses. With this background, we evaluated the activity of nitazoxanide against rotavirus in vitro and in a randomized, doubleblind, placebo-controlled clinical study. Methods: Nitazoxanide and its circulating active metabolite, tizoxanide, were evaluated for activity against rotavirus (Simian rotavirus SA11) in MA104 cell cultures. A randomized, double-blind, placebo-controlled clinical study was conducted in 50 pediatric patients hospitalized with rotavirus gastroenteritis at the Cairo University Children's Hospital. Rotavirus was diagnosed by stool test using ELISA (Ridascreen $囚$, R-Biopharm AG). Patients were randomized to receive nitazoxanide suspension $(100 \mathrm{mg} / 5 \mathrm{~mL}) 7.5 \mathrm{mg} / \mathrm{kg}$ BID for three days or an equivalent quantity of a matching placebo suspension. All patients received optimal supportive care including oral or intravenous rehydration therapy. Administration of the study medication, food intake, adverse events, and number and consistency of stools were monitored and recorded daily. The time from first dose to resolution of illness was recorded for each patient and compared by treatmen group using a survival analysis and Wilcoxon test. Results: In vitro, nitazoxanide and tizoxanide inhibited replication of Simian rotavirus SA-11 with IC50s of $0.5 \mathrm{\mu g} / \mathrm{mL}$ and selectivity indices $>100$. Phase contrast microscopy images showed that the compounds were cytoprotective. Further mechanistic studies indicated that the drug has a selective effect on the synthesis of rotavirus protein VP7. The clinical trial enrolled 50 pediatric patients from 4 months to 7 years of age (median age $=10$ months). The median time from first dose to resolution of illness was 31 hours for the nitazoxanide-treated patients compared to 75 hours for patients randomized to the placebo group $(\mathrm{P}=0.0137)$. There were no significant adverse events. Conclusion: Our study indicates that nitazoxanide therapy reduces the duration of rotavirus diarrhea in hospitalized pediatric patients. Further studies are warranted to confirm our findings.

11

HLA Typing in Patients with Whipple's Disease

Federico Biagi, Myriam Martinetti, Carla Badulli, Michele Di Stefano, Jonia Campanella, Gino R. Corazza

Introduction. Whipple's disease (WD) is a systemic chronic-relapsing disorder due to both environmental and host factors. Although Tropheryma Whipplei is known to be the enviromental factor, the host factors are still unknown. Probably due to the rarity of this condition, the association between HLA and WD was studied in only small samples of patients. The few papers that have been published so far have given conflicting results $[1,2]$. The EU funded European Study Group on WD (QRLT-2001-01049) collected DNA and tissue samples from sixty western European WD patients. Methods. HLA class I and II alleles were identified, at low resolution level, with sequence specific primers and PCR using commercial kits (Pel Freez,Wi,USA). Gene frequencies obtained in patients were compared with those published in German healthy population using EPI5 statistical package. Results. DRBI*13, B*44 and $\mathrm{CW}^{*} 05$ alleles were shown to be significantly more frequent in WD patients. $\mathrm{CW}^{*} 02$ allele was shown to be significantly more frequent in controls. Moreover, odd's ratio was 2.22 for $B^{*} 44$ allele alone $(p=0.04)$ and was 2.8 for $D R B 1 * 13$ allele alone $(p=0.01)$ However, odd's ratio incresed to $12.4(\mathrm{p}=0.000017)$ if we consider the presence of both B*44 and DRB1*13 versus none of them. Conclusions. This is the largest HLA study ever performed in WD patients. B*44 and DRB1*13 alleles may act as risk factors related to the disease. $\mathrm{B}^{*} 44$ and $\mathrm{DRB}^{*} 13$ are independent to each other but if they are present together they enhance the risk factor. The $\mathrm{Cw}^{*} 02$ allele could be a protection factor towards the disease. 1. Feurle GE et al. Eur J Clin Invest 1979;9:385-9. 2. Olivieri I et al. J Rheumatol 2001;28:1294-7. 
Homozygote Mutations of Neurogenin-3 in Children with Congenital Generalized Malabsorptive Diarrhea and Dysgenesis of Enteroendocrine Cells Jiafang Wang, Galen Cortina, Vincent Wu, Bob Tran, Ming-Jer Tsai, Jang-Hyeon Cho, Marvin Ament, William Treem, Ivor Hill, Jorge Vargas, Douglas Farmer, Laurie Reyen, Martin Martin

Background: The neurogenin family of proteins are basic-helix-loop-helix transcription factors that play instrumental roles in cell fate determination of neuronal and endocrine cells. Neurogenin-3 (ngn-3) is expressed in endocrine progenitor cells and is essential for endocrine cell development in the pancreas and intestine. A novel autosomal recessive disorder characterized by severe generalized malabsorption and a paucity of enteroendocrine cells is described. Methods: Genomic DNA from three unrelated patients with rare enteroendocrine cells were screened for mutations of the ngn-3 gene, and then assessed for their ability to alter ngn-3 function using in vitro and in vivo assays. Results: The clinical characteristics of three patients were remarkably similar and characterized by severe generalized malabsorption that required prolonged parental nutrition. In all patients, multiple intestinal biopsies showed that the number of chromogranin A-positive enteroendocrine cells was dramatically reduced, while the Paneth, goblet and absorptive cells were of normal appearance and number. Molecular analysis identified two homozygous mutations in the ngn-3 gene. Each alters a strictly conserved arginine residue at position 93 or 107 in the DNA-binding domain of all neurogenins in species ranging from human to $C$ elegans. Transient transfection studies in non-ngn-3 expressing HeLa cells revealed that both mutant-ngn-3 proteins failed to augment promoter activity of NeuroD, a downstream target of ngn-3. DNA binding assays confirmed that both mutations markedly decreased the ability of ngn-3 to bind to an Ebox element located in the NeuroD promoter. Ectopic injection of wild-type ngn-3 mRNA in Xenopus embryos induced endogenous NeuroD expression, while mutant ngn-3 transcripts failed to change NeuroD levels. Conclusions: A novel disorder characterized by malabsorptive diarrhea and lack of intestinal enteroendocrine cells is presented that represents the first description of a loss-of-function mutation in humans of any member of the neurogenin family of proteins. We propose to name this disorder enteric dyspinetic anendocrinosis.

\section{3}

Colorectal Cancer Survival in Ulcerative Colitis Is Associated with Prior Use of 5-Aminosalicylic Acid But Not Surveillance Colonoscopy Fernando Velayos, Edward V. Loftus, Tine Jess, William J. Tremaine, William J. Sandborn

Background: Colorectal cancer is a serious consequence of long standing ulcerative colitis (UC). Unlike in sporadic cancer, prognostic factors are not well-described and the mortality benefit of surveillance colonoscopy is debated. In sporadic colorectal cancer, decreased tumor expression of COX-2 is associated with improved survival. These data suggest chronic 5-aminosalicylate use may be a rational and novel prognostic factor in colitis-associated cancer. Aim: To test if prior 5-ASA use and surveillance colonoscopy are associated with improved cancer survival in UC. Methods: The study population consisted of all patients with a minimum of 10 years of ulcerative colitis who had colorectal cancer diagnosed between 1976-1999 and were evaluated at least 1 year prior to the diagnosis of cancer (n= 76). 5-ASA use for two years prior to the diagnosis of colorectal cancer was categorized as regular ( $>75 \%$ use), intermittent $(25-75 \%)$, or infrequent $(<25 \%)$. Performance of a dysplasiafree surveillance colonoscopy during this same time period was recorded. Five-year colorectal cancer mortality using the Kaplan-Meier method was derived using medical records and death certificates routinely obtained by our institution. The hazard ratio (HR) between the exposures of interest and cancer mortality were determined using Cox proportional hazards regression adjusted for disease extent, duration, age, year of diagnosis, primary sclerosing cholangitis, family history of cancer, and immunomodulator or prednisone use. Results: Increasing 5-year colorectal cancer mortality was associated with decreasing 5-ASA use (10\% regular vs. $35 \%$ intermittent vs. $63 \%$ infrequent) (log rank $\mathrm{p}=0.002$ ) and the absence of a surveillance exam ( $11 \%$ vs. $34 \%$ ) (log rank $\mathrm{p}=0.2$ ). In the adjusted model, both regular (HR=0.1; 95\%CI:0.01-0.4) and intermittent 5-ASA users (HR=0.3;95\%CI:0.1-0.8) had lower cancer mortality compared to infrequent users. The HR associated with a normal surveillance exam was 0.8 (95\% CI: 0.1-6.7). Regularity of 5-ASA use did not correlate with cancer stage, but both variables were independent predictors of mortality. Conclusion: This is the first study in UC patients to suggest a dose-dependent reduction in 5-year colorectal cancer mortality associated with prior 5-ASA use. Regularity of use did not correlate with cancer stage. Given that prognostic factors in colitis-related cancer are not well described, further research may help to identify novel patient and biologic characteristics to explain our findings. The lack of mortality benefit associated with a dysplasia-free surveillance colonoscopy within 2 years is notable, but likely due to wide variation in technique

\section{4}

Increased Inflammatory Activity Is An Independent Risk Factor for Dysplasia and Colorectal Cancer in Ulcerative Colitis: A Case-Control Analysis with Blinded Prospective Pathology Review

David T. Rubin, Dezheng Huo, Jami A. Rothe, Jeremy Hetzel, Mina Sedrak, Nicole Yadron, Alana Bunnag, John Hart, Jerrold R. Turner

Patients with ulcerative colitis (UC) are at risk for dysplasia and colorectal cancer (D-CRC). Some risk factors, including duration and extent of disease, presence of primary sclerosing cholangitis (PSC) and FH of CRC are known but have no potential for therapeutic intervention. A recent report of increased D-CRC risk with active inflammation in UC was based primarily on patients with pan-UC. To evaluate the risk of D-CRC conferred by inflammatory activity in UC of various extents, we performed a blinded case-control prospective review of biopsies obtained from 1994 to 2005. METHODS: The University of Chicago IBD Endoscopy Database was used to identify all UC patients with D-CRC. Controls were all database patients who matched D-CRC patients by disease extent, disease duration, and age. All biopsies (4742) were scored independently by at least 1 of 2 GI pathologists blinded to patient outcome using a 6 point inflammatory activity scale ranging from uninvolved to erosions; interobserver reproducibility was $86 \%$ ( $\mathrm{k}=0.52)$. Detailed information was collected for 5-ASA and immunomodulator adjusted dose; steroid and folic acid use; smoking status; PSC; and FH of CRC. Relationship between inflammatory activity and risk of D-CRC was assessed by conditional logistic regression in univariate and multivariate models, expressed as odds ratios (OR) with $95 \%$ confidence intervals (CI). RESULTS: $146 \mathrm{UC}$ patients (56 cases, 90 controls) were matched for extent of disease (84\% pancolitis), disease duration (mean $20.2 \mathrm{yrs}$ ), and age (mean $47.5 \mathrm{yrs}$, range 19-70). Cases and controls were similar in numbers of procedures (2.1 vs 2.4) and biopsies ( 30 vs 34), steroid exposure, 5-ASA exposure (cumulative and daily dose), smoking status, and FH of CRC, but differed in immunomodulator exposure ( $14 \%$ vs $33 \% \mathrm{p}=0.02)$ and PSC prevalence $(12.5 \%$ vs $3.4 \%$ $\mathrm{p}=0.09)$. Mean inflammatory activity differed significantly between cases (2.0) and controls (1.6, $\mathrm{p}=0.013)$. This corresponded to an $\mathrm{OR}$ of 1.71 (95\% CI 1.15-3.18) in univariate analysis. Multivariate analysis, adjusting for FH of CRC, smoking status, and 5-ASA and immunomodulator use also showed increased D-CRC risk with increased inflammatory activity (OR 2.73, CI 1.44-5.18; $\mathrm{p}=0.002$ ). Multivariate subset analysis of pancolitis patients was similar (OR 2.79, CI 1.35-5.78; $\mathrm{p}=0.006$ ). CONCLUSIONS: These data confirm an association between inflammatory activity and D-CRC risk in pan-UC. They also establish that inflammatory activity is an independent risk factor for the development of D-CRC in patients with UC of various extents. This has profound implications for therapeutic goals and endpoints as well as future prevention strategies.

\section{5}

Risk Factors for Colorectal Cancer in Crohn's Colitis: A Case-Control Study Corey A. Siegel, Ifat Kamin, Bruce E. Sands

Background and Aims: Exposures associated with increased risk or protection for colorectal cancer (CRC) have been well described in the setting of ulcerative colitis. Few data exist regarding exposures associated with CRC in patients with Crohn's colitis. The aim of this study was to identify exposures that alter risk of CRC in patients with Crohn's colitis. Methods: This was a retrospective matched case-control study. The Research Patient Database Registry (RPDR) at Massachusetts General Hospital (MGH) was searched to identify cases and controls. The RPDR is a database of all patients seen at MGH since 1990. Cases had a confirmed diagnosis of Crohn's colitis involving at least $1 / 3 \mathrm{rd}$ of the colon and a confirmed diagnosis of colorectal adenocarcinoma. Controls were randomly chosen from the same source population and matched 1:1 for gender, age of diagnosis of Crohn's disease and age of diagnosis of cancer. Information extracted from the medical record included details of their history of Crohn's disease, medication use, indication for colonoscopy, family history and smoking history. Paired univariate analysis was performed to develop an odds ratio for each exposure. Results: Twenty-seven patients were found to have Crohn's colitis and CRC. The median age at diagnosis of cancer was 56. The median duration of Crohn's disease before the diagnosis of CRC was 25 years. Prior small bowel resection had an odds ratio of 0.18 (95\% CI: 0.02-0.83, p = 0.03). Having had a colonoscopy for an indication of screening or surveillance was associated with an odds ratio of 0.21 ( $95 \% \mathrm{CI}: 0.04-0.77, \mathrm{p}=0.02$ ), as opposed to having had a colonoscopy for a change in symptoms that yielded an odds ratio of 4.67 ( $95 \% \mathrm{CI}: 1.30-25.33, \mathrm{p}=0.02$ ). Non-significant trends for a protective effect included prior appendectomy (OR 0.30,95\% CI: 0.05-1.17, $\mathrm{p}=0.10)$ and regular 5-ASA use (OR 0.30, 95\% CI 0.05-1.17, $\mathrm{p}=0.10)$. Smoking history was associated with a 4-fold increased risk for colorectal cancer, but this was not statistically significant (OR 4.00, 95\% CI $0.80-38.67, \mathrm{p}=0.11$ ). Prior immunomodulator, oral corticosteroid, folate or calcium use were neither protective nor risk factors for developing colorectal cancer. Conclusions: We found that both ileal resection and having colonoscopy for an indication of surveillance or screening are protective factors against developing colorectal cancer in the setting of Crohn's colitis. These data underscore the importance of CRC surveillance for Crohn's colitis in addition to ulcerative colitis, and should prompt further study in this area

\section{6}

Use of Immunosuppressants Results in a Higher Incidence of Abnormal PAP Smears in Women with Inflammatory Bowel Disease (IBD)

Sunanda V. Kane, Bahareh Khatibi, Deepa Reddy

Background and Aims: Many women with IBD require immunosuppression for management of their disease. Previous work has shown that immunosuppression leads to an increased prevalence of cervical dysplasia as compared to healthy controls. We aimed to examine the relationship between immunomodulator use and the presence of abnormal cervical histology in women with IBD. Methods: Women with a detailed history of serial Pap smears followed at a tertiary IBD center were recruited. Results of Pap smears were recorded in relation to exposure to immunomodulatory agents including prednisone, azathioprine/6MP, methotrexate and infliximab. Abnormal cytology was stratified by degree of dysplasia and lesions were categorized as "low risk" or "high risk" for cancer according to accepted definitions. Variables of interest included disease, type and duration of immunosuppressant use, smoking status, family history for gynecologic neoplasia, marital status and OCP use. Results from the IBD patients were compared to a published historical control population of similar socioeconomic background. Results: Thirty-five patients with a total of 101 Pap smears were included. There were $5 \mathrm{UC}$ patients and $30 \mathrm{CD}$ patients. The prevalence of an abnormal Pap in a woman with IBD was $35.5 \%$, compared to $14.6 \%$ of controls (Odds ratio 3.1, 1.4-6.5, p= 0.005). Table 1 shows the cytomorphology of the groups: there was an increased risk for low risk lesions (OR 4.6, 3.8-6.8, $\mathrm{p}<0.001$ ) but not for high risk lesions (OR 1.8, 0.6$4.8, \mathrm{p}=0.2)$ as compared to controls. Of those Paps done with at least six months exposure to an immunomodulator, $44.6 \%$ vs. $22 \%$ in the non-exposed patients were abnormal (OR $2.9,1.2-4.1, p=0.02$ ). The cytopathology of all abnormal lesions were consistent with HPV 16, 18 infection. Multivariate analysis did not reveal any differences between the groups when controlled for other variables. Conclusions: Women with IBD have a higher risk of an abnormal Pap smear as compared to healthy controls. Patients with immunomodulator use have a higher risk of an abnormal Pap smear than those not exposed, and is associated with HPV infection. Women with IBD should be included in the American College of Obstetrics and Gynecology screening guidelines for immunocompromised individuals. Table 1 


\begin{tabular}{|c|c|c|c|c|}
\hline & "Low risk" (N) & "High risk" $(\mathrm{N})$ & Normal $(\mathrm{N})$ & Total $(\mathrm{N})$ \\
\hline IBD Exposed $(\mathrm{N}=28)$ & 20 & 9 & 31 & 60 \\
\hline IBD Non-exposed $(\mathrm{N}=7)$ & 3 & 5 & 32 & 41 \\
\hline Control $(\mathrm{N}=749)$ & 37 & 72 & 638 & 749 \\
\hline
\end{tabular}

Abnormal PAP Smear, Cervical Dysplasia and Immunomodulator Therapy in Women with Inflammatory Bowel Disease (IBD)

Thangam Venkatesan, Dawn B. Beaulieu, Vladimir Ferrer, Lydia R. Weber, Judy Podoll Josh F. Knox, Jeanne Emmons, Mazen Issa, Kathy A. King, David G. Binion

Background: Immunosuppressive therapy in organ transplantation is associated with increased risk of neoplasia including cervical cancer. The relationship between cervical dysplasia (cervical intraepithelial neoplasia (CIN)) and cervical carcinoma with immunomodulators in the treatment of IBD has not been defined. Given that 5-7\% of U.S. women have had abnormal cervical cells detected on Pap smear, we characterized the spectrum of cervical dysplasia in women with IBD and its relationship with immunomodulator therapy. Methods: This was a retrospective observational study of female pts treated at an IBD center between 2003-2005. Pts with a history of abnormal Pap smear were identified and data regarding specific IBD diagnosis (Crohn's Disease (CD), Ulcerative Colitis (UC)), disease duration, immunomodulator therapy and duration, smoking history and gynecologic procedures for diagnosis/management of cervical dysplasia were recorded. Results: Among 534 women with IBD ( $74 \%$ CD , 26\% UC) there were 25 pts (mean age $38 \pm 10$ y (SD), IBD duration $12 \mathrm{y}$ ) with a history of abnormal Pap smear ( $5 \%$ of total; $22 \mathrm{CD}, 3 \mathrm{UC}$ ), 8 of whom required more extensive evaluation with cervical conization and loop electrosurgical excision procedure (LEEP). Among IBD women with abnormal Pap, history of tobacco use was $68 \%$ compared with $25 \%$ in the total IBD female cohort ( $\mathrm{p}=0.001$ ). Immunomodulators used in treatment of IBD women with abnormal Pap included azathioprine/6MP (40\%), methotrexate (28\%), infliximab alone or in combination with other agents (44\%), and the mean duration of use was $35 \pm 19$ months (SD). In women with abnormal Pap, $20 \%$ had received no immunomodulators. Among women requiring more extensive cervical diagnosis/treatment, $75 \%$ were active smokers and $86 \%$ were receiving immunomodulators for a mean duration of $55 \pm 22$ months (SD). The majority of women (75\%) had abnormal Pap detected prior to initiation of immunomodulators, while $25 \%$ developed CIN after start of these agents. There were 4 pts who had CIN grades I-III detected and treated with LEEP. All of these pts were active smokers and half had severe dysplasia emerge after immunomodulator initiation. There were no cervical cancers and no hysterectomies during a mean follow-up of $5 \mathrm{y}$. Conclusion: Abnormal Pap smears and CIN occur in female IBD pts at rates similar to those described in the general population. Smoking is significantly increased among IBD women with abnormal Pap. Although no cervical cancer was detected, close gynecologic surveillance and smoking cessation, particularly for those IBD women on immunomodulators, is warranted to prevent disease progression.

18

Serum P53 Antibodies (ABS) in Patients with Ulcerative Colitis (UC) Are Associated with the Development of Colorectal Cancer and Screening of P53 ABS By ELISA Is Useful in Cancer Surveillance Program for UC Patients Nagamu Inoue, Shigeo Yoshizawa, Katusyoshi Matsuoka, Hiromasa Takaishi, Haruhiko Ogata, Yasushi Iwao, Makio Mukai, Tomonobu Fujita, Yutaka Kawakami, Toshifumi Hibi

Purpose: Although molecular mechanisms underlying ulcerative colitis (UC)-associated colorectal cancer (CRC) remains incomplete, mutation of $p 53$ gene and cellular accumulation of p53 protein are frequently detected early in the progression of UC-associated CRC and considered to play an important role in carcinogenesis. For early detection of UC-associated CRC, surveillance program using colonoscopy is recommended in UC patients at high risk. However, poor acceptability for surveillance colonoscopy deteriorates the effectiveness of surveillance program and suitable marker for selecting patients at high risk for the developing neoplasms is needed. Here, we attempted to evaluate the usefulness for measurement of anti-p53 antibodies (Abs) by enzyme-linked immunosorbent assay (ELISA) using sera samples from UC patients with or without CRC. Experimental Design: Sera from 286 patients with UC and 63 healthy controls (HC) were obtained. Serum anti-p53 Abs were detected in duplicate with ELISA method. Immunohistochemical detection of $\mathrm{p} 53$ protein overexpression was performed in 13 UC patients who developed dysplasia or CRC. Results: Serum p53 Ab was positive in $15.0 \%$ (43/286) of UC patients while only one subjects (1.6\%) was positive for $p 53 \mathrm{Ab}(\mathrm{p}=0.0014)$. Even if 8 patients with $\mathrm{CRC}$ were excluded, the positivity of serum p53 Abs in UC group (13.3\%) was still significantly higher than that in HC group ( $\mathrm{p}=$ $0.0063)$. In UC patients, $20.8 \%$ (30/144) of patients with disease duration $\geqq 8$ years were positive for serum p53 Abs while $9.2 \%(13 / 142)$ of patients with duration $<8$ years were positive $(\mathrm{p}=0.0076)$. Six $(75.0 \%)$ of 8 patients with UC-associated CRC were positive for serum 553 Abs, which was significantly higher than that in UC patients without CRC $(\mathrm{p}<0.0001)$. All the UC patients with CRC positive for serum $\mathrm{p} 53 \mathrm{Ab}$ were also positive for p53 staining. The percentage of CRC patients was $2.8 \%$ in whole UC group, $5.1 \%$ in the group of patients with extensive disease and longer disease duration, however, it was $20.0 \%$ in the group of patients positive for serum $\mathrm{p} 53 \mathrm{Ab}$ with longer disease duration while the number of the patients with CRC missed in the group were similar. Conclusions: Serological detection of p53 Abs by ELISA is easy to perform and it can be applied as routine clinical procedure. The present study revealed that serum $\mathrm{p} 53 \mathrm{Abs}$ are associated with the progression of CRC in patients with UC and that utilization of serum p53 Ab positivity improved the positive rate of CRCs in the group, suggesting that serological detection of p53 Abs by ELISA is a complementary method for surveillance colonoscopy by selecting patients at high risk for the development of CRC

Tumour Necrosis Factor $\alpha$ Promoter Polymorphisms: Influence On Phenotype and Severity in Childhood IBD and Regression Analysis of the Relative Contribution to Crohn's Disease Phenotype

Johan van Limbergen, Richard K. Russell, Elaine R. Nimmo, Hazel E. Drummond, Linda Smith, Niall Anderson, David C. Wilson, Peter M. Gillett, Paraic McGrogan, Kamal Hassan, Laurence T. Weaver, Michael W. Bisset, Gamal Mahdi, Jack Satsangi

Background \& Aims: The incidence of childhood IBD in Scotland is rising and is amon the highest worldwide. The tumour necrosis factor $\alpha$ (TNF) gene lies within the HLA class III region of chromosome 6 , a region identified as containing an IBD susceptibility gene in genome wide scans. Our aims were to assess the contribution of polymorphisms -1031T/ $\mathrm{C},-863 \mathrm{C} / \mathrm{A},-857 \mathrm{C} / \mathrm{T},-806 \mathrm{C} / \mathrm{T}$ in the TNF promoter region to disease susceptibility and phenotype in childhood IBD. Methods: 277 patients (male/female 148/129, mean age $( \pm$ SD) 10.46 ( \pm 3.26$)$ years at diagnosis) $(174 \mathrm{CD} / 77 \mathrm{UC} / 26 \mathrm{IC})$ were enrolled. TNF promoter polymorphisms were assessed using direct sequencing. Transmission disequilibrium testing (TDT), case-control analysis with 256 controls, detailed genotype - phenotype analysis and multifactorial analysis were performed. Logistic regression was used to assess the relative contribution of NOD2, OCTN, DLG5 and TNF promoter polymorphisms to CD phenotype Results: By case-control analysis, an association was found between TNF -863A and UC/IC $(\mathrm{p}=0.04$ OR $1.77 \mathrm{CI} 1.01-3.11)$ and the CACC haplotype and IC ( $\mathrm{p}=0.01$ OR $2.46 \mathrm{CI} 1.22$ 4.97). TDT analysis was negative. Haplotype analysis showed a negative association of the CCCC-haplotype with small bowel disease and ileal disease in $C D$ patients $(\mathrm{p}=0.0003$ OR 0.24 CI $0.10-0.59$ and $\mathrm{p}=0.003$ OR 0.31 CI $0.13-0.77$ ), irrespective of NOD2 carriage Negative associations were found between the TCCC- haplotype and BMI $<50$ thcentile ( $\mathrm{p}=$ 0.03 OR $0.59 \mathrm{CI} 0.35-1.00$ ) and inflammatory disease behaviour ( $\mathrm{p}=0.03$ OR $0.50 \mathrm{CI} 0.25$ 1.04). Raised CRP ( $\mathrm{p}=0.01$ OR $0.33 \mathrm{Cl} 0.12-0.93$ ) and hypoalbuminaemia ( $\mathrm{p}=0.04 \mathrm{OR} 0.47$ CI 0.19-1.15) were negatively associated with the CCCC-haplotype. Granuloma formation was negatively associated with the CACC-haplotype ( $p=0.04$ OR 0.53 CI $0.26-1.08$ ). Multifactorial analysis controlling for factors implicated on univariate analysis confirmed the negative association of TNF-1031CC with small bowel disease ( $\mathrm{p}=0.009$ OR $0.02 \mathrm{CI}$ 0.00-0.38) Regression analysis controlling for other susceptibility genes (NOD2, OCTN, DLG5) replicated the protective effect of TNF-1031C on small bowel disease ( $p=0.006$ OR $0.02 \mathrm{C}$ 0.00-0.33). Conclusion: These variant alleles of the TNF promoter polymorphisms have a significant effect on disease location, severity and behaviour in the Scottish early onset CD population. The significant negative association of TNF-1031CC with small bowel disease might explain the milder disease phenotype. The protective effect of TNF-1031CC on smal bowel disease persists when controlling for other genes known to be involved in $\mathrm{CD}$ susceptibility and severity.

20

NODI/CARD4 Insertion/Deletion Polymorphism +32656 and IBD: Influence On Susceptibility and Disease Phenotype in the Scottish Childhood Onset Inflammatory Bowel Disease (IBD) Population and Regression Analysis of the Relative Contribution to Crohn's Disease

Johan van Limbergen, Richard K. Russell, Elaine R. Nimmo, Hazel E. Drummond, Niall Anderson, Linda Smith, David C. Wilson, Peter M. Gillett, Paraic McGrogan, Kamal Hassan, Laurence T. Weaver, Michael W. Bisset, Gamal Mahdi, Jack Satsangi

Background \& Aims NOD proteins are intracellular pattern-recognition receptors that influence Toll-like receptor responses and NF-KB activation. NOD2/CARD15 is a known determinant of susceptibility and need for surgery in CD. The NODl gene lies within the $7 \mathrm{pl} 4 \mathrm{IBD}$ locus. Recently, an association was found between the NOD $1+32656^{*} 1$ deletion variant of a complex del*1/ins*2 polymorphism and an earlier age of onset of IBD. The aim of this study was to assess the influence of NODl+32656 on IBD susceptibility and phenotype at diagnosis and follow-up. Patients and Methods 290 children with IBD aged <l6years at diagnosis (192CD/74UC/22IC), their parents and 318 healthy controls were genotyped using TaqMan. Case-control, transmission disequilibrium testing (TDT) and detailed genotypephenotype analyses were performed. The effect of NODl+32656 genotype on age of onset was assessed with the Mann-Whitney test. Regression analysis was used to assess the relative contribution of NOD1, NOD2, OCTN \& DLG5 variants to CD. Results By case-control analysis, a protective association was found between carriage of NOD $1+32656 * 2$ and IBD (43.8\% v $53.1 \%, \mathrm{p}=0.01$, OR $0.69 \mathrm{CI} 0.50-0.95)$ and IC $(26.1 \%$ v $53.1 \%, \mathrm{p}=0.006$, OR $0.31 \mathrm{CI} 0.12-0.81$ ), but not CD/UC. TDT analysis was negative. The calculated Population Attributable Risk to IBD for NODl+32656*1 was $10.6 \%$. NODl+32656 genotype did not influence age at diagnosis in $\mathrm{IBD}, \mathrm{CD}$ or UC. In $\mathrm{CD}$, on unifactorial analysis of allelic frequency, NOD $1+32656 * 2$ was associated with gastric body disease at diagnosis $(38.7 \%$ v $24.2 \% \mathrm{p}=0.02 \mathrm{OR} 1.98 \mathrm{CI} 1.10-3.55)$ and with perianal disease at follow-up(58.3\% v $21.1 \% \mathrm{p}=0.004 \mathrm{OR} 5.23 \mathrm{CI}$ 1.54-17.80). These findings were confirmed on multivariate analysis ( $\mathrm{p}=0.004$ OR $3.42 \mathrm{CI} 1.47-7.93$ and $\mathrm{p}=0.02$ OR $7.31 \mathrm{CI} 1.31-40.86$, respectively) $\mathrm{NOD} 1+32656 * 1$ carriage was protective against IBD surgery $(84.0 \%$ v $95.7 \% \mathrm{p}=0.002$ OR $0.23 \mathrm{CI} 0.08-0.71$ ), with a stronger effect on drainage than resection. In CD, NOD l+32656*1 carriage was lower in NOD2 variant carriers cf. NOD2 wild type $(85.0 \%$ v $94.9 \%, p=0.01)$ providing evidence for epistasis. After stratification, significant protection against IBD surgery only persisted for NOD2 variant carriers (70.6 v 95.7\%, p=0.008 OR 0.11 CI 0.01-1.04) On regression analysis, only NOD2 (1007fs) and OCTN2 contributed to CD susceptibility $(\mathrm{p}=0.02$ OR $9.99 \mathrm{CI} 1.30-77.08$ and $\mathrm{p}=0.01$ OR $2.73 \mathrm{CI} 1.18-6.33$, respectively). Conclusion NOD $1+32656$ variants are weak determinants of susceptibility to early onset IBD NOD $1+32656$ influences $C D$ phenotype at presentation and at follow-up and interacts with NOD2 in determining CD susceptibility and phenotype.

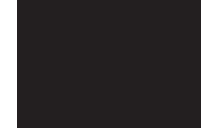


Substantial Differences in Lansoprazole Pharmacokinetics Between Older and Younger Infants and Neonates

Weijiang Zhang, Michael J. Kukulka, Galen Witt, Debra Sutkowski-Markmann, Janine North, Stuart Atkinson

Background: Pharmacokinetic studies were conducted in infants and neonates to establish an appropriate lansoprazole dose to study safety and efficacy in GERD management in pediatric patients up to $l$ year of age. Purpose: Five-day studies were conducted to characterize the pharmacokinetic, pharmacodynamic, and safety profiles of lansoprazole in neonates and infants with clinically evident GERD. Pharmacokinetic data are presented here. Methods: Two phase 1, multicenter, randomized, parallel, open-label, repeated-dose studies were conducted in the United States and Poland. The doses of an investigational formulation of lansoprazole (a microgranule suspension) were $0.5 \mathrm{mg} / \mathrm{kg} /$ day or $1.0 \mathrm{mg} / \mathrm{kg} /$ day in neonates and $1.0 \mathrm{mg} / \mathrm{kg} /$ day or $2.0 \mathrm{mg} / \mathrm{kg} /$ day in infants. Pediatric patients, including 24 infants and 24 neonates (ages were corrected for premature births), received lansoprazole once daily for 5 days. Blood samples (0.2 mL each) were collected on day 1 (hrs $0,1,2,3,4,6,8$, and 12 relative to dosing) and day 5 (hrs 0,2 , and 6 relative to dosing). Results: More than a 5-fold higher dose-normalized lansoprazole plasma total exposure (AUC) was observed in infants with uncorrected age $\leq 10$ weeks compared with infants with uncorrected age $>10$ weeks (Table). The pharmacokinetic parameters from infants $\leq 10$ weeks were similar to those observed in neonates. Conclusions: Lansoprazole pharmacokinetics in pediatric patients older than 10 weeks were substantially different from those in pediatric patients 10 weeks and younger. Additional safety and efficacy studies are necessary to determine appropriate dosing of lansoprazole for infants and neonates.

Mean (CV\%) Preliminary Pharmacokinetic Estimates

\begin{tabular}{|c|c|c|c|c|}
\hline Age Group (n) & $\begin{array}{c}\mathrm{C}_{\max } \\
(\mathrm{ng} / \mathrm{mL})\end{array}$ & $\begin{array}{c}\mathrm{AUC}_{\infty} \\
(\mathrm{ngxh} / \mathrm{mL})\end{array}$ & $\begin{array}{c}\mathrm{t} 1 / 2(\mathrm{~h}) \\
\text { (b) }\end{array}$ & $\begin{array}{c}\mathrm{CL} / \mathrm{F} \\
(\mathrm{L} / \mathrm{hr} / \mathrm{kg})\end{array}$ \\
\hline $\begin{array}{c}\text { Neonates (a) (n=24) } \\
\begin{array}{c}\text { Infants (uncorrected age } \leq 10 \text { weeks) (a) } \\
(\mathrm{n}=3)\end{array}\end{array}$ & $1667(46)$ & $9632(52)$ & 2.27 & $0.16(98)$ \\
\hline $\begin{array}{c}\text { Infants (uncorrected age }>10 \text { weeks) (a) } \\
(\mathrm{n}=21)\end{array}$ & $827.9(51)$ & $1652(46)$ & 0.76 & $0.74(47)$ \\
\hline
\end{tabular}

(a) Normalized to $1.0 \mathrm{mg} / \mathrm{kg} /$ day. (b) Harmonic means are presented.

22

Evaluation of the Utility of Magnetic Resonance Cholangio-Pancreatography (MRCP) in Children Using Endoscopic Retrograde (ERCP), Intra-Operative Or Percutaneous Cholangio-Pancreatography As Gold Standards Neelesh A. Tipnis, Kulwinder S. Dua, John R. Sty, Steven L. Werlin

INTRODUCTION: ERCP is an invasive, highly specialized procedure not available at all pediatric centers. Although MRCP is rapidly replacing diagnostic ERCP in adults, there is little published information on its use in children. AIMS: To evaluate the accuracy of MRCP in children using ERCP, percutaneous fluoroscopic and intra-operative assessment of the pancreatico-hepato-biliary tree as gold standards. METHODS: Records of 32 children (age 0-18 y, 17 male) who underwent MRCP between Jan 2001-July 2005 were reviewed. Results of MRCP, ERCP, percutaneous fluoroscopic or intra-operative studies of the pancreaticohepato-biliary tree and clinical outcomes were evaluated. RESULTS: Of the 32 children, 17 (52\%) had MRCP alone, 11 (36\%) MRCP and ERCP, 3 (9\%) MRCP and intra-operative, and $1 \mathrm{MRCP}$ and percutaneous fluoroscopic evaluation. MRCP results correlated with other evaluative methods in 14/15 cases. There was 1 false positive (anomalous pancreatic duct union) and no false negatives for anatomic abnormalities. 2 children with normal anatomy by MRCPs and ERCP were diagnosed with sphincter of Oddi dysfunction (SOD). MRCP had 100\% sensitivity, $89 \%$ specificity and $86 \%$ PPV for the identification of anatomic abnormalities. In 10 children, MRCP preceded ERCP, intra-operative or percutaneous evaluation (MRCP diagnosis: 5 normal, 2 choledocholithiasis, 1 cong absence of gallbladder, 1 choledochal cyst, 1 anomalous pancreatic duct union). 4 children initially evaluated by MRCP (2 SOD, 2 choledocholithiasis) underwent endoscopic or surgical intervention (stent/ sphincterotomy 3 , cholecystectomy 1). In 5 children where MRCP followed other imaging, only 1 (PSC) had an intervention (sphincterotomy and dilatation). 10/17 children evaluated by MRCP alone had normal findings and all were well with a follow up period of 198-1266 days (median 866 d). 7 children evaluated by MRCP alone had the following diagnoses and abnormal findings: 4 recurrent pancreatitis ( 3 pancreatic parenchymal changes alone, 1 tortuous main pancreatic duct), 2 refractory cholestasis (intrahepatic duct dilation and contracted gallbladder), and 1 ulcerative colitis with autoimmune hepatitis (irregular intrahepatic bile ducts). 5/7 children improved clinically, the child with autoimmune hepatitis is unchanged and 1 child with chronic pancreatitis died from status epilepticus; none of these children had further pancreatico-hepato-biliary interventions. CONCLUSIONS: In this retrospective pilot study, MRCP was sensitive and specific in identifying anatomic abnormalities in the pancreatico-hepato-biliary tree in children. Prospective studies with large cohorts will be required to confirm these findings.

23

Effects of Osmolality, Volume, and Nutrient Density On Gastric Emptying (GE) in Preterm Infants

Robert J. Shulman, Alex Ramirez, William Wong

Background: Impaired GE is an important component of feeding intolerance in preterm infants. However, the factors regulating GE are unclear. We sought to clarify the independent as well as the interactive effects of osmolality, volume, and nutrient density on GE. Design/ Methods: In Study I, 10 infants (birth gestational age: $29 \pm 1$ wk; postnatal age: $28 \pm 15$ $\mathrm{d}$; mean $\pm \mathrm{SD}$ ) received in random order one of four feedings (A,B,C,D; i.e., one per day) to test a single variable on GE (Table). Infants received their usual feeding (human milk or preterm infant formula). Sorbitol was used to increase osmolarity because it does not add energy and has similar effects on GE as glucose (J. Physiol 1960;154:254). In Study II, 7 infants (birth gestational age: $27 \pm 1 \mathrm{wk}$; postnatal age: $34 \pm 14 \mathrm{~d}$ ) received in random order one of two feedings (D, E; i.e., one per day) to test the combined effects of altering osmolality and volume on GE (Table). GE was determined by measuring ${ }^{13} \mathrm{C}$ breath (over $4 \mathrm{~h}$ ) after adding $10 \mu \mathrm{L} / \mathrm{kg}{ }^{13} \mathrm{C}$-octanoic acid $\left(99 \%{ }^{13} \mathrm{C}\right)$ to the feedings to obtain the time it took to empty half of the stomach contents $\left(\mathrm{T}_{1 / 2}\right)$. Results: Cumulative percent dose recovered was similar among feedings for both studies (Study I: $50 \pm 20,59 \pm 15,45 \pm 9$, $57 \pm 22, A, B, C, D$, respectively; Study II: $37 \pm 5,33 \pm 6, \mathrm{D}, \mathrm{E}$, respectively). Study I: $\mathrm{T}_{1 / 2}$ was similar among feedings ( $90 \pm 24,93 \pm 13,85 \pm 22,97 \pm 35 ; A, B, C, D$, respectively). Study II: $T_{1 / 2}$ was significantly faster with feeding E vs D $(102 \pm 15$ vs $125 \pm 35$, respectively; $P=0.035)$. Using results from all infants $(n=17)$ for feeding $D$ (i.e., full strength human milk or formula), $\mathrm{T}_{1 / 2}$ was inversely related to gestational age at birth $\left(\mathrm{P}=0.026, \mathrm{r}^{2}=0.24\right)$ but did not correlate with birthweight, postnatal age, study weight, or type of feeding (human milk vs formula). Conclusions: 1) In contrast to adults, individual alterations in osmolality, volume, and nutrient density do not differ in their effects on GE in preterm infants; 2) Simultaneously decreasing osmolality and increasing volume enhances GE by $25 \%$ and we speculate, may account, in part, for reduced feeding intolerance seen clinically with this regimen (Am J Dis Child 1988;142:730 and Indian Pediatr 1990;27:829);3) Birth gestational age still exerts an influence on GE even at one month postnatal age.

\begin{tabular}{|c|c|c|c|}
\hline $\begin{array}{c}\text { Feeding (human milk } \\
\text { or formula) }\end{array}$ & $\begin{array}{c}\text { Total Osmolality } \\
(\mathrm{mOsm} / \mathrm{kg} \mathrm{H} 2 \mathrm{O})\end{array}$ & $\begin{array}{c}\text { Total Feeding } \\
\text { Volume }(\mathrm{mL} / \mathrm{kg})\end{array}$ & $\begin{array}{c}\text { Total Nutrient Density } \\
(\mathrm{kcal} / \mathrm{mL})\end{array}$ \\
\hline A & 310 & 10 & 0.4 \\
\hline B & 155 & 10 & 0.4 \\
\hline C & 310 & 20 & 0.4 \\
\hline D & 310 & 10 & 0.8 \\
\hline E & 155 & 20 & 0.4 \\
\hline \multicolumn{2}{|c|}{ A vs B = osmolality effect A vs C = volume effect A vs D $=$ nutrient density effect D vs E $=$} \\
combined effect of osmolality and volume
\end{tabular}

24

Corticotropin-Releasing Factor (CRF) Levels Are Elevated During Episodes of Cyclic Vomiting Syndrome

Seth B. Marcus, M Agjabe-Williams, De Grigoriadis, Y Tache, Don Zimmerman, B U. Li

Background: Cyclic vomiting syndrome (CVS) is a severe, recurrent, functional vomiting disorder affecting children and adults. Although the relationship to migraine headaches is well established, no specific pathophysiologic cascade has been elucidated. Based upon animal data demonstrating that CRF alters gastric motility, CRF has been proposed as a potential mediator of vomiting in CVS (Taché, Dig Dis Sci 1999;44(8S):79S). We hypothesized that CRF levels in asymptomatic CVS patients would be similar to control values but elevated during symptomatic vomiting. Methods: We studied three groups of children ages 5-19 years: healthy controls $(n=8)$, asymptomatic $(n=14)$ and symptomatic CVS patients $(\mathrm{n}=10)$ during vomiting episodes. We evaluated the hypothalamic-pituitary-adrenal (HPA) axis at 8 am and 4 pm in controls and asymptomatic CVS patients, and, within the first four hours of an CVS episode. Plasma CRF, ACTH and cortisol were assayed by radioimmunoassay. Results: Diurnal 8 am cortisol levels were significantly higher than at $4 \mathrm{pm}$ in both controls and CVS patients. There were no significant differences between CRF, ACTH and cortisol levels between controls and asymptomatic CVS patients at either 8 am or 4 pm. During vomiting episodes, CRF was significantly $(\mathrm{p}<0.01)$ elevated when compared to 8 am baseline values in controls $(\dagger)$. Conclusions: There is heightened HPA axis activation in children with CVS during active vomiting episodes. Our study provides the first evidence of elevated peripheral CRF values in episodes of CVS and supports the concept that CRF may trigger or mediate vomiting in cyclic vomiting syndrome. Supported by NINS 44187

\begin{tabular}{|c|c|c|c|}
\hline Group & CRF pg/ml & ACTH pg/ml & Cortisol pg/ml \\
\hline Controls & $5.34 \pm 3.74$ & $56.27 \pm 27.70$ & $31.71 \pm 27.97$ \\
\hline CVS-Well & $7.29 \pm 4.83$ & $62.41 \pm 34.58$ & $39.63 \pm 19.66$ \\
\hline CVS-Vomit & $10.34 \pm 3.21$ & $193.44 \pm 229.90$ & $50.07 \pm 27.64$ \\
\hline
\end{tabular}

25

Cholinergic Contractile Responses and Signal Transduction Mechanisms of Gastric Smooth Muscle in M2 and M3 Muscarinic Receptor Subtype Knockout Mice

Alan S. Braverman, Henry P. Parkman, Jurgen Wess, Michael R. Ruggieri

Atropine slows gastric emptying indicating involvement of muscarinic cholinergic pathways. Although the majority of muscarinic receptors in the GI tract are M2 receptors, cholinergic contractions of GI smooth muscle are primarily mediated through the M3 subtype. AIMS: 1) to explore the subtypes of muscarinic receptors mediating cholinergic contractions of the stomach using $\mathrm{M} 2$ and $\mathrm{M} 3$ receptor knockout (KO) mice; 2) to determine contractile signal transduction cascades activated by M2 and M3 receptors. METHODS: Gastric body smooth muscle strips from M2 and M3 KO mice and their wildtype (WT) strains were studied in vitro. The maximal contractile response was determined using $\mathrm{KCl}$. After rinsing, a concentration response curve (CRC) to carbachol was performed in absence or presence of the $\mathrm{M} 3$ receptorpreferring antagonist darifenacin or signal transduction pathway enzyme inhibitors. RESULTS: Contractile responses to $120 \mathrm{mM} \mathrm{KCl}$ between strains were similar $(0.35 \pm 0.07$, $0.36 \pm 0.09,0.38 \pm 0.07$, and $0.44 \pm 0.09 \mathrm{~g}$ for M2 WT, M3 WT, M2 KO, and M3 KO respectively). The maximal contraction to carbachol was decreased in $\mathrm{M} 3 \mathrm{KO}$ mice $(75 \%$ decrease; $\mathrm{p}=0.0005)$ and M2 KO mice ( $29 \%$ decrease; $\mathrm{p}=0.060)$ compared to their WT controls. 10 
nM darifenacin shifted the carbachol CRC to the right for M2 KO, M2 WT and M3 WT strains $(\mathrm{Kb}=2.0 \pm 1.5,0.8 \pm 0.4$ and $4.0 \pm 0.4 \mathrm{nM}$ respectively) with no shift for the $\mathrm{M} 3 \mathrm{KO}$ strain $(\mathrm{Kb}>10 \mathrm{nM})$. This suggests carbachol contractions in WT controls and M2 KO mice were mediated by M3 receptors but by M2 receptors in M3 KO mice. Y27632, an inhibitor of rho kinase (ROCK), reduced carbachol contractions in WT and M2 KO but not in the M3 KO. ET-18-OCH3, an inhibitor of phosphoinositide specific phospholipase C, had no effect on the carbachol response in any strain. D609, an inhibitor of phosphatidyl choline specific phospholipase C (PC-PLC), attenuated contractions in M3 WT and M2 KO. Chelerytherine, an inhibitor of protein kinase $\mathrm{C}$ ( $\mathrm{PKC})$, inhibited contraction only in the $\mathrm{M} 2 \mathrm{KO}$. $\mathrm{H} 89$, an inhibitor of protein kinase A, ROCK and protein kinase $\mathrm{G}$, suppressed contractions in the M2 WT. CONCLUSIONS: Cholinergic contractions of the gastric body are primarily mediated by the $\mathrm{M} 3$ receptor subtype with a smaller component mediated by the $\mathrm{M} 2$ subtype. The M3 subtype activates ROCK, PKC and PC-PLC pathways to mediate gastric smooth muscle contraction. Because M2 mediated contractions in M3 KO mice were unaffected by the enzyme inhibitors, either none of these enzymes are essential for M2 signaling or redundant pathways are activated by gastric smooth muscle M2 receptors. These studies suggest several parallel pathways mediate cholinergic contractions in gastric smooth muscle.

26

Physiological Dependence of Gastric Emptying and Intestinal Motility On 5$\mathrm{HT}_{4}$ Receptors: Analyses in Wild-Type and 5- $\mathrm{HT}_{4}$ Knockout Mice Abigail Cuenca, Min-Tsai Liu, Kerri Holick, Rene Hen, Michael D. Gershon

The 5- $\mathrm{HT}_{4}$ receptor is expressed by neurons in both plexuses of the mammalian enteric nervous system (ENS). These receptors are presynaptic and increase the amplitude of excitatory postsynaptic currents. We have thus proposed that $5-\mathrm{HT}_{4}$ agonists strengthen neurotransmission in prokinetic pathways. Despite the ability of $5-\mathrm{HT}_{4}$ agonists to accelerate intestinal motility and gastric emptying, $5-\mathrm{HT}_{4}$ antagonists do not, by themselves, block the peristaltic reflex. To test the hypothesis that $5-\mathrm{HT}_{4}$ receptors are necessary for normal motility, we studied gastric emptying, small and large intestinal transit in 3 month-old 5$\mathrm{HT}_{4}$ knockout (KO) mice and their wild-type (WT) littermates. Gastric emptying was assessed by instilling $250 \mu \mathrm{l}$ of rhodamine-B-dextran (RD; $10 \mathrm{mg} / \mathrm{ml}$ ) into the stomach and measuring the retained RD fluorescence and that emptied into the small intestine (to obtain the \% of dose emptied). Small bowel transit was determined from the distribution of RD in the small intestine and from video images of small bowel propulsion in vitro. Colonic motility was estimated by measuring the time required to expel a glass bead pushed a distance of $2 \mathrm{~cm}$ up the rectum. Numbers of myenteric neurons (visualized with antibodies to $\mathrm{Hu}$ ) were counted in $\mathrm{KO}$ and WT animals at 10,15 and 20 weeks of age. The rate of gastric emptying in $\mathrm{KO}$ mice $(38.0 \pm 10.2 \%$ emptied; $\mathrm{n}=5)$ was significantly slower than that of WT animals $(75.6 \pm 10.2 \%$ emptied; $\mathrm{n}=5 ; \mathrm{p}<0.05)$. Small bowel transit was slower in $\mathrm{KO}$ (geometric center $=2.2 / 10 ; \mathrm{n}=5)$ than in WT mice $(4.1 / 10 ; \mathrm{n}=5 ; \mathrm{p}<0.05)$ and the time to eject a bead from the colon was also longer in $\mathrm{KO}$ mice $(139.4+15.1 \mathrm{sec} ; \mathrm{n}=4)$ than in their WT littermates $(74.1 \pm 21.7 \mathrm{sec} ; \mathrm{n}=4 ; \mathrm{p}<0.05)$. The numbers of myenteric neurons in the colon were consistently less in KO mice than in their WT littermates (the ratios of WT/ $\mathrm{KO}$ were 1.9 at 10 wks, 1.5 at 15 wks, and 1.7 at 20 wks mean $=1.7 ; \mathrm{p}<0.02$ ). The 5 $\mathrm{HT}_{4}$ agonist, tegaserod, reversibly increased in vitro intestinal propulsion by $27 \%(\mathrm{n}=6$; $\mathrm{p}<0.001$ ); this effect was abolished by the $5-\mathrm{HT}_{4}$ antagonist, GR113808. These observations suggest that $5-\mathrm{HT}_{4}$ receptors are physiologically prokinetic and required for normal gastric emptying, small, and large intestinal motility. Because the number of colonic myenteric neurons is reduced in $\mathrm{KO}$ mice, it is possible that the receptors also exert a neuroprotective effect on enteric neurons; alternatively, the loss of neurons in animals lacking 5- $\mathrm{HT}_{4}$ receptors may be an indirect effect of constitutive slow transit. Supported by Novartis and NIH grants NS12969 and NS15547.

27

Identification of New Variant of Corticotropin Releasing Factor (CRF) Receptor Type $2 \alpha(\operatorname{Crf} 2 \alpha)$ : Differential Distribution and Regulation in the Gastrointestinal (GI) Tract in Rats

Pu-Qing Yuan, S Vincent Wu, Lixin Wang, Yvette Tache

Background: Two types of CRF receptors, CRF1 and CRF2 differentially modulate GI functions. In rodents, CRF $2 \alpha$ is the major splice variant expressed in brain, whereas CRF $2 \beta$ is found in peripheral tissues with high levels in the GI tract and heart where it is subject to down regulation by endotoxin. Aims: 1 . To determine whether CRF $2 \alpha$ mRNA is also expressed in rat GI tract and compares its gene expression pattern with those of CRFI and CRF2 $\beta$. 2. To examine the regulation of CRF $2 \alpha$ mRNA by endotoxin in rat stomach and colon. Methods: Esophagus, lower esophageal sphincter (LES), gastric corpus, antrum, pylorus, duodenum, jejunum, ileum, cecum, proximal colon, distal colon and rectum were collected from 4 SD male naive rats and total RNA was processed for RT-PCR using specific primers for rat CRF1, CRF $2 \alpha$ and CRF2 $\beta$. Rats (5/group) were injected intraperitoneally (ip) with saline $(0.3 \mathrm{ml})$ or lipopolysaccharide (LPS, $100 \mu \mathrm{g} / \mathrm{kg}$ ) and gastric corpus and proximal colon were harvested $6 \mathrm{~h}$ later. Mucosa and submucosa plus muscle layers $(\mathrm{S}+\mathrm{M})$ were separated. Total RNA was processed for RT-PCR of CRF2 $\alpha$. RT-PCR for acidic ribosome protein (ARP) served as a control for RNA quality and normalization. The intensity of bands was quantitatively analyzed using Scion imaging software. Results: CRF $2 \alpha$ mRNA was detected within the whole GI tract and expressed more abundantly at two ends of the GI tract with the highest level in the esophagus and LES, which is similar to that found for CRF $2 \beta$. In addition, a novel splice variant named CRF $2 \alpha-13$ was identified and confirmed by sequencing, which contains an unspliced intron 3 and is predominantly expressed in the mid GI tract with the highest level in the jejunum. The CRF2 $\alpha$ distribution pattern differs from that of CRFl which is expressed at highest levels in the ileum and colon while very low to undetectable levels are found in the esophagus and gastric corpus. CRF2 $\alpha$ is decreased by $84 \%$ and $82 \%$ in mucosa and $90 \%$ and $52 \%$ in S+M of gastric corpus and proximal colon respectively after LPS injection. By contrast, CRF $2 \alpha-\mathrm{I} 3$ is significantly increased by $40 \%$ and $179 \%$ in mucosa and $118 \%$ and $143 \%$ in $\mathrm{M}+\mathrm{S}$ of gastric corpus and proximal colon respectively after LPS injection. Conclusion: CRF2 $\alpha$ and CRF $2 \alpha-13$, a newly identified splice variant, are expressed in rat GI tract with a distribution pattern distinct from that of CRF1 and CRF2 $\beta$. The regulation of CRF $2 \alpha$ mRNA expression by LPS suggests that $C R F 2 \alpha$, like CRF2 $\beta$, may play important roles in LPS-induced alterations of gastric and colonic function. Supported by NIHDDK 57238

\section{8}

Ghrelin Agonist Activity in a Rat Model of Postoperative Ileus and OpioidDelayed Gastrointestinal Motility

Kalina Venkova, Graeme Fraser, Hamid Hoveyda, Beverley Greenwood-van Meerveld

Acute postoperative ileus (POI) is the product of two main mechanisms: (1) disruption of spinal and supraspinal signaling pathways as a consequence of surgical injury and (2) reduction in propulsive gastrointestinal (GI) motility as a side effect of opioid analgesic administered for post-surgical pain. Although opioid antagonists may be used to treat the latter mechanism, no single agent has been reported to potently address both aspects of POI. TZP-101 is a novel ghrelin receptor agonist with selective gastroprokinetic activity The aim of this study was to determine whether TZP-101 could effectively treat both the individual and combined components of acute POI in a rat model. Methods: Delaved GI motility was induced by surgery [i.e. laparotomy and manipulation of the bowel, model 1] pretreatment with morphine ( $3 \mathrm{mg} / \mathrm{kg}$, s.c.) [model 2] or a combination of both [model 3] In all three models, gastric retention was measured 15-min after co-incident, intragastric administration of ${ }^{99 \mathrm{~m}} \mathrm{Tc}$ labeled methylcellulose and i.v administration of TZP-101 (30$1000 \mu \mathrm{g} / \mathrm{kg}$ ) in fasted rats. Results: As expected, gastric emptying of the radiolabeled meal was delayed in all three models in vehicle-treated subjects (Table 1) in comparison to nonoperated, morphine-naive controls $(30.4 \pm 4.7 \%, n=8)$. TZP-101 dose-dependently normalized gastric emptying with similar efficacy and potency in all three models (Table 1). Summary: Selective activation of ghrelin receptors resulted in a complete recovery of impaired gastric emptying induced by surgery alone, morphine alone or a combination of surgery and morphine. The unique finding that there was no diminution of TZP-101 potency in the combination model suggests that TZP-101 activates a common prokinetic pathway to counteract the delay in GI transit consequent to morphine treatment and surgery. Table 1

\begin{tabular}{|c|c|c|c|}
\hline \multirow{2}{*}{$\begin{array}{c}\text { Treatment (6-8 rats per } \\
\text { dose) }\end{array}$} & \multicolumn{3}{|c|}{ Gastric Emptying (\%gastric residue) } \\
\cline { 2 - 4 } & $\begin{array}{c}\text { Surgery (model } \\
\text { 1) }\end{array}$ & $\begin{array}{c}\text { Morphine (model } \\
\text { 2) }\end{array}$ & $\begin{array}{c}\text { Surgery + Morphine } \\
\text { (model 3) }\end{array}$ \\
\hline Vehicle & $73.8 \pm 4.6$ & $67.0 \pm 5.8$ & $70.5 \pm 5.6$ \\
\hline TZP-101 $30 \mu \mathrm{g} / \mathrm{kg}$ & $54.9 \pm 6.6$ & -- & $52.0 \pm 6.9$ \\
\hline TZP-101 $100 \mu \mathrm{g} / \mathrm{kg}$ & $50.0 \pm 7.5^{*}$ & -- & $45.7 \pm 1.5^{* *}$ \\
\hline TZP-101 $300 \mu \mathrm{g} / \mathrm{kg}$ & $43.4 \pm 7.8^{* *}$ & $45.4 \pm 12.4^{*}$ & $37.1 \pm 6.3^{* * *}$ \\
\hline TZP-101 $1000 \mu \mathrm{g} / \mathrm{kg}$ & $28.2 \pm 7.7^{* * * *}$ & $29.8 \pm 6.8^{* * * *}$ & $28.3 \pm 6.7^{* * * *}$ \\
\hline
\end{tabular}

One-way ANOVA with Bonferroni's MCT. ${ }^{*} \mathrm{p}<0.05,{ }^{*} \mathrm{p}<0.01,{ }^{* * *} \mathrm{p}<0.001$ compared to vehicle

\section{9}

The New $\mathrm{CRF}_{1}$ Selective Agonist Cortagine Injected Peripherally and Centrally Stimulates Colonic Motor Function and Induces Diarrhea in Rats: A Relevant Tool for Stress-Related Gastrointestinal Disease Studies Muriel H. Larauche, Karina Pambukchian, Armen Karapetyan, Mulugeta Million, Jean Rivier, Yvette Tache

Background: Corticotropin releasing factor (CRF) binds to two subtypes of receptors: CRF receptor type $1\left(\mathrm{CRF}_{1}\right)$ and type $2\left(\mathrm{CRF}_{2}\right)$. Stress via the release of $\mathrm{CRF}$ and the activation of peripheral and central $\mathrm{CRF}_{1}$ induces alterations of colonic motility and secretion and may be relevant for Irritable Bowel Syndrome (IBS) (Br J Pharmacol. 2004, 141:1321-30). Whereas highly selective agonists for $\mathrm{CRF}_{2}$ exist, no agonist with a similar selectivity for $\mathrm{CRF}_{1}$ was available until the recent development of the new $\mathrm{CRF}_{1}$ agonist: cortagine (binding affinities $2.6 \mathrm{nM}_{\text {for }} \mathrm{rCRF}_{1}$ vs $540 \mathrm{nM}$ for mCRF $\beta$ ) (PNAS 2004;101:9468-73). Aim: To characterize the properties of cortagine on colonic and gastric motor functions in rats and establish its relevance in the study of stress-related gastrointestinal diseases. Methods: Studies were performed in conscious male Sprague-Dawley rats (250-300g). The fecal pellet output (FPO) and diarrhea incidence were monitored for $2 \mathrm{~h}$ after intraperitoneal (IP) injection of cortagine ( 3 and $10 \mu \mathrm{g} / \mathrm{kg}$ ). The central effect of cortagine ( 3 and $5 \mu \mathrm{g} / \mathrm{rat}, 10 \mu \mathrm{l}$ ) was evaluated on FPO and diarrhea incidence after its intracerebroventricular (ICV) injection. The selectivity of IP cortagine $(10 \mu \mathrm{g} / \mathrm{kg})$ for $\mathrm{CRF}_{1}$ was tested on the FPO by injecting the specific $\mathrm{CRF}_{1}$ antagonist, CP154,526 (20 mg/kg) subcutaneously (SC) $30 \mathrm{~min}$ before cortagine, and by evaluating its effects on gastric emptying of solid food. Results: Cortagine at the dose of $10 \mu \mathrm{g} / \mathrm{kg}$, but not $3 \mu \mathrm{g} / \mathrm{kg}$, IP, significantly increased the FPO in rats $(6.9 \pm 1.9$ vs $0.3 \pm 0.3$ pellet/hour in vehicle IP) and induced diarrhea in $43 \%$ of rats within $30-45$ min postinjection. Cortagine IP had no effect on gastric emptying of a chow meal compared to vehicle-treated rats. The increase in FPO induced by cortagine ( $10 \mu \mathrm{g} / \mathrm{kg}$ IP) was significantly reduced by $\mathrm{CP} 154,526$ pretreatment $(3.1 \pm 1.0$ vs $6.9 \pm 1.9$ in cortagine IP). Central cortagine ( 3 and $5 \mu \mathrm{g} / \mathrm{rat}$ ICV) significantly increased the FPO for both doses and induced diarrhea after $60 \mathrm{~min}$ in 14\% and $40 \%$ of responders respectively. Conclusions: The data using the new selective $\mathrm{CRF}_{1}$ agonist cortagine, confirm the involvement of $\mathrm{CRF}_{1}$ at both peripheral and central levels in the stimulation of colonic secretory motor functions leading to diarrhea in rats. They also support the selectivity of cortagine for $\mathrm{CRF}_{1}$ since the solid food gastric emptying known to be CRF 2 mediated (AJP 2002;282:G34-40) is not modified and the increased FPO is abolished by a selective $\mathrm{CRF}_{1}$ antagonist. All together, those data highlight the potential of cortagine as a useful tool in the study of stress-related gastrointestinal disorders, such as IBS. Supported by R01 DK33061.

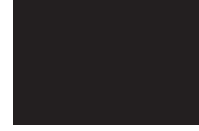


Activation of the RHo-INteracting PRotein P116 ${ }^{\mathrm{RIP}}$ By Src Kinase Inhibits RHOA and Enhances M̄lc Phosphatase Activity: A Mechanism for Inhibition of Acetylcholine-Induced Muscle Contraction By Internalized Cannabinoid $\mathrm{Cb}_{1}$ Receptors

Sunila Mahavadi, Jiean Huang, Karnam S. Murthy

Sustained $\mathrm{MLC}_{20}$ phosphorylation and contraction in smooth muscle are mediated by a $\mathrm{Ca}^{2+}$-independent MLC kinase (ZIP kinase) and concurrent inhibition of MLC phosphatase. The latter is mediated by dual Rho-dependent pathways involving phosphorylation of the regulatory subunit of MLC phosphatase, MYPT1 by Rho kinase, and phosphorylation CPI17, an endogenous inhibitor of MLC phosphatase, by PKC. The endocannabinoid, anandamide, acting via $C B_{1}$ receptors inhibits sustained contraction. The inhibitory mechanism is not known, since our previous studies have shown that $\mathrm{CB}_{1}$ receptors are coupled to an atypical $G$ protein $\left(G \alpha_{i 2} / G \beta_{5} / R G S 6\right)$ and signal only after internalization via a $G$ proteinindependent GRK5/ $\beta$-arrestin/c-Src pathway. Aim: To determine whether inhibition of Rhomediated smooth muscle contraction is dependent on internalization of $\mathrm{CB}_{1}$ receptors by GRK5/c-Src pathway. Methods. Acetylcholine (ACh)-stimulated Rho kinase activity was determined by immunokinase assay in cultured gastric smooth muscle cells in the presence or absence of the $\mathrm{CB}_{1}$ antagonist $\mathrm{AM} 251$, or the c-Src inhibitor PP2, and in cells expressing dominant negative GRK5 (K215R). Association of the Rho-Interacting Protein pl16 $6^{\mathrm{Rip}}$ with RhoA and MYPTl was determined by co-immunoprecipitation. MLC phosphatase activity

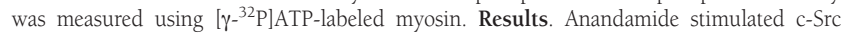
activity, which was blocked by the $\mathrm{CB}_{1}$ receptor antagonist AM251 and the Src-kinase inhibitor PP2, and in cells expressing dominant negative GRK5 (K215R). Acetylcholine stimulated Rho kinase activity and sustained smooth muscle contraction (determined by scanning microscopy); contraction and Rho kinase activity were inhibited by anandamide, and the inhibition was reversed by AM251 and PP2. Anandamide increased the association of pl16 $6^{\text {Rip }}$ with RhoA and MYPT1, and stimulated MLC phosphatase activity; the increase in association and MLC phosphatase activity were blocked by AM25l and PP2. The association of p116 $6^{\text {Rip }}$ with RhoA inhibits RhoA activity and thus blocks the inhibition of MLC phosphatase via Rho kinase, whereas its association with MYPTl stabilizes the binding of MYPTl to the catalytic subunit and thus enhances MLC phosphatase activity. Conclusion. Internalization of $\mathrm{CB}_{1}$ receptors via $\mathrm{GRK} 5$ leads to activation of $\mathrm{c}-\mathrm{Src}$, which increases the association of p116 Rip with RhoA and MYPTl, thereby enhancing MLC phosphatase activity and causing inhibition of sustained muscle contraction. The study provides the first evidence for regulation of p1 $16^{\text {Rip }}$ upon receptor internalization by G protein-independent GRK5.

\section{1}

Effect of Inflammation On the Intestinal Nhe8 Gene Expression

Hua Xu, Jennifer K. Uno, Rongji Chen, Huacong Chen, Fayez K. Ghishan

Introduction: NHE8 transporter is a newly identified NHE isoform from kidney and intestine. This transporter protein is expressed at the apical membrane of the epithelial cells of the renal and intestine, suggesting the possible role of NHE8 in vectorial Na+ transport in both tissues. Although other work has been shown that the $\mathrm{NaCl}$ absorption in the gastrointestinal tract of patients with inflammatory bowel disease (IBD) and in animal models of chronic enteritis is reduced, little is known about the role of $\mathrm{Na}+\mathrm{H}+$ exchange and the involvement of NHE isoforms in the pathogenesis of inflammatory disorders and the mechanism of inflammation-associated diarrhea. Thus, the current study was performed to investigate the involvement of NHE8 in inflammatory disorders. Methods: Rats were injected interaperitoneally with one dose of LPS at $10 \mu \mathrm{g} / \mathrm{g}$ body weight or with equal volumes of vehicle. Twenty hours after injection, rats were sacrificed and jejunal mucosa was harvested. RNA and BBM protein purification were performed from collected jejunal mucosa samples. Semiquantitative PCR analysis was used to detect NHE8 mRNA abundance in control and LPS injected rats. Western blotting was used to detect NHE8 protein expression levels. The NHE8 gene promoter region was subcloned into pGL3 basic vector and used to study the effect of TNF$\alpha(100 \mathrm{ng} / \mathrm{ml})$ on NHE8 gene promoter activity in Caco-2 cells. The endogenous NHE8 gene expression in Caco-2 cells was measured by Real time PCR method. Results: Western blotting indicated a significantly reduction of NHE8 protein by LPS treatment, with phosphoimage units from $0.36+/-0.01$ in control rats to $0.20+/-0.058$ in LPS treated rats. Semiquantitative PCR with rat NHE8 specific primers and $\beta$-actin primers showed that the rat NHE8 mRNA expression was reduced by 40\% in LPS injected rats. Promoter assay indicated that the human NHE8 (hNHE8) promoter construct is active in Caco-2 cells, and TNF- $\alpha$ treatment inhibits the hNHE8 promoter activity by $44 \%$. A similar reduction in endogenous NHE8 gene expression was also observed in TNF- $\alpha$ treated Caco-2 cells. Conclusion: We conclude that the novel NHE isoform, NHE8, is altered by inflammation. The NHE8 expression is inhibited by endotoxemia introduced by LPS or by proinflammatory cytokine TNF$\alpha$ treatment. The mechanism of this inhibition may involve inactivation of the NHE8 promoter. This investigation was supported by NIH grant R37-DK33209.

32

The Role of Ctla-4 On Regulatory T Cell Function in a Mouse Model of Microbially-Driven Inflammatory Bowel Disease

Koichiro Watanabe, Rao P. Varada, Shilu Xu, Theofilos Poutahidis, Masahiro Ohtani, Kristen Clapp, Juri Miyamae, Arlin B. Rogers, Zhongming Ge, Toshio Fujioka, Susan E. Erdman, James G. Fox

Background: We have shown that transfer of $\mathrm{CD} 4^{+} \mathrm{CD} 25^{+} \mathrm{CD} 45 \mathrm{RB}^{\text {low }}$ regulatory $\mathrm{T}$ (Treg) cells inhibits colitis and cancer induced by Helicobacter hepaticus infection in Rag2-/- mice. Regulatory function is mediated by cytokines such as IL-10 and CTLA-4. The latter is a costimulatory molecule, expressed highly on Treg cells, and binds B7 on the antigen presenting cells resulting in the regulation of $\mathrm{T}$ cell proliferation, and is known to be important in inflammatory modulation. Aim: We examined the cell contact-mediated function of Treg cells through CTLA-4 signaling in an H. hepaticus-induced colitis model. Materials and Methods: $\mathrm{CD}^{+} \mathrm{CD} 25^{+} \mathrm{CD} 45 \mathrm{RB}^{\text {low }}$ cells were isolated from donor spleens and mesenteric lymph nodes of H. hepaticus-free $129 \mathrm{SvEv}$ wild type mice, as described previously. Rag2-/$129 \mathrm{SvEv}$ mice received the donor cells i.v. at $3.0 \times 10^{5}$ cells/animal 3 days before infection. H. hepaticus (ATCC 51449) was administered per os every other day for three doses (2.0 $x$ $10^{7}$ bacteria/animal). The recipient mice underwent 2-week administration with either hamster anti-mouse CTLA-4 Mab or control Ig ip, starting at one day before or 6 weeks after $H$. hepaticus infection. Inflammation and epithelial dysplasia were evaluated histologically and scored 0 to 4 as described previously. The mice were euthanized 6 weeks after the last dose of anti-CTLA-4 treatment. Results: $H$. hepaticus infection induced moderate to severe inflammation (pathology score, median[range]: 4 [2-4]), with mononuculear cells and neutrophils, and moderate dysplasia $(2[2-3])$ in the transverse colon. Adaptive transfer of Treg cells led to the inhibition of inflammation (1 [0-4]) and dysplasia (0 [0-4]), matching earlier findings in this model. However, the recipients of Treg cells treated with anti-CTLA4 showed significant weight loss and blood in feces, and developed transmural inflammation (4 [4-4]) with deep ulcerations and severe dysplasia (3 [3-4]) in the colon. This effect was independent of time interval of administration of anti-CTLA-4. Inflammation was not observed in the stomach, ileum, and liver of anti-CTLA-4 treated mice. Conclusion: In vivo administration of anti-CTLA-4 eliminated the inhibitory effect of Treg cells on H. hepaticusinduced colitis and epithelial dysplasia. These data suggest that CTLA-4 signaling is involved in the immune-suppressive function of Treg cells.

Pathology scores of transverse colon

\begin{tabular}{|c|c|c|c|c|c|c|}
\hline \multirow[b]{2}{*}{ Group } & \multicolumn{2}{|c|}{ Antibody administration: None } & \multicolumn{2}{|c|}{$\begin{array}{c}\text { Antibody } \\
\text { administration: One day } \\
\text { before H. hepaticus } \\
\text { infection }\end{array}$} & \multicolumn{2}{|c|}{$\begin{array}{c}\text { Antibody } \\
\text { administration: Six } \\
\text { wekks after H. } \\
\text { hepaticus infection }\end{array}$} \\
\hline & $\begin{array}{c}\text { H. } \\
\text { hepaticus-free } \\
\text { control }\end{array}$ & $\begin{array}{c}\mathrm{H} . \\
\text { hepaticus-infected }\end{array}$ & $\begin{array}{c}\mathrm{H} \\
\text { hepaticus } \\
\text { + Treg } \\
\text { control } \\
\text { Ig }\end{array}$ & $\begin{array}{l}\text { H. hepaticus } \\
\text { + Treg + } \\
\text { anti-CTLA-4 }\end{array}$ & $\begin{array}{c}\mathrm{H} \\
\text { hepaticus } \\
\text { + Treg+ } \\
\text { control } \\
\text { Ig } \\
\end{array}$ & $\begin{array}{c}\text { H. hepaticus } \\
+ \text { Treg + } \\
\text { anti-CTLA-4 }\end{array}$ \\
\hline Inflammation & $0[0-0]$ & $4[2-4]$ & $1[0-4]$ & 4 [4-4] & $0.5[0-3]$ & $3.5[2-4]$ \\
\hline Hyperplasia & $0[0-0]$ & $3[3-4]$ & $0.5[0-4]$ & 4 [4-4] & $0.5[0-2]$ & $3.5[2-4]$ \\
\hline Dysplasia/Neoplasm & $0[0-0]$ & $2[2-3]$ & $0[0-4]$ & $3[3-4]$ & $0[0-1]$ & $2[0-4]$ \\
\hline
\end{tabular}

median [range]

33

TNF and Light Cause Epithelial Barrier Dysfunction in Vivo, But Only TNF Induces Diarrhea: Role of NHE3

Daniel R. Clayburgh, Mark W. Musch, Yang-Xin Fu, Jerrold R. Turner

Acute $\mathrm{T}$ cell-mediated diarrhea is associated with increased mucosal expression of proinflammatory cytokines, including the TNF superfamily members TNF and LIGHT. Either TNF neutralization or myosin light chain (MLC) kinase inhibition prevented T cell-mediated epithelial barrier dysfunction and diarrhea, i.e. net water secretion. We therefore asked whether in vivo administration of either purified TNF or LIGHT was sufficient to induce acute barrier dysfunction and diarrhea. METHODS: C57BL/6 mice were injected i.p. with 5 ug of recombinant murine TNF or LIGHT. In vivo jejunal perfusions were used to determine net water flux and paracellular permeability. Bidirectional ${ }^{22} \mathrm{Na}^{+}$flux was assessed in Üssing chambers. $10 \mu \mathrm{M}$ S3226-sensitivity defined the NHE3 component. Immunofluorescent morphometry on frozen jejunal tissue used antisera against occludin, phosphorylated MLC, NHE3, or PKCa. RESULTS: TNF injection resulted in acute diarrhea within 2 hrs. This was accompanied by epithelial barrier dysfunction and water secretion. The loss of barrier function was characterized by increased epithelial MLC phosphorylation and occludin internalization. Both barrier loss and water secretion could be prevented by MLC kinase (MLCK) inhibition, although water absorption was not fully restored. LIGHT injection caused similar MLCK-dependent barrier dysfunction and occludin internalization, but was not associated with either water secretion or reduced water absorption. Thus, despite quantitatively and qualitatively similar effects on barrier function, TNF and LIGHT had markedly different effects on water flux. TNF, but not LIGHT, blocked $\mathrm{Na}^{+}$absorption due to NHE3 downregulation. This was associated with NHE3 translocation from the brush border to a subapical cytoplasmic pool. In contrast, LIGHT did not cause NHE3 internalization. TNF, but not LIGHT, treatment also caused PKC $\alpha$ translocation to the apical membrane, suggesting that TNF might downregulate NHE3 via PKC $\alpha$-mediated internalization. CONCLUSIONS: These data suggest that both TNF and LIGHT are able to induce in vivo MLCK-dependent epithelial barrier dysfunction and that this barrier dysfunction is necessary for diarrhea. However, only TNF caused diarrhea and only TNF inhibited NHE3-dependent $\mathrm{Na}^{+}$absorption. This suggests that NHE3 downregulation is also required for acute cytokine-meditated diarrhea to occur. These observations explain previous studies showing that neither barrier dysfunction nor $\mathrm{Na}^{+}$malabsorption alone are sufficient to cause diarrhea. TNF may cause diarrhea by both increasing paracellular permeability and reducing the driving force for water absorption, i.e. $\mathrm{Na}^{+}$absorption.

34

Bifidobacterium Animalis Causes Extensive Duodenitis and Mild Colonic Inflammation in Interleukin-10 Knockout Mice James P. Moran, Susan L. Tonkonogy, J.N. Walter, Gerald W. Tannock, Balfour R. Sartor

Backgound/aims. The interleukin-10 knockout (IL-10 KO) mouse has been studied extensively as a genetically susceptible model for IBD. When housed under germ-free (sterile) conditions these mice remain healthy, however, when housed under specific pathogen free (SPF) conditions IL-10 KO mice develop chronic THl-mediated colitis. We have previously shown that the bacterial species Bifidobacterium animalis is more prevalent in the colons of IL-10 KO mice than in wild type (WT) animals colonized with the same SPF fecal microbiota. In the present study we tested this organism for its ability to cause disease by selectively introducing it into germ-free IL-10 KO and WT mice. Methods. Germ-free IL-10 KO and 
WT 129S6/SvEv mice ages 10-26 wks were monoassociated with Bifidobacterium animalis subsp. animalis (ATCC\# 25527). Mice were killed at 11,17 and 23 weeks after colonization. Inflammation was measured by blinded histologic scores (0-4+ range) of the duodenum, cecum and colon and by spontaneous secretion of IL-12p40 by colonic strips cultured for 24 hrs. Bacterial specific T cell responses were measured by IFNy secretion by CD4+ cells isolated from the mesenteric lymph nodes (MLN) of monoassociated mice co-cultured for 72 hrs. with T cell-depleted splenic antigen presenting cells (APC) pulsed with lysates of several bacteria. Results. $B$ animalis was found to colonize the intestines of germ-free WT and IL-10 KO mice to similar levels after 23 weeks (WT duodenum $=6.7 \pm 1.3$ vs. $\mathrm{KO}=6.6 \pm 1.4$; WT distal colon $9.3 \pm 0.5$ vs. $\mathrm{KO}=9.4 \pm 0.3 \log$ colony forming units/g tissue). B. animalis caused marked duodenal inflammation in monoassociated IL-10 KO mice characterized by extensive mononuclear cell infiltration, architectural distortion and thickening. Mild inflammation was detected in the cecum and colon of IL-10 KO animals by histological analysis and by spontaneous release of IL- $12 \mathrm{p} 40$ from colon explants (WT $=0.4 \pm 0.3 \mathrm{vs}$. IL$10 \mathrm{KO}=1.0 \pm 0.5 \mathrm{ng} / \mathrm{ml} \mathrm{p}<0.005$ ). Disease was associated with immune cell activation in IL$10 \mathrm{KO}$ mice as measured by IFNy release from CD4+ MLN cells co-cultured with bacterial lysate pulsed APC $(17.9 \pm 7.4 \mathrm{ng} / \mathrm{ml}$ IFN $\gamma$ for $B$. animalis vs. $4.5 \pm 2.4$ for $B$. infantis vs. $7.0 \pm 3.6$ for B. bifidum). No disease or immune activation was detected in WT animals. Conclusions. Bifidobacteria are considered beneficial members of the gut microbiota and are often included in probiotic products. We show that B. animalis induces marked duodenal and mild colonic inflammation when introduced alone into germ-free IL-10 KO mice, demonstrating a pathogenic role for a bacterial species previously shown to form an increased proportion of the gut microbiota of colitic mice.

35

Leukotriene D4 Modulates Na-Neutral Amino Acid Co-Transport During Chronic Intestinal Inflammation Jamil Talukder, Ramesh Kekuda, Uma Sundaram

Background: Na-neutral amino acid (e.g. alanine) co-transport is present on the brush border membrane (BBM) of the absorptive villus cells in the rabbit small intestine. This co-transport process is inhibited in the chronically inflamed small intestine secondary to a reduction in the affinity of the co-transporter for alanine. Leukotriene D4 (LTD4) is markedly increased in the mucosa during chronic enteritis. Whether the inhibition of Na-alanine co-transport during chronic enteritis is mediated by LTD4 is unknown. Aim: Determine the effect of LTD4 on Na-alanine co-transport in enterocytes. Methods: Rat small intestinal epithelial cells (IEC-18) were grown on transwell plates and confluent monolayers were used for transport experiments at the 10 th day post confluence. $3 \mathrm{H}$-alanine was utilized to determine $\mathrm{Na}$-dependent alanine uptake in IEC-18 cells. Na+/K+-ATPase activity was determined by measuring the inorganic phosphate formation. Total RNA was isolated from IEC-18 cells using Trizol reagent. ASCTI mRNA from both groups was quantified by real-time PCR. Results: Na-dependent $3 \mathrm{H}$-alanine uptake in IEC-18 cells was significantly inhibited by LTD4 $(6.4 \pm 0.1 \mathrm{nmol} / \mathrm{mg}$ protein $/ 2 \mathrm{~min}$ in control and $2.6 \pm 0.1$ in LTD4 treated cells, $\mathrm{p}<0.01$, $\mathrm{n}=3$ ). Since $\mathrm{Na}+/ \mathrm{K}+$-ATPase provides the favorable $\mathrm{Na+}$-gradient for this co-transporter, it was measured and found not to be altered $(26 \pm 6 \mathrm{nmol} / \mathrm{mg}$ protein/min in control and $25 \pm 3$ in treated cells, $\mathrm{n}=6$ ). The effect of LTD4 was reversed by cysteinyl leukotriene receptor antagonist REV5901. Kinetic studies revealed that the mechanism of inhibition of Na-alanine co-transport by LTD4 in IEC-18 cells was secondary to a decrease in the affinity for alanine without a significant alteration in the maximal rate of uptake. Real-time PCR demonstrated that $\mathrm{Na}$-dependent neutral amino acid co-transporter (ASCTl) mRNA was not significantly altered by LTD4 treatment. Conclusions: These studies demonstrate that LTD4 inhibits Naneutral amino acid co-transport in intestinal epithelial cells. This inhibition is not secondary to an alteration in the Na extruding capacity of the cells. The mechanism of inhibition of Na-alanine co-transport in IEC-18 cells by LTD4 is secondary to a decrease in the affinity of the co-transporter for amino acid without a significant change in co-transporter numbers. This mechanism of inhibition of Na-neutral amino acid co-transport is similar to that seen in the rabbit model of inflammatory bowel disease where mucosal levels of LTD4 are known to be elevated. Thus, these results indicate that proinflammatory leukotrienes inhibit Nadependent neutral amino acid co-transport during chronic intestinal inflammation.

\section{6}

Essential Roles for P53 and Puma in T Cell Induced Stem Cell Activation, Proliferation and Crypt Cell Apoptosis

Navdha Mittal, Ramanarao Dirisina, Jennet Manjali, Yueming Tang, Gery R. Grimm, Goo Lee, Navdeep S. Chandel, Terrence A. Barrett

INTRO/AIMS: 533 is linked to oxidative DNA damage and tumor formation in humans with ulcerative colitis. To investigate the effect of inflammation on $p 53$ activation and examine its role in epithelial proliferation and cell death,we utilized the anti-CD3 model of inducing systemic T cell activation. METHODS:Apoptosis was assessed by TUNEL and activated caspase-3(casp-3)staining and Western blot. The induction of apoptosis in cells undergoing proliferation was examined by double staining for casp-3 and BrdU incorporation(8h pulse). p53 levels were assessed by Western blot on isolated epithelial cells,localized by $\mathrm{IHC}$ and its requirement was tested in $\mathrm{p} 53$-/- mice. The induction of BH3-only proteins were detected using RT-PCR and Western blot for Bim, Bid, Bax, Bak and PUMA and their requirements for mediating apoptosis were examined using gene knockout mice. Intestinal stem cells (ISC) were identified by BrdU labeling $\mathrm{x} 48 \mathrm{~h}$ after radiation. Label retaining cells(LRC) were detected as BrdU+ cells 21 days after radiation. RESULTS: Evaluation of TUNEL and Western blot for casp- 3 revealed that $\mathrm{T}$ cell activation increased apoptosis by 17 and 35 -fold respectively with $>85 \%$ lower levels of both markers in p53-/- mice. Notably, apoptosis was unaffected in all BH3-only gene knockout mice except for PUMA-/- mice where it was abrogated (Table). Double staining for BrdU and casp- 3 revealed that all apoptosis in the crypt was restricted to proliferating cells. IHC staining revealed high levels of p53 in epithelial cells localized to the lower and mid-crypt areas. To determine whether this population included stem cells, p53 levels were examined in mice $21 \mathrm{~d}$ after radiation and BrdU labeling of stems (Methods). Results show that $3 \mathrm{~h}$ after anti-CD3, p53 staining localized to two populations: 1) label retaining stem cells at position 4, and 2) proliferating cells in the mid-crypt. Examination of proliferation-associated phospho-cMyc and cyclin D1 revealed that levels increased 3-fold in B6 mice without induction in p53-/- mice. CONCLUSION These data suggest that $\mathrm{T}$ cell-induced crypt cell apoptosis occurs via a p53-dependen pathway that requires the $\mathrm{BH} 3$-only p53 target protein, PUMA to execute rapidly proliferating cells. To our knowledge, this is the first report linking a role for p53 in ISC division to apoptosis. We suspect that this explains the p53-dependent crypt cell proliferation due to T cell activation which may provide clues to the role of $\mathrm{p} 53$ in mediating neoplastic transformation in colitis-induced cancer.

\begin{tabular}{|c|c|c|c|c|c|c|c|}
\hline & C57BL6 & p53--/ & Bid-/- & Bim-/- & Bax-/- & Bak-/- & PUMA-/- \\
\hline TUNEL & ++ & -- & + & ++ & ++ & ++ & -- \\
\hline BrdU & ++ & -- & ++ & ++ & ++ & ++ & ++ \\
\hline
\end{tabular}

37

Comparison of Digital Image Analysis (DIA) and Fluorescent in Situ Hybridization (FISH) in the Detection of Neoplasia Barrett's Esophagus Kenneth K. Wang, Ganapathy Prasad, Navtej S. Buttar, Michel Wongkeesong, Lynn S. Borkenhagen, Sheila Krishnadath, Marlys Anderson, Sarah Papenfuss, Kevin Halling, Thomas Smyrk, Catherine Demars, Lori Lutzke

Biomarkers such as $\mathrm{LOH}$ of $\mathrm{p} 53$, pl6, and DNA ploidy have been shown to be of value in determining cancer risk in Barrett's esophagus. However, they have not previously been used as a tool to detect high-grade dysplasia or cancer in Barrett's esophagus. Aim: To compare the ability of DIA and FISH to detect high-grade dysplasia or cancer in Barrett's esophagus. Methods: Patients referred to the Barrett's Esophagus Unit were prospectivel assessed with both DIA and FISH performed on brush cytology prior to surveillance biopsies that were taken in 4 quadrants every centimeter and assessed by two expert pathologists. The brush cytology specimens were processed by placing the specimens in PreservCyt solution (Cytyc Corp., Boxborough, MA). These were then collected using a ThinPrep 2000 Processor and stained with Feulgen dye and assessed with a CAS 200 digital imaging system. A DNA index was calculated by comparing the intensity of the nuclear staining of the Barrett's cells obtained by brush cytology to that of morphologically benign appearing epithelial cells within the specimen. A DNA index between 0.90 and 1.10 is considered diploid. Values between 1.1 and 1.9 are considered aneuploid. Values between 1.9 to 2 . are considered tetraploid. FISH was performed using a twelve probe set including 12-probe set; p15, 5q21-22, CEP7, 7p12, 8q24.12-13, CEP 9, 9p21, CEP 17, 17p13.1, 17q11.2-12 CEP Y, and 20q13. In order to compare results, we dichotomized the results of DIA an FISH as positive or negative based on the presence of any abnormalities (excluding loss of $\mathrm{Y}$ on FISH since this is almost ubiquitous in Barrett's mucosa and excluding nuclear morphometry on DIA). Using DIA, abnormalities such as increased tetraploidy or aneuploidy would be considered as a positive DIA test Results: A total of 92 patients were assessed of whom 8 had adenocarcinoma, 61 had high-grade dysplasia, 13 had low-grade dysplasia, and 11 patients had no dysplasia on biopsy. patients with high-grade dysplasia or cancer had DIA abnormalities in $47 / 69$ (68\%). This same group of patients had FISH abnormalities in 57/ $69(82 \%)$ of patients indicating that FISH abnormalities appear to be superior to DIA for predicting the presence of high-grade or cancer $(\mathrm{p}<0.05)$ Conclusions: FISH detected genetic abnormalities in cytological specimens correlated significantly better than DIA with the presence of neoplasia in Barrett's esophagus. This test has the potential to improve surveillance in Barrett's esophagus.

38

Cyclin a Immunocytology As a Risk Stratification Tool in Barrett's Esophagus Surveillance

Pierre Lao-Sirieix, Laurence Lovat, Rebecca C. Fitzgerald

Background The incidence of esophageal adenocarcinoma (AC) is increasing rapidly. Endoscopic surveillance of patients with Barrett's esophagus (BE) using biopsies for histopathological assessment is prone to sampling bias, is not cost effective and interpretation of dysplasia is subjective. Alternative methods for surveillance are direly needed. We have previously demonstrated that surface expression of a proliferative marker, $\mathrm{Mcm} 2$, is a marker of progression. However, the detection of patients at risk of progression through detection of $\mathrm{Mcm} 2$ positive cells in cytological specimens lacked specificity. Aims To determine whether cyclin A, which detects proliferating cells in the $S$ and $G 2$ phases of the cell cycle, would be more specific than $\mathrm{Mcm} 2$ for the detection of patients at risk of progression to AC. Methods Archival specimens ( 30 squamous esophagus (SE), 12 gastric antrum (GA) and 9 duodenum (D2), $62 \mathrm{BE}+/$ - dysplasia, $16 \mathrm{AC}$ ) were stained for cyclin A. In addition, 9 patients with 3-13 years follow-up who developed AC were compared with 18 controls matched for age and length of follow-up who did not progress. Endoscopic cytological brushings were taken from a prospective cohort (24 SE $93 \mathrm{BE}+/$ - dysplasia and $36 \mathrm{AC}$ ) and scored blind as cyclin A positive or negative. Results There was no surface expression of cyclin A in contro samples (NE, GA, D2) and its expression at the surface of BE samples correlated with the degree of dysplasia $(p=0.016)$. In the case-control cohort, patient with biopsies expressin cyclin A at the surface were more likely to progress to AC than those who did not (OR $10.0,95 \% \mathrm{CI} 1.5-64.2$ ). The sensitivity and specificity of cyclin A expression in brushings for the detection of high-grade dysplasia (HGD) and AC were $97.9 \%$ and $61.2 \%$ respectively. The associated negative predictive value (NPV) was $98.2 \%$. Comparatively, the sensitivity, specificity and NPV of $\mathrm{Mcm} 2$ for detection of $\mathrm{AC}$ and $\mathrm{HGD}$ patients, in a previous cohort, were $100 \%, 35.6 \%$ and $100 \%$. Using cyclin A nearly doubled the specificity with little impact on the sensitivity or NPV. Conclusions Expression of cyclin A at the luminal surface is a marker of progression to AC. Detection of cyclin A positivity in immunocytology could be used as a first step to stratify BE patients according to their risk of progression. A large clinical prospective study is required to confirm these findings. 
Pathologic Distinction of Barrett S Esophagus from Carditis with Intestinal Metaplasia: A Blinded Interobserver Variability Study

Amitabh Srivastava, Mark Redston, Gregory Y. Lauwers, Donald A. Antonioli, Robert D. Odze, Jonathan N. Glickman

Background: Barrett s esophagus (BE) and carditis with intestinal metaplasia (CIM) have different etiologies, natural history and risk of malignancy. We have previously identified several morphological parameters, listed below, that are useful in distinguishing these two conditions in mucosal biopsies from the gastro-esophageal junction (GEJ) region. The purpose of this study was to evaluate the level of interobserver agreement among expert GI pathologists in recognizing the various morphological parameters and in distinguishing BE from CIM in GEJ biopsies. Design: Routinely processed H\&E stained biopsies from the GEJ region, representing 10 of distal esophagus in $\mathrm{BE}$ and 10 of proximal stomach in CIM, were evaluated by four GI pathologists in a blinded manner without knowledge of the clinical or endoscopic findings. Each pathologist was asked to categorize cases as either BE or CIM, and to score the presence or absence of the following previously reported BE-associated features: crypt atrophy, crypt disarray, incomplete or complete IM, extensive incomplete IM ( $>50 \%$ crypts), squamous epithelium over metaplastic crypts (Sq/IM), hybrid glands, multilayered epithelium $(\mathrm{ME})$ and esophageal gland/ducts (EG/ED). Interobserver agreement was calculated for each morphological parameter and for the overall diagnosis, using kappa ( $\mathrm{k}$ ) statistics for concordance between multiple raters. Interobserver agreement was rated as excellent $(\mathrm{k}>$ $0.80)$, good (0.6-0.8), fair (0.4-0.6) and poor $(<0.4)$. Result: Interobserver agreement was good for the overall diagnosis of $\mathrm{BE}$ vs. CIM $(\mathrm{k}=0.6)$. Three or more observers correctly diagnosed BE or CIM in 15/20 (75\%) cases. The interobserver agreement for each individual morphological parameter ranged from excellent to good for some features (EG/ED: $k=0.83$, Sq/IM: 0.65, incomplete IM: 0.56, crypt atrophy: 0.53), fair for extensive IM (0.47), and poor for the others (complete IM: 0.25 , crypt disarray: 0.21 , and ME: 0.17 ). Interestingly, when none of the $\mathrm{BE}$-associated features were present $(5 / 20$ cases), agreement for a diagnosis of CIM was excellent ( 3 or more observers agreed in all five cases). Similarly, when four or more BE-associated parameters were present in the same biopsy specimen (10/20 cases), three or more observers agreed on the diagnosis of BE in all cases. Conclusion: There is good agreement among expert GI pathologists for distinguishing BE from CIM in GEJ biopsies when using the morphological features outlined above. In fact, a correct diagnosis of $\mathrm{BE}$ is more often reproducible when a combination of these features $(>4)$ are present together in the same biopsy specimen.

40

Biomarkers in the Therapy of Barrett's Esophagus with High-Grade Dysplasia Ganapathy A. Prasad, Kenneth K. Wang, Halling C. Kevin, Shannon Brankley, Navtej S. Buttar, Louis M. Wongkeesong, Lori S. Lutzke, Sarah M. Papenfuss, K K. Krishanadath

Rationale: Photodynamic therapy (PDT) has been shown to be effective in the treatment of high-grade dysplasia (HGD) in Barrett's esophagus (BE), however a substantial proportion of patients do not respond. Aims: To determine if biomarkers known to be important in Barrett's neoplasia can predict response to PDT in BE and HGD/mucosal cancer. Methods: Patients with BE and HGD/mucosal cancer referred for endoscopic therapy to the Mayo Clinic were prospectively studied from 2002-2005. All patients underwent a protocol assessment with EGD, 4 quadrant biopsies every $\mathrm{cm}$, EMR of visible nodules and EUS. Biomarkers were assessed using FISH for region specific and centromeric probes. Biomarkers assessed included:loss of 9p21 (site of p16 gene) and 17p13.1 (site of p53 gene) loci; gains of 8q24(cmyc), 17q (HER2-neu) and 20q13 loci. Respective centromeric probes were used to assess chromosomes 9, 17, 20, and 8. Cells were also assessed for loss or gain of chromosomes (aneusomy and polysomy). Patients were treated with PDT 48 hours following administration of sodium porfimer.Demographic and clinical variables were prospectively collected. Logistic regression was performed to determine predictors of response to PDT (defined as the absence of dysplasia at surveillance biopsies 3 months following PDT). Results: 58 patients have been entered with a mean age 67.6 years (SEM1.38). 54 (93\%) were males. 41 (82\%) had HGD and the remainder had cancer. The mean BE segment length was $5.5 \mathrm{~cm}$ (SEM 0.44). $46(80 \%)$ patients had EMR done before PDT. $31(53 \%)$ of patients was responders at 3 months following PDT. On univariate analysis, BE segment length (OR $0.67[0.53,0.85] \mathrm{p}=$ $0.001), 9 \mathrm{p} 21$ (pl6) loss (OR $0.15[0.05,0.52] \mathrm{p}=0.003)$, were significant predictors of lack of response to PDT. Loss of the 17p13.1(p 53) locus (OR $0.25[0.06,1.15] \mathrm{p}=0.08$ ) was a marginally significant predictor. Age, gender, EMR, and other biomarkers were not significant predictors on univariate analysis. On multivariable analysis, BE segment length and loss of pl6 locus were independent predictors of response to PDT. The presence of polysomy and/ or gain at $8 \mathrm{q} 24 / 17 \mathrm{q} / 20 \mathrm{q} 13$ loci was a marginally significant predictor of response to PDT.(see table) Conclusion: This is the first prospective study which identifies a biomarker, pl6, as predicting response to PDT in BE and HGD/cancer. Identification of biomarkers may help in the selection of appropriate therapy for patients and improve treatment outcomes.

\begin{tabular}{|c|c|c|}
\hline Variable & OR $(95 \% \mathrm{CI})$ & $\mathrm{p}$ value \\
\hline BE length & $0.68(0.53,0.88)$ & 0.004 \\
\hline p16 loss & $0.12(0.05,0.58)$ & 0.008 \\
\hline Polysomy/loci gain & $0.24(0.05,1.11)$ & 0.07 \\
\hline
\end{tabular}

41

DNA Methylation in the Esophageal Mucosa of Patients 5 Or More Years After a Fundoplication for Barrett's Esophagus

John J. Kelly, Eric Smith, Paul A. Drew, Stuart R. Phillis, Andrew R. Ruszkiewicz, Glyn G. Jamieson

Introduction: Barrett's esophagus results from reflux and is a major risk factor for adenocarcinoma of the distal esophagus. Aberrant DNA methylation is frequent in Barrett's esophagus and adenocarcinoma and is thought to contribute to the disease progression. Fundoplication protects the oesophagus from reflux and may result in regression of the Barrett's mucosa, but it is not known what effect it has on methylation in the esophagus. Aim: To test the hypothesis that reflux control by fundoplication in patients with Barrett's esophagus will reduce DNA methylation in the esophageal mucosa. Method: We studied 23 patients with histologically proven Barrett's esophagus preoperatively who had a laparoscopic fundoplication more than 5 years ago. Reflux was measured by 24 hour $\mathrm{pH}$ monitoring. Biopsies at $2 \mathrm{~cm}$ intervals from any columnar epithelium and squamous epithelium were obtained at endoscopy. Methylation was measured in the promoter region of 9 genes - APC, CDKN2A, ID4, TEMMF2, MGMT, RBP1, RUNX3, SFRP1 and TIMP3. Results: The median follow up was 8 years ( 60 - 127 months). Of the 18 patients with no reflux, 17 had an apparent regression of the columnar mucosa. This regression was complete in 9 patients ( 5 long segment and 4 short segment) and partial in 8 patients. Five patients had reflux and persistent Barrett's esophagus. There was significantly less methylation in the columnar epithelium of patients without reflux compared to those with reflux $(\mathrm{p}<0.0001)$. The squamous biopsies from patients without reflux but continuing Barrett's had more methylation than either squamous epithelium proximal to the Barrett's in patients with reflux $(\mathrm{p}=0.005)$, or from patients with complete regression $(\mathrm{p}<0.001$ ). This may reflect the squamous biospies coming from areas in which metaplastic changes are reversing. Conclusions: We observed complete regression to squamous mucosa in $50 \%$ of patients with a fundoplication and no acid reflux. In patients with complete regression the regenerated squamous had minimal methylation which did not differ from the squamous proximal to Barrett's. In patients with partial regression there was significantly less methylation in the columnar lining compared to the patients with continuing reflux, but more methylation in the squamous regenerating region than in the normal esophagus. The significance of the methylation in the partially regressed esophagus warrants further investigation.

42

CDX2 Expression Predicts the Presence of Specialized Intestinal Metaplasia in Columnar Epithelium of the Esophagus Even in the Absence of Goblet Cells Marjon Kerkhof, Dorine A. Bax, Leon M. Moons, Anneke J. van Vuuren, Herman van Dekken, Ernst J. Kuipers, Johannes G. Kusters, Peter D. Siersema

Background: Barrett's esophagus (BE) is a premalignant condition characterized by the presence of specialized intestinal metaplasia (IM) in columnar epithelium. In some patients, patchy areas of IM and cardia-type mucosa (CM) are present in the columnar-lined esophagus, whereas in other patients only CM is detected, which is generally not regarded as premalignant. In contrast to patients with $\mathrm{IM}$, patients with only $\mathrm{CM}$ are currently not advised to undergo endoscopic surveillance. As IM and CM are endoscopically indiscernible, sampling error during endoscopy may result in false-negative diagnoses of IM. Aim: To investigate whether intestinal markers for IM, such as CDX2 (early intestinal marker), MUC2 (goblet cell marker) and villin (late intestinal marker), in the absence of a histological diagnosis of IM (characterized by goblet cells), are predictive for the presence of IM in another part of the columnar-lined esophagus. Methods: A total of 122 sets of biopsies from columnar epithelium of the esophagus from 61 patients ( 42 males, mean age at first endoscopy 56 years) were collected at two different gastroscopies with a medium interval of 24 (range 4156) months. Patients were divided into three groups: 15 patients with IM in both biopsy sets (group 1), 25 patients with in one biopsy set only CM and in the other IM (group 2), and 21 patients with only CM in both biopsy sets (group 3). Haematoxylin and eosin, alcian blue, and periodic acid-Schiff staining was performed to identify the type of epithelium. CDX2, MUC2 and villin expression was determined by immunohistochemistry. Results: IM was present in 55/122 (45\%) samples, which were all positive for CDX2 and MUC2. Villin expression was present in 32/55 (58\%) IM samples. In addition, CDX2 expression was detected in 23/67 (34\%) samples with only CM. CDX2 in CM was more often $(\mathrm{p}<0,05)$ observed in patients of group 2 , in which the other biopsy set was positive for IM (13/25; $52 \%$ ), compared to patients of group 3, in which IM was absent in both biopsy sets (10/ $42 ; 24 \%$ ). The detection of CDX2 in CM significantly increased the chance of finding IM in another biopsy sample of the columnar segment (OR 3.5 (95\% Cl=1.2-10). Thirteen of these $23(57 \%)$ CDX2 positive CM samples were also positive for MUC2, whereas villin expression was detected in $7 / 23(30 \%)$. Conclusion: CDX2 expression in CM, in the absence of goblet cells suggestive for IM, predicts the presence of IM in another part of the columnarlined esophagus. Our results suggest that CDX2 staining should be used as an additional histological marker to determine if endoscopic surveillance is indicated. 
H. Pylori Accelerates the Progression of Dysplasia of the PYLORIC Antrum in Tff2-/- C57bl X Sv129 Infected Mice

James G. Fox, Arlin B. Rogers, Evelyn Kurt-Jones, Timothy C. Wang

Trefoil facter 2 (TFF2) also referred to as spasmolytic polypeptide is upregulated in Helicobacter spp infected gastric tissues of both humans and mice. Recent studies (Kurt Jones et al submitted) demonstrated that this peptide plays a role in regulation of innate immunity similar in some respects to that of IL10. To ascertain TFF2's biological effects in vivo, TFF-/C57BL x Svl29 (n=20) and C57 BL6 x Svl29 mice $(n=20)$ were orally dosed with H. pylori SS1 $\left(10^{9} \mathrm{CFU}\right)$ every other day for a total of 3 doses. Two other control groups of TFF-/and WT mice were sham dosed. Gastric lesions were scored in blinded fashion by a comparative patholgist at 6 and 19 months post inoculation (pi). At 6 mos pi, there was not a statistically significant difference in corpus inflammation between $H$. pylori TFF2-/and WT mice, but their was a significant difference $(\mathrm{p}<0.05)$ for some epithelial criteria (mucosal defects, oxyntic atrophy, hyperplasia and intestinal metaplasia) in the corpus of TFF2-/- vs WT mice. At 19 mos pi, a similar statistical difference in epithelial parameters was noted in the antrum of TFF2-/- vs WT mice $(\mathrm{p}<0.01)$ (See figure below). However, corpus lesions at 19 mos pi did not differ significantly in infected TFF2-/- and WT mice. Importantly, 2 of 9 TFF2-/- H. pylori infected mice developed gastric intraepithelial neoplasia (GIN) of the pyloric antrum and the remaining had high grade dysplasia whereas only one of 11 WT infected mice developed antral GIN and the remaining had only low grade dysplasia $(\mathrm{P}<0.01)$. A gender effect was not noted. In conclusion, TFF2 provides a protective gastric epithelial effect against the progression of premalignant lesions of the pyloric antrum in H. pylori infected mice.

\section{9 month antral lesion scores H. pylori infected mice}

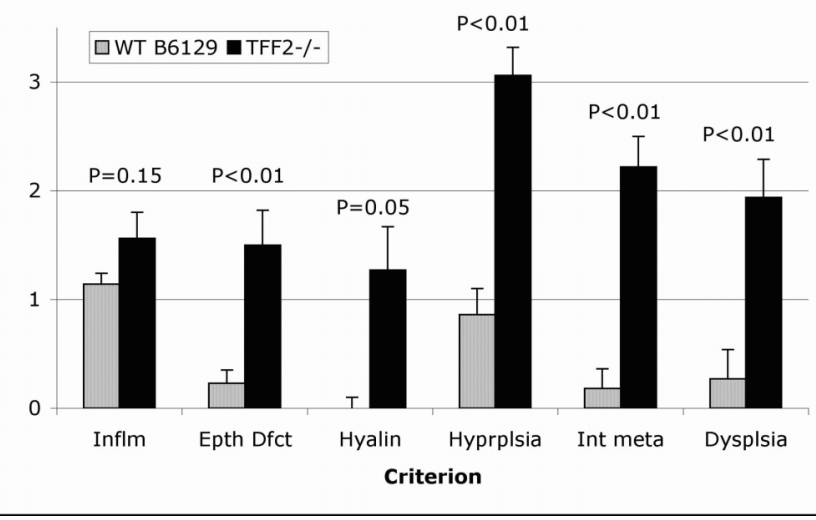

44

Cobral: A Novel Regulator of TFF1 Transcription in Upper Gastrointestinal Adenocarcinomas

Patricia McChesney, Mohammad Razvi, Rong Li, Chrsitopher Moskaluk, Alexander Zaika, Wael El-Rifai

We and others have shown that TFFl mRNA and protein expression is frequently downregulated in upper gastrointestinal adenocarcinoma (stomach, GEJ, and lower esophageal). Although promotor hypermethylation and LOH have been suggested as mechanisms for downregulation of TFFl in gastric cancers, the majority of gastric tumors lack these changes. In this work, we demonstrate that Cofactor of BRCAl (COBRAl), a newly characterized member of the Negative Elongation Factor (NELF) complex, is overexpressed in the majority of primary upper UGCs, and its overexpression correlates with downregulation of TFFl. We have detected overexpression of COBRAl mRNA using quantitative real time RT PCR in twenty-eight primary UGCs (79\%). Immunohistochemical analysis of UGC tissue arrays that contained 70 tumor samples demonstrated moderate-strong staining for COBRAl in 60 tumors (84\%). Simultaneous loss of TFFl expression and overexpression of COBRAl was observed in $42 / 70(60 \%)$ tumors. Using siRNA technology, with gastric cancer cells, we demonstrate that COBRAl inhibition leads to increased TFFl promoter activity and gene expression. Promoter analysis of TFFl indicates that regulation of TFFl by COBRAl is estrogen independent, and that COBRAl can negatively regulate the AP-1 complex at the TFF1 promoter, and thus, downregulate TFF1 expression in gastric cancer cell lines. Electrophoretic mobility shift assay (EMSA) verifies that COBRAl attenuates AP-1 binding to DNA. In conclusion, our results demonstrate a novel transcriptional mechanism that may explain the loss of TFFl expression in the majority of upper gastrointestinal carcinomas. COBRAl acts as a transcription factor binding protein that regulates the activity of AP-1, thus inhibiting expression of TFFl in gastrointestinal cells. These findings suggest that COBRAl/APl is a novel transcription regulatory mechanism with oncogenic potential in UGCs.
Effects of Peroxisome Proliferator-Activated Receptor-Gamma Agonist, Rosiglitazone, On Chemoprevention of Gastric Cancer in Rat Wai K. Leung, Shanying Liao, Zhi-Rong Zeng, Christine Y. Wong, Wai-Kit Chu, Victoria Y. Cheng, Ka-Fai To, Jun Yu, Pin-Jin Hu, Joseph J. Sung

Background: Peroxisome proliferators-activated receptor-gamma (PPAR $\gamma$ ) is a nuclear hormone receptor that regulates gene expression and cellular differentiation. We have previousl shown that PPARy suppresses the growth and induces apoptosis of gastric cancer cells [Leung, Gut 2004]. Aim: To evaluate the in vivo chemopreventive effects of PPARy agonis in an animal model of gastric carcinogenesis. Methods: Gastric tumors were induced in Wistar rats with N-methyl-N'-nitro-N-nitrosoguanidine (MNNG) as described previously [Hu et al, Gut 2004]. MNNG $(100 \mu \mathrm{g} / \mathrm{ml})$ were given in drinking water ad libitum. Fourweek-old male rats were divided into 4 groups (20 in each group) to receive rosiglitazone at 0.2 (Group A), 0.8 (B), 3.2 (C) $\mathrm{mg} / \mathrm{kg} / \mathrm{d}$ or distilled water (positive control; D) by ora gavage for 40 weeks. Rats treated with vehicle alone without MNNG were used as negative control (E). All animals were sacrificed at the end of study. Stomach was harvested and gastric carcinoma was verified by histology. Gene expression profiles of rat gastric tumors treated with rosiglitazone or MNNG alone were analyzed by oligonucleotide microarrays (CodeLink UniSet Rat I Bioarray) that contains 10,000 rat gene targets. Microarray data was processed by the Significance Analysis of Microarrays (SAM) and fold-difference filtering Results: $70 \%$ of MNNG treated (Group D) rats developed gastric adenocarcinoma and none of the control rats fed with vehicle alone (Group E) had stomach cancer. Treatment with rosiglitazone significantly reduced the incidence of gastric cancer development in rats (Group A: $15 \% ; B: 20 \% ; C: 25 \%, P=0.0005)$. The mean tumor volume was also lower in rosiglitazone treated rats (Group A, B and C: $0 \pm 0,1.6 \pm 1.6$ and $16.1 \pm 15.7 \mathrm{~mm}^{3}$ ) as compared to MNNG group ( $\left.50.4 \pm 30.5 \mathrm{~mm}^{3}, \mathrm{P}=0.004\right)$. The best chemopreventive effect was obtained in rats treated with rosiglitazone $0.2 \mathrm{mg} / \mathrm{kg} / \mathrm{d}$. Oligonucleotide array showed that 79 genes were differentially upregulated in rosiglitazone treated gastric tumors when compared to MNNGinduced tumor. These genes were involved in transcription factors (e.g. Stat5A and TGFBR1), signal transduction (LHCGR, NPY5R), metabolism (METla), cell cycle (bcl2) and biosynthesis (HMBS) by Gene Ontology annotation. 54 genes had yet unknown biological function Notably, no gene was found to be significantly downregulated in rosiglitazone treated gastric tumors. Conclusion: Our results showed that the PPAR $\gamma$ ligand rosiglitazone has a potent chemopreventive effect against gastric cancer development in rat. Gene activation appears to play a major role underlying the chemopreventive effects of PPAR $\gamma$ ligands.

46

Chemoprevention of Gastric Cancer By Celecoxib in H. Pylori-Infected Mongolian Gerbils

Seiji Futagami, Kenji Suzuki, Tetsuro Hiratsuka, Tomotaka Shindo, Tatsuhiko Hamamoto, Atsushi Tatsuguchi, Nobue Ueki, Shinji Yoko, Masafumi Kusunoki, Ken Wada, Kazumasa Miyake, Katya Gudis, Taku Tsukui, Choitsu Sakamoto

Aims/Background: A number of studies have shown that the cyclooxygenase(COX)-2/PGE2 pathway plays a crucial role in cancer development and progression. Although, elevated expression of COX-2 had been clearly shown in gastric cancer, it was not clear whether the inhibition of COX-2 in turn lead to the inhibition of gastric cancer occurrence or to the inhibition of pre-cancerous lesions that culminate in gastric carcinoma. Therefore, in this study, we evaluated whether administration of celecoxib, a selective COX-2 inhibitor, contributes to the prevention of not only gastric cancer but also of intestinal metaplasia in MNUpretreated H. pylori-infected Mongolian gerbils. Methods: Fifty-two male Mongolian gerbils were given MNU (N-Methyl-N-nitrosourea) (30ppm) every second week for 10 weeks and divided into three groups: group $1(\mathrm{n}=10)$ received Brucella broth without $H$. pylori; group $2(\mathrm{n}=20)$ received $H$. pylori inoculation at week 7 ; group $3(\mathrm{n}=22)$ given a daily CRF-1 die containing celecoxib $(1500 \mathrm{ppm})$ after $H$. pylori inoculation. At experimental week 54 , al animals were sacrificed. The incidence of gastric adenocarcinoma was determined by histological analysis. COX activity in the gastric tissue was measured by the production of prostaglandin E2 using ELISA methods. Apoptotic index was determined by terminal deoxynucleotidyl transferase-mediated nick end labeling assay. The extent of intestinal metaplasia (Alcianblue PAS), CdX2, and MUC2 expression in the stomach were evaluated semi-quantitatively immunohistochemically. Results: The incidence of gastric adenocarcinoma at sacrifice was $0 \%(0 / 10)$ in group $1,65 \%(13 / 20)$ in group 2 , and $23 \%(5 / 22)$ in group 3. Celecoxib significantly prevented gastric cancer development induced in MNU-pretreated $H$. pyloriinfected Mongolian gerbils. Overall intensity of COX-2 expression measured by immunohistochemistry in intestinal metaplasia, dysplasia, and gastric adenocarcinoma was nearly the same in group 2 and 3 gerbils. However, celecoxib administration significantly reduced COX activity and provoked apoptotic activity in group 3 gerbils. CdX2 was also expressed in intestinal metaplasia, dysplasia, and gastric adenocarcinoma in $\mathrm{H}$. pylori-infected gerbils, while celecoxib also significantly reduced extent of CdX2 as well as MUC2 expression and AB-PAS-positive intestinal metaplasia in H. pylori-infected gerbils. Conclusions: The data presented here suggests that the selective COX-2 inhibitor celecoxib prevents gastric cancer occurrence by disrupting the progression of intestinal metaplasia into gastric carcinoma through its inhibition of $\mathrm{CdX} 2$ expression in MNU-pretreated H. pylori-infected Mongolian gerbils.

47

Female Hormones Provide a Protective Effect in Helicobacter Pylori Induced Gastric Disease in Ins-Gas Mice

Masahiro Ohtani, Zhongming Ge, Alexis Garcia, Arlin B. Rogers, Nancy S. Taylor, Sandy $\mathrm{Xu}$, Koichiro Watanabe, Kristen Clapp, Juri Miyamae, Robert P. Marini, Takeshi Azuma, Timothy C. Wang, James G. Fox

Background: The incidence rate of human gastric cancer in males is approximately 2 times the rate recorded in females. Although some epidemiological studies indicate that female hormones reduce the risk of gastric cancer, this hypothesis is still controversial. In addition,

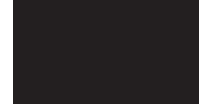


the role of female hormones in gastric carcinogenesis caused by infection with H. pylori has not been evaluated in rodent models. We previously demonstrated that gastric cancer caused by infection with $H$. pylori is male-predominant in INS-GAS transgenic mice (Fox et al, 2003) Aim: The aim of our study was to investigate the effect of female hormones on gastritis and cancer caused by infection with $H$. pylori in INS-GAS mice. Methods: Forty-six (16 males, 30 females) specific pathogen free INS-GAS mice on a FVB background were used in this study. Ovariectomy or sham ovariectomy were performed on female mice at the age of 8 weeks. Eight to 10 week old mice were inoculated three times by oral gavages with $10^{8}$ cfu of $H$. pylori SS1 or broth alone. Mice were euthanized at 16 weeks post-infection. Histological evaluation (inflammation, atrophy, hyperplasia, intestinal metaplasia and dysplasia) were graded on a blinded basis, on a scale from 0 to 4 , by a comparative pathologist Quantitative cultures of H. pylori were performed on gastric tissue (antrum and corpus). Statistical analyses were performed using Mann-Whitney U test. Results: Male mice infected with H. pylori developed corpus gastritis with atrophy, intestinal metaplasia, foveolar hyperplasia and dysplasia. Intact female mice infected with $H$. pylori developed attenuated gastric pathology compared to infected male mice $(\mathrm{P}<0.05)$. In contrast, ovariectomized mice infected with $H$. pylori developed corpus gastritis with atrophy and dysplasia comparable to infected male mice. All lesion scores in ovariectomized mice infected with $H$. pylori were significantly higher than those in infected female mice $(\mathrm{P}<0.05)$. Ovariectomy didn't affect colonization levels of $H$. pylori when compared to other infected groups of mice. Conclusion: Ovariectomy in female INS-GAS mice statistically increased severity of gastric disease caused by infection with $H$. pylori. This finding suggests that female hormones have a suppressive role in development of gastritis and premalignant lesions. A long-term study to investigate the molecular mechanisms responsible for the protective effect of female hormones on development of gastric cancer is in progress.

48

Reciprocal Fundic and Antral Changes in Sonic Hedgehog (SHH) During the Progression from Atrophic Gastritis to Gastric Cancer

Meghna Waghray, Weiqun Kang, Yana Zavros, Juanita Merchant

Objective: Previously we have shown that loss of gastrin results in impaired gastric acid secretion and the development of antral cancer within one year (Oncogene 2005). Prior studies by Beachy and co-workers have shown that sonic hedgehog (Shh) levels are elevated in human gastric cancer cells (Nature, 2003). Therefore, we examined the expression of Shh and relevant signaling molecules in gastrin deficient (G-/-) mice during progression to gastric cancer. Methods: RNA isolated from the antral tissues of G-/- and wild type (WT) mice was subjected to microarray analysis. Stomachs from the 15 month old G-/- and WT mice were separated into fundus and antrum then further compartmentalized into epithelium and mesenchyme via EDTA chelation. The purity of the separation was documented using HK-ATPase and gastrin RT-PCR as fundic and antral epithelial specific markers respectively, with Actg2 and Madcaml as mesenchymal markers. RT-PCR was used to analyze the abundance of Shh and its target genes in specific compartments. Protein prepared from WT and G-/- mice was analyzed for Shh and its target gene patched (Ptc) by western blot. Results: Atrophic changes were detected in the fundus of the G-/- mice, with a subsequent progression of hyperplasia in the antrum. Accordingly, western blot analysis showed reduction of Shh (15-fold) and Ptc (7.5-fold) in the G-/- fundus, but significant elevation of Shh (4-fold) and Ptc (3.2 fold) in the neoplastic antrum. RT-PCR results confirmed reduction of Shh and Ptc in the fundus, and induction in the mesenchymal compartment of G-/ antrum. In addition, Shh target genes BMP7 and sFRPl also increased in the antral mesenchyme. Microarray analysis consistently revealed prominent upregulation of the genes belonging to TGFbeta and Wnt signaling pathways in antral tumors. Conclusion: Atrophic gastritis results in the reduction of hedgehog signaling in the fundus. In the antrum, an elevation in the hedgehog signaling pathway is seen, which may contribute to antral transformation. Thus, the G-/- mouse model may be used to study how spatial and temporal changes in hedgehog signaling and its target genes predispose the stomach to atrophy leading to gastric cancer.

49

Elevated Iron Absorption in the Neonatal Rat Reflects High Expression of Iron Transport Genes in the Distal Small Intestine and Colon David M. Frazer, Sarah J. Wilkins, Therese L. Murphy, Gregory J. Anderson

Background: Neonatal mammals have a very high rate of intestinal iron absorption to ensure an adequate supply of iron to the growing infant. They may absorb up to $100 \%$ of a test dose of radioactive iron during the suckling period, but absorption drops rapidly to adult levels (approximately 10\%) at the time of weaning. Aim: To investigate the molecular basis of this dramatic change in absorption by determining the expression of iron transport genes in the gastrointestinal tract of neonatal rats before and after weaning and correlating gene expression with iron absorption. Methods: All studies were carried out using Sprague-Dawley rats which were weaned when they were 21 days old. Suckling rats were maintained with their mothers. Samples from the duodenum, jejunum, ileum and colon were taken at 10 , 15, 20 and 25 days after birth. Tissue was also taken from adult rats (10 months old) maintained on normal pellet diet. The expression of iron transport genes was determined by ribonuclease protection assay. Intestinal iron absorption was determined by measuring the retention of an oral dose of $59 \mathrm{Fe}$ in whole animals, or by measuring iron transported to the body using cannulated intestinal loops. Results: Iron absorption measurements showed that 10 and 15 day old rats absorbed $87.0 \pm 2.2 \%$ and $84.1 \pm 4.2 \%$ of a test dose respectively, but this reduced dramatically around weaning with 20 day old rats absorbing $50.6 \pm 4.9 \%$ and 25 day old animals absorbing $7.8 \pm 1.7 \%$. The expression of the brush border iron transporter DMTl and reductase Dcytb increased during suckling in the duodenum to reach a maximum just before weaning. After weaning, expression declined to adult levels. Duodenal expression of the basolateral transport components hephaestin and ferroportin remained relatively constant throughout suckling and weaning. Neither of these expression patterns correlates well with iron absorption. However, during the suckling period, all genes studied were expressed at high levels in the colon and to a lesser extent in the ileum. After weaning, only hephaestin was expressed in the distal alimentary tract. Iron absorption by duodenal loops was similar in 15 and 25 day old rats, but absorption by ileal and colon loops was increased 6.6 fold and 3.3 fold respectively in the younger animals relative to their older counterparts. Conclusions: These results suggest that the entire length of the small intestine and the colon may be involved in iron absorption in the neonate and that following weaning the absorption of iron becomes restricted to the duodenum and upper jejunum.

50

Protein Composition of the Pre-Chylomicron Transport Vesicle (PCTV)-CIS Golgi Fusion Complex

Shadab Siddiqi, James Mahan, Charles Mansbach

Vesicle fusion with its target membrane is mediated by specific membrane proteins, soluble NSF attachment protein receptors (SNAREs). SNARE complexes are composed of 1 vesicle SNARE (v-SNARE) and 3 target membrane SNAREs (t-SNAREs) which form a 4 component $\alpha$-helical bundle which by itself can induce membrane fusion. SNAREs select cargo for inclusion in the transport vesicle, attract coatomer proteins (COPII), and target the vesicle to specific acceptor membranes. We have identified vesicle associated membrane protein 7 (VAMP7), a post Golgi SNARE protein, to be uniquely localized to the endoplasmic reticulum (ER) in the intestine (Siddiqi, S, et al. J. Cell Sci., 2005, in press) and have shown that VAMP7 is both concentrated on PCTV and functional in transporting chylomicrons to the cis Golgi. We postulated that VAMP7 functions as a v-SNARE for PCTV and forms a unique SNARE complex with t-SNAREs on the cis Golgi enabling fusion. We have made an antirat VAMP7 specific antibody (Siddiqi, S. J. Cell Sci., 2005) that we used for immunoprecipitation of its binding partners. PCTV were generated from rat ER (Siddiqi et al. J. Cell Sci., 2003). We were able to dock PCTV with cis Golgi, thus stabilizing the SNARE complex, by incubation with cytosol at $4 \mathrm{C}$ in the absence of ATP and $\mathrm{Mg} 2+$ as identified by PCTV becoming iso-dense with the cis Golgi indicating association and the lack of PCTVchylomicron acquisition of apolipoprotein-AI (apo-I). Apo-AI is present in the Golgi but not in PCTV-chylomicrons. Bead-bound VAMP7 antibodies immuno-precipitated the tSNAREs syntaxin5, vtila, and rBetl in a $112 \mathrm{kDa}$ complex disassociated by boiling. Bead bound rBetl antibodies immuno-precipitated VAMP7, syntaxin5, and vtila also in a 112 kDa complex. Antibodies to membrin, GOS28, and Sec22b, ykt6, other v- and t-SNAREs, identified each to be present in docked PCTV-cis Golgi, but at their monomer Mr indicating they were not part of the SNARE complex. Antibodies to each SNARE inhibited fusion of PCTV with cis Golgi. Incubation of PCTV with cis Golgi, cytosol, ATP and Mg2+ at 37 C resulted in chylomicron acquisition of apo-AI as determined by isolating chylomicrons from post-fusion Golgi membranes indicating a fusion event. PCTV were shown not to fuse with liver or kidney cis Golgi indicating the specificity of the fusion process. We conclude that the protein composition of the PCTV-cis Golgi fusion complex is composed of VAMP7, syntaxin5, vtila, and rBetl. We propose that this unique system facilitates the transport of chylomicrons to the cis Golgi which occurs intermittently with fat feeding as compared to the constant transport of newly synthesized proteins

51

Intestinal Threonine Metabolism in Wild Type and MUC2 Deficient Mice Maria Van der Sluis, Maaike Schaart, Barbara de Koning, Henk Schierbeek, Anna Velcich, Ingrid Renes, Johannes van Goudoever

Mucin 2 (Muc2) is the major secretory mucin of the mucus layer covering the intestinal epithelium. Muc2 is a large glycoprotein rich in threonine-proline-serine tandem repeats. Previous studies in neonatal pigs showed that the intestine utilizes $60-90 \%$ of the dietary threonine intake. We therefore hypothesized that dietary threonine is mainly used for intestinal Muc2 synthesis. Our aim was to determine the threonine utilization in the intestine of Muc2 knockout $\left(\mathrm{Muc2}^{-/}\right.$) mice and wild type (Muc2 $2^{+/+}$) mice. Muc2 $2^{-/}$and $\mathrm{Muc2}^{+/+}$mice received a $\mathrm{U}_{-}{ }^{13} \mathrm{C}$ - threonine bolus. Mice were sacrificed 1,3 and 6 hours after threonine administration. The concentration and isotopic enrichment of threonine was measured in small intestine, colon and faeces by GC-(IR)MS. In the gastrointestinal tract $41.5 \%$ of the dietary $\left[{ }^{13} \mathrm{C}\right]$ threonine administered was recovered after 6 hours in Muc2 $2^{+/+}$mice $[33.7 \%$ $\left[{ }^{13} \mathrm{C}\right]$ threonine and $7.7 \%$ as $\left[{ }^{13} \mathrm{C}\right]$ glycine $]$ vs. $44.8 \%$ in the Muc $2{ }^{-1}$ mice $\left[34.3 \%\left[{ }^{13} \mathrm{C}\right]\right.$ threonine and $10.5 \%$ as $\left[{ }^{13} \mathrm{C}\right]$ glycine]. In the Muc $2^{+/+}$mice, $27.5 \%$ was recovered as protein bound $\left[{ }^{13} \mathrm{C}\right]$ threonine in the intestine vs. $25.2 \%$ in the Muc2 $2^{-1-}$ mice (non significant), when the threonine tracer was given as free amino acid. There were no significant differences in the percentage of $\left.{ }^{13} \mathrm{C}\right]$ threonine recovered in small intestinal tissue proteins between Muc $^{+/+}$and Muc2 $2^{-/}$mice after 6 hours $[14.1 \%$ vs $8.0 \%$ respectively]. The percentage of $\left[{ }^{13} \mathrm{C}\right]$ threonine recovered in colonic tissue proteins was significantly higher in Muc ${ }^{-1-}$ mice compared to $\mathrm{Muc}^{+/+}$mice at 6 hours $[0.4 \%$ vs. $0.2 \%$, respectively $\mathrm{p}=0.02] .27 .5 \%$ of the $\left[{ }^{13} \mathrm{C}\right]$ threonine in the faeces was derived from Muc2. Conclusions: Dietary threonine metabolised by the intestine of mice was used for synthesizing constitutive proteins, as well as Muc2. Thus, secretory protein synthesis is an important pathway of intestinally utilized dietary threonine.

52

Conditional Intestinal Lipotoxicity: Functional Superiority of APOB48 and a Survival Advantage for APOB mRNA Editing Yan Xie, Jianyang Luo, Zhouji Chen, Nicholas O. Davidson

Microsomal triglyceride transfer protein (MTTP) and apolipoprotein B (apoB) expression are dominant genetic restriction points in hepatic and intestinal lipoprotein secretion. Homozygous germ-line deletion of either Apob or Mttp in mice results in embryonic lethality Earlier studies suggested a subtle functional advantage in lipid export of apoB48 over apoB100, but Apobec-1-/- mice (apoB100-only) appear to grow normally and intestinal enterocytes appear indistinguishable from wild-type (apoB48) controls. We have bypassed the embryonic lethality associated with targeted Mttp deletion by creating conditional, tamoxifen inducible intestine specific Mttp deletor mice (Mttp-IKO), which demonstrate intestinal triglyceride (TG) and free fatty acid (FFA) accumulation when fed a chow diet. 
We then generated Mttp-IKO mice on an apoB100 (ie Apobec-1-/-) background (AMttpIKO) and examined their response to high fat intake. On a chow diet AMttp-IKO mice manifest dramatic TG and FFA accumulation, similar to Mttp-IKO mice. However, when challenged with a high fat diet $100 \%$ AMttp-IKO mice $(\mathrm{n}=7)$ died by two weeks, while $100 \%$ of the Mttp-IKO mice survived for at least 1 month. There was gross distortion of the villus architecture and altered enterocyte proliferation, suggesting that there is greater conditional intestinal lipotoxicity with Mttp deletion in the background of apoB100 compared to apoB48. To address the relevance of this observation in the perinatal setting, when apoB mRNA editing occurs, and to examine the survival advantage of apoB48, we generated compound heterozygous mice containing one apoB38.9 hypomorphic allele (apoB38.9hypo) and either an apoB48-only allele (apoB38.9hypo/48) or an apoB100-only allele (apoB38.9hypo/100). The apo38.9 hypomorphic allele is functionally equivalent to an apoB null. All animals expressing the apoB38.9hypo/48allele survived the neonatal period while $90 \%$ animals expressing the apoB38.9hypo/100died within 4 days. These findings further confirm a survival advantage for neonatal intestinal apoB48 expression. In summary, the data demonstrate that intestinal Mttp expression is necessary for surviving the high fat challenge encountered during suckling. In addition, our findings of lethal intestinal lipotoxicity in AMttp-IKO mice and perinatal death of the compound apoB38.9hypo/100 mice imply a survival advantage for apoB48. These are the first conclusive data to demonstrate a plausible physiological role for mammalian intestinal apoB mRNA editing.

\section{3}

Intestinal Epithelial Cell-Derived IL-7 Contributes to the Alternation in Intraepithelial Lymphocytes After Total Parenteral Nutrition Hua Yang, Xiaoyi Sun, Daniel H. Teitelbaum

Background: Total parenteral nutrition (TPN) results in profound changes to both intestinal epithelial cells (EC), as well as adjacent intraepithelial lymphocytes (IEL). Such changes may result in alterations in the mucosal immune system and a loss of mucosal barrier function. IL-7 is vital for IEL development and maintenance. Because EC are a rich source of IL-7, and TPN results in marked EC changes, it was hypothesized that TPN would diminish ECderived IL-7 expression, and contribute to the observed IEL changes. Methods: Adult C57BL/ 6J mice received 1) TPN; 2) ad lib feeding (Control); 3) TPN supplemented with exogenous IL-7; or 4) enterally fed mice treated with anti-IL-7 receptor (IL-7R) antibody (Ab). Mice were sacrificed at 7 days after TPN administration. EC-derived IL-7 expression was measured by RT-PCR and Western immunoblot. IEL phenotype, proliferation rate ( $\mathrm{S} / \mathrm{G} 2+\mathrm{M}$ phase), and IL-7R expression in sub-populations were detected with flow cytometry. Antibodies to IL-7 and CD3 (T cell marker) were used for confocal microscopy to observe for co-localization of EC-derived IL-7 to IEL. Results: TPN administration significantly decreased EC-derived IL-7 expression. IL-7R expression was observed in CD4+, CD8+, TCR- $\alpha \beta+$, as well as TCR$\gamma \delta+$ IEL. Significant changes in IEL phenotype and a marked decrease in IEL numbers were noted after TPN administration (See Table). IEL proliferation decreased 1.6-fold with TPN. Further, maturation of the IEL (as measured by the CD44+ IEL) declined with TPN. Blocking IL-7R with anti IL-7R Ab on normal health mice, resulted in several similar changes in the IEL as those observed with TPN administration. To further address the contribution of IL7 to these changes, TPN mice received exogenous IL-7, which significantly attenuated TPNassociated IEL changes, including reducing the decline in IEL proliferation, and significantly prevented the development of TPN-associated IEL phenotypic changes. Finally, confocal microscopy showed a close IEL-EC cross-communication mediated by EC-derived IL-7 expression. Conclusion: TPN leads to a significant decrease in EC-derived IL-7 expression. This decrease in EC-derived IL-7 may be a contributing mechanism accounting for the observed changes of neighboring IEL after TPN administration.

\begin{tabular}{|c|c|c|c|c|c|}
\hline & $\begin{array}{c}\text { IEL number } \\
(\times 106)\end{array}$ & $\begin{array}{c}\text { IEL proliferation } \\
(\%)\end{array}$ & $\begin{array}{c}\text { CD } 8 \alpha \beta+ \\
(\%)\end{array}$ & $\begin{array}{c}\text { CD4+CD8+ } \\
(\%)\end{array}$ & $\begin{array}{c}\text { CD4+CD44+ } \\
(\%)\end{array}$ \\
\hline Control & $8.1 \pm 1.7^{*}$ & $2.8 \pm 0.3^{*}$ & $8.5 \pm 1.0^{*}$ & $4.0 \pm 1.3^{*}$ & $8.1 \pm 2.0^{*}$ \\
\hline TPN & $5.2 \pm 0.8 \dagger$ & $1.7 \pm 0.2 \dagger$ & $3.4 \pm 0.8 \dagger$ & $0.9 \pm 0.7 \dagger$ & $2.5 \pm 1.2 \dagger$ \\
\hline TPN+IL-7 & $12.9 \pm 0.1^{*} \dagger$ & $3.0 \pm 0.4^{*}$ & $9.2 \pm 0.8^{*}$ & $3.2 \pm 0.4^{*}$ & $6.6 \pm 0.5^{*}$ \\
\hline $\begin{array}{c}\text { IL-7R } \\
\text { Blocking }\end{array}$ & $4.2 \pm 0.4 \dagger$ & $1.9 \pm 0.4 \dagger$ & $3.8 \pm 0.6 \dagger$ & $1.3 \pm 0.3 \dagger$ & $3.3 \pm 0.4 \dagger$ \\
\hline
\end{tabular}

Data are expressed as mean \pm SD. Each group $n=6 .{ }^{*} \mathrm{p}<0.05$ compared with TPN group, $\uparrow \mathrm{p}<0.05$ compared with Control

54

Novel Differentially Expressed Genes Revealed By Microarray Analysis of Duodenum in Patients with Disorders of Iron Homeostasis James F. Collins, Zihua Hu, Stuart Rakka, Ellen Kilcourse, Kris V. Kowdley

Background/Aim: Previous studies have demonstrated that genes which encode proteins related to iron transport are induced in the human duodenum by iron-deficiency. We thus performed microarray analyses to identify changes in duodenal gene expression patterns in patients with altered iron homeostasis. Methods: Duodenal tissue was obtained via endoscopy from 2 patients with iron-deficiency ("ID"), 2 patients with iron-overload ("IO"; one with HFE-associated iron-overload $\{\mathrm{HFE}+/+\}$ and one with non-HFE related iron-overload), and 2 patients that had the HFE genotype associated with hemochromatosis $(\mathrm{C} 282 \mathrm{Y}+/+)$ but that did not have iron-overload ["NE"; non-expressers\}. Total RNA was purified from duodenal biopsies and microarray analyses were performed utilizing Affymetrix human genome gene chips (U133A and U133B or U133 Plus 2.0; representing >40,000 human genes). All resulting data were within the quality control parameters established by the manufacturer (Affymetrix). Microarray data were processed by 3 different probe set algorithms (RMA, dChip, MAS 5.0) and analyzed using regularized t-test. Only differentially expressed genes (DEGs) which were common to all three probe set algorithms were considered for further analysis. Results: The following numbers of genes were identified by the different comparisons: 77 (IO vs. ID), 49 (NE vs. ID), and 38 (NE vs. IO). Several genes known to be involved in intestinal iron transport showed increased expression in the ID patients versu the NE and IO groups, including Divalent Metal Transporter 1 (DMT1), Iron-Regulated Gene 1 (IREGl; also called ferroportin), Hephaestin (HP) and Transferrin Receptor l (TfRl) Furthermore, many novel genes were induced in the ID group, including genes that encode proteins involved in intestinal copper and ascorbic acid transport and in regulating gene transcription. Conclusions: This pilot study has identified several novel DEGs in the duodenum of iron-deficient patients. Further research is indicated to more precisely characterize the expression of these novel genes and to decipher their physiological roles during disturbances of iron homeostasis. These studies were supported by NIH Grants 1 R21 DK068349 (JFC) and DK002957 (KVK)

55

Early Immunomodulator Use Improves 18 Month Outcomes in Pediatric Crohn's Disease

Jaya B. Punati, James Markowitz, Trudy Lerer, Jeffrey Hyams, Subra Kugathasan, Anne Griffiths, Anthony Otley, Joel Rosh, Marian Pfefferkorn, David Mack, Jonathan Evans, Athos Bousvaros, Susan Moyer, Robert Rothbaum, Robert Wyllie, Vasundhara Tolia, Maria Oliva-Hemker, Adam Mezoff, Neal Lelieko, J Fernando- Del Rosario, David Keljo, Wallace Crandall

BACKGROUND AND AIM: 6-mercaptopurine (6-MP)/Azathioprine (AZA) have been shown to have steroid sparing effects and to maintain remission in pediatric Crohn disease (CD). We sought to describe 6-MP/AZA use in children with newly diagnosed $C D$, comparing outcomes following early vs. late initiation of therapy. METHODS: Patients $\leq 16$ years of age with newly diagnosed CD were enrolled in a prospective, multicenter (20 site) observational study (The Pediatric IBD Registry). All patients were managed according to the dictates of their individual physicians. Patients who received 6MP/AZA and had $\geq 2$ yrs follow-up were identified. Early use of 6MP/AZA was defined as the initiation of therapy within 3 months of diagnosis and late use was defined as initiation of therapy between 3 and 12 months. Moderate to severe disease was defined by physician global assessment or a Pediatric Crohn Disease Activity Index > 30. RESULTS: Of the 482 children with CD enrolled in the database, 185 ( $60 \%$ male, mean age 11.6 years) had $\geq 2$ years of follow up. Of those 185 children, $94(51 \%)$ started 6-MP/AZA within 3 months of diagnosis, $43(23 \%)$ began between 3 and 12 months, $17(9 \%)$ were started after 12 months and $31(17 \%)$ did not receive 6MP/AZA. Sixty eight percent of patients were categorized as moderate to severe disease at diagnosis. Eighty six percent of those with moderate to severe disease and $49 \%$ with mild disease at initial presentation received 6MP/AZA within the first year. More severe disease activity was predictive of $6 \mathrm{MP}$ use within the first year $(\mathrm{p}<01, \mathrm{OR}=4.6,95 \% \mathrm{CI}=1.7-12.4)$ and showed a trend toward predicting early use $(\mathrm{p}<.06, \mathrm{OR}=2.5,95 \% \mathrm{CI}=1.0-6.5)$. Age, gender, Tanner stage, disease extent and initial laboratory values were not predictive of 6MP/AZA use. Patients with moderate to severe disease treated with early $(n=81)$ and late $(\mathrm{n}=27)$ 6MP/AZA were compared for the outcomes of corticosteroid use, infliximab treatment, hospitalizations and surgery. No differences were noted in the rates of infliximab use or surgery between groups. However at 18 months from diagnosis, the number of patients who received steroids in the previous 3 month period ( $12 \%$ vs. $33 \%, p<.05)$ and the cumulative rate of patients with at least one hospitalization ( $26 \%$ vs. $48 \%, p=.05$ ) were lower in the early group as compared to the late group. CONCLUSIONS: Seventy-four percent of a large inception cohort of children newly diagnosed with $C D$ were treated with 6MP/AZA within the first year and $81 \%$ by the end of 2 years. Early use of 6MP/AZA was associated with lower rates of corticosteroid use and number of patients hospitalized 18 months after diagnosis.

Infliximab As a First Choice Therapy in Children with Newly Diagnosed Crohn's Disease (CD) Promotes Long-Term Sustained Remission and Alters the Course of the Disease

Erminia Romeo, Franca Viola, Gianluigi De Angelis, Alessia Vernuccio, Veronica Pannone, Barbara Bizzarri, Osvaldo Borrelli, Salvatore Cucchiara

Background. Infliximab (IFX), the monoclonal antibody anti-TNF-alpha, is currently indicated for children with CD unresponsive to traditional regimens such as corticosteroids (CS) immunosuppressors, antibiotics, nutritional therapy. IFX has never been used as a first line choice in pediatric patients (pts) with a recent diagnosis of $\mathrm{CD}$. Aim. To compare clinical outcome between pts receiving IFX as initial therapy (group A) and pts treated with a traditional therapeutic approach (group B). All had an active $C D$ recently diagnosed. Methods. 13 pts of group A (range age 5.9-18.0 years) had a baseline i.v. IFX schedule $(5 \mathrm{mg} / \mathrm{kg} /$ dose, 0-2-6 weeks) and 9 of them also underwent IFX retreatment for 12 months every 8 weeks; all also had long term azathioprine (AZA) or methotrexate (MTX). Of 19 pts of group $B$ (range age: 5-17 years), initial therapy consisted of a nutritional course alone in 5 , CS in 14; all received a long term maintenance therapy with AZA (or MTX). At diagnosis all group $\mathrm{A}$ and $\mathrm{B}$ pts had an ileo-colonic mucosal disease, 9 of group A and 3 of group B also had perianal disease (fistulae and/or ulcers). At baseline all underwent ileocolonoscopy with histology and clinical assessment with the PCDAI, that was also measured after remission and thereafter. Results $\left(\right.$ mean $\left._{ \pm} \mathrm{SD}\right)$ : following initial therapy, remission $(\mathrm{PCDAI}<15)$ was achieved in all pts of both groups (A: $9.3 \pm 2.4$ : B: $9.6 \pm 2.1$ p $<0.01$ ); during the following 12 months, disease relapses (PCDAI > 15) were seen in 1 of group $A$ and in 17 of group $B$ pts (Fisher's Exact test: $p<0.01$ ); number of relapses was significantly lower in group A $(0.1 \pm 0.2 .9)$ than in group $B(1.93 \pm 0.9, p<0.01) ; 4$ group $B$ pts also underwent surgery for intestinal resection. At the end of the 12 month maintenance treatment, the PCDAI was markedly higher in group B $(22.3 \pm 8.9)$ than in group A $(11.3 \pm 1.89, \mathrm{p}<0.01)$ and the ileocolon endoscopic score (CDEIS) was markedly lower in group A $6.5 \pm 2.8$ than in group B $12.4 \pm 4.1$. Conclusions. In children with a recently diagnosed CD, the initial therapy either with IFX or traditional regimens induce clinical remission; however, only use of IFX suppresses intestinal inflammation and promotes a more prolonged and stable remission Our data support the immunological view that the use of a potent immunomodulator in early $\mathrm{CD}$ (typically occurring in childhood) may have a great therapeutic benefit and change the disease course. 
Maintenance Therapy with Infliximab Every 8 Weeks Is Superior to Every 12 Weeks in Maintaining Response and Remission in Pediatric Patients with Moderately to Severely Active Crohn's Disease

Jeffrey Hyams, Wallace Crandall, Subra Kugathasan, Anne Griffiths, Allan Olson, Jewel Johanns, Grace Liu, Suzanne Travers, Robert Heuschkel, James Markowitz, Stanley Cohen, Harland Winter, Gigi Veereman-Wauters, George Ferry, Robert Baldassano

Background \& Aims: The REACH study was a multicenter, randomized, open-label study that evaluated infliximab therapy in children with moderately-to-severely active Crohn's disease (CD). Methods: Patients ( $n=112)$ with a Pediatric Crohn's Disease Activity Index (PCDAI) score $>30$ received infliximab $5 \mathrm{mg} / \mathrm{kg}$ at weeks 0,2 , and 6 . Patients responding to treatment at week 10 were randomized to receive infliximab $5 \mathrm{mg} / \mathrm{kg}$ every 8 weeks or every 12 weeks through week 46 and followed through week 54 . The primary endpoint was clinical response at week 10 (decrease from baseline in the PCDAI score $\geq 15$ points, and a total score $\leq 30$, at week 10). Clinical remission was defined as a PCDAI score $\leq 10$ points. The median age was 13 years, the median duration of disease was 1.6 years. Results: At week 10, 99 of $112(88.4 \%)$ patients responded to infliximab $95 \%$ confidence interval: $82.5 \%, 94.3 \%)$ and 66 of $112(58.9 \%)$ patients achieved clinical remission (95\% confidence interval: $49.8 \%, 68.0 \%)$. At week 54,33 of $52(63.5 \%)$ and 29 of $52(55.8 \%)$ patients receiving infliximab every 8 weeks were in clinical response and clinical remission, respectively, compared with 17 of 51 (33.3\%) and 12 of 51 (23.5\%) patients receiving treatment every 12 weeks ( $\mathrm{p}=0.002$ and $\mathrm{p}<0.001$, respectively). The clinical response rates of patients with age $\leq 13$ yrs and $>13$ yrs, with disease duration $\leq$ equal to $1 \mathrm{yr}$ and $>1 \mathrm{yr}$ and with disease in the colon and in the small intestine were similar at week 10. Adverse events, and serious adverse events including serious infections were comparable among patients treated with infliximab every 8 weeks vs patients treated every 12 weeks. Safety findings in children were also comparable to those observed in adults. Conclusions: Response to infliximab approached $90 \%$ at week 10 and was similar regardless of age, disease duration, and location of disease. Maintenance dosing with infliximab every 8 weeks was superior to every 12 weeks dosing in maintaining response and remission.

\section{8}

Natural History of Crohn's Disease in Children : A Population-Based Cohort Study in Northern France

Mamadou Balde, Gwenola Vernier-Massouille, Corinne Gower-Rousseau, Dominique Turck, Jean-Louis Dupas, Olivier Mouterde, Jean-Louis Salomez, Eric Lerebours, Antoine Cortot, Jean F. Colombel

Background and methods: The natural history of Crohn's disease (CD) in children is poorly described and a limited number of population-based studies is available. In a geographically derived incidence cohort diagnosed from 1988 to 2002 we identified all patients with onset of $\mathrm{CD}$ before the age of 17 years to describe their clinical course and morbidity. Results [median (range)] : 424 cases of childhood CD were recorded ( $8 \%$ of all CD cases in Northern France). The mean standardized incidence was 2.3/105. Follow-up information is available to date in 304 children ( $72 \%$ ) (167 M and $137 \mathrm{~F}$ ) for a median follow-up of 69 mos (41107). CD location at diagnosis and last follow-up was both small bowel and colon (65\% and $83 \%$ respectively), colon only (18.5\% and $8.5 \%$ ), small bowel only (15\% and $8 \%$ ). Upper gastrointestinal tract lesions were present in $18 \%$ of patients at diagnosis and in $30 \%$ at follow-up. Anoperineal lesions were present in 30\% of patients at diagnosis and in 52\% at follow-up. $84 \%$ of children required treatment with corticosteroids within the first year after diagnosis. Corticosteroid resistance occurred in $9.5 \%$ and corticosteroid dependence in $51 \%, 11$ mos (4-24) after corticosteroids were started. Immunomodulators were prescribed in 173 children (57\%) after 18 mos (9-33)(all received azathioprine and 42 azathioprine followed by methotrexate) with a response in $68 \%$, intolerance in $13 \%$ and failure in $9 \%$. Sixty-five children $(21 \%)$ received infliximab with a response in $81 \%$. The cumulative probability of resection surgery was $8.1 \%$ at 1 year, $19.5 \%$ at 3 years and $32.2 \%$ at 5 years. Time between diagnosis and surgery was significantly longer in children who received immunomodulators than in those who did not [44(24-181) vs 11.5(4-43); $<<0.01]$. In these two groups, the cumulative probabilities of surgery were $2.4 \%$ and $16.7 \%$ at 1 year $(\mathrm{p}<0.01), 16.7 \%$ and $23.7 \%$ at 3 years (ns) and $31.8 \%$ and $32.9 \%$ at 5 years (ns) respectively. No death occurred in this cohort. Conclusion: In one of the largest available population-based cohorts, childhood-onset $\mathrm{CD}$ was characterized by an extensive disease, frequent anoperineal lesions, a high percentage of patients treated early with steroids followed by a dependence in more than half. Immunomodulators seemed to prolong time to surgery but this effect was not sustained at 5-year follow-up.

\section{9}

Height of Growth Delayed Children with Active Crohn's Disease Improves During Treatment with Infliximab

Anne Griffiths, Jeffrey Hyams, Wallace Crandall, Subra Kugathasan, Allan Olson, Jewell Johanns, Grace Liu, Aparna Raychaudhuri, Suzanne Travers, Robert Heuschkel, James Markowitz, Stanley Cohen, Harland Winter, Gigi Veereman-Wauters, George Ferry, Robert Baldassano

Background \& Objectives: Delayed linear growth is a common problem in children with moderately to severely active Crohn's disease. The effect of infliximab on growth was examined in children with growth delay at the time of entry into the REACH study (a multicenter, randomized, open-label study that evaluated infliximab therapy in children with moderately to severely active Crohn's disease). Methods: Children $(n=112)$ with a Pediatric Crohn's Disease Activity Index score $>30$ received infliximab $5 \mathrm{mg} / \mathrm{kg}$ induction treatment at weeks 0,2 , and 6 . Patients responding to induction treatment assessed by the principal investigators at week $10(\mathrm{n}=103)$ were randomized to receive infliximab $5 \mathrm{mg} / \mathrm{kg}$ every 8 weeks or every 12 weeks through week 46 and followed through week 54 . Height status $z$ scores, which adjust for age and gender, were calculated using the SEER database at baseline, and Weeks 30 and 54 for the subgroup of children with at least a 1 year delay in bone age (as measured by a wrist radiograph) at the time of study entry $(n=38)$. Results: At week 0 , the median height status $z$ score was -1.6 and the mean height status $z$ score was -1.5 . The mean height status $z$ scores were significantly improved from baseline at both Week 30 (mean $=0.3, \mathrm{p}<0.001)$ and Week 54 (mean $=0.5, \mathrm{p}<0.001)$, in the combined infliximab dose groups. At Week 54, the improvement in height status was greater in patients in the every 8 week maintenance treatment group with $43.5 \%$ improving their $z$ score by at least 0.5 standard deviation and $13.0 \%$ improving their $z$ score by at least 1 standard deviation in contrast to $20.0 \%$ and $6.7 \%$ respectively in the q12 week maintenance treatment group. Conclusions: Infliximab significantly improved the height status of children with moderately to severely active Crohn's disease who had a 1 year delay in bone age

60

Inter-Center Variation in the Initial Management of Children with Crohn's

Disease

M Kappelman, A Bousvaros, J Hyams, J Markowitz, M Pfefferkorn, S Kugathasan, J Rosh, A Otley, D Mack, A Griffiths, J Evans, R Grand, C Langton, J Finkelstein

Background and Aims: Recent data suggest that early use of immunomodulators and biologics may prolong remission in children with Crohn disease (CD). Because there are no practice guidelines for the management of pediatric IBD, considerable variation in the timing and usage of available therapies may exist. We performed this study to determine the extent of inter-center variation in the initial treatment of children with $\mathrm{CD}$. Methods: Since 2002, newly diagnosed children with IBD at 20 North American pediatric centers have been prospectively followed and comprise the Pediatric IBD Collaborative Group Registry. Patients are managed according to the usual practices of their physicians, and not by protocol. All patients with $\mathrm{CD}$ and at least 3 months of follow-up were eligible to be included in this study; final analysis was based only on patients from centers contributing $\geq 20$ such patients. Using logistic regression to control for disease severity (Physician Global Assessment), age, gender, and race, we compared initial utilization (within 3 months of diagnosis) of systemic steroids, immunomodulators (6 MP, azathioprine, and methotrexate), 5-aminosalicylates, antibiotics, and infliximab among our study centers. Results: Data from 321 patients (10 centers) were analyzed. $72 \%$ of patients presented with moderate/severe disease; severity did not differ by center. Initial treatment, however, varied greatly. Variation was statistically significant for treatment with prednisone, immunomodulators, 5-aminosalicylates, and antibiotics, and remained significant after adjusting for disease severity and demographic factors. For infliximab, inter-center variation after adjustment approached statistical significance. Table 1 provides the overall proportion of patients receiving each treatment, the range among the centers, and adjusted $\mathrm{p}$ values obtained from logistic regression. Conclusions: Considerable inter-center variation exists for the initial management of children with $C D$, even after adjusting for demographic factors and disease severity at presentation. Efforts to reduce this variation, such as the implementation of clinical practice guidelines, may be an important step in improving the care for these children. Table 1: Proportion of Patients Receiving each Treatment by Center

\begin{tabular}{|c|c|c|c|}
\hline Treatment & $\begin{array}{c}\text { Overall Proportion of patients receiving therapy } \\
(\%)\end{array}$ & $\begin{array}{c}\text { Range } \\
(\%)\end{array}$ & $\begin{array}{c}\text { Adjusted } p \\
\text { value }\end{array}$ \\
\hline Prednisone & 70.6 & $32.1-86.1$ & $<.0001$ \\
\hline Immunomodulator & 58.4 & $28.6-97.1$ & $<.0001$ \\
\hline 5-aminoalicylate & 62.3 & $25-91.7$ & $<.0001$ \\
\hline Antibiotics & 26.4 & $10-67.9$ & $<.0001$ \\
\hline Infliximab & 10.4 & $2.9-22.2$ & .06 \\
\hline
\end{tabular}

61

Endoscopic Ultrasonography and MRI-Pancreatography in the Evaluation of Patients with Suspected Chronic Pancreatitis: Are Both Imaging Techniques Required?

Julio Iglesias-Garcia, Carmen Villalba, Jose Larino-Noia, Enrique Dominguez-Munoz

Endoscopic ultrasonography (EUS) and magnetic resonance (MRI) plus MRI-pancreatography (MRP) are becoming the methods of choice for the diagnosis of chronic pancreatitis (CP) in clinical practice. Whether the information provided by EUS and MRI-MRP is superimposible or complementary is unknown. Aim: To compare and correlate EUS and MRI-MRP findings in patients with clinical suspicion of CP. Methods: 26 consecutive patients (mean age 48 years, range 28-70, 14 male) with clinical suspicion of $C P$ were prospectively evaluated. EUS and MRI-MRP were performed 2 to 7 days apart. EUS was performed under conscious sedation by an endoscopist blinded to MRI findings. Parenchymal and ductal EUS criteria for $\mathrm{CP}$ were evaluated. CP was defined by the presence of $\geq 3$ criteria. Pancreas MRI with gadolinium i.v. and MRP with secretin i.v. were performed by a radiologist blinded to EUS findings. Parenchymal enhancement after gadolinium and ductal changes before and after secretin injection were evaluated. $\mathrm{CP}$ was defined by the presence of at least one parenchymal and one ductal change at MRI-MRP. Data are shown as median and 95\%CI. Concordance and correlation studies (Kappa score, Spearman correlation, chi-square, and Wilcoxon test for paired data) were performed. Results: CP was diagnosed by both EUS and MRI-MRP in 17 patients (65.4\%), whereas the disease was consistently excluded in 4 patients (15.4\%). Therefore, EUS and MRI-MRP provided with the same diagnosis in 21 patients (80.8\%). The MRI-MRP finding was considered as equivocal in the remaining 5 patients, three of them with normal EUS and two with EUS criteria of CP. Diagnostic concordance was highly significant (kappa score $0.60 ; 95 \% \mathrm{CI} 0.36-0.84)(\mathrm{p}<0.00001)$. The number of parenchymal criteria at EUS correlated with the signal intensity ratio at MRI ( $\mathrm{p}=0.008)$. Patients with $\geq 3$ parenchymal EUS criteria have a lower signal intensity ratio at MRI than patients with $\leq 2$ EUS criteria (1.24 [IC95\% 1.07-1.41] vs 1.69 [IC95\% $1.38-2.0$ ]; $p=0.063$ ), and maximal MRI enhancement occurred at the venous phase and the arterial phase, respectively $(\mathrm{p}<0.05)$. Patients with abnormal pancreatic duct at MRP before secretin injection is associated with more EUS ductal criteria $(4.5 ; 95 \% \mathrm{CI} 3.9-5.0)$ than patients with abnormal pancreatic duct 
only after secretin injection $(3.0 ; 95 \% \mathrm{CI} 1.7-4.3)$, and these in turn more than patients with normal duct at MRP $(1.5 ; 95 \% \mathrm{CI} 0.3-2.6)(\mathrm{p}<0.05)$. Conclusion: Both EUS and MRI-MRP are accurate methods to evaluate pancreatic changes in the context of $\mathrm{CP}$. The information provided by both imaging techniques is highly concordant, so only one of them is enough to establish the diagnosis of CP in most cases.

62

Combined Pancreatic Juice IL-8 Concentration and EUS Are Highly Predictive to Diagnose Chronic Pancreatitis

Surakit Pungpapong, Kyung W. Noh, Mohammed Al-Haddad, Michael B. Wallace, Timothy A. Woodward, Massimo Raimondo

Background: The diagnosis of chronic pancreatitis $(\mathrm{CP})$ remains challenging. EUS seems to be highly sensitive, but not specific enough to diagnose CP. Pancreatic juice IL-8 concentration has been proposed as a specific marker for pancreatic diseases. In this study, we aim to compare the diagnostic accuracy of pancreatic juice IL-8 concentration and EUS to diagnose CP. Methods: Seventy-nine patients presenting at our pancreas clinic with abdominal pain suspicious for CP were prospectively enrolled in the study from January 2003 to December 2004. EUS was performed with Olympus radial and linear echoendoscopes. The diagnosis of $\mathrm{CP}$ by EUS was established when 4 or more of the previously established criteria were present. After intravenous secretin $(0.2 \mu \mathrm{g} / \mathrm{kg})$ was administered, pancreatic juice emptied into the duodenum was collected and immediately frozen in liquid nitrogen until IL- 8 concentration was measured by a modified ELISA assay. The diagnosis of CP was made when pancreatic juice IL- 8 concentration was $20 \mathrm{pg} / \mathrm{mL}$ or higher. Final diagnosis of CP was made by the referring gastroenterologist using the composite gold standard: ERCP, histology, CT or MRI, and clinical follow-up (mean 18 months). Results: Thirty-eight patients (mean age 56 years; male $42 \%$ ) were diagnosed with $\mathrm{CP}$, whereas 41 patients were found to have no pancreatic diseases and therefore considered as controls (mean age 49 years; male 29\%). No significant differences were demonstrated in age and gender distribution. The accuracy of EUS to diagnose CP was $80 \%$ with sensitivity and specificity of $71 \%$ and $88 \%$, respectively. The accuracy of pancreatic juice IL-8 concentration was $71 \%$ with sensitivity and specificity of $47 \%$ and $93 \%$, respectively. EUS was significantly more sensitive, but equally accurate and specific to diagnose CP compared to pancreatic juice IL-8 concentration. Combining the findings of EUS and pancreatic juice IL- 8 concentration, the sensitivity and specificity significantly increased to $82 \%$ (either EUS or IL- 8 positive) and $100 \%$ (both EUS and IL-8 positive), respectively. Conclusions: Both EUS and pancreatic juice IL-8 concentration are accurate diagnostic modalities for the diagnosis of $\mathrm{CP}$. Pancreatic juice collection can be performed at the time of EUS. EUS and pancreatic juice IL- 8 concentration are complementary with higher sensitivity and specificity when both are used together. If confirmed in larger series, the combination of EUS with pancreatic juice IL- 8 will be highly predictive of $\mathrm{CP}$

63

Stopping Smoking At the Clinical Onset of Chronic Pancreatitis Reduces the Risk of Developing Pancreatic Calcifications

Talamini Giorgio, Massimo Falconi, Nora Sartori, Marina Mastromauro, Claudio Bassi, Paolo Pederzoli, Italo Vantini

Cigarette smoking is associated with a higher risk of developing chronic pancreatitis and increases the likelihood of developing pancreatic calcifications. It is not known whether stopping smoking is capable of modifying the course of this disease. Methods. We reviewed 701 chronic pancreatitis patients with well known smoking and drinking habits and selected a subgroup of patients with follow-up longer than 6 years and with no calcifications by the end of the 5th year. A total of 360 patients with the above-mentioned characteristics were identified. Three groups of patients were studied: smokers, non-smokers and patients who stopped smoking within 5 years of onset of symptoms of chronic pancreatitis. In the actuarial analysis the 6 th year from onset of symptoms was taken as time 0 . Results. The 360 patients selected comprised 43 females $(11.9 \%)$ and 317 males (88.1\%) with a mean age of 38.7 years (SD 11.4). The median follow-up was 19.0 years ( 25 th percentile $13.0 ; 75$ th percentile 24.0). The chronic pancreatitis was alcohol-associated in 255 patients, familial in 10 , obstructive in 54 , and idiopathic in 41 . At the end of follow-up 212 patients (59.8\%) developed calcifications. Thirty-seven patients stopped smoking. Smokers were 317 (88.1\%) with a mean total of 23.4 cigarettes smoked daily. Drinkers drank a mean amount of 147 grams of alcohol daily. As regards the risk of calcifications, non-smokers and ex-smokers had similar actuarial survival curves and these were significantly different from the curve for smokers $(p<0.003)$. Cox multivariate actuarial analysis supported these data. Considering non-smokers as the reference class, ex-smokers had an Odds Ratio (OR) of 0.56 (0.2-1.4; $\mathrm{p}=\mathrm{ns}$ ), patients smoking $1-10 \mathrm{cig} /$ day an $\mathrm{OR}$ of 1.95 (1.1-3.4; $\mathrm{p}<0.019)$, patients smoking $11-20 \mathrm{cig} /$ day an OR of $1.76(1.1-2.8 ; \mathrm{p}<0.018)$, and those smoking $>20 \mathrm{cig} /$ day an OR of 1.79 (1.1-2.9; $\mathrm{p}<0.019)$. Conclusion. Stopping smoking within 5 years of clinical onset of chronic pancreatitis reduces the subsequent risk of developing pancreatic calcifications.

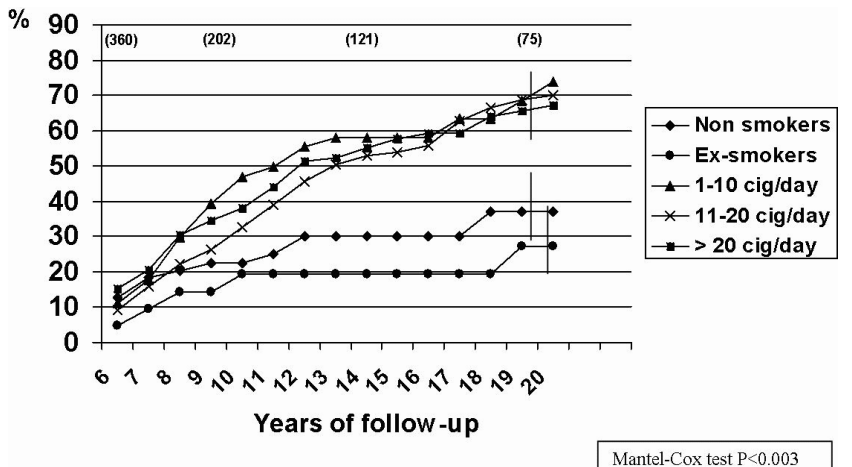

64

Abnormal Pancreatic Duct Morphology Is Highly Suggestive of Pancreatic Acinar Cell Dysfunction

Mansour A. Parsi, Darwin L. Conwell, Gregory Zuccaro, John A. Dumot, John J. Vargo

Background: Chronic pancreatitis is characterized by morphological ductal and parenchymal changes that typically lead to loss of function. The correlation of pancreatic ductal changes and acinar cell function has not been well studied and is poorly understood. Hormone stimulated pancreatic function testing allows comparison of secretory function to radiologic imaging. Aim: To assess whether pancreatic ductal morphology by endoscopic retrograde pancreatography (ERP) can predict pancreatic acinar cell function. Methods: 35 patients with chronic abdominal pain and suspected chronic pancreatitis prospectively underwent an ERP and an endoscopic pancreatic function test (ePFT). The pancreatograms obtained at ERP were scored by 3 experienced endoscopists in a blinded fashion based on the Cambridge classification. The final score was based on consensus among at least 2 endoscopists or if no consensus, based on the average of the 3 scores. The ePFT was performed after continuous intravenous infusion of synthetic CCK (Sincalide $40 \mathrm{ng} / \mathrm{kg} / \mathrm{h}$ ) as previously described. Duodenal fluid was collected with an upper endoscope during endoscopy at $30,40,50$, and 60 minutes during infusion and the peak lipase concentration was determined (Normal > 800,000 IU/L). Results: In 18 patients the pancreatogram showed morphological changes consistent with chronic pancreatitis. The median and interquartile range (IQR) Cambridge score in this group was 3 (2-3) and the median (IQR) peak lipase concentration was 268,700 (148,000-425,350). Seventeen patients had normal pancreatograms with median (IQR) peak lipase of $435,000(328,400-628,000)$. The correlation between the Cambridge score and the peak lipase concentration was 0.42 using the Spearman correlation test $(p=0.01)$. The sensitivity, specificity, and the positive and negative predictive values of ERP for detecting patients with pancreatic insufficiency was 57\%,80\%,94\% and $24 \%$ respectively. Conclusion: There is a poor correlation between the pancreatic duct morphology by ERP and pancreatic acinar cell function by ePFT. However, an abnormal pancreatogram is highly suggestive of pancreatic acinar cell dysfunction with presence of pancreatic insufficiency. For practical purposes, in patients with pancreatic duct abnormality by ERP, pancreatic insufficiency can be assumed. In patients with normal pancreatic duct morphology, a dedicated pancreatic function test is necessary to assess for presence or absence of pancreatic insufficiency

65

Clinical Aspects of Chronic Pancreatitis Associated with Gene Mutations Luca Frulloni, Chiara Scattolini, Giulia Martina Cavestro, Cecilia Pravadelli, Laura Bernardoni, Anna Katsotourchi, Paolo Bovo, Riccardo Manfredi, Rossella Graziani, Giorgio Cavallini

Background: Mutations of CFTR gene, SPINKl gene, and K8 gene have been associated with chronic pancreatitis $(\mathrm{CP})$. The clinical outcome of $\mathrm{CP}$ associated with gene mutations (CPgm) seems to be different from $\mathrm{CP}$ secondary to other aetiologies. Aim of this study was to compare clinical and radiological findings between $\mathrm{CPgm}$ and $\mathrm{CP}$ with negative genetic tests (CPwt). Patients and Methods: We collected clinical and radiological data from all CP patients referred to our Dpt between 2000 and 2005. All patients underwent genetic tests for 18 CFTR gene mutations, N34S mutation on the SPINKl gene and G61C gene mutation on the K8 gene. We study 400 unrelated partners of cystic fibrosis pts as control group for CFTR gene mutations, and 200 blood donors as control subjects for K8 and SPINKl gene mutations. Results: We found 39 out of $143(27.3 \%)$ pts with CPgm, of whom $23(16.1 \%)$ with CFTR gene mutations, 11 (7.7\%) with SPINKl gene mutation and $7(4.9 \%)$ with K8 gene mutation. The relative risk to develop CP was 5.29 (CI 2.64 - 10.6) for CFTR, 16.6 (CI 2.2 - 129.9) for SPINKl and 10.24 (CI 1.25 - 84.2) for K8. The mean age at the onset of the disease was younger in CPgm $(37.3 \pm 17.3 \mathrm{yrs})$ than in CPwt $(44.4 \pm 12.3 \mathrm{yrs})$ ( $\mathrm{p}=$ $0.007)$. Female sex was significantly more frequent in CFTR pts $(59 \%)$ than in SPINK $(30 \%), \mathrm{K} 8(17 \%)$ and CPwt $(27 \%)(\mathrm{p}=0.025)$. Heavy drinkers were more frequently found in CPwt (40\%) than in CPgm (8\%) (p<0.0001), as well as smokers ( $78 \%$ vs $34 \%$; $<<0.0001)$. Pancreatic calcifications were less frequent in CFTR (50\%) pts than in SPINK1 (90\%) and K8 $(83 \%)$ groups $(\mathrm{p}=0.053)$. Exocrine and endocrine pancreatic insufficiency appeared less frequently and later in pts with CFTR and SPINKl than in K8 gene mutations and in CPwt. Conclusions: Clinical and radiological findings and the evolution of the disease are different in CPgm vs CPwt. 
Glucidic Homeostasis Improvement After Endoscopic Ductal Drainage in Chronic Pancreatitis

Pierre Deprez, Helene Devroye, Jean-Paul Thissen, Yves Horsmans, Jean-Francois Gigot, Michel Hermans

Chronic pancreatitis is characterized by progressive parenchymal fibrosis, ductal obstruction and calcification of the pancreas. One of the common complications of the disease is the onset of "secondary" diabetes, which is the result of the progressive destruction of the Langerhans islets. The influence of medical, endoscopic and surgical therapy in chronic pancreatitis on endocrine function has received little attention in clinical research. The aim of our study was to evaluate the impact of endoscopic drainage on endocrine function in chronic pancreatitis. Our hypothesis was that an efficient drainage of the obstructed ducts might improve endocrine pancreatic insufficiency and/or secondary diabetes of these patients. Patients and methods: prospective study including patients with chronic pancreatitis (CTEUS-MRI or ERCP proven) and an indication for endoscopic drainage (pain, duct dilation, pseudocyst). Endocrine function was evaluated by glycosylated hemoglobin levels and HOMA test before and after ( 1 and 3 months) ductal decompression. Inflammatory (WBC and CRP) and nutritional (weight, BMI, fat mass, IGF-1, prealbumin, $\mathrm{Cu}$, Zn, Se levels) parameters were recorded. Results: 34 pts were included between, mean age $54 \mathrm{y} \pm 12$; mean BMI was $21.2 \pm 3.1 \mathrm{~kg} / \mathrm{m} 2$. Etiology was alcohol in 19 and obstructive or genetic or idiopathic in 15. Smoking was present in $56 \%$, calcifying pancreatitis in $73.5 \%$, exocrine insufficiency in $65 \%$ and diabetes in $35 \%$. At the first visit, we observed a significantly worse endocrine function in patients with pancreatitis caused by alcohol and smoking, with calcifying pancreatitis and when exocrine insufficiency was present $(\mathrm{P}<0.01)$. After endoscopic drainage (successful in $81 \%$ of pts), increase in the $\beta$ cell function was significant in the group of patients with less severe (non calcifying) pancreatitis ( $76 \%$ before vs. $101.2 \%$ after drainage, $\mathrm{P}<0.05$ ) and reduction in glycosylated hemoglobin was significant in the diabetic subgroup $(7.1 \%$ before vs. $6.1 \%$ after drainage, $\mathrm{P}<0.05$ ) Conclusion: patients with non calcifying pancreatitis appear to be the favorite candidates for endoscopic drainage which seems to be more efficient on endocrine functional recovery when it is carried out early in the course of the ductal obstruction. However, glucidic homeostasis was also improved in the more severe pancreatitis group with a reduction in glycosylated hemoglobin levels, suggesting that endoscopic drainage might also be a valuable option.

\section{7}

Effect of Acid Inhibition On Reflux Symptoms Depends On Symptom Association Probability

Marissa C. Aanen, Bas L. Weusten, Mattijs E. Numans, Niek J. de Wit, Melvin Samsom

Introduction In patients with heartburn the symptomatic response to a proton pump inhibitor (PPI) is variable. We hypothesized that the highest response to PPI treatment can be expected in patients in whom a proven relationship exists between symptoms and reflux events. Methods Seventy-four heartburn patients were categorized into 4 groups according to presence or absence of pathological reflux, defined as $\mathrm{pH}<4>6 \%$ of the time $(\mathrm{pH}+/ \mathrm{pH}-)$ and positive and negative SAP (SAP+/SAP-). During 7 days off and 13 days on esomeprazole 40 mg daily subjects kept a diary in which overall symptoms $(\mathrm{O})$ and heartburn $(\mathrm{H})$ were scored from 0 [absent] to 5 [very bad]. Data are presented as mean ( 7 days off PPI vs. last 7 days on PPI) and analysis was performed with Kruskal-Wallis tests. Results The 4 groups $(\mathrm{SAP}+\mathrm{pH}+(\mathrm{n}=40) ; \mathrm{SAP}+\mathrm{pH}-(\mathrm{n}=12) ; \mathrm{SAP}-\mathrm{pH}+(\mathrm{n}=10) ; \mathrm{SAP}-\mathrm{pH}-(\mathrm{n}=10))$ were similar in age and gender. Pre-treatment scores were highest in the SAP+pH+ and SAP+pH- subgroups. In all subgroups of patients a reduction in reflux symptoms was seen. The greatest reduction was achieved in $\mathrm{SAP}+\mathrm{pH}+$ subjects $(\Delta \mathrm{O} \mathrm{p}<0.01, \Delta \mathrm{H} \mathrm{p}<0.02)$. The residual symptom scores on treatment were lowest in $\mathrm{SAP}+\mathrm{pH}+$ and $\mathrm{SAP}-\mathrm{pH}+$ subjects $(\mathrm{O} \mathrm{p}<0.01, \mathrm{H}$ ns) and relatively high in the SAP+pH- subjects. Conclusion The best response to PPI treatment was found in patients with positive SAP and pathological reflux. Subjects with a positive SAP and physiological reflux responded less favorably, presumably reflecting the element of visceral hypersensitivity in these patients

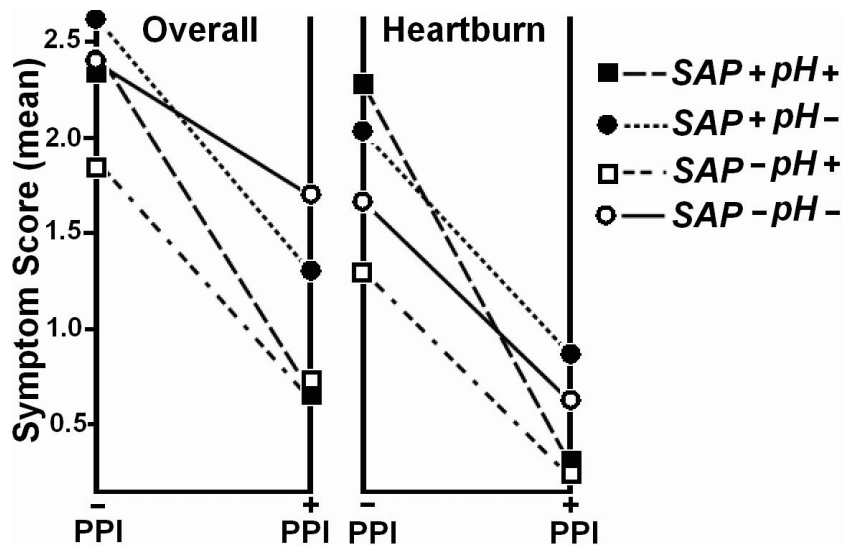

68

Adherence and Persistence to Proton Pump Inhibitors in Daily Clinical Practice

Eva van Soest, Peter D. Siersema, Jeanne P. Dieleman, Miriam C. Sturkenboom, Ernst J. Kuipers

Purpose: The worldwide expenditure on proton pump inhibitors (PPIs) is high and further increasing. The adherence to daily prescriptions is thought to be high, but it is currently not well known how PPIs are actually being used under everyday circumstances. We therefore aimed to analyse the PPI usage pattern by indication in the general population. Methods: We conducted a cohort study using the IPCI database, a general practice research database containing the complete, longitudinal electronic medical records of 600,000 persons throughout the Netherlands. For all persons who had started PPI treatment between 1996 and 2003, persistence (treatment duration) and adherence (medication possession ratio) with treatment were calculated. Potential predictors of persistence and adherence were identified. Results: During the 8-year study period, 17,813 persons started PPI treatment. Persistent use was seen in $25 \%$ of patients after 1 year and in $19.5 \%$ after 2 years. One-year persistence was highest in patients using PPIs for gastroprotection with aspirin (42\%), and if taken for gastro-esophageal reflux symptoms (30.6\%). Fifteen percent of patients with non-reflux dyspepsia or Helicobacter pylori-associated disease used PPIs for at least 1 year. A specialist visit in the year prior to PPI treatment, presence of co-morbidity, increasing age and use of co-medication were associated with longer persistence. Overall, $55 \%$ of patients had an adherence level $\geqq 80 \%$, meaning that they were at least $80 \%$ of time on treatment. Patients using PPIs in combination with aspirin were most adherent $(61.5 \%$ with adherence level $\geqq 80 \%$ ). Adherence increased with age and use of co-medication. Subgroup analysis showed that patients with Barrett's esophagus (BE) were not only more persistent ( $66 \%$ after 1 year), but also more adherent ( $64 \%$ with adherence level $\geqq 80 \%)$ compared to the whole group of PPI users. Conclusions: The majority of patients (75\%) stop PPI use within one year, however at least $15 \%$ of patients with an indication for short-term treatment used PPIs longer than 1 year. Almost half of the patients use PPIs on a non-continuous basis (adherence level $<80 \%$ ), indicating intermittent or on-demand use. The consequences of these usage patterns are probably minor in patients treated for symptom control only. However, infrequent use of PPIs may have a more serious impact if prescribed for other purposes, such as treatment of BE or gastroprotection with non-steroidal anti-inflammatory drugs or aspirin.

69

Positive Association Between Intragastric and Intraesophageal Acid Control and Healing of Los Angeles Grade C and D Erosive Esophagitis: Results of a Prospective, Controlled Clinical Trial

Philip O. Katz, Gregory G. Ginsberg, Paul Hoyle, Mark B. Sostek, John Monyak, Debra G. Silberg

BACKGROUND \& AIM: Acid control has been considered to be a surrogate marker for clinical efficacy (EE [erosive esophagitis] healing and symptom control). However, the relationship between efficacy for EE healing and acid control has not been prospectively established. This study's aim was to assess the relationship between the percentage of time intragastric $\mathrm{pH}$ is $>4$ and the healing of EE, using an acid suppression drug. Also assessed was the relationship between intragastric acid control and gastroesophageal reflux disease (GERD) symptom control. METHODS: In this 28-site, double-blind, exploratory study (D9612L00062), adult patients with endoscopically verified Los Angeles grade C or D EE were randomly assigned to oral esomeprazole 10 or $40 \mathrm{mg}$ once daily for 4 weeks. At treatment day 5, patients underwent a dual-electrode, intraesophageal and intragastric, catheter-based, 24-hour $\mathrm{pH}$ study. The electrodes were placed $5 \mathrm{~cm}$ above and $10 \mathrm{~cm}$ below the proximal border of the lower esophageal sphincter $(15 \mathrm{~cm}$ apart). At 4 weeks, an endoscopist blinded to the $\mathrm{pH}$ study results assessed EE healing status. Investigators scored GERD symptoms before treatment and at 4 weeks, before the final endoscopy, as none (0), mild (1), moderate (2), or severe (3). Analyses included patients who satisfactorily completed the $\mathrm{pH}$ study and final endoscopy, and met predetermined protocol criteria. A t-test was used to compare the percentage of time $\mathrm{pH}$ was $>4$ for healed vs unhealed patients. Spearman rank correlations $(\mathrm{r})$ and tests of significance were done for percentage of time $\mathrm{pH}$ was $>4$ and investigator-assessed symptom scores. RESULTS: Of 103 patients who had evaluable data (mean age, 48.7 years; $65 \%$ men), EE was healed in $69.9 \%$ at 4 weeks. Mean percentages of time intragastric $\mathrm{pH}$ was $>4$ at day 5 in patients with healed and unhealed EE were $61.3 \%$ and $42.1 \%$, respectively $(P=.0002)$. EE healing rates were positively related to the percentage of time intragastric $\mathrm{pH}$ was $>4$. A post hoc analysis showed a significant difference in the 
percentages of time intraesophageal $\mathrm{pH}$ was $>4$ between healed and unhealed patients $(95.2 \%$ and $88.9 \%$, respectively; $P=.0059$ ). Better control of gastric acid was also correlated with lower final heartburn and acid regurgitation symptom scores $(r=-29 \%$ and $-21 \% ; P=.003$ and .032 , respectively). CONCLUSION: This is the first prospective study to show a positive relationship between intragastric and intraesophageal acid control and the clinical outcomes of EE healing and symptom control. These data support the importance of intragastric $\mathrm{pH}$ as a surrogate marker for drug efficacy. Supported by AstraZeneca LP

70

The Effect of Baclofen On Esophago-Gastric Motility and Gastroesophageal Reflux in Children with GER Disease

Taher Omari, Marc Benninga, Ross N. Butler, Lloyd Sansom, John Dent, Geoffrey P. Davidson

INTRODUCTION: The GABA(B) agonist baclofen has been shown to inhibit transient LES relaxation (TLESR) and thereby diminishing gastroesophageal reflux (GER) in adults and animal models. The aim of this study was to evaluate the effect of baclofen on the rate of TLESRs, GER and gastric emptying (GE) in neurologically normal children with GER disease (GERD). METHODS: The efficacy of $0.5 \mathrm{mg} / \mathrm{kg}$ baclofen was evaluated in a randomised double blind placebo controlled trial. Thirty children with GERD ranging from 2-17 years were enrolled. Patients were intubated with a manometric/pH assembly and positioned recumbently. Patients were given a milk drink consisting of $250 \mathrm{ml}$ of full cream cow's milk and then esophageal motility and $\mathrm{pH}$ were measured for two hours (Control period). Baclofen/ placebo was then administered via the assembly and after a one hour a second identical drink was given and esophageal motility and $\mathrm{pH}$ measured for a further two hours (Test period). $13 \mathrm{C} \mathrm{Na-octanoate}(100 \mathrm{mg}$ ) was added to the second meal for breath test measurement of gastric half emptying time (GEt1/2). TLESRs and GER occurring during the control and test periods were analysed using standard criteria and compared. RESULTS: Baclofen produced a significant reduction in the number of TLESRs (mean $7.3 \pm 1.5$ vs $3.6+1.2$ TLESR/ $2 \mathrm{~h}, \mathrm{p}<0.05$ ) and acid GER (mean $4.2 \pm 0.7$ vs $1.7 \pm 1.0$ TLESR+GER/2h, $\mathrm{p}<0.05$ ) during the Test period compared to Control. GE rate was significantly faster in children receiving baclofen compared to placebo (median [IQR] GEt1/2 61 [39, 81] vs $114[67,170]$ min, $\mathrm{p}<0.05$ ). In children who received baclofen, $\mathrm{GEt} 1 / 2$ was also less frequently 'delayed' compared to age matched controls ( $29 \%$ vs $64 \% \%$ in placebo group, $\mathrm{p}=0.066$ ) and $13 \mathrm{CO} 2$ excretion curves used in for the estimation of GE were less frequently multi-peaked (29\%, vs $86 \%$ in placebo group, $\mathrm{p}<0.01$ ). Baclofen did not significantly alter swallowing rate, the patterning of esopageal peristalsis, or LES pressure. CONCLUSION: We conclude that baclofen inhibits TLESR and GER and accelerates GE in children with reflux disease. An acceleration of GE may explain the improvement in symptoms of emesis previously reported in the children with neurological disease and GER who were given baclofen. This trial was supported by the Women's \& Children's Hospital Research Foundation, the JH \& JD Gunn Medical Research Foundation and AstraZeneca R\&D.

\section{1}

Influence of Gastric Motility Modifying Drugs On the Postprandial Acid and Bile Pockets At the Gastro-Esophageal Junction

Raf Bisschops, Daniel Sifrim, Rita Vos, Jozef Janssens, Jan Tack

Gastro-oesophageal reflux (GOR)occurs mainly postprandially and is more pronounced in the distal oesophagus. Fletcher et al (2001) have previously established that a pocket of highly acidic gastric juice is present at the gastro-oesophageal junction (GOJ) after intake of a solid meal in healthy volunteers. Bilitec ${ }^{\circledR}$ studies have shown that a "bile pocket" is also present at the GOJ in some subjects. Factors that determine the location and the extent of the acid and bile pockets have not been identified, but gastric motility is likely to play a role. The aim of the current study was to investigate whether altering gastric motility would affect the location and the extent of postprandial acid and bile pockets in healthy volunteers. Methods: 16 healthy volunteers underwent acid and bile pocket studies on 3 different occasions, at least one week apart. LES position was determined preprandially with pull-through manometry. Dual pH electrode and Bilitec $®$ probe stepwise pull-through $(1 \mathrm{~cm} /$ min, LES-10 to $+5 \mathrm{~cm}$ ) was performed before and 10, 30 and 50 min after a liquid meal (200 $\mathrm{ml}$ Nutridrink $\left.{ }^{\circledR}, 300 \mathrm{Kcal}\right)$. At the start of the meal, saline, the gastrokinetic erythromycin 200 $\mathrm{mg}$ i.v. or the gastric relaxant sumatriptan $6 \mathrm{mg}$ s.c. were administered in a randomized fashion. Results: After saline pre-treatment, a significant $\mathrm{pH}$ drop of on average $2.26 \pm 0.56$ units to a nadir $\mathrm{pH}$ of $2.71+0.68(\mathrm{p}<0.05)$, was present between LES-2 and $+2 \mathrm{~cm}, 10$ minutes after the meal. A similar pattern was found after 30 and 50 min with pH drops of $1.59 \pm 0.40$ and $1.68 \pm 0.42$ units to nadir pHs of $2.02 \pm 0.45$ and $1.74 \pm 0.47$. Erythromycin significantly suppressed the $\mathrm{pH}$ drop at the GOJ at $10 \mathrm{~min}$ to $0.66 \pm 0.17$ units and a nadir $\mathrm{pH}$ of $3.43 \pm 0.89$ ( $\mathrm{p}<0.05$ compared to saline), with comparable patterns at 30 and $50 \mathrm{~min}$ (nadir $\mathrm{pH}$ resp. $4.20 \pm 1.08$ and $4.13 \pm 1.07, \mathrm{p}<0.05$ ). Intragastric $\mathrm{pH}$ at $50 \mathrm{~min}$ was higher after erythromycin $(4.19 \pm 1.05$ vs. $5.40 \pm 1.39, \mathrm{p}<0.05)$. Sumatriptan was associated with a $\mathrm{pH}$ drop of $2.04 \pm 0.54$ and nadir $\mathrm{pH} 2.16 \pm 0.58$ at $10 \mathrm{~min}$ (NS). The position of the acid pocket relative to the LES was not altered by sumatriptan. However, at 30 and 50 min the nadir $\mathrm{pH}$ after sumatriptan was significantly lower (resp. $2.02 \pm 0.54$ and $1.74 \pm 0.47, \mathrm{p}<0.05$ ). A "bile pocket" (segmentary rise in Bilitec $®$ absorbance $>0.14$ ) was seen in $49 \%$ of pullthroughs after saline, compared to $76 \%$ after erythromycin $(\mathrm{p}<0.05)$ and $24 \%$ after sumatrip$\tan (\mathrm{p}<0.05)$. Conclusion: Gastric motility modifying drugs have a major influence on the presence of postprandial acid and bile pockets at the GOJ in health. Enhanced motility disrupts the acid pocket but increases occurrence of a bile pocket, while motor inhibition has the opposite effect.

\section{8}

Intestine Specific Deletion of Math1 Reveals Its Roles in Epithelial Cell Fate Determination and Homeostasis

Noah F. Shroyer, Vincent Y. Wang, Huda Y. Zoghbi

Background: Math1 (Mouse atonal homolog $\underline{1}$; Atoh1) is a basic helix-loop-helix transcription factor essential for formation of Paneth, goblet, and enteroendocrine cells. The aims of this study were to generate and characterize mice that lack Mathl in the intestinal epithelium. Methods: We used homologous recombination in ES cells to generate mice bearing a conditional Math1 allele flanked by loxP sites (Math $1^{\text {flox }}$ ). Math $1^{\text {flox }}$ mice were crossed to Fabpl ${ }^{4 x}$ at -132-cre mice to generate intestine-specific Math1-ablated mice (Math1 $\Delta$ intestine) Histological, immunochemical, and gene expression analysis was performed on small and

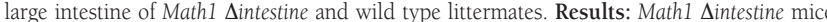
were viable and fertile. Their growth and adult weight was not significantly different than wild type littermates. Analysis of cre-mediated recombination showed a mosaic pattern with $75-80 \%$ deletion of Math1 in the distal ileum, and $80-90 \%$ deletion in the cecum and colon Due to their clonal nature, crypts were either wild type or Math1-ablated, whereas villi were polyclonal showing mixed wild type and Math1-ablated epithelium. Math1-ablated crypts lack all secretory lineages (Paneth, goblet and enteroendocrine cells). Immunostaining revealed loss of transcription factors important for differentiation of the secretory lineages (e.g., Gfil, Ngn3, GATA5, GATA6) We found increased proliferation but unchanged apoptosis in Math1-ablated crypts. Fate mapping with the Math1 lac $Z$ allele suggests that cells fated to become secretory cells undergo a fate switch and differentiate as absorptive enterocytes. Abnormal mucosal architecture was observed in adult Math1 $\Delta$ intestine mice with branched villi in the ileum and branched and cystic crypts in the colon; this phenotype became more prominent in older animals, but survival of animals up to one year was not compromised. Conclusions: Our results confirm that Math1 is required for intestinal secretory cell specification, and that Math1 functions upstream of genes involved in differentiation of the secretory lineages. We further demonstrate that in the absence of Math1, progeny from the intestinal crypt adopt an absorptive fate. We find abnormal proliferation in crypts lacking Math1, and suggest that this aberrant proliferation leads to the abnormal mucosal morphology seen in adult Math1 $\Delta$ intestine mice. Math1 $\Delta$ intestine mice will serve as a usefu model to study the effects of loss of secretory lineages in a normal physiologic milieu This work was supported by HHMI, FDHN, and NIH (DK56338, DK7664, DK63747, and DK71686).

79

Identification of Sonic and Indian Hedgehog Activity During Small Intestine Morphogenesis

William Zacharias, Blair B. Madison, Xing Li, Asa Kolterud, Deborah L. Gumucio

Sonic (Shh) and Indian (Ihh) hedgehog are secreted signaling molecules that are expressed in the small intestinal epithelium during intestinal organogenesis; lack of either Shh or Ih results in marked developmental abnormalities involving both the epithelial and mesenchymal compartments of the intestine. Recent work in our laboratory utilizing animals expressing a secreted form of the pan-Hh inhibitor Hhip (Villin-Hhip) demonstrates that inhibition o the combined Shh and Ihh signal leads to disorganized villus formation, mislocalization of myofibroblasts, and perturbed smooth muscle development. We recently developed two additional mouse models to further examine phenotypes resulting from Hh inhibition and identify crucial Hh target genes. A Villin-flox- $\beta$ gal-flox-Hhip X Villin-Cre double transgenic model recapitulates the Hhip phenotype previously reported, with a significant decrease in differentiated smooth muscle in the cores of the villi and a mislocalization of myofibroblasts in adults. A complementary transgenic Villin-Ihh model exhibits a reciprocal phenotype: significant increase in villus smooth muscle at several adult stages examined. Our results suggest that Hh signaling is crucial not only for the patterning of muscularis externa, as previously demonstrated, but also for the development of smooth muscles within the villus cores and muscularis mucosa. These models, in combination with microarray experiments performed on tissue derived from Hhip mice or on cultured, Hh-treated embryonic smal intestinal mesenchyme, are now being used to identify specific mesenchymal targets of Hh signaling.

80

Specification and Terminal Differentiation of Small Intestinal Endocrine Cells Occurs Independently of Wnt Signaling Yang Wang, Andrew B. Leiter

Wnt signaling is required for the maintenance of intestinal stem cells and their proliferating descendents in the crypts. Wnt loss of function studies indicate that differentiation of the three secretory lineages depends on Wnt signals. Because these studies examined loss of Wnt signals in relatively immature proliferating cells in the intestinal crypts, it is not known at what stage of development Wnt is required for endocrine differentiation in the intestine Prior cell lineage tracing studies indicate that most enteroendocrine cells arise from precurso cells expressing the basic helix loop helix transcription factor, neurogenin 3 (Ngn3). In addition, Ngn 3 null mice fail to develop enteroendocrine cells, suggesting that Ngn 3 is necessary for their differentiation and that Ngn3 expression is a very early event in endocrine specification. To determine whether Ngn3+ cells require Wnt for further differentiation, we knocked out the $\beta$-catenin gene in Ngn3+ cells by crossing Ngn3-Cre recombinase transgenic mice to mice with one null and one conditional allele for $\beta$ - catenin. Examination of the small intestine revealed normal morphology and normal enteroendocrine cells, suggesting that Wnt signaling is not required for endocrine precursor cells to terminally differentiate To further examine the role of Wnt in intestinal differentiation, we took advantage of our previous observations that small numbers of secretory progenitors that give rise goblet and Paneth cells and rare pluripotent cells express Ngn3 yet do not become endocrine cells. We generated mice with conditional deletion of the $\beta$-catenin gene in Ngn3 expressing cells in a Rosa26-LacZ reporter background, allowing us to identify all cells arising from Ngn3

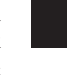


cells expressing Cre recombinase by staining for $\beta$ galactosidase activity. The number of $\beta$ gal positive enteroendocrine cells was the same in control and transgenic mice confirming that Wnt was not required for terminal differentiation of enteroendocrine cells. Likewise, the number of $\beta$-gal+ goblet cells was normal, suggesting that secretory progenitors committed to become either goblet or enteroendocrine cells do not require Wnt for terminal differentiation. However, the numbers of $\beta$-gal+ Paneth cells and crypt-villus strips with all cells $\beta$ gal labeled were reduced by $80 \%$ and $74 \%$, indicating that Wnt was required for Paneth cell differentiation as well as for maintenance of pluripotent cells. We conclude that enteroendocrine and goblet cells, unlike Paneth cells, differentiate independently of Wnt and comprise a distinct subpopulation arising from intestinal secretory progenitor cells.

81

$\beta 1$ Integrins Regulate Wnt Signaling in the Intestinal Epithelium Scott K. Kuwada, Brett Doxey, Phillip Gray, Xiufen Li

We recently reported that conditional deletion of $\beta 1$ integrins in the intestinal epithelial cells (IEC) of mice $(\beta l \mathrm{KO})$ disrupted intestinal homeostasis through a loss of Hedgehog expression and signaling. We report now that the $\beta 1 \mathrm{KO}$ mice demonstrate striking similarities to mice in which Apc was conditionally deleted in the IEC's. Both KO mice demonstrated dysplastic crypts, increased Paneth cell markers, defective enterocyte differentiation, early neonatal malnutrition, and expansion of intestinal stem cells. AIM. To determine the role of $\beta 1$ integrins in the regulation of Wnt signaling in the intestinal epithelium. METHODS: Villin-Cre mice were crossed with $\beta 1$ integrin(lox/+) mice, and, the offspring were backcrossed to generate villin-Cre/ $\beta 1$ integrin(lox/lox) mice. The loss of $\beta 1$ integrin expression was determined by immunoblot (IB) and immunohistochemistry (IHC). Quantitative reverse transcription-polymerase chain reaction (qRT-PCR), IHC, and IB were used to determine the expression levels of Wnt signaling pathway members. RESULTS: The mRNA expression of the Wnt signaling pathway targets Matrix Metalloproteinase-10 and Defensin/ Cryptdin 5 , in the IECs of the $\beta 1 \mathrm{KO}$ mice was increased by 3-10-fold compared with control littermates. Cryptdin 5 protein was localized to Paneth cells in the control mice but was expressed along the entire crypt-villous axis in the $\beta 1 \mathrm{KO}$ mice, however, electron microscopy and expression of enterocyte markers showed that the villous IECs were enterocytes and not Paneth cells. These results demonstrated increased and mislocalized Wnt signaling in the IEC's of the $\beta 1 \mathrm{KO}$ mice. Nuclear $\beta$-catenin was seen in similar numbers of Paneth cells in the crypts of the $\beta 1 \mathrm{KO}$ mice and control littermates indicating that Wnt signaling was not increased proximal to $\beta$-catenin. IHC and IB demonstrated nuclear Tcf4 in a few IECs in the crypt bases of the control mice, but increased nuclear Tcf-4 expression in IECs along the entire crypt-villous axis in the $\beta 1 \mathrm{KO}$ mice. Normal mouse pups were treated every other day with the Hedgehog inhibitor cyclopamine or vehicle for 10 days. Cyclopamine caused increased nuclear Tcf-4 expression along the entire crypt-villous axis. CONCLUSIONS: $\beta 1$ integrins mediate Wnt signaling at the level of Tcf-4 in a Hedgehogdependent fashion. These studies demonstrate the signaling pathways by which the extracellular matrix regulates IEC proliferation

P53-Activated Ndrgl Expression Plays An Important Role in Inhibition of Normal Intestinal Epithelial Cell Proliferation Following Polyamine Depletion Ai-Hong Zhang, Jaladanki N. Rao, Tongtong Zou, Lan Liu, Bernard S. Marasa, Lan Xiao, Jie Chen, Jian-Ying Wang

Normal intestinal mucosal growth requires polyamines that regulate expression of various genes involved in cell proliferation, growth arrest, and apoptosis. Our previous studies have shown that polyamine depletion stabilizes p53 resulting in inhibition of intestinal epithelial cell proliferation, but the exact down-stream targets of induced p53 are still unclear. The NDRGl (N-Myc down-regulated gene 1) gene encodes a growth inhibiting protein, which is primarily regulated at the transcriptional level. The current study tests the hypothesis that induced p53 causes inhibition of normal intestinal epithelial cell proliferation by activating NDRGl transcription following polyamine depletion. Method: Studies were conducted in IEC-6 cells, derived from rat normal intestinal crypts. The NDRGl gene transcription was examined by its promoter luciferase reporter gene assays, and levels of NDRGl mRNA and protein were measured by RT-PCR and Western blot analysis. p53 was knockdown by specific siRNA, and ectopic expression of the NDRGl gene was induced by infection with the recombinant adenoviral vector containing wild-type NDRGl cDNA (AdNDRG1). Results: Polyamine depletion by DFMO, the specific inhibitor of polyamine synthesis, not only activated NDRGl promoter but also increased levels of NDRGl mRNA and protein. The NDRGl promoter activity was increased by 5 -folds on day 6 after treatment with DFMO, while levels of NDRGl mRNA and protein were increased by $\sim 4$-folds, which were completely prevented by exogenous polyamine putrescine. Decreased $\mathrm{p} 53$ expression by specific $\mathrm{p} 53$ siRNA in polyamine-deficient cells prevented the increased expression of NDRGl. Ectopic expression of the NDRGl gene by AdNDRGl infection dramatically inhibited IEC- 6 cell proliferation, but had no effect on apoptosis. Cell number was decreased by $~ 50 \% 48 \mathrm{~h}$ after infection with AdNDRGl. In contrast, there were no significant differences in rates of apoptotic cell death between cells overexpressing NDRGl and cells infected with the adenoviral vector lacking NDRGl cDNA after exposure to TNF $\alpha$ plus cycloheximide. Conclusions: These results indicate that decreasing cellular polyamines increases NDRGl transcription through a p53-dependent mechanism and that p53-induced NDRGl following polyamine depletion plays a critical role in inhibition of normal intestinal epithelial cell proliferation.
Critical Interactions Between Elf and Cdk4 in Regulation of Gastrointestinal Cell Proliferation in TGF- $\beta$ Signaling Pathway

Sang Soo Kim, Hye Jung Baek, Anthony Kang, Yi Tang, Varalakshmi Katuri, M. Blair Marshall, Bibhuti Mishra, E. Premkumar Reddy, Lopa Mishra

TGF- $\beta$ is an important regulator of Gl/S cell cycle progression. Inactivation of TGF- $\beta$ signaling frequently occurs in gastrointestinal cancer. We have shown that disruption of ELF disrupts TGF- $\beta$ signaling through Smad 3 and Smad4. Interestingly, our previous analysis of elf-/- and elf+/- mice showed developmental defects with embryonic lethality and a prominent increase in spontaneous gastrointestinal cancer, respectively. In addition, ELF functions as a Smad3 and Smad4 adaptor. Recently, Smad3 has been shown to be a CDK4 substrate indicating that suppression of Smad3 by CDK4 could represent a mechanism by which tumorigenesis occurs. However, there is no clear evidence for tumorigenic contributions from Smad3 mutations in human and knockout mice. Aim; To gain further insight into the potential regulatory role of ELF in CDK4 function, cell cycle progression, and neoplasia formation, we carried out detailed biochemical analyses and genetic studies Methods and Results; Here we show that CDK4 is activated in cancers from elf+/- mutant mice and ELF interacts with CDK4 by forming a complex containing Smad3 in COS-7 cells. Our further mapping analysis identified the N-terminal actin-binding domain of ELF to be responsible for interactions with CDK4. Interestingly, CDK4 also interacts Smad3 in the presence of ELF upon TGF- $\beta$ stimulation. To ascertain interactions between CDK4 and ELF in tumor formation, we intercrossed elf+/- and CDK+/R24C mice where CDK4-R24C mutations abolish the ability of CDK4 to bind to pl6(INK4a) thus rendering CDK4 constitutively active. Surprisingly, most of the compound heterozygote elf and CDK4-R24Cmice showed embryonic lethality resembling homozygote phenotypes of elf-/- mice with CNS and GI defects. Conclusion; These results show the molecular interactions between ELF and CDK4 are essentially for cell cycle regulation, cell proliferation, and differentiation. This study also suggests that ELF, a major dynamic scaffolding protein, is a key molecule by modulating the interaction of regulatory proteins such as CDK4 and also for transmitting the TGF- $\beta$ signaling pathway, which is essential for cell cycle regulation and tumor suppression.

84

The Basic Helix-Loop-Helix Transcription Factor Mistl Regulates Differentiation of Gastric Zymogenic (Chief) Cells from Their Mucous Neck Cell Precursors

Victoria G. Ramsey, Christopher C. Chen, Jason M. Doherty, Thaddeus S. Stappenbeck, Jason C. Mills

Digestive enzyme secreting zymogenic (chief) cells (ZCs) reside in the bottom of gastric units of mammalian stomachs. The ZC arises from its precursor, the mucous neck cell (NC), which has its own distinct, specialized functions. Loss of differentiated ZCs is a hallmark of precancerous lesions such as atrophic gastritis and intestinal metaplasia. However, the genes that coordinate the trans-differentiation from the NCs to ZCs are not known. To identify candidate genes, we used laser-capture microdissection (LCM) to purify individual ZCs, parietal cells, and surface pit cells from stomach frozen sections. RNA was extracted, amplified, converted to cRNA and hybridized to Affymetrix MOE430v2.0 GeneChips. Using commercially available as well as in-house bioinformatic software, we identified $\sim 30$ transcription factor (TF) transcripts enriched in ZCs vs the other cells. One gene, the basic helixloop-helix TF Mistl, had been previously shown to be expressed in ZCs. In normal mice, NCs and ZCs are thought to differentiate as they migrate. To examine whether Mistl affected this process, we obtained Mistl knockout mice and examined well-oriented gastric units with immunofluorescent markers of $\mathrm{NC}$ and ZC differentiation. In control mice, we observed, as expected, that mean fluorescent intensity/cell (MFI) of the NC marker increased linearly with distance from the stem cell compartment. At the bottom of the neck region of the gastric unit, there was a brief transition where 3-4 cells/gastric unit co-expressed NC and ZC markers. Thereafter, cells shut off the NC marker, expressing only the ZC marker. In Mistl knockouts, neck cell differentiation was normal, but the numbers of $\mathrm{NC}->\mathrm{ZC}$ transitional cells were doubled. There was a ten-fold increase specifically in the numbers of transitional cells co-expressing high levels of the ZC marker while retaining GSII (NC) expression. MFIs of the ZC marker then decreased as cells migrated out of the transition zone, contrasting with control mice whose ZCs increase zymogenic markers as they migrate to the base. Electron microscopic studies corroborated that, in knockouts, the mature zymogenic granules characteristic of terminally differentiated ZCs occurred aberrantly in transitional cells and were then lost as ZCs migrated toward the base. Immunofluorescence showed that Mistl is expressed only in the cells that are aberrant in the knockout: ZCs and late transitional cells. Thus, we propose that Mistl regulates the emergence and maintenance of the ZC lineage. Experiments are underway to determine whether Mistl expression is lost in mouse models of atrophy and in human carcinomas and pre-cancerous lesions.

85

The Risk of Gastric Cancer Correlates with Serum Pepsinogen I / Pepsinogen II Ratio Both in Longitudinal Settings As Well As Cross-Sectional Hirotsugu Watabe, Yutaka Yamaji, Makoto Okamoto, Ryoichi Wada, Hidetaka Doi, Haruhiko Yoshida, Takao Kawabe, Toru Mitsushima, Masao Omata

INTRODUCTION: Serum pepsinogen (PG) is related to gastric atrophy. PG I /PG II ration is a useful marker for gastric cancer. However, the evidence supporting this usefulness is mainly based on the cross-sectional studies. It is unclear that $P G$ is also helpful to predict development of gastric cancer during follow-up. We previously conducted a prospective endoscopic follow-up study to evaluate efficacy of the combination of $\mathrm{H}$. pylori antibody and serum pepsinogen (Watabe $\mathrm{H}$, et al. GUT 2005; 54: 764). We reanalyzed this cohort to evaluate the efficacy of $\mathrm{PG}$ as a predictive marker for gastric cancer in cross-sectional and longitudinal settings in the present study. METHOD: Between March 1995 and February 1997, 10234 subjects (male/female; 7021/3213, mean age; 49.1 18.8 ) participated in the endoscopic mass screening program. PG I and II levels were simultaneously assayed. We 
reclassified the subjects into 3 groups according to PG I / PG II ratio (PGR); Low PGR group (PGR < 3.0; $n=2863$ ), Medium PGR group ( $3.0 \leqq P G R<4.0 ; n=1819)$, and High PGR group (PGR $\geqq 4.0 ; n=5552$ ). In the cross-sectional analysis, the prevalence of gastric cancer was investigated according to PGR groups in the population at the entry. In the longitudinal analysis, we analyzed the cohort of 6983 followed-up subjects for 4.7 years, and investigated the incidence rates of newly development of gastric cancer. Risk ratios adjusted by age and gender were calculated for PGR status. RESULT: At the initial endoscopy, $32(0.3 \%)$ had gastric cancer. Odds ratios compared with High PGR group were 5.8 (95\% CI; 1.5-22.5, p= 0.01 ) in Medium PGR group, and 10.3 (3.0-35.0, $\mathrm{p}=0.0002)$ in Low PGR group, respectively. Among the 6983 subjects with endoscopic follow-up, 43 subjects ( $0.6 \%$ ) developed gastric cancer. The annual incidence rates of gastric cancer were $0.02 \%, 0.12 \%$, and $0.35 \%$ in groups of High, Medium, and Low PGR, respectively. Hazard ratios compared with High PGR were 4.1 (95\% CI; 1.2-14.1, p=0.02) in Medium PGR group, and 10.0 (3.5-28.6, $\mathrm{p}<0.0001)$ in Low PGR group. Older age and male gender were also independent risk factors for both the prevalence and the incidence of gastric cancer. We could establish a super high-risk group, male older than 60 with Low PGR. They had the prevalence of $1.46 \%$ and annual incidence of $0.71 \%$. CONCLUSION: Serum pepsinogen I / pepsinogen II ratio was a correlated marker for the risk of gastric cancer.

86

Gastrin-Induced Pepsinogen Regulates Sonic Hedgehog Processing and Prevents Gastric Atrophy

Yana Zavros, Meghna Waghray, Linda Zhang, Arthur Tessier, Deborah Gumucio, Linda C. Samuelson, Andrea Todisco, Juanita L. Merchant

Objective: Sonic hedgehog (Shh) has been reported to regulate epithelial cell differentiation and maturation in the adult stomach. Shh is highly expressed in the acid-secreting parietal cells. During progression from gastritis to gastric cancer, the parietal cells fail to produce acid and are eventually replaced by mucous secreting cells (atrophy). Gastrin regulates acid production in the parietal cell. Gastrin-deficient (G-/-) mice have impaired acid secretion and over time develop parietal cell atrophy that eventually results in gastric cancer. Since loss of Shh correlates with atrophic gastritis, the hypothesis that gastrin regulates Shh production within the parietal cell was tested. Methods: Shh expression was analyzed by qRT-PCR and western blot using RNA and protein extracted from mucosal scrapings of wild type, G-/-and gastrin-infused G-/- mice. Shh protein expression was analyzed using whole cell extracts (WCE) prepared from canine parietal cells treated with vehicle, gastrin, omeprazole or both. Shh processing was assessed by incubating WCE prepared from treated parietal cells with in vitro translated Shh $45 \mathrm{kDa}$ peptide. To directly test the digestion of Shh protein with pepsin A, the activated enzyme was incubated with in vitro translated Shh protein. Results: Both the $45 \mathrm{kDa}$ precursor and $19 \mathrm{kDa}$ biologically active Shh proteins were absent in the G-/- mice. Restoration of acid secretion after gastrin infusion resulted in Shh processing of the precursor to the $19 \mathrm{kDa}$ form and increased Shh mRNA abundance. Gastrin-treated canine parietal cells showed processing of the $45 \mathrm{kDa}$ precursor in WCE to the secreted $19 \mathrm{kDa}$ biologically active peptide in the media. When acid secretion from the parietal cells was blocked with omeprazole, the effect of gastrin on Shh processing was abolished. WCE collected from gastrin treated parietal cells processed the $45 \mathrm{kDa}$ to $19 \mathrm{kDa}$ fragment, and this processing was blocked with the protease inhibitor pepstatin. Pepsinogen I (PGI) expression was induced by gastrin and inhibited by omeprazole treatment of parietal cells. Digestion of pepsin A clearly showed cleavage of the precursor $45 \mathrm{kDa}$ peptide to a $19 \mathrm{kDa}$ protein within 20 min. Conclusion: Gastrin induces the expression of PGI that under acidic conditions converts to the active protease pepsin A. Pepsin A cleaves the $45 \mathrm{kDa}$ Shh peptide to the soluble secreted biologically active protein. Therefore, the current study links the loss of Shh during atrophic gastritis to the loss of PGI expression. Specifically, low levels of PGI are predictive of the presence of atrophic gastritis, which is the most important predictor of susceptibility to gastric cancer

87

Activation of Hedghog Signaling in GI Cancers

Jingwu Xie, J.N. He, Tao Sheng, Arwen Stelter, Laura Westerberg, Shuhong Huang, Xiaoli Ma, Hongwei Zhang, Robert Whitehead, Patrick Adegboyega, Stan Lemon, Mark Ever

GI cancers are the major causes of cancer-related death worldwide. Patients with GI cancers usually present at late stages and have a poor prognosis. Thus, identification of specific drug targets in the tumor is an essential step to reduce the mortality. Recent studies indicate that abnormal activation of the sonic hedgehog pathway is aasociated with pancreatic cancer, gastric cancer and esophageal cancer. To determine if hedgehog signaling activation can be utilized for diagnosis and treatment for GI cancers, we performed a comprehensive study to assess hedgehog pathway activation in over 200 primary GI cancer specimens, including gastric cancer, esophageal cancer, pancreatic cancer, colon cancer and heptocellular carcinomas. Through analyses of hedgehog target gene expression, we discovered that hedgehog signaling is frequently activated in gastric cancer, esophageal cancer, hepatocellular carcinoma and pancreatic cancers, but rarely in colon cancers. Our studies further indicate that hedgehog signaling activation is associated with tumor stage, differentiation and tumor subtype of gastric cancer. Over-expression of sonic hedgehog (shh) was observed for tumors with activation of the hedgehog pathway, suggesting that elevated Shh expression may be responsible for hedgehog signaling activation in the tumor. Furthermore, activated shh expression was also observed in the mouse HCC derived from HCV-transgenic mice. The significance of hedgehog signaling in GI cancers was demonstrated by the fact that targeted inhibition of hedgehog signaling by either cyclopamine or Shh neutralizing antibodies reduced cell growth of cells with activated hedgehog signaling. Our data indicate that hedgehog signaling is an important abnormality in GI cancers. These data predict that targeted inhibition of the hedgehog pathway may be effective in the prevention and treatment of GI cancers.
Evidence That Integrin-Linked Kinase Is Involved in Murine Colitis-Associated Carcinogenesis

Baljinder Salh, Kiran Assi, David Owen, Shoukat Dedhar

Integrins are transmembrane cell surface receptors that mediate cell-cell and cell-matrix contacts. The recently identified integrin-linked kinase (ILK) is a binding partner of integrins and has been ascribed essential roles in development and angiogenesis. It also impart tumorigenic properties to cells when overexpressed. As we had previously shown dysregulation of this molecule in human colon cancer, we were interested to explore its role in murine tumorigenesis, using a recently described colitis-associated cancer model dependen upon azoxymethane and DSS. To create intestinal ILK knockout animals (ILK-iko), we used Fabp/Cre mice (Cre recombinase expressed under the control of a modified Fabp promoter) and mated them with mice carrying a LoxP-flanked (floxed) ILK gene (ILKflox/flox). (These mice have been used to demonstrate ILK's effects on chondrocytes as well as its role in vascular development). Expression of Cre and excision of ILK were determined in the offspring using PCR. We have previously observed that mice with intestinal epithelial cel ILK knockout develop normally upto 1 year. They exhibited partial reduction in the size of the cecum (approximately 70-80\% of littermate size). They did not exhibit any macroscopic signs of basally increased inflammation. Immunohistochemical analysis revealed that ther was diminished ILK expression in ILK-iko animals. Evaluation of BrDU staining in the knockout animals revealed a significant reduction in cells positive for this parameter a compared to the wild-type animals in both the cecum and colon $(\mathrm{p}<0.001$ for both) confirming ILK's effects on cell proliferation. Following azoxymethane and DSS treatment, we observed fewer total tumors in the ILK knockout animals, especially those $>5 \mathrm{~mm}$ in diameter (wt:7.9 +/- 3.4/animal, ko:2.7 +/- 1.8/animal, $\mathrm{p}<0.05, \mathrm{n}=14$ and 10 animals for the two groups respectively, data represent mean $+/$ SEM). Histology revealed that there were fewer invasive tumors in knockout animals (only 1 compared with 4 in the wild-type group), and this correlated with a reduction in MMP9 expression. We conclude that ILK has a role in intestinal epithelial cell proliferation and provide the first evidence that it influences the development of colitis-associated neoplasia.

89

Annexin II Binds Progastrin and Gastrin Like Peptides, and Is Required for Mediating Growth Factor Effects of Autocrine and Exogenous Gastrins On Colon Cancer and Intestinal Epithelial Cells

Pomila Singh, Hai Wu, Azar Owlia

BACKGROUND. We and others have reported the presence of novel progastrin (PG) preferring receptors on normal and cancerous intestinal cells (AJP 2003). We had earlier identified $\sim 34 \mathrm{KDa}$ proteins on cellular membranes of colon cancer $(\mathrm{CA})$ cells, that were specifically cross-linked to radio-labeled gastrins. It is not known if the $\sim 34 \mathrm{KDa}$ proteins represent the novel PG preferring receptor proteins. AIM. To identify the binding proteins in the $\sim 34$ $\mathrm{KDa}$ band, and analyze the functional significance of the protein(s) thus identified. METHODS. 125I-rhPG and 125G-17 were used for cross-linking the membrane proteins from CA cells, followed by solubilization of the native binding proteins and enrichment by $>1,000$-fold by several steps of purification. The purified 32-36 KDa proteins, specifically cross-linked to 125I-Gastrins, by carbodiimide cross-linker, were subjected to SELDI-TOFMS analysis (using the protein chip system from Ciphergen) and the MALDI-TOF-MS analysis (in the UTMB Biomolecular Research Facility). Peptide masses were researched against the NCBInr database using the ProFound search engine to identify the proteins in the punched out bands of interest. RESULTS. Annexin II was identified as the major protein in the 34 $\mathrm{KDa}$ cross-linked band from CA cells. Since HCT-116 cells express autocrine PG, the in situ association of autocrine PG with Annexin II was confirmed in pull down assays. The binding of PG peptides with Annexin II, was further confirmed in vitro solution binding assays with purified Annexin II and rhPG. Annexin II/rhPG in solution were immunoprecipitated with either anti-PG or anti-Annexin II Antibodies (Abs), followed by Immunoblo analysis with either of the Abs. The results provided the first direct evidence that rhPG and Annexin II bind in solution. Annexin II also functions as a membrane receptor for Tenascin$\mathrm{C}$ (Ten-C). Therefore, the relative binding affinity (RBA) of Ten- $\mathrm{C}$ for displacing the binding of 125I-rhPG to IEC-18 and CA cells was examined. The RBA of Ten-C was similar to that of rhPG, suggesting that the 2 proteins may share binding sites on Annexin molecules. In order to confirm the functional importance of these observations, sense(S) and anti-sense (AS) clones of IEC-18 and HCT-116 cells were generated for the expression of Annexin II the AS clones demonstrated a significant loss in the growth response of the cells to exogenous (IEC-18) or autocrine (HCT-116) PG. CONCLUSIONS. The membrane associated protein Annexin II, specifically binds PG and gastrin-like peptides, and its expression is required for mediating growth factor effects of exogenous and autocrine PG on normal and cancerous intestinal cells.

90

$\mathrm{Cd} 4+\mathrm{Cd} 45 \mathrm{rb}^{\mathrm{Lo}} \mathrm{Cd} 25+$ Regulatory Lymphocytes Protect Against Induction of Helicobacter Pylori Gastritis in C57bl/6 Mice Through An Illo Dependent Mechanism

Chung-Wei Lee, Varada P. Rao, Susan E. Erdman, Arlin B. Rogers, James G. Fox

Mouse models of Helicobacter pylori (Hp)-induced gastritis and associated lesions are valuable for the study of immunopathogenesis of gastric cancer in humans. Gastritis due to $\mathrm{Hp}$ involves $\mathrm{CD} 4+\mathrm{CD} 45 \mathrm{RB}^{\text {hi }}$ effector $\mathrm{T}$ cells both in mice and humans, whereas $\mathrm{T}$ cell populations of $\mathrm{CD} 4+\mathrm{CD} 45 \mathrm{RB}^{\mathrm{lo}} \mathrm{CD} 25+$ phenotype are known to be protective against $\mathrm{Hp}$-induced gastritis. Interestingly, IL10-deficient C57BL/6 mice develop more severe Hp-induced gastritis than wild-type (WT) mice. We hypothesized that IL-10, a potent immunosuppressive cytokine of regulatory $\mathrm{T}$ cells, is critical for the control of gastritis in the host. In the present study, we assessed regulatory function of $\mathrm{CD} 4+\mathrm{CD} 45 \mathrm{RB} \mathrm{B}^{\mathrm{lo}} \mathrm{CD} 25+\mathrm{T}$ cells from WT or IL10-deficient donors in Hp-infected C57BL/6 Rag2-/- mice that have received CD4+CD45RBhi effector T cells (Table 1). Severe gastritis and wasting occurred in Hp-infected Rag2-/- mice 10-12 
weeks post transfer with effector T cells (inflammatory score 2.7+/-0.11). In contrast, Hpinduced gastritis was ameliorated in mice that received co-transfer of effector $\mathrm{T}$ cells and regulatory $\mathrm{T}$ cells from WT donors $(1.0+/-0.15, \mathrm{p}<0.001)$. Suppression of gastritis, however, was not observed when regulatory T cells from IL10-/- donors were used (inflammatory score $2.0+/-0.35, \mathrm{p}=0.146$ ), suggesting that IL10 is critical for regulatory $\mathrm{T}$ cell function of $\mathrm{CD} 25+$ cells in this model. Similarly, adoptive transfer of WT regulatory T cells was effective in ameliorating pan-gastritis observed in mice that have received effector $\mathrm{T}$ cells in the absence of $\mathrm{Hp}$ infection. We observed a significant decrease in $\mathrm{Hp}$ colonization in mice reconstituted with effector $\mathrm{T}$ cells compared to non-transferred control animals. Compared to the non-transferred group, mice that have received co-transfer of effector and regulatory $\mathrm{T}$ cells also had lower levels of $\mathrm{Hp}$ colonization. These data support previous studies demonstrating that mounting an immune response against $\mathrm{Hp}$ reduces Hp colonization in the gastric compartment. These findings highlight the important protective role of IL10competent $\mathrm{CD} 4+\mathrm{CD} 45 \mathrm{RB}{ }^{\text {lo }} \mathrm{CD} 25+$ regulatory $\mathrm{T}$ cells in Hp pathogenesis, and suggest that approaches aimed at selectively enhancing regulatory $\mathrm{T}$ cell function in humans may prevent Hp-induced gastritis and gastric cancer.

Table 1

\begin{tabular}{|c|c|c|c|c|c|}
\hline Group & H. pylori & TE & TR & Inflammation score & Colonization log (CFU/g) \\
\hline Нр & + & - & - & $0.7+/-0.10^{\dagger}$ & $5.73+/-0.24$ \\
\hline $\mathrm{Hp}+\mathrm{TE}$ & + & + & - & $2.7+/-0.11$ & $1.26+/-0.52^{*}$ \\
\hline $\mathrm{Hp}+\mathrm{TE}+\mathrm{wt} \mathrm{TR}$ & + & + & WT & $1.0+/-0.15^{\text {靷 }}$ & $2.77+/-0.89^{*}$ \\
\hline Hp+TE+IL10-I-TR & + & + & IL10-/- & $2.0+/-0.35$ & $2.06+/-1.19^{*}$ \\
\hline
\end{tabular}

"p<0.001 when compared to Hp+TE group, ${ }^{*} \mathrm{p}<0.05$ when compared to Hp+TE+IL10-/-TR group ${ }^{*}<<0.001$ when compared to Hp group

H. Pylori-Induced Phosphorylation of C-Fos Is the Key Regulatory Step in the Activation of Macrophage Apoptosis

Mohammad Asim, Rupesh Chaturvedi, Francoise I. Bussiere, Kshipra Singh, Keith T. Wilson

Background: Inhibition of innate immune response to H. pylori (Hp) is inhibited by induction of macrophage apoptosis. We have reported that: this apoptosis is dependent on generation of polyamines by ornithine decarboxylase (ODC), $\mathrm{ODC}$ induction requires binding of $\mathrm{c}$ Myc to the ODC promoter, and c-Myc expression is mediated by phosphorylation of extracellular signal regulated kinase (ERK) 1/2 and translocation of phospho-ERK1/2 to the nucleus. Our aim was to determine how phospho-ERK1/2 activates c-Myc. Because c-Myc transcription can be regulated by the AP-1 complex, we assessed the involvement of AP-1 member proteins c-Fos, c-Jun, and Jun-D in the response to Hp. Methods: RAW 264.7 macrophages were stimulated with lysates of Hp SS1. We assessed c-Myc, ODC, and c-Fos mRNA expression by real-time PCR, c-Myc and Jun-D protein levels by Western blotting, and c-Fos and cJun phosphorylation by phosphoblotting. Oligonucleotide pull-down assays were performed using an AP-1 consensus binding sequence as a probe. Apoptosis was quantified by flow cytometry (annexin V/PI staining). Results: Hp induced c-Fos mRNA expression that peaked at $20 \mathrm{~min}$. with a $5.8 \pm 1.2$ fold increase $(p<0.01)$ and c-Fos phosphorylation that peaked at $40 \mathrm{~min}$. To determine if c-Fos is a downstream target of phospho-ERK1/2, we tested the effect of inhibition of ERK1/2 with PD98059, and found that while there was no effect on c-Fos expression, this completely blocked Hp-stimulated c-Fos phosphorylation. Inhibition of p38 MAP kinase or c-Jun N-terminal kinase (JNK), with SB203580 and SP600125, respectively, had no effect on c-Fos expression or phosphorylation. Hp also induced c-Jun and Jun-D, and c-Jun phosphorylation. SP600125 blocked c-Jun phosphorylation and JunD protein expression, but did not affect Hp-stimulated c-Myc and ODC levels or apoptosis, indicating that phosphorylation of c-Jun or the expression of Jun-D is not involved, and phosphorylation of c-Fos is the major event in the AP-1 complex formation and the induction of c-Myc. Pull-down assay confirmed the presence of phospho-c-Fos in the AP-1 complex formed in nuclear extracts of $\mathrm{Hp}$-stimulated cells. When cells were transfected with a dominant negative form of c-Fos (Fos-A) there was significant attenuation of Hp-induced c-Myc and ODC expression. In parallel, Fos-A inhibited Hp-stimulated apoptosis by 76.5 $\pm 12.6 \%(p<0.01)$. Conclusions: We have identified that phosphorylation of $c-F o s$ in the nucleus by phospho-ERK1/2 is a critical event in c-Myc/ODC-dependent apoptosis in Hpstimulated macrophages. Activation of early stress response genes of the AP- 1 complex is an important part of the dysregulated immune response to $\mathrm{Hp}$.

92

Site Specific Activation of Focal Adhesion Kinase (FAK) Is a Key Regulator of Helicobacter Pylori Mediated Intracellular Signaling

Fazal H. Tabassam, Yoshio Yamaoka

Background: CagA containing Helicobacter pylori induce cytoskeletal organization, motility and elongation of gastric epithelial cells (e.g. AGS cells). These changes have been related to CagA which following injection into the cell undergoes tyrosine phosphorylation in EPIYA motifs. The initial signaling events resulting in these phenotypic changes remain unknown. There are a number of potential phosphorylation sites on FAK that result in activation of different pathways. Aim: To examine the effect $H$. pylori on site specific activation of FAK. Methods: AGC cells were co-cultured with H. pylori (wild type and its isogenic cag pathogenicity island (PAI) or oipA mutants) in various dose and time courses and phosphorylation status of FAK, Src, Erk1/2 and paxillin was evaluated by immunoblot and fluorescence microscopy analyses. Results: $H$. pylori caused rapid, transient phosphorylation of FAK at residue Y397 and Src at Y418 followed by tyrosine phosphorylation of FAK at Y407, Y576, Y577, Y861 and Y925 in a dose- and time-dependent manner. Activation of FAK and Src induced tyrosine phosphorylation of Erk1/2 at sites T202/Y204 and paxillin at Y31 and Y118. Chemical inhibitors of PI3 kinase and Src blocked the H. pylori mediated tyrosine phosphorylation of FAK, Src, Erk1/2 and paxillin. These results suggest that complete activation of FAK through interactions and activation of Src and paxillin are likely responsible for the regulation of intracellular signaling leading ultimately to cytoskeletal reorganization, motility and phenotypic changes associated with CagA positive $H$. pylori. Interestingly, use of cag PAI or oipA isogenic mutants showed that phosphorylation of FAK at Y397, Y576, Y 577 and Y925 was mediated by OipA. In contrast, phosphorylation of FAK at Y407 and Y861 was induced by the cag PAI, while phosphorylation of Src,Erk 1/2 and paxillin were both virulent factors dependent. These results are consistent with the notion that different upstream effectors and downstream signaling pathways are mediated by different virulent factors. Conclusion: These findings suggest that the virulence factors, cag PAI or OipA, control the phosphorylation of FAK, Src, Erkl/2 and paxillin and thus contribute to actin remodeling enhanced inflammatory responses and various pathological outcomes from $H$. pylori infection. Inhibition of site specific proteins should provide novel therapeutic strategies for interfering signaling at multiple phosphorylation stages and inhibition of various down stream signaling. Supported in part by the Gulf Coast Digestive Disease Center.

\section{3}

Interleukin-1 $\beta$ Antagonizes Helicobacter Pylori-Mediated Down-Regulation of H,K-ATPase $\alpha$ Subunit Gene Transcription

Arindam Saha, Charles E. Hammond, Monika Gooz, Maria Trojanowska, Adam J. Smolka

H. pylori infection of human gastric fundic mucosa perturbs normal acid secretory mechanisms, inducing hypochlorhydria, fundic atrophy, and increasing risk of gastric carcinoma. Gastric acid secretion is mediated by $\mathrm{H}, \mathrm{K}$-ATPase, a $\mathrm{K}^{+}$-stimulated, $\mathrm{H}^{+}$-translocating ATPase in parietal cell apical membranes. We showed previously that $H$. pylori down-regulates $\mathrm{H}, \mathrm{K}-$ ATPase $\alpha$ subunit $(\mathrm{HK} \alpha)$ gene transcription in vitro and also decreases parietal cell proton pump expression, providing a partial mechanistic basis for clinical hypochlorhydria. Given that $H$. pylori infection up-regulates gastric mucosal IL-1 $\beta$ expression and that IL-1 $\beta$ inhibits secretagogue-stimulated acid secretion, we sought to determine the role of IL-1 $\beta$ in $H$. pylorimediated down-regulation of $\mathrm{HK} \alpha$ transcription. Human gastric adenocarcinoma cells (AGS) were transfected with luciferase reporter gene plasmids containing deletion fragments of human $\mathrm{HK} \alpha$ 5'-flanking sequence. Maximal transcriptional activity of $\mathrm{HK} \alpha$ sequences was induced by $100 \mathrm{nM}$ phorbol myristate acetate, the cells were treated ( $24 \mathrm{hr})$ with $\mathrm{H}$. pylori (ATCC 49503) and/or IL-1 $\beta$, and relative light units were normalized against co-transfected H. pylori-insensitive GFP plasmids. H. pylori dose-dependently inhibited transcriptional activity of 2199 bp, 207 bp, 195 bp, and 132 bp HKa deletion fragments. H. pylori-mediated inhibition of 2199 bp HK $\alpha$ activity (29\%) was antagonized by IL-1 $\beta(10 \mathrm{ng} / \mathrm{ml})$. The activity of the smaller deletion fragments was significantly stimulated by IL-1 $\beta(100 \%, 46 \%$, and $35 \%$ respectively), and co-treatment of these transfected AGS cells with $H$. pylori and IL$1 \beta$ did not further decrease HKo activity. Pre-treatment of AGS cells with PD 98059 (a MEK-1 inhibitor) prevented the IL-1 $\beta$ mediated stimulation, but SB203580 (a p38 inhibitor) and SP600125 (a JNK inhibitor) did not. IL-1 $\beta$ mRNA levels in AGS cells were very low and unaffected by H. pylori treatment, and ELISAs of H. pylori-treated and control AGS cell culture supernatants showed no measurable IL-1 $\beta$ secretion in either case. These data indicate that 1 ) an IL-1 $\beta$-dependent positive cis-response element is located between - 177 and -64 $n$ in the HK $\alpha$ 5'-flanking region; 2) IL-1 $\beta$ antagonizes $H$. pylori-induced down-regulation of $H K \alpha$ transcription by up-regulating $H K \alpha$ transcription through an ERK-1 kinase pathway; and 3) neither endogenous AGS cell IL-1 $\beta$ nor exogenous IL-1 $\beta$ mediate $H$. pylori-induced $\mathrm{HK} \alpha$ inhibition at the transcriptional level in vitro. We conclude that IL-1 $\beta$ exerts direct positive regulatory effects on $\mathrm{HK} \alpha$ gene transcription, and that any contribution made by IL-1 $\beta$ in $H$. pylori-mediated hypochlorhydria occurs by indirect, alternative mechanisms.

94

Gastrin and Helicobacter-Infection Synergistically Up-Regulate Expression and Secretion of Interleukin-8 in Gastric Cells Through Distinct Signaling Pathways

Shigeo Takaishi, Alexander Dubeikovskiy, Zinaida Dubeikovskaya, Iva Smirnova, Wandong Ai, Shuiping Tu, Tomoyuki Okumura, An V. Tran, Kelly S. Betz, Timothy C. Wang

Background \& Aims: We have previously described synergistic interaction between Helicobacter felis (H.felis) infection and gastrin in a gastric cancer (INS-GAS) mouse model. The chemokine interleukin-8 (IL-8) has previously been implicated in both inflammation and carcinogenesis. In this study, we investigated the regulation of IL-8 expression in gastric cells by gastrin stimulation and Helicobacter pylori (H.pylori) coculture in vitro, as well as in vivo expression of two chemokines, $\mathrm{Cxcl}$ and $\mathrm{Cxcl} 2$, both IL-8 family members. Methods: AGS-E gastric cancer cell line stably transfected with gastrin/CCK-2 receptor was stimulated in vitro with amidated gastrin G-17 (1-100nM) and/or cocultured with H.pylori strain 60190 (MOI:1-100) for 24 hrs. IL-8 gene expression was analyzed by quantitative realtime RTPCR (Q-PCR), and IL-8 secretion was measured by ELISA. Human IL-8 $2 \mathrm{~kb}$ promoterreporter construct was analyzed by luciferase assay. In vivo, male INS-GAS mice were infected with H.felis for 6 months, and the expression of $\mathrm{Cxcll}$ and $\mathrm{Cxcl} 2$ in stomach was analyzed by Q-PCR. Results: Gastrin stimulation and H.pylori coculture of AGS-E cells upregulated IL-8 gene expression in dose-dependent manner, with a maximum 3- and 2.5fold increase, respectively (100nM and MOI:100). Gastrin plus H.pylori synergistically upregulated IL-8 gene expression 10-fold. In addition, gastrin and H.pylori increased IL-8 secretion from AGS-E cells in dose-dependent manner, with a maximum 15- and 5-fold increase, respectively. Gastrin plus H.pylori increased IL-8 secretion 20 -fold. In IL-8 promoterluciferase assay, either transfection with a dominant-negative IKB- $\alpha$ plasmid or treatment with a NF-KB inhibitor (pyrolidine-dithiocarbamate) resulted in near-complete inhibition of gastrin-induced IL-8 up-regulation, whereas H.pylori-induced up-regulation was not suppressed by these treatments. Murine chemokine Cxcll was up-regulated in stomachs of hypergastrinemic (INS-GAS) mice and H.felis-infected non-transgenic FVB/N mice 2- and 20 -fold, respectively, compared with non-infected FVB/N mice, and the expression was synergistically up-regulated 40-fold in H.felis-infected INS-GAS mice. A closely related chemokines $\mathrm{Cxcl} 2$ also showed a similar expression pattern. Conclusions: Gastrin stimulation and H.pylori infection synergistically up-regulated expression and secretion of IL-8 
through distinct signaling pathways in human gastric cells, and the expression of related chemokines Cxcll and Cxcl2 was markedly increased in H.felis-infected hypergastrinemic mice stomach, a mouse model of gastric cancer. The results suggest a significant role for these chemokines in gastric carcinogenesis

95

Do Intrinsic Sensory Neurons Show Directional Stretch-Sensitivity in Guinea Pig Distal Colon?

Eamonn J. Dickson, Terence K. Smith, Grant Hennig, Nick J. Spencer

Background: We have recently identified that a class of mechanosensory S-interneurons are strongly activated by maintained circumferential stretch and are likely responsible for the activation of a maintained discharge of ascending excitatory and descending inhibitory nerve pathways in the guinea pig distal colon. Aim: Since extrinsic afferent nerve endings have been recently shown to be activated by either circumferential or longitudinal stretch i.e. no directional sensitivity, we sought to determine whether intrinsic sensory neurons (myenteric S-interneurons) that underlie the peristaltic reflex would show a similar phenomenon. Methods: Animals were euthanized humanely and a segment of distal colon excised $(\sim 3 \mathrm{~cm}$ length). The mucosa and submucosa were removed by fine dissection and the colon was mounted (serosal side uppermost) in a Sylgard-lined organ bath containing oxygenated Krebs solution at $37^{\circ} \mathrm{C}$. Simultaneous intracellular recordings were made from pairs of $\mathrm{CM}$ cells at either cut end (20mm apart) under alternating conditions of maintained circumferential or longitudinal muscle stretch. Results: Under slack conditions (i.e. no circumferential or longitudinal stretch) no spontaneous activity was ever observed in CM. Interestingly, under these conditions, transmural stimuli evoked IJPs at both the oral and anal cut ends ( 3 of 3 animals). In 9 of 13 animals, longitudinal stretch ( 2 times slack diameter) evoked IJPs simultaneously at both the oral and anal cut ends in response to transmural stimulation. No synchronized spontaneous activity was ever observed in response to longitudinal axis stretch, although in 4 animals transmural stimulation produced small amplitude $(<5 \mathrm{mV})$ oral EJPs and anal IJPs at the anal cut end. Upon removal of longitudinal stretch in these same animals, the application of circumferential stretch now revealed (in 8 out of the 13 animals) synchronized large amplitude oral EJPs (ascending excitation) and anal IJPs (descending inhibition). In all these 8 animals transmural stimulation now produced robust oral EJPs and anal IJPs at the oral and anal recording sites. Conclusions. In the absence of circumferential stretch, spontaneous or electrical activation of enteric nerve pathways preferentially activates ascending inhibitory and descending inhibitory nerve pathways to the CM layer. Activation of ascending excitatory nerve pathways is only revealed in response to circumferential stretch. Unlike extrinsic afferents, the activation intrinsic sensory neurons that underlies ascending and descending inhibitory pathways shows a high degree directional stretch-sensitivity Supported by NIH RO1 DK45713

96

Co-Transmission from Sympathetic Vasoconstrictor Neurons in Mouse Colonic Submucosal Arterioles and the Effect of Colitis Alan Lomax, Stephen J. Vanner

Maintenance of tissue oxygenation is vital for repairing inflammatory damage and maintaining normal function in the gastrointestinal (GI) tract. The GI microvasculature delivers oxygen to GI tissues and is subject to complex neural control mechanisms. Recent studies have suggested that altered neural control of the microvasculature may contribute to the pathogenesis of inflammatory bowel disease. Therefore, we wished to determine the effects of nerve stimulation on the submucosal microvasculature in the mouse colon and to examine the effect of colitis. Videomicroscopy techniques were employed to measure submucosal arteriolar diameter changes in response to nerve stimulation at 5,10 and $20 \mathrm{~Hz}$. In arterioles that were preconstricted by superfusion of prostaglandin $\mathrm{F} 2 \alpha(400 \mathrm{nM})$, nerve stimulation resulted in frequency-dependent vasoconstrictor responses. The neurally mediated constrictions were abolished by tetrodotoxin $(1 \mu \mathrm{M} ; \mathrm{n}=4)$ and guanethidine $(10 \mu \mathrm{M} ; \mathrm{n}=6)$, indicating that they were due to action potentials in sympathetic nerve terminals. ATP or a related purine is the neurotransmitter at sympathetic neuroeffector junctions in submucosal arterioles in guinea-pig ileum, as sympathetic vasoconstrictions can be abolished by a P2 purinoceptor antagonist. In contrast, superfusion of mouse colon submucosa with two potent P2 receptor antagonists (Suramin at $100 \mu \mathrm{M}$ and PPADs at $30 \mu \mathrm{M} ; \mathrm{n}=18$ combined) reduced but did not abolish the vasoconstriction. The residual vasoconstriction was not affected by the alpha adrenergic receptor antagonist phentolamine $(1 \mu \mathrm{M} ; \mathrm{n}=8)$. However, the alpha 1 receptor antagonist prazosin $(100 \mathrm{nM} ; \mathrm{n}=5)$ abolished the non-purinergic vasoconstriction. We have also examined the effect of TNBS colitis on arteriolar responses to nerve stimulation. Nerve-evoked constrictions of submucosal arterioles from mice with TNBS colitis had a slower onset and offset than arterioles from naive controls and were not reduced by suramin (in 6 of 8 preparations). This appeared to be due to a lack of arteriolar responsiveness to purines, as superfusion of ATP $(10 \mu \mathrm{M})$ resulted in a large vasoconstrictions in control mice ( $n=5 / 5$ arterioles tested) but had no effect in mice with colitis ( $n=9 / 9$ arterioles tested). These data identify co-transmission from sympathetic vasoconstrictor neurons in mouse colon. Moreover, in colitis the sympathetic vasoconstriction is mediated only by the non purinergic component. The lack of a purinergic vasoconstriction may be due to the absence of a post-junctional response to purines during colitis

\section{7}

Enteric Glia (EGC) Support Barrier Functions of Intestinal Epithelial Cells (IEC) Under Physiological and Inflammatory Conditions Christiane Hank, Sebastian Hoff, Michael Pfaffl, Michael Schemann, Anne Ruh

Glial ablation causes epithelial barrier dysfunction in the gut, resulting in fulminant tissue inflammation (Bush e.a., Cell 1998). As alterations of the epithelial barrier contribute to intestinal inflammation, it was our aim to analyse the influence of EGC on epithelial functions during inflammatory conditions. Using a coculture model in which IEC (T84) are grown as mature monolayers in the apical compartment of a transwell filter system with or withou EGC in the basolateral compartment, we mimicked inflammatory conditions by application of the proinflammatory cytokine tumor necrosis factor (TNF) $\alpha$. Tightness of the IEC monolayers was monitored by assessing transepithelial electrical resistances (TER). Full differentiation of IEC was accomplished when TER had reached a stable plateau which usually occurred after 14 days. At this time, IEC were treated for 24hrs with human recombinant TNF $\alpha(10 \mathrm{ng} / \mathrm{mL})$ in the presence or absence of EGC. Subsequently, media were changed and epithelial barrier functions were monitored for another week. To detec major cytokine-induced alterations of paracellular pathways, macromolecular permeability was measured with fluorescein sulfonic acid (478Da) and fluorescein dextran (7kD). Coculturing IEC with EGC significantly enhanced TER within $48 \mathrm{hrs}(\mathrm{p}<0.001)$. In the absence of EGC, a marked drop in TER was observed after TNF $\alpha$ stimulation $(\mathrm{p}<0.02)$, mos likely reflecting tight-junctional alterations because there were no detectable increases in macromolecular permeability at any time-point. The drop in TER was prevented when EGC were present during cytokine exposure $(\mathrm{p}<0.001)$. Forty-eight hrs after cytokine-exposure, all treated IEC showed a significant rise in TER $(\mathrm{p}<0.005)$. This was potentiated in the presence of cytokine-stimulated EGC in the basolateral compartment $(\mathrm{p}<0.001)$. Addition of EGC to IEC after cytokine-treatment significantly augmented TER values after at least $48 \mathrm{hrs}$ of coculture $(\mathrm{p}<0.001)$. Neurotrophin production was measured by qPCR and revealed NGF and NT-3 production by basolateral EGC. We conclude that under physiologica conditions, epithelial tightness is enhanced by EGC. Acute inflammation diminishes epithelia tightness which is restored within $48 \mathrm{hrs}$. The presence of EGC during an acute inflammatory insult prevents disruption of the epithelial barrier and appears to accelerate and potentiate epithelial restoration, suggesting that EGC modulate epithelial barrier functions not only under physiological conditions but also during acute inflammation, potentially via neurotrophins. Supported by Broad Medical Research Program IBD-0105R.

98

An Alpha-7 Selective Nicotinergic Agonist Ameliorates Postoperative Ileus By Inhibiting Activation of Intestinal Macrophages

Wouter de Jonge, Frans O. The, Esmerij P. van der Zanden, Dennis C. van der Coelen, Rene M. van den Wijngaard, Guy E. Boeckxstaens

Background. Post-operative ileus (POI) results from activation of intestinal macrophages due to bowel manipulation during surgical procedures. Vagus nerve-derived acetylcholine inhibits macrophage cytokine production by activating alpha-7 nicotinic receptors presen on these cells. This anti-inflammatory pathway is mediated by transcription factor STAT31 Here, we evaluated the efficacy of nicotinergic agonists to ameliorate POI, and the cellular mechanism behind this effect. Methods. Mice were treated with 1)vehicle, 2)nicotine $(0.4$ $\mathrm{mg} / \mathrm{kg}$ ), 3)AR-R1779 (a specific alpha-7 nicotinic agonist; $10 \mathrm{mg} / \mathrm{kg}$ ) by i.p. injection. Thirty min thereafter mice underwent either control laparotomy (L) or intestinal manipulation (IM) Gastric emptying of a semi-liquid meal was determined by scintigraphic imaging 24 hrs after surgery. Myeloperoxidase (MPO) positive mononuclear cells were quantified in ileal muscularis of the manipulated region. Peritoneal macrophages were pre-treated in vitro with nicotine $(0-10 \mathrm{uM})$, or AR-R1779 $(0-10 \mathrm{uM})$ prior to challenge with LPS $(10 \mathrm{ng} / \mathrm{mL})$ and cell lysates were immunoblotted. Results. In mice treated with vehicle, post-surgical (24hrs) gastric emptying (an indicator of POI) was significantly delayed in IM-mice compared to $\mathrm{L}$ control (gastric retention (\%) at $80 \mathrm{~min}: 13.3 \pm 2.7$ (L) and $38.9 \pm 9.7$ (IM); $\mathrm{p}<0.05$ $\mathrm{n}=8$ ). Treatment with AR-R1779, but not nicotine, accelerated post-operative gastric emptying after IM, compared to saline (gastric retention (\%) at $80 \mathrm{~min}: 30.7 \pm 8.6$ (nicotine), ns, $19.6 \pm 4.4$ (AR-R1779), $\mathrm{p}<0.05 ; \mathrm{n}=8$ ). Nicotine or AR-R17779 treatment did not affect gastric emptying after control L. In saline treated mice, IM was associated with a significant increase in MPO-positive cells in intestinal muscularis $(50 \pm 11$ (L) vs $572 \pm 112$ (IM) cells $/ \mathrm{mm} 2, \mathrm{p}<0.05)$. In mice pretreated with AR-R179, but not nicotine, the number of inflammatory cells recruited after IM surgery was reduced (cells/mm2.231 +33 (AR-R1779) $\mathrm{p}<0.05$, and $395 \pm 50$ (nicotine) $\mathrm{ns}, \mathrm{n}=8$ ). In LPS-stimulated peritoneal macrophages, nicotine and AR-R1779 dose-dependently activated the anti-inflammatory transcription factor STAT3 Conclusion. The alpha-7 nicotinergic receptor agonist AR-R17779 activates STAT3 in intestinal macrophages and reduces manipulation-induced inflammation thereby ameliorating POI. Nicotine at the dose tested fails to reduce inflammation but higher doses may be required. 1. de Jonge,W.J. et al. Stimulation of the vagus nerve attenuates macrophage activation by activating the Jak2-STAT3 signaling pathway. Nat. Immunol. 6, 844-851 (2005).

99

Intestinal Handling During Abdominal Surgery Induces Mast Cell Degranulation and Intestinal Inflammation in Man

Frans O. The, Roelof J. Bennink, Willem M. Ankum, Marrije R. Buist, Matthe P. Burger Sicco van der Heide, Rene M. van den Wijngaard, Wouter J. de Jonge, Guy E. Boeckxstaens

Introduction: Intestinal handling during surgery is increasingly recognized as an importan factor involved in the pathogenesis of postoperative ileus. In mice, we previously showed that mast cell degranulation triggered by intestinal handling results in the release of inflammatory mediators and the influx of inflammatory cells leading to postoperative ileus. To what extent this sequence of events also occurs in man is currently unknown. Aim: 1. To assess if intestinal handling results in mast cell activation and inflammatory mediator release during abdominal surgery and 2. to evaluate whether intestinal handling leads to intestinal leukocyte recruitment. Methods: 1 . In patients undergoing an abdominal hysterectomy, peritoneal lavage fluid was obtained during surgery at three different time points: 1 . immediately after opening of the peritoneum (basal), 2. after initial inspection and gentle handling of the intestine (early) and 3. at the end of the surgical procedure, immediately before closure of the peritoneum (late). Tryptase, ILI $\beta$, IL6, TNF $\alpha$ and IL8 release were determined in the different samples. 2. Another series of patients undergoing an abdominal or a vagina hysterectomy were invited to have an abdominal 99mTc-labelled leukocyte SPECT-CT scan 24 hours before (basal) and after surgery. Clinical recovery (i.e. flatus, defecation and hospital stay) was evaluated in both groups. Results: 1 . Gentle intestinal handling resulted in a significant increase of tryptase, but not of the other mediators, in the early samples compared 
to basal levels $(6.4 \pm 1.7 \mu \mathrm{g} / \mathrm{l} \mathrm{vs} .28 .4 \pm 6.2 \mu \mathrm{g} / \mathrm{l}, \mathrm{p}=0.02, \mathrm{n}=6)$. In the late samples, tryptase $(55.9 \pm 12.8 \mu \mathrm{g} / \mathrm{l}, \mathrm{p}=0.002, \mathrm{n}=6)$, IL6 and IL8 were increased (increase compared to basal IL6 $=1756 \pm 1648 \mathrm{pg} / \mathrm{ml}, \mathrm{p}=0.046$ and IL8 $=2128 \pm 2079 \mathrm{pg} / \mathrm{ml}, \mathrm{p}=0.046, \mathrm{n}=6)$, but not IL1 $\beta$ and TNF $\alpha$. 2. Leukocyte scanning $24 \mathrm{~h}$ after surgery showed an increase of intestinal activity compared to basal in abdominal hysterectomy patients $(\Delta=132.2 \pm 8 \%$ of preoperative scan, $\mathrm{p}=0.01, \mathrm{n}=8)$ but not in vaginal hysterectomy patients $(\Delta=99.4 \pm 12 \%$ of preoperative scan, $\mathrm{n}=8$ ). Clinical recovery was prolonged after abdominal hysterectomy in comparison to vaginal hysterectomy and correlated with the leukocyte activity $(\mathrm{r}=0.58, \mathrm{p}=0.018)$ Conclusion: The current study demonstrates that intestinal handling during surgery activates mast cells resulting in the release of inflammatory mediators (IL-6, IL-8) leading to an influx of inflammatory cells. These results identify mast cells as an important therapeutic target to shorten postoperative ileus.

100

\section{Role of Adaptive Immune Cells in the Functional Response to Intestinal} Nematode Infection

Justin E. Elfrey, Aiping Zhao, Kathleen B. Madden, Thomas L. Sutton, Joseph F. Urban,

Terez Shea-Donohue

Nematode infection induces stereotypic changes in gut function that are dependent on IL13 activation of STAT6 signaling pathways. Recently we showed that smooth muscle and epithelial cells exhibit a constitutive expression of receptors for IL-13 indicating that these cells can respond directly to IL-13. Aim: To determine the contribution of adaptive immune cells to infection-induced changes of intestinal smooth muscle and epithelial cell function. Methods: BALB/c (WT) and SCID mice received IL-13 (10 mg iv) or VEH daily for 6 days prior to study, or were inoculated with Nippostrongylus brasiliensis $(\mathrm{Nb})$ and studied 9 days later. Segments of jejunum were suspended longitudinally in organ baths and stretched to 9.9 $\mathrm{mN}$. Spontaneous contractions (SC) of smooth muscle and responses to 5-HT or acetylcholine $(\mathrm{ACH})$ were recorded. Muscle-free segments of jejunum were mounted in Ussing chambers to measure tissue resistance and changes in $\mathrm{I}_{\mathrm{sc}}$ in response to serosal addition of $\mathrm{ACH}$ or 5-HT. IL-13 gene expression was measured by real-time PCR. Results: Nb significantly increased IL-13 mRNA expression in WT mice(200 fold) and to a lesser extent in SCID mice(18 fold). Exogenous administration of IL-13 had no effect on IL-13 gene expression in WT or SCID mice. In WT mice, both $\mathrm{Nb}$ infection and IL-13 significantly decreased mucosal resistance, inhibited epithelial cell secretion to ACH and 5-HT, enhanced small intestinal smooth muscle responses to 5-HT and $\mathrm{ACH}$ and increased the amplitude of SC (Table). Nb-induced hyposecretion and hypercontractility were not observed in SCID mice. In contrast, these functional changes were observed in SCID mice given exogenous IL-13. Mucosal resistance was unaltered by $\mathrm{Nb}$ or IL-13 in SCID mice. Conclusion: The small upregulation of IL-13 in Nb infected SCID mice is insufficient to impact gut function, but supports an endogenous source of IL-13 such as NK cells. The ability of exogenous IL-13 to alter function in both WT and SCID mice indicates that smooth muscle and epithelial cells respond directly to IL-13.

\begin{tabular}{|c|c|c|c|c|c|c|c|}
\hline & & \multicolumn{3}{|c|}{ Epithelial Cell Function } & \multicolumn{3}{c|}{ Smooth Muscle Tension (N/cm2) } \\
\hline & & ACH & $\mathbf{5 - H T}$ & $\mathbf{R}\left(\Omega^{*} \mathbf{c m}^{\mathbf{2}}\right)$ & ACH & $\mathbf{5 - H T}$ & SC \\
\hline WT & VEH & $95 \pm 8$ & $35 \pm 9$ & $17 \pm 1$ & $16 \pm 1$ & $6 \pm 1$ & $5 \pm 1$ \\
\hline & Nb & $18 \pm 13^{*}$ & $7 \pm 6^{*}$ & $10 \pm 2^{*}$ & $30 \pm 1^{*}$ & $17 \pm 1^{*}$ & $11 \pm 1^{*}$ \\
\hline & IL-13 & $26 \pm 8^{*}$ & $1 \pm 1^{*}$ & $11 \pm 1^{*}$ & $32 \pm 6^{*}$ & $15 \pm 4^{*}$ & $8 \pm 1^{*}$ \\
\hline SCID & VEH & $172 \pm 37$ & $31 \pm 11$ & $13 \pm 1$ & $11 \pm 2$ & $4 \pm 1$ & $5 \pm 1$ \\
\hline & Nb & $100 \pm 16$ & $20 \pm 7$ & $14 \pm 1$ & $11 \pm 2$ & $5 \pm 1$ & $3 \pm 1$ \\
\hline & IL-13 & $59 \pm 12^{\Phi}$ & $14 \pm 5^{\Phi}$ & $12 \pm 1$ & $34 \pm 7^{\Phi}$ & $14 \pm 5$ & $11 \pm 3^{\Phi}$ \\
\hline
\end{tabular}

Epithelial cell function $=\mu \mathrm{A} / \mathrm{cm}^{2} ;{ }^{*} \mathrm{p}<0.05$ vs. WT VEH, $\Phi \mathrm{p}<0.05$ vs.SCID VEH; $[\mathrm{ACH}]$, $[5-\mathrm{HT}]=100 \mu \mathrm{M} ; \mathrm{n} \geq 4$

101

At Motif Binding Factor 1 (ATBF1) Negatively Regulates Transcription of the Muc5ac Gene in Gastric Cancer

Yoshinori Mori, Hiromi Kataoka, Makoto Sasaki, Satoshi Tanida, Tadayuki Oshima, Naotaka Ogasawara, Yutaka Miura, Takashi Joh

Background and aims: MUC5AC belongs to the family of secreted mucins and is expressed in foveolar cells of the stomach. Alterations of mucin expression take place in gastric cancer cells and MUC5AC expression is known to correlate with poor prognosis. However, the mechanism of MUC5AC transcriptional regulation has not been elucidated. AT motif binding factor 1 (ATBF1) is a homeotic transcription factor which was originally found to binds to AT motif in AFP promoter region and negatively regulates it. We reported that the absence of ATBFl is a distinct feature of AFP-producing gastric cancer cells which are characterized by extremely high malignancy (Oncogene 2001). Furthermore, we recently showed that ATBFl is a new candidate of tumor suppressor (Nat. Genet. 2005). In this study, we investigated the transcriptional regulation of MUC5AC by ATBFl. Materials and Methods: 1. We analyzed ATBFl and MUC5AC expression in 123 area of 41 gastric cancer specimen by immunohistochemistry. 2. We explored AT motif like element which ATBFl might bind to in MUC5AC promoter region by computer search. 3. To analyze the transcriptional regulation of MUC5AC by ATBF1, we performed dual luciferase-reporter assay using MKN45 cells. 4. To analyze the transcriptional regulation of MUC5AC by ATBFl in protein level, we examined MUC5AC protein expression in MKN45 cells by transfectiing ATBFl using confocal microscopy. 5. We performed chromatin immunoprecipitation (ChIP) assay to assess the binding of ATBFl to the AT motif like element in MUC5AC promoter region. Results: 1. Inverse correlation between ATBFl nuclear staining and MUC5AC staining was detected (p<.0001) (Table 1). 2. We detected the AT motif like element (-1646 -1634) in MUC5AC promoter region. 3. Dual luciferase-reporter assay demonstrated that ATBF1 suppressed the MUC5AC promoter activity to $35 \%$ (p<.0001). 4. Overexpressed ATBF1 suppressed endogeneous MUC5AC protein expression in gastric cancer cells. 5. Chromatin immunoprecipitation (ChIP) revealed that ATBFl binds to the AT motif like element in MUC5AC promoter region. Conclusion: ATBFl binds to the AT motif like element in MUC5AC promoter region and negatively regulates its transcription in gastric cancer cells. The negative correlation between ATBFl and MUC5AC

\begin{tabular}{|c|c|c|c|}
\hline MUC5AC & positive & negative & total \\
\hline ATBF1 positive & 3 & 30 & 33 \\
\hline ATBF1 negative & 68 & 22 & 90 \\
\hline
\end{tabular}

102

Kras and Hypoxia Can Induce VEGF Through C-Myc in Colon Cancer Yusuke Mizukami, Eva-Maria Duerr, Manish Gala, Won-Seok Jo, Xiaobo Zhang, Yutaka Kohgo, Daniel C. Chung

BACKGROUND: The induction of VEGF is an essential feature of tumor angiogenesis Hypoxia is a potent stimulator of VEGF, and hypoxia inducible factor-1 (HIF-1) is considered to be critical for its induction. However, we have previously demonstrated that induction of VEGF by hypoxia both in vitro or in vivo was preserved in the absence of HIF-lalpha when KRAS signaling was activated. The aim of this study was to clarify the mechanism by which KRAS and hypoxia can upregulate VEGF independently of HIF-1. METHODS: The effect of hypoxia on KRAS effector pathways was determined through Western blotting. Specific inhibitors of ERK, PI3K, Akt, Rho, and ROCK were tested. 5' VEGF promoter deletion constructs were designed in order to localize the region of the promoter responsive to hypoxia. Gel electrophoretic mobility shift assays and site-directed mutagenesis were performed to characterize the novel response element. Silencing of c-Myc was accomplished with siRNA duplex oligonucleotides. RESULTS: In colon cancer cells, hypoxia stimulated multiple KRAS effector pathways including PI3K. Inhibition of Akt did not block the hypoxic induction of VEGF promoter, but the induction was completely abrogated when the PI3K targets Rho and ROCK were inhibited. 5' deletions of the VEGF promoter revealed a region between $-418 \mathrm{bp}$ and $-223 \mathrm{bp}$ from the transcriptional start site that was responsive to hypoxia. Electrophoretic mobility shift assays further defined a fragment between -300 bp and -251 bp that demonstrated a unique shift only in hypoxic conditions. A binding site for c-Myc, a target of ROCK, was identified at -271 bp. Activation of both Rho and c-Myc was demonstrated when colon cancer cells were stimulated by hypoxia, and these effects were enhanced by KRASV12. Finally, the role of c-Myc in the hypoxic induction of VEGF was verified by site-directed mutagenesis of the VEGF promoter and silencing of c-Myc by siRNA. CONCLUSIONS: In colon cancer, VEGF can be synergistically induced by hypoxia and KRAS. A binding site for c-Myc located at -271 bp of the VEGF promoter can mediate this effect in a PI3K/Rho/ROCK-dependent and HIF-independent manner. These findings emphasize the interplay between oncogenic signaling pathways and hypoxia, and highlight the pivotal role of KRAS in angiogenesis in colon cancer.

103

KLF4 Promoter Activity Is Inhibited By the Notch Signaling Pathway Walden Ai, Adolfo Ferrando, Timothy C. Wang

Introduction: Kruppel like factor 4 (KLF4), a transcription factor and tumor suppressor in gastrointestinal tract, is required for goblet cell differentiation in colon. Notch regulates the cell fate decisions of proliferating cells in development and in adult life, including the development of goblet cells. We hypothesized that KLF4 could be regulated by the Notch signaling pathway in goblet cell differentiation. Aim: To examine the regulation of KLF4 promoter by the Notch signaling pathway. Methods: Construction of KLF4 promoter reporters, transient cotransfection and promoter activity assays. Results: $2 \mathrm{~kb}$ human and mouse KLF4 promoters were generated by PCR and cloned into the pGL3-basic vector Transient cotransfection assays in a human gastric cancer cell line (AGSE cells) and a human colon cancer cell line (HCT 116 cells) with a constitutively active Notch receptor (ICN1) construct and a human Hesl (hHesl) construct showed that the human KLF4 promoter activity was inhibited by ICNl and hHesl (50\% inhibition). The mouse KLF4 promoter activity was also inhibited by overexpression of ICNl and hHesl in AGSE cells by the same assay. A series of 5' truncation reporters from the full length 2.0kb human KLF4 promoter were further generated to identify ICNl and hHesl responsive elements in the promoter. While a KLF4 promoter reporter, containing 495bp nucleotide sequence upstream of the transcription start site (-495bp), showed a similar inhibition by hHesl as did the full length KLF4 promoter, further truncation of the promoter to -181bp position totally abrogated the inhibition of the promoter by hHesl, suggesting that the hHesl-responsive element was located between -495bp and -18lbp upstream of KLF4 promoter. Since hHesl is a downstream target of Notch, we tested the possibility that ICN1 inhibits KLF4 promoter through hHesl. Surprisingly, an element responsive to ICN1 was identified between -15lbp and $108 \mathrm{bp}$ upstream of KLF4 promoter by the same cotransfection assays. Conclusions: KLF4 promoter activity was repressed by constitutively active ICNl and hHesl. In the human KLF4 promoter, the responsive element of ICNl is different from that of hHesl, suggesting that Notch inhibits KLF4 gene expression by Hesl dependent and independent mechanisms.

104

Krüppel-Like Factor (KLF5) Mediates the Proliferative Effect of Oncogenic KRAS in Intestinal Epithelial Cells IEC6 Containing An Inducible K-RAS (IECiK-Ras)

Mandayam O. Nandan, Hongmiao Sheng, Jinyi Shao, Vincent W. Yang

BACKGROUND: Krüppel-like factor 5 (KLF5) is a member of the Krüppel-like family of transcription factors. Studies indicate that KLF5 expression is enriched in the proliferating crypt cells of the intestinal epithelium. We previously showed that KLF5 is essential for 
mediating the transforming effect of activated H-Ras in H-Ras-transformed NIH3T3 fibroblasts by accelerating cell cycle progression [Nandan et al. (2004) Oncogene 23, 3404; Nandan et al. (2005) FEBS Lett. 579, 4757l. AIM: To determine the role of KLF5 in K-Rasmediated transformation of intestinal epithelial cells IEC6. METHODS: IEC-iK-Ras cells were established by stable transfection of IEC6 cells with an IPTG-inducible oncogenic K-Ras ${ }^{\text {V12G }}$ [Sheng et al. (2001) Cancer Res. 61, 2670]. PD98059 was used to inhibit MEK/ERK activities. Small interfering RNA (siRNA) specific for KLF5 was used to inhibit KLF5 expression. Several clonal derivatives of IEC-iK-Ras cells were established from colonies that formed in soft agar due to K-Ras induction. Cells were examined for KLF5 expression, MEK/ERK activity, rate of proliferation and transforming potential. RESULTS: The level of KLF5 in IEC-iK-Ras was increased with the induction of oncogenic K-Ras from IPTG treatment and was correlated with both accelerated proliferation and anchorage-independent growth in soft agar. In addition, MEK/ERK activity was significantly elevated in IPTG-treated IEC-iKRas cells. Upon treatment of IPTG-induced IEC-iK-Ras cells with either PD98059 or KLF5specific siRNA, the level of KLF5 protein was significantly reduced. This reduction in KLF5 expression abrogated the increased rate of proliferation of IEC-iK-Ras cells from IPTG induction. Clonal derivatives of IEC-iK-Ras cells, selected from soft agar after prolonged (14 days) IPTG treatment, showed significantly increased basal levels of both K-Ras and KLF5. These cells exhibited an increased rate of proliferation when compared to the unselected parental IEC-iK-Ras cells. The levels of KLF5 were further increased in selected clones after IPTG treatment and were correlated with a further increase in the rate of proliferation. CONCLUSIONS: Induction of K-Ras in IEC-iK-Ras results in elevated KLF5 expression that is accompanied by an increase in cell proliferation and MEK/ERK activities. Inhibition of KLF5 expression by MEK inhibitor or KLF5-speicific siRNA reduces K-Ras-mediated proliferation. Moreover, stably transformed clones of IEC-iK-Ras cells have an even higher rate of proliferation that correlates with further increased levels of K-Ras and KLF5. These results suggest that KLF5 mediates the proliferative effect of K-Ras in intestinal epithelial cells.

\section{5}

CDX2 Promotes Tumorigenicity in the Human Colon Cancer Cell Line Lovo Long H. Dang, Fang Chen, Chi Ying, Sang Y. Chun, Stephanie A. Knock, Henry D. Appelman, Duyen Dang

BACKGROUND: CDX2 is a Drosophila caudal-related homeobox transcription factor that is important for the establishment and maintenance of intestinal epithelial cells (JBC 1994; 269:15229-37). CDX2 is a marker of colon cancer, with strong staining in up to $90 \%$ of colonic adenocarcinomas (Mod Pathol 2004; 17:1392-9). CDX2 heterozygous-null mice develop colonic hamartomas, which have suggested that CDX2 is a tumor suppressor (Nature 1997; 386:84-7). However, several lines of evidence have suggested that CDX2 may not be a tumor suppressor. First, CDX2 does not affect colon cancer xenograft growth (JBC 1998; 273:14030-6). Second, CDX2 is rarely mutated in colon cancer (Br J Cancer 1999; 79: 4404). Third, CDX2 has been identified as the transforming event in a mouse model of leukemia (PNAS USA 2004:101: 817-22). AIM: To determine the functional contributions of CDX2 to colon cancer. METHODS: The CDX2 gene in LOVO cells was sequenced and found to be not mutated. The CDX2 gene in LOVO cells was disrupted using targeted homologous recombination (Nature 2004; 416:1). The resulting CDX2-/- cells are isogenic with their parental cells, with the exception of CDX2 ablation, as confirmed by western blot analyses. In vitro and in vivo assays were used to assess the effects of CDX2 disruption on tumorigenicity. Four independent clones were analyzed. RESULTS: Consistent with its role as a putative tumor suppressor, loss of CDX2 led to a 20-30\% increase in anchorage-DEpendent colony formation on plastic culture plates. In contrast, loss of CDX2 led to a fourfold decrease in anchorage-INdependent colony formation in soft agar. Cell cycle analyses revealed that CDX2 promotes anchorage-INdependent G1 to S progression and prevents anoikis (programmed cell death caused by loss of adhesion). CDX2 promotion of anchorage-independent cell survival was associated with phosphorylation of the anchorage-independent growth kinases ERK and FAK; increased expression of the anti-apoptotic factor Bcl-2; and decreased cleavage of caspase-3 and PARP. In vivo xenograft studies confirmed that loss of CDX2 led to a 5-fold decrease in LOVO xenograft growth. There were no microscopic alterations in tumor histology. CONCLUSIONS. These data directly demonstrate, for the first time, that CDX2 mediates anchorage-independent growth and survival in colon cancer cells. This is consistent with CDX2 mediation of anchorage-independent growth in leukemia cells (PNAS USA 2004;101: 817-22). These data suggest that CDX2 may have a tumorigenic role in a subset of colon cancers in which it is expressed.

106

ZBP-89 Is Essential for Butyrate-Induced P53 Acetylation At Lys320 Longchuan Bai, Juanita Merchant

BACKGROUND: The zinc finger protein ZBP-89 stabilizes $\mathrm{p} 53$ protein and enhances its transcriptional activity. Studies using ZBP-89 haploinsufficient mice demonstrated that ZBP89 is required for $\mathrm{p} 53$ phosphorylation at Ser15, the same site targeted by ATM. Ser15 phosphorylation of $\mathrm{p} 53$ usually precedes its acetylation at C-terminal lysines by acetyltransferases (HATs), including K320. Acetylation of p53 at lysine 320 stabilizes p53, enhancing activation of p53-responsive genes. Since phosphorylation of p53 at Ser15 promotes its association with CBP/p300 HATs, we tested whether ZBP-89 facilitates p53 acetylation at K320. METHODS: Human cell lines HCT116 and ataxia telangiectasia (A-T, ATM defective) fibroblasts were used. Point mutations of $\mathrm{p} 53$ and ZBP- 89 were generated by site-directed mutagenesis. The p2lwafl reporter containing $2.4 \mathrm{~kb}$ of the human $\mathrm{p} 21$ wafl promoter was used. RESULTS: In ATM-defective A-T cells, we found that basal and trichostatin A (TSA)induced p53Ser 15 phosphorylation was attenuated as expected since ATM is the kinase phosphorylating p53Ser15. Moreover, basal and TSA-induced levels of acetylated p53 were reduced. To assess whether this result was due to the absence of TSA-induced ZBP-89 protein, an immunoblot was performed. TSA significantly increased ZBP-89 protein levels in the normal but not the A-T fibroblasts, confirming that regulation of ZBP-89 was proximal to 53 regulation and suggesting a possible role for ATM in ZBP-89 regulation of $\mathrm{p} 53$. Next, we tested whether ATM phosphorylated ZBP-89. Indeed, we found that ATM phosphorylated ZBP-89 and through a series of truncating mutants mapped the ZBP-89 phosphorylation site to Ser202 within the second zinc finger. As ZBP-89 is required for p53Ser15 phosphoryl ation, we further tested whether it is also essential for $\mathrm{p} 53$ acetylation. We found that reduction of ZBP-89 with specific siRNA not only blocked butyrate-induced p53 phosphorylation at Ser15 but also the p53K320 and subsequently diminished butyrate-induction of p21wafl a p53 target gene. Examining further the significance of the ATM phosphorylation of ZBP89 on p53-dependent transcription, Ser202 in ZBP-89 was disrupted by a point mutation. We found that the ZBP-89Ser202 mutation reduced p53 induction of p21wafl expression by $\sim 30 \%$, whereas a zinc finger deletion completely abolished induction. This suggested that additional residues within the ZBP-89 zinc finger domain is likely to cooperate with ATM to regulate p53. CONCLUSIONS: ZBP-89 is essential for butyrate-induced p53 acetylation at Lys320. ATM phosphorylates ZBP-89 at Ser202, which contributes to p53 induction of p2lwafl.

Moderate Alcohol Drinking May Not Be the Risk Factor for Fatty Liver in Health Checkup of Middle-Aged Japanese Tamaki Yamada, Mitsuru Fukatsu, Sadao Suzuki

(Background/Aim) Fatty liver (FL) associated with multiple metabolic disorders was reported to be caused by obesity and alcohol drinking, while alcohol consumption is associated with a lower prevalence of metabolic syndrome (Diabetes Care 2004:27;2954-59. Obes Res 2004:12;1375-85). Therefore, we aimed to determine whether alcohol drinking may be the risk factor for FL by cross-sectional and longitudinal manners in health checkup of the apparently healthy middle-aged Japanese. (Methods) 1) Cross-sectional study: 55,615 participants without hepatitis virus who received health checkup from 1999 to 2003 (men $29,101.49 .3 \pm 11.8$, y.o., women: $26,514.48 .7 \pm 11.4$ y.o.) were enrolled and data at the first visit during this period were used to determine characteristics of drinking habits and the related factors of FL diagnosed by ultrasonography. 2) Longitudinal study: 9,156 participants of both 1998 and 2003 without hepatitis virus (men: 4,750 49 1+9.6 y.o, women: 4.406 . $48.7 \pm 8.9$ y.o.) were enrolled to determine the risk factors for newly diagnosed FL by ultrasonography in 2003. Multiple logistic regression analyses were performed to determine alcohol drinking as the risk factor for FL in men and women, adjusted for age, body mass index (BMI), and smoking in the cross-sectional study and these factors and BMI increase for 5 years in the longitudinal study. (Results) 1) The overall percentages of occasional and daily drinkers were 33.6 and $45.3 \%$ in men and 31.3 and $8.9 \%$ in women, respectively. The average alcohol consumptions in the occasional and daily drinkers were 53.8 and 218.7 $\mathrm{g} /$ week in overall, 58.3 and $222.9 \mathrm{~g} /$ week in men, and 48.1 and $194.2 \mathrm{~g} /$ week in women, respectively. The prevalence of FL were 17.2,23.5, and $10.3 \%$ in overall men, and women. The prevalence of FL in non-, occasional, and daily drinkers were $27.9,27.3$, and $18.6 \%$ in men and $12.2,7.9$, and $5.3 \%$ in women, respectively ( $\mathrm{p}<0.01$ for both). Occasional and daily drinking were negatively associated with FL in both sexes (men: occasional, OR:0.86, 95\%CI:0.80-0.94. daily, OR:0.56, 95\%CI:0.51-0.60. women: occasional, OR:0.78, 95\%CI:0.71-0.87. daily, OR:0.57, 95\%CI:0.46-0.69). 2) FL was newly developed in 13.3 17.2 , and $9.1 \%$ of overall, male, and female participants in 5 years, respectively. Daily drinking was negatively associated with FL in men (daily, OR:0.77, 95\%CI:0.62-0.96). (Conclusions) In health checkup of middle-aged Japanese, the prevalence of FL was significantly lower in the alcohol drinkers of both sexes and daily alcohol drinking was negatively associated with FL in men. Our findings suggested that moderate alcohol drinking may not be the risk factor for FL in these participants.

\section{4}

Role of Uncoupling Protein-2 in Fasting-Induced Metabolic Response and Hepatic Steatosis

Anthony Sheets, Peter Fulop, Zoltan Derdak, Edmond Sabo, Jack R. Wands, Gyorgy Baffy

Background: Uncoupling proteins are mitochondrial inner membrane constituents that mediate regulated proton leak. UCP2 is heavily upregulated in genetically determined and diet-induced fatty liver, but its biological role remains debated. While UCP3, a muscle homologue, is implicated in fatty acid metabolism, a similar function for UCP2 has not been established. Aim: To assess the effect of UCP2 on lipid utilization and disposal in the acute model of fasting-induced steatosis, which avoids potential confounding effects of insulin resistance. This work is an extension of previous findings (Hepatology 36(S1): 486A) Methods: UCP2-/- mice and wild type littermates were fasted up to $72 \mathrm{~h}$ with controls fed on regular diet ( $\mathrm{n}=5$ to 12$)$. We assessed the time course of steatosis (oil-red-O staining and tissue lipid extraction), serum metabolic parameters, and expression of key genes in lipid metabolism. Results: While fasting-induced steatosis was prominent in wild type liver at $24 \mathrm{~h}$ and mostly resolved by $72 \mathrm{~h}, \mathrm{UCP} 2-/$ - livers showed delayed but persistent hepatic fat accumulation $(\mathrm{p}=0.017)$. Hepatic UCP2 $\mathrm{mRNA}$ increased in fasted wild type mice to 3 -fold by $72 \mathrm{~h}(\mathrm{p}<0.001)$. Higher baseline insulin levels typical of UCP2-/- mice plummeted to same levels as wild type mice after fasting. Serum fatty acid levels showed no genotype difference. However, fasting-induced decrease of serum triglycerides was delayed in UCP2-/ mice $(24 \mathrm{~h}, \mathrm{p}=0.024)$. In addition, fasting-induced increase in expression of carnitine palmitoyl transferase I, acyl-CoA carboxylase (alpha), acyl-CoA oxidase, D-bifunctional protein, CYP2El, and microsomal triglyceride transfer protein was blunted in UCP2-/- mice (72 h, p $=0.005$ to 0.048 ). Conclusion: Our findings indicate that lack of UCP2 impairs the effects of fasting on lipid utilization, fatty acid oxidation and VLDL formation, and may cause prolonged fat accumulation in the fasting liver. Accordingly, the role of UCP2 in lipid metabolism may not be ignored. Supported by NIH grant DK-61890.

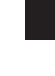




\begin{tabular}{|c|c|c|c|c|c|c|}
\hline STRAIN & GENDER & $\begin{array}{c}\text { BODY WT } \\
(\mathrm{G})\end{array}$ & $\begin{array}{c}\text { LIVER WT } \\
(\mathrm{G})\end{array}$ & $\begin{array}{c}\text { GLUCOSE } \\
(\mathrm{MG} / \mathrm{DL})\end{array}$ & $\begin{array}{c}\text { INSULIN } \\
(\mathrm{NG} / \mathrm{ML})\end{array}$ & $\begin{array}{c}\text { ALT } \\
(\mathrm{U} / \mathrm{L})\end{array}$ \\
\hline $\begin{array}{c}\text { CD1 } \\
\text { aP2DTA }\end{array}$ & $\mathrm{F}$ & $36.3(4.0)$ & $2.7(0.8)$ & $115(28)^{*}$ & $3.3(3.4)^{*}$ & $\begin{array}{c}3233 \\
(695)^{*}\end{array}$ \\
\hline CD1 WT & $\mathrm{F}$ & $36.7(3.3)$ & $2.2(0.3)$ & $86(9)$ & $0.4(0.3)$ & $\begin{array}{c}1705 \\
(1019)\end{array}$ \\
\hline $\begin{array}{c}\text { CD1 } \\
\text { aP2DTA }\end{array}$ & $\mathrm{M}$ & $47.3(4.2)$ & $3.3(0.9)^{*}$ & $95(28)^{*}$ & $2.3(3.0)^{*}$ & $\begin{array}{c}3397 \\
(327)^{*}\end{array}$ \\
\hline CD1 WT & $\mathrm{M}$ & $42.6(6.3)$ & $2.1(0.4)$ & $72(15)$ & $0.3(0.2)$ & $\begin{array}{c}1070 \\
(888)\end{array}$ \\
\hline $\begin{array}{c}\text { C57B } \\
\text { aP2DTA }\end{array}$ & $\mathrm{F}$ & $27.2(1.9)$ & $1.4(0.1)$ & $112(37)^{*}$ & $0.8(0.5)^{*}$ & $3500^{*}$ \\
\hline $\begin{array}{c}\text { C57B WT } \\
\text { C57B }\end{array}$ & $\mathrm{F}$ & $25.6(2.9)$ & $1.3(0.1)$ & $72(11)$ & $0.2(0.1)$ & $\begin{array}{c}1462 \\
(1134)\end{array}$ \\
\hline aP2DTA & $\mathrm{M}$ & $32.9(1.8)$ & $1.7(0.3)$ & $97(25)$ & $0.7(0.3)^{*}$ & $3500^{*}$ \\
\hline C57B WT & $\mathrm{M}$ & $35.9(2.8)$ & $1.7(0.4)$ & $78(16)$ & $0.3(0.1)$ & $\begin{array}{c}2629 \\
(1063)\end{array}$ \\
\hline
\end{tabular}

Low Circulating Dhea Levels in Severe Non-Alcoholic Fatty Liver Disease - A Potential Metabolic Basis of Disease Progression

Michael R. Charlton, Paul Angulo Hernandez, Phunchai Charatcharoenwitthaya, Lawrence Burgart, Samer Gawrieh, Keith Lindor, Kimberly Viker, Anuradha Krishnan

Background: The biological basis of variability in histological progression of NAFLD is not known. More severe non-alcoholic fatty liver disease (NAFLD) is characterized by oxidative stress, insulin resistance and progressive fibrosis, phenomena which can be modulated by dehydroepiandrosterone (DHEA). Aim: We evaluated whether circulating DHEA levels vary between and/or can be used to identify and differentiate patients with mild (simple steatosis and NASH with fibrosis stage 0-2) and more severe (NASH with fibrosis stage 3-4) NAFLD. Methods: Serum samples were obtained prospectively at the time of liver biopsy in 78 patients with NAFLD and 44 controls with other forms of liver disease. Serum levels of DHEA were measured by ELISA. Model building was carried out using logistic regression techniques. Results: DHEA levels in patients with NAFLD are shown in figure 1. All patients with more severe NASH $(n=25)$ had low plasma levels of DHEA $(<0.45$ microg/dl). A model incorporating DHEA (stratified into $<0.45 \mathrm{microg} / \mathrm{dl}>0.45 \mathrm{microg} / \mathrm{dl}$ ), patient age $(<45 \mathrm{vs}$. $>45$ ) and gender (M vs. F) was found to exhibit the greatest area under the receiver operator curve ( $\mathrm{AUC}=0.87$ ). A DHEA level of $>0.45 \mathrm{microg} / \mathrm{dl}$ alone (a level associated with hypoadrenalism) had both sensitivity and negative predictive value of $100 \%$ in distinguishing between mild and severe NAFLD Specificity was $58.5 \%$, and positive predictive value was $47.8 \%$. A relationship between histological severity of NAFLD and DHEA levels was not seen in the control group with cholestatic liver diseases. Conclusions: Patients with more severe NAFLD, as indicated by the presence of NASH with advanced fibrosis stage, can be identified with a high degree of accuracy using a simple model. These data provide novel evidence for relative DHEA deficiency in patients with histologically progressive NASH.

Figure 1

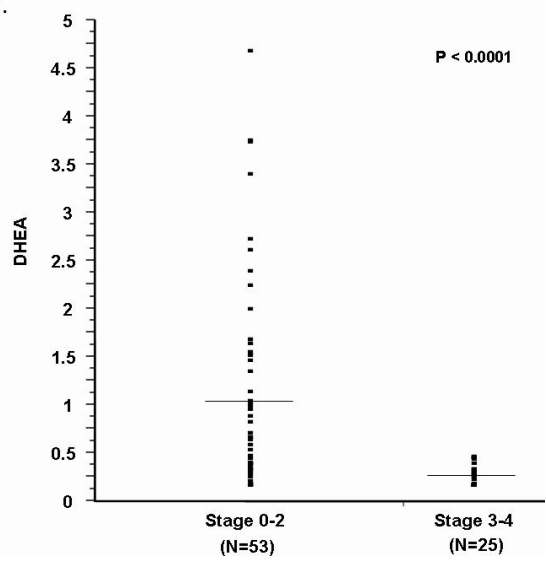

116

Increased Sensitivity to Carbon Tetrachloride Hepatotoxicity in the AP2DTA Mouse Model of Acquired Lipodystropy and Insulin Resistance Shazia Rafiq, Valerie Andersen, Sateesh K. Satchidanand, Reed Graves, Michael D. Sitrin

Introduction: Non-alcoholic fatty liver disease (NAFLD) most commonly occurs in patients with the "metabolic syndrome" characterized by insulin resistance. It is currently hypothesized that progression from NAFLD to nonalcoholic steatohepatitis (NASH) and cirrhosis occurs because hepatitic steatosis or insulin resistance sensitizes the liver to a "second hit" such as an oxidant stress, toxin, virus, etc. Testing of this hypothesis and investigation of the pathogenesis and treatment of NASH has been hampered by lack of appropriate animal models. The aP2DTA transgenic mouse was developed by linking the diptheria toxin A gene to the aP2 promoter that directs adipocyte-specific gene expression. These mice develop adipocyte necrosis and become essentially devoid of fat by 8-9 months, a model of acquired lipodystrophy. The mice have insulin resistance, dyslipidemia, and in some strains fatty liver. Methods: In the present studies, adult aP2DTA mice and wild-type littermates in CD1 and $\mathrm{C} 57 \mathrm{Bl} / 6 \mathrm{~J}$ backgrounds (7-10 per group) were given i.p. injections of $0.8 \mathrm{mg} / \mathrm{kg}$ body weight carbon tetrachloride ( $\mathrm{CCl} 4)$ diluted $1: 4$ in corn oil. After 48 hrs., the mice were sacrificed, blood collected for measurement of blood glucose, insulin, and serum alanine aminotransferase (ALT), and liver tissue obtained for histology. Results: As shown in the Table [mean (SD)], aP2DTA males and females from both strains were insulin resistant, with elevated glucose and insulin levels. aP2DTA CD1 mice, but not aP2DTA C57B1/6] mice, had hepatomegaly due to fatty liver. Transgenic mice from both strains had a significantly greater rise in ALT after CCl4 treatment than wild-type controls. Conclusions: aP2DTA transgenic mice demonstrate greater sensitivity to CCl4-induced hepatoxicity than wild-type controls. This was observed in mouse strains that differ with respect to hepatic steatosis, but have similar degrees of insulin resistance, suggesting that insulin resistance rather than fatty liver is responsible for susceptibility to a hepatotoxin.
Upper limit for ALT $=3500 ; * \mathrm{p}<.05$

117

Vitamin D and Parathyroid Hormone (PTH) Status in Adults with NonCholestatic Chronic Liver Disease

Leon Fisher, Alexander A. Fisher

Background: Liver plays a central role in the metabolism of vitamin D resulting in formation of 25-hydroxyvitamin D [25(OH)D]. In addition to calcium homeostasis, vitamin D affects immune and epithelial cell functions. In chronic liver disease (CLD) vitamin D status has mainly been studied in relation to cholestatic diseases and hepatic osteodystrophy. Objective: To investigate the vitamin D-PTH status in a cohort of patients with non-cholestatic CLD and its relation to the aetiology and severity of underlying disease. Methods: In a tertiary referral center (Canberra, latitude 33'15"S) we studied 94 consecutive outpatients [58 males, 36 females, mean age $48.9+/-12.2$ (SD)] with non-cholestatic CLD due to alcohol use (n= 36), hepatitis $C(n=36)$, hepatitis $B(n=12)$, autoimmune hepatitis $(n=4)$, hemochromatosis $(\mathrm{n}=41)$ NASH $(\mathrm{n}=2) \cdot 45$ patients had cirrhosis. The diagnosis was confirmed by biopsy $(\mathrm{n}=$ 35), clinical and biochemical features. Serum concentrations of $25(\mathrm{OH}) \mathrm{D}$, immunoreactive intact PTH (iPTH), calcium, phosphate, magnesium, creatinine and LFT's were determined in each subject. Results: Serum 25(OH)D concentration was inadequate in $90.2 \%$ of patients, including severe or moderate deficiency $(<25 \mathrm{nmol} / \mathrm{L})$ in $28.3 \%$, mild deficiency $(25-49$ $\mathrm{nmol} / \mathrm{L}$ ) in $39.1 \%$ and hypovitaminosis $\mathrm{D}(50-80 \mathrm{nmol} / \mathrm{L})$ in $22.8 \%$. Secondary hyperparathyroidism (serum iPTH $>6.8 \mathrm{pmol} / \mathrm{L}$ ) was present in $16.1 \%$ of patients. The prevalence of low $25(\mathrm{OH}) \mathrm{D}$ concentrations $(<50 \mathrm{nmol} / \mathrm{L})$ was significantly higher in cirrhotic vs non-cirrhotic patients $(87.5 \%$ vs $46.8 \%, \mathrm{p}=0.0001)$, in subjects with alcohol related CLD compared to other causes $(87.1 \%$ vs $53.6 \% ; \mathrm{p}=0.004)$ and in patients with hyperbilirubinemia ( $>60$ $\mathrm{mmol} / \mathrm{L} ; 100 \%$ vs $56.5 \% ; \mathrm{p}=0.0001)$. Serum $25(\mathrm{OH}) \mathrm{D}$ concentration in patients with ChildPugh class $C$ was significantly lower than in patients with class A $(22.2+/-10.5$ vs 42.1 $+/-13.5 \mathrm{nmol} / \mathrm{L} ; \mathrm{p}=0.0014)$. Of 54 patients with preserved liver function only $9(16.7 \%)$ had a desirable level of $25(\mathrm{OH}) \mathrm{D}(>80 \mathrm{nmol} / \mathrm{L})$. Serum $25(\mathrm{OH}) \mathrm{D}$ positively correlated with serum albumin $(r=0.4835 ; \mathrm{p}<0.0001)$ and negatively with INR $(\mathrm{r}=-0.4382 ; \mathrm{p}<0.0001)$, bilirubin $(r=-0.4057 ; p=0.0001)$ and ALP $(r=-0.3346 ; p=0.0015)$. Conclusions: Most patients with non-cholestatic CLD (90\%) have inadequate serum 25(OH)D concentrations and 1/6 have secondary hyperparathyroidism. Hypovitaminosis D is common even in patients with preserved liver function, and the $25(\mathrm{OH}) \mathrm{D}$ level further decreases in parallel with liver damage. Since vitamin D exerts important calciotropic and non-calciotropic functions, correction of vitamin D-PTH status may prevent osteoporosis, fractures and improve the course of CLD. This requires further investigation.

\section{8}

Protection Against Fatty Liver But Normal Adipogenesis in Mice Lacking Adipose Differentiation Related Protein (ADFP)

Benny H. Chang, Lan Li, Antoni Paul, Vijayalakshmi Nannegari, William C. Heird, Pradip Saha, Lawrence Chan

Adipose differentiation related protein (ADFP or ADRP), also known as adipophilin or ADPH, is a lipid droplet (LD) protein found in most cells and tissues. ADFP expression is strongly induced in cells with increased lipid load. We have inactivated the Adfp gene in mice to better understand its role in lipid accumulation. The Adfp-deficient mice have unaltered adipose differentiation or lipolysis in vitro or in vivo. Importantly, they display a $60 \%$ reduction in hepatic triglyceride (TG), and are resistant to diet-induced fatty liver. To determine the mechanism for the reduced hepatic TG content, we measured hepatic lipogenesis, VLDL secretion, lipid uptake and utilization, all of which parameters were shown to be similar between mutant and wild-type mice. The finding of similar VLDL output in the presence of a reduction in total TG in the Adfp-deficient liver is explained by the retention of TG in the microsomes where VLDL is assembled. Given that lipid droplets are thought to form from the outer leaflet of the microsomal membrane, the reduction of TG in the cytosol with concomitant accumulation of TG in the microsome of Adfp-/- suggests that ADFP may facilitate the formation of new LDs. In the absence of ADFP, impairment of LD formation is associated with the accumulation of microsomal TG but a reduction in TG in other subcellular compartments. We crossed Adfp-deficient mice with the leptin deficient (ob/ob) mice. The double deficient mice maintained similar body weight and adipocity as those of the leptin deficient alone. They, however, have reduced fatty liver and improved insulin sensitivity. Adfp, therefore, plays an important role in liver TG storage and is a key determinant in the development of fatty liver disease. 
119

Altered Gastrointestinal Motility and Enteric Neuronal Apoptosis in Diabetes Is Due to Increased Oxidative Stress

Shareen Iqbal, Andrew Gewirtz, Dean Jones, Shanthi Srinivasan

Background and Significance: Chronic diabetes causes complications such as gastroparesis and altered colonic motility which lead to significant morbidity. These motility related effects have been attributed to decreased intestinal inhibitory neuronal innervation. We have previously demonstrated hyperglycemia-induced apoptosis in enteric neurons. We examined the role of oxidative stress in hyperglycemia-induced apoptosis of enteric neurons and the ability of Glial Derived Neurotrophic factor (GDNF), on modulating this oxidative stress. Methods: In vitro experiments were performed in primary enteric neurons isolated by immunoselection from digested El4 rat embryonic intestines. Apoptosis was assessed using the Hoechst method. Oxidative stress was assessed by dichlorodihydrofluorescein staining intensity. Diabetes was induced using intraperitoneal injection of streptozotocin in C57BL6 mice in WT and GFAP-GDNF overexpressing mice. Gastrointestinal motility was measured by assessing gastric emptying (methylene blue) and intestinal transit. Results: Primary enteric neurons exposed to $20 \mathrm{mM}(24 \mathrm{~h})$ glucose have a significantly higher level of apoptosis (TUNEL method) and levels of reactive oxygen species (dichlorodihydrofluorescein staining intensity) compared to exposure to $5 \mathrm{mM}$ glucose $(\mathrm{P}<0.001)$. GDNF reduced this increased oxidative stress and apoptosis $(\mathrm{P}<0.001) .8$ weeks after inducing diabetes in C57BL6 mice we find evidence of enhanced enteric neuronal degeneration and apoptosis by electron microscopy in diabetic mice compared to controls. Diabetic mice had loss of NADPHdiaphorase staining myenteric neurons, delayed gastric emptying and increased intestinal transit time. The pathophysiological effects of hyperglycemia (apoptosis, loss of inhibitory neurons, motility changes) were reversed in diabetic GFAP-GDNF transgenic mice. Conclusions: Hyperglycemia induces enteric neuronal cell apoptosis through increased oxidative stress. GDNF ameliorates these effects. These studies may provide novel therapeutic strategies to prevent the neuronal injury that occurs in patients with diabetes.

\section{0}

Catalase Overexpression in Transgenic Mice Improves Motor Dysfunction in Dextran Sulfate Sodium (DSS)-Induced Colitis

Weibiao Cao, Zhongmao Guo, Karen M. Harnett, Claudio Fiocchi

We have previously shown that $\mathrm{H}_{2} \mathrm{O}_{2}$ is elevated in the circular muscle layer of ulcerative colitis (UC) patients, and sigmoid circular muscle cells exhibit reduced contraction and $\mathrm{Ca}^{2+}$ signaling in response to the endogenous neurotransmitter neurokinin A (NKA). The $\mathrm{H}_{2} \mathrm{O}_{2}$ scavenger catalase reduces $\mathrm{H}_{2} \mathrm{O}_{2}$ levels and restores the decreased $\mathrm{Ca}^{2+}$ signal and cell shortening to normal levels. These observations suggest that $\mathrm{H}_{2} \mathrm{O}_{2}$ contributes to the motor dysfunction of the sigmoid muscle layer in UC. In the present study we investigated whether overexpression of catalase in transgenic mice could improve motor dysfunction in the model of dextran sulfate sodium (DSS)-induced colitis. Colitis was induced by feeding mice (C57Bl/ $6,20-22$ week old) $1 \%, 1.5 \%, 2 \%$ or 3\% DSS (molecular weight $36000-50000$ ) for 7 days and the animals were sacrificed on the eighth day. Disease activity index (DAI) was determined by scoring changes of weight loss, stool consistency and occult/gross blood in the stools according to well established criteria. In wild-type mice, $2 \%$ and 3\% DSS caused severe bloody diarrhea and a $>15 \%$ weight loss. 1.5\% DSS caused moderate bloody diarrhea and weight loss with good tolerance. $1 \%$ DSS caused neither diarrhea nor weight loss. Therefore, $1.5 \%$ DSS was used in our study. 1.5\% DSS-induced colitis significantly increased the levels of $\mathrm{H}_{2} \mathrm{O}_{2}$ in the distal colonic circular muscle layer of wild-type mice, and significantly decreased NKA-induced contraction and intracellular $\mathrm{Ca}^{2+}$ increase in circular muscle cells. In contrast, in catalase-overexpressing transgenic mice, the DAI of 1.5\% DSS-induced colitis was significantly lower, infiltration of inflammatory cells and mucosal ulceration in the distal colon were less severe, and production of $\mathrm{H}_{2} \mathrm{O}_{2}$ in distal circular muscle layer was significantly lower than in DSS-treated wild-type littermates. In addition, in catalase over-expressing transgenic mice, the reduction in NKA-induced cell shortening and $\mathrm{Ca}^{2+}$ signal in the distal colonic circular muscle cells was significantly lower than wild-type littermates. We conclude that in vivo reduction in $\mathrm{H}_{2} \mathrm{O}_{2}$ levels by endogenously overexpressed catalase improves inflammation and distal colonic motor dysfunction of DSS-induced colitis, confirming a role of $\mathrm{H}_{2} \mathrm{O}_{2}$ in colitis-associated motor dysfunction. Supported by NIDDK R21 DK62775-01

\section{1}

Hydrogen Peroxide $\left(\mathrm{H}_{2} \mathrm{O}_{2}\right)$ Contributes to Decreased Spontaneous Phasic Contractions of Human Sigmoid Circular Smooth Muscle in Ulcerative Colitis Weibiao Cao, Karen M. Harnett, Victor E. Pricolo

We have previously shown that in normal sigmoid circular muscle strips, electrical field stimulation (EFS)-induced contraction is blocked by the NK-2 antagonist Bz-Ala-Ala-D-TrpPhe-D-Pro-Pro-Nle-NH2 (NK-2ra), but not by the NK-1 antagonist FK888, indicating that NK-2 receptors mediate neurally induced contraction of human sigmoid circular smooth muscle. In addition, in vitro spontaneous phasic contractions of normal sigmoid circular smooth muscle are significantly reduced by the NK2 receptor antagonists NK-2ra and MEN10376, but not by atropine or by the NKl antagonist FK888, suggesting that NK-2 receptors are involved in development of spontaneous contractions in human sigmoid colon. In the present study we explored a possible mechanism for reduction of spontaneous phasic contraction in UC. To confirm that neurokinin A (NKA) is an excitatory neurotransmitter in normal sigmoid colon, we measured NKA release before and after electircal field stimulation. In basal condition, normal sigmoid circular muscle released $11.8 \pm 3.1 \mathrm{pg} \mathrm{NKA} / \mathrm{g}$ protein/ min. Electrical stimulation significantly increased the NKA release to $128.3 \pm 29.7 \mathrm{pg} / \mathrm{g}$ protein/ min. Tetrodotoxin $\left(10^{-5} \mathrm{M}, 90 \mathrm{~min}\right)$ significantly decreased basal NKA release as well as EFS-induced NKA release and reduced both spontaneous and EFS-induced contraction. In addition, spontaneous phasic contractions were abolished by $\mathrm{Ca}^{2+}$-ATPase inhibitors thapsigargin and cyclopiazonic acid and significantly decreased by the PKC inhibitor chelerythrine and by the calmodulin inhibitor CGS9343B, suggesting that spontaneous phasic contractions may be mediated by $\mathrm{Ca}^{2+}$ release from intracellular stores and by a PKC-and calmodulin-dependent pathway. These inhibitors had no effect on spontaneous NKA release. In strips from patients with ulcerative colitis, $\mathrm{H}_{2} \mathrm{O}_{2}$ levels were significantly higher than in normal muscle and EFS-induced and spontaneous contractions were significantly lower than in normal muscle. The reduced contraction was partially restored by the $\mathrm{H}_{2} \mathrm{O}_{2}$ scavenger catalase. NKA release, however, was not affected. In addition, $\mathrm{H}_{2} \mathrm{O}_{2}$ significantly affected NKA-induced release of $\mathrm{Ca}^{2+}$ from intracellular stores. We conclude that spontaneous phasic contractions of human sigmoid circular smooth muscle are mediated by NKA-induced calcium release from intracellular stores and activation of calmodulin and PKC. In UC patients, spontaneous phasic contractions are decreased and this decrease is not related to decreased release of NKA but rather to overproduction of $\mathrm{H}_{2} \mathrm{O}_{2}$ affecting sigmoid circular muscle, most likely through depletion of releasable calcium stores. Supported by NIDDK R21 DK62775-01.

Beta-3 Adrenoceptor Agonism Decreases Motility and Induces AntiHyperalgesia: A Novel Target for IBS

Selim Cellek, Ramkumar Thangiah, Anna K. Bassil, Colin A. Campbell, Kelly H. Donaldson, Karen M. Gray, Celestine T. O'Shaughnessy, Jennifer L. Stretton, David E. Uehling, Shanmugam Vivekanandan, Wendy J. Winchester, Gareth J. Sanger, Kevin Lee

Beta-3 adrenoceptor (AR) agonists have previously been shown to relax colonic smooth muscle and to induce the release of somatostatin (SST), a known endogenous analgesi substance. Here we demonstrate that a selective beta-3 AR agonist GW427353 (pEC50 = 8.4 in stimulating cAMP accumulation in $\mathrm{CHO}$ cells expressing human beta-3 AR; Uehling et al. J. Med. Chem. Submitted) decreased electrical field stimulation ( $50 \mathrm{~V}, 0.5 \mathrm{~ms}$ pulse duration, $5 \mathrm{~Hz}$, for $10 \mathrm{~s}$, every $1 \mathrm{~min}$ )-induced cholinergic contractions in human isolated colon circular muscle. The inhibitory effect of GW427353 on cholinergic contractions was reversible with beta-3 AR antagonist SR-59230A (1 $\mu \mathrm{M})$. SST-2 receptor antagonis CYN154806 or desensitisation to SST did not alter the inhibitory effect of GW427353 on cholinergic contractions. GW427353 elicited neurogenic release of SST from human colon strips measured using ELISA; an effect completely inhibited by tetrodotoxin $(1 \mu \mathrm{M})$ or SR59230A (1 $\mu \mathrm{M})$. GW427353 had no effect on carbachol-induced contractions of the human colon. Furthermore, immunofluorescence studies demonstrated that beta-3 AR was colocalised in cholinergic nerve fibres in the human colon with SST. In conscious rats, another beta-3 AR agonist (CL-316243; 0.3-1 mg/kg po) decreased wet weight of faecal matter following oral castor oil administration. Similarly, in a model of hyperalgesia, CL-31624 $(0.03-0.1 \mathrm{mg} / \mathrm{kg}$, po) decreased the number of arches induced by intrarectal administration of mustard oil; an effect reversed by a SST-2 receptor antagonist CYN154806 (10 mg/kg sc). These results suggest that beta-3 AR agonism inhibits cholinergic contractions in human colon, an effect consistent with an ability to reduce intestinal motility via SST-independen mechanisms, while inducing anti-hyperalgesia by releasing SST from enteric nerves. These dual actions of beta-3 AR agonism represent a novel therapeutic target for diarrhoea-predominant IBS.

123

FG7142, a Benzodiazepine Inverse Agonist, Acts Peripherally to Stimulate Colonic Motor Functions in Rats

Muriel H. Larauche, Lixin Wang, Mulugeta Million, Yvette Tache

Background: Stress induces alterations of colonic motility and secretion via the release of corticotropin releasing factor $(\mathrm{CRF})$ and activation of $\mathrm{CRF}_{1}$ receptors $\left(\mathrm{CRF}_{1}\right)$ in rodents and may have relevance to Irritable Bowel Syndrome (IBS) (Br J Pharmacol 2004;141:1321-30). The benzodiazepine inverse agonist FG7142 is an anxiogenic drug acting on $\mathrm{GABA}_{\mathrm{A}}$ receptors It suppresses the inhibitory tone of the GABAergic system on the release of CRF in vivo and in vitro in the paraventricular nucleus of the hypothalamus, a nucleus known to be involved in the colonic motor alterations induced by stress (AJP 2001;280:G173-7). Aim: To determine whether FG7142 administered intraperitoneally (IP) in rats alters colonic motor function and involves the $\mathrm{CRF} / \mathrm{CRF}_{1}$ system as shown in other stress models. Methods: Male and ovariectomized (OVX) female SD rats (250-300g) were used in 4 experiments to monitor: 1) the colonic and hypothalamo-pituitary-adrenal (HPA) axis responses to FG7142 (2, 10 and $20 \mathrm{mg} / \mathrm{kg} \mathrm{IP}$ ); 2) the role of $\mathrm{CRF}_{1}$ in the effects of FG7142 (10 mg/kg IP) by pretreating rats subcutaneously (SC) with the $\mathrm{CRF}_{1}$ antagonist NBI-35965 (30 mg/kg) or intracerebroventricularly (ICV) with the $\mathrm{CRF}_{1 / 2}$ antagonist astressin $\left.(10 \mu \mathrm{g} / \mathrm{kg}, 10 \mu \mathrm{l}) ; 3\right)$ the influence of estrogen on FG7142 (10 mo/kg IP) effect by pretreating OVX females acutely with $17 \beta$ estradiol ( $400 \mu \mathrm{g} / \mathrm{rat} \mathrm{SC}, 18 \mathrm{~h}$ before) and 4) the central/peripheral site of action of FG7142 on the modulation of colonic functions by injecting it centrally $(30,100$ and $300 \mu \mathrm{g} / \mathrm{ra}$ ICV). In each experiment, the fecal pellet output (FPO) and corticosterone plasma levels (CORT) were determined. Results: FG7142 (2,10 and $20 \mathrm{mg} / \mathrm{kg}$ ) induced a dose-related increase in FPO in males $(2.3 \pm 0.5,5.0 \pm 0.7,5.8 \pm 1.0$ pellet/h respectively vs 0 in IP vehicle) and at $10 \mathrm{mg} / \mathrm{kg}, 20 \%$ had diarrhea. FG7142 (20 mg/kg) induced a 3-fold increase in CORT $(342 \pm 24 \mathrm{ng} / \mathrm{ml}$ vs $96 \pm 15 \mathrm{ng} / \mathrm{ml}$ in vehicle). Peripheral NBI-35965, central astressin or $17 \beta$ estradiol did not modify FG7142-induced increased FPO in rats. FG7142 injected centrally did not reproduce the effects of the peripheral administration on colonic functions. Conclusions: The data show that acute systemic administration of FG7142 in male and female rats leads to an increase in defecation and an activation of the HPA axis similar to the well described stress response. The data did not show the $\mathrm{CRF}_{1}$ receptor system involvement, centrally and/or peripherally in the action of FG7142. Estrogen does not seem to influence the effect of FG7142. The absence of effect of centrally-injected FG7142 suggests a primary peripheral site of action that may involve inhibition of the inhibitory enteric GABAergic system. 
Serotonin-Transporter (SERT) Polymorphism Genotype and SERT Expression in Mucosal Biopsies of Patients with Irritable Bowel Syndrome

Christopher Andrews, Michael Camilleri, Adil E. Bharucha, Paula J. Carlson, Irene Ferber, Debra Stephens, Raul Urrutia, Jeroen Aerssens, Leen Thielemans, Hinrich Goehlmann, Ilse van den Wyngaert, Bernard Coulie

Background: A previous study has suggested that irritable bowel syndrome (IBS) with diarrhea (IBS-D) or constipation (IBS-C) is associated with low mucosal levels of serotonin transporter (SERT, encoded by the SLC6A4 gene) mRNA and immunoreactivity. Aims: To compare the mucosal biopsy levels of SLC6A4 mRNA in IBS-C or IBS-D and controls, and to study whether differences in mucosal SLC6A4 mRNA were associated with polymorphisms of the SLC6A4 promoter (SERT-P). Methods: Validated bowel symptom questionnaires characterized the phenotype in 14 patients with IBS-D, 16 IBS-C and 15 healthy asymptomatic controls. Subjects underwent SLC6A4 promoter genotyping and rectal and sigmoid colon mucosal biopsies. Mucosal SLC6A4 mRNA was characterized using RTQ-PCR and compared between groups using ANOVA. Concordance and reproducibility of sigmoid mucosal SLC6A4 mRNA was assessed on duplicates on same day and median 82 days apart. Fishers' exact test compared the proportion of SLC6A4 genotypes in the three groups; the relationship between tissue SLC6A4 mRNA and genotype was explored. Results: Results from sigmoid biopsies taken on the same day and median 82 days apart were highly concordant and reproducible. There were no differences in SLC6A4 mRNA in rectal or sigmoid biopsies between controls and IBS-C; in contrast, IBS-D was associated with significantly decreased SLC6A4 mRNA in the sigmoid, but not in rectal biopsies (Fig.). The proportions of SLC6A4 promoter genotypes were unrelated to the SLC6A4 mRNA levels in the sigmoid mucosal samples. Conclusion: Relative to control tissues, expression of SLC6A4 mRNA in sigmoid mucosal biopsies is decreased in patients with IBS-D and unchanged in IBS-C; the observed differences do not appear to be related to SLC6A4 promoter genotype.

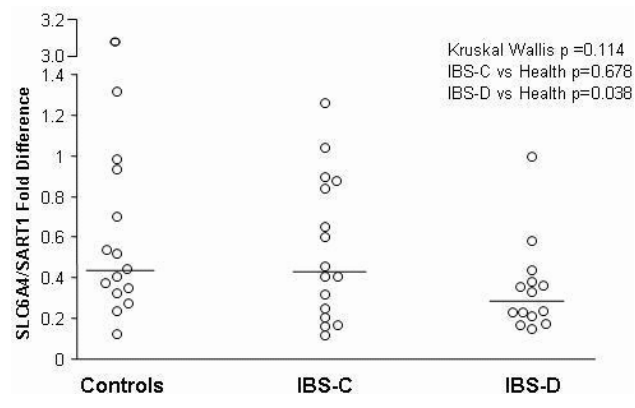

125

Identification and Validation of Serological IBD Biomarkers By a Novel High Throughput Proteomic Approach Using High Density Protein Chip Technology Sean Sullivan, Heng Zhu, Carmen Cuffari, Steve Brant, Shengce Tao, Yueping Chen, Xuhang Li

Background: Anti-microbial or anti-human (autoantibodies), are present in the sera of patients with inflammatory bowel disease (IBD), and may be used as serological biomarkers to enable classification, and may predict disease progression. Herein, we describe a novel highthroughput and highly sensitive proteomic approach to screen and identify new serological biomarkers for IBD. Methods: We developed highly reproducible high-density protein chips, each containing 5800 unique yeast proteins that cover essentially the entire yeast proteome. Each protein was robotically spotted in duplicate on a $75 \times 25 \mathrm{Xl} \mathrm{mm}$ glass slides coated with specific polymer. Each protein chip was used for serological IBD biomarkers screening using individual sera from normal pediatric subjects and IBD patients. Pediatric patients were utilized as this population tends to experience less concomitant disease, and is at relatively earlier stages compared with adult patients. Positive identification was achieved with a GenePix array scanner using goat anti-human antibodies covalently conjugated with fluorescent dyes $\mathrm{Cy} 3$ or $\mathrm{Cy} 5$. The intensity of antibody-protein recognition was quantitatively measured by fluorescent intensity with automatic subtraction of background surrounding each protein spot. Results: 45 protein chips were screened against serum from healthy children and children with IBD. Serum was analyzed from 15 children with active Crohn's ileocolitis, and 15 from patients with active pan-ulcerative colitis (UC), and was compared to healthy controls. Disease activity was defined histologically as moderate to severe for the majority of patients. More than 220 IBD-specific antibodies, which were defined as antibodies that were not present in sera of any normal subject, were identified from serum of patients with UC and Crohn's disease (CD) patients, suggesting a hyper-inflammatory immune response. $20 \%$ of the 220 unique yeast proteins recognized by IBD-specific antibodies have human homologues that can be grouped into 5 major functional classes: ubiquitination, nucleotide-binding/transcription regulation, signal transduction, cell division, cytoskeleton/ vesicular trafficking. > 40 additional antibodies in CD/UC patients were also present in at least one of the normal subjects. Among IBD-specific antibodies, 130 were CD-specific and 40 were UC-specific. Additional 50 antibodies were identified to be present in at least one of the $\mathrm{CD}$ or UC patients studied. A newly designed protein chip, on which all proteins recognized specifically by IBD sera are robotically spotted, is being tested for its accuracy and specificity in diagnosis of IBD and differentiation between $C D$ and UC.
Gastrointestinal Diseases in Chronic Granulomatous Disease Sushil Ahlawat, Jordan Feld, Nadeem Hussain, David Kleiner, Victoria Anderson, Dianne Hilligoss, John Gallin, Harry Malech, Steven Holland, Theo Heller, T. Jake Liang, Jay Hoofnagle

Background: Chronic granulomatous disease (CGD) is a group of hereditary disorders characterized by the impaired intracellular destruction of certain bacteria and fungi. CGD affects multiple organ systems. However, a comprehensive clinical picture of gastrointestinal (GI) tract involvement is lacking. Our aim was to describe the spectrum of GI disease and its predictors among patients with CGD. Methods: 194 patients with CGD have been followed at the clinical center of the NIH for a median of 7.8 years. A retrospective chart review was carried out focusing on the GI tract abnormalities and their treatment. Results: Initial mean age was 12 years. The genetic defect was identified as gp 91 phox in 110, p22phox in 4, p47phox in 55, p67phox in 3, and was unknown in 22 patients. Inflammatory conditions including the obstructive lesions of the GI tract, colitis or enteritis were seen in $42 \%$ of patients. Colitis or enteritis was the most common GI disease in the present series, occurring in $28 \%(n=54)$ of patients. Patients with colitis or enteritis were managed with steroids (78\%), antibiotics such as a combination of ciprofloxacin and metronidazole (30\%), and 5ASA compounds (21\%). Azathioprine or infliximab were used only in 3 patients. Five patients underwent partial bowel resection or fistula repair. Obstructive lesions of the upper GI tract were found in $13 \%(n=26)$ of patients. Gastric outlet obstruction was the most common lesion involving the upper GI tract $(n=20)$. Four patients were noted to have esophageal dysmotility described as achalasia like abnormalities. Steroids were used to manage upper GI tract obstructive lesions in $38 \%(n=20)$ of patients. Three patients had endoscopic dilatation and 1 patient required partial bowel resection. Age and gender were not associated with GI disease; however, Caucasian race including Hispanics was found risk factor for $\mathrm{GI}$ disease $(\mathrm{OR}=2.4 ; \mathrm{CI}, 1.1-5.2 ; \mathrm{p}<0.02)$. $\mathrm{p} 47$ phox genotype had significantly less upper GI disease $(O R=0.38 ; C I, 0.20-0.72 ; p<0.003)$. No significant association was found between GI disease and mortality. Conclusions: Inflammatory colitis or enteritis and the gastric or esophageal outlet obstruction are the most common GI diseases in patients with CGD. Caucasian race appears to be a risk factor for developing GI disease. However, p47 phox genotype appears to be associated with less upper GI disease, though lower GI manifestations of colitis and enteritis are seen in a significant number of these patients and in some cases is the main morbidity associated with their CGD. Mortality was not influenced by GI disease.

127

The Relationship Between Abnormal Innate and Adaptive Immune Function and Fibrostenosis in Crohn's Disease Patients

Andrew F. Ippoliti, S Devlin, H Yang, L Mei, K Papadakis, E Vasiliauskas, G Melmed, O Shaye, P Enyati, G Chen, J Choi, I Nastaskin, Y Patel, E Mehdikhani, K D. Taylor, C Landers, J I. Rotter, S Targan

Bkgd: Allelic variants of NOD2 have been associated in some studies with fibrostenosing (FS) phenotype of Crohn's disease (CD), (Abreu Gastro 2002, Mow Gastro 2004). Multiple antibodies (ab) to commensual gut microorganisms, ASCA, I2, OmpC, and CBir, are positive and at high levels in patients with FS, (Mow Gastro 2002, Targan Gastro 2005). The purpose of this study was to analyze the relationship between NOD2 variants and abs to ASCA, I2, OmpC, and CBir in CD patients with FS. Methods: Serologic, genetic and phenotypic data were examined in $745 \mathrm{CD}$ patients. ASCA and abs to I2, OmpC, and CBir were measured by ELISA. Quartile scores and overall quartile sums (range 4-16) of levels for all abs were calculated as previously described, (Mow Gastro 2004). Genotyping (TaqmanMGB) was performed for 3 Crohn's-associated variants of the NOD2 gene, single nucleotide polymorphisms 8,12 , and 13. Phenotypes were determined by chart review by pairs of observers blinded to serologic and genetic data. Results: FS phenotype was present in $44 \%$ of CD patients. ASCA was present in 51\%, I2 in 58\%, OmpC in 38\%, and CBir in $56 \%$ of patients. NOD2 variants were found in $33 \%$ of patients, $27 \%$ had one allelic variant and $6 \%$ had two. Among the subgroup with FS phenotype $41 \%$ had at least one allelic variant vs $27 \%$ in those without FS, $\mathrm{p}<.0001$ by ChiSquare). There was an increasing prevalence of $\mathrm{FS}$ in patients with $0 \rightarrow 4$ positive abs; $0=14 \%, l=31 \%, 2=49 \%, 3=54 \%, 4=63 \%(p<.0001)$. There was a similar increase in this phenotype when patients were grouped by antibody quartile; $4-6=21 \%, 7-9=31 \%, 10-13=55 \%$, and $14-16=60 \%(p<.0001)$. These results confirm the association of FS with NOD2 variants and ab seropositivity in this cohort. Further, NOD2 variants were more common patients with multiple positive abs and high ab levels. Among the 325 patients with FS the prevalence of NOD2 variants was only $19 \%$ in patients without ab positivity and increased to $60 \%$ when all 4 abs were positive. Similarly, in the lowest quartile group only $19 \%$ had NOD2 variants as compared to $64 \%$ in the highest quartile group. NOD2 variants were independently associated with FS when compared to individual abs; OmpC $\mathrm{p}<.002, \mathrm{I} 2 \mathrm{p}<.03$, CBir $\mathrm{p}<.0003$, ASCA p<.023. However NOD2 variants were not independently associated with FS when compared to multiple positive abs $(\mathrm{p}=.97)$ or when compared to quartile sums ( $\mathrm{p}=46$ ). Concl:While both NOD2 variants and antibody seropositivity are strongly associated with the FS phenotype, it is not an independent association. This suggests that a patient with defects of innate immunity as well as hyperresponsiveness to commensual microbial antigens (adaptive immunity) will frequently develop FS. 
Immune-Mediated Inflammatory Disorder Co-Occurrence Among Inflammatory Bowel Disease Patients in US Healthcare Datasets Between 2001-2002

Don Robinson, Jr, Monica Hackett, Kevin Renahan, Clark Paramore, Kathy Fraeman, Ning Zhao, Stephen Xu, Mohan Bala

Background: In 2005, Bernstein et al and Gupta et al independently reported a higher risk for certain other immune-mediated inflammatory disorders (I.M.I.D.s) among inflammatory bowel disease (IBD) patients in Canadian (Manitoba) and UK healthcare datasets. We report on similar work conducted in two large US healthcare datasets. Methods: Adult patients continuously enrolled from 2001-2002 were identified in two US healthcare claim data sets with initial populations of about 6 and 11 million covered lives, respectively. Patients with ulcerative colitis (UC) and Crohn's disease (CD) were identified based on the presence of one claim with ICD-9 codes corresponding to these diseases. Controls for each disease were matched 3:1 based on age, gender, US Census region and pre-baseline insurance coverage. Odds ratios (OR) for the risk of selected I.M.I.D.s were computed between IBD cohorts and controls; confidence intervals (CI) were estimated using the Mantel-Haenszel method. A sensitivity analysis was conducted that required at least one inpatient or two ambulatory face-to-face medical visits to identify patients with UC and CD. Results: The OR for the risk of selected I.M.I.D.s were consistent across US data sets and IBD sub-types (see Table). CD and UC patients had risks for these I.M.I.D.s that were similar to the UK and Canadian findings. The highest OR was for ankylosing spondylitis; an OR of about 2.00 was seen for rheumatoid arthritis, scleroderma and systemic lupus. These findings persisted in the sensitivity analysis. Conclusion: This US study confirms previous UK and Canadian reports, suggesting a notably higher likelihood for certain other immune disorders among IBD patients. Prospective research is needed to confirm these findings and clarify the relationship between these diseases

Table: Risk for Selected I.M.I.D.s Among CD and UC Patients*

\begin{tabular}{|c|c|c|c|c|}
\hline & \multicolumn{2}{|c|}{ Crohn's Disease } & \multicolumn{2}{c|}{ Ulcerative Colitis } \\
\hline & $\begin{array}{c}\text { Data Set A } \\
(\mathrm{N}=7,401)\end{array}$ & $\begin{array}{c}\text { Data Set B } \\
(\mathrm{N}=9,267)\end{array}$ & $\begin{array}{c}\text { Data Set A } \\
(\mathrm{N}=10,104)\end{array}$ & $\begin{array}{c}\text { Data Set B } \\
(\mathrm{N}=11,220)\end{array}$ \\
\hline $\begin{array}{c}\text { Ankylosing } \\
\text { Spondylitis }\end{array}$ & $9.38(5.40-16.30)$ & $9.03(5.99-13.62)$ & $3.72(2.18-6.35)$ & $7.18(4.69-11.01)$ \\
\hline Diabetes Type I & $0.95(0.57-1.58)$ & $1.19(0.69-2.05)$ & $0.93(0.61-1.42)$ & $0.89(0.54-1.47)$ \\
\hline $\begin{array}{c}\text { Multiple } \\
\text { Sclerosis }\end{array}$ & $1.48(1.00-1.48)$ & $1.59(1.17-1.59)$ & $1.17(0.81-1.68)$ & $1.47(1.11-1.95)$ \\
\hline Psoriasis & $1.40(1.13-1.74)$ & $1.65(1.39-1.96)$ & $1.36(1.14-1.62)$ & $1.37(1.16-1.61)$ \\
\hline $\begin{array}{c}\text { Rhematoid } \\
\text { Arthritis }\end{array}$ & $2.37(2.02-2.77)$ & $2.97(2.56-3.45)$ & $2.16(1.88-2.49)$ & $2.76(2.40-3.18)$ \\
\hline Scleroderma & $2.00(0.97-2.00)$ & $1.73(0.90-3.33)$ & $2.07(1.11-3.86)$ & $2.00(1.14-3.53)$ \\
\hline Systemic Lupus & $2.48(1.76-3.49)$ & $1.93(1.40-2.68)$ & $2.23(1.67-2.98)$ & $1.90(1.42-2.53)$ \\
\hline Asthma & $1.48(1.34-1.64)$ & $1.51(1.39-1.63)$ & $1.48(1.35-1.61)$ & $1.52(1.41-1.64)$ \\
\hline
\end{tabular}

* I.M.I.D.s reported in either the Canadian or UK study and reported as OR (95\%CI)

129

A Panel of Serological Antibodies (gASCA, OMP, ACCA, ALCA and AMCA) Predicts Complicated Disease Course and Surgery in Crohn's Disease Marc Ferrante, Marie Pierik, Liesbet Henckaerts, Nir Dotan, Gary L. Norman, Rom T. Altstock, Iris Dotan, Karolien Claes, Nele van Schuerbeek, Zakera Shums, Benjamin Schooler, Gert van Assche, Paul Rutgeerts, Severine Vermeire

BACKGROUND \& AIMS: Crohn's disease (CD) presents heterogeneously and may often lead to complications needing surgery. Several serological markers (ASCA, pANCA, antiOmpC, anti-I2) have been identified in IBD and are associated with distinct clinical phenotypes. We tested if a panel of new serological markers could predict complicated disease course (as fistulas or strictures) or the need for surgery in Crohn's disease. METHODS: A total of $913 \mathrm{CD}$ patients (mean age 42.3 years, 58\% female) were tested for Anti Saccharomyces cerevisiae Antibodies (gASCA), Outer Membrane Porin antibodies (*Omp), AntiChitobioside Carbohydrate Antibodies (ACCA), Anti-Laminaribioside Carbohydrate Antibodies (ALCA) and Anti-Mannobioside Carbohydrate Antibodies (AMCA) by commercially available ELISA-assays (Glycominds Ltd, Israel and *INOVA Diagnostics Inc., USA) in a blinded way. Based on the cut-off values and the number of positive markers a score ranging from 0 (no serological markers) to 5 (positive for all markers) was developed. Clinical characteristics on smoking, disease location, complications (strictures or fistulas) and the need for surgery, were obtained and analyzed using SPSS. RESULTS: Multivariate analysis using each serological marker separately showed that ACCA, AMCA and Omp were independently associated with need for surgery (all $\mathrm{p}<0.01$ ). gASCA also showed a trend for association with the need for surgery $(\mathrm{p}=0.06)$ and was independently associated with a non-inflammatory behavior $(\mathrm{p}=0.02)$. Assessing the data from a clinical perspective, a complicated disease course (strictures or fistulas) needing surgery was independently associated with gASCA ( $\mathrm{p}=$ $0.002)$, ACCA $(p=0.011)$, Omp $(p=0.008)$, and ileal $(p=0.043)$ as well as anal $(p=0.002)$ involvement. Combining the different markers, 126 patients had a score lower than 1 (group A), 591 had a score between 1 and 3 (group B) and the remaining 196 had a score higher than 3 (group C). A more complicated disease course (presence of strictures or fistulas) was noted with an increasing score (from $35 \%$ to $60 \%$ to $83 \%$ respectively; group A vs. B OR 2.80 (1.73-4.53); group A vs. C OR 9.10 (5.01-16.55)). Very similar findings were found using the need for surgery as outcome (from $33 \%$ to $51 \%$ to $79 \%$ respectively; group A vs. B OR 2.19 (1.35-3.56); group A vs. C OR 7.95 (4.46-14.17)). DISCUSSION: Our results illustrate that serological markers directed against glycan-epitopes (gASCA, ACCA, AMCA) and the outher membrane porins (Omp) are all associated with severe and complicated
130

Both Preoperative pANCA and CBIR1 Flagellin Expression in Ulcerative Colitis (UC) Patients Influence Pouchitis Development After Ileal Pouch-Anal Anastomosis (IPAA)

Phillip Fleshner, Eric Vasiliauskas, Marla Dubinsky, Ling Mei, Carol Landers, Andrew Ippoliti, Konstantinos Papadakis, Stephan Targan

PURPOSE: Although most studies of pouchitis after IPAA for UC consider acute pouchitis (AP) and chronic pouchitis (CP) to be a single entity, several lines of evidence suggest that they are distinct disease processes. Antibody responses to microbial antigens define different groups of patients with IBD. Preoperative high-level pANCA (perinuclear antineutrophi cytoplasmic antibody) expression is associated with CP but not AP development (Fleshner et al., Gut 2001;49:671). The association of serum responses to CBirl flagellin with AP or $\mathrm{CP}$ is unknown. AIM: The aim of this study was to assess the association of preoperative CBirl flagellin and PANCA expression on AP or CP development after IPAA for UC METHODS: Patients were prospectively assessed for the development of clinically and endoscopically proven AP (antibiotic responsive) or CP (antibiotic dependent or refractory to antibiotic therapy). Sera obtained at time of colectomy in 238 colitis patients were analyzed for ANCA and CBir using ELISA. pANCA+ patients were substratified into high-level (>100 $\mathrm{EU} / \mathrm{ml})$ and lower-level $(<100 \mathrm{EU} / \mathrm{ml})$ groups. RESULTS: There were $171 \mathrm{pANCA}+$ patient ( $72 \%)$ and 46 CBirl+ patients (19\%). After a median followup of 47 months (range, 3-153 mos), 72 patients (30\%) developed pouchitis. Median time to diagnosis of pouchitis was 7 months (range, 1-116 mos). Pouchitis developed in 36\% of pANCA+ patients vs. $16 \%$ of pANCA- patients $(\mathrm{p}=0.005), 46 \%$ of CBirl+ patients vs. $26 \%$ of CBirl- patients $(\mathrm{p}=0.02)$ and $54 \%$ of $35 \mathrm{pANCA}+/ \mathrm{CBirl}+$ patients vs. $31 \%$ of $136 \mathrm{pANCA}+/ \mathrm{CBirl}$ - patients $(\mathrm{p}=0.02)$ AP was seen in 43 patients (18\%) and CP seen in 29 patients (12\%). AP developed in 37 pANCA+ patients ( $22 \%)$ vs. 6 pANCA- patients $(9 \%)(\mathrm{p}=0.02)$, and $12 \mathrm{CBirl}+$ patients $(26 \%)$ vs. 31 CBirl-patients $(16 \%)(\mathrm{p}=0.1)$. Overall pANCA and CBirl were not associated with CP development. Twenty-one patients (12\%) were high-level (HL) pANCA+ and 150 patients (88\%) were lower-level (LL) pANCA+. Although AP was not influenced by pANCA level, AP was seen in 38\% of LL pANCA+/CBirl+ patients vs. 18\% LL pANCA+/CBirpatients ( $\mathrm{p}=0.03$ ). CP was seen in $29 \%$ of HL pANCA+ patients vs. $11 \%$ of LL pANCA+ patients $(\mathrm{p}=0.03)$. There was no significant difference in $\mathrm{CP}$ incidence between HL pANCA+/ CBirl+ patients (50\%) and LL pANCA+/CBir- patients (20\%) (p=0.3). CONCLUSION: Both pANCA and CBirl expression are associated with pouchitis after IPAA. AP is influenced by both lower-level pANCA+ expression and CBirl, whereas CP appears to be linked solely to high-level pANCA+ expression. These unique serologic patterns suggest that changes in reactivity to microbial antigens may manifest as different forms of pouchitis after IPAA.

\section{1}

A Dose-Ranging, Double-Blind, Placebo-Controlled Study of Lubiprostone in Subjects with Irritable Bowel Syndrome and Constipation (c-IBS) John F. Johanson, Raymond Panas, P. Christopher Holland, Ryuji Ueno

IBS is a condition that affects nearly 30 million individuals in North America and accounts for $25-50 \%$ of the referrals to gastroenterologists. Lubiprostone is a novel type-2 chloride channel (ClC-2) activator that has been shown to be efficacious and well tolerated in a number of well-controlled clinical trials in subjects with chronic constipation. We present the results from a 12-week dose-ranging study where lubiprostone was tested, for the first time, exclusively in subjects with IBS with constipation (c-IBS), as defined by the Rome Il Criteria. Methods: Approximately 50 subjects were randomized in a double-blind fashion to each of the four treatment groups: placebo $(0 \mu \mathrm{g})$ or 16,32 , or $48 \mu \mathrm{g}$ lubiprostone daily $(8,16$, or $24 \mu \mathrm{g}$ BID). In an electronic diary, subjects recorded data relating to dosing abdominal symptoms (bloating and discomfort/pain), bowel movements (BMs; frequency and straining and consistency ratings), and rescue medication use. Weekly diary questions queried subjects on their assessment of the treatment effectiveness. Trend-tests were used to detect dose-dependent efficacy relationships and a step-down testing procedure was used to make pairwise comparisons between the active lubiprostone and placebo groups in the case of a dose-dependent trend. Safety was assessed by adverse event (AE) incidence rates Results: Significant dose-dependent trends were observed during at least 2 of the 3 months for abdominal discomfort/pain abdominal bloating spontaneous BM frequency (SBM), stool consistency, bowel straining, and assessments of constipation severity. Pairwise comparisons revealed many significant differences between the active groups and placebo. During month 1 and 2, improvements in abdominal discomfort/pain and SBM frequency rates were more than doubled in all lubiprostone groups as compared to placebo. Specifically, at Month 1 , decreases from baseline in abdominal discomfort (based on a 5-point scale) were $0.19,0.45$ 0.40 , and 0.46 points in the placebo, 16,32 , and $48 \mu$ groups, respectively; at Month 2 decreases from baseline were $0.23,0.52,0.53$, and 0.54 points, respectively; and, at Month 3 , decreases from baseline were $0.34,0.56,0.59$, and 0.53 points, respectively. Overall, improvements were typically highest in the $48 \mu \mathrm{g}$ group. With respect to safety, dosedependent trends were also observed. AE incidence and drop-out rates typically increased with increasing dose. Conclusion: Lubiprostone is an efficacious and well-tolerated treatment for c-IBS. Dose-dependent trends were observed with respect to safety and efficacy. 


\section{2}

Effects of Multidose Administration of MD-1100 On Safety, Tolerability, Exposure, and Pharmacodynamics in Healthy Subjects

Caroline Kurtz, Don Fitch, Robert W. Busby, Angelika Fretzen, G. Steven Geis, Mark G. Currie

MD-1100 acetate (MD-1100), a guanylate cyclase-C (GC-C) agonist, is a novel agent that acts in animal models to promote intestinal secretion and transit and to reduce visceral pain. These pharmacological actions are ideal attributes for the treatment of constipationpredominant irritable bowel syndrome (IBS-C) and chronic idiopathic constipation (CIC). Aim: In an effort to better understand the potential utility of MD-1100 for treating gastrointestinal (GI) disorders, we have begun to characterize its safety, tolerability, exposure, and pharmacodynamics in healthy male and female subjects. Methods: A placebo-controlled, double-blind, randomized, multiple dose level study was performed with 48 healthy subjects. Oral doses of 30,100, 300 or $1000 \mu \mathrm{g}$ of MD-1100 or placebo were administered once daily for 7 days. Subjects were assessed for safety, tolerability, MD-1100 systemic exposure, and GI pharmacodynamics using the Bristol Stool Form Scale (BSFS). Subjects recorded stool characteristics including stool frequency, consistency, and ease of passage in diaries for 7 days preceding dosing and for the duration of the 7 day treatment period. Additionally, stool weight was measured during the 7 day treatment period. Results: MD-1100 was well tolerated at all dose levels. There was no detectable systemic exposure to MD-1100 or any evidence of metabolites at any dose level. All subjects completed the study and there were no serious adverse events. Subjects who received MD-1100 reported a loosening of stools during treatment as measured by a statistically significant increase from the baseline in the mean BSFS stool consistency scores at the $30 \mu \mathrm{g}(\mathrm{p}=0.050), 300 \mu \mathrm{g}(\mathrm{p}=0.001)$ and 1000 $\mu \mathrm{g}$ dose levels $(\mathrm{p}=0.001$ ). Mean ease of stool passage scores showed a statistically significant increase from baseline at the $1000 \mu \mathrm{g}$ dose level $(\mathrm{p}<0.001)$. Other dose-related indications of GI pharmacodynamic activity in subjects receiving MD-1100 included increases in stool frequency and stool weight. Conclusion: In this multiple-dose Phase 1 study, MD-1100 was well tolerated across the dose range studied $(30-1000 \mu \mathrm{g})$. There was no detectable systemic exposure to MD-1100. MD-1100 demonstrated dose-related effects on GI pharmacodynamics including stool consistency, ease of passage, stool frequency and stool weight. To date, the preclinical and clinical data in healthy subjects support the potential of MD-1100 for the treatment of GI disorders, including IBS-C and CIC.

\section{3}

Predictors of Premature Antidepressant Discontinuation in Functional Gastrointestinal Disorders (FGIDs): A Survival Analysis Approach Gregory S. Sayuk, Jill E. Elwing, Patrick J. Lustman, Ray E. Clouse

Antidepressants (AD) are a mainstay in the management of moderate to severe FGID, but patient adherence to $\mathrm{AD}$ regimens can be poor. Preliminary data from a placebo-controlled trial of a tricyclic antidepressant (TCA) in women with functional bowel disorders suggest that high degrees of somatic complaints (somatization, SOM) predict subsequent side effects. Other potential risk factors for premature discontinuation have not been determined nor have the preliminary findings been confirmed in open-label treatment of broader subject groups. Methods: 173 consecutive patients $(45.9 \pm 1.2 \mathrm{yr} ; 70 \%$ female) with FGID $(29.6 \%$ IBS) treated with $\mathrm{AD}$ specifically for management of the FGID and followed by an academic gastroenterology practice were studied. Clinical features, $\mathrm{AD}$ type, duration of Rx, side effect type and severity, global AD treatment response (GTR), presence of psychiatric disorder (PSY: anxiety disorder or depression), and degree of SOM were based on systematic chart review. High degree of SOM was present if subjects endorsed $>7$ recent symptoms from the PHQ-15 or scored >median on \# of historical functional GI and non-GI disorders. Survival analysis methods with Cox proportional hazards models were used to determine independent predictors of $\mathrm{Rx}$ discontinuation with 12 mo of $\mathrm{AD}$ initiation. Logistic regression models were used to determine predictors of maximal GTR as well as side effect severity. Results: Subjects were followed for $12.4 \pm 1.4$ mo after AD initiation. At time of discontinuation or censoring, 94.2\% were receiving TCAs with the remainder receiving other agents. High degree of SOM was present in $41.9 \%$ by PHQ- 15 threshold; $34.1 \%$ had history of PSY. In Cox models that included age, sex, FGID dx, PSY, and SOM, both high degree of SOM (by PHQ-15: OR 2.9, 95\%CI 1.4-6.3; by medical history: OR 2.5, 95\%CI 1.3-4.8; $\mathrm{p}<0.01$ for each) and male sex (OR 2.8, 95\% CI 1.1-6.8; $\mathrm{p}=0.02$ ) predicted early discontinuation. The logistic regression models showed that only high degree of SOM predicted poor GTR (OR $3.0,95 \% \mathrm{CI} 1.3-6.9 ; \mathrm{p}=0.005)$, whereas both high degree of SOM and male sex predicted high side effect severity (OR $3.4,95 \%$ CI 1.4-8.7; and OR 2.7.95\% CI 1.03-7.3, respectively; $\mathrm{p}<0.05$ for each). Conclusions: A high degree of SOM, manifested either by recent symptoms or a medical hx of functional disorders, is a strong predictor of poor treatment outcome, side effect severity, and premature Rx discontinuation when AD are used for FGIDs in clinical practice. Male subjects without high degrees of SOM also tolerate AD poorly. Designs for clinical trials and treatment algorithms should consider these observations to optimize adherence to $\mathrm{AD}$ maintenance regimens.

\section{4}

A 10-Day Course of Rifaximin, a Non-Absorbable Antibiotic, Produces a Durable Improvement in All Symptoms of Irritable Bowel Syndrome: A Double-Blind Randomized Controlled Study Mark Pimentel, Sandy Park, Yuthana Kong, Kimberly Low, Soumya Chatterjee, Hyo-Rang Lee

Recent data suggest a relationship between gut bacteria and IBS symptoms such that improvement in IBS is observed after antibiotic treatment. We have recently reported the beneficial effects of rifaximin, a non-absorbed antibiotic, on global improvement in IBS. In this subanalysis, the component symptoms of IBS and their response to rifaximin are determined. Methods: Rome I criteria positive IBS patients were enrolled in a double-blind randomized placebo-controlled study. After a l-week run in, subjects were asked to complete a symptom questionnaire with diarrhea, constipation, bloating and abdominal pain rated on a VAS score from $0-100 \mathrm{~mm}$ after which a lactulose breath test was conducted. Subjects were then randomized to rifaximin $400 \mathrm{mg}$ or placebo tid for 10-days. One week after completion of treatment, subjects repeated their questions. Thereafter, for an additional 9 weeks, subjects completed a weekly symptom questionnaire. The number of subjects with $>50 \%$ improvement in each of the symptom categories was determined over the 10-week follow-up time period. Two endpoints were examined. The first endpoint was one week after completing treatment. The second was the overall 10 -week period. Results: 87 subjects were randomized in the study ( 44 placebo, 43 rifaximin). One week after rifaximin $48.6 \%$ of subjects demonstrated a clinical response with diarrhea ( $>50 \%$ improved) compared to $23.5 \%$ for placebo $(\mathrm{p}<0.05)$. For bloating, $34.9 \%$ of subjects demonstrated relief compared to $18.6 \%$ for placebo $(\mathrm{p}=0.07)$. At this first endpoint, constipation and abdominal pain were not improved. When the proportion of patients with clinical improvement ( $>50 \%$ improvement) was determined for the entire 10-week follow-up period for each symptom, all four symptoms were statistically improved including bloating $(49.2 \pm 6.8$ vs. $22.6 \pm 3.5 \%$, p $<0.0000001)$, diarrhea $(50.6 \pm 5.7$ vs $35.3 \pm 6.3 \%, \mathrm{p}<0.00001)$, abdominal pain $(39.7 \pm 7.4$ vs $28.9 \pm 7.2 \%, \mathrm{p}<0.01)$ and constipation $(35.1 \pm 7.0$ vs $28.1 \pm 5.0 \%, p<0.05)$. Conclusions: Rifaximin treatment results in a significant improvement in the four major symptoms of IBS for 10 weeks after completion of treatment.

135

Tegaserod Improves Multiple Symptoms in Women with Mixed/Alternating Bowel Habits As Well As Those with IBS-C

William D. Chey, Pierre Pare, Andrea Viegas, Gregory Ligozio, Michael A. Shetzline

BACKGROUND: Tegaserod, a promotility agent, is safe and effective in the treatment of irritable bowel syndrome with constipation (IBS-C) and chronic idiopathic constipation. IBS patients with a mixed or alternating bowel pattern (IBS-M), although clinically distinct, share clinical characteristics with the IBS-C group. At present, no medical therapy has been demonstrated to be effective in IBS-M patients. METHODS: A randomized, double-blind, placebo-controlled, multicenter study was performed in women with IBS-C (Rome II criteria) and IBS-M (not IBS-C or IBS-D by Rome II). The primary efficacy variable was patients' assessment of satisfactory relief over 4 weeks' treatment with tegaserod (T) $6 \mathrm{mg}$ b.i.d. or placebo (P). The proportion of patients reporting satisfactory relief for $\geq 3$ out of 4 treatment weeks ( $75 \%$ rule) and improvement during each of the 4 weeks in individual IBS symptoms were also assessed. Treatments were compared using a generalized linear model with logit link. RESULTS: 661 women were randomized (IBS-C 337; IBS-M 324). Baseline symptom assessment clearly distinguished between the IBS-C and the IBS-M cohorts. Statistically significant differences were found between the two cohorts in bowel movement (BM) frequency, stool consistency and straining. Overall, T provided statistically significant improvement in satisfactory relief of IBS symptoms over a 4-week treatment $(\mathrm{OR}=1.75 ; 95 \% \mathrm{CI}$ : $1.35-2.25 ; \mathrm{p}<0.001)$ for the IBS-C and IBS-M cohorts. In the two cohorts, the percentage of patients experiencing satisfactory relief of IBS symptoms ( $75 \%$ rule) was significantly higher for T when compared to P (IBS-C: $43.3 \%$ vs $28.9 \%, p=0.008$ and IBS-M. $52.3 \%$ vs $36.3 \%, p=0.010)$. T was significantly superior $(\mathrm{p}<0.05)$ to $\mathrm{P}$ at improving weekly $\mathrm{BM}$ frequency (IBS-C: T 6.88, P 6.06 and IBS-M: T 9.78, P 8.34), stool consistency score using a 7-point scale (IBS-C: T 3.53, P 2.89 and IBS-M: T 3.95, P 3.49) and days/week with straining (IBS-C: T 3.08, P 3.55 and IBS-M: T 2.42, P 2.87). Adverse event discontinuations were low (IBS-C: T $1.2 \%$ vs P $2.4 \%$ and IBS-M: T $2.5 \%$ vs P $3.6 \%$ ). The most frequent adverse events with discontinuation of study medication were diarrhea (1.1\%) and headache $(0.6 \%)$. No cases of ischemic colitis were reported. CONCLUSION: This study demonstrates that $\mathrm{T}$ is effective and safe in treating the overall symptoms of IBS in patients with a mixed/ alternating bowel habit. Efficacy in this subgroup was similar to that demonstrated in IBS$\mathrm{C}$ patients. This is the first study to identify an efficacious medical therapy for patients with IBS and a mixed bowel pattern.

136

Human Visceral Pain Hypersensitivity Is Not Attenuated By a Selective Oral Neurokinin-1 (NK-1) Receptor Antagonist. A Randomized Double Blind Placebo Controlled Pharmacodynamic Study in Healthy Volunteers Robert P. Willert, Claire Delaney, Anthony R. Hobson, Kirsty J. Hicks, Odile E. Dewit, Qasim Aziz

Introduction: In animal models, use of NK-l receptor antagonists (NKIRA) and of NK-1 knock-out mice have demonstrated a role of Substance-P (SP) in the development of visceral hypersensitivity. Aims: To determine whether SP is involved in the development of human visceral pain hypersensitivity, using a selective NK1RA, in a validated model of acid induced esophageal hypersensitivity [Willert et al, 2004]. Methods: In 13 healthy subjects (6 male; mean age 26 years), sensory and pain thresholds to electrical stimulation $(\mathrm{mA})$ were measured in the proximal esophagus (secondary allodynia) and the foot (somatic control) in a doubleblind randomized 2-period crossover study, pre- and for 4 hours post distal-esophageal acid infusion. These measurements were taken on the 3rd day of dosing with either an oral NKIRA or matching placebo, with 2 weeks wash-out between periods. Plasma drug concentrations were measured concomitantly to the threshold measurements. The study was powered to detect an $18 \mathrm{~mA}$ between treatment difference in post-acid infusion area under the curve compared to baseline (AUCA $[\mathrm{PT}] \mathrm{PO}$ ) in the proximal esophageal pain threshold, as observed in a previous study of an EP-1 antagonist [Sarkar et al,2003]. Results: Pre-acid infusion sensory and pain thresholds did not differ between treatments: mean (SD) for the NKIRA and placebo treatment groups respectively: sensory thresholds: proximal esophagus 7.1 (2.9) and 6.0 (2.3); foot $1.6(0.6)$ and $1.7(0.7)$; pain thresholds: proximal esophagus 37.2 (12.3) and 37.9 (16.7); foot 39.5 (25.3) and 37.8 (23.7). The reduction in pain threshold in the the proximal esophagus post acid infusion was similar between treatments (mean (SD) change from pre- to 4 hours post-acid infusion: -6.1 (6.2) and -5.9 (8.5), NKIRA and placebo respectively); and for AUCD[PT]PO: $95 \%$ confidence interval $[-18,15]$. Plasma concentrations of the NKIRA overlapped the target exposure range. No serious adverse events occurred. Conclusions: The results in this study are in keeping with data in human somatic pain where NK1RA's did not attenuate hyperalgesia, despite convincing data in animal somatic and visceral models. The lack of effect of this NKIRA on baseline sensory 
or pain thresholds in the esophagus or foot is consistent with published data, where NK1 receptor antagonists were anti-hyperalgesic but not analgesic, in non-inflamed states. References: Willert et al Gastroenterology 2004;126(3):683-692 Sarkar et al Gastroenterology 2003;124(1):18-25

137

Cost of Illness of Gastroesophageal Reflux Disease (GERD): Comparison of Medical, Pharmacy, Sick Leave, short- and Long-Term Disability, and Workers' Compensation Claims and Medical Claims By Place of Service Between Employees with and Without GERD

Richard A. Brook, Sara Campbell, Peter Wahlqvist, Mari-Ann Wallander, Nathan L. Kleinman, Ingela Wiklund, James E. Smeeding

OBJECTIVE: To assess the Annual Cost of Illness (CoI) of GERD in an employed population. METHODS: A retrospective analysis was conducted using the Human Capital Management Services Research Reference database of multiple large geographically diverse US based employees with data from 2001 through 2004. Data included medical, pharmacy, payroll and demographic data. The analysis compared the direct medical costs by Place of Service (POS) for a subset of employees where this data was available. ICD-9 Codes were used to distinguish employees with GERD from the non-GERD cohort. The index date in the GERD cohort was defined as the date of first diagnosis during 2001 or later, while the average GERD index date was used in the non-GERD cohort. Regression modeling was used to measure the cost differences between employees with GERD and employees without GERD while controlling for age, job tenure, gender, salary, region, and comorbidities. RESULTS: Data were available for 267,269 employees (4.3\% with GERD). The mean age of employees with GERD was 43 years, and 94\% were full time employees. All of the annual CoI and PoS comparisons (Tables 1 and 2) were statistically different (all $P<0.001$ ). GERD was associated with an annual mean incremental cost of $\$ 3,355$, of which direct medical costs accounted for $65 \%$, prescription drug costs for $17 \%$, and indirect costs in terms of sick leave, short- and long-term disability, and workers' compensation for 19\%. The largest PoS component $(47 \%)$ was in the category "Outpatient Hospital or Clinic". CONCLUSIONS: GERD is associated with substantial direct and indirect costs, of which direct medical costs excluding prescription medications contributed to $65 \%$ of total incremental costs in this study. Table 1. Cost of Illness

\begin{tabular}{|c|c|c|c|c|c|}
\hline & \multicolumn{2}{|c|}{ Employees with GERD } & \multicolumn{2}{|c|}{ Employees without GERD } & \\
\hline Cost Category & $\mathrm{N}$ & Adjusted Mean Cost & $\mathrm{N}$ & Adjusted Mean Cost & $\Delta$ \\
\hline Direct Medical & \multirow{2}{*}{11,653} & $\$ 3,794$ & \multirow{2}{*}{255,616} & $\$ 1,627$ & $\$ 2,167$ \\
\cline { 3 - 6 } Prescription Drug & & $\$ 922$ & & $\$ 364$ & $\$ 558$ \\
\hline Sick Leave & 6,172 & $\$ 498$ & 133,466 & $\$ 353$ & $\$ 145$ \\
\hline Short-term Disability & 5,573 & $\$ 417$ & 118,494 & $\$ 246$ & $\$ 170$ \\
\hline Long-term Disability & 9,611 & $\$ 53$ & 187,191 & $\$ 26$ & $\$ 26$ \\
\hline Workers' Compensation & 10,790 & $\$ 1,194$ & 232,281 & $\$ 906$ & $\$ 288$ \\
\hline & & $\$ 6,878$ & & $\$ 3,522$ & $\$ 3,355$ \\
\hline
\end{tabular}

Table 2. Mean Direct Medical Costs by Place of Service

\begin{tabular}{|c|c|c|c|}
\hline Place of Service & $\begin{array}{c}\text { Employees with GERD } \\
(\mathrm{N}=5,878)\end{array}$ & $\begin{array}{c}\text { Employees without GERD } \\
(\mathrm{N}=80,422)\end{array}$ & $\Delta$ \\
\hline Doctor's Office & $\$ 1,081$ & $\$ 581$ & $\$ 500$ \\
\hline Inpatient Hospital & $\$ 1,222$ & $\$ 562$ & $\$ 660$ \\
\hline $\begin{array}{c}\text { Outpatient Hospital or } \\
\text { Clinic }\end{array}$ & $\$ 1,821$ & $\$ 737$ & $\$ 1,084$ \\
\hline Emergancy Department & $\$ 37$ & $\$ 18$ & $\$ 18$ \\
\hline Laboratory & $\$ 21$ & $\$ 12$ & $\$ 9$ \\
\hline Other & $\$ 85$ & $\$ 38$ & $\$ 47$ \\
\hline & $\$ 4,267$ & $\$ 1,949$ & $\$ 2,318$ \\
\hline
\end{tabular}

138

Nighttime GERD and More Severe GERD Symptomatology Are Associated with Greater Work Productivity Loss

Adam B. Elfant, Stephen M. Lange, Quan V. Doan, Lynda S. Welage, Stephen Brunton, Richard B. Lynn, Robert W. Dubois

OBJECTIVE: To assess the relationship between work productivity loss and GERD-related severity among daytime (DG) and nighttime GERD (NG) cases. METHODS: A survey was conducted among adults. A screening questionnaire assessed frequency and severity of GERD symptoms during the past 12 months and past 7 days and identified GERD cases and controls. GERD was assessed using the validated GERD Symptom $\&$ Medication Questionnaire (GERDSMQ). Symptomatic GERD was defined as GERD-SMQ score $>9$ and $=>1$ episode of heartburn or acid reflux during the past 7 days. Symptomatic NG was defined as individuals with GERD reporting $=>2$ nights with symptoms (whether or not daytime symptoms are present) or 1 night with symptoms (in absence of daytime symptoms). A maximum value from 4 severity ratings of heartburn and acid regurgitation (each based on a 10-point Likert scale) was used as the severity score to stratify patients into the following severity groups: mild (1-4), moderate (5-7) and severe (8-10). Among the DG and NG cases, the severity score was based solely on the 2 nighttime severity ratings and solely on the 2 daytime severity ratings, respectively. GERD-specific productivity losses were assessed using the validated Worker Productivity \& Activity Impairment Questionnaire (WPAI), which measures work time absenteeism and impairment while working. Mean percent reductions in Work Produc tivity Scores (WPS) were calculated for all GERD, NG and DG cases and differences wer assessed using statistical test. RESULTS: 13,231 (21.4\%) of 65,001 invited to participate responded; 1.515 satisfied the study criteria (mean age 44.3 years; $57 \%$ women). Of these, 1002 were symptomatic GERD cases (475 NG vs 469 DG) and 513 were non-GERD controls. More severe symptoms were associated with statistically greater work productivity loss among GERD, DG, and NG cases. Overall, patients with NG suffered significantly greater work productivity loss compared with DG cases $(\mathrm{P}<0.05)$. Patients with NG in moderate or severe strata had statistically greater work impairment than DG cases. CONCLUSIONS: GERDrelated symptom severity is strongly associated with work impairment. Patients with NG reported substantially greater work impairment in every severity stratum compared with DG cases.

GERD-specific Work Productivity Loss

\begin{tabular}{|c|c|c|c|c|}
\hline \multirow{2}{*}{ Severity } & \multicolumn{3}{|c|}{ Mean \% Reduction } & \multirow{2}{*}{ DG vs NG Difference } \\
\cline { 2 - 5 } & GERD* $(\mathrm{n}=1002)$ & $\mathrm{DG}^{*}(\mathrm{n}=469 \Phi)$ & $\mathrm{NG}^{*}(\mathrm{n}=475 \Phi)$ & \\
\hline Mild & 4.6 & 3.3 & 6.6 & 3.3 \\
\hline Moderate & 11.4 & 7.7 & 14.5 & $6.9 \psi$ \\
\hline Severe & 15.3 & 11.5 & 16.4 & $4.9 \psi$ \\
\hline Overall & 9.0 & 5.6 & 12.2 & $6.8 \psi$ \\
\hline
\end{tabular}

* $\mathrm{P}<0.05 \Phi 58$ cases $w /$ missing data excluded $\psi \mathrm{P}<0.05$, comparison of $\mathrm{DG}$ and NG

\section{9}

Gastroesophageal Reflux Disease As a Cause of Death Is Increasing: A Population Based Analysis of Fatal Cases After Conservative and Operative Treatment

Jarmo A. Salo, Tuomo K. Rantanen, Jari V. Rasanen, Eero I. Sihvo

Background: Only few population based analyses of fatal outcome of gastroesophageal reflux disease (GERD) exist. Therefore, we evaluated, using unselected population based data, the mortality of GERD and compared this to the use of health resources. Methods: All death certificates in which esophagitis, esophageal ulcer, stricture, or hiatus hernia were mentioned between January 1st 1987 and December 31st 2000 in Finland were obtained. The medical records of these 306 patients were analyzed. Objective findings and symptoms were recorded to ascertain the diagnosis of GERD and 93 patients had to be excluded due to uncertain diagnosis of GERD. National use of antireflux medication and rate of antireflux surgery (obligatory annual report given by all Finnish hospitals to the Central Registry of Nationa Research and Development Center for Welfare and Health) were assessed by using Finland's administrative databases. Results: From 1987 to 2000 the annual mortality of GERD increased $(\mathrm{p}<0.001$ ) from $0.18 / 100000$ to $0.46 / 100000$. The increase was seen in patients under conservative treatment. Annual mortality of antireflux surgery stayed around 1.9/1000 operations. At the same time, use of $\mathrm{H} 2$-blockers, proton pump inhibitors, and annual rate of antireflux surgery increased significantly $(\mathrm{p}<0.001)$. Of 213 patients whose cause of death was GERD, 185 (87\%) had undergone conservative treatment, 24 (11\%) antireflux surgery, and $4(2 \%)$ diagnostic or therapeutic endoscopy. The most common cause of death in the conservative group was hemorrhagic esophagitis $(85 / 185,46 \%)$ followed by aspiration pneumonia $(46 / 185,25 \%)$, esophageal ulcer perforation $(25 / 185,14 \%)$, spontaneous esophageal rupture $(15 / 185,8 \%)$ and esophageal stricture $(14 / 185,8 \%)$, and at the interventional group gastric or esophageal perforation $(14 / 28,50 \%)$. Conclusions: Regardless of the increased usage of health resources the mortality of GERD, especially under conservative treatment, has increased. The mortality of antireflux surgery seems, however, to be 5 to 10 times greater than estimated mortality of symptomatic erosive esophagitis under conservative treatment.

140

Association Between Erosive Esophagitis and Visceral Fat Accumulation Quantified By Abdominal CT Scan: A Case Control Study Hang Lak Lee, Oh Young Lee, Yong Chul Jeon, Dong Soo Han, Byung Chul Yoon, Ho Soon Choi, Soon Young Song, Jin Bae Kim

Backgroud \& Aims: To date, many studies have reported on the association between obesity and reflux esophagitis, although none of these were conducted systematically. There was no objective data between erosive esophagitis and abdominal fat mass. BMI and other classica anthropometric measure are imperfect estimates of adiposity, particularly in male, mainly because of greater muscle mass. Computed tomography quantifies subcutaneous and internal abdominal fat allowing measurement of visceral fat (VF) and determination of its relation with disease risk. Under these backgrounds, we conducted this study to examine the association of abdominal obesity and reflux esophagitis. Methods: Between May 2004 and October 2005, a total of 100 erosive esophagitis diagnosed by upper endoscopy were included in a prospective manner. Control subjects, age and sex matched, without erosive esophagitis and any reflux symptoms, were included. All of patients and control groups conducted abdominal CT scan. Body fat distribution was assessed by CT with 10 -mm-thick slice at the level of the fourth lumbar vertebra. VF area was defined all of the pixels with attenuation coefficients of adipose tissue. Subcutaneous fat (SF) area was defined as the adipose area between the two defined contours and total fat (TF) area was defined as the sum of VF and SF. Results: Erosive esophagitis patients presented with a significantly higher mean visceral fat area (104.68 39.47 vs. $75.90 \pm 49.10 \mathrm{~cm} 2, p=0.014$ ) than control group. However, there was no association between erosive esophagitis and subcutaneous fat areas ( $109.72 \pm 49.09$ vs. $98.66 \pm 52.43$ $\mathrm{cm} 2, \mathrm{p}=0.379)$ and total fat areas $(214.41 \pm 78.78$ vs. $172.59 \pm 90.49 \mathrm{~cm} 2, \mathrm{p}=0.054)$. Multivariate logistic regression analysis demonstrated that hiatal hernia $(\mathrm{p}=0.0001)$, high body mass index $(\mathrm{p}=0.047)$, high visceral fat areas $(\mathrm{p}=0.0035)$, and smoking $(\mathrm{p}=0.005)$ are independent factors associated with erosive esophagitis. Conclusions: These results suggest a role for visceral obesity, quantified by $\mathrm{CT}$, as a risk factor for erosive esophagitis. 
Characteristics of Patients with Gastroesophageal Reflux Disease (GERD) Who Respond Incompletely to Therapy with Proton Pump Inhibitors Roger Jones, Harley Liker, Phillippe Ducrotte

Objective: Proton pump inhibitors (PPIs) are useful in the treatment of GERD, although a number of patients receiving PPI treatment still experience symptoms. In addition to assessing the frequency and severity of GERD symptoms in the general population, one objective of this large survey was to characterize patients who have incomplete responses to PPI therapy. Methods: People suffering from symptoms of GERD were identified by random telephone screening of $>200,000$ households in the USA, UK, Germany and France. Those who agreed to participate in the survey had face-to-face interviews. Patients diagnosed with GERD who were receiving PPIs were defined as complete responders (no symptoms), well controlled (used a PPI compliantly, experienced an improvement in or decrease in frequency of symptoms, and suffered key GERD symptoms on $\leq 1$ of the past 7 days), incomplete responders (the same definition as well controlled but with symptoms on $\geq 2$ of the past 7 days), or non responders (no improvement or symptoms worsened). Results: Of the 537 PPI users, $23 \%$ were complete responders, $12 \%$ well controlled, $46 \%$ incomplete responders, $9 \%$ non responders, and $10 \%$ other (e.g. responded but were non-compliant). Incomplete responders and non responders had suffered GERD symptoms for significantly more days of the past week (table). Incomplete responders were significantly more likely than more responsive patients to have concomitant anxiety (25\%), migraine (24\%), irritable bowel syndrome (22\%), or chest pain (13\%). They had been receiving their current PPI for significantly longer than well controlled patients ( 2.4 vs 1.6 years). Incomplete responders were more likely than complete responders or well controlled patients to experience sleep disturbed by GERD symptoms (49\% vs $0 \%$ and 30\%). Their symptoms were more likely to impact quality-of-life (QOL) measures (tired or worn out, generally unwell, having worries or fears, difficulty socializing, eating less than usual), they thought about their condition significantly more often, and their perceived seriousness of the condition was significantly higher. Conclusions: Despite good compliance, a large proportion of patients receiving PPIs have incompletely controlled GERD. Interestingly, these incomplete responders are more likely to experience concomitant conditions and to have impaired QOL than responsive patients. Frequency of GERD symptoms (days) in the past week in patients receiving PPIs

\begin{tabular}{|c|c|c|c|c|}
\hline & Complete responders & Well controlled & Incomplete responders & Non responders \\
\hline Heartburn & 0 & 0.7 & 3.9 & 4.7 \\
\hline Acid reflux & 0 & 0.7 & 3.9 & 4.7 \\
\hline Epigastric pain & 0 & 0.7 & 4.7 & 4.9 \\
\hline
\end{tabular}

148

The Extracellular Matrix Component Hyaluronan (HA) Is Increased Over Time in the DSS Model of Colitis, and Precedes Leukocyte Influx. Monocyte Interaction with HA Affects Migration and Gene Expression

Carol A. de la Motte, Sudip K. Bandyopadhyay, Sean Kessler, Scott A. Strong

Background: Hyaluronan (HA) levels are increased in human colonic mucosa involved in active inflammatory bowel disease (IBD), as compared to non-inflamed tissues. HA appears to emanate largely from the muscularis mucosae. Additionally, we have shown the generation of leukocyte adhesive hyaluronan structures in vitro from colonic mucosal smooth muscle cells (M-SMC) treated with virus or stimulated with a viral mimetic, poly I:C (synthetic double stranded RNA), among other cell stressors, but not cytokines. The question of whether hyaluronan accumulation is promoting leukocyte infiltration or is a result of the inflammation has not previously been determined, and a mouse model of colitis was chosen to follow the course of hyaluronan deposition and inflammation in vivo. The impact of monocyte binding to $\mathrm{HA}$ in vitro was also assessed. Methods: 8 wk old C57Bl/6 mice were treated with or without $2.5 \%$ dextran sulfate sodium (DSS) via their drinking water. Individuals were sacrificed periodically over the two-week duration of treatment and the colon tissue fixed, sectioned and stained for histologic analysis and HA detection. HA leukocyte binding structures were generated in M-SMC cultures from surgically resected human colon by treating with or without poly I:C for 18 hours. Isolated peripheral blood monocytes were used to assess the effect of HA on RANTES-driven migration across a M-SMC monolayer. Additionally, gene expression was compared between HA-bound and non-bound monocytes in HA producing M-SMC cultures by Affymetrix gene array. Results and Discussion: DSS-induced colitis mice showed a time dependent change in HA deposition, especially pronounced in the distal colon, which preceded the mucosal influx of leukocytes. In vitro, smooth muscle cultures producing leukocyte adhesive HA impeded monocyte transmigration across M-SMC monolayers in response to RANTES compared to non-producing cultures, suggesting that HA contributes to leukocyte retention in tissue. Additionally, monocyte binding to $\mathrm{HA}$ induced the expression of greater than 8 fold increases in the expression of over 100 genes, many of which are growth factor related and could contribute to the smooth muscle cell hyperplasia typical of IBD pathology. Significance: We have previously reported HA accumulation in the mucosa of inflamed IBD colon, and demonstrated that M-SMC generate HA-based leukocyte adhesive structures in response to viral, as well as endoplasmic reticulum stress inducing agents. The findings that HA deposition precedes inflammation, has an impact on monocyte migration and triggers gene expression supports the idea that HA is an active participant in the pathological process.
The Role of the Pro-Inflammatory Mediator S100a12 in IBD D.R. Foell, Helmut Wittkowski, Dorothee Viemann, Jan Heidemann, Pia Lebiez, Torsten Kucharzik, Johannes Roth

Objectives: S100A12 is a pro-inflammatory protein expressed and secreted by neutrophils S100A12 lacks a leader sequence necessary for trafficking via the endoplasmatic reticulum or the Golgi complex. Binding of released S100A12 to the receptor for advanced glycosylation end products (RAGE) activates endothelium and leukocytes. Blocking S100Al2 activity revealed promising therapeutic effects in various murine models of colitis. In previous studies we found that IBD patients in remission who have residual inflammation not leading to clinically apparent signs of disease activity nevertheless had elevated S100A12 serum levels. Methods: We investigated mechanisms involved in S100al2 secretion. S100A12-induced effects on human endothelial cells (HMECs and HUVECs) and human intestinal microvascular endothelial cells (HIMECs) were analyzed. Signaling pathways leading to pro-inflammatory activation of target cells have been investigated. Sensitivity of S100A12 serum measurements for the detection of relapsing disease activity was analyzed by ELISA serum samples from IBD patients who were followed up prospectively. Results: S100A12 secretion follows an alternative pathway bypassing the classical Golgi-route. Secretion depends upon intact microtubules and protein kinase C (PKC). Erk1/Erk2 and Syk signaling are involved. Stimulation of HMECs and HIMECs with S100A12 increased surface expression of VCAM-1 and ICAM1 , decreased endothelial resistance and promoted transendothelial migration of phagocytes. After S100A12-stimulation, pro-inflammatory genes were upregulated in endothelial cells. S100A12 serum levels were significantly elevated in patients with inactive IBD prior relapses compared to patients in stable remission. Conclusions: Released S100A12 is a mediator which activates immune cells critical to pathogenesis of colitis. Activation of intestinal endothelium by S100A12 leads to enhanced extravasation of neutrophils, thus promoting infiltration of intestinal tissue. The pro-inflammatory properties of S100A12 make this neutrophilic protein an attractive target for anti-inflammatory therapies. S100A12 may provide an ecellent serum marker for the early detection of relapsing colitis.

150

Enhanced Platelet Adhesion Induces Angiogenesis in the IBD Microcirculation Silvio Danese, Franco Scaldaferri, Cristina Graziani, Carol de la Motte, Alessandro Sgambato, Alfredo Papa, Achille Cittadini, Antonio Gasbarrini, Claudio Fiocchi

Platelet (PLT) dysfunction is a prominent feature of inflammatory bowel disease (IBD) and PLTs actively contribute to gut inflammation. We recently reported that angiogenesis is a novel component of IBD pathogenesis, but the cell type(s) promoting angiogenesis in the inflamed mucosa are still undefined. We investigated whether PLTs contribute to induction of angiogenesis in IBD. Unstimulated or thrombin-activated human PLT were overlaid on resting or TNF- $\alpha$-treated human intestinal microvascular endothelial cells (HIMEC), in the presence and absence of VCAM-1, -ICAM-1 or -integrin $\alpha \mathrm{v} \beta 3$ blocking antibodies. Expression of VCAM-1, ICAM-1 and $\alpha v \beta 3$ was analyzed by flow cytometry, adhesion of PLTs to endothelium was measured by fluorescence microscopy, and presence of angiogenic factors (VEGF, bFGF and sCD40L) in culture supernatants was checked by ELISA. A matrigel tubule formation assay was used to evaluate the capacity of PLTs to induce HIMEC angiogenesis. TNF- $\alpha$ strongly up-regulated VCAM- 1 , ICAM- 1 and $\alpha v \beta 3$ expression on HIMEC (all $\mathrm{p}<0.01)$. Unstimulated HIMEC bound few PLT, but adhesion increased significantly $(\mathrm{p}<0.05)$ in TNF- $\alpha$ pre-treated HIMEC, reflecting the greater capacity of inflamed endothelium to recruit cells. When activated PLT were co-cultured with unstimulated HIMEC, a significant $(\mathrm{p}<0.01)$ increase in PLT adhesion was observed, and adhesion further increased when HIMEC and PLTs were concomitantly activated. Adhesion was ICAM-1- and integrin $\alpha v \beta 3$ (both at $\mathrm{p}<0.05$ ), but not VCAM-1, -dependent. VEGF, bFGF and SCD40L were not detected in HIMEC cultures either before or after TNF- $\alpha$ stimulation. In contrast, co-cultures of resting HIMEC with resting PLT contained small amounts of PLT-derived VEGF and SCD40L, which significantly $(p<0.05)$ increased when resting or activated PLT were placed on TNF$\alpha$-pre-treated HIMEC. bFGF was not detected in any of the co-cultures. Finally, activated PLT significantly induced HIMEC tubule formation. PLT adhesion is maximal with TNF$\alpha$-pre-treated HIMEC, a process mediated by ICAM- 1 and $\alpha v \beta 3$, and accompanied by the release of PLT-derived VEGF and $\mathrm{SCD} 40 \mathrm{~L}$. This indicates that, in inflammatory conditions like IBD, the mucosal microcirculation displays an enhanced capacity to recruit PLT which, upon contact, actively release angiogenic factors able to promote angiogenesis in the inflamed mucosal microvasculature.

151

PD-L1/PD-1-Mediated Interaction Between Normal and Ulcerative ColitisDerived Colonic Myofibroblasts and T Cells

Irina V. Pinchuk, Jamal I. Saada, Ellen J. Beswick, Julia Brenmoehl, Giovanni Suarez, Patrick A. Adegboyega, Randy C. Mifflin, Victor E. Reyes, Gerhard Rogler, Don W. Powell

BACKGROUND: Current theories regarding the pathogenesis of ulcerative colitis (UC) suggest that UC represents a disruption of tolerance to intestinal microflora, leading to dysregulation of mucosal CD4+T cell responses and chronic inflammation. Recent evidence indicates that regulation of this response involves, at least in part, $\mathrm{PD}-1 / \mathrm{PD}-\mathrm{Ll}$ negative co-stimulator interactions between T cells and antigen presenting cells (APC). Moreover, strong upregulation of these molecules has been observed in the inflamed colon of with inflammatory bowel disease patients. However, the nature and spectrum of PD-Ll-expressing APCs in normal and inflamed colon are poorly characterized. We recently reported that normal human colonic myofibroblasts (CMFs), which are located in the lamina propria just beneath the epithelial layer, are novel non professional APCs that constitutively express negative (PD-Ll and PD-L2) and positive (ICOSL and B7-H3) costimulators and can suppress proliferation of CD3-activated CD4+T cells. We thus hypothesize that PD-Ll+ CMFs are novel, local negative regulator of $\mathrm{T}$ cells in the colon and that abnormalities in CMF-mediated immune inhibitory signals may contribute to UC pathogenesis. METHODS: We have analyzed expression of PD-Ll by using FACS analysis of acutely isolated and cultured CMFs and 
evaluated PD-Ll involvement in CMF mediated suppression of CD3-activated CD4+ T cells using lymphoproliferation assays and IL-2 ELISA. A combination of real time RT-PCR, Western Blot and FACS analysis was used to compare PD-Ll expression by CMFs from UC patients versus the control group. RESULTS: We demonstrated that 15\% of freshly isolated lamina propria mononuclear cells from normal mucosa constitutively express PD-L1. CMFs represented $\sim 80 \%$ of this PD-Ll-expressing population. In vitro functional analyses demonstrated that CMFs suppress proliferation of CD3-activated CD45RA+CD4+T cells via cellcontact-dependent mechanisms. Blockade of PD-L1 on CMFs enhanced T cell proliferation and IL-2 production. When compared to normal colon, a significant increase in PD-L1, but not PD-L2, was observed on CMFs isolated from UC patients. CONCLUSIONS: These results are consistent with the hypothesis that CMFs expression of negative co-stimulators may favor their suppressive function on the response of activated CD4+ T cells in the colonic mucosa. Our data also indicate that PD-Ll contributes to the CMF-mediated suppressive capacity and suggest that abnormalities in expression of this molecule on CMFs may play a role in UC associated immunopathogenesis. Supported by NIDDK, GRIP and the Gulf Coast DDC, James W McLaughlin Endowment.

\section{2}

Enteric Glia (EGC) Are Activated By Bacterial Products Via Nod and Nuclear Factor-Kappab (Nf-кB) Signaling

Eike Hollenbach, Manfred Neumann, Michael Vieth, Bruce Vallance, Peter Malfertheiner Anne Ruhl

We have previously shown that EGC may participate in intestinal immune responses via phagocytosis, upregulation of MHC II and ICAM-1 in response to proinflammatory signals, MHC-II restricted and antigen-specific stimulation of syngeneic T-cells, and production of cytokines. Here, we have investigated if EGC can be activated by bacterial products like peptidoglycans (PGN), employing an $H$. pylori based model of intracellular translocation of PGN. Sixty min post infection (p.i.) of EGC with a wild type (wt) H. pylori strain possessing a type IV secretion system for PGN translocation, immunoblot analysis revealed a strong

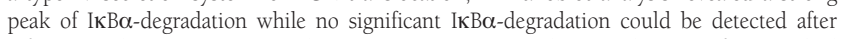
infection with a mutant strain without secretion system. Nuclear translocation of p65 peaked with expected delay at 60-90 min p.i. only in EGC previously infected with wt $H$. pylori. These findings were confirmed by EMSA revealing active p65/p50 and p65/c-Rel heterodimeric NF$\kappa \mathrm{B}$ complexes in EGC infected with wt $H$. pylori only. To further analyse the signaling pathways induced by immune-activation of EGC, we studied upregulation of NOD, MAP kinases p38 and ERK in EGC upon PGN challenge. Because Rip-like interacting caspaselike apoptosis-regulatory protein kinase (RICK) is a critical downstream kinase of NOD and as such key component of a pathway leading to NF- $\mathrm{KB}$ activation, we assessed RICK activation after intracellular PGN translocation by in vitro kinase assays. We found that RICK activity is dramatically upregulated after delivery of PGNs into EGC peaking at $60 \mathrm{~min}$ p.i. While the mutant $H$. pylori strain did not activate RICK, p38 and ERK were activated by both $H$. pylori strains with a maximum at 30 min p.i. Of note, only ERK but not $\mathrm{p} 38$ activation was completely independent of intracellular PGN delivery. Finally, we validated these data in salmonella typhimurium infected mice. Immunohistochemical analysis of murine ceca revealed enhanced nuclear translocation of the transcriptionally active phosphorylated p65 in EGC 24hrs p.i., while NF- $\mathrm{\kappa B}$ activation in macrophages peaked at $48 \mathrm{hrs}$ p.i. Overall, bacterial products induce strong and early responses in EGC with activation of the RICK/ NF- $\mathrm{KB}$ signaling cascade as well as the MAP-kinase pathway. These data implicate EGC as a supplementary immunoregulatory cell-type in the intestine which supports the innate immune system. Supported by Broad Medical Research Program IBD-0105R.

\section{3}

Mesenteric Adipose Tissue Is An Important Source of C-Reactive Protein in Crohn's Disease

Florent Gonzalez, Christel Rousseaux, Laurent Dubuquoy, Xavier Thuru, Cecilia Decourcelle, Luc Gambiez, Alain Saudemont, Christel Neut, Luc Penicaud, Jean-Frederic Colombel, Pierre Desreumaux

Background: C-reactive protein (CRP) is an important biomarker in Crohn's disease. Its production is believed to mainly occur in the liver by the hepatocytes as part of the acute phase response upon stimulation by inflammatory cytokines originating at the site of inflammation. Mesenteric adipose tissue (MAT) hypertrophy is a characteristic feature of $\mathrm{CD}$. There is accumulating evidence that adipocytes from the mesentery may participate in the immune response in $\mathrm{CD}$. We hypothesize that adipocytes from MAT constitute an important extra-hepatic source of CRP in CD. Aim: A)to study in vitro expression and regulation of CRP in adipocytes; B)to quantify the expression of CRP in the MAT and the intestine of patients with $C D$ and ulcerative colitis (UC) and of controls. Methods: A) Primary cultures of human adipocytes, murine 3T3-Ll preadipocytes and mature adipocytes, and HepG2 human hepatocytes were used. CRP mRNA levels were quantified in resting cells and after activation by inflammatory cytokines and bacterial components. B) Ex vivo, CRP expression was quantified in paired biopsy specimens of MAT, subcutaneous adipose tissue, healthy and inflamed ileal and colonic tissues of 29 CD patients, 17 UC patients and 13 controls. CRP was assessed by real-time polymerase chain reaction and immunochemistry. Results: A) CRP mRNA levels were similar in resting mature adipocytes and hepatocytes. Adipocyte differentiation as estimated by morphological features and expression of adipocyte fatty acid binding protein (aP2) was associated with a $21 \pm 2.5$ fold increase of CRP mRNA levels. CRP mRNA expression increased $2290 \pm 350$ fold the resting cell level after stimulation with E. coli $(p<0.01), 50 \pm 5$ fold with LPS $(p<0.01)$ and $20 \pm 3$ fold with TNF $\alpha(p<0.01)$. B) Low and similar CRP mRNA levels were detected in the healthy and inflamed small bowel and colon of CD, UC and controls. CRP mRNA levels were $150 \pm 90$ times higher in MAT and $145 \pm 87$ times higher in the subcutaneous adipose tissues than in the intestine of UC and controls. Patients with $\mathrm{CD}$ were characterized by a dramatic over-expression of CRP mRNA levels in MAT, which were $80 \pm 40(\mathrm{p}<0.05)$ and $1450 \pm 750(\mathrm{p}<0.01)$ times higher compared to CRP levels detected in MAT of UC and controls respectively. No difference was observed between the subcutaneous tissues of $\mathrm{CD}, \mathrm{UC}$ and controls. These results were confirmed and extended using immunohistochemistry. Conclusion: CRP is expressed by adipocytes and is induced by specific stimuli such as E. coli, LPS and TNF $\alpha$. Healthy and inflamed intestine is a minor source of CRP. MAT in CD is characterized by an increased production of CRP which may participate to the elevated plasma levels.

\section{4}

Activation of the Cholecystokinin-1 Receptor Subtype Attenuates Development of Desensitization of Opioid Receptors in Morphologically-Identified Myenteric S-Type Neurons in Guinea-Pig Small Intestine

Yun Xia, Guo-Du Wang, Xiyu Wang, Sumei Liu, Na Gao, Gui-Jun Fie, Hong-Zhen Hu, Jackie D. Wood

Cholecystokinin (CCK) and opioid peptides are well recognized as important signaling molecules in enteric neural control of gastrointestinal motility and secretion. We used intracellular electrophysiological recording methods to investigate how the two neuropeptides interact in the small intestinal myenteric plexus. Bath application of the $\mu$-opioid recepto agonist [Tyr-D-Ala-Gly-MePhe-Gly-ol]enkphalin (DAMGO, 10-500 nM) evoked slowlyactivating hyperpolarization of the membrane potential ranging from 6 to $18 \mathrm{mV}$ in $22 \mathrm{o}$ 38 S-Type neurons in 31 whole-mount myenteric plexus preparations. The hyperpolarizing responses, evoked by DAMGO, were suppressed by the selective mu-opioid receptor antagonist cyprodime $(10 \mu \mathrm{M})$. Desensitization occurred in 16 of 22 S-Type neurons when 200nM DAMGO was applied 4 or 6 times in 10-min exposures within a 2-hour period. Application of the CCK-1 receptor agonist, CCK octapeptide sulphate (CCK-8, $10-500$ $\mathrm{nM}$ ), evoked slowly-activating depolarization of the membrane potential ranging from 8 to $25 \mathrm{mV}$ and augmented excitability reflected by elevated spike discharge in 26 of $32 \mathrm{~S}$-Type myenteric neurons. Pretreatment with the CCK-1 receptor antagonist proglumide $(5-10 \mu \mathrm{M})$ or lorglumide $(5-10 \mu \mathrm{M})$ or the CCK-2 antagonist YM022 (5-10 $\mu \mathrm{M})$ reversed the excitatory responses to CCK8 in 17 of 26 S-Type neurons. Pretreatment with DAMGO suppressed the depolarizing responses to CCK-8. CCK- $8(20 \mathrm{nM})$ acted in converse manner to attenuate the inhibitory action of DAMGO. Co-application of DAMGO and relatively high concentrations of CCK-8 (0.5-2 $\mu \mathrm{M})$ attenuated development of desensitization to DAMGO in 7 of 22 neurons This action of CCK- 8 was reversed by co-application of the CCK-1 receptor antagonists, proglumide or lorglumide, but not by CCK-2 receptor antagonist,YM022. Neuronal labeling confirmed that each S-Type neuron in this study expressed the characteristic morphology of uniaxonal Dogiel Type-I neurons. The results show co-expression of CCK and $\boldsymbol{u}$-opioid receptors by S-Type neurons in the myenteric plexus and suggest that stimulation of the CCK-1 receptor attenuates development of opioid tolerance in the neurons. Consequently, treatment with a CCK-1 receptor antagonist might facilitate desensitization of the neurons to endogenously-released opioid peptides and thereby enhance propulsive motility and secretion by suppressing some of the known inhibitory action of endogenous opioid peptides on enteric neuronal excitability. (Supported by NIH RO1 DK37238 and KO8 DK060468)

155

Endogenous Adenosine Acts At Al Or A3 Inhibitory Receptors to Modulate the Stereotype Neural-Motor Behavior of the Gut Triggered By Histamine Or Distension

Andrey Bozarov, Yu-Zhong Wang, Jun-Ge Yu, Helen J. Cooke, Fievos L. Christofi

Background: Endogenous adenosine acts at high affinity $\mathrm{Al}$ adenosine receptors (AlR) on enteric neurons to inhibit synaptic transmission and enteric reflexes. However, the function of low affinity A3R expressed in gut neurons remains unknown. Aim: The aim was to elucidate the role of A3R in gut neural circuits and reflexes. Methods: The histamine $\mathrm{H}_{2}$ receptor agonist dimaprit was used to elicit a stereotype cyclical increase in short circui current $\left(\mathrm{I}_{\mathrm{sc}}=\right.$ chloride secretion) in coordination with motility. $\mathrm{I}_{\mathrm{sc}}$ was recorded in guinea pig distal colon (in flux chambers under voltage clamp) simultaneously with muscle length in the circular orientation (by sonomicrometry with $1 \mathrm{~mm}$ piezoelectric crystals fixed on serosa $5 \mathrm{~mm}$ apart). Distension reflexes were evoked by serosal removal of $10 \mu \mathrm{l}$ of fluid for $10 \mathrm{sec}$ Results: Dimaprit $(10 \mu \mathrm{M})$ caused cyclical increases in $\mathrm{I}_{\mathrm{sc}}$ of $40 \pm 2.3 \mu \mathrm{A} / \mathrm{cm}^{2}$ at frequencie of $2.4 \pm 0.3$ cycles $/ 5 \mathrm{~min}$ coordinated with contractions measured as intercrystal distance (ICD) of $260 \pm 22.5 \mu \mathrm{m}(\mathrm{n}=6)$. AlR selective antagonists 8-cyclopentyltheophylline (CPT) or 1,3dipropyl-8-(2-amino-4-chlorophenyl)xanthine (PACPX) caused a concentration dependent augmentation of coordinated responses $(1 \mathrm{nM}-10 \mu \mathrm{M}, \mathrm{n}=3) ; 100 \mathrm{nM}$ antagonist caused maximum blockade of AlR and enhanced $\mathrm{I}_{\mathrm{sc}} 133 \%(\mathrm{p}=0.0001)$ and ICD 64\% $(\mathrm{p}=0.0006, \mathrm{n}=$ 6) with $\mathrm{ED}_{50}$ values of $30-50 \mathrm{nM}$. In $100 \mathrm{nM}$ CPT (or PACPX), the selective A3R agonist $\mathrm{N}^{6}$ (3-iodobenzyl)-adenosine-5'-N-methyluronamide (IB-MECA,10 $\mathrm{\mu M}$ ) abolished coordinated responses $\left(\mathrm{IC}_{50}-\mathrm{I}_{\mathrm{sc}}=2.1 \mu \mathrm{M} ; \mathrm{IC}_{50}-\mathrm{ICD}=7.1 \mu \mathrm{M}, \mathrm{n}=6\right)$. In mucosa-submucosa, dimaprit $\mathrm{I}_{\mathrm{sc}}$ responses were abolished by $10 \mathrm{nM}$ AlR agonist 2-chloro- $\mathrm{N}^{6}$-cyclopentyladenosine and

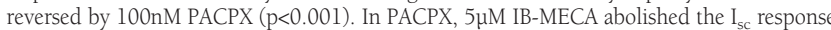
that was reversed by MRS $1191(5 \mu \mathrm{M}-30 \mu \mathrm{M}, \mathrm{p}<0.001)$. In a parallel study, coordinated responses to dimaprit displayed the same sensitivity to AlR and A3R ligands $(n=5)$. The coordinated response to dimaprit was blocked by TTX $(0.1 \mu \mathrm{M}, \mathrm{n}=4)$, and remaining myogenic activity $(254.4 \mu \mathrm{m} \pm 24)$ was reduced $48.4 \%$ by IB-MECA in CPT-treated tissues ( $\mathrm{n}=$ $3, \mathrm{p}<0.0001)$. MRS $1191(1 \mathrm{\mu M})$ enhanced the TTX-sensitive distension-evoked $\mathrm{I}_{\mathrm{sc}}$ response from $50 \pm 8.6$ to $95 \pm 9.6 \mu \mathrm{A} / \mathrm{cm}^{2}(\mathrm{p}=.003, \mathrm{n}=3)$. A3R immunoreactivity occurs in myenteric and submucous neurons of guinea-pig, mouse, rat and human colon ( $\mathrm{n}=4$ each species) Conclusions: Endogenous adenosine acts at inhibitory AlR and A3R to modulate the stereotype neural-motor behavior triggered by the mast cell mediator histamine or a distension reflex. Recent availability of A1/A3R KO mice should provide unequivocal proof of function (NIH grants DK 44179, NCRR 1S10RR11434, DK57016)

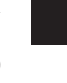


Endothelin Receptors and Actions in Adult Guinea-Pig Enteric Nervous System

Sumei Liu, Xiyu Wang, Xiucai Fang, Na Gao, Chuanyun Gao, Hong-Zhen Hu, Guo-Du Wang, Yun Xia, Jackie D. Wood

The endothelin receptor-B (ETB) and its ligand endothelin-3 (ET-3) have a key role in the development of the enteric nervous system (ENS). Loss of signaling through the ETB receptor leads to failure of colonization of the developing gut by neural crest precursors and results in aganglionic megacolon, which resembles human Hirchsprung's disease. The three endothelin peptides (ET-1, ET-2, and ET-3) and their receptors (ETA and ETB) are known to be expressed in adult rat, guinea-pig and human ENS. However, a functional role for ET receptors and their ligands in the mature ENS has not been examined. We used indirect immunofluorescence, Western blot analysis, intracellular "sharp" microelectrode recording followed by intraneuronal injection of biocytin to study the expression of ET receptors and actions of ETs on morphologically-identified myenteric neurons in the adult guinea-pig small intestine. Exposure of myenteric neurons to ETs depolarized the membrane potential and elevated neuronal excitability in 63\% (59/93) for ET-1, 54\% (21/39) for ET-2, and 53\% (18/34) for ET-3. Both Dogiel Type I neurons with S-type electrophysiological behavior and Dogiel Type II neurons with AH-type electrophysiological behavior responded to ETs. Depolarizing responses to ETs were concentration-dependent with an EC50 of $37.25 \mathrm{nM}$ for ET-1, $43.04 \mathrm{nM}$ for ET-2, and $41.74 \mathrm{nM}$ for ET-3. The selective ETB receptor antagonist IRL 1038, but not a selective ETA receptor antagonist BQ-123, suppressed the actions of ETs. The selective ETB receptor agonist safarotoxin S6c mimicked the excitatory actions of ETs on myenteric neurons. Pre-incubation with U-73122, a phospholipase C inhibitor, suppressed the depolarizing responses to ET exposure. Western blot analysis confirmed expression of ETB receptor protein in the adult guinea-pig and human ENS. Immunoreactivity for the ETB receptor was expressed by the majority of neurons in adult guinea-pig and human small intestine. The results suggest that ETs might act as neuromodulators within the mature ENS to increase neuronal excitability and thereby influence intestinal physiology. Actions of ETs on myenteric neurons were mediated by the ETB receptor subtype coupled with a phospholipase C intraneuronal signaling pathway (Supported by NIH ROI DK37238).

157

Adenosinergic A3 Receptor Regulation in the Human Enteric Nervous System Jacqueline E. Wunderlich, Helen J. Cooke, Zhixiong Chen, Jun Ge Yu, Iveta Grants, Kristen Hinshaw, Scott Melvin, Fievos L. Christofi

Background \& Aim: Little information exists on mechanisms of synaptic transmission and modulation in the human enteric nervous system (ENS). Optical $\mathrm{Ca}^{2+}$ recordings revealed excitatory P2Yl purinergic synaptic transmission in human submucous neurons. Other purinergic receptors including adenosine $\mathrm{Al}$ or $\mathrm{A} 3$ receptors (A3R) are negatively coupled to synaptic transmission in rodent gut. Our aim was to test the hypothesis that A3R contribute to inhibitory purinergic transmission in the human ENS. A3R immunoreactivity is abundant in human submucous neurons. Methods: Microdissected submucosa (SMP) from jejunum of Roux-en-Y patients was loaded with $30 \mu \mathrm{M}$ Fluo-4/AM Ca${ }^{2+}$. Time-series $\mathrm{Ca}^{2+}$ analysis of intracellular $\mathrm{Ca}^{2+}$ levels was done using a Zeiss LSM/REN 410 laser-scanning confocal imaging system. A 3 s fiber tract electrical stimulation (EFS) $(0.1 \mathrm{~Hz}, 0.5 \mathrm{~Hz}, 1 \mathrm{~Hz} 5 \mathrm{~Hz}, 10 \mathrm{~Hz}, 25 \mathrm{~Hz}$ $50 \mathrm{~Hz}, 75 \mathrm{~Hz}, 100 \mathrm{~Hz}$ ) was applied by a $25 \mu \mathrm{m}$ tip titanium electrode placed $0.3 \mathrm{~mm}$ away from the recording ganglion. Drug effects were assessed after $30 \mathrm{~min}$ superfusion in Krebs at $36^{\circ} \mathrm{C}$. Short-circuit current $\left(\mathrm{I}_{\mathrm{sc}}=\right.$ chloride secretion) was recorded in flat sheets of mucosaSMP in flux chambers under voltage clamp. Distension evoked a reflex $I_{s c}$ response by serosal removal of $150 \mu \mathrm{l}$ fluid for $30 \mathrm{~s}$. Results: Data analysis included 319 neurons from 39 SMP from 10 patients. EFS elicited a $\mathrm{Ca}^{2+}$ response in 319 of 531 neurons responsive to high $\mathrm{K}^{+}$-depolarization $(3 \mathrm{~s}, 25 \mathrm{~Hz}$; $\uparrow$ of $66.0 \pm 6.0$ intensity units) that was blocked by

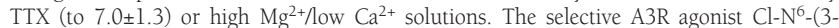
iodobenzyl)-adenosine-5'-N-methyluronamide (Cl-IB-MECA) caused a concentrationdependent inhibition of synaptic $\mathrm{Ca}^{2+}$ responses $(0.1 \mu \mathrm{M}-5 \mu \mathrm{M} ; \mathrm{n}=79 ; \mathrm{p}=.0001)$. The human A3 antagonist MRS 1220 caused a 50-70\% augmentation of the synaptic $\mathrm{Ca}^{2+}$ response (1, $10 \mu \mathrm{M} ; 3 \mathrm{~s}, 25 \mathrm{~Hz} ; \mathrm{n}=62 ; \mathrm{p}=0.0001$ ). In contrast, the rodent A3 antagonist MRS 1191 had no effect $(\mathrm{n}=67, \mathrm{p}>0.05)$. EFS caused a frequency-dependent synaptic $\mathrm{Ca}^{2+}$ response peaking at $25 \mathrm{~Hz}$ and declining at $50 \mathrm{~Hz}-100 \mathrm{~Hz}$. At $5 \mu \mathrm{M}, \mathrm{Cl}-\mathrm{IB}-\mathrm{MECA}$ nearly abolished synaptic $\mathrm{Ca}^{2+}$ responses from $0.1 \mathrm{~Hz}-10 \mathrm{~Hz}(\mathrm{p}<0.001)$ and reduced by $70 \%$ responses between $25 \mathrm{~Hz}-100 \mathrm{~Hz}$ $(\mathrm{p}<0.001)$. The distension-evoked $\mathrm{I}_{\mathrm{sc}}$ response was reduced $29.3 \pm 7.1 \%(\mathrm{p}=0.006)$ by $10 \mu \mathrm{M}$ capsaicin and $1 \mu \mathrm{M}$ TTX reduced it by $63.7 \pm 7.0 \%(\mathrm{p}=0.002, \mathrm{n}=7)$. MRS $1220(2 \mu \mathrm{M})$ enhanced the distension response by $30.7 \pm 1.5 \%(\mathrm{p}=0.0025, \mathrm{n}=3)$ and enhancement was blocked by TTX $(p=0.002)$. Conclusions: Endogenous adenosine may act at low affinity neural A3Rs to inhibit both synaptic transmission and distension reflexes in the human submucosa (DK44179, DK37240, NCRR 1S10RR11434)

\section{8}

Extrinsic Primary Afferent Neurons Stimulate Enteric Mast Cells to Release Histamine As a Paracrine Modulator in the Enteric Nervous System in the Guinea-Pig Intestine

Guo-Du Wang, Xi-Yu Wang, Sumei Liu, Na Gao, Gui-Jun Fei, Hong-Zhen Hu, Yun Xia, Jackie D. Wood

Release of substance P (SP) or calcitonin gene-related peptide (CGRP) from primary afferents evokes excitatory responses in enteric neurons. We tested a hypothesis that SP and CGRP, released from primary afferents, stimulate release of paracrine neuromodulators (e.g., histamine) from mast cells. Mast cells were identified by immuno-reactivity (IR) for tryptase or chymase. A subset of the tryptase-IR cells expressed IR for the neurokinin-1 (NK-1) receptor. Application of SP, CGRP, capsaicin or compound 48/80 increased the concentration of histamine in the bathing medium in a range of $855 \pm 103$ to $979 \pm 114$ ng.gtissue ${ }^{-1}$ from basal levels of $684 \pm 82$ ng.gtissue ${ }^{-1}$ determined by ELISA for intestinal preparations from 8 animals. Stimulation of histamine release by SP was reversed by the selective NK-l receptor antagonist,
RP6758. The selective CGRP antagonist, CGRP8-37 reversed the histamine-releasing action of CGRP and the selective VR-1 receptor antagonist, SB366791, reversed the action of capsaicin. Electrical stimulation of nerve trunks containing primary afferent fibers in the intestinal mesentery stimulated release of histamine from $684 \pm 82$ to $1100 \pm 142$ ng.gtissue${ }^{1}$ in preparations from 8 animals. Pretreatment with an NK-1 or CGRP receptor antagonist, but not a VR-1 antagonist, partially suppressed histamine release evoked by mesenteric nerve stimulation to $826 \pm 96 \mathrm{ng}$. gtissue- $1(\mathrm{n}=6)$. Pre-incubation with the mast cell stabilizing drugs, cromolyn or ketotifen decreased basal release of histamine from $684+82$ to $313 \pm 73$ and $162 \pm 41$ ng.gtissue ${ }^{-1}$ respectively. Either of the mast cell stabilizing drugs significantly reduced the stimulating action of SP, CGRP, capsaicin and electrical stimulation on histamine release. Application of SP, CGRP or histamine evoked membrane depolarization and prolonged discharge of action potentials reminiscent of slow synaptic excitation in submucosal and myenteric neurons. Neither cromolyn nor ketotifen suppressed the neuronal responses to SP, CGRP or histamine. Pretreatment with cromolyn or ketotifen suppressed the slow EPSP-like responses in myenteric neurons that were evoked by electrical stimulation of primary afferents in the mesentery. The results are consistent with the hypothesis that firing of intramural primary afferents releases SP and CGRP at junctions with enteric mast cells. SP and CGRP both act at their receptors on the mast cells to stimulate the release of histamine. Once released, histamine "blankets" the enteric nervous system as a paracrine neuromodulator. (Supported by NIH ROI DK37238, ROI DK57075 and KO8 060468)

159

Perturbing NMDA Receptor - PSD-95 Protein Interactions Prevents Selective Loss of Nitric Oxide Synthase (NOS) Containing Neuron in Rat Gastric Myenteric Plexus

Chun Hsu, Shi-Yi Zhou, Yuanxu Lu, Kwang-Deok Moon, Il Song, Chung Owyang

Defective accommodation and gastroparesis are serious complications of diabetes. We previously showed that in diabetic rats, gastric myenteric NOS containing neurons were selectively damaged through chronic NMDA receptors activation, and that this damage was prevented by NMDA antagonist. We hypothesize that glutamate activation and nitric oxide release are important in damage of NOS containing neurons, and that dissociating NMDA receptor from down stream neurotoxic signal may prevent this deleterious effect. To test this hypothesis, we demonstrated via immunocytochemistry (ICH) studies of whole mount preparations of gastric corpus that glutamate containing neurons were abundant and frequently co-localized with NOS or CHAT containing neurons. Glia cells containing glutamate synthetase were present in close proximity with enteric neurons. These findings suggest that the glutamate system in the stomach enteric nervous system is similar to that in the central nervous system (CNS). In diabetic animals, whole mount gastric corpus ICH of streptozotocin (STZ)-induced diabetic and age matched control rats demonstrated that diabetic animals had a $37.5 \%$ reduction of NOS containing neurons but no reduction of the CHAT and glutamate containing neurons. Glutamine containing glia cells also appeared intact in diabetic animals. To demonstrate that NOS containing gastric neurons are selectively damaged by the Glut-NMDA-PSD95-NOS signal pathway, we first demonstrated via rtPCR that exposure of whole mount preparations of gastric corpus to L-glutamate $1.5 \times 10-3 \mathrm{M}$ for 20 minutes resulted in $17 \%$ increased NOS mRNA expression. We then constructed Tat-NR2B9c, a peptide that readily transduces cellular cytoplasm and perturbs the NMDA receptor-PSD-95 protein interactions, thereby selectively decreasing glutamate mediated downstream neurotoxic signaling without affecting other glutatmate mediated neuronal activities. Exposure of whole mount preparations of gastric corpus to L-glutamate $1.5 \times 10-3 \mathrm{M}$ for 2 hours resulted in $55 \%$ loss of NOS containing neurons. This loss was prevented by Tat-NR2B9c peptide transduction, but not by exposure to Tat $38-48$, a peptide that does not transduce cell membrane. In conclusion, we showed that the gastric myenteric glutamate system is similar to that in CNS and that glutamate leads to increased NOS mRNA expression. We also showed that Tat-NR2B9c, a peptide that selectively interrupts the Glut-NMDA-PSD 95-NOS signal pathway, can prevent loss of glutamate induced NOS containing neurons. This approach to prevent the loss of gastric myenteric NOS neurons may have important therapeutic implications for gastric motility complications of diabetes.

160

Feedback Inhibition of $G \alpha_{I}$ Function By $G \beta \gamma_{I}$ Subunits: A Novel Mechanism Involving Phosphorylation of $G \alpha_{1}$ By G $\beta \gamma$-Dependent Src Kinase, Recruitment to Rgs12, and Inactivation of GTP-Bound $\mathrm{G \alpha}_{1}$ Jiean Huang, Sunila Mahavadi, Karnam S. Murthy

$\underline{R}$ egulators of $\underline{G}$ protein signaling (RGS) accelerate the intrinsic GTPase activity of activated $\bar{G} \alpha$ subunits, and thus determine the intensity of G protein signaling. RGS12, in addition to its RGS domain, contains other domains including a phospho-tyrosine binding (PTB) domain that binds to phospho-tyrosine residues in other proteins. Tyrosine phosphorylation of $\mathrm{G} \alpha$ subunits could promote their association with and inactivation via RGS12. Agoniststimulated dissociation of $G \beta \gamma_{i}$ induces activation of PI 3-kinase and $\mathrm{c}$-Src raising the possibility of Src-mediated phosphorylation of $G \alpha_{i}$ subunits. Aim. To examine whether tyrosine phosphorylation of $G \alpha_{\mathrm{i} 2}$ via a $\mathrm{G} \beta \gamma_{\mathrm{i}} / \mathrm{PI}$ 3-kinase/Src kinase pathway increases RGS12 binding to and inactivation of $\mathrm{G \alpha}_{\mathrm{i} 2}$. Methods. $\mathrm{G} \alpha_{\mathrm{i} 2}$ activity was measured as inhibition of forskolin-stimulated cAMP formation in response to the $\delta$-opioid receptor agonist, DPDPE. Tyrosine phosphorylation of $\mathrm{G}_{\mathrm{i}_{2}}$ was determined by immunoblot analysis and the association of RGS12: $G \alpha_{i 2}$ by immunoprecipitation. Results. Previous studies have shown that $\delta$-opioid receptors are coupled to $G_{i 2}$ in smooth muscle. DPDPE inhibited forskolin-stimulated cAMP formation in cultured gastric muscle cells and increased tyrosine phosphorylation of $G \alpha_{i 2}$. Treatment of the cells with the PI 3 kinase inhibitor LY294002, the Src kinase inhibitor PP2, or expression of a $G \beta \gamma$-scavenging peptide blocked tyrosine phosphorylation of $G \alpha_{i 2}$ and caused further inhibition of cAMP, implying that tyrosine phosphorylation of $\mathrm{Ga}_{\mathrm{i} 2}$ attenuated $G \alpha_{i 2}$ activity. DPDPE also increased RGS12:G $\alpha_{i 2}$ binding; the binding was inhibited by LY294002 or PP2, and in cells expressing G $\beta \gamma$-scavenging peptide. The results suggest tyrosine phosphorylation of $\mathrm{G} \alpha_{i 2}$ by Src kinase facilitated binding of phosphorylated $G \alpha_{i 2}$ to the PTB domain of RGS12, thereby accelerating inactivation of $G \alpha_{i 2}$. This notion was examined by mutation of putative tyrosine phosphorylation sites in $G \alpha_{i 2}$ to phenylalanine 
(Y69F, Y231F and Y321F). Expression of $\mathrm{G} \alpha_{\mathrm{i} 2}$ mutants, (i) blocked DPDPE-induced tyrosine phosphorylation of $G \alpha_{i 2}$, (ii) inhibited the increase in $R G S 12: G \alpha_{i 2}$ binding, and (iii) caused a further decrease in forskolin-stimulated cAMP, providing additional evidence that tyrosine phosphorylation of $\mathrm{G \alpha}_{\mathrm{i} 2}$ increases RGS12 binding and augments $\mathrm{G} \alpha_{\mathrm{i} 2}$ inactivation. Conclusion. This study shows that effectors downstream of $G \beta \gamma_{i}$ promote inactivation of $G \alpha_{i}$. This novel inhibitory feedback mechanism involves activation of Src kinase via $G \beta \gamma_{i}$ and tyrosine phosphorylation of $G \alpha_{i}$, which enhances the binding of phosphorylated $G \alpha_{i}$ to the PTB domain of RGS12 and accelerates inactivation of $G \alpha_{i}$.

\section{1}

Substance P (SP) Induces Ubiquitination and Degradation of the Neurokinin Receptor (NK1R)

Graeme S. Cottrell, Benjamin E. Padilla, D.R. Roosterman, Stella Pikios, Martin Steinhoff, Nigel Bunnett

Little is known about the post-endocytic trafficking of receptors back to the plasma membrane, which mediates receptor resensitization, or to late endosomes and lysosomes, which mediates receptor down-regulation. Low concentrations of SP $(1-10 \mathrm{nM})$ promote $\mathrm{NK}_{1} \mathrm{R}$ endocytosis and recycling, but the effects of sustained stimulation of cells with high concentrations of SP, which may occur during chronic inflammation and pain, on trafficking of the $\mathrm{NK}_{1} \mathrm{R}$ are unknown. We hypothesized that sustained stimulation with high concentrations of $\mathrm{SP}$ causes ubiquitination and degradation of the $\mathrm{NK}_{1} \mathrm{R}$. To examine down-regulation of $\mathrm{NK}_{1} \mathrm{R}$ signaling, we exposed KNRK cells expressing $\mathrm{NK}_{1} \mathrm{R}$ to $\mathrm{SP}(100 \mathrm{nM}, 3 \mathrm{~h})$ or vehicle (control), washed the cells, and then examined SP-induced $\mathrm{Ca}^{2+}$ signaling. Immediately after washing there was complete desensitization and full resensitization took $16 \mathrm{~h}$. This resensitization was abolished by cyclohexamide, suggesting resensitization requires synthesis of new receptors. To examine the importance of ubiquitination in degradation, lysinedeficient mutants of $\mathrm{NK}_{1} \mathrm{R}$ were made $\left(\mathrm{NK}_{1} \mathrm{R} \Delta 5 \mathrm{~K} / \mathrm{R}, \mathrm{C}\right.$-terminal tail lysines and $\mathrm{NK}_{1} \mathrm{R} \Delta 10 \mathrm{~K} /$ $\mathrm{R}$, all intracellular facing lysines). Both mutants were expressed at the plasma membrane, and responded normally to $\mathrm{SP}$ by $\mathrm{Ca}^{2+}$ mobilization. $\mathrm{NK}_{1} \mathrm{R}, \mathrm{NK}_{1} \mathrm{R} \Delta 5 \mathrm{~K} / \mathrm{R}$ and $\mathrm{NK}_{1} \mathrm{R} \Delta 10 \mathrm{~K} /$ $\mathrm{R}$ were completely desensitized after exposure to SP, but the mutants resensitized 4-fold more rapidly than $\mathrm{NK}_{1} \mathrm{R}$. In contrast, $\mathrm{NK}_{1} \mathrm{R} \Delta 324$, which corresponds to a naturally occurring truncated variant of the $\mathrm{NK}_{1} \mathrm{R}$, showed diminished desensitization and enhanced resensitization. SP $(100 \mathrm{nM}, 3 \mathrm{~h})$ induced ubiquitination of $\mathrm{NK}_{1} \mathrm{R}$, determined by immunoprecipitation of the $\mathrm{NK}_{1} \mathrm{R}$ under both native and denaturing conditions and probing Western blots for ubiquitin. In contrast, $\mathrm{NK}_{1} \mathrm{R} \Delta \mathrm{l} 0 \mathrm{~K} / \mathrm{R}$ was completely resistant to ubiquitination. Moreover, whereas SP induced degradation of $\mathrm{NK}_{1} \mathrm{R}$, determined by Western blotting in the presence of cyclohexamide $(41 \pm 9 \%$ reduction after $16 \mathrm{~h}$ with $\mathrm{SP}), \mathrm{NK}_{1} \mathrm{R} \Delta \mathrm{l} \mathrm{KK} / \mathrm{R}$ showed diminished degradation $\left(25 \pm 9 \%\right.$ reduction). To determine the fate of activated $N_{1} R$, we labeled living cells with an antibody to the extracellular epitope tag of the $\mathrm{NK}_{1} \mathrm{R}$, exposed cells to SP (100 $\mathrm{nM}, 3 \mathrm{~h}$ ), washed them, and at various times localized the antibody by immunofluorescence. $\mathrm{SP}$ induced similar endocytosis of $\mathrm{NK}_{1} \mathrm{R}, \mathrm{NK}_{1} \mathrm{R} \Delta 5 \mathrm{~K} / \mathrm{R}$ and $\mathrm{NK}_{1} \mathrm{R} \Delta 10 \mathrm{~K} / \mathrm{R}$. However, whereas $\mathrm{NK}_{1} \mathrm{R}$ remained internalized even after $4 \mathrm{~h}$ recovery, $\mathrm{NK}_{1} \mathrm{R} \Delta 5 \mathrm{~K} / \mathrm{R}$ and $\mathrm{NK}_{1} \mathrm{R} \Delta 10 \mathrm{~K} / \mathrm{R}$ recycled to the plasma membrane. Thus, chronic exposure to SP induces ubiquitination of the $\mathrm{NK}_{1} \mathrm{R}$, which is not required for endocytosis, but is necessary for degradation and down-regulation of the $\mathrm{NK}_{1} \mathrm{R}$. Supported by $\mathrm{NIH}$ grant DK39957.

\section{2}

Up-Regulation of Rgs4 Expression By Interleukin-1 $\beta$ in Colonic Smooth Muscle Is Enhanced By Erk1/2 and P38 Mapk and Inhibited By PI 3-Kinase/ Akt/Gsk3 $\beta$

Wenhui Hu, Fang Li, Sunila Mahavadi, Karnam S. Murthy

Initial $\mathrm{Ca}^{2+}$-dependent contraction of intestinal smooth muscle is inhibited upon exposure of muscle cells to the pro-inflammatory cytokine, interleukin-1 $\beta$ (IL-1 $\beta$ ). We have recently shown that the decrease in contraction reflects up-regulation of RGS4 via a canonical NF$\kappa \mathrm{B}$ pathway resulting in rapid deactivation of $\mathrm{G} \alpha_{\mathrm{q}}$. Exposure of other cell types to IL-1 $\beta$ results also in activation of various protein kinases, including extracellular signal-regulated kinases (ERKl/2) and p38 MAP kinase that could influence directly or indirectly activation of NF-KB and RGS4 expression. Aim. To characterize the role of ERKl/2, p38 MAP kinase, and the PI 3-kinase/Akt/GSK3 $\beta$ pathway in the up-regulation of RGS4 expression in muscle cells by IL-1 $\beta$. Methods. Cultured rabbit colonic smooth muscle cells in first passage were treated with IL- $1 \beta$ for various time periods with or without various kinase inhibitors and RGS4 mRNA expression was determined by real-time RT-PCR. Activities of various kinases and NF- $\mathrm{\kappa B}$ were determined by Western blotting with phospho-specific antibodies. Results. IL-1 $\beta$ caused a transient, rapid phosphorylation (activation) of ERK1/2 and p38 MAPK. IL$1 \beta$ also caused phosphorylation of Akt and GSK3 $\beta$, sequential downstream effectors of PI 3-kinase. Phosphorylation of GSK3 $\beta$ inactivates GSK3 $\beta$. PD98059 (a MEK inhibitor) and SB203580 (a p38 MAP kinase inhibitor) strongly inhibited IL-1 $\beta$-induced RGS4 mRNA expression. In contrast, LY294002 (a PI 3-kinase inhibitor) augmented IL-1 $\beta$-induced RGS4 expression. PD98059 blocked IL-1 $\beta$-induced IKK2 phosphorylation, IKB $\alpha$ degradation, and p65 phosphorylation, whereas SB203580 had no effect, implying that effect of ERK1/2 is exerted on IKK2 or on upstream components of NF-KB signaling, whereas the effect of p38 MAP kinase is not exerted directly on NF- $\mathrm{KB}$. The increase in RGS4 expression caused by LY294002 was accompanied by increase in IKK2 phosphorylation, implying that GSK3 $\beta$, the terminal effector of the PI 3-kinase pathway, which is inhibited upon phosphorylation by Akt acts normally to augment activation of NF-KB. Conclusion. ERK1/2, p38 MAP kinase, and PI 3-kinase/Akt are activated in muscle cells treated with IL-1 $\beta$. ERK1/2 and p38 MAP kinase enhance IL-1 $\beta$-induced increase in RGS4 expression; the effect of ERK1/ 2 reflects its ability to phosphorylate IKK2 and increase NF-KB activity. The PI 3-kinase/Akt/ GSK3 $\beta$ pathway attenuates IL-1 $\beta$-induced up-regulation of RGS4 expression by inhibiting activation of NF- $\mathrm{KB}$
163

Agonist Induced Trafficking of the Motilin Receptor Anna Mitselos, Inge Depoortere, Pieter Vanden Berghe, Theo Peeter

The motilin receptor (MTLR) is a potential therapeutic target for the treatment of hypomotility syndromes. However the MTLR is subject to rapid desensitization (Thielemans et al., 2005) and this may limit the effectiveness of motilin agonists as therapeutic agents. We studied the mechanisms underlying the desensitization process in $\mathrm{CHO}$-cells expressing the motilin receptor. Methods. Agonist induced $\mathrm{Ca} 2+$ luminescence was measured in $\mathrm{CHO}$-cells expressing the $\mathrm{Ca} 2+$ indicator apoaequorin (Euroscreen, Belgium) and the MTLR C-terminally tagged with EGFP (MTLR-EGFP) or the C-terminally truncated MTLR C-terminally tagged with EGFP (MTLRA358-EGFP). Cells were desensitized by incubation for $2 \mathrm{~h}$ with differen concentrations (10-4-10-12M) of motilin prior to a second stimulation with motilin. Recepto phosphorylation was evaluated after preincubation of CHO-MTLR-EGFP cells with inorganic 32P followed by motilin stimulation and receptor immunoprecipitation with anti-EGFP antibody. Internalization of the MTLR-EGFP was visualized by fluorescence microscopy and analyzed with ImagePro software. Organelles were identified using the endosomal marke transferrin Alexa-Fluor 594 and the lysosomal marker cathepsin D. Results. The potency (pEC50) of motilin to induce Ca2+ fluxes and to desensitize (pDC50) was $10.02 \pm 0.09$ and $7.82 \pm 0.04$ resp. Motilin induced a rapid $(\mathrm{Tl} / 2<1 \mathrm{~min}$.) and dose-dependent phosphorylation (pEC50:8.33 \pm 0.13 ) of the MTLR. With fluorescence microscopy maximal internalization in the cytosol was observed after approximately $50 \mathrm{~min}$ of constant exposure to $10-7 \mathrm{M}$ motilin Colocalization of the receptor with the endosomal marker transferrin was already observed 20 min after stimulation with motilin. During the timecourse of internalization, colocalization with the lysosomal marker cathepsin D was never observed. Following stimulation with motilin (10-7M) for $2 \mathrm{~h}$, cells were washed and receptor fluorescence was monitored at 0 , 2, 4 and $8 \mathrm{~h}$. Complete recycling of the internalized MTLR-EGFP to the plasma membrane was observed $8 \mathrm{~h}$ after removal of motilin. C-terminal truncation of the MTLR remarkable reduced the potency of the MTLR (pEC50:8.26 \pm 0.20 ) but motilin was still able to desensitize the receptor $(\mathrm{pDC} 50: 7.48 \pm 0.32)$. Conclusion. Desensitization of the motilin receptor after stimulation with motilin involves receptor phosphorylation followed by sequestration of the MTLR in the endosomal compartiment from where it is recycled back to the plasma membrane. The C-terminus of the receptor is not critical for desensitization.

\section{4}

Motilin Receptor Antagonism Attenuates Naturally Occurring Mmcs and Reverses Motilin-Impaired Gastric Accomodation in Concious Dogs Graeme Fraser, Eric Marsault, Bernard Coulie, Luc Ver Donck

Background: Motilin has been postulated to have a role as an interdigestive hormone regulating antral migrating motor complex (MMC) in various species, including dog and man. A potent and selective antagonist is required to establish motilin's physiological role, but no such antagonist has yet been described. The aim of this study was to investigate the role of motilin in the interdigestive and post-prandial periods in conscious dogs using a novel, potent and selective motilin antagonist (JTZ-2002). Methods: Gastrointestinal motility was measured for $24 \mathrm{~h}$ by telemetry in freely moving Beagle dogs (F) instrumented with strain gauges on antrum and duodenum. In a second series of experiments, fundic accommodation in response to a $200 \mathrm{ml}$ milk meal was measured using a barostat. Results: Following an overnight fast, JTZ-2002 (0.1, 0.3, $1 \mathrm{mg} / \mathrm{kg}$, i.v.) suppressed the regular occurrence of phase III activity of the MMC in a dose-dependent manner. Baseline MMC interval during the $4 \mathrm{~h}$ period before drug was $86 \pm 5 \mathrm{~min}$ and contraction amplitude (CA) was $276.7 \pm 66.0 \mathrm{mN}$ (mean \pm SEM, $\mathrm{n}=6)$. After JTZ-2002 $(1 \mathrm{mg} / \mathrm{kg})$, time to first MMC was $225 \pm 31 \mathrm{~min}$ vs. $99 \pm 15 \mathrm{~min}$ in controls $(\mathrm{p}<0.01, \mathrm{n}=4)$ and $\mathrm{CA}$ was reduced to $5 \pm 2 \%$ of baseline CA over the first hour and recovered to $52 \pm 21 \%$ after 4 h. The 0.1 and $0.3 \mathrm{mg} / \mathrm{kg}$ doses also suppressed MMC activity where CA returned to baseline levels after $3 \mathrm{~h}(\mathrm{n}=2)$. After a standard $75 \mathrm{~g}$ meal, JTZ-2002 $(0.3 \mathrm{mg} / \mathrm{kg}$, i.v., dosed $2 \mathrm{~h}$ after meal $)$ suppressed postprandial CA during the first hour to $58 \pm 4 \%$ of pre-drug baseline $(n=2)$ whereas vehicle treatment maintained CA at $106 \pm 10 \%(\mathrm{n}=4)$. While JTZ-2002 (1 mg/kg, i.v.) did not influence normal fundic accommodation in response to a milk meal (AUC $1 \mathrm{~h} 296 \pm 39$ vs. $365 \pm 26 \mathrm{ml}$.h in control, $\mathrm{p}>0.05, \mathrm{n}=5)$, JTZ-2002 $(0.3 \mathrm{mg} / \mathrm{kg})$ did antagonize the fundic contraction induced by infusion of exogenous motilin $(0.01 \mathrm{mg} / \mathrm{kg} / \mathrm{h}$, i.v. $) ; \mathrm{AUC}_{5 \min }$ of fundic relaxation for vehicle administration was $18.7 \pm 3.3$ vs. $27.3 \pm 3.5$ ml.h after JTZ-2002 $(p<0.05, n=4)$ Conclusions: These data provide the first pharmacological evidence that motilin is an endocrine regulator of the phase III contraction of the MMC and further suggest that motilin also has a role in stimulating post-prandial antral and duodenal contractions. Thus, motilin antagonists may have a role in the treatment of gut motility disorders characterized by increased spontaneous activity in the upper gut and/or poor gastric accommodation.

165

The Orphan Nuclear Receptor Lrh-1 Regulates Intestinal Inflammation Laurent Dubuquoy, Pierre Desreumaux, Johan Auwerx, Kristina Schoonjans

Background: Liver receptor homolog 1 (LRH-1: NR5A2) is an orphan nuclear receptor that controls many metabolic pathways. In the gut, LRH-1 was shown to stimulate intestinal crypt cell renewal and to enhance intestinal tumorigenesis (1-2), but its effect during intestinal inflammation remains unknown. Aim: To investigate the role of LRH- 1 in the regulation o intestinal inflammation in mice and to quantify its expression in patients with inflammatory bowel diseases. Methods: Acute or chronic colitis was induced by administration of TNBS or DSS in Lrh-1+/- mice and their wild type littermates. The intensity of colitis was assessed by macroscopic and histologic inflammatory scores and quantification of colonic myeloperoxydase and inflammatory cytokines. In parallel, LRH-1 mRNA and protein levels were quantified in paired healthy and inflamed small bowel and colon biopsies of untreated IBD patients ( $7 \mathrm{CD}, 7 \mathrm{UC}$ ) and controls ( $\mathrm{n}=9)$ using real-time PCR and western blot. Results: LRHexpression was induced by inflammation in the colon of WT animals. LRH-1 haplo-insufficiency predisposes Lrh-1+/- mice to the development of acute and chronic intestinal inflammation when exposed to TNBS or DSS, as reflected by a significant increase of macroscopic

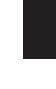


and histological scores and by an induction of local inflammatory parameters. In human intestinal biopsies, LRH-1 levels were similar in healthy mucosa of IBD patients compared to controls. However, a dramatic and similar decrease of LRH-1 mRNA and protein levels was observed in inflamed mucosa of CD and UC patients compared to healthy areas of IBD patients or controls. Conclusions: LRH-1 is an important nuclear receptor to control colon inflammation in mice. Impaired expression of LRH-1 in inflamed mucosa of IBD patients may participate through a defect of epithelium renewal to the pathophysiology of the diseases. 1: Botrugno OA, et al. Mol Cell. 2004 Aug 27:15(4):499-509 2: Schoonjans K, Dubuquoy L, et al. Proc Natl Acad Sci U S A. 2005 Feb 8;102(6):2058-62.

\section{2}

Low Serum Adiponectin Levels in a Transgenic Mouse Model of Hepatitis C Infection

Aymin Delgado, Yoshitaka Kamegaya, Sergio H. Jordan, Saurabh Agrawal, Yoichi Hiasa, Raymond T. Chung

Background and Aims: Recent studies have demonstrated an association between HCV infection and insulin resistance. However, the underlying mechanisms for this association remain unclear. We sought to evaluate whether HCV decreases in vivo levels of adiponectin, an anti-inflammatory adipokine known to induce insulin sensitivity. Methods: Transgenic ( $\mathrm{Tg}$ ) mouse models expressing $\mathrm{HCV}$ core and $\mathrm{HCV}$ core-E1-E2 proteins under the control of the HBV enhancer and albumin promoter, respectively, were created on a hybrid C57BL 6/FVB background as previously described (Kamegaya Y., Hepatology 41: 660-7, 2005). On this background, mice do not develop significant liver pathology. Twenty-eight $\operatorname{Tg}(18$ core, 10 core-El-E2) and 12 littermate controls were evaluated. All mice were 16 to 18 months old. Serum samples were collected following an overnight fast, and immediately frozen at -80C. Adiponectin levels were measured using Luminex ELISA technology. Results: Mean adiponectin levels were $16.45 \mu \mathrm{g} / \mathrm{ml}$ for non- $\mathrm{Tg}$ vs. $13.79 \mu \mathrm{g} / \mathrm{ml}$ for $\mathrm{Tg}$ mice ( $\mathrm{p}=$ 0.2 . There were no differences in adiponectin levels observed between the core and coreE1-E2 Tg animals. The greatest difference in frequencies was observed for the group of adiponectin levels $<10 \mu \mathrm{g} / \mathrm{ml}$ (36\% Tg vs. 9\% non-Tg). Thus, adiponectin was dichotomized at the critical value of $10 \mu \mathrm{g} / \mathrm{ml}$, and low adiponectin was defined as any value below 10 $\mu \mathrm{g} / \mathrm{ml}$. Logistic regression was used to compare the probability of having a low adiponectin level between the $\mathrm{Tg}$ and non-Tg groups. The odds of having a low adiponectin level were $30.4(1.6-583.8)$ times greater $(\mathrm{p}=0.02)$ for the $\mathrm{Tg}$ than the non- $\mathrm{Tg}$ group. Conclusions: $\mathrm{HCV}$ Tg mice were significantly more likely to have low adiponectin levels as compared with non-Tg mice. These data demonstrate an in vivo association between HCV and low adiponectin levels, and suggest that HCV structural protein expression alone may suffice to decrease adiponectin levels. These results provide a plausible mechanism for HCV-associated insulin resistance.

173

The Prevalence of Metabolic Syndrome in Veterans with Chronic Hepatitis C Virus

Prashant K. Pandya, Sharad C. Mathur, Peggy Callahan, Priyank Shah, Dean Reker

Background: In addition to traditional predictors of poor response to antiviral therapy for chronic hepatitis $C$ such as viral load, genotype, race, and advanced fibrosis, recent evidence has implicated obesity related factors such as insulin resistance and hepatic steatosis. The prevalence of insulin resistance and related MS in veteran population with chronic hepatitis $\mathrm{C}$ has not been systematically studied. Methods: We performed a cross-sectional analysis of the prevalence of the metabolic syndrome in chronic hepatitis $\mathrm{C}$ infection. We evaluated 38 consecutive veterans referred for evaluation of chronic hepatitis $C$, who were drinking less than $20 \mathrm{~g}$ alcohol per day (by standard questionnaire). We hypothesized that the metabolic syndrome (MS) would be associated with worse hepatic histology than $\mathrm{HCV}$ infection alone. We collected detailed demographic, anthropometric, serologic, histologic and metabolic parameters in order to compare metabolic, virologic and histologic status between subjects with and without the MS. Insulin resistance was defined as a HOMA-R (fasting insulin $(\mathrm{mU} / \mathrm{L}) \times$ fasting glucose $(\mathrm{mmol} / \mathrm{L}) / 22.5)$ greater than 2.5. MS was defined by the presence of 3 or more ATP III criteria. Additional data included determination of hs-CRP, TNF- $\alpha$, IL- 6 , adiponectin and leptin. Groups were compared using t-test and/or Fisher's exact analysis. Results: The average age, BMI, viral load, ALT and HOMA were $53.3 \pm 7.0,28.0 \pm 6.3,2.57 \times 106,59.4 \pm 38.3$ and $4.7 \pm 7.4$ respectively. All but one of the patients were male. Caucasians and African Americans comprised $71 \%$ and $18.4 \%$ of the cohort. The predominant genotype in the cohort was Type 1 (81\%). Twenty of the 38 veterans studied met the ATP III criteria for the MS. Veterans with the MS had higher BMI $(p=0.001)$, HOMA-R $(p=0.012)$, and leptin $(p=0.008)$, while no differences were seen $(p=$ ns) in age, race, ALT, TNF- $\alpha$, Il- 6 , adiponectin, hs-CRP, viral load, or genotype. Patients with MS had significantly higher prevalence of steatosis $(\mathrm{p}=0.001)$ and fibrosis on biopsy $(p=0.001)$. Conclusion: Metabolic syndrome was present in $53 \%$ of veterans referred with chronic hepatitis $C$. Leptin but not adiponectin was significantly associated with the metabolic syndrome. Veterans with metabolic syndrome have more steatosis and advanced fibrosis. The high prevalence of metabolic syndrome may adversely impact outcomes with antiviral therapy in veterans.
174

Immunohistochemical Expression of Junctional Proteins and Intestinal Permeability Are Altered in Hepatitis C Virus (HCV) Related Chronic Hepatitis ( $\mathrm{HCV}-\mathrm{CH})$

Luigi Martorelli, Sapone Anna, Pasquale Esposito, Laura Demagistris, Michele Russo, Maddalena Generoso, Maridela Carteni', Gabriele Riegler, Franca Ferraraccio, Alessio Fasano

Background/rationale: Altered intestinal permeability values (IP) and intestinal ultra-structural abnormalities have been shown in cirrhotic patients as well as other liver diseases. Aims: To study the IP, serum levels of the intestinal tight junction (tj) modulator zonulin (Z), and the immunohystochemical expression of junctional proteins occludin, claudin, desmoglein and intracellular actin in duodenal mucosa of HCV-CH patients. Methods: The IP was evaluated by the Lactulose/Mannitol (LA/MA) test. Serum $Z$ levels were determined by sandwich-ELISA. Distal duodenum biopsies were obtained by EGD and processed for immunohystochemistry. Staining was obtained with antibodies specific for occludin, claudin4, desmoglein-3, and actin. Twenty-five consecutive naive $\mathrm{HCV}-\mathrm{CH}$ patients were enrolled (12 males, 13 females, mean age $59,9+10,5$, range 35-72); none of them was on interferon treatment. All enrolled patients underwent IP and Z investigation; 9 of them gave informed consent to perform an EGD with duodenal biopsies. Results: The LA/MA mean \pm SD value in the 25 patients studies was $0.025 \pm 0.020$, with 8 patients $(32 \%)$ having LA/MA values higher than the normal cut off $(<0.028)$. The mean $\pm \mathrm{SD}$ value of $\mathrm{Z}$ in the 25 patients resulted $1.27 \pm 1.64 \mathrm{ng} / \mathrm{mg}$ protein, with 10 of them $(40 \%)$ having $Z$ values higher than the normal cut off $(<0.6 \mathrm{ng} / \mathrm{mg}$ protein). There was a correlation between elevated LA/MA and serum zonulin levels. Occludin immunoreactivity at the cell membrane surface resulted diminished in all cases but one (decrement ranging between 50-90\% as compared to normal controls). These changes were paralleled by an increased cytoplasmatic occludin immunoreactivity (2-50\%), suggesting a displacement of the tj protein from the membrane boundary to the cytoplasm. Claudin- 4 expression resulted diminished by $30-60 \%$ in all cases and was associated to a decrease in actin immunoreactivity (45-85\%). Desmoglein immunoreactivity also decreased by $45-70 \%$. Conclusions: Alteration in IP was detected in a one third of the $\mathrm{HCV}-\mathrm{CH}$ patients studied and was associated to elevated serum $\mathrm{Z}$ values. These changes were associated to modification of $\mathrm{tj}$ proteins expression and localization. Our data suggest that a subgroup of $\mathrm{HCV}-\mathrm{CH}$ patients has increased serum $\mathrm{Z}$ and changes in ti proteins distribution compatible with altered IP. These changes may lead to increased non-self antigens access that may influence the course of the liver disease.

175

Novel Defective HCV Clones Influence a Resistance to Antiviral Therapy in Recurrent Hepatitis C After Living-Donor Liver Transplantation

Akio Iwai, Hiroyuki Marusawa, Yasutsugu Takada, Hiroto Egawa, Motoshige Nabeshima, Tsutomu Chiba

Background: Interferon (IFN)- $\alpha 2 b$ plus ribavirin (RBV) combination therapy is widely used for recurrent hepatitis C following liver transplantation; however, the rate of sustained virological response (SVR) in transplant patients has been limited. Recurrent hepatitis $C$ in transplant patients differs from that in immunocompetent individuals in several ways such as immunosuppressive condition and extremely high viral loads. However, it remains unclear whether viral and host factors determine the effectiveness of combination therapy in those patients. Aim: The aim of this study was to identify the host and viral factors associated with a SVR to antiviral therapy in patients with recurrent hepatitis $C$ after living-donor liver transplantation (LDLT). Methods: Twenty-three patients receiving the IFN- $\alpha 2 \mathrm{~b}$ plus RBV combination therapy for recurrent hepatitis C after LDLT were enrolled. The hepatitis C virus $(\mathrm{HCV})$ genome clones circulating in sera of those recipients were cloned by reverse transcription-polymerase chain reaction. Results: Twenty of the 23 patients (87\%) were infected with HCV genotype lb. Before treatment, all patients had serum HCV RNA levels over $100 \mathrm{KIU} / \mathrm{mL}$ by the Amplicor HCV 2.0 assay. Moreover, HCV RNA levels were greater than $850 \mathrm{KIU} / \mathrm{mL}$ (ranging from 850 to $21,200 \mathrm{KIU} / \mathrm{mL}$ ) in 17 cases $(74 \%$ ), which is equivalent to $8.5 \times 10^{5}$ to $2.12 \times 10^{7}$ copies/mL HCV RNA, indicating that a majority of the patients in this study had high baseline viral loads before treatment. SVR was achieved in seven of 23 patients (30\%). Predictive factors for SVR included; (1) $2 \log _{10}$ decline in HCV RNA at two weeks after the start of therapy, (2) disappearance of HCV RNA at four or 24 weeks after the start of therapy, (3) frequency of positivity for HLA-B51, and (4) frequency of negativity for HLA-A26. Since the pretreatment high viral load showed no association with SVR, we asked whether other viral factors played roles in determining response to the combination therapy in transplant recipients. We found several novel defective $\mathrm{HCV}$ clones in four (33\%) of twelve recipients' sera with genotype lb and extremely high HCV RNA loads (over $850 \mathrm{KIU} / \mathrm{ml}$ ). All defective HCV clones had deletions in the envelope region of HCV genome. Interestingly, no patients with defective clones showed a $2 \log _{10}$ decline in HCV RNA at two weeks after the start of therapy. Conclusions: Early decline in serum HCV RNA after treatment was closely associated with SVR. The circulating defective $\mathrm{HCV}$ clones are present and might be responsible for resistance to the combination therapy in patients with recurrent hepatitis after liver transplantation.

\section{6}

Reliable Early Prediction of Viral Relapse By Detection of Minimal Residual Hepatitis C Viremia At Treatment Week 12

Alexandra Bergk, Christoph Sarrazin, Michael von Wagner, Gerlinde Teuber, Peter Buggisch, Viola Weich, Bertram Wiedenmann, Thomas Berg

Introduction: Current treatment guidelines for the therapeutic management of chronic hepatitis $\mathrm{C}$ virus ( $\mathrm{HCV}$ ) genotype 1 infected patients include a week 12 stopping rule for nonresponders defined by a drop of viral load less than $2 \log 10$ in patients treated with pegylated interferon and ribavirin. Nevertheless there has been no convincing prognostic tool to easily and reliably predict viral relapse after the end of 48 weeks of treatment. Aims: Aim of the present study was to evaluate the predictive value of a minimal residual $\mathrm{HCV}$ viremia for 
a relapse in genotype 1 infected patients with early virologic response to therapy. Patients and methods:We retrospectively analyzed viral kinetics at week 12 and response of 773 treatment naive HCV genotype 1 -infected patients using PegIFN $\alpha-2 \mathrm{a} / \mathrm{b}$ and ribavirin treated at our outpatient clinics. For the detection of residual viremia at week 12 a quantitative real-time PCR with a lower limit of detection of $10 \mathrm{IU} / \mathrm{mL}$ (TaqMan) was used. Results: Using standard quantitative HCV-RNA assays at week $1275 \%$ (430/773) of the patients treated with PegIFNa-2a/b and ribavirin showed an early virologic response defined by week 12 viral load drop $\geq 2 \log 10$. By re-analyzing 222 available serum samples of early virologic responders by TaqMan a residual viremia could be detected in 84 out of 222 patients (38\%). The presence of viremia highly correlated with a viral relapse after the end of 48 weeks of treatment $(p<0,001)$. Residual viremia was detectable by real-time PCR at week 12 in only 19 out of 126 sustained responders (15\%) as compared to 69 out of the 96 relapse patients $(72 \%)(\mathrm{p}<0,001)$. A relapse occurred in $78 \%$ of the patients who had detectable HCV viremia at week 12 (68/88) as compared to only 19\% (25 out of 134 patients) if HCV RNA was undetectable at week 12. The relative risk to suffer from a relapse was 3.5 and 0.26 in patients with and without residual hepatitis $C$ viremia at week 12 , respectively $(p<0,001)$. Discussion:Using a highly sensitive real-time PCR the detection of minimal residual hepatitis C viremia at treatment week 12 was in $78 \%$ associated with a viral relapse after the end of therapy and is therefore a valuable prognostic tool for the prediction of individual treatment outcome. Treatment of patients with detectable viremia at week 12 should be individually intensified by either prolonging treatment duration or preventing dose reductions by the application of erythropoetin or GM-CSF.

177

JC Virus Is Present in Adenomatous Polyps and Associated with Microsatellite Instability

Woontae Jung, Meishu Li, Ajay Goel, C. Richard Boland

Background: JC virus (JCV), a human polyomavirus, has been implicated in the pathogenesis of colorectal cancer (CRC), but its role in premalignant lesions is unknown. We have shown that JCV induces chromosomal instability when introduced into diploid cells in vitro, but it is not known how it participates in the early stages of colorectal carcinogenesis. Aims: We tested the hypothesis that JCV is present and expresses $\mathrm{T}$ antigen (T-Ag) in adenomatous polyps, and sought associations with specific forms of genomic instability in those lesions. Methods: DNA was extracted from 32 paraffin-embedded adenomatous polyps. JCV gene sequences were amplified by PCR using primers specific for T-Ag, and confirmed by sequencing. Immunohistochemical (IHC) staining was performed using an anti-T-Ag monoclonal antibody. To test for microsatellite instability (MSI), five mononucleotide repeats (BAT-25, BAT-26, NR-21, NR-24, and NR-27) were coamplified in a pentaplex PCR and analyzed for mutations (change in allele length) in an automated DNA sequencer. Results: JCV T-Ag DNA sequences were found in 91\% (29/32) and T-Ag was expressed in 16\% (5/32) of the polyps. The T-Ag staining was localized exclusively in the nuclei of adenoma cells, but not in the cytoplasm nor in adjacent non-neoplastic cells. The prevalence of MSI-H ( $\geqq 3$ mutations), MSI-L (1-2 mutations), and MSS (no mutations) was 3\% (1/32), 38\% (12/32), and 59\% (19/32), respectively. Among 29 specimens in which JCV DNA was detected, MSI was found in 45\% (13/29), however, all specimens negative for JCV DNA were MSS. Conclusions: This study demonstrates that JCV DNA is detectable in $91 \%$ of colorectal adenomas, expresses $\mathrm{T}$-Ag in $16 \%$, and unexpectedly, is associated with MSI in these lesions.

\section{8}

Double RNA Interference of Dnmtl and Dnmt3b Induces Promoter Demethylation and Reactivation of Tumor Suppressor Genes and Apoptosis in Human hepatocellular carcinoma Cells

Satoshi Kurita, Yoshimasa Saito, Hajime Higuchi, Nobuhiro Nakamoto, Hitomi Horikawa, Hideaki Kanamori, Shinichiro Tada, Hidetsugu Saito, Toshifumi Hibi

[Background \& Aims] DNA methylation plays critical roles in chromatin remodeling and regulation of gene expression in mammalian development and human disease, such as cancer. We have reported that several tumor-suppressor genes are silenced by aberrant DNA methylation of their promoter CpG islands in the tumor tissues from patients with hepatocellular carcinoma (HCC), and that expression levels of DNA methyltransferases (DNMTs) were increased during the progression from chronic hepatitis or liver cirrhosis to HCC. Although both DNMTl and DNMT3b are required to maintain genomic DNA methylation patterns in mammalian cells, their roles in human hepatocarcinogenesis have not been fully understood. To clarify the correlation between DNA methylation and hepatocarcinogenesis, we depleted DNMT1 and DNMT3b in human HCC cells. [Methods] Human HCC cell lines (PLC/PRF/5 and Huh7) were transfected with short interfering RNAs (siRNAs) to specifically downregulate the expression levels of DNMTl and DNMT3b. Expression levels of mRNAs and proteins were examined by real-time RT-PCR and western blotting, respectively. DNA methylation status of the promoter $\mathrm{CPG}$ islands in tumor suppressor genes (pl6, RASSFlA and E-cadherin) was examined by methylation-specific PCR after bisulfite modification. Cell proliferation was measured by MTS assay and the cell nuclei were stained with DAPI. Caspase-3, -8 , and -9 activities were measured by EIA. [Results] Trasnfection of specific siRNA to each DNMT significantly reduced mRNA and protein expression levels of each enzyme, but did not affect DNA methylation status of promoter CpG islands in pl6, RASSFlA and E-cadherin genes. On the other hand, trasnfection of both siRNAs successfully induced demethylation of promoter $\mathrm{CpG}$ islands and reactivation of these genes. Moreover, knockdown of both DNMTs resulted in the significant reduction of cell proliferation, and DAPI staining of the transfected cells showed nuclear condensation and/ or cleavage. Caspase- $3,-8$, and -9 activities were increased in the cells transfected with both siRNAs, indicating that apoptosis was induced by double knockdown of DNMTl and DNMT3b. [Conclusion] These data suggest that DNA methylation of specific genomic regions maintained by DNMTl and DNMT3b plays a critical role in survival of HCC cells, and a simultaneous knockdown of both DNMTl and DNMT3b may be a novel anti-cancer strategy for the treatment of $\mathrm{HCC}$

\section{9}

Role of Braf Mutation in HNPCC Screening Strategies Xavier Bessa, Belen Balleste, Beatriz Bellosillo, Montserrat Andreu, Spanish Gastroenterological Association GI Oncology Group

The selection of patients according to Bethesda guidelines and further genetic testing, is the most efficient strategy for detection of MSH2 or MLH1 gene carriers in patients with newly diagnosed colorectal cancers (CRC)(Piñol V. JAMA 2005). Recently, an oncogenic mutation in BRAF gene has been found in colorectal tumours showing DNA mismatch repair gene deficiency without germinal mutations in the mismatch repair genes. MSI in these patients are due to hypermethylation of MLHl. It has been suggested that BRAF analysis could simply and improve the cost-effectiveness of the genetic testing reducing the number of DNA repair genes sequencing. Objective: To know the prevalence of BRAF mutation in a population sample with CCR and microsatellite instability. To evaluate the differential characteristics according to BRAF mutation. To analyse the costs of BRAF mutation analysis compared to traditional strategies. Methods: Study sample was the 1222 patients with colorectal cancer belonging to EPICOLON study (Piñol V. JAMA 2005). We determine the BRAF mutation in 91 cases with deficiencies in DNA mismatch repair genes (MSI and/or lack of MLH1 or MSH2 inmmunostaging). The BRAF mutation was evaluated by automatic sequencing. Results: BRAF mutations were found in 20 patients (21.4\%) with colon cancer showing deficiencies in DNA mismatch repair genes. None of them showed unambiguous germline mutations in MLHl and MLH2 genes. Only 2 patients (4.8\%) with MSI-L had BRAF mutation. Variables associated with mutation of BRAF were: older age $(78+6$ vs. $67+15, \mathrm{p}<0.002$ ) and right tumour location (OR: 4.69; IC 95\%: 1.01-21.94). In patient fulfilling revised Bethesda guidelines, performing BRAF analysis allowed a small reduction in costs (saving of 700 or 322 euros per detected mutation based on the presence of MSI or abnormal inmunostaging, respectively.) However, if we performed BRAF analysis in patients without previous selection according to these clinical criteria, we achieved a significant minimisation of costs (a saving of 3970 or 1867 euros per detected mutation based on the presence of MSI or abnormal inmunostaging, respectively) Conclusions: Detection of BRAF mutation could simplify and improve the cost-effectiveness of genetic testing for HNPCC, especially in patients whose family history is incomplete or unknown.

180

Loss of Imprinting of the Insulin Growth Factor 2 (IGF2) Gene in Buccal Cells: Correlation with Risk of Colorectal Cancer

Enrique Quintero, Daniel Deniz, Cristina Paz-Cabrera, Alejandro Jimenez, Antonio Z Gimeno, Adolfo Parra-Blanco, David Nicolas, Eduardo Salido

Introduction: Loss of imprinting at the IGF2 gene (IGF2-LOI) is an epigenetic change present in colonic tissue of about $40 \%$ of colorectal cancer (CRC) patients. Aim: to investigate whether IGF2-LOI in the epithelial cells of buccal samples is a surrogate marker that could predict the risk of CRC. Methods: 34 patients with CRC, 60 with colonic adenomas and 62 controls (colonoscopy without pathological findings) were included. LOI was assessed by studying the methylation status of a key CpG island at the IGF2 promoter, using PCRRFLP after bisulfite treatment of DNA (COBRA method). Hypomethylation was taken as a surrogate marker for LOI, which was investigated in DNA from buccal cells, tumor samples, non-neoplastic adjacent mucosal samples, and peripheral blood cells. Results: In buccal cells, IGF2-LOI was detected in 5/62 (8\%) controls, 10/60 (17\%) patients with colonic adenoma and 12/34 (35\%) patients with colorectal carcinoma. In neoplastic tissue, IGF2 LOI was observed in $9 / 43(21 \%)$ samples of carcinoma and $13 / 50$ (26\%) samples of adenomas. No differences in IGF2-LOI were observed in the DNA from peripheral blood cells among the various study groups. Buccal samples of patients with colorectal carcinoma presented a higher relative risk to show IGF2-LOI than the controls (OR 6,21; IC 1,96-19,70, p<0.01) Conclusion: IGF2-LOI in the DNA from buccal cells could be a non-invasive surrogate marker useful for the screening of colorectal cancer in the average-risk population.

\section{1}

Disruption of Activin Signaling Components in Microsatellite Stable (MSS) Colon Cancers

Barbara Jung, Jessica Gomez, Christine M. Caro, Eddy Chau, Temitope Keku, Robert S. Sandler, John M. Carethers

BACKGROUND: Mutations in TGF[beta] receptor 2 (TGFBR2) have been identified in both microsatellite unstable (MSI) and MSS colon cancers, Activin, a growth-suppressive ligand from the transforming growth factor beta superfamily, binds to activin receptor 2 (ACVR2) which in turn phosphorylates activin receptor 1 (ACVR1), causing SMAD2 phosphorylation, association with SMAD4, and translocation to the nucleus affecting gene transcription. We have previously shown that ACVR2 is mutated in one of its two coding polyadenine tracts in more than $80 \%$ of MSI primary colon cancers, leading to loss of expression and increased local tumor growth. Here, we examined if activin signaling is also abrogated in MSS colon cancers. METHODS: Fifty MSS population-based colon cancers were tested for presence of ACVR1 and ACVR2 protein by immunohistochemistry. Loss of ACVRl or ACVR2 was correlated with levels of pSMAD2. Markers flanking the ACVR2 gene locus were used to assess for loss of heterozygosity (LOH) in tumors with protein loss and coding polyadenine tracts of ACVR2, which is the prime site of inactivation in MSI colon cancers, were sequenced. RESULTS: Of 50 primary MSS colon cancer specimens, 5 lost ACVR2, 2 lost ACVR1 and 1 lost expression in both. This correlated with decreased pSMAD2 in 2 cases. One case showed a decrease in PSMAD2 in tumor tissue without concomitant loss of ACVR2 or ACVR1 or TGFBR2. We found no cases with $\mathrm{LOH}$ at ACVR2, nor mutation in the polyadenine tract of the tumors tested, implicating different mechanisms for loss of expression. CONCLUSIONS: Both ACVR2 as well as ACVR1 loss occurs in MSS colon cancers and correlates with decrease in downstream PSMAD2 activation, implicating disruption of activin signaling regardless of the type of genomic instability in colon cancers. The mechanism for ACVR2/ ACVRl inactivation is being analyzed at the genetic level. 
The Role of the Bone Morphogenetic Protein Pathway in Sporadic Colorectal Cancers with Different Microsatellite Instability Status

Liudmila L. Kodach, Sylvia A. Bleuming, Alex R. Musler, Maikel P. Peppelenbosch, Gijs R. van den Brink, Johan A. Offerhaus, James C. Hardwick

Transforming Growth Factor b (TGFb) is thought to play a central role in colorectal cancer (CRC). TGFb receptor 2 (TGFbR2) is the most frequently mutated gene in MSI (microsatellite unstable) cancers, but quite how this leads to cancer is unclear with TGFbR2 knockout mice showing no CRC phenotype. The TGFb superfamily consists of TGFb, Activin and Bone Morphogenetic Protein (BMP) subfamilies. Recently Activin receptor 2 (ACVR2) has been found to be mutated in the majority of MSI cancers. The finding of BMP Receptor la mutations in Juvenile Polyposis suggests that BMPs also play a role in CRC carcinogenesis. Our aim was to investigate the role of BMPs in sporadic CRC. We assessed the expression of BMP receptors and SMADs 1 and 4 in 72 sporadic CRCs (56 Mismatch repair (MMR)proficient and 16 MMR-deficient tumors), using a Tissue Microarray and immunohistochemistry. We also investigated whether BMP receptor type 2 was normally expressed in 4 MSI cell lines and 3 microsatellite stable (MSS) cell lines using immunoblotting. Colon cancer cell lines were further analyzed for mutations in a coding sequence microsatellite (A7) of BMPR2, as well as the known A8 tract in ACVR2 and A10 tract in TGFbR2. MMR-deficient CRCs show frequent loss of expression of BMP receptors $1 \mathrm{~A}, 1 \mathrm{~B}$ and 2 compared with MMR-proficient cancers. The expression of BMPR 2 is altered in $75 \%$ of MMR-deficient and in 25\% ( $\mathrm{p}<0.001)$ MMR-proficient colorectal cancers. Loss of BMPR2 expression is only seen in SMAD4 expressing cancers and not in SMAD4 negative cancers. BMPR2 is mutated at A7 microsatellite sequence in 2 out of 4 MSI colon cancer cell lines and in none of the MSS cell lines. BMPR2 is not expressed at protein level in MSI cell lines, but is normally expressed in MSS cell lines. Our data provide evidence that the BMP pathway is important in sporadic colon cancer. The mutually exclusive nature of BMPR2 and SMAD4 expression loss is as would be biologically expected and strongly suggests that we are not looking at an artefact of immunohistochemistry. This is further supported by our cell line data where we see the same expression pattern. Furthermore, in two cell lines we find a mutation at a microsatellite in BMPR2. The loss of BMPR2 expression in MSI cancer ties up neatly with the known defects in TGFbR2 and ACVR2 expression in MSI cancers and confirms the importance of the TGFb superfamily in the carcinogenesis of CRC. The mouse knockout data would support the hypothesis that while a mutation in one of the SMADs is sufficient to cause neoplasia, mutations at the receptor level in the same pathway must occur in combination

\section{3}

Proteomic Analysis of Immunoisolated H/K-ATPase Containing Tubulovesicles from Human Parietal Cells

Lynne A. Lapierre, Kenya Avant, Catherine M. Caldwell, Janice A. Williams, Amy-Joan L. Ham, Salisha Hill, Adam Smolka, James R. Goldenring

Gastric parietal cells possess an amplified apical membrane dedicated to regulated recycling of the H/K-ATPase to the luminal surface of the stomach. While apical recycling is amplified in parietal cells, this process is critical to polarized secretory processes in most epithelial cells. To understand better the regulation of apical recycling in polarized cells, we used a proteomic approach to characterize the components of $\mathrm{H} / \mathrm{K}$-ATPase-containing recycling vesicles in human parietal cells. Using a monoclonal antibody specific to the a-subunit of the gastric $\mathrm{H} / \mathrm{K}$-ATPase, we immunoisolated vesicles from an enriched tubulovesicle preparation derived from resting fundic mucosa of 4 organ donor stomachs obtained under IRB approval. Proteins were eluted from the immunoisolated vesicle preparations with CHAPS and analyzed by multidimensional-LC-MS-MS mass spectrometry. We identified and validated by western blots many of the components previously identified on immunoisolated rabbit tubulovesicles including Rab1l and Rab25 as well as two previously unrecognized small GTPases, Rab4 and Rab10. Similarly, we confirmed the presence of several proteins involved in vesicle docking and fusion, including syntaxin 3, SCAMPs and VAMP2, previously identified on rabbit tubulovesicles. In addition, we detected several previously unrecognized proteins including VAMP8, syntaxins 7 and 12/13, pantophysin, DP1/TB2 (the human homolog of YOPlp) and Annexins. We also identified the potassium channel component, KCNE2. Immunostaining of human gastric mucosal sections confirmed the presence of each of these proteins in parietal cells and their co-localization with H/K-ATPase on the tubulovesicles. To investigate the role of these proteins in apical recycling, we transfected them as DSRed2 fusions into a GFP-Rabl la expressing MDCK cell line. Both VAMP8 and pantophysin caused a collapse of the GFP-Rablla compartment, while a less dramatic effect was observed in cells transfected with syntaxin 7 or VAMP2. Interestingly, while overexpression of DsRed2pantophysin altered the GFP-Rablla compartment, it did not co-localize with the GFPRablla, but was localized to an adjacent tubular compartment. This study, which represents the first proteomics analysis of an immunoisolated vesicle population from native human tissue, not only identified previously unrecognized proteins present in parietal cell H/ K-ATPase recycling membranes, but also revealed common regulators of epithelial cell apical recycling

\section{4}

Identification of a Novel Negative Feedback Regulation of the ECL Cell During Acid Secretion By the Parietal Cell Using Subtractive Hybridization Microarray Gene Expression Profiling

Nils Lambrecht, Iskandar Yakubov, Cindy Zer, George Sachs

Background: The identification of novel cell-specific genes from gastrointestinal epithelial cells will lead to new therapies for diseases of the gastrointestinal tract. Although much data have been accumulated as to the function of some of the most prevalent proteins expressed in the major epithelial cell types of the gastric mucosa, others have not been suspected or identified. Here we present specific microarray expression profiles of parietal cells and ECL cells of the rat gastric mucosa obtained by a novel microarray based technique - subtractive hybridization. These profiles suggest proteins involved in novel functions in both cell types, which can be further studied by classical physiological model systems. Methods: Rat ECL and parietal cells were purified to more than $95 \%$ homogeneity using a physical separation approach (density centrifugation and counterflow elutriation) followed by FACS based on unique properties of each cell type. RNA from the purified cells was compared to RNA from the whole gastric mucosa using Agilent $22 \mathrm{~K}$ whole genomic expression oligonucleotide microarray. Only genes specific to ECL or parietal cells are present in high amounts with equal abundance of "house keeping" genes present in all cells of the gastric mucosa. This approach is the subtractive hybridization method. Here, we report on proteins involved in regulation of acid secretion and targets were tested by immunohistochemical localization in rat gastric mucosa and by intracellular calcium imaging and aminopyrine uptake in gastrin stimulated intact rabbit gastric glands. Results: 1) Comparison of ECL cell specific and parietal specific genes identified apelin expressed specifically in parietal cells and the apelin binding APJ receptor expressed specifically in ECL cells, confirmed by immunohistochemical localization. 2) Gastrin stimulated intracellular calcium signals in ECL and parietal cells in the gastric glands are abolished in the presence of apelin. 3) Gastrin stimulated aminopyrine uptake in the glands is significantly inhibited in the presence of apelin. Conclusion: Expression of apelin in parietal cells and the inhibitory APJ receptor in ECL cells suggests a novel negative feedback loop between these two cell types during acid secretion. Although we did not study the release of apelin from parietal cells, the fact that apelin abolishes gastrin stimulated ECL cell calcium signals, the hallmark of histamine release in these cells, and inhibits acid secretion in the rabbit gastric gland model, strongly supports the hypothesis, that apelin is released during stimulation of parietal cells and directly down regulates histamine release from ECL cells.

185

MIRP1 (kcne2) Is Essential for Gastric Acid Secretion Torsten K. Roepke, Arun Anantharam, Stephanie M. Busque, Jeffrey B. Young, John P. Geibel, Daniel J. Lerner, Geoffrey W. Abbott, Philipp Kirchhoff

Background: Gastric acid secretion via $\mathrm{H}+/ \mathrm{K}+$-ATPase is coupled to luminal $\mathrm{K}+$ uptake and therefore requires a $\mathrm{K}+$ recycling mechanism across the apical membrane. Several different $\mathrm{K}+$ channels are proposed to contribute to this mechanism. One of them, KCNQ1, is postulated to form functional complexes in parietal cells with the voltage-gated potassium (Kv) channel $\beta$ subunit MiRPl (encoded by kcne2), converting it into a constitutively open 'leak' channel. Mice lacking the KCNQ1 gene exhibit hypochlorhydria and gastric hyperplasia. Here, we directly studied the role of the $\mathrm{Kv} \beta$ subunit MiRPl in gastric acid secretion. Methods: To investigate the role of MiRPl in acid secretion we created kcne2 (+/-) and (-/) mice by replacing the entire kcne2 coding region with the E.coli lacZ gene and a neomycin resistance cassette (neor) flanked by loxP sites using homologous recombination. The effects of kcne2 knockout on gastric acid secretion were characterized using intracellular pH (pHi) measurements of individual parietal cells in freshly isolated murine gastric glands under stimulation with histamine $(100 \mathrm{mM})$ or carbachol $(100 \mathrm{mM})$; and $\mathrm{pH}$ measurements in ex vivo whole stomach preparations. Results: Comparing $\mathrm{pHi}$ in isolated gastric glands, kcne2 $(-/)$ mice have a $90 \%$ reduction in gastric acid secretion $(\mathrm{n}=70$ parietal cells from 5 glands), and kcne2 (+/-) mice show a 50\% reduction ( $\mathrm{n}=140$ cells, 15 glands). Mean ex vivo whole stomach $\mathrm{pH}$ was raised from $\mathrm{pH} 3.75 \pm 0.01$ in wild-type mice to $\mathrm{pH} 4.12 \pm$ 0.28 (+/- mice) and $\mathrm{pH} 5.65 \pm 0.84(-/$ - mice $)(\mathrm{n}=2-3$ whole stomach preparations per group). Further, kcne2 (-/-) mice displayed fivefold enlargement by weight of the stomach, significant glandular hyperplasia due to lengthening of the neck and fundus portions, parietal cell atrophy and hypergastrinemia $(317.38 \mathrm{pg} / \mathrm{ml} \pm 103.87(-/-) \mathrm{vs} .62 .78 \mathrm{pg} / \mathrm{ml} \pm 29.8(+/$ $+)$ ). Nevertheless, kcne2 (-/) mice appear healthy at 12 weeks, and show no significant difference in bodyweight compared to wild-type $(27.19 \mathrm{~g} \pm 1.09$ vs. $26.14 \mathrm{~g} \pm 1.42$ (male); $20.15 \mathrm{~g} \pm 1.87$ vs. $21.76 \pm 0.47$ (female), respectively ( $\mathrm{n}=5$ - 10 per group). Conclusions: Our findings provide first genetic evidence that MiRPl, encoded by kcne2, is required for gastric acid secretion. The data suggest that conversion of KCNQ1 to a leak channel by MiRPl is essential for this process. Furthermore, MiRPl appears important for normal development and structure of mouse gastric parietal cells.

186

Intracellular Signal Transduction Pathways Activated By Growth Factors in Isolated Parietal Cells

Nonthalee Pausawasdi, Mahdi Sareban, Maria Y. Mao, Andrea Todisco

BACKGROUND: EGF and IGF-I exert important regulatory actions on gastric epithelial cell growth and differentiation. We previously reported that while incubation of purified (>95\%) canine parietal cells with EGF for $16 \mathrm{~h}$ induces $\mathrm{H}+/ \mathrm{K}+-\mathrm{ATP}$-ase gene expression through the activation of Akt, prolonged exposure $(>72 \mathrm{~h}$ ) of the cells to EGF leads to parietal cell morphological transformation and to inhibition of $\mathrm{H}+/ \mathrm{K}+-\mathrm{ATP}$-ase gene expression through MAPK-dependent mechanisms. We also observed that BMP-4, a peptide known to promote gastric epithelial cell differentiation, induces $\mathrm{H}+/ \mathrm{K}+-\mathrm{ATP}$-ase gene expression in the parietal cells. AIM: We explored the effects of prolonged exposure $(72 \mathrm{~h})$ of the parietal cells to IGF-I and we compared them to those observed in the presence of EGF. Moreover, we investigated if BMP-4 modulates the actions of EGF and IGF-I in the parietal cells. METHODS: $\mathrm{H}+/ \mathrm{K}+-\mathrm{ATP}$-ase gene expression was examined by Northern blots with a canine $\mathrm{H}+/ \mathrm{K}+-$ ATP-ase alpha-subunit cDNA probe. MEKl, MAPK and Akt activation were measured by western blots with anti-phospho-MEK1, -MAPK and -Akt antibodies. Parietal cell morphology was examined by immunohistochemical staining of the cells with anti-H+/K+-ATP-ase alphasubunit antibodies. RESULTS: Both IGF-I ( $10 \mathrm{nM})$ and EGF (10 nM) changed the morphology of the parietal cells that acquired the appearance of fusiform cells. IGF-I induced the activation of both MEKI and MAPK and this effect was less then that seen in the presence of EGF. IGF-I induced Akt while EGF had no effect. EGF inhibited H+/K+-ATP-ase gene expression, while IGF-I had a modest stimulatory effect. BMP-4 $(20 \mathrm{ng} / \mathrm{ml})$ potentiated the effect of IGF-I on $\mathrm{H}+/ \mathrm{K}+$-ATP-ase gene expression and it blocked the inhibitory actions of EGF BMP-4 inhibited EGF and IGF-I stimulation of both MEKI and MAPK, but it had no significant effect on IGF-I induction of Akt. CONCLUSIONS: In contrast to EGF, IGF-I does not inhibit $\mathrm{H}+/ \mathrm{K}+-\mathrm{ATP}$-ase gene expression, it induces a sustained activation of Akt 
and it only weakly activates the MAPK signal transduction pathway. BMP-4 appears to potentiate the actions of IGF-1 and to inhibit those of EGF. Thus, IGF-I and EGF regulate different programs of parietal cell activation. These results underscore the importance of the Akt signal transduction pathway in the regulation of parietal cell differentiation and they suggest that complex networks of growth factors and regulatory peptides might be needed for the regulation of the biological functions of the gastric parietal cells.

\section{7}

Transgenic Expression of Hedgehog Interacting Protein (HIP) in Parietal Cells Blocks Sonic Hedgehog Activity and Acid Secretion

Yana Zavros, Meghna Waghray, Michael J. Smith, Andrea Todisco, Deborah Gumucio, Juanita L. Merchant

Objective: The hedgehog signaling pathway is critical to normal gastrointestinal development and function. The stomach is the largest source of sonic hedgehog (Shh). In particular, Shh is highly expressed in the acid-secreting parietal cells where it induces $\mathrm{H}^{+}, \mathrm{K}^{+}$-ATPase gene expression and enhances histamine-stimulated acid secretion. Chronic gastritis and parietal cell atrophy associated with an increased risk for the development of gastric cancer. Hedgehog interacting protein (HIP) binds to and sequesters Shh by preventing the interaction with its receptor patched (Ptc). Therefore, we test the hypothesis that loss of the Shh signaling pathway within parietal cells is crucial for the development of parietal cell atrophy. Methods: The parietal cell-specific $\mathrm{H}^{+}, \mathrm{K}^{+}$-ATPase $\beta$ subunit promoter (HK) was used to drive expression of mouse HIP (mHIP) lacking the transmembrane domain which allows HIP to be secreted. $\mathrm{HK} / \mathrm{mHIP}$ mice were sacrificed at 2 months of age and examined for tissue morophology and gastric acid content. Total RNA was prepared from stomachs of $\mathrm{HK} / \mathrm{mHIP}$ mice and their age matched non-transgenic ( $\mathrm{Ntg}$ ) litter mates. RT-PCR was used to analyze $\mathrm{H}^{+}, \mathrm{K}^{+}$ATPase, Shh, Ptcl and Glil mRNA abundance. Protein was also prepared and analyzed by western blotting for Shh expression. Results: Histology revealed that there was a decrease in parietal cell numbers. Gastric acidity was significantly lower in the HK/mHIP $(3.07 \pm$ $0.92 \mu \mathrm{Eq},{ }^{*} \mathrm{P}<0.05$ versus $\left.\mathrm{Ntg}, \mathrm{n}=3\right)$ compared to the $\mathrm{Ntg}(36.4 \pm 2.35 \mu \mathrm{Eq}, \mathrm{n}=3)$ mice. Reduced gastric acidity in the HK/mHIP mice was consistent with reduced $\mathrm{H}^{+}, \mathrm{K}^{+}$-ATPase mRNA abundance. Western blots revealed no differences in the $45 \mathrm{kD}$ a precursor Shh protein in the stomachs of $\mathrm{Ntg}$ and $\mathrm{HK} / \mathrm{mHIP}$ mice that was consistent with no change in Shh mRNA abundance. Interestingly, the processed biologically active $19 \mathrm{kDa}$ Shh peptide was significantly reduced in the HK/mHIP animals. RT-PCR data revealed a loss of the Shh downstream gene targets Ptcl and Glil mRNA in the HK/mHIP animals. Conclusion: Secreted HIP binds to the $19 \mathrm{kDa}$ biologically active Shh peptide that subsequently blocks the hedgehog signaling pathway within the gastric parietal cells. As a consequence there is loss of acid secretion and the development of parietal cell atrophy within 2 months. The $\mathrm{HK} / \mathrm{mHIP}$ mouse model may be used to study how loss of Shh predisposes the stomach to atrophy and the development of gastric cancer

\section{8}

Glycosylation-Mediated Apical Sorting of the Na,K- and Gastric H,K-ATPase $\beta$ Subunits in Polarized Cells

Olga Vagin, Shahlo Turdikulova, George Sachs

Background: The gastric H,K-ATPase is located in tubulovesicles in the resting parietal cell and relocates to the canalicular (apical) membrane upon stimulation of acid secretion. The homologous $\mathrm{Na}$, K-ATPase resides in the basolateral plasma membranes in most polarized cells. The present work was aimed at identifying the role of $\mathrm{N}$-glycosylation in apical sorting of the gastric H,K-ATPase. Methods: Stable cell lines expressing YFP-linked $\beta$-subunits of the $\mathrm{H}, \mathrm{K}$ - or $\mathrm{Na}, \mathrm{K}$-ATPase were developed. Cellular localization of the subunits was examined using confocal microscopy. Apical and basolateral content of the $\beta$-subunit and the rate of endocytosis was determined using surface-selective biotinylation. Results: The YFP-linked $\mathrm{H}$,K-ATPase monomeric $\beta$ subunit was expressed on the apical membranes in three types of polarized cells: kidney-derived LLC-PKl and MDCK cells and parietal cell-derived HGT1 cells. Mutagenic removal of any of the seven N-glycosylation sites from the H,K-ATPase $\beta$ subunit impaired delivery of the protein from TGN to the apical membrane and enhanced endocytosis from the apical membrane in all three cell types. Deletion of sites at N1, N3, N5 and N7 had the most significant effect, suggesting a critical role for these sites in apical sorting of the H,K-ATPase. HGT-1 cells express the endogenous H,K-ATPase and the homologous $\mathrm{Na}, \mathrm{K}$-ATPase $\beta$ subunits on the apical membrane in contrast to MDCK and LLC-PKl cells that lack H,K-ATPase and express the Na,K-ATPase only on the basolateral membrane. HGT-1 cells express the Na,K-ATPase $\beta 2$, but not the $\beta 1$ isoform, while the $\beta 1$ is the major isoform in MDCK and LLC-PKl cells. When expressed in HGT-1 cells, the YFP-linked $\beta 1$ subunit was localized to the lateral surface. The human $\beta 2$ subunit has eight $\mathrm{N}$-glycosylation sites, while the $\beta 1$ isoform has only three. We tested whether the greater number of glycosylation sites in the $\beta 2$ subunit as compared to the $\beta 1$ is responsible for the apical localization of the Na,K-ATPase subunit in HGT-1 cells. Hence, up to five additional $\mathrm{N}$-glycosylation sites homologous to the ones present in the $\beta 2$ subunit were successively introduced in the $\beta 1$ subunit by site-directed mutagenesis. The mutated $\beta 1$ subunits were detected on both apical and basolateral membranes but the fraction of the mutant $\beta 1$ subunit present on the apical membrane increased in proportion to the number of glycosylation sites inserted and reached $80 \%$ of the total surface amount for the $\beta 1$ mutant with five additional sites. Conclusion: The N-glycans linked to the $\beta$ subunit isoforms of P2-type ATPases contain apical sorting information and may be essential for parietal cell sorting of the H,K-ATPase
Expression of Oncogenic Kras in Developing Zebrafish Pancreas: A New Vertebrate Model of Exocrine Pancreatic Cancer

Seungwoo Park, Jon Davison, Steven Leach

The zebrafish has emerged as a powerful vertebrate system for the study of cancer biology In an effort to generate the first zebrafish model of exocrine pancreatic cancer, we created transgenic zebrafish in which ptfla regulatory elements were utilized to target oncogenic Kras to developing zebrafish pancreas. Materials and Methods: Two different BAC transgenes were generated by recombineering a genomic BAC spanning the zebrafish ptfla locus (CH211-142H2). Ptfla coding sequences were replaced by either GFP alone (ptfla:GFP) or by a GFP-Kras(G12V) fusion construct (ptfla:GFP-Kras(G12V)), in order to enable real-time visualization of transgene expression. Transgenic lines were established following injection of modified BACs at the single cell stage. Results: Ptfla:GFP transgene expression faithfully recapitulated the normal developmental pattern of ptfla expression. GFP expression became apparent at 32 hours post fertilization and persisted in virtually all exocrine pancreatic cells through adulthood. In contrast, expression of the ptfla:GFP-Kras(G12V) transgene gradually diminished during development, becoming restricted to an abnormal population of undifferentiated precursor cells. However, widespread expression of the ptfla:GFP-Kras(G12V) transgene reappeared at adulthood, as evidenced by external examination of transcutaneous green fluorescence (Tc-GFP), immunohistochemistry, and RT-PCR. Selective sacrifice of fish with Tc-GFP revealed pancreatic carcinoma with occasional invasion and metastasis into bowel wall, liver, and ovary. Proportions of fish with detectable Tc-GFP increased with advancing age of the fish: $20 \%(6 / 30)$ at 3 months, $41 \%(31 / 75)$ at 6 months, and $67 \%$ (30/45) at 9 months. The extent of detectable Tc-GFP was highly concordant with tumor burden. Tumors displayed a mixed pattern of differentiation, with areas of ductal or squamoid differentiation intermixed with areas of undifferentiated and acinar cell carcinoma. Immunostaining for pan-Ras and GFP showed robust transgene expression confined to tumor cells. Tumor cells also stained positively with Alcian blue, PAS, and mucicarmine, confirming the production of mucin. Similar to human pancreatic cancer, components of the Hedgehog signaling pathway, including Ptc2, Smoothened and Glil, were upregulated in tumor cells. Conclusion: We have generated the first zebrafish model of exocrine pancreatic cancer, in which use of a GFP-Kras fusion protein allows for the non-invasive determination of tumor burden. This model offers a novel platform for high-throughput, in vivo screening for new pancreatic cancer drugs, in a manner not currently achievable in mouse or human.

\section{0}

Reconstitution of P16ink4a in An Orthotopic Pancreatic Mouse Model Inhibits Tumor Growth and Angiogenesis

Petra Schulz, Arne Scholz, Annett Rexin, Peter Hauff, Michael Schirner, Bertram

Wiedenmann, Katharina Detjen, Stefan Rosewicz

Introduction: The tumor suppressor gene pl6INK4a is inactivated in more than $90 \%$ of patients with pancreatic cancer. The biological relevance of $\mathrm{p} 16$ inactivation is well characterized at the cellular level and represents an essential step in the transformation process However, less information is available about effects on cancer progression, metastasis an angiogenesis. We used an in vivo inducible system to reexpress pl6 in an orthotopic model of pancreatic cancer and examined the impact on these clinically relevant aspects of pancreatic cancer tumor biology. Methods: The vectors pcDNA6/TR and pcDNA4/TO-p16 were sequentially transfected into p16 deficient MiaPaCa-2 cells and clones with inducible expression of p16 were selected (MiaPaca-2-TREx-p16). After in vitro characterization of proliferation (Alamar blue assay), colony formation (Human Tumor Clonogenic Assay) and cell cycle analysis (Flow Cytometry) the cells were orthotopically implantated into the pancreas of nude mice. Mice were randomly assigned to treatment and control groups (11 each). Animals in the treatment group received doxycycline (Dox) in the drinking water. Tumor growth, vascularization and metastasis were determined at the time of autopsy. Micro vessel density (MVD) and lymph vessel density (LVD) were determined by IHC based on CD31 and Lyve1 staining, respectively. IHC detection of Human Cytokeratin was used to confirm metastatic cells. Results: Induction of pl6 in MiaPaCa-2 cells in vitro led to a small reduction o proliferation (13\% of the control) and colony formation ( $40 \%$ of the control) as well as to an accumulation of cells in the Gl-phase. In vivo, Dox-induced expression of pl6 was confirmed in 6/11 mice with Mia PaCa-2-TREx-pl6 orthotopic tumors. In these animals, tumor size was reduced to $60 \%$ of the respective controls. In contrast, Dox treatment had no significant effect on tumor size in mice inoculated with MiaPaCa-2-TREx control cells. Induction of $\mathrm{pl} 6$ expression led to a significant reduction of MVD ( $34 \%$ of the control). Similarly lymph-angiogenesis was decreased in the presence of pl6 (36\% of the control) In addition to the decrease in vascularization, tumor cells with reexpression of p16 were less frequently found in lymph nodes ( $27 \%$ of lymph nodes positive as compared to $90 \%$ in controls). Conclusions: In extension to previous reports on antiproliferative effects of p16, we demonstrate that reexpression of the tumor suppressor in an orthotopic model of pancreatic cancer reduced both primary tumor growth and metastasis. Furthermore this is the first study to show an anti-(hem/lymph)-angiogenic effect of pl6 in a clinically relevant model of pancreatic cancer

191

Dissemination of Human Pancreatic Tumor Cells Is Mediated By Caveolae Lidiya Orlichenko, Muyiwa Awoniyi, Mark A. McNiven

BACKGROUND: Pancreatic cancer is a lethal disease with an exceptionally poor prognosis, as pancreatic neoplasms have a marked tendency to invade and metastasize to distal organs. Currently, the molecular mechanisms that make these tumors so aggressive are poorly defined. Recently we have demonstrated that EGF stimulation induces both normal epithelial cells and human pancreatic tumor cells (PANC-1) to form large numbers of endocytic structures termed caveolae at cell borders. Caveolae formation precedes internalization o the cell adherens junction protein E-cadherin (E-Cad), loss of cell-cell contacts, and subsequent cell dissemination and migration. The GOAL of this study was to test if the loss of

\section{(1)}


pancreatic ductular cell-cell integrity during metastasis is mediated by EGF-stimulated caveolae formation. RESULTS: We find that the endogenous levels of both caveolin 1 (Cavl), a coat protein for caveolae, and E-Cad differ among normal epithelial cell lines (MDCK, NRK) and neoplastic pancreatic tumor cell lines (BxPC3, PANC-1, HPAF). Furthermore, cells with decreased Cavl levels, either endogenously or by siRNA knockdown, form few caveolae and maintain the integrity of cell-cell contacts. In contrast, cells over-expressing Cavl initiate an active internalization of E-Cad-containing caveolae vesicles from the cell borders upon EGF stimulation, leading to cell dissemination. Interestingly, these internalized vesicles coalesce into large endocytic structures at the cell center, reminiscent of "caveosomes," and this may facilitate E-Cad degradation. Indeed, SDS-PAGE analysis of E-Cad in cells overexpressing Cavl shows substantial proteolysis of E-Cad. EGF stimulation induces phosphorylation of Tyrl4 of Cavl and, interestingly, expression of a Tyrl4 phosphorylation mutant form of Cavl greatly reduces cell separation, internalization of E-Cad, and subsequent E-Cad proteolysis. Thus, this suggests that phosphorylation of Cavl via the EGF signaling pathway contributes to these processes. CONCLUSION: We find that there is a relationship between the cellular levels of Cavl protein and the capacity of cells to form caveolae, internalize E-Cad, and maintain cell-cell integrity. Cells that express high levels of endogenous or exogenous Cavl protein internalize and subsequently sequester E-Cad into large endocytic structures upon EGF stimulation, whereas cells expressing low Cavl levels, either endogenously or by siRNA knockdown, form few caveolae in response to EGF stimulation and maintain E-Cad-based cell-cell contacts. This study provides new molecular insights into pancreatic tumor cell dissemination and metastasis and the role of caveolae in these processes.

192

The Kinase Mirk/Dyrklb Is Activated Through the Racl-Mkk3 Pathway and Mediates Survival in Pancreatic Ductal Adenocarcinoma

Xiaobing Deng, Kideok Jin, Daina Z. Ewton, Sheena Li, Asghar Naqvi, Stephen E. Mercer, Eileen A. Friedman

Ductal adenocarcinoma of the pancreas is highly resistant to apoptosis induced by chemotherapeutic drugs or by radiation. Mirk/dyrklB is a stress-activated kinase which facilitates the survival of regenerating skeletal muscle cells after injury, and mediates survival in pancreatic ductal adenocarcinoma where it is upregulated. Increased expression of Mirk was seen in pancreatic carcinomas compared with primary cultures of normal ductal epithelium by serial analysis of gene expression. Mirk was detected in the majority of cases of pancreatic ductal adenocarcinoma by immunohistochemistry with elevated expression in $39 \%$, and in fetal pancreatic ductal epithelium, so Mirk may be an oncofetal protein. Mirk was detected in 7 of 8 pancreatic cancer cell lines and was an active kinase in each cell line where it was detected. Mirk was activated as a kinase by signaling from Racl to the MAP kinase kinase MKK3, which in turn phosphorylates Mirk. Rac is a Ras superfamily GTPase which, when activated, functions downstream of Ras oncoproteins to promote cell survival, transformation and membrane ruffling. The constitutively active mutant RaclQL activated Mirk through MKK3, which in turn activated Mirk by phosphorylation. Dominant negative Racl, dominant negative MKK3 and knockdown of MKK3 by RNA interference inhibited the kinase activity of co-expressed Mirk. Mirk knockdown by RNA interference reduced the viability of Pancl cells several-fold and in a dose-dependent manner, demonstrating that Mirk is a major survival-mediating kinase in these cancer cells. Mirk initiated survival signals which inhibited apoptosis in Pancl cells, while Mirk knockdown increased the apoptosis induced by gemcitibine and nocodazole in these cells. Mirk kinase activity in pancreatic cancer cell lines was inversely related to the activity of the survival kinase Akt, which suggested that pancreatic cancer cells relied primarily on either Mirk or Akt for anti-apoptotic signaling. Mirk does not appear to have wide-spread effects on cell viability as others have found that knockout of Mirk/dyrk1B was not lethal to the embryo. It is believed by many investigators that carcinoma cells depend on a few dominant survival signals, while normal cells rely on many survival signals, so there might be a therapeutic window which would allow the targeting of a survival kinase such as Mirk in cancers. Because of its properties and expression pattern, Mirk may represent a novel therapeutic target for pancreatic ductal adenocarcinoma.

193

Pancreas-Specific Inactivation of Relap65 in Two Mouse Models of Pancreatic Tumor

Maryna Lesina, Hana Alguel, Nakhai Hassan, Paxian Stephan, Matthias Treiber, Roland M. Schmid

$\mathrm{NF}-\mathrm{kB} / \mathrm{Rel}$ has been shown to be activated during oncogenesis of the pancreas in several murine tumor models. Its activation has been associated with antiapoptotic and proliferative functions. Therefore inhibition of NF-kB/Rel has been evaluated as therapeutic agents in the treatment of pancreatic cancer. Here we present a mouse in which RelAp65 has been specifically inactivated in the pancreas using the Cre/lox-system. Tissue-specific deletion of RelAp65 in the pancreas enabled us to examine the role of NF-kB/Rel in the development of preneoplastic lesions or pancreatic oncogenesis in two mouse models. The floxed RelAp65 mouse was crossed with the TGF $\alpha$ transgenic model of pancreatic tumor and with the KRASG12D mice representing a model for PanIN lesions. Tissue-specific deletion of RelAp65 was directed to the pancreas by using a p 48 Cre mouse which expressed the Cre recombinase in progenitor cells of the exocrine compartment. This resulted in a truncated RelAp65 protein which is incapable of translocating into the nucleus, binding DNA or even forming dimers as it has been analysed in electromobility shift assays (EMSA). In both mouse models, pancreas-specific inactivation of RelAp65 did not prevent progression of preneoplastic lesions, but promoted development of metaplastic ductal structures. RelAp65 deficient mice overexpressing TGF $\alpha$ under the control of the elastase promoter exerted an accelerated progression to duct-like metaplastic formation and successive loss of acinar cells. Pancreas-specific deletion of NF-kB/Rel and activation of heterozygous KRASG12D displayed lesions similar to intraductal papillary mucinous neoplasms (IPMN) with strong expression of MUC5 in mice at the age of 18 weeks. IPMN-similar formations were numerous with nearly no acinar cells in-between. Acinar cell death in both models was probably due to pronounced apoptosis with higher proliferation rates in duct-like structures. These data suggest that RelAp65 did not prevent pancreatic oncogenesis, but promoted early metaplastic lesions in two mouse models of pancreatic tumor. Moreover, we suggest a distinct role of RelAp65 during pancreatic oncogenesis. While deletion of NF-kB/Rel induced apoptosis in acinar cells, it did promote metaplastic lesions arising from duct like structures with IPMN-like features.

194

Spectral Markers of the Duodenum Can Detect Pancreatic Cancer Randall Brand, Yang Liu, Young Kim, Alexy Kromine, Hemant Roy, Nahla Hasabou, Dhiren Shah, Vadim Backman

Introduction: Due to limitations in pancreatic imaging modalities and lack of good tumor markers, it is not possible to diagnose individuals with pancreatic adenocarcinoma (PC) at an early stage. Our group has developed a novel optics technology, four-dimensional elastic light-scattering fingerprinting (4D-ELF), which allows quantitative assessment of the nanoscale cellular architecture. We have demonstrated that 4D-ELF has unprecedented sensitivity for assessment of the genetic/epigenetic changes of the field effect field of colon carcinogenesis, whereby, uninvolved mucosa accurately predicted the presence of colonic neoplasia. Assessment of the duodenal mucosa by 4D-ELF represents an attractive approach given the profound genetic/epigenetic alterations in the histologically normal duodenal mucosa of PC patients(pts) (Clin Cancer Res 2005:11:573). We, therefore, investigated in this pilot study whether 4D-ELF derived spectral markers from normal appearing duodenal mucosa could detect the presence of PC. Methods: Periampullary biopsies from 28 control pts obtained by endoscopy and 19 PC pts, obtained by pancreatic resection(7) or endoscopy(12), were analyzed for the 4D-ELF derived markers of spectral slope and fractal dimension. The spectral slope evaluates size distribution of intracellular components ranging from macromolecules to small organelles. The fractal dimension reflects the tissue organization at larger length scale, ranging from large organelles to groups of cells. Results: The median age of PC pts and controls were $74(51-87)$ and $57(27-84)$, respectively. $26 \%$ of PC pts and $25 \%$ controls were male. PC stage was I-2, II-8, III-2, and IV-7. There were statistically significant differences in the spectral slope $(\mathrm{p}=0.02)$ and the fractal dimension $(\mathrm{p}=0.003)$ between the controls and PC pts. Diagnostic accuracies are shown in Table. Conclusions: We demonstrate for the first time that 4D-ELF of periampullary biopsies can identify PC. This study provides compelling data to further investigate 4D-ELF's ability to discriminate PC from not only normal pts but pts with other pancreatic diseases. The use of additional spectral markers may increase both our sensitivity and specificity. A probe is currently under development, which would alleviate the need to obtain biopsies, and thereby, allow for an even less invasive manner to predict the presence of PC.

\begin{tabular}{|c|c|c|c|c|}
\hline & Sens & Spec & PPV & NPV \\
\hline Spectal slope & $53 \%$ & $57 \%$ & $64 \%$ & $45 \%$ \\
\hline Fractal Dimension & $53 \%$ & $89 \%$ & $77 \%$ & $74 \%$ \\
\hline Combined & $68 \%$ & $79 \%$ & $79 \%$ & $68 \%$ \\
\hline
\end{tabular}

207

Hemochromatosis Is Associated with Increased Colorectal Cancer Prevalence in a National Sample of US Veterans: A Case-Control Study Alastair D. Smith, Deborah A. Fisher

Introduction: Iron is a pro-oxidant and may promote carcinogenesis. However, studies of patients with iron overload syndromes (genetic susceptibility, dietary iron intake) vary in their conclusion of the potential relationship between iron and colorectal cancer (CRC). Our aim was to determine whether patients with hemochromatosis (HC) were at increased risk of developing CRC compared to subjects without HC. Methods: We examined the national VA inpatient and outpatient databases (October 2003 - September 2005) for a random cross-sectional sample of 118,999 unique patients, aged $\geq 50$. The diagnoses were identified by ICD9 codes: 153, 154 for CRC and 275 for HC. Using logistic regression, we assessed the relationship between $\mathrm{CRC}$ and $\mathrm{HC}$, and calculated the adjusted odds ratio (aOR) with 95\% confidence intervals (CI). Models were adjusted for age, gender and race Results: The study population was 96\% male, and its mean (SD) age was 67 (11) years. The prevalence of CRC was 1\% (1246 patients) and of HC 0.6\% (724 patients). Eighteen patients carried both diagnoses $(0.02 \%)$. There was a significantly increased likelihood of CRC among patients with $\mathrm{HC}$, with aOR of $2.4(95 \% \mathrm{CI} 1.5-3.8 ; \mathrm{p}=0.0004)$. Other predictors of CRC included African American race (versus Caucasian) with an aOR of 1.4 (1.2-1.7; $\mathrm{p}=$ $0.0002)$, male gender with an aOR of $2(1.4-3.3 ; \mathrm{p}=0.0005)$, and age with an aOR of 1.5 $(1.5-1.6 ; \mathrm{p}<0.0001)$ per 10 years. Conclusions: Our results suggest that $\mathrm{HC}$ increases the risk of CRC even when other risk factors, such as age, are considered. Longitudinal analysis of a younger patient sample may support earlier CRC screening for patients with known HC.

208

Patients with Coronary Artery Disease Are At High Risk for Developing Colorectal Cancer and Adenoma: An Interim Analysis of a Prospective Study Annie On On Chan, Man Hong Jim, Kwok Fai Lam, David Cw Siu, Teresa Sm Tong, Fook Hong Ng, Wai Mo Hui, Chi Kuen Chan, Kam Chuen Lai, Wayne Hc Hu, Man Fung Yuen, Yuk Kong Lau, Stephen Lee, Shiu Kum Lam, Benjamin Cy Wong

Background: Identifying high risks groups for developing colorectal neoplasia (cancer and adenoma) $(\mathrm{CN}$ ) allows early detection and hence prevention. We observed in our retrospective study that there was a strong association between patients with $\mathrm{CN}$ and coronary artery disease (CAD), probably due to common risk factors. Aim: To investigate the prevalence of $\mathrm{CN}$ in patients with and without $\mathrm{CAD}$ in those aged $\geq 50$ years in a prospective study and to identify the underlying risk factors. Methods: Patients were recruited for screening colonoscopy after undergoing coronary angiogram, and was defined as CAD+ if there was greater than $50 \%$ stenosis of one of the main arteries, and CAD- if not. Advanced colonic lesion was defined as the presence of cancer, or adenomas with villous component, or with 
moderate to severe dysplasia, or $\geq 1 \mathrm{~cm}$, or $>2$ adenomas. Underlying risk factors were recorded. The calculated sample size was 200 in each arm. An interim analysis was carried out at a sample size of 150 at each arm. Pearson chi-square test was carried out to test for the association between $\mathrm{CN}$ and CAD. To adjust for the factors of age, sex and other risk factors, a random-effects bivariate logistic regression analysis was used. Bayesian analysis was carried out using the software WinBUGS. Intracluster correlation (ICC) is a side product of the model that gives an idea of the strength of the association after adjusting for the explanatory variables. Results: There were 307 patients $(68.7 \%$ male) recruited, $46.3 \%$ of them were CAD+. The prevalence of $\mathrm{CN}$ was $30.3 \%$ vs $19.4 \%(\mathrm{p}=0.027)$, and advanced lesions was $(16.9 \%$ vs $6.7 \%, \mathrm{P}=0.005)$ in patients with and without $\mathrm{CAD}$. There were 5 colorectal cancers $(3.5 \%)$ detected in the CAD+ arm but none in CAD- $(p=0.015)$. All the cancers were asymptomatic except anemia was found incidentally in one patient. Using logistic regression analysis, the variance of the random effects $\sigma 2=1.7$ ( $95 \% \mathrm{CI}: 0.42,4.59$ ), and ICC $=0.32$ ( $95 \% \mathrm{CI}: 0.11,0.58)$, both indicates a strong association between $\mathrm{CN}$ and $\mathrm{CAD}$ after adjusting for the risk factors. Male sex and smoking were risk factors for predicting the occurrence of the two diseases. Conclusion: Patients with CAD+ are at high risk for developing colorectal cancer and adenomas, screening by colonoscopy for these patients is mandatory

209

A Prospective Evaluation and Classification of Extra-Colonic Abnormalities Identified with Computed Tomographic Colonography Screening in Asymptomatic, Average Risk Individuals

Patrick E. Young, Cecilia H. Kim, Priscilla A. Cullen, Duncan S. Barlow, Donald W. Jensen, James W. Kikendall, Peter W. Soballe, Brooks D. Cash

Background: Computed tomographic colonography (CTC) uses CT technology to detect colorectal polyps. While CTC technique differs from dedicated abdominal CT technique, CTC can detect extra-colonic (EC) abnormalities. The nature and outcomes of these findings have not been fully investigated in a proscribed manner. We report herein the prevalence and characteristics of EC findings in patients undergoing CTC for colorectal cancer (CRC) screening over a 12 month period. Methods: 979 asymptomatic, average risk patients underwent CTC for CRC screening from October 2004 to October 2005 using a Phillips 40 slice CT scanner EC and colonic abnormalities were recorded. EC abnormalities were categorized as critical, moderate, or incidental. Critical findings were those that required further therapeutic intervention whereas moderate findings only required additional diagnostic intervention (serial CT scans, etc.). Incidental findings required no further action. The likelihood of detecting EC abnormalities was compared to that of detecting colonic abnormalities using a 2-sided t-test for two percentages. Results: 15.9\% (156/979) patients had 188 EC abnormalities which required further evaluation. The most common of these were coronary artery calcifications $(n=41)$ and non-calcified pulmonary nodules $(n=33)$. A variety of other abnormalities were noted and are delineated in the chart below. $83.3 \%(133 / 188)$ had a single EC abnormality whereas $2.6 \%$ had 3 or more abnormalities. $51.6 \%$ were classified as incidental abnormalities, $33 \%$ were moderate and $15.6 \%$ were critical. The likelihood of detecting EC abnormalities paralleled that of colonic abnormalities $(15.9 \%$ vs. $13.5 \%$, p= 0.13). Location and number of findings $(n=188)$ Pulmonary 42 Coronary 41 Renal 41 Hepatic 16 GU (non-renal) 12 Aortic 10 GI (non-polyp) 7 Biliary 6 Bone 4 Pancreatic 3 Other 6 Conclusions: Extra-colonic abnormalities are detected in approximately $16 \%$ of screening CTC. The majority of these abnormalities are incidental in nature and do not require additional evaluation but more significant abnormalities are encountered and represent nearly $50 \%$ of all EC abnormalities. While early detection of potentially morbid conditions may benefit select individual patients, EC abnormalities identified by CTC may substantially increase the indirect cost of the exam. Future cost-benefit analyses using this type of realworld data and outcomes are needed before routine use of this technology can be endorsed.

210

Colonoscopy (C-scope) in Ambulatory Outpatients in the Va: Should We Protect Elderly Veterans from Unbeneficial Procedures Andy Fan, Stephen J. Sontag, Thomas G. Schnell, Jack Leya

Introduction: Requests for C-scope in elderly Pts with co-morbid diseases are becoming more frequent with the aging of the population. The reasons both for and against performing a C-scope are numerous, and few data are available to justify either approach. In July 2001, the Hines VA GI Section adopted a strictly regulated policy in which inappropriate, unnecessary, or unlikely-to-benefit C-scopes were denied. No published mortality data on this subset of Pts is available. Aim: To determine whether our rigid policy has resulted in adverse outcomes in elderly VA Pts in whom elective C-scopes were requested and denied. Methods: During the program's first 3 years of existence (Sep 2001-Dec 2004), 279 elective outpt requests for C-scope in Pts 80 yrs or older were received and reviewed by the GI fellow. C-scope requests for these Pts were approved ONLY IF the requestor was willing to explicitly document in a separate progress note that the pt was (1) healthy enough to undergo the procedure (ASA class 2 or less) and (2) was expected to live more than 5 years. All consults were reviewed by a GI fellow using pre-determined criteria and, when appropriate, template responses. The fellow's responses were reviewed by a senior GI attending, and the fellow's decision was supported. Results: In the group of 279 Pts, the mean age was 83 years and the mean follow-up was 26 months. 67 of the 279 Pts died (all-cause mortality rate: $24 \%$ ). The mean time to death from the initial C-scope request date was 15 months. Of the 279 requests, 167 (60\%) were approved for C-scope and 112 were denied. Of the 167 approved, 44 (26\%) died; of the 112 denied, 23 (21\%) died. Kaplan Meier survival curves showed no statistical difference between the groups $(\mathrm{p}=0.39)$ Fig 1 . Thus, within a mean of 15 months of the request for elective outpt C-scope in octogenarian Pts, regardless of whether the request was approved or denied, $24 \%$ were dead, and none died from CRC. There was no difference in overall mortality between those who were approved for C-scope and those who were denied. Conclusion: Without even considering the potential for $\mathrm{C}$ scope complications, our data clearly show that the decision to perform or not perform elective C-scope in veterans 80 years or older does not positively influence overall mortality.
With nearly a quarter of the total group dying within 2 years, careful scrutiny must be given to $\mathrm{C}$-scope requests in this group of patients.

\section{1}

Risk for Adenoma Recurrence At the Second Surveillance Exam Douglas Robertson, Carol A. Burke, Leila A. Mott, John A. Baron

Background: Current guidelines for post-polypectomy colonoscopic surveillance are based only on the most recent exam findings. Aim: We determined adenoma recurrence risk ove time in the context of an individual's entire lifetime adenoma history. Methods: Subjects were drawn from 2 adenoma chemoprevention trials. At enrollment, all subjects had one or more adenoma and had undergone clearing colonoscopy. For this analysis, we only included subjects whose qualifying adenoma(s) were their first lifetime adenoma(s). "Firs follow up exam' was defined as the protocol exam occurring either three or four years after the baseline exam (varied by trial). Any exam $\geq 2$ years after the 'first follow up exam' was defined as the 'second follow up exam'. The main outcome of interest was development of an advanced adenoma (tubulovillous or villous histology, high grade dysplasia, or $\geq 1 \mathrm{~cm}$ ) or multiple $(>2)$ adenomas (AdvMult) at the second follow up exam after baseline. Statistical significance was assessed by $\chi 2$. Results: 793 subjects (mean age, 58 years) were identified $62 \%$ were male. Mean time from baseline to first follow up exam was 3.4 years, and from first to second follow up exam, 4.2 years, $82(10 \%)$ subjects developed AdvMult at the second follow up exam. When separately considering high and low risk populations at baseline, outcomes at second follow up exam were significantly influenced by both prior colonoscopy results (see table). For example, those with high risk findings (AdvMult) at both baseline and first follow up exams had a 30\% risk of AdvMult on the second follow up exam. This was significantly higher than the $15 \%$ risk of AdvMult at 2nd follow-up in those with high risk findings at baseline but no adenomas on the first follow up exam(p= .03). Similarly, in a low risk group at baseline ( $<3$ small tubular adenomas), findings at the second follow up exam were significantly influenced by the first follow up exam. In the low risk group at baseline,those with AdvMult at the first follow-up exam were at much higher risk for AdvMult at 2 nd follow-up (15\%) compared to those with no adenomas at the first follow-up (3.6\%) $(\mathrm{p}=.01)$. Conclusion: An individual's entire adenoma history is useful in determining adenoma recurrence risk. Based on findings from both colonoscopies, high and low risk groups can be identified that may lead to more effective surveillance recommendations.

Subjects (\%) With Advanced or Multiple Adenomas on 2nd Follow Up Exam

\begin{tabular}{|c|c|c|c|}
\hline & \multicolumn{3}{|c|}{ 1st Follow Up Exam Findings } \\
\hline Baseline Findings & $\begin{array}{c}\text { No } \\
\text { Adenomas }\end{array}$ & $\begin{array}{c}\text { Non Advanced and } \\
\& \# 8806 ; 2 \text { adenomas }\end{array}$ & $\begin{array}{c}\text { Advanced or }>2 \\
\text { adenomas }\end{array}$ \\
\hline $\begin{array}{c}\text { Non Advanced and \&\#8806;2 } \\
\text { adenomas ( } \mathrm{n}=482)\end{array}$ & $3.6 \%$ & $8.0 \%$ & $15.0 \%$ \\
\hline $\begin{array}{c}\text { Advanced or }>2 \text { adenomas }(\mathrm{n}= \\
311)\end{array}$ & $15.2 \%$ & $14.3 \%$ & $30.0 \%$ \\
\hline
\end{tabular}

212

Comorbidity and Colorectal Cancer Screening Utilization in a National Sample of Veterans Health Administration Patients Deborah A. Fisher, Joseph Galanko, Tara K. Dudley, Nicholas J. Shaheen

Objectives: Quality assessment of colorectal cancer (CRC) screening in the Veteran Health Administration (VHA) system relies on reporting the proportion of patients aged 52-80 years screened. This approach does not assess for comorbid illnesses that may impact a patient's suitability for screening. The Charlson-Deyo score, which can be calculated from administrative data, is a predictor of mortality. Our primary aim was to determine the relationship between comorbidity, as measured by the Charles-Deyo score, and fecal occult blood testing (FOBT) in the national VHA database. Our secondary aim was to test the validity of the Charlson-Deyo score as a predictor of 1-year mortality in VHA. Methods: We examined the national VHA administrative databases (October 2003- February 2005) for a random sample of 86,785 primary care patients, aged $\geqq 50$. Charlson-Deyo score was calculated. The association between Charlson-Deyo score and mortality was assessed using chi squared analysis. Using logistic regression, we also assessed the relationship between comorbidity, and the likelihood of receiving CRC screening. Models were adjusted for age and sex. Results: The sample was $97 \%$ men; mean age was 67 years. Charlson-Deyo score was $45 \%=0,24 \%=1$, $14 \%=2,7 \%=3,3 \%=4,2 \%=5,1 \%=6,3 \% \geqq 7$. Overall there was no significant association between Charlson-Deyo score and whether or not an FOBT was performed. There was a strong and incremental relationship between Charlson-Deyo score and l-year mortality There was no association between FOBT status (performed or not) and 1-year mortality within any of the Charlson-Deyo score categories. Conclusions: In the VHA system, patients with severe comorbidity undergo FOBT screening as frequently as healthy patients. Substantial effort is being directed toward screening patients with an increased likelihood of dying from competing causes. Further, there was no evidence providers selectively screened the healthiest patients within each Charlson-Deyo score category. The current "one-size-fits-all" quality measurement for CRC screening in the VHA may promote inappropriate screening in ill patients, and does not emphasize screening in those with the most life-years at stake.

213

Effect of Helicobacter Pylori (HP) Screening and Treatment in a Population Questionnaire Data for 10,433 Individuals At 5-Years Follow-Up J.N. M. Hansen, Mette Wildner-Christensen, Ove B. Schaffalitzky de Muckadell

Aim: to evaluate the long term effect of screening and treatment of $\mathrm{Hp}$ in a population. Methods: 20,000 individuals aged 40-65 years were identified and randomised to screening and treatment (group 1) or control (group 2). Both groups were followed by a questionnaire 
including the "Gastrointestinal Symptom Rating Scale" (GSRS), and SF36 at year (yr) 0, 1, and 5. The primary endpoint was dyspepsia (an aps-score $>=2$ in GSRS). Results: 12,530 individuals were included. 11,098 were alive and had not moved at yr 5 and 94\% answered the questionnaire (84\% of included individuals). At inclusion $17.5 \%$ were Hp-positive and received Hp-eradication. Dyspepsia decreased more in group 1 than in group 2 in the $5 \mathrm{yr}$ follow-up $(24.9 \%$ to $20.4 \%$ and $21.0 \%$ to $19.6 \%$, respectively $(p<0.01)$ ). Multiple logistic regression analyses were applied in order to evaluate the effect of the intervention adjusting for baseline imbalances (dyspepsia, ASA/NSAID use, and smoking): the effect was significant at yr 1 (OR 1.25 (1.12-1.40)) but not at yr 5 (OR 1.04 (0.93-1.16)). A subgroup analysis showed a larger decrease in dyspepsia in Hp-positive $(27.8 \%$ at yr 0 to $24.5 \%$ at yr 1 and $22.5 \%$ at yr 5 ) than in Hp-negative individuals $(23.6 \%, 19.7 \%$ and $20.0 \%$ at $y r 0,1$, and 5 , respectively). Prevalence of reflux symptoms decreased in both groups during follow-up. The frequency of dyspepsia related visits at GP and sick leave days, and upper endoscopy was lower in group 1 than in group 2 at yr 5 (table). The use of ulcer drugs, NSAID/ASA increased in both groups (table). Conclusion: In this low Hp prevalence area, screening and reatment for $\mathrm{Hp}$ only had a modest long term effect on dyspepsia and dyspepsia related health care use

\begin{tabular}{|c|c|c|c|c|c|c|}
\hline & \multicolumn{3}{|c|}{ Group 1 } & \multicolumn{3}{c|}{ Group 2 } \\
\hline & Year 0 & Year 1 & Year 5 & Year 0 & Year 1 & Year 5 \\
\hline Number & 5749 & 5359 & 4821 & 6781 & 6222 & 5612 \\
\hline Visit GP -month \% & 3.5 & 2.4 & 2.8 & 3.0 & 2.7 & 3.1 \\
\hline Sick Leave -month \% & 2.3 & 2.3 & 1.9 & 1.7 & 2.6 & 2.5 \\
\hline Endoscopy -year \% & & 3.2 & 3.6 & & 2.8 & 4.3 \\
\hline Ulcer drug - month \% & 7.4 & 5.5 & 7.7 & 7.0 & 5.6 & 7.2 \\
\hline ASA/NSAID -month \% & 20.8 & 18.3 & 25.7 & 18.3 & 19.0 & 26.5 \\
\hline
\end{tabular}

214

Determinants of Symptom Severity and Weight Loss in Functional Dyspepsia Lukas van Oudenhove, Vandenberghe Joris, Brecht Geeraerts, Rita Vos, Phillippe Persoons, Sebastien Kindt, Koen Demyttenaere, Jan Tack

Background: Both gastric sensorimotor dysfunction and psychosocial factors have been implicated in symptom generation in functional dyspepsia (FD). The aim of this study is to determine the relative influence of gastric pathophysiological mechanisms and psychosocial factors on symptom severity and weight loss in FD. Methods: In 139 consecutive FD patients (mean age $39.5 \pm 12$ years) from a tertiary referral center, gastric sensorimotor function was studied with a barostat (sensitivity, compliance $\&$ accommodation) and gastric emptying test. Psychosocial factors (depression, anxiety, fatigue, alexithymia, perceived stress and abuse history) and somatic symptom severity/somatization (SSS) were assessed using selfreport questionnaires. Gastric pathophysiological mechanisms, psychosocial factors and SSS were correlated with a dyspepsia severity score (DSS) (obtained from a l0-item symptom questionnaire) and with weight loss. Furthermore, gastric pathophysiological mechanisms and psychosocial factors were correlated with SSS (obtained from a 15-item symptom questionnaire). Multiple linear regression was used to identify independent determinants of DSS, weight loss and SSS. Results: DSS was higher in women and in hypersensitive patients and correlated with age, perception threshold, gastric emptying coefficient, SSS, fatigue, positive affect and the lack of joy factor of the perceived stress questionnaire. Multiple linear regression identified three independent determinants of DSS: age $(\beta=-.05, \mathrm{p}=.06)$, SSS ( $\beta=$ $.41, \mathrm{p}<.0001)$ and lack of joy $(\beta=-.21, \mathrm{p}=.04)$ (model $\left.\mathrm{R}^{2}=.28, \mathrm{p}<.0001\right)$. Weight loss was higher in women and correlated with DSS, SSS, depression, momentary and chronic fatigue and positive affect. In multiple linear regression, gender $(\beta=.76, p=.01)$ and a composite depression-SSS index $(\beta=.60, \mathrm{p}<.0001)$ were determinants of weight loss (model $\mathrm{R}^{2}=.24, \mathrm{p}<.0001$ ). SSS was higher in women, in patients with panic disorder and in patients with chronic fatigue symptoms. SSS correlated with DSS, weight loss, perception threshold, gastric emptying, depression, panic, momentary fatigue, positive $\&$ negative affect and perceived stress. Gastric emptying ( $\beta=-1.71, p=.08)$, DSS $(\beta=.35, \mathrm{p}=.02)$, presence of chronic fatigue $(\beta=4.1, \mathrm{p}<.0001)$ and a composite depression-negative affect-stress index $(\beta=1.52, p<.0001)$ were independent determinants of SSS in multiple linear regression (model $\mathrm{R}^{2}=.64, \mathrm{p}<.0001$ ). In conclusion, symptom severity in FD is determined by psychosocial factors (especially somatization), rather than gastric pathophysiological mechanisms.

215

H.pylori Eradication V Empirical Acid Suppression in Uninvestigated H.pylori Positive Dyspepsia: A Cochrane Collaboration Individual Patient Data MetaAnalysis

Brendan C. Delaney, Michelle Qume, Paul Moayyedi

Introduction: The Dyspepsia Trials Collaborator's Group has been conducting a series of prospective, individual patient data meta-analyses of initial management strategies for dyspepsia for the Cochrane Collaboration. H.pylori test and treat has been shown to be marginally less effective, but more cost-effective than endoscopy-based management, whilst empirical acid-suppression is as effective as endoscopy but less costly. The question has remained over the cost-effectiveness of eradication therapy compared with empirical acid suppression. Methods: Prospective, individual patient data meta-analysis of RCTs comparing H.pylor eradication therapy v placebo eradication plus PPI in the first-line management of H.pylor positive adult patients with dyspepsia in the primary care setting. Original datasets were obtained and costs re-calculated using US healthcare costs for 2004. Patient level net benefits were calculated using the formula, Net Monetary Benefit $(\mathrm{NMB})=$ Effect $\mathrm{x}$ willingness to pay(WTP) - cost. Relative risk of remaining dyspeptic was pooled using a fixed-effect model and NMB using weighted mean difference. A sensitivity analysis on WTP was conducted. Results: Two studies including a total of 571 subjects, met the inclusion criteria, one published and one unpublished. Costs and effects were reported after one year of followup. Eradication therapy was significantly more effective than in preventing epigastric pain (RR 0.83, 95\% CI 0.69-0.89), but not heartburn (RR 0.93, 95\% CI 0.78-1.10). At a wtp of zero, the pooled difference in NMB was $\$ 6.12$ (95\% CI -13.30 to 25.53), at a wtp of $\$ 1000$ per patient symptom free the difference was $\$ 79.07$ (95\% CI 0.84 to 157.31 ) in favour of eradication therapy. Conclusions: The analysis provides support for recent guidelines promoting first line use of H.pylori test and treat for dyspepsia in patients with predominant epigastric pain. Eradication therapy was more effective than placebo plus PPI for the management of uninvestigated epigastric pain, but not heartburn symptoms Eradication therapy was also more cost-effective than PPI at reasonable willingness to pay Existing RCTs are not able to exclude a small residual benefit in heartburn, and data is awaited from ongoing studies.

216

Who Consults with Dyspepsia? A Ten-Year Cross-Sectional Survey Alexander C. Ford, Alastair G. Bailey, David Forman, Anthony T. Axon, Paul Moayyed Introduction: Dyspepsia is a chronic relapsing, remitting disorder, the natural history of which has been studied extensively. However, there are few studies examining factors that influence the likelihood of consulting a family practitioner with symptoms in those who are symptomatic, particularly over a long time period. Methods: The authors performed a tenyear follow-up cross-sectional survey of individuals previously recruited into a community screening and treatment program for Helicobacter pylori. All surviving, traceable participants were contacted, using a validated postal dyspepsia questionnaire. Baseline demographic data, quality of life at study entry (assessed via the psychological and general well-being index), and dyspepsia and irritable bowel syndrome (IBS) symptom data were already on file. Written informed consent was sought to examine primary care records, and data on the use of non-steroidal anti-inflammatory drugs (NSAIDs) and aspirin, and the number of consultations as a result of dyspepsia over the ten-year period were extracted from these by two individuals. Results: Of 8407 individuals originally involved, 4003 (48\%) responded to the questionnaire, and $3266(39 \%)$ gave consent to examination of primary care records. The mean age of included individuals was 55 years, and 1799 (55\%) were female. 1738 (53\%) individuals had dyspepsia at any point during ten-year follow-up, when dichotomized according to questionnaire data. Of these, 729 (42\%) consulted their family practitioner. The mean number of consultations in these individuals was 3.5 (range 1 to 23). Univariate analysis revealed that $H$. pylori infection, female gender, tobacco use, Asian ethnicity, lower socioeconomic status, lower quality of life at study entry, irritable bowel syndrome, and NSAID or aspirin use significantly increased the likelihood of consultation, whilst the opposite applied to regular alcohol use. Following logistic regression analysis, H. pylori infection, lower socioeconomic status, lower quality of life at study entry, and aspirin use remained independent risk factors for consultation. Conclusions: The reasons for consulting a family practitioner with dyspepsia are multi-factorial, but $H$ pylori status, aspirin use, low socioeconomic status, and poor quality of life are independent predictors of consultation.

217

Score \& Scope Versus Test $\&$ Treat Strategies for the Management of Dyspepsia: A Randomised Controlled Trial

Merce Barenys, Rosa Rota, Anna Garcia -Altes, Agueda Abad, Josep Maria Lopez Balaguer, Sergi Romero, Margarita Admetlla

INTRODUCTION .Many studies have tried to address the issue of the best initial work-up in patients with uninvestigated dyspepsia. There are few randomised clinical trials in this field, so recomendations are generally based on decision analysis and favor empirical approach in young patients(1). AIM:To compare the effectiveness and cost of 2 strategies: Score \& Scope and test \& treat METHODS : Randomised controlled trial in a Catalan regional Hospital. Subjects: Uninvestigated young dyspeptic subjects, without alarm symptoms or isolated reflux disease, between 18 - 55 years old. INTERVENTION: Randomisation to: Group SCORE \& SCOPE (S\&S) A previously locally validated scoring system(2) is performed and classifies patients in high or low risk for organic dyspepsia. Group TEST \& TREAT (T\&T) A UB Test $\mathrm{Cl} 3$ for $\mathrm{H}$. Pylori classifies patients in infected or not. Infected patients eradication and non infected treatment with PPI during 1 month. In both groups: Lack of improvement or relapse during follow-up prompted a second breath test in infected patients or an endoscopy in patients with PPI. According to those antisecretory drugs, antibiotics or procinetics were prescribed. A follow-up of 18 months was established. OUTCOME MEASURES Efectiveness. Complete: Rate of assymptomatic patients at the end of follow-up orpartial: Rate of patients with a reduction of symptoms $>50 \%$ on a LiKert scale. Costs. Direct costs only. Drugs, tests and Visits costs considering tariffs (2005)from the Catalan Health Service. RESULTS (See table below) CONCLUSIONS 1. Costs were significantly * lower in the score and scope group than in the test and treat group. 2 . Clinical effectiveness $(>80 \%)$ was similar in both groups although complete resolution of symptoms was superior in the T\& T group. 3. Empirical approaches in dyspepsia can avoid endoscopic procedures in 56\% of young dyspeptic patients. REFERENCES 1.García Altés A, Rota R, Barenys M. Cost-effectiveness of a "score and scope" strategy for the management of dyspepsia. A decision analysis. Eur J Gastroenterol \& Hepatol 2005; 2.Barenys M, Abad A, Pons JMV. Scoring system has better discriminative value than $H$. Pylori testing in patients with dyspepsia in a setting with high prevalence of infection. Eur J Gastroenterol \& Hepatol 2000 Comparison between Score and H.pylori selection

\begin{tabular}{|c|c|c|}
\hline Variables & Group Score & Group T\&T \\
\hline N. patients & 34 & 30 \\
\hline Total cost in euros per patient & 141 & 220 \\
\hline Efecctiveness complete/partial & $9 / 18(80 \%)$ & $16 / 9(83 \%)$ \\
\hline
\end{tabular}

$53 \%$ patients in the T\& T were infected by h.pylori and $23 \%$ in the S\&S were classified as hirgh risk dyspepti 
Three-Fold Increased Risk of Infertility: A Meta-Analysis of Infertility After Pouch Surgery in Ulcerative Colitis

Akbar Waljee, Jennifer. Waljee, Arden Morris, Peter D. Higgins

Background: Increased rates of infertility in females have been reported post ileal pouchanal anastomosis (IPAA) for ulcerative colitis. The reported infertility rates vary substantially. Aims: 1) To perform a systematic review and meta-analysis of the relative risk of infertility post-IPAA to better inform patients. 2) To estimate the rate of infertility post-IPAA. 3) To identify any modifiable risk factors which contribute to infertility. Methods: Medline, EMBASE, Current Contents, meeting abstracts, and bibliographies were searched independently by two investigators. Study selection criteria included the following: 1) publication in full manuscript form in English; 2) data provided from both patients with medically treated colitis and patients post-IPAA for ulcerative colitis; 3) raw data provided on infertility, defined as lack of conception despite trying to conceive for at least 12 months; and 4) data not duplicated in another manuscript. Twenty-eight eligible articles were reviewed, eight studies met criteria, and data from these studies were extracted independently. Consensus was achieved on each data point, and a meta-analysis was performed with the metan command in Stata 9.0. Funnel plots and sensitivity analysis were performed. Results: The initial meta-analysis of eight studies had significant heterogeneity $(\mathrm{p}=0.003)$ due to one study with a very high baseline infertility rate (38\%) in medically treated UC patients. When this study was excluded, the relative risk of infertility shifted from $2.42(1.99-2.95)$ to 3.17 (2.41-4.18) with a heterogeneity chi-squared $=8.00, \mathrm{p}=0.238$. The weighted average infertility rate in medically treated UC was $15 \%$ for all 7 studies, and the weighted average infertility rate was $49 \%$ post IPAA (51\% if the high-baseline study is included). We were unable to identify any technical factors that consistently affected the risk of infertility, including the number of abdominal surgeries, the use of an adhesion barrier, intraoperative lysis of adhesions, the pouch type ( $\mathrm{S}$ vs. J), or the use of pouch anti-adhesives. One study did report increased risk of infertility in cases with intra-operative transfusion, which may be a marker of surgical complexity. Conclusions: IPAA increases the risk of infertility in females with ulcerative colitis by at least three fold, raising the baseline infertility rate in medically treated UC patients from $15 \%$ to $49 \%$. Approximately half of females post-IPAA for UC who attempt conception will fail to conceive in the first 12 months. This provides a basis for counseling patients considering colectomy with IPAA. Further studies to identify modifiable risk factors are needed.

\section{9}

Disease Activity During Pregnancy in Women with Inflammatory Bowel Disease (IBD): A Population Based Cohort Study Uma Mahadevan, Douglas Corley

Introduction: Referral center studies suggest IBD activity during pregnancy is associated with adverse outcomes. Using a sample that approximated the population of Northern California, we evaluated the association between disease activity per trimester and adverse maternal/fetal outcomes. Methods: We conducted a cohort study among members of Northern California Kaiser Permanente. 461 pregnant women with a pre-birth diagnosis of IBD were frequency matched to 495 non-IBD pregnant women for age and hospital of delivery. Maternal demographics, potential confounders (e.g. smoking, alcohol, etc.), IBD activity, medication use, pregnancy outcome and maternal/fetal complications were abstracted from electronic and written prenatal and birth records. Univariate analyses included chi-square and t-tests; multivariate analyses used unconditional logistic regression. All analyses were two tailed Results: The table outlines disease activity per trimester (T). The majority of patients were inactive throughout pregnancy. Disease activity during conception/Tl had an odds ratio(OR) of $1.23(0.93,1.63)$ for an adverse conception outcome (spontaneous (SAB) or therapeutic (TAB) abortion). If $\mathrm{TAB}$ is not included, $\mathrm{OR}=0.81(0.53,1.24)$. Disease activity in a particular $\mathrm{T}$ was not associated with adverse pregnancy outcome (preterm birth, low birth weight, intrauterine growth retardation, small for gestational age, congenital anomalies, APGAR score <7, stillbirth). OR for $\mathrm{Tl}=1.02(0.69,1.51)$; $\mathrm{T} 2=1.20(0.85$, $1.69) ; \mathrm{T} 3=1.41(1.00,1.97)$. Disease activity in $\mathrm{T} 3$ was not associated with adverse labor outcome, OR $0.85(0.58,1.25)$. A flare in a particular $\mathrm{T}$ was not associated with adverse neonatal outcome (NICU admission, jaundice, seizure, mortality). Conclusions: Active disease during conception and $\mathrm{Tl}$ is not associated with a higher rate of spontaneous abortion. Though there is a trend towards an increase in adverse pregnancy outcomes with disease activity in T3, overall and per trimester, there is no significant association with disease activity and adverse pregnancy, labor or neonatal outcome. Few women with IBD experience significant disease activity during pregnancy in a population based setting. While this is reassuring, it may explain the lack of association between disease activity and adverse outcomes in this study.

Disease Activity per Trimester (T)

\begin{tabular}{|c|c|c|c|c|c|}
\hline \% disease activity & & Inactive & Mild & Moderate & Severe \\
\hline Conception & CD UC & 76.574 .8 & 10.719 .7 & 12.15 .1 & 0.70 .3 \\
\hline T1 & CD UC & 69.370 .8 & 13.321 .4 & 16.07 .1 & 1.30 .7 \\
\hline T2 & CD UC & 72.569 .0 & 12.120 .3 & 14.39 .1 & 1.11 .5 \\
\hline T3 & CD UC & 73.365 .1 & 10.525 .9 & 14.06 .9 & 2.32 .1 \\
\hline Post-partum & CD UC & 79.878 .4 & 7.916 .0 & 11.25 .7 & 1.10 \\
\hline
\end{tabular}

220

Risk Factors for Pregnancy Outcome in Patients with Inflammatory Bowel Disease (IBD)

Clemens Dejaco, Sieglinde Angelberger, Thomas Waldhoer, Thomas Haas, Heimo Wenzl, Peter Knoflach, Wolfgang Miehsler, Gottfried Novacek, Harald Vogelsang, Walter Reinisch

Active disease and immunosuppressive treatment during pregnancy in IBD have been critically discussed. We have recently shown that maintenance therapy with azathioprine/6mercaptopurine may be safe throughout pregnancy, whereas the impact of disease activity has not yet been evaluated prospectively. Aim: This prospective study has been performed to assess risk factors for pregnancy outcome in patients with IBD. Patients \& Methods Eighty pregnancies from 58 women with IBD were prospectively followed till birth. For statistical reasons in women with multiple pregnancies one index pregnancy was selected based on the occurrence of an adverse event (referring to abortion, malformation, or perinatal complication) and/or prematurity, defined by birth before the 37 gestational week or by a birth weight of less than $2500 \mathrm{~g}$. If all pregnancies were uneventful, the most recent one was chosen. The impact of the potential risk factors maternal age, nicotine abuse and disease activity on unfavorable pregnancy outcome subsuming adverse events and prematurity, was assessed by using the exact Fisher test as well as by a multivariate logistic regression model Disease activity was defined by the physician's overall assessment based on stool frequency and/or abdominal pain. Results: After exclusion of two patients with voluntary abortion 56 index pregnancies from 43 women with Crohn's disease and 13 with ulcerative colitis were evaluated. Median maternal age was 29 years (21-39), body mass index was 22.8, (16.6. $32.9), 13$ women $(23 \%)$ were smokers. Seventeen patients $(30 \%)$ had active disease at some time during pregnancy. There were 13 abortions, 2 cases with malformation (Turner Syndrome, Fallot's tetralogy) and one serious perinatal complication. Prematurity was found in 10 children. Unfavorable pregnancy outcome and prematurity as independent variables were significantly related to active disease (46\% vs $19 \%$ inactive, $\mathrm{p}<0.04$ and $70 \%$ vs $18 \%$, $\mathrm{p}<0.004$, respectively), but neither to maternal age nor nicotine abuse. Abortion as a single outcome parameter was not associated with any of the investigated potential risk factors Conclusion: This first prospective study clearly demonstrates that active disease during pregnancy represents a significant risk factor for unfavorable birth outcome and support the current treatment guidelines that maintenance of remission during gestation is essential.

221

Rising Hospitalization Rates for Crohn's Disease But Not Ulcerative Colitis in the United States

Meenakshi Bewtra, Chinyu Su, James D. Lewis

Background: Over the last two decades, medical therapy for inflammatory bowel disease (IBD) has witnessed increased use of immunomodulator and biologic therapies. We hypothesized that increasing use of these therapies would result in fewer hospitalizations and surgeries for IBD. This study examines trends in hospitalization and surgery rates for IBD in the United States over a 14-year period. Methods: We performed an analysis of secular trends of hospitalization and surgery rates from Crohn's disease (CD) and ulcerative colitis (UC) using 1990 to 2003 National Hospital Discharge Survey data. Rates were based on the total US population, including patients with and without IBD. We used Spearman's correlation coefficient to examine the association between calendar year and rates of hospitalization or bowel resection surgery. Analyses for hospitalizations required $\mathrm{CD}$ or $\mathrm{UC}$ to be listed as the primary diagnosis. Results: From 1990 to 2003, hospitalization rates per 100,000 people ranged from 9.3 to 17.1 for $C D$ and 8.2 to 12.4 for UC (Figure). Over this period, there was a significant trend for increasing hospitalization rates for $\mathrm{CD}(\mathrm{rho}=0.83, \mathrm{p}=.0002)$ but not UC (rho=0.06, $\mathrm{p}=.83$ ). Over the same period, the annual rates of bowel resection surgeries per 100,000 population ranged from 2.8 to 5.0 for $C D$ and from 1.6 to 3.4 for $\mathrm{UC}$. We did not observe a significant trend in surgery rates over the study period for $\mathrm{CD}$ $(\mathrm{rho}=0.30, \mathrm{p}=.30)$ or $\mathrm{UC}(\mathrm{rho}=-0.31, \mathrm{p}=.28)$. As expected, there was a significant trend for shorter hospitalizations for both $\mathrm{CD}(\mathrm{rho}=-0.98, \mathrm{p}<.0001)$ and $\mathrm{UC}($ rho $=-0.87, \mathrm{p}<.0001)$ Conclusions: Despite advances in therapy, IBD hospitalization and surgery rates in the US have not declined since 1990. Rather, there has been a significant increase in hospitalizations for $C D$, with stable rates of bowel resection surgery for $C D$, hospitalization for $U C$ and surgery for UC. 
CD Hospitalization - UC Hospitalization $\rightarrow$ UC Sugery

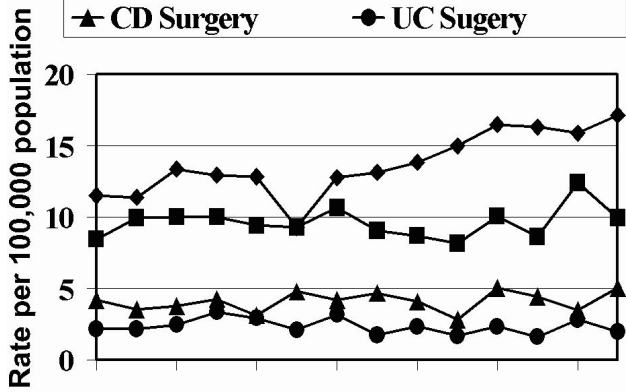

1990199219941996199820002002

222

Steroid Dependency and Steroid Resistance in Crohn's Disease (CD): Is There Any Predictable Risk Factor?

Manuel Barreiro-Acosta, Aurelio Lorenzo, Enrique Dominguez-Munoz

Background: Steroid dependency (SD) and steroid resistance (SR) develop frequently in the context of $C D$ patients. Risk factors for the development of $S D$ and $S R$ in $C D$ are unknown. We hypothesized that some of the known risk factors for $\mathrm{CD}$ may be helpful to predict the development of SD and SR in these patients. Aim: We aimed at evaluating the potential association between different known factors related to $\mathrm{CD}$ and the development of SD or SR. Methods: 188 consecutive patients with CD (111 female, 77 male, mean age 36 years, range 17-76 years) were included. SD was defined as a relapse within 30 days after the end of steroid treatment or after at least two attempts of tapering the steroid dose within the last 12 months. SR was defined as the absence of respond to steroid therapy (minimum $50 \mathrm{mg}$ of prednisolone daily) for more than 7 days. Gender, smoking habits, previous appendectomy, family history of $\mathrm{CD}$, extraintestinal manifestations of the disease, presence of at least one mutant allele in any of the three considered variants of CARD15 gene (R702W, G908R and 1007fs) and presence of TLR4+299 or the CD14-260 sequences variants were analyzed as potential risk factors for SD and SR. Results are shown as percentages and 95\% confidence intervals $(95 \mathrm{CI})$. Association is shown as odds ratio $(\mathrm{OR})$ and $95 \mathrm{CI}$ and analyzed by the Fisher's exact test. Results: Forty patients (21.3\%, 95CI: 15.4-27.1\%) developed SD, whereas SR was observed in 29 (15.4\%, 95CI: 10.3-20.6\%). SD was associated to smoking (OR 3.2; 1.4-7.4) $(\mathrm{p}<0.005)$ and to the presence of extraintestinal manifestations of the disease (OR 3.7; 1.7-7.6) $(\mathrm{p}<0.001)$. In addition, although SD was not related to any of the evaluated gene mutations, it tended to be more frequent among patients with a positive family history of $C D(O R 2.3 ; 0.9-5.6)(p=0.07)$. None of the analyzed factors, except previous appendectomy (OR 3.7; 1.5-9.1) $(\mathrm{p}<0.01)$, were associated to SR. Conclusion: Smoking habit, presence of extraintestinal manifestations and, probably, family history of $\mathrm{CD}$, appear to be associated to steroid dependency. Only a previous appendectomy is related to the development of steroid resistance. Genetic variants of CARD15, TLR4 and CD14 play not role in the development of SD and SR.

223

Aminosalicylate (ASA) Use During Pregnancy Is Not Associated with Increased Adverse Events Or Congenital Malformations (CM) in Women with Inflammatory Bowel Disease (IBD)

Uma Mahadevan, Douglas Corley

Introduction: ASA's are FDA pregnancy category B. Using a sample that approximated the population of Northern California, we evaluated the association between ASA exposure and adverse pregnancy outcomes (AO). Methods: A cohort study among members of Northern California Kaiser Permanente identified 461 pregnant women with a pre-birth diagnosis of IBD frequency matched to 495 non-IBD pregnant women for age and hospital of delivery. Maternal demographics, potential confounders (e.g. smoking, alcohol, etc.), IBD activity, medication use, pregnancy outcome and maternal/fetal complications were abstracted from electronic and written prenatal and birth records. Sulfasalazine (SAS) use was evaluated independently of other ASA's. Bivariate analyses were conducted of ASA and SAS use during conception/trimester $\mathrm{l}(\mathrm{T})$ and T2/T3 versus $\mathrm{AO}$ in all patients and by IBD diagnosis (Crohn's Disease - $\mathrm{CD}$, Ulcerative colitis-UC). A multivariate analysis was done of the risk of $\mathrm{CM}$ taking into account maternal age, current alcohol, ever smoking and race. Results: During conception/T1, 158 women had ASA exposure, 58 SAS and 570 had no exposure. During T2/T3, 137 women had ASA exposure, 47 SAS and 684 had no exposure. Table 1 summarizes the rate of $A O$ in all patients exposed to ASA/SAS and by IBD diagnosis versus unexposed patients. UC Patients who used ASA's in T2/T3 ( $n=94)$ had lower rates of AO than UC patients who did not $(n=197)(67 \%$ vs. $82 \% \mathrm{p}=0.004)$. This same effect was not seen with SAS alone, but was seen with either ASA or SAS use in T2/T3 (70\% vs. $83 \%, \mathrm{p}=0.008)$. Overall, there was no association between ASA or SAS use and CM. (Table 2). There was a trend towards increased CM with SAS exposure during conception/Tl, but this was not statistically significant. Conclusions: The use of ASA and SAS during pregnancy is not associated with an increase in AO. The increase in AO in UC patients not on ASA in T2/ $\mathrm{T} 3$ and the fewer patients on ASA in T2/T3 versus T1 suggests that continuing ASA into T2/T3 may be protective. There is no risk of CM with the use of ASA during pregnancy. There is a trend towards an increased risk in patients on SAS during conception/Tl Table 1: Risk of Adverse Outcome with ASA use

\begin{tabular}{|c|c|c|c|c|c|c|}
\hline & $\begin{array}{c}\text { Conception/T1 } \\
(\%)\end{array}$ & $\mathrm{P}$ & $\begin{array}{c}\mathrm{T} 2 / \mathrm{T} 3 \\
(\%)\end{array}$ & $\mathrm{p}$ & $\begin{array}{c}\text { Any Use } \\
(\%)\end{array}$ & $\mathrm{P}$ \\
\hline ASA CD UC & 788375 & $\begin{array}{c}0.100 .69 \\
0.46\end{array}$ & 707567 & $\begin{array}{c}0.620 .30 \\
0.00\end{array}$ & 767874 & $\begin{array}{c}0.300 .62 \\
0.21\end{array}$ \\
\hline SAS & 79 & 0.24 & 72 & 0.93 & 79 & 0.24 \\
\hline $\begin{array}{c}\text { ASA/SAS CD } \\
\text { UC }\end{array}$ & 8177 & 0.860 .73 & 7570 & 0.240 .01 & 7976 & 0.730 .58 \\
\hline
\end{tabular}

Table 2: Risk of CM with ASA use

\begin{tabular}{|c|c|c|c|c|}
\hline & ASA vs. no ASA (\%) & OR $(95 \%$ CI $)$ & SAS vs. no SAS $(\%)$ & OR $(95 \%$ CI $)$ \\
\hline Conception/T1 & 10.6 vs. 10.7 & $1.07(0.52,2.2)$ & 18 vs. 9.8 & $2.0(0.83,4.87)$ \\
\hline T2/T3 & 12.2 vs. 10.2 & $0.97(0.47,2.0)$ & 15.9 vs. 9.95 & $1.37(0.55,3.4)$ \\
\hline
\end{tabular}

230

Ctla4-IG Abrogates TNBS Colitis By Inducing the Expression of Indoleamine2,3-Dioxygenase

William Stenson, Gregory J. Gurtner, Suzanne Schloemann, Filipe Muhale, Keely G. McDonald, Rodney D. Newberry

Background: CTLA4-Ig is a biological agent consisting of the extracellular domain of Cytotoxic T cell Late Antigen-4 (CTLA-4) fused to the Fc region of IgG. CTLA4-Ig potentially promotes tolerance both via costimulatory blockade as well as through the induction of indoleamine 2,3-dioxygenase (IDO) in professional antigen presenting cells (APCs) including dendritic cells. Objectives: We had previously demonstrated that IDO was induced in trinitrobenzene sulfonic acid (TNBS) colitis and that inhibition of IDO with 1-methyltryptophan(1-mT) resulted in worsening of TNBS colitis. We sought to determine if induction of IDO by administration of CTLA4-Ig could abrogate TNBS colitis, and if so, to determine if IDO played a role in this process. Methods: Intrarectal TNBS and intraperitoneal CTLA4-Ig were administered to SJL/J mice along with 1-methyltryptophan(1-mT), a specific IDO inhibitor. Clinical and histological criteria were assessed to determine colitis severity. IDO protein and mRNA expression were assessed by Western blotting and real time PCR respectively. Results: We found that CTLA4-Ig induced IDO in cultured lamina propria mononuclear cells (LPMNCs) as well as in the murine colon after systemic administration. Immunohistochemistry for IDO in mice given CTLA4-Ig showed staining of pericryptal mesenchymal cells that appeared to be a subset of CDIlc+cells. Intraperitoneal CTLA4-Ig administration prior to and at the time of TNBS administration significantly abrogated colitis both clinically and by histological criteria. At 6 days mice treated with TNBS had a 25\% survival,those receiving CTLA4-Ig+TNBS had a $68 \%$ survival while those receiving CTLA4-Ig+TNBS+1-mT had a $25 \%$ survival $(\mathrm{p}=02)$. Similarly treatment with TNBS was associated with a $17 \%$ weight loss at day 2, treatment with CTLA4-Ig+TNBS was associated with an $8 \%$ weight loss and CTLA4-Ig+TNBS+1-mT was associated with a $16 \%$ weight loss $(\mathrm{p}=.05)$. The results of morphologic and histologic scoring followed the same pattern as survival and weight loss. Colonic IFN $\alpha$ and IFN $\gamma$ mRNA were measured by RT-PCR. Treatment with TNBS resulted in a 2-fold increase in IFN $\alpha$ and a 5-fold increase in IFN $\gamma$, whereas treatment with CTLA4Ig+TNBS resulted in a 2 -fold increase in IFN $\alpha$ but no increase in IFN $\gamma$. Conclusion: This study demonstrates that CTLA4-Ig improves the clinical outcome in TNBS colitis via IDO induction, and suggests a potential therapeutic role for CTLA4-Ig in the treatment of IBD.

231

An Anti-Inflammatory Role for the Heme Oxygenase-1 Pathway in Chronic Th2-Mediated Murine Colitis

Refaat Hegazi, Arthur Barrie, Antonia Sepulveda, Leo Otterbein, Scott Plevy

Introduction: The products of the heme oxygenase-1 (HO-1) pathway, namely, carbon monoxide (CO), biliverdin (BV), and iron, exert immunomodulatory effects in tissue injury and acute inflammation. Our laboratory has recently demonstrated for the first time that $\mathrm{CO}$ also suppresses chronic inflammation utilizing a Thl-mediated mouse model of inflammatory bowel disease (Hegazi R, J.Exp.Med., 2005, In Press). Up to now, we had not yet determined if this novel protective role for the HO-1 pathway in chronic disease is unique to Thl specific disorders. Aim: To determine if the HO-1 pathway also suppresses Th2 mediated chronic inflammation, we assessed the therapeutic potential of $\mathrm{CO}$ and $\mathrm{BV}$ in the T cell receptor- alpha (TCR $\alpha)$ deficient $(-/)$ murine model of colitis. Methods: TCR $\alpha-/$ - mice at 16 weeks of age were exposed to $\mathrm{CO}(250 \mathrm{ppm})(\mathrm{n}=9)$ and compared to air exposed mice ( $\mathrm{n}=9)$. In other experiments, TCR $\alpha-/$ - mice were injected IP with BV $(30 \mathrm{mg} / \mathrm{kg}$ twice weekly) for two weeks $(n=10)$ and compared to a vehicle-injected group $(n=6)$. At the end of the study period, mice were sacrificed and colonic tissue sections assessed for inflammatory changes by a single pathologist blinded to treatment group. Colonic mucosal explants were cultured for 24 hours, and IL-10, IL-1 $\beta$, IL-5, IL-6, IL-12 p40, IL-23 and IL17 protein secretion determined by multiplex ELISA. Results: Significant amelioration of colitis is demonstrated in both the CO-exposed and BV-treated groups compared to their respective controls ( $\mathrm{CO}$ vs. air colitis scores $=0.39 \pm 0.2$ vs. $1.68 \pm 1.02, \mathrm{p}<0.05$; BV vs. vehicle colitis scores $=0.79 \pm 0.43$ vs. $1.54 \pm 0.37, \mathrm{p}<0.05)$. Consistent with the observed histologic improvement, both $\mathrm{CO}$ and BV repress numerous Th2 pro-inflammatory cytokines including IL-1 $\beta$, IL-5, and IL-6 in colonic mucosal explant cultures, while intestinal IL-10 is induced in CO- exposed but not BV-treated mice. In addition, IL-12 p40, IL-23 and IL17 are overexpressed in TCR $\alpha$-/- intestinal tissue and are inhibited by BV. Conclusions: The heme metabolites $\mathrm{CO}$ and BV suppress pro-inflammatory cytokine expression and Th2 mediated colitis in TCR $\alpha$-/- mice. This observation in combination with previous investigations suggests that $\mathrm{HO}-1$ products are ubiquitous anti-inflammatory factors in both acute and chronic inflammation. $\mathrm{CO}$ and BV may act via alternative mechanisms based on the divergent IL-10 levels in these two treatment groups. Lastly, independent of these findings, the observed expression pattern of IL-23 and IL-17 implicates a novel role for this cytokine axis in this disease model. 
Innate Cell-Targeting Therapeutic Effect of IL-22 On Experimental Colitis Ken Sugimoto, Atsuhiro Ogawa, Yasuyo Shimomura, Akira Andoh, Atul K. Bhan, Atsushi Mizoguchi

BACKGROUND \& AIMS: Dysregulated host/microbial interactions result in the development of IBD. Activation of signal transducer and activator of transcription (STAT) 3 has previously been shown to activate innate responses against enteric bacteria to avoid the development of colitis (Immunity 1999,10:39; Nat Med 2002,8:1089). Recently, IL-22 has been identified as a strong activator of STAT3 in innate cells (such as epithelial cells) but not acquired cells (such as T and B cells) (Immunity 2004,21:241). However, the role of IL-22 in the pathogenesis of colitis has not yet been defined. METHODS: In vitro and ex vivo studies using human epithelial cancer cell lines and freshly isolated colonic epithelial cells (CEC) from mice were performed to test the effect of IL-22 on the CEC activation. In addition, in vivo studies using experimental models of colitis were carried out to define the role of IL-22 in the pathogenesis of colitis. RESULTS: IL-22 expression was specifically elicited in the colon under inflammatory conditions observed in experimental colitis models as well as human IBD patients. Interestingly, the receptor specific for IL-22 was constitutively expressed on CEC but not acquired cells. IL-22 efficiently induced the activation of STAT3 and subsequently enhanced the expression of suppressors of cytokine signaling (SOCS) 3 in human colonic cancer cell lines and freshly isolated CEC from mice. Inactivation of STAT3 using siRNA approach abolished the IL-22-mediated upregulation of SOCS3. To test the role of IL-22 in the pathogenesis of colitis, IL-22 activity was neutralized in DSS-induced colitis model by administration of anti-IL-22 antibodies. Neutralization of IL-22 activity significantly delayed the recovery from DSS-induced acute colitis. To explore the therapeutic potential of IL-22 for IBD, we established a new lipid complex-based, pressurized local gene delivery approach to induce the overexpression of IL-22 into the colonic lamina propria (LP). Of note, this approach efficiently delivered the gene encoding IL-22 with GFP as indicated by massive accumulations of GFP+ cells in the colonic LP. Importantly, the overexpression of IL-22 in the colon led to a rapid suppression of colitis in T cell receptor $\alpha$ knockout (TCR $\alpha \mathrm{KO})$ mice that have developed severe colitis. This rapid attenuation of colitis was associated with restoration of CEC homeostasis as indicated by a significant increase in goblet cells. CONCLUSION: We here demonstrate that IL-22 contributes to the suppression of colitis by specifically restoring the CEC homeostasis presumably through the activation of STAT3.

\section{3}

Nemo Binding Domain Peptide Ameliorates Chronic T-Helper-1 (Th1)Mediated Murine Colitis

Shaival H. Dave, Jeremy S. Tilstra, Khaja K. Rehman, Paul D. Robbins, Albert S. Baldwin, Scott E. Plevy

BACKGROUND: The NF- $\mathrm{KB}$ family of transcription factors is a central regulator of chronic inflammation. The phosphorylation of IKB proteins by a specific IKB kinase (IKK) is a key step in NF-KB activation. IKK is a complex of two catalytic subunits, IKK $\alpha$ and IKK $\beta$, and a regulatory subunit, "NF- $\mathrm{\kappa B}$ essential modulator" (NEMO). An N-terminal region of NEMO associates with a hexapeptide sequence within the C-terminus of both IKK $\alpha$ and IKK $\beta$, named the NEMO binding domain (NBD). A cell permeable peptide containing a cationic protein transduction domain (PTD) coupled to NBD (PTD-NBD) disrupts the association of NEMO with IKKs in vitro and blocks NF-KB activation in vivo. As a therapeutic modality, PTD-NBD has the theoretic advantage of selectively inhibiting activated NF- $\mathrm{KB}$, and the potential for local and systemic administration. OBJECTIVES: The purpose of this study is to determine efficacy and mechanism of a novel cell permeable peptide NF-kB inhibitor (PTD-NBD) in murine colitis. METHODS: To determine transduction efficiency, murine bone-marrow (BM) derived macrophages were incubated with two PTDs (8K and HIV-TAT). $\mathrm{NF}-\mathrm{KB}$ activity was evaluated using an NF- $\mathrm{KB}$ luciferase reporter plasmid transfected into RAW264.7 cells. IL-10 deficient (-/-) mice at 10 weeks of age were injected i.p. with PTD$\operatorname{NBD}(1,5$, or $10 \mathrm{mg} / \mathrm{kg}$ and vehicle) daily for two weeks. Colitis severity was scored by a pathologist blinded to treatment group. TNF and IL-12 p40 production were determined by ELISA (mean \pm SD) in supernatants from intestinal explant cultures and LPS $(100 \mathrm{ng} / \mathrm{ml})$ plus IFN- $\gamma(10 \mathrm{ng} / \mathrm{ml})$ activated splenocytes. RESULTS: The PTD peptides transduce BMderived macrophages with close to $100 \%$ efficiency. In RAW264.7 cells, PTD-NBD dosedependently inhibits LPS-induced NF-KB activity. In vivo, IL-10-/- mice treated i.p. with PTD-NBD demonstrate amelioration of colitis (colitis score $1.6 \pm 0.4$ vs. $5.4 \pm 0.9$ ) compared to IL-10-/- mice treated with vehicle. Intestinal explants of treated mice express less TNF $(71 \pm 43 \mathrm{pg} / \mathrm{ml}$ vs. $219 \pm 34 \mathrm{pg} / \mathrm{ml})$ and IL-12 p40 $(270 \pm 11 \mathrm{pg} / \mathrm{ml}$ vs. $650 \pm 56 \mathrm{pg} / \mathrm{ml})$ than controls. Decreased LPS plus IFN- $\gamma$ activated IL-12 p40 is demonstrated in splenocytes cultured ex vivo from PTD-NBD treated IL-10-/- mice. CONCLUSIONS: A cell permeable peptide inhibitor of IKK, PTD-NBD, ameliorates colitis in IL-10-/- mice. Mechanistically, mucosal explants and splenocytes cultured ex vivo for 24 hours demonstrate decreased cytokine expression, suggesting that inhibition is not related to the continued presence of the short-lived PTD-NBD peptide. These results demonstrate that inhibitors of IKK, in particular a PTD-NBD peptide, could be therapeutic in the treatment of colitis.

\section{4}

Cannabinoid Receptor 2 Activation Prevents and Reduces Intestinal Inflammation

Xavier Thuru, Mathias Chamaillard, Meliha Karsak, Emilie Gantier, Edmone Erdual, Caroline Dubuquoy, David Philippe, Claudio Schteingart, Jacek Stalewski, Andreas Zimmer, Pierre Riviere, Pierre Desreumaux

Background: The G protein coupled cannabinoid receptor 2 (CB2) is specifically expressed on immune cells, mediating analgesia and regulation of the immune response. However, the potential regulatory role(s) of CB2 and its ligands during intestinal inflammation remain unknown. Aim: Evaluation of the potential therapeutic effect and the underlying mechanisms of CB2 function in preventing/treating acute and chronic intestinal inflammation using different experimental models of colitis in mice. Methods: Colitis was induced by intrarectal administration of TNBS $(150 \mathrm{mg} / \mathrm{kg}, 5$ days) in CB2-/- mice and their respective wild type littermates (WT). Effects of CB2 ligands were also investigated using a known selective CB2 agonist (JWH-133), a new CB2 ligand (FE 200859) and/or a specific CB2 receptor antagonist (SR144528), given preventively or in a treatment mode in C57/Bl6 mice with acute or chronic colitis induced either by TNBS or DSS. The intensity of colitis was assessed by mortality rates, macroscopic and histologic inflammatory scores and quantification of myeloperoxydase activity. Results: Mutant mice lacking CB2 were highly susceptible to colitis. Consistently, we found suggestive anti-inflammatory properties of the known CB2 agonist JWH-133 $(0.1 \mathrm{mg} / \mathrm{kg} /$ day) during TNBS-induced colitis. The novel specific CB2 ligand FE 200859 administered preventively or in treatment mode was effective at optima dosages ranging from $0.1-1 \mathrm{mg} / \mathrm{kg} / \mathrm{day}$, reducing the mortality and the intensity of colitis in mice with acute and chronic inflammation. Consistently, this effect was CB2-dependen and reversed by the administration of the CB2 receptor antagonist SR144528 (1 mg/kg day). Finally, we showed in vivo that activation of CB2 reduces neutrophil recruitment in the mouse colon, providing an explanation for the anti-inflammatory role of $\mathrm{CB} 2$ in the gut. Conclusion: Modulation of CB2 function and the particular efficacy of the new CB2 ligand FE 200859 open a novel therapeutic strategy for the treatment of intestinal inflammation.

235

Intestinal Colonization with the Helminth Heligmosomoides Polygyrus Abrogates Ill7a Production By Mesenteric Lymph Node Cells Ahmed Metwali, John Leung, M. Nedim Ince, Sarah Winkler, Arthur Blum, Tommy Setiawan, Joseph F. Urban, Joel V. Weinstock, David E. Elliott

Interleukin 17A (IL17, CTLA-8) is a pro-inflammatory cytokine required for autoimmune pathology. IL17A is made by a distinct linage of T cells separate from classical Thl and Th2 cells. IL17A expression is increased in active Crohn's disease and ulcerative colitis. Exposure to intestinal helminths inhibits aberrant inflammation in several mouse models of colitis. Colonization with the helminth Trichuris suis decreases disease activity in patients with Crohn's disease or ulcerative colitis. Because elevated IL17A expression is associated with active IBD, we tested if helminth exposure affects gut-associated T cell IL17A expression Wild-type C57Bl/6 mice were colonized with the helminth Heligmosomoides polygryrus by gastric gavage containing 150 larvae. Controls received no larvae. H. polygyrus is a nematode that resides in the duodenum of mice and inhibits TNBS or IL10-deficient colitis. Two weeks after gavage, mice were euthanized and mesenteric lymph node (MLN) cells isolated for RNA extraction or cell culture. As determined by real-time PCR, freshly isolated MLN cells from $H$. polygyrus-colonized mice expressed 16 fold fewer IL17A mRNA transcripts than MLN cells from control mice $(\mathrm{p}<0.05)$. MLN cells from either group produced no ELISA detectable IL17A when cultured without stimulation or stimulated with LPS (100ng/ $\mathrm{ml})$ or $\mathrm{CpG}$ oligonucleotide $(0.6 \mu \mathrm{g} / \mathrm{ml})$. When stimulated with anti-CD3, MLN cells from worm-free control mice produced copious IL17A $(1.40 \pm 0.23 \mathrm{ng} / \mathrm{ml})$. Identically simulated MLN cells from $H$. polygyrus-colonized mice produced much less IL17A $(0.20 \pm 0.10$, $\mathrm{p}<0.01$ ). Results are from three separate experiments each with 3 to 4 mice per group Conclusion: Helminth colonization inhibits IL17A mRNA expression and TCR-stimulated production by gut-associated lymphocytes. IL17A is a critical cytokine that promotes inflammation. The inhibition of IL17A may explain how helminth exposure can limit colitis. (DK58755, DK49382, and VAMC)

\section{6}

Antrum Mucosal Protein (AMP)-18 Activates Multiple Pathways to Assemble and Stabilize Tight Junctions

Margaret M. Walsh-Reitz, Sreedharan Kartha, Marc Bissonnette, Bogdan Goc, F G. Toback

AMP-18 (gastrokine-1), a novel $18 \mathrm{kDa}$ protein, is cell protective, mitogenic, and motogenic for intestinal epithelial cells. Both recombinant human (rh)AMP-18 and a synthetic 21-mer AMP peptide stimulate accumulation of specific tight junction (TJ) proteins and stabilize perijunctional actin. These actions triggered by AMP-18 suggest that it could not only protec the mucosal barrier, but also speed its recovery after injury in inflammatory bowel disease as we observed in mice with dextran sulfate sodium-induced colitis. To determine if AMP18 is a molecular regulator of TJ protein assembly after TJ disruption, we asked if it activates protein kinase C-zeta $(\mathrm{PKC})$ and generates the multimeric phospho (p)-PKCE/Par6/ Cdc42 ECT2-GTP/Par3 protein complex known to associate with JAM-A and ZO-l proteins needed to form mature TJs. By utilizing immunoblotting, confocal microscopy (CF), and measurements of transepithelial electrical resistance we showed that addition of rhAMP-18 to Caco2/ bbe (C2) cell monolayers induced phosphorylation of PKC $\zeta$ by 10 minutes, followed by the appearance of p-PKC $\xi$ in the detergent-insoluble, cytoskeletal fraction. Furthermore, $\mathrm{CF}$ revealed that cytosolic p-PKC\& colocalized with perijunctional actin, suggesting its translocation to the TJ. AMP-18 also promoted the translocation of Par6, Cdc42, and the GTP-GDP exchange factor ECT2 into the detergent-insoluble fraction of cell monolayer exposed to low-calcium medium (LCM) to disrupt their TJs. ZO-1, ZO-2, and JAM-A also accumulated in TJs after treatment with rhAMP-18. We then asked if the capacity of AMP18 to stabilize the actin cytoskeleton following exposure to the actin-disrupting agent cytochalasin D could be mediated via activation of the p38 MAP kinase (MAPK)/heat shock protein (hsp) 27/actin pathway. Immunoblotting demonstrated that AMP peptide rapidly stimulated phosphorylation of p38 MAPK and hsp27, whereas the specific p38 MAPK inhibitor SB202190 prevented phosphorylation of hsp27 suggesting that p38 MAPK activation is required for hsp27 phosphorylation. CF showed that treatment with AMP peptide increased accumulation of p-hsp27 at 30 minutes and its translocation to perijunctional actin. After TJs in C2 cell monolayers were disrupted by LCM, treatment with AMP peptide resulted in rapid phosphorylation of hsp27 and its translocation along with ZO-1 and ZO2 to actin. These observations suggest that AMP-18 stimulates phosphorylation of both hsp27 and PKC $\zeta$, and that translocation of these phosphoproteins promotes assembly of T proteins and stabilizes the actin filament network to protect mucosal barrier structure and function and speed recovery after injury. 


\section{7}

GTPase Activation in Polarity Complexes Initiates Intestinal Epithelial Defense Responses

Atsuko Fukazawa, Kiyotaka Kurachi, Xiubin Gu, Ben Margolis, Karl Matter, Hans C. Reinecker

Background and aim: The coordination of two evolutionarily conserved multiprotein complexes, Cdc42-Par6-Par3-aPKC and Crumbs-PALS1-PATJ, has been implicated in the regulation of tight junction integrity. GEF-Hl has been identified as a tight junction-associated guanine nucleotide exchange factor for RhoA. The aim of this study was to identify the protein interaction of GEF-Hl in junctional complexes and determine its function as a coordinator for Rho-GTPase dependent signaling pathways in polarized epithelial cells. Materials and Methods: Interaction of GEF-Hl with polarity and tight junctional proteins was assessed by immunoprecipitations and live cell imaging using spinning disc confocal microscopy. Subcellular redistribution of polarity and junctional proteins was assessed in Triton X-soluble and -insoluble protein fractions. Expression vectors were generated for GFP tagged GEF-Hl, mRFP-tagged RhoA, Racl, Cdc42 and their dominant negative or constitutive active mutants. Additional constructs included non-tagged and VSV-tagged GEFH1, HA-tagged PAR3, PAR6 and 14-3-35. Transcriptional activation was assessed by dualluciferase assays using reporter constructs containing NF-KB consensus binding motifs or the human IL-8 promoter. HEK293, Caco-2 and MDCK cell lines were used for the analysis of GEF-Hl functions. Results: We originally identified GEF-Hl as a serine phosphorylated protein in claudin-2 containing tight junctional complexes of human intestinal epithelial cells. The protein interactions responsible for the subcellular recruitment of GEF-Hl changed with epithelial cell differentiation. In nonpolarized epithelial cells, GEF-HI associated with microtubules but shifted into junctional complexes upon polarization and establishment of functional tight junctions. GEF-Hl was found to bind to partition defective (PAR) 3 in a complex with the adaptor 14-3-3६. Formation of this complex inactivated GEF-HI. Increases in cytoplasmic GEF-Hl resulted in the activation of RhoA with subsequent enhanced recruitment of $\mathrm{Cdc} 42$ but not RhoA or Racl into junctional complexes. Interestingly, release of GEF-Hl let to the RhoA dependent activation of NF-KB and the induction of IL-8 promoter activity in epithelial cells. Conclusion: We show that GEF-HI is recruited during epithelial differentiation to become part of the apical polarity complexes. There GEF-HI may serve as a trigger in tight junction responses to challenges to the intestinal barrier for the induction of epithelial repair and defense signals.

\section{8}

The Tumor Necrosis Factor (TNF) Family Member Light Causes ActomyosinDependent Occludin Endocytosis and Barrier Dysfunction Brad T. Schwarz, Fengjun Wang, Yingmin Wang, Yang-Xin Fu, Jerrold R. Turner

LIGHT (Lymphotoxin-like Inducible protein that competes with Glycoprotein-D for binding to Herpes virus entry mediator on T cells) is a member of the TNF superfamily involved in $\bar{T}$ cell regulation during innate and adaptive immune responses. Although related, it is notable that LIGHT and TNF utilize distinct, non-overlapping surface receptors to trigger differing intracellular signals. Mucosal LIGHT expression is increased in human Crohn's disease and, in mice, transgenic LIGHT expression induces Crohn's-like disease. Effects of TNF on intestinal epithelial cells have been linked to barrier dysfunction in Crohn's disease. However, no studies have examined the effects of LIGHT on the epithelial barrier. The aim of this study was to determine if LIGHT is able to disrupt the intestinal epithelial barrier and, if so, to determine the mechanisms involved. METHODS: Caco- $2_{\mathrm{BBe}}$ monolayers were treated with basolateral cytokines (IFNy, 10ng/ml, and/or LIGHT, $50 \mathrm{ng} / \mathrm{ml}$ ) as indicated. Barrier function was measured as transepithelial resistance (TER). Myosin light chain (MLC) phosphorylation and MLC kinase (MLCK) expression were determined by immunoblot. Tight junction protein localization was assessed by deconvolution immunofluorescent microscopy. RESULTS: LIGHT did not affect TER in the absence of IFNy. In monolayers primed with IFNy (24hrs), expression of the LIGHT receptor LT $\beta$ R was upregulated and LIGHT treatment caused TER to fall within 4hrs, with maximal TER loss of $31 \pm 7 \%$ after 8 hrs. LIGHT-induced barrier dysfunction was not accompanied by apoptosis, but was associated with markedly increased MLCK expression and MLC phosphorylation. TER loss correlated with internalization of the tight junction protein occludin. Both TER loss and occludin distribution were restored by specific MLCK inhibition. Occludin endocytosis occurred via a caveolae-mediated pathway based on increased occludin:caveolin-1 colocalization and inhibition by $M \beta C D$, which blocks caveolae-dependent endocytosis. CONCLUSIONS: LIGHT disrupts intestinal epithelial barrier function. This process is distinct from TNF-induced barrier dysfunction, as LIGHT and TNF do not share cell surface receptors. LIGHT-induced tight junction disruption occurs as a result of caveolae-mediated occludin internalization. These data are the first to suggest a role for LIGHT in epithelial barrier loss in Crohn's disease. Moreover, these data imply that MLCK inhibition may be an effective strategy to correct LIGHT-induced barrier dysfunction.

239

Role of Keratin-8 in Intestinal Barrier Function Lixin Wang, Didier Merlin, Shanthi V. Sitaraman

Background and Significance: Keratin 8 (K-8) and keratin-18 (K-18) are the predominant intermediate filament proteins in the small and large intestinal epithelia. Recent studies have shown that Keratin-8 null mice develop spontaneous diarrhea and colitis. Moreover, mutations of keratin-8 may be associated with inflammatory bowel disease. The regulation and function of keratin in the intestinal epithelia is largely unknown. We recently demonstrated that IL-6 induces a transcriptionally-mediated synthesis of K8 and K18. In this study we addressed the role of IL-6-induced K8 expression in barrier function. Methods: Caco2BBE cell line was used to study the effect of IL-6 (100ng/ml) on keratin expression. Paracellular permeability was assessed by apical-to-basal transport of a dextran probe (MW4000) conjugated to fluorescein (percentage of apical flux per $\mathrm{cm} 2 / \mathrm{h}$ ) and expressed as \% of vehicle treated cells. Keratin expression was studied by Northern blot and Western blot. Caco2bbe cells were transiently transfected with scrambled or K8 siRNA and silencing of K8 expression was confirmed by Western blot. Results: IL-6 decreased paracellular flux of FITC dextran compared to vehicle treated monolayer by $31+2 \%$ compared to vehicle treated cells. K8 siRNA abolished the decrease in paracellular permeability induced by IL-6 (scrambled siRNA+1L- $643 \pm 8 \%$ and K8 siRNA+IL-6 $120 \pm 18$ of vehicle treated cells). IL- 6 significantly upregulated mRNA and protein levels of K8, K18 and K19. Confocal microscopy showed a reticular pattern of intracellular keratin localized to the sub-apical region after IL-6 treatment. Keratin was localized to the triton-insoluble fraction after IL-6 treatment. Interestingly, in IL6 treated cells, immunoprecipitation studies showed that K8 associated with apcial junctional proteins, E-cadherin and occludin. Conclusions: Collectively, our data demonstrate that IL6 induces K8 which mediates permeability changes induced by IL-6. We show that K8 regulates barrier function and the effect of $\mathrm{K} 8$ on barrier function may be related to its direct interaction with apical junctional proteins.

\section{0}

WIP1 Protects Hydrogen Peroxide-Induced Colonic Epithelial Barrier Dysfunction By Targeting Claudin-4

Tadayuki Oshima, Takaya Shimura, Fumitaka Fujita, Yoshinori Mori, E.J. Kubota,

Tomonori Yamada, Tsuneya Wada, Naotaka Ogasawara, Satoshi Tanida, Hiromi Kataoka, Makoto Sasaki, Toshiyuki Takeuchi, Takashi Joh

Background and Aim: Tight junctions create a paracellular permeability barrier, and though reactive oxygen species (ROS) have been implicated as mediators of inflammation in inflammatory bowel diseases their effect on the function of colonic epithelial tight junctions remains unknown. In this study, we examined the effect of hydrogen peroxide $\left(\mathrm{H}_{2} \mathrm{O}_{2}\right)$ on colonic mucosal barrier properties and tight junction organization and investigated the role of Wipl, a serine/threonine phosphatase. Methods: Human colonic epithelial cells, Caco2, were used in this study. Epithelial barrier integrity was determined by assessing trans-epithelial electrical resistance (TEER) using a volt-ohm meter with 'chopstick' electrodes and by measuring the paracellular permeability of dextran molecules. To examine the effects of $\mathrm{H}_{2} \mathrm{O}_{2}$ on junctional proteins, immunoblotting and immunofluorescent staining were performed with specific antibodies (claudins-1, -3, -4, and -7). In some experiments, cells were dual stained and samples were analyzed with a confocal laser scanning microscope. The mRNA level was analyzed by real-time PCR. The activation of $\mathrm{P} 38 \mathrm{MAP}$ kinase was detected by a phosphospecific antibody. SB203580 and PD98059 were used as a p38 MAP kinase inhibitor and a MAP kinase kinase (MEK) 1 inhibitor, respectively. Wipl-expressing Caco2 were produced by transfection with adenovirus vector containing the Wipl gene. Results: $\mathrm{H}_{2} \mathrm{O}_{2}(500 \mathrm{uM})$ treatment of colonic epithelial monolayers caused a significant decrease in TEER and a significant increase in dextran permeability. Oxidant-mediated permeability and phosphorylation of p38 MAP kinase were significantly attenuated by SB203580 ( $1 \mu \mathrm{M})$, but not by PD98059 $(10 \mu \mathrm{M})$. Although the amount of tight junction proteins was not altered with $\mathrm{H}_{2} \mathrm{O}_{2}$ stimulation, $\mathrm{H}_{2} \mathrm{O}_{2}$ changed the localization of claudin-4 protein from an NP-40 insoluble fraction to a soluble fraction. Interestingly, levels of claudin-4 mRNA were increased by $\mathrm{H}_{2} \mathrm{O}_{2}$ stimulation. Wipl transfection significantly attenuated phosphorylation of $\mathrm{p} 38$ MAP kinase, oxidant-mediated permeability, and levels of claudin-4 mRNA. $\mathrm{H}_{2} \mathrm{O}_{2}$-induced changes in claudin-4 localization were abolished by SB203580 pretreatment as well as Wip 1 transfection. Conclusions: This is the first study able to demonstrate that the change in claudin-4 localization is important for $\mathrm{H}_{2} \mathrm{O}_{2}$-induced colonic permeability and that Wipl functions to protect colonic mucosal integrity by targeting claudin-4.

241

Mechanism of Prednisolone Modulation of the Intestinal Epithelial Tight Junction Barrier: Role of the Glucocorticoid Receptor Pathway in Promoter Regulation

Michel Boivin, John C. Kennedy, Dongmei Ye, Thomas Y. Ma

Background: Glucocorticoids are the first-line therapeutic agents for inflammatory bowel disease. Glucocorticoid treatment results in the restoration of the intestinal epithelial tight junction (TJ) barrier in patients with active Crohn's disease. Previously we have shown that prednisolone prevents the TNF- $\alpha$ induced increase in Caco-2 TJ permeability. Prednisolone prevented the TNF- $\alpha$ induced opening of the TJ barrier by inhibiting the TNF- $\alpha$ induced increase in expression and activity of the enzyme myosin light chain kinase (MLCK). Glucocorticoids exert anti-inflammatory effects by binding to cytoplasmic glucocorticoid recep$\operatorname{tor}(\mathrm{GR})$ which then translocates to the nucleus to bind its transcription factor motif: the glucocorticoid receptor element(GRE). The aim of this study was to examine the intracellular mechanisms of prednisolone inhibition of the TNF- $\alpha$ induced increase of Caco-2 TJ permeability Methods: Filter grown Caco-2 intestinal epithelial monolayers were used as an in-vitro model of the intestinal epithelial barrier. Results: TNF- $\alpha$ treatment resulted in a significant increase in Caco-2 TJ permeability. Prednisolone had no effect on baseline TJ permeability but prevented the TNF- $\alpha$ induced increase in permeability. Prednisolone caused a rapid cytoplasmic to nuclear translocation of Caco-2 GR as determined by immunostaining. Prednisolone also caused an increase in binding of activated GR to a DNA oligonucleiotide containing a consensus GRE. The known GRE binding antagonist RU-486 inhibited the prednisolone induced increase in GRE binding. TNF- $\alpha$ caused a significant increase in MLCK promoter activity and expression; and prednisolone inhibited the TNF- $\alpha$ induced increase in MLCK promoter activity and expression. Moreover, RU-486 prevented prednisolone inhibition of MLCK activation. A GRE binding motif was identified on the MLCK promoter and an MLCK promoter construct was created with a mutation of the GRE binding site using site-directed mutagenesis. TNF- $\alpha$ also caused an increase in the promoter activity of the GRE-mutated MLCK promoter. However, in the absence of the GRE binding motif, prednisolone no longer prevented the TNF- $\alpha$ increase in MLCK promoter activity. Conclusion: Prednisolone protects against the TNF- $\alpha$ induced increase in Caco-2 TJ permeability by preventing the TNF- $\alpha$ induced activation of MLCK promoter activity and subsequent increase in MLCK expression. Our results show that the prednisolone inhibition of MLCK promoter activity is mediated by prednisolone activation of GR which translocates to the nucleus and binds to the GRE 
site on the MLCK promoter. GR binding to the GRE on the MLCK promoter inhibited MLCK promoter activity.

242

Slow Wave Circus Movements and Reentry in the Canine Stomach in Vivo Wim Lammers, Luc Ver Donck, Betty Stephen, Jan A. Schuurkes, Bernard Coulie

The mechanisms underlying gastroparesis have not been well elucidated but could be based on gastric tachy- or brady- dysrhythmias. Such arrhythmias could be due to focal or reentrant activations. We analyzed the pattern of slow wave propagation in the stomach in an in vivo canine model. Five fasted dogs were anesthetized and after a median laparotomy, the ventral surface of the stomach was exposed in situ. A multi-electrode assembly of 240 electrodes (covering a 4 by $4 \mathrm{~cm}$ area with $2.5 \mathrm{~mm}$ interelectrode distance) was positioned on the serosal surface of the stomach. After a 5-minute stabilization period, electrical activity was recorded for $5 \mathrm{~min}$ from all 240 electrodes simultaneously. The timings of slow waves at each recording site were determined off-line and slow wave propagation maps were constructed. In the majority of cases, slow wave behavior was regular, homogeneous and uniform (4.8 cycles/min). They propagated from corpus to antrum at increasing speed until the impulses terminated in the pyloric region. Occasionally $(n=5)$, the regular slow waves were followed by a burst of 2-3 extra slow waves, occurring at much shorter intervals $(5.5 \mathrm{sec})$ than the regular interval $(12.4 \mathrm{sec})$. Reconstruction of the propagation of these extra slow waves revealed inhomogeneous conduction and propagation blocks, mainly in the transitional area between corpus and antrum. Around these blocks, the slow waves propagated in elliptical loops (long and short diameter 22 and $7 \mathrm{~mm}$ respectively) with an interval of 5-6 seconds. After two to three revolutions, lasting a total of approximately 20 seconds, the slow waves were blocked in the corpus area and the bursts thereby terminated. This is the first demonstration of reentry and circus movement of the slow wave in the stomach. The fact that these fasted and anaesthetized animals were otherwise normal, seems to indicate that reentrant activity in the stomach could be more common than previously recognized.

\section{3}

Diabetes Induces Gender Dependent Changes in Gastric Neuronal Nitric Oxide Synthase (nNOS) Expression, Dimerization and Function Pandu R. Gangula, Maria-Adelaide Micci, John Winston, Raj Kumar, Xiemin Cao, Pankaj J. Pasricha

Introduction: Both idiopathic and diabetic forms of gastroparesis, like many other disorders of gastrointestinal motility, predominantly affect women. However, the biological basis of this gender bias remains completely unknown. Based on the importance of neuronal nitric oxide synthase (nNOS) in the regulation of gastric motility, we explored the effect of gender on its expression and function in healthy and diabetic rats. Methods: Age-matched male and female Sprague-Dawley rats were used. Diabetic animals were studied 12 weeks after streptozotocin ( $55 \mathrm{mg} / \mathrm{kg}$ body weight, i.p.)-induced sustained hyperglycemia. Changes in gastric nNOS mRNA and protein levels were analyzed using real-time PCR and Western immunoblotting respectively. Both $\mathrm{COOH}$ - and $\mathrm{NH} 2$-terminal nNOS antibodies were used to measure changes in expression of both total nNOS protein and the alpha homodimer in normal and diabetic gastric tissues. nNOS dimerization studies were performed using lowtemperature SDS-PAGE. Nitrinergic relaxation (AUC/mg tissue weight) was studied after the application of electric field stimulation (EFS) to fundal, antral and pyloric tissues in an organ bath. Results: As compared with males controls (MC), gastric nNOS mRNA, protein expression and nitrinergic relaxation were significantly $(\mathrm{P}<0.05)$ elevated in healthy female control rats $(\mathrm{FC})$ in all regions of the stomach. The active dimeric form and dimer: monomer ratio (DMR) of nNOS alpha were also higher in females compared to male rats $(\mathrm{P}<0.05)$. Interestingly, diabetic females (FD), but not males, showed significant $(\mathrm{P}<0.05)$ impairment in both nNOS alpha dimerization (Fundus FD 7.97 0.02 vs. FC 22.31 \pm 5.88 ; Antrum FD $15.6 \pm 1.29$ vs. FC $41.6 \pm 3.02$; Pylorus FD $3.4 \pm 1.17$ vs. FC $8.98 \pm 0.82$ ) and nitrinergic relaxation (Fundus FD $-0.26 \pm 0.06$ vs. FC $-0.55 \pm 0.16$; Antrum FD $-0.07 \pm 0.02$ vs. FC $-0.49 \pm 0.19$; Pylorus FD $-0.22 \pm 0.07$ vs. FC $-0.46 \pm 0.09$ ). Conclusions: This study demonstrates significant gender differences in gastric nitrinergic expression, and function in both health and disease. Nitrinergic relaxation is more pronounced in healthy females accounted, in part, by an increased expression of the active dimeric form of nNOS alpha. On the other hand, chronic hyperglycemia causes a greater reduction in active forms of nNOS in females associated with significantly more impairment of nitrinergic relaxation. This study also illustrates for the first time the importance of nNOS dimerization in gastric physiology. While we are still exploring the underlying mechanisms, these findings may provide a biological explanation for the greater vulnerability of females to develop diabetic gastroparesis.

\section{4}

Gender Influences Effect of Cannabinoid Receptor Agonist, Dronabinol, On Human Gastrointestinal Motor Functions

Tuba Esfandyari, Irene Ferber, Duane Burton, Kari Baxter, Alan R. Zinsmeister, Michael Camilleri

Cannabinoid receptors (CBR) are located on cholinergic neurons in the brain stem, stomach and colon. CBR stimulation inhibits motility and increases food intake in rodents. Effects in humans are unclear. Hypothesis: Dronabinol (DRO), a non-selective CBR agonist, retards gastric emptying and colonic transit, and enhances gastric accommodation. Aims: To compare the effects of DRO and placebo (PLA) on gastric emptying, small bowel and colonic transit, gastric volume, satiation and postprandial symptoms in healthy volunteers. Methods: In a double-blind, parallel-group study, 30 volunteers were randomly assigned to DRO $5 \mathrm{mg}$ b.i.d. or PLA, each given for 3 doses. Using validated tests, we measured: on day 1 , scintigraphic transit $\left({ }^{111} \mathrm{In}\right.$-egg meal) and fasting and postprandial gastric volume by ${ }^{99 \mathrm{~m}} \mathrm{Tc}$ SPECT; on day 2, maximum tolerated volume (MTV) and 30-minute post-Ensure ${ }^{\circ}$ symptoms. ANCOVA was used to assess treatment effects, with gender and age as covariates. Planned sample size had sufficient power to detect $25-30 \%$ differences in primary endpoints.
Results (see table): There was an overall significant $(\mathrm{p}=0.018)$ retardation of gastric emptying (GE) with DRO; this was most pronounced in females (PLA 158 \pm 10 min vs. DRO 200 \pm 13 , $\mathrm{p}=0.011)$, but less so in males $(\mathrm{p}=0.18)$. A significant $(\mathrm{p}=0.04)$ interaction of gender by treatment was detected for fasting gastric volume; DRO increased the volume in males $(185 \pm 16$ on PLA vs. $238 \pm 17$ on DRO, p $<0.05)$, but slightly decreased it in females. MTV and postprandial symptoms, small bowel and colonic transit were not significantly differen with DRO and PLA. Conclusion: A non-selective cannabinoid agonist retards gastric emptying in humans; effects are gender related. Effects on satiation of changes in fasting gastric volum with DRO require further study. These data are consistent with the observation that gonada sex steroids influence neuronal responses to cannabinoids (Neuroendocrinol 2001;74:185).

\begin{tabular}{|c|c|c|}
\hline Data mean \pm SEM; ${ }^{*} \mathrm{p}<0.05 ; \# \mathrm{p}=0.08$ & Placebo $(\mathrm{n}=15)$ & Dronabinol $(\mathrm{n}=15)$ \\
\hline Age $(\mathrm{y})$ & $29.2 \pm 1.8$ & $25.7 \pm 1.7$ \\
\hline Gender F:M & $8: 7$ & $8: 7$ \\
\hline BMI $\left(\mathrm{kg} / \mathrm{m}^{2}\right)$ & $25.4 \pm 1.0$ & $25.4 \pm 0.8$ \\
\hline GE $\mathrm{t} 1 / 2$, min & $149.5 \pm 20.1$ & $174.6 \pm 10.9^{*}$ \\
\hline Colonic filling $6 \mathrm{~h}, \%$ & $29.5 \pm 8.2$ & $28.5 \pm 6.6$ \\
\hline Colonic geometric center $24 \mathrm{~h}$ & $2.26 \pm 0.23$ & $2.08 \pm 0.24$ \\
\hline Fasting gastric volume, $\mathrm{ml}$ & $214 \pm 13$ & $221 \pm 11$ \\
\hline Post-meal $\Delta$ gastric volume, $\mathrm{ml}$ & $530 \pm 20$ & $573 \pm 30$ \\
\hline MTV, ml & $1170 \pm 125$ & $1245 \pm 77$ \\
\hline Aggregate symptom score $(\mathrm{max} .400)$ & $164 \pm 21$ & $137 \pm 21$ \\
\hline Fullness score $($ max. 100$)$ & $73 \pm 4$ & $55 \pm 6 \#$ \\
\hline
\end{tabular}

245

Neuropathological and Genomic Changes in the Stomach of Patients with Human Diabetic Gastroparesis

Harsha Vittal, Gianrico Farrugia, Nonko D. Pehlivanov, Matt S. Lurken, Pankaj J. Pasricha

Background: The pathophysiological basis of diabetic gastroparesis is unknown, with almost no data on neuropathological and molecular changes in the stomachs of patients. The aim of this study is to use modern analytical methods to determine morphological and molecular changes in the gastric wall in patients with diabetic gastroparesis. Methods: Full thickness gastric biopsies were obtained laparoscopically from gastroparetic patients undergoing surgical intervention and from comparable disease-free areas of control subjects undergoing other forms of gastric surgery. Samples were processed for histological and immunohistochemical examination as well as mRNA preparation and gene expression analysis using Affymetrix Gene Chips. Results: As part of an ongoing study, specimens have been obtained from two patients with diabetic gastroparesis and two control subjects. Although both patients had severe refractory symptoms with malnutrition, requiring the placement of a gastric stimulator one of them had no detectable abnormalities as compared with controls. This patient had an abrupt onset of symptoms with a short duration of well controlled diabetes and no significant episodes of ketoacidosis or hypoglycemia. By contrast, the other patient had long standing poorly controlled diabetes with numerous episodes of diabetic ketoacidosis and frequent hypoglycemic episodes. Histological examination in this patient revealed increased fibrosis in the muscle layers as well as significantly fewer nerve fibers and myenteric neurons as assessed by PGP9.5 staining. Further, significant reduction was seen in staining for nNOS, VIP and tyrosine hydroxylase as well as for c-KIT. By contrast, staining for substance P appeared to be increased. Analysis of mRNA expression confirmed some of these changes including a 1.9 fold decrease in Kit and 2.2 fold increase in tachykinin precursor 1 and indicated changes in several other potentially relevant genes such as a 1.5 -fold reduction in tryptophan hydroxylase I, a 3.2-fold reduction in heme oxygenase 2 and a nearly 12 fold reduction in the muscarinic M3 receptor. Conclusion: Poor metabolic control in diabetes is associated with significant pathological changes in the gastric wall that affect all major components including muscle, neurons and ICC. However, severe symptoms can also occur in the absence of these changes and may reflect vagal, central or hormonal influences. Gastroparesis is therefore likely to be a heterogenous disorder. Careful molecular and pathological analysis may allow more precise phenotypic differentiation and shed insight into the underlying mechanisms as well as identify novel therapeutic targets

\section{6}

Predictive Factors for Clinical Improvement with Enterra Gastric Electric Stimulation Treatment for Refractory Gastroparesis

Jennifer L. Maranki, Vanessa Lytes, John E. Meilahn, Sean Harbison, Frank Friedenberg, Robert S. Fisher, Henry P. Parkman

Gastric electrical stimulation (GES) treatment for refractory gastroparesis has been available for 5 years. Although studies have investigated the clinical effectiveness of this therapy, the clinical factors that predict a favorable response have not been addressed. The AIMS of this study were to: 1) to analyze the clinical response to GES in patients with refractory gastroparesis; and 2) to determine factors associated with a favorable response to Enterra GES treatment. METHODS: This clinical protocol was conducted prospectively at our institution in patients undergoing Enterra GES (Medtronic, Inc) for refractory gastroparesis under the FDA's Humanitarian Device Exemption program. Symptoms associated with gastroparesis were scored before and at regular intervals after GES implantation using the validated Gastroparesis Cardinal Symptom Index (GCSI) questionnaire with additional questions abou abdominal pain and global clinical response. RESULTS: During an 18 month study period from 3/2004 to 9/2005, 29 patients ( 25 female, 4 male; mean age: 40 years) underwent implantation of Enterra GES. Follow-up data were available for 28 patients, with average follow-up of 148 days. At follow-up, 14 of 28 patients felt improved; 8 remained the same; and 6 worsened. The overall GCSI significantly decreased by $12 \pm 7 \%$ (SEM) $(3.3 \pm 0.2$ to

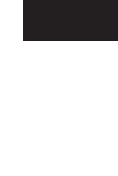


$2.7 \pm 0.2 ; \mathrm{p}<0.05)$ with improvement in the nausea/vomiting subscore $(30 \pm 7 \% ; \mathrm{p}<0.01)$ and the postprandial subscore $(10 \pm 10 \% ; \mathrm{p}<0.05)$, but no improvement in the bloating subscore or abdominal pain. The decrease in the GCSI was greater for the 12 diabetic patients $(18 \pm 11 \% ; \mathrm{p}<0.05)$ than the 16 idiopathic patients $(7 \pm 9 \% ; \mathrm{p}=\mathrm{NS})$. The subgroup of 22 patients with a chief complaint of nausea/vomiting had a greater improvement $(16 \pm 9 \%$; $\mathrm{p}<0.05)$ than the 6 patients with a chief complaint of abdominal pain $(3 \pm 11 \%$; $=N S)$. The 13 patients taking narcotic analgesics at the time of implant had a poorer response with the GCSI slightly increasing by $9 \pm 10 \%$ ( $\mathrm{p}=\mathrm{NS}$ ) compared to the 15 patients who were not, whose GCSI scores decreased by $30 \pm 8 \%(p<0.01)$. There was no effect of gender, BMI, or hemoglobin Alc in diabetics on the clinical response. CONCLUSIONS: Gastric electric stimulation resulted in clinical improvement in $50 \%$ of patients with refractory gastroparesis. Three clinical parameters were identified to be associated with a favorable clinical response: 1) diabetic rather than idiopathic gastroparesis, 2) nausea/vomiting rather than abdominal pain as the primary symptom, and 3) independence from narcotic analgesics prior to stimulator implantation. Knowledge of these three factors may allow improved patient selection for gastric electric stimulation.

\section{7}

Multi-Channel Electrogastrography and Autonomic Nervous System Function in Different Subtypes of Patients with Functional Dyspepsia

Xiaohong Xu, Zhiqiang Song, Zhaolu Ding, Zhifeng Wang, Xiaoyan Chen, Meiyun Ke

Previous studies have shown that patients with functional dyspepsia(FD) had significantly lower percentage of slow wave coupling (\%SWC) in fasting state. However, the effects of test meal on multi-channel Electrogastrography(MEGG) and autonomic nervous system (ANS) in FD patients with different subtypes were not conclusive. The aim of this study was to utilize MEGG and ANS test to investigate the difference of MEGG parameters and ANS parameters between FD patients and healthy subjects(HS). Methods: 62 patients with the diagnosis of FD were enrolled in this study $(16 \mathrm{M}, 46 \mathrm{~F}$, mean age: 43yrs) and divided into dysmotility-like FD group(15M, 35F, mean age: $42 \mathrm{yrs})$ and ulcer-like FD group(1M,11F,mean age:46yrs) based on Rome II criteria. $50 \mathrm{HS}$ were recruited as control (25M, 25F, mean age: 44yrs). MEGG were recorded simultaneously with the electrocardiogram (ECG) recording for $30 \mathrm{~min}$ in the fasting state and $60 \mathrm{~min}$ after a standard $450 \mathrm{Kcal}$ test meal. MEGG parameters included dominant frequency (DF), normal percentage of 2 $4 \mathrm{cpm}$ gastric slow waves $(\% \mathrm{~N})$ and $\%$ SWC. ANS was determined by power spectra analysis of HRV and parameters included sympathovagal balance LF/HF Ratio, mainly sympathetic activity LF and vagal activity HF. Results: 1) Compared with HS, \%SWC was significantly lower in ulcer-like FD group in fasting state $(\mathrm{P}<0.05)$ but not in fed state. No significant difference of \%SWC was shown between dysmotility-like FD group and HS either in fasting state or in fed state. 2) Compared with HS, LF/HF ratio and LF were significantly lower and HF was significantly higher in fasting state in dysmotility-like FD group $(\mathrm{P}<0.05)$, this difference was abolished by test meal in the 1 st $30 \mathrm{~min}$ fed state and the lower LF and higher HF were present in the 2 nd $30 \mathrm{~min}$ fed state again in dysmotility-like FD group. There was no significant difference between ulcer-like FD group and HS in any of those ANS parameters either in fasting state or in fed state. 3) Compared with fasting state, test meal significantly increased the DF in the 3 groups but decreased the $\% \mathrm{~N}$ and \%SWC in both dysmotilitylike FD group and $\mathrm{HS}$ in fed state $(\mathrm{P}<0.05), \% \mathrm{~N}$ only significantly decreased in 1 st $30 \mathrm{~min}$ fed state and \%SWC had no change in fed state in ulcer-like FD group. Test meal significantly increased the LF/HF ratio and LF but decreased the HF in all 3 groups in fed state $(\mathrm{P}<0.05)$. Conclusions: Gastric slow wave coupling was abnormal in patients with ulcer-like FD. Patients with dysmotility-like FD had abnormal ANS function in fasting state and test meal could only correct the abnormal ANS for short time which suggest that different subtypes of FD may have different patterns in ANS test.

\section{8}

Flexible Sigmoidoscopy is Associated with a Reduced Incidence of Distal But Not Proximal Colorectal Cancer (CRC): a Population-Based Cohort Study Linda Rabeneck, James D. Lewis, Lawrence Paszat, Refik Saskin, Therese A. Stukel

Background: Flexible sigmoidoscopy (FS) is used to triage those with distal colon polyps to colonoscopy and those without polyps to repeat FS screening in $5 \mathrm{yr}$. However, it is unclear whether those with a negative FS (without polyps) have a reduced incidence of cancer proximal to the rectosigmoid colon. Objectives: In a population-based cohort study of persons with a negative FS to: 1) estimate the annual incidence of CRC; and (2) identify factors associated with incident CRC. Methods: We identified all individuals 50-80 yr who had a negative FS between 1/1/1996 and 12/31/1998 in Ontario and followed them through $12 / 31 / 2002$. We computed the annual age- and sex- standardized incidence rates (SIRs) for colon (ICD-9 codes 153.0-153.4, 153.6-153.9) and rectum or rectosigmoid (154.0-154.1) cancers for the study cohort and for the remaining Ontario population. We compared the annual incidence of CRC between the study cohort and the remaining Ontario population. Cox proportional hazards models were used to examine patient (age, sex), physician (type, procedure volume) and procedure setting (office, hospital) factors associated with incident cancers among those with a negative FS. Data were obtained from the: 1) Ontario Cancer Registry; 2) Canadian Institute for Health Information database, which has information on all patients discharged from hospitals; 3) Ontario Health Insurance Plan (OHIP), which has information on all claims for physician services; and 4) Registered Persons Database, which is a roster of all permanent residents who are covered under OHIP. Results: Between 1996 and 1998 we identified 45,304 persons who had a negative FS, of whom $58.3 \%$ were women. During follow-up 324 cancers ( 269 colon, 58 rectum/rectosigmoid) were observed in these patients. For rectum/rectosigmoid cancers the annual SIRs following negative FS were significantly lower than in the Ontario population. For example, at $4 \mathrm{yr}$, the SIR following negative FS was 0.24 cancers/ 1000 persons (95\% CI: $0.11-0.45$ ) vs $0.54 / 1000$ (0.51-0.57) in the Ontario population ( $R R=0.44,95 \%$ CI: 0.16-0.73). For colon cancers, the annual SIRs following negative FS were not significantly different than in the Ontario population. For example, at $4 \mathrm{yr}$, the SIR following negative FS was 1.32 cancers/1000 persons (1.00-1.72) compared with $1.34 / 1000(1.29-1.39)$ in the Ontario population (RR= $0.99,95 \%$ CI: 0.72-1.25). Only age was significantly associated with incident CRC following negative FS. Conclusions: Following negative FS the incidence of rectum/rectosigmoid cancers - but not colon cancers - is reduced for up to 4 years. Age, but not sex, is associated with incident CRC following negative FS.

249

A One Year, Randomised, Double-Blind, Placebo Controlled Trial of a Lactobacillus Or a Bidifobacterium Probiotic for Maintenance of SteroidInduced Remission of Ulcerative Colitis

Fergus Shanahan, Francisco Guarner, Atte Von Wright, Terttu Vilpponen-Salmela, Diarmuid O'Donoghue, Barry Kiely, Progid Investigators

Background: Probiotics have been shown to have modest but consistently significant efficacy in attenuating disease severity in animal models of inflammatory bowel disease (IBD) However, the role of probiotics in human IBD is less clear. Aim: to compare two different probiotics vs. placebo in the maintenance of remission of ulcerative colitis following steroidinduced remission. Design: a prospective, balanced, randomised, parallel group, double blind, placebo-controlled trial of $\left(10^{9}\right.$ daily for one year) Lactobacillus salivarius subsp. Salivarius UCC118 or Bifidobacterium infantis 35624 versus placebo, each administered as a rehydrated blended yoghurt powder. Patients were entered within one month of achieving clinical remission of ulcerative colitis following a documented relapse that required steroids to induce remission. Remission was defined as $<3$ bowel movements/day (without frank/ gross blood) out of 7 , while off all steroids. A stable dose of aminosalicylate was the only concomitant medication for colitis permitted. The number of patients in remission at study end was the primary efficacy variable with time to relapse being a secondary criterion. A total of 157 patients were recruited with similar numbers (52-53) per group. Results: Patients' demographic characteristics were similar across the three treatment groups. The extent of colitis which was similar across the groups was left-sided in about one third, limited (proctitis) in one third and pan-colitis in one third. About half of all patients were still in remission at end of the study with no difference across the three study groups. The risk of relapse was not significantly different across the groups. Patient age was the only prognostic factor with a significant $(\mathrm{p}=0.04)$ influence on risk of relapse, with odds for an older patient decreasing over that of younger patients by $2.8 \% / y e a r$ of age. Mean times to relapse were also similar for each study group. Adverse events were uncommon, unrelated to the treatment, and similar across the groups. Conclusions: Prolonged feeding with live probiotics is safe in patients with ulcerative colitis. However, in contrast to studies in animal models using the same probiotics, there was no significant benefit with either probiotic over placebo. Differences in probiotic efficacy between animal and human IBD may reflect the timing of administration and might indicate the importance of manipulating the enteric flora prior to the onset of disease, and/or may relate to differences in disease severity or effective probiotic dose/body weight.

250

Can Aspirin be Reintroduced with Proton Pump Inhibitor Infusion After Endoscopic Hemostasis? A Double Blinded Randomized Controlled Trial Joseph Sung, James Lau, Jessica Ching, Wai-Keung Leung, Yuk-Tong Lee, Justin Wu, Vincent Leung, Francis Chan

Background: Among patients with aspirin-induced bleeding ulcers who received pantoprazole infusion after endoscopic hemostasis, it is uncertain whether aspirin can be reintroduced immediately. Aim: This study aims to investigate whether early reintroduction of aspirin is safe in patients who received intravenous pantoprazole for peptic ulcer bleeding. Method: This double-blind, placebo-controlled, randomized trial included patients who required aspirin for cerebrovascular (CNS) or cardiovascular (CVS) diseases but developed ulcer bleeding. After confirmation of peptic ulcer bleeding (active bleeding, visible vessel or adherent clot), endoscopic hemostasis was applied using epinephrine injection and thermal coagulation. Patients were randomized to receive aspirin $80 \mathrm{mg}$ daily or placebo aspirin daily. All patients received intravenous pantoprazole ( $80 \mathrm{mg}$ bolus injection followed by $8 \mathrm{mg} /$ hour for 72 hours and then oral pantoprazole $40 \mathrm{mg}$ daily until end of follow up at 8 weeks. The primary endpoint was recurrent upper gastrointestinal bleeding within 30 days. Secondary endpoints included blood transfusion, requirement for surgery, hospital stay, 1-month and 2-month mortality, and cardiovascular/cerebrovascular event. Results: In this interim analysis, 113 patients were randomized ( 58 to aspirin and 55 to placebo). The aspirin and placebo groups were comparable in age ( $74.2 \mathrm{yr}$ vs $73 \mathrm{yr}$ ), gender (men: $62 \%$ vs $69 \%$ ), indications for aspirin (CVS:CNS:both 33:19:6 vs 30:19:6), previous history of ulcer (6.9\% vs $3.7 \%$ ) and concomitant use of NSAID ( $12 \%$ vs $11 \%$ ). Patients randomized to aspirin and placebo also have comparable ulcer location (GU:DU $28: 30$ vs $33: 22)$, size $(1.13 \mathrm{~cm}$ vs $1.20 \mathrm{~cm}$ ) and ulcer appearance (bleeding: visible vessel: adherent clot 16:24:18 vs 17:21:17). Recurrent bleeding within 30 days was documented in $11(18.9 \%)$ who received aspirin and $6(10.9 \%)$ who received placebo (Log rank test $\mathrm{P}=0.25$ ). There was no difference in requirement for blood transfusion, surgery and hospital stay. The aspirin-treated patients have lower 1month ( $1.7 \%$ vs $9.1 \%, \mathrm{P}=0.08)$ and 2 -month $(1.7 \%$ vs $14.5 \%, \mathrm{P}=0.012)$ mortality compared to those who received placebo. Conclusion: In the presence of intravenous and oral pantoprazole, immediate reintroduction of aspirin has not substantially increased the risk of recurrent bleeding from peptic ulcers. There is, however, an associated increased mortality with discontinuation of aspirin

251

The Degree of COX-2 Selectivity and Risk of Cardio and Cerebrovascular Events

Neena Abraham, Christine Hartman, Hashem El-Serag, Anita Deswal

Background: To determine if the degree of Cox-2 selectivity of an NSAID influences risk of cardio and cerebrovascular events, we assessed the association between Cox-2 selectivity and myocardial infarction (MI) or non-hemorrhagic stroke (CVA). Methods: A retrospective cohort study of veterans $\geq 65$ years prescribed NSAIDs at the VA from 01/01/00 to 12/31/ 
02. VA pharmacy, inpatient and outpatient data was linked to inpatient Medicare claims files. NSAIDs were categorized as poorly (naproxen, ibuprofen), moderately (celecoxib, etodolac, meloxicam, nabumetone) or highly (rofecoxib, valdecoxib) Cox-2 selective. Each day of observation was assessed for presence of NSAID and degree of selectivity. Validated ICD-9-CM codes were used to define MI and CVA. The incidence of MI and CVA over 180 days was assessed using Cox-Proportional Hazards Models adjusted for gender, race, cardiovascular risk factors, the daily presence of low dose ASA, anticoagulant or antiplatelets, and the propensity score for prescribing a highly Cox-2 selective NSAID. Patients were censored at event, end of follow-up period, last day of exposure or death. Hazard Ratios (HR) with $95 \%$ confidence intervals were calculated. Results: Of 384,322 patients (97.5\% men; $85.4 \%$ white), $79.4 \%$ were prescribed a poorly selective NSAID, $16.4 \%$ were prescribed a moderately selective NSAID and $4.2 \%$ were prescribed a highly selective NSAID. There were 985 cases of MI and 586 cases of CVA in over 145,870 person years of follow-up. Exposure to highly selective agents was associated with the highest rate of MI (12.3 per 1000 person-years) and CVA (8.1 per 1000 person-years). The incidence rates were lower among veterans prescribed a moderately (7.6 MI and 5.4 CVA per 1000 person-years) or poorly selective agent (7.4 MI and 4.2 CVA per 1000 person-years). Periods without NSAID exposure were associated with the lowest risk (3.9 MI per 1000 person-years; $2.2 \mathrm{CVA}$ per 1000 person-years). The prescription of highly selective NSAIDs was associated with a $61 \%$ increase in CVA and a $47 \%$ increase in MI, when compared to poorly selective NSAIDs. In all adjusted models, no NSAID exposure was protective for both $\mathrm{MI}(\mathrm{HR}=0.66 ; 95 \% \mathrm{CI}$ : $0.55-0.80)$ and CVA (HR=0.58; $95 \% \mathrm{CI}: 0.45-0.74)$. Similar results were observed among lower risk patients (those without a prior MI or CVA, a history of revascularization procedures, atrial fibrillation, or evidence of anticoagulant or antiplatelet agents at index NSAID prescription). Conclusions: Among older veterans, the risk of MI and CVA increases with exposure to any NSAID. Highly Cox-2 selective NSAIDs confer the greatest risk of MI and CVA.

252

Notch 1 Acts At Multiple Stages of Endocrine Differentiation to Regulate Endocrine Cell Fate in the Pancreas

Maryann Giel-Moloney, Andrew B. Leite

Notch signaling enables individual cells to adopt cell fates that are distinct from neighboring cells. When Notch receptors on the cell surface bind to cell surface ligands like delta or jagged, expressed by adjacent cells, the receptor is cleaved, releasing the notch-intracellular domain fragment which migrates to the nucleus where it associates with RBP-Jk as a coactivator, resulting in expression of downstream Notch effectors including the transcriptional repressor, Hesl. Notch appears to block endocrine differentiation in the pancreas by inhibiting bHLH transcription factors, including neurogenin3 (Ngn3) which initiates the earliest stages of endocrine specification. A downstream bHLH protein, BETA2/NeuroD is required for normal islet morphogenesis and terminal differentiation of $\beta$ cells. Others have shown that conditional activation of Notch during early pancreagenesis when Ngn3 is first expressed arrested epithelial differentiation, whereas Notch had no effect on the adult pancreas. To further determine at what stage of endocrine differentiation precursor cells still respond to Notch, we used the Cre-loxP system to activate Notch signaling at the onset of BETA2 expression in developing islets. We generated transgenic mice expressing Cre inserted into a BAC containing $115 \mathrm{~kb}$ of $5^{\prime}$ and $78 \mathrm{~kb}$ of 3 ' flanking sequence from the BETA2 locus to ensure its expression would recapitulate that of the endogenous BETA2 gene. BETA2-Cre mice were crossed with ROSA-Notch mice, a line containing a bicistronic transgene with the Notch 1 intracellular domain (NICD) and EGFP inserted into the constitutively expressed ROSA26 locus. Transcription read through of the transgene was prevented by an upstream stop sequence flanked by loxP sites. Expression of Cre recombinase results in excision of the stop sequence leading to permanent, constitutive expression of NICD and EGFP. BETA2Cre X ROSA-Notch mice showed disrupted islet morphogenesis with a marked reduction in insulin staining in the islet core. The cells in the islet center showed staining for EGFP, suggesting that these were BETA2 expressing cells destined to become $\beta$ cells. In addition, many of the EGFP expressing cells formed large aggregates of mixed cell lineages expressing glucagon, somatostatin, and PYY. The present study is the first to show that Notch functions at later stages of pancreatic development. Thus the effects of Notch on the endocrine pancreas depend on both the context and the stage of differentiation much like other organs. We conclude that activation of Notch 1 in BETA2-expressing precursors in the pancreas inhibits $\beta$ cell maturation resulting in cells adopting a non- $\beta$ cell fate

\section{3}

Acetylcholine Stimulation of the Nkccl Transporter Is Calcium-Dependent and Is Rapidly Desensitised By Endocytosis in Human Colonic Crypts: Major Implications for Intestinal Fluid Secretion

Amy Reynolds, Alyson Parris, Richard Tighe, Michael Lewis, Denise Westgarth, Duncan Bell, Crawford Jamieson, Jamie Dearlymple, Joanne Southgate, Kevin Sargen, Martin Philips, Mark Williams

Intestinal fluid secretion is driven by transcellular active transport of chloride. NKCCl mediates basolateral uptake of chloride and is emerging as a master controller of fluid secretion. Acetylcholine (ACh) stimulates a calcium dependent secretory response that is transient in nature. Recent observations in T84 cells point to the importance of NKCCl internalisation as a regulatory mechanism (Del Castillo et al., AJP 2005; 289: C1132-42). AIM: To characterise NKCCl expression along the intact human colonic crypt axis and $\overline{\text { study }}$ its cellular location in response to cholinergic calcium signals. METHODS: Crypts were isolated from tissue biopsies obtained at sigmoidoscopy from healthy-subjects (Ethical approval). Isolated crypts were attached to collagen-coated coverslips and cultured for 24 hours - 7 days in serum-free DMEM ( $5 \% \mathrm{CO} 2 / 37 \mathrm{oC})$. Total NKCCl expression was probed for using a panel of polyclonal antibodies. Labelling was visualised using a FITC conjugated secondary antibody in conjunction with confocal microscopy. For calcium imaging experiments colonic crypts were loaded with the calcium-sensitive dye Fura2-AM. RESULTS: $\mathrm{NKCCl}$ exhibited an expression gradient along the crypt-axis; all cells were $\mathrm{NKCCl}$ positive and labelling predominated at the crypt-base. A dichotomy was evident for NKCCl labelling at the cellular level: one population of cells (approx. 25\%) exhibited labelling on both basal and basolateral membranes, the remaining cells expressed $\mathrm{NKCCl}$ on basolateral membrane only. These characteristics were also evident in crypts stimulated with ACh $(10 \mu \mathrm{M})$ for up to 3 mins. After stimulation for 10 mins, vesicular cytosolic NKCCl labelling juxta latera membranes was apparent. Prolonged exposure ( 30 mins) led to a striking accumulation at the apical pole of NKCCl labelled cytosolic vesicles, with the concomitant loss of membranous NKCCl. Ionomycin mimicked ACh-induced calcium mobilisation from intracellular stores and was sufficient to induce internalisation of $\mathrm{NKCCl}$. Accordingly, inhibition of AChinduced calcium mobilisation by TMB-8 $(100 \mu \mathrm{M})$ abolished NKCCl internalisation. In contrast, chronic stimulation with the cAMP secretagogue forskolin $(100 \mu \mathrm{M})$ did not lead to internalisation of membranous NKCC1. CONCLUSIONS: For the first time we have demonstrated the dynamic regulation of membrane transporter trafficking in the intac human intestinal epithelium. $\mathrm{NKCCl}$ internalisation explains the transient nature of the cholinergic secretory response and identification of the underlying molecular mechanism may be exploited in the development of therapies for secretory diarrhoea. (Supported by the BBSRC)

\section{4}

An Essential Role of Farnesoid X-Receptor in Regulating Intestinal Fibrosis in Murine Model of Chronic Colitis

Piero Vavassori, Andrea Mencarelli, Barbara Renga, Giovanni Rizzo, Antonio Morelli, Mark Pruzanski, Roberto Pellicciari, Stefano Fiorucci

Backgound. The Farnesoid X Receptor (FXR) is a member of the nuclear receptor superfamily involved in cholesterol and bile acid homeostasis and expressed in the liver and gastrointestinal tract. INT-747, a potent FXR ligand, has been demonstrated to reduce $\alpha$ l (I) collagen and TGF $\beta 1$ in hepatic stellate cells and to be beneficial in the treatment of liver fibrosis.Aim. Because Crohn's disease if often characterized by excessive extracellular matrix deposition that can lead to intestinal obstruction, in this atusy we have investigated whether INT-747 may represent a therapeutic option to prevent the intestinal fibrosis in a murine model of chronic colitis. Methods. Chronic colitis was induced in mice with an 8-week administration of increasing amounts of TNBS enemas ( 0.5 to $1.25 \mathrm{mg} /$ mouse). In the first in vivo experiment, $\mathrm{Balb} / \mathrm{c}$ mice were treated with daily intragastric administration of $5 \mathrm{mg} / \mathrm{kg}$ of INT-747 or saline solution. In an second in vivo experiment, chronic colitis was induced in wild-type and FXR knockout mice (C57Bl/6 background). All mice were weekly examined for signs of colitis and systemic inflammation. Each colon was scored regarding macroscopic signs of inflammation and/or fibrosis. Serial paraffin sections of each colon were stained with $H \& E$ to asses the degree of inflammation and with Sirius Red to detect the connective tissue. Colonic expression of $\alpha$ l (I) collagen, fibronectin, $\alpha$ SMA, MMPs and TIMP-1, TNF- $\alpha$ and TGF $\beta 1$ was examined by quantitative RT-PCR. Results. Eight-week administration of TNBS induced in Balb/c mice a chronic colitis predominantly characterized - at macro- and microscopic level - by fibrosis and collagen deposition into the intestinal wall, and increased colonic expression of $\alpha$ l (I) collagen (10 folds when compared to the naive mice), fibronectin, $\alpha$ SMA, TNF- $\alpha$ and TGF $\beta 1$ mRNAs. INT-747 treatment resulted in an almost complete prevention of the intestinal fibrosis, at macro- and microscopic level. In mice treated with the FXR ligand, the colonic expression of $\alpha \mathrm{l}(\mathrm{I})$ collagen, fibronectin, $\alpha \mathrm{SMA}, \mathrm{TNF}-\alpha$ and TGF $\beta 1$ mRNAs was similar to that of naive mice. Differently than Balb/c mice, C57Bl/6 wild-type mice resulted to be insensitive to the chronic administration of TNBS. In contrast, C57Bl/6 FXR knockout mice developed a severe chronic colitis similar to those observed in Balb/c mice. In vitro experiments on intestinal fibroblast cell line confirmed the inhibitory effect of INT-747 on the TGF $\beta 1$-induced collagen production. Conclusion. This study provides evidence that FXR ligands might represent a novel therapeutic option to treat intestinal fibrosis.

255

Role of the mRNA Stability Factor HuR in Inflammatory Bowel Disease and Colon Carcinogenesis

Kristi Bemis-Standoli, Lisa Cox, Vimala Kasa, Melinda Lynch, Hyllaerye Ford, Robert J. Coffey, Dan A. Dixon

Aberrant overexpression of many growth-related immediate-early response genes, including proto-oncogenes, cytokines, and angiogenic growth factors, is commonly observed in inflammatory bowel disease (IBD) and colorectal cancer. A central point in the regulation of these factors occurs through post-transcriptional mechanisms controlling rapid mRNA decay. However, recent evidence reveals that the loss of post-transcriptional regulation is a characteristic feature found in cancer and chronically inflamed cells. To better understand the mechanisms regulating these factors in IBD and colon cancer, we have identified the RNA-binding protein HuR as a potential link. This protein binds to the AU-rich element (ARE) located within the 3'-untranslated region (3'UTR) of many tightly regulated mRNAs and functions as a nuclear-cytoplasmic shuttling protein, yet when overexpressed in cells, HuR interferes with rapid mRNA degradation. Normally expressed at low levels and located in the nucleus, HuR cytoplasmic overexpression was observed in tissues obtained from ulcerative colitis, colon adenomas, adenocarcinomas, and metastases. Overexpression of the HuR target gene COX-2 colocalized with elevated HuR expression. Similarly, HuR levels are elevated in colonic epithelium and correlate with increased COX-2 expression in a murine model of IBD. Elevated cytoplasmic HuR levels are observed in colon cancer cells and promote cancerassociated gene expression. Down-regulation of HuR by siRNA leads to respective downregulation of the HuR targets COX-2, IL-6, IL-8, VEGF, and c-fos. To determine the functional significance of HuR overexpression in vivo, a HuR-transgenic mouse (HuR-Tg) was created to overexpress HuR in GI epithelium. This transgenic utilizes the intestinal-specific A33 promoter to drive expression of a HuR/GFP bicistronic transgene restricted to the small intestine and colon. HuR-Tg mice display normal growth and breeding characteristics. Gross and histological analysis did not reveal any morphological changes in the GI tract of the HuR-Tg mice, however elevated endogenous COX-2 and VEGF mRNA were observed in the colon and small intestine of all HuR-Tg lines. The impact of HuR overexpression on tumorigenesis was further examined through breeding with the APCMin/+ mouse. At 15 weeks of age, HuR-Tg/APCMin/+lines display approximately 2-fold increased tumor burden in both the colon and small intestine compared to APCMin/+control. These findings indicate 
HuR functions in tumorigenesis downstream of a tumor-initiating event and identify this mRNA stability factor as a new molecular target for therapeutic intervention in controlling pathogenic gene expression.

\section{8}

Accuracy of Computed Tomographic Colonography for Colorectal Cancer (CRC) Screening in Asymptomatic Individuals

Brooks D. Cash, Cecilia Kim, Priscilla Cullen, Myra Kim, Cathy A. Dykes, Donald W. Jensen, Duncan S. Barlow, Mark H. Johnston, James W. Kikendall, Peter W. Soballe

Background: Pickhardt demonstrated that computed tomographic colonography (CTC) was comparable to optical colonoscopy (OC) for the accurate detection of adenomas. Our study seeks to validate Pickhardt's findings using the same technique for CTC. Methods: Participants are asymptomatic, average-risk patients referred for colorectal cancer screening who undergo CTC with subsequent care based on CTC interpretation. Patients with polyps $>10 \mathrm{~mm}$ or $>3$ polyps exceeding $6 \mathrm{~mm}$ proceed to same day OC in which all polyps identified by CTC or OC are removed (Category A). Patients with 1 or 2 polyps measuring 6-9 $\mathrm{mm}$ undergo $\mathrm{CTC}$ and clearing $\mathrm{OC}$ at 1 year (Category B) and patients without any polyps $>6 \mathrm{~mm}$ have OC in 5 years (Category C). Fleets Phosphosoda prep is used with oral contrast to tag fluid and stool for digital subtraction. CO2 is used for colon insufflation and a 3-D flythrough serves as the primary mode of assessment by an experienced (>500 CTC) radiologist. Polyp matching adheres to Pickhardt's method of segmental unblinding during OC. Data is presented on a per patient basis and is not histology specific. Results: 666 patients have undergone CTC since inception (mean age 56.0 years; $33.6 \%$ female; $78.5 \%$ Caucasian). $16.1 \%$ (107/666) patients have proceeded to OC; $35.5 \%$ (38/107) for significant CTC findings (category A) and 44.9\% (48/107) randomly selected (categories B/C). For lesions $>10 \mathrm{~mm}$, CTC polyp detection sensitivity and specificity are $94.1 \%$ (95\% CI: $80.3 \%$ to $99.3 \%$ ) and $93.2 \%$ ( $95 \%$ CI: $84.7 \%$ to $97.7 \%$ ), respectively, with positive predictive value (PPV) and negative predictive value (NPV) of $86.5 \%$ (95\% CI: $71.2 \%$ to $95.5 \%$ ) and $97.1 \%$ ( $95 \%$ CI: $90.1 \%$ to $99.7 \%$ ). The $93.6 \%$ accuracy of CTC is comparable to OC ( $p=0.10$ McNemar's Test). For 6-9 mm polyps, the sensitivity, specificity, PPV and NPV of CTC are $65.6 \%$ (95\% CI: $46.8 \%$ to $81.4 \%$ ), $92 \%$ (95\% CI: $83.4 \%$ to $97 \%$ ), $77.8 \%$ (95\% CI: $57.7 \%$ to $91.4 \%$ ), $86.3 \%$ (95\% CI: $76.7 \%$ to $92.9 \%$ ), respectively, with an accuracy of $84.1 \%$ (p= 0.001 ). There are too few patients in group B with evaluable outcomes to comment on the observational history of intermediate sized polyps at this time. Conclusions: These results indicate that CTC and OC have similar accuracy for the detection of polyps $>10 \mathrm{~mm}$ and validate the previous findings of Pickhardt. CTC accuracy is significantly less than OC for polyps 6-9 mm, however the observed accuracy of CTC for these polyps is superior to previous studies. The natural history of intermediate sized polyps remains unclear, but CTC may be a valuable tool in elaborating this information in future longitudinal outcomes analyses.

\section{9}

The Management of Small (6-9mm) Polyps Found By Virtual Colonoscopy: Results of a Decision Analysis

Chin Hur, Daniel C. Chung, Robert E. Schoen, Amy B. Knudsen, G. Scott Gazelle

Background: Although there is a firm consensus that larger $(>9 \mathrm{~mm})$ colonic polyps should be removed, the necessity of polypectomy for smaller polyps is controversial. The majority of polypoid lesions found by VC will be less than $9 \mathrm{~mm}$ in size. Furthermore, recently published VC reporting guidelines suggest that it may be reasonable to manage a 6-9mm polypoid lesion discovered on VC with another VC study 3 years later. The aim of this study was to compare the outcomes of two management strategies for smaller polyps discovered by VC. Methods: A Markov decision-analytic model was constructed to analyze the outcomes of patients with a small polyp on a screening VC. These hypothetical patients were simulated to either: 1) undergo immediate colonoscopy for polypectomy (COLO); or 2) wait 3 years for a repeat VC examination (VC). The time horizon (f/u period) for the model analysis was 3 years and endpoints compared included \# of deaths, \# of cancers (CAs) and CA stage (SEER Summary Staging). Model input (parameter estimates) values were derived from and calibrated to SEER data and published estimates and included: VC false positive rate $=31.9 \%$; POLYPS- Hyperplastic $=39.3 \%$, Adenomas (Low Risk=94.1\%, High Risk $=5.0 \%$, Cancer $=0.9 \%$ ); ANNUAL CANCER MORTALITY RATES- Local $=2 \%$, Regional $=$ $7.5 \%$, Distant (metastatic) $=37 \%$; COLONOSCOPY- Complication rate $=0.1 \%$, Complication Death Rate $=0.01 \%$, Inability to find polyp $=5 \%$. Results: The results (see Table 1 ) are 3 years after the initial VC and the \#s presented are out of 100,000 patients in each strategy. The overall number of CAs in the VC group was $1168(1.168 \%)$ compared to $58(0.058 \%)$ in the COLO group (polypectomy cured the majority of CAs in the polyp). All the deaths in the VC group were from CA (111) vs 6 CA and 10 colonoscopy complications deaths in the COLO group. The stage distribution of the CAs is also presented as they significantly impact both prognosis and treatment. Conclusions: Following a small polyp found on VC for 3 years will result in a greater number of CAs and more deaths than immediate COLO. Table 1. Results (\# out of 100,000 pts)

\begin{tabular}{|c|c|c|c|}
\hline & COLO & VC & Difference* \\
\hline Total CA & 58 & 1168 & -1110 \\
\hline Localized & 21 & 416 & -395 \\
\hline Regional & 8 & 163 & -155 \\
\hline Distant & 3 & 63 & -60 \\
\hline Total Deaths & 16 & 111 & -95 \\
\hline CA Deaths & 6 & 111 & -105 \\
\hline CC** Deaths & 10 & 0 & 10 \\
\hline CC & 100 & 0 & 100 \\
\hline
\end{tabular}

*Neg value $=\mathrm{VC}$ greater $* * \mathrm{CC}=\mathrm{COLO}$ complication

280

MR Colonography Without Bowel Purgation for the Assessment of Adenomatous Colon Polyps: Preliminary Results of a Prospective Cohort Study

Jost Langhorst, Christiane Kuehle, Thomas Zoepf, Michael Nuefer, Waleed Ajaj, Barkhausen Joerg, Guido Gerken, Gustav J. Dobos, Thomas Lauenstein

Background: Colorectal cancer, which arises mostly from preexisting adenomatous polyps, continues to be the second most common cancer. Colonoscopy (in conjunction with histopathological biopsy) is considered the gold standard for the detection of adenomatous polyps. The impact of existing colonic screening techniques strongly depends on the according patients' acceptance. Recently developed MRC techniques based on the tagging of fecal material allow for the visualization of colorectal lesions without prior bowel cleansing. Aim: To compare virtual MR colonography (MRC) to colonoscopy (CC) regarding the detection of adenomatous polyps $>5 \mathrm{~mm}$ and patients' acceptance in a screening population. Methods: 284 asymptomatic patients ( 150 female, 134 male, average age 59 years) underwent MRC. Preparation for MRC included the ingestion of $200 \mathrm{ml}$ of a contrast solution containing $5 \%$ gastrografin, $1 \%$ barium and $0.2 \%$ locust bean gum with every main meal starting two days before the MR examination. MRC was performed on a 1.5 T MR system (Magnetom Sonata, Siemens Medical Solutions, Erlangen, Germany) in patients' prone position. After the placement of a rectal tube, the colon was filled with approximately $2500 \mathrm{ml}$ of warm tap water. CC was performed with a maximum time lag of 4 weeks between both procedures. The overall acceptance was evaluated using a 10-point scale ( $1=$ excellent, $10=$ poor acceptance). Results: Colorectal adenomatous polyps $>5 \mathrm{~mm}$ were found in 33 patients by CC. MRC revealed these lesions with a sensitivity of $78 \%$ and a specificity of $93 \%$. In addition, one inflammatory pseudotumor due to sigmoid diverticulitis and one adenocarcinoma of the sigmoid were detected by both methods. No significant difference was detected between the overall rating for MRC (mean value: 3.4) and CC (mean value: 3.0). Most unpleasant aspects of the examinations were bowel cleansing for $\mathrm{CC}$ and the placement of the rectal tube for MRC. No significant difference was found as for the preference of future examinations: $46 \%$ of the patients would choose MRC, while $43 \%$ preferred CC. Conclusion: Fecal tagging MRC provides a satisfactory accuracy for the detection of relevant colorectal lesions in a screening population. Both MRC and CC have comparable general acceptance levels in a screening population. However, in patients with positive findings, conventional endoscopy is required for polypectomy.

281

CT Enterography in Crohn's Disease: A Prospective Study Demonstrating Inter-Observer Agreement and Correlation with Disease Activity Taft Bhuket, Elaine M. Caoili, Peter D. Higgins, Beth Manoogian, Joel Platt, Paul Sonda, Tahira Khan, Ellen M. Zimmermann

Background: CT Enterography (CTE) yields high resolution images of the bowel and is effective in assessing Crohn's Disease. Performance characteristics including inter-observer variability have not been determined. Correlation of CTE findings with measures of disease activity including the Crohn's Disease Activity Index (CDAI) is lacking. Questions exist as to what CTE adds to standard clinical activity assessment. Aims: 1) To prospectively determine the inter-observer agreement for 10 findings on CTE: 2) To determine the Spearman correlation between CTE findings, CRP, ESR and CDAI; 3) To use CTE findings and lab markers to predict CDAI, and determine whether CTE findings add predictive power to the assessment of disease activity. Methods: 41 patients with Crohn's disease were enrolled for prospective assessment of the CTE, CRP, ESR, and CDAI. CTE was performed per standard protocol with intravenous contrast, oral ingestion of $1500 \mathrm{cc}$ of a low-density barium sulfate contrast, and the use of a multi-detector CT. Two expert radiologists blinded to clinical data independently rated 10 CTE findings, including 5 scaled items (rated 0-3): mucosal enhancement, mesenteric vascularity, bowel wall thickening, stranding, and global assessment. An additional 5 dichotomous findings were rated 0 if absent or 1 if present: extraluminal gas, fistula, fluid, lymphadenopathy, and bowel fat. Agreement analysis was performed with a weighted kappa for scaled variables, and a simple kappa for dichotomous variables. Spearman correlations were calculated for each comparison, and linear regression modeling was used to determine whether lab or radiologic findings could predict the CDAI. Results: 7 findings had substantial inter-observer agreement (0.6-0.8); fluid had an almost perfect kappa of 0.88 , and extraluminal gas and stranding had only moderate agreement $(0.4-0.6)$. The scaled CTE findings correlated well (0.30-0.39) with the CDAI, in comparison to the ESR (0.23) and the CRP (0.35). Extraluminal fluid (0.50) correlated well with CDAI, the only one of the dichotomous variables to do so. A model with ESR and CRP predicted only $17 \%$ of the variance of CDAI, while a model that added bowel thickening and extraluminal fluid to CRP predicted 39\% of the variance ( $\mathrm{p}=0.001$ for the model). Conclusions: CTE findings in Crohn's disease have good inter-observer agreement, the scaled CTE findings correlate better with CDAI than laboratory measures of inflammation, and these findings add to the ability to predict disease 
activity. These finding are consistent with an important role for CTE in assessing Crohn's disease activity and identifying complications.

282

Complete Versus Incomplete Optical Colonoscopy: Predictive Factors Based On Clinical and Virtual Colonoscopy Findings

Meghan Hanson, Perry J. Pickhardt, David H. Kim, Andrew J. Taylor, Patrick R. Pfau, Deepak V. Gopal

Background \& Aims: The purpose of the study was to compare clinical and CT virtual colonoscopy (VC) findings in patients who underwent either complete $\mathrm{OC}(\mathrm{COC})$ or incomplete OC (IOC). Methods: Clinical data and VC examinations were reviewed in 100 consecutive patients who underwent COC immediately after VC and in 85 patients with IOC who were subsequently evaluated by VC. The 3D colon map with automated centerline and 2D MPR images from VC were independently reviewed by two experienced GI radiologists for colonic lengths (total, sigmoid, and transverse), number of acute angle flexures or bends, and presence of advanced diverticular disease. Discrepancies were resolved by consensus. Results: From the clinical perspective, there were significant differences between COC and IOC groups for age (mean, 58.2 vs. 64.2 years; $\mathrm{p}<0.0001$ ), gender (M/F: 60/40 vs. $35 / 50$; $\mathrm{p}<0.05)$, and history of prior surgery $(26.0 \%$ vs. $44.7 \%$; $<<0.01)$. Significant differences were also seen for several evaluated VC factors between COC and IOC groups, including total colorectal length (mean, 167.0 vs. $209.0 \mathrm{~cm}$; p<0.0001), sigmoid length (mean, 48.7 vs. $66.4 \mathrm{~cm} ; \mathrm{p}<0.0001$ ), transverse length (mean, 49.2 vs. $65.3 \mathrm{~cm}$; $\mathrm{p}<0.0001$ ), number of flexures (mean, 9.4 vs. 11.9; p<0.0001), and advanced diverticular disease ( $22.0 \%$ vs. $34.1 \%$; $\mathrm{p}<0.05)$. Conclusions: In addition to patient age, gender, and surgical history, certain anatomic features seen at VC, such as colonic length, number of colonic flexures, and presence of advanced diverticular disease are associated with failure to reach the cecum at OC. These predictive factors may have implications for patients who are sent to OC for polyps detected at VC.

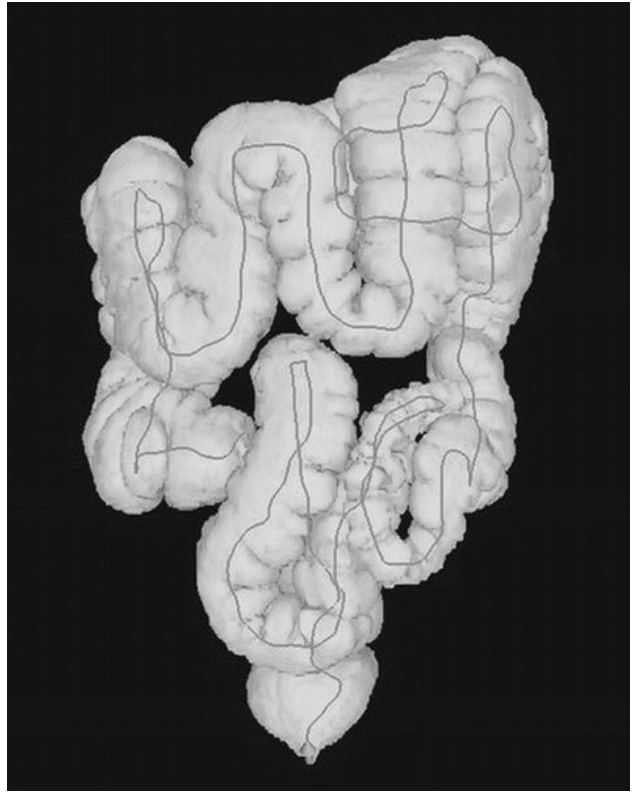

283

Colon Cancer Screening According to Polyp Characteristics: Cost-Effectiveness Analysis with CT Colonography and Endoscopy

Cesare Hassan, Angelo Zullo, Andrea Laghi, Paola Cerro, Franco lafrate, Sergio Morini

Background \& Aims: The relationship between the natural history of each of the different types of adenomatous polyps and the cost-effectiveness of colon cancer (CRC) screening is unknown. A high accurateness of computerized tomographic colonography (CTC) for large polyps has been shown in two meta-analysis, which also confirmed a low sensitivity for $<5$ $\mathrm{mm}$ polyps. Aim of this analysis was to assess the impact of avoiding the removal of diminutive polyps on the cost-effectiveness of CTC in comparison with that of colonoscopy and flexible sigmoidoscopy (FS). Methods: Cost-effectiveness of screening strategies was compared by using computer models of a Markov process, in which a hypothetical population of 100000 persons 50 years of age undergoes CTC, colonoscopy or FS every decade or once in life-time. Outcomes were projected to US national level. Results: Incremental costeffectiveness as compared to no screening (ICER) of CTC, colonoscopy and FS was 7.138\$, $7.407 \$$, and $9.180 \$$, respectively. CTC ICER improved to $4.362 \$$ when avoiding $<5 \mathrm{~mm}$ polyp removal. As compared to such a policy the ICER of CTC and colonoscopy was $118.440 \$$ and $63.900 \$$, whilst FS was dominated. To avoid polypectomy for diminutive lesions in the US population allowed an earning of $60.437 .106 \$$ and $433.584 .633 \$$ in the one-time and in the once every 10 year schedule, respectively. Conclusions: To avoid removal of diminutive lesion appears as the most cost-effective and safe option when simulating a general population screening with CTC. If a high accuracy for large polyps is confirmed, such technique could also be a convenient alternative to colonoscopy

\section{9}

Neoplasia Yield of FOBT Following a Negative Screening FS

Senore Carlo, Segnan Nereo, Andreoni Bruno, Bisanti Luigi, Castiglione Guido, Ferrari Arnaldo, Foco Alberto, Gasperoni Stefano, Malfitana Giuseppe, Pera Angelo, Recchia Serafino, Saracco Giorgio, Zappa Marco

Aims: To estimate the additional yield of neoplasia achievable offering fecal occult blood test (FOBT) to subjects with negative screening sigmoidoscopy (FS). Methods: We conducted a population based multicenter randomized trial comparing four screening protocols: 1 Biennial immunological FOBT, 2) "Once-only" FS, 3) FS followed by biennial FOBT for screenees with negative FS; 4) Patient's choice between FS and FOBT. A random sample of men and women, aged 55 to 64 , was drawn from general practitioners (GP) rosters. GPs were asked to exclude patients with a history of colorectal cancer (CRC), adenomas, inflammatory bowel disease, recent ( $\leq 2$ years) colorectal endoscopy or FOBT, or two first degree relatives with CRC. Eligible subjects were randomized, within GP, and were mailed a persona invitation. The aim of FS was to advance the scope beyond the sigmoid-descending colon junction. Screenees with "high risk" distal polyps (villous component, high-grade dysplasi or cancer, size $\geq 10 \mathrm{~mm}$, or $>2$ adenomas), and those with positive FOBT, were referred for colonoscopy (TC). Only subjects allocated to group 3 are included in this analysis. The most advanced lesion was considered to estimate the detection rate (DR) of the tests and the cumulative DR per persons invited. Results: Out of 20142 people allocated to group 3 , $5569(27.3 \%)$ had a FS and $3218(61.0 \%)$ of those with negative exam attended the invitation for a FOBT two years later. The DR at the initial FS was $0.42 \%$ for CRC $(0.38 \%$ distal $0.04 \%$ proximal) and $4.85 \%$ for advanced adenoma (4.72\% distal; $0.13 \%$ proximal). O 3218 subjects performing FOBT, $115(3.6 \%)$ were positive and $103(89.6 \%)$ of them underwent colonoscopy. The DR was $0.15 \%$ (5/3218) for CRC $(0.02 \%$ distal; $0.13 \%$ proximal) and $0.53 \%(17 / 3218)$ for advanced adenoma $(0.22 \%$ distal; $0.31 \%$ proximal). The cumulative DR per invited persons increased from $011 \%$ to $0.14 \%$ for CRC and from $1.32 \%$ to $1.40 \%$ for advanced adenomas, when considering also the results of FOBT. Conclusions Among people with negative FS the positive predictive value of FOBT is low. FOBT may lead to some increase in the DR of advanced neoplasia in the proximal colon only. The DR for advanced proximal neoplasia $(0.44 \%)$ is about twice as high as among people undergoing a second FOBT screening following a previous negative test in group 1 ( $D R=0.24 \%$ ). Costeffectiveness of this strategy should be evaluated over several screening rounds.

\section{0}

Prevalence of a First Degree Family History of Colorectal Cancer in Persons with a Screen-Detected Colorectal Adenoma in the Prostate, Lung, Colorectal, and Ovarian (PLCO) Cancer Screening Trial

Ajay Pabby, Robert E. Schoen, Paul F. Pinsky, Richard B. Hayes, Joel L. Weissfeld

Objective: To estimate colorectal cancer (CRC) risk in families with an index case of colorecta adenoma, we measured the association between screen-detected distal adenoma and a family history of CRC, as reported by participants in a community-based cancer screening trial. Methods: We selected 55-74 year-old participants in the Prostate, Lung, Colorectal, and Ovarian (PLCO) cancer screening trial who did not report a personal history of cancer colon polyp, diverticular disease, or recent fecal occult blood testing or lower gastrointestinal procedure. Among persons with an adequate $50 \mathrm{~cm}$ screening flexible sigmoidoscopy examination, we contrasted the self-reported CRC family histories of 2257 persons with distal adenoma(s) against the CRC family histories of 19,790 persons without a distal polyp Results: Relative to persons without a distal polyp, a family history of CRC was more prevalent among persons with distal adenoma(s) (adjusted odds ratio (adj OR) 1.43, 95\% confidence interval (CI) 1.25-1.63). The association of adenomas and family history was not any stronger among persons with distal advanced adenoma(s) (adj OR 1.53, 95\% C 1.26-1.86), among persons with more than one distal adenoma (adj OR 1.62, 95\% CI 1.27 2.07), or among younger persons with adenoma(s)(age 55-59) (adj OR $1.52,95 \%$ CI 1.20 1.92). Similarly, the risk for having multiple affected first degree relative with CRC (adj OR $1.56,95 \%$ CI 1.00-2.42), or having a younger onset affected first degree relative ( $<50$ years) (adj OR 1.42, 95\% CI 1.01-1.98), was of a similar magnitude as the risk for any affected relatives. Conclusions: We observed a $40 \%$ increased risk of CRC in first degree family members of subjects with a screen-detected distal colorectal adenoma. However, the usual indicators of increased risk for CRC (presence of advanced or multiple adenomas and younger age of adenoma detection) and enhanced genetic susceptibility to disease (adenoma associated with multiply affected relatives or younger age of relative with (RC) did not magnify the degree of association.

\section{1}

A Prediction Model Based On Age, Gender and Distal Findings Accurately Identifies Black and Hispanic Patients At Low-Risk for Advanced Proximal Colorectal Neoplasia (APN) But Underestimates Absolute Risk Benjamin E. Levitsky, Colin C. Brown, Heeren C. Tim, Paul C. Schroy

Background: Tailoring the use of screening colonoscopy based on the risk of APN has been advocated as a strategy for reducing demand and optimizing cost-effectiveness. A simple, 7 -point scoring system has been proposed that stratifies individuals into low, intermediate and high risk categories (Imperiale et al. Ann Intern Med, 2003:139:959-65). The aim of this study was to determine the validity of this clinical index, which was derived and validated among a mostly White patient population, for Black and Hispanic patients. Methods: Data including age, gender, colonoscopic findings and any pathology results, were collected retrospectively from 1094 consecutive White, 1119 Black and 388 Hispanic asymptomatic average-risk patients undergoing first-time screening colonoscopy. Scores ranging from 0 to 7 were derived for each subject using Imperiale's system, which assigns points for each category of age $(<55 y=0,55-59 y=1,60-64 y=2,>65 y=3)$, gender (female $=0$, male $=1)$ and distal findings (no polyps $=0$; hyperplastic polyps $=1$; tubular adenoma $<1 \mathrm{~cm}=2$; advanced neoplasia =3). 'Advanced' was defined as an adenoma $\geq 1 \mathrm{~cm}$ in size or one with villous histology, severe dysplasia, or cancer. Rate of occurrence of APN was assessed for each risk 
group (low, intermediate, and high) after stratification by race and compared using $\chi 2$ analyses. Results: For Whites, 33\% were classified as low risk (score 0-1), 43\% as intermediate risk (score 2-3) and 24\% as high risk (score 4-7); corresponding rates of APN for each group were $0.8 \%, 3.0 \%$ and $3.8 \%$, respectively. For Blacks, $37 \%$ were categorized as low risk, $47 \%$ as intermediate risk, and $16 \%$ as high risk; corresponding rates of APN were $1.2 \%, 2.5 \%$ and $5.0 \%$, respectively. For Hispanics, $44 \%$ were categorized as low risk, $38 \%$ as intermediate risk, and $18 \%$ as high risk; corresponding rates of APN were $0.6 \%, 2.1 \%$ and $4.5 \%$, respectively. If colonoscopy was performed on everyone, regardless of risk group, the number needed to endoscope (NNE) to detect one patient with APN ranges from 38 for Whites to 41 for Blacks to 55 for Hispanics; conversely, if colonoscopy is performed only for patients in the intermediate and high risk groups, the NNE falls to 31 for Whites, 32 for Blacks and 36 for Hispanics. Restricting the use of colonoscopy for the intermediate and high risk groups only would detect $89 \%$ of APN in Whites, $82 \%$ in Blacks and $86 \%$ in Hispanics but miss $11 \%, 18 \%$ and $14 \%$, respectively $(\mathrm{P}=0.774)$. Conclusions: A prediction model based on age, gender and distal findings accurately identifies Blacks and Hispanics at low risk of APN. Miss rates of up to $18 \%$, however, may limit dissemination of the model into clinical practice.

302

Risk Stratification for Colonic Neoplasia Through Enhanced Backscattering Spectroscopy Analysis of the Endocopically-Normal Rectal Mucosa Hemant Roy, Young Kim, Yang Liu, Michael Goldberg, Nahla Hasabou, Vladimir Turzhitsky, Mohammed Jameel, Eric Elton, Vadim Backman

Background: Risk stratification is critical for designing an efficacious, cost-effective colon cancer screening program. Examination of the rectal mucosa for markers of the "field effect" (e.g. aberrant crypt foci, epithelial proliferation, apoptosis etc.) can reliably predict the occurrence of proximal neoplasia; however, their performance characteristics have been suboptimal for clinical practice. Working with some of the pioneers of light scattering technology (Nature 2000, Nature Med 2001, Gastroenterology 2004, Gut 2005), our group has developed a new generation of optics technology, enhanced backscattering spectroscopy (EBS). EBS can provide heretofore unattainable depth-selective quantitative assessment of the nanoscale architecture of the cell (reflecting subtle genetic/epigenetic events). We have previously reported outstanding sensitivity for the "field effect" in the azoxymethane-treated rat and MIN mouse models of colon carcinogensis (Clin Cancer Res in press). In this study, we assess the ability of novel EBS markers obtained from the rectum to predict colonic neoplasia in humans. Methods: Two rectal biopsies from endoscopically normal mucosa were taken from 88 patients undergoing colonoscopic evaluation at our institution. The fresh tissue was subjected to EBS analysis using an advanced light scattering apparatus. Five spectral markers were chosen that span the spectrum of EBS parameters and values were calculated by an investigator blinded to colonoscopic findings. Results: Spectral markers from histologically normal rectum are detailed in the table below. In general, there were highly significant differences between the values from patients harboring neoplasia when compared to those who were neoplasia free. Importantly, the magnitude of alteration progressively increased with importance of colonoscopic findings (i.e. advanced adenomas> adenomas). With this panel of 5 markers, it was possible to approach $100 \%$ sensitivity for predicting advanced adenomas. Conclusions: We report, for the first time, that novel EBS markers assessed from the rectum were able to accurately predict neoplasia throughout the colon. Our long term goals would be to develop a rectal probe that could be used by the primary care physicians to decide upon timing of colonoscopic screening.

Correlation of EBS Markers from normal rectal mucosa to findings on colonoscopy

\begin{tabular}{|c|c|c|c|c|c|}
\hline & $\begin{array}{c}\text { Spectral } \\
\text { Slope }\end{array}$ & $\begin{array}{c}\text { EBS } \\
\text { Auto-correlation }\end{array}$ & $\begin{array}{c}\text { Enhancement } \\
\text { factor }\end{array}$ & $\begin{array}{c}\text { Principle } \\
\text { component 1 }\end{array}$ & EBS Width \\
\hline $\begin{array}{c}\text { No Neoplasia } \\
\text { (control) }\end{array}$ & 0.0045 & 22005 & 1.103 & -0.007 & 0.459 \\
\hline Adenoma & $\begin{array}{c}0.0036 \\
(\mathrm{p}<0.0001)\end{array}$ & $22245(\mathrm{p}=0.31)$ & $1.098(\mathrm{p}=0.027)$ & $\begin{array}{c}0.016 \\
(\mathrm{p}=0.002)\end{array}$ & $\begin{array}{c}0.469 \\
(\mathrm{p}=0.26)\end{array}$ \\
\hline $\begin{array}{c}\text { Advanced } \\
\text { Adenoma }\end{array}$ & $\begin{array}{c}0.0031 \\
(\mathrm{p}<0.0001)\end{array}$ & $23899(\mathrm{p}=0.006)$ & $1.093(\mathrm{p}=0.041)$ & $\begin{array}{c}0.023 \\
(\mathrm{p}=0.013)\end{array}$ & $\begin{array}{c}0.56 \\
(\mathrm{p}=0.008)\end{array}$ \\
\hline
\end{tabular}

p values compare control versus adenoma or control versus advanced adenoma, respectively

303

A Population Based Study of Effectiveness of a Negative Colonoscopy Harminder Singh, Donna Turner, Lin Xue, Laura E. Targownik, Charles N. Bernstein

Background: There is limited evidence to guide the optimal frequency of repeat endoscopic examination for colorectal cancer (CRC) screening after a negative colonoscopic evaluation. We have established a population based cohort of individuals who had colonoscopic evaluation that did not result in a diagnosis of colorectal neoplasia i.e. a negative colonoscopic evaluation. Methods: Manitoba Health's (the province's single insurer) physician billing claims database was utilized to identify individuals who had a negative colonoscopy between April 1, 1989 and December 31, 2003. Individuals with a history of CRC prior to the index endoscopic evaluation, IBD, resective colorectal surgery, and those with a lower gastrointestinal endoscopic examination of any type within 5 years prior to the index colonoscopy were excluded. The cohort was followed until diagnosis of CRC in the provincial cancer registry, death or migration, whichever came first. Standardized incidence ratios (SIRs) were calculated to compare observed cancer incidence to that expected in the general population. Stratified analysis was performed to determine the duration of the protective effect of negative colonoscopy. Results: There were 35,975 individuals with an initial negative colonoscopy and with 164,733 person- years of follow-up. A negative colonoscopy was associated with statistically significant SIRs of 0.69 at six months, 0.66 at one year, 0.59 at two years, 0.55 at five years and 0.28 at ten years. A subgroup analysis limited to individuals who did not have a repeat lower gastrointestinal endoscopy until they were diagnosed to have CRC was associated with significant SIRs of 0.49 at six months, 0.43 at one year, 0.36 at two years, 0.24 at five years and 0.00 at ten years. There were more right sided cancers (defined as cancers arising from caecum, ascending colon or hepatic flexure) in the colonoscopy cohort compared to all CRC cases diagnosed in the general population ( 76 out of 163 vs. 2884 out of $10197 ; \mathrm{p}<0.001)$. Conclusions: This is the first study to demonstrate the lower likelihood of developing CRC beyond ten years after a negative colonoscopy. There is a need to improve the early detection rate of right sided colorectal neoplasia in the usual clinical practice.

\section{4}

Chromoendoscopic Colonoscopy Detects More Adenomas Than Conventional Colonoscopy: A Randomized Trial of Back-to-Back Colonoscopies Elena M. Stoffel, David H. Stockwell, Daniel P. Normolle, Missy Tuck, Norman Marcon, D. Kim Turgeon, John Baron, Nadir Arber, Robert Bresalier, Sapna Syngal, Dean E. Brenner

Background: Conventional colonoscopy, currently the gold standard for the detection of colorectal neoplasia, misses some neoplastic lesions. Application of contrast dye (chromoendoscopy) may help increase the sensitivity of polyp detection during colonoscopy. Aim: To compare the efficacy of chromoendoscopy vs. standard colonoscopy for detecting adenomas, taking into account the prolonged inspection time of chromoendoscopy. Methods: Fifty subjects with a personal history of sporadic colorectal cancer or adenomatous polyps underwent tandem colonoscopies at one of 5 study centers of the Great-Lakes New England Consortium of the Early Detection Research Network. The first exam for each subject was a "standard" colonoscopy with removal of all polyps found. The second exam was randomly allocated to be either pan-colonic indigocarmine chromoendoscopy or conventional standard colonoscopy with intensive inspection lasting $>20$ minutes. Size, histology, and numbers of polyps detected on each exam were recorded. Results: Seventeen of 50 (34\%) subjects had a total of 40 adenomas detected on the initial conventional colonoscopy; 19 (48\%) adenomas were found in subjects in the chromoendoscopy arm and $21(52 \%)$ in those assigned to intensive inspection. Of the 27 subjects randomized to chromoendoscopy, 12 (44\%) were found to have additional adenomas on second exam, 7 of these did not have adenomas on the initial exam. Among the 23 subjects randomized to intensive inspection, 4 (6\%) had adenomas found on the second exam $(\mathrm{p}=0.07)$, yielding a total of 5 additional adenomas. In the chromoendoscopy group 38 adenomas in total were detected; 19/38 (50\%) adenomas were found on the second exam, as compared with 5/26 (19\%) adenomas in the intensive inspection group $(p<0.01)$. Ten of the 24 adenomas missed on first colonoscopy were characterized as "flat". Of the 19 additional adenomas found during chromoendoscopy, 16 were located in the right colon vs. 2 of 5 for intensive colonoscopy. On average, polyps found on first colonoscopy were larger $(3.74+3.12 \mathrm{~mm})$ than on second colonoscopy $(2.39+1.25 \mathrm{~mm})$. Average inspection time was 29.7 minutes in the chromoendoscopy group, as compared with 20.4 minutes for the intensive inspection group. In multivariable analysis, the use of chromoendoscopy remained a significant predictor for detecting greater numbers of adenomas. Conclusions: Chromoendoscopy detects more adenomas missed by standard colonoscopy than intensive inspection. Because as many as half of colonic adenomas may be missed by conventional colonoscopy, pan-colon chromoendoscopy may increase the yield for neoplastic lesions. Supported by NCI grant CA86400

321

Toll-Like Receptor (TLR) 2 Maintains Tight Junctional-Associated Intestinal Epithelium Barrier Integrity Against Stress-Induced Damage in Vivo Elke Cario, Guido Gerken, Daniel K. Podolsky

Background: Stress stimuli such as toxic injury, inflammation or spontaneous apoptosis induce functional and structural damage of the intestinal epithelial barrier by degradation of morphological integrity of tight (TJ) and adherens (AJ) -junctional proteins. We have recently demonstrated that TLR2 enhances intestinal epithelial barrier integrity through translocation of zonula occludens- $1(\mathrm{ZO}-1)$ via protein kinase $\mathrm{C}$ in vitro. The aim of this study was to determine the functional relevance of TLR2-induced modulatory effects on structural intestinal epithelial barrier integrity against apoptotic or chemical stress-induced damage in vivo. Materials \& Methods: Mice (TLR2 knockouts (TLR2KO; F10), wildtype (WT; C57BL/6J)) were maintained under strict SPF conditions. DSS-colitis was induced by standard protocol (4\%; 5 days). Subcellular distribution of TJ/AJ-associated proteins was assessed by laser confocal microscopy of primary intestinal epithelial cells (IEC) in situ or cultured short-term ex-vivo with or without PCSK (synthetic Pam3Cys-SK4), a known TLR2 ligand. Spontaneous apoptosis was assessed by TUNEL. Results: DSS-induced colitis in TLR2KO was paralleled by dissociation of ZO-l from the lateral TJ membrane, loss of apical symplekin and disorganization of E-cadherin in IEC Compromised intestinal epithelial barrier integrity in TLR2KO resulted clinically in significantly more severe intestinal bleeding and diarrhea than in DSS-WT mice which did not show any significant changes in subcellular TJ structure. Ex-vivo short-term culture of IEC from TLR2KO induced dramatic disruption and depolarization of the epithelial barrier. Membrane-associated ZO-1 was internalized and redistributed to the cytoplasmic center. However, TLR2 stimulation with PCSK preserved intestinal epithelial integrity which correlated with inhibition of apoptosis. PCSK significantly prevented stress-induced injury maintaining consolidated lateral cell-cell contacts in distinct TJ/AJ areas of ex-vivo IEC monolayers from WT, but not in the absence of TLR2. Conclusions: The results of the present study suggest an essential physiological role for TLR2 in preservation of intestinal epithelial integrity against exogenous stress-induced TJ/AJ damage, thus exerting important protective effects on mucosal homeostasis between commensals and host in vivo. 
322

Protection from Lethal Septic Peritonitis By Neutralizing the Biological Function of Interleukin-27

Stefan J. Wirtz, Ingrid Tubbe, Mark P. Birkenbach, Peter R. Galle, Richard S. Blumberg, Markus F. Neurath

Sepsis represents a significant health problem worldwide. At present, there are only a few effective therapeutic approaches for the treatment of septic patients and mortality rates still remain high. Once the host fails to restrict microbes to a local area, they may enter the circulation and trigger an inflammatory response that can be overwhelming and as a consequence fatal to the host. In the present study, we analyzed the contribution of the recently discovered heterodimeric cytokine Interleukin-27 (IL-27) to the development of severe septic peritonitis. First, we analyzed C57BL/6 mice in the clinically relevant cecal ligation and puncture model (CLP) of polymicrobial sepsis. Interestingly, we detected a strong upregulation of IL-27 protein in serum and peritoneal lavage fluid shortly after surgery. Interestingly, IL-27 mRNA is apparently upregulated earlier than that of the subunits of the related IL12 family members IL-12 and IL-23 consistent to in vitro data leading to the suggestion that the biological function of IL-27 precedes IL-12/IL-23 action. Further studies demonstrated that mice deficient for the EBI3 subunit of IL-27 displayed improved long-term survival compared to WT mice, which almost all died within $72 \mathrm{~h}$ post CLP. We observed a similar outcome, when $\mathrm{EBI}^{--}$or WT mice were intraperitoneally injected with $4 \times 10^{8}$ live gram negative bacteria. However, after systemic administration of recombinant single chain IL27 into $\mathrm{EBI}^{-1-}$ mice immediately post CLP, these mice died within 2 days after treatment suggesting that IL-27 is critically involved in sepsis pathophysiology. The reduced mortality in $\mathrm{EBI}^{-1-}$ mice was accompanied by an attenuated proinflammatory cytokine response as indicated by decreased expression of proinflammatory cytokines including IL-6, IL-1 and IL-17. To determine the cellular targets for the early biological functions of IL-27 during sepsis, we analyzed the expression of the IL-27 receptor in cells, which have been implicated in the pathogenesis of the disease. As a result, we determined that not only T and NK cells, but also mast cells, neutrophils and resident peritoneal macrophages co-expressed both receptor chains. Moreover, in the latter cells IL-27 can induce high amounts of proinflammatory mediators. Finally, we evaluated whether neutralizing of IL-27 can be beneficial for CLP subjected mice. A single i.p treatment with a soluble IL-27 receptor fusion protein immediately post CLP resulted in significantly improved survival. Conclusion : Our results indicate that high IL-27 expression is harmful for the septic host and that IL-27 is a novel potential therapeutic target for treatment of sepsis

323

T-Cell Induced Colitis Is Associated with Enhanced Myelopoiesis and Remarkable Neutrophil Infiltration Into Secondary Lymphoid Tissue Dmitry V. Ostanin, Dwain Dsouza, Kathryn L. Furr, Jason Hoffman, Carla M. Brown, Laura Gray, Stephen Pruett, Matthew B. Grisham

Introduction: Numerous studies have focused on the role that the adaptive immune system plays in the pathogenesis of chronic colitis in vivo. However, virtually nothing is known about the role that granulocytes (neutrophils) may play in promoting (or regulating) colonic inflammation in immune-based models of colitis. The objective of this study was to determine how the induction of chronic colitis affects myelopoiesis and the infiltration of neutrophils into secondary lymphoid tissue and the intestinal interstitium. Methods: Adoptive transfer of $5 \times 10^{5} \mathrm{C} 57 \mathrm{Bl} / 6 \mathrm{CD} 4+\mathrm{CD} 45 \mathrm{RB}^{\text {high }} \mathrm{T}$-cells mice into syngeneic recombinase activating gene1-deficient $\left(\mathrm{RAG}_{-} \mathrm{-}^{-/}\right.$) recipients was used to induce chronic colitis whereas transfer of $\mathrm{CD} 4+\mathrm{CD} 45 \mathrm{RB}^{\text {low }} \mathrm{T}$-cells into RAG-1 ${ }^{-1-}$ mice was used to generate healthy controls. Histopathology and flow cytometry were used to assess inflammation and leukocyte numbers in different tissues. Serum protein levels of IL-17 and granulocyte colony stimulating factor (G-CSF) were measured using ELISAs. Results: We found that at 8 weeks following transfer of CD45RB high T-cells, mice developed chronic colitis characterized by massive leukocyte infiltration, crypt abscesses, bowel wall thickening, goblet cell loss and epithelial breaks and erosions. Co-incident with disease was a dramatic leukocytosis in which total circulating leukocytes increased 5-6 fold compared to unmanipulated RAG-1 ${ }^{-/}$mice. Differential counting of the peripheral blood revealed that neutrophils comprised approximately $50 \%$ of the total circulating leukocyte pool in the CD45RB high $\rightarrow R A G-1^{-1-}$ with mononuclear cells (i.e. monocytes, lymphocytes) accounting for the other $50 \%$ of circulating leukocytes. In addition, we found that serum levels of the granulopoietic cytokines IL-17 and G-CSF increased by 4 - and 9-fold, respectively compared to the healthy $\mathrm{CD}_{4} 5 \mathrm{RB}^{\text {low }} \rightarrow \mathrm{RAG}-\mathrm{1}^{-1}$ controls. Corresponding to these dramatic increases in systemic neutrophil production in the CD45RB high $\rightarrow \mathrm{RAG}^{-1}{ }^{-1}$ mice, we observed large and significant increases (30-50-fold) in GR-1 and Mac-1 double positive cells in the spleen, MLNs and colonic lamina propria. Cytospin preparation and differential staining of single cell suspensions prepared from these three tissues confirmed the presence of large numbers of mature neutrophils. Conclusions: T-cell-induced chronic colitis is associated with a remarkable myelopoiesis and infiltration of large numbers of neutrophils into the MLNs, spleen and colonic lamina propria. The role that these granulocytes play in modulating the onset and/or severity of colonic inflammation is currently under investigation. (Supported by DK64023, DK43785 and Yamouchi USA Foundation).

\section{4}

\section{A20 Is Critical for Restricting Basal TLR Signal}

Osamu Hitotsumatsu, Emre Turer, Ruslan Medzhitov, Averil Ma

A20 is a novel protein that is induced by pro-inflammatory NFkB signals in multiple cell types. Prior work from our lab suggested that $\mathrm{A} 20$ is required for preventing spontaneous inflammation and that A20 is critical for terminating TNF induced NFkB signals. More recently, we have found that A20 is also critical for terminating heterologous Toll-like receptor (TLR) induced NFkB signals and limiting the production of inflammatory cytokines by macrophages. We have also acquired provocative data suggesting that A20 is a novel enzyme with two independent ubiquitin modifying functions - including the ability to modify the uibiquitylation status of TRAF6, a key TLR signaling protein. As recent studies suggest that intestinal luminal microbes may tonically stimulate TLR dependent signals in unmanipulated mice living in specific-pathogen free conditions, we are now investigating whether such basal signaling may contribute to the inflammation seen in A20 deficient (A20-/-) mice. We will present preliminary studies suggesting that A20 is indeed required for restricting basal TLR responses in vivo. Specifically, mice that lack both A20 and the common TLR-adapter MyD88 (A20-/- MyD88-/- double mutant mice), show a dramatic amelioration in the cachexia and spontaneous inflammation when compared to A20-/- MyD88+/- mice. These finding contrast with the A20-/- RAG-1-/-, A20-/- TNF-/- and A20-/- TNFR1-/- double mutant mice, all of which develop spontaneous colitis similarly to A20-/- mice. They also highlight the critical role of TLR signals at the apex of physiological inflammation. We will also presen studies using radiation chimera generated from A20-/- MyD88-/- hematopoietic stem cells that will help dissect the role of MyD88 dependent TLR signals on intestinal hematopoietic cells versus stromal cells in this process. We will also present complementary studies using a published cocktail of broad spectrum antibiotics to reduce luminal bacteria and TLR signals in the intestine. These findings emphasize the importance of restricting basal TLR responses in vivo and highlight the critical role of A20 in this process, as well as the importance of TLR signaling in regulating physiologically downstream inflammatory events.

325

Association Between Serum IgG Antibody to C. Difficile Toxin A and Polymorphism of the Interleukin-8 Gene

Zhi-Dong Jiang, Herbert L. Dupont, Kevin Garey, Margaret Price, Gail Granham, Pablo C. Okhuysen, Thanh Dao-Tran, Mark Larocco

Previous studies have shown that defective humoral immune response to C. difficile (CD) toxin $\mathrm{A}$ is associated with the development of more severe $\mathrm{C}$. difficile diarrhea (CDD) and relapse. We have previously shown that single nucleotide polymorphism (AA) in the IL-8 gene promoter region (-25l position) (SNP) is associated with increased susceptibility to CDD in hospitalized patients. The present study was to look at the relationship of serum antibodies to CD toxin A, presence or absence of SNP and levels of fecal IL-8 in a group of hospitalized patients with toxin- $\mathrm{B}$ positive $\mathrm{CDD}(\mathrm{n}=24)$ and $\mathrm{CD}$ toxin- $\mathrm{A} \& \mathrm{~B}$ negative asymptomatic controls $(\mathrm{n}=20)$ matched by age, length of hospital stay, comorbidity and receipt of antibiotics. At enrollment, serum and stool samples were collected from each participate. Serum IgG antibody levels against $C D$ toxin A were measured by an enzymelinked immunosorbent assay (ELISA). CD toxin A antigen was obtained from Remel Inc (Lenexa, KS). Fecal samples were collected from all subjects and studied for level of IL-8 by commercial ELISA. The results are given in the table. In the study, we found an association between $C$. difficile diarrhea, absence of $\operatorname{IgG}$ antitoxin A antibody, presence of AA SNP genotype and fecal IL- 8 . The IgG antitoxin negative CDD patients with AA IL- 8 genotype more commonly had detectable IL-8 in stool samples ( $70 \%$ verses $40 \%, \mathrm{p}<0.001)$ and with significantly higher levels of fecal IL-8 $(820.23 \mathrm{pg} / \mathrm{mL}$ vs. $0 \mathrm{pg} / \mathrm{mL}, \mathrm{p}=0.001)$ than the CDD cases who were IL-8 genotypes AT or TT. The non-diarrhea controls without IgG antitoxin less frequently showed the AA IL- 8 genotype than the CDD cases (29\% verses $67 \%, p=$ 0.035 ). The study provides evidence that host susceptibly to $C$. difficile diarrhea is related both to IL-8 genetics and to absence of a humoral immune response to CD toxin A. Both factors were associated with higher levels of fecal IL-8. Future studies will focus on the relationship between host genotype and immune responsiveness to $\mathrm{CD}$ and its toxins. The study suggests that host genetics, immune responsiveness to toxin A and presence of the fecal inflammatory markers IL-8 may help to define populations at risk for C. difficile or provide important information about disease pathogenesis.

\begin{tabular}{|c|c|c|c|c|c|c|c|c|c|}
\hline \multirow{3}{*}{ Group } & \multirow{3}{*}{$\mathrm{N}$} & \multicolumn{4}{|c|}{$\begin{array}{l}\text { Subjects with IgG Positive to CD } \\
\text { Toxin A }\end{array}$} & \multicolumn{4}{|c|}{$\begin{array}{c}\text { Subjects with IgG Negative to CD } \\
\text { Toxin A }\end{array}$} \\
\hline & & \multicolumn{2}{|c|}{ AA Genotype } & \multicolumn{2}{|c|}{ AT/TT Genotype } & \multicolumn{2}{|c|}{ AA Genotype } & \multicolumn{2}{|c|}{ AT/TT Genotype } \\
\hline & & $\begin{array}{c}\text { Subject } \\
(\%)\end{array}$ & $\begin{array}{c}\text { Fecal } \\
\text { IL-8 } \\
\text { (Median) }\end{array}$ & $\begin{array}{c}\text { Subject } \\
(\%)\end{array}$ & $\begin{array}{c}\text { Fecal } \\
\text { IL-8 } \\
\text { (Median) }\end{array}$ & $\begin{array}{c}\text { Subject } \\
(\%)\end{array}$ & $\begin{array}{c}\text { Fecal } \\
\text { IL-8 } \\
\text { (Median) }\end{array}$ & $\begin{array}{l}\text { Subject } \\
(\%)\end{array}$ & $\begin{array}{c}\text { Fecal } \\
\text { IL-8 } \\
\text { (Median) }\end{array}$ \\
\hline CDD & 24 & $1(4.2)$ & $1(100.6)$ & $\begin{array}{c}8 \\
(33.3)\end{array}$ & $0(0)$ & $\begin{array}{c}10 \\
(41.7)\end{array}$ & $7(858.2)$ & $\begin{array}{c}5 \\
(20.8)\end{array}$ & $2(113.5)$ \\
\hline Asymptomatic & 20 & $0(0)$ & N/A & $\begin{array}{c}3 \\
(14.3)\end{array}$ & $0(0)$ & $\begin{array}{c}5 \\
(23.8)\end{array}$ & $0(0)$ & $\begin{array}{c}12 \\
(57.1)\end{array}$ & $1(142.5)$ \\
\hline
\end{tabular}

Median for fecal IL-8 Concentration (pg/mL)

326

Molecular Characterization of NOD2 and Grim-19 Interactions By Ligand Activation

Jose E. Aguirre, Jesus Yamamoto-Furusho, Nicolas Barnich, Daniel K. Podolsky

Background: The protein product of NOD2/CARD15, the first susceptibility locus linked to Crohn's disease, activates NF-KB and serves an antibacterial function in intestinal epithelial cells following MDP stimulation. Mutations of the LRR regions, including the frameshift mutation 3020insC, results in susceptibility to Crohn's disease due to loss of the defensive function in IECs. Grim-19 (Gene Associated with Retinoid-IFN induced Mortality 19), a binding partner of NOD2, reduces the ability of Salmonella typhimurium to invade intestinal epithelial cells. Aim: To define the interacting regions and binding characteristics of NOD2, the mutant NOD2 proteins associated in Crohn's, and Grim-19 and examine these interactions in response to MDP. Methods: NOD2 and Grim-19 mutants were generated by PCR using Quick Change Site Directed Mutagenesis Kit. The proteins were expressed in COS-7 cells transiently co-transfected with Flag-tagged NOD2 or Flag-tagged truncations of NOD2 and Xpress-tagged Grim-19; protein interactions and expression were checked by immunoprecipitation and western blot analysis. Results: The binding region of NOD2 for Grim-19 is contained within the LRR region and binds to a specific region of Grim-19. Interestingly, mutations in the LRR region associated with the susceptibility to Crohn's disease by linkage 
analysis may disrupt but not eliminate binding to Grim-19. Blau syndrome NOD2 mutations elicit a similar relationship. MDP stimulation of NOD2 and Grim-19 co-transfectants appears to affect binding ability. Conclusion: NOD2 and Grim-19 interactions could be affected by molecular mutations resulting in a lack of ligand activation.

327

Functional Characterization of the Serotonin Transporter (SERT) in Human Intestine

Ravinder K. Gill, Seema Saksena, Sangeeta Tyagi, Ayesha Zaheer, Amika Singla, Waddah A. Alrefai, Pradeep K. Dudeja

Serotonin is an endogenous amine that exerts a wide range of biological effects on mammalian physiology. About $90 \%$ of the whole body content of serotonin is present in the gastrointestinal tract. In the gut, serotonin plays an important role in the regulation of both the gut motility and electrolyte and fluid transport. Since serotonin plays an important role as a signaling molecule, a means for its inactivation must exist in the cells. However, the enzymes that catabolize serotonin are located intracellularly and require serotonin to be internalized before being inactivated enzymatically. Emerging evidence suggests that serotonergic transmission is under the regulation of serotonin transporter (SERT) that serves to inactivate mucosal serotonin by reuptake into epithelial cells through a $\mathrm{Na}+$ and $\mathrm{Cl}$ - coupled mechanism. Recently, the presence of a functional SERT was established in Caco-2 cells at both the apical and basolateral membrane domains. However, the mechanism of inactivation of serotonin has not been characterized in the native human intestine. Therefore, the objective of the present studies was to characterize SERT function and expression along the length of the human intestine. Purified apical (AMV) and basolateral membranes (BLM) were isolated from human small intestinal and colonic mucosa obtained form organ donor intestine. Uptake of $3[\mathrm{H}]$-serotonin and immunoblotting studies were performed in the purified apical and basolateral membrane vesicles utilizing SERT antibodies. Immunoblotting studies detected a protein band $(\sim 70 \mathrm{kDa})$ most prominently in the ileal plasma membranes. The expression of SERT was found to be substantially higher in the ileal basolateral membranes compared to apical membranes. Functional studies suggested that uptake of $3[\mathrm{H}]$-serotonin $(150 \mathrm{nM})$ was significantly increased in the presence of both $\mathrm{Na}+$ and $\mathrm{Cl}$ - in human ileal BBM and BLM vesicles. The uptake was found to be linear with time for up to atleast 1 min in AMVs and BLMs at $30^{\circ} \mathrm{C}$. The $3[\mathrm{H}]$-serotonin uptake was inhibited $(\sim 50 \%)$ by the neuronal SERT inhibitor, fluoxetine $(10-50 \mu \mathrm{M})$ in apical and basolateral membranes. The uptake of $3[\mathrm{H}]$-serotonin exhibited saturation kinetics indicating the presence of carriermediated process for transport of serotonin in ileal AMVs and BLMs. In conclusion, our data suggest that in the human ileum, SERT is functionally expressed at both apical and basolateral membranes. Knowledge of the detailed characterization of SERT-mediated transport of serotonin might have major implications in understanding the pathophysiology of nflammatory bowel disease associated with altered serotonin availability.

328

MAPKAPK2 Is a Critical Signaling Intermediate in NHE3 Activation Following $\mathrm{Na}^{+}$-Glucose Cotransport

Zhihong Hu, Yingmin Wang, Mark W. Musch, Liping Su, Jerrold R. Turner

Villus enterocyte nutrient absorption occurs via precisely-orchestrated interactions among multiple transporters. For example, transport by the apical $\mathrm{Na}^{+}$-glucose cotransporter, SGLT1, triggers a cascade that leads to increased $\mathrm{Na}^{+}-\mathrm{H}^{+}$exchange as a consequence of NHE3 translocation to the apical plasma membrane. This NHE3 translocation requires sequential activation of p38 MAP kinase (MAPK), Akt2, and ezrin. Akt2 directly phosphorylates ezrin, but the precise role of $\mathrm{p} 38 \mathrm{MAPK}$ in this process remains unknown. The aim of this study was to identify the mechanism by which p38 MAPK effects downstream Akt2 activation. METHODS: Caco- $2_{\mathrm{BB}}$ cell monolayers stably transfected with wild type ezrin or dominant negative (N-terminal truncation mutant) ezrin were initially equilibrated in media with mannose in place of glucose. SGLTl-mediated transport was initiated by iso-osmotic replacement with glucose-containing media. Protein phosphorylation was detected by immunoblot. siRNA knockdown was performed by standard approaches. Surface NHE3 expression was quantified by modified whole-cell ELISA. RESULTS: In silico analysis suggested that p38 MAPK could not phosphorylate Akt2 Ser473. However, MAPKAPK2, a direct p38 MAPK effector may phosphorylate Akt2 Ser473 in human neutrophils. We therefore hypothesized that MAPKAPK2 might link p38 MAPK and Akt to effect ezrin-dependent NHE3 translocation. MAPKAPK2 was rapidly phosphorylated at Thr334 an indicator of activation, after initiation of $\mathrm{Na}^{+}$-glucose cotransport. This occurred with kinetics that paralleled activation of p38 MAPK, Akt2, and ezrin. p38 MAPK inhibition attenuated MAPKAPK2, Akt2, and ezrin phosphorylation. Dominant negative ezrin expression had no effect on p38 MAPK, MAPKAPK2 or Akt2 phosphorylation. In contrast Akt2 inhibition blocked ezrin, but not p38 MAPK or MAPKAPK2 phosphorylation, suggesting that MAPKAPK2 could be an intermediate in p38 MAPK-dependent Akt2 activation. siRNA-mediated MAPKAPK2 knockdown inhibited Akt2 and ezrin phosphorylation but did not affect p38 MAPK phosphorylation. MAPKAPK2 knockdown also blocked NHE3 translocation to the apical surface. CONCLUSIONS: These studies show that MAPKAPK2 is a critical intermediate in the signal transduction pathway that causes NHE3 translocation after SGLT1-mediated transport. Moreover, the data show that the role of MAPKAPK2 is to phosphorylate Akt2, thereby linking upstream p38 MAPK o downstream Akt2 and ezrin activation. As $\mathrm{p} 38 \mathrm{MAPK}$ and NHE3 are both activated under hypo-osmotic conditions, these data may also provide a mechanistic basis for understanding NHE3 responses to osmotic stimuli.
329

Distinct Isofroms Encode Apical and Basolateral Intermediate Conductance K (IK) Channels

Christoph Rahner, Christian Barmeyer, Youshan Yang, Frederick J. Sigworth, Henry J. Binder, Vazhaikkurichi M. Rajendran

Basolateral IK channels are generally thought to provide the driving force for agonist (cAMP/ $\mathrm{Ca}+$-)-induced $\mathrm{Cl}$ secretion, while apical IK channels mediate Ca2+-activated $\mathrm{K}$ secretion in rat colon (Am J Physiol 285:Gl85-G196, 2003). Although IK channel-like functions and proteins have been demonstrated in both apical (Ap) and basolateral (Bl) membranes, only a single IK cDNA (designated as IKI) has previously been cloned. In these studies we sought to establish whether both Ap and Bl IK channels are encoded by IKl or by two (or more) distinct isoforms. Two distinct transcripts (1423 bp and $1336 \mathrm{bp}$ ) were identified by RTPCR analyses using mRNA from rat colon and oligos that were designed from the 5 ' and 3' untranslated regions flanking the coding sequence of IK1. The larger one (1423 bp) was identical as IK1, while the second RT-PCR product (1336 bp) was designated as IK2. IK2 differs from IKI by lacking the entire second exon that encodes a continuous 29 aa domain that also represents entire predicted 2 nd membrane spanning domain in IKl. Immunofluoresence studies using an antibody (anti-IKl-2) raised against C-terminal region of IKl localized IK-like proteins in both Ap and Bl membranes of rat colon. Western blot analyses established that anti-IKl-2 antibody recognized 37 and $40 \mathrm{kDa}$ proteins, while immunoprecipitation studies indicate that anti-IKl-2 antibody also precipitated 37 and $40 \mathrm{kDa}$ proteins from rat colonic epithelial cell homogenate. In addition, immuno-EM studies with human colon indicated the IKI-2 antibody also localized IK-like proteins in both Ap and Bl membranes. In contrast, an antibody (anti-IKl) raised against a peptide from the 29 aa motif (that is deleted in IK2) localizes IK-like proteins only in Ap membranes of rat colon. In transiently transfected HEK293 cells, IK2 exhibits Ca2+-dependent K current with intermediate conductance (16 ps). IK-proteins are expressed in surface membrane of IKl-cRNA injected oocytes, but in IK2-cRNA injected oocytes no surface membrane expression was seen. In contrast, surface membrane expression of IK-protein was present in oocytes co-injected with IK2 and MaxiK $\beta$-subunit cRNAs. We conclude that apical and basolateral IK channels are encoded by IK1 and IK2 isoforms, respectively. We speculate that a $\beta$-subunit-like accessory protein is necessary for surface membrane expression of IK2 (but not IK1) proteins. Thus, these studies establish that IKl channel is an Ap membrane protein associated with agonistinduced $\mathrm{K}$ secretion, while IK2 channel is localized to $\mathrm{Bl}$ membrane and provides the driving force for agonist-induced $\mathrm{Cl}$ secretion.

330

Regulation of Kcnq1/Kcne3 By Basolateral Sphingomyelinase (SMase) David Saslowsky, Nori Tanaka, Wayne Lencer

The environment of the human intestine contains a variety of SMases contributed by both host cells as well as the intestinal microbial flora. SMases cleave the phosphorylcholine head group of sphingomyelin (SM) leaving the ceramide component intact in the lipid bilayer. These enzymes are implicated in physiologic processes including cell differentiation, proliferation, apoptosis, and atherosclerosis. Through our studies on the biology lipid rafts, we discovered that conversion of SM to ceramide by SMase on the basolateral membrane of intestinal T84 cells attenuates (by $>50 \%$ ) the cAMP-induced $\mathrm{Cl}$ - secretory response. In contrast, apically applied SMase has no effect on Cl- secretion. Our Aim is to explain the mechanism of SMase action. Methods: $\mathrm{Cl}$ - secretion is measured as a short circuit current (Isc) on intact and semi-permeabilized T84 cell monolayers by standard electrophysiology. Phosphorylation of ERK1/2.JNK, and c-Jun is measured in cell lysates by SDS-PAGE and immunoblot. Results: Basolateral SMase acts rapidly and inhibits the Isc induced by the cAMPagonists vasoactive intestinal peptide, forskolin, and 8-Br-cAMP. In T84 cells permeabilized to monovalent ions by amphotericin B, incubation with basolateral SMase causes inhibition of the cAMP-gated and $\mathrm{Ba} 2+$-sensitive $\mathrm{K}+$ conductance, presumably $\mathrm{KCNQ1/KCNE3.} \mathrm{The}$ $\mathrm{Ca} 2+$-gated $\mathrm{K}+$ conductance, presumably $\mathrm{KCNN} 4$, is only marginally affected. ERKl/2 is not involved as assessed using the chemical inhibitor PD98059 and by assay for phosphoERK1/2. However, SMase induced the phosphorylation of both JNK and the chemical inhibitor of JNK SP600125 dose-dependently reverses the SMase induced block of cAMPinduced Isc. Pharmacological induction of membrane ceramide levels recapitulates the SMaseinduced inhibition of the forskolin secretory response. Similarly, this effect was rescued by inhibition of JNK. Conclusion: Conversion of SM to ceramide by SMase in the basolateral membrane of T84 cells blocks $\mathrm{Cl}$ - secretion by inhibiting the cAMP-gated basolateral $\mathrm{K}+$ channel, presumably KCNQ1/KCNE3. Signal transduction likely occurs by ceramide-induced activation of JNK. This mechanism may allow control of transepithelial Cl- secretion by adjacent eukaryotic and perhaps prokaryotic cell types.

331

Enac Expression By Aldosterone Is Paralleled By Upregulation of Claudin-8 and Increased Barrier Function in Human Colon

Salah Amasheh, Maike Bergs, Maren M. Amasheh, Alexander G. Markov, Anton J. Kroesen, Michael Fromm, Joerg D. Schulzke

Background \& Aims: In colon sigmoideum, the limiting factor for sodium absorption is induction of the epithelial sodium channel $(\mathrm{ENaC})$ by aldosterone. In order to investigate the functional interplay between transport and barrier, we analyzed the regulation of the tight junction in human sigmoid colon during $\mathrm{ENaC}$ induction by aldosterone in vitro. Material and Methods: Epithelial preparations from surgically removed sigmoid colon were mounted in Ussing chambers. Colonic ENaC induction was performed by incubation with aldosterone $(3 \mathrm{nM})$ in vitro. Short circuit current $\left(\mathrm{I}_{\mathrm{SC}}\right)$ and transepithelial resistance $\left(\mathrm{R}^{\mathrm{t}}\right)$ of the tissues was reported and $\mathrm{ENaC}$ induction was determined by final addition of amiloride $\left(10^{-4} \mathrm{M}\right)$. Subsequently, tissues were removed from the chambers and used for Western blotting and laser-confocal microscopy of immunofluorescent stainings of $\mathrm{ENaC}$ and tight junction proteins. Results: Aldosterone stimulated $\mathrm{J}_{\mathrm{Na}}$ by inducing $\mathrm{ENaC}$ in the apical membrane of epithelial cells within $8 \mathrm{~h}$. After subsequent blockade of $\mathrm{ENaC}$ by addition of 
amiloride, $\mathrm{R}^{\mathrm{t}}$ values were higher after aldosterone-treatment compared to respective controls, indicating an additional mechanism tightening the epithelium. Analyses of the tight junction by means of Western blot and immunofluorescence analyses revealed a differential regulation of tight junction molecules. An increase of occludin and claudin-8 during aldosterone incubation was observed, whereas claudin- $1,-4$, and -5 remained constant. Confocal laser scanning microscopy revealed that the increase of occludin was limited to subjunctional regions, whereas claudin- 8 was induced within the tight junctional complex. A separate approach adding amiloride prior to aldosterone in the Ussing chamber experiment revealed no increase of $\mathrm{R}^{\mathrm{t}}$, occludin, and claudin- 8 indicating $\mathrm{ENaC}$ action being part of the signaling pathway. Conclusion: Aldosterone enhances $\mathrm{Na}^{+}$absorption by two independent mechanisms acting in concert, namely increased $\mathrm{ENaC}$-mediated sodium transport and an increase of barrier function. On molecular level of the tight junction, expression of the sealing tight junction protein claudin- 8 is enhanced, which is one of the "tightening" tight junction proteins. In this manner, paracellular back-leak of actively absorbed sodium is diminished by selectively sealing the tight junction for cations. This process may enable a pronounced electrochemical gradient from lumen to plasma and thus contribute to $\mathrm{Na}^{+}$absorption efficiency. Prerequisite for this mechanism is a functional $\mathrm{ENaC}$.

332

Elevated Intracellular Calcium ([Ca2+]i) Inhibits NHE3 Activity By a PDZK1 (NHERF3) Dependent Process in Which NHE3 and PDZK1 Bind Basally and Dissociate with Elevated $[\mathrm{Ca} 2+] \mathrm{i}$

Nicholas C. Zachos, Xuhang Li, Olga Kovbasnjuk, Rafiq Sarkar, Boris Hogema, Hugo de Jonge, Mark Donowitz

The NHERF family (NF) of PDZ proteins is known to play a role in acute regulation of NHE3 activity, including inhibition of NHE3 activity by cAMP, cGMP, and elevated [Ca2+]i. Calcium regulation of NHE3 activity occurs through specific NHERF interactions with NHE3 (NHERF1 = no effect; $\mathrm{NHERF2} \mathrm{=} \mathrm{inhibition;} \mathrm{NHERF4} \mathrm{=} \mathrm{stimulation})$, which involve formation of multi-protein complexes and affect NHE3 trafficking. While specificity of calcium regulation has been demonstrated for NHERFs 1, 2 and 4, the role of NHERF3 is not defined. The current study tested the hypothesis that PDZKI had a role in calcium regulation of NHE3 activity. NHE3 activity was measured using the pH-sensitive dye, BCECF in PS120 cells stably expressing NHE3 alone or with NHERF3. Treatment of PS120/NHE3 cells with the calcium ionophore, 4-Br-A23187 (0.5 $\mu \mathrm{M})$, had no effect on NHE3 activity. However, in PS120/NHE3/NHERF3 cells, elevated [Ca2+]i decreased NHE3 activity by $\sim 50 \%$. This decrease correlated with a similar decrease in NHE3 surface expression $(\mathrm{Ctl}=12 \pm 2 \%)$ after treatment with A23187 $(7+1 \%)$. Confocal microscopy demonstrated NHE3 and NHERF3 co-localize in PS120 cells under basal conditions but dissociate after treatment with A23187. While the pattern of NHE3 localization did not appear to be different than control with elevated [Ca2+]i, NHERF3 formed distinct intracellular clusters which were spatially separate from NHE3. Acceptor photobleaching FRET showed direct NHE3/NHERF3 interaction basally and a complete loss of NHE3 and NHERF3 interaction 15 min after A23187. NHERF3 clusters were also observed with elevated [Ca2+]i in the brush border (BB) of Caco2/bbe cells treated with carbachol $(10 \mu \mathrm{M})$. Under basal conditions, NHE3 and NHERF3 co-localize in the BB of Caco2/bbe cells. However, in Caco2/bbe cells treated with carbachol, NHERF3 formed clusters in the BB while NHE3 appeared to localize to a subapical region immediately below the $\mathrm{BB}$. Thus, the current study demonstrated that (1) Elevated [Ca2+]i decreased NHE3 activity in PS120/NHE3/NHERF3 cells. (2) Calcium inhibition of NHE3 mediated by NHERF3 involves reduced NHE3 surface expression. (3) Elevated [Ca2+]i results in NHERF3 clustering and dissociation from NHE3 in fibroblasts and polarized intestinal epithelial cells. Taken together these results suggest that (1) NHERF3 may anchor NHE3 in the plasma membrane under basal conditions and this interaction is disrupted with elevated $[\mathrm{Ca} 2+] \mathrm{i}$. (2) NHERF3 clustering is associated with NHE3 removal from the plasma membrane thereby decreasing NHE3 activity. (3) NHERF3 does not traffic with NHE3 from the plasma membrane to the recycling endosome.

339

Altered Immunologic Function Determines Symptom Pattern and Severity in Patients with Irritable Bowel Syndrome

Tobias Liebregts, Birgit Adam, Christoph Bredack, Susan Lester, Sarah Downie-Doyle, Alexander Roth, L. Ashley Blackshaw, Gerald Holtmann

Background: There is increasing evidence that immunologic mechanisms play a role for the manifestation of functional gastrointestinal disorders (FGID). Altered immunologic responses may determine the manifestation and severity of symptoms in patients with FGID. We hypothesized that the release of pro-inflammatory cytokines from peripheral blood mononuclear cells (PBMC) is linked to the severity of symptoms in patients with Irritable Bowel Syndrome (IBS). Thus, we aimed (1) to characterize systemic cytokine production, (2) the cellular response to LPS stimulation and (3) to correlate symptom pattern and severity with immunologic function. Methods: PBMC from 15 (alternating-) IBS patients and 20 matched controls were isolated by density gradient centrifugation and cultured for 24 hours in RPMI 1640 supplemented with 10\% FCS. Basal and E. coli LPS (lipopolysacharide)(1-100ng/ml) stimulated cytokine production (TNF- $\alpha$, IL-1 $\beta$, IL- 6 ) of PBMC was measured by enzymelinked immunosorbent assay (ELISA). Abdominal symptoms and psychological co-morbidities were assessed utilizing the standardized Bowel Disease Questionnaire (BDQ) and Hospital Anxiety and Depression Scale (HADS). Results: At baseline TNF- $\alpha(69.2 \pm 4.8 \mathrm{pg} / \mathrm{ml}$ vs. $35.4 \pm 18.2 \mathrm{pg} / \mathrm{ml}), \mathrm{IL}-1 \beta(86.4 \pm 21.7 \mathrm{pg} / \mathrm{ml}$ vs. $147.8 \pm 41.3 \mathrm{pg} / \mathrm{ml})$, but not IL-6 release were significantly higher $(\mathrm{p}<0.05)$ in IBS patients compared to healthy subjects. LPS stimulation caused a dose dependent increase of all cytokines. Interestingly, the LPS induced increase of TNF- $\alpha$ and IL-1 $\beta$ was significantly attenuated in IBS patients compared to healthy controls. In IBS patients baseline TNF- $\alpha$ level was correlated with pain frequency $(r=618, p<0.01)$, intensity $(r=.494, p<0.05)$ and loose $(r=630, p<0.01)$ and watery stools $(r=.445, p<0.05)$ while IL-1 $\beta$ level were linked to constipation $(r=440, p<0.05)$ and lumpy stool $(r=614$, $\mathrm{p}<0.01)$. LPS stimulated IL-1 $\beta$ production was inversely correlated with symptom frequency $(r=-.618, p<0.01)$, intensity $(r=-.599, p<0.05)$ and pain severity $(r=-.524, p<0.05)$. Depression was correlated with the LPS induced TNF- $\alpha$ production $(r=.503, p<0.05)$. Summary and
Conclusion: PBMC of IBS patients are characterized by an increased baseline and impaired LPS- induced release of pro-inflammatory cytokines in-vitro. These alterations of cytokin production are closely linked to symptom severity. Since low-grade inflammation is believed to play a role in IBS patients, the altered cytokine release may reflect an abnormality in the regulation of immune responses of IBS patients.

340

Markers of Inflammation in IBS: Increased Permeability and Cytokine Production in Diarrhoea-Predominant Subgroups

Eugene Campbell, Marguerite Richards, Stephen Foley, Maggie Hastings, Peter Whorwell, Yashwant Mahida, Ian Hall, Keith Neal, Robin Spiller

BACKGROUND: We have previously described increased mucosal lymphocytes in postinfective IBS (PI-IBS) and others have demonstrated low-grade mucosal inflammation and immune activation. It is uncertain if subgroups of IBS differ in their degree, type or site of inflammation. AIMS: To assess evidence of inflammation between IBS subgroups and healthy controls by measuring small bowel (SB) and colonic (C) permeability, stool cytokines, urine neopterin and peripheral blood mononuclear cell (PBMC) cytokine production. METHODS: Fifty-five IBS patients satisfied Rome II criteria for IBS. Twenty-one normal healthy control were also recruited. Gut permeability was measured by Cr51-EDTA method, with timed urine collection for small and large bowel permeability; Stool supernatants and medium from PBMCs after incubation for 24 hours were assayed for cytokines by cytometric bead array. Anxiety and depression were measured by Hospital Anxiety and Depression scale. RESULTS: (Mean \pm SEM) Small bowel permeability was significantly increased in D-IBS, whereas colonic permeability was unchanged. PBMC incubations from D-IBS and PI-IBS had significantly elevated IL-1, IL-10 and TNF levels compared to healthy controls. High sensitivity CRP, urine neopterin and stool cytokines showed no significant differences between patient groups and controls CONCLUSION: Subgroups of IBS have evidence of immune activation and altered gut permeability. IBS patients with diarrhoea show evidence of systemic immune and altered small bowel but not colonic permeability. The increased cytokine profile suggests systemic monocyte activation which may be due to bacterial products absorbed across a permeable small intestine.

Permeability

\begin{tabular}{|c|c|c|c|c|c|}
\hline & HC $n=21$ & D-IBS $n=25$ & PI-IBS $n=15$ & C-IBS $n=15$ & pv HC \\
\hline Age & $34 \pm 3$ & $45 \pm 3$ & $38 \pm 4$ & $42 \pm 3$ & \\
\hline Anxiety & $3 \pm 1$ & $8 \pm 1$ & $7 \pm 1$ & $8 \pm 1$ & all $p<0.02$ \\
\hline Depression & $1 \pm 1$ & $4 \pm 1$ & $7 \pm 1$ & $8 \pm 1$ & all $\mathrm{p}<0.02$ \\
\hline SB Permeability & $0.81 \pm 0.06$ & $1.09 \pm 0.10^{*}$ & $0.87 \pm 0.11$ & $0.73 \pm 0.05$ & $* \mathrm{p}=0.04$ \\
\hline Colonic Permeability & $0.99 \pm 0.06$ & $1.2 \pm 0.15$ & $1.2 \pm 0.12$ & $0.99 \pm 0.18$ & $\mathrm{p}=\mathrm{ns}$ \\
\hline
\end{tabular}

PBMC cytokines

\begin{tabular}{|c|c|c|c|c|}
\hline & HC $n=13$ & D-IBS $n=14$ & PI-IBS $\mathrm{n}=13$ & $\mathrm{p} \vee \mathrm{HC}$ \\
\hline $\mathrm{IL}-1 \beta$ & $383 \pm 171$ & $862 \pm 165 *$ & $1246 \pm 269 * *$ & $* \mathrm{p}=0.01 * * \mathrm{p}=0.005$ \\
\hline $\mathrm{IL}-10$ & $123 \pm 58$ & $327 \pm 54 *$ & $456 \pm 108 * *$ & $* \mathrm{p}=0.005 * * \mathrm{p}=0.008$ \\
\hline TNF- $\alpha$ & $100 \pm 63$ & $196 \pm 60 *$ & $201 \pm 39 * *$ & $* \mathrm{p}=0.04 * * \mathrm{p}=0.01$ \\
\hline
\end{tabular}

341

Decreased Number of $\mathrm{IGA}^{+}$Plasma Cells in the Ascending Colon Mucosa of Patients with Irritable Bowel Syndrome (IBS)

Johan Forshammar, Stefan Isaksson, P-O Stotzer, Hans Strid, Henrik Sjovall, Magnus Simren, Lena Ohman

AIM: Irritable bowel syndrome (IBS) has been suggested be associated with a low grade inflammation in the intestinal mucosa. Food reactions may contribute to the immune activation, since some patients with IBS symptoms benefit from specific elimination diets. Moreover, one of the most frequently recognised immunological features of IBS is increased numbers of mast cells in colonic lamina propria and muscularis propria. However, the profile of plasma cells in the colonic mucosa of patients with IBS has not been investigated previously. METHOD: 11 healthy controls subjects and 11 patients with IBS, defined by the Rome Il criteria, participated in the study. Biopsies were taken during colonoscopy and were then frozen, sectioned and mounted on slides whereupon they were saturated with antibodies with affinity for human $\operatorname{IgM}, \operatorname{IgG}$, IgA and IgE conjugated with horse radish peroxidase (HRP). Positive cells were detected by light microscopy. RESULTS: The number of mucosal IgA ${ }^{+}$plasma cells of the ascending colon $\left(201 \mathrm{cell} s / \mathrm{mm}^{2}\right)$ was significantly lower in IBS patients than in healthy controls $\left(462\right.$ cells $\left./ \mathrm{mm}^{2}\right)(\mathrm{p}=0.039)$. Furthermore, IBS patients had a significantly lower number of IgA ${ }^{+}$plasma cells in the ascending colon $\left(201\right.$ cells $/ \mathrm{mm}^{2}$ as compared to the sigmoid colon $\left(430\right.$ cells $\left./ \mathrm{mm}^{2}\right)(\mathrm{p}=0.04)$. The $\mathrm{Ig} \mathrm{G}^{+}$plasma cell populations did not significantly differ between IBS patients and controls in the ascending colon (166 cells $/ \mathrm{mm}^{2}$ vs 291 cells $/ \mathrm{mm}^{2}$ ) or in the sigmoid colon $\left(285 \mathrm{cells} / \mathrm{mm}^{2}\right.$ vs $174 \mathrm{cells} / \mathrm{mm}^{2}$ ). The $\mathrm{IgM}^{+}$plasma cell populations were comparable in IBS patients and controls in the ascending colon (180 cells/ $\mathrm{mm}^{2}$ vs 240 cells $/ \mathrm{mm}^{2}$ ) and in the sigmoid colon (219 cells/ $\mathrm{mm}^{2}$ vs 264 cells $/ \mathrm{mm}^{2}$ ). Very few $\mathrm{IgE}^{+}$cells were detected in the colonic mucosa and there was no difference between the two subject groups. CONCLUSION: The reduced number of IgA+ plasma cells in the ascending colon mucosa of IBS patients suggests that IBS patients may have an impaired IgA mediated mucosal immune defence. Whether this phenomenon is causally related to symptoms remains unknown and deserves further investigation. 


\section{2}

Intestinal Immune Activation in Post-Infectious Functional Dyspepsia Sebastien Kindt, Alexander Terychnyy, Karel Geboes, Jan Tack

Background: Recent studies have shown that, similar to post-infectious (PI-) irritable bowel syndrome (IBS), functional dyspepsia (FD) symptoms may develop acutely and persist after a gastrointestinal infection. Biopsy studies in PI-IBS have shown signs of persisting lowgrade colonic inflammation and increased counts of enterochromaffine cells (EC). It is unclear whether PI-FD is similarly characterized by low-grade intestinal inflammation. The aim of the present study was to compare signs of inflammation and EC hyperplasia on duodenal biopsies in PI-FD and unspecified-onset (U-)FD. Methods: Duodenal biopsy specimens were obtained at upper g.i. endoscopy in $12 \mathrm{U}-\mathrm{FD}$ and 12 PI-FD patients ( 15 women, mean age $38.8 \pm 3.2$, onset of symptoms on average $10 \pm 3$ months earlier). The mean number of intra-epithelial lymphocytes (IEL) and Chromogranine C (CC) positive cells per villus, presence of focal aggregates of T-cells, increased number of T-cells at the base of the villi, lymphoid aggregates and focal IEL aggregates were compared between both groups with Student t-test, Wilcoxon test and Fisher exact test. Data are shown as mean \pm SEM. Results: The mean numbers of IEL $(20.6 \pm 1.7$ vs. $23.5 \pm 2.3)$ and the mean number of EC cells $(3.6 \pm 0.3$ vs. 3.5 \pm 0.2$)$ per villus did not differ between PI-FD and U-FD. Focal aggregates of T-cells, reminiscent of cryptopatches (CP) in mice, were found in 6/12 PI-FD patients, and in none of 12 U-FD patients $(\mathrm{p}=0.01)$. In mice, $\mathrm{CP}$ are inducible and have been shown to play a major role in gut immune defense (Eberl 2005). No significant differences were reached when comparing in PI-FD and U-FD the presence of increased numbers of T-cells at the base of the villi (respectively $7 / 12$ and 3/12), focal aggregates of IEL (respectively $6 / 12$ and 2/12) or lymphoid aggregates (1/12 in each group). Conclusion: PI-FD is not associated with increased lymphocyte or EC cells counts in villi, but is characterized by the presence of focal T-cell aggregates which persist several months after the acute insult. We speculate that, similar to murine $\mathrm{CP}$, these aggregates indicate ongoing low-grade intestinal immune activation in PI-FD.

\section{3}

Pathogenic Mechanisms of Post-Infectious Functional Gastrointestinal Disorders: Results After 3 Years

Antonia Perello, Monica Perona, Agustin Balboa, Miquel Sans, Ruth Benasayag, Antonio Salas, Marc Perez-Oliveras, Jordi Coderch, Asuncion Garcia, Fermin Mearin

We have previously shown that one year after a Salmonella infectious outbreak, which affected more than one thousand people, 17\% developed functional dyspepsia (FD) and 12\% irritable bowel syndrome (IBS) (Gastroenterology 2005;129:98-104). Aims: To evaluate possible mechanisms related to the development and persistence of post-infectious functional gastrointestinal disorders (PI-FGID) 3 years after the outbreak. These include mucosal inflammation, visceral hypersensitivity and psychological factors. Methods: Participants were recruited from our previous prospective study: 16 patients (cases) suffered PI-FGID (7 FD, 3 IBS and 6 overlap between the two) and 8 control subjects without PI-FGID. We performed upper and lower digestive endoscopy obtaining multiple biopsies in gastric fundus, antrum, right and left colon. Samples were processed for histological study, mast cell quantification and interleukin-1 $\beta$ production (IL-1 $\beta$ ). Genomic DNA was typed for the IL-10 gene polymorphisms. Visceral sensitivity was assessed by the satiety drinking test and rectal barostat. Psychological aspects were evaluated by the SCL-90 and vital events questionnaires. All studies were conducted under blind test conditions for the investigators. Results: Biopsies did not show significant inflammatory signs in the stomach. In the colon 3 cases and 2 controls had an increase cellularity in the lamina propria, also without evident acute inflammatory changes. Mast cell count in the stomach was similar between the two groups $(188 \pm 16$ vs $155 \pm 13)$ so was the level of IL- $1 \beta(2.9 \pm 0.9$ vs $4.9 \pm 1.8)$. In the colon the number of mast cells was also similar between the two groups $(198 \pm 25 / \mathrm{mm} 2$ vs $176 \pm 25 / \mathrm{mm} 2)$ so was the level of IL- $1 \beta(2.6 \pm 0.3$ vs $2.8 \pm 0.9)$. No differences in carriage, genotype and allele frequencies of the IL-10 gene polymorphisms were found. Gastric sensitivity was similar between the groups (maximal volume intake: $676 \pm 87$ vs $605 \pm 118 \mathrm{kcal}$ ). Cases showed a lower pain threshold to rectal distension $(29 \pm 2$ vs $37 \pm 2 \mathrm{mmHg}$; $\mathrm{p}<0.05)$. Scores for anxiety $(0.63 \pm 0.11$ vs $0.28 \pm 0.14 ; \mathrm{p}<0.05)$ and somatization $(1.01 \pm 0.15$ vs $0.45 \pm 0.15 ; \mathrm{p}<0.05)$ were higher in cases than controls. The number of stressful events $(7.2 \pm 1.7$ vs $5.4 \pm 0.8)$ and stress scores for events did not differ between groups $(15.5 \pm 3.1$ vs $11.7 \pm 2.6)$. Conclusion: Three years after salmonellosis there is no evidence of inflammatory changes in the stomach or the colon in patients who developed PI-FGID. Rectal sensitivity is higher than in control subjects whereas gastric sensitivity is normal. Anxiety and somatization levels are increased.

\section{4}

Prognosis of Post-Infectious Irritable Bowel Syndrome (PI-IBS) Four Years After the Walkerton Outbreak of Waterborne Gastroenteritis (GE) John K. Marshall, Marroon Thabane, Amit X. Garg, William F. Clark, Stephen M. Collins Background and Aim: In May 2000, municipal water contamination by E. coli 0157:H7 and Campylobacter species led to a large outbreak of acute GE in Walkerton, Ontario. A prospective cohort study was undertaken to determine the incidence and natural history of PI-IBS in this population. Methods: Local residents who attended study clinics in 2002 and 2003 were invited for follow up in 2004. Standardized interviews included a modified Bowel Disease Questionnaire to diagnose IBS by Rome I criteria. Subjects who were age $>16$ and permanent residents of Walkerton in May 2000 with no prior diagnosis of IBS or inflammatory bowel disease were included. The prevalence and incidence of IBS among participants with and without GE were estimated. Risk factors for IBS in 2004 were assessed by multiple logistic regression. Results: 3280 of 4315 participants enrolled in 2002/2003 returned in 2004. Of 1587 who were eligible for study cohort (57.2\% female; mean age $48.1 \pm 16.5$ ), $1012(63.8 \%)$ reported exposure to GE in May 2000 and 273 (17.2\%) fulfilled Rome I criteria in 2004 (38.1\% IBS-D, 12.1\% IBS-C). Among 302 subjects with GE in 2000 and IBS in 2002/2003, $153(50.7 \%)$ still fulfilled Rome I criteria in 2004. Among 1226 subjects with no IBS in 2002/2003, IBS was detected in 2004 in 80 of 710 (11.3\%) with GE in
2000 versus 23 of 516 (4.5\%) with no exposure to GE (p<0.001)(see Table). Among subjects exposed to GE, independent predictors of IBS in 2004 included female gender, weight loss, abdominal pain and duration of diarrhea during the outbreak. Conclusion: The prognosis of PI-IBS appears favorable, with spontaneous resolution in half of patients. A diarrheapredominant phenotype of PI-IBS remains common. Funded by the Ontario Ministry of Health and Long-Term Care

Prognosis of PI-IBS After Waterborne GE Outbreak

\begin{tabular}{|c|c|c|c|c|}
\hline GE in 2000 & IBS 2002/2003 & IBS Positive in 2004 & IBS Negative in 2004 & Total \\
\hline Yes & Yes & $153(50.7 \%)$ & $149(49.3 \%)$ & 302 \\
\hline & No & $80(11.3 \%)$ & $630(88.7 \%)$ & 710 \\
\hline No & Yes & $17(28.8 \%)$ & $42(71.2 \%)$ & 59 \\
\hline & No & $23(4.5 \%)$ & $493(95.5 \%)$ & 516 \\
\hline Total & Total & $273(17.2 \%)$ & $1314(82.8 \%)$ & 1587 \\
\hline
\end{tabular}

345

Analysis of the IBD2 Locus: Inherited Variants in Gli-1, the Major Effector of the Hedgehog Signalling Pathway, Are Strongly Implicated in Susceptibility to Ulcerative Colitis

Charlie Lees, Elaine R. Nimmo, Gwo-Tzer Ho, Hazel E. Drummond, Albert Tenesa, Malcolm G. Dunlop, Sarah E. Howie, Jack Satsangi

Introduction. The Hedgehog (Hh) signalling pathway is critical to GI tract development, homeostasis and disease ${ }^{1}$. Recapitulation of embryonic Hh signals play vital roles in tissue response to injury and repair, and regulation of $\mathrm{T}$ cells \& macrophages. Expression of pathway components is dysregulated in $\mathrm{IBD}^{2-3}$. Gli-1, the major transcriptional target of the Hh signalling pathway, is located in the IBD2 susceptibility locus $(12 \mathrm{ql} 3.3)^{4}$. Aim. To determine whether inherited variants in Gli-1 contribute to IBD susceptibility, using a genewide haplotype-tagging approach. Methods. Initial mutation screening of the Gli-1 gene was performed in $32 \mathrm{IBD}$ cases. Of 8 SNPs present, 4 tagging SNPs (rs3817474 [tSNP ${ }_{1}$ ], rs $2228225\left[\mathrm{tSNP}_{2}\right], \mathrm{rs} 2228224\left[\mathrm{tSNP}_{3}\right], \&$ rs2228226 [tSNP 4 ]) were identified which defined Gli-1 variation. Five haplotypes (A-E, see table) were present in the Scottish healthy control population ( $\mathrm{HC}, \mathrm{n}=362)$. SNPs were assayed by Taqman in HC, Crohn's disease (CD, $n=$ 335) \& ulcerative colitis (UC, $\mathrm{n}=474$ ). Taqman failures were assayed by direct sequencing. Haplotype \& variant analysis was performed using chi $^{2}$ analysis. Log-likelihood testing assessed the overall contribution of Gli-1 variation to disease susceptibility. Results. Loglikelihood testing demonstrated a highly significant association of the Gli-1 gene with UC $(\mathrm{p}<0.00001)$, but only a modest effect in $C D(p<0.05)$. In $U C$, the rare haplotypes D \& E were significantly associated with susceptibility $(\mathrm{p}<0.0001)$, whilst haplotypes A \& $\mathrm{C}$ were protective (see table). In $\mathrm{CD}$, haplotype $\mathrm{B}$ had a protective effect of borderline significance $(\mathrm{p}<0.05)$. Analysis of individual SNPs revealed significant associations for $\mathrm{SSNP}_{2 \& 4}$, but not $\mathrm{tSNP}_{1 \& 3}$. Carriage of the ' 2 ' allele on $\mathrm{SSP}_{2}$ was associated with IBD $(\mathrm{OR}=1.30, \mathrm{p}=0.049)$. Allelic frequency and carriage of the ' 2 ' allele on $\mathrm{tSNP}_{4}$ was also associated with IBD (OR= $1.22, p=0.043$ and $O R=1.41, p=0.0086$ ). Conclusion. Inherited variants in Gli- 1 are strongly associated with UC. The data presented suggest that rs2228225 ( $\mathrm{tSNP}_{2}$ ) may be in LD with the causal variant. Further detailed analyses of the gene are in progress. References. 1. Lees et al Gastroenterology 2005;129(5):1696-710; 2. Nielson et al Lab Invest. 84(12);1631-42; 3. Lees et al Gastroenterology 2005;128(4):A504; 4. Satsangi et al Nat Genet 1996;14(2):199-202

\begin{tabular}{|c|c|c|c|c|c|c|c|c|c|}
\hline & \multicolumn{4}{|c|}{ HAPLOTYPE } & \multirow{2}{*}{ UC } & \multirow{2}{*}{ CD } & \multirow{2}{*}{ HC } & \multicolumn{2}{|c|}{ UC vs. HC } \\
\cline { 1 - 3 } & tSNP $_{1}$ & $\mathrm{tSNP}_{2}$ & $\mathrm{tSNP}_{3}$ & $\mathrm{tSNP}_{4}$ & & & & OR (CI) & p-value \\
\hline A & 1 & 1 & 1 & 1 & $52.4 \%$ & $58.7 \%$ & $60.6 \%$ & $0.72(0.59-0.87)$ & 0.0009 \\
\hline B & 2 & 2 & 2 & 2 & $26.5 \%$ & $35.9 \%$ & $29.9 \%$ & $0.84(0.68-1.05)$ & 0.14 \\
\hline C & 2 & 2 & 2 & 1 & $3.8 \%$ & $5.1 \%$ & $6.8 \%$ & $0.55(0.35-0.85)$ & 0.0074 \\
\hline D & 1 & 2 & 1 & 1 & $7.4 \%$ & $0 \%$ & $0.5 \%$ & $14.4(5.21-39.5)$ & $<0.0001$ \\
\hline E & 2 & 1 & 2 & 2 & $3.9 \%$ & $0.1 \%$ & $0.6 \%$ & $9.89(3.95-24.8)$ & $<0.0001$ \\
\hline
\end{tabular}

346

IBDChip: A New Strategy to Predict Clinical Course and Development of Complications in Patients with Inflammatory Bowel Disease (IBD) Miquel Sans, Marta Artieda, Tejedor Diego, Carlos Cara, Luis Rodrigo, Manuel Barreiro, Maria Teresa Arroyo, Montserrat Aceituno, Manuel Alvarez-Lobos, Jordi Yague, Juan I. Arostegui, Llorenc Quinto, Laureano Simon, Josep M. Pique, Antonio Martinez, Julian Panes

Background: Accurate prediction of clinical behaviour and response to therapy in IBD patients is, at present, not available. Previous studies have failed to identify single genes as clinically relevant predictive factors, probably because both Crohn's disease (CD) and ulcerative colitis (UC) are polygenic conditions. The IBDchip is a DNA array, allowing the simultaneous study of 61 single nucleotide polymorphisms (SNPs) of 40 genes, which have been selected by their potential impact on IBD, according to the published literature. Aim: To evaluate the usefulness of the IBDchip to predict clinical course and development of complications in IBD patients. Methods: The genetic profile of 579 patients $(335 \mathrm{CD}$ and 244 UC), with $>5$ years of follow-up, was assessed by the IBDchip. The following clinical outcomes were considered: 1) In CD patients: Extension, fistulizing phenotype, perianal disease, stenosing phenotype, extraintestinal manifestations and bowel resection. 2) In UC patients: Extension, extraintestinal manifestations and need for colectomy. Relationship between clinical outcomes and the patient's pattern of SNPs and other clinical characteristics, including gender, age at disease onset and smoking habit, was analyzed by logistic regression. For each clinical outcome the optimal predictive model, based on a weighted combination 
of IBDchip SNPs and clinical characteristics and aimed at resulting in a very high (95\%) specificity, was identified. Results: At abstract submission we have completed the analysis of the IBDchip predictive value for 4 clinical outcomes. The IBDchip was able to discriminate between fistulizing and inflammatory phenotype in CD patients with a specificity (Sp) $95 \%$, sensitivity (Se) $58 \%$, positive predictive value (PPV) $92 \%$, negative predictive value (NPV) $65 \%$, overall accuracy (OA) $74 \%$, and likelihood ratio (LR) 11.7. Need for colectomy in UC patients was predicted with a Sp 95\%, Se 70\%, PPV 66\%, NPV 96\%, OA 93\% and LR 15.3 . The IBDchip was also able to identify extraintestinal manifestations in CD patients with a Sp 95\%, Se 31\%, PPV 82\%, NPV 65\%, OA 68\% and LR 6.7; and in UC patients with a Sp $95 \%$, Se $60 \%$, PPV $79 \%$, NPV $89 \%$, OA $87 \%$, and LR 12.4. Conclusions: The IBDchip is able to discriminate between fistulizing and inflammatory phenotype in $\mathrm{CD}$ patients and to identify a significant proportion of UC patients requiring colectomy or presenting extraintestinal manifestations, with a very high specificity. The IBDchip could be useful in the clinical setting, selecting a subgroup of higher risk IBD patients, who might benefit from specific treatment.

347

Socio-Economic Status Critically Influences Penetrance of the DLG5 113A Variant in Early-Onset IBD: A Novel Gene-Environmental Interaction Richard K. Russell, Hazel E. Drummond, Elaine R. Nimmo, Niall Anderson, Linda Smith, David C. Wilson, Peter M. Gillett, Paraic McGrogan, Kamal Hassan, Lawrence T. Weaver, Michael W. Bisset, Gamal Mahdi, Jack Satsangi

Introduction: The $113 \mathrm{G} \rightarrow \mathrm{A}$ mutation within the discs, large homolog 5 Drosophila (DLG5) gene located on chromosome 10q22 has been associated with susceptibility to Inflammatory Bowel Disease (IBD) in adults, although data are inconsistent across populations. No paediatric studies are described. We studied the contribution of this mutation in detail in a cohort of Scottish patients with early-onset IBD. Patients and methods: 296 patients (197 Crohn's Disease (CD), 73 Ulcerative Colitis (UC), 26 Indeterminate Colitis) with IBD diagnosed at $<16$ years of age were analysed. Parental DNA was collected for Transmission Disequilibrium Testing (TDT) analysis. Clinical, anthropometric and socio-economic data (assessed using a standardised deprivation category DepCat score) were collected for each patient. Results: TDT demonstrated an association of DLG5 $113 \mathrm{~A}$ variants with IBD $(\mathrm{p}=0.045)$, but casecontrol analysis was negative $(\mathrm{p}=0.92)$. Correlations were seen with socio-economic and nutritional status. On unifactorial analysis carriage of $113 \mathrm{~A}$ variants was seen with: 1) higher social class (DepCat score 1 compared to 2-7 and DepCat 1-2 combined compared to 3-7) ( $66.7 \%$ vs. $22.6 \% \mathrm{p}=0.0005$ OR $6.84[1.99-23.55]$ and $37.2 \%$ vs. $22.2 \% \mathrm{p}=0.03$, OR $2.08[1.04-4.17]$ respectively) 2 ) higher (75-91st) weight and height centile ( $56.2 \%$ vs. $23.4 \%$ $\mathrm{p}=0.004, \mathrm{OR} 4.20[1.49-11.81]$ and $48 \%$ vs. $22.4 \% \mathrm{p}=0.006$ OR $3.19[1.36-7.47]$ respectively) 3) males with $\mathrm{CD}(29.3 \%$ vs. $16.9 \%, \mathrm{p}=0.04$ OR 2.04 [1.01-4.11]. Multifactorial analysis demonstrated that higher social class was independently associated with carriage of variants of $113 \mathrm{~A}(\mathrm{p}=0.001, \mathrm{OR}=6.92[2.24-21.33])$. Conclusions: The DLG5 113A mutation is associated with susceptibility to IBD in Scottish children living in relative affluence but not in socioeconomically deprived children, a novel gene-environmental interaction. We suggest socioeconomic status critically influences the expression of disease in children carrying this variant.

\section{8}

TLR5 Polymorphisms Are Associated with OmpC and CBIR1 Expression and with Severity of Crohn's Disease in Ashkenazi Jews

Jerome I. Rotter, Kent D. Taylor, Huiying Yang, Ling Mei, Yoanna Picornell, Thomas R Hawn, Maria T. Abreu, Alan Aderem, Kostas A. Papadakis, Eric A. Vasiliauskas, Alan Ipolitti, Stephan R. Targan

Background. Increasing evidence supports the paradigm that genetic variation contributes to the pathophysiology of $\mathrm{CD}$ by altering the response of the innate immune system to gut microflora. We have reported that serum expression of antibodies to OmpC (E. coli outer membrane porin $C$ ) is heritable and associated with more severe $C D$, and that expression of antibodies to CBirl (bacterial flagellin) identifies a CD subgroup. Since members of the Toll-like receptor (TLR) gene family play roles in pathogen recognition and in activation of the innate immune response, and that TLR5 is activated by flagellin, TLR5 is a candidate gene for $\mathrm{CD}$ and for serum expression of microbial antibodies. Aim: To investigate the association of TLR5 SNPs and serum expression of antibodies to microbial antigens in CD using a case/control design. Methods: In 889 CD patients \& 236 controls, serum expression of OmpC \& CBirl was measured by ELISA, and 3 TLR5 SNPs (R392X, N592S, F616L) were genotyped by TaqMan MGB. Chi-square was used to test for the association of allele with disease, clinical characteristics, \& antibody combinations, and Wilcoxon and logistic regression for antibody expression levels and disease severity. Results: the F616L F allele was associated with the presence of $\mathrm{OmpC}$ expression in Jewish cases (the $\mathrm{F}$ allele was present in $93.3 \%$ of OmpC+, Jewish, CD cases and in $81.2 \%$ OmpC-, p=0.0025; $p=0.0005$ for trend through 3 genotypes). By multivariate analysis, the $\mathrm{F} / \mathrm{F}$ genotype contributed to a higher level of $\mathrm{OmpC}$ expression in both ethnic groups (presence of $\mathrm{F} / \mathrm{F} \mathrm{p}=0.019$; OR of $\mathrm{F} / \mathrm{F}$ versus L/L, $2.0295 \% \mathrm{CI}$ 1.15-3.58). Similarly, the N592S S allele was associated with the presence of OmpC expression in Jewish cases (30.8\% of OmpC+, Jewish, CD cases had the $\mathrm{S}$ allele compared with $19.5 \% \mathrm{OmpC}-, \mathrm{p}=0.02$ ). In contrast, the $\mathrm{N}$ allele was associated with higher levels of CBirl flagellin expression (278 for N/N \& N/S genotype \& 190 for S/ $\mathrm{S}, \mathrm{p}=0.05$ with logistic regression). On multivariate analysis, the $\mathrm{N} / \mathrm{N}$ allele was associated with $\mathrm{CD}$ severity as defined by internal penetrating, perianal perforating and severe fibrostenosis combined (OR 6.88, 95\% CI 1.65-28.6, $\mathrm{p}=0.0059$ ). Conclusion: TLR5 SNPs are associated with the level of OmpC expression, particularly in Jews. The observation that the opposite allele of one of the TLR5 SNPs is associated with CBirl flagellin, a TLR5 ligand, supports the concept that specific variation in genes of innate immune pathways govern variations between individuals in expression of specific antibodies to microbial antigens. The combination of these immune signals then leads to increasing severity of disease

\section{9}

The Familial Mediterranean Fever (FMF) Gene (MEFV) As a Disease Susceptibility Gene in Inflammatory Bowel Disease (IBD)

Alexandra-Chloe Villani, Mathieu Lemire, Edouard Louis, Mark Silverberg, Yannick Renaud, Sebastien Brunet, Catherine Collette, Genevieve Fortin, Cecile Libioulle, Jacques Belaiche, Alain Bitton, Daniel Gaudet, Albert Cohen, Diane Langelier, John Rioux, Paul Rutgeerts, Gary Wild, Severine Vermeire, Thomas J. Hudson, Denis Franchimont

Familial Mediterranean fever (FMF) is a hereditary auto-inflammatory disease caused by mutations in the MEFV gene (Chr.16pl3), which encodes pyrin/marenostrin that, like NOD proteins, belongs to a structural super-family of death domain proteins. Moreover epidemiological studies have revealed a higher prevalence of Crohn's disease (CD) in FMF non Sepharadic Jewish patients. We therefore sought to evaluate MEFV as a susceptibility gene for IBD. Methods: Phase 1: MEFV expression was examined in human biopsies collected from CD patients $(n=7), U C$ patients $(n=6)$, and controls $(n=4)$, using the ABI PRISM 7900HT Taqman system. Phase 2: DNA samples were obtained from 4 centers: Liege (190TRIOS: 156CD, 34UC; 96 UC, 106 controls), Leuven (435TRIOS: 356CD, 79UC), Quebec (125 TRIOS: 95CD; 30UC), and Toronto (190 TRIOS: 131CD, 59UC). The haplotype structure and fine-mapping of MEFV was done by genotyping 65 SNPs over $96.5 \mathrm{~kb}$ using SNPstream UHT, FP-TDI, and sequencing technologies. Standard measures of linkage disequilibrium (LD) were estimated using EM algorithm, and association testing was done using FBAT v1.4.2. and Armitage test for trend. Results: Phase l: CD colonic biopsies showed a significan increase in MEFV expression ( $\mathrm{p}<0.024)$ compared to controls. No significant difference in expression level was observed in UC. Phase 2:LD analysis of MEFV gene showed 2 regions of high LD. Association analysis revealed that the minor alleles of SNPs in exon 2 of MEFV, and SNPs in high LD with them, are significantly associated with UC ( $\mathrm{p}=0.0002)$ in Belgium families (Leuven and Liege), even after correcting for the number of SNPs being tested. Moreover, these associations are consistent across both Belgium centers (Liege: $\mathrm{p}=0.00047$ Leuven: $\mathrm{p}=0.018$ ). In addition, the minor alleles of SNPs located in exon 5, and SNPs in high LD with them, were also found to be significantly associated in both Quebec trios ( $\mathrm{p}=$ $0.0060)$ and Liege case-control $(\mathrm{p}=0.025)$, indicating possible allelic heterogeneity for this gene. No significant associations were observed with the Toronto UC trios. Furthermore weak associations with the major alleles of SNPs located around exon 2 were observed in the $C D$ Belgium families $(p=0.0138)$. Other associations were also observed with SNPs located in exon 5 in the Toronto $C D$ trios $(\mathrm{p}=0.0338)$. These associations were not replicated in Quebec CD trios. Conclusion: The MEFV gene appears to be a disease susceptibility gene for IBD. Interestingly, this study suggests that a gene associated with a rare inflammatory disorder could be implicated in the susceptibility of more common inflammatory diseases, such as IBD

\section{0}

Genotype/Phenotype Analysis of a Panel of Genes in Pediatric Patients with IBD. A Study of the Italian Society for Pediatric Gastroenterology (SIGENP) A Latiano, S Cucchiara, G Vieni, G Guariso, R Berni Canani, E Miele, D de Venuto, S Accomando, C Romano, A Campanozzi, C Bascietto, F Bossa, O Palmieri, Mr Valvano, Vito Annese

Background: The identification of CARD15, OCTN and DLG5 as susceptibility genes for inflammatory bowel disease (IBD), as well other candidate genes like TNF-alpha and MDRl, have suggested that these genes may influence both the predisposition and the clinical course of these disorders. Approximately $10 \%$ and $30 \%$ of patients with IBD are diagnosed under the age of 18 and 21 years, respectively. However, it unclear whether the impact of these genes is of relevance in pediatric onset Crohn's disease (CD) and ulcerative colitis (UC) Aim: To investigate the frequency of a panel of genes variants in an Italian population o pediatric onset IBD and to analyse genotype-phenotype correlations. Patients and Methods: A panel of 11 SNPs of CARD15 (R702W, G908R and L1007fsinsC), IBD5 locus (IGR2198a 1, IGR2096a_1), OCTN (SLC22A4 and SLC22A5), DLG5 (R30Q), TNF-alpha (-308 and 857), and MDR1 (C3435T) genes was assessed in 228 children with IBD (115 with CD and 113 with UC), and 254 unrelated healthy controls. Transmission disequilibrium testing (TDT) on 177 available trios was also performed. Accurate information on clinical subphenotypes (age at diagnosis, disease localization and behaviour, presence of perianal fistulae or extra-intestinal manifestations, need of surgery), response to medical therapy, and subclinical markers (ASCA, ANCA) was available. Results: A significant correlation in CD patient was found with $C A R D 15$ (one variant, $p<0.001, O R=4.3$; two variants $O R=6.3$ ), IBD5 locus (homozygotes $\mathrm{p}=0.028$, OR 1.86), OCTN (SLC22A4, $\mathrm{p}<0.006, \mathrm{OR}=2.17$; $\mathrm{SLC} 22 \mathrm{~A} 5, \mathrm{p}<0.003$, $\mathrm{OR}=1.91), \operatorname{DLG} 5(\mathrm{p}=0.015$ at TDT $)$, and TNF-alpha-308 $(\mathrm{p}=0.003, \mathrm{OR}=2.3)$. At genotype/ phenotype analysis, an excess of OCTN and DLG5 variants was found only in patients with fistulizing behaviour ( $\mathrm{p}<0.04$ and $\mathrm{p}<0.05$, respectively). A trend towards a growth delay at diagnosis was found in $C D$ patients with TNF-alpha-308 variant $(\mathrm{p}=0.05)$. No significant correlation in UC patients was found. Conclusions: Variants of CARD 15, OCTN, DLG5 and TNF-alpha genes are significantly involved in predisposition to $C D$ with pediatric onset; however, only a weak influence on clinical features and response to medical therapy was found.

\section{1}

Absence of Functional Peroxisome Proliferator Activated Receptor- $\alpha$ (Ppar- $\alpha$ ) Increases Tumour Formation in APC Min/+ Mice Anthony Shonde, David R. Bell, Andrew J. Bennett, Christopher J. Hawkey

Background: Peroxisome proliferator-activated receptor-alpha (PPAR-alpha) is expressed at reduced levels in murine and human colon tumours relative to normal mucosal. Apc Min/ + mice dosed with methylclofenapate (a PPAR-alpha ligand) have significantly less polyp area in the small intestine and colon compared to control animalsl. It is not certain that this response is PPAR-alpha specific. We investigated the hypothesis that the absence of functional PPAR-alpha would incease tumour formation in Apc MIN/+ mice. Before studying the effect of pharmacological PPARa ligand methyclofenapate on colon carcinogenesis, we 
examined the role of PPAR-alpha in colon carcinogenesis using Apc Min/+ PPAR-alpha-/mice. Aim: To establish if PPAR-alpha has a tumour suppressive function in $\operatorname{Apc}(\operatorname{Min}) /+$ mice. Method: Once weaned C57BL/6J Apc Min/+ mice and C57BL/6J Apc Min/+ PPAR$\alpha-/$ - mice were fed standard chow (Rat \& mouse standard diet, BEEKAY, Humberside) throughout the study. Mice were weighed weekly and until mice showed signs of anaemia or lost $>20 \%$ of weight at which time they were sacrificed by cervical dislocation. The primary endpoints to be compared brtween groups were the number and location of tumours. Result: Apc Min/+ PPAR- $\alpha-/$ - mice have significantly greater number of tumours in the colon. They also tend to have more tumours in the small intestine but this increase failed to reach significance $(p>0.05)$. The mean number of polyps in the colon was 2.43 (SE 0.31) in Apc Min/+ PPAR- $\alpha-/-$ mice and $0.75(0.25)$ in Apc Min/+ (Min) $\mathrm{p}<0001$. In the small intestine the mean numbers of tumours were $18.00(2.26)$ and 15.92 (1.7) respectively ( $\mathrm{p}=$ ns). Conclusions: PPAR- $\alpha$ has a role in the formation of tumours in Apc Min/+ mice. Deletion of PPAR- $\alpha$ leads to increased polyp number inthe colon. This data allied to the tumour suppressive effects of MCP indicate a tumour suppressive role for PPAR- $\alpha$ in colon cancer which may be clinically exploitable. 1. Potential role for peroxisome proliferator activated receptor- $\alpha$ (PPAR- $\alpha$ ) in preventing colon cancer. Jackson L, Wahli W, Michalik L, Watson SA, Morris T, Anderton K, Bell DR, Smith JA, Hawkey CJ, Bennett AJ. Gut. 2003 Sep;52(9):1317-22.

\section{2}

Mitochondrial DNA (mtDNA) Mutations in Helicobacter Pylori Chronic Gastritis and Gastric Carcinoma

Ceu Figueiredo, Valdemar Maximo, Paula Soares, Jose C. Machado, Raquel Seruca, Fatima Carneiro, Manuel Sobrinho-Simoes

Mitochondrial DNA (mtDNA) is a likely hot spot for mutations in cancer as it is preferentially modified by many carcinogens. mtDNA alterations have been observed in various types of tumors, including gastric carcinoma. Gastric carcinoma is considered a long-term consequence of $H$. pylori chronic gastritis. Therefore, we aimed at elucidating the significance of mtDNA mutations in the context of $H$. pylori chronic gastritis and gastric carcinoma. A total of $150 \mathrm{H}$. pylori infected patients ( 99 with chronic gastritis and 61 with gastric carcinoma) were included in this study. DNA isolated from gastric samples and matched blood or adjacent normal tissue, was screened for mutations in mitochondrial genes encoding NADH dehydrogenase 1, 3, 4, and 5 (ND1, ND3, ND4, and ND5), cytochrome c oxidase subunit I (COI), ATP synthase (ATPase6), and two regions of the displacement loop (D-Loop; D310, corresponding to a mononucleotide $\mathrm{C}$ repeat, and D514, corresponding to a dinucleotide CA repeat) $H$. pylori vacA and cagA genotypes were determined by multiplex PCR and reverse hybridization (LiPA). Overall, mtDNA mutations were more frequent in gastric carcinoma $(28 / 61 ; 46 \%)$ than in chronic gastritis patients $(20 / 99 ; 20 \%)(\mathrm{p}<0.0001)$. D-Loop mutations were more frequently detected in gastric carcinoma $(22 / 61 ; 36 \%)$ than in chronic gastritis patients $(17 / 99 ; 17 \%)(\mathrm{p}=0.008)$. Both gastric carcinoma and chronic gastritis patients harbored D310 mutations (19/61; 31\% vs. 17/99; 17\%, respectively), whereas DLoop D514 mutations were restricted to gastric carcinoma cases $(9 / 61 ; 15 \%)$. In coding genes, mutations preferentially affected complex I genes, and were more frequent in gastric carcinoma $(13 / 61 ; 21 \%)$ than in chronic gastritis $(5 / 99 ; 5 \%)(\mathrm{p}=0.003)$. NDl mutations were present in both groups of patients, whereas ND3, ND5, COI, and ATPase6 mutations were gastric carcinoma specific. Mutations in coding genes were frequently transitions, preferred targets for oxidative DNA damage. Unconditional logistic regression was performed, with both mtDNA mutations and $H$. pylori genotypes being tested in the model. In the final model, both the term for mutations in the D-Loop region, and the term for $\mathrm{H}$. pylori vacA $m$-region were statistically significant. The threshold of statistical significance was not attained for $H$. pylori vacA s-region, $H$. pylori cagA, as well as for mutations in coding genes, probably due to lack of statistical power. Altogether, these results suggest that mtDNA mutations are frequent events in gastric carcinoma, and that these mutations are independent of $\mathrm{H}$. pylori genotypes.

\section{3}

Genome Scanning Analysis Identifies Alx4 Gene Methylation As a Potentia Marker for Colorectal and Other Gastrointestinal Adenocarcinomas Matthias Ebert, Fabian Model, Christoph Rocken, Hans-Ulrich Schulz, Peter Malfertheiner, Cathy Lofton-Day

Background \& Aims: The identification of novel genetic and epigenetic markers indicative of changes in the pathogenesis of colon cancer along with easier to use, more sensitive assay methods may improve the detection, treatment and overall prognosis of this malignancy. Methods: Using Methylation Specific Arbitrarily Primed PCR a fragment of the ALX4 gene that was highly methylated in colon adenomas and cancer was identified. Methylation of ALX4 was analysed in colorectal cancers $(n=47)$ and matched normal colon mucosa ( $n=$ 21), as well as in the liver metastasis of 24 patients with colorectal cancer and 61 other neoplasias, including gastric, esophageal, hepatocellular cancer and cholangiocarcinoma. ALX4 methylation was also analysed in the serum of 30 colon cancer patients. Results: ALX4 was more frequently methylated in primary colorectal cancers (30/47) compared to the normal colon mucosa $(0 / 21)(\mathrm{p}<0.0001)$. In 16 of 24 colon cancer metastasis methylation of ALX4 was also observed. In addition, ALX4 methylation was frequently observed in adenocarcinomas of the esophagus (12/14), stomach (11/15) and bile ducts (4/5), compared to all other cancers $(\mathrm{p}<0.001)$. ALX4 gene methylation was also more frequently found in sera of colon cancer patients compared to non-cancer controls $(\mathrm{p}<0.0001)$ and using a cutoff of $41.4 \mathrm{pg} / \mathrm{ml}$ sensitivity and specificity were $83.3 \%$ and $70 \%$, respectively. Conclusions: Apart from primary and metastatic colorectal cancers, ALX4 is frequently methylated in adenocarcinomas of the gastrointestinal tract. ALX4 gene methylation in sera of cancer patients may, thus, serve as a methylation-specific test for colon and other gastrointestinal cancers.
354

JC Virus T-Antigen Expression Is Associated the Methylator Phenotype in Sporadic Colorectal Cancers

Ajay Goel, Meishu Li, Takeshi Nagasaka, Sung Kwan Shin, Florentine Fuerst, Luigi Ricciardiello, C. Richard Boland

$\mathrm{JC}$ virus (JCV) is a polyomavirus which ubiquitously infects the human gastrointestinal tract, where it has been implicated in carcinogenesis. JCV encodes a "transforming" antigen, Tantigen ( $\mathrm{T}-\mathrm{Ag}$ ), which mediates the oncogenic potential of the virus. Previous in vitro data indicate that JCV can induce chromosomal instability (CIN) in diploid colorectal cancer (CRC) cells. However, JCV can be found in CRCs regardless of whether or not they have CIN. Aim: This study was designed to test the hypothesis that JCV may associate with multiple forms of genomic instability in CRC. Methods: We characterized a cohort of 100 primary unselected sporadic CRCs for microsatellite instability (MSI) and CIN using a panel of microsatellite markers. PCR amplifications were performed for T-Ag DNA sequences and immunohistochemical (IHC) staining was performed to detect T-Ag expression in tissue sections. De novo methylation of the promoter regions of nine tumor suppressor genes implicated in CRC (hMLH1, APC, pl6, pl4, PTEN, TIMP3, RUNX3, HICl and RARß) were studied by a methylation-specific PCR (MSP) assay. Results: JCV T-Ag DNA sequences were found in $77 \%(77 / 100)$ of the CRCs, and 56\% (43/77) of these cancers (or $43 \%$ of the total) expressed T-Ag by IHC. A significant association was observed between T-Ag expression and $\mathrm{CIN}$ in the $\mathrm{CRCS}(\mathrm{p}=0.017)$. Unexpectedly, $\mathrm{T}-\mathrm{Ag}$ expression was slightly more frequent in CRCs with MSI $(8 / 15 ; 53 \%)$ than those without MSI $(35 / 85 ; 41 \%, \mathrm{p}=0.55)$. However, we observed a significant association between T-Ag expression and promoter methylation of multiple genes, including hMLHl $(\mathrm{p}=0.01)$. Conclusions: The significant association between T-Ag expression and promoter methylation in CRC suggests that the methylator phenotype may directly contribute to genomic instability through dysregulation of methylation, or indirectly influence the MSI and CIN pathways. Taken in the context of our prior work, the association between JCV T-Ag expression and multiple pathways of genomic instability in CRC suggests that JCV may be involved in neoplastic transformation through all of the mechanisms that lead to genomic instability in the colon.

355

Activated Neutrophils Cause An Hmlh1-Independent G2-M Arrest and P53ser15 Phosphorylation in Colorectal Cells Christoph Campregher, Gloria Luciani, Christoph Gasche

Background: Chronic inflammation in the GI tract is associated with increased risk of cancer development. This is particularly evident in ulcerative colitis (UC). Exposure of colon epithelial cells to reactive oxygen species generated by activated polymorphonuclear neutrophils (PMNs) and macrophages has been implicated in carcinogenesis. DNA mutations may occur during replication; however, the mismatch repair (MMR) system is thought to detect replication errors and maintain DNA fidelity. Since microsatellite instability is a common finding in colitis tissue (even without dysplasia), a relaxation of the MMR system has been proposed. Because the molecular mechanism of the underlying pathway is incompletely understood, we intended to simulate inflammation-driven mutagenesis in a cell culture model. Methods: A co-culture system was developed using HCTl16 (hMLHl mutated colorectal cancer cells) as target cells and freshly isolated human PMNs as effector cells in 6-well plates separated by a semipermeable membrane (Transwell, Costar). PMNs were activated with PMA for $30 \mathrm{~min}$, ROS was measured by lucigenin-amplified chemiluminescence, and cells were co-cultured at various ratios (1:1 to 1:10) for 24 hours. Cell cycle progression of HCT116 was analyzed by flow cytometry. Changes in levels of MMR proteins (MSH2, MSH3, and MSH6), cell cycle-related protein p21Wafl and phosphorylation of p53Ser15,Chk1Ser345, Chk2Thr68 were detected by Western blotting. Results: PMA activation of PMNs caused an increase in extracellular ROS (about 100-fold). Co-cultivation with PMA-activated PMNs at all ratios caused HCT116 cells to accumulate in the G2-M phase (table). No changes in hMSH2, hMSH3, or hMSH6 were found. However, p53Serl5 was phosphorylated and the expression of p21Wafl, a p53 target gene, was increased. Discussion: In our co-culture model, activated PMNs induced an increase in the G2-M cell cycle phase indicating that the production of ROS caused DNA damage. The G2-M arrest in hMLH1deficient cells points to the activation of an MMR-independent G2-M checkpoint. This seems to involve p53Ser15, a site targeted by ATM or ATR kinases, that might induce expression of p2lwafl, which is an inhibitor of cyclin dependent kinases. Our system will serve as a tool to study the molecular processes that are involved in inflammation-associated carcinogenesis Cell cycle distribution of HCT116 cells

\begin{tabular}{|c|c|c|c|}
\hline HCT116 & G1 (\%) & S (\%) & G2 (\%) \\
\hline w/o PMNs & $18.6 \pm 0.45$ & $53.3 \pm 2.80$ & $28.1 \pm 2.55$ \\
\hline PMNs (-PMA) & $17.0 \pm 0.58$ & $51.3 \pm 2.80$ & $31.8 \pm 3.40$ \\
\hline PMNs (+PMA) & $15.3 \pm 0.57$ & $49.4 \pm 1.20$ & $35.2 \pm 1.50^{*}$ \\
\hline
\end{tabular}

*P $<0.05$ compared to w/o PMNs

356

IL-6, TNF-A and CRP Gene Polymorphisms Are Associated with Increased Colon Cancer Risk in African Americans Compared to Whites in the North Carolina Colon Cancer Study

Temitope O. Keku, Joseph Galanko, Seun Omofoye, Rachel Holston, Janie Peacock, Jeffrey Barnes, Chris Martin, Robert S. Sandler

Background: Among major US ethnic groups, African Americans have the highest incidence and mortality of colon cancer. However, critical questions remain unanswered about genetic susceptibility to colon cancer in African Americans. Cytokines mediate immune and inflammatory responses, which may act in neoplastic pathways. Genetic variants that influence 
cytokines levels may thus affect colon cancer risk. Aim: To evaluate IL-6 (G -174 C), TNFa (G -308 A), CRP (G -1081 A) genetic polymorphisms among African Americans and Caucasian Whites in relation to the risk of colon cancer and assess interactions with lifestyledietary factors. Methods: We analyzed 528 incident colon cancer cases (231 African Americans, 297 Whites) and 836 controls (306 African Americans, 530 Whites) from a large population-based case control study in North Carolina. Controls were frequency matched to cases on age, race and sex. Consenting subjects provided blood specimens, information about lifestyle and diet histories, and anthropometric measurements. IL-6, TNF-a, CRP genotypes were determined by the TaqMan assay. Logistic regression was used to calculate odds ratio (OR) and 95\% confidence intervals (CI) for variant genotypes while controlling for potential confounders such as age, sex, non-steroidal anti inflammatory drugs (NSAIDs), and body mass index (BMI). Results: IL- 6 and TNF-a variant genotypes were significantly associated with increased risk of colon cancer among African Americans, compared to wildtype genotypes (IL-6 GC+CC, OR 1.7, 95\% CI 1.0-2.8; TNF-a GA+AA, OR 1.6, 95\% CI 1.0-2.4), but not in Whites. However, the CRP GA+AA variant genotype showed a modest association with colon cancer in Whites compared to wildtype (OR 1.3, 95\% CI 1.0-1.8). Interestingly, in a combined analysis with all three genes, using common wildtypes as referent, having one or more variants was significantly related to colon cancer risk in both African Americans and Whites (OR 1.3, 95\% CI 1.0-1.6) but this association was stronger for African Americans (OR 1.4, 95\% CI 0.9-2.0; Whites OR 1.2, 95\% CI 0.9-1.7). No interactions were observed with BMI and NSAIDs use. However, the risk of variant genotypes was greater among persons with elevated waist-to-hip ratios (WHRs). The OR for having greater-than-median WHR plus 1 or more variants was 2.2 (95\% CI 1.6-3.2), compared to persons below median WHR having no variants. Conclusion: These results suggest that genetic polymorphisms in IL-6, TNF and CRP strongly contribute to colon cancer susceptibility, particularly in African Americans. Supported by grants from NIH K01CA093654, P30DK34987 and P50CA106991

\section{7}

Gender Effects in Nutrient-Stimulated PYY and Ghrelin Secretion Lukas Degen, Stefanie Graf, Sibylle Oesch, Christoph Beglinger

Background: Ghrelin and peptide YY (PYY) appear to play an important role in appetite regulation, and their release is modulated by food ingestion. Ghrelin increases hunger and food intake, and its levels decrease after eating. PYY decreases food intake and appetite, and its levels increase proportionally to the amount of ingested calories. The aim of the present study was therefore to investigate whether nutrient stimulated responses of ghrelin (suppression) and PYY (release) were modulated by gender. Methods: 20 healthy subjects ( 10 males, 10 females, aged 20-25 years) with normal BMI were studied on two different occasions in a randomised order. After fasting for at least 10 hours, participants ingested the following meals on two different days: 1) $500 \mathrm{kcal}$ meal and 2) $1500 \mathrm{kcal}$ meal. The meals were identical in content (orange juice, ham sandwiches, chocolate mousse, water), but differed in size and calories. Blood was drawn in regular intervals over $240 \mathrm{~min}$ for plasma ghrelin and PYY concentrations. Hormone concentrations were determined by specific radioimmunoassay systems. Results:(Mean \pm SEM) Data were analysed using ANOVA followed by multiple paired t-test with Bonferroni's correction. * indicates significant difference males vs females $(\mathrm{p}<0.05)$. Summary: 1) The two test meals induced a calorie-dependent, significant decrease in ghrelin levels $(\mathrm{p}<0.01)$, and this decrease was modulated by sex. 2) PYY levels were calorie-dependently increased by the two test meals; gender did not affect these responses. Conclusion: 1) The calorie content of a test meal determines the degree of postprandial suppression of ghrelin levels, both in males and females. 2) Healthy normal weight females exhibit a higher ghrelin suppression after meal intake than males. We infer from these data that ghrelin concentrations (unlike PYY) are subject to modulation according to gender. Hormone responses to two test meals

\begin{tabular}{|c|c|c|c|c|}
\hline Parameter & Ghrelin Males & Ghrelin Females & PYY Males & PYY Females \\
\hline Basal levels (pg/ml) & $777 \pm 101^{*}$ & $1062 \pm 66$ & $141 \pm 11$ & $116 \pm 6$ \\
\hline $500 \mathrm{kcal} \mathrm{meal} \mathrm{AUC} \mathrm{(ng/240min)}$ & $179 \pm 26^{*}$ & $232 \pm 18$ & $33 \pm 3$ & $32 \pm 2$ \\
\hline $1500 \mathrm{kcal}$ meal AUC (ng/240min) & $135 \pm 15^{*}$ & $177 \pm 15$ & $42 \pm 3$ & $44 \pm 2$ \\
\hline
\end{tabular}

358

Alterations in Gut PDK4 and Calpain-9 Are Associated with Improved Insulin Sensitivity Following Roux-en-Y Gastric Bypass Surgery

Edward Lin, Ih-Ping Huang, Leena Khaitan, Li Gu, Thomas R. Ziegler, C. Daniel Smith, Nana Gletsu

BACKGROUND: Pyruvate dehyrogenase kinase-4 (PDK4) and Calpains (CAPN) are novel hormones that affect insulin resistance. Skeletal muscle PDK4 downregulates pyruvate dehydrogenase activity which inhibits glucose utilization. CAPN have been shown to improve peripheral glucose transport by facilitating GLUT4 translocation. Insulin resistance is associated with inappropriate levels of PDK4 and CAPN, and restoration of normal expression improves insulin sensitivity. We studied the effects of roux-en-Y gastric bypass surgery (RYGB) on gut PDK4 and CAPN-9 (gut specific) expression and its association with insulin resistance. METHOD: Five severely obese women (BMI 49.7 \pm 1.1 ) were admitted to the General Clinical Research Center for IVGTT studies at baseline and at 6 months following RYGB. Gastric and jejunal mucosa were biopsied from identical sites at baseline and at 6 months. PDK4 and CAPN-9 gene expressions were determined using microarray analysis and independently confirmed using RT-PCR. Results are reported as means \pm SEM. RESULTS: BMI at 6 months was $36.3 \pm 1.6$. Overall gastric and jejunal PDK4 expression was downregulated at 6 months, while CAPN-9 was upregulated. The most significant change was seen in a reduction in gastric PDK4 (densitometry $1.0 \pm 0.1$ to $0.64 \pm 0.06, p=0.01$, and an 8.7 fold decrease by microarray analysis). There was an overall $50 \%$ increase in insulin sensitivity (Si) at 6 months. CONCLUSIONS: This is the first documentation of alterations in gut PDK4 and CAPN-9 expression following RYGB, which are associated with significantly improved insulin sensitivity. These changes in hormone expression may provide an additional mechanism for the resolution of type-2 diabetes observed following RYGB.

\section{9}

Markov Economic Model for Surgery in the Morbidly Obese Donald Garrow, Kit Simpson, Joe Romagnuolo

Introduction: Gastric bypass surgery appears to be an effective tool for weight loss in the morbidly obese, but is an expensive procedure. Some insurance companies and governmen payers have been reluctant to fully fund this procedure. We sought to determine the costeffectiveness of gastric bypass surgery in the morbidly obese. Methods: We used a Markov process to model progression of weight loss and associated costs for both surgical and nonsurgical interventions. The base-case was a 50 year old patient with a body mass index (BMI) $>40$. A third-party payer perspective was used, using direct health care costs and indirect costs related to recovery from surgery. We included literature-derived data concerning BMI-specific direct costs (including physician visits, medication costs, hospitalizations, etc), transition probabilities, and quality of life (utilities), as well as competing agespecific death rates, for both laparoscopic and open gastric bypass surgeries versus diet and exercise with a decision tree. Indirect costs related to time lost recovering from surgery wer considered. Transitions between BMI states were modeled in 1-year transitions, over lifelong time horizon. Downstream health care costs and utilities were discounted at 3\% per year. Incremental cost-effectiveness ratio (ICER) calculations were planned for nondominant strategies. Results: Surgical therapy for obesity was dominant (less costly and higher quality of life), therefore an ICER is not calculable (no tradeoff). The discounted health care cost savings per patient over an expected lifetime for surgery vs. non-surgical therapy was $\$ 21,248$. However, the total procedure cost, ancillary services and hospital stay for the initial surgery took 13 years to recoup. Over the expected lifetime, 1.5 years (discounted) quality-adjusted-life-years (QALYs) were gained per patient for surgery vs. nonsurgical therapy. Sensitivity analyses revealed the model conclusions to be robust. Conclusions: Surgery for the morbidly obese is a cost-effective (in fact dominant) choice for morbidly obese subjects when lifetime healthcare costs are considered.

360

Six Months Supplementation with Conjugated Linoleic Acid Decreases Fat Mass Region-Specifically in Overweight and Obese

Jean-Michel Gaullier, Christian Syvertsen, Jo Halse, Hans O. Hoivik, Marianne O Shea, Alexandra W. Einerhand, Ola Gudmundsen

Conjugated linoleic acid (CLA) isomers are normal constituents of fats derived from ruminan animals and dairy products. Animal studies have demonstrated CLA to positively affect diabetes, osteoporosis, immune function, carcinogenesis, atherosclerosis, and body composition. In humans the effects of CLA are not well established, because thus far only limited amount of long term clinical trials with high numbers of participants have been conducted. The present study aimed to assess the regional effects of CLA on body composition. Additionally, the safety of CLA was evaluated by monitoring adverse events and all changes in blood safety parameters, and insulin sensitivity using means of euglycemic CLAMP. In a randomized double-blind placebo controlled trial 118 overweight and obese subjects (BMI 28-32 kg/ $\mathrm{m} 2$ ) received either $3.4 \mathrm{~g} / \mathrm{d}$ CLA or placebo-olive oil for 6 months. Results obtained on the $\mathrm{PP}$ population demonstrate a significant reduction in body fat mass by $-5.6 \%(\mathrm{P}=0.004)$ for the CLA group when compared to the placebo group. Reduction of the fat mass was already observed after 3 months $(-0.7 \mathrm{~kg}, \mathrm{P}=0.034)$ in the CLA supplemented group, and the reduction was located in the legs and the abdomen of women whereas changes in men occurred essentially in the abdomen. Waist $(-3.1 \mathrm{~cm}, \mathrm{P}<0.001)$ and Waist/Hip ratio $(-0.024$ $\mathrm{P}=0.017)$ also decreased in the CLA supplemented group. Body weight and BMI reduced relative to placebo $(-1.5 \mathrm{~kg}, \mathrm{P}=0.05$ and $-0.6, \mathrm{P}=0.05$ respectively). Individuals with the highest BMI responded best to CLA. Insulin sensitivity was unaffected. In conclusion: this study shows that CLA supplementation for 6 months in healthy, overweight and obese adults affected body fat mass in specific regions of the body and was well tolerated.

Regulation of Glucose-Dependent Insulinotropic Polypeptide (GIP) Expression in Cells of Pancreatic and Intestinal Lineage Lisa I. Jepeal, Michael O. Boylan, M. M. Wolfe

Background: GIP, a potent stimulator of insulin secretion, has been implicated in the regulation of fat metabolism and in the pathogenesis of obesity. Because GIP appears to link nutrient intake with adipocyte function, the regulation of GIP expression may have potentia applications in treating obesity. However, a thorough examination of GIP expression has been hampered by difficulty in isolating GIP-producing enteroendocrine K-cells, widely dispersed within the small intestinal mucosa. The identification of suitable surrogate cell lines would thus be invaluable to facilitate studies examining the regulation of GIP expression Purpose: To elucidate the molecular mechanisms governing the regulation of GIP expression and to identify GIP-producing cell lines to perform such studies. Methods: In addition to the mouse intestinal cell line STC-1, which we and others have shown previously to express GIP, 13 intestinal and pancreatic cell lines were assessed for their suitability for studies examining GIP expression. A bioassay measuring bioactive GIP only was used to screen cells for their ability to secrete intact GIP. RNA encoding GIP and relevant transcription factors were detected by RT-PCR and Northern analysis, and Western analysis was employed to measure the transcription factors. Transient transfection of GIP promoter constructs wa performed to examine GIP promoter activity, and transient transfection of a GATA-4 expression plasmid was done to assess the effects of exogenous GATA-4 expression on GIP mRNA and secreted peptide. Results: Both STC-1 and the pancreatic cell line $\beta \mathrm{TC}-3$ were found to express GIP mRNA and secrete biologically active GIP. However, levels of GIP mRNA and bioactive peptide and the activity of transfected GIP reporter constructs were significantly lower in $\beta \mathrm{TC}-3$ than STC-1 cells. When $\beta \mathrm{TC}-3$ cells were analyzed for transcription factors

. 
known to be important for GIP expression, PDX-1 and ISL-1, but not GATA-4, were detected. Consistent with this result, transient transfection analysis of a GIP promoter construct lacking the GATA-4 binding site did not appear to affect GIP promoter activity in $\beta \mathrm{TC}-3$ cells, although an intact binding site was necessary for high promoter activity in STC-1 cells. Finally, exogenous expression of GATA-4 in $\beta$ TC-3 cells led to marked increases in both GIP transcription and secretion. Conclusion: Both STC-1 and $\beta$ TC-3 cells synthesize and secrete biologically active GIP and thus represent excellent cell lines to characterize GIP expression. Moreover, our data support the hypothesis that GATA-4 may not be requisite for GIP expression, but rather may act as an enhancer that upregulates GIP expression.

362

Nutrient Stimulation of Stomach Apelin Secretion

George H. Greeley, Jason T. Reed, Ella W. Englande

Apelin is the endogenous ligand for the APJ receptor. Apelin is produced in the GI tract and brain, the stomach is a primary production site for enteric apelin. Apelin has been shown to influence food and water intake and cholecystokinin and insulin secretion. The aim of these experiments was to examine the effect of different nutrient types, given directly into the stomach, on apelin levels in the systemic circulation and in the gastric lumen. Methods. Fasted adult SD rats were either given intragastric (IG) $5 \mathrm{ml}$ dextrose (30\%), casein $(5 \%)$, peptone $(2 \%$ ) a fatty acid (corn oil) or a triglyceride mixture. Thirty minutes after IG administration of secretagogues, plasma was collected for extraction and concentration of apelin with C18 Sep Paks. In a separate experiment, the gastric contents were harvested after giving the above IG secretagogues. The solid gastric contents and supernatant were separated by centrifugation. Apelin levels in the supernatants and plasma extracts were measured by an apelin immunoassay. Results. IG administration of all nutrient types increased plasma apelin levels significantly (Table). Plasma apelin levels increased 3- to 14fold when compared to basal levels. The order of magnitude of potency for stimulation of apelin secretion by the various nutrients was triglycerides $>$ casein $>$ amino acids/fatty acid/ peptone > dextrose. IG administration of nutrients also resulted in an elevation of apelin levels in the stomach lumen. In the gastric lumen, apelin levels increased 2- to 3-fold. Conclusions and Discussion. Secretion of gastric apelin into the systemic circulation is activated by all nutrient types. Triglycerides and intact protein (casein) are the most potent secretagogues for stimulation of gastric apelin secretion. Gastric apelin is released into the gastric lumen as well as the systemic circulation. These findings indicate that gastric apelin may influence target tissues by systemic and luminal routes.

\begin{tabular}{|c|c|c|c|c|c|c|}
\hline \multicolumn{7}{|c|}{ Plasma Apelin $(\mathrm{pg} / \mathrm{ml})$} \\
\hline basal & dextrose & peptone & fatty acid & amino acid & casein & triglyceriedes \\
\hline $26 \pm 6^{1}$ & $92 \pm 30^{*}$ & $150 \pm 39^{*}$ & $189 \pm 84^{*}$ & $171 \pm 31^{*}$ & $258 \pm 60^{*}$ & $363 \pm 107^{*}$ \\
\hline
\end{tabular}

$1=$ mean $\pm \mathrm{SEM}^{*}=\mathrm{P}<0.05$ vs basal, $\mathrm{N}=5-6$ rats/group

363

Hypnotic Medications Impair Nocturnal Esophageal Acid Clearance: A DoubleBlind, Placebo-Controlled Study Using Simultaneous $\mathrm{pH}$ Testing and Polysomnography

Ashish P. Shah, Sidney Cohen, Karl Kwok, Susie Rivera, Nikroo Hashemi, Kar Doghramji, Anthony J. Dimarino

BACKGROUND: Nocturnal gastroesophageal reflux (GER) can induce sleep arousals and awakenings, which initiate protective mechanisms to clear acid from the esophagus. Prolonged acid contact with the esophageal mucosa is implicated in the pathogenesis of refluxrelated complications. The effect of hypnotic medications on nocturnal spontaneous acid reflux, acid clearance time (ACT), and simultaneous sleep architecture has not been studied. Hypnotics may impair reflux-induced arousals and awakenings, and delay esophageal acid clearance during sleep. AIM: To evaluate the effects of the hypnotic, zolpidem, on ACT, reflux-induced sleep arousals and awakenings, and other sleep architectural parameters. METHODS: A total of 16 subjects were entered into the study; 8 had GER and 8 did not, based on previous 24-hour $\mathrm{pH}$ testing. Subjects were randomized to zolpidem $10 \mathrm{mg}$ or placebo in a double-blind, placebo-controlled fashion and underwent simultaneous nocturnal $\mathrm{pH}$ testing and polysomnography. Each subject received zolpidem and placebo on two separate sleep study nights. Entry criteria included normal esophageal manometry, no prior use of zolpidem or other hypnotics, and no reported history of a sleep disorder, such as sleep apnea syndrome, periodic limb movement disorder or narcolepsy. Subjects with GER stopped acid suppressive therapy five days prior to the study. RESULTS: The mean age was $47.0 \pm 3.9$ years $($ mean \pm SEM); 9 female, 7 male; mean BMI was $26.6 \pm 1.0$. In all subjects, mean ACT increased significantly from $60.4 \pm 37.0$ seconds (s) with placebo, to $148.2 \pm 76.2 \mathrm{~s}$ with zolpidem $(\mathrm{p}<0.05)$. In non-GER subjects, mean ACT increased from $1.1 \pm 0.3 \mathrm{~s}$ with placebo, to $15.7 \pm 13.4 \mathrm{~s}$ with zolpidem. Likewise, in GER subjects, mean ACT increased from $247.0 \pm 66.4 \mathrm{~s}$ with placebo, to $394.0 \pm 102.7 \mathrm{~s}$ with zolpidem. A reflux-associated sleep awakening occurred more often with placebo $(82 \%)$ than with zolpidem (25\%) Similarly, the reflux-induced arousal index was lower with zolpidem (18/hour) than with placebo (22/hour). Sleep efficiency (total sleep time/time in bed) and sleep latency (time to fall asleep) improved in 10 out of 16 subjects with zolpidem. CONCLUSIONS: 1) Hypnotic medications significantly increase nocturnal esophageal acid exposure by impairing acid clearance in GER and non-GER subjects. 2) Hypnotic medications suppress reflux-induced arousals and awakenings while improving sleep efficiency and latency. These results suggest that hypnotic use may lead to, or worsen damage to the esophagus and may cause false positive results with esophageal $\mathrm{pH}$ testing.
Obesity and GERD: Is Increased Intragastric Pressure the Mechanism of Linkage?

Sudip K. Ghosh, James G. Brasseur, John E. Pandolfino, Peter J. Kahrilas

AIM: Obesity (BMI>30) is epidemiologically linked to GERD, but the causal mechanism is argued. Recent data show that intragastric pressure and, particularly, the median gastroesophageal pressure gradient (GEPG) at inspiration, when reflux events are most likely to occur, are significantly greater in the obese than in the non-obese (Pandolfino JE et al. Gastroenterology; In Press). Reflux volume is also increasingly recognized as a key determinant of GERD. Our aim was to use computer simulations to explore the mechanical significance of the increased GEPG observed in obesity (15 vs $10 \mathrm{mmHg}$ ) on reflux events and reflux volume. METHODS: Anatomic and physiologic properties of the esophagogastric junction (EGJ) were obtained from physiological data and, along with Newton's laws, used to develop a computer model of the EGJ. Simulations were done to examine the sensitivities of: 1) EGJ opening (reflux) to changes in EGJ stiffness and GEPG, and 2) reflux volume to changes in GEPG, assuming stiffness constant. Refluxate volume was calculated for 0.5 seconds of flow. GEPG was varied between the median values of non-obese $(\mathrm{n}=129)$ and obese subjects $(\mathrm{n}=$ 75). RESULTS: Opening of the relaxed EGJ occurred almost instantaneously $(0.02-0.19 \mathrm{sec})$, strongly driven by increases in GEPG but relatively insensitive to changes in EGJ stiffness (Figure I). EGJ radius as well as the refluxate volume showed a strong dependency on changes in GEPG. As GEPG increased from 10 to $15 \mathrm{mmHg}$, reflux volume increased more than two-fold, from 6.7 to $14.1 \mathrm{ml}$ (Figure II). CONCLUSION: Our computer simulations suggest that: 1) opening of the relaxed EGJ is primarily driven by the GEPJ rather than EGJ stiffness, 2) an increased GEPG of the magnitude observed with obesity doubles the volume of reflux and halves the time to initiate reflux, all other variables held constant. These findings suggest that the increased GEPG with obesity may be a key mediator in the causal pathway linking obesity with GERD
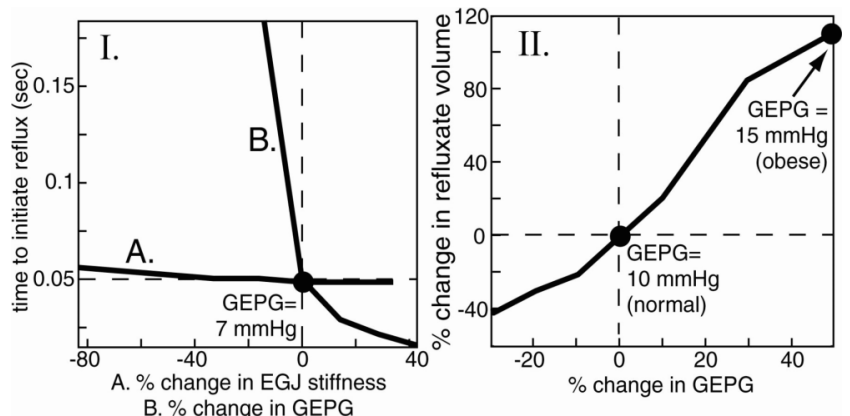

365

Obesity: A Challenge to Esophagogastric Junction Integrity Nimeesh Shah, John E. Pandolfino, Hashem B. El-Serag, Sudip K. Ghosh, Zhang Qing Peter J. Kahrilas

Background and Aims: The aim of the current study was to analyze the relationship between obesity and the morphology of the esophagogastric junction (EGJ) pressure segment using high-resolution manometry. Methods: 285 patients (108 males, ages 18-87) were studied. A solid-state manometric assembly with 36 circumferential sensors spaced $1 \mathrm{~cm}$ apart was placed trans-nasally and simultaneous intra-esophageal and intra-gastric pressures were measured over 6 to 8 respiratory cycles. Separation of the LES and crural diaphragm was quantified by measuring the distance between the two EGJ elements during inspiration. The association between anthropometric variables and pressure values were examined using univariate and multivariate analysis. Results: There was a significant correlation of BMI and waist circumference (WC) with intra-gastric pressure (Inspiration, BMI $(r=0.57)$, WC $(r=$ 0.62) $\mathrm{P}<0.0001$; Expiration, BMI $(\mathrm{r}=0.58), \mathrm{WC}(\mathrm{r}=0.64), \mathrm{p}<0.0001)$ and gastroesophageal pressure gradient (GEPG) (Inspiration, BMI $(\mathrm{r}=0.37)$, WC $(\mathrm{r}=0.43), \mathrm{P}<0.0001$; Expiration, BMI $(r=0.24)$, WC $(r=0.26), p<0.0001)$. Multivariate analysis adjusting for age, gender and the patient type did not alter the direction or magnitude of this relationship. In addition, obesity was associated with separation of the EGJ pressure components (BMI, $r=0.17$, $\mathrm{p}<0.005$; WC, $\mathrm{r}=0.21, \mathrm{p}<0.001)$. Conclusions: Obese subjects are more likely to have EGJ disruption (leading to hiatus hernia) and an augmented GEPG providing a perfect scenario for reflux to occur. Whether or not weight loss can reverse these abnormalities is unknown.

366

Critical Role of Stress in Increased Esophageal Mucosal Permeability Induced by Acid and Pepsin

Ricard Farre Marti, Pieter Vanden Berghe, Kathleen Blondeau, Jan Tack, Jozef Janssens, Daniel Sifrim

In patients with non-erosive reflux disease (NERD), heartburn is thought to occur when acid reaches sensory nerve endings through mucosal dilated intercellular spaces (DIS)(Barlow\&Orlando 2005). Stress or anxiety may increase heartburn perception, and this is attributed to central or peripheral hypersensitivity. In the rabbit esophagus, perfusion of acid and pepsin induces DIS and increases mucosa permeability. In the rat, acute stress increases gastric and intestinal mucosa permeability. We hypothesized that stress might also increase esophageal mucosa permeability and thereby, enhances acid induced esophageal symptoms. Aim: To evaluate the effect of acute immobilization stress (AIS) on esophageal mucosa permeability in rats. Methods: Male Sprague-Dawley rats were handled daily for one-week prior to study. Stressed rats were placed in a harness for $2 \mathrm{~h}$, followed by $2 \mathrm{~h}$ of 
free movements in their home cage. Control rats were maintained in their home cage for $4 \mathrm{~h}$. Rats were euthanized and segments from esophagus and ileum $(15 \times 8 \mathrm{~mm})$ were removed and mounted in diffusion chambers. Mucosal permeability was assessed by measuring the mucosa-to-serosa passage of FITC-dextran $4 \mathrm{KDa}$ (FD4) and $20 \mathrm{KDa}$ (FD20). The tissue was pre-incubated for 30 min with Krebs $\mathrm{pH} 7.2$ or $\mathrm{HCl} \mathrm{pH} 2.0+$ pepsin $1 \mathrm{mg} / \mathrm{mL}$ in the mucosal side. Permeability was expressed in mmols of FD obtained at the serosal side after incubation for 30,60, 90 and 120 min. Results: The stress protocol was effective: AIS increased the fecal output for $4 \mathrm{hr}(4.14+0.7$ vs $10+1.7, \mathrm{~N}=7 \mathrm{P}<0.01)$; increased glucose concentration by $24 \pm 5.4 \%,(\mathrm{~N}=7, \mathrm{P}<0.01)$ and increased small intestinal permeability to FD 20 (ANOVA $P<0.0001$ ). Esophageal mucosa permeability increased in stressed rats only when the tissue was pre-incubated in $\mathrm{HCl}+$ pepsin solution both to FD4 (ANOVA $\mathrm{P}=0.004$, $\mathrm{N}=5, \mathrm{n}=10$ ) and to FD20 (ANOVA $\mathrm{P}<0.0001, \mathrm{~N}=5, \mathrm{n}=10$ ), reaching significant differences at 60,90 and $120 \mathrm{~min}$. The increase at $120 \mathrm{~min}$ was from $0.70 \pm 0.27$ to $3.80 \pm 1.35$ pmols, $(\mathrm{P}<0.01)$. Esophageal mucosa from stressed rats did not show changes in permeability when the tissue was preincubated in normal Krebs solution and esophageal mucosa permeability was not increased by $\mathrm{HCl}+$ pepsin in control rats. Conclusions: In rats, acid-pepsine alone or stress alone do not induce significant changes in esophageal mucosa permeability. In contrast, esophageal acid-pepsin exposure in stressed animals has a dramatic effect on mucosal permeability. These results suggest that stress plays a critical role in the acid-pepsininduced increase in esophageal mucosa permeability. We propose that this mechanism may contribute to stress-influences on heartburn symptoms in man.

\section{7}

Production of IL-6 and IL-1 $\beta$ in GERD-Associated Human Esophagitis: Impact On Esophageal Motility

Florian Rieder, Ling Cheng, Manijeh H. Phillips, Gail A. West, Weibiao Cao, Michael V. Sivak, Piero Biancani, Claudio Fiocchi

Introduction: Gastroesophageal reflux disease (GERD) is associated with abnormal esophageal motility, and the presence of active esophagitis may contribute to motor disturbances. Various inflammatory mediators can alter esophageal muscle contraction in vitro, including the proinflammatory cytokines IL- 6 and IL- $1 \beta$. However, essentially no information is available on the production of such cytokines in inflamed human esophageal tissue and their cellular sources. Therefore, we investigated the presence of these cytokines in esophageal biopsies, their cellular sources, and impact on esophageal motility in vitro. Methods: Endoscopic mucosal biopsies were obtained from the upper, middle and lower third of the esophagus of patients with and without endoscopic evidence of esophagitis. Biopsies were organ-cultured overnight and undernatants recovered for measurement of cytokines. The profile of cytokine production by esophageal epithelial and mesenchymal cells was assessed in supernatants of the human esophageal squamous epithelial cell line HET-1A and primary human esophageal mesenchymal cells isolated from surgical specimens. Cytokines were measured by ELISA and mRNA determined by rtPCR. Super- and undernatants were used for in vitro esophageal muscle contraction assays. Results: IL-6 was present in all control and inflamed biopsy undernatants. IL-1 $\beta$ was detected exclusively in biopsies with severe esophagitis. In esophagitis patients the amount of IL-6 found in the lower third was ten times as high as that of control subjects $\left({ }^{*} \mathrm{p}<0.01\right)$, and also higher $\left({ }^{*} \mathrm{p}<0.01\right)$ than that found in the upper and middle thirds of the same patients. HET-1A cells and primary esophageal mesenchymal cells spontaneously secreted IL-6 in low amounts, which increased remarkably after stimulation with IL-1 $\beta$ or IFN- $\gamma+$ PMA. IL-1 $\beta$ was only detected at the mRNA level. Both the undernatants of esophageal biopsies and supernatants of primary cell cultures reduced in vitro esophageal muscle contraction in response to electrical field stimulation. Conclusion: Production of IL- 6 and IL-1 $\beta$ is significantly elevated in the inflamed esophagus of GERD patients, and esophageal epithelial as well as mesenchymal cells are sources for IL-6. IL-6 directly influences esophageal motor function inducing changes that mimic those found in experimental cat esophagitis. These results suggest a direct link between the inflammatory mediators produced in reflux esophagitis and the motor disturbances associated with this condition.

368

Body Mass Index Is Associated with Gastroesophageal Reflux Symptoms in Both Normal Weight and Overweight Women

Brian C. Jacobson, Samuel C. Somers, Charles S. Fuchs, Ciaran P. Kelly, Carlos A Camargo

Background: Overweight and obese individuals are at increased risk for gastroesophageal reflux disease (GERD). An association between body mass index (BMI) and reflux symptoms among normal weight individuals has not been demonstrated. Methods: In 2000, a supplemental questionnaire was used to determine the frequency, severity, and duration of GERD symptoms among randomly-selected participants of the Nurses' Health Study, a cohort study of 121,700 registered nurses begun in 1976. After categorizing women by BMI as measured in 1998, we used age- and multivariate-adjusted logistic regression models to study the association between BMI and GERD symptoms (heartburn and/or acid regurgitation). Heartburn was defined as "a burning pain or discomfort behind the breast bone in your chest" and acid regurgitation was defined as "a bitter or sour-tasting fluid coming into your throat or mouth." Results: Among 10,545 women who completed the questionnaire (86\% response rate), 6,215 (59\%) reported experiencing at least one episode of GERD symptoms in the previous year and 2,310 (22\%) reported symptoms at least once a week (frequent reflux). The majority (55\%) described their symptoms as moderate in severity, while 303 (4.5\%) thought their symptoms were sufficiently severe to affect their lifestyle. We observed a dosedependent relationship between increasing BMI and frequent reflux symptoms (multivariate P for trend $<0.001$ ). Compared to women with BMI $20-22.49 \mathrm{~kg} / \mathrm{m} 2$, the multivariate odds ratios (OR) were 1.38 (95\% CI 1.13-1.67) for a BMI of 22.5-24.9; 2.20 (95\% CI 1.81-2.66) for a BMI of 25-27.4; 2.43 (95\% CI 1.96-3.01) for a BMI of 27.5-29.9; 2.92 (95\% CI 2.353.62) for a BMI of 30-34.9 and 2.93 (95\% CI 2.24-3.85) for a BMI of 35 or greater. Women in the lowest BMI category $(<20)$ had less risk for frequent GERD symptoms than those with BMI 20-22.49 (multivariate OR 0.67; 95\% CI 0.48-0.93). A similar trend was seen across all degrees of symptom severity and duration and for the association between waistto-hip ratio and GERD. Among women who experienced a gain in weight over the previous 14 years, a dose-dependent increase in risk of symptoms was observed. However risks were significantly decreased among women who lost weight during the same time period, with a nearly $40 \%$ reduction in risk of frequent GERD symptoms among those with a decrease in BMI of more than $3.5 \mathrm{~kg} / \mathrm{m} 2$ (OR 0.64; $95 \% \mathrm{Cl}$ 0.42-0.97 compared to women withou a change in BMI). Conclusion: BMI is directly associated with GERD symptoms in both normal weight and overweight individuals. This suggests that even modest weight gain among normal weight individuals may cause or exacerbate reflux symptoms.

369

Chemicals Formed from the Gastric Acidification of Salivary Nitrite Influence Oesophageal and Gastric Function

Jonathan J. Manning, Angela Wirz, Kenneth E. McColl

Introduction: Saliva contains high concentrations of nitrite derived from the enterosalivary recirculation of dietary nitrate and its reduction by buccal bacteria. Acidic gastric juice converts the nitrite in saliva to varying proportions of nitrous acid and nitric oxide, determined by vitamin $\mathrm{C}$ availability. In vitro nitric oxide (NO) and nitrous acid relax the smooth muscle of the stomach, lower oesophageal sphincter (LOS) and oesophageal body. Neuronally generated NO plays a major role in upper gastrointestinal motility and contributes to the pathophysiology of GORD. Aim: To determine whether luminal administration of NO or nitrous acid alters oesophageal or gastric function. Method: 15 Helicobacter Pylori negative healthy volunteers were studied on 3 separate days. A water-perfused manometry catheter was placed across the lower oesophageal sphincter (LOS) after a meal, to record oesophageal, LOS and gastric pressure and transient lower oesophageal sphincter relaxations (TLOSRs). Two $\mathrm{pH}$ probes were placed in the oesophagus to record reflux events. A submental swallow sensor was also positioned. On each day, one of three solutions was infused, for one hour into the region of the LOS. These were 1) Control solution of hydrochloric acid $\mathrm{pH} 1.0$ $(\mathrm{HCl}), 2) \mathrm{HCl} \mathrm{pHl} .0$ plus nitrite $1.6 \mathrm{mM}$ ie: nitrous acid and 3) $\mathrm{HCl}$ pHl.0 plus nitrite $1.6 \mathrm{mM}$, plus ascorbic acid $5 \mathrm{mM}$ to generate NO. The manometry catheter channels were used as both a delivery system for the test solutions and also as a recording system for the manometry data. Solutions were randomised and double-blinded. Duration of $\mathrm{pH}<4$ was assessed for each pH probe. TLOSR frequency was also quantified as well as the effect on intragastric pressure during the infusion. Results: The NO solution gave a significantly increased oesophageal acid exposure, $62.2 \%$, compared to both control $37.5 \%(p<0.03)$ and nitrous acid, $36.6 \%$ ( $\mathrm{p}<0.002$ ). The frequency of TLOSRs was also significantly increased by the NO solution (5.2/hour) compared to both control $(3.5, \mathrm{p}<0.01)$ and nitrous acid $(3.1, p<0.0001)$. There was also evidence of impaired oesophageal clearance of acid refluxate following the NO infusion. During the nitrous acid infusion the intragastric pressure was $3.4 \mathrm{mmHg}$ lower than the control $(\mathrm{p}<0.03)$ indicating loss of gastric smooth muscle tone. Conclusion: Luminal chemicals formed from the acidification of salivary nitrite influence both oesophageal and gastric motility and may contribute to the aetiology of disorders associated with disturbed gastric and/or oesophageal motility.

381

Cardiovascular Outcomes from a Long-Term Randomized Colorectal Adenoma Chemoprevention Trial of a Once-Daily Dose of Celecoxib

Nadir Arber, Scott Solomon, Janet Wittes, Maria Lechuga, Paola Gerletti, Katie

MacDonald, Craig Eagle, Marc Pfeffer, John McMurray, Robert Fowler, Peter Finn, Ann Zauber, Frank Ruschitzka, Bernard Levin

Background: The cardiovascular safety of specific cyclooxygenase-2 (COX-2) inhibitors is currently of concern, with only limited data available from placebo-controlled clinical trials. Methods: The PreSAP study is an international (32 countries), multi-center (106), placebocontrolled, randomized, double-blind trial designed to measure the effect of three years of treatment with celecoxib on the risk of recurrent adenomatous polyps in individuals with a prior history of polyps. Patients $(\mathrm{n}=1,561)$ with a history of colorectal adenomas were randomized in a 3:2 ratio, 933 to receive celecoxib (400 mg once a day) and 628 to receive placebo. An external adjudication committee reviewed all serious adverse events and death in a blinded fashion, categorizing each event as cardiovascular or non-cardiovascular. Nonfatal cardiovascular events were categorized according to a pre-specified scheme. An externa statistician analyzed the data in an intent-to-treat framework. Results: During a mean of 32 months of observation ( 83 percent and 54 percent of patients had at least 24 or 36 months of safety data, respectively), a composite cardiovascular end point of death from cardiovascular causes, myocardial infarction, stroke, or heart failure occurred in 12 (1.9 percent) and 22 (2.4 percent) of patients in the placebo and celecoxib group, respectively (hazard ratio 1.2.95 percent confidence interval 0.6 to 2.5) Conclusion: Celecoxib at a dose of $400 \mathrm{mg}$ once a day did not significantly increase either the short- or long-term risk of serious cardiovascular events (cardiovascular death, myocardial infarction, stroke, or hear failure) in patients with a history of colorectal adenomas; however, the small number of events limits our ability to exclude a hazard of the magnitude reported for other randomized trials of this class of drugs.

382

Comorbidity and Colorectal Cancer Screening Utilization in a National Sample of Veterans Health Administration Patients Deborah A. Fisher, Joseph Galanko, Tara K. Dudley, Nicholas J. Shaheen

Objectives: Quality assessment of colorectal cancer (CRC) screening in the Veteran Health Administration (VHA) system relies on reporting the proportion of patients aged 52-80 years screened. This approach does not assess for comorbid illnesses that may impact a patient's suitability for screening. The Charlson-Deyo score, which can be calculated from administrative data, is a predictor of mortality. Our primary aim was to determine the relationship between comorbidity, as measured by the Charles-Deyo score, and fecal occult blood testing 
(FOBT) in the national VHA database. Our secondary aim was to test the validity of the Charlson-Deyo score as a predictor of 1-year mortality in VHA. Methods: We examined the national VHA administrative databases (October 2003- February 2005) for a random sample of 86,785 primary care patients, aged $\geqq 50$. Charlson-Deyo score was calculated. The association between Charlson-Deyo score and mortality was assessed using chi squared analysis. Using logistic regression, we also assessed the relationship between comorbidity, and the likelihood of receiving CRC screening. Models were adjusted for age and sex. Results: The sample was $97 \%$ men; mean age was 67 years. Charlson-Deyo score was $45 \%=0,24 \%=1$, $14 \%=2,7 \%=3,3 \%=4,2 \%=5,1 \%=6,3 \% \geqq 7$. Overall there was no significant association between Charlson-Deyo score and whether or not an FOBT was performed. There was a strong and incremental relationship between Charlson-Deyo score and 1-year mortality. There was no association between FOBT status (performed or not) and 1-year mortality within any of the Charlson-Deyo score categories. Conclusions: In the VHA system, patients with severe comorbidity undergo FOBT screening as frequently as healthy patients. Substantial effort is being directed toward screening patients with an increased likelihood of dying from competing causes. Further, there was no evidence providers selectively screened the healthiest patients within each Charlson-Deyo score category. The current "one-size-fits-all" quality measurement for CRC screening in the VHA may promote inappropriate screening in ill patients, and does not emphasize screening in those with the most life-years at stake.

\section{3}

\section{The Impact of Literacy On Colonoscopy}

Rony Ghaoui, Arthur T. Evans, Bashar M. Attar

Background and Aims Approximately 20-30\% of attempted colonoscopies are incomplete because of poor bowel preparation. This problem is not only costly and wasteful, but increases risks and delays diagnosis and treatment. Although literacy skills are necessary for patients to successfully follow home bowel preparation instructions, they are nearly impossible to assess informally. Our aim was to evaluate the impact of literacy on bowel preparation and completion of colonoscopy, with the ultimate objective of redesigning instructions to be more effective, regardless of patients' literacy skills. Methods We performed a prospective cohort study at a large urban public hospital during the summer of 2005 among patients who presented for outpatient colonoscopy. Before the colonoscopy, literacy was assessed using the 7-minute test of functional health literacy. Using structured interviews, we also gathered information on patients' past history, beliefs, and self-reported behaviors on bowel preparation. Results of the colonoscopy were obtained from the procedure reports. Results The sample of 195 subjects included 64\% women, 49\% African Americans, and 40\% with low literacy skills, $25 \%$ of colonoscopies were not completed, primarily $(90 \%)$ because of poor bowel preparation. Low literacy increased the risk of an incomplete exam 4-fold: 12 $\%$ incomplete exams among patients with adequate literacy skills compared to $45 \%$ with low literacy skills $(\mathrm{p}<0.001)$. Other important predictors of an incomplete exam were: eating lunch or dinner the previous day, not taking the bisacodyl ( $\mathrm{p}=0.001$ ), and not finishing the l-gallon polyethylene glycol solution $(\mathrm{p}=0.01)$. Patients with a past history of colonoscopy $(\mathrm{p}=0.03)$ and those who received additional oral instructions from a physician or nurse ( $\mathrm{p}=$ 0.05 ) were more likely to have a complete colonoscopy. Low literacy remained the strongest independent predictor of incomplete colonoscopy after controlling for the contribution of all other factors ( $\mathrm{OR}=8.7$; $95 \% \mathrm{CI}: 3.4-22.1 ; \mathrm{p}<0.001)$. Discussion/Conclusion Low literacy accounts for $30 \%$ of all incomplete colonoscopies. This important effect is independent of age, gender, ethnicity, and even whether oral instructions supplemented the written instructions. In light of these findings, we must redesign our methods of instructing patients about bowel preparation in order to improve the efficiency and effectiveness of colonoscopy

\section{4}

Vertebral Fracture Prevalence in Crohn's Disease: Patients with or Without Decreased Bone Mineral Density

Jesse S. Siffledeen, Kerry Siminoski, Ho Jen, Richard N. Fedorak

Introduction: Vertebral fracture prevalence in patients with Crohn's disease (CD) suggests frequency of between 14 and $22 \%$. However, most studies have limited the patient population to those with low bone mineral density (BMD) and have not examined the occurrence of vertebral fractures in $\mathrm{CD}$ patients with normal BMD. Objective: Our primary objective identified the prevalence of vertebral fractures in a representative cohort of $C D$ patients. Our secondary objective identified clinical and biochemical factors associated with these fractures as risk predictors of vertebral fractures in $C D$ patients. Patients \& Methods: 225 patients with active or quiescent $C D$ were recruited and underwent BMD analysis, as well as thoracic and lumbar spine radiographs. Vertebral fractures were identified by two methods of radiographic assessment: First, quantitative height reduction morphometry on vertebrae T2-L2, (six points on each vertebra), was recorded as the lowest ratio of these measures compared to the greatest posterior vertebral height. Second, spinal fracture index (SFI) assessed vertebral fracture severity by visual estimation of vertebral height reduction and morphologic change. Vertebrae were graded as normal, mildly deformed (Grade 1 : reduction of $20-25 \%$ vertebral height and $10-20 \%$ of projected vertebral area), moderately deformed (Grade 2: $26-40 \%$ height reduction and $21-40 \%$ of projected vertebral area), or severely deformed (Grade 3: > $40 \%$ reduction in height and area). Results: 44/225 (19.6\%) patients demonstrated 84 vertebral fractures $(\mathrm{SFI} \geq 1)$, with a mean age of $40.6 \pm 11.0$ years. Sixty percent reported corticosteroid (CS) use in the preceding year. 16/44 patients with vertebral fractures had normal BMD (19.0\% of all patients with normal BMD). Comparative analysis between patients with or without vertebral fractures did not demonstrate any significant differences in BMD of the lumbar spine or hip, or in reported CS use. Linear regression analysis demonstrated that elevations in body mass index, C-reactive and parathyroid hormone significantly predicted vertebral fractures (SFI grades I-III, $\mathrm{r}=0.415, \mathrm{p}<$ 0.05 and height reduction $>20 \%, r=0.417, p<0.05$ ). Conclusion: This study is the first to demonstrate that vertebral fractures in $C D$ patients occurs with an equal frequency in those with low and normal BMD. The mean age of $C D$ patients with vertebral fractures was much lower than that reported in the general population. The ability of elevations in BMI, CRP and PTH to predict vertebral fractures in CD patients suggests that sedentary lifestyle, inflammatory activity and metabolic bone disease play an important role in their pathogenesis.
Risk of Pancreatic Adenocarcinoma and Hereditaty Pancreatitis: The First National Cohort in France

Vinciane Rebours, Marie Christine Boutron-Ruault, Claude Ferec, Dermot O'Toole, Matthieu Schnee, Frederique Maire, Pascal Hammel, Philippe Ruszniewski, Philippe Levy

Hereditary pancreatitis (HP) is an autosomal dominant disease caused by a mutation in the cationic trypsinogene gene (PRSS1). Chronic pancreatitis (CP) is a known risk factor of pancreatic adenocarcinoma (PA) with a standardized incidence ratio (SIR) of 27 . The frequency of pancreatic cancer in the inherited type of pancreatitis is uncertain. Aims: To evaluate the incidence of the PA in extensive French national series of patients with HP and to search for risk factors. Patients (pts) and methods: A cohort comprising all French HP pts was constituted by contacting all French gastroenterologists and pediatricians (response rate: $84 \%$ ) and genetics laboratories (response rate: $100 \%$ ). Inclusion criteria were the presence of PRSS1 gene mutation, or CP in at least 2 first-degree relatives, or 3 seconddegree relatives, in the absence of another cause of CP (notably, alcohol). CFTR and SPINK 1 gene mutations were also systematically examined. Diagnosis of PA was based on histological examination. Results: The historical cohort included 78 families and 200 pts of whom 181 were alive. It represented 6673 person-years (median number of generation: 3, males: 53\%, chronic alcoholism: $5 \%$, tobacco intoxication: $34 \%$ ). At time of inclusion, the median age was 30 y (range: 1-84). PRSS1 mutations were present in 68\% (R122H: 78\%, N29I: 12\%, others: $10 \%$ ) SPINKl and CFTR mutations were present in $13 \%$ and $2 \%$, respectively. PRSS1 inheritance was maternal for 65\%. 10 PA were diagnosed (6 males) at a median age of 55 y (range: 39-78). The SIR of PA for the cohort, the males, and the females was 87 (42-113), 69 (25-150) and 142 (38-225), respectively. At 50, 60 and $75 \mathrm{y}$, the cumulated risk of PA was $11 \%, 16 \%$ and $49 \%$ for males and $8 \%, 22 \%$ and $55 \%$ for females, respectively. The risk of PA for smokers at age $75 \mathrm{y}$ was $61 \%$ and $70 \%$ for males and females, respectively. 19 patients died, 8 from PA (42\%). The cause of death was known for all the patients except one. Conclusions: Patients with HP have a markedly increased risk of pancreatic cancer (SIR: 87) compared with the general French population. This may be explained in part by the long exposure time to $\mathrm{CP}$. There is no correlation between this risk and the PRSS1 genetic status but the PA is highly linked with the tobacco intoxication. PA is the first cause of death in patients with $\mathrm{HP}$

386

Risk Factors for Esophageal and Gastric Adenocarcinoma

Crane J. Sarah, G R. Locke, Yvonne Romero, William S. Harmsen, Diehl N. Nancy, Alan R. Zinsmeister, Lee J. Melton, Nicholas J. Talley

Background: Some have suggested that increased use of acid suppressing drugs explains the increasing incidence of distal esophageal cancer. Database studies are flawed because the esophagogastric junction (EGJ) and the proximal stomach cannot be discriminated. Hence it is unclear whether these two sites share similar risk factors or reflect different at-risk populations. Aim: To evaluate risk-factors associated with esophageal and gastric adenocarcinoma in a population-based, case-control study. Methods: The data resources of the Rochester Epidemiology Project were used to identify all cases of gastric and esophageal adenocarcinoma in Olmsted County, Minnesota between 1971 and 2000. Cases were categorized as esophageal, EGJ, proximal stomach, distal stomach and diffuse. An age and sex matched control group was identified from Olmsted patients seen for evaluation during the same time period. A complete medical record review was conducted, including histology, radiology, endoscopic, autopsy, operative, inpatient and outpatient records and reports. Risk factors, including a history of gastroesophageal reflux disease (GERD), duodenal or gastric ulcer, acid-suppressing medication use, BMI $>30$, tobacco, alcohol abuse, other cancer diagnosis, family history of esophageal or gastric cancer, previous cholecystectomy and blood type were assessed using a conditional logistic regression analysis, reported as (OR [95\% CI]). Results: A total of 186 cases of esophageal and gastric adenocarcinoma were identified. Of these, 29 were esophageal, 36 were EGJ, 27 were proximal gastric, 75 were distal and 19 were diffuse. Incidence rates in this population demonstrated a significant increase in esophageal adenocarcinoma from 0.4 to 2.5 per 100,000 py over this time period. A history of GERD was significantly associated with esophageal $(\mathrm{OR}=5.5[1.2,24.8])$ and $\mathrm{EGJ}(\mathrm{OR}=13[1.7,99.3])$ cancer but not with proximal gastric cancer. PPI use was not associated with esophagus and EGJ cancer combined $(\mathrm{OR}=5.0,95 \% \mathrm{CI}[0.6,42.8])$. No significant risk factors for proximal or diffuse gastric cancer could be detected, though sample sizes were small. A history of gastric ulcer and a $\mathrm{BMI}>30$ were associated with distal gastric cancer $(\mathrm{OR}=12.0[1.6,92.3])$ and $(\mathrm{OR}=$ 2.7[1.0.6.8], respectively). Conclusions: Esophageal and EGJ adenocarcinoma are significantly associated with GERD. Proximal gastric adenocarcinoma appears to have a different risk factor profile suggesting a different disease pathogenesis. Supported in part by an ACG research award.

\section{6}

Co-Localization of Lysosomal Hydrolases with Digestive Enzyme Zymogens in Acinar Cells During Pancreatitis Is Not Dependent Upon Digestive Zymogen Activation

Gijs J. Van Acker, Eric R. Weiss, Michael L. Steer, George Perides

Background: Digestive enzyme zymogens become co-localized with lysosomal hydrolases within acinar cell cytoplasmic vacuoles and digestive zymogens become activated within acinar cells during the early stages of experimental pancreatitis. We have previously hypothesized that the co-localization phenomenon leads to zymogen activation by facilitating cathepsin B-catalyzed activation of trypsinogen but others have argued that the co-localization phenomenon might be the result, rather than the cause, of zymogen activation. The current studies were designed to resolve this controversy. Methods: Mice were given tail-vein injections of CA- $074 \mathrm{me}(5 \mathrm{mg} / \mathrm{kg}) 1$ hour prior to induction of pancreatitis to inhibit cathepsin B activity and then acute pancreatitis was induced by administration of hourly i.p. caerulein injections $(50 \mu \mathrm{g} / \mathrm{kg})$. The co-localization phenomenon was monitored using the techniques of both sub-cellular fractionation and immunohistochemistry. For the former, pancreas samples were 
subjected to differential centrifugation while the activities of amylase and aryl sulfatase (a lysosomal hydrolase) were tracked using specific substrates. In vivo immunohistochemistry studies were accomplished using confocal microscopy and antibodies directed against amylase and the lysosomal membrane protein LAMP-1. F-actin distribution was determined by phalloidin staining. JNK activation was evaluated by immunoblot and AP-1 activation by EMSA. Results: In vivo cathepsin B activity was completely inhibited by administration of CA-074 me and, under these conditions, supramaximal stimulation with caerulein did not lead to intrapancreatic activation of trypsinogen. In contrast, inhibition of cathepsin B activity and prevention of caerulein-induced trypsinogen activation did not interfere with either the caerulein-induced co-localization phenomenon or with other caerulein-induced responses including redistribution of sub-apical f-actin, activation of JNK, and activation of AP-1. Conclusion: Our results indicate that trypsinogen activation is not a pre-requisite for induction of the co-localization phenomenon or for either f-actin redistribution or pro-inflammatory factor activation. Our studies exclude the possibility that the co-localization phenomenon, f-actin redistribution, and pro-inflammatory factor generation are the result of digestive zymogen activation and they support the hypothesis that the co-localization phenomenon leads to zymogen activation during pancreatitis. Supported by NIH grant R01-31396

407

Transgenically Elevated Basal Levels of Nf- $\kappa \mathrm{B}$ Increase Pancreatic Sensitivity to Injury and Pancreatitis Severity

Baoan Ji, Jian Song, Craig D. Logsdon

Background \& Aims: NFKB activation in pancreatic acinar cells is an early event in experimental pancreatitis models. However, the role of $\mathrm{NFKB}$ in this disease is still uncertain. Pharmacological inhibition has not been able to differentiate the specific effects of NFKB because the inhibitors have non-specific actions. Adenoviral mediated gene transfer of the p65 subunit of $\mathrm{NFKB}$ was previously found to induce pancreatic inflammation, but suffers from the potential non-specific effects of viral infection. Therefore, in the current study, we utilized a transgenic approach to investigate the role of $\mathrm{NF \kappa B}$ activation in pancreatitis. Methods: A transgenic mouse line was created with the CMV-chicken beta actin chimeric promoter followed by loxP sites flanking GFP-Stop and p65 (Floxed-p65). This construct expresses GFP throughout the animal but activation of Cre leads to removal of the GFPStop cassette and allows expression of p65. These animals were crossed with a Pdxl-Cre mouse, to drive the expression of $\mathrm{p} 65$ specifically in the pancreas. Pancreatitis was induced by twelve hourly injections of caerulein $(50 \mathrm{ug} / \mathrm{kg})$ or JMV180 $(1 \mathrm{mg} / \mathrm{kg})$. Serum amylase, pancreatic edema and histology were evaluated. Results: GFP was expressed throughout the floxed-p 65 mice and this expression was greatly diminished in the acinar cells of double transgenic floxed-p65/Pdxl-cre mice indicating efficient recombination. The p65 subunit was expressed in the pancreas from double transgenics as shown by Western blot. Despite obvious over-expression of p65 in acinar cells there were no changes in pancreas size, weight or histology in double transgenic animals. This was likely due to a compensatory negative feedback mechanism involving p 65 mediated increases in IKB- $\alpha$ that was observed in these animals. However, animals with high levels of p65 responded to treatments with caerulein with much greater severity of pancreatitis as indicated by higher levels of edema, inflammatory cell infiltration and acinar cell damage than controls. Furthermore, JMV180, a CCK analog that does not cause pancreatitis in wild type mice, caused pancreatitis in double transgenic mice. Conclusions: Developmental alterations in p65 expression lead to compensatory changes in other pathway regulators. However, elevated levels of basal NFкB determine the sensitivity and severity of the proinflammatory response in acute pancreatitis.

408

Double-Edged Role for Relap65 in Acute Experimental Pancreatitis: Relap65 Protects Local Tissue Damage, But Promotes Systemic Complications Following Acute Pancreatitis

Hana Alguel, Hassan Nakhai, Dieter Saur, Stephan Paxian, Marina Lesina, Matthias Treiber, Roland M. Schmid

NF-kB/Rel activation has been shown to be activated during acute experimental pancreatitis. In several studies the inhibition of NF-kB/Rel activation has been associated with attenuated inflammatory response and severity of pancreatitis. Although previous studies suggest a deleterious effects of activated NF-kB/Rel in acute experimental pancreatitis, there is still disagreement about its effect in acinar and infiltrating cells. RelAp65 knockout mice have been shown to be embryonic lethal due to pronounced apoptosis of hepatocytes. Therefore we used the Cre/lox-system to inactivate RelAp65 (floxed RelAp65) specific in the pancreas and infiltrating cells analysing its role in acute inflammation. Crossing the floxed RelAp65 mouse with the $\mathrm{p} 48 \mathrm{Cre}$ and Lysozyme-Cre mice we were able to generate a mouse producing truncated RelAp65 either in the pancreas or pancreas and myeloid cells. This truncated RelAp65 is incapable of translocating into the nucleus, binding DNA or even forming dimers as it has been analysed in electromobility shift assays. Following cerulein challenge mice lacking functional RelAp65 in the exocrine part of the pancreas develop severe necrotizing pancreatitis which is associated with enhanced necrosis and apoptosis compared to floxed control animals. Severe inflammation in the pancreas lead to systemic complications involving liver as well as lung. Impaired upregulation of $\mathrm{Bcl}-\mathrm{xL}$ and PAP I were responsible for apoptosis and necrosis in the pancreas. Lentiviral mediated transfer of PAP I into the pancreas protected acinar cells from necrosis, but not from apoptosis. Moreover, additional deletion of RelAp65 in macrophages and granuloyctes rescued systemic complications, but did not alter local damage. These data suggest that activation of RelAp65 in acinar cells is a critical event involved in a cascade of mechanisms following acute pancreatitis. Its inactivation increases acinis susceptibility to inflammation-associated apoptosis and necrosis. Therefore RelAp65 in the pancreas protects apoptosis via upregulation of Bcl-xL and necrosis via induction of the acute phase protein pancreatitis-associated-protein I (PAP I) during acute inflammation, but promotes systemic inflammation and lung failure when activated in myeloid cells. Therefore we suggest a double-edged role for NF-kB/Rel during acute pancreatitis.
Proteolytic Cleavage and Inactivation of Human Pancreatic Secretory Trypsin Inhibitor (SPINK1) By Cathepsin B

Thomas Wartmann, Thilo Kaehne, Hans-Ulrich Schulz, Miklos Sahin-Toth, Markus M. Lerch, Walter Halangk

Trypsinogen activation represents an early event in the pathogenesis of acute pancreatitis. We and others have shown that cathepsin B can act as a trypsinogen activating enzyme via limited proteolysis of the trypsinogen activation peptide. Here we have studied whether cathepsin B can also affect the inhibition of activated trypsin by its physiological inhibito SPINKI (PSTI). The human SPINKl gene was cloned into the pET SUMO vector and expressed in the E. coli strain BL21 (DE3). Molecular masses of native and cleaved SPINK were determined by MALDI-TOF mass spectrometry. Cleavage was followed by SDS-PAGE and staining of purified SPINKl protein. Western blotting was used to demonstrate degradation of SPINKl in homogenates of pancreatic tissue incubated with cathepsin B. Activity o SPINKl was measured by inhibition of trypsin in a fluorometric assay. Recombinantly expressed SPINKI had a M.W. of 6,247 Da, and inhibited human cationic as well as anionic trypsin. Incubation of homogenates from human pancreas with cathepsin B resulted in a rapid decrease of the SPINKl protein band on Western blots. This cleavage was insensitive to a benzamidine-type protease inhibitor indicating that SPINKI degradation was not catalyzed by trypsin generated from trypsinogen activation by cathepsin B. Rather, SDS-PAGE demonstrated that the incubation of SPINKI with human cathepsin B led to rapid degradation of the SPINKI protein band. MALDI-TOF mass spectrometry of SPINK1 incubated with cathepsin B at pH 5.5 demonstrated a reduction of the $6.25 \mathrm{kDa}$ peak. The intermediate appearance of peaks at 4.99 and $4.03 \mathrm{kDa}$ indicated cleavage sites at positions R67-Q68 and C32-Y33 of SPINK1. Our results show that cathepsin B can affect trypsinogen activation via two independent mechanisms in pancreatic acinar cells: i) as shown previously via limited proteolysis of the trypsinogen activation peptide and ii) as shown here via inactivation of the physiological trypsin inhibitor SPINKl. The latter of the two mechanisms has the potential to be of much greater consequence since it would promote, unlike the first mechanism, trypsinogen autoactivation in a cascade-like manner.

410

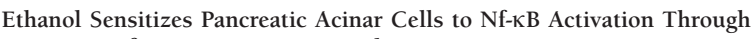
Activation of Protein Kinase C Epsilon

Akihiko Satoh, Anna S. Gukovskaya, Joseph R. Reeve, Ilya Gukovsky, Stephen J. Pandol

Background \& Aims: Although alcohol abuse is the most common cause of pancreatitis, the mechanisms of alcohol's effects on pancreas are poorly understood. Previously we demonstrated that both in vitro and in vivo, ethanol treatment augments the cholecystokinin8 (CCK) induced activation of the transcription factor NF-KB, a key signaling system mediating the inflammatory response of pancreatitis. We further determined that protein kinase $\mathrm{C}$ (PKC) novel isoforms $\delta$ and $\varepsilon$ are involved in NF-KB activation by CCK in pancreatic acinar cells. The aim of the present study was to determine the role for individual PKC isoforms in the sensitizing effect of ethanol on CCK-induced NF-אB activation in pancreatic acinar cells. Methods: Dispersed rat pancreatic acini were treated for $3 \mathrm{~h}$ with and withou $100 \mathrm{mM}$ ethanol (EtOH), and then stimulated with CCK for $30 \mathrm{~min}$. We determined PKC activation and translocation by isoform-specific kinase assays and by measuring the levels of PKC isoforms in membrane and cytosolic fractions with Western blot. NF-KB binding activity was measured by electrophoretic mobility shift assay. To evaluate the roles of individual PKC isoforms in NF-KB activation, we applied PKC isoform-specific peptide inhibitors and activator. Results: As we showed before, NF- $\mathrm{KB}$ activation by a supraphysiologic concentration of CCK $(100 \mathrm{nM})$ was mediated in acinar cells by activation of novel $\mathrm{PKC}$ isoforms, $\delta$ and $\varepsilon$. We found that a physiologic dose of CCK $(0.1 \mathrm{nM})$, which did no activate NF-KB, only activated PKC- $\delta$ but not other PKC isoforms. Acini stimulation with $0.1 \mathrm{nM}$ CCK in combination with a specific peptide activator of PKC- $\varepsilon$ resulted in NF-KB activation, although PKC- $\varepsilon$ activator alone had no effect on NF- $\mathrm{\kappa B}$. These data indicate that activation of both PKC- $\delta$ and PKC- $\varepsilon$ is required for the CCK-induced NF-KB activation in pancreatic acinar cells. We found that ethanol alone activated PKC- $\varepsilon$ in acinar cells without affecting other PKC isoforms. Ethanol by itself did not cause NF- $\mathrm{\kappa B}$ activation; however combined stimulation of acini with ethanol and $0.1 \mathrm{nM}$ CCK resulted in activation of NF$\kappa \mathrm{B}$, as well as of both PKC- $\delta$ and PKC- $\varepsilon$. The NF- $\mathrm{KB}$ activation induced by the combined treatment with ethanol and $0.1 \mathrm{nM} \mathrm{CCK}$ was inhibited by specific inhibitors of either PKC $\delta$ or PKC- $\varepsilon$. Conclusion: Taken together, the results demonstrate that activation of both PKC- $\delta$ and PKC- $\varepsilon$ is required for NF- $\kappa B$ activation. They suggest that ethanol enhances the CCK-induced NF- $\mathrm{KB}$ activation at least in part through activation of PKC- $\varepsilon$.

\section{1}

Lack of the Chemokine Receptor Ccr5 Exacerbates Acute Pancreatitis in Mice Christophe Moreno, Charles Nicaise, Thierry Gustot, Eric Quertinmont, Nathalie Nagy, Hubert Louis, Jacques Deviere

Background: Acute pancreatitis (AP) is an inflammatory disease involving the production of different cytokines and chemokines, and is characterized by leukocyte infiltration. CCR5 is a chemokine receptor for the CC chemokines CCL3/MIP- $1 \alpha$, CCL4/MIP- $1 \beta$ and CCL5/ RANTES. Aims: To investigate the expression of CCR5 ligands and the role of CCR5 in experimental AP in mice. Methods: AP was induced by hourly intraperitoneal injections of caerulein $(50 \mu \mathrm{g} / \mathrm{kg})$ in CCR5-deficient (CCR5-/-) or WT mice. Anti-mouse CC chemokines (CCL2/MCP-1, CCL3/MIP-1 $\alpha$, CCL4/MIP-1 $\beta$ and CCL5/RANTES) monoclonal antibodies were administered 30 minutes before starting caerulein injections. Severity of AP was determined by measuring serum amylases, lipases and IL-6 levels, and by histopathological changes analysis, 6 and 10 hours after starting caerulein injection. Pancreatic gene expression of CCR5 ligands during experimental AP was determined by RT-PCR. Serum levels of CC chemokines and IL-6 were measured by ELISA. Results: Induction of AP resulted in an early significant increase of pancreatic CCL3/MIP-1 $\alpha$ and CCL4/MIP-1 $\beta$ mRNA expression, while CCL5/RANTES mRNA expression occurred later. CCR5-/- mice develop a more severe

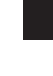


pancreatic injury in comparison with WT mice, as assessed by a more pronounced increase in serum amylases, lipases and IL-6 levels and by more severe pancreatic edema, inflammatory infiltrates and necrosis. CCR5-/- mice also exhibited increased production of CCL2/MCP-1 and CCL4/MIP-1 $\beta$. In vivo neutralization of CCL2/MCP-1 reduced pancreatic damage during AP in CCR5-/- mice, suggesting that increased production of CCL2/MCP-1 is partially responsible for the exacerbation of AP observed in CCR5-/- mice. Conclusions: Lack of the chemokine receptor CCR5 exacerbates experimental acute pancreatitis and leads to increased levels of CC chemokines. This suggests that CCR5 expression can modulate severity of AP.

\section{3}

Influence of Polymorphisms in the NOD1/CARD4 and NOD2/CARD15 Genes On the Clinical Outcome of H.pylori Infection

Philip C. Rosenstiel, Stephan Hellmig, Jochen Hampe, Wolfgang Fischbach, Hany Sahly, Ralph Lucius, Dana Philpott, Stefan Schreiber

Background:Host immune response influences the clinical outcome of H.pylori infection leading to ulcer disease, gastric carcinoma and MALT-lymphoma. A genetic risk profile for gastric cancer has been identified, but genetic susceptibility to develop MALT-lymphoma is still unclear. We investigated the role of NOD1 and NOD2 as intracellular recognition molecules for pathogen-associated molecules in H.pylori infection in vitro and analysed the influence of single nucleotide polymorphisms on susceptibility to ulcer disease and MALTlymphoma. Methods: The influence of NOD1 and NOD2 expression on H.pylori-induced NF-KB activation was assessed using luciferase reporter gene assays. Expression of NOD1 and NOD2 protein in the gastric mucosa was investigated using Western Blot of isolated gastric epithelial cells and by immunohistochemistry of biopsy material. 534 healthy blood donors, $440 \mathrm{H}$.pylori infected patients with chronic gastritis and gastric ulcer and 153 patients with gastric MALT-lymphoma were genotyped for NOD1 and NOD2 polymorphisms by Taqman technology. Associations were tested in a case-control setting. Results: Expression of NOD1 and NOD2 significantly sensitized HEK293 cells to H. pylori- induced NF-KB activation in a cagPAI-dependent manner. In cells carrying the Crohn-associated NOD2 variant R702W the NF-KB response was significantly diminished. NOD1/NOD2 expression levels were induced in the gastric epithelium in H.pylori-positive patients. No mutations were found to be associated with gastritis or gastric ulcer development. However, the R702W mutation in the NOD2/CARD15 gene was significantly associated with gastric lymphoma. Carrier of the rare allele $\mathrm{T}$ had a more than doubled risk to develop lymphoma than controls (OR: 2.4, 95CI: 1.2-4.6; p< 0.044). Conclusions: H.pylori-induced upregulation of NOD 1 and NOD2 in vivo may play a critical role in the recognition of this common pathogen. A missense mutation in the leucine-rich region of CARD15 is associated with gastric lymphoma.

\section{4}

Global Analysis of the Human Gastric Epithelial Transcriptome Altered By $H$. Pylori Eradication in Vivo

Murray B. Resnick, Edmond Sabo, Sung Soo Kim, Young S. Cho, Patricia Meitner, Hyunk K. Kim, Rose Tavares, Steven F. Moss

Analysis of gastric epithelial genes regulated by $H$. pylori (HP) is important in the elucidation of HP molecular pathogenesis. The transcriptional profile of gastric epithelial cell lines cocultured with laboratory HP strains has been investigated previously, as has the global gene expression of whole gastric mucosa in vivo. We aimed to overcome limitations of both prior types of study by determining the effects of HP eradication in vivo on the transcriptional profile of purified gastric epithelial cells extracted from intact human tissue samples (excluding contaminating stromal and inflammatory cells that add noise to gene expression signals), and using each patient as their own control. Methods: Laser capture microdissection (Arcturus) was employed to extract mRNA from formalin-fixed, paraffin-embedded gastric antral foveolar epithelial cells from 12 patients with peptic ulcer disease, before and 1 month after HP eradication. mRNA was reverse transcribed and applied onto Affymetrix cDNA microarray chips customized for mRNA from paraffin and formalin-exposed tissue. Differentially expressed genes were statistically identified, annotated with GeneMapp and MAPPFinder bioinformatics tools and validated by real-time PCR. Results: After removing the concordantly absent microarray signals, 13,817 transcripts decreased and 9,680 increased following HP eradication. Applying cutoff criteria ( $\mathrm{p}<0.02$, fold-change threshold 2.5 ) reduced the sample to 112 significantly differentially expressed genes. Multiple gene function categories were represented including immune response, transcriptional regulation, signal transduction, cell cycle regulation, apoptosis, cell adhesion, growth factors, metabolism, ion channels and structural genes. So far PCR has confirmed results for 10 selected transcripts examined. For specific examples of highly differentially regulated genes see table. Conclusions: This is the first report of the transcriptome of purified gastric antral epithelial cells from HP-infected patients. Studying paired biopsy samples from the same patients before and after HP eradication has confirmed some previous studies while highlighting novel candidate genes involved in HP pathogenesis.

\begin{tabular}{|c|c|c|c|}
\hline Increased by HP & Fold change & Decreased by HP & Fold change \\
\hline NOS 2A & +3.6 & Prothymosin alpha & -6.6 \\
\hline Beta 4 Defensin & +3.3 & Secretoglobulin & -3.3 \\
\hline Histone deacetylase 6 & +2.7 & Somatostatin & -3.3 \\
\hline Survivin & +3.0 & IGF BP 6 & -3.2 \\
\hline Interleukin-8 & +12.8 & Calcyclin & -3.0 \\
\hline Chemokine ligands (gro) 2,3 & $+3.2,+3.9$ & GDDR (=TFIZ1) & -7.1 \\
\hline Chemokine receptors 7,8 & $+2.5,+3.1$ & Gastrokine & -2.5 \\
\hline REG 3 alpha & +6.0 & Osteonectin & -3.0 \\
\hline
\end{tabular}

415

Helicobacter Pylori Induces Gastric Epithelial Cell Invasion in a Cag Pai Dependent Manner

Maria J. Oliveira, Ana C. Costa, Lara Henriques, Gianpaolo Suriano, John Atherton, Jose C. Machado, Raquel Seruca, Marc Mareel, Ancy Leroy, Ceu Figueiredo

Numerous studies have shown that $H$. pylori is able to interact with gastric epithelial cells, activating signaling pathways, modifying host cellular functions, and inducing cell phenotype important for carcinogenesis. One of the less explored cell phenotypes induced by H. pylori is cellular invasion, and little is known about the mechanisms involved in this process. Our aim was to investigate the role of $H$. pylori on gastric epithelial cancer cell invasion, and the mechanisms underlying this process. We also examined whether there were differences between strains in their ability to stimulate cell invasion, especially to assess the role of cag pathogenicity island (cag PAI), CagA, and VacA in cell invasion. We found that H. pylori induced in vitro AGS cell invasion in two well established invasion assays, collagen type I gels and Matrigel filters. H. pylori mediated cell invasion was blocked by the c-Met receptor inhibitor NK4, and by silencing c-Met expression with small interference RNA. Supernatants of cells cultured with $H$. pylori showed increased MMP-2 and MMP-9 activity, which was also suppressed by silencing the c-Met receptor. Studies with different $H$. pylori strains revealed that cell invasion, c-Met tyrosine phosphorylation, and increased MMP-2 and MMP9 activity, were all dependent on the presence of an intact cag PAI. Our findings suggest that $H$. pylori strains with an intact cag PAI activate c-Met and induce high levels of MMP2 and MMP-9 activity, possibly increasing extracellular matrix degradation and leading to subsequent invasion of cancer cells.

416

Spermine Oxidase As the Link Between H. Pylori Caga and Gastric Carcinogenesis

Rupesh Chaturvedi, Mohammad Asim, Francoise I. Bussiere, Kshipra Singh, Robert A Casero, Richard M. Peek, Keith T. Wilson

Background: We have reported that oxidation of polyamines, specifically spermine, by the enzyme spermine oxidase [(SMO) also known as polyamine oxidase 1$]$ is induced by $H$. pylori $(\mathrm{Hp})$ in gastric epithelial cancer cell lines and in the epithelium of gastritis tissues. This causes $\mathrm{H}_{2} \mathrm{O}_{2}$ generation, and the resulting oxidative stress causes apoptosis at MOI 200 and DNA damage at MOI 800. A rodent-adapted strain of Hp, 7.13, has been reported to cause gastric cancer in gerbils, while its parental strain, B128 does not; this has been related to increased translocation of CagA into gastric epithelial cells with 7.13. Our Aim was to determine if the effects of this carcinogenic strain of $\mathrm{Hp}$ are mediated by SMO Methods: We utilized conditionally immortalized mouse gastric epithelial cells (MGECs) for these studies. Cells were exposed to live Hp strains B128, 7.13, and 7.13 cagA- isogenic mutant at MOI 200 and the following were assessed: SMO mRNA by real-time PCR, intracellular $\mathrm{H}_{2} \mathrm{O}_{2}$ levels by flow cytometry using $\mathrm{CM}-\mathrm{H}_{2}$ DCFDA dye, and apoptosis by flow cytometry using annexin V/PI staining. DNA damage was studied in MGECs stimulated with Hp at MOI 200-800, by measuring FITC-8-oxoguanosine binding using flow cytometry. Results: Hp 7.13 induced significantly more SMO mRNA expression than B128, and this induction was prevented by cagA deletion (Table 1). Similarly, 7.13 induced more $\mathrm{H}_{2} \mathrm{O}_{2}$ generation, apoptosis, and DNA damage than B128, and these responses were markedly attenuated with the cagA- strain of 7.13 (Table 1). Notably, significant DNA damage was detected even at MOI of 200 when 7.13 was used. Consistent with the high, low, and absent levels of SMO induced by strains 7.13, B128, and 7.13 cagA-, respectively (Table 1), transfection of MGECs with SMO small interfering RNA inhibited apoptosis induced by 7.13 by $80 \%$, but with B128 stimulation apoptosis was only inhibited by $50 \%$, and with 7.13 cagA- there was no inhibition. Conclusions: The increased levels of apoptosis and DNA damage induced by a carcinogenic strain of $H$. pylori appear to be mediated by CagA-dependent SMO expression and associated $\mathrm{H}_{2} \mathrm{O}_{2}$ generation. Polyamine oxidation may be an important cause of CagAinduced dysregulation of gastric epithelial cell integrity and risk for neoplastic transformation. Table 1

\begin{tabular}{|c|c|c|c|c|}
\hline & Control & B128 & 7.13 & $7.13 \mathrm{cagA}-$ \\
\hline SMO mRNA Levels/Control & $1.0 \pm 0.2$ & $2.9 \pm 0.6$ & $7.7 \pm 1.5^{* *+\dagger}$ & $0.9 \pm 0.2 \S \S$ \\
\hline $\mathrm{H}_{2} \mathrm{O}_{2}$ (RFU) & $319 \pm 63$ & $346 \pm 34$ & $600 \pm 86^{* \dagger}$ & $327 \pm 23 \S$ \\
\hline Apoptosis (\%) & $3.4 \pm 0.5$ & $16.5 \pm 1.5^{* * *}$ & $32.1 \pm 2.9^{* * * \dagger}$ & $16.8 \pm 2.3 * * \S \S$ \\
\hline DNA Damage (RFU) & $4235 \pm 295$ & $7259 \pm 357^{* *}$ & $8470 \pm 210^{* *}$ & $5949 \pm 349 * \S \S$ \\
\hline
\end{tabular}

${ }^{*} p<0.05,{ }^{* *} p<0.01$ vs. control; $\uparrow p<0.05, \uparrow \uparrow p<0.01$ vs. B128; $\S p<0.05, \S \S p<0.01$ vs. 7.13 . 
Innate Immune Response Gene Polymorphisms and Their Role in H. PyloriInduced Gastric Cancer

Georgina L. Hold, Malcolm G. Smith, Mairi H. McLean, Susan Berry, Kenneth E. McColl, Ashley Mowat, Emad M. El-Omar

Background: The nature of the immune response to $H$. pylori infection is determined initially by the innate immune system. This response is orchestrated by a variety of pattern-recognition receptors, most notably Toll-like receptors (TLRs). The family of human TLRs currently comprises 11 members, with at least 5 TLRs namely TLR2, 4, 5, 9 and 11 involved in recognising bacterially derived pathogen-associated molecular patterns. The majority of these TLRs and other innate immune response genes have been shown to contain functional single nucleotide polymorphisms. However, other than TLR4, there has been little data on innate immune response genes in the context of $H$. pylori pathogenesis. Aim: We examined whether SNPs in TLR2 (TLR2 Arg753Gln), TLR5 (TLR5 ${ }^{392}$ Stop), TLR9 (TLR9-1237 T/C) and CD14 (CD14-159 C/T, which is part of the TLR4 signalling complex) influenced the risk of developing gastric atrophy and hypochlorhydria (ATR/HC), the most important precursors of gastric cancer. Subjects \& Methods: We used PCR-RFLP and 5' nuclease assays to genotype the 4 SNP's in 149 healthy gastric cancer relatives (GCR) and 100 population controls. The GCR had detailed assessment of gastric morphology and physiology and were divided on the basis of these measurements into three groups: (1) H. pylori-infected subjects with ATR/HC (2) H. pylori-infected subjects without these abnormalities (No ATR/HC), and (3) H. pylori negative subjects with normal physiology and morphology. Results: An association between carriage of TLR9-1237T/C and CD14-159C/T SNPs and presence of hypochlorhydria and gastric atrophy was identified. Compared to infected subjects who did not develop ATR/HC, the odds ratio for $H$. pylori-induced ATR and HC in subjects with the TLR9-1237T/C was 3.9 (95\% confidence interval 1.7 - 8.6) and CD14-159 C/T was 3.8 (95\% confidence interval $1.4-9.8$ ). Although the functional consequences of the TLR9 promoter polymorphism are unknown, in silico analysis indicates that the SNP disrupts a putative NF-KB binding site. In contrast, the CD14 polymorphism is functional and affects binding of the promoter GC box and also Sp3 proteins. No association was detected between functional polymorphisms in TLR2 and 5 and the presence of premalignant gastric changes. Conclusions: Our results indicate that carriage of the TLR9-1237T/C and CD14-159 C/T mutations predispose $H$. pylori infected subjects to a severe phenotype characterised by hypochlorhydria and gastric atrophy, the two most important precursor lesions of GC. These novel findings call for further evaluation of innate immune gene polymorphisms as important host genetic factors in $H$. pylori-induced gastric cancer

\section{8}

Chemokine Receptors Regulate Intestinal Dendritic Cell Subspecifications in Mucosal Defense

Jan Hendrik Niess, Rosa Maria Salazar-Gonzalez, Xiubin Gu, Mike Pazos, Beth A.

McCormick, Ifor R. Williams, Stephen J. McSorley, Hans-Christian Reinecker

Aim and Methods: Dendritic cells (DCs) play an important role in innate and adaptive mucosal immune responses to intestinal entero-invasive pathogens. To define the intestinal DC subsets responsible for the recognition of intestinal pathogens, we utilized mice in which one or both copies of the gene encoding the chemokine receptors CCR6 or CX3CRl were replaced with a Green Fluorescent Protein (GFP) reporter. The distribution of DC subsets and the interaction of DC subsets with pathogen-specific CD4+ T cells in living intestinal tissues was assessed by $3 \mathrm{D}$ reconstructive confocal microscopy. $\mathrm{T}$ cell activation by $\mathrm{DC}$ subsets was determined by the analysis of CD25 and CD69 expression on T cells after adoptive transfer of congenic Salmonella-specific CD4+ T cells (SM1) during S. typhimurium infection. Results: An extensive CX3CR1+ myeloid-derived mucosal DC system populates the lamina propria of the intestine and Peyer's patches (PPs). Lamina propria DCs depend on CX3CRl to form transepithelial dendrites, which enables the luminal sampling and transport of commensal and pathogenic microbiota to mesenteric lymphnodes (MLN). In contrast, CCR6+ DCs are confined to PPs and absent from the lamina propria of the small intestine. CCR6+ DC were recruited into the dome regions of PPs in response to invasion of the follicle associated epithelium (FAE) by entero-invasive pathogens. Twelve hours after oral S. typhimurium infection CCR6+ DCs formed aggregates with pathogen-specific T cells in PPs leading to the rapid activation and expansion of Salmonella-specific T cells. CCR6 deficient DCs were unable to enter the dome regions of infected PPs and consequently failed to initiate pathogen-specific T cell activation resulting in enhanced susceptibility to oral S. typhimurium infection. Conclusion: PPs contain a DC subset, which can be distinguished from lamina propria DCs by CCR6 expression. CCR6-dependent regulation of this PP specific DC subset is responsible for localized T cell dependent defense activation against enteroinvasive pathogen

\section{9}

Isolated Lymphoid Follicles Contain a Heterogenous T-Lymphocyte Population and Support the Development of IL-10 Producing Cd4+ T-Lymphocytes Keely G. McDonald, Jacquelyn S. McDonough, Caihong Wang, Rodney Newberry

Background: Isolated lymphoid follicles (ILFs) are inducible organized intestinal lymphoid structures that function as inductive sites for mucosal immune responses. The role these structures play in mediating beneficial or harmful intestinal immune responses is unclear. To better understand the function of ILFs within the mucosal immune system, we examined phenotypic and functional characteristics of T-lymphocytes within ILFs. Methods: Splenic, Peyer's patch (PP), lamina propria, and ILF cellular populations were isolated from 3A9 transgenic mice which express a T-cell receptor specific for a peptide of hen egg-white lysozyme presented in the context of I-AK and from C57BL/6 mice receiving LT $\beta$ R-Ig in utero to block Peyer's patch formation. Cellular populations were examined by flow cytometry. Cytokine production was measured following in vitro culture with antigen using ELISA and ELISPOT assays. Antigen specific T-lymphocyte proliferative responses were examined using CFDA incorporation. Results: ILF T-lymphocytes were predominantly, if not exclusively, TCR $\beta+C D 103$ - and either CD4 or CD8 $\alpha$ single positive. CD4+ T-lymphocytes outnumbered CD8+ T-lymphocytes by a ratio of greater than 2:1. Similar to the spleen and Peyer's patch, 20\% of the CD4+ T-lymphocytes expressed Foxp3, however in contrast to other organs, the majority of the Foxp3+ T-lymphocytes in the ILFs were CD25-. ILF CD4+ T lymphocytes had lower levels of CD62L expression when compared with CD4+ T-lymphocytes from the spleen and Peyer's patch. The majority of ILF T-lymphocytes expressed CCR7, while a minority of the ILF T-lymphocyte population expressed CXCR5. Following in vitro culture with antigen T-lymphocytes from ILFs displayed a proliferative capacity equivalen to that of splenic and PP T-lymphocytes. Following in vitro culture with antigen ILF Tlymphocytes produced less IL-4 and IFN $\gamma$, and more IL-10 when compared with splenic and PP T-lymphocytes. Conclusions: ILFs contain a heterogeneous T-lymphocyte population most consistent with the presence of naive T-lymphocytes that have been recently activated a population of $\mathrm{T}$ follicular helper cells, and a population of Foxp3 expressing regulatory T-lymphocytes. When compared with the spleen and PP, ILFs preferentially support the development of IL-10 producing CD4+ T-lymphocytes. These findings suggest that ILFs propagate homeostatic mucosal immune responses in unchallenged animals.

420

Cdld-Dependent Regulation of Bacterial Colonization in the Intestine Edward E. Nieuwenhuis, Tetsuya Matsumoto, Mark Exley, Ytje Simons-Oosterhuis, Margaretha Lambers, Janneke Samsom, Dicky Lindenbergh, Sean Colgan, Andrew Onderdonk, Richard S. Blumberg

The host mechanisms involved in controlling intestinal colonization with commensal bacteria are poorly understood. The cellular locations of CDld expression in the intestine and the known role of CDld in microbial host defense prompted us to examine the role of CDld in regulating the normal colonization of the intestine. In a murine intestinal colonization model under specific pathogen free or gnotobiotic conditions, established by intragastric application of Pseudomonas aeruginosa or Escherichia Coli, CDld-/- mice exhibited markedly reduced intestinal eradication and enhanced translocation of these microorganisms compared with wild-type mice. Furthermore, treatment of mice with $\alpha$-galactosylceramide, a lipid that activates CDld-restricted $\mathrm{T}$ cells, was associated with rapid clearance and prevention of colonization. When raised in germ-free conditions, CDld-/- mice showed a strongly enhanced colonization rate with various bacteria (E coli, Staph. aureus) in comparison to wild-type mice, particularly at early time points ( $109 \mathrm{CFU}$ vs. $103 \mathrm{CFU}$ at $\mathrm{t}=24 \mathrm{~h}$ for $\mathrm{E}$ coli). The differences in colonization were present throughout the entire intestine, but remained over time ( $\mathrm{t}=4$ days) within the jejunum. The small intestine was identified as the main site of CDld-restricted anti-microbial regulation that was further confirmed by applying a small intestinal overgrowth model. These data indicate a role for CDld in regulating mucosal colonization of the intestine with commensal bacteria.

\section{1}

Subclinical Helicobacter Hepaticus Infection Alters Immune Response to Soluble Luminal Antigen

Megan Mcbee, Onyinye I. Iweala, Cathryn Nagler-Anderson, David B. Schauer

Development of mucosal immunity and ease of delivery make oral vaccines desirable However, oral delivery of soluble antigen can lead to tolerance, rather than immunity, which complicates the design and development of oral vaccines. In order to better understand the relationship between oral delivery of antigen and tolerogenic versus immunogenic responses, we investigated subclinical bacterial infection in the intestinal tract of C57BL/6 mice. Previous studies demonstrated that enteric helminth infection acts as an adjuvant, priming for a Th2 biased response to soluble ovalbumin (OVA), a tolerogenic form of dietary antigen. A comparable Thl-biased adjuvant effect was found to be mediated by infection with enterohepatic Helicobacter species by feeding OVA to C57BL/6 mice infected with Helicobacter hepaticus. An 8-week chronic infection with $H$. hepaticus primed for a systemic IFN- $\gamma$ response to intragastrically administered OVA, even though infection of C57BL/6 mice did not lead to overt intestinal inflammation. H. hepaticus infection also influences systemic $\mathrm{T}$ cell responses at distant sites, as increased T-cell proliferation in response to OVA was detected in draining lymph nodes following subcutaneous immunization. Using quantitative RT-PCR, MHC class II expression in mesenteric lymph nodes (MLN) from mice infected with $H$. hepaticus was found to be 2 to 4 -fold lower than in the MLN of uninfected mice between 6 and 48 hours post-inoculation. The apparent decrease in MHC class II expression may be due to an influx of polymorphonuclear leukocytes early in infection. Dendritic cells isolated from MLN immediately prior to and within hours of H. hepaticus inoculation were analyzed by flow cytometry for co-stimulatory molecule expression. Despite immune activation and adjuvant effects, subclinical $H$. hepaticus infection of C57BL/6 mice did not alter OVA-specific IgG2C or IgGl antibody titers. Thus, the ability of enteric pathogens to influence immunologic responses to a model dietary antigen is common to both Thl and Th2 polarizing infections, and does not appear to depend on the establishment of an inflammatory cell infiltrate at the site of antigen uptake. These findings have implications for understanding the basic mechanism of oral tolerance as well as for possible immunomodulatory consequences of subclinical infections with enterohepatic Helicobacter species.

422

Food Hyposensitization Differs in Milk Proteins Belonging to Different Protein-Families

Franziska Roth-Walter, Cecilia Berin, Stephanie Dahan, Giulia Roda, Xian Yang Yio, Hugh Sampson, Lloyd Mayer

Background: Despite the abundance of protein present in food, most allergens belong to limited number of protein families and the factors that determine allergenicity are not known. We investigated the allergenicity of food allergens belonging to different protein families As model allergens, we used the milk proteins b-lactoglobulin (BLG), a-lactalbumin (ALA) 
and casein (CAS) to compare their allergenicity by oral sensitization. Methods: Allergenicity in vivo was tested by orally sensitizing $\mathrm{C} 3 \mathrm{H} / \mathrm{HeJ}$ mice with $1 \mathrm{mg}$ ALA, BLG or CAS plus cholera toxin as mucosal adjuvant once a week for a six-week period ( $\mathrm{n}=12 \mathrm{mice} /$ group). Subsequently, mice underwent a graded oral food challenge. Anaphylactic symptoms and mean body temperature (MBT - negatively correlating with anaphylaxis severity) were recorded. Plasma was analyzed for histamine and serum for IgE and IgA. Splenocytes were re-stimulated with allergen in vitro and their supernatants collected for analysis of interleukin IL-4, IL-5, IL-10 and IFN- $\gamma$. We compared antigen-uptake using polarized Caco-2 cells in vitro. Antigen-uptake was also compared in vivo by feeding $\mathrm{C} 3 \mathrm{H} / \mathrm{HeJ}$ mice with FITClabeled ALA, BLG and CAS and assessing uptake by microscopy. Results: Allergenicity was clearly different between the 3 allergens, with ALA inducing the most severe anaphylaxis (MBT $33.5^{\circ} \mathrm{C}$ ) at the lowest dose of antigen (median oral dose $10 \mathrm{mg}$ ). BLG next, inducing anaphylaxis (MBT $34.2{ }^{\circ} \mathrm{C}$ ) at a median dose of $20 \mathrm{mg}$. Casein induced mild anaphylaxis $\left(36.3{ }^{\circ} \mathrm{C}\right.$ ) only at doses of $50 \mathrm{mg}$ orally or $50 \mathrm{mg}$ administered systemically. Allergenicity correlated with antigen binding of serum $\operatorname{IgE}(800,200$ and $120 \mathrm{ng} / \mathrm{ml}$ antigen in ALA, BLG, and CAS sensitized mice, respectively) but was not equivalent to immunogenicity however, as CAS induced significantly $(\mathrm{p}<0.05)$ higher levels of IL-5, IL-10, IFN-g, and IgA compared to BLG and ALA. IL-4 was undetectable in all cases. To test if allergenicity was related to uptake across the epithelial barrier, transcytosis studies were performed on polarized Caco-2 cells and in vivo in mice. Transcytosis of BLG was 3-fold higher than ALA or CAS, despite their similar molecular weights (14-25 kDa. In mice, there was abundant uptake of BLG, detectable uptake of ALA, and sparse uptake of CAS into the lamina propria. Conclusions: Food proteins of different families elicit significantly different immune responses when given orally with cholera toxin, with a spectrum of allergenic or immunogenic responses. Factors intrinsic to the protein, not related to uptake, are likely to determine the nature of the immune response in the GI tract.

\section{3}

\section{The Ccr6-Ccl20 Chemokine Axis Is Necessary for Food Allergen-Induced} Diarrhea

Adina K. Knight, Shu Zhang, Sergio Lira, Lloyd Mayer, Hugh A. Sampson, Cecilia Berin

Allergic inflammation of the gastrointestinal tract is associated with a Th2 cytokine profile that supports the development and survival of effector cells including mast cells and IgEproducing plasma cells in the lamina propria. CCR6 is present on B cells, memory T cells, and dendritic cells and its ligand CCL20 (MIP3 $\alpha$ ) is highly expressed by intestinal epithelial cells. We hypothesized that the CCR6-CCL20 chemokine axis is critical for the development of gastrointestinal allergic symptoms. Methods: Balb/c mice or CCR6 -/- mice (Balb/c background) were sensitized to ovalbumin (OVA/alum i.p., day 0, 14), followed by feedings of OVA (50 mg, days $28,30,32,34)$. mRNA was isolated from full-thickness jejunum and real time RT-PCR was performed to assess cytokine and chemokine gene expression. Results: Wild-type mice developed severe acute diarrhea beginning 20-30 min after the 3rd feed, and diarrhea recurred at the next feed (53.9\% with diarrhea after feed $3,82.4 \%$ after feed $4, \mathrm{n}=17)$. CCR6 $-/$ - mice were significantly protected ( $\mathrm{p}=0.001$ by survival curve analysis) from development of allergic diarrhea $(9.5 \%$ after feed $3,34.0 \%$ after feed $4, n=21)$. OVAspecific serum IgE was boosted after feeding in CCR $6+/+$ mice, whereas no boosting of OVA-specific IgE was observed after feeding in CCR6 -/- mice. Expression of the Th2 cytokines IL-4 and IL-10 were significantly upregulated in the lamina propria of wild-type mice after 4 OVA feeds in comparison to unfed OVA-sensitized mice $(p<0.05)$, and this upregulation was significantly suppressed in CCR6-/- mice. The absence of CCR6 also impaired the upregulation of the Th2 chemokine/receptor pair CCLl(TCA-3)/CCR8 in the lamina propria $(\mathrm{p}<0.05)$. Flow cytometric analysis demonstrated that CCR6 was constitutively expressed on B cells and dendritic cells in the MLN, and upregulated on CD4+ T cells after activation in vitro. Therefore the absence of CCR6 could be playing a role in the trafficking of multiple cell types from the MLN to the lamina propria in this model. CCR6 did not influence the initial systemic sensitization, as baseline $\operatorname{IgE}$ and cytokine levels prior to feeding were not significantly different between wild-type and CCR6 knockout mice. Conclusions: The CCL20/CCR6 chemokine/receptor pair is necessary for the full development of allergic diarrhea. Absence of CCR6 inhibited not only antigen-specific IgE and development of a local Th2 cytokine milieu, but also chemoattractants known to recruit Th2 cells, demonstrating that CCR6 may function at an early stage in the generation of allergic inflammation of the gastrointestinal tract.

\section{4}

Arachidonic Acid Metabolites Uniquely Regulate Anion: HCO3 Exchangers in the Chronically Inflamed Rabbit Small Intestine

Steven Coon, Uma Sundaram

Background: There are two anion exchangers in rabbit intestinal villus cell brush border membrane (BBM) both of which are inhibited during chronic intestinal inflammation $\mathrm{Cl}: \mathrm{HCO} 3$ exchange is inhibited secondary to an alteration in the affinity for $\mathrm{Cl}$. In contrast, short chain fatty acid (SCFA):HCO3 exchange is inhibited secondary to a decrease in Vmax. Arachidonic acid metabolites (AAM), known to be elevated in the chronically inflamed intestinal mucosa, may regulate these two anion exchangers. Aims: Determine whether AAM mediate the inhibition of villus cell anion exchangers during chronic enteritis. Methods: A rabbit model of chronic enteritis induced by Emeria magna was utilized. Villus cells were isolated from the intestine by a Ca++ chelation technique. BBM vesicles (BBMV) were prepared from villus cells by $\mathrm{Ca++}$ precipitation and differential centrifugation. Rabbits were treated with arachidonyl trifluoromethyl ketone (ATMK), an inhibitor of Phospholipase A2, to prevent the release of arachidonic acid from plasma membrane phospholipids. Results: ATMK treatment stimulated $\mathrm{Cl}: \mathrm{HCO} 3$ exchange in villus cell BBMV from the normal intestine $(540 \pm 60 \mathrm{pmol} / \mathrm{mg}$ protein $/ 15 \mathrm{sec}$ in control and $938 \pm 89$ in ATMK treated, $\mathrm{p}<0.05, \mathrm{n}=5)$ while SCFA:HCO3 exchange was unaffected. ATMK treatment of rabbits with chronic enteritis alleviated the inhibition of $\mathrm{Cl}: \mathrm{HCO} 3$ exchange $(535 \pm 73 \mathrm{pmol} / \mathrm{mg}$ protein $/ 15 \mathrm{sec}$ in control, $172 \pm 24$ in inflamed, and $607 \pm 53$ in inflamed treated with ATMK, $p<0.01, n=4)$. ATMK treatment also alleviated the inhibition of SCFA:HCO3 exchange in the chronically inflamed intestine $(700 \pm 80 \mathrm{pmol} / \mathrm{mg}$ protein/9 sec in normal, $650 \pm 50$ in normal treated with ATMK,
$100 \pm 30$ in inflamed, and $730 \pm 30$ in inflamed treated with ATMK, $p<0.01, n=4)$. Kinetic studies demonstrated that the mechanism of ATMK mediated stimulation of $\mathrm{Cl}: \mathrm{HCO} 3$ exchange in the normal intestine was secondary to a significant increase in the Vmax without an alteration in the affinity for $\mathrm{Cl}$. During chronic intestinal inflammation, the mechanism of reversal of $\mathrm{Cl}: \mathrm{HCO} 3$ exchange by ATMK treatment was secondary to a restoration in the affinity without a change in Vmax. In contrast, the mechanism of reversal of SCFA:HCO3 exchange by ATMK treatment during chronic enteritis was secondary to a restoration of the Vmax without a change in $\mathrm{Km}$. Conclusions. These data indicate that AAM inhibit $\mathrm{Cl}: \mathrm{HCO} 3$ exchange in the normal intestine while SCFA:HCO3 exchange is not affected. Further, AAM mediate the inhibition of $\mathrm{Cl}: \mathrm{HCO} 3$ and SCFA:HCO3 exchange during chronic enteritis. However the mechanisms of inhibition of $\mathrm{Cl}: \mathrm{HCO} 3$ and SCFA:HCO3 exchange by AAM during chronic intestinal inflammation are uniquely different.

425

Vav Proteins Maintain the Colonic Epithelial Barrier Thaddeus S. Stappenbeck, John Liu, Wojciech Swat, Jason Mills

The mammalian intestine performs its primary function, the absorption of water and nutrients, while maintaining a physiochemical barrier directed against luminal microbes. The physical barrier is a single layer of polarized, terminally differentiated epithelial cells at the luminal surface that undergo continuous replacement from a population of proliferative stem cells in underlying crypts. The apical cytoplasm of the surface epithelial cells is highly enriched in cytoskeletal elements that are linked to cell-cell junctions. This transcellular network has long been assumed to be critical for maintaining barrier function. However, testing the components of this barrier in adult animals is difficult as cytoskeletal genes are typically expressed in many tissues and function in cell division. Here we show that expression of Vavs, a protein family of Rho GTPase guanine nucleotide exchange factors that are capable of functionally regulating the cytoskeleton is restricted to the surface barrier cells in a limited region of the intestine. The surface epithelial cells of mice deficient for all three Vavs (Vav1/2/3null) contain an abnormal apical microtubule cytoskeleton that is associated with abnormalities in cell size and cell polarity. Surprisingly, these defects occurred in an absence of any perceptible defects in the actin cytoskeleton and its associated structures (microvilli, apical junctions). Also, the underlying colonic crypts of Vavl/2/3/null mice are normal in appearance, proliferation rate and are not surrounded by excess inflammatory cells in the lamina propria. The surface epithelial defects, in combination with an adaptive immunodeficiency of Vav1/2/3null mice, predispose to mucosal injury of the intestinal lining in the form of numerous focal ulcers. As demonstration that ulcer formation requires an absence of Vav expression in both the epithelium and hematopoietic cellular compartments, bone marrow chimeras of irradiated Vav1/2/3null mice with bone marrow transplants from wild type mice contain abnormal polarity of the surface colonic epithelium but do not develop ulcers. We anticipate these findings will stimulate further discovery into the interactions between the actin and microtubule cytoskeletal networks in epithelial barrier cells and will lead to the recognition of new molecular targets for diseases such as inflammatory bowel disease where gut injury is a fundamental feature of the pathology. This work further demonstrates the interplay of the immune system and surface epithelial cells is required to maintain barrier function.

\section{6}

Distribution of and Alterations in Na-Glutamine Co-Transporters SN1 and SN2 During Chronic Intestinal Inflammation in Rabbits Jamil Talukder, Ramesh Kekuda, Vadivel Ganapathy, Steven Coon, Uma Sundaram

Background: Glutamine, the primary metabolic fuel for enterocytes, is absorbed by Naglutamine co-transporters in the small intestine. System N1 and N2 are two candidates for Na-glutamine co-transport in the rabbit small intestine. However, the distribution of SNl and SN2 in the normal small intestine is not known. Further, how SN1 and SN2 may be altered during chronic intestinal inflammation is also not known. Aim: Determine the distribution of SN1 and SN2 along the crypt-villus axis of the normal rabbit small intestine and alterations during chronic enteritis. Methods: A rabbit model of inflammatory bowel disease induced by Eimeria magna was utilized. Villus and crypt cells were isolated from the rabbit intestine by a Ca++ chelation technique. Uptake studies were done by rapid filtration technique using $3 \mathrm{H}$-glutamine. Total RNA was isolated from enterocytes using Trizol reagent. Real-time quantitative PCR was performed to determine the levels of SN1 and SN2 message using total RNA. Results: Both SN1 and SN2 mRNA are present in villus and crypt cells. SN2 message is about 2-fold higher in crypt than in villus cells while preliminary studies indicate that SN1 message is greater in villus than in crypt cells. Nadependent glutamine uptake is found in both villus and crypt cells in normal intestine, albeit higher in villus as compared to crypt cells $(1551+101 \mathrm{nmol} / \mathrm{mg}$ protein $/ 2 \mathrm{~min}$ in villus and $507 \pm 43 \mathrm{nmol} / \mathrm{mg}$ protein $/ 2 \mathrm{~min}$ in crypt cells, $\mathrm{n}=3, \mathrm{p}<0.01$ ). Na-dependent glutamine uptake was decreased in villus cells from chronically inflamed intestine $(557 \pm 44 \mathrm{nmol} / \mathrm{mg}$ protein/2 min in inflamed villus cells, $\mathrm{n}=3, \mathrm{p}<0.01$ ). The message for $\mathrm{SNl}$ decreased about 3 -fold in villus cells from inflamed rabbit small intestine. In contrast, Na-dependent glutamine uptake increased in crypt cells from chronically inflamed rabbit small intestine (1238 93 $\mathrm{nmol} / \mathrm{mg}$ protein $/ 2 \mathrm{~min}$ in inflamed crypt cells, $\mathrm{n}=3, \mathrm{p}<0.01$ ). Message for SN2 increased about 3-fold in crypt cells from chronically inflamed rabbit small intestine. Conclusions: These data indicate that $\mathrm{SNl}$ is most likely the Na-glutamine transporter in villus cells and it is inhibited during chronic enteritis. In contrast, SN2 is most likely the Na-glutamine cotransporter in crypt cells and it is stimulated during chronic enteritis. Thus, these results indicate that $\mathrm{SNl}$ and $\mathrm{SN} 2$ are differentially distributed in the normal rabbit small intestine. Further, SN1 and SN2 are uniquely regulated in the chronically inflamed rabbit small intestine. 
Epithelial Calcium-Sensing Receptor Activation By Eosinophil Granule Protein Analogs Stimulates Collagen Matrix Contraction

Peter Ngo, R. John MacLeod, Punitha Ramalingam, Sean P. Colgan, Glenn T. Furuta

Background: Eosinophils reside directly adjacent to basolateral surfaces of epithelial cells in eosinophilic gastrointestinal diseases and inflammatory bowel diseases. Recent evidence demonstrates that highly cationic molecules, similar to eosinophil granule proteins, bind the epithelial extracellular calcium-sensing receptor $(\mathrm{CaR})$. We hypothesized that $\mathrm{CaR}$ ligation by eosinophil granule proteins leads to collagen matrix contraction. Aim: To define the molecular mechanisms of $\mathrm{CaR}$ activation by eosinophil granule proteins and its influence on collagen contraction Methods: Caco2 intestinal epithelial cells, AML14.3D10 eosinophils, HEK293 cells not expressing CaR (HEK-wt) or HEK cells stably over-expressing CaR (HEK$\mathrm{CaR})$ were suspended in type 1 collagen gels $(1 \mathrm{mg} / \mathrm{ml})$ and stimulated with the analog of eosinophil major basic protein, poly-L-arginine $(\mathrm{PA}, 1 \mu \mathrm{M})$. Contraction was measured as percent reduction of initial gel surface area. Western blot and electrochemiluminescence (Meso Scale Diagnostics) was performed to quantify phosphorylated ERK 1/2 (pERK) and total ERK 1/2 on lysates of Caco2, Hela, HEK-wt and HEK-CaR cells stimulated with PA, $\mathrm{Ca} 2+(20 \mathrm{mM})$ or Epidermal Growth Factor (EGF 100ng/ml). To assess loss of CaR function, Hela cells were transduced with CaR dominant negative adenovirus (R185Q) or control virus. Results: $\mathrm{Caco} 2$ cells co-cultured with 3D10 eosinophils stimulated greater contraction $(51.5 \pm 1.0 \%)$ than $\mathrm{Caco} 2$ cells alone $(34.3 \pm 2.2 \%)$ or $3 \mathrm{D} 10$ cells alone $(0.7 \pm 3.6 \%)$. Similarly, $\mathrm{Caco} 2$ populated collagen gels treated with $\mathrm{PA}$ augmented contraction compared to untreated Caco2 gels $(53.4 \pm 6.1$ vs. $44.0 \pm 2.5, \mathrm{p}<0.02)$. Mechanistic studies revealed that PA induced pERK in Caco2 and Hela cells within 5 min. Transduction of Hela cells with CaR dominant negative virus attenuated this response compared to control virus. HEK-CaR cells stimulated with PA or $\mathrm{Ca} 2+$ resulted in 9.0 and 11.1 fold increases in pERK respectively, compared to only 1.4 and 2.0 fold in HEK-wt. Response to EGF (CaR independent pERK activation) was similar between HEK-CaR and HEK-wt cells (12.4 and 9.0 fold increase respectively). Similar results were obtained in transiently $\mathrm{CaR}$ transfected HEK cells. HEK-CaR populated gels treated with PA displayed augmented contraction compared to control $(41.2 \% \pm 2.9$ vs. $29.0 \% \pm 3.9, \mathrm{p}<0.001$ ), while PA treated HEK-wt gels displayed no difference in contraction $(28.2 \% \pm 1.9$ vs. $30.3 \% \pm 0.9)$. Conclusion: These results indicate that $\mathrm{CaR}$ activation by eosinophil granule protein analogs stimulates collagen matrix contraction. We speculate that eosinophils activate $\mathrm{CaR}$ to produce the dysmotility and fibrosis seen in intestinal inflammatory conditions.

\section{8}

PI3-Kinase Regulates Immune-Mediated Induction of Intestinal Goblet Cell Gene Expression

Mei-Lun Wang, Sue A. Keilbaugh, Xi C. He, Rao Dirisina, Tanesha Cash-Mason, Terrence A. Barrett, Linheng Li, Gary D. Wu

Background and Aims: Th2 adaptive immune responses, typified by mammalian host responses to intestinal nematode infection, lead to intestinal goblet cell hyperplasia and the induction of goblet cell-specific genes, including TFF3 as well as the $\alpha$ and $\beta$ members of the RELM family of secreted proteins. Although it has been established that TFF3 and RELM $\alpha$ are regulated by STAT 6 through an IL $4 R \alpha$-dependent pathway the immune-mediated mechanisms by which RELM $\beta$ is activated are unknown. In the current study, we use both in vivo and in vitro model systems to elucidate the signaling pathways responsible for the regulation of RELM $\beta$ gene expression. Methods and Results: Goblet cell morphology and RELM $\beta$ expression were studied in mice with conditional inactivation of PTEN, a tumor suppressor gene that inactivates PI3-kinase. Whereas RELM $\beta$ was essentially undetectable in the small intestine of wild-type mice, inactivation of PTEN led to a dramatic induction of RELM $\beta$ expression concurrent with the development of goblet cell hypertrophy. Consistent with this observation, we noted a significant decrease in RELM $\beta$ expression in the LS174T intestinal goblet cell line following treatment with the PI3-kinase inhibitor LY-294002. To investigate the divergent signaling pathways by which RELM $\alpha$ and $\beta$ may be regulated in vivo, we studied their expression in both classic Th2 and generalized T-cell mediated models of intestinal inflammation. Wild-type BALB/c, STAT6-/-, and IL4R $\alpha-/$ - mice were either infected with the intestinal nematode Heligmosomoides polygyrus or received intraperitoneal injections of anti-CD3 monoclonal antibody. As predicted, RELM $\alpha$ induction occurred in an IL4R $\alpha$ and STAT6-dependent fashion in both model systems. In contrast, RELM $\beta$ expression was induced through IL4R $\alpha$ and STAT6-independent pathways. Conclusions: Using RELM $\beta$ as a model, we demonstrate that PI3-kinase pathways are important regulators of goblet cell-specific gene expression. This observation is consistent with the role of PI3kinase signaling in the pathogenesis of goblet cell hyperplasia observed in many juvenile polyposis syndromes as well as recent evidence implicating the role of RELM $\beta$ in insulindependent signaling pathways.

\section{9}

Inability to Regulate Ileal Bile Acid Transporters in a Novel Mouse Model of Necrotizing Enterocolitis

Melissa Halpern, Nazzareno Ballatori, Sarah Doelle, Tara Saunders, Dania Molla Hosseini, Anna Stagner, Jessica Clark, Bohuslav Dvorak

Background: Necrotizing enterocolitis (NEC) is the most common gastrointestinal disease of prematurely born infants. In a neonatal rat model of NEC, we have shown that the accumulation of bile acids (BA) in the distal ileum contributes to the development of NEC, and may be a result of an inability to regulate BA homeostasis. We have recently developed a neonatal mouse model of NEC to further examine the pathophysiology of this disease, but the regulation of ileal BA transporters in this model are unknown. Aims: To examine the regulation of ileal BA transporters in a newly developed neonatal mouse model of NEC. Methods: Newborn, never suckled C57BL/6 mice were fed cow's milk-based formula every 4 hours for 3 days and exposed to asphyxia and cold stress twice daily to develop NEC (NEC, $n=24)$. Dam-fed littermates, also exposed to asphyxia and cold stress, were used as controls ( $\mathrm{DF}, \mathrm{n}=12$ ). Using this method, approximately $70 \%$ of the formula-fed group develop ileal damage consistent with NEC, while none of the DF animals develop disease Ileal tissue from each animal was used for immunohistology and Western Blot techniques to evaluate the apical sodium-dependent bile acid transporter (Asbt), ileal bile acid bindin protein (Ibabp) and the heteromeric organic solute transporter Ost $\alpha$-Ost $\beta$. Results: Asbt, Ost $\alpha$ and Ost $\beta$ were up-regulated in the distal ileum of neonatal mice with NEC compared to DF controls, but were significantly lower than in adults. In contrast, Ibabp was significantly lower in mice with NEC compared to DF mice. Further, animals in the formula fed group that did not develop NEC had significantly greater Ibabp protein levels than those that developed disease. Conclusions: The inability to adequately up-regulate ileal BA transporter may lead to accumulation of BA in the terminal ileum and may contribute to the developmen of experimental NEC in the neonatal mouse model. Supported by NIH Grants HD-39657 and DK-067954

436

Enteroendocrine Precursor Cells Develop Small Intestinal Tumors in Response to Wnt Signaling and Lose This Response During Further Differentiation Yang Wang, Maryann Giel-Moloney, Andrew B. Leiter

Wnt signaling is required to maintain cell proliferation in intestinal crypts. Mutations resulting in increased Wnt signaling are associated with the development of intestinal neoplasms. It has not been established which intestinal cell lineages differentiating from pluripotent cells are capable of responding to abnormal Wnt signals. Two basic helix loop helix (bHLH) transcription factors, Neurogenin 3 (Ngn3) and BETA2/NeuroD, play an important role in cell fate determination and differentiation of enteroendocrine cells. Ngn3 is transiently expressed in the earliest enteroendocrine cell progenitors and required for the specification of intestinal endocrine cells. BETA2 is a factor downstream of Ngn3, which coordinate enteroendocrine cell differentiation at later stage. In the present study, we sought to determine when during differentiation intestinal endocrine cell precursors are capable of responding to Wnt signaling. We used site specific recombination to conditionally activate Wnt signaling in enteroendocrine progenitor cells at either Ngn3 or BETA2 expressing stage. We crossed transgenic mice expressing Cre recombinase under control of bacterial artificial chromosomes (BAC) containing the Ngn3 or BETA2 genes with Catnb mice. Catnb mice have a conditiona $\beta$-catenin allele with loxP sites on either side of exon3, which encodes all GSK3 $\beta$ phosphorylation sites in $\beta$-catenin. Deletion of this exon prevents $\beta$-catenin from forming complexes with APC that target it for degradation. As a result, the stabilized $\beta$-catenin localizes to the nucleus to function as a coactivator of Wnt target genes. Ngn3-Cre X Catnb mice were born with the expected frequency and appeared normal until 4 weeks of age. After 4 weeks, adenomatous polyps appeared with increasing frequency in the small intestine with the eventual development of widespread small intestinal adenocarcinoma, resulting in death by the age of 6-9 months. Tumor cells frequently expressed serotonin and PGP9.5, confirming their neuroendocrine origin, as well as a number of Wnt target genes. In contrast, Wnt signaling initiated at a later stage of differentiation in BETA2-Cre X Catnb mice, failed to induce neoplasms or to alter intestinal differentiation. Our results are the first to demonstrate that precursor cells committed to a specific intestinal cell lineage continue to respond to Wnt signals much like more immature and pluripotent cells in the crypts. In addition we have defined a specific stage later in endocrine differentiation where this response is lost.

437

Effect of Helicobacter Pylori Infection in Experimental Gastric Carcinogenesis of Mongolian Gerbils

Koji Nozaki, Xueyuan Cao, Harunari Tanaka, Yuzuru Ikehara, Tetsuya Tsukamoto, Hayao Nakanishi, Nobuyuki Shimizu, Sachiyo Nomura, Hirokazu Yamaguchi, Yoshio Yamaoka, Michio Kaminishi, Masae Tatematsu

Helicobacter pylori ( $\mathrm{Hp})$ infection facilitates gastric disorders and gastric cancer development. As for in vivo experimental animal models, Mongolian gerbils can be used to study the virulence of $\mathrm{Hp}$ infection because of their high susceptibility to infection with $\mathrm{Hp}$, and the resultant gastritis and gastric cancers. To explore the causal link between Hp infection and gastric carcinogenesis, we studied following experiments, I: Eradication after long-term $\mathrm{Hp}$ infection model; II: Hp infection and $10 \% \mathrm{w} / \mathrm{w}$ high-salt diet administration model; III Eradication model at early, middle, or late period after Hp infection; and IV: Establishmen of gastric cancer cell lines from Mongolian gerbil. I: Long-term Hp infection frequently developed proliferative glands with dysplastic change of constituent cells, and finally with intestinal phenotype with Paneth cells. After eradication, proliferative glands were reduced and gastric lesions in mucosa improved with few remnants of the former injury. Thes proliferative glands are induced solely by $\mathrm{Hp}$ infection and are related to severe gastritis and intestinal metaplasia. II: The interaction of $\mathrm{Hp}$ infection and a high salt diet on gastric carcinogenesis was investigated using gerbils with a combination of $\mathrm{N}$-methyl-N-nitrosourea, inoculation with $\mathrm{Hp}$, and a $10 \%$ high salt diet. A high-salt diet significantly enhanced the effects of $\mathrm{Hp}$ infection on gastric carcinogenesis, and these two factors acted synergistically to promote the development of gastric cancers. III: The timing effect of eradication in gastric carcinogenesis (eradication at the early, middle or late period) was investigated using gerbils with a combination of $\mathrm{Hp}$-infection, eradication and $\mathrm{N}$-methyl-N-nitrosourea-treatment. The tumor incidence at week 75 was significantly higher in animals treated with $\mathrm{Hp}$, and was related to the period of inflammatory status. Eradication at an early stage of inflammation was effective in preventing Hp-enhanced gastric carcinogenesis. IV: Gastric cancer cell lines with different invasive activities, designated $\mathrm{MGCl}$ and $\mathrm{MGC2}$, were established from one original cancer tissue induced in the Mongolian gerbil. The derived cells can be stably attached with $\mathrm{Hp}$, and easily transplanted to the nude mice. Rapid phosphorylation of IkB Erkl/2, and AKT of these cells was observed by IL-1 beta stimulation, and luciferase reporter gene assay revealed the Hp-dependent NF-kB transcriptional activation. Long-term Hp infection strongly enhances gastric carcinogenesis, and eradication of $\mathrm{Hp}$ at an early timing resulted in lower incidence of gastric carcinogenesis, showing that eradication of $\mathrm{Hp}$ can be useful for prevention of gastric cancer 
Aspirin for the Prevention of Recurrent Colorectal Adenomas - Results of the UKCAP Trial

Richard F. Logan, Kenneth R. Muir, Matthew J. Grainge, Nicholas C. Armitage, Vic C. Shepherd, Ukcap Trial Group And

Background: Many observational studies have found regular aspirin use is associated with a reduced risk of colorectal $(C R)$ cancer and 2 randomised trials have shown that aspirin reduces risk of recurrent $\mathrm{CR}$ adenomas, although results were not wholly consistent (Baron et al., N Engl J Med, 2003; Benamouzig et al., Gastroenterology, 2003). We report the preliminary aspirin results of a large factorial trial of aspirin (enteric $300 \mathrm{mg} /$ day) and folate supplements in patients under surveillance for recurrent CR adenomas. Methods: A doubleblind, placebo-controlled randomised trial was carried out in 10 centres ( 9 in the UK and 1 in Denmark). Patients who had one or more adenomas $(>0.5 \mathrm{~cm})$ removed by colonoscopy in the 6 months prior to enrolment were randomised to receive either aspirin (enteric 300 $\mathrm{mg}$ /day) or placebo. All participants were followed-up at intervals of 4 months to assess compliance, with a second colonoscopy arranged for 3 years after the date of trial entry. The primary outcome measure was a histologically confirmed CR adenoma or cancer either at the end examination or during the course of the trial. Results: 945 patients were recruited into the study, of which 853 ( 434 receiving aspirin and 419 placebo) underwent a second colonoscopy and were included in an intention to treat analysis. $56.9 \%$ of randomised patients were male and the mean age at recruitment was 57.8 years. Full compliance with trial medication was reported by 700 patients. In total, 101 (23.3\%) patients receiving aspirin had a recurrent adenoma compared with 120 (28.6\%) patients who received placebo (Relative Risk $=0.81 ; 95 \% \mathrm{CI}, 0.65$ to 1.02). 103 advanced CR adenomas were observed (on the basis of villous/tubulovillous features, size $>1 \mathrm{~cm}$, evidence of severe dysplasia, or CR cancer diagnosis); 39 (9.0\%) of these were in the aspirin group and 64 (15.3\%) in the placebo group (Relative Risk $=0.59 ; 95 \% \mathrm{CI}, 0.40$ to 0.86 ). This advanced group included 11 patients with CR cancers ( 3 aspirin and 8 placebo). Conclusion: Aspirin use ( $300 \mathrm{mg} /$ day) resulted in a 19\% reduction in risk of any colorectal adenoma recurrence and a marked, statistically significant, $41 \%$ reduction in risk of advanced adenoma development.

\section{0}

Association of a Complex Insertion/Deletion Polymorphism of NOD1/CARD4 with Susceptibility to Inflammatory Bowel Disease in the Scottish Population Johan van Limbergen, Charles W. Lees, Elaine R. Nimmo, Richard K. Russell, Hazel E. Drummond, David C. Wilson, Ian D. Arnott, Jack Satsangi

Background \& Aims NOD1/CARD4 and NOD2/CARD15 are intracellular pattern-recognition receptors that influence Toll-like receptor responses and NF-kB activationl. The NODl/ CARD4 gene lies within the 7p14.3 IBD locus2. Recently, the deletion allele NOD1+32656*1 of a complex deletion*1/insertion*2 polymorphism was found to be associated with IBD and CD3. On haplotype analysis, the presence of the NOD 1+32656*2 allele was shown to have a strong protective effect on IBD susceptibility. The aim of this study was to assess the influence of NOD $1+32656$ on IBD susceptibility in a Caucasian population with a low NOD2/CARD15 contribution. Patients and Methods 991 patients with IBD (476 CD, 491 UC, 24 IC) and 318 healthy controls were genotyped for NODl+32656A/C using TaqMan. We performed a case-control analysis using $\chi 2$ (Yates). The Population Attributable Risk (PAR) for the NOD $1+32656 * 1$ allele was calculated. The effect of NOD 1+32656 genotype on age of onset was assessed using the Mann-Whitney test. Results By case-control analysis, the NOD $1+32656 * 1$ deletion allele was associated with susceptibility to IBD (allelic frequency $74 \%$ v $70 \%$ p=0.04 OR $1.23 \mathrm{CI} 1.01-1.49$ and $* 1 * 1$ genotype frequency $54 \%$ v $46 \% \mathrm{p}=$ 0.02 OR $1.35 \mathrm{CI} 1.05-1.74)$ and $\mathrm{CD}(* 1 * 1$ genotype frequency $54 \% \mathrm{v} 46 \% \mathrm{p}=0.03 \mathrm{OR}$ $1.37 \mathrm{CI} 1.03-1.82$ ), but not UC. These data suggest the possibility that NOD $1+32656 * 1$ is a recessive risk allele. Indeed, the $\mathrm{NOD} 1+32656 * 2$ insertion allele has a protective effect on IBD susceptibility (allelic frequency $25 \%$ v $30 \% \mathrm{p}=0.03$ OR $0.82 \mathrm{CI} 0.67-0.99$ ). Carriage of the NODl+32656*2 insertion allele is protective against IBD (45\% v 53\% p $=0.01$ OR $0.74 \mathrm{CI} 0.58-0.96)$ and $\mathrm{CD}(45 \%$ v $53 \% \mathrm{p}=0.02$ OR $0.73 \mathrm{CI} 0.55-0.97)$. The heterozygous genotype is associated with protection against IBD and CD $(39 \%$ v $46 \% \mathrm{p}=0.03$ OR 0.76 CI $0.59-0.98$ and $39 \%$ v $46 \% \mathrm{p}=0.03$ OR $0.75 \mathrm{CI} 0.56-0.99$, respectively). The PAR to IBD of NODl+32656*1 is $13.6 \%$ to IBD and $13.9 \%$ to CD cf. $11 \%$ for NOD2/CARD154. In contrast with a previous report, no effect was seen of NOD $1+32656$ genotype on age of diagnosis of IBD, CD or UC. Conclusion In our population, NODl+32656 variants are weak determinants of susceptibility to IBD and $\mathrm{CD}$, but not UC. We were unable to replicate the previously reported association with age at diagnosis. Our data suggest that the $\mathrm{NOD} 1+32656 * 1$ deletion allele is a recessive risk allele for IBD, although we can not exclude a direct protective effect of the NOD $1+32656 * 2$ insertion allele. References 1. van Heel et al. Eur J Immunol 2005;35:2471-2476 2. Satsangi et al. Nat Genet 1996;14(2):199-202 3. McGovern et al. Hum Mol Genet 2005;14(10):1245-50 4. Arnott et al. Genes Immun $2004 ; 5: 417-25$

442

NOD2 Variants Are Significantly Associated with Sero-Reactivity to Microbial Antigens in Crohn's Disease (CD)

Shane M. Devlin, Andrew Ippoliti, Huiving Yang, Konstantinos Papadakis, Eric Vasiliauskas, Maria T. Abreu, Gil Y. Melmed, Phillip Fleshner, Kent D. Taylor, Ling Mei, Carol J. Landers, Jerome I. Rotter, Stephan R. Targan

Background: Recently a locus on chromosome 3 of the C3Bir mouse (Cdcsl) was identified to confer severe colitis associated with a decrease in innate immune function and an increase in adaptive T-cell responses to commensal bacterial antigens, a link between innate and adaptive immunity. We have previously shown in $C D$ that the number and magnitude of antibody responses to microbial antigens are associated with a more severe disease course. Since functional mutations in the innate immune gene NOD2 are associated with CD, the aim of our study was to determine the relationship of NOD2 variants to the magnitude of $\mathrm{CD}$ associated antibody responses to microbial antigens. Methods: Serologic and genetic data were examined in $748 \mathrm{CD}$ patients. ASCA and antibodies to I2, OmpC, and CBir were measured by ELISA. Antibody sums (AS) and overall quartile sums (QS) (ranging from 416) of levels for all four antibodies were calculated as previously described (Mow et al Gastro 2004:126:414). Genotyping (TaqmanMGB) was performed for 3 CD-associated variants of the NOD2 gene, R702W, G908R, and 1007fs. Results: 1] ASCA was detected in 51\%, antiI 2 in $58 \%$, anti-OmpC in $38 \%$, and anti-CBirl in $56 \%$. 2] 250 of 748 CD patients (33.4\%) had at least one NOD2 variant;206 (27.5\%) having one and 44 (5.9\%) having two. 3] NOD2 variants were present at increasing frequency in patients with reactivity to increasing numbers of antigens. Variants were present in those with $0,1,2,3$ or 4 positive antibodies in $24 \%, 25 \%, 36 \%, 36 \%$, and $46 \%$, respectively (p for trend $<0.0001$ ). 4 ] NOD2 variants were present at increasing frequency in patients with increasing cumulative quantitative immune response as reflected by individual QS ( $\mathrm{p}$ for trend $<0.0001$ ). QS were also clustered into four groups by increasing cumulative quantitative immune response (group $1=4-6$, group $2=7-9$, group $3=10-13$, and group $4=14-16$ ). The frequency of having at least one NOD2 variant in each of the four groups was $22 \%, 29 \%, 35 \%$, and $49 \%$ in groups $1,2,3$, and 4, respectively ( $\mathrm{p}$ for trend $<0.0001$ ). 5] The mean AS (number of positive antibodies) and QS was higher for patients with at least one NOD2 variant versus those with no variant $(2.28+/-1.21 \& 10.70+/-2.99$ vs. $1.90+/-1.23 \& 9.68+/-2.97$, respectively. $\mathrm{p}<0.0001)$. Conclusions: Individuals with $C D$ who have variants of the NOD2 gene as a marker of abnormal innate immunity are more likely to have an increased adaptive immune response to multiple enteric organisms. These data provide a potential pathophysiologic link to similar findings in rodent mucosal inflammation. This should allow disease relevant crossover genetic and functional studies.

\section{3}

Detection of Muramyl Dipeptide Sensing Pathway Defects in Patients with Crohn's Disease

David A. van Heel, Karen A. Hunt, Subrata Ghosh, Kathy King, Simon Gabe, Christopher Mathew, Alastair Forbes, Raymond J. Playford

Background \& Aims: Crohn's disease is strongly associated with double mutations in NOD2/ CARD15. Three common mutations (Arg702Trp, Gly908Arg, Leul007fs) impair innate immune responses to bacterial muramyl dipeptide. Rare NOD2 variants occur, but it is difficult to both identify them and assess their functional effect. We assessed the true frequency of defective muramyl dipeptide sensing in Crohn's disease and developed a rapid diagnostic assay. Methods: An ex-vivo assay was established and validated based on muramyl dipeptide stimulation of peripheral blood mononuclear cell cytokine production. Muramyl dipeptide induced enhancement of IL-8 secretion, and synergistic increase in lipopolysaccharide induced IL-1 $\beta$ secretion were studied. Assay results were compared with NOD2 genotype status (three common mutations and rare variants) in 91 individuals including a prospective cohort of 49 Crohn's disease patients. Results: The assay was highly sensitive and specific for detection of profound defects in muramyl dipeptide sensing caused by double NOD2 mutations (IL-8 P=0.0002; IL-1 $\beta \mathrm{P}=0.0002$ ). Disease state, active inflammation or concurrent use of immunosuppressive medication did not influence results. Healthy NOD2 heterozygotes had modest impairment of muramyl dipeptide induced IL-8 secretion ( $\mathrm{p}=0.003)$. Only 1 of 7 Crohn's disease patients with both a common mutation and a rare variant had a profound muramyl dipeptide sensing defect. Conclusions: Profound defects in muramyl dipeptide sensing were found in $10 \%$ of Crohn's disease patients. Defects were due exclusively to inherited mutations in NOD2. This is the first demonstration of impaired function in NOD2 heterozygotes. The ex-vivo assay has multiple potential applications as a clinical diagnostic tool to distinguish patients with muramyl dipeptide sensing defects, and for research investigation.

444

Genes Regulating the Expression of Antibody to CBIR1 Flagellin in Humans Are Located Within a Syntenic Region to the Major Mouse Colitogenic Locus Cdcs 1

Kent D. Taylor, Huiying Yang, Ling Mei, Carol J. Landers, Yingzi Cong, Kostas A. Papadakis, Eric A. Vasiliauskas, Andrew Ippolitti, Charles O. Elson, Jerome I. Rotter Stephan R. Targan

Background: CBirl flagellin has been identified as an antigen capable of inducing severe colitis in the C3H/HeJBir substrain (Lodes et al 2004, J Clinical Investigation 113: 1296). Recently, a $7.6 \mathrm{Mb}$ region from the C3Bir strain spanning the NfkbI gene on mouse chromosome 3 has been identified as a major modifier of colitis in IL-10 deficient mice (Beckwith et al 2005, Gastroenterology, 2005: 129; 1473). Bone marrow derived cells from C3Bir.il10-/- mice had diminished responses to the TLR5 ligand, CBirl flagellin, but showed increased CD4 T-cell responsiveness to this antigen. We have recently shown that antibodies to CBirl flagellin are also expressed in the serum of a subset of patients with severe Crohns disease (CD) as characterized by small-bowel, internal-penetrating, and fibrostenosing disease features. Aim: To investigate the genetic determinants of CBirl response in human $C D$ by linkage analysis of CBirl expression as a quantitative trait (QTL). Methods: Measurement of CBirl serum levels in 968 individuals in 80 CD-only (no UC affecteds) and 57 Mixed (both CD and UC affected) families was performed by ELISA and a 10cM genome scan was performed with microsatellite markers. QTL analysis was performed using the SOLAR program. Results: The heritability of expression of serum antibodies to CBirl antigen in this study was 0.19 ( $(\mathrm{p}=0.0005)$. A peak multipoint LOD score for CBirl expression was observed for human chromosome 4 at $91 \mathrm{cM}$ (LOD=1.82, marker ATA2A03). This LOD increased with bivariate linkage analysis of expression of both ASCA and CBirl antibodies (LOD= 1.99). This region is syntenic to the mouse chromosome 3 Cdcsl region containing the NfkbI gene. Conclusions: This observation supports the role of genetic variation that determines response to CBirl flagellin in this region in both mouse and human and raises the possibility that genetic variation in the NF-kappa-B gene creates an abnormal innate response to enteric bacterial antigens that results in severe $C D$. For $C D$, these studies will allow crossover studies of human and mouse models to determine whether the same genes regulate similar responses in these species. 
The Characterisation of Distinct Intestinal Gene Expression Profiles in Inflammatory Bowel Disease (IBD) By Microarray Analysis

Colin L. Noble, Alex Abbas, Jennine Cornelius, Ian D. Penman, Ian D. Arnott, Zora Modrusan, Toy Karen, Jack Satsangi, Lauri Diehl

Introduction: Gene expression technology using microarray allows a comprehensive picture of gene expression at the tissue and cellular level. The aim of this study was to investigate differential gene expression in endoscopic colonic biopsies of a well phenotyped cohort of IBD patients and controls. Patients and methods: 67 Ulcerative colitis (UC), 53 Crohn's disease (CD), and 31 normal and 'inflamed non-IBD' controls (HC) were studied. Paired endoscopic biopsies were taken from specific anatomical locations for RNA extraction and histology. Patients symptoms were scored at the time of endoscopy (simple clinical colitis activity index for UC, Harvey-Bradshaw score for CD), medication was noted and phenotypic data were collected by patient questionnaire and case note review. Whole genome arrays were used to analyze 230 biopsies using the agilent platform. Conformation of results was carried out by RTPCR and immunohistochemistry. Results: Comparing 95 UC biopsies to $67 \mathrm{HC}$ biopsies, 1099 genes were up-regulated and 1203 genes were down regulated in the UC samples (1x10-35<p<0.001, Fold change (FC) - 1.8- +5.0). When these genes were grouped by molecular function there were a preponderance of genes associated with deaminase activity ( $p=0.0007$ OR 7.3 ) and protein binding ( $p=0.01$ OR 1.3). When histologically inflamed (18) and non-inflamed (19) UC sigmoid biopsies were examined, 1438 genes were up regulated and 1393 genes were down regulated $(2.0 \times 10-41<\mathrm{p}<0.001$, FC, -11.2+8.4 ). Comparing acutely inflamed (11), chronically inflamed (8) and non-inflamed (11) sigmoid colon UC biopsies, a greater number of differentially expressed genes were associated with organic acid transporter activity ( $\mathrm{p}=0.006$ OR 4.3) and had protein functions in the extracellular region ( $\mathrm{p}=2.5 \times 10-5$ OR 2.3). A subset of 2309 genes were up regulated and 1860 genes were down regulated in CD biopsies (69) when compared to HC biopsies (67)(1x10-45<p $<0.001$, FC $-2.3-+7.2)$. Differentially regulated genes were associated with immune function when biological processes were considered ( $\mathrm{p}=5.1 \times 10-9$ OR 1.72 ), hydrogen ion transporter activity when molecular function was considered $(\mathrm{p}=1.7 \times 10-9$ OR 2.15), ribosomal ( $p=1.2 \times 10-8$ OR 7.7$)$ and mitochondrial $(p=1.8 \times 10-8$ OR 1.79$)$ locations of function. Conclusions: In this large, rigorously phenotyped IBD microarray study, a number of novel gene families have been identified and implicated in the pathogenesis of $\mathrm{CD}$ and $\mathrm{UC}$. These findings have led to the identification of novel potential therapeutic targets

\section{6}

Safety and Efficacy of Pravastatin 80mg/Day in 320 Hypercholesterolemic Patients with Compensated Chronic Liver Disease (CLD): Results of a Prospective, Randomized, Double-Blind, Placebo-Controlled Study James H. Lewis, Mary Jean Fusco, Jeffrey R. Medoff, Mary Ellen Mortensen, Steven Zweig HMG-CoA reductase inhibitors are associated with mild elevations in liver-associated enzymes (LAEs). In pts in clinical trials of cholesterol reduction using pravastatin (Prava)with ALT values up to $1.5 \mathrm{X}$ ULN at entry, no significant hepatic events were reported (Circulation 2002;105:2341), and retrospective studies suggest the safe use of statins in pts with elevated LAEs (Gastroenterology 2004;126:1287). Herein we present the first prospective study of a statin in hypercholesterolemic pts with known CLD. METHODS: Screened adult pts with $>6 \mathrm{mo}$ of well-compensated CLD of any type and LDL cholesterol $>100 \mathrm{mg}$ were eligible for statin treatment. The primary efficacy endpoint was the percentage (\%) chnage from baseline to wk 12 of LDL-C and the primary safety endpoint was the $\%$ of pts with at least one ALT $>2 \mathrm{X}$ for those with a normal baseline or a doubling of ALT for those with elevated baseline ALT (up to 5X)within 36wk of once daily dosing with Prava 80mg. Several CLD were represented, the most common being NAFLD (64\%)and chronic hepatitis C (24\%). Other CLD included PBC, PSC, Wilson dis., autoimmune hepatitis, and hemochromatosis. IRB approval and permission was obtained for all subjects. Enrollment was in a ratio of 1:1 Prava vs placebo (PBO). RESULTS:Prava pts had a clinically significant reduction in total cholesterol (20\%), LDL-C (31\%) and TGs $(9 \%)$ compared to PBO by wk $12(3 \%, 3 \%$, and $6 \%$ increase respectively; $\mathrm{p}<0.0001$ for all 3 comparisons). The $\%$ fall in LDL from baseline at wk 12 for Prava-treated pts was similar for those with mild (100-130mg/dL), moderate(130$160 \mathrm{mg} / \mathrm{dL})$ and severe $\mathrm{LDL}(>160 \mathrm{mg} / \mathrm{dL})$ at baseline $(29.1 \%, 32.3 \%$ and $33 \%$ respectively). Among 160 pts on Prava, only $12(7.5 \%)$ met the ALT safety endpoint vs $20(12.5 \%)$ of 160 $\mathrm{PBO}$ recipients $(\mathrm{p}=\mathrm{NS})$. There was no statistically difference in ALT endpoints at any of the cumulative visits from wk 1 to 36 vs $\mathrm{PBO}$, and the \# pts having sustained ALT elevations for at least 2 consequtive wks was comparable ( $5 \%$ vs $7 \% \mathrm{PBO}, \mathrm{p}=\mathrm{NS}$ ). The time to onset of elevated ALT was also not different between groups (median for both $>36 \mathrm{wk}$ log rank $\mathrm{p}=\mathrm{NS}$ ). The cumulative \# of events in the Prava group regardless of ALT at baseline also was not different from PBO. One pt with NAFLD in the PBO group had transient bilirubin $>2 \mathrm{X}$ ULN w/o ALT elevation that resolved. CONCLUSIONS:This prospective RCT of Prava $80 \mathrm{mg} /$ day was not associated with any excess of ALT or bilirubin elevations compared to $\mathrm{PBO}$ in a variety of CLDs in hypercholesterolemic pts over $36 \mathrm{wks}$ and produced a significant reduction in total cholesterol, LDL-C and TGs, thereby supporting its safety and efficacy in this setting

447

Phosphatidylcholine Transfer Protein (PC-TP) Is Regulated By Peroxisome Proliferator Activated Receptor $\alpha($ PPAR $\alpha)$ and Participates in Ppar $\alpha$-Mediated Hepatic Triglyceride Metabolism

Keishi Kanno, Erez F. Scapa, Wen-Jun Wang, Gabriela Orasanu, Jorge Plutzky, David Cohen

Background: We have demonstrated a regulatory role for PC-TP in hepatic triglyceride metabolism. We have also shown that the Pctp gene promoter contains a binding site for PPAR $\alpha$, a liver enriched ligand-activated transcription factor that regulates hepatic triglyceride homeostasis. Aim: To investigate regulation of PC-TP by PPAR $\alpha$ in vivo and to determine a role for PC-TP in PPAR $\alpha$-mediated regulation of hepatic triglyceride metabolism. Methods To elucidate regulation of PC-TP by PPAR $\alpha$, eight week old male wild type and Pctp-/C57BL/6J ( $\mathrm{n}=6 /$ group) and FVB/NJ ( $\mathrm{n}=8 /$ group) mice, as well as PPAR $\alpha-/-(\mathrm{n}=6)$ and wild type $(n=4) 129 S 3 / S v I m J$ mice were fed $7 d$ chow or chow supplemented with $0.2 \%$ fenofibrate, a known PPARa ligand. Hepatic PC-TP expression was analyzed by quantitative real-time PCR, as well as Northern and Western blot analysis. To identify potential downstream hepatic targets of PC-TP, gene expression profiling was performed using eight week male Pctp-/- and wild type FVB/NJ mice fed 7d fenofibrate-supplemented chow. Total RNA was extracted from liver and used to hybridize Affymetrix Mouse Genome Array GeneChips (one chip per mouse, $n=6 /$ group), each containing 14,000 mouse genes. Differentially regulated genes were identified using Bayesian Analysis of Differential Gene Expression (BADGE) software (Adv Dent Res. 17:104, 2003). Metabolic pathways regulated by PC-TP were identified using Ingenuity Pathways Analysis software (www.ingenuity.com). Results: In chow-fed wild type mice, hepatic PC-TP protein, as well as mRNA levels, were lower in FVB/NJ compared with the C57BL/6J strain. Fenofibrate feeding increased PC-TP mRNA expression and protein levels in both genetic strains. Quantitative real-time PCR revealed a 22 -fold increase of PC-TP mRNA in FVB/NJ mice compared with a 3-fold increase in C57BL 6J mice, and a 6-fold increase in wild type 129S3/SvImJ mice. Fenofibrate did not induce hepatic PC-TP mRNA in PPAR $\alpha$-/- mice, indicating that this response was PPAR $\alpha$-dependent. Based on the potent induction of PC-TP by fenofibrate, FVB/NJ mice were utilized for gene expression analysis. Consistent with a key role in PPAR $\alpha$-mediated triglyceride metabolism, BADGE followed by pathway analysis identified that both fatty acid metabolism (14 genes) and biosynthesis ( 5 genes) were highly regulated by PC-TP expression. Conclusions: PCTP is regulated by PPAR $\alpha$ and plays a key role in coordinating PPAR $\alpha$-mediated hepatic triglyceride metabolism. Because it binds phosphatidylcholines containing polyunsaturated acyl chains, we speculate that PC-TP may provide a link between triglyceride metabolism and hepatocellular membrane composition.

\section{8}

Probiotics Reduce Inflammation in Nonalcoholic Steatohepatitis in An Animal Model of Sedentary Lifestyle

Stephanie H. Abrams, Chantal A. Rivera, Anita Mantri, James Versalovic, Milton J.

Finegold, C. Wayne Smith

Nonalcoholic steatohepatitis (NASH), which includes steatosis; inflammation; and fibrosis, currently affects up to $3 \%$ of the world's population. We have previously reported tha male rodents exposed to hindlimb unloading (HU) have pathology resembling NASH. Thi phenomenon was associated with elevated levels of portal endotoxin and was not found in endotoxin-resistant rodent strains. In a genetic model of NASH, probiotics have been used to modify intestinal flora and have shown a decrease in injury. This study was designed to assess if NASH secondary to sedentary lifestyle (modeled by HU) and a high fat/high sucrose (HF/HS) diet is alleviated by colonization with probiotics. It was hypothesized that, as in other models of steatohepatitis, animals receiving probiotic would have decreased inflammation. To test this hypothesis, male Wistar rats were fed a HF/HS diet (15\% of calories from casein $32 \%$ from corn oil, and $48 \%$ from sucrose) for 3 weeks. Subsequently, half of each group underwent 3 weeks of $\mathrm{HU}$, with the remaining animals housed separately to control for thermoregulation. VSL\#3, a mixture of lyophilized bifidobacteria, lactobacilli, and Streptococcus thermophilus, was given to half of each of the aforementioned groups at a dose of 1.5 X $10^{\wedge} 9$ colonies/rat/day for 5 days. Colonization of stool was confirmed at the end of 5 days, prior to $\mathrm{HU}$, and at the end of 6 weeks. Upon sacrifice, Oil-red O stained liver sections demonstrated that the combination of HF/HS diet and HU caused hepatic fat accumulation and focal necro-inflammatory foci. While treatment with VSL\#3 improved necosis and inflammation, it did not affect steatosis. Consistent with these histological findings, serum ALT levels of VSL\#3 treated HU animals were decreased by more than 3-fold $(\mathrm{p}=0.009)$. Total circulating leukocyte counts were similar among all of the treatment groups; however, circulating neutrophil counts were significantly decreased in VSL\#3, HU animals when compared to control, $\mathrm{HU}$ animals $(\mathrm{p}<0.001)$. As expected, adiponectin levels were significantly increased in the VSL\#3, HU group $(\mathrm{p}=0.02)$, indicating improved insulin resistance in these animals. It was concluded that the combination of high fat diet and sedentary behavior due to HU causes steatosis and inflammation consistent with NASH in male adolescent rats. Consistent with our hypothesis, probiotic treated animals had a decreased inflammatory response.

\section{9}

The Gene Encoding the Nuclear Bile Salt Receptor FXR Is Associated with Cholesterol Gallstone Susceptibility in a Murine Model and in a Mexican Population

Rahel Kress, Jacqueline Rocha, Peter Kovacs, Nahum Mendez-Sanchez, Michael Stumvoll, Joachim Mossner, Henning Wittenburg

Cholesterol gallstone susceptibility includes the combination of predisposing alleles of lithogenic (LITH) genes and environmental factors. Employing quantitative trait locus mapping in a previous intercross of gallstone susceptible PERA/EiJ and gallstone resistant I/Ln] mice, we detected a Lith locus on chromosome 10 named Lith7. The gene encoding the nuclear bile salt receptor FXR (NrIh4) is a positional candidate gene for the Lith7 region. To investigate further an association of Nr1h4 alleles and gallstone susceptibility in mice, we employed real-time PCR to determine mRNA expression levels of Nr1h4 and its downstream genes Cyp7a1 and Cyp8b1 in gallstone susceptible and resistant mice after feeding chow or a cholic acid enriched diet for four weeks. Furthermore, the NrIh4 gene wa sequenced in inbred mouse strains with susceptible and resistant Lith7 alleles. To identify polymorphisms in the orthologous human gene, the 11 exons, the intron-exon boundaries and $1,5 \mathrm{~kb}$ of the 5 ' region of NR1H4 were sequenced in 24 subjects. Subsequently, 80 gallstone carriers and 80 age- and sex matched subjects were genotyped for the polymorphisms detected by sequencing employing the TaqMan method. Significance levels were determined by logistic regression. After feeding chow, the expression of $\mathrm{Nr} 1 \mathrm{~h} 4$ or its downstream genes Cyp $7 a 1$ and Cyp8b1 did not differ between mice carrying the susceptible or resistant allele of the gene. However, the susceptible allele of Nr1h4 contains polymorphisms in the 
coding region that lead to amino acid changes. Following activation of FXR by feeding cholic acid, mice carrying the susceptible $\mathrm{Nr} 1 \mathrm{~h} 4$ allele displayed significantly lower expression levels of Cyp7al and Cyp8b1 compared with animals carrying the wild type allele suggesting an association of impaired cholesterol degradation into bile salts and cholesterol gallstone formation. To translate our findings from inbred mice to men we aimed to identify an association of genetic variation of NR1H4 and gallstone prevalence in a Mexican population. Sequencing detected 7 single nucleotide polymorphisms in the screened population that were all in Hardy-Weinberg equilibrium. Three polymorphisms were associated with cholelithiasis: 5 ' region $-20647 \mathrm{~T}>\mathrm{G}, \mathrm{p}=0,06$, RR 2,38 (95\% confidence interval 0,95 - 5,9); exon3 $1 \mathrm{G}>\mathrm{T}, \mathrm{p}=0,04, \mathrm{RR} 4,0(1,05-15,2)$ and IVS7 $-3 \mathrm{IT}>\mathrm{A}, \mathrm{p}=0,05$, RR 2,11 $(0,99-4,50)$. In conclusion, our results indicate a primary role of FXR in the genetic susceptibility and pathogenesis of cholesterol gallstones. Therefore, the bile salt receptor is an attractive target for the development of new means of prevention and non-surgical management of cholesterol cholelithiasis.

450

Characterisation of the Intrahepatic Immune Response in C5 Bac-Transgenic Mice During Liver Fibrogenesis

Sonja Hillebrandt, Hermann E. Wasmuth, Judith Dahmen, Christian Trautwein, Tilman Sauerbruch, Frank Lammert

Background: BAC transgenesis of the gene for complement factor C5 in C5-deficient FVB/ $\mathrm{N}$ mice leads to increased susceptibility to liver fibrosis and confirms the identity of $\mathrm{C5}$ as the fibrogenic modifier gene identified on chromosome 2 (Hillebrandt et al. Nat Genet 2005:37:835-43). Recently it has been postulated that the fibrosis phenotype in different organs is tightly regulated by the quality of the T-helper (Th)-cell response (Wynn Nat Rev Immunol 2004:4:583-94). The aim of the present study was to characterize the intrahepatic immune regulation related to fibrogenesis in C5 BAC-transgenic mice. Methods: Liver fibrosis in $\mathrm{C} 5 \mathrm{BAC}$-transgenic mice and in the $\mathrm{FVB} / \mathrm{N}(\mathrm{C} 5-/-)$ background was induced by $\mathrm{CCl}_{4}$ challenge for 6 weeks ( $2 \mathrm{ml} / \mathrm{kg} / \mathrm{wk}$ i.p.). For quantification of liver fibrosis, hepatic collagen concentrations and histological stages of fibrosis were determined. The hepatic mRNA expression of regulatory type 1 cytokines IL-12p35, IL-23p19 and IL-27p28 as well as the expression levels of the cytokines IL- 6 and TNF- $\alpha$ were measured by quantitative RT-PCR (Tagman). Results: After challenge with $\mathrm{CCl}_{4}, \mathrm{C} 5 \mathrm{BAC}$-transgenic mice displayed significantly $(P<0.01)$ higher hepatic fibrosis stages $(2.00 \pm 0.10)$ and collagen concentrations $(352 \pm$ $12 \mu \mathrm{g} / \mathrm{g})$ compared to non-transgenic FVB/NJ mice $(1.46 \pm 0.14$ and $227 \pm 10 \mu \mathrm{g} / \mathrm{g}$, respectively). Interestingly, the expression levels of the regulatory cytokines IL-12p35, IL-23p 19 and IL-27p28 were significantly lower in C5-sufficient BAC-transgenic mice in comparison to the $\mathrm{C}$-deficient FVB/N background (all $P<0.05$ ). The reduced expression of these type 1 cytokines was correlated to the severity of liver fibrosis. In contrast, the expression of IL6 and TNF- $\alpha$ did not differ significantly between the strains, indicating that immune activation was not generally suppressed by C5 BAC transgenesis. Conclusions: The reduced expression of regulatory type 1 cytokines of the IL-12 family in fibrosis prone C5-sufficient mice is in line with the paradigm that polarized Th cells regulate organ fibrosis and that a shift from Thl to Th2 cytokine subsets promotes fibrogenesis

451

Efficacy of Leukocyte Esterase Dipstick Test As a Screening Test for Spontaneous Bacterial Peritonitis (SBP)

Worawut Rungsangmanoon, Pradermchai Kongkam, Rungsun Rerknimitr

Introduction: The diagnosis of spontaneous bacterial peritonitis (SBP) is established by a positive ascitic fluid bacterial culture and/or more than 250 cell/mm3 of polymorphonuclear leukocyte (PMN) in ascitic fluid. One of rapid tests for detecting PMN is leukocyte esterase (LE) strip test. Aims: To evaluate efficacy of the LE test as a screening method for SBP. Methods: There were 200 ascitic fluid samples from patients whom SBP was suspected Urine dipstick (Combur10 Test ®M from Roche, Mannheim, Germany) was used as a screening test. And then manual PMN count was referred as a gold standard. Number of PMN more than 250 cells / mm3 by manual count was used as a cut off values. One to three plus LE strip test results were used as a positive result. Results from both tests were compared to determine the efficacy of LE strip test. Results are shown in the table. Results: Prevalence of SBP diagnosed by manual cell count was $21.0 \%$. The sensitivity and specificity of LE test were $88.1 \%$ and $81.0 \%$ respectively. Positive and negative predictive values were $55.2 \%$ and $96.2 \%$ respectively. The likelihood ratio was 4.6 times. Conclusion: LE strip test can be used as a screening test to exclude SBP since it has a high negative predictive value.

\begin{tabular}{|c|c|c|c|}
\hline LE test/ PMN counts & More than or equal to 250 cells / mm3 & Less than 250 cells / mm3 & Total \\
\hline Positive (1-3) & 37 & 30 & 67 \\
\hline Negative (0) & 5 & 128 & 133 \\
\hline Total & 42 & 158 & 200 \\
\hline
\end{tabular}

452

VIP Induces L-Type Calcium Channel Gene Expression in Human Colonic Circular Smooth Muscle Cells: A Novel Role in Regulation of Motility Function

Xuan-Zheng Shi, Barun K. Choudhury, Sheng Yan, Bal M. Mishra, Sushil K. Sarna

Vasoactive Intestinal Polypeptide (VIP) is an established inhibitory neurotransmitter of the NANC neurons. The aim of the present study was to determine whether VIP also mediates gene expression in human colonic circular smooth muscle cells (HCCSMC), and if so what are the molecular mechanisms of this novel function of VIP. Our findings show that VIP induces gene expression of the pore-forming $\alpha_{1 C}$ subunit of L-type $\mathrm{Ca}^{2+}$ channels. Methods:
The experiments were performed on primary cultures of HCCSMC and on freshly isolated human colonic circular smooth muscle strips. Results:Incubation of HCCSMC with $10^{-8}$ to $10^{-6} \mathrm{M}$ VIP for 24 hours concentration-dependently increased $\alpha_{1 \mathrm{c}}$ mRNA and protein levels when compared with medium control. The maximal increase of $\alpha_{1 c}$ mRNA and protein levels at 24 hours were $45 \pm 4 \%$ and $42 \pm 5 \%$, respectively. None of the other neurotransmitters (Acetylcholine, Nitric oxide, ATP, Substance P) at same concentrations produced this effect. VIP $\left(10^{-7} \mathrm{M}\right)$ time-dependently enhanced cellular cAMP and phosphorylated protein kinase A (PKA) VIP also transiently phosphorylated cAMP response element binding (CREB)transcription factor for about one hour. Sequence analyzer identified two variant cAMP response elements, CREl (5'-TGACGTCA-3', -563 to -556 from the transcription start site) and CRE2 (5'-TGACAGCA-3', -176 to -169) on the promoter of human $\alpha_{1 C}$ gene of L-type $\mathrm{Ca}^{2+}$ channels $\left(\mathrm{h} \alpha_{1 \mathrm{c}} \mathrm{lb}\right.$ ). Agarose nucleotide pull down assay showed increased binding of CREB to both response elements in response to $10^{-7} \mathrm{M}$ VIP (CREl>CRE2). The inhibition of CREB by siRNAs blocked VIP-induced binding to CRE1 and CRE2 as well as the increase in expression of $\alpha_{1 C}$. PKA inhibitor H-89, which blocked VIP-induced CREB phosphorylation also inhibited the enhanced gene expression of $\alpha_{1 \mathrm{c}}$. VIP $10^{-7} \mathrm{M}$ increased $\alpha_{1 \mathrm{c}} \mathrm{lb}$ promoter activity by $43 \pm 7 \%$ in HCCSMC. Studies with progressive $5^{\prime}$ deletions of promoter ho $\alpha_{1 \mathrm{C}} \mathrm{lb}$ showed that CRE1 motif, rather than the CRE2 motif, plays a predominant role in regulating VIP-enhanced expression of $\alpha_{1 c}$. Incubation of fresh circular muscle strips for $24 \mathrm{~h}$ with $10^{-7} \mathrm{M}$ VIP enhanced their response to $10^{-6}$ to $10^{-2} \mathrm{M}$ ACh in organ bath, when compared with those incubated with medium only and it also increased the expression of $\alpha_{1 C}$ in tissue. Conclusion: VIP, one of the neurotransmitters of NANC neurons, also regulates gene expression of the pore-forming subunit $\alpha_{1 C}$ of L-type $\mathrm{Ca}^{2+}$ channels that maintains normal human colonic smooth muscle function. This novel function of VIP is PKA-dependent and is mediated by transcription factor CREB.

453

A Novel Innervation of the Crural Diaphragm By the Vagus Richard L. Young, Nicole J. Cooper, L Ashley Blackshaw

The gastroesophageal reflux barrier is secured by both the inner lower oesophageal sphincter (LES) and the external crural diaphragm (CD). During gastroesophageal reflux both transient LES relaxation and CD inhibition occur concomitantly, permitting esophageal common cavity, and acid reflux. Our group has characterised how the LES is controlled by the vagus nerve during reflux events [1] but the neural mechanism that controls relaxation of the $C D$ has not been fully investigated. Recent evidence suggests a novel inhibitory nerve pathway may act on the CD independent of the phrenic nerve [2]. Understanding this pathway and its relation to LES control may provide new insights into the mechanisms of gastroesophageal reflux. The aims of this study were to determine if the vagus provides a motor and/or sensory innervation of the $C D$ in ferrets. METHODS: Restricted volumes of retrograde nerve tracers CTB-FITC and CTB-HRP were injected into the CD, costal diaphragm and LES of 8 ferrets. Brainstem, spinal and vagal tissues were removed after 5 days recovery. RESULTS. CD tracing labeled spinal sensory neurons in dorsal root ganglia and motor neurons in phrenic motor nucleus (C5-7) as expected. In addition we labeled a unique population of vagal sensory (nodose ganglion) and motor neurons (rostral dorsal motor nucleus of vagus). Innervation of the $\mathrm{CD}$ by the vagus was specific for this portion of the diaphragm as tracing from the costal diaphragm did not label vagal structures. Dual-tracing studies from LES and $\mathrm{CD}$ also revealed rare co labeled vagal afferents in the nodose ganglion. CONCLUSIONS: These findings indicate that the $\mathrm{CD}$ is uniquely and specifically innervated by vagal afferents and efferents in the ferret. Moreover, the finding of co-innervation from LES and CD suggests that a single vagal afferent may provide sensory input from both structures. The function and phenotype of vagal afferent and efferent neurons innervating the $C D$ is the subject of ongoing studies in our laboratory. 1. Blackshaw L.A. et al J Auton Nerv Syst 1997 66:179-88 2. Liu J. et al Am J Physiol Gastrointest Liver Physiol 2000 278:G281-8 Supported by: AstraZeneca

\section{4}

Chemical Hypersensitivity in Patients with Functional Dyspepsia Johannes Matiasek, Lorenz Pipal, Johann Hammer

Background: The pathophysiology of functional dyspepsia (FD) is unclear; chemical hypersensitivity might be a causing factor. Capsaicin stimulates the vanilloid receptors (VRI) on afferent neurons, that are involved in chemical nociception in the upper GI tract. Aim: To evaluate the sensitivity for gastric capsaicin in healthy controls and in patients with FD or other GI disorders. Methods: $\mathrm{N}=25$ healthy controls, $\mathrm{n}=30$ patients with functional dyspepsia (FD), $n=17$ with inflammatory bowel disease (IBD), $n=10$ with gastric ulcers or erosions, and $\mathrm{n}=19$ with other GI symptoms swallowed a capsule after an overnight fast containing $0.75 \mathrm{mg}$ capsaicin. Subjects filled out a graded questionnaire evaluating the severity of 9 different upper abdominal symptoms before and 30 minutes after capsule ingestion; an aggregate symptom score was calculated by adding all symptom scores. Data are expressed as mean \pm SEM; p-values $<0.05$ were considered significant. Results: Healthy controls had an aggregate symptom score of $6.3 \pm 2.9$; the median was 6.5 (25\% confidence interval: 4 ). The $75 \%$ confidence interval was 9 and was considered as the upper limit of normal. The FD group had a mean symptom score of $12.2 \pm 5.6$ ( $\mathrm{p}<0.001$ vs. controls). The aggregate symptom scores in the IBD group was $3.9 \pm 4.4$ (NS vs. controls, $p<0.001$ vs. FD), in the ulcer group was $3.3 \pm 4.3$ (NS vs. controls, $\mathrm{p}<0.001$ vs. FD), and in the group with other GI symptoms was $6.1 \pm 6.3$ (NS vs. controls, p<0.001 vs. FD). $60.0 \%$ of the FD group had a positive capsaicin test (aggregate symptom score $>9$ ), $40.0 \%$ were negative. Among the FD group testing positive, $38.9 \%$ had pain-predominant FD, $50 \%$ discomfort-predominant dyspepsia; in 2 subjects data were missing. $33.3 \%$ of the patients with a negative capsaicin test had pain predominant FD, 50\% discomfort predominant FD, in 2 subjects data were missing. Summary: $60 \%$ of patients with FD were hypersensitive against gastric capsaicin, independent whether the predominant symptom was pain or discomfort. Conclusion: Chemical hypersensitivity discriminates functional disorders from healthy controls and patients with other upper GI disorders. The capsaicin test is a simple and non invasive method to detect the subgroup of functional dyspepsia with chemical hypersensitivity. 
Targeting the VRl receptor might be a therapeutic option in a large subgroup of patients with functional dyspepsia

455

Alternatively Activated Macrophages Contribute to Enteric Nematode Infection-Induced Intestinal Smooth Muscle Hypercontractility in Mice Aiping Zhao, Robert M. Anthony, Justin E. Elfrey, Kathleen B. Madden, Rex Sun, William C. Gause, Joseph F. Urban, Terez Shea-Donohu

We showed previously that nematode infection induced an intestinal smooth muscle hypercontractility that depends on IL-13 activating STAT6. Macrophages orchestrate inflammatory responses and have been implicated in nematode infection. Depending on the cytokine environment, macrophages can differentiate into distinct subsets: classically activated macrophages ( $\mathrm{caM} \Phi)$ that are activated by IFN $\gamma$ and are characterized by the secretion of nitric oxide via NOS-2; or alternatively activated macrophages (aaM $\Phi)$ that are induced by Th2 cytokines, IL-4 and IL-13, and are characterized by the upregulation of arginase-I. Aim: To determine the contribution of aaM $\Phi$ to $H$. polygyrus $(\mathrm{Hp})$ infection-induced changes in murine intestinal smooth muscle function. Methods: BALB/c mice were infected with $\mathrm{Hp}$, cured, re-infected $\left(\mathrm{Hp}^{2}\right)$, and studied 14 days later. Groups of infected mice were injected with $0.2 \mathrm{ml}$ of PBS- or clodronate-liposomes to deplete macrophages. Segments of jejunum from mice were suspended longitudinally in organ baths. After equilibration, the amplitude of the spontaneous contractions (SC) was measured over a 2 -min period. Responses to acetylcholine (ACH), electric field stimulation (EFS), and 5-HT were determined. Real-time quantitative PCR was used to measure the mRNA expression of the molecular markers for caM $\Phi$ and aaM $\Phi$ from intestine. Results: $H p^{2}$ infection increased F4/80 mRNA expression (4-fold), a macrophage molecular marker. In addition, infection upregulated the mRNA expressions for aaM $\Phi$ markers including arginase I (144-fold), macrophage mannose receptor (13-fold), MGL1 (5-fold), MGL2 (59-fold), and FIZZ1 (498-fold), but did not affect the expression of NOS-2, a caM $\Phi$ marker, indicating specific accumulation and activation of aaM $\Phi . H p^{2}$ infection induced an intestinal smooth muscle hypercontractility (Table), including elevated responses to ACH, EFS, and 5-HT, and increased amplitude of SC. However, depleting macrophages with clodronate prevented $\mathrm{Hp}^{2}$-infected mice from developing this hypercontractility. Conclusions: $\mathrm{Hp}^{2}$ infection induces the recruitment and activation of aaM $\Phi$ that are required for the induction of intestinal smooth muscle hypercontractility. Thus, aaM $\Phi$ play a major role in IL-13-induced alterations in smooth muscle function that contribute to host defense against intestinal nematode infection.

\begin{tabular}{|c|c|c|c|c|}
\hline & ACH $(0.1 \mathrm{mM})$ & EFS $(20 \mathrm{~Hz})$ & 5 -HT $(0.1 \mathrm{mM})$ & SC \\
\hline Control & $15.7 \pm 1.0$ & $8.2 \pm 1.4$ & $6.4 \pm 1.4$ & $4.7 \pm 1.7$ \\
\hline$H p^{2}$-PBS & $36.8 \pm 4.9^{*}$ & $13.1 \pm 0.9^{*}$ & $13.7 \pm 1.7^{*}$ & $10.0 \pm 2.2^{*}$ \\
\hline$H p^{2}$-clodronate & $22.4 \pm 1.5^{\Phi}$ & $5.2 \pm 0.7^{\Phi}$ & $5.3 \pm 1.3^{\Phi}$ & $3.9 \pm 0.7^{\Phi}$ \\
\hline
\end{tabular}

Values=Tensions in $\mathrm{N} / \mathrm{cm}^{2} ;{ }^{*} \mathrm{p}<0.05$ vs Control; $\Phi \mathrm{p}<0.05$ vs $\mathrm{Hp}{ }^{2}-\mathrm{PBS} ; \mathrm{n} \geqq 4$

456

Timed Feeding Alters Clock Gene Expression in the Murine Stomach and Colon

Willemijntje A. Hoogerwerf, Joshua R. Scott, Tor C. Savidge, Helen Hellmich

Background. Disruption of biological rhythms, such as occurs with shift work or time zone traveling, has been associated with gastrointestinal symptoms. These observations suggest a functional correlation between biological rhythms and gastrointestinal physiology. Biological rhythms are controlled by clock genes. A central clock is located in the suprachiasmatic nucleus of the brain. Clock genes have been identified in peripheral organs as well. Within a peripheral organ, clock genes can control the expression of a subset of tissue-specific genes, which may impact directly on its physiological function. Clock genes synchronize themselves to external time cues such as the light/dark cycle and feeding. We previously reported circadian expression of clock genes in the murine proximal colon. The aim of this study was to determine (1) whether circadian clock gene expression occurs in other parts of the gastrointestinal tract and if so, whether they are synchronized and (2) whether clock genes in the murine intestine can shift their expression patterns in response to a restricted daytime feeding schedule. Methods. Adult male C57BL/6J mice were kept under a $12 \mathrm{hr}$ light/dark cycle (lights on at $7 \mathrm{AM}$ ). Group 1 was fed ad lib for one week. Group 2 and 3 were fed for the first 4 hours of the light cycle for 48 hrs and 1 week respectively. Total RNA was isolated from the stomach body, proximal- mid- and distal colon every 4 hrs starting at $4 \mathrm{AM}$ ( $\mathrm{n}=3$ mice per time-point). A real-time multiplex PCR assay was developed for the detection of the clock genes period (PER2, PER3), cryptochrome (CRY1) and PAS helix-loop-helix transcription factor (Bmall). PCR data were normalized against 18S. Statistical analysis was performed using a 2 way-ANOVA followed by a Tukey test. Results. Peak/ trough ratios for PER2, PER3, CRYl and Bmall in the proximal colon changed from 20.1, 23.3, 2.3 and 10 at baseline to 5.9, 3.2, 5.4 and 5.6 following 48 hrs of restricted feeding, respectively $(\mathrm{P}<0.05)$. Peak expression shifted from $8 \mathrm{PM}$ to $4 \mathrm{PM}, 8 \mathrm{PM}$ to $8 \mathrm{AM}, 4 \mathrm{AM}$ to $12 \mathrm{PM}$ and $4 \mathrm{AM}$ to $4 \mathrm{PM}$, respectively. This pattern persisted following 1 week of restricted feeding. Similar observations were made in the stomach body, mid- and distal colon. Conclusion. Restricted feeding can shift clock gene expression in the murine stomach and colon. The data suggest that the stomach and colon may have a time-keeping capability that allows for the physiological adaptation to environmental stimuli. We speculate that gastrointestinal symptoms may result from the inability of clock genes to make timely adjustments to changes in environmental cues, such as occurs with time zone traveling and shift-work.
Fecal Serine-Protease Activity: A Pathophysiological Marker and Possible Therapeutical Target in Diarrhea-Predominant Irritable Bowel Syndrome Richard Roka, Andras Rosztoczy, Mathilde Leveque, Ferenc Nagy, Janos Lonovics, Rafael Garcia-Villar, Jean Fioramonti, Tibor Wittmann, Lionel Bueno

Background: The pathogenesis of irritable bowel syndrome (IBS) remains only partially understood, and no specific or universally effective patient management procedure has been developed so far. Activation of receptors for proteases located on colonocytes may participate in immune mucosal stimulation. Consequently, our study was designed to evaluate if colonic luminal serine-proteases may be altered in IBS. Methods: Fecal samples of 38 IBS patients (IBS-D: 17; IBS-C: 14; IBS-A:7), 15 patients with ulcerative colitis (UC) and 15 healthy control were studied. Fecal serine-protease activity was determined photometrically using azocasein as a proteolytic substrate; fecal pancreatic elastase-1 and mast cell tryptase content were measured by ELISA. Fecal secretory leukocyte protease inhibitor (SLPI) concentration was determined by ELISA in control subjects and in patients with diarrhea-predominant IBS Results: Fecal serine-protease activity was 3 -fold higher $(\mathrm{p}<0.001)$ in patients with diarrheapredominant IBS $(67.9 \pm 10.8 \mathrm{U} / \mathrm{mg}$ protein), than in both controls $(16.4 \pm 5.0 \mathrm{U} / \mathrm{mg}$ protein and IBS patients with either constipation $(16.0 \pm 7.0 \mathrm{U} / \mathrm{mg}$ protein) or alternating bowe habits $(16.4 \pm 7.1 \mathrm{U} / \mathrm{mg}$ protein). Fecal serine-protease activity was not correlated with the frequency of bowel movements in all groups. Elevated serine-protease activity was also detected in stools of UC patients $(67.4 \pm 15.3 \mathrm{U} / \mathrm{mg}$ protein). No significant difference was observed in fecal mast cell tryptase and pancreatic elastase concentrations between all groups, and in fecal SLPI concentration between controls and diarrhea-predominant IBS patients. Conclusions: Fecal serine-protease activity is markedly elevated in patients with diarrheapredominant IBS. This increase, however is not coupled with changes in either mast cell tryptase or pancreatic elastase concentrations, and may participate in the mucosal immune stimulation described in such patients and subsequently in the genesis of their symptoms

458

Inhibition of the Putative Anion Transporter (PAT-1, SLC26A6) Impairs the Ability of Upper Villous Epithelial Cells to Regulate Intracellular $\mathrm{pH}$ During $\mathrm{H}^{+}$-Dipeptide Transport

Janet E. Simpson, Nancy M. Walker, Manoocher Soleimani, Lane L. Clarke

Carrier-mediated transport of di- and tripeptides via the small intestinal oligopeptide transporter PEPTl accounts for the majority of dietary amino acids absorbed after protein digestion Proton influx during PEPTI di-/tripeptide uptake by intestinal epithelium requires maintenance of intracellular $\mathrm{pH}\left(\mathrm{pH}_{\mathrm{i}}\right)$ to sustain the driving force for peptide absorption. Studies performed in the absence of $\mathrm{CO}_{2} / \mathrm{HCO}_{3}{ }^{-}$in the medium have demonstrated that the apical membrane $\mathrm{Na}^{+} / \mathrm{H}^{+}$exchangers NHE3 and NHE2 can effectively minimize $\mathrm{pH}_{\mathrm{i}}$ acidification during proton di-/tripeptide transport. However, the contributions of acid-base transpor proteins in the regulation of $\mathrm{pH}_{\mathrm{i}}$ during $\mathrm{H}^{+}$-peptide transport have not been fully investigated under physiologic conditions (i.e., in the presence of $\mathrm{CO}_{2} / \mathrm{HCO}_{3}^{-}$). Therefore, we used $\mathrm{BCECF}$ microfluorimetry to measure $\mathrm{pH}_{\mathrm{i}}$ and to investigate the role of acid-base transporters during exposure of the epithelium to the dipeptide glycyl-sarcosine (gly-sar) in Kreb's $\mathrm{HCO}_{3}$ Ringers. Measurements were made in the upper villous epithelium of the murine duodenum because this is a major site of PEPTl activity. Surprisingly, we found that inhibition of luminal $\mathrm{Cl}^{-} / \mathrm{HCO}_{3}{ }^{-}$exchange with either $100 \mu \mathrm{M}$ niflumic acid or application of bilateral $\mathrm{Cl}^{-}$free conditions resulted in a greater proton influx during gly-sar exposure (NFA = $7.05 \pm 0.89 ; \mathrm{Cl}^{-}$free $=-15.93 \pm 0.21 \mathrm{mM} / \mathrm{min} ;$ control $=-0.83 \pm 0.94 \mathrm{mM} / \mathrm{min} ; \mathrm{n}=3$ $\mathrm{p}<0.05)$. Since PAT-1 is the major apical membrane $\mathrm{Cl}^{-} / \mathrm{HCO}_{3}{ }^{-}$exchanger in the upper duodenal villous epithelium, we measured the effect of gly-sar transport on $\mathrm{pH}_{\mathrm{i}}$ in wildtype (WT) and PAT-1 knockout (KO) duodena. Exposure to gly-sar resulted in a greater proton influx in the PAT-1 KO as compared to WT $(-9.54 \pm 3.40$ vs. $-0.07 \pm 0.79 \mathrm{mM} /$ min, $\mathrm{n}=3, \mathrm{p}<0.05)$. The effect of gly-sar on $\mathrm{pH}_{\mathrm{i}}$ was also compared between WT and carbonic anhydrase II (CAII) knockout intestine because previous studies have shown that CAII interacts with PAT-1 and plays a role in facilitating peptide transport in physiological solutions. Gly-sar-induced acidification was significantly greater in the CAII KO intestine $14.37 \pm 1.71$ vs. $-1.68 \pm 1.03 \mathrm{mM} / \mathrm{min}, \mathrm{n}=4, \mathrm{p}<0.05)$. We conclude that PAT-1 Cl$-/$ $\mathrm{HCO}_{3}{ }^{-}$exchanger in conjunction with CAII contributes to protection against intracellular acidification during $\mathrm{H}^{+}$-dipeptide absorption in the upper villous epithelium, perhaps by operating in $\mathrm{Cl}^{-}{ }_{\mathrm{out}} / \mathrm{HCO}_{3}^{-}$in mode as a base-loading mechanism for the epithelial cell. Sup ported by NIH.

459

Glucose-Dependent Insulinotropic Polypeptide (GIP) Stimulates Transepithelial Glucose Transport in Mouse Jejunum in Vitro Selvi Krishnan, M.michael Wolfe, John H. Schwartz, Satish K. Singh

Background: Recent reports have suggested a key role for GIP, a regulatory peptide synthesized by intestinal enteroendocrine K-cells, in the pathogenesis of obesity. Previous studies from our group have demonstrated effects of GIP on nutrient deposition. Thus, we hypothesized that GIP may regulate intestinal glucose transport directly and thereby influence systemic glucose homeostasis. Objective: To characterize the effects of GIP on jejunal glucose transport in vitro. Methods: Stripped proximal jejunal epithelium from fasted mice was mounted in Ussing chambers: The serosal side was bathed with a bicarbonate-Ringer solution containing $5 \mathrm{mM}$ glucose, and the mucosal side, with a solution containing $10 \mathrm{mM}$ 3-O-methyl glucose (3OMG), a non-metabolizable glucose analog. Mucosa-to-serosa fluxes of $30 \mathrm{OMG}$ (Jms3OMG) and short circuit current (Isc) were determined in the presence and absence of $1 \mu \mathrm{M}$ and $10 \mathrm{nM}$ GIP, with and without the inhibitors of glucose transport, phlorizin $(20 \mu \mathrm{M})$ and phloretin $(250 \mu \mathrm{M})$. To examine whether GIP stimulates epithelial cells directly, viable enterocytes isolated by $\mathrm{Ca2}+$-chelation were incubated with GIP for $4 \mathrm{~h}$ after which cAMP was measured by RIA. Results: GIP increased both Isc and Jms3OMG significantly. The increase in Jms3OMG was greater than the change in Isc (68\% vs. $53 \%)$. As such, the GIP-induced increase in Jms3OMG was primarily electrogenic, with a small electroneutral

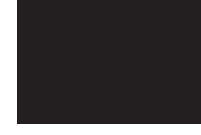


component. The stimulatory effect of GIP on Jms3OMG was concentration-dependent: $1 \mu \mathrm{M}$ and $10 \mathrm{nM}$ GIP increased Jms3OMG by $70 \%$ and $30 \%$ over control, respectively, consisten with receptor activiation. Phlorizin, an inhibitor of SGLTl, abolished the increase in Isc and decreased Jms3OMG by $\sim 65 \%$. These results indicate that stimulation of SGLT1 by GIP only partially accounts for the increase in Jms30MG. Phloretin further inhibited GIP-stimulated Jms3OMG, suggesting that GLUT2 contributes to the effects of GIP on glucose transport. Finally, cAMP, a previously described second messenger of GIP in pancreatic islet beta-cells, was increased 2.5-fold in isolated enterocytes, consistent with a direct effect on these epithelial cells. Conclusion: The present studies are the first to demonstrate direct stimulation of intestinal glucose transport by GIP independent of its insulinotropic properties. GIP stimulates epithelial cAMP and thereby upregulates glucose transport. The GIP-induced increase in glucose transport is mediated primarily by SGLT1 and, to a lesser extent, by GLUT2. The stimulation of multiple jejunal glucose transport processes further supports the hypothesis that GIP plays an important role in glucose homeostasis and the pathogenesis of obesity.

460

Role of Peptide YY in Ileal Brake Induced Satiety and Proximal Gastric Function

My K. Vu, Jeroen W. Maljaars, Izak Biemond, Banafsche Mearadji, Ad Masclee

Activation of the ileal brake with ileal fat perfusion induces satiety and reduces food intake. Peptide YY (PYY) is considered as mediator of the ileal brake. Recent studies indicate that PYY influences satiety but the role of PYY as physiological mediator is under debate. The present study was undertaken to compare the effects of ileal brake activation with ileal fat (endogenous PYY release) versus exogenous PYY infusion on satiety and proximal gastric motor function. Two protocols were performed in 16 healthy volunteers ( 8 per protocol; 7M, 9F; age 22-53 yr). Study A: ileal fat perfusion (Intralipid 20\%, $2 \mathrm{kcal} / \mathrm{min}$ for $3 \mathrm{hrs}$ ) versus saline (control) on two separate occasions. Study B: PYY 3-36 infusion at low and high doses of 15 and $30 \mathrm{pmol} / \mathrm{kg} / \mathrm{hr}$ vs. placebo, on three separate occasions. Studies were performed in the fasting state and in response to a $200 \mathrm{ml}, 300 \mathrm{Kcal}$ liquid meal. Parameters studied were: plasma PYY (RIA), satiety (hunger, fullness, wish to eat, nausea) by visual analog scale (range $0-100 \mathrm{~mm}$ ) and proximal gastric motility (volume by barostat). Results: Ileal fat significantly $(\mathrm{p}<0.05)$ increased plasma PYY from $15 \pm 1$ to $26 \pm 2 \mathrm{pM}$. During PYY infusion plasma levels of $28 \pm 3 \mathrm{pM}$ (with the dose of $15 \mathrm{pmol} / \mathrm{kg} / \mathrm{hr}$ ) and $55 \pm 10 \mathrm{pM}$ (with the dose of $30 \mathrm{pmol} / \mathrm{kg} / \mathrm{hr}$ ) were reached. Ileal fat significantly $(\mathrm{p}<0.01)$ increased satiety (fullness increment $15 \pm 3 \mathrm{~mm}$ ) in contrast to PYY (fullness increment $2 \pm 1 \mathrm{~mm}$ ). In the postprandial state, ileal fat also significantly $(\mathrm{p}<0.01)$ increased satiety (meal+ileal fat vs meal+placebo: fullness increment $25 \pm 3$ vs $12 \pm 3 \mathrm{~mm}$ ) while exogenous PYY did not further increase satiety (meal + PYY vs. meal with placebo : fullness increment $11 \pm 2$ vs. $10 \pm 2 \mathrm{~mm}$ ). The same was true with respect to hunger scores. Fasting gastric volume (barostat) increased significantly ( $<<0.01$ ) in response to ileal fat (from $150 \pm 14 \mathrm{ml}$ to 433 $\pm 54 \mathrm{ml}$ ) but not in response to PYY 3-36 infusion (from $162+18 \mathrm{ml}$ to $180+19 \mathrm{ml}$; n.s.). In all experiments ingestion of the meal resulted in an identical increase in proximal gastric volume to a volume of $400-450 \mathrm{ml}$ at $15 \mathrm{~min}$ postprandially. Postprandial proximal gastric volume at 90 min after meal ingestion was significantly $(\mathrm{p}<0.01)$ higher during ileal fat vs. control $(410 \pm 42 \mathrm{ml}$ vs $183 \pm 20 \mathrm{ml})$ while PYY infusion did not affect volume over control $(230 \pm 19 \mathrm{ml}$ vs $187 \pm 15 \mathrm{ml})$. It is concluded that ileal fat induces satiety and results in proximal gastric relaxation, in contrast to exogenous PYY 3-36 at identical plasma levels. These data do not support a role for PYY as physiological mediator in ileal brake induced satiety or ileal brake induced proximal gastric relaxation.

461

A Comparison of Nasogastric, Nasojejunal and Parenteral Feeding in Acute Stroke Patients with Lower Esophageal Sphincter Dysfunction Richa Dewan, Nitin Gupta, Rahul Anand, Pankaj Mehta, V V. Mittal, Divey Manocha, Ravi Nehru, Kuldeep Kumar

In acute ischemic stroke, aspiration is the 2nd most common cause of mortality (after brain herniation). Lower Esophageal Sphincter (LES) dysfunction may predispose to aspiration in this setting, and there is no prospective trial in acute stroke comparing the various modes of feeding. OBJECTIVE: To compare the effect of Nasogastric (NG), Nasojejunal (NJ) and Total Parenteral Nutrition (TPN) on aspiration and mortality in acute stroke with LES dysfunction. METHOD: 71 patients of Cerebral Infarction with GCS score $\leq 7$, and who failed the Water Swallow Test at 72-96 hours of onset of cerebral infarction were studied. Those with diabetes, known esophageal/jejunal disease, and known aspiration were excluded. Esophageal manometry was performed on entry at $72 \mathrm{hrs}$ post stroke, and patients with resting LES tone of $\leq 4.5 \mathrm{mmHg}$ were included in the study. Baseline parameters included age, sex, site and size of infarct, and $\mathrm{NIH}$ stroke scale and GCS scores. At $72-96$ hours poststroke, they were randomly divided into 3 groups receiving NG, NJ and TPN feeds respectively for next 7 days while being monitored for outcomes of aspiration and death. RESULTS: Of 71 patients, 18 (25\%) developed aspiration pneumonia. 11 of 25 (48\%) on NG feeds, 4 of $23(17 \%)$ on NJ feeds and 3 of $23(13 \%)$ on TPN aspirated. All patients who developed aspiration pneumonia died. Aspiration was comparable in patients on NJ feeds and TPN $(\mathrm{p}=1)$. The patients on NG feeds had significantly higher aspiration pneumonia and related mortality as compared to the other 2 groups $(p=0.027)$. There was significantly more gastric residual volume in the NG feeds group. Diarrhea was more in the NJ group as compared to the TPN group, but this difference was not significant. Metabolic complications were more common with TPN but not significant. A total 29 patients died, 16 of $25(64 \%)$ in the NG group, 7 of 23 (30\%) in NJ group and 6 of $23(26 \%)$ in the TPN group. Of these 29 patients, 18 patients died of aspiration pneumonia. Mortality was significantly higher in NG feeds group ( $\mathrm{p}=0.013$ by Pearson chi square test), as compared to the NJ feeds and TPN groups which were comparable to each other $(\mathrm{p}=1)$. CONCLUSION: In patients of acute stroke with LES dysfunction, Nasojejunal feeding significantly reduces the risk of aspiration pneumonia and mortality as compared to Nasogastric feeding, and is comparable to TPN in terms of reducing aspiration risk.
Effects of Modified Oral Diet and Human Growth Hormone On Nutrient Absorption and Parenteral Nutrition Needs in Adults with Severe short Bowel Syndrome: Results of a Randomized, Double-Blind, Placebo-Controlled Clinical Trial

Concepcion Fernandez-Estivariz, Menghua Luo, Niloofar Bazargan, Li H. Gu, Shanthi V. Sitaraman, Jan-Michael Klapproth, Dean P. Jones, John R. Galloway, Lorraine M. Leader, Thomas R. Ziegler

Background: Limited data exist on gut rehabilitation with dietary changes alone versus diet + growth factors in patients with SBS. We evaluated gut nutrient absorption and PN needs in response to diet change \pm growth hormone $(\mathrm{GH})$ over a 12 -week period in adults with severe SBS. Methods: Stable adults with PN-dependent SBS were admitted to the GCRC for 28 days. Subjects underwent 7-day baseline studies with usual oral diet and PN. Gut absorption (\% of intake) of nitrogen $(\mathrm{N})$, fluid, sodium $(\mathrm{Na})$, potassium $(\mathrm{K})$ and magnesium (Mag) and stool wet weight (WW) was determined in pooled 4-day studies. Body composition was determined by DEXA. During the subsequent 21 days, subjects continued usual PN, but were placed on individualized oral diets, modified daily based on tolerance and stool output. Dietary principles included small frequent feeds, use of oral rehydration solutions and limited simple sugars, lactose, and fat. During this 21-day period, subjects were randomized to receive, in a double-blind manner, sc saline (control; $\mathrm{n}=9)$ or $\mathrm{hGH}(0.1 \mathrm{mg} / \mathrm{kg} / \mathrm{day} ; \mathrm{n}=14)$ daily. Nutrient absorption and DEXA studies were repeated during week 4 (days 25-28). Subjects were discharged home on a 3X/week study drug regimen for 8 subsequent weeks with close outpatient dietary and clinical monitoring. PN was weaned as appropriate, based on serial clinical and biochemical data, diet responses and daily urine and stool output. At week 12 , subjects were readmitted to the GCRC and the studies above were repeated. Results: Control and hGH groups were similar in gender, age, residual gut length and chronic daily PN needs. Both diet modification and hGH were well tolerated. Nutrient absorption and PN weaning were similar over time in the diet $+\mathrm{hGH}$ and the diet + placebo patients (not shown). However, \% change in nutrient absorption from baseline to weeks 4 and 12 markedly improved in the entire study group for $\mathrm{N}$ (week $4+10$ and week $12+10 \%$, respectively), fluid $(+13$ and $+22 \%), \mathrm{Na}(+36$ and $+43 \%), \mathrm{K}(+22$ and $+24 \%), \mathrm{Mag}(+3$ and $22 \%)$ and stool wet weight fell $(-710 \pm 207$ and $-489 \pm 180 \mathrm{~mL})$; all $\mathrm{P}<0.01$ vs baseline Weekly PN needs in the entire study group at week 12 were markedly decreased from baseline $(-7.5 \pm 1.0 / \mathrm{L} ; \mathrm{P}<0.001)$. The change in lean body mass (LBM) by DEXA was significantly greater in the $\mathrm{hGH}$ group at week 4 (control $+0.7 \pm 0.6 \mathrm{vs} h \mathrm{GH}+2.1 \pm 0.4 \mathrm{~kg}$; $\mathrm{p}<0.04$ ) and week 12 (control $-0.8 \pm 0.7$ vs $\mathrm{hGH}+1.8 \pm 0.1 \mathrm{~kg} ; \mathrm{p}<0.04$ ). Conclusion: Use of $\mathrm{hGH}$ did not add to the effects of diet alone to enhance nutrient absorption and PN weaning but maintained LBM. Individualized modified diet is an effective approach to improve nutrient absorption and facilitate PN weaning in adult SBS patients.

463

in Vitro and in Vivo Characterization of the Minimal Promoter of the Human Intestinal Biotin Transporter HSMVT: Adaptive Regulation of the Biotin Uptake Process

Jack C. Reidling, Hamid M. Said

Background: The molecular mechanisms involved in the regulation of biotin transport in mammalian cells are poorly understood. Previous studies established that the human sodium dependant multivitamin transporter, SMVT, is the predominant system involved in biotin uptake in the human intestine. Little is known about the basal transcription activity of the SMVT gene or about possible adaptive regulation of the human intestinal biotin uptake process by dietary substrate levels or the mechanism(s) involved in such a regulation. Our aim in this study was to address these issues. Methods: In vitro characterization of the minimal promoter region of the SMVT gene was performed using human-derived intestinal epithelial Caco-2 cells, mutational analysis and electrophoretic mobility shift assays (EMSA); in vivo characterization was achieved using transgenic mice that express the human SMVT promoter (fused to the luciferase reporter gene). Adaptive regulation was studied by comparing carrier-mediated biotin uptake, SMVT protein and mRNA levels as well as SMVT promoter activity in Caco-2 cells under biotin deficient and control conditions. Results: In vitro studies demonstrated that mutation of specific cis-elements in the SMVT minimal promoter (GKLF, AP2) led to a decrease in activity. Using EMSA specific DNA/protein complexes are being identified and interacting factors determined by oligonucleotide competition and antibody super-shift analysis. In vivo studies using transgenic mice established the physiological relevance of in vitro data by demonstrating activity of the cloned full-length SMVT promoter. In addition, the in vivo studies showed that the pattern of expression of the SMVT promoterluciferase constructs in transgenic mice were similar to our observed RNA expression pattern in native human tissues. Our studies on possible adaptive regulation of biotin in Caco-2 cells showed that maintaining these cells in a biotin deficient condition leads to a significant and specific up-regulation in carrier-mediated biotin uptake which is associated with an induction in protein and mRNA levels of SMVT. In addition, an increase in the activity of the human SMVT promoter was observed in Caco-2 cells maintained under biotin deficient conditions. Conclusions: 1) GKLF and AP2 play roles in driving the basal transcription of the SMVT promoter, 2) the cloned promoter is functional in vivo in transgenic mice, and 3) biotin uptake by Caco-2 cells and expression of SMVT is adaptively up-regulated during biotin deficiency, and this up-regulation is mediated via transcriptional regulatory mechanism(s). [Support:DK58057, DK56061 Jack Reidling NIH NRSA award 1 F32 DK062629].

464

Night-Time Working Increases the Ulcerogenic Potential of H. Pylori Infection Antonio Pietroiusti, Andrea Magrini, Antonio Forlini, Alberto Galante, Luca Coppeta, Umberto Iannaccone, Antonio Bergamaschi

Backgroung. Night-time working has been found to be associated with an increased risk of peptic ulcer disease. Since most peptic ulcers are due to $H$. pylori infection, it is possible that night-time working potentiates the ulcerogenic effect of infection; alternatively, the link 
between peptic ulcer and night-time working might be due to an increased prevalence of infection in these workers. This subject has never been explored until now. Aim of this study was to evaluate the interaction between night-time working and $H$. pylori infection. Patients and Methods. All subjects were selected for endoscopy by a pool of primary care physicians involved in an educational program with our Institution. Entry criteria were: being active worker,persistent dyspeptic symptoms during the last year, presence of $H$. pylori infection according to urea $13 \mathrm{C}$ breath test or stool testing, no previous endoscopic examination, no treatment with gastric antisecretory drugs or antibiotics during the previous month. Regular NSAIDs users were excluded. Before endoscopy, work characteristics of the patients were analyzed by an occupational physician. On this basis, subjects were divided into two broad categories: night-time workers (those performing work at night for at least six times per month from at least one year) and day-time workers (those working during day-time only). 101 consecutive night-time workers, and 247 consecutive day-time workers were enrolled during one year. At endoscopy, three biopsies were taken from the gastric antrum, and three from the body. At each site, H. pylori infection was diagnosed if culture was positive and/or if the organism was concomitantly detected at histology (Giemsa staining) and urease testing. H. pylori cagPAI status was determined by PCR cagA amplification on isolated strains. Results. The prevalence of duodenal ulcer was higher in night-time workers than in day-time workers ( 29 out of $101,28.7 \%$ vs 23 out of $247,9.3 \%$; $<<0.0001$ ), and persisted after multivariate analysis taking into account age, sex, smoking, familial history of peptic ulcer disease, length of work, and social status as possible confounders: odds ratio $4.51,95 \%$ confidence interval 2.08 to 7.23 ). Among day-time workers, duodenal ulcers were strongly related to cagA status (18 out of 99 cagA positive (18.2\%) vs 5 out of 148 cagA negative (3.4\%); $\mathrm{p}<0.05)$, whereas among shift workers they were not: 16 out of 43 cagA positive ( $37.2 \%)$ vs 13 out of $48,(27.1 \%) ; \mathrm{p}=\mathrm{NS})$. Conclusions Shift working increases the ulcerogenic potential of $H$. pylori. Application of a test and treat strategy in these workers may have strong health and economic implications.

465

Effect of Intravenous Application of Esomeprazole $40 \mathrm{Mg}$ Vs Pantoprazole 40 Mg On 24 Hour Intragastric pH in Healthy Adults Dieter Schilling, D.R. Hartmann, Juergen F. Riemann

Background: Intravenous infusion of proton pump inhibitors (ppi) represents an alternative administration route in patients for whom the oral administration is inappropriate. Recently an intravenous (i.v.) formulation of esomeprazole has been developed. There are only rare data about the equal doses of intravenous administration of esomeprazole (ESO) and pantoprazole (PAN). This study compared the effect on intragastric $\mathrm{pH}$ of ESO and PAN Methods: We performed a single center three way cross over study comparing three intravenous - single - shot - treatments (A: esomeprazole $40 \mathrm{mg}$ as a 15-minute infusion; B: pantoprazole $40 \mathrm{mg}$ as a 15 -minute infusion; C: pantoprazole $40 \mathrm{mg}$ as a 3- minute injection) separated by a wash out interval of at least 13 days. Subjects were required to be Helicobacter pylori negative or positive as determined by $13 \mathrm{C}$ Urea Breath test. 24-hour intragastric pH was recorded using a microelectrode (Inggold-bipolar glass) linked to a Digitrapper $\mathrm{pH} 100$. 6 hours after injection or end of infusion a standardized meal was given to the subjects. The time for intragastric $\mathrm{pH}>6,>5$ and $>4$ within the examination time of 24 hours as well as the time of intragastric $\mathrm{pH}>6,>5$ and $>4$ within the first 6 hours after application were analysed. The differences between the three treatments were assesed using the signedrank-test. Results: 28 people (mean age $33.7+/-7.65$ years were enroled into the study. 15 persons were men. 21 Volonteers were H-pylori negative. All treatments were equally well tolerated. For results see table below. Conclusion: Esomeprazole $40 \mathrm{mg}$ i.v. provides a statistically significant better acid suppresion than pantoprazole $40 \mathrm{mg}$ intravenously. Effect of the three treatments on intragastric $\mathrm{pH}$

\begin{tabular}{|c|c|c|c|}
\hline & A $40 \mathrm{mg}$ ESO & B 40 mg PAN INF & C 40 mg PAN INJ \\
\hline Median time [Min.] pH $>4 /$ first6h & $231^{* \#}$ & 57 & 145 \\
\hline Median time [Min.] pH>5/first6h & $160^{* \#}$ & 27 & 92 \\
\hline Median time [Min.] pH>6/first6h & $109 \#$ & 5 & 33 \\
\hline Median time [Min.] pH>4/24 hrs & $818^{* \#}$ & 292 & 450 \\
\hline Median time [Min.] pH>5/24 hrs & $471^{* \#}$ & 126 & 273 \\
\hline Median time [Min.] pH>6/24 hrs & $258^{*}$ & 56 & 105 \\
\hline Median pH/24 hrs & $4.5^{*} \#$ & 2.2 & 2.8 \\
\hline
\end{tabular}

* $\mathrm{p}<0.05$ versus PAN INJ (treatment $\mathrm{C}$ ) \# $\mathrm{p}<0,05$ versus PAN INF (treatment B)

466

Ulcer Healing Properties of Pioglitazone Are Inhibited in Diabetic Rats Peter C. Konturek, Tomasz Brzozowski, Grzegorz Burnat, Stanislaw Konturek

Experimental diabetes mellitus (DM) is associated with a significant increase in the vulnerability of gastric mucosa against different irritants and delay in ulcer healing. Our previous studies demonstrated that PPAR $\gamma$ ligands, which are used in the therapy of DM as insulin sensitizer, accelerate ulcer healing. However, there is no information whether PPAR $\gamma$ ligands influence ulcer healing in diabetic rats. The aim of the present study was 1) to compare the effect of treatment with PPAR $y$ ligand, pioglitazone, on ulcer healing in normal rats and those with streptozotocin (STZ)-induced diabetes mellitus; 2 ) to assess the effect of pioglitazone on the mRNA expression of cyclooxygenase-2 $(\mathrm{COX}-2)$ and interleukin $1 \beta ; 3)$ to investigate the role of endogenous nitric oxide in the healing effect of pioglitazone. Methods: STZ (70 $\mathrm{mg} / \mathrm{kg}$ i.p.) was used to induce DM in rats. 4 weeks after induction of DM, experimental gastric ulcers were induced, and 10 days later, the healing rate and the gastric blood flow (GBF) were measured by planimetry and hydrogen (H2)-gas clearance method, respectively. Following major groups of rats with gastric ulcers were used: (1) vehicle (saline), (2) STZ

alone; (3) pioglitazone (40 mg/kg i.g.); 4) pioglitazone (40 mg/kg i.g.) in diabetic rats; 5) pioglitazone (40 mg/kg i.g.) in combination with L-NNA (20 mg/kg i.p.) and 6) pioglitazone $(40 \mathrm{mg} / \mathrm{kg}$ i.g.) in combination with L-NNA $(20 \mathrm{mg} / \mathrm{kg}$ i.p.) in diabetic rats. The expressio of IL-1 $\beta$ and COX-2 was assessed by RT-PCR. Results: In the diabetic rats, ulcer healing was significantly delayed and accompanied by a reduction in the gastric mucosal blood flow. This prolongation of ulcer healing in diabetic rats was associated with an increase in gastric mucosal expression of IL-1 $\beta$. Administration of pioglitazone significantly accelerated the ulcer healing in normal rats, but this effect was significantly attenuated in diabetic animals. Pioglitazone significantly inhibited the expression of IL- $1 \beta$ and COX-2 mRNA at the ulcer margin of normal rats, but this effect was significantly attenuated in diabetic rats and the expression of these two genes was significantly upregulated. Pretreatment with LNNA abolished the healing effect of pioglitazone and led to a significant delay in ulcer healing. The inhibitory effect of L-NNA on ulcer healing was significantly more pronounced in diabetic rats than in normal animals. Conclusion: Experimental DM dramatically impairs ulcer healing, depending upon the increased release of proinflammatory cytokines. The ulce healing effect of pioglitazone, which is mediated, at least in part, by endogenous NO is significantly attenuated in diabetic rats.

\section{7}

Proton Pump Inhibitor Use Is Associated with the Development of Sporadic Fundic Gland Polyps Mathilde Jalving, Jan J. Koornstra, Jelle Wesseling, Jan H. Kleibeuker

Background: Fundic gland polyps (FGPs) are the most common gastric polyps, occurring in approximately $2 \%$ of the general population (termed sporadic FGPs) and in up to $84 \%$ of FAP patients. Proton pump inhibitors (PPIs) are widely used to inhibit gastric acid secretion. There is conflicting evidence regarding the association between the development of sporadic FGPs and use of proton-pump inhibitors (PPIs) and the influence of PPIs on the development of dysplasia in FGPs. FGPs are relatively rare making a case-control study the most feasible method to determine whether there is a relationship. Methods: In this case-control study, the prevalence and duration of PPI use in a consecutive group of patients undergoing esophagogastroduodenoscopy were assessed. For each patient, sex and age were recorded as well as the duration of PPI use. For each patient with FGPs, the number localization and size of FGPs were assessed. Biopsies were taken from FGPs and normal gastric mucosa. The biopsies were fixed in formalin, embedded in paraffin and stained for hematoxylin and eosin $(\mathrm{H} \& \mathrm{E})$ for routine histology. The presence of dysplasia was evaluated and graded as negative, low-grade or high-grade. Results: Data were obtained from 599 patients, of whom 324 were on PPI therapy. Patients on PPI-therapy were older than patients not using PPIs $(56+13$ vs $5 l+17$; mean age \pm SD). There was no difference in sex distribution between the groups. Histologically confirmed FGPs were identified in 107 patients. Patients with FGPs were older than patients without FGPs $(60 \pm 12$ vs $52 \pm 16$ $\mathrm{p}<0.001)$. PPI therapy was associated with an increased risk of FGPs (OR 2.3, 95\% CI 1.5 - 3.6). Subgroup analysis showed that long term PPI use was associated with an increased risk of FGPs ( $1-4.9$ years use: OR 2.2, 95\% CI $1.3-3.8$ and $>5$ years use: OR 3.8, 95\% CI 2.2 - 6.7) while short-term PPI therapy (<1 year use) was not (OR $1.0,95 \%$ CI 0.5 1.8). Linear regression analysis showed that both age and PPI use were independent predictors of having FGPs ( $p<0.001$ for both). There were no differences in the localization, size or number of FGPs found in patients with and without PPI use. None of the FGPs showed dysplasia. Conclusion: Long-term use of proton-pump inhibitors is associated with an up to 4 -fold increase in the risk of sporadic fundic gland polyps. The risk of dysplasia in these polyps is, however, negligible.

\section{8}

Increased Plasma Ghrelin Levels in Patients with Peptic Ulcer

Hidekazu Suzuki, Tatsuhiro Masaoka, Hiroshi Hosoda, Toshihiro Nishizawa, Yuriko Minegishi, Eisuke Iwasaki, Kenji Kangawa, Toshifumi Hibi

Ghrelin is a novel appetite-promoting peptide secreted primarily from the A-like cells of the gastric fundic mucosa. As gastric inflammation caused by $H$. pylori infection increase in extent, the number of ghrelin-producing A-like cells in the gastric fundus decreases as does the gastric ghrelin content (Gut 53:187, 2004). We previously reported that plasma levels of ghrelin, which correlated well with serum pepsinogen (PG) I and the PG I/II ratio, but not with $H$. pylori seropositivity, decreased with increasing severity of gastric mucosa atrophy in non-ulcerogenic conditions (Hepatogastroenterol. 51:1249, 2004), and that plasma ghrelin levels increased in rats with cysteamine-induced duodenal ulcers (Am. J. Physiol., Gastrointest. Liver Physiol. 289:G138, 2005). The present study was designed to investigate the plasma levels of ghrelin in patients with peptic ulcer disease. Patients and Methods. Two hundred and eighty-six patients with epigastric symptoms who underwent upper GI endoscopy were enrolled for the study. Fasting blood samples were collected prior to the endoscopy after a $12 \mathrm{hr}$ fast. The patients were grouped as follows: chronic gastritis with high PG $(n=197)$, chronic gastritis with low PG ( $n=58)$, duodenal ulcer ( $\mathrm{n}=$ $14)$, and gastric ulcer $(n=17)$. Plasma levels of total and active ghrelin, as well as serum levels of PG I and PG II were measured by radioimmunoassay. Six patients with duodenal ulcer and 7 patients with gastric ulcer were also assessed after ulcer healing. Results. Plasma gherlin levels were significantly lower in patients with low PG-gastritis than in patients with high PG-gastritis, and were significantly higher in patients with duodenal ulcers $(\mathrm{p}<0.05)$ and gastric ulcers $(\mathrm{p}<0.05)$ compared to those without peptic ulcers (chronic gastritis with high PG; $169 \pm 12 \mathrm{fmol} / \mathrm{ml}$, chronic gastritis with low PG; $144 \pm 19$, duodenal ulcers; $232 \pm 62$, gastric ulcers; $212 \pm 51$ ). Similar elevations of plasma ghrelin were found in peptic ulcer patients with and without $H$. pylori colonization. Elevated plasma total ghrelin levels did not change significantly after the ulcer healing. Conclusions. Plasma ghrelin levels were significantly higher in patients with peptic ulcers irrespective of $H$. pylori colonization when compared to patients with chronic gastritis only. Since elevated levels of plasma ghrelin did not decrease after the ulcer healing, the increase in plasma ghrelin was not the results of the ulceration, but would be one of predictive markers for determining the ulcer susceptibility. 
Polymorphisms of TLR-4/TLR-2 and Risk of Helicobacter Pylori Infection and Duodenal Ulcer Disease in Children

Dulciene M. Queiroz, Andreia M. Rocha, Silvia B. Moura, Gifone A. Rocha, Juliana B. Guerra, Paulo Bittencourt, Taciana F. Soares, Ludmila M. Godoi, Luciana R. Almeida

Although host factors linked to the outcome of Helicobacter pylori (HP) infection have been investigated, no study has evaluated yet the role of single nucleotide polymorphisms (SNPs) in genes that encode mediators of the innate immune response such as Toll-like receptor (TLR)-4 and TLR-2 in the risk of HP infection and HP associated diseases in children. The SNPs of genes encoding TLR-2 and TLR-4 are thought to impair the efficiency of bacterial ligant signaling and the capacity to elicit the host defense against microorganisms. Considering that the infection is mainly acquired in childhood, we evaluated 432 children (193 boys, mean age 9.4 years (SD 3.8), range 1-18). Among them, 207 were $\mathrm{HP}+(72$ with DU). cagA status was investigated by PCR. TLR-4 (Asp299Gly) and TLR-2 (Arg753Gln, Arg677Trp) polymorphisms were investigated by PCR-RFLP and confirmed by sequencing. The allele Arg677Trp was not seen in this population. The other alleles were in Hardy-Weinberg equilibrium. No association was seen between the polymorphic alleles and DU ( $\mathrm{p}=0.71$, $\mathrm{OR}=0.78,95 \% \mathrm{CI}=0.33-1.94$ for $\mathrm{TLR}-4$ and $\mathrm{p}=0.32, \mathrm{OR}=1.68,95 \% \mathrm{Cl}=0.45-5.65$ for TLR2). In addition, these polymorphisms did not associate with HP status ( $\mathrm{p}=0.34, \mathrm{OR}=0.63$, $95 \% \mathrm{CI}=0.26-1.49$ for TLR-4 and $\mathrm{p}=0.69, \mathrm{OR}=0.75,95 \% \mathrm{CI}=0.27-2.02$ for TLR-2). However, the polymorphic G allele of TLR-4 was significantly associated with infection with cagA+ strains $(\mathrm{p}=0.02, \mathrm{OR}=9.08,95 \% \mathrm{Cl}=1.21-18.8)$. Our results show that SNPs in TLR-2 and TLR-4 were neither associated with the susceptibility to HP infection nor with the outcome of the infection in childhood. However, TLR-4 SNP was associated with susceptibility to more virulent HP strains. Grants: CNPq/FAPEMIG.

483

Anti-Tissue Transglutaminase Autoantibodies are Deposited in the Small Intestinal Mucosa of Patients with Type 1 Diabetes

Mariantonia Maglio, Francesco Paparo, Adriana Franzese, Raffaella Spadaro, Maria Vittoria Barone, Luciano Rapacciuolo, Virginia Vitale, Linda Cosenza, Riccardo Troncone

Backgroung Anti-tissue transglutaminase (anti-tTg) IgA are coeliac disease (CD) associated autoantibodies. Most evidences suggest that the primary site of their production is the small intestine. The association between CD and type 1 diabetes (TID) has long been established. Signs of gluten sensitivity (organ culture and rectal gluten challenge studies) have been reported also in TlD patients with absence of anti-tTg antibodies in their serum. Aim To investigate the deposition of anti-tTg autoantibodies in the jejunal mucosa of TID patients irrespective of their presence in the serum. Methods By double immunofluorescence staining, anti-tTg IgA deposits were evaluated in jejunal biopsies from 29 TID patients (median age 11 years, range 3-22) with normal mucosa; only in 12 of them serum anti-tTg autoantibodies were present. Ten untreated coeliac patients (median age 8 years, range 1.5-15.5) and 29 patients (median age 5.6 years, range $0.7-18$ ) with normal mucosa and absence of serum anti-tTg served as controls. IgA deposits were detected in green by FITC-labelled rabbit antibodies against human IgA, while tissue transglutaminase was revealed in red by RPElabelled rabbit anti-mouse secondary antibodies, primary antibodies being monoclonal mouse anti-Guinea pig Tg (CUB 7402). The anti-tTg IgA deposits stained yellow; colocalization was further assessed at confocal microscopy. Results Jejunal mucosa from all 10 untreated coeliac patients presented evident anti-tTg IgA deposits in the subepithelial region. 11/17 TID patients without serum anti-tTg antibodies, as well as 10/12 TlD patients with positive serum anti-t $T g$ antibodies showed intestinal anti-t Tg IgA deposits, like classical CD patients, but with a patchy distribution. In the control group only $4 / 29$ patients presented a positive IgA deposit pattern. Conclusion In most of TID patients with absent anti-tTg antibodies in the serum, anti-t $\operatorname{Tg} \operatorname{IgA}$ are deposited at the level of the small intestinal mucosa. This finding confirms recent experimental data obtained by phage display libraries (Florian et al. J Pediatric Gastroenterol Nutr vol 40, 2005: 617). It remains to be estabilished if this phenomenon is related to dietary gluten.

484

Novel Antibody Tests Based On Synthetic Gliadin-Related Peptides Have a Great Yield for Celiac Disease

Emilia Sugai, Horacio Vazquez, Fabio Nachman, Maria L. Moreno, A Czech, Silvia Pedreira, Sonia Niveloni, Roberto Mazure, Zulema Kogan, Juan C. Gomez, Eduardo Maurino, Julio C. Bai

Background/aim: Celiac disease (CD) serology has a key role for screening, diagnosis and follow-up of patients. Although antigliadin antibodies (AGA) were recognized more of 30 yr ago, they have lost enthusiasm by a disappointing low specificity and the identification of more reliable autoantibodies. We aimed to explore the diagnostic value of newly developed peptide antibody assay addressing specific synthetic gliadin-derived deamidated peptides. Materials: We studied serum samples prospectively obtained from a selected population of 92 consecutive patients with newly diagnosed CD and 113 controls (84 with non CD-related disorders and 29 with Crohn's disease). Diagnosis of $C D$ was based on histological criteria (Marsh II enteropathy or greater), CD-related serology and/or response to a gluten-free diet. Patients were re-evaluated after 6-mo $(n=56)$ and 1 -yr $(n=20)$ on treatment. Methods: All patients and controls underwent intestinal biopsy and a set of CD-related serology tests. A newly developed enzyme-linked immunosorbent assay (ELISA) to detect IgA and IgG antibodies against synthetic deamidated gliadin epitopes (AGA II IgA and AGA II IgG) was used (QUANTA Lite Gliadin IgA and IgG II- Inova Diagnostic Inc.; San Diego, CA). Results: At diagnosis, sensitivity and specificity for AGA II IgA were $94.6 \%$ and $99.1 \%$ and for AGA Il IgG $92.4 \%$ and $100 \%$, respectively. Interestingly, the only control subject with a positive AGA II IgA test had other evidences of gluten sensitivity. When patients were assessed at 6-mo and 1-yr after starting on a gluten-free diet, mean absolute values were reduced significantly ( $\mathrm{p}<0.0000$ and $\mathrm{p}<0.001$, respectively). Furthermore, the proportion of patients with positive samples was also significantly reduced at the same periods. Compared with negative predictive values, diagnostic accuracy and likelihood ratios. Compared with antitissue transglutaminase antibodies, AGA II had a similar sensitivity but a greater specificity, predictive values and better likelihood ratios. Considering the IgA isotype, an excellent agreement ( $\kappa$ statistics: 0.92) was shown between AGA II and a-tTG. Conclusions: In our knowledge, this is the first study assessing the value of an ELISA assay to detect antibodies against specific gliadin-related peptides in CD patients. Our data evidenced that the new AGA II tests are reliable tools for diagnosis of $C D$ and suggests a promising diagnostic accuracy that might be very useful in practice.

485

Epidermal Transglutaminase Antibody Differentiates Dermatitis Herpetiformis from Celiac Disease

Federico Biagi, Jonia Campanella, Paola I. Bianchi, Olga Ciocca, Camilla Vassallo, Clara Piccolo, Giovanni Borroni, Gino R. Corazza

Introduction: Dermatitis herpetiformis (DH), the cutaneous counterpart of celiac disease (CD), is a blistering itchy skin disease characterized by IgA granular deposits localized either in the dermal papillae or along the dermo-epidermal junction. These immunocomplexes allow differential diagnosis between $\mathrm{DH}$ and other skin bullous diseases [1]. It has recently been shown that $\mathrm{DH}$ immunocomplexes contain epidermal transglutaminase (eTG) and that sera from patients with DH contain antibodies specifically directed against eTG [2]. We studied whether serum eTG ab discriminates between $\mathrm{DH}, \mathrm{CD}$, and other gastrointestinal and dermathological diseases. Patients and methods: eTG ab were tested in sera from 34 patients with untreated DH (UDH) (18 males, mean age 34, range 18-70 years), 19 patients with treated DH (TDH) (all from UDH group: 9 males, mean age 35, range 22-65 years), 54 patients with untreated $\mathrm{CD}$ ( 14 males, mean age 34, range 4-64 years), 52 gastrointestinal pathologic controls (18 males, mean age 40, range 18-75 years), and 31 dermathological pathologic controls (all affected by skin bollous disease; 12 males, mean age 53, range 1085 years). Samples were tested for eTG ab with an ELISA kit (Immunodiagnostik). Based on the cut off provided by the producer, results were considered positive when optical density was $\geq 18$ arbitrary unit (AU). Results: Average eTG ab levels were $62.9 \pm 37.3 \mathrm{AU}$ in UDH, $14.9 \pm 7.0$ in TDH, $25.7 \pm 30.1$ in untreated CD, $9.8 \pm 4.7$ in gastrointestinal controls, and $6.5 \pm 3.7$ in dermathological controls. So, sensitivity was $97.1 \%$ and specificity was $96.2 \%$. Statistical analysis showed that in UDH eTG levels were significantly higher than in both TDH ( t test, $p<0.0001)$, untreated $C D(p<0.0001)$, gastrointestinal controls $(p<0.0001)$, and dermathological controls ( $\mathrm{p}<0.0001)$. Conclusions: eTG ab can almost perfectly discriminate between $\mathrm{DH}$ and other skin bullous diseases; eTG ab levels decrease in $\mathrm{DH}$ patients on a gluten free diet. Moreover, eTG ab levels are significatively higher in UDH patients than in untreated $C D$ patients. Detection of serum eTG ab is therefore a new tool for the diagnosis of patients with skin bullous diseases. 1. Fry L. Bailliere's Clin Gastroenterol 1995:9:371-93. 2. Sardy M, et al. J Exp Med 2002;195:747-57.

486

Involvement of Systemic Serum Cytokine Levels in the Pathophysiology of Celiac Disease

Lincoln Hernandez, Sindhura Kunduvaram, Anne Lee Roland, Edward Ciaccio, Naiyer Afzal, Govind Bhagat, John Manavalan, Green H. Peter

Celiac disease (CD) is an autoimmune disorder that occurs in genetically predisposed individuals as the result of an immune response to gluten. Although considered to be a local upper GI autoimmune disease it is now known to have widespread systemic implications We tried to define the nature and role of systemic cytokine levels in the pathophysiology of disease. We preformed a multi-plex cytokine assay on 4 different groups of adult patients; Patients with Active CD, patients on a Gluten Free Diet (GFD) with positive TTG IgA Antibodies, patients on a GFD with negative antibodies and those with Refractory Disease (RF). We individually compared all groups with normal controls, and we found those patients with active disease and those on GFD that had positive antibodies had significantly higherlevels of proinflammatory cytokines, such as INF-gamma IL1-B, TNF Alfa, IL-6 and IL- 8 and also Th-2 Cytokines such as, IL-4 and IL-10, as compared to normal controls and patients on GFD without antibodies. Interestingly patients on GFD for less than 1 year had significantly higher levels of both proinflamniatory cytokines and Th2 cytokines as compared to the patients on GFD for more than 1 year. Within the group on GFD for less than a year, at least $75 \%$ had positive TTG antibodies, while those on a GFD for more than 1 year, only $25 \%$ had positive TTG Ig A antibodies. Based on a linear regression analysis there was a statistically significant correlation between the serum levels of TTG Ig A and the levels of Th-1 Cytokines as IL-4 ( $\mathrm{p}<0.025)$ (fig. 1), IL-10 ( $<<0.03$ ) and Inflammatory cytokines such as TNF alpha $(\mathrm{p}<0.006)$, IL $(\mathrm{p}<0.02)$ and IL-8 $(\mathrm{p}<0.0001)$. This is the first study to demonstrate a significant correlation between the levels of TTG Ig A Antibodies and the presence of proinflammatory and Th2 Cytokines in patients with CD. 


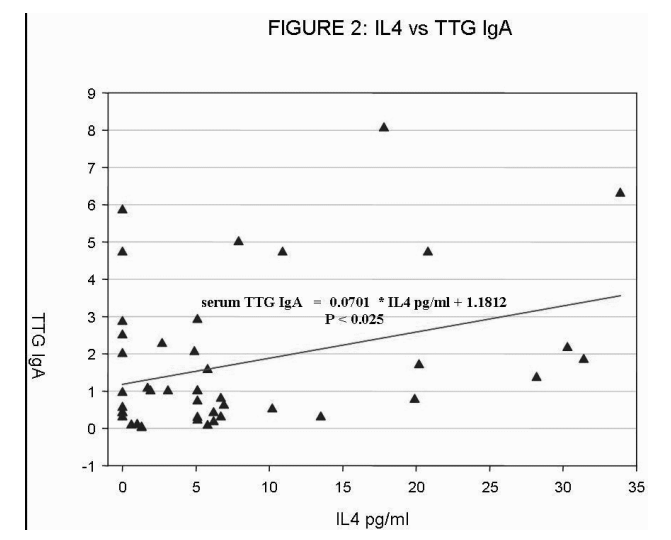

487

Association Studies of Myo9b Genetic Variants in a British Celiac Disease Cohort

Karen Hunt, Alienke J. Monsuur, Parveen J. Kumar, Simon P. Travis, Julian R. Walters, Derek P. Jewell, Raymond J. Playford, Cisca Wijmenga, David A. van Heel

Background and Aims: Development of celiac disease involves an interaction between environmental factors (especially dietary wheat, rye and barley antigens) and genetic factors (there is a strong inherited disease susceptibility). The known HLA-DQ2 and -DQ8 association explains only a minority of disease heritability. A recent study in the Dutch population suggested that genetic variation in the 3' region of myosin IXB (MYO9B) predisposes to celiac disease (Monsuur et al. Nature Genetics 2005 doi:10.1038/ng1680). MYO9B is a Rhofamily GTPase-activating protein involved in epithelial cell cytoskeletal organisation. MYO9B is hypothesised to influence intestinal permeability, and hence intestinal antigen presentation. Methods: Four single nucleotide polymorphisms (rs2305767, rs2305765, rs2305764, rsl457092) were chosen to tag all common haplotypes of the MYO9B 3' haplotype block (exons 15-27). We genotyped 375 celiac disease cases and 1346 controls ( 371 healthy and 975 population-based). All individuals were white UK Caucasian ethnicity. Results: UK healthy control and population control allele frequencies were similar for all MYO9B variants. Case-control analysis showed no significant association of any variant or haplotype with celiac disease. Frequencies of rs 2305764 were $38.7 \%$ in celiac disease and $41.5 \%$ in controls. Conclusions: Genetic variation in MYO9B does not have a major effect on celiac disease susceptibility in the UK population. Differences between populations, a weaker effect size than originally described, or possibly a type I error in the Dutch study, might explain these findings.

488

Long Term Histological Follow-Up of People with Coeliac Disease Gerry Robins, Thomas Fitzgerald, Peter Howdle

Background: Increasing numbers of people are being diagnosed with celiac disease (CD) for which the only treatment is a gluten-free diet [GFD]. There are few studies looking at the long-term histological follow-up of people with CD. Methods: All CD patients, with an index small bowel biopsy and one or more sets of follow-up biopsies and attending a CD clinic in either of two large UK teaching hospitals have their details entered into a Celiac Patient Database [CPD]. Clinical information is entered prospectively where possible and relevant retrospective data extracted from notes, including histological score [HS] (classified as normal, increased intraepithelial lymphocytes [IELs] only, partial villous blunting [PVB], subtotal villous atrophy [SVA] or total villous atrophy [TVA]) and overall compliance with GFD (good, partial, poor). The primary aim of this study is to look at the length of time to histological remission [HR] in CD patients. Correlation between HS, gender, age and compliance was examined by Kendall's rank, Mann-Whitney U or Chi square tests, as appropriate Results: Of the 169 patients ( 125 female, 44 male) mean age at diagnosis was 42.8 (range 0.73-80.32) years with a mean follow up of 6.03 (range 0.13-26.21) years. Median number of sets of biopsies taken was 3 (range 2-12). Nine (all female) patients in the CPD had an initial biopsy reported as normal (i.e. they had latent CD), 12 with IELs, 31 with PVB, 56 with PVA and 61 with TVA. Of the 160 patients with an initial histological abnormality, 58 (43 female, 15 male) patients had a documented complete HR. Only 28 $(48 \%)$ remissions occurred within 2 years, 41 (71\%) within 5 years and the remaining 17 took greater than 5 years to achieve HR. Overall $129(81 \%)$ of these 160 patients showed either HR or some improvement in their HS at least once, but 26 (16\%) showed no change and $5(3 \%)$ a deterioration. There was no association between either gender or age and HR, although there was a significant trend towards faster $H R$ as patients got older $(P<0.01)$. There was a strong correlation $(\mathrm{P}<0.001)$ between good compliance with a GFD and better HS, but not between age and HS, gender and HS or age and compliance. Conclusion: Time taken to histological remission even for CD patients with good compliance for GFD may take longer than many clinicians realise, especially in younger patients.
Risk Factors for Opportunistic Infections in Inflammatory Bowel Diseases: A Case-Control Study

Murat Toruner, Edward V. Loftus, Jean F. Colombel, Robert Orenstein, W S. Harmsen, Alan R. Zinsmeister, William J. Sandborn, Laurence J. Egan

BACKGROUND: Numerous case series have reported opportunistic infections in patients with inflammatory bowel disease (IBD). However, risk factors that predispose IBD patients to opportunistic infections have not been defined. AIMS: We sought to identify clinical factors that were associated with opportunistic infections in IBD patients. METHODS: We conducted a case-control study to identify risk factors for opportunistic infections in IBD patents seen at Mayo Clinic Rochester between 1998 and 2003. Opportunistic infections were defined as infections caused by an organism that does not ordinarily cause disease but that under certain circumstances becomes pathogenic. Liver transplant patients were excluded. Cases were identified by searching the diagnostic index and were only accepted with laboratory confirmation of opportunistic infection, or a pathognomonic clinical presentation. For each case, 2 controls that did not have a history of opportunistic infection were selected, and matched on type of IBD, gender, date and residence. Conditional logistic regression was used to assess associations between putative risk factors and opportunistic infections. RESULTS: One hundred verified cases were identified. Cases and controls were similar with respect to age, disease duration and extent. Corticosteroid, AZA/6MP or infliximab use within 3 months before the infection was associated with a significantly elevated risk of opportunistic infection, odds ratios (OR) 3.4 (95\% CI 1.8-6.2), 3.1 (95\% CI 1.7$5.5)$ and 4.4 (95\% CI 1.2-17.1) respectively. The OR for opportunistic infection associated with the use of any 1 of these drugs (relative to none) was 2.6 (95\% CI 1.4-4.7), and increased when 2 or more drugs were used to $12.9(95 \% \mathrm{CI} 4.5-37.0)$. The use of corticosteroids alone or AZA/6MP alone was associated with OR of 2.2 (95\% CI 1.1-4.8) and 2.5 (95\% CI 1.25.1) respectively, while their use in combination increased the OR to 15.7 (95\% CI 4.1 59.5). The combination of infliximab with AZA/6MP did not significantly increase the risk. The number of subjects receiving infliximab, corticosteroids and AZA/6MP was insufficient for assessment. The most common opportunistic infection with corticosteroids was candidiasis, with AZA/6MP was viral (e.g. cytomegalovirus and Epstein-Barr virus) and with infliximab was histoplasmosis. No patient died from any opportunistic infection. CONCLUSION Corticosteroids, AZA/6MP and infliximab were all independently associated with an increased risk of opportunistic infections, a risk that increased when they were used in combination. The absolute risk remains to be determined, as does any potential benefit of antimicrobial prophylaxis.

490

Safety of Infliximab and Other Crohn's Disease Therapies - Treat ${ }^{\mathrm{TM}}$ Registry Data with Nearly 15,000 Patient-Years of Follow-Up Gary R. Lichtenstein, Russell D. Cohen, Brian G. Feagan, William J. Sandborn, Bruce A. Salzberg, Donny M. Chen, Michelle L. Pritchard, Delma L. Broussard, Robert H. Diamond

Background and Methods: TREAT, a prospective patient registry, was established to study the long-term safety of infliximab (IFX) and other therapies in Crohn's disease (CD). Results: 6273 patients (pts) were enrolled as of 8/05, of whom 3272 received IFX (8314 pt-yrs (py)) ( $88 \% \geq 2$ infusions) and 3001 received other therapies only (6596 py) with a mean followup of 2.7 yrs. More IFX-treated pts had moderate-to-severe (31.5\% vs $10.7 \%, \mathrm{p}<0.0001$ ) or severe-fulminant $(2.6 \%$ vs $0.6 \%, \mathrm{p}<0.0001) \mathrm{CD}$, more had been hospitalized $(27.4 \%$ vs. $19.1 \%, \mathrm{p}<0.0001)$ in the prior year, and more were taking prednisone $(27.2 \%$ vs. $15.9 \%$, $\mathrm{p}<0.0001$ ) or immunomodulators $(49.4 \%$ vs. $31.9 \%, \mathrm{p}<0.0001$ ). Infusion reactions occurred in $4.0 \%$ of 28,281 infusions; severe reactions in $0.10 \%$. Mortality was identical for both IFX and non-IFX-treated pts (0.47 per 100 py vs $0.47 ; \mathrm{RR}=1.0,95 \% \mathrm{CI}=0.64-1.56)$. In Cox proportional hazards analysis, only the use of prednisone $(\mathrm{HR}=2.24, \mathrm{CI}=1.37-3.66, \mathrm{p}=0.001)$ and narcotics $(\mathrm{HR}=2.63, \mathrm{Cl}=1.60-4.32, \mathrm{p}<0.001)$ were associated with increased risk of mortality. The incidence of malignancies in the two groups was similar $(0.58$ per 100 py in IFX pts vs 0.53 in non-IFX pts; RR=1.1, 95\% CI=0.71-1.63), as was the incidence of lymphoma $(0.06$ per 100 py in IFX pts vs 0.05 in non-IFX pts; $R R=1.3,95 \% C I=0.36$ 5.03). The incidence of serious infections (SI) was 1.29 per 100 py within 3 months of an IFX infusion and 0.68 not within 3 months of an IFX infusion (RR=1.9, 95\% CI $=1.34$ $2.70, \mathrm{p}=<0.001$ ). However, Cox analysis showed IFX was not an independent predictor of SI (HR=1.40, 95\% CI=0.95-2.07). The only medications independently associated with SI were prednisone $(\mathrm{HR}=2.01, \mathrm{Cl}=1.40-2.90, \mathrm{p}<0.0001)$ and narcotics $(\mathrm{HR}=2.72,95 \% \mathrm{CI}=$ $1.87-3.96, \mathrm{p}<0.0001)$. One IFX treated patient with known latent TB, who did not receive adequate prophylaxis, developed active TB. Conclusions: IFX safety is similar to that of conventional immunomodulators. Despite having more severe CD, IFX pts had similar rates of mortality, neoplasm and lymphoma as pts not treated with IFX. IFX-treated pts have an increased risk of serious infection but Cox proportional hazard analysis suggests that this increased risk is independently associated with prednisone and narcotic use, and not infliximab therapy.

491

Risks and Benefits of Infliximab for the Treatment of Crohn's Disease Corey A. Siegel, Chin Hur, Joshua R. Korzenik, Bruce E. Sands

Background \& Purpose: Infliximab is effective for the treatment of active Crohn's disease (CD). However, serious potential complications related to infliximab therapy including lymphoma, sepsis and death have been reported. The purpose of this study was to analyze the risks and benefits of infliximab for the treatment of $C D$ with the goal of providing data to aid both physicians and patients in the decision making process. Methods: A decision tree analysis model was constructed to determine the risks and benefits of infliximab when compared to standard therapy in a 35 year old patient with active $C D$. The analysis simulated two cohorts of 100,000 patients each with one arm receiving infliximab while the other remained on standard therapy. To estimate the risks of lymphoma and other complications, 
a systematic review was performed of all published manuscripts specifically reporting infliximab-related adverse effects and with a minimum of 48 -weeks of follow-up. Compiling data from the 6 manuscripts meeting our criteria, at 1 year there were 1.6 lymphomas/1000 patients and 4.0 non-lymphoma related deaths/1000 patients in the infliximab arm. $46 \%$ of these patients were concomitantly taking either 6-MP or azathioprine. The benefits of infliximab were based on data from the ACCENT 1 trial (Hanauer, Lancet 2002). The clinical course of the standard therapy strategy (no infliximab) and quality of life adjustment factors were based on previously published literature (Silverstein et al. Gastroenterology 1999 , Lewis et al. Gastroenterology 2000). Results: Model results show that in 100,000 patients at 1 year, infliximab will lead to 12,216 more patients in remission, 4255 fewer surgeries and 33 fewer deaths from flares of disease. This is at the cost of 201 more lymphomas and 249 more deaths related to complications from infliximab. Overall, the infliximab strategy resulted in more quality-adjusted life years (QALYs) than the standard therapy strategy $(0.77$ QALYs/patient vs 0.75 QALYs/patient). Sensitivity analysis demonstrated that the model results are robust with changes in endpoints occurring only with large changes (or unlikely values) of model input estimates. Conclusions: Despite an increased risk of lymphoma and death associated with use of infliximab, the substantial clinical improvement and saved surgeries due to infliximab results in an increase in quality-adjusted life years. In properly selected patients, the benefits of infliximab could outweigh its risks. These data should help guide decision making and the informed consent process when considering the use of infliximab for the treatment of Crohn's disease.

492

A Safety Evaluation for Progressive Multifocal Leukoencephalopathy (PML) in Greater Than 3,500 Patients with Crohn's Disease (CD), Multiple Sclerosis (MS), and Rheumatoid Arthritis (RA) Previously Treated with Natalizumab in Clinical Trials

William Sandborn, Stephan Targan

Introduction: Natalizumab is a humanized $\lg _{4}$ monoclonal antibody against $\alpha 4$ integrins It was approved by the FDA in November 2004 for the treatment of MS. Phase 3 trials for $\mathrm{CD}$, had completed enrollment, and a Phase 2 trial in RA was ongoing. Clinical trials and marketing were voluntarily suspended in February 2005 based on the reports of 2 patients receiving combination therapy with natalizumab and interferon beta-la in a MS clinical trial who developed PML. Subsequently, a previously diagnosed malignant astrocytoma in a natalizumab-treated patient with previous exposure to azathioprine was reclassified as PML. Thereafter, a safety evaluation was performed in all patients who had recently been treated with natalizumab in clinical trials to screen for PML. Methods: The safety evaluation protocol included informed consent and an urgent additional follow-up visit(s) to include a physical exam, referral to a neurologist, brain MRI, polymerase chain reaction (PCR) analysis of cerebral spinal fluid (CSF) and serum for JC virus. MRI scans of the brain were performed and interpreted at each center. MRI images were also sent to a core laboratory facility for central interpretation by a neuroradiologist with expertise in white matter disease. An independent adjudication committee (IAC) consisting of experts in JC virology, neurological and clinical manifestations of PML, and MRI imaging of neuroinflammation was created to adjudicate all suspected cases of PML and make a final determination of whether the patient had PML. In addition to patients from clinical trials, 4 post-marketing MedWatch reports of suspected PML were also referred to the IAC. Results: Enrollment in the safety evaluation included $87 \%(1,275), 91 \%(2,248)$ and $92 \%$ (296) of all eligible CD, MS, and RA patients, respectively. Neurological exams and MRIs were completed in $>97 \%$ of CD, MS, and RA patients. CSF was analyzed for JC virus in $6 \%$ of $\mathrm{CD}, 16 \%$ of MS, and $4 \%$ of RA patients, and plasma in $88 \%$ of $\mathrm{CD}, 56 \%$ of MS, and $95 \%$ of RA patients. No additional cases were judged by the IAC to have PML. The 4 post-marketing cases referred to the IAC were also judged not to have PML. Conclusions: Results of this analysis suggest that the absolute risk of developing PML during therapy with natalizumab is low (approximately $0.1 \%$ ). It is not yet clear whether any screening strategy for the early detection of PML will be effective. The potential clinical benefit of natalizumab in selected patients with moderate to severe $\mathrm{CD}$ needs to be carefully weighed against the small risk of PML.

493

Incidence of Benign Infections in Inflammatory Bowel Disease (IBD) Patients Treated with Azathioprine (AZA)

Philippe Seksik, Jacques Cosnes, Isabelle Nion-Larmurier, Jean-Pierre Gendre, Laurent Beaugerie

Introduction: It is well established that serious infections may occur in IBD patients treated by immunomodulators and/or biologics. However, there is no data concerning the incidence of benign infections during exposure to AZA. The aims of our study were: 1) to determine the incidence of benign infections (upper respiratory tract infections (ear nose throat infections and bronchitis), herpes lesions and viral warts) in a prospective cohort of IBD patients receiving $A(A Z A+) ; 2)$ to compare this incidence with that observed in IBD patients receiving neither immunomodulators nor biologics (AZA-); 3 ) in the AZA+ group, to test the role of neutrophil and lymphocyte blood counts in the development of benign infections. Patients and Methods: Between September 2003 and November 2004, 230 outpatients were included in a prospective cohort followed up to July 2005. Total observation time was 207 patientyears and mean observation time per patient was $0.9 \pm 0.4$ patient-years (range: $0.2-1.7$ ). Episodes of upper respiratory tract infections, herpes flare ups and viral warts arising or worsening were prospectively noted using a standardized form filled at each visit. In parallel, neutrophil and lymphocyte blood counts were collected. In each individual, the number of benign infections was expressed as the number of events per observation-year (mean $\pm \mathrm{SD}$ ). In the AZA+ group, neutrophil and lymphocyte blood counts were dichotomized around the median values. Results: The incidence of upper respiratory tract infections in the cohort was $2.1 \pm 2.2$ per observation-year. There was no difference between the AZA+ $(n=169)$ and AZA- $(\mathrm{n}=61)$ groups $(2.2 \pm 2.3$ vs. $2.1 \pm 2.1, \mathrm{p}=0.77)$. The incidence of herpes flare-ups was significantly increased in the AZA+ group compared with the AZA- group $(1.0 \pm 2.6$ vs. $0.2 \pm 0.8$ per year, $\mathrm{p}=0.04)$. Similarly, there was significantly more patients with arising or worsening viral warts in the AZA+ group $(17.2 \%(\mathrm{AZA}+)$ vs. $3.3 \%(\mathrm{AZA}-), \mathrm{p}=0.004)$. In the AZA+ group, there was a trend towards an increased incidence of herpes flare-ups in the subset of patients with low lymphocyte count $(\mathrm{p}=0.06)$. Conclusion: This study suggests that the incidence of herpes flare-ups and the appearance or worsening of viral warts are increased in IBD patients receiving AZA. The increased risk of herpes flare-ups seems to be more pronounced in the subset of patients with low lymphocyte count.

494

Increasing Incidence of Clostridium Difficile Infection in Patients with Inflammatory Bowel Disease

Joseph F. Rodemann, Kimberly A. Reske, Erik R. Dubberke, Christian D. Stone

BACKGROUND: In the past decade, rates of nosocomial Clostridium difficile-associated disease (CDAD) have been increasing for all diagnoses. The incidence of $\mathrm{CDAD}$ in patients with inflammatory bowel disease (IBD) has not been examined, however. We analyzed a sevenyear database of inpatient admissions to determine if the incidence of CDAD has also been increasing in Crohn's disease (CD) and ulcerative colitis (UC). Because CD in particular is often treated with outpatient antibiotics effective against $C$. difficile, we hypothesized that the $C D A D$ rate would be lower in $C D$ than in UC. Based on the observation that CDAD is sometimes the cause for admission in IBD, we also hypothesized that $C$. difficile toxin positivity would, on average, occur within 48 hours of admission, which suggests the infection was acquired in the outpatient setting METHODS: An informatics database of 345,808 adult inpatient admissions from 1998 to 2004 at a large tertiary hospital was analyzed for demographic data, length of stay (LOS), presence of a C. difficile infection, and time from admission to a positive $C$. difficile test. Incidence of CDAD was calculated per year for all admissions combined and separately for the primary ICD-9 discharge diagnoses of $C D$ and UC. Cases with insufficient data or indeterminate colitis were excluded. Descriptive statistics and Chi-square testing were used to determine the risk for CDAD in IBD. RESULTS For all years combined, the rates of CDAD for CD and UC were 16 and 39 cases/1000 admissions respectively, compared to 13 cases/1000 for all admissions. The incidence of CDAD increased in all three groups over the time period studied. Comparing 1998 and 2004 data, CDAD incidence doubled for all admissions (8.8 to 16.9/1000 admissions) and CD (9.5 to 22.2/1000 admissions), and tripled in UC (18.4 to 57.3/1000 admissions; Chisquare test for trend in $\mathrm{UC}=3.8, \mathrm{p}=0.05)$. LOS did not differ significantly between the three groups. Compared to CD, the odds ratio (OR) for CDAD in UC was 2.3 (95\% CI: $1.5,3.6)$. Compared to non-IBD admissions, the ORs for CDAD in CD and UC were 1.5 $(1.1,2.1)$ and $3.5(2.5,4.7)$ respectively. The median times in days from admission to a positive $C$. difficile test for $C D, U C$ and all admissions were $0.8,0.9$ and 4.0 respectively. CONCLUSIONS: Over the seven years studied, the incidence of CDAD in IBD has increased and is higher in UC than CD. IBD patients are more likely to be admitted to the hospital with a $C$. difficile infection compared to non-IBD patients. In both CD and UC, C. difficile toxin assays were predominantly positive within 48 hours of admission, suggesting infection was acquired prior to hospitalization.

\section{1}

Munc18b Controls Zymogen Granule Exocytosis in Pancreatic Acinar Cells Patrick Lam, Maria Kauppi, Laura Cosen-Binker, Vesa Olkkonen, Herbert Y. Gaisano

Background: The Sec1/Munc18 (SM) proteins are essential accessory components of molecular machineries required for membrane fusion. SM proteins serve to induce conformational changes to Syntaxins (Syn) to enable Syns to bind their cognate SNARE partners to form secretory-competent SNARE complexes. Pancreatic acinar cells express non-neuronal SM isoforms Munc18b and Muncl8c, which regulate apical and basolateral exocytosis, respectively. Aim: In the present study, we examined Muncl8b interactions with Syn-2 and Syn3 in mediating specific apical exocytotic events in rat pancreatic acinar cells. We employed adenoviral over-expression of Muncl8b mutants with altered affinity and specificity to Syn2 and Syn-3. Results: Wildtype (WT)-Muncl8b, which binds both Syn-2 and Syn-3 with high affinity, and S48D which binds only Syn-2, both inhibited CCK-stimulated amylase secretion, supporting a role for Syn-2 in apical exocytosis. K314L/R315L mutant, with reduced affinity to both Syns, had no inhibitory effect. E59K mutant, which has no detectable binding to either Syn, caused major inhibition in secretion. Electron microscopic examination revealed that WT-Muncl8b induced formation of abnormal multi-leaflet membrane structures at sites of contact between ZGs, which was not observed with the other Muncl8b mutants, which taken together with the secretion results suggest a role for Syn-3 in ZGZG fusion. Subcellular fractionation detected endogenous Muncl8b on ZGs and plasma membranes (PM), and on CCK stimulation, Muncl8b was released from the ZGs while remaining in the PM. Acini over-expressing WT and S48D mutants showed no changes in Munc18b levels in the ZGs or PM. In E59K transfected acinar cells, CCK stimulation caused a paradoxical increase in this mutant Muncl $8 \mathrm{~b}$ association with ZGs and PM fractions. Syn2, Syn-3 and SNAP23 were all found on the ZG and PM fractions, and the level of Syn-3 decreased in the ZG fraction upon CCK stimulation. Conclusion: Our findings support a role for Munc18b interaction with Syn-2 in apical exocytosis, and Syn-3 in ZG-ZG homotypic fusion, which seems to require Muncl8b displacement from ZGs, likely as a mechanism to activate cognate Syn-3. (Funded by National Institutes of Health, Alcohol Beverage Medical Research Foundation, and Finnish Cultural Foundation).

502

Diverse Roles of Class I PI3K Isoforms $\delta$ and $\gamma$ in Cerulein-Induced Pancreatitis

Lars Fischer, Aurelia Lugea, Mohammad Shahsahebi, Bart Vanhaesebroeck, Josef M. Penninger, Helmut Friess, Anna S. Gukovskaya, Stephen J. Pandol, Ilya Gukovsky

Background \& Aims: Phosphoinositide 3-kinase (PI3K) is a key signaling molecule; however, its function in pancreatitis is poorly understood. Class I PI3K isoforms are critical mediators of immunoinflammatory responses. Furthermore, we recently showed that PI3K $\gamma$ regulates key pathologic responses of the pancreatic acinar cell. In this study, using genetic approaches, 
we sought to determine the roles of PI3K $\delta$ and $\gamma$ in various responses of acute pancreatitis in the cerulein (CR) model. Methods: To inactivate PI3K we used mice deficient in PI3Kg catalytic subunit, pl10g, or homozygous for loss-of-function pl10dD910A mutant of PI3Kס. Pancreatitis was induced by hourly injections of $50 \mu \mathrm{g} / \mathrm{kg} \mathrm{CR}$. We measured both early and late responses of pancreatitis at, respectively, $30 \mathrm{~min}$ and $7 \mathrm{~h}$ after the 1st CR injection. Results: Genetic inactivation of PI3K d or $\gamma$ reduced neutrophil infiltration in pancreatitis by $50-60 \%$. Pancreatic necrosis was also inhibited $\sim 2$-fold. However, serum amylase and lipase levels, and acinar cell vacuolization were unaffected in PI3K mutant mice. PI3K d or $\gamma$ inactivation stimulated apoptosis in pancreatitis by up to 2 -fold. Caspase-3 activity did not increase, suggesting that PI $3 \mathrm{~K}$ regulates apoptosis via a caspase-independent mechanism. The lysosomal marker hexosaminidase was activated in pancreatitis, which was inhibited by $\mathrm{PI} 3 \mathrm{~K} d$ or $\gamma$ inactivation by 85 and $65 \%$, respectively. Cathepsin B activity was also inhibited in PI3K mutant mice. These results suggest important roles for both PI3K isoforms in lysosomal activation in CR pancreatitis. In both mutants, premature trypsinogen activation was markedly reduced at both early $(30 \mathrm{~min})$ and late $(7 \mathrm{~h})$ time points, with the late phase completely inhibited in PI3K $\delta$ mutant mice. In pancreatic acini isolated from PI3K mutant mice, trypsinogen activation by $\mathrm{CR}$ was only partially reduced (compared with wild type), suggesting that non-acinar cells may regulate trypsinogen activation in pancreatitis via PI3K. We previously showed that neutrophils regulate trypsinogen activation. Neutrophils isolated from PI3K mutant mice displayed impaired oxidative burst, which might contribute to the observed inhibition of trypsinogen activation. Conclusions: PI3K $\mathrm{d}$ and $\mathrm{g}$ regulate key responses of pancreatitis, i.e., inflammatory infiltration, trypsinogen activation, and multiple pathways of parenchymal cell death (necrosis, apoptosis, and lysosomal activation), but are not involved in other responses, i.e., hyperamylasemia. The results further indicate the role of inflammatory cells in trypsinogen activation in pancreatitis.

\section{3}

Impaired Pancreatic Regeneration in Notch Signaling Ablated Mice During Acute Experimental Pancreatitis

J.N. T. Siveke, Marcel Lee, Clara Lubeseder-Martellato, Christine Koehler, Roland M. Schmid

Introduction: The adult mammalian pancreas has a strong regenerative capacity after severe cell damage such as in acute pancreatitis. The mechanisms for this process, however, are not well known but involve reactivation of pathways activated in undifferentiated pancreatic cells such as Notch, beta-Catenin or expression of Pdxl. Thus, we were interested what effect ablation of Notch signaling may have during the course of acute pancreatitis and what members of the Notch signaling pathway are involved. Ablation of Notch signaling and its effect during the course of acute pancreatitis was studied in two models, in genetically engineered mice with pancreas-specific inactivation of Notchl or Notch2 and in wildtype mice by using the gamma-secretase inhibitor DBZ which inhibits cleavage of active NotchIC. Methods: Conditional pancreas-specific Notchl and Notch2 knockout mice were generated by crossing p $48+/$ Cre mice with floxed Notchl and Notch 2 mice. These mice are alive and show no obvious organ pathology. Notchl and Notch2 knockout and control mice as well as wildtype mice treated with either DBZ or vehicle as control were followed during caerulein-induced acute pancreatitis. Histological examination, mRNA and protein analysis and ChIP assays were performed before, 8 and 72 hours after induction of acute pancreatitis. Results: During the course of acute pancreatitis, we found only modest morphological differences between Notch knockout, DBZ treated and respective control mice $8 \mathrm{~h}$ after caerulein induction. However, $72 \mathrm{~h}$ after induction we found a significant increase in acinar cell death as well as a change in acinar differentiation status in DBZ treated compared to control mice. Interestingly, Notchl but not Notch2 knockout mice revealed similar morphological alterations as DBZ treated mice providing evidence for a pivotal role of Notchl in acinar cell regeneration. The higher rate in apoptosis together with no change in proliferation rate resulted in a significantly smaller pancreas in DBZ treated and Notchl ablated mice. Histomorphologically, an increase in undifferentiated pancreatic cells, expressing Pdxl, beat-Catenin and Clusterin and most likely of acinar origin, was notable. Conclusion: These data suggest that inhibition of Notch signaling during pancreatic regeneration after cellular damage results in an altered regulation of acinar differentiation processes. By comparing Notchl to Notch2 knockout mice, regenerative processes during acute pancreatitis seem to be regulated by Notchl. The observable shift in acinar differentiation status points to an important role for Notch signaling in differentiation processes in the adult mammalian pancreas

\section{4}

Pancreatic Stellate Cells Are Involved in Pancreatic Regeneration Following Caerulein-Induced Acute Pancreatitis in Rats

Filip Sermon, Veronique Andriessen, Daniel Urbain, Albert Geerts

Background and Aims: Pancreatic stellate cells (PSC) play a major role in the fibrogenesis associated with chronic pancreatitis and are responsible for the desmoplastic reaction surrounding pancreatic tumours. The role of these cells in acute pancreatitis has not been investigated until now. Material and Methods: Acute pancreatitis was induced in rats by six $(50 \mu \mathrm{g} / \mathrm{kg})$ intraperitoneal injections of caerulein at hourly interval. Animals were sacrified at different time points between 1 hour and 14 days after the first injection. The severity of acute pancreatitis was assessed by hydrolase serum levels, histology, and water content. Immunostaining for desmin, vimentin, GFAP, $\alpha$-SMA, and Ki-67 were performed at all time points. Results: In normal pancreatic tissue, PSC were easily identified as desmin or vimentinpositive cells with a typical morphology and a periacinar and periductular distribution. After induction of acute pancreatitis, immunostaining for desmin and vimentin showed lack of immunoreactivity in large areas of the pancreatic parenchyma as soon as 1 hour after the first caerulein injection and a complete absence of immunoreactivity 1 hour after the third caerulein injection whereas blood vessels still showed positive staining (internal positive controls). Some PSC could be detected again after 20 hours. From that time point onward, the density of PSC in the pancreatic parenchyma increased gradually exceeding the density of PSC in normal pancreas with a peak (2.7 X normal) at day 2-3. The density of PSC then decreased gradually to the normal value over the next five days. PSC were not only more numerous during the regeneration phase, they also looked bigger with more cytoplasmic processes as compared to controls. The mean increase in cell surface at day 2 was $67 \%$. Dual immunostainings for Ki67 and desmin allowed us to study simultaneously cell proliferation and reinvasion of the pancreas by PSC. The sequence was as follow: 1) 20 hours: reappearance of PSC (desmin+) around the ductuli 2) 23 hours: presence of Ki67+ cells in and around the ductuli 3) 29 hours: increasing number of ductular Ki67+ cells and invasion of the pancreatic parenchyma by desmin+/Ki67- PSC 4) 48 hours: regeneration phase with numerous Ki67+ acinar cells. Taken together, these results suggest that PSC originate from a periductular reservoir. Conclusion: During an episode of acute pancreatitis, PSC proliferate and reinvade the injured pancreatic tissue before acinar proliferation. This reinvasion seems to originate from a periductular reservoir

\section{5}

Nuclear Factor of Activated T-Cells (NFATs) Function As a Downstream Effector of Calcineurin in CCK-Induced Pancreatic Growth Grzegorz T. Gurda, John A. Williams

Proliferation of pancreatic acinar cells has been shown to be regulated by several GI hormones, most notably cholecystokinin (CCK). Studies in our lab have determined that the Ca2+ activated phosphatase calcineurin is one of the key mediators of the trophic effects of CCK but the mechanism by which calcineurin regulates pancreatic growth are not well understood. The present study aims to evaluate whether the Nuclear Factor of Activated T-cells (NFAT) family of transcription factors act as a transcriptional effector of calcineurin. Feeding camostat, a synthetic trypsin inhibitor that induces endogenous CCK release, to mice was used to study the effects of this hormone in vivo, whereas isolated acinar cells directly stimulated with CCK served as an in vitro model. Western blots showed that NFATcl, c2 and c3 were present in exocrine portion of the pancreas and that acute camostat feeding activated these transcription factors, as indicated by increased nuclear abundance of NFATcl and c2 Administration of camostat also induced a transient increase in nuclear NFAT DNA binding activity with a peak at 1 hour, as shown by EMSA. This effect was dependent on calcineurin, as it was blocked by injection of a calcineurin inhibitor FK506. Supershift analysis suggested that NFATcl and $\mathrm{c} 2$ in cooperation with AP-1 family members c-jun and c-fos were the primary components of this transcriptional complex. Camostat also increased mRNA levels of NFAT-dependent MCIP1, but not NFAT-independent homologous genes MCIP2 and MCIP3. More directly, the transcriptional activity of NFAT, as measured by adenovirally delivered NFAT-luciferase reporter, was induced in vitro in a dose-dependent fashion by $10 \mathrm{pM}$ to $\operatorname{lnM}$ of CCK as well as by $0.5 \mu \mathrm{M}$ to $2 \mu \mathrm{M}$ of a calcium ionophore A23187 (serving as a positive control). NFAT-luciferase activity shows a small, but significant 2.5 -fold increase with $10 \mathrm{pM} \mathrm{CCK}$ and peaks at nearly 600 -fold increase with $100 \mathrm{pM} \mathrm{CCK}$. This increase in activity was nearly completely abolished ( $>95 \%$ inhibition) by addition of low doses o calcineurin inhibitors FK506 (1nM) and CsA (10nM). CCK also increased NFAT DNA binding activity in isolated acini, as shown by western blotting and EMSA. Lastly, both CCK and A23187 induced nuclear translocation of a NFATcl-GFP protein delivered by an adenoviral vector into acinar cells, which points to functional activation of this transcription factor. Thus, both in an in vivo physiological model and in isolated acini, CCK is able to induce nuclear translocation and DNA binding of NFATs, indicating that NFATs act as a downstream effector of calcineurin in CCK-induced pancreatic growth.

\section{6}

Impact of Purinergic Signaling On Fibrosis and Angiogenesis in Chronic Pancreatitis

Beat M. Kunzli, Pascal O. Berberat, Eva Csizmadia, Elzbieta Kaczmarek, Keiichi Enjyoji, Helmut Friess, Simon C. Robson

Introduction: Extracellular nucleotides are released from injured cells, bind specific purinergic-type 2 receptors (P2-R) and modulate inflammation and immunity. Ectonucleotidases hydrolyze extracellular nucleotides to regulate P2-R signaling. Studies in null mice show that Cd39/NTPDasel is the dominant ectonucleotidase of the vasculature and immune cells and has a regulatory role in inflammation, vascular thrombosis, tissue remodeling and angiogenesis. Persistent inflammation, venous thrombotic disease, and progressive fibrosis with parenchymal atrophy and angiogenesis characterize chronic pancreatitis (CP). Manifestations of $\mathrm{CP}$ might be therefore linked to disordered purinergic signaling in vivo. Aim: We have explored the impact of deletion of $\mathrm{Cd} 39$ on the development of fibrosis and angiogenesis in experimental $\mathrm{CP}$ and examined expression of $\mathrm{CD} 39$ and $\mathrm{P} 2-\mathrm{R}$ in human pancreatic disease. Methods: Experimental pancreatitis was induced by intraperitoneal injections of cerulein in wild type (wt) mice and Cd39 null mice, both pretreated with Cyclosporin A. Morphologica and immunohistochemical analyses were performed at 3 and 6 weeks to score inflammation, fibrosis and angiogenesis. Expression of CD39, P2X7, P2Y2, metalloproteinases (MMP-2, MMP-9, MMP-13) and inhibitors (TIMP1), TGF- $\beta$, smooth muscle actin (SMA), CD45, procollagen- $\alpha$ and CD31 was determined by immunohistology, validated at RNA and protein level. Comparable studies were done on human CP specimens. Results: Wt mice $(n=19$, died) developed morphological features in keeping with CP with parenchymal atrophy with fibrosis and neo-angiogenesis. Cd39, P2X7, P2Y2 and MMP-9 were upregulated and markedly overexpressed at 6 weeks. Cd39 expression was on vascular endothelium and infiltrated fibrocytes co-expressing Cd45. Cd39 null mice ( $n=16)$ exhibited no mortality and had preservation of pancreatic weights at 6 weeks (wt $0.10 \mathrm{~g} \pm 0.01 \mathrm{vs}$. null $0.15 \mathrm{~g} \pm 0.01 ; \mathrm{p}=$ 0.002 ). Immunopathology revealed minimal fibrotic changes and blunted angiogenesis. Upregulation of P2-R was observed at all time points as for wt, but substantial early induction of MMP-2, MMP-13, TIMP1 and TGF- $\beta$ was noted specifically within Cd39 null tissues Analysis of human CP samples ( $\mathrm{n}=11$ ) also confirmed high level expression of CD39, P2Y2 and P2X7 within fibrotic tissues and vasculature. Conclusions: High expression of CD39 and P2-R is observed in both murine models of pancreatic inflammation and human CP $\mathrm{Cd} 39$ deletion with alterations in purinergic signaling impact experimental pancreatitis, impeding both fibrogenesis and angiogenesis. Our data implicate purinergic signaling in $\mathrm{CP}$ and suggest novel therapeutic avenues. 
Nodose Ganglia Neurons Demonstrate Gluco-Responsiveness Mediated Via Voltage-Dependent Potassium Channels

Gintautas Grabauskas, Shi-Yi Zhou, Il Song, Chung Owyang

We have shown that hyperglycemia inhibits gastric motility. In rats, the inhibitory action is abolished following perivagal capsaicin treatment. Vagal afferent fibers receive and convey the sensory signals that elicit vago-vagal gastric reflexes, and are strategically positioned to mediate rapid changes in gastric motility in response to systemic glucose. Our hypothesis: a subset of vagal nodose neurons is glucoresponsive and expresses ATP-sensitive $\mathrm{K}+$ channels. Immunocytochemistry studies were performed on neurons isolated from rat nodose ganglia. Immunoreactivities of glucotransporters (Glut $1,2,3,4$ ) were detected in a subgroup of neurons which also demonstrated immunoreactivities of the inwardly rectifying $\mathrm{K}+$ channel 6.2 and the sulfonylurea receptor, as well as glucokinase immunoreactivities. Similar findings on gene message were obtained from RT-PCR. Whole-cell patch clamp recordings were done to determine if the excitability of vagal afferent neurons is modulated directly in response to changes in glucose concentration. In 9/15 (60\%) neurons, substitution of mannitol for glucose $(15 \mathrm{mM})$ in the perfusing medium elicited an outward current $(50 \pm 5 \mathrm{pA})$ associated with an increase in membrane conductance (from $1 / 368 \pm 66 \mathrm{M} \Omega$ to $1 / 223 \pm 37 \mathrm{M} \Omega$ ). Responses were fully reversible, and increased in a concentration-dependent manner as external glucose concentration was reduced in $5 \mathrm{mM}$ increments over the range of 15 to $0 \mathrm{mM}$. The current responses to reductions in external glucose concentration were abolished reversibly by tolbutamide $(0.5 \mathrm{mM}, \mathrm{n}=3)$, a specific blocker of the KATP channels. Responses were also prevented when neurons were dialyzed with a high concentration of ATP ( $1 \mathrm{mM}$ in recording pipette solution). By contrast, disruption of glycolytic activity either by 1) substitution of 2-deoxyglucose for glucose in the perfusion medium, $(\mathrm{n}=3) ; 2)$ administration of the glucose transporter blocker phloretin $40 \mu \mathrm{M},(\mathrm{n}=3) ; 3)$ inhibition of glucokinase with glucosamine $5 \mathrm{mM}(\mathrm{n}=6)$, blocked voltage-dependent $\mathrm{K}+$ currents and produced an inward shift in membrane holding current. These results provide the first demonstration that vagal afferent neurons process glucotransporters and KATP channels. These neurons respond in a specific manner to changes in extracellular glucose concentration. The data suggest that glucose induced modifications of neuronal excitability involve modulation of voltage-dependent $\mathrm{K}+$ channels, including the ATP-sensitive $\mathrm{K}+$ channel. These results provide a functional basis for our previous in vivo findings that nodose neurons play a critical role in mediating alterations in gastric motility in response to changes in systemic glucose levels

508

Stimulation of CCK-1 Receptors Activates a TRPC Like Inward Current in GI Vagal Afferent Neurons

Michael Beyak, Annemarie Surprenant, David Grundy

CCK is one of the most important GI hormones regulating short term food intake via an action on vagal afferents. The ionic mechanism by which CCK stimulates gastrointestinal vagal afferents is unclear. TRPC channels are receptor operated cation channels and are thus potential candidates for mediating CCK induced responses. Methods: NGNs innervating the GI tract were labelled by an i.p. injection of fast blue $(4 \%, 10 \mu \mathrm{L}) .2-7$ days later cells were isolated and whole cell patch clamp recordings were performed on labelled neurons 12-48 hours later. Cells were voltage clamped at $-60 \mathrm{mV}$ and drugs applied using a fast flow solution switching system. Antagonists were applied 2-5 min prior to agonists. Extracellular recordings were made from mouse gastric vagal afferents in vitro. Responses to drug superfusion and ramp distension were examined. Results: CCK-8S (1-100nM) induced a concentration dependent, slowly developing inward current in 38/72 (53\%) of labelled nodose ganglion neurons. The amplitude of this current ranged from $10-150 \mathrm{pA}$ (mean $38 \pm 4.5 \mathrm{pA}$ ), and the reversal potential was approximately $+10 \mathrm{mV}$. CCK induced currents were blocked completely and reversibly by the CCK-1 receptor antagonist lorglumide $(1 \mu \mathrm{M} n=5)$. The effect of a number of putative TRP channel blockers on the CCK induced current was examined. Ruthenium red $(3 \mu \mathrm{M})(\mathrm{n}=5) 2-\mathrm{APB}(100 \mu \mathrm{M})(\mathrm{n}=5)$, blocked the CCK induced current. Furthermore, in all CCK sensitive NGNs ( $n=12$ ), and in 24/29 NGNs tested in total $\mathrm{GdCl} 3(100 \mu \mathrm{M})$ activated a large transient inward current. This current was completely and reversibly blocked by 100uM 2-APB $(\mathrm{n}=5)$. Prolonged application (3-5 min) of Gd desensitized the $\mathrm{Gd}$ sensitive response as well as the CCK induced current $(\mathrm{n}=10)$. In vagal afferent recordings, CCK also stimulated gastric vagal afferents (mean increase $55 \pm 9.5$ spikes/ s) and resulted in a marked shift of the tension / response relationship to the left. These effects were prevented with $100 \mathrm{\mu M} 2$-APB. 2-APB also significantly attenuated stretch induced responses $(66 \%$ inhibition, $\mathrm{p}<0.05)$. Conclusions: CCK-1 receptors activate a TRP - like cation conductance in GI - projecting nodose ganglion neurons. All of these cells also express a $\mathrm{GdCl} 3$ activated TRPC4/5 - like current. We speculate that CCK-1 receptors are coupled to the activation of TRPC channels, as has been described for other Gq coupled receptors. TRPC4/5 appear be candidates. This appears to be a major mechanism by which CCK activates gastrointestinal vagal afferents. Antagonism by 2 -APB of both stretch and CCK induced responses in gastric vagal afferents suggests a potential common mechanism by which CCK and mechanical stimuli may interact.

\section{9}

Serotonin Differentially Activates C- and A- Type Vagal Primary Afferent Neurons Which Contain Vanilliod (VR1) and Puringenic (P2X) Receptors Xiaoyin Wu, Chung Owyang, Ying Li

It is well known that serotonin (5-HT) released from intestinal EC cells mediates motility, secretion, and visceral sensation through activation of vagal afferent $\mathrm{C}$-fibers. These $\mathrm{C}$-fibers contain VRl and are capsaicin sensitive. We have also shown that lower intensity electrical vagal stimulation which activates capsaicin-insensitive A-fibers regulates CNS cortical activities stimulated by noxious colorectal sensation. P2X receptors have been reported to be present on vagal nodose neurons and afferent fibers. In this study we investigate whether 5-HT activates both C- and A-type vagal afferent fibers and examine whether 5-HT sensitive vagal afferents contain VRl and P2X receptors. Single neuronal discharges of nodose ganglia neurons innervating the duodenum were recorded in vivo in rats, followed by juxtacellular neurobiotin labeling to characterize VRI and P2X receptor expression. Vagal A- and C-type neurons were identified based on conduction velocities. Luminal perfusion of 5-HT (10$5 \mathrm{M}$ ) elicited an increase in vagal $\mathrm{C}$ - and A-type neuronal discharges in 17/30 and 11/36 neurons recorded respectively (from basal $0.7 \pm 1$ to $29 \pm 5$ and $33 \pm 4$ impusles $/ 20 \mathrm{sec}$ ). Double labeling immunocytochemistry showed that all of the 9 C-type neurons labeled by neurobiotin contained VRl receptors. In contrast, while 7 labeled A-type neurons activated by 5-HT contained P2X receptors, none of them expressed VR1 receptors. In separate studies using perivagal capsaicin-treated rats, luminal 5 -HT (10-5M) stimulated A-type vagal afferent discharges in $8 / 33$ neurons tested. Intra-arterial injection of ATP $(2$ and $10 \mathrm{mg} / \mathrm{kg})$ and the selective P2X receptor agonist $\alpha, \beta$-methylene-ATP ( 5 and $20 \mu \mathrm{g} / \mathrm{kg}$ ) increased neuronal firing in a dose dependent manner. Further, $\alpha, \beta$-methylene-ATP augmented the neuronal responses to luminal 5-HT (10-5M) by $30 \%$. In capsaicin-treated rats, immunohistochemistry studies showed luminal 5-HT significantly increased the number of fos-positive neurons in nodose ganglia and that $94 \%$ of these neurons contain P2X but not VRl receptors. We conclude that intestinal 5-HT differentially activates subpopulations of both A- and C-type vagal primary afferent neurons. Most of 5-HT activated vagal A-type neurons also contain P2X receptors. The synergistic interaction between 5-HT and ATP in the A-type neurons may enhance vagal responses to noxious stimulation. Hence, in contrast to DRG neurons, where P2X and VRl receptors frequently co-localize, these receptors are found on distinct myelinated A-fibers and unmyelinated C-fibers respectively. This suggests that P2X and VRI receptors are involved in mediating distinct 5-HT activated autonomic reflex functions.

510

Acid Exposure Increase TRPV1 Channel Expression in Spinal Afferents Innervating the Esophagus of Cats

Banani Banerjee, Bidyut K. Medda, Jyoti N. Sengupta, Reza Shaker

Background: It is a general notion that proton-gated ion channels play a key role in visceral hypersensitivity. Gastroesophageal reflux disease (GERD) may contribute to esophageal hypersensitivity by acid-induced activation of these channels and one of the potent molecules involved in sensitization could be transient receptor potential vanilloid l (TRPVI). The aim of this study was to evaluate the expression of TRPVl and co-expression with substance P (SP) in the dorsal root ganglia (DRG) and spinal cord receiving input from the esophagus. Method: 4 groups of cats $(\mathrm{n}=3$ in each group) were used in this study. The group 1 (acuteacid) received $0.1 \mathrm{~N} \mathrm{HCl}(\mathrm{pH} 1.2,30 \mathrm{mins} @ 1 \mathrm{ml} / \mathrm{min})$ under awake condition via a chronically implanted catheter in the mid-esophagus. Group 2 (acute-PBS) received $0.1 \mathrm{M}$ PBS ( $\mathrm{pH} 7.4$ ) in similar fashion and; group 3 (chronic-acid) and group 4 (chronic -PBS) received infusion same way for 5 consecutive days. Tissue samples from thoracic (T2-T4) spinal cord and respective dorsal root ganglia (DRG) and esophagi were collected for TRPV1 immunoreactivity (ir), mRNA expression and measurement of protein level. Myeloperoxidase (MPO) content in the esophageal tissue was measured as marker for neutrophil infiltration in inflammation. Result: ELISA absorbance (OD at $460 \mathrm{~nm}$ ) as measured for MPO indicated a 6- and 10-fold increase in MPO in acute-acid $(0.25 \pm 0.05)$ and chronic-acid $(0.51 \pm$ $0.10)$ compared to PBS-treated cats $(0.05 \pm 0.04)$. There was no significant difference in TRPVI mRNA expression in the spinal cord from acute and 5 days acid-treated and PBS infused groups. However, in DRGs TRPVl gene expression was significantly higher in 5 days acid-treated cats, but not in acute treated cats, compared to PBS-treated cats. TRPV1ir cells significantly increased in acute and 5 days acid-treated cats. In addition, it indicated that in acute acid-treated cats 80-90\% TRPV1-ir DRG cells colocalize with substance P (SP) compared to $38-40 \%$ cells in PBS-treated cats. Similarly, a majority of TRPV1-ir cells in chronic-acid exhibited a high level of colocalization with SP (55-65\%) compared to PBStreated cats (25-30\%). Conclusions: The result indicates that acid exposure in the esophagus increases TRPV1-ir cells in the spinal afferents innervating the esophagus. The study provides a strong evidence of involvement of TRPVI channels in esophageal inflammation.

\section{1}

TRPVl Expression and Function in Chronic Pancreatitis and Its Role in Nociceptive Sensitization and Pain

Guang-Yin Xu, John H. Winston, Mohan M. Shenoy, Huaizhi Yin, Pankaj J. Pasricha

Background and Aims: The mechanism of pain in chronic pancreatitis $(\mathrm{CP})$ is poorly understood. We have previously developed a model of trinitrobenzene sulphonic acid (TNBS)-induced chronic pancreatitis that results in nociceptive sensitization in rats manifested by pancreatic hyperalgesia and referred somatic allodynia. The aim of this study was to investigate the molecular substrate of these changes, specifically focusing on the role of the transient receptor potential vanilloid subfamily 1 (TRPV1). Methods: CP was induced in adult rats by a single intraductal injection of TNBS and dorsal root ganglion (DRG) neurons innervating the pancreas were identified by DiI fluorescence labeling. Whole cell patch-clamp recordings were made from acutely dissociated DRG neurons from rats on day 21-26 post treatment with TNBS or vehicle. In separate experiments, cholera B toxin was used to label pancreatic neurons for subsequent laser capture microdissection (LCM) and quantitative PCR as well as immunohistochemistry. In vivo responses to pancreatic electrical stimulation were recorded at baseline and 30 minutes after i.p. injection of SB-366791 (1.3 $\mathrm{mg} / \mathrm{kg}$ in $0.5 \mathrm{ml}$ ), a highly specific small molecule competitive antagonist of TRPVl. Results: Application of capsaicin produced an inward current with an amplitude of $7.0 \pm 2.8 \mathrm{pA} / \mathrm{pF}$ $(n=6)$ in DRG neurons from the control group. TNBS treatment caused a nearly five-fold increase in capsaicin-induced current density $(\mathrm{n}=12, \mathrm{P}<0.02)$. Further, capsaicin-induced responses were observed in $46.2 \%(n=13)$ of all recorded DRG neurons from control and $70.6 \%(\mathrm{n}=17)$ of all recorded neurons from rats with $\mathrm{CP}(\mathrm{P}<0.05)$. As measured by LCM and subsequent RT-PCR, the expression of TRPVI mRNA in pancreas specific DRG neurons increased by $40 \%$ in the neurons of rats with $\mathrm{CP}$ as compared with controls $(\mathrm{P}<0.05)$. Furthermore, the percentage of TRPVI positive DRG neurons innervating pancreas was significantly enhanced after TNBS treatment (control: $59.9 \pm 4.5 \%, \mathrm{n}=4$; TNBS: $82.1 \pm 5.9 \%$, $\mathrm{n}=3, \mathrm{P}=0.01$ ). Systemic administration of the TRPVl antagonist SB-366791 markedly reduced the pain behavioral response to electrical stimulation in rats with $\mathrm{CP}$ to levels similar to that seen in control rats. This was not a non-specific analgesic effect since the compound 
had no effect on pain behavior in control rats. Conclusions: Thus, these experiments suggest that TRPVl sensitization is a specific mechanism contributing to the hyperalgesia of chronic pancreatitis and that TRPVl is a useful target for treatment of pain in this condition (supported by R01 DK62330-01 to P.J.P.)

512

Identification of Mechanically-Activated Pain Pathways from the Gut Wall Xin-Yung Song, Bao-Nan Chen, Marcello Costa, Simon Brookes

Pain is an important symptom of many gastrointestinal disorders, mediated via spinal afferent pathways, but the peripheral endings responsible have not been specifically identified. Intense distension is the most widely used stimulus to elicit pain from naive (non-inflamed) intestines, in animals and humans. Sensory axons in the serosa and mesentery comprise the great majority of spinal afferent endings in small and large intestines (Lynn \& Blackshaw 1999, Brierley et al 2004) and are leading candidates for mechanonociceptors. We identified transduction sites of serosal/mesenteric endings with in vitro electrophysiology, anterograde labelling and immunohistochemistry in guinea pig ileum. Afferent axons in mesenteric nerve trunks responded to strong focal compression $(300-1000 \mathrm{mg}$ ) with blunt probes at punctate "hotspots", as reported in many previous studies. Anterograde tracing labelled many smooth axons-of-passage throughout the mesentery but the only specialised endings were varicose branching axons ("VBAs") on mesenteric arteries. There was a significant association between mechanosensitive "hotspots" and VBAs on mesenteric arteries ( $\mathrm{p}<0.01)$. Serosal mechanoreceptors were also investigated. In preparations where both mucosa and submucosa had been removed, few hotspots could be detected using blunt probes $(5.9 \pm 2.8 \%$ of tested sites, $\mathrm{n}=$ 6). However, in preparations with submucosa attached, but with muscularis externa and serosa removed, numerous hotspots were identified ( $51.3 \pm 8 \%$ of tested sites, $n=10)$, always located on submucosal blood vessels. Our results suggest that mechanosensitive hotspots for "serosal" receptors comprise some of these sensory endings on submucous arteries. Circumferential stretch (10-20g) of the submucosa alone, or of the mesenteries without any of the gut wall, powerfully activated mechanonociceptors, with high thresholds $(10-20 \mathrm{~g})$. The majority of submucous ( $85 \%$ ) or mesenteric hotspots $(68 \%)$ could also be activated by locally applied capsaicin $(1 \mu \mathrm{M})$. We conclude that serosal/mesenteric primary afferents to the gut wall have mechanotransduction sites comprising varicose branching axons on blood vessels in the submucosa or mesentery. They are powerfully activated by strong distension, consistent with a role as mechanonociceptors, in addition to their well established role in vasodilation. 1. Brierley SM et al Gastroenterology. 127(1):166-78, 2004. 2. Lynn PA \& Blackshaw LA J Physiol (Lond). 518.1:271-82, 1999 This work was supported by R01DK56986 from NIH (USA) and by \#275530 from NHMRC Australia.

\section{3}

Dietary Sulfur Amino Acid Supplementation Improves Plasma, Jejunal and Ileal Mucosal Thiol/Disulfide Redox Status and Increases Indices of Intestinal Adaptation After Massive Small Bowel Resection in Rats

Yvonne S. Nkabyo, Li H. Gu, Yancy 0. Mannery, Junqiang Tian, Dean P. Jones, Thomas R. Ziegler

Background: Thiol/disulfide redox status is determined by the glutathione (GSH)/glutathione disulfide (GSSG) and cysteine (Cys)/cystine (CySS) redox couples. This study in a rat model of short bowel syndrome (SBS) determined whether dietary supplementation with the sulfur amino acids (SAA) methionine (Met) and Cys: 1) results in a more reducing thiol/disulfide redox state in plasma and gut mucosa; and 2) enhances adaptive small intestinal growth. Methods: Adult rats underwent mid-small bowel transection (TX) or $80 \%$ mid-jejunoileal resection (RX). After operation, rats in the TX and RX groups were pair-fed complete diets containing adequate SAA (Con; control for diet) or isonitrogenous, isocaloric diets supplemented with SAA (SAA; 300\% of Cys and 180\% of Met content of control diet, respectively). Four groups of animals were studied: TX-Con ( $n=7)$, TX-SAA ( $n=9)$, RX-Con $(n=9)$, and RX-SAA $(n=10)$. Jejunal and ileal mucosal samples were obtained 7 days after surgery and wet weight, DNA and protein content, crypt depth (CD) and villus height (VH) determined. Mucosal and plasma concentrations of thiols (Cys, CySS, GSH and GSSG) were measured by HPLC. Redox potential (Eh in $\mathrm{mV}$ ) of the Cys/CySS and GSH/GSSG couples was calculated using the Nernst equation. Results: Mean daily food intake was not different between groups. Dietary SAA supplementation resulted in a significantly more reducing plasma Cys/CySS redox potential in RX rats (TX-Con $-92 \pm 2$, TX-SAA $-95 \pm 3$, RX-Con $-93 \pm 4$, and RX-SAA $-105+2 \mathrm{mV} \cdot \mathrm{P}<0.05 \mathrm{RX}-\mathrm{SAA}$ vs all other groups). Jejunal and ileal Cys/CySS Eh became significantly more reducing by SAA supplementation in RX rats; jejunum: TXCon $-197 \pm 9$, TX-SAA $-202 \pm 6$, RX-Con $-203 \pm 5$, and RX-SAA $-212 \pm 2 \mathrm{mV}$ (P<0.05 RX-SAA vs all other groups); ileum: TX-Con $-199 \pm 8$, TX-SAA $-199 \pm 4$, RX-Con $-200 \pm 4$, and RXSAA $-210 \pm 3 \mathrm{mV}(\mathrm{P}<0.05 \mathrm{RX}-\mathrm{SAA}$ vs all other groups). A significant increase in adaptive ileal CD $(+20 \%)$ and $\mathrm{VH}(+21 \%)$ occurred in the RX-SAA group vs the RX-Con group $(\mathrm{P}<0.05)$. SAA also significantly increased ileal wet weight $(+20 \%)$, DNA content $(+34 \%)$ and protein content $(+22 \%)$; each $\mathrm{P}<0.05$ RX-Con vs RX-SAA. Ileal mucosal GSH/GSSG Eh in RX-SAA was positively correlated with ileal wet weight $(r 2=-0.48)$, DNA $(r 2=-0.48)$ and protein content $(\mathrm{r} 2=-0.38)$; all $\mathrm{P}<0.05$. Conclusion: Dietary SAA supplementation after massive small bowel resection improves plasma, jenunal and ileal thiol/disulfide redox status in rats. Increased Ileal adaptive growth with dietary SAA appears to be partially dependent on mucosal GSH/GSSG redox status. These data may have translational significance for nutritional approaches to enhance small bowel redox status and adaptive growth in SBS.
Subcutaneous Glucagon-Like Peptide 2 (GLP-2) Alone and in Combination with Oral Cholylsarcosine in the Treatment of short Bowel Syndrome (SBS) Patients

Palle B. Jeppesen, Peter Hoeyerup, Per B. Mortensen

Background: GLP-2 given subcutaneous has been shown to improve intestinal wet weight absorption short bowel syndrome patients, but the effect on macronutrient and energy absorption seems limited. Cholylsarcosine is a synthetic conjugated bile salt that has been shown to increase fat absorption in SBS patients. The combined effect of these two nove treatment modalities has not been evaluated. Methods: 8 short bowel IBD patients received $400 \mathrm{mg}$ TID GLP-2 s.c. for 26 weeks (2F, $6 \mathrm{M} ; 49 \pm 10$ years; small bowel $178 \pm 62 \mathrm{~cm}$; with a colon; 4 on parenteral nutrition or fluids). 72 -hour balance studies were performed at baseline, week 13 and at week 26. At week 26, the patients were given 2 gram TID cholylsarcosine perorally in addition to GLP-2 given subcutaneously in relation to the three main meals. The intake of cholylsarcosine capsules was initiated 48 hours prior to initiation of the balance studies. Access to food and beverages was free, collecting double portions for analysis. Wet weight, energy (bombcalorimetry) and fat (Van de Kamer) absorption were the difference between diet intake and fecal excretion. Results: The results are presented in the table. Conclusions: Subcutaneous administration of GLP-2 significantly decreases feca wet weight excretion by $906 \pm 597$ gram/day thereby improving the relative wet weight absorption by $16 \pm 14 \%$. A decrease in fecal energy excretion was also observed, but due to the trend towards a lower dietary energy intake in relation to GLP-2 treatment, neither the relative or absolute energy absorption changed. The addition of oral cholylsarcosine did not jeopardize the GLP-2 effect wet weight absorption. Although a significant increase in the fat absorption of $27 \pm 27$ gram/day was observed in relation to the combined treatment with GLP-2 and cholylsarcosine compared to baseline, this effect did not translate into an overall improvement in energy absoption.

Results from 72-hour balance studies

\begin{tabular}{|c|c|c|c|c|}
\hline mean +/- SD & Baseline & $\begin{array}{c}\text { Weeks 13 } \\
(\text { GLP-2) }\end{array}$ & $\begin{array}{c}\text { Weeks 26 (GLP-2 + } \\
\text { CS) }\end{array}$ & $\begin{array}{c}\text { ANOVA } \\
\text { p-value }\end{array}$ \\
\hline $\begin{array}{c}\text { Diet Wet Weight } \\
(\mathrm{kg} / \mathrm{d})\end{array}$ & $4.23 \pm 1.59$ & $3.78 \pm 1.25$ & $3.91 \pm 1.29$ & 0.07 \\
\hline $\begin{array}{c}\text { Fecal Wet Weight } \\
(\mathrm{kg} / \mathrm{d})\end{array}$ & $\begin{array}{c}2.76 \pm 1.37 \\
\mathrm{a}\end{array}$ & $1.85 \pm 1.09 \mathrm{~b}$ & $2.09 \pm 1.14 \mathrm{~b}$ & $<0.001$ \\
\hline Wet Weight Abs. (\%) & $35 \pm 17 \mathrm{a}$ & $52 \pm 18 \mathrm{~b}$ & $47 \pm 18 \mathrm{~b}$ & 0.004 \\
\hline Diet Energy (MJ/d) & $15.28 \pm 5.93$ & $13.73 \pm 5.37$ & $13.66 \pm 4.82$ & 0.06 \\
\hline Fecal Energy (MJ/d) & $\begin{array}{c}6.33 \pm 4.20 \\
\mathrm{a}\end{array}$ & $4.84 \pm 3.47 \mathrm{~b}$ & $4.08 \pm 2.87 \mathrm{~b}$ & 0.004 \\
\hline Energy Abs. (\%) & $58 \pm 19$ & $64 \pm 15$ & $65 \pm 25$ & 0.30 \\
\hline Diet Fat (g/d) & $140 \pm 68$ & $132 \pm 68$ & $125 \pm 61$ & 0.12 \\
\hline Fecal Fat (g/d) & $87 \pm 69 \mathrm{a}$ & $69 \pm 57 \mathrm{ab}$ & $45 \pm 42 \mathrm{~b}$ & 0.003 \\
\hline Fat Abs. (\%) & $39 \pm 36 \mathrm{a}$ & $54 \pm 25 \mathrm{ab}$ & $67 \pm 19 \mathrm{~b}$ & 0.002 \\
\hline
\end{tabular}

515

Gut Decontamination and Dietary Glutamine Each Improve Thiol/Disulfide Redox in Rat Jejunal Mucosa Following Combined Partial Small BowelColonic Resection

Junqiang Tian, Li Gu, Prakash Chandra, Menghua Luo, Andrew T. Gewirtz, Ifor R. Williams, Dean P. Jones, Thomas R. Ziegler

Background: Thiol/disulfide redox status is important for cytoprotection and may regulate cell turnover and function. The thiols glutathione (GSH) and cysteine (Cys) are major mediators of cellular and extracellular redox. We studied the effects of luminal bacteria decontamination and dietary L-glutamine (GLN) supplementation on GSH and Cys levels and GSH redox state in residual jejunal mucosa in a rat model of short bowel syndrome. Methods: Adult rats underwent small bowel transection (TX; $n=10$ ), resection of the distal $60 \mathrm{~cm}$ of small bowel + left colon with jejuno-colonic anastomosis (RX; n=9); RX with oral antibiotics (RX-ABX; n=5); or RX with dietary GLN supplementation (RX-GLN; n=6). After operation, TX, RX and RX-ABX rats were pair-fed complete, semi-purified diets; the RXGLN group was pair-fed an isocaloric, isonitrogenous semi-purified diet supplemented with GLN (4\% w/w). RX-ABX rats received an established antibiotic regimen for gut decontamination (neomycin, metronidazole and polymyxin) in drinking water from 3 days preoperatively until tissue collection on day 21. Mucosa of defined segments of jejunum was analyzed for thiol content by HPLC [GSH, glutathione disulfide (GSSG) and Cys concentrations]. Mucosal GSH/GSSG redox potential (Eh; in $\mathrm{mV}$ ) was calculated by the Nernst equation. Results Daily food intake was not different between groups. Jejunal GSH in TX rats was $14.3 \pm 0.7$ $\mathrm{nmol} / \mathrm{mg}$ protein and was unchanged by RX alone (RX $12.8 \pm 0.6 \mathrm{nmol} / \mathrm{mg}$ protein). Jejunal GSH was markedly increased by both oral antibiotics (RX-ABX $39.3 \pm 3.2 \mathrm{nmol} / \mathrm{mg}$ protein $\mathrm{P}<0.001$ vs TX and RX groups) and dietary GLN (RX-GLN $39.7 \pm 14.3 ; \mathrm{P}<0.001$ vs TX and RX groups). Jejunal GSH redox potential (Eh) was unaltered by bowel resection (TX; $243 \pm 2$ vs RX $-242 \pm 2 \mathrm{mV}$; NS), but became significantly more reducing with both oral anti- biotics and GLN supplementation (RX ABX $-263 \pm 4$ and RX-GLN $-259 \pm 5 \mathrm{mV}$; each $\mathrm{P}<0.001$ vs TX and RX groups). Jejunal Cys levels were similar in TX and RX groups (TX $0.9 \pm 0.1$ vs RX $0.9 \pm 0.2 \mathrm{nmol} / \mathrm{mg}$ protein; NS), but were significantly increased by oral antibiotics (RX-ABX $6.2 \pm 1.5 \mathrm{nmol} / \mathrm{mg}$ protein; $\mathrm{P}<0.01$ vs the other 3 groups) and dietary GLN (RX-GLN $2.9 \pm 1.1 \mathrm{nmol} / \mathrm{mg}$ protein; $\mathrm{P}<0.05$ vs TX and RX). Conclusions: Both oral antibiotics and dietary GLN improve important indices of thiol/disulfide redox status in the residual jejunal mucosa of rats following massive small bowel-colonic resection. Our results suggest that: 1) these agents each may have redox-mediated cytoprotective effects in this model; and 2) the presence of luminal bacteria may influence the redox state of the small bowel mucosa. 
The Effect of Severe Malnutrition and Subsequent Refeeding On Gut Mucosal Protein Fractional Synthesis in Human Subjects

Trevor A. Winter, Steven J. O'Keefe, Marie Callanan, Tessa Marks

Objective: Malnutrition has been associated with gut mucosal atrophy, impaired absorption and increased permeability. This study investigated the effect of severe malnutrition, and subsequent re-feeding, on duodenal mucosal architecture, and gastric, and duodenal mucosa protein fractional synthesis (mucosal turnover). Research Methods and Procedures: 23 severely malnourished patients were studied. Endoscopic biopsies were taken from the duodenum for assessment of mucosal architecture. In vivo mucosal turnover was investigated in the gastric body, antrum and duodenum by means of 14C-leucine incorporation into tissue protein following a 4 hour primed/constant IV infusion. The studies were repeated following a period of re-feeding. Results were evaluated in comparison with a group of 22 healthy controls, and illustrated as the mean (standard error of the mean). Statistical analysis was by the Statistica package using analysis of variance (ANOVA), Students t-test and MannWhitney U test as appropriate. Results: The mean body mass index (BMI) of the malnourished patients was $13.29 \pm 0.33 \mathrm{~kg} / \mathrm{m} 2$, compared to $22.67 \pm 0.63 \mathrm{~kg} / \mathrm{m} 2$ in the controls $(\mathrm{P}<0.001)$ Various degrees of villous atrophy were evident in 8 patients (35\%). Mucosal turnover in the controls and malnourished patients were similar (gastric body $36.49 \pm 2.88 \% / d$ vs. $33.41 \pm 3.08 \% / d$; gastric antrum $25.51 \pm 2.20 \% / d$ vs. $24.95 \pm 2.32 \% / d$; duodenum $25.90 \pm 2.58$ $\% /$ d vs. $25.49 \pm 1.99 \% / d$ ). Following re-feeding, the BMI of the malnourished patients increased to $15.87 \pm 0.44 \mathrm{~kg} / \mathrm{m} 2(\mathrm{P}<0.001)$. Villous atrophy was still evident in 3 patients. Mucosal turnover increased significantly (gastric body $51.80 \pm 8.12 \% / \mathrm{d} ; \mathrm{P}=0.02$, gastric antrum $33.44 \pm 3.66 \% / \mathrm{d} ; \mathrm{P}<0.05$, and duodenum $46.27 \pm 80.2 \% / \mathrm{d} ; \mathrm{P}<0.01$ ), with the mucosal turnover of the gastric body and duodenum now significantly greater than the control values $(\mathrm{P}<0.05)$. Conclusions: Despite severe malnutrition, and evidence of villous atrophy, mucosal turnover of the gastric body, antrum and duodenum remained similar to control values. Enteral feeding resulted in a significant increase in mucosal turnover, with improvement in villous atrophy, indicating a trophic response to topical nutrients.

\section{7}

Continuous Enteral Feeding Improves Intestinal Absorption in short Bowel Syndrome Patients

Francisca Joly, Xavier Dray, Sophie Rousseau, Laurence Barbot, Kouroche Vahedi, Nathalie Kapel, Bernard Messing

Background: Continuous enteral feeding (CEF) in the post-operative period may help short bowel syndrome patients (SBS) to be weaned off parenteral nutrition. Past this period, CEF has not been tested either exclusive (ECEF) or partial, i.e. oral + CEF (OCEF) compared to usual oral feeding (OF). It was postulated that absorption could be better with CEF than OF due to motor disturbances in SBS, the latter inducing a decreased contact time between gut and nutrients. Methods: In 16 patients $(7 \mathrm{M} / 9 \mathrm{~F}$, mean age $53 \mathrm{yr}$ ), with SBS constitution $>3$ months and a mean remnant small bowel length of $76 \mathrm{~cm}$ (4 jejunostomy, 12 jejunocolic anastomosis), intestinal absorption were compared on 3 different 7 -d regimen periods: ECEF vs OF ( $n=16)$ vs OCEF (including $1000 \mathrm{Kcal}$ with CEF, $n=9)$. During the last 3 -d of each regimen period, caloric intake and fecal output were measured. Intestinal absorption rates of lipids (Van de Kamers' method), proteins (Kjeldahls' method) and glucids [bomb calorimetry-(proteins + lipids)] were calculated. The study was performed in a median time lag of 33 months after the last surgery. Wilcoxon matched-pairs signed-ranks tests were used for statistical analysis. Results: Total intake were significantly greater with OCEF (3106 $\mathrm{kcal} / \mathrm{d})$ than with CEF $(2407 \mathrm{kcal} / \mathrm{d})$ and OF $(1993 \mathrm{kcal} / \mathrm{d})(\mathrm{p}<0.0001)$. No significant change was seen in stool output between the 3 periods. The caloric absorption rate was significantly greater with ECEF $(82 \%)$ and OCEF $(75 \%)$ than with OF $(65 \%)(\mathrm{p}<0.001)$, Similar significant improvements were observed for both lipids (69\% and 59\% vs $40 \%$ respectively, $\mathrm{p}<0.001$ ) and proteins ( $72 \%$ and $67 \%$ vs $57 \%$ respectively, $\mathrm{p}<0.001$ ). Absolute gain (g per kg body weight) for protein was 1.0 in ECEF and 1.1 in OCEF vs OF (both $\mathrm{p}<0.0001)$. For lipids, gain $(\mathrm{g} / \mathrm{Kg})$ was 0.55 in ECEF and 0.72 in OCEF vs $\mathrm{OF}(\mathrm{p}<0.005)$ Conclusions: in SBS patients, CEF (whether exclusive or not) induces a significant $10-20 \%$ increase in intestinal absorption rates compared to $\mathrm{OF}$ alone, total energy gain being equivalent to $35 \%$ of resting energy expenditure.

\section{8}

Evolution of the Quality of Life of Malnourished Patients Treated with Home Enteral Nutrition

Xavier Hebuterne, Pierre Senesse, Nicole Gaconnet, Patrick Bachmann, Alain Burde, Kamel Arab, Corinne Bouteloup, Erick Beaujard, Laurence Beck, Franck ArnaudBattandier, Stephane M. Schneider

Quality of life (QOL) is lower in home enteral nutrition (HEN) patients than in the general population. As HEN aims at improving QOL, the aim of this study was to assess prospectively the evolution of QOL after the onset of HEN. Methods. All patients able to read, understand, and fill QOL forms and treated with HEN in 23 centers during one year were included in this study QOL was evaluated at home the day of discharge from hospital (M0) and after one (M1) and three months (M3) of HEN. Two validated generic auto-questionnaires (SF36 and EuroQOL) were used. SF-36 explores eight domains: physical functioning (PF), role physical (RP), bodily pain (BP), social functioning (SF), mental health (MH), role emotional (RE), vitality (VT) and general health (GH), ranging from 0 (worst) to 100 (best). EQ-5D includes five questions that permit to calculate a score (0 to 1 ), and a visual analogue scale (VAS) (0 to 100). A subjective assessment questionnaire with 33 questions on HEN daily experience for the patient and his/her relatives, impact on current activities, physical and mental wellbeing and the role of HEN in their evolution was also used. A multivariate analysis was performed to determine discriminating factors associated with the evolution of QOL. Results. 193 patients (132M, 61F; $59 \pm 13$ yrs ; BMI:19.9 \pm 4.4 , loss of BW: $13.9 \pm 10.6 \%$ ) treated with HEN for head and neck or esophagus cancer (66.3\%), digestive disease (12.4\%), neurological disease $(8.8 \%)$ or others, were included. The mean energy input was $1585 \pm 512$ $\mathrm{kcal} / \mathrm{d}, 78 \%$ of patients had a gastrostomy tube, and a pump was used in $70 \%$ of patients EQ5D $(0.56 \pm 0.38$ vs $0.61 \pm 0.30)$ and VAS $(49.5 \pm 19.3$ vs. $55,2 \pm 18,4)$ improved significantly $(\mathrm{P}<0.05)$ at $\mathrm{M} 3$ but not at M1. The evolution of SF-36 domains at M1 and M3 is presented in the table. In multivariate analysis age $>45 \mathrm{yr}$, low performance status and cancer were associated with a lower improvement of QOL. Subjective questionnaires suggested that many burdens were associated with HEN. However, HEN was felt very (49\%), rather $(37 \%)$ or quite $(7 \%)$ beneficial by patients and $92 \%$ of patients felt secure vis a vis HEN Conclusion. Despite burdens due to HEN, health-related quality of life is improved during the first weeks of HEN. This is indicated both by validated QOL markers and by patients' subjective assessment. Discriminating factors associated with a somewhat less effect of HEN on QOL are age, low performance status and cancer.

\begin{tabular}{|c|c|c|c|c|c|c|c|c|}
\hline & PF & RP & BP & GH & VT & SF & RE & MH \\
\hline \multirow{2}{*}{ M1 } & 0.58 & $13.4 *$ & $4.7^{*}$ & -1.25 & $3.09^{*}$ & 1.16 & 9.63 & $-0.50^{*}$ \\
& $-2.4-3.6$ & $7.4-19.5$ & $1.1-8.7$ & $-3.2-0.7$ & $0.4-5.8$ & $-2.7-5.1$ & $3.3-16.0$ & $-0.6--0.3$ \\
\hline \multirow{2}{*}{ M3 } & 3.51 & $16.3^{*}$ & $9.78^{*}$ & 0.37 & $3.09^{*}$ & $6.21^{*}$ & 6.57 & $3.15^{*}$ \\
& $-1.4-8.4$ & $9.1-23.5$ & $5.7-13.9$ & $-2.4-3.1$ & $0.4-5.8$ & $1.8-10.7$ & $-2.7-15.2$ & $0.1-6.2$ \\
\hline
\end{tabular}

Net improvement $(95 \%$ CI) compared with M0. *significantly different from M0

525

Bone Morphogenetic Protein (BMP)-4-Mediated Transformation of Inflamed Squamous Esophageal Mucosa Into Barrett's Esophagus

Jantine van Baal, Francesca Milano, Navtej S. Buttar, Agnieszka M. Rygiel, Floor de Kort, Jacques J. Bergman, Kenneth K. Wang, Maikel P. Peppelenbosch, Kausilia K. Krishnadath

BACKGROUND: To identify genes specifically involved in the transformation of squamous epithelium into Barrett's esophagus (BE), we previously used SAGE to compare the expression profile of BE with normal squamous esophagus (SQ) and gastric cardia mucosa. BMP4 was found to be uniquely expressed in BE. The aim of this study was to investigate the potential role of BMP4 in the metaplastic development of BE. MATERIAL AND METHOD: Immunoblotting was performed on patient biopsies of BE, SQ and inflamed SQ mucosa for the presence of BMP4 and protein members of the BMP pathway i.e. BMP Receptor IA and II, Smad 4, P-Smad 1/5/8 and ID2. Furthermore, primary cell cultures of biopsy specimens of SQ and BE were established. The primary cell cultures of SQ cells were treated with rec. h. BMP4 and the BMP4 pathway was investigated by immunoblotting. Immunohistochemistry was performed for analysis of the Cytokeratin (CK) expression pattern and microarray analysis was done to analyze and compare the gene expression profiles. RESULTS: Immunoblotting revealed BMP4 expression and activation of associated signaling in BE and inflamed SQ mucosa but not in normal SQ (see Fig). Upon treatment of SQ cells with BMP4 the level of P-Smad 1/5/8 was increased, this was blocked through addition of the BMP antagonist Noggin. Phenotipically, a shift of the CK expression pattern, of the BMP4 treated SQ cells towards that of columnar cell type was found with upregulation of CK7 and CK20 and downregulation of CKI0/13. Finally analysis by microarrays demonstrated a shift of the gene expression profile of the BMP4 treated SQ cells towards that of BE cells. CONCLUSION: BMP4 is a keyplayer in the transformation of inflamed esophageal mucosa into BE. We propose that the premalignant metaplasic development of BE is mediated by BMP4 and that inhibition of BMP4 signaling offers a novel avenue for the therapeutic management of this disease.
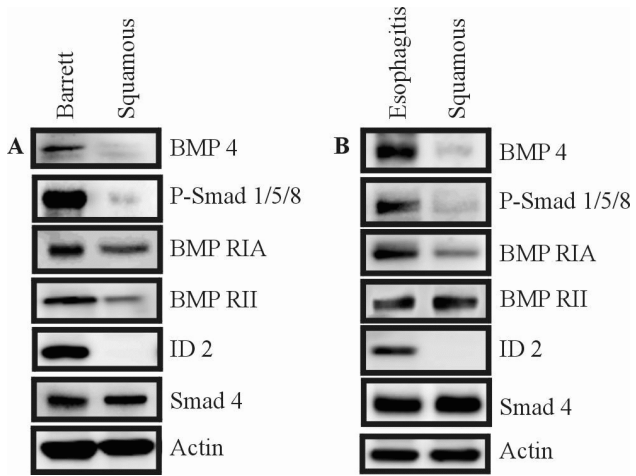

526

A Pro-Inflammatory IL-10/IL-12 Gene Profile Is Associated with An Increased Risk for Developing Barrett's Esophagus

L.M.G. Moons, J.G. Kusters, J van Delft, E.J. Kuipers, R. Gottschalk, W.A. Bode, H. Geldof, A.H.M. van Vliet, J.C.S. Kleinjans, P.D. Siersema

Introduction: The development of a Barrett's esophagus (BE) is paralleled by a shift towards a Th2 type immune response, suggesting a causal relationship. A strong pro-inflammatory response is associated with this shift towards a Th2 response, both in a TNF- $\alpha$ knockout mouse, and in Helicobacter pylori induced gastric carcinoma. Increased expression of proinflammatory cytokines has also been associated with esophageal carcinogenesis, and it is conceivable that this is at least partially based on genetic differences. As patients homozygous for the CCG-haplotype of the anti-inflammatory cytokine IL-10 produce higher IL-10 levels, and the IL-12 C-allele is associated with increased levels of pro-inflammatory cytokine IL12 , the distribution of both genotypes was determined in patients with BE to test whether a genetic based increased pro-inflammatory response is involved in the development of $\mathrm{BE}$ Methods: DNA was obtained from 258 patients with histologically confirmed BE $(100 \%$ Caucasian; mean age $62 \pm 13 ; 68 \%$ male) with a mean BE-segment length of $4.2 \pm 2.3 \mathrm{~cm}$, 
and 247 patients with endoscopically confirmed reflux esophagitis (RE) (100\% Caucasian; mean age $56 \pm 14 ; 54 \%$ male). Gene polymorphisms of IL-12 (A1188C) and IL-10 (C-592A; C-819T, A-1082G) were determined by multiplex SNaP-shot ${ }^{\mathrm{TM}}$ methods. Results: BE patients were less frequent homozygous for the IL-10 CCG-haplotype (21\% CCG versus 79\% Acarriers) as compared to RE patients (30\% CCG versus 70\% A-carrier; $\mathrm{p}<0.015$ ), corresponding to a reduced risk for BE in IL-10 CCG homozygotes [OR 0.61 (95\% CI 0.41-0.91); p= 0.016). The IL-12 C-allele was more frequently detected in BE patients (35\% C-carrier versus $65 \% \mathrm{AA}$ ) as compared to RE patients ( $27 \% \mathrm{C}$-carrier versus $73 \% \mathrm{AA}, \mathrm{p}=0.061$ ), corresponding to an increased risk for $\mathrm{BE}$ in the C-allele carriers [OR $1.4(95 \% \mathrm{CI} 0.98$ 2.10); $\mathrm{p}=0.062$ ). Combining IL-10 and IL- 12 allele data revealed that BE patients more frequently had the pro-inflammatory combination IL-12 C-carrier and IL-10 A-carrier [BE $27 \%$ versus RE $18 \%$. OR $2.5(95 \% \mathrm{CI} 1.40-4.44) ; \mathrm{p}=0.002$ ], and less frequently the antiinflammatory combination IL-12 AA and IL-10GG [BE 12\% versus RE 21\%; OR 0.40 (95\%CI 0.23-0.72); $\mathrm{p}=0.002$ ). Conclusion: Based on the polymorphic IL-10 and IL-12 allele distribution of patients with $\mathrm{BE}$ and RE, BE patients are more likely to have an increased pro-inflammatory response than RE patients in the presence of the same stimulus. This may be an important drive for the inflammatory shift towards the observed Th2 type response in BE, and eventually the development of specialised intestinal metaplasia. Treatment of the inflammatory response may therefore decrease the incidence of $\mathrm{BE}$

527

Origin of Barrett's Epithelium: Development of Columnar Characteristics and Increased Expression of Anti-Apoptotic Proteins in Het-1A Squamous Esophageal Cells Made Resistant to Low $\mathrm{pH}$ and Bile Acids Katerina Dvorak, Melissa Chavarria, Claire M. Payne, Harris Bernstein, Carol Bernstein, Harinder Garewal

Background: Barrett's esophagus (BE) is characterized by the replacement of squamous epithelial cells by metaplastic intestinal-like columnar epithelium. The cell of BE origin remains unclear. We favor the hypothesis that $\mathrm{BE}$ originates from esophageal stem cells as a result of reflux with gastric and bile acids, two components of refluxate potentially important to the pathogenesis of BE. To test this hypothesis we developed esophageal squamous cells lines resistant to low $\mathrm{pH}$ and/or bile acids as in vitro model systems and evaluated them for expression of columnar characteristics and anti-apoptotic proteins. Methods: Normal squamous Het-1A cells were gradually exposed to low $\mathrm{pH}$ (pH 6 or 5) and/or $0.5 \mathrm{mM}$ bile acid cocktail (BA), consisting of equimolar concentrations of glycodeoxycholic, glycocholic, taurocholic, glycochenodeoxycholic and deoxycholic acid. This BA incorporates known bile acids present in the refluxate. Our goal was to develop cell lines able to survive exposure to $\mathrm{pH} 5$ or 6 and/or BA for 3 hours. The resistant cells have thus far been evaluated (1) morphologically using electron microscopy and Giemsa staining in conjunction with brightfield microscopy, (2) for expression of columnar markers, Cdx-2 and villin, by western blot, and (3) for expression of anti-apoptotic proteins, Mcl-1, Bcl-xL, aven and survivin, using western blot and fluorescence microscopy. Results: Over 85 weeks, we have gradually developed several cell lines resistant to different conditions for up to 3 hours, including low $\mathrm{pH}$ alone $(\mathrm{pH} 6,5), 0.5 \mathrm{mM} \mathrm{BA}$, and $0.5 \mathrm{mM}$ BA plus low $\mathrm{pH}(\mathrm{pH} 6,5)$. An untreated long-passage sensitive cell line was also maintained, as a control, to compare with the resistant cells. The resistant lines displayed changes with the most significant altered pattern observed in the cells resistant to $\mathrm{pH} 5$ and $\mathrm{pH} 5$ and BA. These cells were morphologically different in appearance and showed enlarged nuclei, condensed mitochondria and lipid droplets by EM. Increased expression relative to control cells, was observed for columnar differentiation markers, villin and Cdx-2, as well as anti-apoptotic proteins, including Mcl1, survivin, aven and Bcl-xL. Conclusion: Chronic, repeated reflux with gastric acids and bile acids is the major clinical factor for the development of BE. Our studies show for the first time that squamous cells may show characteristics of transdifferentiation after repeated exposures to low $\mathrm{pH}$ and bile acids. These cells also express markers of columnar differentiation and increased levels of antiapoptotic proteins.

528

Pge2 Regulation By Cytosolic Pla2 $\alpha$, a Potential Non-COX-2 Chemopreventive Target, During Carcinogenesis in Barrett's Mucosa

Malini Madhavan, Cathrine Demars, Sarah Papenfuss, Ganapathy Prasad, Lori Lutzke, Marlys Anderson, Louis Wong Kee Song, Kenneth Wang, Navtej Buttar

INTRODUCTION Reflux of gastroduodenal contents into the esophagus increases PGE2 production, which is closely associated with esophageal adenocarcinoma. Cytosolic phospholipase A $2 \alpha(\mathrm{CPLA} 2 \alpha)$ catalyzes the release of arachidonic acid (AA), the substrate for PGE2 production, from peri-nuclear membrane phospholipids. Pharmaceutical inhibitors of CPLA2 $\alpha$ are under development to be used as an anti-inflammatory agent in patients. The AIM of this study was to examine the expression of cPLA2 $\alpha$ during carcinogenesis in Barrett's esophagus and its effect on Barrett's epithelial (BE) cell survival. METHODS Endoscopic mucosal resections from 10 patients that contained squamous, non-dysplastic and dysplastic Barrett's mucosa as well as esophageal adenocarcinoma were selected and stained with anti-cPLA2 $\alpha$ antibody. Semi-quantitative immunohistochemistry was used to assess cPLA2 $\alpha$ expression in the mucosa. BE cell lines were treated with 20 or $40 \mu \mathrm{M}$ of the selective cPLA2 $\alpha$ inhibitor, AACOCF3, with and without AA $(30 \mu \mathrm{M})$ and PGE2 (20 ng/ $\mathrm{ml}$ ). Total cell count, apoptotic cells (Caspase 3 and 7 Immunoassay) and potentially proliferating cells (Ki-67) were counted. RESULTS Dysplastic Barrett's epithelium had higher expression of cPLA2 $\alpha$ than non-dysplastic Barrett's and squamous epithelium. Adenocarcinoma arising in a background of Barrett's mucosa had the highest expression of cPLA2 $\alpha$. The expression was both epithelial and stromal. Compared with control- treated cells, treatment with $40 \mu \mathrm{M}$ AACOCF3 decreased the proliferation of Barrett's epithelial cells by $34 \pm 6.6 \%(\mathrm{p}<0.05)$. Treatment with $40 \mu \mathrm{M}$ AACOCF3 also increased the percentage of apoptotic Barrett's cells by $69 \pm 4.4 \%(\mathrm{p}<0.05)$. Exogenous AA, as well as PGE2 treatment, reversed the effect of inhibition of CPLA2 $\alpha$ by AACOCF3) on BE cell proliferation and apoptosis. CONCLUSION Expression of CPLA2 $\alpha$ increases progressively and appears to be functionally relevant to the process of carcinogenesis in BE. Inhibition of cytosolic PLA2 $\alpha$ appears to be an important chemopreventive approach as it decreases arachidonic acid, which is the substrate on which the COX-2 enzyme acts to generate PGE2. The inhibition of PGE2 synthesis by decreasing the release of arachidonic acid rather than inhibiting COX2 may avoid the thrombo-embolic effects of COX-2 inhibition. With this approach, the supply of arachidonic acid will be decreased to both pro and anticoagulation cascades.

529

P53 Mediates An Anti-Proliferative Effect of Acid in Non-Neoplastic Barrett's Epithelial Cells

Hui Ying Zhang, Linda A. Feagins, Ruben D. Ramirez, Stuart J. Spechler, Rhonda F. Souza

Introduction: The observation that acid has pro-proliferative effects on Barrett's cancer cells and on Barrett's mucosal explants is a basis for the common clinical practice of prescribing high-dose antisecretory therapy routinely for patients with Barrett's esophagus. However, we have found that acid exposure causes an increase in p53 expression that is associated with an anti-proliferative effect in benign, non-neoplastic Barrett's epithelial cells. Therefore we hypothesized that p53 mediates the anti-proliferative effect of acid, and we tested thi hypothesis by observing the effects of p53 inhibition with RNAi on Barrett's cell proliferation after acid exposure. Methods: The retroviral vector PSUPER-siRNA-p53 was generated by digesting the p53 siRNA sequence from the pSUPER.p53 plasmid and cloning the DNA fragment into the pSUPER.retro.neo mammalian expression vector; pSUPER.retro.neo without the insert served as a control. Supernatants containing pSUPER-siRNA-p53 or pSUPER retro.neo viral particles were used to infect telomerase-immortalized, metaplastic Barrett's cells. Expression levels of p53 were determined by Western blotting using $\beta$-actin as a loading control. Equally seeded wells of infected, uninfected, and empty-vector infected cells were placed in acidic media ( $\mathrm{pH}$ 4.0) for two 3-minute exposures, 60 minutes apart. Cell counts were performed 24 hours later. Results: Infection with pSUPER-siRNA-p53 decreased p53 expression levels to $17 \%$ of those found in uninfected cells; p53 expression in cells infected with the empty vector did not differ from uninfected cells. Uninfected and empty vectorinfected cells both exhibited a significant decrease in cell number following acid exposure ( $1374 \pm 24$ to $1159 \pm 29$ SEM and $1400.5 \pm 55$ to $1237 \pm 55$ SEM, respectively, $<<0.05$ ). In contrast, cells containing p53 siRNA exhibited no significant change in cell number after acid exposure. Conclusions: In non-neoplastic Barrett's epithelial cells, multiple acid exposures have an anti-proliferative effect that can be blocked by inhibiting p53 expression. This study elucidates a molecular pathway whereby acid exposure has potentially beneficial, anti-proliferative effects on benign Barrett's epithelial cells and suggests that the common clinical practice of prescribing antisecretory therapy in dosages beyond those required to heal esophagitis could be detrimental.

\section{0}

Acid Exerts An Anti-Proliferative Effect in Non-Neoplastic Barrett's Epithelial Cells By Delaying Cell Cycle Progression

Linda A. Feagins, Hui Ying Zhang, Mizael Quinones, Deena Thomas, Stuart J. Spechler Ruben D. Ramirez, Rhonda F. Souza

Introduction: Acid has been shown to exert pro-proliferative and anti-apoptotic effects on Barrett's cancer cells and on explants of Barrett's epithelium. In a benign, non-neoplastic Barrett's epithelial cell line in contrast, we have found that multiple acid exposures cause a significant decrease in cell numbers that is not due to a decrease in cell viability or to an increase in apoptosis. To further explore the mechanisms underlying the acid-induced decrease in Barrett's cell numbers, we studied whether our cell line maintains the ability to undergo apoptosis and to express p53 and p21 (proteins that may mediate apoptosis and cell cycle arrest), and we studied whether acid induces a decrease in cell proliferation. Methods: Following irradiation of telomerase-immortalized, metaplastic Barrett's cells with varying dosages of UV-B, we determined cell numbers (by cell counting), apoptosis (by immunofluorescence with TUNEL), and expression levels of p53 and p21 (by Western blotting using $\beta$-actin as a loading control). In other experiments, cells were synchronized with a double thymidine block (which arrests them at the Gl/S boundary of the cell cycle) and then placed in acidic media ( $\mathrm{pH} \mathrm{4.0)}$ for two 3-minute exposures, 60 minutes apart. Cells were collected at various time intervals following the second acid exposure, fixed in $70 \%$ ethanol, and stained with propidium iodide for analysis by flow cytometry. Results: Irradiation with $100-400 \mathrm{~J} / \mathrm{m} 2$ of UV-B significantly decreased cell numbers at 24 hours $(\mathrm{p}<0.05)$. Marked TUNEL staining (indicating apoptosis) was found in cells irradiated with 200 and $400 \mathrm{~J} / \mathrm{m} 2$. p53 expression increased in a UV-B dose-dependent manner, wherea p21 expression increased following 50-300 J/m2, but not $400 \mathrm{~J} / \mathrm{m} 2$ of UV-B irradiation. In control cells that were not exposed to acid, the peak fraction of cells in G2/M was seen at 6 hours. In contrast, acid exposure delayed the G2/M peak to 12 hours. Conclusions: Acid decreases proliferation in a benign, non-neoplastic Barrett's epithelial cell line by delaying cell cycle progression. Although acid exposure does not induce apoptosis in these cells, the cells do retain the ability to undergo apoptosis and to express p53 and p21. Our finding of a potentially beneficial effect of acid on Barrett's epithelial cells suggests that the common clinical practice of prescribing antisecretory therapy in dosages beyond those required to heal esophagitis could conceivably be detrimental for patients with Barrett's esophagus.

531

Association Between Central Activation and Pain Reports in Women with IBS Yehuda Ringel, Douglas A. Drossman, J.N. Leserman, Weili Lin, Kathy Wilber, Brandall Y Suyenobu, Steve Berman, Whitehead E. William, Emeran Mayer

Background: Greater pain reporting in response to rectal distention in patients with IBS ("visceral hypersensitivity") may relate to alterations in peripheral (intestinal) and central pain modulatory mechanisms. In previous studies we found that IBS patients with a history of abuse report more pain despite higher (not lower) rectal sensation thresholds, suggesting altered central pain modulation (Ringel, Gut 2004). Aim: 1) To investigate the association between subjective pain reports and regional brain activation using fMRI. 2) To assess the modulating effects of a history of abuse on pain reporting and regional brain activation. 
Methods: Twenty female subjects were studied. Brain fMRI images (BOLD responses) and pain ratings (on a 0-5 pain scale) were obtained during barostat-mediated baseline and painful $(50 \mathrm{mmHg})$ rectal distension. Statistical parametric mapping (SPM2) analysis was used to identify activation differences in 8 predetermined regions of interest (ROI). Correlations between the activation at a specific region and the subjects' reports of pain were assessed by covariate analyses. Results: 1 . Pain scores during rectal distention for all 20 subjects strongly correlated with midcingulate cortex (MCC) activation [corrected p family wise error $(\mathrm{pFWE})=0.045], 2$. Abused $(\mathrm{n}=10)$ patients had significantly greater $\mathrm{MCC}(\mathrm{pFWE}=$ 0.02 ) and posterior cingulate cortex (PCC) $(\mathrm{pFWE}=0.04)$ activation than non-abused ( $\mathrm{n}=$ 9) patients. 3. IBS $(n=9)$ patients showed greater PCC activation compared with non-IBS $(n=10)$ patients $(p<0.05$ by spatial extent and $p F D R=0.06) .4$. The subgroup of patients with IBS and abuse $(n=5)$ scored significantly higher on pain during distention compared to all others ( 4.5 vs. $3.35 \mathrm{p}<0.04$ respectively) and showed significantly greater activation in the PCC (pFWE=0.02) and MCC (pFWE=0.05) and less activation in the perigenual anterior cingulate cortex (pACC) $(\mathrm{pFWE}=0.01)$. Conclusions: We demonstrated a strong correlation between visceral pain reporting and specific regional brain activation, and an enhancing effect of psychosocial trauma on cingulate activation. The greater activation of the MCC, a region activated in response to internal or external perturbation of homeostasis, together with a reduced activation of PACC in the abuse-IBS group explain the greater subjective pain reports in these patients. Supported by DK48351, R24 AT002681, P50 DK64539 (EAM),

532

Non Genomic Transmission of Visceral Hypersensitivity Across Generations After Maternal Separation

Rene M. van den Wijngaard, Olaf Welting, Pieter H. Looijmans, Wouter J. de Jonge, Guy E. Boeckxstaens

INTRODUCTION: Adverse parent-child interactions are associated with an increased risk to develop IBS later in life. Changes in maternal care induced by neonatal maternal separation (MS) in rats lead to altered stress responsiveness in the separated offspring, which can be transmitted across generations in a non-genomic fashion. Earlier, we showed visceral hypersensitivity to colorectal distension (CRD) in response to water avoidance (WA) stress in adult MS rats. Whether this hypersensitivity is also subject to non-genomic transmission is unknown. METHODS: Female rats that underwent MS or were left undisturbed as a pup, were allowed to mate with non-handled $(\mathrm{NH})$ male rats. The subsequent offspring was not subjected to MS. At the age of 3 months the offspring was equipped with EMG electrodes in the abdominal muscles to record the visceromotor response (VMR) to CRD. Visceral sensitivity was assessed by intermittent distention (1, 1.5, $2 \mathrm{ml}$ ) before and 24 hours after WA in male animals only. Post-WA VMR to CRD was expressed as \% of the pre-WA response to $2 \mathrm{ml}$ distension, and the area under the volume/\%VMR curve (AUC) was calculated. $\mathrm{P}<0.05$ is considered statistically significant, pre-WA vs post-WA. Wilcoxon signed rank test. RESULTS: 4 MS female rats yielded 12 non-separated male pups. Nine of these pups became hypersensitive to CRD after WA (AUC $>77$ ). As a group, the post-WA AUC was significantly increased compared to pre WA (fig $1,{ }^{*} \mathrm{P}=0.004$ ), indicating visceral hypersensitivity to CRD. The male offspring ( $\mathrm{n}=6$ ) of the $\mathrm{NH}$ females did not develop visceral hypersensitivity after WA (fig 1). CONCLUSION: We showed that the NH offspring of MS, but not of NH female rats, develops visceral hypersensitivity to CRD after WA later in life. These data suggest that the feature to develop stress-induced visceral hypersensitivity is transferred in a non-genomic behavioral fashion. At present, cross fostering experiments are being carried out to further confirm this hypothesis.

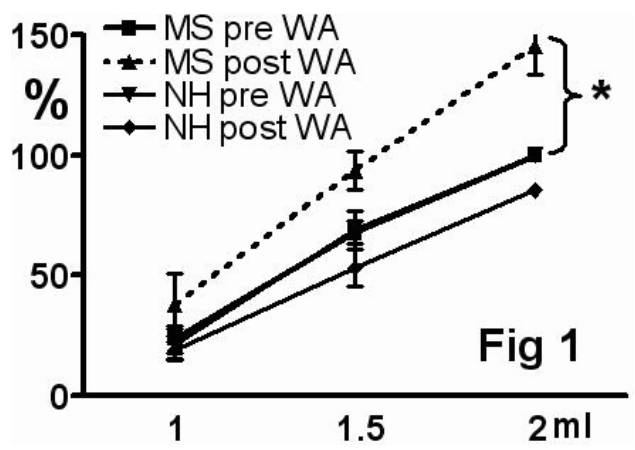

533

Identification of Brain Networks Associated with Visceral Perception and Hypervigilance in Patients with Irritable Bowel Syndrome (IBS) Jennifer S. Labus, Emeran A. Mayer, Steve M. Berman, Brandall Suyenobu, Lin Chang, Bruce D. Naliboff

Background:IBS patients show evidence of hypervigilance and hyperresponsiveness to visceral stimuli. This hypervigilance may play a role in the enhanced perception of visceral stimuli and in the cardinal IBS symptom of abdominal pain. Aims: To identify functional brain networks underlying these responses and especially changes in brain responses associated with perceptual changes over repeated sessions of stimulation. Methods:12 IBS patients(8 females) underwent barostat controlled rectal distension assessments 6 times over a 12month period. During the first and last session, PET(H215O)images were obtained during 2 trials each of resting baseline(BL), aversive rectal distension (INF), and anticipation of an aversive distension(ANT). Multivariate task partial least squares(PLS) was tested for patterns of brain activity related to changing task demands and distributed systems of brain activity operating in IBS patients(seed PLS). Results: IBS symptom severity did not change over the 12 months, but perceptual ratings of the distensions significantly decreased $(\mathrm{p}<.01)$. Task
PLS revealed a pattern of regions that separated BL from ANT across days and included supragenual anterior cingulate cortex(ACC), [Brodmann Area 24], dorsal[46] and ventrolateralprefrontal cortex(PFC) [44/ 47], midbrain, posterior cingulate [31], posterior parietal [40], anterior/mid insula, and hippocampus $(\mathrm{p}<.001)$. Seed PLS identified distributed patterns of activity that were functionally connected with the rostral ACC, an area commonly activated in IBS distension and other experimental pain procedures. Seed PLS revealed two significant networks: one functioning similarly across days and conditions and another that evidenced day-specific functioning. The common network included typical pain regions (thalamus, posterior insula, rostral ACC[24], midcingulate[24/23]), and medial and lateral prefrontal regions (medial PFC[32/10], dorsolateral PFC[46/9]). The day-specific network included somatosensory [2/1/3/4/40], limbic and paralimbic (infragenual[25], amygdala), prefrontal(ventrolateral/medial PFC [11/47]) and midbrain/ brainstem regions. Conclusions: These findings highlight activation of specific networks during actual and anticipated visceral distension. In contrast to the stable neural network which includes the typical pain regions, the day-specific network contains cortico-limbic-pontine circuits. The activity in these circuits may be associated with IBS hypervigilance given that the strength of the functional connections within this circuitry changes following repeated exposure to an aversive visceral stimulus and in conjunction with a decline in perceptual ratings.

\section{4}

Abnormal CNS Response to Anticipation of Visceral Distention in Female Patients with Irritable Bowel Syndrome (IBS) an FMRI Study

Steven M. Berman, Bruce D. Naliboff, Brandall Suyenobu, Lin Chang, Joshua Bueller, Kim Ruby, Michael Minnema, Jean Stains, Emeran A. Mayer

BACKGROUND: Expectation of aversive somatic stimuli results in distinct patterns of brain activation, which can amplify or attenuate afferent signals (Porro et al, 2002). In IBS patients, abnormal brain responses to anticipated visceral discomfort (visceral anxiety) may contribute to altered perceptual and autonomic responses. AIMS: To determine if female IBS patients with constipation-predominant bowel habit (IBS) differ from healthy control subjects (Ctrls) in their brain response to expected visceral discomfort. METHODS: Functional MRI compared brain responses of IBS $(n=14)$ and Ctrls $(n=12)$ to a cue-light which signaled aversive rectal distension in 3-5 s. Subjects received 60-90 15-s distentions. Trials pseudorandomly varied between moderate $(45 \mathrm{~mm} \mathrm{Hg})$, mild $(25 \mathrm{~mm} \mathrm{Hg})$ and sham $(5 \mathrm{~mm} \mathrm{Hg})$ distention. Each trial began with a 15 -s baseline (BL). Data were normalized to a standardized brain image space. SPM2 software generated contrast images contrasting activity during cue (expectation condition) and each level of rectal inflation (inflation condition) to BL for each subject. Within 7 bilateral regions of interest (ROIs) comprising subregions of anterior cingulate gyrus (ACC;3 ROIs), insula (2), amygdala and dorsal brainstem, random effects analyses assessed CNS response to the cue and distensions. RESULTS: Expectation condition: Across all subjects, there were no ROIs significantly activated by the cue light. However, there were BOLD signal decreases, interpreted as deactivations, in insula, amygdala and brainstem. The deactivations were consistent in Ctrls, but not in IBS, producing group differences in right (R) anterior insula $(\mathrm{p}=.009)$, posterior insula $(\mathrm{L}: \mathrm{p}=.098$. $\mathrm{R}$ : $\mathrm{p}=.016), \mathrm{L}$ amygdala $(\mathrm{p}=.044)$ and dorsal pons ( $\mathrm{L}: \mathrm{p}=.005$. $\mathrm{R}: \mathrm{p}=.050$ ). Whole brain exploratory analyses at $\mathrm{p}<.01$ (uncorrected) found the cue activated 291 voxels in IBS but none in Ctrls. In contrast, there were 362 deactivated voxels in IBS compared to 8,251 in $\mathrm{Ctrls}(\mathrm{p}<.001)$. Inflation condition: Both groups showed the expected pain matrix activations during distention, with no significant group differences. CONCLUSIONS: 1. As compared to healthy controls, female IBS patients respond to cued anticipation of uncomfortable visceral distention through less consistent deactivation of brain regions involved in modulation of visceral pain. 2. In contrast, they do not show significant group differences during actual aversive distension. This altered anticipatory response may be related to visceral anxiety. Supported by DK48351, R24 AT002681, P50DK64539 (EAM), NR04881 (BN).

535

A Randomized Controlled Trial of Therapist-Administered vs MinimalTherapist-Contact Cognitive Behavioral Treatment for Moderate to Severe IBS Jeffrey M. Lackner, Kenneth A. Holroyd, Gregory D. Gudleski, Susan S. Krasner, Leonard A. Katz, Catherine D. Powell, Praveen K. Sampath, Rebecca S. Firth, Brian T. Yates

BACKGROUND. While cognitive behavioral therapy (CBT) is regarded as an efficacious treatment for IBS (Gastroenterology, 2003;125(1):19-31), it has not shown a commensurate level of clinical utility because of its cost, limited availability, and labor-intensive demands. Given the prevalence of IBS, the absence of a consistently satisfactory medical option, and a chronic course whose trajectory is subject to strong psychosocial influences, there is an unmet need for a behavioral self management treatment that is less costly to deliver and more transportable yet retains the efficacy of standard, therapist administered CBT. AIMS. To develop and test the feasibility of a largely self administered version of CBT. METHOD. 59 Rome II diagnosed IBS patients (80\% female) were stratified by symptom severity and, after a 4 week baseline, randomized (1:1:1) to 1 of 3, 10-week conditions: (a) 10 session manualized Standard-CBT (S-CBT; $\mathrm{n}=20$ ); (b) 4 session manualized Minimal Contact CBT (MC-CBT, $\mathrm{n}=22$ ); or $(\mathrm{c})$ wait list ( $\mathrm{WL}, \mathrm{n}=17$ ). MC-CBT covered the same range of procedures featured in S-CBT but they were taught through a self-study workbook (Breaking Free of IBS: A Step by Step Guide, Lackner \& Holroyd, 2005) developed expressly for the trial. WL patients' data were obtained before they crossed over (1:1) to an active condition. Primary endpoints were adequate relief from pain and GI symptoms (2 Yes/No items) and global ratings of IBS symptom improvement. Secondary endpoints were distress (BSI), quality of life (IBS-QOL), coping (CSQ, IBS-SEQ), IBS symptom severity (IBS-SS), and cost-efficiency. RESULTS. In intent-to-treat analyses, both CBT versions were significantly $(p<.0001)$ superior to WL at post-treatment in the percentage of participants reporting moderate-tosubstantial improvement in IBS symptoms (MC-CBT $=72.8 \%, \mathrm{~S}-\mathrm{CBT}=73.7 \%, \mathrm{WL}=0.0 \%$ ). A similar pattern was found for the percentage of patients reporting adequate relief of pain $(\mathrm{MC}-\mathrm{CBT}=72.7 \%, \mathrm{~S}-\mathrm{CBT}=73.7 \%, \mathrm{WL}=11.8 \%)$ and $\mathrm{GI}$ symptoms $(\mathrm{MC}-\mathrm{CBT}=63.6 \%$, $\mathrm{S}-\mathrm{CBT}=68.4 \%, \mathrm{WL}=0.0 \%$. MC-CBT patients achieved significant improvements in QOL, IBS symptom severity, distress, and coping (e.g., catastrophizing, self-efficacy to control IBS symptoms), with all pre-post therapy difference $\mathrm{p}$ values $<.01$. Cost effectiveness analyses 
indicated that MC-CBT achieved symptom relief about 5 times as efficiently as S-CBT. 16\% of MC-CBT (vs 15\% of S-CBT) patients dropped out. CONCLUSIONS. Data suggest that a 4-session CBT regimen is a well tolerated, efficient, and clinically effective treatment that provides significant relief from the range of problems of more severe IBS. Supported by NIDDK grant 67878

536

Pain and Depression Predict Improved Health-Related Quality of Life in Severe IBS Treated with Psychotherapy of Antidepressant

Francis Creed, Elspeth Guthrie, Joy Ratcliffe, Lakshmi Fernandes, Barbara Tomenson, Nicholas Read, David Thompson

Objective We have reported previously long-term improved health-related quality of life in patients with severe irritable bowel syndrome (IBS) following treatment with an antidepressant or psychotherapy (1). In this study we assessed the relative importance of improved pain or depression in this finding. Method We assessed 257 patients with severe IBS before treatment and 239 one year after the end of treatment (75 psychotherapy, 85 Paroxetine and 79 treatment as usual) using a visual analogue scale (VAS) of abdominal pain and the Hamilton Depression Rating Scale (HDRS). Linear regression was used to predict change in SF36 physical component score. Structural equations modelling using AMOS tested whether baseline depression (HRSD) score predicted follow-up VAS pain score and vice versa. Results. Preliminary analyses confirmed that VAS pain and HDRS depression scores represented well the dimensions of pain and depression experienced by participants in our sample. Linear regression analysis to predict 1 year follow-up SF36 physical component score (healthrelated quality of life) indicated that pre-treatment VAS pain $(\mathrm{p}<0.001)$ and HDRS depression scores $(\mathrm{p}=0.005)$ and change (follow-up minus baseline) of VAS pain $(\mathrm{p}<0.001)$ and HDRS depression scores $(\mathrm{p}=0.006)$ were all significant independent predictors. Structural equation modelling including baseline and follow-up scores of VAS pain and HDRS depression showed that the overall fit of the model was excellent, chi2 $=2.06, \mathrm{df}=3, \mathrm{p}=0.56$, chi2 $/ \mathrm{df}=0.69$, RMSEA $<0.0005$ and $C F I=1.000$. The standardised regression coefficients for baseline to follow up depression score and VAS pain were $0.36(\mathrm{p}<0.005)$ and $0.38(\mathrm{p}<0.005)$, respectively, demonstrating that each follow up variable is predicted by its own baseline value. The standardised regression coefficient for baseline VAS pain predicting follow-up HRSD was also significant (chi2 $=0.15, \mathrm{p}=0.02$ ), but that for baseline HRSD predicting follow-up VAS pain today was not (chi $2=0.09, \mathrm{p}=0.16$ ). Addition to the model of psychotherapy and SSRI antidepressant treatments as additional variables did not change the model and the same model fits the data in each of the 3 treatment groups, when these were analysed separately. The model held when only patients who also had a psychiatric disorder were included. Conclusion: In patients with severe IBS receiving psychological treatments, both improved pain and depression influences later health-related quality of life but change in depression is not primarily responsible for this result. Other aspects (eg coping with pain and its attribution) are likely to be involved. (1) Gastroenterology 2003; 124 :303-17.

537

Laboratory Parameters in the Diagnosis of Choledocholithiasis: A MetaAnalysis

Jack Braha, Anita Turok, Jian Jun Li, Scott Tenner

The presence of a common bile duct stone warrants intervention to prevent cholangitis and/ or pancreatitis. Due to complications from laparoscopic cholecystectomy including bile leaks, the need for preoperative diagnosis of common bile duct stones has led to increased utilization of imaging, including magnetic resonance cholangiopancreotography (MRCP) and endoscopic retrograde cholangiopancreotography (ERCP). Clinicans often are considering particular laboratory tests in the decision to evaluate the common bile duct in patients with biliary disease. Although several laboratory parameters have been proposed to assist the clinician in determining the risk of a common bile duct stone, there is considerable controversy. The purpose of this study is to determine which laboratory parameters are helpful in predicting the presence of common bile duct stones. In order to overcome the complexity and number of studies that have addressed this issue, a meta-analysis was performed. After a Medline search identified all studies since 1974 that described the role of laboratory parameters in predicting the presence of a common bile duct stone, the papers were analyzed. Only studies that allowed a relative risk calculation were included. Thus, the paper had to include individual data points for the laboratory parameters to be studied. There had to be a group of patients with common bile duct stones and a group of patients without common bile duct stones. The following laboratory parameters were studied: aspartate transaminase (AST), alanine transaminase (ALT), alkaline phosphatase (AP), bilirubin, and amylase. Twenty studies were used in the final analysis. 687 patients with common bile duct stones were compared to 574 patients without common bile duct stones. A weighted mean was calculated with confidence intervals for the results from the combined analysis. The relative risk (RR) for the presence of a common bile duct stone was $3.3(1.2$ - 4.4) for AST, 2.1 (1.1 - 3.5) for ALT, $1.4(0.8-3.3)$ for alkaline phosphatase, and $4.4(2.2-7.7)$ for bilirubin. An amylase of greater than 3 times normal had a RR of $2.2(0.9$ - 3.3). Abnormal liver function tests increase the relative risk of having a common bile duct stone. However, the increased risk appears more significant for the AST, ALT and bilirubin. An elevated alkaline phosphatase and/or amylase is not significantly associated with the presence of a common bile duct stone.

\section{9}

Efficacy of Non-Invasive Hepatic Fibrosis Quantification By Liver Elasticity Measurement in Nonalcoholic Steatohepatitis (NASH) -Comparison of Ultrasonic TRANSIENT ELASTOGRAPHY and Histopathological Diagnosis Yoshitaka Fukuzawa, Tomohiko Ohashi, E.J. Matsumoto, Ken Sato, Minoru Ayada, Naoki Hotta, Akihiko Okumura, Tetsuya Ishikawa, Shinichi Kakumu

[Background and Aim] The global \& golden standard for NASH progression still relies on histopathological diagnosis by liver biopsy, but since this is an invasive test, it causes a large amount of physical and mental stress for patients (particularly elderly patients) and assessment of progression over time (treatment) is difficult. The aim of this study was to compare NASH patients' hepatic fibrosis evaluation using an elastometer to measure elasticity score (ES) with a histopathological diagnosis using a liver biopsy, and examine the efficacy of both methods. [Subjects and Methods] We measured the ES of all NASH patients $(n=38)$ using an elastometer (Fibroscan, ver.3 EchoSens, Paris, France) applying a low-frequency shear wave-elastography. The patients laid on a bed on their backs and the location of measuremen was determined with B-mode ultrasound. The elasticity was automatically calculated in the apparatus and the data were shown as Kilo Pascal (KPa). Measurements were taken 5-10 times for each case and we obtained the average value. We comparatively examined the correlation between the measurement values and hepatic fibrosis progression (Fibrotic Staging, by Brunt classifications) including the control group $(\mathrm{n}=6)$. [Results] Normal control group (group $\mathrm{C}, \mathrm{n}=6)(\mathrm{Mean} \pm \mathrm{SD}, 3.5 \pm 1.3)$, group $\mathrm{S} 0 \quad(\mathrm{n}=8)(3.9 \pm 1.5)$, group $\mathrm{Sl}(\mathrm{n}=9)$ $(6.8 \pm 2.3)$, group S2 $(n=8)(8.4 \pm 3.5)$, group S3 $(n=7)(35.4 \pm 7.8)$, group $S 4(n=6)(59.1 \pm 18.8)$ No significant difference was shown between group $\mathrm{C}$ and group SO, but together with the progression of fibrosis, clear significant differences were evident between groups $(\mathrm{P}<0.05)$. This was particularly notable in the shift in progression from S1-S2 to S3-S4. Further, the average measurement time for each case was $(7.8 \pm 2.8)$ minutes, a significantly shorter time than that with liver biopsy $(\mathrm{P}<0.05)$. [Conclusion] We have shown that using ES obtained by an elastometer, it is possible to assess the progression of hepatic fibrosis in NASH noninvasively, simply, quickly and quantitatively, and it is extremely effective for long-term monitoring, including therapy. It is considered effective (promising) as an alternative test for NASH patients for whom a liver biopsy is not possible, periodically or frequently

540

What Are the Best Cut Offs for the APRI ? Ned Snyder, Rami Hawari, Shu-Yuan Xiao, Daryl Lau, Roger Soloway, John Petersen

Background: The AST/platelet ratio (APRI) is a simple biochemical index for hepatic fibrosis We have found it accurate in retrospective and prospective studies at at our institution. (Gastroenterol 126:A-304. Am J Gastroenterol 99: S100). When first proposed by Wai et al (Hepatology 38:518), the cut offs proposed were $<0.5$ for mild fibrosis, $>1.5$ for significant fibrosis, and $>2$ for cirrhosis. While most investigators have used these cut offs, there is little published data on the accuracy of other cut offs. The ideal cut-off would have a negative or positive predictive value $>90 \%$, and result in a small indeterminate zone. Purpose: We wanted to determine the best cut offs for mild and significant fibrosis and cirrhosis for the APRI in a group of patients enrolled in a prospective study of multiple hepatic fibrosis markers. Methods : We prospectively collected blood from 208 patients with chronic HCV on the same day they underwent pre treatment staging liver biopsy. The liver biopsies were read blindly by one pathologist using the Ludwig Batts criteria for the staging of fibrosis (F0-F4). APRI was calculated as below. ${ }^{*}$ Results: There were 91 patients $(43.8 \%)$ with mild fibrosis (F0-F1), and 117 (56.2\%) patients with significant fibrosis (F2-F4). The ROC curve for the APRI resulted in an AUC of 0.878 (95\% confidence interval 0.822-0.921). The results of cut offs for mild and significant fibrosis are shown in the table below.Utilizing cut offs of 0.42 and 1.2 led to high predictive values and an intermediate zone of $43.3 \%$. The originally proposed cutoffs of 0.5 and 1.5 resulted in a lower negative predictive value, and a similar indeterminate zone (42.3\%). The APRI was not accurate in our patients in predicting cirrhosis. There were 40 patients with Stage 4 disease. At $>1.75,22 / 38(57.9 \%)$ had cirrhosis while at $>2.00,19 / 32$ (59.4\%) had cirrhosis. Conclusion: Our study confirms that the APR is an accurate method to predict the presence or absence of significant fibrosis in patients with chronic $\mathrm{HCV}$. In our patients, 0.42 for mild fibrosis and 1.2 for significant fibrosis appear to be the best cut offs. The use of these cut offs could improve the accuracy of the APRI. The APRI is not as useful in predicting cirrhosis. * APRI = AST/ULN X 100/platelets

\begin{tabular}{|c|c|c|c|c|c|}
\hline APRI & Total & F0-F1 & F2-F4 & NPV & PPV \\
\hline$<0.35$ & 26 & 24 & 2 & 92.3 & \\
\hline$<0.42$ & 45 & 42 & 3 & 93.3 & \\
\hline$<0.50$ & 68 & 55 & 13 & 80.9 & \\
\hline$>1.00$ & 85 & 8 & 77 & & 90.6 \\
\hline$>1.20$ & 73 & 5 & 68 & & 93.2 \\
\hline$>1.50$ & 54 & 5 & 49 & & 90.7 \\
\hline
\end{tabular}

541

Protective Factors for Severe Recurrence of Hepatitis C After Liver Transplantation

Speranta Iacob, Susanne Beckebaum, Vito Cicinnati, Razvan Iacob, Christian Klein, Liana Gheorghe, Cristian Gheorghe, Irinel Popescu, Andrea Frilling, Massimo Malago, Guido Gerken, Christoph Broelsch

INTRODUCTION: Although reinfection of liver grafts is virtually universal, factors influencing disease progression in HCV infected liver transplant (LT) recipients remain controversial and are poorly understood. AIM \& METHODS: The aim of this study was to identify predictive factors for absence of fibrosis at the first liver biopsy showing recurrent hepatitis

histologically confirmed (inflammation and/or fibrosis and positive viral load) based on 1 
year protocol biopsies. We retrospectively assessed the liver biopsies from 109 patients with recurrent hepatitis $C$ and the virus, host (pre and posttransplant), donor and iatrogenic related variables. Univariate and multivariate logistic regression analysis have been conducted RESULTS: We identified 31 (28.44\%) patients without fibrosis at first liver biopsy. There were $71 \%$ and $69.2 \%$ male respectively in the group without and with fibrosis, respectively. The mean age at LT was $53.4+/-9.5$ years and respectively $51.8+/-8.7$ years in the group without and with fibrosis. The following factors were associated with no fibrosis at first biopsy: absence of pretransplant portal vein thrombosis $(\mathrm{p}=0.03)$, cold ischemia time $<400$ minutes ( $\mathrm{p}=0.009$ ), absence of steatosis at first liver biopsy showing histologic recurrence $(p=0.04)$, female donor $(p=0.006)$, hemoglobin serum level of the donor $>10 \mathrm{~g} / \mathrm{dl}(\mathrm{p}=0.02)$ and absence of de novo diabetes mellitus $(\mathrm{p}=0.05)$. Multivariate analysis has identified the following independent predictors for absence of fibrosis in transplanted patients: cold ischemia time less than 400 minutes $(\mathrm{p}=0.02)$ and female gender as donor regardless of the recipient gender $(\mathrm{p}=0.03)$. CONCLUSION: A shorter cold ischemia time and female gender as donor are associated with a less aggressive recurrent $\mathrm{HCV}$ disease.

542

Novel Markers of Cirrhosis and Hepatocellular carcinoma in Hepatitis C Kuntal M. Thaker, Bridget Seymour, Divya Gupta, Kuldip S. Banwait, Mark A. Feitelson, Steven K. Herrine, Victor Navarro

Background and Aim: Previous work has shown that hepatitis B x antigen triggers upregulation of two novel genes (URG\& and URG11), both of which contribute to tumor development in patients with chronic hepatitis B virus (HBV) infection. Anti-URG7 and anti-URG11 have been detected in most patients who develop HBV associated hepatocellular carcinoma (HCC). Since the pathogenesis of HBV and hepatitis $\mathrm{C}$ virus (HCV) associated HCC has some similarities, experiments were designed to test the hypothesis that these antibodies would also be triggered in patients with $\mathrm{HCV}$ related diseases. Methods: Consecutive HCV infected patients seen at the liver clinic from October 2004 to March 2005 were prospectively recruited into four groups: chronic HCV without cirrhosis (Group 1, n=30), HCV with cirrhosis (Group 2, n=29), HCV with HCC (Group 3, n=13), and uninfected controls $(n=8)$. Sera were tested for anti-URG7 and anti-URG1l by specific ELISAs. Results: For anti-URG7, 21/30 (70\%) of group 1; 29/29 (100\%) of group 2; and 11/13 (84.6\%) of group 3 patients were positive ( $\mathrm{p}<0.05$ for group 1 vs. 2 , group 2 vs. 3 ). For anti-URG 11 , $14 / 30(46.7 \%)$ of group $1 ; 19 / 29(65.5 \%)$ of group 2 ; and $6 / 13(46.2 \%)$ of group 3 patients were positive ( $<<0.05$ for group 1 vs. 2 , group 2 vs. 3). Both antibodies were detected in $36.67 \%$ of group $1 ; 65.52 \%$ of group 2 ; and $54.55 \%$ of group 3 patients $(\mathrm{p}<0.05$ for group 1 vs. 2 , group 2 vs. 3). No antibodies were detected in the uninfected controls. Mean ELISA OD values were lower for group 1 (0.0904) as compared to group $2(0.1191)$ with $\mathrm{p}=$ 0.0039 . Mean ELISA OD values were not statistically significant for group 1 versus group 3 , or group 2 versus group 3. There was no relationship between presence and absence of antibodies and amino alanine transferase, alpha-fetoprotein, and hepatitis $C$ viral levels. Conclusions: 1)Anti-URG7 and anti-URG1l are detectable in HCV infected patients, as they are in HBV patients. 2)Up-regulation of anti-URG7 and anti-URGll is not an unique effect of HBxAg as previously believed. 3)No antibodies are present in uninfected controls. 4)Upregulated expression of these two genes in HBV and HCV may have a similar pathogenic mechanism in causing HCC. 5)These antibodies may serve as surrogate markers for cirrhosis and HCC in HCV patients, and may be identified by a simple blood test.

\section{5}

Colonoscopic Screening of First Degree Relatives of Patients with Colorectal Cancer: A Concurrent Case-Control Comparison to First Degree Relatives of Asymptomatic Individuals with Normal Colonoscopic Findings James Y. Lau, Wai-Keung Leung, Priscilla L. Siu, Simon S. Ng, Janet F. Lee, Sara Fung, Raymond Y. Yiu, Joseph J. Sung

Background: First-degree relatives to patients with sporadic colorectal cancer have a higher risk of developing colonic adenomas and cancer. The evidence has been based mostly on cohort studies. There has been controversy over methodology in choosing control subjects. Methods: We invited first degree relatives (aged 40-70) of patients with sporadic colorectal cancers to undergo colonoscopy. In the same period, we also invited control subjects, who were first degree relatives of subjects with normal colonoscopic findings from our asymptomatic screening cohort [Sung Gastroenterology 2003; 124:608-14]. We excluded subjects with known inflammatory bowel disease, suspected FAP or HNPCC, subjects who had undergone colonoscopy in the past 5 years or severe comorbid illnesses that precluded safe colonoscopy. Endoscopists were blinded to family history of subjects. In both studied and control subjects, colonoscopies were performed concurrently. To each examined relative, one control matched for sex, age (t/- 3 yrs), and possible symptom was selected. Results: Between Sep 04 and Nov 05, 163 case and 163 control subjects (mean age of 53, men \% 33) from 128 and 125 families respectively underwent colonoscopies. Prevalence of adenomas in first-degree relatives of patients with colorectal cancer and their matched controls is listed in table 1. Conclusions: Subjects with affected first degree relatives are at increased risk of advanced colonic lesions.

Table 1. Prevalence of adenomas in first-degree relatives of patients with colorectal cancer and their matched controls

\begin{tabular}{|c|c|c|c|c|c|}
\hline Lesion & $\begin{array}{c}\text { Relatives n } \\
(\%)\end{array}$ & $\begin{array}{c}\text { Controls n } \\
(\%)\end{array}$ & $\begin{array}{c}\text { Adjusted } \\
\text { OR }\end{array}$ & $95 \% \mathrm{CI}$ & P \\
\hline All Adenomas & $40(24.5)$ & $25(15.3)$ & 1.8 & $1.0-3.1$ & .05 \\
\hline Adenomas <10mm & $29(19.1)$ & $22(13.8)$ & 1.5 & $0.7-2.5$ & .36 \\
\hline $\begin{array}{c}\text { Advanced neoplasms Size } \geq 10 \mathrm{~mm} \text { Villous } \\
\text { adenomas severe dysplasia/ invasive cancer }\end{array}$ & $\begin{array}{c}11(6.7 \%) \\
1122\end{array}$ & $\begin{array}{c}3(1.8) 31 \\
0\end{array}$ & 3.9 & $1.1-14.1$ & .05 \\
\hline Hyperplastic polyps & $15(9.2)$ & $21(12.9)$ & 0.7 & $0.4-1.4$ & .37 \\
\hline
\end{tabular}

556

Temporal Trend in Relative Risk of Second Primary Colorectal Cancer (CRC) An Impact of Surveillance?

Ananya Das, Amitabh Chak, Gregory S. Cooper

Patients with CRC are at a higher risk for developing a second primary CRC. A number of factors that have potential impact on the risk of second primary CRC such as, survival rate, rate of receipt of surveillance procedures and the overall incidence of colorectal cancers in the western population have changed over the last three decades. However, currently no information is available on the temporal trend in the risk of second primary CRC in patients who survived initial CRC. Objectives: In this study, our aim was to use the Surveillance, Epidemiology and End Results Public-use database to assess whether the relative risks of second primary $\mathrm{CRC}$ has changed in patients with initial primary $\mathrm{CRC}$, and also to compare clinical characteristics of the second primary CRC in patients diagnosed with first primary CRC during two different periods of time. Methods: The database was analyzed to assess the temporal trend in the standardized incidence ratio (SIR) and excess risk of cancer (beyond the expected amount) per 100,000 persons per year for a second primary CRC in these patients. Also, the demographic and clinical features of the second primary CRC was compared in two subgroups based on the year of diagnosis of the first primary CRC: Group A (1973-1977) and Group B (1988-1992). Results: During the period of 1973 to 2002, 216,751 patients developed a primary CRC and over a follow up period of $11,250,687$ person-years, 5,595 of these patients developed a second primary CRC with an SIR of 1.41 (95\% CI, 1.371.44). While the overall age-adjusted incidence rate of first primary CRC declined from 204 per 100,000 in 1992 to 177 in 2002, the excess risk of second primary CRC increased from 85 per 100000 in 1992 to 173 in 2002. Patients in Group A had more advanced disease at diagnosis of the second primary CRC compared to those in Group B. In a Cox proportional hazards model, the period of diagnosis of the first primary $\mathrm{CRC}$ was an independent risk factor for a subsequent primary CRC; the relative hazard of second colon cancer in Group B compared to Group A was 1.18 [ 95\% CI, 1.05-1.31], after controlling for age at diagnosis, gender, race, site and stage of first primary colon cancer. Conclusion: The relative risk of the second primary CRC has increased since early nineties and these subsequent cancers are being diagnosed at an earlier stage. Among other factors, this probably reflects impact of increasing receipt of surveillance procedures.

557

Stated Preferences for Colorectal Cancer Screening Using a Discrete Choice Experiment

Seija K. Kromm, Steven J. Heitman, Robert J. Hilsden, Deborah A. Marshall, S. Elizabeth McGregor, Gillian R. Currie

Background: Uptake of colorectal cancer (CRC) screening may be influenced by the attributes of available screening tests. The purpose of this discrete choice experiment (DCE) was to determine the attributes of CRC screening tests that are most important to the general public and how these attributes might affect uptake. Methods: 218 subjects aged 50-70 (115 men) recruited from the general population participated in a DCE administered as a face-to-face interview involving a series of 8 choices between two hypothetical screening tests and not being screened. Each test was described by 7 attributes: accuracy, need to travel for test, waiting time, test procedure, ability to find polyps, need for follow-up test and testing interval. A series of four focus groups and pilot testing informed the choices of DCE attributes. Responses were analyzed using multinomial logit regression. Results: Respondents preferred to be screened over not being screened $(p<0.001)$. Test accuracy $(p<0.001)$, ability to find polyps $(p<0.001)$ and not having to travel out of the community for the test $(p<0.01)$ were determinants of test choice, in order of importance. Those who had not been screened (56\%) preferred a procedure whose description matched a virtual colonoscopy, all else equal. Respondents who had been screened preferred a procedure whose description matched a colonoscopy, all else equal. The regression coefficients were used to estimate the uptake of existing screening tests. Preference for accuracy and prevention drive uptake when the tests are offered as part of a menu of options. Given a choice between FOBT and colonoscopy at current local wait times for colonoscopy (15 months) and single test FOBT accuracy (50\% of cancers missed), 95\% would choose colonoscopy. With higher FOBT accuracy, as that for annual testing for 10 years ( $25 \%$ of cancers missed), $73 \%$ would choose colonoscopy. Given a choice between colonoscopy and virtual colonoscopy (assuming zero wait time in both cases), $60 \%$ of people would choose colonoscopy. Conclusion: The most important characteristics influencing preferences for screening were test accuracy, ability to find polyps, and the need to travel out of the community for the test. Preferences about the nature of the procedure depended upon screening experience. Predicted uptake of screening was much higher than seen currently. This may be due to the hypothetical nature of the choices, prior screening experience, and the influence of other test features not included in the set of attributes used in the DCE. These findings may be useful in developing education initiatives and a population-based CRC screening program most acceptable to the public. 
Colorectal Cancer Screening in Chinese Immigrants: Who Can Deliver the Message?

Karen E. Kim, Stacy Tong, Eva Lu-Bonn

Background: Colorectal cancer (CRC) ranks third in cancer incidence and mortality in Asian Americans. Studies on colorectal cancer in Asian Americans suggest that screening rates are among the lowest reported. Barriers to colorectal cancer screening (CRS) in immigrants include language, access and absence of routine health care. The effectiveness of ethnic and culturally specific community outreach on colorectal screening in Chinese immigrants has not been previously reported. This study compares the effectiveness of physician directed compared to lay health educator directed CRS programs in Chinese immigrant communities. Methods: Chinese community based organizations were asked to randomize their clients aged 50 and above into two CRS programs. Physician directed programs used Asian, nonChinese speaking physicians coupled with a trained medical interpreter for CRC education and fecal occult blood testing (FOBT), while lay educator programs (LHE) used peer educators for education on CRC and FOBT. All participants completed a translated survey on CRS compliance, CRC risk perception and knowledge gained. The effectiveness of each educational program on CRS behavior was assessed by compliance with fecal occult blood testing (FOBT). Results: 260 participants attended CRC programs. The average age was 54; $55 \%$ were women, the average years in the US was $10.80 \%$ of participants reported speaking Chinese at home and were of limited English proficiency, 5\% of participants in the MD group ( $\mathrm{n}=135$ ) completed the FOBT, compared to $76 \%$ in the LHE group ( $n=125)$. Overall, $30 \%$ were found to have a positive FOBT. $75 \%$ in the MD group and $85 \%$ in the LHE group reported a gain in knowledge about CRC and CRS. Conclusions: In this study, Chinese immigrants were significantly less likely to comply with FOBT when education was delivered by Asian, non-Chinese speaking physicians. In stark contrast, the same program delivered by a peer educator (a respected member of their community) resulted in an extremely high FOBT compliance. Since no significant difference in knowledge gained between the two groups were reported, these differences cannot be attributed to language barriers. Given the high rates of positive FOBT in this population, this study highlights the importance of community based and community directed education in hard to reach populations.

559

Variation in Polyp Detection Rates At Screening Colonoscopy

Thomas F. Imperiale, Elizabeth A. Glowinski, Ching L. Cooper, Gregory N. Larkin,

Faouzi Azzouz, David F. Ransohoff

Background: Wide variation in adenoma detection rates has been found in the U.K. Flexible Sigmoidoscopy Screening Trial. This variation, attributed to endoscopist performance, has been used to justify the need for establishing quality standards for performance of colonoscopy. Purpose: The objectives of this study were to measure, in a program of screening colonoscopy, variation in polyp detection rates (PDRs) and to identify factors associated with PDRs. Methods: We reviewed summary-level data from endoscopists who performed at least 40 first time screening colonoscopies for the Lilly Colorectal Cancer Prevention Program between 9/1995 and 6/2001. Summary-level data include mean age, mean procedure time (MPT), gender distribution, and \% persons with any polyp, any adenoma, any polyp $>=1.0 \mathrm{~cm}$, and multiple polyps. We described the variation in PDRs and used boxplot analysis to identify outliers. Multivariable regression modeling identified factors associated with PDRs and that accounted for some of the variability in PDRs. Results: 2664 screening colonoscopies (1108 [42\%] in women, 1556 in men) were performed by 25 endoscopists, whose number of procedures ranged from 40 to 207. Overall mean patient age was 59 years (mean age range, 56 to $62 \mathrm{yr}$ ), the mean \% women was $42 \%$ (range, 29-52\%), and MPT was 17.1 minutes (range, 9.7-31 min). Mean PDRs, ranges, number of outliers, and outlier values are in the Table. Regression models that included procedure N, mean age, \% women, and MPT accounted for $42 \%$ of the variation in PDRs for any polyp, $56 \%$ of the variation in PDRs for any adenoma, 37\% for large polyps, $36 \%$ for $>1$ polyp, and $46 \%$ for $>1$ adenoma. In all models, only MPT was significantly associated with PDRs. For the endoscopists with the shortest ( 9.7 minutes; $\mathrm{N}=80$ ) vs. longest ( $31 \mathrm{~min} ; \mathrm{N}=56$ ) MPTs, respective PDRs for any polyp, any adenoma, polyps $>=1 \mathrm{~cm},>1$ polyp, and $>1$ adenoma were $20 \%$ vs. $82 \%$, $15 \%$ vs. $43 \%, 0 \%$ vs. $12.5 \%, 7.5 \%$ vs. $50 \%$, and $6.3 \%$ vs. $25 \%$ (all P-values < 0.003 ). Conclusion: PDRs vary widely among screening colonoscopists, though only a few (high) outliers were identified. Variation in PDRs was significantly associated with MPT, but not with age, gender, or the number of procedures. Further research is needed to understand the reasons for variation, and requires attention to patient risk factors, scope withdrawal time, and quality of the prep.

\begin{tabular}{|c|c|c|c|c|c|}
\hline & Any polyp & Any adenoma & Polyp $>=1 \mathrm{~cm}$ & $>1$ polyp & $>1$ adenoma \\
\hline Mean PDR & $36 \%$ & $20 \%$ & $5.3 \%$ & $15 \%$ & $6.8 \%$ \\
\hline PDR range & $13-82 \%$ & $7-44 \%$ & $0-13 \%$ & $1.3-50 \%$ & $1.3-25 \%$ \\
\hline \# Outliers & 1 & 2 & 3 & 1 & 1 \\
\hline Outlier values & $82 \%$ & $43 \%, 44 \%$ & $10 \%, 11 \%, 13 \%$ & $50 \%$ & $25 \%$ \\
\hline
\end{tabular}

560

The Impact of Literacy On Colonoscopy

Rony Ghaoui, Arthur T. Evans, Bashar M. Attar

Background and Aims Approximately 20-30\% of attempted colonoscopies are incomplete because of poor bowel preparation. This problem is not only costly and wasteful, but increases risks and delays diagnosis and treatment. Although literacy skills are necessary for patients to successfully follow home bowel preparation instructions, they are nearly impossible to assess informally. Our aim was to evaluate the impact of literacy on bowel preparation and completion of colonoscopy, with the ultimate objective of redesigning instructions to be more effective, regardless of patients' literacy skills. Methods We performed a prospective cohort study at a large urban public hospital during the summer of 2005 among patients who presented for outpatient colonoscopy. Before the colonoscopy, literacy was assessed using the 7-minute test of functional health literacy. Using structured interviews, we also gathered information on patients' past history, beliefs, and self-reported behaviors on bowe preparation. Results of the colonoscopy were obtained from the procedure reports. Results The sample of 195 subjects included 64\% women, 49\% African Americans, and 40\% with low literacy skills. $25 \%$ of colonoscopies were not completed, primarily (90\%) because of poor bowel preparation. Low literacy increased the risk of an incomplete exam 4-fold: 12 $\%$ incomplete exams among patients with adequate literacy skills compared to $45 \%$ with low literacy skills $(\mathrm{p}<0.001)$. Other important predictors of an incomplete exam were: eating lunch or dinner the previous day, not taking the bisacodyl $(\mathrm{p}=0.001)$, and not finishing the l-gallon polyethylene glycol solution $(\mathrm{p}=0.01)$. Patients with a past history of colonoscopy $(\mathrm{p}=0.03)$ and those who received additional oral instructions from a physician or nurse $(\mathrm{p}=$ 0.05 ) were more likely to have a complete colonoscopy. Low literacy remained the stronges independent predictor of incomplete colonoscopy after controlling for the contribution of all other factors (OR=8.7; 95\% CI: 3.4-22.1; $\mathrm{p}<0.001$ ). Discussion/Conclusion Low literacy accounts for $30 \%$ of all incomplete colonoscopies. This important effect is independent of age, gender, ethnicity, and even whether oral instructions supplemented the written instruc tions. In light of these findings, we must redesign our methods of instructing patients abou bowel preparation in order to improve the efficiency and effectiveness of colonoscopy.

\section{1}

Prevention of Low Dose Aspirin-Associated Gastroduodenal Ulcers and Upper Gastrointestinal Symptoms in Patients Receiving Esomeprazole $20 \mathrm{Mg}$ Per Day Neville D. Yeomans, Angel Lanas, Joachim Labenz, Sander Veldhuyzen van Zanten, Christo van Rensburg, Istvan Racz, Emma Naucler, Lars-Erik Svedberg

OBJECTIVE: To assess whether esomeprazole reduces the incidence of ulcers in patient taking low dose (75-325 mg daily) aspirin (acetylsalicylic acid). METHODS: Male or female Helicobacter pylori-negative patients $\geq 60$ years with a condition requiring daily low dose aspirin, at risk of developing gastroduodenal ulcers were included in this randomized, double-blind, multicenter, placebo-controlled trial. Patients were excluded if they had upper gastrointestinal symptoms, including heartburn, that required treatment, or if they had erosive esophagitis. Patients with Los Angeles Grade A lesions and without reflux symptoms requiring treatment could be included. Patients were randomized to receive either esomeprazole $20 \mathrm{mg}$ or placebo once daily (qd) for 26 weeks. Patients continued to receive the aspirin dose they were taking when enrolled into the study. The primary outcome variable was the presence of gastric and/or duodenal ulcers at endoscopy over the 26-week period. We present data from endoscopy taken at 26 weeks. RESULTS: A total of 991 patients $(57.1 \%$ male, mean age 69.3 years, median aspirin dose $100.0 \mathrm{mg}, 89.0 \%$ taking aspirin for $>4$ weeks) were included in the intent-to-treat population. Eight patients (1.6\%) had developed a gastroduodenal ulcer in the esomeprazole group by 6 months, compared with 27 patients $(5.4 \%)$ in the placebo group $(\mathrm{p}=0.0007)$. This corresponded to a relative reduction of developing an ulcer of $70 \%$ when taking esomeprazole rather than placebo. The related life table estimates were $1.8 \%$ for esomeprazole and $6.2 \%$ for placebo. In patients with Grade A esophageal lesions at baseline and with pre- and post-baseline data, 13 patients $(28.3 \%)$ in the esomeprazole group had Grade A lesions at week 26, compared with 37 patient $(71.2 \%)$ in the placebo group $(\mathrm{p}<0.0001)$. Resolution of investigator-assessed aspirin-associated upper gastrointestinal symptoms (rated as none to severe) was significantly higher with esomeprazole than with placebo for epigastric pain, burning and discomfort, as well as heartburn and bloating ( $\mathrm{p}<0.05$ for all symptoms). Esomeprazole was safe and well-tolerated CONCLUSIONS: Esomeprazole $20 \mathrm{mg}$ qd is more effective than placebo and is well-tolerated in reducing the risk of developing gastroduodenal ulcers and resolving upper gastrointestinal symptoms associated with low dose aspirin use in patients at moderate-to-high risk of developing ulcers. At risk patients taking low dose aspirin may therefore benefit from coadministration of esomeprazole.

562

Effects of Concomitant Aspirin (81 mg qd) On Incidence of Gastric and/Or Duodenal Ulcers in Healthy Subjects Taking Celecoxib Or Naproxen: A Randomized, Placebo-Controlled Trial Jay L. Goldstein, James Aisenberg, Manuela Berger, William E. Dodge

Background: Patients who use nonselective nonsteroidal anti-inflammatory drugs (NSAIDs) or cyclooxygenase-2 (COX-2) selective inhibitors often require concomitant low-dose aspirin (ASA) for cardiovascular (CV) prophylaxis. The impact of the most common prophylactic ASA dose ( $81 \mathrm{mg}$ qd) on the gastrointestinal (GI) safety profile of COX-2 selective inhibitors remains controversial. Methods: In this multicenter, double-blind, placebo-controlled study, healthy subjects (seronegative for Helicobacter pylori) aged 50-75 y were randomized to receive celecoxib $200 \mathrm{mg}$ qd + ASA $81 \mathrm{mg}$ qd, naproxen $500 \mathrm{mg}$ bid + ASA $81 \mathrm{mg}$ qd, or placebo + ASA $81 \mathrm{mg}$ qd for 1 week. The primary end point was incidence of gastric and or duodenal ulcers in subjects treated with celecoxib + ASA compared with naproxen + ASA. Upper GI (UGI) endoscopy was performed at baseline and Day 7 (or early termination). Subjects were excluded if baseline endoscopy revealed $>5$ erosions in the stomach or duodenum; any gastric, pyloric channel, or duodenal ulcer ( $\geq 3 \mathrm{~mm}$ diameter); or an esophageal ulcers/erosions. In addition, subjects with any NSAID use within 2 weeks of enrollment were excluded. Results: A total of 662 subjects (mean age, 58 y) were randomized in a 2:2:1 fashion (celecoxib + ASA, n=267; naproxen + ASA, n=264; placebo + ASA, n= 131). There were no significant differences in baseline demographics between groups. A total of $7 \%$ (18/257) of evaluable subjects randomized to celecoxib had gastric and/or duodenal ulcers compared with $25.3 \%(65 / 257)$ of those on naproxen and $1.6 \%(2 / 129)$ of those on placebo. Statistical pairwise comparisons by Cochran-Mantel-Haenszel test are shown in the Table. For secondary end points, significantly fewer celecoxib-treated subjects had gastric ulcers (GU) or duodenal ulcers (DU) vs naproxen (GU: $5.8 \%$ vs $22.6 \%$; DU $1.2 \%$ vs $7 \% ; P<0.001$ both comparisons). More celecoxib-treated subjects had GUs vs placebo $(P=0.016)$ but there was no significant difference in the incidence of DUs $(1.2 \%$ vs $0.8 \%$ ). Conclusion: In a healthy population taking $81 \mathrm{mg}$ qd ASA, celecoxib co-administration 
results in more gastric and/or duodenal ulcers than aspirin alone but fewer compared with naproxen. In parallel with a similarly designed trial (but with $325 \mathrm{mg}$ qd ASA), these data suggest that daily ASA doses commonly used for CV prophylaxis do not completely negate the UGI benefit of celecoxib compared with naproxen, as measured by endoscopic ulcer rates.

\begin{tabular}{|c|c|c|c|}
\hline & Relative risk & $95 \% \mathrm{CI}$ & $P$ Value \\
\hline Celecoxib + ASA vs naproxen + ASA & 0.28 & $0.17-0.45$ & $<0.001$ \\
\hline Celecoxib + ASA vs placebo + ASA & 4.78 & $1.12-20.32$ & 0.016 \\
\hline Naproxen + ASA vs placebo + ASA & 16.01 & $3.98-64.46$ & $<0.001$ \\
\hline
\end{tabular}

563

Effects of Antisecretory Drugs and Nitrates On the Risk of Ulcer Bleeding Associated with NSAIDs and Anti-Platelet Agents

Angel Lanas, Luis A. Garcia-Rodriguez, Maria T. Arroyo, Fernando Gomollon, Faust Feu, Montse Forne, Sofia Aleman, Enrique Garcia, Luis Bujanda, David Nicolas, On Behalf of AEG Investigators

Background and Aims: After the withdrawal of rofecoxib, an increased prescription rate of some non-selective NSAIDs has been observed, but, according to recent reports, additional prevention strategies are not being followed. In this study we report the effect of antisectory drugs (proton pump inhibitors-PPI; H2-receptors antagonists- H2-RA) and nitrates on the risk of upper gastrointestinal ulcer bleeding (UGIB) associated with NSAID use in clinical practice Methods: Type of Study: hospital-based case-control study with prospective data collection. Setting: A network of 40 general hospitals integrated within the Spanish Association of Gastroenterology. Cases were consecutive patients with UGIB confirmed by endoscopy. Controls matched 2:1 to cases by age ( 5 years range), hospital and month of interview were individuals with an outpatient visit or hospitalised with a primary diagnosis that was neither an indication nor a known contraindication of NSAID or low dose aspirin treatment. The same structured questionnaire was used in all sites. Relative risk (RR) of UGIB was estimated using logistic regression analysis. Results: 2,777 cases and 5,532 controls have been included. Overall, current use of PPI (RR:0.33; 95\%CI:0.27-0.39), H2-RA (RR:0.65; $0.50-0.85$ ) and nitrates (RR:0.52; 0.38-0.70) reduced the risk of developing an UGIB event. The risk reduction was stronger with PPI use among both non-aspirin NSAID (RR: 0.13 ; 0.09-0.19; vs $0.30 ; 0.17-0.53$ with H2-RA and $0.48 ; 0.19-1.24$ with nitrates) and aspirin users (RR: $0.30 ; 0.20-0.40$ vs. $0.40 ; 0.24-0.68$ with $\mathrm{H} 2$-RA and $0.66 ; 0.44-0.98$ with nitrates). Among individual NSAIDs, a similar risk reduction effect with PPI was observed for the 3 most widely used (diclofenac, ibuprofen and naproxen). Among low-dose aspirin users, PPI (RR:0.32;0.22-0.51) and H2-RA (RR: 0.40; 0.19-0.73) use were associated with risk reduction, while nitrates had a weaker effect (RR: $0.69 ; 0.36-1.04$ ). In patients taking clopidogrel, only PPI use was associated with a significant risk reduction (RR: $0.19 ; 0.07-0.49)$. However, among patients taking anticoagulants neither nitrates $(0.67 \cdot 0.33-1.34)$, nor H2-RA $(0.88$; $0.32-2.45)$ or PPI use $(0.67 ; 0.37-1.21)$ were associated with a significant effect on the risk of UGIB event. Conclusion: Treatment with nitrates, H2-RA or PPI is associated with a reduction of the risk of developing UGIB events in patients taking NSAID or aspirin. However, only PPI therapy was associated with a marked and consistent risk reduction among patients receiving all types of agents including non-aspirin anti-platelet agents. Protection was much less apparent in patients on anticoagulant therapy.

564

Gastroprotection Gap: A Rising and Dangerous Omission for Elderly Users of NSAIDs with Arthriti

Gurkirpal Singh, Huijian Wang, Eiichi Tanaka, Alka Mithal, Lauren Gerson, George Triadafilopoulos

Background: GI complications from NSAIDs have been well-recognized since early 1990s, and were significantly reduced by the use of selective cox- 2 inhibitors, concomitant proton pump inhibitors (PPIs) or misoprostol in the early 2000s. Recent media attention on the potential association of cardiovascular events with selective cox-2 inhibitors and market withdrawals resulted in a large decline in the use of these drugs. We report change in prescription patterns in a large cohort of elderly arthritis patients from January 1, 1995 to June 30, 2005. Methods: MediCal, the Medicaid program for California, is the largest Medicaid program in the US, with over 7 million participants per year. All study drugs were available without formulary restrictions or copayments. We studied individuals with physician-diagnosed arthritis who were over 65 years of age and treated with NSAIDs for at least 30 days. Results: Of the total 5,194,765 prescriptions for NSAIDs, 2,634,345 (50.7\%) were for selective cox-2 inhibitors. Among the 2,560,420 prescriptions for non-selective NSAIDs, only 1,215,762 (47.5\%) had concomitant use of PPI or misoprostol. Figure shows the use of selective cox- 2 inhibitors and concomitant PPIs or misoprostol, as a percentage of all NSAID use. The increasing implementation of gastroprotection strategies over the past several years reached a peak in 2004 when the percent of patients not receiving gastroprotection (Gastroprotection Gap) decreased to 14\% from 91\% in 1995 . However this gap more than doubled to $35 \%$ in 2005 , following a decline in selective cox-2 inhibitor use, without a commensurate increase in other gastroprotective therapies. Conclusions: An increasing number of elderly users of NSAIDs are again left without gastroprotection. This trend, if left unchanged, will undoubtedly increase morbidity and mortality from NSAID-related complications to levels unacceptable for optimal medical care and deserves immediate public attention

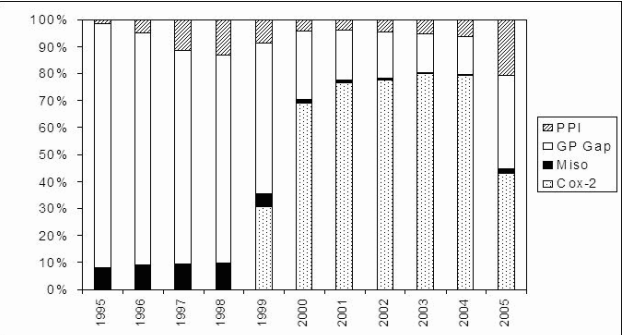

Gastroprotection (GP) gap in elderly patients 1995-2005. PPI=proton-pump inhibitors, miso=misoprostol, cox-2=selective cox -2 inhibitors

565

Improved Gastrointestinal Safety Profile with Lumiracoxib Compared with Naproxen and Ibuprofen in Patients At Least 65 Years Old At Increased Risk of Gastrointestinal Events

Christopher Hawkey, Wilfred Weinstein, Walter Smalley, Gerhard Krammer, Bernhard Mellein, Dominik Richard, Xavier Gitton

Background: Increasing age is a risk factor for nonsteroidal anti-inflammatory drug (NSAID)related gastrointestinal (GI) ulcers and associated ulcer complications. Selective COX-2 inhibitors such as lumiracoxib were developed to reduce the risk of GI events. The Therapeutic Arthritis Research and Gastrointestinal Event Trial (TARGET), the largest published GI outcomes study to date, showed a 79\% reduction in GI ulcer complications (perforation, obstruction or bleeding) for lumiracoxib compared with naproxen and ibuprofen in the non-aspirin population. Here we report additional analyses on the sub-group of TARGET patients $\geq 65$ years of age. Methods: Patients $(n=18$ 325) aged $\geq 50$ years with OA were randomized to receive lumiracoxib $400 \mathrm{mg}$ once daily (od) (4 times the recommended dose for treatment of $\mathrm{OA}$ ), naproxen $500 \mathrm{mg}$ twice daily (bid) or ibuprofen $800 \mathrm{mg}$ three times daily (tid) for 1 year. Randomization was stratified by age $(50-64,65-74, \geq 75$ years) and low-dose aspirin use. The primary endpoint was definite or probable upper GI ulcer complications. Secondary GI endpoints included all definite or probable ulcers (complicated and uncomplicated symptomatic ulcers) and clinically evident major bleeds. Results: In total, 7939 of the TARGET population were $\geq 65$ years (lumiracoxib [ $n=3980$ ] and NSAIDs $[n=3959])$, approximately $31 \%$ of whom took low-dose aspirin. In patients $\geq 65$ years not taking aspirin $(\mathrm{n}=5484)$, there was a significant reduction in ulcer complications in the lumiracoxib group compared with the NSAID group ( $0.29 \%$ vs $1.42 \%$ respectively; $\mathrm{p}<0.0001$ ) and significantly fewer ulcers (complicated and uncomplicated) ( $0.69 \%$ vs $2.62 \% ; \mathrm{p}<0.0001)$ In addition the incidence of clinically evident major bleeds was significantly lower in the lumiracoxib group than in the NSAID group $(0.26 \%$ vs $0.98 \% ; p=0.0012)$. In patients $\geq 65$ taking low-dose aspirin, there were no statistically significant differences between treatments. Conclusion: Lumiracoxib $400 \mathrm{mg}(\mathrm{od})$ delivers a consistent GI safety profile that is superior to the NSAIDs naproxen or ibuprofen in patients $\geq 65$ years not taking aspirin

566

A Prospective, Randomized, Controlled Trial of Clear Liquids Vs. Low-Fat Solid Diet as the Initial Meal After Mild Pancreatitis Brian C. Jacobson, Martha B. Vandervliet, Michael Hughes, Rie Maurer, Katherine D. McManus, Peter A. Banks

Background: Patients recovering from mild acute pancreatitis typically receive a clear liquid diet (CLD) when ready to initiate oral nutrition. The timing of patient discharge frequently depends upon successful advancement to solid food. We hypothesized that initiating feeding with a low-fat solid diet (LFSD) after mild pancreatitis would be well tolerated and would result in a shorter hospital length of stay (LOS). Methods: A sample size of 120 subjects was calculated to have $90 \%$ power to detect a one day difference in LOS between two study arms. Exclusion criteria included narcotic use within 6 hours prior to refeeding, an underlying condition that could itself cause poor oral intake or prolonged LOS, inability to monitor the patient post-discharge, pregnancy, and supervision of a patient's care by a study team member. Patients with mild pancreatitis, defined in accordance with the Atlanta symposium, were randomized to receive either a CLD or a LFSD when the responsible medical team determined refeeding was appropriate. The decision to advance a patient's diet and the timing of discharge were determined by the medical team. Patients were monitored daily for dietary intake, recurrence of pain, need to stop feeding, post-refeeding LOS, and for a total of 28 days post-refeeding to capture symptoms or readmission. Post-refeeding LOS (primary outcome) was compared using a t test and need to stop feeding (secondary outcome) was compared with Fisher's Exact test. Results: We randomized 121 patients: 66 to CLD, 55 to LFSD. Baseline characteristics including age, gender, and cause of pancreatitis were similar in both groups. The number of patients requiring cessation of feeding because of pain, nausea or vomiting was similar in both groups ( $5 \%$ for CLD and $9 \%$ for LFSD; $\mathrm{p}=$ $0.47)$. There was no difference in 28 day re-admission rates between the two arms. By intention-to-treat, the mean LOS after refeeding was similar in both groups $(1.7+/-1.9$ days for CLD and $1.7+/-2.0$ days for LFSD; $\mathrm{p}=0.94$ ). Calorie counts for the first meal consumed were available for $69 \%$ of patients and showed significantly lower calories and grams of fat consumed in the CLD arm than in the LFSD arm (mean $177+/-106$ cal vs $394+/-250 \mathrm{cal} ; 2+/-4 \mathrm{gm}$ fat vs $8+/-7 \mathrm{gm}$ fat; $\mathrm{p}<0.001$ for both comparisons). Perprotocol analyses and analyses restricted to those with calorie counts provided similar results for both diet tolerability and post-refeeding LOS. Conclusions: Initiating feeding with a LFSD was as well tolerated as a CLD, but did not result in a shorter LOS. 
Randomized, Double-Blind, Placebo-Controlled, Trial of High-Dose Intravenous Anti-Oxidant Therapy in Severe Acute Pancreatitis Ajith K. Siriwardena, James M. Mason, Srinivasan Balachandra, Anil Bagul, Simon Galloway, Laura Formela, Jonathon Hardman, Saurabh Jamdar, David Schofield, Pauline Kay, Ioannis T. Virlos

Introduction: Oxygen free-radicals (oxidative stress) mediate acinar injury in acute pancreatitis (AP). In clinical AP, inhibitors of oxidative stress (anti-oxidants) are depleted with extent corresponding to severity. Small studies suggest that anti-oxidant supplementation ameliorates AP but there is no substantive evidence. This study undertakes the first randomised, double-blind, placebo-controlled trial of anti-oxidant therapy in human severe AP. Methods: Patients with a clinical diagnosis of AP were eligible if their admission APACHE II score was $>8$. Principal inclusion criteria were: ability to give informed consent, disease duration $<72$ hours and not on anti-oxidants. Patients were randomised to receive intravenous n-acetylcysteine, selenium and ascorbic acid or three equivalent-volume placebos. All complications were characterized by Atlanta conference criteria. Principal end-point was daily organ dysfunction scores during the first 7 days. A study population of 110 was predicted as sufficient to detect a $25 \%$ improvement in primary end-point at $85 \%$ power. Hospital pharmacy allocated arm by random number generation. Independent monitoring of recruitment/complications was undertaken and stopping criteria were met in 2005 (under-recruitment). Results: 44 patients were randomized ( 23 anti-oxidants vs 21 placebo). Groups were statistically similar in terms of age, gender, etiology, APACHE and organ dysfunction score. Duration of anti-oxidant therapy was median 4 days in both. Anti-oxidant levels fell from admission in placebo but not in the treatment group. Analysis of primary endpoint showed 7 patients to have triggered organ dysfunction during the first 7 days in the anti-oxidant group (7/22 [31\%]) compared to 4 of $21(19 \%)$ in placebo. This difference was not statistically significant $(\mathrm{P}=0.49$ Fisher's exact 2-sided). There was no difference in the incidence of individual organ dysfunction, complications or mortality. Overall 4 patients died (all in the anti-oxidant group) $[\mathrm{P}=0.11$; Fisher's]. Although underpowered to detect change: with 43 patients the original power calculation has a $60 \%$ risk of type II error, further recruitment is unjustified as the probability of detection of any major effect of therapy is exceedingly low. Conclusion: This is the first study to examine the effect of anti-oxidant therapy in a well-characterized population of severe AP using contemporary disease descriptors in a double-blind, placebo-controlled fashion. The results show no evidence of benefit from treatment. Although underpowered, the final analysis suggests that there is unlikely even to be a trend towards benefit from treatment.

\section{8}

Hereditary Pancreatitis Amlodipine Trial (H-PAT): A Phase I Study of a Calcium Channel Blocker in Hereditary Pancreatitis Veronique D. Morinville, Mark E. Lowe, Beth D. Elinoff, David C. Whitcomb

Background: Hereditary Pancreatitis (HP) is a cationic trypsinogen (PRSS1) mutation-mediated form of recurrent acute pancreatitis (AP) with variable complications of fibrosis (i.e. chronic pancreatitis), recurrent and chronic visceral pain, diabetes and pancreatic cancer. Since trypsinogen mutations cluster in the calcium-associated regulator regions, it follows that increased trypsinogen activation in acinar cells during calcium influx after stimulation predisposes these patients to AP attacks. In rats, calcium channel blockers prevent hyperstimulation-associated AP. Thus, a phase 1 study was designed to determine the safety of giving calcium channel blockers to subjects with PRSSI mutations. Methods: Recruited subjects $\geq 6$ years (y) old had a PRSSl mutation, recurrent AP and recurrent abdominal pain. Pancreatic enzymes, acid-blockers and antioxidants had to have been used for $\geq 3$ months. Exclusion criteria included exocrine (elastase $<100 \mu \mathrm{g} / \mathrm{g}$ stool) or endocrine (diabetes mellitus) insufficiency or antihypertensive use. Total study duration was 16 weeks. Weeks 4 to 0 were observational; Weeks $0-12$ were interventional. Amlodipine was given for Weeks $0-11$, including dose escalation and weaning. Dose (2.5, 5 or $10 \mathrm{mg}$ daily) was based on weight (range $0.08-0.17 \mathrm{mg} / \mathrm{kg} / \mathrm{day}$ ). Subjects filled a daily symptom diary including pain level (0-10 scale) and automated blood pressure value. Subjects were clinically assessed at Weeks $-4,0,1,2,6,10,11$ and 12 and telephoned weekly. Laboratory testing was obtained at all visits except Week 11. Subjects filled an SF-36v2 ${ }^{\mathrm{TM}}$ (SF-10 ${ }^{\mathrm{TM}}$ for Children $<14$ y) at Weeks $-4,0,6$ and 10. Data were compared for Weeks -4 to 0 and 6 to 10. Results: Nine subjects signed informed consent ( 4 males; $12-52$ y). Four were excluded during the screening period. Drug was discontinued in one due to development of unilateral lower extremity numbness within 1 day of initiation. Four subjects (12-30 y) completed the study, all with good drug tolerability. Mean blood pressure, standard laboratory tests, physical examination findings and daily pain scores did not clinically significantly differ before and during drug therapy overall, although all subjects reported reduced symptoms. Three subjects reduced pain medication use. Three had slightly improved SF-36v2 ${ }^{\mathrm{TM}}$ and SF-10 $0^{\mathrm{TM}}$ for Children scores. Conclusion: This pilot study suggests amlodipine is generally safe in subjects with $\mathrm{HP}$ and does not increase pain or episodes of acute pancreatitis. Amlodipine's potential benefit must be validated through a larger, blinded, longer-duration study. Supported by NIH/NCRR/GCRC Grant \#M01 RR00056 and the National Pancreas Foundation.

\section{9}

Novel Radiotherapeutic Management of Painful Flare Ups in Chronic Pancreatitis

Luisa Guarner, Xavier Molero, Begona Navalpotro, Jordi Giralt, Juan-Ramon Malagelada

Patients with chronic pancreatitis may present repeated painful flare-ups of pancreatitis and even unrelenting pain. Current management options are limited to analgesics and surgery, in selected cases. We reasoned that anti-inflammatory radiotherapy, which appears to be effective in other inflammation based painful disorders of the body, might prove valuable to severely symptomatic patients with chronic pancreatitis. PATIENTS AND METHODS: We studied prospectively over a 4-year period till Nov. 05, the efficacy of single dose antiinflammatory radiotherapy in 12 consecutive patients with chronic pancreatitis (ethanol related in 7, idiopathic in 4 and cystic fibrosis in one) who fulfilled the following criteria: either 2 flare ups of pancreatitis in the previous 6 months (all 12 patients) and/or continuous pain for more than 3 months (2 of the 12). Median age was 41 years (range 32-80), there were 9 males and 3 females; diagnosis of chronic pancreatitis ( 6 months to 16 years, median 5 years) and number of prior attacks ( 2 to $>15$, median 6 ). Treatment consisted in a single dose of radiation of $8 \mathrm{~Gy}$. Before and after radiation we assessed: exocrine function by fecal elastase, endocrine function by c peptide, quality of life (EuroQol) and clinical outcomes Response was defined as no further pain or flare-ups of pancreatitis. RESULTS: During follow up (median 33 months, range 1 to 48 months) 10/12 patients had no further pain or flare-ups. One patient required a second radiation dose after 1 year and has been wel since (26 months), l patient did not respond and had a pancreaticojejunostomy at 1 month. Before radiation 4 patients had exocrine (fecal elastase $<100 \mu \mathrm{g} / \mathrm{g}$ ) and 2 endocrine (c peptide $<0.50 \mathrm{ng} / \mathrm{ml}$ ) dysfunction. Post treatment 1 additional patient developed exocrin (at 25 months) and endocrine (at 13 months) insufficiency. The responder group (11/12 gained 4 to $20 \mathrm{Kg}$ in weight during follow up (median $6 \mathrm{Kg}$ ) and EuroQol improved significantly from 0.572 before till 0.817 after treatment ( $\mathrm{p}<0.01$ ). CONCLUSION: Radiation treatment of severely symptomatic chronic pancreatitis is effective and could potentially substitute or delay surgery.

570

Correlation Between Coefficients of Fat Absorption and Nitrogen Absorption in Subjects with Cystic Fibrosis-Related Pancreatic Insufficiency Treated with Altu-135

Drucy Borowitz, Christopher Goss, Stacey E. Limauro, Susan Casey, Frederick T. Murray

Background: The largest randomized double-blind parallel dose study of subjects with malabsorption due to cystic fibrosis (CF)-related pancreatic insufficiency (PI) was conducted with ALTU-135, a microbially-produced (bacterial lipase and fungal protease and amylase) pancreatic enzyme replacement therapy, in order to identify the most effective dose to increase oral fat and nitrogen absorption. In this study, one hundred twenty-nine (129) subjects with CF and PI were randomized to one of three treatment arms: Lipase / Protease / Amylase (Units USP/meal or snack): - Arm 1: 5,000 / 5,000 / 750 - Arm 2: 25,000 / 25,000 / 3,750 - Arm 3: 100,000 / 100,000 / 15,000 One hundred twenty-five (125) subjects between the ages of 11 and 55 years of age received study drug. Objective: To identify the relationship between the coefficient of fat absorption (CFA) and the coefficient of nitrogen absorption (CNA) in subjects with CF-related PI using a dose response of pancreatic enzymes with a fixed ratio of 1:1 lipase:protease. Methods: Controlled marker-to-marker stool collections for fecal fat (modified Van de Kamer Method) and fecal nitrogen (Dumas combustion methodology) were conducted. The CFA and CNA were each determined by calculating the mean of two independent fecal fat or nitrogen results from one stool collection. In order to evaluate the relationship between $\mathrm{CFA}$ and $\mathrm{CNA}$, Pearson product moment correlations were conducted for the relationships of baseline CFA with baseline CNA, treatment CFA with treatment CNA, and change in CFA with change in CNA. Results: There were statistically significant positive correlations between CFA and CNA (baseline, treatment, and change) for all three treatment arms as well as for all subjects combined. For all subjects combined correlations between CFA and CNA for baseline $(r=0.8323)$, treatment $(r=0.8642)$, and change from baseline $(r=0.8442)$ were statistically significant $(p<0.0001)$. Conclusions: For subjects with CF-related PI in this study, baseline CFA correlates significantly with baseline CNA, and treatment CFA correlates significantly with treatment CNA. These findings support the efficacy of the 1:1 ratio of lipase:protease in ALTU-135, which is significantly less protease than is present in porcine-derived pancreatic enzyme products.

\section{1}

Resection vs. Extended Drainage in Chronic Pancreatitis: Long-Term Follow Up of a Randomized Trial

Tim Strate, Philipp Busch, Jens-Peter Bruhn, Oliver Mann, Paulus Schurr, Christian Bloechle, Claus Schneider, Emre Yekebas, Jakob R. Izbicki

Objective: To report on the long term follow-up of the randomized trial comparing the pylorus-preserving Whipple's procedure (PPPD) and the organ sparing extended drainage procedure (Frey) in patients suffering from chronic pancreatitis. Background: PPPD has been the standart of operative care in patients suffering from chronic pancreatitis for decades. Organ sparing procedures (like the Frey procedure featuring a limited pancreatic head excision and extended drainage of the pancreatic body and tail) have been proposed to bear significant advantages especially regarding pancreatic functioning and quality of life. These two procedures have been evaluated and published in a randomized controlled trial by our group, however long term results have not been published so far. Methods: 61 patients suffering from chronic pancreatitis were initially allocated to PPPD $(n=30)$ or Frey $(n=31)$. One patient died immediately postoperatively (Frey). This postoperative follow-up included following parameters: Mortality, quality of life (QL), pain (validated pain score), and exocrine and endocrine function. Results: Median follow-up was 7 years. Six patients were not available for follow-up (PPPD $=2 ; F r e y=4)$. There was no significant difference in late mortality $(14 \%(4 / 28)$ vs. $15 \%(4 / 26))$. No significant differences were found regarding QL (global QL $54,17(0-100)$ vs. $50(16,7-83,4))$, pain score $(12,5(0-75)$ vs. 6,25 (0-100)), exocrine$(96 \%$ vs. $85 \%$ ) or endocrine insufficiency ( $67 \%$ vs. $77 \%$ ). Conclusion: After 7 yrs long term follow-up there was no difference regarding mortality, quality of life, pain, exocrine or endocrine insufficiency within the two groups. 
Curcumin, a Promising Drug for Long-Term Maintenance Therapy in Patients with Ulcerative Colitis: Results from a Multicenter, Randomized Double-Blind Placebo-Controlled Clinical Trial

Hiroyuki Hanai, Takayuki Iida, Ken Takeuchi, Masami Yamada, Yasushi Iwaoka, Akira Andoh, Tomoyuki Tujikawa, Yoshihide Fujiyama, Fumitoshi Watanabe, Yasuhiko Maruyama, Keiichi Mitsuyama, Michio Sata, Kazunari Kanke, Hideyuki Hiraishi, Kazuhisa Hirayama, Hajime Arai, Shigehito Yoshii, Masato Uchijima, Toshi Nagata, Yukio Koide

Background and Objective: Curcumin is a plant-derived natural substance present in turmeric. Curcumin is known to have a variety of pharmacological effects including tumor suppressing action, anti-inflammatory and anti-mirobial activities. The pleiotropic effects of curcumin are attributable at least in part to the inhibition of the transcriptional nuclear factor- $\mathrm{\kappa B}(\mathrm{NF}-\mathrm{\kappa B})$. Further, recently we found that treatment with curcumin resulted in the prevention as well as improvement of experimental murine colitis, actions which were associated with the suppression of NF-KB signaling and CD4+ T-cell infiltration in the colonic mucosa. The principal objectives of this clinical trial were to investigate whether, or not curcumin suppresses colitis and maintains remission of quiescent ulcerative colitis (UC) in man. Methods: This report is on the clinical efficacy of curcumin in combination therapy with sulphasalazine (SASP) or 5-aminosalicylic acid (5-ASA). A total of 89 patients with UC in quiescent state were recruited to participate in a double blind, multi-center, randomized trial. Forty-five patients received curcumin plus SASP or 5-ASA (curucumin group) and 44 patients received placebo plus SASP or 5-ASA (placebo group) for 6 months. Results: Seven patients were excluded according to patients' requests to be treated with a steroid or immuno-modulators. Therefore, 43 patients in the curcumin group and 39 patients in the placebo group were eligible for the efficacy analyses. Of the 43 patients who received curcumin, 2 patients (4.55\%) relapsed during 6 months of therapy, while 8 of 39 patients (20.51\%) in the placebo group relapsed $(\mathrm{P}=0.04)$. Recurrent rates evaluated in all patients (intention to treat) also showed a significant difference in favor of the curcumin group ( $\mathrm{P}=$ 0.049). Of particular interest was that curcumin treatment, but not placebo significantly improved the clinical activity index $(\mathrm{CAI} ; \mathrm{P}=0.038)$ and endoscopic index $(\mathrm{EI}, \mathrm{P}=0.0001)$ after 6 months, indicating that curcumin improves the quality of life of patients with UC. Among cytokine mRNA levels examined in the colonic mucosa from patients in remission, IL-8 was well correlated with EI, suggesting that EI score is valuable for assessing intestinal inflammation without biopsy. Conclusions: The results of this study indicate that curcumin may prove to be a promising agent for maintaining remission in patients with UC. Future studies are essential to fully assess the clinical value of curcumin for patients with inflammatory bowel disease

\section{3}

Prediction of Azathioprine Responsiveness in Crohn's Disease By 6Thioguanosine Di- and Triphosphate Levels in Red Blood Cells: A Prospective Study

Markus F. Neurath, Ralf Kiesslich, Ute Teichgraeber, Claudia Fischer, Uta Hofmann

Michael Eichelbaum, Peter Galle, Mathias Schwab

Background and Aims: The mechanism of action of azathioprine is based on the metabolite 6-thioguanosine triphosphate (TGTP) that blocks the guanosine exchange activity of Vav on the small GTPase Racl. Here, we assessed the impact of TGTP levels for monitoring of azathioprine therapy in a prospective study. Methods: Following our initial retrospective study on azathioprine metabolites in 50 patients, we designed a subsequent prospective study. In 79 patients with Crohns disease under long-term azathioprine therapy levels of TGTP and its precursors 6-thioguanosine mono- and di-phosphates (TGMP, TGDP) in red blood cells were determined. Clinical parameters were monitored over time to determine clinical outcome. Results: TGTP levels could be quantified in all patients taking azathioprine and a subgroup of the latter patients showed significant high levels of TGDP. 6-TGN levels showed a significant correlation with TGDP ( $\mathrm{p}<0.0001)$, TGTP ( $<<0.0001)$, and TGDP plus TGTP concentrations, suggesting that active TGTP and its inactive precursor TGDP are the main metabolites within 6-TGN. No TGMP levels could be detected. Patients with high 6TGN and TGTP levels showed on average better response rates than patients with lower 6 TGN levels. A subgroup of patients with higher 6-TGN and elevated TGDP levels showed a worse outcome with lower response and more flares. Finally, patients with low TGTP levels showed more frequently flares and a refractory course of the disease. Conclusions: This is the first prospective study on the azathioprine metabolites TGDP and TGTP in Crohns disease. The data show for the first time that quantification of TGTP can be used to monitor azathioprine therapy in IBD patients. The analysis of TGDP and TGTP levels as well as the metabolite ratio can by used to predict poor response in a subgroup of azathioprine treated patients.

\section{4}

Medical Decision Analysis for Unifocal Low-Grade Dysplasia (LGD) in Ulcerative Colitis (UC)

Geoffrey Nguyen, Kevin D. Frick, Themistocles Dassopoulos

Background Unifocal LGD detected on surveillance of long-standing UC remains a management dilemma between proctocolectomy and continued intense surveillance. Proctocolectomy eliminates the risk of colorectal cancer, and is associated with good health-related quality of life. However, a recent survey of gastroenterologists revealed that only one fifth would refer patients with LGD for colectomy. There are no clinical trials comparing the effectiveness of the two approaches. We therefore used the tools of medical decision analysis to compare the expected costs and effectiveness (measured in quality-adjusted life years, QALY) of total proctocolectomy with ileal pouch-anal anastomosis (TPC-IPAA) and continued surveillance colonoscopy. Methods We developed a larger model that included small Markov models to represent the expected experience of a hypothetical 45 year old male with UC for ten years and with unifocal LGD detected on a surveillance colonoscopy. Initial states in the model include: false positive (no dysplasia) (5\%), true positive with LGD (77\%), or true positive with synchronous but missed colon cancer (18\%). Options for management were TPC-IPAA or continued colonoscopic surveillance. A $3 \%$ annual discount rate was applied and the time horizon was 40 years. The patient was assumed to have a utility value of 0.95 during surveillance and 0.90 after TPC-IPAA. Transition probabilities, including the onset and stage of colon cancer for patients undergoing surveillance, were derived from previous observational studies, and cost estimates were derived from Medicare reimbursement values and those previously reported in the literature. Results For immediate TPC-IPAA, the present value of lifetime expected QALYs was 19.3 and the present value of lifetime expected expenses was $\$ 36,726$. For surveillance colonoscopy, the expected utility was 18.9 QALYs and total expenses were $\$ 51,187$. These results suggest that immediate TPC-IPAA dominates surveillance colonoscopy. A sensitivity analysis showed that for the expected utility states of the interventions to be equal, the utility post-colectomy would have to be as low as 0.72 . At that level, immediate TPC-IPAA would still dominate surveillance as it costs less. Conclusion TPC-IPAA is dominant over surveillance colonoscopy with higher expected QALYs and lower expected lifetime expense. Based on our model, the postcolectomy health utility state would have to be $20 \%$ lower, for colonoscopic surveillance to be considered possibly worthwhile. TPC-IPAA warrants serious consideration in the management of unifocal LGD.

\section{5}

Acute Severe Ulcerative Colitis: A Study of Predictors of Outcome and Efficacy for Cyclosporin and Infliximab

Alissa Walsh, Rebecca Cooley, Di Templeton, Tim Florin, Graham Radford-Smith

Background: Acute severe ulcerative colitis (ASUC) is a life threatening complication of ulcerative colitis which occurs in approximately $15 \%$ of patients with UC. Intensive treatment and early recognition of severe disease have improved outcomes. Several prognostic factors, specifically CRP $>45$ and bowel actions $>8$ on day 3 help to decide further treatment. This may include Cyclosporin (CyA) or Infliximab (INF), or colectomy. CyA and INF are increasingly used for ASUC however there are limited data on efficacy and safety in this setting. Methods: Data were collected prospectively on patients presenting with ASUC from 1996 to 2004. All patients included in the study had ASUC as defined by the Truelove and Witts criteria and received intravenous hydrocortisone. From 2001, intravenous CyA or INF were given to steroid refractory patients. Colectomy was performed in all those not responding to medical therapy. Aims: To determine: 1. Clinical features of patients with ASUC compared to the remainder of the UC cohort. 2. Significant markers of response to therapy 3. Significant predictors of colectomy. 4. Efficacy and safety of CyA and INF in ASUC. Results: There were 63 patients with 80 episodes of ASUC. The proportion of ASUC in our UC cohort was $14.4 \%(63 / 439)$. Significant differences in those with ASUC were younger age of initial diagnosis ( 24.3 y.o vs 31.7 y.0, $\mathrm{p}=0.005$ ) and shorter duration of disease ( 8.2 years vs 11.9 years, $\mathrm{p}=0.008$ ). Significant predictors of response to IV hydrocortisone were number of bowel actions on day 3 of admission (responders(r)/non responders(nr),5/9, p<0.0001) $\mathrm{Hb}$ on admission $(\mathrm{r} / \mathrm{nr}, 129 / 118, \mathrm{p}=0.03)$ and albumin on admission $(\mathrm{r} / \mathrm{nr}, 35 / 31, \mathrm{p}=0.01)$. There was a trend for increased CRP at day 3 in the non responders group $(\mathrm{r} / \mathrm{nr}, 28.7 / 50.9, \mathrm{p}=$ 0.077 ). The only significant predictor of colectomy was CRP on day 3 (56.6 vs $32.7, \mathrm{p}=$ 0.04). CyA and INF delayed colectomy at discharge compared to those that only received IV hydrocortisone ( $28 \%$ vs $43 \%$, p=NS), however there was no difference at 12 months ( $46 \%$ vs $47 \%$ ). Comparison of CyA and INF data (colectomy at discharge $48 \%$ vs $14 \%$ respectively) indicate a better response to INF. Adverse event profiles were very similar ( $18.5 \%$ vs $14 \%$ ) and there were no fatalities in either group. Conclusions: CRP and bowel actions remain the most important predictors of outcome in ASUC. CyA and INF delay colectomy however long term follow up data are disappointing. Other factors are implicated in the risk of developing ASUC, including genetic susceptibility and host intestinal bacteria, which are currently being investigated as cofactors in the development of ASUC.

576

Intravenous Iron Sucrose: A Simple, Safe, and Quick Method to Treat Anemia Secondary to Digestive Diseases

Santiago Garcia Lopez, Fernando Gomollon, Jose Antonio Garcia Erce, Roberto Aramendiz, Beatriz Sicilia, Raquel Vicente

Chronic bleeding from gastrointestinal lesions is the most important cause of Iron Deficiency Anaemia (IDA) in developed countries. IDA cause significant lose of quality of life in our patients. Iron restitution is the main therapy, usually by oral route. However, oral iron faces 2 clinical problems: low adherence due to gastrointestinal upset and slow clinical response. These limitations can be especially relevant in inflammatory bowel disease (IBD) patients. Intravenous (IV) iron can be a goof alternative to improve tolerance and hasten response but concerns about safety persists in many clinicians OBJETIVE: To evaluate safety of IV iron sucrose therapy in gastrointestinal patients PATIENTS AND METHODS: IV iron sucrose in an "outpatient regimen" was used for patients with IDA due to digestive disorders with at least 1 of the following criteria: 1 ) no response or intolerance to oral iron; 2) IBD with severe anaemia (haemoglobin $(\mathrm{Hb})<10.5 \mathrm{~g} / \mathrm{dl}$ ) and/or 3) clinical need of quick recovery of anaemia. Therapeutic approach in all cases follows a predefined protocol: total iron deficiency is calculated according to an accepted formula and it is administered in several sessions of $200 \mathrm{mgr}$ each, once to three times a week. Evaluation of safety is our main objective. Efficacy of every course is evaluated by complete blood count and iron metabolism determinations 15 days after the last session. Hb increase (pre and post-course) is used as main indicator of efficacy. Descriptive data are given and 95\% CI when appropriate RESULTS: We have treated 70 ( 54 IBD, 27 Crohn`s Disease, 18 Ulcerative Colitis, 9 Pouchitis) patients to whom we have administered 148 courses of IV iron (some patients have received $>1$ course), in $>700$ sessions. Security profile is evaluated in all treatments in our series. No severe adverse event happened in our patients. Apart from metallic taste, only 1 isolated mild blood pressure decrease and 1 unexplained fever occurred: two mild side effects in $>700$ treatment sessions $(0.0028 \% ; 95 \% \mathrm{CI} 0-0.01 \%)$. Mean total amount dose of IV iron per course was $920 \mathrm{mgr}$. Average $\mathrm{Hb}$ pre-course was $9.8 \mathrm{~g} / \mathrm{dl}(+/-1.7)$, and post-course $11.7 \mathrm{gr} / \mathrm{dl}(+/-1.5)$, with an average increase of $1.89 \mathrm{~g} / \mathrm{dl}$ (range $-2+5$ '5). In $47 \%$ (95\% CI 36-59\%) of courses $\mathrm{Hb}$ increase exceded $2 \mathrm{~g} / \mathrm{dL}$ CONCLUSIONS: IV iron sucrose has shown to be a simple to use, 
very safe and effective alternative to oral iron in our patients. No significant side-effects were seen. Efficacy appeared to be good, and anaemia could be improved and many times controlled rendering a better quality of life in our patients. IV iron sucrose should become standard treatment in IBD patients with iron deficiency

577

Clinical Course in Crohn's Disease: A Prospective Population Based Follow-Up Study in Southeastern Norway from January 1990 to December 2004 (The IBSEN study)

Inger C. Solberg, Jorgen Jahnsen, Morten Vatn, Bjorn Moum, Idar Lygren

Background: Few prospective studies have described the long-term clinical course in unselected and well-defined Crohn's disease (CD) populations. Aim: To assess the disease outcome in a population based $\mathrm{CD}$ cohort followed prospectively during the first 10 years after diagnosis Methods: A population based inception cohort of $\mathrm{CD}$ patients was recorded in southeastern Norway from 1990 to 1994. This cohort has since inclusion been followed by the same senior gastroenterologists in a standardized way.Statistics: Kaplan-Meier survival plot, Pearson Chi-Square tests and Cox multiple regressions were applied. Results: A complete 10 -year dataset was achieved in 197 of overall $237 \mathrm{CD}$ patients (83\%). Twenty-two patients (9\%) were lost to follow up and 18 patients $(8 \%)$ had died. The cumulative probability of at least one bowel resection was 38\% (95\% CI: 31\%-44\%) after 10 years. Initial location in terminal ileum, age below 40 years, as well as stenosing or penetrating disease behavior at diagnosis were predictive factors concerning the risk of surgery, while smoking habits, familial occurrence of IBD and gender had no influence. Only $10 \%$ of the patients $(n=20)$ stayed relapse-free during the whole study period. There was however, a significant increase in the number of patients with non-relapsing disease in the last 5-year compared to the first 5 -year period $(\mathrm{p}<0.001)$. Still, a chronic intermittent or a chronic continuous course of disease during the entire follow-up were observed in $32 \%(n=63)$ and $19 \%(n=37)$ of the patients, respectively. Furthermore immune-modulating therapy was required in $44 \%$ ( $n=$ 87) during the last 5 years. Conclusion: The cumulative risk of a bowel resection (38\%) was lower than previously reported (55-70\%). This may reflect differences in study design, but also changes in medical treatment and attitudes toward surgery. A significant increase in the number of patients in remission was observed in the last 5-year, which may indicate a decrease in disease activity over time.

590

Increased Activity of Microsomal Triglyceride Transfer Protein, Bile Acid and Cholesterol Synthesis and Trafficking in the Liver of Chilean Hispanics with Gallstones

Juan Francisco Miquel, Juan Castro, Cecilia Galman, Ludwig Amigo, Luz Carreno, Silvana Zanlungo, Mats Rudling, Flavio Nervi

Aim/Background - Gallstone disease is an important, costly health-care problem in Western societies, particularly in the Hispanic population. Bile acids (BA) have long been known to affect triglyceride homeostasis. For example, BA-binding resins induce the production of VLDL triglyceride. We recently showed that BA synthesis was increased in gallstone patients (GS) (Gastroenterology 2004; 126: 741. The aim was now to investigate whether the production of hepatic VLDL, a function associated to BA and cholesterol synthesis, was increased in the liver of GS compared to GS-free patients. Methods - A total of 27 voluntaries subjected to elective surgery, $9(6 \mathrm{~F} / 3 \mathrm{M})$ without GS and $18(13 \mathrm{~F} / 5 \mathrm{M})$ with GS agreed to give a liver biopsy after informed consent. Fasting blood plasma samples were assayed for fatty acids, $7 \alpha$-hydroxy-4-cholesten-3-one (C4) and lathosterol, two indicators of hepatic BA and total body cholesterol synthesis, respectively. We quantified the activity and mRNA content of hepatic Microsomal Triglyceride Transfer Protein (MTTP), a rate limiting protein in VLDL synthesis, as a surrogate of VLDL production. Hepatic lipid levels and mRNA expression of genes responsible for uptake, synthesis, intrahepatic transport of lipids and sterol secretion into bile were determined. Results - Age, body mass index and serum albumin, lipids and fatty acids were similar in both groups. Hepatic histology was normal in all samples. Hepatic cholesterol levels were similar in GS and GS-free patients, whereas hepatic triglycerides were increased by $200 \%$ in GS. MTTP activity increased $70 \%(\mathrm{p}<0.02)$ and mRNA expression $50 \%(\mathrm{p}<0.01)$ in GS. Serum C4 levels were $75 \%$ higher and hepatic CYP7Al mRNA expression was increased by $250 \%(\mathrm{p}<0.01)$ in GS compared to GS-free patients, while CYP27Al was unchanged. Serum lathostherol levels were similar, but hepatic hydroxymethyl-glutaryl-CoA reductase mRNA expression increased by $90 \%(\mathrm{p}<0.01)$ in GS. Expressions of Fatty Acid Binding Protein, Sterol Carrier Protein-2, SR-BI and ABCG8 mRNAs were significantly increased in GS, while LDLR and ABCG5 mRNA were in normal range in GS. Correlation between MTTP mRNA and CYP7Al mRNA was $r=0.52(p, 0.005)$ and between serum $C 4$ and CYP7Al mRNA was $r=0.57$ (p, 0.002). Conclusions - The data show that hepatic MTTP activity and mRNA expression are concomitantly induced, suggesting that VLDL production is increased in GS and further confirm that BA synthesis is induced in GS. Hepatic cholesterogenesis, but not total body cholesterol synthesis is enhanced in GS. The results indicate that hepatic trafficking of cholesterol is increased in GS disease. (Supported by FONDECYT 1030744 and 1040820)

591

Prevention of Cholesterol (Ch) Gallstones By the Potent Ch Absorption Inhibitor Ezetimibe in Gallstone-Susceptible Mice

Helen H. Wang, Piero Portincasa, David Q. Wang

Since biliary Ch hypersecretion is an important prerequisite for Ch gallstone formation, considerable interest has been focused on identifying genetic and physical-chemical determinants of intestinal Ch absorption. Aims: We explored whether $\mathrm{Ch}$ gallstones could be prevented by ezetimibe in doses of $0,2,10,20 \mu \mathrm{g} /$ day in male C $57 \mathrm{~L}$ mice ( $\mathrm{n}=20$ per group) fed a lithogenic diet containing 1 or $2 \%$ Ch plus $0.5 \%$ cholic acid for 8 or 12 wk. Methods: Gallbladder biles and gallstones were examined by microscopy. Gallbladder emptying in response to CCK-8 was measured gravimetrically. Biliary lipid outputs were analyzed by HPLC. Expression levels of the genes for hepatic and intestinal lipid transporters were investigated by real-time PCR. Ch absorption efficiency was determined by mass balance methods. Results: There was a dose-dependent decrease in gallstone prevalence rates from $80 \%, 40 \%, 10 \%$, to $0 \%$ in mice at $8 \mathrm{wk}$ on the lithogenic $(1 \% \mathrm{Ch})$ diet, coupled to significant dose-dependent decrease in intestinal Ch absorption efficiency from $50 \pm 7 \%$, $36 \pm 5 \%, 15 \pm 4 \%$, to $7 \pm 2 \%$. On ezetimibe at $20 \mu \mathrm{g} /$ day, gallstones were prevented in mice fed the lithogenic diet with even higher $(2 \%)$ amounts of $\mathrm{Ch}$ for a longer (12 wk) period. Gallbladder emptying studies showed that fasting gallbladder volumes were larger $(56 \pm 18$ $\mu \mathrm{l})$ on the lithogenic diet than those $(22 \pm 9 \mu \mathrm{l})$ on chow, and that high dietary $\mathrm{Ch}$ induced a-fold increase of residual gallbladder volumes. These lithogenic effects on gallbladder dynamics were fully blocked by ezetimibe at $\geq 10 \mu \mathrm{g} /$ day. Also, biliary Ch outputs and $\mathrm{Ch}$ saturation indices of pooled gallbladder biles were significantly decreased in a dose-dependen fashion from $30 \pm 8$ to $7 \pm 2 \mu \mathrm{mol} / \mathrm{h} / \mathrm{kg}$ and from 1.5 to 0.6 , respectively. Outputs of biliary phospholipid (from $59 \pm 17$ to $27 \pm 14 \mu \mathrm{mol} / \mathrm{h} / \mathrm{kg}$ ) but not bile salts (from $219 \pm 83$ to $187 \pm 65$ $u \mathrm{~mol} / \mathrm{h} / \mathrm{kg}$ ) were significantly reduced. Expression levels of $\mathrm{Npc111}$ in the small intestine and of $A b c g 5 / g 8$ and $A b c b 4$ in the liver were significantly and dose-dependently reduced from $7 \pm 2$ to $0.5 \pm 0.1$, from $5 \pm 2$ to $2 \pm 1$, and from $5 \pm 2$ to $3 \pm 1$, respectively. However, there were not significant changes in mRNA levels of Abcg5/g8, Abcal, Sr-b1 and Slc10a2 in the small intestine and of $A b c b 11$ and $S r-b 1$ in the liver. Conclusions: High efficiency of intestina $\mathrm{Ch}$ absorption is an independent risk factor for $\mathrm{Ch}$ gallstones. By effectively inhibiting intestinal $\mathrm{Ch}$ absorption, ezetimibe can prevent not only $\mathrm{Ch}$ gallstone formation but also the deranged gallbladder motility, mostly due to increased biliary Ch content. Our findings point to an efficacious novel approach for the prevention of $\mathrm{Ch}$ gallstones by inhibiting intestinal Ch absorption in humans.

\section{2}

The Adaptive Immune Response Appears Critical in Promoting Cholesterol Gallstone Formation in a BALB/C Murine Model

Kirk Maurer, Martin C. Carey, James G. Fox

In C57L/J mice, coinfection with Helicobacter hepaticus and $H$. rodentium contributes significantly to cholesterol gallstone formation. One possible explanation for this is modulation of the adaptive immune response by these organisms. To test this hypothesis we studied cholesterol cholelithogenesis in male BALB/C (WT) mice and coisogenic male BALB/c Rag2-/(Rag2) mice which lack functional lymphocytes and are unable to mount an adaptive immune response. Mice were divided into 4 groups: uninfected WT $(n=18)$ and Rag2 $(n=17)$ mice and infected WT $(\mathrm{n}=10)$ and Rag2 mice $(\mathrm{n}=17)$. Infected groups were inoculated at 4-5 weeks of age with both $H$. hepaticus and $H$. rodentium. All groups were fed a lithogenic diet (containing $1.0 \%$ cholesterol and $0.5 \%$ cholic acid) for 8 weeks beginning at $8-9$ weeks of age. At the completion of the study gallbladder bile was analyzed by microscopy. WT mice of either group displayed significant increases in mucin gel, the prevalence of cholesterol monohydrate crystals, sandy stones, and cholesterol gallstones when compared to Rag2 mice (Table 1). Cholesterol gallstone formation in BALB/c mice appears to be less dependent on infection status than in C57L mice. However, infected WT mice demonstrate a nearly significant $(P=0.055)$ increase in cholesterol gallstone formation when compared to their uninfected counterparts (Table 1). These data rigorously demonstrate that the adaptive immune response plays a role in the pathogenesis of cholesterol gallstone formation. It is likely that infection with Helicobacter spp. act to modulate this response leading to an increased prevalence of cholesterol gallstones. Based upon the near absence of mucin gel in Rag2 mice it is likely that the adaptive immune response stimulates excess mucin production creating a pronucleating gel matrix for phase separation of solid crystals leading to cholesterol gallstone formation

\begin{tabular}{|c|c|c|c|c|c|}
\hline $\begin{array}{c}\text { Strain/ Infection } \\
\text { status }\end{array}$ & $\begin{array}{c}\text { Mucin gel } \\
\text { score }\end{array}$ & $\begin{array}{c}\text { Liquid } \\
\text { crystals }\end{array}$ & $\begin{array}{c}\text { Solid } \\
\text { crystals }\end{array}$ & $\begin{array}{c}\text { Sandy } \\
\text { Stones }\end{array}$ & $\begin{array}{c}\text { True } \\
\text { Stones }\end{array}$ \\
\hline WT uninfected & $2.5+/-0.3^{*}$ & $100 \%$ & $78 \% *$ & $67 \% *$ & $39 \% *$ \\
\hline WT infected & $3.4+/ 0.4^{*}$ & $80 \%$ & $90 \% *$ & $80 \% ‡$ & $80 \% * \dagger$ \\
\hline Rag2 uninfected & $0.5+/-0.1$ & $100 \%$ & $6 \%$ & $0 \%$ & $6 \%$ \\
\hline Rag2 infected & $0.8+/-0.5$ & $89 \%$ & $11 \%$ & $11 \%$ & $11 \%$ \\
\hline
\end{tabular}

* $P<0.005$ and $¥ P<0.05$ when compared to Rag2 mice $\uparrow P=0.055$ when compared to WT uninfected mice

Anti-Oxidant Mechanism of Ursodeoxycholic Acid On Gallbladder Muscle Cells from Symptomatic Gallstone Patients

Michele P. Guarino, Ping Cong, Simone Carotti, Rossana Alloni, Michele Cicala, Jose Behar

Bile acids may be involved in the pathogenesis of acute cholecystitis since the hydrophobic chenodeoxycholic acid (CDCA) reproduces in vitro the muscle dysfunction observed in this condition. Gallbladder muscle cells exposed to CDCA show poor contractility in response to agonists caused by oxidative stress as cells generate reactive oxygen species and display lipid peroxidation of plasma membranes. The hydrophilic ursodeoxycholic acid (UDCA) prevents these deleterious effects of CDCA in the animal model of acute cholecystitis. In symptomatic gallstone patients, UDCA reduces the risk of biliary pain and acute cholecystitis and restores muscle cell contractility. These studies were aimed at determining the mechanism of UDCA on gallbladder muscle cells in symptomatic patients with cholesterol gallstones. Methods: A double blind study was performed in 15 patients, 7 were randomized to UDCA and 8 to placebo and treated for 4 weeks prior to cholecystectomy. In the preliminary study, muscle cell contractility resulted to be improved following UDCA and this effect was related to a lower cholesterol content in the plasma membranes. $\mathrm{H} 2 \mathrm{O} 2$, lipid peroxidation, PAFlike lipids, PGE2 and catalase activity were now determined as biochemical markers of oxidative stress and inflammation in gallbladders muscle cells from both groups. Results: 
UDCA treatment decreased the levels of $\mathrm{H} 2 \mathrm{O} 2$ from $13.6 \pm 2.4$ in the placebo treated patients to $4.9 \pm 0.2 \mathrm{mmol} / \mathrm{L}$ per $\mathrm{mg}$ protein $(\mathrm{p}<0.00 \mathrm{l})$. Compared to placebo, UDCA strongly reduced the levels of lipid peroxidation $(2.52 \pm 0.39$ vs $1.29 \pm 0.28 \mathrm{nmol} / 100 \mathrm{mg}$ of protein), of PAF-like lipids ( $29.2 \pm 1.6 \mathrm{vs} 8.6 \pm 1.0 \mathrm{pg} / \mathrm{mg}$ of protein), and the production of PGE2 ( $363 \pm 36$ vs $156 \pm 14 \mathrm{pg} / \mathrm{mg}$ protein), $\mathrm{p}<0.001$. UDCA treatment decreased the activity of catalase, a scavenger of free radicals, from $35.8 \pm 6.4$ to $14.6 \pm 4.6$ units $/ \mathrm{mg}$ protein $(\mathrm{p}<0.001)$. Conclusions: UDCA significantly reduces the biochemical markers of oxidative stress and inflammation in gallbladder muscle cells from symptomatic gallstone patients. It is conceivable that UDCA reduces the oxidative stress by "inactivating" hydrophobic bile acids and this may also explain the prophylactic effects of UDCA on biliary pain and acute cholecystitis in patients with symptomatic gallstones.

\section{4}

Sustained Potential of Soybean Lecithin to Counteract the Progression of Liver Disease and the Premature Mortality Induced By ABCB4 Deficiency in Mice Majorie Martel, Veronique St-Hilaire, Beatriz Tuchweber, Marie-Eve Bourbonnais, Guylaine Bouchard

Mutations in $\mathrm{ABCB} 4$ can lead to progressive familial intrahepatic cholestasis type 3 (PFIC3), characterized by severe portal hepatic fibrosis and ductular proliferation. Although some therapies limiting the progression of the disease are available, transplantation often remains necessary to avoid mortality. Using a murine Abcb4-/- model, we previously established that a diet enriched in soybean lecithin could exert beneficial effects against PFIC-3 in a shortterm experiment. We now tested whether lecithin could provide a sustained protective action against $\mathrm{ABCB} 4$ deficiency-induced liver disease in mice supplemented or not with cholic acid (CA). Methodology: Post-weaning Abcb4-/- mice and wild-type (FVB/J) controls were fed diets containing 0 or $0.025 \% \mathrm{w} / \mathrm{w}$ CA that were supplemented or not with soybean lecithin $(4.0 \% \mathrm{w} / \mathrm{w}$ phosphatidylcholine). Mice were sacrificed after 4 months of dietary intervention as $A b c b 4-/$ - mice receiving CA exhibited advanced health complications and mortality (3/10 mice within 2 weeks). Selected end points were body and liver weights, hepatic serum enzymes and liver histology at sacrifice. A group of $A b c b 4-/-$ mice was also sacrificed at time 0 to establish the extent of the liver disease at the initiation of treatment. Results: Abcb4-/- mice fed CA exhibited a very severe hepatic disease as evidenced by portal inflammation, extensive ductular proliferation, and marked portal fibrosis. Serum ALT, ALP, AST, bilirubin, BS and GGT were also highly elevated. Abcb4-/- mice not receiving CA also displayed significant hepatic disease but in much less advanced stage. Of note, CA administration in $\mathrm{Abcb4+/+}$ mice did not impact adversely any of the parameters followed. Treatment with lecithin abrogated the progression of the liver disease in Abcb4-/- mice, whether they were fed CA or not. In fact, histological features were maintained at a level virtually similar to the one observed in the pre-treatment group. The same was true for serum liver enzymes, except for AST and ALT where lecithin treatment in Abcb4-/- mice not receiving CA only reduced their level of elevation. Lecithin also normalized the body weight gain and the liver to body weight ratio in Abcb4-/- mice fed CA. Conclusion/Speculation: Soybean lecithin is extremely effective to counteract the severely progressing liver disease induced by the absence of ABCB4 in mice. As lecithin safety is well established in human, rapid confirmation of its potential as an anti-cholestatic and anti-fibrogenic agent in PFIC-3 patients but also in other human chronic progressive cholestatic conditions may enable to improve survival and minimize the need for liver transplantation.

\section{5}

Regulation of Fatty Acid Fluxes and Synthesis in the Liver By Phosphatidylcholine Transfer Protein (PC-TP)

Erez F. Scapa, Keishi Kanno, Wen-Jun Wang, David Cohen

Background: PC-TP is a highly specific phosphatidylcholine binding protein that is enriched in liver. We recently showed (Hepatology 42:509A, 2005) that chow fed mice with homozygous disruption of the gene encoding PC-TP (Pctp-/-) develop hepatic steatosis, despite increased hepatic triglyceride secretion rates. Taken together with unchanged plasma concentrations of $\beta$-hydroxybutyrate, which reflect rates of hepatic fatty acid oxidation, these findings suggest that PC-TP expression might limit de novo lipogenesis or the supply of plasma-derived fatty acids. Aim: To explore the molecular basis for hepatic steatosis in Pctp-/- mice by quantifying mRNA expression levels of key genes that control triglyceride and fatty acid metabolism in the liver. Methods: Eight week-old male wild type ( $\mathrm{n}=5)$ and Pctp-/- $(\mathrm{n}=6)$ FVB/NJ mice were maintained on chow. Mice were sacrificed, and liver RNA was extracted and utilized to prepare cDNA for quantitative real-time PCR. Gene amplification was quantified using SYBR green and normalized to ribosomal protein L32 mRNA expression, which was not influenced by genotype. Results: In chow fed mice, the absence of PC-TP expression was associated with a $51 \%$ reduction in mRNA levels of sterol regulatory element binding protein-lc, a transcription factor that promotes hepatic lipogenesis. Accordingly in livers of Pctp-/- mice, we observed significant downregulation of lipogenic target genes: acetyl CoA carboxylase by $41 \%$, fatty acid synthase by $66 \%$ and stearoyl CoA desaturase 1 by $68 \%$. Consistent with our prior observation that plasma $\beta$-hydroxybutyrate concentrations were unchanged in Pctp-/- mice, the absence of PC-TP did not influence hepatic mRNA levels for carnitine palmitoyl transferase I or II, which regulate fatty acid oxidation. In Pctp-/mice, there was a modest $19 \%$ reduction in mRNA of HMG CoA synthase, which promotes ketone body formation. Suggestive of the likelihood that steatosis and increased hepatic triglyceride secretion were primarily attributable to increased uptake of plasma fatty acids by the liver, we observed a $43 \%$ increase in mRNA for fatty acid transport protein 2 . Moreover, there was no change in mRNA for microsomal triglyceride transfer protein, the upregulation of which could alone increase the rate of hepatic triglyceride secretion. Conclusions: In Pctp-/- mice, increased uptake of plasma-derived fatty acids, as opposed to de novo lipogenesis or decreased fatty acid oxidation, appears to account for the overproduction of triglycerides for both storage and secretion. These findings suggest that a key function of PC-TP is the regulation of fatty acid fluxes, as well as de novo synthesis in liver.
Generation of Bacterial-Reactive T Regulatory Cells Able to Inhibit Colitogenic T Cells Brian G. Morris, Langfang Wang, Yingzi Cong, Charles O. Elson

Background and Aims: A set of immunodominant antigens of the bacterial flora has been identified that stimulate pathogenic immune reactions in $\mathrm{C} 3 \mathrm{H} / \mathrm{HejBir}$ mice, a strain that spontaneously develops colitis. We have previously shown that ovalbumin-specific T-regulatory cells can be generated using immature, NF-kB blocked dendritic cells(DCs). The purpose of this study was to generate bacterial antigen-specific Tregs and test their ability to inhibit proliferation of memory $\mathrm{T}$ cells reactive to the same antigen (direct inhibition) or whether such Tregs could inhibit colitogenic Thl cells broadly reactive to the entire cecal bacterial flora (bystander inhibition). Methods: Splenic CD4+ T cells were isolated from C3H/ HeJBir mice immunized with CBirl, an immunodominant bacterial flagellin, and CBir19, an immunodominant bacterial non-flagellin antigen. Primed CD4+ T cells were cultured in the presence of antigen-specific DCs to generate memory T cells(Tmem) or NF-kB blocked DCs to generate regulatory $\mathrm{T}$ cells(Tregs). The DCs were blocked by Curcumin, a specific inhibitor of the NF-KB cascade. CBirl- and CBirl9-specific Tregs were placed in culture along with Tmem or a cecal bacterial antigen(CBA)-specific Thl cell line. $\mathrm{T}$ cell proliferation was measured by ${ }^{3} \mathrm{H}-\mathrm{TdR}$ incorporation. Results: CBirl-and CBir19-specific Tregs both blocked the CBirl Tmem response by 50-75\%, demonstrating the ability to generate bacteria-specific Tregs using curcumin induced NF-KB blockade. These assays also demonstrate direct, antigen specific inhibition as well as bystander, non-antigen specific inhibition. The same CBirland CBir19-specific Tregs also blocked proliferation of CBA-specific Thl cells, a cell line that causes colitis when adoptively transferred into C3H.SCID mice. Both Tregs inhibited proliferation of this Thl line by $75-90 \%$, demonstrating the ability of two immunodominant antigen-specific Tregs, one flagellin and one non-flagellin, to inhibit a colitogenic T cell line in a bystander fashion. Furthermore, the addition of both anti-IL-10RI and anti-TGF $\beta$ together, but neither alone abrogated the inhibition. Conclusions: Bacterial antigen-specific Treg cells can be generated in vitro using NF-kB blocked immature DCs. Furthermore, such Tregs are able to inhibit Tmem and colitogenic Thl cell responses by direct and bystander inhibition in vitro. This inhibition appears dependent on the presence of both IL-10 or TGF$\beta$. We postulate that these Tregs will inhibit colitigenic T cells in vivo as well.

597

The Cd28 Homolog, B and T Lymphocyte Attenuator (BTLA), Is An Important New Target for Immunoregulation in Experimental Colitis Edwin F. De Zoeten, Kenneth Murphy, Jonathan Kaye, Wayne W. Hancock

Many costimulatory receptors and their ligands are now recognized but little is known of their functions in disease, including inflammatory bowel disease (IBD). BTLA, a CD28 homolog induced on activated CD4 and CD8 cells and also expressed by B cells, macrophages and dendritic cells, has two ITIM motifs in its cytoplasmic tail, consistent with an inhibitory function of this surface protein. The ligand for BTLA was recently identified as herpes virus entry mediator (HVEM). We investigated whether BTLA expression contributed to regulation of host immune responses during experimental IBD. Chronic colitis was induced using 3\% dextran sulfate sodium in wild-type and BTLA-/- C57BL/6 mice, and mice were scored daily for weight, bleeding and stool consistency over the next 4 weeks. Colons were evaluated by histology, immunoperoxidase and quantitative real-time PCR (qPCR) for cytokine and costimulatory molecule expression. Spleens, mesenteric lymph nodes, lamina propria cells and intraepithelial lymphocytes were studied by flow cytometry and qPCR. Normal colons contained only low-level BTLA expression, but qPCR analysis of colonic samples from mice treated with DSS showed a marked increase in BTLA expression, and BTLA was localized by immunoperoxidase to infiltrating mononuclear cells. Concomitant flow cytometry studies showed induction of BTLA by T cells and B cells, especially in mesenteric lymph nodes versus spleens, as well as by colonic intraepithelial lymphocytes and lamina propria cells. BTLA-/-mice developed colitis at the same rate but had significantly enhanced and persistent diarrhea $(\mathrm{p}<0.01)$ and bleeding $(\mathrm{p}<0.01)$, compared to WT mice. Moreover, whereas controls regained their weight in 12-14 days, weight loss in BTLA-/- mice persisted over the 28 day period of follow-up $(\mathrm{p}<0.01)$, and comparable findings were noted in wild-type mice treated with DSS and a neutralizing anti-BTLA mAb (but not control IgG). At 28 days, colons of BTLA-/- mice were grossly thickened, hemorrhagic, stiff and adherent to surrounding tissues, whereas wild-type mice had only mildly thickened colons. Compared to controls, colons from BTLA-/- mice had increased expression of multiple costimulatory molecules (ICOS, LIGHT, PD-1, PD-L1) and pro-inflammatory cytokines (IFN-g, TNF-a). Our data show BTLA has an important role in regulation of immune responses during experimental IBD, and suggest that BTLA molecule may be a useful target for IBD therapy. To that end, we are currently testing whether ligation of BTLA, using HVEM-Ig or an agonistic anti-BTLA mAb, can enhance the inhibitory effects of BTLA signaling on activated $\mathrm{T}$ cells and thereby regulate IBD in wild-type mice.

598

Centaurin Beta 1 Protein Down-Regulates Nuclear Factor Kappa B (NF-kB) Activation By NOD2 and NOD1 Pathways

Jesus K. Yamamoto-Furusho, Ramnik Xavier, Tadakazu Hisamatsu, Daniel K. Podolsky

Background: Centaurin Beta 1 (CENTB1) is a GTPase-activating protein belonging to the ADP ribosylation factor family. A yeast two-hybrid screen showed that CENTBl binds NOD2. Nucleotide-binding oligomerization domain (NOD) proteins have been implicated in intracellular recognition of bacterial components such as peptidoglycan containing $\gamma$-Dglutamyl-meso-diaminopimelic acid (iE-DAP) and muramyl dipeptide (MDP) can activate NF-KB. Aims: To study the interaction between CENTB1, NOD2 and NOD1 proteins and its functional role on NF-kB activation. Material and Methods: Yeast two hybrid screening was performed by MATHCMAKER GAL4 Two-Hybrid System 3. Construction of expression plasmids: Xpress-tagged CENTBl mammalian expression vector (pCDNA4/HisMAXCENTB1) was generated by PCR from cDNA of SW480 cells. Real-time RT-PCR was done 
in an ABI Prism 7000 sequence detector using SYBR Green JumpStart detection system. Immunoprecipitation and Western blot: COS7 cells lysate were immunoprecipitated with anti-Flag anti-HA or anti-Xpress monoclonal antibodies and protein A/G sepharose beads. NF$\mathrm{kB}$ activation assay: HEK293 cells were transfected with CENTBl at different concentrations, NOD2 (lng), NOD1 (10ng), pIV luciferase reporter, renilla plasmid, MDP and iE-DAP. Immunostaining was performed in COS-7 cells using Texas-Red-conjugated anti-mouse IgG or FITC-conjugated anti-rabbit IgG antibodies. Invasion assay: Gentamicin protection assay using Salmonella typhimurium was performed in Caco-2 cells. Results: CENTB1 is expressed in several cells from immune system such as Jurkat, THP-1, lymphocytes B and monocytes. Interestingly, colonic epithelial cells lines showed an increased expression of this protein after stimulation with TNF- $\alpha$, MDP and iE-DAP. Expression was also increased in mucosa from Ulcerative Colitis and Crohn's disease patients as compared to normal individuals. Interaction between CENTB1 and NOD2 as well as NOD1 proteins was confirmed by immunoprecipitation. CENTB1 partially colocalized with NOD2 and NOD1 in the cytoplasm of COS-7 cells. CENTBl decreases NF-kB activation in a concentration dependent manner in HEK293 cells co-transfected with NOD2 and NOD1 proteins and stimulated with MDP and iE-DAP respectively. Transfection with CENTB1 siRNA significantly increased the MDP and iE-DAP driven response to NOD2 and NOD1. Conclusion: CENTB1 is a down-regulator of NF-KB activation through NOD2 and NOD1 pathways, suggesting that this protein could play a role in the innate immune response in inflammatory bowel disease.

599

Characterization of Foxp $3+C d 4+C d 25+$ Regulatory T Cells from Mesenteric Lymph Nodes in Human Ulcerative Colitis (UC)

Masayuki Saruta, Qi T. Yu, Allison H. Banham, Konstantinos A. Papadakis

Background: CD4+CD25+ regulatory (Treg) cells can control the development of experimental colitis by regulating effector T cells and innate immune responses in mucosal lymphoid tissues. However, little is known about the presence and/or potential functional impairment of these cells in human inflammatory bowel disease (IBD). We sought to determine the presence and functional characteristics of Treg cells isolated from Mesenteric lymph nodes (MLN) (one of the inductive sites of the mucosal immune system) in human UC. Methods: Colonic MLN were isolated from patients with severe UC that were undergoing surgical resection and lymphocytes were isolated using standard techniques. Phenotypic analysis of CD25+CD4+ T cells isolated from colonic MLN was performed by flow cytometry. Analysis of FoxP3 expression among CD25- and CD25+ subsets was performed by RT-PCR and flow cytometry. Localization of FoxP3+ cells in MLN was performed by immunohistochemistry (IHC). Functional characterization of CD4+CD25+ T cells was performed with proliferation assays following allogeneic (dendritic cells) or polyclonal T cell activation (anti-CD3 plus autologous APC). The effect of CD4+CD25+ T cells on cytokine production by effector CD4+CD25- T cells was performed using the 22-plex Luminex assay. Results: MLN from human UC colon contain a subset of CD4+CD25+ T cells with phenotypic characteristics of Treg cells (expression of CCR4, CD122, HLA-DR, GITR and intracellular CTLA4). FoxP3+ Cells were detected by IHC in the MLN medulla dispersed in the T cell rich areas and occasional FoxP3+cells were present in the follicles. CD4+CD25+ cells express FoxP3 mRNA and protein and potently suppress the proliferation of autologous MLN CD4+ T cells. In contrast to peripheral blood Treg from healthy donors, the CD4+CD25hi and CD4+CD25int MLN T cell subsets exhibit potent suppressor activity in vitro. The suppressor function of CD4+CD25+FoxP3+ cells is not mediated through TGF-beta or IL-10, whereas addition of exogenous IL-2 restores the proliferation of responder CD4+CD25- MLN T cells. In addition to their ability to suppress the proliferation of CD4+CD25- cells, CD4+CD25+ cells potently suppressed the secretion of IL-2, IL-5, IL-13 and IFN-gamma by CD4+CD25- effector T cells. Conclusions: CD4+CD25+ T cells isolated from human colonic MLN in UC display typical features of Treg cells and possess potent suppressor activity in vitro in spite of persistent mucosal inflammation. These data suggest that their suppressor activity is likely compromised in vivo and finding ways to restore their suppressive activity is desirable in order to achieve long-term tolerance in the gut.

600

\section{IL-23 Cross Regulates IL-12 Production in T Cell Dependent Experimental}

\section{Colitis}

Christoph Becker, Heike Dornhoff, Clemens Neufert, Massimo C. Fantini, Stefan Wirtz, Alexei Nikolaev, Hans A. Lehr, A J. Murphy, D M. Valenzuela, G D. Yancopoulos, M Karow, Peter R. Galle, Markus F. Neurath

IL-12 related cytokines are important mediators of cell-mediated immune responses and are implicated in the pathogenesis of chronic inflammatory and autoimmune diseases. We describe a novel mechanism by which crossregulation of IL- 12 by IL- 23 controls colitis. IL23 was found highly expressed in the lamina propria of animals suffering from colitis. Immunohistochemical analysis demonstrated expression of IL-23p19 by infiltrating dendritic cells that had taken up bacteria. To investigate the functional role of IL-23 in experimental colitis, we generated mice in which the coding region for IL-23 was replaced with the reporter gene beta-galactosidase. In unchallenged mice, constitutive reporter gene activity was significantly present only in the ileum and the colon implicating constitutive activity of the IL-23p19 promoter in the latter organs. When mice were challenged with TNBS, unexpectedly, IL-23p19 knockout mice developed more severe colitis than wildtype mice as defined by weight loss, in vivo endoscopy and histology. We next challenged mice with orally administered dextrane sulfate to verify our previous findings. Again, mice deficient for IL-23p19 developed a very severe colitis when compared to wildtype mice. Mice heterozygous for IL-23p19 showed an intermediate course of disease. Colon lysates of heterozygous and knockout mice contained high levels of beta-galactiosidase and reporter gene activity correlated well with the disease severity as determined by the weight loss of the animals. Interestingly, bone marrow derived dendritic cells from knockout mice expressed significantly higher levels of IL-12p35 as compared to wildtype animals. Furthermore, exogenous IL-23 suppressed IL-12p35 mRNA expression in BMDC derived from knockout mice, implicating that mice lacking IL-23p19 may overproduce IL-12. To investigate whether the overproduction of IL-12 may be responsible for the severe colitis observed in IL-23p19 knockout mice, we induced colitis in IL-23p19 knockout mice and subsequently injected a weekly dose of an antibody neutralizing IL-12p40. While in the control group, mice continuously los weight and finally died, in contrast, mice that received anti-IL-12p40 antibody were protected from colitis and survived. This finding was furthermore confirmed by endoscopic examinations of the colon, demonstrating a high colitis score in control mice as compared to antibody IL-12p40 treated animals. Therefore our data strongly suggest a cross regulation of IL-23 and IL-12 in dendritic cells in the regulation of colitis. Our findings question the use of neutrolizing IL-23p19 antibodies in the treatment of colitis.

601

Essential Counter-Regulatory Role of Farnesoid-X-Receptor (FXR) for Intestinal Innate Immunity

Stefano Fiorucci, Barbara Renga, Giovanni Rizzo, Andrea Mencarelli, Moses Disante, Mark Pruzanski, Roberto Pellicciari, Antonio Morelli

Background. The farnesoid X receptor (FXR) is a member of the nuclear receptor superfamily of ligand-activated transcription factors involved in cholesterol and bile acid homeostasis and expressed in the liver and gastrointestinal tract. A growing body of evidence suggest that certain nuclear receptors exerts counter-regulatory function in inflammation. INT-747 (6-Ethyl chenodeoxycholic acid) is a semi-synthetic FXR ligand. Aims. To investigate whether FXR is expressed by cells of innate immunity and regulates inflammation in animal model of colitis. Methods. Human peripheral blood monocytic cells (PBMC) were prepared from healthy volunteers. Endoscopic samples from intact and macroscopically inflamed colon from obtained from patients with Crohn's disease. Colitis was induced in wild type and FXR-/- deficient mice (C57BL6 background) by intrarectal administration of TNBS or by 7 day administration of 5\% DSS in drinking water. Animals were administered with 1,3 or $10 \mathrm{mg} / \mathrm{kg}$ INT747 by gavage for 7 days. Results. FXR is expressed by and exerts counter regulatory effects on cell of innate immunity. Microarray analysis of LPS-induced PBMC derived macrophages reveals that FXR ligands function as negative regulators of NF-KB dependent-genes resulting in a reciprocal regulation of genes involved in innate and adaptive immune response (TNF, IL-1, IL-6, approximately 50-70\% inhibition of mRNA expression) as well as bile acid/cholesterol homeostasis as demonstrated by induction of the FXR regulated gene, SHP. Using two rodent models of colon inflammation, we show that progression of these immune-mediated disorders is severely exacerbated in FXR-/- mice resulting in a reduction of disease activity score of 50-60\% ( $\mathrm{p}<0.01$ versus wild type mice). Colon inflammation in Crohn's disease patients and in rodent models of colitis associates with a significan reduction $(70-80 \%)$ of FXR mRNA expression in the colon. In vivo treatment with INT747 attenuates organ injury and immune cell activation in the TNBS and DSS colitis. At the dose of $3 \mathrm{mg} / \mathrm{kg}$, INT747 significantly increased the colon expression of IBABP, FXR SHP while reduced IL-1, IL-2, IL-6, TNF- $\alpha$ and IFN $\gamma$ mRNA expression and attenuated the disease activity score of $50-60 \%$. FXR is expressed by lamina propria-derived CD1lb+ cells Exposure of these cells to INT-747 significantly reduced cytokine release induced by LPS. Conclusions. These findings identify FXR as a bile acid-dependent regulator of inflammatory gene expression in macrophages, possibly establishing a link between cholesterol/bile acid homeostasis and innate and adaptive immunity in the gastrointestinal tract.

\section{2}

Colon Cancer Specific Antigen-3 and -4 (CCSA-3 and CCSA-4): Novel Serum Based Markers for Detection of Colorectal Cancer (CRC)

Eddy Lehman, Robert E. Schoen, Grant W. Cannon, Robert H. Getzenberg

A blood test for CRC screening would represent a major advance because of the relative ease of implementation and potential to markedly increase patient participation. Using a proteomic approach focused on nuclear matrix proteins specific to colorectal cancer, we developed an ELISA based assay to CCSA-3 and CCSA-4, two novel proteins. Aim: To assess the sensitivity and specificity of these markers. Methods: Serum samples were collected prospectively from patients undergoing colonoscopy, and from subjects with CRC and benign colon conditions prior to surgery. Based on histologic results, subjects were classified into the following categories: normal colonoscopy $(\mathrm{N}=30)$, hyperplastic polyp $(\mathrm{N}=23)$, benign surgical condition $(\mathrm{N}=9)$, and cancer $(\mathrm{N}=28)$. A pilot study was performed to establish cut off points for test positivity (CCSA-3 $(2 \mu \mathrm{g} / \mathrm{ml})$ and CCSA-4 $(0.3 \mu \mathrm{g} / \mathrm{ml})$ ). Results: Mean levels for both CCSA-3 and CCSA-4, respectively, were higher in colorectal cancer (3.2 $1.1 ; .72 \pm .29)$ than in the benign conditions: normal colon $(.66 \pm .3 ; .13 \pm .05)$; hyperplastic polyps $(1.08 \pm .48 ; .13 \pm .76)$; pre-surgical benign $(.58 \pm .13 ; .26 \pm .10)$. At the specified cut off points, CCSA-3 and CCSA-4 each detected $100 \%(95 \% \mathrm{Cl} 88-100)$ of the cancers. The specificity for normal colon, hyperplastic polyps, and benign colon was $98 \%$ (95\%C 88-100) for CCSA-3 and 97\% (95\% CI 86-99) for CCSA-4. Conclusions: This is the first report of a serum based assay able to distinguish subjects with cancer from subjects with a benign colon. Studies in subjects with advanced and non-advanced adenoma are ongoing If substantiated, this would represent a major advance in colorectal cancer screening

603

Apoptosis in Normal Rectal Mucosa Predicts Adenoma Recurrence Ahmad Amin, Joseph Galanko, Christopher Martin, Barbara Schliebe, Robert S. Sandler, Temitope O. Keku

Background Lower levels of apoptosis in the normal rectal mucosa have been shown to be strongly associated with the presence of adenomas elsewhere in the colon (a field effect) in cross sectional studies. It is not known whether apoptosis can predict subsequent adenomas. We evaluated whether apoptosis and other factors at baseline colonoscopy could predic recurrent adenomas. Methods: We evaluated subjects initially enrolled in the Diet and Health Study III (DHS3), a cross sectional study of adenoma risk factors. There were 489 participants enrolled in the original study. The present study was limited to 257 men and women (baseline adenoma status; 129 had adenomas and 128 adenoma free) who returned for follow-up colonoscopy. At baseline, subjects underwent colonoscopy and had biopsies of 
normal mucosa to evaluate apoptosis by morphology on $\mathrm{H} \& \mathrm{E}$ stained sections. The number of adenomas, the size, type and degree of atypia at baseline colonoscopy and the level of apoptosis in normal rectal mucosa were evaluated as predictors of recurrence. T-tests were used to assess relationships between continuous variables and adenoma recurrence. Multiple logistic regression was used to calculate odds ratios (OR) and 95\% confidence intervals (CI). Results: Of the subjects who had adenoma at baseline, $41 \%$ had at least one adenoma at follow-up compared to $15 \%$ of individuals who were adenoma free at baseline. After adjusting for age, race and sex, individuals in the highest tertile of rectal mucosal apoptosis were substantially less likely to have an adenoma at follow-up (OR 0.28; $95 \% \mathrm{CI}, 0.10-0.75$ ) compared to the lowest tertile. The inverse relationship between apoptosis and adenoma status at follow-up was strongest in individuals who were free of adenoma at baseline. Having more than one adenoma at baseline also increased the risk for recurrence (OR 1.61; $95 \% \mathrm{CI}, 1.29,1.87)$. Consistent with the results of previous studies, the presence of an advanced adenoma at baseline also predicted recurrence, but was not statistically significant. No association was observed between adenoma location and recurrence. Conclusion: The results of the study provide new evidence that low apoptosis is strongly associated with increased risk of recurrent adenomas. Apoptosis could potentially be used to identify patients at higher risk for recurrent adenomas. Work was supported by grants from NIH R01 CA44684 and P30 DK 34987

\section{4}

Increased Level and Freqency of Methylation Correlates with a Poor Response to Chemotherapy and Radiation in Esophageal Cancer

James P. Hamilton, Fumiaki Sato, Bruce D. Greenwald, Mohan Suntharalingham, Mark J. Krasna, Martin J. Edelman, Yuriko Mori, Takatsugu Kan, Suna Wang, Bogdan C. Paun, Tetsuo Ito, Yulan Cheng, John M. Abraham, Stephen J. Meltzer

Background and Aims: Multiple studies have shown that promoter methylation of tumor suppressor genes underlies esophageal carcinogenesis. Hypothetically, methylation resulting in tumor suppressor gene inactivation may result in tumors that are unresponsive to chemotherapy and radiation. Accordingly, our aim was to find methylation markers that could be used to predict response to chemoradiation. Materials and Methods: Tumor specimens were obtained prior to treatment from 35 patients enrolled in a uniform chemo-radiation treatment protocol. Methylation-specific quantitative PCR for eleven genes was performed on all samples using the ABI 7700 (Taqman) system. Esophagectomy specimens were used to define response to treatment. Results: 13 (37\%) of 35 patients were responders, and 22 $63 \%)$ of 35 patients were non-responders. The number of methylated genes per patient was significantly lower in responders than in non-responders ( 1.4 vs. 2.4 genes per patient Student's t-test, $\mathrm{p}=0.026$ ). The combined mean level of promoter methylation of $\mathrm{p} 16$, Reprimo, p57, p73, RUNX-3, CHFR, MGMT, TIMP-3, and HPP1 was also lower in responders than in non-responders (Student's t-test, $\mathrm{p}=0.003$; Mann-Whitney test, $\mathrm{p}=0.001$ ). Of particular interest, both the frequency ( 2 , or $15 \%$, of 13 responders vs. 14 , or $64 \%$ of 22 nonresponders; Fisher's exact test, $\mathrm{p}=0.01$ ); and level ( 0.078 in responders vs. 0.313 in nonresponders; Mann-Whitney test, $\mathrm{p}=0.037$ ) of Reprimo methylation was significantly lower in responders than in non-responders. Conclusions: Reprimo methylation occurred at significantly lower levels and frequency in chemoradioresponsive than in non-responsive esophageal cancer patients, suggesting potential clinical application of this single-gene biomarker in defining prognosis and management. In addition, increased methylation of a 9-gene panel correlated significantly with poor responsiveness to chemoradiation.

STANDARDIZED COMBINED MSP VALUES IN

RESPONDERS $V S$. NON-RESPONDERS

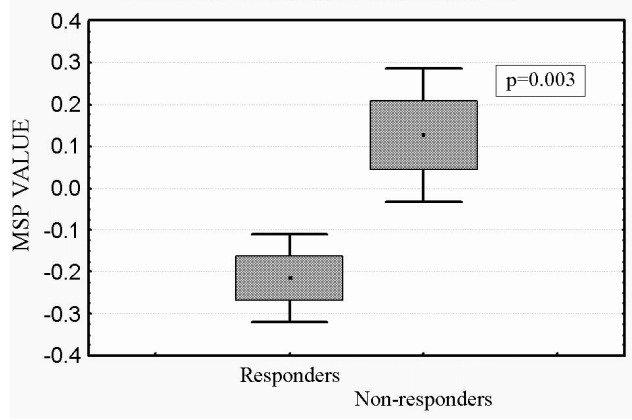

605

Selection and Suitability of Microsatellite Markers for Colorectal Cancer Classification According to Microsatellite Instability Degree

Rosa M Xicola, Xavier Llor, Elisenda Pons, Anna Giros, Miquel A Gassull, Gi Oncology Group Spanish Gastroenterology Association

Introduction: The first set of recommended markers for the identification of microsatellite instability (MSI) in colorectal cancer (CRC) tumors was published in 1998 (Bethesda panel) This included 5 markers, 2 of them mononucleotide repeats (BAT25, BAT26) and 3 dinucleotide repeats. Recently it has been suggested that the use of 5 mononucleotide repeats (NR21, NR22, NR24, BAT25, BAT26) or even just BAT26, could be as effective to detect high degree MSI (MSI-H). A major advantage of using those markers is that they are quasimonomorphic in most populations and there is no need for uninvolved tissue examination Regarding the Bethesda panel, it has also been suggested that when only dinucleotide markers are unstable, it is necessary to check more mononucleotide markers in order to exclude low degree MSI (MSI-L). Objectives:1)To establish if the use of the 5-mononucleotide panel is at least as sensitive as the Bethesda panel for the identification of MSI-H tumors. 2)To establish if it is necessary to study more mononucleotide markers when tumors are MSI-H exclusively due to dinucleotide instability. 3)To establish which are the most useful markers for the identification of MSI-L and MSI-H tumors. Methods: Out of the 1,309 newly diagnosed CRC from the EPICOLON project, 753 were analyzed with the Bethesda panel and 556 with the 5-mononucleotide set. All MSI-L, 100 stable tumors (MSS) and all MSI-H classified as such exclusively through dinucleotide instability with the first panel, were subsequently studied with the 5-mononucleotide set. Results: 1,156 CRC were initially classified as MSS, $105 \mathrm{MSI}-\mathrm{H}$ and $44 \mathrm{MSI}-\mathrm{L}$. The series studied with the 5-mononucleotide set identified a percentage of $9,9 \%$ of tumors as MSI-H while the series studied with the Bethesda markers identified $6,6 \%$ of tumors as MSI-H. Sensibility for MSI-H identification for specific markers was: $91.4 \%$ for BAT26, 93.3\% for BAT25, 94.5\% for NR21 and NR24, and 89\% for NR22. Specificity was $100 \%$ for BAT25, NR22 and NR24. Only 2/105 tumors were MSI-H only for dinucleotide markers and one of them was reclassified as MSI-L with the 5-mononucleotide set. None of the tumors identified as MSI-L with the Bethesda panel was reclassified as MSI-H when adding the study of NR markers. 14 MSI-L tumors were unstable only for NR21 Conclusions: The set of 5-mononucleotide markers (NR21, NR22, NR24, BAT25, BAT26) is the most sensitive and specific for MSI-H identification. Analyzing BAT25, NR21 and NR24 only from tumor tissue we can identify all MSI-H and a significant proportion of MSI-L tumors. If tumors are MSI-H only for dinucleotides, it is necessary to study more mononucleotides to correctly classify them

606

Expression of Multiple Simultaneous Biomarkers in Tissues of Patients with Colitis-Associated Neoplasia

Steven Itzkowitz, Lina Jandorf, Noam Harpaz, Joel Skoletsky, Anthony Shuber

Background: Molecular genetic alterations that are important for sporadic colorectal carcinogenesis have also been implicated in colitis-associated neoplasms (CANs). Few studies have simultaneously analyzed multiple markers in the same tissues of IBD pts. In anticipation of developing a fecal DNA-based assay for detecting CANs, we have begun to analyze tissues from pts with longstanding ulcerative colitis (UC) for expression of the 22 DNA mutations (MuMu-22) currently included in a commercially available fecal DNA test. Aim: To compare the expression of MuMu-22 in cancerous and non-neoplastic tissues of IBD pts and compare this with normal controls (NL). Methods: From the Mount Sinai GI Pathology Database, we identified surgical specimens from 15 UC pts with CRC (one pt had two cancers), and 37 NLs who underwent surgical resection for diverticular disease or volvulus. Average (+/S.D.) duration of UC was $19.8(+/-11.7)$ years. Neoplastic and non-neoplastic tissues were microdissected by an expert GI pathologist and analyzed for MuMu-22 (3 kras, 8 p53, 10 APC, and 1 Bat-26 mutations) using PCR technology as previously described. Results: Among the $37 \mathrm{NLs}$, only one case was positive for a k-ras mutation (Table). In contrast, 7/16 (44\%) UC-cancer specimens had a mutation. Cancers did not have more than one mutation, and, confirming previous observations, APC mutations were rare. Four samples of non-neoplastic tissue had a mutation. Conclusions: Even in a limited sample set, sensitivity of $44 \%$ using this marker panel suggests that a refined marker panel targeting more alterations involved in UC carcinogenesis could lead to a fecal DNA test for pts with UC.

\begin{tabular}{|c|c|c|c|c|c|}
\hline & Any Mutation & APC & Kras & p53 & Bat-26 \\
\hline $\mathrm{NL}(\mathrm{N}=37)$ & $1 / 37(2.7 \%)$ & 0 & 1 & 0 & 0 \\
\hline UC-CRC(N=16) & $7 / 16(44 \%)$ & 0 & 3 & 3 & 1 \\
\hline UC-Non-neoplastic $(\mathrm{n}=15)$ & $4 / 15(27 \%)$ & 1 & 2 & 1 & 0 \\
\hline
\end{tabular}

607

Expression of Galectin-3 and Its Binding Protein (MAC-2BP) Correlate with Progression and Metastasis of Colorectal Adenocarcinoma Kai-Feng Liu, James C. Byrd, Shumei Song, Robert S. Bresalier

Background: Mac-2 BP (90K) is a large oligomeric glycoprotein composed of subunits of approximately $90 \mathrm{kDa}$, identified as a ligand for the carbohydrate binding protein, galectin3 (Gal-3). Immunohistochemical studies of Mac-2 BP expression in colon cancer have not been reported, although previous studies have indicated that serum levels of this protein may have prognostic value in patients with several types of cancer. We have reported that other ligands for Gal-3 such as colon cancer mucin and a $40 \mathrm{kDa}$ aberrantly glycosylated form of haptoglobin may play a role in tumor progression and have diagnostic value in patients with colorectal neoplasia. This study examined the expression of Mac-2BP in colorectal cancers using immunohistochemistry, and correlated its expression and distribution in these tissues with expression of Gal-3 and mucin sub-types. Methods: 33 sporadic colorectal cancers with 11 associated metastases as well as specimens of normal $(n=10)$ and transitional $(n=28)$ mucosa and colorectal adenomas $(n=10)$ were examined immunohistochemically for expression of Mac-2BP and Gal-3, then scored semiquantitatively to take into account distribution and intensity of staining ( $0-3)$. Genetically related colon cancer cells with different metastatic potential were compared by Western blot analysis. Results: Colorectal cancers expressed Mac-2BP in 32 of $33(97 \%)$ cases (mean score $\pm S E, 1.71 \pm 0.14$ ). Expression of this protein in cancers was significantly higher than that in normal mucosa $(0.75 \pm 0.11$, $\mathrm{p}<0.001)$, colorectal adenomas $(0.30 \pm 0.21, \mathrm{p}<0.001)$ and transitional mucosa $(0.54 \pm 0.14$, $\mathrm{p}<0.001)$. Metastases expressed higher amounts of Mac-2 BP than the primary tumors from which they were derived $(2.32 \pm 0.24 ; \mathrm{p}<0.05)$. Mac-2BP was expressed in the cytoplasm of neoplastic tissues and in secreted material, with levels of the ligand paralleling that of Gal-3. In keeping with previous reports, normal tissue expressed predominately nuclear galectin-3. Most colorectal carcinomas (85\%) lost nuclear localization of Gal-3 and expressed the protein only in the cytoplasm (co-localized with Mac-2BP). Some cancers (15\%) expressed both nuclear and cytoplasmic Gal-3. Highly metastatic colon cancer cell line Lim6 expressed higher levels of both galectin-3 and Mac-2BP compared to low metastatic cell line LM12. Mac-2 BP was expressed in mucinous tumors, which strongly expressed MUC2 and MUC5AC, and also in non-mucinous carcinomas. Conclusion: The galectin-3 binding protein Mac-2BP is highly expressed in colorectal cancers and may serve as a marker of neoplastic progression. 
stimulation of colonic motility, these drugs were injected 20 min before the injection of

Early Weaning Stress Predisposes Adult Pigs to Stress-Induced Colonic Barrier Dysfunction: A Novel Model for Stress-Induced Gastrointestinal Disorders in Humans

Adam J. Moeser, Duncan X. Lascelles, Anthony T. Blikslager

Adverse early life experiences may predispose individuals to development of stress-induced gastrointestinal (GI) disorders as adults, often associated with pain. In the neonatal pig, early weaning (less than 21 days) involves multiple psychosocial stressors such as early maternal and sibling separation, abrupt changes in environment and diet, and establishment of a dominance hierarchy with non-littermate pigs. We sought to determine whether early weaning stress predisposes piglets to stress-induced GI dysfunction in adult life. Neonatal piglets were subjected to one of 2 weaning conditions: 1) early weaning at 19 days of age (EW), or 2) weaning at 28 days of age (control). At 63 days of age, the pigs were subjected to a 6-hour period of social stress involving mixing the pigs with unfamiliar pigs (social stress). Colonic tissue and spinal cord was then removed for evaluation. Transepithelial electrical resistance [TER] and mucosal-to-serosal fluxes of ${ }^{3} \mathrm{H}$-mannitol were measured in colonic tissues mounted on Ussing chambers. Ion secretion was determined by measuring short circuit current $\left[I_{\mathrm{sc}}\right]$. Western blot analyses of corticotrophin releasing factor (CRF) receptor 1 and mucosal mast cell staining were performed on colonic tissue. Western blot analyses of COX-1 and 2 on spinal cord segments T12, L2 and L6 were performed. Data were analyzed using a standard l-way ANOVA. Social stress in EW pigs decreased colonic TER by $41 \%$ and induced a 2 -fold increase in mucosal permeability to ${ }^{3} \mathrm{H}$-mannitol $(\mathrm{P}<0.01)$. Social stress did not effect TER and ${ }^{3} \mathrm{H}$-mannitol flux in control pigs. Colon from EW pigs, but not control, exhibited significant elevations in baseline secretory activity following social stress $\left(\Delta \mathrm{Isc}=11 \pm 3 \mu \mathrm{A} / \mathrm{cm}^{2}\right)$. EW, but not control pigs, showed diarrhea following social stress. Western blot analysis revealed enhanced expression of CRF receptor 1 in colonic mucosal homogenates of EW pigs but no change in CRF expression in control pigs. Additionally, mast cells numbers were markedly increased in colonic mucosa of EW pigs following social stress compared with control pigs (cell numbers $=6.6 \pm 0.7 / 10 \mu^{2}$ and $2.8 \pm 1.7 / 10 \mathrm{um}^{2}$ in stressed EW and control pigs, respectively). Spinal cord levels of COX-2, but not COX1 , were elevated in EW pigs compared to control pigs. Results from these studies provide supporting evidence for the validity of this novel porcine model to study human functional GI disorders such as irritable bowel syndrome.

609

Acupuncture Induces Gastric Phase III-Like Contractions Via Ghrelin Release in Conscious Rats

Cindy Chen, Kiyoshi Tsukamoto, Yukiomi Nakade, Theodore N. Pappas, Toku Takahashi

Background; The mechanism(s) of the beneficial effects of acupuncture on GI symptoms still remains unclear. We have previously showed that acupuncture on the hind limb (ST36) changed the fasting gastric motor patterns and elicited strong cyclic contractions (phase III-like contractions) in conscious rats. Acupuncture-induced phase III-like contractions were abolished by atropine, hexamethonium and vagotomy, suggesting the mediation of vagal cholinergic pathways (Am J Physiol 285, R862-72, 2003). Ghrelin is a newly discovered orexigenic peptide originating from the stomach. Peripheral administration of ghrelin induces phase III-like fasting motor activity of the rat stomach (J Physiol 550.1, 227-240, 2003). We hypothesize that acupuncture-induced phase III-like contractions of the rat stomach is mediated via an endogenous ghrelin release. Methods; A strain gage transducer was implanted on the serosal surface of the stomach to record circular muscle contractions. One week after the surgery, the rats were fasted for $24 \mathrm{hrs}$. After the recording of basal gastric motility for 2 hrs, ghrelin (1, 4 and $10 \mu \mathrm{g} / \mathrm{kg}$ ) was administered subcutaneously (sc). Acupuncture needles were inserted to a depth of $5 \mathrm{~mm}$ into the skin and underlying muscles at ST-36 bilaterally. Needles were stimulated by electricity $(0.1 \mathrm{msec}, 1 \mathrm{~mA}$ and $1 \mathrm{~Hz})$ for $1 \mathrm{~min}$. Fasting gastric motility was recorded before, during and after EA in a conscious state. To investigate whether an endogenous ghrelin is involved in mediating acupuncture-induced phase III-like contractions, growth hormone secretagogue receptor (GHS-R) antagonist (400 $\mathrm{nmol} / \mathrm{kg}$ ) was administered $10 \mathrm{~min}$ before EA. It has been demonstrated that ghrelin receptors are located on the vagal afferent. To investigate whether ghrelin- and acupuncture-induced phase III-like contractions are mediated via vagal afferent pathways, we utilized perivagal capsaicin-treated rats. Results; Ghrelin (1-10 $\mu \mathrm{g} / \mathrm{kg}, \mathrm{sc})$ significantly provoked phase III-like contractions in a dose dependent manner in conscious rats. EA at ST-36 also elicited strong phase III-like contractions, which mimicked with ghrelin-induced phase III-like contractions. GHS-R antagonist significantly attenuated the incidence of acupuncture-induced phase IIIlike contractions. Ghrelin $(4 \mu \mathrm{g} / \mathrm{kg}, \mathrm{sc})$ and EA causes no more phase III-like contractions in perivagal capsaicin-treated rats. Conclusions; Acupuncture-induced gastric phase III-like contractions is mediated via an endogenous ghrelin release in conscious rats. Released ghrelin from the gastric mucosa induced by acupuncture primarily acts on vagal afferents to induce phase III-like contractions.

610

Peripherally Administered CRF Stimulates Colonic Motility Via Central CRF Receptors and Vagal Pathways in Conscious Rats

Kiyoshi Tsukamoto, Yukiomi Nakade, Christopher Mantyh, Kirk Ludwig, Theodore N.

Pappas, Toku Takahashi

Background: $\mathrm{CRF}$ (corticotropin releasing factor) is one of the most important factors to the mechanism of stress-induced stimulation of colonic motility. However, it is controversial whether stress-induced stimulation of colonic motility is mediated via central or peripheral CRF receptors. We investigated the hypothesis that peripherally injected CRF accelerate colonic motility through central CRF receptor, but not peripheral CRF receptor. Methods: A strain gauge transducer was sutured on the serosal surface of the proximal colon. Colonic motility was monitored before and after the peripheral injection of $\operatorname{CRF}(3,10,30$ and 100 $\mu \mathrm{g} / \mathrm{kg}$; sc). To study the influence of peripherally administered hexamethonium, atropine, or intracisternal (IC) injection of astressin (a CRF receptor antagonist) on CRF-induced
CRF (30 $\mu \mathrm{g} / \mathrm{kg}, \mathrm{sc})$. To study whether vagus nerve is related to the alteration of colonic motility evoked by CRF, subdiaphragmatic bilateral truncal vagotomy was performed during the operation to install the strain gauge transducers and five days after the operation, the colonic motility was recorded before and after CRF injection (30 $\mu \mathrm{g} / \mathrm{kg}$, sc). In vitro muscle strip study was also performed to investigate the peripheral effects of CRF. Results: Subcutaneous injection of CRF (30-100 $\mu \mathrm{g} / \mathrm{kg}$ ) stimulated colonic motility in a dose-dependent manner The stimulatory effect of peripherally administered CRF $(30 \mu \mathrm{g} / \mathrm{kg}, \mathrm{sc})$ on colonic motility was abolished by truncal vagotomy, hexamethonium and atropine. IC-injection of astressin also abolished the stimulatory effect of $\mathrm{CRF}(30 \mu \mathrm{g} / \mathrm{kg}$, sc) on colonic motility. No contractile responses were observed to CRF (10-9 M -10-7 M) of the muscle strips of the proxima colon in vitro. Conclusions: These results suggested that the stimulatory effect of colonic motility in response to peripheral administration of CRF is mediated by vagus nerve, nicotinic receptors, muscarinic receptors and CRF receptors of the brain stem. It is suggested tha peripherally administered CRF reaches area postrema and activates dorsal nucleus of vag (DMV) via central CRF receptors, resulting in stimulation of vagal efferent and cholinergic transmission of the proximal colon.

611

Pathways Involved in Gut Mucosal Barrier Dysfunction Induced in Adult Rats By Neonatal Maternal Deprivation: CRF and NGF Interplay

Frederick Barreau, Christel Salvador-Cartier, Laurent Ferrier, Jean Fioramonti, Lionel Bueno

Background and aim: The increase in gut paracellular permeability (PP) observed in adult rats submitted to neonatal maternal deprivation involves nerve growth factor (NGF) and mast cell activation. Maternal deprivation triggers an overexpression of NGF at both brain and colonic levels. It also increases hypothalamic corticotropin releasing factor (CRF) expression, but its effect on colonic CRF remains unknown. This study was aimed to determine whether maternal deprivation induces colonic CRF overexpression and whether CRF, NGF and mast cells play an interactive role in alterations of PP induced by maternal deprivation. Methods: Male Wistar pups were either separated, $3 \mathrm{~h}$ per day, from postnatal days 2 to 14 (deprived animals), or left undisturbed with their dam (non-deprived). At 12 weeks of age, adult deprived rats were treated i.p. with a non specific CRF receptor antagonist, alphahelical(9-41)CRF $(250 \mu \mathrm{g} / \mathrm{kg})$, or a specific CRF-Rl receptor antagonist, SSR-125543 (10 $\mathrm{mg} / \mathrm{kg}$ ). Furthermore, adult non-deprived rats were treated by CRF $(50 \mu \mathrm{g} / \mathrm{kg})$, or BrX-537A (a mast cell activator, $2 \mathrm{mg} / \mathrm{kg}$ ) and anti-NGF antibodies $(15 \mu \mathrm{g} / \mathrm{kg}$ ). PP was assessed by oral administration of ${ }^{51} \mathrm{Cr}$-EDTA $(1 \mu \mathrm{Ci} / \mathrm{rat})$, and expressed as the percentage of radioactivity collected in the urine during 24 hours. In addition, rats were sacrificed and colonic strips used for staining of mucosal CRF expression, which was analysed and graded from 0 to 3 according to the degree of immunostaining. Results: Neonatal maternal deprivation increased colonic CRF expression in adult rats, mainly at the bottom of the crypts ( $1.5 \pm 0.2$ vs $0.6 \pm 0.4$ $\mathrm{P}<0.05)$. Alpha-helical CRF(9-41) or SSR-125543 treatment suppressed the increase in PP induced by $\mathrm{MD}(\mathrm{P}<0.05)$. In adult non-deprived rats, CRF increased PP $(3.9 \pm 0.3 \%$ vs $1.8 \pm 0.2 \% ; \mathrm{P}<0.05)$. This increase in $\mathrm{PP}$ was reduced by anti-NGF antibodies treatmen $(2.8 \pm 0.3 \%$ vs $3.9 \pm 0.3 \% ; \mathrm{P}<0.05)$. Similarly, mast cell activation by $\mathrm{BrX}-537 \mathrm{~A}$ increased PP $(4.1 \pm 0.4 \%$ vs $1.8 \pm 0.2 \% ; \mathrm{P}<0.05)$. Anti-NGF antibodies treatment also reduced this increase $(3.1 \pm 0.4 \%$ vs $4.8 \pm 0.4 \% ; \mathrm{P}<0.05)$. Conclusions: Maternal deprivation induces $\mathrm{CRF}$ overexpression in the colon. Since anti-NGF antibodies inhibit the increase in PP induced by both CRF and mast cell activation, it can be concluded that CRF may acts at CRF-Rl receptors to stimulate NGF release from mast cells, which in turn participates to the increase in colonic in PP observed in adult rats that were submitted to neonatal maternal deprivation

612

Imaging Glutamate in Human Swallowing Motor Cortex: Implications for Mechanisms of Neuroplasticity

Salil Singh, Satish Mistry, Samantha Jefferson, Karen E. Davies, Steve R. Williams, Shaheen Hamdy

Background: Activity in the swallowing motor cortex (SMC) can be facilitated by an intervention termed paired associative stimulation (PAS). This technique involves delivery of an electrical stimulus to the pharyngeal muscles followed by a transcranial magnetic stimulus to the SMC. The resultant changes have been termed neuroplasticity but the molecular mechanism remains uncertain. Glutamate, the most abundant excitatory neurotransmitter has been implicated. Aims: To identify the neurotransmitter changes associated with PAS using proton magnetic resonance spectroscopy to measure cortical glutamate concentrations in vivo. Methods: Seven healthy volunteers were studied on two occasions, receiving either real or sham intervention. An electrical pharyngeal stimulus was followed $100 \mathrm{~ms}$ later by a transcranial magnetic stimulus to the SMC. These paired stimuli were repeated every 20 s for 30mins. Post intervention, serial magnetic resonance spectroscopy was undertaken at the stimulated SMC as well as an occipital control site. Data were analysed using jMRU software with metabolite peaks compared across real and sham visits. Results: All subjects showed a reduction in glutamate 1 peak (the most prominent glutamate peak) at the SMC following real stimulation compared to the same site following sham stimulation. Group mean data showed a $27 \pm 6 \%$ reduction in glutamate concentration $(\mathrm{p}<0.004)$. A similar $24 \pm 12 \%$ decrease was confirmed at the glutamate 2 peak $(p<0.05)$ but no significant changes were seen with any other metabolites at the SMC or control sites. Conclusions: Neuroplastic changes induced by PAS are associated with a focal decrease in cortical glutamate. Our data suggest a role for glutamate in the induction of neuroplasticity and give important insights into the molecular basis for plasticity in the human brain. These findings also identify a potential target for pharmacological manipulation. 


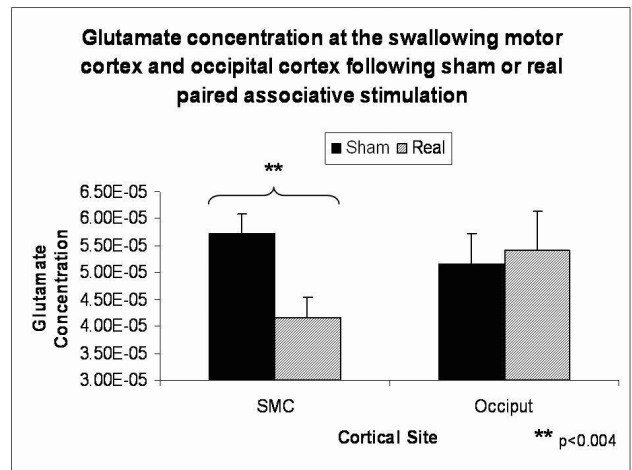

613

Central Glucagon Like Peptide-1 Accelerates Colonic Transit Via a Central CRF and Peripheral Parasympathetic Pathway in Conscious Rats Yukiomi Nakade, Kiyoshi Tsukamoto, Constant Masere, Kirk Ludwig, Christopher R. Mantyh, Theodore N. Pappas, Toku Takahashi

Background: Glucagon like peptide-1 (GLP-1) is a gastrointestinal (GI) regulatory peptide secreted from the small and large intestine in response to absorbed nutrients. Peripheral GLP-1 stimulates insulin secretion on the islet beta cell to decrease blood glucose level. Apart from the role of incretine, GLP-1 is also known to act as a neurotransmitter mediating GI function in the brain. Central administration of GLP-1 inhibits gastric emptying (Am J Physiol 273, G920-927, 1997). However, little is known about the effect of central GLP-1 on colonic motor function. We examined the effects of intracerebroventricular (ICV)-injection of GLP-1 on colonic transit in conscious rats. Methods: Using male SD rats, indwelling silastic cannula was inserted into the cecum and positioned to the proximal colon. ICVcannula was inserted into the right lateral ventricle. One week after the operation, immediately after ICV-injection of GLP-1, radioactive material $(5 \mathrm{lCr})$ was applied via the catheter onto the proximal colon. Ninety minutes after $51 \mathrm{Cr}$ injection, rats were sacrificed and the entire colon was removed and divided into 10 equal segments. The radioactivity of each segment was counted and the geometric center (GC) was calculated as a distribution of $51 \mathrm{Cr}$. To examine the pathway through which central GLP-1 mediates colonic transit, atropine (50 $\mu \mathrm{g} / \mathrm{kg}, \mathrm{IP})$, hexamethonium $(20 \mathrm{mg} / \mathrm{kg}, \mathrm{IP})$, and guanethidine $(5 \mathrm{mg} / \mathrm{kg}, \mathrm{IP})$ were administered 20 minutes before ICV-injection of GLP-1. Truncal vagotomy was performed 1 week before the colonic transit study. To study the relationship between corticotropin releasing factor (CRF) and GLP-1 in the brain, CRF antagonist, astressin $(2.8 \mathrm{nmol})$, was administered by ICV 10 minutes before ICV-injection of GLP-1. Results: GC in vehicle-treated rats was $4.4 \pm 0.3(n=6)$. ICV-injection of GLP-1 $(0.3-3.0 \mathrm{nmol})$ dose-dependently accelerated colonic transit. Maximum effect was observed by $3 \mathrm{nmol}$ of GLP-1 (GC; $7.8+/-0.5, \mathrm{n}=6)$. In contrast, intraperitoneal-injection of GLP-1 (3 nmol) did not accelerate colonic transit $(5.2+/-0.5$ $\mathrm{n}=6$ ). ICV-injection of GLP-1 ( $3 \mathrm{nmol})$-induced acceleration of colonic transit was abolished by truncal vagotomy, atropine and hexamethonium, but not by guanethidine. ICV-injection of GLP-1 ( 3 nmol)-induced acceleration of colonic transit was also abolished by the pretreatmemt with astressin $(2.8 \mathrm{nmol} ; \mathrm{ICV})$. Conclusions: These results indicate that the central administration of GLP- 1 accelerates colonic transit via central CRF and peripheral parasympathetic pathways in conscious rats.

\section{4}

Dual Actions of Neurotensin and Localization of NTS1 Receptors Relative to SK3 Channels and Interstitial Cells of Cajal in Human Ileum Yael Azriel, Fei Shang, Lu Liu, Elizabeth Burcher

Purpose: Neurotensin (NT) is a brain-gut peptide from the ileum, released in response to ingested fats. We studied the mechanisms of action of NT in isolated muscle strips and localized its receptors in relation to various cell types and markers in the human ileum. Methods: Isometric tension recordings were made from longitudinal or circular muscle strip preparations from normal terminal ileum. Discrete concentration responses curves to NT were obtained in the absence and presence of various inhibitors. Inhibitory responses were expressed as a percentage of the height of spontaneous contractions before addition of NT. Ileal sections were incubated with primary antibodies against the NTS1 receptor, SK3 (marker for $\mathrm{Ca}^{2+}$-activated $\mathrm{K}^{+}$channels) and c-kit (marker for interstitial cells of Cajal), then processed for confocal microscopy. Results: In longitudinal muscle, biphasic effects were seen: an initial relaxation and inhibition of spontaneous rhythmic contractions ( $\left.\mathrm{EC}_{50} 2.5 \mathrm{nM}, \mathrm{n}=15\right)$, usually followed by a weak delayed contraction $\left(\mathrm{EC}_{50} 1.6 \mu \mathrm{M}\right)$, with maximum tension only $6.8 \%$ compared to acetylcholine. NT-induced inhibition was unaffected by tetrodotoxin, indomethacin, hexamethonium and L-NAME, but was almost abolished by apamin (100 $\mu M)$. In ileal circular muscle, NT had weak contractile effects $\left(E C_{50} 126 \mathrm{nM}, \mathrm{n}=14\right)$, unaffected by inhibitors. Thus, NT is a substantially more potent inhibitory agent compared to contractile agent in the human ileum. Using RT-PCR, mRNA transcripts were detected for the NTS1 receptor, but not for NTS2. NTS1 immunoreactivity (IR) was observed in myenteric and submucosal ganglia, on mast cells and also as punctate immunoreactivity in longitudinal and circular muscle. There was negligible colocalization of NTS1-IR with c-kit-IR. SK3-IR occurred within and around myenteric ganglia, within muscle and on submucosal arterial blood vessels. Substantial colocalization of NTS1-IR with SK3-IR was seen in the myenteric plexus; approximately $20 \%$ of NTSI-IR in muscle was colocalized with SK3-IR. However the presence of NTS1 receptors in ganglia may be at variance with the functional data showing tetrodotoxin-independent mechanisms. Conclusions: Neurotensin has potent, apamin-sensitive inhibitory actions on ileal longitudinal muscle. Its receptors (NTS1) are found on myenteric and submucosal ganglia as well as the muscularis; colocalization with SK3 was seen. Neurotensin may represent a novel pharmacological target for motility disorders. Supported by NHMRC of Australia.

615

ICC Networks Coordinate Propagation of Longitudinal Muscle Activity and Movements in the Murine Small Intestine

Grant W. Hennig, Peter Bayguinov, Laura Ritchie, Kyu Joo Park, Hyun Tai Lee, Nick J. Spencer, Kent M. Sanders, Terence K. Smith

Background: Rhythmic longitudinal muscle (LM) activity persists in the small intestine of WWv mice that lack slow waves due to the absence of myenteric interstitial cells of Cajal (ICC-MY). The overall frequency of LM activity in WWv mice appears to be similar to wildtype mice, however the coordination of this activity has not been investigated. Aim: We hypothesized that the lack of a pacemaker network in WWv mice would alter the stability and coherence of propagating waves of LM activity and associated motor patterns. Methods: Segments (3-5 cm long) of small intestine were removed, opened along the mesenteric border and pinned mucosa side down in an organ bath maintained at $36^{\circ} \mathrm{C}$ and perfused with Krebs solution. The preparation was loaded with fluo-4 and LM activity was visualized using an upright microscope and CCD camera. In separate experiments to monitor LM movements, the mucosa was removed and small surface markers were applied to the circular muscle (pinned serosal side down) to track the relative movements of the LM using video imaging. Results: The LM of wild-type mice $(n=7)$ displayed cyclic $\mathrm{Ca} 2+$ waves (muscle action potentials) that propagated in a coherent fashion across the field of view ( $1 \mathrm{Ca} 2+$ wave per cycle). In contrast, $\mathrm{Ca} 2+$ waves in the $\mathrm{LM}$ of $\mathrm{WWv}$ mice $(\mathrm{n}=4)$ propagated at varying velocities across the field of view and changed direction from wave-to-wave. Occasionally, a bursting pattern was observed consisting of up to 10 rapid $\mathrm{Ca} 2+$ waves per cycle in WWv mice. Ca2+ waves were blocked with nifedipine $(2 \mu \mathrm{M})$ or nicardipine $(1 \mu \mathrm{M})$, but were unaffected by atropine $(1 \mu \mathrm{M})$. LM motor activity reconstructed from videos of surface markers in wild-type mice often showed regular propagating waves of contraction that traveled for considerable distances along the preparation. LM motor activity in WWv mice was highly erratic and unstable, consisting of "jerk-like" contractions at random locations. Conclusion: While LM in the small intestine remains active in mice lacking ICC-MY, there is little spatio-temporal coordination of this activity. Pacemaker activity in the ICC-MY network promotes regular, ongoing waves of LM activity/contraction that propagate in an orderly fashion. Supported by grants from NIH (NIDDK): R01 DK45713 (TKS), DK41315 (KMS) and Core laboratory Cobre grant P20 RR-18751

\section{6}

Kit-Like Immunoreactivity Localizes in the Myenteric Plexus Region of the Zebrafish Gastrointestinal Tract

Adam Rich, Scott Leddon, Simon Gibbons, Xu Xiaolei, Gianrico Farrugia

Background: Gastrointestinal (GI) motility results from the coordinated actions of enteric neurons, interstitial cells of Cajal (ICC), and smooth muscle cells. The GI tract of the zebrafish has a cellular anatomy that is essentially similar to humans. Previous work by our lab has shown the presence of Kit-like immunoreactivity in the zebrafish GI tract. Kit-like immunoreactivity has been used extensively to identify ICC in the GI tract of other organisms. Characterization of the anatomical location of Kit-positive cells within the tunica muscularis of the GI tract and their relation to enteric nerves and smooth muscle is necessary to classify these cells as ICC subserving similar functions as ICC in other species. Aims: To determine the localization of cells displaying Kit-like immunoreactivity and the relationship to enteric nerves and smooth muscle within the tunica muscularis of the zebrafish GI tract. Methods: Kit protein expression within the zebrafish tunica muscularis was assessed by immunohistochemistry using the anti c-Kit polyclonal antibody, ab16832 (abcam). GI tissues dissected from larvae ( 7 days post fertilization) and from adult zebrafish were fixed in $4 \%$ paraformaldehyde overnight. Transverse sections $(4-8 \mathrm{~mm}$ ) were cut from paraffin-embedded tissues. Enteric neurons and smooth muscle cells were specifically identified using anti a tubulin and anti $\mathrm{Hu}$ C/D (Molecular Probes), and SM22 (abcam), respectively. Results: Immunhistochemistry performed on tissue sections with anti a tubulin revealed a population of enteric neurons between the outer longitudinal layer and the inner circular layer of smooth muscle cells consistent with the enteric myenteric plexus. Kit immunolabeling showed a cell population with Kit-like immunoreactivity in the same region. . Double labeling experiments revealed Kit positive cells adjacent to enteric neurons and smooth muscle within this laver. Intramuscular Kit immunoreactive cellswere not observed, and no Kit immunoreactive cells were observed between the circular layer of smooth muscle cells and the mucosa. Kit-like immunoreactivity did not co-localize with smooth muscle or neural cell markers. Conclusion: These data establish the presence of a cell population with Kit-like immunoreactivity in the myenteric plexus region within the zebrafish GI tract. Cells with Kit-like immunoreactivity were adjacent to enteric neurons, intercalated between enteric neurons and smooth muscle cells within the zebrafish GI tract. The anatomical arrangement of these Kit-positive cells strongly suggest that they are ICC. Supported by NIH DK07158801, NIH-NCRR 12546, and DK52766, DK57061

\section{7}

Involvement of Interstitial Cells of Cajal in Gastrointestinal Dysmotility of Diabetic $\mathrm{Db} / \mathrm{Db}$ Mice

Takahiro Yamamoto, Kenji Watabe, Masanori Nakahara, Koji Isozaki, Tatsuya Kiyohara Syusaku Tsutsui, Shinji Tamura, Yasuhisa Shinomura, Norio Hayashi

[Background] Diabetes mellitus often causes gastrointestinal dysmotility. Neuropathy has been long considered to be a major cause of the diabetic gastroenteropathy. We examined the colon from the patients of diabetes mellitus type 2 and found that the number of ganglion cells did not vary. In contrast, we found that the number of interstitial cells of Cajal (ICCs) decreased. ICCs are considered to be pacemaker cells for the gastrointestinal movement. We recently demonstrated that $d b / d b$ mice (obese due to deficiency for leptin receptor and 
develop diabetic mellitus type 2) could be used as a model of diabetic gastroenteropathy. [Aims] We investigated involvement of ICCs in gastroenteropathy of diabetic $d b / d b$ mice. [Methods] 1) Isometric tension was recorded by tension transducer in intestine. 2) ICCs were detected by fluorescent immunohistochemistry using KIT antibody. The fluorescent image from circular muscle layer and myenteric plexus region in gastrointestinal tract was captured on computer and the area of KIT-positive cells for each region was calculated. 3) Apoptotic cells in gastrointestinal tract were examined by TUNEL assay. 4) mRNA expression of stem cell factor (SCF) in gastrointestinal tract was examined by quantitative real-time PCR. [Results] 1) Isometric tension of intestine was irregular in $d b / d b$ mice when compared with that of $d b /+m$ mice. Since the parameter reflects the autonomic motility, the result suggests malfunction of ICCs. 2) The area of KIT-positive cells both in circular muscle layer and myenteric plexus region from stomach, intestine, and colon decreased in $\mathrm{db} / \mathrm{db}$ mice. 3) There were no apoptotic cells both in circular muscle layer and myenteric plexus region from stomach, intestine and colon in both $d b / d b$ and $d b /+m$ mice. As control, apoptotic immunocytes in submucosal layer were observed in these mice. 4) mRNA expression of SCF from intestine and colon decreased in $d b / d b$ mice. [Conclusions] Irregular isometric tension and decreased number of ICCs suggest involvement of ICCs in gastroenteropathy of diabetic $d b / d b$ mice. Decrease in the number of ICCs may be attributable to the decrease in the supply of SCF from the surrounding tissue.

\section{8}

Jejunal Afferent Hypersensitivity to Mechanical Stimulation in W/Wv Mice Infected with Trichinella Spiralis

Chris D. Keating, Johanna Wheatcroft, Steve Foley, Gulzar Singh, Arleta Reiff.marganiec, Chapman Victoria, Andrew Bennett, Charles Marsden, Robin Spiller, David Grundy

Background: Visceral hypersensitivity is one of the hallmarks of post-infectious IBS. Following Trichinella spiralis infection in mice, there is augmented sensitivity of jejunal afferents responding at low threshold (LT) and high threshold (HT) levels of distensionl. Here, we investigate the role of mast cells in the mechanisms underlying afferent hypersensitivity using mast cell deficient W/Wv mice. Methods: Multiunit recordings from mesenteric afferent bundles supplying in vitro segments of mouse jejunum were made from T. spiralis infected W/Wv and wild type (WT) littermates (=>25 days post infection), and from uninfected controls. Data presented as mean \pm SEM $(\mathrm{N} \geq 8)$ and analysed by one-way ANOVA. Tissue was also processed for immunocytochemistry to determine enterochromaffin (EC) cell numbers. This data is presented as median $(n=>3)$ and compared using Mann Whitney. $P<0.05$ was taken as significant. Results: The WT response to ramp distension of the jejunum consisted of a biphasic increase in afferent firing. The WT increase at LT $(0-10 \mathrm{mmHg})$ levels of distension was comparable in the W/Wv animals $(42.19 \pm 2 \mathrm{imp} / \mathrm{s}$ and $35.44 \pm 1.7 \mathrm{imp} / \mathrm{s}$ respectively, $\mathrm{p}>0.05)$ but was significantly attenuated at HT $(30-50 \mathrm{mmHg})$ levels of distension $(11.2 \pm 1.4$ and $3.4 \pm 1.7 \mathrm{imp} / \mathrm{s}, \mathrm{p}<0.01)$, despite similar pressure-volume curves indicative of compliance. Following infection in the WT there was significant augmentation at both the LT $(58.0 \pm 4.0 \mathrm{imp} / \mathrm{s}, \mathrm{p}<0.05)$ and $\mathrm{HT}(17.1 \pm 1.3 \mathrm{imp} / \mathrm{s}, \mathrm{p}<0.05)$ levels of distension, indicating afferent hypersensitivity. This was associated with an increase in compliance $(287.5 \pm 22 \mu \mathrm{l}$ required for a $50 \mathrm{mmHg}$ distension pressure in infected WT compared to $200 \pm 13 \mathrm{\mu l}$ in uninfected WT, $\mathrm{p}<0.05)$. In contrast, following infection in the W/Wv animals, the hypersensitivity to LT distension was absent $(36.0 \pm 2.75 \mathrm{imp} / \mathrm{s}, \mathrm{p}>0.05)$ while hypersensitivity in the HT response while present, was significantly blunted $(9.9 \pm 1.3 \mathrm{imp} / \mathrm{s}, \mathrm{p}<0.05)$. Compliance remained unchanged in the W/Wv animals. EC cell number was elevated in infected WT animals ( 151 cells $/ \mathrm{mm} 2$ ) compared to the W/Wv (21 cells $/ \mathrm{mm} 2, \mathrm{p}<0.0001)$. Conclusion: Enteric infection with T.spiralis produces a mast cell dependent EC cell hyperplasia, and a sensitization of mechanosensitive afferent neurons. The LT component of this sensitization is mast cell dependent, possibly as a consequence of reduced EC cell number, whilst the HT component appears to be independent of mast cells since it persists, albeit blunted, in the W/Wv animals. The altered baseline sensitivity in the W/Wv mice may indicate a role for interstitial cells of Cajal (ICC) in jejunal mechano-transduction. Funded by the BBSRC 1Keating et al., DDW 2005.

\section{9}

Reduction of Interstitial Cells of Cajal (ICC) in Auerbach's Plexus in Patients with Achalasia: New Insights in Pathogenesis

Ines Gockel, Juergen R. Bohl, Volker F. Eckardt, Theodor Junginger

Background. Interstitial cells of cajal (ICC) form neuronal networks which are spread in submuscular, intramuscular and intermuscular layers of the gastrointestinal tract. They generate pacemaker potentials determinating the spontaneously electrical and mechanical activity of smooth muscle cells. There is a close relationship between the c-kit positive cajal cells and the nitrogen monoxide (NO)-synthetasis as a neurotransmitter of nerve fibres. The aim of our study was to investigate the significance of interstitial cells of cajal in the plexus myentericus in patients with primary achalasia. Patients and methods. In 35 patients with a median age of 55 (14-78) years undergoing surgery for achalasia, staining of the cells of Auerbach's plexus was carried out. In 19 patients, biopsies were taken from the high pressure zone of the lower esophageal sphincter (LES) in the context of a Heller myotomy, whereas in 11 patients the complete esophagus was resected in patients with end-stage achalasia and a decompensated megaesophagus. The tissue samples were fixed in formalin, embedded in paraffin, and investigated microscopically. Apart from conventional staining methods, immunohistochemical examinations were carried out (n-NOS (=neuronal nitrogen monoxide synthetasis), neurofilament, smooth muscle antigen, Desmin, CD 117, S 100, B- and Tlymphocytes, and CD 68). Autopsy samples from patients with normal esophageal function served as controls. Results. The median duration of symptoms was 10 (0.5-63) years. 14 patients had undergone a pneumatic dilation previously, and 10 patients had had previous surgery at the lower esophageal sphincter. The preoperative Eckardt score was 6 (3-10) With a maximum diameter of the esophageal body of $45(25-60) \mathrm{mm}$, the radiologically measured narrowest point at the cardia was 4.5 (1-15) $\mathrm{mm}$. Esophageal manometry displayed a median LES resting pressure of $24.4(12-115) \mathrm{mmHg}$. The present histopathological examination showed a marked reduction up to a complete absence of the ICC in the area of the high pressure zone of the esophagus. Staining with the antibody n-NOS points at a reduced neurotransmission in the LES. Conclusion. The present results suggest that in the pathogenesis of achalasia, especially in the development of the high pressure zone, the deficient or missing function of the cells of cajal play an important role. The associated reduction of the NO-synthetasis, therefore, is possibly responsible for the lack of relaxation of the LES, on account of the missing inhibitory neurotransmission. The reduction of the cajal cell-function also seems to be of relevance even if the cells of Auerbach's plexus are unscathed.

623

Natural Orifice Transluminal Endoscopic Surgery (NOTES) Cholecystectomy: A Transcolonic Survival Study in a Porcine Model Reina D. Pai, Derek G. Fong, Douglas S. Fishman, David W. Rattner, Christopher C. Thompson

Background and Aim: Transgastric cholecystectomy has been reported in two non-survival studies which detail substantial technical limitations and only a 33\% success rate when limited to one gastric exit site despite the use of a multiple channel locking endoscope. The aim of this study was to demonstrate feasibility and evaluate technical limitations of transcolonic approach to cholecystectomy. Methods: Under general anesthesia, adult Yorkshire pigs were prepped with multiple tap water enemas, per-anal instillation of an antibiotic and betadine rinse, and external betadine scrub. A sterile dual-channel endoscope (Olympus ${ }^{\mathrm{TM}}$ ) was introduced through the anus and advanced through a $2 \mathrm{~cm}$, anterior trans-colonic incision created by a needle knife approximately $15-20 \mathrm{~cm}$ from the ana verge. Upon completion of intra-abdominal exploration and identification of all major upper abdominal organs, the cystic duct and artery were dissected and ligated with endoclips. Dissection of the gallbladder away from the liver was achieved using hot biopsy forceps, snare tip, prototype endoscopic scissors and an insulated-tip needle knife. The gallbladder was successfully removed with hot snare cautery. The gallbladder fossa was then lavaged with sterile water, re-examined and additional cautery or endoclips were applied for hemostatsis or closing of defects. At the conclusion of each procedure, the colonic incision was closed using endoloops and/or endoclips. Results: The animals were survived for two weeks followed by elective termination and necropsy. Four of the five animals flourished in the post-operative period demonstrating appropriate feeding and activity patterns as well as stable weights or weight gains. The colonic incision site in all 4 animals healed completely, however external adhesions were appreciated. In the last animal complete closure of the colonic incision site was not possible and a small $4 \mathrm{~mm}$ residual defect remained. The anima was survived for 48 hours but then sacrificed due to concerns of peritonitis. Pathology from all 5 subjects subsequently confirmed the resected organs as gallbladders. Conclusions: The transcolonic approach provides improved visual exposure of the gallbladder and scope stability when compared to the transgastric approach. This study demonstrates the technical feasibility of transcolonic organ resection via a single incision. The one complication appeared secondary to inadequate incision closure and not related to the organ resection. For this approach to be translated to humans, a sterile conduit, secure closure device and better instruments for triangulation are necessary

624

Endoscopic Anastomotic Reduction After Roux-en-Y Gastric Bypass Surgery: A Potential Treatment for Weight Regain

Derek G. Fong, David B. Lautz, Christopher C. Thompson

Background and Aims: The mechanisms leading to weight regain after Roux-en-Y Gastric Bypass (RYGB) are not clearly understood. Dilation of the gastrojejunal (GJ) anastomosis is a structural complication of the surgical pouch that may contribute to weight regain. The aim of this study was to examine our experience with endoscopic anastomotic reduction to effect weight loss after RYGB surgery. Methods: Between November 2003 and June 2005 , thirty-eight endoscopic anastomotic reductions were performed at our institution. These procedures were performed using a Bard EndoCinch suturing device in conjunction with argon plasma coagulation, fibrin sealant, and tissue excoriation with a cytology brush. Fifteen patients with six month follow-up data were identified and included in this analysis. Patients with other upper GI pathology and early iterations of technique were excluded. Results: The GJ anastomotic size prior to reduction ranged from 15 to $30 \mathrm{~mm}$ in diameter (mean size $23.4 \mathrm{~mm}$ ) with the post procedure size ranging from 2 to $15 \mathrm{~mm}$ in diameter (mean size $6.57 \mathrm{~mm}$ ). Eleven of the patients lost weight following the procedure while four of the patients did not experience any weight change and one patient experienced weight gain. Mean percent excess weight loss at six months ranged from a gain of $9.6 \%$ to a loss of $56.8 \%$ (percent mean excess weight loss $14.2 \%$ ). Three of the patient's who did not lose weight were found to have dilation of their GJ anastomosis at follow-up endoscopy and a second reduction was performed within eight months of the initial procedure. Two of the patient's who did not lose weight were known to be noncompliant with dietary restrictions. There were no serious complications from the procedure. Symptomatic complaints from the procedure were self limited and included sore throat, abdominal discomfort, and nausea Conclusions: Endoscopic anastomotic reduction may be a useful treatment option for weight regain in selected patients after RYGB. Endoscopic anastomotic reduction appears feasible and safe, and is associated with variable weight loss. The durability of weight loss and the need for subsequent anastomotic reductions will need to be explored with additional prospective randomized studies.

625

Transcolonic Endoscopic Abdominal Exploration Derek G. Fong, Reina D. Pai, Christopher C. Thompson

Background and Aims: Although a transcolonic endoscopic approach to access the peritoneal cavity has been previously described by our group, the ability to systematically identify abdominal organs with animal survival has not been reported. The aim of this study was to determine the ability to evaluate abdominopelvic organs using a transcolonic approach

. 
in a survival study design. Methods: Six female Yorkshire pigs weighing 25 to $30 \mathrm{~kg}$ were used in this study. Under general anesthesia, the rectum was lavaged with multiple tap water enemas. After endoscopic confirmation that the distal colon was free of particulate matter, a cefazolin suspension was instilled for 10 minutes followed by a betadine lavage. Colonic incision was performed over the anterior colonic wall at a distance of $15 \mathrm{~cm}$ to 20 $\mathrm{cm}$ from the anus. A sterilized Olympus upper endoscope was then passed through the incision and endoscopic abdominal exploration was performed. The incisions site was closed using endoscopic clips, endo-loops, as well as a novel closure device. Results: The peritoneal cavity was accessed through the colonic incision without difficulty. The stomach, liver, gallbladder, spleen, small bowel, and colon were identified in all of the animals in under 3 minutes. The lower pelvic organs were not consistently visualized. All of the animals survived 14 days without apparent sequelae before elective sacrifice. At necropsy, the colonic incision sites were completely closed and well healed from the luminal surface. From the serosal aspect, salpingocolonic and cystocolonic adhesions were identified in 5 of 6 animals. The develpment of adhesions are in contrast to our previous findings with transgastric endoscopy. There was no evidence of organ injury or peritonitis. Conclusions: This study demonstrates the ability of transcolonic abdominal exploration to successfully evaluate major organs in the upper abdomen. In contrast to the transgastric route, a transcolonic approach provides en face orientation to organs in the upper abdomen and allows for better visualization and scope stability. Therapeutic interventions in the upper abdomen including organ resection may therefore be technically easier using a transcolonic approach.

\section{6}

Usefullnes of Endoscopic Mucosal Resection As a Final Diagnostic Method in Gastroesophageal Dysplasias and Carcinomas

Mario Rey

Objective: comparing initial biopsies taken from mucosal tumor lesions with the final result of pathology in mucosectomy. Materials and Methods: 66 patients aged 53.3 old on average, seen to be suffering from 69 mucous lesions by endoscope, were prospectively analyzed 15 lesions in the esophagus and 54 gastric lesions having an average diameter of $12.7 \mathrm{~mm}$ were found. A minimum of 5 biopsies were taken of each lesion; once histological diagnosis had been made, they were removed by endoscope with submucosal infiltration, using endoscopic snare, plastic cap, overtube or IT endoscopic knife. Biopsies and mucosectomies were pathologically classified into being benign, low or high dysplasia and carcinoma and compared to a Spearman's correlation index. Esophageal lesions were type IIa and IIb, around 5 to $20 \mathrm{mms}$ in diameter, half of them being associated with Barrett's esophagus; $76.4 \%$ were localized in the antral part of the stomach. Results: 12 benign lesions were confirmed as were 26 low grade dysplasias, 17 high grade dysplasias and 14 carcinomas; biopsies had $65.2 \%$ correlation $(\mathrm{p}<0.001$ ) with $18.4 \%$ over-diagnosis and $15.9 \%$ underdiagnosis with a 72.4 Spearman coefficient of correlation. Biopsy in esophageal lesions revealed a moderate $69.6 \%$ correlation $(\mathrm{p}<0.01$ ) with $33.3 \%$ over-diagnosis. Better correlation was obtained in the stomach $(87.1 \%, \mathrm{p}<0.001)$ with $14.8 \%$ over-diagnosis and $14.8 \%$ underdiagnosis. Discussion: as serious intra-mucous dysplasias and carcinomas continue to be surgically treated in our area, then establishing that biopsies do not have $100 \%$ correlation with final pathology having greater over-diagnosis could lead to esophagectomies or gastrectomies being practiced on lesser lesions having secondary surgical morbidity-mortality. Conclusions: this article shows that esophageal biopsies in premalign and malign mucosal lesions are only slightly correlated with final pathology, their scores improving in the stomach. Mucosectomy has been shown to be an extremely useful diagnostic tool for surgical decisiontaking regarding this type of lesion.

627

Anxiety and Depression Predict the Likelihood of a Functional GI Disorder (FGID) Separately from Their Relationship to Somatization Gregory S. Sayuk, Jill E. Elwing Patrick J. Lustman, Ray E. Clouse

Psychiatric disorders (PSY), particularly anxiety and depression, are common comorbidities among patients with FGID. Recent studies have shown that somatization (SOM), the reporting of multiple unexplained somatic symptoms, may be responsible for the high rates of PSY in FGID patients via symptom-reporting tendencies, reducing their primary importance. We examined an outpatient clinic sample to determine if PSY and SOM had independent ability to predict FGID diagnoses or whether these features shared a common pathophysiology. Methods: 417 consecutive new outpatient referrals (age $45.9+1.4$ yr $98 \%$ caucasian, $62 \%$ female) to an academic gastroenterology practice from January 2003 to January 2005 were evaluated. Systematic record review was used to determine diagnosis, presence of PSY (recorded anxiety or affective disorder), and degree of SOM; degree of SOM was determined from recent (2 wk) symptom endorsement on a review-of-systems check-list and number of GI and non-GI functional diagnoses recorded in the medical history. High degree of SOM was present if $>7$ key SOM symptoms (based on the PHQ-15) were endorsed on the checklist. Subjects were categorized by primary diagnosis (FGID or structural) after having been followed for $3.5 \pm 0.2$ mo to confirm correct categorization. Logistic regression analysis was used to determine independent predictors of primary diagnosis controlling for potential clinical/demographic confounders. Results: $270(64.7 \%)$ had a FGID primary dx and 147 (35.3\%) a structural GI illness. IBS was the most common FGID dx (29.6\%). FGID pts were more likely female $(70.4 \%$ vs. $46.9 \% ; \mathrm{p}=0.002)$ but were of similar age as structural Gl illness pts $(46.2 \pm 1.2$ vs. $45.6 \pm 1.2 \mathrm{yr} ; \mathrm{p}=0.74)$. Both PSY and high degrees of SOM were more common in FGID patients ( $34.1 \%$ vs. $13.6 \%, \mathrm{p}=<0.001$; and $41.9 \%$ vs. $12.7 \%, \mathrm{p}<0.001$, respectively). The logistic regression analysis revealed that both PSY (OR 2.8; 95\% CI 1.35.7; $\mathrm{p}<0.01)$ and high degree of SOM (OR 3.7; 95\% CI 1.9-7.0; $\mathrm{p}<0.001)$ independently predicted the presence of a FGID over a structural GI illness. A similar analysis using number of GI and non-GI functional illnesses for the SOM measure confirmed the independent contribution of PSY and SOM to a FGID diagnosis ( $\mathrm{p}<0.01$ for each). In each model, female sex had predictive value for FGID independent of PSY and SOM effects. Conclusions: Both PSY (anxiety and depression) and SOM are common in patients with FGID and each independently predicts the likelihood of a functional over structural GI disorder in the outpatient setting. Anxiety and depression thus share a relationship with FGID that is not solely dependent on their linkage to SOM.

628

Pain and Depression Predict Improved Health-Related Quality of Life in Severe IBS Treated with Psychotherapy of Antidepressant Francis Creed, Elspeth Guthrie, Joy Ratcliffe, Lakshmi Fernandes, Barbara Tomenson, Nicholas Read, David Thompson

Objective We have reported previously long-term improved health-related quality of life in patients with severe irritable bowel syndrome (IBS) following treatment with an antidepressant or psychotherapy (1). In this study we assessed the relative importance of improved pain or depression in this finding. Method We assessed 257 patients with severe IBS before treatment and 239 one year after the end of treatment ( 75 psychotherapy, 85 Paroxetine and 79 treatment as usual) using a visual analogue scale (VAS) of abdominal pain and the Hamilton Depression Rating Scale (HDRS). Linear regression was used to predict change in SF36 physical component score. Structural equations modelling using AMOS tested whether baseline depression (HRSD) score predicted follow-up VAS pain score and vice versa. Results. Preliminary analyses confirmed that VAS pain and HDRS depression scores represented well the dimensions of pain and depression experienced by participants in our sample. Linear regression analysis to predict 1 year follow-up SF36 physical component score (healthrelated quality of life) indicated that pre-treatment VAS pain $(\mathrm{p}<0.001)$ and HDRS depression scores $(\mathrm{p}=0.005)$ and change (follow-up minus baseline) of VAS pain $(\mathrm{p}<0.001)$ and HDRS depression scores $(\mathrm{p}=0.006)$ were all significant independent predictors. Structural equation modelling including baseline and follow-up scores of VAS pain and HDRS depression showed that the overall fit of the model was excellent, chi $2=2.06, \mathrm{df}=3, \mathrm{p}=0.56$, chi2/df=0.69, RMSEA $<0.0005$ and $C F I=1.000$. The standardised regression coefficients for baseline to follow up depression score and VAS pain were $0.36(\mathrm{p}<0.005)$ and $0.38(\mathrm{p}<0.005)$, respectively, demonstrating that each follow up variable is predicted by its own baseline value. The standardised regression coefficient for baseline VAS pain predicting follow-up HRSD was also significant (chi2 $=0.15, \mathrm{p}=0.02$ ), but that for baseline HRSD predicting follow-up VAS pain today was not (chi2 $=0.09, \mathrm{p}=0.16$ ). Addition to the model of psychotherapy and SSRI antidepressant treatments as additional variables did not change the model and the same model fits the data in each of the 3 treatment groups, when these were analysed separately. The model held when only patients who also had a psychiatric disorder were included. Conclusion: In patients with severe IBS receiving psychological treatments, both improved pain and depression influences later health-related quality of life but change in depression is not primarily responsible for this result. Other aspects (eg coping with pain and its attribution) are likely to be involved. (1) Gastroenterology 2003; 124 :303-17.

629

Effect of Corticotropin-Releasing Hormone Receptor Antagonist On the Brain Activation By Colonic Distention in Healthy Subjects and Patients with Irritable Bowel Syndrome

Shin Fukudo, Toyohiro Hamaguchi, Michiko Kano, Yasuhiro Sagami, Tomotaka Shoji, Yuka Endo, Masatoshi Itoh, Kazuhiko Yanai, Michio Hongo, Motoyori Kanazawa

Background \& Aims: Determining substance that plays a key role in brain-gut interactions is a crucial step for clarifying pathophysiology of irritable bowel syndrome (IBS). We previously reported that peripheral administration of $\mathrm{CRH}$ antagonist, alpha-helical CRH (ahCRH) improves visceral stimulation-induced increase in colonic motility, abdominal pain, and anxiety in IBS patients (Gut 2004;53:958-64) and that peripheral administration of CRH receptor- 1 antagonist selectively induces similar phenomena in IBS model rats (Gastroenterology 2005;129:1533-43). We tested our hypothesis that peripheral administration of ahCRH modifies brain activation to the colonic distention in normal subjects and IBS patients. Methods: Subjects were 20 healthy subjects and 10 IBS patients. This study was approved by the Ethics Committee and all subjects gave the written informed consent. Barostat bag was inserted to the descending colon with colonoscope. The intracolonic bag was intermittently inflated for $3 \mathrm{~min}$ with no $(0 \mathrm{mmHg})$, mild $(20 \mathrm{mmHg})$, or intense $(40 \mathrm{mmHg})$ stimulation with random order. The same procedure was repeated after the intravenous injection of either placebo in a control subjects $(n=10)$ or ahCRH $(10$ microg/Kg $)$ in the other control subjects $(n=10)$ and IBS patinets $(n=10)$. Radioactive $H 2[15-O]$ saline was injected at the bag inflation and positron emission tomography was performed. Changes in rCBF were analyzed using statistical parametric mapping 2. Results: In controls with $20 \mathrm{mmHg}$-colonic distention, administration of ahCRH significantly inhibited increase in $\mathrm{rCBF}$ at the anterior cingulate cortex (BA32), mid cingulate cortex (BA24), and prefrontal cortex (PFC) compared with that of placebo $(\mathrm{p}<0.0001)$. In controls, the rCBF with 40 mmHg-colonic distention after the administration of ahCRH was significantly less in the right PFC (BA9) than that after the administration of placebo $(\mathrm{p}<0.0001)$. IBS patients showed significantly greater activation in the brain stem to the $20 \mathrm{mmHg}$ and $40 \mathrm{mmHg}$ distention than controls $(p<0.0001)$. Significantly more activated brain regions with 40 mmHg-distention between placebo and ahCRH treatments in IBS patients than that (placebo vs ahCRH treatments) in controls were right anterior insula, right PFC (BAll), and left parahippocampal gyrus. Conclusion: These data suggest functionally crucial role of $\mathrm{CRH}$ in limbic and prefrontal cortices during normal and abnormal visceral perception. Moreover, they may provide rationale of therapeutic challenge for IBS using agents that modify $\mathrm{CRH}$ tone 
Effects of Hypnotherapy On IBS in Different Clinical Settings - Results from Two Randomized, Controlled Trials

Perjohan Lindfors, Peter Unge, Einar S. Bjornsson, Annika Stenman, Patrik Arvidsson, Hasse Abrahamsson, Magnus Simren

Hypnotherapy is considered to be effective in patients with irritable bowel syndrome (IBS) Few randomized, controlled trials exist, and most of the data originate from centers highly specialized in this kind of treatment (Whorwell et al 1984). To be more widely spread the effects of this costly treatment alternative need to be further evaluated in clinical practice. Here we report the results from two randomized, controlled trials on hypnotherapy in IBS in different clinical settings. METHODS: We included 135 patients with IBS refractory to standard management (107 females; mean age 41 (21-68) years). Study 1 was performed in a university hospital specialized in functional GI disorders $(\mathrm{n}=87)$ and study 2 in a county hospital $(n=48)$. In both studies patients were randomized to receive gut-directed hypnotherapy $1 \mathrm{~h} /$ week for 12 weeks performed by specially trained psychologists, or to serve as control group. At baseline, immediately after the treatment period and after 6 and/ or 12 months the treatment effect was evaluated with questionnaires assessing quality of life (IBSQOL / SF-36), anxiety and depression (Hospital Anxiety and Depression (HAD) scale) and GI symptoms (Gastrointestinal Symptom Rating Scale (GSRS)-IBS / GI symptom questionnaire). RESULTS: GI symptoms severity improved after the treatment period in the hypnotherapy group in both study 1 (Cumulative GI symptom score $29 \pm 6.9$ vs. $26 \pm 7.3$ $($ mean $\pm S D) ; p=0.002$ ) and study 2 (GSRS Total score $4.0 \pm 0.9$ vs. $3.4 \pm 0.9 ; \mathrm{p}=0.03$ ), but not in the control groups, respectively. The improvement was more pronounced for abdominal pain, distension and bloating than for the bowel habits. At the one year assessment the improvement was sustained or even enhanced. Anxiety and depression was improved at one year in study 2 compared with baseline (HAD anxiety $8.5 \pm 4.7$ vs. $6.3 \pm 4.5 ; \mathrm{p}=0.009$; HAD depression $6.0 \pm 3.1$ vs. $4.2 \pm 2.8$; $\mathrm{p}=0.005$ ), but no such effect was noted in the hypnotherapy group in study 1 or in the control groups. In both studies we observed clear tendencies towards a better quality of life in the hypnotherapy groups, but not in the control subjects. The total responder rate, in terms of significant improvement of GI symptoms, was higher in the hypnotherapy groups vs. the control groups ( $52 \%$ vs. $32 \% ; \mathrm{p}=0.03$ ) CONCLUSION: In these two randomized controlled trials we have demonstrated that hypnotherapy is a useful treatment alternative with long-term effect in IBS patients refractory to standard management. Hypnotherapy can successfully be conducted in different clinical settings, also outside the highly specialized gastroenterology centers focused on functional GI disorders.

\section{1}

Sex-Specific Differences in a Brain Network Functioning During Anticipation of Rectal Discomfort in Irritable Bowel Syndrome Patients (IBS) Jen Labus, Emeran A. Mayer, Steve Berman, Brandall Suyenobu, Lin Chang, Bruce D. Naliboff

Background: IBS is a chronic functional disorder characterized by abdominal pain associated with altered bowel habits. Evidence supports significant sex-related differences in the autonomic and perceptual response to aversive visceral stimuli in IBS patients. This study aimed to identify and characterize a functional brain network consistent with sex-related differences in general arousal and enhanced threat responsiveness. Methods: 48 IBS patients ( 24 females) received $\mathrm{H} 215 \mathrm{O}$ PET scans during three conditions: resting baseline (BL), rectal distension and ANT. Multivariate task partial least squares (PLS) tested for distributed patterns of brain activity associated with changing condition demands. Seed PLS was used to identify sexrelated and unrelated distributed patterns of activity that were functionally connected with the amygdala, an area that previously has shown sex-dependent activation during anticipation and experience of rectal distension. Based on apriori hypotheses, known neuroanatomical connectivity, and seed PLS results, we specified an amygdala mediated general arousal circuit and tested the effective connectivity of this network using a stacked structural equation modeling approach. Results: Task PLS revealed a significant functional pattern of regions $(\mathrm{p}<.001)$ that optimally separated baseline from anticipation and included bilateral amygdala, prefrontal cortex (PFC) and anterior cingulate cortex (ACC). Seed PLS identified 2 significant networks: one functioning similarly across sex, and another that evidenced sexspecific functioning. The sex-common amygdala network included the thalamus, dorsolateral PFC[Brodmann Area 9/46], rostral ACC[32/24], hippocampus, and mid/posterior insula. The sex-dependent amygdala network included ventro/dorsomedial PFC[10], perigenual ACC subregions[32/24/25] and pontine regions. Significant differences in the strength of the functional connections (effective connectivity) were found in well-characterized amygdala modulatory circuits, including amygdala efferents to the pons, midbrain, and infragenual ACC and afferent input to the amygdala from the supragenual ACC. Conclusions: During anticipation of rectal discomfort, male patients show normal feedback inhibition within a network circuit involving infra- and supra-genual ACC, and amygdala resulting in a suppression of limbic activity. In contrast, female patients show strong connectivity between amygdala and infragenual ACC, but not in feedback inhibition, resulting in stronger infragenual ACC activation. These results are consistent with a greater female responsiveness of a circuit regulating amygdala reactivity to emotionally salient stimuli.

\section{2}

Is Abdominal Distension Related to Delayed Small and Large Bowel Transit in Patients with Constipation Predominant Irritable Bowel Syndrome (IBS-C)? Anurag Agrawal, Peter J. Whorwell, Lesley A. Houghton

Background and Aim: Patients with IBS-C often have slow gastrointestinal (GI) transit (1) and exhibit a diurnal increase in abdominal girth (i.e. abdominal distension) that is related to a feeling of progressive bloating (2). The aim of this study was to investigate whether IBS-C patients with delayed GI transit exhibit greater abdominal distension and more severe bloating than do patients with normal transit. Methods: Abdominal girth was recorded for
24 hours using the validated technique of Ambulatory Abdominal Inductance Plethysmography (3) in 27 IBS-C (Rome II) patients, aged 18-68 years ( 3 male) and 24 healthy voluntee (HV), aged 21-58 years ( 3 male). Within 2 weeks of this recording, both small (SBT) and large (LBT) bowel transit were assessed. SBT was determined from the rise in breath hydrogen after a standard meal (4), and LBT from the number of 3 differently shaped radio-opaque markers ( 24 of each ingested on 3 consecutive days) identified on plain abdominal X-ray 72 hours after ingestion (5). The severity of abdominal bloating was also assessed on a scale of $0-3$, where $3=$ severe. Results: As anticipated, IBS-C patients reported more severe abdominal bloating (IBS: $2.00(1.73,2.26)$, mean $(95 \%$ CI) v HV: $0.33(0.07,0.59) ; \mathrm{p}<0.001)$ and exhibited greater abdominal distension (change in girth from the beginning to end of day: $3.8 \mathrm{~cm}(2.2,5.5) \mathrm{cm}$ v $0.46 \mathrm{~cm}(-1.1,2.0) \mathrm{cm} ; \mathrm{p}=0.007)$ and slower LBT (52 marker $(45,58)$ markers v 35 markers $(28,42)$ markers; $\mathrm{p}=0.003)$ but not SBT (330 min (291, $368) \min v 293 \min (262,325) \mathrm{min} ; \mathrm{p}=0.35)$ compared with HV. Using the 97.5 percentile for LBT in HVs (i.e. > 59 markers), $48 \%$ of IBS-C patients had delayed LBT and these patients exhibited greater abdominal distension $(5.3 \mathrm{~cm}(3.7,6.9) \mathrm{cm})$ than those with normal LBT $(2.4 \mathrm{~cm}(-0.1,5.0) \mathrm{cm} ; \mathrm{p}=0.03)$. Furthermore, there was a direct relationship between LBT and the degree of abdominal distension in IBS-C patients $(r=0.51, p=0.006)$. There wa no difference in bloating severity between patients with $(2.00(1.60,2.39)$ and without (2.00 (1.61, 2.38): $\mathrm{p}=0.63)$ delayed LBT. Conclusion: IBS-C patients with delayed large bowel transit have greater abdominal distension than those with normal transit. Drugs that accelerate transit may therefore be expected to alleviate this often troublesome problem. Refs: (1) Gut 1983; 24: 404-11; (2) Gut 2004; 53 (Suppl III): Al14; (3) Gut 2001; 48 216-20; (4) N Engl J Med 1969; 28: 122-7; (5) Gastroenterol 1987; 92: 40-7.

\section{3}

Effect of 2 Weeks Furazolidone- Or 2 Weeks Metronidazole- Or Combination of lweek Furazolidone+ 1 Week Metronidazole-Based Anti Helicobacter Pylori Regimens in Peptic Ulcer: A Randomized-Controlled Multicenter Trial Y Ajvadi, R Malekzadeh, S Nasseri-Moghaddam, N E Daryani, H Vahedi, N Zendehdel, R Sotoudehmanesh, S Agah, J Mikaeli, A Pourshams, M Khatibian, A Ali Asgari, Sadegh Massarrat

Background:Metronidazole or Clarithromycin combined with Amoxicillin, a Bismuth compound and Omeprazole are commonly used to treat Helicobacter pylori (HP). There is a high bacterial resistance to Metronidazole and high cost for Clarithromycin. Iranian studies show that Furazolidone is effective against HP, with no resistance, but intolerable side-effects are common in 2 weeks therapy. We compared efficacy and side-effects profile of three different anti-HP regimens with 1 or 2 weeks Furazolidone administration. Materials and Methods: Consenting, HP positive peptic ulcer patients were randomly allocated in three groups. Group 1 received Omeprazole 20mg BID+ Amoxicillin 1 g BID + Furazolidone 200 mg BID + Bismuth-subcitrate $240 \mathrm{mg}$ BID for 2 weeks. Group 2 received the same but Furazolidone was replaced with Metronidazole $500 \mathrm{mg}$ BID. Group 3 received regimen 1 for the first week and regimen 2 for the 2 nd week. Patients were followed at weeks 2 and 10. Compliance and side-effects were checked at week 2. 13C Urea breath test (UBT) at week 10 verified HP eradication. Results: 314 patients were enrolled, 299 followed at week 2 and 276 took the UBT. There were 100,102, and 97 patients in groups 1-3 respectively. Seven, 3, and 6 patients discontinued medication prematurely in groups $1-3$ respectively. Side-effects were more common in group 1 ( 7 patient with high fever, 10 vomiting and 19 weakness) than group 2 ( 4 with fatigue) and group 3 (1with fever, 7 fatigue, and 4 vomiting) $(\mathrm{p}<0.05)$. Per protocol eradication rates were $95.2 \%, 83.1 \%$ and $95.3 \%$ in groups 1 to 3 respectively ( $\mathrm{p}=$ 0.005 ). Intention to treat eradication rates were $87.0 \%, 74.5 \%$, and $86.6 \%$ in groups $1-3$ respectively $(\mathrm{p}=0.02)$. Conclusion: Furazolidone given for one week followed by Metronidazole has the same efficacy for HP eradication as Furazolidone for 2 weeks with fewer side effects. Furazolidone-based regimens are superior to Metronidazole based ones.

\section{4}

One-Week Once-Daily Triple Therapy with Esomeprazole, Moxifloxacin and Rifabutin Is Effective in the Treatment of Helicobacter Pylori Resistant to Both Metronidazole and Clarithromycin

Stephan Miehlke, Wulf Schneider-Brachert, Andrea Morgner, Ahmed Madisch, Christian Kirsch, Elke Baestlein, Christian Haferland, Claus Jebens, Holger Knoth, Manfred Stolte, Norbert Lehn

Objectives: Failure of standard triple therapy aiming at eradication of $H$. pylori often leads to post-treatment resistance to metronidazole (MET) and/or clarithromycin (CLA). Subsequent treatment of these patients remains a clinical challenge. We evaluated the efficacy and tolerability of a convenient triple therapy using esomeprazole, moxifloxacin and rifabutin for eradication of $H$. pylori resistant to both MET and CLA in a prospective open-label study. Methods: Consecutive patients with $H$. pylori infection resistant to both MET \& CLA (Etestâ) were treated with esomeprazole $40 \mathrm{mg}$, moxifloxacin $400 \mathrm{mg}$ and rifabutin $300 \mathrm{mg}$, each given orally once daily in the morning for 7 days. Follow-up endoscopy including histology and $H$. pylori culture was performed 6 to 8 weeks after treatment. Results: Between January 2004 and October 2005, 86 patients were enrolled (56 female, 30 male, median age 52 years. 62 patients $(72.1 \%)$ had a history of two or more previous treatment failures. Three patients $(3.5 \%)$ discontinued treatment prematurely due to adverse events). 73 patients $(85 \%)$ are currently available for per protocol efficacy analysis. Successful eradication was confirmed in 58 patients $(79.5 \%)$. In the patients with clinical failure $(n=15)$, post-treatmen resistance to the test drugs rifampicin and ciprofloxacin were detected in 5 and in 3 patients, respectively. Conclusion: One-week once-daily triple therapy with esomeprazole moxifloxacin and rifabutin is effective and safe for eradication of $H$. pylori resistant to both metronidazole and clarithromycin in patients with a history of previous treatment failures. . 
Three and Seven Days Levofloxacin/Azithromycin-Based Triple Therapies As First-Line Treatment for Helicobacter Pylori Eradication

Rosalba Finizio, Enrico C. Nista, Marcello Candelli, Giovanni Cammarota, Giovanni Gasbarrini, Antonio Gasbarrini

Background and aim: The failure of first-line anti-H.pylori therapies is due to primary antibiotic resistance and poor compliance. For this reason it is essential to investigate new antibiotic associations and simple therapeutic schemes. Levofloxacin and azithromycin are broad spectrum antibiotics with long half life administrable in single daily dose that could increase patients compliance. Aim of the study is to compare the efficacy of a 3- and 7days levofloxacin/azithromycin based H. pylori eradication regimen against standard 7-days triple therapy. Material and methods: Ninenty Hp-positive patients (infection was assessed by histology and 13-C UBT) were randomized to receive: Group A (30 pts): levofloxacin (500 mg od), azithromycin (500 mg od) and esomeprazole (20 mg bid) for 3 days; Group B (30 pts): levofloxacin (500 mg od), azithromycin (500 mg od) and esomeprazole (20 mg bid) for 7 days; Group C (30 pts) clarythromicin ( $500 \mathrm{mg}$ bid), amoxicillin ( $1 \mathrm{gr}$ bid) and esomeprazole (20 mg bid). Hp status was re-checked by 13-C UBT six weeks after end of therapies. Results: H. pylori eradication rate in Group A was $86.7 \%$ (26/30 pts), in Group B was $93.3 \%$ (28/30 pts), in Group C was $70 \%(21 / 30)$ in either ITT or PP analysis. Eradication rate of 7 -days levofloxacin/azithromycin-based triple therapy was significantly higher than that observed using standard triple therapy ( $93.3 \%$ vs $70 \%$; $<0.05$ ). Eradication rate of 3-days levofloxacin/azithromycin-based triple therapy was higher even if not significantly than that showed by standard therapy $(86.7 \%$ vs $70 \% \mathrm{p}=0.06)$. Incidence of side effects were lower in groups A and B than in group C. Moreover, prevalence of side effects resulted higher in the group $\mathrm{B}$ than in group $\mathrm{A}$. Conclusions: According to the present data a 7-days levofloxacin/azithromycin-based triple therapy may be considered a high effective therapy for $H$. pylori eradication.

636

Analysis of Effects and Costs of Gatifloxacin, rabeprazole Or LansoprazoleBased Triple Therapies in Eradication of Helicobecter Pylori Infection in China Yingqiang Zhong, Zhongsheng Xia, Qikui Chen, Zhiyong Zeng

OBJECTIVE: The resistance of $\mathrm{H}$. pyroli to antibiotis is more and more severe. Clinically, it is an essential problem of soluting the resistance of $\mathrm{H}$. pyroli to antibiotics to find newer and more effective antibiotic agent. Gatifloxacin is a new and fourth- generation fluoroquinolone, and has comfirmed broad spectum and activity of antimicrobial agent, and lower adverse drug reactions. The present study investigates the effects and costs, adverse drug reactions of gatifloxacin, rabeprazole or lansoprazole-based triple therapies on eradicating Helicobacter pyroli (H. pyroli), so as to find the best one of the schemes. METHODS: Grouping and contrast study were used in present study, and pharmaco- economics was analysed in the ratio of cost and effect $(\mathrm{C} / \mathrm{E})$ of the schemes. 175 patients of primary eradication of $\mathrm{H}$. pyroli were randomized into group A,B,C,D, and 17 patients of second eradication of $\mathrm{H}$. pyroli were into group $\mathrm{E}$. The drugs of group A were gatifloxacin (200mg, bid), metronidazole $(0.4 \mathrm{~g}$, bid) and lansoprazole ( $30 \mathrm{mg}$, bid); Group B were gatifloxacin (400mg, qd), metronidazole (0.4g, bid) and lansoprazole (30mg, bid); Group C were gatifloxacin $(400 \mathrm{mg}, \mathrm{qd})$, metronidazole $(0.4 \mathrm{~g}$, bid) and rabeprazole (10mg, qd); Group D was a control of clarithromycin $(0.5 \mathrm{~g}$, bid), metronida-zole $(0.4 \mathrm{~g}$, bid) and lansoprazole (30mg, bid); and scheme of group E was similar as group A. RESULTS: Eradication rate of $\mathrm{H}$. pyroli was $90 \%, 91.4 \%, 91.7 \%, 84.4 \%$ in group A, B, C, D respectively. There were not significant differences among group $A, B, C$ and $D(P>0,05)$. Eradication rate of $H$. pyroli was $82.4 \%$ in group $\mathrm{E}$. The primary total eradication rate and total eradication rate of $\mathrm{H}$. pyroli with gatifloxacin was $90.1 \%$ and $89.8 \%$ respectively. The mainly adverse drug reactions were nausea, poor appetite, abnormal taste and dizzy of head. A few of reactions were mild diarrhea and lumbago, and few was some rash. There was not phototoxicity, hepatotoxicity and renal toxicity in the objects, but there was transient mildly hyperglycemia. The rate of adverse drug reaction was $12.5 \%, 22.9 \%, 27.8 \%, 39.1 \%$, and $25 \%$ in the group A+E, B, $\mathrm{C}, \mathrm{D}$ and total population respectively. The lowest rate was in group $\mathrm{A}+\mathrm{E}$. There were significances among group $\mathrm{A}+\mathrm{E}, \mathrm{C}$ and $\mathrm{D}(\mathrm{P}<0.001)$, and there was not significance between group $B$ and $C(P>0.05)$. The ratio of $C / E$ was $3.44,3.38,2.77,4.39,3.75$ and 3.45 in group A, B, C, D, E and all groups of gatifloxacin respectively. CONCLUSIONS: Gatifloxacin is a most effective drug of eradicating $\mathrm{H}$. pyroli infection, and has lower adverse drug reactions. Among these schemes, group A and B were better, and group C is best.

\section{7}

\section{In Children Eradication of Helicobacter Pylori Does Not Promote} Gastroespophageal Reflux Disease: A Multichannel pH-Impedance Study Osvaldo Borrelli, Caterina Anania, Letizia Cordischi, Felicia Galos, Marina Aloi, Manuela Cirulli, Valeria Labalestra, Valentina Mancini, Salvatore Cucchiara

Background. Conflicting data on the relationship between Helicobacter Pylori ( $H$. pylori) infection and gastroesophageal reflux disease (GERD) have been reported, and only few studies on this issue have been performed in children. Aim. We aimed at assessing the effect of eradication of $H$. pylori on both acid and non-acid esophageal exposure, as measured with 24-hr multichannel intraluminal impedance and $\mathrm{pH}$ (MMI-pH) monitoring in children without reflux esophagitis. Patients and Methods. 17 consecutive patients (pts) (median age: 10.2 years; range: 4 -15) with H.Pylori infection and no reflux esophagitis were enrolled into the study. Diagnosis was performed by endoscopy with histology and 13C-urea breath test. MMI-pH monitoring was performed before and 8 weeks after eradication of H.Pylori which was achieved using standard triple therapy (omeprazole, amoxicillin and clarithromycin for 14 days) and confirmed by $13 \mathrm{C}$-urea breath test. The MII-pH parameters evaluated were: total numbers of reflux episodes (TN), numbers of acid reflux episodes (AR), numbers of non-acid reflux episodes (NAR), $\%$ of time with esophageal $\mathrm{pH}<4$ (ARI), numbers of long-lasting (> $5 \mathrm{~min}$ ) acid reflux episodes (LAR) and acid clearance time (ACT)(sec). Esophageal acid exposure was considered abnormal when 24-hour ARI was $>5 \%$. Results
$($ mean \pm SD). At baseline ARI was abnormal only in 8 pts; MII-pH TN, AR, NAR, ARI, LAR and ACT were $62.59 \pm 31.92,41.2 \pm 21.3,20.9 \pm 19.8,4.9 \pm 3.1,2.0 \pm 2.3$ and $87.1 \pm 48.9$ respectively. After $H$. Pylori eradication, there was no significant change in all MII-pH parameters evaluated (TN $61.7+35.5 \cdot$ AR $43.7 \pm 27.8$. NAR $17.9 \pm 14.6$. ARI $5.2 \pm 4.1$; LAR: $2.6 \pm 2.4$; ACT: $101.9 \pm 35.1 ; \mathrm{NS}$ ). Of the 8 pts with abnormal ARI, the latter became normal in 4 and persisted abnormal in 4 (in 2 with a reduction of the acid exposure); of the 9 pts with normal baseline ARI the latter became abnormal only in 2. Conclusions. In children with H.Pylori infection, eradication of the organism is not associated with significant change in the pattern of gastroesophageal reflux. Our results fail to show a positive association between anti-H. Pylori therapy and occurrence of de novo GERD

638

Signs of H Pylori Infection in Autoimmune Atrophic Gastritis Patients By Immunoblotting

Edith Lahner, Annalisa Santucci, Dino Vaira, Amelia Pasquali, Roberta Mini, Natale Figura, Gianfranco Delle Fave, Bruno Annibale

Background: Two mechanisms are generally considered able to induce atrophic body gastritis $(\mathrm{ABG})$ : autoimmunity and $H$ pylori infection. The most characteristic clinical presentation of autoimmune $\mathrm{ABG}$ is pernicious anemia (PA). No sign of $\mathrm{H}$ pylori infection is detectable in about $30 \%$ of $A B G$ patients, who are thus considered having autoimmune $A B G$. Western blotting permits an accurate exposure classification to $H$ pylori infection. Aim: to assess the strength of $H$ pylori-ABG relationship by using immunoblotting against specific $H$ pylori antigens to detect past $H$ pylori exposure in patients with autoimmune ABG. Methods: Caseseries consisted of $138 \mathrm{ABG}$ outpatients, classified as autoimmune when histology and ELISA serology were negative, and as $\mathrm{H}$ pylori-related, when histology and ELISA serology were positive (active infection) or only serology was positive (past infection). Immunoblotting against $H$ pylori-specific antigens (CagA, VacA, HspB, UreA and UreB) was performed. Results: $22.5 \%$ of patients had autoimmune $A B G, 21 \%$ had $H$ pylori-related $A B G$ with active infection and $56.5 \%$ with past infection. All autoimmune $A B G$ patients showed immunoblotting seroreactivity against at least one of the $H$ pylori-specific antigens. The number of mean seroreactive antigens of autoimmune ABG patients was similar to those of patients with past $H$ pylori infection $(3.6 \pm 0.2$ vs $3.5 \pm 0.1 \mathrm{~ns})$, but lower than that of patients with active infection $(4.3 \pm 0.2, \mathrm{p}<0.001)$. Seroreactivity against $\mathrm{CagA}$ and $\mathrm{VacA}$ antigens was not different between the three groups $(80.6 \%$ and $93.5 \%$ of autoimmune patients; $86.2 \%$ and $96.6 \%$ in active and $67.9 \%$ and $89.7 \%$ in past $H$ pylori infection, respectively). Some classic features of gastric autoimmunity, as PA and corpus-restricted atrophy were observed more frequently among patients with autoimmune $A B G$, whereas others as positivity to autoantibodies against parietal cells and intrinsic factor and the association of other autoimmune diseses were similarly distributed among patients with autoimmune and $\mathrm{H}$ pylorirelated ABG. Conclusions: $H$ pylori immunoblotting reveals a close relationship between $H$ pylori and autimmune $\mathrm{ABG}$, suggesting a misclassification of exposure and an underestimation of infection in this condition.

657

Intestinal T-Cell Responses to Gluten-Derived Peptides Reveal a Large Repertoire and a Hierarchy of Gluten Epitopes in Adult HLA-Dq2-Positive Celiac Patients

Alessandra Camarca, Robert P. Anderson, Gianfranco Mamone, Mauro Rossi, Gaetano Iaquinto, Nicola Giardullo, Salvatore Auricchio, Riccardo Troncone, Carmen Gianfrani Background: The identification of gluten peptides eliciting intestinal mucosa lesions in coeliac patients has crucial importance for the design of immunological therapies that may augment or replace gluten-exclusion. Methods: We have analysed intestinal T cell responses from 10 adult, HLA-DQ2 + celiac patients (3 untreated, mean age 29.3 years range 30-34; 7 treated, mean age 29.1 range 18-36) to a panel of 21 gliadin (glia) and glutenin (glt) synthetic peptides including: the known alfa-glia 33-mer (57-89)[1], its truncated peptides 13-mer (56-68)[2], 17-mer (57-73)[3] and 3 related polymorphysms, 18-mer (71-89[4] and 25mer (64-89)[4], the glia20[5], glu-5[5], the gamma-glia peptides: DQ2-gamma-I[5], DQ2gamma-II[5] and an elongated 14-mer analogue (105-118)[4], DQ2-gamma-III[6], DQ2gamma-IV[6] DQ2-gamma-V[6], 14-mer (173-186)[4] and glt-1565 peptides. Furthermore, immune responses against two novel peptides, DQ2-omega-1 and glt19-23, and the innate immunity eliciting alfa-glia 31-49 were also assessed. Gliadin-specific, long-term T cell lines (TCL) were obtained by cyclic stimulation of intestinal mononuclear cells with a peptictryptic digest (PT) of gliadin and IL-15 as growth factor. Both proliferation and gammaIFN production were analyzed in response to either native or tTG-deamidated peptides (30ug/ml). Autologous or HLA-DQ2-matched allogeneic EBV transformed-B cell lines were used as APC. Results: Each patient recognized a different set of peptides. Only 5 out of 10 (50\%) of patients recognized 33-mer and all its truncated peptides; 4 of them responded also to DQ2-omega-1, which was recognized by an overall of $5 / 10$ patients, gamma-peptides were recognized by no more than 3 individuals. No response was found to glia20, glu-5, glt19-23, glt-156 and 31-49. The TCL from one patient was unresponsive to all peptides, although it responded very well to PT-gliadin. Interestingly dose curve responses indicate that both 17-mer and 25-mer were as potent as 33-mer in stimulating T-cells. Conclusion: iTCLs from adult HLA-DQ2+ CD patients have diverse specificities for gluten peptides, as previously reported for children patients and confirmed that repertoire of active gluten peptides is larger than previously appreciated. Focusing the exact repertoire of peptides relevant for $\mathrm{CD}$ pathogenesis and their hierarchy would be of great impact for $\mathrm{CD}$ therapy. [1]Shan L. et al. Science 2002; 297:2275 [2]Arentz-Hansen H. et al. J. Exp. Med 2000; 191:603 [3]Anderson RP. et al. Nat Med 2000; 6:337 [4]Mamone G. et al. FEBS Letters 2004; 562:177 [5]Vader W. et al. Gastroenterology 2002;122:1729 [6]Arentz-Hansen H. et al. Gastroenterology 2002; 123:803 
Role of CXCR3 in Gliadin-Mediated Zonulin Release and Increased Intestinal Permeability

Julie Brownley, Ruiliang Lu, Anna Sapone, Bao Lu, Craig Gerard, Stefanie N. Vogel, Alessio Fasano

Background/rationale: The autoimmune process of Celiac Disease (CD) is triggered by gliadin ingestion. Our previous studies have shown that gliadin triggers release of an intestinal tight junction regulator, zonulin, which is associated with an increase in intestinal permeability. These effects are observed only when gliadin was added to the mucosal side of the intestine, suggesting that the protein exerts its effects by interaction with an intestinal luminal receptor. Aims: To establish whether the effect of gliadin on intestinal permeability and zonulin release is mediated by interaction of gliadin with an intestinal luminal receptor and to investigate the identity of this receptor. Methods: Soluble membrane preparations obtained from rabbit small intestine were loaded on an Affi-gel 15 - $\alpha$-gliadin affinity column, incubated for 90 min, washed with PBS containing $0.1 \%$ Triton X-100, and eluted with PBS containing $0.1 \%$ Triton X-100 with increasing $\mathrm{NaCl}$ concentrations. Fractions were collected and subjected to SDS-PAGE and mass spectroscopy analysis. Ex vivo experiments to measure zonulin release and intestinal transepithelial electrical resistance (TEER) changes in response to gliadin exposure were performed using mouse small intestine mounted in microsnapwell chambers. Results: Three main proteins with estimated molecular weights of 93, 100, and $107 \mathrm{kDa}$, were eluted from the gliadin affinity column following adsorption of solubilized intestinal plasma membrane preparations. The bands were subjected to MALDI mass spec fingerprint analysis and the $100 \mathrm{kDa}$ band was identified as the chemokine receptor CXCR3. To determine if CXCR3 plays a role in gliadin-induced increase in intestinal permeability, ex vivo animal studies were conducted on both CXCR3 knock out (KO) and C57BL/6 wildtype (WT) mouse intestinal tissues mounted in microsnapwells. When exposed to gliadin, intestinal segments obtained from WT mice $(n=8)$ released zonulin $(0.34 \pm 0.06$ vs. $0.61 \pm 0.13$ $\mathrm{ng} / \mathrm{mg}$ protein, baseline vs. post-gliadin exposure, respectively; $\mathrm{p}<0.04$ ) and showed a significant TEER decrement $(130.9 \pm 24.7 \Omega . \mathrm{cm} 2$ vs. $80.1 \pm 17.6$, baseline vs post-gliadin exposure, respectively; $\mathrm{p}<0.02$ ). Conversely, intestinal segments obtained from CXCR3 KO mice $(n=18)$ exposed to gliadin failed to release zonulin $(0.36 \pm 0.14$ vs. $0.18 \pm 0.04 \mathrm{ng} / \mathrm{mg}$ protein, baseline vs. post-gliadin exposure, respectively; $\mathrm{p}=$ N.S.) and showed no TEER changes (129.1 $\pm 25.8 \Omega . \mathrm{cm} 2$ vs. $109.2 \pm 27.1$, baseline vs. post-gliadin exposure, respectively; p N.S.). Conclusions: Our data suggest that CXCR3 is a component of the intestinal gliadin receptor and is responsible for zonulin release and increased intestinal permeability.

659

Enteric Glial Protein S100-Beta Modulates Intestinal Mucosa Inflammation in Celiac Disease

Esposito Giuseppe, Carla Cirillo, Daniele De Filippis, Giovanni Sarnelli, Alessandro Iacono, Francesco Paolo D'Armiento, Teresa Iuvone, Rosario Cuomo

The enteric nervous system (ENS) is involved in the homeostasis of the whole gastrointestinal tract. Enteric glia, as part of the ENS, controls the physiological release of neurotrophic factors and participates to the inflammatory responses in animals. In the central nervous system increased expression of astroglial derived $\mathrm{S100} \beta$ protein has been recently associated with the onset and maintaining of inflammation. The role of enteric glial-S100 $\beta$ protein in the gastrointestinal inflammation has never been investigated in humans. We aimed to evaluate the role of $S 100 \beta$ protein in patients with celiac disease that is a well characterized gut chronic inflammation. Duodenal biopsies from 30 patients with celiac disease (15 untreated and 15 on gluten-free diet) and 10 controls were evaluated for S100 $\beta$ protein expression, the iNOS expression and nitrite production. To further test whether S100 $\beta$ was able to modulate the inflammation, biopsies deriving from controls were stimulated for 36-24 hours with exogenous purified $S 100 \beta(0.005-5 \mu \mathrm{M})$ and iNOS protein expression and nitrite were measured. Also, lipid peroxidation and p38-MAPkinase activation were evaluated by adding $S 100 \beta$ alone or in the presence of specific inhibitor of p38-MAPkinase and NFkappa B transcription factor, SB203580 and TLCK, respectively. Results. In untreated celiac patients the $S 100 \beta$ protein expression, the iNOS protein expression and nitrites were significantly increased respectively by $399+7.1 \%, 2537+20 \%$ and $247 \pm 7.3 \%$ as compared to celiacs on gluten-free diet and controls. In cultured duodenal biopsies derived from healthy subjects, incubation with S100 $\beta$ induced a 40 fold increase of iNOS expression and a concentrationdependent increase of the nitrite production $[65.5 \pm 2.5,213.8 \pm 4.8,397.4 \pm 9.0,749 \pm 12.6 \%]$. The effect of $\mathrm{S} 100 \beta(5 \mu \mathrm{M})$ on lipid peroxidation was significantly inhibited by increasing concentration of both SB203580 $(0.03 \cdot 0.3 \cdot 3 \mu \mathrm{M})[29.7 \pm 4.1,49.3 \pm 3.5,72.6 \pm 5.8 \%]$ and $\operatorname{TLCK}(0.01 ; 0.1 ; 1 \mu \mathrm{M})[49.3 \pm 4.8,66.0 \pm 5.1,80 \pm 4.4 \%]$. We found for the first time that $\mathrm{S} 100 \beta$ is increased in the duodenum of patients with celiac disease. This protein seems to modulate duodenal inflammation through induction of iNOS protein expression and nitrite production via p38-MAPkinase activation. Finally, our data highlight the importance of glial cell activation in pathophysiology of celiac disease and more extensively of gastrointestinal inflammation.

660

Challenge of HLA-Dr4, -Dq8, Human CD4 Triple Transgenic NOD Mice with Gliadin in Complete Freund's Adjuvant, Followed By Oral Gluten Tobias Freitag, Grete Sonderstrup, Detlef Schuppan

Introduction: Celiac disease (CD) is a malabsorptive syndrome caused by an uncontrolled immune reaction to ingested gluten proteins. Untreated, $\mathrm{CD}$ patients can develop serious secondary morbidity, such as T cell lymphoma and autoimmune diseases. We aim to develop an in vivo animal model in which non-dietary therapies can be tested. $C D$ is strongly associated with HLA-DQ2 and -DQ8, suggestive of a connection to the CD4 T cell compartment of the immune system. The design of our model is therefore based on HLA-DQ8, associated -DR4 alleles and human CD4 (huCD4). Methods: 4 groups of mice ( $\mathrm{n}=7-8 ; 16$ 24 weeks) transgenic for HLA-DR*0405/DQ8/huCD4, HLA-DQ8/huCD4, HLA-DR*0401/ DQ8/huCD4 or HLA-DR*0401/huCD4 were kept on a gluten-free diet from birth. 4 mice/ group were immunized with $100 \mu \mathrm{g}$ gliadin/CFA i.d. and with 50 $\mathrm{\mu g}$ gliadin/IFA on day 14 From day 14 , all mice were fed a diet containing $3 \mathrm{~g}$ of crude gluten $/ \mathrm{kg}$. On days $56-60$ organs and serum were collected. Splenocytes of individual mice were submitted to red cel lysis and re-suspended at $5 \times 106$ cells/ml in complete RPMI 1640 containing $10 \mu \mathrm{g} / \mathrm{ml}$ gliadin ovalbumine or $3 \mu \mathrm{g} / \mathrm{ml}$ anti-mouse CD3 antibody. After 48 and $72 \mathrm{~h}$ of culture, supernatants were harvested. Results: Mice remained normal over the course of the study. All groups of immunized mice developed high anti-gliadin $\operatorname{Ig} G$ serum titers, without significant differences between the groups with the highest (serum dilution 20,931-fold; HLA-DQ8/huCD4) and lowest (15,810-fold; HLA-DR*0401/huCD4) levels, while titers in non-immunized mice as well as anti-gliadin IgA levels remained low. $\operatorname{IgG} 2 \mathrm{c}$ and $\operatorname{IgGl}$ serum titers did not show significant differences between groups. Production of $\operatorname{IgG} 2 \mathrm{C}$ was greater than IgGl in al groups. Interferon $\gamma$ production in response to gliadin reached high levels, from 12,670 (HLA-DR*0401/DQ8/huCD4) to 24,315 pg/ml (HLA-DR*0401/huCD4) at 48h, in all immunized mice. IL-10 levels ranged from 816 (HLA-DQ8/huCD4) to $1882 \mathrm{pg} / \mathrm{ml}$ (HLADR*040l/huCD4), and IL-2 from 177 (HLA-DQ8/huCD4) to 745 pg/ml (HLA-DR*040l/ DQ8/huCD4). There was no significant production of IL-4. Cytokine levels at $72 \mathrm{~h}$ were invariably lower. While histological analysis of various organs is ongoing, the small intestin was normal. Conclusions: Splenocytes of mice transgenic for HLA-DQ8, HLA-DR*0401, HLA-DR*0401/DQ8 and HLA-DR*0405/DQ8 respond with a Thl pattern to gliadin rechallenge after immunization with gliadin/CFA and oral gluten challenge. The mice do no develop small intestinal lesions. In order to further develop this humanized animal mode of celiac disease, modifications of antigen challenge or the depletion of regulatory $\mathrm{T}$ cells may have to be tested.

661

A 35mer Peptide with T Cell Stimulatory Activity Comparable to Whole Gliadin: A Lead Compound for Peptide Immunotherapy in Celiac Disease? Jason A. Tye-Din, Tim Beissbarth, Robert P. Anderson

Celiac disease (CD) is an HLA-associated enteropathy driven by dietary gluten that is mediated by CD4+ T cells specific for diverse gluten peptides. Studies in other human immune and infectious diseases indicate that antigen-specific immunotherapy and T-cell diagnostics for $\mathrm{CD}$ are likely to utilize peptides that are efficiently and consistently recognized by the majority of gluten-specific T cells in CD. Using comprehensive gluten peptide libraries we have established a hierarchy of gluten peptides with T cell stimulatory activity. AIM: To compare the T cell stimulatory activity of peptides encompassing strings of epitopes ("combitopes") with prolamin fractions from grains toxic in CD. METHODS: Subjects with biopsy proven HLA-DQ2 CD on a gluten free diet consumed either wheat bread $200 \mathrm{~g} / \mathrm{d}(\mathrm{n}=17)$ rye bread $100 \mathrm{~g} / \mathrm{d}(\mathrm{n}=17)$ or barley grains $150 \mathrm{~g} / \mathrm{d}(\mathrm{n}=17)$ for three days. Peripheral blood mononuclear cells (PBMC) collected six days after commencing gluten challenge were incubated in interferon- $\gamma$ ELISPOT assays with deamidated prolamins $(100 \mathrm{mcg} / \mathrm{ml})$, the immunodominant wheat $\alpha$-gliadin peptide QLQPFPQPELPYPQPQP, (which includes two overlapping T-cell epitopes PFPQPELPY and PQPELPYPQ) $(50 \mathrm{mcg} / \mathrm{ml})$, the related $\omega$-gliadin peptide immunodominant after rye or barley ingestion (QPEQPFPQPEQPFPWQP) $(50 \mathrm{mcg} / \mathrm{ml})$, and a 35 mer peptide $(20 \mathrm{mcg} / \mathrm{ml})$ encompassing these $\alpha$ - and $\omega$-gliadin sequences as well as a $\gamma /(0$-gliadin sequence occasionally dominant in HLA-DQ2 CD. RESULTS: PBMC ELISPOT responses to peptides were expressed as a percent of an individual's response to whole deamidated prolamins. Subjects who had a significant response ( $>3 \mathrm{x}$ medium alone $\&>10$ spots/well) to gliadin after wheat challenge (15/17), hordein after barley (13/17) or secalin after rye (13/17) responded to the $35 \mathrm{mer}$ combitope. Following wheat gluten challenge respective responses to the combitope, $\mathrm{p} 57-73$ and DQ2- $\omega$-I were $87 \pm 11 \%($ mean \pm SEM), $51 \pm 5 \%$ and $63 \pm 6 \%$. Following barley challenge they were: $88 \pm 11 \%, 16 \pm 10 \%, 73 \pm 5 \%$, and following rye: $80 \pm 11 \%, 20 \pm 6 \%$ and $70 \pm 12 \%$. CONCLUSIONS: The number of peripheral blood T cells that recognize a 35 mer combitope is equivalent to over $80 \%$ of the total T cell response to deamidated prolamins after in vivo exposure to wheat, rye or barley. This finding indicates substantial redundancy of gluten peptides with T cell stimulatory activity, and simplifies design of peptide immunotherapy and diagnostics.

662

Intestinal Dendritic Cells in Celiac Disease

Antonio Di Sabatino, Karen M. Pickard, John N. Gordon, M R. Beattie, Giovanni Monteleone, Tom T. MacDonald

"Background \& Aims": T-cell activation largely depends on the delivery of costimulatory signals from intestinal dendritic cells (DC) which act as sentinels for incoming food or bacterial antigens. To identify the changes in DC that may underlie the dysregulated T-cel response to gluten in celiac disease (CD), we examined coeliac lamina propria DC in terms of phenotype, activation/maturation state and cytokine production, and investigated the in vitro effects exerted on DC by the immunodominant (p57-68) and the non-immunodominant (p31-43) gliadin epitopes, the latter of which is supposed to activate the innate immune system. "Methods": DC were identified in lamina propria mononuclear cells isolated from untreated CD patients and controls by multicolor flow cytometry as an HLA-DR+ lineagepopulation. Plasmacytoid $(C D 123)$ and myeloid $(C D 11 c)$ receptors, together with activation (CD80/CD86) and maturation (CD83) markers were assessed by flow cytometry. In situ distribution of DC was investigated by confocal microscopy. IFN- $\gamma$, IL-5, IL-12p40, IL-18 IL-23pl 9 and TGF- $\beta$ transcripts were analysed by RT-PCR in sorted DC. After 24-h culture with p31-43 or p57-68 or TNF- $\alpha$, CD83 and HLA-DR expression on DC and COX-2 levels in duodenal biopsies were respectively assessed by flow cytometry and immunoblotting. "Results": A significantly higher number of plasmacytoid DC were found in active CD than in controls. DC from untreated CD patients expressed significantly higher levels of maturation activation molecules, and expressed greater IL-18 and IL-23 transcripts. CD83 and HLA DR expression on coeliac DC were increased after incubation with TNF- $\alpha$, but not p31-43 or p57-68. Organ culture experiments failed to show an increase in mucosal COX-2 levels after treatment with either p31-43 or p57-68. "Conclusions": In active CD, lamina propria DC are activated and express higher number of transcripts of pathologically relevant cytokines. The non-immunodominant gliadin epitope appears to have no effects on DC maturation, HLA-DR expression and COX-2 levels in our hands. 
ELF Phosphorylation By T $\beta$ Ri and Cyclin Dependent Kinases in Cos-7 and Hepg2 Tumor Cell Lines

Nady Golestaneh, Eugene A. Volpe, Jyotshna Kanungo, Stephen R. Evans, Bibhuti Mishra, Harish Pant, Lopa Mishra

TGF- $\beta$ can induce growth inhibition responses during cell cycle progression and escape from this response is a hallmark of many cancer cells. Previously, we demonstrated that TGF- $\beta$ induces phosphorylation and association of ELF (embryonic liver fodrin) with Smad3 and Smad4 resulting in nuclear translocation. However the precise kinases and receptors by which ELF is phosphorylated remain to be determined. Upon phosphorylation, ELF binds to Smad3 and Smad4, the complex translocates to the nucleus and induces the transcription of downstream genes. Strong evidence implicates the TGF- $\beta$ signal transduction in suppression of gastric carcinogenesis. Mice that carry a heterozygous disruption of Smad4 or elf/Smad4 present increased gastric tumors. Aims: 1. To determine the T $\beta$ Rs involved in ELF phosphorylation. 2. To study the role of CDKs in ELF phosphorylation and identify the specific CDK that preferentially phosphorylates ELF. Methods and Results: 1. Cotransfection of Cos-7 cells with ELF and TBR constructs followed by immunoprecipitation, using specific p-ELF antibody raised against S804 revealed that constitutively active TbRI and TbRI receptors significantly phosphorylate ELF, whereas dominant negative TbRII and T $\beta R I I$ do not phosphorylate ELF. Coimmunoprcipitation of ELF and T $\beta$ Rs revealed a robust band confirming the interaction of TbRI with ELF. A weak interaction was also observed between ELF and T $\beta$ RII. However this interaction does not result in ELF phosphorylation 2. Cotransfection of HepG2 tumor cells with ELF/(SBE)4-Lux and TßRI/ (SBE)4-Lux constructs in the presence or absence of TGF-b and TbRI kinase inhibitor (SB 431542) was followed by Luciferase reporter assay. The results revealed that TGF- $\beta$-induced transcriptional activity of ELF and T $\beta R I$ was inhibited up to 3-fold and 6-fold respectively in the presence of $1 \mathrm{mM}$ of inhibitor and further increase in inhibitor concentration did not significantly affect this inhibition. 3. Cotransfection of the COS-7 cells with ELF/CDK4 or CDK5 followed by immunoprecipitation revealed that CDK4 and CDK5 both phosphorylate ELF. However, immuoprecipitation results confirmed direct interaction of CDK4 with ELF whereas CDK5 did not show an interaction. Conclusion: 1.Our results suggest that T $\beta R I$ is required for ELF phosphorylation and this phosphorylation occurs through direct protein interaction. Furthermore, TGF- $\beta$ downstream transcriptional activity requires an active TbRI kinase. 2.CDK4 and CDK5 phosphorylate ELF. The phosphorylation of ELF by CDK4 occurs through direct protein interaction. Further studies are required to fully understand the role of CDK phosphorylation of ELF mediating TGF- $\beta$ signaling pathway

\section{4}

Insulin-Like Growth Factor Binding Protein-3 Regulates Esophageal Epithelial Cell Homeostasis Through Negative Regulation of Insulin-Like Growth Factor Signaling

Munenori Takaoka, Takaomi Okawa, Claudia D. Andl, Carmen Z. Michaylira, Meenhard Herlyn, Hiroshi Nakagawa

Introduction: Esophageal epithelial homeostasis is thought to be regulated by multiple growth factor-mediated signaling pathways. We have recently found that growth factor deprivation induces insulin-like growth factor (IGF) binding protein-3 (IGFBP-3) and that IGFBP-3 is subject to negative regulation by EGF in the esophageal epithelial cells (Takaoka $\mathrm{M}$ et al. Am J Physiol. In press). However, the regulatory role of IGFBP-3 in esophageal epithelial proliferation remains to be elucidated. Methods: Human esophageal cells were grown in serum free medium containing bovine pituitary extract, insulin, and EGF. IGFBP3 was stably transduced with a retroviral vector. To reconstitute the stratified squamous epithelium in vitro (organotypic culture), esophageal cells were seeded upon a type I collagen matrix containing human skin fibroblasts, and exposed to the liquid-air interface. Gene expression was determined by enzyme-linked immunosorbent assay and Western blotting. Western ligand blotting with ${ }^{125}$ I-IGFs was performed to determine the IGFBP-3 binding affinity. Cell proliferation was assessed by ${ }^{3} \mathrm{H}$-thymidine incorporation assay. Cell cycle was analyzed by flow cytometry. The glucose level was measured by colometric glucose oxidization reaction. Results: IGFBP-3 was highly secreted in conditioned media of wild-type or mutant (I56G/L80G/L81G) IGFBP-3 transduced cells $(1.5-2 \mu \mathrm{g} / \mathrm{ml})$ compared with control cells $(2-10 \mathrm{ng} / \mathrm{ml})$. Ectopically expressed wild-type IGFBP-3, but neither the mutant IGFBP3 lacking the binding affinity to IGF, nor empty vector greatly suppressed activation of IGF receptor and AKT by IGF-I, resulting in the impaired Gl/S progression with concomitant failure of induction of $\mathrm{pRB}$ phosphorylation, $60-70 \%$ reduction in cell proliferation, and decreased cellular glucose uptake by $30 \%$. By contrast, insulin fully activated IGF receptor and stimulated cell proliferation regardless of the IGFBP-3 level, implying the lack of negative regulation by IGFBP-3. In organotypic culture, wild-type IGFBP-3 severely impaired epithelial formation of the esophageal cells induced by IGF-I, which was rescued by physiologically exceeding level of insulin. Apoptosis did not occur in IGFBP-3 overexpressing esophageal cells for at least 24 hours even in growth factor deprived condition as cleavage of Caspase3, a sub-Gl fraction and nuclear chromatin condensation were not observed. Conclusions: These results suggest that IGFBP-3 plays a critical role in the regulation of esophageal epithelial formation through inhibition of IGF-I-mediated mitogenic effects and glucose metabolism, and underscore the role of IGF, rather than insulin, in esophageal epithelial homeostasis.

\section{5}

Prostaglandin E2 Stimulates Intestinal Epithelial Proliferation and Migration Through Activation of Intestinal Subepithelial Myofibroblasts George G. Sheng, Jinyi Shao, Randy C. Mifflin, Don W. Powell, Hongmiao Sheng

Cyclooxygenase-2 (COX-2) and its derived prostaglandins (PGs) play critical roles in intestinal inflammation and neoplasia. It has been shown that PGE2 stimulates intestinal epithelial proliferation and neoangiogenesis. Intestinal subepithelial myofibroblasts (ISEMF) are the major stromal cell type in both hyperplastic and neoplastic colonic polyps which express
COX-2 and generate PGE2. In the present study, we show that PGE2 activated ISEMF through Gs protein-coupled EP receptors and the cAMP/PKA pathway. Both primary colonic myofibroblast (CMF) isolates and an established myofibroblast cell line (18Co) expressed a number of growth factors including amphiregulin (AR) and hepatocyte growth factor (HGF). PGE2 exposure robustly increased the expression of AR mRNA in 18Co cells and CMF cultures which normally did not express AR. Levels of AR protein were increased $>10$-fold in PGE2-stimulated 18Co and CMF conditioned media. As a result, 18Co cells stimulated the proliferation of intestinal epithelial cells (RIE) in a co-culture system in which RIE cells were grown in Transwell and 18Co cells were grown in the bottom chamber. Neither PGE2 nor 18Co cells stimulated the proliferation of RIE cells; however, PGE2-activated 18Co cells increased DNA synthesis of RIE cells by $~ 100 \%$. Addition of anti-AR neutralizing antibody significantly attenuated the growth advantage of RIE cells that were stimulated by PGE2treated 18Co conditioned media, indicating the mitogenic effect of 18Co cell-generated AR. Furthermore, PGE2 robustly induced the expression of HGF. HGF protein was detected in $18 \mathrm{Co}$ culture media at a concentration of $\sim 0.4 \mathrm{ng} / \mathrm{ml}$. Addition of PGE2 increased the levels of HGF protein 5-fold by $24 \mathrm{~h}$. In a modified Boyden Chamber Assay, PGE2 did not stimulate the migration of RIE cells and the cells which migrated through the polycarbonate membrane retained a cuboidal appearance. Placing $18 \mathrm{Co}$ cells in the bottom chamber significantly increased the motility of RIE cells. However, when activated by PGE2, 18Co cells strongly stimulated the migration of RIE cells; the number of migrating cells increased by $\sim 100 \%$. It was noted that the migrating RIE cells acquired a widely stretched morphology when co-cultured with PGE2-activated 18Co cells. Addition of anti-HGF neutralizing antibody completely blocked the PGE2/18Co-induced RIE cell migration. These results suggest that autocrine or paracrine PGE2 may stimulate intestinal myofibroblasts to release growth factors which provide a pro-neoplastic microenvironment for the transformation of intestinal epithelial cells.

666

Azoxymethane Protects Intestinal Stem Cells and Reduces Crypt Epithelial Mitosis Through a COX-1 Dependent Mechanism

Courtney W. Houchen, Terrence Riehl, Robert George, Mark Sturmoski, Brian Dieckgraefe, Shrikant Anant

Colorectal cancer is characterized by distinct genetic, morphological, and cellular events. The transformation of a normal mucosal epithelial cell to an invasive colorectal carcinoma occurs via a well-coordinated accumulation of mutations in a series of critical genes. In the gut, tumorigenesis is thought to arise specifically in the stem cell population located at or near the base of intestinal and colonic crypts. Azoxymethane (AOM) is a potent DNA damaging agent and carcinogen that induces intestinal and colonic tumors in rodents. The present study examined the role of cyclooxygenase 1 and 2 in the early response of intestinal stem cells to treatment with AOM. Methods: Wild type (WT ) 10-wk old (C57BL/6J) mice COX-1-/- and COX 2-/- mice on the C57BL/6J background, were treated with AOM (10 $\mathrm{mg} / \mathrm{kg}$ body weight I.P.). Changes in COX-1, COX-2 mRNA and protein as well as prostaglandin E2 (PGE2) were determined at 8 hours after AOM. AOM induced apoptosis and changes in crypt epithelial mitosis were also assessed. The ability of intestinal crypt stem cells to survive $12 \mathrm{~Gy}$ whole body g-irradiation (IR) in treated mice was assessed using a modification of the clonogenic microcolony assay 3.5 days after IR. AOM was administered $8 \mathrm{~h}$ prior to irradiation. Identification of apoptotic cell death, $8 \mathrm{~h}$ after AOM, was assessed using morphologic criteria on hematoxylin and eosin stained sections and confirmed by TUNEL methods. Results: AOM elicited a COX-1 dependent ( 2.5 fold) increase in intestinal stem cell survival. Mice treated $2 \mathrm{~h}$ before AOM with indomethacin $(1.5 \mathrm{mg} / \mathrm{kg})$ but not the COX-2 specific inhibitor NS-398 (lmg/kg), failed to exhibit increased stem cell survival. Furthermore, AOM treatment increased stem cell survival (4-fold) in COX-2 -/- mice but not COX-1 -/- mice confirming an essential role for COX-1 in AOM induced radioprotection. COX-1 mRNA, protein and COX-1 derived PGE2 were increased 8h after AOM treatment. WT mice receiving AOM exhibited increased intestinal crypt apoptosis and a simultaneous reduction in crypt mitotic figures compared to untreated controls. There were no significant differences in baseline or AOM induced intestinal crypt epithelial apoptosis between WT and COX-1-/- mice but there was a complete reversal of the AOM mediated reduction in mitotic figures in COX-1-/- mice. Conclusions: These data suggest that alteration of the cell cycle is an important component of the PGE2 dependent stem cell survival response. Demonstration that AOM elicits a COX-1 dependent stem cell survival response may provide insight into the role of prostaglandins in the early phase of intestinal tumorigenesis in response to DNA damage.

667

The Colorectal Cancer Tumor Suppressor Krüppel-Like Factor 4 Is AntiApoptotic in Response to $\gamma$ Radiation-Induced DNA Damage

Amr M. Ghaleb, Vincent W. Yang

BACKGROUND: Studies indicate that the tumor suppressor p53 is crucial in mediating either cell cycle arrest or apoptosis in response to DNA damage. How a cell decides which of the two pathways to follow is not well understood. We previously demonstrated that the putative colorectal cancer tumor suppressor, Krüppel-like factor 4 (KLF4), is a critical mediator for the cell cycle checkpoint functions of p53 after $\gamma$ radiation-induced DNA damage at both the Gl/S and G2/M transitions [Yoon et al. (2003) JBC 278, 2101; Yoon and Yang (2004) JBC 279, 5035]. AIM: To determine whether KLF4 plays a role in modulating apoptosis following DNA damage caused by $\gamma$ irradiation. METHODS: We examined the responses of the following derivatives of two human colon cancer cell lines and of mouse embryonic fibroblasts (MEFs) to $\gamma$ irradiation: RKO (wild type for p53 and containing negligible endogenous KLF4 levels), EcR/RKO-KLF4 (containing an inducible exogenous KLF4 under the control of the insect hormone, ecdysone); HCT116 KLF4/sh2-2 (with significantly reduced KLF4 levels due to interference by small hairpin (sh) RNA), HCT116 KLF4/sh-vector control; MEF KLF4+/+ (wild-type for KLF4 alleles) and MEF KLF4-/- (null for KLF4 alleles). Cells were irradiated with $12 \mathrm{~Gy}$ of $\gamma$ ray, or not, and maintained in culture for 3 days before analyses. TUNEL staining, cell cycle profile analysis, Western blot, luciferase reporter and chromatin immunoprecipitation (ChIP) assays were conducted. 
RESULTS: Following $\gamma$ irradiation, apoptosis was evident in RKO and uninduced EcR/RKOKLF4 cells but was significantly reduced in EcR/RKO-KLF4 cells treated with ecdysone to induce KLF4. Western blot analyses showed that while levels of p53 and Bax were increased in RKO and uninduced RKO-KLF4 cells by irradiation, those of KLF4 and p2l didn't. Treatment of EcR/RKO-KLF4 cells with ecdysone resulted in increased KLF4 and p21 levels that were accompanied by a reduction in both Bax levels and in apoptosis following irradiation. Similarly, in response to $\gamma$ irradiation, high levels of apoptosis were noted in HCT116 KLF4/sh2-2 cells and MEF KLF4-/-, but not in HCT116 KLF4/sh-vector and MEF KLF4+/ + controls, respectively. ChIP and reporter assays showed that KLF4 bound to the Bax promoter and inhibited the ability of p53 to transactivate the promoter. CONCLUSION: Results of this study suggest that KLF4 exhibits a surprising and unexpected anti-apoptotic function following DNA damage and may therefore be involved in cell fate decision. Potential mechanisms by which KLF4 accomplishes this task are by inducing expression of p21, known to be an inhibitor of apoptosis, and by suppressing Bax expression.

668

Galectin-3 Loss Leads to Pten Down-Regulation and Acquisition of TrailResistance in Human Colon Cancer Cells

Nachman Mazurek, Yun Jie Sun, Kai-Feng Liu, Robert S. Bresalier

Background: Tumor necrosis factor-related apoptosis-inducing ligand (TRAIL) plays an important role in immune surveillance, suggesting a potential role in cancer therapy. Resistance to TRAIL-mediated apoptosis in cancer cells may, however, limit the success of TRAILtherapy. We have reported that galectin-3 (GAL3), a beta-galactoside binding lectin, plays a key role in rendering TRAIL-sensitivity to breast carcinoma cells through induction of PTEN and deactivation of the PI3K/Akt survival pathway. AIM: Identify the molecular mechanisms responsible for TRAIL-resistance in human colon cancer cells. Methods: Human colon cancer cell lines LS174T and LiM6 were used as model systems. TRAIL-induced apoptosis was assessed using a flow cytometry based TUNEL assay and PARP cleavage. The effects of GAL3 and PTEN on TRAIL signaling were examined by transient tranfection of GAL3, and knockdown experiments using GAL3- and PTEN- specific short interfering RNAs and pharmacological inhibitors of PI3K. Results: Exposure of LS174T or LiM6 to TRAIL resulted in 90\% apoptotic cell death. The residual TRAIL-resistant cells (LS-R, LiM6-R) were propagated and maintained under TRAIL selective pressure. Exposure of resistant cells to TRAIL failed to activate caspase-8, indicating that the acquisition of TRAIL-resistance occurred upstream to caspase-8, at the assembly of the death-induced signaling complex (DISC). Western blot analysis, immunohistochemistry and confocal microscopy revealed a marked decrease in GAL3 protein expression accompanied by PTEN-loss and concomitant Akt phosphorylation in LS-R and LiM6-R cells compared to the parental TRAIL-sensitive cells. Silencing of GAL3 in parental LST147 and LiM6 cells resulted in a decrease in PTEN expression, increased Akt phosphorylation, and conferred TRAIL-resistance. Silencing of PTEN in parental cells elicited responses similar to those observed in GAL3 knockdown cells without decreasing GAL3 protein expression, suggesting that GAL3 acts upstream to PTEN. Overexpression of GAL3 in LS-R and LiM6-R cells increased PTEN expression, decreased Akt phosphorylation, and induced TRAIL sensitivity. TRAIL sensitivity was also induced by PI3K inhibitors. TRAIL resistance associated with GAL3 and PTEN loss was not mediated by an increase in intracellular levels of members of the DISC nor by caspase- 8 or its inhibitors. Conclusions: These results further identify GAL3 as a novel regulator of PTEN, and suggest that the acquisition of TRAIL-resistance in human colon cancer cells may be due to nonfunctional assembly of the DISC mediated by loss of GAL3. This may have implications for treatment of TRAIL-resistant tumors.

669

Substance P Mediates Anti-Apoptotic Pathways Through Akt/Protein Kinase B Activation in Human Colonic Epithelial Cells

Hon Wai Koon, Dezheng Zhao, Yanai Zhan, Mary P. Moyer, Charalabos Pothoulakis

Background and Objectives: Substance P (SP) participates in intestinal inflammation via binding to the G-protein coupled neurokinin-1 receptor (NK-1R). Previous reports showed that apoptosis is evident in the colonic mucosal epithelium of rats with dextran sulfateinduced colitis. Here we determined whether SP stimulates anti-apoptotic pathways in colonic epithelial cells. Methods: Human non-transformed NCM460 colonocytes stably transfected with the human NK-1R (NCM460-NK-1R cells) were used for this purpose. MTS assay, TUNEL assay, and PARP cleavage were used to detect apoptosis. Results: SP (10-7M) exposure, time-dependently induced Akt phosphorylation that was evident at 10 min. Pretreatment with the PI3K inhibitor LY294002 $(10 \mu \mathrm{M})$ or the Jak2 inhibitor AG490 $(100 \mu \mathrm{M})$ blocked SP-induced Akt phosphorylation. However, blockade of ERK1/2 and p38 by PD98059 $(25 \mu \mathrm{M})$ and SB203580 $(20 \mu \mathrm{M})$, respectively, did not affect SP-stimulated Akt phosphorylation. Tamoxifen is a well-established inducer of apoptosis, and its effect is mediated by mitochondrial damage and oxidative stress. We found that tamoxifen $(10 \mathrm{uM})$ exposure induced loss of cell viability (MTS assay) and PARP cleavage (Western blot analysis) in colonocytes, while co-administration of SP (10-7M - 10-9M) rescued tamoxifen-induced loss of cell viability and PARP cleavage. SP also reduced tamoxifen-induced nuclear DNA fragmentation evaluated by TUNEL assays in situ. Pharmacological inhibition of PI3K and Akt reversed SP-mediated anti-apoptotic responses on tamoxifen-induced PARP cleavage and reduced cell viability. Conclusion: These are the first results to demonstrate that SP induces Akt phosphorylation and prevents apoptosis in colonocytes. We speculate that SPassociated Akt activation pathways might participate in colonic inflammation and prevention of apoptosis. Supported by grant DK 47343 from the National Institutes of Health.

\section{0}

CREB-Dependent COX-2 Expression By Ang II and EGF in Intestinal Epithelial Cells

Hung Pham, Lee W. Slice

Background. Cyclooxygenase-2 (COX-2) expression plays a major role in a broad range of physiological processes and diseases including human colorectal cancer. Previously, we demonstrated synergistic COX-2 expression by Angiotensin (Ang) II and EGF through distinct signaling pathways requiring small GTPase activity that led to COX-2 promote activation. The aim of this study is to further define the signaling pathways used by Ang II and EGF to express COX-2 that result in prostaglandin $(\mathrm{PG})$ production in intestinal epithelial cells. Methods. IEC-18 cells are a non-transformed epithelial cell line derived from rat ileum that expresses endogenous EGF receptors and type I Ang receptors. COX-2 and GAPDH mRNA was measured using real time RT-PCR. Western blot analysis was used to determine COX-2 expression and the phosphorylation state of signaling proteins. Transient transfection with COX-2 promoter luciferase reporter vectors was used to measure COX-2 promoter activation. Protein-DNA binding was measured using electrophoresis mobility shift assay (EMSA). Immunoassays were used to measure PG levels. Results. Co-stimulation with An II and EGF promoted synergistic induction of COX-2 mRNA and protein and resulted in a high level of PGI2 production. The specific COX-2 inhibitor NS-398 blocked Ang II and EGF-induced PGI2 production. Ang II and EGF induced a transient phosphorylation of ERK p38MAPK and CREB. Co-stimulation with Ang II and EGF prolonged the phosphorylation of ERK, p38MAPK and CREB. The selective p38MAPK inhibitor SB202190 blocked Ang IIdependent CREB phosphorylation and COX-2 expression. The selective MEK inhibitor PD98059 blocked EGF-dependent CREB phosphorylation and COX-2 expression. Inhibition of CREB expression using CREB specific siRNA attenuated COX-2 expression by Ang II, EGF and Ang II \& EGF. Expression of the dominant mutant CREB S133A inhibited COX2 promoter activation by Ang II and EGF. EMSA showed CREB binding to a specific site on the proximal COX-2 promoter. Conclusions. Ang II and EGF synergistically induce both COX-2 mRNA and protein expression in IEC-18 cells that result in PGI2 production Signaling by either Ang II or EGF resulted in transcriptional activation of the COX-2 promoter by phosphorylation of the promoter bound CREB. In addition to $\mathrm{Ca} 2+$ signaling, phosphorylation of CREB was mediated by p38MAPK for Ang II and by ERK for EGF. The synergistic increase in COX-2 expression by Ang II \& EGF correlates with the prolonged phosphorylation of CREB. This suggests that the distinct signaling pathways used by Ang II and EGF in COX-2 expression converge onto the CREB transcription factor. DK061485

671

RNA Interference Reveals a Differential Role of FAK and Pyk2 in Cell Migration, Leading Edge Formation and Increase in Focal Adhesions Induced By LPA in Intestinal Epithelial Cells Xiaohua Jiang, Rodrigo Jacamo, Elena Zhukova, James Sinnett-Smith, Enrique Rozengurt Background: In the gastrointestinal mucosa, cell migration plays a crucial role in the organization and maintenance of tissue integrity but the mechanisms involved remain incompletely understood. Immortalized cell lines derived from FAK-/- mice show decreased rate of migration but these cells have accumulated abnormalities and are fibroblastic rather than epithelial cells. Consequently, the precise role of FAK in cell migration and morphology of intestinal epithelial cells remains poorly understood. Materials and Methods: We used siRNA-mediated depletion of focal adhesion kinase (FAK) protein to determine the role of FAK in woundinduced migration and cytoskeletal organization in the non-transformed intestinal epithelial cells IEC-6 and IEC-18 stimulated with the G protein-coupled receptors (GPCR) agonist lysophosphatidic acid (LPA). Results: Treatment of these cells with FAK siRNA reduced FAK expression by $85 \%$, but did not affect the expression of Pyk2. Knockdown of FAK protein significantly inhibited LPA-induced migration of both IEC-18 and IEC-6 cells. LPA induced reorganization of actin and microtubule cytoskeleton in the leading edge was inhibited in FAK siRNA-transfected cells. Interestingly, in contrast to the FAK-/-cells, which exhibit an increased number of prominent focal adhesions when plated on fibronectin, FAK knockdown IEC-18 cells exhibited dramatically decreased number of focal adhesions in response to both LPA and fibronectin as compared with the control cells. We also used siRNAs to knockdown Pyk2 expression without reducing FAK expression. Depletion of Pyk2 did not prevent LPAinduced migration or cytoskeletal reorganization in IEC-18 cells. Conclusion: Our study shows that FAK plays a critical role in LPA-induced migration, cytoskeletal reorganization and assembly of focal adhesions in intestinal epithelial cells whereas depletion of Pyk2 did not interfere with any of these responses elicited by LPA.

672

Localization of Protein Kinase D2 in Endoplasmic Reticulum and Regulation of Neurotensin Secretion in the Human Bon Endocrine Cell Line J.N. Li, Kathleen L. O'Connor, Srinivasan Rajaraman, Courtney M. Townsend, B. Mark Evers

Protein kinase D2 (PKD2), a novel serine-threonine kinase and member of the PKD family, has been implicated in the secretion of neurotensin (NT), an important gut hormone which regulates intestinal motility, secretion and growth. The purpose of this study was to determine the mechanisms for PKD2-mediated NT release in the human endocrine cell line BON which produces and secretes NT peptide. METHODS. Stable BON cell clones expressing wild type (BON/PKD2WT), kinase dead PKD2 (BON/PKD2KD) or empty vector (BON/ pcDNA3) were established. (i) To further determine the role of PKD2 in NT secretion, BON/ PKD2KD cells were treated with vehicle or PMA and NT release was measured by a NT EIA kit. (ii) To examine the expression of PKD2, BON/PKD2WT and BON/PKD2KD cells were treated with either vehicle or PMA and single or double immunofluorescent staining and confocal microscopic analysis performed. In addition, electron microscopy (EM) was utilized to assess ultrastructural changes. RESULTS. (i) NT release was significantly increased in BON/pcDNA3 cells stimulated with PMA. In contrast, PMA-mediated NT release was inhibited in BON/PKD2KD cells, which is consistent with our previous result showing a

. 
significant increase in NT secretion from BON/PKD2WT cells. (ii) PKD2 localized in the cytosol of BON/PKD2WT cells; translocation to the membrane and large cytosolic vesicles occurred after PMA treatment. Similar vesicles were noted constitutively in BON/PKD2KD cells; these vesicles were not affected by PMA treatment. PKD2 was colocalized with the trans-Golgi network (TGN) as well as the endoplasmic reticulum (ER) in BON/PKD2WT cells. PKD2 disassociated with TGN but associated with the vesicles, which were positively stained by ER markers (PDI and calnexin). In BON/PKD2KD cells, kinase dead PKD2 also colocalized with PDI-stained vesicles which were identified as dilated ER by EM. Decreased secretory granules in BON/PKD2KD cells compared with BON/PKD2WT cells were also noted. CONCLUSIONS. PKD2 plays a crucial role in regulating NT secretion. We also found, for the first time, the unique colocalization of PKD2 with vesicles containing ER components, suggesting the association of PKD2 with ER functions related to NT protein synthesis and transport. Importantly, we identify a significant contribution for the PKD2 isoform in stimulated gut peptide secretion.

673

Pkd3 in Intestinal Epithelial Cells: Activation By Phorbol Esters and G Protein Coupled Receptor Agonists Through Pkc

Jingzhen Yuan, Enrique Rozengurt

BACKGROUND: Protein kinase D (PKD) is the founding member of a novel protein kinase family that also includes PKD2 and PKD3. PKD and PKD2 have been the focus of most studies to date, but very little is known regarding the regulation mechanisms of PKD3 in intestinal epithelial cells. Here, using nontransformed IEC-18 cells, derived from fetal rat intestinal crypt cells, as a model system, we examined whether PKD3 is endogenously expressed and regulated in intestinal epithelial cells through PKC and G protein-coupled receptor (GPCR) signaling. RESULTS: Our results demonstrated that abundant PKD3 was expressed in IEC-18 cells. Treatment of IEC-18 cells with biologically active phorbol ester, phorbol 12,13-dibutyrate (PDBu) led to striking activation of PKD3. PKD3 was also rapidly activated in these cells by GPCR agonists including lysophosphatidic acid (LPA), angiotensin and vasopressin in a concentration-dependent fashion. Either PDB- or the GPCR agonistsinduced PKD3 activation was abrogated by treatment of IEC-18 cells with PKC inhibitors GF-1 and Ro 31-8220. Downregulation of PKCs $\alpha, \delta$, and $\varepsilon$ by chronic stimulation of the cells with PDBu led to complete inhibition of PKD3 activation induced by LPA, angiotensin, and vasopressin, suggesting that the classic and novel PKCs are involved in the GPCRinduced PKD3 activation. To clarify the G protein pathways leading to PKD3 activation induced by the GPCR agonists, we examined the effect of pertussis toxin (PTx, an inhibitor of the Gi) on PKD3 activation comparing with PKD. The results showed that LPA- angiotensin-, and vasopressin-induced PKD3 activation were not prevented by treatment with PTx, suggesting that all the three GPCR agonists induced PKD3 activation via PTx-insensitive Gq pathway. In contrast, exposure of parallel cultures to PTx completely blocked LPA-induced PKD activation but did not affect PKD activation stimulated by angiotensin or vasopressin, indicating that LPA induced PKD activation through a PTX-sensitive pathway involving $\mathrm{Gi}$, whereas angiotensin or vasopressin promoted PKD activation via PTx-insensitive Gq. CONCLUSION: Our results demonstrate that PKD3 is activated in intact IEC-18 cells by biologically active phorbol esters and by the GPCR agonists LPA, angiotensin, and vasopressin through a PKC-dependent signal transduction pathway. These findings reveal a link between PKCs and PKD3, and implied that PKD3, like PKD, also lies downstream of PKCs in a novel signal transduction pathway. In addition, the finding that LPA-induced PKD3 activation is PTX-insensitive suggests an important difference between PKD3 and PKD in the regulation through heterotrimeric $G$ protein pathways.

674

Protein Kinase D2 Potentiates DNA Synthesis Induced By Gq-Coupled Receptors By Increasing the Duration of ERK Signaling in Swiss 3T3 Cells James Sinnett-Smith, Elena Zhukova, Gabriel Kleinman, Xiaohua Jiang, Enrique Rozengurt

Background: Protein kinase D (PKD)/protein kinase $\mathrm{C} \mu$ and two recently identified serine protein kinases termed PKD2 and PKC 7 /PKD3, which are similar in overall structure and primary amino acid sequence to $\mathrm{PKD}$, constitute a new protein kinase subfamily separate from the previously identified PKCs. PKD has been implicated in the regulation of multiple cellular responses but much less is known about the function of PKD2 and PKD3. In particular, the precise role of PKD2 in neuropeptide-induced DNA synthesis and cell proliferation has not been elucidated. One of the major signaling pathways involved in the mitogenic response induced by both receptor tyrosine kinases and G protein coupled receptors (GPCRs) is the ERK cascade. The duration and intensity of ERK pathway activation in response gonists of these receptors is of critical importance for determining specific biological outcomes, including proliferation, differentiation and transformation. The protein products of immediate early genes (e.g. c-Fos) have been proposed to function as molecular sensors of ERK1/2 signal duration. Aim: To examine the role PKD2 plays in DNA synthesis, kinetics of ERK activation and the induction of c-Fos induced by GPCRs in Swiss 3T3 cells. Methods: In order to generate Swiss 3T3 cells stably overexpressing PKD2, cultures of these cells were transduced with retrovirus encoding either wild type PKD2 (Swiss 3T3-PKD2.GFP) or GFP alone (Swiss 3T3-GFP) as control, in which PKD2 and GFP were translated from the same bicistronic mRNA and expressed as two separate proteins. After transduction, cells expressing higher levels of GFP were sorted by FACS, collected and propagated for further studies. Results: Addition of bombesin to Swiss 3T3 cells overexpressing PKD2 potentiated DNA synthesis and induced a striking increase in the duration of ERK1/2 activation as compared with cultures of either control Swiss 3T3 cells. In contrast, the DNA synthesis and the duration of ERK activation in response to epidermal growth factor, which acts via protein kinase C/PKD-independent pathways, were not increased. Furthermore, bombesin promoted a striking increase in phosphorylation (at Ser-374) and accumulation of c-Fos (the c-fos proto-oncogene product) in Swiss 3T3 cells overexpressing wild type PKD2. Inhibition of he sustained phase of ERK/RSK activation abrogated the increase in c-Fos accumulation and DNA synthesis induced by bombesin in PKD2 overexpressing cells. Conclusion: PKD2 facilitates bombesin induced DNA synthesis by increasing the duration of ERK signaling leading to the phosphorylation and accumulation of c-Fos.

675

Induction of Mu Opioid and Cannabinoid Receptors By Probiotics: A New Mechanisms of Actions

Christel Rousseaux, Thuru Xavier, Laurent Dubuquoy, Christel Neut, Caroline Dubuquoy, Arthur Ouwehand, Didier Carcano, Pierre Desreumaux

$\mu$ opioid receptor (MOR) and receptors for cannabinoids ( $\mathrm{CB} 1$ and $\mathrm{CB} 2$ ), which belong to the $G$ protein coupled receptor family, are expressed in the central nervous system where they exert analgesic effect. In the gut, they are expressed by epithelial cells and mediate anti-inflammatory effects. Aim: To evaluate the ability of different strains of probiotics to induce MOR, CB1 and CB2 expression. Methods: In vitro, colonic epithelial cells HT-29 were incubated for 0 to $6 \mathrm{~h}$ with different strains of probiotics or bacteria (100 CFU/cell): L. acidophilus (NCFM), L. salivarius (UCC118), L. paracasei (Lpc 37), a commensal E. coli, and an enteropathogenic E. coli (LF82) strain. To preclude an unspecific effect of bacteria on MOR and CB induction by epithelial cells, the retinoid X receptor (RXR) was quantified. The involvement of NF-KB activation on MOR and CB expression was assessed by a pretreatment of HT-29 cells with a specific inhibitor of NF-KB (CAPE). In vivo, Balb/c mice were orally fed with L. acidophilus NCFM (10e9 CFU) during 15 days. MOR and CB expression was evaluated in the colon of mice at mRNA and protein levels by real time PCR and immunohistochemistry together with a quantification of inflammatory cytokines such as TNF $\alpha, K C$ and IL-1 $\beta$ mRNA. Results: By comparison with resting cells, NCFM $(1 \pm 1$ vs $145 \pm 65, \mathrm{p}<0.01)$ and UCC118 $(1 \pm 1$ vs $28 \pm 8, \mathrm{p}<0.01)$ were the most effective strains to induce rapidly a strong expression of MOR in epithelial cells. This induction was similar to HT-29 cells stimulated by optimal dosage of TNF $\alpha$. Induction of CB1 ( $1 \pm 1$ vs $3.7 \pm 0.8$, $\mathrm{p}<0.05)$ and $C B 2(1 \pm 1$ vs $4.4 \pm 0.98, \mathrm{p}<0.01)$ expression was limited to the NCFM strain. Other probiotic strains or bacteria did not affect MOR or CB expression. RXR mRNA expression remained unchanged in stimulated cells. CAPE did not modify MOR expression level in epithelial cells stimulated by NCFM. In vivo, NCFM administration in mice strongly enhanced colonic levels of MOR mRNA compared to control animals $(24 \pm 0.75$ fold, $p<0.01)$. Induction of MOR, $\mathrm{CB} 1$ and $\mathrm{CB} 2$ expression by colonic epithelial cells was confirmed by immunohistochemistry in mice receiving NCFM and was inversely correlated with a significant $50 \%$ reduction of inflammatory cytokine levels. Conclusion: L. acidophilus NCFM is the most effective strain to induce expression of MOR and CB in colonic epithelial cells. These findings suggest that modulation of the gut microbiota may regulate intestinal inflammation and analgesia through the induction of MOR and $\mathrm{CB}$ expression, which may be used as new markers to screen functional bacterial strains with anti-inflammatory and analgesic properties(patent applications FR04/09966-PCT EP05/010880)

676

Activation of Pkc- $\beta 1$ Isoform - A Novel Mechanism for the Beneficial Effects of Lactobacillus Gg (LGG) On Monolayers of Intestinal Epithelial Cells Ali Banan, Lijuan Zhang, Maliha Shaikh, Christopher B. Forsyth, Jeremy Z. Fields, Ashkan Farhadi, Ali Keshavarzian

Despite a large number of studies of probiotics, major gaps in our knowledge still exist, not the least of which are the interactions of probiotics with gut epithelium and the molecular processes that orchestrate their beneficial effects on the gut barrier. In particular, their mechanisms have not been well-documented in in vitro models of IBD. Because the $\beta 1$ isoform of PKC is key to maintenance of healthy GI epithelial cells, we hypothesized that PKC- $\beta 1$ activation is necessary for LGG-induced protection of the intestinal epithelial barrier function under conditions of oxidative stress. Methods: Cells were transfected to stably overexpress PKC- $\beta 1$ or to under-express it. These clones were preincubated with LGG (10 3 $1012 \mathrm{CFU} / \mathrm{mL}$ ) or a PKC activator (DAG analog, OAG, 0.01-1 uM) before exposure to oxidant (H2O2, 10-500 uM). Naive (un-transfected) cells were treated similarly. We monitored barrier function \{fluorometry\}, tight-junction integrity \{laser confocal microscopy\}, PKC- $\beta 1$ subcellular distribution \{membrane, cytoskeletal, cytosolic\}, PKC- $\beta 1$ subcellular activity \{in vitro kinase assay\}; ZO-1/ZO-2 (immunofluorescence, SDS-PAGE fractionation), $n=3 /$ grp. Results: $\{$ I $\}$ Cells stably overexpressing PKC- $\beta 1$, but not naive cells, were protected from H2O2-induced injury by low doses of LGG or OAG. In oxidant-exposed transfected cells, but not naive cells, pretreatment with a low dose of LGG or OAG was protective: 1) increasing ZO-1/ -2 membrane distribution while decreasing ZO cytosolic distribution; 2) enhancing the architectural integrity of ZO; 3) maintaining barrier permeability (FSA, FD clearances). These effects were prevented by a PKC inhibitor. \{II $\}$ Pretreatment of transfected cells with LGG or OAG caused a large change in subcellular distribution of PKC- $\beta 1$, increasing membrane and cytoskeletal fractions of PKC- $\beta 1$ several fold while decreasing cytosolic fractions, indicating PKC- $\beta 1$ activation. \{III\} In LGG treated cells, PKC- $\beta 1$ was active and complexed with ZO. \{IV\} Anti-sense inhibition of endogenous PKC- $\beta 1$ expression abolished LGG-induced protection, and was associated with less membranous ZO and more cytosolic $\mathrm{ZO}$, unstable ZO cytoarchitecture, and barrier hyperpermeability. Conclusions: We demonstrate a new biologic mechanism that LGG-induced protection against oxidative injury requires PKC- $\beta 1$ activation. The ability of bacteria to promote the normal dynamics of tight junctions and barrier function is a novel mechanism not previously attributed to this PKC isoform. This mechanism suggests a potential target for development of novel therapies for the treatment of a variety of inflammatory GI disorders, including inflammatory bowel disease (IBD) 
Estrogen Modulates Bacterial Colonization in the Gut and Responses to Probiotic Therapy

Brendan Diederichs, Dave Thiel, Francis Cheung, Julia Ewaschuk, Hugo Diaz, Karen Madsen

The pathogenesis of inflammatory bowel disease (IBD) involves a complex interaction between genetic and environmental factors. There is evidence that the magnitude of immune responses in autoimmune diseases are gender-dependent and stronger in females compared with males. Live probiotic bacteria are effective in reducing gut permeability and inflammation; it is unknown how gender affects gut responses to probiotics. We have shown that Bifidobacterium strains release peptide bioactive factors that modulate epithelial resistance in vitro. Aim: The aims of this study were to determine if peptides released from Bifidobacteria retain their bioactivity when administered orally and also if a gender-bias exists in the gut response to these bioactive factors. Methods: B. infantis was incubated overnight in RPMI-1640 media and media centrifuged and filtered to remove live bacteria (CM). Adult $129 / \mathrm{SvEv}$ mice received $\mathrm{CM}(30 \mu \mathrm{l})$ and were sacrificed 6 hrs later for assessment of intestinal permeability in Ussing chambers. Controls received RPMI alone. A separate group of 8 wk old IL-10 deficient mice were treated with CM for 4 wks ( $30 \mu \mathrm{l} / 2 \mathrm{x}$ day). At sacrifice, ileal and colonic tissue were assessed for mRNA levels of TNF $\alpha$, IFN $\gamma$, and TGF $\beta$ by RT-PCR and permeability measured in Ussing chambers. Bacteria adherent to the mucosa were analyzed by culture and DGGE. A separate group of female IL-10 deficient mice were treated with the estrogen receptor antagonist ICI-182780 ( $1 \mathrm{mg} / \mathrm{kg} /$ day sc) \pm CM. Results: CM administration acutely reduced colonic permeability in wild-type mice within 6 hrs of administration. Long-term CM treatment normalized colonic permeability in both male and female IL-10 deficient mice. However, gender differences were evident in the gut immune responses to CM treatment. In males, CM treatment resulted in a significant decrease in intestinal secretion of IFN and TNF and decreased mRNA levels. However, in females, CM treatment resulted in an upregulation of secretion and mRNA levels of IFN and TNF. Furthermore, females demonstrated significantly greater levels of colonic adherent bacteria compared with males, and also exhibited major differences in bacterial strains colonizing the mucosa as compared with males. Blocking estrogen receptors with ICI- 182780 partially attenuated the enhanced cytokine response in females. Conclusion: Estrogen appears to have a significant role in modulating bacterial colonization of the gut and in the gut response to probiotic-based therapies. Peptide bioactive factors from B. infantis retain their biological activity in vivo and are effective in normalizing gut permeability in animal models of colitis.

678

The Probiotic Bacterium E. Coli Nissle 1917 (EcN) Ameliorates Experimental Colitis Via TLR-Dependent Pathways

Anja Grabig, Daniela Paclik, Claudia Guzy, Anja Dankof, Daniel C. Baumgart, Baerbel

Raupach, Bertram Wiedenmann, Ulrich Sonnenborn, Axel Dignass, Andreas Sturm

BACKGROUND: Recent studies demonstrated that bacterial products activate $\mathrm{T}$ cells via Toll-like receptors (TLRs), suggesting a link between the luminal bacterial flora and the immune system. We previously demonstrated that $\mathrm{EcN}$ inhibits activation and proliferation of T-cells through a TLR-2-dependent pathway. We therefore aimed to explore the effects of $\mathrm{ECN}$ in an experimental colitis induced in TLR-2 ko and TLR-4 ko mice METHODS: Acute experimental colitis was induced in wildtype (wt), TLR- 2 ko and - 4 ko mice by adding $5 \%$ DSS to the drinking water. Mice were treated with either $\mathrm{NaCl}$ or EcN twice daily rectally or orally until they were sacrificed on day 8 . Disease activity was quantified macroscopically using an established disease activity index (DAI) and microscopically using the Dieleman score. PBT were isolated, stimulated with CD3 for 3 days and cytokine secretion was determined by CBA analysis. Mucosal recruitment of granulocytes was assessed by MPO activity. Using flow cytometric analysis, IFN- $\gamma$ and TNF- $\alpha$ expression in splenic T cells was determined after stimulation for $6 \mathrm{~h}$ with PMA/lonomycin. RESULTS: Wt and TLR-2 ko mice exposed to DSS developed acute colitis, whereas TLR-4 ko mice developed significantly less inflammation. In wt animals, EcN significantly reduced macroscopic and microscopic disease activity as well as MPO activity compared to controls $(p<0.01)$. Furthermore, secretion of pro-inflammatory and chemotactic cytokines was significantly reduced by $\mathrm{EcN}$ treatment. EcN significantly reduced intracellular expression of TNF- $\alpha$ and IFN- $\gamma$ in splenic T cells. In contrast, in TLR-2 ko and -4 ko mice, DAI, microscopic damage and MPO activity were not significantly modulated by EcN. In TLR- 4 ko mice, TNF- $\alpha$, IFN- $\gamma$, IL- 6 and IL-10 secretion was nearly abolished and no differences between $\mathrm{EcN}$ or control treated mice were observed, whereas MCP-1 secretion was comparable to wt, but not different in control or EcN treated mice. In TLR-2 ko mice, IFN- $\gamma$ secretion was reduced by EcN. MCP-1 secretion was nearly completely abolished and TNF- $\alpha$ and IL-10 secretion were not modulated by EcN. CONCLUSION: EcN significantly ameliorated experimental colitis and reduced proinflammatory and chemotactic cytokine secretion in wt mice. This effect was not observed in TLR-2 or TLR-4 deficient mice, suggesting that both TLR are required to mediate the effects of $\mathrm{ECN}$ on cytokine secretion and modulation of disease activity in experimental colitis. Furthermore, we demonstrated, that TLR-2 and -4 are needed to efficiently secrete pro-inflammatory and chemotactic cytokines in intestinal inflammation.

\section{9}

Lactobacillus Rhamnosus GG (LGG) Soluble Proteins Prevent Tumor Necrosis Factor (TNF)-Induced Epithelial Injury in Colonic Organ Culture Fang Yan, Hanwei Cao, Robert Whitehead, D. Brent Polk

Background. The intestinal epithelial monolayer integrity is essential for maintenance of normal gut function. An increased inflammatory cytokine levels is one of the factors known to disrupt this monolayer in several diseases, such as inflammatory bowel disease (IBD). We reported that the probiotic bacterium, Lactobacillus rhamnosus GG (LGG) prevents cytokine-induced apoptosis in mouse and human colon epithelial cell lines through regulating epithelial cell signaling pathways. Furthermore, we have purified two proteins (p75 and p40) secreted by LGG, which exert anti-apoptotic functions. Therefore, the purpose of this study is to determine the roles of LGG and these soluble proteins on TNF-induced colonic epithelial cell injury. Methods: Colon explants were isolated from 6-8-week old C57BL/6 mice and cultured on the top of inserts in DMEM medium containing $0.5 \%$ FBS. After 6 or 24 hours treatment with LGG, LGG-conditioned-media (LGG-CM), or purified proteins in the absence or presence of TNF $(100 \mathrm{ng} / \mathrm{ml})$, paraffin-embedded sections were prepared to determine tissue injury by H\&E staining, and apoptosis by in situ oligo ligation (ISOL) and active caspase- 3 staining. Polyclonal antibodies against p75 or p 40 were generated and used for immuno-depletion of the respective proteins from LGG-CM. Young adult mouse colon (YAMC) epithelial cells were treated with TNF in the presence or absence of LGG for 24 hours. Culture media were collected and cytokine production detected using a cytokine protein array. Results. Co-culture of colon tissue explants with p75, p40, LGG-CM, or LGG with TNF significantly decreased TNF-induced ulcerative lesions, including mucosal necrosis, and disruption of epithelial integrity. TNF induced colon epithelial apoptosis with $75 \%$ of crypts containing more than 10 apoptotic cells, which was decreased 3-fold by $\mathrm{p} 75$ or p 40 co-treatment $(\mathrm{p}<0.005)$. p75 or p 40 also inhibited TNF-stimulated caspase-3 activation by 4 -fold $(\mathrm{p}<0.005)$. Importantly, LGG-CM inhibitory effects on TNF-induced colon injury and apoptosis were blocked by immuno-depletion of p75 and p40. Furthermore, LGC blocked TNF-induced RANTES and MIP-3b secretion from YAMC cells. Both of these chemokines are known to attract mononuclear inflammatory cells. Conclusion. These studies are the first to identify probiotic bacterial soluble proteins' effects on regulating intestina epithelial homeostasis by inhibiting cytokine-induced apoptosis and chemoattractant production. Importantly, this novel mechanism of probiotic action suggests the potential for application of native bacterial components to prevent TNF-mediated gastrointestinal epithelial injury.

\section{0}

Suppression of Experimental Autoimmune Encephalomyelitis (EAE) By Oral Administration of Lactobacillus Paracasei DSM 13434

Shahram Lavasani, Frida Fak, Balik Dzhambazov, Jan Alenfall, Bjorn Westrom

Background \& Aims: Probiotics has been shown to exert beneficial health effects in intestinal infection and mucosal inflammation by modulating the immune system. In the case of chronic autoimmune diseases, like multiple sclerosis (MS), which is associated with inflammation in the central nervous system, a major goal of treatment is to reduce inflammation. The aim of our study was to investigate whether oral administration of Lactobacillus paracasei DSM 13434 could affect the systemic immune response by suppressing a T cell-mediated chronic inflammation in CNS. To assess this effect we used an animal model for multiple sclerosis, experimental autoimmune encephalomyelitis (EAE). Methods: Female C57BL/6 mice, from 9 to 10 weeks old, were treated daily with L. paracasei DSM 13434 dissolved in the drinking water, at a dosage of $10^{9} \mathrm{cfu} /$ mouse/day throughout the whole experiment. A control group received only water. After 12 days of treatment EAE was induced by immunisation with a myelin oligodendrocyte glycoprotein peptide $\left(\mathrm{MOG}_{35-55}\right)$. Animals were daily scored for the clinical signs of EAE and sacrificed at day 25 post immunisation. Results: Oral administration of L. paracasei DSM 13434 significantly inhibited EAE in mice. This was confirmed by histopathology, demonstrating a decrease of $\mathrm{CD}^{+} / \mathrm{CD}^{+} \mathrm{T}$ cell infiltration in CNS. Autoantigen re-challenge in vitro revealed similar T cell proliferation in L. paracasei DSM 13434 treated and control mice but reduced cytokine response in L. paracasei DSM 13434 treated mice as measured by both IFN- $\gamma$ and TNF- $\alpha$. Investigation of lymphocytes in the mesenteric lymph nodes showed a significantly increased of $\mathrm{CD} 4^{+} \mathrm{CD} 25^{+} \mathrm{T}$ lymphocytes. An increase of plasma IgA was also observed in L. paracasei DSM 13434 treated animals. Moreover, ant CD3 stimulation of spleen cells prior to immunisation showed an increased production of IL-10 and IL-4 cytokines in L. paracasei DSM 13434 treated animals. Conclusions: These experiments showed that oral exposure to L. paracasei DSM 13434 can modulate systemic immune responses and therefore represent a promising approach in the management of autoimmune diseases.

681

Differential Effects of Pdz-Adapterprotein NHERF1, E3karp and Pdzkl Knockout On the Regulation of NHE3 Transport Activity in Native Murine Colonic Epithelium

Ayhan Cinar, Mingmin Chen, Brigitte Riederer, Boris Hogema, Hugo de Jonge, Mark

Donowitz, Edward J. Weinman, Olivier Kocher, Ursula Seidler

Backround \& Aims: The PDZ-adapter proteins NHERFl, E3KARP and PDZK1 have al been shown to bind to the sodium hydrogen exchanger NHE3, and all three proteins are ex-pressed in the brush border membrane (BBM) of murine intestine. In PS120 fibroblasts, NHERF1 or E3KARP coexpression with NHE3 is required for cAMP-mediated inhibition of NHE3 transport activity. We therefore investigated, whether the three NHE3 binding PDZ proteins show functional redundancy, or have differential roles in the native intestinal epithelium. Methods: NHERF1, E3KARP, PDZK1, and NHERF1/PDZK1- deficient mice and their wild type NHE3 transport rates were assessed fluorometrically in the NHE3-expressing surface cell region of BCECF-loaded murine colonic crypts isolated from (wt) littermates. NHE3 BBM abudance was measured by Western analysis of isolated BBM, NHE3 localization was assessed immunohistochemically, and NHE3 mRNA levels were determined by RT-PCR. Results: In NHERFl-deficient surface enterocytes, acid-activated NHE3 transport rate was significantly reduced to $65 \%$ of the rate in wt enterocytes. Significant inhibition of NHE3 transport activity by forskolin was observed in NHERFl-deficient surface enterocytes, but this inhibition was significantly less pronouned than in wt enterocytes $(56 \% \mathrm{vs} .75 \%$, $\mathrm{p}<0.01$ ). A similar reduction in the efficacy of inhibition by 8 -Br-cGMP and ionomycin was also seen. In E3KARP-deficient colonic enterocytes, acid-activated NHE3 transport rate, a well as in-hibition by forskolin and 8-Br-cGMP was identical to wt enterocytes, but the inhibition by ionomycin was completely abolished. In PDZK1-deficient surface enterocytes, acid-activated NHE3 transport rate was strongly reduced to $35 \%$ of that in wt enterocytes, and no significant inhibition of transport rate was seen by any of the above second messengers. Surprisingly, the changes observed in the NHERFl/PDZKl-deficient enterocytes were identical to those in the PDZKl-deficient enterocytes. In NHERFl and E3KARP-deficient intestine, NHE3 BBM abundance was unchanged, whereas it was significantly decreased in PDZK1deficient intes-tine, with a significant upregulation of NHE3 mRNA expression. Conclusions 
The results demonstrate that the PDZ-Proteins NHERF1, E3KARP and PDZKl are important regulators of NHE3 transport activity in native murine intestinal epithelium and appear to serve differ-ent, and partly signal-specific functions.

\section{2}

Modulation of Niemann-Pick C1 Like 1 (NPC1L1) Protein By Cholesterol in Caco2 Cells

Fadi Annaba, Zaheer Sarwar, Ravinder K. Gill, Seema Saksena, Dudeja K. Pradeep,

Waddah A. Alrefai

Niemann-Pick Cl-like 1 (NPClLl) is a novel intestinal cholesterol transporter localized to the brush border membrane of the enterocytes. Studies in knockout mice demonstrated that $\mathrm{NPClLl}$ is an essential intestinal component of cholesterol absorption. Also, NPClLl was shown to be the molecular target of Ezetimibe, the cholesterol absorption blocker used for treatment of hypercholesteremia. Recently, NPClLl expression in mice has been shown to be reduced by cholesterol feeding. However, it is not clear whether the decrease in NPCILl expression by cholesterol occurs via changes in the rate of gene transcription. Therefore, the current studies were designed to investigate the molecular regulation of human NPClL1 expression and promoter activity by cholesterol in human intestinal $\mathrm{Caco} 2$ cell line. The relative abundance of NPCILl mRNA was evaluated by real time PCR. NPCILl gene fragment $(-1741 /+56)$ was amplified from human genomic DNA and cloned into pGL2 vector upstream of luciferase reporter gene. Transient transfection and luciferase assay techniques were employed to assess NPCILl promoter activity. Our results revealed that $24 \mathrm{~h}$ incubation with 5 micro $\mathrm{g} / \mathrm{ml}$ concentration of 25 -hydroxycholseterol significantly decreased the relative abundance of NPClLl mRNA in Caco 2 cells by $56.4 \pm 0.5 \%$ compared to cells treated with vehicle alone. The inhibitory effect of 25-hydroxycholesterol may be mediated by inactivating Sterol Regulatory Element-Binding Protein (SREBP) transcription factor. Therefore, we examined the effect of SREBP activation by depletion of cellular cholesterol on NPCILl expression. Activation of SREBP by incubation with 40 micro M Mevinolin (inhibitor of HMG-CoA reductase) for $24 \mathrm{~h}$ led to a significant increase ( 2 fold) in the relative level of NPC1L1 mRNA compared to vehicle treated control. Similarly, NPC1L1 promoter activity $(-1741 /+56)$ was significantly inhibited $(40.5 \pm 4.5 \%)$ by incubation with 25-hydroxycholesterol and significantly stimulated ( 2 fold) in response to Mevinolin. Sequence analysis of the promoter region revealed a potential binding site for SREBP in a region between nucleotide -506 and -512 . Interestingly, the activity of NPClLl promoter fragment $(-291 /+56)$ lacking SREBP potential biding site was unaffected by 25 -hydroxycholesterol or Mevinolin treatment. Conclusion: Our studies, for the first time, demonstrate the modulation of human NPClLl expression and promoter activity by cholesterol in human intestinal Caco 2 cells. Our findings suggest that NPC1Ll regulation by cholesterol is mediated by SREBP-dependent mechanism.

\section{3}

Evidence for the Association of Apical Ileal Bile Acid Transporter (ASBT) with Lipid Rafts

Waddah A. Alrefai, Zaheer Sarwar, Fadi Annaba, Seema Saksena, Alka Dwivedi, Pradeep K. Dudeja, Ravinder K. Gill

Apical Sodium-Dependent Bile Acid Transporter (ASBT) is responsible for active intestinal absorption of bile acids in distal ileum. Earlier studies demonstrated the involvement of membrane trafficking in the acute modulation of ASBT function. Recent evidence suggests that targeting of membrane transporters to specialized membrane domains such as lipid rafts is crucial for their optimal function. Lipid rafts are cholesterol and sphingolipids rich and resistant to solubilization by detergents. Whether ASBT is present in lipid rafts of plasma membrane is not known. Therefore, the current studies were undertaken to examine the association of ASBT with lipid rafts utilizing Western blotting and Immuno-fluorescent studies. Purified apical membranes (BBMs) were isolated from human ileal mucosa obtained form organ donor intestine. Lipid rats were isolated from Triton-X 100 treated human ileal BBMs based on their low buoyancy and floatation on a discontinuous Optiprep density gradient. Our data showed that ASBT in ileal BBM was detected in the low buoyancy fractions, which were also enriched with alkaline phosphatase, a marker for lipid rafts. hASBT was also detected to some extent in all other fractions of the gradient indicating that only a subpopulation of the transporter on the BBM is associated with lipid rafts. We further investigated the co-localization of ASBT with ganglioside GMl a marker for lipid rafts, in human intestinal $\mathrm{Caco} 2$ cell line, utilizing fluorescent $\mathrm{CtxB}$ which binds to GM1. Confocal microscopy studies showed hASBT protein was primarily localized in the apical membrane and was colocalized with ganglioside GMl-bound to CtxB. Interestingly, depletion of plasma membrane cholesterol by incubation with Methyl $\leq$-cyclodextrin $(\mathrm{M} \leq \mathrm{CD})$ disrupted the association of ASBT with GMl-bound CtxB. Furthermore, treatment with $\mathrm{M} \leq \mathrm{CD}$ decreased Na+-dependent 3H-TC uptake in Caco2 cells and HEK 293 cells expressing ASBT. The inhibition of ASBT function by $\mathrm{M} \leq \mathrm{CD}$ was blocked in the presence of cholesterol and appears to be mediated by a decrease in the Vmax of the transporter. Conclusion: Our studies provide direct evidence for the association of ileal hASBT with multiple pools of BBM of intestinal epithelial cells including lipid rafts and demonstrate that cholesterol content of lipid rafts is essential for the optimal activity of hASBT. Since the luminal bile acid content constantly varies between meals, it is expected that ileal ASBT undergoes rapid adaptive changes to the varying intestinal milieu. These findings suggest a novel mechanism by which hASBT activity is rapidly modulated by alterations in cholesterol content of plasma membrane lipid rafts.
NHE3 Has Restricted Brush Border (BB) Mobility Due to Cytoskeletal Association Which Is Dynamic As Part of Acute Stimulation and Inhibition Boyoung Cha, Anne Kenworthy, Ming Tse, Mark Donowitz

The epithelial BB Na/H exchanger NHE3 is associated with the actin cytoskeleton by binding separately directly to ezrin and indirectly to ezrin via attachment to NHERF family proteins. NHE3 point mutants can separately eliminate the direct (F1) and indirect (F2) ezrin binding. NHE3 mobility in polarized epithelial cell BB is restricted by cytoskeleton binding such that only $30 \%$ of NHE3 in the renal proximal tubule OK cell line is mobile as judged by fluorescence recovery after photobleaching (FRAP) using confocal microscopy to examine the BB. Given that acute NHE3 regulation is partially by changes in trafficking, the hypothesis tested in this study was that the cytoskeleton association of NHE3 was dynamic and changed as part of acute regulation. OK cells stably expressing NHERF2 (to mimic the small intestinal and proximal tubule distribution of NHERF family proteins) had NHE3 acutely stimulated by exposure to LPA(100uM) or inhibited by exposure to serotonin (5HT)(30 M) (both partially act by altering the amount of BB NHE3, with $5 \mathrm{HT}$ acting by elevating $\mathrm{Ca} 2+$ ). With both LPA and 5HT, the BB NHE3 mobile fraction (measured by FRAP/confocal microscopy) rapidly increased to $70 \%$ in a time dependent manner, with maximum mobility $30 \mathrm{~min}$ after ligand exposure and returned to basal mobile fraction by $45 \mathrm{~min}$. Neither LPA nor 5 HT altered NHE3 activity in OK/NHERF2/F2 mutant NHE3 (which does not associate with NHERF proteins). Under these conditions, there were no LPA or $5 \mathrm{HT}$ induced changes in NHE3 mobility. In contrast, LPA caused a normal increase and recovery of NHE3 mobility in the OK/NHERF2/F1 mutant NHE3 (which does not directly bind ezrin). In conclusion: 1) Restricted BB mobility of NHE3 due to cytoskeleton association is dynamic and is increased as part of both acute stimulation and inhibition, which change the amount of BB NHE3. 2) We suggest that acute stimulation of NHE3 by LPA occurs via addition of NHE3 to the $\mathrm{BB}$ and that this addition of NHE3 precedes binding of NHE3 to the actin cytoskeleton via NHERF/ezrin. 3) Acute inhibition of NHE3 by 5HT, which is associated with acute Ca2+ dependent removal of NHE3 from the BB, is associated with an increase in BB mobility of NHE3, although it is not known whether the changes in mobility proceed or follow changes in NHE3 complex formation. These results identify dynamic steps in cytoskeleton involvement with NHE3 regulation which could be manipulated to stimulate or inhibit Na absorption

685

Enteropathogenic E. Coli (EPEC) Infection Reduces Surface Expression of down regulated in adenoma (DRA) Protein in Caco-2 Cells Ravinder K. Gill, Alip Borthakur, Kim Hodges, Jerrold R. Turner, Seema Saksena, Krishnamurthy Ramaswamy, Gail A. Hecht, Pradeep K. Dudeja

Enteropathogenic E. coli (EPEC), an important food-borne enteric pathogen is a common cause of infantile diarrhea. EPEC is neither invasive nor toxigenic but expresses a type III secretion system (TTSS) that allows it to deliver virulence proteins (E. coli secreted proteins; Esp) directly into host cells. We previously demonstrated that alterations in luminal ion absorptive transport processes contribute to the pathophysiology of EPEC-induced diarrhea. Specifically, EPEC infection decreased apical $\mathrm{Cl} / \mathrm{OH}$ - exchange activity in human intestinal epithelial cells via the involvement of TTSS and the effector molecules, EspG and Orf3 (EspG homolog). However, the direct effect of EPEC on anion exchangers responsible for intestinal apical $\mathrm{Cl}-/ \mathrm{OH}-(\mathrm{HCO} 3-)$ exchange process is not known. Increasing evidence suggests the involvement of both SLC26A3 (DRA), (mutated in Congenital Chloride Diarrhea) and SLC26A6 (Putative anion transporter, PAT-1) in mediating luminal $\mathrm{Cl}-/ \mathrm{HCO}-(\mathrm{OH}-)$ exchange in the intestine. Therefore, the current studies were undertaken to ascertain the role of DRA and PAT-1 in the effects of EPEC. Human colonic adenocarcinoma Caco-2 monolayers were infected with EPEC or specific mutants for $30 \mathrm{~min}$ and DRA or PAT-1 protein expression was analyzed by biochemical and confocal microscopic studies. Cell surface biotinylation studies demonstrated that the surface expression of PAT-1 did not change in response to EPEC infection. However, expression of DRA on the plasma membrane was considerably reduced ( $70 \%)$ in EPEC-infected cells, corresponding with decreased Cl-/ $\mathrm{OH}$ - exchange activity. Total cellular DRA was not altered by EPEC infection. Neither nonpathogenic E. coli nor an escN mutant (non-functional TTSS) altered the surface expression of DRA. Confocal immunofluorescence studies showed that DRA protein was predominantly localized to the apical surface of cells infected with non-pathogenic E. coli, however, infection with EPEC caused a marked re-distribution of DRA from the apical membrane to intracellular compartments. Interestingly, deletion of espG, involved in disruption of microtubules, significantly attenuated the removal of DRA protein from the apical membrane as compared to wild type EPEC ( $40 \%$ ). Conclusion: Our data demonstrate that reduction in surface expression of DRA is TTSS-dependent and involves EspG. Alteration in the level of DRA expression at the apical membrane by EPEC demonstrates a novel mechanism for acute modulation of DRA and indicates its regulation by membrane recycling events.

686

Ileal Apical Sodium-Dependent Bile Acid Transporter Activity Is Inhibited By Enteropathogenic E. Coli (EPEC) Infection

Zaheer Sarwar, Ravinder K. Gill, Alip Borthakur, Krishnamurthy Ramaswamy, Pradeep K. Dudeja, Gail A. Hecht, Waddah A. Alrefai

EPEC is a food-borne enteric pathogen and a major cause of diarrhea worldwide. EPEC is non-invasive and non-toxigenic but expresses a type III secretion system (TTSS) through which bacterial virulence proteins are translocated into host cells. The pathophysiology of EPEC-induced diarrhea is multi-factorial. Recent studies showed that EPEC infection modulates intestinal $\mathrm{Na}+, \mathrm{Cl}$ - and short chain fatty acid ion transport processes in human intestinal epithelial cells. In this regard, Apical Sodium-Dependent Bile Acid Transporter (ASBT), responsible for the absorption of the majority of bile acids from the intestinal lumen, has been implicated in diarrhea associated with inflammatory diseases. However, the effect of pathogenic microorganisms that induce diarrhea, such as EPEC, on ASBT activity is not 
known. Therefore, current studies were undertaken to investigate the short-term effects of EPEC infection on ileal ASBT function utilizing the human intestinal Caco2 cell line as a model epithelia. ASBT activity was assessed by the measurement of $\mathrm{Na}$-dependent $3 \mathrm{H}$ taurocholic (TC) luminal uptake. ASBT protein expression was evaluated by immunofluorescence studies. Sodium-dependent 3H-TC uptake in Caco2 cells was significantly inhibited $(64 \pm 1.5 \%)$ following 30 min infection with EPEC but not with non-pathogenic E. coli. Mutation of escN, the gene that encodes the ATPase for TTSS rendering it ineffective, ablated the inhibitory effect on 3H-TC uptake. Also, mutations in genes (espA, espB and espD) encoding proteins of TTSS terminal structure abrogated the observed inhibitory effect of the infection. The inhibition by EPEC was associated with a significant decrease in the Vmax (135 \pm 23 versus $74 \pm 17 \mathrm{pmol} / \mathrm{mg}$ protein $/ 5 \mathrm{~min}$ ) with no changes in $\mathrm{Km}$, suggesting a potential reduction in the luminal membrane ASBT content. Immunofluorescence confocal microscopic studies showed that ASBT localized predominantly to the apical membrane of uninfected cells. EPEC infection, however, significantly decreased the level of ASBT on the apical surface by internalization into intracellular compartments. Conclusion: Our study provides novel evidence that EPEC infection inhibits ASBT activity in a TTSS-dependent manner that results in the removal of ASBT from the luminal surface of intestinal epithelial cells

687

ACE Inhibitor Therapy Is Associated with a Decreased Incidence of Colorectal Cancer

George Makar, James D. Lewis, Yu-Xiao Yang

BACKGROUND Laboratory data suggest that increased angiotensin II may promote the growth of colorectal cancer (CRC) by inducing neovascularization and IGF-1 receptor expression, while angiotensin converting enzyme inhibitors (ACE-I) enhance the antitumor effect of COX-2 inhibition through inhibition of the IGF-1 pathway. Based on these observations, we sought to determine whether ACE-I therapy reduced the risk of CRC in a populationbased cohort. METHODS We conducted a nested case-control study within a cohort of hypertensive patients in the General Practice Research Database (United Kingdom). Cases were subjects with a new diagnosis of CRC. Using incidence density sampling, up to 10 controls per case were selected, matched on age, sex, calendar year and duration of followup. Patients with $<1$ yr of CRC-free follow-up after diagnosis of hypertension and CRC patients with $<1$ yr of ACE-I therapy before their cancer diagnosis were excluded. Conditional logistic regression was used to calculate Odds Ratio's (ORs) for CRC associated with exposure to ACE-I therapy. The primary exposure duration of interest was $\geq 3$ years. We also assessed the effect of escalating durations of exposure. A priori potential confounders adjusted in the model included duration of follow-up after hypertension, number of physician visits within 1 year of index date, BMI, smoking, aspirin/NSAIDs, hormone replacement therapy, folate, calcium, statins, and insulin. A priori testing for effect modification among diabetics was performed, since hyperinsulinemia among diabetics has been shown to increase the risk of colorectal cancer primarily via the IGF-1 system. RESULTS(Table) 1,988 cases were matched with 18,131 controls. Greater than 3 years of ACE-I therapy was associated with a reduction in CRC risk (OR 0.79 (95\% CI, 0.64 -0.96)); a significant duration-response effect was present (Test for trend: $\mathrm{p}=0.02$ ). Larger effects were observed among patients with diabetes (LR test for interaction: $\mathrm{p}=0.06$ ). CONCLUSION Long-term ACE-I therapy was associated with a decreased risk of CRC in this cohort. The magnitude of this effect was greater in diabetics.

Duration of ACE-I Therapy and OR of CRC

\begin{tabular}{|c|c|}
\hline ALL PATIENTS(n=20,119) & ADJUSTED OR \\
\hline $1-3 \mathrm{yrs}$ & $0.99(0.85-1.16)$ \\
\hline $3-5 \mathrm{yrs}$ & $0.87(0.70-1.10)$ \\
\hline$\geq 5 \mathrm{yrs}$ & $0.60(0.41-0.87)$ \\
\hline \multicolumn{2}{|c|}{ DIABETICS(n=771) } \\
\hline $1-3 \mathrm{yrs}$ & $0.72(0.38-1.37)$ \\
\hline$\geq 3 \mathrm{yrs}$ & $0.28(0.10-0.81)$ \\
\hline \multicolumn{2}{|c|}{ NONDIABETICS $(\mathrm{n}=19,348)$} \\
\hline $1-3 \mathrm{yrs}$ & $1.01(0.87-1.19)$ \\
\hline $23 \mathrm{yrs}$ & $0.83(0.68-1.02)$ \\
\hline
\end{tabular}

688

Long-Term Aspirin Use and Mortality: Implications for Chemoprevention Andrew T. Chan, Joann E. Manson, Diane Feskanich, Meir J. Stampfer, Graham A. Colditz, Charles S. Fuchs

Randomized trials have shown that aspirin reduces risk of colorectal adenoma. However, the use of aspirin for widespread chemoprevention has been hindered by uncertainty over its risk-benefit profile. One measure of the overall benefit of aspirin is the relation between long-term aspirin use and total mortality. Methods: We conducted a prospective, nested case-control study of 75,816 women enrolled in the Nurses' Health Study to determine the association between aspirin use and mortality. Women provided data biennially since 1980 and were followed through 2004. Each participant who died was matched with 7 controls alive at the time of her death. For each death, aspirin use was assessed prior to initial diagnosis of cardiovascular disease (CVD) or cancer and during the corresponding time period for matched controls. Results: Over 24 years, we documented 9,477 total deaths. Among women who reported current use of aspirin, the multivariate relative risk (RR) of death from all causes was 0.75 (95\% CI, 0.71-0.81) compared to women who never used. The risk reduction was more apparent for CVD death (RR 0.62; CI, 0.54-0.71) than for cancer death (RR 0.88; CI, 0.81-0.96). The lower risk of cancer death among current users was significant only for colorectal cancer death (RR 0.72; CI, 0.56-0.92). We observed a significant linear relationship between increasing duration of use and decreasing all-cause mortality (Ptrend<.0001). However, for CVD death, much of the benefit was achieved within the first 5 years of use (Ptrend=0.14). In contrast, for all cancer-related mortality, significan risk reduction was not apparent until at least 10 years of use (Ptrend=0.005). The stronges effect of long-term use was observed with colorectal cancer death, with a RR of $0.60(\mathrm{CI}$ $0.41-0.90)$ for $>20$ years (Ptrend=0.02). The benefit associated with aspirin was confined to low and moderate doses. However, we did observe a relationship between increasing dose and lower risk of colorectal cancer death among women who used aspirin $>10$ years ( $\mathrm{P}$ trend $=0.02$ ). Of note, we documented only 41 cases of death related primarily to gastrointestinal bleeding or peptic ulcer disease over the 24 years of follow-up. Conclusions: Aspirin use, at low to moderate doses, is associated with a reduced risk of all-cause mortality, largely due to death from cardiovascular disease. Although aspirin did appear to have a modest benefit against death from cancer, the effect was primarily confined to death from colorectal cancer, and required at least 10 years of use. These data support a need for continued study into long-term use of aspirin in prevention of chronic diseases.

\section{9}

Daily Soluble Aspirin and Prevention of Colorectal Adenoma Recurrence Four Years Results of the APACC Trial

Robert Benamouzig, Jacques Deyra, Antoine Martin, Baktiar Bejou, Jean-Jacques Raynaud, Bernard Girard, Stanislas Chaussade

Aspirin is one of the most promising candidate drugs for colorectal cancer chemoprevention Many case control, cohort and two prospective studies suggested an aspirin protective effect. To clarify this effect, we conducted a prospective, randomized, double-blind, placebocontrolled, multicenter trial (the APACC Study) involving 49 centers in France to compare the efficacy of aspirin as lysine acetylsalicylate 160 or $300 \mathrm{mg} / \mathrm{d}$ vs. placebo on the recurrence of the colorectal adenomas at one and 4 years. A protective effect was previously showed at one year. However the optimal dose and the duration of this chemoprevention remain discussed. We report here the final results at 4 years. Subjects were aged between 18 to 75 years and had undergone a complete colonoscopy with removal of at least one adenoma of more than $6 \mathrm{~mm}$ or at least 3 adenomas irrespective of size. Patients with an history of colorectal cancer, FAP, inflammatory bowel disease and the women likely to be pregnant were excluded. In order to evaluate the tolerance and compliance with the treatment, the 291 eligible patients received $300 \mathrm{mg}$ of lysine acetylsalicylate daily during a 4-week runin period. After this period, 272 patients were randomized: 188 men and 84 women, 58 years, with at least one adenoma of more than $10 \mathrm{~mm}$ in 180 cases and more than 3 adenomas in 90 cases, a personal history of adenoma in 64 cases and a family history of colorectal cancer in 94 cases. After one and 4 years of treatment, 238 and 176 subjects (90 and $65 \%$ of randomized) underwent colonoscopy respectively. The presence of at least one recurrent adenoma at 1 or 4 years was noted in 94 (53\%) of the 176 patients. Aspirin was associated with a reduction in the risk of recurrent adenomas at one year in term of subjects with at least one adenoma of more than $5 \mathrm{~mm}$ or multiple adenomas $(>3)(\mathrm{p}<0,005)$. Aspirin was associated only with lower multiplicity (RR of $0,28, p<0,02)$ at 4 years. This lowe effect observed at 4 years was not related to a lower statistical power related to lost of followup. The effect at one year seems more important with the 300-mg dose while the effect at 4 years seems more important with the lower dose suggesting different transduction pathways according to the aspirin dose. One possible hypothesis could be that the high dose had a direct anti-tumor effect, observed on the polyps left in place, and that low dose would be the "preventive" dose associated with an observed after several years of treatment as suggested by observational studies.

690

All Trans-Retinoic Acid (ATRA) Induces Apoptosis Via P38 and Caspase Pathways in Barrett's Esophagus

Kathy Hormi-Carver, Linda A. Feagins, Stuart J. Spechler, Rhonda F. Souza

Introduction: Retinoids have been used as chemopreventive agents for a number of premalignant conditions. Data suggest that all trans--retinoic acid (ATRA) is one of the safest and best tolerated retinoids and that it exerts pro-apoptotic effects. To explore a potential role for ATRA as a chemopreventive agent for Barrett's esophagus, we studied the effects of ATRA on apoptosis in a non-neoplastic, telomerase-immortalized, metaplastic, Barrett's epithelia cell line. Methods: Telomerase-immortalized metaplastic Barrett's epithelial cells, which are not transformed and which express intact $\mathrm{p} 53$, were grown to $70 \%$ confluence and placed in minimal media overnight. Cells were treated with 1 or $5 \mu \mathrm{M}$ ATRA in the presence and absence of inhibitors to p53 (pSRZ-siRNA-p53), p38 (SB-203580), and the caspase cascade (z-Val-Ala-Asp-fluoromethyl ketone). Twenty-four hours later, apoptosis was determined quantitatively by Annexin $\mathrm{V}$ and qualitatively by immunofluoresence with TUNEL and caspase-3. p53, p38, total p38, and caspase-3 expression were determined by Western blotting using $\beta$-actin as a loading control. Results: Treatment with ATRA significantly increased apoptosis as assessed by Annexin $\mathrm{V}$ in a dose dependent fashion $(0.27 \pm .06$ to $21.60 \pm 1.83 \%$ SEM and $46.12 \pm 6.74 \%$ SEM, respectively, $\mathrm{p}<0.01)$. This finding was confirmed by the finding of abundant apoptotic cells by TUNEL and caspase-3 staining ATRA increased the expression of $\mathrm{p} 53$ protein in a dose-dependent fashion, and also increased the phosphorylation of $\mathrm{p} 38$ and the expression of cleaved caspase-3. The apoptotic effect of ATRA was abolished by treatment with inhibitors of both p38 and the caspase cascade, but not by p53 RNAi. Inhibition of p38 also prevented expression of cleaved caspase-3 suggesting that ATRA activates p38 upstream of the caspase cascade. Conclusions: ATRA induces apoptosis in Barrett's epithelial cells via activation of $\mathrm{p} 38$ and the caspase cascade, but not through p53. This study elucidates molecular pathways whereby ATRA treatment might prevent carcinogenesis in Barrett's metaplasia, and provides the rationale for a clinical trial of ATRA for chemoprevention in Barrett's esophagus. 
A Randomized Controlled Trial of Long Term Rofecoxib On Changes of Gastric Intestinal Metaplasia

Wai K. Leung, Enders K. Ng, Francis K. Chan, Wing Y. Chan, Kui-Fat Chan, Alex C. Auyeung, Candice C. Lam, James Y. Lau, Joseph J. Sung

Background \& Aim: Gastric cancer and its precursor lesion, intestinal metaplasia (IM), frequently express cyclooxygenase-2 (COX-2) at high levels. We tested whether gastric IM would regress with long term use of specific COX-2 inhibitor. Methods: This was a doubleblind, randomized, placebo controlled trial. Individuals with histologically confirmed IM were randomized to receive rofecoxib $25 \mathrm{mg}$ daily or placebo for 2 years. H. pylori infection was eradicated in all patients prior to randomization. None of these patients took NSAID and aspirin. Endoscopy was performed at baseline, end of Year 1 and Year 2 with multiple biopsies taken from the antrum and corpus according to a standard protocol. The severity of histological changes was graded by the updated Sydney Classification (score 0-3). The primary endpoint was the proportion of patients with regression of IM (ie. score lower than baseline by 1 or more). The severity of IM and the proportion of subjects with complete regression of IM were regarded as secondary endpoints. Results: 213 subjects (mean age 51 years, $64 \%$ male) with confirmed IM were randomized: 107 to receive rofecoxib and 106 to placebo. The baseline characteristics and histological results of the two groups were comparable. At Year 2, the COX-2 expression level in the gastric epithelium was significantly lower in patients treated with rofecoxib $(\mathrm{P}=0.027)$. The proportion of subjects with regression of IM did not differ significantly between rofecoxib and placebo groups (antrum: $24.5 \%$ vs $26.9 \%, \mathrm{P}=0.74$; corpus: $4.3 \%$ vs $2.2 \%, \mathrm{P}=0.68$ ). $19.1 \%$ of patients on rofecoxib and $16.1 \%$ of patients on placebo had no IM detectable at the end of 2 -year $(P=0.59)$. There was no significant difference in the severity of IM (Table), acute or chronic inflammation and atrophy between the two groups. Conclusions: Gastric IM failed to regress after two years of treatment with rofecoxib. Our findings may cast doubt on the reversibility of gastric IM by COX-2 inhibition.

IM Scoring at Year 1 and 2

\begin{tabular}{|c|c|c|c|c|}
\hline & \multicolumn{2}{|c|}{ Antrum } & \multicolumn{2}{c|}{ Corpus } \\
\hline & Rofecoxib* $^{*}$ & Placebo* & Rofecoxib* $^{*}$ & Placebo* $^{*}$ \\
\hline Year 1 & $0.71 \pm 0.76$ & $0.72 \pm 0.73$ & $0.11 \pm 0.27$ & $0.12 \pm 0.36$ \\
\hline Year 2 & $0.72 \pm 0.67$ & $0.71 \pm 0.76$ & $0.10 \pm 0.28$ & $0.11 \pm 0.43$ \\
\hline
\end{tabular}

$* \mathrm{P}>0.25$ (rofecoxib vs placebo)

692

Polyphenon E Inhibits Growth of Malignant Human Esophageal Epithelial Cells By Downregulation of CREB and Cyclin D1

Shumei Song, Koyamangalath Krishnan, Kai-Feng Liu, Robert S. Bresalier

BACKGROUND: Green tea polyphenols including epigallocatechin gallate (EGCG) inhibit tumor formation induced by multiple carcinogens in rodent cancer models. Polyphenon E (Poly E) is a decaffeinated well-defined green tea catechin mixture that contains 50\% EGCG and is rapidly prepared. AIM: This study was designed to determine the effects of Poly E on growth of human esophageal epithelial cells and the mechanisms involved in growth regulation by this agent. METHODS: Human esophageal adenocarcinoma cell lines (SEG1, SKGT-4, BIC-1 and BE3) were used as model systems. MTT uptake assays and cell counts were used to monitor cell proliferation, and flow cytometry for cell cycle analyses. Western blots and immunocytochemistry were performed to determine protein expression and activation status (phosphorylation). Transient transfection of CREB and Cyclin D1 promoter constructs were used for promoter activation analyses. RESULTS: Treatment with Poly E potently inhibited the growth of four EA cells in a dose-dependent manner regardless of their p53 status and increased the percentage of cells in the G1 phase of the cell cycle. Poly E blocked transactivation of CREB promoter and phosphorylation of CREB induced by tumor promoters including the bile acids chenodeoxycholic acid and deoxycholic acid. In addition, Poly E significantly decreased cyclin Dl expression and induced hypophosphorylation of pRB in a dose-dependent manner, changes associated with Gl cell cycle arrest. Poly $\mathrm{E}$ also inhibited transcriptional activity of the cyclin Dl promoter. Furthermore, Poly Einduced down-regulation of cyclin Dl was blocked by lactacystin, an inhibitor of the $26 \mathrm{~S}$ proteosome, suggesting that Poly E also downregulates cyclin Dl expression by promoting proteolysis. CONCLUSIONS: The phytochemical polyphenon $\mathrm{E}$ inhibits growth of transformed human esophageal cells by deactivation of CREB transcription factor, and downregulation of cyclin Dl via both transcriptional and post-transcriptional mechanisms. These findings have implications for use of this compound in the prevention and treatment of esophageal adenocarcinoma.

708

Prospective Comparison of Routine Cytology (RC), Digital Image Analysis (DIA), and Fluorescence in Situ Hybridization (FISH) in Patients Undergoing Endoscopic Ultrasound Guided Fine Needle Aspiration (EUS FNA)

Michael J. Levy, Jonathan E. Clain, Amy C. Clayton, Kevin C. Halling, Gavin C. Harewood, Benjamin R. Kipp, Elizabeth Rajan, Lewis R. Roberts, Thomas J. Sebo, Mark D. Topazian, Kenneth K. Wang, Maurits J. Wiersema, Gregory J. Gores

Background: Prior studies indicate enhanced diagnostic accuracy for DIA and FISH versus $\mathrm{RC}$ when evaluating biliary strictures. These tumor markers have never been applied to EUS FNA specimens. We hypothesize that these molecular markers incorporate generic targets capable of identifying a broad spectrum of malignancy. Aim: To compare the diagnostic accuracy of DIA and FISH to RC for a broad spectrum of pathologies sampled during EUS FNA. Methods: We prospectively evaluated pts with known or suspected malignancy by RC, DIA, and FISH. For clinical purposes, presence of malignancy by either DIA or FISH
(DIA/FISH) was considered diagnostic. However for study purposes separate confirmation of malignancy was required for diagnosis. Pts with benign disease were followed at least 12 months and charts were reviewed to determine the accuracy. Results: 39 pts were enrolled and each diagnostic test performed upon samples collected from 42 sites to evaluate lymphadenopathy $(n=19)$, solid pancreatic mass $(n=12)$, cystic pancreatic lesion $(n=7)$, esophageal or gastric wall mass $(n=3)$, and thyroid mass $(n=1)$. Malignancy was detected in 30/42 pts, including esophageal squamous cell carcinoma, esophageal adenocarcinoma, gastric adenocarcinoma, pancreatic adenocarcinoma, pancreatic mucinous cystic neoplasia, intraductal papillary mucinous neoplasia, metastatic forearm sarcoma, small cell lung cancer, non small cell lung cancer, thyroid follicular carcinoma, malignant gastrointestinal stromal tumor, melanoma, adenocarcinoma of unknown primary, and lymphoma. The sensitivity, specificity, and accuracy of DIA/FISH versus RC for detection of malignancy were $97 \%, 100 \%, 98 \%$ versus $87 \%, 100 \%, 90 \%$ respectively. (Table 1 ) No false positive results occurred for DIA or FISH. The one failed diagnosis for DIA/FISH was in a patient with a malignant gastrointestinal stromal tumor. DIA/FISH correctly identified dysplasia or malignancy in 5 pts with cytology interpreted as benign, atypical, or suspicious. The final diagnosis in these pts was pancreatic adenocarcinoma $(n=3)$, esophageal squamous cell carcinoma $(n=1)$, and IPMN $(n=1)$. No complications were identified. Conclusion: Our findings suggest that in pts undergoing EUS FNA that DIA and FISH have a higher diagnostic accuracy than RC. These data suggest that these tumor markers incorporate generic targets as suggested by the high diagnostic sensitivity in this cohort of pts with diverse pathologies. Table 1:

\begin{tabular}{|c|c|c|}
\hline & DIA/FISH & RC \\
\hline Sensitivity & $97 \%$ & $87 \%$ \\
\hline Specificity & $100 \%$ & $100 \%$ \\
\hline Accuracy & $98 \%$ & $90 \%$ \\
\hline
\end{tabular}

709

Screening Colonoscopy with Confocal Laser Endomicroscopy (CLE) for in Vivo Diagnosis of Colorectal Neoplasias

Ralf Kiesslich, Martin Goetz, Arthur Hoffman, Katharina Lammersdorf, Constantin Schneider, Michael Vieth, Manfred Stolte, Peter R. Galle, Markus F. Neurath

Introduction: Confocal laser endomicroscopy (CLE) allows subsurface, microscopic imaging of living cells in colonic tissue in vivo. The aim of the present study was to assess the potential of in vivo confocal laser colonoscopy for prediction of histology during screening colonoscopy for colorectal cancer. Methods: Patients with informed consent designated for screening colonoscopy were enrolled in the study and underwent total colonoscopy with CLE (Optiscan, Australia; Pentax, Japan; excitation of 488nm argon ion laser; detection $>515 \mathrm{~nm}$; optical slice thickness 7 um; lateral resolution 0.7 um; frame rate 0.8 or 1.6 frames/ sec with $1024 \times 1024$ or $1024 \times 512$ pixels). After reaching the cecum, a fluorescent contrast agent (fluorescein) was administered intravenously. During withdrawal of the endoscope, standardized locations (right, transverse colon, rectum) and every circumscript lesion was examined by using the confocal microscope (fluorescence technique), afterwards biopsies were taken. Confocal images were graded according to the confocal pattern classification for the presence of neoplastic changes and compared with final histology. Results: 280 patients were included in the study. 112 colonic lesions (34 non-neoplastic, 65 adenomas, 9 adenomas with high-grade dysplasia, 4 cancers) larger than $5 \mathrm{~mm}$ could be endoscopically diagnosed. By the use of CLE, different cellular structures, capillaries and connective tissue limited to the mucosal layer could be identified. Neoplasia could be prospectively predicted by the use of confocal pattern classification with high accuracy (Sensitivity $98.7 \%$; Specificity; 94.1\%; Accuracy: 97.3\%). All patients developed a transient discoloration of the skin after fluorescein application. However, no further side effects were noted. Conclusions: In this largest prospective study on CLE carried out so far, confocal laser endomicroscopy (CLE) emerged as a safe and well tolerated additional diagnostic option for screening colonoscopy. Endoscopically identified colonic lesions can be microscopically graduated during ongoing colonoscopy and neoplasias can be predicted with high accuracy. Thus, in vivo histology can be used to target endoscopic therapy or biopsies.

710

Spectroscopic Microvascular Assessment from the Endoscopically Normal Mucosa for Colon Adenoma Identification

Hemant Roy, Yang Liu, Young Kim, Michael Goldberg, Nahla Hasabou, Vladimir Turzhitsky, Mohammed Jameel, Eric Elton, Vadim Backman

Developing technology to decrease polyp miss rate(estimated to be $15 \%$ in expert hands)is of great importance from both a clinical and medicolegal perspective. Members of our group have been pioneered light scattering technologies for diagnosis of dysplastic lesions (Nature 2000, Nature Med 2001). We have recently developed four dimensional elastic light scattering fingerprinting (4D-ELF) (Gastro 2004), which allows unprecedented ability to quantitatively probe the mucosal micro-circulation. Using 4D-ELF, we have reported that microvascular blood content was increased in the histologically normal colonic mucosa in experimental CRC models(Gut 2005). In the present study, we assessed whether increased microvascular blood could aid in localization of colonic neoplasia. METHODS One hundred subjects undergoing colonoscopy were recruited and had two biopsies taken from the in the endoscopically-normal mucosa (at least $5 \mathrm{~cm}$ from any adenomas) cecum, mid-transverse colon and rectum. Fresh biopsies were subjected to 4D-ELF analysis Microvascular (within $50 \mu \mathrm{M}$ of tissue surface) were analyzed by an investigator blinded to colonoscopic findings. RESULTS As demonstrated in the figure, microvascular blood content was demonstrated to be markedly elevated in the endoscopically normal mucosa of patients who harbored adenomas in the respective colonic segment (right colon, transverse or left colon). The sensitivity, specificity, positive and negative predictive value of increased microvascular blood was $89 \%, 100 \%$, $100 \%$ and $75 \%$, respectively. CONCLUSIONS: We demonstrate, for the first time, that microvascular blood content in the endoscopically normal mucosa could identify patients 
at risk for colonic neoplasia and aid in localization. In the future, we envision that this approach (with a probe) could determine which colonic segments warrant extra endoscopic scrutiny (including possibly chromoendoscopy)

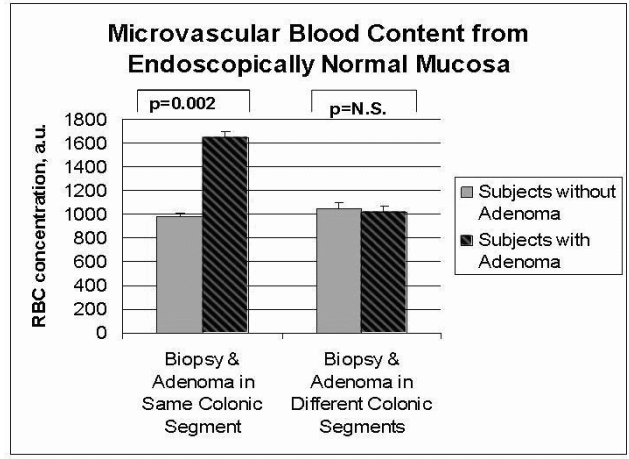

711

Image Registered Gastroscopic Ultrasound (IRGUS): 3D Registration of Endoscopic Ultrasound and CT Improves Efficiency and Structure Identification Over Standard Endoscopic Ultrasound

Christopher C. Thompson, Nicholas Stylopoulos, Raul San-Jose Estepar, Eigil Samset, Randy Ellis, William Brugge, Kirby G. Vosburgh

INTRODUCTION Although Endoscopic Ultrasound (EUS) is a useful tool for the diagnosis and staging of abdominal and thoracic diseases, it has not been maximally adopted by gastroenterologists. This is due in part to the long learning curve and the lack of confidence in ultrasound interpretation. To overcome these hurdles, we have designed and implemented IRGUS, a novel system that links preprocedure CT with real-time EUS imaging. MATERIAL AND METHODS IRGUS aligns preprocedure CT images with real time EUS imaging so that the CT image corresponds to the oblique EUS plane. The operators are also provided with a novel 3D display and important spatial cues that show them how the plane of the EUS is oriented relative to the patient's anatomy. In this study, novice and expert gastroenterologists were asked to perform an in vivo EUS examination of anesthetized pigs, identifying 8 key anatomic structures in a 5-minute period using traditional EUS and IRGUS. A sensor that was mounted on the echoendoscope allowed the tracking and recording of the motion of the scope during the task. These recordings were then used to calculate the efficiency of performing the exam, which is based on a set of kinematic parameters that we have used and validated in previous studies. At the conclusion of the tasks, participants completed a questionnaire. RESULTS Using conventional EUS, novices identified 29\% of the structures and experts $50 \%$, within the allotted time. Using IRGUS these rates increased significantly to $71 \%$ and $80 \%$ respectively $(\mathrm{P}<0.0001)$. IRGUS had the largest impact on the localization and identification of the right lobe of the liver and the right kidney. IRGUS also had a statistically significant benefit in assisting novices to identify the pancreatic body and tail. In the questionnaire, all participants rated IRGUS as superior to EUS. The analysis of kinematic data showed that IRGUS increased efficiency of conventional EUS by improving the economy of movements (path length), the smoothness of motion and the response orientation of the operator. CONCLUSIONS IRGUS is superior to conventional EUS in the localization and identification of anatomic structures and in various kinematic measures. It enhances procedural efficiency of both experts and novices and allows direct CT confirmation of structures and lesions. This novel technology has the capability of expanding the applications of conventional EUS and may allow for broader adoption beyond its current use. Portions of this work are sponsored by the US Department of the Army. The information does not necessarily reflect the position of the government and no official endorsement should be inferred.

\section{2}

Comprehensive Three-dimensional Microscopy of the Porcine and Human Esophagus in Vivo Using Optical Frequency Domain Imaging Melissa Suter, Benjamin J. Vakoc, Norman S. Nishioka, Jerry Evans, Seok H. Yun, Seyedmohammadreza Motaghiannezam, Adrien Desjardins, Brett E. Bouma, Guillermo J. Tearney

Current assessment and diagnosis of patients with Barrett's esophagus involves endoscopy accompanied by the collection of multiple random biopsies. This form of assessment is however invasive and susceptible to sampling errors highlighting the need for improved evaluation techniques. Optical coherence tomography (OCT) has been shown to provide high-resolution cross-sectional images of the esophageal microstructure allowing for accurate differentiation of dysplasia and SIM in patients with Barrett's esophagus. One drawback to traditional OCT is that it is generally restricted to point sampling techniques due to limitations in imaging speed and catheter design. Optical frequency domain imaging (OFDI) is a new imaging technology that is based on frequency domain interferometry and is capable of improving imaging speed over traditional OCT techniques by orders of magnitude without sacrificing image quality. We have developed a catheter based OFDI system that acquires comprehensive three-dimensional microscopic images of the entire distal esophagus. Both porcine and human in vivo studies were performed with the developed OFDI catheter based system. Complete volumetric imaging was performed in $5 \mathrm{~cm}$ longitudinal segments and was consequently analyzed and correlated with histopathology. We anticipate that the introduction of comprehensive volumetric microscopy of the distal esophagus into clinical practice will significantly improve the accuracy of current screening and surveillance methods in patients with Barrett's esophagus and will in turn lead to earlier detection of malignancy.
As the survival rate of esophageal adenocarcinoma is stage dependent, earlier detection may help to significantly reduce the current mortality rate associated with the disease.

713

The Aer-O-Scope ${ }^{\mathrm{TM}}$ Omnivision System, a $360^{\circ}$ Panoramic View of the Colon Douglas Rex, Thomas Roesch, Bernard Levin, Jorje Pfefer, Nadir Arber

The Aer-O-Scope ${ }^{\mathrm{TM}}$ (GI View, Ramat Gan, Israel) is a disposable, miniaturized, self-propelling, self-navigating, endoscope. In a recent human study the cecum was reached in $10 \mathrm{o}$ 12 patients (Vucelic et al, Gastro 2006). The Aer-O-Scope ${ }^{\mathrm{TM}}$ includes an advanced vision system with two simultaneous scanning views that provide complete coverage of the entire surface of the colon. A standard front view system $\left(90^{\circ}\right.$ forward view) is similar to conventional endoscope, while a novel and unique imaging system provides a $360^{\circ}$ panoramic view of the colonic surface, both in front and behind the scanner device (the OmniView) [Fig. 1]. The OmniView mechanism enables detailed inspection of the entire colon surface area, including those areas behind mucosal folds where polyps may be missed. The electrooptical capsule size is $15 \mathrm{~mm}$ OD x $1.5 \mathrm{~mm}$ long containing a CMOS based digital camera Its spatial resolution is greater than $1 \mathrm{~mm}$ within the FOV and the depth of view is from contact up to $\sim 100 \mathrm{~mm}$ which is adequate for all segments of the insufflated colon. The tip is embedded within a low pressure hydrophilic coated vehicle balloon. This balloon has two functions: it is the moving part of the Aer-O-scope, propelled by gas pressure and it also serves as the imaging center of the device. The capsule is covered by a transparen dome containing the optical lenses protruding from the balloon's front end. White LED's with automatic intensity control provide illumination. The OmniVision system was tested in thirty young female pigs. In all of the Aer-O-scope examinations clear and sharp visualization of the colonic mucosa, similar to that obtained during conventional colonoscopy, was achieved. High resolution images from the digital video camera were received, processed and displayed in real time on a PC screen and digitally recorded.

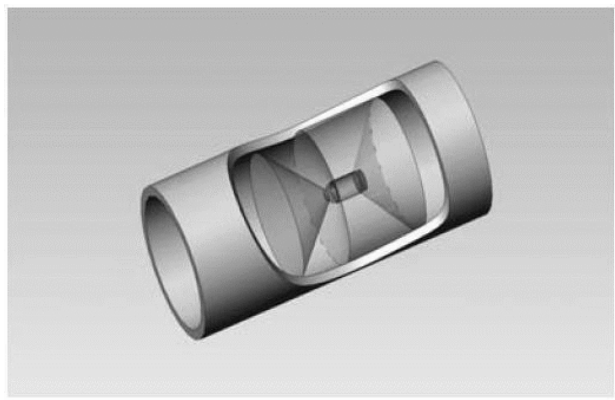

Fig. 1: Simultaneous Frontal and OmniVision Fields of View [FOV]

726

Endothelin-Converting Enzyme 1 (ECE1) Degrades Substance P (SP) and Calcitonin Gene-Related Peptide (CGRP) in Early Endosomes and Thereby Determines the Rate of Receptor Recycling and Resensitization

Graeme S. Cottrell, D.R. Roosterman, Benjamin E. Padilla, Stella Pikios, Laurent Muller Martin Steinhoff, Nigel W. Bunnett

$\beta$-arrestins interact with G-protein coupled receptors at the plasma membrane to mediate desensitization of G-protein signaling and clathrin-dependent receptor endocytosis. However, little is known about the mechanisms that control receptor recycling and resensitization Although neprilysin degrades peptides at the cell-surface to terminate signaling, the role of intracellular peptidases in controlling receptor signaling has not been studied. We hypothesized that ECEl degrades peptides in endosomes and thereby accelerates dissociation of $\beta$ arrestins from receptors to promote recycling and resensitization. We expressed ECEla-dGFP in KNRK cells expressing the neurokinin 1 receptor $\left(\mathrm{NK}_{1} \mathrm{R}\right)$, the receptor for $\mathrm{SP}$, and in HEK cells expressing calcitonin receptor like receptor (CLR) and receptor activity modifying protein 1 (RAMP1), the receptor for CGRP. ECEl isozymes were detected in vesicles that colocalized with early endosomal antigen 1 . When KNRK-NK ${ }_{1} \mathrm{R}-\mathrm{ECEl}$ cells were incubated with Alexa-SP for 30 min at $37^{\circ} \mathrm{C}$, both $\mathrm{NK}_{1} \mathrm{R}$ and SP were detected in early endosomes containing ECEl. Similarly, when HEK-CLR-RAMPl-ECEl cells were incubated with CGRP for $30 \mathrm{~min}, \mathrm{CLR}$ and RAMPl were detected in endosomes containing ECEl. To determine if ECEl degrades SP and CGRP in endosomes, we incubated membranes (5-20 $\mu$ g protein) with SP and CGRP $(25 \mu \mathrm{M})$ at the pH of early endosomes (5.5), and analyzed metabolites by high-pressure liquid chromatography. Membranes from cells expressing ECEl rapidly degraded SP and CGRP, whereas membranes from cells expressing control vector only slowly cleaved these peptides. We exposed cells to SP or CGRP (10 nmol, $10 \mathrm{~min})$, washed and then examined the rate of resensitization of $\mathrm{Ca}^{2+}$ signaling to a second challenge with the same agonist. In KNRK-NK ${ }_{1}$ R cells, resensitization to SP after 30 min was greater in ECEl cells compared to control cells. Similarly expression of ECEl in HEK-CLR-RAMPl cells also accelerated resensitization to second challenge of CGRP. To determine if peptide degradation promoted dissociation of $\beta$-arrestins, which would release receptors for recycling, we localized receptors and $\beta$-arrestins. In vector control cells, SP and CGRP induced the prolonged sequestration of $\beta$-arrestin 1 and 2 in endosomes containing $N_{1} R$ or CLR-RAMPl. Overexpression of ECEl accelerated the dissociation of $\beta$-arrestins from receptors, thereby promoting recycling. Thus, we report a new role for ECEl. ECEl can degrade SP and CGRP in early endosomes to promote the dissociation of the $\mathrm{NK}_{1} \mathrm{R}$ and CLR-RAMPl from $\beta$-arrestins and thereby accelerate recycling and resensitization of the receptors for SP and CGRP. Supported by NIH grant DK39957.

. 
P53 Downregulates Tff2 Expression in Gastric Cancer Cells Through An AP-1 Binding Site

Shuiping Tu, Wandong Ai, Alfred L Chi, Shigeo Takaishi, Zinaida Dubeykovskaya, Timothy Cragin Wang

Background: Spasmolytic polypeptide (SP/TFF2) is a member of the trefoil peptide family, abundantly expressed and secreted by neck cells of stomach. It plays an important role in maintaining mucosal integrity. Expression of TFF2 is upregulated in chronic gastritis, and overexpression of TFF2 is associated with cell migration, resistance of apoptosis, angiogenesis and possibly gastric cancer invasion. Deregulation of $\mathrm{p} 53$ is also frequently observed in preneoplastic conditions of the stomach. However, the relationship between $\mathrm{p} 53$ and TFF2 remains unknown. In this study, we investigated the effect of $\mathrm{p} 53$ on the expression of TFF2 in gastric epithelial cells. Methods: Endogenous mRNA expression of TFF2 was detected by semi-quantitative RT-PCR and quantitative RT-PCR. A series of truncated promoters of human TFF2 gene were generated using a pGL3 luciferase reporter vector (TFF2-Luc reporters). Cells were cotransfected with either wild type p 53 or dominant negative mutant p53 (p53Mut) expression plasmids with TFF2-Luc reporters. Reporter activities were measured by Dual-Luciferase Reporter assay system. Results: TFF2 mRNA was detected in all 5 gastric cancer cell lines tested. Overexpression of p53 inhibited the level of endogenous TFF2 mRNA and p53Mut increased the level of TFF2 mRNA in gastric cell lines. P53 dosedependently inhibited the activity of TFF2 promoter. The temperature-sensitive 553 mutant at the permissive temperature also inhibited the expression of TFF2 mRNA and the activity of TFF2 promoter. Reporter assays showed that p53-depedent inhibition of TFF2 promoter occurred through an upstream -175 -219bp region contains an AP-1-like cis-element. Ectopic expression of c-jun/c-fos activated the TFF2 promoter and p53 inhibited this effect. Furthermore, mutation of AP-1 binding site abrogated c-jun/c-fos-mediated activation and p53-mediated inhibition of TFF2 promoter activity. PMA, a tumor promoter and activator of AP-1, stimulated endogenous mRNA expression and the promoter activity of TFF2, and p53 inhibited the effect of PMA on TFF2. Conclusions: p53 downregulates the expression of TFF2 at the transcriptional level through an AP-1-like cis-acting site. The p53-depedent inhibition of PMA- and c-jun/c-fos-mediated TFF2 activation supports the hypothesis that deregulation of 553 allows oncogenic or inflammation-stimulated upregulation of TFF2. The data suggest the possibility that TFF2 may be a downstream target of $\mathrm{p} 53$. The inhibition of TFF2 by p53 may facilitate damage cells to undergo apoptosis and to inhibit cell invasion and angiogenesis in the chronic inflammation and development of cancer.

728

Rap1 Regulates Neurotensin Secretion Downstream of cAMP/PKA and cAMP/ Epac Pathways in the Human Endocrine Cell Line Bon

J.N. Li, Kathleen L. O'Connor, Courtney M. Townsend, B. Mark Evers

Neurotensin (NT) is a gut peptide localized predominantly to the small bowel that plays an important role in gastrointestinal growth. We have reported that both cAMP/PKA and cAMP/Epac pathways are involved in forskolin (FSK)-stimulated NT secretion, in a novel human endocrine cell line BON; however, the mechanisms regulating cAMP-mediated NT secretion are not known. cAMP regulates secretion of certain hormones via Epac or PKA, but it is not clear if Rapl is involved in their effects. Rapl, a small GTPase, is phosphorylated by PKA; Epac, a guanine nucleotide-exchange protein activated by cAMP, catalyzes the exchange of GTP for GDP and activates Rapl. In the present study, we determined: i) whether Rapl regulates cAMP-stimulated NT secretion, and ii) whether Rapl is a downstream effector of Epac or PKA. METHODS. (i) To determine the role of Rapl in NT secretion, stable BON cell lines expressing constitutively active Rapl (BON/RaplV12) were established. Parental BON cells were transfected with either Rapl siRNA or RaplGAPII plasmid to suppress endogenous Rapl expression or inactivate Rapl activity, respectively. (ii) To determine the cellular localization of Rapl, GFP-tagged Rapl was analyzed by confocal microscopy. (iii) To determine whether Rapl activation was regulated by upstream PKA or Epac, BON cells were treated with PKA or Epac activators (6-Phe-cAMP or 8-pMeOPT-cAMP, respectively) or transfected with wild type Epac or a cAMP binding mutant EpacR279E; in vitro Rapl activity assays were performed. RESULTS. We found that FSK-stimulated NT secretion was significantly enhanced in BON/RaplV12 cells but reduced in BON cells transfected with either Rapl siRNA or RaplGAPII. GFP-tagged, wild type Rapl was expressed in the cytosol and nucleus of BON cells, whereas the constitutively active RaplV12 was predominantly localized to the membrane in addition to the cytosol. The dominant negative RaplN17 was localized in the cytosol associated with granule-like structures. Rapl activity was significantly induced by overexpression of wild-type Epac and Epac activators which increased NT release. In contrast, Rapl activity was inhibited in BON cells stably transfected with EpacR279E, which decreased NT release. Rapl activity was significantly up-regulated by the PKA activator, which also increased NT secretion. CONCLUSIONS. Rapl plays a critical role in cAMPregulated NT secretion. Importantly, we demonstrate, for the first time, that Rapl, acting downstream of both cAMP/PKA and cAMP/Epac pathways, is involved in the regulation of NT hormone secretion, thus identifying these pathways as critical components for stimulated intestinal hormone release.

\section{9}

Mechanisms of Stimulation of Intracellular Calcium in Enteroendocrine STC-1 Cells By Bitter Tastants

\section{Monica C. Chen, Shuping V. Wu, Enrique Rozengurt}

Background: We recently demonstrated the expression of bitter taste receptors of the T2R family and the a subunits of the G protein gustducin (Gogust) and transducins in the rodent GI tract and in GI endocrine cells. However, the intracellular signal transduction cascades initiated by bitter tastants in the enteroendocrine cells have not been explored. Aims: This study was designed to determine calcium signaling pathways initiated by bitter tastants in the enteroendocrine cells. Specifically, we characterized mechanisms of $\mathrm{Ca}^{2+}$ fluxes and mobilization induced by two distinct T2R bitter ligands: denatonium benzoate (DB) and phenylthiocarbamide (PTC), in mouse enteroendocrine cell line STC-1. Methods: Expression of bitter taste signal transducers was determined by RT-PCR and immunocytochemistry. Calcium responses of STC-1 treated with various concentrations of DB or PTC in the presence of ion channel blockers or openers were measured by calcium fluorometry. Results: In addition to previously identified mT2Rs, we detected the expression of Gagust, PLC $\beta 2$, TRPM 5 and L-type calcium channel (Cav1.2 and 1.3) in STC-1 cells. About 95\% of these cells were gustducin-positive, as shown by immunocytochemistry. Both DB and PTC induced a marked increase in intracellular calcium concentration $\left(\left[\mathrm{Ca}^{2+}\right] \mathrm{i}\right)$ in dose- and time-dependent fashion. Depolarization by KCl and L-type calcium channel opener Bay-K8644 mimicked the increase in $\left[\mathrm{Ca}^{2+}\right] \mathrm{i}$ induced by DB and PTC. Chelating extracellular $\mathrm{Ca}^{2+}$ with EGTA blocked the increase in $\left[\mathrm{Ca}^{2+}\right]$ i induced by DB and PTC but did not prevent the effect induced by bombesin. Tharpsgargin at $50 \mathrm{nM}$ blocked the transient increase in $\left[\mathrm{Ca}^{2+}\right] \mathrm{i}$ induced by bombesin, but did not attenuate the $\left[\mathrm{Ca}^{2+}\right]$ i increase activated by DB or PTC. These results indicate that $\mathrm{Ca} 2+$ influx mediates a substantial component of the increase in $\left[\mathrm{Ca}^{2+}\right]$ i induced by DB and PTC in STC-1 cells. Preincubation with the L-type Ca2+ channel blockers nitrendipine or diltiazem inhibited the increase in $\left[\mathrm{Ca}^{2+}\right]$ i elicited by tastants and $\mathrm{KCl}$, indicating that the opening of L-type $\mathrm{Ca}^{2+}$ channels play a major role in mediating the increase in $\left[\mathrm{Ca}^{2+}\right] \mathrm{i}$ in response to DB or PTC in STC-1 cells. Tastants also induced a marked increase in the release of CCK from STC-1 cells, an effect prevented by L-type $\mathrm{Ca}^{2+}$ channel blockers. Conclusion: Our results demonstrated that bitter tastants increase $\left[\mathrm{Ca}^{2+}\right]$ i through $\mathrm{Ca}^{2+}$ influx mediated by the opening of L-type Ca2+ channels in the STC-1 cells. Our data suggest that STC-1 cells provide a model system to study intracellular signal transduction pathways and secretory responses initiated by tastants in enteroendocrine GI cells.

730

Agonists of Protease-Activated Receptor 2 (PAR2) Sensitize Transient Receptor Potential Vanilloid 4 (TRPV4) to Induce Mechanical Hyperalgesia Andrew Grant, Graeme Cottrell, Elizabeth Joseph, Nicola Lissi, Marcello Trevisani Pierangelo Geppetti, Nicole Alessandri-Haber, Jon D. Levine, Nigel W. Bunnett

Proteases that are generated during injury and inflammation, such as trypsins and tryptase, cleave PAR2 on primary spinal afferent neurons to induce hyperalgesia to thermal and mechanical stimuli. Thermal hyperalgesia depends on protein kinase A (PKA) and PKCEmediated sensitization of the ion channel TRPVl, but the mechanisms of PAR2-induced mechanical hyperalgesia are unknown. We hypothesized that PAR2 induces mechanical hyperalgesia by sensitizing TRPV4, which mediates pain responses to mechanical and osmotic stimuli. Since TRPV4 shows preference for $\mathrm{Ca} 2+$ ions, we examined sensitization of TRPV4dependent changes in $[\mathrm{Ca} 2+]$ i in human bronchial epithelial cells (HBE), which naturally express PAR2 and TRPV4, and HEK cells, which express PAR2 and were transiently transfected with human TRPV4. The TRPV4 agonist $4 \alpha$ phorbol 12,13-didecanoate ( $4 \alpha \mathrm{PDD}$, $0.1-10 \mu \mathrm{M})$ and hypotonic stimuli $(-10-20 \%)$ increased [Ca2+]i in HBE and HEK-TRPV4 cells, but not in untransfected HEK cells, suggesting activation of TRPV4. Preincubation with PAR2 activating peptide (AP, SLIGRL-NH2, corresponding to the tethered ligand domain of PAR2, $100 \mu \mathrm{M}, 5 \mathrm{~min}$ ) enhanced the effect of $4 \alpha \mathrm{PDD}$ by $124 \pm 14 \%$ in $\mathrm{HBE}$ and $55 \pm 17 \%$ in HEK-TRPV4 cells, and enhanced the effect of a hypotonic stimulus by $38 \pm 16 \%$ in HBE and $149 \pm 19 \%$ in HEK-TRPV4 cells, indicating TRPV4 sensitization. Inhibitors of PKA (H89, $10 \mu \mathrm{M}$ ) and PKC isozymes (GF109203X, $10 \mu \mathrm{M}$ and Gö6976, 0.1 $\mu \mathrm{M}$ ) inhibited sensitization by $>50 \%$. The inactive reverse peptide sequence (PAR2-RP), and PAR1-AP (TFLLRN-NH2), which do not cause hyperalgesia, had no effect, confirming specificity. Superfusion of slices of rat spinal cord with $4 \alpha \mathrm{PDD}$ or hypotonic solution stimulated the release of the nociceptive peptides substance $\mathrm{P}$ by $>4$-fold and calcitonin gene-related peptide by $>9$-fold. Preincubation with PAR2AP $(100 \mu \mathrm{M}, 20 \mathrm{~min})$ enhanced the effects of $4 \alpha \mathrm{PDD}$ on release of SP and CGRP by 3 -fold and 2 -fold, and enhanced the effects of hypotonic solution of release of both SP and CGRP by 1.5 -fold, compared to PAR2-RP. In wild type mice, intraplantar injection of PAR2-AP increased the frequency of paw withdrawal to mechanical stimulation with a von Frey hair $(0.174 \mathrm{mN})$ by $222 \pm 20 \%$, and enhanced the response to hypotonic stimulus by $189 \pm 23 \%$, indicative of sensitization. These effects were absent from TRPV4-/- mice. Thus, activation of PAR2 sensitizes TRPV4 by PKA and PKC-dependent mechanisms to potentiate the release of nociceptive peptides from primary spinal afferent neurons and cause hyperalgesia to mechanical stimulation. Supported by NIH grants DK57480, DK43207, DK39957.

731

Altered Gastrointestinal Function and Energy Balance in Obestatin Receptor (GPR39) Knockout Mice

Inge Depoortere, Dieder Moechars, Betty de Smet, Benoit Moreaux, Peter King, Kim Cryns, I Goris, Luc Verdonck, Theo Peeters, Bernard Coulie

Obestatin has recently been discovered as the ligand of the orphan GPR39 receptor. Obestatin is encoded by the ghrelin gene and is part of what was known as the ghrelin associated peptide. Functionally obestatin opposes the effects of ghrelin and decreases food intake, body weight gain, gastric emptying and intestinal motility (Zhang et al., Science 310: 996$999,2005)$. To gain insight into the functional role of the obestatin receptor we generated GPR39 $\%$ mice. Methods. Energy balance was investigated by comparing food intake and respiratory quotient (RQ) between wildtype (GPR39 $9^{+/}$) and GPR39 knockout (GPR39-/-) mice. For gastrointestinal function gastric emptying (measured by the ${ }^{14} \mathrm{C}$ octanoic breath test), fecal pellet propulsion and gastric secretion were compared between the two genotypes. Results. Development, growth, immune function and fertility were identical in GPR39-/- and GPR39+/+ littermates. The mature body weight of GPR39-/- mice $(42.3 \pm 1.8 \mathrm{~g})$ was significantly $(\mathrm{P}<0.05)$ higher than that of GRP39 $9^{+/+}$mice $(35.7 \pm 1.9 \mathrm{~g})$ although the growth rate parameters were the same. Under normal ad libitum feeding, both genotypes consumed the same amount of food with normal circadian patterns of spontaneous $24 \mathrm{~h}$ food intake. In contrast, deficiency of the GPR39 receptor led to reduced hyperphagia after fasting. Six hours cumulated food intake after $19 \mathrm{~h}$ fasting was $2.20 \pm 0.29 \mathrm{~g}$ in GPR39 $9^{+/+}$but only $0.96 \pm 0.29 \mathrm{~g}$ in GPR39-1mice $(\mathrm{P}<0.05)$. The RQ was significantly higher in the GPR $39^{-/ /}$mice and this was mainly due to increased RQ values during the light period. Cholesterol levels $(\mathrm{mg} / \mathrm{dl})$ were significantly increased in both male (142.2 \pm 29.0$)$ and female (118.7 \pm 16.2$)$ mutants, compared to wildtype male $(107.3 \pm 37.8)$ and female $(83.5 \pm 24.2)$ littermates. Gastric emptying of a solid meal was 
significantly $(\mathrm{P}<0.005)$ accelerated with a gastric half emptying time of $49.5 \pm 2.2 \mathrm{~min}$ compared to $86.9 \pm 8.4 \mathrm{~min}$ in GPR39 ${ }^{+/+}$mice. A more effective expulsion of distally located pellets (30-75\% of length) was observed in the colon of GPR39-/ mice. Four hour after pylorus ligation, volume of gastric secretion was significantly increased (GPR39-1 : $638 \pm 336$ $\mu \mathrm{l}, \mathrm{GPR} 39^{+/+}: 225 \pm 170 \mu \mathrm{l}$ ), but gastric acid secretion was unchanged. No changes in anxiety were observed between both genotypes but open field test indicated a significant decreased general activity in GPR39-- mice. Conclusion. Our data are mostly in agreement with the described effects of obestatin and suggest that this peptide plays a functional role in the regulation of energy balance and gastrointestinal function through interaction with the GPR39 receptor, probably by counteracting the effects of ghrelin.

732

Proton Pump Inhibitor Plus a COX-2 Inhibitor for the Prevention of Recurrent Ulcer Bleeding in Patients with Arthritis: A Double Blinded, Randomized Trial Francis Chan, Vincent W. Wong, Bing Y. Suen, Justin C. Wu, Wai K. Leung, Yuk T. Lee, James Y. Lau, Joseph J. Sung

BACKGROUND Among patients with a history of NSAID-induced ulcer bleeding, neither NSAIDs plus a proton pump inhibitor (PPI) nor a COX-2 inhibitor is adequate to prevent recurrent ulcer bleeding. AIM To investigate whether PPI plus a COX-2 inhibitor is superior to COX-2 inhibitor alone for the prevention of recurrent ulcer bleeding in patients with previous ulcer bleeding. METHODS Patients with arthritis who presented with NSAIDinduced ulcer bleeding confirmed by endoscopy were eligible if they: 1 . had healed ulcers on follow-up endoscopy, 2. were $H$. pylori negative or eradicated, and 3. required regular NSAIDs. The exclusion criteria were renal failure (creatinine $>2.2 \mathrm{mg} / \mathrm{dl}$ ), concomitant use of low-dose aspirin, steroids or anticoagulants, malignancy and terminal illnesses. The use of prohibited drugs (low-dose aspirin, NSAIDs, steroids, anticoagulants, or other anti-ulcer drugs) during the study period was a protocol violation. RANDOMIZATION After the ulcers had healed, all patients received celecoxib $200 \mathrm{mg}$ b.i.d. They were randomly assigned to esomeprazole $20 \mathrm{mg}$ b.i.d. or its placebo for 12 months. PRIMARY ENDPOINT Recurrent ulcer bleeding determined by an adjudication committee using pre-specified criteria. RESULTS In the intention-to-treat analysis, which included 273 patients (137 receiving celecoxib plus esomeprazole and 136 receiving celecoxib plus placebo). The probability of recurrent ulcer bleeding during the 12 -month period was $0 \%$ in the esomeprazole group and $8.9 \%(95 \% \mathrm{CI}, 4.1$ to 13.7$)$ in the placebo group (log rank test, $\mathrm{P}=0.0004)$. During the study period, $16.1 \%$ of patients in the esomeprazole group and $15.4 \%$ in the placebo group used low-dose aspirin. Among patients who did not use concomitant aspirin, the probability of recurrent ulcer bleeding was $0 \%$ in the esomeprazole group and $7.1 \%$ (95\% $\mathrm{CI}, 2.4$ to 11.8$)$ in the placebo group $(\mathrm{P}=0.004)$. CONCLUSION Among patients with a history of NSAID-induced ulcer bleeding, esomeprazole plus celecoxib was superior to celecoxib alone for the prevention of recurrent ulcer bleeding. This study was not sponsored by pharmaceutical companies.

Baseline characteristics

\begin{tabular}{|c|c|c|}
\hline & $\begin{array}{c}\text { Esomeprazole } \\
\mathrm{n}=137\end{array}$ & $\begin{array}{c}\text { Placebo } \\
\mathrm{n}=136\end{array}$ \\
\hline Men \% & 47 & 49 \\
\hline Mean age (SD) & $70(12)$ & $72(11)$ \\
\hline OA/RA/Others \% & $83 / 3 / 14$ & $90 / 2 / 8$ \\
\hline Smoking \% & 10 & 10 \\
\hline Drinking \% & 10 & 9 \\
\hline Co-morbid illnesses \% & 34 & 35 \\
\hline$>1$ episode of prior ulcer bleed \% & 18 & 19 \\
\hline Previous H. pylori infection \% & 44 & 51 \\
\hline Location of bleeding ulcers prior to enrolment GU/DU/both & $58 / 34 / 8$ & $57 / 36 / 7$ \\
\hline
\end{tabular}

733

In Vivo Diagnosing of Helicobacter Pylori with Confocal Laser

Endomicroscopy - Prospective Evaluation in Comparison with Conventional Histology and Urease Testing

Ralf Kiesslich, Martin Goetz, Arthur Hoffman, Constantin Schneider, Katharina Lammersdorf, Michael Vieth, Manfred Stolte, Peter R. Galle, Markus F. Neurath

Abstract: Confocal laser endomicroscopy (CLE) enables surface and subsurface imaging of living tissue during ongoing endoscopy. Currently, in vivo diagnosis of Helicobacter pylori with acriflavine aided CLE was described. The aim of the current prospective study was the in vivo detection of Helicobacter pylori in patients with dyspepsia and the correlation with histologic findings and urease testing. Methods: Endomicroscopy (Pentax, Tokyo, EC3870 CIFK) of the stomach was performed by using two different contrast stains. Fluorescein was applied intravenously to enable confocal endomicroscopy of the gastric mucosa. Confocal image data were collected at a scan rate of $1.6 \mathrm{sec} /$ frames (1024x1024 pixels) using an optical slice thickness of $7 \mu \mathrm{m}$ (lateral resolution $\leq 1 \mu \mathrm{m}$ ). Subsequently, acriflavine hydrochloride was applied topically onto the surface of the gastric mucosa with the help of a spraying catheter. Acriflavine netted the surface and allowed identification of focal accumulation of Helicobacter pylori at the surface and in deeper layer of the gastric epithelium. Targeted biopsies of the examined areas were performed at the antrum and corpus for urease testing and histology (Warthin-Starry silver). Results: In 11 out of 30 patients Helicobacter pylori infection could be diagnosed by CLE. Accumulated as well as single bacteria with bright contrast could be observed including the distinct shape and flagella of Helicobacter pylori. In addition gastritis could be graduated due to visible vessel and cell alterations. Helicobacter pylori infection was proven in all except one case by histology and urease testing (Sensitivity 90.9\%,
Specificity 96.7\%). The single false positive result was seen in a patient with extremely few germs detected by endomicroscopy. Furthermore, 4 patients were re-examined after eradication. CLE correctly proved the absence of Helicobacter pylori. Conclusions: Endomicroscopy allows simultaneously endoscopic images of the stomach, in vivo histology of gastritis and immediate diagnosis of Helicobacter pylori infection during ongoing upper endoscopy. The diagnostic accuracy is comparable with conventional histology and urease testing.

734

Acidification of Distal Esophagus Increases Cough Reflex Sensitivity in Patients with Gastroesophageal Reflux Disease (GERD) and Chronic Cough Nikoleta Javorkova, Renata Pecova, Silvia Varechova, Michal Demeter, Rudolf Hyrdel, Dusan Balaz, Milos Tatar, Marian Kollarik

BACKGROUND \& AIMS: Gastroesophageal reflux ranks among the most important causes of chronic cough, however, the mechanisms by which gastroesophageal reflux initiates coughing are incompletely understood. It is generally accepted that hyperexcitability of the cough reflex (increased cough reflex sensitivity) results in coughing. Here we address the hypothesis that acidification of distal esophagus increases the cough reflex sensitivity in patients with GERD and chronic cough. METHODS: Eight consecutive patients with GERD complaining of chronic cough (cough persisting for $>8$ weeks) and 18 gender- and agematched patients with GERD without cough were recruited. GERD was confirmed by positive fibroscopy and/or pH monitoring. In a randomized double blind study, saline and acid (HCl $0.1 \mathrm{M})$ were separately infused into distal esophagus via nasoesophageal catheter $(10 \mathrm{ml} / \mathrm{min}$ $15 \mathrm{~min}$ ). The infusions were separated by at least $20 \mathrm{~min}$. Cough reflex sensitivity was determined immediately after completion of each infusion using standard capsaicin inhalation challenge (single-breath inhalation of the aerosols of capsaicin solutions with doubling concentrations, from $0.49 \mu \mathrm{M}$ to $500 \mu \mathrm{M}$ ). The cough reflex sensitivity was expressed as the lowest concentration of capsaicin evoking 2 coughs (C2). Group data were expressed as median (interquartile range). RESULTS: Infusion of acid but not saline into distal esophagus induced mild heartburn and/or chest discomfort in 7/26 patients. In patients with GERD and chronic cough, infusion of acid into distal esophagus increased cough reflex sensitivity [1.95 (0.98-15.6) $\mu \mathrm{M}$ after saline vs. $0.98(0.49-1.95) \mu \mathrm{M}$ after acid, $\mathrm{p}<0.05]$. In contrast, infusion of acid into distal esophagus in patients with GERD without cough had no effect on the cough reflex sensitivity [15.6 (7 81-46.88) $u \mathrm{M}$ after saline vs. 31.25 (4.88-125) uM after acid, $p>0.5]$. CONCLUSIONS: We conclude that acidification of distal esophagu induces the cough reflex hyperexcitability in patients with GERD and chronic cough. We speculate that this phenomenon (likely mediated by vagal nerves) underlies chronic cough due to gastroesophageal reflux. Absence of this phenomenon in patients with GERD who do not complain of cough further supports this notion. Supported by Slovak National Research Grant VEGA 1/2273/05

735

Accelerate Healing of Penetrating Ulcer Using Bone Marrow Cell

\section{Transplantation}

Tsutomu Nishida, Shingo Tsuji, Masahiko Tsujii, Syuji Ishii, Toshiyuki Yoshio, Shinichiro Shinzaki, Satoshi Egawa, Takanobu Irie, Yoshimi Kakiuchi, Masakazu Yasumaru, Hideki Iijima, Hiroaki Murata, Sunao Kawano, Norio Hayashi

Background and Aim: Bone marrow (BM) has been found to be a source of multipoten stem cells that could distribute into various organs including gut. We have reported that BM-derived adult somatic stem cells have a potential to differentiate to myofibroblasts and other types of stromal cells. However, therapeutic potential of BM-derived cells in intractable ulcers has not yet demonstrated. The aim of this study was whether dish attached cultured bone marrow cells promote ulcer healing in mice. Methods: BM cells and gastric myofibroblasts were prepared from GFP-transgenic mice ( $\operatorname{TgN}(\beta$-act-EGFP)Osb). In certain experiments, BM-cells were incubated in plastic dishes and the cells attaching the dishes were collected and designated as cultured BM cells. Gastric ulcers were induced by serosal application of acetic acid on anterior wall of the stomach in C57/B6 mice. Then, the ulcers were implanted with the cultured BM cells $\left(4 \times 10^{5}\right.$ cells), myofibroblasts $\left(4 \times 10^{5}\right.$ cells), and PBS as the control in the serosal surface where gastric ulcers were induced. The animals were sacrificed on day 3 , or 7 . The samples were fixed in $4 \%$ paraformaldehyde/phosphatebuffered saline for $3 \mathrm{~h}$ and mounted in Tissue-Tek OCT compound and frozen in liquid nitrogen. The products of the long and short diameter of ulcer area were represented as ulcer index. The origin and phenotypes of implanted cells were examined by fluorescence immnohistochemistry for GFP, $\alpha$-smooth muscle actin (SMA), vimentine, and other surface marker antigens for stromal cells. Results: Healing of the acetic acid ulcer was significantly promoted by injection of cultured BM cells than the control on day 7 , but not on day 3 (ulcer index $4.4 \pm 1.5$ vs $11.1 \pm 2.8 \mathrm{~mm}^{2}, \mathrm{p}=0.0140$ ). In healing ulcers, $\mathrm{GFP}^{+}$cultured BM cells were observed in submucosal layers. These cells were positive for GFP, $\alpha$-SMA, and vimentine, have spindle shape, and considered to be differentiated mesenchymal cells. On the other hand, healing of the acetic acid ulcer was prolonged in mice implanted with myofibroblasts as compared to the control. GFP+ stromal cells were not seen in ulcered area in mice transplanted with myofibroblast. Conclusions: Cultered BM cells were transplantable to penetrating ulcers. Cultured BM cells could be a new source of mesenchymal cells transplantation to repair intractable gastrointestinal damage. 
Evidence from Linkage Analysis for Susceptibility Genes in Familial Barrett's Esophagus and Esophageal Adenocarcinoma

Yvonne Romero, Joshua P. Slusser, Mariza De Andrade, Julie M. Cunningham, Teresa G. Zais, Debra M. Geno, Gloria M. Petersen, Barrett's esophagus genomic study group

Background: The Barrett’s Esophagus Genomic Study Group comprises 156 physicians from varying practice environments who collaborate to identify Barrett's esophagus (BE) families for genomic analysis. Aim: To map susceptibility genes in familial BE pedigrees by genomewide linkage analysis using BE and esophageal adenocarcinoma (ACA) phenotypes. Methods: Study Group members refer families in which more than two persons are suspected of having long segment BE, with or without ACA. Following informed consent, pathology slides and endoscopy and surgical reports of potential index patients are reviewed. Confirmed index patients provide contact information of living first-degree relatives, or next of kin of deceased relatives. Pathology slides and records on relatives are reviewed to confirm phenotype. To augment phenotype characterization, a separate research project screened family members who had not previously had an upper endoscopy. All available living members of documented BE families were asked to complete a validated symptom questionnaire, and to provide a blood sample. Genotyping of lymphocyte DNA was performed using the ABI PRISM® Linkage Mapping Set version 2.5 using 403 markers equally distributed along the entire autosome at $\sim 10 \mathrm{cM}$ resolution. Two-point and multi-point linkage analyses were performed. Results: 466 families were evaluated for eligibility, and 94 were documented to have multiple affected family members: 2 families had 5 affecteds, 4 families had 4 affecteds, 29 families had 3 affecteds, and 59 had 2 affecteds. Linkage analysis was performed on 278 persons in the 31 most informative families. Under a dominant model with decreased penetrance, the phenotype of BE yielded a multipoint heterogeneity LOD (HLOD) score of 3.07 at $66.9 \mathrm{cM}$, near D2S2259; 2.51 at $79.43 \mathrm{cM}$ between markers D2S391 and D2S337; and 1.57 at $8.64 \mathrm{cM}$ at D2S2211, all on the short arm of chromosome 2. Other promising HLOD scores include: 1.52 on chromosome $12 \mathrm{p}$ at $73.55 \mathrm{cM}$ at D12S368; 1.41 at 43.59 cM near D19S414, and 1.47 at $71.06 \mathrm{cM}$ at D19S902. Linkage remained consistent on chromosomes 2, 12, and 19 when a combined phenotype of BE or ACA was used, with the highest HLOD score being 2.78 at $66.94 \mathrm{cM}$ at D2S2259. Linkage was not identified for either phenotype on chromosome 13 (maximum HLOD= 0.56 ). Conclusion: These results provide strong evidence for major susceptibility genes for familial Barrett's esophagus and esophageal adenocarcinoma.

\section{7}

TRPV-1 Knockout Paradoxically Protects Mouse Gastric Mucosa from Acid/ Ethanol-Induced Injury By Upregulating Compensatory Protective Mechanisms Yasutada Akiba, Tetsu Takeuchi, Misa Mizumori, Paul H. Guth, Eli Engel, Jonathan D. Kaunitz

Capsaicin-sensitive afferent nerves (CSAN) coordinate upper gastrointestinal tract mucosal protective mechanisms through the release of calcitonin gene-related peptide (CGRP) and increasing prostaglandin $(\mathrm{PG})$ synthesis. The acid-sensitive cation channel TRPV-l may serve as a primary acid sensor that activates CSAN in response to luminal acidity. Acute disruption of components of this protective system increases mucosal injury susceptibility. Other putative acid sensors, such as acid-sensing ion channels (ASICs) have been implicated in luminal acid sensing, and PG type E (EPI) receptors are also involved. To evaluate the role of TRPV-1 and effector molecules in gastric mucosal protection, we examined their role in gastric mucosal injury using TRPV-1 knockout (KO) mice, hypothesizing that KO would increase injury susceptibility in a standard gastric injury model. Wild type C57BL/6 (WT) and KO mice were gavaged with $150 \mathrm{mM} \mathrm{HCl} / 60 \%$ ethanol $(0.3 \mathrm{ml})$ in order to produce gastric injury. Pretreatment with the CGRP receptor antagonist hCGRP $_{8-37}(0.3 \mathrm{mg} / \mathrm{kg}$, iv), the nonselective cyclooxygenase (COX) inhibitor indomethacin (Indo, $5 \mathrm{mg} / \mathrm{kg}$, sc), the nitric oxide (NO) synthase inhibitor L-NAME ( $20 \mathrm{mg} / \mathrm{kg}$, sc), the TRPV-1 antagonist capsazepine (CPZ, $10 \mathrm{mg} / \mathrm{kg}$, sc), or the TRPV-1 ligand capsaicin $(10 \mathrm{mg} / \mathrm{kg}$, i.g.) were given to evaluate the role of these components of the capsaicin pathway. Expression of acid-sensing ion channel (ASIC) 1 and ASIC3, and COX-1, COX-2, EP1 receptor, and calca (pro-CGRPQ gene) in the stomach, dorsal root ganglion (DRG) and nodose ganglion (NG) were analyzed by real-time RT-PCR. Unexpectedly, acid/ethanol treatment injured the gastric mucosa of KO significantly less than it did WT. In WT, Indo, hCGRP 8-37, L-NAME and CPZ aggravated whereas capsaicin reduced gastric lesions, confirming the role of afferent nerves in coordinating mucosal protection. In $\mathrm{KO}$, Indo and $\mathrm{hCGRP}_{8-37}$ reversed the protective effect of TRPV1 deletion, producing injury to a degree not different from similarly treated WT. COX-1 mRNA expression increased 4-fold in KO stomach compared with WT. COX-2 and calca mRNA expression were induced and ASIC3 was up-regulated 10-fold in DRG of KO, whereas calca was induced and ASICl was increased 25-fold in NG of KO. EPI was expressed in wild type and KO stomach, but was down-regulated in DRG of KO. The unexpected decrement in mucosal injury observed in TRPV-1 KO can be explained by compensatory upregulation of CGRP release and COX-2 function. In KO stomach, up-regulation of ASICI in NG and ASIC3 in DRG may serve as compensatory, alternative acid sensors in afferent neurons that may coordinate CGRP release and PG synthesis.

\section{8}

ErbB-4 Expression Is Increased in Experimental Colitis and Is Required for Intestinal Epithelial Wound Closure Mark R. Frey, D. Brent Polk

ErbB-4 is the most recently described member of the epidermal growth factor (EGF) receptorrelated receptor tyrosine kinase family. It is expressed in epithelial cells in membrane-bound full-length as well as cytosolic and nuclear cleavage product forms. Studies in other tissues suggest that ErbB-4 regulates cell division and survival; however, only limited data are available on expression and function in the intestine. This study tests a role for ErbB-4 in intestinal epithelial wound healing and response to inflammation. METHODS: Colitis was induced in C57BL/6 mice with 3\% dextran sulfate sodium (DSS) in drinking water for 4 days, followed by 3 days recovery. ErbB-4 expression was assayed by immunohistochemistry on paraffin-embedded tissue or Western blot of colon mucosal scrapings, using antisera specific for total receptor or the cleavable isoform. For wound healing assays, young adult mouse colon (YAMC) cells plated on fibronectin were transfected with ErbB-4 siRNA, subjected to multiple wounds with a rotating silicone tip, and monitored by time-lapse photography. Western blot analysis of cell lysates was performed using phospho-specific and total antibodies for EGF receptor, ErbB-4, FAK, AKT, p38, and ERK. RESULTS: ErbB4 protein expression in mouse colon demonstrated two distinct patterns. Membrane staining for full-length receptor was detected in the upper crypts and surface epithelium of the postmitotic compartment, while nuclear ErbB-4 cleavage product was observed only in the lower crypt. Western blot of mucosal scrapings confirmed the presence of both full-length and cleaved receptor. DSS colitis induced a modest induction of ErbB-4 protein, with staining expanded along the entire length of crypts. During recovery, ErbB-4 expression increased dramatically throughout the renewing epithelial compartment. We therefore asked whether ErbB-4 was required for wound healing in cell culture. In YAMC cells, ErbB-4 siRNA significantly impaired restitution over a $24 \mathrm{~h}$ period. Furthermore, analysis of growth factorinduced signaling showed that loss of ErbB-4 attenuated EGF-stimulated phosphorylation of the adhesion regulator FAK and the anti-apoptotic kinase AKT. CONCLUSIONS: These data represent the first demonstration of spatially segregated expression of different ErbB4 forms in the intestinal epithelium, implying distinct functions for cleaved versus fulllength receptor in this tissue. Our results argue that ErbB-4 plays a role in wound healing and remodeling after inflammation, possibly by regulating growth factor-induced cell survival and migration signals.

739

Gata4 Is Essential for the Maintenance of Jejunal-Ileal Identities in the Adult Mouse Small Intestine

Tjalling Bosse, Christina M. Piaseckyj, Ellen Burghard, John J. Fialkovich, Satish Rajagopal, William T. Pu, Stephen D. Krasinski

The mammalian small intestine is lined by a highly specialized epithelium that displays a wide-ranging, yet tightly regulated functional diversity along its cephalo-caudal axis. Gata4, a member of the zinc finger family of GATA transcription factors, is highly expressed in the mammalian small intestine in proliferating crypt epithelium and mature absorptive enterocytes of the duodenum and jejunum, but is undetectable in distal ileum of adult mice. We confirmed that this pattern is conserved in humans by immunofluorescence analysis on adult intestinal sections. In the present study, we established an inducible, intestine-specific knockout model for Gata4 to test the hypothesis that Gata4 is necessary for the maintenance of jejunal-ileal identities in adult mouse small intestine. An inducible Villin-CreERT2 mouse line was crossed with a mouse line containing Gata4 alleles flanked by loxP sites (Gata4flox). Treatment with Tamoxifen ${ }^{\mathrm{TM}}$ for 5 consecutive days resulted in the inactivation of Gata4 in the jejunum 2 weeks after the last injection. We analyzed the expression of enterocyte genes that are normally expressed in jejunum but not in ileum and found that lactase-phlorizin hydrolase (LPH) and liver fatty acid binding protein (Fabpl) were strongly attenuated as shown by immunostaining for Fabpl and real-time RT-PCR for LPH and Fabpl $(\mathrm{P}<0.05$, $\mathrm{n}=4$ ). In contrast, absorptive enterocyte genes not normally expressed in jejunum, but highly expressed in ileum, such as the apical sodium-dependent bile acid transporter (Asbt) and ileal lipid binding protein (Ilbp), were both markedly induced in the mutant jejunum as indicated by Asbt immunostaining and Asbt and Ilbp real-time RT-PCR $(\mathrm{P}<0.01, n=4)$. Moreover, the goblet cell population was increased $50 \%$ as determined by unbiased counting $(\mathrm{P}<0.05, \mathrm{n}=4)$ and the enteroendocrine cell fate as determined by peptide YY and cholesystokinin mRNA levels were redistributed, all to an ileal-like composition. Consistent with an expansion of secretory cells, Mathl, a known activator of the secretory cell fate, was induced in the jejunum of Gata4 mutant mice $(\mathrm{P}<0.05, \mathrm{n}=4)$. Conclusion: These data demonstrate that Gata4 is a major and essential positional signal that is required for the maintenance of jejunal-ileal identities in the adult mouse small intestine.

740

Sumoylation Modulates Transcriptional Activity of Cdx1 By Direct Interaction Fang Xiao, Eunran Suh

Background: Cdxl plays significant roles in multiple cellular pathways including cell growth, intestinal differentiation, and apoptosis. However, little information is available on how the Cdxl protein is modulated by intracellular and nuclear signaling processes to achieve such a broad range of functions. Sumoylation is one of post-translational modifications, which modulates functions of target protein by regulating protein-protein interactions, translocalization, DNA-protein interactions, and/or protein stability. In the present study, we examined interactions between the $\mathrm{Cdx}$ l protein and Sumo-1, Ubc-9, or PIAS-1, which are members of sumoylation machinery, and determined whether sumoylation modulated biological functions. Methods: GST pull-down assay and co-immunoprecipitation (co-IP) were performed to demonstrate protein-protein interaction in vitro and in vivo, respectively. A series of point mutations, K69R, K69A, K263R, or K263A was generated by using the QuickChange sitedirected mutagenesis kit. Results: The Cdxl protein interacts with Sumo-l conjugating enzyme Ubc9 and E3 ligase PIAS1 in vitro and in vivo. Furthermore, in vitro sumoylation assays has demonstrated that the Cdxl protein is sumoylated by Sumo-1 in Caco2 cells following cotransfection of $\mathrm{Cdxl}$ with Sumo-1. Alignment of the primary amino acid sequence of $\mathrm{Cdxl}$ orthologs from a variety of species demonstrates that there is one evolutionarily conserved somoylation consensus sequence ( $\Psi \mathrm{KXE} / \mathrm{D}$ ), which is located at the amino acid residue K263 of the murine Cdxl protein. A sumoylated form of Cdxl following co-IP with Sumo-1 was detected on wild type Cdxl, but not on K263A/R. This result has indicated that the sumoylation site is at the lysine residue 263 of the $\mathrm{Cdxl}$ protein. To assess the potential functional role of the conjugation of Sumo- 1 to Cdxl, we carried out transfection assays in Caco 2 cells where sucrase-isomaltase promoter-reporter construct was cotransfected with Cdxl in the presence or absence of Sumo-1. The results demonstrate that overexpression of Sumo-1 represses its transcriptional activity in a dose-dependent manner, suggesting that SUMO-mediated transcriptional repression modulates Cdxl function. Conclusion: Cdxl is a new target for Sumo-1 modification and that lysine residue 263 is a sumoylation site of 
Cdxl. In addition, sumoylation represses transcriptional activity of Cdxl, suggesting that sumoylation plays a significant role on modulating Cdxl functions.

741

A Cell Culture System Modelling the Entire Small Intestinal Crypt Villus Axis Katri Lindfors, Essi Myrsky, Heikki Kainulainen, Markku Maki

Introduction: Epithelial cells lining the small intestine represent a highly organised system built up by multipotent stem cells. A process of asymmetric mitosis produces a population of transit amplifying cells that rapidly proliferate and migrate along the crypt villus axis, differentiating into functional mature cells before dying and exfoliating into the intestinal lumen. At present the primary culture of isolated crypts or epithelial cells retaining high viability - not to mention the culture of stem/progenitor cells - is still extremely difficult. The culture of stem/progenitor cells is in part challenging due to the lack of definitive stem cell markers. In addition, long-term cultures (more than 10 days) of small intestinal epithelial cells has proved to be almost impossible. We have developed a long-term cell culture which recapitulates the entire small intestinal crypt villus axis with regard to progenitor cell proliferation as well as terminal differentiation and apoptosis. Methods: Single crypt epithelial cells and small organoids were isolated from mouse small intestine and cultured under specific conditions on collagen with a feeder layer and a specific medium. Immunohistochemical stainings with antibodies against specific proteins involved in either proliferation or differentiation were performed on formalin fixed sections. Results: The isolated small intestinal epithelial cells formed organized clusters that remained viable for at least 10 weeksthe longest period ever published. The clusters were characterized by a large central lumen and a well-deposited basement membrane. Furthermore there were small villus-like structures that protruded into this lumen. A portion of the cells were proliferative as evinced by Ki67 positivity. In addition, the clusters also harbored polarized columnar cells that had differentiated either into absorptive enterocytes as indicated by alkaline phosphatase activity, or goblet cells based on Alcian blue positivity. Additionally, on the basis of EphB3 staining, Paneth cells were also present in these clusters. When these parental clusters were broken into single cells that were cultured under identical conditions, they formed daughter clusters with similar characteristics as the parental ones. Conclusions: We have developed a longterm cell culture system that models the in vivo small intestinal crypt villus axis in terms of epithelial cell proliferation and differentiation. This culture system eases the study of small intestinal progenitor cells, and also research concerning proliferation and differentiation as well as the development of stem cell based therapies for severe small intestinal disorders.

\section{2}

Differences in the Appetite-Stimulating Effect of Orexin, NPY, and Ghrelin Among Young, Adult, and Old Rats

Saeko Akimoto-Takano, Chihiro Sakurai, Setsuko Kanai, Hiroko Hosoya, Minoru Ohta Kyoko Miyasaka

Aging is associated with a progressive decrease in appetite and food intake. The appetitestimulating peptides orexin-A, NPY (neuropeptide Y), and ghrelin are known to play a critical role in food intake. In this study, the stimulatory effect of intracerebroventricular administration of these peptides on food intake was compared among young (4-monthold), adult (11-month-old) and old (24-27-month-old) male Wistar rats. A stainless steel cannula was implanted stereotactically into the left lateral ventricle. After a 7 -day recovery period, different doses of orexin-A ( 0 to $3 \mathrm{nmol})$, NPY and ghrelin (0 to $1 \mathrm{nmol})$ were injected into the left lateral ventricle without anesthesia. Food consumption was measured at 1,2 , and $4 \mathrm{hr}$ after injection. We also examined the plasma levels of acylated and desacyl ghrelin in young and old rats by ELISA. Intracerebroventricular administration of orexin-A and NPY stimulated food intake in young and adult rats, but no effects were observed at any dose in old rats. Ghrelin increased food intake in a dose-dependent manner in all groups, and the effect of ghrelin was reduced with advancing age. Neither the acylated nor the desacyl plasma ghrelin level differed significantly between young and old rats. In conclusion, we observed a decrease in the orexigenic effect of the peptides orexin-A, NPY and ghrelin in old rats, and this reduction may have been responsible for the age-related decrease in food intake.

\section{3}

Controlled Polymeric Delivery of VEGF Enhances Angiogenesis in Developing Intestine

Flavio G. Rocha, Cathryn Sundback, Nicholas Krebs, Kent Leach, David J. Mooney, Stanley W. Ashley, Joseph P. Vacanti, Edward E. Whang

Background: Intestinal ischemia is a common cause of massive bowel resection leading to short bowel syndrome in both children and adults. Our laboratories have previously created a functional neointestine capable of restoring absorptive function. However, the amount of vascular endothelial growth factor (VEGF), a key mediator of angiogenesis, is markedly reduced in the maturing construct compared to native bowel. Therefore, we wanted to deliver VEGF to the neointestine in order to enhance its development. Methods: Rat recombinant VEGF was encapsulated in polymer microspheres by a double emulsion method. Incorporation and release kinetics were determined in vitro using radiolabeled growth factor. Tissue engineered intestine was generated by seeding donor neonatal rat intestinal organoid units onto a cylindrical polymer scaffold. VEGF or empty microspheres were loaded in the lumen of the polymer along with the organoid units and the construct wrapped in the omentum of recipient rats ( 6 animals per group). After four weeks, the animals were sacrificed and the neointestinal cysts harvested for morphometry, VEGF levels, epithelial cellular proliferation, apoptosis and capillary density by ELISA and immunohistochemistry. Results: VEGF was encapsulated in microspheres with 30\% efficiency. Sustained release of VEGF into simulated body fluid occurred over a period of four weeks. Intestinal constructs with encapsulated VEGF were significantly larger $(99.3+0.4 \mathrm{~cm} 3$ vs $25.8+0.2 \mathrm{~cm} 3)$ and $(10.2+3.8 \mathrm{~g}$ vs $3.5+0.5 \mathrm{~g})(\mathrm{p}<0.05)$ than those containing empty microspheres. Tissue VEGF levels were significantly higher in neointestine loaded with VEGF compared to those without growth factor $(106+33 \mathrm{pg} / \mathrm{mg}$ vs $28+34 \mathrm{pg} / \mathrm{mg})(\mathrm{p}<0.05)$ and approached the levels of juvenile native intestine. Epithelial cellular proliferation and angiogenesis were significantly increased in the VEGF-loaded neointestinal constructs $(84.9+7.1 \%$ vs $53.8+13.8 \%$ BrDU staining crypt cells) and $(29.8+4.5$ vs $10.3+1.1$ capillaries/hpf $)(\mathrm{p}<0.05)$; however, apoptosis remained unchanged $(5.0+2.4 \%$ vs $3.8+3.1 \%$ TUNEL staining crypt cells). Conclusion Tissue-engineered intestine responds to sustained delivery of VEGF by increasing microvasculature and epithelial proliferation. This strategy may be used to enhance neointestinal constructs in order to stimulate small bowel development. In addition, these optimized constructs may be able to preserve critical intestinal function after injury.

744

Neonatal FC Receptor for IgG in Intestinal Epithelial Cells Contributes to the Protection Against Epithelium-Associated Pathogen, C. Rodentium Infection Masaru Yoshida, Timothy T. Kuo, Lynn Bry, Jonathan N. Glickman, Steven M. Claypool, Arthur Kaser, Takashi Nagaishi, Darren Higgins, Emiko Mizoguchi, Takeshi Azuma, Atsushi Mizoguchi, Wayne I. Lencer, Richard S. Blumberg

Background: Citorobacter rodentium is a bacterial pathogen that causes a murine infectious colitis equivalent to enterohemorrhagic and enteropathogenic E.coli infection in humans. Importantly, CD4+ T cells, B cells and IgG, but not secretory IgA or IgM, have been shown to play a critical role in the prevention against this infection. The neonatal Fc receptor for $\operatorname{IgG}(\mathrm{FCRn})$ plays a major role in regulating host $\operatorname{IgG}$ levels and transporting $\operatorname{IgG}$ and associated antigens across polarized epithelial barriers (Immunity 2004; 20;769). Aim: We sought to test the possibility that FcRn-mediated transport of $\operatorname{Ig} G$ and bacterial antigen/IgG complexes may be involved in the mucosal immune response to an epithelium-associated pathogen, C. rodentium. Methods and Results: FcRn-/- and littermate FcRn-/+ mice were orally inoculated with C. rodentium. FcRn-/- mice exhibited more body-weight loss, higher bacterial concentrations in the feces, and severer mucosal inflammation in the distal colon Murine $(\mathrm{m}) \mathrm{FcRn}$ and $\mathrm{m} \beta 2 \mathrm{~m}$ double transgenic mice under the control of the intestinal fatty acid binding protein on a $\mathrm{mFcRn}-/$ - mouse background (IFABP-mFcRnTg/m $\beta 2 \mathrm{mTg} / \mathrm{mFcRn}-/$ mice) were developed. As expected, these mice expressed FcRn only in the epithelium and were capable of secreting IgG into the lumen in comparison to FcRn-/- mice which were not. To test whether specific IgG for bacteria is required for the regulation of $\mathrm{C}$. rodentium infection through FcRn-mediated transport of $\operatorname{IgG}, \mathrm{C}$. rodentium was orally inoculated into IFABP-mFcRnTg/m $\beta 2 \mathrm{mTg} / \mathrm{mFcRn}-/$ - and FcRn-/- mice that had or had not received a polyclonal anti-C. rodentium $\operatorname{Ig} G$ antibody. Whereas the nonimmune control antibody had no effect on $\mathrm{C}$. rodentium eradication in $\mathrm{FcRn}$-/- mice or transgenic mice, the pre-administration of specific antibody resulted in less body-weight loss and lower bacterial concentrations in the feces of IFABP-mFcRnTg/m $\beta 2 \mathrm{mTg} / \mathrm{mFcRn}-/$ - mice. This was associated with induction of proliferation of CSFE-labeled OT-II transgenic T cells when mice were infected with C. rodentium expressing OVA. These results indicate that $\mathrm{FcRn}$ expression in intestinal epithelial cells contributes to the protection against $C$. rodentium infection but only in the presence of specific IgG antibodies. Conclusion: Selective expression of FcRn in the epithelium is shown here to be associated with secretion of $\operatorname{IgG}$ into the lumen that allows for defense against an epithelium-associated pathogen. Epithelial-associated FcRn integrates luminal antigen encounters with systemic immune compartments and, as such, provides essential host defense and immunoregulatory functions at the mucosal surfaces.

745

Certolizumab Pegol Administered Subcutaneously Is Effective and Well Tolerated in Patients with Active Crohn's Disease: Results from a 26-Week, Placebo-Controlled Phase III Study (PRECiSE 1)

William J. Sandborn, Brian G. Feagan, Simeon Stoinov, Pieter J. Honiball, Paul Rutgeerts, Juliet A. McColm, Alison Innes, Stefan Schreiber

Background: Certolizumab pegol (CDP870) is a PEGylated Fab' fragment of a humanized monoclonal antibody that neutralizes tumor necrosis factor- $\alpha$. A Phase III, randomized, double-blind, multicenter study assessed efficacy and tolerability of certolizumab pegol in active Crohn's disease (CD). This is the largest long-term induction study of a biologic agen performed to date in $C D$. Methods: Adult patients (pts) with moderate-severe $C D(C D$ Activity Index [CDAI] 220-450 points) were randomized to receive subcutaneous (sc) certolizumab pegol 400mg or placebo (PBO) at Weeks (Wks) 0, 2, 4, then 4-weekly up to Wk 24. Pts were stratified at baseline according to C-reactive protein (CRP) level $(<10 \mathrm{mg} / \mathrm{L}$ or $\geq 10 \mathrm{mg} / \mathrm{L}$ ) and immunosuppressant/corticosteroid use. Safety and efficacy were determined at Wks $0,2,4,6,8,12,16,20,24$ and 26/withdrawal. The co-primary endpoints were the percentages of pts with a clinical response (decrease in $\mathrm{CDAI} \geq 100$ ) at Wk 6 , and at Wks $6 \& 26$, in pts with baseline CRP $\geq 10 \mathrm{mg} / \mathrm{L}$. Secondary endpoints included 70-point response (decrease in $\mathrm{CDAI} \geq 70$, post-hoc analysis), and remission $(\mathrm{CDAI} \leq 150)$ in the $\mathrm{CRP} \geq 10 \mathrm{mg} / \mathrm{L}$ stratum, response and remission in the overall intention-to-treat (ITT) population, and CRP levels (last observation carried forward; LOCF, post-hoc analysis) in both populations. Results: Both primary endpoints showed a significant advantage for certolizumab pegol over PBO. Responses (70- and 100-point) at Wks 4, 6 and Wks $6 \& 26$ were significantly higher $(\mathrm{p}<0.05)$ for certolizumab pegol vs $\mathrm{PBO}$ in the $\mathrm{CRP} \geq 10 \mathrm{mg} / \mathrm{L}$ stratum and the overall ITT population $(\mathrm{n}=659)$ (Table). Remission at Wk 4 was significantly higher for certolizumab pegol vs PBO; there were positive trends at Wk 6 and Wks $6 \& 26$. Median CRP levels were significantly lower for certolizumab pegol vs PBO at Wks 6 and 26 ( $p \leq 0.01$, changes from baseline) (Table). Overall, no safety signals of concern were observed. Conclusions: Monthly certolizumab pegol $400 \mathrm{mg}$ sc demonstrated significant efficacy and was well tolerated compared with $\mathrm{PBO}$ in pts with active $\mathrm{CD}$.

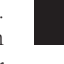




\begin{tabular}{|c|c|c|c|c|}
\hline & \multicolumn{2}{|c|}{ Baseline $\mathrm{CRP} \geq 10 \mathrm{mg} / \mathrm{L}$} & \multicolumn{2}{|c|}{ Overall ITT population } \\
\hline & $\begin{array}{c}\text { PBO } \\
\mathrm{n}=156\end{array}$ & $\begin{array}{l}\text { Certolizumab pegol } \\
400 \mathrm{mg} \mathrm{n}=146\end{array}$ & $\begin{array}{c}\mathrm{PBO} \\
\mathrm{n}=328\end{array}$ & $\begin{array}{l}\text { Certolizumab pegol } \\
400 \mathrm{mg} \mathrm{n}=331\end{array}$ \\
\hline $\begin{array}{c}\text { 70-point response } \\
(\% \text { pts })^{\mathrm{a}}\end{array}$ & & & & \\
\hline $\begin{array}{l}\text { Wk } 4 \text { Wk } 6 \text { Wks } 6 \\
\qquad 26\end{array}$ & $\begin{array}{c}30.333 .1 \\
14.9\end{array}$ & $50.3^{* * *} 46.9 * 29.2^{* * *}$ & $\begin{array}{c}33.737 .8 \\
22.5\end{array}$ & $44.0^{* * *} 46.2^{*} 32.0^{* * *}$ \\
\hline $\begin{array}{c}\text { 100-point response } \\
(\% \mathrm{pts})^{\mathrm{a}}\end{array}$ & & & & \\
\hline $\begin{array}{l}\text { Wk } 4 \text { Wk } 6 \text { Wks } 6 \\
\& 26\end{array}$ & $\begin{array}{c}20.626 .0 \\
12.3\end{array}$ & $32.9 * 37.2 * 21.5 *$ & $\begin{array}{c}21.826 .8 \\
16.0\end{array}$ & $28.7 * 35.2 * 23.1 *$ \\
\hline Remission (\% pts) ${ }^{\mathrm{a}}$ & & & & \\
\hline $\begin{array}{l}\text { Wk } 4 \text { Wk } 6 \text { Wks } 6 \\
\& 26\end{array}$ & $\begin{array}{l}9.716 .9 \\
8.4\end{array}$ & $20.1 * 21.913 .1$ & $\begin{array}{c}11.317 .2 \\
9.8\end{array}$ & $19.5^{* * *} 21.614 .4$ \\
\hline$\underset{(\mathrm{mg} / \mathrm{L})}{\operatorname{Median} \text { CRP LOCF }}$ & & & & \\
\hline Wk 0 Wk 6 Wk 26 & $\begin{array}{l}25.523 .0 \\
27.0\end{array}$ & 28.013 .515 .0 & 8.59 .09 .0 & 7.04 .04 .0 \\
\hline
\end{tabular}

${ }^{*} \mathrm{p} \leq 0.05, * * \mathrm{p} \leq 0.01$ vs $\mathrm{PBO}{ }^{\text {an }} \mathrm{n}$ is decreasing over time

\section{6}

Critical Role of Toll-Like Receptors in Modulating GM-CSF Activity in Rodent Models of Colitis

Stefano Fiorucci, Andrea Mencarelli, Barbara Renga, Eleonora Distrutti, Antonio Morelli, Ed Croze, Sharlene Velicko, Brent Larsen, John Parkinson

Background. Toll-like receptors (TLRs) sense extracellular pathogens and regulate innate immune responses in the intestinal mucosa. Evolving research suggests that Crohn's disease may be caused by a deficiency in cells of the immune system within the mucosal barrier and that this precedes reactive inflammatory processes. GM-CSF (granulocyte macrophage colony-stimulating factor) is a naturally occurring growth factor and part of the innate defense system, which increases the number and activity of white blood cells (neutrophils, monocytes/macrophages, and dendritic cells). Aim. To test the efficacy and immunomodulatory actions of GM-CSF in TNBS and DSS-induced colitis, using wild type and TLR deficient mice. Methods. TNBS-colitis was induced by intrarectal administration of $0.5,1$ or $2 \mathrm{mg} /$ mouse TNBS in $50 \%$ ethanol; DSS-colitis by $5 \%$ DSS in the drinking water. Mice were administered $5 \mu \mathrm{g} /$ mouse/day recombinant yeast-derived pegylated murine GM-CSF (PEGmGM-CSF). Mucosal cytokine content cytokines was assessed by quantitative (q)RT-PCR. Isolated lamina propria (LP) mononuclear cells composition was assessed by flow cytometry. The protective effects of PEG-mGM-CSF were also examined in TNBS-colitis induced in TLR2-/-, TLR4-/-, TLR9-/- and myd88-/-mice. Results. In wild-type mice, PEG-mGM-CSF prevented TNBS- and DSS-induced colitis by all disease endpoints measured: weight loss, inflammation, disease activity/diarrhoea scores and macroscopic/microscopic scores ( $p<$ 0.05). PEG-mGM-CSF reversed established DSS colitis when dosed from days 5-14, attenuating mucosal increases of IL- 6 and IL-1 $\beta$. In TNBS colitis, PEG-mGM-CSF did not alter mucosal TNF- $\alpha$ or COX-2 mRNAs, but increased mucosal levels of IFN- $\gamma$, IL-1 $\beta$ and IL-6 mRNAs ( $\mathrm{P}<0.05$ versus TNBS). Moreover, PEG-mGM-CSF reduced LP CD4+ and CD8+ T lymphocytes, while increasing the number of $\mathrm{CD} 1 \mathrm{lb}+$ myeloid cells and CD19+ B lymphocytes. In TNBS-colitis PEG-mGM-CSF enhanced the antibacterial effector functions of mucosal CD14+ myeloid cells and significantly inhibited bacterial translocation to spleen and lymph nodes. Mucosal protection by PEG-mGM-CSF in TNBS-colitis was preserved in TLR2-/- and TLR4-/- mice, but lost in myd88-/- mice and TLR9-/- mice Conclusions. PEGmGM-CSF prevents DSS- and TNBS-colitis and treats DSS-colitis in mice. Protection by PEG-mGM-CSF in TNBS-colitis is associated with enhanced myeloid antibacterial effector functions, is independent of TLR2 and TLR4, but dependent on the TLR adaptor protein myd88 and the bacterial double-standed DNA ( $\mathrm{CpG}$ )-sensing TLR9. The findings are consistent with the innate immune activating functions of GM-CSF and its use to enhance hostprotective immunity.

747

Natalizumab Induces Sustained Response and Remission in Patients with Active Crohn's Disease: Results from the Encore Trial

Stephan R. Targan, Brian Feagan, Richard Fedorak, Bret Lashner, Remo Panacionne, Daniel Present, Andreas Raedler, Paul Rutgeerts, Zsolt Tulassay, Miroslava Volfova, Douglas C. Wolf, William Sandborn

Purpose: ENCORE (Efficacy of Natalizumab in Crohn's Disease Response and Remission) was a phase 3 , international, randomized, double-blind, placebo (pbo)-controlled trial that evaluated the efficacy and safety of natalizumab induction therapy in Crohn's patients with moderately to severely active disease and objective evidence of active inflammation (elevated C-reactive protein [CRP] levels). Methods: Patients (N=509) with Crohn's Disease Activity Index (CDAI) scores between 220 and 450 (inclusive) and CRP levels greater than the upper limit of normal $(2.87 \mathrm{mg} / \mathrm{L})$ were randomized $1: 1$ and received natalizumab $(300 \mathrm{mg} ; \mathrm{n}=$ 259) or pbo $(n=250)$ infusions at weeks 0,4 , and 8 . Efficacy and safety were assessed at weeks 4,8 and 12 . The primary endpoint examined the ability of natalizumab to induce a clinical response ( $\geq 70$ point decrease in baseline CDAI score) by week 8 that was sustained through week 12. Additional efficacy endpoints included the proportion of patients achieving a clinical remission $(\mathrm{CDAI}<150)$ by week 8 and through week 12 , and the proportion of patients in response or remission at any assessment. Results: A significantly higher proportion of patients in the natalizumab group were in response at both weeks 8 and 12 compared to patients in the pbo group (48\% [124/259] vs. $32 \%$ [81/250], p < 0.001). Response at week 4 (following the first infusion) was 51\% [133/259] for natalizumab vs. 37\% [92/250] for placebo, $\mathrm{p}=0.001$ ), and remained significantly higher in the natalizumab group at all subsequent assessments $(\mathrm{p}<0.001$ for all comparisons with pbo). Remission at both weeks 8 and 12 was also achieved by a higher proportion of patients in the natalizumab group compared with pbo $(26 \%$ [68/259] vs. $16 \%$ [40/250], p=0.002). Patients receiving natalizumab also had superior remission rates at all assessments ( $\mathrm{p} \leq 0.009$ for all comparisons with pbo). The incidence and types of adverse events were similar between groups. Conclusions: Natalizumab induced response and remission by week 8 that was sustained through week 12. The response and remission rates for natalizumab were superior to placebo at all timepoints beginning at week 4 , thus confirming the efficacy of natalizumab as induction therapy. Natalizumab was well tolerated, with adverse events not significantly different than pbo.

\section{8}

GM-CSF Efficacy in Murine DSS Colitis Is Mediated By Changes in the Function of Plasmacytoid Dendritic Cells

Eyad Hanna, Satheesh K. Sainathan, Kumar S. Bishnupuri, Qizhi Luo, Rodney D. Newberry, Courtney W. Houchen, Shrikant Anant, Brian K. Dieckgraefe

Crohn's disease (CD) is a chronic inflammatory condition of the gastrointestinal tract. It is associated with a loss of tolerance to commensal gut flora and an overactive adaptive immune response. Recent knowledge has implicated impaired innate immune clearance in the pathogenesis of $C D$. A recent clinical trial showed efficacy of GM-CSF, a hematopoietic growth factor, in human CD. To understand the mechanism this effect, we studied a murine model of colitis. DSS colitis is a model of impaired mucosal integrity and, therefore, may be ideal to understand the effects of GM-CSF. Administration of pegylated recombinant murine GM-CSF (Peg-rmGM-CSF) significantly ameliorated disease activity in DSS colitis as assessed by weight loss, colon length, histopathological score, stool consistency, and stool hemoccult. In addition, GM-CSF altered the number and function of dendritic cell populations. The therapeutic effect of Peg-rmGM-CSF in DSS colitis was blocked by administration of a monoclonal antibody $(\mathrm{Ab}), 440 \mathrm{c}$, which recognizes a cell surface molecule selectively expressed on plasmacytoid dendritic cells (pDCs). The pDCs are the main interferon (IFN)-producing cell in the intestine. They express toll-like receptor-9, which recognizes bacterial DNA motifs, specifically cytosin-guanosin dinucleotide (CpG). Type-I IFNs (IFN$\alpha$ and $\beta$ ) are produced in response to stimulation with $\mathrm{CpG}$. Addition of monoclonal $\mathrm{Ab}$ $440 \mathrm{c}$, both in vitro and in vivo, blocks IFN- $\alpha$ production in pDCs stimulated with CpG but does not deplete cell numbers. Furthermore, we demonstrated that GM-CSF augments expression of Type-I IFNs, IDO, and IL-10 in the mouse ileum and spleen after stimulation with $\mathrm{CpG}$. Therefore, we hypothesized that the $\mathrm{pDC}$ is involved in the mechanism of therapeutic response of DSS colitis to Peg-rmGM-CSF. Fms-like tyrosine kinase 3 (Flt-3) ligand, another hematopoietic growth factor, is a potent DC growth factor that significantly expands pDC numbers. Accordingly, we examined the effects of Flt-3 treatment on DSS colitis. Flow cytometry data showed that both GM-CSF and more impressively, Flt-3 expand dendritic cell (CDIlc and MHCII positive) populations in the spleen. However, disease activity in animals treated with DSS worsened rather than improved with Flt-3 treatment. Conclusions: The pDC is implicated in the response of DSS colitis to Peg-rmGM-CSF. Expansion of pDC numbers alone is unlikely to explain the therapeutic effects of Peg-rmGMCSF based on the data from Flt-3 treated animals. The data suggests that the response to Peg-rmGM-CSF is mediated through a functional change in $\mathrm{pDC}$ response to bacteria or bacterial products.

749

The Ideal Management of Crohn's Disease: Top Down Versus Step Up Strategies, a Randomized Controlled Trial

Daniel Hommes, Filip Baert, Gert van Assche, Filip Caenepeel, Philippe Vergauwe, Hans Tuynman, Martine de Vos, Sander van Deventer, Larry Stitt, Paul Rutgeerts, Brian Feagan, Geert Dhaens

Current management of moderate-severe Crohn's disease (CD) consists of glucocorticosteroids (GCS), leading to a 26\% 1-year remission rate, steroid dependency and significant toxicity. This study examined if more aggressive induction therapy with infliximab (IFX) and azathioprine (AZA) would lead to higher remission rates, lower toxicity and fewer complications. Methods. $133 \mathrm{CD}$ pts with CDAI > 220, diagnosed < 4years ago and never treated with GCS/immunomodulators/IFX were randomized to (1) top-down (TD) treatment with 3 infusions of IFX (w 0,2 and 6) and AZA 2-2.5 mg/kg/day or (2) step-up (SU) therapy with topical (budesonide $9 / \mathrm{mg} /$ day) or systemic (prednisone $40 \mathrm{mg} /$ day) GCS. In the TD group, relapse was treated with repeat IFX infusions and GCS when they failed to respond to IFX. In the SU group, AZA was added in case of repeated need for GCS or dependency and IFX was only given after failure of immunosuppression. Patients were examined at week $0,2,6,10$ and 14 and every 3 months thereafter until month 24 . The primary endpoint was remission (CDAI $<150$ without GCS and no resection) at month 6 and 12; secondary endpoints included overall treatment success (remission at week 14 and beyond, no resection, no GCS or IFX after week 14), mean CDAI scores, toxicity and prolonged remission up to month 24. Results. Baseline characteristics were similar in both groups (mean CDAI at inclusion 331 TD vs. $306 \mathrm{SU}$ ), 19 pts dropped out before month 12. Induction therapy was successful in $81 \%$ and $73 \%$ of TD and in $60 \%$ and $67 \%$ of SU pts at week 10 and 14 . Remission without GCS and without resection was attained in $60 \%$ (TD) vs. $41 \%$ (SU) $(\mathrm{p}=0.03)$ at 6 months and in $61 \%$ (TD) vs. $50 \%(\mathrm{SU})$ at 12 months $(\mathrm{p}=0.19)$. At 6 months, $31 \%$ of patients in he SU group were still receiving GCS (median dose $26 \mathrm{mg} /$ day) compared with $0 \%$ for TD; at 12 months, $17 \%$ of pts in SU were remained on GCS (median dose $23 \mathrm{mg}$ ) compared with $0 \%$ for TD ( $\mathrm{p}<0.001$ ). At 6 and 12 months $84 \%$ and $93 \%$ of pts were using immunomodulators in the TD group, versus $41 \%$ and $65 \%$ in the SU group (both $\mathrm{p}<0.001)$. Overall treatment success was seen in 29\% TD and 5\% SU pts $(\mathrm{p}<0.001)$. During the first 12 months, $13 \%$ of patients in the SU group needed IFX infusions to induce remission and $41 \%$ of patients in the TD group required at least one further IFX infusion. Serious adverse events were observed in $49 \%$ of TD and $41 \%$ of SU pts (NS). Conclusion. Aggressive induction with IFX + AZA is superior to sequential SU therapy for reducing 
exposure to GCS and attaining overall treatment success. 41\% of TD patients needed further IFX infusions after induction. Complete results until month 24 will be available at DDW

750

Van Gogh-Like Proteinl (Vangl1) Mediates Spatial Organization of Signaling from Itf and EGF Leading to a Polarized Migratory Response of Intestinal Epithelial Cells

Jiri Kalabis, Ian Rosenberg, Hans-Christian Reinecker, Vijay Yajnik, Daniel K. Podolsky

Background: The intestinal trefoil factor (ITF/TFF3) protects intestinal epithelia from a range of insults and contributes to mucosal repair. However, the signaling events that mediate ITF dependent mucosal protection are not well understood. We recently demonstrated that Vangll, a Dlgl binding partner and a member of the planar polarity complex in Drosophila, was phosphorylated in response to ITF signaling and was required for the ITF induced migratory response. The aim of this study was to define mechanisms resulting in directed migration by ITF. Material and methods: GST-Pac and GST-Rhotekin pull down assays were used to demonstrate effects of ITF and EGF on small GTP-ases in HEK293 cells. GFP- or flag-Vangll constructs were used for up-regulation and siRNA for silencing of Vangll. Effects were confirmed by RT-PCR. Western blotting, immunoprecipitation and pull down assay were used to quantitate effects of Vangll on phosphorylation of EGFR or CDC42 activation. Confocal microscopy with anti-phospho EGFR and GFP-Vangl were used to determine spatial organization of ITF signaling. siRNA mediated silencing was used to study effects of small GTP-ases on the ITF induced migratory response in a wound healing assay of HT29 colonic epithelial cells. Results: Vangll is not localized to the leading edge of wound, and contributed to the polarization of migratory response by modulating spatial activation of two signaling pathways. While ITF and EGF stimulated cells increased phosphorylation of EGFR on Y992, overexpression of Vangll decreased Y992 phosphorylation by ITF. The Vangll transfected cells decreased Y992 phosphorylation of EGFR to $50 \%$ of maximal intensity during the initial $10 \mathrm{~min}$ of stimulation. siRNA mediated Vangll downregulation increased Y992 phosphorylation of EGFR following stimulation by both ITF and EGF in intestinal epithelial cells. Spatial interaction between EGFR and Vangll was demonstrated using confocal microscopy. ITF was also found to activate CDC42 as demonstrated by pull down assays, siRNA silencing of CDC42 expression inhibited the ITF induced migratory response of intestinal cells. However overexpression of Vangll inhibited CDC42 activation. Conclusions: ITF induced activation of EGFR and CDC42 signaling is required for ITF dependent migration. Vangll did not associate with the leading edge of migrating cells and had inhibitory activity on EGFR and CDC42. We hypothesize that polarized expression of Vangll together with its inhibitory activity leads to inhibition of the pathways in the posterior compartment of migrating cells and promotes directional migration

\section{1}

\section{Chitinase 3-Like-1 (CHI3Ll) Enhances Potentially Pathogenic Bacterial} Adhesion to Colonic Epithelial Cells in Vitro and in Vivo

Mayumi Kawada, Yuriko Hachiya, Atsuhiro Ogawa, Ken Sugimoto, Emiko Mizoguchi

Background \& Aims: Dysregulated host/microbial interactions play a key role in the development of intestinal disorder. However, the exact mechanisms leading to this dysregulation have not been fully defined. We have recently proposed that Chitinase 3-like-1 (CHI3Ll/ YKL40/HC-gp39) molecule, which is induced on colonic epithelial cells (CECs) and lamina proprial macrophages during inflammatory condition, may play a key role in host/microbial interaction. Studies were performed to further characterize the functional role of $\mathrm{CHI} \mathrm{LL}$ on CECs/bacterial interaction. Methods: Gentamicin protection assay by using S. typhimurium and three different types of (pathogenic, potentially pathogenic and non-pathogenic) Escherichia coli was performed in CMT93 colonic epithelial cell line that was transfected with full-length mouse $\mathrm{CHI} \mathrm{Ll}$ expression vector. To examine the functional role of $\mathrm{CHI} \mathrm{Ll}$ in vivo, anti-CHI3Ll Ab was administrated in S. typhimurium infection model. Results: CHI3Ll, one of mammalian chitinases, has a chitin-binding domain and may efficiently bind to chitin-containing organisms but completely lacks glycohydrolase enzymatic activity. Enhanced CHI3Ll expression by transiently transfection in CMT93 cells significantly facilitated S. typhimurium adhesion ( 4.3 fold) and internalization ( 6.3 fold) one hour after infection compared to mock transfection. Blockade of CHI3Ll activity by anti-CHI3Ll Ab (100 $\mu \mathrm{g}$ / $\mathrm{ml}$ ) or CHI3Ll siRNA significantly reduced the S. typhimurium adhesion and invasion. This blockade also inhibited the adhesion/invasion of two pathogenic E. coli strains (adherent invasive E. coli, and entero-pathogenic E. coli) but not non-pathogenic E. coli strains. CHI3Ll expression in CECs was significantly upregulated in C57BL/6 mice two days after oral inoculation of S. typhimurium. Rabbit anti-CHI3Ll antibody treated C57BL/6 mice exhibited a significantly lower load of the S. typhimurium in mesenteric lymph nodes, spleen, liver and colon. In addition, the typhimurium-induced mortality was significantly reduced by the treatment with anti-CHI3Ll antibody compared to control rabbit IgG: all mice with control rabbit IgG treatment died, whereas about $50 \%$ of mice treated with antiCHI3Ll antibody still survive at 12 days after infection. Conclusions: CHI3Ll plays a pathogenic role in intestinal inflammation by enhancing the adhesion and invasion of intracellular bacteria on CECs. Since neutralization of CHI3Ll activity significantly reduced the bacterial loads in lymphoid organs. Inhibition of CHI3Ll activity would be considered a novel therapeutic strategy for infectious colitis and IBD. Support: NIH DK 64289 and Broad Foundation.

\section{2}

PIAS1 (Protein Inhibitor of Activated Stat1) Interacts with and Up-Regulates Levels and Activities of the Pro-Proliferative Transcription Factor KrüPpel-Like Factor 5 (KLF5)

James X. Du, C Chris Yun, Vincent W. Yang

BACKGROUND: Krüppel-like factor 5 (KLF5) is a zinc finger-containing transcription factor that regulates growth and differentiation of intestinal epithelial cells and fibroblasts, and have been implicated in epithelial cancers. Studies demonstrate that KLF5 promotes proliferation by transcriptionally activating the genes encoding cyclin D1 and Cdc2. AIM: To identify proteins that interact with and regulate the activity of KLF5. METHODS: Yeast two-hybrid screen of a day 17 mouse embryo cDNA library with KLF5 as a bait was conducted to identify KLF5-interacting proteins. Interactions between candidate proteins and KLF5 were verified by co-immunoprecipitation and indirect immunofluorescence microscopy. The effects of the interacting proteins on KLF5's activity were determined by transient cotransfection and dual luciferase assays. RESULTS: Several candidate proteins were identified by the screen. One was protein inhibitor of activated Statl (PIAS1), which was further characterized. PIAS1 is an E3 SUMO (small ubiquitin-like modifier) ligase for a number o transcription regulators, such as $\mathrm{p} 53, \mathrm{Sp} 3$, Statl, and Smad4. KLF5 contains two potential SUMOylation motifs $\Psi$ KXE, suggesting that it is potentially regulated by SUMOylation The interaction between PIAS1 and KLF5 was first verified by co-immunoprecipitation in transfected cells. Consistent with this finding indirect immunofluorescence demonstrated that FLAG-tagged PIASI and HA-tagged KLF5 co-localized to the nucleus and were enriched in subnuclear speckles. Although we obtained no evidence that KLF5 was SUMOylated by PIAS1, we showed that KLF5 physically interacted with SUMO. Transient transfection of PIAS1 and KLF5 in COS-7 cells significantly increased the steady-state levels of each other in co-transfected cells when compared to cells transfected with the individual construct, suggesting that the two proteins stabilize each other. Consistent with the increased level of KLF5, dual luciferase assays with cyclin D1 and Cdc2 luciferase reporters showed that coexpression of PIAS1 and KLF5 led to a significant and synergistic increase in the activities of the cyclin D1 and Cdc2 promoters when compared to cells transfected with PIAS1 or KLF5 alone. Furthermore, cells co-transfected with KLF5 and PIAS1 exhibited enhanced cell proliferation when compared to singly-transfected cells as measured by BrdU incorporation CONCLUSION: The results demonstrate for the first time that PIASI is an interacting partner of KLF5. Interaction between the two proteins significantly increases the levels, and transcriptional and pro-proliferative activities of KLF5. PIAS1 is therefore a biological modifier of KLF5.

Intestinal Barrier Repair Is Impaired Following Ischemic Injury in NHE2Deficient Mice

Adam J. Moeser, Prashant K. Knighot, Lane L. Clarke, Anthony T. Blikslager

The intestinal brush border $\mathrm{Na}^{+} / \mathrm{H}^{+}$exchanger isoforms NHE3 and NHE2 mediate most of the $\mathrm{Na}^{+}$absorption occurring between meals. Although NHE3 appears to be the predominant NHE isoform mediating $\mathrm{Na}^{+} / \mathrm{H}^{+}$exchange in the mammalian intestine, little is known regarding the functional importance of NHE2. We have previously demonstrated an effect of pharmacological blockade of NHE2 in recovery of mucosal barrier function in the ischemiainjured porcine ileum associated with recovery of tight junction structure. To definitively explore the importance of NHE2 in mucosal barrier repair, we studied mice with targeted disruption of the NHE2 gene $\left(\mathrm{NHE}_{2}^{--}\right)$. Ileum from NHE2 ${ }^{--}$mice and their heterozygote $\left(\mathrm{NHE}^{+/-}\right)$and wild-type (WT) siblings was subjected to a 1-hour period of acute mesenteric ischemia in vivo. Ischemic intestinal segments were then allowed to reperfuse/recover for 3 hours after which in vivo plasma-to-lumen clearances of ${ }^{3} \mathrm{H}$-mannitol were performed, as a measure of intestinal permeability. After the 3-hour post-ischemic recovery period, intestinal loops of WT mice exhibited increased permeability compared with non-ischemic control loops ( ${ }^{3} \mathrm{H}$-mannitol plasma-to-lumen clearance $=0.646 \pm 0.2 \mathrm{vs}$. $1.08 \pm 0.3 \mathrm{ml} / \mathrm{hr} \times 100 \mathrm{~g}$ in control and ischemic intestinal loops, respectively, $\mathrm{P}<0.05$ ), indicating a modest residual permeability defect . Similar findings were observed in $\mathrm{NHE}^{+/-}$mice. However, in NHE2 ${ }^{-1}$ mice, plasma-to-lumen clearances were dramatically elevated following the 3 hour recovery period (by approximately 6-fold) compared with clearances observed in non-ischemic control loops $(0.722 \pm 0.19$ vs. $4.52 \pm 1.53 \mathrm{ml} / \mathrm{hr} \times 100 \mathrm{~g}$ in control and ischemic intestinal loops respectively, $\mathrm{P}<0.01)$ and compared with WT and $\mathrm{NHE}^{+/-}$ischemic intestinal loops $(\mathrm{P}<0.01)$ Western blot analysis of the tight junction proteins occludin and claudin-l showed increased expression of both occludin and claudin- 1 in Triton-X soluble fractions in NHE2 $2^{-1}$ mice following the 3 hour recovery period as compared to WT and NHE2 ${ }^{+/-}$mice, suggesting evidence of increases in cytoplasmic expression of these junctional proteins and loss of tigh junction integrity. For claudin-1, there was a corresponding decrease in the Triton-X insoluble fraction. Overall, in vivo permeability data and western analyses of tight junctional protein suggest that $\mathrm{NHE}^{-/-}$mice have impaired mucosal barrier recovery that is characterized by delayed recruitment and assembly of interepithelial tight junctions following ischemic injury

\section{4}

Protective Role of MMP-2 in the Development of Colitis

Pallavi Garg, Anupama Ravi, Vijay K. Matam, Steve Epstein, Andrew Gewirtz, Didier Merlin, Shanthi Sitaraman

Introduction: Matrix metalloproteinases (MMPs) represent a family of proteases that regulate diverse cellular functions. The two known gelatinases, MMP-2 and MMP-9, are upregulated during human as well as animal models of inflammatory bowel disease. We recently demonstrated that epithelial-derived MMP-9 is an important mediator of colitis and targeted deletion of MMP-9 attenuates colitis. In this study we examined the role of MMP- 2 in the development of colitis. Methods: 6-8 week old female and male MMP2-/- mice and age- and sex-matched wild type (WT) mice were obtained from Jackson Laboratories. Colitis was induced by oral administration of $3 \%$ dextran sodium sulfate (DSS) in drinking water. Inflammation was quantitated by clinical (weight loss, blood in stool and diarrhea-total score 12) and histological 
(inflammatory infiltrate, ulceration and crypt damage-total score 11) scores. Another set of mice were administered Salmonella typhimurium (ST) SL 3201 by gavage (25 X $104 \mathrm{cfu} /$ mice) with or without pretreatment with streptomycin ( $\mathrm{n}=10$ per group). Mice were followed for signs of colitis (streptomycin pretreated mice) weight loss and mortality. Results: We observed that total MMP-2 protein as well as MMP-2 activity was upregulated in WT mice treated with DSS or ST compared to WT water using zymography (pro form: $2.5+0.5$ fold and active form $10+3$ fold), Western blotting (5.2+ .07 fold). Interestingly, MMP- $2-/$ - mice were highly susceptible to the development of colitis induced by DSS or ST compared to WT. This was reflected by rapid weight loss, diarrhea, rectal bleeding and death in MMP-

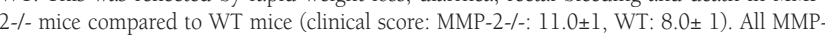
2 -/- mice given DSS died compared to $20 \%$ mortality in WT. Histological assessment suggested massive inflammation and extensive tissue damage among MMP-2 -/- compared to WT mice (Histological score: MMP-2-/-: 10.2 \pm 1 WT: $7.4 \pm 0.5$ ). Conclusions: In contrast to MMP-9, MMP-2 plays a protective role in the development of colitis in two murine models of colitis. Although MMP- 2 and MMP- 9 belong to the family of gelatinases and share common substrates, the lack of collagen V domain in MMP-2 likely alters the substrate specificity of this gelatinase leading to its protective role in the development of mucosal inflammation.

\section{5}

Metalloprotease Cleavage of TGF- $\alpha$ Mediates Oxidant-Induced Intestinal Permeability While Paracrine Hb-EGF/EGFR Signaling Regulates Baseline Intestinal Permeability

Christopher B. Forsyth, Ali Banan, Ashkan Farhadi, Ali Keshavarzian

We have previously identified a novel TNF- $\alpha$ convertase (TACE)-epidermal growth factor receptor (EGFR) dependent pathway regulating intestinal permeability in response to oxidant stress. In this model, oxidant induces activation of TACE resulting in cleavage of surface EGFR ligand precursors such as EGF, HB-EGF,TGF- $\alpha$,and amphiregulin. The ligand then binds to the EGFR initiating ERK1/2 signaling required for increased permeability. Aim: In this study our principal aim was to identify the specific EGFR ligand cleaved by TACE resulting in EGFR-dependent ERK1/2 activation and increased permeability. In addition we sought to identify possible EGFR ligands mediating baseline impermeability and identify mechanisms for regulation of TACE ligand cleavage. Methods: The human Caco-2 cell in vitro model of tissue culture inserts was used for the permeability (FSA dye movement, 18h) as well as cell signaling studies. Confluent cells on glass coverslips were used for microscopy studies. For oxidative stress, cells were exposed to H2O2 (500uM). Cells remained viable. Western blotting of cell lysates from oxidant treated cells was used for phosphoprotein analysis of mitogen activated protein kinases (MAPK) ERKI/2 as well as EGFR phosphorylation. Some cells were pre-treated with specific chemical inhibitors of EGFR and TACE as well as blocking protein (CRM197) to HB-EGF, and blocking antibodies to EGFR, TGF- $\alpha$,and amphiregulin. Immunofluorescent microscopy was used to stain for TACE, EGFR, HB-EGF, and TGF- $\alpha$. Results: Oxidant-induced permeability (5-fold/h) as well as increased EGFR and ERK1/2 phosphorylation. These were blocked $>90 \%$ by EGFR and TACE inhibitors as well as blocking Abs to the EGFR and TGF- $\alpha$. Blocking protein to HB-EGF or Abs to amphiregulin had little effect on oxidant-induced permeability. However, treatment of resting cells with HB-EGF blocker (but not other blockers) increased permeability five-fold. Immunofluorescence images reveal EGFR and HB-EGF localize to the cell periphery in resting monolayers, while TACE and TGF- $\alpha$ stain diffusely. However, oxidant induces translocation of both TACE and TGF- $\alpha$ to the cell-cell contact zones. Conclusions: These data support a significant and new TACE-EGFR model for regulation of intestinal permeability. Our data strongly support TGF- $\alpha$ as the principal EGFR ligand cleaved by TACE to initiate permeability induced by oxidant stress. In addition they support a role for paracrine HB-EGF/EGFR signaling in maintaining baseline impermeability. Co-translocation of TACETGF- $\alpha$ to cell-cell contact zones probably facilitates TACE cleavage of TGF- $\alpha$ to induce permeability.

764

A Combined Regimen of Infliximab and Azathioprine Induces Better Endoscopic Healing Than Classic Step-Up Therapy in Newly Diagnosed Crohn's Disease

Geert R. D'Haens, Daan Hommes, Filip Baert, Martine de Vos, Filip Caenepeel, Gert van Assche, Guy Lambrecht, Jean Claude Coche, Severine Vermeire, Marijke van Camp

Current guidelines for treatment of moderate-severe Crohn's disease (CD) recommend corticosteroids and at a later stage immunosuppressives (IS)such as azathioprine. Often, CD complications develop during the period prior to the initiation of IS, probably because of insufficient mucosal healing with steroid regimens. We examined if a strong induction regimen with anti-TNF therapy in combination with IS given in an early phase of the disease induced better mucosal healing than 'classic' step-up treatment with corticosteroids. Methods: 133 patients with recently diagnosed $C D$ were enrolled in a prospective trial and randomised to receive treatment with 3 infusions of $5 \mathrm{mg} / \mathrm{kg}$ infliximab (IFX) + azathioprine 2-2.5 mg/ $\mathrm{kg} /$ day (TD or 'top-down' treatment) or corticosteroids at standard dosage (SU or 'step-up' treatment). In the latter group, IS was added in case of relapse and need for further steroids; IFX was given if IS failed. Patients enrolled at 8 centers participated in this substudy and had ileocolonoscopy 2 years after enrollment. Results were compared by a blinded investigator with the endoscopy reports at diagnosis. In 5 ileal and colonic segments lesions were scored as follows: $0=$ no ulcers, $1=$ aphthoid ulcers, $2=$ larger ulcers, $3=$ ulcerated stenosis. The primary endpoint was disappearance of all ulcers; the secondary endpoint was percentage of reduction of all CD lesions. Results: 44 pts have reached year 2 and underwent repeat endoscopy. 24 pts received TD therapy and 20 classic SU therapy. The characteristics of the pts and the baseline endoscopies in both groups were comparable. At year 2, all pts in the TD group received IFX and IS vs 45\% IFX and $60 \%$ IS in the SU group. The interval between the last IFX infusion and the second endoscopy was $15.8 \pm 8.7$ months in TD vs $7.44 \pm 4.4$ months in $\mathrm{SU}(\mathrm{p}=0.001)$ and the mean duration of IS was 24 months in TD vs 19 months in the SU group. Complete ulcer disappearance was seen in 17/24 TD pts (71\%) vs in $6 / 20 \mathrm{SU}$ pts $(30 \%)(\mathrm{p}<0.001)$. Mucosal healing was significantly more pronounced in
TD patients (ulcer reduction $88 \%$ versus $47 \%, \mathrm{p}<0.001$; in TD the score went from 5.75 \pm 2.47 to $0.75 \pm 1.60$, in SU from $5.40 \pm 2.41$ to $3.25 \pm 2.97, \mathrm{p}<0.001$ ). Conclusion: the 'top-down' strategy with IFX+IS is superior to induce mucosal healing two years after the start of therapy. This superiority vs classic 'step-up'-therapy is maintained even in patients who receive IFX and IS later on. Since persisting lesions lead to development of complications and healing is associated with a reduction in surgery and hospitalisations, this strategy should be considered in all patients with severe newly diagnosed Crohn's disease.

765

The Treatment with An Oral Spherical Adsorptive Carbon (AST-120) Improves Anal Fistula, PDAI and CDAI Scores - A Randomized Double-Blind PlaceboControlled Trial

Yoshihiro Fukuda, Masakazu Takazoe, Akira Sugita, Tadashi Kosaka, Toshihiko Tomita, Kazutoshi Hori, Ken Fukunaga, Hiroto Miwa, Takayuki Matsumoto, Hiromasa Koizuka, Takashi Sakagami, Takashi Shimoyama

BACKGROUND Crohn's disease (CD) patients are often suffering from anal fistulas, leading difficult lives. The current therapies with various drugs including anti-TNF- $\alpha$ antibodies are effective in most of fistulizing $C D$, but anal fistulas are still remaining unhealed in some patients. Surgical treatment including fecal diversion and stoma is indicated, however, not all of patients can accept it. We conducted a randomized, double-blind, placebo-controlled multi-center clinical trial of an oral spherical adsorptive carbon (AST-120). METHODS CD patients with active fistula under medical treatments were randomly assigned to receive either AST-120 or placebo for 8 weeks. The number and status of draining fistulas were evaluated by the investigators at 0,4 and 8 weeks. The improvement was a reduction of $50 \%$ or more from base line in the number of draining fistulas observed both at 4 and at 8 weeks. The fistula remission was defined by closure of all draining anal fistulas both at 4 and 8 weeks. Severities of symptoms related to CD were graded referring to a patient diary. Also, Crohn's Disease Activity Index (CDAI) and Perianal Disease Activity Index (PDAI) were evaluated. RESULTS Sixty-two patients were evenly assigned to both group and were given AST-120 $(n=27)$ or placebo( $n=30)$. The fistula improvement rate in the AST120 group $(37.0 \%)$ was significantly greater than that in the placebo group $(10.0 \%)(\mathrm{p}=$ 0.025). The fistula remission rate in the AST-120 group (29.6\%) was significantly greater than that in the placebo group $(6.7 \%)(\mathrm{p}=0.035)$. Symptomatic scores such as drainage amount, perianal pain and fecal consistency were significantly improved in the AST-120 group but not in the placebo group. PDAI scores significantly improved both at 4 weeks and at 8 weeks in the AST-120 group compared with those in the placebo group ( $\mathrm{p}=0.004$ and $\mathrm{p}=0.005$, respectively). Also CDAI scores significantly improved both at 4 weeks and at 8 weeks in the AST-120 group compared with those in the placebo group ( $\mathrm{p}=0.007$ and $\mathrm{p}=0.001$, respectively). The AST- 120 treatment was generally well tolerated and was observed no life threatening adverse events. CONCLUSIONS The AST-120 treatment is safe and effective for the control of intractable anal fistulizing $C D$.

766

A Meta-Analysis of the Placebo Outcomes in Clinical Trials Evaluating Maintenance Therapy for Patients with Crohn's Disease

Monina F. Pascua, Gary R. Lichtenstein, James D. Lewis, Colleen Brensinger, Chinyu Su

Background: Placebo-controlled RCTs (PC-RCTs) are the gold standard for evaluating efficacy of maintenance therapy in patients with Crohn's disease (CD). There has been no formal systematic analysis in the literature on the outcome of placebo therapy in maintenance clinical trials for $\mathrm{CD}$ patients in remission. Aim: To estimate the proportion of $\mathrm{CD}$ patients treated with placebo who maintain remission for the duration of the PC-RCTs and to identify factors influencing this rate. Methods: PC-RCTs evaluating CD maintenance therapy were identified from MEDLINE from 1966 through September 2005. Studies evaluating surgicallyinduced remission were excluded. Outcomes were dichotomized into remission vs. active disease at the primary endpoint of the study. Pooled estimate of the proportion remaining in remission was calculated using logistic regression analysis after applying sample weights according to the placebo size. The influence of study design features on this rate was estimated using univariate logistic regression models clustering on study. Results: Of 23 studies fulfilling the inclusion criteria, the pooled estimate of the proportion of placebotreated patients remaining in remission was 0.45 (95\% CI 0.38-0.52), with significant heterogeneity among studies $(\mathrm{p}<0.001)$. These rates were similar if analyses were limited to 20 studies using CDAI and 12 studies using CDAI> 150 plus a minimum point increase as remission definition $(0.44$ and 0.46 , respectively). Factors associated with the placebo remission rate are shown in the Table. The placebo remission rate was independent of the following features: publication years, sample size ratio, drug administration route, number/ frequency of study visits, and age, disease duration/distribution, prior surgery and smoking status of placebo patients. Conclusions: The placebo rate of maintaining remission was $45 \%$ in prior CD trials for maintenance therapy. Study features that influence the placebo remission rate include study duration, trial location, length of remission prior to study, and steroidinduced remission. These factors should be considered when designing future studies to optimize study efficiency.

\begin{tabular}{|c|c|}
\hline Study Features & OR* $(95 \% \mathrm{CI}), \mathrm{P}$-value \\
\hline Location (N Am vs Europe) & $1.75(1.09-2.82) \mathrm{p}=0.02$ \\
\hline Follow-up duration (12 to $\leq 24$ mo vs $<12 \mathrm{mo})$ & $0.57(0.32-1.03) \mathrm{p}=0.06$ \\
\hline Mean duration of remission prior to enrollment $(\geq 4 \mathrm{mo}$ vs $<4 \mathrm{mo})$ & $1.57(1.002-2.46) \mathrm{p}=0.05$ \\
\hline$\%$ of Placebo with prior CD surgery (per 10\% $\uparrow)$ & $1.20(0.98-1.46) \mathrm{p}=0.08$ \\
\hline Steroid induced remission (Y v. N) & $0.59(0.35-1.00) \mathrm{p}=0.05$ \\
\hline
\end{tabular}

* OR >1 indicates the factor was positively associated with a higher proportion of patients in the placebo arm remaining in remission 
Validation of the Crohn's Disease Activity Index As a Measure of PostOperative Disease Recurrence

Thomas D. Walters, A H. Steinhart, C Bernstein, W Tremaine, B Wolff, S Ross, R Parkes, M McKenzie, R S. McLeod

BACKGROUND/AIMS: Objective measures of disease activity are important as endpoints in randomized controlled trials (RCT). The Crohn Disease Activity Index (CDAI) has been used in medical trials with scores $<150$ indicative of remission. Its value in assessing postoperative recurrence is unknown. Taking advantage of an international, prospective, RCT investigating post-operative disease recurrence, we examined the association between a combined clinical/endoscopic assessment of disease activity and the CDAI. We desired to determine the suitability of the CDAI as a single post-operative outcome measure. METHOD/ PATIENTS: 120 patients [38\% male] from 16 surgical units were included in an RCT comparing surgical techniques. All underwent clinical and colonoscopic evaluation 12 months post ileocolic resection, at a mean age of 39.7 yrs [+/- 13.5]. Outcomes were adjudicated by a three person committee and consensus reached. Endoscopic appearance was assessed using the Rutgeerts score [i0-i4]. Symptomatic disease recurrence was defined by the composite of symptom severity warranting therapy and an endoscopic score $>/=$ i2. Eighty eight [73\%] subjects had CDAIs calculated according to established criteria. Comparisons were made using both non-parametric methods and the Receiver Operator Curve (ROC). RESULTS: Thirty five [40\%] patients had evidence of recurrent disease [23\% symptomatic, $17 \%$ endoscopic only] by 12 months. Seven percent had endoscopy scores $>\mathrm{i} 2$ in the absence of symptoms. Median CDAI for symptomatic recurrence was 215 [Interquartile Range (IQR): 116 to 280]; significantly different from asymptomatic subjects [median 70 , IQR 40 to $125, \mathrm{p}<0.001$ ]. The area under the ROC for symptomatic disease and CDAI was 0.80 [ $95 \% \mathrm{CI} 0.67$ to $0.93, \mathrm{p}<0.001]$. Recurrence was best predicted by a CDAI of $>/=$ 148 [sensitivity $70 \%$, specificity $85 \%$, PPV 58\% and NPV 91\%]. CDAI did not differentiate between endoscopic scores $>/=$ or $<\mathrm{i} 2$ in the absence of significant symptoms [median 64 vs $88, \mathrm{p}=0.99]$. CONCULSIONS: This study confirms that a CDAI of 150 is the best cutpoint for assessing disease activity. CDAI is useful in assessing outcome in postoperative RCTs. However, given a PPV $<60 \%$, it does not replace combined clinical/endoscopic evaluation as a single outcome measure in the assessment of post-operative disease recurrence. Supported, in part, by Research Grants from the Canadian Institutes of Health Research (CIHR) and the Crohn's \& Colitis Foundation of America (CCFA).

768

Natalizumab Maintains Remission in Patients with Moderately to Severely Active Crohn's Disease for Up to 2-Years: Results from An Open-Label Extension Study

Remo Panaccione, Jean-Frederic Colombel, Robert Enns, Brian Feagan, Stephen Hanauer, Ian Lawrance, Paul Rutgeerts, William Sandborn, Stefan Schreiber, Stephan Targan, Sander van Deventer

Introduction: Phase 3, randomized, controlled, trials have demonstrated that natalizumab is effective as both induction and long-term maintenance therapy for Crohn's patients with moderately to severely active disease. Patients who responded to 3 monthly natalizumab ( $300 \mathrm{mg}$ ) infusions in the ENACT-1 induction trial were re-randomized to maintenance therapy with 12 monthly infusions of either natalizumab or placebo in the ENACT-2 trial. A significantly higher proportion of patients in the natalizumab group maintained a sustained remission (defined as a Crohn's Disease Activity Index [CDAI] score <150) through 12 months of continuous therapy compared with the placebo group (39\% [5]/130] vs. $15 \%$ [18/120], p < 0.001). This analysis was undertaken to assess the ability of natalizumab to maintain remission for up to an additional year in natalizumab-treated patients who were in remission at month 12 of the ENACT-2 trial. Methods: Patients who completed month 12 of the ENACT-2 trial were eligible to enroll in a phase 3, long-term, open-label, extension (OLE) study. The primary objective of this 2-year study was to examine the long-term safety and tolerability of natalizumab. Secondary efficacy endpoints included evaluation of the ability of natalizumab to maintain remission. The population for this analysis included 146 natalizumab-treated patients who had maintained remission at month 12 of ENACT-2, and subsequently enrolled in the OLE study and received an additional 12 months of natalizumab therapy. Remission rates were calculated using last observation carried forward. Results: Ninety-eight percent (143/146) of patients who had maintained remission at month 12 of ENACT-2 were in remission at baseline of the OLE study. The proportion of these patients in remission following 6 additional infusions of natalizumab in the OLE study was $89 \%$ (130/146) at 24 weeks, and 84\% (122/146) following a total of 12 additional natalizumab infusions (48 weeks). Conclusions: Maintenance therapy with monthly infusions of natalizumab has previously been shown to achieve a sustained remission through 12 months in a significant number of Crohn's disease patients. Patients in this analysis who were in remission after 12 months of natalizumab therapy were highly likely to remain in remission through an additional 12 months of treatment.

769

A Phase I Study: Visilizumab Therapy in Crohn's Disease (CD) Patients Refractory to Infliximab Treatment

Daan Hommes, Stephen Targan, Daniel C. Baumgart, Axel U. Dignass, Lloyd Mayer, James N. Lowder

Purpose: Studies of visilizumab, a humanized anti-CD3 antibody, in ulcerative colitis (UC) patients, show its tolerability and clinical activity at doses ranging from 5 to $15 \mathrm{mg} / \mathrm{kg}$ administered on 2 consecutive days. The current open-label study was designed to evaluate the safety and clinical activity of $10 \mu \mathrm{g} / \mathrm{kg}$ visilizumab doses in patients with moderate-tosevere inflammatory non-penetrating Crohn's disease. Methods: Subjects with a diagnosis of CD, a Crohn's Disease Activity Index (CDAI) > 250, a C-Reactive Protein (CRP) > 4 mg/ $\mathrm{L}$ (above normal), and endoscopic evidence of active inflammatory disease were treated with bolus intravenous visilizumab on days (D) 0 and 1 . A clinical response was defined as a decrease of CDAI at D59 of more than 100 points below the baseline value. Results: The protocol will enroll a total of 18 subjects. To date, a total of 12 subjects have enrolled, 8 of them have been followed for at least 59 days and are discussed below. The median baseline CDAI was 386.5 (308-516). The median baseline CRP was $30.5 \mathrm{mg} / \mathrm{L}$ (2.0-69). All subjects were steroid refractory $(n=3)$ or dependent $(n=5)$ with a median dose of $20 \mathrm{mg} / \mathrm{D}$ prednisone. All subjects had received infliximab; 2 were primary non-responders, 1 had an intolerable infusion reaction and 5 had lost activity after an initial response. Six of the 8 subjects had a day 59 clinical response. The median D59 CDAI was 218 (150-366). Three complete remissions $(\mathrm{CDAI}<150)$ at any time and 1 at D59 were observed. Both primary infliximab failures responded to visilizumab. In visilizumab responders, the CRP decreased quickly after drug administration and it remained low in 2 subjects who achieved clinical remission. Mild to moderate cytokine release syndrome occurred in the majority of subjects. The adverse event (AE) profile of severity, incidence and drug relatedness was similar to that seen in those UC subjects who received $10 \mu \mathrm{g} / \mathrm{kg}$ visilizumab. No lymphoproliferative, malignant or life-threatening AEs were reported. Transient elevations of transaminases were observed in 4 of 8 subjects. Conclusion: Two $10 \mu \mathrm{g} / \mathrm{kg}$ visilizumab doses administered as an IV bolus on consecutive days were tolerated and produced a rapid and sustained improvement in $6 / 8$ steroid and infliximab resistant CD subjects.

770

Is Irritable Bowel Syndrome (IBS) a Diagnosis of Exclusion? A Survey of Primary Care Providers, Gastroenterologists, and IBS Experts Brennan M. Spiegel, Mary Farid, Eric Esrailian, Jennifer Talley, Lin Chang

Background: Published guidelines state that IBS is not a diagnosis of exclusion, and encourage clinicians to make a positive diagnosis using the Rome criteria alone. Yet many clinicians remain unsettled by the prospect of overlooking alternative diagnoses, and instead approach IBS as a diagnosis of exclusion by conducting a series of tests prior to "settling" on a diagnosis of IBS. We measured provider beliefs about whether IBS is a diagnosis of exclusion, and measured testing proclivity in IBS between nurse practitioners (NPs), primary care physicians (PCPs), GIs, and IBS experts. Methods: We developed an online survey consisting of 2 clinical vignettes: 1) Rome-II IBS-D patient, and 2) Rome-II IBS-C patient, both withou alarm features. We developed the vignettes with IBS experts to ensure face validity and comprehensiveness, and subjected the survey to iterative revisions by experts in survey design to ensure acceptable psychometric properties. The survey elicited provider knowledge, attitudes, and beliefs in IBS, including items about testing proclivity and beliefs regarding IBS as a diagnosis of exclusion. We surveyed a random sample of $300 \mathrm{VA}$ NPs, $300 \mathrm{VA}$ PCPs, 300 GIs from the AGA membership rolls, and 45 internationally-recognized IBS experts. Results: The response rate was $31 \%$ (102 NPs, 89 PCPs, 90 GIs, 27 experts). IBS experts were less likely than non-experts to endorse IBS as a diagnosis of exclusion ( $8 \% \mathrm{vs}$ $72 \% ; \mathrm{p}<0.0001)$. Among non-experts, $42 \%$ of GIs and $76 \%$ of both NPs and PCPs shared this belief $(\mathrm{p}<0.01$ for GI vs $\mathrm{NP}+\mathrm{PCP})$. In the IBS-D vignette, experts were more likely than non-experts to make a positive diagnosis of IBS ( $67 \%$ vs. $38 \%$; $<<0.001$ ), to perform fewer diagnostic tests ( 2 vs $4.1 ; \mathrm{p}<0.01$ ), and to expend less cost on testing ( $\$ 297$ vs $\$ 658$ $\mathrm{p}<0.01$ ). These trends were repeated in the IBS-C vignette (+IBS=67\% vs $20 \%, \mathrm{p}<0.01$ tests $=1.4$ vs $2.2, p=0.06$; cost $=\$ 298$ vs $\$ 550, p=0.03$ ). In regression analysis adjusting for provider group, expert status, age, and gender, providers who believed IBS is a diagnosis of exclusion ordered 1.6 more tests and consumed $\$ 364$ more than those who did no $(\mathrm{p}<0.0001)$. Conclusions: Most $(72 \%)$ community providers in this sample believe that IBS is a diagnosis of exclusion, and this belief is associated with increased diagnostic testing and resource utilization. In contrast, "IBS experts" comply more closely with published guidelines to make a positive diagnosis with minimal diagnostic testing. This disconnec between experts and community practice indicates that better dissemination and implementation of IBS guidelines is warranted in order to minimize variation and improve cost-effectiveness of care.

771

Yield of Diagnostic Testing in Patients with Suspected Irritable Bowel Syndrome (IBS): A Prospective, Us Multi-Center Trial

Brooks D. Cash, Allan H. Andrews, Dong H. Lee, John H. Smith, Cecilia H. Kim, Richard Saad, Jennifer K. Rai, William D. Chey

Background: A recent systematic review examining the yield of frequently recommended tests concluded that there was little evidence to support exhaustive testing in suspected irritable bowel syndrome (IBS) pts without alarm symptoms. We report preliminary results from a prospective, multi-center US trial intended to validate these findings. Methods: Consecutive adult pts with suspected non-constipation IBS (Rome II) were invited to participate. Exclusion criteria included alarm symptoms such as hematochezia or weight loss, a family history of organic gastrointestinal (GI) disease, a previous evaluation for IBS, or conditions precluding evaluation. Patients underwent colonoscopy with random biopsies and the following: $C B C$, metabolic panel, thyroid function testing (TFT), antibodies for celiac disease (CD-ab) and inflammatory bowel disease (IBD-ab), and lactase genomics. These latter 3 "non-standard tests" were also collected from asymptomatic pts undergoing screening colonoscopy. Positive CD-ab were confirmed with EGD and small bowel biopsy and IBS symptoms were reassessed 3 months after initiation of a gluten-free diet in confirmed CD. Results: 250 IBS pts (mean age 44.8 years, $66.0 \%$ female) and 201 controls (mean age 53.7 years, $41.8 \%$ female) have been enrolled. $3.2 \%(8 / 250)$ IBS pts were identified with organic disease felt to be responsible for their symptoms. One pt each was diagnosed with IBD, peritoneal carcinomatosis, and sigmoid volvulus. The prevalence of abnormal TFT was $15(6 \%)$ in IBS pts, consistent with the population norm. CD was confirmed in $4(1.6 \%)$ IBS pts and $2(1 \%)$ controls $(\mathrm{p}=0.07)$, however the prevalence of abnormal CD-ab was significantly greater in IBS pts. Conclusions: There does not appear to be a significant difference between IBS pts and asymptomatic controls in organic GI disease diagnosed via colonoscopy and random biopsies or blood tests for thyroid disease, IBD, and lactose intolerance, suggesting that such testing should not be routinely obtained as part of an initial evaluation of IBS pts without alarm features. Abnormal CD-ab, but not biopsy proven CD, 
are more common in IBS pts, perhaps indicative of gluten hypersensitivity. Whether dietary interventions are of benefit in this population remains to be determined.

Abnormal results (\%)

\begin{tabular}{|c|c|c|c|}
\hline Test & IBS $(\mathrm{n}=250)$ & Controls $(\mathrm{n}=201)$ & $\mathrm{p}^{*}$ \\
\hline CD-ab & $18(7.2 \%)$ & $3(1.5 \%)$ & 0.006 \\
\hline ASCA IgA and IgG & $33(13.2 \%)$ & $22(10.9 \%)$ & 0.563 \\
\hline ANCA & $24(9.6 \%)$ & $15(7.5 \%)$ & 0.501 \\
\hline Omp-C & $44(17.6 \%)$ & $18(9.0 \%)$ & 0.009 \\
\hline Lactase nonpersistence & 63 & 51 & 1.0 \\
\hline
\end{tabular}

*Fisher's Exact Test

772

Screening for Celiac Disease (CD) in Patients Fulfilling the Rome II Criteria

for Irritable Bowel Disease (IBS) in a Secondary Care Hospital in the

Netherlands: A Prospective Observational Study

Egbert-Jan Van der Wouden, G. F. Nelis, J. Vecht

Introduction. A few studies showed that screening for $\mathrm{CD}$ in patients with symptoms suggestive of IBS is cost effective, while other studies suggested no benefit. We, therefore, started to screen routinely patients with typical IBS symptoms for celiac disease using antiendomysial antibodies. Methods. Patients attending the outpatient department of our hospital and fulfilling the ROME II criteria for IBS were eligible. All patients received standard care including an interview, physical examination, routine laboratory tests and an endoscopy of the lower gastrointestinal tract. Apart from these tests the treating physician could order any other diagnostic test as considered necessary. Furthermore, in all patients anti-endomysial antibodies were measured according to the instructions of the manufacturer. Results. Between november 2002 and november 2005161 patients (53 males, median age 33 years (range: 16-75)) fulfilling the ROME II criteria were included. 153 patients were finally diagnosed with IBS, 3 with Crohns disease, 2 with hyperthyroidism, 1 with endometriosis, 1 with lactase deficiency and 1 with idiopathic bile salt malabsorbtion. 15 patients were not screened with anti-endomysial antibodies although in 4 of them duodenal biopsies showed normal mucosa. In none of the 146 evaluable patients fulfilling the ROME II criteria $(0 \%, 95 \%$ confidence interval: $0-2.6 \%$ ) anti-endomysial antibodies were positive. In 32 patients the treating physian ordered duodenal biopsies as well, mostly because of predominant diarrheal symptoms. In two of them MARSH 1 was found, in the the other 30 patients duodenal biopsies were normal. Conclusion: Screening for CD with anti-endomysial antibodies in IBS patients in a secondary care hospital in the Netherlands seems to be ineffective.

\section{3}

The Effectiveness of a Model Physician-Patient Relationship Versus Usual Care in Irritable Bowel Syndrome (IBS): A Randomized Controlled Trial Brennan M. Spiegel, Bruce Naliboff, Emeran Mayer, Roger Bolus, Ian Gralnek, Paul Shekelle

Background: Practice guidelines emphasize that the physician-patient relationship is the fundamental cornerstone of treatment in IBS, and that an effective relationship may improve health related quality of life (HRQOL) more than any alternative therapy currently available. However, the components of this relationship have not been operationally defined or prospectively tested, and data suggest that the physician-patient relationship is often sub-optimal in IBS. We performed a study to compare the effect of an intervention aimed at improving the physician-patient relationship in IBS vs. usual care. Methods: We performed a prospective, randomized controlled trial comparing a multi-factorial intervention vs. usual care (i.e. no intervention) in patients with Rome II positive IBS evaluated in the West Los Angeles VA between Jan-Sep 2005. The intervention included 4 components: (1) point-of-care questionnaire designed to rapidly capture patients' agenda, concerns, and HRQOL; (2) MD prompt card outlining a checklist of key components of effective communication in IBS; (3) multi-media patient educational kit, including info pamphlets, dietary card, website listings, and a self-empowerment video; and (4) patient home educational mailings. The intervention did not include specific diagnostic or treatment suggestions. The primary outcome was 3-month change scores on the IBS-QOL - a validated measure of diseasetargeted HRQOL. Secondary outcomes included global symptom relief (percentage scoring $>4$ on 6-point Likert scale), satisfaction with care (7-point scale), and patient assessment of MD interpersonal (IP) skills (IP Skills Rating Form). Results: 63 patients were enrolled (31 intervention, 32 control). The average age was 52, and $33 \%$ were women. There were no significant baseline differences in key covariates between groups. The mean 3-month change scores on the overall IBS-QOL were +2.4 and +12.2 for the control and intervention groups, respectively $(\Delta=9.8,95 \% \mathrm{Cl}=5.2,12.4 ; \mathrm{p}=0.01$; effect size $[\mathrm{ES}]=0.7)$. The $\%$ with global symptom relief was $15 \%$ and $56 \%$ in the control and intervention groups, respectively ( $\mathrm{p}=$ 0.001). Satisfaction with care and assessment of MD interpersonal skills were higher in the intervention group ( $\mathrm{p}<0.03$ for both). Conclusions: These data indicate that a standardized physician-patient relationship improves global outcomes in IBS over and above routine care. The HRQOL impact observed in this study ( $E S=0.7$ ) exceeds the HRQOL impact of the most effective pharmaceutical agents in IBS (ES range $=0.1-0.5$ ), suggesting that medical therapy alone is sub-optimal if not delivered in the context of a supportive physicianpatient interaction
Chronic Treatment-Resistant Constipation in Children: Experience with Radioisotope Transit Studies, Colonic Manometry and Transcutaneous Electrical Stimulation

Bridget R. Southwell, Sebastian K. King, Jonathan R. Sutcliffe, Janet Chase, Susie Gibb, Val J. Robertson, David Cook, Anthony G. Catto-Smith, John M. Hutson

Chronic constipation can be due to dietary defects, avoidance of defecation, withholding, abnormal anal sphincter responses or defects in transit through the colon. New clinical methods (radioisotope transit studies, manometry) should allow different subgroups and etiologies to be separated. This study reports our experience using these methods to analyze 300 children with chronic treatment-resistant constipation presenting to a surgeon at a major tertiary children's hospital in Australia. Methods: Metabolic and genetic disorders were excluded by blood tests and Hirschsprung disease by rectal biopsy. Whole gut transit was measured using gamma camera scintigraphy at $0,2,6,24,30 \& 48$ hrs. $24 \mathrm{hr}$ colonic manometry using 8 channel water-perfused catheter was performed via established appendix stomas. For transcutaneous electrical stimulation using interferential current (IFT), 2 surface electrodes were placed anterior $\& 2$ posterior at the level of the umbilicus with current crossed and applied 3 times/wk for 4 weeks. Results: Children with palpable fecaloma were classified as having anal retention and not sent for nuclear transit studies or biopsies. 120 nuclear transit studies have been performed since 1995. 48hr transit studies clearly separated patients with normal transit $(16 \%)$ or anal retention (11\%) from patients with slow transit in the proximal colon. Slow colonic transit (SCT) consisted of pancolonic slowing (44\%), isolated holdup at the splenic flexure (16\%) and combined small bowel/large bowel slowing (13\%). Colonic manometry was performed on 26 children who had transit studies (18 SCT, 8 non-SCT). SCT children had significantly $(\mathrm{p}<0.05)$ reduced frequency of anterior propagating contractions and no increase in activity post-waking or post-meal compared to non-SCT children with anal retention or normal transit. IFT was applied to 16 children. 8/ 16 were followed by daily diaries. 13/16 increased defecation and 15/16 stopped soiling. The effects lasted more than 3 months post-stimulation. Two patients had colonic manometry before and 6 months after electrical stimulation. Both showed normalized defecation and increased frequency of antegrade propagating contractions into the normal range. Conclusions: Many children with chronic constipation have slow colonic transit independent of outlet obstruction. Clinical assessment using radioisotope transit studies can separate these two groups. SCT is associated with reduced propagating contractions and lack of postprandial increase in activity. Transcutaneous electrical stimulation can increase propagating contractions into the normal range and the effects last months after the stimulation.

775

Prolonged Measurement of Colonic Motility in Slow Transit Constipation Eduard A. Van Hoboken, Hilde Slingerland, Ad A. Masclee

Slow Transit Constipation (STC) is characterized by delayed transit over all colonic segments. Recent studies indicate that decreased phasic colonic motility may be involved in the pathophysiology of STC. Colonic motility is best evaluated by ambulatory colonic manometry. However, using this method the repeatability between two measurement days has been shown to be poor. Therefore ambulatory colonic manometry should be performed over at least several days. Our aim was to investigate colonic motility in STC-patients compared with healthy volunteers (HV) by recording over a prolonged period. Thirteen STC-patients $(12 \mathrm{~F}, 47 \pm 4$ yrs; colonic transit time $115 \pm 13$ hrs (ref: $<69 \mathrm{hrs})$ ) and $12 \mathrm{HV}(10 \mathrm{~F}, 40 \pm 3 \mathrm{yrs})$ underwent 24-hr colonic manometry on two consecutive days. A catheter with 6 solid-state pressure sensors (sl-2 hepatic flexure, s3-4 splenic flexure, s5-6 sigmoid; $10 \mathrm{~cm}$ distance) was positioned endoscopically and clipped to the mucosa. Subjects were ambulatory with standardized resting and eating conditions. Data were averaged over two measurement days. Colonic motility was evaluated by motility index (MI=wave frequency*duration*amplitude; $10^{\wedge} 3 \mathrm{sec} * \mathrm{mmHg}$ ) per hour with MI-values averaged per period (evening-night-daytime). Furthermore several distinct pressure patterns were analyzed, i.e. High-Amplitude Propagated Contractions (HAPC's; $\geq 3$ sensors; $\geq 80 \mathrm{mmHg}$ ), propagated pressure waves ( $\geq 2$ sensors) and retrograde pressure waves $(\geq 2$ sensors; directed orally). MI per hour was sign. $(\mathrm{p}<0.05)$ reduced in STC-patients compared to controls during daytime (14 \pm 2 vs.22 \pm 2 ) and evening $(11 \pm 2$ vs. $19 \pm 4)$. During the night MI was $7 \pm 1$ in both STC and controls. HAPC-frequency per 24 -hr in STC-patients was significantly reduced $(2.2 \pm 0.7$ vs. $5.3 \pm 0.7 ; \mathrm{p}<0.05)$. In STCpatients HAPC's predominantly $(44.2 \%)$ originated at the splenic flexure (s4). In controls HAPC's originated more often $(51.5 \%)$ at the proximal colon ( $\mathrm{s} 1 ; \mathrm{p}<0.05)$. HAPC's propagated over more than three sensors in 30\% of cases in STC-patients versus $52.3 \%$ in HV $(p<0.05)$. Propagated pressure waves occurred less frequently in STC-patients during daytime $(10 \pm 1$ vs $17 \pm 2 / \mathrm{hr} ; \mathrm{p}<0.05)$. Retrograde pressure waves occurred less frequently $(\mathrm{p}<0.05)$ in STC patients during night $(2 \pm 0$ vs $5 \pm 1 / \mathrm{hr})$ daytime $(4 \pm 1$ vs $8 \pm 2 / \mathrm{hr})$ and evening $(3 \pm 1$ vs $7 \pm 1$ / hr). Conclusions. Overall phasic colonic motility is sign. reduced in patients with STC during daytime, but not during nighttime. Furthermore, in STC-patients HAPC-frequency is reduced, HAPC's originate more distally and propagate over a shorter distance. Propagated and retrograde pressure waves occur less frequently in STC-patiens. All of these features may contribute to delayed colonic transit in STC.

776

Overlapping Gene Expression Profiles of Experimental Colitis and IBD Anje Te Velde, Floor de Kort, Ellen Sterrenburg, Inge Pronk, Fiebo Ten Kate, Daniel Hommes, Sander van Deventer

Background: The pathogenesis of Crohn's disease and Ulcerative Colitis is directly related to a dysfunction of innate responses to bacterial products. Mouse models of inflammatory bowel diseases (IBD) are used to unravel the pathophysiology of IBD and to study new treatment modalities, but their relationship to Crohn's disease or Ulcerative Colitis is unclear. Aim: This study was set up to investigate the gene expression profile of the colon of mice with an established colitis and compare this with the expression of genes known to be associated with IBD. Methods: We analyzed the gene expression in three widely used models 
of experimental colitis with microarrays. In two of the models exogenous agents induce the colitis: intrarectal application of TNBS and oral administration of DSS. In the third model the colitis is induced after transfer of a $\mathrm{T}$ cell population (CD4+CD45RBhigh $\mathrm{T}$ cells) that lacks regulatory cells into an immunodeficient host. Agilent mouse TOX oligonucleotide microarrays were hybridised with amplified and labelled cRNA, generated from isolated total colon mRNA. Results: Of 19 genes that are known to be upregulated in IBD: TNF, IFN-gamma, IL-22, Socs3, CCL2, 5, 7 and 20, CXCL5 and 10, MMP3, 7 and 14, Timp1, Reg3gamma and PAP, S100A8, S100A9 and Ptgs2: 2/19 are upregulated in TNBS, 11/19 are upregulated in DSS and 18/19 are upregulated in the CD45RB transfer colitis. Conclusion: Our gene expression profiles indicate that the CD45RB transfer model best resembles human IBD, and this is the only model that is characterized by chronic inflammation. In contrast, the DSS and TNBS model reflect a more acute insult to the intestinal mucosa, either by inflicting epithelial damage (DSS colitis) or by causing a hapten-dependent DTH reaction (TNBS colitis)

777

Combination of QTL and Expression Analyses Revealed Cd14 As a Candidate Gene for IBD in Ill0-Deficient Mice

Maike F. De Buhr, Robert Geffers, Wiebke Hansen, Astrid M. Westendorf, Joerg Lauber, Jan Buer, Hans J. Hedrich, Michael Maehler, Andre Bleich

Background \& Aim: Interleukin-10-deficient, $I 10^{\operatorname{tm} 1 \mathrm{Cgn}}\left(\mathrm{Il10^{-/ }}\right)$, mice serve as a model for inflammatory bowel disease (IBD). The severity of colitis depends on the inbred strain carrying the disrupted $I 110$ gene: $\mathrm{C} 3 \mathrm{H} / \mathrm{HeJBir}(\mathrm{C} 3)$ mice develop severe colitis, whereas C57BL/6J (B6) mice are colitis resistant. Genetic dissection of this susceptibility revealed ten colitogenic quantitative trait loci (QTL). The aim of this study was to identify valuable candidate genes by a combination of QTL mapping and expression analyses. Methods: Affymetrix GeneChips were used to detect gene expression in the colon of C3- and B6$1110^{-/-}$mice and their wildtype (WT) controls. Cd14 gene and CD14 protein expression was determined by real-time RT PCR and western blot analysis in the mouse strains indicated below. Naive peritoneal macrophages were harvested from mice of both $1110^{-/}$strains, cultured, stimulated with cecal bacterial antigens or left unstimulated, and stained with FITC-labeled CDI4 antibody for FACS analysis. Results: Microarray analysis revealed that sixteen genes were differentially expressed between B6- and C3-Il1 $10^{-/}$mice and were located within the QTL intervals. Cd14 showed prominent expression differences between B6- and C3-Il10 $0^{-1}$ as well as between B6 and C3 WT mice, which was confirmed by real-time RT PCR. Western blot analysis revealed strain differences also at the protein level. Cd14 expression rates determined by real-time RT PCR in animals with defective and intact TLR4 signalling $(\mathrm{C} 3, \mathrm{C} 3 \mathrm{H} / \mathrm{HeN}, \mathrm{B} 6)$ made the TLR4 defect of $\mathrm{C} 3$ mice unlikely as the reason for higher Cd14 expression. Lower Cd14 mRNA expression in germfree mice indicates a contribution of the microflora to Cd14 expression. Stimulation of naive peritoneal macrophages with bacterial antigens showed that B6 mice responded with a lower CD14 surface expression than C3 mice. Conclusions: The ultimate goal of QTL mapping is to identify genes underlying a complex trait, which is complicated by large QTL intervals containing multiple candidate genes. By combination of QTL and expression analyses, the number of candidate genes was reduced and Cd14 identified as a promising candidate. Studies are underway to investigate a possible contribution of $C d 14$, which plays an important role in innate immunity, to colitis susceptibility.

\section{8}

Wasp-Deficiency in Mice Leads to Global Regulatory T Cell Dysfunction with IL-10 Deficiency and Aberrant IL-2 Signaling Independent of Immune Synapse Formation

Michel Maillard, Vinicius Cotta-De-Almeida, Pierre Michetti, Cathryn Nagler-Anderson, Scott B. Snapper

Background: Naturally occurring CD4+CD25+Foxp3+ regulatory T cells (Tregs) play a critical role in the prevention of autoimmunity. An imbalance between effector $\mathrm{T}$ cells and regulatory $\mathrm{T}$ cells has been implicated in many murine models of Inflammatory Bowel Disease (IBD). The Wiskott-Aldrich Syndrome (WAS), a primary human immunodeficiency, results from a mutation in the gene encoding the Wiskott-Aldrich Syndrome Protein (WASP), and is associated with autoimmunity including an IBD-like illness. The majority of WASP-deficient (WKO) mice develop colitis; lymphocytes are required and CD4+ T-cells sufficient for colitis induction. In mature T cells, WASP is recruited to the immune synapse where it induces actin polymerization following $\mathrm{T}$-cell receptor signaling. We have recently demonstrated that WKO mice have decreased percentages of CD4+CD25+ Tregs and therefore hypothesized that Treg dysfunction may be central to the pathogenesis of colitis. Using the CD45RB transfer model, we observed a severe dysfunction of WKO Tregs in vivo. The aim of this study was to dissect the molecular mechanisms responsible for Treg dysfunction in WKO mice. Results: We looked at Foxp3 expression in WKO Tregs, a transcription factor that is critical for Treg lineage specification and function. The percentage of CD4+Foxp3+ Tregs in WKO mice was reduced in comparison to WT mice, similar to the reduction in CD4+CD25+ Tregs previously observed. The vast majority (>90\%) of CD4+CD25+ cells were also Foxp3+ in both WKO and WT mice. Correlating with our in vivo findings, we observed a marked reduction in suppression by WKO Tregs employing an in vitro suppression assay. This defect was also observed when using WKO CD4+CD25- cells as responder cells. In vitro pre-activation of WKO Tregs with IL-2 and plate-bound anti-CD3 partially rescued this defect. Finally, we observed a marked decrease in IL-10 secretion by WKO Tregs. We next analyzed actin polarization at the immune synapse by incubating $\mathrm{T}$ regs with antiCD3/anti-CD28 coated beads. Surprisingly, the percentage of WT Tregs with polarized actin was reduced in comparison to WT CD4+CD25- cells. Further, there was no difference in percentage of polarized WKO Tregs in comparison to WT Tregs. Similar findings were observed when spreading of WT or WKO Tregs was analyzed. Conclusion: Treg dysfunction likely plays a role in colitis development in WKO mice. IL-2 and IL-10 deficiency may play a critical role in WKO Treg dysfunction. Our data suggest that WT Tregs do not form an immune synapse and that aberrant synapse formation is not a mechanism accounting for the observed defects in WKO Tregs.
IL-23-Dependent $\mathrm{Cd}^{+} \mathrm{Th}_{\mathrm{IL}-17}$ Cells Mediate Chronic Colitis Charles O. Elson, Yingzi Cong, Terrill McClanahan, Robert Fick, Robert Kastelein

Purpose: IL-23 is increased in IBD lesions but its role is unclear. IL-23 supports a distinc lineage of $\mathrm{T}$ cells producing IL-17 $\left(\mathrm{Th}_{\mathrm{IL}-17}\right)$ that can mediate chronic inflammation. This study was done to define the role of Thl and of $\mathrm{Th}_{\mathrm{IL}-17}$ cells in chronic colitis in mice. Methods: Colitis was induced by transfer of a cecal bacterial antigen (CBA)-specific $\mathrm{C} 3 \mathrm{H} /$ HeJBir (C3Bir) $\mathrm{CD}^{+} \mathrm{T}$ cell line to $\mathrm{C} 3 \mathrm{H} / \mathrm{HeSnJ}$ SCID mice. Anti-CD3 activated polyclonal $\mathrm{CD}^{+} \mathrm{T}$ cells were transferred as a negative control. Cytokines were measured by flow cytometry, ELISA, and real time PCR. Monoclonal anti-IL-23p19 was administered at the same time as or 4 wks after CD4 T cell transfer. Histopathology colitis score was assessed in a blinded fashion. Results: IL-12p40, IFN $\gamma$, IL23p19, and IL-17 mRNA were all elevated in colitic lesions. The pathogenic C3Bir CD4 T cell line contained more cells producing only IL-17 (38\%) than those producing only IFN $\gamma(11 \%)$. A similar high frequency of $\mathrm{Th}_{\mathrm{IL}}$ ${ }_{17}$ cells was observed in CBA-reactive CD4+ $\mathrm{T}$ cells from IL-10 $10^{-1}$ and mdrl $\alpha^{-/-}$mice. C3Bir $\mathrm{CD}^{+}$cell lines producing only IL-17 or only IFN $\gamma$ were generated. After transfer to SCID mice, the $\mathrm{Th}_{\mathrm{L}-17}$ cells induced marked inflammation in a dose dependent manner (Table). Even at a dose as low as $10^{4}$ cells/mouse, $\mathrm{Th}_{\mathrm{IL}-17}$ cells induced more severe disease than did Thl cells at $10^{6}$ cells/mouse. Anti-IL-23p19 mAb given from the time of pathogenic cell transfer abrogated colitis and the associated inflammatory cytokines and chemokines (IL-17, TNF $\alpha$, IL-6, IFN $\gamma$, etc.) compared to mice receiving control antibody. Anti-IL-23pl9 $\mathrm{mAb}$ given to mice 4 wks after Birl4 $\mathrm{T}$ cell transfer was effective at treating active chronic colitis, with virtually complete resolution by $8 \mathrm{wks}$, with concomitant downregulation of cytokines and chemokines in the colon, including IL-17, TNF $\alpha$, IL-6, MIP, and IP-10 among others. Conclusion: Bacterial-reactive $\mathrm{CD}^{+} \mathrm{Th}_{\mathrm{LL}-17}$ cells are potent effector cells in chronic colitis. Inhibition of IL-23p19 is effective in both prevention and treatment of active colitis mediated by $\mathrm{Th}_{\mathrm{IL}-17}$ cells. The IL-23-IL-17 axis is an attractive therapeutic target.

\begin{tabular}{|c|c|c|}
\hline TCell Transferred & \#of cells ransferred & Pathological Score \\
\hline Th17 & $1 \times 10^{6}$ & $82 \pm 17$ \\
\hline & $1 \times 10^{5}$ & $68 \pm 11$ \\
\hline Th1 & $1 \times 10^{4}$ & $38 \pm 11$ \\
\hline & $1 \times 10^{6}$ & $14 \pm 8$ \\
\hline & $1 \times 10^{5}$ & $7 \pm 9$ \\
\hline & $1 \times 10^{4}$ & $7 \pm 9$ \\
\hline
\end{tabular}

780

Colitis in Wild-Type Mice Following Neonatal Bone Marrow Transfer with Mdrla -/- Hematopoietic Stem Cells

Reed A. Dimmitt, Camalla Kimbrough, Robin G. Lorenz

Background: Mice lacking the mdrla gene (p-glycoprotein) develop spontaneous colitis, which is dependent upon the presence of a conventional luminal microbiota. One reported mechanism for this phenomenon is that mdrla-/- intestinal epithelia have a barrier defect, which increases the access of bacterial products to the mucosal immune system. To test this hypothesis, we reconstituted FVB wild-type (FVB) neonatal pups, which should not have this barrier defect, with mdrla-/- hematopoietic stem cells. Methods and Materials: Five day old FVB pups were lethally irradiated in preparation for stem cell transfer. Adult FVB (control) or mdrla-/- mice were used as bone marrow stem cell donors. Following euthanasia the femoral bone marrow was enriched for stem cells using anti-Thyl.2 monoclonal antibody and rabbit complement. Each pup received 106 enriched cells via intraperitoneal injection. The nursing dams were treated with sulfamethoxazole and trimethoprim in the drinking water for 10 days post-transfer. The pups were weaned at 1 month and weighed weekly until sacrifice at 20 weeks. Colonic tissue was harvested for histological analysis and colitis scoring (0-19) and RNA extraction. The expression of various inflammatory mediators was determined using real-time RT-PCR, normalized to $18 \mathrm{~S}$ ribosomal RNA. Results: All the FVB + FVB (n=7) and FVB + mdrla-/- $(n=6)$ mice survived until 20 weeks. There was no significant difference in body weight changes between groups. None of the FVB + FVB mice developed diarrhea and the median colitis score was 0 . In contrast, 4 of the $6 \mathrm{FVB}+$ mdrla- $/$ had diarrhea. The median colitis score of the $6 \mathrm{FVB}+$ mdrla- $/$ - mice was 11.5 (25-75 percentile; 2-14). The inflammatory mediators with the greatest increase in expression in the FVB + mdrla-/- colons were MIP-2, LIX, IL-17, and IFN-gamma. Conclusions: FVB mice reconstituted with mdrla-/- stem cells develop colitis, which appears to be a THl and TH17 mediated process. Thus, FVB mice that have normal intestinal epithelial p-glycoprotein activity still develop disease in the presence of mdrla-/- hematopoietic cells. We believe this model of reconstitution permits the study of the role of p-glycoprotein during the development of mucosal immune function and will provide insight into the mechanism of colitis in the mdrla-/- mouse. Additional reconstitutions (mdrla-/- + FVB and mdrla-/- + mdrla-/-) are underway to further define the disease process. This work was supported by NIH POI DK071176 and R24 DK064400.

781

The Pro- and Anti-Inflammatory Roles of TLRS in a Model of Experimental Colitis

Kyoko Katakura, Sean Fine, Jongdae Lee, Carol Shen, Lars Eckmann, Yukio Sato, Eyal Raz

IL-10 plays an essential role in colonic homeostasis. We explored the protective role of different Toll-like receptors (TLRs), in the absence of IL-10, against colitis induction by dextran sulfate sodium (DSS). While TLR9 ${ }^{-1 /} / \mathrm{IL}-10^{-/-}$double knock-out (DKO) mice

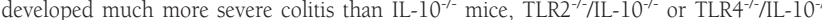
mice showed partial, or complete resistance, respectively, upon DSS challenge. In these

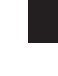


DKO mice, TLR9-deficiency caused higher production of inflammatory cytokine such as TNF- $\alpha$ but lower production of type- 1 IFN and TGF- $\beta$ compared to TLR 2 or TLR 4 deficiency Furthermore, TLR9-//IL-10 ${ }^{-/}$bone marrow-derived dendritic cells (BMDC) exhibited sustained NF- $\mathrm{KB}$ activation in response to mouse colonic microbial extracts and an uncontrolled secretion of TNF- $\alpha$ compared to BMDC from TLR2 $2^{-/} / \mathrm{IL}-10^{-/-}$or TLR $4^{-1 /} / \mathrm{IL}-10^{-/-}$mice. These effects were associated with induction of $\mathrm{A} 20$, a negative regulator of NF- $\mathrm{KB}$ activation. In response to colonic microbial extracts, A20 was strongly induced in TLR2 $2^{-/} / \mathrm{IL}-10^{-/}$, TLR $4^{-/-}$ IL-10 $1-$ BMDC, but not in TLR9 ${ }^{-/} /$IL-1 $10^{-/}$BMDC. These results suggest that TLR9 triggering by microbial DNA suppresses colonic inflammation via the induction of A20 while TLR2 or TLR4 triggering causes inflammation. (Katakura, $\mathrm{K}$ and Fine, $\mathrm{S}$ are cntributed equally.)

\section{2}

Meal Induced Rectal Sensitivity in Patients with Irritable Bowel Syndrome (IBS) May Be Related to a 5-Hydroxytryptamine Mediated Reduction in Rectal Compliance

Lesley A. Houghton, Wendy Atkinson, Catherine Fell, Peter J. Whorwell, Stephen Lockhart, Brian Keevil

Patients with IBS perceive rectal distension, particularly following meal ingestion, more than healthy volunteers (HV) (1). Both distension and the presence of nutrients within the gut releases 5-hydroxytryptamine (5-HT) from the enterochromaffin cells (2), which in the case of the latter, as measured by the concentration found in platelet depleted plasma (PDP), is abnormal in patients with IBS $(3,4)$. As the concentration of PDP 5-HT directly correlates with colonic motility (5), it was the aim of this study to determine whether the increase in perception of rectal distension seen following meal ingestion in IBS patients compared with $\mathrm{HV}$, is related to an abnormal 5-HT mediated reduction in rectal compliance. Methods: Isobaric phasic distension of the rectum was performed one hour after ingestion of a standard meal (12g protein, $65.5 \mathrm{~g}$ carbohydrate, $46 \mathrm{~g}$ fat) in $18 \mathrm{IBS}$ (Rome II) patients, aged 20-51 years ( 6 male) and $12 \mathrm{HVs}$, aged $19-41$ years ( 3 male). Post-prandial PDP 5 -HT concentrations were measured at 30 minutes intervals both 1 hour before (period A) and for 3 hours after the start of the distensions (period B). Results: IBS patients reported pain at lower volume (mean difference from $\mathrm{HV}(95 \% \mathrm{CI})-58.3 \mathrm{ml}(-109.5,-7.0) \mathrm{ml} ; \mathrm{p}=0.027)$ and pressure ($7.8 \mathrm{mmHg}(-16.5,0.9) \mathrm{mmHg} ; \mathrm{p}=0.075)$ thresholds than HVs. The difference in pressure threshold between the two groups however, was smaller than that for volume, and thus probably explains the slightly lower rectal compliance seen in IBS patients $(-1.3 \mathrm{ml} / \mathrm{mmHg}$ $(-3.7,1.1) \mathrm{ml} / \mathrm{mmHg} ; \mathrm{p}=0.264)$. In addition, distension was associated with an increase in PDP 5-HT concentration in IBS patients (ratio B:A 1.48 (1.02, 2.09); $\mathrm{p}=0.04$ ) but not in HVs $(1.09(0.86,1.40) ; p=0.464)$, with this 5 -HT increase in IBS patients negatively correlating with volume threshold $(\mathrm{r}=-0.476 ; \mathrm{p}=0.046)$ and compliance $(\mathrm{r}=-0.337 ; \mathrm{p}=0.171)$ but not with pressure threshold $(\mathrm{r}=-0.014 ; \mathrm{p}=0.955)$. No such correlations were seen in HV (volume threshold $\mathrm{r}=-0.049 ; \mathrm{p}=0.88$, pressure threshold $\mathrm{r}=-0.007 ; \mathrm{p}=0.983$, compliance $r=-0.028 ; p=0.931$. Conclusion: These data are the first to suggest that the increase in rectal sensitivity seen following meal ingestion in IBS patients compared with HV, may be in part related to a 5-HT mediated reduction in rectal compliance. Furthermore, they support the use of 5-HT modulating drugs which increase gut compliance in the treatment of IBS patients with excessive concentrations of PDP 5-HT. Refs (1) Simren et al 2001, (2) Sanger 1996, (3) Houghton et al 2003, (4) Atkinson et al 2006 (in press), (5) Atkinson et al 2005 (DDW)

783

A New Form of Hypervigilance in Irritable Bowel Syndrome (IBS) Patients Monica Garcia Alonso, Enrique Rey, Manuel Diaz-Rubio

Hypersensitivity is well described in IBS. With random protocols, it disappears in some patients, considered as hypervigilant. Some patients show a lower threshold in random than in ascending protocols, and therefore, would be classified as hypersensitive. However, its meaning is unknown. We aim to deep in this perceptual response. MATERIAL AND METHODS: Consecutive patients with IBS (Rome II criteria) were studied with rectal barostat. They underwent a slow ramp distension $(16 \mathrm{mmHg} / \mathrm{min})$ and thresholds was obtained by ascending methods of limits (AML) using a phasic distension protocol. Only patients with disomfort threshold (DT) in AML below the lower limit of the $95 \% \mathrm{CI}$ of control subjects were included. A tracking $(\mathrm{T})$ procedure was performed, providing an initial distension equal to DT in AML followed by random distension phases driven by the report of discomfort to achieve 6 phases after discomfort was reported. DT in T was defined as the mean pressure of discomfort-rated phases. Patients were classified as: Truly Hypersensitivity: DT in T keeps below normal limit; Hypervigilance: DT in T rises above normal limit. We called 'Precipitators' to patients whose discomfort threshold in $\mathrm{T}$ was more than $4 \mathrm{mmHg}$ lower than DT in AML Compliance in AML protocol, DT in ramp distension, AML and T and maximum tolerated distension (MTD) in ramp and AML were compared. Correlation between DT in $\mathrm{T}$ and DT in ramp was done to evaluate the impact of the exclusion of "precipitators". RESULTS: We included 49 consecutive IBS patients hypersensitive in AML protocol (39 women; mean age 49). Table summarizes the results. Precipitators were similar to hypervigilant patients except for defining variables, while both share several differences with truly hypersensitive. DT in ramp and DT in T were not correlated( $\mathrm{r}=0.26$; $\mathrm{p}=\mathrm{ns})$. When precipitators were excluded, DT in ramp correlates to DT in $\mathrm{T}(\mathrm{r}=0.48 ; \mathrm{p}=0.001)$. CONCLUSION Subjects with significant decrease of discomfort threshold in random distension ("precipitators") are closer to hypervigilant subjects than to truly hypersensitive subjects. This perceptual abnormality may have implications in the interpretation of sensitivity studies in IBS.

\begin{tabular}{|c|c|c|c|}
\hline & Truly Hypersensitive $(\mathrm{N}=35)$ & Hypervigilant (N=9) & Precipitators (N=5) \\
\hline DT (AML) & $15.9(1.0)$ & $21.8(4.1)^{*}$ & $18.4(5.4)$ \\
\hline MTD (AML) & $24.1(1.1)$ & $33.8(6.7)^{*}$ & $29.6(7.3)^{*}$ \\
\hline Compliance & $8.7(0.7)$ & $5.6(1.1)^{*}$ & $6.5(0.5)^{*}$ \\
\hline DT (Ramp) & $23.2(1.0)$ & $31.1(10.5)$ & $35.3(4.0)^{*}$ \\
\hline MTD (Ramp) & $29.6(1.3)$ & $37.7(6.7)^{*}$ & $38.6(2.8)^{*}$ \\
\hline DT (Tracking) & $17.2(0.9)$ & $27.9(3.5)$ & $12.8(6.0)+$ \\
\hline DT difference (T-AML) & $1.3(0.7)$ & $6.1(3.8)^{*}$ & $-5.6(1.1)^{*}+$ \\
\hline
\end{tabular}

* $\mathrm{p}<0.05$ vs Truly hypersensitivity $+\mathrm{p}<0.05$ vs Hypervigilant (Dunnet $\mathrm{C}$ Test)

784

Relationship Between Symptoms and Abnormalities in Rectal Sensitivity and Compliance to Distension in Patients with Irritable Bowel Syndrome Kwang Jae Lee, Bo Heon Meong, Jin Hong Kim, Ki Baik Hahm, Sung Won Cho

Background/Aims: Abnormalities in rectal sensitivity and compliance to distension are frequently observed in patients with irritable bowel syndrome (IBS), which is considered to play an important role in the genesis of symptoms. However, their relationship to symptoms is unclear yet. Our aim was to investigate associations between symptoms and the presence of abnormalities in rectal sensitivity and compliance to distension in patients with irritable bowel syndrome. Methods: Twenty-four patients with diarrhea predominant IBS (D-IBS), twenty patients with constipation predominant IBS (C-IBS) and fourteen healthy controls participated in this study. The intensities of the symptoms in the past 4 weeks were scored on a 5-graded Likert scale with descriptive anchors, and the presence of symptoms was determined as the scores $\geq 3$ (moderate). Using a barostat, sequential isobaric rectal distensions were performed before and $30 \mathrm{~min}$ after a meal. Abnormalities in rectal sensitivity and compliance were defined using control means \pm 2 SD. Results: No patients had hyposensitive or hypercompliant rectum to distension, both in the fasting period and in the postprandial period. Hypersensitive rectum to distension was observed in $16 \mathrm{D}$-IBS patients (67\%) and 9 C-IBS patients (45\%) in the fasting period (NS), and in 17 D-IBS patients (71\%) and 9 C-IBS patients ( $45 \%$ ) in the postprandial period (NS). Hypocompliant rectum to distension was observed in 13 D-IBS patients (54\%) and 8 C-IBS patients (40\%) in the fasting period (NS), and in 15 D-IBS patients (63\%) and 8 C-IBS patients (40\%) in the postprandial period (NS). Bloating was more frequent in hypersensitive rectum in the postprandial period compared to normosensitive rectum (50\% vs. $11 \%$ ). Decreased bowel movements were more frequent in normosensitive rectum in the postprandial period compared to hypersensitive rectum ( $61 \%$ vs. $31 \%$ ). Increased bowel movements were more frequent in hypocompliant rectum in the postprandial period compared to normocompliant rectum ( $52 \%$ vs. $24 \%$ ). The other symptoms, including loose stools, incomplete evacuation sense, hard stools, straining, and urgency, did not differ according to abnormalities in rectal sensitivity and compliance to distension. Conclusions: Abnormal sensitivity or compliance of the rectum to distension in the postprandial period is associated with bloating or increased bowel movements. However, most of IBS symptoms do not reflect underlying abnormalities in rectal sensitivity and compliance to distension.

785

Puborectalis Muscle in Nulliparous Women: Functional Assessment Using 3-D Ultrasound Imaging

Milena M. Weinstein, Sung-Ae Jung, Dolores H. Pretorius, Charles W. Nager, Ravinder K. Mittal

Objective: The levator ani and puborectalis muscles have been imaged with 3D ultrasound (US) and measured during rest and with straining, but not with contraction in normal subjects. Our study aim was to determine if measurements of the puborectalis muscle (PRM) are reliable, and if so, do they shorten during pelvic floor contraction when measured with 3-D US. Material and Methods: In a prospective study, 16 nulliparous volunteers with a mean age of 32 (19 - 54yrs) were imaged with a 3D US system (Voluson 730 GE Medical Systems, Milwaukee, WI) using a 5-9 MHz transvaginal transducer probe placed on the perineum. The images were obtained at rest as well as during an anal sphincter squeeze (pelvic muscle contraction). The images were analyzed with the 4D View software program and displayed in a standard, symmetrical orientation in axial, coronal and sagittal planes. The technique used to rotate the 3D image included: 1)rotation of the coronal image into the plane of the PRM, 2)identification and marking of the symphysis pubis in the axial image, 3) rotation of the sagittal image to align symphysis pubis and anorectal angle. Measurements were then made of the anterior-posterior (AP) length, the PRM length, area inside PRM (inner area) and including the PRM (outer area), and PRM area (subtracting outer from inner area) (see Fig. 1). All rotations and measurements were then repeated by a second blinded observer to determine interobserver reliability. Results: see Table 1. Observer 2 data demonstrated the same differences between rest and squeeze. Best interobserver correlation was noted for AP length ( $r=0.70$ for rest and 0.88 for squeeze). Conclusion: 3 $\mathrm{D}$ ultrasound imaging technique can be used reliably to measure PRM length and functional shortening with squeeze

Table 1: Data from one observer

\begin{tabular}{|c|c|c|c|c|c|}
\hline & $\begin{array}{c}\text { AP } \\
\text { length }(\mathrm{cm})\end{array}$ & $\begin{array}{c}\text { PRM length } \\
(\mathrm{cm})\end{array}$ & $\begin{array}{c}\text { Inner } \\
\text { area }(\mathrm{cm} 2)\end{array}$ & $\begin{array}{c}\text { Outer area } \\
(\mathrm{cm} 2)\end{array}$ & $\begin{array}{c}\text { PRM area } \\
(\mathrm{cm} 2)\end{array}$ \\
\hline Rest (mean $\pm \mathrm{SD})$ & $5.0 \pm 0.4$ & $9.3 \pm 0.9$ & $13.9 \pm 1.2$ & $20.7 \pm 1.9$ & $6.9 \pm 1.2$ \\
\hline $\begin{array}{c}\text { Squeeze } \\
(\text { mean } \pm \text { SD) }\end{array}$ & $4.5 \pm 0.4$ & $8.4 \pm 0.8$ & $12.5 \pm 1.2$ & $18.7 \pm 2.0$ & $6.1 \pm 1.0$ \\
\hline P value & $<0.001$ & $<0.001$ & $<0.001$ & 0.001 & $<0.001$ \\
\hline
\end{tabular}




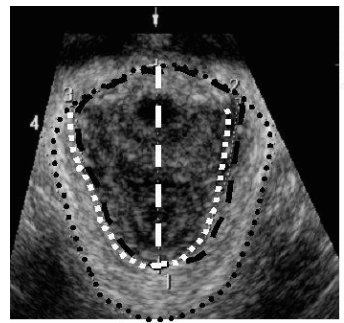

Changes of Rectal Motor and Sensory Response to Distension After Longo's Haemorrhoidectomy: A Barostat Study

Antonio Gianluca Castellaneta, Maura Corsetti, Simone Squillante, Nadine Osman, Pier Alberto Testoni, Sandro Passaretti, Paola de Nardi

The occurrence of faecal urgency, pain and/or increased stool frequency has been reported in up to $31 \%$ of patients treated with Longo's procedure for third and fourth degree hemorroids (Cheetham MJ, 2000). The mechanisms underlying these symptoms are unclear. A previous study using anorectal manometry has demonstrated no changes of rectal function after Longo's procedure but, as stated by the authors, rectal distensibility should have been assessed using the electronic barostat (Fantin AC, 2002). Aim of the study was to assess the rectal motor and sensory response to distension before and after Longo's haemorrhoidectomy using the electronic barostat. Methods: Six patients ( 4 males, mean age $47 \pm 9$ ys) with third and fourth degree haemorroids underwent rectal distensions controlled by an electronic barostat one week before and six months after Longo's procedure. After a conditioning distension, three distensions (a pressure-controlled stepwise ( $4 \mathrm{~mm} \mathrm{Hg} / 2 \mathrm{~min}$ ), a volumecontrolled stepwise $(20 \mathrm{ml} / 2 \mathrm{~min})$ and a ramp at $100 \mathrm{ml} / \mathrm{min}$ ) were performed in each patient. Data $($ mean \pm SD) of rectal distensibility as well as volume and pressure thresholds for sensations of desire to defecate and discomfort were compared before and after surgery by means of the Wilcoxon test. Results: Rectal distensibility was significantly lower after than before surgery during pressure stepwise $(4 \pm 1 \mathrm{ml} / \mathrm{mm} \mathrm{Hg}$ vs $10 \pm 4 \mathrm{ml} / \mathrm{mm} \mathrm{Hg}, \mathrm{P}=0.02)$, during volume stepwise $(0.17 \pm 0.06 \mathrm{~mm} \mathrm{Hg} / \mathrm{ml}$ vs $0.09 \pm 0.03 \mathrm{~mm} \mathrm{Hg} / \mathrm{ml}, \mathrm{P}=0.04)$ and during volume ramp distension $(0.17 \pm 0.02 \mathrm{~mm} \mathrm{Hg} / \mathrm{ml}$ vs $0.07 \pm 0.02 \mathrm{~mm} \mathrm{Hg} / \mathrm{ml}, \mathrm{P}=0.009)$. Discomfort occurred at a significantly lower volume after than before surgery during pressure stepwise $(106 \pm 11 \mathrm{ml}$ vs $199 \pm 79 \mathrm{ml}, \mathrm{P}=0.02)$, during volume stepwise $(100 \pm 40 \mathrm{ml}$ vs $206 \pm 82 \mathrm{ml}, \mathrm{P}=0.03)$ and during volume ramp ( $113 \pm 45 \mathrm{ml}$ vs $220 \pm 80 \mathrm{ml}, \mathrm{P}=0.04)$. Volume threshold for desire to defecate was lower after than before surgery during pressure stepwise $(47 \pm 19 \mathrm{ml}$ vs $139 \pm 55 \mathrm{ml}, \mathrm{P}=0.02)$ while it did not differ during volume stepwise and ramp (NS). Pressure thresholds for desire to defecate and discomfort did not differ before and after surgery during any distensions (NS). Conclusions: Rectal distensibility and volume thresholds for desire to defecate and discomfort decrease after Longo's haemorrhoidectomy. These modifications of rectal function could explain the occurrence of rectal urgency, pain and/or increased stool frequency in some of the patients subjected to Longo's procedure

787

Investigation of Sensori-Motor, Recto Anal Inhibitory and Contractile Reflexes in Rectal Hyposensitivity

Jose M. Remes-Troche, Sherrie de Ocampo, Megan Miller, Jessica Paulson, Satish Sc Rao

Background/Aim: Ano-rectal sensations play an important role in maintaining normal continence and defecation. Recently, we showed that a desire to defecate was associated with a reflex contraction of the anal sphincter -sensori-motor reflex (SMR) (Gastroenterology 2005. 128(2): A38). Whether SMR is altered in patients with rectal hyposensitivity is not known. Our aim was to examine the characteristics of SMR, as well as the recto-anal inhibitory (RAIR) and recto-anal contractile reflexes (RACR) in patients with rectal hyposensitivity (RH). Methods: Rectal sensation and recto-anal reflexes were assessed by performing stepwise balloon distensions in 10-30 cc increments, in $30 \mathrm{RH}$ patients $(\mathrm{M} / \mathrm{F}=7 / 23$, mean age $=45 \mathrm{yr})$ and 23 healthy subjects $(\mathrm{M} / \mathrm{F}=8 / 15$, mean age $=48$ yrs) after placing a 6 sensor probe with a $4 \mathrm{~cm}$ long latex balloon in the rectum. Thresholds for first sensation, desire and urgency to defecate were recorded. Patients were defined as hyposensitive (RH) if at least two of sensory thresholds were greater than 2 SD of normal values. The lowest volume of balloon distension that evoked RAIR, RACR and SMR were recorded. Onset, duration, amplitude, and area under the curve (AUC) for SMR were also analyzed. Results: All subjects showed all 3 reflexes. Unlike healthy controls where a SMR was associated with a desire to defecate, in RH patients, the onset of SMR was not associated with any sensation in $13(43 \%)$ and with a blunted sensation (first perception or fullness) in 17 (57\%). Threshold for SMR was similar between patients and controls, but the amplitude, duration and AUC were higher $(\mathrm{p}<0.05)$ in RH patients (Table). Also, the onset of SMR was prolonged in RH patients who reported no sensation with SMR when compared to patients with blunted sensation during SMR $(13 \pm 9$ vs. $7 \pm 3, p<0.05)$. The minimum balloon volumes that induce RAIR and RACR were significantly higher $(\mathrm{p}<0.001)$ in RH patients $(20 \pm 10 \mathrm{cc}$ and $56.1 \pm 19 \mathrm{cc})$ compared to controls $(12 \pm 6 \mathrm{cc}$ and $32.8 \pm 10 \mathrm{cc})$. Conclusions: Recto-anal reflexes are altered and may play an important role in the pathogenesis of rectal hyposensitivity. The absent or blunted sensory perception in spite of an intact SMR in patients with rectal hyposensitivity suggests that this reflex is mediated by both the ENS and CNS, and that the afferent rectal-brain pathway may be dysfunctional in these patients.

\begin{tabular}{|c|c|c|c|}
\hline & Patients $(\mathrm{n}=30)$ & Controls $(\mathrm{n}=23)$ & $\mathbf{p}$ \\
\hline Volume threshold for inducing SMR (cc) & $92.6 \pm 27$ & $93 \pm 26$ & 0.9 \\
\hline Onset (seconds) & $8.2 \pm 3$ & $9.1 \pm 1.8$ & 0.1 \\
\hline Amplitude (mm Hg) & $\mathbf{3 1 . 7} \pm \mathbf{1 3}$ & $19 \pm 9$ & 0.01 \\
\hline Duration (seconds) & $\mathbf{7} \pm \mathbf{3}$ & $5.5 \pm 1.5$ & 0.04 \\
\hline Area under curve (AUC) & $\mathbf{1 7 7} \pm \mathbf{6 7}$ & $41 \pm 33$ & 0.001 \\
\hline
\end{tabular}

mean \pm SD

788

DX-pH Measurement System: A Sensitive Device for Detecting Liquid and Aerosolized Supraesophageal Gastric Reflux (SEGR)

Gregory J. Wiener, Ross Tsukashima, Colleen Kelly, Erich Wolf, Molly Schmeltzer, Charles Bankert, Lauren Fisk

PURPOSE: Clinical and animal data have documented deleterious effects of acid $\mathrm{pH}<4$ (AGR) and weakly acid pH 4-7 (WAGR) gastroesophageal reflux, even in small amounts, when above UES. Despite recognition of multiple manifestations of SEGR, characterization, identification and response to therapy have evaded understanding due to lack of a suitable detection device. AIM: Evaluate the Dx minimally invasive catheter (Restech) with ionic flow sensor, able to measure $\mathrm{pH}$ in liquid or aerosolized droplets at posterior oropharynx for SEGR detection, using standard $24 \mathrm{hr}$ triple sensor $\mathrm{pH}$ catheter $(24 \mathrm{pH})$ for verification. METHODS: Pts in a GI practice (GW) with chronic symptoms likely due to SEGR, off reflux meds 4-7days, underwent 24pH with 2 esophageal (E) and 1 pharyngeal (P) sensor (Sandhill PHII0-V) positioned with LES Indicator at $5 \mathrm{~cm}>$ LES, $5 \mathrm{~cm}<$ UES and $1 \mathrm{~cm}>$ UES. The $1.5 \mathrm{~mm}$ nasopharyngeal catheter placed at oropharynx behind uvula, above pts discomfor position. Tracings from all 4 synchronized $\mathrm{pH}$ inputs \& pt diary were analyzed graphically on single screen, excluding meals +5 min. SEGR event definition: rapid $\mathrm{pH}$ drops at the Dx sensor, $>3$ S.D. from $60 \mathrm{sec}$ baseline, sequential to drops in $\mathrm{pH}<4$ in $24 \mathrm{pH}$ sensors, then classified as AGR/WAGR. S.I. $<50 \%$ :+. RESULTS: 15 Patients, 5M, 10F, avg. age 57.5 (25-75): 11 cough, 1 loss enamel, 1 sleep apnea, 2 asthma. 4 had normal $24 \mathrm{pH}$. There were 48 Dx SEGR events in 10 pts, being near vertical with gradual return to baseline \& synchronous to $\mathrm{E} \mathrm{pH}$ drops. SEE TABLE 1 . Increase in $\mathrm{pH}$ was $46 \%$ from $\mathrm{E}$ to $\mathrm{P}, 6 \%$ from $\mathrm{P}$ to Dx. These 48 SEGR events were $7.8 \%$ of 616 episodes with $\mathrm{pH}<4$ at lower E. All 5 Dx negative pts had normal $24 \mathrm{pH}$ at E. CLINICAL FOLLOW UP (f/u): 11 pts took PPIs BID (f/u 3-8 mo., avg 6.5) or fundoplication (f/u 4 \& 6 wks). 9 Dx + pts had $60 \%$ Chief Complaint Improvement (CCI), no worsening. 7 of these on PPI had CCI $48.6 \%$ $(0,0,20,50,70,100,100), 2$ had fundoplication with $100 \%$ CCI, post-op Dx without pharyngeal events. 2 Dx negative pts, 1 with CCI $0 \%$ on PPIs, the other $100 \%$ CCI on NO meds. CONCLUSIONS: (A) SEGR exists in oropharynx, detectable by Dx. (B) There is a gradient of increasing $\mathrm{pH}$ from $\mathrm{E}$ to oropharynx, the latter rarely $<4$. (C) Redefinition of significant $\mathrm{pH}$ events above UES as \% or $>3$ S.D. pH drops merits consideration. (D) Clinical management may be influenced by Dx results, further studies should aid understanding of SEGR.

Table 1: Characteristics of Synchronous pH Drops - SEGR

\begin{tabular}{|c|c|c|c|}
\hline pH SENSOR & AGR $(\mathrm{pH} \leq 4)$ & WAGR $(\mathrm{pH}>4)$ & AVERAGE $\mathrm{pH}$ \\
\hline Dx & $6.25 \%$ Avg pH 3.67 & $93.75 \%$ Avg pH 5.65 & 5.52 \\
\hline Pharynx & $25 \%$ Avg pH 3.8 & $75 \%$ Avg pH 5.69 & 5.22 \\
\hline $5 \mathrm{~cm}>$ LES & $66.67 \%$ Avg pH 2.63 & $33.33 \%$ Avg pH 5.47 & 3.58 \\
\hline
\end{tabular}

789

The Measurement of Oesophageal Cross-Section Diameter and Volume Using a Novel Multi-Channel Intra-Luminal Impedance Catheter: In Vitro and in Vivo Validation

Mark Fox, Donald MacLachlan, Amir Thakur, Roy Anggiansah, Terry Wong, Angela Anggiansah

Introduction: Multi-channel intra-luminal impedance (MII) measures fluid movement in the oesophagus and detects non-acid reflux. Larger reflux volumes that cause significant oesophageal distension are more likely to trigger symptoms. A novel MII technique designed to detect variation in volume (vMII) is described. The multi-sensor catheter monitors pressure, $\mathrm{pH}$ and impedance (Gaeltec Ltd, Scotland). Three impedance electrodes are placed at $50 \mathrm{~mm}$ intervals, another is placed $5 \mathrm{~mm}$ from the middle electrode. Impedance measured by closely spaced sensors depends primarily on the conductance of fluid in the lumen; measurements from sensors placed further apart also vary with luminal cross-section diameter (CSD). This data allows the volume of an unknown fluid bolus to be estimated. Aims: (1) To assess the sensitivity of vMII to variation of CSD in vitro. (2) To validate the sensitivity of vMII to variation of bolus volume in vivo. Method: (1) Measurements with the vMII catheter were obtained in cylinders $(0.5-5 \mathrm{~cm}$ CSD) containing water, saline, orange juice (OJ), milk and gastric secretions obtained at endoscopy. (2) Stationary studies were performed in nine healthy volunteers $(6 \mathrm{M}: 3 \mathrm{~F}$, age $21-40)$ in the upright position. The vMII catheter was placed such that the closely spaced impedance electrodes were $5 \mathrm{~cm}$ proximal to the lower oesophageal sphincter. Measurements were obtained for 5 swallows each of 5, 10 and $20 \mathrm{ml}$ for water, OJ and milk. Results: (1) For each fluid, impedance measurements varied inversely with CSD in a logarithmic relationship. The effect was greater for widely spaced than closely spaced electrodes $(p<0.01)$. Impedance was greater for water than other fluids $(p<0.01)$ measurements for OJ, milk and gastric secretions were similar. (2) For each test fluid, impedance significantly decreased as bolus volume increased from 5 to $20 \mathrm{ml}(\mathrm{p}<0.05)$. Again, the relationship was logarithmic and was greater for widely spaced than closely spaced electrodes $(\mathrm{p}<0.01)$. Impedance was less in the distal than proximal oesophagus $(\mathrm{p}<0.01)$ suggesting greater distension of the distal oesophagus after fluid ingestion. Oesophageal CSD and volume were estimated from the impedance measurements obtained from the

. 
widely spaced electrodes by correcting for (1) bolus conductivity as assessed by the closely spaced electrodes (2) reference values from the in vitro measurements. Conclusion: The novel vMII catheter with closely and widely spaced impedance electrodes provides measurements of oesophageal CSD and volume in vivo. Future studies will assess the sensitivity of vMII catheter for the measurement of reflux volume.

\section{0}

Characterisation of Intraluminal Impedance Patterns Associated with Gas Reflux (Belching) in Healthy Volunteers

Michiel P. Van Wijk, Daniel Sifrim, Nathalie Rommel, Marc A. Benninga, Geoffrey P. Davidson, Taher Omari

BACKGROUND: Multichannel Intraluminal Impedance (MII) recording allows assessment of flow through the esophagus and differentiation between liquid and gas contents. MII criteria have been developed for recognition of gas but these have not been validated during known gas reflux in humans. AIMS: To characterize patterns of impedance change associated with gas reflux and to examine the interrelationships between the magnitude of impedance change, luminal diameter and electrode-mucosa contact. METHODS: 10 healthy volunteers (6M:4F, 21-37yrs) were studied using an esophageal MII-manometry catheter. After catheter placement, subjects were asked to drink up to $600 \mathrm{ml}$ of carbonated soft drink. Belching episodes were then recorded for $20 \mathrm{~min}$ and the protocol was repeated. Only belches confirmed manometrically as being triggered by transient LES relaxation and associated with an esophageal common cavity episode were included for analysis. A further 5 subjects (3M:2F, 26-52yrs underwent simultaneous MII and videofluoroscopy(VF) during performance of the same protocol. 'Standard' MII criteria for recognition of gas reflux were an antegrade or synchronous impedance rise of $\geq 50 \%$ in $\geq 2$ channels that exceeded $5 \mathrm{k} \Omega$ in $\geq 1$ channel. VF was analysed blinded from MII results and vice versa. RESULTS: All analysed belches $(n=88)$ were associated with a pattern of impedance rise which was either retrograde $(62.5 \%)$, synchronous(19.3\%) or antegrade(18.2\%). Median (IQR) intraluminal impedance at the onset and peak of gas reflux was $2.0(1.6,2.7) \mathrm{k} \Omega$ and $4.4(2.8,7.5) \mathrm{k} \Omega$ respectively and the median $\%$ increase was $106.6 \%(50.2,200.3)$. Of all belches $31(35.2 \%)$ did not meet the 'standard' MII criteria because the impedance rise was antegrade(18.2\%), did not increase by $\geq 50 \%$ in $\geq 2$ channels $(9.1 \%)$ or did not exceed $5 \mathrm{k} \Omega$ in any channel( $23.8 \%)$. During MII-VF 15 belches were recorded. The magnitude of impedance change increased with increasing luminal diameter during gas reflux $(r=0.718, p<0.0001)$. The magnitude of impedance change was greater when impedance electrodes were not in direct contact with the esophageal mucosa $(7.0(5.4,7.8) \mathrm{k} \Omega)$ compared to when one $(2.4(1.1,5.2) \mathrm{k} \Omega, \mathrm{p}<0.001)$ or two $(1.8(0.8,2.6) \mathrm{k} \Omega, \mathrm{p}<0.001)$ electrodes were CONCLUSIONS: A significant number of gas reflux episodes do not meet current standard impedance-based criteria for their recognition. Factors including the degree of esophageal distension as well as the extent of direct contact between the esophageal mucosa and MII-electrodes influence the magnitude and patterning of impedance change recorded.

791

Effects of Feeding On Esophageal Exposure to Acid Gastroesophageal Reflux During Esophageal Emptying and During Subsequent Mucosal Acid Neutralization in Infants

Frederick W. Woodley, Namhee Kim, Soledad Fernandez, Melissa Metheney, Hayat Mousa

Background: Esophageal pH monitoring (EPM) is limited in its ability to identify mechanisms of esophageal acid exposure; whether due to gastroesophageal reflux (GER) bolus contact or the subsequent neutralization of mucosal acid. Combined EPM and multichannel intraluminal impedance ( $\mathrm{pH} / \mathrm{MII}$ ) can measure durations of volume clearance (VC) and chemical clearance (CC). The effects of feeding on VC and CC of AGER during each stage of the feeding cycle have not been reported. Aims: 1) To assess the extents to which VC and CC influence total esophageal acid exposure. 2) To assess the effects of feeding on VC and CC in infants. Methods: We analyzed EPM/MII tracings from 14 infants ( 9 males, median age 3.5 months) who were referred to our facility for symptoms suggestive of GER. Individual AGER episodes were identified as having occurred during feeding, the first post-prandial hr (lstPP), the second post-prandial hr (2ndPP), or fasting. We measured the duration of VC and CC for each AGER episode using pH/MII. Each outcome variable was expressed as the mean \pm standard error. The study protocol was approved by the Institutional Review Board at Columbus Children's Hospital and all patients were enrolled following parental consent. Results: In nearly $329 \mathrm{hr}$ of combined recording time (median study duration $23.5 \mathrm{hr} / \mathrm{pt}$ ), 331 AGER episodes (median 23/pt) were detected. pH/MII registered $14.9 \mathrm{hr}$ of total acid contact time (median $52.8 \mathrm{~min} / \mathrm{pt}$ ); $2.3 \mathrm{hr}(15 \%)$ was spent during VC and $12.7 \mathrm{hr}(85 \%)$ were spent during $\mathrm{CC}$. CC duration was more prolonged than VC during all phases of feeding and was significantly longer than VC during $2 \mathrm{ndPP}(156.1 \pm 22.3$ vs $26.5 \pm 4.4 \mathrm{~s}$, $\mathrm{p}=.003)$ and fasting $(169.7 \pm 19.4$ vs $26.9 \pm 3.9 \mathrm{~s}, \mathrm{p}=.0005)$. Duration of CC contributed to greater than half of the total acid exposure time and significantly dominated during $2 \mathrm{ndPP}$ $(65 \pm 5.2 \%$ vs $35 \pm 5.1 \%, \mathrm{p}=.004)$ and fasting $(76 \pm 3.2 \%$ vs $24 \pm 3.2 \%, \mathrm{p}=.0002)$. Neither the durations nor the proportion of VC and CC was significantly different during feeding or lstPP. Conclusions: In our infant population, the vast majority of esophageal acid exposure occurred during CC. While esophageal acid exposure during feeding and lstPP phases was comparably attributable to both VC and CC, a significant proportion of the acid exposure during 2ndPP and fasting occured during CC. We speculate that prolonged neutralization of mucosal acid during 2ndPP and fasting is due to reduced primary and secondary peristalsis related to decreased frequency of swallowing during these periods. The shorter duration of CC during feeding is likely due to the buffering effects of swallowed saliva and milk-based formulas during feeds.
792

Four-Day BRAVO pH Recording Combining 48-Hour Periods On and Off Proton Pump Inhibitor Therapy

Pai-Wain C. Lo, Qing Zhang, Nirmala Gonsalves, Ikuo Hirano

Background \& Aims: Bravo monitoring increases the sensitivity of $\mathrm{pH}$ testing by allowing for 48 rather than 24 hour recordings. Controversy exists as to whether $\mathrm{pH}$ studies are optimally done off or on proton pump inhibitor (PPI) therapy. The aim of this study was to determine the feasibility of 4-day $\mathrm{pH}$ recordings using the Bravo $\mathrm{pH}$ system to encompass 2 day periods both before and during PPI therapy. Methods: 16 patients underwent 4-day $\mathrm{pH}$ recordings ( 2 day off; 2 day on) using 2 separate receivers calibrated to a single Bravo capsule. Indications for $\mathrm{pH}$ testing were refractory heartburn, chest pain, and chronic cough. Patients were off PPI therapy for days $1 \& 2$. Rabeprazole $20 \mathrm{mg}$ orally twice a day was administered on days $3 \& 4$. Results: 13 patients completed the 4 day study and were included in the analysis. Off therapy, 5 patients (38\%) had esophageal acid exposure values exceeding $5.3 \%$. On therapy, all patients showed significant and progressive acid reduction (Fig). By day 4, esophageal acid exposure value was $0 \%$ in 8 patients and $<1 \%$ in 5 patients. The symptom index was significantly higher off (median 50\%) than on (median 0\%) PPI therapy $(\mathrm{p}<0.001) .2$ patients with capsule detachment on day 3 and one on day 2 were excluded. Of the excluded patients, two had normal off PPI acid exposure and the lack of day 4 data did not change the overall test interpretation. Conclusions: Prolonged recordings using the Bravo $\mathrm{pH}$ system are feasible and allow for combined testing both off and on therapeutic trial of PPI. The initial 2 day off PPI allows for increased sensitivity and symptom association for the diagnosis of GERD compared with on PPI testing. Such combined studies may allow for acquisition of information useful in the evaluation of refractory and atypical reflux symptoms

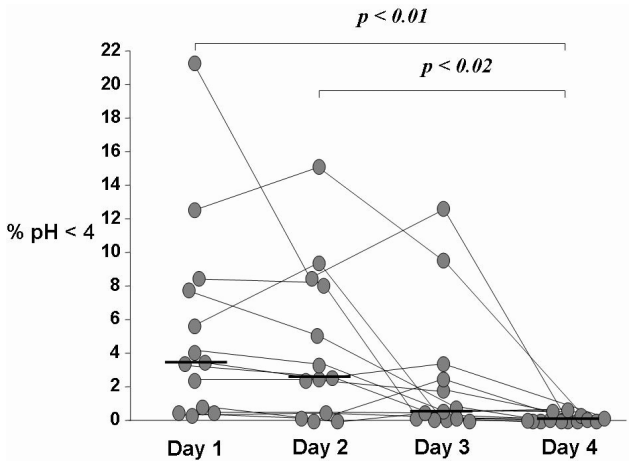

793

Light Microscopy Analisys of Intercellular Space Diameters of Esophageal Epithelium in Nerd Patients: Comparison with Electron Microscopy Mentore Ribolsi, Giuseppe Perrone, Renato Caviglia, Massimo Gentile, Sara Emerenziani, Michele Guarino, Rabbitti Carla, Michele Cicala

Background: Dilation of intercellular space diameters (ISD) of esophageal epithelium detected at transmission electron microscopy (EM) has been shown to represent an objective, sensitive ultrastructural marker of non-erosive reflux disease (NERD). ISD evaluation using the less demanding and more available light microscopy (LM) seems to provide interesting results in GERD patients. Aim/Methods: to compare the gold standard EM against LM in detecting dilation of ISD, two adjacent biopsy specimens, at $5 \mathrm{~cm}$ above $Z$ line, obtained from 12 NERD patients (F8, mean age 43 yrs) and from 7 asymptomatic controls (F4, mean age 32 yrs) were assessed for ISD measure. Computer assisted morphometry was performed on photographs obtained by EM and LM, according to published methods. 100 ISD measures were obtained from each subject. ISD size was also assessed by LM according to a published semi-quantitative score (grade 0: absence of dilated ISD, 1: small size dilation, 2: mild and 3: marked dilation). Results: Mean ISD values and ISD score from patients and controls are shown in the Table. Confidence intervals of mean ISD in patients did not overlap with those in controls both at EM and at LM. A significant correlation between intra-subject mean ISD at EM and at LM was found when considering the study population (r: $0.83, \mathrm{p}<0.01)$ and the patient group; no correlation was found in the control group. Out of 12 patients, 3 presented an ISD score within the normal range (0-1). Conclusions: ISD measures obtained by LM morphometry significantly correlate with those by EM. ISD semi-quantitative score evaluated at LM seems not to be accurate. Light microscopy morphometry seems to be a promising method for assessing ISD of esophageal epithelium in non-erosive gastroesophageal reflux disease. 


\begin{tabular}{|c|c|c|c|}
\hline & \multicolumn{2}{|c|}{ Mean ISD $\mu \mathrm{m}(95 \%$ Confidence Interval) } & ISD score Dilated (2-3 score) (\%) \\
\hline & EM & LM & \\
\hline NERD patients & $1.42(1.37-48)$ & $1.28(1.11-44)$ & $75 \%$ \\
\hline Controls & $0.45(0.39-56)$ & $0.67(0.56-78)$ & $14 \%$ \\
\hline
\end{tabular}

794

A Novel Selective Gene Targeting Approach to Selectively Kill Cancer Cells, in Vitro and in Vivo, with Hyperactive $\beta$-Catenin

Hadas Dvory-Sobol, Diana Kazanov, Eyal Sagiv, Dana Yona, Nadir Arber

Background: The APC or $\beta$-catenin genes are frequently mutated in gastrointestsinal cancers resulting in oncogenic activation of $\beta$-catenin signaling. Recently, we have shown that recombinant adenovirus, that carries the PUMA gene under the control of $\beta$-catenin/Tcf responsive promoter (AdTOP-PUMA), suppressed the growth of human HCT116 and SW480 colon cancer cells. Replacement of AT by GC bases created the negative control adenovirus (AdFOP-PUMA). The PUMA protein (p53 up-regulated modulator of apoptosis) interacts with Bcl-XL and Bcl-2 to induce the release of cytochrome $\mathrm{c}$ from the mitochondria and to induce apoptosis Objective: To further evaluate the efficacy of this model in vitro and in vivo for selective killing of human cancer cells with an activated $\beta$-catenin pathway. Methods: The AdEasy system was used to generate the AdTOP-PUMA and AdFOP-PUMA adenoviruses. In vitro: SW480, HCT116, DLD-1, LS174T and HT29 (colon), HepG2, SK-Hep-1 (liver), AGS (gastric) and Colo357, Panc-1 (pancreas) human cancer cells were infected with the AdTOP-PUMA adenovirus and the vector control AdFOP-PUMA. The combined effect of AdTOP-PUMA and several chemotherapeutic agents (5-FU, doxorubicin, paclitaxel) was also evaluated. Cell viability was measured by methylene blue assay. Protein expression was analyzed by Western blot. Cell cycle and apoptosis were evaluated by FACS analysis. In vivo: $3 \times 10^{3}$ SW480 cells were infected with the AdTOP-PUMA and AdFOP-PUMA, harvested after $24 \mathrm{~h}$, and then inoculated subcutaneously into nude mice. Results: In vitro: The TOPPUMA adenovirus inhibited cells growth in a dose dependent fashion, depending on the level and activity of $\beta$-catenin. The growth of cells with high $\beta$-catenin activity (SW480, DLD-1, HCT116, LS174T, AGS and HepG2 cells) was inhibited, but that of cells expressing low activity levels (HT-29, SK-Hep-1, Panc-1 and Colo357) was not. Growth inhibition was associated with induction of apoptosis. Hepatocellular carcinoma cell line (HepG2) and gastric cancer cell line (AGS), in which $\beta$-catenin is mutated, were very sensitive to AdTOPPUMA therapy. Chemotherapy synergistically enhanced the effect of AdTOP-PUMA. In vivo: AdTOP-PUMA treated mice did not develop tumors (0/8), while AdFOP-PUMA and adenoviral vector control developed (3/8) and (4/7) tumors respectively. Conclusions: This selective gene therapy may be used in cancer therapy. A combination of the adenovirus system with standard therapy may improve the efficacy and reduce the toxicity of chemotherapy in humans.

795

Sox4 Is a $\beta$-Catenin-Interacting, Tumor-Promoting Modulator of Wnt Signaling That Is Upregulated in Colorectal Cancer

Jeffrey Franklin, Emilios Tahinci, Ethan Lee, Loice Jayekumar, Galina Bogatcheva, Tim

Yeatman, Bruce Aronow, Robert J. Coffey

Wnt signaling provides essential functions in embryonic development and in epithelial homeostasis. It is tightly regulated by various effectors to provide stereotypic control of patterning and differentiation of tissues and is often misregulated in colorectal cancer (CRC). We have found that Sox 4 is a unique regulator of Wnt signaling that down-regulates overall Wnt patterning cues in Xenopus embryonic development and in rat intestinal epithelial cells (RIE- 1 cells). At the same time, it increases the stability of $\beta$-catenin in an in vitro proteosomal degradation assay and alters $\beta$-catenin levels in Sox4- overexpressing APC-mutant SW480 cells. In SW480 cells overexpressing Sox 4 , there is enhanced invasion in a Boyden chamber assay and altered cell morphology. Consistent with these effects, we see four-fold increased Sox4 expression in adenomas compared to normal colonic mucosa, and this increased expression persists during CRC progression in 300 CRC tumors. SiRNA directed to Sox 4 in SW480 cells regulates some Wnt effectors but has a greater influence on other signaling pathways (e.g. the Stat pathway, cytoskeletal regulation). Similarly, morpholino knockdown of endogenous Sox4 in Xenopus embryos upregulates Wnt signaling in the embryo phenotypically, by affecting axis formation, without inducing well-known Wnt Sox 4 target genes (like Xnr3). These results imply that Sox4 is not simply a negative regulator of Wnt signaling but can modulate tumor-promoting pathways. Our view of canonical Wnt signaling in cancer needs to be refined to reflect the increasing complexity of interacting transcription factors and their interacting effects. In a single cell, $\beta$-catenin can interact with multiple transcription factors simultaneously. Depending on their relative abundance and activity state, these shifting $\beta$-catenin complexes can influence cell growth in a combinatorial way affecting multiple signaling pathways instead of a binary addition or subtraction of $\beta$-catenin/ TCF-driven expression of targets.

796

Novel Crosstalk of KrüPpel-Like Factor 4 and Beta-Catenin Regulates Normal Intestinal Homeostasis and Tumor Repression

Wen Zhang, Xi Chen, Yoichi Kato, Paul M. Evans, Subo Yuan, Jun Yang, Piotr G

Rychahou, Vincent W. Yang, Xi He, Mark B. Evers, Chunming Liu

Epithelial cells of the intestinal mucosa undergo a continual process of proliferation, differentiation and apoptosis which is regulated by multiple signaling pathways. The Wnt/beta-catenin pathway plays a critical role in this process. Mutations in the Wnt pathway, however, are associated with colorectal cancers. Krüppel-like factor 4 (KLF4) is an epithelial transcriptional factor which is down-regulated in many colorectal cancers. The interplay between Wnt/ beta-catenin signaling and KLF4 in the normal intestine and colon cancers were analyzed in this study. We found that KLF4 interacts with beta-catenin and represses beta-cateninmediated gene expression. Moreover, KLF4 inhibits axis formation of Xenopus embryos. KLF4 also inhibits Wnt/beta-catenin target gene expression in Xenopus embryos and in colon cancer cells. We have established a stable colon cancer cell line that expresses inducible KLF4. We found that KLF4 inhibits xenograft tumor growth in athymic nude mice. Our findings suggest that the crosstalk of KLF4 and beta-catenin plays a critical role in homeostasis of the normal intestine as well as in tumorigenesis of colorectal cancers.

797

$\beta$-Catenin and Nf-кB Differentially Regulate Proliferation in Native Epithelia Shahid Umar, Yu Wang, Joseph H. Sellin

Background: Both $\beta$-catenin and NF-אB have been implicated in our lab as candidate factors in driving proliferation in an in vivo model of hyperproliferation, transmissible murine coloni hyperplasia $(\mathrm{TMCH})$. Although the signaling pathways controlled by these proteins are independent, both inhibitor of NF- $\mathrm{KB}, \mathrm{I} \mathrm{\kappa B} \alpha$, and $\beta$-catenin are regulated by phosphorylation at similar consensus $\mathrm{N}$-terminal serines leading to targeted ubiquitination through SCF $\beta$ TRCP and proteasomal degradation. Aim: To determine whether blocking proteasoma activity in vivo affects $\beta$-catenin and NF-kB-mediated changes in cell census during TMCH Methods: TMCH was induced by Citrobacter rodentium inoculation. Proteasomal activity was blocked in vivo with intra-peritoneal injections of proteasomal inhibitor PS-34l (Velcade ${ }^{\mathrm{R}}$ ) Westerns, immunohistochemistry (IHC) and gel shift assays were performed in distal colonic crypts isolated from normal, days 6 and 12 (peak hyperproliferation /hyperplasia) postinfected mice. Results: The characteristic findings of TMCH were invariably present: a grossly thickened distal colon, elongated crypts without obvious increases in epithelial/submucosal inflammatory cell numbers. Velcade ${ }^{\mathrm{R}}$ treatment to Citrobacter-infected mice led to significant decrease in chymotrypsin-like activity and marked increases in accumulation of polyubiquitinated proteins both at days 6 and $12 \mathrm{TMCH}$, compared to control. Velcade ${ }^{\mathrm{R}}$ however, did not abrogate hyperplasia despite significant inhibition of NF-KB activity due to accumulation of $\mathrm{Ser}^{32,36}$-phosphorylated IкB $\alpha$ in treated samples. Velcade ${ }^{\mathrm{R}}$ also blocked nuclear translocation of activated p65 as IHC failed to detect any nuclear staining for Ser ${ }^{536}$-phosphorylated p65 at either time points. In contrast, proteasomal blockade in vivo did not affect Casein kinase I-mediated phosphorylation of $\beta$-catenin at $\operatorname{Ser}^{45}\left(\beta\right.$-cat $\left.{ }^{45}\right)$. The primary GSK-3 $\beta$ phosphorylation site for degradation $\left(\mathrm{Ser}^{33,37} / \mathrm{Thr}^{41}\right.$ ) in $\beta$-catenin however, did not show accumulation of $\beta$-cat ${ }^{33,37}$ in either untreated or treated samples suggesting a lack of GSK$3 \beta$ action on $\beta$-cat ${ }^{45}$. Indeed, activity assays confirmed temporal inhibition of GSK-3 $\beta$ at these time points. Impairment in linking $\beta$-cat ${ }^{45}$ to subsequent GSK-3 $\beta$-mediated phosphorylation/ degradation stabilized $\beta$-cat ${ }^{45}$ which translocated to the nucleus, interacted with CREB binding protein (CBP) and T-cell factor-4 (Tcf-4) and the complex bound the DNA. Conclusions: 1. Proteasomal inhibition in vivo blocked NF-KB activity but did not abrogate hyperproliferation/hyperplasia. 2. $\beta$-Cat ${ }^{45}$, which forms transcriptionally active complexes with $\mathrm{CBP}$ and $\mathrm{Tcf}-4$, rather than NF-KB, drives proliferation during $\mathrm{TMCH}$.

798

Expression of CDX Proteins Interfere with the Formation of Beta-Catenin/Tcf Complex

Rong J. Guo, Toshihiko Ezaki, Eunran Suh, John P. Lynch

INTRODUCTION: Cdxl and Cdx2 are intestine-specific transcription factors known to regulate proliferation and differentiation. We previously have reported that $\mathrm{Cdx} 1$ and $\mathrm{Cdx} 2$ inhibit the proliferation of human colon cancer cells by inhibiting beta -catenin/Tcf transcriptional activity. Herein we describe studies that further elucidate this mechanism. METHODS Coimmunoprecipitation (CoIP) was performed using anti-c-myc and anti flag tagged techniques to analyze the interaction between beta-catenin/Tcf complex and $\mathrm{Cdx} 1$ and $\mathrm{Cdx} 2$ in 293T and DLD1 cells. GST-beta-catenin pull-down with in vitro-translated Cdx1 or Cdx2 RESULTS: We previously found that $\mathrm{Cdx} 1 / \mathrm{Cdx} 2$ expression dramatically reduced the betacatenin/ TCF transcriptional activity in DLDl and 293T cells. To clarify the mechanism involved, c-myc tagged Tcf4 was transfected with a S33Y beta-catenin and a Cdxl or Cdx2 expression vectors into 293T or DLDl cells, then immunoprecipitated using an anti cmyc antibody. Western blotting of the IP products showed that beta-catenin could not be coimmunoprecipited with TCF4 in the presence of $\mathrm{Cdx} 1$ or $\mathrm{Cdx} 2$ expression. This effect of $\mathrm{Cdx}$ proteins was dose-dependent and consistent with the corresponding inhibitory effect of $\mathrm{Cdx}$ proteins on b-catenin/TCF transcriptional activity. Moreover, expression of a Cdx2 mutant in which a classically characterized transactivation domain (delta $55-134 \mathrm{Cdx} 2$ ) is deleted still had significant effect on inhibiting Topflash activity and interfereing correspondingly with the formation of b-catenin/TCF complex. To explore the possibity of direct proteinprotein interactions between $\mathrm{Cdx}$ and beta-catenin, we performed immunoprecipitations in vivo and GST-pull down studies. Immunoprecipitating Flag-tagged Cdxl or Cdx2 or betacatenin demonstrated a reliable specific interaction between the $\mathrm{Cdx}$ proteins and betacatenin. In the reverse experiment, IP of b-catenin also pulls down $\mathrm{Cdxl}$ or $\mathrm{Cdx} 2$. In contrast, GST-b-catenin could not pull down in vitro translated Cdx proteins, nor could in vitro translated Cdx2 protein disrupt a GST-beta-catenin/TCF4 interaction. This observation suggests that Cdxl and Cdx2 proteins' disruption of the beta-catenin/TCF complex is not a direct effect but is instead mediated by a protein binding both $\mathrm{Cdx}$ and beta-catenin. In summary, we conclude that $\mathrm{Cdx} l$ and $\mathrm{Cdx} 2$ expression reduces b-catenin/TCF transcriptional activity through the disruption of the beta-catenin/TCF complex. Considering the pivotal role of beta-catenin/TCF activity in regulating cell proliferation and carcinogenesis, our findings provide another window for understanding intestinal proliferation and colorectal carcinogensis. 
Overexpression of Human IGFBP-3 Inhibits the Development of Intestinal Tumors in An APC/Min Mouse Model

Irena Kirman, Suvinit Jain, Avraham Belizon, Richard L. Whelan

Deficiency in circulating IGFBP-3 has been linked to the development of various types of tumors in humans. The effect of systemic IGFBP-3 over-expression as a means of overcoming or preventing tumor growth has not been investigated so far. The goal of the current study was to evaluate the development of intestinal tumors in $\mathrm{APC} /$ min mice carrying the human IGFBP-3 transgene. Materials and Methods: 24 wild-type (WT) CDI APC/min mice and 16 $\mathrm{CD} 1 \mathrm{APC} / \mathrm{min}$ mice, males and females, carrying IGFBP- 3 cDNA (IGFBP-3 TG) were maintained until 8 months of age. Genotyping for the min mutation was performed using a PCR reaction, while the presence of human IGFBP-3 in serum from the IGFBP-3 TG mice was confirmed in ELISA. At the end of experiment, the animals were sacrificed, their colons excised, open longitudinally, and then fixed and stained with methylene blue. Tumors and ACF (aberrant crypt foci) were assessed using inverted microscopy and confirmed hitologically. Results: The mean body weight of $\mathrm{CDl}$ males, $36.3 \pm 5.6 \mathrm{~g}$ was comparable to the mean body weight of IGFBP3-TG males, $31.3 \pm 4.5 \mathrm{~g}(\mathrm{p}=0.2)$. Similarly, the mean body weight of $\mathrm{CD}$ l females, $32.3 \pm 4.7 \mathrm{~g}$ was comparable to the mean body weight of IGFBP-3 TG females, $29.9 \pm 2.8 \mathrm{~g}(\mathrm{p}=0.1)$. Visible tumors were found in $1 / 3$ ( 8 out of 24 ) wild-type min mice, but in none of the IGFBP-3 TG mice. The number of ACF per intestine was significantly smaller in IGFBP-3 TG males, $4.1+4.9$, compared to WT min males, $10.7 \pm 7.5(\mathrm{p}<0.002)$. Similarly, significantly less intestinal ACF were found in IGFBP-3 TG min females, 1.7 \pm 2.4 compared to WT min females, $7.0 \pm 4.4$ ( $\mathrm{p}<0.002$ ). Conclusions Over-expression of human IGFBP-3 was associated with a significantly decreased rate of formation of colonic aberrant crypt foci, a forerunner of intestinal malignancies in a min mouse model. Over-expression of human IGFBP-3 may become a strategy to inhibit the development or growth of colonic tumors

806

Molecular Characterization of the Chemopreventive Activity of Pentacylic Triterpene Ursolic Acid

Sandra Ulrich, Bettina Jung, Stefan Buettner, Juergen Stein

Background: Previous studies could demonstrate that ursolic acid (UA), a pentacyclic triterpene found in berries and plants has antiproliferative, antiangiogenic as well as proapoptotic activities on cancer cells. The objective of this study was to elucidate the underlying molecular mechanisms of these chemopreventive effects of UA. Materials and Methods: Caco-2 cells were cultured under standard conditions and were treated with increasing concentrations of ursolic acid $[10-30 \mu \mathrm{M}]$ for different time intervalls. Hypoxia was induced by stimulating Caco-2 cells with $\mathrm{CoCl} 2[100 \mu \mathrm{M}]$. Cell growth was determined by BrdU incorporation and crystal-violet staining. Apoptosis induction was detected by measuring DNA fragmentation. The cell cycle regulating proteins $\mathrm{p} 21^{\mathrm{WAF} 1}$ and $\mathrm{p} 27^{\mathrm{KIP} 1}$, the apoptosis related proteins $\mathrm{BAX}, \mathrm{Bcl}-2$ and TRAIL, as well as the proangiogenic vascular endothelial growth factor (VEGF) were determined by western blot analysis. The VEGF content of the culture supernatants was measured using ELISA. Caspase-3 induction was determined via

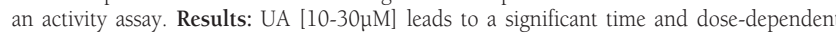
decrease both of cell counts and cell proliferation (Means \pm SE, $n=8 ; * * * p<0.001$ at $30 \mu \mathrm{M}$ ), coincident with the upregulation of the cell cycle inhibitors $\mathrm{p} 21^{\mathrm{WAF} 1}$ and $\mathrm{p} 27^{\mathrm{Kip} 1}$. In addition, ursolic acid leads to a significant induction of apoptosis after $24 \mathrm{~h}$ of incubation (Means \pm $\mathrm{SE}, \mathrm{n}=4,{ }^{* * *} \mathrm{p}<0.001$ at $10 \mu \mathrm{M}$ ), which is mediated by an increase of $\mathrm{BAX} / \mathrm{Bcl}-2$-proteinratio as well as an upregulation of TRAIL protein which meets in an induction of caspase3 activity. VEGF expression and release induced by $\mathrm{CoCl} 2[100 \mathrm{uM}]$ could be significantly counteracted by the treatment with ursolic acid in a time and dose-dependent manner (Means $\pm \mathrm{SE}, \mathrm{n}=2,{ }^{*} \mathrm{p}<0.01$ ). Conclusions: These data demonstrate that ursolic acid mediates its antiproliferative effects via the induction of the cell cycle inhibitory proteins $\mathrm{p} 21^{\mathrm{WAF} 1}$ and $\mathrm{p} 27^{\mathrm{KIP} 1}$. Cell death was induced by activating both intrinsic and extrinsic pathways, leading to activation of proapoptotic caspase-3. Moreover ursolic acid seems to exhibit potent antiangiogenic properties as it counteracts hypoxia-induced VEGF production in colorectal cancer cells.

\section{7}

Novel Curcumin Analog Diphenyl Difluoroketone (L-2395) Mediates AntiColon Cancer Activity Through Epidermal Growth Factor Receptor Signaling Inhibition

Katherine B. Lee, Dharmalingam Subramaniam, Sripathi M. Sureban, Rama P. Ramanujam, Hideg Kalman, Periannan Kuppusamy, Courtney W. Houchen, Brian K. Dieckgraefe, Shrikant Anant

Curcumin is a chemopreventive agent that has been shown to reduce intestinal tumors in murine models. Previously, the water-soluble curcumin analog, diphenyl difluoroketone (L2395, also known as EF24), was shown to induce apoptosis of cancer cells. We have also determined that L-2395 induces apoptosis and inhibits proliferation of HCT-116 and HT29 colon cancer cells. The mechanism, however, of L-2395 anti-cancer activity has not been characterized. This study demonstrates that L-2395 mediates the anti-cancer activity by blocking activation of the epidermal growth factor receptor (EGFR) and subsequent signaling through its downstream cytoplasmic and nuclear targets. HCT-116 human colon cancer cells were treated with EGF $(100 \mathrm{ng} / \mathrm{ml})$ alone, or in the presence of L-2395 $(10 \mu \mathrm{M})$. EGF rapidly induces phosphorylation of EGFR, whereas L-2395 decreases baseline and EGFstimulated EGFR phosphorylation. We next determined the effect of L-2395 on EGFR downstream targets, ERK and AKT. L-2395 suppressed both baseline and EGF-stimulated ERK and AKT phosphorylation in a dose dependent manner. Transcription factor NFkappaB is a downstream target of ERK and AKT activation. Phosphorylation of IkappaB promotes the nuclear translocation of the normally cytoplasmically sequestered NFkappaB subunits p50 (NFKB1) and p65 (RelA). Western blot analyses demonstrated that L-2395 inhibited both baseline and EGF-mediated IkappaB phosphorylation. Moreover, nuclear extracts of L-2395-treated cells show decreased levels of both p50 and p65 proteins, suggesting that the expression of colorectal cancer promoting genes cyclooxygenase-2 (COX-2), interleukin8 (IL-8), and anti-apoptotic mitochondrial gene Bcl- $\mathrm{X}_{\mathrm{L}}$, as well as altering the Bcl-2/Bax ratio. Real time PCR analyses of L-2395-treated cells exhibit reduced baseline and EGFstimulated expression of COX-2 and IL-8. Additionally, EGF-stimulated expression of Bcl$\mathrm{X}_{\mathrm{L}}$ was significantly reduced by L-2395 treatment. Finally, L-2395 suppressed EGF-mediated increase of the anti-apoptotic Bcl-2/apoptotic Bax ratio. These results imply that L-2395 inhibits activation of EGFR and its downstream targets in HCT-116 cells. This L-2395mediated EGFR signaling blockade may account for the growth inhibitory and pro-apoptotic anti-cancer effects of the novel molecule.

808

Red Meat \& Colon Cancer: Dietary Heme Injures Surface Epithelium and Thus Inhibits Apoptosis and Stimulates Cell Proliferation Leading to Crypt Hyperplasia in Rat Colon

Johan de Vogel, Wytske Boersma-van Eck, Aloys Sesink, Denise Jonker-Termont, Jan Kleibeuker, Roelof van der Meer

Background \& Aim: Epidemiologic and animal model studies show that a high intake of heme, present in red meat, is associated with an increased risk of colon cancer. The aim of this study was to elucidate the effects of dietary heme on colonic cell homeostasis in rats. Methods: Rats were fed a purified, Western control diet, or this diet supplemented with 0.5 $\mathrm{mmol}$ heme/kg for 14 days. Fecal water cytotoxicity was determined with a bioassay. Exfoliation of colonocytes was measured as the amount of rat DNA in feces. At day 14 colons were excised and examined histologically after staining with hematoxilin-eosin to score intercryptal colonic surface epithelial damage. In addition to measurement of DNA in feces, caspase- 3 expression and activity were measured in colonic mucosa to determine the rate of apoptosis. Colonic epithelial cell proliferative activity was evaluated with $3 \mathrm{H}$-thymidine or 5-bromo-2'-deoxyuridine (BrdU) incorporation into DNA, both administered $2 \mathrm{~h}$ before sacrifice, or by Ki-67 immunohistochemistry. Results: Dietary heme induced a more than ten-fold increased cytotoxicity of the fecal water and a hundred-fold lower fecal excretion of rat DNA. Colons of heme fed rats showed injured surface epithelium and an approximately $25 \%$ increase in crypt length indicating crypt hyperplasia. Dietary heme inhibited colonic mucosal apoptosis almost completely, whereas at the same time it doubled crypt cell proliferative activity, as shown by all three markers. Conclusions: Our results clearly show that dietary heme causes injury of the colonic surface epithelium, which is overcompensated by inhibition of apoptosis and by hyperproliferation of crypt cells, resulting in crypt hyperplasia. This disturbed epithelial cell homeostasis might explain why a high intake of dietary heme (i.e. red meat) is associated with an increased risk of colon cancer.

809

3,3'-Diindolylmethane (DIM) Induces Apoptosis and Cell Cycle Arrest in Human Colon Cancer Cells

J.N. H. Park, Eun Ji Kim, Dae Young Kwon, Hyun J Choi, So Young Park, Hyun-Kyung Shin, Young-Joon Surh

DIM is a major in vivo derivative of the putative anticancer agent indole-3-carbinol, which is present in cruciferous vegetables and has been reported to have anti-carcinogenic properties. The present study examined the mechanisms by which DIM inhibits human colon cancer cells. To examine the effects of DIM on the growth of HT-29 and HCT116 human colon cancer cells, the cells were cultured with various concentrations $(0-30 \mu \mathrm{mol} / \mathrm{L})$ of DIM. Treatment of cells with increasing concentration of DIM led to a dose-dependent decrease in viable cell numbers in both HCT116 and HT-29 cells. The decrease in cell growth was due to a decrease in cell cycle progression and an increase in apoptosis. Results from DNA flow cytometric analysis revealed that DIM induced G1 and G2/M phase arrests at $12 \mathrm{~h}$ of culture. Western blot analyses of total cell lysates revealed that DIM decreased phosphorylated retinoblastoma proteins (Rb) and E2F-1 and increased hypophosphorylated $\mathrm{Rb}$ levels. DIM decreased cyclin A and Dl and increased cyclin-dependent kinase (CDK) 4 levels in a time-related manner. DIM also increased the protein levels of the CDK inhibitors p21 and p27. In addition, DIM decreased the levels of cell division cycle 2 and cyclin B1. DIM increased annexin-5-stained cells in both HT-29 and HCT116 cells in a dose-dependent manner. Western blot analysis of total cell lysates revealed that DIM induced cleavage of caspase-8, caspase-9, caspase-7, caspase-3, and poly (ADP-ribose) polymerase in both HCT116 and HT-29 cells. In addition, DIM increased the translocation of cytochrome c and Smac/Diablo from the mitochondria to the cytosol in both HCT116 and HT-29 cells. In HCT116 cells, DIM increased truncated Bid levels but did not affect the protein levels of p53, Bcl-2, or Bax. In HT-29 cells, DIM decreased Bcl-2 and increased truncated Bid levels. We have demonstrated that DIM inhibits cell cycle progression and induces apoptosis in human colon cancer cells. These results suggest that DIM inhibits G1 and G2/M phase cell cycle progression by inhibiting CDK activities. The induction of apoptosis by DIM in colon cancer cells may be mediated through changes in mitochondrial events which are associated with the release of cytochrome $\mathrm{c}$ and Smac/Diablo and the activation the caspase pathway through p53-independent pathway.

810

Scutellaria Baicalensis Extract and Constituent Flavonoids Inhibit Proliferation in Pancreatic Cancer Cell Lines

Alexandra Berger, Yantao Niu, Guido Eibl, Vay Liang Go, Diane Harris

Extracts of Scutellaria baicalensis (SB; Chinese Skullcap or Huang Qin) root have traditionally been used to treat inflammation, suggesting inhibition of arachidonic acid (AA) metabolism. Regulation of AA flux through the cyclooxygenase (COX) and lipoxygenase (LOX) pathways modulate pancreatic cell proliferation. Therefore we chose to study the effect of an extract of SB on 6 different human pancreatic cancer cell lines that differ in COX and LOX phenotype; AsPc-1, PANC-1, MiaPaCa-2 are COX-2 negative, while BxPc-3, HPAF2, and CAPAN-2 are 
COX-2 positive. $75 \%$ ethanol extracts of SB (Botanica Biosciences, Ojai, CA) were used (224 mg baicalin/g SB). Pancreatic cancer cell lines (initial 3-5,000 cells) were treated with increasing concentrations $(12.5-200 \mu \mathrm{M})$ of SB extract or selected flavonoids: baicalin, baicalein, wongonin, as well as indomethacin (non-specific COX inhibitor) and nordihydroguaiaretic acid (NDGA; non-specific LOX inhibitor) for $48 \mathrm{~h}$. Levels of SB were matched to baicalin content. Cell viability was assayed using an ATP based cell proliferation assay (CellTiter-Glo, Promega). Expression of 5-lipoxygenase (5-LOX) was assayed by RT-PCR. The SB whole extract was most potent in inhibiting proliferation in the lines positive for 5 LOX expression (AsPC-1, BxPC-3, Capan-2, and HPAF-2; IC ${ }_{50} 46.8-89.8 \mu \mathrm{M}$ baicalin equivalent) and was least effective in the other lines. The whole extract was also generally more potent than baicalin itself. NDGA showed inhibitory activity in most lines (IC 50 23.8$109.0 \mu \mathrm{M}$ ), while indomethacin showed little to no activity in all lines. The results demonstrate that natural compounds with LOX inhibitory activity inhibit proliferation in pancreatic cell lines. These effects appear to be mediated through the 5-LOX and not the COX-2 pathway, as COX-2 negative cells were also responsive to SB. These data suggest that SB extracts may have pancreatic cancer-preventive activity. Supported by NIH AT1535, CA4271, and the AGA Foundation for Digestive Health and Nutrition. $\mathrm{IC}_{50}$ 's for SB in Pancreatic Cell Lines

\begin{tabular}{|c|c|c|c|c|c|c|}
\hline & \multicolumn{7}{|c|}{ Compound } \\
\hline Cell Line & $\underline{\text { SB } *}$ & $\underline{\text { Baicalin }}$ & $\underline{\text { Baicalein }}$ & $\underline{\text { Wogonin }}$ & $\underline{\text { Indomethacin }}$ & $\underline{\text { NDGA }}$ \\
\hline MIAPaCa-2 & 187.0 & --- & --- & 173.5 & --- & 75.1 \\
\hline PANC-1 & 203.4 & --- & --- & -- & --- & 109.0 \\
\hline AsPC-1 & 63.2 & 70.6 & 45.1 & 179.9 & 191.4 & 38.5 \\
\hline BxPC-3 & 48.3 & 67.7 & 34.4 & 63.2 & --- & 23.8 \\
\hline HPAF2 & 89.8 & 121.8 & 57.1 & 174.2 & 199.2 & 42.3 \\
\hline CAPAN-2 & 46.8 & 51.2 & 34.2 & -- & --- & 28.3 \\
\hline
\end{tabular}

$--=50 \%$ inhibition is not reached. $* \mathrm{SB}$ is on $\mu \mathrm{M}$ baicalin basis

811

Bile Acids Activate Epidermal Growth Factor Receptor (EGFR) KinaseDependent Phosphatidylinositol 3-Kinase (PI3K)/AKT Proliferative Signaling in Colon Cancer Cells

Chang Guo, Jean-Pierre Raufman

Recently, we demonstrated that conjugated secondary bile acids transactivate EGFR, activate p44/42 MAP kinase (MAPK) signaling and stimulate colon cancer cell proliferation (Biochem. Pharmacol. 2005;70:1035). In the present study, we used HT-29 and H508 human colon cancer cells to determine the role of EGFR kinase activity and stimulation of PI3K/Akt signaling in mediating the proliferative actions of bile acids. Immunoblotting (IB) of colon cancer cell lysates with specific polyclonal antibodies for phospho-Akt(Ser473) revealed that deoxycholyltaurine (DCT, 10-300 $\mathrm{MM}$ ) caused dose- and time-dependent Akt phosphorylation without altering total cellular Akt. Akt phosphorylation was maximal with $300 \mu \mathrm{M}$ DCT, the highest concentration examined. The time course for DCT-induced Akt phosphorylation was biphasic; initial rapid phosphorylation was maximal at 5 min with a second increase in phosphorylation observed from 20-60 min. Adding either EGFR kinase inhibitors (0.1 $\mu \mathrm{M}$ AGl478 and $2 \mu \mathrm{M}$ PD168393) or PI3K activation inhibitors (30 $\mu \mathrm{M}$ LY294002 and $100 \mathrm{nM}$ wortmannin) abolished DCT-induced Akt phosphorylation. Transfection of HT-29 cells with a dominant negative EGFR mutant (K721M that inactivates EGFR kinase by removing the ATP-binding site) markedly reduced DCT-induced Akt phosphorylation. In contrast, EGFR overexpression by transfection with a wildtype clone increased DCTinduced Akt phosphorylation. These findings provide evidence that in human colon cancer cells, bile acid-induced phosphorylation (activation) of Akt is EGFR kinase- and PI3Kdependent. EGFR kinase (PD168393) and PI3K (LY294002 and wortmannin) inhibitors also reduced DCT-induced proliferation of both HT-29 and H508 cells. Compared to control, incubation with LY294002 $(50 \mu \mathrm{M})$ and wortmannin $(50 \mathrm{nM})$ reduced DCT $(50 \mu \mathrm{M})$ induced proliferation of HT-29 cells by $86.1 \pm 4.6 \%$ and $28.4 \pm 5.0 \%$ (mean $\pm \mathrm{SD} ; \mathrm{n}=4$; $\mathrm{p}<0.01$ ), respectively, and of $\mathrm{H} 508$ cells by $97.6 \pm 1.6 \%$ and $31.9 \pm 9.0 \%$ (mean $\pm \mathrm{SD} ; \mathrm{n}=$ $4 ; \mathrm{p}<0.01$ ), respectively. In contrast to Akt phosphorylation, DCT-induced p44/42 MAPK phosphorylation was not reduced by transfection of HT-29 cells with the K721M EGFR dominant negative mutant or by incubation with an EGFR kinase inhibitor. These findings indicate that DCT-induced activation of p44/42 MAPK is EGFR kinase-independent. Bile acid-induced activation of EGFR kinase-dependent (PI3K/Akt) and kinase-independent (p44/ 42 MAPK) signaling may augment human colon cancer cell proliferation by activation of both anti-apoptotic and pro-proliferative mechanisms, respectively. Collectively, these observations provide mechanisms to explain the in vivo actions of bile acids in promoting colon cancer growth

\section{2}

Treatment of Mild to Moderate Acute Attacks of Distal Ulcerative Colitis with Rectally-Administered E. Coli Nissle 1917: Dose-Dependent Efficacy Harald Matthes, Thomas Krummenerl, Manfred Giensch, Corinna Wolff, Juergen Schulze Introduction: Distal ulcerative colitis (UC) is commonly treated with anti-inflammatory medication, with rectal formulations being particularly successful. Anecdotal clinical observation point to the successful use of enemas containing stool suspensions or probiotic suspensions. Efficacy of oral therapy with probiotic E. coli Nissle 1917 (EcN) for remission maintenance in UC patients has been shown in several studies. Subsequent to a documented phaseI study of EcN enemas which were well tolerated in 80 patients, clinical effectiveness as compared to a placebo was investigated at various dosages to test for efficacy and dosedependency. Study design: A total of 90 patients were enrolled. In a double-blinded study patients were randomly treated with either 40,20 or $10 \mathrm{ml}$ enema containing $10 \exp 8 \mathrm{EcN}$ per $\mathrm{ml}(\mathrm{n}=24,23$ or 23$)$ or a placebo $(\mathrm{n}=20)$. Patients received the study medication once daily over at least 4 weeks. Admission criterion was UC proctitis or proctosigmoiditis with mild to moderate activity (DAI according to Sutherland 4 - 9). After 2 and 4 weeks the clinical DAI (primary objective) as well as data on tolerance were determined. Patients reaching clinical DAI $\leq 2$ within the therapy phase were regarded as responders. Results: According to the (Jonckheere-Terpstra) test for rank correlation evaluating dose-dependen efficacy, 57/90 per-protocol patients (no concomitant acute-phase therapy with aminosalicylates or glucocorticoids, no abrupt stop of acute-phase therapy prior to study commencement, maximal spread of inflammation $45 \mathrm{~cm}$ from anus) were assessed: the responder rates significantly correspond to the applied $\mathrm{EcN}$ doses by increase in ranking $(\mathrm{p}=0.04)$. Remission was achieved by $2 / 11$ patients (18.2\%) receiving a placebo, $3 / 11$ (27.3\%) receiving $\mathrm{EcN}$ $10 \mathrm{ml}, 8 / 18$ (44.4\%) receiving $E_{c N} 20 \mathrm{ml}$ and 9/17 (52.9\%) receiving $E_{c N} 40 \mathrm{ml}$. Time to remission was the shortest in the EcN-40-group, followed by the EcN-20-ml group Assessment of tolerance by patients and by the physician as well as the number of patient with suspected side effects did not reveal significant differences between the treatmen groups. Conclusion: Rectal application of 20 or $40 \mathrm{ml} \mathrm{EcN}$ enemas (10exp8 EcN/ml) for the therapy of mild to moderate distal UC represents an effective and well tolerated alternative to topical use of aminosalicylates or glucocorticoids.

813

Combined Data from Two Pivotal, Randomized, Placebo-Controlled, Phase III Studies Show That SPD476, a Novel mesalamine Formulation Given Once Or Twice-Daily, Is Effective for the Induction of Remission of Mild-to-Moderate Ulcerative Colitis

William J. Sandborn, Michael A. Kamm, Gary R. Lichtenstein, Miguel Gassull, Stefan Schreiber, Lechoslaw Jackowski, Prabhakar Boddu, Natalya Gubergrits

Background: Mesalamine (5-ASA) is a first-line treatment for mild-to-moderate ulcerative colitis (UC). Presently available formulations are associated with inconvenient multiple-daily dosing, which can lead to patient non-compliance, thus reducing drug effectiveness and overall treatment success. Here we report an analysis of combined efficacy and safety data from two, prospective, randomized, multicenter, double-blind, placebo-controlled, phase III studies (SPD476-301 [Study 301] and SPD476-302 [Study 302]) investigating SPD476, a novel, high-strength (1.2g/tablet), once-daily formulation of mesalamine. SPD476 uniquely combines a gastro-resistant polymer film, delaying release of the active drug until the terminal ileum, and MMX Multi Matrix System ${ }^{\mathrm{TM}}$ (MMX) technology, extending consistent delivery of 5-ASA throughout the entire colon. Methods: Eligible subjects had mild-to-moderate UC. Data were combined for subjects receiving SPD476 2.4g/day (given once daily [QD; Study 302] or twice daily [BID; Study 301] combined n=172), SPD476 4.8g/day (given QD [Studies 301 and 302] combined $n=174$ ) or placebo (combined $n=171$ ). The proportion o subjects in remission (primary endpoint: UC disease activity index of $\leq 1$, with a rectal bleeding and stool frequency score of 0 and $\geq 1$ point reduction in sigmoidoscopy score from baseline) at week 8, was compared for the SPD476 groups vs the placebo group. Results: A statistically significantly greater percentage of subjects achieved remission in the SPD476 $2.4 \mathrm{~g}$ /day group compared with the placebo group (37.2 vs $17.5 \%[\mathrm{p}<0.001]$ ) Similarly, a statistically significantly greater percentage of subjects in the SPD476 4.8g/day QD group achieved remission compared with the placebo group (35.1 vs $17.5 \%[\mathrm{p}<0.001]$ ) A total of 10 subjects reported 13 serious adverse events (7 with placebo, 4 with SPD476 2.4g/day BID [aggravated $\mathrm{UC}=1$; pancreatitis $=1$; perianal abscess $=1$; urinary retention $=1$ ] and 2 with SPD476 4.8g/day QD [viral gastroenteritis=1; pancreatitis=1]). Only pancreatitis was considered to be possibly and probably related to study medication. Both cases were resolved following discontinuation of the study drug. Conclusions: These data show that this unique formulation of SPD476, combining a high dose of 5-ASA per tablet and MMX technology, was well tolerated and effective for the induction of remission of mild-tomoderate UC at doses of $2.4 \mathrm{~g} /$ day and $4.8 \mathrm{~g} /$ day given once or twice daily. The efficacy and convenience of SPD476 is anticipated to improve patient compliance and enhance overall treatment success.

\section{4}

Similar Clinical Remission Rates with mesalazine Foam and Enema in Patients with Active Left-Sided Ulcerative Colitis (UC): Results of a Multicentre, Prospective, Randomized, Investigator-Blinded, Comparative Study Antoine Cortot, Denis Maetz, Eric Degoutte, Olivier Delette, Pierre Meunier, Tg Tan, Jean-Brice Cazals, Olivier Dewit, Xavier Hebuterne, Sylvain Beorchia, Bernard Grunberg, Eric Leprince, Geert D'Haens, Sylvie Forestier, Marc Lemann

Aim: To demonstrate that mesalazine foam was at least as effective as mesalazine enema for inducing clinical remission defined as modified Rachmilewitz score (CAI 1-4) inferior or equal to 2 in patients with left-sided active ulcerative colitis treated for 4 weeks. Methods: 375 patients with mild to moderate UC received mesalazine foam $1 \mathrm{~g} / 100 \mathrm{ml} / \mathrm{d}$ or liquid mesalazine enema $\mathrm{lg} / 100 \mathrm{ml} / \mathrm{d}$ for 4 weeks. Inclusion criteria were: newly diagnosed or relapsing active UC, disease extension superior or equal to $5 \mathrm{~cm}$ from anorectal junction to the splenic flexure, CAIl-4 superior or equal to 4 , age superior or equal to 18 years. Oral maintenance treatment at stable doses with mesalazine, azathioprine or methotrexate was permitted. Oral steroids treatment was not allowed. The primary endpoint was clinical remission at week 4. Secondary objectives included rate of endoscopic remission (endoscopic Rachmilewitz score inferior to 4 ) at week 4 , acceptability assessed with VAS, safety and compliance to the treatment. For this non-inferiority study, the equivalence limit was $15 \%$. Results: Patients were comparable in foam and enema groups as far as : duration of UC $(60$ and 64.4 months) duration of the current episode (2.1 months in both groups), \% of relapsing $(68 \%$ and $69 \%), \%$ of proctitis ( $43 \%$ and $46 \%), \%$ of proctosigmoiditis $(51 \%$ in both) and \% of left-sided colitis (6\% and 3\%). Remission rates at 4 weeks in foam and enema groups were $68 \%$ and $73 \%$ in per protocol (PP) analysis (95\% CI: $-15.1 \% ; 4.4 \%$, and $67 \%$ and $70.5 \%$ in intent-to-treat (ITT) analysis (95\% CI: $-13.4 \% ; 5.8 \%$ ). In PP analysis, CAIl-4 was 6.23 and 6.17 at baseline, 2.65 and 2.57 at week 2, 1.87 and 1.67 at week 4 (NS). Endoscopic remission rates were 67.5 and $80 \%$ in $\mathrm{PP}(\mathrm{p}=0.012)$ and $67 \%$ and $77 \%$ in ITT $(p=0.047)$ respectively in foam and enema groups. Global acceptability and compliance 
were similar in foam and enema groups: $82 \%$ and $84 \%, 96 \%$ and $97 \%$ (NS). The enema retention score was better in foam group $(\mathrm{p}<0.001) .11 \%$ and $13 \%$ patients withdrew from the study (NS), $4 \%$ and 3\% due to non-serious AE (abdominal pain and flatulence), $2 \%$ in both groups for worsening. Conclusion: A 4 weeks treatment of $\mathrm{lg}$ mesalazine foam was equivalent for inducing a clinical remission to lg enema in patients with mildly to moderately active left-sided UC. Retention was better with foam. Mesalazine foam is a therapeutic alternative to mesalazine enema for controlling symptoms in such patients.

Four-Week Results of Adalimumab Treatment in Subjects with Fistulizing Crohn's Disease Who Have Failed Response Or Showed Intolerance to Infliximab

J Hinojosa, F Gomollon, P Nos, M Penate, D Ceballos, Ma Gassull

PURPOSE To assess the efficacy and safety of adalimumab, a fully human monoclonal antibody targeting TNF- $\alpha$, for inducing fistula closure in subjects with fistulizing Crohn's disease (CD). METHODS In this open-label multi-center study for the induction of clinical remission and perianal fistula closure, subjects with failed response or intolerance to inflixi$\mathrm{mab}$ and a diagnosis of fistulizing $\mathrm{CD}(\mathrm{CDAI}<220)$ or both luminal (mucosal) and fistulizing $\mathrm{CD}(\mathrm{CDAI}>220)$ were treated with subcutaneous adalimumab: $160 \mathrm{mg}$ at Week $0,80 \mathrm{mg}$ at Week 2, then $40 \mathrm{mg}$ every other week (eow) for 52 weeks. Subjects were routinely assessed for complete or partial ( $\geq 50 \%$ decrease in number of draining fistula compared to baseline) fistulae closure, PDAI score, and adverse events (AE). RESULTS Of 22 subjects treated with adalimumab, all had been treated previously with infliximab: 5 (23\%) had failed response to infliximab, 17 (77\%) were infliximab-intolerant. Subjects had luminal and fistulizing $\mathrm{CD}(45 \%)$ or fistulizing $\mathrm{CD}$ only $(55 \%)$. At baseline, the mean PDAI score overall ( $\mathrm{n}=22)$ was $10.5 \pm 2.8$; the mean number of fistulae was $2.5 \pm 1.2$ in subjects with fistulizing CD only and 3.8 \pm 5.5 in subjects with both luminal and fistulizing CD. Week 4 results are shown in the table. Treatment-related AE were mild to moderate in severity and similar to those observed in studies of ADA in subjects with rheumatoid arthritis. The most common AE were erythema, nausea/dizziness, weakness, and myalgia. One patient dropped out due to non-pruritic erythema. No serious AE's were reported. CONCLUSIONS Adalimumab induced complete or partial fistula closure in these subjects and was well-tolerated. No new safety concerns were found in subjects with fistulizing CD compared to other adalimumab-treated populations

PDAI Scores and Fistula Closure at Week 4 of Adalimumab Treatment

\begin{tabular}{|c|c|c|c|}
\hline & Luminal and Fistulizing $(\mathrm{n}=10)$ & Fistulizing $(\mathrm{n}=12)$ & All $(\mathrm{n}=22)$ \\
\hline Median PDAI & $4.7 \pm 2.2$ & $6.1 \pm 3.6$ & $5.8 \pm 3.1$ \\
\hline Complete closure $\mathrm{n} / \mathrm{N}(\%)$ & $3 / 10(30 \%)$ & $2 / 12(17 \%)$ & $5 / 22(23 \%)$ \\
\hline Partial closure $\mathrm{n} / \mathrm{N}(\%)$ & $4 / 10(40 \%)$ & $5 / 12(42 \%)$ & $9 / 22(41 \%)$ \\
\hline
\end{tabular}

Intention to treat analysis

816

Autologous Hematopoietic Stem Cell Transplantation (HSCT) for Refractory Crohn's Disease (CD), 4 Year Follow Up

Robert M. Craig, Yu Oyama, Larissa Verda, Laisvyde Statkute, Krosnjar Nela, Kathleen Quigley, Richard Burt

Purpose: HSCT is being studied in an effort to ascertain its value in inducing a clinical remission in severe $C D$ Methods. Patients with severe $C D$ who have failed standard therapy (5-ASA, antibiotics, corticosteroids, immunosuppression, and infliximab), whose CDAI was $>250$, were accessed for therapy. Stem cells were mobilized from the peripheral blood using cyclophosphamide $(2.0 \mathrm{~g} / \mathrm{m} 2)$ and G-CSF (10 ug/kg/day), enriched ex vivo by CD34+ selection, and reinfused after immune conditioning with cyclophosphamide $(200 \mathrm{mg} / \mathrm{kg})$ and anti-thymocyte globulin $(90 \mathrm{mg} / \mathrm{kg})$. Clinical variables, small bowel radiography, colonoscopy and T-cell subsets were followed annually following the HSCT. Results: 19 subjects have undergone the therapy successfully. HSCT had no in-patient mortality, and was complicated only by 24-48 hours of culture negative fever. Usually, diarrhea and abdominal pain resolved prior to hospital discharge. Perianal fistulae and perianal disease were slower to resolve, but gradually improved over months to years. Abdominal masses and colonic strictures have resolved. Two patients had progression of their small intestinal strictures following the transplantation and required surgical resection, but their post-operative courses have been uneventful, and they continue to do well off all Crohn's disease medications. Three patients have had major relapses, 2 at one year and 1 at 2 years, requiring re-institution of immunotherapy. One had a minor relapse at 1 year requiring re-institution of infliximab, promoting remission (which was ineffective pre-transplant). Following HSCT, a robust rise in CD4+CD25+(bright) T-cells was observed. There was a smaller rise in these T-cells in those who eventually relapsed. In most subjects, serum albumins, hemoglobins, weights, sedimentation rates, and CRPs have improved or normalized. The patient with the longest follow up, at $41 / 2$ years post $\mathrm{HSCT}$, remains in remission off all CD medication. Conclusion: This preliminary experience in the first 19 patients who have received this unique therapy continues to be encouraging, although 3 have had major recurrences of the disease. Induction of regulatory T-cells may be partially responsible for the salutary effect. A randomized study is in progress.
817

Atorvastatin Decreases Seo Index in Patients with short Duration of Disease in Ulcerative Colitis: A Randomized Placebo-Controlled Clinical Trial Peter D. Higgins, Tahira Khan, John Mapili, Ellen M. Zimmermann

Background: Statin medications have systemic anti-inflammatory effects. Studies in animal models and a case series in humans have shown improvement in colitis with statins. Aims: 1) To determine whether the Seo Index is improved in a randomized controlled 24-week pilot study of daily oral atorvastatin $40 \mathrm{mg}$ in patients with mild to moderate ulcerative colitis; 2) To identify any subgroups of patients most likely to respond to atorvastatin. Methods: Thirty-six patients 18 to 70 years of age with mild to moderately active ulcerative colitis despite a variety of stable medications were randomized to placebo or $40 \mathrm{mg}$ daily oral atorvastatin for 24 weeks. Subjects were allowed to leave the study after a final evaluation if a symptomatic flare occurred. In an intention-to-treat analysis, the final Seo Index when controlled for baseline Seo Index was the primary outcome. A decrease in the Seo Index of $>30$ points was considered clinically significant improvement, and a Seo score of less than 120 points was considered remission. Six pre-specified covariates of disease duration, disease extent, baseline CRP, thiopurine use, steroid use, and pANCA titer were evaluated in a multivariate model to identify subsets of subjects with improved responses. Similar assessments with the IBDQ ( $>205$ for remission, $>20$ change for improvement) were performed to evaluate effects on quality of life. Results: The final Seo Index was 132.1 points in patients randomized to atorvastatin, and 157.6 points in patients on placebo $(\mathrm{p}=0.06)$. The disease duration covariate had a significant interaction with therapy, with longer duration of disease leading to less response. After controlling for this interaction, the final Seo Index was 46 points lower in patients randomized to atorvastatin vs. placebo $(\mathrm{p}=0.02)$. Clinically significant improvement was seen in $40 \%$ of patients on atorvastatin, compared to $25 \%$ with placebo. Clinical remission was achieved in $45 \%$ of patients on atorvastatin, compared to $25 \%$ with placebo. With the IBDQ endpoints, $50 \%$ of patients had clinically significant improvement on atorvastatin vs. $19 \%$ on placebo, and $35 \%$ of patients achieved remission on atorvastatin vs. $31 \%$ on placebo. The atorvastatin group had one significant adverse event: a fall with head injury probably unrelated to drug. No significant elevations in liver tests or creatine kinase occurred. Conclusions: Daily oral atorvastatin $40 \mathrm{mg}$ appears to be moderately beneficial in a subset of patients with shorter duration of disease in this pilot study of atorvastatin for ulcerative colitis. The anti-inflammatory effects of atorvastatin in ulcerative colitis warrant further study.

818

The Seattle Protocol for High-Grade Dysplasia in Barrett's Esophagus-It May Not Be As Good As We Think

Revital Kariv, Thomas P. Plesec, Mary P. Bronner, John R. Goldblum, Mary Oldenburgh, Thomas W. Rice, Gary W. Falk

Background: The optimal management of high-grade dyplasia (HGD) in Barrett's esophagus (BE) remains controversial A biopsy protocol consisting of 4 quadrant jumbo biopsies at $1 \mathrm{~cm}$ intervals plus biopsies of any mucosal abnormalities no matter how trivial (Seattle protocol) is thought to be a reliable method to detect early cancers in HGD patients. However, this protocol has never been validated outside of Seattle. Aim: To validate the Seattle protocol in a cohort of BE patients who underwent esophagectomy for a diagnosis of HGD. Patients and Methods: This is a single center study of 32 consecutive BE patients with a biopsy diagnosis of HGD who underwent esophagectomy between 1999-2005. None had mass lesions suggestive of obvious malignancy at the time of preoperative endoscopy. All biopsies were confirmed by expert GI pathologists prior to surgery. Patients were divided into 2 groups: Group 1 had preoperative surveillance biopsies done according to the Seattle protocol as described above; Group 2 had 4 quadrant biopsies every $2 \mathrm{~cm}$. Postoperative pathology findings confirmed by expert GI pathologists were compared to preoperative findings in both groups. Results: There were 21 patients in Group 1 and 11 patients in Group 2. Age and hiatal hernia size were not significantly different between the 2 groups. Median [IQR]BE length was greater in Group $2(10.0 \mathrm{~cm}[4.0,10.0]$ vs $4.0 \mathrm{~cm}[2.0,5.0](\mathrm{P}=0.01))$. Postoperatively, unsuspected intramucosal cancer was found in 7/21(33.3\%) Group 1 vs 3/11(27.3\%)in Group $2(\mathrm{P}=0.99)$. No patients in either group had a postoperative diagnosis of submucosal cancer or lymph node metastases. Conclusions: Intensive preoperative surveillance biopsies using the Seattle protocol does not reliably predict the presence of intramucosal carcinoma at the time of esophagectomy any better than a less intensive surveillance biopsy protocol. This calls into question the concept that the Seattle protocol consistently detects early cancer arising in $\mathrm{BE}$ patients with $\mathrm{HGD}$.

819

Natural History of High Grade Dysplasia in a Regional Veterans Administration Barrett's Cohor

Gareth S. Dulai, Dennis M. Jensen, Fasiha Kanwal, Brennan M. Speigel, Ian M. Gralnek, Paul G. Shekelle

OBJECTIVES: Published data on the natural history of high grade dysplasia (HGD) in Barrett's esophagus (BE) give widely varying estimates of risk for esophageal cancer. The risk of cancer and related mortality may be greater in prevalent than incident cases due to detection of early stage disease in surveillance or bias. Our primary aim was to describe the natural history of prevalent and incident HGD in a large cohort of BE patients. A secondary aim was to compare outcomes in those with prevalent vs incident cancer. METHODS: Consecutive BE cases from 1988-2002 were identified via pathology databases in a regional VA healthcare system and medical record data were abstracted. The risk of progression to cancer as well as mortality was measured and compared in cases with prevalent vs. incident HGD/cancer using survival analysis. RESULTS: There were 30 cases of prevalent HGD, six of whom developed cancer over 70 patient-years of follow-up. Three of thirteen cases with incident HGD developed cancer over 136 years of follow-up. The crude rate of cancer was 1 in 12 years for those with prevalent vs. 1 in 45 years with incident $\operatorname{HGD}(p=0.085)$. Ten cases with prevalent and five cases with incident HGD died, but only $20 \%$ were due to cancer 
Mortality was 1 in 7 years for those with prevalent vs. 1 in 27 years with incident HGD $(\mathrm{p}=0.005)$. There were 45 prevalent and 11 incident cancers. Cancer was early stage in $16 \%$ of prevalent vs. $45 \%$ of incident cases $(\mathrm{p}=0.03)$. Twenty-eight cases with prevalent and six with incident cancer died. Death was due to cancer in $96 \%$ of prevalent vs. $50 \%$ of incident cases $(\mathrm{p}=0.01)$. Mortality rates were 1 in 4 years for those with prevalent vs. 1 in 16 years for incident cancers $(\mathrm{p}=0.02)$. CONCLUSIONS: In a large cohort study of Barrett's, highgrade dysplasia was associated with a high rate of progression to cancer. The risk of overall mortality was significantly higher in those with prevalent vs. incident high-grade dysplasia and cancer. The reduction in risk may be an effect of surveillance and/or other factors including bias.

820

The Development and Validation of An Endoscopic Grading System for Barrett's Esophagus-the Prague C and M Criteria

Prateek Sharma, David Armstrong, Jacques JGHM Bergman, John Dent, Liebwin Gossner, Yoshio Hoshihara, Janusz A. Jankowski, Ola Junghard, Lars Lundell, Guido Nj Tytgat, Michael Veith

Background and Aims Barrett's esophagus (BE) is a pre-malignant condition for esophageal adenocarcinoma, and its diagnosis relies initially on the endoscopic recognition of a columnar lined distal esophagus (i.e suspected or endoscopic BE). The accuracy of this endoscopic assessment is hampered by a lack of consensus-developed, explicit criteria for the endoscopic diagnosis and grading of its extent. In this study we have developed and validated such criteria. Methods Over several meetings, an international working group agreed on endoscopic diagnosis and grading criteria and developed materials for their formal evaluation by use of video-endoscopic recordings (of the distal esophagus, the gastroesophageal junction (GEJ) and proximal stomach) gathered in a standardized manner in 29 patients. The criteria included assessment of the Circumferential (C) and Maximum (M) extent of the endoscopically visualized BE segment as well as landmarks such as the diaphragmatic hiatus, the GEJ and the proximally displaced squamo-columnar junction. The recordings were scored for multiple criteria by an international panel of 29 endoscopists especially interested in BE (22 of those endoscopists scored $\mathrm{C}$ and $\mathrm{M}$ for all 29 patients). Results The criteria, now named the Prague C\&M Criteria, give explicit guidance on the endoscopic recognition of BE and grading of its extent. Among the 29 patients, the means of the values for ' $\mathrm{C}$ ' ranged from 0 tol $2 \mathrm{~cm}$ and for ' $\mathrm{M}$ ', the range was 0 to $14 \mathrm{~cm}$. The overall reliability coefficients (RC) were excellent for the assessments by the 22 endoscopists of both Circumferential and Maximum extent of the endoscopic BE segment above the GEJ (RC for $C$ value 0.95 and for $M$ value 0.94). For $C$ and $M$ measures, the rates of exact agreement for pair-wise comparisons of individual patient values were $53 \%$ and $38 \%$ respectively whereas the agreement within a $2 \mathrm{~cm}$ interval was $72 \%$ and $76 \%$ respectively. The overall RC for endoscopic recognition of $\mathrm{BE}>1 \mathrm{~cm}$ was 0.72 , but for $<1 \mathrm{~cm}$, the RC was poor at 0.22 . The RC for recognizing the location of GEJ and the diaphragmatic hiatus was 0.88 and 0.85 respectively. Conclusions The Prague C\&M criteria have high overall validity for the endoscopic assessment of visualized BE lengths, when used by endoscopists especially interested in BE. For less than $1 \mathrm{~cm}$ however, use of the criteria did not result in acceptable levels of diagnostic reliability and the causes for this need further research. The reliability of recognizing endoscopic landmarks is excellent. The criteria represent a useful advance in the endoscopic assessment of BE for clinical practice and research

821

Squamous Overgrowth in a 5-Year Randomized Phase III Trial of Photodynamic Therapy Using Porfimer Sodium in Ablation of High-Grade Dysplasia in Barrett's Esophagus

Mary Bronner, Shari Taylor, Bergein Overholt, Kenneth Wang, Steven Burdick, Charles Lightdale, Michael Kimmey, Hector Nava, Michael Sivak, Norman Nishioka, Hugh Barr, Chad Davis, Norman Marcon, Marcos Pedrosa, Michelle Depot

PURPOSE: Squamous overgrowth may obscure the endoscopic extent of Barrett's epithelium. Although squamous overgrowth has never been rigorously studied, it has been speculated that photodynamic therapy (PDT) increased the risk of subsquamous metaplastic glands. Consequently, its true diagnostic significance was assessed following PDT with porfimer sodium plus $20 \mathrm{mg}$ omeprazole BID [PORPDT] versus $20 \mathrm{mg}$ omeprazole BID [O alone] in patients with high-grade dysplasia (HGD) in Barrett's esophagus (BE) in a large randomized trial. METHODS. Patients were randomized (2.1) to PORPDT or to $O$ alone. Patients on PDT received $2 \mathrm{mg} / \mathrm{kg}$ i.v. of POR followed by endoscopic laser light exposure of Barrett's mucosa at a wavelength of $630 \mathrm{~nm}$ within 40-50 hours up to a maximum of 3 courses administered at least 90 days apart. Starting at baseline, every 3 months patients underwent 4-quadrant jumbo biopsies of their pre-treatment Barrett's length until four consecutive quarterly follow-up results were negative for HGD and then biannually up to 5 years or treatment failure. Biopsies were taken at 2 -cm intervals and centrally processed for standardized histologic interpretation by GI pathologists blinded to treatment assignment and patient identity. RESULTS: There were 138 patients and 23,473 total biopsies in PORPDT and 70 patients with 10,160 total biopsies in $\mathrm{O}$ alone. Occurrence of squamous overgrowth was similar for PORPDT relative to $\mathrm{O}$ alone both per patient (31\% vs. 33\%) and per biopsy ( $1.2 \%$ vs. $2.2 \%$ ). In no patient was the highest grade of neoplasia per endoscopy found exclusively beneath squamous mucosa. CONCLUSIONS: This 5-year large randomized trial with a rigorous surveillance biopsy program documents that squamous overgrowth occurrence is similar following either PORPDT or $\mathrm{O}$ alone. This trial confirms that squamous overgrowth is not detrimental to the longer-term safety of PORPDT.
822

New Barrett's Epithelium After the Healing of Erosive Oesophagitis Prospective Endoscopic and Histological Assessments

Ali S. Taha, Margaret Balsitis, Wilson J. Angerson, Frances Gallagher, Christopher G. Morran

BACKGROUND Epidemiological studies have considered a number of risk factors for Barrett's oesophagus including oesophagitis. However, prospective assessments have been lacking We, therefore, aimed at studying the possible development of new Barrett's epithelium in patients with a history of oesophagitis while considering other risk factors including gastroduodenal disease and $H$ pylori. PATIENTS \& METHODS After the endoscopic healing of oesophagitis in 120 patients, 15 (12.5\%) found to have Barrett's oesophagus were excluded The remaining 105 patients were followed-up for the development of new Barrett's epithelium for a median (range) of 52 (38-77) months. They included 67 men (64\%), 38 women (36\%), 39 smokers (37\%), 67 alcohol drinkers (64\%), and had a median (range) age of 52 (1983) years. Baseline endoscopic findings included 41 patients (39\%) with non-erosive and $64(61 \%)$ with erosive oesophagitis, $9(9 \%)$ with gastric ulcers or erosions, $25(24 \%)$ with duodenal ulcers or erosions, and 66 (63\%) with $\mathrm{H}$ pylori gastritis. Eradication therapy was given to 43 patients. Using recent criteria (Coad RA, Shepherd NA. Current Diagnostic Pathology 2003; 9:218-227), histological features diagnostic of Barrett's oesophagus (category 1) included the presence of native oesophageal structures such as oesophageal gland ducts with juxtaposition to glandular mucosa. Less diagnostic or normal categories (2-4) were also noted. The endoscopist and pathologist were not aware of patient's baseline characteristics. RESULTS Diagnostic features (categoryl) of newly developed Barrett's oesophagus were found in 9 patients $(8.6 \%)$ after a median (range) of 52 (35-83) months, with a median (interquartile range) length of $3(1-3.5) \mathrm{cm}$. There was a negative correlation between the length of Barrett's epithelium and the histological categories $(r=-0.28 ; p=0.004)$. Six of the new Barrett's cases developed in those with baseline erosive oesophagitis $(6 / 64,9.4 \%)$ and 3 in non-erosive disease $(3 / 41,7.3 \%)$. Five of 43 patients (12\%) given $H$ pylori eradication therapy developed Barrett's epithelium, compared with 3/23, 13\% whose infection was not treated. Taking all histological categories into account, males had greater tendency to develop Barrett's oesophagus $(\mathrm{X} 2=5.69 ; \mathrm{df}=1 ; \mathrm{p}=0.017)$. No significant tendency was seen with other risk factors including gastro-duodenal abnormalities $\mathrm{H}$ pylori, or eradication therapy CONCLUSIONS New Barrett's epithelium can develop after the exclusion of baseline cases in patients with oesophagitis. It is more likely to develop in men, and does not seem to be influenced by baseline gastro-duodenal disease, $\mathrm{H}$ pylori or its eradication.

823

The Effect of Scheduled Second Endoscopy Against Intravenous High Dose OMEPRAZOLE Infusion As An Adjunct to Therapeutic Endoscopy in Prevention of Peptic Ulcer Rebleeding - A Prospective Randomized Study Philip W. Chiu, Henry Joeng, Catherine Choi, Kwok Hung Kwong, Siu Ho Lam

Background From our randomized study, scheduled second endoscopy reduces rebleeding in bleeding peptic ulcers [GUT 2003.52: 1403-7]. Intravenous high dose omeprazole infusion is another strategy established to reduce rebleeding. This study aimed to compare second endoscopy against intravenous omeprazole infusion in prevention of ulcer rebleeding. Patients and Method We recruited patients who had bleeding peptic ulcer with endoscopic stigmata of acute bleeding, visible vessel or adherent clot and hemostasis achieved on primary endoscopy. One group (2nd OGD) received scheduled second endoscopy 16-24 hours after initial haemostasis, and further therapy applied if endoscopic stigmata persisted as above Another group (PPI) received high dose adjunctive omeprazole infusion. Those patients that developed rebleeding would receive operation if further endoscopic therapy failed. The outcome measures included rebleeding, transfusion, length of stay, and mortality. Results From 2003 to 2005, 335 patients presented with bleeding peptic ulcers. After endoscopic haemostasis, 164 eligible patients were randomized, 84 to PPI group and 80 to 2nd OGD group. $8(9.4 \%)$ in the PPI group and $5(6.3 \%)$ in the 2 nd OGD group sustained rebleeding (Chi square test $\mathrm{p}=0.43 ; \mathrm{RR}=0.63,95 \% \mathrm{CI} 0.19-2.03$ ). There is no difference in the probably of rebleeding within 30 days upon Kaplan Meier statistics (Log rank test $p=0.54$ ) (Fig 1). The number of patients that required surgery for rebleeding was $4(4.8 \%)$ in PPI group and $1(1.3 \%)$ in 2 nd OGD group $(p=0.36 ; R R=0.25,95 \% \mathrm{CI} 0.03-2.32)$. There was no difference in the hospital stay, ICU stay, transfusion or mortality between the two groups. Conclusion Both scheduled second endoscopy and adjunctive high dose omeprazole infusion are effective strategy in prevention of peptic ulcer rebleeding after therapeutic endoscopy

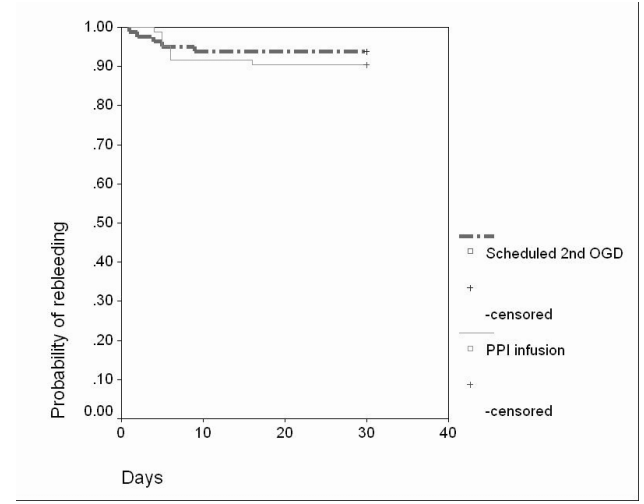

Fig 1 


\begin{tabular}{|c|c|c|c|}
\hline Rebleeding & Wald Chi-square & P-value & Odds-ratio(95\%CI) \\
\hline Gp A vs D & 9.132 & 0.003 & $0.035(.004,30)$ \\
\hline Gp B vs D & 5.812 & 0.016 & $0.185(.047, .72)$ \\
\hline Gp C vs D *NSAID & 6.750 & 0.009 & $0.043(.004, .46)$ \\
\hline NSAID use & 12.250 & 0.001 & $8.101(2.5,26.1)$ \\
\hline
\end{tabular}

Proton Pump Inhibitor Treatment Initiated Prior to Endoscopic Diagnosis in Unselected Upper Gastrointestinal Bleeding: A Cochrane Systematic Review and Meta-Analysis

Stephanie Dorward, Aravamuthan Sreedharan, Grigoris I. Leontiadis, Colin W. Howden, Paul Moayyedi, David Forman

Background The clinical effectiveness of proton pump inhibitors (PPI) in patients with upper gastrointestinal (UGI) bleeding has been evaluated in recent meta-analyses. However, none of these has addressed the role of PPI treatment initiated prior to endoscopic diagnosis in unselected patients with UGI bleeding. Objectives We aimed to systematically review evidence from randomized controlled trials (RCTs) that studied PPI treatment initiated before endoscopy, in UGI bleeding. Methods We performed a search of CCTR, MEDLINE, EMBASE, CINAHL databases and major conference proceedings up to September 2005. RCTs comparing intravenous or oral PPIs with either placebo or H2-receptor antagonists in patients with unselected UGI bleeding were included. Two reviewers independently assessed the eligibility criteria of each study and extracted data regarding outcomes and factors affecting methodological quality. The primary outcome was 30-day mortality; secondary outcomes included 30day re-bleeding and surgical intervention rates, stigmata of recent hemorrhage (SRH) seen at endoscopy, length of hospital stay and blood transfusion requirement. Sub-group analyses were performed for the outcomes in patients with peptic ulcer bleeding. Results Five RCTs were included for review. Four RCTs comprising 1512 patients reported data for all randomized patients. Four RCTs were published in full; one was presented in abstract form. Two RCTs reported adequate concealment of allocation. Meta-analyses were performed for all dichotomous outcomes. Adequate data were not available to perform meta-analyses for continuous outcome measures such as blood transfusion requirements and length of hospital stay. Sensitivity analyses were performed as appropriate and sources of heterogeneity were explored. The main results are presented in the Table. Conclusions PPI treatment initiated prior to endoscopy in unselected UGI bleeding significantly reduced the proportion of patients with SRH at index endoscopy. However, there was no evidence that PPI treatment improved clinically important outcomes such as mortality, re-bleeding or need for surgery. Results

\begin{tabular}{|c|c|c|c|c|c|c|}
\hline Outcome & $\begin{array}{c}\text { Number of } \\
\text { RCTs }(\mathrm{n})\end{array}$ & $\begin{array}{c}\text { Patients } \\
(\mathrm{n})\end{array}$ & \multicolumn{2}{|c|}{$\begin{array}{c}\text { Pooled event rate (\% of } \\
\text { patients) PPI Control }\end{array}$} & $\begin{array}{c}\text { Pooled effect estimate } \\
\text { OR(95\% CI) }\end{array}$ & $\begin{array}{c}\text { P } \\
\text { value }\end{array}$ \\
\hline Mortality & 4 & 1512 & 6.1 & 5.5 & $1.12(0.72-1.73)$ & 0.61 \\
\hline Rebleeding & 3 & 1410 & 13.9 & 16.6 & $0.81(0.61-1.09)$ & 0.17 \\
\hline Surgery & 3 & 1454 & 9.9 & 10.2 & $0.96(0.68-1.35)$ & 0.81 \\
\hline SRH & 4 & 1332 & 37.2 & 46.5 & $0.67(0.54-0.84)$ & 0.0005 \\
\hline $\begin{array}{c}\text { Blood in UGI } \\
\text { tract }\end{array}$ & 3 & 1230 & 20.6 & 27.0 & $0.64(0.32-1.30)$ & 0.22 \\
\hline $\begin{array}{c}\text { Active } \\
\text { bleeding }\end{array}$ & 4 & 1332 & 11.3 & 14.7 & $0.74(0.54-1.02)$ & 0.07 \\
\hline $\begin{array}{c}\text { Blood } \\
\text { transfusion }\end{array}$ & 4 & 1512 & 53.2 & 54.5 & $0.95(0.78-1.16)$ & 0.62 \\
\hline
\end{tabular}

825

7 Or 10fr, with Low Or High Volume Epinephrine? A Single Center Prospective Randomized Control Trial in Acute Non-Variceal Upper Gastrointestinal Bleeding (AUGIB)

Subbaramiah Sridhar, Sherman Chamberlain, Sreeram Parupudi, Sankar Sethuraman, Dharma Thiruvaiyaru, Urias Cuartas, Lynda Roach, Robert Schade

Background: Endoscopic injection of epinephrine $(1: 10,000)$ together with thermocoagulation is considered highly effective for AUGIB. We compared the efficacy of low (10 ml) or high volume $(35 \mathrm{ml})$ epinephrine followed by 7 or 10Fr BICAP probe. Methods: A total of 197 pts., mean age 63.4(SD+16.6) who shared common demographic details with endoscopically confirmed AUGIB (Forrest I $\&$ II) were prospectively randomized to one of 4 effective treatment groups; high vol epi $+10 \mathrm{Fr}(\mathrm{A}, \mathrm{n}=58)$, high vol epi $+7 \mathrm{Fr}(\mathrm{B}, \mathrm{n}=47)$, low vol epi $+10 \operatorname{Fr}(C, n=36)$, \& low vol epi $+7 \operatorname{Fr}(D, n=56)$. All endoscopic procedures were performed within $6-\mathrm{hr}$. of admission by a single endoscopist $(\mathrm{S}, \mathrm{S})$. The outcome variables of interest were: rebleeding incidence, need for surgery, no. of hospital days $\&$ no. \& duration of coagulations. Results: 113 DUs, 77GUs \& 7 MW tears noted. 67 pts. took NSAIDs: $18,17,15 \& 17$ in A, B, C \& D. The no. of rebleeding incidences (<48hrs) were $2,3,4 \& 13$ in gps. A, B, C \& D respectively. Only 1 pt in A required surgery for rebleeding when compared to 6 in $\mathrm{D}$. The mean no of coag \& duration $(5.4,7.7 \mathrm{sec}$ SD $+1.1,2.1$ ) were also low in A when compared to other groups. Using logistic regression analysis, the odds of rebleeding was significantly lower in A when compared to D (ref. to table). Using multivariate ANOVA, ICU \& ward days in non-NSAID users were sig. lower in $A$ than in $\mathrm{D}(\mathrm{P}<0.01)$, but there was no difference between the two groups in NSAID users \& among C, B \& A for all pts. No. of coag. \& duration in sec. were sig. fewer in A when compared to other groups $(\mathrm{P}<0.01)$ in all pts. Conclusion: In our study $10 \mathrm{Fr}$ coagulation probe with high vol. epinephrine was associated with significantly reduced rebleeding risk and lower no. of coag. \& duration. However, there was no sig. difference between the groups with respect to need for surgery for rebleeding. This therapy, when compared to 7 Fr coagulation with low vol. epinephrine, was also superior in reducing hospital stay in non-NSAID users. Use of large probe with high volume epinephrine should be standard therapy when dealing with AUGIB for minimizing morbidity and costs.

Rebleeding Risk (Logistic regression)
*Interaction between NSAID \& groups C vs. D

826

Early Is Good; Is Very Early Better? Does Endoscopy Within 6 Hours of Presentation Improve Outcomes in High-Risk Patients Presenting with Acute Non-Variceal Upper Gastrointestinal Bleeding (ANVUGIB)

Leila Keyvani, Laura E. Targownik, Sanjay K. Murthy, Shanua Leeson

Background: ANVUGIB is a serious cause of morbidity and mortality and is associated with a 5-10\% case-fatality rate. The evidence to date suggests that performance of endoscopy within 24 hours of presentation reduces the risk of rebleeding and mortality in patients presenting with ANVUGIB. However, it is unclear whether performing endoscopy very early within this 24 hour window provides any additional benefit. The benefits of performing very early endoscopy would likely be most pronounced in high-risk patients, including the elderly, those with evidence of shock on presentation, and patients with a history of severe co-morbid illness. Therefore, we sought to determine if there were significant differences in outcomes between high-risk ANVUGIB patients who undergo endoscopies within $6 \mathrm{~h}$ of presentation ("very early") or between $6 \mathrm{~h}$ and $24 \mathrm{~h}$ from presentation. ("early"). Methods: We performed a retrospective review of all patients with ANVUGIB who presented to one of our two tertiary care medical centers in Winnipeg, Manitoba between 1999-2004.We selected the patients who were high risk based on their having a clinical Rockall score >= 3. We separated subjects into two comparator groups based on whether they received endoscopy "very early" ( $<=6$ hrs) or "early" (6-24 hrs). The primary outcome measure was any adverse outcomes (re-bleeding, need for surgery, mortality, readmission within 30 days of ANVUGIB). The secondary outcomes include the length of hospital stay, and receiving a blood transfusion more than $24 \mathrm{~h}$ following the initial endoscopy. Results: 221 patients with a clinical Rockall score $>=3$ underwent endoscopy within 24 hours of presentation. Of these high risk patients, 79 had "very early" endoscopy whereas 142 underwent "early" endoscopy. There were no significant differences in the baseline characteristics between the comparator groups. Patients receiving "very early" endoscopy were significantly more likely to have undergone endoscopic hemostasis ( $50 \%$ "very early" vs. $36 \%$ "early, p=0.01). There were no differences in the incidence of adverse outcomes ( $25 \%$ vs. $22 \%, p>0.2)$. Patients undergoing "very early" endoscopy were significantly more like to have a hospital stay exceeding 5 days (64\% "very early" vs. $49 \%, \mathrm{p}=.032$ ). Conclusion: There is no difference in the rate of adverse outcomes in high risk ANVUGIB patients who undergo "very early" endoscopy and "early' endoscopy, though patients receiving "very early" endoscopy are more likely to require both endoscopic hemostasis and longer stays in hospital. The optimal timing of endoscopy within first 24 hours should be confirmed in well designed randomized controlled trial setting.

827

The Predictive Value of Admission Hematocrit in Patients Hospitalized with Acute Upper and Lower Gastrointestinal Bleeding (GIB) Richard A. Del Rio, Richard C. Wong, Layla Hajjafar, Gregory S. Cooper

Background: The ability of admission hematocrit (HCT) to help identify those patients at risk of death from acute GIB is unclear. Aims: 1) To compare the in-hospital mortality rate of patients with different admission HCT values; and 2) To determine if admission HCT is an independent predictor of mortality. Methods: A retrospective cohort study was performed using 7,909 patients hospitalized with the primary diagnosis of acute GIB. In-hospital mortality rates were compared for patients with lower versus higher admission HCT for specific values: $10,15,20,25,30,35$, and $40 \%$. A multivariate logistic regression model was performed to determine if admission HCT was an independent predictor of mortality after adjusting for: age, sex, race, admission pulse and blood pressure, admission platelet count and prothrombin time, history of GIB, and comorbidities (diabetes, CAD, cirrhosis and chronic renal failure). Results: In-hospital mortality rate was 3.3\% for all GIB, 3.7\% for upper GIB, and $1.8 \%$ for lower GIB. For all GI bleed, low HCT was an independent predictor of mortality for values less than $25 \%$, with the risk of death ranging from $19 \%$ higher for HCT $<25 \%$ to $69 \%$ higher for HCT $<10 \%$ (Table). HCT values of 30,35 and $40 \%$ were not significant predictors of mortality (data not shown). Similar results were found when evaluating upper and lower GI bleed separately (Table). Other predictors of mortality were age, gender, admission pulse and blood pressure, prothrombin time and comorbidities. Conclusion: An admission hematocrit value of less than $25 \%$ appears to be an independent predictor of in-hospital mortality and can be used with other known predictors to help identify patients at risk for poor outcomes.

In-Hospital Patient Mortality Rate for Different Admission HCT Values 


\begin{tabular}{|c|c|c|c|c|}
\hline HCT $(\%)$ & Site & Rate $(\%)$ & OR & $95 \% \mathrm{CI}$ \\
\hline & All & 13.3 & 1.69 & $(0.78,3.70)$ \\
\hline 10 & Upper & 14.3 & 1.67 & $(0.76,3.70)$ \\
\hline & Lower & NP & NP & NP \\
\hline & All & 7.8 & 1.49 & $(1.11,2.00)$ \\
\hline & Upper & 7.3 & 1.35 & $(0.96,1.89)$ \\
\hline 15 & Lower & 10.7 & 2.50 & $(1.27,5.00)$ \\
\hline & All & 5.7 & 1.27 & $(1.06,1.49)$ \\
\hline & Upper & 5.7 & 1.19 & $(1.00,1.41)$ \\
\hline 20 & Lower & 5.4 & 1.69 & $(1.03,2.78)$ \\
\hline & All & 4.6 & 1.19 & $(1.04,1.35)$ \\
\hline & Upper & 5.0 & 1.16 & $(1.00,1.33)$ \\
\hline & Lower & 2.5 & 1.08 & $(0.74,1.35)$ \\
\hline
\end{tabular}

luminal $\mathrm{Cl}^{-}$was removed, the magnitude of cAMP-stimulated $\mathrm{HCO}_{3}{ }^{-}$secretion increased in the DRA(-) duodenum to a level equivalent with the DRA(+) duodenum [cAMP $\triangle$ Jsm DRA()$=1.2 \pm 0.1$ vs. $\operatorname{DRA}(+)=1.2 \pm 0.1 \mu \mathrm{eg} / \mathrm{cm}^{2}{ }^{2}-\mathrm{h}$, ns; $\operatorname{cAMP} \Delta \mathrm{Isc} \operatorname{DRA}(-)=15.0 \pm 2.1 \mathrm{vs}$. $\operatorname{DRA}(+)=13.6 \pm 1.0 \mu \mathrm{eq} / \mathrm{cm}^{2}{ }^{2} \mathrm{~h}, \mathrm{~ns}, \mathrm{n}=3$ ]. It was concluded from these data that under physiological conditions, Slc26a3 (DRA) contributes $\sim 80 \%$ of basal $\mathrm{HCO}_{3}{ }^{-}$secretion and $\sim 40 \%$ of cAMP-stimulated $\mathrm{HCO}_{3}{ }^{-}$secretion. The remaining $60 \%$ of cAMP-stimulated $\mathrm{HCO}_{3}$ secretion is apparently mediated by a CFTR-dependent $\mathrm{HCO}_{3}{ }^{-}$conductance which carries a $\mathrm{HCO}_{3}{ }^{-}$current that is $\sim 12 \%$ of the Isc, i.e., approximately equal to $\mathrm{HCO}_{3}{ }^{-}: \mathrm{Cl}^{-}$permeability ratio of CFTR. Removal of luminal $\mathrm{Cl}^{-}$apparently normalizes the rate of cAMP-stimulated $\mathrm{HCO}_{3}{ }^{-}$secretion across the DRA(-) duodenum by increasing the contribution of the CFTRdependent $\mathrm{HCO}_{3}{ }^{-}$conductance. Supported by NIDDK and CFF.

\section{0}

Intestinal Impactions and Altered cAMP-Stimulated Anion Secretion Across Murine Proximal Colon of Nbcl $\mathrm{Na}^{+}-\mathrm{HCO}_{3}{ }^{-}$Cotransporter Knockout Mice Lara R. Gawenis, Gary E. Shull

Cyclic AMP-stimulated anion secretion in the colon is dependent on apical CFTR and basolateral anion uptake proteins. The NKCCl $\mathrm{Na}^{+}-\mathrm{K}^{+}-2 \mathrm{Cl}^{-}$cotransporter mediates bulk basolateral $\mathrm{Cl}^{-}$uptake, and anion exchanger 2 (AE2) is required for normal transepithelial $\mathrm{Cl}^{-}$and $\mathrm{HCO}_{3}{ }^{-}$secretion. Along with other $\mathrm{Na}^{+}-\mathrm{HCO}_{3}{ }^{-}$cotransporters, basolateral $\mathrm{NBCl}$ is believed to participate in $\mathrm{HCO}_{3}^{-}$uptake during cAMP-stimulated secretion. To test the hypothesis that $\mathrm{NBCl}$ supports transepithelial anion secretion, mice lacking $\mathrm{NBCl}$ were prepared and short-circuit current $\left(\mathrm{I}_{\mathrm{sc}}\right)$ responses to $\mathrm{CAMP}_{\mathrm{i}}$ were measured in muscle-stripped proximal colon mounted in Ussing chambers. $\mathrm{NBCl}$ null mutant $[\mathrm{NBCl}(-)]$ mice exhibited high mortality with no survival beyond 25 days, severe growth retardation, splenomegaly, and intestinal impactions. Surprisingly, the cAMP-induced increase in $\mathrm{I}_{\mathrm{sc}}$ was sharply increased in $\operatorname{NBCl}(-)$ proximal colon $\left(\Delta \mathrm{I}_{\mathrm{sC}} \mathrm{WT}=-187.3 \pm 58.3 \mathrm{vs} . \mathrm{NBCl}(-)=-396.4 \pm 27.3 \mu \mathrm{A} / \mathrm{cm}^{2}\right.$; $\mathrm{n}=5$; $\mathrm{p}<0.005)$. In addition, similar to findings in AE2(-) proximal colon, the $\mathrm{I}_{\mathrm{sc}}$ response to cAMP in $\mathrm{NBCl}(-)$ proximal colon exhibited increased bumetanide sensitivity (increased $\mathrm{NKCCl}$ activity; $\Delta \mathrm{I}_{\mathrm{sc}} \mathrm{WT}=45.5 \pm 11.0 \mathrm{vs}$. $\left.\mathrm{NBCl}(-)=117.2 \pm 6.7 \mu \mathrm{A} / \mathrm{cm}^{2} ; \mathrm{n}=5 ; \mathrm{p}<0.0006\right)$. There were no significant changes in $\mathrm{I}_{\mathrm{sc}}$ response to the distilbene derivative SITS or the residual (post-bumetanide and SITS) cAMP-stimulated $\mathrm{I}_{\mathrm{sc}}$ in $\mathrm{NBCl}(-)$ proximal colon. However, the $\mathrm{I}_{\mathrm{sc}}$ response to amiloride [epithelial $\mathrm{Na}^{+}$-channel $(\mathrm{ENaC})$ activity] was enhanced in $\mathrm{NBCl}(-)$ proximal colon $\left(\Delta \mathrm{I}_{\mathrm{sc}} \mathrm{WT}=4.3 \pm 2.6\right.$ vs. $\mathrm{NBCl}(-)=20.2 \pm 3.3 \mu \mathrm{A} / \mathrm{cm}^{2} ; \mathrm{n}=5$ $\mathrm{p}<0.007)$ and distal colon $\left(\Delta \mathrm{I}_{\mathrm{sc}} \mathrm{WT}=47.4 \pm 6.6 \mathrm{vs} . \mathrm{NBCl}(-)=124.6 \pm 11.8 \mu \mathrm{A} / \mathrm{cm}^{2} ; \mathrm{n}=\right.$ $5 ; \mathrm{p}<0.0004)$. We conclude that the intestinal impactions in mice lacking $\mathrm{NBCl}$ may result from increased $\mathrm{Na}^{+}$absorption via $\mathrm{ENaC}$, while anion secretion may be enhanced to compensate for the imbalance between absorption and secretion by increased $\mathrm{NKCCl}$ activity Current studies are focused on anion substitution experiments and measurement of $\mathrm{HCO}_{3}$ secretion to determine if disruption of $\mathrm{NBCl}$ specifically alters net transepithelial $\mathrm{HCO}_{3}$ secretion. Supported by NIDDK.

\section{1}

Differential Activation of the CFTR Hco3- Conductance By Forskolin and Genistein in Murine Duodenum Biguang Tuo, Ursula Seidler

Background \& Aims: It has been shown that the $\mathrm{Cl}$ - and $\mathrm{HCO} 3$ - conductance of the CFTR anion channel can be selectively activated (Reddy et al, Nature 2004), and that severe forms of cystic fibrosis have mutations that affects their $\mathrm{HCO} 3$ - conductance, whereas mutations which only have a reduced $\mathrm{Cl}$ - conductance have a milder phenotype (Choi, et al. Nature 20001). Targeting the CFTR HCO3- conductance, if possible, may therefore be of grea therapeutic benefit. The flavonoid genistein is known to activate normal and mutant CFTR and has been evaluated in clinical trials. In the present study, we examined the effects of genistein on duodenal $\mathrm{HCO} 3$ - and $\mathrm{Cl}$ - secretion. Methods: Murine duodenal mucosal $\mathrm{HCO} 3-$ and $\mathrm{Cl}$ - secretions were examined in vitro in Ussing chambers by the $\mathrm{pH}$ stat and shor circuit current (Isc) techniques. Results: Genistein markedly stimulated duodenal HCO3secretion and Isc in a dose-dependent manner. Neither cAMP-dependent inhibitors, MDL12330A and H89, nor Ca2+ channel blocker, verapamil, and nor cGMP-dependent inhibitor NS2028 had effect on the action of genistein in duodenum. But, in the CFTR null mice, genistein had no effects on duodenal HCO3- secretion and Isc. After addition o genistein, HCO3- secretion increased $157.79 \%$, and Isc increased $224.09 \%$, whereas, after addition of forskolin, an activator of adenylate cyclase, $\mathrm{HCO} 3$ - secretion increased $196.00 \%$, and Isc increased $604.65 \%$. In Cl--free solutions, $\mathrm{HCO} 3$--free solutions, and both $\mathrm{HCO} 3-$ and $\mathrm{Cl}-$ - free solutions, genistein-induced Iscs were reduced by $41.86 \%, 48.27 \%$, and $89.75 \%$, respectively, whereas forskolin-induced Isc was reduced by $69.23 \%, 18.75 \%$, and $90.89 \%$, respectively Conclusion: Genistein-stimulated duodenal $\mathrm{HCO}$ - and $\mathrm{Cl}$ - secretion occurs via an activation of the CFTR anion channel, but at equipotent concentrations, the relative percentage of $\mathrm{HCO}$ - in the anion secretory response is much larger for genistein than the cAMP-dependent agonist forskolin. This opens the potential for selective drug targeting of the CFTR HCO3- conductance response is much larger. Genistein has a relatively high selectivity to CFTR HCO3- conductance.

832

Epithelial Extracellular Carbonic Anhydrases Facilitate Disposal of Luminal Acid $\mathrm{As} \mathrm{CO}_{2}$ in Rat Duodenal Mucosa

Misa Mizumori, Yasutada Akiba, Tetsu Takeuchi, Caudiu T. Supuran, Paul H. Guth, El Engel, Jonathan D. Kaunitz

In the duodenum, large amounts of concentrated $\mathrm{HCl}$ mix with secreted $\mathrm{HCO}_{3}$. The subsequent neutralization of $\mathrm{H}^{+}$generates $\mathrm{CO}_{2}>500$ Torr, which dissipates in the jejunum, implying that the duodenum absorbs almost all secreted gastric acid and its neutralization products. The mechanism of this high throughput acid disposal, though essential for body acid-base homeostasis, is not well characterized. We hypothesized that duodenal acid disposa )$\left.=0.4 \pm 0.1 \mathrm{vs} . \operatorname{DRA}(+)=2.2 \pm 0.2 \mu \mathrm{eg} / \mathrm{cm}>^{2}-\mathrm{h}, \mathrm{p}<0.05, \mathrm{n}=7-10\right)$ whereas the short(Isc) in DRA(-) duodenum was significantly greater than in DRA(+) duoden [ Isc DRA $(-)=-2.2 \pm 0.3$ vs. DRA $(+)=-0.5 \pm 0.1 \mu \mathrm{eq} / \mathrm{cm}>^{2}-\mathrm{h}, \mathrm{p}<0.05$ ]. During cAMP stimulation with $10 \mu \mathrm{M}$ forskolin, Jsm across the DRA(-) duodenum was reduced by $42 \%$ relative to the DRA $(+)$ duodenum $[$ cAMP $\Delta J \operatorname{sm} \operatorname{DRA}(-)=0.8 \pm 0.1$ vs. DRA $(+)=1.4 \pm 0.1$ $\mu$ eq $/ \mathrm{cm}>^{2}$-h, $\mathrm{p}<0.05, \mathrm{n}=7-10$ ] whereas the $\Delta$ Isc was not different [cAMP $\Delta$ Isc DRA $(-$ )$=6.6 \pm 0.7$ vs. $\left.\operatorname{DRA}(+)=7.0 \pm 0.4 \mu \mathrm{eq} / \mathrm{cm}^{2}-\mathrm{h}, \mathrm{ns}, \mathrm{n}=7-10\right]$. In experiments where 
is facilitated by extracellular ecto-CA isoforms. We thus examined the effect of novel cellimpermeant, specific ecto-CA inhibitors on $\mathrm{CO}_{2}$ diffusion and $\mathrm{HCO}_{3}{ }^{-}$secretion in rat duo-

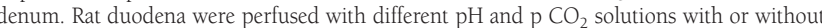
the cell-impermeant benzene sulfonamide CA inhibitors (CAI6a and CAIl4v, 0.1 $u \mathrm{M}$ ), or cell-permeant CA inhibitor methazolamide (MTZ, $1 \mathrm{mM}$ ). Flow-through $\mathrm{pH}$ and $\mathrm{p} \mathrm{CO}_{2}$ electrodes simultaneously and continuously measured perfusate and effluent $\mathrm{pH}$ and $\mathrm{p} \mathrm{CO}_{2}$. Portal venous (PV) $\mathrm{pCO}_{2}$ and $\mathrm{pH}$ were analyzed at the end of the luminal challenge. Furthermore, a ${ }^{13} \mathrm{C}$-labeled high $\mathrm{CO}_{2}$ solution ( $\mathrm{pH} 6.4, \mathrm{pCO}_{2} 260$ Torr) was perfused through duodenal loop, with measurement of $\mathrm{PV}^{13} \mathrm{C} /{ }^{12} \mathrm{C}$. Luminal perfusion with a high $\mathrm{CO}_{2}$ solution $\left(\mathrm{pCO}_{2}=260\right.$ Torr $)$ increased net $\mathrm{CO}_{2}$ loss from the perfusate compared with controls ( $\mathrm{pH} 6.4$ saline, $\mathrm{pCO}_{2} \approx 0$ ) accompanied by portal venous (PV) acidification and $\mathrm{pCO}_{2}$ increase, but with no change of $\mathrm{PV}$ total $\left[\mathrm{CO}_{2}\right]$. Impermeant $\mathrm{CA}$ inhibitors abolished perfusate net $\mathrm{CO}_{2}$ loss, but increased apparent $\mathrm{HCO}_{3}{ }^{-}$secretion, whereas all inhibitors inhibited $\mathrm{PV}$ acidification and $\mathrm{pCO}_{2}$ increase. Furthermore, net $\mathrm{CO}_{2}$ loss during perfusion of a buffer equilibrated with $30 \% \mathrm{CO}_{2}(\mathrm{pH}$ 6.5) was delayed by impermeant $\mathrm{CA}$ inhibitors and was abolished by MTZ. Perfused ${ }^{13} \mathrm{CO}_{2}$ was significantly increased in PV 10 min after the challenge with the increased $\mathrm{pCO}_{2}$ and decreased $\mathrm{pH}$ in $\mathrm{PV}$, inhibited by all inhibitors. $\mathrm{H}^{+}$and $\mathrm{CO}_{2}$ loss from the perfusate were accompanied by increases of $\mathrm{PV} \mathrm{H}^{+}$and tracer $\mathrm{CO}_{2}$, but unchanged $\mathrm{PV}$ total $\mathrm{CO}_{2}$, consistent with transmucosal net $\mathrm{H}^{+}$and tracer $\mathrm{CO}_{2}$ movement, which was inhibited by impermeant $\mathrm{CA}$ inhibitors. Inhibition of extracellular $\mathrm{CA}$ augments $\mathrm{HCO}_{3}{ }^{-}$secretion, normally obscured by high luminal $\left[\mathrm{CO}_{2}\right]$. The duodenum absorbs luminal acid by neutralization with secreted $\mathrm{HCO}_{3}^{-}$to $\mathrm{CO}_{2}$, absorption of $\mathrm{CO}_{2}$, cytosolic conversion of $\mathrm{CO}_{2}$ to $\mathrm{H}^{+}$and transport of cellular $\mathrm{H}^{+}$to the subepithelium and to the PV. Thus, the duodenum, like red cell, absorbs $\mathrm{H}^{+}$as $\mathrm{CO}_{2}$ with constant $\mathrm{CA}$-mediated interconversions between $\mathrm{CO}_{2}$ and $\mathrm{HCO}_{3}$.

833

Role of Intracellular $\mathrm{Ca}^{2+}$ in 5-Hydroxytryptamine (5-HT)-Mediated Duodenal Mucosal Bicarbonate Secretion

Hui Dong, Anders Smith, Alfred Chappell, Kim E. Barrett

Background \& Aim: In previous studies, we have shown that 5-HT likely regulates duodenal mucosal bicarbonate secretion (DMBS) via $\mathrm{Ca}^{2+}$-and cAMP-dependent pathways. However, little is currently known about whether and whereby 5-HT is involved in acid-stimulated DMBS. Thus, the aims of the present study were to demonstrate the physiological relevance of 5-HT in acid-induced DMBS, and to further elucidate the role of the $\mathrm{Ca}^{2+}$ signaling pathway in this process. Methods: short-circuit current ( $\mathrm{I}_{\mathrm{s}}$ ) and bicarbonate secretion across the murine duodenal mucosa were measured. Acid-stimulated 5-HT release from duodenum in vitro was assessed by immunoassay, and acid-stimulated DMBS in vivo was measured using a $\mathrm{CO}_{2}$-sensitive electrode. Cytosolic $\mathrm{Ca}^{2+}$ measurements and siRNA knockdown were also applied to study the 5-HT-mediated $\mathrm{Ca}^{2+}$ signaling pathway further. Results: Acid significantly increased duodenal mucosal release of 5 - $\mathrm{HT}$ from the basal level of $6.4 \pm 0.8$ $\mathrm{pmol} / \mathrm{mg}$ tissue $(\mathrm{pH} 7.4)$ to $16.8 \pm 1.6,14.9 \pm 1.4$ and $16.1 \pm 2.2 \mathrm{pmol} / \mathrm{mg}$ tissue $(\mathrm{pH} 3$, 4,$5 ; \mathrm{n}=5, \mathrm{P}<0.01$ ). SB204070, a selective $5-\mathrm{HT}_{4}$ receptor antagonist, reduced luminal acidstimulated net peak $\mathrm{HCO}_{3}{ }^{-}$secretion in vivo from the control group of $14.8 \pm 3.1 \mu \mathrm{mol} /$ $\mathrm{cm} . \mathrm{h}$ to $7.0 \pm 2.4$ or $1.5 \pm 0.5 \mathrm{umol} / \mathrm{cm}$. h in mice treated with 5 or $10 \mathrm{mg} / \mathrm{kg} \mathrm{SB} 204070$ $(\mathrm{n}=5, \mathrm{P}<0.05$ or $\mathrm{P}<0.01)$, respectively. 5 -HT-inducded $\mathrm{I}_{\mathrm{sc}}$ and DMBS in vitro were abolished by removal of extracellular $\mathrm{Ca}^{2+}$, and significantly attenuated by KB-R7943 (10 $\mu \mathrm{M}$, a selective inhibitor for the reversed mode of $\mathrm{Na}^{+} / \mathrm{Ca}^{2+}$ exchanger (NCX)), thapsigargin $(10 \mu \mathrm{M}$, a selective inhibitor of the endoplasmic reticulum (ER) $\mathrm{Ca}^{2+}$ ATPase), 2-APB(100 $\mu \mathrm{M}$, a permeable inhibitor of $\mathrm{IP}_{3}$ receptors), TRAM-34 (10 $\mu \mathrm{M}$, a selective inhibitor of intermediate $\mathrm{Ca}^{2+}$-activated $\mathrm{K}^{+}$channels $\left(\mathrm{IK}_{\mathrm{Ca}}\right)$ ) or glybenclamide $(300 \mu \mathrm{M}$, an inhibitor of the cystic fibrosis transmembrane conductance regulator (CFTR)). The expression and function of NCX proteins in SCBN cells, a well-characterized nontransformed duodenal epithelial crypt cell line, were confirmed by Western blot analysis and digital $\mathrm{Ca}^{2+}$ imaging. 5-HT directly increased intracellular $\mathrm{Ca}^{2+}$ concentration in SCBN cells and knockdown of $\mathrm{NCXl}$ proteins with a specific siRNA greatly decreased 5-HT-mediated $\mathrm{Ca}^{2+}$ signaling. Conclusions: 5-HT released from the duodenal mucosa in response to acid stimulation may act on 5-HT receptors located on epithelial cells. Activation of 5-HT receptors induces both intracellular $\mathrm{Ca}^{2+}$ release from the ER and extracellular $\mathrm{Ca}^{2+}$ entry via the reversed mode of plasma membrane NCX. An increase in intracellular $\mathrm{Ca}^{2+}$ may activate basolateral $\mathrm{IK}_{\mathrm{Ca}}$ and thereby stimulate DMBS through apical CFTR channels.

\section{4}

The P38 Mitogen-Activated Protein Kinase Regulates Epidermal Growth Factor Receptor Down Regulation

Mark R. Frey, Rebecca S. Dise, Karen L. Edelblum, D. Brent Polk

We have previously shown that epidermal growth factor (EGF)-stimulated young adult mouse colon (YAMC) cell restitution requires p38 mitogen-activated protein kinase (MAPK), and we have proposed that p38 acts as a molecular switch determining whether EGF receptor (R) ligand exposure leads to cell migration or proliferation. Interestingly, p38 alters activation of other EGF-stimulated signaling modules such as ERK MAPK. Therefore, this study tests the hypothesis that p38 influences EGFR expression or activation. METHODS: YAMC cells were given EGF with or without p38-directed pharmacological inhibitors or siRNA. EGFR and the EGFR-directed ubiquitin ligase $\mathrm{Cbl}$ were immunoprecipitated from cell lysates and subjected to Western blot analysis with antibodies specific for EGFR, Cbl, ubiquitin, phosphotyrosine, and individual EGFR phosphosites. Subcellular EGFR localization was determined by laser scanning confocal microscopy. Site specific EGFR mutants were expressed in EGFR-/- mouse colon epithelial cells. RESULTS: The typical near-complete $(>90 \%)$ down regulation of EGFR from YAMC cells after $4 \mathrm{~h}$ EGF exposure was blocked by p38 inhibition. Preserved EGFR retained elevated tyrosine phosphorylation, indicating sustained activity. In addition, depletion of $\mathrm{p} 38$ from the cells led to accumulation of EGFR over a $72 \mathrm{~h}$ period. Pretreatment with cycloheximide showed that p38 does not regulate EGFR downregulation by altering new receptor synthesis. Instead, EGF-induced activation of the ubiquitin ligase $\mathrm{Cbl}$ and subsequent ubiquitination of EGFR were impaired by p38 blockade. Pretreatment of cells with p38 kinase inhibitor completely blocked EGF-induced
Cbl phosphorylation despite robust activation of EGFR and other targets such as ERK MAPK EGF-driven phosphorylation of EGFR on Tyr 1045, a regulatory site for Cbl activation, was also prevented by p38 blockade, while phosphorylation of other specific tyrosines was not. Mutation of EGFR to Y1045F recapitulated the effects of p38 inhibition in that EGFstimulated migration but not proliferation was lost, identifying this tyrosine on EGFR as a key target of p38 signaling. Finally, immunolocalization of preserved EGFR revealed that in the absence of $\mathrm{p} 38$ activity, ligand-stimulated EGFR internalizes properly but accumulates in intracellular vesicles which stain for caveolin- 1 and the early endosome marker EEAl. CONCLUSIONS: Our data show that p38 regulates Cbl-mediated EGFR down regulation and vesicular sorting after ligand binding. These novel results position p38 as a modulator of ligand-stimulated EGFR processing and demonstrate that this processing has a profound impact on the cellular outcome of EGFR signaling.

835

Adaptation Following Massive Small Bowel Resection Requires Math Dependent Intestinal Secretory Lineages

Noah F. Shroyer, Michael A. Helmrath, Vincent Y. Wang, Susan J. Henning, Huda Y. Zoghbi

Background: Small bowel resection (SBR) is a frequent surgical therapy for inflammatory bowel disease and necrotizing enterocolitis. Following SBR, the intestine adapts by increasing villus height and crypt depth. We hypothesized that intestinal secretory cells mediate this adaptive response. Math1 (Mouse atonal homolog 1; Atoh1) is a basic helix-loop-helix transcription factor essential for formation of Paneth, goblet, and enteroendocrine cells. Thus, we studied the adaptive response to SBR in mice that lack Math1 in the intestinal epithelium. Methods: We used the cre/loxP system to generate intestine-specific Math1-

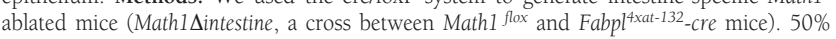
SBR $(\mathrm{N}=38)$ or transection and re-anastamosis (sham operation, $\mathrm{N}=25)$ was performed on Math1Dintestine and wild type littermate male mice. Following a BrdU pulse, distal ileum and proximal jejunum were collected for histology 12 hours, 36 hours, 7 days, and 28 days following surgery. Proliferation, apoptosis, cell number, and linear depth of crypts were

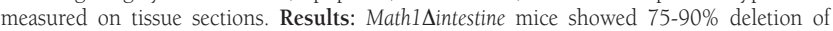
Math1 in the distal ileum, cecum, and colon. Due to their clonal nature, crypts in Math1 $\Delta$ intes tine mice were either wild type or Math1-ablated (lacking secretory cells), whereas villi were polyclonal showing mixed wild type and Math1-ablated epithelium. Math1sintestine mice tolerated the surgery well, with no significant increased mortality after 28 days. Adaptation following SBR, as measured by crypt depth and changes in proliferation, was significantly blunted in Math1-ablated crypts compared to adjacent wild type crypts or to crypts from wild type littermates $(\mathrm{p}<0.01)$. Higher than normal proliferation was observed in unoperated and sham operated Math1-ablated crypts. However, increased proliferation following SBR (as seen in wild type crypts) was not observed in Math1-ablated crypts. Apoptosis was unchanged between genotypes. Conclusions: We observed defective adaptation following SBR in crypts deficient for Math1. Following SBR, Math1-ablated crypts did not increase linear depth whereas adjacent wild type crypts lengthened, indicating the adaptive signal

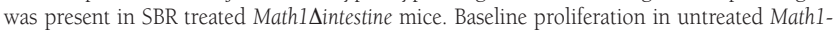
ablated crypts was somewhat increased, but this level of proliferation did not increase after SBR as in wild type crypts. Thus, we conclude that the blunted response to SBR in Math1ablated crypts is due to lack of increased proliferation. Our results implicate Math1-dependent secretory lineages in modulating intestinal homeostasis.

836

Epidermal Growth Factor Stimulates Racl Activation in Intestinal Epithelial Cells Through SRC and PI 3-Kinase to Promote Cell Migration Rebecca S. Dise, D. Brent Polk

Disruption of the epithelial lining of the gastrointestinal tract initiates rapid epithelial cell migration from the wound margins to restore epithelial integrity. We have shown that treatment of young adult mouse colon (YAMC) epithelial cells with epidermal growth factor (EGF) stimulates wound closure. This study tests the hypothesis that EGF activates Rac, a member of the Rho family of small GTPases which regulate migration and differentiation in vivo, to stimulate increased lamellipodia formation and cell migration. Circular wounds were made in confluent monolayers of YAMC cells and the percent wound closure was measured by time-lapse microscopy. Lamellipodia formation and Rac membrane translocation were analyzed in wounded cells using Rac immunofluorescence staining and phase-contrast microscopy. Rac activation was assessed using a GST fusion protein that recognizes only active, GTP-bound Rac followed by Western blot analysis for Rac. EGF treatment of wounded YAMC cells stimulated an increase in lamellipodial extensions and Rac membrane translocation at the wound margin $(\mathrm{p}<0.001)$. EGF stimulated wound closure in YAMC cells was completely inhibited by both a Rac inhibitor and Racl siRNA, confirming that Rac activation is required for EGF stimulated wound closure. Active, GTP-bound Rac was detected in YAMC cell lysates within 30 seconds after EGF treatment until 6 minutes after treatment. EGF receptor (EGFR)-/- mouse colon epithelial (MCE) cells derived from the EGFR knockout mouse failed to activate Rac or stimulate migration in response to EGF treatment. Stable transfection of EGFR-/- MCE cells with wild type EGFR restored EGF stimulated Rac activation and wound closure in these cells, but kinase dead EGFR expression did not, demonstrating a requirement for EGFR kinase activity. A MEK 1/2 inhibitor, U0126, had no effect on Rac activation or migration, while two different PI 3-Kinase inhibitors, LY294002 and Wortmannin, inhibited EGF stimulated Rac activation, lamellipodia formation, and migration. Expression of dominant negative Akt in YAMC cells did not inhibit Rac activation, demonstrating that activation of Akt by PI 3-Kinase is not required. Three different Src inhibitors, CGP77675, PP1, and PP2, inhibited EGF stimulated Rac activation, wound closure, and lamellipodia formation. Treatment of YAMC cells with both LY294002 and CGP77675 ablated Rac activation, suggesting that these kinases work in parallel to activate Rac. These data demonstrate a novel pathway in which EGF activates the small GTPase Rac through two different kinase cascades to promote wound closure in intestinal epithelial cells. 
Insulin-Like Growth Factor-1 Is Essential for Intestinal Growth in Response to Glucagon-Like Peptide-2 in Mice

Philip E. Dube, Catherine L. Forse, Patricia L. Brubaker

BACKGROUND \& AIMS: Glucagon-like peptide-2 (GLP-2) is an intestinal hormone that stimulates intestinal growth through unknown paracrine pathways. We have previously demonstrated that GLP-2 regulates intestinal insulin-like growth factor-1 (IGF-1) secretion in vitro and IGF-1 mRNA transcript expression in vivo. We therefore investigated the role of IGF-1 and the related hormone, IGF-2, as mediators of GLP-2-enhanced mucosal growth in the murine small and large intestine. METHODS: Intestinal growth was assessed in wildtype (Igf1 $1^{+/+}$and $\left.\operatorname{Igf} 2^{+}\right)$, heterozygous $\left(\overline{\operatorname{Ig}} \overline{1^{+/}}\right)$and null $\left(\operatorname{Ig} f 1^{-/}\right.$and $\left.\operatorname{Ig} f 2^{-P}\right)$ mice treated with saline (control, $\mathrm{n}=6-12)$, GLP-2 ( $\mathrm{n}=7-13$ ) or IGF-1 $(\mathrm{n}=3-6)$ for 10 days, followed by measurements of intestinal weight, crypt depth, villus height, mucosal cross-sectional area, proliferation and mRNA expression. RESULTS: Igf $1^{+/+}$mice responded to GLP-2 treatment with increased small and large intestinal weight $(131 \pm 7 \%$ and $138 \pm 12 \%$ of control, $\mathrm{P}<.001$ and .01 , respectively), with concomitant increases in crypt depth in the jejunum and colon $(\mathrm{P}<.05-.001)$, villus height in the jejunum and ileum $(\mathrm{P}<.001)$ and proliferative indices in the jejunum $(\mathrm{P}<.05-.001)$. In contrast, Igf $1^{-/-}$mice did not respond to GLP-2 with respect to either small or large intestinal weights $(110 \pm 9 \%$ and $108 \pm 8 \%$ of control, $P>.05)$ or the other measures of intestinal growth, while $\lg f 1^{+/}$mice demonstrated an intermediate response. Expression of jejunal mRNA transcripts for the GLP-2 receptor and proglucagon were unaffected by genotype or treatment with GLP-2. Igf2+ mice responded to GLP-2 with increased small intestinal weight $(128 \pm 8 \%$ of control, $\mathrm{P}<.05)$, crypt depth in the jejunum and colon $(\mathrm{P}<.05)$, and villus height $(\mathrm{P}<.01)$, mucosal area $(\mathrm{P}<0.05)$ and proliferative indices $(\mathrm{P}<.05-.01)$ in the jejunum. Ig $22^{-P}$ mice responded to GLP-2 with increased small intestinal weight $(115 \pm 5 \%$ of control, $\mathrm{P}<.05)$, crypt depth in the colon $(\mathrm{P}<.01)$, and villus height in the jejunum $(\mathrm{P}<0.05)$, but did not respond with increased mucosal cross-sectional area or proliferative indices. Furthermore, the increase in small intestinal weight in $I g f 2^{-P}$ mice was significantly smaller than that observed in Igf $2^{+}$mice when normalized for body weight $(\mathrm{P}<.05)$. Both $\operatorname{Ig} f 1^{-1-}$ and $\operatorname{Ig} f 2^{-P}$ mice demonstrated normal to enhanced intestinal growth in response to chronic administration of IGF-1, compared to wild-type controls. CONCLUSIONS: IGF-1 is essential for small and large intestinal growth in response to GLP-2, while IGF-2 may be partially involved in the small intestinal growth responses. These findings identify the IGF's as important mediators of the intestinotropic actions of GLP-2.

838

Lack of P21 (WAF1/CIP1) Enhances Intestinal Stem Cell Survival Following Radiation Injury

Courtney W. Houchen, Mark A. Sturmoski, Randal May, Robert George, Brian

Dieckgraefe, Shrikant Anant

The transformation of a normal mucosal epithelial cell to an invasive colorectal carcinoma occurs via a well-coordinated accumulation of mutations in a series of critical genes. The time span between initiation and gross development of tumors creates an enormous challenge in dissecting the critical molecular mechanisms that regulate neoplastic change. In the gut, tumorigenesis arises specifically in the stem cell population located near the base of intestinal and colonic crypts. Identifying and assaying resident intestinal stem cells is quite difficult, because no definitive specific gut stem cell markers have been identified. However, the microcolony assay following g-irradiation (IR) is by definition a functional assay of intestinal stem cell fate and can provide a mechanism for examining the early events of tumorigenesis. The cyclin-dependent kinase inhibitor $\mathrm{p} 21$ (Wafl/Cipl) plays an essential role in cell cycle arrest following DNA damage. While the gene encoding $\mathrm{p} 21$ is rarely mutated in human cancers, a variety of recent studies suggest a tumor suppressor function for $\mathrm{p} 21$. To explore the role of $\mathrm{p} 21$ in the regulation of stem cell fate, we have examined the effects of p21 deletion on intestinal stem cell survival following IR and correlated these findings with expression of the putative intestinal stem cell marker Msi-1. METHODS: Adult wild type(WT) and p21-/- mice were exposed to 13 Gy g-IR. Crypt survival was measured 3.5 days later using the microcolony assay. Crypt viability was confirmed by the presence of $>5 \mathrm{~S}$-phase cells as measured by immunohistochemical detection of BrdUrd incorporation. Msi-1 and p21 mRNA levels were determined by real time PCR. Cell specific expression Msi-1 and p21 protein were determined by immunohistochemical analysis. RESULTS: Higher numbers of surviving crypts (3.5 fold) were detected in p21-/- mice compared to WT littermates. Msi-l mRNA levels measured prior to IR in the small intestines of p2l -/- mice were elevated 2.1 fold compared to WT. Immunohistochemical analysis demonstrated increased Msi-1 protein expression in crypt epithelial cells in both wild type and p21-/- mice 3.5 days after irradiation. CONCLUSIONS: Deletion of p21 in adult mice results in a 3.25 fold increase in stem cell survival following radiation injury compared to WT littermates. These data suggest that functional p21 promotes apoptosis in the stem cell population in response to lethal doses of whole body IR. Furthermore p21 deletion results in a 2-fold increase in Msi1 expression in the crypt. These data provide additional evidence for a potential tumor suppressor function of p21 on intestinal stem cells.

\section{9}

Small Intestinal Adaptation After Partial Enterectomy Is Impaired in Ppar $\delta^{+/}$ Mice

Lihua Wang, Qun Zheng, Deborah C. Rubin, Marc S. Levin

We have shown that retinoic acid (RA) stimulates intestinal adaptation following partial small bowel resection (SBR) by stimulating crypt cell proliferation and by inhibiting apoptosis. RA has been identified as a high affinity agonist of PPAR $\delta$. PPARs are nuclear receptors that serve as sensors of nutritional stimuli and modulators of gene expression. Of the 3 mammalian subtypes, PPAR $\delta$ which is involved in peripheral lipid metabolism, is the most highly expressed in the murine gut. Administration of a PPAR $\delta$ agonist to APC ${ }^{+/ m i n}$ mice increased small intestinal polyp size by inhibiting apoptosis (Nat Med 2004 10:245). These observations suggest that the proadaptive effects of RA may be mediated via PPAR $\delta$. To address the role of PPARס in adaptation, the response after SBR was compared in PPAR $\delta^{+-}$and PPAR $\delta^{+/}$ (WT) mice. PPAR $\delta^{+/}$mice were used due to the reduced viability of PPAR $^{-/-}$mice. Methods C57BL/6 WT $(n=26)$ and PPAR $\delta^{+/}$mice ( $n=24$ gift of R. Evans, Salk Inst.) underwent 50\% SBR or shamresection. At $7 \mathrm{~d}$ post-op the adaptive response was assessed in the remnan gut by quantitation of crypt depths, villus lengths, crypt cell apoptosis (detection of activated caspase 3) and proliferation (5BrdU incorporation). Results: There were no significan differences between shamresected PPAR $\delta^{+/}$and WT mice. After SBR, $\mathrm{PPAR} \delta^{+-}$mice compared to WT mice tended to have reduced crypt depths and villi length in the jejunum and ileum, though the differences were not significant. However, the relative adaptive changes (i.e. resected versus shamresected) were significantly increased in the ileum and jejunum of WT mice, whereas the only significant increase in $\mathrm{PPARS}^{+/}$mice occurred in jejunal cryp depths. Following SBR, PPAR ${ }^{+/-}$compared to WT mice had significantly reduced crypt cel proliferation in the jejunum and ileum and an increased apoptotic index in the ileum (p< $0.05)$. The fold-changes (resected vs shamresected) were also significantly different. Afte SBR, enterocyte proliferation in the jejunum increased by 1.08 fold in resected PPAR $\delta^{+/}$ mice vs 1.32 fold in WT mice. In the ileum the changes were 1.15 fold vs 1.45 fold Apoptotic indices in jejunum increased by 2.13 fold in resected PPAR $\delta^{+/}$mice vs 1.83 fold $(\mathrm{p}<0.05)$ for WT mice and in the ileum the changes were 4.25 fold vs 2.45 fold. Conclusion: After partial enterectomy, PPARS $^{+/}$mice exhibit increased crypt apoptosis and reduced proliferation which result in a blunted morphologic adaptive response. These data sugges that regulation of PPAR $\delta$ target genes contributes to adaptation and are consistent with the hypothesis that the proadaptive effects of RA may partly be mediated via this receptor

840

Regulation of Macrophage TNF Production By MNK Kinases

Mark T. Worthington, Carol A. Chrestensen, Mary G. Harp, Robert Rowlett, Xiao Ming Wang, Fabio Cominelli, Theresa T. Pizarro, Thomas W. Sturgill

Background: Tumor necrosis factor (TNF) is a pathogenic factor in inflammatory bowel disease. In studies of TNF mRNA stability, we found that polyA tail binding PABPl protein blocked tristetraprolin-mediated TNF mRNA degradation and that PABPl is a substrate for the MNK1 kinase. Because MNK phosphorylation of the cap-protein eIF4E increases translation and nucleocytoplasmic transport of another AU-rich element containing mRNA (cyclin D1), we explored the regulation of TNF by MNK in macrophages. Hypothesis: Both the p38 and p42/ERK MAP kinase cascades are required for maximal TNF production. The inducible MNKl and the constitutive MNK2 kinases integrate p38 and ERK signals, although whether the MNKs mediate TNF effects is unknown. We used the RAW264.7 macrophage cell line and bone marrow-derived macrophages from wild-type and SAMPYit/Fc mice to test this hypothesis. SAMPYit/Fc mice are a model of human Crohn's Disease with spontaneous ileitis, perianal disease, and arthritis. Both MNK1 and MNK2 are inhibited by the drug CGP57380 (CGP). Results: Using phosphospecific Western blotting, we found that eIF4Ephosphorylation increases in response to LPS stimulation in RAW264.7 macrophages and is completely suppressed by CGP, without changes in total eIF4E. CGP to $50 \mathrm{uM}$ inhibited RAW264.7 cell TNF production by ELISA in a dose-dependent fashion. Use of a commercia panel of TLR pathway agonists with CGP to $50 \mathrm{uM}$ revealed dose-dependent suppression of TNF by ELISA induced by agonists for TLR2, TLR7, TLR9, TLR6/2, as well as TLR4 (LPS). At $50 \mathrm{uM}$, the drug repressed TNF by ELISA to $0.4 \%$ to $5.7 \%$ of that of control. Mechanistic studies reveal that MNK inhibition sharply limits the pulse of TNF mRNA production after LPS (by 185 rRNA-normalized real-time PCR) and that MNK inhibition accelerates TNF mRNA decay (by actinomycin-inhibited $18 \mathrm{~S}$ rRNA-normalized real-time PCR), suggesting both nuclear and cytoplasmic effects of MNK. We were interested if this is TNF-specific, or whether this extends to other IBD-relevant cytokines. Using a cytometric bead array, CGP inhibits IL6, MCP1/CCL2, and IFNg after LPS stimulation. Interestingly, we saw no IL-10 production with control or unstimulated CPG-treated cells, but elevation of IL-10 in the 4 hour stimulated CPG-treated cells suggests that the overall effect of MNK inhibition may be an anti-inflammatory, Thl-inhibited state. Finally, bone marrow-derived macrophages from wild-type and SAMPYit/FC mice confirm that MNK inhibition has the same effects on TNF production in native cells. Conclusion: MNK inhibitors are promising agents for treating inflammatory diseases like IBD by targeting inflammatory cytokines.

\section{1}

Differential Chromatin Remodeling in Mucosal Diseases Modifies IFNG Expression Rivkah Gonsky, Richard L. Deem, Stephan R. Targan

IFN- $\gamma$ plays an important role in the generation and perpetuation of mucosal inflammation of inflammatory bowel disease. The goal of our studies is to identify mucosa-specific molecular mechanisms involved in regulation of IFNG expression that represent potential targets for selective attenuation of mucosal IFN- $\gamma$ production. Previously, we identified mucosa-specific CD2 cis-elements within the IFNG promoter. Differential IFNG expression may be controlled epigenetically by chromatin remodeling through alterations in histone acetylation and DNA methylation patterns. Histone hyperacetylation of cytokine promoters precedes transcriptional activation and correlates with the hypomethylated status of the promoter. We hypothesized that because LP T cells are inherently activated and poised in-vivo to secrete IFN- $\gamma$ they would display a hyperacetylated and more hypomethylated profile than their PBL counterparts. We evaluated the histone acetylation status of IFNG locus from $-7.6 \mathrm{~kb}$ upstream to $+7.7 \mathrm{~kb}$ downstream using chromatin immunoprecipitation analysis. Selective hyperacetylated histone $\mathrm{H} 3$ and $\mathrm{H} 4$ was revealed following CD2 signaling, in LPMC but not in PBL, with a preference to regions surrounding the coding and downstream regions. Likewise, a more pronounced augmentation of histone acetylation was seen in LPMC compared to PBL following PMA/ionomycin activation. Pyrosequencing was used to examine the CpG methylation status of the -295 to +171 bp IFNG sites in rested CD3+ PB or CD3+ LP T cells. LP T cells displayed a hypomethylated profile within the proximal promoter region compared to PB T cells. No differences were detected in the downstream regions. The hypomethylated profile was reflected in the disease phenotype with decreased methylation in LP T cells from normal or Crohn's mucosa compared with those of UC or PBL. These results demonstrate that compared to peripheral T cells, mucosal T cells exhibit altered histone 
acetylation and methylation of the IFNG promoter. Furthermore, the data suggests that epigenetic modulation of IFNG may play a mechanistic role in disease phenotype and enhanced secretion of IFN- $\gamma$ in the mucosa.

Differential Hypomethylated Profile of IFNG Locus in IBD ( $p$ value)

\begin{tabular}{|c|c|c|c|c|c|c|}
\hline T cell Phenotype & $-295 \mathrm{bp}$ & $-186 \mathrm{bp}$ & $-54 \mathrm{bp}$ & $+122 \mathrm{bp}$ & $+128 \mathrm{bp}$ & $+171 \mathrm{bp}$ \\
\hline PB vs. LP Normal & $<0.001$ & 0.002 & 0.006 & $\mathrm{~ns}$ & $\mathrm{~ns}$ & $\mathrm{~ns}$ \\
\hline PB vs. CD & 0.008 & 0.027 & 0.038 & $\mathrm{~ns}$ & $\mathrm{~ns}$ & 0.015 \\
\hline PB vs. UC & $\mathrm{ns}$ & 0.026 & $\mathrm{~ns}$ & $\mathrm{~ns}$ & $\mathrm{~ns}$ & $\mathrm{~ns}$ \\
\hline
\end{tabular}

ns, not significant

\section{2}

Ceacaml N-Domain Mutation Found in An Ulcerative Colitis (UC) Patient Affects Il2 Secretion

Mee Sook Park, Giulia Roda, Okebugwu Kamalu, Lloyd Mayer

Background - CEACAMI (BGP) is a member of the CEA family of glycoproteins characterized by its expression as a transmembrane molecule. This CEACAM family member is the only one known to be expressed by lymphocytes and has been found on both LPLs and IELs. The presence of a cytoplasmic ITIM and its ability to recruit the phosphatase SHP1 suggests that this molecule might play a regulatory role in T cell activation. Previous studies from our lab support the concept that CEACAM family members are important in immunoregulation in the gut. Defects in expression or function could result in aberrant mucosal immune responses. Methods - CEACAMl specific primers were used to amplify cDNA derived from normal and inflammatory bowel disease (IBD) LPL. The N domain of each PCR product was sequenced and compared to the known sequence in the database. The mutation containing PCR product was put into a plasmid vector. To separate out the mutation containing allelic form, sequencing was carried out using DNA preparations of separate colonies from plasmid transformed bacteria. The wild type (WT) and mutant DNAs were transfected into Jurkat $\mathrm{T}$ cells. These cells were stimulated with Daudi cells and Staphylococcus enterotoxin E (SEE $25 \mathrm{ng} / \mathrm{ml}$ ) with subsequent measurement of IL2 production by ELISA. Results - CEACAMl was expressed equivalently on normal and IBD LPL, and minimally on peripheral blood T cells. Three mutations (Q1->K1, A49->V49, Q89->H89) were found in a PCR product of CDNA from a UC patient. This was verified by sequencing the PCR product of another cDNA sample from the same patient. Sequencing DNA preparations of separate bacterial colonies revealed that the three mutations were all on one allele. We then stably transfected Jurkat cells with the mutant or wild type DNA. Cell growth (3H-thymidine uptake for 6 hours) of mutant and WT transfected cells were $15-25 \%$ and $120-150 \%$ of the vector transfected cells, respectively. Upon stimulation with Daudi cells and SEE for 6 hours, IL2 production by the mutant transfectant was only $10-20 \%$ of the vector transfected control IL-2 production by WT transfected cells was similar to mutant transfectant despite 5-fold higher CEACAMl expression. Conclusion - LPL T cells are unique in their constitutive CEACAMl expression. The CEACAMl mutation found in a UC patient results in markedly reduced IL-2 production. Considering importance of IL-2 for regulatory T cell development as well as immune activation, the relevance of this observation to the disease process remains to be determined.

843

The Vegf-Vegf Receptor (VEGF-R) Pathway Is Up-Regulated and Mediates Mucosal Angiogenesis in Human and Experimental Inflammatory Bowel Disease (IBD)

Silvio Danese, Franco Scaldaferri, Cristina Graziani, Carol de la Motte, Egidio Stigliano, Alessandro Sgambato, Riccardo Ricci, Achille Cittadini, Antonio Gasbarrini, Claudio Fiocchi

A crucial component of several inflammatory conditions is pathological angiogenesis, a process where the VEGF-VEGF-R pathway plays a key mediator role. We recently reported that pathological angiogenesis occurs in IBD and represents a novel component of its pathogenesis. Because essentially no information exists on the VEGF-VEGF-R pathway in gut inflammation, we investigated its expression and activity in both human and experimental IBD. The expression of VEGF-R1 and -R2 in colonic sections of control, Crohn's disease (CD) and ulcerative colitis (UC) mucosa was assessed by immunohistochemestry, and during induction of dextran sodium sulfate (DSS) murine colitis (at day 3, 5, 7 and 10). VEGF levels were quantified by immunoblotting in the same tissues. Using human intestinal microvascular endothelial cell (HIMEC), the capacity of VEGF to induce cell migration was tested in a transwell chemotaxis system, and to induce angiogenesis in a matrigel tubule formation assay. VEGF-Rl expression levels were low and essentially equal in control, CD and UC mucosa. Control healthy mice also displayed very low levels of VEGF-RI and expression did not change with the induction of DSS colitis. In contrast, VEGF-R2 was very strongly up-regulated in both forms of IBD and the same was true during the course of DSS colitis, being particularly evident in newly formed submucosal small vessels. VEGF was detected in normal human mucosa, and was significantly $(\mathrm{p}<0.05)$ higher in $C D$ and $U C$. Moreover, actively inflamed CD and UC contained significantly ( $p<0.05$ ) more VEGF compared to non-inflamed $C D$ and UC tissues. Colitic mice also expressed higher $(\mathrm{p}<0.05)$ VEGF mucosal content. Finally, VEGF contained in mucosal extracts was able to induce HIMEC migration, a process specifically blocked by an anti-VEGF antibody. Recombinant VEGF was a potent inducer of HIMEC tubule formation. The up-regulated expression of VEGF-R2, the inducible form of VEGF-R, in both human and experimental IBD strongly suggests the occurrence of active angiogenesis in the intestinal mucosa during inflammation. In addition, VEGF is aberrantly over-expressed in the inflamed mucosa of IBD patients and colitic mice. Taken together, these results point to a crucial contribution of the VEGF/VEGF$\mathrm{R}$ pathway to the pathological angiogenesis of IBD, and this pathway may represent a novel target for therapeutic intervention.
Intestinal Microsomal Triglyceride Transfer Protein (MTP) Regulates Cdld Function On the Intestinal Epithelium and Protects from Mortality in the Oxazolone Model

Arthur Kaser, Masaru Yoshida, Glenn T. Furuta, Feilin A. Zhu, Nicholas O. Davidson, Sean P. Colgan, Richard S. Blumberg

Introduction. CDld is a MHC class I homolog expressed on myeloid cells, hepatocytes and intestinal epithelial cells, and presents lipid antigens to natural killer T (NKT) cells. This laboratory has previously shown that MTP regulates CDld function. The in vivo function of CDld on the intestinal epithelium is not yet understood. Aim. To assess the role of MTP and CDld in the intestinal epithelium in vivo. Methods \& Results. We crossed Mttpflox/ flox mice with a transgenic mouse expressing a tamoxifen-dependent Cre-recombinase expressed under the control of the villin promotor. Administration of tamoxifen resulted in efficient deletion of Mttp in the intestinal epithelium in the small and large bowel. Deletion of Mttp resulted in decreased CDld immunostaining in the epithelium, suggesting that in the absence of ER-based lipid loading of CDld by MTP, CDld molecules are impaired in their trafficking to the cell surface. Rectal administration of the colitis-inducing hapten oxazolone resulted in $62.5 \%$ mortality within $48 \mathrm{~h}$ in the MTP-deleted mice, while none of the mice in the control groups died within an observation period of 5 days. Severe inflammation with prominent neutrophilic infiltration was observed at early time-points. Microarray gene expression profiling at baseline and $6 \mathrm{~h}$ after oxazolone administration showed upregulation of neutrophil-chemotactic factors and mRNAs primarily expressed in neutrophils in MTP-deleted mice compared to non-deleted littermates. Altogether, these data suggest that MTP regulates a barrier-protective $\mathrm{CDld}$ pathway in the intestinal epithelium. To investigate the mechanistic basis of this protective function, we devised an in vitro reductionist system using the mouse intestinal epithelial cell line MODE-K. Co-culture of the CDld-restricted NKT cell hybridoma 24.7 with MODE-K resulted in release of barrier-protective IL-10, but no IL-12, into the supernatant in the presence of the model glycolipid alpha-galactosylceramide, while replacing MODE-K with CDIlc+ dendritic cells resulted in IL-12 induction, but no IL-10 release. IL-10 and IL-12 release from MODE-K and dendritic cell co-cultures, respectively, was abrogated by anti-CDld antibody. Furthermore, silencing of IL-10 expression in MODE-K confirmed MODE-K cells as the source of IL-10 in the co-culture system. Conclusions. In summary, these latter data implicate that a NKT cell is capable of inducing retrograde signalling of $\mathrm{CDld}$ in intestinal epithelial cells, specifically inducing a barrierprotective transcriptional programme. These data propose a model whereby NKT cells regulate a protective role of the intestinal epithelium via a $\mathrm{CDld} / \mathrm{MTP}$ pathway.

\section{5}

Identification of Inducible Intestinal B Cell Development Pathway Under Inflammatory Condition

Yasuyo Shimomura, Atsuhiro Ogawa, Ken Sugimoto, Mayumi Kawada, Emiko Mizoguchi, Atul K. Bhan, Atsushi Mizoguchi

Background: Intestinal inflammation elicits B cell lymphopoiesis that is often associated with ectopic neolymphoid follicle formation. However, the pathway of intestinal B cell development through this inducible lymphopoiesis has not yet been defined. Methods: For the study of intestinal inflammation-induced B cell development in the colon, $4 \%$ dextran sulfate sodium (DSS) in drinking water was administrated for 4 days and the treatment was terminated by changing DSS water to normal water. Results: DSS-induced inflammation elicits a polyclonal expansion of B cells in the colonic lamina propria (LP) during the recovery (4 days after termination of DSS-treatment) but not acute (on-going DSS treatment) phase of inflammation. Interestingly, the expanded B cells shares several functional and phenotypical similarities with splenic immature transitional type B cells (T1/T2) and do not produced immunoglobulins. Studies utilizing IL-7R knockout (KO) (absence of B2 but not B1 cells), Rac2 KO (absence of marginal zone B cells) and Lyn KO (absence of follicular B cells) mice suggest that the inducible intestinal B cells do not originate from Bl or mature follicular or marginal zone B cells. In addition, B cell receptor (BCR) signaling cascade and T cell help are not required for the inflammation-induced intestinal B cell expansion as indicated by a marked expansion of intestinal B cells during the recovery but not acute phase of DSSinflammation in Btk KO mice (absence of a key molecule in BCR signaling cascade) and monoclonal BCR mice (RAG KO mice crossed with hen egg-lysozome-specific BCR transgenic mice) as well as TCR $\beta \delta$ double knockout mice (absence of T cells). Of note, inflammationinduced intestinal B cell expansion can be induced even in the absence of both spleen and gut-associated lymphoid tissues (splenectomized LT $\alpha \mathrm{KO}$ mice). In addition, the inducible $\mathrm{B}$ cell expansion unexpectedly results in formation of LT $\alpha$-independent aberrant lymphoid clusters that are anatomically and phenotypically distinguishable from resident lymphoid follicle structures (such as colonic patches and isolated lymphoid follicles) within the intestinal LP. The expanded B cells are subsequently maintained through BAFF-R (receptor of B-cellactivating factor of the TNF family) signaling cascade capable of preventing the apoptosis. Conclusion: We herein identify a novel pathway for inducible intestinal B cell development associated with colitis. Importantly, the inducible B cells are characterized by BCR-independent "innate type" B2 (but not B1) phenotype.

846

Directed Expression of Salmonella Avra in Drosophila Melanogaster Reveals Mechanistic Conservation of Innate Immune Repression Rheinallt Jones, Andrew Neish

Background: Enteric pathogens such as Salmonella are important causes of food-borne disease. Many bacterial pathogens influence host cell biology by utilization of preformed effector proteins that are translocated into the cytoplasm of host cells during the initial stages of infection. These effector proteins are thought to function by usurping eukaryotic cellular processes for the benefit of the bacterial life cycle. We have shown S. typhimurium effector AvrA has potent negative effects on the NF-kappaB/Rel pathway in mammalian epithelial cell culture in vitro. Purpose: To evaluate cellular effects of this protein an intact 
eukaryotic organism in vivo, we have employed a genetic model system in Drosophila. Flies possess a well-studied anti-bacterial response exhibiting striking functional and structural homology to human innate immune signaling systems, including NF-kappaB/Rel. Methods: We generated transgenic Drosophila harboring the wild type AvrA gene from S. typhimurium, as well as a version with point mutations in the AvrA catalytic core. The bacterial gene is under transcriptional control of the yeast UAS promoter and can be expressed in a regulated fashion in fly tissue using the bipartite Gal4/UAS expression system. Flies were routinely challenged by microbial infection, which normally results in Rel protein translocation, transcription of antimicrobial effectors and clearance of infection. Nuclear translocation of Rel was detected by immunohistochemistry, anti-microbial gene activation assayed by qRT PCR or reporter gene assay, and immune response determined by survival analysis. Apoptosis was detected by an anti-caspase-3 antibody and by TUNEL staining. Results: We find that expression of AvrA, while having no cytotoxic or pro-apoptotic effects, is a potent inhibitor of NF-kappaB/Rel translocation and transcription of anti-microbial peptides. Expression of AvrA also reduced the ability of bacterially challenged flies to carry out clearance of infection. The catalytically inactive mutant AvrA had no effect in any of there assays, indicating conservation of biochemical function. In further studies, no effects were found on the MAPK pathways as determined using flies harboring lacZ under a MAPK specific promoter. Conclusions: This data reveals that AvrA mediates a highly conserved and specific repression of NF-kappaB/Rel without direct effects on cell survival. This system will allow future forward genetic approaches to elucidate the biochemical function of this and potentially other bacterial proteins

847

Helicobacter Pylori Persistence and Disease Severity Is Modulated By the Induction of Regulatory T-Cells

Rupert M. Kenefeck, Karen Robinson, John C. Atherton

Introduction: Helicobacter pylori $(\mathrm{Hp})$, the major cause of peptic ulcer disease, provokes a vigorous immune response. Despite this, however, most infections are persistent and show highly variable levels of pathology. Regulatory T-cells (Tregs) are found in the Hp infected human gastric mucosa and have been shown to reduce inflammation in animal models. We hypothesize that regulatory T-cells are important in mediating the persistence of Hp infection and modulating disease severity. Methods: Gastric antral biopsies were collected from sixtythree consenting patients attending the Queen's Medical Centre, Nottingham for an upper GI tract endoscopy. Flow cytometry was used to quantify Tregs by staining for the markers CD4 and CD25 and also interleukin-10 (IL-10). Expression of the natural Treg-specific gene FOXP3 was quantified by real-time PCR. Results: Elevated frequencies of CD4+CD25hi regulatory T-cells were detected in the gastric mucosa of $\mathrm{Hp}$-infected compared with uninfected donors $(\mathrm{p}=0.04)$. A 12-fold higher frequency of CD4+CD25hilL-10+ cells was found amongst gastric cells from infected compared to uninfected donors ( $\mathrm{p}=0.002$ ). Up to $95 \%$ of the gastric CD4+CD25hi cells from infected donors were IL-10+ (median 61.3\%; IQR $32.2-83.3 \%$ ), and the proportion of IL10+ Tregs was 18 -fold greater than that obtained with cells from uninfected donors (median 3.5\%; IQR $0.0-24.3 \%$; $=0.002$ ), suggesting that suppression of the normal T-cell response may be IL-10-mediated. Real-time PCR revealed a 7-fold increase in FOXP3 expression in gastric tissues from infected compared to uninfected donors ( $\mathrm{p}=0.003$ ), indicating the presence of natural Tregs during infection. Infected patients were further divided into two groups, according to whether peptic ulcer disease (PUD) was present or absent. The frequency of gastric CD4+CD25hilL-10+ Tregs was significantly elevated in those patients with a normal gastric mucosa (median 2263; IQR 1766-3636) as compared to patients with PUD (median 946; IQR 524-2365; $\mathrm{p}=0.05$ ). In addition FOXP3 transcript levels were also elevated 5 fold in patients without PUD indicating that natural Tregs play an important role in the modulation of inflammatory disease $(p=0.006)$. Conclusion: We show that IL-10-secreting natural Tregs are present in the gastric mucosa of Hp-infected patients and are inversely associated with disease severity. We propose that Tregs not only impede the ability of the immune system to clear infection, but also modulate inflammation and protect against peptic ulcer disease.

848

Salmonella Flagellin Acts As An Avirulence Factor and Limits Disease During Enteric Infection in Vivo

Matam Vijay-Kumar, Huixia Wu, Andrew T. Gewirtz, Andrew Neish

Background: Flagellin, the primary component of bacterial flagella, is a potent activator of inflammatory signaling. We had previously demonstrated that viable aflagellate Salmonella were unable to induce proinflammatory signaling pathways or gene expression in in vitro models, suggesting flagellin is a major proinflammatory determinant of enteropathogenic Salmonella. Purpose: The goal of this study was to evaluate the role of flagellin as a pathogenic determinant in in vivo infection. Methods: We utilized the recently developed streptomycin pretreatment murine model of enteric Salmonellosis. Mice were pretreated with $7.5 \mathrm{mg}$ of streptomycin orally and $24 \mathrm{~h}$ later were orally inoculated with $108 \mathrm{CFU}$ of Salmonella typhimurium (SL3201), an isogenic aflagellate mutant (fliC-/fliB-), or left uninfected. Blood was collected at $6,12,24$, and $48 \mathrm{hr}$ and assayed by ELISA for serum IL-1 and IL-6. Intestinal tissues were harvested at the same times and evaluated by morphology ( $\mathrm{H}$ and E), histochemistry (myeloperoxidase) and immunohistochemistry (active caspase 3). Rat IEC-6 cells were cocultured with the same bacterial strains and apoptosis quantified with fluorescent caspase staining and TUNEL. Transcript levels of anti-apoptotic proteins (IAPs) were measured by qRT-PCR. Results: Consistent with previous in vitro data, aflagellate Salmonella mutants were initially impaired (6-12 hrs) in their ability to upregulate mucosal and systemic inflammatory marker molecules and elicited markedly reduced mucosal inflammation relative to the isogenic WT parent strain. However, these aflagellate bacteria caused markedly exacerbated mucosal inflammation at the later stages of infection ( 24 and 48 hours) and ultimately a more severe clinical course. These phenomena correlated with increased activation of epithelial caspases and subsequent apoptosis. The aflagellate Salmonella also induced strikingly more intense and rapid caspase and apoptotic activation in cultured IEC-6 cells relative to the wild type strain. Aflagellate Salmonella induced significantly less IAP gene expression both in vivo and in vitro. Conclusions: These observations indicate that in Salmonella infection, flagellin plays a dominant role in activation of not only innate immunity but also anti-apoptotic/survival pathways in epithelial cells, controllin caspase activation and thus protecting the host from greater damage. In this situation, flagellin intriguingly acts as an avirulence factor. This notion is consistent with the emergin paradigm that specific TLR ligands may have a fundamental cytoprotective effect during inflammatory stress.

849

Gp96 Is a Colonocyte Plasma Membrane Binding Protein for Clostridium Difficile Toxin A

Xi Na, Ho Kim, Charalabos Pothoulakis, J. Thomas Lamont

Background \& Aims: Clostridium difficile (C. difficile) Toxin A (TxA) is a key mediator of antibiotic-associated colitis in human, causing fluid secretion, epithelial necrosis, and acute inflammation. TxA requires binding to a cell surface receptor prior to internalization, bu the human receptor for TxA has not been identified. The aim of this study was to identify novel plasma membrane binding proteins for TxA and to characterize their role in the TxAmediated inflammatory cascade in colonocytes. Methods: TxA was coupled with biotin and cross-linked to the surface of HT29 human colon cancer cells. The main colonocyte bindin protein for TxA was identified as glycoprotein 96 (Gp96) by co-immunoprecipitation and mass spectrum analysis. The interaction between Gp96 and TxA was further confirmed by fluorescence immunostaining and in vitro co-immunoprecipitation. Cell binding and rounding assays were used to access Gp96 antibody and silencer Gp96 effects on TxA-mediated attachment and cytotoxicity to human colonocytes. Gp96-deficient B cells (E4.126), generated by a frameshift mutagen and wild-type parental cells were used to further delineate the role of Gp96 in TxA mediated signaling. Results: The major TxA binding protein on HT29 cells was Gp96, a member of the heat shock protein family. In resting HT29 cells, this protein is expressed on the cell surfaces as well as in the cytoplasm. Following TxA binding, the TxA- Gp96 complex was translocated from the cell membrane to the cytoplasm. Pretreatment with Gp96 antibody decreased TxA binding to colonocytes and inhibited TxA-induced cell rounding. SiRNA directed against Gp96 reduced Gp96 expression and cytotoxicity in HT29 cells. TxA-induced inflammatory signaling via $\mathrm{P} 38$ and apoptosis as measured by activation of caspase 3 and DNA fragmentation were decreased in Gp96 deficient cells. Conclusions: Human colonocyte Gp96 serves as a plasma membrane binding protein that facilitates cellular entry of the toxin, and participates in cellular signaling events in the inflammatory cascade. Heat shock proteins are chaperone proteins expressed in the endoplasmic reticulum and plasma membrane that bind to proteins, preserve their correct folding. Our data indicate that C. difficile TxA exploits Gp96 as a membrane receptor/co-receptor that facilitates its entry to the cytoplasm.

850

Decay Accelerating Factor (DAF) Is a Cellular Receptor for Helicobacter Pylori Daniel P. O'Brien, Uma Krishna, Judith Romero-Gallo, John Nedrud, M E. Medof, Feng Lin, Raymond Redline, Douglas M. Lublin, Bogdan J. Nowicki, Dawn Israel, Aime T. Franco, Richard M. Peek

Persistent gastritis induced by Helicobacter pylori is the strongest known risk factor for peptic ulcer disease and distal gastric adenocarcinoma. H. pylori induces the activation of NF- $\mathrm{\kappa B}$, and a downstream effect of NF- $\mathrm{KB}$ activation is up-regulation of the gene encoding decayaccelerating factor (DAF). DAF exhibits at least two distinct roles within epithelial cells; protecting cells from complement-mediated lysis, and functioning as a receptor for several microbial pathogens. Adherence of $H$. pylori to gastric epithelial cells is critical for induction of inflammation and injury. We therefore, hypothesized that $H$. pylori uses DAF as a receptor which contributes to pathogenesis. Methods: Chinese hamster ovary $(\mathrm{CHO})$ cells stably transfected with full-length human DAF, individual complement control protein (CCP) domain deletion constructs, or vector control were co-cultured with $\mathrm{H}$. pylori, and adherence was assessed by quantitative culture and immunofluorescence. MKN28 gastric epithelial cells were co-cultured with $H$. pylori strain J166 and isogenic mutants. DAF expression was measured by quantitative real-time RT-PCR, Western immunoblotting, and immunofluorescence. C57/BL6 wild-type (WT) $(\mathrm{n}=14)$ and DAF knockout mice $(\mathrm{n}=13)$ were infected with the rodent-adapted H. pylori strain SSI or broth alone and sacrificed 8 weeks post-challenge. Colonization density was determined by quantitative culture and inflammation was scored from 0-4. Results: $\mathrm{H}$. pylori adhered more efficiently to $\mathrm{CHO}$ cells expressing DAF (12 fold) versus vector alone. Adherence to DAF by a population of clinical $H$. pylori isolates indicated that affinity varies between strains. Studies performed using DAF domain deletion constructs indicated that DAF CCP domains 1, 2, and 3 are essential for $H$. pylori adherence and deletion of CCP-4 reduced binding by 70\%. Co-culture of $\mathrm{H}$. pylori with gastric epithelial cells induced the expression of DAF, and induction was dependant upon a functional cas PAI. In a mouse model of DAF deficiency, H. pylori colonized WT $(n=10)$ and Daf1 $1^{-1-}(n=$ 9) mice equally; however, the intensity of inflammation was significantly attenuated in $H$ pylori-infected Daf1 ${ }^{-/-}$versus WT mice $(\mathrm{p}=0.013)$. Conclusion: These results indicate that $H$. pylori uses DAF as a cellular receptor. Co-culture of $H$. pylori with gastric epithelial cells induces the expression of DAF in a cag PAI-dependent manner, indicating that $\mathrm{cag}^{+}$bacteria may have increased pathogenicity due in part to the induction of DAF as a receptor. $H$. pylori-induced inflammation is significantly attenuated in Daf $1^{-/}$mice, indicating that DAF may regulate $H$. pylori-epithelial cell interactions and pathogenesis.

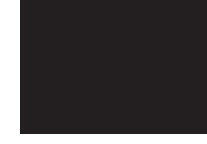




\section{1}

Viable Bacteria Versus DNA Extracts As Anti-Inflammatory Agents in Crohn's Disease

Monica Carol, Marta Llopis, Maria Antolin, Cristina Martinez, Natalia Borruel, Francesc Casellas, Francisco Guarner, Juan-Ramon Malagelada

Background: Other investigators described an immunomodulatory effect of DNA extracts from probiotic bacteria via TLR-9 in animal models and cellular lines. Our group reported a potent anti-inflammatory effect of viable Lactobacillus casei DN-114 001 in ileal tissue from Crohn's disease patients. Aim: To compare the effect of viable L. casei versus bacterial DNA in inflamed ileal mucosa from Crohn's disease patients. Material and methods: Explants of ileal mucosa (20-30 mg) from 4 patients with Crohn's disease were incubated for $18 \mathrm{~h}$ with viable Lactobacillus casei (L. casei, 10E6 CFU/mL), DNA extracts from specific bacterial strains ( $5 \mu \mathrm{g} / \mathrm{mL}$ ), including L. casei (Lc-DNA), Bifidobacterium longum (Bl-DNA), and Bifidobacterium brevis (Bb-DNA), or medium alone (blank). Each condition was run in triplicate $(\mathrm{n}=12)$. Tissue viability (LDH) and $\mathrm{pH}$ of the culture media were monitored; TNF-a levels and recovery of bacteria were assessed in the supernatant at the end of the incubation period. The following additional cytokines: IL-1b, IL-2, IL-6, IFN-g IL-13, IL-4, IL-5, IL-10 were analyzed in L. casei, Lc-DNA and blank conditions. Results: Tissue incubation with viable L. casei and Bl-DNA significantly decreased TNF-a concentration in the supernatant, but

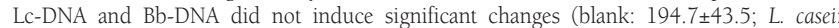
$27.9+15.7 *$ : Lc-DNA: $69.4+16.1$ : Bl-DNA: $32.6+8.2 *$ Bb-DNA: $71.8+40.6$; results are mean \pm sem in $\mathrm{ng} / \mathrm{mL},{ }^{*} \mathrm{p}<0.05$ v.s. blank). Likewise, levels of IL-1b, IL-2, IL-6, and IFN-g were significantly decreased by viable $L$. casei but not by Lc-DNA. On the other hand, IL-13, IL4 , IL-5 and IL-10 were significantly stimulated by both viable L. casei and Lc-DNA. Conclusion: DNA extract form Lactobacillus casei increases the level of antiinflammatory cytokines in Crohn's disease mucosa, but the inhibitory effect of the viable bacteria on Thl cytokines is not reproduced by the DNA extract.

\section{2}

Inhibition of Telomerase By Mutant Template Telomerase RNA and AntiTelomerase Sirna Induces ALT in Immortalized Human Esophageal Epithelial Cells

Michaela Doebele, Alexander von Werder, Christine Fulda, Steffen Heeg, Gitta Goessel, Michael Quante, Hideki Harada, Yasir Suliman, Shang Li, Elizabeth H. Blackburn, Hubert E. Blum, Oliver G. Opitz

Introduction: Immortalization is an important step in the malignant transformation of human cells. Telomere maintenance is essential for immortalization. Immortalized cells maintain their telomeres either through activation of the ribonucleoprotein enzyme telomerase, which contains an intrinsic template RNA moiety (human telomerase RNA; hTER) and the core protein (human telomerase reverse transcriptase) or through utilization of a recombination based alternative mechanism (ALT). Little is known about the regulation and coexistence of these two mechanisms in a single cell. Previously, we could demonstrate that the induction of the respective telomere maintenance mechanism is dependent on cell-cycle kinetics. Now we investigated, whether immortalized cells can switch from one mechanism to the other applying specific genetic telomerase inhibitors. Methods: Cyclin-Dl (EPC-D1) or hTERT (EPC-hTERT) overexpressing cells were generated by retroviral transduction of normal human esophageal epithelial cells (EPC). Furthermore, these cells were transduced with mutated versions of hTER (MT-hTER/AU5, MT-hTER/47A), anti-hTER siRNA or a combination of both using lentiviral gene transfer. Transduced cells were sorted by GFP-coexpression and their growth characteristics, telomerase activity (TRAP-assay), telomere length (PFGETRF) and indirect immunofluorescence (APBs) were assayed. Results: Overexpression of MT-hTER, siRNA and the combination of both rapidly inhibits cell growth in immortalized human esophageal epithelial cells, in contrast to cells transduced with control vectors. A reduction in telomerase activity could be observed whereas the control cells showed a robust activity. TRF analysis revealed heterogeneous telomeres in MT-hTER, siRNA- and MT-hTER plus siRNA-cells, which is characteristic for ALT. In contrast, the control cells displayed stable and homogeneous telomeres correlating with a high level of telomerase activity. In indirect immunofluorescence ALT-associated PML bodies (APBs) could be observed in a higher frequency in telomerase(hTER)-inhibited cells. Summary: Genetically defined immortalized human esophageal epithelial cells are capable to elongate their telomeres using both telomere maintenance mechanisms, namely ALT or telomerase activation. In our telomerasepositive cell populations ALT could be induced by the inhibition of telomerase using a lentiviral delivery system of mutant template telomerase RNA and anti-telomerase shortinterfering RNA. These findings suggest that immortalized cells like cancer cells treated with telomerase inhibitors as potential anti-cancer strategy might find alternative ways to maintain their telomeres.

853

Hypoxia Inducible Factor $1 \alpha$ Regulates Insulin-Like Growth Factor Binding Protein-3 Function in Esophageal Epithelial Cells

Munenori Takaoka, Mangatt P. Biju, Claudia D. Andl, Takaomi Okawa, Carmen Z. Michaylira, Daniel C. Chung, Volker H. Haase, Hiroshi Nakagawa

Introduction: Insulin-like growth factor (IGF) binding protein-3 (IGFBP-3), a key regulator of IGF bioactivity, is induced by cellular stresses such as growth factor deprivation and hypoxia. In normoxia, epidermal growth factor (EGF) potently represses IGFBP-3 mRNA transcription through the Ras-MAPK signaling pathway, while IGFBP-3 protein translation depends upon the PI3K-AKT-mTOR pathway (Takaoka M et al. Am J Physiol. In press). However, it remains unclear how IGFBP-3 is regulated by hypoxia in esophageal epithelial cells. Methods: Immortalized human esophageal cells were exposed to hypoxia $0.2-1 \%$ $\mathrm{O}_{2}$ ). Cells were retrovirally transduced with wild-type (WT) and dominant-negative (DN) hypoxia inducible factor (HIF) $1 \alpha$. Small interfering RNA (siRNA) directed against HIFl $\alpha$ was stably transfected. Gene expression was determined by real-time RT-PCR and Western blotting. Pharmacological inhibitors were used to dissect the signaling pathways involved.
The eukaryotic initiation factor complex was analyzed by the $\mathrm{m}^{7} \mathrm{GTP}$ Cap-binding assay Results: IGFBP-3 mRNA and protein were robustly induced by hypoxia as well as cobalt chloride $\left(\mathrm{CoCl}_{2}\right)$, an inhibitor of oxygen-dependent proteasomal degradation of HIFs, with concomitant induction of HIFl $\alpha$ in esophageal cells. When WT-HIFl $\alpha$ was ectopically expressed and stabilized by hypoxia or $\mathrm{CoCl}_{2}$ in normoxia, induction of mRNA and protein for IGFBP-3 and carbonic anhydrase 9 (CA9), a HIFl $\alpha$ specific target gene, were enhanced ( 2 to 5 -fold) compared with control cells. By contrast, DN-HIFl $\alpha$ and HIFl $\alpha$ siRNA inhibited IGFBP-3 and CA9 expression by $80-90 \%$. Interestingly, EGF failed to fully inhibit HIFl $\alpha$ mediated induction of IGFBP-3. Hypoxia suppressed p44/42 MAPK activity. Moreover, inhibition of MAPK by U0126 (MEKl/2 inhibitor) or growth factor deprivation greatly enhanced hypoxia-dependent induction of IGFBP-3. While LY294002 (PI3K inhibitor) inhibited IGFBP-3 in both hypoxia and normoxia, rapamycin (mTOR inhibitor) appeared to be less efficient to inhibit IGFBP-3 in hypoxia. In concert with this, the $\mathrm{m}^{7} \mathrm{GTP}$ pull-down assay revealed an increased physical association of eukaryotic initiation factor 4E with its inhibitor 4E-BPl in hypoxia compared with normoxia, implying a reduction in rapamycinsensitive (i.e. cap-dependent) translation under a hypoxic condition. Conclusions: In hypoxia, HIFl $\alpha$ plays a critical role in IGFBP-3 induction. Unlike growth factor deprivation, hypoxia may employ cap-independent translation. These novel findings have important implications in how epithelial cells respond to cellular stress during infection, injury and transformation.

854

Reprimo Methylation Is a Potential Early Biomarker of Barrett's-Associated Neoplastic Progression

James P. Hamilton, Fumiaki Sato, Bruce D. Greenwald, Tetsuo Ito, Yuriko Mori, Bogdan C. Paun, Takatsugu Kan, Yulan Cheng, Suna Wang, Carmite Mantzur, John M. Abraham, Stephen J. Meltzer

Background: Reprimo, a novel tumor suppressor gene, regulates p53-mediated cell cylce arrest at the G2 phase of the cell cycle. Accordingly, our aim was to determine the level of Reprimo methylation in normal, premalignant, and malignant human esophageal tissues. Methods: 161 tissue specimens were obtained during endoscopy. Real-time methylationspecific PCR (MSP) for Reprimo was performed using an ABI7700 (Taqman) system. Esophageal cancer cell lines were incubated per protocol. $5 \mathrm{mM}$ 5-Aza-deoxycytidine (5-AzadC) was added every 24 hours for 6 days. Cells were harvested for DNA/RNA extraction on days $0,2,4$, and 6. RT-PCR and MSP was performed using a Taqman. Results: In both Barrett's (BE) and esophageal adenocarcinoma (ADCA), the level and frequency of Reprimo methylation were significantly greater than in normal esophagus (NE) or esophageal squamous cell cancer (SCCA). There was no statistically significant difference between BE and ADCA. In addition, in vitro demethylation with 5-Aza-dC resulted in enhanced mRNA expression of Reprimo. Reprimo methylation was seen in 0 of 19 NE samples (mean MSP: $0.004), 6(14 \%)$ of 44 SCCA (mean: 0.092; SCCA vs. NE, $p=0.157), 8(32 \%)$ of 25 cases of $\mathrm{BE}$ (mean: 0.111; BE vs. NE, $\mathrm{p}=0.019)$, and $44(60 \%)$ of $73 \mathrm{ADCA}$ (mean: 0.214; ADCA vs. NE, $\mathrm{p}=0.002 ; \mathrm{BE}$ vs. ADCA, $\mathrm{p}=0.09$ ). 5 -Aza-dC treatment of ADCA cell line OE33 resulted in reduced methylation of Reprimo (day 0 MSP: 1.2 , day 6: 0.34), as well as increased expression of Reprimo (day 0 mRNA: 0.0002, day 6: 0.002). ROC curve analysis of Reprimo methylation in ADCA vs. NE revealed an area under the curve (AUROC) of 0.812. Conclusions: Reprimo methylation occurs significantly more often in ADCA and BE than in NE or SCCA, suggesting that this is a cell type-specific early event that shows potential as a biomarker for early disease detection.

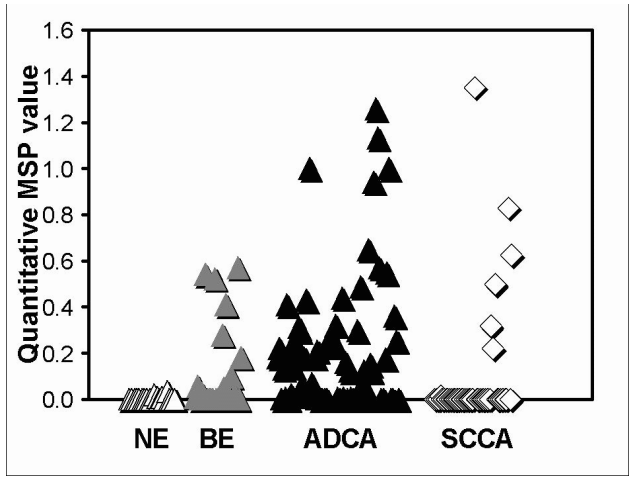

Reprimo Methylation

855

Induction of COX-2 By Unconjugated Bile Acids Involves AP-1, CREB and Reactive Oxygen Species-Mediated Signaling Pathways in Esophageal Adenocarcinoma Cells Shumei Song, Robert S. Bresalier

BACKGROUND: Bile reflux is thought to contribute to esophageal injury and neoplasia. COX-2 is involved in both inflammation and carcinogenesis, and bile acids can increase COX-2 expression. However, the mechanisms by which bile acids enhance COX-2 expression are largely unknown. AIMS: Determine the molecular mechanisms that govern COX-2 regulation by bile acids in esophageal adenocarcinoma (EA) cells. METHODS: Immunoblotting and transient transfection were used to assess the effects of bile acids on COX-2 expression and transcription in SEG-1 EA cells. Specific pharmacological inhibitors, dominant negative (DN) mutant constructs and SiRNA inhibition techniques were used to identify the signaling pathways involved. Flow cytometry and reactive oxygen species (ROS) scavengers $\mathrm{N}$-acetyl cystene and sodium formate were used to examine ROS involvement. RESULTS: 
Chenodeoxycholic acid (CD) and deoxycholic acid (DC), but not conjugated bile acids, were strong activators of COX-2 expression. Increased phosphorylation of AKT and mitogenactivated protein kinasel/2 occurred in unconjugated bile acid-treated cells but not in conjugated bile acid- treated cells. A DN AKT mutant, LY294002 a PI3K inhibitor, and UO126, an inhibitor of MAPK blocked induction of COX-2 by CD and DC. Furthermore, AP-1 protein and activity were increased by $C D$ and DC, and a CMV-driven DN AP-1 vector decreased COX-2 transcription. Phosphorylation of CREB was strongly increased by CD and DC, and inhibitors of AKT and MAKP blocked CREB phosphorylation induced by CD and DC. Silencing of CREB by SiRNA inhibited COX-2 expression. Induction of COX-2 and phosphorylation of CERB were also blocked by reactive oxygen species scanvengers $\mathrm{N}$-acetyl cystene and sodium formate. CONCLUSION: These data suggest that dihydoxy unconjugated bile acids in GE refluxate contribute to COX-2 induction via pathways involving AP- 1 and CREB and ROS-mediated activation of phosphatidylinositol 3-kinase and mitogenactivated protein kinase $1 / 2$. This may explain, in part, the mechanisms by which bile acids contribute to tumorigenesis in the esophagus.

856

Stepwise Endoscopic Resection of the Whole Barrett's Esophagus in Patients with Early Neoplasia Effectively Removes All Genetic Alterations from the Esophageal Epithelium

Femke P. Peters, Jantine W. van Baal, Agnieszka M. Rygiel, Wouter L. Curvers, Wilda D. Rosmolen, Paul Fockens, Fiebo J. Ten Kate, Kausilia K. Krishnadath, Jacques J. Bergman

Background \& Aims Malignant transformation of Barrett's esophagus (BE) is associated with several genetic events that accumulate as the mucosa becomes dysplastic and develops into adenocarcinoma. Stepwise radical endoscopic resection (SRER) of BE with high grade intraepithelial neoplasia (HGIN) or early cancer (EC) is a promising new treatment for BE, which results in complete re-epithelialization of the resected area with neo-squamous epithelium. However, it is not known whether SRER eradicates pre-existing genetic abnormalities. The aim of this study was to prospectively evaluate whether genetic abnormalities as found before SRER are effectively eradicated and absent in the neo-squamous epithelium. Patients $\&$ Methods Nine consecutive BE patients with HGIN or EC who underwent SRER were included in this study. Immunohistochemistry (IHC) for assessing proliferation (Ki-67) and p53 over-expression, and DNA Fluorescent In Situ Hybridization (FISH) were performed on endoscopic resection (ER) specimens of the BE and on biopsies of the neo-squamous epithelium taken during follow up. DNA-FISH was performed to determine aneusomy with centromeric probes for chromosomes 1 and 9 , and with locus specific probes for assessing loss of the tumor suppressor genes pl6 and p53. FISH results were blindly scored by two experts. A minimum of 100 nuclei were scored for each DNA-FISH probe. Lymphocytes in the ER-specimens were used as controls. Results Nine pts were included (3 HGIN, 6 EC). FISH results showed that all pts had genetic alterations in their ER specimens. All pts showed hyperploidy for chromosome 1 and 5 pts for chromosome 9 . Four had loss of chromosome 9. Loss of pl6 was seen in 7 patients, while 8 patients proved to have loss of p53. IHC results showed that 7 pts had intense p53 nuclear staining in $100 \%$ of cells, indicating p53 mutations, while 2 pts were negative for $\mathrm{p} 53$. All pts had Ki-67 positive surface epithelium, indicating an increased proliferation index. In contrary to these pre-treatment findings, all post-treatment biopsy specimens showed a normal diploid signal count for all DNA-FISH probes. Furthermore, in all neo-squamous cases normal IHC staining for Ki-67 and negative staining for P53 was seen. Conclusions Stepwise radical endoscopic resection of the complete Barrett's esophagus with early neoplasia successfully eradicates pre-existing genetic abnormalities such as aneusomy and loss of p16 and p53 and results in neo-squamous epithelium without these genetic abnormalities. This research is financially supported by AstraZeneca BV The Netherlands.

\section{7}

New Establishment of Diagnostic Algorithm of Esophageal Cancer and High Sensitivity Oligo Chip

Yutaka Shimada, Tetsuo Ito, E.J. Tanaka, Motoshige Higashiyama, Atsushi Itami, Go Watanabe, Takatsugu Kan, Fumiaki Sato, Shigemi Matsumoto, Shin'ichi Miyamoto, Michihide Mitsumori, Masashi Kadowaki, Gozoh Tsujimoto, Hideo Akiyama, Akira Myomoto, Osamu Nomura, Hitoshi Nobumasa

Purpose: We have reported that microarray can detect the difference between squamous cell carcinoma and adenocarcinoma (BBRC 2001), prognosis of esophageal cancer (BBRC 2001) and prediction of lymph-node metastasis (Ann Surge Oncol 2004). However, in order to perform tailor-made therapy, the sensitivity of the microarray was inadequate for a biopsy sample. Here we established the algorithm of diagnosis of esophageal cancer, prediction of lymph-node metastasis and prediction of chemo-radiation sensitivity using an ultra sensitive oligo chip. Material and Method: First, we identified esophageal cancer related genes from about 30,000 genes using 100 selected esophageal cancer samples. Secondly, gene expression was normalized by locally weighted scatter plot smooth (LOWESS) and evaluated by support vector machine (SVM) and several bio-informatic analyses. Thirdly, we evaluated 39 biopsy samples by these machines. Fourthly, we tried to predict lymph- node metastasis and chemoradiation sensitivity using the samples which were diagnosed by cancer detection machine. Finally, we successfully established an ultra sensitive oligo chip by nanotechnology, and reevaluated the samples. Results: We selected 22 genes for diagnosis esophageal cancer and 17 genes were newly identified. Accuracy for cancer diagnosis of conventional oligo-chip was $95 \%$ and ultra sensitive oligo-chip was 100\% in learning samples. We also selected 95 genes for prediction of lymph- node metastasis and the accuracy for prediction of lymphnode was $86 \%$ in learning samples and $68 \%$ in biopsy samples. Preliminary evaluation of the accuracy for chemo-radiation was $71 \%$. The sensitivity of our new chip is 100 times more than previous chips and the minimum sample requirement for examination was $0.1 \mu \mathrm{g}$ of total RNA. Conclusion: This technology is able to diagnose esophageal cancer, prediction of lymph-node metastasis and prediction of chemo-radiation-sensitivity using biopsy samples without gene amplification. Thus, this technology may be useful for performing tailor-made therapy to esophageal cancer patients.
Regional Differences in Serotonergic Activity in the Mucosa of the Human Stomach and Duodenum

Niels Van Lelyveld, Jose Ter Linde, Marguerite Schipper, Melvin Samsom

Background \& Aims: The serotonergic system plays an essential role in gastrointestinal motility and sensitivity. In the current study, enteroendocrine (EE) cells, enterochromaffin (EC) cells, and the mRNA levels of several genes encoding components of the serotonergic system were determined in the fundus, antrum, and duodenum. We anticipated that this would elucidate the molecular basis and understanding of the role of serotonin (5-HT) in normal gastroduodenal function. Methods: Mucosal biopsy specimens were collected from the fundus, antrum, and duodenum of 11 healthy subjects. EE cells (chromogranin immunoreactive) and EC cells (5-HT immunoreactive) were counted and the mRNA levels of TPH1 (rate-limiting enzyme of 5-HT synthesis), SERT (5-HT transporter), the 5-HT4 receptor, and the 5-HT3(C) and 5-HT3(E) receptor subunits were quantified by real time RT-PCR Results: The number of EE and EC cells was larger in the duodenum compared to the stomach (all $\mathrm{P}<0.001$ ), however comparable between the antrum and the fundus. The percentage of enteroendocrine cells that were EC cells was larger in the duodenum compared to the fundus $(\mathrm{P}=0.008)$, however comparable between duodenum and antrum and between antrum and fundus. In spite of a larger number of EC cells and a larger fraction of EC cells in the duodenum, TPH- 1 expression in the duodenum was lower compared to the antrum and fundus $(\mathrm{P}=0.017$ and $\mathrm{P}<0.001$ respectively). The expression of SERT was higher in the duodenum compared to the antrum $(\mathrm{P}<0.001)$, and higher in the antrum compared to the fundus $(\mathrm{P}<0.001)$. The relative differences of TPH-1 expression (duodenum versus antrum and fundus: -2.3 and -3.6 respectively) were, although significant, much smaller than the relative differences in SERT expression (duodenum vs antrum and fundus: 19.1 and 457 respectively). The 5-HT4 receptor, the 5-HT3(C), and 5-HT3(E) receptor subunits were more abundantly expressed in the duodenum compared to the stomach (all $\mathrm{P}<0.001$ ) whereas no differences were found between antrum and fundus. Conclusions: 5 -HT synthesis (TPH-1 expression) and 5-HT uptake capacity (SERT expression) do not coincide. 5-HT synthesis varies only marginally between the three regions, whereas the uptake capacity of 5-HT differs tremendously. The higher expression of 5-HT3 and 5-HT4 receptors, and in particularly the higher uptake capacity of 5-HT, suggests a larger 5-HT release in the duodenum, and consequently points to a more prominent role of serotonergic signalling at the mucosal level in the duodenum compared to the stomach.

859

Role of PYLORIC Pressures and Botulinum Toxin (BT) Injection in the Treatment of Diabetic (DM) and Idiopathic Gastroparesis (IG) Katerina Shetler, Linda Anh B. Nguyen, Stephen Bunker, Shelly Parker, William J. Snape

Background: Pylorospasm (basal pyloric pressures >10mmHg; Mearin et al. Gastro 1986) has been implicated in gastroparesis. BT injection into the pylorus has been suggested to reduce symptoms and improve gastric emptying in patients with gastroparesis. It is unclear whether pyloric pressures correlate with gastric emptying. Aims: 1)Correlate baseline pyloric pressures with gastric scintigraphy(GET) in patients with DM and IG. 2)Determine the effect of BT injection on GET in patients with DM and IG. Patients and Methods: 35 patients [(19 DM, age $41.3+10.3$ years) and ( 16 IG age $48.8+13.1)$ l had pyloric manometry and GET 17 of these patients were treated with BT injection into the pylorus and had follow up GET Delayed gastric emptying was defined as gastric retention $>60 \%$ at 2 hours or $>10 \%$ at 4 hours. Pyloric manometry was done during upper endoscopy by marking the pylorus with a hemoclip. A Dent Sleeve was placed across the pylorus using fluoroscopic guidance. The amplitude of the tonic and phasic contractions were measured using the intragastric pressure as a zero point. $100 \mathrm{U}$ of BT was injected into the pyloric sphincter in patients with elevated pyloric pressures. Results: The 4hr GET was higher for DM $(49.9 \pm 21.0 \%)$ than for IG $(33.9 \pm 24.6 \%), p<0.05$. The mean basal pyloric pressure was similar in patients with DM $(15.4 \pm 13.5 \mathrm{mmHg})$ and $\mathrm{IG}(13.8 \pm 7.7 \mathrm{mmHg})$. Baseline pyloric pressures were elevated above a normal pressure of $>10 \mathrm{mmHg}$ in $11(57.9 \%)$ patients with DM and in $10(62.5 \%)$ patients with IG. Correlation between baseline pyloric pressure and 4 hour GET was assessed using a linear regression analysis. The baseline pyloric pressure correlated poorly with 4 hour GET in both groups (DM: $r=-0.012, p=0.48$ and IG: $r=0.07, p=0.4$ ). Of the patients who received BT, there was no difference in basal pyloric pressures $(D M=20.4 \pm 5.1 \mathrm{mmHg}$ vs. $I G=13.8 \pm 2.6 \mathrm{mmHg}, \mathrm{p}=0.26$ ) Repeat GET was done after a mean follow up period o $31.4 \pm 7.2$ days (range: $2-120 \mathrm{~d}$ ). GET did not change after BT injection in the DM group(4hr $66.8 \pm 8.4 \%$ to $76.8 \pm 11.5 \%, p=0.48$ and $2 \mathrm{hr}: 44.6 \pm 7.6 \%$ to $53.6 \pm 12.2 \%, \mathrm{p}=0.054)$. There was a trend toward improved GET in the IG group but it was not statistically significant $(60.3 \pm 6.1 \%$ to $50.5 \pm 7.8 \%$ at 2 hours, $\mathrm{p}=0.34 ; 27.8 \pm 5.9 \%$ to $14.3 \pm 7.7 \%$ at 4 hours, $\mathrm{p}=0.18$. Conclusions: 1)Pylorospasm is present in approximately $60 \%$ of patients with gastroparesis 2)There is no correlation between basal pyloric pressures and gastric emptying. This suggests that other factors affect gastric emptying, especially in the DM group. It would be important to correlate antral activity, pyloric pressures and GET, especially in those patients who do not respond to BT

\section{0}

The Role of the Dopamine-3 Receptor in the Regulation of Gastric Motility Purna C. Kashyap, Maria-Adelaide Micci, Pankaj Jay Pasricha

Background: Enteric neuronal dopamine (DA) inhibits acetylcholine release and gastric motility; this effect has traditionally been thought to be mediated via neuronal D2 receptors (D2R) and the role of newly discovered dopamine receptors such as D3 and D4 has no been explored. The aim of this study was to investigate the modulation of gastric motility by the D3 receptor (D3R). Methods: Adult rat Sprague-Dawley rats were used in the study. D3R expression was measured in pyloric protein extracts by Western blotting. Pyloric relaxation in response to electrical field stimulation (EFS) was assessed in an organ bath in the presence of varying concentrations of a selective D3R agonist (PD 128907 hydrochloride $0.1,1$ and $10 \mathrm{mM}$ ). Gastric emptying was assessed by the phenol red method 20 minutes 
after feeding a non-nutrient methylcellulose meal to rats treated with varying doses of PD 128907 hydrochloride $(0.2,0.5$ or $1 \mathrm{mg} / \mathrm{kg}$ i.p.) or $\mathrm{DA}(1 \mathrm{mg} / \mathrm{kg})$ with and without a selective D3R antagonist (nafadotride 0.1 or $1 \mathrm{mg} / \mathrm{kg}$ i.p.). Control rats were treated with vehicle only (PBS). Results: Western blot analysis confirmed the presence of D3R in the rat pylorus. The D3R agonist PD 128907 significantly $(\mathrm{P}=0.01)$ reduced EFS-induced relaxation of pyloric strips in an organ bath. This effect was dose dependent, showing a greater decrease in relaxation with increasing doses of D3R agonists ( $1 \mu \mathrm{M}$ vs control, $\mathrm{P}<0.05 ; 10$ $\mu \mathrm{M}$ vs control, $\mathrm{P}=0.001$ ). Gastric emptying was also significantly delayed in rats injected with the D3R agonist in a dose-dependent fashion as compared to vehicle (D3R agonist 0.5 $\mathrm{mg} / \mathrm{kg}$ vs vehicle, $\mathrm{P}<0.05 ; 1 \mathrm{mg} / \mathrm{kg}$ vs vehicle, $\mathrm{P}<0.001$ by one-way ANOVA). The D3R antagonist nafadotride partially reversed the effect of DA on gastric emptying. Conclusions: Our data suggest a novel and important role for D3R in the regulation of gastric emptying. D3R activation delays gastric emptying, an effect that may be due to impairment of pyloric relaxation. D3R antagonists therefore hold the promise for being useful agents for the treatment of gastric motility disorders.

861

Slow and Rapid Liquid Nutrient Load Tests in Assessment of Proximal Stomach Function in Healthy Subjects

Ke Zheng, Meiyun Ke, Zhifeng Wang

BACKGROUND: Liquid nutrient load test (LNLT) is a simple and convenient method to evaluate the function of proximal stomach. However, there is no uniform standard for it, especially in the intake rate. AIMS: To compare the rapid (intake rate $100 \mathrm{ml} / \mathrm{min}$ ) and slow (intake rate $15 \mathrm{ml} / \mathrm{min}$ ) LNLT in order to find the most reasonable LNLT. METHODS: Forty Healthy Subjects (HS M:F=1:1, average age $32.2 \pm 2.1 \mathrm{yr}$, BMI $22.7 \pm 0.49 \mathrm{~kg} / \mathrm{m} 2$ ) received both the rapid and slow intake rate of LNLT on two separate days. The caloric density of the nutrient meal was $1 \mathrm{kcal} / \mathrm{ml}$ (Carbohydrate 48\%, Fat 39\%, Protein 13\%). The TVDRI (threshold volume of drink) and MVDRI (maximal volume of drink) were recorded, and the corresponding proximal stomach volume TVPS (threshold volume of proximal stomach) and MVPS (maximal volume of proximal stomach) were measured by B-ultrasound. RESULTS: (1) The results of two different LNLT were shown in table 1. (2) There were correlations between TVDRI and TVPS $(\mathrm{r}=0.387, \mathrm{p}<0.01 ; \mathrm{r}=0.464, \mathrm{p}<0.01)$, and between MVDR and MVPS $(\mathrm{r}=$ $0.645, \mathrm{p}<0.01 ; \mathrm{r}=0.782, \mathrm{p}<0.01$ ) in both slow and rapid LNLTs. (3) TVDRI and MVPS in slow LNLT was significantly smaller than those in rapid LNLT ( $<<0.01)$, but MVDRI was significantly bigger in slow LNLT than that in rapid LNLT $(\mathrm{p}<0.01)$. There was no difference in TVPS between two different LNLTs (NS). (4) TVDRI was much closer to TVPS in slow LNLT than in rapid LNLT. But MVDRI was much closer to MVPS in rapid LNLT than in slow LNLT. (5) All the subjects completed the study and no severe side effects were presented. CONCLUSIONS: Both slow and rapid LNLTs could be used to evaluate the accommodation to a meal and visceral sensitivity of proximal stomach. The visceral sensitivity is related to the accommodation function. TVDRI of slow LNLT is more sensitive in estimating the visceral sensitivity of proximal stomach; MVDRI of rapid LNLT is more accurate in estimating the accommodation of proximal stomach.

Table 1 . Slow and rapid intake rate of LNLT

\begin{tabular}{|c|c|c|c|c|}
\hline Group & TVDRI $(\mathrm{ml})$ & MVDRI $(\mathrm{ml})$ & TVPS $(\mathrm{ml})$ & MVPS $(\mathrm{ml})$ \\
\hline $15 \mathrm{ml} / \mathrm{min}(\mathrm{N}=40)$ & $118 \pm 11^{*}$ & $1113 \pm 40^{*}$ & $86 \pm 6$ & $373 \pm 19^{*}$ \\
\hline $100 \mathrm{ml} / \min (\mathrm{N}=40)$ & $237 \pm 10$ & $1010 \pm 33$ & $95 \pm 7$ & $466 \pm 27$ \\
\hline
\end{tabular}

VS the rapid LNLT, $*: P<0.01$

862

Gastroduodenal Motility Measured in Health and Disease During Transit of An Ambulatory Capsule- Smartpill

Mihaela Podovei, Marek Majewski, Annabel N. Yuen, Braden Kuo, Irene Sarosiek, John Kuhn, Laura Negron, Jack R. Semler, Connor Semler, Richard W. McCallum

Introduction: Differences in GI luminal pressure patterns between healthy normals $(\mathrm{N})$ and patients with gastroparesis (GP)could give insight into pathophysiology. Pressure measurements in the antrum and duodenum have traditionally been performed with invasive indwelling manometry catheters. SmartPill is a wireless ambulatory capsule that measures luminal $\mathrm{pH}$ and pressure as it courses throughout the GI tract after being swallowed. Passage of the indigestible solid SmartPill from the stomach into the duodenum appears to occur near the end or soon after the gastric emptying of a solid meal or during the fasting state when MMCs occur. Aims: To compare pressure patterns between normal subjects and patients with gastroparesis as defined by a previously abnormal gastric scintigraphy. Methods: In 2 centers, healthy subjects and patients swallowed the SmartPill (SP) after an overnight fast together with a standardized meal of $120 \mathrm{~g}$ Eggbeaters, 2 pieces of bread with jam;255 $\mathrm{kcal}, 2 \%$ fat) and $120 \mathrm{cc}$ water. The rapid $\mathrm{pH}$ change from acidic to alkaline ( $>3$ unit rise from baseline gastric $\mathrm{pH}$ ) marked the emptying of the ingested SmartPill from the stomach into the duodenum. The frequency and amplitude of contractions recorded by the capsule were counted in 30 minute intervals from $60 \mathrm{~min}$ before gastric emptying (GE) to $60 \mathrm{~min}$ after the capsule left the stomach. These parameters for Ns and GPs were then compared for each time interval by two-sample unequal variance T test. Results: $21 \mathrm{Ns}(13 \mathrm{M} / 8 \mathrm{~F}$, mean age 30.8) and $16 \mathrm{GPs}((4 \mathrm{M} / 12 \mathrm{~F}$, mean age 40.9 (9 diabetic/7 idiopathic) ) were studied. Mean contraction frequency and pressure amplitudes in both groups for 30 min intervals are summarized in the table below. Gastroparetics had lower gastric contraction frequencies in the time period -60 to $-30 \mathrm{~min}$ (preceding gastric emptying) compared to normals, $\mathrm{p}<0.02$. There were no differences in contraction amplitudes between the 2 groups. Conclusion: 1. This new ambulatory technology represented by SmartPill detected impaired gastric (antral) motility in GP compared to normals. 2. Decreases in frequency in gastroparetics prior to emptying of an indigestible solid may reflect neurogenic abnormalities in Phase II fasting response. 3. Contraction patterns in health and disease throughout the gut can be measured with the SmartPill Capsule.

\begin{tabular}{|c|c|c|c|c|}
\hline & $30-60 \mathrm{~m}$ before GE & $0-30 \mathrm{~m}$ before GE & $0-30 \mathrm{~m}$ after GE & $30-60 \mathrm{~m}$ after GE \\
\hline N Freq (\#contr/min) & 1.2 & 1.3 & 2.0 & 2.0 \\
\hline GP Freq(\#contr/min) & 0.7 & 1.3 & 1.7 & 1.7 \\
\hline P val & 0.01 & 0.93 & 0.46 & 0.44 \\
\hline N Ampl(mmHg) & 26.7 & 39.1 & 21.5 & 16.6 \\
\hline GP Ampl(mmHg) & 29.0 & 49.6 & 25.4 & 17.7 \\
\hline P val & 0.74 & 0.36 & 0.08 & 0.42 \\
\hline
\end{tabular}

863

Relationship Between Proximal and Distal Gastric Motor Activity in Humans Nam Q. Nguyen, Richard H. Holloway, Laura Bryant, Carly Burdstag, Robert Fraser Normal gastric emptying involves not only intact proximal and distal gastric motility but also a coordination of motor activity between the 2 regions. In dogs, fundic volume waves may play a role in transferring contents to the antrum for subsequent emptying. However, data on this relationship in humans are limited to scintigraphic studies. Aim. To determine the relationship between proximal and distal gastric motor activity during fasting and in response to duodenal nutrient stimulation in healthy humans. Methods. Concurrent proximal (barostat) and distal gastric motility (perfused antro-pyloro-duodenal (APD) manometry) were recorded in 10 healthy volunteers $(5 \mathrm{M}: 5 \mathrm{~F}$; age $=28+2 \mathrm{yrs})$. Studies were performed during fasting and two 60-min duodenal infusions of Ensure ( $1 \mathrm{kcal} / \mathrm{min}$ and $2 \mathrm{kcal} / \mathrm{min}$ ), administered in random order separated by a $2 \mathrm{~h}$ "washout" period. Proximal gastric volume (PGV), fundic wave (FW) activity, and number, length and direction of propagation of APD waves were determined. The association between these variables were also assessed. Results During fasting, approximately $32 \%$ FWs were followed by a distally propagated antral wave (PAW) (Table). Associated PAWs were almost all ante-grade $(96+3 \%)$, propagated at least $6 \mathrm{~cm}$ and traversed the pylorus into the duodenum on $57 \pm 7 \%$ of occasions. Duodenal nutrients increased PGV in a dose-dependent fashion. Total FWs and PAWs significantly reduced during $2 \mathrm{kcal} / \mathrm{min}$ but not $1 \mathrm{kcal} / \mathrm{min}$ infusion. The proportion of $\mathrm{FWs}$ followed by a distally PAWs was similar during $1 \mathrm{kcal} / \mathrm{min}(\mathrm{P}=0.89)$ and $2 \mathrm{kcal} / \mathrm{min}(\mathrm{P}=0.43)$ infusions. Although associated PAW during nutrient infusion remained ante-grade $(96 \pm 2 \%)$ and over $6 \mathrm{~cm}$, they were less likely to traverse the pylorus $(36 \pm 7 \% ; \mathrm{P}=0.04)$. Conclusions. In health, proximal and distal gastric motor activities are coordinated during both fasting and after duodenal nutrients. Changes in FWs during duodenal nutrient feedback may influence the rate of gastric emptying of nutrients. These data suggest a close relationship between proximal and distal gastric motility.

\begin{tabular}{|c|c|c|c|c|}
\hline \multirow{2}{*}{$\begin{array}{c}* \mathrm{P}=0.03, \begin{array}{c}\text { vs. } 1 \mathrm{kcal} / \mathrm{min} . \\
\text { expressed mean } \pm \text { SEM }\end{array} \\
\text { Peak increase in proximal gastric volume }(\mathrm{mL})\end{array}$} & & $92 \pm 56$ & & \multicolumn{2}{|c|}{ 2kcal/min } \\
\cline { 2 - 5 } & Fast & Nutrient & Fast & Nutrient \\
\hline Fundic waves (No./10 min) & $15.3 \pm 2.0$ & $11.0 \pm 1.4$ & $15.3 \pm 1.5$ & $\begin{array}{c}8.7 \pm 1.3 \\
* *\end{array}$ \\
\hline PAW (No./10 min) & $6.7 \pm 1.5$ & $5.3 \pm 1.5$ & $8.0 \pm 2.1$ & $\begin{array}{c}4.3 \pm 1.8 \\
* *\end{array}$ \\
\hline PAWs assoc. with FW (No./10 min) & $5.4 \pm 1.0$ & $4.1 \pm 1.1$ & $4.9 \pm 1.2$ & $\begin{array}{c}2.1 \pm 0.9 \\
* *\end{array}$ \\
\hline \% Fundic wave followed by a PAW & $32 \pm 6 \%$ & $33 \pm 7 \%$ & $32 \pm 6 \%$ & $21 \pm 8 \%$ \\
\hline
\end{tabular}

864

Signaling Pathways in TGF- $\beta$ Induced Tumorigenesis in Elf+/-/Smad4+/Mutant Mice Cell Lines and Human Gastric Tumors Nady Golestaneh, Yi Tang, Varalakshmi Katuri, Bibhuti Mishra, Lopa Mishra

TGF- $\beta$ induces antiproliferative gene responses during cell cycle and escape from this response is a hallmark of tumorigenesis. ELF deficiency in mice results in mislocalization of Smad3 and Smad4 truncating TGF- $\beta$ signaling and transcriptional response. Elf $+/ / / S m a d 4+/-$ mutant mice develop gastric tumors and present an ideal model for further analyses of signaling pathways involved in TGF- $\beta$ induced growth inhibition and/or tumorigenesis. Aims: To study the role of TGF- $\beta$ on MAPK signaling pathways in elf $+/ / / S \operatorname{mad} 4+/-$ gastric cell lines and gastric tumors. Methods and Results: 1. Western Blot analysis of ERK pathway members revealed a 15-fold increased expression of activated ERK (P-ERK) in gastric tumors. In the elf+/-/Smad4+/- cells, the basal level of P-ERK (P-p42) expression was 15 fold lower than the wild type control, whereas P-p42 was completely absent in Smad4+/- and elf+/-/ Smad4+/- cells. These mutant cells were not responsive to TGF-b unlike the wild type cells showing an eight-fold decrease in P-p44 and 12-fold decrease in P-p42 expression in the presence of TGF-b induction. 2. Activated JNK (P-JNK) expression was 10 fold higher in Smad4+/- cells compared to the wild type and this expression was responsive to TGF- $\beta$ induction. 3. Activated p38 (P-p38) expression was approximately 10 fold higher in the polyposis and five different human gastric tumors as well as in elf $+/$-/Samd4+/- mutant cells compared to the wild type control. The P-p38 expression was responsive to TGF- $\beta$ and increased by 10 -fold in elf $+/-/$ Smad4+/- cells. 4 . We have also observed a 10 -fold increase in CDK4 expression in Smad4+/- cell lines and this expression was not responsive to TGF$\beta$ induction. Conclusion: 1 . Our results suggest that TGF- $\beta$ may regulate MAPK signaling pathways either directly through elf/Smad4 interaction or indirectly through other downstream protein interactions. 2. Increased level of P-p38 in gastric tumors as well as in elf + /-/ Smad4+/- mutant cells, and higher level of P-JNK expression in Smad4+/- mutant cell lines indicate that disruption of TGF- $\beta$ signaling pathway may induce tumorigenesis through induction of p38 and/or JNK activation. Further studies are needed to delineate TGF- $\beta$ induced signaling pathways during growth inhibition and tumorigenesis. 
CUGBP2. Likewise, RBM3 and HuR levels were significantly higher, while CUGBP2 was

Gastrin-Stimulated Colorectal Cancer Cell Proliferation Is Mediated in Part Through Phosphorylation and Subsequent Degradation of PPAR $\gamma$ Albert J. Chang, Diane H. Song, M. M. Wolfe

Background: We have previously reported that the trophic properties of gastrin in colorectal cancer (CRC) may be mediated in part through a decrease in peroxisome proliferator activated receptor gamma (PPAR $\gamma$ ) activation. Purpose: To elucidate the precise molecular and cellular mechanisms by which gastrin decreases PPAR $\gamma$ activation. Methods and Results: DLD-1 cells, human CRC cells that express high levels of functional CCK-2 (gastrin) receptors, were incubated in the presence of $200 \mathrm{nM}$ amidated gastrin-17 (G-17) for $60 \mathrm{~min}$, after which Western analysis was performed using a phospho-specific antibody recognizing Ser84 phosphorylated PPAR $\%$. Gastrin promoted PPAR $\gamma$ phosphorylation starting at $5 \mathrm{~min}$ and persisting up to $60 \mathrm{~min}$, which coincided with gastrin-induced activation of ERK1/2. We next examined the possibility that ERKl/2 activation might also play a role in gastrinstimulated PPAR $\gamma$ phosphorylation. DLD-1 cells were pre-incubated with the ERK1/2 inhibitor PD98059 for $45 \mathrm{~min}$ prior to the addition of $200 \mathrm{nM} \mathrm{G-17.} \mathrm{Both} \mathrm{gastrin-induced} \mathrm{ERK1/}$ 2 activation and PPARy phosphorylation at Ser84 were abolished by co-incubation with PD98059, suggesting that gastrin enhanced PPAR $\gamma$ phosphorylation at Ser84 is mediated, in part, through ERKl/2 activation. We then examined the possibility that gastrin might attenuate ligand-dependent PPAR $\gamma$ activation by enhancing PPAR $\gamma$ phosphorylation at Ser84. A phosphorylation mutant of PPAR $\gamma$ was generated by altering the Ser84 residue into an alanine residue, and stable DLD- 1 cell lines expressing wild type (wt) and mutant PPAR $\gamma$ were created. While co-incubation with G-17 markedly attenuated PPAR $\gamma$ activation stimulated by the PPAR $\gamma$ agonist rosiglitazone (ROS) in cells overexpressing wtPPAR $\gamma$, gastrin had no effect on PPAR $\gamma$ activation in cells overexpressing mutant PPAR $\gamma$. The mutation of PPAR $\gamma$ at Ser84 also abrogated gastrin-induced degradation of PPAR $\gamma$ in DLD-1 cells. Finally, in the presence of $200 \mathrm{nM}$ G-17, ROS-induced growth suppression was diminished by $64 \%$ ( $\mathrm{p}<0.001$ vs. $5 \mu \mathrm{M}$ ROS alone) in wTPAR $\gamma$ overexpressing cells, whereas in the presence of gastrin, PPAR $\gamma$ growth suppression was minimally affected in cells overexpressing mutant PPAR $\gamma$. Conclusion: The results of these studies demonstrate that the trophic properties of gastrin may be mediated in part by phosphorylation of ERK1/2, which, in turn, phosphorylates PPAR $\gamma$ at the Ser84 residue. The phosphorylation of PPAR $\gamma$ at Ser84 decreases PPAR $\gamma$ transactivational activity and appears to signal PPARy for proteasomal degradation. As a result, PPAR $\gamma$ protein levels are diminished, which attenuates the tumor suppressive effects of PPAR $\gamma$, resulting in enhanced cellular proliferation.

866

\section{Cross Talk Between TGF- $\beta$-IL-6 Pathways Is Important for Suppression of} Hepatocellular Cancer

Varalakshmi Katuri, Yi Tang, Wilma Jogunoori, Anton Sidawy, Bibhuti Mishra, Lopa Mishra

Inter- $\alpha$-trypsin inhibitor-4 (Itih-4) is a liver-restricted member of the serine protease inhibitor family with diverse functions as an anti-apoptotic and matrix stabilizing molecule that are important throughout development and cancer. We first isolated Itih-4 together with ELF, a TGF- $\beta$ adaptor from fore-gut endodermal stem cells. Itih-4 is expressed in early hepatoblasts, is regulated by IL-6 and levels rise eightfold in GADD45 (a P53 target) mutants that develop multiple cancers. The adaptor protein ELF, a beta-spectrin, demonstrated a pivotal role in carcinogenesis through regulation of the TGF-beta pathway. Interestingly, elf $\mathrm{l}^{+-}$mutant mice had increased incidence of hepatocellular cancers (HCC) in elf l- $^{+-}$mutant mice. Aim: To determine the role of TGF- $\beta / \mathrm{ELF}$ as well as IL-6/Itih4 in regulating HCC. Methods and Results: 1. We generated Itih4 deficient mice by targeting exon 2 and part of exon 3 (1.8kb), which is replaced by Neo gene. 2. Genetic studies showed Itih4 deficient mice appear to be normal without any tumor development over the past four years. 3. Elf ${ }^{+/}$mice, known to develop HCC, were crossbred with Itih $4^{-/}$mice. The elf ${ }^{+/}$and elf ${ }^{+/} /$Itih $4^{-/}$offspring were autopsied to determine the development of any abnormalities. Interestingly, hepatocellular cancer incidence is dramatically decreased in elf $+/ /$ Itih $4^{-/}$mice. Only one out of twenty elf $+/ /$ Itih $4^{-/-}$mice developed a small liver tumor at two years compared with nearly two thirds of elf ${ }^{+/}$developing tumors at this age spontaneously. Conclusions: 1 . Loss of IL-6/Itih4 plays critical role in suppression of HCC induced by inactivation of TGF- $\beta$ /ELF pathway. 2 . Our results suggest that TGF- $\beta$-IL- 6 cross talk is important for suppression of HCC, and this model may be important in the development of future therapeutics for hepatocellular cancers.

\section{7}

Posttranscriptional Regulation of COX-2 Gene Expression in Intestinal Tumorigenesis: Role of Novel Protooncogene RBM3

Sripathi M. Sureban, Dharmalingam Subramaniam, Katherine B. Lee, Satish Ramalingam, Aubrey R. Morrison, Courtney W. Houchen, Brian K. Dieckgraefe, Shrikant Anan

RBM3 is a ubiquitously expressed glycine-rich protein that can bind to both RNA and DNA via an amino-terminal RNA binding domain. RBM3 was observed to bind 605 ribosomal subunits, and enhance global protein synthesis. Furthermore, RBM3 was identified as a member of a complex of proteins along with RNA stabilizing protein $\mathrm{HuR}$, that bound to AU-rich sequences (ARE) located in the 3'untranslated region (3'UTR) of cyclooxygenase2 (COX-2) mRNA. However, the functional significance of RBM3 in the complex is not known. We have now identified RBM3 in a yeast two-hybrid screening using HuR as bait. We confirmed RBM3 interactions with HuR and CUGBP2, another RNA stabilizing but translational inhibitor protein using ectopically expressed tagged proteins in HeLa cells. Immunocytochemistry demonstrated that RBM3 colocalized with both HuR and CUGBP2 in the nucleus of transfected cells. Furthermore, bacterially expressed recombinant GSTRBM3 bound to in vitro translated ${ }^{35}$ S-labeled HuR and CUGBP2. Since COX-2 levels are elevated in cancer, we next determined the expression patterns of RBM3, HuR and CUGBP2 in human and murine tumors. Real time PCR analyses of total RNA from human colon cancer tissues demonstrated increased expression of RBM3 and HuR, while decreased expression of lower in intestinal adenomas of $\mathrm{APC}^{\mathrm{min} /+}$ mice compared to the surrounding uninvolved tissues. Finally, HuR and RBM3 expression was significantly induced in HCT116 human colon cancer cell nude mice xenografts. In HCT116 cells in culture, ectopic overexpression of RBM3, HuR and CUGBP2 by transient transfections results in increased COX-2 mRNA expression by at least 2 -fold when transfected alone. In addition, coexpression of RBM3 and HuR further induced COX-2 mRNA levels to greater than 4-fold. In order to determine the mechanism by which RBM3 increased COX-2 mRNA levels, we determined the effect of RBM3 on COX-2 mRNA stability using actinomycin D. The half-life of COX-2 mRNA significantly increased from $1 \mathrm{~h}$ to greater than $4 \mathrm{~h}$ in the presence of RBM3, which was further increased to $8 \mathrm{~h}$ when cotransfected with HuR. These data support a novel protooncogene function for RBM3 whose overexpression results in increased stability of COX-2 mRNA in tumorigenesis.

868

Rack1 Induces Apoptosis of Human Colon Cells By Suppressing SRC Tyrosine Kinase Activity in the Intrinsic and Akt Pathways

Vidya Mamidipudi, Chris Cartwright

Previously we showed that Src tyrosine kinases are activated early in the development of human colon cancer, and are suppressed during intestinal cell differentiation. We identified RACKl as an endogenous substrate, binding partner and inhibitor of Src. We demonstrated that RACKl regulates colonic cell growth in part by suppressing Src activity at the G1 checkpoint. Here we show that overexpression of RACKl induces apoptosis of HT-29 colon carcinoma cells, in part via its inhibitory influence on Src. Depletion of Src has a similar effect. We defined mechanisms whereby RACKl functions in apoptosis. RACKl induces cleavage of caspases 10,9,6 and 3, and the death substrate PARP. In the intrinsic pathway, RACKl overexpression decreases expression of anti-apoptotic Bcl-2, increases expression o pro-apoptotic Bim, and induces translocation of Bim and another pro-apoptotic protein, Bax, from the cytosol to mitochondria. Moreover, RACKl overexpression or Src depletion inhibits Src-mediated cytochrome c oxidase activity, depolarizes the inner mitochondrial membrane and releases cytochrome c. We show that RACKl also induces apoptosis by blocking the Akt cell survival pathway in a Src-dependent manner, and consequently activating caspase 9, the pro-apoptotic factor Bad and the transcription factor FOX03 (which is a potent inducer of apoptosis and Gl arrest). Collectively, our results suggest that RACKl is a tumor suppressor because it inhibits growth and induces death of colon cells. Exploitation of these dual functions could be used to develop new and more powerful and selective strategies for treatment of human colon cancer.

869

Direct Interaction Between RNA Binding Proteins Hur and CUGBP2: Functional Antagonism in Intestinal Epithelial Cells Sripathi M. Sureban, Jacquelyn M. Means, Pavel Rodriguez, Nabendu Murmu, Courtney Houchen, Brian K. Dieckgraefe, Shrikant Anant

Cyclooxygenase- 2 (COX-2), a critical enzyme in prostaglandin synthesis is overexpressed at an early stage in the pathogenesis of colorectal cancer. In addition to transcriptional controls, COX-2 expression is tightly regulated at the post-transcriptional level of mRNA stability and translation by AU-rich sequence elements (ARE) located in the COX-2 3'untranslated region (3'UTR). RNA binding protein HuR binds to the COX-2 ARE and increases mRNA stability and translation, suggesting that HuR plays a role in carcinogenesis in part through induction of COX-2 expression. On the other hand, RNA binding protein CUGBP2 binds to the same COX-2 ARE and also stabilizes the RNA but inhibits its translation, thereby leading to cell apoptosis. HuR and CUGBP2 have high structural similarity consisting of two N-terminal RNA recognition motifs (RRMl and RRM2), a hinge region, and a Cterminal RRM (RRM3). The hinge region in CUGBP2 is longer than that observed in HuR and is rich in alanine and glutamine residues. Nitrocellulose filter binding assays showed that HuR and CUGBP2 bind to the ARE sequences with similar affinities (KD of $27 \pm 4 \mathrm{nM}$ and $34 \pm 4 \mathrm{nM}$ for HuR and CUGBP2, respectively). In in vitro translation assays, HuR increased, while CUGBP2 inhibited COX-2 mRNA translation in a dose dependent manner Furthermore, when both proteins were included in the reaction, COX-2 mRNA translation was inhibited suggesting that CUGBP2 overrides HuR in the translation process. In a yeas two-hybrid screening, with CUGBP2 as bait, HuR was identified as an interacting partner in a CDNA library from human liver cells. This interaction was also observed when the two proteins, expressed in a rabbit reticulocyte lysate cell-free translation system formed heterodimers in vitro. Immunofluorescence studies also showed that HuR and CUGBP2 colocalized in the nucleus of transfected cells. In addition, heterokaryon shuttling assays with HeLa human cells transfected with either FLAG-HuR or FLAG-CUGBP2 and NIH3T3 mouse cells revealed that both proteins shuttled from HeLa to NIH3T3 cell nuclei with similar frequencies. Moreover, immunoprecipitation coupled with RT-PCR demonstrated that both proteins together were associated with COX-2 mRNA, when the two proteins were coexpressed in HeLa cells. These data, taken together suggest a functional antagonism exists between RNA binding proteins HuR and CUGBP2, wherein CUGBP2 interacts with HuR and COX-2 3'UTR to suppress HuR-mediated COX-2 mRNA translation.

870

Inhibition of C-Met As a Therapeutic Strategy for Esophageal Adenocarcinoma Gregory A. Watson, Xinglu Zhang, Michael T. Stang, Steven J. Hughes

Introduction: We have previously demonstrated universal over-expression of the hepatocyte growth factor (HGF) receptor c-Met in esophageal adenocarcinoma (EA), yet the therapeutic potential of c-Met inhibition in EA remains unknown. We hypothesized that inhibition of c-Met signaling in EA would reduce cell proliferation, inhibit motility and invasion, and induce apoptosis. Methods: Three EA cell lines (Seg-1, Bic-1, and Flo-1) previously shown to over-express c-Met were grown in serum-starved media in the presence or absence of 
HGF and the small-molecule, c-Met-specific inhibitor PHA665752. Inhibition of c-Met and known downstream mediators of c-Met signaling (ERK1/ERK2 and PI3K/Akt) were analyzed by immunoblotting. Cell viability over time was assessed by MTT assay and apoptosis was analyzed by FACScan analysis following Annexin V/PI labeling. Motility was evaluated by cell wounding assay and invasion was quantified using the QCM cell invasion assay. Results: c-Met was constitutively phosphorylated in 2 EA cell lines (Bic-1 and Seg-1) known to express HGF, and HGF induced further phosphorylation of c-Met in all 3 EA cell lines. PHA665752 decreased constitutive and HGF-induced phosphorylation of c-Met in a dosedependent fashion, resulting in inhibition of ERK1/ERK2 activity and a 50\% reduction in cell viability $(\mathrm{p}<0.01)$ in all $3 \mathrm{EA}$ cell lines. HGF induced PI3K/Akt activity and stimulated motility and invasion only in Flo-l cells. PHA665752 abolished PI3K/Akt activity, inhibited motility and invasion, and induced apoptosis in this same cell line; inhibition of PI3K activity with LY294002 reproduced the effects of c-Met inhibition. Conclusions: Inhibition of c-Met reduced the proliferation of all $3 \mathrm{EA}$ cell lines but inhibited motility and invasion and induced apoptosis only in a subset of EA cells. Our data suggests that the clinical response of EA to c-Met inhibition may be dependent upon factors other than over-expression of cMet, such as involvement of the PI3K/Akt pathway in c-Met signal transduction.

\section{1}

Reversal of Early Stages of Colorectal Cancer with EGF-Receptor Related Protein (ERRP)

Eva M. Schmelz, Hu Xu, Jianhua Du, Arun K. Rishi, Fazlul H. Sarkar, Adhip P. N. Majumdar

Inactivation of EGFR family members (EGFRs) represents a promising strategy for the development of novel and selective anticancer therapies. Current anti-EGFR therapies target EGFR or HER-2 but not both. Since most solid tumors express several members of EGFRs, identification of inhibitor(s), targeting multiple members of the EGFR family may provide a therapeutic benefit to a broader patient population. EGF-Receptor Related Protein (ERRP), a 53-55 kDa secretory protein, which we have isolated and characterized as a pan-erbB inhibitor that targets multiple members of EGFRs, is a potential therapeutic agent for several epithelial cancers, including colorectal cancer. We have repeatedly observed that ERRP is highly effective in inhibiting growth of SCID mice xenografts of colon cancer cells. However, it remains to be determined whether ERRP can inhibit/regress growth of chemically-induced colonic neoplasia/tumors in immunocompetent mice. As a first step in achieving this goal, we studied the effect of recombinant ERRP on inhibition/regression of aberrant crypt foci (ACF) formed in the colon of mice after dimethylhydrazine (DMH) treatment. ACF are considered to be the precursor of colorectal adenomas and subsequently carcinoma. CFl mice were injected (i.p.) once a wk for 6 wks with DMH ( $30 \mathrm{mg} / \mathrm{kg}$ bodyweight) to induce ACF formation, subsequently divided into two groups and injected (i.p.) once a day for 10 days with recombinant ERRP $(50 \mu \mathrm{g} /$ mouse $)$ or vehicle. Administration of ERRP significantly decreased $(40-50 \%)$ the number of ACF per animal $(\mathrm{P}<0.05)$. The number of ACF per focus was also reduced by $30-40 \%$ after ERRP treatment, compared to the controls $(\mathrm{P}<0.01)$. This was associated with a marked inhibition (50-60\%) of proliferation (PCNA immunoreactivity) and stimulation (60-70\%) of apoptosis (TUNEL assay) in the colonic mucosa. These changes were also accompanied by marked reduction in activation (tyrosine phopshorylation) of EGFR, HER-2 and HER-3 in the colonic mucosa leading to attenuation of NF-kappa-B activity and a marked reduction in COX-2 expression. In contrast, the levels of alkaline phosphatase, a marker of intestinal differentiation, was greatly elevated in the colonic mucosa following ERRP treatment. Taken together, the results suggest that ERRP is effective in regressing the number and the size of existing $\mathrm{ACF}$, which are precursors of colorectal adenomas and colorectal carcinoma. This could partly be attributed to inhibition of EGFRs signaling and induction of differentiation of the colonic mucosa.

\section{2}

Specific Targeting of Tumor Endothelial Cells By a Shiga-Like Toxin-VEGF Fusion Protein As a Novel Treatment Strategy for Pancreatic Cancer Hubert G. Hotz, Joseph M. Backer, Marina V. Backer, Birgit Hotz, Sarah Bhargava, Heinz J. Buhr

Background: Inhibition of angiogenesis is a novel treatment strategy for pancreatic cancer, which is almost resistant against radiochemotherapy. Activated endothelial cells of the tumor vasculature abundantly express receptors for vascular endothelial growth factor (VEGF), a key mediator of tumor neoangiogenesis. The aim of the present study was to specifically target and damage the vasculature of pancreatic cancer by fusing the VEGF isoform VEGF121 to the A-subunit of Shiga-like Toxin I (SLT-VEGF), which inhibits the protein synthesis of target cells. Methods: SLT-VEGF fusion protein containing fragments of the SLT-1 A-subunit fused to human VEGF12 l was expressed in E coli strain Origami(DE3)pLysS and purified to homogeneity. In vivo: 5 million cells of 2 human pancreatic cancer cell lines (AsPC-1/ poorly differentiated, HPAF-2 / moderately differentiated) were injected subcutaneously into nude mice. $1 \mathrm{cmm}$ fragments of the resulting subcutaneous tumors were implanted into the pancreas of 72 other mice. Animals were randomized into control and treatment groups: application of the SLT-VEGF fusion protein $(200 \mu \mathrm{g} / \mathrm{kg}$, every other day ip.) began either 3 days (prophylaxis) or 6 weeks (therapy) after tumor induction. Treatment was continued for 14 weeks or until death of the mice. Volume of the primary tumor (TU-Vol), local infiltration and metastatic spread (dissemination score: D-Score) were determined at autopsy. Microvessel density (MVD) was analyzed in CD31-stained tumor sections. Results: All control animals developed extensive primary tumor growth, local infiltration, and distant metastasis. The effects of SLT-VEGF therapy after delayed onset (therapy) and early begin (prophylaxis) are summarized in the table. Conclusions: SLT-VEGF fusion protein reduces tumor growth and metastatic spread of experimental pancreatic cancer, resulting in increased 14-week survival of the animals. Reduced microvessel density indicates that this effect is mainly due to the toxic effect of SLT-VEGF on endothelial cells of the tumor vasculature. SLT-VEGF therapy was not associated with systemic side effects such as weight loss.

\begin{tabular}{|c|c|c|c|c|c|}
\hline Tumor & Group & TU-Vol $(\mathrm{cmm})$ & D-Score (points) & MVD $(/ 0.74 \mathrm{smm})$ & Survival (n/n) \\
\hline HPAF-2 & Control & $3920 \pm 184$ & $17.0 \pm 1.2$ & $80.5 \pm 4.3$ & $4 / 12$ \\
\hline HPAF-2 & Therapy & $1587 \pm 167^{*}$ & $15.9 \pm 2.7$ & $49.3 \pm 3.5^{*}$ & $9 / 12^{*}$ \\
\hline HPAF-2 & Prophylaxis & $1069 \pm 267^{*}$ & $10.4 \pm 2.2^{*}$ & $45.9 \pm 6.6^{*}$ & $10 / 12^{*}$ \\
\hline AsPC-1 & Control & $1586 \pm 111$ & $19.2 \pm 1.2$ & $69.8 \pm 3.3$ & $2 / 12$ \\
\hline AsPC-1 & Therapy & $492 \pm 72^{*}$ & $18.6 \pm 2.0$ & $48.9 \pm 6.1^{*}$ & $3 / 12$ \\
\hline AsPC-1 & Prophylaxis & $487 \pm 56^{*}$ & $13.2 \pm 2.4^{*}$ & $40.8 \pm 4.3^{*}$ & $9 / 12^{*} \#$ \\
\hline
\end{tabular}

p<0.05: *vs. control, \#vs. therapy

873

Evaluation of Dendritic Cell-Based Vaccination Used in Combination with Gemcitabine in a Murine Model of Pancreatic Carcinoma

Marc Dauer, Christian Bauer, Franz Bauernfeind, Max Schnurr, Stefan Endres, Andreas Eigler

Background: Tumorantigen-specific cytotoxic T lymphocytes (CTL) can be induced in vivo by vaccination with dendritic cells (DC). However, relevant clinical responses are rarely seen in patients with solid cancer after DC-based vaccination. Chemotherapy can sensitize tumor cells to CTL responses and may thus improve the clinical efficacy of DC-based vaccination (Dauer et al., J of Immunotherapy 2005). Here, we evaluated the combined use of a DC-based vaccine and chemotherapy with gemcitabine in a murine model of pancreatic carcinoma. Material and Methods: DC were derived from bone marrow of C57BL/6 mice by in vitro culture with GM-CSF and IL-4. On day 6 of culture, DC were loaded with syngeneic, apoptotic Panc-02 pancreatic carcinoma cells and stimulated with LPS and IFN$\gamma$. For tumor induction, 1 x 10E6 Panc-02 cells were injected s.c. into the flanks of C57BL 6 mice. Therapeutic vaccination was started when tumor size reached 8 to $17 \mathrm{~mm} 2$. For vaccination, $0.3 \times 10 \mathrm{E} 6$ antigen-loaded $\mathrm{DC}$ were administered s.c. into the contralateral flank in weekly intervals. Gemcitabine $(50 \mathrm{mg} / \mathrm{kg}$ ) was given twice weekly. For prophylactic immunization, mice were vaccinated three times prior to tumor challenge. Results: Prophylactic immunization completely prevented tumor development. At day 35 after challenge, tumor size was $79 \pm 10 \mathrm{~mm} 2$ in the control group vs. $0 \mathrm{~mm} 2$ in the DC vaccination group. In the therapeutic setting, combination of DC-based vaccination and chemotherapy improved outcome compared to immunotherapy or chemotherapy alone. Survival at day 58 after tumor challenge was $0 \%$ in the control group, $13 \%$ in the DC vaccination group, $17 \%$ in the gemcitabine group and $50 \%$ in the DC plus gemcitabine group. Discussion: DCbased vaccination showed therapeutic efficacy in a murine model of pancreatic carcinoma. The efficacy of the vaccination could be enhanced by combination with gemcitabine. At present, we analyze whether the combined treatment affects tumor environment and the induced CTL response by histopathological analysis and in vitro-analysis of CTL activity.

874

15d-Pgi 2 Down-Regulates Microsomal Prostaglandin $\mathrm{E}_{2}$ Synthase Type 2 in Colon Cancer Cells

Oliver Schroeder, Yulyana Yudina, Nadine Zahn, Alan Sabirsh, Jesper Haeggstroem, Juergen Stein

Introduction: Clinical, genetic, and biochemical evidence indicates that Prostaglandin (PG) $\mathrm{E}_{2}$ plays a central role in colorectal tumorigenesis. The terminal step in the biosynthesis of $\mathrm{PGE}_{2}$ from arachidonic acid is accomplished by several PGE 2 synthases (PGES). Overexpresion of microsomal PGES type 1 and type 2 (mPGES-1 and mPGES-2, respectively) has been observed in colorectal cancer, whereas the expression of cytosolic PGES remains unchanged in this tumor entity. The cyclopentenone prostaglandin (CyPG) $15 \mathrm{~d}-\mathrm{PG} \mathrm{J}_{2}$ acts both, as a key regulator of negative feedback of the COX pathway in an inflammatory setting and as a potent anti-tumoral agent. We therefore aimed to further determine the potential implication of this $\mathrm{CyPG}$ in $\mathrm{PGE}_{2}$ mediated colorectal cancer promotion. Material and Methods: The human colon cancer cell lines Caco-2 and HCT 116 were challenged with $15 \mathrm{~d}-\mathrm{PGJ}_{2}(0.1-10 \mu \mathrm{M})$ and expression of PGES on mRNA and protein level was analysed by semi-quantitative RT-PCR with the fluorescent dye Pico-Green or immunoblot, respectively. PGES synthase activity after treatment of cells with $15 \mathrm{~d}-\mathrm{PGJ}_{2}$ was determined by RPHPLC. In additional experiments, Caco-2 cells, transfected with a mutant receptor to inhibit wild type PPAR $\gamma$ action, were also treated with $15 \mathrm{~d}-\mathrm{PGJ}_{2}$. Finally, HCT 116 cells were also treated with arsenite $(1-50 \mu \mathrm{M})$ alone and in combination with the antioxidants DTT (2 $\mathrm{mM})$ and $\mathrm{N}$-acetylcysteine $(30 \mathrm{mM})$ and subjected to immunobloting for evaluation of mPGES-2 protein expression. Results: Whereas mRNA expression of CPGES, mPGES-1, COX-1 (expressed in HCT 116) and COX-2 (expressed in Caco-2) remained unchanged following treatment with $15 \mathrm{~d}-\mathrm{PG} \mathrm{J}_{2}, \mathrm{mPGES}-2$ was transiently down-regulated time- and dose-dependently on the mRNA and protein level in both cell lines. Reduced mPGES-2 expression was followed by diminished PGES activity in the microsomal fraction. Comparable effects on the expression of mPGES-2 were observed in PPAR $\gamma$ dominant-negative Caco-2 cells, excluding an involvement of this transcription factor in $15 \mathrm{~d}-\mathrm{PGJ}_{2}$ induced suppression of mPGES-2. Chemically induced oxidative stress by arsenite resulted in a similar downregulation of mPGES-2 protein, which could be completely abolished by the antioxidants DTT and N-acetylcysteine. Conclusion: Our data clearly indicate, that $15 \mathrm{~d}-\mathrm{PGJ}_{2}$ downregulates mPGES-2 expression in Caco-2 and HCT 116 cells without affecting the expression of mPGES-1 or cPGES. The action of $15 \mathrm{~d}-\mathrm{PG} \mathrm{J}_{2}$ occurs to be related to a redox-dependent mechanism. The results obtained may preclude a novel mechanism for the anti-proliferative effects of CyPG and point to mPGES-2 as a promising drug target in colorectal cancer. 
The Oral Multi-Target Tumor Growth Inhibitor ${ }^{\mathrm{Tm}}$, Zk 304709, Inhibits Growth of Pancreatic Neuroendocrine Tumors in An Orthotopic Mouse Model Arne Scholz, Karola Wagner, Martina Welzel, Bertram Wiedenmann, Stefan Rosewicz, Gerhard Siemeister, Katharina M. Detjen

Background: Neuroendocrine tumors of the gastroenteropancreatic system (GEP-NETs) represent a molecularly heterogeneous group of tumors that share a well vascularized phenotype. While biotherapies such as somatostatin analogues and interferon-alpha are well suited to control the hypersecretion syndrome associated with functional NETs, these therapeutic modalities do not provide sufficient control of tumor growth. ZK-304709 is a first in class, oral Multi-target Tumor Growth Inhibitor ${ }^{\mathrm{TM}}$ (MTGI) that induces tumor cell death via inhibition of cell cycle progression and tumor-induced angiogenesis. ZK-304709 is a nanomolar inhibitor of CDKs 1, 2, 4 and 7,VEGF-RTKsl-3 and PDGF-RTK $\beta$, undergoing phase I clinical trials. The multi-targeted profile recommends ZK-304709 as an attractive treatment option in GEP-NETs. Methods: BON and QGP-1 human NET-cell lines were used for in vitro studies. Direct effects of ZK-304709 on NET cell proliferation, survival and cell cycle distribution were determined based on cell numbers, flow cytometry and immunoblotting. For induction of orthotopic NETs in vivo, BON cells were injected into the pancreas of immunodeficient mice. Two weeks after injection, animals were randomly assigned to controlor treatment groups. Animals were sacrificed after nine weeks and primary tumor growth, vascularization and metastatic spread were determined. Apoptosis fraction and micro vessel density (MVD) were examined in situ using TUNEL and immunohistochemical detection of CD34, respectively. Results: ZK 304709 dose dependently inhibited anchorage dependent and anchorage independent growth of NET cell lines. Cell cycle analyses revealed a blockade of cell cycle progression that was consistent with CDK inhibition. In addition, a prominent pre-Gl fraction suggested apoptosis induction, which was confirmed based on detection of the apoptosis specific $85 \mathrm{kDa}$ PARP cleavage product. Oral treatment of mice bearing orthotopic BON human pancreatic NETs with ZK-304709 achieved approx. 80\% reduction of primary tumor growth and reduced the incidence of liver metastases. Tumor samples from ZK-304709 treated animals revealed both an increase in the apoptosis fraction as well as a reduction of MVD when compared to control animals, suggesting that proapototic and antiangiogenic mechanisms contributed to tumor growth inhibition. Conclusion: The multitarget mechanism of action of ZK-304709 attacking tumor cells via inhibition of cell cycle progression and attacking tumor neovascularization via inhibition of VEGF- and PDGFRTKs results in highly efficacious inhibition of growth and metastasis formation of human pancreatic NETs.

876

Life Expectancy, Complications and Causes of Death in Patients with Achalasia: Results of a 33-Year Prospective Follow-Up Investigation Volker F. Eckardt, Tom Hoischen, Gudrun Bernhard

Background and aims: Patients with achalasia often require repeated invasive therapies and may experience multiple complications related to the natural course of this disease and/or its therapy. This study determines the incidence of such complications and causes of death. In addition, survival rates were determined and compared with those obtained in an average population. Material and methods: From 1972 to 2002, 178 consecutive patients (mean age: $47.0 \pm 18.9$ [mean $\pm 1 S D$ ] years, 105 males and 73 females) with newly diagnosed achalasia were admitted to this prospective investigation and followed until 2005 in two-year intervals. The diagnosis of primary achalasia was based on manometric, radiographic and endoscopic findings. Patients regularly underwent structured interviews with regard to their symptoms using a previously described symptom score (Gastroenterology 1992;103:1732) and were reinvestigated if significant changes in health status occurred. Survival rates were determined by Kaplan Meier estimates. We also generated survival data for an average but age and sex matched German population (Bundesamt für Statistik) and compared these findings with those obtained in patients with achalasia. Causes of death were determined from hospital records, informations supplied by private physicians and from death certificates. Results: Complete follow-up was obtained in $98.9 \%$ of all patients. The mean observation period was $10.5 \pm 6.5$ years with a range from 2 to 33 years (in surviving patients). Within this time period, 175 patients underwent a mean of $2.2 \pm 1.8$ invasive procedures (range 1 to 13 ). The mean symptom score was $6.0 \pm 2.2$ at first presentation and $1.5 \pm 1.5$ at last follow-up. Diseaseand therapy-related complications occurred in $18.5 \%$ of all patients, the most common of which were megaesophagus (7.3\%) followed by reflux esophagitis (3.3\%), peptic stenosis $(2.2 \%)$ and large esophageal diverticula (2.2\%). A total of 24 patients $(13.5 \%)$ had died. Causes of death were comparable to those of the control population with the exception of a higher incidence of pulmonary causes in patients with achalasia (12.5 vs. $2.6 \%$ ). The estimated 10 and 20 year survival rates were similar in patients with achalasia (88\% [95\% CI:82-94\%] and 76\% [95\% CI:66-85\%]) and in sex and age matched controls (92\% [95\% CI: $87-97 \%$ ] and $78 \%$ [95\% Cl:67-90\%]). Conclusions: During the course of many years, most patients with achalasia require repeated therapies and experience a significant number of complications. However, causes of death and life expectancy do not differ significantly from those of the average population.

877

Sensory and Biomechanical Properties of the Esophagus After Nissen Fundoplication

Jose M. Remes-Troche, Ranjit Mudipalli, James Maher, Satish Sc Rao

Introduction/Aim: Previously, we have shown that prolonged acid reflux can induce significant sensory-motor changes in the esophageal wall in patients with GERD (Am J Gastroenterol 2001: 96(1):S24). Whether these changes are reversible with definitive therapy for GERD is not known. Our aim was to prospectively assess sensory and biomechanical properties of the esophagus before and after Nissen Fundoplication (NF). Methods: Graded balloon distensions were performed using impedance planimetry to assess sensory and biomechanical properties of the esophageal wall in 6 patients with refractory symptoms and with endoscopic
+ 24-hr pH study evidence of GERD ( $\mathrm{M} / \mathrm{F}=3 / 3$, mean age $=43$ years $)$, and in 12 matched healthy volunteers $(\mathrm{M} / \mathrm{F}=6 / 6$, mean age $=43$ years $)$. GERD patients underwent a baseline evaluation and a repeat evaluation, at least 12 months after surgery (range 13-44 months) Sensory thresholds were evaluated using a 4 point Likert scale. Data were analyzed with ANOVA and paired t test. Results: Before NF, the cross sectional area (CSA) of the esophageal wall was higher $\left(p<0.05^{*}\right.$, Table) and the circumferential wall tension/strain relationship was decreased in patients with GERD compared to healthy controls. After NF, when compared to baseline, CSA and esophageal wall reactivity decreased $(\mathrm{p}<0.05)$ and the wall tension/ strain relationship increased. The biomechanical parameters after NF were similar to healthy controls. Before NF, sensory thresholds were lower in GERD patients compared to controls first perception (mean $\pm \mathrm{SE}, \mathrm{cm} \mathrm{H}_{2} 0 ; 13 \pm 2$ vs $30 \pm 3$, p $<0.01$ ), discomfort ( $34 \pm 4$ vs $54 \pm 4$ $\mathrm{p}<0.01)$ and pain $(49 \pm 7$ vs $62 \pm 2, \mathrm{p}<0.01)$. After NF, there were no changes in these threshold (first perception $=13 \pm 3$, discomfort $=37 \pm 7$, and pain $=53 \pm 6, p=N S$ ) Conclusions: The esophagus has a larger lumen and the esophageal wall is stiffer and less deformable and is hypersensitive in patients with GERD. After NF, the biomechanical abnormalities improved but sensory thresholds were unchanged. Thus, antireflux surgery appears to correct the esophageal biomechanical dysfunction(s) in patients with GERD, but not the underlying hypersensitivity. This finding may explain persistent symptoms in some patients, after Nissen fundoplication

Changes in biomechanical properties in GERD patients and controls

\begin{tabular}{|c|c|c|c|c|c|c|}
\hline Balloon pressure $\rightarrow$ & \multicolumn{3}{|c|}{$10 \mathrm{~cm} \mathrm{H}_{\mathbf{2}} \mathbf{0}$} & \multicolumn{3}{c|}{$40 \mathrm{~cm} \mathrm{H}_{\mathbf{2}} \mathbf{0}$} \\
\hline & Before & After & Control & Before & After & Control \\
\hline $\mathrm{CSA}\left(\mathrm{mm}^{2}\right)$ & $310 \pm 56^{*}$ & $191 \pm 27$ & $119 \pm 30$ & $734 \pm 99^{*}$ & $551 \pm 70$ & $469 \pm 18$ \\
\hline Reactivity $\left(\mathrm{mm}^{2}\right)$ & $84 \pm 16^{*}$ & $56 \pm 10$ & $43 \pm 4$ & $232 \pm 63^{*}$ & $123 \pm 84$ & $199 \pm 28$ \\
\hline Strain & $.35 \pm .1^{*}$ & $.41 \pm .1$ & $.40 \pm .01$ & $1.04 \pm .1^{*}$ & $1.36 \pm .2$ & $1.34 \pm .1$ \\
\hline
\end{tabular}

mean \pm SD $* \mathrm{p} \leq 0.05$ vs after and controls

\section{8}

TRPV1 and P2x3 Expression in Vagal and Spinal Pathways Following AcidInduced Esophagitis in Rats Banani Banerjee, Bidyut K. Medda, Reza Shaker, Jyoti N. Sengupta

Background: Esophageal hypersensitivity is one of the common findings in gastro-esophageal reflux disease (GERD). Transient receptor potential vanilloid 1 (TRPVI)and P2X3 purine receptors contribute significantly in hypersensitivity. The aim of this study was to evaluate the altered expression and co-expression of these two receptor molecules in the spinal and vagal afferents in esophagitis. Method: Four groups of rats ( $\mathrm{n}=3 /$ group) were used in this study. In group 1, 2 and 3, fundus was ligated by suturing the limiting ridge between the fundus and corpus and placing a nalaton ring $2 \mathrm{~cm}$ below the pyloric sphincter to restrict gastric emptying. Group 4 rats (sham control) received a midline abdominal incision, but no fundus ligation. Forty eight hours following surgery group 1 rats were deeply anesthetized and perfused with fixative (4\% paraformaldehyde). Group 2 ( 7 days ligated), 3 (ligated + PPI) and 4 (sham control) were perfused in similar fashion after 7 days. Bilateral nodose ganglia (NG), spinal cord (T2-T4) and respective dorsal root ganglia (DRG) were collected for examining TRPV1, P2X3 and IB4 immunoreactivity (ir). The Group 3 rats received proton pump inhibitors (PPI, omeprazole $20 \mathrm{mg} / \mathrm{kg}$, p.o. Qday) for 7 days starting on the day of surgery. In addition, esophagi were collected from non-perfused rats $(n=2)$ for myeloperoxidase (MPO) assay as a marker of neutrophil infiltration in inflammation. Results: Fundus ligation produced severe inflammation of the distal esophagus. In DRGs, P2X3-ir cells were significantly high $(\mathrm{p}<0.001)$ in $48 \mathrm{hrs}(90.0 \pm 3.2)$ and 7 days $(183.0 \pm 3.2)$ ligated rats compared to sham operated $(47.67 \pm 2.9)$ and PPI-treated $(62.0 \pm 2.9)$ rats. Interestingly, the number of IB4-positive cells in DRGs also increased in ligated rats $(101.67 \pm 3.0 \mathrm{in} 48 \mathrm{hrs}$ and $127.33 \pm 3.8$ in 7 days vs $49.0 \pm 1.7$ in sham, $p<0.001$ ). In NG, TRPVl-ir cells in 7 days ligated rats were significantly higher $(206.67 \pm 5.4$ vs $102.0 \pm 3.8$ in sham, $\mathrm{p}<0.001)$. Similarly, P2X3-ir cells were significantly higher in ligated rats (228.6 \pm 3.2 vs $37.67 \pm 2.60$ in sham) Like in DRG, IB4-positive cells in NG were significantly higher in ligated rats $(127.67 \pm 2.8$ in $48 \mathrm{hrs}, 178.33 \pm 3.8$ in 7 days) compared to sham $(59.0 \pm 1.2)$ and PPI-treated $(84.3 \pm 3.8$ ) groups. Conclusions: This study provides a strong evidence for the involvement of TRPVI and P2X3 channels in reflux-induced changes in vagal and spinal afferents. Results indicate that following inflammation there is a phenotypic change in number of IB4-positive cells in DRG and NG. These changes can be attenuated by PPI. Therefore, it can be speculated that acid can contribute to peripheral sensitization by upregulating ionotropic channels

879

Reduced Pain Perception in Barrett's Esophagus (BE) Patients Is Likely Due to Downregulation of TRPVI Receptors in the Squamous Epithelium Rather Than in the Barrett's Mucosa Itself

Ram Dickman, Pankaj J. Pasricha, John Winston, Mohan Shenoy, Diley Hernandez, Harinder S. Garewal, Ronnie Fass

Background: Patients with BE demonstrate reduced pain perception. It is currently assumed that this is due to the presence of columnar, metaplastic epithelium that is less sensitive to acid. Aims: To compare the distal esophageal expression of TRPVl, a receptor that is gated by protons and is suspected to mediate heartburn sensation, in the squamous and columnar metaplastic epithelium of BE patients to erosive esophagitis (EE) patients and normal controls. Methods: Five normal, healthy controls, 5 patients with $\mathrm{EE}$ and 5 with BE were recruited into the study. All participants underwent an upper endoscopy to ascertain their diagnosis $\mathrm{BE}$ was defined as any columnar-like extension into the tubular esophagus that is positive on biopsy for intestinal metaplasia. In all groups, 2 biopsies were obtained from normal appearing mucosa in the distal esophagus, $6 \mathrm{~cm}>$ esophagogastric junction. Two additiona biopsies were taken from the Barrett's tissue in BE patients. TRPVl expression was analyzed by Western blots. Results: The mean age and M/F ratio was, $49.8 \pm 8.9 \mathrm{yrs}, 3 / 2$ for healthy 
controls, $52.0 \pm 3.5,3 / 2$ for $\mathrm{EE}$ and $62 \pm 4.3,5 / 0$ for $\mathrm{BE}$, respectively. The mean length of the Barrett's mucosa was $5.9 \pm 0.5 \mathrm{~cm}(4.5-7.0 \mathrm{~cm})$. The distribution of erosive esophagitis grading as Grade A - 1, Grade B - 3 and Grade C - 1. Patients with BE had the lowest expression of TRPV1 receptors in the squamous epithelium (0.3771) as compared with EE $(0.4167)$ and healthy controls $(0.5151)(\mathrm{p}<0.05)$. In contrast, the TRPVl receptors expression within the Barrett's tissue was the highest (0.9652) when compared to the TRPVl expression in the squamous epithelium of all other groups $(p<0.05)$. Conclusions: Patients with BE have the lowest TRPVI receptors expression in the distal esophageal squamous epithelium, but at the same time the highest TRPVI receptors expression within the Barrett's tissue when compared to EE patients and healthy controls. It is likely that the observed reduction in pain perception of BE patients is due to downregulation of TRPVI within the esophageal squamous epithelium and not within the Barrett's mucosa.

\section{0}

The Magnitude of Visceral Pain Hypersensitivity After Distal Oesophageal Acidification Correlates with Pre-Study Anxiety State Scores Abhishek Sharma, Qasim Aziz, Claire Delaney, Anthony Hobson

Patients with stress or anxiety at the time of gastrointestinal injury/inflammation (eg gastroenteritis) have a higher risk of developing subsequent Irritable Bowel Syndrome. Anxiety is common in functional chest pain of presumed esophageal origin, where previous injury from acid/bile may play a role, but how they interact is unknown. We have previously shown that oesophageal pain thresholds (PT) are inversely correlated with anxiety scores (1). In addition, we have shown that distal oesophageal acidification induces sensitisation of spinal dorsal horn neurones leading to the development of pain hypersensitivity in the non-acid exposed proximal oesophagus (PO) and chest wall (CW) (2). The effect of anxiety in modulating the magnitude of sensitisation to oesophageal acid has not been studied. Aim: To determine whether the magnitude of pain hypersensitivity after oesophageal acidification correlates with pre-study anxiety state. Methods: In 14 healthy volunteers ( 7 male, 7 female, and age 20-50, mean 34.2yrs) the Spielberger State Anxiety Inventory (SAI), a validated measure of state anxiety, was completed. PT to electrical stimulation (in milliamperes, $\mathrm{mA}$ ) were determined in the PO, CW and foot pre and post a 30-minute distal oesophageal infusion of $0.15 \mathrm{M} \mathrm{HCl}$. Results: Mean SAI score was 30.8 (SD 8.7, range 20-51). Acid infusion resulted in a reduction in PT in the PO $[35.3 \mathrm{~mA}$ (SD $5.8 \mathrm{~mA}$ ) Vs $25.8 \mathrm{~mA}$ (SD 7.7mA), $\mathrm{p}<0.0001]$ and $\mathrm{CW}[31.3 \mathrm{~mA}(\mathrm{SD} 13.5 \mathrm{~mA})$ Vs $27.5 \mathrm{~mA}(\mathrm{SD} 8.7 \mathrm{~mA}), \mathrm{p}=0.04$ ] but not the foot [22.6 mA (SD 11.1mA) Vs $22.3 \mathrm{~mA}$ (SD 9.7mA), $\mathrm{p}=0.8)$. There was a group correlation between baseline spielberger score and maximum decrease in PT post acid (magnitude of sensitisation) in the $\mathrm{PO}(\mathrm{r}=-0.572,95 \% \mathrm{CI}-0.845$ to $-0.059 ; \mathrm{p}=0.03)$ but not the CW ( $\mathrm{r}=0.333,95 \% \mathrm{CI}-0.240$ to 0.734$)$. Subgroup analysis suggested the strength of this correlation was proportional to the baseline Spielberger score. If the SAI score was $<30$ then $\mathrm{r}=-0.001,95 \% \mathrm{CI}-0.665$ to $0.663 ; \mathrm{p}=0.99$. If the SAI score was $>30$ then $\mathrm{r}=$ $0.953,95 \% \mathrm{Cl}=-0.997$ to $-0.448 ; \mathrm{p}=0.01 \mathrm{Vs}$. Furthermore, if $>35$ then $\mathrm{r}=-0.999,95 \%$ CI -0.999 to $-0.956 ; \mathrm{p}=0.0009$. Conclusions: Increased baseline anxiety state is associated with a greater reduction of oesophageal pain threshold after acid infusion. There may be a role for anxiety in modulating the sensory responses to injurious stimuli in the gut. Future studies with larger numbers and more objective physiological measures of stress and anxiety may further help to understand the mechanism by which anxiety influences post injury gut sensitisation. References: [1] Worthen S et al. Gut 2005; 54(suppl 2): A19. [2] Sarkar et al Lancet 2000; 356(9236): 1154-9.

\section{1}

Neural and Non-Neural Pathways in Vanilloid Receptor (TRPV1) -Mediated, Acid-Induced Esophageal Inflammation in the Cat

Ling Cheng, Karen M. Harnett, Weibiao Cao, Claudio Fiocchi, Jose Behar, Piero Biancani

The transient receptor potential channel vanilloid subfamily member-1 (TRPV1), originally described in primary sensory neurons as a receptor for capsaicin and related natural irritants (referred to as vanilloids) such as heat and acids, has recently been demonstrated in nonneural cells. To test whether this acid-sensitive receptor may be the initial mediator of acidinduced inflammation in the cat esophagus, a tubular segment of normal esophageal mucosa was removed and tied at both ends to form a mucosal sac (AJP-GI 289:860-869, 2005). The sac was filled with $0.01 \mathrm{~N} \mathrm{HCl}$ (or normal Krebs' solution for control) and kept in oxygenated Krebs' solution at $37^{\circ} \mathrm{C}$. The medium around the $\mathrm{HCl}$-filled sac (supernatant) was collected after $3 \mathrm{hr}$ incubation and examined for inflammatory mediator content. Compared to control, substance P levels in mucosal tissue and in supernatant increased six-fold in response to $\mathrm{HCl}$, while CGRP levels increased 200 times in the mucosa and 10 times in the supernatant. The HCl-induced increases in substance P and CGRP in mucosa and in supernatant were abolished when the TRPVI receptor antagonist IRTX was added inside the mucosal sac, or when tetrodotoxin (TTX) was added inside the sac and to the supernatant, suggesting that TRPV1-induced production of substance P and CGRP was neurally mediated. PAF levels increased more than three-fold in mucosa and in supernatant in response to $\mathrm{HCl}$, and the increase was blocked by IRTX. The increase in PAF levels in mucosa and supernatant, however, were not affected by neural block with TTX, suggesting that production of PAF may arise from non neural pathways. We then examined the presence of TRPVI receptor mRNA in esophageal mucosa by RT-PCR. We derived primers from conserved regions of mRNA sequences of humans, rat, dog, mouse guinea pig and rabbit and confirmed, through the BLAST database, that the primers were specific for TRPVl. The band produced using these primers was sequenced, obtaining the sequence of cat TRPV1 with $95 \%$ homology to human TRPVl. RT-PCR of mucosa exposed to $\mathrm{HCl}$ for 3 hours showed upregulation of TRPVl cat receptor mRNA. To demonstrate the expression of TRPVl receptor mRNA in non-neural cells, esophageal epithelial cells were enzymatically isolated, sorted by flow cytometry, and a 90-95\% pure cell preparation was obtained. RT-PCR confirmed the presence of TRPVI receptor mRNA in these cat esophageal epithelial cells. We conclude that HClinduced activation of TRPVl receptors in esophageal mucosa results in upregulation of receptor mRNA, neurally mediated release of substance P and CGRP, and non-neurally mediated release of PAF from epithelial cells.
Distinct Clinical Characteristics of Patients with Non-Erosive Reflux Disease (NERD) and Reflux Esophagitis (RE)

Justin Wu, Carrian Cheung, Vincent Wong, Joseph Sung

BACKGROUND: It has been postulated that NERD and RE are two distinct entities of GERD instead of a continuous spectrum. AIM: To compare the clinical characteristics, quality of life, prevalence of concomitant functional GI and psychological disorders between NERD and RE patients. METHODS: We prospectively recruited consecutive patients presenting with weekly attacks of heartburn or acid regurgitation. Exclusion criteria included previous gastric surgery, recent use of NSAID or PPI, and peptic ulcer. All patients completed a selfadministered GI symptom standard questionnaire and a validated, 18-item disease specific quality of life (QoL) questionnaire (HK GERD-QOL: 0-72 points, high marks indicated poor QoL). Concomitant functional dyspepsia (FD) and irritable bowel syndrome (IBS) were diagnosed by Rome II criteria. Psychological disorders (PD) including depression and panic disorder were documented by standard questionnaires. RE and hiatus hernia (HH) were assessed by EGD. Esophageal manometry (EM), Bernstein test (BT) and 24-hour pH metry were performed for all patients. NERD and RE patients were compared for QoL and prevalence of co-morbidities. RESULTS: 214 patients (NERD: 113, RE: 111; mean age: 53.2+/-19.6; male: $49 \%$ ) were studied. Although both groups of patients had comparable age, gender, duration and ratings of symptom severity, NERD patients had significantly higher QoL scores $(54.8+/-16.6$ Vs $42.5+/-13.8, \mathrm{p}=0.02)$ than RE patients. Compared with RE patients, NERD patients also had higher prevalence of $\mathrm{FD}(65 \mathrm{Vs} 42 \%, \mathrm{p}=0.003$ ), IBS ( $44 \mathrm{Vs} 15 \%, \mathrm{p}<0.001$ ), PD ( 9 Vs $1 \%, p=0.04$ ) and positive BT ( 41 Vs $20 \%, p=0.004$ ). However, RE patients had more HH ( 35 Vs 18\%, $\mathrm{p}=0.009$ ), higher esophageal acid exposure (Mean DeMeester score: $12.6+/-8.5 \mathrm{Vs} 8.2+/-9.1, \mathrm{p}=0.01$ ) and more severe esophageal dysmotility (Ineffective esophageal motility: $26 \mathrm{Vs} 13 \%, \mathrm{p}=0.03$; lower esophageal sphincter pressure $<10 \mathrm{mmHg}$ : 29 Vs $11 \%, \mathrm{p}=0.002$ ). CONCLUSION: NERD and RE patients have distinct difference in clinical characteristics. Despite similar severity of reflux symptoms, NERD patients have poorer QoL related to GERD, higher prevalence of other functional GI and psychological disorders, and more sensitive esophagus. These observations suggest visceral hypersensitivity predominates in the mechanism of NERD, while esophageal dysmotility and defective anti-reflux mechanism plays a more important role in RE.

889

The Functional Lumen Imaging Probe (FLIP) for the Evaluation of the Esophagogastric Junction (EGJ) Barry P. McMahon, J.N. B. Frokaer, Peter Kunwald, Donghua Liao, Peter Funch-Jensen, Hans Gregersen, Asbjorn M. Drewes

Despite being one of the most dynamic structures in the human body, knowledge about the esophagogastric junction (EGJ) behaviour is very limited. A functional lumen imaging probe (FLIP) using impedance planimetry technology was used to quantify the competence of the EGJ. The aims of the study were; to verify the efficacy and usefulness of the FLIP for the evaluation of the EGJ in healthy controls (HC); to confirm the location of the FLIP with ultrasound imaging of the EGJ and to compare the ability of FLIP in determining EGJ characteristics in HCs with data on a classical achalasia patient. 8(6 m) mean age 25.5 (2135 ) were recruited for the study. Inclusion criteria $\leq 35 \mathrm{yrs}$, non-smokers, no history of reflux, heartburn or regurgitation. FLIP consisted of a catheter with a $10 \mathrm{~cm}$ long cylindrical bag which could be infused with saline to a diameter of $3.2 \mathrm{~cm}$. After calibration the probe was inserted orally and using manometry the respiratory inversion point was located and used to place the FLIP bag straddling the EGJ. A radial scanning ultrasound miniprobe (UM-3R, Olympus Corp. Tokyo, Japan) was inserted into the balloon to confirm FLIP position. Stepwise distentions allowed ultrasound and FLIP measurements to be made. Then volume controlled distensions to $60 \mathrm{ml}$ at a rate of $45 \mathrm{ml} / \mathrm{min}$ were performed before and after the administration of butylscopolamine and data on 8 CSAs at $4 \mathrm{~mm}$ intervals in the cylindrical bag were recorded along with bag pressure. The ultrasound images verified the probe was actually placed in the EGJ. A variation on the geometry for all 8 HCs was observed across the 8 CSA measurements. Pressure-volume data for the HCs indicated that pressure rose from on average from 0 to $35 \mathrm{~cm} \mathrm{H} 20$ but for the achalasia patient from 0 to $50 \mathrm{~cm}$ $\mathrm{H} 20$ when the bag is filled to a volume of $60 \mathrm{ml}$. However, pressure-CSA data indicated that the minimum CSA measured remained at the lowest measurable CSA for the achalasia patient as the pressure increased while the volunteer CSAs reached $250 \mathrm{~mm} 2$ on average. In all the HCs the narrowest point measured moved proximally during distension. Three dimensional lumen imaging with the FLIP indicated visually the difference between the pattern of the $\mathrm{HC}$ distensions and the pattern of the achalasia patient. FLIP represents a new technique for measuring EGJ competence. The FLIP can be placed successfully in the EGJ using manometry. It can distinguish between normal subjects and an achalasia patient suggesting it may have a use in determining EGJ function in different diseased states. It has the potential to monitor patients before, during after endoscopic and surgical treatments for gastroesophageal reflux disease.

890

Characteristics and Determinants of Long-Term Outcome in Acute Onset Functional Dyspepsia Patients

Sebastien Kindt, Philippe Caenepeel, Joris Arts, Jozef Janssens, Jan Tack

Background: Recent studies have shown that functional dyspepsia (FD) may persist after an acute gastroenteritis, and that post-infectious (PI) origin may be present in up to $20 \%$ of FD patients. The aim of this study was to evaluate the long-term prognosis of PI-FD and to identify factors influencing outcome. Methods: FD patients of presumed PI origin (acute onset with symptoms suggestive of infection) were prospectively followed and evaluated by telephone interview and mailed questionnaires. Patients underwent a full pathophysiological investigation (gastric emptying, barostat, H.pylori status) at inclusion, and follow-up data included FD symptom status, changes in body weight, quality of life (using NDI questionnaire) and trait and state anxiety and depression (using STAI and HADS). Correlation of 
these characteristics to FD status was assessed using Chi-square, parametrical or nonparametrical tests, and logistic regression. Results: Ninety-four PI-FD patients (mean age $46.1 \pm 1.4 \mathrm{y}, 66$ women), with a mean weight loss at presentation of $7.0 \pm 0.8 \mathrm{~kg}$ were recruited. After a mean follow-up of $63 \pm 3$ months, FD symptoms had resolved in $30 \%$ of the patients. There was an increase in weight of $3.0 \pm 1.1 \mathrm{~kg}$ during follow-up, resulting in a residual net loss of $3.5 \pm 1.1 \mathrm{~kg}$ compared to symptom onset. Persisting FD symptoms during long-term follow-up were not influenced by any of the pathophysiological variables assessed, but were significantly associated with body weight at presentation and STAI scores. FD symptom status was also significantly associated with the NDI and the HADS-depression and -anxiety scores. In multivariate logistic regression, only STAI-trait was significantly associated with long-term FD outcome. Conclusion: Five years after the onset PI-FD, the majority of patients are still symptomatic and body weight has not fully recovered. Persisting symptoms are highly associated with a decreased quality of life, anxiety and depression. Trait anxiety is the main determinant of outcome of long-term prognosis in PI-FD

891

Comparison of Outcomes of Gastric Electrical Stimulation (GES) in Diabetic, Postsurgical and Idiopathic Gastroparesis By Applying Intention-to-Treat Analysis

Zhiyue Lin, Irene Sarosiek, Jameson Forster, Richard W. McCallum

Previous reports of GES therapy for refractory gastroparesis were based on perprotocol analysis and did not compare outcomes in different etiologies of gastroparesis. The aims of this study were to compare outcomes of GES therapy among patients with diabetic, idiopathic and postsurgical gastroparesis by applying intention-to-treat (ITT) analysis and to identify possible predictors associated with symptom improvement. Methods: 130 gastroparetic patients (81 diabetic, 29 idiopathic, 20 postsurgical) with GES implantation (Enterra ${ }^{\mathrm{TM}}$ System, Medtronic) for at least 1 year from 4/1998 to 10/2004 were retrospectively assessed. Total symptom scores (TSS) derived from 7 symptom sub-scores, a low-fat 4-hour gastric emptying test (GET), electrogastrogram (EGG), quality of life (QOL) using SF-36 Health Status Survey questionnaire including physical composite score (PCS) and mental composite score (MCS) and weight were compared between baseline and 1-year follow-up among the three etiologies. ITT analysis was performed where the missing data from those who died (6 diabetic and 1 idiopathic patients from non-device related complications.), had device removed $(\mathrm{N}=7)$, had partial data sets $(\mathrm{N}=8)$ within 1 year of GES therapy or lost to followup $(\mathrm{N}=9)$ were imputed using the last observation carried forward method. Results: The median reduction in TSS in the postsurgical and diabetic groups was substantially greater than in idiopathic patients ( $59 \%$ vs. $52 \%$ vs. $34 \%$ ). Mean baseline vomiting score in diabetics was significantly higher $(\mathrm{P}<0.05)$ than for postsurgical but similar to the idiopathic group (3.2 vs. 2.2 vs. 2.9 ). PCS and MCS were significantly improved to similar degrees among the 3 groups. Idiopathic patients reported more abdominal pain than diabetic and postsurgical groups both at baseline and after GES. Diabetic and postsurgical patients gained more weight than idiopathics ( 2.4 vs. 1.5 vs. $0.2 \mathrm{~kg}$ ). $82 \%$ idiopathic patients had an abnormal baseline EGG (dysrhythmia $>30 \%$ or decrease in postprandial power) which was substantially more than the diabetic (56\%) and postsurgical groups (50\%). No significant improvement in GET occurred in any of the 3 groups. 5 patients (4\%) had devices removed within 12 months of GES due to infections in the pulse generator pocket. Conclusions: High-frequency GES is more effective in symptomatically improving gastroparesis in diabetic and postsurgical patients than the idiopathic group who had the poorest response (35\% reduction in symptoms). Idiopathic patients comprise a population presenting with prominent abdominal pain and more EGG abnormalities and require a more thorough evaluation prior to considering GES therapy.

892

Natural History of Functional GI Disorders: A 15 Year Longitudinal Population-Based Study

Smita Halder, G Richard Locke, Cathy D. Schleck, Alan R. Zinsmeister, L Joseph Melton, Nicholas J. Talley

Background: Functional Gl disorders (FGID) fluctuate and transition, yet the long term natural history of FGID is unknown. Aim: To describe the 15 year natural history of FGID in a US population. Methods: This prospective cohort study collected symptom data by mailing multiple validated surveys to random samples of Olmsted County, MN residents over a 15-year period. Each subject received a minimum of 2 surveys. Survey responses were utilized to categorize subjects into 10 mutually exclusive FGID subgroups (see below). Point prevalence (per 100), onset and disappearance rates and transition probabilities were calculated for each FGID. A generalized logits logistic model for transitions among FGID subgroups was used to assess the association (Odds Ratios (OR) [95\% CI]) with age, gender, length of time in the study, education, alcohol use and smoking (at first survey). Results:Overall,14,070 separate surveys were mailed over the 15 year period with several people receiving more than 2 mailings; 8673 responded (68\%). Over the 15 year period the prevalence was stable for IBS-any and AP, but increased four-fold for Dysp. The number of people reporting onset was greater than disappearance for each of the nine disorders. Although the proportions varied by group, in general, one third had resolution of symptoms, one third had symptom stability and one third transitioned to a new group (table). Women had greater odds for maintaining (2.0 [1.2.3.4]), developing (1.8 [1.2.2.6]) and gaining symptoms $(2.0[1.2,3.3])$ over time than men, smokers had decreased odds $(0.4[0.3,0.96])$ for symptom stability; high alcohol consumption was associated with increased odds $(2.4[1.2,5.0])$ for symptom stability. Conclusions: Most people with FGID in the community continue to have symptoms for more than a decade but specific symptoms change over time. Gender, smoking and alcohol use were associated with symptom transition. Supported by an unrestricted grant from Novartis.

\begin{tabular}{|c|c|c|c|c|c|c|c|c|c|c|}
\hline \multirow{2}{*}{$\begin{array}{l}\text { FGID on } \\
\text { initial survey }\end{array}$} & \multicolumn{10}{|c|}{$\%$ of FGID on final survey based on initial disorder } \\
\hline & IBS-both & IBS-C & IBS-D & $\begin{array}{l}\text { IBS-no } \\
\text { C or D }\end{array}$ & $\begin{array}{l}\text { Both } \\
\text { C\&D }\end{array}$ & C & D & Dysp & AP & $\begin{array}{c}\text { No } \\
\text { Symptoms }\end{array}$ \\
\hline $\begin{array}{c}\text { IBS-both } \\
(\mathrm{n}=17)\end{array}$ & 23.5 & 0 & 29.4 & 0 & 0 & 0 & 5.9 & 0 & 11.8 & 29.4 \\
\hline IBS-C $(n=25)$ & 12.0 & 12.0 & 0 & 16.0 & 0 & 8.0 & 8.0 & 0 & 24.0 & 20.0 \\
\hline IBS-D $(n=40)$ & 2.5 & 0 & 20.0 & 17.5 & 0 & 0 & 15.0 & 0 & 7.5 & 37.5 \\
\hline $\begin{array}{l}\text { IBS-no } C \text { or } \\
D(n=76)\end{array}$ & 1.3 & 7.9 & 7.9 & 30.3 & 2.6 & 5.3 & 2.6 & 1.3 & 5.3 & 35.5 \\
\hline $\begin{array}{c}\text { Both C\&D } \\
(n=8)\end{array}$ & 0 & 0 & 12.5 & 0 & 12.5 & 0 & 0 & 0 & 12.5 & 62.5 \\
\hline$C(n=45)$ & 0 & 6.7 & 0 & 4.4 & 4.4 & 22.2 & 0 & 4.4 & 6.7 & 51.1 \\
\hline $\mathrm{D}(\mathrm{n}=87)$ & 3.5 & 1.1 & 5.8 & 4.6 & 2.3 & 4.6 & 28.7 & 2.3 & 4.6 & 42.5 \\
\hline Dysp $(n=4)$ & 0 & 0 & 0 & 25.0 & 0 & 0 & 0 & 0 & 25.0 & 50.0 \\
\hline $\mathrm{AP}(\mathrm{n}=198)$ & 2.5 & 4.6 & 10.6 & 9.1 & 0.5 & 3.5 & 6.6 & 1.0 & 19.2 & 42.4 \\
\hline $\begin{array}{c}\text { No symptoms } \\
(\mathrm{n}=865)\end{array}$ & 1.0 & 1.0 & 3.4 & 8.7 & 1.9 & 3.9 & 7.6 & 1.5 & 7.5 & 63.5 \\
\hline
\end{tabular}

$\mathrm{C}=$ Constipation; D=Diarrhea; AP=Abdominal pain only; Dysp=Dyspepsia

893

The Epidemiology of Episodic Crampy Abdominal Pain: A Population Based Study

Meredythe A. Mcnally, Smita L. Halder, G. Richard Locke, Alan R. Zinsmeister, Cathy D. Schleck, Nicholas J. Talley

Background: Frequent abdominal pain is reported by up to $25 \%$ of the U.S. population. Much is known about IBS and dyspepsia yet detailed epidemiologic data about frequent abdominal pain, in general, and episodic crampy abdominal pain (ECAP), in particular, is lacking. Aim: To describe the epidemiology of ECAP in a population based study. Methods: A valid GI symptom survey was sent in 2003 to 4196 randomly selected residents of Olmsted County, MN, who were previously surveyed between 1988 and 1993. Subjects were asked to report episodes of abdominal pain separate from menstrual cramps and to characterize the pain in detail. After adjusting for age, gender, and education level, separate logistic regression models were used to estimate the odds ratios (OR[95\%CI]) for ECAP (versus frequent non-crampy abdominal pain) of several reported abdominal pain related symptoms. Results: Of those mailed, 2300 responded (55\% response rate) and 2255 had sufficient data available for analysis. Twenty-two percent of the population reported frequent abdominal pain (> 6 episodes per year) and $31.7 \%(6 \%$ overall, $[5 \%, 7 \%])$ described their pain as crampy. Increased odds for ECAP were observed in subjects who reported their pain as moderate (OR 2.3, $[1.3,4.0]$ ) or severe (OR 3.1, $[1.4,6.5])$. The odds for ECAP were smaller in subjects who experienced daily (OR $0.37,[0.2,0.7])$ or multiple weekly episodes of pain (OR 0.3, $[0.1,0.8]$ ) and smaller in subjects who reported pain that lasted more than 2 hours (OR 0.5, $[0.3,0.9])$. The odds for ECAP were significantly increased in subjects reporting pain below the navel (OR 7.7, $[4.3,13.8]$ ) or both above and below the navel (OR 7.9, $[4.2,14.9])$. A decreased odds for ECAP was observed in subjects who experienced improvement in pain with a bowel movement (OR 0.14, [0.1, 0.2]), had bowel movements triggered by pain onset (OR $0.16,[0.1,0.3])$, or experienced loose bowel movements with pain onset (OR 0.12, $[0.1,0.2])$. While the odds for ECAP were decreased in those experiencing pain relief with $\mathrm{H} 2$ blockers or proton pump inhibitors (OR $0.5,[0.3,0.9])$, the odds were increased in those reporting pain relief with antispasmodics (OR 5.4, [1.1, 25.9]). Of those with ECAP, $76 \%$ met Rome II criteria for IBS yet only $8 \%$ of ECAP patients had seen a physician specifically for this pain. Conclusion: ECAP affects $6 \%$ of the population. The multiple predictors of ECAP, when compared to frequent, non-crampy abdominal pain suggest that ECAP may represent a distinct symptomatic entity in the community and should be recognized clinically.

895

Factors Related to Lymph Node Metastasis and Feasibility of Endoscopic Mucosal Resection in Poorly Differentiated Early Gastric Cancer Young Dae Park, Yun Jin Chung, Chang-Min Cho, Wan Sik Yu, Won Young Tak, Seong Woo Jeon

Background: Although most of poorly differentiated early gastric cancer (EGC) have no accompanied with lymph node metastasis (LNM), endoscopic mucosal resection (EMR) is currently not accepted as an alternative treatment to surgery. If patients with low-risk of LNM were defined in this type of EGC, application of EMR would be possible instead of surgery. This study was performed to determine the factors related to LNM in poorly differentiated EGC and the feasibility of EMR. Methods: We investigated 234 patients with poorly differentiated EGC confined to mucosal and submucosal layer who underwent radical gastrectomy with lymph node dissection from January 1999 to September 2005. Several clinicopathological factors were investigated to identify predictive factors for LNM: age, sex, tumor location, type of operation, tumor size, gross type, ulcer findings, depth of invasion and lymphatic invasion. Results: Lymph node metastases were found in 29 patients (12.4\%). In univariate analysis, tumor size $(\mathrm{P}=0.039)$, gross type $(\mathrm{P}=0.008)$, depth of invasion $(\mathrm{P}<0.001)$ and lymphatic invasion $(\mathrm{P}<0.001)$ were significantly related with $\mathrm{LNM}$ in EGC patients. In multivariate analysis, tumor size $(>30 \mathrm{~mm}$ vs. $\leq 15 \mathrm{~mm}$, odds ratio $=4.1, \mathrm{P}=$ $0.05)$, depth of invasion ( $\geq$ SM2 vs. $\leq \mathrm{SM} 1$, odds ratio $=6.23, \mathrm{P}=0.01)$ and lymphatic invasion (odds ratio $=9.14, \mathrm{P}<0.001)$ were associated with LNM. Using tumor size $(>15$ $\mathrm{mm}$ ) and depth of invasion (> SMl) as the predictive factors, no LNM was observed in patients who had neither of these factors. In contrast, the LNM rate was calculated to be $19.5 \%$ in patients who had both factors. Conclusion: Poorly differentiated EGC, which is 
confined to mucosa or SMl layer in depth and less than $15 \mathrm{~mm}$ in size, is good candidate for EMR, given the null possibility of LNM. Feasibility of EMR in poorly differentiated EGC should be evaluated in order to avoid extensive radical gastrectomy.

\section{6}

Non-Cardia Gastric Cancer Remains An Important and Deadly Cancer in the United States: Secular Trends in Incidence and Survival Melvin K. Lau, Anne Le, Hashem B. El-Serag

Background: Non-cardia gastric cancer is not frequently mentioned in the United States. However, it is unclear if previously reported decline in non-cardia gastric cancer has continued, and if detection and management has affected overall survival outside the setting of clinical trial. The recent temporal trends in incidence and survival of this malignancy have not been well examined. Methods: We utilized Surveillance, Epidemiology, and End Results registry (SEER) to identify all cases of non-cardia gastric cancer diagnosed between 1973 and 2002. The yearly age-adjusted incidence rates and the relative survival rates were calculated. Cox proportional hazards ( $\mathrm{PH}$ ) models were used to examine temporal trends (yearly from 1983 to 2003) in mortality risk while controlling for changes in age, race, sex, geographic region (9 SEER regions), cancer stage (localized, regional, distant), and treatment (gastrectomy, gastrectomy and radiation). Results: The age-adjusted yearly incidence rate has declined by $23 \%$ between 1973 and 2002 from 4.3 to 3.3/100,000 (esophageal adenocarcinoma was 2.4 per 100,000 in 2002). However, the incidence of localized non-cardia gastric cancer (invasive neoplasm confined to the organ of origin) remained without change between 0.9 and 1.0 per 100,000 , and has increased slightly for persons 85 and older. The incidence rates in men were double those in women, and 1.6-fold and 2.6-fold higher in Blacks and Other races (mostly Asians), respectively compared to Whites. These differences have remained relatively unchanged during 1973-2002. The 3-year survival was 34\% (95\% $\mathrm{CI}, 32 \%-35 \%)$ in patients diagnosed during 1995-99. The Cox PH analysis shows no significant change in mortality risk for patients diagnosed between 1983 and 2002, both in unadjusted as well as adjusted analyses for all the variables listed above. However, there were significant independent regional and racial variations in survival. For example, compared to Hawaii, the mortality risk for Utah and New Mexico were $19 \%$ and $16 \%$ higher, respectively. Asians had a $17 \%$ lower mortality risk compared to Whites. Conclusions: Despite the overall decline in non-cardia gastric carcinoma, the incidence of local stage disease have remained stable in most ages and even increased in old ages. Unfortunately, there has been no significant improvement in survival during the past 20 years. Moreover, there remain considerable regional as well as racial variations in mortality.

897

Risk Factors for Pancreatic Cancer in Younger and Older Patients Sara H. Olson, Jennifer Simon, Emmy Ludwig, Robert C. Kurtz

Pancreatic cancer (PC) is generally thought of as a disease of aging. The purpose of this analysis is to investigate patterns of weight in adulthood, smoking, family history of cancer, and risk of pancreatic cancer PC in individuals according to age ( $<60 \mathrm{vs}>=60$ years). Methods. Our Familial Pancreatic Cancer Registry is tied to an epidemiologic case-control study of environmental risk, which includes both familial and sporadic PC cases. Data are from this hospital-based case-control study including PC patients as cases $(n=241)$ and controls consisting of well spouses or visitors accompanying patients to an endoscopy clinic $(\mathrm{n}=122)$. All participants were questioned on several potential risk factors for PC, including maximum weight, lifetime smoking habits, family history of cancer, and demographic characteristics. Logistic regression was used to determine adjusted odds ratios (OR) and 95\% confidence intervals (CI). Results. We analyzed data from 241 patients $(97$ aged $<60$ years and 144 aged $>=60$ ) and 122 controls (61 aged $<60$ years and 61 aged $>=60$ ). In the total sample, increased risk was associated with obesity (BMI $>=30)(\mathrm{OR}=2.21,95 \% \mathrm{CI} 1.20$ 4.04); heavy smoking ( $>=15$ pack years) (OR $1.5195 \% \mathrm{CI} 0.88-2.62$ ) and history of $\mathrm{PC}$ in first or second degree relatives (OR 1.91, 95\% CI 0.93-3.92). Results for obesity and smoking were stronger among the younger respondents: adjusted ORs were 3.42 (95\% CI 1.33-8.79) for obesity and 3.62 (95\% CI 1.24-10.6) for heavy smoking; compared to 1.71 (95\% CI 0.73-4.01) for obesity and 1.16 (95\% CI 0.58-2.34) for heavy smoking among older respondents. For family history, there was less difference in risk according to age group, with adjusted OR of 2.02 (95\% CI 0.66-6.17) among younger respondents and 1.54 (95\% Cl 0.57-4.16) among older ones. Family history of other cancers that are found in some families with PC (breast, ovarian, melanoma, colon, and endometrium) were not different between cases and controls overall or when stratified by age group. Conclusions. Our study shows that smoking and obesity, independent risk factors for PC development, appear to be more important in younger PC patients than in older ones. It is possible that in older people variables related to aging such as weakening of immune function or DNA repair capacity, could be more important. While, as expected, family history also increased PC risk, the age related differences were smaller. This study emphasizes the need for aggressive populationbased smoking cessation and weight control programs, especially among younger people.

\section{8}

Detereminant and Outcomes of Curative Internt Surgery (CIS)for Pancreatic Cancer in the United States

Yasser H. Shaib, Jessica A. Davila, Chris R. Nauman, Hashem B. El-Serag

Background: Pancreatic cancer is the fourth leading cause of cancer death in the United States. Curative intent surgery (CIS) is presumed to be the only curative option in the treatment of pancreatic cancer. The determinants and the outcomes of surgical resection are not clear at the population level. Methods: Using data from 9 registries of the Surveillance Epidemiology and End Results (SEER) program we examined the predictors of receiving CIS as well as the survival of patient receiving CIS between 1987-2001. The definition of CIS in SEER has been previously validated and found to be highly accurate. Cox proportional hazard model was used to examine the effect of potential determinants on survival. Results:
We identified 32,348 cases of pancreatic cancer. Of those 3,545 (10.9\%) received CIS. The proportion of patients receiving CIS decreased significantly with age $(p<0.0001)$, was similar across all racial groups (10.8\% in Whites, $11.4 \%$ in Blacks, $11.5 \%$ in Asians and $11.2 \%$ in Hispanics, $p=0.5)$, was slightly higher in males $(11.3 \%$ vs. $10.5 \%, p=0.02)$, decreased with advanced disease stage $(\mathrm{p}<0.0001)$ and progressively increased over time $(7.5 \%$ in $1987-89,9.1 \%$ in $1990-92,10.4 \%$ in $1993-95,12.4 \%$ in $1996-98$ and $13.4 \%$ in 1999 2001, p < 0.0001) and differed significantly across different SEER registries $(9.7 \%$ in San Francisco, 11.8 in Connecticut, $12.5 \%$ in Detroit, $11.7 \%$ in Hawaii, $9.3 \%$ in Iowa, 10.1 in New Mexico, $9.5 \%$ in Seattle, $13.2 \%$ in Utah, $11.4 \%$ in Atlanta, $\mathrm{p}<0.0001$ ). In the multivariable logistic regression analysis, more recent time periods, younger age, early disease stage and geographic location, but not race or gender were independent predictors of receiving CIS (Table). In the Cox survival analysis, younger age, early disease stage, more recent time period, geographic location and receipt of CIS were independent predictors of improved survival and Black race was an independent predictor of shorter survival Conclusions: Early stage disease, female gender, younger age, geographic location and more recent time periods are predictors of receiving CIS. Early disease stage and receipt of CIS are the strongest predictors of improved survival among patients with pancreatic cancer. Black race is an independent predictor of shorter survival.

Predictors of Recieving CIS

\begin{tabular}{|c|c|}
\hline Variable & Odds Ratio $(95 \% \mathrm{CI})$ \\
\hline Age: $-<40$ - 40-54 - 55-64 - $65-74->74$ & $\begin{array}{c}\text { Ref } 0.6(0.5-0.8) 0.5(0.4-0.7) 0.4(0.3-0.5) \\
0.1(0.1-0.2)\end{array}$ \\
\hline Race - White - Black - Asian - Hispanic & $\operatorname{Ref} 0.9(0.8-1.0) 1.0(0.8-1.3) 0.9(0.7-1.1)$ \\
\hline Gender - Male - Female & Ref $1.0(0.9-1.1)$ \\
\hline Stage - Metastatic - Localized - Regional & Ref $15.7(13.7-18.1) 17.9(16.0-20.0$ \\
\hline $\begin{array}{c}\text { Time Period - 1987-89 - 1990-92 - 1993-95 - } \\
1996-98 \text { - 1999-2001 }\end{array}$ & $\begin{array}{c}\text { Ref } 1.4(1.2-1.7) 1.7(1.4-1.9) 2.0(1.7-2.3) \\
2.0(1.8-2.4)\end{array}$ \\
\hline $\begin{array}{l}\text { SEER Registry - Seattle - San Francisco - } \\
\text { Connecticut - Detroit - Hawaii - Iowa - New Mexico }\end{array}$ & $\begin{array}{l}\text { Ref } 1.1(0.9-1.2) 1.6(1.4-1.9) 1.5(1.3-1.7) \\
\quad 1.3(1.0-1.7) 1.2(1.0-1.4) 1.1(0.9-1.3)\end{array}$ \\
\hline
\end{tabular}

899

Risk of Pancreatic Adenocarcinoma and Hereditaty Pancreatitis: The First National Cohort in France

Vinciane Rebours, Marie Christine Boutron-Ruault, Claude Ferec, Dermot O'Toole, Matthieu Schnee, Frederique Maire, Pascal Hammel, Philippe Ruszniewski, Philippe Levy

Hereditary pancreatitis (HP) is an autosomal dominant disease caused by a mutation in the cationic trypsinogene gene (PRSS1). Chronic pancreatitis (CP) is a known risk factor of pancreatic adenocarcinoma (PA) with a standardized incidence ratio (SIR) of 27. The frequency of pancreatic cancer in the inherited type of pancreatitis is uncertain. Aims: To evaluate the incidence of the PA in extensive French national series of patients with HP and to search for risk factors. Patients (pts) and methods: A cohort comprising all French HP pts was constituted by contacting all French gastroenterologists and pediatricians (response rate: $84 \%$ ) and genetics laboratories (response rate: $100 \%$ ). Inclusion criteria were the presence of PRSS1 gene mutation, or CP in at least 2 first-degree relatives, or 3 seconddegree relatives, in the absence of another cause of CP (notably, alcohol). CFTR and SPINK 1 gene mutations were also systematically examined. Diagnosis of PA was based on histological examination. Results: The historical cohort included 78 families and 200 pts of whom 181 were alive. It represented 6673 person-years (median number of generation: 3, males: 53\%, chronic alcoholism: $5 \%$, tobacco intoxication: $34 \%$ ). At time of inclusion, the median age was 30 y (range: 1-84). PRSS1 mutations were present in 68\% (R122H: 78\%, N29I: 12\%, others: $10 \%$ ). SPINK1 and CFTR mutations were present in $13 \%$ and $2 \%$, respectively. PRSS1 inheritance was maternal for 65\%. 10 PA were diagnosed (6 males) at a median age of $55 \mathrm{y}$ (range: 39-78). The SIR of PA for the cohort, the males, and the females was 87 (42-113), 69 (25-150) and 142 (38-225), respectively. At 50, 60 and $75 y$, the cumulated risk of PA was $11 \%, 16 \%$ and $49 \%$ for males and $8 \%, 22 \%$ and $55 \%$ for females, respectively. The risk of PA for smokers at age 75 y was $61 \%$ and $70 \%$ for males and females, respectively 19 patients died, 8 from PA (42\%). The cause of death was known for all the patients except one. Conclusions: Patients with HP have a markedly increased risk of pancreatic cancer (SIR: 87) compared with the general French population. This may be explained in part by the long exposure time to CP. There is no correlation between this risk and the PRSS1 genetic status but the PA is highly linked with the tobacco intoxication. PA is the first cause of death in patients with HP

900

The Short Health Scale - A Valid and Easy to Use HRQOL Instrument in Crohn's Disease

Henrik Stjernman, Christer Granno, Leif Ockander, Gunnar Jarnerot, Curt Tysk, Bjorn Blomberg, Magnus Strom, Henrik Hjortswang

Background: Health related quality of life (HRQOL) is an essential part of IBD health assessment not only in clinical trials but also in quality assurance of routine management. HRQoL can be divided into four aspects: symptoms, function, worry and general well-being We have developed the Short Health Scale (SHS), a HRQoL questionnaire in which the patients score the disease impact on each of these four aspects on visual analogue scales. The SHS has in a previous study shown to be valid, reliable and sensitive for change (responsive) on patients with ulcerative colitis. Aim: To evaluate the SHS on patients with Crohn's disease (CD). Methods: 367 patients with CD completed the SHS and four other HRQoL questionnaires (IBDQ, SF-36, RFIPC, and PGWB) and a symptom diary at their regular outpatient visits. 330 patients completed the questionnaires at a second visit after 6 months or earlier in case of deterioration. In addition, reliability data was obtained with replicate measurements after four weeks from the first visit in 38 patients stable in remission. Results: Patients in remission scored better on all four questions than patients with active 
disease $(\mathrm{p}<0.001)$ and all four questions correlated strongly with the corresponding dimensions of the other HRQoL questionnaires ( $\mathrm{r} s=0.74-0.83$ ) in keeping with a good validity. Reliability was confirmed with high correlations $(\mathrm{rs}=0.66-0.80)$ and intraclass correlation coefficients $(0.65-0.77)$ between test and retest measurements in stable patients. Patients who changed from remission to active disease or vice versa had a significant change in SHS score for all four questions $(\mathrm{p}<0.01)$. Conclusion: SHS is a valid, reliable and responsive HRQoL instrument also in patients with CD. It is easily completed by the patient and demands no further calculation by the investigator. SHS gives a comprehensive overview of the main aspects of the patient's subjective health perception to broaden the basis of clinical decision and to deepen the insights of disease impact on daily life.

901

Differences in Health Care Resource Consumption in a European Multinational Population-Based Inflammatory Bowel Disease Inception Cohort with 10 Years Follow-Up

Selwyn H. Odes, Michael Friger, Hillel Vardi, Frank Wolters, Maurice Russel, Bjorn Moum, Ebbe Langholz, Paolo Bodini, Kostas Katsanos, Juan Clofent, Joao Freitas, Giuseppe D'Albasio, Severine Vermeire, Colm O'Morain, Reinhold Stockbrugger

BACKGROUND/AIMS: Consumption of health care resources in inflammatory bowel disease (IBD) may vary by country and during the time course of disease. The prospectively-incepted, uniformly-diagnosed, population-based cohort of the European Collaborative Study on IBD presents an ideal setting for examining this hypothesis. METHODS: We studied 1321 IBD patients from 9 countries diagnosed during the 2 year inception period October 1, 1991 to September 30, 1993 and followed for 10 years. Data on resource use were obtained retrospectively from medical records in hospitals and community clinics. Resources were grouped for analysis as: diagnostics (consultations with gastroenterologists, general practitioners and surgeons; radiology, endoscopy, pathology), medical and surgical hospitalizations, 5-aminosalicylates (5-ASA), other drugs. Data are means per patient-year (p-y). RESULTS: Resource consumption by IBD patients varied significantly by country (Table), but not in terms of any geographical gradient. Denmark had the highest and Norway the lowest total hospitalization days and diagnostic events. 5-ASA treatment events exceeded those of other drugs in all countries. Rates of medical hospitalization, surgical hospitalization and diagnostics were higher in the lst follow-up year (7.84 days/p-y, 1.58 days/p-y, 7.84 events/p-y) than the next 9 years (2.56 days/p-y, 1.15 days/p-y, 2.56 events/p-y). Treatment events for 5-ASA and other drugs were fairly constant over all 10 years. Associations were noted for medical hospitalization days with diagnostics $(\mathrm{r}=0.93)$, and surgical hospitalization days with 5ASA events $(r=-0.70)$. Compared with Crohn's disease (set at $100 \%)$, consumption in ulcerative colitis of medical hospitalization was $54 \%$, surgical hospitalization $26 \%$, diagnostics $73 \%, 5$-ASA $107 \%$ and other drugs $53 \%$. CONCLUSIONS: This study is the first multinational, population-based, time-dependent characterization of the use of resources in IBD patients. Increased use was predicted largely by country, hospitalization, follow-up year and disease diagnosis.

Mean resource consumption by IBD patients per country

\begin{tabular}{|c|c|c|c|c|c|}
\hline Country & $\begin{array}{c}\text { Medical } \\
\text { Hospitalization* }\end{array}$ & $\begin{array}{c}\text { Surgical } \\
\text { Hospitalization* }\end{array}$ & Diagnostics^ & 5-ASA^ & $\begin{array}{c}\text { Other } \\
\text { Drugs^ }\end{array}$ \\
\hline Denmark & 4.95 & 1.93 & 7.39 & 2.89 & 1.18 \\
\hline Greece & 1.01 & 0.17 & 2.06 & 3.49 & 1.52 \\
\hline Ireland & 2.50 & 1.76 & 3.16 & 2.59 & 1.68 \\
\hline Israel & 2.98 & 0.15 & 5.30 & 3.24 & 1.52 \\
\hline Italy & 1.11 & 0.38 & 1.96 & 4.04 & 0.72 \\
\hline Netherlands & 1.82 & 0.72 & 4.57 & 3.45 & 1.50 \\
\hline Norway & 0.40 & 0.35 & 1.61 & 3.05 & 0.93 \\
\hline Portugal & 2.43 & 2.52 & 4.43 & 2.78 & 1.26 \\
\hline Spain & 2.44 & 0.59 & 3.56 & 3.19 & 1.45 \\
\hline
\end{tabular}

* days/patient-year, ^ events/patient-year

902

Environmental Factors Associated with Ulcerative Colitis: A Large PopulationBased Case-Control Study

Richard B. Gearry, Ann Richardson, Christopher M. Frampton, Judith A. Collett, Andrew Dodgshun, Bruce A. Chapman, Michael J. Burt, Murray L. Barclay

Introduction: The increase in the incidence of ulcerative colitis (UC) in developed countries is likely to be due to exposure to environmental factors in genetically susceptible individuals. Aim:. To identify environmental factors associated with UC in a large population-based cohort. Methods: 1421 IBD patients (668 UC, 715 Crohn's disease (CD)) were recruited, representing over $91 \%$ of people with inflammatory bowel disease in Canterbury, New Zealand (population 464,800). This is part of the Canterbury IBD Project, a populationbased study of genetic and environmental factors associated with IBD. Sex and age frequencymatched controls (599) were recruited at random from the electoral roll. Cases and controls completed a self-administered questionnaire which was posted back to the investigators. Univariate and multivariate analysis was performed. Subjects were encouraged to corroborate answers with family members or child health books. Results: Response rates were 95.2\%, $94.4 \%$ and $84 \%$ for UC, CD subjects and controls, respectively. Age, sex, current and childhood socioeconomic status (SES) and smoking status were included in the multivariate model. Statistically significant adjusted odds ratios with $95 \%$ confidence intervals included: one relative with IBD 2.48 (1.79-3.47), two relatives with IBD 6.80 (3.18-14.58), smoker at diagnosis 0.67 (0.49-0.91), ex-smoker at diagnosis 1.82 (1.40-2.37), appendicectomy $0.45(0.30-0.67)$, breastfed as infant $0.71(0.52-0.96)$, antibiotic use in adolescence 1.71
(1.04-2.81), and having a household vegetable garden in childhood $0.62(0.40-0.87)$. Fur thermore, a duration-response effect was observed for breast-feeding $(\mathrm{p}=0.002)$, high childhood SES was associated with later development of UC ( $\mathrm{p}=0.007)$ and there was a significan relation between increased household crowding early in life and reduced risk of later development of UC $(p=0.01)$. Over sixty additional factors were not significantly associated with UC. Conclusions: In addition to previously recognised factors associated with UC, we report some further environmental factors. The protective effect of breast-feeding suggests that the risk of later developing UC may be influenced by the composition of the faeca flora. The protective effect of increased household crowding suggests that hygiene-related factors may be important early in life. The association with having a vegetable garden suggests that diet early in life may play an important role in $\mathrm{CD}$ aetiology

\section{3}

Mortality in the Kaiser Permanente Northern California Inflammatory Bowe Disease Registry, 1996-2003

Susan Hutfless, Xiaoping Weng, Liyan Liu, James E. Allison, Lisa J. Herrinton

Background \& Aims: We sought to describe the causes of death in a community-based cohort of 9,076 patients with incident and prevalent inflammatory bowel disease (IBD) within the Kaiser Permanente Northern California (KPNC) IBD Registry for the period 1996 through 2003. Methods: The 9,076 IBD patients included persons with at least one inpatien or two outpatient diagnoses recorded in computerized data during 1996-2002 from among the average annual 3.2 million membership of KPNC. Deaths were ascertained from the 2003 California Death Master File, which uses a probability algorithm to assign a single cause of death from all contributing causes listed on the death certificate. To determine the validity of the Master File's classification, chart review was performed on 32 patients whose cause was attributed to IBD (ICD codes 555 and 556). Standardized mortality ratios (SMR) were calculated as a measure of the association between IBD and risk of mortality with exact Poisson 95\% confidence intervals. Results: A total of 545 IBD patients died compared with 470 expected, resulting in 75 excess deaths. All-cause mortality was increased by $15 \%$ among IBD patients ( $95 \%$ CI, 6-26\%), with most of the increase being focused on Crohn's disease $(C D)(40 \%$ increase, $\mathrm{CI}, 10-70 \%)$. The number of excess deaths was greatest for respiratory disease $(\mathrm{N}=29)$, followed by septicemia and other infections $(\mathrm{N}=15)$, colorectal cancer $(\mathrm{N}=14)$, digestive diseases other than IBD $(\mathrm{N}=11)$, and small bowel cancer $(\mathrm{N}=2)$. The relative excesses in risk were as follows: respiratory disease, $50 \%$ excess $(95 \% \mathrm{CI}, 20$ $90 \%$ ); septicemia, 380\% (130-780); other infections, $180 \%$ (50-370); colorectal cancer $100 \%$ (30-190); and digestive disease other than IBD, 50\% (0-100). Two deaths from smal bowel cancer occurred among CD patients, compared with 0.04 expected $(\mathrm{p}<0.001)$. The risk of death from colorectal cancer did not differ appreciably between ulcerative colitis and $\mathrm{CD}$. Conclusions: IBD, especially $\mathrm{CD}$, is related to a substantial increase in mortality. The role of IBD and its treatment in death from respiratory disease is underappreciated and merits greater attention. Ulcerative colitis and $\mathrm{CD}$ were similarly related to death from colorectal cancer.

\section{4}

Lifetime Utilization of Health Care Resources by Patients with IBD in Manitoba: A Profile of Time Since Diagnosis

Teresa Longobardi, Charles Bernstein

Keywords: Lifetime costs, case-control studies, inflammatory bowel disease Objectives: We tested the hypothesis of non-linear longitudinal trends for health-care resource utilization by individuals with inflammatory bowel disease (IBD) in Manitoba. Methods: Administrative databases were used to examine utilization of health-care resources by persons with IBD 5756 cases of IBD in the 2000-2001 fiscal year were separated into incident cases (0-5 years of disease), cases with long-standing disease (6-15 years of disease), and cases with very long-standing disease (16 or more years of disease). A pooled group of 66,406 matched controls represented the general population against which the likelihood of utilization for a number of services was estimated. Odds ratios indicating the likelihood of resource-use within each category of disease duration were computed using logistic regression analysis, controlling for age and gender. Excess demand was inferred for outpatient visits and overnight hospital stays including visits with a specialist, gastroenterologist or surgical visits. IBD cases were stratified by whether they had an IBD-specific diagnosis (a primary or secondary ICD 9-CM diagnosis code) IBD or non-IBD-specific diagnoses. IBD cases were also stratified by the type of IBD, namely Crohn's disease (CD) or ulcerative colitis (UC). Results: Compared with the general population, in the first five years of disease IBD cases were over five times as likely (OR=5.51; $95 \% \mathrm{CI}=(4.92-6.18)$ to have had a gastroenterology visit; over three times as likely to have had an outpatient surgical visit $(\mathrm{OR}=3.53 ; 95 \% \mathrm{CI}=(3.15-3.95))$; and over three times as likely to have had an overnight hospital stay and an overnight stay attended by a specialist $(\mathrm{OR}=3.28 ; 95 \% \mathrm{CI}=(2.85-3.77)$; and $\mathrm{OR}=3.37 ; 95 \% \mathrm{CI}=(2.88-3.95)$ respectively). IBD patients that had a longer duration of disease had significantly lower odd of utilization compared with the incidence cases. Utilization rates appeared to be constan across disease duration categories. There were few differences found in the odds of resourceuse of $\mathrm{CD}$ cases compared to UC cases. Conclusions: Our results suggest within the first five years from disease diagnosis were when the greatest increase in costly resources were used by cases with IBD. We can likely measure the greatest proportion of treatment effects on resource use within a relatively short period.

905

The Impact of Negative Affectivity \& Conscientiousness On Quality of Life \& Symptom Severity in Patients with Inflammatory Bowel Disease Robert Rahimi, Laurie Keefer, Shahriar Sedghi, Ece Mutlu, Ali Keshavarzian

Individual psychological variables can substantially mediate the effects of chronic illness Less is known about psychological factors influencing Inflammatory bowel disease (IBD), chronic inflammatory condition associated with reduced quality of life (QOL) \& severe 
symptoms. Early identification of such factors may influence disease management. The aim of this study was to understand the role that positive $\&$ negative attributes, including conscientiousness $(\mathrm{C})$, neuroticism $(\mathrm{N})$ and hostility $(\mathrm{H})$ may play in symptom severity \& QOL in IBD. Methods: 72 patients w/biopsy-confirmed inactive IBD (37 CD, 35 UC) were studied [mean age 42.29 (19-75); 9.84 (9.54) yrs w/disease]. Psychological questionnaires included the NEO-FFI personality inventory, Buss-Perry Aggression Questionnaire, SF-12 health-related QOL measure \& a global measure of symptom severity. Data was analyzed using SPSS v.12. Multivariate analyses of variance \& regression were performed to identify possible relations between the aforementioned psychological characteristics, QOL \& symptom severity. Results: $\mathrm{C}$ alone predicted better physical QOL $\&$ accounted for as much as $30 \%$ of the variance $(\mathrm{R} 2=.32, \mathrm{t}=2.70, \mathrm{p}=.008)$. There was an interaction effect for $\mathrm{N}$ and $\mathrm{H}$ such that patients w/high levels of both traits exhibited the lowest QOL. There was also an interaction effect of diagnosis such that patients w/CD who had higher levels of $\mathrm{N}$ and $\mathrm{H}$ had the lowest mental health $\mathrm{QOL}(\mathrm{F}=3.20, \mathrm{p}=.048)$. While $\mathrm{N}$ and $\mathrm{H}$ have some degree of overlap $(\mathrm{r} 2=.41, \mathrm{p}<.001)$ they were independent constructs. $\mathrm{C}$ positively mediated the effects of abdominal pain $[\mathrm{F}=2.98, \mathrm{p}=.04]$ but not overall symptom severity. None of the other personality constructs were significantly linked to QOL or symptom severity. Conclusion: Identifying baseline personality constructs relevant to IBD is important for disease management. Our data supported our hypothesis that the presence of high levels of $\mathrm{N}$ and H negatively impacted QOL \& symptom severity in IBD. This seemed to be most notable in patients w/CD. Previous research has linked negative affect to poor health outcomes in a variety of conditions including cardiovascular disease and cancer. As expected, $C$ accounted for $30 \%$ of the variance in predicting general physical well being \& improved symptoms of abdominal pain. $\mathrm{C}$ has also been linked to improved disease management $\mathbb{E}$ adherence to medical regimens in other diseases including diabetes and HIV. C may also be a positive attribute in IBD where adherence is critical in reducing flares. Future studies are needed to determine whether psychosocial interventions can positively affect IBD course and disease outcome.

906

Rapid Reorganisation of the Human Swallowing Motor Cortex to a Virtual Lesion Induced By lhz Repetitive Cortical Stimulation Satish Mistry, John Rothwell, David G. Thompson, Shaheen Hamdy

Background: The cortical control of swallowing is bilaterally innervated but displays interhemispheric asymmetry, with dominant (D) and non-dominant (ND) projections. However the precise relationship between these pathways remains unclear. $1 \mathrm{~Hz}$ repetitive transcranial magnetic stimulation (rTMS) can transiently suppress brain excitability non-invasively (a virtual lesion) with effects on peripheral function. Until now, virtual lesions have not been applied to bilateral systems such as swallowing. Aims: We assessed the effects of a $1 \mathrm{~Hz}$ rTMS-induced virtual lesion on the excitability of D and ND swallowing motor cortex (SMC) and compared this to hand MC. Methods: 7 healthy adult volunteers (4 male, age range 25-46) had EMG excitability measurements from the pharynx and the hand in response to TMS before and up to 60 minutes post $1 \mathrm{~Hz}$ rTMS. Real and sham rTMS paradigms (600 pulses over D SMC) were applied at 120\% of pharyngeal resting motor threshold and randomised to separate days. Data were analysed using a non-parametric ANOVA. Results: Real $1 \mathrm{~Hz}$ rTMS dramatically suppressed D SMC by $36 \pm 6 \%\left({ }^{*} \mathrm{p}<0.006\right)$ immediately and for up to 45 minutes afterwards (Figure 1). Hand MC was similarly suppressed by $41 \pm 13 \%$ $(\dagger p<0.01)$ up to 30 minutes whereas ND SMC showed a transient increase in activity. In contrast, sham rTMS had no effect. Conclusions: An rTMS-induced virtual lesion can induce differential inter-hemispheric suppression and excitation of SMC. These compensatory changes may be relevant to mechanisms of recovery after dysphagic stroke, a likely consequence of the bilateral innervation pattern for swallowing.

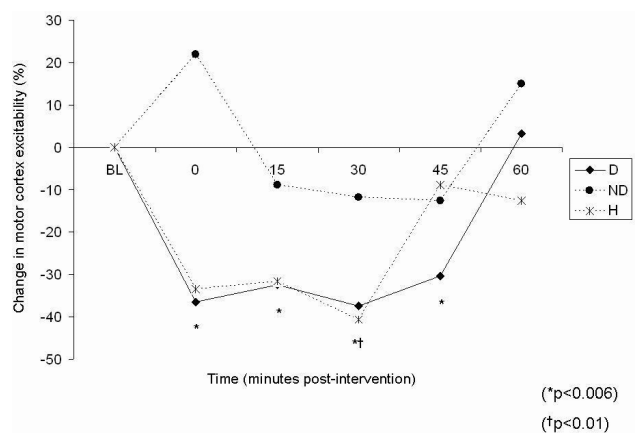

Figure 1: Changes in excitability in swallowing and hand motor cortex following real $1 \mathrm{~Hz}$ rTMS

907

Regional Brain Activation During Gastric Nutrient Infusion in Healthy Volunteers

Brecht Geeraerts, Lukas van Oudenhove, Patrick Dupont, Stijn Dirix, Dominique Vanderghinste, Guy Bormans, Koen van Laere, Jan Tack

Background: Brain regions processing gastrointestinal (GI) sensory information are classically studied using balloon distension of the GI tract, but it is unknown whether more physiological stimuli, including ingestion of a meal, activate similar brain regions. The aim of the present study was to identify brain regions which are activated by gastric nutrient infusion in healthy volunteers. Methods: Brain 150-water PET imaging was performed on a Siemens BGO HR+ in 15 healthy volunteers $(22.8 \pm 2.1$ years, 11 women $)$ during administration of a standardized liquid meal (Nutridrink $\circledR_{1}, 150 \mathrm{kcal} / 100 \mathrm{ml}$ ) through a nasogastric tube. A liquid nutrient bolus was injected 60 seconds prior to each image acquisition. Satiety and abdominal sensation were scored on separate $0-6$ graded scales ( $0=$ no sensation, $6=$ maximal sensation) immediately after each acquisition. Measurements were stopped when abdominal discomfort (score $\geq 5$ ) was reached. To determine maximal activation, baseline activity was subtracted from activity at discomfort intragastric volume. To detect activations correlating with total injected nutrient volume, satiety or upper abdominal sensation, analyses were performed using total volume, satiety score and sensation score as covariate. Data were analysed using Statistical Parametric Mapping (SPM2) software Results: Subtraction analysis showed significant activation $\left(\mathrm{P}_{\text {corrected }}<.05\right)$ of the right superior temporal gyrus and the left cerebellum at discomfort nutrient volume $(783 \pm 65 \mathrm{ml})$. Several key regions of the "visceral sensory network" (including insula, anterior cingulate cortex and somatosensory cortex (SI/ SII), which are activated by intragastric balloon distension (Vandenberghe 2005), were not significantly activated in this study, even at a low threshold $\left(\mathrm{P}_{\text {uncorrected }}<.05\right)$. Several brain regions were found to covary significantly $\left(\mathrm{P}_{\text {corrected }}<.05\right)$ with nutrient volume as well as satiety score and upper abdominal sensation score: bilateral gyrus frontalis medius/inferior and a small part of the right gyrus frontalis superior and gyrus orbitalis (orbitofrontal cortex, BA10/11), left cerebellum and a small part of the gyrus frontalis medialis/gyrus frontalis superior bilaterally (dorsomedial prefrontal cortex, BA8/9). Conclusion: The results of the subtraction analysis question the generalizability of balloon distension studies towards physiological stimuli like food ingestion. The covariate analyses confirm the role of the orbitofrontal cortex in multimodal processing of food-related information (Price 2003).

908

Regional Cerebral Cortical Fmri Activity Volume Is Directly Related to the Intensity of Subliminal Proximal and Distal Esophageal Distension Mark Kern, Adeyemi Lawal, Arthi Sanjeevi, Tanya Rittmann, Candy Hofmann, Reza Shaker

FMRI studies have documented brain activation in response to subliminal stimulation of the esophagus. At perceived levels, cortical activity response was significantly more than at subliminal levels. Presence or absence of a dose response to subliminal stimulations in specific regions of the brain has not been studied. AIM: Determine the fMRI response to a range of subliminal proximal and distal esophageal distensions in the cingulate gyrus and insular cortex. Methods: We studied 12 healthy subjects (age: 18-35) during fMRI of the insula $(n=6)$ or cingulate $(n=6)$. Paradigm-driven scans were performed during intervals of randomly timed rest and 15-seconds of esophageal distension before and after 30 min of distal esophageal acid perfusion $(0.1 \mathrm{~N} \mathrm{HCl}, 1 \mathrm{ml} / \mathrm{min})$. Correlation analysis was used to test the relationship between subliminal stimulus intensity and fMRI activity. Results: For both proximal and distal esophagus, there was always a positive correlation between subliminal distension and the amount of fMRI activity in both the cingulate and insula. The positive correlation between stimulus intensity and fMRI activity was preserved after acid in the distal esophagus (figure). Correlation coefficients were always positive for distal (pre-acid coefficient range:0.44 to0.99 post-acid:0.77 to 0.99 ) and proximal (pre-acid: 0.76 to 0.99 , post-acid: 0.74 to 0.99 ) esophageal distensions. Conclusions: In the subliminal domain of distal and proximal esophageal distension, the magnitude of recruitment in the cingulate and insula is directly related to the intensity of esophageal distension. This dose-dependent response esophagus is preserved after distal esophageal acid exposure. 


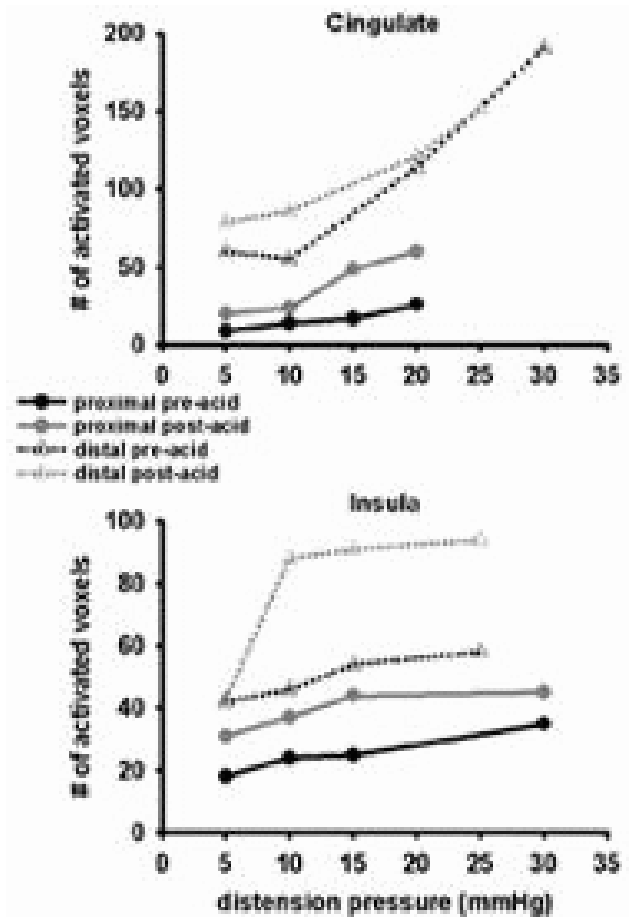

909

Attentional Modulation of Visceral and Somatic Pain

Paul Dunckley, Qasim Aziz, Richard G. Wise, Jonathon Brooks, Irene Tracey, Lin Chang

Background/Introduction. Hypervigilance, an increased state of guardedness, watchfulness or attention, has been proposed as a possible underlying aetiology in irritable bowel syndrome (IBS) and fibromyalgia (FM). As hypervigilance must be a centrally mediated process, functional magnetic resonance imaging of the brain can be used to test this hypothesis. However, a better understanding of the normal attentional mechanisms during visceral pain in health is initially required. The aim of this experiment was to identify the neural correlates underlying the attentional modulation of visceral and matched somatic pain. Methods. 12 healthy, pain-free, right-handed subjects were recruited for the study. Simultaneous electrical pulses and auditory tones lasting 6 seconds were delivered to the subjects during a wholebrain functional scan acquisition. The electrical catheter was placed into the rectum for the visceral scan and onto the lower abdomen for the somatic scan. Subjects were instructed to attend to and count either the auditory tones or electrical pulses. Electrical pulses and auditory tones were delivered at either $2,3.5$ or $4.3 \mathrm{~Hz}$ thereby varying the cognitive demand of the tasks. Pain intensity, unpleasantness and tone/pulse count were recorded after each stimulus. Results. During the $3.5 \mathrm{~Hz}$ and $4.3 \mathrm{~Hz}$ conditions alone, distraction by the auditory tones resulted in a significant reduction in pain report $(\mathrm{p}<0.05)$ in both sensory modalities. There was a marked spatial overlap in the group statistical activation maps during somatic and visceral pain. The typical "pain matrix" was activated. Using pain intensity values as regressors in a group level regression analysis, the left primary somatosensory cortex (SI) increased in activity with increasing pain report, during attention to visceral pain. Bilateral anterior insula cortex activity increased with somatic pain report independent of the direction of attention. Conversely, the primary auditory cortex (AI) increased in activation with decreased pain report. Right dorsolateral prefrontal cortex (DLPFC) activity correlated positively with pain intensity during somatic pain. Conclusions. Pain perception during attentional modulation is reflected in the primary sensory cortex and anterior insula cortex activity and may be influenced in a top-down manner by the right DLPFC during somatic pain. A positive correlation of the right DLPFC, a region more commonly activated in IBS and FM patients than controls in functional imaging studies, with somatic pain intensity suggests that this region biases incoming sensory inputs (nociceptive and auditory) towards nociceptive processing.

910

Structural Brainstem Alterations in Patients with Irritable Bowel Syndrome (IBS)

Joshua Bueller, Steven M. Berman, Brandall Y. Suyenobu, Bruce D. Naliboff, Kashyap Trivedi, Jean Stains, Emeran A. Mayer

BACKGROUND: The periaqueductal gray (PAG) plays a major role in descending pain modulation and central autonomic control. Patients with IBS have enhanced perception of visceral stimuli and alterations in autonomic regulation of gut function. We have reported altered activation of the PAG in IBS patients during aversive visceral stimulation as well autonomic dysregulation due to this stimulus. Thus, IBS, and perhaps other functional disorders, may be partially mediated by altered structure/function of the PAG. AIMS: Compare the amount of gray matter in the PAG between IBS patients and healthy control subjects using voxel-based morphometry (VBM). METHODS: 11 female constipation-predominant (IBS-C) patients and 11 healthy age and sex matched control subjects were scanned on a 1.5T Siemens Sonata camera using a 3D Tl-weighted MPRAGE sequence $(0.5 \times 0.5 \times 1$ $\mathrm{mm})$. VBM was conducted on segmented normalized images after modulation to correct for volume changes. Statistical Parametric Mapping software (SPM2; p<.01) made voxelwise group comparisons. Differences in the amount of gray matter were assessed for normality and significance with SPSS. RESULTS: Gray matter volume in a region including the PAG was significantly larger in IBS patients compared to controls $(t=3.07, p=0.007)$. In patients, the size of the PAG positively correlated with the duration of symptom flare-up ( $\mathrm{p}=0.043$ $r=0.617)$. CONCLUSIONS: This is the first demonstration that functional abnormalities of IBS patients in brainstem regions involved in central autonomic control and endogenous pain modulation may be accompanied by structural abnormalities.

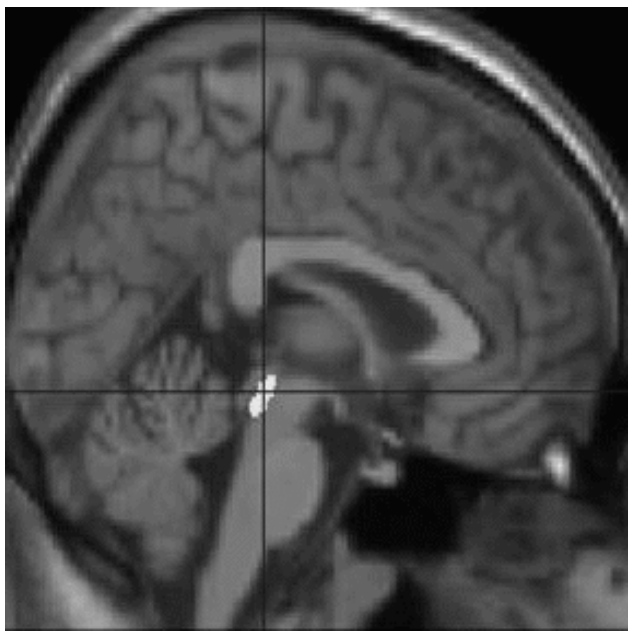

911

The Effect of Esophageal Acidification On Airway Mucociliary Function Ivan M. Lang, Steve T. Haworth, Bidyut K. Medda, Reza Shaker

Gastroesophageal reflux disease (GERD) is associated with symptoms of asthma, i.e. narrowing of the airways and increased airway resistance; but asthma is also characterized by increased airway mucus secretion. However, the effect of esophageal acidification on airway mucociliary function is unknown. AIM: Our aim was to determine the effects of esophageal acidification on measures of airway mucociliary function. METHODS: Cats $(\mathrm{N}=18)$ were anesthetized with alpha-chloralose $(50 \mathrm{mg} / \mathrm{kg}$, IP) and the effects of esophageal acidification on three measures of airway mucociliary function: tracheal mucus secretion, tracheal mucociliary transport, and lung clearance, were determined. In all studies the esophagus was cannulated just below the cricopharyngeus and just above the lower esophageal sphincter and the esophagus was perfused with $0.1 \mathrm{~N} \mathrm{HCl}$ or $0.1 \mathrm{MPBS}$ at $1 \mathrm{ml} / \mathrm{min}$. Tracheal mucociliary transport was quantified $(\mathrm{N}=7)$ by determining the rate of orad movement of a tantalum puck $(1 \mathrm{~mm}$ diameter, $0.3 \mathrm{ug}$ ) using a micro-focal $\mathrm{x}$-ray system. Tracheal mucus secretion was quantified $(\mathrm{N}=6)$ by measuring the hexosamine content of tracheal perfusate $(0.9 \%$ $\mathrm{NaCl}$ at $0.25 \mathrm{ml} / \mathrm{min}$ ) taken every $10 \mathrm{~min}$. Lung clearance was quantified ( $\mathrm{N}=5$ ) by measuring the clearance of Tc99 sulfur colloid from the lungs using gamma camera. RESULTS: We found that esophageal acidification significantly decreased tracheal mucociliary transport by about $50 \%$, increased tracheal mucus secretion by about $350 \%$, and stopped lung clearance (Table). CONCLUSIONS: Esophageal acidification causes a significant increase in mucus secretion that results in a significant decrease in tracheal mucociliary transport and lung clearance. In prior studies it was found that esophageal acidification caused a small increase in airway resistance or small decrease in airway diameters. Therefore, we conclude that the primary airway response to esophageal acidification is increased mucus secretion and given the buffering ability of mucus this physiological response acts to protect the airways from subsequent $\mathrm{H}^{+}$aspiration that may occur during GERD. Supported in part by DK-25731 Table. Effect of esophageal acidification on airway mucociliary function

\begin{tabular}{|c|c|c|}
\hline Airway Mucociliary Function Measures & PBS & HCl \\
\hline Tracheal Mucociliary Transport $(\mathrm{mm} / \mathrm{min})$ & $8.5 \pm 1.2(4)$ & $4.8 \pm 1.2(3)^{*}$ \\
\hline Tracheal Hexosamine Output $(\mu \mathrm{g} / 10 \mathrm{~min})$ & $420 \pm 350(6)$ & $1450 \pm 500(6)^{*}$ \\
\hline Lung Clearance $(1000$ counts/min) & $2.5 \pm 1.2(4)$ & $-0.2(1)$ \\
\hline
\end{tabular}

Values are mean $\pm \mathrm{SE}(\mathrm{N}) . *, \mathrm{P}<0.05$ for a difference between $\mathrm{PBS}$ and $\mathrm{HCl}$.

912

Non-Acid Reflux Events Reaching the Pharynx Are Important Factors Associated with Cough

Robert N. Patterson, Inder Mainie, Gerard Rafferty, Lorcan P. McGarvey, Liam G. Heaney, Radu Tutuian, Donald O. Castell, Brian T. Johnston

Gastroesophageal reflux has been suggested as an aggravating factor in patients with asthma and chronic persistent cough. To date most studies have focused on acid reflux and measured $\mathrm{pH}$ below the UES. The aim of this study was to assess the relationship between cough and reflux both above and below the UES. Patients: 37 respiratory patients were recruited from the respiratory clinic (18 with chronic persistent cough and 19 with stable asthma). Methods Impedance: A combined multichannel intraluminal impedance and $\mathrm{pH}$ probe with impedance measuring segments at 3,5,7 and $9 \mathrm{~cm}$ above the LES and 1 and $2.5 \mathrm{~cm}$ above the UES and $\mathrm{pH}$ sensors $5 \mathrm{~cm}$ above the LES and $1 \mathrm{~cm}$ above the UES was used to monitor patients with respiratory symptoms after having stopped any acid suppressive therapy for at least 7 days 
SAP for cough: Symptom association probability (SAP) was calculated as described by Weusten et al. using a 2-minute association window between symptoms and bolus entry $5 \mathrm{~cm}$ above the LES SAP was considered positive if $>95 \%$. Results (median (IQR)): On average $13 \%$ of impedance episodes reached above the UES. In total only $1-2 \%$ of all these episodes registered on the pharyngeal pH probe. 26 of 37 patients recorded cough. 7 of these $26(27 \%)$ had a positive cough SAP. The +ve SAP group recorded more impedance episodes crossing the UES compared with -ve SAP patients. This was true for both the total number reaching the pharynx $(5(2.5-8.5)$ v. $2(1-4) \mathrm{p}=0.05)$ and when expressed as a \% of all esophageal impedance episodes $(14 \%(12.5-28 \%)$ v. $6 \%(2-12.5), \mathrm{p}<0.02)$. There was no difference between patients with a +ve SAP and those with a -ve SAP in the total number esophageal impedance episodes (37(24-57) v. 31(16.5-48.5)), acidic esophageal impedance episodes (12(7.5-18.5) v. 10(2.5-17)) or esophageal acid exposure time (\%total $<\mathrm{pH} 4)$ (1.7(1.2-4.5) v. 3.8(1.1-10.2)). Conclusion: A positive cough SAP did not correlate with any parameter of esophageal reflux. By contrast, it was significantly associated with reflux reaching the pharynx. These episodes were not detected by pharyngeal $\mathrm{pH}$ monitoring. Pharyngeal non-acid reflux is an important measurement in patients with cough. Weusten BL et al. Gastro 1994:107:1741-5.

\section{3}

The Role of Gastroesophageal Reflux (GER) in Exercise-Triggered Asthma Kathryn Peterson, John Fang, Chistopher Canale, Darin Ryujin, David Young, Wayne Samuelson

Background: Exercise-triggered asthma develops when vigorous physical activity triggers symptoms of cough, wheezing, dyspnea, and/or chest tightness during or directly after exercise. ETA can occur both in people with chronic asthma and in otherwise healthy individuals. In patients prone to symptoms of supra-esophageal reflux, exercise may trigger GER, resulting in shortness of breath and cough. Aims: (1)To determine the prevalence of abnormal $\mathrm{pH}$ in patients with exercise-triggered asthma, (2)To determine whether acid suppression improves respiratory symptoms during exercise in ETA patients. Methods :We performed a randomized, double blind trial of rabeprazole versus placebo in the treatment of patients (both asthmatic and non-asthmatic) with exercise-triggered asthma. Patients reported "heartburn" less than $2 x /$ week. Upon enrollment, subjects underwent underwent 24 hour $\mathrm{pH}$ testing while undergoing 30 minute treadmill program exercising them at predetermined $60-65 \%$ of their VO2max. They were subsequently randomized to rabeprazole $20 \mathrm{mg}$ Qam and placebo QPM, rabeprazole $20 \mathrm{mg}$ po BID, or placebo BID for 12 weeks. At the end of 12 weeks, exercise testing was repeated. Subjects reported whether their treadmill symptoms improved or did not improve at the end of the study. Results: 37 total patients were recruited. A total of 30 patients completed the study in it's entirety (20 asthmatics, 10 non-asthmatics). 22/30 (73\%) subjects had abnormal pH studies. For all subjects, rabeprazole improved symptoms more often than placebo( $\mathrm{p}=0.04)$. In the $\mathrm{pH}+$ group, rabeprazole resulted in even greater improvement $(\mathrm{p}=02)$. Discussion: Acid reflux is common in ETA patients. Many patients with exercise-related respiratory symptoms are under-diagnosed (or misdiagnosed) as chronic asthmatics. In addition, exercise related symptoms improve with the use of acid suppression. Such patients may benefit from an empiric trial of high dose acid suppression.

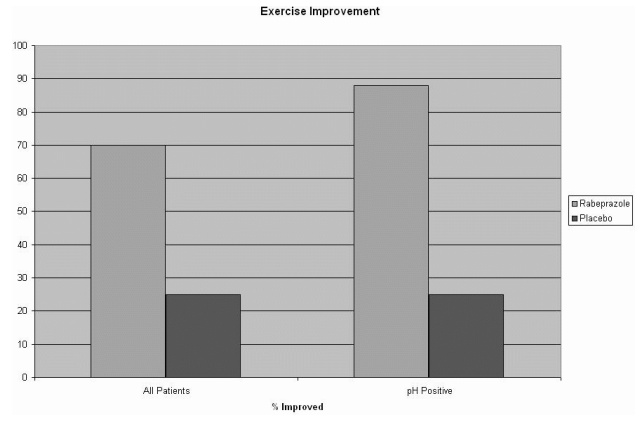

914

What Is the Optimal Initial Therapy Duration for Patients with Suspected GERD-Related Laryngitis?

Michael F. Vaezi, Rocio Lopez, Douglas Hicks, Thomas Abelson, Claudio Milstein

Objective: ENT and GI literatures currently recommend initial PPI therapy of at least 3-4 months in pts suspected with GERD-related laryngitis. However, there are currently no well designed studies in support of such recommendations. Thus, the aim of our study was to assess the optimal treatment response duration for this group of patients. Methods: Pts with suspected GERD-related laryngitis based on chronic throat symptoms and laryngoscopic findings were enrolled. Questionnaire assessed the presence/severity of throat symptoms and heartburn. Symptom severity scored using a scale of 1 to $4[1=$ rare (once a month or less); $2=$ occasional (once a week or less); 3 =frequent (several times a week); $4=$ all the time (several times daily)]. Subjects kept daily symptom diaries of the primary throat symptom and heartburn during 4-months of BID PPI therapy. Greater than 50\% primary symptom improvement from baseline defined symptom response. Results: 47 Patients enrolled (median age: 49 yrs, IQR: 44.0 - 65.0; 76\% Caucasian; 34\% male). Daily symptom diaries were complete in $43(91 \%)$ patients. $34 / 43(79 \%)$ patients responded to therapy. The most common presenting throat symptoms (prevalence, median severity score) included: throat clearing $(84 \%, 2.8)$, hoarseness $(80 \%, 3.0)$, and cough $(71 \%, 2.3)$. Heartburn $(65 \%, 2.4)$ and regurgitation $(55 \%, 2.1)$ were present in nearly half of pts. Maximum $(92 \%)$ symptom response to therapy was achieved by 30 days for the primary throat symptoms (Figure) and by 18 days for heartburn (Figure 2). Conclusions: 1) In patients with suspected GERDrelated laryngitis one month of therapy is adequate to assess response to therapy. 2) In those unresponsive to PPI therapy after one month the likelihood of symptomatic response with continued PPI therapy does not justify the cost.

Primary Symptom Score by Day

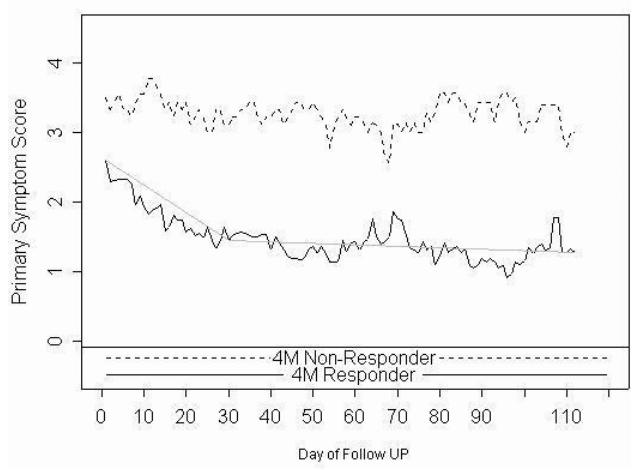

915

Effects of High Dose PPI (rabeprazole) On Laryngopharyngeal Reflux Disease: A Prospective, Double Blind, Randomized Controlled Study Apichart Suramethakul, Permsarp Isipradit, Supinda Saengpanich, Sutep Gonlachanvit

Background: The role of high dose PPI on laryngopharyngeal reflux disease has not been well established. We performed a randomized, placebo-controlled, double blind, single center study to evaluate the effect of high dose PPI in patients with chronic laryngopharyngeal reflux symptoms. Methods: Thirty patients $(8 \mathrm{M}$, age $45 \pm 2 \mathrm{yrs}$, mean $\pm \mathrm{SEM})$ with suspected of having laryngopharyngeal reflux disease for $>12$ weeks(median durations 15 mo(3-120) by ENT doctors were included. Each patient underwent a video laryngoscopy and a dual channel(15 cm apart), $24 \mathrm{hr}$ esophageal $\mathrm{pH}$ testing before receiving either rabeprazole(R) $20 \mathrm{mg}$ bid or placebo(P) for 12 wks. Each laryngopharyngeal reflux symptom including, hoarseness, lump in the throat, cough, clearing throat, sore throat, burning throat, breathing difficulties or chocking, and mucous in the throat was scored 0-5 using modified visual analog scales before and at the end of treatments. Positive $\mathrm{pH}$ monitoring results for lower and upper esophagus were defined as $\%$ time $\mathrm{pH}<4$ at lower esophagus $>4 \%$ and upper esophagus $>1 \%$, respectively. A positive $\mathrm{pH}$ test was defined as a positive result in either lower or upper esophagus. Results: The main symptoms were lump in the throat $(n=20)$, sore throat(4), burning throat(5), and hoarseness(1). 16 patients had weekly typical GERD symptoms. 15, 15, and 18 patients had positive esophageal $\mathrm{pH}$ tests at lower, upper, and

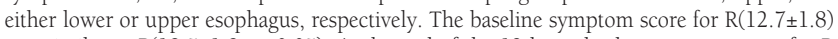
was similar to $\mathrm{P}(13.5 \pm 1.3, \mathrm{p}>0.05)$. At the end of the 12 th week, the symptom score for $\mathrm{R}$ was $4.2 \pm 1.6$, significantly improved compared to $\mathrm{P}(8.7 \pm 1.3, \mathrm{p}<0.05)$. Baseline symptom scores for patients with positive $\mathrm{pH}$ test in $\mathrm{R}$ and $\mathrm{P}$ group were similar(11.4 $\pm 1.9 \mathrm{vs} 14.2 \pm 1.9$, $\mathrm{p}>0.05)$. R significantly improved the symptom scores compared to $P$ at the end of the 12 th wk $(2.9 \pm 1.2$ vs $9.7 \pm 1.7, \mathrm{p}<0.01)$ in patients with positive $\mathrm{pH}$ tests. In contrast, the symptom scores for $\mathrm{R}$ and $\mathrm{P}$ group in patients with negative $\mathrm{pH}$ tests were similar at both baseline $(14.5 \pm 3.4$ vs $12.3 \pm 1.7, \mathrm{p}>0.05)$ and the 12 th wk $(6.0 \pm 3.4$ vs $7.2 \pm 2.4, \mathrm{p}>0.05)$. Reflux finding scores(RFS) evaluated by video endoscopies were improved similarly in both $\mathrm{R}$ and $\mathrm{P}(\mathrm{p}>0.05)$. There was no significant different of RFS improvements after R or P irrespective of the $\mathrm{pH}$ results ( $p>0.05$ ). Conclusions: High dose rabeprazole is superior to placebo for relieving ENT symptoms in patients with laryngopharyngeal reflux disease especially in patients with positive $\mathrm{pH}$ tests, but not in patients with negative $\mathrm{pH}$ tests. The dual channel, 24-hour esophageal $\mathrm{pH}$ monitoring is helpful for identifying patients who will have benefits from high dose PPI therapy.

916

Reg IV Increases Apoptotic Resistance and Crypt Stem Cell Survival in a Radiation-Induced Intestinal Injury Model

Kumar S. Bishnupuri, Qizhi Luo, Satheesh K. Sainathan, Eyad M. Hanna, Courtney W Houchen, Shrikant Anant, Brian K. Dieckgraefe

Background: The human Regenerating (Reg) gene family encodes four small-secreted proteins. Reg IV, a recently identified member of the family is expressed at highest levels in the colonic mucosa and is significantly upregulated in malignancies of the gastrointestinal (GI) tract. Overexpression of Reg IV is also associated with increased resistance of colon tumor cells to chemotherapy. The present study tests the hypothesis that Reg IV increases resistance of GI tumor cells to apoptosis and enhances crypt stem cell survival following radiationinduced intestinal injury. Methods: 6-wk old female C57BL/6J mice were injected IP with vehicle or HPLC purified recombinant human Reg IV (rhR4) ( $4 \mathrm{mg} / \mathrm{kg}$ body weight) either once at $1 \mathrm{~d}$ or daily for $5 \mathrm{~d}$ prior to 6- or $12 \mathrm{~Gy}$ whole body $\gamma$-irradiation (IR). To assess apoptotic cell death, mice were sacrificed $6 \mathrm{~h}$ following IR, and tissue from distal jejunum was stained by Hematoxilin/Eosin and TUNEL methods. To identify GI crypt stem cell survival, a microcolony assay was performed where the mice were administered bromodeoxyuridine (BrdU) at $82 \mathrm{~h}$ following IR and sacrificed $2 \mathrm{~h}$ later. Proliferating crypts in the distal jejunal sections were identified by immunostaining for BrdU. Surviving crypts were counted as those with at least 6 BrdU positive cells. Results: Mice treated with rhR4 $1 \mathrm{~d}$ prior to IR exhibited a significant reduction in the number of apoptotic cells per crypt compared to controls $(1.4 \pm 0.03$ vs. $3.3 \pm 0.12$ in controls, and $1.4 \pm 0.05$ vs. $3.7 \pm 0.11$ in controls for 6- and $12 \mathrm{~Gy}$, respectively). Moreover, the number of cells undergoing apoptosis at crypt cell positions 3 to 6 counted from the base (corresponding to the location of the putative stem cell) was less in rhR4-treated mice than in controls. Further, in the microcolony assay, the rhR4-treated mice had significantly higher numbers of surviving crypts than controls $(25.0 \pm 1.2$ vs. $15.7 \pm 1.2)$. These data suggest that rhR4 may protect 
GI tumor cells including the crypt stem cell population against IR-mediated apoptosis. Similar results for both apoptosis and stem cell survival were observed when Reg IV was administered for $5 \mathrm{~d}$ before IR. Immunostaining of sections and real-time RT-PCR analyses of total RNA isolated from distal jejunum $6 \mathrm{~h}$ following IR demonstrated significantly increased expression of the anti-apoptotic genes, survivin, Bcl-2 and Bcl-XL in rhR4-treated mice. Conclusion: Reg IV appears to serve as an anti-apoptotic mediator in GI tumor cells. Since, Reg IV is significantly upregulated in GI cancers, therapeutic strategies designed to interfere with its activity may enhance the effect of radiation.

917

Hif-1 $\alpha$ Promotes Non-Hypoxic Proliferation in Colon Cancer Cells and Xenografts

Duyen Dang, Fang Chen, Lawrence Gardner, Jordan Cummins, Carlo Rago, Fred Bunz, Sergey V. Kantsevoy, Long H. Dang

BACKGROUND: The hypoxia-induced transcription factor HIF-l $\alpha$ is overexpressed in cancer and its level of expression is correlated with patient mortality (Nat Rev Can 2003:3:72132). HIF-l $\alpha$ mediates cancer cell adaptation to hypoxia by directly transactivating genes important for growth, metabolism, angiogenesis, and metastasis. More recently, cancer genetic alterations have been shown to induce the expression of HIF-l $\alpha$ under non-hypoxic conditions (Embo J 1998:17:5085-94). The role of HIF-l $\alpha$ expression in non-hypoxic cancer cells remains unknown. AIM: We hypothesized that HIF-l $\alpha$ mediates colon cancer cell proliferation under non-hypoxic conditions. METHODS: HIF-l $\alpha$ was genetically disrupted by targeted homologous recombination in HCT116 human colon cancer cells (Nature 2004: 416:1). The resulting HIF-l $\alpha-/$ - cells are isogenic with their parental cells, with the exception of HIF-l $\alpha$ ablation, as confirmed by western blot analyses. The effects of HIF-l $\alpha$ disruption on cell proliferation, glycolysis, and downstream gene expression were determined in vitro. In vivo, the effects of HIF-l $\alpha$ disruption on xenograft growth, microvessel density, blood flow, hypoxia, and proliferation were measured. Two independent clones were analyzed. RESULTS: In vitro, loss of HIF-l $\alpha$ significantly reduced glycolysis, Gl to $S$ cell cycle progression, cell proliferation, and the expression of known downstream genes under both normoxic and hypoxic conditions. In vivo, loss of HIF-l $\alpha$ led to a $~ 40 \%$ decrease in xenograft growth. Surprisingly, there were no alterations in tumor microvessel density as determined by CD31 endothelial staining, or tumor blood flow as determined by Hoescht 33342 diffusion. Moreover, there were no alterations in the extent of the hypoxic tumor compartments as measured by incorporation of the hypoxia marker pimonidazole. Most significantly, loss of HIF- $1 \alpha$ led to $~ 50 \%$ less incorporation of BrdU in the non-hypoxic tumor compartments (BrdU positive, pimonidazole negative regions). Thus, loss of HIF- $1 \alpha$ affected cell proliferation within the non-hypoxic tumor compartments; rather than cell survival in the hypoxic compartments, or tumor angiogenesis. CONCLUSIONS: These data provide the first evidence that HIF-l $\alpha$ promotes cell proliferation in non-hypoxic cells in vitro and in vivo, through induction of aerobic glycolysis and Gl to $S$ cell cycle progression. Surprisingly, while HIF$1 \alpha$ promoted cell proliferation and survival under hypoxic conditions in vitro, its expression did not grossly contribute to the tumor hypoxic compartments in vivo. These data suggest that HIF-l $\alpha$-targeted therapy may be potentially valid in smaller, non-hypoxic tumors.

\section{8}

Expression of the Pro-Proliferative KrüPpel-Like Factor 5 (KLF5) in Intestinal Epithelial Cells Is Induced By Bacterial Infection in Vitro and Correlates with Colonic Crypt Cell Hyperplasia Caused By Citrobacter Rodentium in Vivo Beth B. Mcconnell, Jan-Michael A. Klapproth, Maiko Sasaki, Sengthong Chanchevalap, Jonathan P. Katz, Vincent W. Yang

BACKGROUND: Krüppel-Like Factor 5 (KLF5) is a pro-proliferative transcription factor that is highly expressed in the intestinal tract and is localized to the base of the crypt epithelium where active proliferation occurs. Citrobacter rodentium is a gram-negative bacterium that colonizes the mouse colon, resulting in transmissible murine colonic hyperplasia (TMCH). Previously, we reported that KLF5 expression is increased in intestinal epithelial cells in response to the bacterial component lipopolysaccharide (LPS) [S. Chanchevalap and V.W. Yang (2005) Gastroenterology 128, A33]. Here, we address whether KLF5 expression is induced by bacterial infection in cultured intestinal epithelial cells and whether KLF5 levels are increased in the mouse colon in response to C. rodentium infection. METHODS: Confluent rat intestinal epithelial cells, IEC6, were infected with $C$. rodentium over a $5 \mathrm{hr}$ time course. KLF5 transcript and protein levels were determined by Northern and Western blot analyses, respectively. Mice were infected by oral gavage with $5 \times 10^{8}$ colony-forming-units $C$. rodentium or phosphate-buffered saline (PBS) control. Five infected and control animals were sacrificed at 8,15 or 21 days post-infection and the distal colon harvested and examined by immunocytochemistry using polyclonal antibodies against KLF5 or the proliferative marker, Ki67. RESULTS: In IEC6 cells infected with C. rodentium, KLF5 mRNA expression was induced acutely and transiently, with maximal induction of 2 -fold at 1.5 hrs post-infection. The increase in KLF5 transcript levels was followed by a corresponding increase in KLF5 protein levels. In a mouse model of bacterial infection, animals colonized with $C$. rodentium showed a significant increase in colonic crypt epithelial cell proliferation by day 8 , with crypt height increasing up to 3-fold by day 21 when compared to controls. Expression of KLF5 was correspondingly increased by 3 -fold in the colon of infected animals at day 21 , as measured by the height of KLF5 immunofluorescence signals within the crypt compared to its height in control tissues. The pattern of KLF5 staining in the expanding crypts of infected mice correlated with that of the proliferation marker, Ki67. CONCLUSION: Our study demonstrates that expression of KLF5 is induced in both rat intestinal epithelial cells in vitro and in mouse colon tissues following infection with C. rodentium in vivo. This induction correlates with an expansion of the crypt epithelium, and higher levels of KLF5 are found in the proliferating cells of the crypt. Thus, KLF5 may potentially mediate crypt cell proliferation in mouse colon in response to pathogenic bacterial infection.

\section{9}

Protein Kinase C- $\varepsilon$ Regulates the Apoptosis and Survival of Intestinal Epithelial Cells

Yuning Zhou,, Qingding Wang, B. Mark Evers, Dai H. Chung

Free oxygen radicals are thought to be involved in the pathogenesis of necrotizing enterocolitis (NEC) in premature infants. We have recently found that multiple intracellular signaling pathways, including protein kinase $\mathrm{C}$ (PKC), are activated during oxidative stress-induced intestinal epithelial cell injury. In particular, PKC- $\varepsilon$ isoform has recently been implicated during cellular response to ischemic stress in intestinal epithelia; however, its potential role in NEC is unknown. Therefore, the purpose of this study was to determine the role of PKC $\varepsilon$ in the apoptosis and survival of intestinal epithelial cells during oxidative stress. METHODS Rat intestinal epithelial (RIE)-1 cells were maintained in DMEM with $10 \%$ fetal bovine serum. Cells were plated for $24 \mathrm{~h}$ and then treated with either $\mathrm{H}_{2} \mathrm{O}_{2}(500 \mu \mathrm{M})$ or vehicle. To knockdown the expression of PKC- $\varepsilon$, RIE-1 cells were transfected with small interfering RNAs (siRNA) directed against the PKC- $\varepsilon$ or control siRNA. Treated cells were harvested over a time course and protein extracted. Cell apoptosis was quantitated using a DNA fragmentation ELISA assay. Western blotting was performed to determine PKC- $\varepsilon$, caspase3 activation, cleavage of poly (ADP-ribose) polymerase (PARP), total and phosphorylated protein kinase D (PKD), a novel protein kinase which mediates important cell surviva signals during oxidative stress. RESULTS: Treatment with $\mathrm{H}_{2} \mathrm{O}_{2}$ resulted in the rapid induction of phosphorylation of PKC- $\varepsilon$ phosphorylation without affecting total PKC- $\varepsilon$ expression. $\mathrm{H}_{2} \mathrm{O}_{2}$ significantly increased DNA fragmentation, caspase- 3 activation and PARP cleavage in RIE-1 cells; siRNA directed against PKC- $\varepsilon$ significantly enhanced $\mathrm{H}_{2} \mathrm{O}_{2}$-induced RIE1 cell apoptosis. To delineate the molecular mechanisms involved in the enhanced apoptosis noted by PKC- $\varepsilon$ silencing, we determined the phosphorylation status of PKD, which can be regulated by $\mathrm{PKC}-\varepsilon$. Treatment with $\mathrm{H}_{2} \mathrm{O}_{2}$ increased the expression of phosphorylated PKD and PKC- $\varepsilon$ siRNA completely blocked this induction. CONCLUSIONS: Our results suggest that PKC- $\varepsilon$ plays an anti-apoptotic role in the oxidative stress-induced intestinal cell injury Moreover, PKC- $\varepsilon$ regulates PKD activity and may be essential for the survival of intestinal cells. Delineating the signaling pathways regulating oxidative stress-induced intestinal cell injury will provide a better understanding of the molecular mechanisms contributing to NEC and potentially lead to novel treatment strategies.

\section{0}

Basic Fibroblast Growth Factor-Induced Multidrug Resistance 1 Expression Plays An Important Role in the Protective Effect On Intestinal Epithelial Injury

Minoru Matsuura, Hiroshi Nakase, Satohiro Masuda, Masanori Asada, Satoru Ueno, Hiroshi Kitamura, Satoko Inoue, Norimitsu Uza, Katsuhiro Kasahara, Yasuhiko Tabata, Ken-Ichi Inui, Tsutomu Chiba

Background and aims: Multidrug resistance (MDR) 1 gene product is highly expressed in the epithelial surfaces of intestine, where it constitutes a barrier against luminal antigens and bacteria. We previously reported that rectal administration of basic fibroblast growth factor (bFGF) ameliorated experimental murine colitis (Gastroenterology, 2005). However it remains unclear whether MDRl expression plays an important role in the protective effect of bFGF on intestinal epithelial injury. The aim of this study is to investigate whether bFGFinduced MDRl expression is involved in the protective effect on intestinal epithelial injury in vitro and in vivo. Materials and Methods: 1) Gene expression of MDRla and MDRlb in IEC-6 cells stimulated with and without human recombinant bFGF (HrbFGF; 100 ng/ml) was determined by semiquantitative RT-PCR and western blot analysis. We also examined the signaling pathway associated with bFGF-induced MDRl expression. 2) MDRl expression in the small intestine of FVB/N normal mice treated with $\mathrm{HrbFGF}(5.0 \mathrm{mg} / \mathrm{kg}$, i.p.) was determined by western blot analysis. 3) We investigated the protective effects of bFGF on intestinal epithelial injury using both mdrl knockout mice and FVB/N control mice. Intestinal epithelial injury was induced by X-ray irradiation (12Gy). Mice received HrbFGF (5.0 mg/ $\mathrm{kg} /$ day, i.p.) daily for 5 days just before and after irradiation. Mice were killed at 5 days after irradiation, and histologic findings of small intestine were examined. Results: 1) Gen expression of MDRla and MDRlb in bFGF-treated cells was significantly higher than in non-treated cells (MDRla/GAPDH ratio: $1.21 \pm 0.08$ vs $0.80 \pm 0.08 ; p<0.05$, MDRlb/GAPDH ratio: $1.38 \pm 0.11$ vs $0.71 \pm 0.05 ; p<0.05$ ). bFGF-induced MDRl expression was inhibited by pretreatment with ERK1/2 inhibitor and p38 inhibitor. Similar results were confirmed by western blot analysis. 2) MDRl expression in the small intestine of FVB/N normal mice treated with $\mathrm{HrbFGF}$ was higher than in the non-treated mice. 3) Histologic findings of small intestine in X-ray-irradiated FVB/N mice revealed shortening of small intestinal villus height and destruction of intestinal crypt. In contrast, in FVB/N control mice treated with HrbFGF, an obvious increase of villus height and restoration of intestinal mucosa were observed. However, histologic findings of mdrl knockout mice treated with HrbFGF revealed severe distortion of regenerative crypt and less restoration of goblet cells, compared with bFGF-treated FVB/N control mice. Conclusion: The present study strongly suggests that bFGF-induced MDRl expression plays an important role in the protective effect on intestina epithelial injury.

\section{1}

Toll-Like Receptor (TLR)4 Is Involved in the Epithelial to Mesenchymal Transition (EMT): Implications for Colitis-Associated Cancer Masayuki Fukata, Anli Chen, Suneeta Krishnareddy, Junsuke Maki, Moshe Arditi, Maria Abreu

Background: We have recently demonstrated that mice deficient in TLR4 or its downstream adapter molecule MyD88 have decreased epithelial cell proliferation following DSS induced colitis, resulting in defective epithelial repair. During repair or carcinogenesis, epithelial cells may undergo an EMT characterized by morphological changes, increased expression of fibroblast markers, and increased cell motility. We hypothesized that TLR4 signaling mediates EMT in intestinal epithelial cells (IEC). Methods: The TLR4-expressing IEC (SW480) was 
stimulated with LPS ( $2 \mu \mathrm{g} / \mathrm{ml})$ for 24 hours. EMT was assessed by morphology and wound closure assay. Alterations in expression of epithelial and mesenchymal markers were determined by immunofluorescent staining of E-cadherin and vimentin or integrin $\alpha v \beta 6$, respectively. Carcinogenic phenotype was examined by epidermal growth factor receptor (EGFR) phosphorylation and expression of EGF like amphiregulin (AR) using Western blot and real-time PCR, respectively. Transforming growth factor (TGF- $\beta 1$ ), tumor necrosis factor (TNF- $\alpha$ ), and secreted AR were measured by ELISA. Small interfering RNA (siRNA) against MyD88 was used to confirm the downstream signal involved in this process. Results: Treatment of IEC with LPS resulted in a spindle cell morphology. LPS stimulation significantly increased cell migration rate into a wounded area, and this effect was abolished by MyD88 siRNA. Punctate expression of the epithelial marker E-cadherin decreased after LPS stimulation. By contrast, the fibroblast marker integrin $\alpha v \beta 6$ was detected in the cytoplasm of LPS treated cells but not in untreated cells. Vimentin increased with LPS stimulation. EGFR phosphorylation was observed within 30 min after LPS stimulation and was abolished by MyD88 siRNA. Expression of AR peaked 24 hours after LPS stimulation. TGF- $\beta 1$, a potent inducer of EMT, was significantly induced after 24 hours of LPS treatment. Induction of TNF- $\alpha$ was also observed after 24 hours but was not temporally associated with EGFR phosphorylation. Conclusion: TLR4 signaling results in EMT in IEC in a MyD88-dependent fashion. TLR signaling is critical for epithelial repair but activation of the same pathways may lower the cancer threshold in the setting of chronic injury. Because dysregulated immune responses to commensal bacteria are observed in patients with IBD, our results may provide novel insights into the pathogenesis of colitis-associated cancer in IBD.

922

Optimization of 6-Thioguanine Production By Allopurinol in Inflammatory Bowel Disease Patients Not Responding to Azathioprine/ 6-Mercaptopurine Leads to Improved Disease Activity, Reduced Corticosteroid Requirements and Normalization of Liver Enzymes

Miles Sparrow, Scott A. Hande, Sonia Friedman, Dingcai Cao, Stephen B. Hanauer

Background: At least $40 \%$ of IBD patients do not respond to the immunomodulators azathioprine(AZA) or 6-mercaptopurine(6-MP). Many non-responders have high/normal thiopurine methyltransferase (TPMT) activity and preferentially metabolize 6-MP to 6-methylmercaptopurine(6-MMP). Aim: We describe the clinical response to adjunctive allopurinol, used to shunt 6-MP metabolism towards the active metabolites 6-thioguanine nucleotides (6-TGN), in 6-MP/AZA non-responders. Methods: 21 outpatients (13 CD, 6 UC, 2 IC) from 2 IBD clinics who were clinical non-responders to AZA/6-MP and whose metabolite profiles demonstrated preferential metabolism towards 6-MMP $>6-\mathrm{TGN}$ were included. Subjects were commenced on allopurinol $100 \mathrm{mg}$ daily and the current dose of 6-MP/AZA was reduced by $50-75 \%$. Patients were followed clinically with weekly CBCs and serial 6-TGN and 6-MMP levels. Partial Harvey Bradshaw Index scores (pHBI-Number of bowel movements, pain, well being)) were used to assess response in $C D$ patients, Mayo Scores (without endoscopy) were used to assess patients with UC \& IC. Results: Median doses of AZA(6 patients) pre- and post allopurinol were $200 \mathrm{mg}(50-250)$ and $92 \mathrm{mg}(50-100)$ respectively; and median doses of 6 MP(15 patients) pre-and post allopurinol were $87 \mathrm{mg}(0-125)$ and $51 \mathrm{mg}(25-100)$ respectively. After initiating allopurinol, 6-TGN levels increased from a mean of 187.2 ( \pm SEM) \pm 16.7 to $387.3 \pm 37.4 \mathrm{pmol} / 8 \times 108 \mathrm{RBCs}(\mathrm{p}<0.001)$ while 6 - MMP levels decreased from a mean of $10517.1 \pm 1212.3$ to $1919.3 \pm 423.6 \mathrm{pmol} / 8 \times 108 \mathrm{RBCs}(\mathrm{p}<0.001)$. The addition of allopurinol led to a decrease in WBC from a mean of $8.6 \pm 1.0$ to $6.1 \pm 0.6 \times 108 / \mathrm{L}(\mathrm{p}=$ 0.003). Analysis of disease activity indices showed that the addition of allopurinol led to a reduction in the $\mathrm{pHBI}$ in $\mathrm{CD}$ patients from a mean of $4.9 \pm 1.0$ to $1.5 \pm 0.3$ points $(\mathrm{p}=0.001)$ and in UC patients the Mayo Score decreased from a mean of $4.1 \pm 0.7$ to $2.9 \pm 0.7$ points $(\mathrm{p}=$ $0.13)$. The addition of allopurinol enabled a reduction in prednisone dosage from a mean daily dose of $17.6 \pm 3.9$ to $1.8 \pm 0.7 \mathrm{mg}(\mathrm{p}<0.001)$ and led to normalization of transaminases with mean ALT levels reducing from $101.6+26.9$ to $33.9+5.2 \mathrm{IU}(\mathrm{p}=0.01)$ and mean AST levels reducing from $42.5 \pm 8.1$ to $23.5 \pm 1.6 \mathrm{IU}(\mathrm{p}=0.12)$. Conclusions: The addition of allopurinol to thiopurine non-responders with high/normal TPMT activity and preferential shunting to 6-MMP appears to be an effective and safe means of optimizing 6-TGN production, leading to improved disease activity scores, reduced corticosteroid requirements and normalization of hepatic transaminases. Long-term efficacy and safety related to potential nodular regenerative hepatic changes will be monitored.

923

Continuation of Immunomodulators Is Not Required to Maintain Adequate Infliximab Efficacy in Patients with Crohn's Disease But May Improve Pharmacokinetics

Gert A. van Assche, Gilles Paintaud, Geert D'Haens, Filip Baert, Severine Vermeire, Maja Noman, Herve Watier, Charlotte Magdelaine, Paul Rutgeert

Background. Concomitant immunomodulators at the start of infliximab treatment improve long-term duration of response in parallel with sustained infliximab serum levels. Aims: to investigate the role of immunomodulators in maintaining adequate infliximab trough levels and long-term efficacy in patients beyond 6 months of combined therapy. Methods. 80 patients were randomized to continue (CON, $n=40$ ) or to interrupt (DIS, $n=40)$ immunomodulators (azathioprine or methotrexate) $\geq 6$ months after the start of infliximab ( $5 \mathrm{mg} / \mathrm{kg}$ IV) in combination therapy. All patients were in clinical remission at baseline. Infliximab serum levels were determined q8 wks. (before every infusion) and efficacy and adverse events were assessed at the same time-points. Patients requiring a change in IFX dosing strategy due to a disease flare (increase in $\mathrm{CDAI} \geq 70$ ) or to a de novo fistula, were considered failures but were followed for efficacy and safety. Results. Patients in the 2 treatment groups had comparable overall IFX trough levels (median CON: 3.2 (percentile 25-75: 1.4-5.4), DIS $2.4(0.9-5.3) \mu \mathrm{g} / \mathrm{mL}$ (CON) (median follow up: 83 wks.), but high inter-individual variations were observed. For the change of IFX levels over time we observed a trend towards decrease in the discontinuation group (median change baseline to Wk 24: $+0.32(0.005-1.8)(\mathrm{CON})$, $0.647 \mu \mathrm{g} / \mathrm{mL}(-2.0-1.28)$ (DIS, $\mathrm{p}=0.05)$, baseline to Wk48: $+1.29(0.9-3.0)(\mathrm{CON}),-0.57$ $(-2.2-3.1) \mu \mathrm{g} / \mathrm{mL}$ (DIS, $\mathrm{p}=0.18)$. Fourteen/40 (35\%) (CON) and $13 / 40(32 \%, \mathrm{p}=0.81)(\mathrm{DIS})$ patients required a change in dosing interval. Two more patients in each group failed because of tolerability or lack of compliance. The median time to failure was: CON 17 wks. (25-75 perc.:8-25), and DIS 8 wks. (6.5-25.5) (CON) respectively ( $\mathrm{p}=0.82)$. A $\geq 50 \%$ decrease in infliximab trough levels preceded clinical relapse in only $1 / 13$ (CON) and 4/13 (DIS, p= 0.18 ) patients respectively. IFX was interrupted due to complete loss of response or tolerability in $2 / 40(\mathrm{CON})$ and $4 / 40(\mathrm{DIS}, \mathrm{p}=0.40)$ patients respectively. All others regained clinical response with changes in IFX treatment schedule. Conclusion. Discontinuation of immunomodulators after at least 6 months of combined treatment with systematic IFX maintenance does not affect long term efficacy but may lead to lower trough levels.

924

Adjustment in Infliximab Dose Or Dosing Interval in Crohn's Disease (CD): Experience from the University of Pittsburgh Benjamin Siemanowski, Kevin Kip, Scott Plevy, Miguel Regueiro

BACKGROUND: Infliximab is effective therapy (tx) for CD. Standard dosing includes 3 dose induction $(0,2,6 \mathrm{wks})$ at $5 \mathrm{mg} / \mathrm{kg}$ followed by q8wk maintenance. Pts who lose response often require a dose increase or interval decrease. Up to $62 \%$ of Rheumatoid arthritis pts require a change in infliximab dosing (JMCP2005;11:383-93). The proportion of CD pts requiring a dose adjustment is unknown. OBJECTIVE: To determine the frequency of infliximab dose adjustment in CD pts. METHODS: Using our infliximab infusion database we identified all $C D$ pts who received $>8$ doses (46 wks) of infliximab. Data collected included infliximab dose and dosing interval, concomitant immunomodulators (6MP/AZA/ MTX) at the onset, and presence of a lapse in tx (defined as a $>6$ mos interval between doses at any point). Subgroup comparison of infliximab dosing adjustments was made between pts with and without lapses in tx. RESULTS: There were 293 pts who received infliximab for $\mathrm{CD}$ and 108 who had received $>8$ doses. All 108 pts had an initial response to infliximab and none were primary nonresponders. $51 / 108$ (47.2\%, 95\% confidence interval (CI): $37.5 \%-57.1 \%$ ) had an increase from $5 \mathrm{mg} / \mathrm{kg}$ to $10 \mathrm{mg} / \mathrm{kg}$; median number of doses before change was 5 (range 2-17). 28/108 (25.9\%,95\% CI: 18.0\%-35.3\%) had a decrease in interval from q8 wks to every $4-6$ wks; median number of doses before interval decrease was 7 (range 3-18). 25/108 (23.1\%, 95\% CI: $15.6 \%-32.3 \%$ ) had a change in both dosage and interval, while $3 / 108$ had a change in interval only. Overall, 54/108 (50.0\%, 95\% CI: $40.2 \%-59.8 \%$ ) had a dosage increase and/or an interval decrease. Although the reason for dose adjustment was not available, our practice is to adjust infliximab dosing in pts experiencing sx's and signs (endoscopic recurrence) of CD. The majority of pts (91/108, 84.3\%) were on concomitant immunomodulators; of the 17 not, 9 required a dose adjustment. 58 pts (57\%) had no lapse in infliximab and $43(43 \%)$ pts had at least one $>6$ mos lapse between doses (no data available in $7 \mathrm{pts}$ ). The proportion of pts requiring an increase in infliximab dose or decrease in dosing interval was similar between pts with and without a lapse in tx ( $48.9 \%$ vs. $53.4 \%$, respectively: $\mathrm{p}=0.69)$. Of the 58 pts without a lapse in therapy, $29(50 \%)$ were on concomitant immunomodulators at the time of $0,2,6 \mathrm{wk}$ infliximab induction and q8wk maintenance; 14/29 (48.3\%) of these pts still required infliximab dosing adjustment. CONCLUSIONS: $50 \%$ of CD pts receiving long-term infliximab require an increase in dose or decrease in dosing interval. Concomitant immunomodulators and a q8wk schedule did not prevent the need for infliximab dosing change.

925

Is Infliximab a Durable Therapy for Crohn's Disease? Stephen J. Rudolph, David I. Weinberg, Robert P. McCabe

Background: Long-term use of maintenance infliximab (IFX) therapy for Crohn's disease (CD) is becoming increasingly common. Maintenance infliximab has been shown to be safe and effective up to 12 months. However, evaluation of maintenance treatment beyond 12 months is limited. Many IFX treated patients eventually experience a loss of benefit or an adverse event that results in discontinuation of IFX therapy. The aim of this study was to determine if maintenance of response (MOR) to IFX therapy is durable beyond 12 months, and to examine factors affecting this durability. Methods: A retrospective chart review was performed on $198 \mathrm{CD}$ patients who started IFX with maintenance dosing planned and had at least 30 months follow-up. MOR was defined as continued IFX therapy, without loss of response or adverse event requiring discontinuation. MOR rates were estimated using KaplanMeier analysis. The effect of specific variables on MOR rates was calculated using multivariate Cox proportional hazard regression analysis. Results: An initial response was seen in 174/ $198(88 \%)$ patients. MOR rates at 72 months were estimated to be $66.4 \%$ for initial responders and $58.2 \%$ for all patients. Analysis of the 174 initial responders revealed smoking decreased MOR $(H R=2.63, p=0.005)$, immunomodulators initiated $>3$ months prior to IFX tended to increase MOR (HR=0.50, $\mathrm{p}=0.088)$ and immunomodulators initiated $<3$ months prior to IFX had no effect on MOR ( $\mathrm{HR}=0.80, \mathrm{p}=0.55)$. Analysis of all 198 patients failed to show an impact of smoking on $\mathrm{MOR}(\mathrm{HR}=1.63 \mathrm{P}=0.087)$, while immunomodulators started $>3$ months prior to IFX increased MOR $(\mathrm{HR}=0.50, \mathrm{p}=0.027)$, and those started $<3$ months prior to IFX had no effect $(\mathrm{HR}=0.66 \mathrm{P}=0.17)$ (figure). Conclusions: Long-term maintenance IFX treatment of $\mathrm{CD}$ was durable for most patients. Concurrent immunomodulators may increase the durability of maintenance IFX treatment. Smoking appears to decrease the durability of maintenance IFX treatment. 


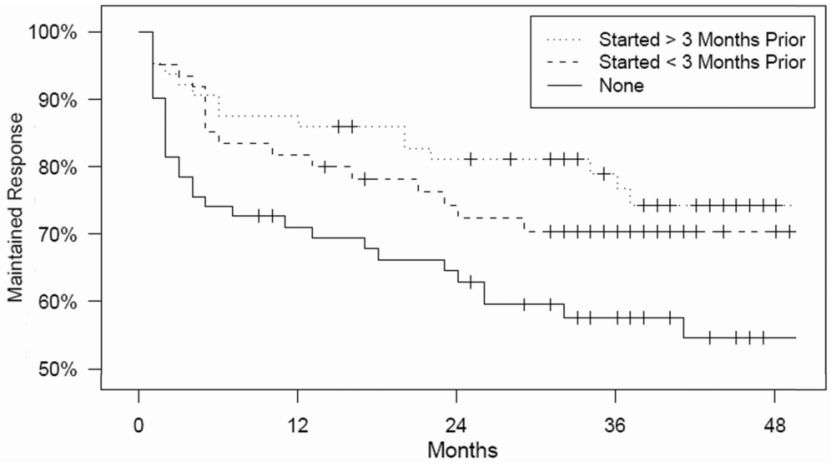

Effect of Immunomodulators on Maintenance of Response in All 198 Patients Treated

926

Long-Term Infliximab Mainteance Infusion Regimens and Rates of Hospitalization, Surgery and Disability in Crohn's Disease

John B. Williams, L:ydia R. Weber, Dawn B. Beaulieu, Mazen Issa, Joshua F. Knox, Jeanne Emmons, Mary F. Otterson, Ossama A. Hatoum, David G. Binion

Introduction: Although infliximab is effective in the management of moderate to severe Crohn's disease (CD), there is no consensus regarding optimal infusion schedules and the need/benefit of maintenance treatment. Concerns regarding cost, and lack of long-term efficacy data beyond $1 \mathrm{yr}$ have diminished enthusiasm for ongoing maintenance treatment. We reviewed our center's database, focusing on rates and incidence of hospitalization, surgery and disability in a cohort of $\mathrm{CD}$ pts, before and after specific long-term infliximab regimens followed beyond 2 years. Methods: This was a retrospective, observational study of $C D$ patients who were treated with maintenance infliximab beyond 2 yrs. Maintenance infliximab infusion regimens were categorized as SCHEDULED (loading and maintenance infusions $\mathrm{q}$ $\leq 8$ weeks) or EPISODIC (all other infusion schedules; i.e. no loading regimen and/or any maintenance infusion interval delayed beyond $8 \mathrm{wks}$ ). Histories of hospitalizations, surgeries and permanent disability were recorded before and after the initiation of infliximab. Results: 56 pts met criteria for long-term maintenance infliximab treatment (31-SCHEDULED maintenance; 25-EPISODIC maintenance). Rates of hospitalization (39\% vs 48\%; $\mathrm{p}=\mathrm{NS}$ ) and surgery ( $55 \%$ vs $48 \%$; $\mathrm{p}=\mathrm{NS}$ ) between the SCHEDULED and EPISODIC pts were identical prior to infliximab initiation. The number of hospitalizations per pt between the SCHEDULED and EPISODIC groups ( 0.548 vs $0.6 ; \mathrm{p}=\mathrm{NS}$ ) were identical. Similar relationships were evident in surgical events prior to initiation of therapy (meanl.839 vs. 1.8; $\mathrm{p}=\mathrm{NS}$ ). After long-term infliximab maintenance treatment, rates of hospitalization between the SCHEDULED and EPISODIC infusion regimens differed significantly ( $16 \%$ vs $64 \% ; \mathrm{p} \leq 0.001)$. Surgical rates favored SHEDULED over EPISODIC as well ( $3 \%$ vs $48 \%$; $\leq 0.01$ ). The cumulative number of hospitalizations after infliximab in SCHEDULED was 5 and EPISODIC was 29. Following infliximab initiation the cumulative number of surgeries in SCHEDULED was 3 and in EPISODIC was 20 in the $>2$ year followup period. Finally, rates of permanent disability were significantly lower in the CD pts treated with long-term SCHEDULED infusions compared with the EPISODIC regimen ( $3 \%$ vs $25 \%$; $\leq 0.03$ ). Conclusions: Significant differences in clinical outcome emerge in $C D$ pts treated with specific infliximab infusion regimens beyond 2 yrs. Scheduled maintenance infusions are superior to episodic infusions schedules, when rates of hospitalization, surgery and disability are used to assess long-term clinical outcome

927

Predictive Factors of Response to Cyclosporine (CsA) in Severe SteroidRefractory Ulcerative Colitis (SSRUC)

Cacheux Wulfran, Lemann Marc, Marteau Philippe, Seksik Philippe, Nion-Larmurier Isabelle, Afchain Pauline, Allez Mathieu, Daniel Fady, Beaugerie Laurent, Gendre JeanPierre, Cosnes Jacques

CSA is an effective rescue therapy in SSRUC and may avoid immediate colectomy in more than fifty per cent of patients. However, the individual response to CsA is poorly predictable. The aim of this study was to identify predictive factors of response to CSA in SSRUC. Methods : 135 patients with SSRUC admitted consecutively in three Paris IBD centers between 1992 and 2004 were included in this retrospective analysis. Data collected on the lst day of CSA therapy were age, gender, smoking habit, UC characteristics, duration of steroid treatment, Truelove and Witts criteria, weight loss, polynuclear neutrophils, lymphocytes and platelets counts, C-reactive protein, serum magnesium, and serum cholesterol. Colonoscopy was performed within two days preceding or following CsA treatment in 118 patients and presence of severe endoscopic lesions (extensive deep ulceration, mucosal detachment, well-like ulcerations, and large mucosal abrasion) was searched for. In order to identify independent predictive factors of colectomy, the variables selected at the 0.20 level by the log-rank test were introduced into a regression Cox model, successively for the whole population and for the patients who have had colonoscopy. Results: The actuarial rate of colectomy was 0.45 at 6 months. Cox analysis in the whole population selected three predictive criteria of colectomy : body temperature $>37,5^{\circ} \mathrm{C}$ [adjusted hazard ratio 1.94 (CI 95\% 1.51-2.49)], heart rate > 90 bpm [adjusted HR 1.86 (1.45-2.38)], and CRP > 45 $\mathrm{mg} / \mathrm{L}$ [adjusted HR 1.70 (1.34-2.16)]. The 6-mo. colectomy rates were 22, 47, 55 and $92 \%$ when $0,1,2$ and 3 of these criteria, respectively, were present. In the 118 patients who underwent colonoscopy, the presence of at least one severe lesion was an independent predictive factor of colectomy (adjusted HR 2.38 [1.80-3.14]). Colonoscopy findings did not change the therapeutic decision in patients without severe clinical or biological criteria $(\mathrm{n}=42)$ nor in those with 3 criteria $(\mathrm{n}=12)$. However, colonoscopy was decisive in patients with 1 or 2 criteria [among 35 patients with severe endoscopic lesions, 25 colectomized $(71 \%)$ vs. 5 among 29 without severe endoscopic lesion $(17 \%), p<0,001]$. In total, the clinical, biological and endoscopic criteria allowed the classification of patients into 2 groups, those with a high risk of colectomy ( $80 \%$ at 6 months) and those with a low risk of colectomy (20\% at 6 months). Conclusion : In patients with SSRUC the combination of simple criteria (fever, tachycardia and elevated CRP) is useful to predict the response to CsA. Colonoscopy is crucial in patients with intermediate clinical and biological severity.

\section{8}

Colonic Irritation in the Rat Sensitizes Urinary Bladder Afferents to PAR-2 and Capsaicin: Role of Neuropeptides in the Overlap of Chronic Pelvic Pain Disorders

Elena E. Ustinova, Michael A. Pezzone

Background: Chronic pelvic pain (CPP) disorders frequently overlap, but the mechanism underlying this phenomenon is still unclear. Proteinase activated receptors (PARs), importan mediators of pain and inflammation (Steinhoff 2000; Fiorucci 2002), can sensitize the capsaicin receptor to induce hyperalgesia (Amadesi 2004). In the bladder, PAR-2 receptors are co-localized in nerve fibers with capsaicin receptors, and upregulation of PAR-2 receptor was shown in cyclophosphamide-induced cystitis (Dattilio 2005). Some data suggest that activation of PAR-2 receptors is mediated by neuropeptide-dependent mechanisms ( $\mathrm{S}$ 2005). Recently we demonstrated that colonic irritation with trinitrobenzenesulfonic acid (TNBS) lead to the development of neurogenic cystitis (Pezzone 2005)as evidenced by the activation of bladder mast cells and increased sensitivity of bladder afferents to capsaicin, substance $\mathrm{P}$, and bradykinin. We hypothesize that colonic irritation causes cross-organ bladder sensitization by way of neuropeptide release from sensory afferents and upregulation of PAR-2 receptors in the bladder. Methods: Single unit afferent activity was recorded from fine filaments of the pelvic nerve in urethane-anesthetized SD female rats 10 days after intracolonic administration of TNBS. Only spontaneously active capsaicin sensitive afferents with receptive fields in the bladder and conduction velocities $<2.5 \mathrm{~m} / \mathrm{s}$ (unmyelinated C-fibers) were studied. Afferents were tested with iv capsaicin and the PAR-2 agonist, SLIGRL. Results: In the control group, $37 \%$ of capsaicin-sensitive afferents were activated with the PAR-2 agonist. In the TNBS group, the number of PAR-2 activated afferents increased to $58 \%$ $(\mathrm{p}<0.01)$. The magnitude of afferent responses to capsaicin and SLIGRL was increased by $270 \%$ and $190 \%$, respectively, in TNBS group as compared to controls. Colonic irritation also significantly increased the responses of bladder afferents to bladder distension. Pretreatment of the animals with capsaicin ( $100 \mathrm{mg} / \mathrm{kg}, 3$ days before TNBS administration) abolished these effects. Conclusions: In rats, colonic irritation sensitizes urinary bladder afferents to capsaicin, PAR-2 agonist and bladder distension. Systemic depletion of sensory afferents abolished these effects suggesting that neuropeptides facilitate the release of endogenous PAR-2 agonists such as mast cell tryptase to upregulate PAR-2 and to produce cross-organ sensitization. These findings add further support to the role of mast cells and their mediators in pelvic afferent cross-sensitization and the overlap of CPP disorders. Supported by $\mathrm{NIH}$ grant DK066658 to M.A.P.

\section{9}

TRPV4 Is Implicated in $\mathrm{PAR}_{2}$-Induced Visceral Hyperalgesia and Allodynia Nicolas Cenac, Christophe Altier, Nathalie Vergnolle

Background and aim: $\mathrm{PAR}_{2}$ activation induces somatic and visceral hyperalgesia and allodynia by sensitizing TRPVl (Transient Receptor Potential Vanilloid) in response to therma but not mechanical stimuli. The TRPV4 ion channel transduces osmotic and mechanical stimuli. In trpv4 null mice, TRPV4 was found to be necessary for normal thresholds in response to noxious mechanical stimuli. We hypothesize that TRPV4 is implicated in PAR induced visceral hyperalgesia and allodynia in response to a mechanical stimulus. Methods: In a first set of experiments, neurons from mice dorsal root ganglia (DRG) were isolated to perform immunostaining of $\mathrm{PAR}_{2}$ and TRPV4. In a second set of experiments, three groups of 8 mice were intrathecally injected with TRPV4 SiRNA and 3 other groups with a contro SiRNA. Three days after the SiRNA treatment, mice were treated with the TRPV4 agonist $4 \alpha \mathrm{PDD}\left(100 \mu \mathrm{M}, 10 \mu \mathrm{l}\right.$ per mouse), or with a non inflammatory dose of $\mathrm{PAR}_{2}$ agonist (10 $\mu \mathrm{g}$ per mouse), SLIGRL. To evaluate visceral nociception in mice, we performed colorectal distention (CRD) 30 min after TRPV4 agonist or 6 hours after $\mathrm{PAR}_{2}$ agonist treatment. CRD was performed by insertion of a balloon catheter into the colon, $5 \mathrm{~mm}$ proximal to the rectum and was increasingly inflated in stepwise $15 \mathrm{mmHg}$ gradation of pressure, from 0 to $60 \mathrm{mmHg}$. The external abdominal oblique muscle contraction is recorded and used as an index of pain sensation. Results: $90 \%$ of the DRG neurons express TRPV4 and $60 \% \mathrm{PAR}_{2}$ $\mathrm{PAR}_{2}$ and TRPV4 coexpress on the same neurons. Intrathecal administration of TRPV4 SiRNA significantly decreases pain sensation in response to CRD compared to control SiRNA groups, for all volumes of distension 3 days after its administration. Intrathecal injection of $4 \alpha \mathrm{PDD}$ caused hyperalgesia and allodynia 30 minutes after its administration in response to CRD. Intracolonic administration of SLIGRL caused hyperalgesia and allodynia 6 hours after its administration in response to CRD. Hyperalgesia and allodynia induced by the treatment with $4 \alpha \mathrm{PDD}$ or with SLIGRL were both significantly inhibited by the pretreatment with TRPV4 SiRNA. Conclusions: Our study shows that treatment with TRPV4 or PAR agonist increased visceral hyperalgesia and allodynia by a TRPV4-related mechanism. Moreover, we have demonstrated that TRPV4 is implicated in the response to CRD in the basal condition.

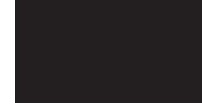


Dexamethasone Prevents Visceral Hyperalgesia Induced By ProteinaseActivated Receptor-2 Agonist Through Modulation of Colonic Mast Cell Activation in Rats

Richard Roka, Afifa Ait-Belgnaoui, Christel Salvador-Cartier, Aurelie Waget, Jean Fioramonti, Lionel Bueno

Background. Recent clinical data suggest that low-grade inflammation plays a role in the genesis of irritable bowel syndrome (IBS). Intracolonic infusion of proteinase-activated receptor-2 (PAR-2) agonist induces a delayed colonic hypersensitivity resulting from alterd gut permeability and mast cell degranulation. The aim of this study was to evaluate if a corticoid such as dexamethasone may prevent PAR-2 activation induced visceral hyperalgesia in rats, and to determine its level of action. Methods. Abdominal contractions provoked by rectal distension were recorded in rats equipped with abdominal intramuscular electrodes. Changes in sensitivity to rectal distension following intracolonic administration of PAR-2 activating peptide (SLIGRL), changes in colonic mucosal rat mast cell proteinase-II (RMCP-II) content, mast cell number and PAR-2 expression were measured after 4 days treatment with dexamethasone ( $1 \mathrm{mg} / \mathrm{day} / \mathrm{rat}$ ip) or its vehicle (water). Effect of short term dexamethasone administration ( $3 x$ during 24 hours) on mast cell degranulator (BRX537A)-induced visceral hyperalgesia was also determined. In vitro, the effects of SLIGRL and mast cell degranulator (C48/80) on permeability of colonic strips from vehicle or dexamethasone treated rats were investigated in Ussing chambers. Effect of short term dexamethasone administration (3x during 24 hours) on mast cell degranulator (BrX537A) provoked visceral hyperalgesia was also determined. Results. Dexamethasone (4 days) diminished the SLIGRL-induced hyperalgesia for all volumes of distension from 0.4 to $1.2 \mathrm{~mL}$ while it had no effect per se on rectal sensitivity. This effect was coupled with reduced in vitro colonic permeability response to $C 48 / 80(0.03 \pm 0.32$ vs. $\left.1.86 \pm 0.46 \mathrm{nmol} / \mathrm{h} / \mathrm{cm}^{2}, \mathrm{p}<0.05\right)$, decreased RMCP-II content $(0.17 \pm 0.01$ vs. $0.25 \pm 0.02$ $\mathrm{ng} / \mathrm{mg}$ of total proteins, $\mathrm{p}<0.05)$ and mast cell number $(15.02 \pm 2.77 \mathrm{vs} .147 .30 \pm 14.38$ mast cell $/ \mathrm{mm}^{2}, \mathrm{p}<0.01$ ). Dexamethasone did not influence colonic mucosal PAR-2 expression and permeability response to SLIGRL. Short term dexamethasone administration prevented BrX 537A induced visceral hypersensitivity ( $4.7 \pm 1.9$ vs. $11.8 \pm 2.0$ contractions $/ 5 \mathrm{~min}, \mathrm{p}<0.01$ a $0.4 \mathrm{~mL})$. Conclusions. Dexamethasone attenuates PAR-2 agonist induced visceral hypersensitivity in rats by reducing colonic mast cell activation but does not prevent the action of PAR-2 agonist on colonic permeability.

931

Agonists of Protease-Activated Receptor 2 (PAR2) Activate Protein Kinases D (PKD) 1-3 Expressed in Primary Afferent Neurons to Sensitize Transient Receptor Potential Vanilloid (TRPV) 1 and 4

Andrew Grant, Lorna Divino, Osvaldo Rey, Enrique Rozengurt, Nigel Bunnett, Silvia Amadesi

Agonists of many G-protein coupled receptors activate PKD, a family of 3 Ser/Thr kinases, by protein kinase $\mathrm{C}$ - (PKC) dependent phosphorylation. PKC isozymes mediate the hyperalgesic effects of agonists of certain receptors, including PAR2, by sensitizing TRPV channels in primary spinal afferent neurons. We investigated if agonists of PAR2 activate PKD in nociceptive neurons and sensitize TRPVl and -4 to cause hyperalgesia. We localized PKDs in rat dorsal root ganglia (DRG) using immunofluorescence and confocal microscopy. Immunoreactive PKD1/2 and PKD3 were detected at the membrane, cytosol and nucleus of neurons and in nerve fibers. Small diameter neurons expressing PKDs expressed calcitonin generelated peptide and bound isolectin B4, markers of nociceptive $C$ fibers. Large diameter neurons expressing PKDs expressed neurofilament $200 \mathrm{kDa}$, a marker of Ad fibers. Neurons expressing PKDs coexpressed PAR2 and PKC epsilon, potential upstream regulators of PKD, and TRPV1, a potential downstream target of PKD. We examined trafficking of PKD in HEK293 cells using PKD-GFPs, and in cultured rat DRG using PKD antibodies. In unstimulated cells, PKD1-3 were cytosolic. PAR2 activating peptide (AP, $100 \mu \mathrm{M})$ and the phorbol ester PMA (positive control, $1 \mu \mathrm{M}$ ) induced membrane translocation of PKDs within $5 \mathrm{~min}$. To confirm enzyme activation, we used antibodies to phospho-PKD-S $S^{744 / 748}$ and $S^{916}$. In unstimulated cells, there was minimal detectable PKD phosphorylation. PAR2-AP and PMA increased PKD-S $S^{74 / 748}$ and $S^{916}$ at the plasma membrane in HEK293 cells and neurons. PKD phosphorylation in HEK293 cells was confirmed by western blotting. To determine if PKD contributes to sensitization of ion channels, we examined PAR2-induced sensitization of TRPV-mediated $\mathrm{Ca}^{2+}$ signals in HEK293 cells. Pretreatment with PAR2-AP $(100 \mu \mathrm{M}, 5$ min) sensitized responses to the TRPVl agonist capsaicin (10 nM) and the TRPV4 agonist 4a-phorbol 12,13-didecanoate $(0.1 \mu \mathrm{M})$. The PKD inhibitor, Gö6976 $(0.1 \mu \mathrm{M})$ prevented sensitization of both channels. To assess the role of PKD in hyperalgesia, we measured the latency of paw withdrawal (LPW) of mice to radiant heat. Mice received intraplantar injections

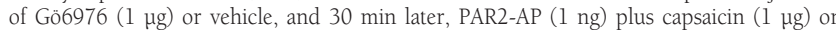
vehicles. $3 \mathrm{~h}$ later, the LPW was 12.05s in mice receiving Gö6976/vehicle vs. 8.51s in mice receiving vehicle/PAR2-AP-capsaicin. This hyperalgesic effect was not observed in mice receiving Gö6976/PAR2-AP-capsaicin, whose LPW was 12.31s. Thus, PAR2 agonists activate PKD1-3 in nociceptive neurons, where PKD may sensitize TRPV channels to mediate hyperalgesia. Supported by NIH grants DK57480, DK43207.

\section{2}

Effects of Proteinase-Activated Receptor-2 $\left(\mathrm{PAR}_{2}\right)$ Deficiency in Ischemia and Inflammatory Responses in Mouse Intestine Eric Hyun, Patricia Andrade-Gordon, Nathalie Vergnolle

$\mathrm{PAR}_{2}$ is a member of a G-protein coupled receptor family that is activated by various proteinases including trypsin, coagulation factor VIIa/Xa, and human mast cell tryptase. Considering that the activation of $\mathrm{PAR}_{2}$ elicits pro-inflammatory responses in various tissues including mouse colon, together with the fact that high levels of trypsin and tryptase are present in the colon of inflammatory bowel disease (IBD) patients, it is reasonable to assume that $\mathrm{PAR}_{2}$ plays a significant role in the pathogenesis of colitis. Therefore, we investigated the effects of $\mathrm{PAR}_{2}$ deficiency in mice models of intestinal inflammation using $\mathrm{PAR}_{2}$-deficient
( $\mathrm{PAR}_{2} \mathrm{KO}$ ) mice. Colitis was induced in C57BL6 wildtype and $\mathrm{PAR}_{2} \mathrm{KO}$ mice by administration of $2.5 \%$ DSS (in drinking water) or TNBS ( $1 \mathrm{mg}$ or $2 \mathrm{mg}$ in $100 \mu \mathrm{l}$ of ethanol, intracolonically) Seven days after colitis induction, intravital microscopy was performed on selected colonic venules to assess the role of $\mathrm{PAR}_{2}$ on leukocyte rolling, adherence and recruitment. Intestinal inflammation was also induced by ischemia/reperfusion via clamping of the superior mesenteric artery for 30 minutes, where immediately after intravital microscopy was performed on selected intestinal venules. Various inflammatory parameters including maximum bowel thickness, myeloperoxidase (MPO) activity and macroscopic damage score were measured. Lastly, the survival rate and weight loss of the animals were also assessed. $\mathrm{PAR}_{2} \mathrm{KO}$ with ischemia/reperfusion-, DSS-, or TNBS-induced intestinal inflammation displayed significant lower numbers of adherent leukocytes to the vessel wall, and vessel dilation compared to wildtype animals. Concurrently, MPO activity in $\mathrm{PAR}_{2} \mathrm{KO}$ was significantly lower compared to wildtype mice in all three models of intestinal inflammation. Colitis induced by DSS or TNBS elicited higher damage score and bowel thickness in wildtype mice than $\mathrm{PAR}_{2} \mathrm{KO}$. We observed a rate of $64 \%$ mortality in wildtype mice, whereas only $8 \%$ was observed in $\mathrm{PAR}_{2} \mathrm{KO}$ animals. Lastly, severe weight loss commonly associated with the TNBS-induced colitis was more readily seen in wildtype mice than $\mathrm{PAR}_{2} \mathrm{KO}$. In conclusion, deficiency in $\mathrm{PAR}_{2}$ modulated the recruitment of leukocytes in the intestinal tissues and attenuated the inflammatory responses in the mouse colon. Furthermore, reduction in numerous inflammatory parameters, weight loss and mortality rates in $\mathrm{PAR}_{2} \mathrm{KO}$ compared to wildtype animals indicate a pro-inflammatory role of $\mathrm{PAR}_{2}$ in the intestine. Thus, this study shows $\mathrm{PAR}_{2}$ activity and $\mathrm{PAR}_{2}$ activating proteases as possible therapeutic targets in the treatment of inflammatory diseases in the intestine.

\section{3}

Signaling By Protease-Activated Receptors PAR1 and PAR2 in Smooth Muscle Reflects Differential Activation of $G$ Proteins: $G_{Q}, G_{1}$ and $G_{12}$ By PARl and $\mathrm{G}_{\mathrm{Q}}, \mathrm{G}_{\mathrm{I}}$ and $\mathrm{G}_{13}$ By PAR2

Wimolpak Sriwai, Jiean Huang, Karnam S. Murthy

Extracellular proteases generate intracellular signals by activating a family of four cloned $G$ protein-coupled protease-activated receptors (PARl-PAR4). PAR receptors couple variously to pertussis toxin (PTx)-sensitive and -insensitive G proteins. Their expression, $G$ protein coupling, and signaling in smooth muscle are not known. Aim. To determine the expression of PARs and characterize their signaling pathways in rabbit gastric muscle cells. Methods. PAR protein expression was determined by immunoblot and $G$ protein activation by $\left[{ }^{35} \mathrm{~S}\right] \mathrm{GTP} \gamma \mathrm{S}$ binding to activated $\mathrm{G} \alpha$ subunits. PI hydrolysis, cAMP formation, and Rho kinase activity were measured in freshly dispersed muscle cells and in cultured muscle cells transfected with various $G \alpha$ minigenes. Muscle contraction was measured by scanning micrometry. Results. Immunoblot analysis demonstrated expression of PAR1 and PAR2 but not PAR3 or PAR4 in smooth muscle. The PARl agonist SFLLR activated $G_{q}, G_{12}$, and $G_{i 3}$, but not $G_{i 1}, G_{i 2}, G_{13} G_{5}$ or $G_{2}$, whereas the PAR2 agonist SLIGRL activated $G_{1}, G_{13}, G_{i 1}$, and $G_{i 2}$, but not $G_{13}, G_{12}, G_{s}$ or $G_{z}$. Both PARl and PAR2 agonists stimulated PI hydrolysis and Rho kinase activity and inhibited cAMP formation in a concentration-dependent fashion. PARl-stimulated PI hydrolysis was abolished in cells expressing $G \alpha_{q}$ minigene, but was not affected in cells expressing $\mathrm{G} \alpha_{i}$ minigene or in cells treated with PTx. PAR2-stimulated PI hydrolysis was partly inhibited in cells expressing $\mathrm{G} \alpha_{\mathrm{q}}(62 \%)$ or $\mathrm{G} \alpha_{\mathrm{i}}(35 \%)$ minigene and in cells treated with PTx $(30 \%)$. Thus, although it activated $G_{i 3}$, PARl stimulated PI hydrolysis exclusively via $G \alpha_{q}$, whereas PAR2 stimulated PI hydrolysis via both $G \alpha_{q}$ and $G \beta \gamma_{i}$. PARlstimulated Rho kinase activity was abolished in cells expressing $G \alpha_{12}$ minigene, whereas PAR2-stimulated Rho kinase activity was abolished in cells expressing $G \alpha_{13}$ minigene. Both PAR1 and PAR2 agonists induced a transient initial contraction that was selectively blocked by inhibition of PI hydrolysis with U73122 and MLC kinase activity with ML-9. The sustained contraction by the PARl agonist was preferentially inhibited by the PKC inhibitor bisindolylmaleimide (78\%) and to minor extent by the Rho kinase inhibitor Y27632 (18\%), whereas sustained contraction by the PAR2 agonist was preferentially inhibited by Y27632 $(80 \%)$ and to a minor extent by bisindolylmaleimide (14\%). Conclusion. PARl and PAR2 are differentially coupled to $G$ proteins. PARl stimulate PI hydrolysis via $G_{q}$ and PAR2 via $G_{q}$ and $G_{i}$. Both receptors activate RhoA, PARl via $G_{12}$ and PAR2 via $G_{13}$, but this distinctive coupling results in different effects on Rho-dependent sustained contraction.

934

Induction of Autophagy, a Novel Effect of H. Pylori Vacuolating Toxin Vaca Mauricio R. Terebiznik, Cristina L. Vazquez, Karl Torbicki, Noboru Mizushima, Tamotsu Yoshimori, Maria I. Colombo, Nicola L. Jones

Autophagy is an evolutionarily conserved mechanism for the degradation of cellular components in the cytoplasm. It has multiple physiological functions including protein degradation, organelle turnover and autophagic type II cell death. In addition disruption of autophagy has also been implicated in carcinogenesis. Although bacteria and viruses are vulnerable to autophagy, several intracellular pathogens have developed strategies to utilize this pathway for their own benefit. Current evidence indicates that $H$. pylori can invade epithelial cells in the gastric mucosa and survive inside large vacuolar compartments. However, relatively little is known about the biology of H. pylori invasion and survival in host cells. In AGS cells invaded with $H$. pylori, bacteria containing vacuoles originate through the fusion of late endosomal-lysosomal organelles by a mechanism based on the ability of the VacA toxin to hijack the small GTPase Rab7 at the membrane of the bacterial compartment. Since the scission of Rab7 from its functional locations disrupts the host's endocytic pathway, we hypothesized that $H$. pylori could induce a nutritional stress that triggers autophagy. To assess this possibility, we monitored the outcome of autophagy by expressing the autophagic protein markers Rab24 and LC3 in AGS cells. H. pylori infection caused the recruitment of GFP-LC3 and Rab24 to a sub-population of intracellular compartments where H. pylori resides. To further confirm this observation, we utilized embryonic fibroblast cells defective for autophagy (atg5-/- MEFs). In atg5-/- MEFs H. pylori-containing vacuoles were not labeled with LC3 indicating that autophagy is responsible for the recruitment of LC3 to the H. pylori vacuolar membrane. Importantly, the engagement of the autophagic pathway was totally dependent on the presence of the bacterial toxin VacA since autophagy was not detected 
when isogenic VacA mutant bacteria were utilized. Furthermore, our findings indicate that autophagy contributes to vacuolation since the vacuolating phenotype was impaired in autophagy deficient mutant cells infected with $H$. pylori or exposed to VacA positive bacterial supernatants. Our results describe a novel interaction of $H$. pylori with the host cell. Since modulation of autophagy has been linked with carcinogenesis these findings may have relevance for the development of $H$. pylori associated pathologies.

935

Regulation of Urease Activity in Helicobacter Pylori Acid Acclimation David Scott, Elizabeth A. Marcus, Hong Wu, George Sachs

Background: Helicobacter pylori, a neutralophile, is dependent on urease for gastric habitation by a process now defined as acid acclimation. The urease gene cluster consists of soluble intrabacterial urease apoenzyme (UreA + UreB $=\sim 15 \%$ of bacterial protein), UreI, a proton gated inner membrane located urea channel and the cytoplasmic $\mathrm{Ni}^{2+}$ insertion proteins, $\mathrm{UreE} / \mathrm{G}$ and $\mathrm{UreF} / \mathrm{H}$ that are essential for activation of the UreA/UreB apoenzyme. Only about $30 \%$ of the soluble urease is active at neutral $\mathrm{pH}$. When $\mathrm{H}$. pylori encounters an acidic environment that decreases periplasmic $\mathrm{pH}$ to 6.1 or less, UreI opens, permitting urea entry to the cytoplasm where it is hydrolyzed by urease to $2 \mathrm{NH}_{3}$ and $\mathrm{CO}_{2}$ for cytoplasmic buffering. $\mathrm{NH}_{3}$ and $\mathrm{CO}_{2}$ diffuse into the periplasm where $\mathrm{NH}_{3}$ consumes entering $\mathrm{H}^{+}$by conversion to $\mathrm{NH}_{4}{ }^{+}$and $\mathrm{CO}_{2}$ is converted to $\mathrm{HCO}_{3}{ }^{-}$, effectively buffering the periplasmic $\mathrm{pH}$ to 6.1 . Perhaps, under continued or greater acid stress, the inactive urease apoenzyme is activated to enhance acid acclimation. Aims: This study investigated the mechanism of activation of the urease apoenzyme and its possible role in acid acclimation under acidic conditions. Methods: Urease activity, synthesis and cellular location were determined after incubation at pH 5.5 and 7.4 in the absence or presence of urea, chloramphenicol, dimethylglyoxime and UreI for up to 4 hours. Urease location was observed by post-sectioning immunoelectron microscopy. UreI mutants were constructed by homologous recombination. Results: Total bacterial urease activity increased 3 to 5 fold at pH 5.5 in the presence of the protein synthesis inhibitor, chloramphenicol, but not in the absence of $\mathrm{Ni}^{2+}$ or UreI. There was an accompanying 3 fold increase in survival to acid shock $(\mathrm{pH} 2.5)$. Urease shifted from a predominately cytoplasmic localization at neutral $\mathrm{pH}$ to the inner membrane at $\mathrm{pH} 5.5$, dependent on the presence of UreI. Native gel electrophoresis showed UreB and UreI association in the membrane fraction. Conclusions: The data suggest that at neutral $\mathrm{pH}$ only about $1 / 3$ of the total bacterial urease contains the $\mathrm{Ni}^{2+}$ required for enzyme activity. A decrease in medium $\mathrm{pH}$ increases the amount of active urease by $\mathrm{Ni}^{2+}$ insertion into apoenzyme, independent of de novo urease synthesis. $\mathrm{Ni}^{2+}$ insertion and urease $\mathrm{pH}$ dependent redistribution was UreI dependent. Therefore, UreI in addition to its urea channel activity appears to play a role in acid acclimation by serving as a membrane anchor for urease and its $\mathrm{Ni}^{2+}$ insertion accessory proteins to increase the amount of active urease, thus increasing acid survival likely at more extreme conditions of acidity.

\section{6}

Dupa-Positive H. Pylori Strains Are Highly Prevalent in Brazil and Are Not Associated with Duodenal Ulcer in Brazilian Adults and Children

Dulciene M. Queiroz, Luciana I. Gomes, Gifone A. Rocha, Taciana F. Soares, Andreia M. Rocha, Ludmila M. Godoi

Helicobacter pylori (HP) infection is associated with clinical outcomes as different as distal gastric carcinoma (GC) and duodenal ulcer (DU). Although HP virulence factors have been associated with clinical complications of the infection, they do not distinguish between DU and GC. Recently, Lu et al. (2005) described a new putative HP virulence marker (dupA) associated with an increased risk for DU and reduced risk for GC in Japan and Korea, and with a reduced risk for GC in Colombia. Since differences between the association among HP virulence markers and HP-associated diseases have been demonstrated around the world, we investigated the presence of dupA in a large number of HP strains isolated from Brazilian patients. We evaluated $487 \mathrm{HP}$ strains from children (38 DU, 100 gastritis) and adults (125 DU, 143 gastritis, 81 GC). Bacterial DNA was PCR amplified for cagA (Peek et al., 1995) and jhp0917-0918 (Lu et al., 2005). We constructed primers located at the jhp0917 3' region (sense primer) and jhp0918 5' region (antisense primer) to test again jhp0917-0918-negative strains and for sequencing 30 jhp0917-0918-positive strains (10 randomly selected from each group of disease) in order to detect the insertion of $\mathrm{C}$ or $\mathrm{T}$ (after position 1385) in the jhp0917 3' region that characterizes dupA. Data were analyzed with the SPSS statistical package. Both genes were present in 422(86.7\%) and absent in 37(7.6\%) strains. jhp0917 or jhp0918 was present in 22(4.5\%) and 6(1.2\%) strains, respectively. Since an insertion of $\mathrm{T}$ or $\mathrm{C}$ was observed in $90 \%$ of the jhp0917-0918 positive strains, the presence of the two genes was considered to be dupA+. Strains with only one gene were not included in the analysis. Almost all samples from children with $(38 / 38,100 \%)$ or without $(98 / 100,98.0 \%)$ DU were dupA+, without any difference between the disorders $(\mathrm{p}=1.0)$. No association was also observed among the strains from adults with gastritis $(89.8 \%), \mathrm{DU}(86.6 \%, \mathrm{p}=0.6)$ and GC $(89.0 \%, p=0.9)$. Conversely, cagA + status was independently associated with DU (in adults and children) and with GC in logistic analysis. Curiously, dupA- and cagA-positive status were associated with each other $(\mathrm{p}=0.02)$. When children and adults were compared, the presence of dupA was significant higher in children, even when only the group of gastritis was analyzed $(\mathrm{p}=0.003)$ which may indicate that the bacteria lost 1 or 2 genes along the adult life. In conclusion, in Brazil, dupA was not associated with GC or DU in both adults and children. Similarly to that seen with other HP virulence markers, there are wide regional differences in the distribution of dupA that is highly frequent in Brazilian strains.
937

Phase Variations of Helicobacter Pylori Outer Membrane Protein Expression in Infected Mongolian Gerbils

Tomoyuki Ohno, Saori Fujimoto, Hiroaki Ogiwara, Stefan Odenbreit, Rainer Haas, David Y. Graham, Yoshio Yamaoka

Background: The outer membrane proteins (OMPs) of Helicobacter pylori (H.pylori) are thought to play important roles in colonization, adherence, resistance to the gastric milieu and protection from the host immune response. BabA, SabA and OipA are OMPs putative virulence factors that undergo phase variations. Phase variations have been observed both in vitro laboratory passages and rhesus monkey models. However, it remains unknown whether the phase variations were limited to monkeys or whether phase variations played a role in gastric damage. Methods: Six week male Mongolian gerbils were inoculated with strain TN2GF4 and sacrificed after 1, 3 and 18 months. At least five infected gerbils were used in each time period. At least 10 single colonies were picked up from each gerbil and the expression of BabA, BabB, SabA and OipA was determined by immunoblot. The ability to induce interleukin-8 from gastric epithelial cells (MKN45 cells) co-cultured with $H$ pylori obtained by expansion of each single colony were determined by an enzyme linked immunosorbent assay. Results: Challenged strain TN2GF4 expressed functional BabA, BabB and OipA and did not express SabA. All isolates recovered from challenged gerbils at 1 month expressed BabA, BabB and OipA proteins. At 3 months BabA/BabB expression was absent from one gerbil and was present in less than $25 \%$ of recovered colonies from the other 4 gerbils. By 18 months, BabA/BabB expression was mostly absent except for two colonies from one gerbil. OipA was expressed in all isolates at all time periods. SabA expression was not observed in any isolate from challenged gerbils. In vitro IL-8 levels were independent of the expression of BabA and BabB. Conclusions: We confirmed that phase variations of BabA and BabB occurred in infected gerbils. BabA and BabB may be important for colonization but continued expression is either not required or possibly confers a disadvantage for survival possibly as the gastric milieu changes. Supported in part by the Gulf Coast Digestive Disease Center.

\section{8}

Role of H. Pylori Adhesins in Colonization of the Primate Stomach Andre Dubois, Hui Liu, Cristina Semino-Mora, Steven Mog, Thomas Boren

H. pylori adherence to gastric epithelial cells is critical for gastric colonization and life-long persistence in the primate stomach. Adherence depends on the $\mathrm{ABO} / \mathrm{Le} \mathrm{e}^{\mathrm{b}}$ blood group antigen binding BabA adhesin and on the sialic acid binding SabA adhesin that binds to the host's sialyl-Lewis glycosphingolipid. To explore the role of these adhesins, we inoculated $11 \mathrm{H}$ pylori negative rhesus monkeys with $10^{7} \mathrm{CFU}$ of either a J99babA::cam sabA::kan double mutant (N=5; DM; Science 2002;297:573) or wild type (WT) H. pylori (N=6). Videogastroscopies were performed at regular intervals for 6 months after inoculation and biopsies were cultured and analyzed by RT-PCR. The mRNA expression of $\mathrm{H}$. pylori-specific $16 \mathrm{~S}$ rRNA and monkey IL-1 $\beta$ and IL-8 (copies/100 ng RNA) were determined using an absolute real-time quantitative RT-PCR(TaqMan) method, standard curves of cloned cRNA, and normalization with monkey $18 \mathrm{~S}$ rRNA. In situ hybridization (FISH) was used to detect sabA and babA expression in WT and DM strains and in the gastric mucosa. All WT-infected monkeys were culture+ and RT-PCR+. The DM inoculate and all post-inoculation biopsies were negative for both sabA and babA. Culture of all DM post-inoculation biopsies were negative and RT-PCR detected the DM strain in only two monkeys and only at 7 days after inoculation. Gastritis scores and IL-1 $\beta$ and IL-8 expression were lower than in WT controls but increased significantly in the corpus of the stomach (figure) but not in the antrum. These observations suggest that either SabA or BabA or both adhesins are required for successful establishment of colonization and persistent H. pylori infection. Ongoing experiments with $b a b A$ and sabA single mutants explore whether the presence of one of the two adhesins is sufficient for gastric colonization by $\mathrm{H}$. pylori. 


\section{DM - Gastric corpus}
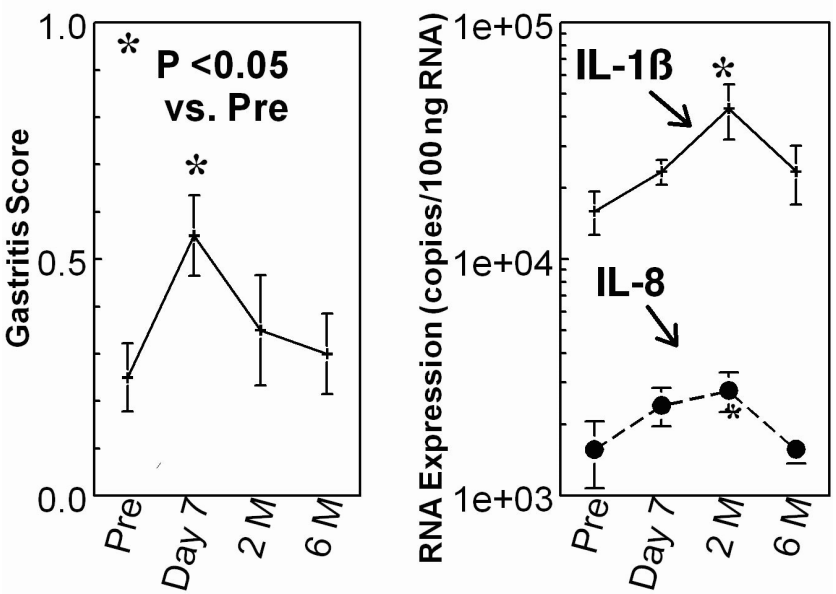

939

Helicobacter Pylori Vaculating Cytotoxin Inhibits Duodenal Bicarbonate Secretion By a Histamine-Dependent Mechanism Biguang Tuo, Bettina Gebert, Rainer Haas, Michael P. Manns, Ursula Seidler

Background\&Aims: Helicobacter pylori (HP) infection reduces the secretory capacity of the duodenal mucosa for bicarbonate (Hogan et al. 1996), and CagA/VacA positive HP water extract strongly decreases murine duodenal $\mathrm{HCO}$ - secretion in vitro within one hour (Tuo et al 2004). In this study, we investigated the effect of HP vacuolating cytotoxin (VacA) on duodenal mucosal bicarbonate secretion, and the underlying mechanisms for the VacAinduced reduction in $\mathrm{HCO} 3$ - secretion. Methods: Bicarbonate secretion by murine duodenal mucosa was examined in vitro in Ussing chambers. HP extracts from a VacA producing strain (P12) and an isogenic mutants lacking VacA (P12 $\Delta$ vacA) were used (Gebert et al 2003). Results: VacA-positive extract reduced PGE2-stimulated duodenal HCO3- secretion by $48.9 \%(\mathrm{P}<0.0001)$, whereas the VacA-negative extract only slightly reduced PGE2-stimulated bicarbonate secretion by $17 . \%(\mathrm{P}>0.05)$. Preincubation with the $\mathrm{H} 2$-receptor antagonist ranitidine, but not with the $\mathrm{Hl}$ receptor antagonist diphenhydramine or the $\mathrm{H} 3$ receptor antagonist thioperamide, significantly attenuated the inhibitory effect of VacA-positive extract on PGE2-induced bicarbonate secretion partially. The VacA-positive HP extract stimulated duodenal mucosal histamine release concentration-dependently. After incubating $15 \mathrm{~min}$ with VacA-positive HP extract, duodenal mucosal histamine release markedly increased, it reached maximal levels at $45 \mathrm{~min}$, and this corresponded to the development of the reduction in PGE2-mediated HCO3- secretion. Conclusion: Hp VacA inhibits PGE2-stimulated duodenal epithelial bicarbonate secretion in part via the release of mucosal histamine, which in turn inhibits HCO3- secretion in an H2-receptor dependent mechanism. HP VacA inhibition of duodenal bicarbonate secretion might therefore be an important pathogenic factor contributing to HP related duodenal mucosal injury.

940

Single Screening Colonoscopy in Octogenarians: A Markov Decision Analysis Grace Shih, Yu Xiao Yang

BACKGROUND: The benefits of a single colorectal cancer screening colonoscopy (SC) in previously unscreened patients over 75 years old are unknown. AIMS: (1) To determine the effect of single SC on the life expectancy of octogenarians versus no SC. (2) To determine the cost effectiveness of single SC in octogenarians versus no SC. METHODS. We constructed a lifetime Markov cost-effectiveness model with one year cycle length. Patients cycled between no neoplasia, polyp, unresectable cancer, resected cancer, and death. Age adjusted mortality from the US census was used. Transition probabilities, quality of life measurements, and costs were obtained from systematic literature review. Costs of differing colonoscopies were obtained from Medicare data. We assumed that all patients undergoing SC had 100\% adherence rate to follow up colonoscopies and/or surgeries if polyps or neoplasia were found. Otherwise, all other patients received no further colonoscopies. Success rate in completed SC was 90\% (Lukens et al., Am J Gastro 2002). Cancer rates were based on SEER data. Mortality rate from colonoscopy was $0.01 \%$ (Winawer et al, Gastro 1997). We analyzed the model for the starting ages of $75,80,85$, and 90 . Sensitivity analysis was also performed. RESULTS: SC provides marginal benefit in quality adjusted life years (QALY) for all age groups (75yrs, $0.03 \mathrm{yrs} ; 80 \mathrm{yrs}, 0.03 \mathrm{yrs} ; 85 \mathrm{yrs}, 0.02 \mathrm{yrs} ; 90 \mathrm{yrs}, 0.006 \mathrm{yrs})$. The incremental cost-effectiveness ratios are $\$ 3006, \$ 3576, \$ 14787$, and $\$ 59631$ respectively. Sensitivity analysis shows no threshold values for success rate for all ages. Threshold values for cost of colonoscopy ranged from $\$ 497$ in 75 year olds to $\$ 156$ in 90 year olds. Cost of colonoscopy accounted for at least $99 \%$ of model uncertainty. SC sensitivity and success rate account for $<1 \%$. CONCLUSIONS: A single SC in octogenarians provided marginal benefit of QALY. Success rate in this population did not greatly affect the benefit of single $\mathrm{SC}$. The younger octogenarian is the most ideal candidate in this population, while single $\mathrm{SC}$ in older octogenarians became less cost effective.
941

Isolated Lymphoid Follicle Formation Is Augmented and Aberrant with Aging Keely G. Mcdonald, Jacquelyn S. Mcdonough, Caihong Wang, Rodney Newberry

Background: Isolated lymphoid follicles (ILFs) are organized lymphoid structures in the murine intestine that act as inductive sites for the generation of adaptive mucosal immune responses. Intestinal lymphoid aggregates, the human counterpart to ILFs, are increased with inflammation and have been proposed to be the sites for the initiation or propagation of the inappropriate immune responses seen in IBD. With aging,the mucosal immune system undergoes immunosenesce resulting in a decrease in protective immunity and a predisposition toward inappropriate immune responses. Given these associations we examined the presence, composition, and function of ILFs in aged mice. Methods: Two month old (young) and twenty month old (old) Balb/c mice from the national institute of aging colony were examine for the presence of isolated lymphoid follicles using whole mount and immunohistochemical techniques. Cellular populations from ILFs, spleens, and Peyer's patches were examined using flow cytometry. Immunoglobulin production by ILF cellular populations was examined using ELISA. Results: Old mice had a greater than three fold increase in the numbers of ILFs per small intestine when compared with young mice. The cellular composition of ILFs from old mice was also altered when compared with young mice. ILFs from old mice had a decreased population of B-lymphocytes, $30 \%$ of the cellular population, and an increased population of T-lymphocytes, $50 \%$ of the cellular population, when compared with young mice. No differences were observed in the ILF dendritic cell population between the two groups. In addition the ILF cellular population from old mice contained CD4+ CD8 $\alpha+$ (double positive) TCR $\beta+$ T-lymphocytes, which comprised up to $30 \%$ of the ILF T-lymphocyte population. This double positive T-lymphocyte population was not observed in the spleen or Peyer's patches of old mice. ILFs from old mice had a smaller population of Foxp3 expressing T-lymphocytes when compared with young mice. ILF cellular populations from young and old mice produced predominantly IgA. IgG production was observed in ILF populations from old mice, but not from ILF populations from young mice. Conclusions: ILF formation is increased with aging and ILFs from aged mice have an abnormal cellular composition which includes a substantial population of $\mathrm{CD} 4+\mathrm{CD} 8 \alpha+$ (double positive) TCR $\beta+$ T-lymphocytes. In contrast to young mice, ILFs from old mice produce IgG. These findings suggest that aberrant ILF formation, composition, and function may contribute to immunosenescence of the mucosal immune system.

942

Age and the Risk of Failure of Medical Therapy in Inflammatory Bowel Disease (IBD): A Case-Control Study

William J. Tremaine, Lawrence J. Timmons, Edward V. Loftus, Darrell S. Pardi, William J. Sandborn, William S. Harmsen, Prabin Thapa, Alan R. Zinsmeister

Background \& Aims: There are few data on the response to medical therapy for Crohn's disease and ulcerative colitis with respect to the patient's age. We examined age as a risk factor for failure of medical therapy, as defined by the need for surgery for control of symptoms of IBD. Materials and Methods: This was a single center case-control study of patients seen at a tertiary referral center from 1994-2001. Case subjects were all patients seen at our institution from MN and the surrounding 5 states (WI, IA, ND, SD, IL) with ulcerative colitis or Crohn's disease who required an initial operation for disease refractory to medical therapy. Controls were matched 1:1 based on gender, IBD subtype, date of first visit to our center $( \pm 2 \mathrm{yr})$, time interval from IBD diagnosis elsewhere prior to visit to our facility ( $\pm 3 \mathrm{yr}$ ), and duration of follow-up based on the time from diagnosis to surgery for case subjects. Cases and controls were excluded if the indication for surgery was dysplasia or malignancy, or if IBD was not diagnosed until surgery. The association with age, disease extent, smoking history, and medication use vs. case/control status was assessed using multiple variable conditional logistic regression to estimate the Odds Ratios (OR [95\% CI]) for being a case (undergoing surgery). Results: Among 132 Crohn's patients, the median age was $30.6 \mathrm{yr}$ (range, 8.9 -88.5). Older patients had smaller odds for surgery (OR per 5 years $=0.86[0.75,0.98])$. Alternatively, patients $\geq 50 \mathrm{yr}$ had smaller odds than patients $<50 \mathrm{yr}$ to fail medical therapy (OR $0.13[0.02,0.69])$. The failure rate of medical therapy was not significantly associated with disease distribution (ileitis, ileocolitis, colitis), use of 5-ASA, or use of thiopurines. Among 234 ulcerative colitis patients, the median age was $37.5 \mathrm{yr}$ (4.5- 82.9). Patients $\geq 50 \mathrm{yr}$ had smaller odds than patients $<50 \mathrm{yr}$ to fail medical therapy (OR $0.49[0.26,0.92])$. The failure rate of medical therapy was not related to disease extent or cigarette smoking. Ulcerative colitis patients who had taken 5-ASA were at increased odds for surgery (OR 10.4 [1.1, 99 1]) as were patients who had taken azathioprine (OR $5.0[2.2,11.5])$, or 6 -MP (OR $3.0[1.1,8.1])$. Conclusions: Failure of medical therapy for Crohn's disease and for ulcerative colitis was more common in those younger than age 50 , irrespective of disease distribution. There was a suggestion that among current smokers with Crohn's disease, older age was associated with increased odds for failed medical therapy, but this interaction was not stable across different age break points. This study was supported by research funds from $P \& G$ Pharmaceuticals.

943

Incidence, Risk Factors, and Mortality Associated with Fecal Incontinence in Older Individuals

Carline Quander, Martha C. Morris, Julia Bienias, Jocelyn Carter, Denis Evans

OBJECTIVE: In a large, biracial community study of older persons, we estimated the mortality risk associated with fecal incontinence and investigated risk factors associated with newly acquired symptoms of fecal incontinence. METHODS: As part of a 1993-2004 longitudinal study of community residents aged 65 years and older, study participants were administered comprehensive in-home interviews at baseline and at 3-year follow-up. The interviews included a wide range of questions regarding demographics, medical history, medication use, the presence of fecal incontinence, and assessments of physical disability, cognitive function, and depressive symptoms. RESULTS: Fecal Incontinence was reported by 585 of 6,099 participants $(9.6 \%)$ at the baseline. The prevalence of fecal incontinence was associated 
with increased mortality (hazard ratio $=1.8$ for a person of average age (75), 95\% CI: 1.6, 2.1) in a Weibull hazards model. Among 3976 participants unaffected by fecal incontinence at the baseline and who participated in the 3-year follow-up, fecal incontinence was reported by $336(8.5 \%)$. We examined risk factors for developing these symptoms over 3 years in multiple logistic models that included terms for age, sex and race and their interactions. There were statistically significant interactions between race and sex and also between race and age, such that the risk of incident fecal incontinence was higher with older age and black race and was highest among older black women. In addition, higher incidence of fecal incontinence was associated with diabetes ( $\mathrm{OR}=1.6 ; 95 \% \mathrm{CI} ; 1.2,2.1)$, stroke $(\mathrm{OR}=2.3$; $95 \% \mathrm{CI} ; 1.7,3.2)$, use of anti-psychotic (OR=3.6; 95\% CI; 1.6, 8.0) and anti-parkinsonian medications (OR=5.2; 95\% CI; $2.2,12$ ), physical disability (OR=1.6; $95 \% \mathrm{CI}: 1.4,1.7)$, and number of depressive symptoms $(\mathrm{OR}=1.1 ; 95 \% \mathrm{CI} ; 1.1,1.2)$. Lower incidence of fecal incontinence was associated with higher cognitive scores ( $\mathrm{OR}=0.6 ; 95 \% \mathrm{CI} ; 0.5,0.7)$. CONCLUSION: Fecal incontinence among older persons is associated with increased mortality, probably at least partially because of its association with multiple markers of poor health. The risk of developing this condition may be associated with a history of diabetes or stroke, certain medications, symptoms of depression, and physical or cognitive impairment.

\section{4}

Colorectal Cancer Screening Colonoscopy in Elderly Patients with Dementia: a Decision Analysis

Grace Shih, Keith Obstein, Jerry Johnson, Yu-Xiao Yang

BACKGROUND: The benefits of colorectal cancer screening colonoscopy (SC) in the elderly population with varying degrees of dementia are unknown. AIM: (1) To determine the effect of SC on the life expectancy of patients with varying degrees of dementia. (2) To determine the cost effectiveness of SC in patients with dementia. METHODS: We constructed a lifetime Markov cost-effectiveness model with one year cycle length. Patients cycled between no neoplasia, polyp, unresectable cancer, resected cancer, and death. Age adjusted mortality rates based on mini-mental status in dementia patients (score of 18-21 was mild/moderate; $<18$ severe) were used (Larson et al, Ann of Int Med 2004). Transition probabilities, quality of life measurements, and costs were obtained from systematic literature review. Costs of differing colonoscopies were obtained from Medicare data. We assumed that all patients undergoing SC had 100\% adherence to follow-up colonoscopies and/or surgeries. Cancer rates were based on SEER data. Mortality rate from colonoscopy was $0.01 \%$ (Winawer et al, Gastro 1997). We analyzed the model for the starting ages of $65,70,75$, and 80 . Sensitivity analysis was also performed. RESULTS: SC provides marginal benefit in quality adjusted life years (QALY) for all age groups of patients with varying degrees of dementia (Table). The incremental cost-effectiveness ratios (ICER) are shown in the Table. Sensitivity analysis shows a threshold value of $\$ 364$ for the cost of colonoscopy in patients aged 65 but $\$ 200$ for age 80 . Age and severity of dementia accounted for $58 \%$ and $41 \%$ of model uncertainty, respectively. Sensitivity and cost of colonoscopy accounted for the other $1 \%$. CONCLUSIONS: Colorectal cancer SC in patients with varying degrees of dementia provided a marginal benefit of survival although at relatively low costs. Age is slightly more important than dementia severity in choosing whether to undergo SC.

\begin{tabular}{|c|c|c|c|c|}
\hline Age & MMSE & SC: QALY & no SC:QALY & ICER \\
\hline 65 & $18-21$ & 10.56 & 10.49 & $\$ 1035$ \\
\hline & $<18$ & 8.92 & 8.88 & $\$ 1602$ \\
\hline 70 & $18-21$ & 8.43 & 8.39 & $\$ 6177$ \\
\hline & $<18$ & 7.01 & 6.98 & $\$ 8532$ \\
\hline 75 & $18-21$ & 7.09 & 7.05 & $\$ 9814$ \\
\hline & $<18$ & 5.48 & 5.46 & $\$ 17256$ \\
\hline 80 & $18-21$ & 4.00 & 3.99 & $\$ 38092$ \\
\hline & $<18$ & 3.39 & 3.38 & $\$ 52046$ \\
\hline
\end{tabular}

945

Age-Related Rise in Colorectal Adenomas Is Associated with Increased EGFReceptor Expression

Sudha Reddy, Sabeena Misra, Richard Jaszewski, Hu Xu, Edi Levi, Nadeem Ullah, Adhip P. N. Majumdar

Although the incidence of colorectal and other gastrointestinal cancers increases with advancing age, reason for this increase is poorly understood. Earlier studies from this and other laboratories have demonstrated that at least in Fischer-344 rats, aging is associated with increased proliferative activity and decreased apoptosis in the colonic mucosa (Mech. Ageing Dev. 122: 1849-1864, 2001). Morphological studies of the colonic mucosa of human volunteers have also revealed that cell proliferation in the young is confined to the lower twothirds of the crypt, while with aging there is a major shift from the base to the middle and upper-third of the gland, a pattern that is commonly seen in colorectal cancer. Although the regulatory mechanisms for the age-related rise in mucosal proliferation and reduction in apoptosis have not been fully delineated, we have reported that these events are associated with increased expression and activation of EGF-Receptor (EGFR) and reduction in EGFReceptor Related Protein (ERRP), a negative regulator of EGFR (Dig. Dis. Sci. 48: 856-864, 2003). An inverse relationship of EGFR and ERRP has been observed in colorectal cancer (Cancer Lett. 213: 249-255, 2004). To determine whether aging predisposes the colon to the processes of carcinogenesis, we examined the relationship between aging and formation of colonic adenomas, the most frequent premalignant colorectal lesion. In our study there were 177 patients with colorectal adenomas in different age groups. Our data show that the number of polyps formed increase with advancing age (age in years: 1 polyps in $<51$ yrs; 2 polyps in $51-60 ; 3$ polyps in $61-70$ yrs; 4 polyps in $71-80 ; 5$ polys in 80 yrs $=0.95$ ). The age-related rise in polyp formation was associated with a concomitant increase in EGFR expression in the macroscopically normal rectal mucosa. The levels of phosphorylated (active) form of EGFR was also higher in macroscopically normal rectal mucosa from 60-70 years old patients with colonic adenomas, when compared with their younger counterparts. On the other hand, levels of ERRP, a recently identified pan-erbB antagonist (Mol. Cancer Ther 4: 435-442, 2005), were found to decrease with advancing age in patients with colorecta adenomas. We also observed that the expression of cyclooxygenase-2 (COX-2) increases with aging in patients with adenomas. In conclusion, there is a positive relationship between advancing age and polyp formation. This is associated with increased expression of EGFR and COX-2 and downregulation of ERRP.

946

The Effects of Obesity and Weight Loss Surgery On Symptoms of Gastroesophageal Reflux (GER)

Milan E. Folkers, Tom Cloward, Ted Adams, John Fang, Mae Go, Kathryn Peterson

Background: Obesity is an increasing epidemic in developed countries and has been associated with an increased risk of hypertension, hyperlipidemia, diabetes, coronary artery disease, stroke and gastroesophageal reflux disease. The use of bariatric surgery as an option to trea obesity has increased dramatically in the past several years and has been shown to be effective in treating obesity and its co-morbidities. To our knowledge, no one has assessed the effects of Roux En Y gastric bypass surgery for morbidly obese patients on GER in a prospective, controlled manner. Aim: To assess the effects of Roux En Y gastric bypass on reported "heartburn" and acid regurgitation in morbidly obese patients. Methods: In conjunction with the Utah Obesity Study, 1159 participants were recruited into three groups: a surgica group of 415 patients who underwent bariatric surgery, a nonsurgical group of 420 patients who were denied or refused surgery, and a community group consisting of 321 morbidly obese patients randomly selected from the community. These patients were administered a GER questionnaire upon initial enrollment and at their 2 year follow-up period. The questionnaire assessed severity and frequency of heartburn and acid regurgitation as well as the presence of supra-esophageal manifestations of reflux. Results: General linear model analysis was used to compare changes in reported reflux and heartburn among subjects within the surgical treatment $(n=144)$, non-surgical treatment $(n=22)$ and community control $(n=263)$ groups, controlling for age, sex, smoking status, BMI, apnea, apnea score, use of PPI drugs, use of antacids and baseline reflux and heartburn respectively. Significant differences were observed for both reflux $(p=0.005)$ and heartburn $(p<0.002)$, with paired analyses showing significant differences between surgery and control groups $(\mathrm{p}=0.001$ for reflux and $\mathrm{p}$ $<0.001$ for heartburn). Discussion: From this analysis, gastric bypass surgery provides symptomatic relief of heartburn and reflux up to 2 years post-operatively. This finding remains significant even after controlling for BMI, suggesting reduction of reflux symptoms related to the surgical procedure rather than the associated weight loss. Future analysis will include extra-esophageal symptoms of reflux in these patients.

947

The Tantalus ${ }^{\mathrm{TM}}$ System for Obesity: Effect On Gastric Emptying of Solids Claudia P. Sanmiguel, Scott A. Cunneen, Edward H. Phillips, Edy E. Soffer

Background: Gastric electrical stimulation (GES) is currently investigated for the treatmen of obesity. The TANTALUS System delivers gastric contractility modulation (GCM) signals in synchrony with gastric slow waves, resulting in significant augmentation of gastric contractions during food intake. We hypothesized that such modulation of contractile activity may affect gastric emptying and plasma ghrelin levels. Aim: To test the effect of GCM of the gastric antrum on gastric emptying of solids and ghrelin levels. Methods: 12 obese subjects were implanted with 2 pairs of antral electrodes and an implantable pulse generator (IPG TANTALUS TM).Gastric emptying test (GE) for solids was performed twice, on separate days, in each subject, starting few weeks after implantation: 1) control, before the start of stimulation, and 2) with stimulation, after device was turned on. Blood samples for ghrelin, were taken at baseline, and at 15,30,60 and 120 min after the test meal. Results as mean $+\mathrm{SD}$, analysis by $\mathrm{t}$-test and $\mathrm{p}<0.05$. Results: 11 females, 1 male, age: $39.1 \pm 8.9$ years, BMI:41.6 $\pm 3.4,3$ subjects with type 2 diabetes. One diabetic patient did not complete GE test because of technical issues. GCM significantly accelerated gastric emptying: retention at 2 hours $18.7 \pm 12.2 \%$ vs. $31.9 \pm 16.4 \%$, stimulation vs. control respectively, $\mathrm{p}=0.008$. T $1 / 278.3 \pm 23.5$ vs. $95 \pm 31.7 \mathrm{~min}$, stimulation vs. control respectively, $\mathrm{p}=0.04$. Mean results for gastric emptying were within normal at both baseline and stimulation. Meal ingestion induced only minimal, insignificant reduction in ghrelin levels. There was no significant difference in AUC of ghrelin between control and stimulation. Conclusions: 1) After GCM stimulation, there is significant acceleration of gastric emptying of solids in obese patients, without affect on ghrelin levels. 2) The obese subjects did not exhibit the significant, meal-induced reduction in ghrelin 


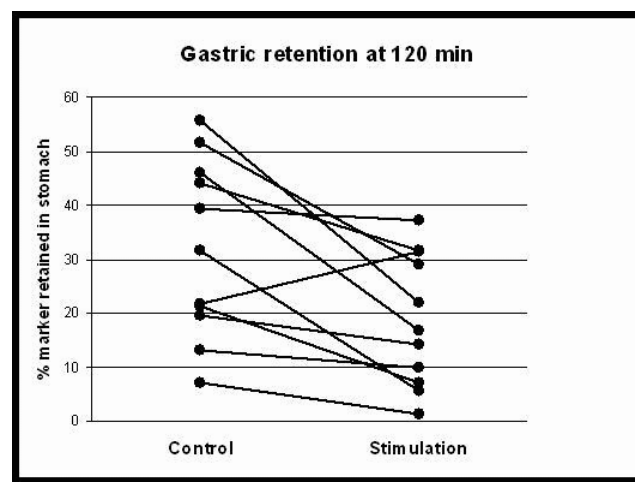

948

Endoscopic Management of Upper Gastrointestinal Hemorrhage Following Laparoscopic Roux-en-Y Gastric Bypass

Laith H. Jamil, Clara Jackson, Kevin Krause, David Chengelis, Michael C. Duffy

Laparoscopic Roux-en-Y Gastric Bypass (LRYGBP) is increasingly being used for the management of morbid obesity. Upper gastrointestinal hemorrhage (UGIH) has been reported as an infrequent complication, ranging from $1 \%-3.8 \%$, and previous publications addressing management have been small case series, with reluctance to perform upper endoscopy (EGD) in the immediate postoperative period for fear of complications. We sought to determine how frequently UGIH complicates LRYGBP and whether endoscopic management is successful in controlling hemorrhage. Methods A retrospective chart review of all patients (pts) who underwent LRYGBP from 11/2001 to 7/2005 at William Beaumont Hospital was conducted with hemorrhage as a complication identified by standard coding criteria. UGI hemorrhage was defined as bleeding from the surgical site, and/or hematemesis and/or melena, and/or hematochezia with significant hemodynamic changes (increase in heart rate $>20$ BPM, decrease in systolic blood pressure $>20 \mathrm{mmHg}$ ), and/or significant drop in hemoglobin $(>2$ $\mathrm{gm} / \mathrm{dl}$ ). Results Of 933 pts who underwent LRYGBP, $30 \mathrm{pts}$ (3.2\%) developed postoperative UGIH. EGD was performed in $27 / 30$ pts $(90 \%)$. Almost all patients $(25 / 27,93 \%)$ were found to be bleeding from the gastrojejunostomy (GJ) staple line. The other 2 pts had an adherent clot on the GJ site. All pts had endoscopic signs of recent bleeding, including 13 pts with blood oozing from the GJ site, 7 pts with a bleeding vessel, 1 pt with a visible vessel in a cratered ulcer on repeat endoscopy, and the remainder with adherent clots at the GJ site. Endoscopic intervention was performed in 24/30 (80\%) with epinephrine injection and heater probe cautery being used most commonly. Endoclips were used in 2 patients. Endoscopic therapy was successful in controlling all hemorrhage, with 5 patients (17\%) requiring a second EGD for rebleeding. No patient required surgery to control hemorrhage. Blood transfusions were given in 14/30 (47\%) with an average of 2.6 units $\mathrm{PRBC} / \mathrm{pt}$ among those requiring transfusion. One patient aspirated during the endoscopic procedure with respiratory compromise and subsequent anoxic encephalopathy and died 5 days postoperatively. Twenty-one patients (70\%) developed UGIH in the intraoperative or immediate postoperative period (<4 hrs postop). Conclusions: 1. UGIH complicates LRYGBP in a small but significant number of patients. 2. Bleeding usually occurs at the GJ site. 3 . EGD is safe and effective in controlling hemorrhage with standard techniques. 4. UGIH occurs most commonly in the immediate postoperative period and is best managed in the operating room with the patient intubated to prevent aspiration.

949

Pilot Study of Preoperative Weight Loss in Super Morbid Obese Patients: The Additional Effect of Endoscopic Intragastric Balloon Placement Gianluca Bonanomi, Simona Di Caro, Mario Traina, Ilaria Tarantino, Giselle G. Hamad, Madelyn H. Fernstrom

Background: Preoperative weight loss is often needed to reduce operative risk. Furthermore, adherence to dietetic and behavioral instructions is a determinant of patient compliance and outcome for bariatric surgery. Methods: Twenty consecutive patients suffering from super morbid obesity (BMI $\geq 50 \mathrm{~kg} / \mathrm{m} 2$ ) were randomized for a 6 -month balanced hypocaloric diet, either alone (group A) or associated with endoscopic intragastric balloon placement (group B). Mean age was 37.2 years (range: $21-55$ ) in Group A and 38.9 (range: $24-58$ ) in group B. Mean BMI was $56.5 \mathrm{~kg} / \mathrm{m} 2$ (range: $51-61$ ) in group A and 58.4 (range: $50-69$ ) in group B. There was no significant difference in age, BMI, gender, and comorbidities between the 2 groups. All patients received counseling and follow-up by a dietitian and were evaluated for possible bariatric surgery after 6 months. Results: Total mean weight loss after 6 months was $7 \mathrm{~kg}$ (range: $0-21)$ in group A and $19 \mathrm{~kg}$ (range: $8-32$ ) in group B ( $<<0.05)$. Mean $\%$ excess weight loss was $12 \%$ in group A and $32 \%$ in group B. Five patients in group A and no patients in group B experienced less than $5 \mathrm{~kg}$ weight loss after 6 months. One patient in group B developed pulmonary embolism that required early balloon removal. Nine out of ten patients with intragastric balloon and five out of ten patients on diet alone experienced a significant improvement of comorbidities and have undergone uneventful bariatric surgery. Patients who failed preoperative weight loss were submitted to additional dietetic and behavioral counseling or were referred for intragastric balloon placement. Conclusion: The results of this pilot prospective randomized study suggest that preoperative intragastric balloon placement in super morbid obese patients results in significant short term weight loss and comorbidities improvement as compared to diet alone.
Pre-Operative Endoscopic Screening Is Not Cost-Effective in Patients Undergoing Bariatric Surgery

Cindy Mong, Jacques van Dam, Lauren B. Gerson, John M. Morton, Myriam J. Curet, Subhas Banerjee

Background: Bariatric surgery patients undergo pre-operative screening endoscopy. EGD in morbidly obese patients carries an increased risk due to high rates of sleep apnea. The yield of EGD in detecting clinically significant lesions in this patient population has not been well studied. The purpose of this study was to examine the yield of screening EGD in the bariatric patient population and to determine its cost effectiveness. Methods: Patients undergoing bariatric surgery between Aug '02 and July '05 at our institution were identified from a database. Clinical, endoscopic and pathological data were analyzed. The prevalence of endoscopic findings of moderate (erosive esophagitis, Barrett's esophagus, erosive gastritis, peptic ulceration, benign upper GI neoplasms) and high (UGI cancer/lymphoma) clinical importance were determined. The cost of endoscopy and biopsy/pathology were determined by Medicare reimbursement and institutional billing records. Results: A total of 270 patient records were examined. At the time of analysis, complete records were available for 217 . $188(87 \%)$ of these were female and $167(75 \%)$ were Caucasian. The median age was 43 yrs (range 20-64 yrs) \& the median BMI 47 (range 35-75). 71 (33\%) patients had sleep apnea documented by a sleep study. Only $26(12 \%)$ patients had endoscopic findings of moderate clinical importance [see table], leading to alteration in surgical technique (subtotal gastrectomy) in a single patient. ${ }^{*} 17$ of these patients (65\%) had a prior history of GERD or dyspepsia. The cost of detection per clinically important lesion was $\$ 7343$. Conclusions: 1. Lesions of moderate significance were found in only 26 (12\%) of 217 patients, leading to alteration of surgical technique in only a single patient. No findings of high clinical significance were noted in any patient. 2 . The detection cost per significant lesion with screening EGD is high at $\$ 7343$. As acid reflux is essentially cured by Roux-en-Y gastric bypass and most cases of peptic ulcer disease are caused by $H$. pylori, a more cost effective approach might be to perform H.pylori stool antigen testing in all patients (with eradication therapy for all testing positive), reserving preoperative EGD only for patients with 'red flag' symptoms.

Table 1

\begin{tabular}{|c|c|}
\hline Lesions at EGD & $\mathrm{n}(\%)$ \\
\hline Erosive Esophagitis & $10(5 \%)$ \\
\hline Barrett's Esophagus & $4(2 \%)$ \\
\hline Erosive Gastritis & $4(2 \%)$ \\
\hline Gastric Ulcer & $7(3 \%)$ \\
\hline Duodenal Ulcer & $2(0.9 \%)$ \\
\hline Gastric Carcinoid & $1(0.5 \%)$ \\
\hline UGI Cancer/ Lymphoma & $0(0 \%)$ \\
\hline
\end{tabular}

951

Vagal Blocking for Obesity Control (VBLOC): Concordance of Effects of Very High Frequency Blocking Current At the Neural and Organ Levels Using Two Preclinical Models

Katherine S. Tweden, Mehran Anvari, Michael D. Bierk, Charles J. Billington, Michael Camilleri, Christopher N. Honda, Mark B. Knudson, David E. Larson, Richard R. Wilson, James W. Freston

BACKGROUND: Vagal blocking using intermittent, very high frequency current is being studied for obesity management. The targeted physiologic effects are to: (1) inhibit gastric accommodation leading to early satiation and reduced food consumption; (2) inhibit gastric contractile activity leading to prolonged satiety; and, (3) inhibit pancreatic exocrine secretion (PES) leading to reduced calorie absorption. Historically, surgical vagotomy for refractory ulcers caused transient anorexia and weight loss by mechanisms that are unclear. AIM: To evaluate the effects of vagal blocking using intermittent, very high frequency current on pancreatic function and neural activity as measured by inhibition of PES and compound action potential (CAP) propagation, respectively. METHODS: In an acute porcine model using non-vagolytic (chloralose) anesthesia, electrodes were placed on the anterior and posterior vagal trunks at the esophagogastric junction. PES was measured by direct cannulation of the pancreatic duct. PES, as well as heart rate (HR), blood pressure (BP) and blood glucose, were measured before, during and after very high frequency vagal blocking at $5000 \mathrm{~Hz}$. In an acute rat model, three pairs of bipolar hook electrodes were placed in series for stimulation, blocking and recording, respectively, on either the cervical vagus or sciatic nerves. The effect of block at $5000 \mathrm{~Hz}$ on the amplitude of electrically evoked fast and slow CAP waves was measured following 5 minutes of blocking at times $0,1,2,3,4$, 5,10 and 15 min post-block. Data are expressed as relative response (post-amplitude/preamplitude $) \times 100$. RESULTS: Vagal blockade decreased PES $>80 \%(0.35 \pm 0.06$ vs. $0.06 \pm 0.01 \mathrm{ml} / \mathrm{h}, \mathrm{n}=6, \mathrm{p}=.005$ ). PES returned to baseline values within $19 \pm 8 \mathrm{~min}$ post-block. Immediately after block. CAP amplitude (fast and slow waves) was reduced to $3 \pm 2 \%$ of baseline. CAP recovered to $50 \%$ by $2 \mathrm{~min}$ and $90 \%$ by $10 \mathrm{~min}(\mathrm{n}=9)$. HR, BP and glucose remained unchanged. CONCLUSIONS: 1. Vagal blocking reversibly inhibits pancreatic exocrine secretion and compound action potential propagation. 2 . The effectiveness of vagal blocking using very high frequency current was corroborated at the neural and organ levels in two preclinical models. 3. Physiologic changes caused by intermittent vagal blocking may offer potential for treating obesity without long-term loss of efficacy. 
Resectability of Pre-Symptomatic Pancreatic Cancer and Its Relationship to Onset of Diabetes: A Retrospective Review of CT Scans and Fasting Glucose Values Prior to Diagnosis

Mario Pelaez-Luna, Naoki Takahashi, Joel G. Fletcher, Suresh T. Chari

Background/Aim: Pancreatic cancer $(\mathrm{PaC})$ is frequently unresectable at diagnosis. The timeline of progression of $\mathrm{PaC}$ from resectable to unresectable disease is unknown. Glucose intolerance and diabetes (DM) occur in up to $80 \%$ of $\mathrm{PaC}$ and new-onset DM may be a marker of early $\mathrm{PaC}$. Our purpose was to determine the resectability of $\mathrm{PaC}$ on $\mathrm{CTs}$ done prior to clinical diagnosis and correlate resectability with onset of DM. Methods: $20 \mathrm{PaC}$ patients with DM who had $\geq$ labdominal CT scans prior to diagnosis of $\mathrm{PaC}$ (total 45 CT scans) were included in the study. Most CTs were performed for non specific abdominal pain or for other reasons different from $\mathrm{PaC}$ related symptoms. CTs were reviewed by one radiologist and pancreatic changes characterized as negative for pancreatic mass, pancreatic ductal changes (dilatation, abrupt termination), resectable, relative unresectable or unresectable $\mathrm{PaC}$. Fasting blood glucose (FBG) values obtained at and prior to diagnosis were available in 14 patients. Date of onset of DM was defined as date of first FBG $\geq 126 \mathrm{mg} / \mathrm{dl}$ with a prior normal FBG. Interval between onset of $\mathrm{DM}$ and diagnosis of $\mathrm{PaC}$ was noted. Results: At diagnosis of $\mathrm{PaC}$, $8(57 \%)$ patients were deemed resectable; however, successful surgical resection was possible only in $3(21 \%)$ cases. 23 CTs in 20 patients were done $>6$ months prior to diagnosis; 18 were normal and 5 had pancreatic ductal changes or resectable mass. At the onset of DM, 7 patients had normal pancreas, 5 had resectable and 2 had unresectable $\mathrm{PaC}$. By the time of $\mathrm{PaC}$ diagnosis $3 / 7$ cases with previous normal pancreas had unresectable disease after a median interval of 13 months. Among those with resectable $\mathrm{PaC}$ on previous scans, 4 were still resectable at diagnosis of $\mathrm{PaC}$ and 1 was unresectable. Conclusions: Resectability of $\mathrm{PaC}$ can be significantly improved if detected $>6$ months prior to clinical diagnosis. Onset of $\mathrm{PaCDM}$ predates clinical diagnosis of $\mathrm{PaC}$ by $>12$ months. Detecting asymptomatic $\mathrm{PaC}$ in subjects with new-onset diabetes can lead to improved resectability.

\section{3}

Ductal Pancreatic Cancer Development of the Pancreas Among Intraductal Papillary Mucinous Neoplasm

Minoru Tada, Takashi Sasaki, Natsuyo Yamamoto, Yosuke Nakai, Takeshi Tsujino, Naoki Sasahira, Hiroyuki Isayama, Takao Kawabe, Masao Omata

Background Intraductal papillary mucinous neoplasm of the pancreas (IPMN) is a benign or low-grade malignant tumor. Branch duct type IPMN is expected to have more favorable clinical course than main duct type. Therefore, some of them are closely and carefully followed-up without resection. In this study, we prospectively examined incidence and types of neoplasm developing in the pancreas by follow-up of the patients with IPMN. Methods Eighty patients (50-87 years old, average 67), who were diagnosed as asymptomatic branch duct type IPMN without characteristics of malignant features that mural nodules were not observed within the cystic lesion, were followed-up as outpatients up to 8 (average 4) years. Clinical examinations, blood tests and imaging diagnosis (US, EUS, CT or MRCP) were performed twice a year. In addition, incidence of pancreatic cancer was compared with expected number of pancreatic cancer death that was calculated with age-gender matched population. Results Malignant pancreatic neoplasms were detected in 4 patients. Two arose from the cystic wall that was previously detected. These cases were consistent with malignant IPMN. In the other two patients, ductal pancreatic carcinoma developed. In one patient whose tumor was found smaller than $2 \mathrm{~cm}$, it was arising in the apparently different site from the cystic lesions. The other patient who was found at advanced stage died with extremely rapid tumor progression accompanying liver metastasis. Overall incidence of malignancy was $1.25 \%$ per year. The relative risk for pancreatic cancer was 30 times higher compared with the expected incidence of pancreatic cancer death in general population according to Japanese statistics. Conclusion Pancreatic cancer may develop in two different manners in patients with IPMN: One originated from cystic wall, and the other was conventional ductal pancreatic cancer that developed apart from cystic lesion.

954

Discovery of Novel Diagnostic Approach for Pancreatic Cancer Joo Kyung Park, Yong Bum Yoon, Yong Tae Kim, Ji Kon Ryu, Jun Kyu Lee, Won Jae Yoon, Sang Hyub Lee

Introduction Pancreatic cancer remains one of the most deadly human cancers. The imaging studies, fine needle aspiration cytology (FNAC) or biopsy (FNAB), and ERCP guided brush cytology are usually performed as a method of diagnosis. However, the reliability of cytologic analyses of fine needle aspirates remains unsatisfactory, with a diagnostic accuracy of $\leq 80 \%$, and even worse in brush cytology. The purpose of this study was to develop a novel diagnostic approach based on genetic and epigenetic characteristics of pancreatic cancers. Especially, aberrant methylated genes have been identified in surgical specimen, and NPTX2 were found more than $90 \%$. Therefore, we tried to prove NPTX2 as a useful diagnostic marker. Methods The 50 patients with pancreatic cancer were enrolled prospectively between 2004 and 2005. They underwent computed tomography, FNAC or FNAB, and ERCP guided bile and pancreatic duct brush cytology. Genomic DNA was extracted using phenol-chloroform extraction method in cytology samles. DNA methylation patterns in the CpG islands of the target genes were determined by chemical modification of unmethylated, but not methylated, cytosines to uracils, and subsequent PCR amplification using primers specific for either methylated or modified unmethylated DNA. The bisulfite-modification was performed. The NPTX2 CpG island was examined by real-time PCR amplification using oligonucleotide primers and a TaqMan probe specific for a fully methylated bisulfite converted portion of the NPTX2 gene. The $\beta$-actin primers and probe set, designed specifically for bisulfite-converted DNA, were used as a reference gene. Results The fifty patients were all pathologically confirmed with definite diagnosis of pancreatic cancer. In brush cytology, the 5 out of 31 patients (16\%) had the pathologic reports of positive for malignant cells, and another 5 patients had atypical cells. The rest of 21 patients had the negative for malignant cells. In real-time PCR, aberrant methylation of NPTX2 was detected in 27 out of 31 patients (87\%). In FNAC, the 9 out of 19 patients (47\%) had the pathologic reports of positive for malignant cells, and 3 patients had the atypical cells. The rest of 7 patients had the negative for malignant cells. In real-time PCR, aberrant methylation of NPTX2 was detected in 19 patients (100\%). Conclusion Our diagnostic approach resulted in $87 \%$ accuracy in brush cytology and 100\% in FNAC. The aberrant methylation of NPTX2 gene can be expected to become a valuable new tool to improve the reliability in conjunction with conventional diagnostic procedures in pancreatic cancer.

955

Tumour-M2-Pyruvate Kinase (Tu-M2-PK): A Novel Biomarker of Adverse Prognosis in Pancreatic Cancer

Priyantha Siriwardana, James M. Mason, Kolitha Goonetilleke, Nicolas K. King, Michael W. France, Ajith K. Siriwarden

Introduction A wide range of human tumours express the enzyme pyruvate kinase, component of aerobic glycolysis pathways. Of the four different isoforms found in mammalian tissue, the M2 isoenzyme is strongly over-expressed in cancer states. A commercially available enzyme-linked immunosorbent assay (ELISA) kit has allowed for the reliable detection an quantification of the enzyme tumour-M2-pyruvate kinase (Tu-M2-PK) in blood. This study tests the hypothesis that Tu-M2-PK is a prognostic marker in pancreatic cancer. Methods Between July 2002 and March 2004, 104 patients were admitted to the hepato-pancreaticobiliary service of a University Hospital with suspected pancreatic cancer and gave informed consent for participation. CA 19-9 and Tu-M2-PK levels in blood were assayed prior to resection or pancreatic biopsy. 23 Jaundiced control patients had evidence of the absence of cancer at ERCP and 44 non-jaundiced controls underwent elective cholecystectomy. The principal outcome measures were estimation of the sensitivity and specificity of Tu-M2-PK and CA 19-9 individually and in combination for the pre-operative diagnosis of periampullary cancer and for prediction of survival. Of the 104 pts, 49 had jaundice. Subsequent Histopathologic findings confirmed pancreatic/peri-ampullary malignancy in 76 . Survival data (up to 3 years follow-up with a median of 271 [2-1129] days) in patients with confirmed cancer were used to gain further understanding of the test findings. Results The mean plasma Tu-M2-PK level for patients with histologically-proven malignancy was $40.5(26.4) \mathrm{U} / \mathrm{m}$ and for all non-cancer patients was 29.9 (20.9) U/ml. This difference was statistically significant (Mann-Whitney U=1163, P = 0.006). However, Tu-M2-PK with an AOC of 0.623 is unlikely to provide a useful discriminant test for pancreatic cancer although Tu-M2-PK test findings were unaffected by the presence of jaundice. Tu-M2-PK correlated with poor differentiation and metastatic disease. Multivariate Cox regressions modelling determinants of survival with Tu-M2-PK dichotomised at the optimal cut-off $(27 \mathrm{ml} / \mathrm{U})$ and fitted as a binary variable in the model revealed that patients with values above the cut-off were dying 2.6 times faster than those with values below $(95 \% \mathrm{CI}: 1.3$ to 5.3$)$. THis difference was statistically significant at the $\mathrm{P}<0.01$ level and was sustained throughout the 3 -yr follow-up on Kaplan-Meier survival analysis. Conclusion In a tertiary referral practice setting an elevated Tu-M2-PK over 27 units/ml was a strong independent predictor of poor survival and this is worthy of incorporation into future management algorithms

\section{6}

Evaluation of Hedgehog (HH) Pathway Expression in Cell Lines and Endoscopic Ultrasound-Guided Fine-Needle Aspirate (EUS-FNA) Biopsies of Pancreatic Adenocarcinoma

Adam Steg, Selwyn Vickers, Mohamad Eloubeidi, Amy Petersen, Wenqan Wang, Andra Frost, Martin Johnson

INTRODUCTION: Pancreatic adenocarcinoma is characterized by an unusual resistance to radiation and chemotherapy. Recent studies suggest that activation of the $\mathrm{HH}$ signaling pathway is involved in the initiation, maintenance and progression of pancreatic adenocarcinoma and that targeting this pathway could potentially be used as a novel therapeutic strategy OBJECTIVE: The goals of this study were to: 1) examine HH expression in EUS-FNA biopsies obtained from patients diagnosed with pancreatic adenocarcinoma; and 2) determine the molecular basis of response to the $\mathrm{HH}$ pathway antagonist cyclopamine in pancreatic cancer cell lines. METHODS: The expression of $46 \mathrm{HH}$ pathway-associated genes was examined in 11 EUS-FNA biopsies and 11 pancreatic cancer cell lines (AsPC-1, BxPC-3, CFPAC1 HPAF-2, MiaPaCa-2, Panc-1, Panc 2.03, Panc 6.03, Panc 8.13, Panc 10.05 and S2013 using Taqman Low Density Array (TLDA). To test for response to cyclopamine, cells were cultured in medium containing different concentrations $(1,2,3.5,5,7.5,10,12.5,15$, $17.5,20,22.5,25,27.5$ or $30 \mu \mathrm{M}$ ) of cyclopamine. After 96 hours of treatment, cell viability was calculated using an MTS colorimetric assay relative to vehicle control. Linear regression analysis was performed to determine the dosage at which $50 \%$ inhibition of cell growth was achieved (IC50). RESULTS: Among the HH ligands examined, Sonic and Indian demonstrated the highest average mRNA levels in tumor compared to uninvolved pancreas (336 and 162 fold respectively). Among the $\mathrm{HH}$ receptors, Patched 1 and 2 , expressed 1.48- and 5.38fold higher, respectively, while Smoothened, the target of cyclopamine, demonstrated a 1.67-fold decrease in expression in tumor. The glioma-associated oncogenes, GLI-1, -2 and 3 , transcription factors which have been correlated with $\mathrm{HH}$ pathway activity, expressed 4.18-, 10.18- and 3.34-fold higher in tumor, respectively. The response to cyclopamine varied among the cell lines with IC50's ranging from a low of $7.62 \mu \mathrm{M}$ for BxPC-3 cells to a high of $42.91 \mu \mathrm{M}$ for $\$ 2013$ cells with a trend toward low Smoothened and high Glycogen Synthase Kinase 3-beta (GSK3B) mRNA levels in sensitive cell lines as compared to resistan cell lines. CONCLUSION: These data suggest $\mathrm{HH}$ pathway expression varies among both pancreatic tumor samples and cell lines and that Smoothened and GSK3B may be indicators of response to cyclopamine. This characterization could, ultimately, be used to develop a criteria for the selection of pancreatic cancer patients who would benefit from $\mathrm{HH}$ inhibitor therapy.

\section{.}


Prevalence of Pancreatic Cancer Risk Factors and Perceptions of Cancer Risk Among Individuals with An Inherited Predisposition to Pancreatic Cancer Kerry B. Dunbar, Marcia I. Canto

BACKGROUND: Individuals with a family history of pancreatic cancer (PC) have increased risk for developing the disease. Little is known about the prevalence of risk factors for sporadic PC in high risk individuals and their perceptions of their cancer risk. This information is important for possible future intervention trials. AIM: To identify potentially modifiable risk factors, such as smoking, obesity and physical inactivity and to study perceptions of cancer risk among high risk individuals with a genetic predisposition for PC. METHODS: Cancer of the Pancreas Screening (CAPS) Studies 1\&2 (1998-2002) prospectively screened adult high risk patients from familial PC kindreds with at least 2 affected first-degree relatives for early pancreatic neoplasia using CT and EUS. Adult control subjects without a personal or family history of PC and without suspicion of pancreatic disease were concurrently enrolled. Risk factor information was prospectively obtained through a standardized questionnaire, including race, weight, height, personal and family history of neoplasia, medical history, tobacco use, medication use, alcohol use, physical activity and perception of cancer risk. RESULTS: Age, race and gender were comparable among the 113 high patients and 129 normal risk controls studied. However, high risk individuals were more likely be overweight or obese (BMI>25) compared to controls ( $64.6 \%$ vs. $43.9 \%, \mathrm{p}=0.008$ ) but not more likely to be inactive (i.e. total physical activity $<4$ hours/week) (61.5 vs. $85.7 \%$ ). Exposure to cigarette smoke was similar in high risk and control subjects; $46 \%$ had ever smoked cigarettes, $86 \%$ had passive smoke exposure, but only $8.9 \%$ were current smokers. High risk subjects were also more likely to be current regular alcohol drinkers than controls ( $31 \%$ vs. $19 \%$, $\mathrm{p}=0.03$ ) but their exposure to aspirin, folic acid and HMG-CoA reductase inhibitors, which have been associated with a potential protective effect for PC development, were similar to controls. High risk individuals were more likely to be moderately to extremely worried about PC $(86 \%)$ and to perceive their lifetime risk for PC as moderately to extremely high (91\%), but their perceived risk for other cancers was no different from controls $(\mathrm{p}=0.09)$. CONCLUSIONS: Individuals with a family history of PC possessed other potentially modifiable risk factors of PC (overweight, inactivity, smoking). They were significantly more worried about their PC risk compared to controls. This high level of concern presents an opportunity to encourage lifestyle modifications and study interventions that could potentially modify their risk for PC

958

P130cas and CRK May Regulate Human CACO-2 Intestinal Epithelial Cell Migration Via the Small GTP Binding Protein Racl and the GuanineNucleotide Exchange Factors Dock180 and C3g

Matthew A. Sanders, Marc D. Basson

We have previously observed that Caco-2 intestinal epithelial cell spreading, migration and lamellipodial extension on the extracellular matrix protein collagen IV requires Src-dependent phosphorylation of the adaptor protein p130Cas and also requires the adaptor protein Crk, which binds to phosphorylated p130Cas. We hypothesized that focal adhesion kinase (FAK), which contains a potential binding site for the p130Cas SH3 domain, may also be required for collagen IV-dependent phosphorylation of p130Cas and its subsequent interaction with Crk. Additionally, we hypothesized that Caco-2 spreading and migration may require the guanine-nucleotide exchange factors DOCK180 and C3G, which bind to Crk in some cell types. Transfection of Caco-2 cells with a FAK-specific small interfering RNA (siRNA) reduced FAK protein by more than $90 \%$ and strongly inhibited $(47 \pm 4 \%$ inhibition, $\mathrm{n}=3, \mathrm{p}<0.01)$ phosphorylation of p130Cas $\operatorname{Tyr}(\mathrm{P}) 249$, which is an important Crk binding site in some cell types. siRNAs specific to DOCK180 or C3G reduced levels of each protein by more than $80 \%$. DOCK180 siRNA inhibited Caco-2 spreading on collagen IV by $17 \pm 3 \%(n=3$, $\mathrm{p}<0.05)$. C3G siRNA did not significantly inhibit Caco-2 spreading by itself. However, combined C3G and DOCK180 siRNA inhibited cell spreading by $38 \pm 5 \%(\mathrm{n}=3, \mathrm{p}<0.05$ compared to control siRNA or individual DOCK180 and C3G siRNAs). Additionally, combined C3G and DOCK180 siRNA transfection significantly inhibited Caco-2 migration on collagen IV $(32 \pm 5 \%$ inhibition, $\mathrm{n}=4, \mathrm{p}<0.01)$ and similarly to pl30Cas or Crk siRNA transfection noticeably inhibited lamellipodial extension. Since DOCK180 can serve as a guanine-nucleotide exchange factor for the small GTP-binding protein Racl in some cell types, we therefore next examined the role of this protein in Caco-2 spreading and migration on collagen IV. The Racl specific inhibitor NSC23766 strongly inhibited Caco-2 migration $(66 \pm 5 \%$ inhibition, $\mathrm{n}=3, \mathrm{p}<0.01)$ and lamellipodial extension on collagen IV, as well as cell spreading $(42 \pm 9 \%$ inhibition, $n=6, p<0.01)$. We confirmed the specificity of this result using siRNA specific for Racl. Transfection with siRNA to Racl reduced Racl protein by more than $80 \%$ and similarly to NSC23766 Racl siRNA inhibited Caco-2 spreading on collagen IV by $39 \pm 3 \%(n=6, p<0.001)$. Taken together, these results suggest that collagen IV may regulate Caco-2 migration via FAK and Src-dependent p130Cas phosphorylation and binding to Crk and subsequent signaling through the guanine-nucleotide exchange factors DOCK180 and $\mathrm{C} 3 \mathrm{G}$ to the small GTP-binding protein Racl.

\section{9}

Novel Roles of IGF-1 in Gastric Ulcer Healing: Activates G-Actin Polymerization and RE-Epithelialization Via Cross-Talk Between COX2 and PI3K Signaling Pathways

Thomas Nguyen, Jianyuan Chai, Tetsuya Tanigawa, Andrzej S. Tarnawski

Background \& Aims: Insulin-like growth factor-1 (IGF-1) promotes cell survival in a variety of cells and may play a role in gastric ulcer healing. It is unknown whether IGF-1 regulates re-epithelialization during gastric ulcer healing, and if so, what are the molecular mechanisms and signaling pathways of this action. We examined effect of gastric ulceration on expression and cellular localization of IGF-1 in gastric mucosa in vivo, and determined in in vitro studies in gastric epithelial RGMl cells the effects of exogenous IGF-1 on G-actin polymerization into F-actin, epithelial cell migration and Cox2 expression. We also examined dependence of these processes on PI3K and MAPK signal transduction pathways. Methods: Gastric ulcers were induced in rats by focal serosal application of acetic acid. Specimens of ulcerated and non-ulcerated mucosa were obtained sequentially following ulcer induction for analysis of IGF-1 and Cox-2 mRNA and protein expression by RT-PCR, ELISA and immunohistochemistry, respectively. To evaluate the signaling pathways mediating IGF-1 actions and the role COX-2, we used specific inhibitors of PI3- kinase, MEK1 kinase and Cox2. IGF-1-induced RGMl cell migration was evaluated using standard "scratch wound assay" in the presence IGF-1 with or without PI3-kinase inhibitor (LY294002), MEKl kinase inhibitor (PD98059) or COX-2 inhibitor (NS-398). Results: Gastric ulceration increased tissue IGF-1 concentration by $226 \%, 305 \%$ and $254 \%$, respectively, at 4,6 and 12 days (all $p<0.001$ ). Immunohistochemical staining demonstrated increased IGF-l expression in epithelial cells at the ulcer margin base, and later in the cells re-epithelializing granulation tissue. Treatment of RGMI cells with exogenous IGF-1 (10-50 $\mu \mathrm{g} / \mathrm{ml})$ produced a dramatic increase in $\mathrm{G}$-actin polymerization into F-actin, $\sim 800 \%$ increase in cell migration ( $\mathrm{p}<0.001),>200 \%$ increase in cell proliferation ( $\mathrm{p}<0.05$ ), and a significant increase in COX-2 expression. Inhibition of PI3kinase activity abolished IGF-1-induced actin polymerization, RGMl cell migration and proliferation and COX-2 protein expression. MEK/MAPK inhibitor reduced IGF-1-induced activation of all above processes by $30-50 \%$. Conclusions: 1) Gastric ulceration activates IGF-1 gene in epithelial cells of the ulcer margin. 2) In vitro, IGF-1 stimulates in gastric RGMI cells, in a PI3-kinase dependent manner: G-actin polymerization into F-actin, cell migration and proliferation and induces COX-2 expression. 3) A strong IGF-1 expression in the cells migrating onto granulation tissue and re-epithelializing gastric ulcer indicates its critical role in autocrine regulation of this process.

960

Role of 15-Deoxy Prostaglandin J2 and NRF2 in Protection of Gastric Mucosa Against Oxidative Stress During H.pylori Infection in Mice

Akinori Yanaka, Ken Itoh, Masafumi Tauchi, Hideo Suzuki, Takeshi Shibahara, Hirofumi Matsui, Akira Nakahara, Ichinosuke Hyodo, Masayuki Yamamoto

Background: Persistent H.pylori (Hp) infection causes gastritis, ulcers, and gastric cancer. However, cells can withstand stresses by inducing a variety of antioxidant enzymes, thereby prevents gastric mucosa from oxidative injury. 15-deoxy PGJ2 (15-DPGJ2), which is endogenously generated by COX-2 in inflamed tissues, affords strong cytoprotective activity against oxidative stress (Ann N Y Acad Sci. 2003;993:208-16). Although PGJ2 family has been shown to exert anti-inflammatory action by activating PPAR $\gamma$ and/or by inhibiting NF-kB, the exact mechanisms by which15-DPGJ2 protects cells against oxidative stress remains unclear. We have previously reported that nrf2 (NF-E2 p45-related factor-2), a transcription factor, which regulates cellular response against oxidative stress, plays an important role in induction of antioxidant enzymes in gastric mucosa during Hp infection (AGA 2003). In the present study, we aimed to determine if endogenously generated 15-DPGJ2 contributes to gastric mucosal protection via up-regulating nrf2-dependent antioxidant enzymes. Methods: Standardized mice model of Hp infection were set up by inoculating C57/BL6 female mice (nrf2+/+ and nrf2-/-) with Hp Sydney Strain; SS1. Mice were maintained with high salt diet $(7.5 \% \mathrm{NaCl})$. Some mice were treated with either NS-398 and/or 15-DPGJ2. Mice were sacrificed at 4, 8, and 16 wks later. Degree of gastritis was evaluated by updated Sydney system. DNA damage was estimated by measuring mucosal level of 8-OHdG. Expressions of TNF- $\alpha$, IL- $1 \beta$, COX-2, and antioxidant enzymes (GST, and HO-1) were analyzed by real time RT-PCR. Mucosal level of 15-DPGJ2 was evaluated using ELISA. Results: 1. In nrf2+/ + mice, $4 \mathrm{w}$ treatment with $\mathrm{Hp}$ + high salt diet caused severe inflammation in corpus mucosa, accompanied by marked elevation of TNF- $\alpha$, IL-1 $\beta$, COX- 2 and $8-O H d G$, while expressions of 15-DPGJ2, GST and HO-1 were not prominent. 2. During 8-16 w treatment, mucosal inflammation improved over time, accompanied by a gradual decrease in levels of TNF- $\alpha$, IL-1 $\beta$, COX-2, and 8-OHdG. In contrast, 15-DPGJ2, GST and HO-1 were more up-regulated than those at $4 \mathrm{w}$. 3. Treatment with NS-398 exacerbated the corpus gastritis at $4 \mathrm{w}$, and inhibited subsequent improvement of gastritis, whereas administration of exogenous 15DPGJ2 in combination with NS-398 rescued the worsening effect of NS-398 on gastric mucosa. 4. The effects of NS-398 and 15-DPGJ2 were completely abolished in nrf2-/- mice treated with $\mathrm{Hp}$ and high salt. Conclusion: These results suggest that endogenous 15-DPGJ2 protects gastric mucosa against oxidative stress during Hp infection, at least in part by upregulating nrf2-dependent antioxidant enzymes.

961

Protein Kinase C-Delta (Pkc- $\delta$ ) Isoform Is a Key Regulator of NF-kB and Its Endogenous Modulator I-kb During Oxidative Stress Injury: A Crucial ProInflammatory Mechanism in Gut Epithelium

Ali Banan, Lijuan Zhang, Maliha Shaikh, Jeremy Z. Fields, Ashkan Farhadi, Christopher B. Forsyth, Ali Keshavarzian

NF-kB activation \& gut barrier injury are required factors in the pathogenesis of inflammatory GI disorders. Using GI cells, we found that oxidative stress activates NF-kB (by degrading its modulator $\mathrm{IkB} \alpha) \&$ leads to disruption of the intestinal barrier. But, the underlying molecular mechanisms are unclear. Since PKC- $\delta$, a member of the novel family of PKC isoforms, is key in cellular injury, we investigated whether inhibition of PKC- $\delta$ expression is required for protection against oxidant-induced IkB- $\alpha$ degradation, NF-kB activation \& barrier disruption. Methods: We used GI cell clones transfected to either overexpress or underexpress PKC- $\delta$. Monolayers of these unique GI clones were then incubated with PKC activators or inhibitors \pm oxidant (H2O2). Naive, wild type (WT) cells were treated similarly. We then assessed cell survival \{LIVE/DEAD assay\}, monolayer barrier permeability \{fluorometry\}, tight junction integrity \& assembly \{laser confocal microscopy, PAGE fractionation\}, PKC- $\delta$ subcellular activity \& distribution (immunoprecipitation, in vitro kinase\}, IkB $\alpha$ levels $\{$ PAGE\}, NF-kB (p50/p65) subunit activities \{ELISA, EMSA\}, n=6/grp. Results: $\{A\}$ Cells transfected to underexpress PKC- $\delta$ or WT cells pretreated with the PKC$\delta$ inhibitor (rottlerin) were protected against oxidative damage as indicated by: PKC- $\delta$ inactivation, IkB $\alpha$ stabilization, NF-kB (p50/p65 subunits) inactivation, occludin assembly (increased membrane fraction, decreased cytosolic fraction), occludin cytoarchitectural integrity, \& monolayer barrier stability. All measures of protection against NF-kB induced by 
inhibition of PKC- $\delta$ were synergistic with protection by growth factor (EGF). $\{B\}$ Sense transfection to induce expression of full-length endogenous PKC- $\delta$ caused IkB $\alpha$ degradation $\&$ NF-kB activation, and led to occludin \& barrier hyperpermeability, paralleling oxidantlike affects in WT cells. $\{C\}$ Most of the overexpressed PKC- $\delta$ resided in membrane \& cytoskeletal fractions, indicating constitutive activation. In both transfected clones \& oxidanttreated WT cells, PKC- $\delta(75 \mathrm{kDa})$ was active and found with $\mathrm{IkB}(37 \mathrm{kDa})$, forming novel endogenous PKC- $\delta$ :IkB complexes. $\{\mathrm{D}\}$ PKC- $\delta$ expression $\&$ activation prevented all measures of EGF protection against oxidant-induced IkB/NF-kB activation \& monolayer barrier instability. Conclusions: Our findings support novel pathophysiologic mechanisms in GI cells that: 1) Activation of PKC- $\delta$ is necessary for cellular $\&$ barrier injury due to the stress of $\mathrm{I}-\mathrm{kB} / \mathrm{NF}-\mathrm{kB}$ activation; 2) PKC- $\delta$ appears to be a unique intracellular modulator of I-kB; 3) Protection of barrier integrity can occur through inactivation of PKC- $\delta$ and of I-kB/NF-kB.

962

Telomere Shortening and Senescence Differentially Affect Gastric Acid and Duodenal Bicarbonate Secretory Capacity in Aging Mice

Biguang Tuo, Zhenyu Ju, Brigitte Riederer, Regina Engelhardt, Michael P. Manns, Lenhard Rudolph, Ursula Seidle

Background\&Aims: The incidence of non-H.pylori-related duodenal ulcers strongly increases with age, but the study of its pathophysiology has been hampered by the lack of suitable animal models. There is a growing evidence that telomere shortening and senescence influence human aging. We therefore wondered how telomere shortening may affect the aggressive and protective ion secretory processes in the gastroduodenal mucosa of late generation telomerase deficient (mTERC-/-) mice with shortened telomeres. Methods: Murine duodenal mucosal bicarbonate secretion and gastric acid secretion were examined in vitro in Ussing chambers. Duodenal mucosal CFTR and Slc26a6 expression levels were analyzed by quantitative RT-PCR. Results: In aged late generation mTERC-/- mice with shortened telomeres, forskolin-stimulated bicarbonate secretion and Isc were reduced by $43 \%$ and $47 \%$ respectively, compared with age-matched wt mice with long telomere reserves $(\mathrm{P}<0.0001)$. In contrast, forskolin-stimulated gastric acid secretion was not significantly altered $(P>0.05)$. Correspondingly, duodenal mucosa of mTERC-/- showed villous atrophy in the duodenum, whereas gastric mucosal histology was not altered compared to wt controls. Interestingly, the ratio of CFTR and Slc26a6 mRNA expression in relation to cytokeratin 18 was not altered, demonstrating a balanced atrophy along the whole crypt length with no shift in the expression of villous (SLC26A6) vs crypt-predominant (CFTR) transporters. p21 is a downstream effector of senescence signaling resulting in cell cycle arrest. In order to investigate whether the defect in bicarbonate secretion in mTERC -/- mice was due to $\mathrm{p} 21$ dependent senescence induced by telomere shortening, we analyzed duodenal mucosal bicarbonate secretion in late generation mTERC-///p21-/- double knockout mice. In mTERC-//p21-/mice duodenal atrophy was rescued compared to mTERC-/- single knockout mice and forskolin-stimulated bicarbonate secretion and Isc were not different from wt controls $(\mathrm{P}>0.05)$. Conclusion: Telomere shortening and senescence results in mucosal atrophy and reduced bicarbonate secretory capacity in the duodenum of aging TERC-/- mice. In contrast, the stomach with its longlived glandular cells displays normal histology and acid secretory function. This suggests that telomere shortening and senescence has a differential effect on gastroduodenal ion transport functions, resulting in an imbalance between aggressive and protective secretions and thus predisposing to duodenal ulcer formation

963

Short Food Deprivation Abolishes Orexin-a Induced Intracellular Calcium Signaling in Acutely Isolated Duodenal Enterocytes

Gunnar Flemstrom, Magnus Bengtsson, Kari Makela, Gunilla Jedstedt, Markus Sjoblom, Karl-Heinz Herzig

BACKGROUND \& AIM: Neural pathways including reflexes within the enteric nervous system as well as stimuli elicited within the central nervous system and transmitted to the intestine by autonomic nerves are key elements in the control of small intestinal secretion. Some appetite regulating hormones, including orexin- $\mathrm{A}$ and orexin-B are produced in the hypothalamus but also expressed in the intestinal submucosa. Interactions between central nervous and intestinal actions of these hormones would seem of particular interest. We have compared effects of Orexin-A on enterocyte intracellular calcium [Ca2+]i signaling in enterocytes acutely isolated from duodenum in continuously fed and overnight food deprived animals. METHODS: Rats had continuous access to their regular food supply, or were overnight deprived of food (but not water). Animals were sacrificed at 8 am and the duodenal mucosa was cut into $0.5-1.0 \mathrm{~mm}$ pieces followed by mild digestion (collagenase/dispase) to yield clusters (10-100 cells) of duodenal enterocytes. Clusters were loaded with fura-2, mounted in a perfusion chamber and $[\mathrm{Ca} 2+] \mathrm{i}$ was measured with fluororescence imaging. RESULTS: Orexin-A (1-100 nanomol/L) in a dose-dependent manner induced [Ca2+]i signaling in duodenal enterocytes isolated from continuously fed animals. There was a rise in $[\mathrm{Ca} 2+]$ i to a peak level, and the response was similar to those observed with carbachol or cholecystokinin. In contrast, there were no responses to orexin- $\mathrm{A}$ in enterocytes from overnight food deprived animals. Even at a high concentration ( 1 micromol/L), the muscarinic agonist atropine did not affect [Ca2+]i signaling induced by orexin-A.The orexin (OX1) receptor antagonist SB-33487 was used at concentrations of 1-100 nanomol/L). The highest concentration of SB-33487 alone induced [Ca2+]i signaling, similar to that observed with orexin-A suggesting that the compound is a partial agonist. At the lower concentration of 10 nanomol/L, SB-33487 inhibited the response to 10 nanomol/L of orexin-A but did not abolish that to 100 nanomol/L of the peptide. CONCLUSION: The orexogenic peptide orexin-A induces $[\mathrm{Ca} 2+]$ i signaling in duodenal enterocytes from fed animals and short fasting abolishes the response. These findings are in line with absence of an electrolyte secretory response to orexin-A in fasted animals and strongly suggest that enterocyte OXI receptors are involved in regulating intestinal secretion. Supported by the Swedish Research Council and the Finnish Academy

\section{4}

Increased Life-Time Risk for Colorectal Cancer in Patients with Loss of Genomic Imprinting of Insulin-Like Growth Factor 2: A Survival Analysis Marcia Cruz-Correa, Ronghua Zhao, Dana Sands, Eric Weiss, Juan Nogueras, Steven Wexner, Francis M. Giardiello

Background: Loss of genomic imprinting (LOI) of the IGF2 gene is independently associated with colorectal cancer (CRC), but underlying carcinogenic mechanisms remain unclear. Whether LOI-positive individuals have a higher life-time risk for CRC than LOI-negative individuals is unknown. This study investigated the incidence rate and life-time risk of developing CRC in patients whose LOI-status was known. Methods: We performed PCR on gDNA and RT-PCR on RNA extracted from normal peripheral blood lymphocytes (PBL) of prospectively recruited individuals. After digestion by Apa I, the PCR and RT-PCR products were run on $2 \%$ agarose gel and the gel photos were quantitatively analyzed to determine the informative status and IGF2 imprinting status, respectively. Incidence rates of CRC were calculated by person-year analysis using Kaplan-Meier survival estimates and cox regression as appropriate. Results: A total of 245 informative patients were recruited including 130 normal individuals ( 73 males; mean age $60.0 \pm 12.6$ years), 71 patients with colorecta polyps ( 33 males; age $62.8 \pm 7.7$ years) and 44 with CRC ( 18 males; age $61.9 \pm 12.3$ years) The incidence rates for CRC were 2.3 and 6.0 per 1000 person-years for LOI-negative and LOI-positive patients, respectively (Hazard Risk 3.03, 95\% CI 1.62-5.7). Using age-atdiagnosis as a time variable in a gender-adjusted cox regression model, LOI-positive patient presented with CRC at earlier age compared to LOI-negative patients $(\mathrm{p}=0.001)$. Conclusion Individuals with LOI of IGF2 as noted in PBL had an increased life-time risk for and earlier age of diagnosis of CRC, compared to LOI-negative patients. These findings suggest a distinct carcinogenic pathway for LOI-positive patients, which may have implications in screening and risk assessment. Support: NIH grants K07 CA092445 and P50 CA-62924-10, The John G. Rangos, Sr. Charitable Foundation, The Cancer Research and Prevention Foundation, The Clayton Fund

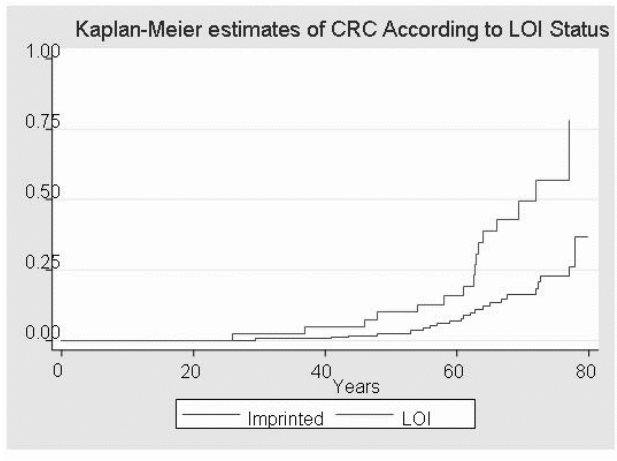

965

Risk of Colorectal Cancer in Juvenile Polyposis

Lodewijk A. Brosens, Anne C. Tersmette, Linda M. Hylind, Christine Iacobuzio-Donahue, Katharine Romans, Jennifer Axilbund, Arnout van Hattem, G. Johan A. Offerhaus, Francis M. Giardiello

Background: Juvenile polyposis is an autosomal dominant disorder characterized by the development in childhood of juvenile polyps primarily in the colorectum but also elsewhere in the gastrointestinal tract. Juvenile polyposis is associated with colorectal cancer, but the risk is unclear. Therefore, we determined the relative and absolute risk of colorectal cancer in juvenile polyposis patients. Methods: The study population consisted of 73 patients with juvenile polyposis from the Johns Hopkins Polyposis Registry contributing 1625.2 person years of follow-up. This included 35 males ( 738.9 person-years) and 38 females (886.3 person-years). The incidence rates of colorectal cancer in these patients with juvenile polyposis were compared with the general U.S. population through person-year analysis using SEER data with adjustment for population. Results: In juvenile polyposis patients the relative risk (RR) of colorectal cancer was 34.5 times (95\% confidence limits [CL], 15.068.5) the general population. Similar risks were noted in both males (RR 36.2; CL, 13.0 76.8) and females (RR 30.1; CL, 3.5-103). The cumulative lifetime risk for colorectal cancer was $39.4 \%$. The mean age of diagnosis of colorectal cancer was 40.4+/-10.7 (SD). Other gastrointestinal tumors were not observed in this cohort. Conclusion: Patients with juvenile polyposis are at high relative and absolute risk for colorectal cancer. Vigilant colorecta cancer surveillance of these patients starting at young age is imperative. Supported by The John G. Rangos, Sr. Charitable Foundation, The Clayton Fund, The Netherlands Digestive Disease Foundation, NIH grants K07 CA092445, CA 53801, and P50 CA 62924-10.

966

Prospective Results of Surveillance Colonoscopy in Autosomal Dominant Familial Colorectal Cancer Families with and Without Lynch Syndrome Isis Dove-Edwin, Andrea de Jong, Joanna Adams, Lara Lipton, Peter Sasieni, Hans Vasen, Huw Thomas

Background \& Aims: Lynch syndrome is an autosomal dominant predisposition to colorectal cancer caused by mutations in DNA mismatch repair genes; colorectal cancer risk is high. Few studies have addressed colorectal cancer risk in individuals from dominant families without mismatch repair deficiency. We sought to establish whether they are also at increased risk by examining the incidence of advanced neoplasia during surveillance Methods: In this prospective cohort study, carried out at two tertiary centres, 126 individuals from 98 families with a dominant colorectal cancer history had BAT26 testing of colorectal cancers to classify

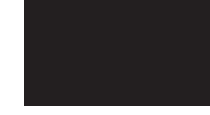


them as Lynch syndrome (microsatellite unstable) or non-Lynch syndrome (microsatellite stable). Results of colonoscopy in 291 at-risk family members were compared. Results: 29 families were classified as Lynch syndrome and 69 as non-Lynch syndrome. 785 colonoscopies were undertaken. High risk adenomas ocurred in 7/91 (7.7\%) of Lynch syndrome individuals and 17/200 (8.5\%) of non-Lynch syndrome individuals chi-sq=0.01, p=0.92. Cancer was observed in $4 / 91$ (4.4\%) of Lynch syndrome individuals, none occurred in nonLynch syndrome Fisher's exact chi-sq $=7.0460 \mathrm{p}=0.017$. Multiple adenomas were only seen in non-Lynch syndrome 9/200 (4.5\%) Fisher's exact chi-sq=4.2257, p=0.040. Forty percent (6/15) of individuals with multiple adenomas developed a high risk adenoma at some point during surveillance $\mathrm{OR}=12.0$ (95\% CI $(2.4,59.4)) \mathrm{p}$-value $=0.002$, adjusting for age, sex and extent and centre solely within the non-Lynch group. Conclusions: Individuals with an autosomal dominant family history of colorectal cancer with and without evidence of Lynch syndrome are at equal risk for the development of high risk adenomas during surveillance, but colorectal cancer was only seen in Lynch syndrome. Therefore non-Lynch syndrome individuals do require colonoscopic surveillance, but the interval could be lengthened because risk of (interval) cancer is low. Lynch syndrome individuals require short surveillance intervals as is the recommended practice.

967

The Genotype-Phenotype Relationship of Polyposis Families Reveals Clinically Distinct Subgroups

Kevin J. Monahan, Volikos Emmanouil, Robin K. Phillips, Thomas J. Huw, Andrew R. Silver, Ian P. Tomlinson

Background: Familial adenomatous polyposis (FAP) is an autosomal dominant condition predisposing to multiple polyps of the large bowel. However, not all patients with polyposis carry germline mutations in the APC (adenomatous polyposis coli) gene or other known genes which predispose to this condition. Aims: The genotype-phenotype relationship of polyposis kindreds without identified mutations in known genes was determined in order to characterise features of a previously undescribed polyposis syndrome. The yield using stringent mutation detection methods was also determined. Patients: A cohort of sixty-two unrelated patients recorded at St Mark's Hospital did not have mutations in the APC or MYH genes identified using standard techniques in diagnostic genetics laboratories. Methods: The coding and splice site regions of the APC gene were directly sequenced. Exon copy number changes were detected using a relatively new technique called multiplex-ligation probe amplification (MLPA). Haplotype analysis was performed using six flanking microsatellite markers, and two intronic single-nucleotide polymorphisms (SNPs) within APC. The MYH gene was screened for mutations using single-stranded conformational polymorphism (SSCP) analysis and sequencing. Results: Nineteen kindreds were found to have either previously undetected APC mutations or exon copy-number changes. Four patients were found to carry a biallelic MYH mutation. Therefore approximately $37 \%$ of patients had mutations found not previously identified by diagnostic genetics laboratories. Five novel mutations of APC were identified, including a duplication of exon 4 of APC found using MLPA. Duplication mutations have not previously been identified. Genotype-phenotype analysis revealed a difference in polyp number between mutation positive $($ no.=23) and mutation negative (no.=39) groups ( $\mathrm{p}<0.003, \mathrm{t}$ test). A subgroup analysis of the mutation negative group (based on family history) shows that there is a less penetrant condition among those with evidence of recessive inheritance of the trait. There is a later age of diagnosis of polyposis ( 41 years versus 31.8 years, $p=0.007, t$ test), lower rate of colorectal and extra-colonic cancers ( $\mathrm{p}=0.002$ and 0.017 respectively, Fisher's exact), and lower rate of duodenal polyposis ( $\mathrm{p}<0.0001$, Fisher's exact). Conclusions: The mutation negative group is likely to consist of a heterogeneous group of patients with mutations in different predisposition genes for polyposis. A subgroup analysis indicates that those kindreds with a recessive type of inheritance pattern have a less severe phenotype and may represent a distinct syndrome.

968

Association of Y Chromosome Haplotypes with Barrett's Esophagus and Esophageal Adenocarcinoma in the Dutch Caucasian Population Agnieszka M. Rygiel, Sjoerd Repping, Leon M. Moons, Sander Ouburg, Franceska Milano, Jantine W. van Baal, Jacques G. Bergman, Servaas A. Morre, Peter Siersema, Cornelis L. Mulder, Johannes G. Kusters, Maikel P. Peppelenbosch, Jan M. Hoovers, Kausilia K. Krishnadath

BACGROUND: Barrett's esophagus (BE) is a metaplastic condition of the distal esophagus, which predisposes for the highly malignant esophageal adenocaricoma (EAC). It is known that BE and EAC have a 3-7 times increased incidence in males especially in the Caucasian population. Interestingly the loss of $Y$ chromosome is one of the most consistent aberration in $\mathrm{BE}$ and occurs already in the stage of metaplasia and has a high frequency in dysplasia. HYPOTHESIS: We hypothesized that certain Y chromosome haplotypes may be associated with increased or decreased susceptibility for BE and EAC development. MATERIAL and METHODS: To test this hypothesis, we have analyzed a set of $6 \mathrm{Y}$ chromosome linked polymorphisms to define 7 major $\mathrm{Y}$ chromosome haplotypes (A,B-C, DE, $\mathrm{F}(\mathrm{xJ}, \mathrm{xK}), \mathrm{K}(\mathrm{xP})$, J, $\mathrm{P}(\mathrm{xRla}), \mathrm{Rla})$ in the Dutch Caucasian patients presenting with $\mathrm{BE}(\mathrm{n}=286)$, EAC $(\mathrm{n}=$ $116)$ and esophagitis without $\mathrm{BE}(\mathrm{n}=109)$. Their $\mathrm{Y}$ haplotype profile was subsequently compared with matched for age ( $>50$ years old), BE free Control population $(n=90)$. RESULTS: The frequency of $Y$ haplotypes in the investigated populations is shown in the table below. We found significant difference in frequencies of the P(xRla) and DE haplotypes between the patient groups and the control population. The $\mathrm{P}(\mathrm{xRla})$ haplotype had a significantly higher frequency in BE patients (62\%) vs. Control (44\%) (OR- 2.0, p<0.001). In contrast the frequency of the DE haplotype was significantly lower in patients with $\mathrm{BE}(3 \%)$ and EAC $(1,7 \%)$ vs. Control (14,5\%)(OR- 0.19, p<0.001 and OR-0.10, p<0.0001, respectively). Also the DE haplotype was underrepresented in esophagitis $(7,3 \%)$ vs. EAC (1.7\%) (OR$0.22, \mathrm{p}<0.05)$. CONCLUSIONS: Our results suggest that the $\mathrm{P}(\mathrm{xRla})$ haplotype is associated with increased susceptibility for BE. In contrast the DE haplotype is associated with decreased predisposition to both BE and EAC. We postulate that haplotypes $\mathrm{P}(\mathrm{xRla})$ and DE might be linked to the variants of genes which predispose and protect against BE/EAC development, respectively. Together with other markers, this may help identify those patients who are at risk for BE or might benefit from surveillance.

Y chromosome haplotypes frequency (\%) in BE, Esophagitis, EAC and Contol population.

\begin{tabular}{|c|c|c|c|c|}
\hline Y hapoltype & Control & Esophagitis & BE & EAC \\
\hline P(xR1a) & 44,4 & 50,4 & 62 & 57,7 \\
\hline F(xJ,xK) & 22,2 & 25,7 & 28,7 & 32,7 \\
\hline J & 10 & 8,2 & 2,8 & 3,4 \\
\hline A-B or C & 0 & 0 & 0 & 0 \\
\hline DE & 14,4 & 7,3 & 3,1 & 1,7 \\
\hline K(xP) & 3,3 & 2,7 & 1,4 & 2,6 \\
\hline R1a & 5,5 & 2,7 & 2,1 & 1,72 \\
\hline Nr of cases & 90 & 109 & 286 & 116 \\
\hline
\end{tabular}

969

Biallelic and Monoallelic Germline Myh Mutations and Colorectal Cancer (CRC): Correlation with Family History of CRC and Related Cancers Francesc Balaguer, Sergi Castellvi-Bel, Victoria Gonzalo, Antoni Castells, . GI Oncology Group of the Spanish Gast. Association

Background: Germline mutations in the base-excision-repair MYH gene have been associated with the development of multiple colorectal adenomas and $\mathrm{CRC}$ with an autosomal recessive inheritance. In that sense, different studies have shown that biallelic germline mutations account for less than $1 \%$ of the total of CRC. However, the risk of CRC among monoallelic MYH gene mutation carriers is controversial, with a suggested weakly penetrant autosomal dominant inheritance pattern. Aims: 1. To establish the prevalence of germline MYH gene mutations in newly diagnosed CRC patients. 2 . To evaluate the association of monoallelic MYH mutations with family history of CRC and related cancers (endometrial, gastric, ovarian, hepatobiliary, small bowel, or transitional cell carcinoma of the renal pelvis or ureter) Patients and methods: Between November 2000 and October 2001, all newly diagnosed CRC patients in 20 hospitals were included in the EPICOLON study, a prospective, multicenter, nation-wide survey aimed at establishing the incidence and characteristics of familial forms of CRC in Spain. Mutational analysis of the MYH gene was performed in two steps: 1. screening for the recurrent missense mutations Y165C (494A>G; exon 7) and G382D (1145G $>\mathrm{A}$; exon 13) by allelic discrimination using TaqMan technology; 2 . in heterozygotes for any of these variants, PCR-based single strand conformation polymorphism analysis of the 16 exons of the MYH gene. Abnormal migration shifts on SSCP were then sequenced. Results: Pathogenic biallelic MYH germline mutations were detected in 2 out of $518(0.4 \%)$ evaluated patients, one patient being homozygote for $\mathrm{Y} 165 \mathrm{C}$ and the other compound heterozygote (Y165C/G382D). Eleven additional patients (2.1\%) were heterozygotes for either Y165C or G382D. In 4 out of these 11 patients, two known MYH gene polymorphisms (Q324H in three and V22M in one) were identified. On the other hand, compared with non-carriers, carrying a germline Y165C or G382D mutation was neither associated with family history of CRC ( $9.1 \%$ vs. $18.7 \%$, respectively; $\mathrm{p}=0.36)$ nor other related cancers ( $27.3 \%$ vs. $31.6 \%$, respectively; $\mathrm{p}=0.52$ ). Conclusions: Biallelic MYH gene mutations account for less than $1 \%$ of all CRC cases. Moreover, monoallelic MYH gene mutations seem not to be associated with family history of CRC or other related neoplasms.

\section{S1097}

Are Women Choosing Careers in Academic Gastroenterology? Lauren B. Gerson, Dayna Early, Gail Hecht, Linda Lee, Kenneth McQuaid, Theresa Pizarro, Sarah Street, Cynthia Yoshida, Kay Twomey

Background: Representation of women in the AGA and in Gastroenterology leadership positions has not increased significantly in the past decade. We performed a national survey in order to determine factors that influence women to pursue academic or practice careers. Methods: An on-line survey was administered to all AGA members between 2004-2005 including physicians in private practice, academic centers, and GI fellows. The survey inquired about career satisfaction, the effects of gender on career advancement, and integration of family and career. Results: There were a total of $424(14.8 \%)$ responders from 2856 invited physicians. $92(22 \%)$ were in private practice, $233(55 \%)$ in academic careers, and $99(23 \%)$ trainees. There were 237 (56\%) women and 187 (44\%) men; equal numbers of women $(157 / 237)$ and men $(108 / 187)$ were in academic careers $(p=0.07)$ Significantly more women (45\%) compared to men ( $8 \%$ ) stated that gender affected their career advancemen $(\mathrm{p}<0.0001)$ regardless of the practice setting. There was no significant difference between the number of men or women who had obtained the rank of associate or full professor ( $\mathrm{p}=$ NS). Men were more likely to have held leadership positions. ( $p<0.001)$ Sixty-three $(58 \%)$ men and $63(40 \%)$ women in academics responded to questions regarding career satisfaction: there were no significant differences regarding satisfaction of salary levels, work load expectations, academic lifestyle, or balance between work and family. Men in academics tended to report higher overall career satisfaction $(\mathrm{p}=0.06)$. Women were more likely to describe the tenure process as difficult $(\mathrm{p}=0.01)$ and the promotional process as unfair $(\mathrm{p}<0.001)$. There were no difference between genders regarding mentorship, however both men and women were more likely to have had male mentors $(\mathrm{p}<0.001)$. When asked when fellows decide to enter private practice, men were more likely to make this decision during residenc $(\mathrm{p}=0.02)$. Women in private practice were as satisfied as men regarding their financial compensation, balance between work and family, and ability to be a shareholder in a group practice $(\mathrm{p}=\mathrm{NS}$ ). Significantly more men compared to women had children and were married $(p<0.001)$. There was no significant difference between the genders for hours worked per week. All respondents perceived that women in internal medicine residency programs were less likely to choose GI as a career option because of lack of role models and difficulty with 
family life issues. Conclusions: Compared to their male counterparts, female gastroenterologists perceived that gender affected their career advancement and were less likely to hold leadership positions.

\section{S1098}

A Survey Assessing Attitudes Towards Privacy and Research

Subhas C. Ganguli, Yousra Al-Rweli, Christine Vanderkooy, Karen Miranda, Lehana Thabane

INTRODUCTION: Legislation has recently been passed in Canada and Ontario to increase the privacy of patients and their health records. However, patient views on privacy and its applications to health research are poorly known. It was the aim of this study to assess patient views towards privacy and health research. METHODS: A convenience sample of 450 outpatients in waiting areas at an Ontario teaching hospital (St Joseph's Hospital, Hamilton, ON) were asked to complete a paper-based survey to assess their views on privacy and health research. RESULTS:The average age of respondents was 51 , and $61 \%$ were female. Purpose of visit was to see a doctor in $76.5 \%$, for a test in $14.6 \%$ and for another reason in $6.2 \%$. Patient were more willing to be contacted about studies into the cause of their disease if they had previously given explicit consent to be contacted $(52.7 \%$ vs $45.9 \%$; $\mathrm{P}<0.01)$. Patients were more likely $(\mathrm{P}<0.01)$ to want to be contacted about adverse sideeffects of drugs (AEs) which they were currently taking (93.8\%) than to be asked to be involved in studies of their disease causation (45.9\%) or new therapies (49.6\%). Most also felt that a computerized database could be kept for notification about drug side-effects without their explicit permission (53.4\%) but not for studies assessing disease causation $(36.0 \%)$ or new therapies $(37.9 \%)(\mathrm{P}<0.01)$. When asked about Ethics Board (REB) approved access to their charts for retrospective review of drug AEs, $50.8 \%$ of patients felt they did not have to be notified, while $37.1 \%$ preferred to be contacted and $12.1 \%$ felt this was essential. When asked about future possible uses of their chart for REB approved studies, $42.2 \%$ of patients agreed that notification by letter (with option to not be involved) was acceptable, while $30.1 \%$ felt they should be contacted to give blanket consent for any future studies and $17.3 \%$ wanted to be contacted for each access to their chart. Opinions on this question correlated with degree of skepticism towards healthcare providers $(\mathrm{P}<0.01) .90 .3 \%$ of patients agreed that medical research is beneficial; this was weakly correlated with their satisfaction with the amount of time their physician spent explaining their medical condition to them $(r=0.14 ; P<0.01)$. CONCLUSIONS: Most patients want to be contacted about new side-effects of their drug therapy and are willing to have their charts used for retrospective reviews of this; many also want to be contacted about studies looking at the cause or new therapies for their disease

\section{S1099}

Three Month Nutritional Supplementation Improves Both Muscle Function and Quality of Life in Malnourished GI Patients - A Prospective Randomised Controlled Trial

Kristina Norman, Henriette Kirchner, Manuela Freudenreich, Herbert Lochs, Matthias Pirlich

Introduction: Malnutrition is a common problem in hospitalized GI patients and is generally associated with higher morbidity and mortality. Nutritional status frequently worsens during hospitalization and both weight loss and muscle dysfunction cause higher risk of readmission and loss of general functionality. Aim: We therefore investigated the effect of a three month post hospital nutritional intervention with a high protein oral supplementation on body composition, muscle function and quality of life in malnourished GI patients. Methods: Seventy-seven malnourished patients with benign gastrointestinal disease were randomized to receive either a high protein supplement $(400 \mathrm{ml}, 150 \mathrm{kcal}$ and $10 \mathrm{~g}$ protein/100ml) in addition to their normal food or dietary counselling for 3 months. Nutritional status was determined with the Subjective Global Assessment, body composition by bioelectrical impedance, muscle function with hand grip strength and peak flow. Quality of life (QoL) was assessed by the validated Medical Outcomes Study 36-item Short-Form Questionnaire (SF 36). All measurements were made at baseline and after 3 months. Results: Age, body cell mass (BCM), muscle function (hand grip and peak flow), gender distribution and QoL did not differ between intervention $(n=37)$ and control group $(n=40)$ at baseline. Oral supplementation increased nutritional intake by $706 \pm 127 \mathrm{kcal} / \mathrm{d}$ and $46 \pm 9 \mathrm{~g}$ protein $/ \mathrm{d}$ in the intervention group. Body weight and BCM improved significantly in all patients after 3 months, but to a slightly greater extent in the intervention patients. However, both hand grip strength, peak flow as well as the physical component of SF 36 improved significantly only in the intervention patients and remained unchanged in the controls. Conclusion: A three month intervention with high protein oral supplements improves nutritional status and functional parameters in malnourished GI patients. This leads to an improvement of quality of life. Oral supplements should therefore be included in the standard therapy of malnourished GI patients.

\begin{tabular}{|c|c|c|c|}
\hline Mean Change & Intervention patients & Controls & P value \\
\hline Body weight $(\mathrm{kg})$ & $5,4 \pm 4,3$ & $4,9 \pm 2,3$ & n.s. \\
\hline BCM $(\mathrm{kg})$ & $1,9 \pm 3,7$ & $1,1 \pm 4,2$ & n.s. \\
\hline Handgrip strength $(\mathrm{kg})$ & $5,2 \pm 6,8$ & $0,8 \pm 5,1$ & 0.007 \\
\hline Peak flow $(1 / \mathrm{min})$ & $61,4 \pm 102,9$ & $22,7 \pm 73,1$ & n.s. \\
\hline SF 36 -PCS (\%) & $34,8 \pm 12,6$ & $4,3 \pm 14,3$ & 0.013 \\
\hline
\end{tabular}

Abbr.: BCM= Body cell mass; SF 36 PCS= Physical Component Summary of Quality of Life
$\$ 1100$

Overweight and Obesity Protect Against Inguinal Hernia in the United States Population

Constance E. Ruhl, James E. Everhart

Inguinal hernias are common, but few studies have investigated their risk factors. Therefore we examined the relationship of potential risk factors with the occurrence of inguinal hernia in a large national population-based prospective study. Methods. In the first National Health and Nutrition Examination Survey, 1971-75, interview and examination data were collected on possible risk factors for inguinal hernia. Participants were followed through 1992-93 for inguinal hernia as identified by a hospital diagnosis (ICD-9-CM 550) or physician diagnosis Ninety-six percent of the baseline cohort was recontacted. Hazard rate ratios for inguinal hernia according to levels of potential risk factors were calculated using Cox proportiona hazards analysis. Results. A total of 5,316 men and 8,136 women were followed for a median of 18.2 years (range $0.02-22.1$ ). Because of the much higher cumulative incidence at 20 years, and hence number of cases, in men (14\%, 500 cases) than women $(2.1 \%, 120$ cases), further analysis was restricted to men. In both age-adjusted and multivariate analysi (Table), factors associated with a higher incidence $(\mathrm{p}<0.05)$ of inguinal hernia among men included middle and older age, white race, lower BMI, and doctor-diagnosed hiatal hernia Factors unrelated to inguinal hernia included smoking, physical activity, constipation, body fat distribution, chronic cough, chronic obstructive pulmonary disease, and alcohol intake Conclusion. In the U.S. population, inguinal hernia is a common condition among men that increases substantially with aging. The lower risk among overweight and obese men was an unexpected finding that bears further study

\begin{tabular}{|c|c|c|c|}
\hline Variable & $\begin{array}{c}\text { Cumulative incidence of inguinal } \\
\text { hernia (\%) }\end{array}$ & $\begin{array}{c}\text { Hazard } \\
\text { ratio }\end{array}$ & $\begin{array}{c}95 \% \text { confidence } \\
\text { interval }\end{array}$ \\
\hline $24-39$ years & 7.3 & 1.0 & \\
\hline $40-59$ years & 14.8 & 2.2 & $1.7-2.8$ \\
\hline $60-74$ years & 22.8 & 2.8 & $2.2-3.6$ \\
\hline White race & 15.1 & 1.0 & \\
\hline Black race & 8.4 & 0.58 & $0.42-0.79$ \\
\hline BMI $<25 \mathrm{~kg} / \mathrm{m}^{2}$ & 15.6 & 1.0 & \\
\hline BMI $25-<30 \mathrm{~kg} / \mathrm{m}^{2}$ & 14.0 & 0.79 & $0.66-0.95$ \\
\hline $\begin{array}{c}\text { BMI \&\#8807;30 } \\
\mathrm{kg} / \mathrm{m}^{2}\end{array}$ & 8.3 & 0.51 & $0.36-0.71$ \\
\hline No hiatal hernia & 13.6 & 1.0 & \\
\hline Hiatal hernia & 26.9 & 1.8 & $1.2-2.7$ \\
\hline
\end{tabular}

\section{S1101}

Obesity, Physical Activity, and the Long-Term Risk of Peptic Ulcers Edward Lew, Nancy R. Cook, Martin J. Blaser, Guillermo Perez-Perez, J. Michael Gaziano

Background: Recent studies report that the incidence of peptic ulcers unrelated to Helicobacter pylori or the use of nonsteriodal anti-inflammatory drugs (NSAIDs) is rising, suggesting that other factors are likely involved. To date, it remains unclear whether obesity and physical activity affect ulcer formation. Methods: We prospectively examined the associations of increasing body mass index, physical activity and the development of peptic ulcers among 21,426 men in the Physicians' Health Study. Information about these factors as well as other atherosclerotic risk factors and NSAID use were assessed at baseline and periodically updated during follow-up. Proportional hazards models with time-varying predictors were used to calculate relative risks (RR). In a separate nested case-control study within the cohort, we also examined case subjects who developed ulcers and controls (1:4) matched by age and follow-up interval. Stored blood samples obtained at study entry were assayed for antibodies against $H$. pylori. NSAID use was determined up to date of the ulcer for cases or to the respective follow-up date for controls. Odds ratios (OR) with 95\% confidence intervals (95\% $\mathrm{CI}$ ) were calculated by conditional logistic regression analysis. Results: The mean age of the participants was $53.7+/$ - (standard deviation) 9.5 years. During a 12.5 -year follow-up, we identified 562 new peptic ulcers. Increasing body mass index (RR, 1.033 per unit kg/m2 increase; 95\% CI, 1.006 - 1.060) elevated the risk of developing peptic ulcers, while controlling for NSAID use, older age, cigarette smoking and other atherosclerotic risk factors. In contrast, regular exercise at least once a week (RR, 0.798; 95\% CI, 0.662 - 0.961) was inversely related to ulcers. In the nested case-control study, we examined 183 case subjects who developed peptic ulcers and 730 matched controls. As expected, H. pylori infection (OR, 2.160; 95\% CI, 1.511 - 3.088) and increasing NSAID use (OR, 1.663 per month of NSAID use during the year; $95 \% \mathrm{CI}, 1.274-2.171)$ were associated with an elevated risk of peptic ulcers. Increasing body mass index (OR, 1.061 per unit $\mathrm{kg} / \mathrm{m} 2$ increase; $95 \% \mathrm{CI}$, 1.002 - 1.124) also elevated ulcer risk, whereas regular exercise at least once a week (OR, $0.677 ; 95 \%$ CI, 0.460 - 0.995) was inversely related to ulcers while controlling for $\mathrm{H}$. pylori, NSAID use, and other atherosclerotic risk factors. Conclusions: Obesity increases ulcer risk whereas regular exercise at least once a week is associated with a decreased risk of peptic ulcers, independent of $H$. pylori and NSAID use. Further studies are needed to confirm these findings.

\section{S1102}

Alcohol and Symptomatic Gallstone Disease: A Swedish Twin Study Despina Katsika, Catherine Tuvblad, Curt Einarsson, Hanns-Ulrich Marschall

Background and aim: With data from the Swedish Twin Registry, we recently estimated the overall contributions of genetic and environmental factors for the development of symptomatic gallstone disease (GD; Hepatology 2005). We now aimed to define the risk of alcoho 
consumption on the development of GD. Materials and Methods: The risk of alcohol consumption as a specific environmental factor can be estimated from associations within twin pairs discordant for disease in which the influence of genetic and early environmental factors is substantially reduced. For this purpose, the Swedish Twin Registry was linked to the Swedish Inpatients and Causes of Death Registries for GD and GD-surgery related diagnoses for twins born between 1888 and 1958 to identify twins with GD. Data of alcohol consumptions, as evaluated from self-reports, were provided by the Registry. Consumption was defined as moderate, $600-1.800 \mathrm{~g} /$ month for females and 900-2.400 g/month for males, or high, $>1.800 \mathrm{~g} /$ month for females and $>2.400 \mathrm{~g} /$ month for males. The follow-up time was 1970-2002. The cohort analyses were based on 23.636 twins of the same gender and with known zygosity. The within-pair analyses then included 1.411 twin pairs discordant for GD. Odds ratios (OR) and 95\% confidence intervals (CI) were calculated by logistic regression. Results: In the twin cohort analyzed, both moderate and high alcohol consumption was associated with a lower prevalence of GD as compared to no alcohol consumption (OR: 0.83; 95\% CI: 0.66-1.04, and OR: 0.83; 95\% CI: 0.66-1.03, respectively). These negative associations remained consistent in within-pair analyses and reached statistical significance for high alcohol consumption: Moderate alcohol consumption, MZ: OR: 1.39; 95\% CI: 0.99 1.95, DZ: OR, 1.00; 95\% CI, 0.80-1.25; high alcohol consumption: MZ: OR, 0.68; $95 \%$ CI, 0.48-0.95; DZ: OR: 0.96; 95\% CI: 076-1.20. There were no differences between monozygotic (MZ) and dizygotic (DZ) twins. Conclusion: The data from the Swedish Twin registry are consistent with data from some epidemiological studies that alcohol consumption "protects" against symptomatic GD

\section{S1103}

Differential Time Trends in the Incidence of Cancer of the Esophagus in the Netherlands 1989-2001

Mark Van Blankenstein, Caspar W. Looman, Peter D. Siersema, Ernst J. Kuipers, Jan W. Coebergh

Background: In common with other industrialized countries, The Netherlands has been experiencing a rising incidence of esophageal adenocarcinoma since the 80 's. The country of 16 million inhabitants is well served by a comprehensive system of Regional Cancer Registries to which both hospital medical record departments and histology departments report all malignancies. The Netherlands Cancer Registry in turn collates these data from all 9 Regional Cancer Registries. Aims: To investigate whether similar time trends were present in the annual incidence rates of the two types of esophageal cancer, i.e. adenocarcinoma of the esophagus (ACE) and the gastric cardia (AGC) and in addition, to compare them with those of squamous cell carcinoma of the esophagus (SCC), which is considered to have another etiology. Methods: We analyzed the annual European Standardized Rates (ESR) for AGC, ACE and SCC in The Netherlands over the 13 year period 1989 to 2001, as provided by the Netherlands Cancer Registry. Results: In contrast to the highly significant annual rises in the ACE incidence rates of $6.5 \%$ for males and $2.6 \%$ for females, the annual incidence rates of SCC and AGC remained stable, with the exception of an actual significant decline in the annual AGC incidence rates for men. Conclusions: The etiological factors driving the rising incidence of ACE in The Netherlands appear to have been absent in both AGC and SCC. This finding provides confirmation for their differing etiologies, expected in SCC, but unexpected in AGC. Some of the decline in AGC incidence rates in men may be explained by diagnostic drift on the part of clinicians from AGC to ACE. In view of the recently reported 20 year delay in the age of onset of Barrett's esophagus (the precursor for ACE) in females as compared to males (1), the current far lower female ACE incidence may well be mirroring the male ACE incidence rates 20 years ago. (1) van Blankenstein et al, Am J Gastroenterol 2005;100:568-576.

\begin{tabular}{|c|c|c|c|c|c|c|}
\hline $\begin{array}{c}\text { Esophageal } \\
\text { Tumor }\end{array}$ & Gender & $\begin{array}{c}\text { Numbers } \\
\text { Observed }\end{array}$ & $\begin{array}{c}\text { ESR per } \\
100000\end{array}$ & $\begin{array}{c}\text { Annual } \\
\text { Change }\end{array}$ & $\begin{array}{c}95 \% \text { CI Annual } \\
\text { Change }\end{array}$ & p Values \\
\hline SCC & $\begin{array}{c}\text { Males } \\
\text { Females }\end{array}$ & 36162295 & 3.851 .9 & $-0.6-0.6$ & $\begin{array}{c}-1.4 ;+0.3 \\
-1.7 ;+0.5\end{array}$ & 0.200 .25 \\
\hline AGC & $\begin{array}{c}\text { Males } \\
\text { Females }\end{array}$ & 42631258 & 4.480 .94 & $-1.0-0.1$ & $\begin{array}{c}-1.8 ;-0.2 \\
-2.4 ;+0.5\end{array}$ & 0.010 .19 \\
\hline ACE & $\begin{array}{c}\text { Males } \\
\text { Females }\end{array}$ & 37991289 & 4.00 .9 & $+6.5+2.6$ & $\begin{array}{c}+5.6 ;+7.5 \\
+1.1 ;+4.1\end{array}$ & $\begin{array}{c}<0.001 \\
0.002\end{array}$ \\
\hline
\end{tabular}

\section{S1104}

Eosinophilic Esophagitis in Adolescence Juan F. Villalona, Thomas M. Rossi, Marilyn R. Brown, Ashok Shah, Phillip Katzman, Carol French

INTRODUCTION A large number of adolescent patients undergo endoscopic evaluation for esophageal symptoms by pediatric and adult gastroenterologists. Eosinophilic esophagitis (EE) should be included in the differential diagnosis of adolescents with esophageal symptoms. METHODS Forty-eight charts were selected for review from the Division of Pediatric Gastroenterology and Nutrition at the Golisano Children's Hospital at Strong, Rochester, NY. Inclusion criteria were age (12-18 years) and the diagnosis of EE. Sex, age at presentation, age at onset of symptoms, specific symptoms, pH probe test results, RAST tests, biopsy results and endoscopic findings were observed. RESULTS Thirty patients met the current diagnostic criteria for EE (15-20 eosinophils/HPF in the esophagus). The 18 excluded had fewer than 15 eosinophils/HPF. Of the 30 with EE, $22(73 \%)$ were male and $8(27 \%)$ were female. The mean age was $16.5 \pm 2.5$ years. The mean age at onset of symptoms was 11.8 \pm 3 years. The mean age at presentation was $12.5 \pm 3$ years. Predominant symptoms were dysphagia $22(73 \%)$, food impaction $10(33 \%)$, abdominal pain $9(30 \%)$, and heartburn 8 (27\%). A pH probe study was done in $22(73 \%)$ patients, of which $6(20 \%)$ demonstrated gastroesophageal reflux. Nineteen $(63 \%)$ reported food, medications or environmental allergies. Radioallergosorbent test (RAST) was done in 22 (74\%) of cases and of those, 14 (64\%) were positive. Milk was the most common allergen. The most common endoscopic findings were linear grooves $(50 \%)$, white spots $(27 \%)$ and rings (17\%). Five (17\%) patients had mid-esophageal strictures. One patient had normal an appearing esophagus. Biopsies from the mid- and proximal esophagus revealed greater than 20 eosinophils/HPF in 26 (87\%) cases. Aggressive acid-blockage therapy alone did not improve the symptoms of EE. All patients responded clinically to elimination of the food allergen and fluticasone. Mast cell stabilizers and leukotriene receptor antagonists and proton-pump inhibitors were used for maintenance. CONCLUSION Pediatric and adult gastroenterologists should be aware of this disorder, as they will be treating many adolescents with esophageal symptoms. Dysphagia and food impaction were common presenting symptoms. Interestingly, food impaction was associated with mid-esophageal strictures in five patients. The characteristic endoscopic (furrowing, white spots and trachealization) and biopsy ( $>15$ eosinophils/HPF, especially at the mid- and proximal esophagus) findings are diagnostic of $\mathrm{EE}$. It is important to recognize this disorder early so that proper treatment may be started and complications can be avoided. Treatment is easy and usually effective.

\section{S1105}

The Natural Course of Acute Idiopathic Pancreatitis Paul G. Lankisch, Nils Breuer, Anja Bruns, Bettina Weber-Dany, Patrick Maisonneuve, Albert B. Lowenfel

The natural course of acute idiopathic pancreatitis is unknown. The aim of our study was to determine the incidence of relapses and to identify any factors associated with these recurrences. Patients and Methods: We identified 106 patients with a first attack of acute idiopathic pancreatitis admitted to our hospital from 1987 - 2004. Alcohol consumption was less than $40 \mathrm{~g}$ of pure alcohol/day in all patients, and in none of them could biliary disease or other causes of acute pancreatitis be identified as the underlying etiology. All living patients were interviewed by telephone and questionnaire to obtain information on their health status, lifestyle, and family health history. For patients who died after the first acute pancreatitis the cause of death was obtained from their general practitioners. Results: 1. Diagnosis: The etiology proved to be different than originally thought (idiopathic) in two patients in whom a pancreatic carcinoma diagnosed during follow-up was responsible for the first attack. 2. Mortality: 38 (37\%) patients died, 9 (9\%) during the first attack and 29 (28\%) from unrelated causes. None of the latter group experienced any relapse. 3. Followup: Of the 66 living patients 64 could be contacted, and 8 of these had had recurrent attacks ( 4 patients: 1 attack, 3 patients: 2 attacks, 1 patient: 3 attacks). The etiology of these relapses was again unknown with the exception of one attack which occurred following alcohol abuse. In none of the patients did relapsing pancreatitis progress to chronic pancreatitis. One patient developed pancreatic cancer 15 years after a single attack of acute pancreatitis. 4. Recurrence risk: Among living patients, recurrence was more frequent in those with a family history of pancreatic disease (acute pancreatitis, pancreatic cancer, or diabetes mellitus; $\mathrm{p}=0.047)$ and in smokers, although the latter association was not statistically significant ( $\mathrm{p}=$ 0.17). However, 3 of the 4 patients, who had multiple relapses, were smokers. Neither the APACHE II score on admission for the first attack, nor the Imrie and Ranson score after 72 hours, nor the result of a contrast-enhanced computed tomography (Balthazar score) correlated with the recurrence rate. Conclusion: The relapse rate in patients with acute idiopathic pancreatitis is low.In the case of recurrence, a change of etiology is very unlikely. There is no progression from acute to chronic pancreatitis in this etiological subgroup. Patients who have experienced acute idiopathic pancreatitis are advised to stop smoking and to undergo appropriate imaging procedures 3 months after the attack in order to identify the eventual presence of an pancreatic carcinoma.

\section{S1106}

Prospective Study of Time-Related Morphological Changes in Patients with Intraductal Papillary Mucinous Tumours of the Pancreas (IPMT) Confined to the Branch Ducts (BD)

Pierre Emmanuel Rautou, Anne Couvelard, Marie Pierre Vullierme, Dermot O'Toole, Laurent Palazzo, Alain Sauvanet, Frederique Maire, Olivia Hentic, Pascal Hammel,

Philippe Ruszniewski, Philippe Levy

Compared to main pancreatic duct (MPD) involvement, IPMT involving exclusively the BDs bears a low risk of malignancy that may allow patients follow-up (FU) without surgery. However to date the long-term safety of conservative management has poorly been evaluated. Aim of the study: Assess time-related morphological changes and risk of malignancy inpatients with BD-IPMT. Patients (pts) and methods: All consecutive pts seen for BD-IPMT were prospectively followed from 1999 to 2005. IPMT diagnosis relied on histological confirmation or was highly probable on radiological findings (cystic lesions communicating with MPD on a least 2 imaging procedures). Pts with at least 1 criterion of malignancy (BD diameter $>30 \mathrm{~mm}$, cyst wall thickening $>2 \mathrm{~mm}$ or mural nodule, $\mathrm{n}=13)$ or with $\mathrm{FU}<\mathrm{lyr}(\mathrm{n}=65)$ were excluded. Surveillance included CT, MRI and EUS, at least annually. Results: 102 pts (M: 22, median age at diagnosis: 64 (30-82 years) were included (histological confirmation in 20 pts and highly probable diagnosis in 82). Diagnosis was incidental, due to pancreatic pain, acute pancreatitis or others in 63,23, 13 and 3 pts, respectively. Median number of dilated $\mathrm{BD}$ was 2; with a median size of the largest BD of 15 (3-30) $\mathrm{mm}$. Diffuse BD involvement was present in 12 pts. Median follow-up was 28 (12-80) months from diagnosis with 3 (1-15) imaging procedures/pt. Morphological changes occurred in 12 pts after 26 (5-61) months: size $>30 \mathrm{~mm}$, cyst-wall thickening $>2 \mathrm{~mm}$, involvement of the MPD in 3, 7 and 2 pts, respectively. Surgery, performed in $3 / 12$ pts found 1 invasive carcinoma, 1 highgrade dysplasia (HGD) and 1 low-grade dysplasia (LGD). The 9 remaining pts were not operated on due to minor BD changes, associated severe co-morbid conditions or refusal. Close follow-up during 0-24 months (EUS every 6 months) revealed no further modifications Among the 90 pts without morphological changes, 10 underwent surgery ( 7 for recurrent acute pancreatitis, 3 for miscellaneous raisons). At pathological examination, hyperplasia, LGD and HGD were found in 3,6 and 1 pts, respectively. Uneventful follow-up was 28 (12-80) months in the remaining 80 pts. Conclusion: In the absence of criteria suggestive of malignancy at diagnosis, BD-IPMT exhibit a low rate (12\%) of morphological changes during a more than 2 years FU period. These data support conservative management of BD-IMPT in those without criteria of malignancy. However, careful monitoring is mandatory, since malignancy cannot be excluded and should be assessed in long-term studies. 
S1107

Prevalence of High-Intensity Proton Pump Inhibitor Use in Manitoba: A Population Based Analysis

Laura E. Targownik, Colleen J. Metge, Stella Leung

Proton pump inhibitors (PPIs) are among the most commonly prescribed drugs in North America. While PPIs are often recommended to be used at standard doses, PPIs are often prescribed at higer doses when standard doses are ineffective, though the incremental benefit of increasing the PPI dose in limited. We therefore sought to determine the prevalence of high intensity PPI use and to describe the characteristics of high-intensity users. Methods: We chose to determine the prevalence of high-dose chronic PPI use on July 1st, 2003. We categorized individuals as being chronic PPI if they had been using PPIs for more than $80 \%$ of the ninety days before July 1st, 2003. A chronic user was considered high dose if he was using on average more than 1.5 standard doses of PPIs daily for at least one-half of the ninety days before July lst, 2003. We compared age, gender, burden of comorbid illness, socioeconomic status, history of upper GI events and of endoscopic investigations between high-intensity and standard intensity PPI users. The burden of comorbid illness was determined by enumerating the number of unique aggregated diagnostic groups (ADGs) for which the enrollee received care in the year prior to July lst, 2003. Results. Out of 825,429 enrollees, we identified 117,201 individuals who had filled at least one prescription for a PPI, of whom 26,943 (22.9\%) had were chronic users as of July lst, 2003. 3.394 (12.6\%) of chronic PPI users could be classified as high-intensity users by our definition High intensity PPI users were of similar age to standard intensity users (both groups $=63.5$ yrs.) and the groups had a similar percentage of females (62.5 "high dose" \% vs $60.2 \%$ "standard dose"). Other differences between high-dose and standard dose users are summarized in Table 1. Conclusions. Approximately 12\% of PPI users are using these medications at higher than standard doses. High-intensity PPI use is associated with a greater burden of comnorbid illness and use of other medications known to promote GI complications. We intend to perform further analyses with this comprehensive database to characterize the population of high-intensity PPI users and to gain further insights into the clinical and economic outcomes associated with high-intensity PPI use Comparison of High Dose vs. Standard Dose PPI Users

\begin{tabular}{|c|c|c|c|c|c|c|c|}
\hline & $\begin{array}{c}\% \text { with } \\
\text { over 5 } \\
\text { ADGs }\end{array}$ & $\begin{array}{c}\% \\
\text { using } \\
\text { ASA }\end{array}$ & $\begin{array}{c}\% \text { using } \\
\text { NSAIDs }\end{array}$ & $\begin{array}{c}\text { \% using } \\
\text { warfarin }\end{array}$ & $\begin{array}{c}\text { \% using } \\
\text { SSRI }\end{array}$ & $\begin{array}{c}\% \text { with Hx } \\
\text { of HP } \\
\text { treatment }\end{array}$ & $\begin{array}{c}\% \text { with Hx of } \\
\text { UGI } \\
\text { admission }\end{array}$ \\
\hline $\begin{array}{c}\text { Standard Dose } \\
\text { PPI Users } \\
(\mathrm{n}=26,943)\end{array}$ & 18.15 & 7.14 & 21.89 & 4.13 & 12.45 & 4.71 & 3.96 \\
\hline $\begin{array}{c}\text { High Dose PPI } \\
\text { Users } \\
(\mathrm{n}=3,394)\end{array}$ & 27.99 & 7.98 & 24.16 & 5.8 & 15.88 & 12.46 & 5.27 \\
\hline $\mathrm{p}$-value & $<0.001$ & 0.01 & $<0.001$ & $<0.001$ & $<0.001$ & $<0.001$ & $<0.001$ \\
\hline
\end{tabular}

\section{S1108}

Relevance of Phase Angle Percentiles in Hospitalized Patients Tatjana Schuetz, Matthias Pirlich, Kristina Norman, Hebert Lochs

Rationale: Evaluation of prognostically relevant changes of nutritional status is still controversial. Phase angle $(\mathrm{PhA})$ is calculated from resistance and reactance measured by bioelectric impedance analysis (BIA) and is an indicator for membrane integrity. We hypothesized that $\mathrm{PhA}<10$ th percentile (U. Kyle et al., Clin Nutr 2004; 23: 758) reflects clinically relevant malnutrition. Methods: In 1313 patients (697 m, 616 f; age 20-75) consecutively admitted to hospital ( $\mathrm{n}=861$ medical, $\mathrm{n}=452$ surgical) $\mathrm{PhA}$ was measured by BIA (Nutrigard-2000M, Data Input, Germany). BMI, Subjective Global Assessment (SGA), the number of drugs and length of hospital stay (LOS) were assessed. Results: In 665/1313 (50.6\%) patients PhA was below the age- and gender-matched 10th percentile. These patients were older, more frequently malnourished and took more drugs as surrogate parameter for severity of disease than patients with $\mathrm{PhA}>10$ th perc (table). In 627/1265 (49.6\%) patients with a BMI $>18.5$ $\mathrm{kg} / \mathrm{m} 2 \mathrm{PhA}$ was $<10$ th percentile. Patients with a low PhA had a significantly longer LOS. In all patients significant $(\mathrm{p}<0.001)$ correlations between PhA and age $(r=-0.427)$, BMI $(r=$ $0.162)$, number of drugs $(\mathrm{r}=-0.313)$ and LOS $(\mathrm{r}=-0.182)$ were calculated. Conclusions: Measurement of PhA with the 10th percentile as cut-off might be a simple tool to detect patients with clinically relevant malnutrition.

\begin{tabular}{|c|c|c|c|c|}
\hline & Age (years) & SGA B+C & No. of drugs & LOS (days) \\
\hline $\mathrm{PhA}<10$ th percentile & $57.1 \pm 13.3 * *$ & $31.6 \% * *$ & $3.4 \pm 3.2 * *$ & $11.1 \pm 10.8 *$ \\
\hline $\mathrm{PhA}>10$ th percentile & $53.6 \pm 13.6$ & $6.2 \%$ & $2.3 \pm 2.6$ & $9.3 \pm 7.4$ \\
\hline
\end{tabular}

PhA: phase angle; SGA: Subjective Global Assessment; LOS: lengsth of stay **p<0.001; * $\mathrm{p}<0.05$

\section{S1109}

Rectal Bleeding in Children: Findings of Pancolonoscopy

Lissy D. Ridder, Anna V. Lingen, Jan Taminiau, Marc A. Benninga

Introduction Limited reports are available evaluating the value of endoscopy and histopathology in children referred to a tertiary hospital for rectal bleeding. In our hospital, it is policy to perform pancolonoscopy under general anaesthesia in children presenting with severe and/or prolonged rectal bleeding. In case of suspicion of inflammatory bowel disease (IBD) gastroscopy is performed and intubation of the terminal ileum is attempted. In all cases biopsies are taken for histopathological examination. We have conducted a retrospective cohort study of this policy to obtain information on endoscopic diagnosis and complications. Methods All records of children undergoing a colonoscopy because of prolonged and/or severe rectal bleeding over an 8-year period (January, 1998 - November 2005) were reviewed Results 151 Colonoscopies were performed in 140 paediatric patients ( 66 boys). Ten patients underwent two and one patient three colonoscopies because of persistent, unexplained bleeding. The mean age at time of the first colonoscopy was 9.3 years (range 10 days- 17.8 years). Diagnoses after the first colonoscopy were; normal $(n=29)$, lymphonodular hyperplasia $(n=7)$, infectious $(n=5)$, anal fissure or haemorrhoid $(n=10)$, aspecific proctitis $(n=10)$, IBD $(n=49)$, polyps $(n=25)$, polyposis coli $(n=1)$, allergic colitis $(n=2)$, neutropenic colitis $(n=$ $1)$, enterocolitis $(n=1)$, ulcus $(n=5)$, Meckel's diverticulum $(n=2)$, sarcoidosis $(n=1)$ and amyloidosis $(n=1)$. The ileum was intubated in $76 \%$ of the procedures performed. In al but 3 patients who were diagnosed IBD the ileum was intubated (93\%). In 70 out of 140 patients gastroscopy was performed, in these patients IBD was part of the differential diagnosis. In 25 patients 27 polyps were found. All but one were juvenile polyps, one patien had an inflammatory polyp. One patient had 3 polyps, all other patients had one polyp The polyps were located in the rectum $(n=11)$, sigmoid $(n=10)$, descending colon $(n=5)$ or transverse colon $(n=1)$. Therefore, in case a sigmoidoscopy was performed as first procedure, $22 \%$ of the polyps would have been missed. No complications occurred either during or after colonoscopy or anaesthesia. Conclusions Colonoscopy under general anaesthesia is a safe and important tool in diagnosing rectal bleeding in children. However, in approximately $20 \%$ of the patients no macroscopic or microscopic cause could be identified to explain rectal bleeding. In case of persistent bleeding diagnostic procedures such as Meckel's scan and/or video capsule endoscopy should be considered. Pancolonoscopy as firs diagnostic procedure in children with prolonged and/or severe rectal bleeding is advisable

\section{S1110}

Prevalence of Celiac Disease Among Blood Donors in Sao Paulo City - The Most Populated and Multi-Ethnic Center of Brazil

Marilia L Alencar, Carmen L. Ortiz-Agostinho, Ieda Nishitokukado, Aderson Omar M. Damiao, Clarice P. Lemos, Andre Z. Leite, Thales Brito, Dalton A. Chamone, Aytan M. Sipahi

Background: Celiac disease is a permanent enteropathy caused by the ingestion of gluten and leads to an immunologically mediated inflammation of the small intestine mucosa. Th prevalence of celiac disease varies among different nations and ethnic backgrounds, and its diversity is determined by genetic and environmental factors. More recently, several serological population screenings have shown that the prevalence of the disease is more common than previously imagined. Sao Paulo city is one of the biggest cities in the world, with a large population and with an important history of migratory flow from other countries, mainly European ones, and from other Brazilian cities, also with an important miscegenation. Since Sao Paulo city can be thought as a reflection of ethnic and social characters of the Brazilian population, we have decided to study the prevalence of celiac disease in Sao Paulo city. The aim of the present study was to assess the prevalence of unrecognized adult celiac disease in Sao Paulo and correlate it with further information about ancestry of the population studied. Methods: We have measured the prevalence of celiac disease by means of a survey of serum markers (IgA transglutaminase antibody-guinea pig and IgA endomysium antibodyhuman umbilical cord) in 4,000 volunteers donors in the central Blood Bank in Sao Paulo, "Pro-Sangue Foundation". The antibody-positive subjects were asked to undergo smallbowel biopsy. Results: There were 1896 female and 2104 male with mean age of 33 years In the ancestry evaluation of the 4,000 volunteers, we had $68 \%$ subjects with Brazilian origin and the remaining from other countries, mainly Italy, Portugal, Spain and Germany Of the 4,000 subjects, fourteen subjects had positive tests, with results of the two antibody tests concordant. Eight subjects were positive for IgA-class tissue transglutaminase only. The prevalence of serum markers positivity for celiac disease is at least 1 case in 286 healthy blood donors. Most donors diagnosed with celiac disease were asymptomatic and when symptomatic the most common symptoms were flatulence and constipation. So far, in 8 positive patients, duodenal biopsies were performed and in all the patients the diagnosis of celiac disease was confirmed (Marsh criteria). Conclusion: Among apparently healthy blood donors, the prevalence of serum markers positivity for celiac disease was similar to that seen in the United States and Europe.

\section{S1111}

\section{Intrafamilial Clustering of Atrophic Gastritis}

Yoshihisa Urita, Susumu Ishihara, Kenichiro Arai, Jun Kondo, Tatsuo Akimoto, Hiroto Kato, Noriko Hara, Hideyuki Koshino, Yoshiko Honda, Yoko Nagai, Kazushige Nakanishi, Nagato Shimada, Motonobu Sugimoto, Kazumasa Miki

Background: Many previous papers desclibed marked intrafamilial clustering of Helicobacter pylori (H.pylori) infection. Although H.pylori is an important cause of atrophic gastritis, it has been unknown whether all family members with H.pylori infection have advanced atrophic gastritis. Only a part of infected patients go on to develop atrophic gastritis, suggesting that factors other than H.pylori, such as environmental and host genetic factors, may contribute to the progression of atrophic gastritis. Serum pepsinogen (PG) is a known marker of gastric mucosal status, including mucosal atrophy. We therefore investigate the intrafamilial clustering of atrophic gastritis using serum PG method. Patients and Methods: Forty-six H.pylori-infected men (40-59 years) whose both parents had a positive serology for H.pylori were studied. Venous blood samples were obtained after overnight fasting and serum PGI and PG2 concentrations were measured. If PGl/2 ratio was less than 3, the patient was defined to have atrophic gastritis. Results: Atrophic gastritis was found in 20 patients $(43 \%)$. The PG1/2 ratio of their fathers and mothers were significantly lower $(2.0$ $+/-1.1,2.1+/-1.0$, respectively), compared with patients without atrophic gastritis $(3.2+/-$ $1.8,2.6+/-1.0$, respectively). When the $\mathrm{PGl} / 2$ ratio of patients is lower than that of their parents, atrophic gastritis is considered to progress more markedly than their parents. This marked progression was found in 17 patients (37\%) although atrophic gastritis progress with age. These results suggest that $37 \%$ of H.pylori-infected patients had more progressive atrophic gastritis than expected from the PGl/2 ratio of their parents. There may be other factors affecting the progression of atrophic gastritis in H.pylori-infected patients. Conclusions: The intrafamilial clustering of atrophic gastritis suggests the influence of genetic factors in 
H.pylori-infected patients, whereas one third of H.pylori-infected patients were more influenced by environmental factors than genetic factors.

\section{S1112}

Effective Management of Pancreatic Cancer Is Dependent On the First Symptoms

Marek Benes, Julius Spicak

Introduction: The prognosis of pancreatic cancer is dismal, and effective treatment is dependent on correct and early diagnosis and staging. Aims and methods: The aim of our multicentric study was to analyze the effectiveness of the diagnostic process reflected in adequate treatment. We prospectively analyzed the initial symptoms, length of the diagnostic process, and use of imaging methods with consequent application of adequate therapy. Results: A total of 110 patients with pancreatic cancer being managed within the last 12 months were enrolled. The most frequent first symptom was dyspepsia (43 patients), followed by pain (38) and jaundice (29). While, overall, hyperglycemia was documented at the beginning of the diagnostic period in 30 patients $(27.2 \%)$, it was present in $23(77 \%)$ of those with dyspepsia. Radical resection was performed in $26(23.6 \%)$ patients, exploratory laparotomy in 16 $(14.5 \%)$, palliative surgery in 38 , and chemotherapy in 21 patients. Mean time from the onset of the first symptoms to presentation (time I) was $38 \pm 30$ days. Mean time of the diagnostic process to specific treatment (time II) was $3.5 \pm 3.4$ months (1-24 months). Mean time I in patients presenting with dyspepsia was $44.8 \pm 25$. 1 days, in patients presenting with jaundice or pain $30.2 \pm 28.9$, and in those who underwent resection $26.1 \pm 24.3$ days. Mean time II in these groups was $3.9 \pm 4.5,2.7 \pm 2.1$, and $2.1 \pm 1.4$ months, respectively. Ultrasound was the most frequently used imaging method (180 examinations) followed by CT (127), ERCP (110), endosonography (35), and MR (7). The mean number of imaging methods used per patient was $4.6 \pm 1.5(2-11)$. Fine needle biopsy was performed in 18 patients. The most frequent algorithm of use of imaging methods was ultrasound followed by $\mathrm{CT}$ and ERCP. Conclusion: The decision-making process undertaken preoperatively is not sufficiently effective, as reflected by the inadequately high rates of exploratory laparotomy. The length of the process of diagnosis is dependent on the first symptoms, which can influence resectability. The diagnostic process was significantly shorter in resected patients. Dyspepsia with hyperglycemia have been shown to serve as a warning sign, and when this combination appears at an adequate age, intensive search for pancreatic cancer should be initiated.

\section{S1113}

Gastrointestinal Carcinoid Tumors: A Problem of Increased Magnitude and Diminished Support

Irvin M. Modlin, Geeta N. Eick, Mark Kidd, Manish C. Champaneria

A major medical misconception is that carcinoid tumors (CTs) of the GI tract are rare, indolent neoplasms. On the contrary, the diagnosis of GI CTs is increasing rapidly and many exhibit aggressive behavior with metastasis at presentation. To clarify the magnitude of the problem, we initially analyzed the NCI SEER registry (1973-1999) and the earlier ERG and TNCS series (Cancer 2003, 15:934-59). The greatest incidence of CTs is in the GI tract $(67.5 \%)$ and the bronchopulmonary system $(25.3 \%)$. Within the GI tract, most CTs occur in the small intestine (39.3\%). Evaluation of SEFR 2005 showed that small intestinal CTs have increased $740 \%$ over a 3 -decade period from $27 / \mathrm{yr}$ to $273 / \mathrm{yr}$ (same as ovarian carcinoma). Iowa and Connecticut have the highest incidences of all continental US states $(0.82 / 100,000)$ and the North-Eastern seaboard harbors $\sim 20 \%$ of all GI CTs. SEER 2005 recorded 562 gastric carcinoids (GCs) over the last 50 yrs, comprising $4.61 \%$ of all CTs. In the endoscopic era, the incidence of GCs has risen from $3.5 \%$ of all CTs to $6 \%$, a $\sim 75 \%$ increase over the last decade with the greatest increase $(800 \%)$ in white females. Overall GCs increased 2,700\% over a 3-decade period from 4/yr to 86/yr. These marked increases reflect varying rules of registration among databases, improvements in diagnosis and increased awareness. Nevertheless, physicians are increasingly faced with lesions they know little of, and for which limited therapeutic options are available. A major reason is the limited visibility afforded CT disease and the lack of funding for its study. Thus, only 3-5 CT meetings are held annually compared to between 30-40 to discuss GERD and Barrett's esophagus and its relationship to esophageal adenocarcinoma. Analysis of SEER 2005 reveals that the latter has increased 12 -fold since 1973 yet been declared an "epidemic" in contrast to CT, which is labeled an orphan disease. A review of Federal research funding in 2005 demonstrates that almost 600 grants have been awarded to study carcinoma in contrast to only 2 for $\mathrm{CT}$ or neuroendocrine tumor disease. Thus $\$ 110$ million is spent annually on carcinomas vs $<\$ 300,000$ for CTs. Research dollars for carcinoma range from $\$ 189 /$ patient/ yr (pancreas) to $\$ 85$ (colorectal) while carcinoids receive only $\$ 34$. Current epidemiological data indicate the prevalence of GI CTs is far higher than previously thought $(>0.82 / 100,000$ vs $0.05 / 100,000$ ) and the incidence is rising (700-2700\% depending on organ site since 1973). The lack of awareness, knowledge and research in the area of CT is fundamentally different to that of other GI cancers and merits more attention by the medical and scientific community.

\section{S1114}

Assessment of ACGME Core Competencies in Gastroenterology Trainees: Results of a National Survey

Derek Patel

Background: In 2003, the Accreditation Council for Graduate Medical Education (ACGME) established a competency-based standard for medical training. Training programs must demonstrate that their graduates are competent in six "core" areas. The current state of trainee competency evaluation in Gastroenterology (GI) fellowship programs is not known. Aims: To assess the current practices and opinions regarding the evaluation of ACGME core competencies in GI trainees. Methods: A web-based survey was emailed to the program directors of all United States (including Puerto Rico) adult GI fellowship programs. The survey consisted of 14 questions about program demographics, evaluation methods and program directors' attitudes toward the core competencies and competency assessment tools. Results: 155 GI fellowship training programs were identified. Valid email addresses were obtained for the directors of 147 programs. 72 (49\%) of the contacted program directors completed the survey. $81 \%$ of the respondents administer university-based training programs. $89 \%$ report having at least 5 total trainees in their program. $92 \%$ of responding fellowship directors describe themselves as "very familiar" with the six ACGME core competencies. However, only $44 \%$ feel that the trainee evaluation methods currently used in their program are "adequate." The assessment tools used most widely are procedure logs (used by $97 \%$ of programs), standardized evaluation forms (96\%), written examinations (67\%) and trainee portfolios (47\%). A majority ( $56 \%$ ) of the responding program directors favor the required use by all programs of a set of standardized assessment tools. $72 \%$ of respondents believe that professional organizations or the ACGME should be responsible for developing such tools. Conclusions: GI fellowship program directors are knowledgeable about ACGME core competencies and the assessment tools used to evaluate trainee competency. Yet, only a minority of program directors rate their programs' current evaluation methodology as being adequate. Potential solutions include greater leadership by professional societies and the ACGME in developing assessment tools, and the mandatory use of a set of standardized assessment tools.

\section{S1115}

Development of a For-Profit Free Standing Endoscopy Center (EASC) As Part of An Academic Gastroenterology Division

Ann Ouyang, Lisa S. Poritz, Abraham Mathew, Thomas J. McGarrity, Thomas R. Riley

We describe a successful for-profit EASC venture within an academic medical center. The project was developed with voluntary participation by faculty in the Divisons of GI and colorectal surgery. Start-up costs were financed $60 \%$ by physicians (controlling group) who formed an LLC (limited liability company), 20\% by a management LLC formed by a private practitioner with expertise in EASC development and management with key members of his organization, and $20 \%$ by Nittany Healthcare Systems, Inc. (Nitt Health), a for-profit corporation affiliated with Penn State University. A win-win arrangement recognizing expertise, contribution and needs of all partners was achieved. The management group provided expertise and time instead of money and developed the budget, negotiated leases and contracts with third-party payers, and hired the architect, contractors and staff. Nitt Health provided "name recognition" as most patients are directly referred, and agreed to take over the EASC if physicians were to leave without sufficient replacement investors. Nitt Health would then become controlling partner. Physicians were able to design and control a facility providing efficient and pleasant service to patients for routine procedures, generate wRVU's efficiently while at the EASC, and have opportunity for financial gain with clearly defined and limited risk. Buy-in and buy-out terms are relatively cheap with all profit being paid to investors quarterly. The Dept of Medicine gained increased professional income and contributed by loaning the cost of the physician legal fees to Nitt Health who agreed to pay back from profit when available. The Dept recognized that the EASC would help in recruiting faculty. The EASC has existed for 2 yrs and is now profitable. The GI Division increased the number of procedures/clinical FTE by $30 \%$ during the first year and by an additional $10 \%$ during the second year. The hospital unit decreased its total number of procedures by only $3 \%$ while the total number of endoscopic procedures (both EASC and inpatient) increased by $30 \%$ by the second year of EASC operation. While the adult GI service decreased its activity in the hospital unit, the unit has accommodated an increase in activity by other services. The colorectal surgeon doubled her colonoscopy numbers with the same time commitment. There is a negative impact on fellows' procedure with a decrease in available colonoscopies by $1 / 3$, however the numbers still allow fellows to perform over twice guideline numbers. We conclude that it is feasible to develop a for-profit enterprise within the Department of Medicine if a win-win situation can be developed for all parties involved.

\section{S1116}

Patients with IBS Have Greater Treatment Satisfaction with Tegaserod Compared with Placebo Pierre Pare, Carlos Francisconi, Andrea Viegas, Christopher R. McBurney BACKGROUND: Irritable bowel syndrome (IBS) is a chronic medical disorder that significantly impacts on patients' lives. However, the impact of medical treatment of IBS is often not clear. Tegaserod, a promotility agent approved for use in patients with irritable bowel syndrome with constipation (IBS-C) and chronic constipation, has demonstrated clinical efficacy in multiple studies. In this study, we investigated the use of tegaserod in patients with IBS and how this therapy impacts on patients' perception of treatment satisfaction. METHODS: Women ( $\leq 65$ years) meeting Rome II criteria for IBS, excluding those with IBS with diarrhea (IBS-D) and/or a recent episode of diarrhea, were randomized in a doubleblind, placebo-controlled, multicenter study. After a 2-week baseline, patients were treated with tegaserod ( $6 \mathrm{mg}$ b.i.d.) or placebo for 4 weeks. After the treatment period, a questionnaire assessed patients' expectations including satisfaction with treatment, symptom relief, and willingness to re-use and recommend treatment with tegaserod at the end of the study. Data were analyzed using Cochran-Mantel-Haenszel tests. RESULTS: 661 women with IBS were randomized (tegaserod $[\mathrm{n}=339]$, placebo $[\mathrm{n}=322]$ ), and satisfaction data were available for 329 tegaserod and 322 placebo patients. Patients who received tegaserod were more likely to be satisfied (tegaserod $76 \%$, placebo $65.3 \%, p=0.021$ ) compared with placebo. More tegaserod users reported their treatment expectations were met or exceeded (tegaserod $61.2 \%$ vs placebo $47.8 \%, \mathrm{p}<0.001$ ). When satisfaction was compared with prior IBS medical therapy, $60 \%$ (vs placebo $45 \%, p=0.032$ ) of tegaserod patients reported greater relief of symptoms with tegaserod. In addition, the majority of patients treated with tegaserod would recommend it to others with IBS (tegaserod $71 \%$ vs placebo $61 \%, \mathrm{p}=0.007$ ). There was not a statistically significant difference for greater future utilization of study medication between treatment groups. CONCLUSIONS: In this controlled study of IBS subjects, patients were more satisfied with tegaserod as a treatment for their symptoms related to IBS-C than placebo, and the majority of IBS patients who received tegaserod would continue to use this promotility agent and recommend the therapy to others with IBS. 


\section{S1117}

High Prevalence of Abnormal Endoscopic Findings in Patients with NonGERD Dyspepsia

Nimish Vakil, Sander Veldhuyzen van Zanten, Nigel Flook, Nicholas J. Talley, Karsten Lauritsen, Torre Persson, Johan Brun, Tore Lind, Elisabeth Bolling-Sternevald, Ewa Bjorck OBJECTIVE: To determine the prevalence and nature of abnormal endoscopic findings in patients with non-GERD dyspepsia. METHODS: Patients aged 18-70 years with pain or burning centered in the upper abdomen were enrolled for possible inclusion in an international, multicenter trial (Study SD-NED-0021) of acid suppression. Patients with predominant symptoms or with a diagnosis of gastroesophageal reflux disease (GERD) were excluded (heartburn >once a week), as were patients taking proton pump inhibitors (PPIs) or $\mathrm{H}_{2}$ blockers as maintenance therapy within the preceding 6 months. Patients with alarm symptoms or with a history of non-steroidal anti-inflammatory drug use were also excluded. Helicobacter pylori status was determined using a ${ }^{13} \mathrm{C}$ urea breath test. We present data from patients who were not randomized because of abnormal findings at baseline esophagogastroduodenoscopy (EGD). RESULTS: 2868 patients were enrolled, of which 2741 were eligible for study entry and underwent an EGD. The most frequent endoscopic findings, plus malignancies, are presented in the table. Three patients had an esophageal malignancy (aged 30, 53 and 65 years) and 3 had a gastric malignancy (aged 50, 51 and 58 years). CONCLUSIONS: 1. Abnormal endoscopic findings are common in patients with nonGERD dyspeptic symptoms. 2. Most lesions are treatable with $H$. pylori eradication or acid suppressive therapy, supporting recent guidelines for the empirical medical management of dyspeptic patients. 3. Although uncommon, upper gastrointestinal malignancies will be missed despite a careful evaluation of alarm features. 4. Empirical age cutoffs for early endoscopy e.g. 60 years in the AGA guideline will detect some but not all of these patients.

\begin{tabular}{|c|c|}
\hline & Frequency (\%) \\
\hline Total patients enrolled & 2868 \\
\hline Erosive esophagitis & $407(14.8 \%)$ \\
\hline Barrett's esophagus & $13(0.5 \%)$ \\
\hline Esophageal ulcers & $19(0.7 \%)$ \\
\hline Gastric erosions & $169(6.1 \%)$ \\
\hline Gastric ulcers & $62(2.3 \%)$ \\
\hline Gastritis/duodenitis & $98(3.6 \%)$ \\
\hline Duodenal ulcers & $75(2.7 \%)$ \\
\hline Malignancies & $6(0.2 \%)$ \\
\hline
\end{tabular}

\section{S1118}

Tegaserod Significantly Reduces Work Productivity Loss and Daily Activity Impairment in Patients with IBS Versus Non-Tegaserod-Treated Patients Pierre Pare, Robert Balshaw, Shideh Khorasheh, Martin Barbeau, Suzanne Kelly, Christopher R. McBurney

BACKGROUND: Abdominal pain/discomfort, bloating and altered bowel function are gastrointestinal (GI) dysmotility and sensory symptoms related to irritable bowel syndrome (IBS). Dysmotility symptoms are associated with impaired quality of life (QoL) and work productivity. LOGIC (Longitudinal Outcomes study of GI symptoms in Canada) is an ongoing, prospective, observational study designed to evaluate the treatment patterns and health outcomes of patients with IBS symptoms in Canada. METHODS: 1.555 subjects with IBStype symptoms were enrolled from 147 community and specialist medical sites across Canada. Clinical data and patient-reported outcomes were collected at baseline and at Months $1,3,6,9$ and 12 . Work productivity and daily activity impairment were assessed using the WPAI:IBS. Tegaserod (T) subjects were defined as those taking $\geq 7$ days of therapy or $\geq 7$ tablets, non-tegaserod (Non-T) subjects did not report T use; all other subjects were excluded from the analysis. RESULTS: Baseline data were obtained from 1,555 patients of whom 1,372 could be identified as T subjects $(n=360)$ or Non-T subjects $(n=1,012)$. These patients had a mean age of 46 years and mean disorder duration of 12 years and $86 \%(1,182)$ were women. Self-reported bowel patterns were predominantly constipation (40\%) and mixed/ alternating (38\%). Of the T patients, $216(60.5 \%)$ were working, as were $598(59.4 \%)$ of the Non-T patients. There was no improvement in any item of the WPAI for the Non-T group between baseline and Month 6 for the work productivity components (there was a decrease in daily activity impairment). In the T group, there was an improvement in all WPAI items between baseline and Month 6 . In addition, the T group had a bigger improvement than the Non-T group in all items of the WPAI: absenteeism $(2.8 \%)$, presenteeism $(8 \%)$, overall productivity impairment $(8.2 \%)$ and daily activity impairment $(12.4 \%, \mathrm{p}<0.001)$. Averaged over a 40-hour working week, the T group reduced work productivity loss by 3.28 hours more than the Non-T group. CONCLUSIONS: In this study, after 6 months, T users had 3.28 hours of increased productivity per week when compared to Non-T users. Furthermore, the $\mathrm{T}$ users had a significant improvement in daily activity compared to the Non-T users. This is the first prospective observational study to show that $\mathrm{T}$ has a positive impact on work productivity as well as daily activities in the setting of usual care.

\section{S1119}

Canadian IBS Observational Study Shows Tegaserod Patients Have Greater Improvements in QOL As Compared to the Control Group

Pierre Pare, Robert Balshaw, Shideh Khorasheh, Martin Barbeau, Suzanne Kelly, Christopher R. McBurney

BACKGROUND: Abdominal pain/discomfort, bloating and constipation are gastrointestinal (GI) symptoms related to irritable bowel syndrome (IBS). These symptoms are associated with impaired quality of life (QoL) and work productivity. LOGIC (Longitudinal Outcomes
Study of GI Symptoms in Canada) is an ongoing, prospective, observational study designed to evaluate the treatment patterns and health outcomes of patients with IBS symptoms in Canada. AIMS AND METHODS: LOGIC enrolled 1,555 patients at 147 community and specialist physician sites across Canada. Clinical data and patient-reported outcomes were collected at baseline and at Months 1, 3, 6, 9 and 12. QoL was assessed with the IBS$\mathrm{QOL}$. Tegaserod treated subjects ( $\mathrm{T}$ ) at baseline and non-tegaserod subjects (Non-T) were compared. Unadjusted mean QoL at baseline (BL) and Month 6 were compared between groups using the Wilcoxon rank-sum test. The within-subject slope of the QoL scores was compared using a mixed model analysis of variance. The model compared the average slope of the T and Non-T subjects after adjusting for IBS severity (measured by the Total Symptom Severity score, TSS), usual bowel pattern (normal, constipated, diarrhea, alternating), duration of symptoms, gender, history of tegaserod use, and baseline IBS-QOL. RESULTS: O the 1,555 subjects enrolled, 1,372 were identified as either T-users $(n=360)$ or Non-T-user $(\mathrm{n}=1012)$. At baseline, T subjects had a mean IBS-QOL score of 61.38 compared to NonT subjects with a mean of 68.39 ( $\mathrm{p}<0.001$ ). By Month 6 , the unadjusted mean scores were 67.30 for $\mathrm{T}$ and 71.43 for Non-T patients, $\mathrm{p}=0.037$. After adjusting for baseline covariates, $\mathrm{T}$ subjects showed significantly greater improvement in IBS-QOL scores over the first 6 months of the study (mean improvement of 1.04 vs. 0.42 points per month, $\mathrm{p}=0.004$ ) CONCLUSIONS: The LOGIC study is an ongoing, prospective, observational study that will follow patients for 1 year. This analysis of the LOGIC study followed treatment in a rea world setting with physicians treating patients on an individual basis. Subjects using T a baseline showed more improvement in their IBS-QOL scores than the Non-T users at 6 months. This is the first prospective observational study to show that $\mathrm{T}$ has a positive impact on health-related quality of life in the setting of usual care.

\section{S1120}

Longitudinal Outcomes Study of GI Symptoms in Canada (LOGIC): Tegaserod Versus Usual Care Comparison At Baseline

Pierre Pare, Robert Balshaw, Shideh Khorasheh, Martin Barbeau, Suzanne Kelly, Christopher R. McBurney

BACKGROUND: Some GI motility disorders may be chronic and episodic, such as irritable bowel syndrome (IBS), and can be characterized by recurrent abdominal pain and altered bowel habit. Tegaserod, a selective 5- $\mathrm{HT}_{4}$ receptor agonist, is effective and well tolerated in patients with symptoms of abdominal pain, bloating and constipation, associated with IBS. LOGIC is an ongoing prospective observational study designed to evaluate treatmen patterns and health outcomes in patients with IBS symptoms in Canada. AIMS AND METHODS: The study is designed to characterize quality of life ( $\mathrm{OoL}$ ), healthcare resource utilization and productivity of patients with symptoms associated with IBS over a 1-year period. A total of 1,555 subjects were enrolled at 147 centers. The present analysis compares the characteristics of patients receiving tegaserod at baseline with those not receiving tegaserod (Non-tegaserod) RESULTS: Baseline data were obtained from 1,555 patients, of which 1,372 could be identified as tegaserod $(n=360)$ or Non-tegaserod $(n=1,012)$. The mean age in both groups was 46 years. The majority of the patients were female (86\%) and Caucasian (88.3\%) Significant but minor differences between the groups were noted for gender, ethnicity and the mean duration of IBS. The patients' self-reported bowel habits indicated that IBS-C was more common in the tegaserod group (38.6\%), and IBS-A (30.3\%) in the Non-tegaserod group $(\mathrm{p}<0.0001)$. The group receiving tegaserod had a significantly $(\mathrm{p}<0.01)$ higher percentage of subjects reporting more severe bowel movement-related symptoms at baseline based on frequency, intensity and resulting distress when compared with Non-tegaserod. In addition, the group receiving tegaserod reported significantly more physician visits in the month prior to the baseline visit. DISCUSSION: Baseline data from this ongoing, prospective observational study indicate that the severity of symptoms influences the choice of treatment. This study will follow patients for 1 year, providing insight into the assessment, treatment and outcomes of IBS symptoms. However, future analyses of data gathered in this study will need to take into account the baseline differences presented here.

\section{S1121}

Tegaserod Significantly Reduces Work Productivity Loss and Daily Activity Impairment in Colombian Patients with IBS with Constipation

Maria T. Galiano, Jairo A. Martinez, Ciro Crispin, Giovanny de Vuono, Ramon J. Espinoza, Jacobo Feris, Carlos Gomez, Belen Mendoza, Jaime Obregon, Federico Pelaez, Adriana Varon, Maria C. Eslava, Claudia Deaza, Claudia J. Ramirez, Amparo Castro

BACKGROUND: Irritable bowel syndrome with constipation (IBS-C) symptoms are responsible for a negative impact on patients' quality of life as well as affecting aspects like work and personal daily activities. Tegaserod (T) is a selective, serotonin type 4 (5-HT4) receptor agonist with proven efficacy and safety in patients with IBS-C. AIMS: One of the objectives of this study was to assess the impact of $\mathrm{T} 6 \mathrm{mg}$ b.i.d. on work-related productivity and daily activity impairment in a working population of patients (pts) with IBS-C. METHODS: Pts (men and women $<65$ years old who met Rome II criteria for IBS-C) were assessed during this open-label trial, with a 2 -week baseline period and a 12 -week treatment period with T $6 \mathrm{mg}$ b.i.d. Absenteeism, presenteeism, and overall work productivity loss (combined absenteeism plus presenteeism) and daily activities due to IBS symptoms during the previous 7 days were measured with the validated Work Productivity and Activity Impairment Questionnaire for IBS (WPAI-IBS). The questionnaire was performed on days -7 and at baseline as well as during days $14,28,56$ and 84 of the treatment period. Results are presented as a comparison between the baseline period (no treatment), and the end of T treatment (after 12 weeks of therapy). Analysis was by intention to treat. RESULTS: 120 pts with IBS-C were included in the trial. Mean age was 37 years (range 19-62) and $97.5 \%$ of pts were women. At the end of $\mathrm{T}$ treatment, and in comparison with baseline, T significantly reduced absenteeism by $6.26 \%(\mathrm{p}<0.01)$, presenteeism by $10.9 \%(\mathrm{p}<0.03)$, overall work productivity loss by $9.33 \%(\mathrm{p}<0.02)$, and daily activity impairment by $12.69 \%(\mathrm{p}<0.005)$. Assuming a 40-hour workweek, T reduced work productivity loss by 2.1 hours. In comparison with baseline, $\mathrm{T}$ treatment was superior, providing relief from every symptom, such as abdominal pain/discomfort, abdominal bloating, bowel movements and overall IBS symptoms evaluation 
(p values $<0.05$ ). CONCLUSIONS: T treatment significantly improved work-related productivity and reduced the number of daily activity hours lost due to IBS-C. These benefits were maintained at 12 weeks. This is the first Latin American study to show that one IBS therapy has a significant positive impact on work productivity as well as on daily activities.

\section{S1122}

Impact of $0.5 \mathrm{Mg} \mathrm{Qd}, 1 \mathrm{Mg}$ Qd and $1 \mathrm{Mg}$ Bid Alosetron Hydrochloride On Quality of Life (QOL) and Productivity in Women with Chronic, Severe Diarrhea-Predominant IBS (D-IBS)

Susan Gordon, Vanessa Ameen, Marquita West, Amy Heath, Teresa Perschy, Eric Carter

Objective: IBS negatively impacts physical, social, and psychological well-being and results in lost social activity and work time. The 5HT3 receptor antagonist alosetron at a dose of $1 \mathrm{mgBID}$ is indicated for women with chronic, severe D-IBS who have not responded adequately to conventional therapy. This dose regimen improves IBS-related QoL and lost productivity in women with severe D-IBS but is associated with constipation. The objective of this study was to determine the effect of lower alosetron doses on IBSQoL and productivity in patients with chronic, severe D-IBS (protocol S3B30040). Methods: 705 women with severe D-IBS were equally randomized to 12 wks of placebo, $0.5 \mathrm{mgQD}$, $1 \mathrm{mgQD}$, or $1 \mathrm{mgBID}$ alosetron, followed by a $4 \mathrm{wk}$ follow-up period. IBSQoL and workplace/household/leisure activity time questionnaires were administered at Randomization and wk12/Final Visit. Changes from baseline in scale-specific scores were computed for 9 QoL domains: emotional, mental health, sleep, energy, physical functioning, food, social functioning, physical role, sexual relations. Pairwise treatment comparisons were adjusted for the 9 QOL domains using the general multiple Simes procedure described by Hommel. To assess productivity, changes from baseline in the amount of time prevented from doing normal activities due to IBS symptoms were compared. Results: With regard to IBSQoL, all alosetron regimens showed improvement on multiple domains vs. placebo on the IBSQoL questionnaire. Treatment differences for the alosetron groups were statistically significant compared with placebo $(p<0.038)$ on all domains, with the exception of: sexual relations for all three alosetron doses, physical functioning in the $1 \mathrm{mg}$ QD and $1 \mathrm{mg}$ BID groups, and emotional and sleep in the $1 \mathrm{mg}$ QD group. With regard to productivity, lost workplace activity(hrs), social/ leisure and household activity(days) were reduced from baseline in all three alosetron treatment groups. Significant treatment differences $(\mathrm{p}<0.05)$ over placebo were seen in the $0.5 \mathrm{mgQD}$ and $1 \mathrm{mgBID}$ regimens in lost workplace and social/leisure activities. Conclusion: Lower doses of alosetron also show improvement in multiple IBS specific QoL measures and productivity in women with chronic, severe D-IBS.

\section{S1123}

The Clinical Characteristics of Patients with Idiopathic Constipation with a Positive Family History

Annie On On Chan, Wai Mo Hui, Kwok Fai Lam, Gigi Leung, Wayne Hc Hu, Chi Kuen Chan, Man Fung Yuen, Shiu Kum Lam, Benjamin Cy Wong

Background: We observed that there is familial aggregation in patients with idiopathic constipation, which may be due to underlying genetic factors or intrafamilial environment. The clinical characteristics between those with and without a positive family history of constipation have not been studied. Aim: To investigate the clinical characteristics of patients with idiopathic constipation with and without a positive family history. Methods: 230 consecutive patients with idiopathic constipation satisfying Rome II criteria were recruited. A Rome II questionnaire on constipation was given to the patients' families to identify if there was any family members with idiopathic constipation. Results: There were 104 and 126 patients with (at least one lst degree relative) and without a positive family history, respectively. The patients in the two groups were comparable in mean age $(\mathrm{p}=0.3)$, and sex distribution ( $p=0.98$ ). Using the method of Analysis of Interval-Censored/Grouped Failure Time Data, patients with positive family history had a younger age of onset (median: 1120 years vs $21-30$ years, $\mathrm{p}=0.0042$ ). Moreover, patients with positive family history were less likely to be married $(60.6 \%$ vs $75.4 \%, \mathrm{P}=0.016)$, had longer duration of constipation $(19.8 \pm 14$ vs $16.3 \pm 13, \mathrm{p}=0.042)$, more often associated with digital evacuation ( $26 \%$ vs $13.5 \%, \mathrm{p}=0.017$ ), more complications eg symptomatic hemorrhoids, anal fissure and rectal prolapse ( $44.2 \%$ vs $31 \%, \mathrm{p}=0.038)$, less precipitating factors leading to the onset of constipation $(17.3 \%$ vs $30.2 \%, p=0.024)$, and fewer associated psychological disorders ( $2.9 \%$ vs $11.9 \%, \mathrm{p} 0.011)$ Conclusions: Patients with idiopathic constipation and with a positive family history showed different clinical characteristics. This may be related to the early age of onset of the symptoms, which may in turn, gives clues to the underlying etiology.

\section{S1124}

The Impact of Non-GERD Dyspepsia On Productivity While At Work and in Regular Daily Activities - Results from One Study in Endoscoped (STARS I) and One Study in Uninvestigated (STARS II) Dyspeptic Patients

Peter Wahlqvist, Tore Persson, Johan Brun, Nigel Flook, Karsten Lauritsen, Nimish Vakil, Sander Veldhuyzen van Zanten, Katarina Halling, Nicholas Talley

OBJECTIVE: To assess the impact of dyspepsia on productivity while at work and in regular daily activities in endoscoped (STARS I study) and in uninvestigated (STARS II study) dyspeptic patients. METHODS: Dyspeptic patients (with symptoms of pain or burning centered in the upper abdomen) were enrolled into 2 randomized, placebo-controlled, double-blind, multinational studies of similar design. During the week before start of treatment, patients rated dyspeptic symptoms daily using a 4 grade scale $(0=$ none to $3=$ severe) and only those with a sum symptom score of 5 or more during the last 7 days of the runin period were included in the study. In STARS I, all patients underwent an endoscopy at baseline, and those with abnormal findings were excluded. Before start of treatment, patients completed the Reflux Disease Questionnaire (RDQ) to assess symptom severity in three dimensions (heartburn, regurgitation, dyspepsia), and the Work Productivity and Activity Impairment (WPAI) questionnaire to assess disease-specific absence from work, reduced productivity while at work, and reduced productivity while carrying out regular daily activities. Both questionnaires use a recall period of 1 week. RESULTS: Productivity results were obtained from 568 and 452 patients (of which 372 and 344 were employed) in the STARS I and II studies, respectively. Overall, patients reported that dyspeptic symptoms had a substantial impact on productivity (Table). This impact was slightly more pronounced in the endoscoped than in the uninvestigated patients. However, because these were two different populations the reasons for this difference are unclear. Mean number of workhours lost per employed patient and week due to absence from work and reduced productivity while at work was 11.1 hours $(1.9+9.2)$ in endoscoped patients and 8.7 hours $(2.0+6.7)$ in uninvestigated patients. Results on reduced productivity were more strongly correlated with the RDQ dyspepsia dimension $(r=0.2$ to 0.4$)$ than with the other dimensions ( $r=$ 0.1 to 0.2 ), which lends support to the observed productivity loss mainly being related to non-GERD dyspepsia. CONCLUSION: Non-GERD dyspepsia has a substantial impact on productivity while at work and in regular daily activities in both endoscoped and uninvestigated patients.

Productivity results, mean values and $95 \%$ confidence intervals (CI)

\begin{tabular}{|c|c|c|c|c|}
\hline Productivity variable & \multicolumn{2}{|c|}{ STARS I } & \multicolumn{2}{c|}{ STARS II } \\
\hline & Mean & $95 \%$ CI & Mean & $95 \%$ CI \\
\hline Work hours absent & 1.9 & $(1.2 ; 2.6)$ & 2.0 & $(1.2 ; 2.8)$ \\
\hline Percent reduced productivity at work & 26.4 & $(24.0 ; 29.0)$ & 19.8 & $(17.7 ; 21.9)$ \\
\hline Hours lost due to reduced productivity while at work & 9.1 & $(8.2 ; 10.1)$ & 6.7 & $(5.9: 7.4)$ \\
\hline Percent reduced productivity in daily activities & 33.0 & $(31.0 ; 35.0)$ & 26.8 & $(24.8: 28.8)$ \\
\hline
\end{tabular}

\section{S1125}

Randomized, Double-Blind, Placebo-Controlled Crossover Trial of Famotidine in Patients with Functional Dyspepsia

Mototsugu Kato, Takeshi Yoshida, Tamotsu Hata, Yuji Ono, Shouko Ono, Manabu

Nakagawa, Souichi Nakagawa, Yuichi Shimizu, Masahiro Asaka

Background: histamine 2-receptor antagonists were used as a first therapy against functional dyspepsia (FD). Meta-analyses on the treatment of FD have demonstrated the effectiveness of H2RAs. Most reports to date have examined reflux-like FD, and few FD studies have been conducted based on the Rome II criteria. Reflux-like FD includes endoscopy-negative GERD and tends to respond better to antisecretory drugs. Also few clinical studies with famotidine for FD have been reported. Aim: To evaluate the effectiveness of famotidine for FD patients. Methods: A multi-center, randomized, double-blind, placebo-controlled crossover trail was conducted. Patients diagnosed with FD by the Roma II criteria were included. Subjects were randomized into two groups, and received either famotidine or placebo as the first four weeks medication. After a one-week washout period, they were switched to the other regimen for another four weeks. Evaluation was made prior to the start of study, upon completion of the first drug cycle, and the second drug cycle, by GSRS(Japanese version) for the seriousness of abdominal symptoms, and by SF-36(Japanese version) for the level of QOL. Results: Nineteen patients out of 21 enrolled patients successfully completed this study. Significant improvement in GSRS scores was observed in abdominal pain $(\mathrm{P}=0.007)$, indigestion, and reflux syndrome after famotidine treatment. GSRS abdominal pain scores were lowest following the administration of famotidine in both treatments. In patients treated first with famotidine, there was a rebound in increase of abdominal pain scores in the following period of placebo administration. Also QOL scores showed significant improvement in body pain, vitality, and general health perceptions after famotidine treatment. There was no improvement of symptoms and QOL scores after administration of placebo. Conclusions: Famotidine was effective for improving symptoms and $\mathrm{QOL}$ in FD patients. In the situation of high placebo response, crossover design is advantageous to identify true drug responders and nonresponders.

\section{S1126}

Association of Perfectionism with Psychophysiological Background of Functional Dyspepsia and Gastroesophageal Reflux Disease Compared to Anorexia Nervosa

Masahiro Ochi, Kazunari Tominaga, Toshiya Iketani, Kaori Kadouchi, E.J. Sasaki, Masatsugu Shiba, Toshio Watanabe, Yasuhiro Fujiwara, Nobuhide Oshitani, Kazuhide Higuchi, Nobuo Kiriike, Tetsuo Arakawa

Background and Aims: Anorexia, early satiety, bloating, and belching were common complaints for patients with functional dyspepsia (FD), gastroesophageal reflux disease (GERD), and anorexia nervosa (AN). Although showing similar abdominal symptoms, the difference in psychophysiological background among these diseases remains unknown. Perfectionism has been associated with the pathogenesis of eating disorder and identified as a predisposing factor in patients with AN. In this study, we analyzed the relationship of perfectionism as the psychophysiological background in patients with FD and GERD, compared to that in patients with AN. Methods: FD patients according to Rome II criteria (N=154, 59\% female, mean age 49.2 years), GERD patients ( $N=21,48 \%$ female, mean age 53 years), AN patients according to DSM-IV criteria ( $\mathrm{N}=101,100 \%$ female, mean age 23 years) were recruited in this study. Number of healthy controls was $130 ; 81 \%$ female, mean age 26.2 years. We used the Frost's Multidimensional Perfectionism Scale (FMPS) to explore the association of perfectionism, which comprises 35 items in the form of statements with a Lickert-type 5point response format from strongly disagree (1) to strongly agree (5). These 35 items are divided into 6 sub-scales tapping 6 dimensions of perfectionism: concern over mistake (CO); personal standards (PS); parental expectations (PE); parental criticism (PC); doubts about actions (DA); organization (OR). Results: Scores of all sub-scales expect for OR in AN patients were higher than those of controls $(\mathrm{p}<0.05)$. PC scores of FD patients were also significantly higher than that of controls, but lower than those of AN patients as well as all other subscales expect for OR. On the other hand, CO and PC scores of GERD patients were significantly higher than those of both controls and FD patients, whereas CO and DA scores of GERD 
patients were lower than those of AN patients. Conclusion: Continuous long-term mental stress such as parental criticism (PC) was suggested to be critical for some eating disorders. But perfectionism identified as a predisposing factor in AN was not closely related to the psychological background of FD, but might be in part involved in the psychophysiological background of GERD.

\section{S1127}

Outcomes of Recurrent Abdominal Pain (RAP) in Children and Responses to a Multidimensional Measure for RAP (MM-RAP): A 2 Years Follow-Up Study Hoda M. Malaty, Michael Takenticho, Kennard J. Fraley, Suhiab Abudayyeh, David Y. Graham, Kimberley Omalley, Mark A. Gilger

Background: Recurrent abdominal pain (RAP) is a common problem in children and adolescents. Evaluation of children with RAP continues to challenge investigators due the lack of a psychometrically sound measure for RAP. Aims: 1) to characterize the course of children with RAP 2) to evaluate the diagnostic outcomes of RAP, 3) to compare responses of a newly developed measure for RAP across the different diagnostic categories of RAP. Methods: We conducted a prospective 2 year study of children diagnosed with RAP during their first visit to the Pediatric Gastroenterology Clinic at Texas Children's Hospital and who were followed-up. At their initial visit, the eligible child/ parent completed a multi-dimensional measure for RAP (MM-RAP) consisting of 4 scales (pain intensity scale, symptoms scale, disability scale, and satisfaction scale). The final diagnosis was defined as the diagnosis at their last visit. Outcomes were classified into 3 categories: functional RAP, organic RAP, and Gastroesophgeal Reflux Disease (GERD). The responses of the MM-RAP were analyzed by One-Way ANOVA analysis among the 3 RAP outcome groups. Results: One hundred and forty one children ages 4 to 18 years ( $44 \%$ boys) participated. The follow-up ranged from 4-130 weeks (mean $=50$ weeks). The final diagnoses were functional RAP in 46\%, organic RAP in $24 \%$.and GERD in $30 \%$. Neither age nor sex predicted the outcome. The total scores for the pain intensity scale were significantly higher among children with functional RAP than organic RAP or GERD $(19.7(+3.5)$ vs. $14.2(+4.7)$ and $13.1(+4.2)$; respectively, $\mathrm{p}=001)$. Although the non-pain symptoms total score did not differ between the three outcome groups, independent items within the scale, i.e. diarrhea, vomiting, and heartburn were significantly higher among children with organic RAP and GERD compared to functional RAP. The scores for pain disability and health satisfaction scales were similar among the three outcome groups. Conclusions: The MM-RAP discriminated between functional and organic RAP across the studied population. Children with functional RAP report more intense pain than children with organic RAP or GERD. Neither the disability scale nor the satisfaction scale differentiated between the functional and organic RAP

\section{S1128}

Rikkunshito Improves Induction Therapy for Depression with SSRI Takakazu Oka

Background: Gastrointestinal (GI) symptoms such as nausea and vomiting by serotoninspecific reuptake inhibitors (SSRI) are critical issues that impair QOL of patients with depressive disorder and that may result in discontinuation of drug use. Rikkunshito, a Japanese Kampo medicine, has been reported to improve gastric emptying by functioning as a serotonin-3 receptor antagonist and improve GI symptoms in patients with functional dyspepsia. Aim: To assess if Rikkunshito reduces GI symptoms when treating depressed patients with SSRI by a randomized controlled study. Methods: Forty-three patients who met DSM-IV criteria for major depressive disorder (19 to 75 years; mean age 40.2 years) were randomly allocated to one of the following two groups: SSRI alone (fluvoxamine 50 to $150 \mathrm{mg}$ ) group ( $\mathrm{n}=2 \mathrm{l}$ ) or SSRI plus Rikkunshito $7.5 \mathrm{~g}$ combination group $(\mathrm{n}=23)$. The treatment period was 8 weeks. The following parameters were compared between the two groups. (1) GI symptoms were compared by GSRS (Japanese edition). (2) Depressive symptoms were compared by self-rating depression scale (SDS). (3) The number of patients who required discontinuation of SSRI treatment because of adverse reactions. Results:(1) In the SSRI alone group, GI symptoms significantly worsened. In the SSRI plus Rikkunshito combination group, no significant changes were observed in the GSRS scores including total GI symptoms, reflux symptoms, abdominal pain and dyspepsia symptoms. Scores of dyspepsia symptoms in the SSRI plus Rikkunshito combination group were significantly lower than those in the SSRI alone group $(\mathrm{p}<0.05)$. (2) In the SSRI alone group, SDS scores significantly improved in the 8th week. In the SSRI plus Rikkunshito combination group, the scores improved significantly in the 4 th and 8 th weeks $(p<0.05)$. There were no differences in antidepressive effect between the groups. (3) SSRI treatment was discontinued because of adverse reactions in $47.6 \%(10 / 21)$ in the SSRI alone group and in $19.4 \%(4 / 22)$ in the SSRI plus Rikkunshito combination group $(\mathrm{p}<0.05)$. Conclusions: In the SSRI plus Rikkunshito combination group the number of patients who discontinued SSRI was less than that in the SSRI alone group. This study suggests that Rikkunshito is beneficial for reducing SSRI-induced GI symptoms.

\section{S1129}

Blood-, Breath- Or Gene-Test for Detection of Lactose Malabsorption? A Comparison of Three Methods

Per G. Farup, Oistein Hovde

Introduction: Genetic testing for lactose malabsorption (LM) is a new diagnostic method that supplements commonly used blood- and breath- tests. Aims and Methods: The study compares blood- breath- and gene-tests for detection of LM. Patients with suspected LM were included. LM was assessed with a combined blood- and breath- test. A gas chromatograph (Model SC MicroLyzer, Quintron Instrument) was used for the breath tests. Samples for $\mathrm{H} 2$ and $\mathrm{CH} 4$ were measured before and 30, 60, 120 and 180 min after intake of $25 \mathrm{~g}$ lactose, and samples for blood glucose were taken before and after 15, 30, 60 and 90 minutes. Peak values of $\mathrm{H} 2$ breath excretion $>20 \mathrm{ppm}$ above the lowest preceding value, peak $\mathrm{CH} 4$ excretion $>12 \mathrm{ppm}$ above baseline, and/or combined $\mathrm{H} 2$ and $\mathrm{CH} 4$ increase $>15$ ppm were considered a positive breath test for LM, and increase in blood glucose $<1.0$ $\mathrm{mmol} / \mathrm{l}$ as a positive blood test. The combined test was repeated $10-18$ months later and supplemented with a gene test in patients with a discrepancy between the results of the first blood- and breath- tests. The "correct" diagnosis was based on an overall evaluation of all the tests. Results: 60 patients (men/women: 17/43) with a mean age of 39 years (range 18 - 71), most of them with symptoms of functional gastrointestinal disorders, were included 46 out of 49 patients with a normal breath test had a normal blood test, and 5 out of 11 patients with a pathological breath test had a pathological blood test (agreement $85 \%$, kappa $0.44, p=0.004)$. LM was considered to be "correct" in $7(12 \%)$ of the patients. Referring to the "correct" diagnosis, 4 false positive breath tests normalised when repeated, 2 out of 3 false positive blood tests normalised, 2 false negative blood tests became positive when repeated, and one gene test was false negative. The table gives the relation between correct diagnosis and the first breath- and blood-tests and the gene test. The sensitivity and specificity of the breath-, blood-, and gene- test were $100 \%$ and $92 \% ; 71 \%$ and $94 \%$; and $50 \%$ and $100 \%$ respectively. Conclusion: All the tests are suitable for identifying patients with LM, but none of them are perfect. The breath test seems to be best suited for daily practice, but positive tests should be repeated. There were no false negative breath tests, and all false positive tests normalised when the test was repeated.

The relation between the "correct" diagnosis and the first breath- and blood- tests and the gene test (number of patients)

\begin{tabular}{|l|c|c|c|c|c|c|}
\hline & \multicolumn{2}{|c|}{ Breath test } & \multicolumn{2}{c|}{ Blood test } & \multicolumn{2}{c|}{ Gene test } \\
\hline & Positive & Negative & Positive & Negative & Positive & Negative \\
\hline Correct +ve & 7 & 0 & 5 & 2 & 1 & 1 \\
\hline Correct -ve & 4 & 49 & 3 & 50 & 0 & 7 \\
\hline
\end{tabular}

S1130

Sacral Neurostimulation in Fecal Incontinence - Is Clinical Response Associated with Improved Anal Sphincter Pressures Or Anorectal Perception? Heiko Fruehauf, Daniel Pohl, Oliver Goetze, Franc H. Hetzer, Dieter Hahnloser, Werner Schwizer, Michael Fried, Radu Tutuian

BACKGROUND: Sacral Neurostimulation (SNS) is a promising emerging treatment option for patients with fecal incontinence (FI). However, the mode of action is insufficiently understood and the effects on anal sphincter pressures and anorectal perception remain controversial. AIMS: To evaluate the effect of SNS on anorectal sphincter pressures and rectal sensation in patients with FI. METHODS: 15 consecutive patients $(12 \mathrm{~F}$; mean age 64 , range 38-86 years) with idiopathic $(n=2)$, neurogenic $(n=2)$ or structural $(n=11)$ Fl refractory to conservative therapy, underwent anorectal manometry and sensory testing before and 6 months after definitive neurostimulator implantation in our institution. The Wexner score (Dis Colon Rectum 36:77-97: 1993), bowel habit diaries, anal resting pressure (RP), squeeze pressure above RP, initial sensation, urgency to defecate and maximal tolerated volume to balloon distention before and 6 months after neurostimulator implantation were compared using paired t-test. Data are presented as mean \pm SEM. RESULTS: During SNS FI improved in all 15 patients (Table). Six months after SNS implantation we found a decrease $(p<0.001)$ in the Wexner score of $9 \pm 1$ points, a $55 \%$ reduction in urgency episodes $(\mathrm{p}<0.02)$ and a $77 \%$ reduction in uncontrolled bowel movements $(\mathrm{p}=0.087)$ per day. Anal manometry revealed an increase of $14 \pm 4 \mathrm{mmHg}(\mathrm{p}<0.01)$ in the squeeze pressure even though the latter remained below the lower limit of normal in 10/15 patients. SUMMARY: Clinical improvement after SNS implantation is associated with an increase in external anal sphincter pressure, whereas internal sphincter pressures and anorectal perception remain unchanged. CONCLUSION: SNS is an effective treatment option in patients with fecal incontince.

\begin{tabular}{|c|c|c|c|}
\hline Parameter (normal values) & Pre SNS & Post SNS & p-value \\
\hline Wexner score & $14 \pm 1$ & $5 \pm 1$ & $<0.001$ \\
\hline Urgent bowel movements per day & $1.43 \pm 0.40$ & $0.70 \pm 0.29$ & $<0.02$ \\
\hline Uncontrolled bowel movements per day & $0.21 \pm 0.07$ & $0.05 \pm 0.02$ & 0.087 \\
\hline Resting pressure $(>40 \mathrm{~mm} \mathrm{Hg})$ & $35 \pm 5$ & $37 \pm 7$ & 0.413 \\
\hline Squeeze pressure rise $(>50 \mathrm{~mm} \mathrm{Hg})$ & $35 \pm 8$ & $49 \pm 8$ & $<0.01$ \\
\hline Initial sensation $(<30 \mathrm{ml})$ & $45 \pm 8$ & $41 \pm 5$ & 0.427 \\
\hline Urge to defecate $(150 \pm 20 \mathrm{ml})$ & $93 \pm 16$ & $87 \pm 8$ & 0.673 \\
\hline Maximum tolerated volume $(250 \pm 20 \mathrm{ml})$ & $171 \pm 22$ & $153 \pm 18$ & 0.332 \\
\hline
\end{tabular}

\section{S1131}

Familial Aggregation in Constipated Subjects in a Tertiary Referral Center Annie On On Chan, Wai Mo Hui, Wayne Hc Hu, Gigi Leung, Chi Kuen Chan, Man Fung Yuen, Shiu Kum Lam, Benjamin Cy Wong

Background: Clinical observation showed that there is family aggregation in constipated subjects, but formal data are lacking. This prompted us to conduct a formal family study in constipated subjects Methods: Constipated subjects (probands) were identified according to the Rome II and Chinese constipation questionnaire criteria, healthy subjects were chosen as controls. Living first degree relatives (parents, siblings and children) and spouses from both groups were identified. The questionnaire on Rome II criteria was given to the relatives either through the index subjects or by mail. The questionnaire was received by mailing back or through the index subjects. Any non-responders were chased. Results: There were 132 probands with constipation and 114 controls identified. The Rome II questionnaire was sent to a total of 677 relatives of the probands and 591 of the controls. The respond rate was $100 \%$. Relatives were comparable in mean age, sex distribution, family size and marital status in the two groups. Constipation prevalence was $16.4 \%$ in probands' relatives 
versus $9.1 \%$ in controls' relatives ( $p<0.001$ ). Among them, only $6 \%$ vs $9.3 \%$ of the relatives reporting constipation were spouses of the probands and controls $(\mathrm{p}=0.5)$. Probands' relatives were at increase risk to have constipation than controls' (odds ratio 1.86 for at least on member with constipation $((\mathrm{CI}) 1.11,3.14)$, odds ratio 3.54 for two or more member with constipation (CI: 1.71, 7.36)). Conclusions: Familial aggregation of constipation occurs, supporting a genetic or intrafamilial environment component.

\section{S1132}

The Epidemiology of Irritable Bowel Syndrome and Its Relationship with Gastroesophageal Reflux Disease in the General Population: Results from the Loiano-Monghidoro Study

Rocco Maurizio Zagari, Paolo Pozzato, Silvia Damian, Maria Luisa Bianchi, Liza Ceroni, Lorenzo Fuccio, Maria Eugenia Minardi, Mari-Ann Wallander, Saga Johansson, Bahman Farahmand, Enrico Roda, Franco Bazzoli

Purpose: We aimed to study the relationship between irritable bowel syndrome (IBS), gastroesophageal reflux disease (GERD) and esophagitis in the general population. Methods: A representative sample of 1533 adults from two Italian villages was approached between 2000 and 2004 to undergo gastrointestinal symptom assessment and endoscopy (SavaryMiller classification). We recorded the frequency of 18 different gastrointestinal symptoms during the previous 12 months using a validated questionnaire. Based on the Rome Il criteria, IBS was defined as abdominal pain or discomfort associated with at least two of the three following features: abnormal stool frequency; urgency or feeling of incomplete evacuation; relief of abdominal pain by defecation. GERD was defined as reflux symptoms at least monthly with or without esophagitis. Odds ratios (OR) and 95\% confidence intervals (CI) were calculated by unconditional logistic regression models. The odds ratio estimates were adjusted for age and gender and for other variables included in the models. Results: Data were obtained from 1069 individuals representative of the general population (a response rate of $70 \%$ ). The overall prevalence of IBS and GERD was $39.6 \%$ and $45.3 \%$, respectively. Female gender (OR: 2.8; CI: 2.0-3.9) and the presence of dyspepsia (OR: 2.7; CI: 2.0-3.7) increased the risk of IBS. The risk of IBS was also significantly increased in individuals with GERD. This association between IBS and GERD was stronger in individuals who had GERD without esophagitis (OR: 2.5; CI: 1.8-3.4) than in those who had GERD with esophagitis (OR: 1.8; CI: 1.1-2.8). Individuals with both IBS and GERD were more likely to be younger, have a higher body mass index, and have a prior history of peptic ulcer disease than those with IBS but no GERD. In the subgroup of individuals with IBS and GERD, those with esophagitis were more likely to have a hiatus hernia (OR: 3.8; CI: 1.6-9.0), but less likely to have dyspeptic symptoms (OR: 0.04: CI: 0.01-0.12), than those without. Conclusion: Our results show an association between IBS and GERD, especially in young, overweight individuals with a prior history of peptic ulcer disease. The association between IBS and GERD was stronger in individuals with GERD who did not have esophagitis than in those who had esophagitis. An increased awareness of the comorbid occurrence of different gastrointestinal conditions may result in better diagnosis and treatment for individuals affected by both IBS and GERD.

\section{S1133}

The Community Prevalence of Abdominal Pain and Cramps: A Multi-National Survey

Guido N. Tytgat, Luiz G. Paulo, Stefan Mueller-Lissner, Eamonn M. Quigley, Richard G. Locke, Eckhard Schaefer, Iris Helfrich

Background: Abdominal pain and cramps are common symptoms of functional disorders of the gastrointestinal tract. These ailments are widespread in the general population in various countries worldwide. Aim: To describe the demographics, clinical features and management of abdominal pain and cramps in the community. Methods: Interviews were carried out in Argentina, Belgium, Brazil, Germany, Italy, Japan, Mexico, UK, and USA in two phases. In the first phase approximately 1,000 people in each country were randomly selected from the general population. The second phase consisted of interviews with a subsample of approximately 200 subjects in each country. Acid-related complaints and menstrual pain were excluded. Data were analysed using descriptive statistics and logistic regression. Results: In the first phase, 10,005 respondents (55.5\% women) were interviewed. Females were more likely to suffer from abdominal pain and cramps than males (OR: 1.25, C.I. 1.21-1.30). Those aged less than 60 years were also more likely to complain of these symptoms (OR: 1.40, C.I. 1.27-1.53). South American countries reported the highest prevalence of symptoms (39.9\%; OR: 2.32, C.I. 2.13-2.53) and Japan (10.5\%) the lowest. A total of 1,717 subjects ( $65 \%$ females) were included in the second phase, carried out in all countries except Japan. The frequency of episodes of abdominal cramps and pain differed from country to country, being by far the highest in the US, followed by Brazil, Belgium and the UK. The average age at onset of symptoms was similar in all countries (27-31 years). Frequency of drug usage was highest in the US and lowest in Germany and Italy. In most countries, the intensity of different episodes was considered similar. The incidence of consultation of a physician for these symptoms varied considerably across countries, from $74 \%$ in Italy to less than 50\% in Mexico and the UK. The use of medication was higher in the US and in Latin America (87-91\% of all sufferers) than in Europe (68-77\%). With the exception of Mexico, OTC products dominated. Antispasmodics were most popular in Latin America and Italy, and antacids in Germany and the UK. Conclusions: Although prevalence and severity of abdominal symptoms seemed similar and common in the general population especially among young to middle-aged females throughout the world, the rates of health care seeking and medication usage were quite different between the countries. These findings have important implications for multi-national studies of functional disorders. This research was funded by Boehringer Ingelheim, Ingelheim am Rhein, Germany.

\section{S113}

Provider Beliefs Regarding Diagnostic Testing in IBS Exhibit Extreme Variation and Diverge from Guidelines

Brennan M. Spiegel, Mary Farid, Eric Esrailian, Jennifer Talley, Lin Chang

Background: Data indicate that diagnostic testing is generally low yield in IBS, yet many clinicians remain unsettled by the prospect of overlooking alternative diagnoses. We measured provider beliefs about the appropriateness and yield of common tests in IBS. Methods: We developed an online vignette survey that elicited provider beliefs about the appropriateness (using 9-point RAND Appropriateness Scale [RAS], where 1-3=inappropriate, 4-6=unsure, $7-9=$ appropriate) and diagnostic yield of common tests in IBS. We calculated the RAND Disagreement Index (DI) for each set of ratings. The DI is a validated measure of provider variation, where $\mathrm{DI}>1.0=$ extreme variation, and $\mathrm{DI}<1.0=$ acceptable variation. We surveyed a random sample of 300 VA NPs, 300 VA PCPs, 300 GIs from the AGA membership, and 45 recognized IBS experts. Results: The response rate was 31\% (102 NPs, 89 PCPs, 90 GIs, 27 experts). Table 1 shows the RAS and DI results of the IBS-D vignette. Experts only rated celiac sprue testing and $\mathrm{CBC}$ as appropriate (mean RAS>6), and breath testing as inappropriate. Non-experts rated most tests as appropriate. Experts were consistent in their disfavor of breath testing and favor of $\mathrm{CBC}$ and sprue testing $(\mathrm{DI}<1.0)$, but exhibited extreme variation for all other tests. Non-experts revealed a mix of extreme and acceptable variation across tests. Compared to published data, $45 \%$ of experts vs. $69 \%$ of non-experts overestimated the yield of diagnostic testing $(\mathrm{p}<0.004)$. There were similar results in the IBS-C vignette (data not shown). Conclusions: Despite the dissemination of guidelines regarding diagnostic testing in IBS, there remains extreme variation in beliefs among both experts and non-experts. Compared to experts, non-experts consistently over-estimate the appropriateness and yield of testing. This suggests that better dissemination of IBS diagnostic guidelines is warranted in order to minimize extreme variation and improve cost-effectiveness of care. Table RAS and DI Results. Mean RAS $>6=$ "Appropriate," and DI>1="Extreme Variation."

\begin{tabular}{|c|c|c|c|c|}
\hline & Mean Expert RAS & Expert DI & Mean Non-Expert RAS & Non-Expert DI \\
\hline CBC & 7.4 & -1.4 & 7.5 & -0.9 \\
\hline Celiac Sprue Ab & 6.8 & -3.1 & 4.8 & 1.7 \\
\hline ESR & 5.4 & 4.1 & 5.4 & 1.8 \\
\hline Chem Panel & 4.7 & 1.1 & 6.9 & -6.2 \\
\hline TSH & 4.8 & 1.8 & 6.2 & -12 \\
\hline Stool WBC & 4.8 & 1.8 & 6.6 & -6.2 \\
\hline Colonoscopy & 4.7 & 1.6 & 5.9 & 7.0 \\
\hline O\&P & 4.6 & 1.6 & 6.6 & -6.2 \\
\hline C\&S & 4.1 & 1.6 & 6.2 & 1.6 \\
\hline Flexsig & 3.8 & 1.1 & 5.1 & 1.7 \\
\hline Breath test & 2.3 & 0.5 & 4.1 & 1.0 \\
\hline
\end{tabular}

S1135

Does the Use of Complementary and Alternative Medicines (CAM) Predict a Non-Organic Gastrointestinal Disorder?

Shital Amin, Subhadip Ghosh-Ray, Niall van Someren, Kalpesh Besherdas, Steven Mann

Background: Many studies have highlighted the increase in use of CAM in adults in the developed world. Adults with gastroenterological complaints are also known to be frequent users of CAM. Reports suggest that up to $50 \%$ of patients with IBS or inflammatory bowel disease use some form of CAM. Aim: To review the use of CAM in consecutive new patients seen in a general gastroenterology outpatient setting in one National Health Service Hospital Trust. Method: Consecutive new patients referred to our clinics (NVS, KB, SM) completed a questionnaire in the waiting room before the consultation. The questionnaire was in the form of a tick box enquiry looking at demographic data, use of CAM (15 options) and symptoms at presentation (19 options). Data were collected prospectively and re-analysed after appropriate diagnostic work-up. Patients were divided into two groups according to the final diagnosis: organic $(\mathrm{O})$ or non-organic $(\mathrm{NO})$ disease (functional dyspepsia, functional abdominal pain, or functional colonic pain). Non-parametric analysis was used to evaluate any correlations. Results: 154 patients (F:78 [51\%]) completed the questionnaire. The distribution of diagnoses was O:75/154 (49\%; F:43 [57\%]), NO:79/154 (51\%; F:35 [44\%]). 22 patients had used CAM (14\%; F:9 [63\%]). The distribution of diagnoses amongst CAM users was O:10/22 (45\%; F:3 [30\%]), NO:12/22 (55\%; F: 6 [50\%]). Use of CAM was not associated with a diagnosis of non-organic disease $(\mathrm{p}=0.67)$. The age distribution of CAM users was $15-29$ years: $13.6 \%, 30-49$ years: $32 \%, 50-69$ years: $41 \%, 70+$ years: $13.6 \%$. The commonest therapies used were herbal or dietary supplements. $50 \%$ of CAM users reported a 'modest' or 'significant' improvement in symptoms. Conclusion: There is a perception in gastroenterology that most CAM users will have a non-organic underlying disorder. Our data has failed to support this notion. CAM users are a heterogeneous group, with an equal distribution of organic and non-organic conditions represented.

\section{S1136}

Making a Diagnosis of Gastroparesis Reduces Diagnostic Cost and Unnecessary Surgeries in Patients with Recurrent Nausea and Vomiting Chadwick W. Hatfield, William D. Chey, David Barthel, Jack Semler, Steve Harrell, John Wo

Gastroparesis (GP) is a disorder defined by delayed gastric emptying. Symptoms of GP are non-specific and the diagnosis is often overlooked. Extensive medical resources may be spent for its diagnostic workup. AIM: To determine the extent of medical utilization in diagnosing GP in subjects with recurrent nausea and vomiting. METHODS: Potential subjects 
were identified at University of Louisville and University of Michigan from Jan 2001 through Dec 2003 using ICD billing codes for GP, miscellaneous gastric, nausea, vomiting, and nausea with vomiting. Outpatient records were reviewed in chronological order. Study criteria were nausea and vomiting $\geq 3$ months and documented gastroparesis by delayed gastric scintigraphy or residual solid food by EGD despite overnight fast. Those $<18$ years old and non-ambulatory individuals were excluded. Diagnostic tests were separated into 3 categories: endoscopic, radiographic (including scintigraphy), and motility. Type and number of each diagnostic test and surgical procedure was obtained before and after making the diagnosis of GP. Cost of diagnosis was calculated using Medicare 2005 physician and hospital outpatient payment schedule for Kentucky and Michigan. Mann-Whitney U, Wilcoxon signed-rank and paired McNemar's tests were utilized. RESULTS: 240 charts were reviewed. 141 subjects (median age $46 \mathrm{yrs}, 79 \%$ female) met study criteria. Results are shown in table. There was a high variability in total diagnostic cost among the subjects. Gender, age, and etiology of GP did not significantly affect the total diagnostic cost. Cost associated with endoscopies was significantly greater than radiographic and motility testing $(\mathrm{p}<0.01)$. Total diagnostic cost decreased significantly after the diagnosis of GP was made. Prior to diagnosis of GP, 56 subjects (40\%) had cholecystectomy, compared to only 1 of the remaining 85 subjects (1\%) after diagnosis. 23 subjects (16\%) had laparoscopy/laparotomy before diagnosis of GP, compared to 3 subjects (2\%) after diagnosis. CONCLUSIONS: Diagnostic cost of GP in patients with chronic nausea and vomiting was quite variable. Establishing a diagnosis of GP significantly reduced the cost associated with testing and number of unnecessary surgeries unrelated to GP.

\begin{tabular}{|c|c|c|c|c|}
\hline \multirow[b]{2}{*}{$\begin{array}{l}\text { Diagnostic } \\
\text { category }\end{array}$} & \multicolumn{2}{|c|}{ Before diagnosis of gastroparesis } & \multicolumn{2}{|c|}{ After diagnosis of gastroparesis } \\
\hline & $\begin{array}{c}\# \text { of } \\
\text { tests/subject } \neq\end{array}$ & Cost/subject $\dagger$ & $\begin{array}{c}\# \text { of } \\
\text { tests/subject } \neq\end{array}$ & Cost/subject $\dagger$ \\
\hline Endoscopic & $1(0-9)$ & $\$ 645(\$ 0-\$ 5,234)$ & $1(0-10)$ & $\$ 550(\$ 0-\$ 5,974)$ \\
\hline Radiographic & $2(0-15)$ & $\$ 492(\$ 0-\$ 6,090)$ & $1(0-20)$ & $\$ 246(\$ 0-\$ 2,973)$ \\
\hline Motility & $0(0-4)$ & $\$ 0(\$ 0-\$ 998)$ & $0(0-3)$ & $\$ 0(\$ 0-\$ 997)$ \\
\hline Total & $4(1-21)$ & $\begin{array}{c}* \$ 1,581 \\
(\$ 246 \$ 8,480)\end{array}$ & $2(0-23)$ & $\begin{array}{c}* \$ 1,035 \\
(\$ 0-\$ 6,824)\end{array}$ \\
\hline
\end{tabular}

$\dagger$ Median (range); * $\mathrm{p}<0.01$

\section{S1137}

Quality of Life Survey in Patients with Functional Gastrointestinal Disorders: An Asian Study

Daphne Ang, Hock-Foong Lui, Widjaja Luman

Aim: Health-related quality of life (HRQoL) is an increasingly important outcome in patient care. Data on $\mathrm{HRQOL}$ in Asians with functional gastrointestinal disorders are lacking. Functional dyspepsia (FD) and irritable bowel syndrome (IBS) are commonly encountered functional gastrointestinal disorders. This study quantified and compared the impact of FD and IBS on HRQoL in subjects seen at a tertiary referral hospital; and compared patients' scores against previously published normative United States(US) data, using the well validated, generic Medical Outcomes Study 36-item Short-Form Health Survey (SF-36)form. Methods: 185 patients seen at a tertiary referral centre in Singapore who fulfilled Rome II criteria for FD or IBS were invited to participate. Patients completed a baseline questionnaire at clinic visits that included demographics; duration and severity of symptoms and the SF36 questionnaire forms. The mean scores for each of the 8 domains of the SF- 36 questionnaire for the FD group were compared against the IBS group; scores for both groups were compared against previously published US data of normal controls. Unpaired student's t-test was used for statistical analysis. Results:Completed questionnaire forms were obtained in $62 \mathrm{FD}$ patients (45 females; mean age $34.2+/-8.5$ years; Chinese $83.9 \%$ )and 42 IBS patients (26 females; mean age 38.0 +/- 11.2 years; Chinese $83.3 \%$ ). Patient demographics; symptom duration and severity did not differ between the 2 groups. Mean scores (standard deviation $\mathrm{SD}$ in parentheses) are presented in Table 1. No significant differences in mean scores for all 8 individual SF-36 domains occurred between FD and IBS groups. Differences between FD group and US norms were significant in all domains $(\mathrm{p}<0.05)$ except for physical functioning and role function (emotional). Differences between IBS group and US norms were significant for all domains ( $\mathrm{p}<0.05)$ except role function (physical) and vitality. Conclusion: The baseline HRQoL in both FD and IBS groups were significantly lower in most domains compared to US norms. Both FD and IBS showed similar reduction in HRQOL in this Asian study. Table 1

\begin{tabular}{|c|c|c|c|}
\hline SF36 & FD & IBS & US controls \\
\hline Physical Functioning & $80.2(23.3)$ & $76.3(25.4)$ & $84.2(23.3)$ \\
\hline Social Function & $71.5(23.2)$ & $75.9(22.9)$ & $83.3(22.7)$ \\
\hline Role Function (physical problem) & $60.9(42.9)$ & $71.4(36.4)$ & $81.0(34.0)$ \\
\hline Role Function (emotional problem) & $73.7(48.0)$ & $66.7(43.6)$ & $81.3(33.0)$ \\
\hline Mental health & $62.6(18.0)$ & $64.2(17.9)$ & $74.7(18.1)$ \\
\hline Vitality & $50.7(18.4)$ & $55.1(19.5)$ & $60.9(21.0)$ \\
\hline Pain & $56.5(23.2)$ & $60.8(21.0)$ & $75.2(23.7)$ \\
\hline General Health Perceptions & $53.7(19.2)$ & $53.5(16.9)$ & $72.0(20.3)$ \\
\hline
\end{tabular}

\section{S1138}

The Impact of Sleep Disruption On Quality of Life (QOL) in IBD \& IBS Laurie Keefer, Ali Keshavarzian, Ranjbaran Ziba, Laura Benson, Edward J. Stepanski

Chronic sleep disruption is a risk factor for disease \& linked to inflammation \& altered immune function. Sleep c/o are common in IBS; less is known about sleep in IBD. We examined sleep patterns in IBD \& IBS \& relations w/QOL. Meth: 16 biopsy-proven inactive IBD (8CD ,8UC), 9 IBS \& 7 controls (C) completed the Pittsburgh Sleep Quality Index (PSQI) Epworth Sleepiness Scale (ESS), IBDQ, SF-12 \& overnight polysomnography (PSG). Mean ages of IBD,IBS \& $C$ were 41.4(13.3), 52.7(12.1) \& 34.3(9.4). PSGs were scored manually in 30 s epochs w/standard criteria. One-way ANOVAs w/post-hoc tests were performed for $\mathrm{b} / \mathrm{w}$ group comparisons; linear regression identified assoc b/w sleep \& QOL. Res: IBS \& IBD groups did not differ from each other on subjective parameters but differed from $\mathrm{C}$ on impaired sleep quality, duration, efficiency, disruption $\&$ global PSQI scores. IBS reported the most sleep disruption, followed by IBD, followed by C (Table 1). IBS \& IBD did not differ on objective sleep parameters but both differed from C; IBS exhibited less total sleep time and reduced sleep efficiency. IBD fell numerically between IBS \& $C$ on most parameters (Table 2). PSQI global linked to IBDQ $(\mathrm{R} 2=.63, \mathrm{p}=.00)$, SF12-physical $(\mathrm{R} 2=.25, \mathrm{p}=$ $.01) \&$ mental health $(\mathrm{R} 2=.43, \mathrm{p}=.00)$. IBD \& IBS share similar sleep $c / 0$, objectively \& subjectively; c/o are linked to QOL. IBS reported the most disturbance followed by IBD and C. CNS hyperarousal \& cognitive attributions may contribute to sleep c/o in IBS; sleep may affect IBD in additional ways ie, immuno-inflammatory pathways or increased risk to flare.

\begin{tabular}{|c|c|c|c|c|c|}
\hline Quest. & IBD & IBS & $\mathrm{C}$ & $\mathrm{F}(2,26)$ & $\mathrm{p}$ \\
\hline Qual & $1.36(.63) \mathrm{a}$ & $1.88(.84) \mathrm{b}$ & $.29(49) \mathrm{ab}$ & 11.05 & $.00^{*}$ \\
\hline Latency & $1.29(1.07)$ & $1.63(1.30)$ & $.57(1.13)$ & 1.63 & .22 \\
\hline Duration & $1.14(.77)$ & $1.75(.71) \mathrm{b}$ & $.57(.53) \mathrm{b}$ & 5.24 & $.01^{*}$ \\
\hline Effic. & $.79(1.12)$ & $1.50(1.20) \mathrm{b}$ & $0(0) \mathrm{b}$ & 4.14 & $.03^{*}$ \\
\hline Disrupt & $1.36(.63)$ & $2.00(.76) \mathrm{b}$ & $.86(.69) \mathrm{b}$ & 5.35 & $.01^{*}$ \\
\hline Med Use & $1.21(1.25)$ & $1.63(1.41)$ & $.14(.38)$ & 3.25 & .06 \\
\hline Day Impair & $1.86(1.40)$ & $2.88(2.17)$ & $1.14(1.21)$ & 2.22 & .13 \\
\hline PSQI Global & $9.00(4.84) \mathrm{a}$ & $13.25(4.83) \mathrm{b}$ & $6.71(2.23) \mathrm{ab}$ & 9.05 & $.00^{*}$ \\
\hline ESS & $8.09(5.17)$ & $10.00(6.7)$ & $6.71(2.23)$ & .70 & .51 \\
\hline Naps/mo & $5.71(8.2)$ & $2.25(2.87)$ & $0(0)$ & 2.30 & .12 \\
\hline
\end{tabular}

if share letter $\mathrm{p}<.05$

\begin{tabular}{|c|c|c|c|c|c|}
\hline PSG & IBD & IBS & C & F(2, 26) & $p$ \\
\hline S1 Time & $418.55(55.67)$ & $365.65(66.44) \mathrm{a}$ & $464.18(19.26) \mathrm{a}$ & 5.49 & $.01 *$ \\
\hline Effic & $84.44(5.06)$ & $78.0(13.43) \mathrm{a}$ & $92.76(4.73) \mathrm{a}$ & 5.05 & $.01 *$ \\
\hline Onset Lat. & $20.86(17.99)$ & $20.34(29.20)$ & $18.80(29.77)$ & .02 & .99 \\
\hline$\% \mathrm{~S} 1$ & $12.38(5.21)$ & $14.03(9.14)$ & $6.46(5.11)$ & 2.22 & .13 \\
\hline$\% \mathrm{~S} 2$ & $50.32(10.34)$ & $48.93(12.32)$ & $53.48(3.71)$ & .31 & .74 \\
\hline$\%$ SWS & $16.54(8.41)$ & $15.60(8.51)$ & $14.80(8.87)$ & .09 & .91 \\
\hline$\%$ REM & $20.73(5.24)$ & $21.43(7.27)$ & $25.24(2.36)$ & 1.23 & .30 \\
\hline REM Lat. & $125.50(61.52)$ & $115.65(58.36)$ & $105.30(30.90)$ & .26 & .77 \\
\hline ArsIIndex & $17.89(12.10)$ & $18.73(10.56)$ & $8.70(3.55)$ & 1.29 & .29 \\
\hline
\end{tabular}

if share letter $p<.05$

S1139

Targeted Esophageal Biopsies: What You See Is What You Get (WYSIWYG) Or Not?

Robert H. Riddell, Vijay Mann, Paul Moayyedi, Sjo Veldhuyzen van Zanten, Janusz Jankowski, Marc Bradette, Jacob Louw, Garth Noad, Robert J. White, David Armstrong

Background: Evaluation of esophageal epithelial histology for features of gastroesophageal reflux disease (GERD) has shown regional variations related to the distance from the Z-line, anterior or posterior location and proximity to mucosal breaks (MB). Discrepancies between histological and endoscopic findings for MB and Barrett's esophagus (BE) have been attributed to deficits in endoscopic recognition of lesions but discrepancies may also indicate the difficulty of acquiring targeted biopsies in the esophagus. If the latter is true, the accuracy 
of biopsies from the Z-line (readily recognized at endoscopy) and MB should be equivalent. Aim: To assess the accuracy of biopsy acquisition from the esophagus in GERD patients. Methods: An ongoing 6-month, open-label, multicentre trial planned to recruit patients from tertiary care with heartburn and erosive esophagitis (EE: $n=50$ ), nonerosive reflux disease (NERD: $\mathrm{n}=150$ ) and heartburn non-dominant dyspepsia (HND: n= 150). At baseline endoscopy, biopsies were obtained from 7 sites, including 1 each from the Z-line anterior \& posterior locations, and, in EE patients, from the edge of an MB. Histological assessment evaluated the accuracy of acquisition of biopsies by determining the proportions of (a) Z line biopsies that contained both columnar mucosa $(\mathrm{CM})$ and squamous epithelium (SE) and (b) MB biopsies that showed features of an erosion or MB. Results: To date, 145 patients have been enrolled - EE: 50, NERD: 50, HND: 45. Evaluable Z-line biopsies are available from 145 patents ( 290 biopsies) and MB biopsies from 49 patients ( 49 biopsies). In 26/145 patients (18\%), both Z-line biopsies showed both CM and SE and in another $45(31 \%)$, one Z-line biopsy showed CM and SE; overall, 97/290 (33\%) of biopsies were acquired accurately from the Z-line. In 3/49 (6\%) patients, histology showed fibrinopurulent exudates consistent with an erosion and in another $12(24 \%)$, there were features of regeneration overall, 15/49 (31\%) were acquired accurately from an MB. Conclusions: "WYSINotWYG" Although the accuracy of biopsy acquisition from the Z-line and MB was comparable, acquisition of targeted biopsies in the esophagus was accurate in only about one-third of cases; this appears to be related to technical difficulties in targeting specific lesions rather than failure to recognize the lesions although a true mismatch between the endoscopic and histological features or mucosal breaks cannot be excluded. These results have implications for the conduct of clinical studies, for accurate biopsy acquisition from the lower esophagus and cardia, for endoscopic surveillance in patients with BE and, possibly, for endoscopy training

\section{S1140}

The Clinical Impact of Prolonged (48 and 96-hour) Oesophageal $\mathrm{pH}$ Monitoring By the BRAVO ${ }^{\mathrm{TM}}$ System

Mark Fox, Amir Thakur, Roy Anggiansah, Angela Anggiansah, Terry Wong

Introduction \& Aims: The day-to-day variability of $\mathrm{pH}$ recordings is high; thus prolonging the duration of $\mathrm{pH}$ studies is likely to improve the reliability and diagnostic yield of the investigation. The optimal duration of $\mathrm{pH}$ monitoring is unknown. The catheter-free $\mathrm{Bravo}^{\mathrm{TM}}$ system is designed to record intra-oesophageal pH over 48 hours; however the capsule remains in place for an average 5-6 days. This provides an opportunity to examine variability in $\mathrm{pH}$ recordings over extended periods, and the impact of prolonged recordings on the diagnostic yield of gastro-oesophageal reflux disease (GORD). Methods: 56 consecutive patients (22M:34F; 48 (35-61) years) with reflux symptoms referred for $\mathrm{pH}$ monitoring were studied. 43 patients were monitored for 2 days and 13 patients over 4 days (returning after 48-hrs to download $\mathrm{pH}$ data and replace batteries). The day-to-day variability of $\mathrm{pH}$ measurements was assessed. Patients were classified with normal or abnormal acid exposure $(>4.2 \% \mathrm{pH}<4 / 24 \mathrm{hr}$ ) during each test period. The increase in diagnostic yield for GORD by prolonging $\mathrm{pH}$ recording from 24 to 48 and 96 -hours was calculated. Kappa (k) values assess diagnostic agreement between test periods. Results: Two-day recordings were available for $47 / 56$ patients (incomplete recording $(\mathrm{n}=8)$, early detachment $(\mathrm{n}=1)$ ). Four-day $\mathrm{pH}$ studies were available for $12 / 13$ patients (day 3 detachment $(n=1)$ ). There was no difference in mean acid exposure day $1-4(8.2 \%, 7.8 \%, 7.9 \%, 7.3 \%$ respectively, $\mathrm{p}=0.61)$; however within patient day-to-day variability was high, $\pm 30 \%$ relative to the mean. Pathological acid exposure was present in 29/46 day 1 and 27/46 day 2 (12 patients changed diagnostic classification). Cumulative diagnostic yield increased from 29/46 (48\%) day 1 to $34 / 46$ (74\%) day 2. Diagnostic reproducibility of $24-\mathrm{hr} \mathrm{pH}$ recording was fair $(\mathrm{k}=0.45)$. Within patient variability between 48 -hr test periods was lower than that for 24-hr test periods $\pm 16 \%$ vs. $\pm 30 \%$ relative to the mean $(\mathrm{p}<0.01)$. Only $1 / 12$ patients changed diagnostic classification from the first to the second 48-hr test period. Diagnostic reproducibility of 48 -hr $\mathrm{pH}$ recording was very good $(\mathrm{k}=0.76)$. Similar results were obtained for alternative diagnostic 'pH values' for GORD and the DeMeester score. Conclusions: Increasing the duration of $\mathrm{pH}$ monitoring from 24- to 48-hrs significantly improves the reliability of clinical measurement and raises the diagnostic yield of GORD by $25 \%$. Preliminary evidence suggest that prolonging $\mathrm{pH}$ studies to 96 -hrs provides little further improvement in diagnostic yield 48-hr pH monitoring provides important advantages for the investigation of reflux symptoms in clinical practice.

\section{S1141}

Multichannel Intraluminal Impedance and pH (MII-pH) Is Accurate in Assessing Esophageal Transit Abnormalities

Amine Hila, Amit Agrawal, Wojciech Blonski, Inder Mainie, Janice Freeman, Donald O. Castell

BACKGROUND: 24-hr MII-pH esophageal monitoring allows detection of both acid and non-acid gastroesophageal reflux (GER) episodes. The MII-pH catheter is $2 \mathrm{~mm}$ in diameter and contains 6 impedance segments placed 3, 5, 7, 9, 15 and $17 \mathrm{~cm}$ above LES, plus a pH electrode at $5 \mathrm{~cm}$. MII and esophageal manometry (MII-EM) allows both functional and manometric evaluation of the esophagus. MII-EM catheter is $4.5 \mathrm{~mm}$ in diameter and contains 4 impedance segments $(5,10,15$ and $20 \mathrm{~cm}$ above LES) and 5 pressure transducers (at LES and $5,10,15$ and $20 \mathrm{~cm}$ above LES). $5 \mathrm{cc}$ saline boluses are given in the recumbent position when performing an MII-EM study. In our laboratory, all patients have an MII-EM study performed prior to MII-pH study. However, this is not true everywhere. AIM: To assess accuracy of swallows given prior to ambulatory MII-pH monitoring in detecting esophageal transit abnormalities. METHODS: 37 consecutive patients ( 27 females; mean age=53yrs) presenting for MII-EM and 24-hr MII-pH study, also received 10 saline swallows in the recumbent position at the beginning of the MII-pH study. Impedance for these swallows was assessed for the presence of complete or incomplete transit, defined as bolus entry at $17 \mathrm{~cm}$ and bolus exit at 15,9 and $5 \mathrm{~cm}$, defined by a rise in impedance above $50 \%$ between baseline and the nadir, and remaining above this value for at least 5 seconds. Out of 10 swallows, presence of 8 or more complete swallows satisfies MII-EM criteria for diagnosis of complete transit. These findings were blindly compared to those of the 10 saline swallows on the MII-EM study. RESULTS: The MII-pH swallows gave identical transit diagnosis to the MII-EM in 35 patients (95\%). In 1 patient, diagnosis changed from incomplete transit ( 7 complete swallows) on MII-EM to complete ( 8 complete swallows) on MII-pH, and from complete transit ( 10 complete swallows) on MII-EM to incomplete ( 7 complete swallows) on MII-pH in the other patient. With both methods there were 27 patients with complete transit and 10 with incomplete transit. Thus, MII-pH swallows had a sensitivity of $90 \%$ and a specificity of $97 \%$ for detection of esophageal transit abnormalities. Comparing swallow diagnosis for each patient, we found that there was a highly significant correlation $(\mathrm{p}<0.0001)$ for the number swallows with of complete (Pearson $\mathrm{r}=0.9$ ) and incomplete bolus transits $(\mathrm{r}=0.9)$. CONCLUSION: Use of 10 saline swallows at the beginning of MII-pH studies is both accurate and highly sensitive and specific for detection of esophageal transit abnormalities. This may provide a method for assessment of esophageal function when an MII-EM study is not planned prior to MII-pH monitoring.

\section{S1142}

The Diagnostic Value of the Proton Pump Inhibitor Test for Gastroesophageal Reflux Disease: A Population-Based Study

Marissa C. Aanen, Bas L. Weusten, Mattijs E. Numans, Niek J. de Wit, Andre J. Smout

Introduction General practice guidelines often suggest as diagnostic tool for GERD the symptomatic response to a short course of proton pump inhibitor (PPI test). Our aim was to re-determine the diagnostic abilities of the PPI test by using the most relevant reference test, the symptom association probability (SAP), in a primary care population. Methods Subjects suspected of GERD who had heartburn complaints for at least twice a week during at least 3 months were recruited from primary care, either during consultation or by advertisement. After a $24 \mathrm{~h} \mathrm{pH}$ recording with calculation of the SAP, subjects started using $40 \mathrm{mg}$ esomeprazole once daily for 13 days. The PPI test was considered positive when the subjects reported that their symptoms were adequately suppressed. Sensitivity, specificity and positive likelihood ratios (LR) were determined daily for each PPI test day. Results A total of 90 subjects were included (1/3 consultation, 2/3 advertisement). Successful $24 \mathrm{~h} \mathrm{pH}$ recording was accomplished in 84 subjects and the SAP was calculable in 74 . The median age was $51 \mathrm{yr}$ (IQR $41-62$ ), and $62 \%$ was male. The SAP was positive in $70 \%$ of the subjects. The sensitivity of the PPI test was $92.2 \%(87.4-93.5)$ and the specificity was $28.6 \%$ (19-33.3). The LR was low (1.3 (1.2-1.4)) and fairly constant during the 13 days (figure) Conclusion The sensitivity of the PPI test for diagnosing GERD is high, but its specificity low. The low LRs observed from day 1 to day 13 indicate that the PPI test does not change the high pre-test probability (70\%) of GERD found in primary care patients with heartburn. Thus the additional value of short term treatment with a PPI for diagnosing GERD in primary care is relatively poor even with the most adequate reference test SAP.

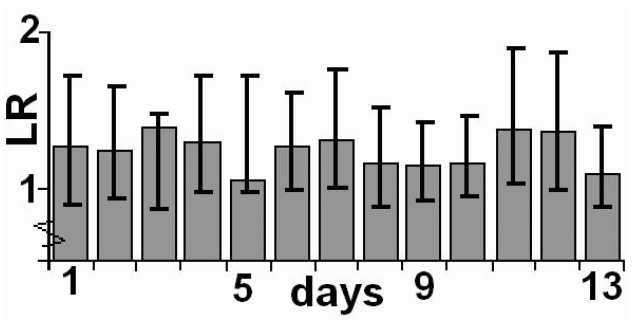

S1143

Non Acid Reflux in Children By Long Term pH-Impedance Study, - An Analysis of Practice Based Data

Sudipta Misra, Zahangir Khaled, Amy Lee

Introduction: Due to lack of control population, no pediatric norms are available for nonacid refluxes detected by Multichannel intraluminal impedance (MII). We present data from our practice. Method: Prospective data collection, automated and manual analysis of study data in children undergoing MII (Sandhill Scientific, USA) for clinical indications. The $\mathrm{pH}$ port of the impedance catheter was placed, under fluoroscopy, at 3 and $5 \mathrm{~cm}$ above the diaphragm in young $(<2$ years) and older children respectively. Informed consent was obtained from the caregivers. All frequency data were normalized for occurence/24 hours. Mann-Whitney U and Aspin Welch tests, percentiles and correlations were used for data analysis. Results: - Three of the 142 children (Table) in the study were excluded from acid reflux analysis for probe failure. Percentile norms of the refluxes were calculated (Table). Numbers of liquid, mixed and gas MII refluxes and numbers of those of respective refluxes migrating to the proximal esophagus (PM) had excellent correlations $(r=0.81$ to $0.93, r 2=$ 0.79 to 0.86 ). Good correlation was found between number of acid reflux episodes and percentage of acid reflux $(r=0.74, r 2=0.55)$. No correlation could be established between non-acid refluxes (total and PM) and acid reflux indices. 2-18 years group had fewer incidences of some non-acid refluxes (Table), less episodes of long $\mathrm{pH}$ refluxes than the other two groups $(\mathrm{p}<.05)$ and less number of liquid (total and PM) refluxes on antacids. Though children on antacids ( $\mathrm{n}=82$ ) had less liquid refluxes, their acid reflux indices did not differ from those who were not $(\mathrm{n}=57)$. Conclusion: Older children had fewer non-acid refluxes with or without antacids, Number of non-acid refluxes migrating to the proximal esophagus appears to be a function of total number of respective types of refluxes. Acid reflux indices were independent of numbers of non-acid refluxes, suggesting a possible role of $\mathrm{pH}$ only (without detectable refluxate in the esophagus) refluxes in acid exposure. Non Acid Reflux in Children (Episodes/ 24 hours) 


\begin{tabular}{|c|c|c|c|c|c|c|}
\hline & \multicolumn{2}{|c|}{$<6 \mathrm{MO}(\mathrm{n}=51)$} & \multicolumn{2}{c|}{6 Mo-2 Yrs $(\mathrm{n}=31)$} & \multicolumn{2}{|c|}{$2-18$ Yrs $(\mathrm{n}=60)$} \\
\hline & $\begin{array}{c}50 \text { th centile } \\
(95 \% \mathrm{Cl})\end{array}$ & $\begin{array}{c}\mathrm{iQ} \\
\text { range }\end{array}$ & $\begin{array}{c}50 \text { th centile } \\
(95 \% \mathrm{CI})\end{array}$ & $\begin{array}{c}\text { QQ } \\
\text { range }\end{array}$ & $\begin{array}{c}50 \text { th centile } \\
(95 \% \mathrm{Cl})\end{array}$ & $\begin{array}{c}\text { IQ } \\
\text { range }\end{array}$ \\
\hline Liquid & $38.6+(26.3-47.1)$ & 46.1 & $28.2(17.3-33)$ & 28.9 & $24.5+(14.6-31)$ & 24.3 \\
\hline Mixed & $14(7.7-22.2)$ & 20.5 & $28.2 *(12.6-30.9)$ & 23.6 & $10.4 *(8-12.9)$ & 15.4 \\
\hline Gaseous & $20.1(11.7-28.3)$ & 36.2 & $16.2(7.9-22.1)$ & 18.5 & $13.7(9-17.9)$ & 18.8 \\
\hline $\begin{array}{c}\text { Liquid with } \\
\text { Prox Migr }\end{array}$ & $19.5 @(11.5-31.8)$ & 34.9 & $16.7 * *(11.9-24)$ & 17.2 & $10.7 * * / @(6.5-15.8$ & 16.9 \\
\hline $\begin{array}{c}\text { Mixed with } \\
\text { Prox Migr }\end{array}$ & $8.9(4.5-14.5)$ & 13.8 & $18.2++(6.6-20.3)$ & 16.7 & $4.3++(2.7-7.9)$ & 11.6 \\
\hline $\begin{array}{c}\text { Gas with } \\
\text { Prox Migr }\end{array}$ & $11.1(7.1-19.5)$ & 28 & $9.9(5.1-16)$ & 14.7 & $8.3(5.6-11.6)$ & 17.2 \\
\hline
\end{tabular}

still be improved by considering also meal time and acid exposure on just the most distal channel. Gas reflux episodes are common in pediatric patients although their clinical significance need to be further clarified. Symptom correlation with reflux episodes highly benefits from MII-pH analysis.

\section{S1146}

Symptoms of Both Acid and Non-Acid Reflux Occur Almost Exclusively in the Upright Position

Amit Agrawal, Amine Hila, Wojciech Blonski, Radu Tutuian, Inder Mainie, Marcelo Vela, Donald O. Castell

Background: 24 hour multichannel intraluminal impedance and $\mathrm{pH}$ (MII-pH) esophagea monitoring allows detection of both acid and non-acid gastroesophageal reflux (GER) episodes. The MII-pH catheter contains 6 impedance segments placed 3, 5, 7, 9, 15, and 17 $\mathrm{cm}$ above the LES, plus a $\mathrm{pH}$ electrode at $5 \mathrm{~cm}$ allowing detection of cephalad progression of refluxate. Symptom index (ratio of times the symptom occurs when the $\mathrm{pH}$ is less than 4 to the total \# symptoms) documents the relationship between patient's symptoms and actual reflux episodes during the study. A positive symptom index can define an abnormal study even with normal esophageal acid exposure or normal number of non-acid reflux episodes. Aim: To assess symptom index in the upright versus recumbent position for acid and non-acid reflux using 24 hour MII-pH. Method: 75 consecutive MII-pH studies (49 females; mean age 47 years; range 8 months - 85 years) on PPI therapy (qd or bid) performed in our laboratory were analyzed. The total number of symptoms reported and positive symptom index (Positive symptom index with reflux is defined as positive $>50 \%$ ) was calculated in the upright and recumbent positions. We analyzed the data for typical GERD symptoms (heartburn, regurgitation and chest pain) and atypical symptoms (cough and throat clearing). Results: There were 47 (63\%) negative and 28 (37\%) positive MII-pH studies. The total number of symptoms events reported in the $75 \mathrm{MII}-\mathrm{pH}$ studies were 1139 $247(22 \%)$ were positive for non-acid reflux and only 34 (3\%) positive for acid reflux. 990 $(87 \%)$ events occurred in the upright position and $149(13 \%)$ in the recumbent position Upright symptoms accounted for $33(97 \%)$ positive with acid reflux episodes and $230(93 \%)$ positive non-acid reflux episodes versus only $(\mathrm{p}<0.05)$ l $(3 \%)$ symptom event positive with acid reflux episode and 17 (7\%) symptom events with non-acid reflux episodes in the recumbent position. Thus, $95 \%$ of reflux-positive symptoms occur in the upright position. Conclusions: Many patients experience symptoms associated with acid and non-acid reflux despite PPI therapy. Almost all symptoms with either acid or non-acid reflux occur in the upright position versus the recumbent position.

\section{S1147}

reflux exposure; symptom correlation to proximal reflux, and \% discordance between $\mathrm{pH}$ and impedance. Results: LPR patients had a 33\% increase in duration of proximal bolus contact time when compared to those with GERD(27.28 vs $18.27 \mathrm{sec} p=0.04)$. There was a trend towards a greater number of proximal events in the LPR group (ave. 21 vs 16 events) with percent total events reaching proximal esophagus of 40.7 vs $34.6 \%$. LPR patients had a $21 \%$ increase in total liquid reflux events reaching the proximal esophagus, but gaseous reflux events were not increased. Patients with LPR had a 30\% increase in symptomcorrelation to gaseous events compared to GERD. The PPV in diagnosing LPR is three fold for impedance when compared to 24 hour $\mathrm{pH}$. $\mathrm{pH}$ testing had a 4 fold sensitivity for diagnosing GERD in patients with typical symptoms. Conclusion: Impedance testing reveals that patients with LPR symptoms have a significant increase in proximal esophageal reflux contact time with a trend towards increased number of total and proximal esophageal reflux events compared to patients with GERD. Impedance was useful in reconsidering the diagnosis of LPR in patients with normal pH studies. We conclude that impedance-pH monitoring is a promising complimentary analytical tool which aids our ability to more confidently diagnose LPR through evaluation of proximal esophageal reflux characteristics.

\section{S1145}

MII-pH Monitoring in Infants and Children: What We Can Get from the Autoscan?

Silvia Salvatore, Bruno Hauser, C Luini, S Arrigo, Yvan Vandenplas

Multichannel esophageal intraluminal impedance (MII-pH) is an emerging pH-independent diagnostic tool for gastroesophageal reflux (GER) in both pediatric and adult subjects. The MII may detect any kind of reflux episodes: acid and non-acid and with fluid, gas or mixed content and thus offering the possibility of a more precise correlation between episodes of reflux and symptoms. The new software versions provide automatic analysis that offers wideranging data and a considerable advantage in the trace reading time. Patients and Methods: 40 pediatric patients $(20 \mathrm{~F}-20 \mathrm{M}$, mean age $( \pm$ SD) $72 \pm 66$ months, range $1-161$ months) with suspected GER were submitted to prolonged MII-pH study (Sandhill Scientific, MII-pH). Automatic reading through Autoscan measurements analysis (Bioview Analysis software version 4.9) was used twice for each patients: once, as suggested by the manufacturer, for episodes associated with bolus movements (meal excluded) (Autoscan) and then for bolusindependent episodes with falls of $\mathrm{pH}$ below threshold of 4 (meal included) (pH-Autoscan: a reading more similar to normal $\mathrm{pH}$-study). All traces with artefacts were previously excluded from the study. Acid beverages were not allowed during the registration time. Results: The mean duration of MII-pH was $21 \pm 1$ hours. Total episodes of reflux detected by Autoscan

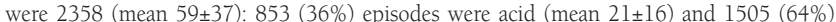
non acid (mean 38 \pm 29 ): 1045 (44\%)(mean 26 \pm 26 ) liquid, 549 (23\%)(mean 14 \pm 12 ) mixed and $765(32 \%)$ (mean 19 \pm 23 ) gas refluxes. PH-Autoscan analysis detected 1519 acid episodes (mean $38 \pm 27$ ) representing $64 \%$ of total episodes reported by Autoscan. Conversely, Autoscan detected $56 \%$ of acid episodes reported by pH-Autoscan analysis. Thirty-six (90\%) among 40 subjects presented symptoms during the registration. In 15/36 (42\%) subjects only nonacid symptom correlation was present. In 4/36 (11\%) subjects only (acid) pH-Autoscan showed a correlation with symptoms. In $17 / 36(47 \%)$ both acid and non-acid reflux episodes were associated with symptoms. Conclusion: Using Autoscan analysis we found that non acid reflux prevails on acid reflux episodes although a more complete automatic analysis (including all acid esophageal exposure, pH-Autoscan) provides nearly 1/3 further acid episodes. The new MII-pH software automatic analysis is useful and time-sparing but might

Typical and Atypical GERD Symptoms Refactory to PPI Therapy Are Produced By Different Mechanisms

Inder Mainie, Radu Tutuian, Amine Hila, Amit Agrawal, Wojciech Blonski, Donald O. Castell

Background: Refluxate with a pH > 4 ('non-acid' reflux, NAR) cannot be accurately detected on standard esophageal pH monitoring. Combined multichannel intraluminal impedance and $\mathrm{pH}(\mathrm{MII}-\mathrm{pH})$ detects gastroesophageal reflux (GER) at multiple levels of the esophagus independent of $\mathrm{pH}$. Aim: To clarify the relationship of typical and atypical GERD symptoms to reflux episodes (acid and NAR). Methods: All patients with persistent symptoms despite proton pump inhibitor (PPI) therapy referred to the esophageal laboratory underwent combined MII-pH monitoring. Prior to the study, patients and/or the referring physician were contacted to ensure the patient had been on at least twice daily PPI for at least a month or more prior to testing. Upon completion of the manometry a $2.1 \mathrm{~mm}$ MII-pH cathteter was passed transnasally and the esophageal $\mathrm{pH}$ sensor was positioned $5 \mathrm{~cm}$ above the LES. The configuration of the catheter allowed monitoring changes in intraluminal impedance at 3 $5,7,9,15$ and $17 \mathrm{~cm}$ above the LES. The pH was monitored at $5 \mathrm{~cm}$ above and $10 \mathrm{~cm}$ below the LES. A positive SI (+SI) was declared if $\geq 50 \%$ (i.e. at least half of symptoms associated with reflux). Typical GERD symptoms were heartburn, regurgitation or chest pain. Atypical symptoms were cough, hoarseness, abdominal discomfort, nausea, dysphagia, throat clearing, mucous, globus, and wheeze. Results: From a total of 200 patients (Female: 98; mean age 44 ; range 3 months to 85 years); 172 patients had symptoms during test day; typical GERD symptoms ( $\mathrm{n}=99$ ), atypical symptoms $(\mathrm{n}=73$ ). Table shows MII-pH SI for the primary typica and atypical symptoms $(\mathrm{p}<0.01)$. Conclusion: MII-pH monitoring separates types of reflux causing symptoms. Typical GERD symptoms on PPI therapy are more likely to be associated with a positive symptom index, primarily non-acid, whereas atypical symptoms are more likely to be associated with no reflux.

\begin{tabular}{|c|c|c|c|}
\hline Symptoms (\# Patients) & +SI for NAR & +SI for acid & -SI \\
\hline Typical (99) & $47(48 \%)$ & $11(11 \%)$ & $41(41 \%)$ \\
\hline Atypical (73) & $14(19 \%)$ & $2(3 \%)$ & $57(78 \%)$ \\
\hline
\end{tabular}

\section{S1148}

Interobserver Variation in the Magnification Endoscopy Findings of Non Erosive Reflux Disease

Rupa Banerjee, Nalini M. Guda, Santosh Darisetty, Manu Tandan, Sandeep Lakhtakia, Rajesh Gupta, Venkat G. Rao, Nageshwar D. Reddy

Background: Magnification endoscopy after contrast enhancement with acetic acid or staining with methylene blue has been able to detect minimal mucosal changes in patients with non erosive reflux disease (NERD). However the interobserver variability of this new technology in the assessment of NERD has not been studied. Patients and methods: 10 patients with reflux symptoms and 10 healthy volunteers with a normal standard endoscopy were prospectively evaluated. High resolution magnification endoscopy was undertaken and data collected as still images (at the 12, 3, 6 and 9-oclock positions) and digital videotapes concentrating 
specially on the tops of the folds. The evaluation criteria used followed the published pilot observations: a) pinpoint/ comma shaped capillaries above the $Z$ line b) punctuate erythema proximal to the $\mathrm{Z}$ line $\mathrm{c}$ ) increased vascular markings distal to the $\mathrm{Z}$ line $\mathrm{d}$ ) loss of palisade pattern of blood vessels above $\mathrm{Z}$ line e) triangular indentations into squalmo-columnar mucosa by villiform columnar mucosa and f) islands of squamous epithelium distal to the $\mathrm{Z}$ line. Examples of magnification endoscopic examinations were shown and agreed upon by 5 experienced endoscopists. The recordings were then evaluated by them in a mixed and blinded fashion and results obtained as per the criteria above. Levels of agreement were evaluated and expressed with kappa statistics using the SPSS software. Results: With regard to the criteria selected for evaluation, pin point blood vessels, loss of palisade pattern of vessels and triangular indentations of villiform mucosa were seen more frequently in reflux patients than in healthy controls $(\mathrm{p}<0.05)$. Interobserver variability was also low with a kappa value of 0.71 and 0.63 for presence of pinpoint vessels and the villiform triangular indentations respectively. However the variability was high (all kappa $<0.4$ ) for the presence of punctuate erythema, increased or decreased vascular markings and presence of islands of squamous mucosa just distal to the $\mathrm{Z}$ line. Conclusion: There are some magnification endoscopic features seen more frequently in patients with reflux with low interobserver variability. These criteria may be used as prospective markers for NERD on magnification endoscopy.

\section{S1149}

Correlating Impedance (Imp) Falls from Above to Below 1000 Ohms with Barium Presence and Amount At the Impedance Site: Findings from Simultaneous Videoesophagram, Impedance Monitoring, and Manometry Francisco J. Marrero, Hala Imam, Steven Shay

Videoesophagram is the gold standard for bolus transit evaluation. Imp is a new method that measures bolus transit. Aim. 1) Correlate Imp falls from above to below $1000 \mathrm{ohms}$ with barium presence and amount at the Imp site by concurrent videoesophagram. 2) Compare Imp falls due to failed clearing of a swallowed bolus vs Imp falls between swallows after successful bolus clearance, e.g., gastroesophageal reflux. Method. 17 normal volunteers and 63 patients with a variety of esophageal disorders were the study population. A catheter with both an Imp electrode pair $(2.5 \mathrm{~cm}$ between the electrodes) and a pressure transducer at four sites (5,10,15 and $20 \mathrm{~cm}$ above LES) was passed per nares. Six $10 \mathrm{cc}$ boluses of $45 \%$ barium mixed with $0.9 \% \mathrm{NaCl}$ were swallowed at about 20 sec intervals while recumbent. A timing device accurate to $.03 \mathrm{sec}$ displayed a simultaneous signal on the impedance waveform monitor and the fluoroscopy screen. For each Imp fall from above to below $1000 \mathrm{ohms}$, the lowest Imp value (Imp nadir) was determined. This Imp nadir value was correlated with simultaneous fluoroscopy screen findings. If barium was present, barium area was calculated in square millimeters $\left(\mathrm{mm}^{2}\right)$. Results. 38 subjects had $169 \mathrm{Imp}$ falls from above to below 1000 ohms. Mean (SEM) Imp fall was $1107 \pm 50$ ohms and mean Imp nadir was $565 \pm 17$ ohms. 97\% (164/169) of Imp falls had barium present. There was good correlation ( $\mathrm{r}=82$, $\mathrm{p}<.001$ ) between Imp nadir and barium area (see table). The Imp nadir value of $600 \mathrm{ohms}$ best distinguished minimal and small barium areas from moderate and large barium areas. Imp values of $<400$ ohms were nearly always associated with a large barium area. Barium area and Imp nadir were similar for 85 Imp falls due to noncleared swallowed boluses compared to 84 Imp falls between swallows after successful bolus clearance. Barium area and Imp nadir were also similar in the 38 Imp falls in 8 normal subjects compared to 131 Imp falls in 30 symptomatic patients. Conclusions. 1) This study validates that impedance falls from above to below 1000 ohms denote bolus presence, whether the Imp fall is from a noncleared swallowed bolus or occurs between swallows. 2) The lowest impedance value reached during the impedance fall has a good correlation with bolus volume Table. Correlation of the amount of barium with the lowest impedance value at an impedance site

\begin{tabular}{|c|c|c|c|c|}
\hline \multirow{2}{*}{ Barium area in millimeters $^{2}$} & \multicolumn{4}{|c|}{ Impedance nadir (ohms) } \\
\cline { 2 - 5 } & $800-999$ & $600-799$ & $400-599$ & $<400$ \\
\hline$\& \# 8806 ; 30$ & 20 & 7 & 0 & 0 \\
\hline $31-150$ & 9 & 23 & 5 & 0 \\
\hline $151-300$ & 5 & 8 & 16 & 2 \\
\hline$>301$ & 1 & 2 & 23 & 48 \\
\hline
\end{tabular}

$*$ rho=.82; $<<0.001$

\section{S1150}

Evaluation of Cyp2c19 Genetic Polymorphism On the Accuracy of ProtonPump Inhibitor (PPI) Test in the Diagnosis of Gastroesophageal Reflux Disease (GERD)

Jaw-Town Lin, Yi-Chia Lee, Hsiu-Po Wang, Han-Mo Chiu, Shih-Pei Huang, Ming-Shiang $\mathrm{Wu}$

Background: Although the PPI test can provide diagnostic value in patients with symptoms suggestive of GERD, the role of cytochrome (CYP) $2 \mathrm{Cl} 19$ genetic polymorphism and optimal dosage of PPI are unclear in a population with low prevalence of extensive metabolizers. Aim: To evaluate whether the CYP2C19 genotype can alter the diagnostic accuracy of rabeprazole-based PPI testing in a Chinese population. Patients \& Methods: Patients presenting with symptoms suggestive of GERD were randomized to receive $20 \mathrm{mg}$ or $40 \mathrm{mg}$ rabeprazole after a diagnostic endoscopy. Their symptom responses were assessed with a daily, five-graded scale during a two-week treatment. The PPI test was considered positive if the symptom score improved more that 50\% from the first baseline score after treatment. DNA from peripheral blood leukocytes were was genotyped with PCR-RFLP technique with allele-specific primers. Results: Of the 97 patients ( 56 female, 41 male; mean age: 49.1 yrs; range: $83-25$ yrs.) who completed the study, 50 (51.6\%) were classified as erosive and 47 $(48.4 \%)$ as non-erosive. Their demographics and outcomes are tabulated and stratified. The 20mg testing provided a sensitivity of $73.1 \%$ and a specificity of $66.7 \%$, which were similar with those of the $40 \mathrm{mg}$ testing $(85.7 \%$ and $73.1 \%$, respectively). In a multiple logistic regression with GERD diagnosis as a dependent variable, the presence of variant $\mathrm{CYP} 2 \mathrm{Cl} 9$ alleles did not show any significant effect $(\mathrm{P}=0.28)$. Conclusions: Both PPI regimens were sensitive and specific for diagnosing GERD and the accuracies were unrelated to the CYP2C19 genotypic status.

\begin{tabular}{|c|c|c|c|}
\hline Characteristics & 20mg rabeprazole $(\mathrm{n}=50)$ & 40mg rabeprazole $(\mathrm{n}=47)$ & $\mathrm{P}$ \\
\hline Male \% & 42 & 42.6 & 0.96 \\
\hline Mean age (SD) & $47.2(14.5)$ & $51.2(11.4)$ & 0.13 \\
\hline Smoking \% & 18 & 12.8 & 0.48 \\
\hline Drinking \% & 22 & 27.7 & 0.52 \\
\hline Body mass index (SD) & $23.7(3.5)$ & $23.7(3.4)$ & 0.96 \\
\hline Endoscopic esophagitis \% & 51 & 43.5 & 0.46 \\
\hline Positive response \% & 54 & 53.2 & 0.94 \\
\hline
\end{tabular}

S1151

Minimal Change Esophagitis Symptomatically Resembles Functional Dyspepsia, But Is Pathophysiologically Similar to GERD

Yasuyuki Shimoyama, Motoyasu Kusano, Osamu Kawamura, Shiko Kuribayashi, Masaki Maeda, Atsuto Nagoshi, Hiroaki Zai, Tatsuya Higuchi, Fumitaka Moki, Tsutomu Horikoshi, Munetoshi Toki, Masatomo Mori

BACKGROUND AND AIMS: Minimal change (MC) esophagitis is endoscopically characterized by prominent erythema that does not show clear demarcation from whitish cloudiness of the lower esophageal mucosa obscuring the longitudinal blood vessels. These findings do not fit the criteria for mucosal breaks in the Los Angeles classification (LA) of esophagitis. We characterized the symptoms and pathophysiology of patients with MC esophagitis. SUBJECTS AND METHODS. The subjects were 347 patients who attended the Gastroenterology Outpatient Department of Gunma University Hospital. All patients underwent upper GI endoscopy to rule out organic disorders. The diagnosis was as follows: 39 patients with MC esophagitis, 85 with GERD (LA $\geqq$ grade A), 195 with functional dyspepsia (FD) who had no abnormalities apart from endoscopic gastritis, and 28 with other conditions. A 50item questionnaire requiring only "yes" or "no" answers was completed before endoscopic examination, which consisted of 10 questions relating to reflux-like symptoms, 12 to dysmotility-like symptoms, six to ulcer-like symptoms, and 11 to psychosomatic symptoms. The chi-square test was used to compare the percentage of positive replies to each question among the patients with MC esophagitis, GERD, and FD. We performed 24-hour pH monitoring ( $\mu$ Digitrapper, Sweden) at $5 \mathrm{~cm}$ above the LES in 7 patients with MC esophagitis, 14 patients with GERD, and 7 normal controls (C). RESULTS. An affirmative answer to the questions about "nausea" and "heavy stomach" was given in the order of FD>MC esophagitis $>$ GERD. There was a significant $(\mathrm{p}<0.05)$ differences between FD and MC esophagitis, and between MC esophagitis and GERD. "Heartburn" and "regurgitation" were significantly more common in GERD patients than in MC or FD patients. "Fatigue" and "anxiety" were significantly more common in FD patients than in those with GERD, but not than in those with MC esophagitis. By pH monitoring, the total number of GER episodes in MC patients $(61.9 \pm 16.4 / 24$ hour, mean \pm SE) was significantly greater than in the $C$ group $(11.7 \pm 4.6)$, and was similar to that in LA grade $\mathrm{A}+\mathrm{B}(79.0 \pm 23.2)$. The $\%$ time at $\mathrm{pH}<4$ for MC esophagitis patients $(3.8 \pm 1.1)$ was significantly larger than that in the $\mathrm{C}$ group $(0.53 \pm 0.27)$, while it was similar to that in the LA A+B group $(3.3 \pm 1.2)$, and smaller than that in LA grades C $(15.9 \pm 4.3)$ and D $(58.4 \pm 10.5)$. CONCLUSIONS: Patients with MC esophagitis can be pathophysiologically classified as GERD. Therefore, proton pump inhibitors should be administered as the initial treatment, although the symptoms of MC patients mimic those of patients with functional dyspepsia.

\section{S1152}

Association Between Non Acid Reflux and Symptoms. Multicenter Ambulatory 24-Hour pH-Impedance Study in 150 Patients Off and On Proton Pump Inhibitors (PPI) Therapy

Frank Zerbib, Sabine Roman, Alain Ropert, Stanislas Bruley des Varannes, Philippe Pouderoux, Ulriikka Chaput, Francois Mion, Jean Paul Galmiche, Daniel Sifrim

Combined ambulatory pH-impedance studies allow detection of both acid and non acid gastro-esophageal reflux (GER). However, the clinical relevance of nonacid reflux remains to be determined in clinical practice. The aim of this study was to evaluate the diagnostic yield of this technique in patients with suspected GER symptoms on and off PPI therapy. Patients and methods. Patients from 7 academic centers with symptoms possibly related to GERD have been included in this retrospective study. Reflux events were detected visually using impedance (Sandhill, CO, USA) and then characterized by pHmetry as acid (AR), or nonacid (NAR) reflux, i.e. weakly acidic, or weakly alkaline (Gut 2004:53:1024-31). A symptom index $(\mathrm{SI}) \geq 50 \%$ was considered indicative of a positive association. Results. A total of 150 patients was included, 103 females (mean age $52 \pm 14$ years, range 16-84). In the group of patients off PPI ( $\mathrm{n}=79,57$ females, mean age $53 \pm 12$ years), 4 had no symptoms during the study, SI was $\geq 50 \%$ in $44(58.7 \%)$, for AR in $25(33.3 \%)$, for NAR in 9 $(12.0 \%)$, and for both $10(13.3 \%)$. The most frequently associated symptoms with GER were regurgitation ( $n=15$ patients for $A R, n=4$ for NAR), heartburn ( $n=10$ for $A R, n=3$ for NAR) and cough ( $n=8$ for AR, $n=3$ for NAR). In the group of patients on PPI ( $n=71,46$ females, mean age $51 \pm 15$ years), 10 had no symptoms during the study, SI was $\geq 50 \%$ in $26(42.6 \%)$, for AR in $3(4.9 \%)$, for NAR in $20(32.7 \%)$, and for both $3(4.9 \%)$. The most frequently associated symptoms with GER were regurgitation ( $n=4$ for $A R, n=13$ for NAR), heartburn ( $n=1$ for $A R, n=5$ for NAR) and cough ( $n=8$ for $A R, n=3$ for NAR). Conclusion. By the means of 24-hour pH-impedance studies, NAR can be associated with symptoms (mainly regurgitation and heartburn) in 25\% and 38\% of patients off and on PPI respectively. 
S1153

The Relevance of Duodenogastroesophageal Reflux and Its Diagnosis Ulrich Muller, Patrick Darb-Esfahani, Thomas Fiedler, Sarah Johanssen, Daniel Kottler, Heike Muller, Herbert Lochs, Winfried A. Voderholzer

Introduction: Duodenogastroesophageal reflux (DGER) is a common finding in patients suffering from gastroesophageal reflux (GER) symptoms and has been accused to be an important factor in the pathogenesis of Barrett's esophagus. However, data on large samples are rare. Aim: To elucidate whether or not DGER results in specific symptoms or Barrett`s esophagus. Methods: We conducted a retrospective analysis of patients undergoing semiquantitative esophageal 24h-bilirubin measurement (Bilitec ${ }^{\mathrm{TM}}$ ) for evaluation of GERD between 2000 and 2005 . All patients were evaluated by a standardized reflux symptom questionnaire (which included heartburn, regurgitation, dysphagia, odynophagia, nausea, vomiting, cough, asthma, sore throat, thoracic pain, problems with nose and ears, abdominal pain, and other problems) and underwent esophageal manometry, 24hour pH-metry (partially double probe measuring), and esophagogastroduodenoscopy (EGD). Pathologic DGER was defined as the percentage time with bilirubin absorption $>0.2$ exceeding $10.7 \%$. Results: We included 217 patients $(54.4+12.9$ years; 95 female, 139 male). Sixty-three of them had isolated acid reflux, 20 isolated biliary reflux, 70 combined reflux, and 64 had no reflux at all. There was no significant difference between patients in each group with regard to the development of erosive esophagitis (ERD)or Barrett's esophagus. In $42 \%$ of patients a pathologic DGER was found (mean DGER $31.0 \pm 17.6 \%$ ). On EGD, DGER positive and negative patients did not differ with respect to ERD (37.8\% of all patients with DGER vs. $26.5 \%$ without, p= 0.056) nor with respect to short segment Barrett (10.9\% with DGER vs. $9.8 \%$ without DGER, $\mathrm{p}=0.68$ ) or long segment Barrett $(13.9 \%$ with DGER vs. $8.3 \%$ without DGER, $\mathrm{p}=$ 0.13). As expected, patients with ERD $(n=122)$ had a significantly higher DeMeester Score than patients without esophageal erosions $(65.3 \pm 60.0$ for ERD vs. $30.6 \pm 36.7$ for NERD, p= 0.0001). However, ERD-patients suffered from significantly more DGER than NERD-patients $(17.6 \pm 18.4 \%$ for ERD vs. $10.1 \pm 15.5 \%$ for NERD, $p=0.004)$. DGER correlated with distal esophageal acid exposure time $(\mathrm{r}=0.432 ; \mathrm{p}<0.0001)$ but not with proximal reflux $(\mathrm{r}=0.206 ; \mathrm{p}=$ 0.07 Spearman-rho test). Symptom evaluation did not find any association of DGER with reflux symptoms. Conclusions: Our data confirm the positive correlation of DGER with acid GER. Although DGER can be found in almost half of the patients with reflux symptoms there is no symptom indicative of DGER. Our study does not support the hypothesis that DGER is a relevant factor in the development of Barrett's esophagus.

\section{S1155}

Esophageal Manometry Findings in Patients with GERD-Like Symptoms Unresponsive to Proton Pump Inhibitors Obtained By 24-Hour $\mathrm{pH}$ and Motility Recordings

Susan N. Ramdhaney, Venkat Pavan R. Kancharla, Manojkumar Singh, Vijaya Boyella, Yatin Zhang, Hulya Levendoglu

Many patients (pts) with GERD-like symptoms have neither significant acid reflux nor respond to proton pump inhibitor (PPI) treatment. The cause of these symptoms is not known. We performed 24-hour esophageal manometry and $\mathrm{pH}$ testing in a total of $247 \mathrm{pts}$ with GERD-like symptoms unresponsive to PPIs (176 female, 81 male), age ranging from 21 to 96 years, to evaluate if changes in motility and $\mathrm{pH}$ parameters may explain these symptoms. Testing was performed with specially designed manometry and $\mathrm{pH}$ probes after the location of LES was established by a stationary manometry. Composite acid and alkaline reflux scores were calculated. We found in the 52 pts with no reflux (A), 69 pts with increased acid reflux (B), 20 pts with both increased acid and alkaline reflux (C) and 106 pts with increased alkaline reflux (D) that baseline mean gastric $\mathrm{pH}$ was appropriately high in $\mathrm{D}>\mathrm{C}>\mathrm{A}>\mathrm{B}$ and overall $24 \mathrm{hr}$ wave activity was higher in $\mathrm{A}>\mathrm{B}>\mathrm{C}>\mathrm{D}$. Contribution of peristaltic activity to overall esophageal contractions was decreased to $<66$ percent in all groups (no significant difference) and the \% of simultaneous and isolated waves correspondingly increased in all groups. However, $\mathrm{C}$ and D pts had significantly increased simultaneous waves in the distal $13 \mathrm{~cm}$ of esophagus compared to $B(p=0.006, p=0.06)$ and $A(p=0.04$, $p=0.02$ ) respectively. In addition, isolated contractions in the distal esophagus were increased in $\mathrm{D}$ pts $(\mathrm{p}=0.08)$ and the \% repetitive contractions were increased in the distal esophagus in $C$ and D pts ( $p=0.04, p=0.02$ ) when compared to A. Although B pts had increased repetitive waves more than $A$ pts, this was not significant $(p=0.08)$. In addition median amplitude of waves in the distal esophagus in D pts was significantly increased compared to $A(p=0.009)$ and $B(p=0.008)$ and the duration of waves also was somewhat prolonged. Based on these findings, we may conclude that GERD pts unresponsive to PPIs are a heterogenous group of pts having no reflux, acid, alkaline or combined acid and alkaline reflux. Irrespective of reflux scores all patients have decreased primary peristalsis and increased simultaneous and isolated contractions. Overall, total esophageal contractions were significantly decreased in alkaline reflux patients when compared to normal. In addition, patients with alkaline reflux or combined reflux show more disturbed motility in the distal esophagus regarding the increased repetitive waves and the amplitude of contractions. Therefore, in GERD patients unresponsive to PPIs, symptoms are most likely related to motility abnormalities rather than the presence or absence of acid or alkaline reflux or both.

\section{S1156}

Ambulatory Esophageal Impedance-pH Monitoring in GERD Suspected Patients: A Visual Analysis of Recording Segments Preceding the Occurrence of Symptoms Is Needed

Sabine Roman, Stanislas Bruley des Varannes, Philippe Pouderoux, Ulriikka Chaput, Francois Mion, Jean-Paul Galmiche, Frank Zerbib

Esophageal impedance-pH monitoring allows the detection of acid and non acid reflux. Direct visual analysis of impedance recordings is however time consuming and represents the main limitation to the diffusion of this technique. The aim of this tudy was to compare "visual" results with those provided by the automatic analysis (autoscan) of the BioviewR software (Sandhill, CO, USA) for the diagnosis of GERD. Subjects and methods. 73 suspected
GERD patients (52 females, mean age 50.4 years, range 16-84) underwent ambulatory 24 hour esophageal impedance-pH monitoring in 5 academic centers. Reflux episodes wer detected and characterized as acidic $(\mathrm{pH}<4)$, weakly acidic $(4 \leq \mathrm{pH}<6.5)$ and weakly alkaline ( $\mathrm{pH} \geq 6.5$ ) (Gut 2004:53:1024-31). Each recording was analysed twice i.e. visual analysis (V) using the BioviewR software system and automatic analysis using the autoscan (AS) function of that software. Symptoms were considered as associated with acid or non acid reflux provided that the symptom index (SI) was $\geq 50 \%$. Results were expressed as median and compared using the Wilcoxon test. Agreement between both analyses was evaluated with Kendall's coefficient (W). Results. Autoscan analysis detected more reflux episodes than visual analysis ( 50 vs $44, p<0.001)$. Weakly acidic ( 13 vs $9, p<0.001)$, weakl alkaline ( 3 vs $2, p=0.011$ ), liquid ( 19 vs $15, p=0.049$ ), pure gas ( 14 vs $7 \mathrm{p}<0.001$ ) and proximal reflux events ( 20 vs $9, \mathrm{p}<0.001$ ) were also significantly more often detected by AS analysis. Agreement between V and AS analysis was good $(\mathrm{p}<0.01$ ) for all parameters (W $>0.72$ ). During impedance-pH monitoring, 65/73 patients reported symptoms. Agreemen between $\mathrm{V}$ and AS analysis to detect a positive symptom-reflux association was good in 48 patients $(73.8 \%)$. In the 17 remaining patients, AS analysis over estimated SI in 11 cases (64.7\%). Conclusion. Despite good agreement with visual analysis, automatic analysis overestimates the occurrence of reflux episodes, especially weakly acidic, pure gas and the proximal extension of reflux episodes. Automatic analysis allows a correct assessment o symptom-reflux association in $75 \%$ of GERD suspected patients. Visual analysis of recording periods that precede marker event activation is mandatory to avoid overestimation of symptom index.

\section{S1157}

Clinical Impact of Esophageal Impedance-pH Measurement in Children Results from the German Pediatric Impedance Group (G-PIG)

Tobias G. Wenzl, Denisa Pilic, Thorsten Frohlich, Ulf Pfeifer, Michael Welter, Henrik Kohler, Anjona Schmidt-Choudhury, Heino Skopnik

Gastroesophageal reflux (GER) is frequent in children. Pathology arises from associated symptoms, e.g. dysphagia and esophagitis, but also extraesophageal symptoms such as chronic cough, bronchitis and apnea. The old diagnostic standard, $\mathrm{pH}$ monitoring, can only detect acidic $(\mathrm{pH}<4)$ episodes. Non-acidic GER and laryngopharyngeal reflux (LPR) is concealed. In 2005 the German Pediatric Impedance Group (G-PIG) was formed to standardize and further advance the use of intraluminal impedance (IMP) combined with $\mathrm{pH}$ measurements in children. Methods: All four G-PIG centers perform combined 24-hour esophagea impedance-pH measurements in children presenting with symptoms suggestive of reflux and an indication for a long-term reflux study. We use a single catheter with 6 impedance channels and 1 distal pH sensor, with the impedance channels spanning from the pharynx to the distal esophagus. Data is recorded with a portable device (Sleuth, Sandhill Scientific USA). Results: So far, combined routine studies were performed in 201 children (median age 3.5 years, range $0-16$ years). Patient age varied remarkably depending on the presenting symptoms: gastrointestinal symptoms, $\mathrm{n}=89$, median age 6.5 (range 0 -16 years); pulmonary symptoms, $\mathrm{n}=93$, median age 2.3 (range $0-15$ years); neurological symptoms, $\mathrm{n}=19$ median age 1,1 (range $0-8$ years). In 152 patients $(75 \%) \mathrm{pH}$-data showed normal findings However, in 92 patients with non-acidic GER/LPR documented by IMP an association with the individual symptoms could be demonstrated. Conclusions: This is the largest pediatric series and represents first data on the collaborative use of multiple intraesophageal impedance$\mathrm{pH}$ measurements in clinical routine in children. Overall, combined studies have significant clinical impact in all participating centers. They improve the detection of reflux and symptom association, and allow specific assignment of treatment options. The role of extraesophageal reflux symptoms is being increasingly recognized, especially in infants and young children With a portable recording device the combined measurement of $\mathrm{pH}$ and impedance will replace isolated $\mathrm{pH}$ monitoring and become the new standard for complete GER / LPR detection. Normative data and validated automated analysis are the most important tasks for the near future.

\section{S1158}

Effects of Test Meal On Multi-Channel Electrogastrography and Autonomic Nervous System Function in Patients with Gastroesophageal Reflux Disease Xiaohong Xu, Zhaolu Ding, Zhifeng Wang, Xiaoyan Chen, Meiyun Ke

Most previous studies applied single channel electrogastrography in patients with gastroesophageal reflux diseases(GERD) and spatial information of gastric myoelectrical activity was missing in those studies. The aims of this study were to utilize multi-channel electrogastrography(MEGG) and power spectra analysis of Heart Rate Variability(HRV) to investigate the responses of gastric myoelectrical activity and autonomic nervous system(ANS) to test meal in patients with GERD and study whether there is any correlation between MEGG parameters and ANS parameters in patients with GERD. Methods: 16 patients with the diagnosis of GERD were enrolled in this study (11M, 5F, mean age: 47yrs) and 16 healthy subjects (HS) matched for age and gender were recruited as control (11M, 5F, mean age: 47yrs). MEGG was recorded simultaneously with the electrocardiogram (ECG) recording for $30 \mathrm{~min}$ in the fasting state and $60 \mathrm{~min}$ after a standard test meal (450 Kcal). MEGG parameters included dominant frequency (DF), dominant power (DP), the normal percentage of 2-4cpm gastric slow waves $(\% \mathrm{~N})$ and the percentage of slow wave coupling (\%SWC). ANS was determined according to the parameters of power spectra analysis of HRV which was derived from the ECG recording and the parameters included sympathovagal balance LF/HF Ratio, mainly sympathetic activity LF and vagal activity HF. Results: 1)Compared with fasting state, test meal significantly increased the DF but decreased the \% N and \% SWC in both GERD patient and $\mathrm{HS}$ in the $1 \mathrm{st} 30 \mathrm{~min}$ and $2 \mathrm{nd} 30 \mathrm{~min}$ fed state $(\mathrm{P}<0.05)$,DP was not significantly altered by test meal in both groups in fed state. However, there is no significant difference between lst $30 \mathrm{~min}$ and $2 \mathrm{nd} 30 \mathrm{~min}$ fed state for DF, \%N and \%SWC. 2) Compared with fasting state, test meal significantly increased the LF/HF ratio and LF but decreased the HF in both groups in the 1st 30min after meal $(\mathrm{P}<0.05)$; however, the increase of $\mathrm{LF} / \mathrm{HF}$ ratio and LF but decrease of HF were still present in HS whereas absent in patients with GERD in the 2nd $30 \mathrm{~min}$ fed state. There is no significant difference between $1 \mathrm{st} 30 \mathrm{~min}$ and $2 \mathrm{nd} 30 \mathrm{~min}$ fed state for those ANS parameters. 3) There was no significant difference between GERD 
patients and HS in any of those MEGG and ANS parameters neither in fasting nor in fed state. 4) There was no significant correlation between MEGG parameters and ANS parameters neither in fasting nor in fed state in both groups. Conclusions: Both patients with GERD and HS may have similar response to test meal in MEGG test. GERD patients could not maintain the response to test meal longer in ANS test suggests GERD patients may have impaired ANS regulation to test meal.

\section{S1159}

Multichannel Intraluminal Esophageal Impedance Predicts Early Persistent Symptoms Following Fundoplication

Matthew Shane, Julia P. Shane, Morgenthal B. Craig, C Dan Smith, Edward Lin, Vickie Swafford, Leena Khaitan

Background: Esophagogastric fundoplication (EGF) is now considered the procedure of choice in the surgical management of patients suffering from gastroesophageal reflux disease (GERD). Up to 20\% of patients will have worsening of their symptoms after surgical therapy, yet preoperative manometric findings alone are not predictive of poor symptomatic outcomes. Combined multichannel intraluminal impedance and esophageal manometry (MII-EM) is a technique that allows simultaneous evaluation of esophageal bolus transit in relation to manometric changes. This study evaluates the use of preoperative MII-EM in predicting which patients will have persistent symptoms following EGF. Methods: From 12/02 - 11/ 05, all patients referred for reflux disease who did not have outside manometry underwent MII-EM. Reflux symptoms were scored pre-operatively and post-operatively using a standard questionnaire that has been used in our institution for over 12 years. Data was analyzed using an ordered probit logistical model using STATA- 9 statistical software (Stata Corporation, College Station, Texas). Results: We retrospectively identified 71 patients who had undergone MII-EM at our institution prior to fundoplication. Of those, 55 (77\%) had both pre-operative and post-operative symptom score data available for review, with a mean follow-up of 3 months. Though fundoplication was associated with a significant overall improvement in symptom scores $(\mathrm{p}<0.001), 9$ patients $(16 \%)$ had either no effect or worsening of their total symptom scores. Manometry alone was not a statistically significantly factor in determining outcome $(\mathrm{t}=-0.88)$. However, abnormal MII was found to be an independent, statistically significant factor in determining worsening post-operative symptoms $(\mathrm{t}=1.86)$. The predicted probability of having undesirable post-op symptoms is strongest when both the impedance and manometry demonstrate abnormal findings (60.64\%). Conclusion: Esophageal manometry is an independently significant factor in predicting persistent postoperative symptoms. Combined MII-EM is able to predict which patients will have persistent symptoms in the early postoperative period following EGF. Further investigation is needed to determine its value in predicting long- term results after fundoplication

\section{S1160}

Accuracy of a Comprehensive Histopathological Score for the Diagnosis of Patients with Gastroesophageal Reflux Disease

Sharad Mathur, Ajay Bansal, Srinivas R. Puli, Amit Rastogi, Prateek Sharma

Background: The role of histology in the diagnosis of GERD remains controversial, in part due to the conflicting results in recent publications after the initial promising studies. Besides the evaluation of traditional features such as basal cell hyperplasia and papillary elongation. recently, evaluation of dilated intercellular spaces (DIS) has also been proposed to be a marker for GERD. Aim: To compare the accuracy of a comprehensive histopathological score in patients with GERD (both erosive esophagitis- EE and non erosive reflux diseaseNERD) and control subjects. Methods: Subjects with and without GERD symptoms as documented by 2 validated GERD questionnaires (GERQ and RDQ) were enrolled in the study. Upper endoscopy was then performed and the distal esophagus was carefully examined for the presence of erosive esophagitis. Two biopsies were obtained from the distal esophagus, $1 \mathrm{~cm}$ above the normal SCJ and in case of patients with EE, away from the areas of erosions. All biopsies were stained with $H \& E$ and were evaluated by a single blinded pathologist for basal cell hyperplasia, length of papillae, DIS, eosinophils, neutrophils and necrosis and an overall histopathological score was calculated (range: 0-12). The scores between EE/NERD group and controls were compared by an unpaired $t$ test. Sensitivities and specificities of this score were calculated by plotting ROC curves. Results: A total of 72 subjects were enrolled in the study- 36 GERD patients (23 EE, 13 NERD) and 36 controls. The mean age was $60 \mathrm{yrs}$ and included 67 males. The mean histologic scores (including all the above mentioned parameters) were as follows: EE patients: $5.4 \pm 2.3$ ( $\mathrm{p}=0.009$ vs. controls), NERD patients: $6.5 \pm 1.8$ ( $\mathrm{p}=0.0002$ vs. controls) and controls: $3.9 \pm 1.9$. Based on ROC curves, in the NERD group, a cutoff score of 5.3 gave a sensitivity of $75 \%$ and specificity of $87 \%$ for the diagnosis whereas in the EE group, a cutoff value of 4.5 had a sensitivity of $74 \%$ and specificity of $70 \%$ for the diagnosis. Conclusion: Using a comprehensive histopathological scoring system based on a combination of multiple histological findings, GERD patients (NERD and EE) can be accurately discriminated from non-GERD subjects. The objectivity of this score may prove to be a useful tool in clinical practice and research studies to further improve the diagnosis of GERD.

\section{S1161}

Esophageal and Intragastric pH Monitoring Using a Wireless System (BRAVO) to Evaluate Esophageal Acid Exposure and Gastric Acid Secretion

Ayaka Seza, Takeo Odaka, Michiko Kobayashi, Taketo Yamaguchi, Syunn Genn, Takuto Suzuki, Hiromitsu Saisho

Background Recently, a new catheter-free pH monitoring system (Bravo) has become available. However, only a few reports on normal values for esophageal exposure using wireless system have been seen. The aims of this study were to establish the cut off level for the diagnosis of GERD patients, to discriminate the patients with reflux from those with nonerosive reflux disease (NERD) and to examine the differences in gastric acid secretion between with and without reflux. Methods Fifteen patients with non-erosive reflux disease and fifteen healthy volunteers underwent 48 -hr esophageal and gastric $\mathrm{pH}$ monitoring using the Bravo system. Two $\mathrm{pH}$ capsules were positioned under direct endoscopic visualization. One was positioned at $5 \mathrm{~cm}$ above the squamocolumner junction, and the other at the posterior wall of lower gastric body. The signals from both capsules were recorded by separate receivers synchronized for time and pH data was analyzed in Excel spreadsheets. We used \% time $\mathrm{pH}<3, \%$ time $\mathrm{pH}<3.5, \%$ time $\mathrm{pH}<4, \%$ time $\mathrm{pH}<4.5$ to assess esophageal acid exposure and mean gastric $\mathrm{pH}$ to assess intragastric acidity. Results The cut-off level (mean+2SD) for the diagnosis of GERD as calculated from the esophageal $\mathrm{pH}$ of controls were as follows: $1.49 \%$ for $\%$ time $\mathrm{pH}<3,2.30 \%$ for $\%$ time $\mathrm{pH}<3.5,3.44 \%$ for $\%$ time $\mathrm{pH}<4,7.89 \%$ for $\%$ time $\mathrm{pH}<4.5$. The cut-off levels for the diagnosis of GERD were lower than those previously reported. In all parameters, the detection rates of the patients with reflux to those with NERD were $53.3 \%(8 / 15)$. The mean gastric pH did not differ between NERD patients with and without reflux or between them and controls. Conclusions Normal values for esophageal acid exposure were lower with the Bravo wireless system than with conventional catheter $\mathrm{pH}$ system in healthy volunteers. The detection rates of the patients with reflux from those with non-erosive gastritis disease using the Bravo wireless system were higher than those by conventional methods. Intragastric acidity did not differ between NERD patients with and without reflux or between them and controls.

\section{S1162}

Use of BRAVO Wireless pH Monitoring Placed in the Columnar Segment of Barrett's Esophagus to Assess Antisecretory Therapy

Jorge Uribe, Marianna Dejongh, Matthew Gideon, Philip O. Katz

INTRODUCTION: Normalization of esophageal acid acid exposure is felt by many to be desirable in patients with Barrett's esophagus(BE). Documentation of esophageal acid control with catheter-based $\mathrm{pH}$ monitoring is limited to a 24 hour period and may not be reflective of consistency of control of intraesophageal pH day to day. The electrode is placed $5 \mathrm{~cm}$ above the lower esophageal sphincter which may not accurately reflect acid exposure in the most distal columnar segment in patients with BE. BRAVO capsule allows for 48-hour monitoring, provides a better estimate of normal daily activities and most importantly, can be placed closer to the gastroesophageal junction, providing information on esophageal acid exposure in the distal esophagus. The reliability of BRAVO pH recordings placed in the columnar segment in BE while on antisecretory therapy is unknown. AIM: Determine if the BRAVO capsule will provide reliable information on esophageal acid exposure when placed in the columnar epithelium, and to assess variability in esophageal acid exposure over 2 days in esophageal $\mathrm{pH}$ on esomeprazole $40 \mathrm{mg}$ twice daily in patients with BE. METHODS: Data was prospectively collected on patients with known BE of at least $2 \mathrm{~cm}$. Bravo capsule was placed one $\mathrm{cm}$ above the top of the gastric folds in columnar mucosa during scheduled surveillance upper endoscopy. All patients were on antisecretory therapy with esomeprazole $40 \mathrm{mg}$ BID for at least 7 days prior. Studies reviewed for number of hours of recorded data and time esophageal $\mathrm{pH}<4$ (normal $<4.2 \%$ total) Analysis was performed as normal vs. abnormal acid exposure on days 1 and 2 separately, both days combined and for worst day when only one day was abnormal. RESULTS: 25 studies were performed, of which only one provided less than 48 hours of data (damaged external recorder due to patient non compliance), 24 studies analyzed for $\mathrm{pH}$ control (table) No statistically significant differences (Fisher exact test) between different analytic methods. CONCLUSIONS:1)The BRAVO capsule records consistent and reliable data when placed $1 \mathrm{~cm}$ above the top of the gastric folds in the columnar segment of patients with Barrett's 2)Esomeprazole $40 \mathrm{mg}$ BID resulted in consistent acid control over 2 days using normal/abnormal as endpoint 3)While more data are required 24 hours of $\mathrm{pH}$ monitoring may be sufficient to determine the adequacy of esophageal acid control in Barrett's patients on high dose antisecretory therapy. This study was supported by a grant from ISS program of Astra Zeneca.

\begin{tabular}{|c|c|c|c|c|}
\hline Acid Exposure & Day 1 & Day 2 & Worst day & Combined \\
\hline Normal & 20 & 21 & 19 & 21 \\
\hline Abnormal & 4 & 3 & 5 & 3 \\
\hline
\end{tabular}

S1163

Lower Esophageal Recording of Acid Exposure Better Explains Symptoms in Patients with Non-Erosive Reflux Disease

Ram Dickman, Jimmy M. Bautista, Wai-Man Wong, Rajan Bhatt, Joy N. Beeler, Isaac B. Malagon, Sara Risner-Adler, Ronnie Fass

Background: Patients with non-erosive reflux disease (NERD) demonstrate the lowest esophageal acid exposure profile and percent symptoms associated reflux as compared to the other gastroesophageal reflux disease (GERD) groups. Aim: To determine if lower esophageal acid exposure recordings at $1 \mathrm{~cm}>$ lower esophageal sphincter (LES) is greater and better explaining symptoms in NERD patients than recording at $6 \mathrm{~cm}>$ LES as compared to the other GERD groups. Methods: Consecutive patients with classic heartburn symptoms were enrolled into the study. Patients were evaluated by a demographics questionnaire and the validated GERD Symptom Checklist. Upper endoscopy was performed to evaluate the presence of esophageal erosions and Barrett's esophagus. Ambulatory pH testing was performed using a commercially available 4 -sensor $\mathrm{pH}$ probe located $5 \mathrm{~cm}$ apart. The distal sensor was placed $1 \mathrm{~cm}$ above the lower esophageal sphincter. Results: A total of 64 patients completed the study. Of those, 21 patients had non-erosive reflux disease $(55.7 \pm 15 \mathrm{yrs}$, M/F 16/5), 20 erosive esophagitis (52.1 $\pm 12 \mathrm{yrs}, \mathrm{M} / \mathrm{F} 14 / 6)$ and 23 Barrett's esophagus $(64.0 \pm 14 \mathrm{yrs}, \mathrm{M} / \mathrm{F} 18 / 5)$. All patient groups demonstrated a significant increase in mean $\%$ total time $\mathrm{pH}<4$ (NERD $-10.4 \pm 1.4$ vs. $7.9 \pm 1.1$, EE $-15.4 \pm 3.0$ vs. $11.7 \pm 2.5$ and $\mathrm{BE}-26.0 \pm 2.9$ vs. $17.3 \pm 2.4, \mathrm{p}=0.009)$ and mean $\%$ upright time $\mathrm{pH}<4$ (NERD 15.4 \pm 2.0 vs. $11.4 \pm 4.9 . \mathrm{EE}-19.5 \pm 4.2$ vs. $13.1 \pm 2.7$ and $\mathrm{BE}-23.9 \pm 2.9$ vs. $15.8 \pm 2.2$ $\mathrm{p}=0.016)$ at the $1 \mathrm{~cm}>$ LES versus $6 \mathrm{~cm}>$ LES sensor. Mean \% supine time $\mathrm{pH}<4$ was significantly increased only in BE patients $(29.2 \pm 4.3 \mathrm{vs} .19 .3 \pm 3.4, \mathrm{p}<0.001)$ at the 1 $\mathrm{cm}>$ LES versus $6 \mathrm{~cm}>$ LES sensor. There was a twofold increase in the number of NERD 
patients with a positive symptoms associated reflux and only NERD patients demonstrated a significant increase in the \% symptoms associated reflux from $6 \mathrm{~cm}$ to $1 \mathrm{~cm}>$ LES (NERD $50 \pm 14$ vs. $75 \pm 16, p=0.01$, EE $84 \pm 7.9$ vs. $94 \pm 4.1, p=N S, B E-64 \pm 10$ vs. $68 \pm$ $10, \mathrm{p}=\mathrm{NS}$ ). Conclusions: All GERD groups demonstrated a significant increase in esophageal acid exposure at the lower $\mathrm{pH}$ sensor $(1 \mathrm{~cm}>\mathrm{LES})$, primarily due to short upright reflux events. Only in NERD patients the lower $\mathrm{pH}$ measurements explained better GERDrelated symptoms.

\section{S1164}

Multichannel Intraluminal Impedance (MII-pH) for the Evaluation of Heartburn in Subjects Both On and Off PPI Therapy

Ellen Stein, Sharmila Anandasabapathy, Barry W. Jaffin

BACKGROUND: Patients with persistent heartburn symptoms on maximal doses of PPI (proton pump inhibitor) therapy are a therapeutic and diagnostic challenge. Through better characterization of reflux composition and patterns in these individuals, MII-pH may better guide therapy. We used 24 hour MII-pH in symptomatic individuals both on and off PPI therapy to determine what reflux characteristics are associated with heartburn episodes. METHODS: Subjects with frequent, daily symptoms of heartburn both OFF $(n=23)$ and ON $(\mathrm{n}=20)$ twice-daily PPI therapy underwent 24-hr MII-pH study. In all individuals, upper endoscopies revealed no Barrett's or esophagitis, and manometries revealed no functional abnormalities. Symptom indices (SI)were calculated in all patients. Patients with positive SI both $\operatorname{OFF}(n=13)$ and $\mathrm{ON}(\mathrm{n}=5)$ PPI therapy were further evaluated to determine which reflux characteristics were associated with heartburn sensation. RESULTS: Subjects both OFF and ON therapy were well matched in terms of age, study duration, and bolus clearance time. Both mean \% acid exposure and total number of reflux events were lower in subjects ON PPI therapy (Table 1). Among patients with a positive SI, 59\% had heartburn symptoms related to acid reflux. Full column reflux was more likely to occur in subjects OFF PPI therapy and was a significant predictor of heartburn sensation. (P value $=0.015$ ) CONCLUSION: In individuals with chronic, daily heartburn, reflux-symptom correlation was higher in individuals OFF PPI therapy and was associated with both acidity and height of refluxate. In patients ON PPI therapy, overall acid exposure was low and the total number of reflux events was markedly reduced. Although a majority of patients with a positive SI had symptoms associated with acid reflux,approximately $41 \%$ of individuals has a positive non-acid SI and all these subjects were on PPI therapy. In this subset of patients, escalating PPI dosage may be ineffective and alternate therapies need to be considered.

Table 1: Mean Characteristics in Subjects OFF and ON PPI therapy

\begin{tabular}{|c|c|c|}
\hline Treatment & OFF & ON \\
\hline Age(years) & 47.6 & 46.5 \\
\hline Gender & $55 \%$ female & $67 \%$ female \\
\hline Study Duration & $22.2 \mathrm{hrs}$ & $21.9 \mathrm{hrs}$ \\
\hline BCT & $15 \mathrm{sec}$ & $14 \mathrm{sec}$ \\
\hline \% Acid exposure & $9.34 \%$ & $1.53 \%$ \\
\hline Total \# of reflux events (mean) & 71.5 & 37.8 \\
\hline
\end{tabular}

\section{S1165}

Prolonged Wireless pH-Metry in Community Practice: Does the Longer Sampling Time Improve the Yield of $\mathrm{pH}$ Testing?

Saeid Goshtasbi, Nimish Vakil, David Carron, Michael Molloy, Steven Lipscomb

Introduction: It has been suggested that wireless $\mathrm{pH}$ metry improves the yield of $\mathrm{pH}$ testing by prolonging the sampling duration but the available studies are small in size and are from expert centers. The aim of this study was to determine if the yield of $\mathrm{pH}$ testing was increased by a 48 hour sampling period in 135 consecutive patients in a community setting. Methods: (Mean+SEM; comparisons by Wilcoxon test). The records of 135 consecutive patients who underwent wireless pH-metry (Bravo, Medtronics) between 2003 and 2006 were analyzed using a pre-determined data abstraction tool. Results: The principal indications were noncardiac chest pain $=(\mathrm{n}=18)$, atypical ENT presentations $(\mathrm{n}=28)$ and symptoms refractory to PPI therapy (n=37). 116 of $135(86 \%)$ patients had received a trial of PPI therapy before $\mathrm{pH}$ metry and 55 (41\%)had received double dose PPI therapy. The wireless capsule failed to attach in 2 cases (1.5\%). This was recognized endoscopically and a second capsule was successfully attached in both cases. Day 1 recordings were obtained in all 135 patients and day 2 recordings were obtained in 134 patients. Receiver error resulted in incomplete recordings in 1 patient $(1 \%)$ in whom day 2 data were lost. The mean number of reflux episodes on day 1 was $37+4$ and on day 2 (median 27, IQR=35) was $35+4$ (median 21 , $\mathrm{IQR}=46 ; \mathrm{p}=0.025$ ). The total time $\mathrm{pH}$ was $<4$ was $54+9 \min ($ median $=21, \mathrm{IQR}=55)$ on day 1 and $52+10$ (median=21, IQR=58; $\mathrm{p}=0.07$ ) on day 2 and the fraction of time $\mathrm{pH}$ was $<4$ on day 1 was $3.9+0.6 \%$ (median=1.5, IQR=4) on day 1 and $4.2+0.8$ (median=1.6, IQR= 3.8 ) on day $2(\mathrm{p}=0.39)$. Assuming a normal acid exposure of $4.4 \%$ (Scand J Gastroenterol. $2005 \mathrm{Jul} ; 40(7): 768-74), 48$ patients (35\%) had an abnormal recording (either day 1 or day 2 , or both days). 28 patients had an abnormal recording on only one of the days (day $1=$ $17 / 28(61 \%)$, day $211 / 28(39 \%))$. Most procedures were performed in the morning. Conclusions: 1. In routine US practice, wireless $\mathrm{pH}$-metry is generally performed in patients who have failed acid suppressive therapy. Normal values for this population are lacking and are an important need. 2. Distal acid exposure is not greater on day 2 as has been claimed in some smaller studies. 3. Many patients are diagnosed by abnormalities on only one of the study days suggesting that the longer sampling time improves the yield with wireless pH-metry.

\section{S1166}

Reproducibility of pH Profiles in 48hour Wire Free Capsule Esophageal pH Monitoring

Joanna Gray, Aileen Sutherland, Grant M. Fullarton

The wire-free capsule (WFC) is a recognised alternative to conventional wire-based catheter systems in esophageal pH monitoring. Although WFC has improved patient acceptability in terms of comfort, the benefit and reproducibility of a $48 \mathrm{hr}$ recording period remains unclear. In addition,the presence of an esophageal implant may affect oesophageal motility which could alter $\mathrm{pH}$ profiles over a prolonged period. The aim of this study was to assess the reproducibility of esophageal $\mathrm{pH}$ profiles on day 1 and day 2 of WFC esophageal pH monitoring. Methods 48 patients undergoing WFC esophageal $\mathrm{pH}$ monitoring as part o their GERD assessment were studied. The WFC (BRAVO, Medtronic) were all secured by a single endoscopist under sedation with propofol. The WFC was secured $6 \mathrm{~cm}$ above the endoscopically identified squamocolumnar junction. For each patient $48 \mathrm{hr}$ esophageal pH profiles were recorded and the parameters assessed were: percentage of total time esophagea $\mathrm{pH}<4$, number of long refluxes and the duration of long refluxes for day 1 and 2 . Results All patients had successful endoscopic placement of the WFC with only 2/48 patients having dislodgement of their electrode prior to completion of the recording period. The percentage of total time esophageal $\mathrm{pH}<4$ was $5.5(0.1-41.5)$ (median, range) on day 1 and $5.1(0-43)$ on day 2. Rank correlation coefficient $\mathrm{R}=0.7(\mathrm{p}<0.001)$ (Figure). The number of long refluxes on day one was $3(0-9)$ and $3(0-21)$ on day $2(R=0.7, p<0.003)$ The duration $(\mathrm{sec})$ of these refluxes was $12(0-115)$ on day 1 and $12(0-250)$ on day $2(\mathrm{R}=0.6, \mathrm{p}<0.002)$. Conclusions Esophageal $\mathrm{pH}$ profiles demonstrate close correlation between day 1 and 2 of the $48 \mathrm{~h}$ recording period with the WFC system. This suggests that the WFC system does not induce capsule related reflux changes, at least during the initial $48 \mathrm{hr}$ recording period.

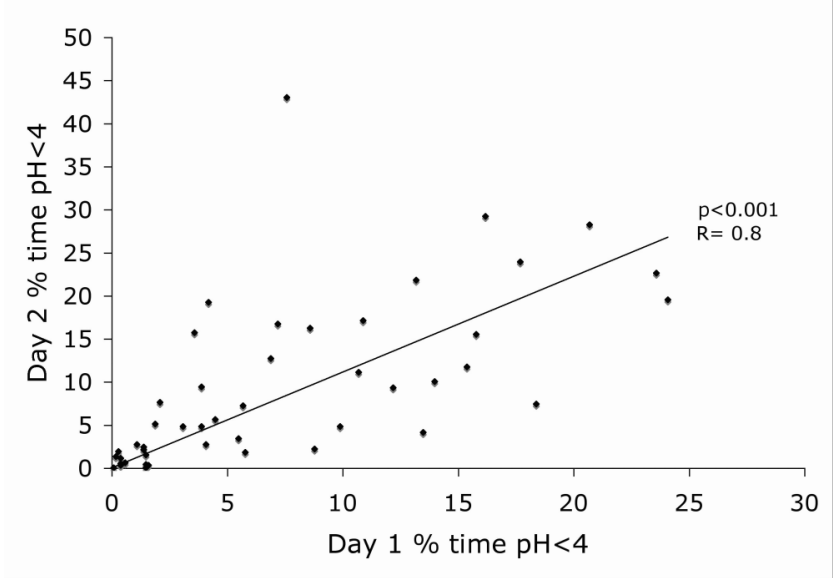

S1167

Magnification Endoscopy Changes of Minimal Change Esophagitis: Effect of Rabeprazole Therapy

Rupa Banerjee, Manu Tandan, Rajesh Gupta, Santosh Darisetty, Sandeep Lakhtakia, Venkat G. Rao, Nageshwar D. Reddy

Introduction: More than $60 \%$ of patients suffering with gastroesophageal reflux disease show no or minimal changes on standard endoscopy (NERD). Magnification endoscopy after contrast enhancement with acetic acid is able to detect subtle mucosal alterations. The primary aim of this study was to identify and evaluate these changes before and after ora Rabeprazole therapy as possible predictors of NERD. Methods: Consecutive patients with GERD as identified by a questionnaire and normal standard endoscopy were included in the study after informed consent. These patients were examined by high resolution magnification endoscopes (Olympus GIF Q 160Z). Subsequently these patients were treated 20mg Rabeprazole/day for four weeks and re-examined by magnification endoscopy. Data was collected as still images and digital videotapes concentrating on the tops of the folds. Potential criteria for minimal change esophagitis were identified according to pilot observations in prior studies: a) comma shaped dilated intrapapillary capillary loop (IPCL) above the Z line b) increased vascular markings distal to the $\mathrm{Z}$ line c) loss of palisade pattern of blood vessels above $Z$ line d) triangular indentations into squalmo-columnar mucosa by villiform columnar mucosa and e) islands of squamous epithelium distal to the $\mathrm{Z}$ line. Images and videos were coded and examined by the investigator and results validated by two independent observers Results: 18 patients with reflux symptoms and normal standard endoscopy were included in the study. Frequency of these changes and response to PPI are shown (Table 1). Conclusions: Some of the changes of minimal change esophagitis on magnification endoscopy may resolve with Rabeprazole therapy. These reversible changes may represent true endoscopic markers of minimal change esophagitis

Results

.

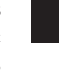




\begin{tabular}{|c|c|c|}
\hline Criteria & Before PPI & After PPI \\
\hline a & 14 & 1 \\
\hline b & 15 & 12 \\
\hline c & 7 & 0 \\
\hline d & 16 & 10 \\
\hline e & 8 & 4 \\
\hline
\end{tabular}

Table 1

\section{S1168}

Gastrin-17 (G-17) As a Sensitive Serological Bio-Marker for Diagnosis of Gastro-Esophageal Reflux Disease (GERD) Independently of H. Pylori Status Lucas G. Cavallaro, Antonio Nouvenne, Andrea Maini, Roberta Merli, Libera Fanigliulo, Daniela Basso, Mario Plebani, Veronica Iori, Giulia Martina Cavestro, Marta Maino, Giorgio Nervi, Angelo Franze', Francesco Di Mario

Background: The prevalence of GERD is $25 \%$ of the general population and is due to an excess of acid into the oesophagus. High basal acid output (BAO) can determine very low Gastrin 17 (G-17) levels due to the negative feedback relating antrum and corpus mucosa. Serum Pepsinogens I and II (SPGI and SPGII) are surrogate markers of maximal acid output (MAO) and gastric inflammation respectively. Aim: To evaluate G-17, sPGI and II levels in patients with GERD compared to patients with other acid-related disorders.. Patients and Methods: 124 consecutive outpatients ( $60 \mathrm{M}$, mean age: $39 \pm 9.4$ years, range 21-59) with upper GI symptoms and not under PPI treatment were enrolled. This population was divided, on the basis of upper GI endoscopy and gastric histology, predominant symptoms and medical history, into: 84 with GERD 42 (17 Hp+ve) with Esophagitis (ERD); 42 (19 Hp+ve) with Non Erosive Disease (NERD), 42 with Other Acid-related Disorders 6 ( $5 \mathrm{Hp}+\mathrm{ve}$ ) with Duodenal Ulcer (DU), 28 (14 Hp+ve) with Gastritis (GAS) and 6 (3Hp+ve) with Gastric Ulcer (GU). In all patients H. pylori status was assessed and, a blood sample was taken to evaluate serum G-17 and sPGs levels (EIA, Biohit, Helsinki, Finland). Mann-Whitney U test was used to compare the means of serological parameters into the different clinical subgroups. Results: The mean of G-17 levels showed a marked decrease in group GERD vs Other acidrelated disorders (GERD: $2 \pm 2$, Other: $5 \pm 3$ pmol/, p $<0.001$ ). No difference was found between ERD and NERD according to G-17 and sPGs. Notably all $\mathrm{Hp}+\mathrm{ve}$ patients showed significantly increased sPGII levels (Hp+vel5 $\pm 4 ;$ Hp-ve $9 \pm 3$ pmol/, p $<0.001$ ). GERD patients confirmed significant lower G-17 levels also in this Hp+ve subcohort (GERD 2.3 2 ; Other $5 \pm 2$ pmol/ $1, \mathrm{p}<0.001)$. Decreasing levels of sPGI were observed from DU $(124 \pm 33 \mathrm{pmol} / \mathrm{l})$, to GAS (109 $\pm 36 \mathrm{pmol} / \mathrm{l})$, to GERD patients $(93 \pm 35 \mathrm{pmol} / \mathrm{l})$, to GU $(82 \pm 35 \mathrm{pmol} / \mathrm{h})$. Conclusion: G17 could be useful, in the first approach, to the management and diagnosis of GERD, independently of $H$. pylori status, highlighting a new risk factor of acid exposure. On the other hand, Pepsinogens are good serological biomarkers to assess gastric hypersecretion and mucosal inflammation.

\section{S1169}

Evaluating the Day to Day Variability Detected in the 48 Hour Ambulatory BRAVO pH Data

M. Babitha Reddy, Gregory Haber

Introduction: The ambulatory Bravo $\mathrm{pH}$ test offers extended esophageal acid exposure recording time with improved patient comfort and compliance in esophageal pH testing. However, the initial reports of data obtained from the two day ambulatory Bravo $\mathrm{pH}$ test illustrate significant day to day variability in the results. Aim: To retrospectively review all consecutive Bravo pH studies from a single institution from January 2003 through August 2005 and to calculate the discordance in positive studies. We also assessed the possible impact of sedation, studied while on and off proton pump inhibitors (PPI), and time of day the study commenced. Methods: Bravo pH results from January 2003 through August 2005 were obtained from the computerized records of the endoscopy unit from a single institution. Sixty nine studies were excluded due to insufficient recording time (less than 36 hours) or insufficient collected data due to patient diary violations. Of 175 included studies, 57 were performed on PPI and 118 off PPI. Discordance was defined as a positive study in only one of the two days recorded and concordance defined as positive studies in both days recorded. Early morning studies were defined as studies completed between 8am and 10am and late afternoon studies were defined as studies completed between $2 \mathrm{pm}$ and $5 \mathrm{pm}$. A positive study was defined as $\mathrm{pH}<4,>5.3 \%$ of the recorded time in each $24 \mathrm{~h}$ time interval. Results: Two hundred forty four Bravo $\mathrm{pH}$ results were reviewed and 175 studies were included. Of these, 100 (mean age 50) were female. Among the 118 patients off PPI, 50\% of studies were positive, 16 only on Day 1, 9 only on Day 2, and 34 on both days with a discordance rate of $42 \%$. Discordance rates were similar in all groups with the exception of the supine, $74 \%$, vs. upright, $34 \%$ position. Among the 57 patients on PPI, 19\% of studies were positive 4 only on Day 1, 3 only on Day 2, and 4 on both days with a discordance rate of $63 \%$. This was similar in all groups. The overall discordance rates were higher in patients on PPI $(63 \%)$ versus off PPI $(43 \%)$ and this was true for all subgroups. The overall discordance rates among all groups were higher than previously reported. Conclusions: This large study of Bravo $\mathrm{pH}$ data collected from a single institution demonstrates day to day variability greater than with previously published data. Percentage of positive studies was similar in all groups. The discordance rates are higher on PPI vs. off PPI. The discordance rates were higher in the supine position on PPI than the other subgroup analysis.

\section{S1170}

Time Esophageal $\mathrm{pH}<4$ Significantly Overestimates the Prevalence of Pathologic Esophageal Reflux in GERD Subjects Treated with Proton Pump Inhibitors (PPIs)

Lauren B. Gerson, Peyman Sahbaie, George Triadafilopoulos, Winston Young, Jerry D, Gardner

Background: A recent study concluded that $50 \%$ of GERD patients have pathologic esophageal acid exposure despite PPI treatment that abolished all GERD symptoms (Am I Gastroenterol 2004; 99:991-96). We considered the possibility that this high prevalence of pathologic esophageal acidity occurred because data were analyzed in terms of time esophageal $\mathrm{pH}<4$. Aim: To compare the prevalence of pathologic esophageal reflux assessed by calculating time $\mathrm{pH}<4$ and integrated acidity from the same records. Methods: Raw data from 49 esophageal $\mathrm{pH}$ recordings in GERD patients from the study cited above were analyzed. Three records were omitted for technical inadequacies. The postprandial and nocturnal periods were 900-2200 and 2200-900, respectively. Receiver operator characteristic (ROC) analyses of results for integrated esophageal acidity based on 24-hour ambulatory esophageal $\mathrm{pH}$ recordings from 26 normal subjects and 57 GERD subjects who reported heartburn at least 4 times/week for at least 6 months (Aliment Pharmacol Ther 2003; 17:955-964) were used to determine cut-off points that defined pathologic integrated esophageal acidity (mmol.hr/L) as 24-hour $>8.1$; postprandial $>1.85$, and nocturnal $>6.8$. Similar analyses showed that pathologic time $\mathrm{pH}<4(\%)$ was defined as 24 -hour $>4.3$; postprandial $>6.8$, and nocturnal $>2.35$. Results: For the 24-hour period, the prevalence of pathologic esophageal reflux measured as time $\mathrm{pH}<4$ was $43 \%$ [95\% CI; 29\%, 57\%] and was significantly higher $(\mathrm{P}=0.006$; Fisher's Exact Test) than that measured as integrated acidity $(15 \%[5 \%, 25 \%])$. For the nocturnal period, the prevalence of pathologic esophageal reflux measured as time $\mathrm{pH}<4(35 \%[21 \%, 49 \%])$ was also significantly higher $(\mathrm{P}=0.012)$ than that measured as integrated acidity $(11 \%[2 \%, 20 \%])$. For the postprandial period, the prevalence of pathologic esophageal reflux measured as time $\mathrm{pH}<4(28 \%[15 \%, 41 \%])$ was not significantly different $(\mathrm{P}=0.821)$ from that measured as integrated acidity $(33 \%[19 \%, 47 \%])$. Conclusion: The present results indicate that in GERD subjects treated with a PPI until they are asymptomatic, assessing esophageal acidity with time $\mathrm{pH}<4$ significantly overestimates the prevalence of pathologic esophageal acid exposure compared to integrated acidity. The 3-fold overestimate of the prevalence of pathologic nocturnal but not postprandial esophageal reflux occurs because time $\mathrm{pH}<4$ fails to fully quantify effects of PPIs on esophageal acidity and as a result, gives artificially high values for esophageal acid exposure.

\section{S1171}

Endoscopic Placement Under Sedation Does Not Affect pH Monitoring By the BRAVO ${ }^{\text {TM }}$ System

Amir Thakur, Mark Fox, Roy Anggiansah, Wong Terry, Angela Anggiansah

INTRODUCTION \& AIMS: The Bravo ${ }^{\mathrm{TM}}$ system is designed to provide catheter-free, intraoesophageal $\mathrm{pH}$ measurements over 48 hours. Early observations have suggested that oesophageal acid exposure is less during the first than the second 24 hour period.[Pandolfino AmJG 2003] This study examined whether endoscopic placement under sedation has effects on $\mathrm{pH}$ measurement, and if present whether these effects are a direct consequence of the procedure or caused by a reduction in oral intake. METHODS. 56 consecutive patients (median age 48 (35-61) yrs) referred for $\mathrm{pH}$ monitoring were studied. The Bravo ${ }^{\mathrm{TM}}$ capsule was placed using a standard gastroscope under moderate sedation (midazolam 3-5mg, fentanyl $0-50 \mathrm{mcg}$ ) on an afternoon list and measurements began one hour after the procedure. 'Refluxogenic' test meals (1000kcal) were provided to $12 / 56$ patients for lunch and dinner throughout the study. Acid exposure during the 3, 6 and 24 hours after endoscopy on the first day were compared to the same time periods on the second test day. Data is presented as the median \pm SEM. RESULTS: Complete 48-hour $\mathrm{pH}$ data was available for $47 /$ $56(85 \%)$ patients (incomplete recording $(n=8)$, early detachment $(n=1)$ ). Pathological acid exposure $(>4.2 \% \mathrm{pH}<4 / 24 \mathrm{hr})$ was recorded on at least one day in $34 / 47(74 \%)$ patients. Acid exposure was similar after endoscopic placement on day 1 than the same period on day 2 at 3 -hrs $8.7 \pm 1.4 \%$ vs. $7.3 \pm 1.6 \%(\mathrm{p}=0.28)$, at 6 -hrs $8.8 \% \pm 1.8 \%$ vs. $8.0 \% \pm 1.5 \%(\mathrm{p}=$ $0.71)$ and over $24-\mathrm{hr} 8.2 \pm 1.4 \%$ vs. $7.8 \pm 1.3 \%(\mathrm{p}=0.61)$. Moreover $\mathrm{pH}$ measurements during the first 3 hours after endoscopy were not significantly different to those during any other time period ( $\mathrm{p}=0.58$ by repeated measures ANOVA). Acid exposure was also comparable in the postprandial periods $(11.5 \pm 1.6 \%$ vs. $11.2 \pm 1.5 \%, \mathrm{p}=\mathrm{ns})$ and during the first and second nights / supine periods $(7.5 \pm 1.4 \%$ vs. $6.9 \pm 1.5 \%$, p=ns). Similar results were found in the subset of patients that received set meals. Moreover the reproducibility of $\mathrm{pH}$ measurements between day 1 and day 2 over 3, 6 and 24 hours was not significantly improved by the provision of set meals. CONCLUSIONS: Endoscopic placement of the Bravo ${ }^{\mathrm{TM}}$ capsule under moderate sedation had no impact on $\mathrm{pH}$ recordings. Providing patients with set meals did not alter the reproducibility of $\mathrm{pH}$ measurements. The interpretation of $\mathrm{pH}$ recordings obtained by the Bravo ${ }^{\mathrm{TM}}$ system need not take the effects of endoscopy on acid reflux into account.

\section{S1172}

Wireless Vs Traditional pH Monitoring: Better Tolerability and Lower Impact On Daily Activities and Food Intake Ausilia Grigolon, Ivana Bravi, Paolo Cantu', Roberto Penagini

Background and aim Catheter-based esophageal pH monitoring is traditionally considered the gold standard for the diagnosis of gastroesophageal reflux disease (GERD). Patients often report discomfort and limitations of daily habits during the test, however few objective data are available. The new wireless technique may overcome these limitations. Aim of our study was to evaluate the tolerability and impact on daily activities and food intake of traditional catheter based and of the new wireless pH monitoring. Methods One-hundred and twentyfive consecutive patients with suspected GERD, referred for ambulatory esophageal $\mathrm{pH}$ monitoring were enrolled. Seventy-height patients $(36 \mathrm{M}, 53+2 \mathrm{yr})$ underwent $24 \mathrm{~h}$ traditional $\mathrm{pH}$ monitoring (Slimline; Medtronic) and 47 patients (21 M, $45+3 \mathrm{yr}$ ) $48 \mathrm{~h}$ wireless (Bravo; 
Medtronic) $\mathrm{pH}$ monitoring. The traditional $\mathrm{pH}$ catheter was passed transnasally and positioned in the oesophagus with the $\mathrm{pH}$ step-up method, whereas the $\mathrm{pH}$ capsule was positioned transorally during endoscopy. Discomfort during placement of the $\mathrm{pH}$ electrodes and during the whole $\mathrm{pH}$ monitoring were evaluated by two $100 \mathrm{~mm}$ visual analogue scales. Limitations of food intake and of daily activities were evaluated by two standardized questionnaires (score 0-3: 0 = no limitation; 3 = food intake limited during all meals/activities severely limited). Results (means + SEM). See table. Successful pH study was completed in all patients except one in the Bravo group for early detachment of the capsule. $\mathrm{pH}$ data were captured by the receivers for $98.0 \%+1.5$ and $94.5+1.0$ of time during traditional and wireless monitoring respectively. Fewer patients reported discomfort $(\mathrm{p}<0.01)$, limitation of food intake and of daily activities during wireless compared to traditional monitoring (21\% vs $46 \% \mathrm{p}<0.05,17 \%$ vs $65 \% \mathrm{p}<0.01$ respectively). Twenty-six $\%$ of the patients had chest complaints during wireless monitoring. Conclusions Traditional catheter-based $\mathrm{pH}$ monitoring is reasonably well tolerated; it induces, however, important limitations of food intake and daily activities, which may impair diagnostic accuracy. The Bravo wireless $\mathrm{pH}$ monitoring is technically successful, better tolerated and, above all, has minor impact on food intake and daily living. Whether this translates in better diagnostic accuracy remains to be evaluated.

\begin{tabular}{|c|c|c|}
\hline & Traditional & Wireless \\
\hline Discomfort at placement $(\mathrm{mm})$ & $32+3$ & $31+4$ \\
\hline Discomfort during whole test $(\mathrm{mm})$ & $36+3$ & $22+3 *$ \\
\hline Limitation of food intake & $0.9+0.1$ & $0.4+0.1 \dagger$ \\
\hline Limitation of daily activities & $1.2+0.1$ & $0.2+0.1 *$ \\
\hline
\end{tabular}

$* \mathrm{p}<0.01, \uparrow \mathrm{p}<0.05$ vs traditional technique (unpaired Student's $\mathrm{t}$ test)

\section{S1173}

Esophageal pH Testing in Patients Symptomatic On PPI Therapy Barbara Ann M. Mackalski, Alexandra Ilnyckyj

Background: The clinical utility of $24 \mathrm{hr} \mathrm{pH}$ testing in the setting of PPI therapy is unclear. Pivotal data reports $\mathrm{pH}$ tests are predictably normal in $92 \%$ of patients $*(1)$. Aim: To describe $24 \mathrm{hr} \mathrm{pH}$ findings in patients undergoing testing for the indication of GERD unresponsive to PPI therapy. Methods: The St. Boniface General Hospital is a tertiary care centre located in the province of Manitoba, Canada. Our laboratory performs approximately $100 \mathrm{pH}$ tests yearly. Requests are limited to specialists; gastroenterologists or GI/thoracic surgeons. Clinical indication and medical therapy are documented by the referring specialist on a standardized form. All patients are contacted prior to the test by a dedicated GI nurse to confirm medical regimen and reinforce importance of drug compliance prior to and during the $\mathrm{pH}$ study. $\mathrm{pH}$ tests are performed using standardized technique and analyzed using Esophagogram software (Medtronic, Inc). Charts were reviewed and data extracted by a GI fellow. Results: A retrospective review of 415 consecutive patients referred over a $31 / 2$ yr period, January 2002- June 05 inclusive identified 107 patients (35\% male) meeting study criteria. $61 / 107$ had typical symptoms and 61/107 were on BID dosing. Test results were classified as abnormal by applying 3 different criteria for abnormal reflux parameters: \#1) DeMeester score $>14.7$ \#2) \% time $\mathrm{pH}<4$ : total $>5.5 \%$, upright $>8.3 \%$, supine $>3.0 \%$ and \# 3$) \%$ total time $\mathrm{pH}<4:>1.6 \%$. Results are tabulated below. Conclusion: A significant number of patients in a specialty practice who remain symptomatic on PPI therapy, including BID dosing, will have abnormal $\mathrm{pH}$ parameters. Therefore the role of acid in their symptoms cannot be excluded. *1. Charbel S, Khandwala F, Vaezi M. The Role of Esophageal pH Monitoring in Symptomatic Patients on PPI Therapy. Am J Gastroenterol 2005;100:283-89. Abnormal pH tests by criteria/dose

\begin{tabular}{|c|c|c|c|}
\hline PPI dose & criteria 1 & criteria 2 & criteria 3 \\
\hline OD & $14 / 4630.4 \%$ & $11 / 4624 \%$ & $22 / 4648 \%$ \\
\hline B.I.D & $18 / 6129.5 \%$ & $15 / 6124.5 \%$ & $31 / 6151 \%$ \\
\hline TOTAL & $32 / 10730 \%$ & $26 / 10724 \%$ & $53 / 10749.5 \%$ \\
\hline
\end{tabular}

\section{S1174}

Multichannel Intraluminal Impedance and pH (MII-pH) Esophageal Testing Is More Specific Than $\mathrm{pH}$ Alone for Diagnosing Acid Gastroesophageal Reflux Amine Hila, Amit Agrawal, Wojciech Blonski, Janice Freeman, Donald O. Castell

BACKGROUND: 24-hr multichannel intraluminal impedance and pH (MII-pH) esophageal monitoring allows detection of both acid and non-acid gastroesophageal reflux (GER). The MII-pH catheter contains 6 impedance segments placed 3, 5, 7, 9, 15 and $17 \mathrm{~cm}$ above the LES, plus a pH electrode at $5 \mathrm{~cm}$ allowing detection of cephalad progression of refluxate. Standard 24-hour $\mathrm{pH}$ monitoring detects changes in $\mathrm{pH}$, and defines reflux as drops in $\mathrm{pH}$ below 4. The new MII-pH technology has shown that some of these $\mathrm{pH}$ detected episodes are actually due to swallowing acid foods. AIM: To assess accuracy of $\mathrm{pH}$ monitoring alone in detecting acid reflux compared to MII-pH. METHODS: Review of 60 consecutive ambulatory MII-pH studies recorded off acid suppressing therapy performed from 6/03 to $8 / 05$ (36 females; mean age $=52.7$ yrs; range: $16-77$ yrs). All studies were initially read by exclusively analyzing the $\mathrm{pH}$ tracing for acid reflux episodes ( $\mathrm{pH}$ drop $<4$ ), excluding the meal periods. Symptom association with acid reflux was also evaluated. Subsequently, all studies were blindly read again analyzing MII-pH detected acid reflux episodes (pH drop $<4$ and an MII detected reflux episode), excluding the meal periods. Symptom association with MII-pH acid reflux was also evaluated. A symptom was positively associated with acid reflux if $\geq 50 \%$ of symptoms occurred within 5 minutes of the start of an acid reflux episode. Normal values for $\%$ time $\mathrm{pH}<4$ were: total $<4.2 \%$; upright $<6.3 \%$; recumbent $<1.2 \%$. RESULTS: The table below shows the sensitivity and specificity of $\mathrm{pH}$ alone compared to MII-pH for detection of acid GER using either \% time $\mathrm{pH}<4$ alone or symptom association alone, and including both as diagnostic criteria. The upright, recumbent and total \% time
$\mathrm{pH}<4$ was significantly higher when using $\mathrm{pH}$ alone compared to MII-pH (Wilcoxon signed ranked test; $p<0.0001)$. In these 60 patients, MII-pH detected 1133 acid reflux episodes while $\mathrm{pH}$ alone detected 2307 episodes (Wilcoxon signed ranked test; $\mathrm{p}<0.0001$ ). 81\% o the acid GER episodes exclusively detected by $\mathrm{pH}$ were associated with a swallow as demonstrated by MII. Of the 60 patients, 13 (22\%) were diagnosed as having an abnormal 24 hour $\mathrm{pH}$ test, that proved to be negative for acid GER when the MII was included in the evaluation. CONCLUSION: Use of $\mathrm{pH}$ alone for detection of acid reflux is very sensitive but lacks specificity compared to MII-pH. pH alone may over diagnose abnormal acid reflux in up to $22 \%$ of tested patients.

\begin{tabular}{|l|c|c|c|}
\hline \multirow{2}{*}{} & \multicolumn{3}{|c|}{ Diagnostic Criterion } \\
\cline { 2 - 4 } & $\%$ time $\mathrm{pH}<4$ & Symptom association & $\%$ time $\mathrm{pH}<4$ and Symptom association \\
\hline Sensitivity & $100 \%$ & $100 \%$ & $100 \%$ \\
\hline Specificity & $68 \%$ & $67 \%$ & $58 \%$ \\
\hline
\end{tabular}

\section{S1175}

Diagnostic Value of Oesophageal Physiology Studies - An Eight Year Prospective Study Samir Mehta, Alexander P. Boddy, Richard Lowndes, Michael Rhodes

Introduction Physiology studies are an aid in the diagnosis and management of patients who suffer from gastro-oesophageal symptoms. The purpose of this study was to evaluate the diagnostic value of oesophageal $\mathrm{pH}$ and manometry studies at a university hospita physiology unit over a nine-year period. Methods Between July 1996 and October 2004 there were 1116 referrals for $24 \mathrm{hr}$ oesophageal $\mathrm{pH}$ / manometry to investigate four cardinal symptoms. These were symptomatic gastro-oesophageal reflux $(n=802)$, atypical chest pain $(n=137)$, dysphagia $(n=153)$, and achalasia $(n=74)$. Results Studies were successfully completed in $92.4 \%$. Reasons for failure were patient intolerance (3.3\%) and recording malfunction (4.3\%). Results are shown in the table: Conclusion Symptoms alone are a poor predictor of abnormal oesophageal function. 24 hour $\mathrm{pH}$ and manometry studies are valuable in defining the physiological abnormality in order to allow appropriate treatment.

\begin{tabular}{|c|c|c|c|c|c|}
\hline Symptom subtype & $\begin{array}{c}\text { \% with } \\
\text { spasm }\end{array}$ & $\begin{array}{c}\text { \% with } \\
\text { disordered } \\
\text { peristalsis }\end{array}$ & $\begin{array}{c}\text { \% with } \\
\text { abnormalities of } \\
\text { lower oesophageal } \\
\text { sphincter }\end{array}$ & $\begin{array}{c}\text { \% with } \\
\text { abnormal } \\
\text { reflux }\end{array}$ & $\begin{array}{c}\text { \% with } \\
\text { normal } \\
\text { findings }\end{array}$ \\
\hline $\begin{array}{c}\text { Reflux } \\
\text { (Heartburn/Regurgitation) }\end{array}$ & 18.3 & 50.7 & 37.3 & 51.1 & 11.9 \\
\hline Atypical chest pain & 33.9 & 32.2 & 19.0 & 23.9 & 37.2 \\
\hline Dysphagia & 47.0 & 57.6 & 12.9 & 30.0 & 7.6 \\
\hline Achalasia & 40.4 & 82.5 & 66.7 & $\begin{array}{c}\text { No pH } \\
\text { studies } \\
\text { performed }\end{array}$ & 1.8 \\
\hline
\end{tabular}

\section{S1176}

Heartburn and Regurgitation - Does That Mean I Have GERD? Inder Mainie, Radu Tutuian, Amine Hila, Amit Agrawal, Donald O. Castell

Background: Typical symptoms such as heartburn and regurgitation are believed to be associated with gastroesophageal reflux (GERD). Aim: To clarify if patients presenting with heartburn or regurgitation have abnormal reflux. Method: Patients were evaluated for typical symptoms of heartburn and regurgitation. All were studied off therapy with PPIs discontinued for a minimum of 5 days prior to the study. The $2.1 \mathrm{~mm}$ dual electrode antimony $\mathrm{pH}$ catheters were placed after an overnight fast 5 and $20 \mathrm{~cm}$ above the manometrically located proximal border of the lower esophageal sphincter (LES). Each study was analyzed for percent time $\mathrm{pH}<4$ in the distal (normal values: upright $<6.3 \%$; recumbent $<1.2 \%$ ) and proximal (normal values: upright $<1.3 \%$; recumbent $=0$ ). The symptom index was calculated by dividing the number of symptoms preceded by a reflux episode within a 5 minute window by the total number of symptoms (postive if $\geq 50 \%$ ). Results: $\mathrm{pH}$ studies from 450 patients off therapy (303 female; mean age 53; range 11-88) between July 2002 and October 2004 were analyzed. The mean duration of ambulatory studies was 22 hours and 34 minutes; range 20 - 24 hours. 220 patients had abnormal reflux (121 upright; 156 recumbent and 57 both) 127 patients recorded heartburn, 14 patients recorded regurgitation and 14 recorded both. See table below. Conclusion: These results indicate that typical symptoms are very non-specific in the diagnosis of GERD 


\begin{tabular}{|c|c|c|c|c|}
\hline $\begin{array}{c}\text { Symptoms } \\
\text { (Patients \#) }\end{array}$ & $\begin{array}{c}\text { Positive SI and } \\
\text { abnormal reflux }\end{array}$ & $\begin{array}{c}\text { Positive SI and } \\
\text { normal reflux }\end{array}$ & $\begin{array}{c}\text { Negative SI and } \\
\text { abnormal reflux }\end{array}$ & $\begin{array}{c}\text { Negative SI and } \\
\text { normal reflux }\end{array}$ \\
\hline Heartburn (127) & $55(43 \%)$ & $13(10 \%)$ & $19(15 \%)$ & $40(32 \%)$ \\
\hline $\begin{array}{c}\text { Regurgitation } \\
\text { (14) }\end{array}$ & $5(36 \%)$ & $3(21 \%)$ & $4(29 \%)$ & $2(14 \%)$ \\
\hline Both (14) & $9(65 \%)$ & $3(21 \%)$ & $1(7 \%)$ & $1(7 \%)$ \\
\hline
\end{tabular}

regarding possible over estimations of acid exposure that may occur when estimates are based solely on EPM.

\section{S1179}

Gastroesophageal Reflux Disease in Tehran

Mehdi Nouraie, Maryam Asadi, Hadi Razjouyan, Siavosh Nasseri-Moghaddam

Introduction: GERD is a common, chronic and relapsing disorder with a rising incidence in the western world. The prevalence of gastro-oesophageal reflux disease (GERD) in Asian populations is reported to be lower than that in the west. Population-based data on the prevalence and symptom profile of gastroesophageal reflux disease (GERD) in developing Caucasian countries is lacking. Aim: Our aim was to determine the prevalence of gastrointestinal symptoms and clinical spectrum of GERD in Tehran, Iran and their association with patient characteristics. Methods: A two step screening telephone survey of 1700 simple random Tehran household telephone number using a validated questionnaire was performed. In each answered call a second rapid survey was done to select a random subject aged 1865. Several patient characteristics (age, education, sex) and history of acid regurgitation and heartburn during the last week and three months were questioned. Results: Of the 1700 selected numbers 278 did not answer nor had eligible case, and 220 refused to participate. A total number of 1202 subjects ( $42 \%$ males, mean age: 36 years, range: 18-65 yr) were surveyed. The prevalence of heartburn occurring monthly, weekly, and daily was $4.7 \%$ (95\% CI: 3.5-6.0), $1.6 \%$ (95\% CI, 1.0-2.5), and $0.05 \%$ (95\% CI: 0.02-1.2), respectively. The corresponding figures for acid regurgitation were $15.6 \%$ (95\% CI, 13.6-17.7), $5.7 \%$ (95\% CI, 4.4-7.1) and 1.5 (95\% CI,0.9-2.4) respectively. The prevalence of GERD, defined as heartburn and/or acid regurgitation experienced daily, weekly and monthly was $1.9 \%$ ( $95 \% \mathrm{CI}, 1.2-2.9), 6.8 \%(95 \% \mathrm{CI}, 5.4-8.3)$ and $18.4 \%(95 \% \mathrm{CI}, 16.2-20.6)$. There was no relationship between the prevalence of GERD and either sex, age or education. CONCLUSIONS: Monthly GERD symptoms occur in $18.5 \%$ of the general population in Tehran. Acid regurgitation is more common (4-5 times) than heartburn. Sex, age and level of education do not affect the prevalence of GERD symptoms. phageal junction image (first Z line image) to the first stomach image. Statistical analysis using multiple variance analysis (MANOVA) was used between multiple groups and a Ttest analysis was used between two groups. Results: There was no significant difference ( $>0.05)$ between any of the groups in total esophageal transit time. (See Table 1) There was a significant difference $(\mathrm{p}<0.05)$ in the time the capsule took to pass from the GE Junction into to the stomach in the Barrett's esophagus group vs. the other groups. (See Table 2) Conclusions: Total esophageal transit time of the PillCam ESO ${ }^{\mathrm{tm}}$ was the same in patients with Barrett's esophagus, reflux esophagitis, and NERD compared with normals. However patients with Barrett's esophagus had significantly slower transit times of the PillCam ESO ${ }^{\mathrm{tm}}$ from the first GE Junction image to the first image of the stomach. This suggests that there may be a motility disorder predisposing to the formation of barrett's esophagus.

Table 1: Total Esophageal Transit Time (min)

\begin{tabular}{|c|c|c|c|c|}
\hline & Average & Std & Min & Max \\
\hline Esophagitis & $3.64^{*}$ & 1.95 & 0.15 & 7.18 \\
\hline NERD & $3.92^{*}$ & 2.93 & 0.02 & 13.87 \\
\hline Normal & $2.96^{*}$ & 2.65 & 0.23 & 6.63 \\
\hline Barrett's & $4.06^{*}$ & 4.41 & 0.02 & 27.63 \\
\hline
\end{tabular}

*p $>0.05$

Transit Time From 1st GE Junction Image $\rightarrow$ First Stomach Image (min)

\begin{tabular}{|c|c|c|c|c|}
\hline & Average & Std & Min & Max \\
\hline Esophagitis & $0.62^{\ddagger}$ & 1.29 & 0 & 5.58 \\
\hline NERD & $0.28^{\ddagger}$ & 0.78 & 0 & 3.77 \\
\hline Normal & $0.14^{\star}$ & 0.21 & 0 & 0.63 \\
\hline Barrett's & $1.56^{\ddagger}$ & 3.51 & 0 & 21.62 \\
\hline
\end{tabular}

$\mathrm{p}<0.05$

S1178

Acid Gastroesophageal Reflux Reports in Infants: A Comparison of Esophageal $\mathrm{pH}$ Monitoring and Multichannel Intraluminal Impedance Measurement Techniques

Frederick W. Woodley, Hayat M. Mousa

Background: Over the past decade, several groups have reported successful use of the combined esophageal $\mathrm{pH}$ monitoring (EPM) and multichannel intraluminal impedance (MII) for measuring gastroesophageal reflux (GER) frequency and duration. While previous studies have compared the sensitivity of these two techniques in detecting the frequency of GER, the variable abilities of these two techniques to assess distal esophageal acid GER (AGER) exposure has not been reported. Aim: To test the differences between EPM and pH/MII in detecting acid reflux. Methods: We reviewed parallel EPM and pH/MII reports from 14 infants ( 9 males, median age 3.5 months) who were evaluated over a 24 hr period. Results: Significantly fewer AGER were detected by pH/MII $(25.1 \pm 4.0 /$ patient $)$ compared to EPM $(99.9 \pm 18.3 /$ patient,$p=.001)$. Significantly more time was spent in AGER when determined by EPM $(156.6 \pm 30.4 \mathrm{~min} /$ patient versus $66.6 \pm 15.9 \mathrm{~min} /$ patient, $\mathrm{p}=.002)$. The mean percentage of total study time spent below pH 4 was $9.7 \pm 1.7 \%$ using EPM and $4.7 \pm 1.1 \%$ using $\mathrm{pH} / \mathrm{MII}(\mathrm{p}=.002)$. The mean duration of an individual AGER event was $154.8 \pm$ 20.0 s using $\mathrm{pH} / \mathrm{MII}$ and $94.6 \pm 15.6 \mathrm{~s}$ using EPM $(\mathrm{p}<.0001)$. Of the total AGER episodes detected by EPM, $71.8 \%$ could not be confirmed by pH/MII. Conclusions: Compared to $\mathrm{pH} / \mathrm{MII}$, EPM detects acid exposure that is significantly greater in AGER frequency and duration. Detection of significant numbers of "EPM-only" AGER episodes raises concerns

\section{S1180}

Validation of Structured Scoring Using the La Classification for Esophagitis and Barrett's Esophagus in Asian Patients

Reuben Km Wong, Khay Guan Yeoh, Kok Ann Gwee, Khek Yu Ho

Background The Los Angeles (LA) classification is commonly used to grade reflux esophagitis. Aim To determine the inter-observer agreement and validity of the LA Classification in scoring the severity of esophagitis in Asian patients. Methods Video endoscopy clips were selected from 44 consecutive patients who participated in reflux esophagitis studies. The videos were randomly arranged and annonymised, and viewed by 3 experienced endoscopists blinded to the diagnoses. The endoscopists were required to independently complete a worksheet to score the presence, severity and distribution of esophagitis, including Barrett's esophagus. Each video was subsequently replayed, discussed and a consensus reached on the findings. Statistical analysis was performed using kappa correlation for inter-observer agreement as well as for individual endoscopists against the final consensus grade. Results 10 video clips were excluded for poor image quality or inadequate visualization of the esophageal-gastric junction. 34 clips were scored and analyzed (final consensus LA classification: no esophagitis 5 (15\%), grade A 21 (62\%), grade B 7 (21\%), grade C 1 (3\%), respectively). Twelve patients (35\%) had endoscopic Barrett's esophagus. There was good agreement on the presence of esophagitis (mean $\mathrm{k} 0.79$, range 0.72 to 0.82 ). Observer agreement on the LA grade against the consensus grade was moderate to strong (mean $\mathrm{k}$ 0.76 , range 0.72 to 0.79 ). Evaluation of the extent of esophagitis showed good $k$ values using the alternative approach of grouping by circumferential extent (mean $\mathrm{k} 0.86$, range 0.64 to 0.97 ) at the lower categories of severity. There was good agreement on the presence

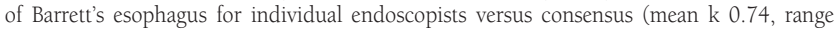
0.75 to 0.80 ) while between endoscopists the mean $\mathrm{k}$ was 0.52 (range 0.46 to 0.62 ). There was a clear trend of increasing inter-observer agreement as viewings progressed, with superior kappa correlation after 15 cases. Conclusions Scoring using the LA classification for esophagitis and the concurrence on the presence of Barrett's esophagus showed moderate to strong agreement amongst Asian endoscopists. Our results also suggested that an alternative method of scoring esophagitis based on proportion of circumferential involvement was reliable. A learning effect was observed and reliable scoring was achieved after 15 patients.

S1181

One Week rabeprazole Trial Is Not Enough to Diagnose Patients with GERDRelated NCCP

Jeong Hwan Kim, Poong-Lyul Rhee, Hee Jung Son, Jae J. Kim, Jong Chul Rhee

Background: Noncardiac chest pain (NCCP) presents as a frequent diagnostic challenge, with patients tending to use a disproportionate level of health care resources. Gastroesophageal reflux disease (GERD) is the most frequent cause of NCCP. Empirical trial with high-dose proton pump inhibitor (PPI) has been shown to be a sensitive tool for diagnosing patients with GERD-related NCCP. However, the optimal duration of PPI trial is defined poorly. Therefore, we aimed to determine the optimal duration of an empirical trial of highdose rabeprazole in detecting patients with GERD-related NCCP Methods: Patients who were referred by a cardiologist after a comprehensive evaluation, with at least two episodes per month of unexplained chest pain as the predominant symptom, were enrolled into this study. Patients completed a daily diary assessing severity and frequency of chest pain as the predominant symptom during 14 days for a baseline symptom assessment. Then, the following evaluations were undergone: upper endoscopy to determine esophageal mucosal disease followed by 24-h esophageal $\mathrm{pH}$ monitoring to assess acid exposure. Patients were then assigned to rabeprazole $20 \mathrm{mg}$ orally twice daily during 14 days. Patients completed the same daily diary throughout the PPI treatment. The rabeprazole empirical trial was considered diagnostic if chest pain score improved $\geqq 50 \%$ than baseline. The assessment of chest pain 
score was performed by day 7 and by day 14. Results: Of the 28 patients with NCCP that were enrolled, 12 (43\%) had erosive esophagitis and/or pathologic acid exposure (GERDpositive) and $16(57 \%)$ had both tests negative (GERD-negative). The rabeprazole empirical trial was considered significantly higher diagnostic in patients with GERD-positive NCCP $(n=9,75 \%)$ than in patients with GERD-negative NCCP $(n=6,38 \%)$ on two weeks PPI trial $(\mathrm{P}<0.05)$. However, there was no significant difference between the two groups on one week PPI trial (GERD-positive vs. GERD-negative $=58 \%$ vs. $38 \%, P=0.24)$. Conclusions: The rabeprazole empirical trial was highly diagnostic in detecting patients with GERD-related NCCP. The optimal duration of the rabeprazole empirical trial in diagnosing patients with GERD-related NCCP was determined as two weeks in this study.

\section{S1182}

Reflux Episodes Are Similar in Asymptomatic African Americans and Non Hispanic Whites

Kenneth Vega, Christina Lambiase, Louis Lambiase

African Americans develop Barrett esophagus at a much lower rate than non Hispanic Whites. This suggests there may be less esophageal acid reflux in African Americans (AA) as opposed to Non Hispanic Whites (W). Very little is known about the prevalence of acid reflux in AA. Further, $24 \mathrm{hr} \mathrm{pH}$ testing has only been validated in $\mathrm{W}$. There is no data establishing normal ranges for $24 \mathrm{hr} \mathrm{pH}$ testing AA. AIM. To determine the normal parameters for 24 hr esophageal $\mathrm{pH}$ probe measurements in AA. Further to compare those values to those obtained from W to determine any differences. Methods: $24 \mathrm{hr} \mathrm{pH}$ probes were performed in asymptomatic AA and $\mathrm{W}$ patients who were classified based on self reported ethnicity. Values for Number of reflux episodes, number of reflux episodes longer than 5 minutes, length of time (minutes) of the longest reflux episode and the total time (minutes) the $\mathrm{pH}$ was less than 4 was recorded. Data was recorded in two channels the distal placed $5 \mathrm{~cm}$ from a manometrically determined lower esophageal sphincter (LES) and the proximal, which was $15 \mathrm{~cm}$ above the LES. Means were compared using an Independent Sample t test. Results: See Tables. $33 \mathrm{AA}$ and $29 \mathrm{~W}$ patients were studied. There were no significant differences between AA and W for all parameters. Conclusion: Normal $24 \mathrm{hr} \mathrm{pH}$ probe values for AA are reported. There appears to be no difference from those values found in W. This would suggest, that if the racial difference in Barrett esophagus incidence is due to differences in esophageal acid exposure, that difference does not occur in asymptomatic patients.

Proximal Channe

\begin{tabular}{|c|c|c|c|c|}
\hline & Number of Episodes & Number $>5$ min & Longest (minutes) & Total time $\mathrm{pH}<4$ (minutes) \\
\hline AA & 34.27 S.D. 29.03 & 0.27 S.D. 0.88 & 3.3 S.D. 8.7 & 13.03 S.D. 3.46 \\
\hline W & 31 S.D. 36.35 & 0.28 S.D. 0.99 & 4.31 S.D. 9.34 & 14.48 S.D. 4.13 \\
\hline & $\mathrm{p}=.77$ & $\mathrm{p}=.99$ & $\mathrm{p}=.662$ & $\mathrm{p}=.787$ \\
\hline
\end{tabular}

Distal Channel

\begin{tabular}{|c|c|c|c|c|}
\hline & Number Episodes & Number $>5$ min & Longest episode (minutes) & Total Time $\mathrm{ph}<4$ (minutes) \\
\hline AA & 62 S.D. 43.01 & 0.54 S.D. 1.02 & 7.63 S.D. 14.62 & 29.54 S.D. 32.84 \\
\hline W & 50 S.D. 37.30 & 0.8 S.D. 1.57 & 7.00 S.D. 10.22 & 23.47 S.D. 33.13 \\
\hline & $\mathrm{p}=0.377$ & $\mathrm{p}=0.536$ & $\mathrm{p}=0.886$ & $\mathrm{p}=0.579$ \\
\hline
\end{tabular}

\section{S1183}

Ginger-Ale Swallow Test to Locate the BRAVO pH Capsule in the Esophagus Kozhikot Kumaran

BACKGROUND AND AIM: Bravo capsule ambulatory esophageal pH testing has become the standard procedure in evaluating symptoms related to gastroesophageal reflux disease. Aim of this study is to use ginger-ale swallow to verify the location of Bravo $\mathrm{pH}$ capsule in the esophagus. Occasionally the $\mathrm{pH}$ capsule may become detached from the wall of the esophagus soon after it is implanted, making the test invalid. A sustained acidic pH below 3 will suggest the capsule is in the stomach, requiring the need to implant a second capsule to continue with the test. However, if there is no acid in the stomach due to the use of PPI, bile reflux or other conditions, the $\mathrm{pH}$ measurement alone will cause incorrect localization. To circumvent this problem, we come up with the "ginger ale swallow test". METHOD: Standard EGD procedures established for the Bravo $\mathrm{pH}$ tests were followed in all patients. Prior to the implantation of the $\mathrm{pH}$ capsule in the esophagus, we routinely aspirated the gastric juice using the endoscope for $\mathrm{pH}$ determination. Ginger-ale swallow test was performed soon after the patient recovered from sedation and was able to swallow liquids. While observing the $\mathrm{pH}$ reading in the Bravo monitor, each patient was allowed to swallow about two ounces of room temperature ginger-ale ( $\mathrm{pH}$ 2.8) followed by a swallow of water, or the patient's own saliva. If the capsule was located in the esophagus, a transient drop in the $\mathrm{pH}$ reading below 4 was observed as the bolus of ginger-ale passed through the distal esophagus, followed by the rise in $\mathrm{pH}$ to the esophageal $\mathrm{pH}$ level of about 6 . A sustained low $\mathrm{pH}$ below 4 indicated the capsule location in the stomach, requiring the need for the implantation of a second capsule for the successful completion of the study. RESULTS: Out of the 40 consecutive tests performed, six patients $(15 \%)$ had no acidic $\mathrm{pH}$ in the stomach and the ginger-ale swallow test helped to verify the location of the capsule in all 5 patients. In one patient (3\%), a second capsule had to be placed in the esophagus as the first capsule fell off the esophagus because the $\mathrm{pH}$ reading after the placement of the first capsule was indicative of its dislodgement into the stomach. This was confirmed by direct endoscopy. In one case (3\%) the capsule did not slough off the esophageal wall even after 30 days as verified by radiology, and was retrieved safely by the endoscope. No complications occurred in any of these patients with ginger- ale test. CONCLUSION: Ginger-ale swallow test is very simple, safe and useful in testing the proper location of the $\mathrm{pH}$ capsule after endoscopic placement and prior to the patient discharge.

\section{S1184}

Normal Values for Ambulatory 24-H Combined Impedance-pH Monitoring On Acid Suppressive Therapy

Radu Tutuian, Inder Mainie, Amit Agrawal, Janice Freeman, Donald O. Castell

Background: Combined multichannel intraluminal impedance and $\mathrm{pH}(\mathrm{MII}-\mathrm{pH})$ is increasingly used to monitor patients with persistent reflux symptoms on proton pump inhibitor (PPI) therapy. The normal range of the number of reflux episodes "on therapy" $(<73)$ was determined by extrapolating data from healthy volunteers "off therapy" (Shay et al. Am Gastro 2004) and by assuming that PPI primarily change the $\mathrm{pH}$ of the refluxate withou affecting the total number of reflux episodes as seen during post-prandial studies (Vela e al. Gastroenterology 2001). Aim: Evaluate the effects of twice daily PPI on reflux 24-h parameters during combined MII-pH monitoring and define normal values "on therapy". Methods: Healthy volunteers underwent combined MII-pH monitoring before and after dosing with esomeprazole $40 \mathrm{mg}$ bid AC for 5 days. The 8 channel combined MII-pH probe recorded impedance data at $3,5,7,9,15$ and $17 \mathrm{~cm}$ above the LES and $\mathrm{pH}$ data $5 \mathrm{~cm}$ above and $10 \mathrm{~cm}$ below the LES. In each subject we counted the total number of MIIdetected reflux episodes, the number of acid reflux episodes (MII-detected reflux episodes with intraesophageal $\mathrm{pH}<4$ ) and the number of non-acid reflux episodes (MII-detected reflux episodes with intraesophageal $\mathrm{pH}>4$ ). Results: Twenty healthy volunteers $(12 \mathrm{~F}$, age mean 32y, range 18-67y) completed baseline and "on therapy" impedance-pH monitoring There was a statistically significant decrease in the total number of reflux episodes and the number of acid reflux episodes at 5 and $15 \mathrm{~cm}$ above the LES during dosing with esomeprazole compared to baseline. The number of non-acid reflux episodes was unchanged during dosing with PPI compared to baseline. Conclusions: Acid suppressive therapy reduces the number of acid reflux episodes but not the number of non-acid reflux episodes. The revised upper limit of normal for reflux episodes on PPI therapy $(<48)$ should improve the sensitivity of identifying patients with an abnormal number of reflux episodes on acid suppressive therapy

\begin{tabular}{|c|c|c|c|}
\hline & Baseline $(\mathrm{N}=20)$ & Esomeprazole 40mg bid (N=20) & -value \\
\hline Total @5cm above LES & $42 \pm 19$ & $22 \pm 13$ & $<0.001$ \\
\hline Acid @ $5 \mathrm{~cm}$ above LES & $23 \pm 14$ & $2 \pm 5$ & $<0.001$ \\
\hline Nonacid @ $5 \mathrm{~cm}$ above LES & $19 \pm 7$ & $20 \pm 12$ & 0.73 \\
\hline Total @ 15cm above LES & $14 \pm 10$ & $7 \pm 6$ & 0.006 \\
\hline Acid @ 15cm above LES & $10 \pm 8$ & $1 \pm 2$ & $<0.001$ \\
\hline Nonacid @ $15 \mathrm{~cm}$ above LES & $4 \pm 3$ & $6 \pm 6$ & 0.29 \\
\hline
\end{tabular}

Data are presented as mean $\pm \mathrm{SD}$; $\mathrm{p}$-value for paired Student T-test

\section{S1185}

Discontinuation of Proton Pump Inhibitors in Patients On Long-Term Therapy: A Double-Blind, Placebo-Controlled Trial

Einar Bjornsson, Hasse Abrahamsson, Magnus Simren, Niklas Mattsson, Pia Agerforz, Anders Kilander

Background: The proportion of PPI users on long-term therapy who could discontinue PPIs without developing symptoms is unknown. It has been suggested that acid rebound followin treatment with PPIs can make it more difficult to stop PPIs due to exacerbation of symptoms. We aimed to study what proportion of long-term PPI users is able to discontinue their medication and whether tapering down the PPIs makes it easier to stop PPIs. Method: Patients buying PPIs on prescription at the pharmacy were asked to complete a questionnaire concerning the indication for the medication and their symptomatology. Patients using PPIs for at least two months, without a history of peptic ulcer or esophagitis were asked to participate and examined with upper endoscopy. If an ulcer or esophagitis was observed the patient was excluded. Remaining patients were randomized double-blindly to taper down or continue with a constant dosage of omeprazole for three weeks. Thereafter, all patients discontinued PPI therapy. Results: A total of 115 patients underwent endoscopy, 19 patients were excluded due to organic GI disease (16 had esophagitis). Of the 96 enrolled (median age 63 years, 52 females), who had used PPI for a median time of 48 months (IQR 22-87; range 4-180 months), 23 (24\%) had helicobacter pylori, 75 (78\%) had GERD and 21 (22\%) other indications. A total of 26 patients (27\%) did not use PPIs during the year after discontinuation. A total of $31 \%$ randomized to tapering discontinued PPIs whereas $22 \%$ of those who did not taper PPIs could discontinue therapy (NS). Sex, age, duration of PPI therapy or HP status did not predict PPI requirement. GERD patients were more prone to resume PPI therapy than those with other indications. Only 16 (21\%) of GERD patients were off PPIs compared with $48 \%$ of patients without GERD ( $<<0.05)$. GERD patients resumed their PPI therapy after a median time of 18 days (4-34; range 4-193) whereas the non-GERD patients resumed therapy 95 days (17-114; range 22-203) after the three week study drug period $(\mathrm{p}<0.0001)$. Serum gastrin was higher at baseline in GERD patients who resumed PPIs vs. those who did not ( $59 \mathrm{pmol} / \mathrm{L}$ (36-81) vs. 38 (25-57); $\mathrm{p}=0.03$ ). GERD and serum gastrin were independent predictors of PPI requirement. Conclusions: Discontinuation of PPIs was successful in $27 \%$ of long-term PPI users. Patients with GERD were less likely to discontinue PPIs than those with non-GERD. Tapering down the PPIs did not significantly increase the possibility of a successful discontinuation. Hypergastrinaemia appeared to be an important predictor of PPI requirement in GERD patients. 
Predictors for Treatment Failure of On-Demand Proton Pump Inhibitor (PPI) Therapy in Gastroesophageal Reflux Disease (GERD)

Justin Wu, Carrian Cheung, Vincent Wong, Joseph Sung

BACKGROUND: It is unclear whether on-demand PPI is equally effective as step-down therapy in patients with non-erosive reflux disease (NERD) and reflux esophagitis (RE). AIM: To compare the efficacy of on-demand PPI therapy in NERD and RE patients and identify the predictors for treatment failure. METHODS: We prospectively recruited consecutive patients with weekly attacks of heartburn or acid regurgitation. HP eradication was completed before recruitment. Exclusion criteria included high grade (B or above) RE, previous gastric surgery, recent use of NSAID or PPI, and peptic ulcer. All patients completed a self-administered questionnaire for baseline assessment of reflux symptom. Diagnosis of concomitant functional dyspepsia (FD), irritable bowel syndrome (IBS) and psychological disorders (PD) were recorded. RE and hiatus hernia (HH) were assessed by EGD. Esophageal manometry (EM), Bernstein test (BT) and 24-hour pH metry were performed for all patients. Esomeprazole $20 \mathrm{mg}$ daily were given for 8 weeks after baseline assessment. Patients with complete symptom resolution were then enrolled to open-label, on-demand Esomeprazole $20 \mathrm{mg}$ for 26 weeks. Treatment failure was defined as need of Esomprazole in $>/=75 \%$ of time for satisfactory symptom control at 26 weeks. NERD and RE patients were compared for treatment failure rates after 26-week on-demand PPI therapy. Potential predictors (age, gender, RE, $\mathrm{HH}$, baseline reflux severity, FD, IBS, PD, positive BT, abnormal $\mathrm{pH}$ study, ineffective esophageal motility (IEM: failed peristalsis $>/=30 \%$ ) and weak lower esophageal sphincter $(\mathrm{LESP}<10 \mathrm{mmHg})$ ) were determined using multivariate logistic regression model. RESULTS: 182 patients (NERD: 113, RE: 69; mean age 52+/-16.8, male: 48\%) were studied. NERD patients had significantly higher prevalence of FD $(65 \mathrm{Vs} 42 \%, \mathrm{p}=0.003)$ and positive BT (4l Vs 20\%, $\mathrm{p}=0.004$ ) than RE patients. Both groups had comparable symptom resolution rate after 8-week daily PPI (NERD Vs RE: 92.0 Vs $97.1 \%, \mathrm{p}=0.16$ ). However, NERD patients had significantly higher treatment failure rates after 26-week on-demand PPI therapy (36.3\% Vs $20.3 \%, p=0.023$ ) than RE patients. Multivariate analysis showed that daily reflux symptom (OR: 2.1, $\mathrm{p}=0.001)$, FD (OR: 1.7, $\mathrm{p}=0.02)$ and positive $\mathrm{BT}(\mathrm{OR}: 1.6, \mathrm{p}=0.03)$ were the significant predictors for treatment failure of on-demand PPI. NERD was not a predictor (OR: 1.1, p=0.73). CONCLUSION: Daily reflux symptom, concomitant FD and positive BT are associated with failure of on-demand PPI therapy in GERD patients. Although NERD apparently has higher treatment failure rate, it is actually confounded by higher prevalence of FD and esophageal sensitivity.

\section{S1187}

Proton Pump Inhibitor Therapy for Suspected GERD - Related Chronic Laryngitis: Is It Effective? A Meta-Analysis

Mohammed A. Qadeer, Rocio Lopez, J. Pieter Noordzij, David L. Steward, Maria Suurna, Thomas Havas, Wo John, Colin W. Howden, Michael F. Vaezi

Background: Chronic laryngitis (CL) suspected to be GERD-related is increasingly diagnosed and usually treated with proton pump inhibitors (PPIs). Since placebo-controlled trials have reported mixed results, we performed and report the first meta-analysis of the randomized controlled trials (RCTs). Methods: Literature search was conducted using Medline, Cochrane database and Embase. Individual authors were contacted if only abstracts were available without published manuscripts. RCTs were included if they used PPIs, and enrolled patients with chronic laryngeal symptoms but excluded if patients with only chronic cough or chest pain were enrolled and if the model suggested significant study heterogeneity. The primary endpoint was at least $50 \%$ reduction in the primary symptom or overall symptom score. Each study was assessed for quality and subjected to sensitivity analysis. Heterogeneity was evaluated by Q statistic and a random-effects model was used was used to calculate the summary odds ratio (OR). Results: Overall, seven RCTs qualified and included 325 patients (186 in the PPI group and 139 in placebo group). There was no significant heterogeneity $(\mathrm{X} 2=7.63 ; \mathrm{p}=0.27)$ and the quality scores ranged from moderate to high. None of the individual RCTs achieved statistical significance. The summary OR in favor of PPIs was 1.49 (95\% CI 0.82-2.69) (Figure 1; Forest plot). Sensitivity analysis did not change the summary OR significantly suggesting that the result was robust to the exclusion of individual RCTs. Conclusion: 1) This meta-analysis of RCTs suggests that there is no significant difference between PPIs and placebo for the treatment of suspected GERD-related CL. 2) The dichotomy between clinical practice and the results of this meta-analysis suggests over diagnosis of GERD-related CL

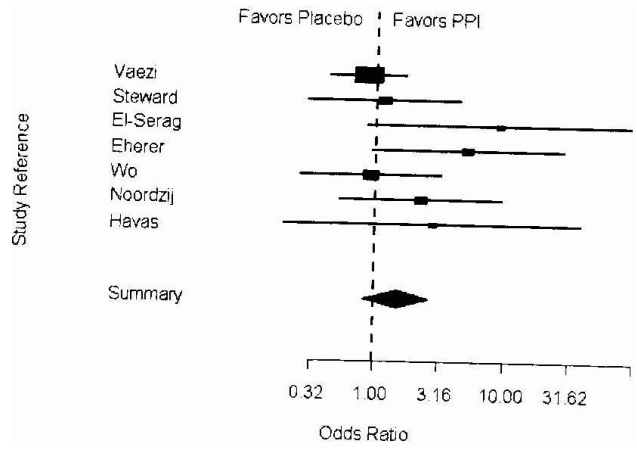

Forest plot displaying individual and summary odds ratio.
Involvement of Cannabinoid Receptors in the Triggering of TLESRs in Healthy Subjects

Hanneke Beaumont, Magnus Ruth, Jorgen Jensen, Anders Lehmann, Guy Boeckxstaen

Background/ Aims: Transient Lower Esophageal Sphincter Relaxations (TLESRs) are the main mechanism underlying gastroesophageal reflux and are a potential target in the treatment of gastroesophageal reflux disease. Previous animal experiments have showed that activation of cannabinoid $(\mathrm{CBl})$ receptors reduces the occurrence of TLESRs. To determine whether these receptors also control TLESRs in humans, we evaluated the effect of the CB receptor agonist delta-9-tetra-hydrocannabinol (THC) on meal induced triggering of TLESRs in healthy volunteers. Methods: The effect of THC (marinol ® 10 and $20 \mathrm{mg}$, p.o.) was evaluated on the occurrence of postprandial TLESRs in a randomized double blind placebo controlled study. On 3 different study days, 18 healthy volunteers (19-51, M) underwent combined esophageal manometry / pH metry using a 10 channel water perfused sleeve catheter. TLESRs were evoked by meal ingestion. Basal LES pressure was determined as visual means of one minute periods every 15 minutes. Samples for plasma concentration measurements were collected. For statistical analysis, paired Student's t-tests were used and data is presented as mean $\pm S E$, except the number of TLESRs which are presented as median and interquartile range. Results: All subjects tolerated the lowest THC dose. However, due to nausea and vomiting in the first hour after meal intake, only 8 subjects completed the $20 \mathrm{mg}$ THC session. Meal ingestion resulted in an increase in TLESRs mainly during the first postprandial hour. This increase was dose dependently inhibited by THC compared to placebo (placebo 8.0 (6.0-9.3); THC 10 mg 5.0 (3.8-7.0); THC 20 mg 2.5 (0.0- 7.3), ( $<<0.018)$ ). No significant effect was observed in the second and third postprandial hour. In addition, THC $10 \mathrm{mg}$ significantly reduced basal LES pressure at every measured time point during the first postprandial hour compared to placebo (mean LES pressure at 45 min after meal intake: placebo $12 \pm 2 \mathrm{mmHg}$; THC $10 \mathrm{mg} 6 \pm 1 \mathrm{mmHg}(\mathrm{P}<0.011)$; THC $20 \mathrm{mg} 5 \pm 1 \mathrm{mmHg}$ (NS)). The number of acid reflux episodes did not differ between THC and placebo (placebo $4 \pm 1$; THC $10 \mathrm{mg} 4 \pm 1$; THC $20 \mathrm{mg} 3 \pm 1$ (NS)). Conclusion: THC significantly reduces basal LES pressure and inhibits the increase in TLESRs evoked by meal ingestion. Based on these findings, we conclude that $\mathrm{CB}$ receptors are involved in the triggering of TLESRs in humans.

\section{S1189}

How Do Consumers Take "Over-the-Counter" Omeprazole?

Nicholas J. Shaheen, Karina Berenson, John Doyle, Kurt Brown

BACKGROUND \& AIM: Over-the-counter omeprazole (Prilosec OTCTM [POTC]; Procter $\&$ Gamble), the only available OTC proton pump inhibitor in the United States, is intended for the treatment of frequent heartburn ( $\geq 2 \mathrm{~d} / \mathrm{wk}$ ). Product directions instruct patients to take 1 tablet once a day for 14 days and not repeat the 14-day courses more often than every 4 months, unless directed by a physician. The aim of this study was to assess the actual dosing patterns and acid suppressive medication use in consumers purchasing POTC. METHODS: POTC users were identified and recruited via telephone and the Internet among subjects who reported the purchase of pharmaceutical items at US retail outlets. Those subjects agreeing to participate were administered an Internet-based questionnaire consisting of questions about demographics, reasons for use, patterns of use, insurance status, and perceived knowledge about gastroesophageal reflux disease (GERD). RESULTS: Of the 1076 eligible POTC users identified, $93.5 \%$ agreed to participate. The mean (SD) age was 51.7 (12.0) years, and $63 \%$ were women; $88 \%$ reported having health insurance ( $52 \%$, managed care; $16 \%$, Medicare; $11 \%$, indemnity; 3\%, Medicaid; $9 \%$, subject uncertain; $8 \%$, other), and $91 \%$ of these insured subjects reported having prescription drug coverage. Fifty-five percent of subjects began taking POTC $>1$ year ago. Most subjects taking POTC $(80 \%)$ were taking the drug every day. On the days POTC was used, subjects took an average of 1.25 pills per day. Of POTC users, 33\% had not received a previous diagnosis of GERD from health care providers. Of the subjects who had not previously received a GERD diagnosis, $77 \%$ were taking POTC every day; these subjects used the drug an average of 23 weeks per year. In the past 12 months, 53\% of POTC users reported antacid medication use; 37\% reported use of an $\mathrm{H} 2$-receptor antagonist; and 32\% reported use of a prescription proton pump inhibitor. CONCLUSIONS: Consumers who had not received a GERD diagnosis and who bought POTC used it for a longer time and at doses higher than recommended. Polypharmacy for GERD was common in POTC users. The public's use of POTC varied substantially from what is recommended by the manufacturer. Supported by AstraZeneca

S1190

Probiotics As Prevention of Infections in GERD-Affected Children Taking Gastric Acidity Inhibitors

Roberto Berni Canani, Gianluca Terrin, Silvia Salvatore, Pia Cirillo, Annalisa Passariello, Riccardo Muscariello, Maria Concetta Siani, Francesco Porcaro, Alfredo Guarino

Background. We have demonstrated that gastric acidity (GA) inhibitors therapy, including histamine-2 receptor antagonists and proton pump inhibitors, increases the risk of acute gastroenteritis (AG) and community-acquired pneumonia (CAP) in gastroesophageal reflux disease (GERD)-affected children (Pediatrics, 2006 in press). These effects are at least in part attributable to modifications in intestinal microflora composition. Probiotics are able to positively influence intestinal microflora composition and to limit the risk of infection. We aimed to test the hypothesis that probiotics could reduce the risk of infection deriving from GA-inhibitors therapy. Participants and Methods. We conducted a prospective, randomized, case-controlled study in children (age range 4-36 months) consecutively referred for GERD and treated with GA inhibitors. Exclusion criteria were: history of GA inhibitors therapy in the previous 4 months, H.pylori infection, diabetes, chronic lung or heart diseases, immunodeficiency, allergy, congenital motility gastrointestinal disorders, neuromuscular diseases or malnutrition. GA-inhibitors (omeprazole, $1 \mathrm{mg} / \mathrm{kg} /$ day) were prescribed by the physicians for 8 weeks. Enrolled patients were randomly assigned to one of the following treatment groups: Group 1, received a commercially available probiotic mixture (YOVIS, 
Sigma Tau, Italy) containing at least 290 billion viable lyophilized bacteria per gram (S.salivarus subsp.thermophilus, B.breve, B.infantis, B.longum, L.acidophilus, L.plantarum, L.casei, L.delbrueckii subsp. Bulgaricus, S.faecium), $500 \mathrm{mg}$ /day given orally for 8 weeks; Group 2 , received placebo powder containing starch which looked identical to the study agent for 8 weeks. Main Outcome Measures. Number of patients presenting AG or CAP during a 8 weeks follow up period. Results. 70 subjects were enrolled. Eight were excluded (6 in Group 1, 2 in Group 2) within the first 2 weeks from the enrollment because insufficient compliance. We obtained data in 62 subjects: 32 in Group 1, and 30 in Group 2. The two groups were comparable for demographics characteristics and for AG and CAP incidence in the previous 8 weeks. Rate of subjects presenting AG (30\% vs. $9 \%, p=0.041)$ and CAP $(37 \%$ vs. $6 \%, p=$ 0.004 ) was significantly increased in the placebo group compared to probiotics-treated children. In addition, the number of patients taking antibiotics was significantly higher in the placebo group ( $37 \%$ vs. $6 \%, p=0.004$ ). Conclusions. Probiotics could be able to limit the risk of infections in GA-inhibitors treated children. Our results support the importance of microflora as crucial factor to prevent infectious diseases in children.

\section{S1191}

Exposure of the Esophagus to Acid in Patients with Reflux Treated with Tegaserod

M Bafutto, C Carvalho Aquino, A S. Borges

BACKGROUND: Studies have shown that tegaserod (T), a selective 5-HT 4 agonist, enhances production of salivary buffers, reduces post-prandial gastroesophageal reflux and improves gastric emptying. ${ }^{1-3}$ As the direct effect of $\mathrm{T}$ on acid exposure is unknown, this study evaluated esophageal acid exposure in patients with reflux treated with T. METHODS: At baseline (pre-T), 14 patients with reflux ( 3 male, 11 female) underwent 24-hour esophageal pHmetry (proximal electrode placed $20 \mathrm{~cm}$ above the lower esophageal sphincter (LES), distal electrode placed $5 \mathrm{~cm}$ above the LES). Patients received T $6 \mathrm{mg}$ b.i.d. for 7 days. pHmetry was repeated after the first $\mathrm{T}$ dose on Day 7. Medications that may interfere with acid secretion and/or gastric motility were not permitted. Electrode measurements were collected after food, and in standing and supine positions. Esophageal clearance ( $\mathrm{min} / \mathrm{reflux}$ ) was recorded and pathologic acid reflux episodes (defined as $\mathrm{pH}<4$ ) were evaluated using Castell parameters (proximal electrode) and the DeMeester score (distal electrode). Statistical analyses were performed using paired $t$ tests. RESULTS: T significantly reduced the number of supine acid reflux episodes, measured at the distal electrode (pre- vs post-T, mean[SEM]: $18.43[5.13]$ vs $5.57[1.18] \mathrm{p}=0.022$ ). This difference almost reached significance at the proximal electrode $(2.79[0.81]$ vs $0.93[0.45] \mathrm{p}=0.056)$ and there were non-significant trends for fewer reflux episodes post-T measured standing after food and overall. Compared with pre-T, T significantly reduced the overall duration of esophageal exposure to acid (29.50[7.56] vs $10.43[3.52] \mathrm{p}=0.03,106.07[13.88]$ vs $61.07[14.10] \mathrm{p}=0.03$, proximal and distal electrodes, respectively) and duration of supine esophageal exposure to acid (14.57[7.84] vs $2.36[1.53] \mathrm{p}=0.138 ; 39.43[11.02]$ vs $13.00[3.93] \mathrm{p}=0.033$, proximal and distal electrodes, respectively). Non-significant trends for reduced duration of reflux episodes post-T were also observed for standing and post-prandial measurements. Clearance of esophageal acid was more rapid after $\mathrm{T}$ compared with pre- $\mathrm{T}$, the difference being significant at the proximal electrode (3.88[1.04] vs $1.41[0.22] \mathrm{p}=0.022,1.96[0.18]$ vs $1.57[0.31] \mathrm{p}=0.288$ for proximal and distal electrodes, respectively). CONCLUSIONS: T reduced the number of acid reflux episodes, duration of esophageal exposure to acid and esophageal clearance of acid. T may improve esophageal acid clearance through its prokinetic properties and therefore may be of clinical benefit in patients with reflux. 1 Degen. Aliment Pharmacol Ther 2001;15:1745-51 2 Jaworski. Dig Dis Sci 2005;50:357-65 3 Kahrilas. Aliment Pharmacol Ther 2000;14:1503-9

\section{S1192}

Predictors of Endoscopic Remission After Healing and Maintenance Therapy for Reflux Esophagitis: Results from the Expo Study

Joachim Labenz, David Armstrong, Sofia Andersson, Stefan Eklund, Andreas Leodolter

Objective: The EXPO study compared esomeprazole and pantoprazole treatment for the healing and maintenance of remission in patients with reflux esophagitis (RE). This analysis of management (healing plus maintenance) identified factors predicting endoscopic remission at the end of the management period. Methods: In a multicenter, randomized, doubleblind study (SH-NEG-0008), patients with endoscopically confirmed RE and symptoms of gastroesophageal reflux disease (GERD) were randomized to once-daily (qd) esomeprazole $40 \mathrm{mg}(\mathrm{n}=1562)$ or pantoprazole $40 \mathrm{mg}(\mathrm{n}=1589)$ for $4-8$ weeks. Patients without moderate/ severe symptoms of heartburn and acid regurgitation, and with healed RE, were then randomized to receive maintenance treatment with esomeprazole $20 \mathrm{mg}$ qd $(\mathrm{n}=1377)$ or pantoprazole $20 \mathrm{mg}$ qd ( $\mathrm{n}=1389)$ for 6 months. Factors affecting the likelihood of remission were examined in patients who were randomized to the same medication during both the healing and maintenance phases (esomeprazole: $n=772$, pantoprazole: $n=797$ ) Proton pump inhibitor (PPI), age, severity of RE, Barrett's esophagus, hiatus hernia, Helicobacter pylori status, BMI, dysphagia, gender, epigastric pain, acid regurgitation, severity of heartburn and history of GERD symptoms were investigated in univariate analyses using logistic regression. The covariates identified as significant $(\mathrm{p}<0.05)$ were included in the multivariate analysis. Remission was defined as absence of mucosal breaks according to Los Angeles grading at final endoscopy. Results: Univariate analyses identified PPI choice, age, RE severity, presence of Barrett's esophagus, $H$. pylori status and BMI as significant prognostic factors for remission of RE. Multivariate analysis confirmed that all of these factors, with the exception of Barrett's esophagus, had an independent effect on remission at the end of RE management treatment (Table). Conclusion: PPI choice of esomeprazole, older age, less severe baseline RE, positive H. pylori status and BMI less than 30 all have a positive influence on management of RE. Estimated odds ratio for factors affecting endoscopic remission (ITT population)

\begin{tabular}{|c|c|}
\hline Prognostic factor & Odds ratio for remission $(95 \% \mathrm{CI})$ \\
\hline Esomeprazole treatment (vs. pantoprazole) & $1.61(1.30-2.00) * * * *$ \\
\hline Age (per year) & $1.02(1.01-1.02)^{* * * *}$ \\
\hline LA grade A+B GERD (vs. C+D) & $1.90(1.49-2.42) * * * *$ \\
\hline H. pylori-positive (vs. negative/missing) & $1.37(1.07-1.75)^{*}$ \\
\hline BMI <30 (vs. $\geq 30)$ & $1.36(1.07-1.71)^{*}$ \\
\hline
\end{tabular}

$* \mathrm{p}<0.05 ; * * * \mathrm{p} \leq 0.0001$

S1193

The Effect of Repeated Doses of Esomeprazole On Pharmacodynamic and Pharmacokinetic Variables in Infants Aged 1 to 24 Months with GERD Taher Omari, Geoff Davidson, Patrik Bondarov, Emma Naucler, Catharina Nilsson-Pieschl, Per Lundborg

AIM: To measure the effects of repeated doses of esomeprazole on the pharmacokinetics (PK), intragastric $\mathrm{pH}$, intraesophageal $\mathrm{pH}$ and reflux episodes in patients, aged 1-24 months, with gastroesophageal reflux disease (GERD) symptoms. METHODS: In this single-center, randomized, single-blind study (SH-NEC-0001) infants with symptoms of GERD (irritability/ crying, vomiting, choking/gagging) and $\geq 5 \%$ time with intraesophageal $\mathrm{pH}<4$ received oral esomeprazole $0.25 \mathrm{mg} / \mathrm{kg}$ or $1 \mathrm{mg} / \mathrm{kg}$ for 8 days. On day 7 or 8 blood samples $(0.6 \mathrm{ml})$ were taken before, and $0.5,1,1.5,2,3,4$ and 6 hours after dosing in patients aged $\geq 3$ months and $1,2,3,4$,and 6 hours after dosing for patients aged $\geq 1$ month to $<3$ months. 24-hour esophageal and gastric $\mathrm{pH}$ were measured at baseline and on day 7 or 8 using a dual $\mathrm{pH}$ probe. RESULTS: 50 infants were randomized to treatment. The mean percentage of time with intragastric $\mathrm{pH}>4$ at baseline was $30.5 \%$ and $28.6 \%$ in the esomeprazole $0.25 \mathrm{mg} / \mathrm{kg}$ and $1 \mathrm{mg} / \mathrm{kg}$ groups, respectively. After treatment, the mean percentage of time with intragastric $\mathrm{pH}>4$ was significantly higher with esomeprazole $1 \mathrm{mg} / \mathrm{kg}(69.3 \%)$ than with esomeprazole $0.25 \mathrm{mg} / \mathrm{kg}(47.9 \%)(\mathrm{p}<0.001)$. The mean percentage of time with intraesophageal $\mathrm{pH}<4$ at baseline was $11.6 \%$ and $12.5 \%$ in the esomeprazole $0.25 \mathrm{mg} / \mathrm{kg}$ and $1 \mathrm{mg} / \mathrm{kg}$ groups respectively. Both esomeprazole dosages decreased the percentage of time with intraesophageal $\mathrm{pH}<4$, to means of $8.4 \%$ and $5.5 \%$, respectively. On day $7 / 8$, the number of reflux episodes longer than 5 minutes decreased from a mean of 6 at baseline to 3 and 2 with esomeprazole $0.25 \mathrm{mg} / \mathrm{kg}$ and $1 \mathrm{mg} / \mathrm{kg}$, respectively. For both dosages there was large interindividual variability in the PK variables. Numerically there was a larger-than-dose proportional increase in AUC, AUCtao and steady state maximum concentration (CSSmax) between dosages, although this was not statistically significant. Both dosages of esomeprazole were well-tolerated. CONCLUSIONS: Repeated administration of $0.25 \mathrm{mg} / \mathrm{kg}$ and $1 \mathrm{mg} / \mathrm{kg}$ of esomeprazole resulted in a dose-related increase in the percentage of time with intragastric $\mathrm{pH}>4$, and a reduction in the percentage of time with intraesophageal $\mathrm{pH}<4$, in infants aged 1-24 months with GERD. Pharmacokinetic results

\begin{tabular}{|c|c|c|}
\hline Variable & Esomeprazole dose & Estimated geometric mean $(95 \% \mathrm{CI})$ \\
\hline AUCt $(\mu \mathrm{m} * \mathrm{~h} / \mathrm{L})$ & $0.25 \mathrm{mg} / \mathrm{kg}(\mathrm{n}=17)$ & $0.23(0.11-0.48)$ \\
\hline & $1 \mathrm{mg} / \mathrm{kg}(\mathrm{n}=18)$ & $1.79(0.89-3.64)$ \\
\hline AUCtao $(\mu \mathrm{m} * \mathrm{~h} / \mathrm{L})$ & $0.25 \mathrm{mg} / \mathrm{kg}(\mathrm{n}=9)$ & $0.65(0.27-1.57)$ \\
\hline & $1 \mathrm{mg} / \mathrm{kg}(\mathrm{n}=7)$ & $3.51(1.28-9.59)$ \\
\hline $\mathrm{CSSmax}(\mu \mathrm{mol} / \mathrm{L})$ & $0.25 \mathrm{mg} / \mathrm{kg}(\mathrm{n}=17)$ & $0.17(0.09-0.31)$ \\
\hline & $1 \mathrm{mg} / \mathrm{kg}(\mathrm{n}=17)$ & $0.85(0.45-1.60)$ \\
\hline $\mathrm{t}_{1 / 2}(\mathrm{~h})$ & $0.25 \mathrm{mg} / \mathrm{kg}(\mathrm{n}=9)$ & $0.77(0.55-1.08)$ \\
\hline & $1 \mathrm{mg} / \mathrm{kg}(\mathrm{n}=8)$ & $0.95(0.66-1.35)$ \\
\hline
\end{tabular}

\section{S1194}

A Comparison of Clinical, Demographic, and Endoscopic Findings Among Patients with Erosive and Non-Erosive Reflux Disease

Sachin Wani, Ajay Bansal, Priya Maddur, Sandi Hall, Srinivas R. Puli, Amit Rastogi, April Higbee, Prateek Sharma

Background: Gastroesophageal reflux disease (GERD) is a common disorder associated with a huge economic burden. Erosive esophagitis is present in 30-40\% of GERD patients with the remaining 60-70\% classified to have non erosive reflux disease (NERD). The factors associated with and that could possibly predict NERD in patients with reflux symptoms are not known. Aims: The aim of this study was to compare clinical, demographic, and endoscopi findings among patients with NERD and EE. Methods: Consecutive patients presenting to the GI endoscopy unit (for their first upper endoscopy ever) for the evaluation of GERD were asked to complete a validated GERD questionnaire (GERQ). The questionnaire documents the duration of GERD symptoms (heartburn, acid regurgitation) and their frequency and severity over the past year. Patient demographics (age, gender, ethnicity), clinical features (smoking, $\mathrm{ETOH}, \mathrm{PPI}$ use, BMI, family history) and endoscopic findings (EE, Barrett's, hiatus hernia) were recorded. NERD and EE patients were compared using a univariate analysis (chi square and t-test) and thereafter multiple logistic regression analysis was performed to determine factors significantly different between the 2 groups. Results: 451 patients with NERD and 248 patients with EE were analyzed. The mean ages of the NERD and EE groups were $58.9+12.6$ and $55.1+12.9$ years respectively $(\mathrm{p}=0.0001)$, and included $80 \%$ Caucasians in each group. By univariate analysis, compared to EE patients, NERD patients were significantly more likely to be older $(p=0.002)$, non-smokers $(p=0.005)$, use PPIs $(p=<0.0001)$ but with less frequent $(\mathrm{p}=0.0009)$ and severe $(\mathrm{p}=0.003)$ heartburn symptoms. On the other hand, EE patients were more likely to have nocturnal symptoms $(\mathrm{p}=0.0006)$ and hiatus hernia $(p=<0.0001)$. Logistic regression analysis demonstrated a significant correlation between EE 
with the presence of hiatus hernia (OR: 3.8, 95\% CI: 2.4-5.8, p<0.001) and nocturnal GERD (OR: $2.4,95 \%$ CI: 1.3-4.5) while patients with NERD were more likely to be older (mean age 58.9 years) and using PPIs (OR: 2.3, 95\% CI: 1.3-3.9). Conclusion: Presence of a hiatus hernia appears to be an independent risk factor for EE. Prior to endoscopy, NERD patients are more likely to use PPIs (OR 2.3) indicating that PPI use may be responsible for healing of low grades of EE and thereby increasing the prevalence of NERD. Another possible explanation is referral bias- NERD patients, who are more likely to fail PPI therapy compared to EE patients, may be referred more often for endoscopy.

\section{S1195}

\section{Inappropriate Use of Proton Pump Inhibitors in a Hospital Setting in the} Netherlands

Evelyn P.M. van Vliet, Henk J.A.M. Otten, Ernst J. Kuipers, Pieter D. Knoester, Arjan Rudolphus, Henk C. Hoogsteden, Peter D. Siersema

Background: Guidelines suggest that proton pump inhibitors (PPIs) should be used for the treatment of acid-related disorders and the prevention of nonsteroidal anti-inflammatory drug (NSAID)-associated gastrointestinal symptoms. Information on the use of PPIs in a hospital setting is not widely available. The aim of this study was to assess prescription patterns of PPIs on two medicine wards. Methods: We prospectively assessed the number of patients on PPIs and the indications for use of PPIs on two pulmonary medicine wards, one from a university (240 patients) and one from a regional (60 patients) clinic in The Netherlands. Results: On admission, 88/300 (29\%) patients already used PPIs. During hospitalization, use of PPIs was terminated in $3(1 \%)$ patients, whereas PPIs were initiated in $42(14 \%)$ patients, resulting in $127(42 \%)$ patients using PPIs. Indications for PPI use were in 33/127 (26\%) patients an acid-related disorder, in $75(59 \%)$ patients the prevention of medication-associated complications (mainly complications of NSAIDs, corticosteroids and antibiotics), and in 19 (15\%) patients another reason. In 72/127 (57\%) patients on PPI therapy, PPIs were used for a registered indication, whereas in $55(43 \%)$ patients, a nonregistered indication for PPI use was present (overuse). The reasons for overuse were the prevention of (non-registered) medication-associated complications in 41/55 (75\%) patients, or another reason in $14(25 \%)$ patients. Contrary to overuse, 21/300 (7\%) patients were not prescribed PPIs despite the presence of a registered indication (i.e., an acid-related disorder (mainly reflux esophagitis) or the prevention of NSAID-associated gastrointestinal symptoms) (underuse). The only statistically significant difference between the university and the regional pulmonary medicine ward was the number of patients with underuse of PPIs ( $5 \%$ vs. $13 \%$, resp.) $(\mathrm{p}=0.03)$. The number of patients on PPIs on admission $(31 \%$ vs. $22 \%)(\mathrm{p}=0.15)$ and during hospitalization $(45 \%$ vs. $33 \%)(\mathrm{p}=0.12)$, and the number of patients with overuse ( $20 \%$ vs. $13 \%)(\mathrm{p}=0.26)$ were not significantly different between the university and the regional unit. Conclusion: More than $40 \%$ of patients on pulmonary medicine wards in The Netherlands are on PPIs. More than $40 \%$ of these patients use PPIs for an unregistered indication. As it is likely that the same is true for other hospital departments, physicians working in hospitals need to be educated on the present guidelines for PPI use.

\section{S1196}

Intragastric Acidity After H.Pyori Eradication: Is More Suppression Needed? Yuhong Yuan, Marroon Thabane, Mohammad Yaghoobi, Jenny Liu, Richard H. Hunt

Background: Some studies suggest that PPIs suppress gastric acid secretion more effectively in H.pyori infected than in uninfected subjects. This may increase the risk of developing reflux symptoms in some patients and whether eradication can reduce the proportion of the time with $\mathrm{pH}$ above a given threshold is not clear. This study reviewed the effects of H.pyori eradication on 24 hour intragastric $\mathrm{pH}$ values in H.pyori infected healthy volunteers during PPI therapy. Methods: Medline (1984-2005) was comprehensively searched to identify English language clinical trials investigating the effects of H.pyori eradication on 24 hour intragastric $\mathrm{pH}$ in healthy volunteers during PPIs therapy. Keywords included "H.pyori", "eradication", "pH metry" or "gastric acid", "PPI(s)". For trial eligibility, 24 hour $\mathrm{pH}$ values had to be obtained by $\mathrm{pH}$ metry. $\mathrm{pH}$ parameters of day 5-8 (steady state) after PPI therapy were compared before and after eradication. Pooled mean estimates and corresponding 95\% Cl for $\mathrm{pH}$ parameters were estimated using a random effects model. Analysis was performed using Review Manager 4.2.8. Results: Of the 7 relevant papers retrieved, two were excluded because gastric acidity was measured by gastric aspiration or only 8 hour nighttime $\mathrm{pH}$ data was reported. The remaining 5 included trials had a pooled total sample of 65 subjects. The sample size per trial ranged from 10 to 18. PPIs included omeprazole ( $4 \mathrm{arm}, 20 \mathrm{mg}-40 \mathrm{mg} /$ day) and lansoprazole ( $1 \mathrm{arm}, 30 \mathrm{mg} /$ day). At steady state on PPI therapy, after eradication of H.pyori, $\mathrm{pH}$ decreased significantly with 24 hour mean $\mathrm{pH}$ compared to the pre-eradication period [weighted mean difference (WMD) $=1.38(95 \% \mathrm{Cl}=0.92$ $183)$, no significant heterogeneity was observed $(\mathrm{p}=0.13)$. Only two studies reported $\%$ time $\mathrm{pH}>4 / 24 \mathrm{hrs}$, and this decreased significantly after eradication $(\mathrm{WMD}=31.96,4.05-$ 59.88 ), although there was significant heterogeneity for these two studies $(\mathrm{p}<0.01)$. Conclusion: H.pyori eradication diminishes acid suppression by PPIs, resulting in a 24 hour $\mathrm{pH}$ decrease of $1-2 \mathrm{pH}$ units and likely reduces holding time above any given target $\mathrm{pH}$ threshold. Whether this "statistically significant" difference has clinical significance on PPI treatment in patients with reflux disease needs further research.

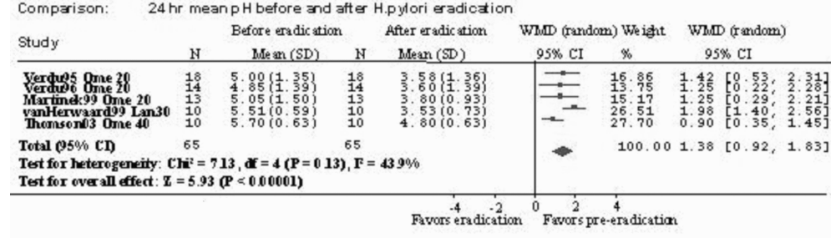

S1197

Maintenance Therapy with Continuous and On-Demand Rabeprazole (RAB) 20mg in Uninvestigated Gastroesophageal Reflux Disease

David G. Morgan, Michael F. O'Mahony, William F. O'Mahony, Jean Roy, Julie Dinniwell, Fernando Camacho, Farah A. Husein-Bhabha

This multicentre, randomized study evaluated 6 months of RAB 20mg daily continuous therapy (COT) with RAB $20 \mathrm{mg}$ on-demand (ODT) maintenance therapy in uninvestigated GERD patients demonstrating satisfactory heartburn (HB) control during the final week of a 4-week treatment period with daily RAB 20mg. Acute phase ( $\mathrm{n}=331$ ): Excellent HB control was seen after 4 weeks of RAB $20 \mathrm{mg}$, with $78 \%$ experiencing no or mild $\mathrm{HB}$, and $93 \%$ rating HB "satisfactorily" or "completely" controlled. Maintenance phase $(n=268)$ : There were significantly more HB-free days with COT $(90 \%)$ compared to ODT $(65 \%$; $<0.0001)$ Defining good symptom control as no more than 2 episodes/week with maximum severity rated mild, more patients in COT experienced approximately 5 days/week ( $>70 \%$ of days) with no or mild $\mathrm{HB}(\mathrm{COT}=98 \%$, ODT $=86 \% ; \mathrm{p}=0.0004)$ and rated $\mathrm{HB}$ for $>70 \%$ of weeks "satisfactorily" or "completely" controlled (COT=94\%, ODT=84\%; $\mathrm{p}=0.0098$ ). A significantly larger proportion of COT subjects experienced $\leqq 2$ days of $\mathrm{HB}$ with maximum severity rated mild (COT $=84 \%, O D T=41 \% ; \mathrm{p}<0.0001)$. There was overall improvement in quality of life (QOL) from baseline to end of treatment for both COT and ODT groups based on GSAS and PAGI-QOL scores. Significant differences were noted between treatment groups, with the COT group experiencing fewer and less severe symptoms (GSAS), and greater improvement in all PAGI-QOL sub-scores (excepting relationships). More patients were "satisfied" or very satisfied" with overall treatment effect of COT regimen (92\%) vs. ODT $(79 \% ; \mathrm{p}=0.0070)$; however, proportions rating overall effect of regimen on HB control as "good" or "very good" were similar between groups (COT $=89 \%, \mathrm{ODT}=83 \%, \mathrm{p}=\mathrm{NS}$ ). The ODT group experienced a greater mean number of $\mathrm{HB}$ episodes $(\mathrm{COT}=7, \mathrm{ODT}=26)$ and longer mean episode duration $(\mathrm{COT}=1.4, \mathrm{ODT}=4.4$ days; $\mathrm{p}=0.0319)$ compared to COT. Overall mean daily antacid usage was low $(\mathrm{COT}=0.1, \mathrm{ODT}=0.3$ tablets; $\mathrm{p}=0.0023)$. Both treatment regimens were safe and well tolerated. Conclusions: HB control was better with COT maintenance therapy, with less frequent, less severe episodes, and greater improvement in QOL compared to ODT. Patients indicated satisfaction with HB control with both regimens. While continuous treatment with rabeprazole is better overall for symptom control, on demand therapy may be an acceptable treatment option for GERD maintenance in selected patients. Funded by an unrestricted grant from Janssen-Ortho Inc. Toronto, Canada.

\section{S1198}

A Randomized, Comparative Study of Three Doses of Azd0865 and Esomeprazole for Healing of Reflux Esophagitis

Peter J. Kahrilas, John Dent, Karsten Lauritsen, Peter Malfertheiner, Hans Denison, Stefan Franzen, Pelle Lundborg

Purpose: AZD0865 is a member of a new class of acid-suppressing agents, potassiumcompetitive acid blockers, with a more rapid onset of action and greater potency than proton pump inhibitors. The objective of the study was to compare the efficacy and tolerability of 3 doses of AZD0865 and esomeprazole $40 \mathrm{mg}$ in esophagitis patients. Methods: A total of 1521 patients with reflux esophagitis according to the LA classification, and with heartburn of moderate or severe intensity for $\geq 4$ days/week, were randomized to AZD0865 25, 50, or $75 \mathrm{mg}$, or esomeprazole ( $40 \mathrm{mg}$ ) once daily for up to 8 weeks. The primary endpoint was endoscopically assessed healing of esophagitis by the 3 doses of AZD0865 at 4 weeks. Healing was also assessed at 2, 4, and 8 weeks for each AZD0865 dose and esomeprazole, as was control of heartburn. In a subgroup of patients, 24-h ambulatory intraesophageal/ intragastric $\mathrm{pH}$ monitoring was performed on day 14 of therapy. Safety and tolerability were also assessed. Results: Esophagitis severity was balanced across patient groups, with approximately $25 \%$ of patients having LA grade C or D esophagitis. After 4 weeks of treatment, healing rates of $76.9 \%$ (95\% CI: $72.4-81.1 \%$ ), $78.2 \%$ (95\% CI: $73.7-82.3 \%$ ), and $81.1 \%$ (95\% CI: $76.7-84.9 \%$ ) were reported with AZD0865 25, 50, and $75 \mathrm{mg}$. A comparable healing rate of $81.9 \%$ (95\% CI: $77.6-88.7 \%$ ) was observed with esomeprazole $40 \mathrm{mg}$ at 4 weeks. Healing rates were also similar between treatment arms at 2 and 8 weeks. When stratified by baseline LA grade, there were no significant differences in healing rates between AZD0865 doses and esomeprazole $40 \mathrm{mg}$. Sustained absence of heartburn was reported by $50 \%$ of patients within 7-9 days of starting treatment with AZD0865, and within 6 days of starting treatment with esomeprazole $40 \mathrm{mg}$. No significant differences for either intraesophageal or intragastric $\mathrm{pH}$ were seen on day 14 for any AZD0865 dose versus esomeprazole $40 \mathrm{mg}$. Overall, AZD0865 and esomeprazole were well tolerated, headache, nausea, and diarrhea being the most frequent adverse events. Serious adverse events occurred infrequently and were not considered to be treatment related. Reversible increases in liver transaminases were observed in a small number of patients receiving AZD0865. Conclusion: Despite theoretical advantages related to pharmacokinetics and pharmacodynamics, treatment with AZD0865 $25 \mathrm{mg}, 50 \mathrm{mg}$, and $75 \mathrm{mg}$ was unable to provide greater efficacy than esomeprazole $40 \mathrm{mg}$, either in terms of esophagitis healing or in the speed and degree of heartburn control. Both agents were in general well tolerated. 
S1199

H2 Receptor Antagonists Do Not Diminish the Acid Inhibitory Effects of Omeprazole

Lars Fandriks, Anders Pettersson, Nimish Vakil

Background: Relief from acid-related reflux symptoms is directly correlated to speed of onset and duration of acid suppression. Theoretically, the ideal pharmacological approach for ondemand treatment should be to combine the rapid onset of action of a histamine-2 (H2) receptor antagonist with the more profound and long-lasting effect of a proton pump inhibitor (PPI) to relieve recurrent symptoms. However, as proton pump inhibitors are administered as prodrugs and require an acidic milieu for activation, concern exists as to whether an increase in intragastric $\mathrm{pH}$ in response to $\mathrm{H} 2$ blockers, may decrease the efficacy of proton pump inhibitors. Aims: To determine if co-administration of an $\mathrm{H} 2$ receptor antagonist decreases the efficacy of acid inhibition with omeprazole. Methods: $H$ pylori negative healthy volunteers ( $n=6)$ were studied in an open, randomized, crossover trial $(n=6)$ with a 2 week washout between treatments. Intra-gastric $\mathrm{pH}$ was monitored on day 1 and day 8 after daily administration of omeprazole $20 \mathrm{mg}$ (Ome); famotidine $10 \mathrm{mg}$ (Famo) or a combination of these (Ome+Famo) Standardized meals were administered on the test days. Results: Time required for the $\mathrm{pH}$ to rise to $>4$ for at least 10 minutes after ingestion of medication as well as fraction of time $\mathrm{pH}>4$ during the entire daytime was significantly better $(\mathrm{p}<0.05)$ with combination therapy than Ome on day 1 . No differences between the treatments were found after 8 days treatment. Fraction of time $\mathrm{pH}>4$ during nighttime was not different between treatments at any of the test days. Conclusion: 1. A combination of an $\mathrm{H} 2$ receptor antagonist and a PPI raises gastric $\mathrm{pH}>4$ in less than one hour and is comparable to potassiumcompetitive acid blockers 2 . Acid control over the entire daytime period is significantly better on day 1 with combination therapy 3 . $\mathrm{H} 2$ receptor antagonists do not inhibit activation or efficacy of omeprazole on day 1 or day 8 challenging long held beliefs on acid inhibition Duration in minutes after ingestion (mean \pm SEM) to sustained intragastric $\mathrm{pH}>4$ :

\begin{tabular}{|c|c|c|c|}
\hline & Ome & Famo & Ome+Famo \\
\hline Day 1 & $161 \pm 14$ & $92 \pm 27$ & $52 \pm 11$ \\
\hline Day 8 & $50 \pm 9$ & $79 \pm 25$ & $49 \pm 12$ \\
\hline
\end{tabular}

$\%$ time (mean \pm SEM) $\mathrm{pH}>4$ during daytime (0900 to 2300)

\begin{tabular}{|c|c|c|c|}
\hline & Ome & Famo & Ome+Famo \\
\hline Day 1 & $24 \pm 3.0$ & $34 \pm 7.5$ & $42 \pm 6.7$ \\
\hline Day 8 & $54 \pm 3.2$ & $28 \pm 6.1$ & $60 \pm 3.1$ \\
\hline
\end{tabular}

\section{S1200}

Nocturnal Gastric Acidity After Bedtime Dosing of Proton Pump Inhibitors in Patients with Nighttime GERD Symptoms

Philip O. Katz, David Ballard, Frauke K. Koch, Barry Goldlust, Jacqueline M. Major, Theresa C. Gautille, Bonnie Hepburn

Introduction: Many patients with nighttime GERD have incomplete relief when treated with proton pump inhibitors (PPIs) once daily before breakfast. Dosing before dinner or twice daily (before breakfast and dinner) decreases nocturnal gastric acidity when compared to morning dosing; however, optimal control of symptoms and acid remain clinical challenges. Previous data showed immediate-release omeprazole suspension (IR-OME) dosed at bedtime reduced nocturnal acidity to a greater degree than pantoprazole dosed before dinner. Aim: To evaluate the effect of bedtime dosing with IR-OME $40 \mathrm{mg}$ (Zegerid ${ }^{\circledR}$ ), esomeprazole $40 \mathrm{mg}$ (ESO) and lansoprazole 30mg (LAN) on nocturnal gastric acidity. Design: Open label, 3period, randomized crossover (with washouts) evaluating IR-OME, ESO, and LAN given daily at 2200 for 7 days (self-administered on days 1-5; in the clinic with standardized meals at 0800,1200 , and 1700 on days 6 and 7). Gastric pH was measured over 24 hrs beginning at 0700 on day 7. Primary endpoint: Nocturnal acid breakthrough (NAB) (gastric $\mathrm{pH}<4$ for $>1$ continuous hour between 2200 and 0600). Secondary endpoints: Percent time gastric $\mathrm{pH}>4$ median gastric $\mathrm{pH}$. Results: Forty-nine patients were analyzed (51\% male, median age 31 years). Primary endpoint: Percentage of patients with NAB was less with IR-OME (61\%) vs. ESO (92\%) and LAN (92\%), p<0.001, both. Percent time gastric $\mathrm{pH}>4$ (between 2200-0600) was comparable for IR-OME and ESO (53\% vs. 55\%), both superior to LAN (34\%), $\mathrm{p}<0.001$. Median gastric $\mathrm{pH}$ and percent time gastric $\mathrm{pH}>4$ were substantially different when evaluated in cumulative $2 \mathrm{hr}$ intervals from 2200 to 0600 (Table). Conclusions: Bedtime dosing with immediate-release omeprazole results in a substantial reduction in NAB compared to ESO and LAN, and provides more rapid control of nighttime $\mathrm{pH}$. Nighttime ( $8 \mathrm{hr}$ ) acid control with immediate-release omeprazole is comparable to that with ESO and superior to that with LAN. These data suggest that bedtime dosing with immediate-release omeprazole may be an effective strategy for managing patients with nighttime heartburn.

\begin{tabular}{|c|c|c|c|c|}
\hline & $\mathbf{2 2 0 0 - 2 4 0 0 ~ \mathbf { ~ }}$ & $\mathbf{2 2 0 0 - 0 2 0 0 ~}$ & $\mathbf{2 2 0 0 - 0 4 0 0 ~} \mathbf{~ 2 2 0 0 - 0 6 0 0 ~ h}$ \\
\hline IR-OME 40mg & & & & \\
\hline \% Time Gastric $\mathrm{pH}>4$ & 32.3 & 51.9 & 62.6 & 53.4 \\
\hline Median Gastric pH & 3.54 & 4.34 & 4.35 & 4.04 \\
\hline LAN 30mg & & & & \\
\hline \% Time Gastric $\mathrm{pH}>4$ & $0.0^{*}$ & $12.0^{*}$ & $26.6^{*}$ & $34.2^{*}$ \\
\hline Median Gastric pH & $1.25^{*}$ & $1.51^{*}$ & $1.76^{*}$ & $2.09^{*}$ \\
\hline ESO 40mg & & & & \\
\hline \% Time Gastric $\mathrm{pH}>4$ & $0.1^{*}$ & $30.1^{*}$ & 46.5 & 54.9 \\
\hline Median Gastric $\mathrm{pH}$ & $1.43^{*}$ & $2.37^{*}$ & 3.49 & 4.85 \\
\hline
\end{tabular}

* $\mathrm{P}<0.001$ when compared to IR-OME, Wilcoxon signed-rank test.

S1201

The Effect of Acid Suppression On Subjective Sleep, Gastric Myoelectrical Functioning, and Cardiac Autonomic Function in Patients with Erosive GERD Chien-Lin Chen, Terry Bj Kuo

Background: Autonomic dysfunction and sleep disturbances have been observed in patients with reflux disease or non-erosive esophageal reflux disease (Gastroenterology 2001;120:A629 and 2005;128:A518). Aim: This study was to investigate whether acid suppression changes the functional characteristics regarding to heart rate variability, subjective sleep, and gastric myoelectrical activity with a non-nutrient water load in patients with reflux esophagitis. Methods: Twenty-eight patients with erosive esophagitis underwent heart rate variability as well as electrogastrography with a 5-min water load and a subjective sleep questionnaire. All patients were studied before and after esomeprazole $40 \mathrm{mg}$ daily for 8 weeks. Subjects were also divided into mild or moderate esophagitis in order to determine any variation in response related to the severity of esophagitis. Results: Sleep disturbance occurred in $56 \%$ of patients with reflux esophagitis. The reflux symptom scale significantly improved from 4.2 at baseline to 1.0 at week $8(\mathrm{P}<0.001)$. The global Pittsburgh Sleep Quality Index score decreased from 5.0 at baseline to 3.6 at week $8(\mathrm{P}<0.01)$. A significant increase in postprandial gastric $3 \mathrm{cpm}$ (normal) activity was observed at week $8(\mathrm{P}<0.05)$. No significant changes were seen in any heart rate variability parameters or the volume of water load. A significant improvement in subjective sleep was observed in mild esophagitis $(\mathrm{P}=0.03)$ while a significant increase in postrandial gastric $3 \mathrm{cpm}$ (normal) activity occurred in moderate esophagitis $(\mathrm{P}<0.05)$. Conclusions: Acid suppression improves not only sleep disturbances but also gastric myoelectrical functioning. Improvement in sleep appears to be inversely related to the severity of erosive esophagitis.

\section{S1202}

Gerdyzer ${ }^{\mathrm{TM}}$ - A New Scale for Patient-Reported Outcome of HRQOL in GERD Shows Excellent Psychometric Criteria. Validation Trial in 396 Patients with Erosive Esophagitis

Juan R. Malagelada, Kenneth R. Devault, Olivier Chassany, Holger Schmitt, Ursula Gebauer, Gerald Holtmann

PURPOSE: Health-related quality of life (HRQoL) is a key parameter for the evaluation of treatment effectiveness in chronic conditions. To assess HRQoL, no GERD specific, reliable, sensitive, and validated questionnaire existed so far. Therefore GERDyzerTM a self-assessed, multidimensional, short, and easy to understand questionnaire was designed for use in international GERD clinical trials. Here, we report data from the validation trial in erosive esophagitis (EE). METHODS: GERDyzer ${ }^{\mathrm{TM}}$ covers 10 dimensions of HRQoL: general wellbeing, pain/discomfort, physical health, energy, daily activities, leisure activities, social life, diet/eating/drinking habits, mood, and sleep. Each dimension is illustrated by a pictogramlike drawing, simplifying the communication with the patients, and assessed by a Visual Analogue Scale. A multinational clinical trial with 396 EE patients (LA grade A-D, pe protocol) was performed. After 7 days pre-treatment, patients were treated with pantoprazole $40 \mathrm{mg}$ od for 28 days. Patients filled in GERDyzer ${ }^{\mathrm{TM}}$ on days -7 and 0 of the pre-treatment phase, and after 7,14 , and 28 days of treatment. For scale development a factor analysis was performed. Weights were determined by optimizing the non-parametric effect size fo the total score. Psychometric analyses included internal consistency, test-retest reliability, and responsiveness. Construct validity was evaluated by comparison with ReQuest TM and it's subscales, the Gastrointestinal Symptom Rating Scale (GSRS), and the Psychological General Well Being (PGWB) index scale. RESULTS: Factor analysis showed consistency of the dimensions and no reduction of dimensions was necessary. Further psychometric characteristics are summarized in the table CONCLUSIONS. This trial demonstrates that GERDyzerTM clearly exceeds all psychometric requirements of a valid tool to assess patient reported outcome. High internal consistency (Cronbach's alpha 0.94) and test-retest reliability, as well as large responsiveness and good construct validity were shown. GERDyzer ${ }^{\mathrm{TM}}$ is a validated and sensitive instrument that reliably assesses treatment-induced changes in HRQoL in GERD patients with erosive esophagitis.

GERDyzer ${ }^{\mathrm{TM}}$ : Psychometric parameters 


\begin{tabular}{|c|c|}
\hline Internal Consistency (Cronbach's alpha) & 0.94 \\
\hline Test-retest reliability (intraclass-correlation-coefficient) & 0.91 \\
\hline Responsiveness (non-parametric effect size) & 1.37 \\
\hline Construct validity (Pearson correlation coefficient): & \\
\hline GSRS & 0.60 \\
\hline PGWB & -0.57 \\
\hline ReQuest & 0.67 \\
\hline ReQuest & 0.65 \\
\hline ReQues-GI (gastrointestinal) & 0.62 \\
\hline
\end{tabular}

\section{S1203}

Establishment of New Oral Treatment for Nerd Patients

Akinori Koide, Taketo Yamaguchi, Hidehiko Koyama, Takeo Odaka, Yoshihiro Koma, Kenichi Nakajima, Izumi Shinbo, Hitoshi Maeda, Masato Ai, Yoshiharu Tokoro, Hiromitsu Saisho

We reported a study which compared PPI plus Rikkun-shi-to (Liu-jun-zi-tang: Japanese kampo medicine) combination treatment with PPI alone treatment in patients with unidentified complaints in the upper gastrointestinal tract including GERD patients at this meeting last year. This study was a randomized control group study, which demonstrated that the improvement effect especially on endoscopy-negative GERD (hereinafter, NERD) was significantly greater in the Rikkun-shi-to combination group than that in the PPI alone group. [Gastroenterology128:A530,2005 Gastroenterology126:A440,2004]. Objective: Randomized control study among three groups of NERD patients to evaluate each treatment effect by PPI alone, Rikkun-shi-to alone and PPI plus Rikkun-shi-to combination. Methods: Seventytwo NERD patients who showed no response to PPI treatment in PPI test were randomly allocated to one of the following three groups: PPI (Omeprazole $20 \mathrm{mg}$ ) continuous treatment group, Rikkun-shi-to alone group and PPI plus Rikkun-shi-to combination group. The treatment period was 4 weeks. Evaluation method: Using the GSRS (Gastrointestinal Symptom Rating Scale, Japanese edition), score improvement in 4 GSRS parameters of all gastrointestinal symptoms before and after administration, reflux symptoms, abdominal pain and dyspepsia, and differences in GSRS scores ( $\triangle$ GSRS scores) among three groups ( $\Delta$ GSRS score) were compared. Results: There were no differences in background factors among the three groups. Evaluation results: 1) In PPI alone group $(n=24)$, significant changes in GSRS scores of all parameters were not observed before and after administration. 2) In Rikkunshi-to alone group ( $\mathrm{n}=21$ ), significant improvement in GSRS score of the parameter "dyspepsia" was observed ( $\mathrm{p}<0.05)$. 3) In PPI plus Rikkun-shi-to combination group ( $\mathrm{n}=27$ ), significant improvements were observed in GSRS scores of the following three parameters: all gastrointestinal symptoms and reflux symptoms ( $\mathrm{p}<0.05$, respectively), and dyspepsia symptoms $(\mathrm{p}<0.01)$. Comparing $\triangle \mathrm{GSRS}$ scores among the groups, scores of the 2 parameters of reflux symptoms and dyspepsia symptoms in PPI plus Rikkun-shi-to combination group were significantly lower than those in PPI alone group and Rikkun-shi-to alone group ( $\mathrm{p}<0.05)$. Conclusion: We investigated oral treatment for NERD patients who had no response to PPI. The results indicated that PPI plus Rikkun-shi-to combination treatment provided greater effect than PPI alone and Rikkun-shi-to alone treatments in terms of patients' QOL improvement.

\section{S1204}

A Randomized, Comparative Efficacy Study of Three Doses of Azd0865 and Esomeprazole in Patients with Non-Erosive Reflux Disease John Dent, Peter Kahrilas, Jan Hatlebakk, Nimish Vakil, Hans Denison, Stefan Franzen, Goran Hasselgren

Purpose: AZD0865 belongs to a new class of acid-suppressing agents, potassium-competitive acid blockers, with a more rapid onset of action and greater potency than proton pump inhibitors. This study compared the efficacy and tolerability of 3 doses of AZD0865 with esomeprazole $20 \mathrm{mg}$ in patients with non-erosive reflux disease. Methods: In this 4-arm parallel group, double-blind, multicenter, dose-finding comparative study, 1469 patients with heartburn $\geq 4$ days/week of moderate or severe intensity were randomized to AZD0865 25,50 , or $75 \mathrm{mg}$ or esomeprazole $(20 \mathrm{mg})$ once daily for 4 weeks. The primary endpoint was time to sustained absence of patient-reported heartburn (time to the first 7 consecutive days free of heartburn) for the 3 doses of AZD0865. Secondary endpoints included the comparative efficacy of each AZD0865 dose and esomeprazole for other patient-reported symptoms. 24-h ambulatory intraesophageal/intragastric $\mathrm{pH}$ was performed in a subgroup of patients on day 14 of therapy. Safety and tolerability were also assessed. Results: Following 4 weeks of treatment, the time to sustained absence of patient-reported heartburn was similar between the 3 doses of AZD0865, and also between each dose of AZD0865 and esomeprazole. The cumulative incidence of sustained absence of heartburn was also similar across all groups (e.g. $62-70 \%$ on day 21). A comparable proportion of patients were free from heartburn during the first, second, and fourth weeks of treatment with each dose of AZD0865 and with esomeprazole $20 \mathrm{mg}$ (45-53\% during the 4th week). No significant differences were observed with respect to other symptom variables between the AZD0865 doses and esomeprazole. AZD0865 $75 \mathrm{mg}$ was significantly more effective than esomeprazole with respect to time with intragastric $\mathrm{pH} \geq 4$ and 24-h median intragastric $\mathrm{pH}$, but not for time with intraesophageal $\mathrm{pH}<4$, as assessed at day 14 . There were no significant differences between AZD0865 doses on these pH parameters. Both agents were well tolerated. Adverse events of headache, diarrhea, and nausea were reported most frequently in the study. Serious adverse events were infrequent in all treatment groups, and assessed to be unrelated to study drug. Reversible increases in liver transaminases were observed in a small number of AZD0865-treated patients. Conclusions: The fast onset of acid inhibition documented previously with AZD0865 did not translate into faster or more effective control of heartburn in patients with non-erosive reflux disease. Esomeprazole $20 \mathrm{mg}$ was at least as effective at controlling heartburn as the three doses of AZD0865. Both agents were well tolerated overall

\section{S1205}

Impact of Esophagitis Classification in Evaluating Healing After Therapy with Proton Pump Inhibitors (PPIs)

Mohammad Yaghoobi, Sara Padol, Yuhong Yuan, Richard H. Hunt

Background: The Hetzel-Dent (HD), Savary-Miller (SM), and Los-Angeles (LA) classifications describe the severity and healing of erosive esophagitis (EE) in clinical trials with PPIs. Whether the reported healing rates are comparable between studies using different classifications has not been evaluated. The aim of this study was to review systematically the literature to compare EE healing rates assessed by HD, SM or LA classifications. Methods: A recursive, English language literature search in PubMed and Cochrane databases to August 2005 was performed using keywords: (o) esophagitis, reflux, GERD, and PPI(s) (and individual names of PPI). Double-blind RCTs comparing a PPI with the other PPIs, H2-RAs or placebo using endoscopic assessment of healing of EE by HD, SM or LA, or their modified classification at 4 or 8 weeks, were included in the study. Abstracts, studies without raw ITT data for retrieval or clear definition of classification and duplicate publications were excluded. Data of healing rate under the same PPI, and same EE grade was pooled and compared using Fisher's exact test or Chi-square when appropriate. Results: Fifty-three studies were included from 256 potential citations. Of these, 25 compared PPI with another PPI, 5 with placebo, and 23 with H2-RAs. Study subjects in PPI arms $(n=79)$ were 41829 (7205 in 16 studies with HD, 3874 in 26 studies with SM, and 27670 in 11 studies with LA). There was insufficient data for rabeprazole and esomeprazole to compare as they were evaluated by only one scoring system. When data from all PPIs was pooled regardless of EE grades, SM (74\%) healing rate was significantly higher than LA (71\%) and $\mathrm{HD}(68 \%)$ at 4 weeks while LA $(90 \%)$ was significantly higher than SM $(84 \%)$ and $\mathrm{HD}(83 \%)$ at 8 weeks $(\mathrm{P}<0.001)$ The proportion of different grades was not separately available in all therapeutic groups but for pantoprazole where the distribution of EE grades was similar for both LA and SM subgroups. When analyzing data for PPI and dose, LA showed a higher healing rate for omeprazole $20 \mathrm{mg} / \mathrm{d}$ and pantoprazole $40 \mathrm{mg} / \mathrm{d}$ (significant at 8 weeks; $\mathrm{P}<0.01$ ), while SM was significantly higher for omeprazole $40 \mathrm{mg} / \mathrm{d}$ and lansoprazole $30 \mathrm{mg} / \mathrm{d}$ at 4 and 8 weeks $(\mathrm{P}<0.001)$. Healing rate under individual $\mathrm{EE}$ grade was not always available or robust enough for meaningful analysis. However, a non-consistent difference between systems remained. Conclusion: There is a significant, but not always consistent, difference in EE healing rates in PPI users reported by HD, LA or SM classifications. The possible difference between grading classifications should be considered when interpreting or comparing EE healing rates from different studies.

\section{S1206}

An Exploratory, Randomised, Controlled, Parallel-Group, Open-Label Study to Evaluate Different Dosing Regimens of rabeprazole in Controlling Nocturnal Heartburn (NT HB) Symptoms in Uninvestigated Symptomatic Gastroesophageal Reflux Disease (s-GERD)

David G. Morgan, Subhas C. Ganguli, Michael F. O Mahony, Jean Roy, Fernando Camacho, Bruno Teixeira, Farah A. Husein-Bhabha

This exploratory study evaluated three RAB regimens for control of NT HB symptoms in sGERD patients with predominant NT HB, and no more than mild, infrequent daytime (DT) HB symptoms on standard proton pump inhibitor or histamine-2 receptor antagonist therapy. Eligibility was determined by NT and DT scores during the 14-day run-in period (score = summation of frequency X severity of episodes for the defined period); patients also had to indicate a lack of satisfaction with regards to their current acid suppressive therapy. Randomized patients $(n=48)$ received either RAB $20 \mathrm{mg}$ taken once daily prior to breakfast (QAM), or RAB 20mg taken once daily prior to dinner (QPM), or RAB 10mg taken twice daily in morning and evening prior to meals (BID) for 8 weeks. Diary assessments included heartburn occurrence and severity (daily), RAB and rescue antacid usage (daily), and ratings of heartburn control and satisfaction (weekly). Results: Mean $( \pm$ SD) NT HB scores at 4 weeks were QPM: $6.9 \pm 8.0, Q A M: 8.1 \pm 10.4$ and BID: $12 \pm 12.6(\mathrm{p}=0.2330)$. There was significant improvement in mean NT HB scores at 4 and 8-weeks from baseline for all regimens, with larger changes observed for QAM and QPM regimens. There was an overall decrease in NT antacid usage at both 4 and 8-weeks for QAM and QPM regimens; however, the change in NT antacid usage from baseline was significant at 4 weeks for the QAM and QPM regimen, but only significant at 8 weeks for the QAM regimen. The majority of patients in all regimens rated NT HB control "satisfactorily" or "completely controlled" at 4- and 8-weeks, as well as rated satisfaction with NT HB control as "satisfied" or "very satisfied" at these time points; no differences were observed between regimens. PAGI-SYM, PAGI-QOL and WPAI-GH mean scores improved in a similar manner for all regimens, at all time-points, compared to baseline. The between treatments comparisons indicated that subjects in the QPM regimen tended to report lower scores than the QAM group, and both groups, in turn, reported lower scores than the BID group. Compliance with each regimen was excellent $(>/=95 \%)$. All RAB regimens were safe and well tolerated. Conclusions: The results suggest that the QAM, and possibly the QPM regimen, may provide better nighttime symptom control with a corresponding improvement in QOL and productivity than a BID RAB regimen. Further studies are needed to provide more conclusive evidence and also to further investigate the phenomenon of NT $\mathrm{HB}$. This research was funded by an unrestricted grant from Janssen-Ortho Inc., Toronto, Canada. 
S1207

On-Demand Maintenance Therapy in Patients with Esophagitis and NonErosive Reflux Disease (NERD): A Prospective, Randomized, Crossover Comparative Study of rabeprazole Versus Lansoprazole Miguel Sobrino-Faya, Marta Iglesias-Rey, Enrique Dominguez-Munoz

Background: On-demand therapy with proton pump inhibitors (PPI) has been shown to be a cost-effective approach for long-term maintenance therapy of NERD. Whether on-demand PPI therapy is suitable for esophagitis patients is unclear. In this context, comparative studies between different PPI are lacking. Aim: To evaluate the efficacy of on-demand therapy in patients with low-grade esophagitis and NERD, and to compare in this context the effect of rabeprazole and lansoprazole. Methods: 63 patients (mean age 46 yrs, 35 male) with GERD were consecutively included in this prospective, randomized, open-labelled, crossover, comparative study, once severe esophagitis (grades C or D) was excluded. Patients were randomized to receive lansoprazole $30 \mathrm{mg}$ /day or rabeprazole $20 \mathrm{mg} /$ day for 8 weeks. Patients with complete symptom relief and esophagitis healing were treated on-demand with the same PPI for 8 weeks and then changed to the other PPI for further 8 weeks. Discontinuation because of unwillingness to continue, incomplete heartburn control or endoscopic relapse was considered as failure of on-demand therapy. Quality of life (QoL) was quantified by the QOLRAD questionnaire, and PPI and antacids consume was recorded. Results: After 8week daily PPI therapy, 15 patients were not suitable for on-demand schedule because of persistence of esophagitis and/or symptoms $(\mathrm{n}=11)$, or protocol violation $(\mathrm{n}=4)$. Among the 48 patients who received on-demand maintenance therapy, 32 (66.7\%) completed the study and were willing to continue under on-demand treatment. Discontinuation occurred in 11 out of 22 patients (50\%) with previous esophagitis, mainly because of endoscopic relapse, compared to 5 out of 26 NERD patients $(19 \%)(\mathrm{p}=0.15)$. Most of these patients $(87.5 \%)$ discontinued on-demand therapy during the first 8 weeks, both under rabeprazole and lansoprazole. In total, 11 out of 43 patients discontinued during on-demand rabeprazole and 5 out of 39 during on-demand lansoprazole (n.s.). Consume of PPI (mean 1 dose every 5 days) and antacids (mean 1 dose every 12 days) was similar for lanzoprazole and rabeprazole. QoL score (basal 96.5 [31.0-140.1]) improved markedly after 8 weeks of daily PPI intake (34.5 [25.0-83.4]; $\mathrm{p}<0.001$ ). At the end of the 16-weeks on-demand therapy, QoL (54.5 [25.0-113.3]) remained better than basal $(\mathrm{p}<0.001)$, but impaired compared to daily PPI intake $(\mathrm{p}<0.01)$. Conclusions: On-demand maintenance therapy is effective in most patients with NERD, but it appears to be inadequate for patients with low-grade esophagitis. In this context, rabeprazole and lansoprazole appear to be equally effective.

\section{S1208}

Control of Transient Lower Esophageal Sphincter Relaxations (TLESRs) and Reflux By a Histamine H2 Receptor Antagonist (H2RA) Nizatidine (NIZ) in

\section{Healthy Subjects}

Katsuhiko Iwakiri, Makoto Kotoyori, Yoshinori Hayashi, Yuriko Tanaka, Noriyuki

Kawami, Choitsu Sakamoto, Richard H. Holloway

Background: A study in Japan has found that NIZ $150 \mathrm{mg}$ bid, was more effective than other H2RAs in treating reflux disease even though the NIZ group included more severe reflux esophagitis (RE) compared with other H2RAs. As it has been reported that there is no difference in the level of acid suppression among H2RAs it is possible that the NIZ has other effects on acid reflux besides acid suppression. We have previously reported that there is no difference between healthy subjects and patients with RE in the rate of either TLESRs or acid reflux during TLESRs at $2 \mathrm{~cm}$ above the LES. In this study, we have evaluated the effect of NIZ on the rate of TLESRs and the rate of acid reflux during TLESRs at $2 \mathrm{~cm}$ above the LES in healthy subjects. Methods: In 10 healthy subjects, esophageal motility and $\mathrm{pH}$ study were measured, with the subjects in the sitting position, for 3 hours after a $692 \mathrm{kcal}$ meal on 2 separate days at least 1 week apart. On one occasion, participants received an oral dose of $150 \mathrm{mg}$ of NIZ 60 minutes before the meal and on the other placebo. Both studies were preceded by a week of treatment with either NIZ (150mg, bid) or a placebo. The order of treatment was randomized. Results: The rate of TLESRs, the rate of acid reflux during TLESRs and esophageal acid exposure time after receiving NIZ were significantly lower than after receiving a placebo. Conclusions: NIZ significantly inhibits gastroesophageal reflux episodes by inhibiting both the rate of TLESRs and acid reflux during TLESRs. These findings suggest that NIZ may be useful for mild esophagitis which is characterized by mild excessive esophageal acid exposure from reflux that occurs primarily during TLESRs.

\begin{tabular}{|c|c|c|}
\hline & Nizatidine & Placebo \\
\hline TLESRs (No./3h) & $16.5(16.0-19.0) *$ & $22.0(17.0-22.0)$ \\
\hline$\%$ TLESRs with acid reflux & $24.7 \%(12.5-76.4) *$ & $74.4 \%(54.2-76.9)$ \\
\hline Esophageal acid exposure & $0.2 \%(0.1-1.0) * *$ & $2.8 \%(1.1-3.7)$ \\
\hline
\end{tabular}

median (interquartile range), ${ }^{*} \mathrm{p}<0.05,{ }^{* *} \mathrm{p}<0.01$ vs. placebo

\section{S1209}

Comparison of the Effects of Fasting Morning, Fasting Evening, and Fed Bedtime Administration of Tenatoprazole On Intragastric $\mathrm{pH}$ in Healthy Volunteers: A Randomized Three-Way Crossover Study

Alan B R. Thomson, Patrick Cohen, Herve Ficheux, Florence Domagala, Michel Homerin

Background/aims: The effectiveness of marketed benzimidazole-based proton pump inhibitors (PPIs) is influenced by meals and administration time. Product labelling generally recommends that they should be taken fasting before breakfast. Poor night-time acid control and persistence of night-time symptoms often result in twice a day dosing. Tenatoprazole is a novel imidazopyridine-based PPI with a 7-fold prolonged plasma half-life; it demonstrated a prolonged effect and a significantly better control of night-time acid secretion than esomeprazole at treatment initiation, 7 -day steady state and 3 and 5 days after treatment withdrawal.
This study aimed at comparing the effects of tenatoprazole on intragastric acidity in healthy volunteers, when administered before breakfast, before dinner and at bedtime. Methods This randomized 3-period crossover study included 12 Helicobacter pylori-negative subjects, who were given tenatoprazole $40 \mathrm{mg}$ qd either fasting at 7:00 am, fasting at 7:00 pm or fed at 9:30 pm for 7 consecutive days. Subjects washed out for 2 weeks between periods. Intragastric $\mathrm{pH}$ was monitored for 24 hours on day 7 of each of 3 periods. Results: On day 7, the median 24-hour $\mathrm{pH}$ was $4.7 \pm 0.5,5.1 \pm 0.6$ and $4.7 \pm 0.5$ after breakfast, dinner and bedtime dosing, respectively ( $\mathrm{p}=0.11$ ), whereas the median night-time $\mathrm{pH}$ was $4.2 \pm 1.2$, $5.0 \pm 1.0$ and $4.4 \pm 0.8$, respectively $(\mathrm{p}=0.13)$. The mean percentage of time spent over $\mathrm{pH} 4$ in 24 hours was $62 \pm 11,72 \pm 13$ and $64 \pm 11$ after breakfast, dinner and bedtime dosing respectively (NS), and $54 \pm 17,68 \pm 18$ and $56 \pm 10$ during night-time $(\mathrm{p}=0.06)$. Nocturna Acid Breakthrough (NAB) incidence was reduced from $100 \%$ at baseline to $83 \%, 55 \%$ and $75 \%$ after $7.00 \mathrm{am}, 7.00 \mathrm{pm}$ and $9.30 \mathrm{pm}$ dosing, respectively ( $\mathrm{p}=0.18$ ), and mean NAB duration dropped from $6.2 \pm 3.4$ to $2.8 \pm 2.2,1.0 \pm 1.2$ and $2.2 \pm 1.8$ hours $(\mathrm{p}<0.05$ ). Conclusion 7-day administration of $40 \mathrm{mg}$ tenatoprazole once-a-day exerts a profound control of intragastric acidity expanding over night-time, with little effect of food intake and time of dosing. Clinical trials are in progress, which are aiming at demonstrating the clinical advantages of the improved flexibility of the treatment with once-a-day tenatoprazole.

\section{S1210}

High Dose Versus Standard Dose Proton Pump Inhibitors in the short Term Management of Reflux Esophagitis

Mostafizur Khan, Jose Santana, Clare Donellan, Cathy Preston, Paul Moayyedi

Introduction: Proton pump inhibitors (PPIs) are the most effective medical therapy for GERD. Some GERD patients, however, do not respond to standard dose PPI therapy and a common practice is to double the dose. The efficacy of this strategy is uncertain and is also unclear whether there is any difference between different types of PPIs. We therefore evaluated the efficacy of double dose versus standard dose PPI therapy in patients with esophagitis in a Cochrane systematic review. Methods: We conducted a search of electronic databases (Medline, Cinhal, Embase and Cochrane controlled trials register) to May 2005. We also performed a recursive search of literature from identified articles. All randomized control trials (RCTs) in adult patients $(\geq 18$ ) with endoscopically proven esophagitis comparing standard dose with double dose PPI for 4 - 8 weeks were eligible. Eligibility check and data extraction were performed by two independent reviewers and discrepancies if any were resolved by a third reviewer. Results were expressed as relative risk of esophagitis persisting in the double dose versus standard dose PPI groups. Meta analysis was performed using a DerSimonian and Larid random effects model (Rev-Man version 4.2.8 and Stata version 8). Results: We identified 14 articles evaluating 11,497 esophagitis patients. Overall esophagitis persistence in high dose group (22.6\%) was lower than in standard dose group. (28.7\%), but the difference was not statistically significant. (RR of persistence at 4 weeks $=0.89$, $95 \% \mathrm{CI}=0.76$ to 1.04$)$ with a number needed to treat of $33(95 \% \mathrm{Cl}=$ benefit 14 to harm 100). Overall symptoms persisted in $25.6 \%$ patients in the group taking a high dose of PPIs, compared to $23.1 \%$ in the standard dose group and this difference was not statistically significant $(\mathrm{RR}$ of persistence at 4 weeks $=1.06,95 \% \mathrm{Cl}=0.90$ to 1.25$)$ with a number needed to harm of $100(95 \% \mathrm{CI}=$ harm 25 to benefit 50$)$. A subgroup analysis of trials that evaluated esomeprazole $40 \mathrm{mg}$ once daily showed statistically significant effect on esophagitis healing ( $\mathrm{RR}$ of esophagitis persistence $=0.75,95 \% \mathrm{Cl}=0.62$ to 0.9 ) compared to standard dose PPI therapy. There was statistically significant heterogeneity between studies (ch square $=18.2, \mathrm{df}=4, \mathrm{P}=0.001$ ) and this was not explained by the quality of trial, grade of esophagitis or the type of PPI evaluated in metaregression model. There was no statistically significant difference between any of the other PPIs. Conclusion: These data do not support the practice of doubling the dose of PPI therapy in patients that do not respond to standard dose therapy. An exception to this may be esomeprazole $40 \mathrm{mg}$ once daily.

\section{S1211}

Predictors of Resolution of Heartburn in Patients with Reflux Esophagitis: Results from the Healing Phase of the Expo Study

Joachim Labenz, David Armstrong, Sofia Andersson, Stefan Eklund, Andreas Leodolter

Objective: The aim of this analysis was to identify factors predicting sustained resolution of heartburn in patients with reflux esophagitis (RE) after 4 weeks of healing therapy with a proton pump inhibitor (PPI) during a multicenter, randomized, double-blind trial (the EXPO study; SH-NEG-0008). Methods: Patients with endoscopically confirmed RE and symptoms of gastroesophageal reflux disease (GERD), including heartburn for $\geq 4$ days of the last 7 days before enrollment (overall moderate or severe), were randomized to once-daily esomeprazole $40 \mathrm{mg}(\mathrm{n}=1562)$ or pantoprazole $40 \mathrm{mg}$ (1589) for 4-8 weeks. PPI, age, severity of RE, Barrett's esophagus, hiatus hernia, Helicobacter pylori status, BMI, dysphagia, gender, epigastric pain, acid regurgitation, severity of heartburn and duration of GERD symptom history were investigated in univariate analyses using logistic regression. The covariates identified as significant $(\mathrm{p}<0.05)$ were included in the multivariate analysis. Resolution of heartburn was defined as no heartburn during the last 7 days prior to the 4-week visit. Results: Univariate analyses identified choice of PPI, age, H. pylori status, absence of dysphagia, epigastric pain and acid regurgitation, gender and duration of GERD symptom history ( $>5$ yrs vs $\leq 5 \mathrm{yrs}$ ) as significant prognostic factors for heartburn resolution at week 4. With the exception of dysphagia and history of GERD symptoms, multivariate analysis confirmed that all of these factors were predictive of resolution of heartburn (Table). Conclusion: PPI choice of esomeprazole, older age, positive H. pylori status, male gender, lack of epigastric pain and lack of acid regurgitation all have a positive influence on sustained resolution of heartburn during RE healing treatment.

Odds ratio estimates for factors affecting heartburn resolution at 4 weeks (ITT population) 


\begin{tabular}{|c|c|}
\hline Prognostic factor & Heartburn resolution $(95 \% \mathrm{CI})$ \\
\hline Esomeprazole treatment (vs. pantoprazole) & $1.32(1.12-1.54)^{* * *}$ \\
\hline Age (yrs) & $1.01(1.01-1.02) * * * *$ \\
\hline H. pylori-positive (vs. negative/missing) & $1.44(1.19-1.74)^{* * * *}$ \\
\hline Gender (male vs. female) & $1.35(1.15-1.60)^{* * *}$ \\
\hline Epigastric pain (no vs. yes) & $1.50(1.25-1.79)^{* * * *}$ \\
\hline Acid regurgitation (no vs. yes) & $1.32(1.04-1.67)^{*}$ \\
\hline
\end{tabular}

${ }^{*} \mathrm{p}<0.05 ;{ }^{* * *} \mathrm{p}<0.001 ;{ }^{* * * *} \mathrm{p} \leq 0.0001$

\section{S1212}

Predictors of Heartburn Remission in Patients with Reflux Esophagitis: Results from the Maintenance Phase of the Expo Study

Joachim Labenz, David Armstrong, Sofia Andersson, Stefan Eklund, Andreas Leodolter

Objective: This analysis aimed to identify factors predicting remission of heartburn in patients with reflux esophagitis (RE) during maintenance therapy with a proton pump inhibitor (PPI). Methods: During a multicenter, randomized, double-blind trial (the EXPO study; SHNEG-0008) patients with endoscopically confirmed RE and symptoms of gastroesophageal reflux disease (GERD) were randomized to once-daily (qd) esomeprazole $40 \mathrm{mg}$ or pantoprazole $40 \mathrm{mg}$ for $4-8$ weeks. Patients without moderate/severe symptoms of heartburn and acid regurgitation, and with healed RE, were randomized to receive maintenance esomeprazole 20 $\mathrm{mg}$ qd ( $\mathrm{n}=1377)$ or pantoprazole $20 \mathrm{mg}$ qd $(\mathrm{n}=1389)$ for 6 months. PPI, age, severity of RE, Barrett's esophagus, hiatus hernia, Helicobacter pylori status, BMI, dysphagia, gender, epigastric pain, acid regurgitation, severity of heartburn and history of GERD symptoms were investigated in univariate analyses using logistic regression. The covariates identified as significant $(\mathrm{p}<0.05)$ were included in the multivariate analysis. Remission was defined as absence of heartburn at clinical visits throughout the maintenance phase. Results: Univariate analyses identified choice of PPI, age, H. pylori status, absence of dysphagia, absence of epigastric pain, length of history of GERD symptoms and severity of heartburn as significant prognostic factors. With the exception of dysphagia, multivariate analysis confirmed that all of these factors had an independent effect on maintaining resolution of heartburn (Table). Conclusion: PPI choice of esomeprazole, older age, positive H. pylori status, lack of epigastric pain, moderate severity of heartburn (as opposed to severe) and a shorter history of GERD symptoms all have a positive influence on the chance of heartburn remission during maintenance therapy.

Odds ratio estimates for factors affecting heartburn remission (ITT population)

\begin{tabular}{|c|c|}
\hline Prognostic factor & Remission of heartburn $(95 \% \mathrm{CI})$ \\
\hline Esomeprazole treatment (vs. pantoprazole) & $1.76(1.43-2.17)^{* * * * *}$ \\
\hline Age (per year) & $1.02(1.01-1.03)^{* * * * *}$ \\
\hline H. pylori-positive (vs. negative/missing) & $1.67(1.30-2.15)^{* * * * *}$ \\
\hline Epigastric pain (no vs. yes) & $1.40(1.11-1.76)^{* *}$ \\
\hline$\leq 5$-yr GERD history (vs. $>$ yrs) & $1.37(1.11-1.69)^{* *}$ \\
\hline Moderate heartburn (vs. severe) & $1.35(1.67-1.10)^{* *}$ \\
\hline
\end{tabular}

${ }^{* *} \mathrm{p}<0.01 ; * * * \mathrm{p} \leq 0.0001$

\section{S1213}

\section{Assessment of Therapy and the Patients' QOL in Gastro-Esophageal Reflux} Disease in Japan: A Cross-Over Trial

Hiroyuki Okada, Ryuta Takenaka, Yoshiro Kawahara, Tatsuya Toyokawa, Yuuichi Mouri, Yasuhiro Nagahara, Masahiro Takatani, Junji Shiode, Jouichiro Horii, Satoru Yagi, Takao Tuzuki, Daisuke Tanioka, Jun Katoh, Hirofumi Kawamoto, Yasushi Shiratori

Purpose: To compare the efficacy of the proton pump inhibitor (PPI) with that of $\mathrm{H} 2-$ receptor antagonist (H2-RA) and to evaluate the patients' QOL in the Japanese patients with gastro-esophageal reflux disease (GERD). Methods: A cross-over trial was conducted on Japanese patients with heartburn who received either PPI (20 mg of rabeprazole) or H2-RA (300 $\mathrm{mg}$ of ranitidine) for 8 weeks.. If the symptom did not resolve with the initial therapy, the drug was replaced by the other drug 2 weeks after completion of the initial therapy and administered for 8 weeks. If the symptom recurred after relief by the initial drug, the other drug was administered. The patients were asked about their condition using SF-36 questionnaire before and after treatment with the respective drugs. Results: One hundred patients who completed the cross-over trial patients (Los Angeles grade A in 31, B in 27. $\mathrm{C}$ in 3, D in 1, and $\mathrm{N}$ in 38) were the subjects of our analysis. Heartburn resolved in 81 patients $(81 \%)$ with PPI and 58 patients (58\%) with $\mathrm{H} 2$-RA, and neither drug was effective in $7(7 \%)$. Treatment with PPI was significantly more effective than that with H2RA in 29 patients aged under 50 years $(72 \%$ versus $45 \% ; \mathrm{P}<0.05)$ and 36 patients aged from 50 to 64 years ( $83 \%$ versus $58 \% ; \mathrm{P}<0.05$ ), whereas the efficay rate was not significantly different between both drugs in 35 patients aged $>$ or $=65$ (83\% versus $66 \% ; \mathrm{P}=0.1$ ). Although PPI was significantly more effective than H2-RA in 54 patients without $\mathrm{H}$. pylori infection (84\% versus $53 \%: \mathrm{P}<0.05)$, the efficacy rate was not significantly different in 46 patients with $H$. pylori infection ( $73 \%$ versus $60 \% ; \mathrm{P}=0.18$ ). In $\mathrm{SF}-36$, the scores of the scale for eight items contributing to the degrees of physical health and mental health (sex- and age-adjusted deviation) were lower than those of the general population at the baseline and recovered by treatment. Especially in the patients aged under 50 years, the scores concerning body pain and general health, among the eight items, were much inferior to those of the general population before treatment. Conclusions: PPI is more effective than H2RA in relieving the symptom of GERD, especially for the younger and the middle aged patients and those without H. pylori infection. However, in Japan where GERD is commonly mild, similar efficacy is observed in the elderly patients or those with $H$. pylori infection.

\section{S1214}

A Meta-Analysis of Efficacy of Esomeprazole Compared with Other Proton Pump Inhibitors On Erosive Esophagitis Hong Gao, Tianshu Liu, Naiqing Zhao, Jiyao Wang

Objective Meta-analysis was performed to evaluate the efficacy of esomeprazole, an S-isomer of omeprazole, with other proton pump inhibitors on erosive esophagitis(EE). Methods Medline, EMBASE, CBM and AstraZeneca's internal database were searched for abstracts and papers(-Sep 2005). Search terms and strategy were((gastroesophageal reflux) OR (esophagitis OR oesophagitis )) AND esomeprazole. The criteria for selection of trials for inclusion were a study design that was a randomized controlled trial or retrospective control test, direct comparison of esomeprazole $40 \mathrm{mg}$ with standard healing dose of any proton pump inhibitor, which contained endoscopic healing data or resolution rate of symptom such as heartburn based with intention-to-treat population at 4 weeks and/or 8 weeks. The articles whose Jadad scores were below or equal to 2 were excluded. Meta analysis was performed with the method of General Base Variance. If the heterogeneity was significant the methods of Der Simonian and Laird with the randomized effect model were adopted. If $\mathrm{p}$ value was above 0.05 fixed effect model was adopted. The substraction healing rates of EE of the study group and the control group was used as the effect size. The calculation of resolution rates was the same. The sensitivity analysis was performed according to the different PPIs that were used as control drugs. The microsoft of statistics was STATA 7.0. Results Eight trials including 18147 patients comparing the efficacy of esomeprazole and omeprazole, lansoprazoel, pantoprazole on EE were identified. The average difference of healing rate of 4 weeks (healing rate of esomeprazole - that of other PPI) was $8.8 \%$ and $95 \% \mathrm{CI}$ was $5.9 \% \sim 11.8 \%$ $(\mathrm{P}<0.001)$. The average difference of healing rate of 8 weeks (healing rate of esomeprazole that of other PPI) was $6.0 \%$ and $95 \% \mathrm{CI}$ was $4.0 \% \sim 8.1 \%(\mathrm{P}<0.001)$. The average difference of resolution rate of 4 weeks (resolution rate of esomeprazole - that of other PPI) was $7.2 \%$ and $95 \% \mathrm{CI}$ was $4.0 \% \sim 10.4 \%(\mathrm{P}<0.001)$. According to the test of heterogeneity the including studies were heterogeneous. We classified the studies according to the different control drugs. Test of heterogeneity showed that the studies comparing esomeprazole with omeprazole or lansoprazole respectively were homogeneous. Fixed effect model was adopted. The result of meta-analysis suggested that the healing rates of 4weeks, 8weeks on EE and the resolution rate of heartburn of esomeprazole were higher than those of omeprazole and lansoprazole(p<0.01). Conclusion Esomeprazole exhibits better efficacy on erosive esophagitis than the other PPIs such as omeprazole and lansoprazole.

\section{S1215}

Multivariate Analysis of Factors Affecting the Healing of Reflux Esophagitis in Japan

Ken Ariizumi, Shuichi Ohara, Tomoyuki Koike, Shigeyuki Kobayashi, Hatushi Yamagishi, Atushi Miura, Yoshifumi Inomata, Yasuhiko Abe, Masashi Kawamura, Yasushi Kitagawa, Katsunori Iijima, Akira Imatani, Tooru Shimosegawa

Background and aims: PPIs are acid suppressors that are being used to treat reflux esophagitis(RE), though some patients are unresponsive to PPIs. This study was designed to investigate what kind of factors were related to the healing of RE in treatment with PPIs. Methods: Subjects were 135 Japanese patients who had endoscopically diagnosed RE of grade A to D by the Los Angeles(LA) classification (male/female:78/57; mean age 60.3 314.7 years). They were treated with daily oral administration of $30 \mathrm{mg} \operatorname{LPZ}(\mathrm{n}=41)$ or $10 \mathrm{mg}$ RPZ(n=94). At 8 weeks after the start of treatment, healing of RE was evaluated endoscopically. Gender, age, body mass index(BMI), presence of hiatus hernia, grade of RE, H.pylori infection status, alcohol drinking and smoking were investigated by the univariate analysis and logistic regression analysis. Results: At 8 weeks after the start of treatment, the overall healing rate of RE was $91.1 \%(123 / 135)$. Healing rates in the respective factors were as follows: male and female were $91.0 \%(71 / 78)$ and $91.2 \%(52 / 57)$, hiatal hernia $(+)$ and $(-)$ were $89.7 \%(104 /$ $116)$ and $100 \%(19 / 19)$, H.pylori positive and negative were $90.8 \%(79 / 87)$ and $91.7 \%(44 /$ 48), drinking habit(+) and (-) were $90.4 \%(47 / 52)$ and $91.6 \%(76 / 83)$, smoking habit(+) and (-) were $87.9 \%(29 / 33)$ and $92.2 \%(94 / 102)$, LA classification grade A,B,C and D were $94.7 \%(36 / 38), 90.3 \%(65 / 72), 87.0 \%(20 / 23)$ and $100 \%(2 / 2)$. By the univariate analysis, there were no significant differences in gender, presence of hiatus hernia, grade of RE, H.pylori infection status, alcohol drinking and smoking. BMI of the healed and unhealed patients was $24.9 \pm 3.6$ and $24.2 \pm 3.9$, respectively. Ages of the healed and unhealed patients were $59.6 \pm 14.7$ and $68.0 \pm 7.6$, respectively. There were no significant differences in the age and BMI for the healed and unhealed patients. By logistic regression analysis, age was revealed to be the significant factor that affect the healing rate of RE. Conclusions: Age is the significant factor that affect the healing rate of RE in Japan. p-value (Logistic regression analysis) 


\begin{tabular}{|c|c|}
\hline gender & 0.95 \\
\hline age & 0.01 \\
\hline hiatal hernia & 0.054 \\
\hline H.pylori infection & 0.36 \\
\hline LA classification & 0.72 \\
\hline alcohol & 0.54 \\
\hline smoking & 0.12 \\
\hline BMI & 0.37 \\
\hline
\end{tabular}

\section{S1216}

A Quality of Life in Patients with EE and Nerd Treated by Rabeprazole-An One-Year's Multi-Center Open Study

Xiaohong Liu, Meiyun Ke, Zhiqiang Song, Jinyan Luo, Yaozong Yuan, Xiaohua Hou, Youling Zhu, J.N. Sun, Hui Zha

Background: GERD significantly affects a patient's quality of life (QOL). The QOL severity and responsiveness to PPI have been considered different between erosive esophagitis (RE) and non-erosive reflux disease (NERD), but few studies have compared the long-term outcomes. Objectives: To compare the health-related quality of life (HRQoL) in the patients with EE and NERD treated with rabeprazole (RAB, Pariet) in an one-year's multi-center open study. Methods: From 2002.12 to 2003.6, 80 patients with EE diagnosed by endoscopy, and 40 patients with NERD defined as a negative endoscopic finding, the Demeester scores $>14.27$ and reflux symptoms score $>6$, were enrolled in. All patients were treated with RAB (10mg bid ac) for 8wks. The erosions healed endoscopically in EE patients and the reflux symptoms score decreased $>75 \%$ in patients were divided into maintenance (10mg qd) and on-demand therapy groups, and fellowed up 52w. The impacts on HRQoL (SF36) and GERD-HROoL were assessed before and after therapy. Results: Cure rate of EE was 93.4\% after 8w treatment. At baseline, HRQoL in NERD was impaired greater than in EE. After therapy, the qol was improved in all subscales in EE group, however, it was much lower in NERD group. The scale of GERD-HRQoL improved significantly in EE than in NERD group. $52 \mathrm{w}$ followup was available in $67 \mathrm{EE}$ and 31 NERD patients. There was not significant difference between maintenance and on-demand therapies in EE and NERD. The improvement of HRQoL at $8 w$ was still kept during $52 w$ in EE. In NERD the HRQoL, as well as the reflux symptoms, was upgraded gradually and close to EE (NS). Conclusions: NERD causes a more significant impairment in the qol than EE, which can be attenuated partly after $8 \mathrm{w}$ treatment with RAB, unlike the satisfactory results in EE. But in NERD HRQoL was improved gradually in long-term PPI maintenance, therefore On-demand might be suitable to NERD patients.

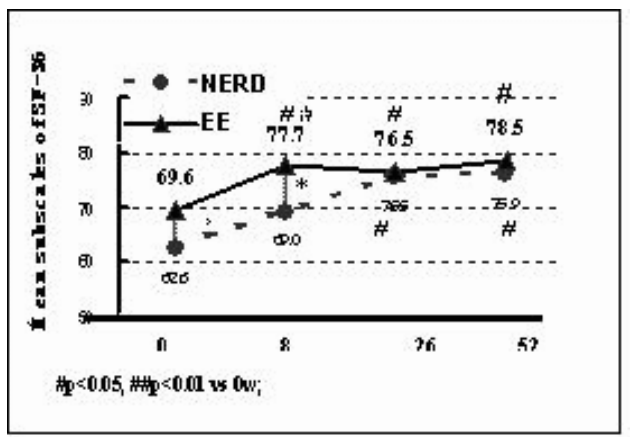

\section{S1217}

Night-Time Heartburn As a Possible Indicator of Degree of Heartburn Severity, Frequency and Response to Standard Acid Suppressive Regimens

David G. Morgan, Subhas C. Ganguli, Michael F. O'Mahony, Jean Roy, Fernando

Camacho, Bruno Teixeira, Farah A. Husein-Bhabha

Night-time heartburn (NT HB) is common in patients with symptomatic GERD (s-GERD), and is often an indicator of nocturnal acid reflux events. A better understanding of NT HB has implications for the management of s-GERD patients. As part of a randomized, controlled, open-label study evaluating different dosing regimens of rabeprazole (RAB) in controlling NT HB symptoms, this pre-randomization phase identified patients with clinically important NT HB (as defined by a NT HB score $>/=4$ (score = summation of frequency X severity of episodes), with adequate daytime (DT) HB control, and who were not satisfied with their current therapy. Patients screened for the presence of NT HB symptoms despite acid suppressive therapy (proton pump inhibitor (PPI) or histamine receptor antagonist (H2RA)) for minimum of 4 weeks were enrolled. Patients continued their current regimen during the 14-day pre-randomization period. Data are presented for 128 enrolled patients $(60 \%$ female, mean age 52 years, mean duration of heartburn 12 years, mean duration of NT HB symptoms 8 years); 120 patients met diary compliance requirements. All primary PPI or H2RA therapy at entry was at recommended doses for s-GERD treatment. Dosing was reported most commonly as morning before breakfast for PPIs, and morning and evening pre-meals for H2RAs. Clinically important NT HB was observed in 87\% (104/120) of patients; of these, $48 \%(50 / 104)$ had predominantly NT HB (with none, or mild, infrequent DT HB) and $52 \%$ (54/104) had both NT HB and clinically important DT HB (moderate or greater DT symptoms and/or more than 3 DT episodes in 7 days; DT score $>5$ ). Patients with clinically important NT HB (group A) experienced significantly greater frequency and severity of both NT and DT HB than those with predominantly DT HB or good overall DT and NT HB control (group B): NT= mean of 9.5 episodes, mean score of 19.2 for A (vs. 1.0 episodes, mean score of 1.2 for B), DT= mean of 6.2 episodes, mean score of 9.5 (vs. 1.4 episodes, mean score of 1.9 for B); all p-values $<0.05$. Group A also needed antacids for significantly longer durations to manage DT and NT symptoms. Conclusions: Of patients self-identifying with nocturnal symptoms at study entry, the majority had bothersome, clinically important nighttime heartburn despite acid suppressive therapy. A large proportion of these patients have both frequent and severe daytime and nocturnal symptoms, suggesting that the presence of night-time heartburn may require more detailed questioning about symptoms and a differen therapeutic approach than standard regimens. Funded by an unrestricted grant from JanssenOrtho Inc. Toronto, Canada.

\section{S1218}

Comparison Between the Combined Analysis and the Demeester Score to Predict Reponse to PPI Therapy

Inder Mainie, Radu Tutuian, Amine Hila, Amit Agrawal, Donald O. Castell

Background: Distal esophageal acid exposure can be quantified by either the DeMeester score (DS) or percentage time $\mathrm{pH}<4$. In addition to these parameters symptom index (SI) is used to evaluate the association of symptoms and acid reflux. Combined analysis (CA) including the SI and percent time $\mathrm{pH}<4$ provides a more complete interpretation of $\mathrm{pH}$ monitoring Aim: To compare the accuracy of the CA to the DS for interpretation of pH monitoring. Method: $\mathrm{pH}$ studies from 450 patients (303 female; mean age 53; range 11 88 ) between July 2002 and October 2004 were analyzed. DS (normal < 14.7), percent time $\mathrm{pH}<4$ (normal; upright $<6.3 \%$, recumbent $<1.2$ ) and SI measured, (positive SI $(+\mathrm{SI}) \geq$ $50 \%$ ). Symptom responses to PPIs were assessed in patients with discordant DS and CA using a chart review or contact by phone. Results: Mean duration of monitoring was 22 hours and 34 minutes. $245(54 \%)$ patients had an abnormal study using the combined analysis. $27(11 \%)$ of the abnormal studies had a positive SI but normal \% time $\mathrm{pH}<4$ $180(40 \%)$ patients had a DS > 14.7. Comparing DS to CA, the sensitivity was $73 \%$ and the specificity was $99.5 \% .67$ patients had discordance between DS vs CA, 66 patients had a normal DS and an abnormal CA. 41 patients had abnormal reflux; 13 (32\%) of the 41 patients had a positive SI and $16(39 \%)$ of the patients had no symptom during the study. 25 patients had a positive SI but normal levels of reflux. 56 of the $66(85 \%)$ discordant patients were followed-up by chart review or phone call and 10 patients were lost to followup. $43(77 \%)$ of 56 patients improved with a PPI (including two with benefit from a fundoplication), 5 (9\%) patients had never been prescribed a PPI and 3 of these patients were still symptomatic. 8 (14\%) reported no improvement with a PPI. Of the 25 patients with a positive SI and normal levels of reflux, follow up was available for 19. Symptom improvement was reported by 17 patients, one with a fundoplication and 16 currently taking a PPI. Conclusions: Interpreting the results of $\mathrm{pH}$ monitoring based solely on the composition DS misses patients with an "acid sensitive esophagus", a group of patients who benefit from PPI therapy.

\section{S1219}

Does the Absence of Erosive Esophagitis Really Predict a Worse Response to Proton Pump Inhibitor (PPI) Therapy in Patients with Gastroesophageal Reflux Disease?

William D. Chey, Borko Nojkov, Richard Saad, Jennifer Rai, Susan A. Adlis, Michael J. Shaw

Background: It has been suggested that patients with nonerosive reflux disease (NERD) respond less well to PPI therapy than those with erosive esophagitis (EE). However, the evidence to support this possibility comes largely from pooled trial data acquired in different populations. There have been few trials which have directly compared the impact of PPI therapy in patients with NERD and EE from the same population. Aims: To compare the impact of PPI therapy on symptom severity and quality of life in patients with NERD and EE. Methods: Patients with heartburn at least twice per week referred for EGD were recruited. Those taking a PPI within 2 weeks of EGD were excluded. Eligible patients completed validated surveys including: Digestive Health Symptom Index (DHSI - upper \& lower GI symptoms), Reflux Disease Questionnaire (RDQ-severity and frequency of GERD symptoms) QOLRAD (disease-specific QoL), and SF-12 (generic QoL). All patients were treated with open label rabeprazole sodium (Aciphex, Eisai Inc.) $20 \mathrm{mg} / \mathrm{d}$ x 8 wks after which follow-up surveys were completed. Results: 99 patients have participated to date. 32 (32\%) had EE and 67 had NERD Overall, PPI therapy led to highly significant improvements in GERD and dyspeptic symptoms, disease specific QoL, and generic QoL. Baseline symptom severity and response to therapy was not different between those with EE and NERD. Improvements in QoL were also similar between EE and NERD (see table). Conclusions: Contrary to currently accepted dogma, this open label trial which simulated clinical practice did not identify differences in the baseline or post-PPI therapy symptom severity or QoL between patients with EE and NERD. This study was supported by an unrestricted grant from Janssen Pharmaceutica, a unit of Ortho-McNeil and Esai Inc. 


\begin{tabular}{|c|c|c|c|}
\hline & EE & NERD & p-value \\
\hline RDQ-GERD & & & \\
\hline Baseline & 17.5 & 16.2 & 0.51 \\
\hline FU & 5.8 & 5.8 & 0.98 \\
\hline p-value & $\angle 0.0001$ & $\angle 0.0001$ & \\
\hline RDQ-Dyspepsia & & & \\
\hline Baseline & 10 & 8.8 & 0.36 \\
\hline FU & 3.3 & 3.2 & 0.90 \\
\hline p-value & $\angle 0.0001$ & $\angle 0.0001$ & \\
\hline QOLRAD-Total & & & \\
\hline Baseline & 3.7 & 3.9 & 0.45 \\
\hline FU & 5.1 & 5.2 & 0.62 \\
\hline p-value & $\angle 0.0001$ & $\angle 0.0001$ & \\
\hline
\end{tabular}

trend in tumor volume reduction at the highest dose, but the changes were not statistically significant. Serum calcium concentrations were normal in all groups of mice, except in those receiving $0.02 \mu \mathrm{g}$ molar equivalents of the most potent Gemini analog. Conclusion: The results from these studies demonstrate that vitamin D deficiency may accelerate colorectal cancer growth and that novel Gemini analogs of $1,25(\mathrm{OH})_{2} \mathrm{D}_{3}$ may potentially represent an effective new approach for the prevention and treatment of colorectal cancer

\section{S1222}

Angiotensin Converting Enzyme Inhibitors Reduce the Incidence of Colon Cancer: A Study of Half a Million US Veterans Vikas Khurana, Hanmanth R. Bejjanki, Gloria Caldito

AIM: To investigate the effect of Angiotensin Converting Enzyme (ACE) Inhibitors use in reducing the incidence of colon cancer in the US veteran population. BACKGROUND: ACE Inhibitors are commonly used antihypertensive and nephroprotective agents. Vascular Endothelial Growth Factor (VEGF) is believed to play a major role in angiogenesis in human tumors. Blocking the VEGF inhibits angiogenesis and suppresses tumor growth. ACE inhibitors cause suppression of VEGF in experimental models, leading to their anticancer effect. ACE Inhibitors have been noted to suppress tumor growth by inhibiting tumor angiogenesis in several animal and experimental models. DESIGN: The VISN 16 database contains clinical and demographic information about all veterans (>1.4 million patients) cared for in the South Central VA Health Care Network (eight states in south central United states). The data was queried from Oct 1998 to June 2004, using a retrospective case control design. Statistical analysis was performed using SAS software version 9.0 (Chicago, IL) Multiple logistic regression analysis was used with calculation of odds ratios and 95\% confidence intervals. The data was adjusted for age, race, gender, BMI, smoking, alcohol use, diabetes and statin use. Patients were placed in the ACE inhibitor user group if they were using ACE inhibitors prior to the diagnosis of colon cancer. RESULTS: A total of 483,733 patients were included in the analysis. 184,743 (38.2\%) of those were using ACE inhibitors. Colon and rectal cancer (ICD-9 codes 153 and 154) was seen in 6697 (1.4\%) ACE inhibitor users were $47 \%$ less likely to develop colon cancer (Odds ratio $0.47 ; 95 \%$ $\mathrm{CI} 0.45-0.50, \mathrm{p}<0.0001)$. The protective effect of ACE inhibitors was independent of statin use. Other significant covariates include age (OR 1.054, 95\% CI for OR 1.052, 1,056, p $<0.0001$ ); prior statin use (OR $0.454,95 \%$ CI for OR $0.427,0.482, \mathrm{p}<0.0001$ ) and diabetes (OR 1.605, 95\% CI for OR 1.510, 1.706, p<0.0001). CONCLUSION: ACE inhibitors are associated with a $47 \%$ reduced incidence of colon cancer after controlling for age, race, gender, BMI, smoking, alcohol use, diabetes and statin use. The limitations of our data are the VA population, the database and the fact that this is a case control study. The dose, duration and type of ACE inhibitor used were not factored into the analysis. reated with AOM $(20 \mathrm{mg}$ ip/kg-body wt, 80 rats) or saline (20 rats) weekly for 2 wks and sacrificed after 44 wks. Tumors were harvested and graded for histology. Activation levels of colonic epithelial ErbB2, AKT and ERK were quantified by Western blotting using phosphoactive antibodies. Statistical comparisons in tumor incidence were calculated by Fisher exact test and BrdU incorporation (\% total cells) and kinase activations compared by Student's ttest. Results: AOM and Gefitinib treatments were well tolerated. Gefitinib significantly reduced overall tumor incidence ( $21.6 \%$ vs. $71.5 \%, \mathrm{p}<0.05)$, and the percentage of adenomas and cancers (adenoma incidence $20 \%$ vs. $60 \%$, cancer incidence $8 \%$ vs. $50 \%, \mathrm{p}<0.05$ ). Gefitinib also inhibited tumor growth $(17 \pm 3 \mathrm{mg}$ vs. $80 \pm 16 \mathrm{mg}, \mathrm{p}<0.05)$. Compared with control, AOM significantly increased levels of EGFR $(2.9 \pm 0.3$ fold, $\mathrm{p}<0.05)$, pErbB2 $(2.8 \pm 0.9$ fold, $\mathrm{p}<0.05)$, effectors $\mathrm{pERK}(10.0 \pm 3.0$ fold, $\mathrm{p}<0.05)$ and $\mathrm{pAKT}(2.9 \pm 0.3, \mathrm{p}<0.05$ fold $)$, and target cyclin Dl (2.1 \pm 0.3 -fold, $\mathrm{p}<0.05)$, whereas Gefitinib significantly inhibited upregulation of EGFR (35\% inhibition) and cyclin D1 (41\%), and activations of ErbB2 (64\% inhibition), ERK ( $71 \%$ ) and AKT (56\%). Conclusions: Gefitinib significantly reduced AOM tumor incidence, tumor growth and progression. Concomitantly, Gefitinib limited cyclin D1 up-regulation and activation of ErbB2, ERK and AKT in colonic epithelium. These studies demonstrate that EGFR signaling participates in AOM-induced colonic carcinogenesis and that Gefitinib inhibits colonic tumor development. Further studies to explore EGFR inhibition in patients at increased risk for colon cancer should be explored.

\section{S1221}

Prevention and Treatment of Colorectal Cancer in Mice with Vitamin D and Its Gemini Analogues

Min Yao, Catherine S. Spina, Vin Tangpricha, Wei Zhou, Hubert Maehr, Milan Uskokovic, Luciano Adorini, Michael F. Holick, M. M. Wolfe

Background: It has been recognized that persons who live at higher latitudes and who are vitamin $\mathrm{D}$ deficient are at a significantly higher risk for dying from many commonly encountered malignancies, including colorectal cancer (CRC). Purpose: To determine whether CRC tumor growth is enhanced in mice fed a vitamin $\mathrm{D}$ deficient diet and whether treatment with novel, active Gemini analogs of $1,25(\mathrm{OH})_{2} \mathrm{D}_{3}$ might inhibit the growth of CRC in a vitamin D sufficient state. Methods and Results: Groups of BALB/C mice were transplanted subcutaneously with $10^{3} \mathrm{MC}-26$ cells, a mouse CRC cell line, and were fed either a vitamin D-deficient or -sufficient diet. Serum calcium levels did not differ between the two groups. In contrast, whereas the serum concentration of $25(\mathrm{OH}) \mathrm{D}$ was undetectable in mice fed the vitamin D-deficient diet, levels were normal $(65.6 \pm 15 \mathrm{nmol} / \mathrm{L})$ in animals administered a vitamin D-sufficient diet. Starting at day 9, the tumor volume in mice fed a vitamin Ddeficient diet was significantly larger, and at the end of the period of observation (day 19), tumors in these mice were $64.7 \%$ larger compared to tumors in animals administered a vitamin D-sufficient diet $\left(560 \pm 110 \mathrm{~mm}^{3}\right.$ vs. $\left.340 \pm 70 \mathrm{~mm}^{3}, \mathrm{P}<0.05\right)$. In separate experiments, mice transplanted with MC-26 cells were fed a low calcium diet and were treated with placebo or one of several novel 19-nor-Gemini (vitamin D) compounds. $24 \mathrm{~h}$ after tumor cell implantation, the mice received one of the Gemini vitamin D analogs or vehicle 3 times weekly. The administration of these Gemini analogs produced a dose-dependent decrease in tumor volume. Treatments with a less potent Gemini analog at the dose level of $0.02 \mu \mathrm{g}$ molar equivalents, compared to $1,25(\mathrm{OH})_{2} \mathrm{D}_{3}$, reduced the mean tumor volume by $41 \%$ $(\mathrm{P}<0.05)$, compared to the control group. At the same time point, mice treated with the most potent Gemini analog, administered at the 10 -fold lower dose of $0.002 \mu \mathrm{g}$ molar equivalents, demonstrated a $52 \%$ reduction in the mean tumor volume $(\mathrm{P}<0.05)$, compared to the control group. Animals that received $1,25(\mathrm{OH})_{2} \mathrm{D}_{3}$ at 0.002 and $0.02 \mu \mathrm{g}$ showed a

\section{S1223}

Celecoxib and Curcumin Synergistically Inhibit the Growth of Colorectal Cancer in Rat Model

Baruch Shpitz, Nis Giladi, Eyal Sagiv, Diana Kazanov, Menachem Moshkowits, Aharon Halak, Nadir Arber

Background: Colorectal Cancer (CRC) is one of the leading causes of cancer death in the western world. Multiple studies have indicated that specific COX-2 inhibitors may prevent CRC. However, the long-term use of COX-2 inhibitors is not toxic free and may be limited due to its gastrointestinal and cardiovascular side effects. The chemopreventive efficacy of the phytochemical, curcumin has been demonstrated in several in vitro and animal models. Previously we had shown that physiological doses of celecoxib $(5 \mu \mathrm{M})$ that can be achieved in human serum, and curcumin $(10-15 \mu \mathrm{M})$ synergistic inhibit cancer cell growth in vitro (Clin Cancer Res 2005). Aim:To examine the chemopreventive effect of this combination in 1,2-dimethylhydrazine (DMH) rat model. Methods: Forty male rats (F344), age eight weeks, were subcutaneously injected in the back with DMH $100 \mu \mathrm{g} / \mathrm{kg}$, twice, two weeks apart. Eight rats were not injected and served as the negative control group for aberrant crypt foci $(\mathrm{ACF})$ formation. One week following the second DMH injection the rats were randomly divided into four groups (10 rats in each group): a) AIN-076 control diet. b) AIN-076/curcumin (0.6\%); c) AIN-076/celecoxib (0.16\%); d) AIN-076/celecoxib (0.16\%) and curcumin $(0.6 \%)$. After one month the rats were sacrificed and their colon dissected. ACF were identified by their characteristic elongated slit-shaped lumen, surrounded by thickened epithelium that was more intensively stained with methylene blue than the surrounding normal crypts. Results: There were no ACF in the non DMH injected rats. The average number of ACF per rat colon was $64.2 \pm 3$ in the control group, $39 \pm 5$ and $47 \pm 10$ for the curcumin and celecoxib treated group respectively. In the group that had received both curcumin and celecoxib, there were $29 \pm 15$ ACF. This reduction of $27 \%, 39 \%$, and $62 \%$ in the number of ACF per rat for the different treatment groups was statistically significant (all $\mathrm{P}<0.05)$. Conclusions: in vivo, curcumin potentiates the growth inhibitory effect of celecoxib in the DMH rat model. This is clinically important as this dose of celecoxib (equivalent to $200 \mathrm{mg}$ ) can be achieved in human serum following standard anti-inflammatory dosing. Phase III study in CRC patients has been initiated. All patients are receiving standard therapy and curcumin (4 gr), celecoxib (200 mg), their combination or placebo.

\section{S1224}

A Randomised Study of the Effects of N-3 Fatty Acid Supplementation On COX-2 Expression in Barrett's Epithelium

Samir Mehta, Alexander P. Boddy, J.N. Cook, Elizabeth K. Lund, Virginia Sams, Ian T. Johnson, Michael Rhodes

Introduction Evidence from animal and in vitro studies indicates that n-3 fatty acids may inhibit carcinogenesis, and epidemiological studies suggest a reduced risk of oesophageal cancer in populations with high consumption of fish. One of the possible mechanisms for this chemopreventive effect is the suppression of arachidonic acid derived eicosanoid biosynthesis. In this study we have determined the effects of dietary supplementation with 
the n-3 fatty acid eicosapentaenoic acid (EPA) on eicosanoid levels $\left(\mathrm{PGE}_{2}\right.$ and $\left.\mathrm{LTB}_{4}\right)$ and the enzyme cyclo-oxygenase-2 (COX-2) in human Barrett's oesophagus. Methods Fifty patients with known Barrett's oesophagus underwent an upper GI endoscopy. Biopsies were taken at a recorded level from the area of Barrett's. 30 patients were then randomised to EPA supplementation (1.5g/day) for 6 months; 20 patients acted as controls. At the end of this period patients were re-endoscoped and biopsies taken at the same level. Tissue samples taken before and after supplementation were analysed for mucosal lipid profile, $\mathrm{PGE}_{2}$, and $\mathrm{LTB}_{4}$ levels. We also assessed COX-2 levels by immunoblotting and by a specific enzymelinked immunosorbent assay (ELISA). Results There was a significant increase in mucosal EPA content after dietary supplementation ( 6 months vs. baseline: $2.4 \%$ vs. $0.8 \%$ of total lipid content; $\mathrm{p}<0.001$ ), and a reduction in mucosal n-6 and n-9 fatty acid levels. There was no change in the untreated controls. EPA supplementation had no significant effect on the levels of $\mathrm{PGE}_{2}$ in Barrett's (6 months vs. baseline: 36.1 vs. $33.1 \mathrm{pgs} / \mathrm{\mu g}$ protein) or on $\mathrm{LTB}_{4}$ levels (12.6 vs. $11.8 \mathrm{pgs} / \mu \mathrm{g}$ protein). The enzyme COX-1 was highly expressed in all patients, but in contrast to recent reports, COX-2 was only present at very low levels, except in a single patient with histologically confirmed moderate dysplasia. Immunoblotting revealed two bands for COX-2; a band at $62 \mathrm{kDa}$ which was significantly reduced following intervention with EPA $(\mathrm{P}<0.05)$ and a weaker band only present in some patients at the expected $72 \mathrm{kDa}$ mark. The COX-2 ELISA confirmed a reduction in mucosal COX-2 in Barrett's tissue after EPA supplementation $(\mathrm{p}<0.05)$. Conclusion Supplementation with EPA significantly altered the lipid profile of Barrett's mucosa but failed to reduce eicosanoid levels. The down-regulation of COX-2 expression by EPA may result in chemopreventive effects through alternative mechanisms

\section{S1225}

Smokers Have a Higher Risk for Significant Colorectal Neoplasia and At a Younger Age Than Those Who Never Smoked: Implications for Screening Guidelines

Joseph C. Anderson, Brendan J. Wiggins, Zvi A. Alpern, Carol A. Martin, Patricia Hubbard-Ells

Background:Age and family history are the main risk factors considered when stratifying for colorectal cancer screening. In a screening population, smoking and age were the most predictive factors for colorectal neoplasia (Anderson et al AJG 98:2777-83,2003). One expert has suggested that smokers should be considered a high risk group but also stated sufficient data did not exist to justify earlier screening for smokers (Giovannucci et al, Dig Liv Dis $36: 643-5,2004)$. The goal of our study was to determine if smokers are at risk for colorectal neoplasia at a younger age. Methods:Asymptomatic patients ( $>40$ years old) undergoing screening colonoscopy are queried with respect to age, gender,BMI, family history, smoking history (pack years and year quit), medications, alcohol use, aspirin/NSAID use, exercise, fruit/vegetable and red meat intake. Endoscopic findings and pathology are entered into the database. Subjects were divided:1)Never smokers 2)Current smokers or patients with $>10$ pack-years, still smoking or having quit recently 3)Low exposure smokers: $<10$ pack years or who having quit over 10 years ago. Significant colorectal neoplasia $(\mathrm{SCN})=$ villous tissue, high grade dysplasia, large tubular adenomas or $>2$ adenomas. Results: 2466 patients were screened. Current smokers had a higher prevalence $(67 / 478 ; 14 \%)$ for SCN than never smokers $(115 / 1385 ; 8.3 \%)(\mathrm{p}<0.001)$ or low exposure smokers $(44 / 566 ; 7.8 \%)(\mathrm{p}<0.01)$. Current smokers had a higher risk than never smokers for SCN(OR=1.84;95\% Cl=1.352.49; $<0.001)$. The average age for smokers with SCN $(58.1 ; 95 \%$ CI: 55.5-60.6) was lower than never smokers with SCN $(62.2 ; 95 \%$ CI:60.0-64.5) $(\mathrm{p}=0.04)$. There was no difference other than age between these two groups, including gender, BMI, family history and alcohol use. Smoking is a risk factor for SCN in younger (most who are not eligible for screening) subjects as well(see Table). Conclusions: 1)Current smokers have a higher risk and prevalence of SCN than in never smokers. 2)SCN occurs in current smokers 4 years earlier than in those who never smoked suggesting an etiological association between smoking and colorectal neoplasia. 3) Our results strongly support the notion current smokers are at high risk for colorectal neoplasia at a younger age and probably should be screened at an earlier age than non-smokers.

Prevalence and Risk of Significant Neoplasia in the 40 to 55 Age Group

\begin{tabular}{|c|c|c|c|}
\hline Smoking Status & Prevalence (\%) & OR & $95 \%$ CI \\
\hline Never smokers & $32 / 576(5.6 \%)$ & 1.0 & 1.0 \\
\hline Current smokers & $25 / 205(12,2 \%)$ & 2.36 & $1.36-4.09$ \\
\hline
\end{tabular}

p<0.003; OR, after controlling for BMI, Fam Hx, gender

\section{S1226}

Growth Factors Dictate the Inhibitory Response of Target Cells to the Dietary Agent, Curcumin

William Rengifo-Cam, Pomila Singh

Background. Progastrin (PG) and insulin-like growth factors (IGFs) exert proliferative and anti-apoptotic effects on colon cancer cells via endocrine/paracrine/autocrine routes (Singh P, 2000). Dietary agents such as curcumin inhibit the growth of transformed cells and inhibit colon carcinogenesis at the initiation, promotion and progression stages (Kawamori T et al, 1999). It is not known if curcumin exerts inhibitory effects in the presence of growth factors such as PG and IGF-II that are known to be expressed by colorectal cancers. Aim. To examine anti-prolifeative and pro-apoptotic efficacy of curcumin in the presence or absence of PG and IGF-II and to delineate relative phosphorylation of specific signaling molecules. Methods. IEC-18 cells are responsive to both PG and IGF-II, and were therefore used in these studies. Routine assays were used for examining the end points of growth, apoptosis and phosphorylation of kinases. Results. PG (0.1nM) and IGF-1 (10nM) significantly increased the proliferation of IEC cells by $\sim 2.5$ fold $(n=4-6)$. 10uM-100uM curcumin decreased cell proliferation in a dose dependent manner from 50 to $80 \%$. IGF-II was significantly more effective in reducing the inhibitory effects of curcumin on cell growth, than PG. Similarly the pro apoptotic effects of curcumin on IEC cells (activation of caspase 9), was also reversed to control values in the presence of IGF-II, but only partially reversed in the presence of PG. Relative levels of phosphorylated Akt, NFkB, Src, p42/44 (ERK) and p38 (MAPK) on treatment with curcumin, in the presence or absence of the growth factors was in the order of, IGF alone $>$ PG alone $>$ IGF+curcumin $>$ PG+curcumin $>$ serum free control $>$ curcumin alone. The loss in the relative levels of phosphorylated NFKB, Src and p42/44 (ERKs), in response to curcumin, was reversed most effectively in the presence of IGF-II. Summary 1. IGF-II is $\sim 1.5$ and $>4$ fold more effective in protecting the IEC cells against the antiproliferative and pro-apoptotic effects of curcumin, respectively. 2. Several kinases/transcription factors mediate protective effects of the growth factors against curcumin, including $\mathrm{NF \kappa B}, \mathrm{Src}$ and p42/44 (ERKs). Conclusion: These results suggest that intestinal tumors, growing in response to autocrine or circulating IGFs are likely to be significantly less responsive to the growth inhibitory effects of curcumin than tumors growing in response to PG. Therefore the growth factor profile of the tumor cells may dictate the response of the tumor cells to growth inhibitory effects of dietary agents, such as curcumin.

\section{S1227}

Vitamin C Suppresses H. Pylori Colonization But Has NO Effect On Helicobacter Pylori-Induced Gastritis in Vitamin C-Deficient L-Gulono- $\gamma-$ Lactone Oxidase Knock-Out (Gulo-/-) Mice

Chung-Wei Lee, Xiang-Dong Wang, Arlin B. Rogers, Timothy C. Wang, James G. Fox

Epidemiological studies link vitamin C (VC) deficiency to more severe Helicobacter pylor (Hp)-associated gastritis and a higher risk of gastric cancer, and suggest that high dietary VC intake may decrease the risk of stomach cancer. Vitamin C-deficient L-gulono- $\gamma$-lactone oxidase knock-out (Gulo-/-) mice on a C57BL/6 background were used as a model to examine the role of VC supplementation in Hp-associated gastric lesions. Gulo-/- and C57BL 6 (WT) mice were experimentally infected with Hp, and Gulo-/- were supplemented with either low $(0.033 \mathrm{~g} / \mathrm{L})$ or high $(3.3 \mathrm{~g} / \mathrm{L}) \mathrm{VC}$ in their drinking water. Mice were killed at 16 or 32 weeks post infection (WPI). In Gulo-/- mice, plasma and gastric VC contents were positively correlated with the dietary VC supplementation (Table 1). Hp colonization at 16 WPI measured by quantitative PCR was significantly lower in the low or high VC supplemented groups compared to WT mice (log CFU/g: $5.491+/-0.303,5.374+/-0.272$, and $6.681+/-0.434$, respectively; $\mathrm{p}<0.001$ ), suggesting a bactericidal effect of the VC or an enhancement of the host inflammatory response to Hp. However, the Hp-specific, Thlassociated IgG2c response was depressed in Gulo-/- mice with low VC or high VC supplementation as compared with that of WT mice at 32 WPI (anti-Hp outer membrane protein IgG2c: $0.274+/-0.065,0.441+/-0.103$, and $0.867+/-0.202$, respectively $\mathrm{p}<0.01)$. In addition, no histopathological differences in stomach lesion scores were observed among these groups with different treatments at both 16 and 32 WPI. The data indicate that the restoration o VC status of Gulo-/- mice by dietary VC supplementation alone does not protect against $H$. pylori-induced gastritis and associated lesions. These findings also support previous in vitro data demonstrating that $\mathrm{VC}$ has bactericidal activity against Hp. To delineate the relationship among gastritis, Hp colonization, and immunogenicity of Hp in VC-supplemented Gulo- $/$ mice, further studies are needed.

Table 1

\begin{tabular}{|c|c|c|c|c|c|c|}
\hline Group & Treatment & $\begin{array}{c}H . \\
p y l o r i\end{array}$ & $\begin{array}{c}\text { Plasma VC } \\
(\mu \mathrm{g} / \mathrm{mL}), 16 \\
\text { WPI }\end{array}$ & $\begin{array}{c}\text { Stomach VC } \\
(\mu \mathrm{g} / \mathrm{g}), 16 \mathrm{WPI}\end{array}$ & $\begin{array}{c}\text { Plasma VC } \\
(\mu \mathrm{g} / \mathrm{mL}), 32 \\
\text { WPI }\end{array}$ & $\begin{array}{c}\text { Stomach VC } \\
(\mu \mathrm{g} / \mathrm{g}), 32 \mathrm{WPI}\end{array}$ \\
\hline 1 & $\begin{array}{c}\text { Low VC, } \\
\text { Gulo-/- }\end{array}$ & - & $0.71+/-0.039^{*}$ & $19.02+/-2.084^{*}$ & $0.95+/-0.004^{*}$ & $26.65+/-2.046^{*}$ \\
\hline 2 & $\begin{array}{c}\text { Low VC, } \\
\text { Gulo-/- }\end{array}$ & + & $0.69+/-0.033^{*}$ & $15.60+/-1.126^{*}$ & $0.91+/-0.030^{*}$ & $21.79+/-1.456^{*}$ \\
\hline 3 & $\begin{array}{c}\text { High VC, } \\
\text { Gulo-/- }\end{array}$ & - & $11.10+/-0.838$ & $155.88+/-18.498$ & $15.20+/-1.580$ & $155.10+/-10.73$ \\
\hline 4 & $\begin{array}{c}\text { High VC, } \\
\text { Gulo-/- }\end{array}$ & + & $11.10+/-1.078$ & $107.96+/-17.395^{\dagger}$ & $15.34+/-1.547$ & $193.8+/-10.07^{\dagger}$ \\
\hline 5 & $\begin{array}{c}\text { No VC, } \\
\text { WT }\end{array}$ & + & $10.87+/-0.803$ & $154.60+/-9.658$ & $11.48+/-0.600$ & $190.70+/-22.72$ \\
\hline
\end{tabular}

* $\mathrm{p}<0.001$ when compared to Gulo-/- mice with high VC supplementation or WT mice $\mathrm{p}>0.05$ when compared to group 3

\section{S1228}

Regulation of RAS in Colon Cancer Cells By Chemopreventive Ursodeoxycholic Acid Sharad Khare, Mehdi Baluch, Cory Holgren

Background: A number of potential mechanisms have been proposed for bile acid-induced colonic tumor promotion. While the predominant primary bile acids are tumor promoters, we have shown that another bile acid ursodeoxycholic acid (UDCA) has chemopreventive effects in AOM-induced colonic carcinogenesis [Cancer Research, 54: 5071-5074, 1994 Cancer Epidemiology, Biomarkers and Prevention, 11: 1316-1321, 2002]. More recently, we showed UDCA also suppressed the development of tumors with ras mutations or tumors with activated wild type Ras, the gene product encoded by ras [Cancer Research, 63: 3517 $3523,2003]$. We now investigated the mechanism of wild type Ras inhibition by UDCA in HCA-7 cells, an adenocarcinoma colon cancer cell line. Methods: HCA-7 cells were grown in McCoy's $5 \mathrm{~A}$ media containing 10\% fetal calf serum. Phosphorylation status of c-Met-R, EGF-R, IGF-1R and EphA2 was assesed by immunoprecipitation and Western blotting. Ras activation was measured by a pull-down assay utilizing Raf1-RBD agarose. Expression of Ras-GAP and co-association of Sos-1, Grb2 and Shc was assessed by immunoprecipitation and Western blotting. Results: Ras-GTPase activating protein (ras-GAP), a negative regulator of 2 lras was poorly expressed in HCA-7 cells. However, we noted a significant expression of an alternative splicing product, which has a molecular mass of $100 \mathrm{kDa}$. UDCA had no 
significant effect on expression of this gene. Phoshorylation status of receptor tyrosine kinases (RTKs) c-Met-R, EGFR, IGF-1R and EphA2 was assessed. Sos-1, a well-known Ras-guanine nucleotide exchange factor, which favors ras activation, was constitutively associated with growth factor receptors. UDCA significantly decreased Sos-1 association with c-Met-R and suppressed wild type Ras activation. As a control, deoxycholic acid (DCA), a tumor promoting bile acid dramatically increased Sos- 1 association with c-Met-R in a time and concentration dependent manner and activated wild type Ras in HCA-7 cells. Grb2 is intrinsically complexed with the Sos and an enhanced Grb2-Sos association with Shc upon DCA stimulation was observed. Summary: These data demonstrate that bile acids can function through RTK. Further, for the first time, a molecular mechanism of Ras regulation by UDCA is investigated.

\section{S1229}

Vitamin D Receptor (VDR) and 25-Hydroxyvitamin D3-1 $\alpha$-Hydroxylase $(1 \alpha(\mathrm{OH})$ Ase $)$ in Normal and Malignant Human Colon

Damien Matusiak, Robert Carroll, Richard Benya

Although considerable evidence exists to support the use of vitamin D to prevent and/or treat colorectal cancer (CRC), toxic hypercalcemia limits the routine use of bioactive vitamin D. Yet recent studies have shown that colonic epithelial cells express mRNA for $1 \alpha(\mathrm{OH})$ ase an enzyme that converts non-toxic pro-vitamin D [25(OH)D3] to its bioactive form. However, nothing is known about the cellular expression of $l \alpha(\mathrm{OH})$ ase and the VDR in the earliest histopathological structures associated with malignant transformation such as aberrant crypt foci $(\mathrm{ACF})$ and polyps, nor is anything known as to the expression of these proteins in CRC as a function of tumor cell differentiation or metastasis. METHODS: 10 colorectal cancers (CRC) were randomly selected from our GI Tumor Bank, containing 53 separate and distinct regions that were well differentiated, 66 that were moderately differentiated, and 32 that were poorly differentiated. $l \alpha(\mathrm{OH})$ ase and VDR expression was determined using specific antibodies, and the amount of staining quantified as previously described [J Histochem Cytochem 2003; 51: 205]. RESULTS: $1 \alpha(\mathrm{OH})$ ase was present at equal high levels in normal colonic epithelium as in ACF's, polyps, and CRC irrespective of tumor cell differentiation. In contrast, VDR levels were low in normal colonic epithelial cells; were increased in ACF's, polyps, and well differentiated tumor cells; and then declined with increasing dedifferentiation. Both $l \alpha(\mathrm{OH})$ ase and VDR levels were negligible in tumor cells metastasizing to regional lymph nodes, irrespective of the differentiation of the tumor found in either the primary or metastatic lesion. Finally, since the VDR acts by translocating to the nucleus after binding ligand, we assessed the relative amounts of VDR expressed in nuclear and cytoplasmic regions of each tumor cell. While the ratio of nuclear to cytoplasmic VDR exceeded 4 in normal colonic epithelial cells, indicating that most of this protein was in the nucleus, this ratio dropped to $<1.0$ for even ACF's, the earliest lesion associated with CRC formation. Thus an ever-declining percentage of VDR translocates, or is able to translocate, to the nucleus of colonic epithelial cells that are not histologically normal. CONCLUSIONS: Non-toxic forms of vitamin D may be useful in CRC chemoprevention. In contrast, the absence of $l \alpha(\mathrm{OH})$ ase and VDR in metastases, and the decreased ability of the VDR to translocate to the nucleus in even the earliest histopathological lesions associated with malignant transformation, suggests that vitamin D may not be useful in CRC chemotherapy.

\section{S1230}

Regulatory Mechanism for Folic Acid Induced Inhibition of Recurrence of Colorectal Adenomas

Nataliya Razumilava, Saadia Siddiqui, Edi Levi, Hu Xu, Nadeem Ullah, Martin Tobi, Richard Jaszewski, Adhip P. N. Majumdar

Accumulating murine as well as epidemiologic and case controlled human studies suggest that folic acid may be an effective chemopreventive agent for colorectal neoplasia. Indeed, data from our recently completed double-blind, placebo controlled clinical trial on 177 patients with colorectal adenomas $(\geq 0.5 \mathrm{~cm}$ ) [who had undergone polypectomy] demonstrated that daily consumption of supplemental folic acid ( $5 \mathrm{mg} /$ day) for 3 years significantly decreased the recurrence of adenomas (Gastroenterology 128: A-93, 2005). Although the regulatory mechanisms for folic acid mediated decreased recurrence of polyps are not fully understood, we hypothesize that this could partly be the result of inhibition of proliferation and/or stimulation of apoptosis. We further hypothesize that EGF-receptor (EGFR), which regulates the growth related process, is critically involved in regulating folic acid-mediated changes in proliferation and/or apoptosis in the colonic mucosa. Methods: Of 177 patients enrolled in the study, 94 subjects completed the 3-year study: 46 in folic acid group and 48 in placebo. Mucosal biopsies, taken from macroscopically normal rectal mucosa ( $\leq 10$ $\mathrm{cm}$ anal verge) at baseline and at 1- and 3-year follow-up colonoscopy, were fixed in buffered-formalin, subsequently paraffin-embedded, sectioned (4-5 micron) and analyzed immunohistochemically for changes in (a) proliferative activity by measuring PCNA immunoreactivity, (b) apoptosis by TUNEL assay, (c) EGFR and (d) EGFR-Related Protein (ERRP), which we have identified and characterized as a negative regulator of EGFR. Results: At baseline, no significant difference in any of the above mentioned parameters was observed between the placebo and folic acid consumed groups. However, consumption of supplemental folic acid for 3 years resulted in a statistically significant $(20-30 \% ; \mathrm{P}<0.0009)$ reduction in prolifetative activity without an apparent change in the rate of apoptosis in the colonic mucosa. Folic acid mediated reduction in mucosal proliferative activity in the colonic mucosa was associated with a marked reduction in the relative abundance of EGFR. This was accompanied by a concomitant increase in ERRP expression. Conclusion: Our data suggest that folic acid exerts a chemopreventive effect on colorectal neoplasia, as demonstrated by inhibition of recurrence of adenomas. This could be attributed to reduction in proliferation. EGFR appears to play a critical role in regulating this process.
S1231

A New Aminoacid Derivative of Ursodeoxycholate, N-L-Glutamyl-UDCA (UDCA-Glu) to Release Selectively UDCA in the Rat Colon

Carlo Clerici, Danilo Castellani, Stefania Asciutti, Elisabetta Nardi, Giorgio Gentili, Roberto Pellicciari, Kenneth Setchell, Nancy O'Connell, Vittorio Giuliano, Fabio Chistolini, Olivia Morelli, Mattia Clementi, Monia Baldoni, Antonio Morelli

Introduction: Ursodeoxycholic acid (UDCA) showed a protective effect against colon cancer development in rats and the recurrence of high-grade dysplasia adenomas in humans. After oral administration, UDCA is mainly absorbed in the small intestine and a small amount is directly delivered in the colon. In our search for new strategies for the selective release of UDCA in the colon, we synthesized UDCA-glutamate (UDCA-Glu) that utilizes the selectivity of peptide bond cleavage exhibited by brush border enzymes to increase the concentration of UDCA in the colon. Methods: 30 Fisher male rats were utilized in 3 experiments (A, B, C). A: 10 animals received via an intragastric probe a daily dose of UDCA $(10 \mathrm{mg} / \mathrm{kg} / \mathrm{die})$ or UDCA-Glu (at the equivalent dosage of $10 \mathrm{mg} / \mathrm{kg} /$ die of UDCA) for 5 days; after sacrifice, the small intestine and the colon were removed to study fecal intestinal tract bile acid composition. B: 10 animals received UDCA $(10 \mathrm{mg} / \mathrm{kg} / \mathrm{die}$ ) or UDCA-Glu (at the equivalent dosage of $10 \mathrm{mg} / \mathrm{kg} /$ die of UDCA) for five days; fecal samples were collected everyday. All fecal samples collected in the A and B experiments were analyzed by GC-MS and FAB-MS in order to study quantitative and qualitative fecal bile acid composition. C: 10 animals were given an intraduodenal bolus of UDCA $(10 \mathrm{mg} / \mathrm{kg} / \mathrm{die})$ or UDCA-Glu (at the equivalent dosage of $10 \mathrm{mg} / \mathrm{kg} / \mathrm{die}$ of UDCA) in order to study bile flow. Bile samples were collected by an external biliary fistula every $15 \mathrm{~min}$ for $330 \mathrm{~min}$ and then weighted in order to determine bile flow. Result. A: In the resected small intestine significantly higher UDCA level (unconj. $1.50 \pm 0.32 \mathrm{mg}$ ) was found in UDCA treated rats as compared with UDCA-Glu group (unconj. $0.75 \pm 0.12 \mathrm{mg}$ ) where high concentration of UDCA-Glu (unconj. $2.08 \pm 0.10$ $\mathrm{mg}$ ) were detected. In the resected large intestine instead significantly higher amount of

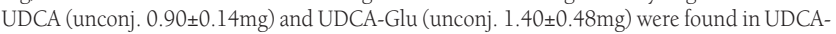
Glu treated rats in respect to UDCA group (unconj. UDCA $0.50 \pm 0.15 \mathrm{mg}$ ). B: the analysis of total fecal bile acid recovery confirmed higher UDCA concentration (unconj. $3.39 \pm 0.30 \mathrm{mg}$; conj. $6.40 \pm 1.03 \mathrm{mg}$ ) in rats treated with UDCA-Glu as compared to those treated with UDCA (unconj. $2.27 \pm 0.11 \mathrm{mg}$; conj. $0.04 \pm 0.01 \mathrm{mg}$ ). C: UDCA treated animals maintained bile flow better than UDCA-Glu group where there was an evident decrement in bile flow after 3 hours from the administration of both compounds. Conclusion: Conjugation of UDCA with glutamic acid limits its absorption in the small intestine and leads to increased colon delivery and fecal excretion of UDCA. UDCA-Glu could be a promising pro-drug to deliver UDCA in the colon.

S1232

NSAIDs, COX-2 Inhibitors, LES Relaxing Drugs and Esophageal Cancer: A Case- Control Study Satish Ranka, Jenny M. Gee, Ian T. Johnson, J.N. Skinner, Andrew R. Hart, Michael Rhodes

Aim: To examine the association between the use of NSAIDs, beta adrenergic agonists, theophylline and calcium channel blockers with the risk of esophageal cancer (EC). Introduction: The incidence of EC has doubled in the last three decades. It has an overall 5 year survival rate of less than $5 \%$ and an effective preventive strategy is therefore desirable NSAIDs are known to affect some of the crucial metabolic pathways that control tumor development. Beta agonists, aminophylline and calcium channel blockers, relax the lower esophageal sphincter (LES) which may increase gastro-esophageal reflux. This may in turn increase the risk of esophageal adenocarcinoma (EAC). Methods: Between 1999 and 2004 411 patients with primary neoplasm of the esophagus and cardia were matched with 1644 controls with non-melanotic skin lesions. Each case was matched for age, sex and year of hospital admission. Data on use of NSAIDs, beta adrenergic agonists, aminophylline and calcium channel blockers was collected. Results: The mean age of patients with EC was 71 years and the commonest site of disease was the lower 1/3rd of the esophagus. Smoking was more common in cases as compared to controls (OR 3.18, 95\% CI 2.23 - 4.12). Alcohol intake was similar in cases and controls (OR 0.90, 95\% CI $0.70-1.21$ ). The odds ratios (OR) and 95\% confidence intervals (CI) for exposure to different drugs in cases versus controls are as follows: Aspirin 0.38, (0.26-0.50); other NSAIDs 0.29, (0.19-0.42); COX-2 inhibitors $0.35,(0.16-0.91)$, beta agonists 3.6 (95\% CI 2.6 to 4.9$)$, aminophylline 2.1 (95\% CI 1.1 to 4.1 ) and calcium channel blockers 2.9 (95\% CI 2.1 to 4.1). Conclusion: Esophageal cancer has a negative association with NSAIDs and a positive association with drugs which relax the LES. Any regular use of NSAIDs may have a protective role for patients at high risk of EC such as those with Barrett's esophagus and widespread use of drugs which relax the LES may be a contributing factor in increasing the incidence of EAC in this population.

S1233

Effects of Rofecoxib, a COX-2 Selective Inhibitor, in Patients with Barrett's Esophagus: Final Results of a 6-Month Randomized Controlled Trial Angel Lanas, Federico Sopena, Javier Ortego, Eva Barrio, Luis Bujanda, Angel Cosme, Adolfo Parra, Javier Alcedo, Angel Ferrandez, Eduardo Bajador, Josep M. Pique

Background: COX-2 is overexpressed in Barrett's esophagus (BE). Short-term treatment with COX-2 inhibitors reduce cell proliferation in BE. Aim: To determine the long-term effect of a COX-2 inhibitor on several cellular mechanisms involved in the neoplastic progression of BE. Methods: Multicenter randomized controlled trial of BE patients computer allocated either to take their usual PPI alone or combined with rofecoxib $25 \mathrm{mg}$ /day for 6 months. Exclusion criteria: presence of high-grade dysplasia \& contraindication to take rofecoxib No NSAID/ASA use was allowed. Multiple biopsy specimens of BE and duodenum were obtained at baseline and after treatment. All patients were asymptomatic on their PPI ( $90.3 \%$ standard doses)and were monitored at 0,1,3 and 6 months. The effect of Rofecoxib on cell proliferation (Ki-67; mibl) in BE glands was the primary end point. Cell apoptotis, as well 
as COX-2, cycline D1, and p53 expression were also measured blinded by inmmunohistochemistry by the same pathologist. BE cell proliferation and apoptotic index (number of $(+)$ cells/number of cells within the gland x100) was determined by a computer assisted technique (Image J, $\mathrm{NIH} ; \mathrm{USA}$ ) in all specimens. Expression was graded from $0=$ No expression to $2=$ moderate-intense expression. Chi square, parametric and non-parametric test were used for data analysis. Only data for those who underwent both initial and final endoscopy are presented. Results: Of 62 patients with BE (length $=4.3 \pm 2.1$; age $=58.8 \pm 13.7 ; 77.4 \%$ males), 8 did not finalize the study; 6 due to adverse events and 2 voluntarily. Low grade dysplasia was present in 19,4\% of patients at baseline. Adverse events were present in 4 patients on the rofecoxib arm and 2 on PPI alone. Compliance was high ( $89.1 \pm 22.2 \%$ of pills). Rofecoxib+PPI treatment was not associated with reduction of cell proliferation index $(40.4 \pm 13.04 \%$ at baseline vs. $46.1 \pm 15.1 \%$ after treatment). In the same way, rofecoxib $+\mathrm{PPI}$ treatment did not affect the expression of cycline D1 or p53 and did not affect the incidence of low-grade dysplasia ( $24.2 \%$ at baseline vs. $15.2 \%$ after treatment). Unlike PPI alone, rofecoxib+PPI treatment decreased COX-2 expression (moderate-intense expression in $92,8 \%$ of patients at baseline vs $50 \%$ after treatment; $\mathrm{p}<0.01$ ) and increased the apoptotic baseline index (3. $1 \pm 2.7 \%$ o) in cells of BE glands $(215 \pm 122 \%$ vs. $67.8 \pm 32 \%$ increase in PPI alone treated; $\mathrm{p}<0.05)$ Conclusions: The addition of rofecoxib $25 \mathrm{mg}$ /day to PPI therapy does not affect cell proliferation index of BE cells, p53, or cycline Dl expression, but it increases cell apoptosis and reduces COX-2 expression after 6 months of therapy.

S1234

The Increase of 15-LOX-1 Expression On Carcinoma Cells May Enhance the Sensitivity to NSAIDs-Induced Apoptosis in Colorectal Cancers Masahiro Yoshinaga, Hiroyuki Murao, Yosuke Kitamura, Koutaro Koga, Satoru Tsuruta, Hisato Igarashi, Hidefumi Higashi, Kazuhiko Nakamura

Background: Non-steroidal anti-inflammatory drugs (NSAIDs) can prevent the colorectal cancer (CRC), but its effect is limited. To overcome the limitation, the molecular features in CRCs from patients who have been treated with NSAIDs for a long period need to be elucidated. Recent studies show the involvement of 15-lipoxygenase-1 (15-LOX-1) in NSAIDs-induced apoptosis in colorectal carcinoma cells. Therefore, we compare the 15LOX-1 expression in CRCs from NSAID users with that from NSAID nonusers and certify the role of 15-LOX-1 in the NSAIDs-induced apoptosis of colorectal carcinoma cells. Methods: In 22 CRC surgical samples from NSAID users who had been constant for more than 5 years and 28 CRC surgical samples from NSAID nonusers, the expressions of 15-LOX-1, cyclooxygenase-2 (COX-2), beta-catenin, and p53 were analyzed using immunohistochemistry. To identify the level of apoptosis in two groups, the terminal deoxynucleotidyl transferase (TdT)-mediated dUTP nick-end labeling (TUNEL) assay was performed for samples. The effects of the transient transfection of 15-LOX-1 cDNA on indomethacin-induced apoptosis were examined in HCT-116 cells. The effects of adding a major metabolite of 15LOX-1, 13-S-hydroxyoctadecadinoic acid (13-S-HODE) on indomethacin-induced apoptosis were also examined in HCT-116 cells. The level of apoptosis was determined by the analysis of the floating-cells ratio and DNA gel electrophoresis. Results: In tumor cells, the expression of 15-LOX-1 on CRCs from NSAID users was significantly decreased compared with those from NSAID nonusers $(2 / 22$ vs $12 / 28, \mathrm{p}<0.05)$; however, the expressions of other molecules were not significantly different between two groups. In adjacent normal mucosal cells, the expressions of all molecules were not different between two groups. The level of TUNEL scoring in samples from NSAID users was similar to those from NSAID nonusers. One hundred $\mu \mathrm{M}$ of indomethacin induced less apoptosis in mocked HCT-116 cells, whereas the same concentrations of indomethacin enhanced the level of apoptosis in 15-LOX-1transfected HCT-116 cells. 13-S-HODE also increased the level of indomethacin-induced apoptosis in HCT-116 cells dose-dependently. Conclusion: Results suggest that the increase of 15-LOX-1 expression on carcinoma cells may enhance the sensitivity to NSAIDs-induced apoptosis in CRCs from patients who are treated with the compounds.

\section{S1235}

Combination Treatment with Curcumin and Quercetin of Adenomas in Familial Adenomatous Polyposis

Marcia Cruz-Correa, Daniel A. Shoskes, Patricia Sanchez, Rhongua Zhao, Linda M. Hylind, Steven D. Wexner, Francis M. Giardiello

Background: Familial adenomatous polyposis (FAP) is an inherited disorder with development of hundreds of colorectal adenomas and eventual colorectal cancer. Regression of adenomas in FAP occurs with NSAIDs and COX-2 inhibitors, but these drugs can have side effects. We evaluated the combination of diet derived nonprescription supplements curcumin and quercetin to regress adenomas in FAP patients. Methods: Five FAP pts with prior colectomy (4 with retained rectum, 1 with ileal anal pouch) received curcumin $480 \mathrm{mg}$ and quercetin $20 \mathrm{mg}$ orally three times a day. Number and size of polyps were assessed at baseline and after therapy. Wilcoxon sign-rank test was use to determine differences in number and size of polyps. Treatment side effects and medication compliance were evaluated. Results: All five patients had a decreased polyp number and size from baseline after a mean of 6 months of treatment with curcumin and quercetin. The mean percent decrease in number and size of polyps from baseline was $60.4 \%(\mathrm{p}<0.05)$ and $50.9 \%(\mathrm{p}<0.05)$, respectively. Patient 1 (figure) had initial regression of polyps between 0 and 3 months of therapy but was noncompliant with treatment between 3 and 6 months. After reinstruction, the patient continued on therapy (from month 6 to 9) with reoccurring polyp regression. Minimal adverse side effects and no laboratory abnormalities were noted. Conclusion: The combination of curcumin and quercetin appears to reduce the number and size of ileal and rectal adenomas in patients with familial adenomatous polyposis without appreciable toxicity. This is the first report of efficacy of these compounds for adenoma regression in humans, but randomized controlled trials are needed to validate these findings. Supported by The John G. Rangos, Sr. Charitable Foundation, The Clayton Fund, NIH grants K07 CA092445, CA 53801, and P50 CA 62924-10.

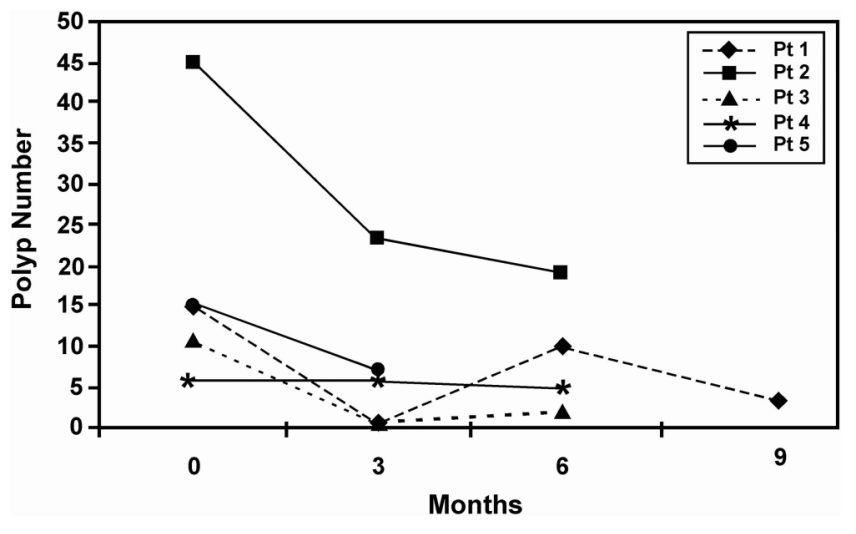

S1236

Induction of C-Terminal SRC Kinase (CSK) By NSAIDs: A Novel AntiProliferative Target in the Chemoprevention of Colorectal Cancer Dhananjay Kunte, Ramesh Wali, Jennifer Koetsier, Hemant Roy

Background: While the NSAIDS protect against human colorectal cancer (CRC), the molecular mechanisms remain unclear with a myriad of potential molecular targets. We have recently shown that the tumor suppressor gene C-terminal Src kinase (CSK) (a Src inhibitor) is down-regulated early in colon carcinogenesis, thus inducing Src which further leads to increased cell proliferation. Given that the CSK/Src axis impacts on many pathways that are implicated in NSAID-chemoprevention (control of apoptosis, proliferation, invasiveness and angiogenesis), we investigated the effect of NSAIDS on the Src/CSK axis. Methods: AOMtreated Male Fisher 344 rats were fed with AIN-76 diet either with or without 320 ppm sulindac for 10 weeks after which they were sacrificed. After sacrifice, the levels of CSK and p-Src-527 were monitored by western blotting and immunohistochemistry. In order to determine the role of CSK in the anti-proliferative activity of NSAIDs, we genetically engineered HT-29 cells for knockdown of CSK gene expression using the CSK-specific shRNA vector. The responsiveness of wild type HT-29 cells and the CSK shRNA construct to sulindac sulfide treatment $(100 \mu \mathrm{M}$ for $72 \mathrm{~h})$ was assessed by western blotting. Src activity was measured using a tyrosine kinase assay kit. Results: Treatment with sulindac resulted in marked increase in CSK (174\% of control, $\mathrm{p}<0.01)$ with corresponding increase in Src 527 (the inhibitory phosphorylation site) in the AOM-rats. In the CRC cell line HT-29, CSK was induced ( $144 \%$ of vehicle, $\mathrm{p}=0.014)$ by sulindac sulfide $(100 \mu \mathrm{M}$ for $72 \mathrm{~h}$ ) with corresponding increase in Src 527 (141\% of vehicle, $\mathrm{p}=0.021$ ) and a marked decrease in Src activity (30\% $\mathrm{p}<0.05$ ). In the CSK shRNA stable construct, sulindac sulfide did not induce CSK and the antiproliferative effect of sulindac sulfide (reduction in PCNA) was reduced by $42 \%$ as compared to wild type HT-29 cells. Furthermore, decrease in the E-cadherin phosphorylation (an in vivo indicator of decreased Src activity) was reduced by 39\% ( $\mathrm{p}=0.023)$ in the CSK shRNA construct than the wild type. Conclusion: We demonstrate, for the first time, that CSK was modulated by NSAIDS in both the AOM-treated rat and HT-29 cells. Importantly, blocking CSK-induction resulted in partial abrogation of the anti-proliferative effect of NSAIDS. Thus, this report provides compelling evidence that CSK induction is responsible, at least partly, for the chemopreventive effects of NSAIDS.

Effect of CSK status on anti-proliferative activity of sulindac sulfide.

\begin{tabular}{|c|c|c|}
\hline & HT-29 & CSK stable construct \\
\hline CSK induction & $44 \%$ & $2 \%$ \\
\hline PCNA suppression & $47 \%$ & $20 \%$ \\
\hline
\end{tabular}

S1237

The Effect of Helicobacter Pylori Eradication On Incident of Gastric Cancer Keiji Ogura, Yoshihiro Hirata, Ayako Yanai, Wataru Shibata, Tomoya Ohmae, Yuzo Mitsuno, Shin Maeda, Hirotsugu Watabe, Yutaka Yamaji, Makoto Okamoto, Haruhiko Yoshida, Takao Kawabe, Masao Omata

[Aim] The association between chronic Helicobacter pylori infection and development of gastric cancer is well established epidemiologically. The possibility of prevention for gastric cancer by eradicating $H$. pylori infection is recently investigated by several research groups and still controversial. The aim of this study is to explore the possibility of reduction in gastric cancer incidence by eradication of $H$. pylori infection. [Methods] Three-hundred four patients with persistent $\mathrm{H}$. pylori infection and $404 \mathrm{H}$. pylori-eradicated patients were annually examined for gastric cancer development by endoscopy for 3.1 years and 3.2 years, respectively. [Results] During the study period, thirteen and six patients were diagnosed as having gastric cancer in each group. The cumulative incidence of gastric cancer was statistically different between the groups ( $\mathrm{p}=0.019$; log-rank test). The hazard ratio by $H$ pylori eradication was 0.335 by Cox's proportional-hazard model $(\mathrm{p}=0.047)$. Differentiated gastric cancer was found in 11 patients in persistent infection group and in three in eradicated group, respectively. The incidence of differentiated cancer was significantly different ( $\mathrm{p}=$ $0.017)$ between the groups, but that of undifferentiated cancer was not $(\mathrm{p}=0.847)$. [Conclusions] The results of the present study support the preventive effect on gastric cancer by eradication of $\mathrm{H}$. pylori. The eradication of $\mathrm{H}$. pylori may not prevent the incidence of undifferentiated type of gastric cancers. 
(\%) Cumulative incidence of gastric cancer 20

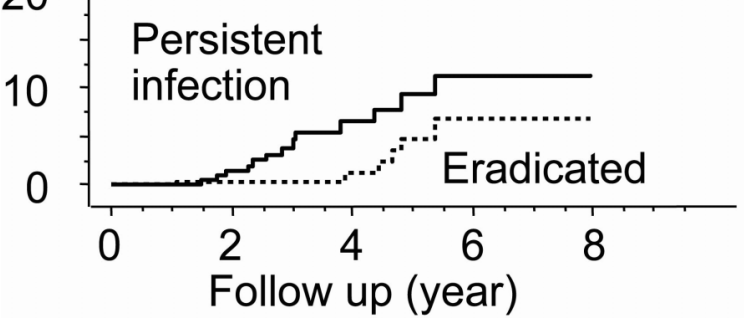

S1238

Mesalamine Downregulates Oncogene C-MYC and Genes Encoding Transcription Factors and Signaling Transduction Molecules Involved in Cell Survival and Proliferation in Colon Cancer Cells: Molecular Basis for Chemopreventive Action

Eric Chu, Jianyuan Chai, Andrzej S. Tarnawski

BACKGROUND: Patients with ulcerative colitis have increased risk of developing colorectal carcinoma depending on duration and extent of disease. Cohort and case-control studies suggest that mesalamine (5-ASA) may exert a chemopreventive effect. Recent metaanalysis demonstrated a protective association between 5-ASA and cancer or the combined endpoint of cancer/dysplasia. Mesalamine shares close molecular similarity with aspirin and may share chemopreventive properties comparable to aspirin and NSAIDs. Limited studies have suggested that mesalamine may promote apoptosis and inhibit proliferation, but the molecular mechanisms involved in 5-ASA reducing cancer risk remain incompletely explained. AIMS: To determine the effect of mesalamine on 96 genes of a cancer pathway gene array. METHODS: Human colon cancer Caco-2 cells were treated with vehicle or mesalamine (4 $\mathrm{mM}$ or $40 \mathrm{mM}$ ) for 2 and 5 hours. RNA was isolated from the cells and used as templates for hybridization with a cancer pathway gene array. The following studies were performed: 1) mRNA expression by gene array, 2) protein expression by Western blot analysis, 3) subcellular localization by immunohistochemistry, and 4) apoptosis by Annexin V-FITC binding to membrane of cells undergoing apoptosis. RESULTS: Two-hour treatment with mesalamine $4 \mathrm{mM}$ and $40 \mathrm{mM}$ downregulated expression of genes encoding transcription factors and signaling transduction molecules such as Akt ( $61 \%$ and $158 \%$, respectively), c-Ets2 ( $74 \%$ and $77 \%$, respectively), and c-Myc ( $50 \%$ and $89 \%$, respectively). In addition, the gene encoding apoptosis regulator Bcl-x was decreased by $~ 34 \%$ and $89 \%$, respectively. Five-hour treatment with mesalamine $40 \mathrm{mM}$ significantly decreased protein expression of c-Myc $\sim 3$ fold $(\mathrm{p}<0.05)$ compared to cells treated with mesalamine $4 \mathrm{mM}$ or control. Immunohistochemistry likewise demonstrated reduced expression of both Akt and c-Myc in cells treated with $40 \mathrm{mM}$ mesalamine. Annexin V staining demonstrated increased apoptosis in cells treated with mesalamine compared to controls. CONCLUSIONS: 1) Mesalamine downregulates genes encoding anti-apoptotic factors, transcription factors, and signaling transduction molecules involved in survival and proliferation in human colon cancer cells. 2) The pro-apoptotic and growth inhibitory effects of mesalamine are dose-dependent. 3) Expression of c-Myc protein is significantly reduced by high dose mesalamine. 4) Binding of Annexin $\mathrm{V}$ to membranes of cells undergoing apoptosis is a novel quantitative method for detection of apoptosis in colon cancer cells.

\section{S1239}

Efficacy and Safety in a Phase I Safety Trial of Celecoxib in Children with FAP Patrick M. Lynch, Carol Burke, Ernie Hawk, Craig Eagle, Mabel Woloj, James Church, Hennie Hasson, Sherri Patterson, Elizabeth Half, Gregory D. Ayers

Celecoxib has been approved by the FDA for adjunctive use in FAP, but safety and efficacy in children with FAP is unknown. We undertook a 3-month, Phase I trial to establish the highest safe dose of celecoxib in children with FAP between the ages of 10-14 years. 3 cohorts of 6 children had a 4:2 drug:placebo assignment, escalating from 4 to 8 to $16 \mathrm{mg} /$ $\mathrm{kg} / \mathrm{d}$. The \% change in adenoma burden was compared between baseline and end of study. Colonoscopy to cecum was performed after PEG or PO4 prep. Number, size and location of all polyps were documented by the endoscopist and recorded on DVD. Sedation: Propofol +/- narcotic/benzodiazepine, with anesthesiology support. Results: 18 subjects completed drug dosing and underwent 3-month colonoscopy. Mean age of subjects: 12.3 years. Racial composition was 15/18 (83\%) Caucasian, with 2 African American and 1 Asian. The \% female was $56 \%$. Overall, mean number of polyps at baseline was 31 . At 3 months there was a $50 \%$ increase in polyp count in placebo subjects, while in the highest dose celecoxib group a $50 \%$ reduction was seen $(\mathrm{P}=0.011)$ Fig. Adverse events(AE): $10 \mathrm{AEs}$, all grade 1 , were reported in 6 placebo subjects. In the 10 celecoxib subjects, of $16 \mathrm{AE}$ 's, 14 were grade I and 2 grade II (both GI) No overall difference in AEs was seen between placebo any of the 3 doses. No Cardiovascular events were recorded. No complications of colonoscopy or anesthesia were noted. Conclusions : Celecoxib dose of $16 \mathrm{mg} / \mathrm{kg} / \mathrm{d}$ significantly reduced the number of colorectal polyps in children with FAP. Celecoxib in dose of $16 \mathrm{mg} / \mathrm{kg} / \mathrm{d}$, which corresponds to the adult dose of $400 \mathrm{mg}$ BID is safe and well tolerated by children with FAP. Colonoscopy with propofol-based anesthesia is very well tolerated by children in age range 10-14 Supported by NCI/NIH contract N01-CN-05126-02 (P Lynch, PI), and by Pfizer Pharmaceuticals

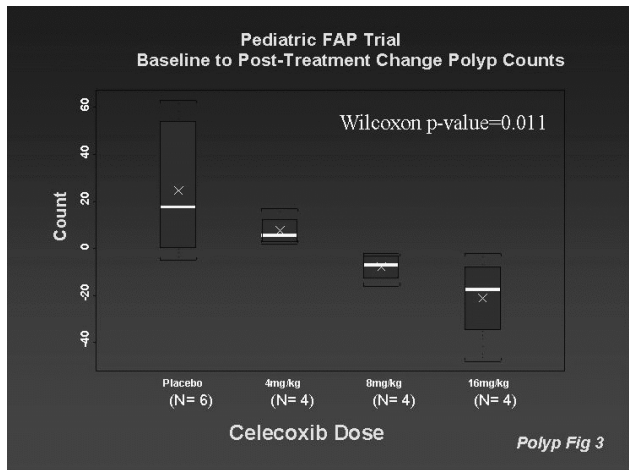

S1240

Combination of Cetuximab and Trastuzumab Cooperatively Reduce Cell Growth and Augment NSAID-Mediated Cell Death in Human Colon Cancer Cells

Elizabeth Half, Ho Yee Yeung, Yunjie Sun, Frank A. Sinicrope

BACKGROUND. Up-regulation of EGFR (ErbB-1) is detected in a majority of human colorectal cancers and its selective inhibition modestly reduces tumor growth rates. ErbB-2 is the preferred heterodimerization partner of EGFR. Cyclooxygenase-2 (COX-2) is a transcriptional target and downstream effector of both EGFR and ErbB-2. We studied the effects of antiEGFR and anti-ErbB-2 antibodies alone, together, and in combination with NS398 (selective COX-2 inhibitor), on cell viability and cell death in human colon cancer cells. METHODS HCA-7 cells were stimulated with heregulin or EGF and then incubated with monoclonal antibodies against EGFR [cetuximab, $20 \mu \mathrm{g} / \mathrm{ml}$ (Imclone)] and/or ErbB-2 [trastuzumab, 10, $20 \mu \mathrm{g} / \mathrm{ml}$ (Genentech)] \pm NS398 (50-200 $\mu \mathrm{g} / \mathrm{ml}$, Sigma). p-ERK, ERK-1, and COX-2 proteins were analyzed by immunoblotting. Cell viability was determined by MTS assay and total cell death by propidium iodine + annexin-V labeling. RESULTS. Both cetuximab (C)and trastuzumab (T) inhibited EGF- and heregulin-stimulated ERK activation and COX-2 expression that was further suppressed by co-treatment compared to trastuzumab alone.(C) or (T) produced a $26.3 \%$ or $14.5 \%$ mean reduction in cell viability, respectively, and their combination did so by $66.1 \%$ relative to untreated cells (Fig. 1). While ineffective individually, the antibody combination increased total cell death by $78.2 \%$ relative to untreated cells. NS398 at $100 \mu \mathrm{g} / \mathrm{ml}$ reduced cell viability by $27 \%$ and the addition of (C) or (T) further decreased it by $46 \%$ and $41 \%$, respectively. NS398-mediated growth inhibition was not further suppressed by both antibodies compared to either alone. CONCLUSION. Combined inhibition of EGFR and ErbB-2 signaling pathways inhibit downstream p-ERK and COX-2 to cooperatively decrease colon cancer cell viability. Furthermore, a COX-2 inhibitor may augment the efficacy of these molecularly targeted antibodies against colon cancers.

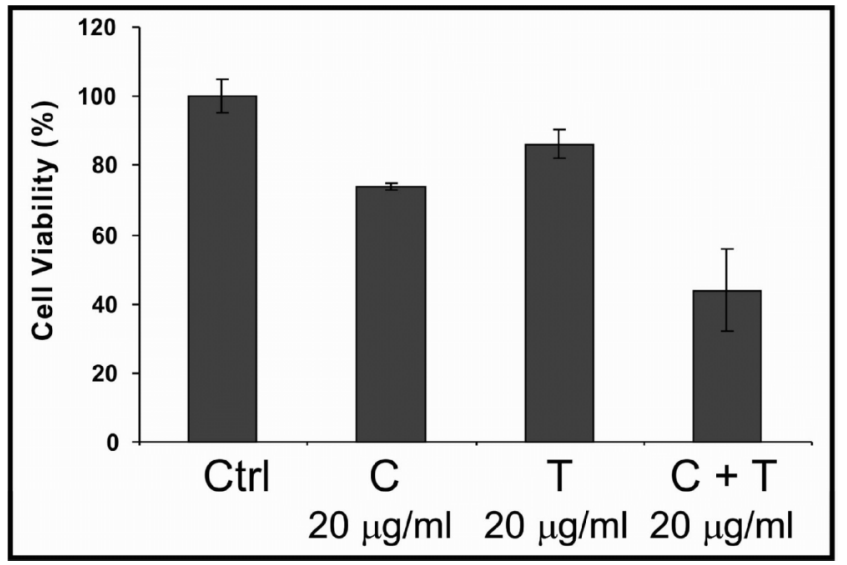

S1241

The Salicylate Derivative 5-Aminosalycilate (5-ASA) Affects Cell Cycle Progression in Colorectal Cancer Cells By Activating a P53- and MMRIndependent, Reversible Replication Checkpoint

Gloria Luciani, Christoph Campregher, Christoph Gasche

BACKGROUND: Colorectal cancer (CRC) is the second leading cause of cancer death in the Western world. Individuals with inflammatory bowel disease are particularly at risk of developing CRC. Findings from epidemiological, animal, and laboratory studies suggest that 5-ASA protects from the development of CRC by altering cell cycle progression and by inducing apoptosis. Further, our previous results indicate that 5-ASA improves replication fidelity in colorectal cells (Cancer Res 65:3993, 2005), an effect that is active in reducing mutations, independent of its anti-inflammatory properties. We hypothesized that 5-ASA restrains cell cycle progression by activating checkpoint pathways that would improve genomic stability. AIM: We aimed to investigate the molecular mechanisms by which 5ASA prevents tumor development and progression in colorectal cancer cell lines. METHODS: Colorectal cancer cells with different genetic backgrounds such as HT29 (p53 mutant), Hct116 (hMLHl mutant and p53 wt), Hctl16p53-/- (hMLH1 mutant and p53 null), 
Hctl16+chr3 (hMLHl and p53 wt) and LoVo (hMSH2 mutant and p53 wt), were treated with 5-ASA (0-40mM, Sigma) for 2-72hr. Cell cycle progression was investigated by flow cytometry with propidium iodide and BrdU staining. Phosphorylation and DNA binding of cell cycle checkpoint proteins (p53Ser15, ATMSer1981, ATR, Claspin, Chk1Ser345, Rad17Ser645, RPA, and H2AXSer139) was analyzed by Western blotting. RESULTS: 5-ASA at concentrations between 10 and $40 \mathrm{mM}$ affects cell cycle progression within 48 hours. It causes cells to accumulate in S-phase indicating that it interferes with DNA replication. This effect was independent of the hMLHl, hMSH2 and $\mathrm{p} 53$ status as it was observed to a similar extent in all cell lines under investigation. Moreover, wash-out experiments demonstrated reversiblity within 24 to 48 hours. Although p53 did not have a causative role, p53Ser 15 was strongly phosphorylated. Proteins involved in the ATR-dependent S-phase checkpoint response (Chkl and Rad17) were also phosphorylated, but not ATM CONCLUSIONS: Our data demonstrate that 5-ASA causes cells to reversibly accumulate in S-phase and activates an ATR-dependent checkpoint. These findings are independent of the mismatch repair complex and of p53. The observed p53 phosphorylation thus may be a downstream event of ATR activation. The reversible activation of replication checkpoint(s) may slow down DNA replication and lead to the improvement of DNA replication fidelity, both of which increase the maintenance of genomic stability and counteract carcinogenesis.

\section{S1242}

Statins Increase the Risk of Colorectal Adenomatous Polyps

Gulam Khan, Nazia Qazi, Swarnalatha Asokan, Jyothi Tummala, Jian Jun Li, Scott Tenner

Statins are the most commonly prescribed medications in the United States. As inhibitors of 3-hydroxy-3-methyl glutaryl CoEnzyme A Reductase, they are effective lipid lowering agents. Recently, several studies have suggested that similar to aspirin and non-steroidal anti-inflammatory drugs, statins may decrease a persons risk of developing colorectal cancer. Statins have been shown to inhibit the growth of colon cancer cell lines. A case-control study found a decreased incidence of colon cancer in persons taking statins (NEJM 2005; 352: 2184-92). In order to determine if statins prevent the development of adenomatous polyps, the following study was performed. A consecutive series of patients who underwent screening colonoscopy at a single institution were studied. Demographic information was collected, including statin use, aspirin and NSAID use, family history of colon cancer. The physician performing the colonoscopy was not aware of whether the patient was taking a statin. One thousand patients were included in the study, 401 male, 599 female, age 61.7 +/- 7.9 years. There were 317 African American, 352 caucasion, 150 Asian and 179 Hispanic patients. Three hundred four patients were using statins, mean duration of statin use 23.3 +/- 13.8 months. During colonoscopy, adenomatous polyps were found in 304 patients. The patients reporting the use of a statin were significantly more likely to have adenomatous polyps $(118 / 227)$ compared to patients not taking a statin $(186 / 452), \mathrm{p}<0.01$. This association remained significant after adjustment for other factors, including family history of colon cancer, gender, age, aspirin and NSAID use. There was no association between statin use and the size, number or location of the polyps. We conclude that the use of a statin is associated with an increased incidence of adenomatous polyps. Given the widely accepted "adenoma to carcinoma sequence", our study does not support the prior finding that statin use decreases the risk of colon cancer. Although other factors in our patient population may be involved, our study suggests that persons taking statins are at an increased risk of colorectal neoplasia.

\section{S1243}

Can Calcium Chemoprevention of Adenoma Recurrence Substitute for Colonoscopic Surveillance Or Extend Surveillance Intervals?

Aasma Shaukat, Murtaza Parekh, Joseph Lipscomb, Uri Ladabaum

BACKGROUND: Most colorectal cancers (CRC) arise from adenomas. Current post-polypectomy management consists of colonoscopic surveillance. Calcium may decrease adenoma recurrence by $30 \%$ and large $(>=1 \mathrm{~cm})$ adenoma recurrence by $50 \%$. AIMS: To examine the potential cost-effectiveness of calcium chemoprevention post-polypectomy, and to explore whether it could substitute for surveillance or extend surveillance intervals. METHODS: We constructed a Markov model of post-polypectomy adenoma recurrence and CRC development, calibrated to data from prospective chemoprevention trials of fiber, calcium, antioxidants and aspirin. We modeled 4 scenarios for 50 yr-old patients immediately after polypectomy: 1) natural history (NH) with no further intervention; 2) elemental calcium 1200mg/ day (CALCIUM); 3) surveillance from age 50-80 every 5 yrs, or 3 yrs for large adenoma (SURV); 4) calcium + surveillance (CALCIUM+SURV). Patients were followed until age 100 or death. The model accounted for missed adenomas. We assumed that $90 \%$ of CRC arise from adenomas. Base case inputs included: relative risk (RR) of recurrence with calcium vs. no calcium of 0.80 for small adenoma and 0.65 for large adenoma at 3 yrs; calcium cost $\$ 12 / y r$; colonoscopy sensitivity $95 \%$ for CRC, $90 \%$ for large polyp, $85 \%$ for small polyp; colonoscopy cost $\$ 820$ ( $\$ 1,200$ with polypectomy). We estimated discounted (3\%/yr) life$\mathrm{yrs} / \mathrm{pt}$ and cost/pt. BASE CASE RESULTS: CALCIUM was more effective than NH (18.654 vs $18.642 \mathrm{life-yr} / \mathrm{pt}$ ) and was cost-saving ( $\$ 2350 \mathrm{vs} \$ 2450 / \mathrm{pt}$ ). However, SURV was significantly more effective than CALCIUM (18.729 life-yr/pt) at an incremental cost of $\$ 22,100$ / life-yr gained. CALCIUM+SURV yielded a very small benefit (0.0003 incremental life-yrs/ pt) compared with SURV, at a substantial incremental cost of $\$ 421,000 /$ life-yr gained. Depending on the willingness to pay, surveillance intervals could potentially be extended (e.g. surveillance every 5 yrs, or 3 yrs for large adenoma cost $\$ 325,000 /$ life-yr gained compared with surveillance every 6 yrs, or 4 yrs for large adenoma). This extension was possible even without calcium use. SENSITIVITY ANALYSIS: Decreasing the RR of recurrence with calcium to 0.67 for small adenoma and 0.52 for large adenoma increased the cost of SURV vs. CALCIUM to $\$ 24,100$ and decreased the cost of CALCIUM+SURV vs. SURV to $\$ 182,300$. At a calcium cost of $\$ 6 / y r$, CALCIUM+SURV cost $\$ 23,000 /$ life-yr compared with SURV. CONCLUSION: Post-polypectomy calcium chemoprevention is unlikely to be a reasonable substitute for surveillance, or to allow extension of surveillance intervals. It may be cost-effective in patients unwilling or unable to undergo surveillance.

\section{S1244}

Sensitivity and Specificity of Immunochemical, Automated Developed, Fecal Occult Blood Test (OC-Sensor) for Detecting Significant Colonic Neoplasia Zohar Levi, Rachel Hazazi, Alex Vilkin, Paul Rozen, Amal Waked, Eran Maoz, Shlomo Birkenfeld, Yaron Niv

Background: Guaiac fecal occult blood test is the standard screening test for colorectal cancer (CRC). Automated-developed, immunochemical occult blood test (I-FOBT) is human $\mathrm{Hb}$ specific, eliminates diet restriction and allows choosing an optimal Hb threshold for followup colonoscopy. Aim: To evaluate performance characteristics of an I-FOBT by comparing it to colonoscopy. The end-point being the number of colonoscopies needed to identify significant neoplasia, CRC \&/or advanced adenomatous polyps (AAP). Methods: 1,000 consecutive patients undergoing colonoscopy, either for non-bleeding symptoms or for screening/surveillance, also prepared 3-daily I-FOBTs before colonoscopy. These were examined by the OC-Sensor automated instrument (Eiken, Japan), using Hb threshold of $100 \mathrm{ng}$ $\mathrm{ml}$ to determine positivity (Vilkin A et al, Am J Gastroenterol 2005, in press). Two patients required colonoscopy to detect one significant neoplasia. Results: Table Conclusions: OCSensor I-FOBT has good sensitivity and very high specificity for CRC \& AAP. The suitability and cost effectiveness for average risk population CRC screening should be evaluated. Results: I-FOBT versus total colonoscopy

\begin{tabular}{|c|c|c|c|c|}
\hline I-FOBT (n) & Colonoscopy & & & \\
\hline & Normal & AAP & CRC & \\
\hline Negative (884) & 849 & 33 & 2 & \\
\hline Positive (116) & 58 & 43 & 15 & \\
\hline Analyses & & & & \\
\hline CRC or AAP & Sensitivity & Specificity & NPV & PPV \\
\hline I-FOBT & 0.62 & 0.94 & 0.96 & 0.50 \\
\hline
\end{tabular}

AAP - advanced adenomatous polyp CRC - colorectal cancer I-FOBT - immunochemical occult blood test NPV - negative predictive value PPV - positive predictive value

\section{S1245}

Type 2 Diabetes Mellitus: The Impact On Colorectal Adenoma Risk in Women Jill E. Elwing, Feng Gao, Nicholas O. Davidson, Dayna S. Early

Background \& Aims: Increased risk for colorectal cancer (CRC) has been associated with type 2 diabetes. Despite several studies linking insulin resistance to increased CRC risk there is limited data on colorectal adenoma risk in diabetic women. We hypothesized that diabetic women would have increased rates of colorectal adenomas relative to a group of non-diabetic women. Methods: 100 women with type 2 diabetes mellitus $(60.0 \pm 9.5 \mathrm{yr}, 41 \%$ Caucasian, $10 \%$ with a first degree relative with CRC, BMI $34.4 \pm 8.0,29 \%$ on insulin therapy) and 500 non-diabetic, hormone status matched controls $(59.0 \pm 9.2 \mathrm{yr}, 68 \%$ Caucasian, $7 \%$ with a first degree relative with CRC, BMI 28.5 \pm .1) were selected consecutively from women undergoing screening colonoscopy at an outpatient university endoscopy center. Adenomas were defined as any adenoma, or advanced adenoma (villous or tubulovillous features, size $>1 \mathrm{~cm}$ or high grade dysplasia). A multivariate model was used to determine the independent effects of diabetes on colorectal adenoma incidence. Results: Diabetic as compared with non-diabetics had increased rates of any adenoma (37\% vs. $24 \%$; OR1.8, $95 \%$ CI: $1.2-2.9$; $\mathrm{p}=0.009$ ) and advanced adenomas ( $14 \%$ vs. $6 \%$; OR $2.4,95 \%$ CI: $1.2-4.7 ; \mathrm{p}=0.009) .245$ obese compared with 321 non-obese subjects had increased rates of any adenoma (32\% vs. 22\%; OR 1.8, 95\% CI: 1.3-2.6; $\mathrm{p}=0.001$ ). Obese diabetics compared with non-obese, nondiabetics had increased rates of any adenoma ( $42 \%$ vs. $23 \%$, OR $2.6,95 \% \mathrm{CI}: 1.5-4.6$; $\mathrm{p}=$ $<0.001$ ) and advanced adenomas ( $19 \%$ vs. $7 \%$; OR 3.5, $95 \%$ CI: 1.7-7.4; $\mathrm{p}=<0.001$ ). Multivariate analysis (including age, race, diabetes, hypertension, hypercholesterolemia, and BMI showed that adenomas and advanced adenomas were independently predicted by diabetes (OR $1.8,95 \% \mathrm{CI}$ : $1.1-2.9$ and OR $2.2,95 \% \mathrm{CI}: 1.004-4.6$ respectively: $\mathrm{p}<0.05)$ and adenomas by age $(\mathrm{p}=0.08)$. Discussion: Women with type 2 diabetes mellitus had higher rates of colorectal adenomas as compared with lean and non-diabetic women. This finding adds to the evidence that type 2 diabetes is an important factor in the progression of the adenomacarcinoma sequence.

\section{S1246}

Endometrial Cancer: The Impact On Colorectal Adenoma Risk in Women Jill E. Elwing, Feng Gao, Dayna S. Early, Nicholas O. Davidson

Background \& Aims: Colorectal cancer (CRC) and endometrial cancer occur in the same individual more frequently than by chance alone. We hypothesized that patients with endometrial cancer would have increased rates of colorectal adenomas compared to a contro group without previous gynecologic malignancy. Methods: 60 endometrial cancer patients $(61 \pm 9.0 \mathrm{yr}, 97 \%$ Caucasian, $3 \%$ with a first degree relative with CRC, $18 \%$ diabetic, BMI $32.4 \pm 9.1$ ) and 600 hormone status matched controls without a history of gynecologic malignancy $(59.1 \pm 9.6 \mathrm{yr}, 66 \%$ Caucasian, $7 \%$ with a first degree relative with CRC, $17 \%$ diabetic, BMI 29.4 \pm 7.5 ) were selected consecutively from women undergoing screening colonoscopy at an outpatient university endoscopy center. Adenomas were defined as any adenoma or advanced adenoma (villous or tubulovillous features, size $>1 \mathrm{~cm}$ or high grade dysplasia). A multivariate model was used to predict the independent effect of endometrial cancer on colorectal adenomas. Results: Increased rates of colorectal adenomas (42\% vs. 26\%; OR 2.0, 95\% CI: 1.2-3.5; $\mathrm{p}=0.01$ ) and advanced colorectal adenomas ( $17 \%$ vs. $8 \%$; OR $2.5,95 \%$ CI:1.2-5.2 $\mathrm{p}=0.02$ ) were found in women with a history of endometrial cancer as compared to controls. Subjects with both endometrial cancer and diabetes or with both endometrial cancer and obesity, had highly significant increased rates of adenomas (OR 5.4 95\% CI 1.6-18.9; $\mathrm{p}=0.007$ and OR 3.2, 95\%CI: 1.6-6.4; $\mathrm{p}=0.001$, respectively) and advanced 
adenomas (OR 8.4, 95\%CI: 2.3-30.1; p=0.005 and OR 3.8, 95\%CI: 1.5-9.6, p=0.009, respectively). Multivariate analysis (including age, race, diabetes, hypertension, hypercholesterolemia, BMI and endometrial cancer) showed that colorectal adenomas and advanced adenomas were independently predicted by endometrial cancer (OR 1.8, 95\% CI: 1.04-3.3; $\mathrm{p}=0.04$ and $\mathrm{OR} 2.2,95 \% \mathrm{CI}: 0.99-4.9 \mathrm{p}=0.05$, respectively) as well as diabetes $(\mathrm{p}=0.03$, $\mathrm{p}=0.007)$ and age $(\mathrm{p}=0.04, \mathrm{p}=0.05)$. Discussion: Women with a history of endometrial cancer had higher rates of colorectal adenomas as compared with controls. Women with both endometrial cancer and diabetes or obesity had higher rates of colorectal adenomas than did subjects with each risk factor alone. An association between adenomas and endometrial cancer could have an impact on CRC screening recommendations.

\section{S1247}

Impact of Systematic 18FDG-PET On Colo-Rectal Cancer Relapse Diagnosis and Management: Results of a Randomized, Controlled Multicenter Two Years Follow Up Study

Iradj Sobhani, T. Andre, T. Aparicio, C. Gornet, E. Itti, D. Le Guludec, R. Lebtahi, M. Lemann, M. Meignan, F. Mentre, E. Mitry, F. Montravers, Y. Panis, R. Parc, P. Piedbois, S. Retout, P. Rougier, J.N. Talbot, E. Tiret, C. Vaylet

BACKGROUND: A rapid diagnosis procedure of colo-rectal cancer relapse during the survey period represents a therapeutic challenge. Imaging procedures and increasing carcinoembryonic antigen (CEA) level are tools available to attempt this challenge. We evaluated the interest of additional systematic positron emission tomography (18FDG-PET) scan as a means of relapse diagnosis in colorectal cancer. PATIENTS \& METHODS. Patents with rectal or colon cancer $(\mathrm{N}=135)$ of stage pTNM III or IV were randomised when all tumours were surgically extirpated (R0) and adjuvant chemotherapy ended to be followed in conventional -Con-arm (clinical, biological marker every three months, Chest X ray, abdominal and pelvic CT Scan at 9 th and 15th month) or PET arm (conventional procedures plus PET -18FDG at 9th and 15th month). The end point was relapse confirmed by histology or "a new decision therapy" during the follow up survey including surgery, chemotherapy, and palliative therapy. RESULTS. At 2 years follow up, 129 patients (age 32 to 82 ys; median 58) were analyzed and 6 excluded from the analysis because of missing data. Two arms were balanced in terms of number of patients (64 in PET and 65 in Con arm) included, age, sex ratio, pTNM stage radio- and chemotherapy at baseline. All imaging procedures were performed in a blinded way by independent doctors. When at least one of examinations was suggestive of relapse, patient was considered in relapse if histology confirmed it. When biopsy or surgery was not performed, the time course of tumour evolution was criteria for relapse only if this was followed by a "new decision therapy". Up to 24 month, tumour relapsed in 38 patients ( 21 in PET vs 17 in Con; $\mathrm{P}=0.3$ ) as documented by pathology and/ or time course of tumour evolution a "new decision therapy", and 81 patients were considered in remission. There was more surgery and biopsy procedures for pathology examination in PET arm with therapy decisions made $10.3+/ 2.4$ months from baseline in PET arm (vs 14.8+/- 4.9 in Con arm; $\mathrm{p}=0.02$ ). This was accompanied with three false positive cases in PET arm with 18FDG-PET being the only examination showing single abdominal tracer accumulation and no tumour found (two after laparoscopy and 1 up to the end of survey period). At the same time, 18FDG-PET failed to detect peritoneal carcinoma in two cases shown by CT Scan and CEA elevated marker. In three additional cases, 18FDG-PET revealed unexpected tumours (1 gastric GIST, 2 pulmonary primitive tumours). Conclusion. The addition of systematic 18FDG-PET may influence therapy strategies and management of patients with high risk colo-rectal cancer relapse.Supported by PHRC-CEDIT.

\section{S1249}

Impact of Initial Polypectomy Versus Follow-Up Surveillance Colonoscopy On Colorectal Cancer Incidence in Post-Polypectomy Patients

Ann G. Zauber, Iris Vogelaar, Marjolein van Ballegooijen, Rob Boer, Deborah Schrag, Dik Habbema, Sidney J. Winawer

INTRODUCTION. The National Polyp Study (NPS) demonstrated that detection and removal of adenomatous polyps reduced subsequent colorectal cancer (CRC) by $76 \%$ to $90 \%$. This overall incidence reduction was achieved with initial polypectomy together with surveillance colonoscopy (SC) for patients with adenomas in a RCT comparing surveillance intervals at 1,3 and 6 years versus 3 and 6 years post polypectomy. Of importance is how much the incidence reduction was due to the initial vs the SC and which patients may benefit from SC. METHODS. The MISCAN-Colon micro-simulation model was used to assess the separate effects of initial and SC by the patient's adenoma characteristics. A population of adenoma patients of the same age, sex and baseline adenoma characteristics of the NPS followed over 10 years was simulated for three scenarios - 1) no initial colonoscopy and no SC 2) initial colonoscopy but no SC and 3) initial colonoscopy and SC. The surveillance effect was the difference in the percent developing CRC with and without SC and assessed by the patient characteristics of multiplicity ( 1 or 2 versus 3 or more) and size ( $<=0.5 \mathrm{~cm}$ (small), 0.6-0.9 $\mathrm{cm}$ (medium), or $>=1.0 \mathrm{~cm}$ (large)) of the adenomas at initial colonoscopy. RESULTS. $90 \%$ of the impact of colonoscopic intervention is due to the initial colonoscopic polypectomy rather than to SC for the 10 years post polypectomy. SC provides the largest benefit for the $20 \%$ of NPS patients with 3 or more adenomas where SC reduces the CRC rate by $1.3 \%$. SC has a smaller effect for those with 1 or 2 adenomas regardless of size with $0.4 \%$ to $0.7 \%$ reduction. CONCLUSIONS. The initial colonoscopy rather than SC provides the largest impact on CRC incidence in the 10 years following initial polypectomy. For those with 3 or more adenomas, SC at 3 year intervals shows a benefit. Based on these results the surveillance interval for those with only 1 or 2 adenomas could be extended to 5 to 10 years. Tailoring subsequent surveillance recommendations to the baseline colonoscopy findings can achieve the benefits of colonoscopic polypectomy and a reduction in the number of surveillance colonoscopies required with their associated risks.

\section{S1250}

Results of Mass Screening with Fecal Occult Blood Test in 4 Pilot Areas in France

Jean-Francois Bretagne, Vincent Dancourt, Bernard Denis, Gerard Durand, Jean Faivre, Gaud Mallard, Philippe Perrin, Christine Piette, N Touillon

Aim: mass screening of colorectal cancer with biennially fecal occult blood test (FOBT) has been recommended by health authorities in France. Twelve first pilot areas so-called "departments" were selected to implement screening for the general population in 2003. Here we report the pooled data of the first round in 4 "departments" (Ille et Vilaine, Côte d'or, Saône et Loire, Haut-Rhin). Methods: the campaign has been organized according the schedule of conditions defined by health organization. General practitioners (GPs) were trained in small groups. The round began by sending an information letter to each invited subject along with an information brochure. GPs offered the test free of charge to the eligible patients seen at their office. For patients who did not consult or did not complete the test, the coordination centre sent a new invitation letter before mailing the test and when necessary a reminder letter. Hemoccult II tests were processed without rehydration. Results: the target population comprised of 688984 men and women aged between 50 and 74 years before exclusion: 65042 individuals were excluded because of history of colorectal adenoma or cancer, family history of colorectal cancer, if they underwent a complete colonoscopy in the last 5 years or for others reasons. 320419 asymptomatic individuals had been screened corresponding to a participation rate of $51.4 \%$. FOBT results were positive in 9291 subjects $(2.5 \%)$ and non analysable in $4.1 \%$ of cases. A complete colonoscopy was performed in $82.4 \%$ of people referred for a positive test $(n=6901)$. According to the most advanced lesion $9.5 \%$ of subjects had colonic carcinoma and $32.5 \%$ had adenoma. Among the latter group, $51.9 \%$ had adenoma larger than $1 \mathrm{~cm}$ and $30.3 \%$ had adenoma with high grade dysplasia (in situ carcinoma were classified as high grade dysplasia). Carcinoma were diagnosed within resected adenomas in $37 \%$ of cases and were classified T0-Tl in 56.6\% of cases. Six cases of perforation (0.8 p.1000 examinations) resulted from colonoscopy. Conclusions: the mass screening with FOBT in France reproduces the results of the French controlled trial (Gastroenterology 2004;126:1674). These data should encourage health authorities to be quick to implement FOBT screening in the whole population.

\section{S1251}

Risk Factors for Advanced Colorectal Neoplasia in Women: Comparison of the Concern and VA 380 Populations

Brooks D. Cash, Andrew Flood, David Weiss, Arthur Schatzkin, David Lieberman, Philip S. Schoenfeld

Background: VA Cooperative Study 380 data identified several important risk factors for advanced colorectal neoplasia (i.e., adenomas $>10 \mathrm{~mm}$, villous adenomas, adenomas with high-grade dysplasia or carcinoma), including family history of colorectal cancer, current smoking or current alcohol use. However, generalizability of these findings is limited since $97 \%$ of the study population was male. The CONCeRN study of asymptomatic, averagerisk women who underwent screening colonoscopy identified significant differences between men and women in the anatomic distribution of colorectal neoplasia. The current analysis examines risk factors for advanced neoplasia in women and contrasts these results to men from VA 380. This is the first study to report risk factors for advanced neoplasia and risk factors for proximal and distal adenomas for women undergoing screening colonoscopy. Methods: Prior to colonoscopy in both studies, patients completed standardized, validated questionnaires to assess potential risk factors for advanced neoplasia. Responses were evaluated using univariate and multivariate logistic regression models to identify associations between risk factors and advanced neoplasia and adenomas (any size). Results: Among 1463 women, $4.9 \%$ had advanced neoplasia and $20.4 \%$ had adenomas (any size). Women in the CONCeRN study demonstrated significant associations between advanced neoplasia and African-American race $(\mathrm{OR}=2.06 ; 95 \% \mathrm{CI}: 1.07-3.94)$, current or past regular tobacco use $(\mathrm{OR}=1.64 ; 95 \% \mathrm{CI}: 1.01-2.66), \mathrm{BMI}>35 \mathrm{~kg} / \mathrm{m} 2(\mathrm{OR}=2.73 ; 95 \% \mathrm{CI}: 1.18-6.31)$, and NSAID use (OR $=0.48 ; 95 \%$ CI: 0.28-0.85) while men from VA 380 did not demonstrate these associations. Conclusions: Several risk factors appear to be associated with an increased risk of advanced colonic neoplasia in women including a family history of CRC, African American race, a history of current or past regular tobacco use, and BMI $>35 \mathrm{~kg} / \mathrm{m} 2$, while a history of regular NSAID use appears to be protective in women. Several of these risk factors appear to be more important for women than men.

Risk Factor Associations in CONCeRN and VA 380 


\begin{tabular}{|c|c|c|c|c|}
\hline $\begin{array}{c}\text { Risk Factor } \\
\text { (OR;95\% CI) }\end{array}$ & $\begin{array}{c}\text { Any adenoma } \\
\text { CONCeRN }\end{array}$ & $\begin{array}{c}\text { Advanced adenoma } \\
\text { CONCeRN }\end{array}$ & $\begin{array}{c}\text { Any adenoma } \\
\text { VA 380 }\end{array}$ & $\begin{array}{c}\text { Advanced } \\
\text { adenoma VA 380 }\end{array}$ \\
\hline $\begin{array}{c}\text { Family history } \\
\text { CRC }\end{array}$ & $1.67 ; 1.19-2.33$ & $1.96 ; 1.08-3.55$ & $1.40 ; 1.12-1.76$ & $1.63 ; 1.17-2.28$ \\
\hline African American & $1.38 ; 0.89-2.15$ & $2.06 ; 1.07-3.94$ & $1.16 ; 0.89-1.51$ & $0.81 ; 0.51-1.31$ \\
\hline $\begin{array}{c}\text { Current or past } \\
\text { regular tobacco } \\
\text { use }\end{array}$ & $1.22 ; 0.94-1.58$ & $1.64 ; 1.01-2.66$ & $1.37 ; 1.14-1.65$ & $1.27 ; 0.96-1.69$ \\
\hline $\begin{array}{c}\text { Regular NSAID } \\
\text { use }\end{array}$ & $0.65 ; 0.49-0.87$ & $0.48 ; 0.28-0.85$ & $0.85 ; 0.72-0.99$ & $0.80 ; 0.62-1.04$ \\
\hline BMI >35 kg/m2 & $1.76 ; 1.08-2.86$ & $2.73 ; 1.18-6.31$ & $1.36 ; 1.00-1.82$ & $1.13 ; 0.72-1.78$ \\
\hline HRT & $0.75 ; 0.58-.098$ & $0.68 ; 0.42-1.10$ & na & na \\
\hline
\end{tabular}

Temporal Trends in Colorectal Procedure Use Following Colorectal Cancer Resection

Gregory S. Cooper, Jonathan D. Payes

Introduction: Colorectal procedures, including flexible sigmoidoscopy (FS), colonoscopy $(\mathrm{COL})$ and barium enema $(\mathrm{BE})$ are commonly performed for surveillance care in colorecta carcinoma survivors. Given temporal trends in the use of colorectal screening tests in the general population, we hypothesized that similar patterns would be observed in cancer survivors. Methods: Using the linked SEER-Medicare database, we identified all patients $\geq$ 65 years with an initial diagnosis of colorectal carcinoma between 1992 and 1999. Patients with distant stage or unstaged disease or who did not undergo surgical resection were excluded. Using Medicare claims data, the use of FS, COL and BE within 1 year and 3 years after diagnosis was measured in patients who survived through the end of that follow up period. Results: A total of 35,447 eligible patients survived through 1 year of follow up and 28,278 patients survived through 3 years. Within one year of diagnosis, $18.9 \%$ underwen FS, $59.5 \%$ had COL, $14.5 \%$ had BE and $69.6 \%$ had one or more of the procedures. Corresponding rates for the 3 -years after diagnosis were $24.8 \%, 81.3 \%, 17.6 \%$ and $86.9 \%$, respectively. Over the 8 year study period, there was a progressive decrease in the proportion of patients with FS within 1 year of diagnosis (20.9\% 1992 to $16.1 \% 1999, \mathrm{p}<0.0001$ by chi-square for trend) and BE within 1 year of diagnosis (19.1\% 1992 to $10.0 \% 1999$, $\mathrm{p}<0.0001)$. Similar temporal trends were observed for $\mathrm{FS}$ within 3 years of diagnosis $(28.0 \%$ 1992 to $20.2 \% 1999, \mathrm{p}<0.0001)$ and BE within 3 years of diagnosis $(23.9 \% 1992$ to $11.4 \%$ $1999, \mathrm{p}<0.0001)$. In contrast, the use of COL remained unchanged for 1 year after diagnosis (58.9\% 1992 to $60.5 \% 1999, \mathrm{p}=0.1)$ and increased somewhat 3 years after diagnosis $(78.4 \%$ 1992 to $82.9 \% 1999, \mathrm{p}<0.0001)$. Overall procedure use within 1 year decreased somewhat $(71.7 \% 1992$ to $68.2 \% 1999, \mathrm{p}<0.0001)$ but 3 year rates were unchanged over time $(86.9 \%$ 1992 to $86.5 \%$ 1999, p=0.7). Conclusions: Consistent with temporal trends in the cancerfree population, the use of FS and BE in colorectal cancer survivors decreased through the 1990's. Although COL use increased somewhat with time, even in the most recent time period, approximately $20 \%$ of patients did not undergo this procedure within 3 years of diagnosis and $10-15 \%$ underwent none of the colorectal procedures. Further studies should identify subgroups that are least likely to undergo surveillance testing as well as those with potential overuse of procedures. notification strategies on population participation in FIT-based CRC screening Populatic 2400 people aged 50-74 randomly selected from the electoral roll of the Australian Electora Commission (compulsory registration for all aged $>18$ years) who had not previously received an invitation to participate in organized FIT screening. Methods: Those selected were randomly allocated to receive (1) a standard invitation letter explaining the concept, value, risk for CRC and method of screening (Control); (2) an invitation with additional risk messages (Risk); (3) an invitation with additional advocacy messages from past participants with screen detected curable CRC (Advocacy) or (4) first, an advance notification letter introducing Control letter messages, followed after 2 weeks by a standard invitation (Notification). All invitations were sent from a central screening facility, were of similar length and included an FIT kit (InSure $®)$. Participation was compared by Chi2. Results: Participation rates were: Control: 237/600, 39.5\%, Risk: 242/600, 40.3\%, Advocacy: 216/600, 36.0\%, Notification: 290/600, 48.3\%. Participation was significantly greater than Control in the Notification group only $(\mathrm{p}=0.003)$. This effect was apparent as early as 2 weeks post mail-out $(25.2 \%$ versus $18.2 \%, p=0.004)$. Age and sex were not associated with response to the notification letter. Conclusions: An advance notification letter sent prior to mailing CRC screening invitations significantly increased participation. Invitation letters incorporating enhanced risk or lay advocacy messages had little effect on participation. Advance notification letters are a simple and inexpensive way to increase participation in organized CRC screening programs. As the advance notification letter contained simple messages covering the magnitude, risk and curability of CRC, it is likely that increased participation was due to greater awareness and readiness to participate in CRC screening.

\section{S1253}

Colonoscopy with An Indigo Carmine Capsule: A Randomized Controlled Trial

Adolfo Parra-Blanco, David Nicolas, Antonio Z Gimeno, Livia Rezende, Marta Carrillo, Juan Adolfo Ortega, Margarita Menacho, Rosa Mari Rodriguez-Hernandez, Lourdes Rodriguez-Perez, Cristina Rodriguez-Galito, Enrique Quintero

Introduction: pancolonic indigo carmine (IC) applied with a catheter during colonoscopy has shown a slight benefit in the detection of polyps. In non-randomized studies oral IC delivered in a capsule showed an increased detection of flat and depressed lesions. Aim: to assess whether the indirect application of IC increases the detection of colonic polyps and flat lesions during colonoscopy. Methods: 551 patients undergoing colonoscopy were randomized in Group I: Polyethylene Glycol Solution ( $3 \mathrm{~L}$ ) immediately followed by an IC capsule (200 mg) ( $\mathrm{n}=265$ patients), or Group II: Polyethylene Glycol Solution (3 L) ( $\mathrm{n}=$ 286). Four experienced endoscopists performed the colonoscopies. The following variables were recorded: baseline features (age, gender, indication), presence of polyps, presence of flat or protruding polyps, total number of polyps, number of flat or protruding polyps, insertion and withdrawal time. The study was aproved by the local Ethical Commitee. Results: patients in Group II were significantly younger $(53 \pm 20$ vs $57 \pm 17, \mathrm{p}<0.05$ ). There were no differences in the distribution of gender or indication for colonoscopy, nor in the presence of polyps (global, protruding or flat). The mean number of polyps and the mean number of protruded polyps per patient were not statistically different between Groups I and II $(1.4 \pm 1.9$ vs $1.1 \pm 1.7$, and $0.8 \pm 1.4$ vs $0.7 \pm 1.3$, respectively $)$. However the mean number of flat lesions per patient with IC capsule was significantly greater than in those receiving the standard prep $(0.63 \pm 1.2$ vs $0.44 \pm 0.93 ; \mathrm{p}=0.04)$. No differences were registered between Groups I and II in insertion time $(13.0 \pm 8.3$ vs $13.5 \pm 9.1)$ or withdrawal time $(22.9 \pm 14.5$ vs $23.1 \pm 15.0)$. Conclusions: the administration of an IC capsule after Polyethylene Glycol Solution increases the detection rate of flat lesions during colonoscopy, with no apparent impact on procedure duration.

\section{S1255}

A Prospective Study of Community Screening Colonoscopy in African American Women (AAW) and Latino Women (LW): Barriers, Compliance, Results and Satisfaction

Moshe Shike, Alvaro Genao, Ann Zauber, Margaret Burke, Mark Schattner, Winsome Grant, Valerie Cuyjet, Shelby Samuel, Franklin Marsh, Lianne Russo, Rian Rodriguez, Harold Freeman

Survival of colon cancer patients is substantially lower among minorities, mainly because of late diagnosis. There is little information on screening colonoscopy (SC) in minority women. The only SC study specifically of women (CONCeRN) was performed in a noncommunity setting and included a very small \% of minorities. We report on a community based prospective study of SC in AAW and LW. Women having mammography at a breas center in Harlem NY were offered to participate in a SC study and those willing signed consent. The study involved completing questionnaires, and undergoing a colonoscopy. The rationale and alternatives were explained by a bilingual college graduate in English or Spanish. Colonoscopy and pathology review were performed at the local hospital. The study was offered consecutively to 5,673 women 50y or older (approximately $90 \%$ minorities); 3,057 were excluded because of prior screening or colon examination (a screening program existed prior to the study ), 2005 refused due to: wanting to discuss with own physician (585), lack of insurance (415), wanting colonoscopy but not study (368), already scheduled for colonoscopy (158), fear (147), medical problems (29), inadequate knowledge (18), prefer FOBT/Sig (15), miscellaneous (270). Of 611 women who consented, 317 (mean age 57y range: $50-85)$ attended pre-colonoscopy medical evaluation by a nurse and completed colonoscopy, 60 are pending, 234 dropped out due to: insurance (58), lack of time (33), colonoscopy elsewhere (32), fear (31), medical problems (24), lost to follow up (24), miscellaneous (32). Colonoscopy findings: $70 \%$ normal, $18 \%$ had non-adenomatous polyps, $12 \%$ had adenomas, 1 colorectal cancer. Adenoma prevalence was comparable among AAW and LW and slightly less than in predominantly white military women in the CONCeRN study, while hyperplastic polyp prevalence was comparable in the 3 groups. There were no colonoscopy related complications. Payment for colonoscopy: insurance $158(50 \%)$, cash $21(6 \%)$, philanthropy $138(44 \%)$. Those covered with philanthropic funds would not have had the procedure otherwise. A total $89 \%$ of women were satisfied and would have colonoscopy again, $4 \%$ would not, $7 \%$ were unsure. Conclusions: The overwhelming majority of minority women in this prospective study were interested in screening colonoscopy (but not necessarily in study)after an explanation by a non-medical person. Lack of insurance is a major barrier. Screened women were pleased with colonoscopy. Colonoscopy findings are comparable to those in white women in tertiary centers studies. The results suggest that colonoscopy is feasible and acceptable for screening of AAW and LW in the community.

\section{S1256}

Results and Cost of a Population-Based Biennial Faecal Occult Blood Colorectal Cancer Screening Program

Bernard Denis, Philippe Perrin, Jean Francois Vies, Jean Paul Milleliri, Frederic Vagne, Guy Ventre, Jean Marc Boyaval, Fernand Planchon

Despite strong evidence that screening reduces both colorectal cancer (CRC) mortality and incidence, CRC screening tests remain underutilized. Aim: To report the results and cost of a population-based biennial faecal occult blood (FOBT) CRC screening program in the French area of Haut-Rhin (710 000 inhabitants), an area with one of the highest CRC incidence in Europe. Methods: All 188,438 residents aged 50-74 years were invited by mail 
for a CRC screening using a non rehydrated FOBT (Hemoccult II). FOBTs were first provided by the GPs and then directly mailed to persons who didn't comply after 2 invitations. The processing of FOBTs was centralized. Results: 87,790 people (46.6\%) completed a FOBT and 18,995 (10.1\%) were excluded for recent CRC screening, high risk of CRC or concurrent severe disease, so that adjusted participation rate was $51.8 \%$. Participation was higher in women $(54.2 \%)$ than in men $(49.4 \%)(\mathrm{p}<0.001)$ and ranged from $45.4 \%$ to $59.8 \%$ according to districts. Participation was lower below 60 years $(47.5 \%)$ than after $(56.6 \%)$. FOBT positivity rate was $3.3 \%$, higher in men $(3.9 \%)$ than in women $(2.8 \%)(\mathrm{p}<0.001)$. To date 2,408 colonoscopies were performed. The positive predictive value was $10 \%$ for CRC (women $7.2 \%$, men $12.5 \%$ ), $20.8 \%$ for advanced adenomas (women $13.4 \%$, men $26.9 \%$ ) and $42.2 \%$ for neoplasia (women $30.6 \%$, men $51.8 \%$ ). Detection rates for neoplasia and CRC were 11.6 and 2.8 per 1,000 people screened. $27.2 \%$ of CRC were in situ, $50 \%$ of invasive CRC were stage I and $23.4 \%$ stage II. The rate of proximal advanced neoplasia increased with age ( $16.5 \%$ below 65 years, $25.4 \%$ after) but didn't differ with gender. Flexible sigmoidoscopy alone would have missed $21.4 \%$ of people with advanced neoplasia, without significant difference according to age and gender. The overall cost of this biennial screening program (without the fees related to colonoscopies) was $\$ 2.7$ million: fixed cost was $\$ 1.88$ million ( $\$ 5$ per year per eligible person) and variable cost $\$ 0.82$ million ( $\$ 4$ per screened person). The cost per screened person was $\$ 30$ and the cost to find an early-stage neoplasia (advanced adenoma or in situ CRC or stage I CRC) was $\$ 4,300$. Conclusion: Participation and diagnostic yield of randomised controlled trials of FOBT screening are reproducible at a reasonable cost in the real world through an organized population-based program involving GPs. Efforts should be made to enhance the participation of men and people below 60 years.

\section{S1257}

Prevalence of Anal Dysplasia in a French Population

Isabelle Etienney Sarra Vuong. Fady Daniel, Benoit Mory, Milad Taouk, Samy Sultan, Christian Thomas, Josee Bourguignon, De Parades Vincent, Nathalie Meary, Andre Balaton, Patrick Atienza, Pierre Bauer

Studying anal cytology to detect intraepithelial dysplasia has been demonstrated to be useful in highly selected populations with a high risk of epidermoid cancer of the anal canal. We have demonstrated the acceptability and feasibility in France of taking anal canal smears during consultations. The aim of this study was to determine the frequency of abnormal smears in a wide sample of patients consulting for anorectal symptoms. Patients and methods: An anal smear was systematically proposed during a consultation and carried out, after obtaining informed consent, using a Dacron brush, without disinfection or using a retractor. It was immediately put into suspension in a liquid phase (Thin Prep®). Results: 205 patients (112 women, 93 men) were included between 12 July and 7 October 2005. A history of human papilloma virus (HPV) anal lesions was found in $13 \%$ of the cases. A history of HPV gynecological lesions or conization was noted respectively in $5 \%$ and $4 \%$ of the women. Twenty-four (12\%) patients were infected by the human immunodeficiency virus (HIV) while 174 were not, and the serological status was unknown for 7 patients. Following the consultation, pathology was linked to HPV in $23(11 \%)$ of the cases (evolving lesions $\mathrm{N}=$ 12 , follow-up of condylomas without clinical lesion $\mathrm{N}=5$, follow-up of dysplasia $\mathrm{N}=3$ and monitoring of irradiated anal canal cancer without recurrence $N=3$ ). Ninety-nine percent of the smears were able to be analyzed ( 2 smears were acellular). Four cases of low grade anal intraepithelial neoplasia (AIN) were found, all in HIV patients with a history of condylomatous lesions, 40 smears were interpreted as atypical squamous cells of undetermined significance (ASCUS) and 159 were normal. The sensitivity of the smears was $67 \%$ and the specificity $81 \%$, with a positive predictive value of $18 \%$ and a negative predictive value of $97 \%$. The prevalence in this population of AIN was 19\% in HIV seropositive men, and 15\% in the case of a past history of condyloma. The factors associated with an abnormal smear (AIN or ASCUS) were a history of anal condylomatous lesions OR 4.9 [2.1-11.5], HIV seropositivity OR 4.0 [1.6-9.9] and smoking OR 2.1 [1.1-11.5]. In women the frequency of abnormal smears was not significantly increased where there was a history of HPV gynecological lesions or conization. Conclusion: This work confirms the raised frequency of AIN in HIV seropositive men, but also where there is a history of condyloma, which confirms the necessity for regular monitoring and screening of these patients at risk.

\section{S1258}

Potential Usefulness of Detecting Cyclooxygenase 2 and Matrix Metalloproteinase 7 Messenger RNA in Feces for Colorectal Cancer Screening Tetsunari Takai, Shigeru Kanaoka, Ken-Ichi Yoshida, Mutsuhiro Ikuma, Masayoshi Kajimura, Naoyuki Miura, Haruhiko Sugimura, Akira Hishida

Background: Colorectal cancer (CRC) is one of the most common causes of death in Western world. To reduce the mortality, it is important to point out the lesions at early stage and to resect them before metastasis. We reported that Fecal COX-2 assay, one of RNA-based stool assays, is useful for detecting CRC (Gastroenterology 2004; 127: 422-427). It is reported that matrix metalloproteinase 7 (MMP-7) is upregulated in colorectal cancer and adenoma. To raise the sensitivity of the assay keeping $100 \%$ specificity, we chose MMP- 7 as a combination target for Fecal COX-2 assay. Methods: Standard histological techniques were used to classify adenoma or malignancy at I to IV stages according to TNM classification. We purified RNA from routinely collected stool samples and screened mRNA using COX-2 and MMP7 specific nested RT-PCR as previously described, consequently compared the results with those of a single immunochemical FOBT (Magstream 1000/HemSp; FUJIREBIO, Inc., Tokyo, Japan) on same stool samples. Results: Stool samples from 65 patients with CRC, 13 patients with advanced adenoma, and 31 control patients without neoplastic disease were studied. The specificity of Fecal MMP-7 assay as well as Fecal COX-2 assay was 100\% (95\% confidence interval $[\mathrm{CI}], 88.8-100 \%$ ), while that of IFOBT was $87.1 \%$ (95\% CI, $70.1-96.4 \%)$. The sensitivity of RNA-based stool assay, combination of MMP-7 and COX-2 as targets, was $90.8 \%$ (95\% CI, 80.9-96.5\%) for CRC, and $69.2 \%$ (95\% CI, 38.6-90.9\%) for advanced adenoma, while that of IFOBT was $73.0 \%(95 \% \mathrm{CI}, 60.2-84.6 \%)$ and $30.8 \%(95 \% \mathrm{CI}, 9.1-$ $61.4 \%$ ), respectively. COX-2 and/or MMP-7 mRNA was detected in $84.6 \%$ of stage I $(n=$ 13), $96.8 \%$ of stage II $(n=31), 85.7 \%$ of stage III or IV $(n=21)$, while IFOBT was positive in $41.7 \%$ of stage I, $80.0 \%$ of stage II, $81.0 \%$ of stage III or IV. Noteworthy, COX-2 and/ or MMP-7 mRNA was detected in 17 of 22 stool samples from patients with curable cancer or advanced adenoma who had negative IFOBT. When the size of cancer and adenoma was limited from 1 to $3 \mathrm{~cm}$, the sensitivity of RNA-based stool assay was $78.6 \%(59.0 \%-91.7 \%)$, while that of IFOBT was $35.7 \%(18.6 \%-56.0 \%)$. Finally, the sensitivity of this assay was $87.7 \%$ (95\% CI, 78.0-94.9\%) for advanced adenoma to stage II cancer, while that of IFOBT was $60.0 \%$ (95\% CI, $45.8-73.1 \%$ ). Conclusions: RNA-based stool assay is superior to a single IFOBT in term of detecting colorectal cancer and advanced adenoma, especially curable tumors. These results strongly suggest that this assay would be useful for CRC screening.

\section{S1259}

Age-Associated Mutation of the DCC Gene in Patients with Normal Colonic Mucosa

Nolan E. Perez, Satish B. Maryala, Yingjie Yu, Adhip P. Majumdar

PURPOSE Although the incidence of colorectal and other gastrointestinal cancers increases with advancing age, the underlying molecular mechanisms are not fully understood. Since accumulation of genetic abnormalities is thought to drive the adenoma-carcinoma sequence towards colorectal cancer (CRC) development, we hypothesize that aging may render target cells more susceptible to carcinogenesis through mutation of tumor suppressor genes. Mutations of tumor suppressor genes are frequently observed in patients with colonic dysplasia or neoplasia. Deleted in Colon Cancer (DCC), a tumor suppressor gene involved in regulating cell to cell interactions, has been shown to have allelic deletions in colorectal and other gastrointestinal cancers. Previously, we reported that the incidence of mutations of several tumor suppressor genes, including DCC, is higher in the gastric mucosa of subjects over 60 years of age (Am J Physiol Cell Physiol 2001:282;G932-G936). To determine if this age-related phenomenon occurs in the colon, we tested normal (non-dysplastic and nonneoplastic) mucosa for the presence of DCC gene mutations in patients of various ages. Furthermore, we obtained samples from the proximal (ascending and transverse) and distal (descending and rectosigmoid) colon to ascertain whether aging increases susceptibility to mutation in a location-specific manner. METHODS Patients undergoing screening or diagnostic colonoscopy were offered enrollment in our study if they were at average risk for $\mathrm{CRC}$ and were not taking chemopreventive agents. If no dysplasia or neoplasia was found at endoscopy (i.e. no adenomas or cancers), random biopsies were obtained from the ascending, transverse, descending and rectosigmoid colon. DNA analysis by PCR amplification was performed for detecting mutations in the DCC gene. RESULTS We enrolled 27 patients in our study. The age ranged from 31 to 81 years, with a mean age of 58 years. The vast majority (81\%) of subjects were male. In patients younger than 60 years, 2 of 17 (12\%) patients had mutations in the proximal colon and 3 of $17(18 \%)$ had mutations in the distal colon. Among patients older than 60 years, 4 of $10(40 \%)$ had mutations in the proximal colon and 2 of $10(20 \%)$ had mutations on the distal colon. CONCLUSION Aging is associated with an increased rate of DCC gene mutation, especially in the proximal colon. Although more studies are needed, we postulate that age-related mutations in tumor suppressor genes predispose towards the development of colorectal cancer by creating a field defect.

\section{S1260}

Long-Term Safety and Efficacy of Lubiprostone for the Treatment of Chronic Constipation in Elderly Subjects

Ryuji Ueno, Raymond Panas, Aimee Wahle, Yan Zhu, P. Christopher Holland

Constipation is a common and bothersome gastrointestinal condition that is especially prevalent among the elderly (those $\geq 65$ years of age). Lubiprostone is a novel type- 2 chloride channel (ClC-2) activator that has been studied to show long-term safety and efficacy for the treatment of chronic constipation in 3 open-label clinical trials. In order to create an adequate pool of elderly subjects, data were combined from these three studies, resulting in 163 elderly subjects (aged $\geq 65$ years) and 715 non-elderly subjects (aged 18-64). Safety was assessed by adverse event (AE) incidence rates and efficacy was evaluated by improvements in subject assessments of constipation severity, abdominal bloating, and abdominal discomfort. All assessments were based on 5-point severity scales where $0=A b s e n t$ and $4=$ Very Severe. Results revealed that, with regard to safety, slightly fewer elderly subjects report adverse events compared to non-elderly subjects (121/163, $74.2 \%$ vs $573 / 715,80.1 \%$ ). Notably, the incidence rate for the most common AE, nausea, was markedly decreased in elderly subjects as compared to non-elderly subjects (17.8\% vs $29.4 \%)$. With regard to longterm efficacy, improvements in assessments of constipation severity, abdominal bloating, and abdominal discomfort were all statistically significant at all post-baseline time points from Week 1 to Week 48 for both elderly and non-elderly subgroups ( $<<0.0001$ ). Amongst the elderly, constipation severity was improved by an average of 1.11 points at Week $1(\mathrm{~N}=$ 108), 1.32 points at Week $24(\mathrm{~N}=81), 1.21$ points at Week $48(\mathrm{~N}=58)$, and 0.97 points for the last on-drug measurement $(\mathrm{N}=159)$. Abdominal bloating was improved by an average of 0.76 points at Week $1(\mathrm{~N}=108), 0.99$ points at Week $24(\mathrm{~N}=81), 1.05$ points at Week $48(\mathrm{~N}=58)$, and 0.71 points for the last on-drug measurement $(\mathrm{N}=159)$. Abdominal discomfort was improved by an average of 0.49 points at Week $1(\mathrm{~N}=108), 0.75$ points at Week $24(\mathrm{~N}=81), 0.79$ points at Week $48(\mathrm{~N}=58)$, and 0.52 points for the last on-drug measurement $(\mathrm{N}=159)$. We conclude that lubiprostone is a well-tolerated and efficacious long-term treatment for chronic constipation in the elderly.

\section{S1261}

Risk Analysis of Gastrointestinal Surgery in Elderly Patients Over 80 Years Old: Usefulness of the Possum Scoring System Tsuneo Tanaka, Yasuhiro Matsugu

Aim: It is known well that the function of various organs is compromised at advanced ages However, since the degree of dysfunction varies among different organs, preoperative and precise evaluation of the reserved capabilities of individual organs is difficult. The present study was undertaken to evaluate the operative risk of elderly patients (over 80 years old) 
scheduled to undergo surgery. Materials \& Methods: The subjects of this study were 1,326 patients with gastrointestinal disease who underwent surgery between January 2002 and June 2005. The underlying disease was benign in 471 (36\%) cases and malignant in 855 cases $(64 \%)$. Emergency surgery was performed on 205 cases (15\%) and elective surgery on 1121 cases $(85 \%)$. They were divided by age into three groups: a younger group (below 69 years, $n=834$ ), an older group ( $70-79$ years, $n=367$ ) and the oldest group (over 80 years, $n=125$ ). The presence/absence of preoperative complications, hematological parameters and magnitude of operative stress were compared among these three groups. For risk assessment using the POSSUM system, data on 12 parameters related to physiological score (PS) and 6 parameters related to operative severity score (OS) were derived from the database or individual patients. The risk of individual patients was rated on a three-category scale (lowrisk:12-19, moderate-risk:20-24 and high-risk:more than 25) on the basis of PS scores. Results: The percentage of patients having preoperative complications of the respiratory, cardiac and neurological systems was significantly higher in the oldest group than in the younger group. Hemoglobin, albumin, prothrombin time, sodium and urea levels were significantly lower in the oldest group than in the younger group. The PS was $15.6 \pm 3.7$ $($ mean \pm SD)in the younger group, $19.9 \pm 4.4$ in the older group, and $22.9 \pm 4.6$ in the oldest group. The percentage of patients rated as having high-risk increased as age advanced. Postoperative complications were seen in 339 cases (25.6\%), and death during hospital stay occurred in 23 cases $(1.7 \%)$. The hospital death rate and the incidence of postoperative complications were significantly higher in the high-risk group than in the low- and moderaterisk groups. Conclusion: Elderly patients over 80 years old often have preoperative complications and compromised organ function. The PS score as assessed using the POSSUM is useful for evaluation of operative risk in elderly patients.

\section{S1262}

Efficacy and Safety of Lubiprostone for the Treatment of Chronic Constipation in Elderly vs Non-Elderly Subjects

Ryuji Ueno, Taryn R. Joswick, Aimee Wahle, Yan Zhu, P. Christopher Holland

Constipation is a common and bothersome gastrointestinal condition that is especially prevalent among the elderly (those $\geq 65$ years of age). Lubiprostone is a novel type- 2 chloride channel (ClC-2) activator (AJP Cell Physiol 287:C1173-Cl183, 2004) that has been shown to be efficacious and well tolerated in a number of well-controlled clinical trials of 3-4 weeks duration (Gastroenterology 122:A315, 2002; Gastroenterology 124:A38, 2003; Am J Gastroenterol 100:S324, S328 and S329, 2005). We have examined pooled results from these trials so that subgroups of elderly and non-elderly subjects can be compared. Methods: In order to create an adequate pool of elderly subjects, data were combined from three well-controlled clinical trials. Spontaneous bowel movement (SBM) frequency rates, stool consistency ratings, and bowel straining assessments were compared between treatment groups (placebo and lubiprostone $48 \mu \mathrm{g} / \mathrm{day}$ ) via inferential statistics and used to assess efficacy. Mean improvements over placebo are compared between elderly and non-elderly subjects. Adverse event $(\mathrm{AE})$ incidence rates are also compared between treatment groups and age groups. Results: The pooled elderly subgroup consisted of 31 placebo and 26 lubiprostone subjects. Mean changes from baseline in SBM rates were significantly improved among lubiprostone elderly subjects as compared to their placebo counterparts during Weeks 1,3 , and 4 ( $\mathrm{p} \leq 0.0286$ ). Improvements during each of the 4 weeks ranged from 4.6 to 5.4 additional SBMs per week for elderly lubiprostone subjects compared to 1.29-2.27 SBMs per week for elderly placebo subjects. Results were similar for stool consistency and bowel straining ratings. With regard to safety, fewer elderly lubiprostone subjects experienced AEs (46.2\%) compared to their placebo counterparts (61.3\%). Conclusion: Lubiprostone is an efficacious and well-tolerated treatment for chronic constipation in the elderly.

\section{S1263}

Etiology and Outcome of Acute Pancreatitis in Infants and Toddlers Leena Kandula, Mark E. Lowe

BACKGROUND: The incidence of acute pancreatitis (AP) in children has increased in western countries. Even so, little is known about the causes and outcome in infants and toddlers with AP. AIM: To determine the spectrum of etiological factors and outcome of AP in infants and toddlers. METHODS: We reviewed medical records of children < 3 years of age admitted to Children's Hospital of Pittsburgh with a diagnosis of AP (ICD-9 577.0) between January 1995 and December 2004. AP was defined as three fold elevation in serum amylase (normal, $<90 \mathrm{IU} / \mathrm{L}$ ) or lipase (normal, < $200 \mathrm{IU} / \mathrm{L}$ ) levels, with clinical signs and symptoms or radiological findings consistent with the diagnosis. Demographic, clinical, laboratory and diagnostic data were collected. Patients were classified as idiopathic if no identifiable cause was found. Severity of the disease was based on Atlanta criteria. The study was approved by the University of Pittsburgh Institutional Review Board. RESULTS: Of 90 cases with a diagnosis of AP, 73 ( 35 male, 38 female) met the diagnostic criteria. Mean age was 19.3 +/- 9 months (range: 1 week to 35 months). Common presenting symptoms included vomiting in $30(41 \%)$, irritability in $29(39.7 \%)$ and abdominal pain in $19(26 \%)$ cases. AP was associated with multisystem disease in $42(57.5 \%$ ) cases (Sepsis, 27; hemolytic uremic syndrome, 7 ; shock and multi organ failure, 8 ). No single organism predominated in the patients with sepsis. AP was associated with trauma in $6(8.2 \%)$, pancreaticobiliary disease in 7 (9.5\%) (Gall stones, 5; choledochal cyst, 1; annular pancreas, 1), liver transplantation in $2(2.7 \%)$, cystic fibrosis in $1(1.3 \%)$ and was idiopathic in $15(20.5 \%)$ cases. Mean peak serum amylase level was $325 \mathrm{IU} / \mathrm{L}$ (range: $<30$ to $1533 \mathrm{IU} / \mathrm{L}$ ) and mean peak serum lipase level was $7188 \mathrm{IU} / \mathrm{L}$ (range: 607 to $45,640 \mathrm{IU} / \mathrm{L}$ ). Abdominal ultrasound was done in 32 $(43.8 \%)$ and was useful in making a diagnosis in 8 cases. Abdominal CT was done in 21 (28.7\%) and was diagnostic in 8 cases. MRCP was done in $3(4.1 \%)$ and was diagnostic in 1 case. Only one patient underwent ERCP and sphincterotomy. AP was mild in 63 (86.3\%), severe in $3(4.1 \%)$, and death from complications unrelated to pancreatitis occurred in 7 cases $(9.5 \%)$. Of 3 patients with severe AP, two had pancreatic necrosis following trauma and one had a pseudocyst. 4 patients $(5.5 \%)$ had surgery involving the pancreas (pancreatic resection, 2; pseudocyst drainage, 1; annular pancreas repair, 1). CONCLUSIONS: In this age group, AP is most commonly associated with multisystem disease. In contrast to common teaching, biliary disease is also an important etiology. Severe AP was most often associated with trauma

\section{S1264}

Use of Clostridium Botulinum Toxin for the Treatment of Obstructive Symptoms Or Enterocolitis in Patients with Non-Relaxing Internal Anal Sphincter: A Long-Term Outcome Study

Bruno P. Chumpitazi, Steven J. Fishman, Samuel Nurko

Background: Obstructive symptoms or enterocolitis may occur due to a non-relaxing interna anal sphincter (IAS) in children with IAS achalasia or in those with postoperative Hirschsprung's disease (PHD). Clostridium botulinum toxin injections (BoTox) to the IAS are used as therapy, but there is limited information about their long-term effects. This study evaluates the long-term outcome of children with non-relaxing IAS who received BoTox therapy for obstructive symptoms or enterocolitis. Methods: Retrospective review of symptomatic children with non-relaxing IAS who received BoTox. Both BoTox and rectal biopsies were generally done under the same anesthetic procedure. Long-term outcome was classified into 4 groups (excellent, good, fair, poor) based on symptoms, incontinence, laxatives, rectal therapies, or surgery. Results: 55 children received BoTox; 28 had IAS achalasia, and 27 PHD. Mean follow-up was $23 \pm 3$ months. Mean age at the time of first BoTox was $5 \pm 0.5$ years. Improvement after the first BoTox was seen in $44(77 \%)$ and predicted an excellen or good long-term clinical outcome $(\mathrm{p}<0.01)$. Mean duration of response after the first BoTox was $4.6 \pm 0.9$ months. 35 children received more than one BoTox $(2.3 \pm 0.2$ injections patient). After BoTox there was a reduction in the \% patients using rectal therapies (47 vs $25 ; \mathrm{p}<0.008$ ), and in the hospitalization rates for enterocolitis ( 2.4 admissions/year vs. 0.4 admissions/year; $\mathrm{p}=0.02$ ). Five children ( $9 \%$ ) developed transient fecal incontinence, and 1 $(2 \%)$ had rectal pain after the injection. Seven $(12 \%)$ underwent myectomy. Long-term outcome is shown in the table. In those with a good/excellent outcome, the response was maintained for $12 \pm 3$ months after the last BoTox. Five (18\%) children with postoperative HD were found to have residual aganglionosis, a predictor of failure to respond to BoTox $(\mathrm{p}<0.01)$. There was no statistical difference comparing the response between IAS achalasia and HD. Conclusions: BoTox is an effective and safe therapy for children with non-relaxing IAS. An initial improvement after the first BoTox predicted an excellent/good long-term outcome. No difference was seen in the response regardless of the etiology of the nonrelaxing IAS. Because patients with residual aganglionosis do not respond to BoTox, it is important to perform a rectal biopsy in symptomatic patients with PHD.

\begin{tabular}{|c|c|c|c|}
\hline & IAS Achalasia $\mathrm{n}=27$ & PHD* $\mathrm{n}=22$ & All $\mathrm{n}=49$ \\
\hline Excellent & $2(7 \%)$ & $4(18 \%)$ & $6(12 \%)$ \\
\hline Good & $17(63 \%)$ & $6(27 \%)$ & $23(47 \%)$ \\
\hline Fair & $3(11 \%)$ & $7(22 \%)$ & $10(20 \%)$ \\
\hline Poor & $5(18 \%)$ & $5(23 \%)$ & $10(20 \%)$ \\
\hline
\end{tabular}

* Those with residual aganglionosis are excluded.

\section{S1265}

Fanconi Syndrome in Patients with Microvillous Inclusion Disease Karin M. Van Dael, Sven C. van Ijzendoorn, Leonie J. Pierik, D.R. J. Reijngoud, Hilda M. Keuning, Arend Karrenbeld, Marius C. van den Heuvel, Edmond H. Rings

Introduction: Microvillous inclusion disease (MID) is a rare congenital defect of the brush border of the intestinal epithelium and characterized by periodic acid-Schiff (PAS)-positive secretory granules within the apical cytoplasma of the enterocytes and microvillous inclusions in these cells as observed by electron microscopy. Furthermore, a variable degree of villous atrophy of the intestinal villi is seen. MID leads to lifelong intestinal failure for which total parental nutrition is required. Better homecare parental nutrition and early intestinal transplantation is suggested to be of benefit to change clinical outcome. As enterocytes are characterized by microvilli, we hypothesize that proximal renal tubular epithelial cells share a similar defect leading to clinical symptoms earlier not recognized because of the short life span of these children. Therefore we aimed to study renal function and the renal morphology at the light microscopic and electron microscopic level, with emphasis on the proximal tubuli, in children with MID. Materials and Methods: Two children, aged 2 years, with confirmed intestinal MID, who were evaluated for intestinal transplantation, were studied. Renal function was analyzed and kidney biopsies were evaluated by light and electron microscopy. Results: Both children had a persistent and progressive metabolic acidosis in conjunction with glucosuria, hyperaminoaciduria, beta-2-microglobinuria and tubular solute losses as part of a Fanconi syndrome. They also developed severe carnitine deficiency and rickets as result of impaired phosphate reabsorption. They were growth retarded, despite total parenteral nutrition and exclusion of enteral feeding. The kidney biopsies did not reveal histological abnormalities at the light microscopy level, although PAS-positive granules at the apical membrane of the tubular epithelial cells were seen. At electron microscopy, normal architecture of the proximal tubule cells was seen, although the microvilli seemed less well organized per cell compared to controls. In contrast to enterocytes, no intracellular microvillous inclusions in these cells were observed. Conclusions: Proximal tubular dysfunction (Fanconi syndrome) can be part of MID. Growth retardation, which is often seen in children with MID, may also be dependent on this renal dysfunction. Fanconi syndrome should be taken into account in the management of children with MID before as well as after intestinal transplantation. Microvillous inclusions, the characteristic finding in enterocytes in MID, were not observed in the proximal tubular epithelial cells in the two studied biopsies

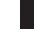




\section{S1266}

The Role of Capsule Endoscopy in the Evaluation of Patients with Unexplained Growth Failure

Libia Moy, Jeremiah Levine

Background: Poor weight gain and growth can be caused by innumerable medical, nutritional, behavioral, psychological and environmental disease states. Although we know that 15 to $40 \%$ of children with Crohn's disease have growth failure, the diagnosis is not always readily apparent. Negative findings on routine serologic, radiographic and endoscopic diagnostic studies can result in significant delays before appropriate therapy can be instituted. Lack of a definitive diagnosis also frequently leads to additional and potentially costly testing in an attempt to identify the etiology of the poor growth. The aim of this study is to evaluate the role of the Capsule Endoscopy (CE) as part of the evaluation of older children and adolescents with unexplained growth failure. Methods: We retrospectively reviewed the records of all children with unexplained growth failure who underwent $C E$ at Schneider Children's Hospital between August 2002 and November 2005. Results: Eight patients (4 males and 4 females, mean age of $12.1 \pm 3.5$ year old, mean weight of $30.6 \pm 9.4 \mathrm{Kg}$ ) had $\mathrm{CE}$ studies to review. Laboratory studies including $\mathrm{CBC}$, biochemistry profiles and celiac serologies were negative in all subjects. Only 1/8 had an elevated ESR. All had normal small bowel series perfomed prior to the CE. All had both endoscopically and histologically normal esophagogastroduodenoscopy and colonoscopy as well. In 5/8 subjects, multiple small bowel apthous ulcerations consistent with Crohn's disease were identified by CE. Two subjects had normal CE and one had an incomplete CE study due to delayed passage of the capsule. Conclusions: Sixty three percent of older children and adolescents with unexplained growth failure and normal small bowel series were found to have Crohn's disease involving the small intestine. To avoid further costly testing for possible endocrine or psychiatric causes of poor growth, $\mathrm{CE}$ should be considered as part of the armamentarium in evaluating children with severe unexplained growth failure

\section{S1267}

Infliximab's Growth Related Effects for Children and Adolescents with Crohn's Disease

Sean D. Sullivan, Alexandra Eidelwein, Richard Thompson, Carmen Cuffari, OlivaHemker Maria

Introduction: Growth impairment commonly complicates the clinical course of pediatric Crohn's disease (CD). Corticosteroids, a mainstay in moderate to severe $\mathrm{CD}$ are also known to impair linear growth in children. Infliximab has been proven to be an effective steroid sparing anti-inflammatory agent.There are however, limited studies assessing infliximab's potential for growth promoting effects in pediatric $\mathrm{CD}$. Aim: The goal of this study was to evaluate the effect of infliximab on growth parameters before and after the initiation of therapy in children and adolescents with refractory CD. Methods: Medical records were reviewed of pediatric patients follwed at the Johns Hopkins Children's Center who received at least three infliximab infusions between March 2000 and April 2005. Data collected included: patient demographics, $\mathrm{CD}$ phenotype, number of infliximab infusions, and cumulative corticosteroid dose for the 6 months preceding and following the initial infliximab infusion. Growth parameters were recorded including: weight and height corrected for age using standardized Z-scores. BMI and BMI Z-score. Clinical data was analyzed utilizing the student's t-test and logistic regression analysis. Results: 59 patients were identified (56\% Male). The mean age of the patients was 15 years \pm 4.2 years. Indications for infliximab use included corticosteroid and immunomodulator refractory luminal disease (44 pts), perianal disease $(9 \mathrm{pts})$ and both $(6 \mathrm{pts})$. From this population 24 children less than 15 years old were evaluated. The average number of infliximab infusions was 6 (range 3-9). 17 patients received corticosteroids during the study period. Height Z-scores increased during 12 months of infliximab therapy with a trend toward significance, $p=0.065$. For the population of patients whose baseline height $Z$ score was $<0$, an increase in height $z$ score to 0.9 was noted at 12 months, $p=0.0089$. A trend toward significance at 12 months was noted with BMI Z score increasing in those patients whose baseline BMI Z score was $<$ zero, $\mathrm{p}=0.06$. The difference between the pre- and post-infusion corticosteroid dose was $20.9 \mathrm{mg} / \mathrm{kg}, \mathrm{p}=0.0014$. Logistic regression analysis in the patients receiving corticosteroids demonstrated a significant increase in height independent of steroid exposure, $\mathrm{p}=0.004$ Conclusions: Infliximab therapy was associated with signficiantly increased growth in pediatric patients with $C D$ and with height $Z$ scores less than zero. This benefit appeared to be independent of corticosteroid exposure. Larger prospective controlled studies are needed to confirm these findings.

\section{S1268}

Gastrointestinal (GI) Inflammation in Children with Recurrent Abdominal Pain (RAP) Is Not Related to Pain and Stooling Pattern Robert J. Shulman, Danita Czyzewski, Monica Jarrett, Lonnie Zeltzer

Objective: Recent histologic studies have suggested evidence of low grade inflammation in many adult patients with irritable bowel syndrome (IBS). Additionally, fecal calprotectin concentration, a marker of GI inflammation has been reported to be abnormal in up to $30 \%$ of adults with IBS (Gastroenterology 2002;123:450). Whether the same is true for children with RAP, a condition that affects $10-15 \%$ of school age children and bears many physiological similarities to IBS in adults (e.g., functional pain, visceral hyperalgesia) has not been studied. Study Design: Children (age 7-10 yr) were identified initially by chart review in pediatrician's or pediatric gastroenterologist's offices. Children with RAP met Rome II criteria for functional abdominal pain or IBS. Non-RAP Control subjects were recruited through the same pediatrician's offices. Further screening was done with parents by phone. After instruction in the home by research assistants children saved a stool for measurement of calprotectin concentration and kept a 2-wk diary to record abdominal pain frequency and severity and number and character of stools. Results: Groups were comparable in age: RAP: $8.5 \pm 1.1$ vs C: $8.7 \pm 1.0$; mean \pm SD. Fecal calprotectin concentration was greater in children with RAP than in Controls (Table). Differences between RAP and Controls also were noted for mean pain score, maximum reported pain, and number of pain episodes, though not for number of stools or number of days with no stool. However, more stools were hard balls in RAP children (19\% vs 7\%, RAP vs Control). Regression analyses revealed no relationship between fecal calprotectin concentration and abdominal pain frequency and severity and number and character of stools for the groups combined or separately. Conclusions: 1) Children with RAP have evidence of GI inflammation as measured by fecal calprotectin concentration; 2) Pain and stooling pattern do not appear to be related to calprotectin concentration; 3) GI inflammation as measured by fecal calprotectin concentration does not appear to play a significant role in pain and bowel symptoms in children with RAP.

\begin{tabular}{|c|c|c|c|}
\hline & RAP $(\mathrm{n}=73)$ & Control $(\mathrm{n}=32)$ & $\mathrm{P}$-Value \\
\hline Fecal calprotectin $(\mu \mathrm{g} / \mathrm{g}$ stool $)$ & $58.8 \pm 75.1$ & $38.8 \pm 23.0$ & 0.046 \\
\hline Mean pain score & $13 \pm 14$ & $5 \pm 6$ & 0.004 \\
\hline Maximum pain score & $56 \pm 30$ & $31 \pm 35$ & 0.001 \\
\hline Number of pain episodes & $7.2 \pm 8.5$ & $2.1 \pm 3.6$ & 0.002 \\
\hline
\end{tabular}

S1269

Cyclooxygenase 2 Expression in Polyps from a Juvenile Polyposis Syndrome Patient with Mutant Bmprla

Jayde Kurland, Stayce Beck, Carol Solomon, Oscar Bran, John M. Carethers, Sherry C. Huang

Background: COX2 expression is increased in colorectal cancers, and has been reported to be upregulated in Peutz-Jegher polyps. To determine if germline and somatic loss of BMPRIA in polyps from a Juvenile Polyposis Syndrome (JPS) kindred have altered COX2 expression, we characterized a JPS kindred for BMPRIA germline mutations, and examined the polyps for BMPR1A expression and COX2 expression. Methods: DNA analysis for BMPRlA was performed on a JPS kindred. Multiple polypectomies were performed and several polyps showed adenomatous change. Genomic DNA was extracted from polyp material for Loss of Heterozygosity $(\mathrm{LOH})$ analyses with microsatellite markers. Immunohistochemistry was performed on sections using antibodies for BMPR1A and COX2. Results: The kindred possessed a germline BMPRIA missense mutation. In polyp domains containing cystic and adenomatous epithelium, no LOH was observed using markers near the BMPRIA locus Immunostaining indicated decreased expression of phospho-SMADl (pSMADl), functionally downstream of the mutant BMPRIA receptor in the cystic epithelium, with further reduction in adenomatous portions within the polyp. COX2 immunostaining, normally not expressed in the colon, was present and greater in the lamina propria compared to the epithelium, and further increased in the adenomatous portions of the polyp. . Conclusion: Decreased expression of pSMADl in the cystic epithelium with further reduction in the adenomatous area, and an inverse relationship with the presence of COX2 expression in polyps from the BMPRlA heterozygote, suggests a potential mechanism for adenomatous pathogenesis in these hamartomatous polyps. This may imply that COX2 inhibitors could be a means for chemoprevention in this syndrome.

\section{S1270}

CT Enterography in Children with Crohn's Disease: Correlation with Inflammatory Markers and Disease Activity

Mark A. Naddaf, Peter Higgins, Peter Strouse, Pamela Brown, Ellen M. Zimmermann

Background: CT enterography (CTE) produces high resolution images of the bowel. CTE is currently used to evaluate adults with Crohn's disease $(C D)$; no reports in pediatric patients exist. CTE provides information similar to small bowel follow through (SBFT), but CTE adds detail of the bowel wall and surrounding tissue that may help guide treatment. We retrospectively reviewed CTEs performed on pediatric patients. We evaluated whether CTE findings correlated with clinical disease activity and markers of systemic inflammation, and whether CTE detected novel findings not available from SBFT. Methods: We reviewed records from 19 patients with known or suspected CD who underwent CTE. The five findings evaluated included mucosal enhancement with contrast, luminal narrowing, bowel wall thickening, mesenteric fat stranding, and increased vascularity. A Modified Harvey Bradshaw Index (HBI) clinical activity score, the erythrocyte sedimentation rate (ESR), and the $\mathrm{C}$-reactive Protein (CRP) level were taken from medical records. Spearman correlations were calculated for each comparison. Results: 10 patients had known CD based on positive biopsy or prior SBFT. 9 patients had bowel symptoms but had negative biopsies and no prior Crohn's diagnosis. In the CD cohort, the mean age was 14.3 years. HBI mean was 6.6 (range 0 to 12). 8 had CD in the small bowel and 2 had colonic disease. CTE demonstrated $\mathrm{CD}$ in all patients. Increased vascularity, luminal narrowing and mucosal enhancement was detected in 3 patients, mesenteric fat stranding in 1 patient. Fistulae and sacroiliitis were identified in 1 patient. Bowel wall thickening was detected in 6 patients. HBI correlated with the following CTE findings (rho): bowel thickening $(0.75, \mathrm{p}=0.01)$, fat stranding $(0.41$ $\mathrm{p}>0.05)$, and lumen narrowing $(0.42 \mathrm{p}>0.05)$. CRP correlated with the following CTE findings: bowel wall thickening $(0.84, \mathrm{p}=0.01)$, vascularity $(0.62)$, and fat stranding $(0.41)$ ESR correlated with the following CTE findings: vascularity $(0.65)$ and fat stranding $(0.41$ $p>0.05)$. No abscesses were seen in these 10 subjects. There were no false negative exams. In the group with no CTE findings for CD, 4/9 ultimately had UC and 5/9 had functional pain. Conclusion: CTE provides exquisite images of the bowel lumen and surrounding tissues. There was a significant correlation between bowel thickening and CRP, as well as $\mathrm{HBI}$; CRP and increased vascularity. A trend toward significant correlation with ESR was also demonstrated. CTE is able to identify findings in CD not seen in SBFT. This may indicate that CTE can predict inflammation vs. non-inflamed (fibrotic) bowel making it extremely useful in guiding managemen 
S1271

Growth Failure Persists Despite Current Treatment Paradigms in Pediatric Crohn's Disease

Marian D. Pfefferkorn, Jeffrey Hyams, Georgine Burke, James Markowitz, Joel Rosh, David Mack, Anthony Otley, Subra Kugathasan, Jonathan Evans, Athos Bousvaros, Susan Moyer, Robert Rothbaum, Robert Wyllie, Vasundhara Tolia, M Oliva-Hemker, Adam Mezoff, Neal Leleiko, Fernando Del Rosario, Wallace Crandall, David Keljo, Robert Baldassano, Anne Griffiths

Background/Aim: Impairment of linear growth is a recognized complication of pediatric Crohn Disease (CD), but prevalence data in the era of increased immunomodulatory and biologic therapies are sparse. We analyzed the prevalence, severity, and predictors of growth abnormalities in a contemporary population of newly diagnosed children with CD. Methods: Clinical, laboratory, and growth data were gathered from the Pediatric IBD Collaborative Research Group Registry database. This prospective observational study, established in January 2002 at 20 US/Canadian centers, records outcomes data on children diagnosed at $<16$ years of age but does not dictate treatment regimens. Results: We identified 175 children who were Tanner I-III at diagnosis and for whom at least 1 year follow-up was available (mean age $11 \mathrm{yr} \pm 2.7 \mathrm{SD}, 63 \%$ male). At diagnosis, $73 \%$ of subjects had moderate/severe $\mathrm{CD}$ by physician global assessment and the rest were mild. At 1 year, $57 \%$ were inactive, $33 \%$ mild, and $10 \%$ moderate/severe. Treatment in the first year included immunomodulator (82\%), corticosteroid (77\%), 5-ASA (75\%), infliximab (37\%) and nutritional therapy (28\%). Height velocity $\mathrm{Z}$ score at diagnosis $(\mathrm{n}=105)$ was $-3.7 \pm 4.0 \mathrm{SD} ; 75 \%$ were below -1.8 . For the entire sample of 175 , mean ht $Z$ score at diagnosis was $-0.5 \pm 1.2 \mathrm{SD} ; 10 \%$ were below 2.0. At 1 year, mean ht $Z$ score remained at $-0.5 \pm 1.1 \mathrm{SD}$ and $28 \%$ grew $<4 \mathrm{~cm} / \mathrm{yr}$. Mean height velocity $\mathrm{Z}$ score over the year post-diagnosis was $-2.0 \pm 2.5 \mathrm{SD}$. Height $\mathrm{Z}$ scores improved over the first year for subjects with baseline ht $Z$ scores $<-1$ and decreased for all others (mean difference 0.15 and -0.10 , respectively, $\mathrm{p}<.0004$ ). Factors at diagnosis associated with improved ht velocity $\mathrm{Z}$ scores at 1 year $(\mathrm{F}=6.0, \mathrm{p}<0.0001)$ were low baseline height $\mathrm{Z}$ score, low Hgb, high ESR, and moderate to severe disease. Conclusion: Despite the use of aggressive medical therapy during the first year after diagnosis, children with CD remain shorter and have a depressed growth velocity compared to healthy, age-matched peers. While current therapy modestly helps the most severely affected children, the growth of many others may continue to decline. Longer follow-up will determine whether ongoing therapy ultimately results in improved linear growth

\section{S1273}

Comparison of Quantitative Calcaneal Ultrasound and Dxa in Children with Inflammatory Bowel Disease

Manisha Harpavat, Qianyu Dang, David Keljo, Susan Greenspan

Background: Quantitative Ultrasound (QUS) of the calcaneum provides a radiation-free and inexpensive assessment of bone density and microstructure. Purpose: The purpose of this study was to compare DXA measurements of the whole body and lumbar spine to QUS measurements obtained from the calcaneus. Methods: DXA and QUS measurements were obtained simultaneously. Bone mineral content (BMC, grams) and BMD (grams/cm2) measurements of the whole body and lumbar spine were assessed by DXA (Lunar Prodigy). Broadband ultrasound attenuation (BUA; $\mathrm{dB} / \mathrm{MHz}$ ) was measured in the posterior part of the calcaneus at the region of interest (ROI) by QUS (Quidel QUS-2). Data analysis was performed by Pearson's correlation. Receiver operator curves (ROC) were constructed to determine how well BUA discriminated between normal and diseased populations. Results: 70 children (46 with IBD and 24 healthy controls) underwent a total of 219 measurements over 12 months. The IBD group and healthy control group did not differ in terms of age, gender, or race. Correlation coefficients between DXA and QUS measurements ranged from 0.70-0.84 and were comparable between the two groups. Positive significant correlations were found between calcaneal BUA and whole body (BMC and BMD) and lumbar spine (BMC and BMD) for both groups. The area under the curve of a range of BUA values in detecting reduced bone mass (BMD $z$ score $\leq-1$ ) and severely reduced bone mass (BMD $z$ score $\leq-2$ ) of the whole body and lumbar spine was calculated from the ROC and is shown in Table 1. Conclusion: These results indicate that QUS measurements of the calcaneus positively correlate with DXA measurements of the whole body and lumbar spine. From the ROC area under the curve, QUS appears to be a useful screening tool in determining which patients have severely reduced bone mass (BMD z score $\leq-2$ ) and require further evaluation with DXA

Table 1 .

\begin{tabular}{|l|c|}
\hline & Area under the curve \\
\hline whole body BMD z score $\leq 1$ & 0.731 \\
\hline whole body BMD z score $\leq 2$ & 0.831 \\
\hline lumbar spine BMD z score $\leq 1$ & 0.672 \\
\hline lumbar spine BMD z score $\leq 2$ & 0.713 \\
\hline
\end{tabular}

\section{S1274}

Abnormalities of the Enteric Nervous System, Smooth Muscle and Interstitial Cells of Cajal in Children with Colonic Motility Disorders

Maartje M. van den Berg, Hayat M. Mousa, Carlo Di Lorenzo, Marc A. Benninga, Guy E. Boeckxstaens, Mark Luquette

Background: Colonic manometry studies in children with severe defecatory disorders often reveal abnormal contraction patterns. These patterns have traditionally been classified into myopathic, neuropathic or mixed types. Current study evaluates the relation between colonic manometry findings and abnormalities of the colonic enteric nervous system (ENS), smooth muscle and interstitial cells of Cajal (ICC). Methods: Colonic specimens from surgical resections or full-thickness biopsies were assessed from a cohort of children who underwent colonic manometry testing prior to surgery. Manometry and histopathologic findings of colonic segments were correlated. Following immunohistochemistry stainings were used to identify abnormalities of the ENS: Neuron Specific Enolase, Calretinin, Neurofilament (neuronal markers), Synaptophysin (synaptic vesicles marker), Tyrosine hydroxylase (adrenergic pathway marker), S-100 (glial cell marker), GFAP (glial fibrillary acidic protein). ICC were stained with CD117 (c-kit), and smooth muscle markers were SMA (alpha actin) and MSA (gamma actin). Results: Eight colonic specimens from 5 patients with clinical symptoms of dysmotility were evaluated. One patient was previously diagnosed with Hirschsprung's disease and had persistent symptoms of dysmotility after resection of the aganglionotic segment. Four patients had severe constipation of unknown origin. Manometry showed an absence of colonic contractions in 3 colonic segments and low amplitude propagating contractions (LAPC) in 2 segments, compatible with a myogenic pattern of dysmotility These specimens showed abnormalities of the ENS and a marked decrease or absence of ICC, but no abnormalities in smooth muscle markers (SMA and MSA). Simultaneous contractions, indicative of a neuronal disorder were seen in 2 segments, Histopathology showed an elevated level of GFAP positive cells, loss of ICC and normal smooth muscles One segment from a patient with severe symptoms of colonic dysmotility and who had normal high amplitude propagating contractions showed minimal abnormalities on histopathology including an elevated GFAP level and reduced ICC staining. In total 7/8 specimens showed elevated levels of GFAP positive cells, suggestive of neuronal damage. Conclusions: Although a myogenic pattern was observed on colonic manometry, abnormalities in ICC or the ENS but not in smooth muscle markers were found. Loss or absence of ICC and elevated levels in GFAP were prevalent findings in pediatric colonic motility disorders, irrespective o the manometric pattern. Caution should be used when predicting the type of underlying neuromuscular disorder based on colonic manometry

\section{S1275}

Predictors of Maternal Responses to Child Gastrointestinal Symptoms Shelby L. Langer, Lynn S. Walker, Joan M. Romano, William E. Whitehead, Rona L. Levy

Background: Prior research and social learning theory suggest that inappropriate GI illness behaviors may be maintained at least in part by social reinforcement processes. Wellintentioned parents may respond to their child's somatic complaints with solicitous behaviors and discourage activity, potentially fostering illness vs. wellness behavior (Levy et al., 2004). Thus, identifying factors that are associated with solicitousness (or the converse, minimization) is important in determining when interventions to reduce solicitousness may be most useful. Aims: The purpose of this study was to examine predictors of maternal responses to children's abdominal pain symptoms, specifically: protectiveness (limiting the child's activities, giving treats), encouragement/ monitoring (encouraging activity while monitoring symptoms) and minimization (expecting normal responsibilities, criticizing illness behavior) Methods: Participants were 450 mother/ child dyads: $46 \%$ of the mothers had a diagnosis of Irritable Bowel Syndrome. Demographics were as follows: child mean age $=11$ (SD $=$ 2.6), $52 \%$ female, $80 \%$ Caucasian; maternal mean age $=42(S D=6.2), 80 \%$ Caucasian Children rated the severity of their abdominal pain in the past 2 weeks. Mothers completed the Adult Responding to Children's Symptoms Inventory, the Brief Symptom Inventory and the Children's Pain Beliefs Questionnaire. The latter assessed maternal perceptions regarding the severity of the child's condition. Hierarchical linear regression was used to examine demographic and psychosocial predictors of maternal response. Results: Fifty- four percent of children reported having abdominal pain in the past 2 weeks. Minimization was predicted by low perceived condition severity $(\mathrm{p}=.047)$ and, counter to prediction, maternal IBS status $(\mathrm{p}=.005)$ and higher maternal anxiety $(\mathrm{p}=.029)$. Encouragement/ monitoring was predicted by younger child age $(\mathrm{p}=.002)$, male child gender $(\mathrm{p}=.014)$, high perceived condition severity $(\mathrm{p}=.013)$ and higher maternal somatization $(\mathrm{p}=.017)$. Protectiveness was predicted by absence of a father in the home $(\mathrm{p}=.020)$ and a gender $\mathrm{x}$ condition severity interaction $(p=.018)$. Mothers were protective of girls regardless of condition severity, but more protective of boys believed to have a more serious condition. Conclusions These findings offer implications for clinical intervention. Physicians should be aware when risk factors for solicitousness exist and, when appropriate, encourage parents to maintain normal child responsibilities. This could result in increased wellness behavior and reduced disability. Supported by grant ROI HD36069.

\section{S1276}

Nuclear $\beta$-Catenin in Germline Pten Mutated Hamartomatos Polyps Jashdeep Dhoot, John M. Carethers, Sherry C. Huang

Background: The mechanisms involving intestinal hamartomatous polyp transformation towards adenoma or adenocarcinoma are not known. In sporadic colon cancers, the canonical Wnt signaling pathway when altered will lead to nuclear localization of the proto-oncogene $\beta$-catenin. GSK3 $\beta$ is a regulator of the Wnt pathway, and is phosphorylated by PI3 kinase and presumed antagonized by PTEN, which negatively regulates PI3 kinase. Here, we examined polyps from germline PTEN-mutated hamartomatous syndrome patients to discern any regulation of Wnt signaling. Methods: Eight polyps from 3 PTEN germline mutated hamartomatous polyposis patients were analyzed for tissue expression of PTEN, $\beta$-catenin total and phosphorlyated GSK3 $\beta$, and APC by immunohistochemistry. We then compared the various proteins and their expression patterns. Results: All 8 hamartomatous polyps lacked PTEN expression in the epithelial components of the polyps. Within the epithelium, full length APC was expressed, but $\beta$-catenin was localized to the nucleus. Phosphorylated GSK3 $\beta$, which blocks cytoplasmic degradation of $\beta$-catenin, was upregulated compared to total GSK3 $\beta$ in the epithelium. Conclusions: Hamartomatous polyps from germline PTENmutated patients have lost PTEN expression and show nuclear $\beta$-catenin with upregulated phosphorylated GSK3 $\beta$ in the epithelial components of their polyps despite expressing full length APC. This suggests that loss of PTEN might affect Wnt signaling in favor of the nuclear localization of $\beta$-catenin as a potential pathway for adenoma or adenocarcinoma transformation

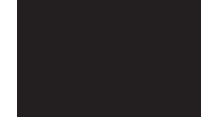




\section{S1277}

Azathioprine Maintains First Remission in Newly Diagnosed Pediatric Crohn's Disease: A Retrospective Study

Gerald J. Jaspers, Henkjan J. Verkade, Johanna C. Escher, Lissy de Ridder, Jan A. Taminiau, Edmond H. Rings

Background: 6-Mercaptopurine has been shown to spare corticosteroid use and to maintain remission in pediatric Crohn's disease (CD; Gastroenterology 2000;119:895-902). We evaluated retrospectively to what extent azathioprine (AZA), the active metabolite of 6-mercaptopurine, was used in newly diagnosed pediatric $C D$ patients and its relation to maintenance of remission. Methods: We reviewed from 3 pediatric centers the data of children with newly diagnosed CD (period 1998 - 2003, follow-up > 18 months). Two definitions of active disease (initial or relapse) were used: Pediatric Crohn's Disease Activity Index (PCDAI) > 10 , or systemic corticosteroid use. Remission was defined as PCDAI $\leq 10$ without use of corticosteroids. Treatments were compared with respect to initial therapy, duration and severity of active disease, and maintenance of first remission. Results: 88 children (55M/ $33 \mathrm{~F}$, age $12 \pm 3$ yr., mean \pm SD) were included. Only 2 patients received AZA within 1 month after diagnosis. During the first period of active disease, 33 patients received AZA (38\%), while at the end of the follow-up period ( $38 \pm 17 \mathrm{mo}$.) 72 patients $(82 \%)$ had been prescribed AZA. In patients diagnosed after 2000, AZA was prescribed at 233 days after diagnosis, compared with 686 days in patients diagnosed between 1998-2000 (median values, $\mathrm{p}<0.05$ ). At initial presentation, a larger fraction of AZA patients had moderate-severe disease activity (AZA vs. non-AZA: $75 \%$ vs. $52 \%$; $\mathrm{p}<0.05$ ) and were prescribed corticosteroids ( $89 \%$ vs. $58 \% ; \mathrm{p}<0.005)$. Initial therapy with respect to aminosalicylates $(\sim 85 \%)$ and enteral nutrition $(\sim 27 \%)$ did not differ in AZA and non-AZA patients. The first period of active disease, according to corticosteroid use, was longer in AZA patients (232 vs. 168 days; $\mathrm{p}<0.005$ ). In patients who had used corticosteroids during initial disease activity, the median maintenance of the first remission in AZA patients was 544 days (PCDAI) or 575 days (corticosteroidfree) compared with $254(\mathrm{p}=0.08)$ and 259 days $(\mathrm{p}<0.05)$ in non-AZA patients, respectively. In 12 months after reaching the first remission, a profoundly larger fraction of AZA patients had maintained a corticosteroid-free period, compared to non-AZA patients (15/26 vs. 8 / 28; $\mathrm{p}<0.05$ ). Conclusion: This study demonstrates a beneficial effect of AZA in pediatric $\mathrm{CD}$ with respect to maintenance of first remission. AZA therapy has become more frequently used for pediatric $C D$, but its initiation within one month after diagnosis is still rare. Our data support a more prominent role for AZA therapy in pediatric CD.

\section{S1278}

Outcome of Moderate to Severe Pediatric Crohn's Disease: Effect of Infliximab Therapy

Joel R. Rosh, Jeffrey Hyams, Trudy Lerer, James Markowitz, Marian Pfefferkorn, David Mack, Anthony Otley, Subra Kugathasan, Jonathat Evans, Athos Bousvaros, Susan Moyer, Robert Rothbaum, Robert Wyllie, Vasu Tolia, Maria Oliva-Hemker, Adam Mezoff, Neal Leleiko, Fernando Delrosario, Wallace Crandall, David Keljo, Robert Baldassano, Anne Griffiths

Background: There are little data on the long-term clinical outcome of moderate to severe pediatric Crohn disease (CD) patients who receive infliximab (IFX). Aim: To compare clinical outcomes in moderate to severe pediatric $C D$ patients who do and do not receive IFX. METHODS: Since January 2002, 20 US/Canadian pediatric GI centers prospectively enrolled 692 newly diagnosed children with IBD in an observational registry. All subjects were managed according to the dictates of their physicians, not by standard protocols. A database search identified children with moderate to severe $C D$ at diagnosis who had at least 18 months of follow-up. IFX use was categorized as: No IFX (NI), early (within 3 months of diagnosis = EI), and late (between 3-12 months from diagnosis = LI). Clinical and demographic characteristics were compared among the three groups at baseline and at 18 months after diagnosis. RESULTS: Of 482 CD subjects 262 had at least 18 months of follow-up (62\% male, age range $1.4-16 \mathrm{yrs}$, mean $11.7 \pm 2.8 \mathrm{yrs}) .181$ (69\%) presented with moderate to severe disease by Physician Global Assessment (PGA) or PCDAI $>30.136(75 \%)$ were NI. $45(25 \%)$ received IFX with $26(14 \%)$ EI and $19(11 \%)$ LI. There were no significant differences in mean age, gender, disease location, or baseline lab values (hemoglobin, sedimentation rate or serum albumin) among the three IFX groups. Baseline mean PCDAI was similar in EI $(40.4 \pm 16.6)$, NI $(34.7 \pm 14.3)$ and LI $(30.8 \pm 12.0)$. Use of steroids as the initial induction agent was similar in all groups ( $85 \%$ overall). Immunomodulators were used within 3 months of diagnosis in $88 \%$ of EI vs. $53 \%$ LI and $56 \% \mathrm{NI}(\mathrm{p}<0.01)$. Rapid clinical deterioration prior to IFX was common in EI demonstrated by $58 \%$ hospitalized and $23 \%$ with IBD surgery within 90 days of diagnosis and before the start of IFX therapy ( $\mathrm{p}<0.05 \mathrm{vs}$. LI and NI for surgery). 18 month outcome was similar among groups with PGA of inactive/mild in $86 \% \mathrm{EI}$ and $76 \% \mathrm{LI}(\mathrm{p}=\mathrm{NS}) .18$ month PCDAI values were also similar in those with available data: $\mathrm{EI}=7.7 \pm 6.3 \cdot \mathrm{LI}=9.3+13.8 \cdot \mathrm{NI}=9.2+10.5(\mathrm{p}=\mathrm{NS})$. CONCLUSIONS: $1.25 \%$ of children presenting with moderate to severe CD received IFX within 12 months of diagnosis. 2. Neither baseline clinical disease activity (PCDAI) nor traditional laboratory parameters were predictive of earlier use of infliximab therapy. 3 . Despite poor early response to non-biologic therapies in some patients, current patterns of infliximab use allow for a marked reduction in clinical disease activity at 18 months after diagnosis in the majority of pediatric patients who presented with moderate to severe CD.

\section{S1279}

Longitudinal Effect of Corticosteroids in Pediatric Crohn's Disease Manisha Harpavat, Qianyu Dang, David Keljo, Susan Greenspan

Background: Corticosteroids have been implicated as a major cause of reduced bone mass and delayed growth in children with Crohn disease (CD). Longitudinal studies examining the effect of corticosteroids on bone mass acquisition and growth in children with $\mathrm{CD}$ are lacking. Aim: To observe the effects of corticosteroids on bone mass acquisition and growth in a group of steroid naive children newly diagnosed with $C D$ compared to healthy age, gender, and race matched controls. Methods: 6 otherwise healthy steroid naive children newly diagnosed with $\mathrm{CD}$ and 6 age, gender, and race matched controls underwent DXA measurements at baseline, 6 , and 12 months. After baseline measurements were obtained, the $6 \mathrm{CD}$ subjects were started on corticosteroids (equivalent to $2 \mathrm{mg} / \mathrm{kg}$ prednisone) for an average of 74.2 days (range 55-99). After completing the corticosteroid course none of the patients received corticosteroids again during the 12 months. Bone mineral content (BMC, grams) and bone area (BA,cm2) measurements of the whole body (WB)and lumbar spine (LS) were recorded. Results: 6 CD patients (2 male) and 6 healthy controls were studied. Results of measured variables are in Table 1. The mean Pediatric Crohn's Disease Activity Index score at baseline was 32.1 (range 12.5-55), at 6 months $7.1(0-17.5)$, and at 12 months $8.8(0-20)$. Height velocity was decreased in the CD group at 6 months compared to the control group but was similar between the groups by 12 months $(4 \pm 2.4$ $\mathrm{cm} / \mathrm{yr}$ CD group vs $3.7 \pm 3.5 \mathrm{~cm} / \mathrm{yr}$ control group). WB BMC and BA at baseline was lower for the CD group but at 12 months had increased to similar values as the control group. LS BMC and BA at baseline was lower for the CD group, declined even further at 6 months, but by 12 months had increased to similar values as the control group. Conclusion: In this group of 6 previously steroid naive children newly diagnosed with $C D$ it appears that despite initial corticosteroid use, bone mass acquisition and growth after 12 months was comparable to healthy age, gender, and race matched controls. Bone mass acquisition at the LS, which is composed of trabecular bone was affected at 6 months but WB, which is composed of $80 \%$ cortical bone was not affected. It is possible that corticosteroid use in children newly diagnosed with $\mathrm{CD}$ may only have a transient impact on bone mass acquisition and growth. A larger sample size is needed to confirm the observed findings. Table 1.

\begin{tabular}{|c|c|c|}
\hline & CD & Controls \\
\hline age at baseline & $12.5 \pm 3.8$ & $12.5 \pm 4$ \\
\hline baseline Tanner stage & 2.8 & 3 \\
\hline bseline BMI z score & $-0.2 \pm 1.7$ & $0.8 \pm 1.2$ \\
\hline baseline height z score & $0.6 \pm 1.3$ & $0.5 \pm 0.8$ \\
\hline
\end{tabular}

\section{$\mathrm{S} 1280$}

Symptoms Refactory to PPI Therapy Associated with Non-Acid, Acid Or No Reflux in Children

Inder Mainie, Radu Tutuian, Amine Hila, Amit Agrawal, Donald O. Castell

Background: Gastroesophageal reflux in children has been diagnosed as the cause of systemic illnesses in children. Combined Multichannel Intraluminal Impedance (MII) detects all types of gastroesophageal reflux (GER), (non-acid reflux, (NAR) or acid reflux) at multiple levels of the esophagus independent of $\mathrm{pH}$. Aim: To clarify the relationship of typical and atypical GERD symptoms to reflux episodes (acid and NAR). Method: Starting January 2002, all patients with persistent symptoms despite proton pump inhibitor (PPI) therapy referred to the esophageal laboratory underwent combined MII-pH monitoring. Prior to the study, parents and/or the referring physician were contacted to ensure that the patient had been on at least twice daily PPI for at least a month or more prior to testing. In pediatrics less than 2 years the $\mathrm{pH}$ was placed $2 \mathrm{~cm}$ above the LES with impedance measurements at $2,3.5,5,6.5,8$ and $9.5 \mathrm{~cm}$ above the LES. In pediatrics $(2-6 \mathrm{yrs})$ the $\mathrm{pH}$ was placed $3 \mathrm{~cm}$ above the LES with impedance measurement at $3,5,7,9,11$ and $13 \mathrm{~cm}$ above the LES and in the 6-10 years the $\mathrm{pH}$ was at $4 \mathrm{~cm}$ above the LES with impedance measurements 4,6,8,10,12 and $14 \mathrm{~cm}$. Studies were recorded for 24 hrs. While taking PPI therapy bid, the symptom index was defined as the number of symptoms associated with reflux divided by the total number of symptoms and was positive (+SI) if $\geq 50 \%$. Typical GERD symptoms were heartburn, regurgitation or chest pain. Atypical symptoms were cough, nausea, wheezing, hoarseness and throat clearing. Results: Fifty-six children (Female 18; mean age 3 years; range 5 weeks - 15 years). Forty-six (82\%) children recorded symptoms during test day; typical GERD symptoms $(n=10)$, atypical symptoms $(n=36)$. Table below shows MII-pH SI for the primary typical and atypical symptoms $(\mathrm{p}<0.01)$. Conclusions: Typical symptoms in children were more likely associated with reflux (primarily non-acid) whereas atypical symptoms were usually associated with no reflux.

\begin{tabular}{|c|c|c|c|}
\hline Symptoms (\# Patients) & +SI for NAR & +SI for Acid & - SI \\
\hline Typical (10) & $6(60 \%)$ & $1(10 \%)$ & $3(30 \%)$ \\
\hline Atypical (36) & $11(30 \%)$ & $1(3 \%)$ & $24(67 \%)$ \\
\hline
\end{tabular}

\section{S1281}

Do Abnormalities of Peristalsis Result in Abnormal Esophageal Clearance? the Use of Esophageal Function Testing (EFT), a Novel Technology to Evaluate Dysphagia in Children

Rachel L. Rosen, Julia Fritz, Nurko Samuel

Background: The gold standard for the evaluation of dysphagia in children is esophageal manometry (EM). However, recent studies in adults have suggested that patients with abnormal EM may have normal bolus transit. The recent availability of EFT (combined esophageal manometry and impedance) provides an instrument to correlate esophageal transit (measured by impedance) and pressure (measured by manometry). This is the first report of the use of EFT to correlate esophageal peristalsis and bolus transit in children. Methods: EFT tracings of all children with dysphagia studied in the motility unit were reviewed. Patients with achalasia were excluded because of inability to calculate bolus transit. EFT was performed with a solid state catheter that combines pressure transducers and impedance rings. Bolus presence time, total bolus transit time, and standard esophageal manometry variables were measured for both liquid (LS) and viscous swallows (VS). Swallows were considered ineffective if the amplitude in the distal esophagus was less than $30 \mathrm{mmHg}$. Results: EFT was completed in 11 children with dysphagia. Based on EM, 5 had nonspecific motor disorders and 6 had normal peristalsis. 268 swallows were recorded. 15\% 
of swallows had ineffective peristalsis and $23 \%$ of swallows had incomplete bolus clearance. More than $80 \%$ of swallows classified as ineffective peristalsis had complete bolus transit. Bolus transit varied between LS and VS, with an abnormal transit being more frequent in those with ineffective peristalsis after VS ( $25 \%$ of VS) compared to LS ( $15.8 \%$ of LS). Furthermore, despite the convention that distal, low amplitude contractions are pathologic, only $19.4 \%$ of swallows with low amplitude, distal contractions had incomplete bolus transit. When any esophageal contraction amplitude was less than $30 \mathrm{mmHg}$, complete bolus transit was significantly reduced (table). Conclusions: This is the first report of the use of EFT in children. Esophageal boluses are effectively cleared in more than $80 \%$ of ineffective swallows suggesting that manometry may be inadequate to assess esophageal function in children. EFT provides new insight into the relationship between esophageal function and pressures and may redefine the approach to children with esophageal motility disorders. Relationship between esophageal pressure and bolus clearance

\begin{tabular}{|c|c|c|c|}
\hline & $\begin{array}{c}\text { Normal amplitude in all } \\
\text { esophageal segments }\end{array}$ & $\begin{array}{c}\text { Abnormal amplitudes in 1+ } \\
\text { esophageal segments* }\end{array}$ & $\begin{array}{c}\text { Abnormal Amplitude in } \\
\text { distal 2 segments }\end{array}$ \\
\hline $\begin{array}{c}\text { Complete Bolus } \\
\text { Transit }\end{array}$ & $125(93.3 \%)$ & $81(60.4 \%)$ & $25(80.6 \%)$ \\
\hline $\begin{array}{c}\text { Incomplete } \\
\text { Bolus Transit }\end{array}$ & $9(6.7 \%)$ & $53(39.6 \%)$ & $6(19.4 \%)$ \\
\hline
\end{tabular}

*chi-square, $\mathrm{p}<0.0001$

\section{$\mathrm{S} 1282$}

Worries About Childhood Functional Abdominal Pain: The Fathers Perspective

Nader N. Youssef, Miranda van Tilburg, Annette Langseder, William E. Whitehead

BACKGROUND: In childhood functional abdominal pain (FAP), healthcare utilization is often initiated by the affected child's mother. Addressing the maternal concerns and providing education during office evaluations often is part of the planned therapeutic intervention for childhood FAP. To date, there is little data on what concerns fathers have about their children who suffer from FAP. This lack in knowledge combined with less common access to fathers may prevent paternal concerns from being adequately identified and addressed. AIM: To investigate if concerns differ between fathers and mothers of children being evaluated for FAP. SUBJECTS \& METHODS: Prior to an initial evaluation at a tertiary pediatric gastroenterology center, 48 mother-father dyads of children (mean age 9.6 years, ranging from 4 to 18 years) suffering from FAP completed the Parental Worries about RAP Questionnaires (PWRQ). The PWRQ is a validated and reliable tool that measures parental worries. It consists of 4 subscales: (1) Fear to ignore symptoms-worries surrounding the willingness to ignore or focus on symptoms (6 items), (2) Desire for care- frustration with doctors for failing to understand, treat, or diagnose ( 7 items), (3) Worry about coping- feeling uncertain or unable to cope with symptoms ( 7 items), and (4) Exacerbating factors- recognizing the role of stress and family (5 items). Responses are scored on a 1 (not at all worried) to 5 (very much worried) scale. Scores on items were added to create a subscale score. A diagnosis of FAP was made utilizing the Pediatric Rome Criteria. RESULTS: Paired T-test revealed no differences between groups. (see Table) CONCLUSIONS: Mothers and fathers of children with functional abdominal pain do not differ in their concerns and worries. Acknowledgement that concerns are shared by both parents may be an important issue to address during initial evaluation of childhood FAP. Involving fathers from the very beginning of planned educational and therapeutic interventions for childhood FAP may be a necessary feature to ensure full acceptance of diagnosis and treatment plan of childhood FAP. [Supported by grant R24 DK67674.

PWRQ Scores

\begin{tabular}{|c|c|c|c|}
\hline & Mothers & Fathers & SIG. \\
\hline Fear to Ignore Symptoms & 8.9 & 9.8 & $\mathrm{~ns}$ \\
\hline Desire for Care & 16.6 & 16.0 & $\mathrm{~ns}$ \\
\hline Worry about Coping & 22.7 & 22.3 & $\mathrm{~ns}$ \\
\hline Exacerbating Factors & 11.7 & 11.8 & $\mathrm{~ns}$ \\
\hline
\end{tabular}

$\mathrm{S} 1283$

Gastro-Esophageal Reflux Disease in Young Children: Incidence and Presentation Patterns in a Population-Based Birth Cohort

Denesh K. Chitkara, Heiko de Schepper, Amy Weaver, Mary Jo Rucker, Slauvica Katusic, Giles R. Locke, Nicholas J. Talley

Background:Gastro-esophageal reflux disease (GERD) is a common complaint of early childhood and affected children can continue to experience symptoms later in life. However, little is known about the natural history, and patterns of presentation of this disorder in young children. Aims: 1. To estimate the incidence of GERD presentation in a population based birth cohort from birth to 5 years, and 2. To examine the patterns and proportion of medical visits for GERD in this age group. Methods: A birth cohort of all children born between 1976 and 1982 to mothers who were residents of Rochester, MN, and who remained in the community until age 5 years $(\mathrm{N}=5718)$ was considered for this study. The vital status of each member of the birth cohort during the 1995-1996 school year was established. HICDA diagnostic codes for GERD for the birth cohort members were identified. The medical records were reviewed to confirm a medical visit primarily for GERD, the date of presentation, number of medical visits, and specific symptoms and the frequency and duration of overall symptoms. Results: Of the 5506 birth cohort members, the overall age- and sex-adjusted incidence was 0.9 per 1000 person-years (py). The age-specific incidences were similar for girls (0.52 (95\% C.I., 0.14-0.92) per 1000 py) and boys (1.27 (95\% C.I., 0.68-1.85) per 1000 py). The mean age of initial GERD diagnosis was 7.3 months, and $90 \%$ of patients presented within the first year of life. Both typical (vomiting, regurgitation, and symptoms after meals) and atypical symptoms (cough and asthma/reactive airway disease) of GERD were reported during the incident visit (Table). Twenty percent of children with GERD had documented symptoms for greater than 1 month, and $24 \%$ of children had 3 or more medical visits for GERD by 5 years of age. Conclusion: This is the first examination of the incidence of GERD in children using a population based birth cohort. In young children who present for symptoms, GERD accounts for a high number of repeat medical visits. Supported by an unrestricted research grant to the Dyspepsia Center from Novartis, and the Mayo Family Endowment in Gastroenterology at Children's Hospital Boston.

Individual symptoms during the incident visit in children with GERD from birth to 3 months and 3 months to 1 year

\begin{tabular}{|c|c|c|}
\hline & Birth-3 months (n=10) & 3 months-1 years $(\mathrm{n}=7)$ \\
\hline Vomiting & $100 \%$ & $100 \%$ \\
\hline Regurgitation & $90 \%$ & $100 \%$ \\
\hline Post-prandial symptoms & $30 \%$ & $71 \%$ \\
\hline Cough & $20 \%$ & $14 \%$ \\
\hline Vomiting blood & $20 \%$ & $0 \%$ \\
\hline Asthma & $20 \%$ & $0 \%$ \\
\hline
\end{tabular}

\section{S1284}

ASCA Positivity Independently Predicts Oral Crohn's Disease in a Large Early Onset IBD Population

Richard K. Russell, Brian Ip, Marian C. Aldhous, Hazel E. Drummond, Ian D. Arnott, Margaret McDougall, David C. Wilson, Peter M. Gillett, Paraic McGrogan, Kamal Hassan, Lawrence T. Weaver, Michael W. Bissett, Gamal Mahdi, Jack Satsangi

Introduction: Anti-Saccharomyces cerevisiae antibodies (ASCA) have been proposed to be useful in the diagnosis of Crohn's Disease (CD). ASCA status and its relationship with disease phenotype was determined in a cohort of Scottish early onset IBD patients. Patients and Methods: ASCA status (IgA, IgG) was determined in 301 Inflammatory Bowel Disease (IBD) patients [197 CD, 76 Ulcerative Colitis (UC), 28 IC] using an Enzyme-Linked Immunosorben Assay kit. ASCA data from 78 healthy adult controls was also available. ASCA status was matched with phenotypic features of IBD at diagnosis. Results: CD patients had a higher prevalence of ASCA antibodies compared to UC patients and healthy controls (41.6\% vs $15.8 \%, \mathrm{p}=0.0001$ Odds Ratio $(\mathrm{OR})=3.80[1.93-7.50]$ and $41.6 \%$ vs. $7.7 \% \mathrm{p}<0.0001$ OR $8.56[3.55-20.62]$ respectively). ASCA status predicted CD with sensitivity of $41.6 \%$ and specificity of $88.6 \%$. In CD patients univariate analysis demonstrated a positive ASCA was associated with oral $(68.0 \%$ vs. $38.6 \%, p=0.008$ OR 3.39 [1.38-8.34]) and perianal $(50.6 \%$ vs. $35.2 \%, p=0.04$ OR $1.89[1.04-3.44])$ disease location, raised CRP $(48.9 \%$ vs. $24.5 \%$, $\mathrm{p}=0.006$ OR $2.95[1.36-6.37])$, hypoalbuminaemia (51.8\% vs. $29.0 \%, \mathrm{p}=0.01 \mathrm{OR}$ $2.28[1.19-4.37])$, the need for surgery (55.1 vs. $36.6 \%, p=0.03$ OR $2.11[1.10-4.06])$ and the presence of granulomata ( $47.7 \%$ vs. $28.8 \%$, $\mathrm{p}=0.02$ OR $2.25[1.13-4.48]$ ). On a Multivariate binary logistic regression analysis, oral Crohn's disease location was an independent predictor for a positive ASCA status (adjusted p = 0.001, OR 22.22[3.41-142.86). Conclusions: ASCA prevalence is $41.6 \%$ in Scottish early onset CD patients. Phenotypic analysis demonstrated association with a number of factors most notably the presence of oral Crohn's disease.

\section{S1285}

Detection and Characterization of Hepatic Metastases from Gastrointestinal Endocrine Tumor with Contrast-Specific US Modes and a Sulfur HexafluorideFilled Microbubble Contrast Agent

Claude D. Marcus, Hedia Brixi-Benmansour, Louis Job, Viviane Ladam-Marcus, Stephanie Lagarde, Guillaume Cadiot

Purpose : To assess wether detection and characterization of liver metastases in patients with gastrointestinal endocrine tumor could be improved by using ultrasonographic (US) contrast-specific modes after injection of sulfur hexafluoride-filled microbubble a new contrast agent as compared with unenhanced US. Material and Methods : 37 focal hepatic lesions in 10 patients with a history of liver metastases from gastrointestinal endocrine tumor were examined using US and enhanced US after contrast injection. Lesion vascularity and the kinetics of contrast enhancement within the lesions at the arterial phase, portal phase and late phase were analysed. Findings from enhanced and non enhanced US were compared with each other and with MRI as the reference method. Results : 25 of the 37 lesions (68\%) were identified by using unenhanced US. Lesions were hypoechoic in 11 of the 37 cases, hyperechoic in 9 of the 37 cases, heterogeneous in 5 cases. The number of detected lesions increased from 25 (68\%) with unenhanced US to $37(100 \%)$ after contrast agent injection ( $\mathrm{p}<0.01$ ). 17 of the 37 lesions (46\%) enhanced markedly during arterial phase (Fig. 1) 21 of the 37 lesions $(57 \%$ ) showed a central hypoechoic pattern during portal phase. 37 of the 37 lesions (100\%) demonstrated diffuse homogeneous hypoechoic appearance to the adjacent liver during the late phase. Conclusion: The use of contrast-specific modes with a sulfur-hexafluorid contrast agent led to improved detection and characterization of liver metastases from endocrine tumors in comparison with unenhanced US. 


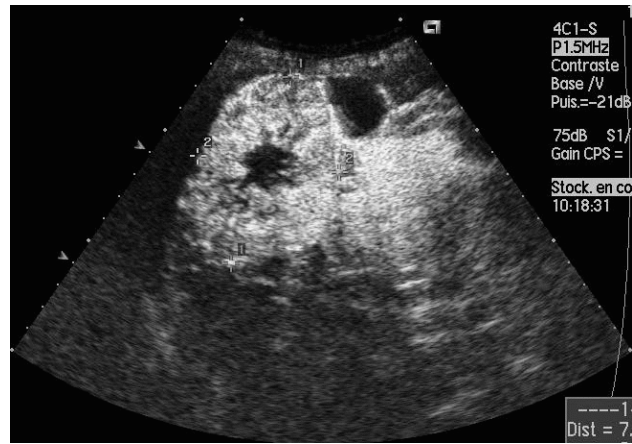

Figure 1 : US view at arterial phase after contrast agent injection shows diffuse heterogeneous enhancement of the hepatic metastase

\section{S1286}

Non-Invasive in Vivo Visualization of Pancreatic Cancer Signature with Conditionally Replicative Adenovirus System

Julia Davydova, Tatyana Gavrikova, Seiji Yamamoto, Amy Nelson, Masato Yamamoto

Recent elucidation of cancer biology has identified series of pancreatic cancer molecular signatures. Non-invasive imaging of such signatures may enable tumor detection and determination of disease spread, and may provide a powerful method assisting clinical decision making process. The realization of such strategy requires a system which is selective and potent. We have been developing infectivity enhanced conditionally replicative adenoviruses (CRAds) as a selective and potent therapeutic modality for the treatment of GI cancers, and reported several CRAds for pancreatic cancer including cyclooxygenase-2 (Cox-2) promotercontrolled CRAd (RGDCox2CRAdF, Gastroenterology. 2003;125(4):1203-18). In this project, we applied CRAd structure for non-invasive imaging of Cox-2 status (one of pancreatic cancer molecular signatures), and tested its in vitro and in vivo functionality. We incorporated either the enhanced green fluorescent protein (EGFP) or the firefly luciferase (Luc) into the E3 region of Cox-2 CRAds under the control of the adenovirus (Ad) major late promoter, which turns on only when the virus is replicating, to enable reporter gene expression in replication-restricted manner. The vectors were tested in pancreatic cancer cell lines, Cox2 status control cell lines (positive: A549, weak: A431, negative: BT474), and human Ad non-permissive mouse hepatoma cell line (BNL ING A.2). In vitro, reporter signal from replicative imaging vectors was more than 100 -fold higher than CMV promoter based nonreplicative positive control vector. On the contrary, no signal was seen in BNL cell. When Cox-2 control cells were infected with the Cox-2 CRAds with Luc, the signals showed clear correlation with Cox-2 status of the cells. When an Ad fiber modification (Ad5/3) was applied for the vectors, the signals from Ad redistant Hs766T cell was dramatically enhanced. When Cox-2 CRAd with Luc reporter was injected into subcutaneous xenograft of Cox-2 control cell lines, the luminescence reflected the Cox-2 status of the cell lines. In contrast, the signals with vectors without control mechanism did not show any correlation to Cox2 status. When the fiber modified Cox-2 CRAd with Luc reporter was injected into Hs766T pancreatic cancer xenograft, the tumor exhibited strong signal comparable to the vector without control elements showing non-controlled full replication. These data indicated that CRAds strategy can provide potent and selective imaging of a cancer molecular signature. Since this structure has versatility for combination with a number of control mechanisms, it has a great potential for imaging of various cancer molecular signatures.

\section{S1288}

Comparison of Dynamic High Field Magnetic MR-Colonography (3-Tesla) for Detection and Characterization of Colorectal Lesions Compared with Conventional Colonoscopy (CC): A Feasibility Study

Peter Netzer, Juergen M. Gschossmann, Christoforos Stoupis, Wojtek Bielecki, Hanno Hoppe, Bettina Saar

Background \& Aim: CT colonography (CTC) has shown high accuracy in detection of colorectal lesions $>10 \mathrm{~mm}$ and was better tolerated than CC (1). However, pts are exposed to considerable doses of ionizing radiation. MR-colonography (MRC) may not only be an alternative screening method for detection of colorectal lesions but may also be able to characterize these lesions. The aim of this study was to evaluate the feasibility of dark lumen MRC for detection and characterization of colorectal lesions using the newest MR technology ( 3 Tesla) and parallel imaging technique (iPAT). Patients and Methods: Fifty pts ( $22 \mathrm{~m}$, age 24-86 yrs) underwent dark-lumen MRC prior to CC using a 3 Tesla unit (Trio, Siemens, Germany). After colon distension with tap water, a high resolution dynamic Tlw 3D-GRE sequence using PAT (iPAT: GRAPPA x2, TR/TE $2.53 / 1.03 \mathrm{~ms}$, flipl4, voxel $1.6 \times 1.0 \times 1.8$ mm3, matrix $250 \times 384$, FOV $400 \mathrm{~mm} 2$, TAl8-20 s) was performed. Measurements were repeated before and 35, 75 and $110 \mathrm{~s}$ after bolus injection of Gadoteridol $0.1 \mathrm{mmol} / \mathrm{kg}$ (Prohance, Bracco, Italy). Images were reviewed by a radiologist blinded to the patient's history. If findings were discrepant, a second look CC was performed after unblinding. In a later workup, the dynamic behavior of the colorectal lesions was calculated by the signalto-noise ratio (SNR) over the time. These findings were compared with the histological workup. Results: MRC and CC were successfully performed in all pts wt any complications. CC showed 46 polyps in 24 pts (size: $3-45 \mathrm{~mm}$ ) Additionally, 4 carcinoma were found. All malignant lesions were correctly identified by MR-colonography. MRC missed 10 polyps, all smaller than $5 \mathrm{~mm}$ in size with the exception of one flat adenoma at the ileocecal valve which was thinner than $5 \mathrm{~mm}$ but $20 \mathrm{~mm}$ in size. The patient-based sensitivity for MRC was $92 \%$, specificity was $96 \%$. There were no false positive results. Residual stool could be differentiated from colorectal lesions. All malignancies showed an early and intensive increase of SNR and a mild wash-out phenomena. Hyperplastic polyps showed continuous increase of signal intensity of the colonic wall. Several extracolonic findings were detected by MRexamination. Conclusion: MRI with a stronger magnetic filed (3-Tesla) is suited for MRC and provides comparable results to $\mathrm{CC}$ for detection of colorectal lesions $>5 \mathrm{~mm}$ with the exception of flat adenomas $<5 \mathrm{~mm}$ of thickness. Analysis of the dynamic behavior of the colorectal lesions may be helpful to distinguish between neoplastic and non-neoplastic lesions.

\section{S1289}

Magnetic Resonance Imaging Predicts Circumferential Resection Margin Status in Patients Receiving Pre-Operative Chemoradiotherapy for Rectal Cancer Laura Hancock, Colm P. Forde, Jay B. Bradbury, Fergus Gleeson, Neil J. Mortensen

Introduction: Accurate tumour re-assessment after down-sizing pre-operative chemoradiotherapy (CRT) for locally advanced middle and lower rectal cancer provides valuable prognostic information prior to surgical resection. Aims: 1. To assess the accuracy of post CRT magnetic resonance imaging (MRI) in predicting circumferential resection margin status 2. To assess the accuracy of MRI in staging chemoradiation down-sized rectal tumours, Methods: Patients from a single tertiary referral centre who received pre-operative CRT for biopsy-proven rectal cancer between January 2003 and February 2005 were identified from a database. All patients received long-course CRT (50Gy over 5 weeks and 5-fluorouracil/ leucovorin) prior to surgery. A comprehensive MRI reporting proforma was completed for each patient and results were compared directly with histological specimens. Results: 50 patients were eligible for the study. The overall accuracy for predicting circumferential resection margin status was $76 \%$ (38/50, $P=0.006$, sensitivity $=96.9 \%$, specificity $=73.8 \%$, negative predictive value $=96.9 \%)$. For T staging MRI was accurate in $52 \%(26 / 50)$ and for nodal staging MRI was correct in 60\% (30/50). Venous invasion was predicted in 66\% (33/ $50, P=0.04)$. A radiological rectal cancer regression grade was derived and this correlated with histological rectal cancer regression grade in 65\% (32/49). Conclusions:This study demonstrates that post CRT MRI is accurate in predicting the circumferential resection margin status which provides useful prognostic information prior to surgery.

S1290

3d Ultrasonography Is a Valid and Accurate Measure of Liquid Gastric Emptying in Healthy Subjects

Diana Gentilcore, Trygve Hausken, Michael Horowitz, Karen L. Jones

Scintigraphy represents the current 'gold standard' for measurement of gastric emptying (GE), but is relatively expensive and associated with a radiation burden. Recent studies suggest that three-dimensional (3D) ultrasonography may allow a precise measure of GE, given its capacity for accurate volume calculations of the stomach - 3D ultrasonography has been shown to correlate well with gastric volume measurements in an in-vitro porcine model (1) and gastric barostat volumes in humans (2). The objective of this study was to compare and validate measurements of GE of low- and high- nutrient drinks with 3D ultrasonography. Ten healthy subjects (6M, 4F; age 18-35 yr) had concurrent measurements of GE by scintigraphy and 3D ultrasonography after ingestion of $500 \mathrm{ml}$ (low nutrient) beef soup (12 $\mathrm{kcal}$ ) and $300 \mathrm{ml}$ (high nutrient) dextrose (314 kcal) labeled with $20 \mathrm{MBq} 99 \mathrm{mTc}$-sulfur colloid, on two separate days. Results are shown (mean \pm SEM). There was no significant difference between scintigraphic and ultrasonographic measurements of GE (Figure); $50 \%$ emptying times (T50) (soup: scintigraphy $-27.7 \pm 4.8$ min vs ultrasonography $-23.8 \pm 4.8$ min; dextrose: scintigraphy $-122.2 \pm 13.3 \mathrm{~min}$ vs ultrasonography $-131.9 \pm 10.2 \mathrm{~min}$ ) There was a close correlation between the scintigraphic and ultrasonographic T50's for both soup $(r=0.92, P=0.0005)$ and dextrose $(r=0.88, P=0.0007)$. For the $T 50$, the limits of agreement were $-15.2 \mathrm{~min}$ and $+8.1 \mathrm{~min}$ for soup (mean difference $-3.6 \mathrm{~min}$ ) and $35.3 \mathrm{~min}$ and $+47.6 \mathrm{~min}$ for dextrose (mean difference $+6.2 \mathrm{~min}$ ). We conclude that 3D ultrasonography has good agreement, and correlates closely, with scintigraphy in the measurement of GE of low- and high- nutrient liquids. 1. Gilja et al. (1997). Gastroenterology 113, 38-49. 2. Tefera et al. (2002). Gut 50, 153-158.
Soup

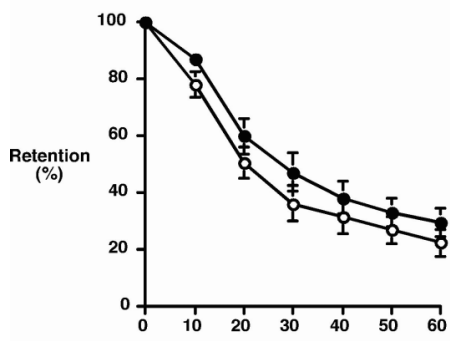

\section{Dextrose}

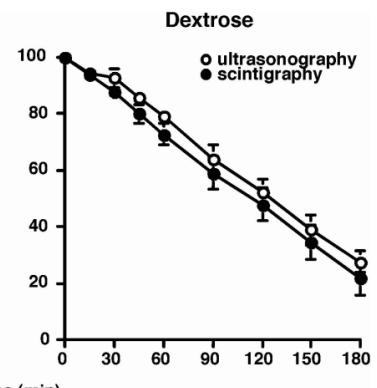

S1291

Quantitative and Non-Invasive Assessment of Postprandial Intragastric Dilution As Assessed By Magnetic Resonance Imaging (MRI) Reto Treier, Andreas Steingoetter, Oliver Goetze, Peter Boesiger, Michael Fried, Werner Schwizer

Introduction: Quantitative assessment of postprandial intragastric dilution and mixing is of major importance for a detailed understanding of transport and emptying mechanisms in the gastrointestinal tract. In gastric MRI, non-invasive determination of mixing and dilution processes of contrast marker labelled test meals can be achieved by detecting Tl relaxation times, using the mathematical interrelation between $\mathrm{Tl}$ and marker concentration. In this pilot study a fast $\mathrm{Tl}$ mapping technique for quantitative $\mathrm{Tl}$ determination in the human stomach was developed and evaluated in healthy subjects. Methods: Two fasting, healthy 
volunteers were imaged in prone body position using a $1.5 \mathrm{~T}$ whole-body MRI scanner (Intera, Philips Medical Systems, Best, The Netherlands). After drinking the labeled test meal

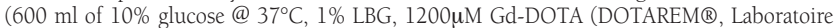
Guerbet, France)) and attaching a test meal sample (1000uM Gd-DOTA) as reference to the back of the subjects, Tl mapping (6 transversal image slices) were performed every 5 min over $40 \mathrm{~min}$. Mean \pm SD Tl values of the sample and gastric contents were calculated within ROIs of $7 \times 7$ pixels (see figure). In addition, $\mathrm{Tl}$ of the sample was determined using as standard inversion recovery (IR) spectroscopy sequence. Results: The figure shows a transverse MRI image (left) and the corresponding calculated Tl map (right) 40 min after drinking, both with outlined stomach wall and defined ROIs. Calculated Tl values of the sample are in good agreement with IR T1 values (Tlmaps@10min: 149 \pm ms, @ 40min: $152 \pm 5$ and IR: $157 \pm 4 \mathrm{~ms})$. Test meal was diluted over $40 \mathrm{~min}$ as observed by a $25 \%$ increase in $\mathrm{Tl}(\mathrm{t}=10 \mathrm{~min}: 197 \pm 26 \mathrm{~ms} ; \mathrm{t}=40 \mathrm{~min}: 247 \pm 28 \mathrm{~ms}$ ) corresponding to a local dilution of $20 \%$. Discussion: This new and fast Tl mapping technique allows the three-dimensional determination of intragastric dilution within $20 \mathrm{~s}$ (1 breathhold). This is the first method allowing non-invasive assessment and quantification of gastric secretion in humans.

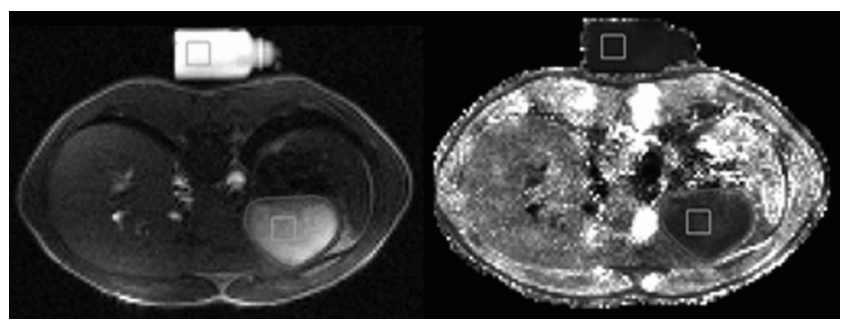

S1292

Gadobenate Dimeglumine Enhanced MRI Evaluation for Acute Bile Duct Injury: A Pilot Study

Paari Vijayaragavan, Piyal Nag, Ajay Sharma, Sadiq S. Sikora, Vinay K. Kapoor, Sunil K. Jain, Rakesh K. Gupta

Introduction Acute bile duct injuries (BDI) usually require a gamut of investigations such as ultrasonogram,CT scan, MRCP, angiography, etc for a complete evaluation. Gadobenate dimeglumine (Multihance $\left.{ }^{\circledR}\right)$ contrast enhanced (CE) MRI was evaluated as a single investigation in the workup of acute BDI. Patients and methods Eleven patients with post cholecystectomy BDI underwent T2-MRCP and serial Tl weighted FSPGR sequences before and till 2 hours after intravenous administration of Gadobenate dimeglumine. MR angiography of the hepatic artery and portal vein was obtained and the FSPGR images were reconstructed to get contrast enhanced (CE) T1 MRCP. Biliary and vascular anatomy and bile leak in T1 and T2 MRCP was noted and correlated with other investigations. Results Conventional MRCP revealed BDI of Bismuth type III in 4, type II in 3 and 2 each of type IV and V. In all patients type of injury on CE MRCP correlated with conventional MRCP although good quality CE MRC revealing all the segmental ducts could be obtained in only 5 patients. Biliary leak was detected on TI FSPGR images in 10 out of the 11 patients; this was demonstrated as contrast leak into biloma $(n=7)$, extravasation of contrast in drainage tube $(n=2)$ and extravasation from duodenum $(\mathrm{n}=1)$. Post contrast T2 MRCP images showed evidence of bile leak in 6 patients seen as serial reduction in the intensity of intra abdominal fluid collections. The reconstructed TI FSPGR images detected the origin of bile leak as to be from hilum in 5 , duodenum in 1 and indeterminate in 4 patients. MR angiogram revealed normal portal vein in all and a right hepatic artery injury in one patient. The study demonstrated complete loss of biliary continuity in 9 which was corroborated by fistulogram in 4, ERC in 2 and operative finding in 3 patients. Of the 6 patients with suggestion of internal fistula on CE $\mathrm{MRCP}$, it was confirmed in 4 out of 5 patients who were operated upon. On retrospective review, CE MRCP provided complete information in $9(81 \%)$ patients without the need for any further work up. Percutaneous drainage for ongoing bile leak $(n=3)$ and avoidance of ERC because of loss of biliary continuity $(n=5)$ were the therapeutic decisions influenced by the study. Conclusion Gadobenate dimeglumine enhanced MRI evaluation is a promising and effective single investigation in the workup of acute BDI.

\section{S1293}

Stereoscopic High-Resolution MRCP As 3D Anatomical Information of the Cystic Duct Prior to Cholecystectomy

Tetsuya Yamagishi, Karl H. Hoehne, Takao Itoi, Takashi Kawai, Kimihiko Abe

Purpose: In conventional MRCP(c-MRCP), difficulties may occur to obtain firm anatomical information of the cystic duct. This presentation shows that the combination of highresolution(HR) data and stereoscopic visualization leads to a decisive improvement for obtaining the three-dimensional(3D) extent of the cystic duct. Materials and Methods: Thirtyeight patients(16 males and 22 females: $38-76$ year-old) with cholecystolithiasis underwent HR and c-MRCP on the same day prior to cholecystectomy(5-24 days before operation). (1). Stereoscopic HR-MRCP: The source images (total 88-96 slices) were collected with thin section $(0.8 \mathrm{~mm})$ by a respiratory-gating with PACE(Prospective Acquisition CorrEction), which is one of the 3D data acquisitions using navigator triggering. Stereoscopic maximum intensity projection(MIP) images were created by a commercially available 3D rendering software(VOXEL-MAN). The resulted images were displayed as a movie with omni-direction and viewed with Red/ Green glasses for stereoscopic visualization. (2). c-MRCP: 2D RARE single-shot were performed with thick slab $(5-7 \mathrm{~cm})$ around the vertical axis/ every 10 -degree. (3). Estimating the observer performance: Ten board certified radiologists and endoscopists specializing the pancreaticobiliary region independently compared HR-MRCP with c-MRCP. (i). The accuracy for the detection of anatomic variants of the cystic duct insertion into CBD [a. Proximal, b. Right lateral, c. Anterior spiral, d. Posterior spiral, e. Low medial and f. Low lateral] was evaluated. Any inter-observer discrepancy was fully discussed and standardized. In all cases, the final anatomical information of the cystic duct was obtained by endoscopic retrograde cholangiogpaphy or cholangiography during operation. (ii). The confidence value for making diagnosis defined as a four-point questionnaire: definite, probable, suspected and poor. Results: The cystic duct insertion pattern was $a=14, b=10, c=1, d=4$, $\mathrm{e}=2$ and $\mathrm{f}=7$ cases by cholangiography. The diagnostic accuracy was 92 -percent by stereoscopic HR-MRCP versus 56-percent by c-MRCP. All observers evaluated the morphologica delineation of the cystic duct was decisively improved in HR-MRCP and diagnostic confidence was obviously higher than c-MRCP. Stereoscopic visualization was also helpful to get ric of the signal overlay by surrounding organs(stomach, intestine) and/ or cystic lesions of the liver, pancreas and kidneys, which leads reduced burden for film-interpret. Conclusion Stereoscopic HR-MRCP could be considered as a useful alternative modality to cholangiography in almost all cases, prior to cholecystectomy, because of its high accuracy and diagnostic confidence.

\section{S1294}

Impact of Operators Experience On Value of High-Resolution Transabdominal Ultrasound (US) in the Diagnosis of Choledocholithiasis: A Prospective Comparison Using Endoscopic Retrograde Cholangiography (ERC) As Gold Standard

Steffen Rickes, Gerhard Treiber, Klaus Moenkemueller, Ulrich Peitz, Antal Csepregi, Stefan Kahl, Anika Vopel, Kathleen Wolle, Ebert P.A. Matthias, Sabine Klauck, Peter Malfertheiner

Aim of the study: The aim of this study was to evaluate the accuracy of US in the diagnosis of common bile duct stones depending on the operators experience. Patients and methods: From April 2003 through November 2004, 126 patients (pts.) referred because of suspicion of the presence of common bile duct stones were included in the study. Two pts. were excluded because they refused ERC. Consequently, the study comprised 124 pts. US (2-5 $\mathrm{MHz}$ sector scanner, Siemens Elegra, Erlangen, Germany, and HDI 5000, Philips Ultrasound, Bothel, WA, USA) was performed by operators unaware of the results of other imaging procedures. The definitive diagnosis was established by means of ERC. Chi-square test was used to detect significant differences. Results: Thirty-five of 124 pts. were investigated by experienced examiners. Twenty-seven of 35 pts. (77\%) were found to have stones at ERC. Bile duct stones were found correctly with US in 22 of 27 pts. (sensitivity $82 \%$ ). Of the 8 pts. without stones at ERC one false-positive diagnosis was made with US (specificity 88\%). In conclusion, correct diagnoses were observed in 29 of 35 (accuracy 83\%) pts. investigated by experienced examiners. Eighty-nine of 124 pts. were investigated by less experienced examiners. Fifty-four of 89 pts. (61\%) were found to have stones at ERC. Choledocholithiasis was found correctly in only 25 of 54 pts. (sensitivity 46\%). Of the 35 pts. without stones at ERC 3 false-positive diagnosis were made with US (specificity 91\%). In conclusion, correct diagnoses were observed in 57 of 89 pts. (accuracy 64\%) investigated by less experienced examiners ( $<<0.05$ in comparison to the results of experienced examiners). Conclusions US done by experienced examiners has a high accuracy in the diagnosis of choledocholithiasis. Therefore, a good training and continued experience are prerequisites for a successful detection of bile duct stones with US. Under these conditions further expensive and invasive methods may not be necessary.

\section{S1295}

Comparison of Secretin-Stimulated Dynamic Magnetic Resonance Cholangiography and Operative Cholangiography in the Diagnosis of Pancreaticobiliary Reflux

Hosoki Takuya, Tokuda Yukiko, Sakai Mio, Sai Hidemi, Kashiwasaki Masaki, Nakamor Masaji, Michida Tomoki

Simultaneous opacification of the pancreatic duct at cholangiography was considered abnormal, suggesting pancreaticobiliary maljunction (PBMJ). However, this radiographic finding has not shown to be a direct diagnostic test for pancreaticobiliary reflux (PB reflux). Secretinstimulated dynamic magnetic resonance cholangiopancreatography (SS-dynamic MRCP) has been reported to be useful for diagnosing PB reflux. The purpose of this study is to evaluate SS-dynamic MRCP findings in patients with suspected PBMJ at operative cholangiography METHODS: Fifteen controls and thirty-three cholecystectomy patients were prospectively examined by SS-dynamic MRCP. The patients were divided into two groups; fourteen patients without evidence of PBMJ (Group 1) and nineteen patients with suspected PBMJ (Group 2) at operative cholangiography. PBMJ was suspected when simultaneous opacification of the pancreatic duct ( $>2 \mathrm{~cm}$ in length) occurred during continuous contrast injection at the cholangiography. The patients with anomalously long PB junction were excluded from this study. SS-dynamic MRCP was performed using a breath-hold, single-shot turbo spin-echo T2-weighted sequence. The optimal MRCP section was repeated 35 times at 10-sec interval after secretin injection; the acquisition time was $4 \mathrm{~s}$ per image. The signal intensity (SI) of the extrahepatic duct was compared between the controls and patients for the degree and direction of SI change, i.e. whether increase in SI change occurred in an upstream to downstream direction, or vice versa (retrograde direction). The retrograde SI change of the extrahepatic duct after secretin injection was judged as the presence of PB reflux. Mean SI was measured in each proximal, middle, and distal third of the extrahepatic duct, and also on the corresponding images of serial imaging sets. Peak time (PT) was defined as an interval between the time of the secretin injection and that at which a first peak SI was obtained RESULTS: In all controls, no SI change was noted in any part of the extrahepatic duct over the observation period, providing no apparent PT. In 2 (14\%) of Group 1 and 14 (74 \%) of Group 2, the extrahepatic duct showed retrograde SI increase and sequential delay in occurrence of the PT from its distal third to its proximal third; the incidence was significantly greater in Group 2 than in Group 1 ( $\mathrm{p}<0.005)$. In Group 2, mean PT (s) +-SD was 113+ $57,145+-59$, and $190+-47$ at the distal, middle, and proximal third of the extahepatic duct, respectively. CONCLUSION: In patients with suspected PBMJ at operative cholangiography $\mathrm{PB}$ reflux was demonstrated to occur by SS-dynamic MRCP. 
S1296

MRI Studies of Blood Flow in the Superior Mesenteric Artery (SMA) Adam D. Jeays, Richard Gillott, Rodney Hose, Patricia Lawford, Samuel Jacob, Paul Spencer, Karna D. Bardhan

Introduction Chronic ischaemia of the small intestine is uncommon. It is hypothesised that this may be related to the nature of flow in the SMA and the dynamic response to calorific load. To investigate this, SMA blood flow was studied during fasting and following ingestion of a controlled calorific load. Method Flow in the SMA was assessed in 18 healthy volunteers (11 male, 7 female, mean age 36) by 1.5 Tesla magnetic resonance (MR) imaging. ECGgated, flow-sensitive (phase-contrast) sequences were acquired with high temporal resolution (21 frames per heartbeat) over the cardiac cycle. Images were obtained after overnight fasting and 30 minutes after ingestion of a $600 \mathrm{Kcal}$ Scandishake $\mathrm{B}^{\circledR}$ milk-shake. A period of 30 minutes post-prandial was chosen as it coincides with peak flow (as determined from Doppler flow studies $\left.{ }^{[1]}\right)$. Velocity fields in the SMA were monitored throughout the cardiac cycle and mean flow calculated throughout diastole. Results During systole, flow disturbances, indicative of high velocity flows, were seen in 2 subjects after fasting. After feeding, this increased to 15 subjects (including the original 2). For this reason, only flow results from the diastolic portion of the cardiac cycle were considered. At 30 minutes post-prandial, mean diastolic flow was $153 \mathrm{ml}$ per cycle $(\mathrm{n}=18, \mathrm{SD} 67.5$, range 41.6-262), compared to $40.4 \mathrm{ml}(\mathrm{n}=18, \mathrm{SD} 14.9$, range 8.1-78.4) in the fasted case. This equates to a 3.8 fold mean rise in flow after feeding $(n=18, S D 2.2$, range $0.8-9.8)$ - In the cohort scanned twice, mean diastolic flow was 2.2 times that of the fasted value $(n=5, S D 0.3$, range 1.8-2.5) on the first visit, and 3.1 times that of the fasted value ( $\mathrm{n}=5, \mathrm{SD} 0.9$, range 2.1-3.9) on the second visit. Discussion \& Conclusions 1. Diastolic flow in the SMA increases markedly in response to calorific load. 2. The velocity of blood in the SMA during systole is sufficient to cause instabilities in the flow: the resulting loss of MR signal prevents the quantitative interpretation of velocity data at these high flows. 3. For most subjects, flow during systole is undisturbed following fasting. 4 . The change in flow regime over these time-scales may have important effects on wall shear stress, endothelial cell morphology and risk of atherosclerosis. ${ }^{[1]}$ Small intestinal blood flow in man: The effects of age and caloric loading. PA Spencer et al. Gut 2000;46(S2):A67-W139 This work was funded by the Bardhan Research E Educational Trust, Registered Charity No. 328452

\section{S1297}

In Vivo Magnetic Resonance Imaging of Experimental Mouse Colitis and Association to Inflammatory Activity

Anna E. Larsson, Silvia Melgar, Rehnstrom Erika, Erik Michaelsson, Lennart Svensson, Hockings Paul, Lars E. Olsson

BACKGROUND AND AIMS: Various imaging techniques such as endoscopy and computed tomography are used to monitor ulcerative colitis and Crohn's disease. Magnetic resonance imaging (MRI) is emerging as an alternative because it is non-invasive and free from ionising radiation. Today, biomarkers of inflammation are needed that can be used from mice to man in e.g. testing of putative therapeutic drugs. Several clinical studies have shown that contrast enhanced Tl weighted (Tlw) and T2 weighted (T2w) MRI can identify pathological bowel segments in patients with inflammatory bowel disease. The aim of the present study was to investigate if MRI can provide biomarkers of inflammation in an experimental mouse colitis model. METHODS: Acute and chronic colonic inflammation was chemically induced by exposing mice to dextran sulphate sodium (DSS). A control group of healthy mice was also included. MRI was used to assess colon wall thickness and also the T2w signal, which mainly reflects presence of oedema. Additionally, MRI was used to measure gadodiamideenhanced Tlw signal in the colon wall, reflecting presence of inflammation. Inflammatory scoring, based on stiffness (0-2), oedema (0-3), visible ulcerations ( $0-1)$ and thickness $(0-$ $4)$, was performed ex vivo to assess inflammation. Haptoglobin and interleukin- $1 \beta$ served as systemic and local inflammatory markers, respectively. RESULTS: DSS exposed animals had significantly higher haptoglobin and interleukin- $1 \beta$ levels, and also inflammatory scores compared to healthy animals. The colon wall thickness was significantly increased in inflamed compared to healthy animals, and a positive correlation between colon wall thickness and inflammatory scores was observed. Moreover, the contrast enhanced Tlw signal was significantly increased in acutely inflamed mice compared to healthy mice. The T2w signal in the inflamed animals correlated positively with haptoglobin levels and colon wall thickness. CONCLUSION: Our results show that colon wall thickness, measured with MRI, as well as contrast-enhanced $\mathrm{Tl} \mathrm{w}$ and $\mathrm{T} 2 \mathrm{w}$ imaging of the colon wall are potential biomarkers in characterizing inflammation. Future studies will reveal if these putative biomarkers may be useful in evaluation of candidate drugs in longitudinal studies.

\section{S1298}

Diagnostic Accuracy of CT Colonography Evaluated By Surgically Resected Specimen As the Gold Standard: Is Optical Colonoscopy Adequate Gold Standard?

Shinichiro Okada, Yutaka Kawamura, Junichi Sasaki, Fumio Konishi

BACKGROUND and AIM Recently, CT colonography (CTC) has been introduced as a new and promising modality for the diagnosis of colorectal polyps. The accuracy of CTC is important to determine its indication, feasibility and clinical significance, however, the evaluation of its 'true' diagnostic ability is thought to be difficult because the optical colonoscopy (OCS), the gold standard for the presence of polyps, associates with overlooking even if done back-to-back. The aim of this study is to clarify the 'true' diagnostic ability of CTC using surgically resected specimens which we believe are the most reliable source of the presence of polyps. MATERIALS and METHODS CTC was performed for 150 consecutive patients who were scheduled to elective surgery for colorectal cancer, which was defined as the index lesion. All patients underwent preoperative OCS. Usually about $20 \mathrm{~cm}$ of the large intestine was resected with $10 \mathrm{~cm}$ distal and proximal margin. Of these 40 patients had simultaneous lesions in the resected specimen and were included in this study. Location (distance from the index lesion), configuration and diameter of the lesions were compared to the results of CTC and OCS. RESULTS There was no morbidity associated with CTC and OCS. In 6 patients, tumorous stricture interfered with OCS ability to examine the colon proximal to the index lesion. Fifty-six polyps were found in surgical specimens. Of these, 9 lesions could not be evaluated by OCS because of the stenosis and these lesions were excluded from the comparison between CTC and OCS. Sensitivity of CTC and OCS were $82.1 \%$ / 66.0\% $(\mathrm{P}=0.12)$, respectively, and the positive predictive value (PPV) were $88.5 \%$ / $100 \%(\mathrm{P}=0.13)$, respectively. For 9 lesions located in the colon proximal to the stricture, sensitivity and PPV of CTC were $89 \%$ and $80 \%$, respectively, which were not significantly different from those for the lesions in the distal colon. CONCLUSION The diagnostic accuracy of OCS using surgical specimens as the gold standard was unexpectedly low. Therefore, it is thought to be inadequate to use OCS as the gold standard for the evaluation of diagnostic accuracy of CTC. Although not statistically significant, diagnostic ability of CTC appeared to be better than that of OCS. Our study revealed that CTC is a reliable modality for the diagnosis of colorectal neoplasm.

\section{S1299}

MRCP and MRI Findings in Autoimmune Pancreatitis

Pong Yui Chen, Terumi Kamisawa, Go Kuwata, Yuyang Tu, Hitoshi Nakajima, Naoto Egawa, Kouji Tsuruta, Atsutake Okamoto, Noriko Kamata

Background and Aim: The characteristic radiological findings of autoimmune pancreatitis (AIP) include irregular narrowing of the main pancreatic duct (MPD) on ERCP and enlargement of the pancreas on CT and US. Recently, MRCP. We evaluated MRCP findings in conjunction with MR images in AIP patients. Patients and Methods: The subjects were 9 patients with AIP who had MRCP, ERCP, CT, and US. Seven patients had elevations of serum IgG4. On ERCP, the irregular narrowing of the MPD (diffuse $(n=1)$, segmental-head $(n=3)$, segmentally skipped-head and body $(n=3)$, segmental-tail $(n=2))$ and enlargement of the pancreas was seen in all patients. All patients were given steroid treatment and responded both morphologically and serologically. MRCP and MR images obtained before and after steroid therapy were examined and compared to 10 cases of pancreatic head cancer. Results: On MRCP, the narrowed portion of the MPD noted on ERCP was not visualized, while the noninvolved segments of the MPD were visualized. Two segmentally skipped MPD lesions in the head and body of the pancreas were not visualized in 3 patients. Although upstream dilatation of the proximal MPD was detected in 6 patients, the degree of dilatation was milder than in cases of pancreatic carcinoma. Stenosis or obstruction of the lower bile duct was detected in 8 patients; 3 of these patients also showed stenosis of the intrahepatic bile duct. MR images showed enlargement of the pancreas with decreased signal intensity on T1-weighted MR images compared with that in the liver, increased signal intensity on T2weighted MR images, and, in 3 patients, a hypointense capsule-like rim. After steroid therapy, the previously not visualized portion of the MPD was seen, along with improvements of the upstream dilatation of the proximal MPD and of the bile duct stenosis. Pancreatic enlargement decreased, and the abnormal signal intensity on both T1- and T2-weighted MR images became isointense. Conclusion: The irregular narrowing of the MPD on ERCP was not visualized on MRCP, while the other region of the MPD was visualized. MRCP cannot differentiate the irregular narrowing of the MPD seen with AIP from the stenosis of the MPD seen with pancreatic carcinoma; therefore, ERCP is necessary to make the diagnosis of AIP. However, the MRCP findings of skipped, non-visualized MPD lesions, accompanied by a lesser degree of upstream dilatation of the MPD, might suggest the presence of AIP. These MRCP findings in conjunction with MR imaging of pancreatic enlargement that shows abnormal signal intensity on T1- and T2-weighted MR images are useful in supporting a diagnosis of AIP.

\section{S1300}

Alterations in I-Kappa B Kinase (I-kK) Pathways Predict the Instability of Mucosal Claudin Isotypes (1-5) in Patients with Inflammatory Bowel Disease: A Novel Gut Barrier Dependent Mechanism for the Pathophysiology of Human IBD

Ali Banan, Maliha Shaikh, Lijuan Zhang, Jeremy Z. Fields, Ece Mutlu, Ashkan Farhadi Christopher B. Forsyth, Ali Keshavarzian

Mucosal NF-kB hyperactivation and tight-junctional instability ( $\&$ intestinal barrier disruption) appear to be early hallmarks of IBD. However, the mechanisms underlying gut barrier instability in vivo and their contribution to tissue injury remain unclear. In vitro studies suggest that the mechanism may depend on oxidative stress, NF-kB signaling \& instability of barrier proteins. However, these crucial events are only poorly characterized in human IBD. Aims: We determined whether in the inflamed IBD mucosa (1) there is oxidation and degradation of various Claudin isotypes (22-25 kDa tight-junctional Claudin isoforms, -1, $2,-3,-4,-5$ ); (2) dysregulated I-kK / NF-kB signaling (I-kK isotypes $\alpha / \beta, \mathrm{IkB}, \mathrm{NF}-\mathrm{kB}$ subunits, iNOS) \& tissue oxidative stress. (3) These variables are comparable (correlate) with markers of tissue inflammation \& injury. Methods: Mucosal biopsies from IBD patients (ulcerative colitis, Crohn's Disease, specific colitis) and healthy/normal controls were used to measure: oxidation \& instability of Claudin isotypes (1-5); levels / phosphorylation / activation of the I-kK pathway; markers of mucosal oxidation / injury (protein carbonylation / nitration, NO levels). Results: Barrier tight-junctional Claudin-1, Claudin-2, Claudin-3, Claudin-4, and Claudin-5 were largely carbonylated, nitrated and destabilized in inflamed IBD mucosa, but not so in normal-appearing mucosa from the same patients. Inflamed mucosa also showed marked increases in phosphorylation of I-kK- $\alpha / \beta$ isotypes and of IkB $\alpha$; degradation of IkB $\alpha$; hyperactivation of I-kK- $\beta$ and of NF-kB (p50/p65 subunits). Other mucosal oxidative injury markers such as iNOS, NO metabolites, and protein nitration were also increased. These changes were not seen in normal-appearing, non-inflamed, IBD mucosa. Furthermore, degraded barrier claudin proteins and hyperactivated I-kK-NF-kB were mainly found within the epithelial layer, and strongly correlated with IBD disease severity. Conclusions: Our findings suggest that underlying human IBD pathophysiology are pivotal changes in barrier function and in I-kK signaling. This novel mechanism is one in which hyperactivation of an I-kK-NF-kB driven pathway is required for mucosal barrier protein oxidation and instability. These crucial barrier altering events could lead to initiation and/ 
or perpetuation of human IBD flare-up. \{Supported by NIDDK60511 \& NCCAM01581; NIAAA13745\}.

\section{S1301}

Urinary Neopterin Excretion Is a Good Non Invasive Biomarker for Monitoring Crohn's Disease Activity

Stephane Nancey, Armand Perret-Liaudet, Driffa Moussata, Helene Navarro, Ivan Graber, Bernard Renaud, Dominique Kaiserlian, Bernard Flourie

Background : Crohn's disease (CD) is a chronic, relapsing T cell-mediated inflammatory bowel disease. Neopterin is a biochemical marker released from human macrophages upon stimulation with IFNg produced by activated $\mathrm{T}$ cells. Urinary neopterin excretion has been shown to be increased in autoimmune diseases like systemic lupus erythematosus, rheumatoid arthritis and psoriasis where the highest levels of neopterine were detected during active diseases. Our objective was to test the usefulness of urinary neopterin in Crohn's disease and its relation with disease activity. Patients and Methods : $90 \mathrm{CD}$ patients (49F/41M) and 100 healthy volunteers $(54 \mathrm{~F} / 46 \mathrm{M})$ provided a single urinary sample $(20 \mathrm{~mL})$. CD patients were under treatment with corticosteroids (19\%), 5ASA (10 \%), immunosuppressors (40 $\%$ ) and/or infliximab (55\%). CD activity was assessed by the Crohn's Disease Activity Index (CDAI). C reactive protein level was performed by routine dosage. Urinary neopterin was measured by HPLC and results were expressed in $\mathrm{mmol} / \mathrm{L}$ corrected for urinary creatinin. Results : Urinary neopterin excretion (mean +/- SD) was significantly higher in the 46 patients with active $C D(C D A I>$ or $=150)$ than in the 44 patients with quiescent $C D$ $(\mathrm{CDAl}<150)$ and in the 100 healthy volunteers (respectively $395+/-221,145+/-48$ and $200+/-25 \mathrm{mmol} / \mathrm{L} ; \mathrm{p}<0.01$ ). Urinary neopterin excretion was well correlated with CDAI (Spearman test, $\mathrm{r}=0.78 ; \mathrm{p}<0.001$ ) and the correlation was better than that found between $\mathrm{CRP}$ and CDAI (Spearman test, $\mathrm{r}=0.49 ; \mathrm{p}<0.001$ ). Conclusion : Urinary neopterin excretion is a good indicator of $\mathrm{CD}$ activity and might help clinicians to monitor $\mathrm{CD}$ patients. Ongoing study are performed to assess its value in predicting relapse of $C D$.

\section{S1302}

Prognostic Significance of Endoscopic Remission in Patients with Active Ulcerative Colitis Treated with Oral and Topical mesalazine: Preliminary Results of a Prospective, Multicenter Study

Gianmichele Meucci, Renato Fasoli, Simone Saibeni, Daniela Valpiani, Renzo Gullotta, Enrico Colombo, Renata D'Inca', Maddalena Terpin, Giovanni Lombardi

Background: it is generally recommended that the treatment of active ulcerative colitis should be continued until complete healing of endoscopic lesions. However, the evidence supporting this recommendation is scanty. Aims: 1) to assess the rate of patients with active ulcerative colitis that achieve clinical but not endoscopic remission after treatment with oral plus topical mesalazine 2) to compare the rate of relapse in patients with clinical/endoscopic remission and those with only clinical remission Methods: patients with active mild or moderate ulcerative colitis extending beyond the rectosigmoid junction were eligible for the study. Patients requiring systemic steroids, those with previous or ongoing immunosuppressive treatment and those with proctitis were excluded. All patients underwent baseline colonoscopy and were then treated with mesalazine, $4 \mathrm{gr} /$ day orally and $2 \mathrm{gr} /$ day per rectum for 6 weeks. Those achieving clinical remission underwent a second colonoscopy: afterwards, all received maintenance treatment with oral mesalazine, $2 \mathrm{gr} /$ day orally. A one year clinical follow-up was then planned. Clinical remission was defined as normal frequency of bowel movements with formed stools, no abdominal pain and no blood in the stools. Endoscopic remission was defined as normal appearing mucosa or only mild redness and/or friability, without either ulcers or erosions. A formal assessment of concordance among endoscopists in evaluating endoscopic activity was carried out before starting the study. Results: so far, 78 patients ( 47 males, mean age 48 years, range 19-77) have been enrolled at 9 participating institutions. After six weeks of oral and topical treatment with mesalazine, 59 of them (75\%) were clinically in remission. Endoscopic activity was still present in only five of them (8.5\%). After a mean follow-up of 8,7 months, 15 patients $(26 \%)$ relapsed. The cumulative rate of relapse at one year was 23\% in patients with clinical and endoscopic remission and $80 \%$ in patients with only clinical remission ( $\mathrm{p}<0.0001$ ). Conclusions: persistence of endoscopic activity is a rare event in patients with active ulcerative colitis achieving clinical remission after a 6-week treatment with oral plus topical mesalazine, but is a very strong predictor of early relapse

\section{S1303}

Serological Markers Are Associated with Severity of Disease and Need of Surgery in IBD Patients

Shuwen Xue, Joanne M. Stempak, Abdul A. Elkadri, Gordon R. Greenberg, Tom D. Walters, Anne M. Griffiths, Hillary Steinhart, Mark S. Silverberg

IBD is a complex disorder with a spectrum of clinical manifestations. Recent data demonstrate that serological markers are associated with disease behaviour, severity and complications. AIM: To further elucidate the association of IBD behavior with serological markers. METHODS: Subjects were recruited primarily from the MSH and HSC IBD Centres in Toronto. Sera of unrelated patients with CD $(n=359), U C(n=185)$ and healthy subjects ( $\mathrm{HC}, \mathrm{n}=62$ ) were tested for the presence of the following antibodies: pANCA, ASCA, OmpC and Flagellin (Prometheus Laboratories, San Diego, CA). Review of medical charts was performed to document clinical information including diagnosis, age of diagnosis, disease location, behaviour (for $\mathrm{CD}$, fistulizing, fibrostenotic or inflammatory) and need of surgery. RESULTS: The seroprevalence of pANCA, ASCA, anti-OmpC and flagellin was $15 \%, 62 \%$, $38 \%$ and $67 \%$ in $\mathrm{CD} ; 62 \%, 12 \%, 28 \%$ and $31 \%$ in UC; and $5 \%, 7 \%, 2 \%$ and $18 \%$ in $\mathrm{HC}$ ( $\mathrm{p}<0.0001$ for all markers). ASCA, anti-OmpC and flagellin have high specificity ( $89 \%$, $79 \%$, and $72 \%$ ) for CD individually and specificity rises to $98 \%$ for those positive for all three markers. pANCA has high specificity $(86 \%)$ for UC. ASCA, anti-OmpC and flagellin are associated with early age at diagnosis of $\mathrm{CD}$ ( 20 vs $25, \mathrm{p}=0.0001 ; 20$ vs $23, \mathrm{p}=0.03 ; 21$ vs $24, \mathrm{p}=0.02$ respectively); need of surgery ( $61 \%$ vs $45 \%, \mathrm{p}=0.003 ; 74 \%$ vs $43 \% \mathrm{p}<0.0001$, $61 \%$ vs $49 \% \mathrm{p}=0.04$ respectively). ASCA and anti-OmpC are also associated with highe number of surgeries ( 1.5 vs $1.1, \mathrm{p}=0.004 ; 2.1$ vs $0.9, \mathrm{p}<0.0001$ ). ASCA, anti-OmpC and flagellin are associated with more aggressive disease behaviour (fibrostenotic/fistulizing) in CD (see Table). Anti-OmpC is associated with higher rate of colectomy in UC (58\% vs $29 \%$ $\mathrm{p}=0.0003$ ). CONCLUSIONS: The presence of ASCA, anti-OmpC and flagellin seropositivit is associated with more aggressive disease behaviour and the requirement for surgical intervention. These markers are useful to identify those who should be considered for earlier and more aggressive therapy.

\begin{tabular}{|c|c|c|c|c|c|c|}
\hline & \multicolumn{2}{|c|}{ ASCA } & \multicolumn{2}{|c|}{ OmpC } & \multicolumn{2}{|c|}{ Flagellin } \\
\hline & positive & negative & positive & negative & positive & negative \\
\hline fibrostenotic & $28 \%$ & $21 \%$ & $32 \%$ & $21 \%$ & $30 \%$ & $16 \%$ \\
\hline fistulizing & $32 \%$ & $15 \%$ & $32 \%$ & $21 \%$ & $27 \%$ & $23 \%$ \\
\hline inflammatory & $40 \%$ & $64 \%$ & $36 \%$ & $58 \%$ & $43 \%$ & $61 \%$ \\
\hline$p$ value & \multicolumn{2}{|c|}{$<0.0001$} & \multicolumn{2}{|c|}{$=0.0002$} & \multicolumn{2}{|c|}{$=0.003$} \\
\hline
\end{tabular}

S1304

A Unique Inflammatory Mechanism in Man: Dysregulation of An EGF-R and PKC Isoform [PKC- $\zeta$, PKC- $\beta 1]$ Dependent Pathway Appears to Underlie Mucosal Oxidative Stress, NF-kB Activation and Gut Injury in Inflammatory Bowel Disease (IBD)

Ali Banan, Maliha Shaikh, Lijuan Zhang, Ece Mutlu, Jeremy Z. Fields, Ashkan Farhadi, Christopher B. Forsyth, Ali Keshavarzian

NF-kB activation $\&$ oxidative stress are key features of human IBD. Our in vitro studies suggest that dysregulation of a pathway dependent on EGF-Receptors \& PKC isoforms PKC- $\beta 1$ \& $\%$ or PKC- $\zeta$ (Zeta) - leads to increased oxidative stress \& inflammation. Nonetheless, molecular mechanisms involving these factors are not well-characterized for human IBD Aims: We sought to determine whether: $[A]$ deactivation of EGFR \& tyrosine kinase activity and deactivation of PKC- $\zeta$ and/or PKC- $\beta 1$ occur in inflamed IBD mucosa; [B] EGF-R and PKC isoform activity correlate with markers of gut inflammation \& injury. Methods: We used pinch biopsies from IBD patients (UC, CD, specific colitis) \& normal controls, and measured: EGF-R \& tyrosine kinase activity (tissue distribution/expression); PKC-ᄃ/ PKC $\beta 1$ (levels/activity/distribution); markers of gut inflammation \& oxidative injury (NF-kB IkB $\alpha$, iNOS, NO, protein/cytoskeletal oxidation/instability). Results: Compared to normal mucosa, inflamed IBD mucosa showed marked decreases in EGF-R distribution/expression and tyrosine kinase activity, indicating EGF-R deactivation. PKC- $\beta 1$ (78 kDa) and PKC- $\zeta$ $(72 \mathrm{kDa}$ ) were down-regulated $\&$ deactivated (both were reduced in membrane $\&$ cytoskeletal mucosal fractions). In parallel, we found large increases in NF-kB activation (p50/p65 subunits), IkB $\alpha$ phosphorylation, iNOS upregulation, oxidant (NO, O2) generation, and protein carbonylation \& nitration. In normal appearing (non-inflamed) IBD mucosa, smaller changes in oxidative markers \& EGF-R / PKC isoforms were seen. Tissue structural proteins were largely oxidized in inflamed mucosa, less so in normal mucosa. Mucosal EGF-R, PKC $\zeta$ and PKC- $\beta 1$ deactivation correlated positively with tissue inflammation, oxidative injury $\&$ IBD severity. Most of the changes in EGF-R, PKC, NF-kB/iNOS \& oxidation were seen within the epithelial layer. Conclusions: 1) The EGF-R $\rightarrow$ PKC- $\zeta$ / PKC- $\beta 1$ pathway is downregulated and deactivated in $\mathrm{IBD}$ mucosa while the $\mathrm{NF}-\mathrm{kB} \rightarrow \mathrm{NO}$ oxidative damage pathway is upregulated. Tissue structural proteins also exhibit oxidative damage and instability when EGF-R is deactivated. 2) Measures of tissue inflammation, oxidative injury and IBD disease severity inversely correlate with mucosal EGF-R/ PKC. 3) Marked deactivation of EGF$\mathrm{R} \rightarrow \mathrm{PKC}-\zeta$ / PKC- $\beta 1$ signaling and activation of $\mathrm{NF}-\mathrm{kB} \rightarrow \mathrm{NO}$ signaling (\& structural protein oxidation) are only seen in inflamed mucosa. These findings suggest that a novel EGF-R and PKC isoform dependent mechanism underlies upregulation of NF-kB $\rightarrow$ iNOS signaling and that these key processes are required for mucosal inflammation and IBD flare up in man. \{Supported by NIDDK60511 \& NCCAM01581; NIAAA13745\}.

\section{S1305}

Positive CARD15 Mutation SNP12 and ASCA Predict Increased Disease Severity and Surgical Cost in Crohn's Disease

Selwyn H. Odes, Michael Friger, Hillel Vardi, Severine Vermeire, G Claessens, X Bossuyt, Lene Riis, Frank Wolters, Maurice Russel, Ole Hoie, Patrizia Politi, Ioannis Mouzas, Colm O'Morain, Juan Clofent, Estela Monteiro, Andrea Messori, Pia Munkholm, Reinhold Stockbrugger

BACKGROUND: CARD15, a susceptibility gene in Crohn's disease, is associated with ileal pathology, intestinal stenosis and more frequent surgery. Anti-Saccharomyces cerevisiae antibody (ASCA), a serological marker for Crohn's disease, is associated with ileal and more complicated disease. AIM: We hypothesized that the presence of ASCA and CARD15 mutations could predict the cost of health care in Crohn's disease. METHODS: Crohn's disease patients in the EC-IBD community-based inception cohort (Denmark, Greece, Israel, Italy, Netherlands, Norway, Portugal, Spain) had blood drawn for ASCA IgG and IgA, SNP8 SNP12 and SNP13 (all tests done in Leuven). Days in medical hospitalization (M-days) days in surgical hospitalization (S-days) and drug use (corticosteroids, immuno-suppressives, infliximab) were calculated as markers of disease severity. Costs were determined of total health care, medical hospitalizations (M-hosp), surgical hospitalizations (S-hosp) and drugs. Data were regarded as parametric and calculated as means, with statistical analysis by ANOVA. Since health costs are often skewed, the data were also considered as non-parametric and calculated as medians; this part of the analysis was by Median Test with Yates' Continuity Correction. Results are expressed in days or euros per patient-year (€/p-y). RESULTS: Patients were followed for mean 8.3 [SD 2.6] years. SNP12 positive patients had longer admissions and greater costs for total, medical and surgical hospitalizations, and drugs than SNP12 negative patients (Table, means). ASCA positive patients had longer surgical admissions and surgical hospitalization costs than ASCA negative patients. SNP13 positive patients had 
greater drug costs than SNP negative patients. SNP8 showed no differences. By Median Test SNP12 and ASCA retained significance for surgical admissions and cost. CONCLUSIONS: A positive ASCA test or the presence of the CARD15 mutation SNP12 was associated with longer and costlier surgical admissions. Genetic mutations and ASCA can forecast more severe disease and higher costs in Crohn's disease.

\begin{tabular}{|c|c|c|c|c|c|c|c|}
\hline Marker & $\mathrm{n}$ & M-days & S-days & Total Cost & M-hosp & S-hosp & Drugs \\
\hline & & days/p-y & days/p-y & $€ /$ p-y & $€ / \mathrm{p}-\mathrm{y}$ & $€ / \mathrm{p}-\mathrm{y}$ & $€ / \mathrm{p}-\mathrm{y}$ \\
\hline SNP12 pos & 9 & $6.37 *$ & $4.17 * \wedge$ & $4696 *$ & $2380 *$ & $1781 * \wedge$ & $585 \#$ \\
\hline SNP12 neg & 212 & 2.51 & 1.45 & 2311 & 937 & 385 & 230 \\
\hline ASCA pos & 68 & 2.76 & $2.54 \& \wedge \wedge$ & 2627 & 1031 & $1134 \& \wedge$ & 292 \\
\hline asca NEG & 165 & 2.79 & 1.17 & 2377 & 1037 & 542 & 216 \\
\hline SNP13 POS & 15 & 3.51 & 2.43 & 2919 & 1317 & 1100 & $525 @$ \\
\hline SNP13 neg & 207 & 2.70 & 1.50 & 2366 & 1006 & 681 & 225 \\
\hline
\end{tabular}

ANOVA: * SNP12 pos vs neg $\mathrm{P}<0.01$, \# SNP12 pos vs neg $\mathrm{P}<0.02$, \& ASCA pos vs neg $\mathrm{P}=$ 0.001 , @ SNP13 pos vs neg P<0.05. MEDIAN TEST: ^ SNP12 pos vs neg $\mathrm{P}<0.05, \wedge \wedge$ ASCA pos vs neg $\mathrm{P}=0.001$

\section{S1306}

99mtc-Infliximab Scans Measure Disease Extent and Activity in Inflammatory Bowel Disease (IBD)

Guru Iyngkaran, Chris Tsopelas, Suresh Sivanesan, David Hetzel, Francis D. Bartholomeusz

In IBD there is accumulation of Tumour Necrosis Factor (TNF $\alpha$ ) in inflamed bowel that allows for therapy with anti TNF drugs such as Infliximab. We developed a stable kit of $99 \mathrm{mTc}$ labeled Infliximab $(99 \mathrm{mT}$ c-inf) and demonstrated by in vitro tests with ELISA and stimulated human lymphocytes that it retained $>90 \%$ biological activity. In patients with rheumatoid arthritis there was uptake of $99 \mathrm{mTc}$-inf in inflamed joints but not in normal bowel. AIM: To measure bowel uptake of $99 \mathrm{mTc}$-inf in patients with active IBD and to compare this with the extent and severity of inflammation noted on recent colonoscopy, $\mathrm{CT}$, labeled leukocyte scans and $\mathrm{C}$ reactive protein (CRP). METHOD: Subjects with active IBD were studied. The CRP was measured and Disease Activity Index (DAI) was calculated for Ulcerative Colitis (UC) and Crohn's disease (CD). Whole body scintigraphy with SPECT imaging of the abdomen was performed at 1 and 3 hours following intravenous injection of $350 \mathrm{MBq}$ of $99 \mathrm{mTc}$-inf (1mg Infliximab). Scans were read blind to clinical information. The $99 \mathrm{mTc}$-inf uptake was calculated using regions of interest and uptake in inflamed bowel compared to non inflamed bowel (counts/pixel). Extent of bowel uptake was compared to colonoscopy, radiology or leukocyte scans. Degree of bowel uptake was compared to CRP RESULTS. Ten patients were studied (6 UC and $4 \mathrm{CD}$ ). The $99 \mathrm{mTc}$-inf scans showed uptake in inflamed small and large bowel at 1 and 3 hours. In UC extent of disease on the scan correlated with colonoscopy in 4 patients. There was 1 under and 1 overestimation. In $C D$ the scan matched other investigations in 1 patient and there was 1 under and 2 overestimations. Patients with higher CRP had more bowel uptake of $99 \mathrm{mT} \mathrm{c}$-inf $(\mathrm{p}=0.0086$ ) CONCLUSION: There is specific accumulation of $99 \mathrm{mTc}$-inf in inflamed bowel in IBD patients. If confirmed in a larger group of patients the scans may have potential to localize disease, assess activity and possibly predict response to infliximab therapy.

\begin{tabular}{|c|c|c|c|c|}
\hline Patient(UC) & CRP & DAI & Disease on 99mTc-inf scan & Disease site \\
\hline $22 \mathrm{M}$ & 34 & 11 & TDSR & TDSR \\
\hline $20 \mathrm{~F}$ & 58 & 10 & DSR & DSR \\
\hline $18 \mathrm{M}$ & 21 & 9 & TDS & ATDSR \\
\hline $69 \mathrm{M}$ & 320 & 9 & ATDS & ATDS \\
\hline $38 \mathrm{~F}$ & 26 & 8 & TDSR & TDSR \\
\hline $37 \mathrm{~F}$ & 65 & 9 & DSR & SR \\
\hline
\end{tabular}

\begin{tabular}{|c|c|c|c|c|}
\hline Patient (CD) & CRP & CDAI & Disease on 99mTc-inf scan & Disease site \\
\hline $33 \mathrm{M}$ & 120 & 192 & DS & ATDS \\
\hline $50 \mathrm{M}$ & 69 & 61 & IC & I \\
\hline $51 \mathrm{M}$ & 58 & 102 & IC & I \\
\hline $40 \mathrm{M}$ & 10 & 85 & $\mathrm{I}$ & $\mathrm{I}$ \\
\hline
\end{tabular}

R=Rectum, S=Sigmoid, D=Descending, T=Transverse, A=Ascending, C=Caecum, I=Ileum

S1307

Nodular Regenerative Hyperplasia and Sinusoidal Dilatation of the Liver in a Non-Thiopurine Using IBD-Cohort

Nanne K. De Boer, Henriette Tuynman, Elisabeth Bloemena, Johan Westerga, Chris J. Mulder, Miquel A. Cuesta, Stefan G. Meuwissen, Carin M. van Nieuwkerk, Adriaan A. van Bodegraven

Introduction The use of thiopurines (azathioprine, 6-mercaptopurine and 6-thioguanine) has been associated with the induction of nodular regenerative hyperplasia (NRH) and sinusoidal dilatation (SD) of the liver in IBD patients. However, it is unclear whether IBD itself is not a risk factor in inducing these histological liver abnormalities. Aims \& Methods Between 1977 and 2005 liver histology was obtained from IBD patients, independent of laboratory results or clinical signs, who underwent gastrointestinal surgery indicated for their IBD. The liver biopsy specimens from patients using thiopurines were excluded in order to asses the prevalence of NRH and SD of the liver in a non-thiopurine using IBDcohort from a referral centre. The liver specimens (minimal staining: H\&E, reticuline and Gomorri trichrome) were evaluated by two blinded liver pathologists. Pathohistological outcomes were related to disease and patient characteristics. Results Eighty-five patients (M:37\%/F:63\% and CD:62\%/UC:38\%) underwent 92 surgical procedures during which liver tissue (wedge:23\%/needle:77\%) was obtained. Median age at biopsy was 36 years (range $15-73$ years) and median duration of disease was 5 years (range $0-56$ years). Severe SD (> $2 / 3$ of the specimen) was observed in $3,3 \%$, moderate SD ( $1 / 3$ to $2 / 3$ of the specimen) in $8,7 \%$, slight SD ( 0 to $1 / 3$ of the specimen) in $21,7 \%$ and no SD in $66,3 \%$ of all biopsies, respectively. A significant difference was observed in type of IBD when considering both severe and moderate $\mathrm{SD}$ (combined $\mathrm{N}=12$ ) as a histological relevant abnormality. Significantly more patients were diagnosed with $\mathrm{CD}(\mathrm{N}=10)$ compared to $\mathrm{UC}(\mathrm{N}=2)(\mathrm{P}=0,022)$. In addition, there was a significant difference in gender as relatively more patients, with at least moderate $\mathrm{SD}$, were male $(\mathrm{P}=0,012)$. One liver specimen was excluded for evaluation on $\mathrm{NRH}$ as it was not properly stained. Nodular regenerative hyperplasia was detected in $5,5 \%$ of biopsies $(\mathrm{M}: 2(2 \mathrm{XCD})$ and $\mathrm{F}: 3(\mathrm{XCD}))$. The age at biopsy was significantly correlated with NRH $(\mathrm{r}=-0,256, \mathrm{P}=0,014)$. Conclusion Nodular regenerative hyperplasia $(5,5 \%)$ and sinusoidal dilatation of the liver (13\% (at least moderate)) were relatively common pathohistological findings in our non-thiopurine using IBD-cohort. The reported association between thiopurine use and NRH and SD should be weighed against the background prevalence of these two histological liver abnormalities in the IBD population.

S1308

The TNF/Adam 17 System: Implication of an ADAM 17 Haplotype in the Clinical Response to Infliximab in Crohn's Disease

Vinciane Dideberg, Emilie Theatre, Frederic Farnir, Severine Vermeire, Paul Rutgeerts, Martine de Vos, Jacques Belaiche, Andre van Gossum, Denis Franchimont, Edouard Louis, Vincent Bours

Infliximab, a chimeric anti-TNF- $\alpha$ antibody induces a clinical response in 70\% of Crohn's Disease (CD) patients. The response to infliximab therapy could be partially determined by genetic factors. The implication of both transmembrane and soluble forms of TNF- $\alpha$ in the mechanism of action of infliximab has been demonstrated. ADAM17 is a member of the adamlysin family of metalloproteinase that cleaves many membrane proteins, including the TNF- $\alpha$. This enzyme thus plays a predominant role in controlling the ratio of soluble TNFa versus transmembrane TNF- $\alpha$, which may be important for the response to Infliximab. The aim of our work was therefore to explore the role of TNF and ADAM-17 variants in the response to infliximab in Crohn's Disease. Patients and methods: the response to infliximab was evaluated in 222 Caucasian CD patients with a luminal $(n=160)$ or fistulizing $(n=62)$ form of the disease. Clinical and biological responses were based on the evolution of CDAI score as well as fistulae drainage and CRP levels. The entire TNF gene was sequenced on the complete cohort. Twelve single nucleotide polymorphisms (SNPs) spanning the ADAM 17 locus were studied. Haplotype blocks were determined using the Haploview v3.2 software and the haplotypes were rebuilt using Phase v2.1 software. Results: a clinical response was observed in $64 \%$ of the patients and biological response in $77.1 \%$ of patients. We identified 21 SNPs in the TNF gene. No association was found between TNF gene SNPs or haplotypes and the clinical or biological response to infliximab. One haplotype block was defined in the ADAM 17 gene, spanning from the rs2001658 to the rs11684747 SNP (including 11 of the 12 SNPs). One haplotype in this block was associated with a clinical response to infliximab in $\mathrm{CD}$ patients (adjusted $\mathrm{P}<0.02$ ). There were 16 patients homozygote mutant for this haplotype: all had a clinical response and a decrease of at least $40 \%$ in CRP after infliximab. Conclusion: our results exclude, with a reasonable power, any implication of the TNF gene in the response to infliximab in $\mathrm{CD}$, but reveal a potential role of the ADAM 17 gene in this response.

\section{S1309}

A Translational Strategy Suggests a Future Direction for Inflammatory Bowel Disease (IBD) Therapy: Changes in Tissue \& Cellular Lambda $(\lambda)$ Isoform of PKC May Underlie Oxidative Stress \& Inflammatory Processes in Intestinal

\section{Mucosa}

Ali Banan, Maliha Shaikh, Lijuan Zhang, Ece Mutlu, Ashkan Farhadi, Jeremy Z. Fields, Christopher B. Forsyth, Ali Keshavarzian

Oxidative injury to gut mucosa is a major pathophysiological mechanism in IBD. Despite nearly two decades of attempts at preventing \&/or reversing IBD flare up, there remains a lack of effective therapeutic regimes. This is, in turn, due to incomplete understanding of abnormal intracellular processes that cause IBD. Aims: Using a unique translational strategy involving both intestinal epithelial cells and mucosal pinch biopsies from patients, we determined whether: (a) cellular PKC- $\lambda$ is crucial in oxidative stress-induced iNOS and NO upregulation and consequent protein oxidation \& monolayer instability; (b) activation of PKC- $\lambda$ occurs in inflamed IBD mucosa; (c) activity of PKC isoforms correlate with markers of tissue inflammation \& oxidative injury (iNOS, NO, protein oxidation, etc). Methods: We used tissue pinch biopsies from IBD patients (UC, CD, specific colitis) \& normal controls as well as innovative gut epithelial clones transfected to overexpress PKC- $\lambda$ or to inhibit its expression. We monitored barrier integrity of monolayers of intestinal cells, tissue / cellular protein oxidation, tissue / cellular structural protein stability \{e.g., actin\}, PKC- $\lambda$ tissue/ subcellular activity \& distribution, iNOS activity / expression, NO levels, cytoskeletal oxidation \& nitration. Results: [A] In cells, PKC- $\lambda$ expression \& activation led to oxidative stresslike conditions including iNOS upregulation, $\mathrm{NO}$ overproduction, general protein nitration / oxidation, structural protein (actin) oxidation \& disassembly, \& monolaver instability. Dominant mutant inhibition of cellular PKC- $\lambda$ activity protected against iNOS and oxidative stress injury. [B] In tissue IBD biopsies, inflamed mucosa showed increased expression $\&$ activation of the $74 \mathrm{kDa}$ PKC- $\lambda$ (redistributed into mostly membrane + cytoskeletal mucosal fractions with only a small cytosolic fraction) as well as increased iNOS upregulation, NO production, \& protein oxidation (carbonylation / nitration), paralleling in vitro findings. In 
normal appearing (non-inflamed) IBD mucosa, smaller changes in oxidative markers \& PKC$\lambda$ were found. [C] Importantly, both mucosal \& cellular PKC- $\lambda$ activity correlated positively with oxidative injury \&/or with IBD disease severity. Conclusions: These translational findings based on both in vitro and in vivo conditions indicate a novel pathophysiological mechanism in IBD. One in which activation of a PKC- $\lambda$ dependent mechanism is required for cellular $\&$ tissue oxidative injury. If this unique mechanism underlies mucosal inflammation \& flareup in IBD, it could provide a novel target for future anti-IBD drugs.

\section{S1310}

Interaction Between Homocystein Metabolism and TPMT Activity in IBD Patients Under Azathioprine

Xavier Roblin, Alain Chartier, Veronique Ducros, Gaelle Hardy, Aurelie Durand, Germain Bessard, Bruno Bonaz

Polymorphism in the methylenetetrahydrofolate reductase (MTHFR) gene is able to modulate TPMT activity in patients under azathioprine (AZA) (1). Indeed, a defect of methylation, as represented by hyperhomocysteinemia, can decrease TPMT activity and conversely increase active AZA metabolite levels, particularly 6-thioguanine nucleotides (6-TGN). Aims: 1) to compare the prevalence of hyperhomocysteinemia between 2 groups of IBD patients under AZA. Group A: patients under AZA (dose $<2 \mathrm{mg} / \mathrm{kg} / \mathrm{d}$ ) with a pharmacologic profile which evokes a MTHFR gene mutation without any identified mutation: low level of methylated mercaptopurine metabolites (6-MMP+6-meTIMP $<1500$ pmoles/8x108 erythrocytes) associated to a high 6-TGN level (> 550 pmoles/8x108 erythrocytes), twice controlled, without TPMT gene mutation. Group B: IBD controls. 2) to analyse 6-TGN level modifications after folate and/or vitamin B12 supplementation. Methods: consecutive IBD patients under AZA followed in our department between March and September 2005 were included. Homocysteinemia, 6-TGN and methylated mercaptopurine metabolites (6-MMP + 6-meTIMP) levels were systematically evaluated. Patients with TPMT genotype mutation were excluded. Results: 70 patients ( 20 and 50 in groups $A$ and B respectively) were included ( 50 CD, 20 UC; mean age: $41.4 \pm 16.2$; sex ratio $\mathrm{M} / \mathrm{W}: 0.9)$. Hyperhomocysteinemia $(>15 \mu \mathrm{mol} / \mathrm{l})$ was observed in $18(90 \%)$ and $22(44 \%)$ patients of group A and B respectively $(\mathrm{p}<0.05)$. After folate $(\mathrm{n}=$ 16) and/or vitamin B12 ( $n=12$ ) supplementation of the 20 patients (group A), 6-TGN levels of these patients significantly decreased from $680 \pm 120$ pmoles to $360 \pm 90$ pmoles $(\mathrm{p}=$ 0.02 ) while methylated mercaptopurine metabolites increased from $880 \pm 120$ pmoles to $2400 \pm 680$ pmoles $(\mathrm{p}=0.04)$. Conclusion: hyperhomocysteinemia, whatever its origin, is able to decrease TPMT activity as represented by an increase of 6-TGN levels. In that case, folate and/or vitamin B12 supplementation decreases 6-TGN levels. These data have potential clinical implications: a) prevention of 6-TGN myelotoxicity, b) possibility of AZA loss efficacy after vitamin supplementation, c) systemic evaluation and treatment of hyperhomocysteinemia in case of heterozygote TPMT gene mutation. (1) Arenas M et al. Clin Chem. 2005; 51: $2371-2374$

\section{S1311}

A Potential Role of Soluble Angiopoietin-2 and Angiopoietin Receptor TIE-2 in Inflammatory Bowel Disease

Ioannis Koutroubakis, Costas Xidakis, Konstantinos Karmiris, Aekaterini Sfiridaki, Ermioni Kandidaki, Elias Kouroumalis

Background: Angiogenesis has been suggested to play an important role in inflammatory bowel disease (IBD). Aim of the present study was to evaluate the serum markers of angiogenesis angiopoietin-2 (Ang-2) and soluble angiopoietin receptor Tie-2, in patients with ulcerative colitis (UC) and Crohn's disease (CD). Methods: Ang-2 and Tie-2 serum levels were measured in 160 inflammatory bowel disease patients (79 ulcerative colitis and 81 Crohn's disease) and in 80 matched healthy controls (HC) using commercially available enzyme-linked immunosorbent assays. Ang-2 and Tie-2 levels were correlated with disease activity, type, localization and treatment. Results: Median serum Ang-2 levels were significantly higher in both UC patients and CD patients compared with healthy controls $(\mathrm{P}<0.05)$ (Fig 1). Similarly median Tie-2 levels were significantly higher in both UC patients and $\mathrm{CD}$ patients compared with healthy controls $(\mathrm{P}<0.001)$ (Fig 2). IBD patients with early disease (diagnosis during the last two years) had median serum Ang-2 significantly higher compared with patients with late disease (diagnosis more than two years) $(\mathrm{P}=0.04)$. On the other hand the levels of Tie-2 were significantly lower in patients with early disease compared with patients with late disease $(\mathrm{P}=0.02)$. $\mathrm{CD}$ patients with active disease had significantly higher levels of Ang-2 compared to non-active disease ( $\mathrm{P}=0.02)$. A significant correlation between serum Ang- 2 and serum Tie-2 levels was found $(r=0.19, P=0.01)$. No other significant associations of these markers with the disease characteristics were found. Conclusions: Serum Ang-2 and Tie-2 levels are elevated in patients with inflammatory bowel disease. These data suggest that these markers may mediate angiogenesis and vascular permeability and may participate in the restoration of the mucosa of IBD patients. Further studies of these markers in conjunction with other angiogenesis factors in the gut tissues of IBD patients are needed.

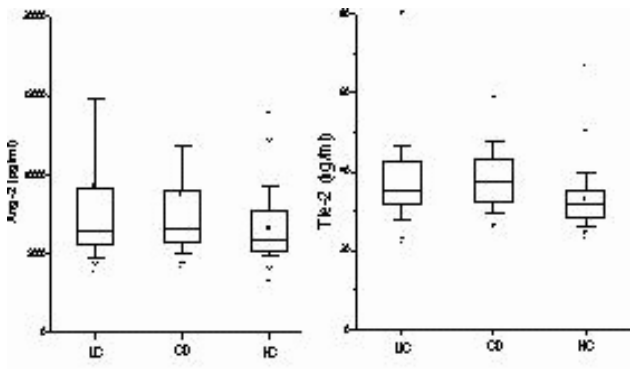

S1312

Feasibility and Patient Acceptance of a Home Telemanagment System in Inflammatory Bowel Disease

Raymond K. Cross, Joseph Finkelstein

Introduction: Telemanagement is a telemedicine system designed to assist clinicians trea and monitor patients according to current guidelines, assist in educating patients, and assis patients in following self-care plans. Telemanagement has been successfully implemented in chronic diseases similar to IBD. Our objectives were to test the feasibility and acceptance of a telemanagement system for IBD (HAT). Methods: HAT consists of a laptop, a weight scale, and a web portal. The laptop administers questions on symptoms, medical adherence, medication side effects, delivers an educational curriculum, and measures body weight. The results are obtained weekly and physicians can view the results on the web portal. Patients were recruited from the IBD clinic at the University of Maryland and the Veterans Affairs, Baltimore. Disease activity, quality of life, IBD knowledge, and satisfaction with IBD services was assessed at baseline and after 6 months. Attitudinal surveys were obtained at the end of the study. Results: 25 patients completed the 6-month study. $89 \%$ of subjects were adherent with weekly self-testing. Attitudes toward HAT were very good. $95 \%$ of subjects said that self-testing was not complicated. $90 \%$ reported that answering the symptom diary and medication side effects questions were not difficult. Subjects reported that self-testing took very little time and that testing did not interrupt their usual activities. $70 \%$ of patients felt safer using the system and only $10 \%$ of the subjects did not want to use the self-testing program in the future. At completion of the study, patient satisfaction with IBD care services was very good. $71 \%$ reported that almost all their medical needs were met and $86 \%$ reported that they were very satisfied with their IBD care compared to $40 \%$ and $70 \%$ respectively at baseline. Mean self-reported compliance with IBD medications was $90 \%$ throughout the study. Disease activity, quality of life, and patient knowledge improved compared to baseline. See Table 1. Discussion: HAT can be easily implemented and the technology is feasible and accepted in patients with IBD. HAT improved patient knowledge and trends to improvement in disease activity and quality of life were seen. Further studies are needed to determine if HAT improves outcomes compared to routine care.

Table 1: Improvement in Clinical Outcomes After Use of HAT

\begin{tabular}{|c|c|c|c|c|}
\hline End Point & Variable & Baseline & 6 -month Visit & P value \\
\hline Clinical Disease Activity & Harvey Bradshaw Index & $3.8+/-4.3$ & $2.2+/-3.6$ & 0.09 \\
\hline & ESR & $25.7+/-34.4$ & $16.1+/-17.9$ & 0.06 \\
\hline & CRP & $11.3+/-25.9$ & $3+/-4$ & 0.13 \\
\hline Quality of Life & SIBDQ & $49.2+/-15.1$ & $55.6+/-14.5$ & 0.06 \\
\hline Patient Knowledge & CCKNOW & $9.2+/-5.3$ & $12+/-4.4$ & 0.0007 \\
\hline
\end{tabular}

\section{S1313}

The MDR1 G2677T Polymorphism Is Associated with Gastrointestinal Intolerance to Azathioprine

Konstantinos Katsanos, Marc Ferrante, Marie Joossens, Sofie Joossens, Liesbet Henckaerts, Marie Pierik, Karolien Claes, Nele van Schuerbeek, Afroditi Katsaraki, Gert van Assche, Epameinondas V. Tsianos, Paul Rutgeerts, Severine Vermeire

BACKGROUND \& AIM: Some of the azathioprine (AZA) side effects are associated with TPMT gene. We studied if the MDRl gene is also associated with side effects of AZA in inflammatory bowel disease (IBD) patients. METHODS: A total of 1815 IBD records were reviewed for intake of AZA and possible reasons for discontinuation. All patients were genotyped for TPMT (G238C, G460A and A719G) and MDR1 (G2677T/A and C3435T) using PCR-RFLP. RESULTS: We identified 775 IBD patients receiving AZA of whom 229 (29.5\%) were discontinued (disAZA) (163 Crohn's and 66 ulcerative colitis), after a mean of $20.5 \pm 24.5$ months (range 1 week-129 months). The mean AZA daily dose was $2.16 \pm 0.84 \mathrm{mg} / \mathrm{Kg} /$ day. The main reasons for discontinuation of AZA were inefficacy in 56 (24.4\%) and side-effects in 142 (62\%) patients (table). The MDR1 G2677T mutation was more prevalent in the disAZA group $(70.3 \%$ and $46 \% \mathrm{~T}$ allele frequency) compared to the AZA tolerant group (63.4\% and 39\% T allele frequency) [p=0.028, OR 1.59 ( $95 \% \mathrm{CI} 1.05$ 2.41)]. Using multivariate analysis, an independent association was found with gastrointestinal and general intolerance $[\mathrm{p}=0.009$, OR 3.99 (95\% CI 1.33-11.93)]. No significant differences for any of the TPMT variants were found between the AZA and disAZA group. We also could not find an association between TPMT variants and specific side effects to AZA including bone marrow toxicity although the 1 patient developing leucopenia 1 week after start carried 2 mutations (TPMT*3B/*3C). CONCLUSIONS: In this large retrospective analysis on the use of AZA in IBD, AZA was discontinued in $24.4 \%$ of patients because of inefficacy and in another $62 \%$ due to side effects. Whereas no association was observed for TPMT variants, patients carrying the MDRl-G2677T polymorphism more often had to interrupt AZA because of gastrointestinal or general intolerance. These findings now need 
prospective validation but suggest a role for MDRl/P-gp in the mechanism of action of azathioprine.

\begin{tabular}{|c|c|c|}
\hline Reasons for AZA interruption in IBD & No patients $(\mathrm{n}=229)$ & $\%$ \\
\hline Gastrointestinal or general intolerance & 40 & 17.5 \\
\hline Pancreatic toxicity & 24 & 10.5 \\
\hline Hepatotoxicity & 22 & 9.7 \\
\hline Bone marrow toxicity & 16 & 7.0 \\
\hline Severe infection & 14 & 6.1 \\
\hline Flu-like symptoms & 13 & 5.7 \\
\hline Skin allergy & 11 & 4.8 \\
\hline Cancer/precancerous/lymphoma & 4 & 1.7 \\
\hline Rare causes & 2 & 0.9 \\
\hline Death from other than IBD cause & 1 & 0.4 \\
\hline Long-term disease remission & 12 & 5.2 \\
\hline Ineffectiveness/non-response & 56 & 22.2 \\
\hline Pregnancy & 14 & 6.1 \\
\hline
\end{tabular}

S1314

Comparative Study of New Rapid Bedside Fecal Calprotectin Test with An Established ELISA to Assess Intestinal Inflammation in a Prospective Study Yogesh Shastri, Nada Povse, Jurgen Stein

Background: Estimation of fecal concentration of the neutrophil granulocyte-derived protein calprotectin has been proposed as a screening non invasive test to assess intestinal inflammation of the gut. However, most assays for detecting calprotectin are Enzyme Linked Immunosorbent Assay (ELISA) based thus require a suitable well equipped laboratory i.e. an ELISA reader, limiting their widespread use because of logistics and higher costs. The biggest advantages of this new bedside IFOBT are its simplicity, rapidity (needs just about $5 \mathrm{~min}$ ), convenience, no need of cumbersome ELISA readers and the fact that it can be performed at the patient bedside or in physician's office by any health care personnel. Patients and Methods: 301 patients (139 males and 162 females) underwent estimation of fecal calprotectin using a commercial quantitative ELISA (Immunodiagnostik, Bensheim, Germany) and the rapid bedside test (PreventID® CalDetect, Preventis, Bensheim, Germany), which is a semiquantitative immunochromatographic rapid test using monoclonal antibodies. There age ranged from 7 months till 91 years (median being 40 years). The study duration was from January 2005 till November 2005. Both these tests were performed by an experienced technician who was blinded to the patient's clinical profile. As per the manufacturer the cutoff calprotectin level for both the tests was $15 \mathrm{ng} / \mathrm{mL}$. Results: The patient's test characteristics as shown in Table 1. The sensitivity and specificity of the new bedside tests for diagnosing CD and UC were $96.3 \%$ and $94.0 \%$ as against quantitative ELISA of $96.0 \%$ and $99 \%$ (not statistically significant) respectively. Conclusion: This new bedside fecal calprotectin assay has proved to be an accurate, simple, convenient, noncumbersome tool as compared to the routine ELISA based test and can replace the cumbersome ELISA fecal calprotectin estimation. Table 1. Test characteristics in 301 consecutive patients

\begin{tabular}{|c|c|c|c|}
\hline Diagnosis (No) & Calprotectin level & ELISA test & Bedside test \\
\hline $\mathrm{CD}(109)$ & $<15 \mathrm{ng}>\mathrm{mL}>15 \mathrm{ngmL}$ & 3106 & 4105 \\
\hline $\mathrm{UC}(46)$ & $<15 \mathrm{ng}>\mathrm{mL}>15 \mathrm{ngmL}$ & 145 & 244 \\
\hline Cystic fibrosis (41) & $<15 \mathrm{ng}>\mathrm{mL}>15 \mathrm{ngmL}$ & 1625 & 1922 \\
\hline Diarrhea (34) & $<15 \mathrm{ng}>\mathrm{mL}>15 \mathrm{ngmL}$ & 1024 & 1321 \\
\hline Others (21) & $<15 \mathrm{ng}>\mathrm{mL}>15 \mathrm{ngmL}$ & 912 & 1110 \\
\hline Controls (50) & $<15 \mathrm{ng}>\mathrm{mL}>15 \mathrm{ngmL}$ & 482 & 473 \\
\hline
\end{tabular}

\section{S1315}

A Panel of Anti-Glycan Antibodies (gASCA, ALCA, ACCA and AMCA) in the Diagnosis and Differential Diagnosis of IBD

Marc Ferrante, Marie Pierik, Liesbet Henckaerts, Nir Dotan, Rom T. Altstock, Iris Dotan, Marie Joossens, Sofie Joossens, Konstantinos Katsanos, Karolien Claes, Nele van Schuerbeek, Gert van Assche, Paul Rutgeerts, Severine Vermeire

BACKGROUND \& AIMS: Serological markers have been proposed in IBD for distinction between ulcerative colitis (UC) and Crohn's disease (CD), and also contribute to disease stratification in $\mathrm{CD}$. Given the sub-optimal accuracy of the available markers in daily clinical practice, we tested a new panel of antibodies directed against glycan-epitopes in IBD and controls. METHODS: A total of 1225 IBD patients ( 913 CD, 272 UC, 40 IC), as well as 200 healthy controls and 113 patients with non-IBD gastrointestinal inflammation (GIcontrols) were tested for Anti Saccharomyces cerevisiae Antibodies (gASCA), Anti-Laminaribioside Carbohydrate Antibodies (ALCA), Anti-Chitobioside Carbohydrate Antibodies (ACCA) and Anti-Mannobioside Carbohydrate Antibodies (AMCA) by commercially available ELISA-assays (Glycominds Ltd, Israel) in a blinded way. Using the manufacturers conditions and reconstruction of ROC curves, a cut-off value of 50 (gASCA), 70 (ALCA) or 90 (ACCA and AMCA) units was defined for positivity. Based on the antibody titres of gASCA, ALCA and AMCA a combined serology score (CSS) was computed, ranging from 0 (all 3 markers negative) to 3 (all 3 markers positive), allowing for intermediate titres (which were given a score of 0.5 ). Accuracy (sensitivity, specificity, positive and negative predictive value) of the individual markers, as well as the combined panel and CSS were calculated using SPSS.
RESULTS: The accuracy of the individual markers is depicted in table 1 and for the combined score in table 2. Among the 398 gASCA negative CD patients, 28 were ALCA positive and 50 were AMCA positive. DISCUSSION: Our data show that CD is associated with an adaptive immune response against various glycan-epitopes. Although all antibodies studied in this panel have a high specificity for IBD and CD in particular, gASCA is the preferred antibody given its higher sensitivity. However, the use of a combined serological score, based on gASCA, ALCA and AMCA, further increased the specificity and may have an additional value in differentiating IBD from other causes of abdominal complaints. Table 1

\begin{tabular}{|c|c|c|c|c|c|c|c|c|}
\hline \multirow{2}{*}{ Disease } & \multicolumn{2}{|c|}{ gASCA } & \multicolumn{2}{c|}{ ALCA } & \multicolumn{2}{c|}{ ACCA } & \multicolumn{2}{c|}{ AMCA } \\
\cline { 2 - 8 } & Sens & Spec & Sens & Spec & Sens & Spec & Sens & Spec \\
\hline CD vs. UC & $\mathbf{5 6 \%}$ & $\mathbf{9 0} \%$ & $\mathbf{1 8} \%$ & $\mathbf{9 3} \%$ & $\mathbf{2 1 \%}$ & $\mathbf{8 5} \%$ & $\mathbf{2 8} \%$ & $\mathbf{8 2} \%$ \\
\hline IBD vs. control & $\mathbf{4 5 \%}$ & $\mathbf{9 9} \%$ & $\mathbf{1 5} \%$ & $\mathbf{9 9} \%$ & $\mathbf{1 9 \%}$ & $\mathbf{8 6} \%$ & $\mathbf{2 6 \%}$ & $\mathbf{9 2} \%$ \\
\hline IBD vs. GI-control & $\mathbf{4 5 \%}$ & $\mathbf{9 8} \%$ & $\mathbf{1 5} \%$ & $\mathbf{9 9} \%$ & $\mathbf{1 9} \%$ & $\mathbf{8 4} \%$ & $\mathbf{2 6} \%$ & $\mathbf{9 3} \%$ \\
\hline
\end{tabular}

Table 2

\begin{tabular}{|c|c|c|c|c|c|c|c|c|}
\hline \multirow{2}{*}{ Disease } & \multicolumn{2}{|c|}{ CSS \&\#8807; 1.0} & \multicolumn{2}{|c|}{ CSS \&\#8807; 1.5} & \multicolumn{2}{|c|}{ CSS \&\#8807; 2.0} & \multicolumn{2}{|c|}{ CSS \&\#8807; 2.5} \\
\hline & Sens & Spec & Sens & Spec & Sens & Spec & Sens & Spec \\
\hline CD vs. UC & $74 \%$ & $61 \%$ & $54 \%$ & $83 \%$ & $37 \%$ & $93 \%$ & $20 \%$ & $97 \%$ \\
\hline IBD vs. control & $66 \%$ & $86 \%$ & $45 \%$ & 99\% & $30 \%$ & $100 \%$ & $16 \%$ & $100 \%$ \\
\hline IBD vs. GI-control & $66 \%$ & $82 \%$ & $45 \%$ & $98 \%$ & $30 \%$ & $100 \%$ & $16 \%$ & $100 \%$ \\
\hline
\end{tabular}

S1316

The Positivity of Systemic Cytomegalovirus (CMV) Viral Load Is Highly Predictive of Associated CMV Colitis in Patients Hospitalized for Exacerbation of Ulcerative Colitis Or Crohn's Disease with Colonic Involvement Sophie Buyse, Joel Gozlan, Corinne Amiel, Nadia Hoyeau, Isabelle Nion-Larmurier, Philippe Seksik, Jacques Cosnes, Jean-Pierre Gendre, Laurent Beaugerie

Latent infection with CMV and Epstein-Barr Virus (EBV) is observed in the majority of healthy adults. In patients with Crohn's disease, transient systemic reactivation of EBV infection may occur in the setting of severe intestinal inflammation and/or treatment by immunosuppressants [1]. The aims of this pilot study were to assess whether significant reactivation of systemic CMV infection in patients admitted for active IBD colitis is: i) mostly observed under immunosuppressant therapy; ii) associated with concomitant reactivation of EBV infection; iii) predictive of associated CMV colitis. Patients and Methods: Between January 2004 and October 2005, 33 patients (16 males, 17 females), aged from 16 to 68 years (mean: 36)), admitted for moderate to severe attacks of ulcerative colitis (UC, $n=19$ ) or Crohn's ileocolitis or colitis (CD, n=14) were studied. All the patients had within the 48 hours following admission a quantification of systemic CMV and EBV viral load by real time PCR and endoscopic evaluation of colonic lesions with biopsies. CMV inclusions were searched for by standard histology and immunohistochemistry. Results: The positivity of CMV viral load (above the level of 400 copies $/ 10^{6}$ leukocytes, threshold of reproducible positivity of the technique) was observed in $12(36 \%)$ patients (2(14\%) of CD patients vs. $10(53 \%)$ of UC patients $(\mathrm{p}=0.06)$ ). All 12 patients with positive CMV viral load were receiving high doses of steroids $(n=7)$ or immunosuppressants $(n=5)$ at the time of viral load determination. Eleven (92\%) of the patients with positive CMV viral load had a concomitant increase ( $>400$ copies $/ 10^{6}$ leukocytes) of EBV viral load. Eight patients had CMV colitis; all of them had a positive CMV viral load. The positive predictive value of CMV colitis in patients with positive CMV viral load was $67 \%$. The negative predictive value of CMV colitis in patients with negative CMV viral load was $100 \%$. Conclusions: A significant positivity of systemic CMV viral load ( $>400$ copies $/ 10^{6}$ leucocytes) at admission of patients with active IBD colitis: is mostly observed in UC patients receiving steroids or immunosuppressants; is associated in most cases with a concomitant reactivation of systemic EBV infection, suggesting a status of transient immunodeficiency; is highly correlated with the risk of concomitant CMV colitis. [1] Reijasse D et al. Inflamm Bowel Dis 2004;10: 85.

\section{S1317}

Hyperhomocysteinemia, Folate Deficiency, and Carcinogenesis in IBD Patients Xavier Roblin, Jean Marc Phelip, Veronique Ducros, Jean Luc Faucheron, Bruno Bonaz

In the general population, it is generally admitted that folate deficiency, which induces hyperhomocysteinemia, increases the risk to develop cancerous or precancerous lesions (1). To our knowledge, no data are available in IBD patients. Aim: to evaluate, in a cohort of IBD patients, risk factors of colonic carcinogenesis, in particular folate and homocysteinemia levels. Methods: IBD patients with carcinogenic lesions discovered under colonoscopy (low or high grade adenoma, low or high grade dysplasia, colorectal cancers) were included and compared to the whole population of IBD patients with a normal colonoscopy performed during the same period. Patients with primary sclerosing cholangitis and/or with a family history of colorectal cancers were excluded. The following parameters were collected: age, sex, type-length-activity-extent of the disease, treatment, smoking, vitamin B12-folate-homocysteinemia levels. A univariate analysis and then a multivariate analysis were performed after adjustment according to the main parameters. Results: 110 patients ( $37 \mathrm{UC}, 73 \mathrm{CD}$; mean age: 44.7 years; sex ratio: 1) were included. Twenty two carcinogenic lesions were isolated: 10 polyps ( 3 with high grade dysplasia, 7 adenomatous without dysplasia), 6 low grade dysplasia, 2 high grade dysplasia, 4 colorectal cancers. In univariate analysis, risk factors of carcinogenesis were: active smoking $(\mathrm{p}=0.03)$, folate level $<145 \mathrm{pmol} / \mathrm{l}(\mathrm{p}=$ $0.011)$, hyperhomocysteinemia $>15 \mu \mathrm{mol} / \mathrm{l}(\mathrm{p}=0.002)$, length of the disease $>10$ years $(\mathrm{p}=0.006), \mathrm{UC}(\mathrm{p}=0.02)$. In contrast, immunosuppressives, pancolitis, age of onset and 
clinical activity of the disease were not risk factors. A high correlation between hyperhomocysteinemia, folate deficiency and carcinogenic risk was observed. In multivariate analysis, patients with hyperhomocysteinemia and folate deficiency had 15 times as much carcinogenic risk as patients with normal homocysteinemia whatever the folate status and the length of the disease $(p<0.01)$. Patients with an isolated hyperhomocysteinemia had 2 times as much carcinogenic risk than patients with normal homocysteinemia whatever the folate status; however the difference was not significant $(\mathrm{p}=0.07)$. Conclusion: our data suggest that in IBD patients with a normal homocysteinemia, the carcinogenic risk is negligible whatever the length of the disease. Conversely, in case of hyperhomocysteinemia, folate status is important to appreciate the carcinogenic risk. Complementary studies on a large scale are necessary to confirm these data which suggest modifications in the carcinogenic surveillance in IBD patients. (1) Pufulete M. et al Gastroenterology 2003; 124: 1240-48

\section{S1318}

Faecal M2-Pyruvate Kinase; A Novel, Non-Invasive Marker of Ileal Pouch Inflammation

Matthew W. Johnson, Susan Maestranzi, David H. Dewar, Alastair Forbes, Roy Sherwood, Paul J. Ciclitira, John R. Nicholls

There is an over expression of the dimeric M2 pyruvate kinase (M2-PK) isoform seen with the rapid cell turnover of tumour cells. The concentration of this dimeric M2-PK in the plasma and tumours of patients with gastrointestinal (GI) cancers correlates well with the staging and metastatic capability of the tumour cells. We postulated that faecal dimeric M2PK would also be increased in inflamed ileal pouches with concentration levels correlating to the degree of inflammation. 54 stool samples were taken from 49 ileal pouch patients. 41 had ulcerative colitis (UC) and 8 had familial adenomatous polyposis (FAP) coli. Pouchitis was defined as an objective pouchitis score (OPS) of $\geq 5 / 12$ (endoscopic score of $\geq 3 / 6$ and an acute histological score of $\geq 2 / 6$ ) or a pouch disease activity index (PDAI) of $\geq 7 / 18$. Faecal M2-PK was determined by a quantitative sandwich-type enzyme immunoassay (ScheBo ( Biotech UK). Values of $>4 \mathrm{U} / \mathrm{ml}$ represent positive results. Statistical analysis was performed using Mann Whitney unpaired t-test ( $\mathrm{p}$ values) and Spearman rank coefficient ( $\mathrm{r}$ values). The well validated inflammatory bowel marker, faecal Calprotectin, was also measured ("PhilCal ELISA New Method", Nycomed Pharma AS). Using the OPS, 6 patients were diagnosed with UC pouchitis (+ pre-pouch ileitis), 13 had UC pouchitis alone, 27 had a healthy UC pouch, 1 had FAP pouchitis (+ pre-pouch ileitis) and 7 had an uninflamed FAP pouch. Respective median M2-PK values (U/ml) for these groups were 49.5, 12, 2.2, 19.5, and 1 , with mean [95\% confidence intervals] of 52.7 [6.5-99], 46 [8.6-83.3], 11.4 [2.6$20.2], 19.5$, and $1.4[0.4-2.3]$. Statistically significant differences were noted between the inflamed and non-inflamed pouches $(\mathrm{p}<0.0001)$, but not between pouchitis patients with and without pre-pouch ileitis $(\mathrm{p}=0.4)$ or between healthy UC pouches and non-inflamed FAP pouches $(\mathrm{p}=0.4)$. The M2-PK values correlated significantly with the OPS $(r=0.63$, $\mathrm{p}<0.0001)$, PDAI $(\mathrm{r}=0.64, \mathrm{p}<0.0001)$, endoscopy $(\mathrm{r}=0.56, \mathrm{p}<0.0001)$, acute histological $(\mathrm{r}=$ $0.68, \mathrm{p}<0.0001)$ and neutrophil scores $(\mathrm{r}=0.66, \mathrm{p}<0.0001)$, but poorly with the clinical score $(r=0.22, p=0.1)$. The receiver operating characteristic analysis demonstrated a sensitivity and specificity of $70.6 \%$ and $80 \%$, respectively. Similar results were seen using the PDAI, albeit with a slightly lower specificity. The M2-PK and faecal Calprotectin results correlated closely $(r=0.87, \mathrm{p}<0.0001)$. Dimeric faecal M2-PK has become established as a screening tool for cancer. We have, however, shown that M2-PK is also a novel and sensitive marker of bowel inflammation, which could prove useful in differentiating between healthy and inflamed ileal pouches.

\section{S1319}

Low Molecular Mass Polypeptide 2 (LMP2) Expression Is Enhanced in the Intestine of Patients with Inflammatory Bowel Disease

Leo R. Fitzpatrick, Jeffrey Small, Kevin McKenna, Lisa S. Poritz, Walter A. Koltun

Low molecular mass polypeptide 2 (LMP2) is an inducible immunoproteasome subunit that plays a role in antigen presentation, as well as in the NF- $\mathrm{KB}$ signaling pathway. To date, the expression of LMP2 has not been investigated in patients with IBD. Aims: The primary goal of this study was to examine LMP2 expression in IBD patients. A secondary goal was to determine if there was an association between LMP2 expression and histological injury. Methods: Resected intestinal samples from 19 patients ( $\mathrm{n}=1$ to 5 per patient) were used in this study. These patients included 5 non-IBD controls ( 2 colon cancer, 1 constipation, 1 FAP, 1 diverticulitis), 7 patients with Ulcerative Colitis (UC) and 7 patients with Crohn's Disease $(C D)$. Intestinal samples were taken from both the colon and ileum. In control patients, samples were taken from grossly normal areas of the bowel. Samples of intestine were processed for immunohistohemistry, using a specific polyclonal antibody to LMP2. The percent area of LMP2 staining was determined on coded slides, with a $25 \mathrm{~mm}$ ocular grid. From the same colonic sections, histology scores were determined on a 0 to 40 point severity scale. Intestinal LMP2 expression was also determined by western blot, in a subset of patient samples. Western blots were quantified by a relative densitometry analysis. Results: LMP2 expression in control patients was confined primarily to epithelial cells in the upper $1 / 3$ of the crypts and on the mucosal surface. In contrast, prominent LMP2 expression was observed throughout the crypts, as well as in leukocytes within the lamina propria and submucosa. By histochemistry, percent LMP2 expression values were: $17 \pm 2$ (control), 45 \pm 2 (UC) and $52 \pm 3(\mathrm{CD})$. Overall, the percent intestinal LMP2 expression in IBD patients was significantly greater $(\mathrm{p}<0.01)$ than in the control patients. In specific areas within the colon (proximal, mid, distal), LMP2 expression was always significantly greater in patients with IBD. Similarly, enhanced expression of this proteasome subunit was found in the ileum of patients with CD. Interestingly, increased intestinal LMP2 expression (36\%) was found in macroscopically normal areas from IBD patients. Western blot analysis confirmed that intestinal LMP2 expression was enhanced in patients with IBD. Specifically, LMP2 was increased by 3.2 fold in UC patients and 4.0 fold in $C D$ patients. A significant correlation $(\mathrm{r}=0.674, \mathrm{p}=0.0016)$ was found between LMP2 expression and histological damage in these 19 patients. Conclusions: Enhanced LMP2 expression was evident in the intestine of IBD patients. LMP2 may be a potential marker for predicting and/or following the severity of IBD
S1320

High Prevalence of Sarcopenia in Patients with Crohn's Disease: Association with Osteopenia

Rima Al-Jaouni, Jerome Filippi, Stephane M. Schneider, Xavier Hebuterne

Rationale: Reduction of bone mineral density (BMD) is frequent in patients with Crohn's disease (CD) and is associated with an increased risk of fractures. Many factors that are known to be associated with osteopenia are also known to decrease muscle mass. The aim of the present study was to evaluate the prevalence of sarcopenia in patients with $C D$ in remission, and to determine if sarcopenia was associated with osteopenia. Methods: $67 \mathrm{CD}$ patients (CDAI<150; $C R P<10 \mathrm{mg} / \mathrm{L})(37 \mathrm{~F} / 30 \mathrm{M} ; 37 \pm 13$ years; BMI $21.2 \pm 3.6)$, and 39 volunteers (24F/15M; $40 \pm 14$ years; BMI 22.1 \pm 2.4 ) have been studied. Appendicular muscle mass (AMM) and BMD were measured by using DEXA (Hologic). BMD was expressed as $\mathrm{g} / \mathrm{cm} 2$ and $\mathrm{T}$ score. The number of patients with osteopenia was assessed according to the WHO classification. AMM was expressed in absolute $(\mathrm{kg})$, and prevalence rate of sarcopenia was determined according to the following definition: AMM/ht $2<$ young normal minus $2 \mathrm{SD}$ Results: AMM was lower in patients than in controls $(6.0 \pm 1.1$ vs. $6.4 \pm 1.1 ; \mathrm{P}=0.02)$. A positive correlation was found between AMM and BMD $(r=0.50 ; P=0.0001)$. Prevalence of sarcopenia was $61.2 \%(19 \mathrm{M} / 22 \mathrm{~F})$ in patients and $20.0 \%(4 \mathrm{M} / 4 \mathrm{~F})$ in controls $(\mathrm{P}<0.01)$. Prevalence of osteopenia was $30.0 \%$ in patients and $5.1 \%$ in controls. The prevalence of osteopenia was $43.0 \%$ in patients with sarcopenia, and $8 \%$ in patients without sarcopenia $(\mathrm{P}<0.001)$. BMI was lower $(\mathrm{P}<0.05)$ in patients with sarcopenia $(20.4 \pm 3.7)$ than in patients without sarcopenia $(22.5 \pm 3.0)$; a negative correlation was found between AMM and disease duration $(r=0.27$ $\mathrm{P}=0.03$ ) but not with treatments. Conclusions: The prevalence of sarcopenia is high in young patients with CD. Sarcopenia is strongly associated with osteopenia. These two phenomena may have synergic deleterious effects and may share similar mechanisms. Our findings suggest that the routine determination of both BMD and AMM by DEXA may be helpful in $\mathrm{CD}$ patients

\section{S1321}

Whole Blood Gene Expression Differentiates Between Active Disease and Remission in Patients with Crohn's Disease

Robert Burakoff, Scott Hande, Sonia Friedman, Frederick Makrauer, Peter Banks, Martha Vander Vliet, S Mohr, C.C. Liew

Introduction: Current methods for determining disease activity in patients with Crohn's disease are invasive, time-consuming, and non-specific. A blood test serving as a surrogate marker for disease activity is desirable. The differential expression of RNA in whole blood has been used in previous studies to discriminate between disease states unrelated to IBD. Since circulating blood cells are exposed to the inflammatory mediators of active Crohn's disease, their pattern of gene expression may be surrogate markers for Crohn's disease activity. Methods: 38 patients with documented Crohn's disease were enrolled. CDAI score were obtained, as well as total RNA extracted from $10 \mathrm{~mL}$ of whole blood. Remission was defined as a CDAl<150. Affymetrix U133Plus 2.0 GeneChips (Chondrogene) were used to screen RNA samples for genes differentially expressed between patients with active Crohn's disease and patients with disease in remission. These gene arrays were also compared with those of 9 healthy controls. Chip interpreters were blinded to diagnosis and disease activity. Logistic regression analysis was used to identify linear combinations of genes that displayed significant changes in expression level by quantitative RT-PCR. Results: 22 patients had Crohn's disease in clinical remission (CDAI range -7-145), and 16 patients had active Crohn's disease (CDAI range 155-568). A pattern of differential RNA expression distinguishing active Crohn's disease from both remission and healthy controls was seen on the basis of 108 genes $(\mathrm{p}<0.01)$. Among the genes with reduced expression in active Crohn's were those for occludin ( $\mathrm{p}=0.003)$ and integrin $\mathrm{a} 4(\mathrm{p}=0.007)$. The genes encoding vimentin and $\mathrm{b}$-glucosidase were among those over-expressed (both $\mathrm{p}<0.001$ ). Gene expression patterns and CDA scores correlated poorly within the subgroup of 16 patients with active disease. Two distinct gene expression patterns were seen in patients with Crohn's in remission. The clinical distinction between these remission groups remains to be determined. Conclusion: The pattern of gene expression in circulating blood cells is different in patients with active Crohn's disease compared with patients in remission and healthy controls by cluster analysis Several of the genes aberrantly expressed in patients with active disease have previously been implicated in the pathophysiology of Crohn's disease. Gene arrays of circulating blood cells may provide surrogate markers for disease activity, as well as identify inflammatory mediators in patients with Crohn's disease. Whole blood gene array may also be useful for predicting disease behavior or response to therapy in Crohn's disease.

\section{S1322}

Biomarkers for Visilizumab Therapy of Intravenous Steroid Refractory Ulcerative Colitis (IVSR-UC)

Jacky Woo, James Sheridan, Stephen Keller, Jordan Hiller, Yu L. Chang, James Lowder, Vladimir Vexler

Background: Visilizumab (Nuvion®), a humanized low FcR-binding IgG2 anti-CD3 monoclonal antibody, is currently being tested for the treatment of IVSR-UC. In clinical studies to date $\sim 60 \%$ IVSR-UC subjects have responded to visilizumab treatment. Clinical responses are durable and persist long after visilizumab is cleared from the circulation. In this study, potential associations between clinical response and an array of possible blood biomarkers were evaluated. Methods: As part of a phase I/II dose range-finding study of visilizumab in IVSR-UC, IV bolus doses of visilizumab were administered on day 1 and day 2 at $5,7.5$, 10 , or $12.5 \mathrm{mg} / \mathrm{kg}$ dose. Treated subjects with a MTWSI score that dropped below 10, with at least a 3 points decline, were classified as responders. Absolute numbers of $C D 3+C D 4+$ and CD3+CD8+ T cells, CD3-CD56+CD16+ NK cells and CD3-CD 19+ B cells were measured by flow cytometry (TruCOUNTÔ). Serum cytokines, chemokines, and growth factors were measured using a validated 16-plex Luminex assay. Cell-mediated immunity (CMI) was assessed with ImmuKnowTM assay (Cylex®), which reflects the immuno-competence of peripheral CD4+ cells. Results: Of 76 visilizumab-treated subjects evaluated, 51 subjects

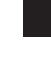


responded to treatment. No statistically significant associations of response with respect to changes in $\mathrm{CD} 3+\mathrm{CD} 4+\mathrm{T}, \mathrm{NK}$ and $\mathrm{B}$ cell counts, or $\mathrm{CMI}$ were observed. In contrast, absolute $\mathrm{CD} 3+\mathrm{CD} 8+\mathrm{T}$ cell counts were significantly higher on Day 30 in the responder group compared to non-responders. In addition, an increase in absolute CD3+CD8+cell counts on Day 30 was associated with improvement in mucosal healing. Although levels of many cytokines, chemokines, and growth factors were elevated in the first hours after visilizumab dosing, only IP-10 demonstrated a sustained increase. Further, between days 30 and 60 post-treatment, serum IP-10 levels were significantly higher in responders compared with non-responders. Conclusion: Increases in peripheral CD8+ T cell counts and serum IP-10 levels are observed long after visilizumab is cleared from the circulation and are associated with clinical response to visilizumab in IVSR-UC subjects. These efficacy biomarkers could be associated with durable clinical response to visilizumab and will be further evaluated with the aim of better understanding the mechanism of visilizumab.

\section{S1323}

A High Level of Methylated Mercaptopurine Metabolites Is a Cause of Myelotoxicity in IBD Patients Under AZA/6-MP

Xavier Roblin, Alain Chartier, Joanna Pofelski, Gaelle Hardy, Germain Bessard, Bruno Bonaz

High levels of methylated mercaptopurine metabolites (6-methyl mercaptopurine: 6-MMP 6-methylthioinosine monophosphate: 6-meTIMP) are potentially involved in AZA/6-MP hepatotoxicity or myelotoxicity $(1,2)$. Aim: to evaluate in a cohort of IBD patients under AZA/6-MP if the levels of 6-thioguanine nucleotides (6-TGN) or methylated mercaptopurine metabolites are correlated with hepatotoxicity or myelotoxicity of the compounds. Methods: consecutive IBD patients under AZA/6-MP followed in our department between March and September 2005 with 6-TGN and total methylated mercaptopurine metabolite (6-MMP and 6 -meTIMP) dosages were included. Hepatotoxicity was defined as transaminases $>3 \mathrm{~N}$ and/ or alkaline phosphatases $>3 \mathrm{~N}$ and/or GammaGT $>3 \mathrm{~N}$. Myelotoxicity was defined by a leucocyte count $<3000 / \mathrm{mm} 3$ and/or neutrophils $<1500 / \mathrm{mm} 3$ and/or platelets $<100$ 000/ $\mathrm{mm} 3$. AZA/6-MP imputability was retained after elimination of the other causes. Patients with TPMT gene mutation, either homo- or heterozygote were excluded. Results: 66 patients (20 UC, 46 CD; median age: 42.6 years, sex ratio: 1) were included. Fifteen cases of myelotoxicity were observed (10 leucopoenia, 7 neutropenia, and 2 thrombopenia). If the median 6-TGN level (pmoles/8x108 erythrocytes) was comparable in the groups of patients with and without medullary involvement [374 pmoles (260-620) vs 352 pmoles (230580); $\mathrm{p}=\mathrm{ns}]$, methylated mercaptopurine metabolite levels (pmoles/8x108 erythrocytes) were significantly higher in case of myelotoxicity [16450 pmoles (3922-26680) vs 4750 pmoles (1980- 8420); $\mathrm{p}=0.03$ ]. After logistic regression on the following parameters: age, sex, IBD type, 6-TGN and methylated mercaptopurine metabolite levels, only a methylated mercaptopurine metabolite level $>10000$ pmoles was isolated as a risk factor of myelotoxicity [OR: 18.5; IC95: 1.7-220; $\mathrm{p}=0.025]$. Four cases of hepatotoxicyty were observed without any relation with 6-TGN and/or methylated mercaptopurine metabolite levels. Conclusion: a high level of methylated mercaptopurine metabolites (6-MMP and 6-meTIMP > 10000 pmoles/8x108 erythrocytes) is highly associated (x18) with myelotoxicity in IBD patients under AZA/6-MP. Consequently, early monitoring of methylated mercaptopurine metabolites should be of interest to isolate high-risk patients. (1) Dubinsky M et al. Gastroenterology 2000; 118: 705-713. (2) Hindorf U et al. Gut 2005 (in press).

\section{S1324}

Immunomodulatory Therapy with Azathioprine Is Accompanied By a 28-Fold Increase in Concentrations of Mucosal Bacteria in Patients with Ulcerative Colitis and Indeterminate Colitis

Alexander Swidsinski, Yvonne Doerffel, Vera Loening-Baucke, Laura P. Hale, Franz Theissig, Hartwig Andree, Herbert Lochs

Background: The efficacy of the immunosuppressant drugs azathioprine and 6-mercaptopurine has been well established in the therapy of inflammatory bowel diseases (IBD). However, its use has been complicated by a high incidence of serious adverse drug reactions such as bone marrow and liver toxicity, pancreatitis and gastrointestinal disturbances. The impact of azathioprine on the mucosal flora is unknown. Aims: To compare the concentrations and spatial organization of mucosal bacteria in IBD patients treated with azathioprine and/or 5 aminosalicylic acid (5-ASA) using fluorescence in situ hybridization with $16 \mathrm{~S}$ rRNA-targeted probes. Methods: The mucosal bacteria were investigated in multiple colonoscopic biopsies from patients with ulcerative colitis or indeterminate colitis. Three different theraputic regimes were compared: azathioprine alone $(\mathrm{n}=20)$, azathioprine plus 5-ASA $(\mathrm{n}=20)$, and 5-ASA alone $(\mathrm{n}=20)$. The groups did not differ in age, gender disease duration, or concomitant prednisolone treatment. None of the patients had a history of antibiotic treatment in the last 12 months. Results: A steady increase in concentrations of mucosal bacteria $(\mathrm{p}<0.001)$, the percent of the intestinal surface covered with adherent biofilms $(p<0.001)$, and the amenability of mucosal bacteria to FISH (a measure of ribosomal content and thus metabolic activity) $(\mathrm{p}<0.01)$ was seen in patients treated with azathioprine alone compared to those treated with 5-ASA alone The concentrations of adherent Bacteroides and Enterobacteriaceae was also higher in azathioprine-treated groups (74\% versus 52\%). Despite this increase in bacteria, the histological signs of the inflammation and the number of leukocytes within mucus was lower in azathioprine treated patients. The combined azathioprine/5-ASA group took an intermediate position for all investigated parameters. Conclusions: Azathioprine reatment effectively controls inflammation in IBD but has minimal to no influence on the disturbed mucosal flora that is characteristic of IBD. Future longitudinal studies are in progress to determine whether azathioprine treatment may enhance the development of adherent bacterial biofilms by suppressing the local inflammation.

\section{S132}

Bowel Sonography for the Diagnosis and Grading of Post-Surgical Recurrence of Crohn's Diseas

Antonio Rispo, Luigi Bucci, Francesco Sabbatini, Giovanni de Palma, Roberto Grassia, Alessandro Compagna, Anna Testa, Dario D'Antonio, Fabiana Castiglione

BACKGROUND: Post-surgical recurrence (PSR) is very common in patients with Crohn's disease (CD) and previous surgery. Endoscopy is crucial for the diagnosis of PSR, also showing high prognostic value. Bowel sonography (BS) is accurate for CD diagnosis but its role in PSR detection and grading is poorly investigated. AIM: to evaluate the diagnostic accuracy of BS for PSR compared to the endoscopical Rutgeerts's grading system. PATIENTS AND METHODS: From March 2002 to October 2005 we prospectively performed endoscopy and $\mathrm{BS}$ in $45 \mathrm{CD}$ patients with previous bowel resection to gain evidence of possible PSR. Endoscopy and BS were executed 1 year after surgery, with PSR diagnosis and grading made in accordance with Rutgeerts's. BS was considered suggestive for PSR in presence of bowel wall thickness (BWT) $>3 \mathrm{~mm}$. Diagnostic accuracy was calculated by StatsDirect software. Also, a ROC curve was constructed in order to define the best cut-off of BWT to discriminate mild from severe PSR (grade 0-2 vs 3-4 of Rutgeerts's). Cohen's k measure was applied to test the consistency between endoscopy and BS for the grading of PSR. RESULTS: 24 out of the $45 \mathrm{CD}$ showed an endoscopical evidence of PSR (53\%). A severe PSR was present in 16 patients $(66 \%)$. Sensitivity, specificity, positive and negative predictive values of BS were $80 \%, 93 \%, 92 \%$ and $80 \%$, respectively. All the patients with a false negative BS presented a mild PSR at endoscopy. On ROC curve, a BWT $>5 \mathrm{~mm}$ showed sensitivity, specificity, positive and negative predictive values of $94 \%, 92 \%, 90 \%$ and $96 \%$ for the diagnosis of severe PSR, with a remarkable agreement with endoscopy $(k=0.89)$. CONCLUSIONS: BS shows good sensitivity and high specificity for the diagnosis of PSR in CD, with a BWT $>5 \mathrm{~mm}$ strongly indicative of severe endoscopical PSR. Accordingly, BS could replace endoscopy for the diagnosis and grading of PSR in many cases.

\section{S1326}

Effectiveness of Darbepoetin Alfa in Combination with Intravenous Iron Sucrose in Patients with Inflammatory Bowel Disease and Refractory Anemia Ioannis Koutroubakis, Konstantinos Karmiris, Sokratis Makreas, Costas Xidakis, Georgia Tsiolakidou, Elias Kouroumalis

Background: The combination of intravenous iron and recombinant human erythropoietin (EPO) has been proved to be effective in the treatment of refractory anemia in patients with inflammatory bowel disease (IBD). Darbepoetin alfa (DPO) has a three fold longer terminal half-life than EPO. The purpose of this pilot study was to determine whether DPO is also effective for the treatment of refractory anemia in IBD. Methods: As cases with refractory anemia were considered patients who had received one course of intravenous iron sucrose (mean total iron dose $1.2 \pm 0.4 \mathrm{~g}$ ) with poor response (no increase of hemoglobin, or increase $<2.0 \mathrm{~g} / \mathrm{dl}$ ) during a six month of follow up. Twenty IBD patients ( 9 ulcerative colitis and 11 Crohn's disease) and refractory anemia received intravenous iron sucrose (total iron dose $1.3 \pm 0.5 \mathrm{~g}$, range $0.7-1.9$ ) and DPO at the single, weekly dose of $0.9 \mu \mathrm{g} / \mathrm{Kg}$ s.c. for 4 weeks. Serum erythropoietin, ferritin, tranferrin, soluble tranferrin receptor (sTfR), C-reactive protein and interleukin 6 (IL-6) were measured at baseline and after treatment. Results: Hematopoietic response (increase of hemoglobin $>$ or $=2.0 \mathrm{~g} / \mathrm{dl}$ ) was observed in 15 out of the 20 patients $(75 \%)$. The mean hemoglobin concentrations increased from $9.48 \pm 0.82$ $\mathrm{g} / \mathrm{dl}$ at baseline to $12.71 \pm 1.12 \mathrm{~g} / \mathrm{dl}$ after treatment $(\mathrm{P}<0.0001)$. Mean corpuscular volume (MCV) and serum ferritin levels were also significantly increased whereas mean CRP levels and endogenous erythropoietin levels significantly decreased after treatment $(\mathrm{P}<0.05)$. CRP, IL6 and ferritin levels were higher in responders compared to non-responders. However, the differences did not reach statistical significance due to the small number of non-responders. Patients on therapy with infliximab had also a trend for a prompt response but the difference compared to patients without infliximab was not statistically significant $(\mathrm{P}>0.05)$. DPO treatment was well tolerated without adverse events. Concerning iron treatment only one patient experienced an adverse event (facial rash). Conclusions: Our results suggest that darbepoetin, in conjunction with intravenous iron sucrose, may be of use in patients with inflammatory bowel disease and severe anemia refractory to iron therapy. The use of darbepoetin at higher doses might be more effective than in the present study Further randomized studies directly comparing darbepoetin alfa with recombinant human erythropoietin for the treatment of refractory anemia in IBD should be conducted.

\section{S1327}

Hepatotoxicity of Long-Term and Low-Dose 6-Thioguanine in IBD Patients Nanne K. De Boer, Lennard P. Gilissen, Luc J. Derijks, Gijsbertus Den Hartog Berend D. Westerveld, Leopold G. Engels, Adriaan A. van Bodegraven, Elisabeth Bloemena, Chris J. Mulder

Introduction Initial data on short-term efficacy and toxicity of 6-thioguanine (6-TG) in IBDpatients who were intolerant or refractory to azathioprine (AZA) or 6-mercaptopurine (6MP) were promising, however these have been challenged by recent reports concerning its potential hepatotoxic effect (nodular regenerative hyperplasia (NRH) and veno-occlusive disease (VOD)). Nonetheless, the hepatotoxicity of 6-TG may be a dose-dependent phenomenon. Aims \& Methods In the Netherlands 6-TG therapy was generally initiated in a standard dosage of $20 \mathrm{mg} /$ day, which is significantly lower than reported dosages in literature (40 to $80 \mathrm{mg} /$ day). We conducted a study in IBD-patients treated with 6-TG for at least 30 successive months to evaluate liver histology, its relationship with 6-TG use, laboratory parameters (including 6-TGN measurements and TPMT genotyping), and abdominal ultrasonography. Results Thirty patients (F:13/M:17 and CD:17/UC:13) underwent a liver biopsy after using 6-TG (median dosage $20 \mathrm{mg}$ ) for a mean period of 39 months (range 30-53 months). 6-TG therapy was initiated due to intolerance to AZA/6-MP ( $\mathrm{N}=26)$, de novo ( $\mathrm{N}=$ $3)$ and refractory to AZA/6-MP $(\mathrm{N}=1)$. No clinically relevant changes in liver tests and whole blood counts (no thrombocytopenia) were observed over time. The mean 6-TGN level was 580 pmol/8x10E8 RBC (SD 296, range 106-1199). TPMT genotyping $(\mathrm{N}=21)$ revealed only 
one patient with a mutant TPMT allele (6-TGN: $1092 \mathrm{pmol} / 8 \times 10$ E8 RBC). Ultrasonography showed two cases of steatosis $(7 \%)$, but no splenomegaly. The mean cumulative dose of 6 TG was $22645 \mathrm{mg}$ (range $12150-44400 \mathrm{mg}$ ). Histological evaluation revealed: no abnormalities $(N=15,50 \%)$, slight regenerative changes $(N=7,23 \%)$, slight steatosis $(N=3,10 \%)$, slight fibrosis ( $\mathrm{N}=2,7 \%$ ( $1 \mathrm{x}$ alcohol abuse)), suspicion of $\operatorname{VOD}(\mathrm{N}=1,3 \%)$, steatosis+mild fibrosis $(\mathrm{N}=1,3 \%$ (apparently NASH)) and primary sclerosing cholangitis+hemochromatosis $(\mathrm{N}=1$, $3 \%$ ). No cases of NRH were detected. The 6-TGN level, cumulative dose and TPMT status were not correlated with pathohistological findings. Conclusion In the majority of this cohort of IBD-patients, long-term use of low-dose 6-TG (20 mg/day) did not induce clinically relevant histological liver abnormalities. Hepatotoxicity due to 6-TG use may indeed be dependent on the dosage of 6-TG. Nonetheless, rigorous and frequent monitoring (including a liver biopsy) of IBD patients using low-dose 6-TG as a rescue drug remains mandatory.

\section{S1328}

Endoscopic Scores of Postoperative Recurrence in Crohn's Disease: A Reproducibility Study

Philippe Bulois, Hugues Coevoet, Laurence Martin, Jean-Yves Mary, Yoram Bouhnik, Arnaud Bourreille, Guillaume Cadiot, Franck Carbonnel, Benoit Coffin, Bernard Duclos, Jean-Louis Dupas, David Laharie, Edouard Louis, Philippe Marteau, Jacques Moreau, Jean-Claude Soule, Jean-Frederic Colombel, Marc Lemann, Getaid The

Assessment of endoscopic activity is of growing importance in Crohn's disease (CD) both in clinical practice and in therapeutic trials. The Rutgeerts's score (RS) is considered as the standard to evaluate endoscopic recurrence after surgery. The Crohn's Disease Endoscopic Index of Severity (CDEIS) is a prospectively developed and validated score widely used in $\mathrm{CD}$ trials, but not in this specific setting. The aim of this prospective study was to compare the two scores and to evaluate their reproducibility in assessing postoperative (po) endoscopic recurrence. Methods. Endoscopic data of 72 patients participating in two multicentre randomized clinical trials for the prevention of endoscopic recurrence after curative ileocolonic resection were used for the purpose of this study. During each procedure, endoscopic recurrence was graded using the RS ranging from i0 (no lesion) to i4, and the po-CDEIS was calculated for two segments (ileum and anastomosis). Endoscopies were recorded on videos in 39 patients, and each record was re-evaluated by 4 of 13 independent blinded observers using a balanced incomplete block randomization. The reproducibility of the two scores was calculated using the kappa estimates for the RS (agreement was considered as poor, moderate, good and excellent for kappa coefficients $<0.4,0.4-0.6,0.6-0.8$ and $>0.8$, respectively), and the intraclass correlation coefficient for the po-CDEIS. Results. In the whole population $(\mathrm{n}=72)$, a significant correlation was found between the RS grade and the median po-CDEIS score $(\mathrm{p}<0.001)$, but with overlap, especially for the i2 and i3 grades. In the subset of 39 patients used to assess the interobserver reproducibility, the kappa estimates were 0.51 (95\%CI: $0.38-0.64), 0.16(0.02-0.20), 0.40(0.30-0.50), 0.19(0.03-0.35)$, and $0.72(0.61-0.83)$ for the i0, il, i2, i3 and i4 grades of the RS, respectively. The kappa estimate was $0.57(0.43-0.71)$ for the RS globally, $0.45(0.23-0.66)$ for a score $>11$, and 0.64 $(0.44-0.83)$ for a score $>\mathrm{i} 2$. The intraclass correlation coefficient for the po-CDEIS was 0.52 (95\%CI: 0.36-0.67; $<<0.001$ ). Conclusion. The Rutgeerts score and the po-CDEIS correlate for assessment of endoscopic postoperative recurrence but the reproducibility of the two scores is moderate. Definition of endoscopic recurrence based on the sole use of a cutoff value is questionable.

\section{S1329}

Inherent Resistance to 6-Thioguanine (6-TG) Induced Apoptosis Correlates with Disease Activity in Children with IBD

Ryan S. Carvalho, James A. Mahoney, Maria M. Oliva-Hemker, Carmen Cuffari

Introduction: The pathogenesis of IBD may represent an inherent failure to suppress the normal immune response (Neurath et al. Trends in Immunology 2001) to antigenic stimulation. Although most immunosuppressive drugs, including azathioprine are purported to facilitate drug induced T-lymphocyte apoptosis in adult patients with Lupus and IBD, there is little data in children (Ringheanu et al. IBD 2004). Aim: To demonstrate the inherent resistance of peripheral T-cell lymphocytes to 6-TG induced apoptosis in children with IBD compared to healthy volunteers. Methods: Peripheral blood was collected in CPT Ficoll tubes. PBMCs were isolated and suspended in RPMI 1640 at a concentration of lmillion cells $/ \mathrm{ml}$. Cells were then stimulated with Concovin A at a concentration of $5 \mathrm{mcg} / \mathrm{ml}$ and incubated with 6TG (6 mcM) for 72-96 hours (Tiede et al. J Clin Invest 2003). Simultaneous controls were incubated without the drug. Only double positive cells for Annexin V PE for PS and TOPRO-3 for DNA were quantified as positive for apoptosis. The cells were also gated for CD3 after staining with FITC-CD3. Results: The mean (SEM) percentage of apoptosis was significantly $(\mathrm{p}<0.004)$ higher in healthy volunteers [31.7 (3.6)] compared to children with IBD [13.4 (0.9)] at baseline, and after in vitro exposure to 6TG [59.4 (6.2) vs 47 (4.5), $\mathrm{p}<0.05$ ). This trend of apoptosis was also mirrored by CD3 gated Annexin V positive T lymphocytes and was also shown to be higher than that observed in adult patients with IBD (Fig 1). Conclusions: Inherent resistance to apoptosis may explain the immune pathogenesis of IBD in children. While inter-patient differences in 6-TG induced T-cell lymphocyte apoptosis may correlate with clinical responsiveness to azathioprine therapy, future studies are needed to determine if the current trend in starting anti-metabolite therapy sooner correlates with an overall improved maintenance of clinical response.

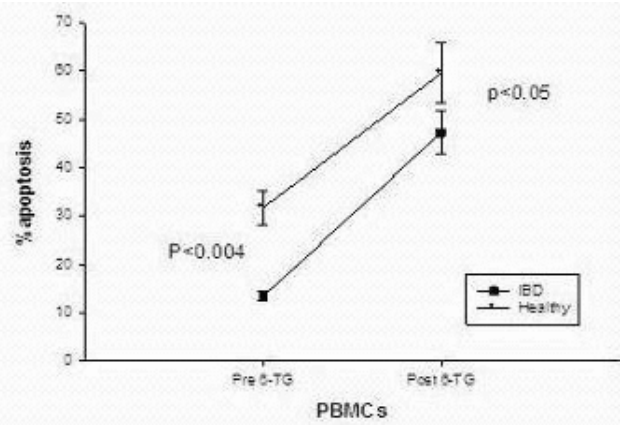

S1330

IgM Anti $\alpha$-Enolase Antibody As a Serologic Marker in Patients with Intestinal Bechet's Disease: Relation to Clinical Course

Sung Jae Shin, Tae Il Kim, Byung Chang Kim, Sooyoung Park, Jae Yong Jang, Kwang Hoon Lee, Chang Hwan Choi, Won Ho Kim

Background/Aim: Intestinal Behcet's disease (BD) is a chronic inflammatory bowel disease such as Crohn's disease (CD) and ulcerative colitis (UC). But, unlike CD (ASCA) and UC (pANCA), a serologic marker for intestinal BD is not well known. Recently, IgM anti $\alpha$ enolase antibody, one of the anti-endothelial cell antibodies, was detected in sera of BD patients. It serves as a plasminogen receptor on the surface of endothelial cells, and modulates pericellular and intravascular fibrinolysis. The aim of this study is to evaluate the prevalence of IgM anti $\alpha$-enolase antibody in patients of intestinal BD and inflammatory bowel disease, and it's clinical correlations. Methods: One hundred patients with intestinal BD, 100 patients with Crohn's disease, 100 patients with ulcerative colitis and 23 healthy controls were included for serologic test. IgM anti $\alpha$-enolase antibody was detected by ELISA. The positivity of IgM anti $\alpha$-enolase antibody was defined as an optical density greater than the mean plus 3SD for the 23 normal control sera. In 80 patients with intestinal BD, who were followed for mean 88.8 months after the diagnosis, the various parameters such as demography subtype of $\mathrm{BD}$, colonoscopic findings and treatment modality were collected from medical records retrospectively. Because there was no activity index for intestinal BD, the HarveyBradshaw index (HBI) for $\mathrm{CD}$ was used for disease activity estimation of intestinal BD Results: The prevalence of IgM anti $\alpha$-enolase antibody was $63 \%$ in intestinal BD, $31 \%$ in Crohn's disease, $40 \%$ in ulcerative colitis and $0 \%$ in healthy controls. The positivity rate of IgM anti $\alpha$-enolase antibody was significantly higher in female patients with intestinal BD (male vs. female; $57.4 \%$ vs. $81.8 \%, \mathrm{p}=0.022$ ). Also, it was higher in complete or incomplete BD than suspicious BD $(77.5 \%$ vs. $51.6 \%, \mathrm{p}=0.033)$. IgM anti $\alpha$-enolase antibody $(+$ patients had higher mean HBI score than $(-)$ patients $(5.60$ vs. $4.61, p=0.003)$ and the leve of IgM anti $\alpha$-enolase antibody correlated with the HBI score $(r=0.224, p=0.046)$. Steroid treatment and cumulative steroid use rate for intestinal as well as extra-intestinal lesions were higher in IgM anti $\alpha$-enolase antibody (+) patients than (-) patients ([81.5\% vs. 53.8\%, $\mathrm{p}=0.031]$, [p=0.022], respectively). However, there was no correlation between IgM anti $\alpha$ enolase antibody expression and the frequency of intestinal operation due to disease flareup. Conclusions: Prevalence of IgM anti $\alpha$-enolase antibody was $63 \%$ in intestinal BD Patients with IgM anti $\alpha$-enolase antibody-positive intestinal BD showed higher disease activity and steroid use rate.

\section{S1331}

Crohn's Disease in Pregnant Women and Nursing Mothers: Which Drugs Are Safe? A Multidisciplinary European Expert Panel (EPACT)

Christian Mottet, Pascal Juillerat, Christian Felley, Vincent Wietlisbach, Valerie Pittet, Jean-Jacques Gonvers, Robert W. Dubois, John-Paul Vader, Bernard Burnand, Florian Froehlich, Pierre Michetti

Introduction: High-grade evidence (randomized controlled trials) is lacking regarding the use of drugs in pregnant and nursing Crohn's Disease (CD) patients. Explicit panel-based safety criteria may therefore provide the best available alternative for clinical decision-making Methods: An international multidisciplinary expert panel (EPACT, European Panel on the Appropriateness of Crohn's Disease Therapy) from 12 European countries (10 gastroenterologists, 3 surgeons, 2 general practitioners) convened in Lausanne, Switzerland, to judge the appropriateness of $C D$ therapy, including safety of therapy for pregnant $C D$ patients, using a combined evidence- and panel-based method (RAND Appropriateness Method). Treatment options for $\mathrm{CD}$ in these patients before conception and in the first trimester, in late pregnancy, as well as during nursing, were submitted to the panelists for an individual rating on a 9point scale: 1 = very unsafe, 9 = extremely safe. For the analysis, a median of 7-9 was considered as safe (S); 4-6 equivocal (E); 1-3 unsafe (U). Results: Panel results are shown in the following table with the FDA pregnancy categorization of drug risk to the fetus in the last column, from "Category A" = safest to "Category D" = fetal risk. Conclusion: Mesalazine, sulfasalazine and prednisone were considered safe during pregnancy and nursing. The panel position was, however, cautious and mainly based on label recommendations despite an accumulation of evidence from databases on the safety of AZA/6-MP and infliximab before conception and during the first trimester. These safety criteria are freely available on the internet (www.epact.ch) and may be helpful in clinical decision-making. 


\begin{tabular}{|c|c|c|c|c|}
\hline & $\begin{array}{c}\text { Before conception } \\
\text { +first trimester }\end{array}$ & $\begin{array}{c}\text { Late } \\
\text { pregnancy* }\end{array}$ & Nursing & $\begin{array}{c}\text { FDA category of } \\
\text { drug risk }\end{array}$ \\
\hline Mesalazine & $\mathrm{S}$ & $\mathrm{S}$ & $\mathrm{S}$ & $B$ \\
\hline Sulfasalazine & $\mathrm{S}$ & $\mathrm{S}$ & $\mathrm{S}$ & $B$ \\
\hline $\begin{array}{c}\text { Antibiotics } \\
\text { (ciproxine/metronidazole) }\end{array}$ & $\mathrm{U}$ & $\mathrm{E}$ & $\mathrm{U}$ & $\begin{array}{c}C \text { ciproxine } B \\
\text { metronidazole }\end{array}$ \\
\hline Budesonide & $\mathrm{E}$ & $\mathrm{E}$ & $\mathrm{E}$ & $C$ \\
\hline Prednisone & $\mathrm{S}$ & $\mathrm{S}$ & $\mathrm{S}$ & $B$ \\
\hline AZA/6-MP & $\mathrm{E}$ & $\mathrm{S}$ & $\mathrm{E}$ & $D$ \\
\hline Infliximab & $\mathrm{E}$ & $\mathrm{E}$ & $\mathrm{E}$ & $B$ \\
\hline Ciclosporine & $\mathrm{U}$ & $\mathrm{U}$ & $\mathrm{U}$ & $C$ \\
\hline
\end{tabular}

* from the end of the first trimester. NB: methotrexate (FDA category X, demonstrated teratogenicity) was considered contraindicated and was therefore not subjected to vote.

\section{S1332}

Visilizumab Treatment Promotes Morphological Recovery, Reduces Inflammatory Markers and Affects T Cell Subsets in Mucosa of Ulcerative Colitis Patients

Lili Zhang, Mien Sho, Mian Su, Jim Lowder, Jacky Woo, Debbie Law

Introduction: In the 291-408 open-label Phase I/II trial, 60\% of subjects with intravenous steroid refractory ulcerative colitis (IVSR-UC) improved upon treatment with visilizumab, a humanized anti-CD3 antibody. The effects of visilizumab treatment on morphology and inflammatory cells/marker expression in the mucosa of subjects were assessed. Methods: Subjects with endoscopically diagnosed UC and an MTWSI > 11 who failed to respond to at least 5 days of i.v corticosteroids were eligible for the study. Response was defined as an MTWSI less than 10 with a drop of at least 3 points. Colonic biopsies were collected at baseline and Day 30. Formalin-fixed samples from 14 subjects underwent blinded histological evaluation. Hemotoxylin \& eosin-stained sections were scored using a system that assesses both morphological changes and the severity of inflammation. Immunohistochemistry (IHC) was used to examine the expression of inflammatory markers and cell types in serial sections stained with antibodies specific for Ki67, STAT1, CXCR3, NFkB, CD3 and CD8. Results: Histological and morphological changes associated with inflammation (such as increased numbers of mononuclear cells (MNC) and neutrophils, crypt destruction, and the presence of ulcers/erosions), were present in all baseline biopsies. By Day 30, biopsies from 9 of 11 clinical responders showed histological improvement, with mucosal healing observed in 2 of 11 subjects. No histological improvement was observed in 3/3 samples from non-responders. Based on a qualitative evaluation, the expression of inflammatory markers was decreased in Day 30 samples from most responders. Ki67, a marker of proliferating cells showed the greatest reduction. In contrast, biopsies from all 3 non-responders showed increased or unaltered staining for inflammatory proteins. Further analysis of the T cells present within the biopsies showed that $\mathrm{CD} 3+\mathrm{T}$ cell numbers were reduced in the majority of responders but remained static or increased in the 3 non-responders. Interestingly, the percentage of the remaining $\mathrm{T}$ cell population that was $\mathrm{CD} 8+$ increased after treatment with visilizumab but only in responders. Conclusions: The histological evaluation of biopsy samples pre and post-visilizumab treatment demonstrates a decrease in histological disease activity in the majority of clinical responders. Clinical response to visilizumab also coincides with reduction of mononuclear cell proliferation in the lamina propria (assessed by Ki67 staining); reduction in other inflammatory markers; and a relative increase of CD8+ cells within the remaining $\mathrm{CD} 3+\mathrm{T}$ cell population in the colonic mucosa.

\section{S1333}

Validation of the New "Phical" Faecal Calprotectin Test As a Diagnostic Tool for Pouchitis

Matthew W. Johnson, Susan Maestranzi, David H. Dewar, Alastair Forbes, Ingvar Bjarnason, Roy Sherwood, Paul J. Ciclitira, John R. Nicholls

One of the hallmarks of pouchitis is the polymorphonuclear granulocyte (PMN) infiltration of the mucosa, thought to be driven by dysbiosis. Calprotectin is a stable antimicrobial protein produced by activated PMN. The use of the new "PhiCal" faecal Calprotectin assay is already well established as a diagnostic tool in inflammatory bowel disease. We aimed to validate its use in pouchitis. 54 stool samples were taken from 49 ileal pouch patients. The samples were processed and analysed using a qualitative type enzyme linked immunosorbant assay ("PhilCal ELISA New Method", Eurospital, Italy). 41 had ulcerative colitis (UC) and 8 had familial adenomatous polyposis (FAP) coli. Pouchitis was defined as an objective pouchitis score (OPS) of $\geq 5 / 12$ (endoscopic score of $\geq 3 / 6$ and an acute histological score of $\geq 2 / 6$ ) or a pouch disease activity index (PDAI) of $\geq 7 / 18$. Statistical analysis was performed using Spearman Rank and Mann-Whitney. The faecal Calprotectin concentration correlated well with the OPS $(r=0.68, p<0.0001)$, PDAI $(r=0.7, p<0.0001)$, endoscopy score $(r=0.6$, $\mathrm{p}<0.0001)$, acute histological score $(\mathrm{r}=0.71, \mathrm{p}<0.0001)$ and neutrophil score $(\mathrm{r}=0.68$, $\mathrm{p}<0.0001)$, but correlated poorly with patient's clinical symptoms $(\mathrm{r}=0.3, \mathrm{p}=0.03)$. Using the OPS, 6 patients were diagnosed with UC pouchitis (+ pre-pouch ileitis), 13 with UC pouchitis alone, 27 had a healthy UC pouch, 1 had FAP pouchitis (+ pre-pouch ileitis) and 7 had a healthy FAP pouch. Respectively, median faecal Calprotectin values $(\mathrm{mg} / \mathrm{kg}$ ) for these groups were $865,145,56,305$ and 9 , with means [ $95 \%$ confidence intervals] of 1073 [188.5-1958], 888.8 [174.1-1603], 147 [71.8-222.2], 305 and 12.86 [5.1-20.6]. Statistically significant differences were noted when comparing inflamed and non-inflamed pouches $(\mathrm{p}<0.0001)$. The pre-pouch ileitis group had higher values than those with pouchitis alone but this proved non-significant $(\mathrm{p}=0.2)$. Using the upper normal limit for calprotectin as $50 \mathrm{mg} / \mathrm{kg}$, the receiver operating characteristic analysis revealed a sensitivity of $53 \%$, a specificity of $90 \%$ and a false negative rate of $<5 \%$. Using a higher threshold of $92.5 \mathrm{mg}$ $\mathrm{kg}$ a sensitivity of $76.5 \%$ and a specificity of $90 \%$ were demonstrated. Similar results were obtained using the PDAI, albeit with a slightly lower specificity level. The new "PhiCal" faecal Calprotectin test is a useful non-invasive diagnostic tool for differentiating between healthy and inflamed ileal pouches. This simple test could be use to rationalise the management of ileal pouch patients before exposing them to the potential risks of empirical antibiotic therapy and endoscopic investigations with biopsies.

\section{S1334}

CBIRl Serves As a Multi-Functional Predictor of Inflammatory Bowel Disease in a Six-Marker System

Augusto Lois, Leonard Eggleston, Susan Carroll, Esther Oh, Mary Barry, Bruce Neri

The flagellin-like antigen CBirl was originally identified in the sera from $\mathrm{C} 3 \mathrm{H} / \mathrm{HeJBir}$ mice with colitis and was associated with the development of inflammatory bowel disease (IBD). IBD comprises at least two different diseases, Crohn's disease (CD) and ulcerative colitis (UC). Anti-CBirl antibodies have been found in humans diagnosed with IBD and especially in patients with CD. Other markers associated with IBD in humans include antibodies against several antigens from normal intestinal microbial flora. The identification of these antibodies has provided the means to develop diagnostic tests that identify patients with IBD and that can distinguish UC and CD. The development of a sensitive and robust ELISA assay that quantitatively measures the levels of anti-CBirl IgG antibodies from human serum is described here. The IgG-CBirl ELISA assay was combined with five other known IBDassociated markers to create a new six-marker system. The markers consisted of $\operatorname{IgG}$ antibodies to CBirl, IgA to the E. coli associated outer membrane protein C (OmpC), IgA and IgG anti-Saccharomyces cerevisiae mannan antibodies (ASCA-IgA and ASCA-IgG), anti-neutrophil antibodies with DNase-sensitive perinuclear staining. The contribution of CBirl to the diagnosis of IBD and to the differentiation of $\mathrm{UC}$ and $\mathrm{CD}$ was then assessed. IBD-predictive models were created using classification and regression trees (C\&RT). The best model showed that CBirl is useful in this six-marker system to classify CD patients and also to identify a large percentage of the UC population. The C\&RT model was tested in an 813 patient cohort with $60 \%$ IBD prevalence and equivalent numbers of CD and UC samples. The C\&RT model predicted IBD with a sensitivity of $88 \%$ and specificity of $81 \%$ and correctly predicted normal, CD and UC patients from the levels of CBirl in 3 separate nodes (decision points of the classification tree). For example, if the levels of ASCA IgG are below the model's cut-off (22 Elisa Units [EU]), ANCA ELISA levels are above 14.1 EU and CBirl is <80 EU, the model correctly identified most of the UC samples in the cohort. However, if the levels of CBirl were above $80 \mathrm{EU}$, the model correctly predicted CD. Conclusion: CBirl was included in a new six-marker system that utilizes a classification tree model for data analysis. The levels of CBirl are useful for decision making at various points of the classification tree indicating a broad role for CBirl in the prediction of IBD

\section{S1335}

A New Six-Marker System That Includes CBIR1 Has Greatly Improved Prediction Accuracy for Inflammatory Bowel Disease and for Differentiation of Ulcerative Colitis and Crohn's Disease Compared to Commonly Used Testing with ANCA and ASCA

Augusto Lois, Leonard Eggleston, Susan Carroll, Esther Oh, Mary Barry, Bruce Neri

Correct and quick diagnosis of Inflammatory Bowel Disease (IBD) can be a challenging undertaking for physicians. Serological testing has become an important component of the process to diagnose IBD. In this report, CBirl was included in a new six-marker system analyzed by a novel algorithm which significantly improved the accuracy of IBD diagnosis. The six-marker system includes IgA to the E. coli-associated outer membrane protein C (OmpC), IgA and IgG anti-Saccharomyces cerevisiae mannan antibodies (ASCA-IgA and ASCA$\operatorname{Ig} G$ ), anti-neutrophil antibodies detected in alcohol-fixed neutrophils (ELISA) with a DNasesensitive perinuclear stain and IgG antibodies to CBirl. The new algorithm and IBD sixmarker system was validated in multiple cohorts consisting of normal individuals, Irritable Bowel Syndrome (IBS) patients, UC patients and patients with CD (IBD prevalence ranged from. 60\%-63\%). Analysis of the performance of the test system showed significant improvement in the sensitivity and the specificity of IBD detection and the differentiation of CD and UC. Greater than 90\% sensitivity and specificity was attained for IBD prediction in an 813 patient cohort including UC and CD and normal and IBS samples. The positive predictive value of the IBD six-marker system was greater than $90 \%$. The negative predictive value was slightly lower but greater than $75 \%$. Receiver Operating Characteristic (ROC) analysis indicated that the assay was accurate with an area under the curve above 0.9 . Detection of CD was substantially increased with a sensitivity of $88 \%$ and specificity above $90 \%$. UC detection increased similarly to a total sensitivity of $88 \%$ and specificity $>90 \%$. IBD detection using cut-off values with ANCA and ASCA alone or in combination has very poor sensitivity $(<40 \%)$. Conclusion: Inclusion of CBirl in a new six-marker system with a novel algorithm dramatically improved the accuracy of IBD diagnosis

\section{S1336}

Small Intestine Contrast Ultrasonography (SICUS) Versus Videocapsule Endoscopy (VCE) for Assessing Crohn's Disease Post-Operative Recurrence: A Prospective Longitudinal Study

Livia Biancone, Emma Calabrese, Rosa Maria Bozzi, Carmelina Petruzziello, Sara Onali, Micaela Cretella, Anna Caruso, Alessandra Geremia, Francesco Pallone

Background. Small intestine contrast ultrasonography (SICUS) is a non-invasive technique able to detect small bowel lesions in Crohn's Disease (CD). Videocapsule endoscopy (VCE) allows the visualization of the small bowel inner surface. As $C D$ patients require endoscopic assessment after surgery, non-invasive techniques able to detect the postoperative recurrence may well be of use. The usefulness of SICUS and VCE for assessing the postoperative recurrence of $C D$ is unknown. Aims. We aimed to investigate, in a prospective, longitudinal study, the usefulness of SICUS in comparison with VCE for assessing the postoperative recurrence of $\mathrm{CD}$ patients (pts) 1 year after ileo-colonic resection, when using conventional 
colonoscopy (CC) as gold standard. Methods. $14 \mathrm{CD}$ pts (8 M, age range 25-64 yrs) undergoing ileo-colonic resection for CD were prospectively followed up from July 2003 to November 2005. After surgery, all patients underwent clinical assessment (CDAI) every 3 months for 1 year. At 1 year, CD recurrence was assessed by 3 independent investigators by SICUS and CC, followed by VCE. CD recurrence was assessed by CC (Rutgeerts' score) SICUS was performed after PEG ingestion $(375 \mathrm{ml})$. VCE was performed by using the M2A Given $(11 \times 26 \mathrm{~mm})$ (median excretion time $28 \mathrm{hrs}$ ). Lesions compatible with recurrence assessed by SICUS and VCE were recorded. Results. No pts. showed side effects. At 1 year, all 14 patients were inactive $(C D A I<150)$. In 1 pt, VCE was not performed due to an anastomotic stricture detected by CC. Comparison between the 3 techniques was therefore made in 13/14 pts. CC detected CD recurrence in 12/13 pts. Lesions compatible with recurrence were detected by SICUS in 13/13 pts ( 1 false positive) and by VCE in the same $12 / 13$ pts showing endoscopic recurrence (12 true positives). CC detected recurrence at both the anastomosis and PA-ileum in 9/13 pts and at anastomosis only in 3/13 pts. Recurrence was detected by SICUS at both the anastomosis and PA-ileum in 10/13 (1 false positive) and at the anastomosis only in the same $3 / 13$ pts detected by CC ( 3 true positives). VCE detected CD recurrence at both the anastomosis and PA-ileum in $7 / 13$ pts (2 false negatives) and at the anastomosis only in 5/13 pts (2 false positives). VCE therefore appeared to appropriately detect the presence, but not the site of $\mathrm{CD}$ recurrence, which appeared better defined by SICUS. Conclusions. Present findings from a prospective longitudinal study suggest that SICUS and VCE represent alternative non-invasive technique for assessing $\mathrm{CD}$ recurrence in patients under regular follow-up after ileo-colonic resection. VCE use however requires the exclusion of stricturing recurrence.

\section{S1337}

Alterations in Composition and Activity of Faecal Microbiota During AntiTNF- $\alpha$ Antibody Treatment in Crohn's Disease: A Pilot Study H.M.C. Van Nuenen, C.J. van der Woude, K. Venema, G. Welling, E.J. Kuipers

Crohn's disease (CD) is a multifactorial disease. Gastrointestinal microorganisms and their metabolites may play a role in CD. We aimed to determine whether anti-TNF- $\alpha$ monoclonal antibody therapy for $\mathrm{CD}$ has an effect on the composition of their faecal microbiota. It was hypothesized that if the microbial composition correlates with clinical disease activity, microbial metabolites possibly do as well, given their relationship with the microbial composition. Therefore, the second aim of this study was to investigate the possible use of microbial metabolites as markers for disease activity in CD. The study population consisted of 23 subjects divided over three groups: healthy individuals, IBD patients in remission $(\mathrm{CDAI}<100)$ and patients with active $C D$ (CDAI 107-265). The latter patients were studied twice: immediately before and two weeks after a single, first infusion of anti-TNF- $\alpha$ antibody. Microbial composition was determined using the classical plating technique and fluorescent in situ hybridisation (FISH), and the following potential disease activity markers were determined in the faecal samples: dry matter, $\mathrm{pH}$, short-chain fatty acid, branched-chain fatty acids, and lactate. Anti-TNF- $\alpha$ antibody therapy led to an increase of the density of the faecal bacterial population (plating: $1.1 * 108 \mathrm{CFU} / \mathrm{g}$ before treatment, $2.4 * 108 \mathrm{CFU} / \mathrm{g}$ after treatment; $\mathrm{p}=0.07$, FISH: 3.5*109 CFU/g before treatment, $3.0 * 1010 \mathrm{CFU} / \mathrm{g}$ after treatment; $\mathrm{p}=$ 0.07 ), as well as an increase in faecal D-lactate compared to L-lactate ( 0.5 before treatment, 1.3 after treatment; $\mathrm{p}=0.07$ ), and to an increase of faecal dry matter ( $11.0 \%$ before treatment, $17.3 \%$ after treatment; $p=0.3$ ). By comparing the patients with active $C D$ with the healthy controls and the IBD patients in remission, an increase in total number of faecal bacteria, in faecal D:L lactate ratio, and in faecal dry matter seem to correlate overall with a lower severity of disease activity, and thus with health status (healthy controls versus active CD: $\mathrm{p} \leq 0.04$ for all three parameters). The detected increase in the number lactobacilli in the active $\mathrm{CD}$ patients upon therapy $(8.0 * 103 \mathrm{CFU} / \mathrm{g}$ before treatment, $7.8 * 104 \mathrm{CFU} / \mathrm{g}$ after treatment; $\mathrm{p}=0.06$ ) can be of interest because lactobacilli were also present in lower amounts in IBD patients in remission than in healthy controls (IBD remission: 6.1*104 CFU/g, healthy controls: $5.2 * 108 \mathrm{CFU} / \mathrm{g}$ after treatment; $\mathrm{p}=0.01$ ). Active $\mathrm{CD}$ is characterized by i) a significant lower total number of bacteria; ii) a significant lower D:L lactate ratio; and iii) a significant lower dry matter than found in healthy individuals. These faecal parameters may be used as clinical disease activity markers of $C D$.

\section{S1338}

Fdg-PET Imaging of Murine Experimental Colitis

Thorsten Vowinkel, Sven Hermann, Markus Loeffler, Rudolf Mennigen, Emile Rijcken, Markus Utech, Matthias Bruewer, Norbert Senninger, Michael Schaefers, Christian F Krieglstein

Introduction: Detection of disease activity in inflammatory bowel diseases (IBD) like ulcerative colitis is of crucial importance for the management of the disease. Non-invasive quantification of the severity of colitis would thus be advantageous. The aim of the present study was to assess the possible value of positron emission tomography (PET) in identifying intestinal inflammation in murine experimental colitis. Methods: Colitis was induced by feeding wildtype (wt) Balb/c mice 5\% dextran sulfate sodium (DSS) in drinking water for 7 days. Disease activity index (DAI) was used to score colitis clinically. On day 0, 4 and 6 during the course of DSS administration PET images were taken using the high-resolution 32module quadHIDAC small-animal PET scanner. Sixty minutes prior to scanning $10 \mathrm{MBq}$ fluorine-18-fluoro-deoxyglucose (F-18-FDG) was injected intravenously. The photodetection technique is based on gas multiwire proportional chambers offering a submillimeter spatial resolution (FWHM with resolution recovery). Results: DSS-induced colitis was characterized by a median weight loss of $17 \%$ from the original weight and high disease activity index values. Healthy controls showed a non-specific variation of bowel uptake of F-18-FDG throughout the entire colon. With increased severity of the disease F-18-FDG accumulation at day 6 was most prominent in the distal and left sided colon, which are the characteristic sites of inflammation in the DSS colitis model. Separate PET scanning of the resected and flushed colon showed the majority of FDG accumulation within the intestinal wall. However, in some DSS-colitic mice the FDG signal was variable with respect to location, degree and time course of FDG-uptake in the bowel. Conclusions: Our study demonstrates that similar to the clinical situation DSS colitis in mice can be monitored non-invasively over time by
FDG-PET examination. Further morphological and functional studies regarding site and relevance of FDG-uptake into the colonic wall will follow. These may facilitate interpretation of FDG-PET scanning in IBD patients.

\section{S1339}

Faecal S100A12 Is a Useful Non-Invasive Screening Test in Children with Gastrointestinal Symptoms Suggestive of Inflammatory Bowel Disease Marc A. Sidler, Steven T. Leach, Andrew Day

Introduction: We have recently shown that faecal levels of the calcium-binding protein S100A12 are increased in children with IBD and that this test provided a sensitive and specific non-invasive marker of gastrointestinal inflammation in children when $10 \mathrm{mg} / \mathrm{kg}$ was used as a cut-off. We hypothesised that faecal Al2 offers enhanced utility over standard tests to distinguish IBD from non-inflammatory bowel disease (such as functional disorders) when used as a screening test. Methods: Stools were obtained from 37 children (2.2 to $16 \mathrm{yrs})$ with gastrointestinal symptoms and suspected IBD before undergoing upper gastrointestinal endoscopy and colonoscopy. Faecal levels of A12 were measured by immunoassay. Result were correlated with standard blood tests and histological findings. Results: In children with IBD $(n=20)$ faecal A12 level were elevated $(143.5 \pm 122.2 \mathrm{mg} / \mathrm{kg})$ compared to the non$\operatorname{IBD}$ group $(\mathrm{n}=17,6.8 \pm 14.5)(\mathrm{p}<0.0001)$. When $<10 \mathrm{mg} / \mathrm{kg}$ was used as upper reference limit faecal Al2 had sensitivity of $100 \%$ and specificity of $89 \%$, which were superio compared to the sensitivities and specificities of standard blood tests (ESR, CRP, PLT albumin). Serum A12 levels were not different between the two groups. There was no linear correlation between A12 and the standard tests. Conclusion: Faecal S100A12 is a highly sensitive and very specific non-invasive marker of gut inflammation in children presenting with gut symptoms. S100A12 may be an additional simple test to screen and select children who warrant further invasive and laborious procedures such as endoscopies for investigation of their symptoms. Further larger studies are required to confirm these findings.

\section{S1340}

Higher Human Beta-Defensin 2 Levels in Feces in Active Ulcerative Colitis and Ileal But Not Colonic Crohn's Disease Compared to IBS Jost Langhorst, Sigrid Elsenbruch, Andreas Rueffer, Andreas Michalsen, Gustav J. Dobos Background: Antimicrobial peptides such as human defensins appear to be part of the innate immune system, and provide non-specific mucosal defense in the intestinal barrier against microorganism. The expression of human beta-defensins (HBD) is induced by pro-inflammatory cytokines and by microorganism. A HBD-2 deficiency is proclaimed for the intestinal mucous membrane of patients with Crohns disease (CD). Thus far, the expression of betadefensins has been quantified in the intestinal mucosa by using polymerase-chain reaction. This is the first study to investigate fecal measurements of HBD-2. Aim: To investigate the levels of HBD-2 in feces in patients with active Inflammatory Bowel Disease (IBD) and Irritable Bowel Syndrome (IBS). Methods: Fecal specimens were collected from a total of 47 patients: 17 IBS patients and 30 IBD patients (15 ulcerative colitis (CU); 15 CD). Disease status was addressed in each patient by ileocolonoscopy and histopathology. Fecal HBD-2 was measured by ELISA and reported as $\mathrm{ng} / \mathrm{ml}$ feces. Results: The mean \pm S.D. (standard deviation) level of $\mathrm{HBD}-2$ for the 17 IBS pts was $74,2 \pm 54.7$. Compared to IBS, in active UC (proved by endoscopy and histopathology) $121,5 \pm 73,1(\mathrm{p}<.05)$ and in active CD $125,8 \pm 79,5(\mathrm{p}<.05)$, the levels were significantly increased. The $8 \mathrm{CD}$ pts with active ileal disease $149,1 \pm 75,7 ;(\mathrm{p}=0.01)$ but not the $5 \mathrm{CD}$ pts with active colonic disease 88,6 $\pm 78,1(\mathrm{p}=0.64)$ showed significantly elevated HBD-2 levels. Conclusion: This is the first study to present preliminary data on HBD-2 levels in feces in pts with IBD and IBS. The results indicate significantly elevated levels in pts with active UC and active ileal but not colonic CD compared to IBS pts. The lower expression of HBD-2 in colonic CD in feces may indicate different responses of the mucosal innate defence, although the functional significance remains to be established.

\section{S1341}

Inherent Differences in 6-Thioguanine (6-TG) Induced Apoptosis May Predict Clinical Responsiveness to Induction and Maintenance 6-Mercaptopurine (6MP) Therapy Independent of 6-TGN Metabolite Levels in Children with Crohn's Disease

Ryan S. Carvalho, James A. Mahoney, Maria M. Oliva-Hemker, Carmelo Cuffari

Introduction: Although the measurement of 6-TGn metabolite levels has proved to be helpful in monitoring toxicity to 6-mercaptopurine (6-MP) therapy, newer metabolites (TGTP and TGDP) have been shown to better correlate with clinical response. Furthermore, the lack of consensus on applying therapeutic 6-TGn drug monitoring in clinical practice may in part be explained by inherent differences in anti-metabolite drug susceptibility (Carvalho et al. Gastroenterology 2004). Aim: To correlate in vitro sensitivity of peripheral blood mononuclear cells (PBMCs) to 6TG induced apoptosis in children with IBD naive to, and either on induction or maintenance 6-MP therapy. Clinical response will also be correlated with erythrocyte 6-TGn metabolite levels (Prometheus Labs). Methods: Peripheral blood was drawn from children with IBD naive to, or on maintenance (>6months $+/$ - mesalamine) 6MP therapy in CPT Ficoll tubes. PBMC's were isolated and suspended in RPMI 1640 at a concentration of 1 million cells $/ \mathrm{ml}$. Cells were stimulated with Concovin A at a concentration of $5 \mathrm{mcg} / \mathrm{ml}$ and incubated with 6TG at a dose of $6 u \mathrm{M}$ for 72-96 hours. Simultaneous, controls were incubated without 6-TG. Flow cytometry was used to measure apoptosis Only double positive cells for Annexin V PE for PS, and TOPRO-3 for DNA were quantified as positive for apoptosis. The cells were also gated for CD3 after staining with FITC-CD3 Remission was defined as a PCDAI $<10$; response a decrease of 12 points from baseline. Results: Clinical responsiveness to either induction or maintenance 6-MP therapy correlated well with 6-TG induced apoptosis, independent of erythrocyte 6-TGn metabolite levels Despite presumed therapeutic 6-TGn $(>240)$ metabolite levels, the mean apoptosis in patient with active disease ( $\mathrm{PCDAI}>30$ ) was lower than in patients either in remission or responsive 
to 6MP therapy (Table 1). Conclusion: 6-TG induced apoptosis correlates with clinical responsiveness to either maintenance or induction 6-MP therapy independent to erythrocyte 6-TGn metabolite levels in children with Crohn's disease. Future studies are needed to determine if susceptibility to 6-TG induced T-cell lymphocyte apoptosis can be used clinically to predict responsiveness to anti-metabolite therapy.

\begin{tabular}{|c|c|c|}
\hline & Inactive Disease (PCDAI $<10)$ & Active Disease (PCDAI $>30)$ \\
\hline \% Apoptosis & & \\
\hline Maintenance 6-MP & $58.8 \%$ & $43.5 \%$ \\
\hline Induction 6-MP & $55.6 \%$ & $28.8 \%$ \\
\hline
\end{tabular}

\section{S1342}

Urinary PGE-M, a Metabolite of PGE2: A Novel Biomarker of Crohn's Disease Activity

Jeffery C. Johnson, David A. Schwartz, Prashant R. Joshi, Jason D. Morrow, Robert J. Coffey, Robert D. Beauchamp, Nipun B. Merchant

INTRODUCTION: The available clinical measures of Crohn's disease (CD) activity can be overly influenced by functional symptoms. Placebo response rates in clinical trial are high. What is needed is a simple, non-invasive biologic measure of disease $C D$ activity. Cyclooxygenase-2 (COX-2) is involved in prostaglandin E2 (PGE2) synthesis and is expressed in epithelial inflammatory conditions and some cancers. We have developed an assay to quantify the major urinary metabolite of PGE2, PGE-M. PGE-M has been previously shown to be elevated in the urine of patients with advanced colorectal neoplasia relative to controls. The purpose of this study was to determine whether urinary PGE-M levels correlated with Crohn's disease activity METHODS: Twenty-eight patients with a known diagnosis of Crohn's disease were prospectively enrolled. Disease activity was quantified using the Harvey-Bradshaw index (HBI). Urine specimens and blood for $\mathrm{C}$-reactive protein were collected at the time of colonoscopy. Similarly, urine specimens were obtained from 72 healthy individuals who were endoscopically proven to have no neoplastic or inflammatory colorectal disease. Urine PGE-M levels were determined by mass spectroscopy. PGE-M levels were correlated with HBI and serum C-reactive protein (CRP) levels. RESULTS: The median urine PGE-M level for patients with Crohn's disease was $21.94 \mathrm{ng} / \mathrm{mg} \mathrm{Cr}$ (IQR 7.99 - 90.24) compared to 7.2 $\mathrm{ng} / \mathrm{mg} \mathrm{Cr}(\mathrm{IQR} 1.55$ - 31.5) for the healthy patients (Wilcoxon rank-sum $\mathrm{p}<0.0001$ ). There was a trend toward a positive correlation between PGE-M level and HBI (Spearman's rank correlation $p=0.064)$. Furthermore, urinary PGE-M levels were able to segregate several patients with a high functional component to their symptoms, but normal endoscopy (and low PGE-M levels). There did not appear to be a relationship between PGE-M level and Creactive protein $(C R P)$ level (Spearman's rank correlation $\mathrm{p}=0.68$ ) nor was there a correlation between CRP levels and DAS. (Spearman's rank correlation $p=0.71$ ) CONCLUSIONS: Urine PGE-M levels are significantly elevated in patients with Crohn's disease relative to healthy individuals. There appears to be a trend towards a correlation between urinary PGE$\mathrm{M}$ levels and Crohn's disease activity as measured by the HBI. These findings suggest that this assay may be a useful objective measure of disease activity.

\section{S1343}

Bioequivalence of 4 G Pentasa* (mesalamine) Daily; Dosed As 4xlg Sachets Or 2 × 2 X 1 g Sachet

Peggy Gandia, Isabelle Idier, Musse Foldager, Georges Houin

Aim Compare the kinetics of mesalamine and its main metabolite (acetyl-mesalamine) after repeated oral administration of $4 \mathrm{~g} / \mathrm{d}$ during 8 days according 2 schedules ( $4 \mathrm{~g}$ od and $2 \mathrm{~g}$ bid). Material and method A randomized, open, single center, two-way crossover study was conducted in 30 healthy male subjects aged from 18 to 50 with a BMI from 18 to $28 \mathrm{~kg}$ $\mathrm{m} 2$, to compare plasma pharmacokinetic profile of a new dosing regimen of mesalamine $(1 \times 4 g)$ with a reference dosing ( $2 \times 2 \mathrm{~g}$ with $12 \mathrm{~h}$ between the two administrations). The two dosing schedules were administered during 8 days and separated by a drug free wash-out period of 2 weeks. Plasma concentrations of mesalamine and its main metabolite, 5-acetylmesalamine, were determined on day 1 and day 8 from 0.5 to $21 \mathrm{~h}$ post dose by a sensitive and validated HPLC assay. The concentrations were used to assess pharmacokinetic parameters such as the Cmax, Tmax, and AUCO 24h. Bioequivalence was determined by comparing the AUCO-24h of mesalamine (primary criterion) and 5-acetyl-mesalamine after the once daily with the twice daily dosing at day 1 and day 8 . The two ways of administration were considered bioequivalent if the CI90\% of the ratio of the AUC0-24h $(1 \times 4 \mathrm{~g} / 2 \times 2 \mathrm{~g})$ was between 0.80 and 1.25. A clinical and biological examination was performed on the healthy volunteers throughout the study to determine tolerability of the treatment. Results The oral administration of mesalamine was well tolerated with both the once and twice daily dosing regimens. The values of the pharmacokinetic parameters and the ratio of the AUCO-24h with the corresponding $\mathrm{C1} 90 \%$ are shown in the table. Discusion and conclusion Bioequivalence was demonstrated for mesalamine and 5-acetyl-mesalamine for a once and twice daily dosing regimen of $4 \mathrm{~g}$ PENTASA at day 1 and for mesalamine on day 8 . At steady state, one oral administration of $4 \mathrm{~g}$ induces a slight decrease in the AUC0-24h of 5 acetylmesalamine. Once daily versus twice daily dosing of $4 \mathrm{~g}$ mesalamine were equivalent. * PENTASA is the registered trademark of Ferring b.v.

\begin{tabular}{|c|c|c|c|c|c|c|c|}
\hline & & & $\begin{array}{c}\text { Cmax } \\
(\mathrm{ng} / \mathrm{mL})\end{array}$ & $\begin{array}{c}\text { Tmax } \\
\text { (h) }\end{array}$ & $\begin{array}{c}\text { AUC0-24 } \\
(\mathrm{h} . \mathrm{ng} / \mathrm{mL})\end{array}$ & ratio & CI90\% \\
\hline mesalamine & $\begin{array}{c}\text { day } \\
1^{*}\end{array}$ & $1 \times 4 \mathrm{~g}$ & 8561.36 & 1.5 & 35657 & 0.96 & $0.85-1.08$ \\
\hline mesalamine & $\begin{array}{c}\text { day } \\
1^{*}\end{array}$ & $2 \times 2 \mathrm{~g}$ & 5103.51 & 13.0 & 36456 & 0.96 & $0.85-1.08$ \\
\hline mesalamine & $\begin{array}{c}\text { day } \\
8^{*}\end{array}$ & $1 \times 4 \mathrm{~g}$ & 9742.51 & 1.5 & 50742 & 0.88 & $0.81-0.96$ \\
\hline mesalamine & $\begin{array}{c}\text { day } \\
8^{*}\end{array}$ & $2 \times 2 \mathrm{~g}$ & 6803.70 & 13.0 & 57519 & 0.88 & $0.81-0.96$ \\
\hline 5-acetyl-mesalamine & $\begin{array}{c}\text { day } \\
1^{*}\end{array}$ & $1 \times 4 \mathrm{~g}$ & 7659.10 & 2.0 & 55217 & 0.89 & $0.81-0.97$ \\
\hline 5-acetyl-mesalamine & $\begin{array}{c}\text { day } \\
1^{*}\end{array}$ & $2 \times 2 \mathrm{~g}$ & 5527.30 & 14.0 & 61147 & 0.89 & $0.81-0.97$ \\
\hline 5-acetyl-mesalamine & day 8 & $1 \times 4 \mathrm{~g}$ & 8002.75 & 2.0 & 68509 & 0.82 & $0.77-0.88$ \\
\hline 5-acetyl-mesalamine & day 8 & $2 \times 2 \mathrm{~g}$ & 6350.10 & 14.0 & 82363 & 0.82 & $0.77-0.88$ \\
\hline
\end{tabular}

* significant bioequivalence

\section{S1344}

A Prospective Assessment of Clinical Activity Indices vs Calprotectin in Patients in Remission with Crohn's Disease and Ulcerative Colitis. Are We Understating the Level of Occult Inflammation?

Caitlin O'Mahony, John K. Collins, Eamonn Quigley, Liam O'Mahony, Fergus Shanahan

Introduction \& Aims: Clinical activity indices for Crohn's disease (CDAI) and ulcerative colitis (UCAI) are used in clinical trials but rarely in clinical practice and have dubious value for patients thought to be in remission. The level of calprotectin in feces is a marker of the presence of acute inflammation in the gut and has been proposed as a surrogate disease activity test in inflammatory bowel disease (IBD). The purpose of this study was to assess calprotectin levels in patients following steroid-induced remission and to compare with activity indices and systemic biomarkers. Methods: Fecal samples were collected from patients with $C D$ before $(n=60)$ and after $(n=20)$ steroid-induced remission and from those with UC after steroid-induced remission $(n=129)$ and at time of relapse $(n=24)$. Controls included patients with irritable bowel syndrome (IBS, $n=41)$, resected colon cancer $(n=78)$ and healthy volunteers $(\mathrm{n}=34)$. Calprotectin was quantified by ELISA $(\mathrm{mg} / \mathrm{Kg})$. Results: As expected, fecal calprotectin was significantly increased in active CD $(1098 \pm 141)$ and active UC $(1010 \pm 209)$ compared with all disease controls and healthy volunteers $(<40, \mathrm{p}<0.001$ for both). However, after induction of remission, calprotectin for CD $(850 \pm 191)$ and UC $(714 \pm 78)$ was still significantly elevated ( $<<0.001$ for both), despite normal activity indices. No significant correlation between fecal calprotectin and systemic markers including CRP soluble IL-2 receptor- $\alpha$ and soluble IL-6 receptor was found. Conclusion: Elevated levels of calprotectin during clinical remission show that conventional measures and systemic markers of disease activity of IBD seriously underestimate the degree of inflammation present in patients with so-called remission. This is of therapeutic relevance particularly in relation to proposals for more aggressive use of immunomodulatory agents to prevent long term complications of IBD

\section{S1345}

Utility of Fecal Calprotectin in Differentiating Inflammatory Bowel Disease (IBD) from Recurrent Abdominal Pain (RAP) in Children Robert J. Shulman

Background: It often is difficult to differentiate IBD from RAP in children. Fecal calprotectin concentration has been proposed as a marker to identify gastrointestinal inflammation and it may be useful in distinguishing organic disease (i.e., IBD) from normals. However, there are scant data regarding its utility in distinguishing IBD from RAP in children. We hypothesized fecal calprotectin concentration would discriminate between IBD, RAP, and Control children without disease. Values in children (as compared to infants) do not change with age. Design/Methods: Children were identified from pediatrician (RAP and Controls) and pediatric gastroenterologist (RAP and IBD) records. Parents were contacted and screened further by phone for eligibility. Children with RAP met Rome II criteria for functional abdominal pain or irritable bowel syndrome. The Pediatric Crohns Disease Activity Index (PCDAI) and the Ulcerative Colitis Clinical Activity Index (UCCAI, BMJ 1989;298:82) were calculated. A random stool was collected for determination of fecal calprotectin concentration. Results: Mean (SD) ages were IBD: $13.6 \pm 2.8 \mathrm{yr} .(\mathrm{n}=52)$; RAP: $8.5 \pm 1.1(\mathrm{n}=75)$; Control: $8.7 \pm 1.0(n=44)$. Fecal calprotectin concentration was significantly different among the groups (ANOVA, $\mathrm{p}<0.0001$ ) as well as between groups: IBD: $1113 \pm 1009 \mu \mathrm{g} / \mathrm{g}$ stool; RAP. $59 \pm 75$. Control: $36 \pm 21$. IBD vs RAP or Control, P $<0.001$; RAP vs Control, P 0.021 ). Fecal calprotectin correlated weakly with the PCDAI (score: $24 \pm 13 ; \mathrm{P}=0.028$, $\mathrm{r}^{2}=0.11, \mathrm{n}=36$ ) but not for the Clinical Activity Index (score: $4.6 \pm 3.4 ; \mathrm{P}=0.1, \mathrm{n}=$ 16). Sensitivity, specificity, positive predictive value, and negative predictive values are shown in the Table (normal <50 $\mu \mathrm{g} / \mathrm{g}$ ). Conclusions: Fecal calprotectin concentrations: 1 ) are elevated in children with IBD and RAP compared with controls; 2) are greater in RAP than controls; 3) appear to be useful as a screening test in children being evaluated for IBD whether they have RAP or not; 2 ) are somewhat specific for RAP compared with Controls but lack sensitivity and negative predictive value. (Supported by a grant from the CCFA) 


\begin{tabular}{|c|c|c|c|}
\hline & IBD vs RAP* & IBD vs Control* & RAP vs Control \\
\hline Sensitivity & $0.88(0.74-0.95)^{* *}$ & $0.88(0.74-0.95)$ & $0.3(0.2-0.42)$ \\
\hline Specificity & $0.70(0.58-0.80)$ & $0.83(0.70-0.94)$ & $0.83(0.68-0.93)$ \\
\hline Positive Predictive Value & $0.67(0.54-0.78)$ & $0.88(0.74-0.95)$ & $\mathbf{0 . 7 5}(0.55-0.89)$ \\
\hline Negative Predictive Value & $0.89(0.77-0.96)$ & $0.85(0.70-0.94)$ & $\mathbf{0 . 4 2 ( 0 . 3 1 - 0 . 5 3 )}$ \\
\hline
\end{tabular}

$* \mathrm{P}<0.001 * * 95 \%$ Confidence Interval

\section{S1346}

Prescribing Patterns and Awareness of Adverse Effects of Infliximab: A Health Survey of Gastroenterologists

Raymond K. Cross, Meaghan Donovan, Kevin Lunney, Olivia Carter-Pokras

Introduction: Infliximab is an effective treatment for Crohn's disease. To our knowledge there are no studies that have examined infliximab prescribing patterns and awareness of adverse drug reactions among gastroenterologists in the community. We sought to determine the prescribing patterns and awareness of adverse drug reactions to infliximab among gastroenterologists in Maryland and Washington, D.C. Methods: We developed a questionnaire to address 3 topic areas: demographics, infliximab prescribing practices, and awareness of adverse drug reactions to infliximab. Cognitive interviews were performed with 3 members of the gastroenterology faculty at the University of Maryland (UM) to assess the content validity of the questionnaire. The survey was then mailed to gastroenterologists in Maryland and Washington, D.C. identified from an existing UM database. The survey was mailed to all nonrespondents 2 weeks later. The survey with a $\$ 2$ dollar incentive was mailed to all remaining nonrespondents 3 months later. Results: 336 gastroenterologists with valid addresses were identified. 96 (29\%) surveys were returned after all three mailings had been completed. $40 \%$ of respondents used infliximab often and $46 \%$ used infliximab sometimes. $48 \%$ of respondents infused infliximab either in their office or in a shared infusion center. Only $67 \%$ of respondents would prescribe infliximab loading $(0,2,6$ week infusions) followed by every 8 weeks maintenance infusions and only $48 \%$ would use an immunomodulator prior to infusing infliximab. $92 \%$ would order a purified protein derivative (PPD) prior to infliximab treatment and $87 \%$ would not prescribe infliximab to a patient with a positive PPD. Surprisingly, $12 \%$ would not prescribe the drug to a sexually active woman. Respondents reported that infusion reactions occur in $12.9+/$ - $14.4 \%$ of infliximab infusions and $89 \%$ would restart an infliximab infusion at a lower rate when confronted with a mild acute infusion reaction. Respondents had little knowledge on the incidence of adverse events associated with infliximab use. Respondents underestimated the risk of serious infection, demyelinating syndromes, malignancy, and death. Conversely, they overestimated the risk of worsening heart failure. Conclusions: Considerable variation exists among gastroenterologists in regards to prescribing patterns of infliximab. Gastroenterologists are unaware of the frequency of adverse events associated with infliximab and report high rates of infusion reactions. Physician education regarding infliximab use and awareness of infliximab related side effects is needed to ensure that gastroenterologists follow current clinical guidelines.

\section{S1347}

Gastroduodenitis Associated with Ulcerative Colitis

Kazutoshi Hori, Yoshio Ohda, Nobuyuki Hida, Chizuko Hayashi, Toshihiko Tomita, Yongmin Kim, Kazuhiko Nakajima, Noriyasu Yamamoto, Yoshio Jinno, Takashi Sakagami, Yoshihiro Fukuda, Takayuki Matsumoto, Hiroto Miwa

Background \& Aims: Ulcerative colitis (UC) is traditionally confined to the colorectum; however, there are several case reports showing upper gastrointestinal involvement. The aims of this study were to create criteria of gastroduodenitis associated with UC (GDUC) and to examine the prevalence and characteristics of this condition. Methods: A prospective, single center study was performed on $148 \mathrm{UC}$ patients (84 male, 93 pancolitis and 100 postcolectomy patients). They underwent esophagogastroduodenal endoscopy with biopsies. The endoscopic and histological findings were compared between UC and 296 non-UC patients. The endoscopic terms of ulcerated mucosa, friable mucosa, granular mucosa and multiple aphthae were defined as follows: mucosa with extensive ulcerations; mucosa with erosions and with contact or spontaneous bleeding; mucosa with multiple white spots without a red halo; and multiple white spots surrounded by a red halo, respectively. Results: Duodenal ulcerated/friable and gastroduodenal granular mucosa resembling UC were observed in 9 patients, although these findings were never seen in controls. Crypt abscess or neutrophilic cryptitis were histologically recognized in 8 of 9 patients. The remaining one patient had focally enhanced gastritis. All of these histological findings were observed significantly more frequently in UC compared to control. Gastroduodenal multiple aphthae were seen in 9 patients, which were significantly less seen in controls (3/296). Since multiple aphthae were seen in other disorders and granular mucosa required distinguishing mucosa with white spots such as duodenal lymphangiectasia, GDUC was defined as follows: multiple aphthae or granular mucosa with one or more of the above histological findings, or ulcerated/friable mucosa with or without histological evidence. The diagnosis of GDUC was made in 17 (11.5\%) patients. The characteristics of GDUC were found to be more extensive colitis, less dosage of prednisolone, higher prevalence of pouchitis and longer postoperative periods compared to non-GDUC patients. The former two characteristics were significant risk factors for developing GDUC in a multivariate analysis. Conclusions: The gut inflammatory reaction in UC may not be restricted to the large intestine.

\section{S1348}

Local Injection of Infliximab in Crohn's Disease Recurrence During Endoscopy: A Prospective Longitudinal Study

Livia Biancone, Micaela Cretella, Carmelina Petruzziello, Claudio Tosti, Palmieri Giampiero, Sara Onali, Alessandra Geremia, Emma Calabrese, Francesco Pallone

Background. Local injection of Infliximab in Crohn's Disease (CD) gut lesions may reduce the risk of side effects, the dose and increase the efficacy of the drug. In a longitudinal study, we assessed the feasibility and safety of local Infliximab injection into the postoperative recurrence of $C D$ patients prospectively followed up for at least 1 year. Materials and Methods. In an open-label study, $8 \mathrm{CD}$ patients (3 M, age range 35-82 yrs) requiring colonoscopy were prospectively followed up for at least 1 year after local injection. Inclusion criteria: 1. Localized $(<5 \mathrm{~cm}) \mathrm{CD}$ recurrence; 2 . Inflammatory pattern; 3 . Inactive $\mathrm{CD}$ $(\mathrm{CDAl}<150) ; 4$. Written informed consent. The endoscopic degree of recurrence was graded according to Rutgeerts et al. (score 0-4). All endoscopies were recorded, with pictures taken from the peri-anastomotic area. Biopsies $(n=3)$ were taken from involved and uninvolved areas for histology. In all patients all the involved area (anastomosis and/or neo-terminal ileum) was injected with Infliximab (median $20 \mathrm{mg}$; range 8-60) during the first colonoscopy. A control endoscopy to assess the safety and efficacy of injection was performed: at 2 weeks in 4 patients ( 3 performing a second injection followed by a control endoscopy at 6 weeks) at 4 weeks in 4 patients ( 2 performing a second injection followed by a control endoscopy at 8 weeks. Results. No patients showed local or systemic side-effects or clinical relapse in the short- and long-term (median follow up 20 months, range 14-21) after local injection. At control endoscopy after the first local Infliximab injection, the endoscopic score of CD recurrence was reduced in 3 out of 8 and unchanged in 5 out of 8 patients, showing macroscopic healing in 2 out of 8 patients. The median endoscopic score did not significantly reduce after treatment (T0: 2 , range $1-3 ; \mathrm{Tl}$ and $\mathrm{T} 2: 1$, range $1-3 ; \mathrm{T} 0$ vs $\mathrm{T} 1 \mathrm{p}=0.08 ; \mathrm{T} 0$ vs $\mathrm{T} 2 \mathrm{p}=0.1 ; \mathrm{T} 1$ vs $\mathrm{T} 2 ; \mathrm{p}=0.1$ ). However, the number and extent of the endoscopic lesions reduced in 7 out of $8 \mathrm{CD}$ patients after the first local injection. The histologic score reduced in 4 , unchanged in 1 and increased in 3 out of 8 patients after local injection. All patients currently maintain clinical remission. Conclusions. This is the first study assessing the feasibility and safety of local injection of Infliximab into CD recurrence. Results indicate that local Infliximab injection into limited $C D$ recurrence is feasible and safe, requiring a low dose. Present findings suggest the need of controlled trials to assess the efficacy of this new and safe endoscopic treatment in CD.

\section{S1349}

Tacrolimus Enema Treatment for Left-Sided Colitis: A Safety Study Jolanda M. Van Dieren, Edward E. Nieuwenhuis, Ernst J. Kuipers, Janneke van der
Woude

Introduction: Systemic treatment with tacrolimus is effective in the treatment of inflammatory bowel diseases (IBD), both ulcerative colitis and Crohn's disease. However, this type of systemic treatment can be associated with severe side-effects, such as liver- and renal function disoders, insulin hyposensitivity, hypertension and myelosuppression. Topical application of tacrolimus is effective in dermatological disorders. Aim: to investigate the safety of loca application of tacrolimus in patients with left-sided colitis. Materials \& Methods: Patients with left-sided colitis refractory to local steroid treatment or systemic steroid-, azathioprineor cyclosporin treatment were treated with one tacrolimus enema daily for four weeks. An enema contained 2 or $4 \mathrm{mg}$ of tacrolimus in a $150 \mathrm{ml}$ aqueous solution. Endoscopic assesement was performed and Disease Activity Index (DAI) was determined prior to and after the treatment period. Tacrolimus whole blood trough levels ( $24 \mathrm{~h}$ after application of the enema) were measured weekly. Bloodcounts, liver enzyme tests, renal function tests, glucose, CRP and blood pressure were determined prior to, halfway and at the end of the treatment period. Results: Eight patients were included in the study. One patient refused to continue the study-medication halfway the treatment period. None of the patients reported adverse effects or showed any laboratory function disorders during or after treatment with the tacrolimus enemas. Furthermore, through levels did not exceed $4,2 \mathrm{ng} / \mathrm{ml}$ in patients either receiving $2 \mathrm{mg}(\mathrm{n}=4)$ or $4 \mathrm{mg}(\mathrm{n}=2)$-enemas/day. These levels are not associated with profuse systemic immunosuppression. Patients who were refractory to at least three immunomodulatory therapies (5-ASA, steroids, methotrexate, azathioprine, ciclosporin infliximab) $(n=5)$ also showed no improvement of DAI on treatment with tacrolimusenema treatment after 4 weeks. However, the two patients who had not been treated ye with multiple but only with a single or double immunomodulatory therapy responded well to tacrolimus enemas. One patient showed an improvement of DAI of 10 points to 2 and another patient showed improvement from 4 points to 0 . Conclusion: Tacrolimus enemas 2 or $4 \mathrm{mg}$ seem to be a safe therapeutic option for left-sided colitis. None of the patients developed side-effects, laboratory function disorders or tacrolimus whole blood levels that may establish systemic immune suppression. Moreover, tacrolimus enemas may be an effective early treatment for patients with left-sided colitis. However, larger studies and placebo-controlled studies are required.

\section{S1350}

Clinical Significance of Mucosal Suppressors of Cytokine Signaling (SOCS) Expression in Ulcerative Colitis

Yoshihiro Miyanaka, Shinji Tanaka, Yoshitaka Ueno, Kyoko Yoshioka, Tsuyoshi Hatakeyama, Masaru Shimamoto, Kazuaki Chayama

Background and Aim: Ulcerative colitis (UC) is a chronic immune mediated disorder whose pathogenesis and etiology are largely unknown. The suppressor of cytokine signaling (SOCS) is a family of molecules that act as a negative regulator for cytokine signaling. The role of SOCS has been demonstrated in several murine experimental colitis models. Our present study was undertaken to investigate the clinical importance of SOCS expression in UC. Method: Biopsy specimens were obtained endoscopically from the rectum of 62 patients with UC (36 men; age 13-76years). The patients were classified endoscopically according to Matts grade (grade 1 to 4 ). The disease duration was 6.1years. Biopsy specimens were 
obtained for histological analysis and the detection of mRNA. Mucosal SOCS1 and SOCS3 mRNA expressions were quantified in the samples using the competitive reverse transcriptionpolymerase chain reaction (RT-PCR). GAPDH was used as an internal control for the efficiency of RT-PCR and amount of RNA. Result: SOCS3 mRNA expression was significantly increased in the inflamed mucosa of UC, compared with the uninflamed mucosa. The expression was well correlated with the degree of both endoscopic and histologic inflammation. There was no relationship between SOCS3 expression and disease duration. The treatment with corticosteroids did not affect the SOCS3 expression. Interestingly, among the patients with inactive mucosa, the group that had relatively low level of SOCS expression demonstrated higher remission rate in one year $(\mathrm{P}<0.005)$. In contrast, the expression of SOCS1 mRNA was not correlated with the activity of colonic mucosa nor prognosis. Conclusion: The present study suggests that increased expression of mucosal SOCS3 but not SOCS1 may play a crucial role on the development of colonic inflammation of UC.

\section{S1351}

Diagnosis of Inflammatory Bowel Disease: Which Laboratory Parameter Helps the Most?

Alain Schoepfer, Michael Trummler, Beatrice Seibold, Petra Seeholzer, Frank Seibold

Objective: The differentiation of inflammatory (IBD) versus irritable bowel disease (IBS) can be a clinical challenge. Several laboratory parameters in feces and blood have been evaluated for this purpose. Hitherto it is not determined which of these parameters helps the most. Material and methods: A prospective blinded cohort study was done in 56 patients (24 Crohn's disease (CD), 12 ulcerative colitis (UC), 20 IBS), in which the diagnosis of either IBD or IBS was established based on complete conventional clinical, laboratory and endoscopic workup. In all patients we additionally measured the fecal leucocyte markers Calprotectin (EIA) and Lactoferrin (EIA), Hexagon-OBTI (specific for human haemoglobin), LEUKOTest (lactoferrin latex-agglutination) and the antibodies ASCA, PAB and PANCA. Data are given as sensitivity (SE), specificity (SP), statistical analysis was done with the unpaired t-test, chi-squared test and ROC-analysis. Results: SE and SP for Hexagon-OBTI in differentiating IBS vs IBD was $39 \%$ resp. $87 \%(p=0.04)$, for LEUKO-test $44 \%$ resp. $61 \%(p=0.004)$. Mean calprotectin in IBS was $25 \mathrm{ug} / \mathrm{mL}$, in IBD $275 \mathrm{ug} / \mathrm{mL}(\mathrm{p}<0.0001)$, mean lactoferrin in IBS was $0.03 \mathrm{ug} / \mathrm{mL}$, in IBD $0.34 \mathrm{ug} / \mathrm{mL}(\mathrm{p}<0.0001)$. ROC-Analysis: for Calprotectin (cut-off $50 \mathrm{ug} / \mathrm{mL}$ ) the SE was $94 \%$, the SP $90 \%$, for Lactoferrin (cut-off $0.056 \mathrm{ug} / \mathrm{mL}$ ) the SE was $91 \%$ and the SP $100 \%$. Mean CRP in IBS was $5.6 \mathrm{mg} / \mathrm{L}$, in IBD $16.2 \mathrm{mg} / \mathrm{L}(\mathrm{p}=0.1084)$. Mean leukocytes in IBS were $6 \mathrm{G} / \mathrm{L}$, in IBD $7.7 \mathrm{G} / \mathrm{L}$. Using the CD (ASCA+/PAB+/pANCA-) and UC (pANCA+/ASCA-/PAB-) specific antibodies did not allow to differentiate between IBS and IBD due to a low SE (56\%) and SP (50\%). However, ASCA+/PAB+/pANCA- had a high SP $(100 \%)$ for CD and a SE of $58 \%(\mathrm{p}=0.001)$. The SE resp. SP of pANCA+/ASCA-/PAB- for UC was $33 \%$ resp. $88 \%(p=0.14)$. Conclusions: In contrast to lactoferrin-latex agglutination test and Hexagon-OBTI, EIA for lactoferrin or calprotectin are highly accurate for discrimination of IBD versus IBS. ASCA, pANCA and PAB are not useful as tests for discrimination of IBS vs IBD, however they aid to differentiate between CD and UC. ASCA+, PAB+ and pANCA- help to diagnose $C D$, whereas pANCA+, ASCA- and PAB- will lead to the diagnosis of UC.

\section{S1352}

Nature and Frequency of Incidental Extraintestinal Lesions Found On Magnetic Resonance Enteroclysis (MR-E)

Hans Herfarth, Michael Grunert, Frank Klebl, Stefan Feuerbach, Juergen Schoelmerich, Gerhard Rogler, Andreas G. Schreyer

In addition to lack of radiation, the cross sectional imaging technique of MR-E offers the intriguing advantages to detect extraenteric lesions compared to the conventional smal bowel follow through. MR-E is currently recommended for the diagnostic work up in patients with suspected or proven inflammatory bowel disease (IBD). The aim of this study was to determine the occurrence of extraenteric findings in a large cohort of patients undergoing MR-E and to classify the clinical significance of these findings. Methods: We retrospectively analyzed 1154 MR-E performed in 1006 patients referred to our radiological department between 1999-2005. The reasons for referral were suspected or proven IBD $(n=710)$, further diagnostic work-up for small bowel disease because of non-specific abdominal symptoms (SBD; $\mathrm{n}=182$ ) or suspected small bowel malignacies (SBT; $=114$ ). All extraenteric findings were reviewed by a radiologist and a gastroenterologist and were classified as having high, moderate, or low significance for further diagnostic or therapeutic procedures. Results: The average age of all patients was $40 \pm 16(M e a n \pm S D)$ years $(y)(I B D 35 \pm 13 y$; SBD $49 \pm 16 y$; SBT $57 \pm 15 y$ ). A total of 1050 extraenteric findings were detected in 574 patients $(57 \%$; IBD $53 \%$; SBD 65\%; SBT 68\%). Of these findings 117 (11\%) were judged as having a high, 212 $(20 \%)$ a moderate and 721 (69\%) a low significance (see table 1). The most common highly significant lesions were extraintestinal tumors, metastases or masses $(n=70)$, pleural effusions $(n=13)$ and hydronephrosis or ureteral obstruction $(n=16)$. Conclusions: MR-E reveals a substantial number of extraenteric findings, indicating the advantage of this imaging modality. However, the occurrence of extraenteric findings with a high clinical significance in patients with IBD is low probably mainly due to the younger age of this patient group at the time of the diagnostic work-up.

Table 1: Occurrence and clinical significance of extraenteric findings on MR-E

\begin{tabular}{|c|c|c|c|c|}
\hline Dx (patients with findings; $\mathrm{n})$ & Total findings (n) & High $\mathrm{n}(\%)$ & Moderate $\mathrm{n}(\%)$ & Low $\mathrm{n}(\%)$ \\
\hline IBD (378) & 632 & $26(4 \%)$ & $136(22 \%)$ & $470(74 \%)$ \\
\hline SBD (118) & 221 & $26(12 \%)$ & $39(18 \%)$ & $156(70 \%)$ \\
\hline SBT (78) & 197 & $65(33 \%)$ & $37(19 \%)$ & $95(48 \%)$ \\
\hline
\end{tabular}

\section{S1353}

Local Mucosal Parameters in IDB Do Not Correlate with Systemic Parameters Sabine Buhner, Luzia Valentini, Susanne Hengstermann, Lennart Schaper, Carsten Buning, Tatjana Schuetz, Thomas Koernicke, Martina Werich, Brigitte Winklhofer-Roob, Herbert Lochs

Background: Fecal calprotectin (CP) and intestinal permeability have been described as subclinical markers in IBD. A detailed analysis and correlation with systemic parameters was done. Methods: Gastrointestinal permeability was measured using an oral sugar test in 55 persons with IBD and 9 healthy controls. Urinary sugar recovery was quantified using HPLC. Different sugars served as permeability markers for different gut segments; sucrose (suc): gastroduodenum; lactulose/mannitol ratio (PI): small intestine; sucralose (sucra): colon. $\mathrm{CP}$, an index for local intestinal inflammation was assed by ELISA. Additionally full blood count and C-reactive protein (CRP) were analyzed. For classification of the disease activity a $C D A I>150$ in $C D$ (active: $C D A$; remission: $C D R$ ) and a CAI>4 in UC (active: UCA; remission: UCR)were used. Results: Gastric and small intestinal permeability were significantly increased in CD but not in the UC groups (table). Colonic permeability tended to be increased in CDA but also in UC groups. CP was significantly higher in all patient groups compared to controls. In contrast, CRP was increased only in CDA. Gastric and small intestinal permeability values were highly correlated with $\mathrm{CP}$ values in both $\mathrm{CD}$ groups. Neither CP nor PI and Suc clearly correlated with the CDAI, CAI (except CP in UCR group), or with plasma CRP (only in CDA), or with other blood count parameters. Conclusions: Data stress the importance of both, mucosal permeability and $\mathrm{CP}$ as markers for local and early changes in IBD. Both parameters reflect inflammatory changes more sensitively than activity indices or CRP

\begin{tabular}{|c|c|c|c|c|c|}
\hline & Control $(\mathrm{n}=9$ & $\operatorname{CDR}(n=24)$ & $\operatorname{CDA}(\mathrm{n}=9)$ & UCR $(n=14)$ & UCA $(\mathrm{n}=8)$ \\
\hline Age (yrs) & $38(21-53)$ & $37(19-57)$ & $33(19-55)$ & $46(24-70)$ & $40(22-55)$ \\
\hline CDAI or CAI & & $55(32-99)$ & $252(216-323)$ & $3(1-4)$ & $9(7-12)$ \\
\hline $\mathrm{CP}(\mathrm{mg} / \mathrm{l})$ & $0.01(0.00-0.00)$ & $18.0(6.6-33.7)^{a}$ & $70.6(2.0-112.3)^{a}$ & $17.6(2.1-25.5)^{\mathrm{a}}$ & $26.8(22.7-57.3)^{a}$ \\
\hline Suc $(\%)$ & $0.17(0.10-0.24)$ & $0.22(0.14-0.43)$ & $0.37(0.18-1.15)^{a}$ & $0.19(0.12-0.26)$ & $0.14(0.05-0.20)$ \\
\hline $\operatorname{Man}(\%)$ & $15.0(0.1-19.0)$ & $15.6(12.4-19.9)$ & $15.8(12.7-21.6)$ & $14.4(11.2-18.1)$ & $14.9(13.4-15.9)$ \\
\hline Lac $(\%)$ & $0.27(0.21-0.31)$ & $0.43(0.34-0.67)^{\mathrm{a}}$ & $1.14(0.45-2.77)^{a, b}$ & $0.29(0.24-0.41)$ & $0.30(0.17-0.38)$ \\
\hline PI (\% Lac/\%Man) & \begin{tabular}{|c|}
$0.016(0.015-0.020)$ \\
\end{tabular} & $0.028(0.017-0.044)$ & $0.069 \underset{\mathrm{a}, \mathrm{b}}{(0.030-0.158)}$ & $\begin{array}{c}0.022 \\
(0.015-0.029)\end{array}$ & $\begin{array}{c}0.020 \\
(0.012-0.024)\end{array}$ \\
\hline Sucra $(\%)$ & $0.20(0.00-0.63)$ & $0.37(0.00-1.10)$ & $0.61(0.52-1.81)$ & $0.61(0.00-0.80)$ & $0.52(0.07-1.02)$ \\
\hline CRP (mg/) & $1.0(0.6-1.8)$ & $2.0(1.0-5.1)$ & $13.4(1.9-37.1)^{\mathrm{a}}$ & $1.5(0.7-7.2)$ & $1.3(0.6-5.7)$ \\
\hline
\end{tabular}

Except for the age (means (range) all data were given in median (0.25-0.75 percentile); a $\mathrm{p}<0.04$ compared to control; $b$ : $\mathrm{p}<0.03$ between CDR and CDA; n.s. between UCR and UCA; Kruskal-Wallis-test / Mann-Whitney-test and alpha-adjustment by Bonferroni procedure

\section{S1354}

Efficacy and Endoscopic Prediction of Clinical Response to Leukocytapheresis in Patients with Corticosteroid Refractory Or Dependent Ulcerative Colitis: Results with Two Different Leukocytapheresis Systems

Kenji Watanabe, Nobuhide Oshitani, Noriko Kamata, Hirokazu Yamagami, Kazuhide Higuchi, Takayuki Matsumoto, Tetsuo Arakawa

Background and Objective: Ulcerative colitis (UC) is empirically treated with conventional medications (salicylates, corticosteroids, 6-mercaptopurine) and recently with biologics. Use of these drugs is associated with side effects, which add to the disease complications. However, UC is believed to be perpetuated by inflammatory cytokines for which peripheral blood leukocytes (PBL) are major sources. Accordingly, recently PBL have become major targets of therapy. Two systems are available for depleting excess and activated PBL, GCAP (Adacolumn), which selectively depletes granulocytes and monocytes together with a small fraction of lymphocytes (FcgR and complement receptors bearing leukocytes) and LCAP (Cellsoeba), which non-selectively depletes all leukocyte populations. We have investigated the efficacy of GCAP and LCAP and the use endoscopic findings to predict efficacy, especially in corticosteroid refractory and dependent cases. Methods: Thirty-four patients, age 19-52 years and duration of UC 1-12 years were treated. Twenty-seven had total colitis, 7 had left-sided colitis; 24 were drug refractory and 10 were steroid-dependent. Each patient received one or two GCAP $(n=21)$ or LCAP $(n=13)$ sessions/week for 5 weeks, with colonoscopy performed before and after each session. Efficacy was evaluated by measuring changes in clinical activity index after each session. The predictive factor we evaluated was endoscopic findings, defined by Truelove Activity Index, with a decrease of $>10$ points or $<13$ points as marked improvement and 9 to 4 points as an improvement. A marked improvement was when all ulcers healed; an improvement was when some ulcers healed to scars but open ulcers remained. Results: Twenty-five patients $(73.5 \%)$ had a clinical response and most steroid-dependent patients or patients who had steroid side effects were successfully treated. Thirteen of 24 steroid-refractory patients had a clinical response and 18 of 25 patients who were on steroids tapered their steroid dose. Efficacy was seen about 2 weeks after the start of treatment. Endoscopic improvements were seen in 14 of $16(87.5 \%)$ patients with shallow ulcers or erosions throughout the colon and 10 of 15 with deep ulcers, but 2 with massive 
ulceration of the whole mucosa did not respond. These findings support the use of endoscopy in clinical treatment decision-making. During follow-up, 9 patients underwent colectomy due to relapse and exacerbation of UC. Conclusion: GCAP or LCAP is valuable for refractory or steroid-dependent UC; most patients can reduce steroid dose or be spared of additional medication. Endoscopic findings are valuable in predicting clinical effectiveness.

\section{S1355}

Correlation Between Endoscopic Disease Activity and High Sensitivity CReactive Protein, Serum Amyloid A, and Plasma and Fecal Calprotectin in Children with Inflammatory Bowel Disease

Ulrika L. Fagerberg, Lars Loof, Johan Lindholm, Lars-Olof Hansson, Yigael Finkel

Objectives: Elevated plasma concentrations of acute phase proteins, e.g. serum amyloid A (SAA) and C-reactive protein (CRP), are common in inflammatory processes. Calprotectin, a neutrophil-derived protein, is another marker of inflammation. Increased fecal calprotectin excretion has previously been found in inflammatory bowel disease (IBD) but little is known about plasma calprotectin concentrations in children with IBD. The aims of this study were to evaluate the importance of SAA, CRP and plasma calprotectin as markers of endoscopic disease activity in children with IBD and to compare these results with fecal calprotectin and routine blood tests. Methods: Fecal and blood samples were collected from thirty-one children with IBD (Crohn disease $\mathrm{n}=22$, ulcerative colitis $\mathrm{n}=8$, indeterminate colitis $\mathrm{n}=1$ ) when investigated with complete colonoscopies including multiple biopsies. Serum Amyloid A (N Latex SAA®) and CRP (hSCRP®) were analyzed using BNII analyzer (Dade Behring). Calprotectin was measured in plasma and fecal samples with an enzyme-linked immunosorbent assay method (Calprest ${ }^{\circledR}$, Eurospital). The concentrations of the inflammatory markers were correlated to a histopathological, combined scoring of extent and severity of inflammation in eight colonic segments for each patient. Results: The following median values were found: $2.4 \mathrm{mg} / \mathrm{L}$ for SAA (range 0,2-385.0 mg/L), $0.61 \mathrm{mg} / \mathrm{L}$ for hsCRP (range 0.03-80.0 $\mathrm{mg} / \mathrm{L}$ ), $348 \mathrm{mg} / \mathrm{L}$ for plasma calprotectin (range 78.0-4550 microg/L) and $264 \mathrm{mg} / \mathrm{kg}$ for fecal calprotectin $(5.9-1198 \mathrm{mg} / \mathrm{kg}$ ). The correlations between the inflammatory markers and the combined extent and severity score of histological inflammation were $r=0.39$ ( $p=$ $0.029)$ and $r=0.44(p=0.014)$ for SAA respectively hsCRP. For plasma calprotectin the correlation was even stronger $(\mathrm{r}=0.58, \mathrm{p}=0.001)$ but not as good as for fecal calprotectin $(\mathrm{r}=0.74, \mathrm{p}<0.001)$. The correlations were also calculated for erythrocyte sedimentation rate $(\mathrm{r}=0.66, \mathrm{p}<0.001)$, platelet count $(\mathrm{r}=0.57, \mathrm{p}=0.001)$, plasma albumin $(\mathrm{r}=-0.54, \mathrm{p}=0.002)$, plasma orosomucoid $(r=0.40, p=0.027)$ and haemoglobin in blood $(r=-0.27, p=0.138)$ for comparison. Conclusions: Fecal calprotectin seems to be a more reliable test compared to SAA, hsCRP, plasma calprotectin and routine inflammatory markers in blood for estimation of histological inflammatory activity in children with IBD.

\section{S1356}

Usefulness of the ${ }^{13} \mathrm{CO} 2$ Breath Test in Patients with Active and Quiescent Ulcerative Colitis Given $\left[1-{ }^{13} \mathrm{C}\right]$-Butyrate Rectally

Kimitoshi Kato, Yukimoto Ishii, Shigeaki Mizuno, Satoshi Asai, Masahiko Sugitani, Tadashi Kohno, Sigekatu Takahashi, Sachiko Komuro, Maho Iwamoto, Shunpachi Miyamoto, Noriko Nakajima, Takero Mazaki, Yasuyuki Arakawa

BACKGROUND: Impaired ability to metabolize butyrate has been implicated in ulcerative colitis (UC). We investigated whether measuring the metabolic rate by breath test, after rectal $\left[1-{ }^{13} \mathrm{C}\right]$-butyrate administration to $\mathrm{UC}$ patients, is a potential noninvasive method of evaluating inflammatory activity as an adjunct to colonoscopy and histology. METHODS: $\left[1-{ }^{13} \mathrm{C}\right]$-butyrate enemas were administered to 22 patients with active UC ( 8 mild, 12 moderate and 2 severe), to 16 with quiescent UC and to 15 control subjects. The clinical activity index (CAI) scores are based on the Lichitiger symptom score. The rate of ${ }^{13} \mathrm{CO} 2$ production for four hours, was measured by breath test using an infrared spectrometric analyzer. RESULTS: During the period from 15 to 210 minutes after the enema, the quantity of expired ${ }^{13} \mathrm{CO} 2$ in the active UC group was significantly lower than those of the quiescent $\mathrm{UC}$ and control groups. Cumulative ${ }^{13} \mathrm{CO} 2$ production at 240 minutes showed significant negative correlations with $\mathrm{CAI}(\mathrm{r}=-0.65, \mathrm{P}<0.0001)$, the endoscopic activity index $(\mathrm{r}=$ $0.63, \mathrm{P}=0.0001)$ and the histological score $(\mathrm{r}=-0.71, \mathrm{P}<0001)$. A significant increase in the ${ }^{13} \mathrm{CO} 2$ production rate in the active as compared to the quiescent stage was found in six UC patients, in whom clinical remission was achieved, in accordance with improvements in CAI, the endoscopic activity index, histology and fecal butyrate concentrations. Correlation analysis showed a significant inverse relationship between the histological score and the cumulative ${ }^{13} \mathrm{CO} 2$ production rate $(\mathrm{r}=-0.857, \mathrm{p}<0.01)$ in these six UC patients. All UC and control subjects tolerated the $\left[1-{ }^{13} \mathrm{C}\right]$-butyrate enema well. There were no adverse events in any group. CONCLUSIONS: Measuring in vivo butyrate metabolism can be done safely, without invasion of the colonic mucosa, when butyrate is used as the ${ }^{13} \mathrm{C}$ compound Application of this breath test following a ${ }^{13} \mathrm{C}$ butyrate enema in active and quiescent UC appears to be a promising and reliable method for evaluating disease activity and metabolic changes associated with improvement of inflammation.

\section{S1357}

Comparison of CRP, Clinical Activity Indices and Fecal Lactoferrin with Disease Status in Ileocolonoscopy of Patients with IBS and IBD Jost Langhorst, James Boone, Andreas Rueffer, Andreas Michalsen, Gustav J. Dobos Background: Determining the presence of intestinal inflammation is the main criteria for the discrimination of Irritable Bowel Syndrome (IBS) from Inflammatory Bowel Disease (IBD) and for the tailoring of therapy. Clinical indices have proven too complex and time-consuming for daily routine practice. On the other hand, single serologic parameters such as $\mathrm{C}$-reactive protein (CRP) are hampered by a low sensitivity and specificity for intestinal inflammation. Neutrophil-derived proteins in feces such as lactoferrin have been suggested as possible biomarkers for assessing intestinal mucosal inflammation in IBD. Aim: To investigate the correlation of CRP, clinical activity indices and fecal lactoferrin (FLA) to ileocolonoscopy and histopathology in patients (pts) with IBS and IBD. Methods: Elevated serum CRP (>= $1 \mathrm{mg} / \mathrm{dl})$ and FLA ( $>=7 \mu \mathrm{g} / \mathrm{ml})$ were investigated as indicators of intestinal inflammation in a total of 47 patients: 17 IBS patients and 30 IBD patients (15 ulcerative colitis (CU); 15 Crohns disease (CD)). Disease activity was determined for CU with the Colitis Activity Index (CAI - Rachmilewitz-Index; a score of $>5$ indicating active disease) and for CD with Crohns Disease Activity Index (CDAI; a score of $>150$ indicating active disease). Disease status was addressed and classified in each patient by ileocolonoscopy and histopathology using a score of $0-3(0=$ no inflammation to $3=$ high inflammation $)$. Results: A linear relationship was observed between patient groups stratified by endoscopy scores 0 to 3 with CRP levels $($ mean \pm SE) of $5.4 \pm 2.4,1.4 \pm 0.5,0.7 \pm 0.2$ and $0.5 \pm 0.2(\mathrm{r} 2=0.76)$ and LFA of $978 \pm 263$ $424 \pm 196,72 \pm 13$ and $34 \pm 8.5(\mathrm{r} 2=0.89)$, respectively. Serum CRP reached baseline levels for 6 of 10 pts (range 0 to 0.8 ) of group 1. The FLA remained elevated for 10 of 21 pts (range 15 to 103$)$ of group 0 . A significant difference $(\mathrm{p}<0.02)$ was achieved with LFA between endoscopy groups $3 / 1,3 / 0$, and $1 / 0$ ( $\mathrm{p}$-values $=0.021,0.016$ and 0.004 ). No significan difference was observed for FLA between groups 3 and 2 or for CRP with any of the groups. $\mathrm{CDAI}$ scores did not discriminate between the $\mathrm{CD}$ patient groups stratified by endoscopy score. There was no sufficient number of CAI scores between groups for statistical analysis Conclusion: These results are the first for comparing LFA together with CRP to endoscopy score. The FLA showed the highest sensitivity followed by serum CRP for indicating presence of intestinal inflammation in IBS and IBD pts. The CDAI index did not reflect disease activity within our $C D$ population. Further studies are needed to determine the role of FLA in daily practice for monitoring disease activity in IBD

\section{S1358}

A Prospective, Blinded Assessment of Distal Colonic Mucosal Concentrations of 5-ASA and NAC-5ASA in UC Patients Treated with Either Balsalazide (Colazal) Or a pH-Dependent mesalamine (Asacol)

Asher Kornbluth, Carmen Cuffari, James George, Peter Legnani, Michele Hunt, Elliot Freid-Boxt, John Young

Introduction: Mesalamine containing compounds are the first line therapies in patients with UC. Different mesalamine compounds have varying release systems to deliver 5-ASA to the colonic mucosa. Balsalazide is a prodrug containing 5-ASA with an azo bond to 4-aminobenzoic acid, an inert carrier. Asacol is an Eudragit coated mesalamine compound designed to release mesalamine at a $\mathrm{pH}$ of 7.0 However, little is known about mucosal concentrations of 5-ASA, and its primary metabolite Nac-5ASA, in UC patients taking these medications. Objective: The aim of this study was to determine distal colonic mucosal concentrations of 5-ASA and Nac-5ASA in UC patients treated with either balsalazide, or pH-dependant mesalamine. Methods: UC patients undergoing sigmoidoscopy or colonoscopy for either assessment of disease activity, or dysplasia surveillance were invited to participate, if they had taken at least $90 \%$ of their prescribed 5-ASA dose, and were not on any topical mesalamine therapies for at least the prior 2 weeks. Disease and endoscopic activity was assessed with the Mayo Clinic Disease Activity Index (DAI). Patients underwent routine 4 quadrant biopsies for assessment of histologic activity, by a pathologist blinded to the patient's drug treatment, at 5,15 , and $25 \mathrm{~cm}$ from the anal verge. Four quadrant biopsies were also taken from each patient at 5,15 and $25 \mathrm{~cm}$ from the anal verge for measurements of 5-ASA and Nac-5ASA concentrations by HPLC, by an investigator blinded to the patient's drug treatment. Mucosal concentrations of 5-ASA and Nac-5ASA were measured as micrograms/gm of dry tissue weight and adjusted for dose of mesalamine in the parent compound (balsalazide $6.75 \mathrm{~g} / \mathrm{d}=$ ph-dependent mesalamine $2.4 \mathrm{~g} / \mathrm{d}$ ). Results: 13 patients treated with a mean of $6.75 \mathrm{~g} / \mathrm{d}$ of balsalazide (containing $2.4 \mathrm{~g} / \mathrm{d}$ of $5-\mathrm{ASA}$ ) were compared with 17 patients treated with a mean of $3.74 \mathrm{~g} /$ day of $\mathrm{pH}$-dependent mesalamine, respectively. Patients in the balsalazide and ph-dependent mesalamine groups had similar DAI scores (4 vs. 2) endoscopy subscores (1.7 vs. 1.1) and histologic scores (1.2 vs. 0.7). Although interpatient variability was high for both treatment groups, mean mucosal 5-ASA concentrations were $102 \%$ greater with balsalazide than ph-dependent mesalamine at $5 \mathrm{~cm}, 84 \%$ greater at 15 $\mathrm{cm}$ and $102 \%$ greater at $25 \mathrm{~cm}$, respectively. Similar differences were seen for mucosal Nac5ASA concentrations at each site. Conclusions; UC patients treated with oral balsalazide had a mean of $96 \%$ greater distal colonic mucosal concentrations of 5-ASA than patients treated with similar oral doses of ph-dependent mesalamine.

\section{S1359}

Infliximab Significantly Increases Proportion of Overweight/Obese Crohn's Disease Patients Receiving Longterm Maintenance Therapy Mazen Issa, Lydia R. Weber, Sushrut V. Shidham, Dawn B. Beaulieu, David G. Binion

Background: At present, $65 \%$ of Americans are overweight (body mass index (BMI) $>25$ ) of which $21 \%$ are obese (BMI >30). Active CD is associated with weight (WT) loss and malnutrition, which has been linked to increased TNF $\alpha$ production during chronic inflammation. Treatment of refractory CD patients (pts) with the anti-TNF $\alpha$ antibody infliximab has resulted in improved health with resolution of inflammation and WT gain has been described. We characterized the effect of anti-TNF $\alpha$ therapy on WT gain and BMI in U.S. CD pts receiving long-term therapy (ly or greater). Methods: This was a retrospective observational study of CD pts receiving maintenance infliximab at a single center between 1999-2005. In addition to demographic data and height, serial measures of WT, $C$ reactive protein (CRP) and quality of life (SIBDQ) were recorded prior to infliximab initiation and at 6, 12 and 24 months into maintenance therapy. BMI was calculated at start and at time of last dose. Results: Data from 75 pts (30 male, mean age $40.4 \pm 14 y$ (SD), 45 female, mean age $39.6 \pm 17 y$ (SD)) were available for analysis. WT gain in the combined cohort from the start of infliximab therapy to last dose was $5.3 \pm 2 \mathrm{~kg}(\mathrm{SD})$ and BMI increased from $24.2 \pm 6$ to $26.0 \pm 6.3$ at the time of last dose. Among females, mean WT gain peaked at 6 months $(1.96 \mathrm{~kg})$, which decreased at 12 months to $0.74 \mathrm{~kg}$ above the starting level. Mean CRP (mg/dL) in women started at 1.66 and decreased to 0.6 at 6 months and was 0.65 at 12 months. In males, there was an increase in mean WT from $81.9 \mathrm{~kg}$ at the start to $86.7 \mathrm{~kg}$ at 6 months to 88 $\mathrm{kg}$ at 12 months. In male pts the BMI significantly increased from 25 to 27.6 (last dose; $\mathrm{p}=<0.04)$. In male pts the CRP decreased from 2.02 at start to 0.5 at 6 months and 0.6 at 12 months. In women, SIBDQ improved from 45 (start) to 57 (6 months) to 65 (12 months) 
In men, SIBDQ improved from 49 (start) to 54 (both 6 and 12 months). The percentage of total overweight pts at start was $35 \%$, which increased to $51 \%$ at the time of last dose $(p=<0.05)$, and the percentage who met criteria for obesity increased from $13 \%$ to $24 \%$. Conclusion: WT gain is significant in U.S. CD pts treated with maintenance infliximab, with half of pts becoming overweight beyond 1 year. Women had peak WT gain by 6 months (approximately $6 \%$ of total body WT) which plateaued, while they continued to experience clinical improvement with infliximab over 1 yr. Male pts experienced approximately $10 \%$ increase in body WT and continued to gain over time, while their peak benefit from infliximab was at 6 months. Given health concerns with obesity, WT gain should be monitored in CD pts benefiting from long-term anti-TNF $\alpha$ therapy

\section{S1360}

Elevated C-Reactive Protein On Admission Predicts Colectomy in Hospitalized IBD Colitics with and Without Clostridium Difficile Co-Infection

Vladimir Ferrer, Mary F. Otterson, Sarah Lundeen, Lydia R. Weber, Jasmohan S. Bajaj, Ossama A. Hatoum, David G. Binion

Background: Inflammatory markers provide objective data which may help gauge severity of inflammation in inflammatory bowel disease (IBD: Crohn's Disease (CD) and Ulcerative Colitis (UC)). C-reactive protein (CRP) is a serologic measure of acute phase response which elevates during IBD exacerbation. Recent data suggests that increased Clostridium difficile (C. diff) co-infection may precipitate severe IBD flare, often leading to colectomy. There is limited data correlating objective clinical or serologic markers and need for colectomy in IBD pts with C. diff co-infection. We correlated objective clinical and serologic inflammatory markers in hospitalized IBD colitis pts with outcome (i.e. need for colectomy) with a specific focus on C. diff co-infection. Methods: This was a retrospective observational study involving consecutive IBD colitics hospitalized with acute exacerbation of UC or CD between 200405. Multiple C. diff ELISA toxin A/B assays were performed on all inpts until a positive result was obtained. Clinical parameters at admission including heart rate (HR) and temperature (T) were recorded. Measures of inflammatory activity including white blood cell count (WBC), platelets (PLT), erythrocyte sedimentation rate (ESR), and C-reactive protein (CRP) from the first value on admission were analyzed. Outcome measures included successful medical therapy (pts discharged for > lmonth) or need for colectomy. Results: Thirty-one consecutive BD colitics ( $58 \%$ UC, 42\% CD) admitted with acute exacerbation were analyzed. Mean age of the pts was $40 \pm 17 y$ (SD). C. diff was detected in $55 \%(17 / 31)$. Thirteen pts (42\%) underwent colectomy due to clinical deterioration, 7 of which had concomitant C.diff. Duration of disease was similar between colectomy and successful medical therapy groups. The CRP ( $\mathrm{mg} / \mathrm{dL}$; normal<0.5) was elevated in all pts who underwent colectomy (mean 6.7 \pm 3.6 (SD)) and was significantly lower in pts with successful medical therapy (mean 2.9 \pm 3.1 (SD); $<<0.002$ ). CRP $\geq 3 \mathrm{mg} / \mathrm{dL}$ correlated with colectomy in $87 \%$ of pts, while CRP $<3$ correlated with colectomy in 13\%. No significant correlation was found between ESR, PLT, WBC, HR or T at presentation with clinical outcome. Co-infection with C. diff alone did not influence colectomy rate. Conclusions: CRP above $3 \mathrm{mg} / \mathrm{dL}$ was predictive of colectomy in IBD colitics admitted for acute exacerbation with a sensitivity and specificity of $87 \%$ and $88 \%$, respectively. WBC, PLT, ESR, HR, T or C. diff status failed to correlate with clinical outcome. These data suggest that CRP offers predictive value in IBD pts admitted with severe colitis, identifying a high risk group for surgery

\section{S1361}

North American Inflammatory Bowel Disease (IBD) Experts and Community Providers Are Consistent in Their Use of Diagnostic Tests in Crohn's Disease (CD)

Eric Esrailian, Brennan M. Spiegel, Laura E. Targownik, Marla C. Dubinsky, Stephan R. Targan, Ian M. Gralnek

Background: Diagnostic decision-making in $\mathrm{CD}$ is often uncertain, and this uncertainty often leads to a potentially arbitrary sequence of diagnostic testing with various modalities. Although published guidelines in CD are intended to minimize variation in care, it is unknown whether current diagnostic decision-making complies with guidelines. We therefore measured testing proclivity in $\mathrm{CD}$ between established IBD experts and community gastroenterologists. Methods: We developed a survey consisting of 5 clinical vignettes: 1) patient with undiagnosed $C D, 2$ ) patient with newly diagnosed $C D$, 3) presentation of perianal fistula, 4) patient with steroid refractory $\mathrm{CD}$, and 5) patient status post ileocolonic anastomosis for ileal/colonic $\mathrm{CD}$. The survey elicited provider beliefs about the appropriateness of available diagnostic tests in $C D$. We measured appropriateness using the 9-point RAND Appropriateness Scale, where 1-3=inappropriate, 4-6=unsure, $7-9=$ appropriate and "endorsed." We surveyed 55 recognized North American IBD experts and a random sample of 91 GIs from the AGA membership. For each respondent, we itemized the total number of diagnostic tests endorsed and diagnostic cost consumed per vignette, and calculated a mean " $\mathrm{CD}$ Cost Index" $(\mathrm{CDCI})$ for each respondent, defined as the total consumption across the 5 vignettes $/ 5$. We then performed multivariable regression to identify independent predictors of $\mathrm{CDCI}$. Results: Table 1 displays the results. Community providers outspent experts in the new presentation of $\mathrm{CD}$. In contrast, experts outspent community providers in the evaluation of a perianal fistula - a direct result of significantly higher $(\mathrm{p}<0.001)$ endorsement by experts of EUS and exam under anesthesia. Regression analysis revealed that expert status did not independently predict overall resource use, and that only provider age was a predictor of $\mathrm{CDCI}$ (each 10-year increase $=\$ 179$ more per vignette). Conclusions: With the notable exception of perianal fistula, experts and non-experts are consistent in their use of diagnostic tests in $\mathrm{CD}$. This lack of systematic differences in diagnostic testing between community and expert IBD physicians suggests that the dissemination of "best practice" guidelines in $\mathrm{CD}$ may have successfully standardized care.

Table 1. Diagnostic resource use in experts vs community providers

\begin{tabular}{|c|c|c|c|}
\hline Vignette & \$Consumed by Experts & \$Consumed by Non-Experts & p-value \\
\hline Undiagnosed CD & 1401 & 1274 & 0.28 \\
\hline New CD Dx & 869 & 1201 & 0.04 \\
\hline Perianal Fistula & 3973 & 1403 & $<0.0001$ \\
\hline Steroid-refractory & 1145 & 909 & 0.12 \\
\hline S/P IC Anastomosis & 398 & 542 & 0.21 \\
\hline
\end{tabular}

S1362

DLG5 R30q Does Not Predict Response to Infliximab Therapy in Hungarian Patients with Crohn's Disease

Simon Fischer, Peter L. Lakatos, Karolien Claes, Laszlo Lakatos, Istvan Altorjay, Tamas Molnar, Karoly Palatka, Maria Papp, Janos Osztovits, Paul Rutgeerts, Hungarian LBD Study Group, Severine Vermeire, Janos Papp

AIM: Recent data from Germany have suggested that specific haplotypic variants of the DLG5 gene on chromosome 10q23 are associated with susceptibility to inflammatory bowel disease (IBD). Since DLG5 has been implicated in maintaining intracellular signal transduction and cell-cell contact by binding to vinexin, it might be hypothesized that DLG5 affects inflammatory responses and, as a consequence, also response to anti-inflammatory therapy, through influencing intestinal permeability and controlling the entry of luminal antigens into the mucosa. In this study, we investigated the possible association between common DLG R30Q variant allele (impairs protein's scaffolding function) and response to infliximab therapy in Hungarian CD patients. METHODS: 52 unrelated CD patients (m/f: 21/31, age: 34.1 \pm 11.1 years, duration: 7.6 \pm 5.0 years). DLG5 R30Q was tested by PCR-RFLP. Clinical phenotypes - familial disease, age, age-at-onset, duration, location, disease behavior, perianal involvement, extraintestinal manifestations (arthritis, PSC, ocular and cutaneous), need for steroid or azathioprine, steroid resistance, need for surgery or multiple resections and smoking habits - were determined by questionnaire. Comparisons between groups were made by $\chi 2$ test using SPSS13.0 software. RESULTS: $28.8 \%$ of CD patients treated with infliximab carried the 113A. Disease location was ileal in 6 , colonic in 18 and ileocolonic in 28 patients. In addition, two patient had upper GI involvement. Disease behavior was inflammatory in 25 and penetrating in 27. Perianal complications were observed in 27 $51.9 \%$ ) of the patients. $48.1 \%$ of patients had joint manifestations and the rate of cutaneous and ocular manifestations was also high (13.5\% and $15.4 \%)$. Steroids $(98.1 \%)$ and azathioprine $(92.3 \%)$ were prescribed to almost all patients. The indication for infliximab was fistulizing disease in 18 patients and inflammatory, non-responsive to conventional therapy in 34. Overall, no association was found between the carriers and non-carriers of the $113 \mathrm{~A}$ variant allele and short term response (assessed at week 8) to infliximab induction therapy (dose $5 \mathrm{mg} / \mathrm{bwkg}$; no response or worsening: $20.0 \%$ vs. $27.0 \%$, partial response (CDAI decrease by $\geq 70$ points and/or $\geq 50 \%$ decrease in the number of draining fistulae) : $46.7 \%$ vs. $43.3 \%$, remission (CDAI $<150$ or closure of all fistulas): $33.3 \%$ vs. $29.7 \%$ ). CONCLUSION DLG5 R30Q variant allele did not predict the response to infliximab therapy in this cohort of Hungarian $\mathrm{CD}$ patients.

S1363

Comparative Evaluation of the Diagnostic Accuracy of Fecal Markers in the Identification of Intestinal Inflammation: Appraisal for Monoclonal Calprotectin

Oliver Schroeder, Marc Naumann, Yogesh Shastri, Nada Povse, Juergen Stein

Introduction: In recent years, fecal leukocyte-derived markers such as calprotectin, lactoferrin, and PMN-elastase have been established as noninvasive diagnostic tools to test patients for gastrointestinal inflammation as a cause of chronic diarrhea. The aim of this study was to evaluate the clinical accuracy of various fecal marker tests to detect patients with inflammatory bowel disease (IBD) as compared with the non-inflammatory condition, irritable bowel syndrome (IBS). Material and Methods: 103 consecutive patients with chronic diarrhea and/ or abdominal pain who were scheduled for colonoscopy to rule out IBD were included into the study. A full medical history was obtained and a physical examination was performed. All patients underwent hematological and biochemical investigations, antibody testing for coeliac disease as well as a lactose- $\mathrm{H}_{2}$ breath test before endoscopic evaluation. In addition, each participant provided one stool sample $(5 \mathrm{~g})$ for determining calprotectin monoclonal and polyclonal antibody testing, PMN-elastase and lactoferrin within one week prior to colonoscopy. Results: From the enrolled participants, a total of 11 patients had to be excluded from further study participation because of not meeting the diagnosis of either IBD or IBS 41 patients with Crohn's disease, 20 patients with ulcerative colitis, and 31 IBS patients were recruited. The mean $( \pm$ SD) fecal calprotectin concentrations determined with the monoclonal and polyclonal antibody antibody assay were $257 \pm 427 \mu \mathrm{g} / \mathrm{g}$ and $593 \pm 423$ $\mu \mathrm{g} / \mathrm{g}$ in patients with IBD, and $7 \pm 6 \mu \mathrm{g} / \mathrm{g}$ and $33 \pm 50 \mu \mathrm{g} / \mathrm{g}$ in patients with IBS. The corresponding mean $( \pm \mathrm{SD})$ lactoferrin and PMN-elastase concentrations were $85 \pm 112 \mu \mathrm{g} /$ $\mathrm{g}$ and $115 \pm 169 \mathrm{ng} / \mathrm{ml}$ in patients with IBD, and $1 \pm 2 \mu \mathrm{g} / \mathrm{g}$ and $8 \pm 9 \mathrm{ng} / \mathrm{ml}$ in patients with IBS. The diagnostic accuracy of each individual test is present in Fig. 1. Conclusions: Among the four different assays evaluated monoclonal based calprotectin was found to be the most accurate stool marker to detect IBD vs. IBS, which makes it an extremely useful addition to the diagnostic armamentarium in patients with chronic diarrhea and abdominal pain. This method can prove to be useful not only in research settings, but also in clinical settings.

Diagnostic accuracy of fecal stool markers to detect IBD 


\begin{tabular}{|c|c|c|c|c|}
\hline & Cal mAb & Cal pAb & Lactoferrin & PMN-elastase \\
\hline Sensitivity & $91 \%$ & $88 \%$ & $42 \%$ & $31 \%$ \\
\hline Specificity & $95 \%$ & $85 \%$ & $100 \%$ & $100 \%$ \\
\hline NPV & $86 \%$ & $81 \%$ & $50 \%$ & $46 \%$ \\
\hline PPV & $97 \%$ & $91 \%$ & $100 \%$ & $100 \%$ \\
\hline
\end{tabular}

when assessing significance estimates of subsequent genotype-phenotype studies in IBD. Studies of intra-rater reliability are underway and results will be presented.

\section{S1366}

Relationship Between 6-Mercaptopurine Dose and 6-Thioguanine Nucleotide Levels in Patients with Inflammatory Bowel Disease

Ahmed Morales, Sandhya Salguti, Chuan L. Miao, James D. Lewis

S1364

Beneficial Behavioural Effects of IBD Therapy and Gluten/Casein-Free Diet in An Italian Cohort of Patients with Autistic Enterocolitis Followed Over One Year

Federico Balzola, Daniela Clauser, Alessandro Repici, Marina Caldognetto, Claudio Barletti, Anna Sapino, Cristiana Barbera, Pierluigi Calvo, Dario Reggio, Fabrizio Gennari, Antonello Nonnato, Marco Forni, Isabella Morra, Marina Gandione, Roberto Rigardetto, Mario Rizzetto

Autism diagnoses alone have risen from 1:5000 in 1980 to a frightening 1:120 today, that cannot only be the result of better diagnostic variation or recognition of the disease. A substantial proportion of pts with regressive/late onset autism showed evidence of a previously unrecognized IBD, labelled autistic enterocolitis characterized by lymphoid nodular hyperplasia and chronic ileo-colitis. Although improvements in autistic behaviour has been reported when gluten and casein exclusion diets was introduced, no data is available on the effect of specific bowel therapy on the psychiatric symptoms. We aimed to evaluate the effects of specific IBD therapy together with the gluten/casein free diet on specific gastrointestinal and autistic symptoms in a cohort of regressive/late onset autistic pts with autistic enterocolitis, followed over 12 months. Among the 85 consecutive autistic pts observed, 40 presented with severe constipation or diarrhoea. In 12 of them (male, median age 16 yrs, 4-30) an endoscopy and wireless capsule examination were performed because of the permission of the parents and then enrolled in the follow-up study. Macroscopic lesions were observed in $55 \%$ of the pts in both the upper and lower gastrointestinal tract. Microscopic involvement was observed in $70 \%, 60 \%$ and in $100 \%$ of pts in the upper gastrointestinal tract, in the terminal ileum and in the colon respectively, with different histological grade. Mesalazine (8 pts), steroids (2 pts) and 6-mercaptopurine (2 pts) were prescribed according to the histological/clinical score. Casein-free diet and successively glutein-free diet were introduced within the first 3 months by a dietician. A Behavioural Summarized Evaluation (BSE, a score with 29 behavioural items with a degree of frequency validated with a statistical correlation with the Childhood Autism Rating Scale) was used to blindly evaluate the pts at entrance and after 12 months. After 1 year treatment, $80 \%$ of pts with severe constipation or diarrhoea had normal stool movements and in $75 \%$ an evident reduction of abdominal pain was also observed. The total BSE score showed a global improvement after 12 months that ranged from 10 to $32 \%(p=0.00388)$ in all the pts evaluated. When selected symptoms were compared before and after the treatment a reduction of deep anxiety (53\%), agitation (35\%), mood disturbance (24\%), etero-aggressivity (14\%), self-aggressivity (13\%) as well as an improvement of attention (77\%) and sleep (44\%) were also observed ( $\mathrm{p}=0.009796)$. In conclusion, these results proved for the first time a beneficial effect of the combination of drug and dietetic therapy on both bowel and psychiatric symptoms in autism.

\section{S1365}

Reliability of IBD Phenotyping Within the Niddk IBD Genetics Consortium (IBDGC)

Hillary Steinhart, Themistocles Dassopoulos, Geoffrey C. Nguyen, Alain Bitton, Gillian P. Bromfield, L. Philip Schumm, Yahong Wu, Abdul A. Elkadri, Miguel Regueiro, Benjamin Siemanowski, Esther A. Torres, Frederico J. Gregory, Sunanda Kane, Laura E. Harrell, Denis Franchimont, Steven R. Brant, John D. Rioux, Huiying Yang, Richard H. Duerr, Mark S. Silverberg, Judy H. Cho

Background: In 2002, the NIDDK IBDGC began collecting DNA and phenotypic data from large numbers of IBD subjects in order to provide a resource to accelerate the search for disease susceptibility genes. Inclusion of phenotypic information into genetic studies has become a required norm. However, no studies have been performed thus far on the reliability of phenotypic determinations in both Crohn's Disease (CD) and ulcerative colitis (UC) using primary records. The purpose of this study was to determine the inter-rater reliability for the determination of basic phenotypic characteristics. Methods: The medical records of 5 IBD patients were selected from each of the 6 NIDDK IBDGC Genetic Research Centers (GRC). Selection of records was carried out so as to ensure an appropriate distribution of $\mathrm{IBD}$ disease types (CD/UC/indeterminate colitis) and $\mathrm{CD}$ behaviors. The entire medical records were de-identified and 20 were reviewed by 2 phenotypers at each of the 6 GRCs. Phenotypers rated medical records according to disease type, disease location or extent, $C D$ disease behavior, extraintestinal manifestations, year of diagnosis and need for surgery ( 240 separate evaluations of 20 datapoints each). The inter-rater reliability for each of the variables was expressed as the kappa statistic with a $95 \%$ confidence interval. Results: Agreement for disease diagnosis was excellent $(\kappa=0.84 ; 95 \%$ CI $0.70,0.97)$. Agreement for individual $\mathrm{CD}$ locations (jejunal, ileal, colorectal, perianal) was good with $\kappa$ between 0.65 and 0.69 for each. Agreement for esophagogastroduodenal location was fair $(\kappa=0.30)$ but this appeared to be due to the difficulty determining presence or absence of disease when specific evaluation had not been performed. Agreement for UC disease extent was very good $(\kappa=$ 0.72 ; $95 \% \mathrm{CI} 0.56,0.94)$ and for CD behavior was very good $(\kappa=0.69 ; 95 \% \mathrm{CI} 0.46$, 0.88). Insufficient numbers of extraintestinal manifestations were present to allow precise estimates of reliability for determination of those complications. Conclusions: Detailed definitions of phenotypic characteristics of disease diagnosis, CD behavior and disease location/ extent provided very good inter-rater agreement. Further evaluation of agreement regarding less common manifestations or complications is required. These results provide a basis for assessing which phenotypic measures are robust to interobserver variability. They also provide explicit empiric estimates of the phenotyping error rates which should be considered

Background: 6-Thioguanine nucleotides (6TGN) are active metabolites of azathioprine (AZA) and 6-mercaptopurine (6MP). Studies document higher remission rates for patients with higher 6TGN levels. However, controlled trials of AZA/6-MP have traditionally used fixed or weight-based doses to evaluate their efficacy, and many physicians prescribe AZA/6MP using $\mathrm{mg} / \mathrm{kg}$ dosing regimens without measuring $6 \mathrm{TGN}$ levels. We sought to determine the relationship between AZA/6MP dose and 6TGN levels. Methods: Cross-sectional study of patients treated for IBD with AZA/6MP at a tertiary care hospital who had 6TGN levels measured. Patients with low or intermediate TPMT activity were excluded $(\mathrm{N}=13)$. AZA dose was converted to 6MP equivalents. The relationship between dose and 6TGN levels was assessed with the Spearman correlation coefficient. We also used logistic regression after dichotomizing the $6 \mathrm{TGN}$ level as above or below $230 \mathrm{pmol} / 8 \times 10^{8} \mathrm{RBC}$ to adjust for potential confounders (age, sex, disease type, disease activity, use of oral 5-ASA, use of oral steroids). For patients with multiple measurements, only the patient's first measuremen was used in the analyses. Secondary analyses excluded any measurements where the 6TGN level was less than $100 \mathrm{pmol} / 8 \times 10^{8} \mathrm{RBC}(\mathrm{N}=28)$ and/or patients without TPMT testing $(\mathrm{N}=$ 46). Results: 134 patients met our inclusion criteria ( 55 UC, 75 CD, 4 indeterminate colitis; mean age $36.5 \pm 11.9 ; 53 \%$ in remission; median duration of AZA/6MP therapy 48 weeks, range 2 to 832 weeks; median dose $1.09 \mathrm{mg} / \mathrm{kg}$, range .32 to $2.10 \mathrm{mg} / \mathrm{kg}$ ). There was a weak but statistically significant correlation between $6 \mathrm{TGN}$ levels and the absolute dose (rho=.18 $\mathrm{p}=.04)$ and the dose in $\mathrm{mg} / \mathrm{kg}(\mathrm{rho}=.19, \mathrm{p}=.03)$. The correlation between $\mathrm{mg} / \mathrm{kg}$ dosage and 6TGN levels was slightly stronger in those using concomitant 5-ASA medications (rho=.24, $\mathrm{p}=.02$ ). Results of the logistic regression analyses adjusted for 5-ASA and disease activity are shown in the table. Adjustment for other potential confounders did not change the unadjusted odds ratio (OR) by more than $10 \%$. Secondary analyses gave similar results (data not shown). Conclusions: AZA/6MP dose is weakly associated with 6TGN levels. Less than $50 \%$ of patients prescribed $6 \mathrm{MP}$ dose of $1 \mathrm{mg} / \mathrm{kg}$ or greater have $6 \mathrm{TGN}$ levels above 230 pmole $/ 8 \times 10^{8} \mathrm{RBC}$. Use of standard $\mathrm{mg} / \mathrm{kg}$ dosing regimens will result in low $6 \mathrm{TGN}$ levels in the majority of patients.

\begin{tabular}{|c|c|c|}
\hline 6MP Dose $(\mathrm{mg} / \mathrm{kg})$ & \% with $6 \mathrm{TGN}>230 *$ & Adjusted OR $(95 \% \mathrm{CI})$ \\
\hline$<1$ & $23 \%$ & ref \\
\hline 1 to $<1.5$ & $33 \%$ & $1.7(0.7-4.0)$ \\
\hline 1.5 or higher & $44 \%$ & $2.7(0.7-9.6)$ \\
\hline
\end{tabular}

*pmole $/ 8 \times 10^{8} \mathrm{RBC}$

\section{S1367}

Does Faecal Calprotectin Predict Risk of Relapse in Crohn's Disease Patients Lacking Objective Clinical Evidence of Inflammatory Disease Activity? David A. Watts, Norman Anderson, Gordon Brydon, Jack Satsangi, Subrata Ghosh

Background: Marked elevation of faecal calprotectin ( $>200 \mathrm{ug} / \mathrm{g})$ has been shown to be especially valuable in predicting risk of relapse in quiescent Ulcerative Colitis. Our aim was to evaluate the predictive value of even modest elevation of faecal calprotectin for clinica relapse in Crohns disease in asymptomatic patients in clinical remission(GroupA) or in those symptomatic patients lacking objective evidence of inflammatory disease activity (GroupB).Method: 25 patients (median age 39y)were enrolled in Group A and 44 patients (median 35y) in Group B. Informed consent obtained from all patients prior to faecal sampling. Quantitative assay of calprotectin made by ELISA. Baseline Creactive protein (CRP) and ESR were recorded. Relapse rates analysed by Kaplan Meier survival. Results: Group A median calprotectin was $12 \mathrm{ug} / \mathrm{g}$ (1-302) with significant correlation to ESR ( $\mathrm{p}=0.03)$ and CRP $(p<0.0001) .2$ patients were lost to follow up. 23 patients were followed up for a median of 48 months $(12-48) .76 \%(n=19)$ had normal $(<35 \mathrm{ug} / \mathrm{g})$ calprotectin (median 3ug/ g)and remission rates at $12,24,36$ and 48 months were $83 \%, 76 \%, 76 \%$ and $60 \% .5$ patients had elevated calprotectin and followed up for mean 31.2 months (24 - 48 months). No relapse was seen in this group during follow up. No statistical difference in relapse rate was demonstrated between the 2 groups $(\mathrm{p}=0.22)$. Median calprotectin in group $\mathrm{B}$ was 63 .lug g (1-472). Significant correlation to $\operatorname{ESR}(\mathrm{p}=0.03)$ and $C R P(p=0.01\}$ was shown. Clinical outcome: $66 \%(n=28)$ had normal calprotectin (median 8ug/g)and 34\% ( $n=16)$ had raised calprotectin(median $90 \mathrm{ug} / \mathrm{g}$ ). $43 / 44$ patients entered follow up. Mean follow up in those with normal calprotectin was 43.1 months (12-48). Remission rates for those with norma calprotectin at $12,24,36,48$ months was $78 \%, 63 \%, 40 \%, 40 \%$. In those with high calprotectin mean follow up was 41.2 months (12 - 48). Remission rates in this group were $56 \%, 38 \%$, $25 \%$ and $17 \%$ at $12,24,36$ and 48 months. The difference between the relapse rates failed to reach statistical significance $(\mathrm{p}=0.068)$. Conclusion: Faecal calprotectin correlates with ESR and CRP at times of both clinical remission and uncertain clinical activity. A small proportion of Crohns disease patients in clinical remission have elevated faecal calprotectin but this data did not demonstrate a higher rate of relapse of disease in either those with raised faecal calprotectin whilst in clinical remission or in symptomatic patients where clinical evidence of disease activity is lacking. The conflict with previously published data may reflect the modest elevations in calprotectin seen in this study and further studies may be warranted. 


\section{S1368}

Orofacial Granulomatosis: Experience from a Combined Oral Medicine/ Gastroenterology Clinic

Carlo Nunes, Michael P. Escudier, Pepe Shirlaw, Stephen Challacombe, Jeremy D. Sanderson

Background: Orofacial granulomatosis $(\mathrm{OFG})$ is a rare chronic inflammatory disease of unknown aetiology sharing histological features with gut Crohn's disease (CD). Presentation is characterized by lip swelling but the clinical spectrum includes mucosal cobblestoning mucosal tags, and deep linear intra-oral ulceration. We have been running a combined Oral Medicine/ Gastroenterology clinic since 1995 dedicated to patients with OFG. Previous studies addressing the clinical features and natural history of $\mathrm{OFG}$ are limited. Objectives: To gain insight into the overall presentation and clinical features in patients with OFG referred to a dedicated OFG clinic. Methods: In a retrospective study, case notes of patients with OFG attending a combined Oral Medicine/ Gastroenterology clinic were reviewed. Data were extracted for age of onset, sex, clinical features, blood parameters, concurrent $C D$ and patch testing. A concurrent diagnosis of CD was established by standard criteria. Results: Results were available on 73 patients of whom $36(49 \%)$ were female. Median age of disease onset was 24 years. Thirty-six percent were referred by maxillo-facial surgeons and only $15 \%$ by gastroenterologists. Fifteen $(21 \%)$ had concurrent CD. Lip swelling $(69 \%)$ and buccal involvement $(75 \%)$ were the most common sites involved followed by gingivae and floor of the mouth. Mucosal tags (47\% vs. $28 \%$ ) and cobblestoning (40\% vs. $19 \%$ ) were more common in patients with concurrent $\mathrm{CD}$ as were haematinic deficiencies (53\% vs. $29 \%$ ) and raised inflammatory markers (60\% vs. $17 \%$ ). Those with OFG alone (29\% vs. 9\%) were more likely to be patch test positive. No consistent allergen was identified from patch testing. Conclusion: OFG affects predominantly young adults with lip and buccal involvement the most common clinical presentation. Mucosal tags and buccal cobblestoning are more likely in those with concurrent $\mathrm{CD}$. Tests for cutaneous sensitivity are more often positive in $\mathrm{OFG}$ than $\mathrm{CD}$ in keeping with the view that $\mathrm{OFG}$ has an allergic component.

\section{S1369}

Anti-Laminaribioside and Anti-Chitobioside Antibodies As Predictors of Crohn's Disease

Eran Israeli, Itamar Grotto, Miri Blank, Liat Anafi, Eran Goldin, Yehuda Shoenfeld

Background: Anti Saccharomyces cerevisiae mannan antibodies (ASCA) are associated with Crohn's disease (CD). We have previously shown that ASCA were present in the sera of patients many years before clinical diagnosis, with an increase of antibody levels as the time to diagnosis was approached. Novel anti-glycan antibodies, anti-laminaribioside -IgG (ALCA) and anti-chitobioside -IgA (ACCA), were found to be specific for CD. A combined panel of these antibodies improved sensitivity and specificity for diagnosis of $\mathrm{CD}$ in established patients. We assessed the onset and development of the aforementioned antibodies before diagnosis of CD. Methods: Utilizing the Israeli Defense Force (IDF) Serum Repository, we identified sera samples from 28 persons obtained an average of $64 \pm 55$ months before onset of $\mathrm{CD}$, as well as 52 sera from controls matched for gender, age and day of recruitment. Sera were tested for gASCA-IgG, ALCA and ACCA. Results: 12/28 (43\%) of the study group were positive when combining a positive gASCA or ALCA vs. 1/52 (2\%) among matched controls ( $\mathrm{p}<0.001$ ). ACCA was positive in $12 / 28(43 \%)$ of the study group but also in $24 /$ $52(46 \%)$ of matched controls. The average level of all three antibodies in sera obtained from patients after $C D$ diagnosis was higher then in sera obtained from patients before $C D$ diagnosis (the study group). This difference reached statistical significance only for gASCA ( $107.6 \pm 72.9$ Uv $.45+53.5 \mathrm{U}$ respectively, $\mathrm{p}=0.04)$. Conclusions: A panel combining gASCA and ALCA has an improved sensitivity for predicting CD. References: 1) Israeli E et al. Anti-Saccharomyces cerevisiae and antineutrophil cytoplasmic antibodies as predictors of inflammatory bowel disease. Gut 2005;54:1232-6. 2) Altstock RT, et. al. Gastroenterology 2005; 128;A-303

\section{S1370}

Fecal Calprotecin Is a Reliable Surrogate Marker for Endoscopic Activity in Crohn's Disease

Geert R. D'Haens, Filip Baert, Maja Noman, Gert van Assche, Marijke van Camp, Gust van Olmen, Doreen Iwens, Patrick Hanaway, Amy Peace-Brewer, Severine Vermeire, Paul Rutgeerts

Introduction: Fecal calprotectin has been proposed as a marker of inflammatory activity in inflammatory bowel disease (IBD). The test was shown to correlate with disease activity in children and found to be a predictive marker of relapse in patients with both ulcerative colitis (UC) and Crohn's disease (CD)(Buhn, 2001, Costa, 2005). In addition, normal calprotecin levels have been associated with absence of endoscopic lesions (Røseth 2004). Since endoscopic healing has become a goal of treatment in $\mathrm{CD}$, we examined the correlation between fecal calprotectin levels and clinical and endoscopic activity in a cohort of $C D$ patients. Methods: $106 \mathrm{CD}$ patients who were scheduled for colonoscopy at three IBD referral hospitals were invited to deliver a sample of feces immediately prior to the start of their bowel cleansing. All indications for colonoscopy were accepted. Patients also completed a CDAI, Euroquol and IBDQ and had a blood test for serum CRP, albumin, Hct and thrombocytes. All gave informed consent. Six endoscopists experienced in IBD completed the scoring sheets for SES-CD and CDEIS immediately after the procedure. All stool samples were frozen and sent to Genova Diagnostics for measurement of calprotectin with PhiCal Assay (ELISA)and lactoferrin, both neutrophilic markers in the feces. Correlation analysis was done with Spearman Rank tests. Results: $106 \mathrm{CD}$ pts (57 male, age 39 \pm 13 , age at diagnosis $28.6 \pm 12.3 \mathrm{yrs}$ )were studied. The median fecal calprotectin was $142 \mathrm{ug} / \mathrm{gram}$ (IQR 42-775). The Calpro values correlated in decreasing order with: $\operatorname{SES}-\mathrm{CD}(\mathrm{r}=0.47, \mathrm{p}<0.0001)$, CDEIS $(r=0.44, p<0.0001)$, CRP $(r=0.43 p<0.0001)$ and thrombocytes $(r=0.32, p<0.001)$. Poor correlations were seen with the clinical scores CDAI $(r=0.13)(\mathrm{NS})$ and IBDQ $(\mathrm{r}=-$ 0.22 )(NS). Fecal Calpro- en lactoferrin levels correlated at $r=0.43(p<0.0001)$. Conclusion: fecal calprotectin and lactoferrin levels correlate significantly with endoscopic disease acticity in $C D$ and poorly with clinical scores. Whether the test can replace other commonly used biological markers remains te be determined.

\section{S1371}

Fecal Excretion of Human Deoxyribonucleic Acid in Long Term Follow-Up of Ulcerative Colitis

Francesc Casellas, Natalia Borruel, Antonio Torrejon, Encarnacion Varela, Maria Antolin, Francisco Guarner, Juan-Ramon Malagelada

Several indices evaluate disease activity in ulcerative colitis (UC), but most of them have drawbacks to its application and discrepancies between them are frequent. Assuming that luminal desquamation of epithelial and inflammatory cells increases in damaged colonic mucosa, we have previously shown that fecal excretion of human DNA is an index of mucosal damage. However, it has not been established the potential role of fecal DNA measurement in the follow-up of patients with UC. AIM: To determine the stability of fecal DNA in inactive UC and its value as an objective marker of relapse. METHOD: Prospective study in UC patients in clinical remission treated with oral mesalamine followed for 12 month or until clinical relapse (Clinical Activty Index $>7$ ) with determinations of fecal calprotectin by ELISA and fecal human DNA concentration by quantitative PCR of the human betaglobine gene. RESULTS: 54 with clinically inactive UC (median CAI 0.0, hemoglobin 13.9 $\mathrm{g} / \mathrm{dl}$, ESR $8.0 \mathrm{~mm} / \mathrm{h}$ ) were included. During the follow-up, 23 patients (42.5\%) relapsed, showing a median increase in CAI from 1.0 to 8.0 ( $\mathrm{p}<0.01)$. Levels of fecal DNA remained unchanged in patients with stable inactive $\mathrm{UC}$, ranging between $1.4 \mathrm{E}+03$ and $8.4 \mathrm{E}+04 \mathrm{cop} /$ $\mathrm{mg}$ (25 to 75 percentils) during the one-year follow-up. In contrast, a significant increase in concentration of fecal DNA was observed in patients who relapsed $(2.5 \mathrm{E}+05[5.9 \mathrm{E}+04$ $2.8 \mathrm{E}+06] \mathrm{cop} / \mathrm{mg}$ at relapse vs $3.9 \mathrm{E}+03[1.1 \mathrm{E}+03-4.4 \mathrm{E}+04]$ at entry, $\mathrm{p}<0.01)$. Fecal calprotectin levels were stable in patients in remission, ranging between 41 and 1356 microg/ $\mathrm{g}$ (25 to 75 percentils). Relapsing patients showed a significant increase in fecal calprotectin (2327.0 [810 - 4314] microg/g at relapse vs 389.5 [103 - 1388] at entry, p<0.01). CONCLUSIONS: Human fecal DNA concentration remains stable in patients with inactive UC treated with mesalamine, but increases significantly during relapse. Determination of human fecal DNA may be a new objective instrument in the follow-up of UC patients.

\section{S1372}

A Rapid One-Hour Infusion of Infliximab Is Well-Tolerated

Micha Rojany, Jacob Wegelin, Walter Trudeau, Thomas Prindiville

Background: Infliximab is indicated for use in moderate to severe Crohn's disease and has been effective for the induction and maintenance of remission in both fistulizing and inflammatory disease. However, infusions are not without risk with approximately $4 \%$ of infusions associated with an infusion reaction. Aim: To determine if patients with Crohn's disease would be able to tolerate a rapid 1 hour infusion safely with an acceptable rate of infusion reactions. Methods: We retrospectively reviewed the charts of all adult patients with Crohn's disease who received Infliximab infusions between January 2003 and September of 2004. All patients started with a standard induction therapy at 0, 2, and 6 weeks followed by maintenance therapy every 8 weeks. Patients without history of any moderate or severe infusion reaction, or severe systemic disease were eligible to receive a l-hour infusion of Infliximab. All patients were monitored for infusion reactions that were categorized as acute or delayed and further classified as mild, moderate, or severe. For group comparison, logistic regression was used to compare the probability of an infusion reaction between groups with gender and age as covariates. For analysis within individual patients, generalized linear mixed models were fit to assess the chance of a reaction by rate with sex, age, and immunomodulator-use as covariates. Results: There were a total 731 infusions with 547 standard infusions and 184 rapid infusions studied. None of the patients were on steroid therapy. 49 patients received only standard infusions and 49 received at least one rapid infusion ( 40 M 58 F). $92 \%$ (87/95) of patients were on immunomodulators. The mean number of infusion reactions was 0.4 reactions per patient. $11 / 49(22 \%)$ patients in the standard group and $14 / 49(29 \%)$ in the rapid group had at least one infusion reaction ( $>0.5$, chi-square test). The rapid infusion group had a total of 14 infusion reactions ( 7 mild, 7 moderate). 9/49 (18\%) patients in the rapid infusion group returned to standard infusions due to infusion reactions. In the group comparison neither age nor sex was associated with infusion reactions ( $>$. 10). Individual analysis revealed younger men undergoing rapid infusions had a higher probability of infusion reactions ( $\mathrm{p}<0.05$ ). Older women receiving rapid infusions also had a higher rate of infusion reactions but this was not statistically significant $(\mathrm{p}=0.10)$. Conclusion: Appropriate patients tolerated a rapid 1-hour infusion. Patients could be offered a rapid infusion as an alternative to conventional 2 to 3 hour infusions.

\section{S1373}

Calprotectin and Lactoferrin Decrease with Maintenance Infliximab Administration in Luminal Crohn's Disease Ron Palmon, Steven Brown, Thomas A. Ullman, Patrick Hanaway, Lloyd F. Mayer

Non-invasive markers of inflammation have previously been shown to be useful in the diagnosis and assessment of disease activity in patients with inflammatory bowel disease (IBD). Two fecal markers, calprotectin (CL) and lactoferrin (LA), have been the most extensively studied. However, few studies have actually compared the reliability of these measurements in patients undergoing maintenance therapy with infliximab (IFX). Methods: We initiated a prospective study measuring fecal calprotectin $(\mathrm{CL})$ and lactoferrin (LA) in 17 patients with Crohn's disease receiving maintenance IFX treatment for luminal but not fistulizing disease and compared the levels with a Harvey Bradshaw disease activity index (HBI). Stool samples and HBI were collected at the time of treatment (baseline) and every 2 weeks afterward for a total of 8 weeks. Results: Median CL at baseline was $343 \mathrm{ug} / \mathrm{g}$ (range 16-2500). ( $C L>250 \mathrm{ug} / \mathrm{g}=$ active IBD). At week 2, CL decreased from baseline in 13 of the 16 patients with available levels ( $\mathrm{p}=.02$ by Sign test). Median CL at this time interval was $179 \mathrm{ug} / \mathrm{g}$ (a 48\% decrease from baseline). By week 4, CL had risen back toward baseline 
values, with median CL of $297 \mathrm{ug} / \mathrm{g}$. A sustained drop in LA was seen after IFX treatment: Baseline median LA was $52.2 \mathrm{ug} / \mathrm{g}$ (range 4-1724). A decrease in LA at week 2 was noted in 12 of 16 patients with available levels ( $\mathrm{p}=0.01$ by Sign test). Median LA at this time interval was $31.8 \mathrm{ug} / \mathrm{g}$ (a 39\% decrease from baseline). Median LA at week 4 remained decreased at $32.1 \mathrm{ug} / \mathrm{g}$. Median HBI was 6 at baseline reflecting relatively mild disease; median HBI was 3.5 at week 2 and remained low at an HBI of 3 at week 4. Conclusions: $\mathrm{CL}$ and LA levels parallel successful IFX treatment, likely reflecting decreased enteric inflammation. The rise in CL but not LA at week 4 suggests that this may be a more sensitive indicator of a subclinical rebound in inflammatory activity, although further evidence is required to confirm this. Our results suggest that non-invasive fecal inflammatory markers may be useful in monitoring $\mathrm{CD}$ patients receiving IFX therapy.

\section{S1374}

Crohn's-Like Disease Following Ileal-Pouch Anal-Anastomosis (IPAA) for Ulcerative Colitis (UC) Is Associated with IBD Serology Seroconversion John B. Averette, Steve F. Polyak, John F. Valentine

INTRODUCTION: Total colectomy with IPAA is the current recommended therapy for severe, medically refractory UC. IPAA is not recommended for patients with Crohn's disease (CD) due to the high rate of complications, including fistulae, strictures, abscesses, and pouch failure. Serum perinuclear anti-neutrophil cytoplasmic antibody (pANCA) is associated with UC, with a sensitivity of $60-80 \%$ and specificity of $90 \%$. Antibodies to Saccharomyces cerevisiae (ASCA) have been associated with CD, with a sensitivity of $60-70 \%$ and specificity of $91 \%$. Some patients develop CD or Crohn's-like disease following IPAA. We previously reported a series of 11 patients with IPAA CD-like complications of which 45\% were ASCA $(+)$. Unfortunately, we did not have pre-colectomy serologies. METHODS: We report 4 cases of patients diagnosed with UC based on clinical, endoscopic, and pathologic criteria that developed CD-like features following IPAA. Each patient had no significant expression of ASCA antibodies at the time of diagnosis or immediately following total colectomy with IPAA for refractory UC. Following surgery, these patients developed CD-like complications and therefore IBD serologies were reassessed. RESULTS: Two patients were male and two were female. Their ages at diagnosis of UC ranged from 15 to 22 years. Time from diagnosis to surgery ranged from two months to 25 years after diagnosis. CD-like complications developed ten to twenty-five months after the IPAA. The CD-like disease that developed following IPAA for UC included ulceration in the ileum above the pouch $(\mathrm{N}=3)$, jejunal ulceration $(\mathrm{N}=1)$, persistent anal fissure $(\mathrm{N}=1)$, perianal fistula $(\mathrm{N}=1)$. None of the patients had granulomatous inflammation. The initial and follow up IBD serologies are shown in the table. CONCLUSIONS: This is the first report of IBD serology seroconversion in patients with IPAA. Our findings show that expression of ASCA antibodies can change over time and that following IPAA for UC, the development of CD-like pathology is associated with ASCA seroconversion. The significance of this finding is unclear. ASCA antibodies may be a marker of small bowel inflammation, or may indicate that the patient has developed CD. Our findings question the stability of serological expression of pANCA and ASCA over long periods of time. Larger, more detailed examination of this patient population will hopefully clarify these issues.

IBD Serologies

\begin{tabular}{|c|c|c|c|c|c|c|c|c|}
\hline Patient & $\begin{array}{c}\text { Baseline } \\
\text { pANCA }\end{array}$ & $\begin{array}{c}\text { Followup } \\
\text { pANCA }\end{array}$ & $\begin{array}{c}\text { Baseline } \\
\text { ASCA } \\
\text { IgA }\end{array}$ & $\begin{array}{c}\text { Followup } \\
\text { ASCA } \\
\text { IgA }\end{array}$ & $\begin{array}{c}\text { Baseline } \\
\text { ASCA } \\
\text { IgG }\end{array}$ & $\begin{array}{c}\text { Followup } \\
\text { ASCA } \\
\text { IgG }\end{array}$ & $\begin{array}{c}\text { Baseline } \\
\text { OmpC }\end{array}$ & $\begin{array}{c}\text { Followup } \\
\text { OmpC }\end{array}$ \\
\hline 1 & 14.1 & $<12.1$ & $<20$ & 34.8 & $<40$ & $<40$ & $\begin{array}{c}\text { Not } \\
\text { Done }\end{array}$ & 16.5 \\
\hline 3 & 144.3 & 74.2 & $<20$ & 23.4 & $<40$ & $<40$ & $\begin{array}{c}\text { Not } \\
\text { Done }\end{array}$ & $<16.5$ \\
\hline 4 & $<12.1$ & $<12.1$ & $<20$ & 28.6 & $<40$ & 28.9 & $<16.5$ & $<16.5$ \\
\hline
\end{tabular}

All units in $\mathrm{EU} / \mathrm{mL}$

\section{S1375}

Can High-Sensitive C-Reactive Protein Be a Biomarker for Patients with Ulcerative Colitis?

Yoshiaki Takeuchi, Fumihiko Nozu, Katsuhito Arai, Michio Imawari

Introduction: Although there is no clear gold standard, much effort has been made to evaluate severity of ulcerative colitis (UC) by using indices including symptoms, characteristics of stool, biomarkers, and endoscopic findings. Among them, symptoms reported by patients appear to be appropriate, however, symptoms of mild UC is often similar to those of irritable bowel syndrome in quiescent UC patients. Therefore, more reliable and practical measures are required. One of the conventional and non-invasive biomarkers, C-reactive protein (CRP) has been used for assessment of inflammatory bowel disease, and its usefulness has recently been documented mostly in Crohn's disease and not in UC. High-sensitive CRP (HS-CRP) can detect as low as $0.2 \mathrm{mg} / \mathrm{L}$ quantitatively and could be a potent biomarker to assess underestimated mild UC. The aim of this study is to evaluate whether high-sensitive CRP can be a biomarker for UC patients. Methods: Twenty UC patients with conventional CRP being less than $5 \mathrm{mg} / \mathrm{L}$ were recruited in this study. Patients were defined as symptomatic if one of the followings was observed; bloody stool, four or more of bowel movements, lower abdominal pain, rectal urgency, and continuous unformed stool. Biomarkers including complete blood cell count, erythrocyte sedimentation rate, albumin, CRP, and HS-CRP were obtained. Fifteen subjects underwent colonoscopic examination within two weeks of sampling of biomarkers. Fischer's exact test was used to assess associations of HS-CRP. All patients gave written informed consent and this study was approved by ethical committee in our institution. Results: Ten out of 20 subjects had co-morbid diseases such as cardiovascular diseases and primary biliary sclerosis. Eight out of 20 patients were symptomatic and all of them consistently presented inflamed colonic mucosa (Matt's grade 1) by colonoscopy. While
7 of 8 symptomatic patients had increased HS-CRP, 7 out of 12 asymptomatics also showed elevated HS-CRP $(P>0.05)$. Among 7 asymptomatic subjects with increased HS-CRP, 6 had co-morbid diseases, suggesting that HS-CRP increased independent of UC activity. Even when subjects with co-morbid diseases were excluded. HS-CRP was not associated with symptoms. No other biomarkers were associated with increased HS-CRP. Conclusions: In UC patients, increased HS-CRP was not associated with disease activity, rather appeared to be influenced by co-morbid diseases. HS-CRP could not be a biomarker to assess minimal inflammatory change in UC patients.

\section{S1376}

Serologic Markers As Quantitative Predictors of Inflammatory Bowel Disease Activity - Clarifying the Cart and the Horse

David M. Poppers, Brian P. Bosworth, Douglas M. Weine, Quanhong Ni, Ellen J. Scher

Background: Inflammatory Bowel Disease (IBD) comprises a spectrum of chronic illness, and is classically categorized as Crohn's Disease (CD) or ulcerative colitis (UC), with significant overlap between these diseases. There has been great interest in using serologic markers (primarily anti-Saccharomyces cerevisiae antibodies (ASCA) and perinuclear antineutrophil cytoplasmic antibody (pANCA)) to distinguish between UC, CD, and indeterminate colitis (IC). ASCA is often detected in the serum of CD patients, and pANCA is associated with UC. Other data suggest that ASCA is more related to small intestinal/fibrostenotic disease, and pANCA associated with colitis. There has been no published association between severity of disease and quantitative levels of these markers. Aim: We conducted a prospective study to determine if alterations in serologic titers for PANCA and ASCA are associated with degree of disease activity. Methods: A blinded, prospective cohort study of 19 patients with CD, UC, and IC from 1998-2005 was conducted. These patients were maintained on aminosalicylates, antibiotics, immunomodulators (azathioprine, 6-MP, cyclosporine), or infliximab, or combinations thereof. Patients were evaluated endoscopically and by established disease activity indices, and with measurement of serum ESR, pANCA, ASCA (IgA/lgG), and OmpC. Results: For 19 patients with a mean follow-up of 4.8 years, quantitative pANCA and ASCA titers correlate with IBD activity particularly in cases of marked colitis, and with ESR and endoscopic activity. Most strikingly, lower pANCA levels were associated with a decline in the Harvey Bradshaw Index (HBI, $\mathrm{p}=0.03$ ), with a trend toward lower Sutherland Index $(\mathrm{SI}, \mathrm{p}=0.09)$. This was most notable with the addition of 6-MP, cyclosporine, or infliximab in colitis patients, with decreases in PANCA that parallel those in HBI/SI and ESR. Although not statistically significant, a similar trend was noted for OmpC, a marker associated with smal bowel inflammation. There was likely a trend toward association between lower ESR and lower SI $(\mathrm{p}=0.12)$, and lower $\mathrm{HBI}(\mathrm{p}=0.08)$. A trend toward lower ASCA IgA with decreased ESR was seen as well. Conclusions: This study demonstrates a relationship between IBD activity and titers of serologic markers. It is intriguing to postulate that PANCA and other markers are surrogates for disease activity, and may predict therapeutic response to immunomodulator or biologic therapy. Future studies evaluating novel markers, including $\mathrm{OmpC}$ and flagellin/CBirl, may enhance the specificity of UC/CD discrimination, and stratify therapeutic responses, to tailor therapy for more severe cases.

\section{S1377}

Lymphopenia Cd4 Counts in Patients with Inflammatory Bowel Disease and Low

Darrell G. Finlay, Samir Nath, Andrew Dupont, Joseph H. Sellin

Introduction: Dysregulation of the immune response plays a major role in the pathogenesis of IBD. In addition, many medical therapies for IBD are aimed at modulating the immune response. To our knowledge, there are no described alterations in T-cell lineages in patient with IBD. We describe a series of patients with documented IBD who had a profound lymphopenia and very low CD4 count. Aims: i) Assess the frequency of decreased lymphocyte count in patients with IBD. ii) Identify factors that may be associated with lymphopenia. Methods: We performed a retrospective and prospective chart review of 100 consecutive patients with IBD at 2 tertiary referral centers. Routine complete blood counts were obtained from 3 reference laboratories. Lymphopenia was defined as less than 850 lymphocytes/cc, which was the strictest criterion from the 3 different laboratories. The data were analyzed by performing chi-square analyses using the NCSS statistical software package. Results: $21 /$ $100(21 \%)$ of patients were lymphopenic, (total lymphocyte count less than 850 lymphocytes/ cc). Of those 21 patients, all were HIV negative. Flow cytometry performed on 4 patients revealed an abnormally low CD4 count (range 97-249). The CD4/total lymphocyte ratios ranged from $0.28-0.54$ for these 4 patients. $1 / 4$ patients with a low CD4 count had an opportunistic infection (herpes zoster). We examined factors that may be associated with lymphopenia. $86 \%$ of lymphopenic patients were on 6 -MP/Azathioprine versus $48 \%$ of patients having a normal lymphocyte count while on 6-MP/Azathioprine $(\mathrm{p}=0.002)$. No differences were found for other medications, including 5-ASA, prednisone and Infliximab There were no differences in disease distribution (UC vs. CD) in the lymphopenic vs. the non-lymphopenic patients. Similarly, there were no gender differences between the 2 populations. Conclusions: The prevalence of lymphopenia in patients on 6-MP/Azathioprine is surprisingly high. In addition, all patients who underwent flow cytometry had a low CD4 count. Use of immunosuppressives, but not other medications, appears to be associated with the development of lymphopenia. The clinical significance of this remains to be determined, but may warrant additional monitoring to identify risk factors for opportunistic infections. 
S1378

Does Preoperative Wireless Endoscopic Capsule Predict Long-Term Outcome After Ileal Pouch-Anal Anastomosis (IPAA)?

Stefanie J. Schluender, Shahab Mehdizadeh, Eric A. Vasiliaukas, Marla Dubinsky, Konstantinos A. Papadakis, Andrew Ippoliti, Simon Lo, Stephan Targan, Phillip Fleshner PURPOSE: The extent of preoperative small bowel (SB) mucosal inflammation in ulcerative colitis may be an important predictor of outcome after IPAA. Although small bowel followthrough (SBFT) is the traditional method for assessing SB inflammation, wireless capsule endoscopy (WCE) appears to be more sensitive for the assessment of mucosal lesions than radiological testing. The purpose of this study was to investigate the value of preoperative WCE in predicting long-term outcome of IPAA in patients with ulcerative colitis or indeterminate colitis. METHODS: 24 patients undergoing WCE before having an IPAA over the 4year period ending October 2005 were identified. Findings on WCE were classified as positive (erosions, ulcers, erythema), negative, or incomplete. SBFT was done in 20 patients and was classified as positive if ulcerations, mucosal blunting, or wall thickening was identified. Long-term outcome was assessed prospectively and included acute pouchitis (AP), chronic pouchitis (CP), or the development of Crohn's disease (CD). Patients with AP, CP or CD were considered to have pouch inflammation (PI). RESULTS: WCE was positive (WCE+) in 8 patients (33\%), negative (WCE-) in 12 patients $(50 \%)$ and incomplete in 4 patients (17\%). After a median length of followup of 15 months in the 20 patients with a complete WCE, AP was seen in 3 patients $(15 \%)$, one patient $(5 \%)$ developed $\mathrm{CP}$ and one patient $(5 \%)$ developed CD. Within WCE+ patients, 1 (13\%) developed AP and none developed CP or CD. Within WCE- patients, 2 (17\%) developed AP, l (8\%) developed CP, and $1(8 \%)$ developed CD. PI rate for WCE+ patients $(13 \%)$ was lower than WCE- patients (33\%) ( $\mathrm{p}=\mathrm{NS}$ ). SBFT was positive (SBFT+) in 3 patients (15\%) and negative (SBFT-) in 17 patients (85\%). Within SBFT+ patients, 1 (33\%) developed AP and 1 (33\%) developed CP. Within SBFT- patients, 2 (12\%) developed AP and $1(6 \%)$ developed CD. The PI rate for SBFT+ patients $(67 \%)$ was higher than for SBFT- patients $(18 \%)(\mathrm{p}=0.1)$. There was no significant difference in overall PI rate between WCE + and SBFT+ patients $(\mathrm{p}=0.1)$. CONCLUSION: Early observations from this study suggest that in UC or IC patients undergoing IPAA, preoperative WCE+ does not appear to be associated with pouchitis. WCE may not confer any advantage over SBFT in assessing the risk of pouch inflammation after IPAA for patients with UC or IC

\section{S1379}

Ulcerative Colitis in Patients with Primary Sclerosing Cholangitis (PSC-UC) A Specific Subgroup of Inflammatory Bowel Disease

Pavel Drastich, David Kamenar, Julius Spicak

BACKGROUND: Primary sclerosing cholangitis (PSC) is commonly associated with inflammatory bowel disease (IBD), particularly with ulcerative colitis (UC) in $55-75 \%$ cases. PSC UC patients experience the extensive milder course of colonic disease, but other clinical symptoms and endoscopic pictures have not been well described. AIM: To retrospectively evaluate endoscopic features, clinical course and therapeutical management of PSC-UC patients in comparison with PSC free UC patients. PATIENTS AND METHODS: A cohort of 45 PSC-UC patients (mean age $36.8 \pm 12.3$ years) and 47 UC patients (mean age $42.7 \pm 15.1$ years) were enrolled. Patients were from the hospital database (IKEM, Prague, Czech Republic)) and they underwent total colonoscopic evaluation since January 2004 till November 2005. The bowel disease duration was comparable in both groups (mean 6 years in PSCUC group and 7 years in UC group). Chi-square test, Fischer's exact test and stepwise logistic regression were used for statistical analysis. RESULTS: PSC-UC patients are suffering from more extensive disease (pancolitis) than UC patients ( $76 \%$ vs $49 \%, \mathrm{P}<0.01$ ), but the disease is more often in remission/mild course than in moderate/severe course $(75.6 \%$ vs $50 \%, \mathrm{P}<0.01$ and $24.4 \%$ vs $50.0 \%, \mathrm{P}<0.01$, respectively). UC patients were more likely to find post-inflammatory polyps $(46.8 \%$ vs $20 \%, \mathrm{P}<0.01)$ and left-sided colitis was a typical picture of these patients ( $42.6 \%$ vs $4.4 \%, \mathrm{P}<0.001$ ). The right-sided active colitis without rectal involvement was almost unique feature of PSC-UC patients $(20.0 \%$ vs $2.1 \%, \mathrm{P}<0.01)$ PSC-UC patients required less azathioprin therapy $(8.9 \%$ vs $31.1 \%, P<0.01)$ for disease management. Two groups had no significant difference in use of aminosalicylates. Number of patients undergoing colectomy because of disease activity did not differ between the two groups (2/47 in UC group and 3/45 in PSC-UC group). There was no difference in frequency of backwash ileitis and dysplasia of colonic mucosa was find in just 2 patients from both groups. Correct prediction based on multivariate analysis (pancolitis, milder disease, rightsided colitis, absence of post-inflammatory polyps and no azathioprin therapy) was $85 \%$. CONCLUSION: PSC-UC patients may represent a specific IBD subgroup. Characteristic picture of these patients is pancolitis with mild activity or active right-sided colitis with rectal sparing. In contradistinction to UC patients, post-inflammatory polyps are rarely detected and azathioprin was significantly less need for treatment of these patients.

\section{$\mathrm{S} 1380$}

A Cost Effectiveness Analysis of High Dose Oral mesalazine for the Treatment of Moderately Active Ulcerative Colitis: A Decision Analytic Model J Borrill, K Bodger, A Bhatt

Objective: To conduct an economic evaluation of high dose oral mesalazine as a lst line treatment for patients with moderately active ulcerative colitis (UC). Design: A decision tree model was constructed to examine the patterns of treatment, resource consumption and the cost impact of treating a moderately acute episode of UC with a $4.8 \mathrm{~g}$ /day dose of mesalazine $800 \mathrm{mg}$ MR tablets (P\&G Pharmaceuticals) compared to the currently marketed conventional dose of $2.4 \mathrm{~g}$ /day mesalazine $400 \mathrm{mg}$ MR tablets (P\&G Pharmaceuticals, UK). Differences in treatment effects were obtained from a pooled analysis of two, phase III clinical trials. Resource use data was derived from a UK cost of illness study that assessed the direct costs of IBD in secondary care. These data were supplemented with information from the literature and expert opinion. A secondary analysis was conducted in which oral prednisolone (40mg/ day) was used as the comparator treatment. The analysis was conducted from the perspective of the NHS using a time horizon of six months. A hypothetical cohort of 1,000 patients with moderately active $\mathrm{UC}$ was passed through the model. Results are expressed as hospitalisations avoided, incremental cost of treatment and the incremental cost per quality-adjusted life year (QALY). Results: Treatment success at six weeks was achieved in $72 \%$ of patients treated with mesalazine $4.8 \mathrm{~g}$ /day compared to $59 \%$ in the mesalazine $2.4 \mathrm{~g} /$ day group $(\mathrm{p}=0.0034$ ) The model projected that fewer patients in the mesalazine $4.8 \mathrm{~g} /$ day treatment arm would undergo surgery ( $5 \%$ versus $7 \%$ ) or intensive intravenous steroid or immunosuppressive therapy ( $7 \%$ versus $10 \%$ ) compared to those in the $2.4 \mathrm{~g} /$ day treatment arm. Over six months, the mean expected cost per patient was $£ 1,300$ for the mesalazine $4.8 \mathrm{~g} / \mathrm{day}$ treatment group compared to $£ 1,640$ for the 2.4 g/day group - a cost saving of $£ 340$ per patient. The difference in health related quality of life between the treatment groups was negligible. This resulted in an incremental cost per QALY that fell comfortably within the cost-effectiveness threshold adopted in the UK. Sensitivity analysis demonstrated that the results of the cost-effectiveness analysis were robust to changes in both clinical and economic parameters. Similar findings were observed when mesalazine $4.8 \mathrm{~g} / \mathrm{day}$ was compared to oral prednisolone. Conclusion: From the perspective of the UK healthcare system, mesalazine $4.8 \mathrm{~g} /$ day is a cost-effective option for the management of moderately active UC. This result provides an economic argument for clinicians to adopt this treatment strategy in the given patient population

\section{S1381}

Exposure to Diagnostic Medical Radiation in Patients with Inflammatory Bowel Disease: An Additional Risk for Bowel Cancer Evan Newnham, Anusha Surender, Eliza Hawkes, Peter Gibson

Awareness of the level of radiation exposure associated with medical imaging and its carcinogenic risk is poor. Cumulative doses of diagnostic medical radiation (DMR) of 50 to 100 $\mathrm{mSv}$ delivered to, for example, the colon and bladder increase the risk of cancer in those organs. Patients with IBD, who are already at increased risk of colon cancer, often undergo medical imaging involving exposure to these and other radio-sensitive organs, yet the level of exposure has not been documented. Aims: To evaluate the exposure from DMR within a population of patients with IBD and to identify subgroups at higher risk of exposure Methods: DMR exposure was documented by review of medical records (hospital and referring doctor) of 100 consecutive patients attending a hospital-based IBD Clinic. Only verified imaging procedures were included. Effective radiation doses delivered to colon and bladder were calculated using published tables of average exposure for individual test types, and were expressed as total and yearly exposure. Predictors of exposure were also determined. Results: The studied population had a median age of 38 (16-84) years. 43 were men. 34 had ulcerative colitis, 1 indeterminate colitis and 65 Crohn's disease (12 ileal, 38 ileocolonic, 19 colonic). 32 had undergone disease-related surgery. 4 had not been exposed to DMR, and 35 had no abdominal exposure. The major DMR procedures were plain abdominal Xray, $\mathrm{CT}$ abdomen and pelvis and small bowel barium studies. The median exposure to the colon was $20(0-424) \mathrm{mSv}$ and to the bladder $22(0-460) \mathrm{mSv}$. 25\% had exposure already in the at-risk range and, in 12, it exceeded $150 \mathrm{mSv}$. Patients with Crohn's disease had significantly higher radiation exposure (median $30 \mathrm{mSv}$ ) than those with ulcerative colitis $(3 \mathrm{mSv}(\mathrm{p}<0.001$, rank sum test)). The only other predictor of high levels of exposure to DMR was prior surgery. Conclusion: DMR exposure in patients with IBD is high, often above at-risk levels, particularly in Crohn's disease and in the setting of surgery. In an often immunosuppressed population with a chronic illness that already has an elevated risk of bowel cancer, careful consideration should be given to the indication for medical imaging of the abdomen, especially as it is often needed intermittently over the life of the patient.

\section{S1382}

The Granulocyte and Monocyte Adsorption Apheresis (GCAP) Therapy Changes Surgical Indication for Patient with Ulcerative Colitis Tetsushi Kinugasa, Masahide Kuroki, Takayuki Shirakusa, Yuichi Yamashita

There is no specific medical care for ulcerative colitis (UC), and approximately one third of its patients undergo operative treatment. Recent evidence suggests that peripheral blood granulocytes and monocytes/macrophages play a major role in the exacerbation of UC. Recently, granulocyte and monocyte adsorption apheresis (GCAP) has been shown to be safe and effective for active ulcerative colitis in Japan. Our objective was to investigate whether the one of surgical indication factor is after GCAP therapy in patients with steroidrefractory and -dependent UC. METHODS: Eighteen with active ulcerative colitis were studied, of whom 5 had relapsing-remitting ulcerative colitis, 13 had chronic continuous. GCAP was performed with an Adacolumn filled with cellulose acetate beads. Patients received up to 10 apheresis sessions over 12 weeks; one session being for 60 min at $30 \mathrm{~mL} / \mathrm{min}$. Eighteen patients received 5 apheresis sessions over 4 weeks. RESULTS. Sixteen of 18 patients showed an improvement in symptoms (abdominal pain, rectal bleeding, and diarrhea) and could avoid re-administration of steroids after GCAP. At entry, the mean dose of prednisolone was $32.7 \mathrm{mg} /$ day per patient and was reduced to $17.2 \mathrm{mg} /$ day after 10 sessions. Of 18 patients treated with GCAP, 7 (38.8\%) obtained remission (UCDAI < or $=4), 6(33.3 \%)$ showed a clinical response, and $4(22.2 \%)$ remained unchanged and one patient $(0.5 \%)$ was getting worse. Two patients (11.1\%) who hardly showed the improvement of symptom and UCDAI score even after additional GCAP therapy underwent colectomy. GCAP therapy was well tolerated and no serious side-effects were observed. CONCLUSIONS: The findings of this study suggest that GCAP may be a useful alternative therapy for patients with UC, and the efficacy of GCAP may also be useful for one of the surgical operative indication factor. 
S1383

In Vivo Measurement of Colonic Butyrate Metabolism in Patients with Active Ulcerative Colitis

Damian McKay, Stephen J. Kirk, Keith R. Gardiner, Helen Kemp, Wolfram MeierAugenstein

Background. Butyrate, a short chain fatty acid, is a by-product of bacterial fermentation of non-digestible carbohydrates within the gut lumen and is the preferred fuel source for colonic epithelial cells (colonocytes). In vitro work has shown that ulcerative colitis (UC) may be characterised by a defect in the colonocyte metabolism of butyrate. Aim: To assess if colonocyte butyrate metabolism improves with treatment of active ulcerative colitis. Methods. Patients with a flare up of histologically proven UC were recruited from the Royal Victoria Hospital. Following informed consent baseline measurements were performed including clinical scoring questions (St Mark's score, simple clinical colitis index), blood for inflammatory markers and urine for microalbuminuria. These measurements were repeated after an 8 week treatment period. BMI was calculated to adjust individually for $\mathrm{CO} 2$ production. A $100 \mathrm{ml}[1-13 \mathrm{C}]$ butyrate enema was administered to 4 patients before and after the eight week treatment period. Breath samples were obtained every 10 minutes for the first hour and every 15 minutes for a further 2 hours. The breath samples were then transported to the Environmental Engineering Research Centre to measure amount and appearance of $13 \mathrm{CO} 2$ released from [1-13C] butyrate by isotope ratio mass spectrometry (IRMS). Results. There was a marked improvement in butyrate metabolism after the treatment period. The time to maximum $13 \mathrm{CO} 2$ production decreased by a mean of $17.1 \% \mathrm{p}=0.022(4.09-25.9)$ indicating increased rate of butyrate metabolism. The total percentage dose of $13 \mathrm{CO} 2$ recovered in the breath samples increased by a mean of $20.4 \% \mathrm{p}=0.031$ (2.73-28.59). These results correlated well with improvements in both clinical scoring systems and inflammatory markers. Conclusions. Butyrate oxidation by colonic epithelial cells appears to be defective in active ulcerative colitis. Metabolism to $\mathrm{CO} 2$ improves after treatment of an acute flareup and correlates with improvements in clinical markers of the disease

\section{S1384}

Treatment of Small Bowel Inflammatory Stricturing Crohn's Disease with Infliximab : An Open Pilot Study

Edouard Louis, Jacques Boverie, Filip Baert, Olivier Dewit, Martine Devos, Geert D'Haens Stricturing small bowel Crohn's disease (CD) may involve both fixed fibrotic changes and an inflammatory component. While the fibrotic changes are not likely to respond to antiinflammatory drugs, this is not the case of the inflammatory component. Steroids may transiently improve these patients but there is, in the majority of cases, a need for surgery at short or mid term. Efficacy of infliximab in these patients is not documented since it was an exclusion criteria for pivotal trials. We embarked an open pilot study to assess the safety and efficacy of infliximab in patients with stricturing subobstructive CD. Patients and methods: to be included, patients had to harbour a documented and symptomatic small bowel stricture in the context of $\mathrm{CD}$. They had to be refractory to steroid and or immunosuppressor, have no need for immediate surgery and abdomen CT had to prove the absence of peritoneal abscess. They also had to have active inflammation assessed by a CRP $>8 \mathrm{mg} /$ l. These patients were treated with infliximab $5 \mathrm{mg} / \mathrm{Kg}$ and followed up at $\mathrm{wl}$, w2, w4 and w8 on a clinical, biological and abdomen CT scan (w8) basis. Results: 6 patients were included and then the study was prematurely stopped because the safety thresholds of more than 2 surgeries within the first 5 patients was reached. Only two patients completed the 8 week protcol, with a positive response to infliximab and improvement of inflammation confirmed by the CRP and the abdomen CT scan. Two patients had to be operated early (both 10 days after infliximab) because of clinical worsening. Two patients first did well but worsened after one month and were operated 35 ad 42 days after infliximab, respectively. There was no complication of surgery in the 4 operated patients. From a clinical point of view, the two patients requiring early surgery were the only two with an abdominal mass at baseline. From a radiological point of view, these two patients had either a small intramural ileal abscess or intraperitoneal fluid at baseline. One of the two patient who worsened one month after infliximab also had an intramural jejunal abscess, while the two patients who positively responded to infliximab had no such feature. Other abdominal CT scan parameters or clinical and biological parameters were not associated to evolution after infliximab infusion. In conclusion a small subset of patients with subobstructive small bowel stricturing CD may respond to infliximab. The presence of an abdominal mass before treatment may represent a contra-indication to infliximab in this situation. The presence of intra-mural small bowel abscess or intraperitoneal fluid may predict failure of treatment.

\section{S1385}

Serum Protein Signatures Determined By Mass Spectrometry (SELDI-ToF) Accurately Identifies Patients with Inflammatory Bowel Disease By Support Vector Machines with Linear Kernels

Venkataraman Subramanian, Devika Subramanian, Sanjeev Krishna, Daniel Agranoff, Richard Pollok

Introduction: Inflammatory bowel disease (IBD) is an idiopathic chronic disease of the gastrointestinal tract with many debilitating sequelae some of which be life threatening. Diagnosis of IBD often depends on invasive tests. Non invasive serological testing such as ASCA and p-ANCA are insufficiently specific or sensitive for routine diagnostic use. We aimed to develop a novel diagnostic approaches based on differences in protein signatures in sera obtained from individuals with IBD and controls using surface enhanced laser desorption and ionisation time of flight (SELDI-ToF) mass spectrometry (MS). Methods: We studied protein signatures from 55 patients with IBD and 56 controls (normal and patients with other inflammatory pathology). Protein signatures were obtained using a CM10 chip (weak cation exchange chip), and analysed with SELDI-ToF MS. Preliminary analysis was performed using 'p' value determination of integrated peaks. Statistical and neural network analysis was performed using support vector machine (SVM) techniques utilizing linear function kernels. Significant peaks used for discrimination were selected using recursive feature elimination. SVM analysis was done using a 10 fold cross validation technique. Results: Comparing IBD and controls using SVM analysis with a linear function kernel using 10 peaks selected by recursive feature elimination, we obtained an accuracy of $91 \%$, sensitivity of $83 \%$ and specificity of $92 \%$. The peaks selected by SVM analysis were also significantly discriminative when used in individual peak analysis. Conclusions: Using protein signature of patients with IBD and controls we have developed a model of classification which has high accuracy and is more informative than currently available serological tests. Characterisation of discriminant peaks is currently underway.

\section{S1386}

Inflammatory Bowel Disease Phenotype in British Children of South Asian Origin

Nadeem A. Afzal, Lang Hans-Joerg, Sarah Mills, Michael Green, Sandhia Naik, Nigel Meadows, Ian Sanderson, Nick Croft

Aims: In British children ulcerative/indeterminate colitis (UC/IC) (not Crohn's Disease) has been reported to be relatively more common in South Asian than Caucasian families (Sawczenko A, Lancet 2000). Majority of children of South Asian origin in the UK are of Indian (Hindu) origin, most of the remainder being of Pakistani/Bangladeshi (Muslim) parents. In this study we compare the disease phenotype in British children of parents from South Asian regions. Methods: We studied consecutive children with IBD from two paediatric gastroenterology units, from East London were Caucasian and mostly Bangladeshi/Pakistani patients, from Leicester were mostly of Indian origin. We examined the distribution and pattern of inflammatory bowel disease, age at diagnosis, macroscopic and histological details of the endoscopy and presenting features with height and weight and diet. Results: 177 Caucasian and 54 children of South Asian origin were studied. 36 children were of Bangladeshi/Pakistani origin and 18 of Indian origin. The proportion of patients with Crohn's Disease (58\%) and UC/IC (42\%) patients were identical in children of Caucasian and Bangladeshi/ Pakistani origin (MWU, $\mathrm{p}=\mathrm{ns}$ ). The children of Indian origin however had a higher proportion of UC/IC (67\%) (MWU, p=0.03). A comparison of the Bangladeshi/Pakistani children with the Indian children showed no significant difference in sex distribution ( $58.1 \%$ males), duration of symptoms $(0.71 \mathrm{yrs}, 1 \mathrm{SD}=1.41 \mathrm{yrs})$, BMI SDS at presentation $(-0.45,1 \mathrm{SD}=1.3)$ use of azathioprine $(66.1 \%$ ) and age of presentation (Mean $10.9 \mathrm{yrs}, 1 \mathrm{SD}=4.1 \mathrm{yrs}$ ). No differences were found in duration of symptoms, age of presentation, Ht SDS and Wt SDS at presentation in different IBD types in South Asian children (KW, p=ns). Vegetarian diets were commoner in Indian families. Conclusions: British children of Bangladeshi origin with IBD have equal proportion of Crohns (58\%) and UC/IC (42\%) to Caucasian children However children of Indian origin have a higher incidence of UC/IC (67\%) as reported in adults and implied by previous epidemiological studies. Our data suggest IBD phenotype in British children of South Asian origin varies by the country of their parents birth. This implies important genetic differences between these countries and demonstrates that studies need to define the ethnicity more precisely both for genetic and epidemiological research Both environmental (e.g. dietary) and genetic factors could explain these differences.

\section{S1387}

Crp and ESR Do Not Predict Disease Activity in Inflammatory Bowel Disease (IBD) Kapil Dhawan, Qin Li, Elizabeth Shenkman, John F. Valentine

BACKGROUND: C-reactive protein (CRP) and erythrocyte sedimentation rate (ESR) are among the most widely used markers for IBD. Several recent clinical trials of biologic agents in IBD only showed significance in a subgroup with elevated CRP. ESR was not evaluated. The primary objective of our study was to examine the relationship between CRP and ESR and health-related quality of life (QoL) among a cohort of patients with IBD. Methods: A convenience sample of patients with Crohn's disease (CD) or ulcerative colitis (UC) at an academic health center completed the SIBDQ and the HBI and had laboratory testing for CRP and ESR. 55 patients with CD and 11 patients with UC were included in the analyses Patient gender, disease duration, and steroid use were included as covariates. Outcomes for those with CD were the scores on the Short Inflammatory Bowel Disease Questionnaire (SIBDQ) and the Harvey-Bradshaw Index (HBI). The outcome variable for those with ulcerative colitis (UC) was the SIBDQ score. Multivariate regression models examining the relationship between the serologic makers and scores on the SIBDQ and HBI were developed using PROC GLM and PROC MIXED in SAS. RESULTS: The mean age was $42.10+/-15.96 .57 \%$ females; $43 \%$ males. The mean SIBDQ score was $47.26+/-14.57$ and the mean HBI was $4.75+/-5.16$. Mean CRP values were $9.11+/-12.46$ and mean ESR was $33.30+/-28.75$. On average the patients had IBD for $12.24+/-10.40$ years. CRP and ESR were included in the same statistical models together as predictor variables and separately. ESR and CRP were not significantly related to SIDBQ scores in patients with $C D$ in any of the models. Males with $C D$ have higher SIBDQ scores than females when controlling for other covariates $(\mathrm{P}=$ 0.04 ) in the model. Gender, disease duration and CD location were not significant. Patients taking prednisone reported significantly lower SIBDQ scores than those taking Entocort ( $\mathrm{p}=$ $0.03)$. ESR and $C R P$ were significantly related $(P<0.0001), S I B D Q$ and $H B I$ were inversely related $(\mathrm{P}<0.0001)$. Among patients with $\mathrm{UC}$, gender was significantly associated with the SIBDQ scores with males having higher scores than females $(\mathrm{p}=0.04)$. The use of prednisone was marginally significant and associated with lower SIBDQ scores $(\mathrm{p}=0.06)$. Curiously, in the combined group, 16 patients had elevated CRP $>5$ and an ESR $<30$ and 11 had elevated ESR $>30$ and a CRP $<5$, some of these were markedly elevated. Conclusions: CRP and ESR do not correlate with the SIBDQ or the HBI. Better biomarkers for disease activity are needed. In CD, Use of systemically available corticosteroids significantly decreased SIBDQ scores.

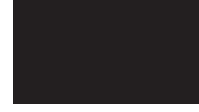


$\$ 1388$

Correlation of CT Enteroclysis with Surgical Pathology in Patients with Crohn's Disease

Michael V. Chiorean, Dean T. Maglinte, Romil Saxena, Attila Nakeeb, Jun Ying

Background: The response to therapy in patients with Crohn's disease (CD) is dependent on the underlying pathology. As opposed to endoscopic mucosal biopsies, CT enteroclysis (CTE) can detect transmural disease. The ability of CTE to differentiate pathological lesions in $\mathrm{CD}$ is unknown. Aims: To determine the accuracy of CTE compared to pathology in patients who underwent surgery for $C D$ and to assess the correlation of specific $C T E$ findings with the pathological diagnosis. Methods: CTE findings from 45 adult patients who underwent surgery for CD between 2002 and 2005 were retrospectively reviewed. The sensitivity, specificity and accuracy of CTE compared with the gold-standard surgical pathology were determined by using a composite inflammatory and fibrostenotic score. Several CTE (mucosal and mural enhancement, wall thickness, mesenteric stranding, stenosis, proximal dilation) and pathology findings (wall thickness, ulceration, stenosis, degree of activity, smooth muscle and connective tissue proliferation) were used to grade the inflammatory activity (0-3) and fibrostenosis (0-2 for CTE and 0-3 for pathology). The correlation between specific CTE findings and surgical pathology was assessed using Spearman correlation and cumulated logit model. Results: Ten patients were excluded (no CD diagnosis or malignancy in the specimen). Of the 35 remaining patients, $63 \%$ were female and $80 \%$ were white. Average age was 39 and disease duration 10 years. All had small bowel involvement. The indications for CTE/surgery were: refractory disease in 20, bowel obstruction in 11, fistula/ abscess in 9; 16 patients had previous surgery. The average interval between CTE and surgery was 8 weeks (0-43). The sensitivity, specificity and accuracy of CTE for fibrostenosis was $80 \%, 65 \%$ and $71 \%$ respectively. There was good correlation between CTE and pathology for both inflammatory $(\mathrm{r}=0.6)$ and fibrostenotic disease $(\mathrm{r}=0.6, \mathrm{p}<0.001)$. The inflammatory score correlation varied with the CTE-surgery time interval while the fibrostenotic score did not. Wall thickness, mural enhancement and fat stranding on CTE were positively associated with the pathology inflammatory score [OR 2.8 $(\mathrm{p}=0.1), 7.1$ and $13.7(\mathrm{p}<0.02)]$. Conclusions: CTE can reliably differentiate between inflammatory and fibrostenotic lesions in patients with small bowel $C D$. Since the management of patients with $C D$ is dependent on the underlying pathology, prospective studies evaluating the impact of CTE on therapeutic outcomes in patients with $\mathrm{CD}$ are warranted.

\section{S1389}

Accuracy of Small Intestine Contrast Ultrasonography (SICUS) in the Preoperative Assessment of Small Bowel Lesions in Crohn's Disease Nadia Pallotta, Naima Abdulkadir Hassan, Danila Guagnozzi, Enrico Corazziari

Small intestine contrast ultrasonography (SICUS) enables to visualize the entire small bowel after the ingestion of iso-osmolar polyethylene glycol (PEG) solution. It has been shown that the diagnostic accuracy of SICUS is comparable to that of a radiological examination, in detecting the presence, number, extension, and sites of small bowel lesions even in patients with undiagnosed small bowel diseases (1). AIM. To evaluate the diagnostic accuracy of SICUS to assess the number, site, and extension of small bowel lesions in patients submitted to surgery for Crohn's disease (CD). Fifteen consecutive patients submitted to surgery for $\mathrm{CD}$ were investigated (F 7: age range 18-70 years). Real-time US was performed preoperatively using a 3.5-MHz convex and a $5-\mathrm{MHz}$ linear array transducer (Toshiba Tosbee, Japan) after the ingestion of $375 \mathrm{ml}$ of PEG oral solution (Promefarm, Italy). After the contrast was seen flowing through the terminal ileum into the colon, a retrograde followthrough assessment of the entire small bowel was performed visualizing, in a caudalcranial sequence, the contrast-filled ileal and jejunal loops. Preoperative SICUS findings were compared with surgery findings. RESULTS. In all patients preoperative SICUS correctly detected the lesions and their location as identified at surgery. The mean extension of small bowel lesions was $28.5 \pm 14.2 \mathrm{~cm}$ at surgery, $29.4 \pm 11 \mathrm{~cm}$ at SICUS (not significant versus surgery). In the assessment of number and site of lesions the concordance index between surgery and SICUS was $0.85(\mathrm{P}<0.001)$. CONCLUSIONS. Compared to surgical findings the diagnostic accuracy of preoperative SICUS in detecting small bowel lesions is high. Preoperative SICUS can be usefully employed to plan surgical treatment in Crohn's disease patients. 1) Pallotta et al. Ultrasound Med Biol. 2001;27:335

\section{S1390}

Efficacy of Adacolumn Selective Leukocytapheresis in Patients with Steroid Naive and Steroid Dependent with Moderate to Severe Ulcerative Colitis Tomotaka Tanaka, Hideharu Okanobu, Abbi R. Saniabadi, Aki Kohgame, Hiroki Imagawa, Yoshito Takemura, Yoshio Kuga, Takashi Moriya, Toshihide Ohya

Background and Objective: In most patients with moderate to severe ulcerative colitis (UC), corticosteroids may promote remission, but their side effects can be new complications. However, UC is believed to be exacerbated, and perpetuated by inflammatory cytokines like TNF-alpha, IL-1beta, IL-6 and IL-8 which are also released by granulocytes and monocytes/ macrophages. Therefore, these leukocytes might be appropriate targets for therapy of inflammatory bowel disease. Based on this thinking, recently an Adacolumn has been developed for depleting excess and activated peripheral blood granulocytes and monocytes/macrophages by selective leukocytapheresisis (SLA). This study was to assess the efficacy of SLA with the Adacolumn in patients with moderate to severe active UC, steroid naive versus steroid dependent patients. Methods: Twenty-nine consecutive patients, 20 male and 9 female, mean age 43.3 years, range 18-70 years with moderate to severe active UC were treated. Ten had total colitis and the rest had left-sided colitis. Similarly, 17 of 29 patients were steroid naive the other 12 had steroid dependent UC. SLA was done with the Adacolumn, each patient received one session/week for 5-10 weeks. None of the 29 patients received new or additional medications within 4 (steroid) to 8 (immunosuppressant/immunomodulator) weeks prior to entry or during SLA treatment. However, the steroid dose could be tapered in responders to SLA. Further, during endoscopy, biopsy specimens were taken from a randomly selected 10 patients for assessing the mucosal level of granulocytes. Results:
At entry, the mean clinical activity index (CAI) was 12.7, range 10 to 14 (by Rachmilewitz). Remission was defined as $\mathrm{CAI}=$ or $<4$. Efficacy assessments including endoscopy performed one week after the last Adacolumn session showed a remission rate of $82.8 \%$ (24 of 29 patients); this included 17 of $17(100 \%)$ steroid naive patients and $7(58.3 \%)$ steroid dependent cases. For all 24 responders, the average remission time was 11.6 months and 15.4 months for the 17 steroid naive patients. Biopsy revealed massive infiltration of granulocytes into the colonic mucosa in patients with active disease. Adacolumn leukocytapheresis was associated with a marked reduction of granulocytes in the mucosa in patients who achieved remission. Conclusions: In our hospital, 100\% of steroid naive patients responded to Adacolumn leukocytapheresis treatment, while just over half of steroid dependent patients responded. Accordingly, we believe that steroid naive patients should be given Adacolumn leukocytapheresis as a first line medication. Large controlled studies are warranted to strengthen our observations.

\section{S1391}

Diagnostic Value of Faecal Calprotectin in Unselected Outpatients Referred for Colonoscopy: A Multicenter Prospective Study

Gianmichele Meucci, Renata D'Inca', Roberto Maieron, Nicoletta Orzes, Maurizio Vecchi, Daniela Visentini, Giorgio Minoli, Elisabetta Dal Pont, Maurizio Zilli, Elvio Benedetti, Tiziana Virgilio, Elio Tonutti

Background and aims: Faecal levels of calprotectin are increased in several inflammatory and neoplastic diseases of the gastrointestinal tract. However, the role of faecal calprotectin measurement in clinical practice is still ill defined. We assessed faecal calprotectin in consecutive outpatients referred for colonoscopy, in order to evaluate its reliability in identifying patients with significant colorectal disease. Methods: Outpatients undergoing colonoscopy at 5 participating institutions were eligible for the study. Patients were asked to collect a faecal sample the day before examination. Written informed consent was obtained. Demographic and clinical data were collected by means of a standard questionnaire. Faecal samples were tested at a single laboratory by means of a commercially available kit (Calprest, Eurospital SpA, Trieste) Results: Eight-hundred seventy patients (males 413, mean age 59.1 years, range 16-89) were enrolled. The rate of caecal and ileal intubation were $91 \%$ and $24 \%$ respectively. Mean levels of calprotectin were significantly higher in patients with neoplastic and inflammatory disorders when compared with subjects with a normal colonoscopy of with trivial endoscopic findings (such as uncomplicated diverticular disease). Calprotectin levels above the upper normal limits ( $>50 \mathrm{mg} / \mathrm{Kg}$ ) were detected in $88 \%$ of patients with colorectal cancer, $85 \%$ of those with inflammatory conditions and $56 \%$ of those with inactive ulcerative colitis but also in $39 \%$ of patients with normal or trivial endoscopic findings Sensitivity, specificity, PPV, NPV and accuracy for detecting "any organic disease" and colorectal cancer respectively are shown in the table. In patients referred for chronic diarrhea, sensitivity and negative predictive value were $100 \%$ in detecting either any organic disease or cancer. In patients referred for symptoms of "suspected functional origin" (abdominal pain, constipation etc) sensitivity and negative predictive value for colorectal cancer were respectively $85 \%$ and $99 \%$.Conclusions: In unselected outpatients referred for colonoscopy, a single measurement of faecal calprotectin is not sufficiently accurate to identify those with significant disease. However, a normal result can help in ruling out organic disease among patients with diarrhea and those with abdominal pain and/or constipation.

\begin{tabular}{|c|c|c|}
\hline & any disease & cancer \\
\hline sensitivity & $86 \%$ & $88 \%$ \\
\hline specificity & $59 \%$ & $55 \%$ \\
\hline positive predictive value & $25 \%$ & $7 \%$ \\
\hline negative predictive value & $96 \%$ & $99 \%$ \\
\hline accuracy & $63 \%$ & $56 \%$ \\
\hline
\end{tabular}

\section{S1392}

Costs of Crohn's Disease According to Severity States

Isabelle Jaisson-Hot, Pascale Guerre, Fady Daniel, Jean-Frederic Colombel, Eric Lerebours, Bernard Duclos, Arnaud Bourreille, Jean-Louis Dupas, Guillaume Cadiot, Yoram Bouhnik, Jacques Moreau, Philippe Marteau, Antoine Cortot, Louis Descos, Cyrille Colin, Marc Lemann, Bernard Flourie, The Getaid And

Introduction : A multicenter, prospective, observational study has been undertaken aiming to estimate costs of care and clinical evolution in Crohn's disease. Methods : 342 patients ( $37 \mathrm{yr}$ mean age, $65 \%$ female) collected data for one year relating to direct and indirect costs (invalidity and sick leave). Patients were stratified into disease severity states using the classification of Silverstein et al (Gastroenterology 1999;117:49-57) modified by recent therapeutic evolutions: REM0 : Remission without medication, REM1 : Remission with minor medication, MILD : mild symptoms and minor medication, REP : drug-responsive to a treatment with oral corticosteroids or immunosuppressive medications or Infliximab, DEP drug-dependent to the same treatments, REF : drug-refractory or intolerance, SURG : surgery within 3 months, REMPS : postsurgical remission. Costs were estimated from the perspective of the French health insurance system. Results : Means (medians) for direct and indirect costs by disease states are shown in table and the contribution of each cost item to total expenditure in figure. As expected there was an incremental increase in costs with worsening disease severity. Interestingly, drug-dependent patients (DEP) cost more than refractory patients (REF), particularly for hospitalizations. Furthermore, indirect costs including work disability took a significant part of total costs, especially for patients REF and MILD. Conclusion: Our data quantify the contribution of different services to the overall economic burden of the disease, taking into account its natural history. The great impact of indirect costs must be considered in economic appraisal for such chronic disease. 


\begin{tabular}{|c|c|c|c|c|c|c|c|c|}
\hline & REM0 & REM1 & MILD & REP & DEP & REF & SURG & REMPS \\
\hline $\begin{array}{c}\text { Direct } \\
\text { costs }\end{array}$ & $359(62)$ & $553(305)$ & $549(390)$ & $1297(290)$ & $2795(2021)$ & $1482(491)$ & $8452(8069)$ & $2121(357)$ \\
\hline $\begin{array}{c}\text { Indirect } \\
\text { costs }\end{array}$ & $254(0)$ & $276(0)$ & $542(0)$ & $330(0)$ & $607(0)$ & $752(0)$ & $821(514)$ & $512(0)$ \\
\hline
\end{tabular}

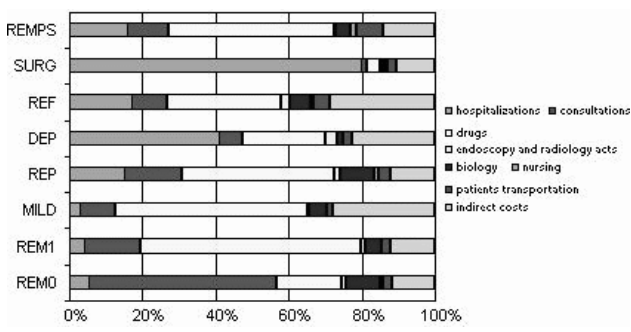

S1393

Accuracy of Four Fecal Assays in Inflammatory Colonic Disease Alain Schoepfer, Michael Trummler, Beatrice Seibold, Petra Seeholzer, Frank Seibold Objective: Several fecal markers have been evaluated for the differentiation of functional (IBS) versus inflammatory bowel disease (IBD). The aim of this study was to evaluate the accuracy of four different fecal markers in the differentiation of patients with IBS, IBD and other forms of inflammatory colonic disease (OCD). Material and methods: Included were 23 patients with IBS, 36 with IBD (24 Crohn's disease, mean CDAI 103, 12 ulcerative colitis), the group of OCD included 8 patients with infectious colitis, 5 ischemic colitis and 5 with medication-induced colitis. Diagnosis was established based on conventional clinical, laboratory and endoscopic workup. Blinded stool samples of all patients were measured for fecal leucocyte markers Calprotectin (EIA), Lactoferrin (EIA), Hexagon-OBTI (specific for human haemoglobin) and LEUKO-Test (lactoferrin latex-agglutination test). Results are given as sensitivity (SE) and Specificity (SP). Statistical analysis was done with Fisher's exact test, t-test and ROC-analysis. Results: SE for Hexagon-OBTI in discrimination of IBS vs IBD was $39 \%$, SP $91 \%$ ( $\mathrm{p}=0.015$ ), SE for IBS vs OCD was $67 \%$, SP $86 \%$ ( $\mathrm{p}<0.001)$. Two IBS patients with positive Hexagon-OBTI took daily Aspirin. SE for LEUKO-test in discrimination of IBD vs IBS was $44 \%$, SP was $91 \%(\mathrm{p}=0.004)$. LEUKO-test was positive in $75 \%$ of patients with infectious colitis. SE for LEUKO-test for IBS vs OCD was $43 \%$, SP 91\% ( $\mathrm{p}=0.014)$. No viable differentiation of IBD vs OCD was possible using Hexagon-OBTI and LEUKOtest. Mean calprotectin in IBS was $25 \mathrm{ug} / \mathrm{ml}$, in IBD $275 \mathrm{ug} / \mathrm{ml}$ ( $\mathrm{p}<0.0001)$, in OCD 35lug/ $\mathrm{ml}(\mathrm{p}=0.39$, IBD vs OCD). Mean lactoferrin in IBS was $0.03 \mathrm{ug} / \mathrm{ml}$, in IBD $0.34 \mathrm{ug} / \mathrm{ml}$ ( $\mathrm{p}<0.0001$ ), in OCD 0.33 ( $\mathrm{p}=0.97$, IBD vs OCD). ROC-Analysis IBS vs IBD: for Calprotectin (cut-off $50 \mathrm{ug} / \mathrm{ml}$ ) the SE was $94 \%$, SP $90 \%$, for Lactoferrin (cut-off $0.056 \mathrm{ug} / \mathrm{ml}$ ) the SE was $91 \%$ and the SP $100 \%$. ROC-Analysis IBD vs OCD for Calprotectin: SE 57\%, SP 53\%, for Lactoferrin SE 52\%, SP 42\%. Conclusions: In contrast to Hexagon-OBTI and LEUKO-test, EIA for fecal Calprotectin and Lactoferrin are good tools for differentiation of inflammatory colonic disease vs non inflammatory disease such as IBS. However, the differentiation of IBD vs other inflammatory colonic disease such as infectious colitis is not possible with these tests. Calprotectin may be increased in patients with Crohn's disease even in remission. IBS patients may however have positive leukocyte fecal markers due to the intake of Aspirin or NSAID. Endoscopic workup remains crucial for diagnosis of colonic disease.

\section{S1394}

Health Related Quality of Life in Crohn's Disease By Severity States Isabelle Jaisson-Hot, Pascale Guerre, Fady Daniel, Jean-Frederic Colombel, Eric Lerebours, Bernard Duclos, Arnaud Bourreille, Jean-Louis Dupas, Guillaume Cadiot, Yoram Bouhnik, Jacques Moreau, Philippe Marteau, Antoine Cortot, Louis Descos, Cyrille Colin, Marc Lemann, Bernard Flourie, The Getaid And

Introduction : A multicenter, prospective, observational study has been undertaken aiming to evaluate the Health Related Quality of Life (HRQOL) of patients with Crohn's disease and clinical evolution. Methods: Every 3 months during one year, patients were examined, disease severity state and HRQOL were assessed with the Short Form-36 (SF-36) and with the Rating Form of Inflammatory bowel disease Patient Concerns (RFIPC). In addition, an alternative method of evaluating HRQOL outcome was used with utility analysis by means of the Time Trade-Off (TTO): this method assessed patient preference and measured the number of years of life in the current health state exchanged for a shorter life in good health. Patients were stratified into disease severity states using the classification of Silverstein et al (Gastroenterology 1999;117:49-57) modified by recent therapeutic evolutions: REM0 Remission without medication, REMI : Remission with minor medication, MILD : mild symptoms and minor medication, REP : drug-responsive to a treatment with oral corticosteroids or immunosuppressive medications or Infliximab, DEP : drug-dependent to the same treatments, REF : drug-refractory or intolerance, SURG : surgery within three months, REMPS : post-surgical remission. Results : 342 patients ( $37 \mathrm{yr}$ mean age, $65 \%$ female) were followed for one year. The remission state gathering REM0, REMl and REMPS resulted in significantly higher SF-36 and RFIPC scores in comparison with the state REP, then with chronically active disease represented by the states MILD, DEP and REF. The SF-36 score for the state MILD was the worst for all subscales, whereas the states REF and DEP had the worst scores for the RFIPC. Contrary to what was observed with the SF-36, the TTO score for the MILD state was the least impaired. Patients with severe disease (DEP and SURG) assigned lower TTO scores, ie. had the lowest acceptance of their health state. Even patients in remission (REM0, REMl and REMPS) had a measurable decrease in TTO scores relative to their health. Only a slight degree of correlation was present between the TTO and RFIPC scores, reflecting the importance of taking into account patient preference. Conclusion : SF-36 and RFIPC order the health states in the predicted manner according to the clinical state and intensity of medical or surgical treatment, except for the MILD state. Time Trade-Off scores reveal that even if patients are in remission, their experiences of disease and treatment lead them to accept to trade off many years of life in their current health state in exchange for perfect health.

\section{S1395}

Projected Clinical Course of Crohn's Disease

Isabelle Jaisson-Hot, Pascale Guerre, Fady Daniel, Jean-Frederic Colombel, Eric

Lerebours, Bernard Duclos, Arnaud Bourreille, Jean-Louis Dupas, Guillaume Cadiot,

Yoram Bouhnik, Jacques Moreau, Philippe Marteau, Antoine Cortot, Louis Descos, Cyrille Colin, Marc Lemann, Bernard Flourie

Introduction : A multicenter observational study was conducted with 3 main objectives : to evaluate disease clinical course and to estimate patient preferences and costs of illness Methods: Every 3 months during one year, patients were examined and the disease severity state was defined according to the classification of Silverstein et al (Gastroenterology 1999:117:49-57) modified by recent therapeutic evolutions: REM : Remission (without or with minor medication, or postsurgical), MILD : mild symptoms and minor medication, REP : drug-responsive to a treatment with oral corticosteroids or immunosuppressive medications or Infliximab, DEP : drug-dependent to the same treatments, REF : drug-refractory or intolerance, SURG : surgery within 3 months. The probabilities of transition into other states were estimated from the data using a maximum likelihood estimate approach assuming a Markov model for transitions among disease states. Results : 342 patients ( $37 \mathrm{yr}$ mean age, $65 \%$ female) were followed for one year and 1332 quarterly Case Report Forms (CRF) were analyzed. The maximum likelihood ratio estimates of the 3-month probabilities of transition between disease states are shown in table. According to the projected distribution of ten future years by disease state, most of future clinical course consisted in drug-responsive state disease (range : 57.27 to 71.58 months, for patients in initial states REM and REP respectively). Conclusion: Our prospective data provide an evaluation of disease clinical course, taking into account the potential impact of new treatment such as TNF alpha antagonists. Unlike previous retrospective results showing that remission was the prominent state of clinical course, our data show that most of the projected clinical course is spent in the drug-responsive state: this may reflect an increasing use of immunosuppressors at leas in the present cohort. Our data will also be useful for modelling a life time cost-utility analysis with an analytic Markov decision model.

\begin{tabular}{|c|c|c|c|c|c|c|}
\hline \multirow{2}{*}{ Initial state } & \multicolumn{6}{|c|}{ Subsequent state } \\
\cline { 2 - 7 } & REM & MILD & REP & DEP & REF & SURG \\
\hline REM & 0.8244 & 0.0653 & 0.0877 & 0.0031 & 0.0130 & 0.0064 \\
\hline MILD & 0.5372 & 0.3398 & 0.1085 & 0.0018 & 0.0085 & 0.0041 \\
\hline REP & 0.0473 & 0.0064 & 0.8538 & 0.0095 & 0.0557 & 0.0272 \\
\hline DEP & 0.0396 & 0.0034 & 0.5790 & 0.1756 & 0.1391 & 0.0632 \\
\hline REF & 0.0652 & 0.0042 & 0.5089 & 0.0451 & 0.2683 & 0.1084 \\
\hline SURG & 0.2118 & 0.0139 & 0.5555 & 0.0108 & 0.0858 & 0.1221 \\
\hline
\end{tabular}

S1396

Malnutrition in Quiescent Inflammatory Bowel Diseases Cannot Be Diagnosed with Standard Tools

Susanne Hengstermann, Luzia Valentini, Carsten Buening, Lennart Schaper, Thomas Koernicke, Ralf J. Schulz, Matthias Pirlich, Tanja Schuetz, Herbert Lochs

Background The aim of this project was to assess the nutritional and functional status as well as body composition in IBD patients in clinical remission and compare the results to healthy controls. Methods Body composition and nutritional status (bioelectrical impedance analysis, BIA; anthropometry; subjective global assessment, SGA) were assessed in 52 (46f/ $6 \mathrm{~m}, 37 \pm 10$ yrs) IBD patients (33 Crohn's disease, CDAI $65 \pm 41 ; 19$ ulcerative colitis, CA $3.3 \pm 1.8$ ) and 52 pair-matched healthy controls $(46 \mathrm{f} / 6 \mathrm{~m}, 37 \pm 11$ yrs, body mass index, BMI $\pm 0.3 \mathrm{~kg} / \mathrm{m} 2$ ). Functional status was compared by hand dynamometry (Jamar. Preston) and by evaluating two non-disease specific questions $(\mathrm{Q} 1, \mathrm{Q} 6)$ of the inflammatory bowel disease questionnaire (IBDQ). Results In total, $88 \%$ of IBD patients were well-nourished according to standard parameters as BMI, SGA, serum albumin $>40 \mathrm{~g} / \mathrm{L}$ and no recent weight loss However, muscle mass was significantly reduced and fat mass was significantly increased in IBD compared to controls (see table). Hand grip strength correlated with body cell mass $(\mathrm{r}=0.615, \mathrm{p}<0.01)$, and was reduced in IBD patients. Additionally, IBD patients felt more tired (IBDQ, Q1) and less energetic (IBDQ, Q6) than controls (see table). No significant difference was found between patients with Crohn's disease and patients with ulcerative colitis. Discussion: Our study shows for the first time that patients with quiescent IBD and with seemingly normal nutritional status have an abnormal body composition and reduced muscle strength. This explains tiredness and fatigue in these patients. 


\begin{tabular}{|c|c|c|c|}
\hline & IBD $(\mathrm{n}=52)$ & Controls $(\mathrm{n}=52)$ & $\mathrm{p}$ \\
\hline Body Mass & & & \\
\hline BMI/kg/m2 & $22.3 \pm 2.0$ & $22.3 \pm 2.0$ & n.s. \\
\hline Arm circumference/cm & $27.5 \pm 2.5$ & $27.5 \pm 1.8$ & n.s. \\
\hline Muscle Mass & & & \\
\hline Lean body mass/kg & $44.3 \pm 6.3$ & $47.0 \pm 7.3$ & 0.001 \\
\hline Body cell mass/kg & $22.1 \pm 4.0$ & $24.3 \pm 4.6$ & 0.001 \\
\hline Arm muscle area/cm2 & $32.0 \pm 9.3$ & $37.7 \pm 9.7$ & 0.004 \\
\hline Fat Mass & & & \\
\hline Fat mass/kg & $18.2 \pm 4.0$ & $16.3 \pm 4.5$ & 0.006 \\
\hline Triceps skinfold/cm & $1.8 \pm 0.6$ & $1.3 \pm 0.7$ & 0.001 \\
\hline Functional capacity & & & \\
\hline Handgrip strength/N & $31.0 \pm 8.0$ & $35.2 \pm 9.8$ & 0.001 \\
\hline IBDQ-Tiredness & $3.9 \pm 1.5$ & $5.2 \pm 1.2$ & 0.001 \\
\hline IBDQ-Energy & $4.8 \pm 1.2$ & $5.7 \pm 0.6$ & 0.001 \\
\hline
\end{tabular}

common disease-causing variants (DCVs), R702W, G908R and Ll007FS in our patients. We identified several other variants, of which the mutant allele at the non-synonymous SNP, P268S was statistically more significantly prevalent in the patients compared to the controls $(\mathrm{P}=0.0066)$. Haplotype studies did not suggest any significant difference. Earlier studies have proposed P268S to be a polymorphism and in phase with the R702W, G908R and L1007FS mutations, the common disease causing variants (DCVs), but we did not have any such observations. Conclusion: Crohn's disease in Indian population is associated with different mutations in CARD15 gene and the P268S variant may be playing an important causal role or may be in linkage disequilibrium with some other variant in any other exon of CARD15 gene.

S1399

Is It Possible to Differentiate Crohn's Disease from NSAID-Enteropathy By Capsule Enteroscopy?

Laurence P. Maiden, Bjarni Thjodleifsson, Sam Adler, Winfreid Voderholzer, Ingvar Bjarnason

Background: Introduction of wireless Capsule Enteroscopy (CE) added a new dimension into the investigation of small bowel disease. It is emerging as a first line investigation for the detection of small bowel Crohn's disease. It also appears to be the investigation of choice for the diagnosis of NSAID-enteropathy, which affects over 50\% of patients receiving these drugs long-term. Furthermore NSAID-enteropathy is associated with serious outcomes (bleeding, perforation and obstruction) as frequently as NSAID-gastropathy. Indeed it has been suggested that certain NSAID-induced small bowel damage may be indistinguishable from that of Crohn's disease. Aim: To assess if it is possible to differentiate between Crohn's disease and NSAID-enteropathy by CE. Methods: The pathology recorded by CE from 18 patients with established small bowel Crohn's disease and 13 with NSAID-enteropathy were localised and quantitated by an established damage score (incorporating reddened (inflamed) folds, denuded areas, petechiae and mucosal breaks (erosions and ulcers)). Four experienced clinicians who had no knowledge of the clinical or demographic details of the patients read the images independently. Each made a diagnosis of Crohn's disease or NSAID-enteropathy ('definite' or 'likely'), based on their overall impression of the images. Results: There was a high rate of concurrence (over 95\%) between the investigators regarding the localisation and type of damage. The number of denuded areas and petechia did not differ significantly between the diseases $(\mathrm{p}<0.1)$. The median number of mucosal breaks (median 10 (range 1 - 26) for Crohn's disease and 2 (range 1-18) for NSAIDs) differed significantly ( $p<0.05$ ) between Crohn's disease and NSAID treated patients. However 3 (23\%) patients with NSAIDenteropathy were incorrectly labelled as 'definite Crohn's disease' ( 3 of the 14 diagnosed as 'definite Crohn's disease') and 3 (17\%) patients with Crohn's disease were incorrectly labelled as either 'definite' ( 1 of 9 diagnosed as 'definite') or 'likely' (2 of 4) NSAID-enteropathy. Conclusions: CE images of the small bowel show that NSAID-enteropathy has a similar range of pathology as seen in patients with Crohn's disease which further corroborates the importance of the small bowel in the overall gastrointestinal damage of NSAIDs. In the absence of a clinical history experienced CE image readers arrive at an incorrect diagnosis in $20 \%$ of cases

S1400

Age At Diagnosis and Disease Course in Pediatric Crohn's Disease Neera Gupta, Melvin B. Heyman, Alan G. Bostrom, Oren Abramson, Barbara S. Kirschner, Harland S. Winter, Benjamin D. Gold, George D. Ferry, Stanley A. Cohen, Robert N. Baldassano

Background: Analysis of data from our multicenter registry revealed that older age at diagnosis of inflammatory bowel disease is associated with increased risk for intestinal resection in pediatric patients with Crohn's disease (CD) (Gastroenterology 2005; A-319). We determined whether additional differences exist in the course of disease by age at diagnosis in pediatric patients with $C D$. Methods: We conducted a retrospective, cohort study using the Pediatric IBD Consortium Registry. Data collected between Jan 2000 and Nov 2003 were analyzed in 989 pediatric patients with $C D$ to compare disease course by age at diagnosis of disease using Cox Proportional Hazards Modeling. Age at diagnosis was dichotomized into 0-5 years and 6-17 years. Results: Mean follow-up time was 3.6.3.1 years in 989 patients; 98 (9.9\%) were $0-5$ yrs and $891(90.1 \%)$ were $6-17$ yrs of age at the time of diagnosis. $43.3 \%$ of patients between $6-17$ yrs at diagnosis were found to be ASCA positive compared with $10 \%$ of patients $0-5$ yrs at diagnosis $(\mathrm{p}=.003)$. The table shows the risk of developing the listed complications and the risk of being treated with specified medications in the 6-17 y/o compared with the 0-5 y/o age group. Conclusions: A higher proportion of older children (6-17 y/o at diagnosis) were found to be ASCA positive compared with children in the 05 y/o age group. Additionally, children who are 6 yrs or older at diagnosis of IBD appear to have a more severe course of disease and are at increased risk for being treated with 5ASA's, immunosuppressants, immunomodulators, and biologic agents compared with younger children $(<6 \mathrm{y} / \mathrm{o})$. Further studies are needed to determine whether diagnosis of IBD at a younger age may alter the severity of the course of $C D$ or represent a less severe disease phenotype. Supported in part by NIH Grants DK007762 and DK060617.

CARD15 Gene in Patients of Crohn's Disease in India : Preliminary Evidence of a Variable Association

Nikita Thakur, G R. Chandak, Rupa Banerjee, Rajesh Gupta, Sandeep Lakhtakia, Manu Tandan, Venkat G. Rao, Nageshwar D. Reddy

INTRODUCTION : Linkage studies in the western population have implicated several genomic regions as likely containing IBD susceptibility genes, with some observed uniquely in $\mathrm{CD}$ or UC or others common to both. Three major coding regions SNPs (cSNPs), R702W (Exon 4), G908R (Exon 8) and L 1007fs (Exon 11) together account for about 28-33\% of $\mathrm{CD}$ patients compared to $7-18 \%$ in the control population. There is no study on the genetic basis of Crohn's disease in the Indian Sub Continent. Methods: We sequenced the three exons including the splice-site junctions of CARD15 gene bearing the R702W, G908R and L1007FS mutations in 68 well-characterized CD patients and 130 unrelated controls. The allele and genotype frequencies were calculated for each SNP and to deviation from the Hardy-Weinberg equilibrium was investigated by Markov simulation based goodness of fit test. Subsequently, haplotypes were generated with the Expectation-maximization algorithm and compared between patients and controls. Results: We did not find any of the three 


\begin{tabular}{|c|c|c|c|}
\hline Variable & Hazard Ratio & $95 \% \mathrm{CI}$ & $\mathrm{p}$ Value \\
\hline Complications & & & \\
\hline Abscess & 5.12 & $1.59-16.5$ & .006 \\
\hline Fistula & 2.67 & $1.15-6.15$ & .022 \\
\hline Stricture & 2.16 & $0.99-4.69$ & .052 \\
\hline Perianal Fissure & 2.24 & $0.97-5.19$ & .06 \\
\hline Growth Failure & 1.92 & $.058-6.32$ & .28 \\
\hline Medications & & & \\
\hline 5-ASA's & 1.37 & $1.07-1.74$ & .01 \\
\hline 6MP/Aza & 2.40 & $1.74-3.32$ & $<.001$ \\
\hline Methotrexate & 3.23 & $0.98-10.6$ & .054 \\
\hline Infliximab & 2.60 & $1.46-4.64$ & .001 \\
\hline Steroids & 1.41 & $1.10-1.81$ & .006 \\
\hline Antibiotics & 1.91 & $1.38-2.66$ & $<.001$ \\
\hline
\end{tabular}

microparticle formulation has been developed which has great potential for the therapy of IBD. The enhanced solubility of the encapsulated drug should allow a more rapid dissolution in the viscous luminal contents of the colon. Furthermore, the slower colonic transit expected for the microparticle formulation over a conventional tablet formulation should facilitate topical anti-inflammatory effect to a large area of inflamed mucosa in patients with active IBD. A clinical trial in human volunteers, correlating GI transit with pharmacokinetic analysis is planned to prove the in-vivo performance of the $\mathrm{pH}$-sensitive microparticles.

\section{$\mathrm{S} 1403$}

Efficacy of Mesalazine in the Treatment of Symptomatic Diverticular Disease Giuseppe Comparato, Libera Fanigliulo, Lucas G. Cavallaro, Giovanni Aragona, Giulia Martina Cavestro, Stefania Liatopoulou, Angelo Franze', Cecilia Carloni, Veronica Iori, Marta Maino, Giorgio Nervi, Francesco Di Mario

Background and aim. In uncomplicated diverticular disease, treatment is aimed to relieve the symptoms in symptomatic patients. Our aim was to evaluate efficacy of mesalazine in relieving symptoms in patients with symptomatic and uncomplicated diverticular disease of the colon. Materials and methods. Two hundred and sixty-eight consecutive eligible outpatients (122 male, 146 female; age 66.1 years, range 31-81 years) were enrolled in fou different schedules according to a randomisation list: Group Rl (66 patients) rifaximin 200 mg bid; Group R2 (69 patients) rifaximin $400 \mathrm{mg}$ bid; Group M1 (67 patients) mesalazine $400 \mathrm{mg}$ bid; Group M2 (66 patients) mesalazine $800 \mathrm{mg}$ bid. Each treatment was administere for 10 days every month for 12 months. Clinical evaluation was performed at admission and at 3-months intervals for 12 months considering 11 clinical variables (upper and lower abdominal pain/discomfort, tenesmus, diarrhoea, abdominal tenderness, fever, general illness, nausea, emesis, dysuria) scored as $0=$ no symptoms, $1=$ mild, $2=$ moderate, $3=$ severe. The Global Symptomatic Score (GSS) was calculated by the sum of each symptom score. Results After 12 months follow-up, Group M2 showed fewer frequency rate of lower abdominal pain $(\mathrm{p}<0.0001)$, bloating $(\mathrm{p}<0.001)$, tenesmus $(\mathrm{p}<0.0001)$, diarrhoea $(\mathrm{p}<0.01)$, fever $(p<0.0001)$, general illness $(p<0.02)$, nausea $(p=0.002)$, dysuria $(p=0.04)$, bleeding $(p=$ 0.01 ). Mean GSS was lower in Group M2 after 6 months $(\mathrm{p}<0.001$ ) and 12 months ( $p<$ 0.0001 ) respectively. Patients treated with mesalazine (Groups M1 and M2 together considered) showed a lower GSS if compared to subjects treated with rifaximin (Groups Rl and R2 together considered) after $6(\mathrm{p}<0.001)$ and 12 months $(\mathrm{p}<0.0001)$, respectively. Conclusion. Cyclic administration of mesalazine is effective in obtaining symptom relief in uncomplicated diverticular disease of the colon. Some symptoms improved more with mesalazine $800 \mathrm{mg}$ bid than with the other schedules.

\section{S1404}

Cronkhite-Canada Syndrome: Clinical Outcomes and Molecular Feature Neal K. Osborn, Schuyler Sanderson, Thomas Smyrk, Linda Murphy, David Ahlquist. Lisa Boardman

Background \& Aims: Cronkhite-Canada Syndrome (CCS) is an acquired condition involving diffuse gastrointestinal polyposis. The polyposis, associated diarrhea, and characteristic mucocutaneous findings may resolve with immunosuppressive treatment. However, few data are available on long-term outcomes and little is known about the underlying molecular mechanisms. Methods: From a patient registry, clinical records and archival tissue from 9 well-characterized CCS patients were evaluated. Using paraffin-imbedded tissue, microarrays were constructed of CCS polyps and controls which included polyps from patients with Juvenile Polyposis Syndrome (JPS) and sporadic Juvenile Polyps as well as normal mucosa from stomach, small bowel, and colon. The antibodies for four DNA Mismatch (MMR) repair proteins (hMLH1, hMSH2, hMSH6, hPMS2) and other colorectal cancer related protein including APC, and IgG-4 were applied. Results: Among CCS patients treated with steroids, $85 \%$ achieved symptomatic remission; however, nearly all relapsed within 3-6 months of stopping or tapering steroids. Two patients sustained remission on long term maintenance with the immunomodulatory agent azathioprine. During the course of follow-up on the 9 CCS patients, $5(56 \%)$ developed adenomatous polyps, 2 (22\%) colorectal cancer, 2 (22\%) oropharyngeal polyps, and 1 (11\%) cecal carcinoid tumor. Among CCS polyps, immunohistochemistry staining for MMR and APC proteins was normal. In contrast, IgG4 expression was significantly higher $(>5$ cells/HPF vs $<5)$ in CCS compared with JPS $(p=0.001)$. The other normal controls and CCS adenomas and CCS cancers were uninvolved. Conclusions: Clinical manifestations of CCS may respond to steroids, and remission may be maintained by sustained immunosuppression. The incidence of colorectal neoplasia appears to be high with CCS. Neither MMR nor APC pathways are affected in the non-neoplastic polyps in CCS. IgG4 expression was increased in CCS polyps compared with those in JPS, supporting an underlying inflammatory process with CCS.

\section{S1405}

Prevalence of Disaccharidase Deficiency in Symptomatic Patients with Existing Gastrointestinal Illnesses

Charles W. Randall, Carlo M. Taboada, Russell D. Havranek, Franz Zurita, David Stump, Gary Gossen, Victor Ostrower, Susan Ryan, Janet Ford

Introduction In this study we followed patients with a variety of underlying GI illness whose symptoms were not completely controlled by conventional therapy. We sought to determine if lactose or fructose intolerance contributed to their continued symptoms. Methods 127 patients were seen in our clinic with known GI disorders. 116 had a functional syndrome (IBS, Functional Nausea, Functional Diarrhea, Gas/Bloat Syndrome \& NUD); 2 had celiac disease; 3 had inflammatory bowel disease; 2 had motility disorders; 2 had lymphocytic colitis; 1 with severe diverticular disease and 1 with intestinal carcinoid. All patients underwent lactose and fructose hydrogen breath testing. For those testing positive appropriate dietary management and follow-up were arranged. Patients with organic or inflammatory illnesses were all evaluated to determine disease activity and all were in a quiescent phase Results A total of 63 patients tested positive for disaccharidase deficiency. 24 were lactose the production of Eudragit $\mathrm{S}$ (soluble $\mathrm{pH}>7$ ) microparticles containing the corticosteroids Electron microscopy revealed that all microparticles were spherical, non-aggregated an non-porous with size $<50 \mu \mathrm{m}$. Microparticles displayed $\mathrm{pH}$-responsive release of prednisolone and budesonide, leaking a negligible amount of drug after 2 hours incubation in acid, while releasing it rapidly at colonic $\mathrm{pH}(\mathrm{pH}$ 7.4). This is due to the large surface area of the microparticles and the improved solubility of the drug, which is encapsulated as a molecula dispersion. Drug release rate increased with drug loading. The incorporation of a waterinsoluble polymer (Eudragit RS or ethylcellulose) into the Eudragit $S$ microparticles produced an extended-release profile at pH 7.4. Conclusions. A novel, colon-specific corticosteroid 
intolerant; 29 were fructose intolerant and 10 were intolerant to both. 12 patients thought to have had a functional disorder had their diagnoses changed. These patients had been resistant to standard therapy and responded well to dietary management. Only 4 patients had no improvement or unsatisfactory results from dietary changes. Conclusions 1.- $49.6 \%$ of patients with known GI disorders but persistent symptoms tested positive for disaccharidase deficiency. 2.- $19 \%$ of patients with a disaccharidase deficiency had been misdiagnosed as having a functional disorder. 3.- Dietary management was efficacious in controlling symptoms in $93.6 \%$ of patients. 4.- Testing for lactose and fructose intolerance is safe and noninvasive and leads to a positive outcome for patients

\section{S1406}

Chronic Drug Consumption and Risk of Microscopic Colitis (MC): A CaseControl Study

Fernando Fernandez-Banares, Maria Esteve, Jorge Espinos, Merce Rosinach, Montse Forne, Josep Maria Viver

$\mathrm{MC}$ is a rare disease of unknown etiology. It has been described that some drugs could cause or worsen the disease, suggesting a high likelihood of inducing MC for 8 drugs (acarbose, aspirin, lansoprazole, NSAIDs, ranitidine, sertraline, ticlopidine and venotonic drugs). However, the scientific evidence is limited. Aim: Case-control study to investigate the possible association of chronic drug consumption with MC. Methods: Groups of cases were: Group 1 - 39 patients with collagenous colitis (CC); Group 2 - 39 patients with lymphocytic colitis (LC); and Group 3 - 52 patients with chronic watery diarrhea of functional characteristics (CD). The control group was formed by 97 age- and sex-matched subjects (56 patients of the major outpatient surgery unit, ophthalmologic and sinonasal surgery, and 41 accompanying persons). At diagnosis, drug consumption history of at least 2 weeks of duration was registered. Drug consumption in the control group was similar to that described in the elderly ( $>65$ yrs) population in Spain. An age- and sex-adjusted logistic regression analysis was used, and the OR (95\% CI) was calculated. Results: Drug consumption was: $89.7 \%$ CC, $92.3 \%$ LC, $76.9 \%$ CD and $76.3 \%$ Control ( $<<0.05$ LC vs control). The mean daily number of drugs by person was: CC, $2.67 \pm 0.37$; LC, 3.79 $\pm 0.44 ; C D, 2.67 \pm 0.36$; Control, $2.13 \pm 0.22$ ( $p=0.04$ LC vs Control, ANOVA adjusted by age and sex). The following associations as compared to control group were observed: Group 1 (CC) - Consumption of NSAIDs (do not include low doses of aspirin) (46.2 vs 23\%; $\mathrm{p}=0.008$; OR=2.9, 1.3-6.4), selective serotonin reuptake inhibitors (SSRI) (18 vs 1\%; $\mathrm{p}=0.005$; OR=21, 2.5-177); Group 2 (LC) - SSRI ( 28 vs $1 \% ; p=0.001 ; O R=37.7,4.7-304$ ), beta-blockers ( 13 vs $3 \% ; p=0.043$; $\mathrm{OR}=4.79,1.04-20$ ), statins ( 13 vs $3 \% ; \mathrm{p}=0.044 ; \mathrm{OR}=4.6,1.04-20$ ), biphosphonates ( 8 vs $0 \% ; \mathrm{p}=0.022$ ); Group $3(\mathrm{CD})$ - SSRI ( 15 vs $1 \% ; \mathrm{p}=0.01 ; \mathrm{OR}=16.2,2-135)$, statins ( 11.5 vs $3 \% ; \mathrm{p}=0.025 ; \mathrm{OR}=5.4,1.2-24)$. Among SSRI there was an association with the use of sertraline only in LC (15.3 vs $0 \% ; \mathrm{p}=0.0005)$. Conclusions: 1 . Drug consumption in LC was higher than in the age-matched control population. 2. Although it is not possible to conclude if drugs are the cause or only worsen MC, the observed associations suggest that consumption of these drugs might have a role in the pathogenesis of the disease. 3 . There were differences between CC and LC in the drug associations found. As the $95 \% \mathrm{CI}$ of the $\mathrm{OR}$ were wide, further studies in larger samples are required to confirm the strength of the observed associations.

\section{S1407}

Mucosal Healing in the Small Intestine Detected By Wireless Capsule Endoscopy

Ruediger Schoo, Carsten Buening, Johann Ockenga, Jutta Wirth, Jutta Weber, Guido

Schachschal, Herbert Lochs, Voderholzer Winfried

Background: Wireless capsule endoscoyp may be useful in the follow up of small intestinal Crohn's disease (CD) in order to detect healing of mucosal lesions. However, data on the follow up of patients with small intestinal CD are rare. Aim of our study was healing during standard therapy. Methods: Patients with active CD (CDAI > 200) were enrolled. Before the WCE- procedure all patients underwent gastroscopy, colonoscopy, small bowel radiology (CT-enteroclysis), and abdominal ultrasound. After exclusion of relevant strictures $(<1 \mathrm{~cm}$ diameter) by $\mathrm{CT}$ enteroclysis and a regular preparation like for colonoscopy, the patients underwent wireless capsule endoscopy. All capsule videos were evaluated by a standardized form that gave information about specific lesions for $\mathrm{CD}$ (cobblestonine, fissural ulcers, aphthes, erythema, strictures). After that, standard medical therapy was initiated. After 2 months, WCE colonoscopy, and ultrasound were repeated. Results: So far, 14 patients have been examined ( $\mathrm{f} / \mathrm{m}=7 / 7,35,1 \pm 11.7 \mathrm{yrs}$ ). All Patients had a clinical improvement (CDAI $1: 316 \pm 108,64$, CDAI 2: 139,43 \pm 87.9 ) after therapy with glucocorticoids and mesalazine (10 patients), azathioprine (2), infliximab (1) and MTX (1). After 2 months of therapy, there was no change with respect to the colonoscopic activity. Moreover, there was no change in kind and frequency of Crohn typical lesions seen in WCE in patients who received glucocorticoids. The patient on MTX had pathological lesions all over the small intestine. After treatment the extent was limited to the terminal ileum and the proximal lesions had disappeared. The one patient who were on azathioprine showed no change. The other patient had almost a complete disappearance of lesions. The patient who received infliximab had a remission of about $50 \%$ of his ulcerations. The only significant change was a minor change of results of abdominal ultrasound $(p=0,034)$. Conclusions: WCE is a suitable method for examining mucosal healing in $\mathrm{CD}$. Standard therapy using glucocorticoids does not result in mucosal healing in the small intestine, although in patients who received an immunmodulatory or immunsuppressive medication (infliximab and azathioprine) the lesions got rare.
S1408

The Significance of Raised C Reactive Protein in Patients with Late RadiationInduced Bowel Symptoms

Usman Khalid, Andrew R. Norman, Jervoise Andreyev

Background: The production of CRP occurs in the liver as part of the acute phase response. An elevated CRP is a valuable marker of disease activity in Crohn's disease, predicts colectomy in ulcerative colitis and outcome following myocardial infarction and in multiple myeloma. It is sometimes elevated in patients with chronic gastrointestinal symptoms following pelvic radiotherapy, but its significance in this context has not been evaluated. Aims and methods: To investigate the significance of an elevated CRP with new gastrointestinal symptoms starting after pelvic radiotherapy. All new patients at first presentation to one gastroenterologist had their CRP measured routinely. The diagnoses reached following systematic investigation of their symptoms were grouped into categories and correlated with the level of the CRP using logistic regression analysis. Results: Since 2001, 159 patients presenting 0-34 years after the end of radiotherapy (median 3 years) with new gastrointestinal symptoms had their CRP checked. 91 were women aged 22-84 (median 61 years), 68 were men aged 31-84 (median 69.5 years). 5 had been treated for anal, 2 bladder, 36 cervical, 36 endometrial, 2 ovarian, 56 prostate, 6 rectosigmoid, 5 seminoma, 7 vagina and 4 other cancers. 131 patients had a normal CRP $(<10 \mathrm{mg} / \mathrm{l})$. In the 28 with an elevated CRP, it ranged from 10-79 mg/l (median 18). 8 categories of diagnosis were recorded for patients (more than one diagnosis possible). These included advanced neoplasia $(n=18)$, small bowel disease $(n=43)$, large bowel disease $(n=42)$, ano-rectal disease $(n=70)$, infection $(n=5)$, chronic pancreatitis $(n=$ $6)$, drugs $(n=7)$ and normal/other $(n=40)$. On univariate analysis, younger age, $p=0.035$ (OR 0.96 95\% CI 0.94-0.99), presence of advanced neoplasia, $\mathrm{p}=0.01$ (OR 3.85 95\% CI 1.34-11.1) and the presence of chronic pancreatitis, $p=0.026$ (OR $8.1395 \%$ CI 1.29-51.23) were statistically associated with an elevated CRP. Gender, years since treatment, tumour primary site, small and large bowel disease, ano-rectal disease, infection, drugs or normal/ other was not associated with abnormal CRP. Multivariate analysis was not performed in view of the size of the cohort. Conclusions: An acute inflammatory response as manifested by a raised $\mathrm{C}$ reactive protein is not a prominent feature of chronic radiation-induced toxicity in the gastrointestinal tract. An elevated CRP in patients with gastrointestinal symptoms not present before radiotherapy should raise the possibility of neoplasia or the rare condition, radiation-induced pancreatic insufficiency.

\section{S1409}

Autoimmune Enteropathy Successfully Treated with Tacrolimus: A Case Report of Two Adult Patients Sami Mustajoki, Martti Farkkila

Background Autoimmune enteropathy (AIE) is a rare autoimmune disease of unknown etiology characterized by refractory diarrhea, villous atrophy of small intestine, and the presence of serum antibodies against enterocytes. Although various immunosuppressive medications have been used, there is no established treatment for AIE. In this report we describe two adult patients suffering from AIE treated with tacrolimus. Patient 1 A 60-yearold female with previously diagnosed migraine and psoriasis was referred to hospital with a 6-week history of diarrhea leading to severe hypokalaemia and weight loss of $10 \mathrm{~kg}$. Colonoscopy revealed no findings macroscopically but histologically there was active nonspecific inflammation in terminal ileum and right-sided colon. In duodenal biopsies there was total villous atrophy and highly active inflammation. Capsule endoscopy finding suggested diffuse villous atrophy in whole small intestine. Serological markers for celiac disease were negative and the patient was negative for HLA haplotype DQ2. Anti-enterocyte antibodies were positive (titer 1:10). Prednisone $40 \mathrm{mg} / \mathrm{d}(0.8 \mathrm{mg} / \mathrm{kg} / \mathrm{d}$ ) resulted in rapid improvement in clinical status, but tapering the dose caused a clinical relapse. Azathioprine had to be discontinued because of abdominal pain, and tacrolimus $3 \mathrm{mg} / \mathrm{d}(0.06 \mathrm{mg} / \mathrm{kg} / \mathrm{d})$ was introduced, adjusting the dose to reach plasma concentration of $10 \mathrm{ug} / \mathrm{l}$. Within 10 days bowel function returned to normal and after five weeks duodenal biopsies showed only mild and partial villous atrophy and no inflammation. Patient 2 A 23-year-old previously healthy male had suffered for 5 months from watery diarrhea, fever and weight loss of 18 $\mathrm{kg}$. In duodenal biopsies subtotal villous atrophy and inflammation was found. Villous atrophy and active inflammation was also detected in terminal ileum. Serological markers for celiac disease and HLA haplotype DQ2 were negative. With prednisone $60 \mathrm{mg} / \mathrm{d}$ (1.2 $\mathrm{mg} / \mathrm{kg} / \mathrm{d}$ ), azathioprine $50 \mathrm{mg} / \mathrm{d}$ and gluten-free diet the clinical response was dis-appointing and progression of villous atrophy and inflammation was seen at biopsies. Eventually, antienterocyte antibodies were positive (titer 1:10) and tacrolimus was started with starting dose of $10 \mathrm{mg} / \mathrm{d}(0.2 \mathrm{mg} / \mathrm{kg} / \mathrm{d})$, aiming at plasma concentration of $10 \mathrm{ug} / \mathrm{l}$. After 4 weeks the patient was asymptomatic and after 9 months duodenal biopsies showed normal villous structure. Also anti-enterocyte antibodies were converted negative. Tacrolimus was ceased without deterioration of symptoms. Two years later, hypothyreosis was diagnosed. Conclusion Based on our experience, tacrolimus is effective, safe and well tolerated treatment for autoimmune enteropathy.

\section{S1410}

Non-Specific Mucosal Microscopic Abnormalities Can Affect the Terminal Ileum and Different Sites of the Large Bowel in Patients with Functional Bowel Disorders

Adriana Marcheggiano, Alfredo Covotta, Carlo Iannoni, Danilo Badiali, Rosanna Cantarini, Marco Paoletti, Nadia Pallotta, Enrico Corazziari

Non-specific microscopic abnormalities (NSMA) of the colonic-rectal mucosa may occur in IBS patients. It is not known, however, whether NSMA affect one or more sites of the large bowel and terminal ileum in patients with irritable bowel syndrome (IBS) and other Functional Bowel Disorders (FBD). AIM of this study was to evaluate the histological pattern of the mucosa of the large bowel and terminal ileum in patients referred for FBD to a third level referral center. PATIENTS. Thirty-four consecutive patients (22 F; age range 22-70 yrs), 
with normal serum biochemistry, $\mathrm{CBC}$, thyroid function, EMA, and normal ileum-colonoscopy were evaluated. Sixteen patients matched the diagnosis of IBS; five of functional diarrhea (FD), and thirteen of unspecified functional bowel disorders (UFBD). Biopsies of the terminal ileum $(n=2)$ cecum $(n=1)$, ascending $(n=1)$, transverse $(n=1)$, descending $(n=1)$, sigmoid $(\mathrm{n}=1)$ colon, and rectum $(\mathrm{n}=1)$ were stained with H-E for microscopic assessment by a pathologist unaware of the clinical diagnosis. RESULTS. Histological abnormalities were found in 9 (26.5\%) patients: 3 IBS, 2 functional diarrhea and 4 UFBD. Increased lymphoplasmacytic infiltrate and granulocyte clusters were present at the level of a) the ileum only in 1 IBS patient, and in 2 UFBD patients; b) the cecum only in 1 IBS patient. Altered crypt architecture was present in the rectum only in 1 UFBD. Collagenous colitis was present in 1 IBS, 2 functional diarrhea, and 1 UFBD, patient. CONCLUSIONS. In a subgroup of patients with Functional Bowel Disorders, IBS and non-IBS, non-specific microscopic abnormalities can be present at the level of the terminal ileum and different sites of the large bowel. Nonspecific microscopic abnormalities of the ileo-colonic-rectal mucosa and collagenous colitis may be equally present in patients with different Functional Bowel Disorders. Biopsies of multiple sites of colon, terminal ileum, and rectum are required to detect Non-specific microscopic abnormalities in patients with functional bowel disorders

\section{S1411}

Management of Acute Pancreatitis: A Survey of Internal Medicine Residents Sameer Barkatullah, Ami Shah, Aziz Aadam, Sri Komandur

PURPOSE: We conducted a survey to evaluate the current understanding of the management of acute pancreatitis (AP) among internal medicine residents. The survey was conducted to assess the efficacy of current teaching patterns and to improve overall patient care at a large urban teaching center. METHODS: We administered a 23-question survey to 58 Internal Medicine residents. The questions were multiple choice and centered on basic aspects of management of AP. These included appropriate use of laboratory testing and radiologic imaging, assessment of severity, use of antibiotics, nutrition, and indications for surgical intervention. RESULTS: Approximately $50 \%$ of the surveys collected were completed by first year residents and the other $50 \%$ by senior residents. Though $38 \%$ could not identify the initial appropriate laboratory testing, an even greater proportion, $62 \%$ of residents, did not recognize Hematocrit as the most important predictor of severity on admission. About $25 \%$ felt that administration of empiric antibiotics for all cases of AP were the standard of care. Furthermore, $21 \%$ felt the diagnosis of AP was made by identifying inflammation on CT scan. In the setting of acute biliary pancreatitis, only $66 \%$ identified RUQ ultrasound as the preferred initial imaging modality, and only $59 \%$ could identify the appropriate timing and utility of ERCP. A larger gap of knowledge was seen in identification and management of severe pancreatitis. The majority of residents (81\%) underestimated the incidence of pancreatic necrosis in the setting of severe pancreatitis. As for early feeding, only $36 \%$ of residents felt that it decreased morbidity and mortality in necrotizing pancreatitis; $31 \%$ preferred total parenteral nutrition to enteral feeding. Perhaps most surprising, only 55\% correlated necrotizing pancreatitis with Systemic Inflammatory Response Syndrome (SIRS). Finally, $66 \%$ of all residents could not recognize appropriate indications for surgical intervention in AP. As for the use of prognostic indices, only about two-thirds used Ranson's criteria occasionally, while $52 \%$ reported never using the APACHE II score to assess severity. Overall only $21 \%$ of residents felt very confident in managing acute pancreatitis. CONCLUSION: The morbidity and mortality of AP continues to be a problem. In addition, there continues to be a large amount of resources utilized for this cohort of patients. The lack of understanding and inconsistent management of severe pancreatitis is alarming. A dedicated educational effort is needed at the resident level in order to begin to decrease the morbidity of AP as well as to curtail ineffective use of costly resources.

\section{S1412}

Evaluation On a Mexican Cohort of a UK Computer Model Developed to Predict Severity of Acute Pancreatitis

Callum B. Pearce, Jose A. Gonzalez-Gonzalez, D.R. A. Garcia-Cantu, Steve R. Gunn, Colin D. Johnson

Background: It has previously been reported that a computer model using 8 variables (age, $\mathrm{C}$-reactive protein $(\mathrm{CRP})$, respiratory rate, $\mathrm{pO} 2$ on air, arterial $\mathrm{pH}$, serum creatinine, white cell count and GCS) created using a UK cohort of patients with acute pancreatitis (AP) can provide accurate prediction of severity using data obtained on admission to hospital. This model has up to now only been tested on UK patients. Aim. To evaluate this model on a retrospectively collected dataset previously obtained in a different geographical and social setting (Monterrey, Mexico). Method: Clinical and biochemical admission data were collected from 202 patients admitted with a diagnosis of AP between October 2002 and November 2004. The data were evaluated using a computer model (BSG March 2004), and the area under the receiver-operating characteristic curve (AUC) was used to compare the accuracy of this model with CRP and CREED systems (a system commonly used in Mexico) in predicting severity in AP. Severe AP was defined by the Atlanta criteria. Results: 42 patients (20.8\%) developed severe disease, and 3 died (1.5\%). 11 patients $(5.4 \%)$ developed renal failure by the Atlanta criteria, and 23 respiratory failure (11.4\%); 146 patients had a biliary aetiology (72.3\%), 25 alcohol (12.4\%), 11 hypertriglyceridaemia (5.4\%), 14 idiopathic $(6.9 \%)$ and 6 post-ERCP (3\%). The UK computer model predicted severity in the Monterrey patients with an AUC of 0.81 , (identical to the figure obtained previously in UK patients). This represents a sensitivity and specificity for prediction of severity of 0.80 and 0.77 at the optimum cut-off point. This compares favorably with the CREED system (AUC 0.70) and was significantly $(p=0.001)$ better than admission CRP (AUC 0.76) in the same group of patients. Comments: This system combines scoring of early systemic disturbance and admission CRP value for prediction of severe AP. The score is simple to use, can give a prediction within a few hours of admission to hospital, and is more accurate than admission CRP or APACHE-II. The validation of the model on a separate cohort of patients from a different background confirms the merit of the computer-assisted approach. A prospective multi-center study using the Southampton computer system is required.

\section{S1413}

Trends in the Epidemiology of Acute Pancreatitis (AP): A Systematic Review Dhiraj -. Yadav, Albert B. Lowenfels

Background and Aims: To systematically review trends in the epidemiology of first attacks of AP based on reported population-based studies. Methods: From Medline we retrieved 18 full length English language peer-reviewed original articles published during 1966-present with population-based information on the epidemiology of first attacks of acute pancreatitis We examined time-tends using linear regression analysis. Results: Nine studies were from the UK and nine were from other European centers. The annual incidence per 100,000 for first attacks of AP and gallstone pancreatitis increased significantly over the last 4 decades ( $\beta=0.5, p<0.001$ and $\beta=0.39, p=0.004$ respectively) after controlling for the site of study (UK vs. non-UK). The incidence of gallstone pancreatitis was similar in UK and non-UK studies $(\beta=-0.19, p=0.93)$, while the incidence of alcoholic pancreatitis was higher in non-UK studies $(\beta=4.44, \mathrm{p}<0.001)$. AP incidence and mortality increased with age. Gallstone pancreatitis was more common in females and alcoholic pancreatitis in middle-aged males AP case fatality (\%) decreased from $15-21 \%$ earlier to about $5 \%$ presently (FIGURE), but the overall population AP mortality rate 100,000 over time was unchanged $(\beta=0.01, p=$ 0.13). Recurrent AP was milder with a substantially lower mortality. Conclusions: The incidence of AP has increased significantly over the last few decades. The differences in the incidence and etiology of AP present between and within countries reflect differences in the prevalence of risk factors. The AP case fatality rate but not the population-based mortality rate decreased over time.

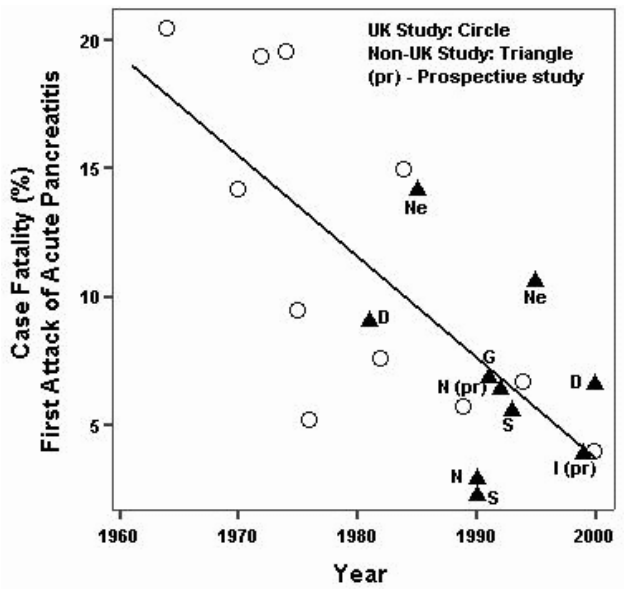

S1414

Predictors of Length of Hospitalization for Acute Biliary Pancreatitis in a Tertiary Medical Center

Sameer Barkatullah, Aziz Aadam, Garth Swanson, Ami Shah, Sri Komanduri

BACKGROUND: Several factors in the management of acute biliary pancreatitis (ABP) have been studied for their value in predicting morbidity and mortality. In addition to these outcomes, factors that provide insight into expected length of hospitalization (LOH) meri evaluation. We retrospectively reviewed the management of $\mathrm{ABP}$, with attention to the predictive value of various standard practices on the $\mathrm{LOH}$ at a large urban teaching center METHODS: Patients with ABP were identified by evidence of epigastric pain, elevated lipase and liver function tests (LFT), and abnormal transabdominal ultrasound demonstrating cholelithiasis or choledocholithiasis with or without ductal dilatation. A total of 75 admissions for ABP were reviewed between between January 1, 2002 and December 31, 2004. APACHE II scores and percent change in lipase, alkaline phosphatase (AP), total bilirubin, aspartate aminotransferase (AST), and alanine aminotransferase (ALT) values were calculated at admission, 48 , and 72 hours. Time to ERCP, cholangiogram findings, and empiric antibiotic usage were also recorded. RESULTS: The mean age was 56 years, with female preponderance $(65 \%)$, and a mean $\mathrm{LOH}$ of 6.8 days. 51 patients underwent ERCP. Those who had ERCP within 72 hours had a significantly shorter LOH compared to those undergoing ERCP after 72 hours (5.8 vs. 9.9 days) $(\mathrm{p}<0.05)$. In addition, those who had filling defects within the biliary tree on ERCP had higher APACHE II scores than those without filling defects at 72 hours $(5.38$ vs. 3.44$)(p<0.05)$. Fluid balance, changes in lipase or LFTs did not reliably predict $\mathrm{LOH}$ at any time interval. Patients receiving empiric antibiotics had a greater mean LOH than those on no antibiotic therapy ( 8.4 vs. 6.5 days). This difference approached statistical significance. CONCLUSION: Our data suggests that early ERCP ( $<72$ hours) in select patients with ABP decreases length of hospitalization. This is in accordance with previous data suggesting improved outcome with early ERCP in this cohort. Interestingly, patients with a filling defect on cholangiogram had significantly worse pancreatitis at 72 hours than those with a negative cholangiogram. This supports the concept of performing ERCP in ABP to prevent ongoing pancreatic injury due to biliary obstruction. We found no correlation between trends in serum lipase or liver function tests, or empiric antibiotic usage and length of hospital stay. Our data suggests that early ERCP, when appropriate, significantly decreases $\mathrm{LOH}$ in ABP. The practice of drawing daily labs and administering empiric antibiotics, however, does not change length of stay, and should be reassessed. 
Hemoconcentration Is An Independent Risk Factor for Severe Acute Pancreatitis

Young S. Oh, Da Hea Seo, Christian D. Stone

BACKGROUND: Hemoconcentration (hematocrit $[\mathrm{HCT}]>44$ ) at initial presentation to the hospital has been shown to be associated with severe acute pancreatitis (SAP). However, it has not been evaluated as an independent risk factor for SAP in a multivariate model. We analyzed a large database of inpatient admissions for acute pancreatitis to determine if hemoconcentration was an independent risk factor for SAP after controlling for other known risk factors. METHODS: A database of 629 adult admissions for an ICD-9 diagnosis of acute pancreatitis was reviewed for demographic data, initial and subsequent HCT values, and outcome measures. Risk factors for SAP considered included age $\geq 50$, obesity (BMI $\geq 30$ $\mathrm{kg} / \mathrm{m} 2$ ), morbid obesity (BMI $\geq 40$ ), Black race, male sex, initial HCT $>44$, and increase in HCT after 24 hours. SAP was defined as presence of acute renal failure, need for ICU admission, or necrotizing pancreatitis on radiographic imaging. Admissions for less than 3 days were excluded because, in a subset chart review, such cases were frequently confounded by the presence of chronic pancreatitis and/or failed to meet clinical criteria for acute pancreatitis. Multivariate logistic regression was used to determine the risk for SAP. RESULTS: The adjusted odds ratio (aOR) for SAP for initial HCT $>44$ was 1.8 ( $95 \%$ confidence interval: $1.1,3.4)$. The adjusted OR for increase in HCT after 24 hours was $0.9(0.6,1.8)$. Other factors found to confer a risk for SAP were Age $\geq 50[\mathrm{aOR}=2.3(1.5,3.5)]$ and morbid obesity $[\mathrm{aOR}=3.4(1.4,7.9)]$. The aORs for male sex, obesity and Black race were elevated but not statistically significant. CONCLUSIONS: Hemoconcentration with an initial HCT > 44 is an independent risk factor for SAP. Worsening hemoconcentration as manifested by an increase in HCT after 24 hours was not an independent risk factor in our model. Age $\geq 50$ and morbid obesity were also independent risk factors for SAP. These findings may be clinically useful in assessing severity of acute pancreatitis upon initial presentation.

\section{S1416}

Prognostic Factors of Life-Threatening Local Complications in Patients with Severe Acute Pancreatitis

Akiyoshi Hagiwara, Takehiko Tarui, Hideaki Goto, Takeaki Matsuda, Yoshihiro

Yamaguchi, Shuji Shimazaki

Objective: The objective of this study was to determine prognostic factors of arterial injuries and gastrointestinal perforation, which are life-threatening local complications associated with severe acute pancreatitis. Materials and Methods: 31 severe acute pancreatitis patients with Ranson score $\geq 3$ were included in this study (female: 8 , male: 23 , aged $54.6 \pm 18.3$ years). Study parameters included: Ranson score, APACH II score and CRP on administration (Day 1) and Day 7, and enhanced CT findings observed on Day 1 (1st) and Day 7-10 (2nd). CT findings were assessed using CT severity index (Balthazar EJ et al.). Results: Local complications developed in 11 of 31 patients. These complications consisted of 6 cases of arterial injuries (ruptures of celiac artery $[n=1]$, middle colic artery $[n=1]$, great pancreatic artery $[\mathrm{n}=1]$, gastroduodenal artery (GDA) $[\mathrm{n}=1]$, occlusion of splenic artery that caused splenic necrosis $[n=1]$, ruptures of both GDA and splenic vein $[n=1])$ and 5 cases of gastrointestinal perforation (stomach $[n=1]$, duodenum $[n=2]$, colon $[n=2]$ ). Three patients died in association with massive bleeding from GDA $(n=1$, Day 51$)$ or perforation of the colon ( $\mathrm{n}=2$, Days 17 and 162). Ranson and APACH II scores on Day 1 were $5.3 \pm 2.1$ and $12.9 \pm 8.7$ for patients who developed complications ( $C$ group) and $4.3 \pm 1.1$ and $9.8 \pm 5.1$ for patients who did not (Non-C group), and no statistical significance was found. APACH II scores and CRP on Day 7 were significantly higher in the $C$ group compared to the NonC group (APACH II scores: $11.7 \pm 6.2$ vs. $5.1 \pm 3.4, \mathrm{p}=002$, CRP: $20.5 \pm 6.6 \mathrm{mg} / \mathrm{dL}$ vs. $8.6 \pm 5.8$ $\mathrm{mg} / \mathrm{dL}, \mathrm{p}<0.01)$. APACH II scores and CRP of all patients in the $\mathrm{C}$ group were $\geq 5$ and $\geq 9.2 \mathrm{mg} / \mathrm{dL}$, respectively. Severity index of $1 \mathrm{st}$ and 2 nd CT were significantly higher in the $C$ group compared to the Non-C group ( 1 st CT: $7.3 \pm 2.4$ vs. $3.4 \pm 1.9, p<0.01,2$ nd $C T$ : $7.6 \pm 2.3$ vs. $3.0 \pm 1.6, \mathrm{p}<0.01$ ). All patients in the $\mathrm{C}$ group had $\geq 4$ severity indices. APACH II scores and CRP in the $\mathrm{C}$ group continued high values after Day 7. The 2nd CT showed severe fat inflammation in the same site of the perforation, i.e. the inflammation of transverse mesocolon in transverse colon perforation. These inflamed and fat-accumulated lesions gradually turned into localized low density areas. Conclusion: Life-threatening and localized complications can occur if the APACH II score, CRP, CT severity index are high on Day 7 and CT after Day 7 shows persistent and severe fatty inflammation.

\section{S1417}

The Prevalence of Pancreatic Serine Protease Inhibitor Kazal Type 1 (SPINK1) and Cationic Trypsinogen Gene (PRSS1) Mutations in Polish Patients with Chronic Alcoholic Pancreatitis (CAP)

Ewa I. Malecka-Panas, Anita Gasiorowska, Renata Talar-Wojnarowska, Beata Smolarz, Hanna Romanowicz-Makowska

We have earlier demonstrated that in more than 90\% Polish patients, excessive alcohol consumption represents the main cause of chronic pancreatitis (CP), however only around $5 \%$ alcoholics develop this diseases. The exact mechanism of CAP pathogenesis is not fully elucidated yet. Mutations of the PRSS 1 and SPINK 1 have been mostly implicated in hereditary and idiopathic $\mathrm{CP}$, but their presence in other types of this disease have also been reported. The aim of the study was to determine the frequency of PRSSI and SPINKI mutations in patients with chronic alcoholic pancreatitis (CAP) as well as to investigate their relation to the clinical course of the disease. The diagnosis of CAP was based on clinical data, ultrasound and computed tomography. DNA was isolated from 37 patients with chronic alcoholic pancreatitis as well as 46 healthy subjects. The N211 mutations of PRSS1 have been detected with ASA-PCR and N34S mutation of SPINKI - with PCR-RFLP. Overall 9 (24.5\%) - among them five homozygous and four heterozygous - N34S SPINK1 mutations in CAP group have been detected. The same mutations (one - homozygous, two - heterozygous) have also been found in three $(6.5 \%)$ control subjects. PRSS1 mutations have been detected in five (13.5\%) patients with CAP and only in those with accompanying SPINKl mutations. In addition the same mutation concerned two (4.3\%) controls. In patients with CAP and the presence of at least one of the mutations evaluated, pancreatic calcifications have been detected significantly less frequently $(\mathrm{p}<0.05)$ that in patients with no mutations. In addition complications requiring endoscopic or surgical treatment $(\mathrm{p}<0.01)$ have been also less frequently noted in CAP with detected mutations compared with the remaining CAP group. No relations have been found between those mutations presence and the demographic data, as well as other clinical features of the CAP patients. Those preliminary data suggest the high incidence of SPINKI and PRSS1 mutations in Polish population generally, as well as in CAP patients. It may be speculated that those mutations contribute to the development of chronic pancreatitis in patients with alcohol overindulgence. On the other hand the clinical course of CAP seems to be more severe in patients with no SPINKI and/or PRSS1 mutations detected.

\section{S1418}

A Novel PRSS1 Mutation (Arg-121 -> Thr) Highlights the Surface Exposed Region PRSS1 A121-R122-V123 As a Hotspot in the PRSS1 Gene and Indicates a Unreported Phenotype

Peter Felderbauer, Juergen Schnekenburger, Rainer Lebert, Tobias Meister, Kerem Bulut,

Frank Schmitz, Wolfram Domschke, Wolfgang E. Schmidt

Introduction: Since the discovery of the mutations in the cationic trypsinogen gene (PRSS1) that cause hereditary pancreatitis the understanding of genetic risk factors for chronic pancreatitis $(\mathrm{CP})$ extended. In the specific region of the gene where the first $\mathrm{R} 122 \mathrm{H}$ mutation was detected subsequently two more mutations, R122C and V123M were detected that resulted in a similar phenotype. Here we report a family with a novel A121T mutation in the PRSSI gene that differs from the previously described mutations in several aspects. Methods: We collected blood samples for clinical chemistry and mutational screening of all family members who accepted genetic testing. Coded DNA samples of the patients were analysed for PRSS1 mutations using melting point curve analysis and DNA sequencing. Properties of the mutated sequence were analyzed by molecular modelling. Results: The sequencing revealed a novel $\mathrm{G}$ to $\mathrm{A}$ transition at position 133279 of the published genomic sequence (\#U66061 GenBank). The mutation results in an amino acid substitution of Arginine by Threonine at position 121 (A121T) of the cationic trypsinogen. 5 additional mutation carriers could be identified among the relatives while only one patient developed $\mathrm{CP}$. The molecular modelling of PRSS1 A121T revealed with high reliability an impaired autodigestion at R122 comparable to the recently reported R122C mutation. Conclusion: The novel PRSS1 A121T mutation emphasizes that the surface exposed region PRSS1 A121-R122V123 represents a hotspot for hereditary pancreatitis associated trypsinogen mutations. Molecular modelling and recently published studies of in vitro enzyme activities of PRSS1 mutated in this region indicate a higher stability against PRSS1 autodegradation and a gain of function as a cause of hereditary pancreatitis.

\section{S1419}

Natural History of Chronic Hereditary Pancreatitis (CHP)

Vinciane Rebours, Marie Christine Boutron-Ruault, Claude Ferec, Dermot O'Toole, Matthieu Schnee, Frederique Maire, Pascal Hammel, Philippe Ruszniewski, Philippe Levy The prevalence and natural history of CHP in France (population: 60 millions), occurring largely due to mutations of cationic trypsinogen gene (PRSS1), remains ill documented. Aim of the study: To assess genetic, epidemiological, clinical and morphological characteristics of CHP as part of a large extensive national survey. Patients (pts) and methods: A cohort comprising all French CHP pts was constituted by contacting all French gastroenterologists and pediatricians (response rate: $84 \%$ ) and genetics laboratories (response rate: $100 \%$ ). Inclusion criteria were the presence of PRSS1 gene mutation, or chronic pancreatitis in at least 2 first-degree relatives, or 3 second-degree relatives, in the absence of another cause of pancreatitis (notably, alcohol). CFTR and SPINK 1 gene mutations were also systematically examined. Results: The cohort comprised 78 families and 200 pts, 181 of whom were alive (mean number of generations/family: 3; men 53\%, chronic alcoholism: 5\%, smokers 34\%). PRSS 1 gene mutations were found in $68 \%$ of the pts (R122 H: $78 \%$, N29I: $12 \%$, others: 10\%). SPINK 1 and CFTR gene mutations were found in 13 and $2 \%$ of the pts, respectively. Maternal inheritance was observed in $65 \%$. Median age at first symptom and at inclusion in the study, was 10 (1-73) and 30 (1-84) years, respectively. $17 \%$ of the pts were symptomfree (penetrance: $83 \%$ ). Median delay between first symptoms and diagnosis was 9 years; CHP manifestations were pancreatic pain $(83 \%)$, acute pancreatitis $(69 \%)$, pseudocysts (23\%), cholestasis (3\%), pancreatic calcifications (61\%, median age of onset 23 years), steatrorrhea (34\%), diabetes mellitus (26\%) and pancreatic adenocarcinoma (5\%, median age 55 years). Exocrine and endocrine insufficiency occurred at 29 and 38 years (median), respectively. No differences in clinical and morphological data according to genetic status were observed. Long-term ( $>3$ months) analgesics intake, endoscopic treatment and surgery were necessary in 10,16 and $23 \%$ of the pts, respectively. 19 pts died, including 10 from $\mathrm{CHP}$, at a median age of 60 years. Conclusion: The numbers of French families and pts with CHP include at least 78 and 180 respectively. PRSS 1 gene mutations are found in 2/ 3 of pts with a $83 \%$ penetrance. Mutation type is not correlated to clinical/morphological expression. Major differences between CHP and chronic alcoholic pancreatitis, apart from age at onset, include a lesser frequency of cholestais and pancreatic insufficiencies.

\section{S1420}

Pancreatitis in Primary Hyperparathyroidism-Related Hypercalcaemia Is Strongly Associated with the N34s Mutation in the SPINK1 Trypsin Inhibitor Gene

Peter Felderbauer, Volker Fendrich, Elias Karakas, Kerem Bulut, Nikolaus Ansorge, Frank Schmitz, Detlef Bartsch, Wolfgang E. Schmidt

Background\&Aims: Primary hyperparathyroidism (pHPT) related hypercalcaemia is considered to represent a risk factor for the development of pancreatitis. We therefore explored whether mutations in genes that were previously identified to increase the risk for pancreatitis 
co-exist in a cohort of 826 patients with pHPT prospectively studied between 1987 and 2002. Methods: Among 826 patients with pHPT, 38 patients were identified with pancreatitis (4.6\%). DNA was available of 25 patients ( 12 females and 13 males). These individuals and 25 patients with pHPT without pancreatitis, pair-matched for age, sex and other risk factors were analysed for mutations in the serine protease inhibitor Kazal type I (SPINK1) gene (N34S) and the cationic trypsinogen gene (PRSS1) (N29I, R122H) by melting curve analysis and DNA sequencing. Results: 4 of 25 patients with pHPT and pancreatitis carried the N34S missense mutation in the SPINKl-gene (16\%) (OR 9.33; confidence interval 1.6-54.3; P < 0.0014 ), while all 25 matched controls (pHPT without pancreatitis) showed no mutation in SPINKI or PRSS1 genes. Mean serum calcium levels in pancreatitis patients [3.1 mmol/ l] did not differ significantly from the mean of the entire cohort $[3.0 \mathrm{mmol} / \mathrm{l}]$ or $\mathrm{pHPT}$ patients without pancreatitis $[2.76 \mathrm{mmol} / \mathrm{l}]$. Conclusions: Pancreatitis in primary hyperparathyroidism is highly associated with the N34S SPINKI mutation

\section{S1421}

The Impact of Genetic Variants in TPMT, ITPA, MTHFR, SPINK1 and PRSS1 On Azathioprine-Induced Pancreatitis

Angel J. Lopez, Mattias Schwab, Heiko Witt, Azhar Ansari, Clemens Dejaco, Elke Schaeffeler, Alexander Teml, Harald Vogelsang, Peter Ferenci, Walter Reinisch

Background: Azathioprine (AZA)-related pancreatitis develops in about 3\% of patients with inflammatory bowel diseases (IBD). The pathogenesis of this idiosyncrasy is still unknown. The role of functional relevant genetic variants in representative candidate genes has not been investigated so far in a large patient cohort as underlying mechanism. Methods: Fiftythree IBD-patients (Crohn's disease $n=49$, Ulcerative coltis $=3$, indeterminate colitis $n=$ 1) with documented AZA-related pancreatitis were included. 54 IBD-patients treated with AZA without development of pancreatitis were recorded as controls. Genotyping for TPMT (*2, *3A-D), ITPA (C94A, IVS2+A21C), MTHFR (C677T), SPINK1 (N34S, P55S) and PRSS1 (A16V, K23R, N29I, R122H) was performed. Results: Heterozygosity for TPMT was found in 4 patients of the control group (all * $1 / * 3 A$ ) and in one patient with pancreatitis, resp. showing a novel TPMT mutation in exon 10. One case of compound heterozygosity for ITPA (C94A and IVS2+A21C) was observed both in the pancreatitis and the control group. One homozygous mutant patient for IVS2+A21C was observed in the pancreatitis group only. Genotyping of SPINK1 revealed 3 heterozygous carriers, 2 in the control and 1 in the pancreatitis group; no mutation was found for PRSS1. Whereas a trend of significance was seen in the case of homozygous mutant patients for MTHFR C677T ( 7 patients with pancreatitis vs. 2 controls; $\mathrm{p}=0.08$ ), the distribution of heterozygous patients was comparable in both groups $(\mathrm{p}=0.77)$. No difference in polygenetic variance was found between both groups. Conclusions: On the basis of our patient population variants of genes related to thiopurine metabolism (TPMT, ITPA) as well as to susceptibility for chronic pancreatitis are unlikely to explain AZAinduced pancreatitis. An association between MTHFR C677T and AZA-induced pancreatitis cannot be excluded. Further investigations are warranted.

\section{S1619}

Novel Mechanisms for Regulation of Grk2 Activity: Inhibition of Grk2 Via Preferential Association with Pkc-Phosphorylated Rkip (Raf Kinase Inhibitory Protein) and Stimulation of Grk2 Via Tyrosine Phosphorylation of Ğrk2 By C$\overline{\mathrm{S}} \mathrm{rc}$

Jiean Huang, Sunila Mahavadi, Karnam S. Murthy

Smooth muscle of the gut expresses mainly VPAC 2 receptors coupled via $G_{s}$ to stimulation of cAMP and activation of cAMP-dependent protein kinase (PKA). $\mathrm{VPAC}_{2}$ receptor desensitization is mediated by GRK2. PKA augments $\mathrm{VPAC}_{2}$ receptor phosphorylation, internalization and desensitization via feedback phosphorylation of GRK2. Since GRK2 activity can also be regulated by other kinases such as PKC and c-Src, contractile agonists that activate these kinases could cross-regulate $\mathrm{VPAC}_{2}$ receptor desensitization. Aim. To determine the mechanisms by which $A C h$ acting via $\mathrm{m} 3$ and $\mathrm{m} 2$ receptors regulates $\mathrm{GRK} 2$-mediated $\mathrm{VPAC}_{2}$ receptor desensitization. Methods. VPAC 2 receptor phosphorylation, internalization (decrease in ${ }^{125}$ IlVIP binding) and desensitization (inhibition of cAMP response) were determined before and after treatment of cultured gastric muscle cells with VIP. Results. VIP-induced VPAC 2 receptor phosphorylation, internalization and desensitization were absent in cells expressing dominant negative GRK2(K220R), implying that the effects were exclusively mediated by GRK2. Stimulation of PKC by PMA or by ACh in the presence of the $\mathrm{m} 2$ receptor antagonist

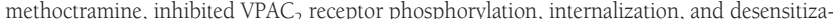
tion; inhibition was blocked by the PKC inhibitor bisindolylmaleimide. In the presence of the $\mathrm{m} 2$ receptor antagonist, ACh induced phosphorylation of RKIP (Raf Kinase Inhibitory Protein), increased RKIP:GRK2 association and decreased RKIP:Raf association, suggesting that upon phosphorylation by PKC RKIP dissociates from its known target Raf to associate with, and block the activity of GRK2. In muscle cells expressing RKIP (S153A), which lacks the PKC phosphorylation site, RKIP phosphorylation was blocked and the effect of ACh and $\mathrm{PMA}$ on $\mathrm{VPAC}_{2}$ receptor phosphorylation, internalization, and desensitization were abolished. In contrast, $\mathrm{ACh}$ in the presence of the $\mathrm{m} 3$ antagonist 4-DAMP, induced tyrosine phosphorylation of GRK2 and augmented $\mathrm{VPAC}_{2}$ receptor phosphorylation and internalization; the augmentation was blocked by the PI 3-kinase inhibitor, LY294002 or by the Src kinase inhibitor, PP2, suggesting that m2-mediated tyrosine phosphorylation of GRK2 increased GRK2 activity. Conclusion. This study identified two novel mechanisms for crossregulation of $\mathrm{VPAC}_{2}$ receptor internalization by $\mathrm{ACh}$. Acting via $\mathrm{m} 3$ receptors, ACh stimulated PKC, which phosphorylated RKIP and promoted its association with and inhibition of GRK2. Acting via $\mathrm{m} 2$ receptors, ACh activated $\mathrm{c}-\mathrm{Src}$, which induced tyrosine phosphorylation of GRK2 and augmented GRK2 activity.
S1620

A Truncated FAK Lacking the Ferm Domain Displays High Catalytic Activity But Retains Responsiveness to Adhesion-Mediated Signals

Rodrigo O. Jacamo, Enrique Rozengurt

Background: The non-receptor tyrosine kinase p1 25 focal adhesion kinase (FAK) is a multidomain protein that localizes to regions of the cell that attach to the extracellular matrix, called focal adhesions. FAK contains three major domains, an $\mathrm{N}$-terminal domain, a central catalytic domain, and a C-terminal domain. The N-terminal domain of FAK displays homology with FERM domains and it has been proposed to repress the catalytic activity of the enzyme by intramolecular auto-inhibition, a mechanism of negative regulation in many protein kinases Upon integrin-mediated cell adhesion, the cytoplasmic domain of integrins is thought to interact with the FERM domain of FAK, relieves FERM-mediated auto-inhibition and thereby activates the catalytic activity of the enzyme. However, the precise role of the N-terminal region in the regulation of the autophosphorylation of FAK in response to cell-adhesion signals remains to be clarified. Aim: To determine the role of the FERM domain in the regulation of FAK phosphorylation at Tyr-397, the major autophosphorylation site. Methods We generated a truncated FAK lacking a region of the N-terminus corresponding to 38 amino acids (FAK 384 ). HEK 293 cells or FAK-/- cells were transfected with either wild type FAK or FAK $\triangle 384$ and the role of the FERM domain in the regulation of FAK phosphorylation at Tyr-397 was determined in attached cells as well as in response to cell-adhesion signals. Results: FAK 384 showed a striking increase in phosphorylation, as compared with wild type FAK, in lysates of HEK 293 cells and FAK-/- cells. In addition, immunoprecipitates of FAK $\Delta 384$ showed a marked increase in Raytide phosphorylation as compared with immunoprecipitates containing wild type FAK. The striking increase in catalytic activity of FAK $\Delta 384$ provides further support to the notion that the FERM domain of FAK represses the catalytic activity of the enzyme by intramolecular auto-inhibition. A salient feature of our results is that suspension of either HEK 293 cells or FAK-/- cells transfected with FAK $\Delta 384$ also resulted in a striking, time-dependent decrease in the phosphorylation of this truncated form of FAK. The phosphorylation at Tyr-397 of wild type FAK and FAK 4384 was restored by replating the cells onto fibronectin-coated dishes. Conclusions: we propose a two-step model of regulation of the catalytic activity of FAK in response to integrinmediated cell adhesion. We hypothesize that maximal phosphorylation of FAK at Tyr-397 requires both the release from FERM-mediated auto-inhibition and as well as the clustering of FAK molecules in focal adhesions and to bring their catalytic domains into close proximity.

\section{S1621}

Rage Activates NfкB Through a Protein Kinase C Dependent Mechanism Maren K. Fuentes, Thiruvengadam Arumugam, Emina H. Huang, Craig D. Logsdon

Background: Previously, we found that S100P plays a major functional role in oncogenesis acting via the Receptor for Advanced Glycation End-products (RAGE). One importan mechanism induced by S100P interaction with RAGE is NFKB activation. However, currently the signaling cascade between RAGE and NFKB is unknown. In this study, we sought to identify the mechanism of RAGE activation of NFKB. Methods: The influence of exogenously added S100P and dominant negative RAGE was analyzed in NIH-3T3 cells on NFKB activation by EMSA and a luciferase reporter assay. Chemical inhibitors of broad spectrum Protein Kinase C (PKC. GF109203x) and specific isoforms of PKC (Rottlerin: PKC $\delta, \theta$ - specific and Gö6976: PKC $\alpha, \beta, \gamma$ - specific) were tested on S100P activation of NFKB. Cell fractionation and western blotting were used to determine PKC translocation after S100P activation of RAGE. Results: S100P activated NFKB in NIH-3T3 cells in a concentration - dependent manner. This activation was blocked by expression of a dominant negative RAGE indicating that the effects of S100P are mediated by RAGE. GF109203x inhibited S100P activation of NFKB in a concentration-dependent manner. Furthermore, rottlerin, but not Gö6976, also blocked RAGE activation of NFKB. S100P stimulated the translocation of PKCS from the cytosol to the membrane, indicating an activation of this isoform of PKC by RAGE. Conclusions: We have discovered a novel signaling cascade involving PKC in the S100P/RAGE activation of NFKB in NIH-3T3 cells. Therefore, blocking or altering this cascade may be useful for new therapeutic approaches to gastrointestinal cancer.

\section{S1622}

Protein Kinase C Negative Regulation of G-Protein Coupled Receptor-Induced Epidermal Growth Factor Receptor Transactivation

Chintda Santiskulvong, Enrique Rozengurt

Background: GI peptides stimulate DNA synthesis and cell proliferation in a variety of cultured cells, and thus are implicated as growth factors. Upon binding to their cognate G-protein coupled receptors (GPCRs), GI peptides stimulate multiple signaling pathways including Gaq-mediated activation of protein kinase C (PKC). Another critical mitogenic pathway is the GPCR-induced epidermal growth factor receptor (EGFR) tyrosine kinase activation, termed EGFR transactivation. While GPCRs positively regulate the EGFR via transactivation, our results demonstrate that PKC mediates a feedback mechanism that negatively regulates EGFR transactivation. Aim: To characterize the role of PKC in GPCRinduced EGFR transactivation. Methods: EGFR activation was assessed by immunoprecipitating the EGFR and immunoblotting with an anti-phosphotyrosine mAb. Extracellular signalregulated kinase (ERK) $1 / 2$ and Akt activation were determined by immunoblotting whole cell lysate with an anti-pThr202/pTyr204 ERK1/2 mAb or anti-pSer473Akt mAb, respectively. Results: Treatment of rat intestinal epithelial IEC-18 cells with the PKC inhibitors GF109203X (GF1) or Ro31-8220, or PKC downregulation via phorbol 12, 13 dibutyrate (PDB) chronic (24h) exposure resulted in a striking increase in angiotensin II-induced EGFR transactivation. Feedback inhibition of GPCR-induced EGFR transactivation by PKC was found in multiple model systems, including carbachol in human colonic epithelial T84 cells, bombesin in Rat1 fibroblasts stably transfected with the bombesin receptor, and neurotensin in the human pancreatic cancer cell line Panc-1. PKC inhibition also increased GPCR-induced EGFR transactivation-dependent downstream signaling, including ERK $1 / 2$ and Akt activation. In contrast, PKC activation by acute (1 min) exposure to PDB inhibited GPCR-induced EGFR 
transactivation and Akt activation. Acute PDB treatment also inhibited transforming growth factor- $\alpha$ (TGF- $\alpha$ )-induced EGFR phosphorylation, and heparin-bound-EGF- and TGF- $\alpha$ induced Akt phosphorylation. Conclusions: Our results indicate that PKC mediates a feedback mechanism in the crosstalk between GPCRs and the EGFR, regulating the intensity of GPCRinduced EGFR transactivation.

\section{S1623}

Secretion, Production and Biological Activity of the Human C-Terminal Flanking Peptide of Progastrin

Kelly Smith, Oneel Patel, Shamilah Lachal, Ian Jennings, Bruce Kemp, John Burgess, Graham Baldwin, Arthur Shulkes

Since the biological activity of gastrin-gly (Ggly) and progastrin was first described, there has been intense interest in the physiological and pathophysiologic roles of these peptides. However it is not generally appreciated that they constitute relatively small proportions of the final gastrin products in the antrum and in most gastrinomas and colorectal carcinomas (CRC). In the present study, we have analysed using region specific radioimmunoassays the relative amounts of progastrin, gastrin-amide (Gamide), Ggly and the C-terminal flanking peptide of progastrin (CTFP) in the antrum from normal subjects and in resected CRC. The plasma concentrations in normal subjects and patients with CRC were also determined. Finally, the biological activity of CTFP in vitro was assessed. The identity of the antral Cterminal flanking peptide of progastrin was confirmed as the hexapeptide progastrin75-80 by HPLC and mass spectroscopy. The CTFP hexapeptide is by far the major stored form of progastrin in antrum (CTFP $8690 \pm 2010$, Gamide $1970 \pm 440$, Ggly $21 \pm 5$, progastrin $430 \pm 140 \mathrm{pmol} / \mathrm{gm}, \mathrm{n}=6)$ and resected CRC (CTFP $19 \pm 2$, Gamide $0.07 \pm 0.01$, Ggly $0.03 \pm 0.02$, progastrin $0.22 \pm 0.09 \mathrm{pmol} / \mathrm{gm}, \mathrm{n}=10$ ). Furthermore, the CTFP circulates at $800 \pm 18$ pmol/, 50 -fold higher in concentration compared with Gamide. Circulating CTFP is released by a meal in a similar fashion to Gamide but is not elevated in patients with gastrin-secreting tumours or CRC. The biological activity of CTFP was determined using a variety of cell lines and readouts. Contrary to previous suggestions, CTFP is biologically active with a similar potency to Ggly. CTFP stimulated the proliferation of gastric and colonic cancer cell lines and the proliferation and migration of a non-transformed gastric cell line and activated early stimulation of the mitogen-activated protein kinase pathway. In summary, the CTFP is the major stored and secreted form of progastrin-derived peptides in the antrum and circulation of normal subjects and in the tumours of patients with CRC. Taken together with the findings that CTFP is biologically active, we suggest that CTFP is not an inactive metabolite of progastrin processing but a bioactive peptide with potential roles in the normal and diseased gastrointestinal tract.

\section{S1624}

Feedback Inhibition of RHOA By $\mathrm{G}_{12}$-Coupled PARl Receptors in Smooth Muscle Is Mediated Via Inhibitory Phosphorylation of $G_{12}$ By PKC Wimolpak Sriwai, Wenhui Hu, Karnam S. Murthy

The protease-activated receptors PARl and PAR2 activate RhoA via $G_{12}$ and $G_{13}$, respectively, in smooth muscle. Both receptors initiate dual RhoA-dependent pathways involving activation of Rho kinase and protein kinase $\mathrm{C}(\mathrm{PKC})$. In vitro studies in cell lines have shown that PKC phosphorylates and thus inhibits $G \alpha_{12}$ but not $G \alpha_{13}$, raising the possibility of feedback inhibition of Rho activity upon phosphorylation of $G \alpha_{12}$ by PKC. Aim. To characterize the pathway for feedback inhibition of RhoA upon its activation by PARl receptors. Methods. RhoA activation was measured as increase in Rho kinase activity. The role of PKC was evaluated using the active phorbol ester, PMA, and the PKC inhibitor, bisindolylmaleimide. Phosphorylation of $G \alpha_{12}$ and $G \alpha_{13}$ was determined by immunoblot analysis in cells prelabeled with ${ }^{32} \mathrm{P}$. Results. Rho kinase activity stimulated by the PARl agonist, SFLLR, but not by the PAR2 agonist, SLIGRL was augmented by bisindolylmaleimide implying that PKC inhibited Rho activity stimulated via PARl receptors only. In support of this notion, pretreatment with PMA inhibited Rho kinase activity stimulated by PAR1 but not by PAR2 agonists. The PARl agonist induced $G \alpha_{12}$ phosphorylation, which was abolished by bisindolylmaleimide and $\mathrm{C} 3$ exoenzyme suggesting that phosphorylation is mediated by PKC derived from activation of RhoA. The effect of the PKC inhibitor on Rho kinase activity was additive to that of the PKA inhibitor, PKI, suggesting that PARl receptors also induce an inhibitory pathway involving PKA. Both PARl and PAR2 can activate the canonical NF-KB pathway leading to degradation of IKB $\alpha$ and release of the catalytic subunit of PKA from its binding to IкB $\alpha$; PKA in turn inhibits RhoA via phosphorylation of RhoA at Serl88. This notion was corroborated in cells expressing PKA phosphorylation-site deficient RhoA(S188A) or dominant negative mutant of IKK2(K44A). The effect of the PKC inhibitor on Rho kinase activity was augmented in cells expressing RhoA(S188A) or IKK2(K44A). Thus, PARl agonists cause feedback inhibition of RhoA via two mechanisms: one mechanism involves PKA induced phosphorylation of RhoA at Ser188; the other involves phosphorylation of $G \alpha_{12}$ upstream of RhoA. Conclusion. This study identified a novel PARl-specific pathway for feedback inhibition of RhoA involving phosphorylation of $\mathrm{G} \alpha_{12}$.

\section{S1625}

C-Type Natriuretic Peptide Receptor (NPR-C) in Human Pancreatic Alpha Cells: Identification, Localization and ANP Mediated Changes in Glucagon Secretion

William R. Gower, Kim D. Moore, Matthew Burgess, Abdel Alli, Gay M. Carter, Hirohito Ichii, Camillo Ricordi

Background: There is evidence for the occurrence of immunoreactive atrial natriuretic peptide (ANP) and specific binding sites for ANP in glucagon-containing $\alpha$ cells of the pancreas. Identification of the specific receptor subtype and the localization of the receptor to a specific islet cell type in the human pancreas have not been reported. The aims of this study were to localize ANP, natriuretic peptide receptor subtypes A (NPR-A) and C (NPR-C) expression in normal and neoplastic human islets and to assess the NPR function in clonal islet cells.
Methods: Cell type specific localization of ANP, NPR-A and NPR-C proteins was examined by immunohistochemistry of normal and neoplastic human pancreas as well as the mouse clonal $\alpha$ cell line, $\alpha$ TC6. Presence of ANP and NPR transcripts and proteins were determined by RT-PCR and immunoblot analysis. aTC6 cells ( $3 \times 105$ /well) were serum deprived, incubated in Krebs-Ringer bicarbonate buffer (KRB) containing $25 \mathrm{mM}$ glucose (high) for $2 \mathrm{~h}$ at $37 \mathrm{C}$, then incubated in KRB containing high or low $(2.8 \mathrm{mM})$ glucose with or without ANP or cANF for 90 minutes. KRB was removed and assayed for glucagon by RIA and cells were lysed for total protein. Results: Immunoreactive NPR-C, but not ANP or NPR-A, was detected in human islets of Langerhans and $\alpha$ TC6 cells. No immunostaining was observed in the exocrine pancreas or ductal structures. Double-staining revealed that the NPR-C receptors were expressed mainly in the glucagon-containing $\alpha$ cells. Immunoblot results demonstrated a $66 \mathrm{kDa}$ protein corresponding to NPR-C in protein extracts of isolated human islets and $\alpha \mathrm{TC} 6$ cells. NPR-C expression in human islets and $\alpha \mathrm{TC} 6$ cells was confirmed by RT-PCR. NPR-C expression was also detected by immunohistochemistry in glucagonoma but not in insulinoma or gastrinoma. ANP and cANF, a specific agonist for NPR-C, had no effect on basal glucagon secretion from $\alpha \mathrm{TC} 6$ cells $(0.36 \mathrm{pg} / \mu \mathrm{g} / 90 \mathrm{~min})$. Low glucose induced a $444 \pm 5 \%$ increase in glucagon secretion. ANP and cANF both produced a dose-dependent $(0.1 \mathrm{nM}-1.0 \mu \mathrm{M})$ inhibition of glucose-stimulated glucagon release from aTC6 cells. Maximal effects were observed at $1.0 \mu \mathrm{M}$ ANP $(65 \pm 5 \%$ inhibition; $\mathrm{p}<0.001)$ and $1.0 \mu \mathrm{M} \mathrm{CANF}(71 \pm 4 \%$ inhibition; $\mathrm{p}<0.001)$. Conclusion: The NPR-C receptor is expressed in normal and neoplastic human $\alpha$ cells. NPR-C mediates ANP inhibition of glucosestimulated glucagon release from mouse clonal pancreatic $\alpha$ cells. These findings suggest a role for natriuretic peptides in the regulation of glucagon secretion. This work was supported by grants from the Veterans Administration and NIH.

\section{S1626}

Apical EGFR Phosphorylation Induced By Basolateral EGF Is Not Due to Transcytosis of Phosphorylated Basolateral EGFR to the Apical Membrane Nelly E. Avissar, Liana Toia, Harry C. Sax

Introduction: Epidermal Growth Factor (EGF) is a major trophic factor for the intestine, and supports repair and adaptation after massive enterectomy. In human polarized enterocytes EGF receptor (EGFR) is predominantly basolateral (Bl), while in injured intestine and in cancer it is redistributed to the apical (Ap) side. The physiological functions induced by activation of Ap EGFR differ from those of Bl EGFR. The main sources of EGF for the enterocytes are luminal (Ap). It is believed that when the membrane barrier is compromised, luminal EGF reaches Bl EGFR through which it exerts most of its physiological effects. We have found that addition of $\mathrm{Bl} \mathrm{EGF}$ to $\mathrm{C} 2 \mathrm{BBel}$ (a subclone of Caco-2) results in phosphorylation of Ap and Bl EGFR to the same degree, implying that functional Ap EGFR is also physiologically important. Our objective was to investigate whether the appearance of phosphorylated EGFR on the Ap membrane is due to transcytosis of the Bl EGFR or to cytosolic signal transduction pathways. Methods: Confluent $\mathrm{C} 2 \mathrm{BBel}$ were biotinylated on the $\mathrm{Bl}$ or Ap membrane, either before or after exposure to Bl EGF (intervals of $30 \mathrm{sec}-10 \mathrm{~min}$ ). Biotinylated proteins were isolated by avidin precipitation and subjected to Western analysis for total and site-specific phosphorylated EGFR. Results: Na+K+ ATPase, a Bl protein, was enriched in $\mathrm{Bl}$ membrane (over 80 fold), indicating successful isolation of the two membrane domains. There was a delay of $1 \mathrm{~min}$ in the appearance of phosphorylated Ap EGFR compared to $\mathrm{Bl}$ EGFR for the cells that were biotinylated after EGF exposure. This implies that the phosphorylation of Ap EGFR is dependent on that of Bl EGFR. When EGFR was biotinylated on the Ap membrane before addition of EGF to the Bl membrane, the degree of Ap EGFR phosphorylation was identical to that of Bl EGFR. This indicates that the phosphorylated EGFR on the Ap membrane is residential as opposed to the transcytosis of phosphorylated Bl EGFR. All the EGFR receptor site-specific tyrosines tested were phosphorylated by Bl EGF to the same degree on the two membrane domains, indicating activation of EGFR intrinsic tyrosine kinase on both membranes. Conclusion: Ap EGFR phosphorylated by $\mathrm{Bl}$ EGF is not Bl EGFR which reached the Ap membrane, but rather the residential Ap EGFR which is phosphorylated by Bl EGF through as yet unidentified signal transduction pathway. The latter, most probably, activates Ap EGFR tyrosine kinase. The fact that Ap EGFR is phosphorylated even by Bl EGF implies an important physiological function of the receptor when in this membrane location. Funded by NIH 5 ROl DK47989-10.

\section{S1627}

Roles of Progesterone (PG) Receptors A and B On Relaxation and Contraction of Muscle Cells from Human and Guinea Pig Colon

Arun Goswami, Ping Cong, Piero Biancani, Jose Behar

We have previously shown that colonic muscle cells from female patients with chronic constipation have an impaired contraction induced by agonists that act on $\mathrm{G}$ protein coupled receptors such as CCK-8 and Ach and G protein dependent such as GTP $\gamma S$. This impairment is due to the dual PG action on $G$ proteins: down regulation of $G q / 11$ that mediates contraction and up regulation of Gs proteins that mediate relaxation and increased mRNA levels of PG receptors. These $\mathrm{G}$ protein abnormalities are consistent with the effects observed in colonic muscle cells treated with PG for $6 \mathrm{~h}$. The present studies were aimed at determining a] whether the up regulation of Gs proteins causes an increased relaxation in response to VIP; and b) whether each PG receptor A and B affects a different signal transduction stimulated by PG. Muscle cells were obtained from human and guinea pig descendingsigmoid colons. Permeable colonic muscle cells were pretreated with buffer (controls) or specific antibodies against A or B receptors followed by treatment with PG $10^{-7} \mathrm{M}$ for 6 h. Muscle cell relaxation was defined as per cent inhibition by VIP $\left(10^{-9}\right.$ to-5 $\left.\mathrm{M}\right)$ of the contraction induced by diacylglycerol $\left(10^{-5} \mathrm{M}\right)$. Antibodies against $\mathrm{PG}$ receptor A blocked the PG increased relaxation induced by VIP compared to controls $(\mathrm{p}<0.01$ ); whereas, antibodies against $P G$ receptor $B$ had no effect on VIP relaxation but blocked the PG inhibition of the contraction induced by CCK-8 $\left(10^{-8} \mathrm{M}, \mathrm{p}<0.001\right)$. PG increased c'AMP formation induced by VIP. PG however did not affect the relaxation induced by dibutyryl-c'AMP that bypasses receptors and $G$ proteins. Western blot analysis revealed that antibodies against PG A receptor blocked the PG induced up regulation of Gs observed in control muscle cells $(\mathrm{p}<0.01)$ but had no effect on the down regulation of $\mathrm{Gq} / 11$ proteins. In contrast, antibodies against PG 
B receptors blocked the down regulation $G q / 11$ proteins induced by PG in muscle cells treated with buffer $(p<0.01)$. It is concluded that $P G$ increases the relaxation induced by VIP by up regulating Gs proteins. And, each of the two actions of PG are mediated by different receptors: PG A receptors, by mediating the up regulation of $G$ s, increase the sensitivity to VIP and PG B receptors, by mediating the down regulation of $\mathrm{Gq} / 11$, impairs the contraction induced by CCK-8.

\section{S1628}

Properties of Human TRPAl, a Mechanosensitive Channel and Putative Nociceptor

Jiahui Lin, Kirk Hillsley, Pieter Peeters, Jeroen Aerssens, David Grundy, Bernard Coulie, Ronald H. Stead

Background: TRPAl is reported to play a role in the transduction of mechanosensitive pain. We have shown that both gut projecting nodose and dorsal root ganglia (DRG) neurons express TRPA1. As the availability of specific pharmacological tools to study this channel are limited, our aim was to characterize the properties of human TRPAl channels expressed in a stably transfected cell line as a prelude to studies in native sensory neurons supplying the GI tract. Methods: HEK 293 cells were stably transfected with human TRPAl cDNA. TRPAl expression was induced by overnight tetracycline (1000-5000 ng/ml) incubation and cells were plated onto Petri dishes for whole cell patch clamp recordings at room temperature and a holding potential of $-80 \mathrm{mV}$, unless otherwise noted. Regular extracellular Krebs solution contained $2.5 \mathrm{mM} \mathrm{Ca}^{2+}$, whilst the pipette was filled with $\mathrm{Cs}^{+}$-rich intracellular solution. Allyl isothiocyanate (AITC) and benzyl isothiocyanate (BITC) were used as TRPAl agonists, and the effects of putative TRPAl antagonists ruthenium red (RR), gadolinium $\left(\mathrm{Gd}^{3+}\right)$, amiloride and gentamicin were also tested. Results: AITC and BITC evoked dosedependent inward currents in HEK-TRPAl cells. BITC $(3 \mathrm{mM})$ evoked a peak amplitude of $-602.2 \pm 48.4 \mathrm{pA}(\mathrm{n}=23)$ and activation time of $17.8 \pm 1.4 \mathrm{~s}(\mathrm{n}=23)$. The peak amplitude of the inward current evoked in response to either BITC or AITC was decreased in the absence of extracellular calcium, but the activation time was not affected. In the absence of extracellular calcium, RR $(50 \mu \mathrm{M}), \mathrm{Gd}^{3+}(100 \mu \mathrm{M})$, amiloride $(500 \mu \mathrm{M})$ and gentamicin $(50 \mu \mathrm{M})$ all inhibited the response to AITC $(10 \mu \mathrm{M})$. RR had an IC 50 of 1.55 $\mu \mathrm{M}$. However, in the presence of physiological concentrations of $\left[\mathrm{Ca}^{2+}\right]_{\mathrm{o}}(2.5 \mathrm{mM}), \mathrm{RR}$ and amiloride still inhibited TRPAl currents but to a lesser extent, whilst $\mathrm{Gd}^{3+}$ and gentamicin had no TRPAl antagonist effect. Conclusions: These data demonstrate that TRPAl channels are potently activated in a calcium-dependent manner by the pungent organic chemicals AITC and BITC, and inhibited by RR and amiloride. As TRPAl is known to be expressed on DRG and nodose neurons, it will be important to evaluate the properties of TRPAl channels in sensory neurons innervating the GI tract, in order to reveal its function in physiological and/or pathophysiological conditions.

\section{S1629}

Identification of Peptide-Ligand Binding Domains of the Growth Hormone Secretagogue Receptor, Compared to the Motilin Receptor Bunzo Matsuura, Teruhisa Ueda, Maoqing Dong, Laurence J. Miller, Sachiko Utsunomiya, Hidetaka Matsui, Yoichi Hiasa, Morikazu Onji

The Class I family of guanine nucleotide-binding protein ( $G$ protein)-coupled receptors that includes receptors for motilin, ghrelin, and growth hormone secretagogue (GHS) has substantial potential importance as drug targets. Understanding of the molecular basis of hormone binding and receptor activation should provide insights helpful in the development of such drugs. We previously reported that perimembranous residues within the unique second extracellular loop domain of the motilin receptor are critical for peptide ligand, motilin, binding and biological activity (J Biol Chem 277: 9834, 2002). In the current work, we focused on the second extracellular loop of the GHS receptor, and identified functionally important residues. For this purpose, GHS receptor constructs were prepared that included sequential deletions ranging from one to ten amino acid segments and replaced with alanine in this domain using site-directed mutagenesis methods. Each construct was transiently expressed in COS cells, and characterized for ghrelin-stimulated intracellular calcium responses and ghrelin radioligand binding. Cys198 was critical for ghrelin binding and calcium signaling. Moreover, perimembranous residues in the second extracellular loop domain, Val184 and The211, were also critical for ghrelin binding and activity, same as the motilin receptor. These results suggest that Cys in the second extracellular loop in this group of Class I G protein-coupled receptor is likely involved in the highly conserved and functionally important disulfide bond, and that the perimembranous residues contribute peptide ligand binding and signaling.

\section{S1630}

Caveolin Regulates Acetylcholine-Induced Translocated Pkc $\alpha$-Hsp27-RHOA Complex On the Membrane of Rabbit Colonic Smooth Muscle Cells Sita Somara, Jeffrey R. Martens, Khalil N. Bitar

Background: Caveolae are abundant cell surface microdomains in which multiple cellsignaling pathways converge through interactions with caveolin to regulate excitation contraction coupling in smooth muscle. The cell surface stability of caveolae is dependent on cytoskeletal organization which is in turn modulated by HSP27. Objective: Does caveolin play a role in docking of acetylcholine-induced translocated PKC $\alpha$-HSP27-RhoA complex on membrane during colon smooth muscle contraction? Methods: SMC were isolated from rabbit colon. Caveolae-enriched lipid raft fractions were isolated from colon SMC for the studies. Isolated SMC from rabbit colon were transfected with dominant negative calveolin1 (cavl). Results: 1) Rabbit colon SMC probed with antibodies for cav1, cav2, and cav3 showed the presence of cavl and cav2; 2) Western blot analysis shows that cavl and PKC $\alpha$ co fractionate in caveolae-enriched lipid raft fractions. 3) acetylcholine induced: i) a significant and sustained increase in the association of cavl with PKC $\alpha(166.39 \pm 3.57 \%$ at $30 \mathrm{sec}$ and $172.46 \pm 2.25 \%$ at $4 \mathrm{~min})$; ii) a significant and sustained increase in the association of cavl with RhoA ( $179.28 \pm 0.33 \%$ at $30 \mathrm{sec}$ and $184.03 \pm 2.59 \%$ at $4 \mathrm{~min})$; iii) a significant and sustained increase in the association of cavl with HSP27 (156.16 $\pm 0.77 \%$ at $30 \mathrm{sec}$ and $164.06 \pm 3.68 \%$ at $4 \mathrm{~min})$; 4) SMC transfected with dominant negative cavl showed inhibition of acetylcholine-induced: i) association of cavl with RhoA (111.36 $\pm 5.58 \%$ at $30 \mathrm{sec}$ and $116.22 \pm 6.45 \%$ at $4 \mathrm{~min})$; ii) association of cavl with PKC $\alpha(104.74 \pm 3.51 \%$ at $30 \mathrm{sec}$ and $112.00 \pm 0.25 \%$ at $4 \mathrm{~min})$; iii) association of cavl with $\mathrm{HSP} 27(108.30 \pm 0.92 \%$ at $30 \mathrm{sec}$ and $115.54 \pm 5.03 \%$ at $4 \mathrm{~min}$ ) suggesting the importance of caveolin in docking of the PKC $\alpha$-HSP27-RhoA complex on the membrane. Summary: Colonic SMC express cavl and cav2, but not cav3. Acetylcholine stimulation of colonic SMC induced a significant and sustained increase in the association of cavl with PKC $\alpha$, RhoA and HSP27. Caveolaeenriched membrane fractions showed the presence of PKC $\alpha$ in the fractions enriched with caveolae and cavl suggesting that PKC $\alpha$ may translocate to caveolae on the membrane in the particulate fraction. Introduction of dominant negative cavl into colon SMC showed reduction in acetylcholine-induced association of cavl with PKC $\alpha$, RhoA and HSP27. Conclusions: During colonic smooth muscle contraction, caveolin is essential for maintenance of caveolae on the plasma membrane which in turn are crucial for the docking of translocated signaling complex PKC $\alpha$-HSP27-RhoA.

\section{S1631}

Orexin-a, An Appetite Hormone, Attenuates Gastric Ischemia-Reperfusion Injury Via of Prostaglandin/Cyclooxygenase Pathway, Vagal and Afferent Sensory Nerves

Tomasz Brzozowski, Peter C. Konturek, Robert Pajdo, Slawomir Kwiecien, Wladyslaw Bielanski, Atsukazu Kuwahara, Jin Kato, Stanislaw J. Konturek, Wieslaw W. Pawlik

Orexin-A (OX-A) detected in hypothalamic nuclei, enteric nerves and endocrine cells of GI tract, stimulates both, food intake and gastric seretory activity, but its role in the mechanism of gastroprotection has been little studied. We tested whether OX-A affects gastric ischemiareperfusion injury and whether prostaglandins $(\mathrm{PG})$ and neural factors could contribute to this protection. OX-A was applied intraperitoneally (i.p.) or intracerebroventricularly (i.c.v.) and gastric mucosal lesions were induced by $30 \mathrm{~min}$ of gastric ischemia (by clamping of celiac artery) followed by $3 \mathrm{~h}$ of reperfusion without or with A) inhibition of cyclooxygenase (COX)-1 activity by SC-560 (5 mg/kg i.g.) or COX-2 activity by rofecoxib (10 mg/kg i.g.) B) cutting of vagal nerves (bilateral vagotomy) and C) functional ablation of sensory nerves by capsaicin (125 mg/kg s.c.) or inhibition of vanilloid receptor (VR-1) by capsazepine (10 $\mathrm{mg} / \mathrm{kg}$ i.g.). The area of gastric lesions was measured by planimetry, the gastric blood flow (GBF) was determined by $\mathrm{H}_{2}$-gas clearance technique and gastric mucosal samples were collected for measurement of mucosal generation of PGE2. Venous blood was withdrawn for the measurement of plasma OX-A (RIA) and gastric biopsy samples were collected for the determination of COX-1-, COX-2- and CGRP mRNAs by RT-PCR. Exposure to I/R produced typical gastric bleeding erosions without influencing OX-A mRNA expression and its plasma level. OX-A (1-60 ug/kg i.p ) or (100-2500 ng/rat i.c. v ) dose-dependently attenuated gastric lesions induced by $\mathrm{I} / \mathrm{R}$. The protective effects of OX-A were accompanied by the significant rise in plasma OX-A level and an increase in the GBF and $\mathrm{PGE}_{2}$ generation The protective and hyperemic effects of OX-A were completely abolished by vagotomy and significantly attenuated by rofecoxib but not by SC-560 treatment. Capsaicin denervation or capsazepine treatment also significantly reduced the lesions caused by I/R and this effect was restored by addition to exogenous CGRP (10 $\mu \mathrm{g} / \mathrm{kg}$ s.c.) to OX-A in capsaicin-denervated rats. The CGRP mRNA was downregulated in gastric mucosa of $I / R$ animals and this effect was counteracted by administration of ghrelin. COX-1 mRNA was unchanged while COX2 mRNA was absent in vehicle-control gastric mucosa but it was upregulated in OX-A treated animals. We conclude that OX-A attenuates I/R injury by the mechanism involving endogenous PG, possibly derived from COX-2 pathway and activation of vagal and sensory nerves releasing vasodilatatory neuropeptides such as CGRP.

\section{S1632}

The Inhibitory Effect of Substance P On HCO3- Secretion from the Guinea Pig Microperfused Pancreatic Ducts

Peter Hegyi, Zoltan Rakonczay, Tamas Takacs, Gyula Papp, Janos Lonovics, Andras Toth, Andras Varro, Mike Gray, Barry Argen

Background. We have previously shown using sealed, non-perfused, ducts that Substance $\mathrm{P}(\mathrm{SP})$ inhibits $\mathrm{HCO} 3$ - secretion by inhibiting $\mathrm{Cl}$--dependant $\mathrm{HCO}$ - efflux on the luminal membrane of the duct cell (Hegyi et al. Am J Physiol Cell Physiol 285.C268-C276, 2003). However, in that study we had no direct evidence that SP affected a luminal anion exchanger. To address this issue we examined the effects of luminal H2DIDS on the inhibitory effect of SP in microperfused ducts. Methods. Small intra/interlobular pancreatic ducts were isolated from guinea pigs. We microperfused the ducts allowing direct access to the luminal membrane of the duct cell. The rate of $\mathrm{HCO} 3$ - secretion was determined by measuring the initial rate of intracellular acidification (using BCECF) following sudden block of basolateral NaHCO3 cotransporters and $\mathrm{Na}+\mathrm{H}+$ exchangers with H2DIDS $(500 \mu \mathrm{M})$ and amiloride $(200 \mu \mathrm{M})$ respectively. The buffering capacity of duct cells was estimated and the rate of transmembrane $\mathrm{H}+$ flux, $\mathrm{J}(\mathrm{H}+)$, was calculated. Since $\mathrm{H}+$ and $\mathrm{HCO} 3$ - will largely be derived from $\mathrm{H} 2 \mathrm{CO} 3$, we have assumed that $\mathrm{J}(\mathrm{H}+)$ is equivalent to $\mathrm{J}(\mathrm{HCO} 3)$. All the experiments were performed in $\mathrm{HCO} 3$--buffered Ringer at $37 \mathrm{oC}$. Results. Rates of HCO3- secretion measured using the inhibitor stop method were about 2 fold higher in microperfused ducts as compared to sealed, non-perfused, ducts. Application of luminal H2DIDS $(0.5 \mathrm{mM})$ caused intracellular $\mathrm{pH}$ to alkalinise and, like SP, inhibited basal and secretin-stimulated HCO3- secretion. SP did not inhibit secretion further when H2DIDS was present in the lumen, suggesting that SP and H2DIDS both inhibit the same transporter on the luminal membrane of the duct cell. Conclusions. SP inhibits an H2DIDS-sensitive HCO3- transport step at the luminal membrane of the duct cell, most likely a member of the SLC26 family anion exchanger. This work was supported by The Wellcome Trust and OTKA. 
S1633

A Novel Model to Study Gastric Pressure in Response to Gastric Content Infusion in Conscious Mice: Effect of Methylatropine, Win 55,212-2 and $\mathrm{N}^{\mathrm{G}_{-}}$ Nitro-L-Arginine Methyl Ester

Pieter Janssen, Maria Astin Nielsen, Per-Goran Gillberg, Leif Hultin

Introduction The stomach accommodates food without major intragastric pressure (IGP) increase. The study of transgenic mice could be of importance in understanding the mechanisms behind gastric accommodation and gastric muscle tone regulation in general. We present a novel model to study the relationship between gastric content and IGP in conscious mice. Methylatropine (unspecific muscarinic receptor antagonist), $N^{G}$-nitro-L-arginine methyl ester (L-NAME; nitric oxide synthase inhibitor) and WIN55,212-2 (unspecific cannabinoid receptor agonist), known to affect gastric muscle tone, were used to validate the model. Material \& Methods All experiments were performed in 6 female C57/Bl mice. After a 5-hour fast, a test-meal infusion system and a catheter to measure IGP were connected to a gastric fistula. The test-meal was non-nutritious and contained lgr barium sulphate/ $\mathrm{ml}$ in a 3\% hydroxypropyl methylcellulose solution. Drugs or their vehicles were injected subcutaneously before the start of the experiment (methylatropine and L-NAME were dissolved in saline, WIN55,212-2 in 5\% ethanol, 40\% solutol and 55\% saline). After a 15 min stabilisation period, test-meal was infused at a preset speed of $0.125 \mathrm{ml} / \mathrm{min}$ until $0.6 \mathrm{ml}$ infused. X-ray sequences were taken to estimate the gastric volume and emptying. Area under the curve (AUC) was assessed from the IGP vs, gastric volume relationship until $0.4 \mathrm{ml}$ gastric volume. Results (mean \pm SEM) were compared using a paired t-test $(\mathrm{P}<0.05$ considered significant). Results IGP increased with increasing gastric volume to a maximum of $12.0 \pm 2.4$ and $11.9 \pm 0.8 \mathrm{mmHg}$ after saline and WIN55,212-2 solvent treatment. Methylatropine (1 $\mathrm{mg} / \mathrm{kg})$ and WIN55,212-2 $(0.5 \mathrm{mg} / \mathrm{kg})$ significantly reduced the AUC, whereas L-NAME (30 mg/kg) significantly increased the AUC. At maximal injected volume, gastric emptying was $31.6 \pm 3.0 \%$ and $28.4 \pm 3.3 \%$ after saline and WIN55,212-2 solvent treatment vs. $37.8 \pm 8.2 \%$ (WIN55,212-2 treatment), $7.4 \pm 2.4 \%$ (methylatropine treatment) and $14.4 \pm 1.5 \%$ (L-NAME treatment), the latter 2 being significantly different from control situations. Discussion \& Conclusion For the same infused volume, methylatropine and WIN55,212-2 reduced IGP while L-NAME increased IGP. Furthermore, we showed that methylatropine and LNAME treatment reduced gastric emptying. This novel model can be used to study gastric emptying and the relationship between gastric volume and intragastric pressure in conscious mice.

\section{S1634}

Fasting Small Bowel Motility Reinstated By Ghrelin Infusion in Rats Ylva Haglund, Celestine O'Shaughnessy, Gareth J. Sanger, Per M. Hellstrom, Erik Naslund Background: Ghrelin has been shown to exert prokinetic properties in both man and rats by increasing gastric emptying and decreasing the interval between activity fronts during the migrating motor complex (MMC). The aim of this study was to see if ghrelin would facilitate the return of fasting motility after food intake and if the effect of ghrelin on MMC holds over an 6 hour infusion period. Methods: Male Sprague-Dawley rats ( $\mathrm{n}=7,300 \mathrm{~g}$ ) were used for the study. Three electrodes were implanted on the serosa of the small intestine 5 (D), 15 (J1), 25 (J2) cm from the pyloric sphincter and tunnelled onto the neck of the rat. A catheter placed in the forestomach was also tunnelled to the neck. An intravenous catheter was placed in the jugular vein for drug administration. After one week the studies commenced. After an overnight fast, fasting small bowel motility was recorded for at least $60 \mathrm{~min}$ and $4 \mathrm{MMC}$ activity fronts were recorded. A bolus of $2 \mathrm{~mL}$ of Isosource (1 kcal/ $\mathrm{mL}$ ) was administered intragastrically over $3 \mathrm{~min}$. Ten min later, either saline or ghrelin (1000 pmol/ $/ \mathrm{kg} / \mathrm{min}$ ) was infused IV and continued until a regular fasting motility pattern was resumed (one propagated activity front). In a second set of experiments, after a $60 \mathrm{~min}$ control period, ghrelin $(1000 \mathrm{pmol} / \mathrm{kg} / \mathrm{min})$ or saline was administered over 6 hours. Data shown as mean \pm SEM. Results: The interval between activity fronts propagated from $D$ to $\mathrm{J} 2$ during fasting was $15.6 \pm 0.8 \mathrm{~min}$ (measured at $\mathrm{J} 2$ ). After feeding and during infusion of saline, the first propagated activity front was seen $39.4 \pm 1.9$ min after administration of Isosource, compared to $33.4 \pm 1.9$ min at $J 2$ during ghrelin infusion $(p<0.05)$. With prolonged administration of ghrelin, the interval in between the activity fronts was significantly decreased compared to saline. However, this effect was transient and after the first hour there was no significant difference. Discussion: Ghrelin significantly hastens the return of fasting motility after food intake by $6 \mathrm{~min}$ in rats. The effect of ghrelin on the MMC seems to be transient and suggests a physiological role of ghrelin in which ghrelin acts transitionally to reinstitute the basal motility pattern, which is then maintained by other factors not yet revealed. However, this effect of ghrelin may promote a rapid gastric emptying, along with a rapid intestinal transit in order to prepare the gut for the next meal.

\section{S1635}

The Gastrointestinal Activity and Peripheral Selectivity of Alvimopan, Adl 080011, and Naloxone in Mice

Scott Armstrong, Christina C. Sandlund, Carrie Richardson, Tina Pham, Adrienne Winans, David T. Beattie

Alvimopan, a peripherally restricted $\mu$-opioid receptor antagonist, is in development for the treatment of opioid-induced bowel dysfunction and post-operative ileus. Based on the similar $\mu$-opioid antagonist potencies of alvimopan and its metabolite, ADL 08-0011, and the higher systemic exposure of the latter after oral dosing of the parent (Foss et al., 2005), it is likely that ADL 08-0011 is responsible for much of the efficacy attributed to alvimopan. In this study, the gastrointestinal (GI) and CNS activities of alvimopan, ADL 08-0011, and the centrally penetrant $\mu$-opioid receptor antagonist, naloxone, were determined in mice. In a Gl model of acute morphine exposure, morphine ( $3 \mathrm{mg} / \mathrm{kg} \mathrm{s.c.)}$ ) or vehicle was dosed 15 minutes after test agent or vehicle. A charcoal meal was dosed orally and GI transit measured 60 minutes later. To assess CNS activity, test compounds were dosed prior to morphine treatment and subsequent analgesia testing (paw lick latency on a hot-plate). In additional studies, mice were treated for 7 days with morphine ( $45 \mathrm{mg} / \mathrm{kg} /$ day s.c.) or vehicle via an osmotic minipump. Test agent or vehicle was then administered (s.c.) prior to assessment of GI and CNS-mediated responses (i.e. stool output, incidence of soft stools, number of jumps and ambulatory activity). Alvimopan, ADL 08-0011 and naloxone inhibited morphinemediated reductions in GI transit ( $\mathrm{ID}_{50}$ values of $0.03,0.02$ and $0.5 \mathrm{mg} / \mathrm{kg}$, respectively) and morphine-mediated increases in paw lick latency ( $\mathrm{ID}_{50}$ values of $6,0.7$, and $0.02 \mathrm{mg} /$ $\mathrm{kg}$, respectively). The peripheral selectivities (i.e. the ratios of $\mathrm{ID}_{50}$ values for reversal of analgesia and delayed GI transit) for alvimopan, ADL 08-0011, and naloxone were 200, 35 and 0.02, respectively, Following chronic morphine exposure, alvimopan, ADL 08-0011 and naloxone increased defecation and had a similar incidence of soft stools. Naloxone and ADL 08-0011 (both $10 \mathrm{mg} / \mathrm{kg}$ ), but not alvimopan $(0.01-10 \mathrm{mg} / \mathrm{kg}$ ) produced a statistically significant ( $\mathrm{p}<0.05$, ANOVA and Dunnett's post-hoc test) increase in jumping behavior and ambulatory activity. It is concluded that the rank order of peripheral selectivity in mice is alvimopan > ADL 08-0011 > naloxone. The clinical significance of these data, particularly the lower peripheral selectivity of ADL 08-0011 versus alvimopan, remains to be determined. Foss, J.F. (2005). Clin. Pharm. Therap., 77, 74.

\section{S1636}

The Activity of Alvimopan, Adl 08-0011 and Methylnaltrexone in the Guinea Pig Ileum

David T. Beattie, Madhavi Cheruvu, Shana Johnson

The peripherally-restricted $\mu$-opioid receptor antagonists, alvimopan and methylnaltrexone are in development for the treatment of opioid-induced bowel dysfunction and post-operative ileus (Camilleri, 2005). In this preclinical study, the activities of alvimopan, its active metabolite, ADL 08-0011, and methylnaltrexone were investigated. Ilea were isolated from guinea pigs treated for 7 days with morphine ( $45 \mathrm{mg} / \mathrm{kg} /$ day s.c.) or vehicle $(0.24 \mathrm{ml} /$ day s.c.) via an osmotic minipump, and mounted in tissue baths containing Krebs-Henseleit solution $\left(37^{\circ} \mathrm{C}\right)$. The effects of test agents on spontaneous mechanical activity, and electrical field stimulation (EFS)-evoked contractions were recorded isometrically. In morphine-naïve ilea, alvimopan and ADL 08-0011 increased, while methylnaltrexone (each $1 \mathrm{nM}-10 \mu \mathrm{M}$ ) decreased, the amplitude of EFS-evoked contractions (mean $\mathrm{pIC}_{50}$ or $\mathrm{pEC}_{50}$ values $=8.1$, 7.5 and 6.4 , respectively). The standard $\mu$-opioid receptor agonists, endomorphin-1, morphine and DAMGO, attenuated the EFS-mediated contractions. Alvimopan, ADL 08-0011 and methylnaltrexone antagonized endomorphin-1-mediated reductions in EFS-evoked contractions (mean $\mathrm{pA}_{2}$ values $=9.6,9.4$ and 7.7, respectively). In ilea isolated from morphinenaive animals, methylnaltrexone reduced the spontaneous activity, while alvimopan and ADL 08-0011 (each at $1 \mathrm{nM}-10 \mu \mathrm{M}$ ) produced small increases. In ilea isolated from guinea pigs treated chronically with morphine, methylnaltrexone, alvimopan and ADL 08-0011, each increased spontaneous contractile activity. The stimulatory activity of alvimopan and ADL 08-0011 in morphine-naive ilea is consistent with antagonism of endogenous inhibitory $\mu$-opioid tone although a non-specific effect cannot be ruled out at present. The enhanced excitatory activity in tissue from morphine-treated guinea pigs may reflect $\mu$-opioid receptor inverse agonist activity (Wang et al., 1994). The inhibitory activity of methylnaltrexone in morphine-naive ileum is consistent with $\mu$-opioid receptor partial agonist activity. Following chronic morphine exposure, methylnaltrexone exerts a stimulatory effect on ileal tone, although to a lesser extent than that of alvimopan and ADL 08-0011. The clinical relevance of the different intrinsic activity profiles of alvimopan, ADL 08-0011 and methylnaltrexone, remains to be determined. Camilleri, M. (2005). Neurogastroenterol. Motil., 17, 157-165. Wang, D. et al. (1994). Life Sci., 54, 339-350.

\section{S1638}

The Effects of a Ghrelin Receptor Agonist On Motility Reflexes and Visceral Pain in Vivo

Yasutake Shimizu, Ed C. Chang, Anthony D. Shafton, Gareth J. Sanger, Jason Witherington, John B. Furness

Purpose: Ghrelin is an endogenous peptide messenger that occurs in greatest amount in the stomach, where it is located to mucosal endocrine cells. It accelerates gastric emptying, but has little effect on the upper small intestine. Ghrelin receptor mRNA is expressed in enteric neurons of human and rat colon, as well as endocrine-like cells in the colonic mucosa (Dass et al., 2003). Initial studies showed a lack of effect on colonic motility (Trudel et al., 2002). We have re-assessed the effects of ghrelin receptor agonism using CP-464709, a non-peptide ghrelin receptor agonist. Methods: Sprague-Dawley rats, 300-400 g, were anaesthetised with $\alpha$-chloralose and ketamine and colonic intraluminal pressure was recorded. In other experiments, visceromotor responses to colorectal distension (VMR) were measured by recording the electromyograph from the upper abdominal muscle. Fecal output was measured from conscious rats housed in a quiet environment. Results: CP-464709 (5 - $20 \mathrm{mg} / \mathrm{kg} / \mathrm{hr}$, intravenous) caused a 3 -fold increase in amplitudes of propulsive contractions that was associated with increased propulsion of colonic contents. The contractions induced by the agonist were large and regular, but it had no direct effect on the muscle. The effect of the ghrelin agonist was sustained for as long as the infusion was maintained, ( $>30 \mathrm{~min})$, indicating that there is not significant desensitisation of the receptor. The effect was slow to wash out. Subcutaneous injection ( $5 \mathrm{mg} / \mathrm{kg}$ bolus) to unanaesthetised rats caused increased fecal output for a period of four hours. In the first hour, fecal output was 5 times greater than control, and the cumulative $4 \mathrm{hr}$ output was 2.2 times control. In contrast to effects on the colon, CP-464709 had little effect on jejunal motility. CP-464709 (20 mg/kg/hr) caused some arousal from anaesthesia, and this resulted in a decrease in the threshold intra-colonic pressure to elicit the VMR. If the arousal was suppressed by further anaesthesia, or lower doses of the drug $(5-10 \mathrm{mg} / \mathrm{kg} / \mathrm{hr}$ ) were used, the VMR threshold was unaltered by CP464709. Conclusion: A ghrelin receptor agonist, in conscious or anaesthetized rats, is a powerful and selective colokinetic with no apparent adverse side effects. Dass NB., et al. Neuroscience, 120, 443-453 (2003). Trudel L., et al., Am J Physiol., 282, G948-G952. (2002). 


\section{S1639}

Tissue Distribution of the Motilin and Ghrelin Receptor Along the Human Gastrointestinal Tract

E.J. Takeshita, Bunzo Matsuura, Maoqing Dong, Laurence J. Miller, Hidetaka Matsui, Yoichi Hiasa, Morikazu Onj

Motilin has been recognized as an important endogenous peptide regulator of gastrointestinal (GI) motor function in mammals, acting at the motilin receptor. Ghrelin has been demonstrated to regulate secretion of growth hormone, and to stimulate gastric motility in rodents, acting via a structurally-related receptor. While there have been extensive physiological and pharmacological studies of motilin and ghrelin action, the differences in GI motor function of these peptides and the differences in tissue distribution of these receptors in human are not yet clear. The aims of this study were to explore the distribution of motilin and ghrelin receptors along the human GI tract, and to establish the molecular nature of the human motilin receptor. Post-mortem and surgical human tissue specimens with no hemorrhage, necrosis, or tumor were obtained from lower esophagus, stomach, duodenum, jejunum, ileum, colon, and rectum. Tissues were divided as total layer and as separated layers of mucosa and muscle. Using the method of real-time polymerase chain reaction after reverse transcription, we analyzed levels of expression of mRNA for motilin and ghrelin receptors and examined their molecular identities. Specimens from stomach, small intestine, and colon were studied for expression of motilin and ghrelin receptor by immunohistochemistry. The long form type of motilin receptor (GPR38A), but not the short form (GPR38B), was expressed in all parts of the GI tract we studied. Motilin receptor mRNA was more heavily expressed in muscle layer than in mucosal layer, and motilin receptor immunoreactivity was present on muscle cells and myenteric plexus. In contrast, ghrelin receptor mRNA was expressed equally in all parts of the GI tract and in both the mucosal and muscle layer. Motilin receptor expression of the specimens from diabetic patients was same as that from non-diabetic patients. These data suggest that motilin has a specific role in regulating human GI motility, and motilin agonists such as peptide motilide and non-peptidyl erythromycin may be useful prokinetic drugs for dysmotility not only of the upper GI tract but also of the lower GI tract.

\section{S1640}

5ht ${ }_{2 b}$ Receptors Are Expressed On Neurons and Non-Neuronal Cells in the Human Gastrointestinal Tract, Rat Colon and Rat DRG

Elizabeth C. Colley, Catherine J. Streutker, Kirk Hillsley, S M. Kelly, Gareth Hicks, Ronald H. Stead

Background: The $5 \mathrm{HT}_{2 \mathrm{~B}}$ receptor $\left(5 \mathrm{HT}_{2 \mathrm{~B}}\right)$ appears to function in the maturation of neurons in the mouse GI tract, although $5 \mathrm{HT}_{2 \mathrm{~B}}$ does not seem to be functional in adult murine intestinal neurons. In contrast there is evidence for functional $5 \mathrm{HT}_{2 \mathrm{~B}}$ receptors in the adult human gut, suggesting that $5 \mathrm{HT}_{2 \mathrm{~B}}$ receptors participate in enteric neuronal relays. However, the extent and cellular distribution of $5 \mathrm{HT}_{2 \mathrm{~B}}$ receptors in the human GI tract is not fully defined. Methods: Formalin-fixed paraffin embedded (FFPE) samples of rat colon and DRG, and human esophagus, stomach, small and large bowel, and rectum, obtained from the non-involved regions of resection specimens, with consent, were stained using immunocytochemistry [ICC] and in situ hybridization [ISH]). Specific antibodies employed were raised against: human/rat $5 \mathrm{HT}_{2 \mathrm{~B}}$ (LifeSpan, WA), chromogranin $\mathrm{A}$ (Dako, CA) and serotonin (Immunostar, WI) For ISH, 5HT PC3 and KU812 cells were employed as positive and negative controls, respectively. Rat DRG were back-labelled by IP injections of Alexa Flour 488 conjugated cholera toxin subunit $\mathrm{B}$ (CTB488; Invitrogen, ON) and subsequent localization with anti-CTB. Results: $5 \mathrm{HT}_{2 \mathrm{~B}}$ was localized by both ICC and ISH in some epithelial cells, EC cells, muscle cells (muscularis mucosa, muscularis propria and vascular), most neurons (both submucous and myenteric) and infiltrating inflammatory cells (resembling eosinophils) throughout the gut as shown in the table. Small diameter DRG neurons were $5 \mathrm{HT}_{2 \mathrm{~B}}-\mathrm{IR}$ and some co-localized with CTB. Serotonin and chromogranin confirmed the EE/EC cell staining for 5-HT 2 . PC3 cells were positive and KU812 were negative using both $\mathrm{IHC}$ and $\mathrm{ISH}$. Conclusions: $5-\mathrm{HT}_{2 \mathrm{~B}}-\mathrm{IR}$ was localized to EE/EC cells and intrinsic neurons; and was also expressed on primary afferent neurons in DRG. This suggests that $5 \mathrm{HT}_{2 \mathrm{~B}}$ ligands might modulate endocrine cell, muscle and both intrinsic and extrinsic afferent neuronal activity in the gastrointestinal tract.

\begin{tabular}{|c|c|c|c|c|}
\hline Site (n) & Epithelium & EE cells & Neurons & Muscle \\
\hline Oesophagus (3) & + & n.a. & + & + \\
\hline Stomach (9) & + & ++ & + & + \\
\hline Small Bowel (12) & + +- & +++ & + & ++ \\
\hline Large Bowel (18) & $+/-$ & +++ & + & ++ \\
\hline Rectum (2) & $+/-$ & +++ & + & ++ \\
\hline
\end{tabular}

\section{S1641}

Ca2+ Influx Induced By Galr2 Stimulation in Smooth Muscle Cells of Rat Small Intestine Is Mediated By the Activation of a Receptor Operated Channel Laura Anselmi, Salvatore L. Stella, Ingrid G. Jaramillo, Catia Sternini, Nicholas Brecha

Background and Aims: Galanin modulates a variety of physiological functions in the gastrointestinal (GI) tract, including regulation of transmitter release and modulation of GI motility and secretion. Galanin has both stimulatory and inhibitory effects on GI motility. Galanin effects are mediated by the activation of three distinct receptors, galanin receptor 1 (GalRl), GalR2 and GalR3. GalR1 and GalR3 have been shown to inhibit adenylyl cyclase activity via Gi/Go proteins, whereas GalR2 couples predominantly to phospholipase $C$ through a Gq protein, implicating an important role for $\mathrm{Ca} 2+$. We have shown that GalR1 and GalR2 are the most abundant GalR subtypes in the GI tract, and we have found that GalR2 is highly expressed by smooth muscle, suggesting that GalR2 mediates the direct effect of galanin on smooth muscle. The aim of this study was to investigate the effect of GalR2 activation on Ca2+ influx in cultured smooth muscle cells using galanin 2-11, an agonist with high selectivity for the GalR2. Methods: Smooth muscle cells were cultured from small intestine using rats from 6 to 10 days old. [Ca2+]i changes were monitored using the $\mathrm{Ca} 2+$ sensitive dye, fluo-3 with confocal microscopy. Results: Galanin 2-11 resulted in a dosedependent (0.01-1.0 $\mu \mathrm{M})$ increase in [Ca2+]i. Phospholipase C (PLC) antagonist, U-73122 $(2 \mu \mathrm{M})$ inhibited the galanin 2-11 mediated $[\mathrm{Ca}+] \mathrm{i}$ increase indicating that $\mathrm{Ca} 2+$ release induced by GalR2 activation is mediated by a PLC pathway. Removal of extracellular Ca2+ abolished the galanin 2-11 mediated $[\mathrm{Ca} 2+]$ i increase, whereas it did not change $\mathrm{Ca} 2+$ release induced by ATP $(10 \mu \mathrm{M})$, suggesting that $\mathrm{Ca} 2+$ influx in response to galanin $2-11$ was not derived from intracellular stores. Since inhibition of the PLC-mediated pathway can suppress the galanin 2-11 evoked [Ca2+]i increase without involving intracellular stores it is likely that the effects of galanin 2-11 are mediated by a receptor operated channe (ROC) at the plasma membrane. To test this hypothesis, SKF-96365, an inhibitor of Ca2 influx mediated by ROC, was added to galanin 2-11. SKF-96365 $(50 \mu \mathrm{M})$ prevented the galanin 2-11 induced [Ca2+]i increase supporting our hypothesis. Conclusions: These findings support the hypothesis that GalR2 mediates the postjunctionally excitatory effect of galanin on smooth muscle and suggest that PLC mediated Ca2+ release induced by activation of GalR2 is due to the opening of a ROC. Supported by NIH grants DK57037 and 41301.

\section{S1642}

Protease-Activated Receptor-1 (PAR1) and PAR2 Mediate Relaxations in Lower Esophageal Sphincter

Shih-Che Huang

Protease-activated receptor-1 (PAR1), PAR2 and PAR4 activation can alter the gastrointestinal motility. To investigate effects mediated by PARs in the lower esophageal sphincter, we measured contraction and relaxation of transverse strips from the guinea-pig lower esophagea sphincter caused by three PAR-1 agonists, thrombin, TFLLR-NH2 and SFLLRN-NH2, three PAR-2 agonists, trypsin, SLIGKV-NH2 and SLIGRL-NH2, as well as two PAR4 agonists, GYPGKF-NH2 and AYPGKF-NH2, according to the procedure published previously (Regulatory Peptides 2005;127:27-35). In resting lower esophageal sphincter strips, thrombin did not cause any relaxation or contraction whereas TFLLR-NH2 and SFLLRN-NH2 caused moderate concentration-dependent relaxation. In carbachol-contracted strips, thrombin caused mild whereas TFLLR-NH2 and SFLLRN-NH2 caused marked concentration-dependent relaxation. These indicate the existence of the PARI mediating relaxation. The TFLLR$\mathrm{NH} 2$-induced relaxation was not affected by tetrodotoxin or atropine, suggesting a direct effect on the lower esophageal sphincter circular muscle. On the other hand, in resting lower esophageal sphincter strips, trypsin caused moderate concentration-dependent relaxation whereas SLIGRL-NH2 and SLIGKV-NH2 did not cause any relaxation or contraction. In carbachol-contracted strips, trypsin caused marked whereas SLIGRL-NH2 and SLIGKV-NH2 caused mild concentration-dependent relaxation. These indicate the existence of the PAR2 mediating relaxation. The trypsin-induced relaxation was not affected by tetrodotoxin or atropine. These again suggest a direct effect on the lower esophageal sphincter circular muscle. In contrast, GYPGKF-NH2 and AYPGKF-NH2, the PAR4 agonists, FSLLR-NH2, a PARl inactive control peptide, as well as VKGILS-NH2, a PAR2 inactive control peptide, did not cause any relaxation or contraction in resting or carbachol-contracted lower esophageal sphincter strips. Taken together, these results demonstrate that PAR1 and PAR2 mediate relaxations in the guinea-pig lower esophageal sphincter.

\section{S1643}

Delaying Gastric Emptying and Enhancing Cholecystokinin Release and Satiety By Using Acid Stable Fat Emulsions

Luca Marciani, Martin Wickham, Gulzar Singh, Debbie Bush, Barbara Pick, Eleanor Cox, Annette Fillery-Travis, Richard Faulks, Charles Marsden, Penny A. Gowland, Robin C. Spiller

Background: Consumer preferences are leading to a steady increase in consumption of convenience foods which often contain highly palatable fat as an emulsion. We have previously shown that oil/water emulsions can rapidly separate in the acid gastric environment leading to layering of fat. This caused rapid emptying of the resulting low fat aqueous phase with rapid reduction of gastric distension and satiation. We hypothesised that stabilising an emulsion would prevent intragastric fat layering and, by ensuring prolonged exposure of duodenal receptors to fat emulsion, maximise cholecystokinin (CCK) release, delay gastric emptying and prolong satiety. Aims: To investigate in healthy volunteers the effect of modifying the intragastric acid stability of equicaloric fat emulsions on gastric emptying CCK levels and satiety. Methods: 9 healthy male volunteers attended on 2 separate days, having fasted overnight. They were fed $500 \mathrm{~mL}$ of one of two olive oil-in-water emulsion test meals with equal fat content $(675 \mathrm{kcal})$ and equal droplet size distributions, but opposite acid stability. One remained intact when exposed to the stomach acidic conditions ("acidstable") and the other ("acid-unstable") broke rapidly on being acidified yielding a layer o oil floating above an aqueous phase. Serial measurements of gastric volumes (using magnetic resonance imaging, MRI), plasma CCK (using radioimmunoassays) and satiety feelings (using self-assessment questionnaires) were made for 10 hours. Results: (mean \pm SEM) MRI images showed a layering of oil which became apparent soon after ingestion of the unstable emulsion but was not seen with the acid stable emulsion. This was associated with a faster gastric half-emptying time for the acid-unstable $(72 \pm 13 \mathrm{~min})$ compared with the acid-stable emulsion meal $(171 \pm 35 \mathrm{~min}$ ), Wilcoxon's $\mathrm{p}<0.008$. The acid- unstable emulsion released less CCK than the acid-stable one (AUC $531 \pm 111$ versus $1095 \pm 244 \mathrm{pmolmin} / \mathrm{L}$ ), $\mathrm{p}<0.001$. An overal exponential correlation between decreasing hunger with increasing CCK plasma level was found ( $\mathrm{r}=0.91)$, with the acid-stable emulsion being associated with greater satiety and higher CCK levels. Conclusion: Rendering fat emulsions stable to gastric acid is a simple way of enhancing satiety by a combination of delaying gastric emptying and enhancing blood levels of CCK. Modifying food production methods to manipulate the post-prandia delivery of fats to the intestine, the gut peptides response and hence satiety, could aid in the prevention as well as treatment of obesity. Funded by BBSRC. 
S1644

Effects of Cholecystokinin-8s (CCK-8s) On Identified Pancreas-Projecting Vagal Motoneurons

Shuxia Wan, R. Alberto Travagli

It has long been known that cholecystokinin acts in a paracrine fashion to activate peripheral vagal afferent fibers that increase pancreatic exocrine secretion via a vago-vagal brainstem circuit. Recent evidence, though, suggests that the locus of the CCK-8s action on the gastrointestinal (GI) system is not restricted to peripheral afferent vagal fibers, but also affects brainstem structures directly. Within the brainstem, neurons of the dorsal motor nucleus of the vagus (DMV) provide the preganglionic parasympathetic efferent fibers that innervate the GI tract, including the pancreas. The aims of the present study were to investigate whether DMV neurons were responsive to exogenously applied CCK-8s and, if so, the mechanism of action. Whole cell patch clamp recordings were made from pancreas-projecting DMV neurons identified by prior application of the retrograde tracer, DiI on the pancreas body. Perfusion with CCK-8s induced a concentration-dependent excitation in approximately $50 \%$ of pancreas-projecting DMV neurons $(\mathrm{N}=90)$. The threshold of the excitatory response was $\operatorname{lnM}$, the EC50 was $30 \mathrm{nM}$ and Emax was $100 \mathrm{nM}$. At $100 \mathrm{nM}$, CCK-8s induced a $12 \pm 1.4 \mathrm{mV}$ depolarization $(\mathrm{HP}=-70 \mathrm{mV})$ and increased the firing rate from $3 \pm 2$ to $121 \pm 20$ a.p. $/ \mathrm{min}(\mathrm{HP}=-60 \mathrm{mV})$. The CCK- $8 \mathrm{~s}$ induced depolarization was reduced from $9 \pm 1$ to $6.8 \pm 1.8 \mathrm{mV}$ by TTX $(l \mu \mathrm{M})$ suggesting that the effects were mediated both directly, via an effect on the DMV membrane, and indirectly, via an effect on local synaptic circuits. Furthermore, the CCK-8s mediated excitation was prevented by the CCK-A antagonist, lorglumide $(1 \mu \mathrm{M})$, while the CCK-B agonist, CCK-ns (100nM) had no effect, indicating the involvement of CCK-A receptors only. Experiments conducted in the voltage clamp configuration indicated that CCK-8s induced a $70 \pm 7 \mathrm{pA}$ inward current $(\mathrm{VH}=-50 \mathrm{mV})$ that reversed at $-106 \pm 3 \mathrm{mV}$ and was accompanied by a $50 \pm 15 \%$ increase in input resistance. These data suggest that the effects of CCK-8s were mediated by a closure of a potassium conductance. Indeed, CCK$8 \mathrm{~s}$ reduced both the amplitude and the time constant of decay of the calcium-dependent potassium conductance, SK, by $30 \pm 3.4 \%$. When tested with pancreatic polypeptide (PP, which reduces pancreatic exocrine secretion), cells that responded to CCK-8s with an excitation were, instead, inhibited by PP. These data indicate that CCK-8s may control pancreas-exocrine secretion via an effect on pancreas-projecting DMV neurons. Supported by NSF grant 0456291

\section{S1645}

Ghrelin Reduces Mechanical Sensitivity of Gastroesophageal Vagal Afferents in Ferrets and Mice

Amanda J. Page, James Slattery, Tracey A. O'Donnell, Stuart Brierley, Catherine Milte, Rhianna Laker, L A. Blackshaw

Background: Ghrelin is a peptide, produced by endocrine cells in the stomach, which is an important regulator of food intake. Ghrelin is thought to act by inhibiting basal activity of vagal afferents (Gastroenterology 2002; 123:1120-8), although direct evidence is lacking on mechanism and which vagal afferents are responsible. Aim: Determine the effect of ghrelin on the mechanically induced activity of gastroesophageal vagal afferent subtypes in the ferret and mouse, and expression of ghrelin receptors. Methods: Single fiber recordings of gastroesophageal vagal mechanoreceptors were made in ferret and mouse using standard electrophysiological techniques (Page et al J. Neurosci 1999; 19: 8597-8602). Ghrelin was applied to the peripheral afferent endings of ferret and mouse gastroesophageal vagal afferents and their responses to graded mechanical stimuli investigated. These afferents have been previously characterized as tension, mucosal and tension/mucosal (TM) receptors according to their responsiveness to circumferential tension or mucosal stroking or both (J.Physiol 1998; 512: 907-16). Results: Ghrelin (1, 3 \&10nM) reduced the response of 2 out of 6 ferret mucosal receptors. This inhibition was reversed on wash out. The ferret TM $(n=10)$ responses to mucosal stroking were also significantly reduced in the presence of ghrelin ( 1 , 3 and $10 \mathrm{nM} ; \mathrm{p}<0.05)$. The response of ferret tension receptors $(\mathrm{n}=4)$ and TM receptors $(\mathrm{n}=$ 10) to circular tension were unaffected by ghrelin. In the mouse, ghrelin $(3 \mathrm{nM} ; \mathrm{p}<0.05)$ significantly and dose-dependently reduced the response of tension receptors $(n=10)$ to circular tension. However, at the higher dose of $10 \mathrm{nM}$ this reduction in response was reversed, possibly indicating action at other receptors. The GHS receptor was detected in the nodose ganglion by RT-PCR. Conclusion: These data indicate that ghrelin selectively inhibits mechanosensitivity in separate populations of afferents in two species. Its potency suggests that ghrelin acts as an endogenous modulator of peripheral mechanosensory function. Supported by National Health and Medical Research Council of Australia \& AstraZeneca.

\section{S1646}

Effects of Cholecystokinin-8s (CCK-8s) Microinjections in the Dorsal Vagal Complex of Deafferented Rats On Pancreatic Exocrine Secretion Zhongling Zheng, R. Alberto Travagli

Cholecystokinin acts in a paracrine fashion to activate peripheral vagal afferent fibers and increase pancreatic exocrine secretion via a vago-vagal reflex. Recent evidence, however, suggests the CCK-8s may also affect brainstem structures directly. The aim of the present study was to investigate whether microinjection of CCK-8s in the dorsal vagal complex (DVC) modulated pancreatic exocrine secretion even in rats that had undergone vagal deafferentation. Sprague-Dawley rats (250-400g) were anesthetized before the common bilepancreatic duct was cannulated at the sphincter of Oddi and pancreatic secretion collected every $10 \mathrm{~min}$. The DVC was exposed via a dorsal approach and microinjections were made according to the co-ordinates of Paxinos $\&$ Watson. Rats were divided into 5 groups: 1)Microinjection of PBS (60nl) 2)Microinjection of CCK-8s (450pmoles in 60nl PBS) 3)Microinjection of CCK-8s one week after unilateral surgical vagal deafferentation 4)Microinjection of CCK-8s one week after unilateral surgical vagal deafferentation and contralateral subdiaphragmatic vagotomy 5)Acute cervical vagotomy (without microinjection) The volume of pancreatic juice secreted was never observed to alter in any of the experimental parameters (approximate secretion was 280 $\mathrm{\mu l} / 10$ minutes). In control rats (PBS injection; $\mathrm{N}=3$ ) or rats that were underwent acute vagotomy $(\mathrm{N}=3)$, the total protein secreted remained stable at $270 \pm 39 \mu \mathrm{g}$ every $10 \mathrm{~min}$ for $120 \mathrm{~min}$ without significant variations. Following CCK-8s microinjection in the DVC, protein secretion increased from $276 \pm 41$ to $715 \pm 173 \mu \mathrm{g} / 10 \mathrm{~min}$ (i.e. a $220 \pm 70 \%$ increase; $N=5 ; P<0.05$ ), the increase was larger when CCK-8s was injected in the left side of the DVC (from $315 \pm 60$ to $1130 \pm 35 \mu \mathrm{g} / 10 \mathrm{~min}$ ) vs the right side (from $250 \pm 60$ to $440 \pm 62 \mu \mathrm{g} / 10 \mathrm{~min}$ ). Protein secretion returned to baseline levels within $30 \mathrm{~min}$. In deafferented rats, microinjection of CCK- 8 s increased protein secretion from $215 \pm 34$ to $507 \pm 36 \mu \mathrm{g} / 10 \mathrm{~min}$ (i.e. a $185 \pm 14$ increase; $\mathrm{N}=10 ; \mathrm{P}<0.05$ ) while in rats that underwent deafferentation plus subdiaphragmatic vagotomy, microinjection of CCK-8s increased protein secretion from $200 \pm 33$ to $318 \pm 67 \mu \mathrm{g} / 10 \mathrm{~min}$ (i.e. a $154 \pm 18 \%$ increase; $\mathrm{N}=5 ; \mathrm{P}<0.05$ ). These data indicate that, as well as activating peripheral vagal afferents, CCK-8s increases pancreatic exocrine secretion via an action in the DVC. Furthermore, the effects of CCK-8s are still present, although reduced, following sensory vagal deafferentation. In physiological conditions, CCK-8s acting in the DVC could originate either be from the circulation (postprandial plasma concentration is approximately $10 \mathrm{pM}$ ) or from local neuronal projections. Supported by NSF grant 0456291

\section{S1647}

Hydrogen Sulfide Is Synthesized in Enteric Neurons and Acts As a ProSecretory Mediator in the Guinea-Pig and Human Gut Rudolf Schicho, Dagmar Kruger, Florian Zeller, Thomas Frieling, Isao Ishii, Hideo Kimura, Michael Schemann

Hydrogen sulfide $\left(\mathrm{H}_{2} \mathrm{~S}\right)$ has been recently identified as a gasotransmitter in the brain and modulates various organ functions. Its role and action in the gastrointestinal tract, however, especially in human tissue, is less elucidated. Immunohistochemistry was used to explore expression of the two $\mathrm{H}_{2} \mathrm{~S}$ producing enzymes cystathionine- $\gamma$-lyase (CSE) and cystathionine$\beta$-synthase (CBS) in the human and guinea-pig enteric nervous system (ENS). Ussing chamber technique was used to study effects of $\mathrm{H}_{2} \mathrm{~S}$ on ion secretion ( $\mathrm{I}_{\mathrm{SC}}$ ) in human and guinea-pig mucosa/submucosa preparations. Neuroimaging with potentiometric dyes was performed to study $\mathrm{H}_{2} \mathrm{~S}$ effects on neural activity of guinea-pig submucous ganglia. Double labeling with the pan-neuronal marker anti-Hu revealed CSE-IR in almost all nerve cell bodies of submucosal ganglia of guinea-pig ileum $(99.4 \pm 0.4 \%)$ and colon $(99.7 \pm 0.1 \%)$ and human colon $(96 \pm 0.7 \%$ ) (3 patients/animals, $20-25$ ganglia each). CSE-IR was also observed in almost all myenteric neurons of guinea pig stomach, ileum and colon and in myenteric interstitial cells of Cajal as indicated by co-localisation with c-kit antibody. All CSE-IR myenteric and submucous neurons were also CBS-IR and vice versa. Ussing chamber experiments revealed that serosal application of the $\mathrm{H}_{2} \mathrm{~S}$ donor $\mathrm{NaHS}(0.1-1 \mathrm{mM})$ significantly increased $I_{S C}$ in guinea-pig ileum and colon $\left(9 \pm 2 \mu \mathrm{A} / \mathrm{cm}^{2}\right.$ for $0.1 \mathrm{mM}, 20 \pm 4 \mu \mathrm{A} / \mathrm{cm}^{2}$ for $1 \mathrm{mM})$. Likewise, NaHS $(0.2-2.5 \mathrm{mM})$ evoked an increase in $\mathrm{I}_{\mathrm{SC}}$ in human ileum and colon preparations $\left(5 \pm 2 \mu \mathrm{A} / \mathrm{cm}^{2}\right.$ for $0.5 \mathrm{mM}, 13 \pm 6 \mu \mathrm{A} / \mathrm{cm}^{2}$ for $\left.2.5 \mathrm{mM}\right)$. The EC50 was $0.21 \mathrm{mM}$ for guinea-pig and $0.51 \mathrm{mM}$ for human tissue which corresponds to reported physiological $\mathrm{H}_{2} \mathrm{~S}$ concentrations in the blood. In all experiments NaHS evoked a transient desensitizing response that lasted from 1-8min. The increased $\mathrm{I}_{\mathrm{SC}}$ was bumetanide-sensitive and therefore due to increased chloride secretion. In human and guinea-pig, ruthenium red $(30 \mu \mathrm{M})$ significantly potentiated the NaHS evoked secretion which was now sustained lacking desensitization. Neuroimaging of guinea-pig submucous neurons revealed that perfusion of $500 \mu \mathrm{M}$ NaHS evoked a profound activation consisting of action potential discharge in 16 out of 46 neurons. This effect also desensitized within $5 \mathrm{~min}$. Our results demonstrate for the first time that both $\mathrm{H}_{2} \mathrm{~S}$ producing enzymes are expressed in human and guinea-pig enteric neurons. This together with the excitatory action on submucous neurons suggests that $\mathrm{H}_{2} \mathrm{~S}$ is a novel signaling molecule in the ENS. We describe a novel function of $\mathrm{H}_{2} \mathrm{~S}$ as a prosecretory mediator in the guinea-pig and human intestine.

\section{S1648}

Role of Endocannabinoids in Gastroprotection Against Acute Gastric Lesions Michal Pawlik, Tomasz Brzozowski, Peter C. Konturek, Anetta Targosz, Danuta Drozdowicz, Greg Burnat, Stanislaw J. Konturek, Wieslaw W. Pawlik, Eckhart G. Hahn

Anandamide, an endocannabinoid acting via $\mathrm{CBl}$ and vanilloid (VR-1) receptors affects gastric motility and gastric emptying and exerts anti-inflammatory action in the gut but the possibility that endocannabinoids applied peripherally or centrally can contribute to the mechanism of gastric mucosal defense against acute gastric lesions, has not been studied. We compared the effect of intraperitoneal (i.p.) and intracerebroventricular (i.c.v.) administration of anandamide and an agonist of CBI and VR-1 receptors, arvanil, on gastric acid secretion in rats equipped with gastric fistula (GF) (series A) and on acute gastric lesions induced by water immersion and restraint stress (WRS) (series B) in rats with or without AM-25l (1 mg/kg i.p.), the selective antagonist of CBl receptors, capsazepine $(10 \mathrm{mg} / \mathrm{kg}$ i.g.), an antagonist of VR-1 receptors and in those with capsaicin-denervation (125 mg/kg s.c.). At $3.5 \mathrm{~h}$ after the end of WRS, the number and area of gastric lesions was determined by planimetry, the gastric blood flow $(\mathrm{GBF})$ was examined by $\mathrm{H}_{2}$-gas clearance method and the plasma gastrin levels and $\mathrm{PGE}_{2}$ generation were assessed by specific RIAs. The expression of mRNA for cyclooxygenases (COX-1 and COX-2) were evaluated in mucosal biopsy samples of stressed and non-stressed rats by RT-PCR. Exposure to WRS produced typical gastric bleeding lesions while significantly increasing gastric acid output in GF rats and decreasing the GBF (by 35\%) as compared to baseline and these effects were further enhanced in animals with capsaicin-denervation. Anandamide $(2.5-50 \mu \mathrm{g} / \mathrm{kg}$ or $50-500 \mathrm{ng} / \mathrm{rat}$ i.c.v), decreased significantly gastric acid secretion; the dose inhibiting the gastric acid output by $50 \%\left(\mathrm{ID}_{50}\right)$ being $10 \mu \mathrm{g} / \mathrm{kg}$ and $200 \mathrm{ng} / \mathrm{kg}$, respectively. Pretreatment with anandamide (20 $\mu \mathrm{g} / \mathrm{kg}$ i.g. or $250 \mathrm{ng} / \mathrm{kg}$ i.c.v.) or arvanil (20 $\mu \mathrm{g} / \mathrm{kg}$ i.p.), significantly reduced WRS-induced gastric lesions while elevating significantly the GBF and plasma immunoreactive gastrin levels and enhancing COX-2 expression and $\mathrm{PGE}_{2}$ generation in the gastric mucosa. These protective and hyperemic effects of anandamide and arvanil were significantly attenuated by pretreatment with AM 251 or capsazepine and completely abolished by capsaicin-denervation and further restored by the addition of exogenous CGRP $(10 \mu \mathrm{g} / \mathrm{kg} \mathrm{s.c.})$ to cannabinoids in capsaicin-deactivated animals. We conclude that central and peripheral cannabinoids exhibit gastroprotective activity against stress damage due to activation of $\mathrm{CB} 1$ and VR-1 
receptors, antisecretory activity and an increase in the gastric microcirculation mediated by neuropeptides released from sensory nerves such as CGRP and activation of PG/COX2 pathway.

\section{S1649}

The Role of Calcitonin Receptor-Like Receptor in Colonic Inflammation and Motility

Matthew S. Clifton, Julia J. Hoy, Prema S. Idumalla, Stephen Dada, Carlos U. Corvera Nigel W. Bunnett, Aditi Bhargava

Calcitonin gene-related peptide (CGRP) mediates neurogenic inflammation and pain and controls intestinal motility. The CGRP receptor is a hetero-dimer of calcitonin receptor-like receptor (CLR) and receptor associated modifying protein 1 (RAMPl), which are expressed in enteric nerves and primary spinal afferent neurons. However, the specific role of CLR in colonic inflammation and motility is unknown. We used RNA interference to silence the CLR gene and to determine its role in control of colonic inflammation and motility. To silence CLR, we injected double stranded RNA to rat CLR or to globin (control) into 2 sites in the colonic wall of anesthetized rats ( $n=4-6 /$ group). To examine the spatial and temporal down-regulation of CLR, we collected tissues at 1-9 days after treatment and localized CLR by immunofluorescence. In control animals, CLR was detected in nerve fibers within the myenteric and submucosal plexuses and in the mucosa. CLR RNAi down regulated immunoreactive CLR in enteric neurons within $2-3 \mathrm{~cm}$ of the site of injection within 1 day, and down regulation persisted for 7-9 days. To examine the effects of this down regulation on colitis, we administered an enema of trinitrobenzene sulfonic acid (TNBS) in 50\% ethanol or ethanol alone (control) into the colon, and examined inflammation after 72 hours. In animals receiving control RNAi, TNBS induced edema, mucosal destruction, and increased submucosal infiltration of polymorphonuclear cells, as determined by staining with hematoxylin and eosin. The histological appearance and myeloperoxidase (MPO) levels were similar in animals treated with control RNAi/TNBS enema and saline/TNBS enema. In animals receiving CLR RNAi, histological examination indicated exacerbated inflammmmation, and MPO activity demonstrated a two-fold increase over control, supporting the histological findings. To assess motility, we measured isometric contractions of longitudinal colonic muscle suspended in an organ bath 72 hours after RNAi treatment. In animals treated with control RNAi, CGRP abrogated contractions entirely, and decreased resting muscular tone. In animals treated with CLR RNAi, muscle tone decreased, but slow wave contractions of varying amplitude persisted. Thus, RNAi can be used to specifically down regulate CLR in the colon. Silencing of CLR exacerbated the TNBS-induced colitis, suggesting a protective role for CGRP and its receptor in colonic inflammation. CLR RNAi also abolished the effects of CGRP on colonic motility, which confirms that CLR mediates these effects of CGRP. Supported by NIH grant DK39957.

\section{S1650}

Neonatal Lipopolysaccharide Increases the Severity of TNBS-Induced Colitis in Adult Rats

Niall P. Hyland, Sarah J. Spencer, Quentin J. Pittman, Keith A. Sharkey

Early life events are recognized as having long-term health sequealae. We previously showed that infection in adults can alter the course of colitis. However, the long term effects of neonatal exposure to an infection, which has previously been shown to alter parameters of adult physiology, remain to be investigated with regard to the gastrointestinal tract. The aim of this study was to investigate the effect of neonatal LPS on enteric physiology and

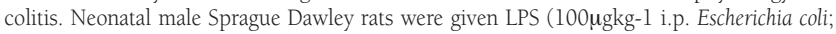
nLPS) or a similar volume of pyrogen free saline (nSaline). Eight to ten weeks later both nLPS and nSaline treated rats received either saline (aSaline) or TNBS (aTNBS) intra-rectally and were allowed to recover for three days. Alterations in body mass and body temperature were monitored daily. Additional animals were housed in metabolic cages and food/water and fecal/urine output was recorded daily. Colonic segments were mounted in Ussing chambers, and responses to veratridine $(30 \mu \mathrm{M})$ or capsaicin $(1 \mu \mathrm{M})$ assessed. Adjacent segments were fixed and processed for mast cell histochemistry or were snap frozen for assessment of myeloperoxidase (MPO) activity. Serum was collected for measurement of corticosterone (CORT) and tumour necrosis factor $\alpha$ (TNF $\alpha$ ). Neonatal LPS treated animals had more severe colitis than animals given saline injections as neonates. This was seen as lower body weight (nLPS-aTNBS weighed $\sim 25 \%$ less compared with nSaline-aTNBS), a significantly increased macroscopic tissue damage score $(8.7 \pm 1.1 \quad(n=8)$ nSaline-aTNBS, $12.5 \pm 0.4 \quad(n=8)$ nLPS-aTNBS) and increased MPO activity. These animals were also hypothermic in response to TNBS compared to nSaline-aTNBS animals. The reductions in body weight were not accompanied by a change in food intake. Colonic segments from all experimental groups taken from areas adjacent to those of the most severe damage displayed no significant difference in basal short-circuit current or resistance nor any difference in response to capsaicin or veratridine. Forskolin $(10 \mu \mathrm{M})$ and bethanechol $(10 \mu \mathrm{M})$ were used as internal controls and no difference in secretory response between nLPS and nSaline treated groups was observed. Serum CORT was unaltered in all groups, however, TNF $\alpha$ was significantly increased in nLPS-aTNBS compared with nSaline-aTNBS and control animals. A single neonatal exposure to LPS produces long-term alterations in the response to colitis. This change is independent of alterations in submucosal secretomotor and epithelial function, but is associated with increased circulating TNF $\alpha$, suggestive of an altered immune response in adults exposed to neonatal infection.

\section{S1651}

Role of CRF Family of Neuropeptides in Colonic Inflammation Julia J. Hoy, Matthew S. Clifton, Prema S. Idumalla, Aditi Bhargava

Corticotropin-releasing factor (CRF) and its family of neuropeptides play a pivotal role in a variety of physiological processes that include coordinating endocrine responses, immune cell activation and gastrointestinal (GI) motility. Spatial distribution pattern of urocortins
(Ucn) and their high affinity receptor CRFR2 in the brain is well established, however, their distribution pattern in the colon is less clear. In this study, we tested the hypothesis that final inhibitory or stimulatory outcomes of GI inflammation depend on the differing spatia distribution (within the GI tract) and temporal expression levels of CRF family of ligand and their receptor (that include CRF, urocortins and two CRF receptors). The distribution pattern of Ucn2 and CRFR2 in the proximal and distal colon was established using immunohistochemistry (IHC). Colonic inflammation was induced by a trinitrobenzene sulfonic acid (TNBS) enema ( $30 \mathrm{mg} / \mathrm{animal}$ ). Male Sprague-Dawley rats were treated as follows ( $\mathrm{n}=4-6$ group): saline injection, $50 \%$ ethanol vehicle enema; saline injection, TNBS enema; doublestranded RNA (dsRNA) injection, TNBS enema. DsRNA against CRF, Ucn2, CRFRl and CRFR2 were injected separately. The descending colon was harvested 72 hours after administration of dsRNA and TNBS enema. Tissues were analyzed by hematoxylin and eosin staining for histologic damage and IHC for inhibition of expression. Concomitant changes in inflammation were measured using a myeloperoxidase (MPO) assay. Urocortin 2 expression wa localized to the epithelial cells of the mucosal crypts, nerve fibres of the submucosal, and the myenteric nerve plexus of both the proximal and the distal colon. CRFR2 expression was most evident in the myenteric nerve plexus in the colon. As expected, TNBS enema resulted in exacerbated tissue edema, mucosal destruction, and increased submucosal infiltration of polymorphonuclear cells on histologic staining when compared to control saline and $50 \%$ ethanol administered rats. Interestingly, colon-specific inhibition of CRF or CRFRl did not alter the course of TNBS-induced inflammation; histological staining and MPO activity were comparable to rats that received a TNBS enema only. Colonic inhibition of CRFR2 resulted in tissue protection and a significant decrease in MPO activity. Surprisingly, and in contrast to CRFR2, colonic inhibition of Ucn2 exacerbated inflammation that was reflected in enhanced MPO activity. Thus, manipulation of the CRF system with RNAi provides an effective starting point for understanding their GI functions, perhaps contributing to the development of novel therapeutic treatments for inflammatory intestinal diseases.

\section{S1652}

Role of Endogenous Ghrelin in Acute and Chronic DSS-Induced Colitis in Mice

Betty de Smet, Theo Thijs, Dieder Moechars, Larissa Polders, Bernard Coulie, Theo L. Peeters, Inge Depoortere

Ghrelin inhibits the synthesis of proinflammatory cytokines, and the ghrelin receptor is expressed in different immune cells, including T lymphocytes. To assess a possible antiinflammatory role of ghrelin in intestinal inflammation, we induced colitis with DSS in wildtype (WT) and ghrelin knockout (KO) mice and compared disease activity and colonic contractility. Methods. $38 \mathrm{KO}$ (developed in collaboration with Lexicon Genetics) and 40 WT mice were divided in 4 groups. Control group, acute group ( $3 \%$ DSS for 5 days), recovery group (3\%DSS for 5 days, $\mathrm{H} 2 \mathrm{O}$ for 5 days), chronic group (3\%DSS for 5 days, $\mathrm{H} 2 \mathrm{O}$ for 21 days). Disease activity index (DAI) was determined as the average of the scores for weight loss, stool consistency and bleeding. Neutrophil infiltration was determined by MPO measurements. In colonic strips we evaluated smooth muscle and neural excitatory and inhibitory responses induced by electrical field stimulation. Results. Disease activity: During colitis body weight loss was less pronounced and stool consistency was less loose in KO mice, resulting in a decreased DAI score for the $\mathrm{KO}$ mice. The onset of neutrophil infiltration (MPO; U/mg tissue) was delayed from the acute phase in WT mice (WT: $0.84 \pm 0.21, \mathrm{KO}$ $0.28 \pm 0.08, \mathrm{P}<0.05)$ to the recovery phase (WT: $1.38 \pm 0.27, \mathrm{KO}: 1.31 \pm 0.18$ ) in $\mathrm{KO}$ mice and was normalized in both genotypes during chronic colitis (WT: $0.54 \pm 0.18$, KO: $0.43 \pm 0.11$ ). Functional studies: Neural cholinergic-tachykinergic responses were increased during the acute period (WT: $+16 \pm 7 \%, \mathrm{KO}:+67 \pm 4 \%, \mathrm{P}<0.05$ ), decreased during the recovery period (WT: $-39 \pm 3 \%, \mathrm{KO}:-57 \pm 2 \%, \mathrm{P}<0.05$ ) and again enhanced during the chronic phase (WT $+111 \pm 16 \%, \mathrm{KO}:+68 \pm 7 \%, \mathrm{P}<0.05)$. Changes in the neural responses were paralleled by changes in the smooth muscle response to ACh. NANC inhibitory responses were reduced in WT mice to a similar extent during the different phases of colitis (acute: 20\%, recovery: $21 \%$, chronic: $18 \%$ reduction). In KO mice the onset of loss of inhibitory responses was delayed (acute: $8 \%$, recovery: $28 \%$, chronic: $18 \%$ reduction). In inflamed WT mice the inhibitory effect of suramin on NANC inhibitory responses was reduced from $26 \pm 2 \%$ to $17 \pm 2 \%$ (acute), $15 \pm 1 \%$ (recovery) and $21 \pm 1 \%$ (chronic), while in KO mice suramin was only less effective during the recovery phase $(8 \pm 1 \%)$. Conclusion. In contrast with wha was expected, absence of ghrelin decreases the disease activity index, delays neutrophil infiltration and delays the onset of contractility changes from the acute until the recovery period. Our data are at variance with a protective role of ghrelin in intestinal inflammation.

\section{S1653}

Glial Cell Line-Derived Neurotrophic Factor Promotes Pancreatic Beta Cell Survival

Simon Mwangi, Alpana Limaye, Shanthi Srinivasan

BACKGROUND: Glial cell line-derived neurotrophic factor (GDNF) is a factor that plays a critical role in the development and survival of the enteric nervous system, but its role in pancreatic $\beta$ cell proliferation and survival has not been established. Recent studies have demonstrated several similarities between $\beta$ cells and neuronal cells raising the possibility that neurotrophic factors such as GDNF could play a role in $\beta$ cell growth and survival. We evaluated the role of GDNF on $\beta$ cell survival, and determined the signal transduction pathways involved focusing on the PI3K signaling pathway. METHODS: All experiments were performed in the $\beta$-TC-6 pancreatic $\beta$ cell line. In addition receptor mRNA expression was also assessed in laser capture microscopy-isolated mouse pancreatic $\beta$ cells. GDNF receptor family $\alpha \mathrm{l}$ (GFR $\alpha 1$ ) mRNA expression was assessed by RT-PCR. The expression of the c-Ret and GFRal receptors was assessed by immunofluorescence microscopy. Apoptosis in response to GDNF withdrawal or thapsigargin treatment was evaluated by immunoblotting for cleaved caspase-3 as well as by cleaved caspase-3 immunofluorescence with Hoechs staining. Akt phosphorylation was assessed by immunoblotting. RESULTS: GFR $\alpha 1$ mRNA was present in both $\beta$-TC- 6 cells and pancreatic $\beta$ cells. Expression of c-Ret and GFR $\alpha$ I receptors was also seen by immunocytochemistry in $\beta$-TC- 6 cells. $\beta$-TC- 6 cells cultured for $72 \mathrm{~h}$ in the absence of GDNF had a much higher level of apoptosis (caspase-3 cleavage

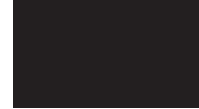


immunoblot) compared to those cultured in the presence of GDNF ( $\mathrm{P}<0.0001)$. Addition of thapsigargin $(24 \mathrm{~h})$ resulted in a significant increase in $\beta$-TC- 6 cells apoptosis $(21.5+2.3$ $\%$ compared to $8.6+1.2 \%+$ in control cells, cleaved caspase 3 immunostaining, $\mathrm{p}<0.0001$ ) In the presence of $\mathrm{GDNF}(100 \mathrm{ng} / \mathrm{ml})$ resulted in a significant reduction of thapsigargininduced apoptosis $(6.4 \pm 1.5 \%, \mathrm{P}<0.0001)$. Analysis of the involvement of the PI3K/Akt signaling pathway in GDNF-mediated $\beta$ cell survival revealed that GDNF promoted the phosphorylation of Akt at its C-terminus ser 473 residue, and that this phosphorylation was inhibited in the presence of the PI3K inhibitor, LY294002. CONCLUSIONS: We have demonstrated that the receptors for GDNF are expressed in pancreatic $\beta$ cells, and that GDNF can protect against $\beta$ cell apoptosis. These results suggest that GDNF plays a role in pancreatic $\beta$ cell survival.

\section{S1654}

Peripheral Nociceptin Prevents Stress-Induced Gastric Damage in Rats Carlo Polidori, Maurizio Massi, Lucia Silvotti, Valeria Albonetti, Daniela Grandi, Giuseppina Morini

Nociceptin (N/OFQ) has been shown to be widely expressed within the CNS and to modulate stress-related effects and behaviors by acting at central level. The purpose of the current study was to evaluate the distribution of N/OFQ in rat gastrointestinal tract and the possible influence of peripheral N/OFQ on acute gastric damage caused by cold-restraint stress in rats. N/OFQ immunoreactivity was present in the regions of the gastrointestinal tract examined: stomach, distal ileum and distal colon. Immunoreactivity appeared to be localized in the myenteric plexus. Cold exposure in restraint conditions for $3 \mathrm{~h}$ resulted in the formation of macroscopically visible hemorrhagic lesions in the glandular portion of the stomach, lesion index being $23.85 \pm 4.85$. On histological examination lesions were characterized by extensive areas of edema and hemorrhage and by focal necrosis. N/OFQ, administered, 0.03 to $1 \mu \mathrm{g} / \mathrm{kg} / \mathrm{h}$ ip, dose-dependently and significantly reduced macroscopic and histologic lesions, with a maximal reduction of $63 \%$ achieved with the highest dose. The selective N/ OFQ receptor agonist, [(pF)Phe4Aib7Arg14Lys15]N/OFQ-NH2 (UFP112), 0.01 to $0.3 \mu \mathrm{g} /$ $\mathrm{kg} / \mathrm{h}$ ip similarly inhibited the formation of gastric lesions by cold-restraint stress, having an higher potency as compared with N/OFQ. Protective effect of N/OFQ was abolished by the concurrent administration of the N/OFQ receptor antagonist, [Nphel,Arg14,Lys15]N/ OFQ-NH2 (UFP 101), $30 \mu \mathrm{g} / \mathrm{kg} / \mathrm{h}$ ip This dose of UFP 101 itself was inactive. Subdiaphragmatic vagotomy reduced gastric lesions induced by cold-restraint and in vagotomized rats the protective effect by N/OFQ was no longer evident. Present results indicate that N/OFQ is expressed in rat gastrointestinal tract and that the peptide reduces the damaging effect of stress on rat gastric mucosa, suggesting a role of peripheral N/OFQ in modulating stressrelated gastric effects.

\section{S1655}

In Vivo Goblet Cell Responses to An Enteric Bacterial Pathogen in a Mouse Model of Infectious Colitis

Kirk S. Bergstrom, Caixia Ma, Waliul Khan, Bruce A. Vallance

Background \& Aims. Intestinal goblet cells (IGCs) are specialized epithelial cells that secrete the mucin Muc2 into the intestinal tract, forming the protective mucus layer. IGCs play an important role in host defense against intestinal parasitic nematodes in association with a T helper (Th) 2 immune response. In contrast, the role of IGCs in host defense against enteric bacterial infections is unclear. Using the Citrobacter rodentium $(\mathrm{Cr})$ mouse mode of enteric bacterial infection, which causes mild diarrhea and colitis in association with a Thl immune response, we sought to characterize IGC responses in vivo, and assess their role in host defense against this infection. Methods. C57BL/6 mice were infected with Cr, and distal colons were isolated at early (days 4-6) and later (days 10-14) time points and analyzed for bacterial numbers as well as changes in mucin biochemical properties, IGCs numbers and morphology, \& Muc2 mRNA expression. The role of IGC-derived Muc2 in host defense was determined by infecting Muc2 -/- mice with $\mathrm{Cr}$. Moreover, the role of the host immune system in mediating IGC responses was assessed by $\mathrm{Cr}$ infection of RAG-1 -/ mice that lack $\mathrm{T} \& \mathrm{~B}$ cells. Results. The distal colons of $\mathrm{Cr}$ inoculated mice were heavily infected by day $4 \mathrm{pi}$. At the same time, histological staining revealed the increased expression of neutral mucins and an overall decrease in mucin sulfation, as well as greater numbers of mature IGCs compared to uninfected controls. Muc2 -/- mice were highly susceptible to $\mathrm{Cr}$ infection, succumbing by day $6 \mathrm{pi}$, in association with severe crypt ulceration and increased bacterial numbers in the colon, mesenteric lymph nodes, and spleen compared to WT mice that all survived. At later time points (day 10-14 pi), as the infection persisted in WT mice, there was a dramatic reduction in mature IGCs, while the mucins that remained were virtually all sulfated. These IGC changes correlated with a decrease in Muc2 mRNA expression and an increase in Thl cytokines. In contrast, RAG-1 - - - mice had abundant mature IGCs at these later time points, and did not show a significant reduction in Muc2 mRNA expression, although mucins were predominantly sulfated. Conclusion. IGCs undergo dynamic morphological, biochemical, and Muc2 gene expression changes in vivo during $\mathrm{Cr}$ infection, which appear to be mediated by the adaptive immune system. Moreover, Muc2 production by IGCs plays an important role in host defense early in infection, most likely by limiting bacterial translocation out of the infected colon. Taken together, these results indicate that IGCs are active players in host defense during infection by enteric bacterial pathogens.

\section{S1656}

Activation of Toll-Like Receptor 3 Protects Against DSS-Induced Colitis Matam V. Kumar, Hui Xia, Vasantha Kolachala, Shanthi V. Sitaraman, Andrew S. Neish, Andrew T. Gewirtz

BACKGROUND: Mimetics of bacterial DNA, given orally or subcutaneousy, protect mice from experimental colitis via a toll-like receptor (TLR)-9 dependent mechanism. GOAL: To investigate whether synthetic viral RNA, poly $(\mathrm{I}: \mathrm{C})$, which is also a potent immunomodulator might also affect murine colitis and, if so, define whether such effects were mediated by TLR3, which is one of at least three known receptors for this viral RNA analog. METHODS: Mice (8-week old female C57BL6, IL-10KO, or TLR3KO) were administered 1.5\% dextran sodium sulfate (DSS) in drinking water for 7 days. Two hours before treatment with DSS, mice were given PBS, 20 micrograms of poly(I:C) subcutaneously, or 100 micrograms of poly $(\mathrm{I}: \mathrm{C})$ intragastrically. Disease activity was assessed by measuring weight loss, occult blood, colon shortening, histopathologic analysis of $H \& E$ stained tissue, and measurement of colonic levels of myeloperoxidase activity, cyclooxygenase-2 and inducible nitric oxide synthase protein. Furthermore, inflammation was serologically assessed by measuring keratinocyte derived chemokine (KC), serum amyloid A and by ELISA and lipocalin-2 by SDSPAGE immunoblotting. RESULTS: In wild type (C57BL6) mice, subcutaneous administration of poly(I:C) dramatically protected against DSS induced colitis as assessed by every parameter analyzed. In contrast, intragastric administration of poly $(\mathrm{I}: \mathrm{C})$ offered no protection in this colitis model nor did its administration activate the innate immune system as assessed by serologic parameters. Subcutaneous poly(I:C) protected against DSS-induced colitis equally well in C57BL6 and IL-10KO mice indicating that this anti-inflammatory cytokine is not required for such protection. Protection against colitis given by poly(I:C) treatment was ablated in TLR3KO indicating the protective action of this viral RNA analog was mediated by TLR3. CONCLUSIONS: Activation of TLR3 on cells that are accessible by systemic, but not oral, administration of synthetic viral RNA results in protection against the acute inflammation that can ensue upon damage of the gut epithelium. Thus, this viral RNA analog, which is under clinical trials for other inflammatory disorders (e.g. lupus), may also have therapeutic value for inflammatory bowel disease.

\section{S1657}

Abnormally Differentiated Subsets of Intestinal Macrophage Induce IL-12 Hyperproduction in Response to Bacteria. -Etiology of Thl Dominant Colitis in IL-10 Deficient Mice-

Nobuhiko Kamada, Tadakazu Hisamatsu, Susumu Okamoto, Kiyoko S. Akagawa, Toshifumi Hibi

BACKGROUND: Disorders in enteric bacteria recognition by intestinal macrophages (Mphi) are strongly correlated with the pathogenesis of chronic colitis; however its precise mechanisms remain unclear. The aim of the current study was to elucidate the roles of Mphi in intestinal inflammation by using an IL-10-deficient (IL-10-/-) mouse colitis model METHODS: GM-Mphi and M-Mphi were generated from bone-marrow (BM) CD1lb+cells with GM-CSF and M-CSF, respectively. Colonic lamina propria Mphi (CLPMphi) and splenic Mphi were isolated from colon lamina propria mononuclear cells or unfractionated splenocytes by CDIlb+ cell separation. These Mphi were then stimulated with heat-killed whole bacteria antigens. Cytokines production from stimulated Mphi was assessed by cytometric beads array and quantitative RT-PCR. RESULTS: GM-Mphi and M-Mphi from IL-10-/- mice were similar to those from wild-type (WT) mice, at least in terms of morphology and cell surface phenotypes. M-Mphi from IL-10-/- mice produced abnormal large amounts of IL12 and IL-23 upon stimulation with heat-killed whole bacteria antigens, whereas M-Mph from wild-type (WT) mice produced large amounts of IL-10 but neither IL-12 nor IL-23. In contrast, IL-12 production by GM-Mphi was not significantly different between WT and IL-10-/- mice. In ex-vivo experiments, cytokine production ability of CLPMphi but not splenic Mphi from WT mice was similar to that of M-Mphi, and CLPMphi but not splenic Mphi from IL-10-/- mice also showed abnormal IL-12p70 hyperproduction upon stimulation with bacteria. Surprisingly, the abnormal IL-12p70 hyperproduction from M-Mphi from IL10-/- mice was improved by IL-10 supplementation during the differentiation process. CONCLUSION: These results suggest that CLPMphi and M-Mphi act as anti-inflammatory Mphi, and suppress excess inflammation induced by bacteria in WT mice. In IL-10-/- mice, however, such Mphi subsets differentiated into abnormal phenotype under IL-10 deficien environment, and bacteria recognition by abnormally differentiated subsets of intestinal Mphi may lead to Thl-dominant colitis via IL-12 and IL-23 hyperproduction. Our data provide new insight into the intestinal Mphi-gut flora relationship in the development of colitis in IL-10-/- mice

\section{S1658}

Irf-1 Mediates Innate Immune Response of Intestinal Epithelial Cells By Regulating the Concerted Expression of Immunosubunits and Tlr3 Atsushi Yoshioka, Shigeru Oshima, Kiichiro Tsuchiya, Shin Namiki, Ryuuichi Okamoto, Tetsuya Nakamura, Takanori Kanai, Mamoru Watanabe

Background \& Aims: We have previously shown that IRF-1, a downstream factor of interferon(IFN) signaling, transcriptionally regulates production of interleukin(IL)-7, a central cytokine of the adaptive immune system, within the intestinal mucosa. However, it is suggested that intestinal epithelial cells (IECS) also play indispensable roles in the innate immune system, and that dysregulation of the innate immune response is critically required for the pathogenesis of inflammatory bowel diseases(IBDs). In the present study, we analyzed the possibility whether key molecules of the innate immune system might be target genes of IRF-1 in IECs. Methods: Human IEC-derived cells were used to perform cDNA expression array hybridization under induced expression of IRF-1 by Tet-on system. Northern blot or real-time PCR was conducted to confirm altered expression level of the genes identified. Functional roles of IRF-1 in transcriptional regulation for a part of its target genes were verified using reporter assays, chromatin immunoprecipitation(ChIP) or si-RNA mediated gene silencing. To ascertain the roles of IRF-1 in vivo, induction of the target gene expression upon IFN- $\gamma$ treatment in IRF-1 deficient mice was analyzed. Results: Expression profiling and subsequent analysis of related genes by Northern blot revealed that 5 out of 16 components of the immunoproteasome were up-regulated by forced expression of IRF-1. All these subunits were confirmed to be up-regulated also by IFN- $\gamma$ stimulation on IECs. Of these subunits, transcriptional expression of LMP-7, a catalytic subunit of the immunoproteasome, was newly identified to be directly regulated by IRF-1 via distinct IRF-E element exising in the promotor region of LMP-7 gene. Futhermore, critical role of IRF-1 in IFN- $\gamma$ inducible concerted expression of immunosubunits was confirmed in vivo using IRF-1 -/- mice Additionally, analysis for mRNA expression of TLRs revealed that TLR3 was significantly 
up-regulated both by IFN- $\gamma$ stimulation and by forced expression of IRF-1 in IECs. Conclusions: These results indicated that IRF-1 plays critical roles for innate immune response in IECS via concerted regulation of mRNA expressions of both immunoproteasomes and TLR3.

\section{S1659}

Regulation of IL-10 Production Induced By NOD2/CARD15 and TLR2 Pathways in Human Monocyte-Derived Dendritic Cells J.N. Li, Matija Hedl, Morassa Mohseni, Kholil Qato, Betul Yilmaz, Samuel Bracamonte, Clara Abraham, Judy Cho

The innate immune system utilizes both NOD2 and Toll-like receptors (TLRs) to recognize various microbial components. Defining the functional relationship between these two pathways is of major interest. It has been previously reported that treatment of monocytic cells with muramyl dipeptide (MDP) and Pam3Cys, ligands for NOD2 and TLR2 respectively, demonstrates synergy in IL-10 production. In this study, we utilized primary monocytederived human dendritic cells (MDDCs) to identify inter-individual differences and define mechanisms of regulation of IL-10 by MDP and Pam3Cys. In MDDCs from 26 healthy individuals, we observed a $1.47 \pm 0.20($ MDP $1 \mathrm{ug} / \mathrm{ml}), 3.26 \pm 0.25$ (Pam3Cys $1 \mathrm{ug} / \mathrm{ml})$ and $7.57 \pm 1.1$-fold induction (MDP $1 \mathrm{ug} / \mathrm{ml}$ plus Pam3Cys $1 \mathrm{ug} / \mathrm{ml}$ ) of IL-10 treatment demonstrating a synergistic effect between these two pathways. However, significant interindividual differences were observed in IL-10 responses. Synergistic responses were defined as a greater than $30 \%$ induction with combined MDP and Pam3Cys treatment compared to the additive effects of either treatment given individually. $50 \%, 69 \%$, and $42 \%$ of individuals demonstrated MDP-responsiveness, Pam3Cys-responsiveness, and synergistic responses, respectively. These classifications were consistently observed within individuals tested on multiple occasions. Surprisingly, neither genetic variants in the IL-10 promoter (-1082 and 592) nor within NOD2 heterozygotes (Leul007fsinsC, G908R and R702T) correlated with IL-10 responses. Importantly, in synergistic responders, we observed a marked induction of SP-1 activation by electromobility shift assay (EMSA) with combined MDP and Pam3Cys compared to either agent alone. In contrast, synergistic induction of NF-kB by EMSA was not observed. As expected, individuals not demonstrating an MDP/Pam3Cys-synergistic IL10 response showed no synergistic activation of either SP-1 or NF-kB. Finally, MDP and Pam3Cys-synergistic induced IL-10 production was significantly reduced by p38 (70\%), JNK $(60 \%)$ and Erk (40\%) inhibitors. The capacity to mediate synergistic responses in IL10 production between the NOD2 and TLR2 pathways varies between individuals and is associated with synergistic activation of SP-1

\section{S1660}

Enteroinvasive E. Coli Activate P38, ERK and Jnk MAP Kinase Phosphorylation and Functionally Regulate Intestinal Epithelial Cell Proinflammatory Chemokine Production By Signaling Through NODl Yukiko Miyamoto, Martin F. Kagnoff

Nodl is an intracellular pattern recognition molecule (PRR) that can be activated in the cytoplasm of intestinal epithelial cells (IECS) by enteroinvasive bacteria or specific peptidoglycan components of Gram negative bacteria, independent of activating membrane-associated Toll-like receptors. We reported before that enteroinvasive $E$ coli (EIEC) signaling through

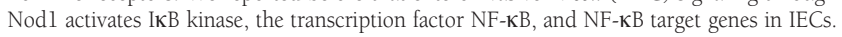
Since many NF- $\mathrm{\kappa B}$ target genes are co-regulated by the activation of MAP kinases, we sought to determine if signaling of EIEC through cytoplasmic Nodl activates MAP kinases and, if so, whether this has functional implications for regulating epithelial cell functions that are important in innate immunity. Methods: Dominant negative (DN) Nodl and a control empty vector (EV) were stably or transiently expressed in human intestinal epithelial cell lines. Cells were infected with EIEC. P38, ERK and JNK MAP kinase phosphorylation were determined by immunoblotting. Production of the neutrophil chemoattractant, CXCL8, was measured by ELISA. Internalized EIEC were counted 1 and $24 \mathrm{~h}$ after infection. Results: EIEC infection significantly activated phosphorylation of p38, ERK, and JNK MAP kinases in human IECs. EIEC stimulated CXCL8 production was significantly inhibited by pretreatment of IECs with the specific MAP kinase inhibitors PD98059 and SB203580, which inhibit ERK and p38 activation, respectively. Importantly, MAP kinase activation in response to EIEC infection was significantly inhibited in cells that express DN Nodl but not empty vector, and this was paralleled also by significant inhibition of CXCL8 production. Although EIEC signaling through Nodl activated major cellular signaling pathways important for cell survival, proliferation and the production of proinflammatory mediators, a lack of signaling through Nod 1 had little or no effect on the proliferation and survival of internalized EIEC. Conclusion: EIEC signaling through the cytoplasmic PRR Nodl is not restricted to NF- $\mathrm{\kappa B}$ activation, but importantly also includes the activation of p38, ERK and JNK MAP kinases. Activation of MAP kinases through Nodl in turn can regulate the expression of host epithelial cell genes important in innate host defense. Pathogenic enteric bacteria that can activate Nodl in IECs have the ability to trigger cytoplasmic signaling pathways that parallel those activated via TLR's. This provides the host with an important mechanism for rapidly activating innate immunity and other key host responses during infection with pathogenic gramnegative bacteria that otherwise bypass signaling of membrane-associated TLR's.

\section{S1661}

Toll-Like Receptor (TLR4) Is Essential for Expression of Antimicrobial Peptides and Prevention of Bacterial Translocation in the Gut Suneeta Krishnareddy, Masayuki Fukata, Anli Chen, Arielle Klepper, Maria Abreu

Background: We have recently demonstrated that mice deficient in TLR4 have increased signs of colitis and bacterial translocation to mesenteric lymph nodes compared to wildtype (WT) mice following dextran sodium-sulfate (DSS)-induced injury. We have also shown that TLR4 regulates $\beta$-defensin 2 expression in vitro. We hypothesized that the expression of $\beta$-defensins and $\alpha$-defensins (cryptdins) in the gut is dependent on TLR4 signaling in vivo. Methods: TLR4-/- and WT mice were given 2.5\% DSS for 7 days then sacrificed.
Bacterial translocation was assessed using homogenized mesenteric lymph nodes and spleens Expression of $\beta$-defensins, pan-cryptdins (the primers used detect cryptdins 1, 4, and 5 ) and TLRs $1,2,3,5,6$ and 9 in the colon and small intestine were analyzed by real-time PCR. Results: All TLR4-/- mice exhibited bacterial translocation to mesenteric lymph nodes and spleen, whereas only $25 \%$ of WT mice experienced bacterial translocation to MLN $(17 \%)$ or spleen $(33 \%)$ and had significantly lower counts. The expression of $\beta$-defensin 2 was significantly lower ( $\mathrm{p}=0.01$ ) in TLR4-/- mice compared to WT mice. Cryptdin expression in the ileum of TLR4-/- mice was also significantly decreased $(\mathrm{p}=0.02)$ compared to WT mice (Figure 1). We next addressed whether the defect in defensin expression was related only to the deficiency in TLR4 or could be due to other TLR defects. Rather than a decrease in expression of other TLRs, all the TLRs tested, 1, 2, 3, 5, 6 and 9, were higher in TLR4-/mice compared with WT mice. Conclusion: We describe an important link between intestina antimicrobial peptide expression and TLR4 signaling in vivo. Despite increases in expression of other TLRs, no alternate pathway compensates for the lack of TLR4. Functionally the outcome may be a lack of containment of luminal bacteria in the setting of epithelial injury and an adaptive immune response to commensal bacteria.

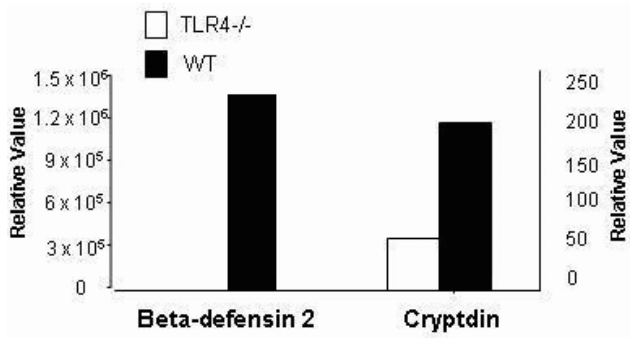

Figure 1

S1662

Akap13 Specifically Mediates NF-kB Signaling Through TLR2

Oren Shibolet, Shan Qin, Tobias Mueller, Ian Rosenberg, Ramnik Xavier, Daniel K. Podolsky

Background: Expression cloning screens identified A-kinase anchoring protein 13 (AKAP13) as an activator of NF-kB. AKAP13 is a scaffolding protein with guanine exchange facto (GEF) activity. Examining microarray data sets using a pre-neighborhood enrichment approach further suggested that AKAP13 may function as a regulator of Toll-like receptor (TLR) signaling pathways. Aim: to examine AKAP13 expression in intestinal epithelial cell (IEC) lines and to evaluate its potential role in NF-kB activation through different TLR signaling cascades. Methods: AKAP13 expression in IEC and human intestinal biopsies was assessed by real time RT-PCR. Functional assays comprised of NF-kB activation assays and ELISA for secreted IL-8 and MCP-1 in native HEK293 or stable HEK-TLR2 and Hela-TLR4 cell lines. These cell lines were transiently transfected with AKAP13-GFP expression vector or AKAP13 SiRNA followed by stimulation with specific TLR ligands. Phosphorylation of MAP kinases, P-38, JNK and ERK was assessed using phosphorylation assays. Results: Caco2, SW480, HT29, T84 and HCT116 IEC lines and human intestinal cells constitutively express AKAP13 mRNA. Overexpression of AKAP13 in Hek293 cells, caused a 100 21 -fold increase in NF-kB activation as compared to empty vector while a point mutation interfering with AKAP13 GEF activity caused a 50\% reduction in NF-kB activation as compared to wild type AKAP13. AKAP13 SiRNA but not scrambled SiRNA caused a significant $65 \pm 8 \%$ decrease in NF-kB activation in response to the TLR2 ligand Pam3CSK4 but not to the TLR4 ligand LPS in stable TLR2 and TLR4 cell lines respectively. This correlated with a $90 \%$ and $75 \%$ decrease in secreted IL-8 and MCP-1 respectively in the HEK-TLR2 but not the Hela-TLR4 cell line. SiRNA to AKAP13 in HEK-TLR2 significantly decreased phosphorylation of JNK and P-38 but not of ERK1 and 2 following stimulation. Conclusion: AKAP13 may play a role in TLR2-but not TLR4-mediated NF-kB activation. This activation may be mediated through AKAP13 GEF function, and identifies AKAP13 as a possible drug target in inflammatory processes mediated via TLR2. The findings suggest that different GEF or scaffold proteins may be involved in conferring specificity to innate immune responses through TLRs

\section{S1663}

Modulation of Lipopolysaccharide-Activated Human Dendritic Cells By Muramyl Dipeptide Promotes the Development of T Helper-Type 2 Cells Relevance to Pathogenesis of Crohn's Disease

Matt Butler, David van Heel, Rakesh Chaudhary, Raymond Playford, Subrata Ghosh

Background and aims: Sensing of commensal micro-organisms via toll-like receptors (TLRs) appears to be essential for generating intestinal homeostasis in healthy individuals. Conversely, Crohn's disease is characterized by an inappropriate T helper-type l (Thl)-mediated adaptive immune response towards enteric flora. The nucleotide-oligomerisation domain-2 (NOD2; CARD15) protein mediates cellular responses to the bacterial peptidoglycan subunit, muramyl-dipeptide (MDP), and activation via this receptor enhances TLR-mediated inflammatory cytokine responses from macrophages and dendritic cells (DCs). It is currently unclear how defects in bacterial sensing via NOD2 lead to adaptive immune response overdrive and Crohn's disease. We thus investigated how MDP signalling, via NOD2, influences activation of DCs via TLRs. DC phenotype and function was determined and stimulation of T lymphocytes was assessed. Methods: We generated monocyte-derived DCs from healthy human donors and stimulated them for 24 hours with highly purified (lacking MDP activity) lipopolysaccharide (LPS $0.1-100 \mathrm{ng} / \mathrm{ml})$ in the presence or absence of MDP $(100 \mathrm{ng} / \mathrm{ml} \mathrm{MDP}$ Lys 18 romurtide). The cell-surface antigens, cytokine production, and T cell stimulatory capacity of these cells was then assayed by FACS analysis, ELISA and mixed lymphocyte reaction with allogeneic T lymphocytes. Results: MDP was found to act synergistically with 
LPS to amplify the production of cytokines IL-8, TNF $\alpha$, and IL-10 whilst leaving secretion of the Thl-inducing cytokine, IL-12p70, unaffected. Additionally, presence of MDP during the LPS response enhanced DC expression of key co-stimulatory molecules such as CD80 (B7.1). These changes profoundly altered the cytokine profile of naive, allogeneic, CD4+ T cells in response to activated DCs - deviating them away from a LPS-induced Thl response and towards a Th2 response dominated by IL-5 and IL-13. Conclusion: This study demonstrates, for the first time, that human DC responses to LPS can be modulated by MDP and peptidoglycan resulting in protective, rather than inflammatory, Th2 responses. These results may help explain why Crohn's disease patients, carrying loss of function mutations in the NOD2 protein, are unable to generate a balanced/homeostatic adaptive T cell response towards normal enteric flora.

\section{S1664}

Probiotic Components Induce the Expression of Cathelin-Related Antimicrobial Peptide Expression in Rat Macrophages: Its Role in Protection Against Polymicrobial Sepsis

Xiao Wang, Heng-Fu Bu, Roxanne Y. Williams, Xiao-Di Tan

Cathelin-related antimicrobial peptide (CRAMP) is an effector molecule of the innate immune system. It is expressed in phagocytes such as macrophages. Probiotics have been well studied for its beneficial role in enhancing the host innate immune function. However, molecular mechanisms through which probiotics modulate immunity have not been elucidated yet. Recently, we found that oral delivery of a cell-free probiotic component (PC) results in protection against cecal ligation and puncture (CLP)-induced sepsis (data will be presented in Experimental Biology 2006 Meeting). In the present study, we further investigated whether PC modulates CRAMP expression in vivo and in vitro. Briefly, we gavaged rats with PC for 5 days, isolated peritoneal macrophages (MФs), and measured CRAMP gene expression with real-time RT-PCR. We found that CRAMP is constitutively expressed in MФs. Treatment with PC in vivo resulted in a marked up-regulation of CRAMP gene expression in the residential peritoneal MФs. Furthermore, we demonstrated that PC directly induces CRAMP mRNA (up to 6-fold) in MФs in vitro. The effect was in a dose dependent manner (1-100 $\mu \mathrm{g} / \mathrm{ml}$ ). In contrast, treatment of MФs with LPS ( $1 \mu \mathrm{g} / \mathrm{ml}$ for up to $24 \mathrm{hrs}$ ) only showed a mild transient effect on CRAMP expression. The data suggested that PC is a potent enhancer on CRAMP-related innate immune capacity in macrophages. To further explore the physiological role of PC-induced CRAMP gene expression capacity, we examined whether the protective effect of PC against CLP-induced sepsis is correlated with CRAMP expression in tissues. To this end, rats were divided into groups of normal control, sham-surgery, CLP, and "PC + CLP". Three days after surgeries, we isolated RNA from the liver and measured CRAMP expression with real-time PCR. Compared to the normal control group, CRAMP expression was decreased in the liver of sham or CLP groups. In contrast, CRAMP gene expression did not change in the group "PC + CLP". These findings indicated that trauma or CLP causes inhibition of CRAMP expression, whereas PC treatment preserves CRAMP gene expression in tissues. In conclusion, our data suggested that PC up-regulates CRAMP gene expression capacity, which may mediate the beneficial effects of probiotics. Study funding: IDPA, NIH, Batts Family

\section{S1665}

Polyamines Are Required for Expression of Toll-Like Receptors in Intestina Epithelial Cells and Involved in Regulation of Innate Immune Response Jie Chen, Jaladanki N. Rao, Tongtong Zou, Lan Liu, Ai-Hong Zhang, Douglas J. Turner, Jian-Ying Wang

The Toll-like receptors (TLRs) allow mammalian intestinal epithelium to detect various microbes and activate the innate immune system after infection. Intestinal epithelial cells (IECs) express TLR2 and TLR4, which are highly regulated in response to TLR ligands. However, the exact mechanisms involved in control of TLR expression in IECs remain unknown. Polyamines are implicated in a wide variety of biological functions, and regulation of cellular polyamines has been recognized to be a central convergence point for multiple signaling pathways driving epithelial cell functions. The current study tests the hypothesis that polyamines regulate TLR expression in IECs and are involved in modulation of the innate immunity. Methods: Studies were conducted in IEC-6 cells (line of rat intestinal epithelial cells) and Caco-2 cells (line of human colon epithelial cells). Cellular polyamines were depleted by DFMO, the specific inhibitor of polyamine synthesis, while TLR activation was induced by challenge with lipopolysaccharide (LPS). Levels of TLR2 and TLR4 mRNAs and proteins were examined by real-time RT-PCR and Western blot analysis. Results: Depletion of cellular polyamines by DFMO decreased basal levels of TLR2 mRNA and protein, but had no effect on expression of TLR4 in both IEC-6 and Caco-2 cells. When normal cells (without DFMO treatment) were challenged by LPS ( $5 \mu \mathrm{g} / \mathrm{ml}$ ), TLR2 expression was increased as indicated by an induction of its mRNA and protein. Levels of TLR2 mRNA increased $1 \mathrm{~h}$ after exposure to LPS, peaked at 2-4 h ( $\sim 5$-folds the control value), and then began to decrease. The increases in TLR2 mRNA were paralleled by increases in its protein. This LPS-induced expression of TLR2 was completely prevented by polyamine depletion. There were no significant changes in levels of TLR2 mRNA and protein when polyaminedeficient cells were challenged by LPS. Exposure of IEC- 6 and Caco- 2 cells to LPS did not increase TLR4 expression regardless of the presence or absence of cellular polyamines. Decreased expression of TLR2 in polyamine-deficient cells was associated with dysfunction of epithelial barrier as shown by an increase in paracellular permeability. Conclusions: These results indicate that polyamines are necessary for expression of TLR2 in intestinal epithelial cells and play an important role in regulation of the innate immunity.

\section{S166}

TLR-Specific Cytokine Production By Preadipocytes and Adipocytes - Further Evidence That Adipose Tissue Is Linked to Innate Immunity

Arvind Batra, Jeannette Pietsch, Thorsten Stroh, Rainer Glauben, Besir Okur, Inka Fedke, Martin Zeitz, Britta Siegmund

Background: The biological significance of mesenterial fat hypertrophy in Crohn's disease is not understood. Recent data indicate that adipose tissue serves not only as energy storage, but is a potent producer of a variety of cytokines, including IL-6, IL-10, TNF- $\alpha$ as well as leptin. In addition, we could previously demonstrate the expression of Toll-like receptors (TLR) on preadipocytes and adipocytes. In order to evaluate whether bacterial translocation from the gut could result in an activation of adipocytes and preadipocytes, the responsiveness to and leptin-dependency of TLR-specific stimulation was studied. Methods: Leptin- (ob/ $o b)$, leptin-receptor $(d b / d b)$-deficient and WT preadipocytes originally isolated from mesenterial fat as well as 3T3Ll cells and adipocytes from all cell lines were stimulated with ligands for TLR2,-3, $-4,-5,-7$ or TLR9 and supernatants were tested for IL-10, IL- 6 and TNF- $\alpha$ The signaling pathways involved were analyzed. Results: TLR-specific stimulation of both preadipocytes and adipocytes resulted in an induction of IL- 6 synthesis. In WT preadipocytes significant IL-6 release was induced by TLR4-specific stimulation whereas ob/ob cells responded to ligands for TLR2, -3 , and -5 as well. In addition, in $d b / d b$ cells the responsiveness was further extended by TLR7 and -9 . However, stimulation of cultured preadipocytes and adipocytes did not induce IL-10. Production of TNF- $\alpha$ was only moderate and solely detectable following stimulation of BL6 and $d b / d b$ cells via TLR4 and -7. Remarkably, maturation to adipocytes resulted in a significant increase in TLR susceptibility. In particular WT adipocytes responded to all TLR ligands tested. Notably, in the absence of leptin or leptin signaling the IL-6 concentration was 10 to 30 times higher than in WT cells, thus further underlining the regulatory function of leptin in the immune system. Signaling studies revealed the PI3 kinase pathway as regulatory intersection for this hypersensitivity in $o b / o b$ or $d b / d b$ cells. Conclusion: Our data provide strong evidence that TLR-ligands can induce production of pro-inflammatory cytokines in adipocytes as well as preadipocytes. An observation that further strengthens the evidence that adipose tissue is linked to innate immunity and which might be of particular importance in IBD where increased bacterial translocation to the mesenteric fat might directly induce an inflammatory response.

\section{S1667}

IL-10 Production By Both Antigen Presenting Cells and Cd4 $4^{+} \mathrm{T}$ Lymphocytes Maintains Mucosal Homeostasis

Bo Liu, Lisa Holt, R Balfour Sartor

IL-10 deficient mice develop TH1- mediated colitis and IL-10- producing regulatory T cells suppress colitis, implicating IL-10 in maintaining mucosal homeostasis. However, the relative role of IL-10 produced by antigen presenting cells (APC) vs. T lymphocytes in immunoregulation is unknown. We studied this question by transferring T cells into IL-10 normal or deficient recipients. Methods: SPF (specific pathogen free) Rag2-/-IL-10-/- and Rag2-/-IL$10+/+$ mice were generated and injected i.p. with $5 \times 10^{5}$ splenic CD4 ${ }^{+} \mathrm{T}$ cells from germ free (GF) and SPF 129SvEv (WT) or IL-10-/- mice. Recipients were killed 6-8 wk after transfer. Colitis was evaluated by blinded histological score and by IL-12p40 and IFN- $\gamma$ production by unfractionated MLN cells stimulated with cecal lysates. CD $4^{+} \mathrm{T}$ cell reconstitution was documented by FACS. APC function was assayed in vitro with $\mathrm{CD}^{+} \mathrm{T}$ cell coculture. Results: Rag2-/-IL-10-/- mice reconstituted with SPF WT CD4+ T cells developed moderate colitis and immune activation (IFN- $\gamma$ and IL- $12 \mathrm{p} 40$ production by cecal bacterialstimulated MLN cells) and severe disease with transferred SPF IL-10-/- CD4+ T cells (Table). Rag2-/-IL-10+/+ recipients developed moderate colitis and mild immune activation with transferred IL-10-/- CD4 ${ }^{+} \mathrm{T}$ cells and no disease with IL-10+/+ cells. CD4+ $\mathrm{T}$ cells from GF IL-10-/- mice induced more severe colitis and immune activation in Rag2-/-IL-10-/- recipients than in Rag2-/-IL-10+/+ recipients (histology score $9.3 \pm 0.3$ vs. $5.1 \pm 0.3$; IFN- $\gamma$ secretion 10.7 fold increased, $21.4 \pm 2.5 \mathrm{ng} / \mathrm{ml}$ vs. $2.0 \pm 0.4$; IL-12 production increased 13 fold, $3.9 \pm 0.3 \mathrm{ng} / \mathrm{ml}$ vs. $0.3 \pm 0.03 ; \mathrm{P}<0.001)$. GF WT CD4+ $\mathrm{T}$ cells transferred to Rag2-/-IL$10-/$ - mice induced more colitis and increased IFN- $\gamma$ production $(13.1 \pm 0.4 \mathrm{ng} / \mathrm{ml}$ vs. 4.3 $\pm 0.8, \mathrm{P}<0.001)$ and IL-12 secretion $(2.2 \pm 0.2 \mathrm{ng} / \mathrm{ml}$ vs. $0.2 \pm 0.03, \mathrm{P}<0.001)$ vs. Rag2-/IL-10+/+ recipients. IL-10-/- CD4+ T cells co-cultured with IL-10-/- APC induced more IFN$\gamma$ production $(1112 \pm 147 \mathrm{pg} / \mathrm{ml}$ vs. $391 \pm 25, \mathrm{p}<0.05)$ and IL-12p40 secretion (1353 \pm $259 \mathrm{pg} / \mathrm{ml}$ vs. $619 \pm 19, \mathrm{P}<0.05)$ than with IL-10+/+ APC. Conclusion: IL-10 produced by APC plays a key role in regulating intestinal inflammation by inhibiting pathogenic $\mathrm{T}$ lymphocyte responses that cause disease. IL-10 secreting $\mathrm{CD}^{+}{ }^{+} \mathrm{T}$ cells are not sufficient to regulate mucosal homeostasis in the absence of IL-10 produced by APC

\begin{tabular}{|c|c|c|c|c|c|c|}
\hline \multirow{2}{*}{$\begin{array}{c}\text { Source of } \\
\text { CD4+ T } \\
\text { cells }\end{array}$} & \multicolumn{2}{|c|}{ Rag2-/-IL-10-/- recipient (n=8) } & \multicolumn{3}{c|}{ Rag2-/-IL-10+/+ recipient (n=8) } \\
\cline { 2 - 7 } & $\begin{array}{c}\text { IFN- } \gamma \\
\text { production by } \\
\text { MLN (ng/ml) }\end{array}$ & $\begin{array}{c}\text { IL-12 } \\
\text { production by } \\
\text { MLN (ng/ml) }\end{array}$ & $\begin{array}{c}\text { Histology } \\
\text { Score }\end{array}$ & $\begin{array}{c}\text { IFN- } \gamma \\
\text { production by } \\
\text { MLN (ng/ml) }\end{array}$ & $\begin{array}{c}\text { IL-12 } \\
\text { production by } \\
\text { MLN (ng/ml) }\end{array}$ & $\begin{array}{c}\text { Histo. } \\
\text { Score }\end{array}$ \\
\hline $\begin{array}{c}\text { SPF } \\
\text { IL-10-/- }\end{array}$ & $18.0 \pm 2.9 *$ & $2.9 \pm 0.5 *$ & $\begin{array}{c}10.7 \pm 0.4 \\
\wedge\end{array}$ & $3.2 \pm 0.4$ & $0.9 \pm 0.1$ & $5.8 \pm 0.7$ \\
\hline SPF WT & $4.6 \pm 1.2^{*}$ & $2.2 \pm 0.2^{*}$ & $\begin{array}{c}4.1 \pm \\
0.3^{\wedge \wedge}\end{array}$ & $0.8 \pm 0.6$ & $0.4 \pm 0.1$ & $2.4 \pm 0.4$ \\
\hline
\end{tabular}

$\left({ }^{*} \mathrm{P}<0.001\right.$ vs. Rag2-/-IL-10+/+ recipients; $\wedge \mathrm{P}<0.001 \&{ }^{\wedge} \wedge \mathrm{P}<0.05$ vs.Rag2-/-IL-10+/+) 


\section{S1668}

Pathological Role of Mucosal Mast Cells in a Mouse Model of Food Allergy: New Insights from Phosphatidylinositol-3 Kinase Deficient Mice Toshihisa Kodama, Takeshi Yamamoto, Naho Utsunomiya, Hirofumi Kuramoto, Makoto Kadowaki

Although gastrointestinal allergic disorders have dramatically increased in prevalence over the last several decades, the underlying mechanisms of food anaphylaxis have yet to be fully defined. An essential question is to determine the specific cells and mediators responsible for these disorders. METHODS: A murine model of food allergy was developed using BALB/ c mice. For induction of food allergy, a group of mice was primed with ovalbumin (OVA) and aluminum hydroxide gel as adjuvant by intraperitoneal injection, and the occurrence of diarrhea following repeated oral challenges with OVA in the primed mice was recorded. Next, the colon of the food allergy mice was removed and used for myeloperoxidase (MPO) activity analysis and for quantitative measurements of inflammatory cytokine mRNA. Furthermore, we examined the expression of mucosal mast cells in colon by immunohistochemical analysis with an antibody against mouse mast cell protease-1 (mMCP-1; a marker of mouse mucosal mast cell). Moreover, we made the food allergy experiments with phosphatidylinositol-3 kinase (PI3K) deficient mice (BALB/c background) selectively lacking gastrointestinal mast cells, to compare severity of allergic symptoms and expression of mucosal mast cells. RESULTS: Exposure of the primed mice to repeated oral OVA challenges developed allergic diarrhea $[85.4 \pm 8.5 \%$ (mean \pm S.E.) after six OVA challenges] and hypertrophy in the colon associated with 2.4-fold increased MPO activities in the colon and with dramatically increased IgE levels in sera. Th2 cytokine production (IL-4, IL-5, and IL-10) increased in the food allergy mice compared with non-treated mice, whereas IFN- $\gamma$ (Thl cytokine) was not detected even in the food allergy mice, suggesting that immune response is skewed toward Th2 in the food allergy mice. All of the primed PI3K $(+/+)$ mice developed allergic diarrhea after six oral OVA challenges, while the primed PI3K (-/-) mice did not develop allergic diarrhea at all. In the proximal colon, a number of mMCP-1 positive cells continued to increase between one and six OVA challenges, inducing extensive mucosal mast cell hyperplasia. In the food allergy experiments with PI3K $(+/+)$ mice, OVA challenge induced a large number of $\mathrm{mMCP}-1$ positive mast cells in the proximal colon. In contrast, there were only few mMCP-1 positive mast cells in the proximal colon of OVA-challenged PI3K (-/-) mice, while IgE levels in sera of OVA-challenged PI3K (-/-) mice was not lower than those in PI3K (+/ + ) mice. CONCLUSIONS: Mucosal mast cells and PI3K pathway in the colon play a critical role in the onset of Th2-mediated food anaphylaxis.

\section{S1669}

Neutrophils Mediate Barrier Function in Cryptosporidium Parvum Infection Leah M. Zadrozny, Stephen M. Stauffer, Martha U. Armstrong, Samuel L. Jones, Jody L. Gookin

Cryptosporidium parvum (CP) is a minimally invasive pathogen of intestinal epithelium that results in villous atrophy, nutrient malabsorntion, diarrhea, and diminished barrier function. Influx of neutrophils (PMN) is a consistent feature of human cryptosporidiosis. However, the role of PMN in disease pathogenesis and their impact in limiting the extent of cryptosporidial infection are unknown. The purpose of this study was to determine the effect of PMN on epithelial defense and barrier function in $\mathrm{CP}$ infection. Neonatal piglets were infected with $10^{8} \mathrm{CP}$ oocysts and euthanized 4 days post-infection at which time sections of ileum were removed for ex vivo study. PMN infiltrates and lipid peroxidation were quantified by myeloperoxidase (MPO) activity and TBARS, respectively. Severity of infection was quantified by histology, daily body weight and fecal consistency. PMN infiltrates were inhibited by treatment with anti-CD18 (1mg/kg IV) or isotype control $\operatorname{lgG}(1 \mathrm{mg} / \mathrm{kg}$ IV). Superoxide was scavenged by treatment with pegylated superoxide dismutase (PEG-SOD 2000 IU/kg IV) or PEG $(0.001 \mathrm{mg} / \mathrm{kg}$ IV). Treatments were initiated at infection (day 0 ) and given daily until euthanasia. Ileal mucosa was mounted in Ussing chambers for measurement of transepithelial electrical resistance [TER] and flux of ${ }^{3} \mathrm{H}$-mannitol. $\mathrm{CP}$ infection resulted in significant villous atrophy, lipid peroxidation (nmolTBARS/mg protein=17.4 \pm 1.8 cont; *36.3 $\pm 6.4 \mathrm{CP}, \mathrm{n}=5-6$ ) and influx of PMN. Treatment with anti-CD18 antibody significantly inhibited PMN influx (MPOactivity/gram tissue $=21.7 \pm 6.8 \mathrm{CP}+\mathrm{IgG} ; * 8.9 \pm 1.9 \mathrm{CP}+$ anti-CD18) but did not attenuate lipid peroxidation or villous atrophy. Treatment with PEG-SOD significantly decreased PMN influx into CP infected mucosa (MPO activity/gram tissue=21.7 $1.6 \mathrm{CP}+\mathrm{PEG} ; * * 9.9 \pm 2.1$ $\mathrm{CP}+\mathrm{PEG}-\mathrm{SOD})$. PMN depletion had no adverse effect on infection severity, diarrhea, body weight or survival. Barrier function was significantly diminished by depletion of PMN (TER $\Omega \mathrm{cm}^{2}=71 \pm 4 \mathrm{CP}+\mathrm{IgG}: * 58+3.4 \mathrm{CP}+$ anti-CD 8$)\left(\mathrm{J}{ }^{3} \mathrm{H}-\mathrm{mann}=0.23 \pm 0.03 \mathrm{CP}+\mathrm{IgG}\right.$. $* 0.31 \pm 0.05 \mathrm{CP}+$ anti-CD 18). There was no difference in $\mathrm{PGE}_{2}$ synthesis by mucosa from piglets treated with IgG or anti-CD18 and barrier function could not be restored by treatment with $\mathrm{PGE}_{2}+\mathrm{PGI}_{2}$. The present studies demonstrate significant infiltration of PMN into $\mathrm{CP}$ infected mucosa and identify superoxide as an important stimulus for this event. PMN do not contribute to peroxidative injury of $\mathrm{CP}$ infected mucosa and have no influence of the severity of epithelial infection or diarrhea. The presence of PMN in CP infected mucosa promotes barrier function by a mechanism independent of mucosal $\mathrm{PGE}_{2}$ synthesis.

\section{S1670}

Persistent Activation of Gastric Innate Immunity in MHC Class II-Deficient Mice Induces Gastric Mucosal Hyperplasia Via Up-Regulation of Gastrin Toshiro Fukui, Akiyoshi Nishio, Kazuyuki Saga, Masahiro Kido, Keiichi Kiriya, Tsutomu Chiba

Background and aim: Major histocompatibility complex (MHC) class II-deficient mice have decreased CD4+ T cells, thus making them immunologically similar to patients with acquired immunodeficiency syndrome (AIDS). These patients have hypertrophic gastric folds, which are usually associated with opportunistic infection. MHC class II-deficient mice also have gastric mucosal hyperplasia. To clarify the mechanism of gastric mucosal hyperplasia, we investigated the pathophysiology and the role of the innate immunity in the stomach of MHC class II-deficient mice. Methods: MHC class II-deficient mice were maintained under specific-pathogen-free conditions. Stomachs from 1, 2, 3, 4, 5, and 6-month-old mice were examined histologically and immunohistochemically (using anti-B220, CD4, CD8, CD11b Ki-67, gastrin, and Reg-I $\alpha$ antibodies). Gene expression of interleukin- $1 \beta$, interleukin- 6 , interferon- $\gamma$, tumour necrosis factor- $\alpha$, Toll-like receptor 2 , Toll-like receptor 4 , cyclooxygenase-2, and myeloperoxidase activity in the gastric mucosa was investigated. Serum gastrin levels and gastric acidity were measured, and bacterial culture of the stomach was performed. To clarify the roles of hypergastrinemia in the gastric mucosa, a gastrin receptor antagonist (AG04lR) was administered to MHC class II-deficient mice, Results: Certainly, CD4+ T cells, but not CD8+ or B220+ cells, decreased in the spleen of MHC class II-deficient mice. MHC class II-deficient mice had a diffusely thick corpus mucosa with infiltration of CDIlbgranulocytes and macrophages. Anti-Ki67 staining demonstrated expansion of the proliferating neck zone and, furthermore, the upper and the lower Ki-67 negative zone. Gene expression of interleukin-1 $\beta$, interferon- $\gamma$, Toll-like receptor 2 , Toll-like receptor 4 , and cyclooxygenase- 2 were up-regulated, and myeloperoxidase activity was increased. Only a small amount of non-pathogenetic bacteria was detected in the stomach. Compared with wild type mice, serum gastrin levels and Reg-I $\alpha$-positive cells in the gastric mucosa increased in MHC class II-deficient mice despite normal gastric acidity. After treatment with AG041R the gastric mucosal thickness in MHC class II-deficient mice was significantly reduced compared with the non-treated mice. Conclusion: Persistent activation of innate immunity in the gastric microenvironment of MHC class II-deficient mice induced hyperplasia of the gastric mucosa, and hypergastrinemia appears to be involved in the hyperplasia of the gastric mucosa partly through Reg-I $\alpha$ production, suggesting a pathophysiology similar to the gastric changes in patients with AIDS.

\section{S1671}

Human Trefoil Factor Family-3 Induces Monocyte Production of IL-10 and Inhibits T Cell Proliferation

Xianyang Yio, Jiang Yio, Jie-Yu Zhang, Ling Shao, Giulia Roda, Stephanie Dahan, Mark Babyatsky, Steven Itzkowitz, Lloyd Mayer

Background: Trefoil factor family-3 (TFF3), a small peptide expressed by intestinal epithelial cells, is involved in promoting mucosal healing by inducing epithelial cell migration and inhibiting apoptosis. TFF3 knockout mice are highly susceptible to colitis caused by acute injury. TFF3-producing intestinal epithelial cells are located in close proximity to lymphocytes and previous studies demonstrated that TFF 3 could induce monocyte migration. Little is known about whether TFF3 might affect other monocyte functions. Aim: To test whether TFF3 affects functions of monocytes, including migration, antigen presentation and cytokine production. Methods: Recombinant human TFF3 (hTFF3) was produced. Mixed lymphocyte reactions (MLR) and antigen specific (candida) lymphocyte proliferation were performed in the presence or absence of varying concentrations of hTFF3. Normal peripheral blood monocytes were treated with hTFF3 and cytokine production was analyzed by RT-PCR and ELISA Results: Similar to previous observations, hTFF3 (20-40 ug/ml) induced peripheral blood monocyte migration. T cell proliferation was significantly inhibited in an MLR using monocytes/DCs pretreated with hTFF3 at doses of 40 to $100 \mathrm{ug} / \mathrm{ml}$. Similarly, hTFF3 inhibited candida-specific T cell proliferation. hTFF3 also inhibited LPS-induced IL6, IL1 beta and TNF-alpha production by peripheral blood monocytes and a human monocyte hybridoma, clone 63 (RT-PCR). Importantly, hTFF3 induced IL-10 production by peripheral blood monocytes by ELISA and RT-PCR. In fact, hTFF3 treatment resulted in dramatic 20 to 120 fold increases in IL-10 production (28.3-159.3 pg/ml in untreated cells vs. 3309 $3465.6 \mathrm{pg} / \mathrm{ml}$ in treated cells). Furthermore, addition of anti-IL-10 receptor antibody reversed the inhibitory effects of hTFF3, suggesting that the induction of IL-10 secretion in monocytes likely mediates TFF3's inhibitory effect. Conclusions: TFF3 is able to regulate immune responses by altering cytokine production in monocytes. This inhibition of lymphocyte proliferation most likely occurs via induction of IL-10 secretion. TFF3 may therefore contribute to the overall suppressed immunologic tone in the gut and contribute to an antiinflammatory immune response. (Sponsored in part by the AGA Centocor Excellence in IBD Clinical Research Fellowship and CCFA grant)

\section{S1672}

Characterization of Potential Role of NADPH Oxidase Organizer 1 (NOXO1) in Oxygen Radical-Dependent Immune Responses of Large Intestinal Epithelial Cells

Kazuhito Rokutan, Yuki Kuwano, Kumiko Tominaga, Hideyuki Sasaki, Kensei Nishida, Kiyoshi Masuda, Atsuo Sekiyama, Shigetada Teshima-Kondo

Reactive oxygen species regulate a variety of pathophysiological processes including hormone biosynthesis, regulation of cell growth, atherosclerosis, and host defense. NADPH oxidase 1 (Noxl) is known as a novel superoxide-generating enzyme predominantly expressed in surface mucous epithelial cells of the human colon. We have already shown that human colonic epithelial cells (T84 cells) induces Noxl and up-regulates superoxide production in response to flagellin from Salmonella enteritidis (J Immunol, 2004) or IFN-gamma (Am J Physiol, 2005). Although flagellin, IL-1-beta, IFN-gamma, and tumor necrosis factor (TNF) alpha similarly induced Noxl, TNF-alpha was the most effective activator for up-regulation of superoxide generation by T84 cells. Recently, a novel adaptor protein, Nox organizer 1 (NOXO1), has been identified and recognized as one of the essential factors required for Noxl activity. Interestingly, NOXOl was induced only when treated with TNF-alpha, suggesting a crucial role of NOXO1 in the cytokine-triggered oxygen burst response of the cells. To elucidate the mechanisms for TNF-alpha-stimulated transcription of the NOXO1 gene, we cloned 5'-franking region of the human $\mathrm{NOXOl}$ gene. The promoter region contain putative binding sites for several transcriptional factors, such as nuclear factor-kappaB, Spl AP-1, and Ets. The promoter assay and EMSA indicated that AP-1 mainly regulated TNFalpha-dependent transcription of the NOXOl gene. In fact, AP-1 oligonucleotide decoys completely blocked the TNF-alpha-stimulated NOXOl transcription and supreoxide production. TNF-alpha is known as a key mediator involved in the pathogenesis of inflammatory bowel diseases particularly in Crohn's disease. We also confirmed that the expression of Noxl and NOXOl was significantly up-regulated in the inflamed tissues of Crohn's disease. Thus, our results suggest that dysregulation of Noxl-related innate immune response may 
result in chronic, persistent inflammation and may play a pivotal role in the pathogenesis of Crohn's disease

\section{S1673}

Inhibition of Interleukin-1 $\beta$ Release from Monocytes By Human $\alpha$-Defensins Jishu Shi, Shelly Aono, Wuyuan Lu, Xueyou Hu, Christine Dyskstra

Although it has been well established that mutations in Nod2 increase susceptibility to Crohn's disease (CD), the role of Nod2 in the pathogenesis of CD remains elusive. Recent studies on human patients and Nod2 knock-in mice have demonstrated that variants of Nod2 are associated with decreased intestinal expression of antimicrobial $\alpha$-defensins but excessive secretion of interleukin $1 \beta$ (IL-1 $\beta$ ). However, mechanisms by which Nod2 mutations lead to the up-regulation of IL-1 $\beta$ secretion have yet to be established. The present study was designed to test our hypothesis that reduced intestinal expression of human $\alpha$ defensins is responsible for the over-production of IL-1 $\beta$ in patients with CD. LPS-activated $(20 \mathrm{ng} / \mathrm{ml}, 2 \mathrm{~h}),{ }^{35} \mathrm{~S}$-methionine-labeled (1 h) human monocytes were treated with human $\alpha$-defensin HNP-1 or HD-5 in the presence or absence of $1 \mathrm{mM}$ ATP for $3 \mathrm{~h}$. Media and cell-associated fractions were harvested separately and IL-1 $\beta$ was recovered from each by immunoprecipitation. The resulting immunoprecipitates were analyzed by SDS-PAGE and autoradiography. In the absence of $1 \mathrm{mM}$ ATP, LPS-activated monocytes released about $10 \%$ of ${ }^{35}$ S-labeled proIL- $1 \beta(31 \mathrm{kDa})$ into the media, but no mature IL-1 $\beta(17 \mathrm{kDa})$ was detected in extracellular milieu. The release of proIL-1 $\beta$ from LPS-activated monocytes was completely blocked by $10 \mu \mathrm{g} / \mathrm{ml}$ of HNP-1 or HD-5. Addition of $1 \mathrm{mM}$ ATP to LPS-activated monocyte culture led to the release of $90 \%$ of newly synthesized proIL-1 $\beta$ from the cell and more than $90 \%$ of that was processed to mature IL-1 $\beta$ and detected in the media. At physiological concentrations ( $5 \mu \mathrm{g}$ to $100 \mu \mathrm{g} / \mathrm{ml}$ ), both HNP-1 and HD-5 blocked ATPmediated proIL-1 $\beta$ release in a dose-dependent manner. These data have demonstrated that $\alpha$-defensins may function as negative regulators of the posttranslational processing and release of IL-1 $\beta$. In addition to their antimicrobial activity, defensins may play an important role in intestinal inflammation by controlling the production of IL-1 $\beta$. Therapeutic approaches aimed at blocking IL-1 $\beta$ production by defensins could lead to the reduction of intestinal inflammation and tissue destruction in inflammatory bowel disease.

\section{S1674}

What Is the Function of Metaplastic Paneth Cells?

Jan Wehkamp, Nita Salzman, Bo Shen, Marko Estrada, Christian Leutenegger, Charles L. Bevins

Introduction: Paneth cells (PCs) are secretory epithelial cells of the small intestinal mucosa and a major source of antimicrobial peptides including the $\alpha$-defensins HD5 and HD6, lysozyme, and sPLA2. In the small intestine, these antimicrobials likely protect from ingested pathogens and may regulate the intestinal microflora. In the presence of inflammation, PCs can be also found outside the small intestine, in a well described process termed PC metaplasia. Since virtually nothing is known about the function of metaplastic Paneth cell, this study aimed to directly test the possible effects of metaplastic Paneth cells on the luminal crypt microbiota. Methods: We analyzed colonic samples from FvB mice with a dextran sodium sulfate (DSS)-induced experimental colitis. Mice were treated with DSS (1-2.5\%) and monitored at various time-points by anti-lysozyme immunohistochemistry to detect metaplastic Paneth cells in the colonic crypts. Individual crypts, either positive or negative for metaplastic Paneth cells from the same mouse were separately isolated using laser capture microscopy. The isolated crypts were used for extraction of bacterial DNA. For quantification of bacteria we used a real-time TaqMan assay which detects bacterial 16S RNA (common motif), as well as one assay for quantification of fungi. Results:. In contrast to mice with experimental colitis, we could not identify metaplastic Paneth cells in the colon of untreated healthy mice. Using laser capture microscopy, we could isolate detectable amounts of bacterial DNA in colonic crypts. In the presence of metaplastic Paneth cells $(n=3)$, the crypts contained significantly lower numbers of bacterial DNA $(\mathrm{p}=0.03)$ and fungi DNA $(\mathrm{p}=0.04)$ as compared to crypts without Paneth cells from the same mouse colon $(n=3)$. Conclusions: The presence of metaplastic Paneth cells has a functional impact on the microbiota of colonic crypts. This suggests that a function of Paneth cell metaplasia is to recruit antimicrobial defenses to sites of inflammation, and as such it represents a novel mechanism of mucosal innate immunity. Current studies are examining the bacterial species within the crypts to determine if the metaplastic Paneth cells selectively alter the composition of the local microflora.

\section{S1675}

Interleukin-8 (IL-8) Is a Critical Signal in Intestinal Epithelial CellMacrophage Cross-Talk

Akhil Maheshwari, Lesley E. Smythies, Ronnie Clements, Yolanda Hartman, Meg Mosteller-Barnum, Phillip D. Smith

Background: Macrophages are an important host defense cell in both non-inflamed and inflamed intestinal mucosa. We published recently (JCI 2005; 115: 66) that intestinal extracellular matrix (stromal) products downregulate the inflammatory, but not host defense activity of monocytes newly recruited to the mucosa, thereby inducing an intestinal macrophage function and phenotype profile. The mechanisms involved in the recruitment of blood monocytes are not known. Since intestinal epithelial cells (IECS) produce IL-8 upon exposure to a variety of bacterial products, we hypothesized that IL-8, a known chemoattractant and activator of neutrophils, also acts as an important signal in the 'cross-talk' between IECs and mononuclear phagocytes in the mucosa. Therefore, we investigated whether IL-8 recruited and activated blood monocytes and/or intestinal macrophages and whether IL-8 enhanced the cells' phagocytic activity. Methods: Blood monocytes were purified by magnetic bead (MACS) separation or counterflow centrifugal elutriation. Human intestinal macrophages were isolated by enzyme digestion of jejunal tissue followed by elutriation. Lamina propria stroma $(1 \mathrm{gm} / \mathrm{mL})$ was cultured in RPMI for 24 hrs to harvest stroma-conditioned media (S-CM). S-CM IL-8 concentrations were measured by ELISA $(n=7)$. Monocyte migration to IL-8 and S-CM was measured in a fluorescence-based microchemotaxis assay. Monocyte and macrophage phagocytic activity for FITC-labeled latex microbeads and GFPexpressing E. coli was measured before and after treatment with IL-8 for 1 hr. Results: We first showed that blood monocytes and intestinal macrophages express CXCR1 $(65 \pm 15 \%$ of macrophages, $51 \pm 13 \%$ of monocytes) and CXCR2 (13.8 $\pm 6 \%$ of macrophages, $59.4 \pm 16 \%$ of monocytes), the cognate receptors for IL-8. IL-8 was detected in S-CM (208-1018 pg/mg protein). Monocytes, but not macrophages, displayed migratory activity to IL-8 and S-CM. S-CM-induced monocyte chemotaxis was significantly inhibited by anti-IL-8 antibody and also by pre-treatment of monocytes with S-CM to ligate the IL-8 receptors. In addition, IL- 8 treatment also increased the phagocytic activity of monocytes as wells as intestinal macrophages (up to $300 \%$ ). Conclusion: IL-8 is a potent chemoattractant for blood monocytes and an activator of phagocytic activity of both monocytes and intestinal macrophages. The ability of stromal IL-8 to recruit blood monocytes supports the role of this cytokine in the 'cross-talk' between IECs and mononuclear phagocytes in the intestinal mucosa. These findings are the first to expand the IL-8-neutrophil paradigm to include the mononuclear phagocyte system.

\section{S1676}

Expression of Human Defensin-5 in Inflammatory Bowel Disease Tissue David A. Elphick, Patrick Tighe, Susan Liddell, Yashwant R. Mahida

Introduction: Human defensin-5 (HD-5) is a major antimicrobial peptide which is present in the lumen in its mature form but stored in normal small intestinal Paneth cells in its precursor form. Alterations in the expression of HD-5 in Paneth cells of Crohn's small intestine or metaplastic Paneth cells of colon affected by inflammatory bowel disease (IBD) may lead to impaired mucosal innate immunity. Methods: Surgical resection specimens of terminal ileum (6 normal, 7 Crohn's disease) and colon [4 normal, 2 Crohn's disease \& 5 ulcerative colitis (UC)] were studied. Tissue sections were used for immunohistochemistry. Paneth cell-containing epithelial crypts were detached from mucosal strips with EDTA. HD5 was purified from acid extracts of epithelial crypts using cation-exchange chromatography and C18 RP-HPLC, using anti-HD-5 antibody to identify fractions containing the alphadefensin. Purified HD- 5 was characterised by mass spectrometry and N-terminal amino acid sequencing. HD-5 isolated from normal and IBD tissue was also studied by acid-urea Western blot analysis. Genotyping for the 3 single nucleotide polymorphisms (SNPs) in the NOD2 gene that have been associated with Crohn's disease (SNP8/R702W, SNP12/G908R, SNP13/ $1007 \mathrm{fs}$ ) was also performed. Results: Immunohistochemical studies showed that HD-5 was expressed predominantly in Paneth cells in normal terminal ileum and in IBD tissue, but absent in normal colon. On acid-urea Western blot analysis of purified HD-5 from normal small bowel (6), Crohn's small bowel (7) and IBD colon (7), identically sized immunoreactive bands were seen. Purified HD-5 from the different tissue types had a similar elution profile on the C18 RP-HPLC column (39\% acetonitrile). Identical N-terminal sequence (ESLQERADEAT, the precursor form) was found for HD-5 extracted from Crohn's small intestine (2), $\mathrm{UC}$ colon (2) and normal terminal ileum. In addition to detecting the mass predicted for the precursor form $(8102 \mathrm{Da})$ in all the purified HD-5 samples tested, preparation from one Crohn's sample also contained similar amounts of HD- 5 precursor truncated by 6 amino acids (sequence: ADEAT, mass $7360 \mathrm{Da}$ ). The mature form of HD- 5 was not detected in any of the samples studied. One Crohn's disease patient was homozygous for SNP13, one heterozygous for SNP8 and one heterozygous for SNP12. Conclusions: In our studies to date, in Paneth cells of Crohn's small intestine and in metaplastic Paneth cells of IBD colon, there was no evidence of processing of HD-5 to the mature form. Significant amounts of a truncated form of HD-5 was identified in Paneth cell extracts of one patient with ileal Crohn's disease.

\section{S1677}

Human Defensin Alpha 1 (DEFAl) Expression and Interaction Are Associated with Nucleotide Oligomerization Domain 2 (NOD2/CARD15)

Jesus K. Yamamoto-Furusho, Daniel K. Podolsky

Backgroud: DEFAl has a broad spectrum of antimicrobial activity against bacteria, fungi, and some enveloped viruses by perforating the cell membrane through formation of multimeric pores. NOD2 is a cytoplasmic protein expressed in several cell types and leads to Nuclear Factor kappa B (NF-אB) activation as a result of intracellular recognition of muramyl dipeptide (MDP) from bacteria. Aims: To determine the effect of Nod2 on DEFAl expression and to evaluate interaction between these two proteins. Methods: Immunoprecipitation and Western blot: HCT116 cells lysate were immunoprecipitated with anti-NOD2 or anti-DEFA1 polyclonal antibodies and protein A/G sepharose beads. NF-kB activation assay: HEK293 cells were transfected with DEFAl at different concentrations, NOD2 (lng), pIV luciferase reporter, renilla plasmid and MDP-LD. Real-time RT-PCR was done in an ABI Prism 7000 sequence detector using SYBR Green JumpStart detection system. Immunostaining was performed in COS-7 cells using Texas-Red-conjugated anti-mouse IgG or FITC-conjugated anti-rabbit IgG antibodies. Invasion assay: Gentamicin protection assay using Salmonella typhimurium was performed in Caco-2 cells. Results: Expression of DEFAl was minimal in Caco-2 and HEK293 cells which lack endogenous NOD2 protein in contrast to high expression of DEFAl in cells with NOD2 endogenous expression such as HCT116 and SW480 cells. A direct interaction between DEFAl and NOD2 proteins was confirmed by immunoprecipitation. DEFAl colocalized with NOD2 in the cytoplasm of COS-7 cells. DEFAl increases the NF-kB activation concentration-dependant in HEK293 cells co-transfected with NOD2 and DEFAl constructs and stimulated with MDP-LD. Transfection with DEFAl siRNA significantly decreased the MDP driven response to NOD2. The invasive ability of Salmonella typhimurium showed antibacterial activity. DEFAl mRNA expression was increased in involved areas from mucosa of $U C$ and $C D$ patients as compared to control patients. Conclusion: Human DEFAl associated with NOD2 mediated NF-KB activation in colonic epithelial cell lines. 
S1678

Cyclooxygenase-2 Regulates Toll-Like Receptor 2 On Intestinal Epithelial Cells Shinichiro Shinzaki, Hideki Iijima, Satoshi Egawa, Shuji Ishii, Takanobu Irie, Tsutomu Nishida, Yoshimi Kakiuchi, Masakazu Yasumaru, Hiroaki Murata, Tatsuya Kanto, Masahiko Tsujii, Shingo Tsuji, Sunao Kawano, Norio Hayashi

Background: COX-2 expression and COX-2-derived prostaglandins (PGs) are reported to affect immune function, but the contribution of COX-2 to regulate mucosal immunity in inflammatory bowel disease(IBD) is undefined. On IECs, Toll-like receptors (TLRs) are expressed and play an essential role on the innate immune system by activation of specific signaling pathways and production of inflammatory cytokines and chemokines. Although TLRs, e.g.,TLR2, TLR4 and TLR5 that recognize luminal bacterial surface components, are reported to contribute to the pathogenesis of IBD, the mechanisms how the expression of TLR and TLR-mediated signaling are regulated are still unclear. Aim: We investigated the possibility that COX-2 regulates the TLR expression and TLR-mediated signaling pathways. Method: The expressions of TLR mRNA were determined by quantitative RT-PCR in two intestinal cell lines, Caco-2 which express low baseline level of COX-2 and HT-29 which express large amount of COX-2. These cells were modified to overexpress or inhibit the production of COX-2 by stable transfection of COX-2 or knock down by RNA interference (RNAi). COX-2-transfected Caco-2 (Caco-2-COX-2) cells and the mock cells were stimulated by Pam3CSK4, selective ligand for TLR2, and production of interleukin(IL)-8 in the culture supernatant was measured by ELISA Caco-2 cells were stimulated by prostaglandin(PG)E2, and expression of TLR2 mRNA and IL-8 production was evaluated. Results: The level of TLR2 expression was significantly higher in Caco-2-COX-2 cells when compared with Caco2 -mock cells. In addition, the TLR2 expression was significantly decreased when COX-2 was knocked down by RNAi in the HT-29 cells in comparison to the original HT-29 cells. Treatment of Caco-2-COX-2 cells with etodolac, a selective COX-2 inhibitor, resulted in decrease of TLR2 expression, whereas treatment of Caco-2 cells by PGE2 increased TLR2 expression in a dose dependent manner. Ligation of TLR2 by Pam3CSK4 in Caco-2-COX2 cells which express high TLR2 induced significantly increased level of IL-8 production than in Caco-2-mock cells. Production of IL-8 by stimulation of TLR2 was significantly increased by the presence of PGE2 and the effect was dose-dependent of PGE2. Conclusion: These results suggest that COX-2 and PGE2 regulate the expression of TLR2 and its downstream IL-8 production in the IECs. Controlling the TLR2 pathway by COX-2 or PGE2 will be therapeutic benefit for IBD.

\section{S1679}

Neutrophil Activation and Neutrophil Extracellular Trap Formation in Inflammatory Bowel Disease

Gareth Parkes, Simon Clare, David Goulding, Neil Rayment, Barry Hudspith, Jonathan Brostoff, Gordon Dougan, Jeremy Sanderson, Liljana Petrovska

INTRODUCTION: Neutrophils play key role in killing bacteria at the early stages of infection. Contact with microbes activates neutrophils and induces formation of neutrophil extracellular traps (NETs) which are involved in entrapment of bacterial and prevention of bacterial spread, and neutralisation of the virulence factors. However, various models of injury suggested that neutrophils are a major contributor to gastrointestinal injury. NET formation has been demonstrated in vitro and in murine models of colitis but never in vivo in humans. AIMS: To investigate neutrophil activation and NETs formation in active inflammation in patients with Crohn's and Ulcerative Colitis. METHODS: Snap-frozen colonic biopsies were taken from 10 patients with UC and Crohn's diseas from inflammed and uninflammed areas and 5 subjects with a normal colon, undergoing colonoscopy. Cryosections were stained by two-colour immunofluorescence to determine the expression of activation molecules on neutrophils. Bacteria colocalised with NETs were detected in cryosections with fluorescent in situ hybridization (FISH) and with $16 \mathrm{~S}$ ribosomal (r)RNA-targeted probes for all bacteria and specific probes for the major representatives of anaerobic microflora (bifidobacteria, Bacteroides, clostridia, atopobia) and aerobic microflora (Enterobacteriaceae, enterococci, streptococci, lactobacilli). RESULTS: Actively inflamed IBD mucosa showed formation of neutrophilic extracellular traps expressing myeloperoxidase and DNA. Disease controls (uninflammed mucosa) and normal individuals showed no NET formation. Active formation of NETs co-localised with the luminal bacteria in the actively inflamed mucosa. NET formation was also confirmed by scanning electron microscopy which confirmed bacterial colocalisation. CONCLUSIONS: Neutrophil extracellular traps are formed and co-localise with luminal bacteria in actively inflamed mucosa in IBD patients. The possible effect of NETs on prevention or induction of gut mucosal injury is a field that warrants further investigation.

\section{S1680}

The Anti-Inflammatory Flavonoid Luteolin Attenuates Spontaneous Colitis in IL-10-/- Mice But Worsens Chemical-Induced Colitis in Nf-kBegfp Transgenic Mice

Thomas Karrasch, Byung-Ik Jang, Christian Jobin

Introduction: The flavonoid luteolin has anti-inflammatory properties both in vivo and in vitro. Recently, we showed that luteolin prevents LPS induced NF-KB signaling and proinflammatory gene expression through inhibition of IKK activity. However, the impact of this flavonoid on experimental models of colitis is unknown. Aim: To establish the therapeutic value of luteolin in the dextran sodium sulfate (DSS) and in the IL-10-/- model of acute and chronic experimental colitis, respectively. Methods: NF-KBEGFP mice (7/group) were fed 3\% DSS in drinking water or water control for 5 days, and luteolin was administered intragastrically ( $1 \mathrm{mg} / \mathrm{Kg}$ body weight) daily. In a different set of experiments, germ-free IL10-/- mice (10/group) were transfered to a specific pathogen free environment and fed a diet chow containing 2\% luteolin or isoflavone free control chow (AIN-76) for 4 weeks. The colons were resected and imaged using a CCD camera specific for EGFP. MAPKp38, RelA and H3Ser 10 phosphorylation were evaluated in isolated primary enterocytes by Western blotting using specific phospho-antibodies. IEC18 cells were grown to confluency and subjected to standard wounding. Results: NF-KBEGFP mice exposed to luteolin showed a trend toward worse DSS-induced colitis (disease activity index, histological scores, IL-12p40 secretion) compared to non-treated mice. In addition, EGFP expression (NF-אB activity) was increased in luteolin-treated mice as compared to control as visualized by CCD fluorescence macroscopy. Interestingly, confocal microscopy showed that EGFP positive cells were mostly located in the lamina propria and not in the epithelium. Enhanced MAPKp38 and H3S1O phosphorylation in enterocytes isolated from DSS-exposed mice was attenuated in luteolin treated NF-אBEGFP mice compared to control treated mice. Interestingly, in vitro experiments showed that restitution was attenuated $(63 \%$ of control, $\mathrm{p}<0.001)$ in luteolin-treated IEC18 cells, suggesting a negative impact of this flavonoid on the wound-healing response. In contrast, luteolin ( $2 \%$ diet) prevented the development of spontaneous colitis in IL-10-/ mice (histological evaluation, macroscopy) compared to AIN-76 fed mice. In addition, RelA phosphorylation was decreased in colonic tissues isolated from luteolin-fed IL-10-/- mice. Conclusion: We conclude that flavonoids such as luteolin may represent an attractive adjuvan in the treatment of immunologically driven colitis. However, caution should be exerted in gastrointestinal disorders involving a compromised barrier function or dysregulated woundhealing response.

\section{S1681}

Novel Role for Chronic Stimulation of NOD2 in Mediating Tolerance to Bacterial Products Matija Hedl, J.N. Li, Judy Cho, Clara Abraham

The Toll-like receptor (TLR) and nucleotide-binding oligomerization domain (Nod) family of proteins are critical for the recognition of bacterial products by the immune system which frequently leads to pro-inflammatory responses. Loss-of-function Nod2 polymorphisms have been associated with Crohn's disease. To date, studies have examined consequences of acute stimulation of Nod2. As the intestine is an environment of tolerance to chronic commensa bacterial exposure, we hypothesized that long-term stimulation of Nod2 might contribute to enteric tolerance to bacteria by suppressing inflammatory responses from innate immune receptors. We find that pre-treatment of primary human monocyte-derived macrophages with muramyl dipeptide (MDP), a ligand specific for Nod2, significantly decreased the production of the pro-inflammatory cytokines TNF-alpha, IL-8 and IL-1-beta upon Nod2 as well as TLR4 and TLR2 re-stimulation in a time-dependent fashion. Importantly, TNFalpha-induced production of proinflammatory cytokines remained intact in these same cells, demonstrating selectivity in Nod2-mediated tolerance pathways. MDP-stimulated macrophages from Crohn's disease-relevant Leul007insC Nod2 homozygote individuals were deficient in their ability to cross-tolerize to subsequent treatment with TLR2 or TLR4 ligands. The Nod2-induced tolerance is not the effect of Nod2, TLR2 or TLR4 downregulation. Significantly, intestinal macrophages exhibit tolerance to MDP-induced cytokine production, but are still able to phagocytose bacteria. These results illustrate a novel role for Nod2 in mediating tolerance to bacterial products.

\section{S1682}

Extracellular Pressure Stimulates Macrophage Phagocytosis Via a Pathway Involving Phosphoinositide 3-Kinase, Akt2, FAK, ERK, and P38 Hiroe Shiratsuchi, Marc D. Basson

Increased intestinal pressure is characteristic of chronic inflammatory bowel disease, and changes in tissue pressure are associated with infection and inflammation. We have previously demonstrated that low extracellular pressure $(20 \mathrm{mmHg})$, equivalent to normal interstitia tissue pressure, increases phagocytosis by primary human monocytes and macrophages and PMA-differentiated human monocytic THP-l cells, in part by FAK and ERK inhibition an p38 activation. PI-3K (phosphoinositide 3-kinase) is known to be required for macrophage phagocytosis through Fc-receptors, but whether the effect of pressure on complement receptor-mediated phagocytosis requires PI-3K is not known. Furthermore, relatively little is known about the role of PI-3K downstream kinases such as Akt in modulating macrophage phagocytosis. Using PMA-differentiated THP-1 macrophages, we studied the contribution of PI-3K, and the downstream kinase Akt to the pressure-increased macrophage phagocytosis that occurs through complement receptors independent of Fc-receptors. Exposure to extracellular pressure induced p85 PI-3K translocation from cytoplasmic to membrane fraction and increased Akt phosphorylation by $36.1 \pm 12.0 \%$ in THP-1 macrophages. Pretreatment with the PI-3K inhibitor LY294002 (40 $\mu \mathrm{M}$ ) or Akt inhibitor IV (cell-permeable benzimidazol compound) $(10 \mu \mathrm{M})$ abrogated increased phagocytosis by pressure but did not affect basa phagocytosis. Basal Akt phosphorylation was inhibited $90 \%$ by LY294002 and 70\% by Akt inhibitor IV and both inhibitors prevented Akt activation by pressure. LY294002 decreased basal FAK-Y397 phosphorylation by 20\% but increased basal ERK and p38 phosphorylation 1.5 fold. LY294002 prevented pressure-induced inhibition of FAK-Y397 and ERK phosphorylation and activation of $\mathrm{p} 38$. Transfection with AktlSiRNA or Akt2SiRNA reduced total Aktl and Akt2 expression by $40 \%$ and $50-60 \%$, respectively. Akt1SiRNA tended to reduce basal, but did not affect pressure-induced THP-1 macrophage phagocytosis. On the other hand, Akt2SiRNA did not affect basal phagocytosis, but abrogated pressure-mediated phagocytosis. Changes in tissue pressure during infection and inflammation may regulate macrophage phagocytosis by activation of PI-3K, which activates Akt2, inhibits FAK and ERK, and activates p 38 in response to this stimulus. In contrast, basal phagocytosis may be more closely regulated by Aktl.

\section{S1683}

High Fat Diets Exacerbate Dextran Sulfate Sodium (DSS) Induced Colitis in Mice By Altering Non-Classic Nkt Cells and Regulatory T Cells Xiong Ma, Zhiping Li

BACKGROUND: Among current theories of the pathogenesis of inflammatory bowel disease (IBD), the balance between effector T cells and regulatory T cells (Treg) plays an importan role in regulating mucosal inflammation. In addition, environmental factors, such as diet, also contribute to the susceptibility for IBD. Epidemiologic studies have implicated that high 
fat diet is a risk factor of IBD. The aim of this study was to understand the mechanisms by which high fat diet regulates intra-epithelial T cells, and in turn change the susceptibility to mucosal inflammation. METHODS: Wild type C57BL6 mice were fed commercial high fat diets (59\% of calories from fat) for 6 weeks. Control mice received an isocaloric volume of normal diets that contains less fat ( $12 \%$ of calories from fat). Intraepithelial lymphocytes (IEL) were isolated from the large intestine by Percol gradient. The IEL phenotype and cytokines profile were analyzed by flow cytometry. Then, mice were subjected to $2.5 \%$ (w/ v) DSS in drinking water. Animal weight, rectal bleeding and colonic inflammation were monitored. RESULTS: Mice fed high fat diet have increased colonic NKT cells. Unlike classic invariant NKT cells that are primarily either CD4+ or CD4-CD8- and restricted by CD1, the colonic NKT cells are mostly CD8+ and CDl independent. They express tumor necrosis factor (TNFa) and interferon (IFN $\gamma$ ), which are increased in high fat diet mice. Mice fed high fat diet also have decreased colonic Treg cells (CD4+CD25+Foxp3+) with less proliferation, as reflected by decreased BrdU incorporation. High fat fed mice have more severe DSS-induced colitis, as reflected by more weight loss, worse rectal bleeding and more severe colonic inflammation. Depletion of colonic NKT cells, or adoptive transfer of Treg cells improves colitis in these mice. Foxp3-/- mice that lack Treg cells also have increased colonic CD8+NKT cells. Adoptive transfer of Treg cells does not reduce CD8+NKT cells in high fat fed mice. However, adoptive Treg cell transfer does reduce CD8+NKT cell expression of TNFa and IFN $\gamma$ in mice fed high fat diets. CONCLUSIONS: High fat diets alter colonic NKT cells and Treg cells and lead to increased susceptibility to DSS-induced colitis. This may provide one mechanism how environmental factors, i.e. diet, may cause increased the susceptibility for IBD.

\section{S1684}

Alterations in Human Defensin-5 (HD-5) Expression Following Gastric Bypass Surgery

David A. Elphick, Magnus Sundbom, Yashwant R. Mahida, Robert N. Cunliffe, Tore Midtvedt, Lars Engstrand, Sven Gustavsson, Lars-Goran Axelsson

Introduction. After Roux-en-Y gastric bypass surgery all ingested food will pass directly (from the esophagus) into the small bowel intestine. Thus, it provides a novel human model to investigate small bowel mucosal innate immunity, in which there is loss of gastric acidmediated protection against orally-acquired microorganisms. We have studied changes in jejunal mucosal HD-5, which is an antimicrobial peptide normally produced by Paneth cells. Methods. Mucosal samples were obtained from 18 female patients (24 - 54 yrs), from the same segment of jejunum during and after (at endoscopy) gastric bypass surgery. Samples were used for bacterial culture and immunohistochemistry using anti-HD-5 antibody. The number of immunoreactive cells per crypt and villus were determined and expressed as mean (SD). Results. No bacteria were cultured from any of the peri-operative jejunal samples but colonies of bacteria normally present in the pharynx were identified during culture of all post-operative jejunal biopsies ( 1 - >100 colonies). Paneth cell numbers per crypt were unchanged after gastric bypass [4.16(0.71) v 4.24(0.78)]. However, following surgery, there was an increase in: HD-positive intermediate cells per crypt $[0.25(0.41)$ v $1.12(0.66)$, $\mathrm{p}<0.01)$, HD-5 staining enterocytes per crypt $[0.31(0.09) \mathrm{v} 1.38(1.10), \mathrm{p}<0.01]$, HD-5 staining material in the crypt lumen [crypt lumens: $5.0(10.9) \%$ v $68.1(27.9) \%, \mathrm{p}<0.01$ ] and HD-5 immunoreactivity coating the luminal surface of villus enterocytes [villi sampled: $15.0(31.0) \%$ v $67.5(42.0) \%, p<0.01]$. Conclusions. 1. Bacteria normally resident in the pharynx were present in the proximal jejunal mucosa following Roux-en-Y gastric bypass surgery. 2. After gastric bypass, there was increased secretion of HD-5 and an increase in HD-5 expressing intermediate cells and enterocytes in the crypt. 3. The increase in HD-5 expression in the jejunal mucosa following gastric bypass surgery is likely to be secondary to exposure to orally-acquired microorganisms.

\section{S1685}

The Role of Endogenous and Pharmacological Ppary Agonists On Restoration of Colonic Homeostasis After Acute Stress-Induced Inflammation and Dysfunction

Javier R. Caso, Angel Ponferrada, Luis Alou, David Sevillano, Maria L. Gomez-Lus, Arturo L. Colon, Ignacio Marin-Jimenez, Maria A. Moro, Ignacio Lizasoain, Pedro Lorenzo, Pedro Menchen, Enrique Cos, Juan C. Leza, Luis Menchen

Background and aims: Psychosocial stress has been implicated in the clinical course of gastrointestinal diseases such irritable bowel syndrome and inflammatory bowel disease, but the mechanisms implicated and the effects of stress on the normal colon are not yet fully understood. Methods: Male young-adult Wistar rats were exposed to immobilisation stress ( 6 hours periods every day during 1,5 or 10 days). After that, colon was dissected and processed for biochemical and protein expression studies, including myeloperoxidase (MPO) activity, inducible nitric oxide synthase (NOS-2) and cyclooxygenase (COX-2) expression and activities $\left(\mathrm{NO}_{\mathrm{x}}^{-}\right.$and $\mathrm{PGE}_{2} / 15 \mathrm{~d}-\mathrm{PGJ}_{2}$ respectively), peroxisome proliferator activated receptor gamma (PPAR $\gamma$ ) expression, immunoglobulin A (IgA) levels and nuclear factor kappa B (NFKB) nuclear translocation. Mesenteric lymph nodes, spleen and liver were processed and cultured to assess bacterial translocation. Colonic permeability to ${ }^{51} \mathrm{Cr}$-EDTA was also evaluated. Results: After 1 day of immobilisation stress, significant weight loss, increase in MPO activity, NFKB nuclear translocation, increase in NOS-2 expression and activity and in COX-2 expression were observed in the colonic mucosa. All these parameters returned to baseline values after 10 days of stress exposure, in parallel to an increase, starting at day 5 , of anti-inflammatory prostaglandin $15 \mathrm{~d}-\mathrm{PG}_{2}$ levels and PPAR $\gamma$ expression. IgA levels in homogenised colonic mucosa were significantly decreased after 1 day of stress, returning to baseline values after 10 days. No bacterial translocation was observed in control animals, but it reached maximal levels in terms of colony forming units per gram of tissue after 1 and 5 days of immobilisation stress, in parallel to an increase in colonic permeability to ${ }^{51} \mathrm{Cr}$-EDTA. In order to study the role of the COX/PPAR $\gamma$ antiinflammatory pathway as a possible homeostatic mechanism in the stressed colon, several groups of animals received ip injections of the physiological PPAR $\gamma$ ligand $15 \mathrm{~d}_{-} \mathrm{PGJ}_{2}(120 \mathrm{mg} / \mathrm{kg})$ and the synthetic PPAR $\gamma$ ligand, rosiglitazone $(3 \mathrm{mg} / \mathrm{kg})$ at the onset of stress. Both $15 \mathrm{~d}-\mathrm{PG} \mathrm{J}_{2}$ and rosiglitazone prevented the increase in colonic permeability and bacterial translocation induced after acute immobilisation stress. Conclusions: Induction of the anti-inflammatory pathway $15 \mathrm{~d}-\mathrm{PGJ}_{2}$ / PPAR $\gamma$ in rat colon in vivo during sustained, repeated immobilisation stress seems to counteract initial colonic inflammation and dysfunction induced by acute stress. Furthermore, these findings open the possibility for preventing accumulation of oxidative/nitrosative species and subsequent colonic damage after stress exposure.

S1686

Structural Requirements of Recombinant Intestinal Mucin Muc3 EGF-Lik Cysteine-Rich Domains for Cell Migration, Anti-Apoptosis, and Intestinal Wound Healing Effects

Laurie Shekels, Rachel Moor, Kenji Takamura, David Evans, Robert L. Heinrikson, Samuel B. Ho

The intestine expresses the related membrane-bound mucins, MUC3, MUC4, MUC12 and MUC17, that have 2 cysteine-rich epidermal growth factor motifs in their extracellular domains separated by a linker region. Muc3 cysteine-rich domains expressed as a GSTfusion protein has been shown to stimulate cell migration in vitro independent of ErbB receptor stimulation (Gastro 2004; 126: A65). The effect of purification tags, individual Muc3 EGF domains and linker region on activity is unknown. Aim: To determine structural factors responsible for the functional properties of Muc3 cysteine-rich extracellular domain Methods: Full length cysteine-rich domains $(\mathrm{m} 3 \mathrm{EGFl}, 2)$ were expressed in E-coli as GST or His tagged proteins. Truncated GST fusion proteins containing only the first or second Muc3 EGF-like domain (m3EGFl or m3EGF2) were synthesized. Proteins were tested for effect on cell migration and anti-Fas induced apoptosis. Intestinal wound healing was tested by inducing colitis with a 5\% acetic acid enema followed by protein treatment ( 2 rectal doses at 12 and 24 hrs post acetic acid). Results: Full length His or GST-tagged m3EGF1,2 form large aggregates $(>200 \mathrm{kD})$ in solution under non-reducing conditions. Reduction resulted in monomeric proteins $(54 \mathrm{kD}$ and $30 \mathrm{kD}$, respectively). m3EGF2, but not $\mathrm{m} 3 \mathrm{EGF1}$, formed a large MW complex in solution that could be reduced. Similar to GST-tagged m3EGF1,2, His-m3EGF1,2 stimulated cell migration 4.1-4.7 fold ( $<<0.013$ ); and reduced fas-mediated apoptosis by $86 \%$ ( $\mathrm{p}<0.0001$ ). Enemas with 100ug His-m3EGF1,2 accelerated healing of acetic acid colitis. Neither m3EGF1 nor m3EGF2 stimulated cell migration, inhibited apoptosis or accelerated healing of colitis. Tyrphostin inhibition of EGF receptor had no effect on the activity of GST- or His-tagged m3EGFl,2. However, m3EGFl, 2 activity is reversed by genestein inhibition of tyrosine phosphorylation and by wortmanin inhibition of PI3 kinase. Conclusion: Both GST- and His-tagged Muc3 cysteine-rich EGF-like domains form disulfide-dependent aggregates in solution and stimulate cell migration, inhibit apoptosis, and accelerate healing of colitis. In contrast, individual Muc3EGF domains do not have biologic activity. The His-Muc3EGF1,2 recombinant protein requires tyrosine phosphorylation and PI-3 kinase stimulation for activity, however, activity is independent of EGF receptor phosphorylation. These data indicate that distinct structural features are needed for the cytoprotective activities of recombinant Muc3 extracellular cysteine rich proteins

S1687

Macrophage-Mediated Protection Against Dextran Sulfate Sodium-Induced Colitis

Joseph E. Qualls, Alan M. Kaplan, Willem de Villiers, Donald A. Cohen

The role of macrophages (MФs) in the development of Dextran Sulfate Sodium (DSS)-induced colitis was evaluated in $\mathrm{C} 57 \mathrm{BL} / 6, \mathrm{BAL} / \mathrm{C}$, and $\mathrm{CB}$ 17/SCID mice. Intrarectal treatment with clodronate-encapsulated liposomes locally depleted colonic MФs and increased severity of DSS colitis in all strains of mice. MФ-depleted mice displayed increased diarrhea and rectal bleeding during colitis compared to non-depleted mice. Inflammatory cell infiltration in colon tissue and loss of mucin from goblet cells was greater in $\mathrm{M} \Phi$-depleted mice during colitis compared to non-depleted mice. Expression of interleukin (IL)-6 and IL-10 from colon explants of DSS-treated mice was also greater in MФ-depleted mice. Histological evaluation of colons showed an increase in neutrophils in the MФ-depleted mice compared to those with normal M $\Phi$ levels. Moreover, $\mathrm{KC}$ chemokine expression and myeloperoxidase (MPO) activity were also increased in colon explants from diseased, MФ-depleted mice compared to diseased, non-depleted mice. Depletion of neutrophils from DSS-treated, MTdepleted mice, reduced the severity of colitis back toward levels seen in DSS-treated, M $\Phi$ intact mice. These data indicate that colonic MФs are protective during the induction of DSS-colitis and that this protection may, in part, involve the regulation of neutrophil influx and/or activity during inflammation.

\section{S1688}

Regulatiion of Wnt Pathway By TLR9 in Intestinal Epithelial Cells Ji-Hun Mo, Jongdae Lee, Yu-Tsueng Liu, Raz Eyal

Activation of Toll-like receptors (TLRs) via intestinal microbes plays an important role in maintenance of colonic homeostasis. We have found that apical and basolateral activation of TLR9 in intestinal epithelial cells(IECs) induce different immune response. While basolateral activation of TLR9 signals inflammatory response, apical signaling of TLR9 delivers a dominant anti-inflammatory response. To find out underlying genetic changes of these domain specific TLR9 signaling, we performed cDNA microarray analysis of IECs after apical and basolateral stimulation with TL R9 ligand. Microarray analysis revealed that apical and basolateral TLR9 signaling induce distinct sets of genes with some common genes. Interestingly, genes related to regulation of transcritption were highly induced in apically stimulated cells. In apically stimulated cells, Fzd5 (Frizzled5), a receptor of Wnt signaling, was the most highly inducible gene. In accordance with this, Fzd5 mRNA level along with its target genes, cryptdins, was down regulated in small intestines of TLR9-/- mice compared to WT mice. Fzd5 is involved in maturation of paneth cells in intestinal crypts that produce anti-microbial peptides, such as cryptdins. Our data suggest that bacterial DNA of intestinal microbes 
activates apical TLR9 to induce certain genes in IECs, some of which are involved in intestinal homeostasis.

\section{S1689}

Chemokine-Induced Aggregation of Peritoneal Macrophages Triggers Peritoneal Adhesion

Taeko Dohi, Akiyoshi Hoshino, Yuki I. Kawamura, Noriko Toyama-Sorimachi, Kenji Yamamoto

Peritoneal adhesion is a significant complication of surgery and intestinal inflammation. In spite of the large number of surgical operations performed daily, the mechanism for peritoneal adhesion, including peritoenal immune response to surgical stress, is not fully understood. Further, In Crohn's disease, transmural ulcerations with fissures or fistulas are the most important pathological findings. The characteristic changes in the serosal surface, includes fat wrapping, correlates directly with overall extent of the stricture of the intestine; the depth of lymphoid aggregate penetration, and the number of granulomas in the underlying intestinal wall. These results suggest that inflammation in Crohn's disease provoke abnormal responses in the connective tissues of the peritoneal cavity. However, peritoneal inflammation in the inflammatory bowel diseases has been poorly studied at the cellular and molecular levels. Here we revealed that peritoneal macrophages (PMF) aggregated at the site of injured peritoneum in murine models of post-operative and post-inflammatory peritoneal adhesion. The chemokine receptor CCR8 was upregulated in the aggregating PMF when compared with naive PMF, while other chemokine receptors were downregulated. The upregulation of CCR8 was also observed in PMF, but not in bone marrow-derived macrophages, treated with inflammatory stimulants including bacterial components and cytokines. Importantly, CCLl, the ligand for CCR8, a product of both PMF and mesothelial cells following inflammatory stimulation, was the strongest enhancer of CCR8 expression. The presence of CCL1 upregulated the expression of integrins in PMF and induced formation of PMF and mesothelial cell aggregates in vitro. Neutralization of CCLl in vivo prevented the formation of peritoneal adhesion in both colitis-associated and post-operational adhesion models. Our study now establishes the presence of a positive feedback system for recruitment of PMF via CCLl/ CCR8, which triggers peritoneal immune responses and adhesion, and offers potential avenues for therapy.

\section{S1690}

Visilizumab Induces Apoptosis of Mucosal T Cells from Ulcerative Colitis Patients in Vitro

Qi T. Yu, Masayuki Saruta, Konstantinos A. Papadakis

Background: Visilizumab (Nuvion ${ }^{\circledR}$ ), a humanized low FcR-binding IgG2 anti-CD3 monoclonal antibody, is efficacious in inducing clinical and endoscopic remission in patients with intravenous steroid-refractory ulcerative colitis (IVSR-UC). Exposure of activated, but not resting lymphocytes, to visilizumab in vitro led to the induction of $\mathrm{T}$ cells apoptosis. We hypothesized that visilizumab may induce apoptosis of lamina propria (LP) T cells obtained from patients with active UC. Methods: LP lymphocytes were isolated from surgical samples collected from 14 patients with moderate to severe UC. Peripheral blood (PB) mononuclear cells were collected from the same patients. Apoptosis of LP or PB T cells in response to visilizumab treatment in vitro was assessed with annexin-V staining. Inhibitors of caspases 3,8 , and 9 were used to determine their effect on visilizumab-induced apoptosis. Caspase 3/7 activity was measured using a luminescent assay. Results: Visilizumab induced dosedependent apoptosis of LP T cells reaching maximal effect at a therapeutic concentration of $100 \mathrm{ng} / \mathrm{ml}$ (Figure; mean +/- SD; $\mathrm{n}=14$ ). In marked contrast, visilizumab did not induce apoptosis of PB T cells $(n=12)$. Addition of a caspase 3 or -8 inhibitor completely abrogated the apoptotic effect of visilizumab in LP T cells. Addition of a caspase 9 inhibitor or antiFasL Abs had no significant effect on the visilizumab-induced apoptosis, suggesting that the mitochondrial pathway of apoptosis or the Fas/FasL pathway are not involved in this process. Moreover, visilizumab treatment of LP T cells caused caspase 3 activation as assessed by a colorimetric assay. Conclusions: Visilizumab in vitro induced apoptosis of LP, but not PB, $\mathrm{T}$ cells isolated from moderate to severe UC patients. The visilizumab-induced apoptosis is mediated through caspase- 8 and caspase- 3 activation and does not involve the Fas/ FasL pathway

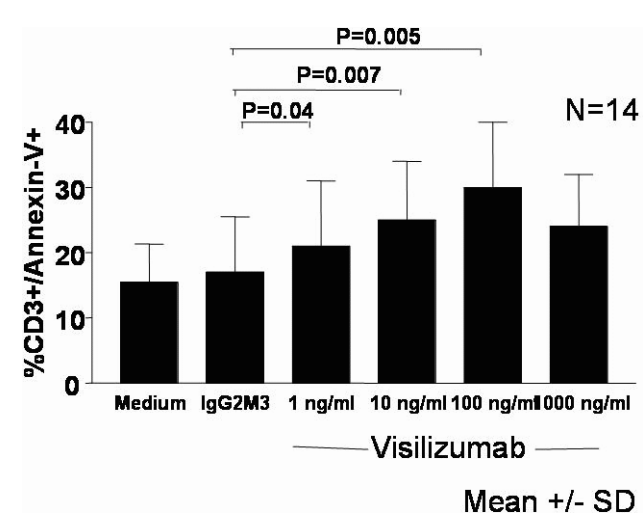

S1691

Diagnosis and Grading of Intestinal Acute Graft-Versus-Host Disease Following Allogeneic Stem Cell Transplantation By Colonoscopy Karin Potthoff, Juergen Finke, Jan Harder, Robert Thimme, Hartmut Bertz, Matthias Egger, Annette Schmitt-Graeff, Wolfgang Kreisel

Objective: Diagnosis and grading of acute intestinal graft-versus-host disease (GvHD) are based on clinical symptoms, e g diarrhoea and abdominal cramping, and are verified by histology. Since acute GvHD grade $\geq 2$ requires intensive immunosuppression, a correct diagnosis is crucial. At time of therapeutic decision making, however, histologic results often are pending. Previously we had found that macroscopic findings such as redness and granulation of the mucosa, aphthous lesions, erosions, ulcerations, confluent defects, mucosal bleeding and loss of epithelium can be regarded as criteria for intestinal involvement of acute GvHD. Depending on the extent of these findings in sigmoidoscopy we had established a score from grades 1 to 4 (Kreisel W et al., Eur J Gastroenterol Hepatol 1994; 6: 723729). Using these criteria we investigated whether colonoscopy is an appropriate method for a rapid and reliable diagnosis of acute intestinal GvHD. Patients and methods: We retrospectively evaluated 35 colonoscopies in 35 successive patients with acute intestinal GvHD following allogeneic stem cell transplantation. The median age was 57.6 years (range 34.5-68.4 years). They were transplanted for AML ( $n=6)$, AML/MDS ( $n=9)$, MDS $(n=6)$, CML $(n=1)$, NHL $(n=5)$, CLL $(n=1), M M(n=2)$ and MPS $(n=3)$. All patients received fludarabine, BCNU and melphalan for conditioning. GvHD prophylaxis consisted of cyclosporine $A$ and mycophenolate mofetile $(n=29)$ or mini-MTX $(n=6)$. In case of unrelated donor grafts ( $\mathrm{n}=13)$, anti-T-cell globulin ATG-S was additionally administered Colonoscopies were evaluated using the described criteria, and a comparison between macroscopy, histology and clinical grading was performed. Results: In no patient colonoscopy induced a complication such as major bleeding, perforation, or infections. In all 35 patients with clinically suspected GvHD $\geq 2$ and histologically proven intestinal GvHD $\geq 2$ macroscopy showed aphthous lesions (grade 3) and/or ulcerations/confluent mucosal defects (grade 4). The sensitivity of macroscopic examination of the colon including the terminal ileum for correct diagnosis of GvHD $\geq 2$ amounted to $100 \%$. Conclusions: (1) Colonoscopy can be safely performed even in patients with high-grade GvHD. (2) Macroscopic grading is a reliable method for objective diagnosis and grading of acute GvHD $\geq$ grade 2 following allogeneic PBSCT. (3) Macroscopic evaluation can be obtained immediately, whereas histology may take one or two days.

\section{S1692}

Regulation of Paneth Cell Alpha Defensin Genes By Microbial Stimuli Suresh Sivanesan, Carolyn Koh, Sanjay Lala, Satish Keshav

Mutations in the NOD2 gene are associated with an increased risk of Crohn's disease affecting the ileum and the gene is expressed in Paneth cells residing in ileal crypts. These cells mediate antibacterial host defence, partly via their production of alpha defensins. Recent data suggest that alpha defensin levels are reduced in Crohn's disease, particularly in the presence of mutations in the NOD2 gene. We investigated regulation of the human alpha defensin 5 (HD5) gene in vitro in colonic epithelial cells, to determine the effect of treatment with bacterial products such as lipopolysaccharide (LPS) and muramyl dipeptide (MDP) Cell lines were treated with MDP and LPS, followed by real time RT PCR quantification of HD5 mRNA. A defensin gene reporter construct was also transfected in the cell lines and reporter activity measured after exposure to MDP and LPS. Treatment with LPS and MDP did not increase the expression of HD5 mRNA, which is expressed at extremely low constitutive levels, although the same stimuli did induce the expression of pro-inflammatory cytokines and matrix metalloproteinase 7 (MMP7). Using the reporter gene construct, some activation of the HD5 promoter was seen in response to MDP treatment, although the magnitude was lower than that of a canonical nuclear factor kappa B promoter construct. These findings suggest that microbial stimuli that readily induce inflammatory changes do not regulate HD5 gene expression to the same extent. The regulation of HD5 expression appears complex and mainly constitutive. However, sensitive probes of promoter function suggest some regulation of the defensin genes, which may not be apparent at the level of steady-state mRNA concentration. This may contribute to the observed reduction in HD5 expression in inflamed tissue in Crohn's disease. 


\section{S1693}

CpG Oligodeoxynucleotides (CpG-ODN) Stimulate IGA Secretion in Intestinal Mucosal B-Cells

Stefan H. Blaas, Manuela Gunckel, Juergen Schoelmerich, Gerhard Rogler

Background: Bacterial DNA motives (such as $\mathrm{CPG-ODN)}$ ) induce a response of the innate immune system via ligation of Toll-like-receptor-9 (TLR9). Recent results indicate a role of TLR9 activation in inflammatory bowel disease. In murine intestinal mucosa CpG-ODN has pro-inflammatory effects and contributes significantly to chronic intestinal inflammation when given after the induction of inflammation, whereas CpG-ODN may ameliorate colitis when given in a prophylactic setting. In humans tonsillar B-cells have been reported to express TLR-9, especially when activated, and to respond to CpG-ODN. Whether B-cells in the human intestinal mucosa also express TLR-9 and whether their function can be influenced by CPG-ODN was unknown so far. Methods: Mucosal B-cells were isolated from surgical specimens of patients with chronic inflammatory bowel disease and from controls by collagenase digestion followed by magnetic cell sorting using anti-CD19 antibody armed magnetic beads (Dynabead-Detachabead $®$ system, Dynal Biotech, Warrington, USA). TLR9 mRNA and protein expression was quantified by Taqman ${ }^{\circledR}$ PCR and Western blot respectively. IgA secretion was measured by enzyme linked immunoassay (ELISA) after stimulation of isolated B cells with CpG-ODN, GpC-ODN or LPS. Results: Flow cytometric analysis of the isolated lamina propria mononuclear cells showed a purification of $73 \%(+/-22 \%)$ CD19 positive cells. By quantitative RT-PCR and by Western blot TLR- 9 expression in this cell population was demonstrated. The expression of TLR-9 was not further induced by exposure to CpG-ODN or LPS. CpG-ODN significantly uprequlated secretion of IgA to 141 $\%(+/-23 \%)$ when compared to unstimulated controls. GpC-ODN (as a control motiv) also upregulated IgA secretion to $111 \%$ (+/- $5.9 \%$ ), but significantly lower than CpG-ODN. There was no change of IgA secretion upon LPS stimulation. Discussion: These results demonstrate that CD19 positive mucosal lymphocytes express TLR-9 and induce release of IgA upon stimulation with CpG-ODN. This suggests that innate immune responses are also involved in human mucosal IgA secretion. However, as our purification of mucosal B-cells was not $100 \%$, an indirect mechanism can not completely be excluded.

\section{S1694}

PLC $\beta$ Is Necessary for the Maintenance of Basal Integrity of Tight Junctions, While PLC $\gamma$ Is Involved in EGF-Mediated Protection of Tight Junctions from Acetaldehyde

Takuya Suzuki, Ankur Seth, R.K. Rao

A growing body of evidence indicates that tight junctions are dynamically regulated by a variety of intracellular signaling pathways. Previous studies showed that EGF prevents acetaldehyde-induced disruption of tight junctions (TJ) in Caco-2 cell monolayers. In the present study, we investigated the role of PLC activity in EGF-mediated prevention of acetaldehyde-induced disruption of TJ. Methods: Caco-2 cells on Transwell inserts were incubated with acetaldehyde $(400 \mu \mathrm{M})$ in the absence or presence of EGF $(20 \mathrm{nM})$ and U73122 (3-30 $\mu \mathrm{M})$ or ET-18-OCH3 $(2-20 \mu \mathrm{M})$. Permeability was evaluated by measuring TER and FITC-inulin flux. Redistribution of TJ-proteins, occludin and ZO-1, from the junctions was analyzed by confocal microscopy. Release of TJ-proteins from the actin cytoskeleton was evaluated by immunoblot analysis of Triton-insoluble fraction. Activation of PLC $\beta$ and PLC $\gamma$ was determined by immunoblot analysis for phospho-PLCs in plasma membrane and cytoskeletal fractions. Cells were transfected with siRNA for PLC $\beta$ and PLC $\gamma$ (or control RNA) to determine the role of specific PLC isoform. Association of active PLC with EGFR was determined by co-immunoprecipitation. Results: Administration of U73122 by itself reduced TER, increased inulin flux in a dose-dependent manner; this was associated with redistribution of occludin and ZO-1 from the intercellular junctions. U73122 (PLC $\beta$-selective inhibitor) up to $30 \mathrm{uM}$ did not affect EGF-mediated protection of TJ from acetaldehyde. On the other hand, ET-18-OCH3 (PLCy-selective inhibitor) dose-dependently prevented EGF-mediated protection of TJ from acetaldehyde, while ET-18-OCH3 by itself produced no significant effect on basal TJ permeability. ET-18-OCH3 also prevented EGF-mediated prevention of acetaldehyde-induced decrease in detergent-insoluble TJ proteins, Thr-dephosphorylation of occludin and redistribution of occludin and ZO-1 from the junctions. PhosphoPLC $\gamma$ was not detectable in control cells, but EGF rapidly increased p-PLC $\gamma$ in plasma membrane and EGFR-immunocomplex, which was blocked AG1478, a EGFR tyrosine kinase inhibitor. A considerable level of phospho-PLC $\beta 1$ was present in control cells, but it was unaffected by EGF. Conclusion: These results indicate that PLC $\beta$ may play a role in the maintenance of basal integrity of TJ, while PLC $\gamma$ is involved in EGF-mediated protection of TJ from acetaldehyde Support by DK55532 and AA12307.

\section{S1695}

Mechanism of Heat Stress Modulation of Occludin Protein Expression: Key Regulatory Role of Heat Shock Factor-1

Karol Dokladny, Dongmei Ye, John C. Kennedy, Robin Matuk, Thomas Y. Ma

Previous studies from our laboratory suggested that the heat stress (HS)-induced increase in occludin protein expression may be an important protective mechanism regulating the HS-induced increase in Caco-2 tight junction (TJ) permeability. The intracellular mechanisms that mediate the HS-induced increase in occludin protein expression are unknown. The aim of this study was to elucidate the intracellular mechanisms that mediate the HS-induced upregulation of occludin protein expression. Methods: Filter-grown Caco-2 intestinal epithelial monolayers were used as an in-vitro intestinal epithelial model system. Results: Increasing incubation temperatures $\left(37-41^{\circ} \mathrm{C}\right)$ resulted in a temperature and time-dependent drop in Caco-2 trans-epithelial resistance and increase in paracellular permeability to inulin over the 24-h experimental period. HS $\left(41^{\circ} \mathrm{C}\right)$ caused a marked increase (3-fold increase) in occludin protein expression and increase in junctional localization of occludin. In the following studies, the possible intracellular mechanisms that mediated the HS-induced increase in occludin expression were examined. $\mathrm{HS}\left(41^{\circ} \mathrm{C}\right)$ induced increase in occludin expression was proceeded by an increase in occludin promoter activity and occludin mRNA expression. HS did not affect the rate of occludin protein degradation as assessed by the 35S-methionine pulse-chase, indicating that occludin expression was due to an increase in promoter activity and transcription and not a decrease in occludin degradation. $\mathrm{HS}\left(41^{\circ} \mathrm{C}\right)$ produced a rapid (within minutes) activation and cytoplasmic-to-nuclear translocation of heat shock factor-1 (HSF-1); and inhibition of HS-induced activation of HSF-1, with a known HSF-1 inhibitor quercetin $(100 \mu \mathrm{M})$, completely prevented the HS-induced upregulation of occludin gene transcription and protein expression. Moreover, quercetin inhibition of HS-induced HSF-1 activation and occludin protein expression was accompanied by a marked greater increase in Caco-2 TJ permeability. Conclusion: Our results indicate that the HS-induced increase in occludin protein expression and junctional localization were due to an increase in occludin promoter activity and gene transcription. Our findings also suggested that the HS-induced HSF-1 activation played a key regulatory role in the upregulation of occludin gene transcription, occludin expression, and protection against the HS-induced disruption of the intestinal TJ barrier.

\section{S1696}

Dynamic Movement of the Tight Junction Protein Claudin 2 in the Lateral Membrane of Live Human Intestinal Epithelial Cells Samantha Wilson, Guy R. Sander, Barry C. Powell

Introduction: The intestinal epithelium maintains a selective barrier to molecules and pathogens. Access through the intercellular space is regulated by tight junctions that create permeable seals near the apical surface of epithelial cells. To probe the nature of the tight junction we used fluorescent protein technology to monitor the dynamics of the tight junction protein, claudin 2, in the membrane of human intestinal epithelial cells. Methods: Various forms of claudin-2, either singly tagged or doubly tagged with the CFP and YFP spectral variants fused at either end were created and transiently transfected into T84 cells. The dynamics of claudin-2 expression was analysed by confocal microscopy and fluorescence recovery after photo-bleaching (FRAP). Results: All claudin-2 fusion proteins were able to incorporate into the lateral membrane of polarized cells. Confocal microscopy revealed a vesicular-like, cytoplasmic pool of protein restricted to a horizontal plane in the upper portion of the cell, suggestive of protein destined for import into the membrane. Using FRAP to investigate the dynamics of tagged claudin-2 in the plasma membrane of live cells, claudin-2 was visualized moving into the membrane within 15 seconds of photo-bleaching. Approximately $52 \%( \pm 7 \%)$ of the tagged protein was found to be mobile, with maximum recovery by $\sim 4$ minutes. The diffusion time was 56 seconds $( \pm 19 \mathrm{~s}$ ). Conclusion: These data provide insight into the dynamics of claudin-2 and indicate that rapid exchange of tight junction proteins can take place within the lateral plasma membrane of intestinal epithelial cells.

\section{S1697}

LPS-Induced Alteration of Colonic Paracellular Permeability Is Linked to Mast Cell Dependent Increase of Luminal Protease Activity

Raphael Moriez, Helene Eutamene, Vassilia Theodorou, Jean Fioramonti, Lionel Bueno

Lipopolysaccharide (LPS)-induced sepsis is associated with gut immune stimulation and change in colonic paracellular permeability (CPP). Increased CPP is the primary event triggering mucosal barrier dysfunction but the mechanisms involved remain unknown. The aim of this study was to evaluate the role of mucosal mast cells (MMC) and colonic luminal serine protease activity (PA) in CPP alterations induced by LPS. Methods: First, 6 groups of 10 male Wistar rats equipped with an intracolonic catheter received respectively, $\mathrm{NaCl}$ 0.9\%, LPS from E.coli 0111:B4 (1mg/kg, IP), MMC stabilizer doxantrazole (20 mg/kg, IP), doxantrazole + LPS, MMC degranulator BRX 537A (2mg/kg, IP) or its vehicle (DMSO). CPP was measured by intracolonic injection of $51 \mathrm{Cr}$-EDTA $(250 \mu \mathrm{L}, 0.7 \mu \mathrm{Ci})$ and expressed as percent of radioactivity collected in the urine for $6 \mathrm{~h}$. Secondly, on 6 similar groups, PA and rat mast cell protease II (RMCP-II) levels were measured in the colonic content $3 \mathrm{~h}$ after LPS administration (maximal effects of LPS on CPP) using azocasein as a proteolytic substrate and ELISA respectively. Results of PA were expressed in optical density and RMCP II levels were expressed in $\mathrm{ng} / \mathrm{ml}$ of supernatant. Thirdly, the effects of colonic content on CPP were assessed on proximal colon mounted into Ussing chambers. Colonic content of control or LPS-treated animals (incubated or not with the protease inhibitor SBTI, $100 \mathrm{mg} / \mathrm{L}$ ) was added at the mucosal compartment. After $30 \mathrm{~min}$, fluorescein isothiocyanate-labeled 4-KDa dextran was measured at the serosal side. Results were expressed as the flux of dextran crossing the epithelial barrier $(\mathrm{nmol} / \mathrm{min} / \mathrm{cm} 2)$. Results: LPS increased the $0-6 \mathrm{~h}$ colonic permeability of $51 \mathrm{Cr}$-EDTA from $0.65 \pm 0.08 \%$ in controls to $1.46 \pm 0.13 \%(\mathrm{P}<0.05)$. Three hours after LPS, colonic intraluminal PA was higher $(\mathrm{P}<0.05)$ in LPS than in controls $(0.57 \pm 0.11$ vs. $0.21 \pm 0.03)$. Also, RMCP-II level was higher from that observed in controls $(+364 \%, P<0.05)$. Previous administration of doxantrazole suppressed $(P<0.05)$ the LPSinduced release of RMCP-II into the colonic lumen, the increase of luminal PA, and the alteration of CPP. Moreover, BRX 537A mimicked the effects of LPS i.e. CPP increase, release of RMCP-II and increase of PA. Colonic content of LPS-treated rat added in Ussing chamber increased the dextran flux across the tissue compared with colonic content of saline-treated rats $(1.56 \pm 0.13$ vs. $1.14 \pm 0.11 ; \mathrm{P}<0.05)$. Previous treatment with SBTI abolished the effects of colonic content of LPS-treated rat on CPP alteration. Conclusion: Taken together our data evidence the key role of proteases, released from mast cells into the colonic lumen, on LPS-induced alterations of CPP. 


\section{S1698}

Expression of the Tight Junction Protein Claudin-2 in Resting and Proliferating Intestinal Epithelial Cells Is Inversely Regulated By TNFAlpha Joachim Mankertz, Shida Tavalali, Bernd Hillenbrand, Heinz Schmitz, Alfred H. Gitter, Peter Florian, Michael Fromm, Joerg D. Schulzke

Background and aims: Inflammatory bowel diseases are associated with a dysfunction of the intestinal epithelial barrier. Decrease of transepithelial electrical resistance (TER) is paralleled by an increase of apoptotic leaks and a reduction of tight junction strands in ulcerative colitis. TNFalpha treatment of the intestinal epithelial cell line HT-29/B6 shows similar effects on apoptosis, TER and tight junction strands. The tight junction protein claudin-2 is upregulated in ulcerative colitis. Aim of the study was to investigate expression, stability, and intracellular localization of claudin-2 in the presence of TNFalpha and interferon-gamma (IFNgamma). Methods: Resting and proliferating HT-29/B6 cells were incubated with TNFalpha and IFNgamma. TER and conductivity were monitored. Claudin-2 protein and mRNA expression was measured by immunoblotting and real-time PCR, respectively. Subcellular localization of claudin- 2 was determined by confocal microscopy. Claudin2 promoter activity was analyzed by reportergene assay. Results: Claudin-2 protein expression increased $(159 \pm 17 \%, \mathrm{p}<0.05$ vs control) in resting epithelial HT-29/B6 cells after incubation with TNFalpha for $24 \mathrm{~h}$ which was paralleled by a decreased TER $(31 \pm 2 \%, \mathrm{p}<0.001$ vs control), while IFNgamma had no effect on protein expression. Claudin-2 mRNA stability was not affected by TNFalpha. The combination of both cytokines had no effect on tight junction localization of claudin-2. Conductance scanning revealed an enhancement of transand paracellular conductivity after cytokine treatment which led to a change from tight to leaky epithelium. Proliferating cells, which contain an increased amount of claudin-2 compared to resting cells, exhibited a decrease in claudin-2 protein expression $(36 \pm 19 \%, \mathrm{p}<0.05$ vs control) after incubation with TNFalpha which can be explained by reduced promoter activity ( $55 \pm 2 \%, \mathrm{p}<0.001 \mathrm{vs}$ control). In the same manner, accumulation of claudin- 2 in resting cells is due to an enhanced gene expression, since claudin-2 specific mRNA expression was elevated in the presence of TNFalpha $(163 \pm 14 \%, \mathrm{p}<0.01$ vs control). Conclusion: In contrast to occludin and other tight junction proteins sealing the epithelial barrier, claudin2 expression was increased in response to TNFalpha in resting HT-29/B6 cells resembling a tight epithelium. Since claudin-2 forms cation-selective pores, its up-regulation leads to an increased permeability of the epithelial barrier. This is an important protective mechanism but also contributes to diarrhea in inflammatory bowel disease, where pro-inflammatory cytokines as well as claudin-2 protein expression are found to be elevated.

\section{S1699}

The TNF-Alpha Convertase (TACE) Regulates Intestinal Permeability Induced By Oxidant Stress Through An EGFR and MAPK-Dependent Pathway

Christopher B. Forsyth, Ali Banan, Ashkan Farhadi, Ali Keshavarzian

Inflammatory bowel disease (IBD) is a chronic intestinal inflammatory disease affecting more than a million Americans. One key component of IBD pathogenesis is increased intestinal permeability to proinflammatory luminal contents. Increased permeability is thought to result from actions of proinflammatory cytokines (TNF- $\alpha$, IFN- $\gamma$ ) and oxidant stress on intestinal epithelial cells due to inflammation. Pre-treatment of epithelial cells with growth factors binding to the epidermal growth factor receptor (EGFR) has been shown to protect against oxidant stress induced permeability. Data from others has also recently shown oxidant signaling through the EGFR via a metalloprotease dependent pathway. Aim: Our goal was to determine whether metalloprotease-mediated EGFR signaling was involved in regulation of oxidant-induced intestinal permeability and investigate possible mechanisms. Methods: The human Caco-2 cell in vitro model of tissue culture inserts was used for the permeability (FSA dye movement, 18h) as well as cell signaling studies. For oxidative stress, cells were exposed to $\mathrm{H} 2 \mathrm{O} 2(500 \mathrm{uM})$. Cells remained viable. All data represent at least three experiments. Permeability data are means from triplicate wells. Western blotting of cell lysates from oxidant treated cells was used for phosphoprotein analysis of mitogen activated protein kinases (MAPKs: ERK1/2, JNK1/2, p38) and AKT as well as EGFR phosphorylation. Cells were treated with oxidant \pm inhibitors to EGFR, TNF- $\alpha$ converting enzyme (TACE), all three MAPKs, PI3K, and Src. These inhibitors were also tested for effects on permeability. Results: Oxidant-induced a 90 -fold increase in permeability overnight. Signaling studies showed oxidant induced phosphorylation of the EGFR, ERK1/2, JNK1/2, p38, and AKT. Inhibitors of EGFR (kinase and blocking Ab), ERK1/2, and TACE blocked oxidant induced permeability by greater than $90 \%$ while effects of other inhibitors were variable. Both EGFR and TACE inhibitors eliminated ERK1/2 phosphorylation. Conclusions: These studies identify a significant and novel pathway regulating oxidant-induced intestinal permeability. The proposed mechanism involves oxidant-induced TACE metalloprotease cleavage of EGFR surface ligands such as EGF that then bind to and transactivate the EGFR. This transactivation results in TACE/EGFR-dependent ERK1/2 activation which is required for the increase in permeability. Studies are currently underway to identify targets for ERKl/2 in this mechanism as well as specific EGFR ligands cleaved by TACE in response to oxidant stress.

\section{S1700}

Pronounced Mucosal Barrier Dysfunction of the Whole Gut After Cardiac Surgery

Sabine Buhner, Christian von Heymann, Jan-Peter Braun, Michael Sander, Lisa Weymann, Pascal Dohmen, Martina Werich, Herbert Lochs, Claudia Spies

Introduction: A failure of the intestinal barrier function is supposed to enhance post-surgical complications. Cardiopulmonary bypass (CPB) operations are a risk factor in this matter, however, mucosal permeability, as an index of barrier function has not been studied in detail. Methods: We measured gastrointestinal permeability using an oral sugar test 24hrs before and 6hrs after isolated coronary artery bypass graft surgery under conditions of normothermic CPB. Urinary sugar recovery was quantified using HPLC. The different sugars served as markers for permeability of different intestinal segments; sucrose: (suc) gastroduodenum; lactulose/mannitol ratio (PI): small intestine; sucralose (sucra): colon. Plasma IL-6 and IL-10 were measured by ELISA. 41 patients (4F/37M; mean age: 63 years (38-78 years) were studied and compared to healthy controls (29F/14M; age: 42 years (17-77 years)) Results: Before surgery patients showed normal gastric and small intestinal permeability patterns which did not differ from values of the control group (table). However, 6 hrs after surgery both parameters substantially increased about 10-fold. Also colonic permeability was higher compared to controls (table). Interestingly, the disaccharide markers (suc, lac sucra) permeating via paracellular tight junctions increased, whereas mannitol, which is permeating mainly transcellularly decreased. Mannitol absorption is known to correlate wel with intestinal surface area. IL-6/IL-10 ration, an index for post-surgical outcome, increased and highly correlated with PI after surgery ( $\mathrm{p}=0.003$, Spearman-Rho). Conclusions: Cardiac surgery with $\mathrm{CPB}$ impairs mucosal barrier of the whole gut. A functional reduction of the active intestinal surface combined with a reduced energy supply of mucosal tight junctions might be underlying mechanisms. An uncontrolled macromolecule uptake and inadequate stimulation of the immune system may predispose for postoperative complications.

\begin{tabular}{|c|c|c|c|c|}
\hline & Controls ( $n=43)$ & \multicolumn{2}{|c|}{ Patients $(n=41)$} & \\
\hline & & Before Surgery & 6 hrs after Surgery & $\mathrm{p}<0.0001$ \\
\hline Sucrose (\%) & $0.13(0.09-0.20)$ & $0.09(0.00-0.18)$ & $0.74(0.34-1.53)$ & $\mathrm{a}, \mathrm{b}$ \\
\hline Mannitol (\%) & $15.33(11.75-19.28)$ & $16.62(11.68-23.81)$ & $4.70(1.99-6.74)$ & $\mathrm{a}, \mathrm{b}$ \\
\hline Lactulose (\%) & $0.29(0.22-0.37)$ & $0.37(0.18-0.56)$ & $0.88(0.41-1.63)$ & $\mathrm{a}, \mathrm{b}$ \\
\hline PI (\%Lac/\%Man) & $0.018(0.015-0.024)$ & $0.021(0.016-0.028)$ & $0.243(0.080-0.541)$ & $\mathrm{a}, \mathrm{b}$ \\
\hline Sucralose (\%) & $0.28(0.00-0.63)$ & & $3.07(2.24-4.47)$ & $\mathrm{a}$ \\
\hline IL-6/L-10 Ratio & & $0.053(0.029-0.080)$ & $0.235(0.112-0.360)$ & $\mathrm{b}$ \\
\hline
\end{tabular}

median (0.25-0.75 percentile); ns between controls and patients before surgery (MannWhitney); a: differences between controls and patients after surgery (Mann-Whitney); b: differences between before and after surgery (Wilcoxon-Test for paired data).

\section{S1701}

LPS from the Commensal Microflora Participates to Stress-Induced Colonic Epithelial Barrier Dysfunction in Mice Julien Demaude, Jean Fioramonti, Laurent Ferrier, Lionel Bueno

Introduction. Intestinal epithelial barrier dysfunction has been described in inflammatory bowel diseases and irritable bowel syndrome in humans. In mice, an acute stress increase colonic paracellular permeability, involving the mucosal immune system (mast cells, T cells) and the commensal microflora. In addition, bacterial wall components, as lipopolysaccharides (LPS) from gram-negative bacteria, are powerful activators of the immune system, through the binding to specific receptors. The aim of our study was to determine whether LPS is involved in the participation of the flora in stress-induced colonic epithelial barrier impairment. Material and Methods. Colonic paracellular permeability was determined by measuring the passage of ${ }^{51} \mathrm{Cr}$-EDTA, instilled into the colonic lumen, across the epithelium for two hours in male Swiss mice. Four groups of mice (1-4) were submitted to a mixed acoustic and restraint stress for two hours, before paracellular permeability measurement. Four other groups (5-8) were used as unstressed controls. Groups 1 and 5 received no treatment. Other groups were treated through drinking water before permeability measurement, as follows: groups 2 and 6 received non absorbable antibiotics, ampicillin $(0.05 \%)$ and neomycin $(0.10 \%)$ for ten days to reduce bacterial microflora; groups 3 and 7 received antibiotics for 10 days and LPS from E coli $\mathrm{K} 235(120 \mu \mathrm{g} / \mathrm{mL})$ the last four days; groups 4 and 8 received only LPS for four days. Results. Acute stress increases colonic paracellular permeability to ${ }^{51} \mathrm{Cr}$-EDTA $(2.2 \pm 0.1 \%$ vs. $1.1 \pm 0.1 \%, \mathrm{P}<0.001)$. This effect was abolished by antibiotics $(0.7 \pm 0.1 \%)$, and restored in mice treated with both antibiotics and LPS $(2.8 \pm 0.2 \%)$. Treating animals with LPS alone exacerbated the effects of stress $(4.1 \pm 0.2 \%)$. In unstressed mice, antibiotics associated or not with LPS did not affect permeability $(0.8 \pm 0.1 \%$ without LPS; $1.5 \pm 0.1 \%$ with LPS, P>0.05 vs. controls). An increased permeability was observed in unstressed animals receiving LPS $(2.5 \pm 0.2 \%)$. Conclusions. Our results confirm the pivotal role of the commensal microflora in stress-induced colonic permeability alterations. In addition, we provide evidence that LPS mimics the action of the microflora in antibioticstreated mice. Thus, we suggest that LPS mediates the effects of the flora on colonic epithelial cell reactivity to stress.

\section{S1702}

Enteropathogenic E. Coli-Induced Activation of Myosin Light Chain Kinase and Early Alteration of Tight Junction Protein Localization Are ESPFIndependent

Michelle M. Muza-Moons, Andrew W. Weflen, Rajani Kanteti, Jerrold R. Turner, V.K. Viswanathan, Gail Hech

Enteropathogenic E. coli (EPEC) is a significant cause of infantile diarrhea and mortality worldwide. Disruption of intestinal epithelial tight junctions (TJ) contributes to EPEC pathogenesis. EPEC decreases transepithelial electrical resistance (TER), a measure of TJ barrier function. The EPEC pathogenicity island harbors genes encoding attachment factors, type III secretion (TTS) proteins, and effector molecules. TJ disruption by EPEC has been correlated in part with myosin light chain kinase (MLCK) activation and phosphorylation of regulatory myosin light chain (MLC) inducing contraction of the perijunctional cytoskeletal ring. The effector molecule EspF is largely responsible for the decrease in TER. The aim of this study was to determine the role of EspF in MLCK activation and further assess the effect of cytoskeletal contraction on TJs. Ratiometric comparison of phosphorylated to total MLC was performed using a phosphospecific antibody. Cultured intestinal epithelial cells (T84, $\mathrm{CaCo}-2$ and MDCK) were infected with wild type (wt) EPEC and specific mutants and the level of phosphorylated MLC (P-MLC) was compared to uninfected monolayers. The effec of a specific cell-permeant MLCK peptide inhibitor, PIK $(330 \mu \mathrm{M})$ was also studied. WT EPEC $(0.93 \pm 0.4$ densitometric ratio), but not the TTS deficient strain $\Delta \operatorname{escN}(0.29 \pm 0.04$, 
$\mathrm{n}=3$ ), enhanced P-MLC. Mutation of espF did not significantly alter the level of P-MLC as compared to wt EPEC $(0.70 \pm 0.4 ; \mathrm{p}>0.10, \mathrm{n}=3)$. While PIK significantly attenuated the decrease in TER by wt EPEC $(-73 \pm 10$ to $-55 \pm 8 \%)$, this inhibitor abolished the EspFindependent portion of the TER decrease $(-36 \pm 15 \%$ vs $-3 \pm 7 \%$, - and + PIK, respectively) supporting that EspF is not responsible for MLCK activation. To assess the impact of MLCK activation on TJ structure, monolayers were stained for ZO-1 and occludin at early time points post-infection. These studies revealed a "crinkle" or "zigzag" pattern at early times (1-3 hrs) consistent with MLCK activation and prior to severe alteration of TJ structure. This subtle phenotype was not seen in uninfected monolayers or those infected with nonpathogenic E. coli or the escN mutant. PIK prevented the "crinkle" phenotype indicating that this alteration is also a consequence of MLCK activation. Screening of a panel of EPEC mutant strains revealed that the effectors Map, Tir, and EspG are not responsible for MLCK activation. We conclude that activation of MLCK and the associated "crinkle" phenotype of TJs induced by EPEC infection are TTSS-dependent/EspF-independent processes and represent key early events in EPEC-mediated TJ disruption.

\section{S1703}

The Active Motif of ZOT, At1002, Increases ZO-1 and Myosin 1 Beta Serine Phosphorylation, Their Interaction, and Intestinal Permeability Manjusha Thakar, Tarcisio Not, Simeon Goldblum, Alessio Fasano

Background: Zonula occludens toxin (Zot) is a protein elaborated by Vibrio cholerae that modulates intestinal epithelial tight junctions (tj) in a PKC-dependent manner. Zot structurefunction analysis and studies on its trafficking within V. cholerae identified a conserved 6 mer protease activated receptor (PAR) motif (named AT1002) that retains Zot activity for tj's. Aim: To determine whether: ATl002 1) modulates tj both in vivo and in vitro; (2) Increases serine phosphoralytion and/or affects interactions between tj integral membrane components and their scaffolding partners. Methods: In presence and absence of AT1002 (1) Transepithelial intestinal resistance (TEER) on rat small intestine mounted in snap wells was monitored. (2) The in vivo intestinal permeability of rat intestine was studied by dual sugar test with HPLC technique. (3) Cell lysates from rat epithelial cells (IEC 6) were studied by phosphoserine immunoblotting and co-immunoprecipitation assays. Results: AT1002 decreased TEER in vitro in a dose- and time-dependent manner. AT1002 also increased intestinal permeability in vivo, while three different control scrambled peptides failed to do so. AT1002 (100 mM) increased serine phosphoralytion of ZO-1 in a time-dependent fashion coincident with decrease in TEER kinetic. A phosphoserine-containing protein communoprecipitated with ZO-1 and was identified as Myosin 1 beta by MS/MS. ZO-1-myosin 1 beta protein-protein interaction increased following AT1002 exposure. These results were confirmed by reciprocal co-immunoprecipitation experiments. Conclusions: 1) AT1002 retains the biological activity of full length Zot both in vivo and in vitro; 2) the AT1002induced increased intestinal permeability was coincident with serine phosphorylation of ZO-1 3) AT1002 increases serine phosphoralytion of myosin 1 beta and its association with ZO-1. To our knowledge, this is the first observation of myosin 1 beta and ZO-1 serine phosphorylation and interaction coincident with increase in intestinal tj permeability.

\section{S1704}

Disturbed Cytokine Response to Mycobacterium Avium Subspecies Paratuberculosis Is Dysregulated in Patients with Crohn's Disease Shomik Sibartie, John Keohane, Paul Scully, Shaun O'Neill, Jim O'Mahony, Liam O'Mahony, Fergus Shanahan

Background: Mycobacterium avium subspecies paratuberculosis (MAP) has been a source of controversy since it was first suggested as a possible cause for Crohn's disease. While a number of studies have focused on identification of the organism in Crohn's disease tissues and others have assessed the serologic response to MAP, few studies have examined the cellular immune response to MAP. Aim: To compare the cellular response to Mycobacterium avium subspecies paratuberculosis between Crohn's disease patients and healthy volunteers. Methods: Peripheral blood mononuclear cells (PBMCs) were isolated from 24 Crohn's disease patients and 20 healthy volunteers. After in-vitro co-incubation for 72 hrs with MAP ATCC 43019 at several concentrations, supernatants were harvested and analysed for IL2, IL-4,IL-6,IL-8,IL-10,TNF- $\alpha$ and IFN- $\gamma$ using cytometric bead analysis. PBMCs stimulated with Salmonella typhimurium (ST)were used as positive controls. Results: Compared to healthy volunteers, PBMCs from Crohn's disease patients secreted higher levels( $\mathrm{p}<0.05)$ of IL- $6(4265 \pm 260$ vs. $2865 \pm 386 \mathrm{pg} / \mathrm{ml})$,TNF- $\alpha(2190 \pm 247$ vs. $1200 \pm 228 \mathrm{pg} / \mathrm{ml})$ and IL$10(265 \pm 53$ vs. $81 \pm 12 \mathrm{pg} / \mathrm{ml})$ upon exposure to MAP(1 MAP bacterial cell:1 PBMC) but showed no difference when exposed to ST. There was a lower IFN- $\gamma$ response from Crohn's disease patients to $\operatorname{MAP}(322 \pm 125$ vs. $1658 \pm 424 \mathrm{pg} / \mathrm{ml})$ but this also occurred in response to ST. The ratio of IFN- $\gamma$ to IL-10 was significantly lower for Crohn's disease patients' PBMCs exposed to MAP compared to healthy volunteers $(\mathrm{p}<0.05)$ but not for ST. There were no differences in IL-8, IL-2 and IL-4 levels. Prior BCG vaccination and concurrent immunosuppressives had no impact on the levels of cytokines secreted. Conclusion: A diminished Th1 response to MAP in Crohn's disease patients may allow for prolonged intracellular survival of MAP in phagocytic cells. This might account for the increased frequency of MAP detection in patients with Crohn's disease but does not imply cause and effect.

\section{S1705}

Intestinal Permeability Is Associated with Severity of Disease in Surgical ICU Patients

F Hietbrink, Mgh Besselink, Mbm de Smet, W Renooij, Lma Akkermans, Lph Leenen

Increased intestinal pemeability is proposed to be the motor of sepsis in surgical critical care patients. It is thought that the surgical stress and hypoxia induce a loss of intestinal barrier function and therefore predispose for bacterial translocation. Sugar absorption tests have produced a wide variation in results. Poly-ethylene glycols (PEGs) of various molecular mass have been used as markers for intestinal permeability in non critically ill patients. PEG
400 can freely pass the intestinal mucosal barrier, larger PEGs can only pass when intestinal permeability is compromised. We investigated whether the intestinal permeability, as measured with PEGs, is associated with the severity of disease in surgical ICU patients. Thirtyseven surgical ICU patients were included (mean APACHE II score $14 \pm 5.5$ ). The patients ingested a solution of 5g PEG 400, 1.5g PEG 1500, 5g PEG 4000 and 10g PEG 10000 dissolved in $100 \mathrm{ml}$ water within the first 48 hours of admission. Urine was collected in the following 12 hours and analyzed for PEG by HPLC. The test was repeated every other day during the first two weeks of ICU stay. PEG values from the ICU patients were compared with control values from an earlier validation study. Severity of organ failure was assessed by the SOFA score. Data are presented as the intestinal permeability index (IPI for PEGi = (PEGi / PEG 400) x 100\%). Statistical analysis was performed using t-test and logistic regression. The IPI for PEG 1500 increased from $1.79( \pm 0.39$ mean \pm SEM $)$ in controls to $3.90( \pm 1.60)$ in surgical ICU patients on admission ( $\mathrm{P}=0.031)$. The IPI for PEG 4000 increased from $0.20( \pm 1.59)$ in controls to $0.52( \pm 0.16)$ in surgical ICU patients on admission $(P=0.040)$. A significant increase in the IPI for PEG 1500 on day 4 was found between patients who developed severe organ failure and patients who did not $(P<0.034)$. The IPI for PEG 1500 correlated with the SOFA score $(\mathrm{P}=0.012)$ as it changed during the period of ICU stay, when corrected for stomach retention and creatinin levels. In conclusion these data suggest an association between intestinal permeability and the course of disease, because 1) intestinal permeability of surgical intensive care patients was increased compared to healthy controls, 2) intestinal permeability on day 4 correlated with the development of organ failure and 3) changes in intestinal permeability correlated with changes in severity of disease over time.

\section{S1706}

Stress Associated Changes in the Composition of Luminal Microbiota in the Human Jejunum

Cristina Martinez, Javier Santos, Carmen Alonso, Mar Guilarte, Laura Ramos, Maria Vicario, Maria Antolin, Francisco Guarner, Juan-Ramon Malagelada

Background: Several evidences support the relevance of intestinal flora stability in the promotion of intestinal health whereas life stress has been linked to the development of mucosal inflammatory disorders in the gut. However, the composition of jejunal comensal microbiota and the effect of stress on the luminal microbiota have not been fully elucidated. Aims: 1) To investigate whether the small bowel bacterial populations can be analyzed by molecular approaches; and 2), to characterize the composition and dynamics of bacterial populations of intestinal microbiota of healthy subjects under acute stress. METHODS: In 10 healthy volunteers, a $20-\mathrm{cm}$ jejunal segment was isolated and constantly perfused at 5 $\mathrm{mL} / \mathrm{min}$. Intestinal effluents were collected at baseline and every $15 \mathrm{~min}$ for $1 \mathrm{~h}$ after hand immersion in cold water for $15 \mathrm{~min}$. Total DNA was isolated from intestinal effluents at baseline (A) and $45 \mathrm{~min}$ after stress (B). The total predominant bacterial community was profiled by targeting the V6-V8 region of the 16S ribosomal DNA by polymerase chain reaction (PCR) and separating the resultant amplicons by denaturing gradient gel electrophoresis (DGGE). After electrophoresis, gels were silver stained. Obtained profiles were compared using the BioNumerics software package and similarity indices (SI) calculated from densitometric curves using the Pearson product moment correlation coefficient. RESULTS: The total bacterial DGGE profiles showed that each subject harbours a specific microbiota with a low interindividual SI at baseline, but, remarkably, this SI was significantly ehhanced after stress (A: $40.8 \pm 11.7 \%$. vs. B: $54.4 \pm 12.5 \%$; $<<0.05$ ). Moreover, one specific band that was nearly undetectable in A samples appeared with high intensity in B samples. On the other hand, the intraindividual composition of the microbiota varied notably after cold stress. The SI of B sample versus A sample of each subject was in average $42.9 \pm 28.5 \%$. In addition, the diversity of the microbiota, estimated by the number of bands, decreased after cold stress (A: $26 \pm 8 ; B: 19 \pm 5 ; \mathrm{p}<0.05$ ), CONCLUSIONS: PCR-DGGE fingerprinting is a useful technique to detect dominant bacterial populations in intestinal effluents. Composition of jejunal microbiota is altered shortly after acute stress by specific changes in the small bowel microflora. The present findings contribute to our understanding of gastrointestinal disorders caused or aggravatted by life stress

\section{S1707}

Ginkgo Biloba Protects Against Intestinal Epithelial Barrier Hyperpermeability By Stabilizing Tight-Junctional Proteins: Role of NF-kB Inactivation, Occludin and Claudin

Ali Banan, Lijuan Zhang, Maliha Shaikh, Ashkan Farhadi, Christopher B. Forsyth, Philip Engen, Ali Keshavarzian

NF-kB activation $\&$ barrier disruption are key in the pathogenesis of inflammatory GI diseases. Several traditional Complementary \& Alternative Medicine (CAM) systems recommend putative anti-inflammatory herbs such as Ginkgo Biloba (GB) for Inflammatory Bowel Disease (IBD). Despite claims, it is not established whether this herb has anti-inflammatory effects, is beneficial in IBD, and can prevent barrier disruption. Also, GB's mechanism of action has not been directly evaluated in an IBD model. Aims: To determine if NF-kB inactivation is involved in GB-mediated protection of tight junction \& barrier proteins against oxidative injury. Methods: Human intestinal monolayers (Caco-2 cells) were incubated in injurious doses $(100 \& 500 \mathrm{uM})$ of $\mathrm{H} 2 \mathrm{O} 2$ or $\mathrm{HOCl} \pm \mathrm{GB}(1-100 \mathrm{ug} / \mathrm{mL})$ or NF-kB modulators. Other studies were done in IkB mutant cells where NF-kB was inactivated. We assessed: barrier function (fluorometry), occludin / claudin integrity (laser confocal microscopy, SDSPAGE), NF-kB activity (EMSA, ELISA), NF-kB modulator, IkB (PAGE), n=6/grp. Results: GB pretreatment $(10 \mathrm{ug} / \mathrm{mL})$ prior to oxidant led to: 1 ) decreased IkB degradation, 2) reduced NF-kB nuclear translocation, 3) decreases in nuclear NF-kB activation, 4) maintenance of normal occludin \& claudin-1 architecture, \& 5) enhanced barrier integrity comparable to controls. These protective effects were synergistic with effects of IkB stabilizers (MG-132, Lactacystin). Further confirming a role for NF-kB in GB protection: [A] preincubation with any of several NF-kB inhibitors (e.g., PDTC) or stabilization of IkB by a dominant mutant transfection synergized with the above protective effects by GB. [B] Pretreatment with potentiators of NF-kB activation [PAO, PV] prevented GB maintenance of barrier integrity $\&$ stabilization of tight junction structure during oxidant insult. [C] IkB stabilization due 
to mutant transfection, similar to GB, protected barrier integrity and occludin \& claudin-1 stability. Indeed, the disruptive effects of oxidants on the barrier were largely prevented by mutant IkB induced stabilization while they were exaggerated by inhibitors of phosphoseryl/ threonyl protein phosphatase (i.e., NF-kB potentiators). [D] Similar to the blunting effects of protein phosphatase, the NF-kB potentiators PAO \& PV prevented protection by GB or growth factor (EGF), \& abolished decreases in NF-kB caused by these protective agents. Conclusions: Our findings suggest that GB has unique anti-inflammatory effects and supports our hypothesis that GB protects against oxidant-induced intestinal epithelial hyperpermeability by stabilizing tight junctions through the inactivation of NF-kB via stabilization of IkB.

\section{S1708}

Fluorometric Detection of Specific Binding of Cy3-Labeled Norwalk Virus-Like Particles to Caco-2 Cells

Anne M. Hutson, Sue E. Crawford, Mary K. Estes

BACKGROUND: Noroviruses are the major cause of epidemic acute nonbacterial gastroenteritis worldwide, and Norwalk virus (NV) is the prototype norovirus strain. Detection of noroviruses in the environment (i.e. groundwater and in sewage treatment) is difficult because of the genetic divergence and antigenic differences among strains. Additionally, studying NV infection is hindered by the lack of a cell culture system or a small animal model supporting replication. However, the NV capsid protein expressed in the baculovirus system produces virus-like particles (VLPs) which are structurally and antigenically similar to $\mathrm{NV}$ virions and may be used as a surrogate to study virus attachment to and entry into cells and viral movement in the environment. Previously, 35S-NV VLPs showed specific binding to and internalization into Caco-2 cells, a human cell line with enterocyte-like properties but is not permissive to NV replication. Metabolic radiolabeling of native NV is not feasible, and radiolabeled VLPs are not useful for evironmental tracking studies. Thus we hypothesized that fluorescently labeled NV VLPs can be used to track particle travel in the environment and in cells. METHODS: We have developed a non-radioactive method of labeling purified NV VLPs with the monovalent Cy3 fluor. RESULTS: Fluorescently labeled 35S-Cy3-NV VLPs demonstrated specific binding to Caco-2 cells identical to that of 35SNV VLPs. Furthermore, 35S-Cy3-NV VLPs were visualized bound to the cells. Interestingly, visual scoring of cell-associated fluorescence was more sensitive than that of the fluorometer. By fluorometry, the detection of Cy3-labeled VLPs was over 1000-fold less sensitive than that of radiometric methods. CONCLUSIONS: Cy3-labeled NV VLP specific binding to Caco-2 cells can be detected by fluorescent microscopy and fluorometry. Developing a nonradioactive method to label and track NV VLP in the environment and in cell binding may help elucidate modes of NV transmission and identify blocks in NV replication in cultured cells

\section{S1709}

Irsogladine, An Activator of Gap-Junctional Intercellular Communication, Suppresses Paracellular Permeability of Human Intestinal Epithelial Cell Monolayers Through Up-Regulation of Claudin-4

Hidekazu Morita, Tatsuro Katsuno, Aihiro Hoshimoto, Kazuki Hatakeyama, Yasuo Suzuki, Yasushi Saito

Background: We reported previously that human intestinal epithelial cell monolayers overexpressing connexin 26 , a component of gap junctions, show significantly reduced paracellular permeability accompanied by up-regulation of claudin-4, a component of tight junctions. (Morita H., et al. Exp Cell Res 2004). This finding indicated that enhancing gap-junctional intercellular communication (GJIC) may suppress paracellular permeability accompanied by altered expression of tight junction proteins. Aim: The present study was performed to examine the effects of irsogladine [2,4-diamino-6-(2,5-dichlorophenyl)-s-triazine maleate], which is known to activate GIJC without influencing protein expression of connexins, on the paracellular permeability of human intestinal epithelial cell monolayers. Methods: Paracellular permeability of Caco-2 human intestinal epithelial cell monolayers was monitored by measuring mannitol permeability and was chemically disrupted by treatment with oleic acid and taurocholic acid. Western blotting analyses were performed to evaluate the protein levels of occludin, claudin- $1,-4$, and $\beta$-actin. Results: Mannitol permeability of untreated monolayers was $1.1 \pm 0.1 \%$, and was markedly increased by chemical disruption ( $42.2 \pm$ $5.2 \%)$. Intriguingly, irsogladine treatment dose-dependently decreased mannitol permeability of chemically disrupted monolayers, with a significant decrease in permeability $(11.7 \pm$ $1.2 \%$ ) on treatment with $10^{-4} \mathrm{M}$ irsogladine. To determine the mechanism by which irsogladine exerts this effect, we utilized $18 \alpha$-glycyrrhetinic acid, an agent to interfere specifically with GJIC. Addition of an excess amount of $18 \alpha$-glycyrrhetinic acid to monolayers cotreated with $10^{-4} \mathrm{M}$ irsogladine again lead to a remarkable increase in permeability (40.1 \pm $3.8 \%)$. These observations indicate that the treatment with irsogladine suppresses paracellular permeability of human intestinal epithelial cell monolayers through activation of GJIC. We next evaluated protein levels of components of tight junctions. Claudin-4 protein level was significantly increased in epithelial cells treated with $10^{-4} \mathrm{M}$ irsogladine, although no changes were observed in those of occludin or claudin-l. Addition of an excess amount of $18 \alpha$ glycyrrhetinic acid to monolayers co-treated with $10^{-4} \mathrm{M}$ irsogladine again decreased the protein level of claudin-4. Conclusion: Activation of gap-junctional intercellular communication by irsogladine leads to a significant decrease in paracellular permeability of human intestinal epithelial cell monolayers partly through up-regulation of claudin-4.

\section{S1710}

Bile Enhances Barrier Function By a COX-2 Dependent Mechanism in Porcine Duodenum

Jenna G. Wooten, B. D. X. Lascelles, Adam J. Moeser, Vanessa L. Cook, Dianne Little, Anthony T. Blikslager

Previous studies suggest bile acids have a protective function in the duodenum, possibly related to neutralizing $H$. pylori and by reducing the deleterious effects of stomach acid. In preliminary studies, bile acids enhanced barrier function of porcine duodenum, suggesting alternate protective effects. Because prostanoids have a positive effect on porcine intestina barrier function, we postulated deoxycholic acid would enhance duodenal barrier function in a COX-2-dependent manner. Porcine intestinal mucosa was mounted on Ussing chambers and transepithelial electrical resistance (TER), a sensitive measure of barrier function, was calculated from voltage clamp measurements. Deoxycholic acid (DCA) was added in concentrations of $1 \mathrm{mM}-1 \mu \mathrm{M}$ to the luminal surface of porcine duodenal, stomach, and jejunal mucosa. No effect of DCA was noted following application to stomach and jejunum. DCA induced a dose-dependent increase in TER in the duodenum, significant at the $1 \mathrm{mM}$ dose Both the non-selective COX inhibitor, indomethacin, and a selective COX-2 inhibitor, deracoxib, blocked the increase in TER, suggesting COX-2 is involved in DCA-induced increased duodenal barrier function. Western analyses revealed increased expression of COX2 in bile treated tissues compared to untreated tissue. We have previously shown that one mechanism for prostaglandin-mediated elevation of barrier function is blockade of electroneutral sodium channels. Therefore, we applied the sodium channel blocker, amiloride $(1 \mathrm{mM})$, to the luminal surface of duodenal mucosa. Amiloride simulated the effects of $1 \mathrm{mM}$ DCA, and had no additive effects when added in combination with DCA, suggesting a similar action of amiloride and DCA. These results indicate that DCA enhances barrier function in duodenum in a COX-2 dependent fashion, possibly via prostenoid-induced inhibition of amiloride-sensitive sodium channels. These findings may in part explain the protective role of bile acids in duodenal barrier function.

\section{S1711}

Transcriptional Expression of Tight Junction Proteins in Celiac Disease and Other Disorders with Compromised Intestinal Barrier Function

Maria G. Clemente, Amit Tripathi, Anna Sapone, Maria P. Musu, Paolo Usai, Stefano de Virgiliis, Alessio Fasano

Background. Intestinal epithelial tight junctions (TJ) play a crucial role in regulating the paracellular pathway. The relationship between the increased intestinal permeability observed in several acute and chronic diseases and the expression of TJ-associated proteins is not well established. Aim of this study was to investigate the expression at transcriptional level of 3 transmembrane TJ proteins, occludin, claudin-1, claudin-2 and of 2 scaffold TJ proteins, zonula occludens-1 (ZO-1) and myosin 9-beta in intestinal biopsies from patients affected by celiac disease (CD), Crohn's disease, peptic ulcer disease and Type 1 diabetes (TID) Methods. Samples of small intestinal mucosa were taken from the second/third portion of the duodenum from subjects with active $C D, C D$ on gluten free diet (GFD), peptic ulcer disease, Crohn's disease, TID and TID-relatives and healthy subjects. After total RNA extraction and cDNA synthesis, quantitative real time polymerase chain reaction assays with SYBR Green was performed. Data were normalized by usingl8S rRNA as standard. Results. A significant down-regulation of all 5 genes studied was observed in $C D$ when compared to the controls. Specifically, occludin expression decreased by $84 \%(\mathrm{p}=0.001), \mathrm{ZO}-1$ by $>99 \%(\mathrm{p}=0.01)$, claudin -1 by $98 \%(\mathrm{p}=0.01)$, claudin -2 by $>99 \%(\mathrm{p}=0.02)$, and myosin 9 beta by $90 \%(p=0.01)$. Interestingly, the transcriptional level of all the 5 genes returned within normal limits in $\mathrm{CD}$ subjects following the implementation of a gluten free diet. In peptic ulcer disease, 3 out of 5 genes were found down regulated as follows: ZO-1 by $>99 \%$ $(\mathrm{p}<0.01)$, claudin- 1 by $>99 \%(\mathrm{p}=0.01)$, and claudin -2 by $>99 \%(\mathrm{p}=0.02)$. In Crohn's disease, only occludin was found down regulated by $95 \%(\mathrm{p}<0.01)$, while no transcriptional changes were observed in TID and their relatives. Conclusions. The down-regulation of intestinal TJ transmembrane or/and intracellular components observed in CD, Crohn's disease, and peptic ulcer diseases might explain the increased intestinal permeability reported in these pathological conditions. Conversely, our data suggest that the increased intestinal permeability reported in TID is probably associated to derangement of TJ protein-protein interaction as previously reported. The meaning of TJs disruption in the pathogenesis of the pathological conditions studied warrants further investigation. The normal transcriptional level observed in $C D$ patients on a gluten free diet suggests that the down-regulation of TJs in $C D$ is reversible and secondary to gliadin exposure rather than to a genetic predisposition.

\section{S1712}

Intestinal Permeability and Effect of NSAIDs On Permeability in Irritable Bowel Syndrome Patients Compared to Healthy Subjects Angele Kerckhoffs, M Samsom, M de Smet, Lma Akkermans, W Renooij

Post infectious Irritable Bowel Syndrome (IBS) patients have intestinal inflammation. Intestinal permeability is increased in some of these patients. In inflammatory bowel disease NSAIDs enhance intestinal permeability. The aim of our study was to investigate intestinal permeability in IBS patients and healthy subjects and to test the effect of NSAIDs on permeability. Permeability was measured as urinary excretion of orally administered polyethylene glycols (PEGs) of increasing molecular weights. A standard lactulose mannitol (LM) excretion test was used for comparison. We determined intestinal permeability in 15 healthy subjects and 14 IBS patients, diagnosed according to Rome II criteria, before and after 2 days naproxen $(750 \mathrm{mg} /$ day) using a PEG test and an LM test. Subjects drank a solution of 5 g PEG 400, 1.5 g PEG 1500, 5 g PEG 4000 and 10 g PEG 10000 in $100 \mathrm{ml}$ water. Urine was collected at 2-hour intervals for 24 hours. PEGs were analyzed by HPLC, and $\mathrm{LM}$ by routine clinical chemical analysis. Intestinal permeability was evaluated from areas under the PEG -excretion time curves $(A U C) \pm S E M$; data from IBS patients and healthy subjects were statistically evaluated using Mann Whitney $U$ test. $P<0.0125$ was considered significant (Bonferroni correction). Results from before and after NSAIDs ingestion were 
statistically evaluated by the Wilcoxon test. LM ratio above 0.03 indicates increased intestinal permeability. For statistical analysis of the LM test the Chi square test was applied. Differences were considered significant at $\mathrm{p}<0.05$. AUC of PEG 4000 in IBS patients $(5.9 \pm 2.4 \mathrm{mg} / \mathrm{ml})$ is significantly $(\mathrm{p}=0.006)$ smaller than in healthy subjects $(16.1 \pm 3.9 \mathrm{mg} / \mathrm{ml})$. No significant differences were found in AUCs of PEGs 400, 1500 and 10000. After NSAIDs ingestion a significant increase of AUC of PEG 4000 to $26.4 \pm 6.2 \mathrm{mg} / \mathrm{ml}$ was found in healthy subjects. After NSAIDs ingestion by IBS patients, AUC of PEG $400(18050 \pm 3140 \mathrm{mg} / \mathrm{ml})$, PEG 1500 $(430 \pm 140 \mathrm{mg} / \mathrm{ml})$ and PEG $4000(390 \pm 19 \mathrm{mg} / \mathrm{ml})$ were significantly increased compared to AUC of PEG $400(10440 \pm 2290 \mathrm{mg} / \mathrm{ml})$, PEG $1500(160 \pm 30 \mathrm{mg} / \mathrm{ml})$ and PEG 4000 $(6 \pm 2 \mathrm{mg} / \mathrm{ml})$ before NSAIDs ingestion. In the absence of NSAIDs, the LM ratio was increased in 1 out of 15 healthy subjects and in 3 out of 13 IBS patients, which was not significantly different. In conclusion, intestinal permeability for PEGs 4000 is decreased in IBS patients compared to healthy subjects. NSAIDs compromise intestinal permeability to a greater extent in IBS patients than in healthy subjects. This suggests that prostaglandin synthesis and thereby the tight junctions are more affected by NSAIDs in IBS patients than in healthy subjects.

\section{S1713}

Sub-Population Specific Deficits of Vagal Gastric Mechanosensitivity in C-Kit Deficient Wwv Mutant Mice

Michael Beyak, Sean Ward, David Grundy

$\mathrm{W} / \mathrm{Wv}$ mutant mice (W/Wv+/-), deficient in the receptor tyrosine kinase c-kit have a loss of interstitial cells of Cajal as well as intramuscular arrays (IMA), a type of putative vagal mechanosensor. They also display altered meal intake patterns, either a consequence of disturbed motility and/or altered afferent nerve function. Aim: To examine mechanosensitive (MS) vagal afferent (VA) nerves innervating the proximal stomach of $\mathrm{W} / \mathrm{Wv}+/$ - mice. Methods: Extracellular recordings were performed in vitro from VAs innervating a $1 \mathrm{~cm} 2$ flat sheet preparation of mouse gastric fundus from 6 male $\mathrm{W} / \mathrm{Wv}+/$ - mice, using wild type littermates (5), and wt C57Bl6 (5) mice as controls. Nicardipine ( $3 \mathrm{uM})$ was used to prevent smooth muscle contraction. Mechanical stimuli were applied using a motorized tissue stretcher attached to the edge of the preparation, connected to a strain gauge. Stretch was applied $0.1 \mathrm{~mm} / \mathrm{s}$ to a maximum tension of $10 \mathrm{~g}$. MS single units were discriminated using the Spike 2 software package. Results: Stretch increased VA firing in C57. WWv wt and W/Wv +/ VAs. Responses did not differ between WWv wt and C57, and thus these were pooled as a control group. Compliance was similar between $\mathrm{W} / \mathrm{Wv}+/$ - and control preparations (mean compliance $1.52 \pm 0.25$ vs. $1.65 \pm 1.5 \mathrm{~mm} / \mathrm{g}, \mathrm{p}=0.5$ ). A total of 18 mechanosensitive single units from W/Wv +/- and 34 from WWv wt / C57 animals were examined. Response properties at tension levels just above threshold were examined. In control preparations 2 distinct populations could be identified. One with a very low threshold $(<\lg )$, reaching greater than $67 \%$ max firing rate at $2 \mathrm{~g}$ of stretch (called LT units) and another with higher thresholds $(>1 \mathrm{~g})$ reaching less than $33 \%$ of max firing rate at $2 \mathrm{~g}$ stretch (called MT units). Of 34 control single units examined, 13 were classified as LT, 14 as MT and 7 unclassified between these. Of 20 single units from W/Wv +/- , 15 were classed as LT, 3 as MT and 2 unclassified ( $\mathrm{p}<0.05$, Fisher's exact test). These differences were reflected in whole nerve discharge patterns. W/Wv + /- preparations reached $74.5 \pm 5.2 \%$ max firing rate at $2 \mathrm{~g}$ stretch, control preparations reached only $55 \pm 8.5 \% \max (\mathrm{p}<0.05)$. Conclusions: Gastric VAs in W/ $\mathrm{Wv}+$ /- mutants are remarkably homogeneous in their stimulus response properties, reaching maximal firing rates at low levels of stretch, consistent with previously described IGLEs. Control animals have another class of MS units, tuned to encode more moderate stimulus intensities. The differences in VA responses are not due to alterations in compliance. We hypothesise that these MT units represent the IMA ending, which are significantly reduced in $\mathrm{W} / \mathrm{Wv}+/$ - mice.

\section{S1714}

Botulinum Toxin a Inhibits Gastric PYLORIC Smooth Muscle By Acetylcholine Independent Muscle Hyperpolarization

Xue D. He, Bo Yang, Madhu Prasad, Raj K. Goyal

Objectives: Botulinum toxin A (BoTA) has been shown to have inhibitory effect on the contractility of the gastrointestinal smooth muscles including the sphincters; and the toxin has been clinically used in the treatment of disorders such as achalasia, gastroparesis and other gastrointestinal motility disorders. The inhibitory effect of BoTA is generally thought to be due to inhibition of acetylcholine release due to cleavage of SNAP 25 in the nerve terminals by the toxin. Recent studies, however, suggest that BoTA may also directly suppress smooth muscle contractility. However, the mechanism of the inhibitory action is not known. We report here that BoTA acts by causing profound membrane hyperpolarization. Methods: Intracellular recordings were made in circular smooth muscle cells of mouse antrum using standard techniques. Effect of BoTA was investigated by incubation of these strips with the toxin for up to 3 hours in order to allow its maximal effect on the cells. Results: Muscle strips without treatment with atropine or nifedipine were very excitable. BoTA (10nM) treatment within $3 \mathrm{sec}$ reduced the excitability and stabilized the tissue. Atropine had a similar effect, suggesting that BoTA acted promptly to suppress cholinergic excitation. Continued BoTA treatment (after $30 \mathrm{~min}$ ) caused hyperpolarization that reaches its peak ($75 \pm 0.2 \mathrm{mV}$ ) after $3 \mathrm{hrs}$. Atropine treatment had no effect on the membrane potential. BoTA induced hyperpolarization was not suppressed by tetrodotoxin pretreatment, suggesting that the hyperpolarizing effect of BoTA was not neutrally mediated. BoTA induced hyperpolarization may be due to opening of potassium channels. However, the hyperpolarizing effect of BoTA was not antagonized by $\mathrm{K}+$ channel blocker TEA $(10 \mathrm{mM})$. In contrast, hyperpolarization caused by chromokalim $(10 \mu \mathrm{M})$ was antagonized by TEA. We also performed whole cell patch clamp studies on isolated smooth muscle cells and found that BoTA had marked inhibitory effect on calcium activated BK currents. Conclusions: These observations indicate that BoTA causes marked inhibition of smooth muscle involving mechanisms that are independent of its effect on acetylcholine release from excitatory nerve terminals and opening of the BK channels in the smooth muscle. BoTA may cause hyperpolarization of the smooth muscle cells by suppressing other than BK potassium, chloride or cation channels.

\section{S1715}

Less Expressions of Neuronal Nitric Oxide Synthase and Substance P in Gastric Interstitial Cells of Cajal in Patients with Diabetes Mellitus

Hirohiko Iwasaki, Masayoshi Kajimura, Satoshi Oosawa, Mutuhiro Ikuma, Takahisa Furuta, Shigeru Kanaoka, Yoshihiko Sato

Background and Aims: Interstitial Cells of Cajal (ICC) in gastrointestinal tract plays a central role in gastrointestinal motility. Gastrointestinal motility is known to be impaired in patients with long-standing diabetes mellitus (DM). However, there were few studies on whether this motility disorder is associated with the impairment of ICC in DM patients. The present study was undertaken to evaluate the density of ICC, neuronal nitric oxide synthase (nNOS), substance P (SP) in gastric antrum in patients with DM and its relevance to the disease severity. Materials and Methods: Paraffin-embedded gastric specimens obtained from 51 control and 36 diabetes male patients with gastric cancer were used for immunohistochemical staining by C-KIT and mast cell tryptase specific antibodies. Further, fresh frozen gastric specimens obtained from 6 control and 5 diabetes male patients with gastric cancer were used for immunofluorescence staining by C-KIT, nNOS and SP specific antibodies, and CKIT, nNOS and SP densities were compared between control and diabetes patients. Results: ICC density in inner circular muscle (CM) in patients with severe DM $(66.1 \pm 23.3 / 2.5 \mathrm{~mm} 2)$ was less than that in control( $147.4 \pm 11.4 / 2.5 \mathrm{~mm} 2)$. In addition, the immunofluorescence study also demonstrated that C-KIT, nNOS and SP expressions in antrum $(2.59 \pm 0.35$, $0.41 \pm 0.18,0.07 \pm 0.01 \%$ ratio) was decreased in patients with DM compared to control(4.24 $\pm 0.56,2.06 \pm 0.33,0.52 \pm 0.06 \%$ ratio). Further, the co-localized density of C-KIT and nNOS or SP in patients with DM(C-KIT and nNOS $0.05 \pm 0.02$, C-KIT and SP $0.00 \pm 0.00 \%$ ratio) was decreased compared to control $(0.44 \pm 0.10,0.14 \pm 0.05 \%$ ratio). Conclusions: In patients with DM, there were less expressions of nNOS and SP densities in gastric ICC concomitant with deficiency of the ICC, suggesting that the presence of functional impairment of ICC may be relevant to dysmotility symptoms observed in patients with DM.

\section{S1716}

Gastric Electrical Stimulation Entrains Gastric Slow Waves (GSWs) Via Nitrergic Pathways in Rats

Yue Yu, Guangfu Yin, Wei Qian, Xiaohua Hou

Background \& Aims: Gastric electrical stimulation (GES) had been paid more attention as a therapeutic modality for gastroparesis. But its mechanism was still unknown. The aim of our study was to investigate effects of nitrergic pathway on the entrainment of GSWs during the gastric stimulation. Methods Sixty four male Wistar rats with two pairs of electrodes were enrolled in this study. Firstly, four groups of 8 were used to investigate the effects of L-NAME on the GSWs in vivo, including control with saline(ip), three groups with various concentration L-NAME $(50 \mathrm{mg} / \mathrm{kg}, 25 \mathrm{mg} / \mathrm{kg}, 12.5 \mathrm{mg} / \mathrm{kg}$, ip, respectively). Then, using immunohistochemistry staining, the nerves containing nNOS were studied between the rats $(n=8)$ with GES and controls $(n=8)$. Lastly, expression of nNOS mRNA in rats $(n=8)$ with GES was compared in rats without stimulation $(n=8)$ using RT-PCR methods. Results: (1) The lower dose of L-NAME $(12.5 \mathrm{mg} / \mathrm{kg}, 25 \mathrm{mg} / \mathrm{kg}$, respectively) had no influence on the GSWs, but the higher dose of L-NAME $(50 \mathrm{mg} / \mathrm{kg})$ induced gastric dysrhythmia. Moreever GES normalized gastric dysrhythmia with higher energy of pacing stimuli $(878.1 \pm 11.4$ vs $537.5 \pm 91.6 \mathrm{~ms} \times \mathrm{mA}, \mathrm{p}<0.001$ ). (2) In GES group, many nNOS positive fibers were observed in the every layers of antrum, nNOS-positive ganglial cells and neuronal bodies were easily found in the antral myenteric plexuses (AMP), but a few nNOS positive fibers were observed, and nNOS -positive ganglial cells and neuronal bodies were difficultly found in AMP in control group. The staining of the nNOS positive products in GES rats was stronger than in control. (3) RT-PCR investigetion showed the levels of nNOS mRNA expression in GES rats were much higher than in controls $(1.18 \pm 0.47$ vs $0.81 \pm 0.39, \mathrm{p}<0.001)$. Conclusion: Normalization of the L-NAME-induced gastric dysrrhythmias should have a high energy gastric stimulation, which facilitate release of $\mathrm{NO}$ in antral myoenteric plexus. It shows nitrergic pathways may be partly involved in the GES-induced entrainment on GSWs.

\section{S1717}

Botulinum Toxin a Inhibits Slow Waves in Mouse Gastric Antrum By a Mechanism That Is Independent of Its Hyperpolarizing Effect Xue D. He, Raj K. Goyal

Objectives: Botulinum toxin A (BoTA) has been shown to have marked inhibitory effect on gastric antrum and pylorus and has been clinically used in the treatment of gastroparesis. Although BoTA is thought to act by suppressing acetylcholine release from nerve terminals, recent studies suggest that BoTA may directly suppress smooth muscle contractility. We have shown that BoTA inhibits antral smooth muscle by causing profound membrane hyprpolarization. We report here that BoTA also suppresses slow waves in the mouse antrum. Methods: intracellular recording were made in circular smooth muscle cells of mouse antrum using standard techniques. Effect of BoTA was investigated by incubation of these strips with the toxin for up to 3 hours in order to allow its maximal effect on the cells. Results: BoTA $(10 \mathrm{nM}$ ) hyperpolarized the resting membrane potential (RMP) by $23 \mathrm{mV}$ (control: $52 \pm 1.4 \mathrm{mV}$ vs BoTA treatment: $75 \pm 2.2 \mathrm{mV}, \mathrm{P}<0.001, \mathrm{n}=7$ in 5 animals) and nearly abolished the slow waves after 3 hours of incubation. The amplitude of slow wave was 17 $\pm 1.4 \mathrm{mV}$ in control and $0.64 \pm 0.3 \mathrm{mV}$ after BoTA $(\mathrm{P}<0.001)$. The frequency of slow wave was $3.14 \pm 0.3 \mathrm{cycle} / \mathrm{min}$ in the control and $0.4 \pm 0.2 \mathrm{cycle} / \mathrm{min}$ after BoTA treatment $(\mathrm{p}<$ 0.001 ), respectively. Atropine treatment had no effect on RMP or the slow waves. The effect of BoTA on the slow waves could have been secondary to hyperpolarization of the RMP. To determine whether the effect of the toxin on the slow waves was due to associated hyperpolariztion, we investigated the effect of conditioning hyperpolarization using the Tomita bath technique. Conditioning hyperpolarization of membrane from $-56 \mathrm{mV}$ to -81 $\mathrm{mV}$ did not significantly affect the slow waves. The amplitude and frequency of slow waves were $11 \pm 0.4 \mathrm{mV}$ and 6 cycles $/ \mathrm{min}$ in the control and $10 \pm 0.5 \mathrm{mV}$ and 6 cycles/min after the conditioning hyperpolarization, respectively. On the other hand, potassium channel opener chromokalim $(10 \mu \mathrm{M})$ caused membrane hyperpolarization $(-15 \mathrm{mV})$ and also partially 
suppressed slow waves. We then examined the effect of recovery of the BoTA or chrormokalim induced hyperpolarization by TEA $(10 \mathrm{mM})$ on the slow waves. Recovery of BoTA induced hyperpolarization did not recover the slow waves, but the recovery of chromokalim induced hyperpolarization recovered the slow waves. Conclusions: These studies suggest that BoTA causes hyperpolarization of the smooth muscles and loss of slow waves in gastric antrum by two independent mechanisms.

\section{S1718}

Effect of Infused Gastric Content On Gastric Distribution, Gastric Pressure, and Blood Pressure in Conscious Rats with Normal and Impaired Gastric Accommodation

Pieter Janssen, Maria Astin Nielsen, Per-Goran Gillberg, Leif Hultin

Introduction The stomach accommodates food without major intragastric pressure (IGP) increase. Impairment of gastric accommodation was associated with abnormal food distribution in the stomach (overloading the antrum) and causes symptoms in functional dyspeptic patients. Previously we showed that Wistar Kyoto (WKY) rats have impaired gastric accommodation compared to Sprague Dawley (SD) rats. We set out to compare these strains in a novel model in which we can measure IGP and gastric distribution in response to testmeal infusion. Blood pressure was measured as a marker for visceral sensation/pain. Material $\&$ Methods All experiments were performed in 6 weight matched female SD and WKY rats. 2 weeks before the start of the experiments telemetric bloodpressure devices and gastric fistulas were implanted. After an overnight fast, a test-meal infusion system and a catheter to measure IGP were connected to the gastric fistula. After a $20 \mathrm{~min}$ stabilisation period, test-meal (non-nutritious; containing $0.5 \mathrm{gr}$ barium sulphate $/ \mathrm{ml}$ in a $3 \%$ hydroxypropyl methylcellulose solution) was infused at a preset speed of $0.25 \mathrm{ml} / \mathrm{min}$ for $2 \mathrm{~min}$, thereafter $2 \mathrm{ml} / \mathrm{min}$ until $9 \mathrm{ml}$ infused. Mean arterial blood pressure during infusion was compared to the mean blood pressure before the start of the infusion. Forestomach, corpus and antrum area, calculated as $\%$ of the total stomach area, were evaluated from X-ray pictures continuously taken during the experiments. Results are expressed as mean \pm SEM. Results In SD rats IGP increased linearly to $11.2 \pm 0.6 \mathrm{mmHg}$ at $4 \mathrm{ml}$ infused and remained stable at this level during further infusion. The IGP in WKY rats, however, increased linearly during the whole test-meal infusion to a maximum of $18.9 \pm 1.9 \mathrm{mmHg}$. Blood pressure during infusion increased $6 \pm 2 \%$ in SD rats and $11 \pm 3 \%$ in WKY rats. With increasing gastric content, the relative forestomach and antrum area increased to respectively $55 \pm 2$ and $16 \pm 1 \%$ in SD rats vs. $48 \pm 1$ and $20 \pm 2 \%$ in WKY rats, whereas the relative corpus area decreased to $30 \pm 3$ and $32 \pm 2 \%$ in SD and WKY rats respectively. Discussion \& Conclusion This novel rat model can be used to study gastric distribution, intragastric pressure and cardiovascular reflexes in response to increasing gastric content. For the same infused volume, WKY rats showed increased IGP and blood pressure, and the content was more distally distributed as compared to SD rats. This data suggests that increased sensitivity to gastric content is associated with impaired gastric accommodation and can be linked to distal stomach overload but also with increased intragastric pressure.

\section{S1719}

Esophageal-Gastric Relaxation Reflex: Dual Control of Peripheral Nitergic and Cholinergic Transmission

Gerlinda E. Hermann, Alberto Travagli, Richard C. Rogers

Esophageal distension produced by swallowing food elicits a powerful proximal gastric relaxation. This gastroinhibitory control by esophageal afferents involves neural pathways between esophageal distension-sensitive neurons in the nucleus tractus solitarius, pars centralis (cNTS) and virtually all levels of the dorsal motor nucleus of the vagus (DMV)[1]. We have shown recently that cNTS responses are excitatory and primarily involve tyrosinehydroxylase immunoreactive (TH-IR)cells [2], while the DMV response involves both an $\alpha 1$ excitatory and an $\alpha 2$ inhibitory response [3]. In the present study, an esophageal balloon distention $(160 \mathrm{uL}$ ) was used to evoke fundic relaxation (esophageal-gastric reflex $=\mathrm{EGR}$ ) as measured by an extraluminal strain gauge aligned with the circular smooth muscle of the fundus. We investigated the peripheral pharmacologic basis for this reflex. Systemic administration of atropine reduced the amplitude of the gastric relaxation to $52.0+4.4 \%$ of the original EGR, while L-NAME reduced it to $26.3+7.2 \%$ of the original EGR. Concomitant administration of atropine and L-NAME reduced the amplitude of the gastric relaxation to $4.0+2.5 \%$ of control. This reduction in amplitude of induced EGR is quite comparable $(4.3+2.6 \%)$ to that seen when the animal is pretreated with the nicotinic ganglionic blocker, hexamethonium [See Figure below]. In the presence of bethanechol, the amplitude of the esophageal-distention induced gastric relaxation was increased to $177.0+10.0 \%$ of control. In this pretreatment condition, administration of L-NAME reduced the amplitude of the gastric relaxation to $19.9+9.5 \%$. Thus far, our data provide a clear demonstration that gastroinhibitory control by the esophagus is mediated by vago-vagal reflexes causing activation of inhibitory nitrergic (NANC) and withdrawal of excitatory cholinergic transmission to the fundus. l. Rogers RC, Hermann GE, and Travagli RA. JPhysiol 514: 369-383,1999. 2.Rogers RC, Travagli RA, and Hermann GE. Am J Physiol 285: R479-R489, 2003. 3. Martinez-Pena y Valenzuela I, Rogers RC, Hermann GE, and Travagli RA. Am J Physiol 286: G333-339, 2004. Supported by NIH grants DK 56373 and 52142

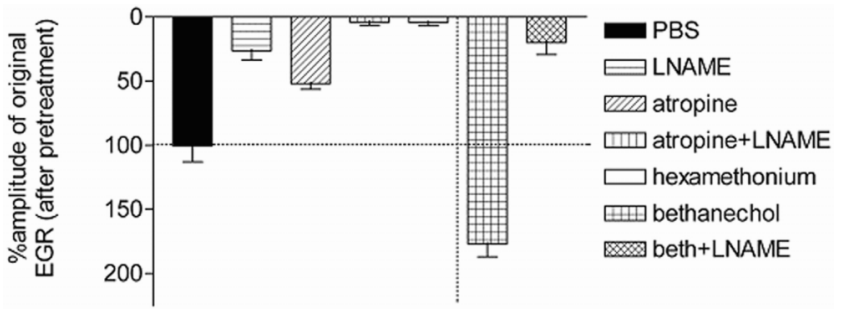

S1720

Opposite Effects of Clonidine and Sumatriptan On Postprandial Gastric Volume Response and Liquid Emptying As Assessed By MRI in Health Monika A. Kwiatek, Andreas Steingoetter, Dieter Menne, Oliver Goetze, Geoffrey S. Hebbard, Peter Boesiger, Miriam Thumshirn, Werner Schwizer, Michael Fried

INTRODUCTION: Gastric barostat has shown relaxation of human proximal stomach after clonidine, an $\alpha_{2}$-adrenergic, and sumatriptan, a 5- $\mathrm{HT}_{1 \mathrm{~B} / \mathrm{D}}$ receptor agonists. While clonidine does not affect solid gastric emptying, sumatriptan prolongs and induces a lag phase after ingestion of solids and liquids, respectively. The physiological intragastric volumetric responses under non-invasive conditions are uncertain. AIM: To assess the characteristics of accommodation and gastric emptying of liquid following pharmacologic modulation of proximal stomach motility by clonidine and sumatriptan. METHODS: Using 1.5T whole body magnetic resonance scanner, 9 healthy fasted volunteers were examined on three separate days directly after $\left(\mathrm{t}_{0}, \mathrm{V0}\right)$ consuming a $500 \mathrm{~mL}, 200 \mathrm{kcal}$ glucose drink labeled with $500 \mu \mathrm{M}$ Gd-DOTA in right decubitus position. Medications were administrated at the start of liquid ingestion in double-blind randomized order: (a) placebo $(0.9 \% \mathrm{NaCl}) \mathrm{IV}$, (b) clonidine $(0.01 \mathrm{mg} / \mathrm{min}$, max. dose $0.1 \mathrm{mg}$ ) IV, (c) sumatriptan (6mg) SC. Intragastric volumes total gastric (TGV) and meal volumes (MV), were assessed at $5 \mathrm{~min}$ intervals for $90 \mathrm{~min}$ RESULTS: In all treatments, gastric relaxation $\left(\Delta \mathrm{TGV} \mathrm{t}_{-5} \rightarrow 0 \mathrm{~min}\right)$ was similar. The stomach expanded to a volume greater than the ingested meal $(p<0.001)$. Subsequent emptying was immediately accelerated by clonidine $(\mathrm{p}=0.001)$ and delayed by sumatriptan $(\mathrm{p}<0.001)$ Decreases in TGV and MV paralleled over the entire postprandial period $(\mathrm{p}=0.07)$. CONCLUSION: During non-invasive volume measurements, initial gastric relaxation is not amplified by clonidine and sumatriptan, implying that initial relaxation is determined by meal volume load. In contrast to reports after solid ingestion, clonidine accelerates liquid emptying in health. Despite differential postprandial effects, both medications preserve the relationship between total gastric and meal volumes.

\begin{tabular}{|c|c|c|c|}
\hline & Placebo & Clonidine & Sumatriptan \\
\hline$\Delta$ TGV t$_{-5 \rightarrow 0 \min }(\mathrm{ml})$ & $768 \pm 44$ & $771 \pm 35$ & $738 \pm 48$ \\
\hline TGV V0 $(\mathrm{ml}) \mathrm{MV} \mathrm{T}_{50}(\mathrm{~min})$ & $881 \pm 24$ & $889 \pm 24$ & $817 \pm 24^{*}$ \\
\hline
\end{tabular}

mean \pm SEM; ${ }^{*} \mathrm{p}<0.05$ vs. placebo; $\mathrm{T}_{50}$, half-emptying time

\section{S1721}

Clonidine Increases Postprandial Distal Antral Motility and Occlusion in Contrast to Sumatriptan - Assessment By MRI in Health Monika A. Kwiatek, Anupam Pal, Andreas Steingoetter, Dieter Menne, Oliver Goetze, James G. Brasseur, Geoffrey S. Hebbard, Peter Boesiger, Michael Fried, Werner Schwizer

INTRODUCTION: Clonidine, an $\alpha_{2}$-adrenergic, and sumatriptan, a $5-\mathrm{HT}_{1 \mathrm{~B} / \mathrm{D}}$ receptor agonists cause relaxation of the proximal stomach detectable by gastric barostat. Whilst clonidine has no effect on solid gastric emptying, sumatriptan prolongs solid emptying and induces a lag phase after ingestion of liquids. The accompanying antral motility patterns are uncertain, especially for liquid meals and in non-invasive conditions. AIM: To assess the propagation and geometry of distal antral contraction waves (ACWs) following pharmacologic modulation of gastric motility by clonidine and sumatriptan. METHODS: Using 1.5T whole body magnetic resonance scanner, 9 healthy fasted volunteers were examined in right decubitus position on three separate days directly after $\left(\mathrm{t}_{0}\right)$ consuming a $500 \mathrm{~mL}, 200 \mathrm{kca}$ glucose drink labeled with 500uM Gd-DOTA. Medications were administrated at the start of liquid ingestion in double-blind randomized order: (a) placebo $(0.9 \% \mathrm{NaCl}) \mathrm{IV}$, (b) clonidine $(0.01 \mathrm{mg} / \mathrm{min}$, max. dose $0.1 \mathrm{mg}$ ) IV, (c) sumatriptan $(6 \mathrm{mg}) \mathrm{SC}$. ACWs were assessed with dynamic MR scan sequences (duration: $167 \mathrm{~s}$ ) at $5 \mathrm{~min}$ intervals, between measures of gastric emptying (GE), for 90min. RESULTS: Clonidine accelerated ( $\mathrm{p}=0.001$ ) and sumatriptan delayed GE $(\mathrm{p}<0.001)$. Initially $\left(\mathrm{t}_{0} \rightarrow 15 \mathrm{~min}\right)$, clonidine increased the number of accompanying ACWs, while a prolonged ( $\mathrm{t}_{0} \rightarrow 40 \mathrm{~min}$ ) reduction was seen after sumatriptan (both $\mathrm{p}<0.04)$. Propagation speed $(2.6 \pm 0.3 \mathrm{~mm} / \mathrm{s})$ of ACWs was not affected. Under placebo, the immediate $\left(\mathrm{t}_{0}\right)$ maximal occlusion increased over time $(\mathrm{p}<0.01)$. Both drugs enhanced this maximal occlusion $(\mathrm{p}<0.01)$, which was maintained and steadily diminished under clonidine and sumatriptan, respectively $(\mathrm{p}<0.02)$. In all treatments, ACWs had the highest amplitude and the smallest width close $(0-1 \mathrm{~cm})$ to the pylorus (all $\mathrm{p}<0.01)$. SUMMARY: Clonidine and sumatriptan have distinct effects on ACWs. Intensification of ACWs caused by clonidine may contribute to faster liquid emptying. Reduction in antral motility by sumatriptan enhances the delay in emptying. CONCLUSION: Stimulation of adrenergic and serotonergic pathways plays a significant role in modulation of liquid emptying by inhibiting motility of the proximal stomach and alteration of antral contractility 


\begin{tabular}{|c|c|c|c|}
\hline & Placebo & Clonidine & Sumatriptan \\
\hline Max. occlusion (\%) at t0 & $60 \pm 3$ & $77 \pm 2^{*}$ & $66 \pm 2^{*}$ \\
\hline$\Delta$ Max. occlusion (\%/min) & $0.08 \pm 0.02$ & $0 \pm 0.04^{*}$ & $-0.11 \pm 0.03^{*}$ \\
\hline Max. amplitude (cm) at 10 & $0.75 \pm 0.05$ & $0.90 \pm 0.02^{*}$ & $0.71 \pm 0.03$ \\
\hline Min. width (cm) at t0 & $1.00 \pm 0.05$ & $0.98 \pm 0.02$ & $0.90 \pm 0.03^{*}$ \\
\hline GE T $_{50}(\mathrm{~min})$ & $70 \pm 11$ & $44 \pm 7^{*}$ & $98 \pm 7^{*}$ \\
\hline
\end{tabular}

mean \pm SEM; *p<0.05 vs. placebo; T50, half-emptying time

\section{S1722}

Chronic Alcohol Consumption Impairs Nitrergic Inhibition in the Mouse Small Intestine

Monika Krecsmarik, Benedicte Y. de Winter, Joris G. de Man, Maria Bagyanski, Luc van Nassauw, Eva Fekete, Paul A. Pelckmans, Jean-Pierre Timmermans

Introduction: Chronic ethanol administration has been shown to alter gastrointestinal motility in alcoholic patients and laboratory animals but the cellular mechanisms underlying altered smooth muscle contraction are largely unknown. Part of the nitric oxide (NO) producing neurons constitute an important enteric neuronal population playing an essential role in regulating the downstream inhibitory phase of peristalsis. Therefore, we aimed at investigating the effect of chronic ethanol consumption on the survival rate and functioning of these neurons and its implications on smooth muscle contractility and gastrointestinal transit. Methods: Mice received a gradually increasing concentration of ethanol in water with a final concentration of $20 \%$, while the control group received isocaloric sucrose solution. Using PGP9.5- and NOS-immunostaining, a quantitative analysis of the total number of myenteric small intestinal neurons and of the proportion of nitrergic myenteric neurons was performed after 4 and 6 weeks of alcohol treatment. The effect of ethanol treatment on nitrergic relaxations to electrical field stimulation (EFS) and exogenous $\mathrm{NO}$ of jejunal circular muscle strips was investigated in vitro. Gastric emptying $(G E)$ and small intestinal transit were measured in vivo after intragastric gavage of Evans blue and expressed as \%GE and geometric center (GC) respectively. Results: The number of NOS-IR neurons did not change in the duodenum, however, it decreased significantly in the jejunum by $17-20 \%(\mathrm{P}<0.01-\mathrm{P}<0.001)$ after 4 and 6 weeks of alcohol treatment, respectively. The total number of neurons remained unchanged. In jejunal circular muscle preparations, the nitrergic nerve-mediated relaxations to 1,2 and $4 \mathrm{~Hz}$ EFS were significantly decreased after 5 weeks of ethanol treatment compared to controls: from $63.79 \pm 4.54 \%$ to $30.95 \pm 7.89 \%$; from $81.25 \pm 3.85 \%$ to $55.71 \pm 6.04 \%$; from $84.64 \pm 2.96 \%$ to $67.92 \pm 5.96 \%$ respectively $(\mathrm{P}<0.05)$ while relaxations to NO remained unchanged. GE and GC were significantly increased after 5 weeks of ethanol treatment from $26.4 \pm 5.2 \% \mathrm{GE}$ and $2.1 \pm 0.1 \mathrm{GC}$ in controls to $40.6 \pm$ $4.1 \% \mathrm{GE}$ and $2.5 \pm 0.1$ in ethanol-treated mice. Conclusions: These results are suggestive of an adaptive, region- and time-dependent, quantitative and functional change in nitrergic innervation within the murine enteric nervous system in response to chronic alcohol consumption. These changes may partially account for the observed increased gastrointestinal transit.

\section{S1723}

Botulinum Toxin a Inhibits Inhibitory Junction Potentials in Gastric PYLORIC Smooth Muscles Raj K. Goyal, Xue D. He

Objectives: Botulinum toxin A (BoTA) has been shown to have inhibitory effect on the contractility of the gastrointestinal smooth muscle including the sphincters and the toxin has been clinically used in the treatment of disorders such as achalasia, gastroparesis and other gastrointestinal motility disorders. The inhibitory effect of BoTA is generally thought to be due to inhibition of acetylcholine release due to cleavage of SNAP 25 in the nerve terminals by the toxin. However, it is not clear whether BoTA also inhibits the release of the inhibitory neurotransmitter. We report here that BoTA also suppresses the inhibitory junction potentials (IJPs) in the mouse gastric antrum. Methods: Intracellular recordings were made in circular smooth muscle cells of mouse antrum using standard techniques. Tetrodotoxin sensitive junction potentials were produced by EFS (trains of 10 pulses of 5.0 msec and $30 \mathrm{~mA}$ at $20 \mathrm{~Hz}$ ). Effect of BoTA was investigated by incubation of these strips with the toxin for up to 3 hours in order to allow its maximal effect on the cells. Results: BoTA (10nM) promptly suppressed cholinergic excitability of the muscles strips that was followed by membrane hyperpolarization that reached a peak effect $(-75 \mathrm{mV})$ in 3 hours. No excitatory junction potentials (EJPs) were observed at any time after the toxin treatment. However, the IJP amplitude was $20 \mathrm{mV}$ in the control period; they were progressively suppressed with continued toxin exposure and were abolished at 3 hours. The suppression of the IJP by BoTA could be secondary to the associated membrane hyperpolarization or the suppression of the release of inhibitory neurotransmitters or both these effects. However, recovery of the BoTA induced hyperpolarization by TEA $(10 \mathrm{mM})$ did not recover the IJP. Conclusions: The studies show that BoTA not only causes smooth muscle membrane hyperpolarization but also suppresses the release of the excitatory as well as the inhibitory neurotransmitters form the nerve terminals. However, BoTA is more effective in suppressing the excitatory than in suppressing the inhibitory neurotransmission.

\section{S1724}

Neurally Mediated Chronotrophic Effects On Gastric Pacemaking Is Limited By Cholinesterase Actviity

Abigail Forrest, Sean M. Ward, Yulia Baguinov, Brian A. Perrino, Fiona Britton, Rebecca Partain, Kenton M. Sanders

Background: Acetylcholine (ACh) released from enteric motor neurons causes chronotropic effects on slow wave frequency in the distal stomach. Methods: We used electrophysiological, molecular (PCR) and protein chemistry (Western blots, immunohistochemistry) techniques to determine the relative expression of AChEsterase (AChE) in gastric corpus and antrum and the effects of AChE inhibition on gastric slow wave frequency. We also compared AChE expression and the effects of neostigmine (AChE inhibitor) in muscles of wildtype and W/ $W^{V}$ mice that lack specific classes of ICC. Results: Neostigmine had chronotropic actions on corpus and antrum muscles. These effects were blocked by tetrodotoxin and atropine, demonstrating that spontaneous release of ACh from nerve terminals is efficiently metabolized by AChE and the chronotropic effects of ACh are kept in check by metabolism. In simultaneous intracellular recordings from the corpus and antrum, neostigmine significantly increased slow wave frequency in both regions but did not lead to functional uncoupling between the corpus and antrum, suggesting that there is balanced ACh metabolism in both regions. These effects were also blocked by atropine. Chronotropic effects of neostigmine were absent in $W / W^{V}$ mice that lacked intramuscular ICC (ICC-IM). Immunohistochemical studies found AChE-like immunoreactivity occurred in a network of nerve fibers in both corpus and antrum but not in ICC-IM. Quantitative PCR and Western blots showed that transcriptional and translational expression of AChE were not significantly different in wildtype and W/WV muscles from either the corpus or antrum. The enzymatic activity of the AChE was also comparable in antral and corpus muscles from wildtype and $W / W^{V}$ mice. Conclusion: Potent chronotropic effects are provided by muscarinic stimulation of gastric muscles. Efficient ACh hydrolysis is required to maintain basal slow wave frequency. Release and metabolism of ACh appears balanced between the proximal and distal stomach with no apparent heterogeneity in cholinergic regulation of slow wave frequency, which if unbalanced, could result in functional uncoupling. Neurons appear to be the major source of AChE in gastric muscles, so loss of ICC-IM does not impact ACh metabolism. Lack of effect of AChE inhibition in W/WV mutants is likely due to the breakdown in cholinergic neurotransmission when synapses between cholinergic neurons and ICC-IM are lost. Imbalances in AChE expression or function in disorders such as gastroparesis, might contribute, however, to inappropriate ACh metabolism and changes in the chronotropic effects of muscarinic stimulation (Supported by DK40569).

\section{S1725}

Abatement Effect of Daikenchuto, One of the Japanese Traditional Herbal Medicine (KAMPO MEDICINE), On the Morphine-Induced Slowing of Gastrointestinal Motility

Nakamura Tomonori, Mizuki Hayashi, Keiko Onoue, Shizuko Tsuchiya, Shingo Yano, Kageyoshi Ono

Backgrounds: Morphine-induced constipation is one of the most severe adverse reactions of drugs. A large number of patients who suffer from carcinomatous strong pain are troubled with the heavy constipation by morphine. The herbal medicine Daikenchuto (DKT) is a mixture of three herbs, Zanthoxylum Fruit, Ginseng Root and Dried Ginger Rhizome, and recently has been applied to post-operative paralytic ileus in Japan. In our recent study, DKT has been shown to abate morphine-induced slowing of gastrointestinal (GI) transit in mice. Aim: We investigated the mechanism in eliciting stimulatory effect of DKT on the GI motility. Methods: Morphine hydrochloride ( $2 \mathrm{mg} / \mathrm{kg}$ ) was i.p. administered in ddY mice (male, 6 weeks old) to make the GI disorder model. DKT (100,300 and $1000 \mathrm{mg} / \mathrm{kg}$ ) was orally administered $60 \mathrm{~min}$ before the onset of experiments. GI transit rate and rectal excretion time were measured by modified charcoal meal test and glass bead test, respectively. In the isolated ileum experiments, the contractile responses to autonomic drugs were measured using a Magnus apparatus. Results: The slowing of intestinal transit by morphine was definitely recovered by DKT in a dose-related manner. Similarly, the rectal excretion time was improved by DKT. In in vitro experiments, DKT transiently contracted the isolated ileum; this effect was mediated largely through cholinergic pathway. To elucidate the longlasting effect of DKT on the isolated ileum, the responses to constrictors were measured after a short-term exposure of the ileum to DKT. As a result, the contractile sensitivity to a submaximal dose of acetylcholine, serotonin or substance P was significantly augmented for more than $60 \mathrm{~min}$ after DKT exposure. These responses were also replicated by a exposure to hydroxyl-sanshool (HS), the active component of Zanthoxylum Fruit. The potentiation by HS was partially blocked by capsazepin, a VRI antagonist. To confirm the enhancement of GI motility by HS in in vivo experiments, pretreatment with $\mathrm{HS}(3 \mathrm{mg} / \mathrm{kg}$, p.o.) was conducted $90 \mathrm{~min}$ before application of mosapride $(0.1 \sim 1 \mathrm{mg} / \mathrm{kg}$, p.o.), a selective 5-HT4 agonist. The intestinal transit rate was significantly accelerated by the combination of HS and mosapride. Conclusion: DKT improves morphine-induced slowing of GI transit in mice. The underlying mechanism therein may be due to long-lasting effect of DKT in augmenting the contractile response to endogenous constrictors such as acetylcholine, serotonin and substance $\mathrm{P}$ in the GI tract.

\section{S1726}

How Common in Rapid Gastric Emptying in Gastroparesis? Comparison of Area Under the Curve, Total Gastric Emptying and 1 Hour Values in Patients and Normal Volunteers

Thomas L. Abell, Warren Starkebaum, Nighat Abidi, An Liu

Introduction: Rapid gastric emptying has been described not only in patients with nausea and vomiting and gastroparesis but also in obesity and dyspepsia. We investigated the use of 3 measures of rapid gastric emptying in a large group of gastroparetic patients compared to normal controls. Patients: From a pool of 214 consecutive patients with the symptoms of gastroparesis we reviewed the results of standardized solid gastric emptying tests and 
compared the results to a groups of normal controls as previously described (AJG 95: 145614622000). Patients were 39 male, 175 femalef mean age 42 years, with diagnosis of 42 Diabetes Mellitus, 26 Post Surgical and 146 Idiopathic disease. Methods: Gastric emptying was compared by 3 models: Area under the emptying curve (AUC, rapid $<0.257$ ), total gastric emptying (TGE: the sum of 1,2 and 4 hours; rapid $<47$ ), and \% remaining at 1 hour $(<37 \%)$ for rapid and/or at 4 hours ( $>10 \%)$ for delayed emptying, using data and techniques previously described (AGJ2004;99:S45 and NGM 2005; 17: 470). Results: Measurable gastric emptying could be determined for 4 hour emptying in 146 patients; 87 patients had complete 1,2 and 4 hour emptying. 44 patients had delayed emptying by 4 hour $\%>10 \% .7$ patients (8\%) had rapid gastric emptying by AUC and 8 pts (9\%) had rapid emptying by the TGE values; and $16(18 \%)$ pts had rapid emptying by the 1 hour value alone. Conclusion Rapid gastric emptying of a solid meal is not uncommon in patients with the symptoms of gastroparesis. The AUC and TGE models identified approximately the same number of patients with rapid emptying, while the $1 \mathrm{hr}$ model identified approximately twice as many patients. Using the three techniques described to determine rapid gastric emptying awaits prospective use in other patients, including dyspepsia and obesity. Standardized measure for rapid gastric emptying may be useful in evaluating therapeutic outcomes for these disorders.

\section{S1727}

Delayed Liquid and Solid Gastric Emptying in Non-Dyspeptic Patients with Irritable Bowel Syndrome

Stamatiki Kritas, Lauren V. Garritt, Lesley M. Stevenson, Geoff P. Davidson, Ross N. Butler

Introduction: Irritable Bowel Syndrome (IBS) is the most common functional gastrointestinal disorder affecting $15-20 \%$ of the population worldwide. The causes of IBS are unknown and patients present with a range of recurrent and chronic symptoms. Many factors are currently thought to be involved in the pathophysiology of IBS, such as psychological status, gut hypersensitivity and low-grade inflammation. Impaired intestinal transit has also been reported in IBS patients, however, the contribution of gastric motility, specifically gastric emptying, has only been evaluated in IBS patients with concurrent functional dyspepsia. Studies investigating gastric emptying in IBS patients without dyspepsia have all been contradictory. The current study evaluated both liquid (GE-L) and solid (GE-S) gastric emptying in IBS patients utilising ${ }^{13} \mathrm{C}$ breath testing. Methods: All subjects fulfilled the ROME II criteria and all with concurrent dyspepsia were excluded. After an overnight fast and a baseline sample, 53 IBS patients $(37.3 \pm 2.0 \mathrm{yrs}, 70 \% \mathrm{~F})$ and 15 healthy controls $(59.4 \pm 3.7 \mathrm{yrs}, 67 \% \mathrm{~F})$ underwent a GE-L breath test by ingesting $200 \mathrm{~mL}$ full cream cow's milk. On a separate occasion, 37 IBS patients $(36,1+2.1 \mathrm{yrs}, 76 \% \mathrm{~F})$ and 11 healthy controls $(55.6 \pm 5.9 \mathrm{yrs}, 55 \% \mathrm{~F})$ ingested a standard pancake for a GE-S breath test. Both meals (liquid and solid) contained $100 \mathrm{mg}{ }^{13} \mathrm{C}$-sodium octanoate salt and breath samples were collected at intervals before and after ingestion of each meal for 240 and 360min, respectively. Breath samples were analysed for ${ }^{13} \mathrm{CO}_{2}$ content and gastric half emptying times were calculated from the resulting ${ }^{13} \mathrm{CO}_{2}$ excretion curves. Results: Gastric emptying time was significantly longer in IBS patients compared to controls for both the liquid ( $62.1 \pm 6.8 \mathrm{~min}$ vs. $40.6 \pm 3.0 \mathrm{~min}$, $\mathrm{p}=0.001)$ and solid meal $(87.1 \pm 8.34 \mathrm{~min}$ vs. $66.2 \pm 4.4 \mathrm{~min}, \mathrm{p}=0.030)$. Conclusions: IBS patients without concurrent dyspepsia had significantly delayed liquid and solid gastric emptying compared to controls. This suggests the potential involvement of altered gastric motility in the pathophysiology of IBS. Further studies are required to evaluate the effectiveness of gastrokinetic drugs as potential therapies for IBS

\section{S1728}

Relationships Between Proximal Gastric Relaxation and Gastric Emptying in Humans: Roles of Meal Volume and Calorie Content

Karla Lourenco, Larissa Sanches, Luiz Troncon, Ricardo B. de Oliveir

BACKGROUND- Proximal gastric relaxation (PGR) and gastric emptying (GE) rate are thought to exert mutual influences on each other. Nevertheless, the relationships between PGR and GE in humans have not been much studied. AIMS - We investigated the relationships between PGR and GE of liquid meals with different volume and calorie content. METHODS Three liquid meals labeled with $99 \mathrm{~m}$ Technetium-phytate were administered to 15 healthy volunteers in a random order in separate days (meal A: $60 \mathrm{ml}, 57 \mathrm{Kcal}$; meal B: $60 \mathrm{ml}$, $137.5 \mathrm{Kcal}$; meal C: $360 \mathrm{ml}, 57 \mathrm{Kcal}$ ). During meal ingestion and for the next $10 \mathrm{~min}$, images of the proximal stomach were acquired by dynamic scintigraphy (one frame.sec-1) for PGR measurement. Thereafter, anterior and posterior images of stomach were acquired in static mode at 10 min intervals up to $70 \mathrm{~min}$ GE assessment. PGR was expressed as the percentage of total radioactivity retained in the proximal stomach. GE was expressed as the percentages of total radioactivity emptied from the total stomach. RESULTS - Ten min after the low volume, low calorie meal (A), PGR was low (median; range-7.6\%:2.3-41.5\%). Increasing both ingested calorie content (meal B) and volume (meal C) was associated with enhanced PGR (meal B - 15.4\%; 1.4-31.3\%; p>0.50 vs. meal A; meal C - 16.1\%; 7.0-41.8\%; p=0.021 vs. meal A). Although there were no significant differences between meals regarding GER (median; range - meal A: 34.1\%; 27.9-70.6\%; meal B: 25.2\%; 16.9-51.2\%; meal C: $29.1 \%$; $18.9-67.9 \% ; \mathrm{p}>0.05)$. A strong $(\mathrm{r}=0.76)$ and significant $(\mathrm{p}=0.01)$ positive correlation between PGR and GE was found for meal B. No significant correlation between PGR and GE was observed with the meal A $(r=0.11 ; p=0.75)$ e meal $C(r=0.03 ; p=0.93)$. CONCLUSIONS In humans, both meal calorie content and volume enhances proximal gastric relaxation within the early pos-prandial period of liquid meals, but only calorie-induced relaxation seems to depend on the rate nutrients leave the stomach.
S1729

Glucagon Alters Frequency and Propagation Velocity of the Gastric Slow Wave Magnetic Field

Leonard A. Bradshaw, Jared A. Sims, Katie McDonald, William O. Richards

Alterations in the gastric slow wave may have important implications for the diagnosis of certain diseases. Previous studies have shown that gastroparesis causes slow wave frequency changes reflected in electrogastrography (EGG) [1]. We have previously used magnetogastrography (MGG) to measure spatial and temporal parameters of the gastric slow wave noninvasively [2]. In this study, we hypothesized that spatiotemporal slow wave changes induced by hyperglycemia would be reflected in the MGG. We measured the MGG in 20 normal human volunteers using a multichannel Superconducting QUantum Interference Device (SQUID) magnetometer. After a 20-minute baseline recording, we injected glucagon ( $9 \mathrm{mcg} / \mathrm{kg} \mathrm{IV}$ ) and recorded for an additional 40 minutes. The gastric slow wave was eviden in MGG recordings by visual analysis of waveforms. We used FFT and AR analysis to compute the frequency spectrum, and we assessed the gastric slow wave frequency and amplitude as the dominant peak in the spectrum. In addition, we used the FFT spectrum to compute the percentage of power distribution (PPD) in frequency ranges denoted as bradygastric $(<2 \mathrm{cpm})$, normogastric $(2-4 \mathrm{cpm})$ and tachygastric $(>4 \mathrm{cpm})$. We also used the spatiotemporal information from the MGG to compute surface current densities (SCDs) that allowed us to evaluate the slow wave propagation velocity. MGGs reflecting the gastric slow wave were measured in all 20 subjects. The slow wave frequency dropped from 2.9 $\pm 0.1 \mathrm{cpm}($ mean $\pm \mathrm{SEM})$ to $2.4 \pm 0.2 \mathrm{cpm}$ post-glucagon $(\mathrm{p}<0.05)$. There was no change in slow wave amplitude after glucagon injection $(2.6 \pm 0.4 \mathrm{pT}$ to $2.0 \pm 0.2 \mathrm{pT}, \mathrm{p}=0.09)$, but we did note a significant decrease in the normogastric PPD $(63.0 \pm 3.0 \%$ to $41.5 \pm$ $3.2 \%, p<0.01$ ) and a concomitant significant increase in bradygastric PPD. We observed an increase in tachygastric PPD that was not significant. Further, gastric slow wave propagation velocities computed from SCD maps decreased post-glucagon from $7.9 \pm 0.7 \mathrm{~mm} / \mathrm{s}$ to 4.2 $\pm 2.1 \mathrm{~mm} / \mathrm{s}(\mathrm{p}<0.05)$. The multichannel MGG noninvasively measures changes in the gastric slow wave caused by glucagon. We are now evaluating diabetic patients with hyperglycemia to identify slow wave abnormalities that may be associated with diabetic gastroparesis. References [1] Abell TL, Malagelada JR. Glucagon-evoked gastric dysrhythmias in human shown by an improved electrogastrographic technique. Gastro 88(6): 1932-1940, 1985. [2] Bradshaw LA, Ladipo JK, Staton DJ, Wikswo JP, Jr., and Richards WO. The human vector magnetogastrogram and magnetoenterogram. IEEE Trans. Biomed. Eng., 46(8): 959-970, 1999

S1730

Gastric Emptying in Diabetic and Non-Diabetic Mice

Kyoung M. Choi, Jin Zhu, Simon J. Gibbons, Gary J. Stoltz, Steven A. Vernino, Joseph H. Szurszewski, Gianrico Farrugia

Background: Diabetic gastroparesis is a complex disorder associated with delayed gastric emptying (GE). Mouse models are very helpful in studying the complications of diabetes. The conventional method for measuring GE in mice requires sacrificing multiple animals at given time points. Changes in GE in individual mice over time and during developmen of diabetes however cannot be studied by this technique. Aims: Apply a non-invasive ${ }^{13} \mathrm{C}$ octanoic acid-based technique to determine GE changes in non-obese diabetic mice (NOD/ $\mathrm{LtJ}$ ) over time and as diabetes develops and to compare their GE to a closely related strain, NOR, often used as controls. Methods: A modification of the technique described by Symonds (Clin Exp Pharm Physiol, 2000) was used. $200 \mathrm{mg}$ of egg yolk containing ${ }^{13} \mathrm{C}$-octanoic acid was used as the test meal. Non-restrained mice were placed in specifically designed chambers with a constant air flow. The amount of ${ }^{13} \mathrm{CO}_{2}$ in exhaled breath was measured using an Infra Red Isotope Analyzer (IRIS, Wagner, Germany) over $240 \mathrm{~min} .{ }^{13} \mathrm{C}$-octanoic acid is rapidly absorbed in small intestine, metabolized in liver to $\mathrm{CO}_{2}$, and exhaled giving a measure of GE. Data were fit by non-linear regression and gastric half-emptying time ( $\mathrm{T}_{1}$ 2) calculated using an inverse gamma function. $T_{1 / 2}$ was determined in three age groups of non diabetic mice (blood glucose $<155 \mathrm{mg} / \mathrm{dl}$ ); $9-15,16-30$ and $31-45$ weeks and weekly after development of diabetes $(>250 \mathrm{mg} / \mathrm{dl})$. Results: $\mathrm{T}_{1 / 2}$ (min, mean \pm SEM) was unchanged in the different age groups and between NOD and NOR mice ( $P>0.05$ all groups, ANOVA, see table). $\mathrm{T}_{1 / 2}$ ( $\mathrm{min}$ ) was not significantly different between pre-diabetic mice (glucose level: $\left.156-249 \mathrm{mg} / \mathrm{dl}, \mathrm{T}_{1 / 2} 83 \pm 9, \mathrm{n}=10\right)$ and mice diabetic for 1 week $(75 \pm 4, \mathrm{n}=17)$. However $\mathrm{T}_{1 / 2}$ was significantly faster after 2 weeks of diabetes $(49 \pm 6, \mathrm{n}=7, \mathrm{P}<0.05)$. After $3-5$ week of diabetes the $T_{1 / 2}$ increased back to baseline values $(73 \pm 8, n=17, P>0.05 \mathrm{c} / \mathrm{w}$ prediabetic mice). Conclusion: Gastric emptying is unchanged with increasing age in NOD and NOR mice. NOR mice have similar GE as non diabetic NOD mice, which confirms that NOR mice are useful controls in the study of diabetic gastroparesis. In the early weeks afte development of diabetes, GE is accelerated, not delayed. Therefore, care must be taken to measure the actual rates of GE when studying the histological and physiological changes associated with the early stages of diabetes. This work is supported by NIH grants DK68055 and DK57061.

\begin{tabular}{|c|c|c|c|}
\hline & $9-15$ weeks & $16-30$ weeks & $31-45$ weeks \\
\hline NOD (non diabetic) & $92 \pm 7, \mathrm{n}=33$ & $84 \pm 6, \mathrm{n}=28$ & $72 \pm 8, \mathrm{n}=6$ \\
\hline NOR & $87 \pm 5, \mathrm{n}=30$ & $101 \pm 8, \mathrm{n}=12$ & $86 \pm 9, \mathrm{n}=8$ \\
\hline
\end{tabular}

\section{S1731}

Effects of short-Term L-Arginine Administration On Proximal Stomach Tone and Gastric Emptying of a Polymeric Enteral Diet in Humans Guillaume Savoye, Yassine Jemaa, Gregory Mosni, Celine Savoye-Collet, Paola Morcamp, Mickael Bouin, Philippe Denis, Philippe Ducrotte

Backgrounds and aims: Endogenous nitric oxide (NO) plays an important role as nonadrenergic, non-cholinergic inhibitory neurotransmitter in GI tract. Our study aim was to investigate the effect of "short-term" l-arginine (L-Arg) administration, as a source for NO, 
on proximal stomach tone and gastric emptying of a polymeric enteral diet in humans. Methods: Eight healthy volunteers were studied in a randomized double blind cross-over study after respectively 15 grams L-Arg, 30 grams L-Arg or placebo administered in the stomach through a gastric tube. The drug administration was followed by a polymeric diet infusion $(500 \mathrm{ml} / 500 \mathrm{Kcal})$ at a $250 \mathrm{ml} /$ hour rate. The gastric tone variations were recorded with an electronic barostat, gastric emptying was concomitantly assessed by repeated ultrasound measurements of antral area and symptoms were recorded throughout the experiment. Results: L-Arg administration was associated with more frequent proximal stomach relaxations and significantly higher increases in barostat bag volumes at both dosage, 30 grams $(117 \pm 16 \mathrm{ml})$ and 15 grams $(67 \pm 15 \mathrm{ml})$ as compared to placebo $(46 \pm 11 \mathrm{ml}), \mathrm{p}<0.05$. The 30 grams L-Arg challenge was associated with a significantly lower increase in maximal barostat bag volumes in response to the polymeric diet $(80+20 \mathrm{ml})$ as compared to $161+17$ $\mathrm{ml}$ for placebo challenge and $126 \pm 30 \mathrm{ml}$ for 15 grams L-Arg challenge. Antral area were not different at any time of measurement between the 3 challenges. Bloating and diarrhea were observed after 30 grams L-Arg administration in 5 subjects out of 8 . Conclusion: Short erm L-Arg administration was able to induce a proximal stomach relaxation that allowed a secondary response to enteral feeding only at a 15 grams dosage. This 15 grams dosage was as well tolerated as the placebo and was associated with no significant changes in gastric emptying patterns.

\section{S1732}

Tumor Necrosis Factor (TNF) Positively Modulates Vagal Afferent Excitability Within the Solitary Nucleus: An in Vitro Live-Cell Imaging Study Richard C. Rogers, Gerlinda E. Hermann

Cancer, autoimmune disorders, chronic infection, and traumatic injury are all associated with the development of gastric stasis, nausea, anorexia and emesis. These co-morbidities are present even though the primary disease process may not directly involve the gastrointestinal tract. Production of the cytokine TNF is a common feature in these disease processes and TNF is widely known to cause gastric stasis and other symptoms just mentioned. Our previous work has shown that: a) TNF can produce gastric stasis thru action directly on gastric vago-vagal reflex neuronal circuitry in the dorsal medulla; an area outside the brainvascular diffusion barrier, b) TNF causes a dramatic increase in the activity of solitary nucleus [NTS]neurons known form part of the relaxatory gastric reflex control mechanism, and c) TNF activation of NTS neurons requires intact glutamatergic neurotransmission and the effects of gastric vagal afferent stimulation on NTS neuron activity is amplified by TNF [1]. These data, coupled with the recent finding that the principal locus for TNF receptors in the dorsal medulla is on afferent vagal fibers [2], strongly suggests that TNF controls gastric function by modulating vagal afferent neurotransmission within the NTS. To study this phenomenon directly, we devised a new technique for imaging the activity of vagal afferent varicosities within the NTS [3]. Vagal afferent fibers and terminal varicosities were preloaded with calcium green dextran. In vitro slice preparations of the medulla were examined with a confocal microscope. These fine vagal afferent fibers and terminal varicosities could be readily activated by direct electrical stimulation [opening voltage gated cation channels], exposure to capsaicin [opening VRl ligand gated cation channels] or exposure to ATP [opening P2X3 ligand gated cation channels]. While TNF alone had little direct effect on vagal afferent calcium signals, TNF significantly increased the vagal afferent terminal calcium levels generated by the other stimuli. These data support the hypothesis that TNF deranges gastric function by increasing the excitability of vagal afferents controlling gastroinhibitory vago-vagal reflexes. 1. Hermann GE, Holmes GM, Rogers RC. Curr Pharm Des. 2005;11(11):1391-409. 2. Hermann GE, Hebert SL, Van Meter MJ, Holmes GM, Rogers RC. Brain Res. 2004 Apr 9;1004(1-2):156-66. 3. Rogers RC, Nasse JS, Hermann GE. J Neurosci Methods. 2005 Aug 11 [EPUB ahead of print] Supported by NIH grants DK56373 and 52142

\section{S1733}

Predictors of Symptom Non-Responders to High-Frequency Gastric Electrical Stimulation for Refractory Gastroparesis

Zhiyue Lin, Irene Sarosiek, Jameson Forster, Richard W. McCallum

Chronic high-frequency gastric electrical stimulation (GES) has been shown to improve gastroparetic symptoms and quality of life (QOL) in up to $70 \%$ of patients with refractory gastroparesis (Gastroenterology 2003;125:421-8). Little is known about factors associated with treatment failure. Clinical and gastric function data (gastric emptying test, GET; electrogastrogram, EGG) were extracted from a retrospective analysis of 87 gastroparetic patients (48 diabetic, 20 idiopathic and 19 postsurgical) who completed GES therapy (EnterraTM System, Medtronic) for at least 1 year. Total symptom score (TSS) (sum of severity of nausea, vomiting early satiety, bloating, postprandial fullness, epigastric pain and burning using a 5-point scale, $0=$ non, $4=$ extremely severe), quality of life (SF-36 Health Status Survey questionnaire including physical composite score (PCS) and mental composite score (MCS)) and GET (4-hour scintigraphy of a low-fat meal) were examined at baseline and 1 year follow-up. A non-responder had $<25 \%$ reduction in TSS and a responder was defined as having a $\geqq 50 \%$ reduction in TSS after 1 year of GES therapy. Results: Overall the nonresponder rate was $23 \%$ (10\% in diabetic vs. $16 \%$ in postsurgical vs. $35 \%$ in idiopathic subgroup). Non-responder rates were similar for men (22\%) and women (23\%) and age was not a factor. Compared to responders of GES therapy, non-responders had similar mean baseline vomiting (2.6 vs. 3.2) and nausea scores (3.3 vs. 3.6) but a lower TSS (17.6 vs. 20.1, P<0.05). Also non-responders had less improvements in mean gastric retention both at 2 hours $(+5.4$ vs. $-8.7 \%)$ and at 4 hours $(+4.0$ vs. $-10.5 \%)$, less improvement in mean PCS $(+2.0$ vs. $+12.4, P<0.05)$ and gained less weight $(-0.4 \mathrm{~kg}$ vs. $+3.7 \mathrm{~kg})$ than responders. $67 \%$ of non-responders had an abnormal EGG (dysrhythmia $>30 \%$ or decrease in postprandial EGG power) vs. $33 \%$ in responders. Non-responders had more baseline mean tachygastria both in the fasting state vs. responders $(26 \%$ vs. $11 \%, \mathrm{P}<0.05)$ and in the fed state $(24 \%$ vs. $11 \%, P<0.05)$ and less increase in postprandial EGG power $(-1.3 \mathrm{~dB}$ vs. $1.6 \mathrm{~dB}, \mathrm{P}<0.05)$. Conclusions: The best symptom improvement achieved by high-frequency GES therapy in refractory gastroparesis is in diabetics (10\% treatment failure rate) and postsurgical (16\% failure) while the worst results are in idiopathics (35\% non-responder rate). Baseline EGG abnormalities are predictors of poor symptomatic response to GES therapy and may be useful in developing a patient profile to optimize selection and expectations for GES therapy.

\section{S1734}

Visceral Specific Analgesic Action of Mosapride Citrate, a Gastroprokinetic Drug, and Its Metabolite Against Gastric Distension Through a 5-HT 4 and 5$\mathrm{HT}_{3}$ Receptor in Conscious Rats Hiroshi Kaneko, Toshihiro Konagaya, Shinichi Kakumu

BACKGROUND: Mosapride citrate is a new gastroprokinetic agent with selective 5- $\mathrm{HT}_{4}$ receptor agonistic properties. We have reported that mosapride not only enhances gastric emptying, but also inhibites the visceromotor response by gastric distension in conscious rats, in which gastric distension-induced pain behavior and abdominal muscle contractions can be evaluated (AGA 2005). In the present study, receptors involved in the analgesic action of mosapride and a selectivity of mosapride-induced analgesic action were examined. METHODS: Male Wistar rats were anesthetized with pentbarbital and a flexible latex balloon was surgically placed into the stomach, then a force transducer was implanted on the abdominal muscle in order to measure its contractions estimated as an index visceromotor response. Measurement was performed at least 4 days after surgical operation. Gastric distension was performed by slow air-infusion into the balloon. The distension-induced abdominal muscle contractions were measured $1 \mathrm{hr}$ before and $l$ or $2 \mathrm{hr}$ after intragastric (ig) administration of morphine hydrochloride, mosapride and granisetron hydrochloride (5- $\mathrm{HT}_{3}$ antagonist), or a mosapride metabolite, $\mathrm{Ml}$ (5- $\mathrm{HT}_{3}$ antagonistic property) subcutaneously (sc). Effect of SB-207266 (a specific 5-HT 4 antagonist, sc) on mosapride-induced changes in visceromotor response was examined. The hot plate test was performed by placing a rat on aluminum plate at $50 \mathrm{C}$ and measuring a hind paw licking latency 30 min before and 1 or $2 \mathrm{hr}$ after mosapride or morphine ig administration. RESULTS: When infusion of air volume amounted to $8-12 \mathrm{~mL}$, abdominal muscle contractions were induced prior to occurrence of a typical pain behavior (writhing phenomenon). Morphine and mosapride (3-10 mg/kg, ig) dose-dependently inhibited the abdominal muscle contraction induced by gastric distension. An inhibitory effect of mosapride on gastric distension-induced visceral pain was significantly, but not completely, inhibited by SB-207266. Granisetron and Ml, at a lesser potency, also significantly inhibited the muscle contractions. Morphine, but not mosapride, significantly prolonged the licking latency. CONCLUSIONS: Mosapride, 5-HT receptor agonist, inhibited the visceromotor response by gastric distension in conscious rats, The anti-visceral pain response of mosapride might be mediated via both $5-\mathrm{HT}_{4}$ receptor activation and antagonistic action against $5-\mathrm{HT}_{3}$ receptor by its metabolite. In addition, mosapride mediated analgesic action looks specific against visceral, but not somatic, pain. (This study was performed by the collaboration with Seto Y \& Yoshida N, Ph.D. at Dainippon Sumitomo Pharmaceutical Co., Ltd, Japan.)

\section{S1735}

Effects of Postgastric ${ }^{13} \mathrm{C}$-Acetate Processing On Measurement of Liquid Gastric Emptying Rates - A Systematic Investigation in Health Oliver Goetze, Heiko Fruehauf, Monika A. Kwiatek, Daniel Pohl, Miriam Thumshirn, Werner Schwizer, Michael Fried

Background and Aims: Uniform postgastric processing of the gastric emptying marker ${ }^{13} \mathrm{C}$ acetate $(\mathrm{AC})$ is an unverified assumption behind its use to measure gastric emptying. We have performed a systematic investigation of the effects of intraduodenally (i.d.) administered volume, caloric density, $\mathrm{AC}$ doses and administration rates on ${ }^{13} \mathrm{CO}_{2}$ exhalation. Methods: ${ }^{13} \mathrm{CO}_{2}$ recovery was assessed in 18 volunteers after i.d. administration of $\mathrm{A}(\mathrm{n}=6)$ : $\mathrm{Al}$ : randomized boluses of $50 \mathrm{mg} \mathrm{AC}$ in $50,100,200 \mathrm{ml}$ water injected over $1 \mathrm{~min}$ every $\mathrm{lh}$; A2: randomized boluses of $12.5,25,50,100 \mathrm{mg} \mathrm{AC}$ in $50 \mathrm{ml}$ water injected over $1 \mathrm{~min}$ every $\mathrm{lh} ; \mathrm{B}(\mathrm{n}=6)$ : infusion of $100 \mathrm{mg} \mathrm{AC}$ in $200 \mathrm{ml}$ water at $0.5,1,2.5 \mathrm{mg} \mathrm{AC} / \mathrm{min}$. C $(\mathrm{n}=6)$ : infusion of $50(100,200) \mathrm{ml}$ Ensure ${ }^{\circ}$ plus $+150(100,0) \mathrm{ml}$ water $+100 \mathrm{mg} \mathrm{AC}$ at $0.75(1.5,3.0) \mathrm{kcal} / \mathrm{min}$; all at $2 \mathrm{ml} / \mathrm{min}$ and $1 \mathrm{mg} \mathrm{AC} / \mathrm{min} .{ }^{13} \mathrm{CO}_{2} /{ }^{12} \mathrm{CO}_{2}$ ratio was determined by continuous molecular correlation spectroscopy (BreathID $®, 1$ sample/3min) Results were expressed as percentage, cumulative percentage doses of ${ }^{13} \mathrm{C}$ recovered and time to peak (PDR $[\% / h]$, cPDR [\%], $\mathrm{t}_{\text {peak }}[\mathrm{min}]$ ). For study A cPDR/mg of administered $\mathrm{AC}[\% / \mathrm{mg}]$ over $1 \mathrm{~h}$ was calculated. Results: $\mathrm{Al}:{ }^{13} \mathrm{CO}_{2}$ recovery and kinetics were similar for different i.d. volume boluses. A2: Increasing doses resulted in decreasing cPDR/mg of AC (12.5 mg: $2.18 \pm 0.78 ; 25 \mathrm{mg}: 0.73 \pm 0.20 ; 50 \mathrm{mg}: 0.38 \pm 0.10 ; 100 \mathrm{mg}: 0.18 \pm 0.02$, all in $\% / m g, p<0.001)$ and increasing $t_{\text {peat }}(14.5 \pm 2.4 ; 11.9 \pm 2.6 ; 14.22 \pm 4.1 ; 20.3 \pm 3.8$ min, $\mathrm{p}<0.05)$. B: During constant i.d. infusion increase of ${ }^{13} \mathrm{CO}_{2}$ excretion over time was only reflected in a linear fashion for the first $40 \mathrm{~min}$, thereafter ${ }^{13} \mathrm{C}$-recovery followed zero order kinetics, in particular for lower AC administration rates. C: cPDR after $3 \mathrm{~h}$ as well as ${ }^{13} \mathrm{CO}_{2}$ excretion kinetics were similar for all caloric loads. Conclusion: Contrary to previous assumptions gastric emptying rates, as measured by ${ }^{13} \mathrm{C}$-acetate breath test, are modulated by non linear ${ }^{13} \mathrm{CO}_{2}$-exhalation kinetics. This is caused by differences in postgastric ${ }^{13} \mathrm{C}$ acetate processing, dependent on the rate of duodenal marker delivery and the total marker dose administered. Our results show that lack of steady state ${ }^{13} \mathrm{CO}_{2}$ excretion by ${ }^{13} \mathrm{C}$-marker redistribution to compartments independent of gastric emptying affect the accuracy of gastric emptying measurements by the ${ }^{13} \mathrm{C}$ acetate breath test.

S1736

Dietary Free Glutamate Modulates Gastric Emptying Rate in Humans Hiroaki Zai, Motoyasu Kusano, Yasuyuki Shimoyama, Masaki Maeda, Atsuto Nagoshi, Tatsuya Higuchi, Shiko Kuribayashi, Osamu Kawamura, Masatomo Mori

Background and Aim: Free glutamate activates the taste receptors in the oral cavity and excites taste nerves to elicit a unique taste 'umami'. Recently calcium sensing receptor sensitive to free amino acids was also found in the gastric mucosa. Although these reports suggest that glutamate acts as a modulator of gastric function, there is no evidence about its effect on gastric emptying. Therefore, we studied the effect of dietary free glutamate on 
gastric emptying rate in humans. Methods: Two types of control liquid test meal (400 kcal / $400 \mathrm{ml}$ ) labeled with $100 \mathrm{mg}$ of $13 \mathrm{C}$ sodium acetate (Cambridge Isotope Laboratories Inc., USA) were used. One meal (protein-rich diet) consisted of 12.5\% dextrin (TK16, Matsutani Chemical, Janan) and $12.5 \%$ casein-calcium (EN9N, DMV Japan); another (carbohydrate diet) contained $25 \%$ dextrin. Monosodium L-glutamate $(0.5 \% \mathrm{w} / \mathrm{v}$, Ajinomoto Co.,Inc., Japan) was added to a control liquid test meal. All meals were flavored with the non-caloric sweetener aspartame (Ajinomoto Co.,Inc., Japan) and plum odor (GIV010790, Givaudan Japan) to mask the taste of glutamate. To investigate whether the presence of nutrients influences the effect of glutamate, $400 \mathrm{ml}$ of water was also tested. After taking a test meal or water, continuous $13 \mathrm{C}$ breath tests (Breath ID System. Oridion Medical Ltd., Israel) were performed. Results: (1) Effect of adding glutamate to the protein-rich meal $(n=10)$ : glutamate significantly accelerated gastric emptying rate. The gastric emptying coefficient (GEC) defined by Ghoos et al (glutamate $+/-$ ) was $3.05 \pm 0.24 / 2.69 \pm 0.28$ (mean \pm S.D., p $<0.01$, multiple ANOVA). The $t 1 / 2$ (glutamate $+/$-) was $153.0 \pm 34.6 / 212.7 \pm 102.6$ minutes (mean \pm S.D., $\mathrm{p}<0.05$ ). The cummulative $\%$ dose at 180 -min was $59.2 \pm 9.8 / 45.8 \pm 14.4$ (mean \pm S.D., $p<0.05$ ). (2) Effect of adding glutamate to the carbohydrate meal $(n=5)$ : glutamate had no significant effect on gastric emptying. (data not shown) (3) Effect of adding glutamate to water $(n=9)$ : glutamate had no significant effect on gastric emptying. (data not shown). Conclusions: This report shows for the first time that dietary free glutamate modulates gastric emptying in humans. Glutamate facilitated the gastric emptying of a protein-rich meal without influence on that of water or carbohydrate meal. These data suggest that free glutamate is an important substance for efficient protein digestion and absorption. The reevaluation of the GI function from the standpoints of chemical sense will help to understand better the pathophysiology of dyspeptic symptoms.

\section{S1737}

Validation of a Novel Symptom-Predominant Classification for Gastroparesis John M. Wo, Jamie Studts, Jennifer L. Koopman, Luwa Cai, Robert Cacchione, Steven P. Harrell

Symptom presentation of gastroparesis (GP) is very diverse. We previously proposed a classification for GP, but prospective studies are needed for validation. AIMS: 1) To determine the concordance of a symptom-predominant classification of GP between physician and patient. 2) To validate physician's classification with a patient self-administrated questionnaire. METHODS: Subjects with symptoms of gastroparesis for $>2$ months and documented delayed gastric emptying were prospectively enrolled. Physician classified each subject based on presenting chief complaints: a) vomiting-predominant GP (vomiting with retching and nausea), b) dyspepsia-predominant GP (discomfort centered in upper abdomen characterized by fullness, early satiety, bloating or nausea), or c) regurgitation-predominant GP (effortless regurgitation of acid or undigested food). Subjects also classified themselves independently from physician. Patient Assessment of GI Disorders-Symptom Severity Index ${ }^{\mathrm{TM}}$ (PAGI-SYM) questionnaire (0 to 5, six subscales) was administered. Cohen's kappa (k) and analysis of variance were used. Receiver operating characteristic (ROC) curves were constructed with sensitivity and specificity of each PAGI-SYM subscale to differentiate subjects into symptompredominant subgroup. Area under the curve (AUC) was used to compare the subscales. RESULTS: 100 GP subjects (87F, mean 48 yrs) were enrolled. There was a 78\% concordance between physician and subject's classification of GP with substantial correlation ( $\mathrm{k}=0.64)$. Results are shown in table. PAGI-SYM nausea/vomiting subscale (AUC=0.79) was the best in differentiating subjects into vomiting-predominant GP, and heartburn/regurgitation subscale (AUC $=0.73$ ) was the best in differentiating regurgitation-predominant GP. No subscale was adequate to differentiate dyspepsia-predominant GP. CONCLUSIONS: There was a substantial agreement between physician and patient using a symptom-predominant classification of GP. Vomiting and regurgitation-predominant GP corresponded very well with patient's assessment of symptom severity, but not for dyspepsia-predominant GP.

\begin{tabular}{|c|c|c|c|}
\hline $\begin{array}{c}\text { PAGI-SYM } \\
\text { subscales (Mean } \pm \\
\text { SD) }\end{array}$ & $\begin{array}{c}\text { Vomiting-predominant } \\
\text { GP }(\mathrm{n}=29)\end{array}$ & $\begin{array}{c}\text { Dyspepsia-Predominant } \\
\text { GP }(\mathrm{n}=49)\end{array}$ & $\begin{array}{c}\text { Regurgitation-Predominant } \\
\text { GP (n=22) }\end{array}$ \\
\hline Nausea/vomiting & $* 3.4 \pm 1.4$ & $1.6 \pm 1.2$ & $2.0 \pm 1.6$ \\
\hline $\begin{array}{c}\text { Post-prandial } \\
\text { fullness/early } \\
\text { satiety }\end{array}$ & $* * 3.7 \pm 1.2$ & $3.0 \pm 1.3$ & $3.0 \pm 1.3$ \\
\hline Bloating & $3.4 \pm 1.3$ & $3.0 \pm 1.4$ & $2.9 \pm 1.5$ \\
\hline $\begin{array}{c}\text { Upper abdominal } \\
\text { pain }\end{array}$ & $2.8 \pm 1.3$ & $2.8 \pm 1.1$ & $2.6 \pm 1.4$ \\
\hline $\begin{array}{c}\text { Lower abdominal } \\
\text { pain }\end{array}$ & $2.1 \pm 1.2$ & $1.9 \pm 1.5$ & $1.9 \pm 1.5$ \\
\hline HB/regurgitation & $2.5 \pm 1.1$ & $1.6 \pm 1.3$ & $\dagger 2.9 \pm 1.2$ \\
\hline Total score & $\dagger 2.9 \pm 1.0$ & $2.2 \pm 0.9$ & $2.7 \pm 1.1$ \\
\hline
\end{tabular}

*p $<0.01$ vs dyspepsia and regurgitation-predominant GP; ** $\mathrm{p}<0.05$ vs dyspepsia-predominant GP; $\uparrow \mathrm{p}<0.01$ vs dyspepsia-predominant GP

\section{$\mathrm{S} 1738$}

The Role of 13c-Octanoic Acid Breath Testing in the Assessment of Gastric Emptying in Critically Ill Patients

Nam Q. Nguyen, Marianne Chapman, Max Bellon, Laura Bryant, Carly Burgstad, Dylan F. Bartholemeuz, Ross Butler, Geoff Davidson, Robert Fraser, Richard Holloway

Delayed gastric emptying and subsequent feed-intolerance is common in critically ill patients and a simple, reliable technique that assesses gastric emptying is therefore needed. Although scintigraphy remains the gold-standard, it is technically demanding in these patients, involves radiation and can interfere with patient care. The 13C-octanoic breath test (13C-BT) is a simple non-invasive, non-radiation technique that has been validated in healthy subjects and diabetic patients. Although a number of studies have reported slow gastric emptying and beneficial effects of prokinetics with 13C-BT, the test has not been validated in the critically ill. Aim. To correlate 13C-BT with scintigraphy for assessing gastric emptying of a liquid meal in critically ill patients. Methods. Gastric emptying of $100 \mathrm{ml}$ of Ensure was assessed prospectively in 14 mechanically ventilated patients (11M; 63+4 yrs; APACHE II $22 \pm 2$ ), using concurrent $13 \mathrm{C}$-BT and scintigraphy. $13 \mathrm{C}$ levels were measured in end expiratory breath samples collected from the ventilator outlet. Samples were taken immediately before the test meal, every 5 min during the first hour and every 15 min thereafter for further $3 \mathrm{~h}$. The gastric emptying coefficient (GEC, normal=3.2-3.8) and gastric half emptying time $(\mathrm{t} 1 / 2(\mathrm{BT})$; normal<120min) were calculated using standard techniques. Scintigraphic measurements were performed over $4 \mathrm{~h}$ to determine $\mathrm{t} 1 / 2(\mathrm{Sct})$ and $\%$ meal retention at 60 120,180 and 240min. Results. Seven patients with normal gastric emptying on scintigraphy $(\mathrm{tl} / 2(\mathrm{Sct})<60 \mathrm{~min})$ had normal $13 \mathrm{C}-\mathrm{BT}$ results. Amongst the 7 patients with prolonged $\mathrm{tl} /$ 2(Sct), 13C-BT was abnormal in 5 and normal in 2 patients. In those with normal 13C-BT but abnormal scintigraphy, the abnormality was mild with $60 \mathrm{~min}<\mathrm{tl} / 2(\mathrm{Sct})<100 \mathrm{~min}$ and $46+2 \%$ of the meal was retained at $120 \mathrm{~min}$ (normal $=22+4 \%$ ). Compared with scintigraphy, $13 \bar{C}$-BT had a sensitivity of $71 \%$ and specificity of $100 \%$. A moderate correlation was found between $\mathrm{t} 1 / 2$ (Sct) and $\mathrm{tl} / 2(\mathrm{BT})(\mathrm{r}=0.68 ; \mathrm{P}=0.008)$ and $\mathrm{GEC}(\mathrm{r}=0.63 ; \mathrm{P}=0.02)$. Meal retention at all time points on scintigraphy correlated positively with $\mathrm{t} 1 / 2(\mathrm{BT})(\mathrm{r}=0.7-0.8 ; \mathrm{P}<0.001)$ and negatively with GEC ( $\mathrm{r}=-0.6-0.7 ; \mathrm{P}<0.001)$. The highest correlation achieved was between $\mathrm{t} 1 / 2$ (BT) and $\%$ meal retention at $120 \mathrm{~min}(\mathrm{r}=0.86 ; \mathrm{P}<0.0007)$. Conclusion. In critical illness, there is a good correlation between $13 \mathrm{C}$-BT and scintigraphy. 13C-BT appears to be a reliable measure of delayed gastric emptying in these patients.

\section{S1739}

\section{The Effect of Food On Electrical and Mechanical Activity of the Canine}

\section{Antrum}

Claudia P. Sanmiguel, Masanobu Hagiike, Scott A. Cunneen, Edward H. Phillips, Jeffrey L. Conklin, Edy E. Soffer

Background: Gastric electrical and mechanical activities are modulated by food, however, the pattern of food-induced changes and the mechanisms governing them are not wel defined. Aim: To characterize food-induced changes in gastric antral activity and to explore underlying mechanisms for such changes. Methods: Experiment 1- intended to test the effect of food on gastric signals. Two bipolar electrodes were implanted in the antrum of 7 mongrel dogs for simultaneous recording of slow wave rate, in events /min (SWR), and contractions assessed by electrical impedance (AI) in ohms. A strain-gauge transducer was implanted in 3 dogs to correlate changes in AI with contractions. The meal consisted of a preload of $100 \mathrm{~g}$ of dry food followed in $10 \mathrm{~min}$, by $900 \mathrm{~g}$ of the same food, to lengthen meal duration. SWR and AI were determined during 4 periods of $5 \mathrm{~min} / \mathrm{each}$ (baseline, end of preload, satiety, $10 \mathrm{~min}$ after satiety). Experiment was repeated 5 times/dog. Experiment 2-acute study, under anesthesia with 3 tests:1) esophageal balloon distension 2) fundic balloon distension at volumes of $50-400 \mathrm{ml}$, using a balloon of $800 \mathrm{ml}$, and 3) nitroglycerin infusion $0.5 \mathrm{microg} / \mathrm{kg} / \mathrm{min}$ for $10 \mathrm{~min}$. results as Mean $\pm \mathrm{SD}$. Results: Experiment 1 - frequency of antral contractions by strain-gauge correlated well with frequency of contractions as measured by $\mathrm{AI}, \mathrm{R}=0.76$. SWRs at baseline, preload, satiety and post satiety were: $5.0 \pm 0.3,4.9 \pm 0.4$, $3.8 \pm 0.65$, and $4.4 \pm 0.47$ respectively. SW amplitudes (ohms) at the same periods were: $5.3 \pm 1.4,8.5 \pm 2.1,12.2 \pm 4.3$ and $12.0 \pm 5.3$ respectively; $\mathrm{p}<0.05$ for satiety and post satiety vs. baseline for both measurements. Both changes peaked around the time of satiety. Mean time interval between satiety and slowest SWR was $80 \pm 40 \mathrm{sec}$. Amplitude of contractions remained elevated for $212 \pm 110 \mathrm{~min}$ after the meal. Experiment 2- fundic distension caused a transient decrease in SWR at lower volumes, but the decrease was sustained at the $400 \mathrm{ml}$ volume, $3.9 \pm 0.5$ vs. $3 \pm 0.8$, baseline vs. distension, respectively, $p=0.002$. NTG significantly reduced SWR: $4.0 \pm 0.7$ vs. $3.5+0.8$, baseline vs. infusion, $p=0.007$. Esophageal distension did not affect SWR. Conclusions: Food ingestion causes a significant decrease in SWR and significant enhancement of antral contractions that peak at satiety. This suggests a possible causal relationship that can be potentially useful when considering gastric electrical stimulation for modulation of satiety signals. Both gastric distension and nitrergic pathways affect SWR.

\section{S1740}

Variability of the 13c-Acetate Breath Test for Gastric Emptying of Liquids in Healthy Children

Bruno Hauser, Jean de Schepper, Vicky Caveliers, Silvia Salvatore, Alessandro Salvatoni, Yvan Vandenplas

Objective: Scintigraphy is considered as the "gold standard" for measuring gastric emptying (GE). The 13C-acetate breath test (13C-ABT) offers an attractive alternative to measure GE of liquids as it is non-radioactive. The aim of this study was to assess the variability of the 13C-ABT for GE of liquids in healthy children using non dispersive infrared spectrometry (NDIRS). Methods: The 13C-ABT was repeated at least 2 times in 21 healthy children (6 girls, 15 boys), aged between 6.2 and 16.4 years, 2 to 7 days apart. After an overnight fast,

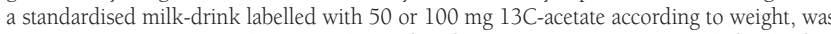
administered. Breath samples were taken before feeding, at 5 minutes intervals for the first 40 minutes and at 10 minutes intervals for the following 140 minutes after feeding. Breath samples were analyzed using NDIRS, and 13C-recovery was used to calculate values for gastric half emptying time (t1/2), time of peak 13C-exhalation or gastric lag phase (tlag) and gastric emptying coefficient (GEC). Intraindividual variability of the parameters $t 1 / 2$ tlag and GEC were expressed as coefficient of intrasubject variation (CV). Results: The median $\mathrm{CV}$ of $\mathrm{tl} / 2$ was $8.3 \%$ (range $\mathrm{CV}$ 1.6-16.2 \%) (interindividual range $\mathrm{tl} / 2$ : $65-112$ minutes; intraindividual range $\mathrm{t} 1 / 2: 4-33$ minutes). The median $\mathrm{CV}$ of tlag was $16.6 \%$ (range CV 2.0-26.6\%) (interindividual range tlag: 31-76 minutes; intraindividual range tlag: 1-35 minutes). The median CV of GEC was $4.3 \%$ (range CV 0.8-15.7\%) (interindividual range GEC: 3.81-4.89; intraindividual range GEC: 0.08-1.31). The CV's of $t 1 / 2$, thag and GEC were independent of age, gender, weight, height and measured values of $t 1 / 2$, tlag and GEC. Conclusions: The 13C-ABT using NDIRS is an easy, non-invasive and non-radioactive 
procedure with a large intraindividual variation for measuring GE of liquids in healthy children, but comparable to the variation reported with other techniques.

\section{S1741}

Effects and Mechanisms of Gastric Electrical Stimulation On Gastric Tone in Rats

Yanquan Luo, J.N. Zhang, Jiande Chen

Background: Our previous studies have shown that gastric electrical stimulation (GES) with sufficient output inhibits gastric tone and this effect is mediated via the nitrergic pathway. However it is unknown whether this effect and mechanism are species specific. The aim of this study was therefore to investigate the effect and mechanism of gastric electrical stimulation (GES) in rats. Methods: Seventeen female SD rats were divided into two groups, eight rats were given GES without L-NNA (nitric oxide synthase inhibitor) as control, nine rats were given GES with L-NNA $(10 \mathrm{mg} / \mathrm{kg})$. Laparotomy was performed under general anesthesia; a pair of electrodes was sutured on the serosa of the lesser curvature; a balloon for gastric tone recording was placed into the proximal stomach. The control session included $30 \mathrm{~min}$ baseline, followed by 30 min with GES; the L-NNA session included 30 min baseline, 30 min immediately after a bolus of L-NNA, and $30 \mathrm{~min}$ with GES. GES was performed using pulse trains with train on time of 2 seconds and off time of 3 seconds and pulse width of $3 \mathrm{~ms}$, amplitude of $6 \mathrm{~mA}$ and frequency of $40 \mathrm{~Hz}$. Gastric tone was measured by a computerized barostat at 4-5 mmHg (MDP $+2 \mathrm{mmHg}$ ). Results: 1) In the control session, GES significantly increased the proximal gastric volume from baseline $0.36 \pm 0.12 \mathrm{ml}$ to $0.47 \pm 0.19 \mathrm{ml}$ (paired t-test, $\mathrm{p}<0.05)$; 2) L-NNA markedly reduced the proximal gastric volume from $0.41 \pm 0.06$ $\mathrm{ml}$ at baseline to $0.28 \pm 0.09 \mathrm{ml}$ (ANOVA $\mathrm{P}<0.05$ ); subsequent $\mathrm{GES}$ was still able to increase the proximal gastric volume from $0.28 \pm 0.09 \mathrm{ml}$ to $0.45 \pm 0.10 \mathrm{ml}$ (ANOVA p<0.05). Conclusions: GES significantly inhibits proximal gastric tone, not like in canine, this inhibitory effect is not blocked by the nitric oxide synthase inhibitor L-NNA, further studies should be done to figure out pathways other than nitrergic pathway which mediate the inhibitory effect of GES on gastric tone in rats.

\section{S1742}

Variability of the 13c-Octanoic Acid Breath Test for Gastric Emptying of Solids in Healthy Children

Bruno Hauser, Jean de Schepper, Vicky Caveliers, Silvia Salvatore, Alessandro Salvatoni, Yvan Vandenplas

Objective: Scintigraphy is considered as the "gold standard" for measuring gastric emptying (GE). The 13C-octanoic acid breath test (13C-OBT) offers an attractive alternative to measure $\mathrm{GE}$ of solids as it is non-radioactive. The aim of this study was to assess the variability of the 13C-OBT for GE of solids in healthy children using non dispersive infrared spectrometry (NDIRS). Methods: The 13C-OBT was repeated at least 2 times in 19 healthy children (6 girls, 13 boys) aged between 6.8-17.1 years, 2 to 7 days apart. After an overnight fast, a standardised pancake labelled with 50 microliter $13 \mathrm{C}$-octanoic acid was administered. Breath samples were taken before the feeding and at 15 minutes intervals for the following 240 minutes after the feeding. Breath samples were analyzed using NDIRS, and 13C-recovery was used to calculate values for gastric half emptying time (t1/2), time of peak 13C-exhalation or gastric lag phase (tlag) and gastric emptying coefficient (GEC). Intraindividual variability of the parameters $\mathrm{t} 1 / 2$, tlag and GEC were expressed as coefficient of intrasubject variation (CV). Results: The median CV of $\mathrm{t} 1 / 2$ was $12.9 \%$ (range CV 2.5-29.6 \%)(interindividual range tl/2: 99-204 minutes; intraindividual range $\mathrm{tl} / 2$ : 5-84 minutes). The median $\mathrm{CV}$ of tlag was $12.4 \%$ (range CV 0.8-27.8\%) (interindividual range tlag: 65-146 minutes; intraindividual range tlag: 1-50 minutes). The median CV of GEC was $10.9 \%$ (range CV 3.4-30.2 \%) (interindividual range GEC: $2.32-3.88$; intraindividual range GEC: 0.17-1.35). The CV's of t l/2, tlag and GEC were independent of age, gender, weight, height and measured values of $\mathrm{tl} / 2$, tlag and GEC. Conclusions: The 13C-OBT using NDIRS is an easy, noninvasive and non-radioactive procedure with a large intraindividual variation for measuring $\mathrm{GE}$ of solids in healthy children, but comparable to the variation reported with other techniques.

\section{S1743}

Gastric Electrical Stimulation for Obesity: Is There a Need for a New Generation Device?

J.N. Zhang, J D Z Chen

Background: Gastric electrical stimulation (GES) using the commercially available implantable device has been applied for treating obesity with controversial outcomes. It is known that all commercially available devices are designed for nerve or cardiac stimulation and the width of stimulation pulse is limited to below $1 \mathrm{~ms}$. Since the stomach is composed of smooth muscles, one wonders whether a wider pulse is needed for GES. The aim of this study was to compare the peripheral and central effects of GES with a pulse width currently used in clinical trials and a wider pulse in rats. Methods: GES with 2 sets of parameters (GES1: the parameters currently used in clinical trials for the treatment of obesity: $6 \mathrm{~mA}$ $0.3 \mathrm{~ms}, 40 \mathrm{~Hz}, 2 \mathrm{~s}$-on, 3s-off; GES2: same as GES1 except increased pulse width of 3ms) was applied via a pair of gastric serosal electrodes near to the less curvature of the rat stomach. Exp. 1 was performed 8 anesthetized fasting rats. A balloon was inserted to the proximal stomach for assessing gastric volume by barastat at baseline and during GES. Exp. 2 was designed to record extracellular potentials of single neurons in the PVN and VMH of anesthetized rats $(\mathrm{N}=58 / 52)$. Neurons were identified as gastric distension-responsive (GD$\mathrm{R}$ ) if they responded to gastric distension (GD, 3 5ml, 10 30s), then GES1 and GES2 were performed for one minute respectively. Results: 1. GES2 but not GES1 reduced gastric tone The gastric volume was increased from $0.38 \pm 0.07 \mathrm{ml}$ to $0.49 \pm 0.07(\mathrm{P}<0.05)$ with GES1. 2 . In the PVN, 104 GD-R neurons were tested with GES, $79.0 \%$ and $91.4 \%$ of these neurons were responsive to GES1 and GES2 respectively (P=0.029, GES1 vs.GES2); In the VMH, while there was no difference between GES1 and GES2 in the activation of the number of neurons ( $63.6 \%$ vs. $73.3 \%, P>0.05$ ), the percentage of increase in neural activity was higher with GES2 than GES1 (increase of $343.6 \pm 89.2 \%$ vs. $97.4 \pm 33.7 \%, P<0.05$ ). Conclusions: The peripheral and central effects of GES are dependent on stimulation pulse width. While the pulse width used in current clinical trials is effective in activating neurons in the hypothalamus, GES with wider pulses are more effective both peripherally and centrally. As GES-induced gastric distention is known to be associated with reduced food intake, it is anticipated that GES with wider pulses is more effective in treating obesity. Accordingly, there may be a need for a new generation device capable of generating wider pulses.

\section{S1744}

A New Method to Improve Liquid Nutrient Load Test Zhiqiang Song, Meiyun Ke, Zhifeng Wang, Lin Xu

BACKGROUND: Liquid nutrient load test (LNLT) may show the reduced intake volume which may result from gastric visceral hypersensitivity or impaired accommodation, so it is not exact to express gastric visceral sensitivity with the index of intake volume. The intra-gastric pressure (IGP) is the main factor inducing gastric mechanical sensation. If we determine IGP by manometric catheters during LNLT, the gastric sensitivity can be more exactly showed by the intra-gastric pressure level. Subjects with a lower IGP at the time of maximal intake volume (MIV) will be considered as those having gastric visceral hypersensitivity. When IGP of patients are same as those of healthy subjects (HS), the reduced intake volume can be deduced to result from impaired accommodation. AIMS: To evaluate liquid nutrient load test combined with determination of the intra-gastric pressure in HS and patients with functional dyspepsia (FD). METHODS: Thirty-two HS (M:F 1:1, mean age $43 \mathrm{yr}$ ) and 52 patients with FD (M:F 1:1.6, mean age 45yr) participated in the study. Following an overnight fast of at least 10 hours, a water-perfusion catheter was introduced through nasal cavity into stomach. The side-hole was positioned in the proximal stomach. Subjects lied on a test bed with a hemi-supine posture. After a 15 minute equilibration period, subjects drank liquid nutrient $(1.5 \mathrm{Kcal} / \mathrm{ml}$, Carbohydrate $48 \%$, Fat $39 \%$, Protein $13 \%, 50 \mathrm{ml} / \mathrm{min}$ ) until MIV with synchronous intra-gastric pressure monitoring. So both MIV and IGP at the time of MIV could be obtained. The study was repeated in $16 \mathrm{HS}$ on separate days. RESULTS: (1) IGP and MIV in HS group are $4.0-6.3 \mathrm{mmHg}$ and $530-886 \mathrm{ml}$ (M) $420-690 \mathrm{ml}(\mathrm{F})$, respectively. Twice studies showed quite similar results and the interclass correlation coefficient of IGP and MIV are $0.953(\mathrm{P}<0.01)$ and $0.795(\mathrm{P}<0.01)$, respectively. (2) The correlation coefficient between MIV and IGP is weak $(r=0.357, P=0.045)$. (3) No statistical differences are found in IGP divided by sex, age and body mass index $(P>0.05)$. (4) The proportion of gastric hypersensitivity in FD $(<4.0 \mathrm{mmHg})$ accounts for $37 \%$; the proportion of impaired accommodation (male $<530 \mathrm{ml}$, Female $<420 \mathrm{ml}$ ) accounts for $20 \%$. (5) Most subjects could tolerate the test well. CONCLUSIONS: The new method of liquid nutrient load test combined with determination of intra-gastric pressure could differentiate gastric visceral hypersensitivity from impaired accommodation. It is simple and clinical feasible with less influencing factors, good repeatability and tolerability.

\section{S1745}

Evidence for Glucoresponsiveness of Gastric Vagal Afferents Mediated By Katp Channels: Immunocytochemistry and Electrophysiology Studies Shi-Yi Zhou, Yuanxu Lu, Chung Owyang

Hyperglycemia plays an important role in the modulation of gastric motor function by delaying gastric emptying. We reported hyperglycemia stimulates vagal afferent pathways which in turn activate vagal efferent cholinergic pathways synapsing with intragastric NOcontaining neurons to mediate gastric relaxation. The mechanism by which glucose is "sensed" in the circulation and evokes neurally mediated reflexes is unknown. We investigated if glucose modulates the activity of vagal afferents in the stomach and if the neurons express KATP channels. Immunocytochemistry studies performed on histological sections of rat nodose ganglia show immunostaining of glucose transporter Glut $1,2,3,4$. The same group of neurons also displays immunoreactivities of the inwardly rectifying $\mathrm{K}+$ channel 6.2 (Kir 6.2) and the sulfonylurea receptor (SURI) as well as glucokinase. These findings were confirmed by Western analysis, which shows glut 1 and 3 as the predominant proteins. Double immunohistochemical staining shows most of these neurons contain glutamate as a neurotransmitter. To determine if the glucose transporters and KATP channels are transported axonally, western analysis was performed on vagal nerve trunks and showed the presence of Glut 1, 2, 3, 4 as well as Kir 6.2 and SUR1 protein. To determine whether gastric vagal afferents can respond to changes in glucose concentration, in vitro isolated stomach-vagus nerve preparations were used in electrophysiological studies. This preparation eliminates any influence of CNS and systemic factors, thus unequivocally showing glucose action at the vagal afferent level. Thin nerve trunks $(\sim 1 \mu \mathrm{m})$ from the distal cut end of afferent fibers were sampled from the ventral branch of the gastric vagal trunk. The average of a 5 minute total spike count before each treatment was normalized as $100 \%(\mathrm{Q}=1)$. Following glucose perfusion $(250 \mathrm{mg} / \mathrm{dl}, 20 \mathrm{sec}), 9 / 21$ units $(43 \%)$ responded to glucose $(\mathrm{Q}=3.1$ excitations). The response was dose dependent $(150-300 \mathrm{mg} / \mathrm{dl})$ and inhibited by glucosamine $(250 \mathrm{mg} /$ $\mathrm{dl})$, a glucokinase inhibitor $(\mathrm{Q}=1.05)$, and the KATP channel inhibitor tolbutamide $(500 \mu \mathrm{M})$ $(\mathrm{Q}=1.3)$. In contrast, the non-metabolizable 2-deoxyglucose $(250 \mathrm{mg} / \mathrm{dl})$ had no effect. These studies demonstrate for the first time that subsets of neurons in the nodose ganglia and gastric vagal afferents are glucoresponsive. Both the glucotransporters and KATP channels are transported axonally to the gastric vagal afferents which can be activated by high glucose levels through modulation of KATP channels. This provides the functional basis for our previous in vivo finding that gastric relaxation evoked by hyperglycemia is mediated via vagal afferent pathways. 


\section{S1746}

Impaired Acid-Induced Esophageal Protective Reflexes During Rem Sleep Gregg S. Gagliardi, Ashish P. Shah, Karl Doghramji, Sidney Cohen, Anthony J. Dimarino

Background: Nocturnal acid gastroesophageal reflux (GER) events induce sleep arousals and awakenings, which leads to esophageal acid clearance. However, the effect of sleep stage on these events has not been investigated. Since respiratory changes (apnea induced arousals) are markedly suppressed in REM sleep, it is possible that acid induced arousals and awakenings may be suppressed as well. Aim: The aim of this study was to investigate the effect of sleep stage on acid GER induced arousals and awakenings using simultaneous 24 $\mathrm{h} \mathrm{pH}$ recording and polysomnography. Methods: The records of 16 patients with and without documented reflux disease, who had previously undergone simultaneous polysomnography and overnight esophageal $\mathrm{pH}$ analysis, were reviewed. Sleep stage was scored using established polysomnographic parameters. An acid GER event was considered to have occurred when the $\mathrm{pH}$ recording was $<4.0$. Awakenings and arousals were reflux associated if they occurred during the reflux event and up to 5 minutes after the $\mathrm{pH}$ had returned to $\geq 4.0$. The corresponding sleep stages and whether or a not an arousal or an awakening took place was recorded. All subjects were studied without the influence of sleep hypnotics. Results: A total of 33 nocturnal acid reflux events were recorded. In early stage 1 and 2 sleep a total of 13 reflux events resulted in 12 arousals or awakenings (92.3\%). In REM sleep there were 6 GER events recorded and only 1 (16.7\%) was associated with an arousal or awakening $(\mathrm{p}<0.01)$. Fourteen events occurred while the patients were awake. There were no reflux events in stage 3 or 4 sleep. Conclusion: 1) Nocturnal acid GER events occurring in early stage 1 and 2 sleep were associated with an arousal or awakening which has been shown to result in an acid clearance response. 2) During REM sleep, GER induced arousals or awakenings were markedly suppressed, thus impairing this protective esophageal reflex. These findings suggest that the depth of sleep during a GER episode may be an important factor in producing arousals and awakenings. Since the duration of REM sleep is not clinically regulated, the importance of nocturnal acid suppression becomes critical.

\section{S1747}

Sustained Hyperexcitability of Mouse Colonic Nociceptive Dorsal Root Ganglia (DRG) Neurons Activated By Proteinase Activated Receptor 2 (PAR2) Is Mediated By a PKC and ERK1/2 Dependent Pathway Ahmed Kaysii, Silvia Amadesi, Nigel Bunnett, Stephen Vanner

The release of proteases from intestinal mast cells has been implicated in the genesis of abdominal pain associated with conditions such as irritable bowel syndrome. We have recently shown that activation of PAR2 on nociceptive DRG mouse neurons evokes sustained excitability increase in these neurons (Gastrol28:S1469,2005) but the signaling pathway underlying this novel finding is unknown. This study examines the role of PKC and ERKl/ 2 pathways using whole cell recordings of mouse nociceptive neurons (i.e. $<40 \mathrm{pF}$ ) and immunohistochemical techniques. DRG neurons exhibit PAR2 immunoreactivity and a subpopulation colocalize CGRP and TRPV1. The effects of PAR2 activation on neuronal excitability were established in current clamp by measuring membrane potential, rheobase and input

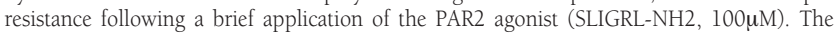
sustained depolarization (mean $>7 \mathrm{mV}$ for over $30 \mathrm{~min}$ ), decreased rheobase (>50\% for more than $30 \mathrm{~min}$ ), and increase in input resistance ( $>50 \mathrm{~min}$ ) evoked by SLIGRL-NH2 were completely blocked at 3 and 10 min when the PKC inhibitor Calphostin $(1 \mu \mathrm{M} ; \mathrm{n}=5)$ was first applied to the bath. Similarly, when cells were incubated for $3 \mathrm{hr}$ with the ERKl/2 inhibitor PD98059 $(100 \mu \mathrm{M} ; \mathrm{n}=6)$ the SLIGRL-NH2 effects were abolished. The reverse peptide LRGILS-NH2 and PKC and ERK1/2 inhibitors alone had no effect. The selectivity of the ERKl/2 inhibitor was studied by examining its effects on the PKA agonist Forskolin $(1 \mu \mathrm{M})$. Forskolin depolarized neurons at $3($ mean $=6 \mathrm{mV})$ and 10 (mean $=7 \mathrm{mV})$ min and these responses were not altered by PD98059. To demonstrate that PAR2 activation phosphorylates ERK1/2, immunofluorescence of antibodies staining phosphorylated ERKl/ 2 (pERK) in isolated rat and mouse DRG neurons was studied using confocal microscopy. In unstimulated neurons, there were low levels of staining for pERK. Phorbol-12-myristate13-acetate (PMA;100nM) or SLIGRL-NH2 $(100 \mu \mathrm{M})$ induced detection of pERK in the vicinity of the plasma membrane within 1-5 min, suggesting activation of ERK in the vicinity of ion channels. These treatments also induced translocation of PKC $\varepsilon$ and PKA catalytic subunit to the plasma membrane, where they too may regulate channel activity. Together, these studies confirm the presence of PAR2 on mouse nociceptive neurons innervating the colon and establish that activation of PAR2 on mouse colonic neurons can phosphorylate ERK1/ 2. Inhibition of PAR2-mediated hyperexcitability of these neurons by PKC and ERK1/2 antagonists suggests signaling through this pathway underlies the sustained actions of PAR2. SUPORTED BY NIH GRANTS DK57480, 43207 and by the CIHR.

\section{S1748}

Nicotinic Agonists Activate Inward Currents in Isolated GI Projecting Nodose Ganglion Neurons

Michael Beyak, Annemarie Surprenent, David Grundy

Background: Nicotine suppresses food intake in both animals and humans. We have previously reported that nicotinic agonists stimulate gastric vagal afferents and potentiate mechanosensitive responses (Beyak et al DDW 2004). The ion channel subtypes underlying these responses are unknown. The aim of the present study was to examine the ion channels underlying the vagal afferent response to DMPP in GI projecting nodose ganglion neurons. Methods: GI - projecting nodose ganglion cells NGNs were labelled with a single i.p. injection of fast blue $(10 \mu \mathrm{L}, 4 \%)$. 2-7 days later, nodose ganglia were harvested, and dissociated. Conventional whole cell patch clamp recordings were performed using potassium based internal and sodium based external solutions. Pharmacological agents were applied using a fast flow solution switching system. Results: The nicotinic agonist DMPP (3-300 $\mu$ M) depolarized a subpopulation of GI nodose ganglion neurons. In voltage clamp experiments at $60 \mathrm{mV}$ this was associated with the development of an inward current, the effect having an EC50 of $32 \mu \mathrm{M}$. DMPP induced currents were seen in $70 \%$ of labelled NGNs and in $55 \%$ of non labelled NGNs. Sensitivity to DMPP was not significantly related to cell size. Superfusion of mecamylamine $(10 \mu \mathrm{M})$ completely blocked the effect of $30 \mu \mathrm{M}$ DMPP $(\mathrm{n}=6)$. Howeve the selective $\alpha 7$ antagonist, methyllycaconatine (MLA, 100nM) had no effect $(150 \pm 15.5 \mathrm{pA}$ control vs $135 \pm 14.5 \mathrm{pA}$ MLA $\mathrm{p}>0.05, \mathrm{n}=5)$. The $\alpha 7$ agonist, choline chloride $(3 \mathrm{mM}) \mathrm{did}$ not activate an inward current $(n=5)$. DMPP sensitive NGNs were examined with respect to sensitivity to ATP $(100 \mu \mathrm{M}, \mathrm{n}=29)$, 2Methyl 5 -HT $(100 \mu \mathrm{M}, \mathrm{n}=23)$, and CCK $(100 \mathrm{nM}, \mathrm{n}=$ 31). DMPP sensitivity was highly predictive of CCK sensitivity, 15/21 DMPP sensitive cells responded to CCK, while only $1 / 10$ DMPP insensitive cells responded to CCK ( $\mathrm{p}=0.02$ Fisher's exact test) DMPP sensitivity was not correlated with ATP or 2-methyl 5-HT sensitivity. Conclusions: Consistent with its effect on gastric and intestinal vagal afferents, the nicotinic agonist DMPP activates an inward current in GI projecting nodose ganglion cells. Sensitivity to mecamylamine and insensitivity to MLA is highly suggestive of $\alpha 3 \beta 4$ receptor subtype. The high incidence of CCK and DMPP co-sensitive cells suggests that nicotinic receptors are expressed on vagal afferents involved in satiety, consistent with previous studies demonstrating an inhibitory effect of nicotine on food intake.

\section{S1749}

Estrogen Modulates Visceral Sensitivity in Adult Rats Exposed to Neonatal Colon Irritation $(\mathrm{CI})$

J.N. Wang, Chunping Gu, Xin Peng, Kirsten Garner, Elie D. Al-Chaer

A number of chronic pain syndromes are more prevalent in women than in men, suggesting a gonadal steroid influence. In previous studies, we showed that visceral hypersensitivit varies with the levels of circulating sex hormones. In this study, we examined the effects of estrogen on visceral hypersensitivity in two groups of rats: rats with neonatal colon irritation $(\mathrm{CI})$ and control rats (non-CI). When the rats reached 3 months of age, ovariectomy $(\mathrm{OVX})$, orchiectomy $(\mathrm{OCX})$ or sham operations were performed. The rats were then given 20 days to recover. Visceral hypersensitivity was assessed by recording the electromyographic (EMG) responses to graded colorectal distension (CRD) in rats mildly sedated with isoflurane (1\%). CRD was induced by rapidly inflating a latex balloon to $20,40,60$ or $80 \mathrm{mmHg}$ for 10 s, with a 5 min interval between distentions. OVX and OCX rats were injected with

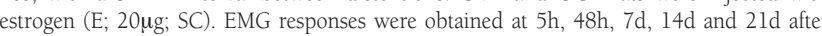
E injection and compared with those obtained from OVX, OCX, sham female or intact male rats. Serum E levels were also measured at each time point using enzyme immunoassay. Our results show: 1) In non-CI rats, E given to sham-OVX reduced EMG responses to $60 \%$ $(60 \mathrm{mmHg})$ of the responses of controls (vehicle injection) $48 \mathrm{~h}$ after the injection in proestrus and estrus. In metestrus and diestrus, EMG response was reduced to $70 \%(60 \mathrm{mmHg})$ of the control response 7 days after the injection $(n=6, p<0.05)$. However, in intact male rats, EMG responses to CRD increased to $58.43 \%(60 \mathrm{mmHg}, \mathrm{n}=3, \mathrm{p}<0.05) 48 \mathrm{~h}$ after $\mathrm{E}$, and the effect lasted for $14 \mathrm{~d}$. 2) In OVX rats, the sensitivity to CRD was significantly decreased to $90.25 \%$ (in control rats) or $91.95 \%$ (in $\mathrm{CI}$ rats) $(60 \mathrm{mmHg}, \mathrm{n}=8, \mathrm{p}<0.001$ ) by comparison to sham-OVX, and the serum estrogen levels in $\mathrm{CI}$ rats also decreased $(16.11 \%, \mathrm{n}=4 \mathrm{p}<0.05)$ 2ld after OVX compared to intact CI female rats. In OCX rats, the sensitivity to CRD significantly decreased to $69.56 \%(80 \mathrm{mmHg})$ in $\mathrm{CI}$ rats $(\mathrm{n}=4, \mathrm{p}<0.05)$ but not in contro rats compared to sham-OCX. 3) Estrogen injected after OVX or OCX in both $\mathrm{CI}$ and non$\mathrm{CI}$ rats restored EMG responses to the levels seen in intact rats. The present data suggest that estrogen has a significant role in modulating viscerosensitivity in both $\mathrm{CI}$ and non-Cl female or male rats. The loss of gonadal hormones in $\mathrm{CI}$ and non-CI gonadectomized rats decreases viscerosensitivity, but estrogen replacement leads to recovery (in female rats) and some hypersensitivity (in male rats). (Supported by NIH / NS40434)

\section{S1750}

An Investigation Into the Role of 5HT in Mediating Jejunal Afferent Hypersensitivity to Mechanical Stimulation in Trichinella Spiralis Infected Mice

Chris D. Keating, Michael Beyak, Annemarie Surprenant, David Grundy

Background: Following Trichinella spiralis infection in mice, there is augmented sensitivity of jejunal afferents responding at low threshold (LT) and high threshold (HT) levels of distension. Here, we investigate the role of $5 \mathrm{HT}$ in the mechanisms underlying afferent hypersensitivity. Methods: Experiments were performed on T. spiralis infected mice ( $>30 \mathrm{~d}$ post infection) and uninfected controls (WT). Gut projecting nodose ganglion neuron (NGNs) from infected and control mice were labelled with an IP injection of fast blue (4\%, 10uL) and whole cell patch clamp experiments were performed on labelled cells isolated 2-7 days later. The response to the 5-HT3 agonist, 2-methyl-5HT was examined using voltage clamp electrophysiology. Multiunit recordings were also made from mesenteric afferent bundles supplying in vitro segments of mouse jejunum taken from infected animals and uninfected controls. Data is presented as mean \pm SEM $(\mathrm{N}=>6)$. Data analysed by one way ANOVA or by Students t-test. P $<0.05$ was taken as significant. Results: In post infected animals, isolated NGNs were more excitable than controls with a lower rheobase $(60 \pm 8.0$ vs $100.9 \pm 21 \mathrm{pA}, \mathrm{p}=0.03)$ and an increased number of spikes generated at two times rheobase $(5.6 \pm 0.7$ vs $3.4 \pm 0.7, p=0.04)$. The proportion of $\mathrm{NGNs}$ responding to $100 \mathrm{uM} 2$-methyl5HT was comparable between control and infected groups (10/21 vs $13 / 20, \mathrm{p}=0.3$ Fishers exact test). However the current density of the 2-methyl-5HT induced current was significantly smaller in infected animals $(3.1 \pm 0.7 \mathrm{pA} / \mathrm{pF}$ vs $6.6 \pm 1.7 \mathrm{pA} / \mathrm{pF}, \mathrm{p}=0.04)$. In in-situ experiments, the WT response to ramp distensions of the jejunum consisted of a biphasic increase in afferent firing. In infected animals there was a significant augmentation of afferent discharge at both LT $(0-10 \mathrm{mmHg})$ and HT $(10-50 \mathrm{mmHg})$ levels of distension. In WT animals $30 \mu \mathrm{M}$ granisetron inhibited the LT response (from $28 \pm 4 \mathrm{imp} / \mathrm{s}$ to $22 \pm 3.4 \mathrm{imp} / \mathrm{s}, \mathrm{p}=0.016$ ) leaving the HT response unaffected $(24.6 \pm 3 \mathrm{imp} / \mathrm{s}$ inhibited to $23.1 \pm 3 \mathrm{imp} / \mathrm{s}, \mathrm{p}=0.49)$. However in infected animals $30 \mathrm{uM}$ granisetron inhibited both the LT $(37.53 \pm 4 \mathrm{imp} / \mathrm{s}$ inhibited to $28 \pm 5 \mathrm{imp} / \mathrm{s}, \mathrm{p}=0.046)$ and HT response $(42.5 \pm 6 \mathrm{imp} / \mathrm{s}$ inhibited to $35.4 \pm 6 \mathrm{imp} / \mathrm{s}$ $\mathrm{p}=0.013)$. Conclusion: $T$. spiralis infection causes an increase in the excitability of gastrointes tinal sensory nerves and a sensitisation of LT and HT mechano-sensitive afferent fibres that is in part dependent upon the release of $5 \mathrm{HT}$ acting via $5 \mathrm{HT} 3$ receptors. Furthermore, there is a functional down regulation of $5 \mathrm{HT} 3$ receptors at the cell membrane in response to $\mathrm{T}$ 
spiralis infection, which may represent an adaptive response to increased bioavailability of $5 \mathrm{HT}$.

\section{S1751}

Nociceptor Excitability Is Associated with Suppression of a-Type Potassium Currents: Insights Into the Pathogenesis of Pain in Chronic Pancreatitis Guang-Yin Xu, John H. Winston, Mohan M. Shenoy, Huaizhi Yin, Pankaj J. Pasricha Background and Aims: The pathogenesis of pain in chronic pancreatitis (CP) is poorly understood. We have previously developed a model of trinitrobenzene sulphonic acid (TNBS)-induced CP that results in nociceptive sensitization characterized by referred somatic allodynia and pancreatic hyperalgesia in rats. The aim of this study was to further explore the cellular and ionic mechanisms underlying this sensitization, particularly focusing on changes in potassium channel conductance, a critical factor in determining the responsiveness of neurons to stimulation. Methods: $\mathrm{CP}$ was induced in adult rats by a single intraductal injection of TNBS. Dorsal root ganglion (DRG) neurons innervating the pancreas were identified by Dil fluorescence labeling. Whole cell patch-clamp recordings were made from acutely dissociated DRG neurons from rats on day 21-26 after TNBS or vehicle treatment. Results: Pancreas-specific neurons from thoracic $\left(\mathrm{T}_{9-13}\right)$ DRGs of rats with $\mathrm{CP}$ displayed more depolarized resting potential than those from controls $(\mathrm{P}<0.02)$, and tended to fire spontaneously as compared with the much more quiescent neurons from controls. TNBSinduced $\mathrm{CP}$ also resulted in a dramatic reduction in current threshold or rheobase (controls: $0.6 \pm 0.5 \mathrm{nA}, \mathrm{n}=17 ;$ TNBS: $0.2 \pm 0.1 \mathrm{nA}, \mathrm{n}=20, \mathrm{P}<0.05)$ and a significant increase in the number of action potentials evoked at twice rheobase $(\mathrm{P}<0.05)$. Under voltage-clamp conditions, neurons from both groups exhibited transient A-type $\left(I_{\mathrm{A}}\right)$ and sustained outward rectifier $\mathrm{K}^{+}$currents $\left(\mathrm{I}_{\mathrm{K}}\right)$. Compared to controls, the $\mathrm{I}_{\mathrm{A}}$ rather than $I_{\mathrm{K}}$ density was significantly reduced in the TNBS group (control: $2.1 \pm 0.3 \mathrm{pA} / \mathrm{pF}, \mathrm{n}=6$; TNBS: $1.3 \pm 0.2 \mathrm{pA} / \mathrm{pF}, \mathrm{n}=9, \mathrm{P}<0.05$ ), without a change in cell diameter. The steady-state inactivation curve for $I_{\mathrm{A}}$ was displaced by $\sim 45 \mathrm{mV}$ to more hyperpolarized levels after TNBS treatment. Conclusions: These data suggest that $\mathrm{CP}$ increases the excitability of pancreas-specific DRG neurons by suppressing $I_{\mathrm{A}}$ density, thus identifying for the first time a specific molecular mechanism underlying chronic visceral pain and sensitization in CP. Further work is ongoing to identify the molecular correlation of this change in current as well as the factor(s) driving it (Supported by R01 DK62330-01 to P.J.P.).

\section{S1752}

Involvement of a 5-HT3 Receptor in the Capsaicin-Induced Inhibitory Duodeno-Gastric Reflex in Man George Karamanolis, Rita Vos, Josef Janssens, Jan Tack

Recently, we demonstrated that acute intra-duodenal administration of capsaicin, a TRPV1 receptor agonist, decreases proximal gastric tone and increases sensitivity to proximal gastric distention, thereby mimicking the effects of intraduodenal acid perfusion. We have previously shown that the acid-induced duodeno-gastric inhibitory reflex is partly mediated via a 5HT3 receptor (Karamanolis DDW 2005). The aim of our study was to investigate involvement of 5-HT3 receptors in the capsaicin-induced duodeno-gastric inhibitory reflex. Methods: Eight healthy volunteers ( 3 men, mean age 27) were studied on 2 occasions, at least one week apart. An infusion tube was positioned in the second part of the duodenum, and a barostat bag was located in the gastric fundus. Proximal stomach tone and sensitivity to distension were assessed before and after duodenal infusion of $1.25 \mathrm{ml}$ of red pepper solution, after pretreatment with saline or the 5-HT3-receptor antagonist ondansetron $8 \mathrm{mg}$ i.v. in a randomized, double-blind, cross-over fashion. Assessment of nine epigastric symptoms, on 100-mm visual analogue scales (VAS), were obtained from all volunteers. Results are given as mean \pm SEM and compared by Student's t-test and ANOVA. Results: After capsaicin instillation, a significant relaxation of the proximal stomach occurred (volume increase $133 \pm 26$ $\mathrm{ml}$; intragastric balloon volume $319 \pm 24 \mathrm{ml}$ ). Ondansetron pretreatment significantly inhibited the intra-duodenal capsaicin-induced inhibition of proximal stomach tone (volume increase $57 \pm 14 \mathrm{ml}, \mathrm{p}=0.005$; intragastric balloon volume $255 \pm 16 \mathrm{ml}, \mathrm{p}=0.06$ ). Capsaicin increased the slope $(34 \pm 5$ vs. $45 \pm 7 \mathrm{ml} / \mathrm{mmHg}, \mathrm{p}<0.001)$ and intercept ( $38 \pm 7$ vs. $99 \pm 21 \mathrm{ml}, \mathrm{p}=0.02$ ) of the gastric pressure-volume curve during stepwise distentions, and this was inhibited by ondansetron (slope $23 \pm 3$ vs. $47 \pm 11 \mathrm{ml} / \mathrm{mm} \mathrm{Hg}, \mathrm{NS}$; intercept $71 \pm 6$ vs. $86 \pm 32 \mathrm{ml}$, NS). Pretreatment with ondansetron did not change the capsaicin-induced symptom scores and the perception scores or perception and discomfort thresholds during gastric distention. Conclusion: 5-HT3-receptors are involved in the capsaicin-induced duodeno-gastric inhibitory motor reflex. However, ondansetron did not alter gastric sensitivity to distention and severity of symptoms induced by duodenal capsaicin instillation.

\section{S1753}

\section{Sensory Innervation of Intestinal Blood Vessels}

Bao-Nan Chen, Xin-Yung Song, Marcello Costa, Simon Brookes

Spinal afferent neurons provide a powerful vasodilator input to blood vessels to the small and large intestine which can be experimentally activated by the neurotoxin, capsaicin. Recent studies from our laboratory have suggested that some varicose branching endings of afferent axons (VBAs) on blood vessels also function as high threshold mechanotransduction sites, sensitive to localized distortion. The distribution, morphology and immunohistochemistry of afferent VBAs on mesenteric blood vessels was characterised. Anterograde labelling with biotinamide labelled VBAs on mesenteric vessels, in which TH immunoreactivity was present in $52 \pm 13 \%$ which are probably sympathetic efferents. CGRP-immunoreactivity was detectable in $19 \pm 6 \%$ of biotinamide labelled VBAs. Mechanotransduction sites have previously been reported to be associated with arterial branch points: anterogradely labelled VBAs were significantly closer to branch points $(303+46 \mu \mathrm{m}, \mathrm{n}=5)$ than randomly generated sites $(427+57 \mu \mathrm{m}, \mathrm{p}<0.05)$. Varicose, branching nerve fibres lacking both TH and CGRP, made up $24 \pm 8 \%$ of all biotinamide labelled axons, indicating that some afferent axons may lack CGRP immunoreactivity. Counts of CGRP and TRPvl (capsaicin receptor) immunoreactivity on axons on mesenteric arteries supported this suggestion. Of all axons labelled by one or both antisera, $85.9+2.0 \%$ had both CGRP and VR1, 3.6+1.3\% had CGRP without VRl and $10.5+2.3 \%$ had VRI without CGRP. Retrograde tracing with the carbocyanine dye, Dil, labelled thoraco-lumbar spinal afferents which provide the major spinal afferent pathway to the intestines. Of Dil-labelled neurons in dorsal root ganglia, 26.9+8.0\% contained CGRP and VRl immunoreactivity, 3.1+0.6\% contained CGRP alone and the remaining $69.9+7.6 \%$ contained neither marker confirming that the gut receives innervation by spinal afferent neurons which lack CGRP or TRPvl. We also investigated afferent axons on submucous blood vessels. Of axons labelled by one or both markers, $91.8+1.4 \%$ contained CGRP with VR1, $6.9+2.3 \%$ contained CGRP without VRl and $1.3+1.2 \%$ contained VRl without CGRP. These results reveal that mesenteric and submucous arteries are innervated by sensory nerve fibres, which make branching endings significantly associated with arterial branch points. A population of these vascular primary afferents, visualised using anterograde or retrograde tracing, appears to be non-peptidergic and may not have been identified previously using immunohistochemical methods. This work was supported by R01-DK56986 from NIH (USA) and \#275530 from NHMRC Australia.

\section{S1754}

Effects of C.Difficile Toxin B On the Electrical Activity of the DRG Neurons Denis G. Colomb, Minho Kang, Hamid I. Akbarali

Clostridium difficile toxins, the causative agents of pseudomembranous colitis, are glucosyltransferases that inactivate small GTPases of the Rho family. In addition to the initial activation of the enterocytes, the pro-inflammatory effects of the toxins may also involve sensory neurons. It is however not known whether the toxins can directly affect the electrical properties of the sensory neurons. Single neuronal cells were enzymatically isolated from the rat L6-S2 dorsal root ganglia. In current clamp mode, C. difficile toxin B (TxB; $5 \mathrm{ug} / \mathrm{ml}$ ) produced a significant hyperpolarization of the DRG cells by approx. $10-15 \mathrm{mV}(\mathrm{p}<0.05$; $\mathrm{n}=5$ ) after 10 mins of perfusion, indicating that $\mathrm{TxB}$ selectively modulates ion channels maintaining resting potential. Under voltage clamp conditions with high $\mathrm{K}+$ intracellular pipette solution, hyperpolarization from a holding potential of $-60 \mathrm{mV}$ (10 mV steps) activated time- and voltage independent inward instantaneous $\left(\mathrm{I}_{\text {inst }}\right)$ current and, at step potentials negative to $-80 \mathrm{mV}$, an additional slow activating component $\left(\mathrm{I}_{\text {slow }}\right)$, characteristic of the hyperpolarization activated cyclic nucleotide gated cation channels (HCN) $(n=55)$. Both $\mathrm{I}_{\text {inst }(\mathrm{HCN})}$ and $\mathrm{I}_{\text {slow }(\mathrm{HCN})}$ had reversal potential of approx. $-25 \mathrm{mV}$ with significant correlation between the two components $\left(r^{2}=0.62 ; \mathrm{p}<0.0001\right)$. The HCN blockers, ZD7288 $(10 \mathrm{uM})$ and $\mathrm{CsCl}(1 \mathrm{mM})$ abolished the slow component but did not alter the resting membrane potential or the instantaneous current. In contrast, TxB perfusion resulted in a time-dependent inhibition of the instantaneous current component of the HCN channel concomitant with hyperpolarization but did not significantly affect the slow component. $\mathrm{I}_{\text {inst }(\mathrm{HCN})}$ was inhibited by approx. $50 \%$ after $8-10$ mins of perfusion. Within this time frame the morphology of the cells and the cell capacitance remained unchanged. $\mathrm{I}_{\text {inst }(\mathrm{HCN})}$ was not affected by either heat-inactivated TxB or rundown over 10 mins. The specificity of TxB on the $\mathrm{I}_{\text {inst }(\mathrm{HCN})}$ component of $\mathrm{HCN}$ channels was further confirmed in heterolougsly expressed human $\mathrm{HCN}-1$ (HEK cells). These data demonstrate that 1 ) the $\mathrm{I}_{\text {inst }(\mathrm{HCN})}$ component, but not $\mathrm{I}_{\text {slow }(\mathrm{HCN})}$ of the $\mathrm{HCN}$ channel contributes towards the resting membrane potential of the DRG neurons, 2) TxB induces hyperpolarization of the DRG soma by specific inhibition of the $\mathrm{I}_{\text {inst }(\mathrm{HCN})}$ and 3) TxB affects the electrical excitability of the sensory neurons separate from its effects on cell morphology. Furthermore, the ability to differentially block $\mathrm{I}_{\text {inst(HCN) }}$ and $\mathrm{I}_{\text {slow }(\mathrm{HCN})}$ suggests that separate gating mechanisms are involved in the two components of the HCN channel. Supported by NIH DK46367, DK069628 and US Navy HSCP(DC).

\section{S1756}

Involvement of the Acid-Sensing Ion Channel ASIC3, But Not ASIC2, in Acid Hypersensitivity Associated with Gastritis in Mice

Peter Holzer, Thomas Wultsch, Evelin Painsipp, Anaid Shahbazian, Rainer Waldmann

Introduction and Aims: Gastric acid is a potentially noxious stimulus, but the molecular sensors relevant to gastric acid nociception await to be identified. The acid-sensing ion channels ASIC2 and ASIC3 are expressed by vagal and spinal afferent neurons supplying the stomach. Here we examined whether knockout of the ASIC2 or ASIC3 gene modifies vagal afferent signaling of a gastric acid insult and whether ASIC2 or ASIC3 contributes to the gastric acid hypersensitivity associated with gastritis. Methods: The gastric mucosa of mice was challenged with $0.25 \mathrm{M} \mathrm{HCl}(0.02 \mathrm{ml} / \mathrm{g})$ given via an esophageal catheter. Two hours later the brainstem was removed and activation of neurons in the nucleus tractus solitarii (NTS) visualized by c-Fos immunocytochemistry. In order to induce gastritis, iodoacetamide $(0.1 \%)$ was added to the drinking water 7 days before the gastric $\mathrm{HCl}$ challenge experiment. Results: Exposure of the gastric mucosa to $\mathrm{HCl}(0.25 \mathrm{M})$ caused a 2.7 -fold $(\mathrm{P}<0.01)$ increase in the number of $\mathrm{c}$-Fos positive neurons in the NTS. Relative to wild-type mice, the gastric acid-evoked c-Fos expression in the NTS remained unchanged in ASIC3 knockout mice. In contrast, ASIC2 knockout enhanced the c-Fos response to $\mathrm{HCl}$ challenge by $33 \%(\mathrm{P}<0.01)$. Exposure of wild-type mice to iodoacetamide $(0.1 \%)$ in the drinking water induced gastric inflammation as revealed by an increase in myeloperoxidase activity. In addition, iodoacetamide-pretreated mice exhibited gastric acid hypersensitivity as revealed by a $41 \%$ increase $(\mathrm{P}<0.01)$ in the number of NTS neurons expressing c-Fos in response to gastric $\mathrm{HCl}$ challenge. The iodoacetamide-evoked acid hypersensitivity was completely absent in ASIC3 knockout mice but was fully conserved in ASIC2 knockout mice. Wild-type, ASIC2 knockout and ASIC3 knockout animals did not differ in the number c-Fos positive neurons in the NTS following intragastric administration of saline. Conclusions: The current data indicate that ASIC3 expressed by vagal afferents plays a major role in acid hypersensitivity associated with experimental gastritis but does not participate in the acid surveillance of the normal gastric mucosa. To the contrary, ASIC2 appears to dampen acidevoked input from the stomach to the NTS, an action that is likely to take place in the brainstem because it does not interfere with iodoacetamide-evoked sensitization of the stomach to acid. Supported by the Jubilee Funds of the Austrian National Bank, the Zukunftsfonds Steiermark and the Austrian Scientific Research Funds. 
S1757

Enhanced Anterior Cingulate Cortex (ACC) Nociceptive Transmission in Visceral Hypersensitive Rats Is Restricted to Visceral Afferent Input Jun Gao, Xiaoyin Wu, Chung Owyang, Ying Li

The anterior cingulate cortex (ACC) mediates affective processing of sensory information Abnormal processing of visceral sensory signals by the ACC may aggravate symptoms of irritable bowel syndrome. We recently reported an enhanced response of the ACC to colorectal distension(CRD) in visceral hypersensitive (VH) rats characterized by increased spontaneous activity of CRD-excited neurons, decreased CRD pressure threshold, and increased response magnitude. Cross-sensitization of visceral and somatic sensory pathways has been reported. Noxious somatic afferent input from the hind limb enhances visceral hyperalgesia in the rat, and this sensitization occurs at the spinal cord. In this study we investigated whether cross-sensitization of visceral and somatic pathways occurs at the level of the ACC in VH rats. We recorded single ACC neuronal discharges in response to CRD in sham treated (control) and $\mathrm{VH}$ rats induced by intraperitoneal injection of egg albumin followed by 3 days of periodic CRD. Neurons in the rostral ACC which exhibited an excitatory response to $50 \mathrm{mmHg}$ CRD were identified. Responses of these neurons in rats subjected to noxious transcutaneous electrical stimulation (TCES) of the hind paw were recorded. Nine of 16 neurons in control and 12 of 20 neurons in VH rats were activated exclusively by CRD but not to TCES. The second group of CRD-excited ACC neurons (7/16 in control and $8 / 20$ in $\mathrm{VH}$ rats) responded to both TCES and CRD. To determine whether crosssensitization of visceral and somatic sensory pathways occurs in the ACC of VH rats, we performed single neuron recording in the ACC neurons which only responded to both CRD and cutaneous noxious heat stimulation of the hind paw. Compared with ACC neurons in control rats, ACC neurons in VH rats show enhanced firing rate in response to CRD (50 $\mathrm{mm} \mathrm{Hg}$ ) (increased from $0.83 \pm 0.1$ to $1.7 \pm 0.2 \mathrm{impulses} / \mathrm{sec}$ in control vs $1.66 \pm 0.15$ to $4.0 \pm 0.4$ impulses/sec in VH rats). In contrast, proportional increases in ACC firing rates in response to cutaneous heat stimulation was similar between control and VH rats (from basal $0.85 \pm 0.06$ to $3.2 \pm 0.4$ at $46^{\circ} \mathrm{C}$ and to $6.4 \pm 0.5$ impulses $/$ second at $52^{\circ} \mathrm{C}$ in control rats, and from basal $1.67 \pm 0.25$ to $4.4 \pm 0.45$ at $46^{\circ} \mathrm{C}$ and to $7.8 \pm 0.9$ impulses/second at $52^{\circ} \mathrm{C}$ in $\mathrm{VH}$ rats). We conclude that $\mathrm{ACC}$ has functionally distinct regions subserving visceral and somatic sensory pathways. In subgroups of ACC neurons which respond to visceral and cutaneous stimulation, there is no cross-sensitization between visceral and somatic pain. In contrast to the spinal cord, enhanced ACC nociceptive transmission in visceral hypersensitive rats is restricted to visceral afferent input.

\section{S1758}

Responses to Mechanical Stimuli in Low-Threshold Slowly-Adapting Rectal Mechanoreceptors After TNBS-Induced Inflammation in the Guinea Pig

Penny A. Lynn, Marcello Costa, Simon Brookes

The effects of TNBS-induced inflammation on specialised, low threshold, slowly adapting rectal mechanoreceptors were investigated in the guinea pig. Under isoflurane anaesthesia, $300 \mu \mathrm{l}$ saline or $300 \mu \mathrm{l}$ of TNBS $(15 \mathrm{mg} / \mathrm{ml})$ in $30 \%$ ethanol, was instilled $7 \mathrm{~cm}$ from the anal sphincter. Six or 35 days later, animals were humanely killed and single unit extracellular recordings made from rectal nerve trunks in flat sheet preparations attached to a mechanical tissue stretcher, while monitoring muscle tension. TNBS caused macroscopic ulceration of the rectal mucosa at 6 days which fully resolved by 35 days. Basal motility and low-threshold stretch-sensitive afferent discharge were unaffected by TNBS treatment at six days (saline control $n=14, N=9$, TNBS-treated $n=27, N=12$ ) or 35 days $(n=9, N=5$ and $n=10, N=5$ respectively) post-treatment. Integrated mean tension, contraction frequency and amplitude evoked by circumferential stretch were unaffected by TNBS at either 6 days $(n=9)$ or 35 days $(n=5)$. Responses of low-threshold rectal mechanoreceptors to stretch were similarly unaffected by TNBS treatment at 6 days $(n=12)$ or 35 days $(n=11)$ post-treatment. The threshold for von Frey hair evoked firing $(0.1-1.1 \mathrm{mN})$ was significantly reduced $(\mathrm{p}<0.01)$ at 6 days but not at 35 days (NS, $n=5)$. Responses to more intense von Frey hair stimulation $(0.3-1.1 \mathrm{mN})$ were otherwise unaffected by TNBS pre-treatment. The inflammatory mediator PGE2 (1 uM) activated low-threshold stretch-sensitive afferents $(\mathrm{p}<0.05)$ in TNBS-treated preparations ( 6 days) with firing closely associated with evoked contractions. PGE2 (luM) also activated 5 stretch-insensitive afferents $(\mathrm{p}<0.01)$. Capsaicin (luM) did not activate lowthreshold stretch-sensitive afferents $(n=8, p=0.87$ ) but excited stretch-insensitive afferents, independent of contractions $(n=5, p<0.01)$. Neither PGE2 nor capsaicin activated stretchsensitive $(n=6)$ or stretch-insensitive afferents $(n=4)$ in control (saline-treated) animals. The mechanical thresholds of specialised, low threshold, slowly adapting rectal mechanoreceptors of the guinea pig rectum are slightly but significantly reduced in TNBS inflammation, but suprathreshold responses are unaffected. Other classes of afferents are sensitised to PGE2 and capsaicin following TNBS treatment, as is smooth muscle contractility, but low threshold mechanoreceptors are not directly affected. These results indicate differential modulation of various classes of colonic afferents by inflammation. This work was supported by R01DK56986 from NIH (USA)

\section{S1759}

Colonic Serotonergic Signaling Molecule and Receptors mRNA Alteration in Rats with Acute or Chronic Restraint Stress

Yan Sun, Gengqing Song, Fengling Liu, Wei Qian, Xiaohua Hou

Background \& Aims: Serotonin (5-HT) is a critical signaling molecule in the gut and plays an important role in initiating peristaltic, secretory, and nociceptive reflexes. Stressful factors could induce gut motility and sensation dysfunction. The aim of this study was to investigate the effects of acute and chronic partial restraint stress (PRS) on 5-HT synthesize enzyme, 5-HT transporter and 5-HT receptors messenger RNA (mRNA) in colon tissues of rats. Methods: 35 male Sprague-Dawley rats were divided evenly into five groups including control without PRS, acute PRS for $2 \mathrm{~h}$, acute PRS with 7-day recovery, chronic PRS for $2 \mathrm{~h}$ a day in 3 consecutive days, chronic PRS with 7-day recovery. Typtophan hydroxylase $(\mathrm{TpH}), 5$-HT transporter, and 5-HTlA and 5-HT4 receptors mRNA in colon tissues were detected by using reverse transcription- polymerase chain reaction (RT-PCR) method. The mast cells in colon tissues were observed by using immunohistochemistry staining. Results: (1) Compared with the rats without PRS, the $\mathrm{TpH}$ and 5-HT transporter mRNA were significantly reduced in the rats with chronic PRS $(0.69 \pm 0.13$ vs. $1.23 \pm 0.36$ and $0.63 \pm 0.18$ vs. $1.20 \pm 0.35$ respectively, $\mathrm{P}<0.05)$ and chronic PRS with 7 -day recovery $(0.70 \pm 0.12$ vs $1.23 \pm 0.36$ and $0.56 \pm 0.15$ vs. $1.20 \pm 0.35, \mathrm{P}<0.05$ ). (2) For the TpH and 5 -HT transporte mRNA expressions, there were no significant differences among the rats with acute PRS or acute PRS with 7-day recovery or the rats without PRS $(P>0.05)$. (3) In comparison with the rats without PRS, the 5-HTIA and 5-HT4 receptors mRNA were significantly higher in the rats with acute and chronic PRS (5-HTlA receptor: $0.42 \pm 0.09$ vs. $0.22 \pm 0.16$ and $0.80 \pm 0.20$ vs. $0.22 \pm 0.16$ respectively $\mathrm{P}<0.05 ; 5$-HT 4 receptor: $0.52 \pm 0.19$ vs. $0.32 \pm 0.15$ and $0.93 \pm 0.31$ vs. $0.32 \pm 0.15$ respectively $\mathrm{P}<0.05$ ). In the rats of 7 days after the acute or chronic PRS, the 5-HTIA and 5-HT4 receptor mRNA expressions were still higher than the rat without PRS $(\mathrm{P}<0.05)$. (4) There was no significant difference in the mast cell count in colon tissues among all groups $(\mathrm{P}>0.05)$. Conclusions: These results show that chronic PRS inhibits serotonergic signaling, whereas the acute PRS does not affect serotonergic signaling in colon tissues. Both acute and chronic PRS increase 5-HT receptors expression. These findings suggest that different changes of 5-HT signaling molecules and receptors may underlie the altered gut motility and sensation under acute or chronic stress conditions.

\section{S1760}

Nippostrongylus Brasiliensis (NB)-Infected Mice Exhibit Chemical But Not Mechanical Hypersensitivity

Kirk Hillsley, Christine McCaul, Pieter Peeters, Jeroen Aerssens, David Grundy, Bernard Coulie, Ronald H. Stead

Background: $\mathrm{Nb}$ infection results in a transient inflammation and subsequent increases in mucosal innervation, mast cells and mast cell-nerve contacts. Previous studies have reported hypersensitivity to intestinal distension, but chemosensitivity has not been investigated. This study determined if $\mathrm{Nb}$ infection had any effect on mechanosensitive and chemosensitive responses in the murine jejunum. Methods: Balb/c mice were infected with $500 \mathrm{~L} 3 \mathrm{Nb}$ larvae or saline (sham) and the following three separate studies performed 3-4 weeks later. Aortic blood pressure was recorded in response to phasic $(30 \mathrm{~s})$ balloon distension with up to 0.9 $\mathrm{ml}$, which evoked an intraluminal pressure (IP) of $115.9 \pm 6.5 \mathrm{mmHg}$. Mesenteric afferent nerve activity and IP were recorded in response to either ramp balloon distension up to 60 $\mathrm{mmHg}$, or to a $2.5 \mathrm{~min}$ intraluminal perfusion $(0.15 \mathrm{ml} / \mathrm{min})$ of $50 \mathrm{mM}$ hydrochloric acic $(\mathrm{HCl})$. Data are expressed as mean \pm SEM and analysed with 2-way ANOVA or unpaired t-tests. Results: Pressor responses were elicited by phasic distension. There was no significant difference in the pressor responses elicited in sham $(n=18)$ and infected $(n=13)$ animals $(\mathrm{p}=0.87$, see Table 1$)$. Ramp distension evoked an increase in afferent nerve activity However, afferent responses in $\mathrm{Nb}$ infected mice $(\mathrm{n}=49)$ were not significantly different ( $\mathrm{p}=0.94$, see Table 1 ) from sham mice $(\mathrm{n}=48)$. In response to $\mathrm{HCl}$ perfusion there was a rapid increase in afferent activity (acute response) that plateaued within $5 \mathrm{~min}$ (prolonged response), and a gradual IP increase that peaked within $5 \mathrm{~min}$. There was an increase in both the afferent and IP responses to $\mathrm{HCl}$ in infected $(\mathrm{n}=26)$ compared to sham $(\mathrm{n}=25)$ animals as detailed in Table 2. There was no significant direct correlation between any changes in nerve activity and IP. Conclusions: Nb infection evokes jejunal hypersensitivity to chemical but not mechanical stimuli. It is postulated that the Nb-induced inflammation mainly impacts the mucosa which alters the sensitivity of chemosensitive afferents, whilst having little impact on deeper mechanosensitive afferents.

Table 1

\begin{tabular}{|c|c|c|c|c|c|c|}
\hline Distension volume $(\mathrm{ml})$ & 0.125 & 0.25 & 0.375 & 0.5 & 0.75 & 0.9 \\
\hline Sham $(\Delta \mathrm{mmHg})$ & $2.1 \pm 1$ & $3.3 \pm 1.2$ & $5.8 \pm 1.8$ & $10.7 \pm 2.1$ & $18.2 \pm 2.3$ & $20.7 \pm 1.8$ \\
\hline $\operatorname{Inf}(\Delta \mathrm{mmHg})$ & $1.7 \pm 0.6$ & $2.8 \pm 0.6$ & $6.2 \pm 1.4$ & $11.3 \pm 2.2$ & $18.4 \pm 1.9$ & $21.3 \pm 1.5$ \\
\hline Pressure $(\mathrm{mmHg})$ & 10 & 20 & 30 & 40 & 50 & 60 \\
\hline Sham $(\Delta \mathrm{imp} / \mathrm{s})$ & $19.8 \pm 2.2$ & $41.6 \pm 3.3$ & $56 \pm 3.8$ & $68.2 \pm 4.9$ & $79.2 \pm 5.4$ & $87.2 \pm 4.6$ \\
\hline $\operatorname{Inf}(\Delta \mathrm{imp} / \mathrm{s})$ & $20.7 \pm 2.4$ & $40 \pm 3.4$ & $55 \pm 3.8$ & $68 \pm 4.2$ & $80 \pm 4.6$ & $88 \pm 5.6$ \\
\hline
\end{tabular}

Table 2

\begin{tabular}{|c|c|c|c|c|}
\hline Measurement & \multicolumn{2}{|c|}{ Nerve $(\Delta$ imp/s $)$} & \multicolumn{2}{c|}{ IP $(\Delta \mathrm{mmHg})$} \\
\hline Response period & Acute & Prolonged & Acute & Prolonged \\
\hline Sham & $41.6 \pm 4.9$ & $27.3 \pm 3.6$ & $0.09 \pm 0.08$ & $0.34 \pm 0.06$ \\
\hline Inf & $53 \pm 3.8$ & $40.5 \pm 4.2$ & $0.18 \pm 0.09$ & $1.28 \pm 0.31$ \\
\hline p value & 0.07 & 0.02 & 0.46 & 0.005 \\
\hline
\end{tabular}

S1761

Effects of Intraluminal Local Anesthetic On Duodenal Glucose Sensing in Humans

Reawika Chaikomin, Selena Doran, Michael Horowitz, Chris Rayner

Enteral administration of glucose modifies gut sensation, diminishes hunger, and slows gastric emptying by suppressing antral motility and stimulating pyloric pressures. The mechanism of small intestinal glucose sensing is unclear. We studied 8 healthy males on two days each, in random order. After an overnight fast, a catheter was positioned with a sleeve sensor across the pylorus, 6 sideholes in the antrum and 7 sideholes in the duodenum Benzocaine, or vehicle alone, was given into the proximal duodenum as an initial bolus, followed by a continuous infusion for 105 minutes ( $t=-15$ to $90 \mathrm{~min}$ ). Glucose was also infused into the proximal duodenum at $3 \mathrm{kcal} / \mathrm{min}$ for 90 minutes ( $\mathrm{t}=0$ to $90 \mathrm{~min}$ ). Sensations of hunger, bloating, and nausea were assessed at frequent intervals with visual analog scales. Antral, duodenal, and isolated pyloric pressure waves $>10 \mathrm{mmHg}$ were 
counted, together with propagated sequences of duodenal waves. Data are mean \pm SEM. Sensations of bloating and nausea were markedly less with benzocaine when compared to vehicle $(\mathrm{P}<0.05$ for each), with no difference in hunger. In contrast, the suppression of antral waves (19 \pm 9.6 for benzocaine v $2.88 \pm 1.3$ for vehicle) and stimulation of pyloric

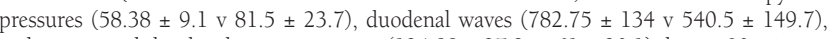
and propagated duodenal wave sequences $(124.88 \pm 27.3$ vs $61 \pm 20.1)$ during 90 minutes of intraduodenal glucose infusion did not differ between the two days. We conclude that nerves in the duodenal mucosa mediate unpleasant sensations induced by enteral glucose, while feedback on appetite and gastroduodenal motility is conveyed by alternative mechanisms, such as gut peptides.

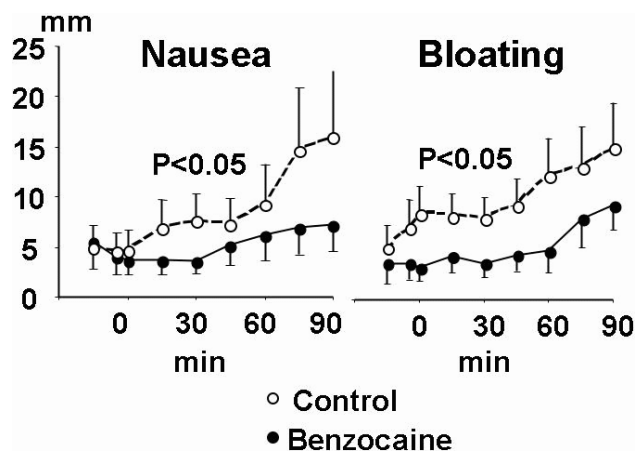

S1762

Adenosine Activates a Subset of Nociceptive Vagal Sensory Nerves in Esophagus

Fei Ru, Marian Kollarik

BACKGROUND \& AIMS: Clinical studies suggest that adenosine acting on the peripheral sensory nerves in esophagus contributes to the pathogenesis of pain in a proportion of patients with non-cardiac chest pain (NCCP) originating from esophagus (Dig Dis Sci. 47:2763). We have demonstrated in guinea pig that in addition to classically described non-nociceptive low threshold mechanosensors ("tension receptors") vagus nerves supply esophagus with nociceptive sensory nerves distinguished by the capacity to discriminate noxious stimuli (J. Physiol 563: 831). Here we address the hypothesis that adenosine selectively activates vagal esophageal nociceptors. METHODS: Single cell RT-PCR was performed from the individual primary sensory neurons enzymatically dissociated from the guinea pig vagal nodose ganglia. Extracellular single-unit recordings were made from the nodose ganglion neurons with the mechanosensitve receptive fields in the esophagus using ex vivo isolated vagally innervated guinea pig esophagus preparation. RESULTS: The adenosine $\mathrm{Al}$ receptor mRNA was detected by the single cell RT-PCR in 10/17 nodose neurons. Consistent with our previous studies, two types of vagal nodose nerve fibers were identified by electrophysiology in esophagus: capsaicin-sensitive C-fibers (conduction velocity $<1.2$ $\mathrm{m} / \mathrm{s}, \mathrm{n}=16$ ) in which nerve activity increased linearly with increasing esophageal distention up to $100 \mathrm{mmHg}$ (nociceptive C-fibers), and capsaicin-insensitive nodose A-fibers (conduction velocity $2-10 \mathrm{~m} / \mathrm{s}, \mathrm{n}=9$ ) in which response to esophageal distention saturated at $<60 \mathrm{mmHg}$ ("tension receptors"). Adenosine (100uM, 10 min) and the selective adenosine $\mathrm{Al}$ receptor agonist CCPA $(1 \mu \mathrm{M}, 10 \mathrm{~min})$ evoked action potential discharge in 6/9 (the peak frequency $3 \pm 1 \mathrm{~Hz}$ ) and $7 / 9$ (the peak frequency $7 \pm 2 \mathrm{~Hz}$ ) nociceptive nodose C-fibers, respectively. In contrast, adenosine $(100 \mathrm{\mu M}, 10 \mathrm{~min})$ had no effect on the non-nociceptive nodose A-fibers ( $n=7, p>0.1$ ), although the positive control stimulus, the P2X receptor agonist $\alpha, \beta$-methyleneATP $(30 \mu \mathrm{M}, 10 \mathrm{~min}$ ) evoked robust activation (peak frequency $15 \pm 2 \mathrm{~Hz}$ ) in these A-fibers. CONCLUSIONS: Adenosine activates a subset of vagal esophageal nociceptors, but not nonnociceptive low threshold mechanosensors ("tension receptors"). Our data suggest that the response of esophageal vagal nociceptors to adenosine is at least partially mediated by the adenosine $\mathrm{Al}$ receptor. We speculate that this pathway may contribute to the perception of pain in patients with NCCP

S1763

Somatic Hypersensitivity in the Irritable Bowel Syndrome Baharak Moshiree, Roger B. Fillingim, Kristy Phelan, Joseph Riley, Nicholas G. Verne

Introduction: We have shown that Irritable Bowel Syndrome (IBS) patients display hypersensitivity in response to nociceptive thermal stimuli (Verne et al., Pain, 2001:93:7-14). These findings differ from previous studies that indicate IBS-associated hypersensitivity is limited to the gut. Aim: The aim of the present study is to examine the differences in sensitivity to ischemic pain in IBS patients compared to controls. Methods: Ten female patients (mean age: $29.5+/-8.7$ ) with IBS and 26 healthy female controls (mean age: $28+/-8.1$ ) completed the study. The right arm was exsanguinated and then occluded with a standard blood pressure cuff inflated to $240 \mathrm{mmHg}$. Subjects then performed 20 hand-grip exercises of 2 sec duration at 4-sec intervals at $50 \%$ of their maximum grip strength. They then rated pain intensity on 0-100 Mechanical Visual Analogue Scale (M-VAS) every 60 seconds. Subjects continued for 15 minutes or until the pain became intolerable. Group differences for threshold and tolerance were determined using a series of 1 -way ANOVA tests. Results: IBS patients had a shorter ischemic tolerance $(239$ secs $)$ compared to controls $(444$ secs $)(\mathrm{F}=4.310, \mathrm{p}=$ 0.46). There were no group differences for ischemic threshold $(p=0.476)$. Conclusions: Our data suggests that a more widespread alteration in central pain processing in IBS patients may be present as they display enhanced hypersensitivity to experimental ischemic pain. These new findings also suggest a dysfunction in inhibitory pain systems in IBS patients as ischemic (deep) pain is under tonic inhibitory control. Supported by VA Merit Review and a NIH ROI-NS053090.
S1764

Peripheral Sensitization and the Role of P2X Receptors in the Pathogenesis of Visceral Hypersensitivity in a Rat Model of Irritable Bowel Syndrome Guang-Yin Xu, Mohan M. Shenoy, John H. Winston, Huaizhi Yin, Pankaj J. Pasricha

Background and Aims: Although sensitization to mechanical stimulation of the colon is common in irritable bowel syndrome (IBS), its pathogenesis and underlying molecular mechanisms remain unknown. Recent work in somatic and other visceral models suggest a role for purinergic signaling mediated by the P2X receptor family. We have previously described a rat model of IBS associated with persistent hypersensitivity to colorectal distention (CRD), induced by neonatal intracolonic acetic acid (AA). In this study, we used this model to determine the presence of peripheral sensitization and to examine a role for $\mathrm{P} 2 \mathrm{X}$ signaling in the pathogenesis of the same. Methods: IBS was induced by colonic injection of AA in neonatal rats at P10. Balloon distention of the colon was performed at 8 weeks of age and nocifensive responses measured on a behavioral scale. Dorsal root ganglion (DRG) neurons innervating the colon were labeled by injection of Dil fluorescence into the colon wall one week before electrophysiological studies consisting of whole cell patch-clamp recordings from acutely dissociated DRG neurons. Results: Colonic sensory neurons from rats with AA-induced hypersensitivity showed a dramatic reduction in current threshold or rheobase (control: $0.55 \pm 0.07, \mathrm{n}=32 ; \mathrm{AA}: 0.2 \mathrm{l} \pm 0.03, \mathrm{n}=27, \mathrm{P}<0.05$ ) and a significant increase in the number of action potentials evoked at twice rheobase (control: $1.16 \pm 0.07, \mathrm{n}=32$; AA: $1.84+0.09, \mathrm{n}=25, \mathrm{P}<0.01)$. Under voltage-clamp conditions, application of ATP $(20 \mu \mathrm{M})$, a $\mathrm{P} 2 \mathrm{X}$ receptor agonist, produced an inward current with an amplitude of $6.0 \pm 1.3 \mathrm{pA} / \mathrm{pF}(\mathrm{n}=$ 20) in colonic neurons from control rats; this was increased two-fold in colonic neurons from rats with hypersensitivity $(\mathrm{n}=17, \mathrm{P}<0.05)$. These currents were completely blocked by the P2X antagonist TNP-ATP (100 nM) in both groups. Further, systemic administration of TNP-ATP $(50 \mathrm{mmol} / \mathrm{kg}$, i.p.) markedly reduced the pain behavioral response to colon distension in rats with hypersensitivity. Conclusions: These data suggest that exposure of the colon to a transient and mild noxious stimulation in the neonatal period can result in permanent hypersensitivity and peripheral sensitization. Our findings also suggest that P2X receptors may play an important role in the pathogenesis of hypersensitivity to mechanical distention of the colon, thus identifying a specific neurobiological target for therapy

\section{S1765}

Localization and Characterization of the Mechanosensory Innervation of the Rat Pancreas

Ann C. Schloithe, Charmaine M. Woods, Joesph S. Davison, Laurence A. Blackshaw, James Toouli, Gino Saccone

Background: Afferent innervation of the pancreas is poorly understood despite the fact that management of pancreatic pain is a major clinical problem. Aim: To map and characterize mechanosensitive afferents in the normal rat pancreas. Methods: A novel isolated pancreas preparation that included the pancreatic duct (PD), common bile duct (CBD) and the splanchnic nerves was obtained from anaesthetized (Nembutal; 60mg/kg i.p.) Sprague Dawley rats $(n=14)$. The CBD was cannulated. The preparation was placed in a 2 chamber organ bath. One chamber accommodated the pancreatic tissue in physiological solution and the second chamber contained the nerve fibers in paraffin oil. Extracellular nerve recordings were obtained using fibers isolated from the left splanchnic nerve. Stimuli consisted of blunt probing (graded and non-graded), CBD/PD distension ( $44 \mathrm{mmHg}$ ), or electrical stimulation (ES; 20V, 0.5ms duration). Results: Discharge from 197 fibers was recorded, of which 57\% displayed spontaneous activity. Each fiber contained between 1 and 5 spontaneously firing units with a mean discharge rate of $2.52 \pm 0.32 \mathrm{imp} / \mathrm{sec}$. Sixty one mechanosensitive receptive fields were identified and were mainly associated with arteries/blood vessels (33/ 61), particularly the gastric, splenic, and hepatic arteries, and the parenchyma (22/61) Receptive fields were also associated with pancreatic tissue near lymph nodes (4/61), CBD $(1 / 61)$ and duodenal mesentery (1/61). 13\% of the receptive fields received innervation from 2 or 3 separate nerve fibers in 7 preparations and $70 \%$ of these receptive fields were located on blood vessels. All mechanosensitive responses were slowly adapting and 33\% displayed continuing discharge after termination of the stimulus. Graded responses recorded from 27 units revealed that $60 \%$ of the units displayed a response threshold less than $10 \mathrm{~g}$. $25 \%$ and $15 \%$ of the units had a response threshold of between 10 and $20 \mathrm{~g}$ or greater than $20 \mathrm{~g}$, respectively. Importantly, CBD/PD distension did not evoke any discharge from spontaneous or quiescent units. Non-mechanosensitive receptive fields, stimulated by ES only, were located in pancreatic tissue (7/17), arteries/blood vessels (9/17) or CBD/PD (1/ 17) and their function is yet to be established. Conclusion: Mechanosensitive nerves are associated with several structures in the pancreas, particularly the vasculature and parenchyma though, notably not with the major duct system. Our continuing studies aim to establish the role of pancreatic afferents in physiological and pathophysiological signalling. This study was supported by the NH\&MRC of Australia (grant \# 229901) and the FMC Foundation

\section{S1766}

Angiotensin As a Potential Mediator of Mast Cell Nerve Interactions in the Esophagus

Shaoyong Yu, Kevin Kwong, Bradley Undem

Introduction: Mast cells are pro-inflammatory cells found in the wall of the esophagus, and their numbers increase during various inflammatory disorders of the esophagus. We previously reported that activation of mast cells causes a substantive increase in excitability of esophageal vagal nodose C-fiber terminals in guinea pig esophagus. Here we begin to address the hypothesis that angiotensin may contribute to mast cell nerve interactions in the esophagus. Aim: We hypothesize that esophageal mast cells contain renin, and that the angiotensin presumed to be formed by the released rennin increases the excitability of nodose neurons. Methods: We used standard immunohistochemical techniques to identify cells in the esophagus that store renin. Mast cell tryptase was used as a marker for mast cells. The effect of angiotensin-II on vagal sensory neuronal excitability was evaluated using 
standard patch clamp recording techniques on neurons acutely dissociated from adult guinea pigs. Results: In 7 animals an average of $10 \pm 2$ cells per cross section of guinea pig esophagus were strongly positive for rennin. The average number of mast cells per cross section of the esophagus was $27 \pm 3$. Among the renin positive cells, $87 \pm 1 \%$ also contained mast cell tryptase. Angiotensin-II (0.1. $\mu \mathrm{M})$ increased the electrical excitability of 4 out of 5 vagal sensory neurons. In these cells, studied in current clamp mode, angiotensin significantly increased the number of action potentials evoked by a $500 \mathrm{msec}$ constant current pulse (100pAmp) from $2.5 \pm 0.8$ to $42 \pm 0.8$ Conclusion: Consistent with recent reports in cardiac tissue (PNAS 101: 13607, 2004), a substantial percentage of esophageal mast cells contain renin. If this renin is released upon mast cell activation it may convert angiotensinogin to angiotensin I. Either mast cell chymase or angiotensi converting enzyme may then convert angiotensin-I to angiotensin-II, a mediator that we show here to be effective at increasing excitability of vagal sensory nerves.

\section{S1767}

Potentiation of Transient Receptor Potential Vanilloid Receptor 3 (TRPV3) Sensing Function By Ethanol

Hong-Zhen Hu, Chumbo Wang, Michael X. Zhu, Jackie D. Wood

Ethanol can act as a noxious irritant and induce inflammatory responses (e.g., gastritis) in the gastrointestinal tract. The transient receptor potential (TRP) channels, expressed by intramural spinal and vagal sensory afferents, become targets for ethanol. For example, the TRP vanilloid receptor Type 1 (i.e., the capsaicin receptor) is sensitized by ethanol ( $\mathrm{Br} J$ Pharmacol 2004;141:1313-20). TRPV3 is another member of the vanilloid receptor family, which is associated with inflammation and thermal sensitivity. We now present evidence that ethanol directly influences TRPV3 function when the receptor is expressed in either HEK293 cells or Xenopus oocytes. Esposure to ethanol significantly increased the amplitude of the TRPV3-activated trans-membrane ionic current that was evoked by the agonist, 2 APB, in Xenopus oocytes injected with mouse TRPV3 cRNA. Ethanol (100 mM and $1 \mathrm{M})$ increased the responses to $100-\mu \mathrm{M} 2$-APB to $245.7 \pm 38.6 \%(n=4)$ and $476.9 \pm 123.6 \%(n=$ 4) of the current in the absence of ethanol. Similarly in HEK293 cells, transfected with mouse TRPV3 DNA, ethanol (100 mM and 1M ) increased the amplitude of 2-APB-evoked current to $239.2 \pm 12.5 \%$ and $547.6 \pm 82.3 \%(n=5)$ of the current prior to ethanol treatment. Increasing the temperature from $23^{\circ}$ to $40^{\circ} \mathrm{C}$ evoked an outwardly rectifying current in TRPV3-expressing oocytes and ethanol increased the amplitude of the heat-activated current to $550 \%$ of the current in the absence of ethanol treatment. The potentiation by ethanol was unchanged by removal of extracellular calcium or intracellular injection of the calcium chelator, BAPTA, in the TRPV3-transfected oocytes. The results suggest that ethanol directly modulates the behavior of thermal sensitive TRPVI and TRPV 3 channels. TRPV3 might also be involved in ethanol-evoked sensitization of primary gastrointestinal afferents in parallel with sensitization of TRPV1. (Supported by NIH ROI DK37238 and ROl DK57075)

\section{S1768}

In Vivo Electrophysiological and C-Fos Study of Pelvic Primary Afferent Nerve Fibers Encoding Colorectal Mechanosensory Signalling in Healthy Female Wistar Rats

Heiko U. de Schepper, Benedicte Y. de Winter, Luc van Nassauw, Jean-Pierre

Timmermans, Paul A. Pelckmans, Joris G de Man

Introduction: The pelvic nerve innervates the distal colon via the lumbosacral portion of the spinal cord (DRG L6-S1). Afferent pelvic neurons are involved in both innoxious and noxious mechanosensation and in extrinsic autonomic gut reflexes. Our aims were to investigate the global behaviour of these pelvic neurons relative to their response threshold $(\mathrm{TH})$ and conduction velocity (CV). Methods: We implemented an in vivo setup for measurement of pelvic afferent nerve signals. Pentobarbital-anaesthetized $(60 \mathrm{mg} / \mathrm{kg}$, i.p.) female Wistar rats were fitted with a silver electrode wrapped around the pelvic nerve. A balloon $(5 \mathrm{~cm})$ inserted in the colorectum allowed phasic colorectal distensions (CRD, 20-40-60$80 \mathrm{mmHg}$ at $4 \mathrm{~min}$ intervals). The lumbosacral spinal cord was exposed and the S1 dorsal root was identified and teased into fine strands. These were individually draped over a bipolar platinum electrode in a liquid paraffin bath $\left(37^{\circ} \mathrm{C}\right)$. Fibers responding to $\mathrm{CRD}$ were identified and stimulus-response curves were constructed. The CV was calculated from the response time of spinal nerves to pelvic nerve stimulation ( $0.5 \mathrm{~ms}$ puls, $5-8 \mathrm{~V})$. Immunohistochemical c-fos staining was performed in DRG S1 in rats with and without prior CRD (80 $\mathrm{mmHg}$ ). Results: We identified 9 pelvic afferent neurons responding to CRD, most with an initial rapid dynamic burst followed by a lower, more sustained discharge rate. $66.7 \%$ (6) of these showed spontaneous activity $(0.7 \pm 0.4 \mathrm{~Hz}) .55 .6 \%$ (5) of these fibers encoded the entire pressure range studied and were identified as low TH $(\leq 10 \mathrm{mmHg})$, while $44.4 \%$ (4) showed a high $\mathrm{TH}$ of activation ( $\geq 28 \mathrm{mmHg}$ ). The firing rate to every CRD intensity studied was significantly higher for low TH fibers compared to high TH fibers (at 60-80 mmHg: $12.2 \pm 5.1 \mathrm{~Hz}$ compared to $5.7 \pm 0.8 \mathrm{~Hz}, \mathrm{P}<0.05$ ). Of the 9 pelvic neurons studied, $55.6 \%(5)$ were identified as unmyelinated C-fibers $(\mathrm{CV}<2.5 \mathrm{~m} / \mathrm{s})$ and $44.4 \%(4)$ as thinly myelinated $A \delta$-fibers $(C V>2.5 \mathrm{~m} / \mathrm{s})$. A $\delta$-fibers showed a higher firing rate to $\mathrm{CRD}$ compared to C-fibers (at $60-80 \mathrm{mmHg}$ : $14.6 \pm 5.9 \mathrm{~Hz}$ compared to $5.26 \pm 0.87 \mathrm{~Hz}$ ). C-fos staining showed an upregulation of S1 neuronal activity after noxious distension. Conclusions: Both electrophysiology and c-fos staining demonstrated the involvement of S1 primary afferent fibers in sensing CRD. Our preliminary results showed 4 groups of neurons responding to $\mathrm{CRD}$, and are as such in concert with previous reports (Sengupta \& Gebhart, J Neurophysiol 1994). In our study however, Aס- and C-fibers were equally distributed in contrast to the $\mathrm{C}$-fiber predominance reported by others. In addition, we found that A $\delta$-fibers responded to $\mathrm{CRD}$ with a higher discharge rate than $\mathrm{C}$-fibers.

\section{S1769}

Chronic Mild Stress-Induced Visceral Hypersensitivity to Rectal Distension in Rats: Role of Protenase-Activator Receptor-2 and Mast Cell Yong Sung Kim, Suck Chei Choi, Moon Young Lee, Young Woo Sohn, Yong Leol Oh, Geom Seog Seo, Tae Hyeon Kim, Chang Soo Choi, Young Mi Lee, Yeon-A Choi, Sang Wook Kim, Myung Gyu Choi, Yong-Ho Nah

Background/Aim: Visceral hypersensitivity is one of the important pathophysiologic mechanism of irritable bowel syndrome (IBS), but the causes underlying this mechanism are not entirely understood. The increased number of mast cells and their proximity to the colonic nerve in IBS patients were recently reported. One study suggests that proteases from degranulated mast cells cleave protenase-activated receptor (PAR)-2 located on submucosal neuron to cause acute and long-lasting neuronal hyperexcitability (Reed DE et al, 2003). This study examined whether mast cell numbers and PAR-2 expression in a rat colon are changed after 6 weeks of chronic mild stress (CMS) protocol that induces visceral hypersensitivity and reflects a sustained human stress situation. Method: Adult Sprague-Dawley male $(\mathrm{N}=5)$ ra (200 g) spent 6 weeks under CMS protocol that has been previously described (Willner et al, 1987). Control group $(\mathrm{N}=5)$ spent the same period under normal conditions. After CMS protocol, we assessed the electromyographic response of abdominal muscles to colorecta distension (CRD) by inflation of a balloon $(0.2,0.4,0.6,0.8,1.0,1.2 \mathrm{~mL}$, ascending-limits phasic distension) using a Fogarty embolectomy catheter. Both the control and CMS rats were killed by decapitation and their colons were then removed. Three colonic pieces (proximal, mid, distal) from each group were stained with toluidine blue to quantify the number of mast cells and immunostaining was performed using anti PAR-2 antibody to quantify the number of PAR-2 expressed cells. Result: The threshold of abdominal muscle contraction to CRD was lower in the CMS group than in the control group $(0.39 \pm 0.09$ $\mathrm{mL}$ vs $0.58 \pm 0.09 \mathrm{~mL}, \mathrm{p}<0.01$ ). Mast cells were found mainly in the submucosal area. The number of mast cells increased higher in the CMS group than in the control group $(3.47 \pm 1.64$ / $\mathrm{mm} 2$ vs $0.55 \pm 0.39 / \mathrm{mm} 2, \mathrm{P}<0.05)$. The number of mast cells in each area significantly increased in the CMS group, but were more prominent in the distal colon $(\mathrm{P}<0.05)$. PAR2 expressed cells were found primarily in the absorptive cells and the lamina propria. The number of PAR-2 expressed cells was higher in the CMS group than in control group $(9.07 \pm 3.37 / \mathrm{mm} 2$ vs $1.53 \pm 0.69 / \mathrm{mm} 2, \mathrm{P}<0.01)$. PAR-2 expressed cells significantly increased in the proximal and distal colon of the CMS group as apposed to the control group $(\mathrm{P}<0.05)$, but there was no statistical difference within CMS group according to area. Conclusion: Chronic mild stress enhances abdominal muscle response to colorectal distension in rat and an increased number of mast cells with up-regulation of PAR-2 in the colon may contribute to visceral hypersensitivity.

\section{S1770}

The Role of TRPVI in the Etiopathogenesis of Visceral Hypersensitivity in a Rat Model of Irritable Bowel Syndrome

John Winston, Mohan Shenoy, Swaroop Pendyala, Pankaj Pasricha

Background: Visceral hypersensitivity is a hall mark of many functional bowel disorders but the molecular mechanisms involved in its initiation remain unknown. The transien receptor potential vanilloid 1 channel (TRPV1), expressed on the majority of colonic extrinsic afferent neurons, is a key molecule in the activation and sensitization of these neurons Although up-regulation of TRPVI expression has been noted in patients with IBS, little is known about its ability to initiate long-lasting visceral hypersensitivity in functional bowe disorders. We addressed this question in a rat model of IBS based upon neonatal exposure of the colon to a non-injurious solution of acetic acid. As adults (8-12 weeks), these rats display hypersensitivity to graded colorectal distention (CRD) in the absence of structural or histological abnormalities. Aims: Our aim was to determine whether pre-treatment with TRPVI inhibitors prior to neonatal acetic acid treatment prevented development of persisten sensitization to CRD. Methods: Ten days old rat pups received TRPVl antagonists, SB366791 or iodo-resiniferatoxin (I-RTX) or vehicle subcutaneously 30 minutes prior to colon acetic acid infusion, and were tested for sensitivity to CRD at 8 weeks of age. Responses to CRD were measured by observation of the abdominal withdrawal reflex (AWR) and by electromyographic (EMG) measurements of visceromotor responses obtained from electrodes implanted in the external oblique muscle. Results: Pretreatment with $1.3 \mathrm{mg} / \mathrm{kg}$ SB-366791 prior to acetic acid infusion in 10 days old pups significantly reduced hypersensitivity of eight weeks old rats to graded CRD as assessed by EMG $(F=7.3, p=0.014)$ and by AWR $(\mathrm{F}=11.0, \mathrm{p}<0.001$. In a second independent experiment, $2.6 \mathrm{mg} / \mathrm{kg}$ SB-366791 significantly decreased sensitivity to $C R D$ by AWR grade $(F=35.0, p<0.001)$ and by EMG $(F=5.4 \quad p=0.042)$. A similar pretreatment with $2 \mu \mathrm{mol} / \mathrm{kg}$ I-RTX also significantly reduced hypersensitivity to graded CRD by AWR grade $(\mathrm{F}=11.1, \mathrm{p}=0.004)$ and by EMG $(\mathrm{F}=7.18, \mathrm{p}=0.044)$. SB-36679 was not a general blocker of neuronal activity as shown by a lack of effect on primary colonic afferent recordings in response to $C R D$ in normal rats. Conclusion: Pretreatment with two TRPVI antagonists, SB-366791 and I-RTX prior to acetic acid infusion in 10 days old pups significantly attenuated the development of colonic hypersensitivity in adult rats. These data suggest a critical role of TRPVI activation in the development of persistent visceral hypersensitivity in our model. We speculate that changes in colonic $\mathrm{pH}$ in the neonatal period could activate TRPVI and result in IBS in susceptible individuals.

S1771

Expression and Action of Corticotropin Releasing Factor (CRF) Receptor 2 and Its Ligands On the Vagal Afferents

Lixin Wang, Vincent S. Wu, Yuhua Wang, Yvette Tache

Background: CRF and urocortins (Ucn) administrated peripherally inhibit gastric emptying and food intake mainly through CRF receptor $2\left(\mathrm{CRF}_{2}\right)$ (AJP 1999:276:G867 and AJP 2001:281:R1401). Ucn 1 binds to both $\mathrm{CRF}_{1}$ and $\mathrm{CRF}_{2}$, while Ucn 2 and Ucn 3 bind selectively to $\mathrm{CRF}_{2}$. A CRF 2 selective antagonist has recently been reported to bind to the rat nodose ganglia, suggesting the presence of $\mathrm{CRF}_{2}$ in vagal afferents (BJP 2002;136:896) Aim: To identify the expression and action of CRF ligands and receptors in the vagal afferents

. 
Methods: The nodose ganglia from adult male SD rats were processed to detect expression of CRF, urocortins and CRF receptors by reverse transcription and polymerase chain reaction (RT-PCR). The peptide locations in the nodose ganglia and the vagal nerves were determined by fluorescent immunohistochemistry using $\mathrm{CRF}_{2}$ antibody (Santa Cruz Biotechnologies), CRF and urocortin antibodies (CURE). In an isolated gastric-vagus nerve preparation in vitro (AJP 2000;279:E695) from fasted rats, the gastric vagal afferent responses to intra-arterial injection of vehicle (saline, $0.1 \mathrm{ml}$ ), human Ucn 2 (hUcn 2; $0.025 \sim 1.2 \mu \mathrm{g}$ ) or $\mathrm{CRF}_{2}$ antagonist, astressin $-\mathrm{B}(1.5$ or $6 \mu \mathrm{g})$, were recorded from the ventral gastric vagal nerve filaments. Results: $\mathrm{CRF}_{2} \alpha, \mathrm{CRF}_{2} \beta$, Ucn 1 and Ucn 2 mRNAs were detected by RT-PCR in the nodose ganglia, while CRF or $\mathrm{CRF}_{1}$ mRNAs were not expressed and the Ucn 3 band was weak. $\mathrm{CRF}_{2}$-immunoreactivity (IR) was observed in neurons and fibers of the nodose ganglia and vagal nerves, while no CRF- or Ucn l-IR was detected in non-colchicine treated rats. 35 out of 45 units with initial basal activity had an average of $0.90 \pm 0.15$ spikes $/ \mathrm{s}$, 27 out of $35(\sim 77 \%)$ units responded to hUcn 2 intra-arterial injection, and astressin ${ }_{2}-\mathrm{B}$ pretreatment blocked the effect. Conclusions: Vagal afferents express $\mathrm{CRF}_{2}$ and urocortins, and Ucn 2 induces a $\mathrm{CRF}_{2}$-mediated increase in vagal afferent activity. These data reveal a possible site of action for CRF-related peptides involved in regulating food intake and gastric motility. Supported by VA Merit Award (YT) and NIH R01 DK 33061 (YT)

\section{S1772}

Fatty Acids and Latex Microspheres Activate Discrete Cellular Sensing Mechanisms

Anthony D. Jackson, Andrew J. Higgins, Maynard Case, John McLaughlin

Background: The enteroendocrine cell line STC-1 responds to fatty acids (FA) by secreting biologically active CCK, via an elevation of intracellular calcium, [Ca2+]i. This therefore represents an appropriate model of FA sensing. Previous work has suggested that the formation of aggregates by FA in physiological media is an important factor in lipid sensing by STC-1 cells. Synthetic latex microspheres (LM) of similar size to FA aggregates also stimulate STC-1 cells causing CCK secretion, raising the possibility that these are sensed by the same mechanism as FA. The cellular mechanism(s) underlying FA sensing remain largely uncharacterized although one candidate is the newly assigned FA receptor, GPR40, first described in pancreatic beta cells, but which is also expressed by STC-1 cells. Aims: To evaluate (1) if LM activate GPR40, and (2) if other epithelial cell models sensitive to LM are also FA sensitive. Methods: RT-PCR was undertaken for GPR40 in STC-1 and the neuroendocrine cell line, PC12. Wild type (WT) PC12 cells, PC12 cells stably transfected with mGPR40, CaCo-2 cells (intestinal epithelial model) and MDCK cells (renal tubule epithelial model) were challenged with FA (linoleic acid, C18:2) and LM. Real-time changes in $[\mathrm{Ca} 2+]$ i were measured using fura-2 loaded cells and fluorescence microscopy. Results: RT-PCR confirmed expression of GPR40 in STC-1 but not PC12 cells. PC12 FA sensitivity was increased by GPR40 overexpression: linoleic acid stimulated PC12-WT and PC12 mGPR40+ cells, with the magnitude of [Ca2+] i response being increased 2.5-fold in mGPR40+ cells $(\mathrm{P}=0.005)$. PC12-WT cells responded to LM, but LM sensitivity was not increased in mGPR40+ cells. Furthermore, the $[\mathrm{Ca} 2+]$ i response to LM was entirely abolished in the absence of extracellular calcium, whilst the FA-induced $[\mathrm{Ca} 2+]$ i response was preserved. Finally, a $[\mathrm{Ca} 2+]$ r response was induced by LM in MDCK and $\mathrm{CaCo}-2$ cell lines, but not induced by FA. Conclusions: Cell stimulation by LM is not via GPR40, nor is it a property restricted to FA-sensitive epithelial cells. This indicates that LM are not mimicking FA aggregates by activating specific FA sensing mechanism(s). The mechanisms by which LM gate intracellular calcium entry remain to be elucidated. Nonetheless, LM induce CCK secretion in enteroendocrine cells so may provide a novel method by which to modify upper gastrointestinal function or satiety, employing a non-nutrient signal that is retained within the lumen of the GI tract. Supported by BBSRC

\section{S1773}

Gonadal Hormones Modulate Stress-Induced Visceral Hypersensitivity in Rats J.N. Wang, Kirsten Garner, Elie D. Al-Chaer

Experimental and clinical studies underline the existence of sex-related differences in visceral sensitivity. In previous studies, we showed that estrogen has a significant role in modulating viscerosensitivity both in female and male rats. In this study, we examined the effects of gonadal hormones on stress-induced visceral hypersensitivity in intact and gonadectomized rats. Ovariectomy $(\mathrm{OVX})$, orchiectomy $(\mathrm{OCX})$ or sham operations were done at 3 months, and rats were given 20 days to recover. Visceral hypersensitivity was assessed by recording the electromyographic (EMG) responses to graded colorectal distension (CRD) in rats mildly sedated with isoflurane (1\%). CRD was induced by rapidly inflating a latex balloon to 20 , 40,60 or $80 \mathrm{mmHg}$ for 10s, with a $5 \mathrm{~min}$ interval between distentions. Water avoidance stress (WAS) was carried out in a plexiglass box [46 cm (h) $\times 25 \mathrm{~cm}$ (1) $\times 25 \mathrm{~cm}$ (w)]. The rat was placed on an $8 \mathrm{~cm} \times 8 \mathrm{~cm}$ square island located in the middle of the box and

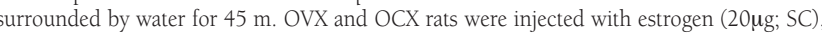
and $48 \mathrm{~h}$ after the injection the rats were subjected to WAS. EMG responses were obtained immediately after WAS. Our results showed: 1) In intact males subjected to WAS, EMG responses to CRD were significantly increased to $98.18 \%(20 \mathrm{mmHg}) ; 99.82 \%(40 \mathrm{mmHg})$; $68.75 \%(60 \mathrm{mmHg}) ; 63.77 \%(80 \mathrm{mmHg})(\mathrm{n}=6, \mathrm{p}<0.05)$ of the responses of control rats (without WAS). In OCX rats subjected to WAS, EMG responses were increased only in response to $80 \mathrm{mmHg}$ CRD to $53.19 \%(\mathrm{n}=8, \mathrm{p}<0.05)$ as compared with the responses of non-WAS OCX rats. 2) In cycling female rats subjected to WAS, EMG responses to graded CRD increased during diestrus and metestrus to $68.15 \%(40 \mathrm{mmHg})$ and $59.7 \%(60 \mathrm{mmHg})$ $(\mathrm{n}=10, \mathrm{p}<0.05)$ of the responses of controls (without WAS); but WAS had no significant effect during proestrus and estrus. OVX rats did not exhibit EMG hyper-responsiveness to CRD after WAS. 3) Estrogen injection increased EMG responses to 20 and $40 \mathrm{mmHg}$ CRD in intact male rats subjected to WAS [99.9\% (20mmHg); 77.11\% (40mmHg); $\mathrm{n}=6, \mathrm{p}<0.05]$. 4) When coupled with estrogen injection, WAS in OVX or OCX rats had no significant effect on EMG responses compared to OVX or OCX rats treated with estrogen but not subjected to stress. Our data suggest that gonadal hormones, particularly estrogen, are involved in stress-induced visceral hypersensitivity. Studies are under way to determine the mechanisms and the role of testosterone and other hormones, if any. (Supported by $\mathrm{NIH} / \mathrm{NS} 40434)$.

\section{S1774}

Estrogen Modulates 5-Hydroxytryptophan Induced Visceral Hypersensitivity in Awake Female Rats

Ching-Liang Lu, Full-Young Chang, Pei-Yi Liu, Shou-Dong Lee

Background: 1) Irritable bowel syndrome (IBS) is more prevalent in women, suggesting a sex hormone influence. 2) We have demonstrated that estrogen modulates mustard oil induced visceral hypersensitivity. (Gastroenterology 2005; S1472). However, marked mucosal inflammation developed in this model, a feature not seen in IBS. 3) Serotonin is important in visceral pain regulation. 5-hydroxytrptophan (5-HTP, the precursor to 5-HT) can cause colorectal hypersensitivity in rats. (Br J Pharmacol 1995; 114:558) 4) We hypothesize that sex hormone can regulate the 5-HTP-induced visceral hypersensitivity. Method:Female rats were prepared for abdominal muscle electromyography, which was recorded in response to phasic colorectal balloon distension at 20,40 and $60 \mathrm{mmHg}$. The visceromotor reflex was measured after subcutaneous injection of 5 -HTP $(10 \mathrm{mg} / \mathrm{kg})$. A hypersensitivity index (HI), the ratio of the EMG intensity between 5-HTP injection and vehicle, was used to represent the magnitude of visceral hypersensitivity. The effect of sex hormone on 5HTP-induced visceral hypersensitivity was evaluated by comparing the HI value between ovariectomized (OVX) or shame-OVX rats. The roles of sex hormones in 5-HTP-related visceral hypersensitivity were evaluated by treating OVX rats with 17- $\beta$-estradiol (E, $10 \mu \mathrm{g} /$ $\mathrm{cc} / \mathrm{kg}$ ), progesterone $(\mathrm{P}, 20 \mathrm{mg} / \mathrm{cc} / \mathrm{kg}$ ), $\mathrm{E}+\mathrm{P}$, or vehicle (sesame oil). The distal colorectum was excised for grading inflammation. Results: 1) In intact rats, 5-HTP treated rats had higher $\mathrm{HI}$ than controls (enhanced visceral hypersensitivity) (HI value: $20 \mathrm{~mm} \mathrm{Hg}: 8.61 \pm 1.04$ vs. $0.88 \pm 0.05 ; 40 \mathrm{mmHg}: 2.80 \pm 0.36$ vs. $0.99 \pm 0.3 ; 60 \mathrm{mmHg}: 2.23 \pm 0.21$ vs. $0.90 \pm 0.10$, $\mathrm{p}<0.05)$; 2) The 5-HTP-induced hypersensitivity was abolished by ovariectomy. (HI value, shame-OVX vs. OVX; $20 \mathrm{mmHg}: 9.41 \pm 1.31$ vs. $0.87 \pm 0.09 ; 40 \mathrm{mmHg}: 3.88 \pm 1.04$ vs. $1.10 \pm 0.14 ; 60 \mathrm{mmHg}: 2.62 \pm 0.47$ vs. $1.04 \pm 0.13, \mathrm{p}<0.05)$. 3) OVX rats pre-treated with $E$ or $\mathrm{E}+\mathrm{P}$, but not $\mathrm{P}$ alone, exhibited significantly higher $\mathrm{HI}$ levels after 5-HTP injection (HI value: vehicle vs. E vs. $\mathrm{P}$ vs. $\mathrm{E}+\mathrm{P}: 20 \mathrm{mmHg}, 0.78 \pm 1.80$ vs. $9.05 \pm 1.84$ vs. $0.69 \pm 0.17$ vs. $10.25 \pm 3.07 ; 40 \mathrm{mmHg}: 0.99 \pm 0.11$ vs. $3.10 \pm 0.60$ vs. $0.70 \pm 0.08$ vs. $2.82 \pm 0.36 ; 60 \mathrm{mmHg}$ : $0.85 \pm 0.05$ vs. $1.92 \pm 0.30$ vs. $0.79 \pm 0.05$ vs. $2.00 \pm 0.18$, p $<0.05)$. 4) No or very mild inflammation was noted in the colonic mucosa after 5-HTP injection. Conclusion: 1) In the absence of mucosal inflammation, systemic injection of 5-HTP can induce visceral hypersensitivity in awake female rats. 2) Estrogen, but not progesterone, is important in mediating the 5HTP-induced visceral hypersensitivity. 3) Our results may partly explain the higher female IBS prevalence and the relatively good response of 5HT drugs in treating IBS

\section{S1775}

The Role of NGF in Sensitization of the Nociceptive Response to Acute Pancreatitis

Liansheng Liu, John Winston, Huaizhi Yin, Bryan Kosler, Shelley Allen, Dave Dawbarn, Pankaj Pasricha

Background: The transient receptor potential vanilloid 1 channel (TRPV1), expressed on the majority of pancreatic afferent neurons, is a molecular integrator of nociception and inflammatory pain. We have previously shown that pancreas-specific DRG neurons exhibit increased TRPVl currents in a model of acute painful pancreatitis induced by L-arginine, and that nerve growth factor (NGF) appears to recruit silent nociceptors, increasing the number of capsaicin responsive pancreas-specific DRG neurons. The aim of this study was to further assess the effect of NGF antagonism on TRPVI activity in pancreatic primary sensory afferents as measured directly in an in vivo model. Methods: Pancreatitis was induced with two i.p injections of L-arginine, one hour apart. Control animals received the same volume of saline. Three days after the injection, rats were treated with either vehicle or the NGF sequestering peptide segment of the trkA receptor (trkA-Ig2; REN 1820) once a day for 5 days $(100 \mathrm{mg} / 0.5 \mathrm{ml} / \mathrm{rat}$, i.p), respectively. After the last treatment, primary sensory afferent activity was measured directly from the left pre-ganglionic great splanchnic nerve (GSN). GSN afferent activity was recorded continuously before and after infusion of $0.05 \mathrm{mg} / 0.3 \mathrm{ml} / 50 \mathrm{sec}$ capsaicin or saline into the pancreatic duct. Five groups of rats were tested: Controls + saline infusion; Controls + CAP infusion; Acute Pancreatitis + CAP infusion; Acute Pancreatitis w/ vehicle + CAP infuse; and Acute pancreatitis w/REN 1820 treatment + CAP infusion. Results: CAP infusion significantly increased GSN activity in control animals compared to saline infusion $(152 \pm 9.6$ vs $102 \pm 4.9, \mathrm{p}<0.01)$. GSN activity in response to CAP infusion was significantly increased in acute pancreatitis $(199 \pm 14, \mathrm{p}<0.05)$. In rats with pancreatitis, REN 1820 treatment significantly attenuated GSN response to CAP infusion compared to vehicle treated rats $(174 \pm 10.8$ vs $215 \pm 8.9, \mathrm{p}<0.01)$. Conclusions: These studies demonstrate that pancreatitis enhanced TRPVl activity on splanchnic nerve endings in the pancreas suggesting that TRPVI sensitization may play a role in the pain associated with acute pancreatitis. This enhanced activity is mediated in part by NGF, providing another potential therapeutic analgesic target.

\section{S1776}

Correlation of Anterior Insula Activity with Sensations of Satiation in Humans Using Continuous FMRI

Mihaela Podovei, Wei Qiu, Annabel N. Yuen, Itzhak Aharon, Mark Vangel, Braden Kuo

Background: The Insula is critical to visceral sensory functions, involved in fundamental GI functions such as feeding. Continuous fMRI imaging of the brain can demonstrate the dynamic changes in regions within the brain in response to a constantly evolving stimulus such as feeding. Aim: To evaluate the correlation of changes in Anterior Insular (AI) activity in response to the changes in gastric sensation from feeding in comparison to changes in brain activity in the motor cortex (MC) which serves as a control because it is less involved in visceral sensation. Methods: 10 healthy subjects (2F/9M, mean age 28 yrs) had a $14 \mathrm{~F}$ 
NG tube placed and, after a $5 \mathrm{~min}$ baseline period, Ensure (1Kcal/cc) was instilled at $60 \mathrm{cc} /$ min till subjects reached maximal satiation. Continuous fMRI activity over the whole brain was recorded using a 3T Siemens scanner $\left(\mathrm{TR} / \mathrm{TE} / \mathrm{q}=2.5 \mathrm{~ms} / 39 \mathrm{~ms} / 90^{\circ}\right.$, slice thickness $=5 \mathrm{~mm}$, inter-slice interval $=1 \mathrm{~mm}, \mathrm{FOV}=200 \mathrm{~mm}, 64 * 64$ matrix, whole brain coverage). Multi-echo PACE sequences were used to compensate for scanner drift. The data was preprocessed for motion correction, Gaussian blur with FWHM $=5 \mathrm{~mm}$ by AFNI. EROICA was used to identify clusters of $\mathrm{AI}$ and $\mathrm{MC}$ activity. In each subject, the average CNS activity time course for the Right $\mathrm{AI}$ and Left MC was smoothed using a cubic spline algorithm with $\mathrm{df}=6$ and the curve was compared to the satiation symptom curve. Activation of the left MC was expected due to periodic right hand movement occurring to document symptom ratings. For each subject, the time courses of $\mathrm{AI}$ and $\mathrm{MC}$ were correlated with the satiation curve and the correlation coefficients (CC) were calculated. A mean CC was generated for each $\mathrm{AI}$ and MC. Student T-test was used to compare the 2 correlation groups: right AI and the satiation curve, left MC and the satiation curve. Results: The mean time to maximal satiation was $30 \mathrm{~min}$ (range 12-58 min). The mean correlation between right AI and the satiation curve was 0.70 ( $95 \% \mathrm{CI} 0.54$ to 0.86$)$ and these correlations were significant $\mathrm{p}<0.01$. The mean correlation between the left MC and the satiation curve was- 0.13 (95\% CI -0.65 to 0.38 ) and these correlations were not significant, $\mathrm{p}=0.58$. The correlations of AI activity to satiation were different from the correlations of $\mathrm{MC}$ activity to satiation, $\mathrm{p}<0.003$. Conclusion: Brain activity within the Anterior Insula is strongly correlated with the satiation symptom curve, while there is no correlation between the left motor cortex and the satiation curve, which was used as control. Continuous fMRI imaging can demonstrate dynamic but disparate CNS activity in different regions of the brain during a visceral stimulus such as feeding.

\section{S1777}

Distribution and Immunohistochemistry of the Mouse Spinal and Vagal Pancreatic Afferents

Kenneth Fasanella, Julie A. Christianson, Ratsamy S. Chanthaphavong, David C. Whitcomb, Brian M. Davis

Background: Dysfunction of primary afferents plays a central role in pain suffered by patients with chronic pancreatitis. To begin developing a mouse model we defined the normal innervation of the mouse pancreas for both spinal and vagal afferents. These afferents are heterogeneous and can be grouped based on the presence or absence of neuropeptides (e.g. CGRP, SP). Peptidergic afferents (CGRP+) typically bind nerve growth factor (NGF), a trophic factor that has been linked to inflammatory hyperalgesia. Non-peptidergic afferents bind the isolectin B4 (IB4). A third population expresses the GFR 33 receptor and binds the growth factor artemin that has recently been implicated in inflammatory pain. Methods: 8week-old male $\mathrm{C} 57 \mathrm{Bl} / 6$ mice were anesthetized and the pancreatic head was injected with CTB-555. In some mice the pancreatic tail was also injected with CTB-488. After sacrifice and perfusion with $4 \%$ paraformaldehyde, dorsal-root ganglia (DRG) and nodose ganglia (NG) were dissected and processed for stereological cell counts. Tissue was also labeled with antisera detecting CGRP, TRPVI, and GFR 33 and stained with IB4. Results: Spinal pancreatic afferents were distributed between $\mathrm{T} 5$ and $\mathrm{T} 13$, with the greatest concentration in T10-T12. The DRG projections were symmetric with equal numbers coming from right and left ganglia. In contrast, the innervation from the left NG was significantly greater than the right $(\mathrm{p}<.05)$. For both spinal and vagal afferents there was significantly greater innervation of the pancreatic head relative to the tail $(\mathrm{p}<.05)$. The total number of retrogradely-labeled afferents in the nodose was very similar to total number of DRG afferents. The neurochemical phenotype of spinal afferents was dominated by CGRP- and TRPVl-positive neurons. In the DRG, 79\% of TRPVl-positive and 49\% of CGRP-positive neurons were GFR $\alpha 3$-positive. In the NG, only $2 \%$ of labeled afferents were positive for GFR $\alpha 3$. Conclusions: These studies show that equal number of vagal and spinal afferents innervate mouse pancreas and that the innervation is concentrated at the head. As seen in previous rat studies, the vagal innervation is asymmetric. The majority of pancreatic afferents were peptidergic, and likely respond to changes in the level of NGF. The identification of GFR $\alpha 3$-positive spinal pancreatic afferents indicates that many of these cells respond to artemin. Artemin is a relatively new member of the GDNF family and recent data from our labs show that it can potentiate the TRPVI responses in cultured sensory neurons and that it increases following inflammation. Thus, both NGF and artemin may play important roles in chronic pancreatitis pain.

\section{S1778}

Target-Derived Nerve Growth Factor (NGF) Regulating Spinal Sensory Axon Outgrowth to the Embryonic Mouse Gut

Wen Jiang, Andrew J. Furley, Karna Bardhan, David Grundy

Introduction: The factors that determine how extrinsic sensory nerves find their targets within the gut wall during development are poorly understood. We aimed to examine how target-derived factors influence sensory innervation to the embryonic gut. Methods: Dorsal root ganglia (DRG) from both thoracic (T8-13) and lumbar (L) regions were co-cultured with gut in collagen gel culture (Tessier-Lavigne et al., Nature 366, 1988). In experiment set 1 , individual T- or L-DRG were cultured in medium containing NGF $(20 \mathrm{ng} / \mathrm{ml})$ either alone, or with a segment of fresh or fixed (and washed) esophagus or colon from mice at embryonic (E) days 13, 14 and 16. In experiment set 2, T-DRG and esophagus were cocultured in the absence of NGF or with anti-NGF antibodies $(400 \mathrm{ng} / \mathrm{ml})$. After 2 days in culture, DRGs were stained for neurofilament and assessed blind for directed axon outgrowth, comparing length, angle and density of neurites outgrowth in the quadrant nearest the gut with that in the distal quadrant. Data are expressed as the number of DRG showing directed growth (attractive, repulsive and non-response). Results: Axon outgrowth was symmetrical when DRG was cultured either alone or with fixed gut. However, directed outgrowth was detected when co-cultured with unfixed gut (Table). In the absence of NGF, axon outgrowth was not detected at E13 (n=3), but was apparent at El4 $(n=17)$ and El6 $(n=9)$. Axon outgrowth was absent from either E14 ( $n=7)$ or 16-DRG $(n=3)$ when the medium contained anti-NGF antibody. Conclusion: The embryonic gut secretes NGF in a temporally regulated fashion which may guide sensory innervation. Supported by the Bardhan Research and Educational Trust

The number (\%) of DRG showing directed axon outgrowth when co-cultured with gut

\begin{tabular}{|c|c|c|c|c|c|}
\hline \multirow{2}{*}{ DRG region } & \multirow{2}{*}{ Response (\%) } & \multicolumn{2}{|c|}{ E14 } & \multicolumn{2}{|c|}{ E16 } \\
\cline { 3 - 6 } & & Esophagus & Colon & Esophagus & Colon \\
\hline \multirow{2}{*}{ T-DRG } & Attraction & $27(96)^{*}$ & $4(16.7)$ & $18(100)^{*}$ & $8(66.7)$ \\
\cline { 2 - 6 } & Repulsion & $1(4)$ & $8(33.3)$ & 0 & $4(33.3)$ \\
\hline \multirow{2}{*}{ L-DRG } & Attraction & $21(91.3)^{*}$ & $3(15.8)$ & $19(95)^{*}$ & $1(5.9)$ \\
\cline { 2 - 6 } & Repulsion & 0 & $10(52.6)$ & 0 & $14(82.4) \psi$ \\
\hline
\end{tabular}

*: Different region of gut had significant effect on directed axon growth from either T- or L-DRG at E14 and 16 (P<0.005, Chi-square Test). $\psi$ : At E16 when co-cultured with colon, the directed neurite response from T- and L-DRG is significant difference $(P<0.005$, Fisher Exact Test)

\section{S1779}

Effect of a 1 Week Trial of Capsaicin P.O. On Intestinal Mechano- and Chemonociception

Johann Hammer

INTRODUCTION: Perception evoked by jejunal application of capsaicin is similar to distension induced perception but does not involve mechanoreceptors. Oral ingestion of capsules containing red pepper powder induced painful sensations during the first week in patients with functional dyspepsia; patients who did not discontinue capsule ingestion experienced improvement of their symptoms after a 5 week trial. AIM: To evaluate the effect of one week p.o. application of capsaicin on jejunal mechano- and chemonociception in healthy subjects. METHODS: 13 healthy subjects (age: $24 \pm 2$ yrs., $5 \mathrm{M}, 8 \mathrm{~F}$ ) ingested capsules containing $0.5 \mathrm{mg}$ capsaicin tid $(n=8)$ or placebo $(n=5)$ for 7 days. To test jejuna mechano- and chemonociception before (day 1) and after (day 8) capsule ingestion, subjects swallowed a tube with an infusion site at the ligament of Treitz and a latex balloon $7 \mathrm{~cm}$ distally to the infusion site. Balloon distensions with preset volumes of air $(24 \mathrm{ml}$ and $40 \mathrm{ml})$ were performed, followed by infusion of a capsaicin solution $(40 \mu \mathrm{g} / \mathrm{ml}$ ) at a rate of $25 \mathrm{ml} /$ min for 60 minutes or until severe sensations arose. A graded questionnaire evaluated quality and severity of 6 different sensations and was filled out during distensions and every 10 min during capsaicin infusion and when capsaicin infusion was stopped. Infusion was stopped when a score of 9 (out of 13) was reached at either one of the sensations. Aggregate perception scores were calculated by summing the scores for each type of sensation RESULTS: Infusion of capsaicin induced feeling of pressure, cramps or pain; the descriptive quality of sensations and the location on the abdomen were similar to sensations induced by distension. In subjects receiving capsaicin capsules, aggregate perception scores at $24 \mathrm{~m}$ distension volumes were 4.2 (day 1, before capsaicin ingestion) and 3.2 (day 8 , after capsaicin ingestion), respectively (NS). At $40 \mathrm{ml}$ distension volume, aggregate perception scores were 10.7 (day 1) vs. 8.4 (day 8$)(\mathrm{p}<0.05)$. The duration of capsaicin infusion until first sensation was reported was $12.0 \mathrm{~min}$ (day 1) and $8.4 \mathrm{~min}$ (day 8) (NS), discomfort thresholds occurred after $41.5 \mathrm{~min}($ day $\mathrm{l})$ and $33.0 \mathrm{~min}($ day 8$)(\mathrm{p}<0.05)$. In the control group, mechano- and chemonociception was not significantly different on study days 1 and 8. SUMMARY: 1 week ingestion of capsaicin sensitized chemonoceceptors while sensitivity of mechanonociceptors for distension decreased. CONCLUSION: Painful sensations during the first week of treatment with capsaicin can be attributed to a sensitising effect of capsaicin on vanilloid receptors. Repeated application of capsaicin over a one week period desensitizes mechanoreceptors

\section{S1780}

NMDA Receptors Differentially Regulate the Excitability of Distinct Classes of Splanchnic Afferent Neurons Innervating Rat Distal Colon David Adelson, James A. McRoberts, Yuhua Wang, Emeran A. Mayer

BACKGROUND: NMDA receptors (NMDARs) are expressed on the peripheral processes of spinal afferent neurons innervating visceral organs. We hypothesized that peripheral NMDAR activation may play a role in normal pain transmission and/or in the development of peripheral sensitization by altering afferent terminal sensitivity to mechanical stimuli. AIMS: To determine if a) NMDAR activation directly excites inferior splanchnic nerve afferent (ISA) fibers, and b) if such activation alters ISA responses to subsequent colorectal distention (CRD) METHODS: ISA activity was recorded from the distal cut end of inferior splanchnic nerve strands innervating a $5 \mathrm{~cm}$ segment of SD rat distal colon maintained in an in vitro organ bath as previously described (Gut 2005; PMID: 15985561). Basal CRD responsiveness was determined using consecutive graded CRD $(0.1-0.5 \mathrm{ml}, 20 \mathrm{sec}$ duration, 10 min interval between $(R D)$ via inflation of an intraluminal balloon. Next, graded doses of the potent, selective NMDAR agonist, trans-ACBD (tACBD, 0-32 $\mu \mathrm{g}$ ) were given inter-arterially (i.a.), followed 2 min later by a single CRD $(0.4 \mathrm{ml})$. Lastly, NMDA $(40 \mu \mathrm{g})$ was given i.a., after which the receptive field was located precisely. Single unit activity was obtained by sorting waveforms present in multi-unit recordings using Spike 2 software. RESULTS: 43 single units from 10 preparations were recorded. Of 25 units whose activity was influenced by NMDAR agonists, 5 response patterns were identified: 1) 10/25 units were chemoreceptive, responding only to trans-ACBD or NMDA, but not to CRD. 2) 7/25 units gave slowlyadapting mechanoreceptive responses to graded CRD. 5/7 of these did not respond to tACBD ia but increasing doses of the agonist caused a dose-related decrease in responses to subsequent CRD. 3) $3 / 25$ units initially unresponsive to CRD became responsive to CRD after i.a. tACBD. In $2 / 3$ of these, low doses of tACBD increased CRD responsiveness, but higher doses progressively inhibited responses to $\mathrm{CRD}$. The 3rd unit gave increasing responses to CRD with increasing doses of tACBD. 4) 2/25 units had spontaneous oscillatory bursting behavior where increasing doses of tACBD altered the pattern of ongoing activity. 5) 3/25 units were chemo- and mechano-responsive, responding to tACBD and/or NMDA directly, and to CRD with increased activity during and after CRD. CONCLUSIONS: NMDAR activation has differential effects on distinct classes of colonic ISA fibers, and may play an important 
role in shaping the pattern of sensory input from the viscera to the CNS under a variety of normal and pathological conditions. Supported by NIH grants DK 58173 and 1 P50 DK64539

\section{S1781}

Genetic Differences Determine Inflammatory Response and Development of Visceral Hyperalgesia in a Rat Model

Birgit Adam, Chris Tsopelas, Tobias Liebregts, F Dylan Bartholomeusz, Andrew Ruszkiewicz, Gerald Holtmann

Background: Transient inflammation is a risk factor for the manifestation of Irritable Bowel Syndrome (IBS) and causes long- lasting changes of visceral sensitivity. Although gastrointestinal infections are common, only a proportion of patients develop post-infectious IBS. We therefore hypothesized that genetically determined differences influence inflammatory response and visceral sensory function. Aim: To characterize the inflammatory response and acute and long-term effects of a transient colitis on visceral sensory function in two different rat strains. Methods: Colitis was induced by single colorectal instillation of Trinitrobenzenesulfonic acid (TNBS; $5 \mathrm{mg} / \mathrm{kg}$ ) plus ethanol $(70 \%)$ or saline (control) in male Fisher 344 (F) and Lewis (L) rats. At $2 \mathrm{hr}$ and 5, 14 and 28 days after induction of colitis colonic tissue samples were analyzed by a blinded pathologist and blood samples assayed for IL-2 plasma levels via ELISA. A subset of rats was injected intravenously with 99mTc-labelled leukocytes (LWC) for qualitative (images) and quantitative (bio-distribution) scintigraphic assessment of colitis at the various study days. To assess visceral sensitivity colorectal distensions (CRD) were performed before and 5, 14 and 28 days after induction of colitis and the visceromotor response (VMR) to CRD quantified by electromyographic recordings. Results: TNBS caused an acute transient inflammation in both strains with a peak at day 5 and histological healing at day 14. Compared to $\mathrm{L}$ rats this inflammatory response was significantly $(\mathrm{p}<0.05)$ lower in F rats based on histological data and LWC assay. IL-2 was increased in both strains prior to occurrence of mucosal lesions and remained elevated at day 14 in $\mathrm{L}$ but not in $\mathrm{F}$ rats. VMR to CRD was significantly enhanced $(\mathrm{p}<0.01)$ on day 5 in TNBS treated rats $(\mathrm{L}+\mathrm{F})$ compared to saline controls and returned to baseline level at day 14. At day 28 VMR was significantly increased in L rats but not in F rats compared to controls. Summary: TNBS caused a transient inflammation with mucosal lesions, leukocyte infiltration and increased IL-2 secretion, which was significantly less severe in F rats compared to $\mathrm{L}$ rats. The VMR to $C R D$ was enhanced during acute inflammation but only in $L$ rats developed a postinflammatory visceral hyperalgesia. Conclusion: Compared to F rats, L rats develop a long lasting visceral hyperalgesia and more severe inflammation following topical administration of TNBS. Thus the presumably genetically determined difference in the susceptibility for a post-inflammatory visceral hyperalgesia appears to be associated with the intensity of the mucosal inflammation and the systemic immune response.

\section{S1782}

Developing a High Through-Put, Minimally Invasive Test of Gastrointestinal Sensitivity

Sepeedeh Saleh, Barbara J. Unsworth, Qasim Aziz, Anthony Hobson

Background: Studies of gastrointestinal (GI) sensitivity in both health and functional gastrointestinal disorders (FGD) reveal a broad spectrum of sensory responsiveness suggesting the presence of distinct GI pain phenotypes within the population. Conventional approaches to GI sensory testing require invasive intubation techniques which increase anxiety and reduce tolerability, often adversely affecting results. The aim of this research was to conduct a feasibility study to develop a minimally-invasive, high through-put acute GI pain model which would allow us to study the large numbers of subjects needed to adequately define GI pain phenotypes. Methods: 3 different types of dried chilli flakes (Caribe, Arbol and Habanero) were purchased from an internet site. These were rated by the source as 3,8 and 10 out of 10 respectively on a rating scale for the strength of chilli based on the Scoville unit scale for capsaicin. Gelatin capsules containing $250 \mathrm{mg}$ of chilli flakes or placebo capsules were given orally in a double blind, randomized manner, to 20 subjects. The capsules comprised: $1 \times 3 / 10$ capsule, $1 \times 8 / 10$ capsule, $2 \times 8 / 10$ capsules, $1 \times 10 / 10$ capsule and placebo. Self-administered questionnaires were used to obtain a psychophysiological profile over a two-hour period. Outcome measures included pain, anxiety, nausea and unpleasantness. Results: Compared to placebo, 2 x 8/10-strength capsules and 1 x 10/10-strength capsule produced significant increases in levels of pain (VAS AUC $=301 \pm 325$ and $985 \pm 777$ versus $29 \pm 55, \mathrm{p}=0.001$ and 0.0005 respectively) and unpleasantness (VAS AUC $=223 \pm 872$ and $871 \pm 207$ versus $14 \pm 39, p=0.003$ and 0.002 respectively), as measured using area under the curve (AUC) of visual analogue scores. In addition, one 10/10-strength capsule produced a significant increase in nausea levels $(370 \pm 802$ versus $5 \pm 13, \mathrm{p}=0.03)$ when compared to placebo. There were no significant changes in anxiety levels throughout the experiments. Conclusions: This is an effective, simple and minimally-invasive technique for human Gl sensory testing which was well tolerated by all subjects. 100 studies were performed over a 6 -week period proving that this approach can be used as a high through-put screening technique. The methodology of the study can now be refined so that future studies could target the release of specific doses of capsaicin to any region of the GI tract using specialized drug delivery systems.

\section{S1783}

Real-Time Release of Serotonin from Mouse Ileum Paul P. Bertrand

Introduction: Serotonin (5-HT) containing enterochromaffin (EC) cells detect chemical and mechanical stimuli in the intestinal lumen and respond with release of 5-HT. Recent electrochemical studies have shown that the real-time release of 5-HT from the mucosa is a dynamic process linked with other enteric reflexes ${ }^{1,2}$. The aim of this study was to characterize 5HT release from mouse ileum and determine whether electrochemical techniques can be applied to tissues other than guinea pig (GP) ileum. Methods: Mice (C57BL/6; n=7) or guinea pigs (Hartley; $n=9$ ) were anesthetized with isoflurane, then killed by severing the carotid arteries and spinal cord. Full thickness ileum was loosely pinned, mucosa up, in a bath of warmed physiological saline. Electrochemical recordings were made using $7 \mu \mathrm{m}$ carbon fiber electrodes. Data are presented as concentration of 5-HT (calculated from the current at $+350 \mathrm{mV}$ and the current produced by $5 \mu \mathrm{M} 5-\mathrm{HT}$ ); time to peak (seconds); the potential (mV) of oxidation maxima during voltage ramps $(\sim 0.47 \mathrm{mV} / \mathrm{s}$; from -0.5 to $+1 \mathrm{~V})$; all data are mean \pm SEM. Results: In mouse ileum, basal release of 5 -HT was $5.5 \pm 2.5 \mu \mathrm{M}$ $(\mathrm{n}=6)$. Compression of the mucosa $(0.8 \pm 0.3 \mathrm{~g} ; \mathrm{n}=7)$ with the electrode stimulated further 5 -HT release for a total of $9.8+3.8 \mu \mathrm{M}$ (peak: $3.8 \pm 1.1 \mathrm{~s} \cdot \mathrm{n}=6$ ) as compared to GP ileum (basal: $3.7 \pm 1.4 \mu \mathrm{M}$; compression: $9.1 \pm 3.1 \mu \mathrm{M} ; \mathrm{n}=9$ ). Similar release was also observed in GP distal colon $(n=2)$ though these data were not quantified. In mouse ileum, with the electrode in the mucosa, oxidation maxima occurred at $+335 \pm 10 \mathrm{mV}$ as compared to exogenous 5 -HT $(+330+10 \mathrm{mV} ; \mathrm{n}=6)$ and GP ileum $(+360+40 \mathrm{mV} ; \mathrm{n}=7)$. A second, unidentified peak occurred at $739 \pm 21 \mathrm{mV}$ in 5 of 7 preparations (versus GP ileum: 720 $\pm 20 \mathrm{mV} ; \mathrm{n}=7)$. Brief application of acetylcholine $(0.1$ to $1 \mathrm{mM})$ elicited robust release of 5-HT $(4.4 \pm 1.8 \mu \mathrm{M}$, peak: $2.0 \pm 1.6 \mathrm{~s} ; \mathrm{n}=4)$ that was concurrent with contraction of the circular muscle as has been reported for GP ileum. Conclusion: The release of 5-HT from EC cells of the mouse ileum is similar to that of the GP ileum. This suggests that electrochemical techniques can extract out data about basic release mechanisms that are applicable across tissues. This is the first step in the process of readying these techniques for use on human tissue. Supported by NH\&MRC (Australia) and Dept of Physiology \& Cell Biology, University of Nevada. 1. Bertrand, P. P., 2004. Real-time detection of serotonin release from enterochromaffin cells of the guinea pig ileum. Neurogastro Mot. 16, 511-514. 2. Bertrand, P. P., 2004 Coordination between motility and serotonin release in guinea pig ileum. Gastro. 126(4): A32 Suppl. 2.

\section{S1784}

Effect of Pathogenic Lipopolysaccharide Translocation On Human Colonic Smooth Muscle Cell Contraction and Toll-Like 4 Receptor Expression Michele Pier Luca Guarino, Carola Severi, Simone Carotti, Gianpiero Ferraguti, Webiao Cao, Alessia Cicenia, Anna Maria Altomare, Rossana Alloni, Sara Emerenziani, Renato Caviglia, Michele Cicala

Gastrointestinal stasis induced by lipopolysaccharide (LPS) may be associated with muscle dysfunction. In animal models, LPS suppresses contractility by production of inflammatory mediators by macrophages, but a direct effect of LPS on muscularis cannot be excluded. A recent study demonstrated that LPS translocates across colonic epithelial cells in normal and LPS-infected rats. LPS is recognized by Toll-like 4 receptors (TLR4) that have been shown to be expressed by intestinal myofibroblasts. AIM: To define if colonic mucosa exposure to pathogenic LPS may affect muscle cell contractility and if this effect could be related to LPS translocation and mediated by TLR4. METHODS: From fresh specimens of human colon, the mucosa and submucosa were removed and sealed between two chambers, with the luminal side of mucosa facing upward and covered with $5 \mathrm{~mL}$ of Krebs solution, and the submucosal side facing downward into $20 \mathrm{~mL}$ of Krebs. Chambers were oxygenated and kept at $37^{\circ} \mathrm{C}$. LPS (Sigma) obtained from the pathogenic strain of Escherichia coli 0111:B4 or normal buffer were added to the luminal side of the mucosa and kept for 30 minutes. Enzymatically isolated smooth muscle cells (SMCs) were exposed for 30 minutes to buffer from the submucosal chamber of mucosa either pre-exposed to normal buffer (nbuffer) or to LPS (LPS-buffer). Acetylcholine (ACh) dose-response (10-12 to 10-5M) was obtained for SMCs. To test whether LPS translocates across the mucosa, 3-deoxy-d-manno2-octulosonic acid (KDO), a constituent of bacterial LPS, was detected by means of spectrometry. TLR4 expression was tested by immunohistochemistry using a specific biotinated TLR4 antibody (Mouse IgG HTA125, BD Biosciences). RESULTS: SMCs exposed to n-buffer presented a dose-response to ACh (Maximal contraction: $32 \pm 8 \%$ with $1 \mu \mathrm{M} \mathrm{ACh;} \mathrm{ED50} \mathrm{at}$ $0.3 \mathrm{nM}$ ) similar to untreated SMCs. Exposure to LPS-buffer inhibited ACh-induced contraction, with $62.9 \pm 12 \%$ inhibition of maximal contraction, and ED50 at $6 \mathrm{nM}$. Detection of KDO in the LPS-buffer showed that, after $30 \mathrm{~min}$ of mucosa exposure to LPS, $30.1 \pm 6 \%$ of KDO crosses the mucosa. When SMCs were directly exposed to LPS at a dose consistent with the KDO concentration detected in the LPS-buffer, a significant impairment of contraction $(72+4 \%)$ was also observed and this was associated with a progressive increase of cell surface TLR4 expression whose presence was rather low on unstimulated SMCs. CONCLUSIONS: After acute exposure of colonic mucosa to pathogenic LPS, a relevant inhibition of SMC contractile response is observed. It is conceivable that the translocated LPS could play a role in the impaired muscle contractility of SMCs, mediated by TLR4 binding.

\section{S1785}

Chronic Intestinal Pseudo-Obstruction: Evidence for Autoimmune Activation of Fas and Autophagy

Roberto De Giorgio, Rosanna Cogliandro, Giovanni Barbara, Vincenzo Stanghellini,

Umberto Volta, Chunfang Guo, Roberto Towns, John W. Wiley

Background: Chronic Intestinal Pseudo-obstruction (CIP) is a debilitating GI motility disorder often of unknown etiology. We examined the hypothesis that sera from a well characterized population of CIP patients contain autoantibodies that bind to neurons and activate the death receptor complex Fas/FADD and the putative cytoprotective autophagy pathways. Methods: Patients were screened based on their clinical presentation, serologic tests and diagnostic studies including radiology and gastroduodenal manometry. Sera from 26 (20 F, $6 \mathrm{M}$; age range 22-62 yrs) CIP patients were screened for the presence of anti-neuronal antibodies using immunofluorescence (intensity scored as:,+++ and +++ ) and Western blot analysis (which was negative for classic onco-neural proteins, including $\mathrm{Hu}$ and Yo proteins). Seven out of $26(27 \%)$ tested positive for anti-neuronal antibodies. Subsequently, cultured human neuroblastoma (SH-SY5Y) cells were exposed to sera from patients without $(-), \mathrm{n}=$ 3 or with $(+), n=2 ;(++), n=3$; or $(+++), n=2$ anti-neuronal antibodies (1:10 dilution for 16$20 \mathrm{~h}$ ). Binding of autoantibodies to the surface of SY5Y cells was evaluated using anti-human IgG and IgM antibodies linked to a fluorescence marker. Activation of Fas was examined using anti-FADD (Fas-activated Death Domain) immunoreactivity (IR) and Western blot analysis. Stimulation of autophagy was monitored using the specific marker for autophagosomes, anti-LC3-II IR, colocalization with mitochondria using anti-mitochondrial antibody 
(AMA) and Western blot analysis. Immunoglobulins (Ig) were removed by exposing sera to protein $\mathrm{L}$ agarose beads. Results: The three patients that tested negative for anti-neuronal antibodies demonstrated no evidence for binding of autoantibodies (either IgG or IgM) to the surface of SY5Y neurons, activation of Fas/FADD or autophagy. The patients with + , ++ , or +++ anti-neuronal antibodies demonstrated increased binding of both $\operatorname{IgG}$ and IgM autoantibodies that correlated with the intensity of anti-neuronal IR. Activation of FADD and autophagy also correlated with the intensity anti-neuronal IR. The increased levels of anti-FADD and anti-LC3 IR diminished with serial dilution (1:10-1:250) of ++ and +++ sera. Exposure of sera to protein L beads markedly decreased binding of Ig, activation of FADD and autophagy. Conclusion: Sera from a subpopulation of patients with CIP contain autoantibodies that bind to neurons and activate Fas and the cytoprotective autophagy pathways. These observations support the novel concept that humoral autoimmunity targeting neurons contributes to the pathophysiology of CIP

\section{S1786}

Intracolonic Oil of Mustard Induces An Inflammatory Ileus with Concurrent Alterations in Small Intestinal Secretomotor Function

Craig R. Schneider, Nathaniel H. Wallace, Jeffrey M. Palmer, Kristen M. Chevalier,

Beverley A. Moore, Paul R. Wade, Pamela J. Hornby, Edward S. Kimball

Background: Ileus is frequently associated with abdominal surgery and inflammatory situations which do not necessarily involve a direct assault to the small bowel. However, the mechanisms and pathways leading to ileus have yet to be fully elucidated. We have developed a model wherein intracolonic application of $0.5 \%$ oil of mustard $(\mathrm{OM})$ in $30 \%$ ethanol produces a severe, acute colitis in mice over a 3-day period. This colitis is associated with hypersecretion of mucus and fluid accumulation in the proximal colon, and delayed transit in the small intestine, both indicative of altered intestinal secretory and motor functions. Purpose: To characterize the motor and secretory responses in the small intestine during active OM-induced colitis. Methods and Results: At 48 hours post-OM administration, a time when colon inflammation is maximal but small intestinal damage is absent, 30 minute carmine dye transit was decreased in the small intestine $(69.5 \pm 5.5 \%$ transit for OM vs $85.0 \pm 4.4 \%$ transit for untreated controls, $\mathrm{p}<0.05)$. Measuring small bowel transit changes with oral FITC-dextran demonstrated that the geometric center of distribution of the FITCdextran marker was shifted $22 \%(\mathrm{p}<0.0004)$ toward a more proximal location in OMtreated mice. An irregular pattern of fluorescence distribution indicated dysregulated propulsive motility in the small bowel. Altered neurosecretory function in the small intestine during OM-induced colitis was evident at 24 and 48 hours. Full-thickness jejunal tissues mounted in Ussing-type flux chambers showed significantly increased responses (4.8-fold and 3.5fold above age-matched controls for 24 and 48 hour samples; $\mathrm{p}<0.0005)$ to electrical field stimulation (EFS: $80 \mathrm{~V}, 0.5 \mathrm{~ms}, 10 \mathrm{~Hz}, 5 \mathrm{~s}$ ). Conclusions: The evidence obtained is consistent with the activation and recruitment of trans-enteric signaling pathways from the inflamed colon to the small intestine resulting in significant functional alterations in both propulsive motility and secretory activity. This model will be useful for understanding how inflammatorymediated changes in the colon can result in small intestinal dysfunction, and how this relates to similar symptoms which appear in ileus.

\section{S1787}

Impact of Humoral Autoimmunity in Severe Gut Dysmotility: Actions of Anti$\mathrm{Hu}$ On Enteric and Visceral Sensory Neurons

Luigia Talamonti, Qin Li, Michael Beyak, Marcello Trevisani, Klaus Michel, Barbara Campi, Giovanni Barbara, Pierangelo Geppetti, David Grundy, Michael Schemann, Roberto De Giorgio

The role of autoimmunity in gut dysmotility is poorly understood. Our aim was to identify mechanisms through which anti-Hu anti-neuronal antibody ( $\mathrm{A}-\mathrm{Hu} \mathrm{IgG}$ ) modulates gut function. A-Hu IgG was isolated by affinity chromatography from sera of patients. Specificity of A-Hu IgG was verified by selective staining of enteric neurons. A-Hu-free serum served as control. Four groups of experiments were performed to examine effects on enteric and visceral sensory neuronal function. First, calcium imaging of cultured guinea-pig myenteric neurons revealed that $\mathrm{A}-\mathrm{Hu}$ (1:50), but not $\mathrm{A}$-Hu-free serum, increased the intracellular calcium concentration in $90 \%$ of the tested neurons (total number of 55), a response dependent on extracellular calcium. Tetrodotoxin $(0.3 \mu \mathrm{M})$, nifedipine $(1 \mu \mathrm{M})$, w-conotoxin $(0.1 \mu \mathrm{M})$ and the receptor-operated calcium channel blocker SKF $96365(30 \mu \mathrm{M})$ significantly inhibited the A-Hu induced calcium mobilization by $91 \%, 73 \%, 76 \%$, and $88 \%$, respectively. Second, fast neuroimaging of freshly dissected LMMP preparations revealed that microejection of A-Hu (400ms, 1:20, 22 ganglia, 4 guinea-pigs) but not A-Hu-free serum activated $17.3 \pm 6.2 \%$ of myenteric neurons causing an immediate spike discharge or increase in spontaneous firing lasting several minutes. Third, local perfusion of A-Hu but not A-Hufree serum significantly increased firing in 15/16 vagal mechanosensitive fibers innervating the mouse gastric fundus. The effect peaked at $76 \pm 11 \mathrm{~s}$ and had a rapidly desensitizing component ( $\mathrm{t} 1 / 2$ 10.2s) as well as a sustained response. DMPP and ATP sensitive and insensitive hotspots responded. The response was similar in calcium-depleted buffer (22.9 \pm 5.2 spikes/s vs. control 19.2 \pm 3.7$)$. Fourth, in patch clamp studies on back-filled nodose neurons A-Hu evoked an inward current in 6/18 cells which reversed around $+10 \mathrm{mV}$, consistent with a non-selective cation conductance. Conclusion: A-Hu IgG isolated from patients with severe gut motility dysfunction causes activation of both enteric neurons and sensory vagal afferents. These data suggest that humoral autoimmunity may generate abnormal immune-neural signaling in enteric and vagal mechanosensory neurons which contribute to the neuropathophysiology of severe gastrointestinal motility disorders

\section{S1788}

Neuro-Immune Interactions and Anorectal Motility in Children with FoodAllergy Related Constipation

Osvaldo Borrelli, Giovanni Di Nardo, Giovanni Barbara, Roberto De Giorgio, Cesare Cremon, Sandra Lucarelli, Tullio Frediani, Vincenzo Stanghellini, Salvatore Cucchiara

Background. Food allergy in childhood has been suggested as a trigger of chronic constipation (CC), but the underlying mechanism has not previously been investigated. Intestinal mas cells (MC) are thought to be involved both in food hypersensitivity and in the pathophysiology of abnormal sensory-motor function of the gut. Aim. To assess in children with CC, recta mucosal MC, neuroimmune interactions and anorectal motility before and after an elimination diet. Patients and Methods. 25 children (median age: 4.4 years; range: 0.5 - 11 years), fulfilling the Rome II criteria for functional constipation underwent anorectal manometry (ARM) and suction rectal biopsy before and after a 8 week elimination diet. Manometric parameter were: anal basal pressure (ABP, $\mathrm{mmHg}$ ), percentage of relaxation $(\% \mathrm{R})$, residual pressure $(\mathrm{RP}, \mathrm{mmHg})$ of internal sphincter. $\mathrm{MC}$ and nerve fibers were immunohistochemically identified with tryptase and neuronal specific enolase. Quantification of MC and MC-NF (MC within $10 \mu \mathrm{m}$ of nerve fibers) was performed using a computer-assisted analysis system. Results. (mean \pm SD). 15 of 25 children were responsive (Resp) clinically to the elimination diet, 10 did not (non-Resp). Baseline ARM showed a significant difference in ABP, \%R, RP between Resp $(64.3 \pm 13.9,80.8 \pm 11.6,13.3 \pm 9.6)$ and non-Resp $(51.3 \pm 12.0,93.4 \pm 5.5$ $3.2 \pm 2.8, \mathrm{p}<0.001)$. After diet a significant decrease in $\mathrm{ABP}$ and RP, and a significant increase in \% R was observed only in Resp ( $49.6 \pm 14.8,92.1 \pm 7.1,4.3 \pm 4.2$ [p<0.001 vs baseline]; nonResp: $47.7 \pm 12.4,92.0 \pm 8.0,3.1 \pm 3.2 ;$ NS). At baseline Resp showed an increased percentage of lamina propria occupied by MC $(8.05 \pm 3.0)$ and MC-NF $(5.14 \pm 2.54)$ as compared with nonResp $(4.2 \pm 1.3$ and $1.8 \pm 1.8 ; \mathrm{p}<0.001)$. After elimination diet only Resp had a significant reduction of MC $(3.3 \pm 1.1)$ and of MC-NF $(1.6 \pm 0.8)(\mathrm{p}<0.01)$ as compared to non-Resp $(3.5 \pm 2.1$ and $1.3 \pm 1.0 ; \mathrm{NS})$. A significant correlation was found between ABP, MC and MC NF (r: 0.56 ; r: 0.59; $\mathrm{p}<0.001$ ), as well as between RP, MC and MC-NF (r: 0.47 , r: 0.55 $\mathrm{p}<0.001$ ). We also found a significant inverse correlation between \% R, MC and MF-NF (r: $-0.35, \mathrm{p}<0.05 ; \mathrm{r}:-0.44, \mathrm{p}<0.01$ ). Conclusions: In children with functional constipation related to food allergy anorectal dysmotility is sustained by abnormal rectal MC density and spatial interactions between MC and nerves. These variables are significantly affected by an elimination diet.

\section{S1789}

Gastrointestinal Dysmotility in 5-Fluorouracil (5-FU)- Induced Inflammatory Intestinal Damage

Pedro M. Soares, Jose Mauricio S. Mota, Gerly Anne C. Brito, Miguel A. Nobre E Souza, Armenio A. Santos, Ronaldo A. Ribeiro, Marcellus H. Souza

BACKGROUND \& AIMS: 5-FU induced- intestinal damage is an important problem associated of cancer chemotherapy. It is characterized by mucosa epithelial ulcerations, and clinica manifestations of abdominal pain, nauseas and diarrhea. It was described that inflammatory intestinal damage could induced gastrointestinal dysmotility. Our hypothesis is that 5-FU could induce intestinal inflammation and gastrointestinal dysmotility. METHODS: Wista rat (250-350g) received one dose of 5-FU (150 mg/Kg, ip), or saline (ip). After 1, 3, 5, 15 or $30 \mathrm{~d}$, the rats were sacrificed and pieces of duodenum, jejunum and ileum were removed for measure microscopic epithelial damage, and neutrophil infiltration (MPO activity). After $3 \mathrm{~d}$ of a single $5-\mathrm{FU}$ injection, other groups of rats were fed by gavage $(1.5 \mathrm{ml})$ with a standard test meal ( $5 \%$ glucose solution with $0.05 \mathrm{~g} / \mathrm{ml}$ phenol red) and sacrificed 10,20 or 30 min later. Dye recovery from the gastric contents (GDR), and from 5 intestinal segments (IDR) were measured by spectrophotometry. Three $\mathrm{d}$ after a 5-FU or saline treatment mechanical response of the gastric fundus and duodenum, pre-contracted by carbacho (CCh) (10-10 to 10-4 M), were recorded with an isometric force transducer and computerized data acquisition system. RESULTS: 5-FU induced a significant $(\mathrm{p}<0.05)$ epithelial intestinal damage (vilus/crypts reason reduction) in all segments (duodenum-control $=3.2 \pm 0.1 \mu \mathrm{m}$ 5 -FU=0.8 $\pm 0.2 \mu \mathrm{m}$; jejunum-control $=2.1 \pm 0.2 \mu \mathrm{m}, 5$-FU=1.0 $\pm 0.3 \mu \mathrm{m}$ and ileum-control $=$ $1.2 \pm 0.0 \mu \mathrm{m}, 5-\mathrm{FU}=0.8 \pm 0.1 \mu \mathrm{m})$, and a increased in MPO (U/mg) activity (duodenumcontrol $=1.9 \pm 0.2,5-\mathrm{FU}=12.2 \pm 1.1$; jejunum-control $=2.4 \pm 0.3$, in $5-\mathrm{FU}=11.3 \pm 1.4$ and ileum-control=1.4 $\pm 0.2,5-\mathrm{FU}=10.1 \pm 2.0$ ), with the greater effect after 3 days. After 15 days, there was a complete repair of 5-FU induced- intestinal damage. After 3 days of 5 FU administration, we observed a significant $(\mathrm{p}<0.05)$ increase in GDR $(\%)$ after 10 (control= $41.4 \pm 5.4,5-\mathrm{FU}=75.6 \pm 6.2), 20$ (control $=42.2 \pm 4.4,5-\mathrm{FU}=88.7 \pm 10.6)$ and $30 \mathrm{~min}$ (control $=29.0 \pm 10.3,5-\mathrm{FU}=76.2 \pm 12.2$ ). On the other hand, IDR were significant $(\mathrm{p}<0.05)$ decreased in the 5 intestinal segments, at all post-prandial intervals. 5-FU induced a significant $(\mathrm{p}<0.05)$ increase in stomach $(\% \mathrm{KCl})($ control $=202.6 \pm 13.2,5-\mathrm{FU}=290.8 \pm 36.2)$ and duodenum (control $=27.5 \pm 7.5,5-\mathrm{FU}=89.4 \pm 21.0$ ) smooth muscle contraction at the $\mathrm{CCh}$ 10-5M. CONCLUSION: Our data suggest that the 5-FU intestinal inflammation induced a gastrointestinal dysmotility. It could explain some of the gastrointestinal symptoms associated of cancer chemotherapy. FINANCIAL SUPPORT: CNPq

\section{S1790}

The Effect of Colonic Mucosal Mediators from Patients with Irritable Bowel Syndrome On Tritiated ACh Release and Motor Responses in Strips of Human Colon

Giovanni Barbara, Gianluigi D'Agostino, Elisabetta Cervio, Barbara Balestra, Cesare Cremon, Roberto De Giorgio, Vincenzo Stanghellini, Roberto Corinaldesi, Marcello Tonini Background \& Aims: Colonic mucosal low grade inflammation and mast cell infiltration has been recently suggested as a mechanism leading to symptom generation in patients with irritable bowel syndrome (IBS) (Barbara et al Gastroenterology 2004:126:693-702). This study was designed to assess the impact of colonic mucosal mediators from patients with IBS on electrically stimulated excitatory motor responses and $3 \mathrm{H}-\mathrm{ACh}$ release in strips of human colon. Methods: Mucosal biopsies were obtained from the descending colon of IBS patients and healthy controls. Mediators spontaneously released by mucosal biopsies were 
collected in HANKS medium and their effect on human colonic strips was assessed in vitro. Longitudinal strips were mounted isometrically and stimulated electrically (EFS). The effect of mucosal mediators was assessed on EFS-induced cholinergic contractile responses (delivered as trains of 5 pulses at $1 \mathrm{~Hz}, 0.1 \mathrm{~ms}, 45 \mathrm{~V}, 120 \mathrm{~s}$ apart) in absence and presence of the prostaglandin (PG) inhibitor BW A868C. Tissue neuronal ACh stores were labeled with [methyl-3H] choline (74 KBequerel/ml) under EFS. After a $120 \mathrm{~min}$ washout period, superfusion fluid was collected. Strips were stimulated 2 times (S1 and S2) by 600 pulses delivered in $5 \mathrm{~s}$ trains at $20 \mathrm{~Hz}(0.1 \mathrm{~ms}$ duration, $90 \mathrm{~V} / \mathrm{cm}, 15 \mathrm{~s}$ apart), and the resting and stimulated 3H-outflow was measured and expressed as S2/S1 ratio in comparison to the equivalent ratio obtained in their absence (control). Results: In human colonic strips, EFS evoked neurogenic, cholinergic contractile responses that were abolished by tetrodotoxin TTX: $1 \mu \mathrm{M})$ and atropine $(1 \mu \mathrm{M})$. Application of mucosal mediators inhibited motor responses by approximately $20 \%$, through a mechanism partly antagonized by the PG inhibitor BW A868C (3 $\mu \mathrm{M})$. EFS-induced 3H-outflow was abolished by TTX $(1 \mu \mathrm{M})$ indicating its neuronal origin. IBS mucosal mediators enhanced 3H-ACh release by about $30 \%$, whereas mediators obtained from healthy controls enhanced $3 \mathrm{H}-\mathrm{ACh}$ release by only 10\%. Conclusions: Colonic mucosal mediators from patients with IBS inhibited colonic excitatory motor responses via a mechanism partly sensitive to BW A868C, indicating a direct, cAMP-mediated inhibitory effect of prostaglandin (PGE2) on smooth muscle cells. Conversely, colonic mucosal mediators increased $3 \mathrm{H}$-ACh release from cholinergic motor neurons. It is likely that PGE2 participates also in this response. The net inhibitory effect of mucosal mediators on cholinergic neuromuscular responses may derive from a prevalent cAMP-mediated effect on smooth muscle cells compared to intrinsic cholinergic motor neurons.

S1791

Effect of Hexamethonium and Different TRPV1 Receptor Antagonists On Impaired Gastric Emptying Induced By Experimental Colitis in Rats Heiko U. de Schepper, Joris G. de Man, Paul A. Pelckmans, Benedicte Y. de Winter

Introduction: Patients with inflammatory bowel disease often suffer from impaired gastric emptying (GE). We previously demonstrated a delay in GE in rats with experimental colitis in the absence of inflammatory changes at the level of the stomach. Our current aims were to investigate whether neuronal transmission and transient receptor potential vanilloid type 1 (TRPVI) were involved in the colitis-induced gastroparesis by using hexamethonium, capsaicin and different TRPVI antagonists. Methods: Distal colitis was induced in male Wistar rats by a colorectal enema of $30 \mathrm{mg}$ trinitrobenzene sulphate (TNBS) in 50\% ethanol $72 \mathrm{~h}$ prior to experiment. GE was measured $30 \mathrm{~min}$ after intragastric instillation of a semiliquid bolus of Evans blue. All drugs were administered intraperitoneally $1 \mathrm{~h}$ earlier. Capsaicin pretreatment was started two weeks prior to experiments. Results: (table) TNBS-colitis reduced GE significantly from $38.4 \pm 3.6 \%$ in controls to $22.7 \pm 4.4 \%$ in TNBS-treated rats $(\mathrm{P}<0.05, \mathrm{n}=12)$. Hexamethonium $(20 \mathrm{mg} / \mathrm{kg})$ reduced $\mathrm{GE}$ in controls but restored it to normal values in TNBS-treated rats. Capsaicin $(125 \mathrm{mg} / \mathrm{kg})$ had a pronounced inhibitory effect on GE in control rats, which was not seen in rats with colitis. The TRPV1 receptor antagonists capsazepine $(10 \mathrm{mg} / \mathrm{kg})$, iodoRTX $(755 \mu \mathrm{g} / \mathrm{kg}), \mathrm{SB} 366791(0.5 \mathrm{mg} / \mathrm{kg})$ and AMG9810 $(30 \mathrm{mg} / \mathrm{kg})$ did not resolve the colitis-induced gastroparesis, iodoRTX even reduced GE compared to its vehicle. Of the vehicles used, DMSO tended to increase GE at concentrations exceeding $40 \%$, while PEG tended to decrease GE. Conclusions: Acute TNBS-induced colitis reduces GE in the absence of gastric inflammation. This "long distance" effect is generated through neuronal pathways as evidenced by the beneficial effects of hexamethonium, a neuronal transmission inhibitor. The capsaicin experiments suggest that extrinsic afferent neurons are part of this pathway. We found no evidence for a role of TRPV1 receptors, although the antagonists available to date may lack specificity or ideal pharmacokinetic properties for an in vivo setup.

\begin{tabular}{|c|c|c|c|c|c|}
\hline Saline $22.7 \pm 4.4 \%$ & $\begin{array}{c}\text { 10\%eth, } \\
\mathbf{1 0 \%} \text { Tw80 } \\
19.2 \pm 2.3\end{array}$ & $\begin{array}{c}\text { 20\% DMSO } \\
26.9 \pm 6.4\end{array}$ & $\begin{array}{c}\text { 45\% DMSO } \\
50.0 \pm 6.6\end{array}$ & $\begin{array}{c}\text { 40\% DMSO } \\
24.8 \pm 3.1\end{array}$ & $\begin{array}{c}\text { PEG } \\
16.4 \pm 5.8\end{array}$ \\
\hline $\begin{array}{c}\text { Hexamethonium } \\
35.8 \pm 4.4^{*}\end{array}$ & $\begin{array}{c}\text { Capsaicin } \\
11.5 \pm 3.8\end{array}$ & $\begin{array}{c}\text { Capsazepine } \\
31.2 \pm 4.7\end{array}$ & $\begin{array}{l}\text { IodoRTX } \\
19.1 \pm 4.9^{*}\end{array}$ & $\begin{array}{c}\text { SB366791 } \\
23.9 \pm 4.6\end{array}$ & $\begin{array}{c}\text { AMG9810 } \\
16.1 \pm 3.6\end{array}$ \\
\hline $\mathrm{n}=12$ & $\mathrm{n}=9-12$ & $\mathrm{n}=7$ & $n=3-5$ & $\mathrm{n}=6$ & $\mathrm{n}=5$ \\
\hline
\end{tabular}

Table: The effect of hexamethonium, capsaicin and different TRPVl receptor antagonists and their respective vehicles on GE in TNBS-treated rats. GE is presented as a mean percentage \pm s.e.m. ${ }^{*} \mathrm{P}<0.05$, significantly different from vehicle-treated rats (unpaired Student's t-test). iodoRTX=iodoresiniferatoxin, eth=ethanol, Tw80=Tween80, DMSO=dimethyl sulfoxide, PEG=polyethylene glycol

\section{S1792}

Increased Spontaneous Serotonin Release from Jejunum of Trichinella Spiralis Infected Mice

Stephen Foley, Gulzar Singh, Johanna Wheatcroft, Arleta Reiff-Marganiec, Christopher Keating, Victoria Chapman, Andrew Bennett, Charles Marsden, David Grundy, Robin Spiller

Background. Post-infectious IBS is associated with elevated post-prandial 5-HT release. Mice infected with Trichinella spiralis develop increased enterochromaffin (EC) cell counts in the duodenum and jejunum. EC counts increase by 14 days after infection and this persists to 56 days, long after acute inflammation has resolved. Methods. Jejunal samples were taken from $\mathrm{NIH}$ mice 14, 28 and 56 days after gavage with $300 \mathrm{~T}$ spiralis larvae, or from uninfected animals. Jejunal dissection in ice cold buffer was performed immediately after cervical dislocation, and $1 \mathrm{~cm}$ strips taken. Mucosal samples were cut into strips, weighed and either snap frozen in liquid nitrogen for 5HT and 5HIAA content, or incubated in gassed Krebbs buffer (containing $10 \mathrm{uM}$ pargyline, $1 \mathrm{mM}$ N-AcetylCysteine) for 10 minutes. The $5 \mathrm{HT}$ released was then assayed by HPLC. Data presented are the mean of 3 samples per animal. Relative expression of Serotonin Transporter (SERT) and cytokeratin 20 (CK20) were assayed using
Real Time Quantitive PCR (Taqman). Results. 5-HT levels were significantly elevated at day 14. Release of 5-HT was very elevated at day 14 , and remained significantly above contro values at day 28. SERT mRNA expression was significantly reduced at day $14(\mathrm{p}<0.01)$, but CK20 expression was also significantly reduced $(\mathrm{p}<0.01)$, and SERT expression relative to CK20 expression was not significantly different suggesting that epithelial cell loss is the major mechanism for downregulation of SERT in this model. Conclusion. 5-HT is elevated in the jejunum of T. spiralis infected mice, paralleling the increased EC cell count. Spontaneous release of 5-HT is also significantly elevated and remains so at day 28 . The ratio of 5 HIAA to 5-HT is depressed at day 14 , reflecting reduced 5-HT re-uptake due to reduced SERT expression. This work was supported by the BBSRC

Results: (Mean $\pm \mathrm{SEM})(\mathrm{n}=12$ per group)

\begin{tabular}{|c|c|c|c|}
\hline & Control & Day 14 & Day 28 \\
\hline 5-HT content (pmol/g) & $21.1(1.9)$ & $45.5(6.2) \#$ & $29.3(1.48)$ \\
\hline 5-HIAA/5-HT ratio & $0.73(0.05)$ & $0.33(0.11)^{*}$ & $0.64(0.06)$ \\
\hline 5-HT release (pmol/g/min) & $0.07(0.003)$ & $0.44(0.04) \#$ & $0.15(0.01)^{*}$ \\
\hline
\end{tabular}

$\# \mathrm{p}<0.001 * \mathrm{p}<0.05$

\section{S1793}

Protective Effect of Emodin On Severe Acute Pancreatitis Through Induction of Apoptosis and Suppression of Cytokines Expression Via Inhibition of Nf- $\mathrm{\kappa B}$ Activation

Weiyan Yao, Yaozong Yuan

INTRODUCTION: As a major component of the widely used Chinese herb rhubarb, emodin has been used in the remedies for many diseases including inflammation and cancers. It has been indicated that emodin is a potent inhibitor of NF- $\mathrm{KB}$ activation and thus could be useful in treating inflammatory diseases. AIMS: This study is aimed to assess the effectiveness of emodin in the treatment of severe acute pancreatitis (SAP), and to clarify whether NF$\kappa \mathrm{B}$ signaling is one of the pathways involved. METHODS: SAP was induced by injection of $5 \%$ sterile sodium taurocholate into bilio pancreatic duct, while control group was only given sham operation. Emodin was administrated once at the dose of $1 \mathrm{mg} / \mathrm{kg}$ after the initiation of pancreatitis. Sera and pancreatic tissue were collected at 6 and 12 hours after the onset of operation. The level of serum amylase, lipid and histology were evaluated. The activation of NF- $\mathrm{KB}$ was determined by electrophoretic mobility shift assays (EMSA). Since the promoter regions of TNF- $\alpha$, intercellular adhesion molecule-1 (ICAM-1), and IL- 6 genes contain NF-KB binding sites and these cytokines are involved in the pathology of SAP, the pancreatic tissue and serum level of these molecules were studied by ELISA. Apoptosis was studied by TUNEL and electron microscope methods. The level of cIAP-1 and caspase 3 was determined by western blot. RESULTS: Taurocholate pancreatitis is characterized by massive necrosis, haemorrhage, and severe leucocyte infiltration in the pancreas. Treatment with emodin significantly attenuated SAP, as determined by serum amylase, lipid and histological assessment of edema, vacuolization, inflammation and necrosis $(\mathrm{p}<0.01)$. Moreover, treatment with emodin markedly inhibited NF-KB DNA-binding activity and the expression level of cIAP-1 in pancreatic tissue ( $<<0.01$, respectively vs SAP). This attenuation was associated with decreased expression of TNF- $\alpha$, IL- 6 and ICAM- 1 in the pancreatic tissues and serum $(p<0.05)$. Compared with the SAP group, apoptosis index of pancreatic acinus cells and the expression level of activated caspase 3 in pancreatic tissue were more significantly increased in emodin-treated group both at $6 \mathrm{~h}$ and $12 \mathrm{~h}$ ( $\mathrm{p}<0.05$,respectively vs SAP). CONCLUSION The result of this study indicated that administration of exogenous emodin attenuates the severity of SAP. The mechanism may be due to inhibition of NF- $\mathrm{KB}$ activation, which then suppress the expression of cytokines and induce apoptosis through regulating cIAP-1 and capspase 3 expression.

\section{S1794}

Evidence of Region-Specific Activation of Cell Injury Mechanisms in Dorsal Root Ganglion Neurons in DBTC-Induced Pancreatitic Rats Shuangsong Hong, Chunfang Guo, John W. Wiley

Background: Chronic pancreatitis is a progressive disease that results in irreversible destruction of exocrine pancreatic tissue and extensive fibrosis. It is unknown whether mechanisms associated with cell injury are activated in dorsal root ganglia (DRG) neurons innervating the pancreas in chronic pancreatitis. Dibutyltin dichloride (DBTC) has been reported to induce chronic pancreatitis with fibrosis in rats. We hypothesized that cell injury mechanisms would be activated in DRG neurons in DBTC-induced pancreatitis rats in a region-specific manner. Methods: Pancreatitis was induced in male Sprague-Dawley rats by DBTC ( $8 \mathrm{mg} /$ $\mathrm{Kg}$ ) injection via the tail vein. In vitro studies were performed using DRGs from control and chronic pancreatitis (2-4 weeks) rats. The following markers were examined: vanilloid receptor 1 (VRl); substance P (SP); substance P receptor (NKl-R); the large DRG, A-fiber marker NF200; the small DRG C-fiber marker peripherin; 8-OHdG (marker for oxidative stress); cytochrome c, activated caspase 3 \& caspase-activated DNase (CAD) (markers for apoptosis); and $\mu$-calpain (a marker for oncosis). Age-matched healthy rats served as controls. Results: Compared with the healthy controls, rats receiving DBTC demonstrated a significant increase in the levels of VR1, SP and NKI-R protein in lower thoracic DRG neurons between 2-4 weeks after treatment, but not in lower lumbar DRGs. The up-regulation of these proteins was colocalized in both small, peripherin-immunoreactive $C$ - and A- $\delta$ fiber neurons and large NF200-immunoreactive A- $\beta$ fiber DRG neurons. The number of cells that were immunoreactive for both VRl and SP (or NKl-R) was significantly increased as well in these DRG neurons. Immunohistochemistry staining showed an increased immunoreactivity of the oxidative stress marker 8-OHdG and markers of activation of the apoptosis cascade, cytosolic cytochrome $\mathrm{c}$ and activated caspase 3 in DRG neurons from the DBTC-injected rats. Increased colocalization of cytochrome $\mathrm{c}$ and activated caspase 3 was observed in thoracic DRGs compared to lumbar DRGs in DBTC-treated rats, and untreated controls. Western blot analysis demonstrated a significant increase in the levels of $\mu$-calpain and CAD in thoracic 
DRG neurons in DBTC-receiving rats, whereas the levels of $\mu$-calpain and CAD did not change in lumbar DRG neurons. Conclusion: DBTC-induced pancreatic rats demonstrate region-specific activation of mechanisms associated with cell injury including increased levels of VRl receptor, and activation of the apoptosis cascade and oncosis.

\section{S1795}

Robust Mouse Reg-2 Induction in Response to Keratin Mutation As a Cytoprotective Mechanism in Acute Pancreatitis

Pavel Strnad, Bihui Zhong, Diana M. Toivola, Xuhuai Ji, Harry B. Greenberg, M B. Omary

Background: The major cytoskeletal intermediate filaments in the pancreas and liver are keratins 8 (K8) and 18 (K18). Their function differs in that liver K8/K18 are essential cytoprotective proteins after an injury challenge while pancreatic K8/K18 appear to be dispensable. This functional dichotomy is based on findings in pancreatic injury models in K8-null and wild-type mice, and suggests that K8-null pancreata may undergo compensatory regulatory or gene expression changes. Aims: We used gene expression profiling to test the hypothesis that cytoprotective pancreatic genes are induced as a consequence of keratin filament mutation. Methods: Total RNA was isolated from K8-null and wild-type mouse pancreata, reverse transcribed then labeled with Cy3 (wild-type) and Cy5 (K8-null). Immunofluorescence staining and real time PCR were used to confirm the mouse microarray $(42,624$ spots/slide) findings. Array results were analyzed using cluster/treeview programs. Transgenic lines included those lacking acinar cell keratins (K8-null), those with membrane associated but no cytoplasmic keratins (K18-null), those with collapsed punctate filaments due to K18 point mutation (R89C), those that express mutant K18 at two phosphorylation sites (S52A or S33A), and those that lack ductal keratin 19 (K19). Results: Members of the Reg gene family, particularly Reg-2, had the most dramatic up-regulation in K8-/- pancreata (15 fold) which was confirmed by quantitative RT-PCR. The function of Reg- 2 is unknown but it has $75 \%$ amino acid identity with Reg- 1 (also called pancreatitis-associated protein I; confusingly Reg-l is sometimes termed Reg-2 in non-murine species). Anti-peptide antibodies to Reg2 were generated and confirmed the preferential pancreatic expression of Reg- 2 and its marked transcriptional induction at the protein level. Reg-2 but not Reg-1 is dramatically induced when tested in the two pancreatitis models, caerulein and CDD. Reg- 2 is also basally overexpressed in K18-null and R89C but not in K19-null mice, while Reg-1 is unaltered. Interestingly S52A but not S33A phosphorylation-mutant mice also manifest Reg-2 upregulation. Conclusion: Mouse Reg-2 is a likely compensatory cytoprotective factor when the pancreatic acinar cell keratin cytoskeleton is perturbed by mutation or ablation. Reg-2 up-regulation associates with keratin phosphorylation in a site-specific fashion. Our results combined with the structural relatedness of Reg-l, which is trophic in neuronal, pancreatic and other tissues, to Reg-2 suggests a similarly trophic role for Reg- 2 in mouse pancreas.

\section{S1796}

Prophylactic Probiotics Reduce Bacterial Translocation and Improve Outcome in Experimental Pancreatitis

L. Paul van Minnen, Harro M. Timmerman, Femke Lutgendorff, Andre Verheem, Wil Harmsen, Sergey R. Konstantinov, Hauke Smidt, Maarten R. Visser, Ger T. Rijkers, Hein G. Gooszen, Louis M. Akkermans

BACKGROUND Infection of pancreatic necrosis by gut bacteria is a major cause of morbidity and mortality in patients with severe acute pancreatitis. The use of prophylactic antibiotics to prevent infectious complications remains a topic of debate. Furthermore, increased concerns regarding the widespread use of prophylactic antibiotics associated complications, such as antibiotics resistance, have been reported. Prophylactic probiotics have been suggested as an alternative to the use of prophylactic antibiotics. Aim of this study was to determine if prophylactic use of a specifically designed multispecies probiotic mixture (Ecologic 641) can reduce bacterial translocation or improve clinical outcome in a rat model of acute pancreatitis. METHODS Male Sprague-Dawley rats $(n=48)$ were randomly allocated into three groups: 1) controls (sham operated, no treatment), 2) pancreatitis, probiotics treated or 3) pancreatitis, placebo treated. Acute pancreatitis was induced by intraductal bile salt infusion (glycodeoxycholate, $15 \mathrm{mM}$ ) followed by pancreatic hyperstimulation (intravenous cerulein, $5 \mu \mathrm{g} / \mathrm{kg} / \mathrm{hr}$, for 6 hours). Probiotics or placebo were administered daily via a permanent gastric cannula, from five days prior to until seven days after induction of pancreatitis. The clinical response to pancreatitis was assessed using a health score. Surviving animals were sacrificed at day seven, and tissue samples for microbiological and quantitative real-time PCR analysis of bacterial translocation were taken. RESULTS Probiotic treatment significantly reduced bacterial overgrowth of potential pathogens in the duodenum $(3.5 \pm$ $0.32 \log 10$ colony forming units (CFU)/gram vs. $5.0 \pm 0.69$, for probiotics vs. placebo, respectively, $\mathrm{P}<0.048)$, resulting in significantly reduced bacterial translocation to extraintestinal organs (Spleen: $2.43 \pm 0.30 \mathrm{CFU} / \mathrm{g}$ vs. $3.71 \pm 0.53, \mathrm{P}=0.039$. Liver: $2.75+0.47$ vs. $4.56 \pm 0.77, \mathrm{P}=0.049$. Pancreas: $3.11 \pm 0.52$ vs. $5.38 \pm 0.96, P=0.042)$. Real-time $\mathrm{PCR}$ demonstrated reduced bacterial loads in mesenteric lymph nodes $(5.90 \pm 0.44$ cells/g vs. $7.00 \pm 0.14, P=0.043)$ and pancreas $(6.70 \pm 0.25$ vs. $7.71 \pm 0.28, P=0.013)$ of rats treated with probiotics. Accordingly, health scores of surviving rats treated with probiotics were higher throughout the experiment. Probiotics significantly reduced late phase mortality ( $\geq 48$ hrs, $0 / 13$ vs. $4 / 15, P=0.049$ ). CONCLUSIONS This experiment demonstrates that bacterial translocation during acute pancreatitis can be reduced by prophylactic multispecies probiotics. Accordingly, probiotics improve the clinical course and outcome of acute pancreatitis.
S1797

A Novel Possibility for Gene Transfer Into the Duct Cells Using NonReplicating Pseudorabies Virus Variants

Maria Kovacs, Zoltan Rakonczay, Balazs Ordogh, Tamas Takacs, Janos Lonovics, Andras Varro, Andras Toth, Gyula J. Papp, Mike A. Gray, Barry E. Argent, Zsolt Boldogkoi, Peter Hegyi

Background. Several types of viral vectors are currently in use for gene transfer, but there is no available method to transfect intact pancreatic ducts. Viral invasion of cells may lead to cytotoxicity, which may question the validity of any observations. Our aim was to establish a model system for transferring genes into the pancreatic duct cells, which did not lead to cell damage. Methods. The non-virulent Ka-RREpOlacgfp (KEG) genetically modified strain of pseudorabies virus (PRV) containing the gene for GFP was used in this study. Small intra/ interlobular ducts were isolated from the pancreas of guinea pigs. Ducts were incubated in McCoy's based culture medium containing KEG virus at four different titers (107, 108, 109 and 1010 plaque forming units/ml) for 6 hours. The rate of HCO3- secretion was determined as described previously (Hegyi et al. Am J Physiol Cell Physiol 285:C268-C276, 2003). Vira structural proteins were visualized by immunohistochemistry. Native GFP expression and immunofluorescence signals were recorded by a confocal laser scanning microscope. Results. We observed that KEG virus infected practically all accessible epithelial cells within the duc when used at a titer of $1010 \mathrm{pfu} / \mathrm{ml}$ after 24 hours as judged by the appearance of GFP Importantly, we did not observe any cytotoxic effects of KEG and could not detect vira antigens on the cell surface, indicating the lack of viral replication. KEG did not affect ductal HCO3- secretion at any of the titers tested. Conclusions. The high efficiency of PRV infection in the pancreatic ductal epithelium may open the possibility for gene transfer and gene therapy of the duct cells using non-replicating PRV variants. This work was supported by The Wellcome Trust and OTKA.

\section{S1799}

SiRNA for Collagen-Specific Chaperone, Heat Shock Protein 47 Prevents Collagen Secretion from Pancreatic Stellate Cells in Rats

Masashi Taguchi, Shiro Watanabe, Hiroshi Asaumi, Yoshikuni Nagashio, Yoko Nomiyama, Makoto Otsuk

Heat-shock protein 47 (HSP47) is a collagen-binding stress protein and is assumed to act as a collagen-specific molecular chaperone during the biosynthesis and secretion of procollagen in the living cell. Chronic pancreatitis is characterized by loss of functional tissue and its replacement by extracellular matrix (ECM), but the molecular mechanism of this process remains unclear. We examined the expression for HSP47 in pancreatic fibrosis by repeatedinjection of cerulein in mice. HSP47 protein was markedly induced and its distribution was spindle-shaped and inflammatory cells by western bolt analysis and immunostaining respectively. Serial section showed these spindle-shaped cells also positive for alfa-smooth muscle actin (alfa-SMA), which was the marker of activated-pancreatic stellate cells (PSCs) We isolated PSCs from the pancreas of Wister rat using collagenase digestion and centrifugation with Nycodentz gradient. Isolated PSCs were also positive for HSP47. Using small interfering RNA (siRNA) transfection into PSCs, we knocked down HSP47 protein levels and analyzed collagen secretion, cell proliferation, alfa-SMA expression and, transforming growth factor-beta l(TGF-beta 1), collagen type 1 and fibronectin mRNA, assessed by Sirius red dye binding assay, BrdU incorporation, western blot analysis, and real-time PCR, respectively. Collagen secretion from PSCs was significantly reduced compared with control but there were no significant differences for cell proliferation, alfa-SMA expression and ECMrelated gene expression. Our results indicated the involvement of HSP47 in the progression of pancreatic fibrosis and that inhibition of HSP47 expression might merit further clinical investigation for the treatment of chronic pancreatitis.

\section{S1800}

Engulfment of Apoptotic Acinar Cells By Pancreatic Stellate Cells Regulates Pancreatic Fibrogenesis

Junko Tahara, Kyoko Shimizu, Keiko Shiratori

Background and Aims: Pancreatic stellate cells (PSCs) are known to play a pivotal role in fibrogenesis in pancreatic diseases. We have previously reported finding that engulfment of apoptotic polymorphonuclear neutrophils by PSCs is regulated by cytokines and PPAR $\gamma$ ligand. The aim of the present study was to investigate whether engulfment of apoptotic acinar cells affects pancreatic fibrogenesis. Methods: Rat PSCs were cultured and characterized as myofibroblast-like cells morphologically. Rat pancreatic acinar cells were incubated for 48 hours to induce apoptosis, and after allowing them to interact with PSCs for 12-48 hours, MTT assay was performed. $\alpha$-SMA expression was detected by Western blot analysis Cell morphology was examined by light microscopy. Annexin V-FITC and propidium iodide staining or detection of DNA fragmentation was used to identify cell death. The culture medium of PSCs that were cultured with apoptotic acinar cells and of PSCs not cultured with pancreatic acinar cells (control PSCs) was collected, and the TGF- $\beta$ concentration of the culture medium was measured by ELISA. Results: Most aged acinar cells were undergoing apoptosis, and a large number of apoptotic acinar cells were engulfed by PSCs. When PSC were cultured with apoptotic acinar cells for 12 hours, the number of living PSCs and expression of $\alpha$-SMA significantly decreased compared to the control PSCs. PSCs cultured with acinar cells showed DNA fragmentation and were Annexin V-positive, suggesting that phagocytosis of apoptotic acinar cells induced apoptosis of PSCs. When PSCs were cultured with apoptotic cells for 48 hours, the growth of the PSCs returned to the same level as that of the control PSCs, and their level of $\alpha$-SMA expressions was higher than in the contro PSCs. There was no difference between the concentrations of TGF- $\beta$ in the medium of the control PSCs and the PSCs that had engulfed acinar cells. Conclusions: Engulfment of apoptotic acinar cells by PSCs may be essential to remove damaged acinar cells and PSCs themselves to limit the early stage of pancreatic inflammation. When PSCs were further cultured with apoptotic acinar cells for 48 hours, engulfment of apoptotic cells activates PSCs to increase fibrogenesis. Phagocytosis of apoptotic acinar cells by PSCs may be an

.

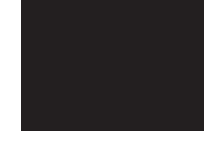


important mechanism in the regulation of inflammation and fibrogenesis in pancreatic diseases.

\section{S1801}

Role of the Cholinergic System in Alcohol-Induced Pancreatitis

Aurelia Lugea, Jose Nieto, Melissa Daghighian, Akihiko Satoh, Stephen J. Pandol

Background \& Aims: Although alcohol abuse can lead to pancreatitis in humans, the mechanisms underlying ethanol-induced pancreatic injury are poorly understood. We have shown that ethanol sensitizes rat pancreas to the inflammatory and acinar cell injury caused by cholecystokinin (CCK). This sensitization, in particular, results in pathologic responses with low doses of CCK or cerulein that by themselves do not cause pancreatitis. CCK actions on human and rodent pancreas include interactions with the cholinergic system. We hypothesized that the cholinergic system contributes to the ethanol-sensitizing effects on pancreatic injury. In the present study we assess whether atropine blocks ethanol-mediated sensitization of pancreatitis in rats, and whether ethanol causes inflammatory responses in cholinergicstimulated rat pancreatic acini. Methods: Male Wistar rats were pair-fed control or ethanolcontaining Lieber-DeCarli diets for 6 weeks. Low doses of cerulein $(0.5 \mu \mathrm{g} / \mathrm{kg})$ or saline were given as 4 hourly i.p. injections. Half of the animals received i.p. atropine $(40 \mu \mathrm{g} / \mathrm{kg})$ as cholinergic blocking agent prior to and during cerulein treatment. Animals were sacrificed $1 \mathrm{~h}$ after the last cerulein injection and blood and pancreatic samples were analyzed for parameters of pancreatitis. Dispersed rat pancreatic acini were incubated for 3 hours with $100 \mathrm{mM}$ ethanol and then stimulated with $1 \mathrm{mM}$ carbachol or vehicle. After $30 \mathrm{~min}$, cells were collected and NF-kB binding activity was measured by EMSA. Results: Neither carbachol nor ethanol stimulation led to NF- $\mathrm{KB}$ activation in dispersed pancreatic acini. However, these two factors in combination induced marked NF- $\kappa \mathrm{B}$ activation, suggesting a sensitizing effect of ethanol to inflammatory effects of cholinergic agonists. Similar sensitizing effects were observed in rats fed ethanol. In these animals, low doses of cerulein caused an episode of acute pancreatitis characterized by increase in blood lipase levels, marked vacuolization of acinar cells and intra-pancreatic trypsin activation. Cerulein was used at low doses that induce only minor effects in control fed animals. Interestingly, the stimulatory effects of ethanol feeding on cerulein pathologic responses in pancreas were abolished by atropine treatment. In particular, atropine blocked the increases in blood lipase and trypsin activation and markedly reduced acinar cell vacuolization. Conclusions: Ethanol sensitizing effects to pancreatic inflammatory and injury are mediated at least in part by the cholinergic system. Ethanol may elicit cholinergic involvement via central or peripheral neural systems and/or muscarinic signaling within pancreatic acinar cells.

\section{$\mathrm{S} 1802$}

Colony-Stimulating Factor Release from Human Pancreatic Periacinar Myofibroblasts

Osamu Inatomi, Akira Andoh, Yuki Yagi, Shigeki Bamba, Kazunori Hata, Takao Saotome, Atsushi Takayanagi, Nobuyoshi Shimizu, Yoshihide Fujiyama

Backgrounds and aims: Colony-stimulating factors (CSFs) play an important role in controlling the proliferation of hematopoietic progenitor cells and enhancing the functional activities of mature myeloid effector cells. CSFs are produced by variety of cells, including monocytes, macrophages, endothelial cells, fibroblasts and stromal cells in response to various inflammatory stimuli. In cases of acute or flare-up chronic pancreatitis, a marked elevation of circulating leukocyte counts is frequently observed. However, the local secretion of CSFs in pancreas remains unclear. In this study, we focused on CSFs secretion in periacinar myofibroblasts (PMFs) isolated from human pancreas. Materials and Methods: Primary cultures of PMFs were prepared according to the method reported by Saotome et al. The cells were cultured in DMEM containing $10 \%$ FBS. All culture media were supplemented with $50 \mathrm{U} / \mathrm{ml}$ penicillin and $50 \mathrm{ug} / \mathrm{ml}$ streptomycin. The studies were performed on passage 2-6 myofibroblasts isolated from 3 resection specimens. CSFs secretion was determined by ELISA, and CSFs mRNA expression was evaluated by Northern blotting. The activity of secreted CSFs on hematopoietic progenitor cells was examined by methylcellulose colony assay. Results: Unstimulated PMFs secreted a small amount of granulocyte (G)- and granulocyte/macrophage (GM)-CSF, and a considerable amount of macrophage (M)-CSF. Interleukin (IL)-1 $\beta$ and tumor necrosis factor (TNF)- $\alpha$ induced a marked increase in G- and GM-CSF release, and weakly but significantly enhanced M-CSF release. The effect of IL-1 $\beta$ and TNF- $\alpha$ were detected in dose- and time-dependent manner. The combination of IL-1 $\beta$ plus TNF- $\alpha$ induced a synergistic increase in G- and GM-CSF release. Adenovirus-mediated transfer of a stable form of I $\kappa \alpha$ markedly inhibited the effects of IL-1 $\beta$ and TNF- $\alpha$. Conditioned medium of IL- $1 \beta$ - and TNF- $\alpha$-treated PMFs enhanced granulocyte and/or macrophage colony formation. Conclusion: The present study demonstrated that human PMFs are local site for CSF biosynthesis. CSFs derived from PMFs may play an important role in the pathophysiology of acute or chronic pancreatitis.

\section{S1803}

The Stimulatory Effect of Various Extracellular Matrices On Rat Pancreatic Stellate Cells

Hiroshi Asaumi, Masashi Taguchi, Mitsuo Tashiro, Shiro Watanabe, Yoko Nomiyama, Makoto Otsuki

INTRODUCTION: Pancreatic stellate cells (PSCs) synthesize and secrete extracellular matrices (ECMs) and cytokines, and contribute to the progression of pancreatic fiborisis. Secreted cytokines are known to stimulate PSCs by themselves. In fibrotic tissue, PSCs are surrounded by various pathological ECMs such as type I collagen. Although integrin $\beta 1$ receptors is known as major adhesion receptors to ECMs, it is not clarified that what types of integrin $\beta 1$ receptors PSCs have. We therefore examined the presence of integrin $\beta 1$ receptors and the interaction between PSCs and various types of ECMs. METHODS: PSCs were isolated from rat pancreas, and the presence of integrin $\beta 1$ receptor family in PSCs was investigated by reverse transcription-polymerase chain reaction (RT-PCR) and Western blotting. [2] We examined the reaction of PSCs against various ECMs as follows; (i) adhesion ability, (ii) collagen secretion into the medium, (iii) various gene expressions and (iv) phospholyration of focal adhesion kinase (FAK) by incubatin PSCs on various types of ECM-coated dishes (type I collagen, type IV collagen and fibronectin). RESULTS: We detected mRNA and protein expression of integrin $\beta 1$ receptor family in PSCs. PSCs attached to each ECMcoated dish in a concentration-dependent manner. Incubation of PSCs on type I collagencoated dish (COL I) significantly increased collagen secretion into the medium and gene expression of type I procollagen compared with that on type IV collagen-coated dish (COL IV) and fibronectin-coated dish (FN). Gene expression of fibronectin significantly increased in order of COLI, FN, and COL IV. COL I and FN significantly increased gene expression of transforming growth factor (TGF)- $\beta 1$, TGF- $\beta$ type II receptor, platelet derived growth facotr- $\alpha$ and $\beta$ receptors compared with COL IV. Adhesion of PSCs to COL I rapidly increased phosphorylation of FAK, reaching the peak level at $1 \mathrm{~h}$. PSCs on COL IV also significantly increased FAK phosphorylation compared with suspended PSCs, the level was less than that on COL I. CONCLUSION: These results clearly demonstrated that type I collagen stimulates PSCs to produce ECMs, cytokine and cytokine receptors through integrin $\beta 1$ receptors. These results suggested that type I collagen may contribute to the progression of pancreatic fibrosis

\section{S1804}

Genome Wide Analysis and Global Gene Expression of 50 Cases of Pancreatic Endocrine Neoplasm (PEN) with Clinical and Pathological Correlation Laura H. Tang, S M. Chung, L Song, Jr Shia, P J. Allen, L H. Blumgart, M F. Brennan, M I. D'Angelica, R Dematteo, H Fong, W R. Jarnagin, D P. Jaques, A Viale, D S. Klimstra

While PEN is less aggressive than its pancreatic adenocarcinoma counterpart, synchronous and metachronous metastases occur in 40-60\% cases. There has been no ideal prognostic classification system based on clinical and pathologic criteria. We studied molecular features of 50 PENs with clinical followup of $66 \pm 7$ months. Comparative genomic hybridization $(\mathrm{CGH})$ was performed using an oligonucleotide based microarray with an average resolution of $35 \mathrm{~kb}$, and gene expression was analyzed with Affymetrix HG-U133A-2. Significantly altered genes were further validated by quantitative RT-PCR. Overall chromosomal aberrations observed in $>80 \%$ of PENs included losses at lp $36,3 \mathrm{p} 21.2,10 \mathrm{q} 24.3,11 \mathrm{p} 11.22$-pl2, $11 \mathrm{q} 13,16 \mathrm{p} 13.12-12.3,16 \mathrm{q} 22,17$-telomeres, and 22q12.3-13.2; and gains at 14q12. When tumors were stratified by size, larger tumors had relatively more chromosomal gains than losses. Similarly, metastases exhibited more complex chromosomal aberrations than their corresponding primaries. Supervised clustering analysis of gene expression was performed in subgroups of PENs (Table 1). Genes with $>5$ fold alterations within each subgroup were identified. Representative genes related to angiogenesis and tumor suppression were evaluated in primary PENs with $(n=13)$ or without $(n=17)$ metastasis, and with 5 cases of hepatic metastases. The amount of target genes, normalized to an endogenous reference, was significantly down regulated (19 folds) in PENs associated with distant metastases when compared to those without metastasis ( $\mathrm{p}=0.015)$; and it was further decreased by 2304 fold in hepatic metastases $(\mathrm{p}=0.004)$. In conclusion, we have identified precise DNA aberrations in PENs using high resolution and sensitivity CGH. Potential genes related to prognosis may provide a more consistent and accurate biological and pathological assessment of PENs. Table 1. Comparison of Alterations in Gene Expression Between Subgroups of PEN.

\begin{tabular}{|c|c|c|c|c|}
\hline \multirow{2}{*}{ Tumor Subgroups } & \multirow{2}{*}{$\Delta$ in Fold } & \multicolumn{2}{|c|}{ \# of Genes } & \multirow{2}{*}{ p Values } \\
\cline { 3 - 4 }$\uparrow$ Regulated & $\downarrow$ Regulated & \\
\hline Functional vs. Non-functional & $21-1677$ & 5 & 11 & $0.000021-0.0065$ \\
\hline Small vs. Large $(2.0 \pm 0.2 \mathrm{~cm}$ vs. $9.3 \pm 1 \mathrm{~cm})$ & $5-41$ & 2 & 14 & $0.000006-0.014$ \\
\hline Without Metastasis vs. With & $5-34$ & 6 & 12 & $0.00013-0.0048$ \\
\hline Primary vs. Metastasis & $5-34$ & 15 & 14 & $0.00037-0.022$ \\
\hline
\end{tabular}

\section{S1805}

Sustained Akt Phosphorylation Is Required for Anti-Apoptotic Effect of Insulin-Like Growth Factor-I in Pancreatic Cancer Cells: Roles of Phosphatases

Jong Kyun Lee, Mouad Edderkaoui, Peggy P. Hong, Patrick Truong, J. David Lambeth, Andrea Berti, Stephen J. Pandol, Anna S. Gukovskaya

Background and Aims: Insulin-like growth factor-I (IGF) plays a major role in protecting pancreatic cancer $(\mathrm{PaCa})$ cells from apoptosis. Pro-survival effects of IGF are mediated via activation of phosphatidylinositol 3-kinase (PI3K) and the downstream Akt kinase. Growth factors are known to cause a rapid and transient Akt activation, whereas inhibition of apoptosis occurs hours later. In this study we sought to determine the mechanisms underlying this temporal relationship, and in particular, the roles of phosphatases and reactive oxygen species (ROS) in the anti-apoptotic effect of IGF in PaCa cells. Methods: In MIA PaCa-2 and PANC-1 cells cultured with and without IGF $(50 \mu \mathrm{g} / \mathrm{ml})$, we measured apoptosis with Cell Death ELISA; Akt activation (i.e., phosphorylation), by Western blot; intracellular ROS, by flow cytometry; and protein tyrosine phosphatase (PTP) activity and oxidation, by enzymatic and electromobility shift (EMSA) assays. Results: In both cell lines, IGF induced biphasic phosphorylation of Akt: in addition to a rapid (minutes) and transient lst phase, there was a slow (24-72 h) and sustained 2nd phase. PI3K inhibitors LY294002 and wortmannin prevented both phases of Akt phosphorylation as well as the anti-apoptotic effect of IGF. Importantly, the pro-survival effect of IGF was also prevented by inhibiting the 2nd phase of Akt phosphorylation alone (LY294002 added 30 min after IGF). Thus, sustained Akt phosphorylation is necessary for the IGF-induced inhibition of apoptosis. Transfection experiments identified the phosphatase LMW-PTP as a negative regulator of sustained Akt phosphorylation in PaCa cells. Using a recently developed non-reducing EMSA, we found that IGF stimulated LMW-PTP oxidation thus inhibiting its activity. Previously, we have shown that IGF activates NADPH oxidase Nox4 in PaCa cells. NADPH oxidase inhibitor DPI and transfection with Nox4 siRNA plasmid both prevented IGF-induced 
oxidation of LMW-PTP and stimulated its activity. Conclusions: The results demonstrate that the signaling pathway mediating the pro-survival effect of IGF starts with activation of NADPH oxidase (Nox4); leading, in turn, to oxidation (i.e., inhibition) of LMW-PTP by ROS produced by NADPH oxidase; and resulting in sustained activation of the anti-apoptotic PI3K/Akt. This mechanism represents a target for novel therapeutic strategies to promote death of PaCa cells and thus overcome their resistance to treatments.

\section{S1806}

Pancreatic Tumor Cell Adhesion and Migration Is Regulated By

Phosphorylation of the Actin-Binding Protein Cortactin

Anne E. Kruchten, Eugene W. Krueger, Mark A. McNiven

BACKGROUND: Neoplastic pancreatic ductular cells disseminate and invade peripheral tissues rapidly, making this cancer particularly aggressive and difficult to treat. Currently, the molecular machinery that supports this active, actin-based, metastatic process is poorly understood. Cortactin is an actin-binding protein involved in cell migration and metastasis. In addition, cortactin is a substrate of the tyrosine kinase Src, and both of these proteins are upregulated in pancreatic cancer. Therefore, together cortactin and Src may participate in actin reorganization that occurs during pancreatic ductular cell migration and subsequent metastasis. Further, phosphorylation of cortactin by Src could modulate the effects of cortactin on actin reorganization and dynamics that occur during cell migration. For example, in vitro, Src phosphorylation of cortactin has been shown to negatively affect actin polymerization. In vivo, however, Src phosphorylation of cortactin has been shown to promote actin-dependent cell migration and to enhance the metastatic capacity of cells. In addition to being phosphorylated by Src, cortactin is also serine phosphorylated by the kinase ERK. In contrast to the negative effects of Src phosphorylation of cortactin on in vitro actin polymerization, ERK phosphorylation of cortactin enhances in vitro actin polymerization. GOAL: This study aims to determine the contributions of Src- and ERK-mediated cortactin phosphorylation on actin assembly in metastatic human pancreatic cells. RESULTS: Expression of a cortactin mutant mimicking the Src-phosphorylated state (Y384/429/445E) resulted in a dramatic extension of lamellipodia, structures rich in actin and indicative of a migratory phenotype, whereas expression of the phospho-blocking mutant (Y384/429/445F) inhibited lamellipodia formation. To test how Src-mediated tyrosine phosphorylation of cortactin promotes lamellipodial extension while inhibiting actin polymerization, as suggested by in vitro data, the effects of cortactin mutants on focal adhesions, dynamic actin-based structures that undergo turnover during cell migration, were examined. Interestingly, cells expressing cortactin Y384/429/ $445 \mathrm{E}$ had numerous focal adhesions with a decreased amount of cortactin and exhibited faster spreading on fibronectin, suggesting a more migratory phenotype. CONCLUSION: We suggest that tyrosine phosphorylation of cortactin plays a role in focal adhesion turnover and thereby affects cell motility. These findings provide novel insights into the role of cortactin phosphorylation in pancreatic cancer cell migration through the regulation of focal adhesion turnover

\section{S1807}

Suppression of C-Terminal Src Kinase (CSK) Leads to Increased Cell Proliferation in Pancreatic Cancer

Dhananjay Kunte, Jennifer Koetsier, Ramesh Wali, Randall Brand, Hemant Roy

Background: While various molecular changes in pancreatic cancer progression are known, the specific signaling events underlying the aggressive growth and proliferation are unclear. The proto-oncogene $\mathrm{Src}$ is a member of the Src family of non-receptor tyrosine kinases (SFKs) that control proliferation, apoptosis, invasiveness and angiogenesis. Src is commonly activated in pancreatic cancer; however the mechanisms remain unclear. One potential means of activating Src is through downregulation of its inhibitor, C-terminal Src Kinase (Csk). We, therefore, investigated the role of the CSK tumor suppressor gene in pancreatic carcinogenesis. Methods: The levels of CSK and Src-527 (the inhibitory phosphorylation site of (SK) in the pancreatic tissues of the normal and adenocarcinoma patients were assessed by immunohistochemistry. In order to study the role of CSK in pancreatic cancer, we genetically engineered human pancreatic cancer cell line MIA PaCa2 for CSK gene knockdown by stably transfecting the CSK-specific shRNA vector using standard methods. Protein samples from the wild type and the CSK shRNA construct were processed for western blotting to study the molecular effects of CSK-inhibition. Results: CSK was suppressed in the pancreatic adenocarcinoma with corresponding decrease in the Src-527. In the MIA $\mathrm{PaCa} 2$ cells, the CSK stable construct showed 54\% decrease in CSK levels and significant increase ( $133 \%$ of the wild type, $\mathrm{p}<0.05$ ) in the cell proliferation marker PCNA. CSK gene knockdown also resulted in the activation of the members of the MAP kinase pathway, viz., MAP Kinase Kinase (MEK, 201\% of control, p<0.05) and MAP Kinase (MAPK) (. Conclusion: We demonstrate that CSK is suppressed with corresponding Src activation in pancreatic cancer tissue. Loss of CSK in pancreatic cancer cell line leads to increased cell proliferation through activation of the MAP kinase pathway.

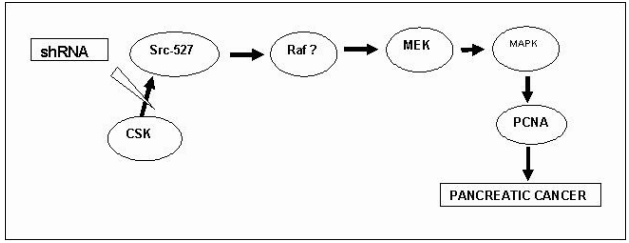

Possible mechanism for CSK-regulated cell proliferation in pancreatic cancer
S1809

Implication of S100p in Pancreatic Cancer As An Early Developmental Marker and a Therapeutic Target

Kenoki Ohuchida, Kazuhiro Mizumoto, Eishi Nagai, Hiroyuki Konomi, Koji Yamaguchi, Masao Tanaka

Backgrounds. Recent microarray analyses identified S100P as a candidate diagnostic marker or therapeutic target of pancreatic cancer. Aim. To investigate the role of S100P in pancreatic cancer. Method. We examined S100P mRNA expression in pancreatic cancer cell lines, pancreatic tissues, cells microdissected from pancreatic tissue, and pancreatic juice from patients with pancreatic diseases. The effect of interference with S100P on pancreatic cancer cells was investigated by in vitro and ex vivo RNA interference experiments inhibiting S100P mRNA expression. Results. S100P mRNA expression was significantly greater in tumora than in non-tumoral tissues. In pancreatic juice, S100P expression differed significantly between patients with carcinoma and those with non-neoplastic diseases. In receiver operating characteristic curve analyses, S100P was a good marker for preoperative diagnosis of pancreatic cancer. Microdissection-based mRNA analyses revealed that S100P expression differs significantly between normal duct and pancreatic intraepithelial neoplasia (PanIN) but no between PanIN and invasive ductal carcinoma. In vitro and ex vivo S100P inhibition decreased cell proliferation and possibly the invasive potential of pancreatic cancer cells. Conclusion

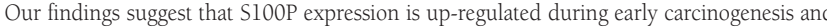
correlates with cell proliferation and possibly cell invasion. Quantitative S100P mRNA assessment is a promising approach to evaluating the risk of pancreatic cancer.

\section{S1810}

Corn Derived Carbohydrate Inositol Hexaphosphate (IP6) Inhibits Barrett's Adenocarcinoma Growth By Pro- Apoptotic Mechanisms

David W. Mcfadden, Dale R. Riggs, Barbara J. Jackson, Cynthia Cunningham

INTRODUCTION: Inositol Hexaphosphate (IP6) is a naturally occurring polyphosphorylated carbohydrate found in food sources high in fiber content. IP6 has been reported to have significant inhibitory effects against a variety of primary tumors including breast and colon. The effects of IP6 have not been evaluated in Barrett's adenocarcinoma. We hypothesized that IP6 would significantly inhibit cell growth rate of Barrett's adenocarcinoma in vitro. METHODS: Two Barrett's-associated adenocarcinoma cell lines, SEG-1 (wild type p53) and BIC-1 (mutant p53), were treated with corn derived IP6 at 0.5, 1.0, and 5.0mMolar concentrations. After 72 hours, cell viability was measured by MTT assay. Apoptosis and necrosis was evaluated by the Annexin V FITC assay. Statistical analysis was performed by ANOVA. RESULTS: Reductions $(\mathrm{p}<0.01)$ in cellular proliferation was observed in both cell lines. In the BIC cells, $5 \mathrm{mM}(80.0 \%)$ and $1.0 \mathrm{mM}(65.4 \%)$ of IP6 decreased cell growth $(\mathrm{P}<0.001)$. In the SEGl cells, $0.5 \mathrm{mM}(55.8 \%), 1.0 \mathrm{mM}(93.1 \%)$ and $5 \mathrm{mM}(86.2 \%)$ of IP6 decreased cell growth $(\mathrm{P}<0.001)$. IP6 increased late apoptotic activity $(\mathrm{p}=0.046)$ in the BIC cells. In the SEG-1 cells, early apoptosis $(\mathrm{p}=0.038)$, late apoptosis $(\mathrm{p}=0.002)$ and necrosis $(\mathrm{p}=0.036)$ were all increased by IP6 treatment CONCLUSIONS: Treatment of Barrett's adenocarcinoma with the common dietary polyphosphorylated carbohydrate IP6 significantly decreased cellular growth by pro- apoptotic mechanisms. Our findings suggest that IP6 has the potential to become an effective adjunct for Barrett's adenocarcinoma. Further in vivo and human studies are needed to evaluate safety and clinical utility of this agent in patients with Barrett's adenocarcinoma.

\section{S1811}

Correlation of DNA Ploidy Analysis By Digital Image Analysis and Flow Cytometry in Barrett's Esophagus

Kenneth K. Wang, Ganapathy Prasad, Navtej S. Buttar, Michel Wongkeesong, Peter Rabinovitch, Thomas Smyrk, Lynn Borkenhagen, Sarah Papenfuss, Catherine Demars, Lori Lutzke

Flow cytometry has been shown to be an important biomarker for cancer risk in Barrett's esophagus. Digital image analysis (DIA) is another method to obtain similar information that requires a much smaller sample of cells for analysis. Aim: To compare the results of DIA and flow cytometry in a consecutive series of patients with Barrett's esophagus and high-grade dysplasia. Methods: Patients referred to the Barrett's Esophagus Unit with highgrade dysplasia were prospectively studied with DIA and flow cytometry. All patients underwent four quadrant biopsies taken every centimeter with histological results reviewed by two expert pathologists. DIA was performed on cytological specimens that were Feulgen stained and then assessed with a CAS 200 digital imaging microscope. A minimum of 50 cells were assessed for DIA. Flow cytometry was performed on histological specimens at the University of Washington. A minimum of 20,000 cells were assessed for flow cytometry In cases with dichotomous results between DIA and flow cytometry, fluorescent in situ hybridization was performed to determine if genetic abnormalities did exist in p16, p53, c-myc, 20q, and Her-2. Results: 18 patients were assessed, 15 with high-grade dysplasia and 3 with carcinoma found on biopsy. In one case, there was inadequate specimens available for flow analysis. Of the remaining 17 cases, DIA and flow cytometry made concordant assessments of DNA ploidy in $82 \%$ of the cases, $(p>0.3)$. These included 5 cases that were diploid and 9 that were aneuploid. There were three discordant pairs that included one that was near diploid on DIA and aneuploid on flow, this case was completely normal on FISH. Two cases were tetraploid on DIA and diploid on flow, Both cases were abnormal by FISH, with loss of heterozygosity of both $\mathrm{p} 53$ and pl6. Conclusions: DNA ploidy by DIA or flow cytometry were reasonably concordant. In the discordant cases, examination of other biomarkers suggest that DIA may have correlated better with genetic abnormalities. This study suggests that DNA ploidy determined by DIA on cytology may be as useful as flow cytometry in determining cancer risk. 
S1812

Accuracy of Wireless Capsule Endoscopy for the Detection of Barrett's Esophagus

Prateek Sharma, Amit Rastogi, Romeo Esquivel, Krishna Gurram, Sachin Wani, Ajay Bansal, Srinivas R. Puli, April Higbee, Lisa Camargo, Richard Sampliner

Introduction: The initial step in the diagnosis of Barrett's esophagus (BE) requires an upper endoscopy to document the presence of a columnar lined distal esophagus (suspected BE), followed by biopsies from the columnar segment. The availability of a wireless esophageal capsule (Pillcam ESO) allows the recording of images from the esophagus and can be potentially used as an office based screening tool in patients with GERD. Aim: To compare esophageal capsule to standard upper endoscopy for the detection of endoscopic Barrett's esophagus. Methods: This study was conducted at 2 sites utilizing standardized methodology, data collection and analysis. Patients with chronic GERD and BE were prospectively evaluated; all patients initially underwent capsule endoscopy followed by standard upper endoscopy. The esophageal capsule is similar to the small bowel capsule, but acquires images from both ends ( 2 cameras) of the device at a rate of 7 frames/sec/camera. The quality of the images were graded from 1-5 (not scoreable-excellent). Capsule images were analyzed by investigators blinded to the upper endoscopy findings; sensitivity and specificity of these findings were then analyzed using the standard endoscopy findings as the gold standard. Results: Eighty-four patients were initially enrolled in the study, 3 were unable to swallow the capsule, whereas images from 3 patients could not be evaluated. Data from 78 patients were available for analysis; mean age of $56.9+12.3$ years; 71 males. By standard endoscopy, BE was suspected in 41 patients; 27 with short $\mathrm{BE}(<3 \mathrm{~cm})$ and 14 with long $\mathrm{BE}(>3 \mathrm{~cm})$. The mean BE length was $3.17 \mathrm{~cm}$. The sensitivity and specificity of esophageal capsule endoscopy for the detection of suspected BE were $73 \%$ and $86 \%$ respectively; sensitivity and specificity for the detection of short $\mathrm{BE}$ were $67 \%$ and $86 \%$ and for long BE were $86 \%$ and $86 \%$ respectively. The quality of the images did not contribute to the false positive/negative results. No adverse events were noted using capsule endoscopy. Conclusions: Esophageal capsule endoscopy can be safely performed in the majority of GERD patients undergoing screening upper endoscopy. The sensitivity and specificity of capsule endoscopy for the diagnosis of suspected BE are high especially for long BE. Future studies should test inter observer variability and the learning curve in the reading of images and steps to further improve the diagnostic accuracy capsule endoscopy.

\section{S1813}

Does Degree of Acid Exposure Differ Between Distal Versus Proximal Esophagus in Patients with Barrett's Esophagus (BE)? Prateek Sharma, Ajay Bansal, Sachin Wani, Srinivas R. Puli, April Higbee, Amit Rastogi Introduction:BE pts have been shown to have abnormal esophageal acid exposure by catheter $\mathrm{pH}$ systems with probe placed $5 \mathrm{~cm}$ above LES. However, it is not known if acid exposure is higher in distal vs. proximal esophagus of BE pts using a wireless pH system. Aim:In BE pts, compare acid exposure in very distal esophagus ( $1 \mathrm{~cm}$ above anatomic GEJ) to conventional location ( $6 \mathrm{~cm}$ above GEJ) and to a group of controls. Methods:Pts with confirmed $\mathrm{BE}$, off PPI therapy for 7 days were enrolled. Under endoscopic vision, $2 \mathrm{pH}$ capsules were placed 1 and $6 \mathrm{~cm}$ above GEJ. Controls [subjects without GERD symptoms on 2 validated GERD questionnaires (GERQ and RDQ)] were also studied. All subjects were encouraged to continue usual activities and diet. pH tracings were reviewed. Only tracings consistent with esophageal and not gastric cardia placement and with 16 hrs of data were used for analysis. Proximal and distal pH values in $\mathrm{BE}$ pts were compared using paired t-test and Wilcoxon summed ranks test. Correlation between $\mathrm{pH}$ (at both locations) and BE length was performed using Pearson's test and scatter plot. Mean pH values of BE pts were also compared to controls with unpaired t-test. Results: $23 \mathrm{BE}$ pts were enrolled (all Caucasian males) - mean age 65.9 yrs (range:40-81) and mean BE length $3.68 \pm 2.7 \mathrm{~cm}$. Data from 19 pts could be analyzed. Within BE group, $\%$ time $\mathrm{pH}<4$ in 24 hrs was significantly higher in distal vs conventional position, Table 1 . This was independent to BE length.Also, esophageal acid exposure in BE pts was significantly higher at both locations compared to controls, Table 2. Conclusions:BE pts have significantly higher acid exposure in distal compared to proximal esophagus, independent of BE length. Its implications on preferential location of dysplasia/cancer in distal vs. proximal esophagus needs to be evaluated. This issue has special relevance given the significant rise in GEJ adenocarcinoma. Table 1: Acid Exposure in the Proximal vs. Distal esophagus in BE patients

\begin{tabular}{|c|c|c|c|c|c|}
\hline & \multicolumn{2}{|c|}{ Percent time $\mathrm{pH}<4$} & & \\
\hline & Proximal & Distal & paired t test & Wilcoxon summed ranks test & unpaired t test \\
\hline Total & 9.89 & 18.64 & $\mathrm{p}<0.02$ & $\mathrm{p}<0.09$ & $\mathrm{p}<0.05$ \\
\hline Upright & 12.15 & 21.69 & $\mathrm{p}<0.02$ & $\mathrm{p}<0.09$ & $\mathrm{p}<0.05$ \\
\hline Supine & 6.31 & 11.93 & $\mathrm{p}<0.16$ & $\mathrm{p}<0.14$ & $\mathrm{p}<0.13$ \\
\hline
\end{tabular}

Table 2: Comparison of Percent times $\mathrm{pH}<4$ in $\mathrm{BE}$ vs. Controls at both locations

\begin{tabular}{|c|c|c|c|c|}
\hline Location & & $\mathrm{BE}$ & Controls & $\mathrm{p}$ (unpaired t test) \\
\hline \multirow{3}{*}{ Esophageal } & Total & 14.56 & 4.21 & 0.01 \\
\cline { 2 - 5 } & Upright & 16.74 & 4.94 & 0.005 \\
\cline { 2 - 5 } & Supine & 11.57 & 0.74 & 0.03 \\
\hline \multirow{3}{*}{ GEJ } & Total & 29.16 & 10.4 & 0.01 \\
\cline { 2 - 5 } & Upright & 30.88 & 10.26 & 0.003 \\
\cline { 2 - 5 } & Supine & 26.2 & 8.54 & 0.07 \\
\hline
\end{tabular}

S1814

The Burden of Upper Gastrointestinal Endoscopy

Michelle E. Kruijshaar, Peter D. Siersema, Marjon Kerkhof, Ewout W. Steyerberg, MarieLouise Essink-Bot

INTRODUCTION: Upper gastrointestinal (GI) endoscopy is an invasive diagnostic procedure that causes pain, discomfort and psychological distress in patients. Regular endoscopic surveillance, as recommended for patients with Barrett's esophagus (BE), may therefore be experienced as burdensome by patients. To what extent it is experienced as burdensome and whether the burden decreases when patients get used to it is unknown. AIM: We prospectively assessed the burden of upper GI endoscopy in BE patients under regular surveillance and in a control group of patients with non-specific upper GI symptoms, and investigated whether these groups perceived the burden of endoscopy differently. METHODS: A total of 394 patients (180 BE patients and 214 patients with non-specific GI symptoms) filled out questionnaires one week before, on the day of, one week after and one month after upper GI endoscopy. Inclusion criteria were a BE segment of at least $2 \mathrm{~cm}$ for the BE group, and dyspepsia without alarm symptoms for the control group. Four variables were assessed: 1) pain and burden experienced during endoscopy, 2) symptoms, 3) psychological distress levels (Hospital Anxiety and Depression scale and Impact of Event Scale), and 4) perceived risk of developing a malignancy. RESULTS: Patients with non-specific GI symptoms were diagnosed with a hiatal hernia (45\%), non-specific gastritis $(25 \%)$, reflux esophagitis $(20 \%)$, and other $(10 \%)$. Of all patients, only $16 \%$ experienced pain from the endoscopy. However, $87 \%$ reported it to be burdensome. Apart from an increase in sore throat (47\% after endoscopy versus $22 \%$ before) the procedure did not cause symptoms. Patients' distress levels were increased in the week prior to the endoscopy compared to the week after. BE patients experienced significantly more burden from the endoscopy (e.g. $7 \%$ reported no burden versus $20 \%$ of the control group) but reported lower distress levels compared to the control group. Patients with a higher risk perception did not report a higher burden or more psychological distress. Having had more prior endoscopies was associated with lower distress levels as measured by the impact of event scale. CONCLUSION Upper GI endoscopy is burdensome for many patients and causes moderate psychological distress. Getting used to the procedure may reduce distress but increases the burden experienced from the procedure in BE patients. Recommendations for endoscopic surveillance should take into account the burden and distress of upper GI endoscopy for patients.

\section{S1815}

Increased Microvascular Density Associated with Progression of Barrett's Esophagus: A New Target for the Next Generation of Endoscopic Technology Andrew Ross, Lucas Campbell, Stefan Seewald, Nib Sohendra, Lynne Stearns, Charles Dye, Amy Noffsinger, John Hart, Irving Waxman

Background: Angiogenesis has been found to be an important factor in the growth malignant neoplasms, including esophageal adenocarcinoma (EC) arising from Barrett's esophagus (BE). When examined using new generation endoscopes employing narrow band imaging (NBI) technology, irregularities in the mucosal vascular pattern have been found to be predictive of $\mathrm{BE}$ with advanced histopathology (high grade dysplasia or intramucosal adenocarcinoma). The aim of the current study was to determine whether microvascular density (MVD) is associated with progression of BE to EC, thus providing a possible "proof-of-principle" for the use of novel imaging technologies such as NBI in the diagnosis and possible treatment of BE with advanced histopathology. Methods: Specimens from patients who had undergone complete Barrett's eradication endoscopic mucosal resection (CBE-EMR) for the treatment of high grade dysplasia (HGD) or intramucosal adenocarcinoma (IMC) were selected. CBEEMR was directed by both white light endoscopy and NBI. Foci of BE, low-grade dysplasia (LGD), HGD and EC were selected from each specimen and were stained with anti-CD31. Following digital scanning, MVD was assessed using Chromavision ${ }^{\circledR}$ software. Mucosa from the gastric cardia served as control (NC). Results: 178 foci were evaluated. Progression from normal mucosa to EC was associated with a significant trend of increasing MVD ( $\mathrm{p}=$ 0.003). Statistically significant differences in MVD (Table) were found between BE and EC $(\mathrm{p}=0.043), \mathrm{LGD}$ and $\mathrm{EC}(\mathrm{p}=0.042), \mathrm{NC}$ and HGD $(\mathrm{p}=0.007)$ and between NC and $\mathrm{EC}(\mathrm{p}=$ 0.002). Measurement of MVD from a significant number of additional mucosectomy specimens will be completed over the next several months. Conclusions: An increase in MVD is associated with neoplastic progression of BE suggesting that neovascularization plays an important role in the metaplasia-dysplasia-carcinoma sequence in BE. Significant differences in MVD between the various pathologic stages of this sequence suggest that imaging technologies such as NBI may be able to accurately identify foci of advanced histopathology based on the appearance of the mucosal vascular pattern. Such technologies hold promise for advances in the diagnosis, surveillance and treatment of BE.

$\mathrm{P}$-value comparison of MVD between histopathologic foci 


\begin{tabular}{|c|c|c|c|c|}
\hline & NC & BE & LGD & HGD \\
\hline BE & 0.11 & -- & -- & -- \\
\hline LGD & 0.10 & 0.83 & -- & -- \\
\hline HGD & 0.007 & 0.19 & 0.59 & -- \\
\hline EC & 0.002 & 0.043 & 0.042 & 0.14 \\
\hline
\end{tabular}

S1816

Cancer Risk in Barrett's Oesophagus: A Meta-Analysis Titus Thomas, Keith R. Abrams, John De Caestecker, Richard J. Robinson

Background: The risk of cancer in Barrett's oesophagus (BO) is uncertain with studies showing a variable annual incidence (from 1/52 to 1/694). Recent reports have suggested regional variations in cancer incidence in the West. However no formal meta-analysis has been performed. Aims and Methods: We aimed to determine by meta-analysis the incidence of oesophageal cancer in patients undergoing surveillance for $\mathrm{BO}$ and to examine geographical variation. A MEDLINE, EMBASE and Pub Med search of all English articles from 1966 to 2004, using the key words "Barrett's oesophagus", "Oesophageal cancer", "surveillance", "short segment Barrett's" (SSBO) was done. References in retrieved papers and relevant review articles were scrutinised to identify papers missed on the initial search. Studies with patients who had histological confirmation of $\mathrm{BO}$ on surveillance, documented follow up data and cancer as the outcome measure were included. Heterogeneity between studies was calculated using the homogeneity statistic $(\mathrm{Q}$ value) and if significant $(\mathrm{p}<0.05)$ a random effects model of meta-analysis was used. Short Segment BO was defined as length of $<3 \mathrm{~cm}$. Results: 42 articles were included in the analysis for conventional $\mathrm{BO}$ and an additional 7 articles were included for SSBO. The overall cancer prevalence was $11.4 \%$ (95\% CI 7.6$17.3 \%)$. The overall incidence rate for cancer was $8 / 1000$ person-years duration of follow up [pyd] (95\% CI, 6-10). There was minimal geographical variation, with the incidence rate in UK being $7 / 1000$ pyd (95\%CI 4-12), USA, 8/1000 pyd (95\% CI 5-13) and Europe,8/ 1000 pyd (95\% CI 5-12) The overall cancer incidence in SSBO was 5/1000 pyd (95\% CI 2-12). There was a non-significant increase in cancer risk in conventional BO compared to SSBO (OR 1.6, 95\% CI 0.56-4.91, p=0.30). Similarly there was a non-significant increase in cancer risk in patients who had medical therapy compared to surgical treatment (OR $1.2,95 \% \mathrm{CI}$ 0.29-5.0, $\mathrm{p}=0.78$ ). There was a non-significant reduction of Barrett's cancer incidence over time $(\mathrm{p}=0.11)$ Conclusion: We found less geographical variation $\mathrm{BO}$ cancer risk than previously suggested between US and UK and a non-significant increase in the risk of cancer in conventional BO vs SSBO. The cancer incidence in Barrett's oesophagus has decreased with time although this is not significant.

\section{S1817}

Fourier Transform Infrared (FTIR) Analysis of Esophagus Using Attenuated Total Reflectance (ATR) for Quantitative Diagnosis of Low Grade Dysplasia Thomas D. Wang, Peyman Sahbaie, Tarun Bhandari, James Crawford, Lisa Dixon, Shai Friedland, George Triadafilopoulos, Roy Soetikno, Christopher Contag

Background/Aims: There is significant interobserver variability in the histological diagnosis of low grade dysplasia from Barrett's that may be improved with use of Fourier transform infrared (FTIR). FTIR measures the spectral absorption of infrared light by tissue, producing a molecular fingerprint that can provide quantitative concentrations of endogenous molecules using attenuated total reflection (ATR). Methods: FTIR spectra were acquired ex vivo from fresh biopsy specimens of esophagus $(n=63)$ from patients with Barrett's $(n=25)$ over the spectral range 950 to $1800 \mathrm{~cm}-1$ using ATR. The concentrations of DNA, protein, glycogen, and mucin were determined quantitatively using a partial least square fit to a calibration set from pure components. The corresponding histology was reviewed independently by two pathologists. Results: The mean concentrations of DNA, protein, glycogen, and mucin for glandular mucosa $(\mathrm{n}=43)$ were $0.09 \pm 0.03,0.66 \pm 0.10,0.05 \pm 0.03,0.20 \pm 0.06 \mathrm{mg} \%$, respectively, and the average values for dysplasia $(n=20)$ were $0.13 \pm 0.04,0.71 \pm 0.10$, $0.02 \pm 0.02,0.14 \pm 0.05 \mathrm{mg} \%$, respectively. Low-grade dysplasia $(\mathrm{n}=16)$, determined from the first pathologist, could be distinguished from glandular mucosa on FTIR by increased DNA ( $p=0.02)$ and decreased mucin $(p<0.001)$. These results agreed with the second pathologist with a sensitivity of $87.5 \%$ and a specificity of $88.7 \%$. Conclusions: FTIR spectra can be quantitatively measured with ATR and reduced to principle components, DNA, protein, glycogen, and mucin. Theses concentrations can provide adjunctive information for pathologists to distinguish low-grade dysplasia in the setting of Barrett's.

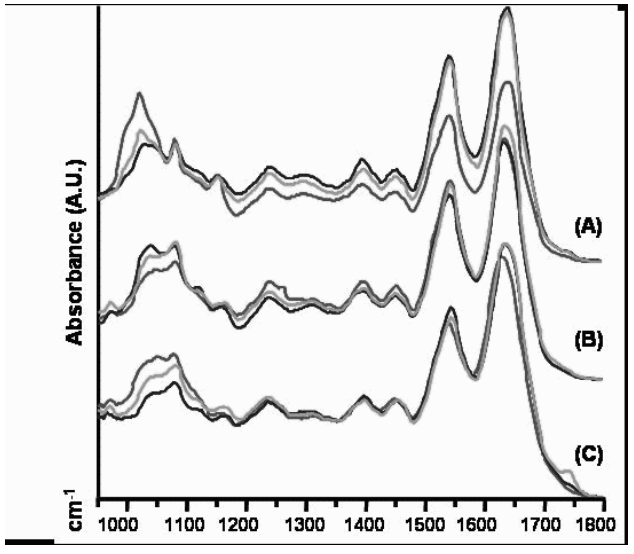

Range of FTIR spectra of esophagus (A) squamous, (B) Barrett's, (C) low grade dysplasia

S1818

Her-2/Neu in Barrett's Esophagus: A Comparative Study Between Histology, Immunohistochemistry and Molecular Technique (FISH)

Elisa Rossi, Domenico Della Casa, Vincenzo Villanacci, Guido Missale, Michele Ghedi, Gianpaolo Cengia, Renzo Cestari

Introduction: HER-2/neu is a protooncogene frequently overexpressed in breast cancer and recently found to be overexpressed in other carcinomas and in Barrett's esophagus (BE) Immunohistochemistry and Fluorescence In Situ Hybridization (FISH) are conventionally used for HER-2 testing in carcinomas, but a single assay is not yet accepted as a "gold standard" in BE. The aim of our study was to evaluate the correlation between histopathologic characterstics and gene espression/amplification along the sequence BE, low grade dysplasia (LGD), high grade dysplasia (HGD) and adenocarcinoma. Methods: we collected 50 endoscopic esophageal biopsies in 42 men and in 8 women with a previous diagnosis of BE. Histopathologic evaluation was carried out using Hematoxylin \& Eosin staining. Paraffin embedded tissue was studied using the HercepTest kit for immunohistochemistry (HercepTest DAKO) and PathVysion kit (PathVysion HER-2 DNA Probe Kit, Vysis) for FISH. HercepTest was scored $0,1+, 2+$ and $3+$ depending on $\%$ membrane staining (cutoff $10 \%$ ), and assessment of gene amplification was based on the ratio between the copy number of HER2/ neu and 17 chromosome. The number of chromosome 17 and HER2/neu signals was scored based on 60 non overlapping nuclei, and HER-2/neu was judged as amplified when the ratio was >2. Results: $21 \mathrm{BE}, 4 \mathrm{LGD}, 12 \mathrm{HGD}$ and 13 cancer of the esophagogastric junction were observed. There was a positive correlation (correlation coefficient 0.88 ) between gene amplification and protein overexpression. In no cases with HercepTest scoring 0 or $1+$ was gene amplification detected, but this was present in $20 \%$ of cases scoring $2+$ and in all cases scoring $3+$. Her-2/neu amplification or overespression was never observed in BE. Coefficient of correlation between the Her-2/neu gene and protein was lower for LGD and HGD when compared to histology ( 0.51 and 0.68 respectively). Gene amplification and overespression was observed in 7 out of 13 adenocarcinomas. Conclusion: Our results suggest that HER2/neu amplification/overexpression may be considered as a marker of progression from $\mathrm{BE}$ to dysplasia. The FISH technique may represent an important and useful diagnostic tool to integrate the result of HercepTest for selecting patients for immunotherapy.

S1819

Patients with Barretts Esophagus Experience Less Reflux Complaints Ahmed Madisch, Stephan Miehlke, Susanne Sell, Bernd Wigginghaus, Manfred Stolte

Background: Barrett's epithelium is assumed to be more resistant to aggressive stomach contents. Thus, it can be postulated, that sensory perception may be less in patients with Barrett's oesophagus (BE). Aim: to assess the severity of reflux complaints in consecutive patients presenting with Barrett's esophagus and reflux esophagitis. Methods: Prospectively consecutive patients with BE (diagnosed by histology) were interviewed by standardized questionnaires comprising both current and history of reflux complaints (heartburn, acid regurgitation). Patients with a reflux esophagitis diagnosed by endoscopy served as a control group. Results: 181 patients with BE (60 female, mean age 58.4 years) and 85 patients with reflux esophagitis ( 36 female, mean age 50.6 years) were included in the analysis. Severity and frequency of current reflux complaints were significantly more pronounced in patients with reflux esophagitis than in patients with $\mathrm{BE}(\mathrm{p}<0.05)$. In both groups younger patients ( $<60$ years) suffer from more severe and frequent reflux complaints than the older patients $(p<0.05)$. Endoscopy in the past was performed in $76.1 \%$ of reflux esophagitis patients due to reflux complaints compared to $47.9 \%$ in BE patients ( $\mathrm{p}<0.0001$ ). The history of reflux complaints were comparable in both groups $(\mathrm{p}>0.05)$. Conclusion: Patients with $\mathrm{BE}$ suffer less from reflux complaints than patients with reflux esophagitis. Thus, reflux complaints do not seem to be suitable for symptom-based screening of patients with BE. 
S1820

Abnormal Expression of Biomarkers in Incompletely Ablated Barrett's Esophagus

Katerina Dvorak, Lois Ramsey, Claire M. Payne, Richard Sampliner, Lisa Camargo, Ronnie Fass, Harris Bernstein, Carol Bernstein, Anil Prasad, Harinder Garewal

Background \& Aims: Barrett's esophagus (BE) is a premalignant lesion of the esophagus in which squamous epithelium is replaced by metaplastic columnar epithelium (intestinal metaplasia, IM). Ablation of IM and its replacement by squamous epithelium is achievable by combining a endoscopic ablation method with acid suppression. We previously found that when there is complete ablation, the neo-squamous epithelium is normal histologically and in biomarker expression. However, squamous islands observed after prolonged use of PPIs were associated with abnormalities in p53 expression and Ki-67 labeling at the new squamous junctions. The aim of the present study was to extend these observations to areas of new squamo-columnar junctions resulting from incomplete ablation produced by reinjury and proton pump inhibitors (PPIs) in patients with non-dysplastic (ND) BE prior to ablation. This report extends and confirms our previous observations and includes two new biomarkers, DNA repair enzyme, Ref-1 and cyclooxygenase-2 (COX-2), Methods: Twenty-one nondysplastic BE cases with incomplete ablation were evaluated for the expression of Ref-1, p53, COX-2 and proliferation (Ki-67) by immunohistochemistry. We focused on junctional areas, i.e. biopsies displaying both squamous epithelium and IM, as we previously found abnormal biomarker expression localized at the junctions. Results: Pre-ablation biopsies that were ND showed the expected normal staining patterns in columnar epithelium, i.e. negative or mild staining for Ref- 1 and p53 expression, normal Ki-67 labeling and rare positive COX2 staining of interstitial cells. In contrast, post-ablation biopsies demonstrated abnormal staining patterns in the glandular area at the new squamo-columnar junctions. Increased expression of Ref- 1 was detected in 6 out of 19 (32\%) patients. In 13 of 21 post-ablation cases $(62 \%)$, increased Ki-67 staining was observed in BE glands at squamo-columnar junctions. Similarly, in 8 of 21 post-ablation junctions (38\%) there was increased p53 staining, and in 8 out of 21 patients (38\%) there was increased COX-2 expression in columnar epithelium. Conclusions: Our findings of increased expression of cancer-associated biomarkers in BE patients with incomplete endoscopic ablation raise a cautionary note regarding this procedure. We hypothesize that newly formed junctions contain cells experiencing increased stress and are thus more susceptible to genomic damage.

\section{S1821}

Systematic Review of Adenocarcinoma Risk in Barrett's Esophagus with Particular Reference to short Segment Barrett's Esophagus and Intestinal Metaplasia

Jose Santana, Mostafizur Khan, Nimish Vakil, Paul Moayyedi

Introduction: Surveillance is recommended for Barrett's esophagus as this condition is associated with an increased risk of esophageal adenocarcinoma. The magnitude of the risk is uncertain as a previous systematic review suggested there was publication bias in the literature. Furthermore definitions of Barrett's have changed to include short segment Barrett's esophagus (SSBE) and to exclude those in whom intestinal metaplasia (IM) is not present regardless of length of the Barrett's segment. We have therefore conducted an up to date systematic literature review to identify current esophageal adenocarcinoma risk and assess the risk in those with SSBE and those without IM. Methods: An electronic database search (Medline and Embase) was conducted to July 2005. Eligibility and data extraction was performed by two independent reviewers. Rates of conversion to esophageal adenocarcinoma were synthesised using a random effects model (DerSimonian and Laird). Funnel plots were produced for each comparison, and Egger's test for funnel plot asymmetry was used to investigate whether or not the results were affected by publication bias. Results: 46 papers were eligible evaluating 11,056 cases of Barrett's esophagus in 42,880 patient-years followup, with 255 cases of esophageal cancer. The overall incidence ratio was $0.75 \%$ per year ( $95 \% \mathrm{CI}=0.56 \%$ to $1.0 \%$ per year). There was no funnel plot asymmetry $(\mathrm{p}=0.19)$ in these data. Nine papers compared the esophageal cancer risk in long segment Barrett's (LSBE) versus SSBE. There were 66 cancers in 5295 patient years follow-up in the LSBE group compared with 9 cancers in 1311 patient years follow-up in the SSBE group $(0.67 \%$ per year increased risk in LSBE group: $95 \% \mathrm{CI}=0.39$ to $0.94 \%$ ). Three papers compared the esophageal cancer risk in Barrett's with and without IM at baseline. There were 41 cancers in 7834 patient years follow-up in the IM group compared with 3 cancers in 1966 patient years follow-up in the no IM group $(0.59 \%$ /year increased risk in IM group: $95 \% \mathrm{CI}=0$ to $1.2 \%)$. Conclusion: This up to date systematic review suggests the annual esophageal adenocarcinoma risk in Barrett's patients is $0.75 \%$ ( $95 \% \mathrm{CI}=0.56 \%$ to $1.0 \%$ ) and this is not subject to publication bias. Current practice of including SSBE but excluding patients without IM regardless of length of Barrett's is illogical given the relative cancer risks.

\section{S1822}

Loss of Goblet Cell Differentiation Occurs with the Progression of Dysplasia in Barrett's Esophagus

Amitabh Srivastava, Jason L. Hornick, Xiaohong Li, Carissa Sanchez, Brian J. Reid, Robert D. Odze

Background: Barrett's esophagus (BE) is defined by the presence of intestinalized epithelium [i.e., goblet cells (GC)] in the esophagus. It is commonly believed that this type of epithelium forms the "field" upon which dysplasia develops. However, the specific role of GC's is the pathogenesis of dysplasia in $\mathrm{BE}$ has never been evaluated. The aim of this novel study was to evaluate GC density in relationship to dysplasia, and to correlate this feature with outcome in a large prospective surveillance cohort of BE patients that began in 1983. Design: 3778 routinely processed biopsies from $15 \mathrm{l} \mathrm{BE}$ patients (M/F ratio;134/17, mean age; 62 years, mean length of $\mathrm{BE} ; 6.5 \mathrm{~cm}$, mean surveillance duration; 55.9 months), 63 of whom eventually developed cancer, were evaluated for the number (\#) of GC's per crypt by counting all GC's in each biopsy and dividing this number by the total number of crypts per biopsy specimen, in both dysplastic and non-dysplastic epithelium separately. Non-dysplastic epithelium, both adjacent to and distant from dysplasia, was also evaluated. The mean \# of GC/crypt ("GC density") was correlated with the degree of dysplasia, and with cancer development, using a Cox regression model to account for follow-up intervals and censored data. Result: The total GC density in non-dysplastic crypts (5.1) was significantly higher than in dysplastic crypts $(0.4, \mathrm{p}<0.0001)$. Interestingly, the GC density in non-dysplastic epithelium from patients without dysplasia (5.63) was significantly higher than in non-dysplastic epithelium from patients with either low-grade dysplasia (LGD) (4.58) or high-grade dysplasia (HGD) (4.65) as their maximum dysplasia diagnosis ( $\mathrm{p}<0.01$ for both comparisons). GC density in non-dysplastic crypts did not correlate highly with risk of carcinoma (relative risk; 0.98, $\mathrm{p}=0.09$ ). Conclusion: Loss of GC differentiation is associated with the progression of dysplasia in BE. GC density in non-dysplastic crypts is not a useful predictor of cancer risk in BE.

S1823

Histological Evaluation of Resection Specimens Obtained At 244 Endoscopic Resections in Barrett's Esophagus

Femke P. Peters, Lianne P. Brakenhoff, Wouter L. Curvers, Wilda D. Rosmolen, Fiebo J. Ten Kate, Kausilia K. Krishnadath, Paul Fockens, Jacques J. Bergman

Aim To study the histological evaluation of endoscopic resection (ER) specimens of early neoplastic lesions in Barrett's esophagus (BE). Patients and methods Routine histological evaluations of ER specimens obtained at all ERs in BE performed between '00-'05 were evaluated. Specimens were pinned down on paraffin (mucosal side up) and immediately fixed in $2 \%$ formalin. No attempts were made to reconstruct the specimens after piece-meal resection. Specimens were cut in $2 \mathrm{~mm}$ slices. Standard H\&E staining was performed on $4 \mu \mathrm{m}$ slides and, if necessary, supplemented with p53 and Ki-67 immunohistochemical staining. All evaluations were performed by two experienced pathologists. Results 244 ERs were performed for low-grade intraepithelial neoplasia (LGIN) in a dysplasia associated lesion or mass $(n=1)$, high-grade intraepithelial neoplasia (HGIN) (n=82), early carcinoma (EC) $(n=53)$ or removal non-dysplastic $B E(n=108)$ for stepwise radical endoscopic resection (SRER). In 194 ERs a piece-meal resection was performed: 97 for HGIN/EC and 97 for SRER with median number of 3 resections (IQR 2-5). A total of 921 specimens were obtained. Histology of the specimens revealed no dysplasia in 48 ERs (all SRER), LGIN in 42, HGIN in $89, \mathrm{Tl} \mathrm{m}$ in 50 , and Tlsm in 15 ERs. Fifty cancers ( $77 \%$ ) were classified as well differentiated (G1). Moderately (G2) or poorly (G3) differentiated lesions were found in $8(12 \%)$ and 7 (11\%) of cancers, respectively. G2-3 cancers significantly more often invaded the submucosa than the G1 cancers $(10 / 15$ vs. $5 / 50, p<0.001$ ). One G3 Tlm3 (penetration into second muscularis mucosae layer) tumor showed lymph vessel invasion. In none of the 921 resection specimens vascular invasion was observed. Specimens of 14 ERs (5\%) had a deeper margin positive for neoplasia. Significantly more G2-3 cancers were irradically removed (6/15 vs $6 / 50 ; p=0.024)$. Of the attempted en-bloc resections, only 8 out of 52 specimens $(22 \%)$ had lateral margins that were found to be free of HGIN/EC. Mechanical and thermal artefacts interfering with the histological evaluation were seen in 61 (23\%) of the procedures. Conclusions Most endoscopically resected early neoplastic lesions in BE contain HGIN or G1 mucosal cancers, and have a deeper margin free of neoplasia. Submucosal invasion is associated with a poorer differentiation grade and invasion into lymph vessels or blood vessels are rare findings. The majority of ERs require piece-meal resection and in cases where an en-bloc resection is attempted, only the minority of the ER specimens have lateral resection margins free of dysplasia. In addition, artefacts affect histological evaluation in a significant percentage of specimens.

\section{S1824}

Flow Cytometry As a Marker of Occult Advanced Neoplasia in Barrett's Esophagus

Wai-Kit Lo, Timothy Kuo, Jason Hornick, Chinweike Ukomadu, John M. Poneros

Background: Previous studies suggest that flow cytometric abnormalities may predict malignant potential better than histology in patients with Barrett's esophagus (BE). Aim: To compare the DNA content in non-dysplastic BE (NDBE) specimens from patients with and without esophageal adenocarcinoma (ADC) or high-grade dysplasia (HGD). The hypothesis being tested is that the fraction of cells in S-phase from NDBE specimens from patients with $A D C$ or HGD is higher than the S-phase fraction from NDBE specimens from patients without coincident ADC/HGD. Methods: 135 biopsies were obtained from 24 patients with known BE undergoing EGD for BE surveillance (2/24) or BE-associated ADC undergoing EUS tumor staging (22/24). Biopsies were taken as per standard BE surveillance protocol, consisting of 4-quadrant "jumbo" biopsies every $2 \mathrm{~cm}$ of BE. Biopsies were bisected; half was sent for flow cytometric analysis and half for routine histopathologic interpretation. All histologic samples were reviewed by a single gastrointestinal pathologist. Data were stratified according to the pathologic diagnosis of each sample and the highest histologic grade of dysplasia (or carcinoma) identified in each patient. Differences in cell cycle populations were calculated using a Student's t-test with two-tailed distribution assuming equal variance and independence between specimens. Results: After refining the cell sorting technique, 79 samples contained cells that were sufficient for cytometric analysis; these were collected from 18 different patients. Significantly higher fractions of cells in S-phase were found in NDBE samples from patients with a history of ADC/HGD ( $n=15$, avg. 27.3\%), compared with NDBE from patients without a history of ADC/HGD ( $\mathrm{n}=11$, avg. 20.8\%) $(\mathrm{p}=0.03)$. There was also a difference between the combined S-phase and G2 (tetraploid) fractions in NDBE samples from patients with ADC/HGD ( $\mathrm{n}=15$, avg. 35.3\%) compared with those without ADC/HGD ( $n=11$, avg. 29.4), although this did not reach statistical significance ( $\mathrm{p}=$ 0.057). S-phase fractions were significantly elevated in ADC samples ( $n=13$, avg. $28.8 \%$ ) compared with NDBE without a history of $\mathrm{ADC} / \mathrm{HGD}(\mathrm{p}=0.04)$. However, in patients with $\mathrm{ADC} / \mathrm{HGD}$, there was no significant difference in the S-phase fractions between ADC samples and NDBE specimens. Conclusion: S-phase fractions of cells as measured by flow cytometry were significantly different in NDBE specimens in patients with and without ADC/HGD. These preliminary findings suggest that flow cytometry may be useful in the detection of coincident advanced neoplasia in patients with $\mathrm{BE}$. 


\section{S1825}

Prevalence of Barrett's Esophagus in a Population Not Primarily Referred for Gastro-Esophageal Reflux Disease

Brechje C. van Eijck, Leonieke M. Wolters, Herman van Dekken, Ernst J. Kuipers, Peter D. Siersema

Background: Cancer of the esophagus, particularly esophageal adenocarcinoma (EAC), is the fastest growing malignancy worldwide. Barrett's esophagus (BE) is an important risk factor for EAC. As it is largely unknown what the prevalence of BE is in asymptomatic individuals, we aimed to study the prevalence of $\mathrm{BE}$ in a population which was not primarily referred for upper gastrointestinal (GI) complaints. Methods: A total of 250 patients undergoing routine, non urgent colonoscopy were included in this study. All patients underwent an upper GI endoscopy prior to colonoscopy. All patients filled out the Gastrointestinal Symptom Rating Scale (GSRS). Biopsies were taken from the Z-line $(n=4)$, and, if BE was suspected, additional 4-quadrant biopsies were taken. Also, biopsies were taken from antrum $(\mathrm{n}=2)$ and corpus $(\mathrm{n}=2)$ to detect H. pylori infection. All biopsies were reviewed by an experienced GI pathologist. Results: In 22 of 250 patients (9\%, 95\% CI $5.6-13.0 \%$ ), endoscopical BE (20 short-segment (SS) $(\leq 2 \mathrm{~cm})$ and 2 long-segment $(\mathrm{LS})(>2 \mathrm{~cm}))$ was found and histologically confirmed (intestinal metaplasia). In these 22 patients, 18 had no dysplasia and 4 had low-grade dysplasia. In another 15 patients (6\%), an endoscopic diagnosis of SSBE was histologically not confirmed. In 12 patients (5\%), intestinal metaplasia of the cardia was found without endoscopical evidence of columnar epithelium in the distal esophagus. Two patients $(1 \%, 95 \% \mathrm{CI} 0.1-2.9 \%$ ) were diagnosed with EAC (a T2NOM0 and T2N1Ml tumor respectively). H. pylori was found in 50 of 250 patients (20\%). BE patients (mean \pm SD: $59.6 \pm 9.6 \mathrm{yrs}$ ) were older than patients without $\mathrm{BE}(51.7 \pm 14.6 \mathrm{yrs})$ $(\mathrm{p}=0.015)$, but gender was not different. In addition, no significant differences were found between subjects with and without BE with respect to smoking (33\% vs. $26 \%$, respectively), alcohol intake ( $66 \%$ vs. $61 \%$ ), use of proton pomp inhibitors ( $18 \%$ vs. $18 \%$ ), use of NSAID/ aspirin $(23 \%$ vs. $16 \%)$, H. pylori infection ( $27 \%$ vs. $19 \%)$ or prevalence of a BMI $>25(57 \%$ vs. $41 \%$ ). Gastrointestinal symptoms, including reflux symptoms, were not different between patients with BE and those without (31\% vs. 33\%). Conclusions: BE was detected in $9 \%$ and EAC in $1 \%$ of patients not primarily referred for evaluation of GERD symptoms. A major question that arises from this prospective study is whether endoscopic screening for BE should be applied to the general population in an effort to identify patients with BE and offer them endoscopic surveillance to detect neoplastic changes at an earlier and therefore more curable stage.

\section{S1826}

Suppression of Acid Reflux with Double-Dose Esomeprazole for 6 Months Does Not Alter DNA Methylation in Patients with Barrett's Esophagus Eric Smith, Paul A. Drew, Awni Abu-Sneineh, William C. Tam, Andrew R. Ruszkiewicz, Mark N. Schoeman, John Dent, Richard H. Holloway

Introduction: Barrett's esophagus is a sequela of longstanding gastroesophageal reflux and is a major risk factor for adenocarcinoma. Aberrant DNA methylation is frequent in Barrett's esophagus and adenocarcinoma and may contribute to the disease progression. Aim: To test the hypothesis that short term high-level acid reflux control by esomeprazole $40 \mathrm{mg}$ bd in patients with Barrett's esophagus will reduce DNA methylation in the esophageal mucosa. Method: Eighteen patients with histologically proven Barrett's esophagus on standard doses of PPI (omeprazole $20 \mathrm{mg}$, lanseprazole $30 \mathrm{mg}$ or pantoprazole $40 \mathrm{mg}$ ) were studied before and after 6 months treatment with esomeprazole $40 \mathrm{mg}$ bd. Esophageal acid exposure was measured by 24 hour $\mathrm{pH}$ monitoring before and 2 months after starting esomeprazole. Biopsies were obtained at $2 \mathrm{~cm}$ intervals from the columnar epithelium and from the adjacent squamous epithelium of the esophagus before and after 6 months of treatment. In each biopsy methylation was measured in the promoter region of 9 genes - APC, CDKN2A, ID4, HPPl, MGMT, RBP1, RUNX3, SFRP1 and TIMP3. Results: Nine patients of the 18 patients had excessive ( $\mathrm{pH}>4$ of $>5 \%$ of the time) acid reflux on standard PPI therapy. Esomeprazole $40 \mathrm{mg}$ bd effectively eliminated acid reflux in 16 of 18 patients $(\mathrm{pH}<4,<1 \%)$ and substantially reduced it in the other 2 patients although it remained increased $(6.5 \%$ and $11.7 \%)$. There was significantly more methylation in the columnar epithelium of all patients compared to the squamous epithelium $(\mathrm{p}<0.0001)$ both before and after 6 months of treatment. However, there was no statistical difference in the methylation in the columnar epithelium of patients who had reflux compared to those that did not have reflux, either before commencement or after 6 months of treatment. Conclusions: Effective control of acid reflux with esomeprazole $40 \mathrm{mg}$ bd neither increased nor decreased the amount of methylation in the panel of relevant gene promoters tested, either in patients whose reflux was controlled at the start of the trial, or those with reflux at the start of the trial which was reversed by the treatment. These findings suggest the need for further study to determine the benefit of the control of acid exposure in preventing or reversing abnormal DNA methylation in Barrett's esophagus, and the possible development of adenocarcinoma. (Supported by Grant-in Aid from AstraZeneca)

\section{S1827}

Impaired Gallbladder Motility in Patients with Barrett's Esophagus Ferenc Izbeki, Andras Rosztoczy, John Y. Sebit, Janos Lonovics, Tibor Wittmann

Background: Although duodenogastric biliary reflux has been demonstrated to occur more frequently in patients with different motility disorders of the gallbladder and biliary reflux is claimed to have a pathogenetic role in the development of Barrett's esophagus (BE) in patients with gastroesophageal reflux disease (GERD), the gallbladder function in patients with $\mathrm{BE}$ is yet to be studied. Therefore the aim of the present study was to investigate the gallbladder motility in patients with Barrett's esophagus. Materials and methods: Three groups were studied: 18 healthy volunteers, 14 patients with GERD and 24 patients with $\mathrm{BE}$. The diagnosis of $\mathrm{BE}$ based on endoscopic and histological evaluation of the patients. All patients with GERD had abnormal 24 hours esophageal $\mathrm{pH}$ monitoring, while those with BE had abnormal Bilitec results as well. Each subject in the three groups underwent abdominal ultrasound (US) examination of the gallbladder. Gallbladder volume was calculated by the ellipsoid method. The US examination was done after an overnight fast. The gallbladder volume was measured in fasting state (BV) and after consumption of a standardized test meal at 10 minutes intervals for 90 minutes. Parameters, such as (1) Ejection volume (EV) the difference between BV and residual volume (RV); (2) Ejection fraction $(\mathrm{EF} \%)$ the difference between BV and RV expressed as percentage BV; (3) Ejection rate (ER $\% / \mathrm{min}$ ) EF divided by time requested to reach the RV were calculated. Results: All of the studied parameters were similar in patients with GERD and in controls. Patients with BE had significantly higher BV $(24.7 \pm 3.5 \mathrm{ml}$ vs. $21.6 \pm 1.9 \mathrm{ml}), \mathrm{RV}(9.8 \pm 2.9 \mathrm{ml}$ vs. $5.1 \pm 0.7 \mathrm{ml})$ and lower EF $(61.0 \pm 7.2 \%$ vs. $76.1 \pm 2.1 \%)$, ER $(1.07 \pm 0.29 \% / \mathrm{min}$ vs. $1.39 \pm 0.21 \% / \mathrm{min})$ values than healthy controls. Patients with BE had also significantly higher RV $(9.8 \pm 2.9 \mathrm{~m}$ vs. $6.1+0.9 \mathrm{ml})$ and lower $\mathrm{EF}(61.0 \pm 7.2 \%$ vs. $72.9 \pm 4.3 \%)$ than those with GERD. Conclusion Our results support the notion that patients with BE have a more complex gastrointestina motility disorder involving the gallbladder also than those with uncomplicated GERD.

\section{S1828}

Prospecive Evaluation of the New Prague Classification for Barrett's Esophagus Andreas Leodolter, Peer Oberueber, Dietmar Zielinski, Michael Vieth, Manfred Stolte, Jochen Labenz

An endoscopic classification of Barrett's esophagus (BE) does not exist. In 2004 an international consensus group proposed the Prague classification for endoscopic assessment of BE The aim of the present study was to evaluate the performance and validity of the new classification in clinical practice. Methods: 110 consecutive patients $(65 \mathrm{~m}, 45 \mathrm{f})$ with endoscopic suspicion of columnar epithelium lined lower esophagus, attending our endoscopy unit, entered the study. The esophageal findings were printed and the endoscopist classified the findings according to the Prague classification: measurement of the circumferential (C) and maximal extension of the columnar epithelium above the top of the gastric folds. Biopsies were taken for histological assessment at the stomach, columnar epithelium of the lower esophagus, and from the squamous epithelium $2 \mathrm{~cm}$ above the Z-line. Furthermore al patients answered a standardized questionnaire (GERD symptoms, past GERD symptoms, concomitant diseases, smoking, ethanol intake). Results: In 47 patients (43\%) the endoscopic suspected BE was confirmed by histology. Symptom pattern was not different between patients with or without confirmed BE. Even in short tongues $(<5 \mathrm{~mm})$ the probability of detecting intestinal metaplasia was around $30 \%$, comparable to the detection rate in tongues up to $20 \mathrm{~mm}$. In patients with tongues of $20 \mathrm{~mm}$ or circumferential extension the likelihood of histological confirmation of $\mathrm{BE}$ is high. Conclusion: The new Prague classification is useful for standardized endoscopic assessment of BE in the daily clinical practice. In almost all patients with circumferential extension or tongues of columnar epithelium of more than $20 \mathrm{~mm}$, specialized intestinal metaplasia was found on histology, whereas only one third of the patients had true BE if the tongues were less than $20 \mathrm{~mm}$.

\begin{tabular}{|c|c|c|}
\hline $\begin{array}{c}\text { Maximal extension of columnar } \\
\text { epithelium }(\mathrm{M})\end{array}$ & $\begin{array}{c}\text { Prevalence of } \\
\text { BE }\end{array}$ & $\begin{array}{c}\text { Prevalence of BE in those w/o } \\
\text { circumferential segment (C=0) }\end{array}$ \\
\hline$>0-5 \mathrm{~mm}$ & $9 / 29(31 \%)$ & $9 / 29(31 \%)$ \\
\hline $6-10 \mathrm{~mm}$ & $15 / 43(35 \%)$ & $14 / 41(34 \%)$ \\
\hline $11-20 \mathrm{~mm}$ & $7 / 19(37 \%)$ & $6 / 17(35 \%)$ \\
\hline$>20 \mathrm{~mm}$ & $16 / 19(84 \%)$ & $3 / 5(60 \%)$ \\
\hline $\begin{array}{c}\text { Circumferential segment of columnar } \\
\text { epithelium (C) }\end{array}$ & $2 / 4(50 \%)$ & \\
\hline$>0-5 \mathrm{~mm}$ & $5 / 5(100 \%)$ & \\
\hline $6-20 \mathrm{~mm}$ & $8 / 9(89 \%)$ & \\
\hline$>20 \mathrm{~mm}$ & & \\
\hline &
\end{tabular}

S1829

Is C-Myb mRNA Over Expression a Good Molecular Marker for Low-Grade Dysplasia in Barrett's Esophagus (BE)?

Christo van Rensburg, Caroline Daniels, Colleen Wright, Martin Kidd, Nico de Villiers, Maritha Kotze

Background The subjective histological evaluation of mucosal biopsy specimens to predict cancer risk in $\mathrm{BE}$ is an ongoing concern and thus the development of molecular markers that can objectively identify these patients is highly desirable. To date, the most promising markers seem to be molecular abnormalities (mutations and loss of heterozygosity) of pl6 and $\mathrm{p} 53$, although DNA ploidy has potential use. In a pilot study significantly more ( $\mathrm{p}=$ 0.03 ) patients $(9 / 13)$ with low grade dysplasia showed over expression of c-myb mRNA compared with patients $(9 / 26)$ with only Barrett's metaplasia (BM). Currently immunostaining for $\mathrm{p} 53$ has a potential, but undefined role in the histological assessment of dysplasia in biopsy specimens of BE. Objective To evaluate the role of c-myb mRNA over expression as an adjunct in differentiating low-grade dysplasia in BE from BM and inflammatory atypia and to correlate these findings with TP53 immuno-staining of the biopsy specimens. Patients and Methods Two expert gastro-intestinal patologists reviewed and reached consensus on the histological diagnoses. The c-myb mRNA expression in the Barrett's epithelium was determined by RT-PCR. The histological specimen for TP53 immuno-histochemistry was stained with a rabbit polyclonal antibody (NCL-p53-CM1, Novocastra Laboratories Ltd), and scored by using a semi-quantitative scale from 0 to $3+$ as follows: 0 , fewer than $1 \%$ o cells staining; 1 , up to $10 \%$ of cells staining, primarily in the lower third of the crypts; 2 more than $10 \%$ but fewer than $50 \%$ staining; and 3, more than $50 \%$ of cells staining. Results A total of 55 Barrett's patients of whom 36 had BM and 19 with mild dysplasia (BD) were evaluated. Over expression of c-myb mRNA occured in 39\% (14/36) of the BM patients and $63 \%$ of the mild $\mathrm{BD}(12 / 19 ; \mathrm{p}<0.05)$. The results of the consensus histological diagnoses correlated with p53 immuno-staining intensity are summarized in table 1. Compared to BM, more specimens of patients with mild BD stained positive for TP53 (64 vs. 84\%) 
However, TP53 staining of $2+$ and $3+$ intensity was similar in both groups (58\%). Conclusion In this study c-myb analysis enhanced the diagnostic assessment of low-grade dysplasia in BE significantly. TP53 immuno-staining though positive in more than $80 \%$ patients with mild $\mathrm{BD}$, does not help to differentiate mild BD from BM.

Table 1. Results of intensity of TP53 staining of Barrett's oesophagus specimens. Data are given as number (percentage).

\begin{tabular}{|c|c|c|c|c|c|c|}
\hline ConsensusHistological Category & & & & & & \\
\hline & Grade 0 & Grade 1 & Grade 2 & Grade 3 & Total Positive & Total \\
\hline Barrett'sMetaplasia & $13(36 \%)$ & $2(6 \%)$ & $18(50 \%)$ & $3(8 \%)$ & $23(64 \%)$ & 36 \\
\hline Barrett'slow-grade dysplasia & $3(16 \%)$ & $5(26 \%)$ & $7(37 \%)$ & $4(21 \%)$ & $16(84 \%)$ & 19 \\
\hline
\end{tabular}

\section{S1830}

No Uniform Approach for the Treatment of High Grade Dysplasia and Early Carcinoma in Barrett's Esophagus Patients

Wouter L. Curvers, Henk Festen, Wim Haameteman, Gerard A. Meijer, Frans T. Peters,

Pieter D. Siersema, H W. Tilanus, Jacques J. Bergman

Introduction Barrett's esophagus (BE) is a premalignant condition for which endoscopic surveillance is recommended. The last few years the effectiveness of endoscopic surveillance programs in BE patients has been questioned. In order to develop new guidelines the Dutch Barrett's Registration Foundation sent out a questionnaire to all Dutch gastroenterologists and internists performing endoscopy to assess the current status of surveillance and management of BE patients in the Netherlands. Methods A questionnaire consisting of 42 questions on the general organisation of Barrett's surveillance programs and daily management of BE patients was sent to 337 endoscopists. A reminder was send to all endoscopists who had not responded after 6 weeks. For all endoscopists who had not responded after the reminder the addresses were checked and a third mailing was sent. Finally, all endoscopists who had not complied after the third mailing received again an email with the request to fill out the questionnaire. Results The response rate was $86 \%$. Ninety-six percent of the responders supported Barrett's surveillance. Barrett's surveillance was mainly conducted according to the current ACG guidelines. Co-morbidity and age played an important role in the decision to initiate Barrett's surveillance and in the treatment choice for high grade dysplasia (HGD) and early carcinoma (EC) in BE. There was, however, no uniform approach in the management of patients with HGD and EC. Surgical resection was often the treatment of choice despite of the good results that are currently achieved with endoscopic treatment of these lesions. If confronted with a 55-year old patient with HGD, 30\% chose for surgical resection, whereas 91\% chose surgical resection if the same patient had a $1 \mathrm{~cm}$ nodule with adenocarcinoma. Fifty-four percent chose for surgery in a 75-year old patient with adenocarcinoma in a 1 cm nodule. Of all responders, 39\% would treat patients with HGD and EC in a center with no specific expertise in this field and without consulting the available multidisciplinary Barrett's advisory organs in The Netherlands. Conclusion Endoscopic surveillance for BE is widely accepted in the Netherlands. The treatment of patients with HGD and EC in BE, however, varies considerably. There is a need for more awareness of the possibilities of endoscopic treatment of HGD and EC in BE patients. Until proven otherwise, these type of patients should probably be treated in centers with ample experience in endoscopic treatment. New guidelines for the treatment of HGD and EC need to be developed. Astra Zeneca BV, Netherlands, supported this study with an unrestricted grant.

\section{S1831}

Immunohistochemical Evaluation of COX-2, CDX2 and CDC2 in Barrett's Esophagus-Dysplasia-Adenocarcinoma Progression

Vincenzo Villanacci, Elisa Rossi, Domenico Della Casa, Paolo Lancini, Luigi Minelli, Guido Missale, Claudia Zambelli, Renzo Cestari

Introduction: Barrett's esophagus (BE) is a pre-neoplastic condition and early detection of dysplasia in Barretts's progression to cancer is the key point in clinical practice. Gastrointestinal pre-neoplastic conditions can be assessed using COX-2 (Ciclooxigenase-2), CDX2 (caudal type homeobox transcription factor 2) and CDC2 (cell division cycle 2) expression, and the aim of our study was to evaluate COX-2, CDX2 and CDC2 expression in the sequence BEdysplasia-adenocarcinoma (ADC) as a tool for early detection of dysplasia in BE. Methods: We studied 46 esophageal biopsies (18 BE, 15 dysplasia and $13 \mathrm{ADC}$ ) by comparing histopathologic characteristics (Hematoxylin \& Eosin) with COX-2, CDX2 and CDC2 immunoistochemical expression. Monoclonal antibodies were used according to manufacturer instructions. Results: COX-2 expression was overexpressed in all 3 conditions and did not correlate with BE-dysplasia-ADC sequence, but it was however significantly correlated with the degree of differentiation (grading) in ADC. Percentage of nuclear CDX2 positive cells decreased from $71 \%$ to $35 \%$ and $17 \%$ for $\mathrm{BE}$, low grade (LGD) and high grade dysplasia (HGD), respectively. In ADC $30 \%$ of cells were CDX2 positive, a value unaffected by cancer grading. Percentage of $\mathrm{CDC} 2$ positive cells significantly increased from $2 \%$ in $\mathrm{BE}$, to $6 \%$ in LGD and to $49 \%$ in $\mathrm{HGD}(\mathrm{p}<0.05)$. In ADC $63 \%$ of cells were CDC2 positive, and positivity was significantly correlated $(\mathrm{p}=0.003)$ with cancer grading. Conclusions: Our results show an uniform COX-2 expression in all samples analysed, with slight differencies in ADC. By contrast with COX-2, CDC2 expression was increased and CDX2 was decreased during progression from BE to ADC (Figure), suggesting that these markers might be useful in clinical practice for early detection of HGD.
S1832

Meta-Analysis of Bleeding with Low-Dose Aspirin and Clopidogrel in Randomized Controlled Trials

Kenneth McQuaid, Loren Laine

BACKGROUND: Prior meta-analyses of low-dose aspirin have shortcomings: doses outside the accepted 75-325mg range are included, concurrent anticoagulant use is allowed, duration of therapy is not considered, all CV prevention studies are not included, the most clinically relevant results (absolute increase in incidence, number needed to harm (NNH)) are not provided. METHODS:PUBMED and Cochrane CCTR were searched for RCTs of aspirin (75-325 mg qd) and/or clopidogrel therapy for prevention of CV disease. Primary endpoints were major bleeding (author-defined, or led to hospitalization, transfusion, or death) and intracranial bleeding. RESULTS: 25 studies were included. Study quality was good: Jadad score $(0-5)$ was $\geq 3$ in 23 studies and $\geq 4$ in 19. Aspirin vs. Placebo: Meta-analysis of placebo-controlled aspirin trials is shown in the Table. The weighted incidence of major GI bleeding with placebo was $0.12 \%$ per year. The absolute increase in incidence with aspirin above placebo was $0.12 \%$ per yr $(95 \% \mathrm{CI}, 0.07-0.19 \%$ per yr) and $\mathrm{NNH}$ at 1 yr was 833 (526-1429). Aspirin vs. Clopidogrel: No study compared clopidogrel with placebo. One RCT $(\mathrm{N}=19,485)$ compared clopidogrel with aspirin $325 \mathrm{mg}$. The RRs of major GI bleeding (RR $1.45 ; 1.00-2.10$ ) and all GI bleeding (RR $1.34 ; 1.11-1.61$ ) were increased in patients taking aspirin. The absolute increase in incidence of major GI bleeding was $0.12 \%$ per yr $(0.00-0.28 \%$ per yr) with an NNH at 1 yr of $833(95 \% \mathrm{CI}, 357-\infty)$. The cost to prevent one major GI bleeding episode from aspirin in 1 yr by substituting clopidogrel would be $\$ 1,216,180$. Aspirin + Clopidogrel vs. Either Alone: Two RCTs $(\mathrm{N}=20,161)$ revealed a decreased risk with aspirin alone ( $R R=0.56 ; 0.39-0.80)$ and with clopidogrel alone ( $R R=$ $0.34 ; 0.23-0.51)$. CONCLUSIONS: Low-dose aspirin doubles the risk of major GI bleeding compared to placebo, but the absolute increase in annual incidence is modest at 1.2 patients per thousand. An increased risk with "higher" low-dose vs. "lower" low-dose aspirin was not seen. Aspirin increases major GI bleeding by 1.5 -fold vs. clopidogrel, but the absolute increase is small and switching to clopidogrel to avoid a major GI bleeding episode is not likely to be cost-effective. Combined therapy significantly increases risk vs. either therapy alone. META-ANALYSIS OF ASPIRIN VS. PLACEBO RCTS

\begin{tabular}{|c|c|c|}
\hline ENDPOINTS & $\begin{array}{c}\text { SUBJECTS: } \\
\text { ASPIRIN/PLACEBO }\end{array}$ & RR (95\% CI) \\
\hline $\begin{array}{c}\text { Major GI Bleeding (14 studies) (Lower } \\
\text { Dose: } 75-162.5 \mathrm{mg} \text { ) (Higher Dose: } \\
>162.5-325 \mathrm{mg})\end{array}$ & $\begin{array}{c}28,686 / 28,71914,778 / 14,842 \\
2,871 / 2,843\end{array}$ & $\begin{array}{c}2.07(1.61-2.66) 2.22 \\
(1.61-3.06) 2.35 \\
(0.98-5.66)\end{array}$ \\
\hline Any GI Bleeding (11 studies) & $25,036 / 25,024$ & $1.62(1.25-2.09)$ \\
\hline Fatal GI Bleeding (12 studies) & $25,964 / 25,993$ & $1.23(0.45-3.41)$ \\
\hline Dyspepsia (5 studies) & $12,510 / 12,496$ & $1.09(0.97-1.22)$ \\
\hline Any major bleeding (9 studies) & $26,673 / 26,712$ & $1.71(1.41-2.08)$ \\
\hline Intracranial bleeding (11 studies) & $27,671 / 27,712$ & $1.65(1.12-2.44)$ \\
\hline
\end{tabular}

S1833

Association of Upper Gastrointestinal Bleeding and Nonsteroidal AntiInflammatory Drugs in Japan: A Case-Control Study

Choitsu Sakamoto, Kentaro Sugano, Shinichi Ota, Susana Perez, David Kaufman, Tsugumichi Sato, Kiyoshi Kubota, Akira Terano

Background \& Aims: Upper gastrointestinal bleeding (UGIB) is a common problem for all nonsteroidal anti-inflammatory drugs (NSAIDs). There have been only a few epidemiological studies of this relationship in Japan, where the prevalence of $H$. pylori infection is high. In this study we estimated the association between UGIB and non-aspirin NSAIDs (NANSAIDs) as well as other risk factors including $H$. pylori. Methods: Cases of UGIB due to duodenal or gastric ulcer or gastritis were identified in 14 study hospitals in various areas of Japan. The final eligibility was judged by 3 GI specialists. For each case, 2 controls were identified from the same district from population registries. Information on drugs and other risk factors was obtained from 175 cases and 347 controls by telephone interviews after informed consent was obtained. Anti-H. pylori antibody in the urine was measured in a single laboratory for all of the cases and 225 controls. Conditional logistic regression was used to estimate risk adjusted for various risk factors Results: The odds ratio (OR) of UGIB was 5.5 (95\% CI: 2.5-11.9) for aspirin and 6.1 (95\% CI: 2.7-3.4) for other NSAIDs (NANSAIDs). The OR for regular use was higher than for occasional use both for aspirin (7.7 vs. 2.0) and NANSAIDs (7.3 vs. 4.1). Compared with light alcohol consumption ( $<1$ drink or 14 gram of ethanol per day), the multivariate OR was 1.2 (95\% CI: 0.6-2.5) for 1-3 drinks per day, and 2.8 ( $95 \%$ CI: 1.3-5.8) for $\geq 4$ drinks per day. Compared with no smoking, the OR was significantly increased, at 3.2 (95\% CI: 1.6-6.3) for <20 cigarettes per day and 3.2 (95\% CI: 1.9-5.4) for $\geq 20$ cigarettes per day. The OR for history of non-bleeding gastric or duodenal ulcer was 2.2 (95\% CI: 1.3-3.7). When 175 cases and 225 controls with known $H$. pylori status were subdivided according to the use of NSAIDs (aspirin plus NANSAIDs) and $H$. pylori status, relative to a baseline of no use of NSAIDs and negative $H$. pylori infection, the crude OR for the use of NSAIDs and negative H. pylori was 4.9 (1.8-13), that for no use of NSAIDs and positive H. pylori was $5.4(2.8-10)$ and that for use of NSAIDs combined with positive H. pylori was 10.4 (4.6-23). The relative excess risk due to the interaction between $H$. pylori infection and NSAIDs was only 1.2 (95\% CI -5 8-8.1). Conclusions: NSAIDs increase the risk of UGIB in Japan as in Western countries, with a similar magnitude of association. Heavy drinking, cigarette smoking and previous history of non-bleeding gastric or duodenal ulcer also increased the risk of UGIB. There was no evidence of biological interaction between NSAIDs and $H$. pylori infection. 
Impact of Aspirin and Comedications On Healing of Biopsy-Induced Gastroduodenal Erosions

Gerhard Treiber, Michael Vieth, Thomas Wex, Peter Malfertheiner

Background: Aspirin and non-aspirin NSAIDS are known to induce lesions such as erosions and ulcers in the upper GI tract. However, less is known about the interplay of different comedications with aspirin in terms of healing of preexisting lesions. Other drugs are thought either to aggravate (steroids, Cox-2 inhibitors, clopidogrel) or to speed up (proton-pump inhibitors) healing of erosions. Prospective data on this topic are scarce. Methods: Prospective, randomised, multiple cross-over, placebo-controlled trial in 10 healthy volunteers (HV; H.pylori negative). HV and investigators were blinded for the comedications, which were given during the first 2 weeks: placebo, clopidogrel $75 \mathrm{mg} / \mathrm{d}$, rofecoxib $25 \mathrm{mg} / \mathrm{d}$, prednisolone $10 \mathrm{mg} / \mathrm{d}$, or esomeprazole $20 \mathrm{mg} / \mathrm{d}$. Subsequently, aspirin $100 \mathrm{mg} / \mathrm{d}$ was added to the same ongoing comedication during another 2 weeks. Between each treatment phase there was a 4 weeks recovery period without any medication. Upper GI endoscopy with 10 biopsies each from the duodenal bulb, gastric antrum and corpus was performed at one treatment phase: before, after 2 and after 4 weeks of scheduled drug intake. The number of visible (=unhealed) erosions (due to preceeding biopsies) was the primary outcome measure, either expressed as mean Lanza score or cumulative number of erosions (all individuals pooled: possible maximum number of $10 \times 10=100$ erosions per region). Results: In the corpus few erosions were seen (data not shown). Data for the antrum und bulb region were shown in the table below. Conclusions: Compared to placebo, esomeprazole accelerates healing in the duodenal bulb even in the presence of aspirin, but not in the antrum. Aspirin alone and in combination with steroids produce the highest number of lesions in the duodenal bulb. In the antrum, aspirin plus rofecoxib is the worst combination in terms of healing of lesions.

Persistance of Biopsy induced Erosions expressed either as Lanza Score or Cumulative Number of Erosions due to different Treatments

\begin{tabular}{|c|c|c|c|c|}
\hline Treatment Regimen & $\begin{array}{c}\text { Antrum Mean } \\
\text { Lanza Score } \\
\text { SD }\end{array}$ & $\begin{array}{c}\text { Bulb Mean } \\
\text { Lanza Score } \pm \\
\text { SD }\end{array}$ & $\begin{array}{c}\text { Antrum - } \\
\text { Cumulative Number } \\
\text { of Erosions }\end{array}$ & $\begin{array}{c}\text { Bulb - Cumulative } \\
\text { Number of } \\
\text { Erosions }\end{array}$ \\
\hline Placebo & $0.9 \pm 0.7$ & $0.3 \pm 0.4$ & 17 & 3 \\
\hline Placebo+Aspirin & $1.6 \pm 0.8$ & $1.3 \pm 1.3 *$ & $56 *$ & $71^{*}$ \\
\hline Esomeprazole & $1.3 \pm 0.8$ & $0.3 \pm 0.5 * *$ & $38 *$ & $4 * *$ \\
\hline Esomeprazole+Aspirin & $1.8 \pm 0.4$ & $0.5 \pm 0.5 * *$ & 53 & $6 * *$ \\
\hline Clopidogrel & $1.2 \pm 0.9$ & $0.8 \pm 0.6$ & 33 & $14^{* * * *}$ \\
\hline Clopidogrel+Aspirin & $1.3 \pm 1.2$ & $1.3 \pm 0.9$ & 42 & $37^{*} * * *$ \\
\hline Prednisolone & $1.5 \pm 0.7$ & $0.9 \pm 0.6$ & $37 *$ & $17 * *$ \\
\hline Prednisolone+Aspirin & $1.4 \pm 0.7$ & $1.8 \pm 0.9$ & $32 * *$ & 69 \\
\hline Rofecoxib & $1.2 \pm 0.6$ & $0.8 \pm 0.6$ & $25 * *$ & $18 * *$ \\
\hline Rofecoxib+Aspirin & $1.7 \pm 1.1$ & $0.8 \pm 0.7$ & 73 & $25 * *$ \\
\hline
\end{tabular}

* significant differences vs. placebo, ${ }^{* *}$ significant differences vs placebo+aspirin

\section{S1835}

Rebamipide Compared with Misoprostol for Nonsteroidal Anti-Inflammatory Drugs Associated Gastrointestinal Toxicity Prevention

Soo-Heon Park, Chul-Soo Cho, Oh-Young Lee, San-Ren Lin, Yao-Zong Yuan, Zhao-Shen Li, Hong-Chuan Zhao, Udom Kachintorn, Chomsri Kositchaiwat, Comson Lertkupinit

Background: Nonsteroidal anti-inflammatory drugs (NSAIDs) had gastrointestinal side effects such as dyspepsia, peptic ulceration, hemorrhage, and perforation. Misoprostol and proton pump inhibitors (PPI) were used in the prevention of gastroduodenal injury. Rebamipide increase gastric mucus and stimulate the production of endogenous prostaglandins. The prophylactic effect of rebamipide on NSAIDs induced gastrointestinal complication is unknown. The aim of this study was to evaluate the NSAIDs induced cumulative incidence of gastrointestinal complication as peptic ulcer or bleeding in the rebamipide and misoprostol treated groups in a randomized, multicenter, controlled trial. Methods: Four hundred ten patients were enrolled from Korea, China, and Thailand. Patients were randomized two groups and took either conventional NSAID plus rebamipide $100 \mathrm{mg}$ t. i. or misoprostol 200 ug t.i.d. for 12 weeks. Compliance for NSAIDs and test drugs were checked every 2 weeks and less than $85 \%$ of patients were excluded. Safety test such as CBC and stool occult blood were checked every 4 weeks. Gastric mucosal damage was evaluated by modified Lansa score. Results: The age, sex, type of NSAIDs, and Helicobacter pylori infection rates were not different between the two groups. The numbers of patients for per protocol analysis were 176 in rebamipide group and 156 in misoprostol group. The prevalence of active gastric ulcers were $7 / 176(3.9 \%)$ in the rebamipide group and 3/156(1.9\%) in misoprostol group (RR: $0.47,90 \% \mathrm{CI}=0.13-1.41, \mathrm{P}=0.2847$ ). The prevalence of peptic ulcers were $8 /$ $176(4.5 \%)$ in the rebamipide group and $7 / 156(4.4 \%$ ) in misoprostol group (RR: $0.98,90 \%$ $\mathrm{CI}=0.40-2.36, \mathrm{P}=0.9796)$. The cumulative incidence of dyspeptic patients was significantly higher in misoprostol group than rebamipide group (142/203 vs. 132/207, $\mathrm{P}=0.0136)$. Conclusion: Rebamipide could prevent NSAID induced peptic ulcer as effective as misoprostol in patients on a long-term NSAID therapy. Considering that dyspeptic symptoms are frequent reason both for withdrawal of NSAIDs and for co-therapy with another drug, rebamipide is reasonable drug for the prevention of NSAID induced peptic ulcer and dyspepsia because of its therapeutic effect and safety.
Upper Gastrointestinal Bleeding in Hemophiliacs: Incidence and Relationship to Non-Steroidal Anti-Inflammatory Drugs

M. Elaine Eyster, Shonda M. Asaad, Silvia E. Cohn, Mary-Anne Ardini, James J. Goedert

The use of non-steroidal anti-inflammatory drugs (NSAIDs) in persons with hemophilic arthropathy is controversial because of bleeding concerns, especially upper gastrointestinal (UGI) bleeding. PURPOSE: To determine the incidence of UGI bleeding and its relationship to the use of conventional nonselective non-steroidal anti-inflammatory drugs (nsNSAID or COX-1 inhibitors) and cyclooxygenase selective (COX-2) inhibitors in persons with hemophilia (PWH). METHODS: From May 2002 through May 2005, all PWH enrolled at the 51 centers participating in the second Multicenter Hemophilia Cohort Study were queried annually about use of COX-1 and COX-2 inhibitors in the previous month and for up to or more than 12 months. These questions were repeated when a UGI bleed occurred UGI bleeding was defined as hematemesis, detection of occult blood in the stools with endoscopically verified ulcer, or melena accompanied by a drop in hemoglobin of at leas 2 grams or requiring red cell transfusion. Cox models were used to estimate relative hazards (RH) with $95 \%$ confidence intervals (CI) for fixed and time-dependent covariates postulated as risk factors. RESULTS: During a mean of 17.4 months (range 2-34), 2285 participants, ages 13 to 89 (mean 36.5) were followed for a total of 3309 person-years (py). Forty-two (42) experienced a UGI bleeding event, for an annual incidence of $1.27 \%$. Identified sites were ulcer (11), gastritis (4), varices (5), Mallory Weiss tears (8), esophagitis (1), and angiodysplasia (1). Likelihood of bleeding was significantly increased in those who used COX-1 inhibitors for < 1 month ( $\mathrm{n}=3$ events; OR 3.66; 95\% CI 1.1-11.9), but not for extended periods of time ( $\mathrm{n}=2$ events). Likelihood of bleeding was not increased in those who used COX-2 inhibitors ( $\mathrm{n}=6$ events). In the multivariable model, likelihood of bleeding was significantly and independently increased with age $>46$ yrs ( $\mathrm{RH} 3.4 ; 95 \% \mathrm{CI} 1.1-10.5$ ), platelet counts of $<100,000 / \mathrm{cu} \mathrm{mm}$ (RH $2.4 ; 95 \% \mathrm{CI} 1.0-5.5)$ and hepatic decompensation (RH 3.9; 95\% CI 1.5-10.1). Adjusted for these, use of a COX-1 inhibitor was not significantly associated with bleeding (RH 2.7; 95\% CI 0.7-11.5). CONCLUSIONS: The annual incidence of clinically important UGI bleeding events in PWH was $1.27 \%$, similar to the $1-2 \%$ rate reported in non-hemophilic users of nsNSAIDs. After 3309 py,use of a COX-1 inhibitor for $<1$ month was associated with a significant increase in UGI bleeding in univariate analysis, although older age, thrombocytopenia and hepatic decompensation were major risk factors Use of a COX-2 inhibitor was not associated with UGI bleeding.

\section{S1837}

How Reliable Is the Clinical History of NSAID Use in Patients Who Present with Upper Gastrointestinal Hemorrhage?

John M. Runnels, Anil Minoch

There is paucity of literature regarding the reliability of clinical history for assessing the use of nonsteroidal anti-inflammatory drugs (NSAIDs) in patients presenting with upper Gl hemorrhage. Platelet function testing using the PFA-100 has been used to detect the effects of NSAIDs on platelets. We hypothesized that surreptitious use of NSAIDs occurs only in a small minority of such patients. Study Design Adult hospitalized patients undergoing endoscopy for evaluation of melena, hematemsis or coffee ground emesis were included in the study. Exclusion criteria included hematocrit less than $25 \%$ or platelet count less than $100,000 / \mathrm{mm} 3$, as these factors cause invalid measurements by the platelet function analyzer. Results Sixty three patients met inclusion criteria. Sixteen patients were excluded due to anemia and thrombocytopenia as above. The average (SD) age of patients was 53.65(15.55) years. Caucasians formed $40 \%$ of the study population, whereas the remaining were AfricanAmericans. Patients were predominantly males (64\%). Twenty one percent of the patients tested had positive PFA-100 measurements that suggested NSAID use. However, $60 \%$ of those with positive PFA-100 test had denied a history of prior NSAID use. Conversely, only $25 \%$ of the patients that gave a history of prior NSAID usage had positive PFA-100 test results confirming prior NSAID use. Conclusions Clinical history is not a reliable indicator of prior NSAID use in patients presenting with upper gastrointestinal bleeding. Alternate testing to evaluate for surreptitious use of NSAIDs should be considered in patients with bleeding due to non-HP, non-NSAID ulcers.

\section{S1838}

Use of GI Prophylaxis in Patients Discharged from Hospital On Ulcerogenic Medicines

Gregory A. Cote, John P. Norvell, John R. Rice, Michael Postelnik, Colin W. Howden

Background: Gastrointestinal (GI) hemorrhage is responsible for 200,000-400,000 admissions in the United States annually. Up to twenty million Americans take nonsteroidal antiinflammatory drugs (NSAIDs) on a regular basis. NSAIDs are responsible for $>30 \%$ of admissions due to GI hemorrhage. Misoprostol reduces the number of upper GI events due to NSAIDs; proton pump inhibitors (PPIs) reduce the incidence of NSAID-related ulcers seen at endoscopy. Despite this, compliance with clinical practice guidelines to minimize medication-induced upper GI events may be poor. Aim: To measure the utilization of appropriate GI prophylaxis in patients discharged from hospital on ulcerogenic medicines Methods: We performed a retrospective chart review of all patients discharged from the medical service over a 3-month period on aspirin, NSAIDs, warfarin and/or corticosteroids Data were collected on PPI or misoprostol administration at the time of discharge. Comorbidities were documented, with particular attention on known risk factors for GI hemorrhage (age, previous hemorrhage, type of ulcerogenic medicine). Other indications for acid suppressive therapy were also documented. Results: 480 patients were discharged between January and March 2005 on aspirin, NSAIDs, warfarin, corticosteroids or some combination 142 were excluded for having a current indication for PPI therapy. Of the 338 remaining patients, 154 (46\%) were prescribed GI prophylaxis: 119 (35\%) received daily PPI, 2 (1\%) misoprostol, 15 (4\%) $\mathrm{H}_{2} \mathrm{RA}$ and $18(12 \%)$ twice daily PPI. Of the 154 patients discharged on prophylaxis, $98(64 \%)$ had been taking it before admission. The 338 patients were also divided into four groups based on risk factors for GI hemorrhage: age, Charlson co-morbidity

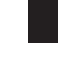


index, and type of medication. $24 \%$ of low-risk ( $n=33$ ), 36\% of moderate risk ( $n=99), 48 \%$ of high-risk $(n=107)$, and $60 \%$ of very high-risk $(n=99)$ patients received some form of prophylaxis. Patients were also divided by Charlson score; $40 \%$ with scores of $0-1,47 \%$ with scores $2-4$, and $51 \%$ with scores $>5$ received prophylaxis. Conclusions: Although there was a trend towards higher use of prophylaxis in the highest risk patients, only $60 \%$ were discharged on appropriate therapy. Further, $24 \%$ of low-risk patients were prescribed prophylaxis without a clear indication. Although hospitalization offers an excellent opportunity to recognize and manage patients at high risk of developing GI hemorrhage, adherence with current guidelines was suboptimal. Educational efforts should be directed at residents in training to help them recognize risk factors for GI hemorrhage and understand indications for prophylaxis.

\section{S1839}

NSAIDs and Induction of Erosive Esophagitis

Gerhard Treiber, Charlotte Fonga, Peter Malfertheiner

Background: Non-steroidal antiinflammatory drugs (NSAIDS) are widely used and known to induce lesions in the stomach and duodenum. The relationship between NSAIDS and erosive esophagitis (EE) is less clear. A Medline search revealed less than 20 publications, all of them retrospective. Methods: To investigate a possible relationship between NSAIDS use and the presence of EE, we conducted 2 studies: 1) retrospective case-control study of 416 patients with fully available individual information about the dose and duration of aspirin and non-aspirin NSAIDS, unselected of symptoms. 2) prospective study in 20 healthy volunteers (HV, normal baseline endoscopy, no hiatal hernia, H.pylori negative, refraining from alcohol, smoking, and other drug intake throughout the study period of 8 weeks); all of them were treated by naproxen $500 \mathrm{mg}$ bid for the first 2 weeks, followed by a 4 weeks recovery period, and then rechallenged to naproxen. All HV were endoscoped at the beginning and at the end of drug intake, biopsies were taken from the bulb, antrum, and corpus at each endoscopy. The primary outcome measure in both studies was the presence of EE. Results: In the retrospective study, the OR for aspirin to induce EE was 0.80 [95\%-CI: 0.37 1.72, $\mathrm{p}=0.71]$, while for non-aspirin NSAIDS the OR was 1.24 [95\%-CI: 0.45-3.55, $\mathrm{p}=$ $0.59]$, corresponding to a prevalence of $15.8 \%$ for EE in patients with aspirin intake vs. $22.7 \%$ with non-aspirin intake. Cofactors like age, H.pylori status, and smoking were similiar between NSAIDS users and non-users. In the prospective study, 5/20 (25\%) HV developed EE denovo ( $L A-A \mathrm{n}=4, \mathrm{LA}-\mathrm{B} \mathrm{n}=1$ ), all of them were asymptomatic. 15 HV have been retreated with naproxen, and the same 4 individuals who had EE during the first course showed EE during the second course. No one developed EE during the second course only. Mean Lanza score for biopsy induced erosions were similiar for all regions in the stomach and bulb. For those $5 \mathrm{HV}$ with EE the cumulative number of biopsy induced erosions in the bulb and in the corpus were higher (RR-bulb: 2.0 [1.4-2.9], $\mathrm{p}=0.0004$; RR-antrum: 1.2 [0.8-1.7], $\mathrm{p}=$ 0.55, and RR-corpus: 3.0 [2.1-4.2], p<0.0001) than in those 15 without EE. Conclusions: EE is associated with NSAID intake, patients with non aspirin NSAIDS are more likely to have EE than aspirin users. Our prospective data indicate, that even in an asymptomatic, low-risk population (such as HV), naproxen reproducibly induces EE in $25 \%$ of investigated individuals (similiar proportion to our retrospective data). The presence of EE due to NSAIDS is also associated with more impaired healing of biopsy induced lesions in the bulb and corpus, but not in the antrum.

\section{S1840}

H. Pylori Infection Is No Longer the Major Cause of Peptic Ulcer in Copenhagen County

Bo Sondergaard, Peter Bytzer

INTRODUCTION: Peptic ulcer disease is almost always caused by infection with $H$. pylor or by ulcerogenic drugs or by a combination of these. Earlier studies have found that at least $85 \%$ of duodenal ulcers and at least $50 \%$ of gastric ulcers are associated with $H$. pylori infection. However, more recent data indicate that the frequency of $H$. pylori infection in peptic ulcer disease is decreasing and the prevalence of idiopathic ulcers, not associated with $H$. pylori or with NSAIDs, may be increasing. AIMS \& METHODS: We aimed to examine the prevalence of $H$. pylori infection and the intake of ulcerogenic drugs, NSAIDs and aspirin (ASA) in patients diagnosed with peptic ulcer disease at an endoscopy center. Case forms for patients with an endoscopic diagnosis of uncomplicated peptic ulcer during the years 2002 and 2003 were identified by searching patient lists. Patients were classified a being $H$. pylori infected if a biopsy-based rapid urease test, a urea breath test or histopathology was positive. Use of ASA (any dose), NSAIDs and proton pump inhibitors during the previous month was registered. RESULTS: 134 patients ( 66 males) were investigated. Mean age was 70 years, range $12-94$ years. $38 \%$ were smokers. 83 patients had a gastric ulcer, 42 patients had a duodenal ulcer and nine patients had both a gastric and a duodenal ulcer. In six patients $H$. pylori status could not be determined. A total of 66/134 (49\%) peptic ulcer patients were infected with $H$. pylori. H. pylori was more common in patients with gastric ulcer compared to patients with duodenal ulcer $(61 \%$ vs. $42 \%, P=0.0024)$. Four of the nine patients with combined ulcers were infected. A total of 48/83 gastric ulcer patients had used either NSAIDs (11), ASA (30) or both (7). Six of these 83 patients were classified as having idiopathic ulcer disease. A total of 28/42 duodenal ulcer patients had used either NSAIDs (7), ASA (13) or both (8). Six of these 42 patients were classified as having idiopathic ulcer disease. 10/83 gastric ulcer patients used proton pump inhibitors, three of these patients were $H$. pylori negative subsequently confirmed by urea breath test. $5 / 42$ patients with duodenal ulcer used proton pump inhibitors, three of these patients were H. pylori negative subsequently confirmed by urea breath test, and two patients were lost to follow-up - these were not classified as having idiopathic peptic ulcer. CONCLUSION: The frequency of $H$. pylori infection in patients with peptic ulcer disease is rapidly decreasing Use of NSAIDs and aspirin is now the major causative factor for duodenal ulcer in Copenhagen County.

\section{S1841}

Physicians' Approaches to the Use of Gastroprotective Strategies in Low-Risk Non-Steroidal Anti-Inflammatory Drug (NSAID) Users

Sanjay K. Murthy, Laura E. Targownik, Sangeeta Kauldher

Introduction: Gastroprotective (GP) strategies are recommended for NSAIDs users who are at increased risk for GI bleeding. We aimed to characterize the factors which predict overuse of GP strategies in low risk NSAID users. Methods: We distributed a questionnaire to all family physicians and general internists in Manitoba. The questionnaire contained a clinical vignette describing a low-risk hypothetical patient with osteoarthritis who was a candidate for NSAID therapy. Respondents were asked whether they would prescribe this patient a GP strategy and to estimate the risk of that patient developing a GI complication on NSAIDs alone, as well as the risk reduction afforded by using either a concomitant PPI or a COX2 selective NSAID. Respondents were then separated into two groups depending on whether they inappropriately recommended using a GP strategy in the low-risk scenario. Results: We received 340 responses (response rate (28.3\%) of which 278 supplied analyzable data. $35 \%$ of respondents inappropriately recommended a GP strategy for the low risk subject inappropriate prescribers were more likely older, farther out from medical school graduation, and in family practice. Inappropriate prescribers were more likely to overestimate the risk of GI bleeding (Table 1); this was strongly predictive of GP strategy recommendation in logistic regression. Conclusions: A substantial proportion of physicians recommended GP strategies for low-risk patients. These physicians tended to overestimate the risk of GI bleeding in low risk NSAID users. Improving the knowledge base of physicians in these areas may lead to a decrease in inappropriate utilization of GP strategies for low-risk patients Comparison of risk estimates for NSAID-related GI complications between appropriate and inappropriate prescribers

\begin{tabular}{|c|c|c|c|}
\hline & $\begin{array}{c}\text { Appropriate Prescribers } \\
(\mathrm{n}=161) \text { Median (IQR) }\end{array}$ & $\begin{array}{c}\text { Inappropriate Prescribers } \\
(\mathrm{n}=87) \text { Median (IQR) }\end{array}$ & $\mathrm{p}$-value \\
\hline NSAIDs alone & $4 \%(2-7.5)$ & $8 \%(4-12.5)$ & $<0.001$ \\
\hline COX-2 Inhibitor & $2 \%(1-5)$ & $3 \%(1-5)$ & $<0.001$ \\
\hline NSAID + PPI & $1.5 \%(1-3)$ & $2 \%(1-5)$ & 0.032 \\
\hline $\begin{array}{c}\text { Relative Risk Reduction } \\
\text { with PPI }\end{array}$ & $50 \%(33.3-66.6)$ & $60 \%(50.0-80.0)$ & 0.001 \\
\hline $\begin{array}{c}\text { Relative Risk Reduction } \\
\text { with COX-2 inhibitor }\end{array}$ & $40 \%(20.0-53.3)$ & $55.5 \%(37.5-70.0 \%)$ & $<0.001$ \\
\hline
\end{tabular}

\section{S1842}

Increasing Prescription of Proton Pump Inhibitors in Patients Admitted to the Verona University Hospital and Decreasing Risk of Peptic Ulcer Giorgio Talamini, Marina Tommasi, Valeria Amadei, Benedetta Pajusco, Anna Fratucello, Alessandro Lechi, Giovanna Scroccaro, Italo Vantini

$\mathrm{H} 2$-antagonists and proton pump inhibitors are the top-ranking prescribed drugs and constitute a substantial economic burden for the national health-care system. This study evaluates the prescribing trends of these drugs and their impact on the risk of peptic ulcer in hospitalised patients. Methods: From January 2001 to June 2004, the number of prescriptions of H2antagonists and proton pump inhibitors in each medical or surgical department of Verona University Hospital was monitored on a monthly basis, distinguishing between iv and oral administration. Over the same period we prospectively recorded the number of upper gastrointestinal endoscopies per department for inpatients with a definite diagnosis of gastric or duodenal ulcer. The following variables were analysed in these patients: gender ratio, age, indication to endoscopy, urgent endoscopy, use of non-steroidal anti-inflammatory drugs, presence of Helicobacter pylori, previous surgery, smoking and drinking habits. Results: A significantly decreasing trend in $\mathrm{H} 2$-antagonist prescriptions $(\mathrm{r}=-0,84 ; \mathrm{p}<0.001)$ and an increasing trend in proton pump inhibitor prescriptions $(\mathrm{r}=0.97 \cdot \mathrm{p}<0.001)$ were observed. The endoscopic incidence of duodenal ulcers decreased linearly from 2001 to 2004 as follows: $6.5 \%(94 / 1439)$ in 2001, $5.6 \%(82 / 1473)$ in 2002, $4.5 \%(63 / 1411)$ in 2003 , and $3.1 \%(22 / 702)(\mathrm{p}<0.001)$ in 2004. Gastric ulcer incidence, gender, age, indication to endoscopy and use of nonsteroidal anti-inflammatory drugs showed no significant change. The prevalence of Helicobacter pylori also remained constant over the three and a half year period, and about $32 \%$ of patients were HP-positive $(p=N S)$. There was a significant inverse correlation between the increase in proton pump inhibitor administration and the decreasing incidence of duodenal ulcer (Spearman correlation $\mathrm{r}=-0.68 ; \mathrm{p}<0.02$ ). Multivariate analysis identified only proton pump inhibitor use (coeff. $-0.91 ; \mathrm{p}<0.001$ ) and non-steroidal antiinflammatory drug use (coeff. $0.48 ; \mathrm{p}<0.035$ ) as factors capable, respectively, of reducing and increasing the risk of duodenal ulcer. Conclusions: In the study period we recorded a marked decrease in the use of $\mathrm{H} 2$-antagonists and an increase in proton pump inhibitor prescription. The significant reduction in duodenal ulcer endoscopic incidence in hospital is associated with the increased prescription of proton pump inhibitors in inpatients.

\section{S1843}

INOS Inhibition Increases HIF-1 $\alpha$ in Mesenteric Macrophages and Reduces the Inflammatory Response to Aspirin

Irene Diez, Carlos Hernandez, Elsa Quintana, Sara Calatayud, Juan V. Esplugues, Maria D. Barrachina

A role for hypoxia inducible factor-l $\alpha$ (HIF-l $\alpha)$ in inflammation has been reported. Nitric oxide, an endogenous mediator associated to inflammatory process, has been shown to play a dual role on HIF-l $\alpha$ stabilization. Aim: To analyze, in the mesentery of aspirin(ASA)treated rats, the role of nitric oxide on HIF- $1 \alpha$ expression and the evolution of the inflammatory response Methods: Fasted rats received a single dose of $1400 \mathrm{~W}(5 \mathrm{mg} / \mathrm{kg}$, s.c., iNOS selective inhibitor) or saline ( $1 \mathrm{ml} / \mathrm{kg}$, s.c.), $10 \mathrm{~min}$ before ASA (150 mg/kg, p.o.) or vehicle 
( $1 \%$ carboxymethylcellulose, p.o.) and 6 or $24 \mathrm{~h}$ later we analyzed in mesenteric windows: a) leukocyte-venular endothelial cell interactions by intravital microscopy; b) iNOS or HIF$1 \alpha$ immunostaining. Results: ASA induced, $6 \mathrm{~h}$ later, an increase in the number of rolling leukocytes $(59 \pm 6)$ and leukocyte adhesion to the endothelium $(18 \pm 3)$ which was significantly $(\mathrm{p}<0.05)$ prevented by inhibition of iNOS-derived NO synthesis (rolling:36 \pm 8 and adhesion:10 \pm 3 ). Twenty-four hours after ASA, no differences in both parameters were observed among the different experimental groups. Quantitative analysis of migrated polimorphonulear cells (PMNs), in an area of the mesentery $(4 \mathrm{~mm} 2)$, revealed a significant increase ( $764 \pm 89$ vs $379 \pm 142) 6 \mathrm{~h}$ after ASA, which was maintained at $24 \mathrm{~h}(933 \pm 143$ vs $191 \pm 15.1)$. Pretreatment with $1400 \mathrm{~W}$ significantly reduces the number of migrated leukocytes $(342 \pm 88$ ) induced by ASA at $24 \mathrm{~h}$ while it lacked of any effect at $6 \mathrm{~h}$. Immunohistochemical analysis showed iNOS expression in both PMNs and macrophages of the mesentery of ASA-treated rats. HIF-1 immunoreactivity was observed in $25 \%$ of PMNs, mainly around the vessels, irrespective of the treatment and the time analyzed. Whereas no differences were observed at $24 \mathrm{~h}, 6 \mathrm{~h}$ after treatments, the percentage of tissue macrophages showing HIF-l $\alpha$ immunoreactivity was significantly $(\mathrm{p}<0.05)$ increased in rats receiving $1400 \mathrm{~W}+\mathrm{ASA}(66 \pm 2 \%)$ compared with control $(15 \pm 6 \%)$ or ASA $(19 \pm 8 \%)$. Conclusions: In ASA-treated rats, inhibition of iNOS-derived NO synthesis increases HIF-l $\alpha$ expression in macrophages of the mesentery and shortens the inflammatory response. HIF-l $\alpha$, by increasing the macrophage phagocytic capacity, could be involved in the resolution of inflammation.

\section{S1844}

A Critical Role of SRF in Myofibroblast Differentiation During Experimental Esophageal Ulcer Healing

Jianyuan Chai, Manith Norng, Andrzej Tarnawski

SRF is a transcription factor that regulates genes involved in cellular response to stimulations of growth factors released during tissue injury. The aims of this study were to examine the effect of local overexpression or knockdown of SRF on myofibroblast differentiation during esophageal ulcer healing and mechanisms involved. METHODS: 1) In vivo study: Esophageal ulcers were induced in rats by topical application of acetic acid onto the serosal surface of

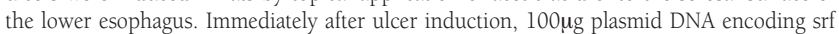
or antisense srf was injected into submucosa adjacent to the induction site. Esophageal tissues were obtained at day 1, 3, 6,9 and 14 after treatment. 2) In vitro study: Rat connective tissue fibroblasts were transfected with the same plasmids used for in vivo study, treated with recombinant TGF $\beta 1$. Myofibroblast differentiation was assessed by expression of smooth muscle $\alpha$-actin (SM $\alpha$-actin) and actin stress fiber formation. Smoothelin was the marker used to eliminate smooth muscle cells from the whole population of SM $\alpha$-actin-positive cells. RESULTS: 1) Overexpression of SRF in esophageal ulcers by gene therapy significantly increased SM $\alpha$-actin expression by $73 \pm 7 \%$ at day 6 and knockdown of SRF reduced the amount of SM $\alpha$-actin protein by $51 \pm 4 \%$ (both $\mathrm{P}<0.01$ ). Overexpression of SRF increased myofibroblast population in granulation tissue by $198+23 \%$ per mm2 and knockdown of SRF reduced the number of myofibroblasts by $52 \pm 10 \%$ per $\mathrm{mm} 2$ (both $\mathrm{P}<0.005$ ). 2) Esophageal ulceration significantly induced TGF $\beta 1$ expression through the entire 14-dayperiod monitoring $(\mathrm{P}<0.001)$. It reached a peak at day 6 (38-fold). 3) TGF $\beta 1$ treatment significantly increased SRF mRNA expression by $78.5 \pm 9.3 \%, 186.8 \pm 15.2 \%$ and $164.4 \pm 22.5$ $\%$ at 2,6 and 12 hrs, respectively, and protein expression by $144 \pm 35 \%, 4-, 6-, 6-$ and 5 fold at 2, 6, 12, 24 and 48 hrs, respectively (all $\mathrm{P}<0.01$ ). EMSA analyses indicated that TGF $\beta 1$ treatment also significantly enhanced SRF protein binding activity to sm $\alpha$-actin SRE in parallel to the elevation in SRF protein expression. 4) Overexpression of SRF induced both SM $\alpha$-actin expression and actin stress fiber formation, while knockdown of SRF prevented TGF $\beta 1$-induced myofibroblast phenotype in fibroblasts. CONCLUSIONS: 1) Local overexpression of SRF during esophageal ulcer healing promotes myofibroblast differentiation; 2) Esophageal ulceration stimulates TGF $\beta 1$ expression and myofibroblast differentiation; 3) TGF $\beta 1$ induces SRF activation in fibroblasts; and 4) SRF is required for myofibroblast differentiation.

\section{S1845}

Effect of Inhibition of Cyclooxygenase-2 and 5-Lipoxygenase Or Antagonism of the Lipoxin A4/Annexin-1 Receptor On Indomethacin-Induced Gastric Mucosal Damage in Rats

Brigitta M. Peskar, Bernhard A. Peskar, Nadia Sawka

BACKGROUND: Metabolism of arachidonic acid by aspirin-acetylated cyclooxygenase (COX) -2 and 5-lipoxygenase yields 15(R)-epi-lipoxin (LX) A4. In the rat stomach, aspirin increased formation of 15(R)-epi-LXA4 which attenuated aspirin-induced damage whereas inhibitors of COX-2 or the LXA4 receptor antagonist BOC 1 aggravated aspirin-induced injury (Fiorucci et al. 2002). In the presence of indomethacin anti-inflammatory mediators are produced from eicosapentaenoic acid by a COX-2- and 5-lipoxygenase-dependent mechanism (Serhan et al., 2000). We examined whether these enzymes and the LXA4/annexin1 receptor might also be involved in counteracting the injurious effect of indomethacin on gastric integrity. METHODS: Conscious rats (5-9/group) were treated with indomethacin $(10 \mathrm{mg} / \mathrm{kg}$, po) with or without co-administration of the COX-2 inhibitor rofecoxib (10 mg/ $\mathrm{kg}$, ip), the 5-lipoxygenase inhibitor A63162 (20 mg/kg, sc) or the LXA4/annexin-1 receptor antagonist BOC $1(2 \times 10 \mu \mathrm{g} / \mathrm{kg}$, ip). For comparison, rats were treated with aspirin (150 $\mathrm{mg} / \mathrm{kg}$, po) with or without co-treatment with rofecoxib (10 mg/kg, ip) or A63162 (20 mg/kg, sc). Mucosal damage was assessed after $5 \mathrm{~h}$ using a lesion index (LI). RESULTS: Indomethacin induced damage with a LI of $10+-1$ which was increased by co-administration of rofecoxib (LI 19+-2.3, p<0.001), A63162 (LI 19+-2, p<0.001) or BOC 1 (LI 20+-3.1, p<0.01). Aspirin caused damage with a LI of $12+-1.6$ that was aggravated by co-treatment with rofecoxib (LI $25+-1.5, \mathrm{p}<0.001$ ) or A63162 (LI 20+-1.3, $\mathrm{p}<0.001$ ). Neither rofecoxib nor A63162 nor BOC 1 in the doses used damaged the gastric mucosa when given alone. CONCLUSIONS: 1) The findings confirm a previous report (Fiorucci et al., 2002) that aspirin-induced gastric damage is increased by inhibition of COX-2 and interference with the LXA4 system. 2) Gastric injury caused by indomethacin is aggravated by inhibition of COX-2, 5-lipoxygenase or antagonism of the LXA4/annexin-1 receptor to an extent comparable to aspirin suggesting that ulcerogenic doses of both aspirin and indomethacin lead to formation of endogenous protective mediators that limit gastric mucosal injury. 3) Formation of protective mediators occurs via acetylated COX-2 in the presence of aspirin but enzyme acetylation is obviousl not a prerequisite for the indomethacin effect. 4) The formation of protective mediators in the presence of an ulcerogenic dose $(10 \mathrm{mg} / \mathrm{kg})$ of indomethacin differs from the protection conferred by a low dose $(1 \mathrm{mg} / \mathrm{kg})$ of indomethacin in gastric ischemia-reperfusion injury since this type of protection is observed even in the presence of the COX-2 inhibitor celecoxib (Sawka et al., unpublished data).

\section{S1846}

A Novel Mechanism for NSAID Enhanced Proteasomic Degradation of Survivin

Shiun-Kwei Chiou, Michael K. Jones, Andrzej Tarnawski

Background: Nonselective NSAIDs such as indomethacin cause gastrointestinal damage Apoptosis is one of the mechanisms. Survivin, a dual functioning protein, inhibits apoptosi and facilitates mitosis. Survivin is expressed in normal human and rat gastric mucosa in surface epithelial and progenitor cells. Previous studies demonstrated that NSAIDs target survivin in inducing gastric injury by promoting proteasomic degradation of survivin protein in the gastric mucosa. Evidence supporting this shows that suppression of survivin expression by siRNA induces injury of gastric mucosal, RGM-l cells. In this study we confirmed whether 1) indomethacin treatment increases ubiquitination of survivin and examined:2) molecula mechanisms involved in survivin protein degradation. Methods: Rat gastric epithelial, RGM1 cells derived from normal gastric mucosa were treated with either $20 \mathrm{mM}$ of the $26 \mathrm{~S}$ proteasome inhibitor, MG-132, or $0.5 \mathrm{mM}$ indomethacin with $20 \mathrm{mM} \mathrm{MG}-132$ for 3 hours. The MG-132 proteasome inhibitor does not interfere with protein ubiquitination, and was used to prevent rapid survivin degradation following ubiquitination. Survivin protein ubiquitination was assessed by immunoprecipitation with anti-survivin antibodies followed by Western blotting with anti-ubiquitin antibodies. To determine the ubiquitin ligase involved in degradation of survivin, cIAP 1, APC and XIAP expression was suppressed in RGM-1 cells by transfection of siRNA oligomers. Survivin protein levels in cells maintained in serum free culture with and without siRNA suppression were monitored by Western blotting at 24, 48 and 72 hours post transfection. Results: 1) Indomethacin plus MG-132 treatmen increased survivin ubiquitination by $3.8+0.82$ fold $(\mathrm{p}<0.05)$ compared to MG-132 only treatment. 2) In RGM-l cells transfected with non-suppressing control RNA, survivin protein level significantly decreased to $0.013+0.001$ fold $(\mathrm{p}<0.05)$ by 48 hours in serum free medium, compared to 0 hours. 3) XIAP suppression maintained survivin protein levels a $1.1+.2(\mathrm{p}<0.05), 0.72+0.09(\mathrm{p}<0.05)$ and $0.55+0.1(\mathrm{p}<0.05)$ fold for 24,48 and 72 hours, respectively, compared to 0 hours, while cIAP 1 and APC suppression by siRNA did not increase survivin protein stability. 4) Maintainance of survivin protein levels in XIAP suppressed cells was not due to upregulation of survivin mRNA expression. Conclusions 1) Indomethacin treatment promotes ubiquitination and proteasomic degradation of survivin in gastric epithelial cells, which likely facilitates gastric epithelial cell injury. 2) XIAP may be an ubiquitin E2 ligase involved in NSAID induced survivin degradation.

\section{S1847}

The Role of Cholinergic Hypersensitivity On NSAID-Induced Gastric Damage Changcheng Wang, Ireneusz T. Padol, Richard H. Hunt

Purpose: Flinders sensitive line (FSL) rats exhibit an increased cholinergic responsiveness in vivo when compared to their counterparts, Flinders resistant line (FRL) rats. Our previous study demonstrated that basal gastric acid secretion was significantly higher in FSL than in FRL rats (Gastroenterology 2004 Suppl 2; 126: A480). In this study, we aimed to determine whether there are differences in NSAID-induced gastric damage between these two strain and if differences in gastric physiology play a role. Methods: The gastric damage index ( $\mathrm{mm}$ ) was assessed macroscopically $6 \mathrm{~h}$ after oral administration of indomethacin $(5 \mathrm{mg} / \mathrm{kg}$ and $20 \mathrm{mg} / \mathrm{kg}$ ) in FSL and FRL rats. In another set of rats, acid secretion was measured by pylorus ligation $3 \mathrm{~h}$ after oral indomethacin. Volume $(\mathrm{ml})$ and acidity $(\mathrm{mEq} / \mathrm{L})$ of gastric juice was measured and acid output ( $\mu \mathrm{Eq} / 3 \mathrm{~h})$ calculated. Results: The gastric damage index was significantly higher in FSL than in FRL 6h after oral administration of $5 \mathrm{mg} / \mathrm{kg}$ and $20 \mathrm{mg} / \mathrm{kg}$ indomethacin ( $12.4 \pm 3.0$ vs $3.4 \pm 1.1, p=0.009$ and $27 \pm 5.7$ vs $12.1 \pm 3.0, p=0.03$; respectively) Baseline, acidity, acid output and gastric juice volume were significantly higher in FSL than in FRL $(70.34 \pm 1.1$ vs $42.65 \pm 9.68, p=0.006 ; 213.47 \pm 29.14$ vs $92.82 \pm 36.26, p=0.02$ and $3.02 \pm 0.4$ vs $1.74 \pm 0.39, \mathrm{p}=0.04$; respectively). Administration of $5 \mathrm{mg} / \mathrm{kg}$ indomethacin resulted in acidity markedly higher than baseline values for both strains (FSL: $91.2 \pm 10.54$ vs $70.34 \pm 1.1, p=0.008$ and FRL: $80.48 \pm 7.57$ vs $42.65 \pm 9.68, p=0.023$ ), while volume and acid output were not. After administration of higher dose indomethacin $(20 \mathrm{mg} / \mathrm{kg})$, acidit was still significantly higher than baseline in FRL rats $(73.28 \pm 4.3$ vs $42.65 \pm 9.68, p=0.025)$. However in FSL rats, acidity, acid output and gastric juice volume were lower than baseline values suggesting extensive damage to gastric mucosa $(51.97 \pm 14.78$ vs $70.34 \pm 1.1, p=0.056$ $80.77 \pm 23.39$ vs $213.47 \pm 29.14, p=0.03$ and $1.54 \pm 0.08$ vs $3.02 \pm 0.4, p=0.055$ respectively) Conclusion: The greater NSAID-induced gastric damage in FSL rats correlates closely to its innate hypersecretion of gastric acid. Indomethacin-induced higher acidity may play a predominant role in gastric damage in both strains. The observed differences between FSL and FRL rats provide the basis for an animal model of cholinergic hyperresponsiveness to NSAID-induced gastric pathology.

\section{S1848}

Role of Cyclooxygenase-2 and Lipoxygenases in the Protective Effect of Indomethacin and Acetaminophen in Ischemia-Reperfusion Injury of the Rat Stomach Nadia Sawka, Bernhard A. Peskar, Brigitta M. Peskar

BACKGROUND: Aspirin leads to formation of protective 15(R)-epi-lipoxin (LX) A4 via acetylated cyclooxygenase (COX) -2 and further metabolism by 5 -lipoxygenase (LO) (Fiorucc et al., 2002). Serhan et al. (2000) have described that in the presence of indomethacin and 
acetaminophen arrays of anti-inflammatory lipid mediators are produced from eicosapentaenoic acid via COX-2-dependent oxygenations and 5-LO. We examined whether indomethacin and acetaminophen protect against gastric ischemia-reperfusion (I-R) injury. METHODS: In male Wistar rats (4-6/group), I-R was induced by clamping the celiac artery for $30 \mathrm{~min}$ followed by reperfusion for $60 \mathrm{~min}$. Gastric damage was assessed by calculation of a lesion index (LI). Drugs were given sc. RESULTS: I-R alone induced minor damage (LI 3+-0.3). Pretreatment $(30 \mathrm{~min})$ with the COX-2 inhibitor celecoxib $(3 \mathrm{mg} / \mathrm{kg})$ markedly increased damage (LI 41+-1.3, p<0.001). Administration of indomethacin ( $1 \mathrm{mg} / \mathrm{kg}$ ) or acetaminophen $(50 \mathrm{mg} / \mathrm{kg}) 30 \mathrm{~min}$ before or after celecoxib protected against the damage-aggravating effect of celecoxib (LI 14+-2.7 and 7+-0.5, respectively, $\mathrm{p}<0.001$ each). The protective effects of indomethacin and acetaminophen were reversed by pretreatment $(30 \mathrm{~min})$ with low doses of inhibitors of 5-LO (A63162), 12-LO (baicalein) or 15-LO (PD146176) $(2.5 \mathrm{mg} / \mathrm{kg}$ each) or the LXA4/annexin 1 receptor antagonist BOC $1(0.15 \mu \mathrm{g} / \mathrm{kg})$. These doses had no effect on I-R damage when given alone. The inhibition of the protective effects of indomethacin and acetaminophen against celecoxib-induced injury induced by pretreatment with LOinhibitors or BOC 1 was significant ( $\mathrm{p}$ at least $<0.02$ ), damage showing a LI ranging from $27+-7.5$ to $41+-1.1$. In additional experiments, higher doses of A63162 (5 mg/kg) or BOC $1(1 \mu \mathrm{g} / \mathrm{kg})$ instead of celecoxib were used to aggravate I-R damage (LI $41+-2.6$ and 38+2.2 , respectively). Indomethacin $(1 \mathrm{mg} / \mathrm{kg})$ and acetaminophen $(50 \mathrm{mg} / \mathrm{kg})$ also significantly $(\mathrm{p}<0.001$ ) protected against the damage-aggravating effects of $\mathrm{A} 63162$ and BOC 1 (LI 8+1 and 7+-1, respectively, for indomethacin and LI 6+-0.4 and 5+-0.3, respectively, for acetaminophen). CONCLUSIONS: 1) Acetaminophen and a low dose of indomethacin exert gastroprotection in that they counteract the damage-aggravating effects of celecoxib, the 5LO inhibitor A63162 and the LXA4 receptor antagonist Boc l in I-R. 2) The inhibition by indomethacin and acetaminophen of celecoxib-induced I-R damage is reversed by inhibitors of LO or blockade of the LXA4 receptor. 3) The findings show that the protective activity of these non-steroidal anti-inflammatory drugs is not mediated by COX-2 as it operates when COX-2 is inhibited but is modulated by LO activity.

\section{S1849}

Is Cyr61 Required for Myofibroblast Differentiation? An Exploration of the Role of Cyr61 in Gastric Ulcer Healing

Jianyuan Chai, Manith Norng

Myofibroblast differentiation is an important event for healing of any wounds including gastric ulcer. Cyr61 is a growth factor-induced secreted protein but is associated with extracellular matrix. It is reported that Cyr61 promotes fibroblast adhesion and migration during cutaneous wound healing. The aim of this study was to explore the role of Cyr61 in gastric ulcer healing, focusing on myofibroblast differentiation. METHODS: 1) In vivo study: Gastric ulcers were induced in rats by serosal application of acetic acid. Normal and ulcerated gastric tissues were harvested at 1, 3, 7, 14 and 30 days after ulcer induction for analyses of Cyr61 mRNA by Northern blotting and protein expression by Western blotting and immunohistochemistry; 2) In vitro study: Rat connective tissue fibroblasts were treated with either recombinant Cyr61 or Cyr61 siRNA, and examined for Integrin activation, focal adhesion assembly, and myofibroblast phenotype. Cells were also treated with recombinant TGF $\beta 1$ as a positive control. RESULTS: 1) Gastric ulceration induced two peaks at day 1

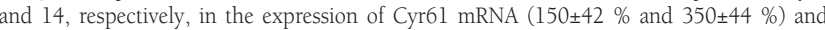
protein ( 3 fold and 16 fold) (all $\mathrm{P}<0.01$ ). The first peak reflected inflammatory response and angiogenesis, while the second peak (greater) concentrated in the extracellular matrix of granulation tissue. Ulceration also massively increased the number of myofibroblasts in the same temporal-and-spatial manner as Cyr61. 2) After 48-hour treatment with siRNA, Cyr61 deficiency in cultured fibroblasts severely impaired cell adhesion as indicated by the reduced number of cells on cover-glasses $(62 \pm 7 \%$ less than control, $\mathrm{P}<0.001)$ and focal adhesion complexes. 3) Exogenous Cyr61 treatment induced expression of smooth muscle $\alpha$-actin in fibroblasts, increased expression of adhesion molecules including Integrin $\alpha 6$ by $63 \pm 10 \%$ and Talin by $204 \pm 32 \%$ (both P < 0.005 ), phosphorylation of FAK by $75 \pm 12 \%$ and Paxillin by $138 \pm 17 \%$ (both P $<0.005$ ), and translocation of Vinculin. 4) Knockdown of Cyr61 in fibroblasts did not prevent TGF $\beta$-induced expression of smooth muscle $\alpha$-actin but prevented TGF $\beta$-induced phosphorylation of FAK and Paxillin and formation of focal adhesion complex. Conclusions: 1) Gastric ulceration induces Cyr61 expression in granulation tissue; 2) Cyr61 is essential for focal adhesion assembly in fibroblasts; and 3) Cyr61 induces myofibroblast phenotype in fibroblasts, but is not required for TGF $\beta$-induced myofibroblast differentiation.

\section{S1850}

Evaluation of the Structural Properties and Activities of a Purified PC-NSAID Preparation

Lenard M. Lichtenberger, Elizabeth Dial, Vasanthi Jayaraman, Z.hong Zhou, Ramanan Krishnamorti, David E. Volk, David G. Gorenstein, Upendra Marathi

Background: It has been determined that the GI toxicity of nonsteroidal anti-inflammatory drugs (NSAIDs) can be significantly reduced in animals and human subjects if the drugs are pre-associated with phosphatidylcholine (PC). The development of the PC-NSAID technology has been restricted somewhat by our inability to purify PC-NSAIDs, suggesting that the GI protective activity and therapeutic efficacy could be further optimized if such a technique could be developed. Objective \& Methods: We have attempted to develop a method of purifying PC-NSAIDs based upon the ability of NSAIDs to induce a remarkable increase in the solubility of PC in the polar solvent, acetone. In the absence of NSAIDs, PC has a limited solubility in acetone $(<0.1 \mathrm{~g} / \mathrm{ml})$. In contrast, if the NSAID, ibuprofen is predissolved in acetone to a concentration of $\mathrm{lg} / \mathrm{ml}$, the solubility of PC in the polar solvent is increased $>2 \mathrm{~g} / \mathrm{ml}$. Removal of the acetone by evaporation results in a viscous (70 poise) oil that is highly enriched in PC-ibuprofen. This purified oil-based PC-NSAID product was subjected to: physico-chemical; structural; and biological examination. Results: A strong molecular interaction was observed between ibuprofen and PC involving both the aromatic nucleus and the carboxyl group of the NSAID, and the choline head group and the carbonyl ends of the acyl chains of the PC of the acetone-prepared oil, as determined by Fourier transform infrared (FTIR) and proton, carbon and phosphorous nuclear magnetic resonance
(NMR) spectroscopy. Both dynamic light scattering and transmission electron microscopy, revealed the presence of spherical macromolecular assemblies having a diameter between 100$150 \mathrm{~nm}$. In comparison to unmodified ibuprofen, the enriched PC-ibuprofen preparation: significantly reduced (70-80\%) GI bleeding in both acute and chronic rodent ulcer models; accelerated the healing of acetic acid-induced gastric ulcers in rats; and had significantly greater analgesic activity in an acute model of joint inflammation. Conclusion: A method has been developed to prepare an enriched preparation of PC-NSAIDs related to changes in the solubility of the components in the polar solvent, acetone. We have used a number of physico-chemical and structural techniques to localize the association of ibuprofen and $\mathrm{PC}$ at an atomic level, and its macromolecular assembly characteristics. Our findings that the enriched PC-ibuprofen product has enhanced GI safety and analgesic activity in rodent model systems, suggest that future clinical evaluation of this PC-NSAID preparation is warranted. (Supported by NIH grant R42 DK063882 and the Texas Higher Education Coordinating Board)

\section{S1851}

Effects of Esomeprazole Sodium On Glutathione Levels in the Gastric Mucosa of Rats Treated with Indomethacin

Manuela Verri, Gianfranco Natale, Federica Boschi, Ornella Pastoris, Marcello Tonini

Background: In the clinical setting, proton pump inhibitors are effective in preventing and healing non steroidal anti-inflammatory drug (NSAID)-induced gastroduodenal lesions by acting as antisecretory agents. Besides gastric acid, oxidative stress induced by tissue antioxidant depletion is a potential mechanism for gastric mucosal damage in NSAID gastropathy. It has been proposed that benzimidazole derivatives may exert their protective action also through antioxidant mechanisms. Aim: To examine the effects of esomeprazole sodium on the levels of glutathione, a major endogenous antioxidant compound, in the gastric mucosa of rats treated with indomethacin. Methods: Male albino Wistar rats, 200-225 g body weight ( $\mathrm{n}=8 /$ group), received one of six treatments by intragastric gavage: $1 \%$ methocel as vehicle (controls), esomeprazole 30 or $60 \mu \mathrm{mol} / \mathrm{kg}$, indomethacin $100 \mu \mathrm{mol} / \mathrm{kg}$, esomeprazole 30 or $60 \mu \mathrm{mol} / \mathrm{kg}$ and indomethacin $100 \mu \mathrm{mol} / \mathrm{kg}$. In rats treated with esomeprazole and indomethacin, esomeprazole was administered 30 minutes before indomethacin. The animals were sacrificed 4 hours after indomethacin administration, and the stomachs were rapidly removed and processed for the evaluation of mucosal levels (expressed as $\mu \mathrm{mol} / \mathrm{g}$ wet tissue) of total (tGSH), reduced (GSH) and oxidized (GSSG) glutathione. Results: An histomorphometric evaluation revealed that esomeprazole markedly reduced mucosal injuries induced by indomethacin. Esomeprazole alone (30 or $60 \mu \mathrm{mol} / \mathrm{kg}$ ) did not significantly affect the mucosal concentration of $\mathrm{tGSH}, \mathrm{GSH}$ and GSSG, whereas indomethacin caused a significant reduction of tGSH and GSH contents $(0.371 \pm 0.024$ and $0.349 \pm 0.025, \mathrm{P}<0.05)$ and a significant increase in GSSG levels $(0.022 \pm 0.003, \mathrm{P}<0.05)$ compared to controls $(0.817 \pm 0.042$ $0.805 \pm 0.043,0.012 \pm 0.001$, respectively). Esomeprazole $30 \mu \mathrm{mol} / \mathrm{kg}$ partly reversed the effect of indomethacin on tGSH, GSH and GSSG levels $(0.598 \pm 0.036,0.584 \pm 0.035,0.014 \pm 0.001$, respectively, $\mathrm{P}<0.05)$. Esomeprazole $60 \mu \mathrm{mol} / \mathrm{kg}$ reversed the effects of indomethacin on glutathione levels $(0.846 \pm 0.049,0.830 \pm 0.048,0.015 \pm 0.002$, respectively, $\mathrm{P}<0.05)$ up to control values. Conclusions: Our findings indicate that esomeprazole dose-dependently counteracts the effects of indomethacin on gastric mucosal glutathione levels. This supports the view that the protective effects of esomeprazole against indomethacin-induced gastric damage are partly dependent on a restored bioavailability of mucosal sulfhydryl compounds.

\section{S1852}

The Host Specific Differences in the Shunt Toward Lipoxygenase(LOX) Pathway Caused By the Inhibition of Cyclooxygenase(COX) Correlate with the Differences in the Extent of NSAID-Induced Gastric Damage Ireneusz T. Padol, Changcheng Wang, Victoria Seto, Richard H. Hunt

Premise: Arachidonic acid is metabolized universally via two distinct pathways; cyclooxygenase (COX) and lipoxygenase(LOX). The products of the LOX pathway such as leukotrines and free radicals are involved in the induction and/or augmentation of gastric damage. It has been postulated that selective and non-selective NSAIDs, in addition to inhibition of the COX pathway, increase arachidonic acid levels, thus causing a "shunt" of metabolism toward the LOX pathway. Our study aimed to examine the role of this shunt on the extent of NSAID induced gastric damage using two strains of mice. Balb/c and C57BL/6 mice were chosen as these strains posess distinctive differences in relation to gastric acid secretion, including NSAID induced gastric damage, prostanoid metabolism (PLA2) and expression of EP3 receptors (AJP 2005, 288, G1110). Methods: Mice from both strains were treated intraperitoneally with two doses of indomethacin, 4 and $40 \mathrm{mg} / \mathrm{kg}$, and aspirin, 25 and 250 $\mathrm{mg} / \mathrm{kg}$. 3 hours later stomachs were scored for mucosal damage and blood and gastric samples collected for determination of PGE2, TBX2 and LTB4 which served as markers of COX and LOX pathways. Results: We observed less gastric damage in C57BL/6 mice than in Balb/c when lower doses of NSAIDs were used, 16.4 vs $7.6 \mathrm{~mm}, \mathrm{p}=0.0003$ for $4 \mathrm{mg} / \mathrm{kg}$ of indomethacin and 8.8 vs $5.2 \mathrm{~mm}, \mathrm{p}=0.001$ for $25 \mathrm{mg} / \mathrm{kg}$ of aspirin respectively. Strain related differences in fasted and non-fasted serum and/or gastric prostanoid levels were noted. Serum levels of PGE2 and LTB4 were higher in Balb/c mice than in C57BL/6 mice 1.9 vs $1.1 \mu \mathrm{g} / \mathrm{ml}, \mathrm{p}=0.05$ and $247.7 \mathrm{vs} 70.4 \mathrm{pg} / \mathrm{ml}, \mathrm{p}=0.02$ respectively, while tissue levels of LTB4 were higher in non-fasted C57BL/6 mice than in Balb/c mice, 19.7 vs $3.2 \mu \mathrm{g} / \mathrm{g}$, $\mathrm{p}=$ 0.05. NSAIDs caused remarkable inhibition of PGE2 in the stomachs from both mice strains. However, simultaneous induction of LTB4 was seen in stomachs from Balb/c mice but not in C57BL/6 mice which had less NSAID induced gastric damage. Discussion and Conclusion: Our study demonstrates that the LOX shunt, associated with inhibition of the COX pathway, results in higher levels of LTB4, with concurrent inhibition of PGE2 production in the stomach but not in blood. Results from NSAID induced gastric damage confirmed our previous findings that Balb/c mice are more prone to NSAID gastropathy than C57BL/6 mice (Gut 2001, 49(Suppl. 3) A1737). We suggest that the "COX to LOX shunt" is associated with a greater extent of NSAID induced gastric damage. This implies a potential relevance of host genetics on NSAID-induced mucosal damage in the clinical setting 
S1853

A Cause-Effect Relationship Between Deficiency of Prostaglandins and Expression of Cyclooxygenase-2 in Rat Stomachs Following Administration of Nonsteroidal Anti-Inflammatory Drugs

Yuji Mashita, Yoshikazu Kubo, Ryo Hatazawa, Koji Takeuchi

Background/Aim: We previously reported the up-regulation of cyclooxygenase (COX)-2 expression in the rat stomach after administration of indomethacin or SC-560 (a selective COX-1 inhibitor), and this process is associated with gastric hypermotility induced by COX1 inhibition by these agents. However, it is possible that this event occurs simply as a compensatory phenomenon resulting from prostaglandin (PG) deficiency due to COX-1 inhibition. In the present study, we investigated a cause-effect relationship between PG depletion and COX-2 expression in rat stomachs after the administration of nonsteroidal anti-inflammatory drugs (NSAIDs). Methods: Male SD rats were used after $18 \mathrm{~h}$ fasting. Various NSAIDs (indomethacin, naproxen, dicrofenac, frulbiprophen, aspirin) were given $\mathrm{SC}$ or $\mathrm{PO}$, and the gastric lesion, $\mathrm{COX}-2$ mRNA expression and $\mathrm{PGE}_{2}$ content were determined 4 h later. Gastric potential difference (PD) and motility were also measured. Results: All NSAIDs tested, except aspirin, decreased the mucosal $\mathrm{PGE}_{2}$ content, increased gastric motility, and caused gastric lesions with the up-regulation of COX-2 expression, irrespective of the route of administration. Aspirin induced damage and COX-2 expression in the stomach when given $\mathrm{PO}$ but not $\mathrm{SC}$, although the mucosal $\mathrm{PGE}_{2}$ content was similarly decreased by this agent on both routes of administration. Gastric motility was not affected by aspirin given either PO or SC, while gastric PD was markedly decreased by aspirin only given PO. Supplementation of $\mathrm{PGE}_{2}$ prevented the expression of COX-2 in the stomach following PO or SC administration of indomethacin but had no effect on the COX-2 expression seen after $\mathrm{PO}$ administration of aspirin. The COX-2 expression induced by indomethacin was also inhibited by atropine, while omeprazole had no effect. In addition, SC-560 but not rofecoxib caused a decrease in $\mathrm{PGE}_{2}$ content and an increase in gastric motility as well as COX-2 expression, without induction of any damage in the stomach. Conclusion: These results suggest that the expression of COX-2 in the stomach after NSAID treatment may be due to the mucosal irritative stimuli caused by the physico-chemical property of drug (aspirin, PO) or the abnormal stomach contraction due to COX-1 inhibition (other NSAIDs) ), rather than being a result of PG deficiency. Luminal acid does not play a role in the up-regulation of COX-2 in the stomach following NSAIDs, inhibition of COX-1.

\section{S1854}

Transcriptome Analysis for Cytoprotective Actions of Rebamipide Against Indomethacin-Induced Gastric Mucosal Injury in Rats

Yuji Naito, Masaaki Kuroda, Katsura Mizushima, Tomohisa Takagi, Osamu Handa, Satoshi Kokura, Norimasa Yoshida, Toshikazu Yoshikawa

Background: Nonsteroidal anti-inflammatory drugs such as indomethacin are known to induce gastric mucosal injury including apoptosis. We have reported that rebamipide, a gastroprotective drug, suppresses indomethacin-induced mucosal injury in human and rats (Dig Dis Sci 50:104, 2005, 43:83, 1998). However, the mechanisms of the cytoprotective actions of rebamipide have not been fully addressed.Aim: We determined mRNA expression profile of the gastric mucosa treated with indomethacin in rats, and investigated the cytoprotective effects of rebamipide against indomethacin-induced injury by a high-density oligonucleotide array. Materials and Methods: We used the Rat Toxicology U34 GeneChip array ( 1,031 probes). Gastric epithelial cells were identified on cryostat sections $(8 \mu \mathrm{m})$ of the specimens obtained from the rat stomach, and the cells were isolated by laser-assisted microdissection. Using the small sample target labeling assay protocol, we obtained a sufficient amount of biotinylated cRNA to perform the GeneChip analysis from the small amount of gastric epithelial cells. Data analysis was performed with a GeneChip Operating Software and Ingenuity Pathway Analysis. Results: Among 1,031 probes, the expression of 160 probes (15.5\%) showed at least a 2.0-fold up-regulation (158 probes) and down-regulation (2 probes) $2 \mathrm{~h}$ after indomethacin administration in comparison with the vehicle-treated rats. Genes involved in redox-related enzymes (superoxide dismutase 1, carbonyl reductase 1, glutathione peroxidase 3, glutathione S-transferese, etc.) and transcription regulators (c-fos oncogene, etc) were included. By the pathway analysis of the up-regulated 123 probes among 158 probes, 5 networks were found to be significant in that they had more of the identified genes present than would be expected by chance. The network 1 contained the majority of these genes, had a highly significant score, and consisted of known clusters of cell death, cancer, and endocrine system disorders. We succeeded in listing 10 genes that were up-regulated by the treatment with indomethacin and that were down-regulated by rebamipide, including growth arrest and DNA damage-induced 45 (GADD45 $\alpha$ ). The induction of GADD $45 \alpha$ mRNA by indomethacin and its inhibition by rebamipide was also confirmed by real time PCR. Conclusions: These data obtained from transcriptome analysis on gastric epithelial cells in vivo demonstrate that cell death, especially apoptosis, pathway is involved in the pathogenesis of indomethacin-induced gastric mucosal injury, and that inhibition of apoptosis-related genes is possibly important for the cytoprotective effect of rebamipide against this injury.

\section{S1855}

Effects of Selective Cyclooxygenase-2 Inhibitors On Healing of Experimental Gastric Ulcers and Mucosal Expression of Cyclooxygenase Isoforms Matteo Fornai, Luca Antonioli, Rocchina Colucci, Gianfranco Natale, Fabio Baschiera, Nunzia Bernardini, Chiara Ippolito, Corrado Blandizzi, Mario Del Tacca

Introduction. Selective cyclooxygenase-2 (COX-2) inhibitors were developed as novel gastric sparing anti-inflammatory drugs. Recent evidence suggests an involvement of COX-2 in gastroprotection, while its role in ulcer healing is currently debated. This study investigates the effects of COX-2 inhibitors on healing of experimental gastric ulcerative lesions. Methods. Male Wistar rats were subjected to induction of gastric injury by intragastric administration of absolute ethanol ( $1 \mathrm{ml} / 200 \mathrm{~g}$ body weight) or subserosal injection of acetic acid $(20 \%$, $40 \mu \mathrm{l}$ ). Treatments with COX inhibitors started 4 hours or 4 days after induction of lesions with ethanol or acetic acid, respectively. Indomethacin ( $1 \mathrm{mg} / \mathrm{kg} / \mathrm{day}$, COX-1/COX-2 inhibitor, indoleacetic derivative), valdecoxib ( $1 \mathrm{mg} / \mathrm{kg} /$ day, COX-2 inhibitor, sulphonamide derivative), DFU ( $5 \mathrm{mg} / \mathrm{kg} /$ day, COX-2 inhibitor, methylsulphonic derivative) or drug vehicle were administered orally for 1,3 or 7 days. At the end of treatments, stomachs were processed for: a) histomorphometric evaluation of ethanol-induced injury (\%); b) measurement of acetic acid-induced ulcer area $\left(\mathrm{mm}^{2}\right)$; c) COX-1 and COX-2 mucosal expression by RT PCR and immunohistochemistry. Results. Histomorphometric analysis displaved a necrotic damage affecting $37.2+6.2 \%, 30.5+2.9 \%$ and $18.1+1.0 \%$ of mucosa at day 1,3 and 7 after ethanol administration, respectively. Treatment with indomethacin or DFU delayed healing of ethanol-induced gastric lesions, the mucosal damage accounting for $37.1 \pm 1.6 \%$ and $32.0 \pm 3.4 \%$ at day 7 , respectively. Valdecoxib did not affect the time course of mucosa healing $(16.1 \pm 1.0 \%$ at day 7$)$. Acetic acid injection was followed by development of single chronic ulcers, macroscopically evident after 1,3 or 7 days from onset of treatments with COX inhibitors. At these times, ulcer areas in controls were $59.3 \pm 4.3,57.4 \pm 4.8$ and $18.7 \pm 4.0$ $\mathrm{mm}^{2}$, respectively. Indomethacin and DFU delayed ulcer healing at day 7 (48.3 \pm 3.6 and $57.4 \pm 4.2 \mathrm{~mm}^{2}$, respectively), while valdecoxib did not $\left(16.7 \pm 3.5 \mathrm{~mm}^{2}\right)$. RT-PCR and immunohistochemistry displayed a COX-2 induction in tissue areas adjacent to ethanol- or acetic acid-induced lesions, whereas COX-1 was unaffected. At day 7 the enhancement of COX-2 induction was higher in animals treated with valdecoxib than in those receiving indomethacin or DFU. Conclusions. Valdecoxib does not appear to interfere with healing of gastric lesions, at variance of other COX-2 inhibitors endowed with different chemical structures. The recruitment of COX-2-independent mechanisms by valdecoxib is likely to account for the lack of effects on ulcer healing, and the concomitant enhancement of gastric COX-2 expression

\section{S1856}

Indigestible Solid Components of Food and Intestinal Hyper-Motilities Play An Important Role in the Formation of Intestinal Ulcers Induced By Indomethacin in Cats and Dogs

Hiroshi Satoh, Sachiko Shiotani, Noriko Otsuka

BACKGROUND: In the last AGA meeting we reported that dietary fiber (DF) such as cellulose played some role in the formation of intestinal ulcers and that diets with no- or less DF could prevent indomethacin (IM)-induced intestinal ulcers in all of rats, cats and dogs. In the present study, the role of DF (soluble or insoluble ) and intestinal motility in IM-induced intestinal ulcers was investigated in both cats and dogs. METHODS: Both male and female cats (2.5-3.5 kg) and dogs ( $7-15 \mathrm{~kg})$ were used (4-6 animals in each group). Role of DF Several types of diets containing various percentages of DF were given to animals twice a day during the experiment. IM was administered orally once a day after a morning meal for 3 days. The animals were sacrificed 24 hrs after the final dose of IM, and mucosal lesions in the small intestine were examined. Role of motilities: In this study, the gastrointestinal motilities were recorded in conscious cats implanted with force transducers in the stomach, duodenum, jejunum and ileum. RESULTS: Role of DF: In both cats and dogs given dry food containing 4 and $6 \% \mathrm{DF}$, resp., IM $(3 \mathrm{mg} / \mathrm{kg})$ produced many lesions in the middle and lower intestine; the mean lesion areas (MLA) were $8.4 \pm 2.7 \mathrm{~cm} 2$ and $31.3 \pm 2.5 \mathrm{~cm} 2$ resp. The lesions were markedly decreased in both animals given canned diets containing no DF (MLA: $0.1 \pm 0.1 \mathrm{~cm} 2$, and $0.4 \pm 0.2 \mathrm{~cm} 2$, resp., $\mathrm{p}<0.05$ ). Whereas the lesions were increased significantly in animals given the canned diet supplemented with 3 or $10 \%$ cellulose (insoluble fiber), but not by addition of 3 or $10 \%$ pectin (soluble fiber). Role of motilities Atropine $(0.1$ and $0.3 \mathrm{mg} / \mathrm{kg}$, s.c., $\times 2 /$ day, for 3 days) almost completely blocked the ulcer formation caused by IM in both animals given dry foods. IM (1-3 mg/kg, p.o.) dosedependently increased the gastrointestinal motilities in cats, and the effects were blocked by atropine $(0.3 \mathrm{mg} / \mathrm{kg}$, s.c.). CONCLUSIONS: It could be concluded that both indigestible solid components of food and intestinal hyper-motility play an important role in the formation of intestinal ulcers by IM, probably by causing physical damage in the surface mucosal cells under a decreased condition of the surface mucus induced by IM.

\section{S1857}

CDNA Microarray Analysis Reveals Anti-Inflammatory Effects of Lansoprazole, a Proton Pump Inhibitor, Against Indomethacin-Induced Intestinal Mucosal Injury in Rats

Hiroshi Ichikawa, Norimasa Yoshida, Toshimitsu Okuda, Kazuhiro Kamata, Hirokazu Kajikawa, Hisato Tsuboi, Takahiro Suzuki, Osamu Handa, Tomohisa Takagi, Satoshi Kokura, Yuji Naito, Toshikazu Yoshikawa

It is well known that indomethacin produces gastrointestinal inflammation and ulceration through a diverse number of mechanisms. We have reported that lansoprazole have antiinflammatory effects as well as inhibition of acid secretion. The purpose of the present study is to evaluate the biological effects of lansoprazole by using cDNA microarray analysis in experimental rat models on indomethacin-induced enteritis in vivo. Male Wistar rats wer administered indomethacin $(10 \mathrm{mg} / \mathrm{kg})$ s.c., killed $3 \mathrm{~h}$ and $24 \mathrm{~h}$ later under deep ether anesthesia, and both the jejunum and ileum were removed. Lansoprazole was given subcutaneously $(5 \mathrm{mg} / \mathrm{kg})$ just after following indomethacin administration. To estimate the severity of the intestinal mucosal damage by indomethacin, we measured the concentrations of thiobarbituric acid-reactive substances (TBA-RS), myeloperoxidase (MPO) activity and the content of cytokine-induced neutrophil chemoattractant-1 (CINC-1) in the intestinal mucosa. To investigate the molecular mechanisms underlying the effects of lansoprazole on this model, we examined gene expression profiles in mucosal cells which were collected by laser capture microdissection method. Preparation of cRNA and target hybridization was performed according to the Affymetrix GeneChip Eukaryotic Small Sample Target Labeling Assay Protocol (Version II). The gene expression profile was determined by the the GeneChip (Rat toxicology U34 array, Affymetrix) microarray system. Single administration of indomethacin at $10 \mathrm{mg} / \mathrm{kg}$ provoked severe hemorrhagic lesions in the small intestine, mostly the jejunum and ileum. The concentrations of TBA-RS, the levels of MPO activities and the content of mucosal CINC-1 in the intestinal mucosa significantly increased $24 \mathrm{hr}$ after indomethacin administration and these increased levels were significantly inhibited by the treatment with lansoprazole. Microarray analysis revealed that lansoprazole restored the increased expression 
of genes for enzymes involved in oxidative stress, such as metallothionein, catalase and superoxide dismutase, in mucosal cells after $3 \mathrm{hr}$ injection of indomethacin. Lansoprazole also suppressed the upregulated expression of genes for cytochrome c oxidase, $\mathrm{N}$-acetyltransferase- 2 and transferrin. Furthermore, some genes for enzymes involved in anti-apoptosis, protein folding and DNA-binding protein were downregulated by indomethacin treatment, and lansoprazole was able to restore these changes. These observations clearly illustrate that lansoprazole protects against intestinal injury induced by indomethacin. It was suggested hat oxidative stress and apoptosis were involved in the pathogenesis of indomethacininduced enteritis

\section{S1858}

Poor Metabolizer Genotype Status of Cytochrome P450 2c19 Is a Risk Factor for Developing Gastric Cancer in Japanese with Helicobacter Pylori Infection

Mitsushige Sugimoto, Takahisa Furuta, Naohito Shirai, Akiko Nakamura, Mutsuhiro

Ikuma, Masayoshi Kajimura, Haruhiko Sugimura, Akira Hishida, Tskashi Ishizaki

Backgrounds and aims: Cytochrome P450 2C19 (CYP2C19) polymorphism, one of the isoforms of CYP enzymes, is a clinically important enzyme responsible for the metabolism of a number of therapeutic drugs, such as proton pump inhibitors. CYP2C19 is associated with the development of lung, liver or esophageal cancer by detoxification of carcinogen or activation of pro-carcinogen. The present study was designed to clarify the association between CYP2C19 genotypes and susceptibility to gastric cancer in Japanese patients with H. pylori infection. Methods: We determined CYP2C19 genotypes (CYP2C19*1, *2 and *3) in $111 \mathrm{H}$. pylori-positive patients with gastric cancer and $315 \mathrm{H}$. pylori-positive controls without gastric cancer consisting of patients with gastritis only or peptic ulcer. CYP2c19 genotypes were classified into the three groups; homozygous extensive metabolizers (homEMs), heterozygous EMs (hetEMs), and poor metabolizers (PMs). Frequencies of CYP2C19 genotypes and serum pepsinogen I and II levels, a biomaker of gastric atrophy, in the gastric cancers and controls were compared. Results: Frequencies of homEMs, hetEMs, and PMs were $31.5 \%, 42.3 \%$ and $26.2 \%$ in the gastric cancer patients and $38.1 \%, 47.0 \%$ and $14.9 \%$ in the controls, respectively. The PM genotype of CYP2C19 was associated with an increased risk for developing gastric cancer with the age and sex-adjusted odds ratio (OR) of 1.975 (95\% confidence interval [CI]: 1.068 - 3.649), especially for diffuse-type gastric cancer (OR; 3.385, CI; 1.187 - 9.648). There was no significant association between CYP2C19 genotypes and serum pepsinogen I levels or pepsinogen I/I ratios, although serum pepsinogen I level in patients with gastric cancers were significantly decreased. Conclusions: In H. pylori-positive Japanese, the PM of CYP2C19 appeared at an increased risk for developing gastric cancer, especially diffuse-type. The preset study results sugget that the CYP2C19 genotyping test is useful for screening individuals with a higher risk for developing gastric cancer when infected with $\mathrm{H}$. pylori and that CYP2C19-related H. pylori-positive PMs should undergo the eradication therapy for the prevention of gastric cancer or would require an intensive follow-up care to scrutinize the possible future gastric cancer development.

\section{S1859}

Induction of Aberrant Methylation in Gastric Mucosae By H. Pylori Infection, and Its Association with Risk of Single and Multiple Gastric Cancers Takeshi Nakajima, Takao Maekita, Ichiro Oda, Takuji Gotoda, Daizo Saito, Masao Ichinose, Toshikazu Ushijima

Purpose: CpG methylation is a stable, but plastic, modification on DNA. Aberrant methylation of promoter $\mathrm{CpG}$ islands (CGIs) of tumor-suppressor genes is deeply involved in some cancers, including gastric cancers. Here, we focused on induction of aberrant DNA methylation in gastric mucosae by Helicobacter pylori (HP) infection, and possible association between the fraction of cells with aberrant methylation in gastric mucosae and risk of single and multiple gastric cancers. Material and Methods: Twenty-six healthy volunteers (HVs), 30 cases with a single well-differentiated gastric cancer, and 32 cases with multiple welldifferentiated cancers were recruited. The average age of HVs was matched to those of cancer cases. Biopsy materials were collected from three standard points of non-cancerous gastric mucosae. HP infection status was analyzed by the culture method. Methylation levels (fractions of methylated DNA molecules among all the DNA molecules) were quantified by realtime methylation-specific PCR of seven regions, i) promoter CGIs of FLNc, HAND1, THBD, HRASLS and LOX, and ii) exonic CGIs of p4lARC and p16. Results: (1) When methylation levels were compared between HP-negative and -positive HVs, HP-positive individuals were shown to have 3.0- to 14.3- fold higher average methylation levels than HP-negative individuals in all the seven regions. (2) Among the HP-negative individuals, FLNc and HAND1 showed step-wise increases of methylation levels in cases with a single cancer (1.3- and 3.0fold, respectively), and cases with multiple cancers (5.0- and 3.6- fold, respectively), compared with HVs. Even when stratified into cases with mild atrophy and severe atrophy, FLNc showed step-wise increases in cases with a single cancer (1.9- and 4.8-fold, respectively) and cases with multiple cancers (4.8- and 15.8-fold). (3) In the HP-positive individuals, methylation levels were increased highly and to various degrees in HVs and cancer cases. (4) Methylation levels were highest in HP-positive individuals (HVs and cancer cases), and followed by HP-negative cancer cases, most of whom were considered to have past exposure to HP infection. Conclusion: Current HP infection potently induced DNA methylation in gastric mucosae. The lower methylation levels in HP-negative gastric cancer cases suggested that active HP infection induces both temporary and permanent methylation. It was considered that the permanent levels can be observed in HP-negative individuals, and that higher levels are associated with single and multiple gastric cancers. Methylation levels in noncancerous gastric mucosae are a promising risk marker for single and multiple gastric cancers.
S 1860

Identification of Increased Fibrinopeptide a Serum Protein Levels in Gastric Cancer Sera By Magnet Bead Assisted Maldi-Tof Mass Spectrometry Matthias Ebert, Soren Deininger, Claudia Knippig, Christoph Roecken, Peter Malfertheiner

Gastric cancer is the second most common malignancy and prognosis remains dismal. The reasons for the poor prognosis are the lack of sensitive serum markers for early detection and screening of high-risk individuals as well as the limited treatment options in advanced cancer stages. Using MALDI TOF mass spectrometry after prefractionation of sera with magnet hydrophobic C8 coated beads sera from 14 patients with gastric cancer and 14 healthy controls mass spectra were generated. A peptide fragment was found to be highly elevated in cancer sera and was identified as fibrinopeptide A. In order to confirm proteome analysis of gastric cancer sera we then screened a larger series of patients with gastric cancer $(n=99)$, high-risk individuals $(n=13)$ and normal controls $(n=111)$ for fibrinopeptide A serum levels. Interestingly, the mean logarithmic concentrations of serum fibrinopeptide A levels were significantly higher in cancer patients (mean $3.636 \pm 0.3738 ; \mathrm{p}<0.0001$ ) and high-risk individuals (mean $3.569+0.4722 ; \mathrm{p}<0.05$ ) compared to normal controls (mean $3.303 \pm 0.4012$ ). In contrast, we observed no association of fibrinopeptide A levels with tumor stage, tumor location, presence of regional or distant metastasis, and Lauren type of gastric cancer. In conclusion, MALDI TOF mass spectrometry of prefractionated gastric cancer sera allows the identification of potential serum markers for gastric cancer and may lead to the development of serum based tests for screening of high-risk individuals.

\section{S1861}

Identification of Human Esophageal Cancer Sub-Types By Serum Protein Analysis Using Maldi-Tof Mass Spectroscopy

Timothy P. Millar, Yuliya V. Karpievitch, Elizabeth G. Hill, Charles E. Hammond, Brenda J. Hoffman, Adam J. Smolka

The majority of patients presenting with symptoms of esophageal cancer (EC) have advanced disease. In patients with potentially resectable disease, the cure rate is still extremely low due to local recurrence and metastatic disease. The best hope of improving survival is early detection and effective therapeutic intervention. This study tested the hypothesis that characteristic protein and/or peptide markers are associated with EC, and that mass spectroscopic human serum analysis can provide differential protein/peptide molecular signatures that discriminate among healthy controls and two major EC subtypes. Sera were obtained from 26 patients undergoing upper endoscopy and/or endoscopic ultrasonography; nine [6 white males (wm), 2 black males (bm), 1 white female (wf)] were diagnosed with esophageal adenocarcinoma (AC), eight ( $3 \mathrm{wm}, 3 \mathrm{bm}, 2 \mathrm{bf}$ ) with squamous cell carcinoma $(\mathrm{SC})$, and nine ( $5 \mathrm{wm}, 3 \mathrm{wf}, 1 \mathrm{bf}$ ) were normal. Sera were treated with reversed-phase ligand (C8)-derivatized superparamagnetic particles from which adsorbed hydrophobic serum proteins/peptides were subsequently eluted. Aliquots of serum eluates were randomized, mixed with $\alpha$-cyano-4-hydroxycinnamic acid matrix and applied to a target plate for matrix-assisted laser desorption ionization-time of flight mass spectroscopy (MALDI-TOF) (Voyager STR, $337 \mathrm{~nm} \mathrm{~N} 2$ laser, linear mode geometry, $25 \mathrm{kV}$ ). Quadruplicate mass spectra spanning an $\mathrm{m} / \mathrm{z}$ window of 800-20,000 Da were acquired for each eluate on 3 separate days. Peak detection and quantitation was restricted to masses $>2000 \mathrm{Da}$ to avoid bias introduced by non-biological matrix signal. Analysis was further restricted to quality spectra (high signal/noise ratio across the $\mathrm{m} / \mathrm{z}$ window), resulting in a contribution of 11 spectra per subject (286 spectra total). After suitable data preprocessing, $110 \mathrm{~m} / \mathrm{z}$ peaks were identified as potential biomarkers. A supervised learning algorithm (random forests) was applied to biomarker peaks to classify patients' disease status. Eight out of nine AC patients (sens $=89 \%$, spec $=88 \%$ ), six out of eight SC patients (sens $=75 \%$, spec $=89 \%$ ), and eight out of nine controls (sens $=89 \%$, spec $=100 \%$ ) were correctly classified. The nine most discriminatory peaks $(\mathrm{m} / \mathrm{z}=3285,2031,4348,3384,8760,8771,6392,3885$, and $4573 \mathrm{Da})$ are targets of further biochemical analysis. We conclude that patient serum samples can be processed using magnetic microsphere-based technology to yield complex, stable, and reproducible MALDI-TOF spectra, statistical analysis of which provides a quantitative assessment of correlation between specific patterns of protein/peptide expression and the presence or absence of two subtypes of esophageal cancer.

\section{S1862}

Improved Performance of a Non-Invasive Fecal DNA (fDNA) Test to Screen for Colorectal Cancer (CRC)

Steven Itzkowitz, Lina Jandorf, Randall Brand, Linda Rabeneck, Paul Schroy, Stephen Sontag, David Johnson, Joel Skoletsky, Sanford Markowitz, Anthony Shuber

Background: Proof of principle that fecal fDNA testing can be a useful technique to screen asymptomatic average-risk individuals over age 50 for CRC was recently established in a multicenter study (MCS) (NEJM 351:2704,2004). That prototype assay (PreGenPlus Version 1; PV1) analyzed 22 DNA mutations (MuMu-22) and DNA integrity (DIA), resulting in 52\% sensitivity (SENS) for CRC and 94\% specificity (SPEC). The SENS of the DIA component was lower than expected due to DNA degradation during specimen shipping. Subsequently, a Version 2 (PV2) assay was developed which incorporates an improved method for stabilizing DNA with buffer (Diagn Mol Pathol 14:183,2005), better extraction of DNA by gel-capture (J Mol Diagn 6:386,2004), and new biomarkers including vimentin methylation (V29; JNCI 97:1124,2005). Aim: To determine the SENS and SPEC of PV2 in pts with CRC and those with normal colonoscopies (NL). Methods: Pts in this prospective multicenter study were consented after colonoscopic findings of either a NL colon $(n=122)$ or CRC $(n=40)$. NLs had no personal or family hx of CRC/polyps. Approx. 6-14 days after colonoscopy (prior to surgery for the CRC group), pts prepared a single stool sample to which they immediately added buffer. Stools were shipped by express mail, homogenized, and DNA was extracted by sequence-specific gel-capture. MuMu-22 and DIA (PV1) analyses were performed as in the MCS. Methylation-specific PCR was performed after sodium bisulfite treatment. Results: Compared to PVl-MCS data, the addition of buffer and gel-capture technology to the 
same marker panel (PVl-New) increased SENS to $72.5 \%$ for CRC [Dukes' Stages A:B:C:D= $88: 80: 53: 100 \%]$. The SENS of even part of the DIA assay (DY) alone was $65 \%[\mathrm{~A}: \mathrm{B}: \mathrm{C}: \mathrm{D}=$ 63:70:53:100\%] and V29 alone was 72.5\%[A:B:C:D=75:80:73:50\%]. The optimal combination, V29+DY, resulted in 88\% SENS [A:B:C:D=75:90:93:100\%]. DY detected more distal CRC $(\mathrm{p}=0.03)$, whereas V29 detected prox. and distal CRC equally well. V29+DY had a SPEC of $82 \%$. NLs with false-positive V29+DY were older than those with negative V29+DY (mean age 62.7 vs. $57.4, \mathrm{p}=0.01$ ). Conclusions: Compared with the prototype PVl assay, an improved and simplified fDNA test demonstrates higher SENS for CRC (87.5\%) with only a modest decline in SPEC. Many false-positives may simply be due to methylation associated with normal aging. The inclusion of fewer markers will make the test easier and less expensive.

\begin{tabular}{|c|c|c|c|c|c|}
\hline & $\begin{array}{c}\text { Prototype } \\
\text { PV1-MCS }\end{array}$ & $\begin{array}{c}\text { Optimized } \\
\text { PV1-New }\end{array}$ & $\begin{array}{c}\text { DIA } \\
\text { (DY) }\end{array}$ & $\begin{array}{c}\text { Vimentin } \\
\text { (V29) }\end{array}$ & $\begin{array}{c}\text { V29+DY } \\
(95 \% \mathrm{CI})\end{array}$ \\
\hline Buffer & - & + & + & + & + \\
\hline $\begin{array}{c}\text { Gel } \\
\text { capture }\end{array}$ & - & + & + & + & + \\
\hline Sensitivity & $51.6 \%$ & $72.5 \%$ & $65 \%$ & $72.5 \%$ & $\begin{array}{c}87.5 \% \\
(77.4-97.6)\end{array}$ \\
\hline Specificity & $94.4 \%$ & $90.2 \%$ & $92.6 \%$ & $86.9 \%$ & $\begin{array}{c}82.0 \% \\
(75.2-88.8)\end{array}$ \\
\hline
\end{tabular}

\section{S1863}

Elevated Genomic Instability in Aberrant Crypt Foci (ACF) Is Associated with Tobacco Use

Sadir Alrawi, Heliodoro Medina, Merril Dayton, John Gibbs, Daniel Stoler, Garth Anderson, Robert Carroll

Background: ACF are the earliest identifiable neoplastic lesions in the colon. We have shown age and tobacco uses are independently linked to ACF number (CGH: March 2005). The progression of adenoma to cancer is accompanied by progressive accumulation of mutations, in tandem with increasing genomic instability (GI). The ability of tobacco carcinogens to induce genomic damage is well established. To determine if this damage was evident as early as the ACF in colorectal tumor progression, we evaluated genomic damage, chromosomal aberrations, and specific mutations within ACF in an age-matched population of smokers and non-smokers. Methods: ACF were identified and biopsied using magnification chromoendoscopy. DNA was extracted from ACF and adjacent normal tissue. Chromosomal and intrachromosomal instability was evaluated by BAC-array Comparative Genomic Hybridization $(\mathrm{aCGH})$ and Inter-Simple Sequence Repeat PCR (ISSR PCR) respectively. The Genomic Instability Index (GII), a measure of genome-wide inter-SSR DNA damage, was calculated for each specimen. K-ras mutation was evaluated by diagnostic PCR amplification and BstNI restriction digestion (sens 1/1000 copies). Colon cell lines with and without known K-ras mutations were used as positive and negative controls. P53 and B-RAF mutations were evaluated by automated sequencing of PCR amplified DNA using fluorescent dideoxy termination nucleotides. Results: $20 \mathrm{ACF}$ from 12 smokers (mean age 61.7yr) were compared to $13 \mathrm{ACF}$ from 8 non-smokers (mean age $60.2 \mathrm{yrs}$ ) Sex distribution was similar in both groups (12M: 8F). The CGH profiles obtained for ACF showed no significant deviation from normal diploid DNA nor were there any significant differences in CGH profiles of smokers and nonsmokers. Genomic instability was detectable in $78 \%$ of ACF by ISSR PCR with a mean genomic instability indices of $3.9 \%$ in smokers and $2.2 \%$ in nonsmokers $(p=0.017)$. K-ras codon 12 mutations were uniformly absent in all the ACF tissue evaluated, regardless of smoking status. No p53 mutations were identified in $20 \mathrm{ACF}$ samples. Two B-RAF mutation in exon 11 and exon 15 from ACF were identified. The exon 11 mutation (T1405C) was synonomous but the 2 nd was a serine 600 to tyrosine mutation in the activation segment of the B-RAF protein. Conclusion: DNA damage associated with smoking is an early event and detectable within colorectal ACF prior to the occurrence of adenoma or carcinoma. Kras and B-RAF mutations were found at low frequency. The mutational profile of individual ACF remains distinct from the profiles of adenoma and cancer suggesting most of these lesions may not be direct precursors of adenoma or cancer.

\section{S1864}

Nestin, a Novel Angiogenesis Marker Correlates with Poor Prognosis of Colorectal Cancer Patients

Nobuhisa Teranishi, Toshiyuki Ishiwata, Noritake Tanaka, Kiyonori Furukawa, Hideyuki Suzuki, Tomoko Seya, Hayato Kan, Hiroyuki Tsuruta, Satoshi Matsumoto, Yukihiro Akiya, Seiichi Shinji, Akihisa Matsuda, Kazumitsu Cho, Jyunpei Sasaki, Masao Kawamoto, Tetsuro Matsunobu, Yuri Fujiwara, Takashi Tajiri, Zenya Naito

(Purposes) Angiogenesis is an important factor in the proliferation and metastasis of colorectal cancer. Microvessel density(MVD) in colorectal cancer cell nests is reported to be closely related to clinicopathological factors and patient survival. CD34 and Factor VIII are commonly used as an endothelial cell marker of tumor vessels. However, these markers identify not only newly formed small blood vessels, but also pre-existing large blood vessels. Nestin, a class VI intermediate filament protein, has recently received attention as a marker that can detect the endothelial cells of newly formed microvessels. In the present study, examined the expression of nestin in colorectal cancer tissues, and compared the expression level with CD34 and Factor VIII-related antigen. Moreover, the sizes of nestin-positive and CD34positive vessels were compared in subcutaneous cancer tumors formed in nude mice. (Materials and methods) 1)One hundred-five colorectal cancers cases operated at Nippon Medical School Hospital were employed in this study. Immunohistochemical analysis was performed using serial tissue sections with CD34, nestin, and Factor VIII. Proliferating endothelial cells were immunohistochemically, detected by PCNA antibody. 2)MVD was determined according to the international consensus report. Briefly, five areas where the density of positive blood vessels was highest in the tumors ware selected. Then, the areas were photographed at X200 magnification, and the blood vessels were counted. A clinicopathological factors and prognose were compared between two groups: higher or lower than median of MVD. 3)HCT 15 cells, a human colon adenocarcinoma cell line, were subcutaneously implanted into th dorsum of male BALB/C nu/nu mice. When the tumor size grew to $3 \mathrm{~cm}$ in a diameter, the tumor was harvested. These specimens were prepared for immunofluorescence stainin using CD34 and nestin antibody. (Results) 1)Immunohistochemically, nestin was localized in the endothelial cells in small blood vessels, while CD34 and Factor VIII were localized in mature blood vessels in human colorectal cancer tissues and subcutaneous tumor from nude mice 2)Ratio of co-localization of PCNA and nestin in the same vascular endothelia cells was higher than co-localization of PCNA and CD34 in endothelial cells $(\mathrm{p}=0.0021)$. 3)There was no correlation with nestin-positive blood vessels or clinicopathological factors in colorectal cancer. 4)Regarding disease-free survival, the prognosis was worse for the highly nestin-positive MVD group ( $\mathrm{p}=0.047$ ). (Conclusion) Nestin is considered a more sensitive marker of proliferating endothelial cells in colorectal cancer tissues.

\section{S1865}

Overexpression of Calcium Binding Protein S100a4 in Barrett's Tumorigenesis Ok-Jae Lee, Seung-Mo Hong, Abbes Belkhiri, Christopher Moskaluk, Wael El-Rifai

Background and Aims. The role of S100A2 in carcinogenesis has drawn a complex picture showing down-regulation or overexpression in different tumors. The biologic function of S100A2 in carcinogenesis has not been elucidated to date. S100A4 is a candidate as a molecular marker for metastatic potential with high prognostic significance. In order to evaluate the role of S100A2 and S100A4 in tumorigenesis of upper gastrointestinal adenocarcinoma, we have investigated the mRNA expression level and protein expression of S100A2 and S100A4 in lower esophageal, gastroesophageal junctional (GEJ), and gastric adenocarcinoma tissues. Materials \& Method. Quantitative real-time RT-PCR was performed in 75 frozen tissue samples of lower-esophageal, GEJ, and gastric adenocarcinomas for the expression of S100A2 and S100A4 mRNA. Immunohistochemistry for the expression of S100A2 and S100A4 proteins was also performed on tumor tissue array that contained 206 loweresophageal, GEJ, and gastric adenocarcinomas. Results. Quantitative real-time PCR analysis revealed high levels of S100A2 and S100A4 mRNA in $96.0 \%$ and $77.1 \%$ of lower-esophageal, GEJ, and gastric adenocarcinomas samples as compared with normal mucosae samples Immunohistochemical analysis showed moderate to strong expression of S100A2 protein in $65.5 \%$ of lower esophageal, $61.2 \%$ of GE junctional, and $46.2 \%$ of gastric adenocarcinomas. Overexpression of S100A2 was not related to any clinic-pathological variables. S100A4 protein was overexpressed in $76.6 \%$ of all cancer tissues in comparison to normal mucosa. The overexpression of S100A4 was more frequently observed in the presence of $B E(p=$ 0.0316). On the other hand, we noticed a change of S100A2 expression location from nuclear (normal tissues) to both cytoplasmic and nuclear in the majority of dysplasia and tumors. Conclusions. S100A2 and S100A4 are overexpressed in upper gastrointestinal adenocarcinomas, and the overexpression of S100A4 is related to Barrett's tumorigenesis. S100A2 undergoes a nucleo-cytoplasmic translocation which may occur at early stage of tumorigenensis.

\section{S1866}

Multiple Primary Cancers After Endoscopic Treatment of Esophageal Carcinoma

Iizuka Toshiro, Yahagi Naohisa, Hoteya Syu, Yamamoto Takashi

Objective: Early detection and the wide use of endoscopic mucosal resection (EMR) have dramatically increased survival time in patients with superficial esophageal carcinoma that carries the risk of multiple primary cancers during the follow-up after EMR. We evaluated our patients with multiple primary cancers who underwent EMR of esophageal carcinoma and searched for early markers of malignancies. Subjects and methods: Of the 167 patients who underwent EMR of esophageal carcinoma between January, 1998 and August, 2005 , 156 patients, who have been followed up to date, were included in this study. On the basis of clinical characteristics and timing of EMR, we investigated the site, frequency, and treatment method of multiple primary cancers. Results: Multiple primary cancers were found in 57 patients (37\%). Before EMR of esophageal carcinoma, 56\% of 27 patients had developed cancers in the stomach and $15 \%$ in the laryngopharynx. Synchronous cancers were detected in 22 patients, including $68 \%$ with gastric cancer and $23 \%$ with hypopharyngeal cancer After EMR, the incidence of gastric cancer was $33 \%$ as well as that of oro- and hypopharyngeal cancer. EMR of gastric cancer was performed before, with, and after treatment of esophageal carcinoma at a rate of $27 \%, 53 \%$, and $100 \%$, respectively. For oro- and hypopharyngeal cancer, the rates of EMR performed with and after esophageal carcinoma EMR were $40 \%$ and $100 \%$, respectively. Discussion Approximately one-third of patients who underwen EMR of esophageal carcinoma developed multiple primary cancers, mostly in the stomach, regardless of the timing of EMR. Oro- and hypopharyngeal cancer was observed more frequently at the time of or after esophageal carcinoma EMR. The presence of esophageal carcinoma could thus be a significant early marker for multiple primary cancers.

\section{S1867}

Expression of iNOS in Pre-Neoplastic and Neoplastic Conditions of Gastric Mucosa After Helicobacter Pylori (H.pylori) Infection: A Molecular and Immunohistochemical Study in Tissue Microarrays (TMAs)

Andreas Karameris, Evangelos Tsiambas, Sofia Gazi, Kyriakos Gerontopoulos, George Vilaras, Dimitrios Stefanou, Theodore Rokkas

Background: Reactive oxygen and nitrogen species may play a crucial role in human carcinogenesis. The reactive nitrogen species are derived from the synthesis of NO, stimulated by iNOS, in a variety of cell types including activated macrophages and neutrophils. Recent studies have revealed that Helicobacter pylori (H.pylori) infection leads to the formation of nitrotyrosine, which may contribute to DNA damage and apoptosis in gastric mucosa. Aim To analyze the expression of iNOS by immunohistochemical and molecular methods in H.pylori infected preneoplastic and neoplastic conditions of the gastric mucosa using Tissue 
Microarrays (TMAs), in an effort to correlate the possible role of active nitric species and H.pylori infection in the progression of gastric carcinogenesis. Material and Methods: 45 formalin fixed, paraffin embedded gastric carcinoma cases were studied for determination of the expression of iNOS (25 H.pylori+ and 20 H.pylori-). Intestinal metaplasia (IM) type I were observed in 5 H.pylori+ cases, IM II in 8 and IM III in 12 of them. Controls included 5 H.pylori- IM I, 6 IM II and 10 IM III cases. The TMArrayer apparatus (Chemicon, USA) was used for the construction of TMAs. iNOS expression in molecular level was determined by differential-PCR in microcore samples taken by TMArrayer and properly analyzed with an Image Analysis System (DIS-200, Digital Image Systems, Hellas). The expression of iNOS molecule was studied by immunohistochemistry using a polyclonal anti-IgG human iNOS antibody (Santa Cruz, USA) and the evaluation of the result was done by DIS-200. Results: 12/13 of intestinal and 10/12 of diffuse H.pylori+ gastric carcinomas overexpressed iNOS as indicated by differential PCR and immunohistochemistry. 2/5 IM I, 8/12 IM II and 7/8 IM III H.pylori+ cases were also expressed increased iNOS levels as well as 5 diffuse and 5 intestinal type H.pylori- carcinomas. In addition 0/5 H.pylori- IM I, 1/6 H.pylori- IM II and 2/10 H.pylori- IM III cases were also expressed high iNOS levels. A statistical significant difference concerning the expression of iNOS between H.pylori+ $\mathrm{Ca}$ and H.pylori- $\mathrm{Ca}(<0.01)$, between IM II and III $(<0.05)$, as well as between H.pylori+ and H.pylori- IM II and III $(<0.01)$ was also observed. Conclusion: Our data suggest that high iNOS expression in gastric mucosa is of significant importance for the initiation and promotion of gastric carcinogenesis. The observation that high iNOS levels in IM type II and III correlated well with H.pylori infection, may be a strong evidence for long term follow up.

\section{S1868}

Stool $\alpha$-Defensin Assay for Colorectal Cancer Screening: Feasibility Analysis Aravind Sugumar, Hongzhi Zou, Jonathan Harrington, Kristie K. Klatt, David A. Ahlquist

Tumor-specific proteins represent a class of candidate stool markers with potentially lowcost application to colorectal cancer (CRC) screening. In a recent report, selected $\alpha$-defensins (HNP1-3) were over-expressed by CRC tissue and elevated in sera of patients with CRC (Gastroenterology 2005;129:66-73). To date, these markers have not been reported in stool. AIM: To evaluate the neoplasm-specificity of HPN1-3 defensins and explore their discriminant value as stool markers for CRC. METHODS: Protein and mRNA expression of human HPN1-3 defensins were initially evaluated in CRC cell lines (SW480, HT29) and white blood cells (WBC); mRNA expression was additionally assessed in microdissected $\mathrm{CRC}$ and normal colon epithelium. Alpha-defensin protein levels were assayed by ELISA with HPN1-3 kit (HyCult Biotechnology, Cat\# HK317); mRNA expression was quantified by real time RT-PCR. Using a modified HPN1-3 kit assay, protein extracts from freezerarchived stools were assayed in blinded fashion from 30 colonoscopically normal subjects, 20 patients with CRC, 10 with colorectal adenomas $>1 \mathrm{~cm}, 10$ with inflammatory bowel disease (IBD), and 10 with upper GI cancers. Stool HPNl-3 defensin levels were correlated with levels of the WBC protein, lactoferrin (Enzyme Immunoassay for Human Lactoferrin, Oxford Biomedical Research, Cat\# FR 18). RESULTS: Defensin proteins were not detected in extracts from CRC cell lines, but levels were extremely high $(>4000 \mathrm{ng} / \mathrm{ml})$ in WBC extracts. mRNA expression levels of HPN1-3 defensins were comparably low in CRC cell lines, microdissected CRC and normal colon epithelium, but $>1000$-fold higher in WBC Mean stool HPNl-3 defensin protein levels were $14 \mathrm{ng} / \mathrm{ml}$ in normal subjects, 37 in patients with adenomas, 80 with CRC, 44 with upper GI cancer, and 92 with IBD. At $100 \%$ specificity, sensitivity of stool defensin was $0 \%$ for adenomas, $20 \%$ for CRC, $0 \%$ for upper GI cancer and $20 \%$ for IBD; at $97 \%$ specificity, sensitivities were $30,25,30$, and $60 \%$, respectively; and at $90 \%$ specificity, sensitivities were $50,40,60$ and $70 \%$, respectively. Stool defensin levels correlated with stool lactoferrin levels $\left(r^{2}=0.45, p<0.001\right)$. CONCLUSIONS: Based on our findings, HPN1-3 $\alpha$-defensins are specific for WBC and not expressed by CRC cells. Stool $\alpha$-defensins are readily assayed and often elevated with CRC and other GI neoplasms. However, these inflammatory markers are also elevated in IBD stools and correlate well with known markers of inflammation.

\section{S1869}

Recovery of RUNX3 Expression in Long-Term Follow-Up After H. Pylori Eradication

Takayuki Motoyoshi, Hideyuki Konishi, Shunji Tachibana, Kouichi Soga, Chohei Sakakura, Masao Noda, Shoji Mitsufuji, Keisho Kataoka, Takeshi Okanoue

Background: It is clear that H. pylori eradication improves an inflammation of gastric mucosa, but scientific evidence is still insufficient in view of its preventive effect for gastric carcinogenesis. Human runt-related transcription factor gene 3 (RUNX3) is known to be a growth regulation factor of gastric epithelial cells. Recent knowledge has shown that in the majority of gastric cancers RUNX3 expression is reduced by hypermethylation not only at the tumor but also at the surrounding intestinal metaplasia. RUNX3 expression is therefore proposed to be a surrogate biomarker for gastric premalignant lesion. Aim: To examine RUNX3 expression in gastric mucosa before and after $H$. pylori eradication therapy, and to compare histological findings with RUNX3 expression. Materials and Methods: A retrospective review of 52 patients of $\mathrm{H}$. pylori-positive gastritis was performed. We classified them in short-term follow-up group (less than one year) and long-term follow-up group (from one to twelve years, mean 4.4-year) after $H$. pylori eradication. We evaluated their histological findings by the updated Sydney System and compared them with RUNX3 expression in gastric mucosa. Immunohistochemical analysis was performed on paraffin-embedded tissue samples using RUNX3 monoclonal antibody (given by Prof. YOSHIAKI ITOH in Institute of Molecular and Cell Biology, National University of Singapore). Results: After H. pylori eradication, inflammation in gastric mucosa was obviously improved both in short-term and long-term follow-up groups $(\mathrm{p}<0.001)$, while mucosal atrophy and intestinal metaplasia was not. RUNX3 expression in gastric mucosa recovered in few cases of short-term follow-up group and most cases of long-term follow-up group $(\mathrm{p}<0.05)$. In both groups, milder atrophy and less intestinal metaplasia cases showed more recovery of RUNX3 expression $(\mathrm{p}<0.01)$ Conclusions: RUNX3 expression recovered long-term follow-up after $H$. pylori eradication especially in milder atrophy and less metaplasia cases. From the point of view of RUNX3 expression, long-term follow-up may be necessary to prevent the development of gastric cancer after $H$. pylori eradication.

\section{S1870}

Pentasialo- and Hexasialotransferrin Isoforms As a Diagnostic Tool of Colorectal Cancer

Thierry Codden, Franz J. Legros, Jean-Pol Henry, Anne Lenaerts, Vincent Nuyens, Sylvianne Delaunay

Background: Malignant neoplasia is known to increase sialylation of most hepatic glycoproteins. This remains debated about the most important glycoprotein, transferrin(Tf). The aim of the study was to determine the ability of serum sialoTf isoforms to detect colorectal cancer. Methods: We have used a Capillary Zone electrophoresis (PACE 5000 series, Beckman Coulter, CA) method (CEofix CDT kit, Analis, Namur, Belgium), as a tool to carefully discriminate Tf glycosilated isoforms following their charge/mass ratio generated by their electronic charge of the terminal sialic acids of $\mathrm{Tf} \mathrm{N}$-glycan chains allowing for separation of the serum asialo- to hexasialotransferrin isoforms. $0.02 \mathrm{~mL}$ of serum was analyzed. Protein absorption was determined at 215 nanometers. Circulating Tf levels (percentages) were drawn from printed electropherograms. From October to November 2005, blood measurements of Tf were performed in 57 consecutively patients referred for screening colonoscopy. Malignant neoplasia was assessed by histological examination. Results: The mean levels of penta- and hexasialoTf were significantly $(\mathrm{p}<0.0001)$ higher in sera of colorectal cancer patients $(\mathrm{n}=$ 12) (Table 1). At a threshold level averaging $20 \%$ of total level, colorectal cancers appeared to be separated from non cancerous patients $(\mathrm{n}=45)$ with a sensitivity of $100 \%$ and a specificity of $97.5 \%$ of the sum (penta- plus hexasialoTf \%). Conclusion: These preliminary results fit with theory about sialylation of glycoproteins in malignant neoplasia, and offer the opportunity of developing a reliable rapid seric detection $(<15 \mathrm{~min})$ of colorectal cancer, requesting blood puncture and $0.02 \mathrm{~mL}$ Capillary Zone electrophoresis of serum.

Table 1. Mean percentages \pm SD of disialo- to hexasialoTf (P2 to P6) in normal colonoscopy and in colorectal cancer patients.

\begin{tabular}{|c|c|c|c|c|c|c|}
\hline Patients & P2 & P3 & P4 & P5 & P6 & P5+P6 \\
\hline Normal colonoscopy $(\mathrm{n}=45)$ & $1.1 \pm 0.1$ & $4 \pm 2.7$ & $74.2 \pm 1.9$ & $14.5 \pm 0.2$ & $2.9 \pm 0.5$ & $17.4 \pm 0.3$ \\
\hline Colorectal cancer $(\mathrm{n}=12)$ & $0.9 \pm 0.2$ & $3.4 \pm 1.5$ & $73.6 \pm 6.7$ & $17.3 \pm 1$ & $3.6 \pm 0.4$ & $20.9 \pm 0.8$ \\
\hline Statistical significance & & & & $\mathrm{p}<0.0001$ & $\mathrm{p}<0.0001$ & $\mathrm{p}<0.0001$ \\
\hline
\end{tabular}

\section{S1871}

Expression of CDX2, Villin, Sima and Apomucins in Small Intestinal Adenocarcinoma: A Comparison with Colorectal Adenocarcinoma Megan Q. Zhang, Zong-Ming E. Chen, Jon H. Ritter, Pei Hui, Fan Lin, Hanlin L. Wang

Background: Aberrant expression of intestinal markers CDX2, villin and small intestinal mucinous antigen (SIMA), and apomucins MUC1, MUC2 and MUC5AC, has been observed in colorectal adenocarcinoma (CRC). Little is known, however, regarding their expression in small intestinal adenocarcinoma (SIA). Methods: Formalin-fixed, paraffin-embedded tissue sections from 31 primary, non-ampullary SIAs and 47 CRCs were immunohistochemically evaluated for the expression of CDX2, villin, SIMA, MUC1, MUC2 and MUC5AC. A tumor was considered negative if $<5 \%$ of the tumor cells exhibited nuclear staining for CDX2, apical staining (with or without cytoplasmic staining) for villin, or cytoplasmic staining (with or without luminal staining) for SIMA, MUC1, MUC2 and MUC5AC. Positive staining was recorded as diffuse if $>50 \%$ of the tumor cells stained, or focal when $5-50 \%$ of the tumor cells exhibited immunoreactivity. Results: As shown in the Table, all the six proteins were variably expressed in SIA and $\mathrm{CRC}$, but the frequencies of expression were significantly higher in $\mathrm{CRC}$ with the exception of $\mathrm{MUCl}$ which was expressed at a similar frequency in SIA and CRC. SIA also more frequently exhibited a focal staining pattern for CDX2, villin, SIMA, MUC2 and MUC5AC, whereas a diffuse staining pattern was evident in CRC. Coexpression of CDX2 and villin was observed in 11 of 26 (42\%) SIAs, in contrast to all (100\%) CRCs $(\mathrm{P}<0.0001)$. Conclusions: The frequency of CDX2, villin, SIMA, MUC2 and MUC5AC expression is significantly lower in SIA compared with CRC. These observations support our recent observations that SIA is immunophenotypically distinct from CRC despite their morphologic similarity. These results also suggest a potential value as diagnostic immunomarkers in the distinction between SIA and CRC when a metastasis is encountered. Table. Differential expression of CDX2, villin, SIMA, MUC1, MUC2 and MUC5AC in SIA and $\mathrm{CRC}$ 


\begin{tabular}{|c|c|c|c|}
\hline Marker & SIA & CRC & P Value \\
\hline CDX2 & $18 / 26(69 \%)$ & $46 / 46(100 \%)$ & 0.0001 \\
\hline Villin & $15 / 26(58 \%)$ & $46 / 46(100 \%)$ & $<0.0001$ \\
\hline SIMA & $13 / 24(54 \%)$ & $38 / 41(93 \%)$ & 0.0003 \\
\hline MUC1 & $17 / 31(55 \%)$ & $25 / 47(53 \%)$ & 0.8864 \\
\hline MUC2 & $13 / 31(42 \%)$ & $35 / 41(85 \%)$ & 0.0001 \\
\hline MUC5AC & $7 / 22(32 \%)$ & $39 / 47(83 \%)$ & $<0.0001$ \\
\hline
\end{tabular}

Tumor M2-PK and Immunochemical FOBT: Valuable and Sensitive Tests for Screening for Colorectal Cancer

Sanna Mulder, Monique E. van Leerdam, Rob J. Ouwendijk, Hanneke J. van Vuuren, Jan Francke, Ernst J. Kuipers

Introduction: Screening for colorectal cancer (CRC) reduces morbidity and mortality from this very common disease. Although biochemical fecal occult-blood testing (FOBT) is the only non-invasive screening method proven to be effective in reducing CRC mortality, the test has limited sensitivity. The ideal test for CRC screening should combine a high sensitivity with an acceptable specificity. In this respect, immunochemical FOBT performs better than biochemical tests. An alternative promising method is the determination of tumor pyruvat kinase isoenzyme type M2 (Tu M2-PK) in stool samples. This may be a valuable new screening tool for CRC. Aim: To compare the accuracy of faecal Tu M2-PK test with immunochemical FOBT in patients with CRC or adenomas. Methods: Out-clinic patients above 18 years of age referred for colonoscopy were asked to provide one stool specimen A subgroup of 123 subjects was analyzed, including 44 with invasive CRC, 35 with adenomas, and 44 matched controls with a normal colonoscopy. A commercially Tu M2-PK ELISA (Schebo Biotech) was used with a cutoff level of $4 \mathrm{U} / \mathrm{ml}$. Two immunochemical FOBT tests Immo-care-C (CARE diagnostica) and OC-LIGHT (Eiken Chemical) were used for the qualitative detection of faecal occult blood. Results: In subjects with CRC, the Tu M2-PK test was positive in 37 of 44 (84\%) patients, the Immo-care detected 40 (91\%) and the OCLight identified 41 (93\%). In subjects with adenomas, the Tu M2-PK test was positive in 9 of $35(26 \%)$, the Immo-care detected $16(46 \%)$ and the OC-Light identified $14(40 \%)$ Specificity in subjects with negative findings on colonoscopy was $91 \%$ for the Tu M2-PK test and 95\% for both the Immo-care and the OC-Light. Conclusion: Both the Tu M2-PK and the immunochemical FOBT appear valuable and sensitive tests for screening for CRC. The performance of these tests is similar. None of these tests is sensitive enough for detection of advanced adenomas.

Results of three tests identified that were not in the TPP. Principal component analysis demonstrated that these global plasma protein profiles were able to clearly distinguish between tumor-bearing ApcMin and wild-type littermates. Leave-one-out cross-validation analysis established that global TPP and global PGP profiles were able to correctly predict tumor-bearing animals in $89 \%$ and $100 \%$ of cases, respectively. Wilcoxon's ranksum test $(\mathrm{p}<0.05)$ was used on both global TPP and PGP profiles to identify significant differentially expressed proteins in the tumorbearing versus control cohorts. Leave-one-out cross-validation analysis confirmed that the significant differentially expressed proteins from both the TPP and the PGP were now able to correctly predict tumor-bearing animals in 100\% of cases. ELISA of the plasma confirmed the presence of the most significantly upregulated protein. Analysis of the significant differentially expressed proteins indicated that they might derive from both the tumor site and the host response. Their biological significance will be discussed. As ApcMin mice present with predominantly small bowel adenomas, we have also initiated plasma proteomic studies in Apc $\Delta 580$ mice that present with a greater number of large bowel carcinomas. We used an approach called Multidimensional Protein Identification Technology (MudPIT) in which peptides are separated over a strong cation exchange column prior to LC-MS/MS analysis. In these studies, we have identified well over 500-600 plasma proteins. These findings will be discussed. In summary, we have identified plasma proteins from both the TPP and PGP that segregate tumor-bearing from control mice. These results have direct clinical implications for CRC screening. Further studies are needed to validate and investigate the biological significance of these findings.

\section{S1873}

Clinical Significance of Prostaglandin E Synthase Expression in Gastric Cancer Tissue

Katya Gudis, Atsushi Tatsuguchi, Ken Wada, Tetsuro Hiratsuka, Kazuhiro Nagata, Seiji Futagami, Teruo Kiyama, Takashi Tajiri, Choitsu Sakamoto

Background: Several lines of evidence suggest that the COX-2/PGE 2 pathway plays a crucial role in cancer progression. In fact, elevated expression of cyclooxygenase-2 (COX-2) has been found in various types of human cancer, including gastric cancer, and the selective inhibition of COX-2 has been shown to inhibit gastric cancer growth in animal models. Prostaglandin E synthase (PGES) functions downstream of COX-2 for $\mathrm{PGE}_{2}$ biosynthesis. To date, at least three distinct isoforms of PGES have been cloned; one cytosolic PGES (cPGES), and two microsomal fractions, mPGES-1 and mPGES-2. Although there have been reports concerning the expression of mPGES-1 in gastric cancer, the clinical significance of each of the three different prostaglandin E synthases remains unclear. Aims: To investigate mPGES-1, mPGES-2, and CPGES expression in gastric cancer tissue, and to examine the relationship of their expression to various clinicopathological factors and patient prognosis. Patients and Methods: A total of 96 gastric cancer patients who had undergone surgery were examined. mPGES-1 and mPGES-2 mRNA and protein expression levels were examined by real-time polymerase chain reaction (PCR) and Western blot analysis, respectively. cPGES protein expression levels were examined by Western blot analysis. MPGES-1 localization was analyzed immunohistochemically. Immunostaining results for mPGES-1 were compared with clinicopathological factors and COX-2 expression. Results: Real-time PCR and Western blot analysis of mPGES-1 mRNA and protein expression showed significantly higher expression levels in gastric cancer tissues than in paired normal tissues. In contrast, mPGES-2 mRNA and protein expression levels did not differ significantly between cancer and paired normal tissues. cPGES protein expression levels were also not significantly different between cancer and paired normal tissues. Immunoreactivity for mPGES-1 was predominantly observed in the cytoplasm of cancer cells in 42 cases (44\%), with occasional weak mPGES1 immunoreactivity seen in stromal cells such as fibroblasts and macrophages. mPGES-1 expression correlated with vessel invasion, lymph node metastasis, depth, stage, and COX2 expression. The prognosis was significantly worse in patients with mPGES-1-positive tumors than in those with mPGES-1-negative tumors. Conclusions: mPGES-1 protein may have an important role in tumor progression in conjunction with COX-2, and may be a biologic marker of prognostic significance in gastric cancer.

\begin{tabular}{|c|c|c|c|c|c|c|}
\hline & \multicolumn{3}{|c|}{ CRC $(\mathrm{n}=44)$} & \multicolumn{3}{c|}{ Adenoma (n=35) } \\
\hline & Sens. & PPV & NPV & Sens. & PPV & NPV \\
\hline M2-PK & $84 \%$ & $90 \%$ & $85 \%$ & $26 \%$ & $69 \%$ & $61 \%$ \\
\hline Immo-care & $91 \%$ & $95 \%$ & $91 \%$ & $46 \%$ & $89 \%$ & $69 \%$ \\
\hline Hemodia Latex & $93 \%$ & $95 \%$ & $93 \%$ & $40 \%$ & $88 \%$ & $66 \%$ \\
\hline
\end{tabular}

PPV/NPV: Positive / Negative predicting value

S1875

The Expression of HER2 in Helicobacter Pylori Infected Intestinal Metaplasia and Gastric Cancer

Noriko Nakajima, Yoko Ito, Kiyoshi Yokoyama, Satoshi Hayakawa, Shigeaki Mizuno, Kimitoshi Kato, Noriko Kinukawa, Nemoto Norimichi, Arakawa Yasuyuki, Eric R. Lacy

Gastric cancers arise through a multistep process from chronic gastritis(CG) and intestinal metaplasia(IM) and finally to invasive gastric cancer(GC). Long-term infection with Helicobacter pylori(HP) is one of the causalities for GC. Overexpression of HER2 gene and protein, a transmembrane glycoprotein with tyrosine kinase activity, and a structure similar to EGF receptor, has been reported in many adenocarcinomas of breast, esophagus and also stomach. HER2 is a successfully exploited target molecule in biomolecular therapies of solid tumors IL-16, a pleiotrophic cytokine, may be involved in the pathophysiological process of chronic inflammatory diseases. Thus it could be a factor in the final development of cancer. Aim:To determine:1)changes in the expression of HER2 in IM and GC in gastric biopsies infected with HP, 2)the effect of HP infection and IL-16 on epithelial cell proliferation and HER2 expression in gastric cells in vitro. Methods:HP infected gastric biopsies, were classified by histological findings as a)CG+, b)IM or c)GC CG without HP infection(CG-) was used as a control. For In vitro studies, AGS cells were incubated with combinations of IL-16 and HP. Cell proliferation was studied by BrdU uptake. The expression of Bcat was studied by ABC, ELISA and RT-PCR. Results:IL-16 expression was detected in all HP infected gastric mucosa. In CG+, HER2 protein(HER2-p) expression increased significantly $(p<0.05)$ (CG$: 5.58 \pm 3.37 \%$ vs. CG $+7.56 \pm 3.36 \%$ ). In HP infected mucosa, the HER2-p expression was significantly higher in $\mathrm{IM}(12.01 \pm 7.46 \%)$ and $\mathrm{GC}(13.01 \pm 6.57 \%)$ than in $\mathrm{CG}+$. In vitro studies:HP alone significantly decreased cell proliferation. Co-incubation of AGS cells with HP and IL-16 increased BrdU uptake and HER2-p only at the higher IL-16 concentration. At 24hrs, co-incubation with IL-16 decreased the expression of HER2 mRNA in HP infected AGS cells. Conclusions: The expression of HER2 in long-term HP infected gastric mucosa may indicate an early stage in carcinogenesis, because it appears before the histologically evident tumor. Short term in vitro exposure of cells to HP did not have this effect suggesting a chronic and multi-factorial process. In vitro cell culture using AGS cells:

\begin{tabular}{|c|c|c|c|}
\hline$\%$ of AGS only & $H P 10^{5} \mathrm{cfu} / \mathrm{ml}$ & $H P+\mathrm{IL}-1610^{-10} \mathrm{M}$ & $H P+\mathrm{IL}-1610^{-9} \mathrm{M}$ \\
\hline BrdU uptake 24h & $83.34 \pm 15.96^{*}$ & $84.88 \pm 6.61$ & $89.1 \pm 15.36^{* * *}$ \\
\hline HER2 protein 24h & $86.85 \pm 13.60^{*}$ & $89.99 \pm 6.50$ & $97.00 \pm 11.60^{* * *}$ \\
\hline HER2 mRNA 6h & $102.43 \pm 27.23$ & $104.63 \pm 36.30$ & $115.13 \pm 39.40$ \\
\hline HER2 mRNA 24h & $99.36 \pm 20.14$ & $86.41 \pm 19.95^{* *}$ & $85.12 \pm 32.65^{* *}$ \\
\hline
\end{tabular}

All data was expressed as $\%$ of AGS cells only. *=significant difference from AGS only **= significant difference between HP only and HP+IL-16 p<0.05 was taken as statistically significant. 
S1876

Analysis of Mucin Expression Patterns Is Useful for Accurate Diagnosis of Gastric Borderline Lesions in Endoscopic Biopsy Samples

Hideki Minematsu, Yasuharu Saito, Rie Kakinoki, Akira Andoh, Ryoji Kushima, Yoshihide Fujiyama

BACKGROUND: Recommendation for diagnosis and treatment of gastric borderline lesions remains controversial. To elucidate those factors helpful for an accurate diagnosis of gastric borderline lesions, we examined the mucin expression patterns in endoscopically-resected and forceps-biopsy samples. METHODS: Sixty-three gastric lesions were histopathologically diagnosed as borderline lesions based on endoscopic forceps biopsy. All of these patients underwent endoscopic resection, and were divided into group A (final diagnosis an adenocarcinoma) and group B (final diagnosis as an adenoma). Immunostaining for MUC2, 5AC, 6 and $\mathrm{CD} 10$ were performed. The mucin phenotype classification was determined according to the criteria described by Tsukashita et al[1]. Similar evaluation was performed in 28 forceps-biopsy samples. RESULTS: The percentage of lesions with redness was significantly higher in group $\mathrm{A}$ than in group $\mathrm{B}(\mathrm{P}<0.05)$. The percentages of a complete gastric phenotype (positive for MUC5AC and MUC6) plus a mixed phenotype (gastric predominant type) were significantly higher in group A $(58.0 \%)$ than in group B $(21.8 \%)(\mathrm{P}<0.05)$. The percentage of a complete intestinal phenotype (positive for MUC2 and CD10) was significantly higher in group B (68.8 \%) than in group A $(19.4 \%)(\mathrm{P}<0.05)$. Similar results were also observed in 28 samples of forceps-biopsy, which were histologically diagnosed as borderline lesion. The percentage of a Ki-67 diffuse-immunostaining pattern was significantly higher in group A than in group $B(P<0.05)$. The positive ratio of $\mathrm{p} 53$ expression was $29.2 \%$ in group A and $4.3 \%$ in group $\mathrm{B}$, respectively, and was significantly higher in group $\mathrm{A}(\mathrm{P}<0.05)$. CONCLUSION: Determination of immunostaining for a mucin phenotype in forceps-biopsy samples may be useful for an accurate diagnosis of gastric borderline lesions.

\section{S1877}

Alpha Fetoprotein Is a Marker of Aggressive and Progressive Neuroendocrine Tumours

Tahir H. Shah, Mani Bhogal, Aneesha Noonan, Niroshan Sivathasan, Caroline WitneySmith, Tejal Amin, Daniel Hochhauser, Martyn E. Caplin

Background: Tumour markers are substances related to the presence or progression of a tumour. Alpha Fetoprotein (AFP) is already well established as a marker for germ cell tumours and hepatocellular carcinoma (HCC). Neuroendocrine tumours (NET) are usually slow growing, although, a significant proportion follow an aggressive course. NETs are, in part, characterised by an ability to secrete hormones and biogenic amines, some of which, e.g. Chromogranin A, have been shown to correspond to disease state. Aims: To evaluate the role of AFP as a potential tumour marker in NETs. Methods: 419 patients were randomly selected from the Royal Free \& University College Medical School NET unit database. Retrospective analysis of AFP, histological grade and radiological progression was conducted. These variables were correlated temporally. Raised AFP was defined as a level $>50 \%$ above the upper limit of normal. Patients in which another recognised cause of raised AFP was identified were excluded. Radiological status was determined using the "Response Evaluation Criteria In Solid Tumours" (RECIST) and divided into: regressive, stable, and progressive disease. Tumour grade was based on standard histological classification and cell proliferation markers (MIB-1), being divided into low, intermediate and high. Results: AFP was raised in 23 of 419 patients with results available: 13 patients with metastatic NETs of unknown origin; 9 patients with pancreatic NETs; and 1 patient with gastric carcinoid. One patient with HCC was excluded. AFP is raised in $5.25 \%$ of the population and was associated with tumour progression in $78 \%$ of patients as compared with $24 \%$ in the AFP negative population ( $p<0.001$ ). High-grade tumours represented $87 \%$ of the raised AFP group against $31 \%$ in the AFP normal group $(\mathrm{p}<0.001)$. Conclusion: In the subset of NETs in which it is raised AFP is a marker of aggressive disease. It is associated with both histologically aggressive and clinically progressive disease. These patients are 3 times more likely to show radiological tumour progression and high grade histology compared to those with normal AFP levels. The next step is to quantify the changes in AFP and correlate these with changes in disease state in the patients. This prospective analysis will allow us to determine the role of AFP as a clinical parameter for monitoring tumour behaviour in individual patients.

\section{S1878}

Fluorescence in Situ Hybridization (FISH) Analysis of Endoscopic Brushings Obtained from Barrett's Esophagus (BE) Patients with Esophageal Adenocarcinoma (EA)

Shannon Brankley, Aaron Harwood, Mona Legator, Lori Lutzke, Larry Morrison, Kenneth Wang, Micheal Campion, Halling Kevin

Background: FISH is a technique that utilizes fluorescently labeled DNA probes to detect chromosomal abnormalities in cells. Recent studies suggest that FISH can be used to detect cells that have chromosomal abnormalities consistent with a diagnosis of dysplasia or adenocarcinoma in cytologic brushing specimens from patients with Barrett's esophagus. The goal of this study was to assess the types of chromosomal alterations that are detected by FISH in endoscopic brushing specimens obtained from patients with biopsy proven EA. Methods: One hundred thirty endoscopic brushing specimens taken from 127 patients under surveillance for Barrett's esophagus were assessed by FISH. Eighteen (14\%) of the 127 patients had a biopsy or endoscopic mucosal resection specimen showing EA. Twenty-one brushings were obtained from these 18 patients. FISH was performed using probes to $8 \mathrm{q} 24$ (C-MYC), 9p21 (P16), 17q11.2-q12 (HER-2), and 20q13. One hundred consecutive noninflammatory, non-squamous cells were analyzed per specimen. A specimen was considered positive for abnormality if: 1) $\geq 5 \%$ of the cells demonstrated polysomy (i.e. gains of two or more of the four probes), 2 ) $\geq 5 \%$ of the cells exhibited trisomy (i.e. three copies) of the $8 \mathrm{q} 24,17 \mathrm{q} 11.2$, or the $20 \mathrm{q} 13$ probe, or 3$) \geq 10 \%$ of the cells exhibited hemizygous $9 \mathrm{p} 21$ deletion (i.e. deletion of one of the 9p21 probes). Results: The types of chromosomal alterations detected by FISH are shown in the Table. All 18 patients were found to have one or more abnormality by FISH. Conclusions: DNA ploidy and cytogenetic studies have previously shown that virtually all EA are hyperdiploid with extra copies of most of the chromosomes. Thus, cells from these tumors should have polysomic or trisomic signal patterns when examined with multi-color FISH probe sets such as the one used in this study. This study reveals that the majority of the endoscopic brushing specimens did exhibit polysomic (16 cases) or trisomic ( 2 cases) signal patterns. Only one patient had a FISH result that was not consistent with the surgical pathology diagnosis of EA. The brushing specimen from that patient exhibited hemizygous 9p21 deletion and an absence of polysomy. Hemizyogus 9p2l deletion is a finding that is consistent with low-grade dysplasia but not adenocarcinoma. This study suggests that FISH analysis of endoscopic brushing specimens may be an accurate method for determining if a patient has EA.

\begin{tabular}{|c|c|c|}
\hline FISH Abnormality & Number of Patients & Percent of Specimens \\
\hline Polysomy & 15 & $83.5 \%$ \\
\hline Polysomy and Trisomy 8q21 & 1 & $5.5 \%$ \\
\hline Trisomy 20q13 & 1 & $5.5 \%$ \\
\hline Hemizygous 9p21 Deletion & 1 & $5.5 \%$ \\
\hline
\end{tabular}

S1879

EGFR Proteins Are Highly Expressed in Pseudomyxoma Peritonei Elizabeth E. Half, Russell R. Broaddus, Kai Feng Liu, Laura A. Lambert, Cathy Eng, Paul F. Mansfield, Robert S. Bresalier

Background. Pseudomyxoma peritonei (PMP) is characterized by intrabdominal accumula tion of large amounts of mucin secreted by adenocarcinomas (primarily of the appendix) Complications due to mucin accumulation is the major cause of morbitidy and mortality in patients with this disease. It is known that both COX-2 and epidermal growth factor receptor (EGFR) are widely expressed in CRC and inhibition of these pathways may be effective treatments. We have previously demonstrated that MUC2 and MUC5AC can be suppressed by COX-2 or EGFR inhibitors in human colon cancer cells. There is currently no effective treatment for patients with PMP. Aim. To determine COX-2, EGFR, and HER2 protein expression in human PMP tumors. Method. COX-2 $(n=40)$, EGFR $(n=30)$, and HER-2 ( $n=16)$ protein expression was analyzed in formalin fixed, paraffin-embedded PMP specimens from adenocarcinomas of the appendix. COX-2 was also analyzed from specimens of normal appendix $(\mathrm{n}=10)$. Protein expression was determined by immunohistochemistry using specific antibodies. Staining intensity $(0-3+)$ and percent tumor cell positivity were determined. A positive result was defined if $>10 \%$ of tumor cells had an intensity of $>2$. Results. EGFR protein expression was detected in 27 of 30 (87\%) tumors. Strong (3+) diffuse ( $>75 \%$ ) membranous EGFR staining was detected in 15 of 30 (50\%) of the cases. COX-2 expression was detected in only 3 of the $40(7.5 \%)$ cases of PMP that were studied. None of the normal specimens expressed COX-2. Only 2 of $16(12.5 \%)$ tumors were positive for HER-2. Conclusion. Our results indicate that in contrast to positive expression in colon cancer, COX-2 is rarely expressed in PMP. EGFR is, on the other hand, frequently expressed in PMP tumors, and inhibition of EGFR signaling may be a useful strategy in the treatment of PMP.

\section{S1880}

Expression of the Tight Junction Proteins Cingulin, Occludin and Claudin-7 in Human Gastrointestinal Tract Cancers

Ulrich-Frank Pape, Ines Eichhorn, Lutz Langbein, Werner W. Franke, Bertram Wiedenmann

Tight junctions (TJ) are composed of various specific proteins which are differentially expressed along the normal human gastrointestinal tract (GIT). The expression of TJ-molecules in GIT-cancers and potential diagnostic implications have not yet been analyzed systematically. We have therefore studied the expression of occludin, cingulin and claudin- 7 in human GIT-cancers ( $\mathrm{n}=92)$ by immunofluorescence microscopy using specific antibodies and compared it to expression patterns in the corresponding normal GIT-epithelia. We found expression of cingulin and occludin in all adenocarcinomas of the GIT (esophagus, stomach, pancreas, liver, bile ducts, colorectum, neuroendocrine carcinomas) preferably at luminal sites of glandular tumor structures. When no lumen was present the TJ-proteins were localized mostly to the lateral cell membrane or diffusely within the cytoplasm. With loss of differentiation grade cingulin and occludin expression semiquantitatively decreased or were even absent from the neoplastic tissues. Interestingly, while claudin-7 cannot be found in the normal human gastric mucosa it was found to be expressed in all gastric adenocarcinomas with intermediate to strong positivity $(n=12)$ suggesting a role of claudin7 as a marker of malignancy in these neoplasms. In hepatocellular carcinomas (HCC; $n=8$ ) claudin-7 was found only weakly if at all expressed while a strong expression was found in primary bile duct cancers $(\mathrm{n}=5)$ which correlates with the expression pattern in normal tissues. Also of interest, high-grade malignant neuroendocrine carcinomas (NEC, n=8) demonstrated almost no expression of occludin, cingulin or claudin-7 while low-grade malignant NEC $(n=17)$ expressed these TJ-proteins to a considerable stronger degree suggesting these TJ-molecules maybe useful in pathological differential diagnosis of these tumors. In all other GIT-cancers claudin-7 expresssion did not appear significantly different from occludin or cingulin, however, in accordance with the subcellular localization in normal tissues claudin7 was localized to the lateral cell membrane rather than to the luminal site of epithelial structures and with loss of differentiation a cytoplasmic claudin- 7 staining was more apparent. In summary, TJ-protein expression can be found not only in normal but also neoplastic human GIT-tissues and it seems to correlate with the differentiation status at least in some GIT-cancers to a significant degree, in particular in NEC. Furthermore, claudin-7 may be an interesting molecule for histopathological evaluation of gastric cancers and primary liver cancers for further differentiation of malignancy or of derivation of primary liver cancer. 
S1881

Interphase Fish As a Biomarker of Gastric Cancer Risk in Helicobacter Pylori Multifocal Intestinalized Pangastritis

Byung-Wook Kim, Mary P. Bronner, Mee-Yon Cho, Bo-In Lee

Background and aim: The only known marker of increased cancer risk in H. pylori-gastritis is intestinal metaplasia (IM) of gastric mucosa, but IM is common and cancer develops in only a fraction. The aim of this study is to identify better biomarkers of gastric cancer in patients with intestinalized H. pylori-pangastritis. Method: Single endoscopic antral biopsies with IM that was negative for dysplasia were studied in seven non-progressor (NP) patients without gastric cancer and eight progressor (P) patients with gastric cancer. Interphase nuclear preparations of epithelium isolated from stroma were analyzed by array-based comparative genomic hybridization (a-CGH) and interphase fluorescence in situ hybridization (FISH) using $18 \mathrm{q} 21.1$ and $4 \mathrm{q} 32.3 \mathrm{BAC}$ probes and a commercially available 9p21 probe. Results: A-CGH using high density 6000 BAC arrays did not reveal significant differences between P and NP patients, nor did $4 q 32.3$ or $9 \mathrm{p} 21$ FISH ( $>0.05$ ), each using random non-dysplastic antral biopsies. Total FISH abnormalities (gains + losses) for 18q21.1, however did distinguish $\mathrm{P}$ and NP patients $(\mathrm{p}<0.01$ ). (Figure) Conclusion: Interphase FISH for $18 \mathrm{q} 21.1$ offers promise as a biomarker of cancer risk in $\mathrm{H}$. pylori intestinalized pangastritis, even on single random non-dysplastic antral biopsies.

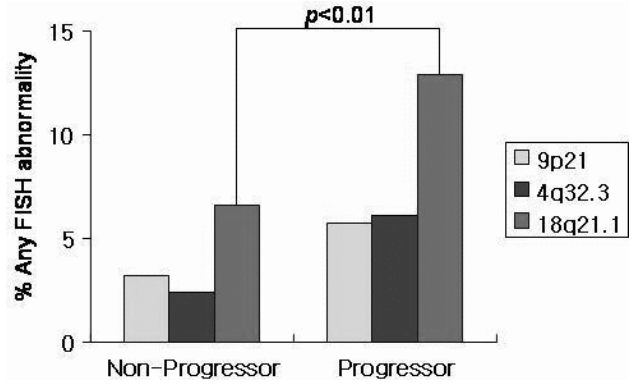

\section{S1882}

Trans-Activation of IGF-R By Fibronectin Protects from Apoptosis in Pancreatic Cancer Cells

Mouad Edderkaoui, Izumi Ohno, Peggy Hong, Stephen J. Pandol, Anna S. Gukovskaya

Background \& Aims: Pancreatic cancer is very fibrotic. Fibronectin and IGF-1 receptor (IGF$\mathrm{R}$ ) are two major pro-survival environmental factors in pancreatic cancer. We hypothesize that fibronectin trans-activates IGF-R in pancreatic cancer cells. The aim of this study is to investigate the mechanism of IGF-R trans-activation by fibronectin and its effect on apoptosis in pancreatic cancer cells. Methods: Human pancreatic carcinoma cells MIA PaCa-2 and PANC-1 were cultured for up to $48 \mathrm{~h}$ on polyHEMA (to prevent extracellular matrix protein deposition and cell attachment) or on fibronectin. The expression and phosphorylation of IGF-R, Akt, integin-linked kinase (ILK), protein tyrosine phosphatase (PTP) SHP-2, Bcl-2, and $\mathrm{Bcl}_{\mathrm{XL}}$ were assessed by Western blot analysis. Interaction between proteins was assessed by imunoprecipitation and Western assay. Apoptosis was measured by DNA fragmentation using Cell Death ELISA kit, caspase activity, and AnV/PI staining. Results: The IGF-R inhibitor AG-1024 as well as transfection with antisense IGF-R oligonucleotides prevent the prosurvival effect of fibronectin in pancreatic cancer cells. Inhibiting IGF-R with both pharmacological and molecular approaches abolished fibronectin-induced activation of downstream anti-apoptotic signaling, in particular, PI3-K activation and expression of anti-apoptotic protein $\mathrm{Bcl}_{\mathrm{xL}}$. We show that fibronectin trans-activates IGF-R by stimulating its phosphorylation. The fibronectin-induced activation of IGF-R is independent of IGF-1. IGF-R was phosphorylated in cells cultured on fibronectin and in the presence of IGF-1 neutralizing antibody. Our immunoprecipitation data indicate that fibronectin stimulates complex formation between its receptor $\beta 3$ integrin, ILK and SHP-2. This complex prevents the SHP- 2 from dephosphorylating IGF-R. Conclusion: IGF-R mediates the pro-survival effect of fibronectin in pancreatic cancer cells. Trans-activation of IGF-R by fibronectin results in activation of downstream PI3-K and $\mathrm{Bcl}_{\mathrm{xL}}$ expression. Fibronectin trans-activates IGF-R by up-regulating its phosphorylation. Fibronectin interacts with integrin $\beta 3$ inducing recruitment of ILK and SHP-2. The later prevents SHP-2 from dephosphorylating IGF-R. The results suggest SHP2 and ILK as potential therapeutic targets to regulate apoptosis in pancreatic cancer cells. The results also suggest that targeting IGF-R, as well as the SHP-2 and ILK, would prevent anti-apoptotic effects of both IGF and fibronectin in pancreatic cancer cells.

\section{S1883}

High-Resolution Genomic Profiling in Human Pancreatic Carcinoma By Genechip Mapping 100k Array

Lian-Jie Lin, Fumihiko Kanai, Motohisa Tada, Yasuo Tanaka, Keisuke Tateishi, Masashi Sanada, Yasuhito Nannya, Seishi Ogawa, Masao Omata

Background and Aim: Pancreatic carcinoma is characterized by aggressive tumor growth and high metastatic rate resulting in an extremely poor prognosis. The pancreatic adenocarcinoma genome harbors multiple amplifications and deletions, pointing to the existence of numerous oncogenes and tumor suppressor genes driving the genesis and progression of this lethal cancer. The initial comparative genomic hybridization (CGH) firstly enabled the genomewide detection of copy number changes, but at low resolution ( $\sim 20 \mathrm{Mb}$ ). In this study, using high-density single nucleotide polymorphism (SNP) array, we sought to chart the molecular genetic events in this cancer with the goals of improving early detection and providing new therapeutic targets. Materials and Methods: A total of 25 established cell lines derived from pancreatic carcinomas or their metastases were included in the investigation.
We isolated high-quality genomic DNA from these cell lines and analyzed for high-level amplification, homozygous deletion and loss of heterozygosity (LOH) using the Affymetrix GeneChip Mapping 100K high-density oligonucleotide arrays, which contain 116,204 SNPs with a mean spacing of $23.6 \mathrm{~kb}$. We used the software named Copy Number Analyzer for GeneChip (CNAG) as our original data analysis tool, which automates the detection of copy number and LOH. Then we performed the PCR, quantitative real-time PCR and fluorescence in situ hybridization (FISH) to verify the results of array analysis. Results: Twenty-seven regions of high-level amplification $(n>4)$ were found in at least two cell lines, and the most frequent copy number gains were found in chromosome region $12 \mathrm{pl} 2.1-12 \mathrm{p} 11.23$, found in 6 cell lines. The size of minimal common amplicon was $114 \mathrm{~kb}$. Thirty regions of homozygous deletion were detected in at least one cell line, and the most frequently region was 9p21.3, found in 12 cell lines. The size of minimal deletion was $17 \mathrm{~kb}$. In addition to known genetic abnormalities, such as amplification of K-ras gene and deletion of p16 gene, high- level amplification region on $3 q 25$ and homozygous deletion region on $8 q 23$ were newly identified. LOH on chromosome arm 18q, 9p, 17p, 13q, 6q, 3p, 6p, 22q, $9 \mathrm{q}$, and $12 \mathrm{q}$ was observed with high frequency. Conclusion: The high-resolution mapping array can help us detect the copy number alterations to understand the pathogenesis of pancreatic cancers, and provide a highly efficient entry point for cancer gene discovery. And the novel amplified and deleted regions discovered in this study may become the target for early diagnosis and therapy in pancreatic cancer.

\section{S1884}

Biochemical and Functional Characteristics of Primary and Secondary Tumors in a Spontaneous Model of Pancreatic Adenocarcinoma Richard J. Aspinall, Sara Weis, Kevin Kuo, Paul J. Pockros, David A. Cheresh

INTRODUCTION: Micrometastases are already present at diagnosis in the vast majority of patients with pancreatic adenocarcinoma and are an important target for future anticancer therapies. We wished to see if cells from either primary or metastatic lesions display distinct biochemical or functional characteristics. In particular, we examined whether abnormal SRC family kinase activity was associated with a metastatic phenotype. We used a genetic model of spontaneous metastatic ductal adenocarcinoma characterized by a pancreas-specific G12D K-ras mutation and pl6Ink4a/Arf knockout (Aguirre et al, Genes Dev 2003). METHODS: Pdx-Cre/InkArf-lox mice were bred with LSL-Kras G12D mice to produce offspring with early PanIN lesions and spontaneous pancreatic cancer. Animals with established carcinomas were sacrificed, primary tumors were excised and internal organs (lymph nodes, lung, liver brain, heart, kidneys, spleen, peritoneum) harvested to isolate secondary tumors. Tissue specimens were minced, subjected to a collagenase digestion and cells grown in sterile medium with $10 \%$ serum. Proliferation, adhesion and migration assays were performed on matrix proteins including collagen, vitronectin and fibronectin. Signaling profiles of starved and growth factor-stimulated cells were obtained using immunoblotting for basal and stimulated levels of FAK, p-FAK, SRC, P-SRC, B-catenin, MEK, p-MEK and ERK2. Cells were stained on coverslips with similar immunohistochemistry antibodies before confocal microscopy. Functional and biochemical profiles were also assessed in the presence of novel small molecule inhibitors of SRC, MEK and RAF. RESULTS: All mice developed spontaneous pancreatic cancer. None survived beyond 12 weeks. Primary tumors ranged from $0.8-4 \mathrm{~g}$ Metastases were mainly in lymph nodes (retroperitoneal, mesenteric and para-aortic) bu also in the liver, lungs, peritoneum and soft tissue. A total of 25 cell lines were created most with primary and secondary pairs from the same mouse. Differential signaling patterns were seen on Western blotting with identification of lymph node pairs showing enhanced activated P-SRC in the metastases. Secondary lines from lymph node and liver metastases appeared less responsive to SRC inhibitors than paired primaries. DISCUSSION: Pairs of primary and secondary tumors demonstrating important biochemical and functional differences can be identified. We are currently examining whether cells with elevated basal SRC levels have increased tumorigenicity or are more likely to metastasize in vivo. We are also exploring whether SRC activity profiling can predict the response to SRC inhibitor drugs.

\section{S1885}

New Technologies for Nanoscale Cell Surface Analysis Reveal Potential for New Diagnostic Tools in Gastrointestinal Tumor Detection Ilona Bredebusch, Bjoern Kemper, Daniel Carl, Gert von Bally, Wolfram Domschke, Juergen Schnekenburger

Introduction: The diagnosis of pancreatic tumors is impaired by low amount of available material for molecular in vitro diagnostics and the limited applicability of novel technologies in early in vivo diagnosis. These obstacles can be overcome by the application of nanoscale surface analysis technologies in the measurement of morphological and physical properties of cellular systems. Here we applied new cell surface analysis technologies to pancreatic tumor cells modified in the expression of the tumor suppressor gene E-Cadherin. Methods: Fixated pancreas tumor cell lines were dehydrated and dried for scanning electron microscopy (SEM). Cells were coated with platinum and carbon and analyzed using a standard SEM For atomic force microscopy (AFM) and digital holographic analysis sub confluent cells on glass slides or tissue culture plates were cultured for $24 \mathrm{hrs}$ and analyzed in vivo at room temperature and normal atmosphere. Digital holography could reconstruct a 3 dimensiona image of the morphology of living pancreas tumor cells and gastrointestinal tumor specimen. Results: E-Cadherin regulates cell-cell contacts and is frequently downregulated during tumor metastasis. E-Cadherin reexpression in metastatic pancreas tumor cell lines resulted in an increased capacity of colony formation, reestablishment of cell-cell contacts and an inhibition of the metastatic potential. Most interestingly SEM analysis of E-Cadherin reexpressing cell lines revealed an altered morphology, the cells showed a flattened phenotype indicating a rearrangement of the actin cytoskeleton. Atomic force microscopy and digital holography on living cells demonstrated a decrease in cell height of more than $60 \%$. The measurement of the surface elasticity of tumor tissue and surrounding healthy tissue from freshly isolated gastrointestinal specimen using digital holography showed a significantly higher elasticity of tumor tissue. Conclusion: These results demonstrate that in principle the correlation of protein expression data, biological cell features and cell surface properties can generate knowledge for the determination of pathophysiological cell features directly from cell surface 
analysis. Digital holography can be used for marker free and non invasive complex tissue analysis. The future application of marker free and partly non invasive nanoscale analysis methods like digital holography offers novel endoscopical in vivo diagnostics for the identification of malignant lesions based on physical cell properties as cell surface elasticity.

\section{S1886}

Granulocyte Colony Stimulating Factor Receptor Promotes $\beta 1$-Integrin Mediated Adhesion and Invasion of Pancreatic Cancer Cells

Arup Chakraborty, Zhimin Tong, Kai-Feng Liu, Robert Bresalier, Sushovan Guha

BACKGROUND: Pancreatic cancer $(\mathrm{PaCa}$ ) is a devastating disease with strong invasive potential. Understanding the molecular events that contribute to invasiveness of pancreatic cancer $(\mathrm{PaCa}$ ) cells is critical to the development of novel therapeutic strategies for this potentially fatal disease. $\beta 1$-integrin plays an important role in adhesion and invasion of multiple cancer cells including PaCa. Several in vitro studies have suggested that granulocyte colony-stimulating factor (G-CSF) can promote cancer growth. Aberrant expression of GCSF and its corresponding receptor (G-CSFR) are reported in multiple transformed epithelial cell types including bladder, breast, and colorectal carcinoma cells. The role of G-CSF/GCSFR in cancer development, however, is not clear. We recently demonstrated that G-CSFR signaling pathways potently promote $\beta 1$-integrin-mediated adhesion and invasion of bladder cancer cells. AIM: Determine the expression and role of G-CSF/G-CSFR in mediating adhesion and invasion of $\mathrm{PaCa}$ cells. METHODS \& RESULTS: G-CSFR is expressed in multiple $\mathrm{PaCa}$ cells, with lower expression in Capan-2, BxPC-3, and AsPC-1 cells (well to moderately differentiated $\mathrm{PaCa}$ ) and higher expression in MIA PaCa-2 and PANC-1 cells (moderate to poorly differentiated $\mathrm{PaCa}$ ) by RT-PCR and western blot analyses. G-CSF production as measured by ELISA was higher in MIA PaCa-2, BxPC-3, and PANC-1 as compared with Capan-2 and AsPC-1. Using flow cytometry and western blot analyses, we demonstrated that G-CSF potently induced expression of bl-integrin in PaCa cells. Further, G-CSFRmediated increased expression of $\beta 1$-integrin is abrogated by G-CSF-specific antibody or G-CSF-specific siRNA in PaCa cells. G-CSFR signaling increased adhesion of PaCa cells to HUVEC, which was potently blocked by a $\beta 1$-integrin-specific antibody. G-CSF/GSFR signaling pathways significantly increased invasiveness of $\mathrm{PaCa}$ cells, which was reduced either by attenuating G-CSF production (G-CSF-specific antibody and G-CSF-specific siRNA) or by interfering with G-CSFR signaling (transient transfection of truncated G-CSFR, GR19). Neutralization of $\beta 1$-integrin with a specific antibody completely blocked G-CSFR-mediated invasion of PaCa cells. We also confirmed aberrant G-CSFR expression in resected pancreatic tumors with G-CSFR-specific immunohistochemistry of formalin-fixed paraffin sections. CONCLUSION: These data suggest that autocrine/paracrine G-CSF/G-CSFR signaling significantly contributes to $\beta 1$-integrin-mediated adhesion and invasion of $\mathrm{PaCa}$ cells. This novel finding may explain, in part, the mechanisms by which $\beta 1$-integrin contributes to major invasive potential of $\mathrm{PaCa}$ cells.

\section{S1887}

Gene Expression Profiles of Pancreatic Neuroendocrine Tumors and Gastrointestinal Carcinoids

Eva-Maria Duerr, Yusuke Mizukami, Andrew L. Warshaw, Matthew H. Kulke, Daniel C. Chung

Pancreatic neuroendocrine tumors (PNET) and gastrointestinal carcinoids (GI-carcinoids) are rare tumors of neuroendocrine origin that share many common features. One particular difficulty is distinguishing benign from malignant tumors based on histology alone. Although some features such as tumor size, angioinvasion and proliferative activity of more than $2 \%$ are suggestive of malignancy, the only definitive criterion is the presence of distant metastases. Molecular markers may potentially provide a more accurate means of predicting tumor behavior as well as distinguishing PNETs from GI-carcinoids. Methods: The gene expression profiles of 19 PNET samples ( 5 benign, 6 low-grade malignant, 8 malignant) and 6 malignant GI-carcinoids were investigated using the Affymetrix Human Genome U133 Set. Quantitative RT-PCR was performed to confirm gene expression levels. Results: Affymetrix data were analyzed utilizing the DChip software package. When GI-carcinoids and malignant PNETs were analyzed together, 269 genes were dysregulated. 25 of these genes were upregulated in GI-carcinoids only. One of them was c-Ret, an oncogene frequently mutated in MEN2A and familial thyroid cancer. Quantitative RT-PCR confirmed the selective upregulation of c-Ret in carcinoids. Sequencing did not reveal any mutations in tumor samples and no gene rearrangements were identified. When PNETs only were analyzed, 2 clusters emerged. One "malignant" cluster comprised 7/8 malignant PNETs, 1/5 benign PNETs and 2/6 low-grade malignant PNETs. The remaining $1 / 8$ malignant and $4 / 6$ low-grade malignant tumors clustered in a second "benign" group with $4 / 5$ benign tumors. 330 genes were differentially regulated. Among the upregulated genes in the "malignant" cluster were IGFBP3 and TM4SF4 both of which regulate cell proliferation. Quantitative RT-PCR confirmed a 3-fold and 16-fold upregulation, respectively. Other upregulated genes included Nuclear Prelamin A recognition factor, Caldesmon 1 and Adenylate Cyclase 1. Conclusion: Molecular profiling can distinguish GI-Carcinoids from PNETs. Within the group of PNETs, histologically lowgrade malignant tumors appeared to cluster with benign tumors on a molecular level. Thus, a molecular classification of neuroendocrine tumors may provide a more accurate tool to predict tumor behavior, as well as provide novel insights into tumor pathogenesis.

\section{S1888}

Resveratrol and Embelin Increase Apoptosis and Decrease Proliferation in Pancreatic Cancer Cells Through Regulation of IAPS

Mouad Edderkaoui, Izumi Ohno, Stephen J. Pandol, Anna S. Gukovskaya

Background \& Aims: Pancreatic cancer is a very aggressive disease that is resistant to different radio and chemo-therapies. One reason for that is the resistance of pancreatic cancer cells to apoptosis because of the over-expression of anti-apoptotic proteins such as survivin and XIAP. Survivin and XIAP belong to the IAPs family (inhibitor of apoptosis proteins), which prevent caspase activation and subsequent execution phase of apoptosis. Recently, two phytochemicals have been shown to inhibit different IAPs. Resveratrol, which is present in grapes and in a variety of medicinal plants was recently shown to decrease the expression levels of IAP-1/2 and survivin. Embelin, phytochemical derived from the Japanese Ardisia herb prevents XIAP inhibition of caspases without affecting its expression. Methods: Human pancreatic carcinoma cells MIA PaCa- 2 and PANC-1 were cultured for up to $72 \mathrm{~h}$ in the presence or absence of resveratrol and/or embelin. The expression of IAP1, IAP2, survivin and XIAP were assessed by Western blot and analysis. Apoptosis was assessed by measuring DNA fragmentation using Cell Death ELISA kit, AnV/PI staining, and caspase activity. Proliferation was assessed by measuring H3-thymidine incorporation. Results: Resveratrol dose-dependently $(5-200 \mu \mathrm{M})$ increases apoptosis in MIA PaCa-2 and PANC-1 cells. This effect was associated with decrease in the expression level of IAP1/2 and survivin. Embelin stimulated apoptosis at doses from 5 to $50 \mu \mathrm{M}$ in MIA PaCa-2 and PANC-1 cells. Both resveratrol and embelin stimulated caspase activation. They also decreased proliferation of pancreatic cancer cells. Combination of resveratrol and embelin increases apoptosis and decreases proliferation to a greater extent than the phytochemicals alone in pancreatic cancer cells. For example, at $48 \mathrm{~h}$ resveratrol $(50 \mu \mathrm{M})$ increases DNA fragmentation 4 times, embelin $(20 \mu \mathrm{M})$ increase it twice, and combination of both phytochemicals increases apoptosis by $\sim 7$ times. Conclusion: IAPs are key pro-survival proteins in pancreatic cancer. In previous studies, we showed that caspase blokade is a major mechanism for the low apoptosis rate in pancreatic cancer. Thus, stimulation of caspase activity by inhibiting IAPs with resveratrol and embelin is of particular clinical importance for pancreatic cancer. Inhibition of IAPs with resveratrol and embelin not only increases apoptosis, but also decreases proliferation. Combination of resveratrol and embelin could be used as a potential treatment for pancreatic cancer.

\section{S1889}

Identification of Novel Differential Proteomic Biomarkers in Pancreatic Cystic Neoplasm Fluid

James J. Farrell, Puneet Souda, Babak Hasanzadeh, Julian Whitelegge, Kym Faull

Background: Preoperative differentiation of premalignant and malignant(mucinous) from benign (serous) pancreatic cysts is difficult. We applied proteomic technologies to identify novel proteins in pancreatic cyst fluid which may more accurately predict pathology preoperatively. Methods: Pancreatic cyst fluid was collected by EUS-FNA from patients with pathologically confirmed cysts. Cyst fluid proteomic analysis with 2-D electrophoresis (2DE) and 2 dimensional liquid chromatography (PF2D) was performed.PF2D fractions were digested and spots from 2D gels were excised and digested. Mass Spectrometry was used to identify unique differential proteins using LC-MS/MS and MALDI-TOF. Results: 42 pancreatic cyst fluid samples were collected. Protein concentration ranged from $1-5 \mathrm{mg} / \mathrm{ml}$. 5 samples (2 mucinous and 3 serous cysts) were analyzed, showing a striking differential protein profile between serous and mucinous fluid(by PF2D in both dimensions) Overlay of the 2-D maps of serous and mucinous cysts suggest many proteins unique to each cystic neoplasm (Fig. 1). Over 700 proteins fractions/sample were collected. Using 2-DE we have already identified over 20 proteins using LC-MS/MS. Conclusions: This is the first report detailing the proteome of pancreatic cystic neoplasm fluid. Differential protein expression was found between pancreatic mucinous and serous cyst fluid. Identification of specific protein patterns may result in highly accurate serum based or cyst fluid based biomarkers.

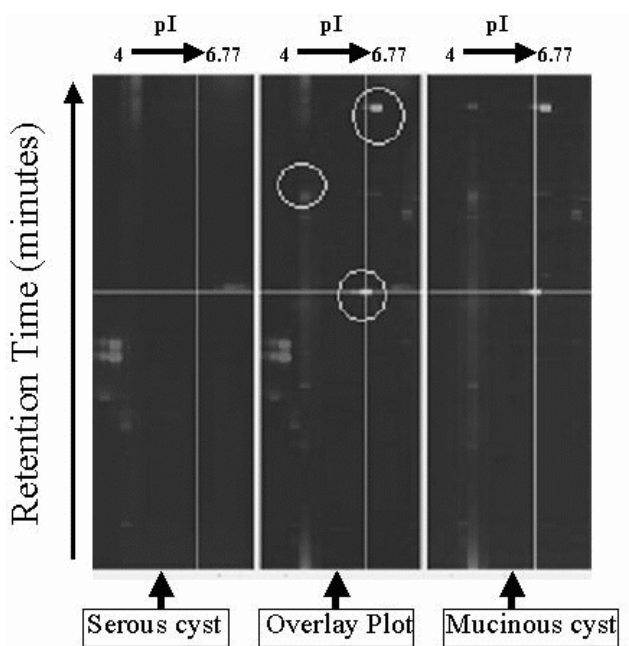

Fig. 1. Overlay of cyst fluid two-dimensional protein profiles. Prominent peaks observed in pancreatic mucinous cysts but not in pancreatic serous cysts are circled.

$\mathrm{S} 1890$

Interleukin-13 Receptor Alpha2 Expression and Its Targeting By IL-13 Cytotoxin in Pancreatic Ductal Adenocarcinoma

Takeshi Shimamura, Syed R. Husain, Raj K. Puri

Patients with pancreatic ductal adenocarcinoma (PDA) have a median survival of less than six months from diagnosis. Due to its late detection, rapid progression and resistance to chemo- and radiotherapy, median survival is marginally improved with current standard therapies. We previously demonstrated that IL-13 cytotoxin comprised of IL-13 and a truncated form of Pseudomonas exotoxin (IL13-PE38QQR) eradicates IL-13Ra2-overexpressing glioblastoma, AIDS-kaposi's sarcoma, and head and neck tumors in mice. Based on our 
pre-clinical studies, IL13-PE38QQR is being tested in a phase III clinical trial in patients with recurrent glioblastoma. We now report for the first time that 36 of 77 (47\%) tumor samples from PDA patients also express moderate to high density IL-13Ra2 as determined by immunohistochemistry. In contrast, IL-13Ra2 expression was not detected in the ductal or acinar cells of normal pancreas $(n=13)$. The RT-PCR and FACS analysis of eight PDA cell lines revealed that three (HS766T, CaPan-1, and Miapaca-2) of them were strongly positive for IL-13Ra2 expression. No IL-13Ra2 was detected in normal pancreas ductal cells. As expected, these three IL-13Ra2 positive cell lines were quite sensitive to IL13-PE38QQR, exhibiting a 50\% protein synthesis inhibition compared to control cells (IC50) over a range of $>0.1-80 \mathrm{ng} / \mathrm{ml}$. Normal pancreatic ductal cells and HUVEC were not sensitive (IC50= $>1000 \mathrm{ng} / \mathrm{ml}$ ). The incidence of IL-13Ra2 expression in almost half of the clinical samples makes PDA a good candidate for IL13-PE38QQR therapy.

\section{S1891}

Inhibition of Bcl-2/Bcl-Xl Proteins Stimulate Apoptosis and Inhibit Proliferation of Pancreatic Cancer Cells

Izumi Ohno, Mouad Edderkaoui, Patrick B. Truong, Irina V. Odinocova, J.N. K. Lee,

Hiromitsu Saisho, Anna S. Gukovskaya, Stephen J. Pandol

Background and Aims: Inhibition of anti-apoptotic Bcl-2 and Bcl-xL proteins is a novel strategy for cancer treatment. Two inhibitors of Bcl-2/Bcl-xL, BH3I-2' and HA-14, have recently become available and were shown to stimulate death in several types of cancer cells. Pancreatic cancer $(\mathrm{PaCa}$ ) cells are notoriously resistant to apoptosis and express high levels of both Bcl-2 and Bcl-xL. The aim of the present study was to determine the effects of the Bcl-2/Bcl-xL inhibitors on apoptosis and proliferation of PaCa cells, and the mechanisms underlying these effects. Methods: MIA PaCa-2 and PANC-1 cells were cultured with and without BH3I-2' and HA-14 for up to 48 hours. We measured apoptosis with Cell Death ELISA and Annexin V/PI staining (flow cytometry); cytochrome c release, by Western blot; reactive oxygen species (ROS), with the fluorescent dye DCF; and proliferation, by ${ }^{3} \mathrm{H}$ thymidine incorporation. Mitochondria were isolated from $\mathrm{PaCa}$ cells by differential centrifugation. In isolated mitochondria, we measured the effects of BH3I-2' and HA-14 on $\Delta \Psi \mathrm{m}$, respiration and ROS using, $\mathrm{TPP}^{+}$and Clark electrodes and the fluorescent dye AmplexRed, respectively. Results: BH3I-2'and HA-14 markedly stimulated apoptosis and inhibited proliferation in $\mathrm{PaCa}$ cells. For example, in MIA PaCa-2 cells cultured for $48 \mathrm{~h}$ with $10 \mu \mathrm{M}$ BH3I-2' apoptosis was 7-fold higher and proliferation 8-fold lower than in control cells. The inhibitors stimulated apoptosis and suppressed proliferation in the same range of concentrations (1-100 $\mathrm{MM}$ ) at which they inhibited Bcl-2/Bcl-xL activities, as measured by fluorescence polarization assay. At the molecular level, BH3I-2' and HA-14 stimulated cytochrome $\mathrm{c}$ release and inhibited mitochondrial ROS production, resulting in a decrease in intracellular ROS. The observed stimulation of cytochrome $\mathrm{c}$ release, a key mediator of apoptosis, represents one mechanism through which the $\mathrm{Bcl}-2 / \mathrm{Bcl}-\mathrm{xL}$ inhibitors induce apoptosis in $\mathrm{PaCa}$ cells. Another mechanism whereby $\mathrm{Bcl}-2 / \mathrm{Bcl}$-xL inhibitors induce death responses could be through inhibition of ROS. Indeed, we recently showed that ROS play a pro-survival role in $\mathrm{PaCa}$ cells by activating anti-apoptotic pathways such as PI3K/Akt. ROS are also known to mediate proliferation of cancer cells. Conclusions: Specific Bcl-2/ Bcl-xL inhibitors induced apoptosis and inhibited proliferation in PaCa cells. The underlying mechanisms include stimulation of cytochrome $\mathrm{c}$ release and inhibition of intracellular ROS. The results indicate that inhibiting $\mathrm{Bcl}-2 / \mathrm{Bcl}-\mathrm{xL}$ is a promising approach to suppress tumorigenesis and stimulate apoptosis in $\mathrm{PaCa}$ cells, thus overcoming pancreatic cancer resistance to treatments.

\section{S1892}

Engineering Diphteria Toxin in Pancreatic Cancer Cells: A Promising Tool for Therapy

Paola Fogar, Filippo Navaglia, Eliana Greco, Alessia Stranges, Carlo-Federico Zambon, Alessandra Falda, Daniela Basso, Sergio Pedrazzoli, Mario Plebani

Backgrownd and aim: Gene therapy using diphteria toxin (DT) is a promising tool for cancer cell killing. It causes a rapid cell death catalysing ADP-ribosylation of elongating factor 2 and kills cells by an apoptosis-mediated pathway. Our aim was to test the ability of the DT catalytic subunit (fragment A comprising 1-193 aa residues) (DTA) to kill 5 different pancreatic cancer cell lines, four obtained from primary tumors (BxPC3, PANCl, PSN1 and MIAPaCa2) and one from hepatic metastasis (CAPAN1). Methods: DTA was subcloned in an eukariotic expression vector $(\mathrm{pRc})$ under the control of a constitutive promoter (RSV). Lipofectamine 2000 was used to transfer the DNA vector into the cells. The efficiency of transfection was evaluated by FACS analysis, using a FITC-oligonucleotide as tracer, 24 hours after transfection. The efficiency of transcription was evaluated by FACS analysis and western blotting after 48 hours from transfection with a reporter gene (GFP) cloned in pRc. Study design was: 1. transfection of 250.000 cells for $6 \mathrm{~h}$ with 4 ug DNA; 2. after $24 \mathrm{~h}$ cells were seeded in quadruplicate in a 96-well plate; 3 . cell growth was evaluated daily for 3 days by the cell viability XTT test. Results: More than $50 \%$ of cells were efficiently transfected GFP transcription was recorded in four out of the five cell lines studied: CAPAN1 did not translate the inserted gene. In agreement this cell line was resistant to DTA gene transfer. A complete growth inhibition was achieved after DTA gene transfer in BxPC3, $\mathrm{PANCl}, \mathrm{PSNl}$ and MIAPaCa2 (figure). Conclusions: DTA expression is lethal for pancreatic cancer cells and this supports the potential use of this toxin for pancreatic cancer gene therapy. The lack of gene translation found in CAPAN-1 cells, a metastatic cell line, might be consequent to the absence of specific transcription factors recognizing the RSV promoter.

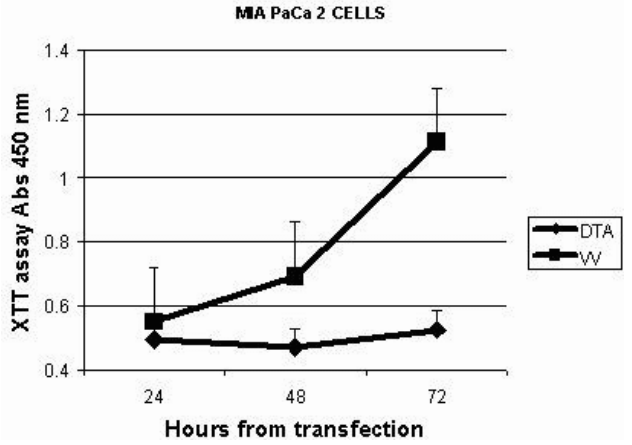

S1893

Opposing Actions of Prostaglandin $\mathrm{E}_{2}$ and Prostaglandin $\mathrm{E}_{3}$ On Pancreatic Cancer Cell Proliferation and Invasion

Hirozumi Sawai, Makoto Satake, Sascha Hasan, Howard A. Reber, Oscar J. Hines, Guido Eibl

Background and Aim: Epidemiologic studies suggest that fish oil, rich in n-3 polyunsaturated fatty acids (PUFA), possesses anti-tumor activity. Increased consumption of n-3 PUFAs appears to alter the composition of membrane fatty acids resulting in the generation of a modified prostaglandin profile. While the n-6 PUFA arachidonic acid (AA) is the substrate for the formation of prostaglandins of the 2-series $\left(\mathrm{PGE}_{2}\right)$, the n-3 PUFAs eicosapentaenoic acid (EPA) and docosahexaenoic acid (DHA) are converted to prostaglandins of the 3-series $\left(\mathrm{PGE}_{3}\right)$. The aim of the present study was to evaluate the effects of AA and EPA, as well as of $\mathrm{PGE}_{2}$ and $\mathrm{PGE}_{3}$, on the growth and invasion of pancreatic cancer cells in vitro. Methods and Results: AA dose-dependently $(0-10 \mu \mathrm{M})$ increased the growth of pancreatic cance $(\mathrm{PaCa})$ cells within 24 hours as determined by the MTT assay and cell count, and stimulated cell invasion as measured by the Matrigel invasion assay. This effect was restricted to $\mathrm{PaCa}$ cells that expressed the COX-2 enzyme, e.g. BxPC-3. In contrast, EPA dose-dependently $(0-10 \mu \mathrm{M})$ decreased proliferation and invasion of COX-2 positive PaCa cells. While AA dose-dependently increased $\mathrm{PGE}_{2}$ production of $\mathrm{COX}-2$ positive $\mathrm{PaCa}-2$ cells, EPA decreased the formation of $\mathrm{PGE}_{2}(\mathrm{p}<0.01)$. $\mathrm{PGE}_{2}$ dose-dependently $(0-10 \mu \mathrm{M})$ enhanced proliferation and invasion, whereas $\mathrm{PGE}_{3}(0-10 \mu \mathrm{M})$ reduced these parameters in BxPC-3 cells $(\mathrm{p}<0.01)$. PGE signals by binding to EP receptors, of which 4 subtypes have been described (EP $\mathrm{EP}_{4}$ ). The effects of $\mathrm{PGE}_{2}$ and $\mathrm{PGE}_{3}$ on cell growth and invasion were inhibited by a combined $\mathrm{EP}_{1} / \mathrm{EP}_{2}$ antagonist (AH6908) and a selective $\mathrm{EP}_{4}$ antagonist (ONO-AE3-208). In contrast, selective $\mathrm{EP}_{1}$ and $\mathrm{EP}_{3}$ antagonists (ONO-8711 and ONO-AE3-240, respectively) had no inhibitory effect. The selective $\mathrm{EP}_{2}$ agonist butaprost $(0-10 \mu \mathrm{M})$ increased $\mathrm{PaCa}$ cell invasion Conclusions: The n-6 PUFA AA and $\mathrm{PGE}_{2}$ stimulated, whereas the n-3 PUFA EPA and $\mathrm{PGE}_{3}$ reduced $\mathrm{PaCa}$ cell growth and invasion in vitro. The effects of $\mathrm{PGE}_{2}$ and $\mathrm{PGE}_{3}$ were mediated by the $\mathrm{EP}_{2}$ and $\mathrm{EP}_{4}$ receptors. Our findings support the notion that diets rich in $n-3$ PUFAs may be therapeutic in the modulation of pancreatic cancer biology.

\section{S1894}

RNAI-Based Knockdown of Bcl-2 Sensitises Pancreatic Cancer to Gemcitabine

\section{Treatment}

Matthias Ocker, Daniel Neureiter, Gabi Sass, Steffen Zopf, Marion Ganslmayer, Kinya Okamoto, Eckhart G. Hahn, Christoph Herold

Background: Pancreatic cancer is a tumor with high intrinsic chemotherapy resistance. This has been linked to the overexpression of anti-apoptotic bcl-2. Knockdown of bcl-2 by siRNA inhibits proliferation and induces apoptosis in pancreatic cancer cells in vitro and in vivo (Ocker et al., Gut 2005;54:1298-308). We now investigated if and to what extend the knockdown of bcl-2 sensitises pancreatic cancer cells to gemcitabine treatment in vitro and in vivo. Methods: Knockdown of bcl-2 was achieved in YAP C pancreatic cancer cells by transfecting specific siRNA or shRNA constructs. After $72 \mathrm{~h}$, different concentrations o Gemcitabine were added and apoptosis was determined by propidium iodide staining Changes in protein and mRNA levels were determined by western blotting and quantitative RT-PCR. 5 x106 cells were injected subcutaneously into male NMRI mice. Mice were treated intraperitoneally with low dose Gemcitabine $(8 \mathrm{mg} / \mathrm{kg}$ every 4 th day) and siRNA against bcl-2 or GFP. Tumor growth was determined daily and verified by immunohistochemistry, qRT-PCR and western blotting after 21 days. Results: Gemcitabine inudeced apoptosis at $0.1 \mu \mathrm{M}$ or higher but was ineffective at $0.01 \mu \mathrm{M}$ or below. The pre-transfection of bcl-2 specific siRNA and the addition of $0.01 \mu \mathrm{M}$ Gemcitabine lead to a time-dependent induction of apoptosis, reaching $50 \%$ after $72 \mathrm{~h}$ of Gemcitabine treatment. Apoptosis was not induced in combinations of mock or control siRNA transfections with $0.01 \mu \mathrm{M}$ Gemcitabine. In vivo, $8 \mathrm{mg} / \mathrm{kg}$ every 4th day Gemcitabine alone or in combination with siRNA against GFP lead to a $50 \%$ reduction in tumour size after 21 days compared to saline controls. Combination of Gemcitabine with bcl-2-specific siRNA lead to a stable tumor size for 21 days, with tumor size of $12.5 \%$ of untreated controls. Conclusions: RNAi-mediated knockdown of bcl2 sensitizes human pancreatic cancer cells to Gemcitabine-induced apoptosis in vitro and delays growth of YAP C xenografts in nude mice. This strategy might contribute to the treatment of pancreatic cancer. 
S1895

Nobel Function of Synthetic Serine Protease Inhibitor (FUT-175) As Apoptosis Inducer of Pancreatic Cancer

Tadashi Uwagawa, Paul J. Chiao, Katsuhiko Yanaga

Background and Aims:Prognosis of pancreatic cancer is extremely poor. Most patients are diagnosed unresectable mainly due to locally advanced or metastatic disease. Furthermore, chemotherapy and radiotherapy have largely failed to improve survival. Advances in molecular biology have lead to improved understanding of pancreatic cancer. A number of studies suggest that constitutive nuclear factor- $\mathrm{KB}$ (NF- $\mathrm{\kappa B}$ ) plays a key role in the growth of pancreatic cancer. In an attempt to identify a novel and effective therapeutic agent for pancreatic cancer, we studied the role of synthetic serine protease inhibitor, FUT-175 that has already been used clinically for pancreatitis or disseminating intravascular coagulation (DIC) in Japan. Methods: The human pancreatic adenocarcinoma cell line MDAPanc-28 was treated with FUT-175, then assays were performed to investigate the effects of FUT-175 on pancreatic cancer cells. Results: FUT-175 induced apoptosis of human pancreatic cancer cells in a time- and dose-dependent manner. FUT-175 inactivated the antiapoptotic activity of NF$\kappa \mathrm{B}$ by blocking IKK-mediated IкB $\alpha$ phosphorylation. Simultaneously, FUT-175 activated the proapoptotic caspase- 8 pathways by upregulating the expressiom of tumor necrosis factor receptor-1 (TNFRl) and induced apoptosis in pancreatic cancer cells. FUT-175induced activation of caspase- 8 was suppressed by RNA interference-mediated inhibition of TNFRl expression, suggesting that FUT-175-induced activation of caspase-8 is TNFRldependent. Furthemore, expression of transcription factor PEA3 was upregulated by FUT175, which was essential to FUT-175-mediated TNFRl expression. These results demonstrated a possible mechanism by which FUT-175 may both suppress the NF-KB antiapoptic activity and induce TNFRl-mediated apoptosis. Conclusion: As suggested by the dual function of FUT-175 as NF-אB inhibitor and TNFRl-mediated caspase- 8 activator, this synthetic serine protease inhibitor may become a novel therapeutic agent for pancreatic cancer.

\section{S1896}

Spontaneous Production of Anti-Mitochondrial Antibodies in Keratin-8 Deficient Mice

Diana M. Toivola, Aida Habtezion, Linxin Y. Zhang, William H. Robinson, Paul J. Utz, Eugene C. Butcher, M B. Omary

Background: Keratin 8 and $\mathrm{K} 18$ (K8/K18) are obligate non-covalent heteropolymers that constitute the intermediate filament cytoskeleton of hepatocytes. Mice that lack K8 or K18 are highly susceptible to liver injury and $\mathrm{K} 8$ mutations in humans predispose to end-stage liver disease, including that caused by autoimmune liver disease. K8-null, but not K18-null, mice also develop an ulcerative colitis phenotype, which together with the liver findings suggest an association between the epithelial cell cytoskeleton and autoimmune manifestations. Aim: We tested the hypothesis that K8-null mice produce autoantibodies that may ultimately play a pathogenic role in the liver and/or intestinal phenotype of these mice. Methods: Sera from wild-type (WT), K8-null and K18-null mice were analyzed for the presence of autoantibodies by immunoblotting total tissue lysates of normal mouse liver, pancreas and colon as well as lysates of human and mouse cultured cell lines from various tissue origins. The autoantibodies and organelle-selective markers were examined by immunofluorescence staining and by immunoblotting of subcellular fractions. Results: We identified one major autoantibody that was present in nine of fifteen $(60 \%)$ sera from K8-null mice but not in sera of WT mice. Presence of the autoantibody is age and disease severity related since the positive K8-null sera were present in all mice 8 months or older, but not in younger mice. The autoantibody was also found in four of ten (40\%) sera from one-year-old K18null mice, thereby suggesting that the origin of this autoantibody is related to the liver rather than colon pathology. The autoantibody recognizes a ubiquitous $42-48 \mathrm{kD}$ protein that partitions in a non-ionic detergent fraction, and is present in all normal tissues and cell lines tested, as well as in livers and colons of K8-null mice. Immunofluorescence staining and subcellular fractionation of cultured cells and tissues showed that the autoantigen is located primarily in the mitochondria. Conclusion: K8-null liver injury is associated with age-enhanced anti-mitochondrial autoantibody production as seen in patients with primary biliary cirrhosis. The $42-48 \mathrm{kDa}$ ubiquitous autoantigen is a mitochondrial protein which is detergent soluble, and conserved across human and mouse species. Differences in the frequency of the autoantibodies in K8 versus K18 null mice may be related to geneticbackground or other phenotype-related differences. Characterization of the autoantigen may unmask a similar autoantibody response in patients with liver disease who harbor K8 mutations.

\section{S1897}

Expression of PD-1, PD-L1 and PD-L2 Within Liver of Autoimmune Liver Diseases

Norikazu Mataki, Kentaro Kikuchi, Toshihiro Kawai, Yoshikiyo Okada, Chie Kurihara Chikako Watanabe, Shunsuke Kohmoto, Ryota Hokari, Atsushi Kawaguchi, Shigeaki Nagao, Toshiro Kondo, Kazuro Itoh, Hiroshi Miyakawa, Soichiro Miura

Background: PD-L1 (also B7-H1) expressed on macrophages and PD-L2 (also B7-DC) expressed on dendritic cells, are ligands for programmed death-1(PD-1), which is a member of the CD28 superfamily of co-stimulatory molecules and plays an inhibitory role in regulating activated T cell, B cell and myeloid cell in the periphery. According to previous reports, they are regulated by different mechanism, with the relatively greater role of interferongamma (IFN- $\gamma$ ) in stimulating PD-Ll expression and role of interleukin-4 (IL-4) in stimulating PD-L2 expression (Eur J Immunol 33:2706, 2003, PNAS 100:5336, 2003). Recently there have been several reports about changes of expression and the role of PD-1 and its ligands in autoimmune diseases. Impaired regulation of this system may cause disruption of selftolerance leading to autoimmunity. Therefore, we examined the expression of PD-1, PD-L1 and PD-L2 in the liver with autoimmune liver disease. Methods: Liver tissue was obtained from patients with autoimmune liver diseases (autoimmune hepatitis $(\mathrm{AIH}) \mathrm{n}=8$, primary biliary cirrhosis $(\mathrm{PBC}) \mathrm{n}=12$ ) and chronic hepatitis type $\mathrm{C}(\mathrm{CH}-\mathrm{C}, \mathrm{n}=12)$ as controls with informed consent. All of patients with autoimmune liver diseases were underwent neither corticosteroid nor ursodeoxycholic acid and $\mathrm{CH}-\mathrm{C}$ patients were not underwent interferon therapy. Immunohistochemical study was performed by using antibodies against PD-1, PD$\mathrm{Ll}$, and PD-L2. mRNA of PD-1, PD-L1, PD-L2, IFN- $\gamma$ and IL-4 were determined by realtime PCR. Results: PD-1 positive cells were mainly observed in portal area. PD-L1 and PDL2 positive cells were observed in both portal and sinusoidal area, but relatively dominant in sinusoidal area. We found that the expression of PD-1, PD-L1 and PD-L2 were low in $\mathrm{PBC}$. In $\mathrm{AIH}$, although the activity of inflammation in the liver was more severe than $\mathrm{CH}$ $\mathrm{C}$, expression levels of PD-1 and PD-L2 was lower than those of $\mathrm{CH}-\mathrm{C}$. To compare the relative expression to PD-1, PD-L1 and PD-L2 was lower in autoimmune liver diseases, especially in AIH. Moreover, expression levels of both PD-L1 and PD-L2 were correlated with the level of IFN- $\gamma$ but not with IL-4, and PD-L1/IFN- $\gamma$ ratio and PD-L2/IFN- $\gamma$ ratio were decreased in autoimmune liver diseases compared with $\mathrm{CH}-\mathrm{C}$, especially in $\mathrm{AIH}$. Conclusions: These results indicated that PD-1/PD-L1 and PD-L2 system was modulated in autoimmune liver diseases. We speculate that low ability of antigen presenting cells to express PD-L1 and PD-L2 in response to IFN- $\gamma$ in the liver indirectly affects to the disruption of self-tolerance, leading to the autoimmne liver diseases.

\section{S1898}

The Role of Regulatory T (TR) Cells On Liver Pathology in the Graft Versus Host Reaction (GVHR) Model Mouse

Teruo Miyazaki, Mikio Doy, Akira Honda, Tadashi Ikegami, Bernard Bouscarel, Yasushi Matsuzaki

Background/Aim An autoimmune GVHR mouse model presents similar histological and immunological observations as those encountered in human liver diseases, such as primary biliary cirrhosis. Recently, the $\mathrm{Tr}$ cells, including $\mathrm{CD} 4^{+} \mathrm{CD} 25^{+} \mathrm{Tr}$, IL-10 receptor (R) ${ }^{+} \mathrm{Tr} 1$, $\mathrm{CDI}^{+}{ }^{+} \mathrm{NKT}$ cells have been shown to participate in the maintenance of immunologic selftolerance. The aim of this study was to investigate the time-dependent relationship between the progression of autoimmune lesions and that of the Tr cells in hepatic tissue from GVHR mouse. Method The GVHR mice $(\mathrm{Fl}: \mathrm{bm} / 2 \times \mathrm{B} 6)$ were induced by intravenous injection of parental B6 spleen $\mathrm{CD}^{+} \mathrm{T}$ cells. Normal received placebo. The mice were sacrificed either immediately (G0), or at 2, 4, and 8 weeks later (G2, G4, G8). Furthermore, a group of GVHR mice received an injection of anti-IL-10 monoclonal antibody at G0, and were kept alive for 2 weeks. The respective hepatic mRNA expression level was quantitatively analyzed by cDNA macroarray. Both the state and the profile of hepatic infiltrated inflammatory cells were evaluated by HE stain and immunohistochemistry (IHC) using specific lymphocyte surface markers (CD3/4/8/20/25/45ro/161, IL-10R). Results The lesions (number, area) with inflammatory cells around bile ducts were significantly increased at G2 compared to those at G0 and normal. However, these lesions were decreased significantly and gradually over time up to G8. The mRNA expression levels of Thl (INF- $\gamma$, IL-2) and Th2 (IL-4, IL-10) cytokines and the Tr cell surface markers (CD25, IL-10R) at G2 were higher than at G0, and these high gene expression levels were maintained up to G8. The expressions of all examined surface markers including Tr cells were detectable by IHC stain in the GVHR mouse at the various times, except at G0. The expression of the IL-10R ${ }^{+}$cells was maintained in G4 although the expression of other surface marker positive cells were gradually decreased along with the improvement of hepatic lesions from 2 to 8 weeks. Furthermore, the inflammatory lesions in the anti-IL-10 antibody-injected GVHR mouse were significantly aggravated compared to those at G2 without antibody injection. Conclusion This study confirms that the autoimmune hepatic lesions in the GVHR model reached a peak at 2 weeks following the donor T cell-injection, but this condition is transient and improved gradually over time. Furthermore, the pathological improvements of the GVHR mice are associated with the expression of $\operatorname{Tr}$ cells, especially the $\operatorname{Trl}$ cells. Therefore, these findings strongly support the expression and activity of Tr cells as key factors in the induction of immunologic selftolerance in autoimmune liver diseases.

\section{S1899}

Long Term Follow-Up of Autoimmune Pancreato-Cholangitis - Insights Into the Natural History of the Disease

Henk R. van Buuren, Menno A. Brink, Sjoerd D. van der Werf, Maarten A. Meijssen,

Maarten G. Thomeer, Annette Fritscher-Ravens

Background: Autoimmune pancreatito-cholangitis (APC) is a recently noted disease in which there is an enlarged pancreatic head, endo- and exocrine pancreatic insufficiency, pancreatic and bile duct strictures with lymphoplasmocytic inflammation and fibrosis. There is usually a good response to steroids. However, little is known about its natural history, especially, when not treated with corticosteroids. Our aim was to study the natural course by reviewing cases, in which APC started more than 10 years ago. Materials and Methods: Since 1988 40 cases with APC were recognized and treated in multiple centres and clinically followed up. Of these, 13 patients (12 male, mean age 58.7years) were followed for 10-18 years (mean 12 years) with regular evaluation of their general condition and liver status. Results: 10 patients had a laparotomy for presumed pancreatic malignancy, 1 refused surgery and 2 had no surgery. Only 2 of the patients were treated with corticosteroids at the time of presentation, 5 patients had no steroids during the entire period, 1 had intermittent shortterm treatment. 5 patients started treatment 6-14 years after onset of symptoms. During the follow-up 6/13 pts developed other autoimmune related diseases including Mb. Sjoergren $(n=3)$, interstitial nephritis $(n=3)$, hypothyroidism $(n=2)$, retroperitoneal fibrosis $(n=1)$, and rheumatoid arthritis (1). One untreated patient developed calcifications in his pancreas. 7/ 13 patients developed liver cirrhosis, one had a liver/kidney transplant after 11 years, one is currently on the transplant list. All of these patients did either not undergo treatment, had only intermittent short-term corticosteroids or treatment after $>8$ years following presentation. None of the 2 patients with initial therapy developed liver cirrhosis. One patient died without autopsy to confirm liver cirrhosis. Conclusion: The development of liver cirrhosis in 54\% of the patients with long-term follow-up (all patients without treatment within the first 8 years of presentation) is remarkable. The development of liver cirrhosis was independent of any symptoms and resulted in liver transplantation in 2/13 patients. These data present new insights into the natural history of APC and suggest that it is worthwhile treating the 
underlying autoimmune process even in the symptom free interval in order to avoid the development of liver cirrhosis and other autoimmune related diseases.

\section{S1900}

Cyclosporine-a Versus Prednisolone for Induction of Remission in Patients with Autoimmune Hepatitis: Interim Report of a Randomized Controlled Trial Siavosh Nasseri-Moghaddam, Reza Malekzadeh, Saeid Karimian

Background: Autoimmune Hepatitis (AIH) is classically treated with corticosteroids (CS). In addition to being ineffective in $13 \%$ of cases, they are associated with significant side-effects, especially among young ladies. Alternative treatments are poorly described. Cyclosporine-A (CA) has been used successfully for refractory cases. We have reported effectiveness of CA in treatment naive $\mathrm{AIH}$ patients in an open-label study previously. Here we report the interim analysis of an RCT comparing CS and CA for induction of remission in treatment naive AIH patients. Methods: Consenting treatment naive AIH patients in whom all other known metabolic and viral diseases had been ruled out are being enrolled. Baseline demographic, clinical and paraclinical information are recorded and the patients undergo a liver biopsy if feasible. Then they are randomized (centrally) to receive either prednisolone orally (50mg for 2 weeks, $40 \mathrm{mg}$ for 2 weeks, 30mg for 6 weeks and tapering by $5 \mathrm{mg}$ every 6 weeks, group-A) or CA (3mg/kg/d divided equally in two doses for one year, group-B). Both groups receive Azathioprine $(1-2 \mathrm{mg} / \mathrm{kg}$ ) from week 30 . Patients are followed regularly (initially at 2 week intervals and then at 6 week intervals). Clinical data, adverse events (AE), and paraclinical data (AST, ALT, INR, Bilirubin, CBC, Creat, UA) are recorded at each visit. Primary endpoints include induction of remission, treatment failure, and serious adverse events necessitating discontinuation of the assigned treatment. Results: Up to now 28 patients (15 in group-A, 18 females, mean age $27.6+/-10.6$ years) have been enrolled. The two groups are comparable demographically and regarding their clinical and paraclinical characteristics. Mean AST and ALT has decreased significantly in both groups with treatment. Mean post treatment values for these variables are not different in groups A \& B (61.0 vs 61.0 for AST, 41.8 vs. 50.4 for ALT, p: NS). Of these, 22 have completed the course of treatment. One patient in the CS group developed intractable sepsis and passed away at week 46 . There was one treatment failure in each group. Another patient in the CA group responded initially but her liver enzymes did not decrease to below twice upper limit of normal. She did not improve further after switching to CS. AE were more common in the CS group $(\mathrm{p}<0.05)$. Coclusions: Our interim data show that CA is at least as effective and safe as CS for induction of remission in AIH patients. AE are more common with CS. CA may be considered as first line treatment for $\mathrm{AIH}$

\section{S1901}

Evidence That An ATP-Binding Cassette (ABC)G5/G8-Independent Pathway Determines Cholesterol (Ch) Gallstone Formation in Mice

Helen H. Wang, David Q. Wang

A major progress in search of the canalicular Ch transporter came from the discovery that mutations in the genes encoding human ABCG5/G8 transporters cause sitosterolemia. It has been shown that $A B C G 5 / G 8$ traffic together to the canalicular plasma membrane in cultured polarized hepatocytes and overexpression of the human $A B C G 5 / G 8$ genes in mice promotes Ch transport: biliary $\mathrm{Ch}$ secretion and saturation are increased markedly. Furthermore, Abcg5/g8 are mapped to murine chromosome 17 and are the candidate gene for Lith9. Aims: We explored whether Ch gallstone formation may be determined by an ABCG5/G8independent pathway. Methods: Male ABCG5/G8 (-/-) and wild-type (WT) mice ( $\mathrm{n}=20$ per group) were fed a lithogenic diet containing $1 \% \mathrm{Ch}$ and $0.5 \%$ cholic acid for $8 \mathrm{wk}$, and concomitantly administered by gavage with the LXR agonist T0901317 at 0 or $20 \mathrm{mg} / \mathrm{kg} /$ day. Gallstones and gallbladder biles were examined by microscopy. Lipid compositions and hepatic outputs of biliary lipids were analyzed by HPLC. Secretion rates of biliary $\mathrm{Ch}$ were determined in mice perfused via the jugular vein with $\mathrm{HDL}$ labeled with $5 \mu \mathrm{Ci}$ of $[14 \mathrm{C}] \mathrm{Ch}$. Expression levels of the genes encoding hepatic lipid transporters were investigated by real-time PCR. Results: At 8 wk on the lithogenic diet, gallstone prevalence $(25 \%)$ was reduced in mice with disruption of the Abcg $5 / g 8$ genes compared with that (80\%) in WT mice. Abcg5/g8 (-/-) mice showed a significant decrease in biliary Ch secretion rates $(8 \pm 3$ $\mu \mathrm{mol} / \mathrm{h} / \mathrm{kg}$ ) and $\mathrm{Ch}$ saturation indices (1.2\%) of pooled gallbladder biles compared with WT mice $(\mathrm{Ch}=31 \pm 8 \mu \mathrm{mol} / \mathrm{h} / \mathrm{kg}$ and $\mathrm{CSI}=1.6 \%)$. Outputs of biliary phospholipid $(34 \pm 12$ $\mu \mathrm{mol} / \mathrm{h} / \mathrm{kg})$ but not bile salts $(202 \pm 49 \mu \mathrm{mol} / \mathrm{h} / \mathrm{kg})$ were reduced markedly in $(-/-)$ mice compared with WT mice (phospholipid $=59+14$ and bile salts $=217+56 \mu \mathrm{mol} / \mathrm{h} / \mathrm{kg}$ ). The total amounts of radioactivity recovered in hepatic biles at $12 \mathrm{~h}$ after perfusion were significantly lower $(7 \pm 3 \%)$ in $(-/-)$ mice than in WT mice $(21 \pm 6 \%)$. Treatment of the LXR agonist significantly upregulated expression levels of $A b c g 5 / g 8$ but not $A b c b 4$ and $A b c b 11$ in the liver and promoted biliary $\mathrm{Ch}$ secretion $(45+12 \mu \mathrm{mol} / \mathrm{h} / \mathrm{kg})$ in WT mice but not in (-/-) mice $(10 \pm 4 \mu \mathrm{mol} / \mathrm{h} / \mathrm{kg})$. Also, gallstone formation was accelerated in LXR agonist-treated WT mice. Expression levels of Sr-b1,Abca1, Npc1l1, Abcg1, and Abcg4 in the liver were comparable in (-/-) and WT mice. Conclusions: Disruption of Abcg5/g8 significantly reduces but does not eliminate hepatic secretion of $\mathrm{Ch}$. Biliary $\mathrm{Ch}$ secretion and $\mathrm{Ch}$ gallstone prevalence in (-/-) mice are decreased by $\sim 70 \%$, suggesting that the ABCG5/G8-independent pathway also plays an important role in biliary $\mathrm{Ch}$ secretion and $\mathrm{Ch}$ gallstone formation.

\section{S1902}

Advanced Glycation Endproducts in the Gallbladder: The Influence of Diabetes and Diet

Abhishek Mathur, Swartz-Basile Deborah, Lu Debao, Hayder Al-Azzawi, Attila Nakeeb, Henry A. Pitt

Introduction: Diabetes and a high carbohydrate diet are independent risk factors for gallstone formation. Advanced glycation end products (AGE) are produced from interactions between sugars and proteins, lipids or nucleic acids. Once produced, AGE form covalent bonds with various proteins, thus contributing to diabetic complications. We have previously demonstrated that leptin-resistant diabetic (Lep db) mice and leptin-deficient diabetic (Lep ob) mice have impaired gallbladder motility. In addition, we have shown that AGE accumulates in the gallbladder of Lep $\mathrm{db}$ mice. As a raised serum glucose is a prerequisite for AGE formation, a high carbohydrate diet should cause an increase in AGE formation. However the effect of dietary carbohydrates on AGE accumulation is not known. Therefore, we tested the hypothesis that 1) gallbladder AGE would be increased in diabetic leptin-deficien compared to non-diabetic mice, and 2) within each strain AGE would accumulate more in mice fed a high carbohydrate diet. Methods: At eight weeks of age 12 non-diabetic C57BL $6 \mathrm{~J}$ and 12 leptin-deficient diabetic Lep ob female mice were fed a $45 \%$ carbohydrate diet while an additional 12 non-diabetic and 12 diabetic mice were fed a $75 \%$ carbohydrate die for 4 weeks. At 12 weeks of age all animals were wighed and then underwent cholecystectomy. Bile was collected and measured, and the gallbladders were snap frozen in liquid nitrogen Gallbladders were pooled ( $\mathrm{n}=2$ per pool) and the presence of AGE was determined by competetive ELISA using monoclonal antibody raised against $\mathrm{N}$-(carboxymethyl)lysine(CML), an established biomarker for AGE. Data were analyzed by analysis of variance, Student's t test and Tukey test. Results: The results for mouse weight, gallbladder volume and AGE concenteration in the gallbladder in non-diabetic and diabetic mice on the $45 \%$ and $75 \%$ carbohydrate $(\mathrm{CHO})$ diets are presented in the table. Conclusions: These data suggest that 1) AGE is increased in the gallbladder of diabetic leptin-deficient mice, 2) gallbladder AGE is not influenced by dietary carbohydrates and 3) gallbladder volume is altered by both diabetes and diet. We conclude that the accumulation of advanced glycation end products in the gallbladder may play a role in the propensity for diabetics to form gallstones

\begin{tabular}{|c|c|c|c|}
\hline & Mouse weight $(\mathrm{mg})$ & Gallbladder volume & AGE $(\mu \mathrm{g} / \mathrm{mg})$ \\
\hline Non-diabetic $45 \%$ CHO & $16.9 \pm 0.14$ & $10.7 \pm 0.5$ & $213.8 \pm 28.0$ \\
\hline Non-diabetic 75\% CHO & $17.0 \pm 0.30$ & $7.76 \pm 0.4 \S$ & $232.6 \pm 31.0$ \\
\hline Diabetic 45\% CHO & $48.5 \pm 0.85^{*}$ & $24.68 \pm 1.3 \Delta$ & $358 \pm 55.1 *$ \\
\hline Diabetic 75\% CHO & $44.2 \pm 0.82 * \dagger$ & $6.99 \pm 0.6$ & $313.5 \pm 43.8$ \\
\hline
\end{tabular}

${ }^{*} \mathrm{p}<0.01$ vs Non-diabetic, $\uparrow \mathrm{p}<0.01$ vs Diabetic $45 \% \mathrm{CHO}, \S \mathrm{p}<0.05$ vs Non-diabetic $45 \%$ $\mathrm{CHO}, \Delta \mathrm{p}<0.01$ vs Other groups

\section{S1903}

Construction of a Partial Full-Length Prairie Dog cDNA of Sodium Hydrogen Exchanger Isoform-3 (NHE3)

Ramakrishnan Ramugounder, Gregg J. Johannes, Mohammad Z. Abedin

Background \& Aims: Gallbladder Na+ and water absorption is linked to gallstone formation Gallbladder $\mathrm{Na}+$ transport is predominantly mediated through apically restricted $\mathrm{Na}+\mathrm{H}+$ exchangers NHE2 and NHE3. We have recently shown that gallbladder NHE is up-regulated prior to cholesterol crystal formation. The mechanisms by which gallbladder NHE become altered are not known. The aim of the present study was to clone and construct the cDNA coding for gallbladder NHE3 in prairie dog. Methods: Total RNA of prairie dog gallbladder was reverse transcribed, and amplified by PCR using three-different length of NHE3-specific primers designed on the basis of conserved sequences among human, rabbit, and rat. These PCR-products were pooled, amplified using three-different blunt-end NHE3-specific primers $(683 \mathrm{bp}+652 \mathrm{bp}+463 \mathrm{bp}=1798 \mathrm{bp})$, ligated, and amplified again by PCR using NHE3-specific primers of $1798 \mathrm{bp}$ length. The PCR-product was analyzed by $1.0 \%$ agarose-gel and the predicted 1798bp NHE3 cDNA was purified and cloned using 2.1 PCR-vector and sequenced. Results: We have cloned the NHE3 mRNA from prairie dog gallbladder $(1.798 \mathrm{~kb}$ of the $2.213 \mathrm{~kb}$ rat-NHE3 open-reading-frame). The cDNA-sequence of $1798 \mathrm{bp}$ was found to be 89,89 and $88 \%$ homologous to the corresponding sequences of NHE3 in human, rabbit and rat, respectively. The deduced amino acid sequence of 599 residues was 94, 94 and 97\% identical to the human, rabbit and rat NHE3. Conclusion: This NHE3 cDNA-construct may be used for in-vitro study to examine the mechanisms of gallbladder NHE regulation during gallstone formation.

\section{S1904}

Estrogen Receptor Alpha Is Expressed By Gallbladder Epithelium in Prairie Dogs

Ross M. Goren, Misbah Zaheer, Ramakrishnan Ramugounder, Mohammad Z. Abedin

Background \& Aims: Gallstone disease is one of the most common and costly digestive diseases. The incidence of gallstones is 2 to 3 times higher in females than in males. Female sex hormones have been implicated as contributory factors to the promotion of gallstone by increasing biliary cholesterol saturation and decreasing gallbladder (GB) motility. The sex hormones mediate its effects by binding to specific estrogen receptors (ER), which appear to regulate gene expression through activation or repression of transcription. Alterations in ER expression may in part account for the increased incidence of gallstones in females. Aim of the present study was to isolate and clone ER-alpha in GB epithelium. Methods: Total RNA was isolated from male and female prairie dog GBs, reverse transcribed, and then amplified in PCR using ER-alpha specific primers. An aliquot of the PCR product was analyzed by $1 \%$ agarose gel and the predicted 360 base pair ER-alpha cDNA was cloned using 2.1 PCR vector, and subsequently sequenced by Sanger method. Results: RT-PCR analysis demonstrated the GB expression of 365 base pair ER-alpha CDNA in both male and female prairie dogs. The cDNA sequence was 92,92 and $93 \%$ homologous to the corresponding ER-alpha sequences in mouse, rat and human, respectively. Deduced AA sequence of 121 residues was $100 \%$ identical. Studies are in progress to examine the gender differences in GB ER-alpha abundance and will be reported. Conclusion: These data will provide an important tool to study the role of estrogen receptors in gender differences in gallstone pathogenesis. 
S1905

Amorphous Calcium Carbonate is the in Vitro Unstable Precursor of the $\mathrm{CACO}_{3}$ Polymorphs Vaterite, Calcite and Aragonite, But Is Stable in Gallstones Containing $\mathrm{CACO}_{3}$ from Leeds UK and Galveston TX Roger D. Soloway, D.R. Taylor, Mark D. Stringer, Jinguang Wu

Background: Amorphous $\mathrm{CaCO}_{3}$ (ACC) has been formed in vitro to serve as a standard for use in Fourier transform infrared (FT-IR) spectroscopy of gallstones. ACC forms small, ovoid solids, 1-3 $\mu$ in diameter, which, in vitro, in a matter of hours, convert to $15 \mu$ spheroids of vaterite or $15 \mu$ cubes of calcite, at room temperature and average humidity. Newly synthesized vaterite is stable in vitro at $100^{\circ} \mathrm{C}$ for at least 7 days, but at $25^{\circ} \mathrm{C}$ converts to calcite within hours. Each of these $\mathrm{CaCO}_{3}$ polymorphs has been identified in gallstones, years after formation, suggesting that the in vitro unstable forms have been stabilized in vivo by proteins. Aim: To examine gallstones containing large amounts of $\mathrm{CaCO}_{3}$ to determine if ACC could be identified and if ACC interaction with other stone components could be identified as the basis for stabilization. Methods: A particle from a stone from Leeds containing $94 \%$ $\mathrm{CaCO}_{3}$ and a particle from a stone from Galveston containing $20 \% \mathrm{CaCO}_{3}$ were examined by FT-IR microspectroscopy and by Scanning Electron Microscopy (SEM). FT-IR methodology was based on serial subtraction of standard spectra from the original spectra containing mixtures of $\mathrm{CaCO}_{3}$ forms. Remainder spectra were examined for peak shifting to demonstrate binding. Components were also identified using unique peaks detected using deconvolution and curve-fitting computer programs on the original spectra. Results: The stone from Leeds, containing more than $90 \% \mathrm{CaCO}_{3}$ and the stone from Galveston containing $20 \% \mathrm{CaCO}_{3}$, as a layer within a cholesterol stone, both contained calcite as the major component and ACC as the minor component. After both ACC and calcite were subtracted from the original spectrum, a small amount of altered amorphous carbonate with a major peak at $1400 \mathrm{~cm}^{-}$ ${ }^{1}$ remained together with protein peaks. This indicated that a proportion of ACC had bound to protein which may have served both as a template and a stabilization structure. Spectra will be presented. Conclusions: 1. Amorphous $\mathrm{CaCO}_{3}$ (ACC) has been identified in unique, virtually pure $\mathrm{UK} \mathrm{CaCO}$ gallstones and US cholesterol stones with $\mathrm{CaCO}_{3}$ layers. 2. This data shows that a portion of ACC is altered by binding to protein. 3. Protein in these stones may act to nidate and stabilize ACC which would otherwise be unstable. 4. This probably occurs on the AAC precipitate surface, allowing the centers to remain as unaltered and otherwise unstable ACC

\section{S1906}

Prevalence of Gallbladder Disease Among Persons with Viral Hepatitis and Cirrhosis

Hyun-Jin Kim, Gyeong-A Jung

Background/Aim: Gallbladder stone is one of the major digestive diseases, and has a high morbidity in adults. In Korea, gallstone prevalence has reported about 2.2 to $3.5 \%$. The risk factors of gallbladder disease(GBD) related to gallstone are age, obesity, rapid weight hypertriglyceridemia, diabetes mellitus, women, smoking, etc. Moreover, cirrhosis is well known for the risk factors of GBD. But, relationship between chronic viral hepatitis and GBD is not exactly known. Some studies suggest that Hepatitis virus infection facillitates gallstone formation. The purpose of the present study was to compare with prevalence of GBD in chronic viral hepatitis $C$, chronic viral hepatitis $B$, and the cirrhosis patients who was progressed from viral hepatitis in long period. Methods: A retrospective analysis was made of patients who underwent abdominal ultrasonography in Gyeongsang National University Hospital from 2000 January to 2004 December for hepatitis C virus infection(420 patients) and hepatitis B virus infection(1,397 patients). Each patients was devided into chronic viral hepatitis and cirrhosis. In a control group, a total of 4,094 persons undergoing routine health screening enrolled. Gallbladder disease was defined as ultrasound-documented gallstones or evidence of cholecystectomy. Results: The $12.1 \%$ (41/338 persons) among chronic viral hepatitis C had a GBD, and this was significantly higher prevalence than 5.6\% (228/4094 persons) in control group $(\mathrm{p}<0.001)$. The odds of GBD (Male=3.026, Female=1.624) among chronic viral hepatitis $C$ was significantly higher compared with control. In chronic viral hepatitis $B$, prevalence of GBD was $9.1 \%$ ( $48 / 526$ persons $)(p=0.001)$. The prevalence of GBD was $20.7 \%$ in Anti-HCV positive cirrhotics, $19.7 \%$ in HBsAg-positive cirrhotics, and $33 \%$ in alcoholic cirrhotics. Conclusion: In chronic viral hepatitis patients, prevalence of GBD was significantly higher than control group and GBD was much more development in chronic viral hepatitis C. Chronic viral hepatitis as well as viral hepatitis related cirrhosis is considered as risk factor of GBD.

\section{S1907}

\section{Genetics of Gallstone Disease - Who Should Be Investigated?}

Clemens Schafmayer, Juergen Tepel, Sandra Freitag, Heike Wolken, Michael Wittig, Ilka Vogel, Herrmann Dittrich, Juergen Belz, Rainer Quaeschling, Hofjat Schekarriz, Volker Mendel, Werner Neugebauer, Friedrich Kallinowski, Anton Schafmayer, Jiri Klima, Henry Voelzke, Ulrich R. Foelsch, Fred Faendrich, Michael Krawczak, Stefan Schreiber, Jochen Hampe

Background: A genetic contribution to the aetiology of gallstones has been recognized as early as 1925. Quantitative trait mapping in mice has since identified up to nine gene loci for bile stone susceptibility. In humans, by contrast, data on gall stone risk loci are scarce, possibly due to the lack of high-risk groups facilitating efficient genetic studies. Aims: To identify human subpopulations suitable for the successful genetic investigation of gallstones and to obtain reliable estimates of the power of such studies. Patients and Methods: A total of 2474 patients who had undergone cholecystectomy were recruited in Northern Germany. Demographic data and family histories were obtained via questionnaires and telephone interviews. Potential predictors of the presence of gallstones and of a history of cholecystectomy were investigated using Cox regression analysis, including frailty terms. Results: The relative risk (RR) of siblings was significantly associated with the age of disease onset in the index patient $(\mathrm{p}<0.001)$. If the disease in the index patient presented before 20 years of age, the sibling hazard ratio is 111.8 (95\% CI: 17.8 - 700) as compared to the case in which the index patient got affected at the population median age of onset of 65 years. Hazard ratio estimates for age groups $20-30$ years and $30-40$ years were $20.5(\mathrm{CI} 8.0-50.5)$ and 11.8 (CI 5.8 - 23.7), respectively. Conclusions: The increased gallstone risk among siblings of patients with an early age of onset of the disease further supports a genetic contribution to gallstone aetiology. Early onset patients and their families are thus showing a stronger genetic contribution and represent a useful high-risk population for the identification of major disease genes.

\section{S1908}

Analysis of the Risk Factors Helicobacter Infection, Overweight, Sex, and Age in Gallstone Disease and Gallbladder Carcinoma in Germany

Ulrich R. Bohr, Doerthe Kuester, Frank Meyer, Thomas Wex, Morena Stillert, Antal Csepregi, Hans Lippert, Albert Roessner, Peter Malfertheiner

Background \& Aims: Recently, Helicobacter infection of the hepatobiliary system has been proposed as a novel risk factor in the pathogenesis of gallstones and biliary tract malignancies Colonization of the biliary tract by Helicobacter species was reported predominantly for regions with a high incidence of gallbladder carcinomas. In Germany gallstone disease is frequent, but the incidence of gallbladder carcinoma is relatively low. This prompted us to investigate if Helicobacter infection of the biliary tract is present in the German population. Methods: Gallbladder tissue from 99 patients having undergone cholecystectomy (43 male, 56 female, age range 7-94 years, mean age 59.1 years) were investigated: 57 patients with gallstone disease (GSD), 20 cases of adenocarcinoma of the gallbladder (GBC), and 22 patients without malignancies or gallstones. The presence of Helicobacter spp. was investigated by culture, immunohistochemistry, and a group-specific PCR targeting the 16S rRNA and detecting all currently known Helicobacteraceae. Helicobacter was cultured by plating dissected gallbladder mucosa onto selective agar and incubation under microaerophilic conditions. PCR was performed with DNA obtained from native or paraffin-embedded tissue. Immunohistochemistry was done using two different anti-Helicobacter pylori-antibodies with crossreactivity to other Helicobacter species. Results: Of the 99 cases investigated, only one patient with GSD was PCR-positive for Helicobacteraceae. Here, sequence analysis of the $16 \mathrm{~S}$ rRNA showed closest homology to the $16 \mathrm{~S}$ rRNA sequence of H. ganmani. Helicobacteraceae were not detected by culture or immunohistochemistry. Statistical analysis of the clinical data showed a significantly higher body mass index in patients with GSD compared to controls $(p<0.05)$. Prevalence of GSD in female patients was higher than in male patients $(79.1 \%$ vs. $63.9 \%$; odds ratio $=2.14,95 \%$ confidence interval $0.71-6.56$ ). Mean age of patients with GBC was significant higher than for GSD $(p<0.01)$ or control patients $(p<0.005)$, while there was no difference between GSD and controls. Conclusions: These data suggest that Helicobacteraceae play no predominant role in the pathogenesis of gallstone formation and gallbladder carcinoma in the German population. However, the low prevalence of Helicobacteraceae in the gallbladder mucosa of German patients may explain in part the relatively low prevalence of $\mathrm{GBC}$ compared to other populations with a high risk of $\mathrm{GBC}$.

\section{S1909}

Cholelithiasis and Biliary Sludge in Children: Predisposing Diseases and Mode of Presentation

Denise Herzog, Guylaine Bouchard

The rising prevalence of gallstone disease (GD) in the adult population of industrialized countries is a cause of concern. European pediatric prevalences range from 0.13 to $1.9 \%$. Aim: to assess the mode of presentation, predisposing diseases, and the prevalence of GD and biliary sludge (BS) in a pediatric population of a tertiary care center. Method: Retrospective review of abdominal ultrasounds recorded at Sainte Justine Hospital over a period of 24 months $(8 / 2003$ to $8 / 2005)$ in patients $<19$ years of age. Patients were grouped into children $<2$ and $>2$ to $<19$ years of age. Results: A total of 9203 abdominal ultrasounds were reviewed. GD was found in 127 (1.3\%), with 107 (1.1\%) new diagnoses, BS in 84 $(0.91 \%)$, and post-cholecystectomy status in 26 patients. During this period 57 cholecystectomies for gallbladder disease were performed. In total, 48 (46\%) patients had idiopathic GD presenting with abdominal pain (32 girls). Of these 48, 29(27.6\%)had associated pancreatitis, cholestasis, or choledocholithiasis; cholecystectomy was done in 28 (58\%), 24 girls (86\%). In hemolytic disease (HD), cystic fibrosis, oncologic disease or organ transplantation patients GD was detected during routine abdominal ultrasound. Preventive cholecystectomy was carried out in 9/19 patients with HD. Post-cholecystectomy status was noted in 14 HD patients and 12 with idiopathic GD, (5 boys (19\%). BS was mainly seen in patients requiring total parenteral nutrition (TPN) $(67 \%<2$ years, $72 \%>2$ years of age). In 10 patients sludge was found to preceed the formation of gallstones. Conclusion: Mode of presentation, age and gender distribution and prevalence of GD found in these 2 years retrospective study resembles that found in a 10 year-retrospective European study (Wesdorp et al). Idiopathic GD represents $46 \%$ of gallstones and is mainly found in adolescents. The complication rate in our patients with gallbladder disease is similar to that of adult patients. GD in children under two years of age was found to be associated with infections and prolonged use of TPN. Prevalence of Gallstones and Biliary Sludge in Pediatric Patients according to Disease and Age

\begin{tabular}{|c|c|c|c|c|c|c|c|c|}
\hline & HD & CF/H & OT & IC & SM & I & Total & Gender (male) \\
\hline Gallstones (median, CI 95\%) & & & & & & & & \\
\hline$<24$ months (4m, 3.5-8.3) & & 13 & & 12 & 7 & & 22 & $11(50 \%)$ \\
\hline $2-19$ years (13 y, 11.4-13.1) & 19 & $10 / 7$ & 10 & 7 & 4 & 48 & 105 & $37(35 \% 9$ \\
\hline Biliary Sludge & & & & & & & & \\
\hline$<24$ months (2 m, 2.1-5.5) & 1 & 3 & & 8 & 10 & 3 & 27 & $16(59 \%)$ \\
\hline $2-19 y e a r s ~(13 y, 9.8-13.1)$ & 12 & 3 & 11 & 9 & 6 & & 57 & $24(42 \%)$ \\
\hline
\end{tabular}

$\mathrm{HD}=$ hemolytic disease, $\mathrm{CF} / \mathrm{H}=$ cystic fibrosis + hepatobiliary diseases, $\mathrm{OT}=$ oncologic/ transplantation, IC=infectious/congenital, $\mathrm{SM}=$ neonatal surgery/malformation, I= idiopathic 
Aminoguanidine Impairs Gallbladder Contractility

Hayder Al-Azzawi, Abhishek Mathur, Debao Lu, Deborah Swartz-Basile, Attila Nakeeb, Henry A. Pitt

INTRODUCTION: Diabetes is associated with an increased incidence of gallstones. Advanced glycation endproducts (AGE) have been found to be increased in the tissues of diabetic patients and animals. As a result, AGE have been implicated in diabetic complications including nephropathy, retinopathy, neuropathy and atherosclerosis. Data from our laboratory have shown that leptin-resistant diabetic mice have higher AGE concentrations in their gallbladders, increased gallbladder volume, and decreased gallbladder contractility compared to non-diabetic mice. Aminoguanidine is an agent that prevents the formation of AGE in tissues and, therefore, prevents diabetic complications. However, no data are available on the influence of aminoguanidine on gallbladder motility. Therefore, we tested the hypothesis that the addition of aminoguanidine to the diet of leptin-deficient diabetic mice would improve gallbladder contractility. METHODS: 20 six-week old female leptin-deficient diabetic mice were fed a $60 \%$ carbohydrate, $1 \%$ cholesterol lithogenic diet for 6 weeks. Half of the animals had aminoguanidine $0.7 \%$ added to their diet. Both at 6 and 11 weeks, all mice were fasted overnight and underwent ultrasonography of the gallbladder under inhalation isoflurane anesthesia. Gallbladder volume before (resting volume) and $45 \mathrm{~min}$ after an intraperitoneal injection of $2 \mathrm{nmol} / \mathrm{kg}$ cholecystokinin (residual volume) were determined using the Visual Sonics Vivo 660TM High Resolution Imaging System. Gallbladder ejection fraction was determined from the gallbladder volumes obtained at 0 and 45 minutes. At 12 weeks of age, all the mice were fasted overnight and underwent cholecystectomy. Bile was aspirated and examined under polarized light microscopy for cholesterol crystals, and gallbladder dry weight was measured. RESULTS: Results for gallbladder resting volume (ul), gallbladder residual volume $(\mu \mathrm{l})$ and ejection fraction (\%) at 1lweeks, and gallbladder dry wt (mg) are presented in the table. Cholesterol crystal numbers did not differ between groups. Data were analyzed by Student's t test. CONCLUSION: These data suggest that in leptin-deficient diabetic mice fed a lithogenic diet, aminoguanidine 1) increases residual gallbladder volume, 2) decreases gallbladder ejection fraction and 3) increases gallbladder weight. Therefore, we conclude that aminoguanidine may increase the propensity for gallstone formation by enhancing gallbladder stasis

\begin{tabular}{|c|c|c|c|c|}
\hline & Resting Volume & Residual Volume & Ejection Fraction & GB Dry Wt. \\
\hline Control & $61 \pm 7$ & $3 \pm 0.5$ & $96 \pm 0.4$ & $2.0 \pm 0.1$ \\
\hline Aminoguanidine & $82 \pm 12$ & $32 \pm 13^{*}$ & $61 \pm 14 \dagger$ & $2.6 \pm 0.2 \dagger$ \\
\hline
\end{tabular}

*p $<0.05$ vs Control, $\dagger \mathrm{p}<0.03$ vs Control

S1911

The Gut Microflora Is Involved in Ethanol Metabolism and Ethanol-Induced Impairment of the Epithelial Barrier in Rodents

Laurent Ferrier, Florian Berard, Laurent Debrauwer, Chantal Chabo, Philippe Langella, Lionel Bueno, Jean Fioramonti

Background. Hepatic toxicity of alcohol is associated with endotoxemia and increased intestinal permeability. Since the colon is the main reservoir of endotoxins, impairment of the epithelial barrier may lead to endotoxemia. Alcohol diffuses easily from blood to colonic lumen, and colonic bacteria are able to metabolize alcohol into acetaldehyde, known to be a potent mast cell activator and to impair the intestinal barrier. This study aimed at investigating whether alcohol metabolism by the colonic flora may be responsible for the alcohol-induced impairment of the colonic mucosal barrier. Methods. Male Wistar rats were given an acute intragastric administration of ethanol $(1.5,3.0$ and $4.5 \mathrm{~g} / \mathrm{kg})$, or isocaloric amounts of dextrose (controls). A group was treated with non absorbable antibiotics (ampicillin and neomycin; ATB) in drinking water for ten days. The involvement of mast cells was assessed by treating another group with the mast cell stabilizer, doxantrazole. Gut paracellular permeability (GPP) was assessed by the passage of orally administered ${ }^{51} \mathrm{Cr}$-EDTA into urine, and ethanol and endotoxin blood levels were measured. Ethanol metabolism by microflora was evaluated by measuring acetaldehyde in colonic contents. Finally, colonic strips from rats pretreated or not with doxantrazole, were mounted in Ussing chambers to measure permeability to dextran. Results. An acute administration of 3.0 and $4.5 \mathrm{~g} / \mathrm{kg}$ ethanol increased GPP $(3.2 \pm 0.2 \%$ and $3.4 \pm 0.4 \%$, respectively, vs. $2.2 \pm 0.2 \%$ for dextrose, $\mathrm{p}<0.05)$, which was significantly reduced either by doxantrazole or ATB treatment. Two hours after intake, ethanolemia was $17.4 \pm 0.2 \mathrm{mmol} / \mathrm{L}$, and reached $34.3 \pm 0.2 \mathrm{mmol} / \mathrm{L}(\mathrm{p}<0.001)$ under ATB treatment. Portal endotoxins were increased 90 minutes after ethanol intake $(789.3 \pm 73.7$ $\mathrm{IU} / \mathrm{L}$ vs. $382.2 \pm 34.2 \mathrm{IU} / \mathrm{L}, \mathrm{p}<0.01$ ). Under $\mathrm{ATB}$, endotoxemia returned to basal values. Acetaldehyde lumenal concentration within the colon $(22.2 \pm 1.5 \mu \mathrm{mol} / \mathrm{L})$ reached 132.6 $\pm 31.6 \mu \mathrm{mol} / \mathrm{L}(\mathrm{p}<0.05)$ and only $86.2 \mu \mathrm{mol} / \mathrm{L} \pm 10.9$ under ATB treatment. In Ussing chambers, $17 \mathrm{mmol} / \mathrm{L}$ ethanol were ineffective. Acetaldehyde dose-dependently increased dextran flux from $120 \mu \mathrm{mol} / \mathrm{L}$ concentration, its effects being inhibited by doxantrazole. Conclusion. The increase in alcoholemia observed under ATB treatment indicates an important role of colonic bacteria in alcohol metabolism in rats. Colonic epithelial barrier alterations by acute ethanol intake involve colonic bacterial metabolism of ethanol into acetaldehyde, which in turn induces mast cell degranulation. These alterations may be responsible for an excessive lumen-to-blood endotoxin crossing, frequently observed in patients suffering of alcoholic liver disease.
Accuracy of Indices Utilizing Common Lab Values in Predicting Hepatic Fibrosis and Cirrhosis in Hepatitis C Patients in a Community Setting Sedat Ekici, Jeffrey Kim, Brian Wolfman, Toomas Sorra, Adnan Khdair, Irwin Grosman Several indices utilizing commonly available lab tests have been proposed for predicting the presence of significant fibrosis or cirrhosis in patients with chronic hepatitis C (CHC) However, their applicability outside the setting of referral centers has not been well studied. Aim: To assess the accuracy of the aspartate aminotransferase (AST)-platelet (PLT) ratio index (APRI), AST/alanine aminotransferase (ALT) ratio (AAR), age-PLT index (API) (Poynard et al. J Viral Hepatol 1997;4:199-208) and the Hepatitis C Antiviral Long-Term Treatment Against Cirrhosis Trial model (HALT-CM) (Lok et al. Hepatology 2005;42:282-292)in predicting significant fibrosis or cirrhosis in our cohort. Methods: Records for 149 consecutive patients with $\mathrm{CHC}$ who underwent biopsy at our institution from 2000 to 2005 were reviewed. Only treatment naive patients, 18 or older, with no other concurrent liver disease or HIV were included. Lab values, including AST, ALT and international normalized ratio (INR) were obtained. All lab values were within 6 months of the biopsy date. APRI was calculated using the formula: ((AST/upper limit of normal)/PLT)x100. AAR equaled AST/ ALT. API and HALT-CM were calculated using published formulas. Fibrosis (F) was staged using the modified Ishak scale (F0 to F4), with $\mathrm{F} \geq 2$ representing significant fibrosis and F4 denoting cirrhosis. Published cut-off values for cirrhosis and significant fibrosis were used to determine sensitivity, specificity, positive predictive value (PPV) and negative predictive value (NPV) for each test. Results: 107 patients who met the criteria were identified; 54 were male. The mean age was $50 \pm 7.5$; the mean fibrosis score was $1.8 \pm 0.9 ; 63 \%$ had significant fibrosis and $5 \%$ had cirrhosis. Sensitivity, specificity, PPV and NPV for each index are presented on the table. Conclusions: Both API and APRI $>1.5$ have low sensitivity but high specificity and PPV for significant fibrosis. APRI $>2.0$ was both sensitive and specific for cirrhosis. The APRI and HALT-CM could be used to reliably exclude cirrhosis. None of the indices studied had a high PPV for cirrhosis, most likely due to its low prevalence in our cohort. The AAR was not a useful predictor of cirrhosis.

\begin{tabular}{|c|c|c|c|c|c|}
\hline & & Sensitivity $(\%)$ & Specificity $(\%)$ & PPV(\%) & NPV(\%) \\
\hline \multirow{3}{*}{$\begin{array}{c}\text { Prediction of Significant } \\
\text { Fibrosis }\end{array}$} & APRI $>0.5$ & 78 & 68 & 80 & 64 \\
\cline { 2 - 6 } & APRI $>1.5$ & 25 & 95 & 89 & 43 \\
\cline { 2 - 6 } & API $\geq 6$ & 36 & 93 & 89 & 46 \\
\hline \multirow{4}{*}{ Prediction of Cirrhosis } & APRI $>1.0$ & 100 & 75 & 16 & 100 \\
\cline { 2 - 6 } & APRI $>2.0$ & 100 & 92 & 38 & 100 \\
\cline { 2 - 6 } & HALT-CM $\geq 20 \%$ & 100 & 37 & 8 & 100 \\
\cline { 2 - 6 } & HALT-CM $\geq 50 \%$ & 60 & 83 & 16 & 97 \\
\cline { 2 - 6 } & AAR $\geq 1.0$ & 40 & 71 & 6 & 96 \\
\hline
\end{tabular}

S1913

Serum Hyaluronic Acid (HA), Ykl-40, Fibrospect-II (FS-II) Test and Digital Quantification of Fibrosis (DQF) Correlate with Ishak Fibrosis Scores in Patients with Chronic Hepatitis C (HCV)

Preeti Mehta, Robert Ploutz-Snyder, Jyotirmoy Nandi, Robert A. Levine

Liver biopsy has limitations of sampling variation and interobserver bias. Direct serum markers of fibrosis such as HA, YKL-40 and FS-II (HA, TIMP-1 and $\alpha-2$ macroglobulin) have been used as surrogate markers to assess liver fibrosis. We reported that DQF discriminates between varying stages of liver fibrosis in $\mathrm{HCV}$ and has excellent interobserver reliability (Liv Intl 2005; 25: 1142). AIMS: To correlate the accuracy of HA, YKL-40, FS-II and DQF parameters with Ishak fibrosis scores. METHODS: Ishak scores, DQF, HA, YKL-40 and FSII were evaluated retrospectively in 96 patients with HCV. Mean liver biopsy length was $\geqq 15 \mathrm{~mm}$ having $\geqq 5$ portal tracts. Statistical analysis was performed using SPSS (v.13.0.1)software. The Spearman's Rho statistic assessed the correlational relationships among Ishak score and the above parameters. Receiver operator characteristic (ROC) curves evaluated the accuracy of these parameters when compared to Ishak stages of fibrosis, as well as between incremental steps of the Ishak scale. Area Under the Curve (AUC) and fals positive rates were determined for each predictor of fibrosis. Variable sample sizes for some of the analyses are due to pending data. RESULTS: All four diagnostic parameters were highly correlated with one-another $(\mathrm{p}<.01)$ and with Ishak scores (Table 1). ROC curves discriminated patients into various fibrosis categories (Table 2). The areas under the ROC curves were significant, indicating excellent diagnostic abilities for all four measures to detect the broad categories of the Ishak (4-6) vs (0-3), and Ishak (4-6) vs (2-3). All three parameters, except YKL-40, were able to differentiate between Ishak (2-3) vs (0-1). DQF showed the highest AUC of all measures tested for discriminating patients into these broad Ishak categories. CONCLUSION: Our data supports the overall ability of serum markers to discriminate between clinically relevant stages of fibrosis/cirrhosis.

Table 1. Spearman's Rho Correlation Matrix Among Diagnostic Parameters

\begin{tabular}{|c|c|c|c|c|c|}
\hline & Ishak & FS-II & DQF & YKL-40 & $\mathrm{HA}$ \\
\hline Ishak n & & $.762 * 68$ & $.878 * * 96$ & $.537 * 62$ & .77962 \\
\hline FS-II n & $.762 * * 68$ & & $.674 * 67$ & $.630 * * 66$ & $.853 * * 65$ \\
\hline DQF n & $.878^{* *} 96$ & $.674 * 67$ & & $.371 * 62$ & $.686^{* * *} 62$ \\
\hline YKL-40 n & $.537 * * 62$ & $.630^{* *} 66$ & $.371 * 62$ & & $.643 * * 67$ \\
\hline $\mathrm{HA} \mathrm{n}$ & $.779 * * 62$ & $.853 * * 65$ & $.862 * 6 * 62$ & $.643 * 67$ & \\
\hline
\end{tabular}

Correlation significant at the $* \mathrm{p}<.01$ and $* * \mathrm{p}<.001(2$-tailed). $\mathrm{n}=$ number of patients tested. Table 2. Area Under ROC Curves for 4 Parameters Discriminating Between Fibrosis Stages 


\begin{tabular}{|c|c|c|c|c|c|}
\hline $\begin{array}{l}\text { Ischak } \\
\text { Scores }\end{array}$ & $\begin{array}{c}\text { Discriminating } \\
\text { Categories }\end{array}$ & AUC & $P$ value & $\begin{array}{l}\text { False Positive } \\
\text { Rate/90\% } \\
\text { Sensitivity }\end{array}$ & $\begin{array}{l}\text { False Positive } \\
\text { Rate/75\% } \\
\text { Sensitiviy }\end{array}$ \\
\hline $\begin{array}{c}4-6 \text { vs } 0-3 \\
n=58\end{array}$ & & & $\begin{array}{l}.000 .000 \\
.000 .000\end{array}$ & 613. 355.226 .355$. & .161.194.290.258 \\
\hline $\begin{array}{c}0-1 \text { vs } 2-3 \\
n=31\end{array}$ & $\begin{array}{l}\text { FS-II DQF } \\
\text { YKL-40 HA }\end{array}$ & $\begin{array}{l}.746 .873 \\
.579 .789\end{array}$ & $\begin{array}{l}.023 .001 \\
.465 .007\end{array}$ & .833 .500 .833 .667 & .417.333.667. 417 \\
\hline $\begin{array}{c}2-3 \text { vs } 4-6 \\
n=46\end{array}$ & $\begin{array}{l}\text { FS-II DQF } \\
\text { YKL-40 HA }\end{array}$ & $\begin{array}{l}.845 .876 \\
.770 .828\end{array}$ & $\begin{array}{l}.000 .000 \\
.002 .000\end{array}$ & .474.368.421. 737 & .263 .316 .368 .421 \\
\hline
\end{tabular}

\section{S1914}

Co-Stimulatory Expansion of Hepatitis C Virus-Specific T Cells: Exploring the Potential for Immunotherapy in Chronic HCV Infection

Jennifer Coleclough, Yun Li, Mark Bonyhadi, Bruce Levine, Barbara Vance, Robert H.

Vonderheide, Kyong-Mi Chang

Background: Hepatitis C virus (HCV) persists with impaired capacity for virus-specific T cells to expand and produce antiviral effector cytokines. Since IFN-alpha therapy does not generally increase HCV-specific T cell response (Kaplan et al, Hepatology 2005), approaches to enhance the number and function of HCV-specific T cells may complement the effectiveness of antiviral therapy. Aim: Based on the efficient T cell expansion demonstrated in-vitro with CD3/CD28 costimulation, we asked if functional HCV-specific T cells may be efficiently expanded in-vitro from peripheral blood mononuclear cells (PBMC) of chronically $\mathrm{HCV}$ infected patients with CD3/CD28 costimulation and T cell growth factors (IL2, IL15). Method: PBMC from chronically HCV-infected (HCV Ab+/RNA+) and spontaneously HCVrecovered subjects (HCV Ab+/RNA-) were stimulated as follows: 1) rIL2 and HCV-derived peptides; 2) rIL2 and HCV peptides with anti-CD3/anti-CD28-conjugated costimulatory beads; 3) rIL2, HCV peptides, anti-CD3/anti-CD28 beads and rIL15. HCV-specific T cell expansion, phenotype and cytokine profile were monitored by FACS with MHC-peptide tetramers specific for $3 \mathrm{HCV}$ and 1 influenza-derived HLA-A2 restricted CD8 epitopes, intracellular cytokine staining and CFSE. Result: Compared to rIL2 and peptide alone, overall expansion of cells in culture was enhanced by about 5 fold with CD3/CD28 costimulatory beads and 10 fold with additional rIL15 (median fold expansion in 2 weeks: 0.5 vs 2.5 vs 5.8). While highest HCV-specific CD8 T cell enrichment was observed with rIL2 and peptide alone, total number of expanded HCV-specific CD8 T cells tended to be greater with CD3/ CD28 costimulation, especially with rIL15, due to increased total T cell expansion in the latter 2 conditions. Yet, compared to patients with spontaneous HCV clearance, expanded $\mathrm{HCV}$-specific CD8 T cells demonstrated poor production of antiviral cytokines (IFNg, TNFa). Conclusion: We conclude that HCV-specific CD8 T cells can be expanded from chronic HCV patients using CD3/CD28 costimulation and that this expansion may be further enhanced with rIL15. However, despite their increased expansion, HCV-specific CD8 T cells did not show efficient antiviral cytokine production. Further studies are ongoing to explore strategies to enhance expansion and function of HCV-specific T cells and mechanisms of T cell dysfunction in HCV persistence. *This study is supported by RO-1 AI47519; XCyte Therapies Inc; Philadelphia VAMC; NIH/NIDDK Center of Molecular Studies in Digestive and Liver Diseases P30DK50306 and its Molecular Biology and Cell Culture Core Facilities. J.A.C is supported by the AGA Student Research Fellowship.

\section{S1915}

Metabolic Syndrome in Veterans with Chronic HCV Infection Correlates with NAFLD Activity Score

Prashant K. Pandya, Priyank Shah, Peggy Callahan, Dean Reker, Sharad C. Mathur

Background: Several retrospective studies have estimated that histopathologic features of NAFLD are present in $4-7 \%$ of patients chronically infected with HCV and are associated with parameters of the metabolic syndrome (MS). Given the increased prevalence of insulin resistance and type 2 diabetes, we systematically evaluated the the clinical and laboratory parameters of MS and correlated these with concomitant histologic findings of NAFLD. Methods: Demographic, anthropometric, serologic, and metabolic parameters were studied in a cross-sectional study of treatment naive veterans with chronic hepatitis $\mathrm{C}$. Insulin resistance was defined as a HOMA > 2.5. MS was defined by the presence of 3 or more ATP III criteria. Serum levels of TNF- $\alpha$, IL-6, adiponectin and leptin were measured. Liver biopsies were evaluated for degree and distribution(zone III) of steatosis, ballooning degeneration, mixed inflammation, and perisinusoidal fibrosis to calculate a NAFLD activity score(NAS) as recently proposed to assess the likelihood of NAFLD. Chronic hepatitis C inflammatory activity and fibrosis were determined according to the modified hepatic activity index (HAI). Histologic findings and NAS were correlated with clinical and laboratory markers of MS using Spearman correlation coefficient. Results: Liver biopsies from 35 veterans (69\% Caucasian and $17 \%$ African American; $97 \%$ male) were evaluated. The average age and BMI were $53.1 \pm 7.2$ and $28.0 \pm 6.0$ respectively. 18 of the 35 veterans(51\%) met criteria for MS. No histological features suggestive of NAFLD were present in 13 of 17(76\%) veterans without MS, compared to 6 out of $18(33 \%)$ with MS ( $p<0.05$, Chi-square). Findings highly suggestive of NAFLD (NAS $\geq 5$ ) were seen only in the group with MS(17\%). In univariate analysis, the presence of MS showed statistically significant association with steatosis ( $\mathrm{p}=0.002)$ and NAS ( $\mathrm{p}=0.016)$ but not with ballooning degeneration or perisinusoidal fibrosis ( $\mathrm{p}=\mathrm{NS}$ ). The degree of ballooning degeneration showed an increasing trend with increasing HAI score, but this was not statistically significant. In addition, the NAS was associated with TNF- $\alpha(\mathrm{p}=0.012)$ and $\operatorname{HOMA}(\mathrm{p}=0.005)$ but not with leptin, adiponectin, and IL-6( $\mathrm{p}=\mathrm{NS})$. Conclusion: In chronic hepatitis $C$ the presence of MS is significantly associated with steatosis and NAS. Findings highly suggestive of NAFLD were observed at in $17 \%$ of veterans, which is 2-3 times higher than reported previously. In the presence of chronic hepatitis $\mathrm{C}$, zone III perisinusoidal fibrosis and ballooning degeneration do not appear to be specific for NAFLD and may be modulated by the activity and stage of chronic hepatitis.

\section{S1916}

Chronic Hepatitis C May Be the Most Common Association for Hepatic Granulomas

Juan G. Martinez, Shu-Yuan Xiao, Ned Snyder

Background: Hepatic granulomas have been reported in up to $10 \%$ of liver biopsie, most of which today are performed for staging of chronic hepatitis C (HCV).Increased frequency of hepatic granulomas may be associated with both mild and severe forms of HCV. It has been postulated that HCV may have a role in granuloma formation. (Yamatamoto. HepatoGastroenterol 42: 291; Emile,Human Pathology 24:1095 ; Goldi. Histopathology 28: 265) Purpose:We reviewed the cases of hepatic granulomas at our institution the last decade to determine 1) the causes of hepatic granulomas 2) whether hepatic granulomas are associated with HCV. Methods: Following institutional review board approval, we used the data from our laboratory information system to identify all patients with the word "granuloma" or "granulomatous" in their final diagnosis or the pathologist's description. When appropriate, reports, patient's charts, and slides were reviewed, and information on special stains and cultures was obtained. Lipogranulomas were excluded from our study. The diagnosis of HCV was based the presence of serum HCV RNA. Results: 31 patients with hepatic epitheloid and/or giant cell granulomas were identified. Special stains for at least AFB and fungi were performed on 24, and tissue cultures on 5 . Twelve (38.7\%) of the patients had HCV. Among the patients with $\mathrm{HCV}$, three had other associations (HBV, sarcoidosis, or HIV). Overall, 9 patients had HCV only, 3 sarcoidosis, 3 histoplasmosis, 2 HIV only, 2 mycobacterium avium complex, 2 primary biliary cirrhosis, 1 Q fever, 1 mucormycosis, 1 tuberculosis, 1 coccidiomycosis, 1 Hodgkin's Disease, 1 cryptococcus, 1 chronic hepatitis B (HBV), 1 HCV/ $\mathrm{HBV}$, and 2 with no associations. Polarizing light revealed crystalloid foreign bodies in only one of the 12 patients with $\mathrm{HCV}$. Conclusions: HCV is the most common association with hepatic granulomas found on liver biopsy at our institution. This may partly reflect that a majority of our liver biopsies were done for chronic HCV. Most of our HCV patients had a history of intravenous drug use, and the granulomas could also result from foreign material. However, we were only able to identify a crystalline substance in one patient. While granulomas are uncommon in HCV and may sometimes be associated with other problems, the finding that $29 \%$ of our cases of hepatic granulomas had HCV as their only association poses the question whether granulomas are part of the histological spectrum of chronic HCV. Granulomas may be another manifestation of the immunological abnormalities seen in $\mathrm{HCV}$.

\section{S1917}

Helicobacter Pylori Infection Does Not Have a Significant Impact On the Natural History Or Response to Treatment of Chronic HCV Christopher J. Christensen, Gagan K. Sood, Diana Little, Maher Azzouz

Introduction: Helicobacter Pylori infection(HP) is seen with increased frequency in patients with chronic $\mathrm{HCV}$ infection. The impact of chronic HP on immune and cytokine response, and its relationship to several chronic diseases such as coronary atherosclerosis, lymphoma, cholelithiasis, chronic fatigue, cerebral ischemia and migraine are frequently recognized in recent medical literature. We studied the impact of HP on the natural history of HCV infection including degree of histopathological changes, response to treatment and the incidence of cholelithiasis. Methods: Patients treated for HCV through our hepatitis clinic(1999-2005) were retrospectively evaluated. 158 patients were included, 78 patients(53\%) had evidence of HP infection(group 1) and 70 patients(47\%) were negative(group 2). Data collected and compared on the two groups included race, sex, age, BMI, serum ferritin, genotype, AST, ALT, HCV RNA level, ETOH history, and Diabetes Mellitus(DM). Liver biopsies were compared regarding histological activity and fibrosis stage. Gallstones were documented by imaging and treatment response was compared between the two groups. Results: The two groups had no statistically significant differences with regard to ETOH history, prevalence of DM, HCV genotype or RNA level. Mean age for group 1 was lower $(49.81+/-5.86)$ vs $(51.86+/-6.54) \mathrm{p}<0.046$. HP prevalence was higher in the African American AA patients, 52/78 (70\%) of group 1 compared to $22 / 70(30 \%)$ of group 2 (chi square $18.32, \mathrm{p}<0.001$ ). Laboratory parameters were similar in the two groups including ALT ( 92.69 vs 97.23 ) AST ( 72.08 vs 70.64), and ferritin ( 289 vs 289 ). No significant histological differences were noted between the two groups with portal inflammation score (1.83 vs $1.74, \mathrm{p}=0.49)$, interface hepatitis score ( 1.80 vsl.76, $\mathrm{p}=0.78)$, lobular hepatitis score ( 1.46 vs $1.46, \mathrm{p}=0.96)$ and (fibrosis score 1.68 vs $1.8, \mathrm{p}=0.59$ ). Although gallstones were noted less frequently in HP infection $21 \%$ vs $29 \%$, this was not statistically significant(p= $0.23)$. Our sample data analysis revealed a correlation between AST and fibrosis $(r=0.40)$. Conclusion: Our sample did not demonstrate any significant impact of HP infection on the natural history of chronic HCV or response to treatment. HP infection was more prevalent in younger and African American patients. A larger size sample would be needed to make a conclusion regarding the need for HP testing and treatment prior to the initiation of antiviral therapy in patients with chronic $\mathrm{HCV}$ and its impact on the formation of gallstones.

\section{S1918}

Mortality Among Subjects Positive for Anti-Hepatitis C Virus Antibody in a Hyperendemic Area of Japan: A Prospective Study

Keiko Toyama, Hirofumi Uto, Chinatsu Hujimoto, Joji Kuroki, Katsuhiro Hayashi, Kenji Nagata, Yuka Takahama, Akio Ido, Sherri O. Stuver, Hirohito Tsubouchi

Background/Aim: Chronic hepatitis C virus (HCV) infection is a major health problem worldwide. HCV is a common cause of fatal liver diseases, including liver cirrhosis and hepatocellular carcinoma (HCC). However, the natural course of HCV infection is not fully understood. The aim of this study is to analyze mortality among subjects positive for anti-HCV antibody in a community-based study in a HCV hyperendemic area in Japan. METHODS: This study enrolled 816 anti-HCV antibody positive subjects who were followed from 1995 through 2004. Information on cause-of-death was obtained from death certificates. Serum $\mathrm{HCV}$ core antigen ( $\mathrm{HCV} \mathrm{CAg}$ ) was quantified in all subjects by fluorescence enzyme immunoassay (FEIA), and the presence of HCV RNA was determined by RT-PCR in subjects whose 
HCVcAg levels were below the FEIA detection threshold in 1995. Subjects positive for $\mathrm{HCV}$ CAg or HCV RNA were considered to have chronic HCV infection. Subjects negative for HCVCAg and HCV RNA were considered to have cleared their HCV infection. RESULTS: Of the 816 subjects studied, 557 were chronic HCV carriers in 1995 (median age, 65 years), and 260 appeared to have cleared their HCV infection (median age, 64 years). Although alanine aminotransferase (ALT) levels were significantly higher in those with chronic HCV infection than in those with prior HCV infection, there were no significant differences in age, gender, alcohol intake, prior history of surgical operations, or blood transfusions between the two groups. The cumulative incidence of mortality was $23.9 \%$ for the chronic HCV carriers and $16.5 \%$ for those with prior infection $(\mathrm{P}=0.044)$. In addition, the risk of death from HCC or other liver diseases in the chronic HCV subjects was $4.8 \%$ and $4.3 \%$, respectively. In contrast, no HCC deaths were observed in the subjects with prior $\mathrm{HCV}$ infection, and the risk of death from other liver diseases was $0.8 \%$. Furthermore, the risk of non-liver causes deaths, including cardiovascular diseases was not associated with the chronic HCV carriers. In the chronic-HCV group, there were no differences in age, gender, HCV serotype, alcohol intake, prior history of surgical operations, or blood transfusion between death from liver disease including HCC and from non-liver causes. Conclusions: The results of this prospective study show a strong effect of chronic HCV infection on liver-related mortality during a 10year follow-up. In addition, the risk of mortality was increased in subjects with chronic $\mathrm{HCV}$ infection compared to subjects with prior $\mathrm{HCV}$ infection.

\section{S1919}

Serum Alanine Aminotransferase Flares in Hepatitis C Virus Carriers with Normal Or Persistently Normal Alanine Aminotransferase Levels in a Hyperendemic Area of Japan

Hirofumi Uto, Kazunori Kusumoto, Katsuhiro Hayashi, Satoru Hasuike, Mayumi Kodama, Kenii Nagata, Keiko Toyama, Yuka Takahama, Akio Ido, Sherri O. Stuver, Hirohito Tsubouchi

Background: Since 1993, we have been analyzing hepatitis C virus (HCV) antibody-positive residents in a hyperendemic HCV area of Japan. In this community-based population, we previously reported that abnormal alanine aminotransferase (ALT) levels ( $>=35 \mathrm{IU} / \mathrm{L}$ ) increased the rate of HCC by almost four-fold in comparison to normal ALT levels. However, late ALT reactivation can occur after many years in some normal or persistently normal ALT (PNALT) individuals, which may lead to progressive liver disease. The clinical features of HCV carriers with normal ALT or PNALT levels ( $<=34 \mathrm{IU} / \mathrm{L})$ have not been fully elucidated. In the present analysis, we evaluated the occurrence of serum ALT flares in HCV carriers with normal ALT or PNALT levels within our study population. Subjects and Methods: We initially evaluated 544 seropositive study subjects who were HCV core antigen- or HCV RNA-positive six months or more after their initial anti-HCV testing and were judged as having persistent HCV infection in 1995. We examined the first occurrence of an ALT elevation, or flare (ALT >= $35 \mathrm{IU} / \mathrm{L}$ ), over the follow-up period of August 1996 through February 2005. An additional analysis focused on the subgroup of $159 \mathrm{HCV}$ carriers who had at least four annual ALT measurements available between 1993 and 2000 and who had PNALT during that time period. For these subjects, the risk of an ALT flare from July 2001 through February 2005 was examined. Results: In 1995, serum ALT levels were $<=19 \mathrm{IU} /$ $\mathrm{L}$ in $116(21 \%)$ of the $544 \mathrm{HCV}$ carrier subjects and 20-34 IU/L in $206(38 \%)$ subjects. Among these 322 subjects with a normal ALT level, an ALT flare occurred in 42 (36\%) subjects in the first group and $148(72 \%)$ subjects in the second group over a follow-up period of almost 10 years. Serum ferritin levels in 1995 were significantly higher in subjects with an ALT flare than in subjects without an ALT flare [138.6 \pm 98.2 vs. $97.0 \pm 63.8 \mu \mathrm{g} / \mathrm{dl}$; $\mathrm{P}<0.001]$. Among the $159 \mathrm{HCV}$-infected subjects who were found to have PNALT through 2000, the cumulative risk of an ALT flare was calculated to be $31.6 \%$ between 2001 and 2005. In addition, although there were no differences in age, gender, alcohol intake, blood transfusion or HCV core antigen levels between PNALT subjects who did or did not experience a subsequent ALT flare, a significant association was observed between serum ferritin levels measured before 2000 and ALT flares [114.7 \pm 56.7 vs. $86.4 \pm 55.9 \mu \mathrm{g} / \mathrm{dl} ; \mathrm{P}=0.019]$ Conclusions: HCV carriers with normal or persistently normal ALT in this population experienced ALT flares. Serum ferritin levels appeared to be a potential predictor of such ALT flares.

\section{S1920}

A Study to Assess Expertise and Training in Transcutaneous Liver Biopsy Amongst Trainee Gastroenterologists in the United Kingdom Peter F. Marden, Ben Colleypriest, John D. Linehan

Introduction: The U.K. Joint Committee of Higher Medical Training (JCHMT) in Gastroenterology Curriculum Feb 2005, states trainees should be skilled in liver biospy. Increasingly transcutaneous liver biopsy in the UK is conducted under ultrasound guidance by radiologists. This raises the possibility that gastroenterology trainees are neither exposed to the procedure or trained in performing liver biopsy despite the stated curriculum aims. This is due to diminishing training oportunities and a move away from biopsying without ultrasound guidance. Aim :This study aims to determine the level of training and competence in transcutaneous liver biopsies amongst trainees in gastroenterology in a U.K. training region (Wessex deanery).This comprises of 13 hospitals covering a population of approximately 4 million people. Methods: All gastroenterology trainees in the region received a questionnaire aimed at assessing their training in liver biopsy and knowledge of the current British Society of Gastroenterology guidelines on the use of liver biopsy in clinical practice. Results: Thirty one trainees were eligible for the study and $77 \%$ responded. None currently worked in a trust where liver biopsies were routinely conducted by a gastroenterologist. Only 54\% had practical experience of liver biopsy under supervision and 29\% without supervision. Just $25 \%$ had a written record of this training. None had conducted a liver biopsy in the last twelve months. Only $4 \%$ knew the pre-procedure platelet count quoted as safe in the BSG guidelines, although $94 \%$ knew the safe level of INR. With regards to pain post procedure and significant haemorrhage only $4 \%$ and $16 \%$ respectively knew guideline figures. Only $25 \%$ could quote accurate mortality figures post liver biopsy. No respondent had received training in transabdominal ultrasound although $45 \%$ felt gastroenterologists should be trained in conducting liver biopsies. Conclusion: This study suggests U.K trainees in gastroenterology have a low level of practical and theoretical knowledge with regards to transcutaneous liver biopsy. Only a more comprehensive survey can determine if this is a national phenomenon. If stated JCHMT curriculum aims are to be addressed, training in transabdominal ultrasound scanning may need to be compulsory for gastroenterology trainees. At present this is not the case, in contrast to equivalent gastroenterology training programmes throughout the rest of Europe or in the USA.

\section{S1921}

Prevalence of HCV Risk Factors in Hispanic-American Subpopulations Stacey B. Trooskin, Maricruz Velez, Steven K. Herrine, Simona Rossi, David J. Axelrod, Robert Winn, Scott McNeal, Victor J. Navarro

HCV disproportionately affects Mexican Americans. The epidemiology of HCV has not been evaluated as thoroughly in other Hispanic-American subpopulations. Therefore, as part of a larger prospective cohort study to investigate barriers to care for HCV among minority patients, we aimed to assess differences in the prevalence of HCV risk factors that may exis among Hispanic-American subgroups. Methods: New patients, 18 or older, were prospectively enrolled at four primary care practices in Philadelphia. These practices included two federally qualified health centers serving predominantly minority populations and two university-based primary care practices, one family medicine practice and one internal medicine practice. Demographic information, including race/ethnicity, was ascertained from all participants as well as risk factors for HCV acquisition, both conventional (injection drug use (IDU), transfusion prior to 1992, health care worker needle stick) and non-conventiona (tattoos, piercings, incarceration, had a spouse or significant other with HCV). Results: O the 1415 patients enrolled in this study, 468 participants identified themselves as being Hispanic; 235 were born in Puerto Rico (PR) or mainland US. The remaining 233 participants were born in 19 other countries. A small percentage of individuals born in each of the following countries reported having a risk factor for HCV acquisition: $14.8 \%$ of those born in the Dominican Republic ( $\mathrm{n}=81), 10.2 \%$ of those born in Mexico $(n=49), 10.5 \%$ of those born in Colombia ( $n=19), 15.8 \%$ of those born in Guatemala $(n=19), 8.3 \%$ of those born in Honduras $(n=12)$, and $12.5 \%$ of those born in Ecuador $(n=8)$, and $14.3 \%$ of those born in Peru $(n=7)$. Comparatively, $45 \%$ of individuals born in PR and $50 \%$ of Hispanics born in mainland US reported having an HCV risk factor; $39 \%$ of Whites ( $n=512)$ and $48 \%$ of Blacks ( $\mathrm{n}=435$ ) reported having a risk factor for HCV acquisition. Both groups had similar prevalence of non-conventional risk factors. However, prevalence differed between the two groups with respect to prevalence of conventional risk factors, specifically IDU. IDU wa more commonly reported among Hispanics born in the US or PR. In a logistic regression model, Hispanics born in the US were more likely to have a risk factor (conventional or non-conventional) than Hispanics born outside the US ( $p<.0001$ ). This relationship remained after controlling for age and sex. In conclusion, Hispanics born in the US or PR have a higher prevalence of conventional HCV risk factors, specifically IDU, as compared to Hispanics born in other countries.

\section{S1922}

Bone Mineral Density Correlates to Insulin-Like Growth Factor I, in NonCirrhotic Osteopenic Hepatitis C Patients with Low Dairy Calcium Intakes $(\mathrm{n}=23)$

Marcel H. Van Gemert, Cmj van Nieuwkerk, Jc Roos, E Bloemena, Ac van Doorn, Cjj Mulder

Dystrophic bone metabolism is frequently associated with chronic hepatitis C (HCV). Its etiology cannot be attributed solely to one mediating determinant. We postulated that chronic hepatitis C patients exhibiting associative osteopenia will have serum markers reflective of their bone pathology. Our goal was to correlate these markers to bone mineral density (BMD) and determine the prevalence and extent of non-cirrhotic related osteopathy. Concurrently we assessed dietary calcium and vitamin D intake status. Our study investigated 23 consecutive patients undergoing treatment for chronic active $\mathrm{HCV}$ infection. BMD was determined by means of a Dual Energy X-ray Absortiometry scan; hepatic histopathology by biopsy. A dietary questionnaire was completed by each patient to assess daily dairy calcium and vitamin D intake. Biochemical values of the following were taken at the time of BMD measurements: alkaline and acidic phosphatase, 25OHD, osteocalcin, PTH, TSH, IGF-I, and serum calcium. $73.9 \%$ of patients had a significant reduction in BMD defined as a T-score $<-1$. The majority of patients were both non-cirrhotic and osteopenic. Of this osteodystrophic group, 4 patients had T-scores <-2.5, indicating osteoporosis; one was cirrhotic. Mean PTH values of osteodystrophic patients were significantly lower as compared to normal-BMD patients $(3.94 \mathrm{pmol} /$ $\mathrm{l}, \mathrm{p}=0.027,95 \% \mathrm{CI}: 3.12-4.98$ vs. $6.83 \mathrm{pmol} / \mathrm{h}, \mathrm{CI}: 3.73-12.49)$. IGF-I correlated significantly with $\mathrm{BMD}$ at the lumbar spine $(\mathrm{r}=.434, \mathrm{p}=0.044)$. Osteocalcin was shown to have a positive correlation with both alkaline phosphatase $(\mathrm{r}=548, \mathrm{p}=0.007)$ and PTH $;(\mathrm{r}=645, \mathrm{p}=0.001)$ The average daily calcium intake, based on dairy products was found to be significantly low overall when compared to the mean recommended daily allowance (RDA) of $1050 \mathrm{mg}$ $\mathrm{Ca} /$ day for standard population groups 19 through 70 years of age (patient ages; range 2666 years, group mean $635.2 \mathrm{mg} / \mathrm{Ca}, \mathrm{p}<0.001$ ). The proportion of patients failing to achieve the minimum calcium- RDA for their individual age group was $86.9 \%$. The osteodystrophic$\mathrm{HCV}$ group had lower calcium intakes than normal BMD-HCV patients; 600 vs $734 \mathrm{mg} / \mathrm{Ca}$ day respectively. Further investigations into chronic HCV as it pertains to osteopenia and or osteoporosis need to establish calcium intake status, precluding causal link judgments. Thus far this simple determinant of bone health in the multifactorial spectrum of viral hepatic osteodystrophy has been overlooked. As well as body mass indices, the specific nutritional calcium intakes warrant more consideration. Dystrophic bone metabolism is not exclusive to cirrhotic-HCV patients. Reduced BMD can be found with earlier hepatic impairment. 
S1923

The Influence of Cigarette Smoking On Response to Treatment with Pegylated Interferon Alfa-2b and Ribavirin in Patients with Chronic Hepatitis $C$ Mary P. Pauly, Alan J. Sheinbaum, Jean-Luc I Szpakowski, Joanna B. Ready, Robert S. Brown, Bradley Freilich, Nezam Afdhal, Paul Kwo, John Santoro, Scott Becker, Ira Jacobson, Louis Griffel, Clifford Brass

Background: Cigarette smoking has been associated with increased inflammation and fibrosis in patients with HCV. There is little data on the effect of cigarette smoking on response to antiviral therapy patients with HCV. Aim: To study the characteristics and response to therapy of HCV infected patients who smoked cigarettes and were treated with pegylatedinterferon (PEG IFN) alfa-2b and ribavirin (RBV), compared with those who did not smoke cigarettes. Methods: The WIN-R study randomized 4913 patients to either PEG IFN alfa$2 \mathrm{~b}$ and RBV $800 \mathrm{mg}$ (FD) or RBV 800-1400 mg (WBD) (Jacobson, AASLD 2005). Patients with genotype 1 and 4 were treated for 48 weeks. Patients with genotype 2 or 3 were further randomized to 24 vs 48 weeks of therapy. Sustained viral response (SVR) was the primary endpoint with primary efficacy analysis including only those patients who were over $65 \mathrm{~kg}$. Results showed that weight based dosing resulted in significantly greater SVR, especially in those with genotype 1 , and that 24 weeks of therapy for genotype 2 and 3 was as effective as 48 weeks. During enrollment, it was noted whether patients were smokers or non-smokers. Smoking data was available on 2859 of $4223(67.7 \%)$ patients included in the primary efficacy analysis. We evaluated the influence of smoking on SVR. Results: Of patients with available data, there were 893 ( $31.3 \%$ ) smokers and 1966 (68.7\%) non smokers. SVR for smokers and non smokers in each group is noted in table 1. Conclusions: Cigarette smokers with genotype 2 and 3 had lower SVR than non smokers. This difference was not seen in the patients with $\mathrm{HCV}$ genotype 1

SVR for Smokers and Non-Smokers by Group

\begin{tabular}{|c|c|c|c|c|c|}
\hline & smokers & & non-smokers & & \\
\hline & $\begin{array}{l}\text { SVR number/total } \\
\text { smokers }\end{array}$ & $\begin{array}{c}\text { SVR } \\
\text { percent }\end{array}$ & $\begin{array}{l}\text { SVR number / total } \\
\text { nonsmoker }\end{array}$ & $\begin{array}{c}\text { SVR } \\
\text { percent }\end{array}$ & $\begin{array}{c}\mathrm{p} \\
\text { value }\end{array}$ \\
\hline G1 FD & $77 / 274$ & 28.10 & $181 / 626$ & 28.87 & 0.8157 \\
\hline $\begin{array}{c}\text { G1 } \\
\text { WBD }\end{array}$ & $81 / 260$ & 31.15 & $218 / 647$ & 33.69 & 0.4619 \\
\hline G23 FD & $88 / 172$ & 51.16 & $218 / 349$ & 62.46 & 0.014 \\
\hline $\begin{array}{l}\text { G23 } \\
\text { WBD }\end{array}$ & $106 / 187$ & 56.68 & $227 / 343$ & 66.18 & 0.031 \\
\hline
\end{tabular}

S1924

Knowledge Deficits Concerning Hepatitis C Among Racial and Ethnic Minorities: A Prospective Study

Stacey B. Trooskin, Maricruz Velez, Steven K. Herrine, Simona Rossi, David J. Axelrod, Robert Winn, Scott McNeal, Victor J. Navarro

Background: HCV disproportionately affects racial and ethnic minorities. However, these groups have less knowledge of HCV-related issues than their White counterparts (Buffington, 2000). We therefore aimed to further characterize differences in knowledge of HCV that may exist among minority patients in primary care settings. Methods: New patients, 18 or older, were prospectively enrolled, at four primary care practices (PCPs) in Philadelphia. The PCPs included two federally qualified health centers, primarily serving minorities and two university-based PCPs, one family medicine and one internal medicine. Demographics including race/ethnicity were ascertained and an 11 question survey was administered, in English or Spanish, querying general knowledge of HCV "True" "False" and "I don't know" were used as responses to all statements. Log-linear regression was used assuming a gamma distribution, to assess the relationship between race and knowledge, measured by percentage of correct answers. Results: Of the 1657 patients enrolled in this study, there were 507 Hispanics, 691 Whites, and 459 African Americans (AA). Whites were most likely to choose the correct answers. Race overall and all comparisons by race remained significant after adjusting for age and education $(\mathrm{p}<0.0001)$. Among Hispanic subpopulations, those born in the U.S. (including Puerto Rico), had a similar percentage of correct answers compared to AA. Non-US born Hispanics knew significantly less than their US born counterparts ( $\mathrm{p}=$ .0005); this relationship was significant after adjusting for education and age. Non-US born Hispanics living in the United States for five years or more were less likely to have misconceptions regarding HCV than individuals who have been living in the US for less than five years. Discussion: AA and Hispanics know significantly less about HCV than Whites; even greater knowledge deficits exist among Hispanics born outside the US and Puerto Rico. Culture, language and literacy level appropriate HCV educational interventions are needed, particularly for those who have recently immigrated to the US.

\section{S1925}

Lack of Adherence As a Critical Factor for Non-Sustained Virological Response to Combination Therapy of Interferon and Ribavirin in Chronic Hepatitis C Patients of Genotype 2 Infection

Yoshiaki Iwasaki, Daisuke Tanioka, Hiroshi Ikeda, Kouichi Takaguchi, Haruhiko Kobashi, Yasuyuki Araki, Minoru Tomita, Noriaki Hashimoto, Kazuhisa Yabushita, Masaharu Ando, Tomonori Senoh, Yasuhiro Makino, Kohsaku Sakaguchi, Yasushi Shiratori

Background and Aims: Hepatitis $\mathrm{C}$ virus genotype is the most important variable influencing outcomes of interferon treatment for patients with chronic hepatitis $C$, and patients infected with genotype 2 or 3 have the best response rate. However, some of the patients of genotype 2 infection can not achieve a sustained response to combination therapy of interferon and ribavirin. Factors associated with non-sustained virological response to combination therapy in these patients were analyzed. Methods: We consecutively enrolled 121 chronic hepatitis $C$ patients of genotype 2 infection; 69 men and 52 women, age (mean \pm SD): $55 \pm 11$ years old. Interferon alpha-2b therapy was conducted daily for two weeks, followed by three times per week for 22 weeks, and ribavirin (600 or $800 \mathrm{mg}$ ) was administered daily. Patients were assessed for efficacy, safety, and tolerance by monitoring hepatitis $C$ virus status and adverse events including laboratory abnormalities every 2-4 weeks. Sustained virological response and dose modification and premature discontinuation of the combination therapy due to adverse events or laboratory abnormality were the endpoints. Results: Of the 121 study patients, dose reduction and discontinuation of therapy was required in 51 (42\%) and $14(12 \%)$ patients, respectively Seventy-four (61\%) patients achieved 80\%-adherence, while 47 (39\%) could not. Univariate logistic regression analysis revealed gender (male) ( $95 \%$ CI of Odds ratio: 1.25-5.62), neutrophil count ( $\geq 2500 / \mu \mathrm{L})(1.10-4.95)$, creatinine clearance ( $\geq 100 \mathrm{mg} /$ day) (1.09-5.10), body weight ( $\geq 60 \mathrm{~kg}$ ) (1.05-4.72), and hemoglobin level $(\geq 14 \mathrm{~g} / \mathrm{dL})(1.05-4.72)$ at baseline to be associated with adherence to therapy. A sustained virological response was achieved in $93(77 \%)$ patients. Univariate analysis revealed that adherence to therapy was the only factor significantly associated with the achievement (Odds ratio $(95 \% \mathrm{CI}): 3.97(1.63-9.71), \mathrm{P}=0.002)$. A sustained virological response was achieved in $86 \%(64 / 74)$ and $62 \%(29 / 47)$ of patients with and without $80 \%$-adherence, respectively $(\mathrm{P}=0.003)$. Univariate analysis revealed that adherence to therapy was still associated with the achievement, even when analyzed in the adherent patients. Conclusions: These results demonstrate an importance of adherence to combination therapy even in chronic hepatitis C patients of genotype 2 infection. Thus, it is suggested that a further improvement in response to combination therapy could be expected in these patients by improving adherence to combination therapy.

\section{S1926}

Successful Treatment with High Dose Consensus Interferon and Ribavirin of Patients with Chronic Hepatitis C Who Are Resistant to PEG-Interferon and Ribavirin Therapy

Kenneth D. Rothstein, Ramesh Koka, Angel Fernandez, Holly Hargrove, Shailender Singh, Victor Araya, Santiago J. Munoz

Background: The majority of nonresponder and relapser patients with chronic hepatitis C are unable to achieve a sustained virologic response (SVR) with the combination of PEGInterferon (PEG-IFN) and ribavirin (RBV), especially those who have genotype 1 and advanced disease. Consensus interferon (Interferon alfacon-1, CIFN) is a bio-optimized alfa interferon that exhibits increased in-vitro antiviral activity than the naturally occurring alfa interferons $2 \mathrm{a}$ and $2 \mathrm{~b}$. Improved response rates have been reported with high-dose CIFN therapy and RBV for patients who have failed to respond to PEG-IFN / RBV. Aim: Evaluate efficacy and safety of high-dose daily CIFN and RBV in HCV patients who failed therapy with PEG-IFN / RBV. Methods: Patients who had been treated with PEG-IFN/ RBV for HCV but did not obtain a SVR were eligible for treatment if they: 1) tolerated treatment with PEG-IFN / RBV, and 2) had advanced liver disease. Patients were given 27 ug of CIFN daily and RBV $400 \mathrm{mg}$ BID during the first four weeks, followed by 18 ug daily and ribavirin $400 \mathrm{mg}$ BID daily for the next eight weeks. At 12 weeks, CIFN was decreased to $15 \mathrm{ug}$ daily while RBV was increased to 1,000-1,200 mg daily for 36 weeks. Results: Thirty eight patients have been enrolled in the study to date, $74 \%$ male with a mean age of 51 years. $97 \%$ had genotype $1.79 \%$ of patients had stage 3 fibrosis or greater, of which $55 \%$ of patients had cirrhosis. $82 \%$ of patients were nonresponders. 22 patients $(62 \%)$ have achieved an early virologic response (EVR) at 12 weeks. 16 patients (56\%) were undetectable at 24 weeks and 8 patients (42\%) achieved an End-of-Treatment response(EOT). Of the 17 patients who have completed 72 weeks of treatment, 9 patients discontinued therapy, 8 patients achieved EOT response, 3 of these patients (18\%) have achieved a Sustained Virological Response (SVR). $50 \%$ of patients required growth factors, $29 \%$ required Epopoetin alfa, Filgastrim $5 \%$ and both $16 \%$. Conclusion: This regimen was well tolerated with dose reduction and discontinuation rates similar to other nonresponder trials using PEG-IFN and RBV. For HCV patients with advanced fibrosis who have previously failed therapy with PEGIFN and RBV, high dose CIFN and RBV combination therapy is an effective option.

\begin{tabular}{|c|c|c|c|c|}
\hline $\begin{array}{c}\text { HCV RNA VIRAL } \\
\text { LOAD }\end{array}$ & $\begin{array}{c}\text { WEEK 12 } \\
(\mathrm{N}=38)\end{array}$ & $\begin{array}{c}\text { WEEK 24 } \\
(\mathrm{N}=34)\end{array}$ & $\begin{array}{c}\text { WEEK 48 } \\
(\mathrm{N}=33)\end{array}$ & $\begin{array}{c}\text { SVR } \\
(\mathrm{N}=17)\end{array}$ \\
\hline$>2$ LOG DECREASE & $62 \%$ & $44 \%$ & ---- & --- \\
\hline UNDETECTABLE & $39 \%$ & $56 \%$ & $42 \%$ & $18 \%$ \\
\hline
\end{tabular}

S1927

High Dose Induction Therapy with Consensus Interferon (CIFN) in Patients with Chronic Hepatitis C Is Safe and Highly Efficient: An Open-Label Pilot Study

Thomas Witthoeft, Diether Ludwig

Abstract: Treatment options for patients with chronic hepatitis $C$ are limited. Before introduction of peoylated interferons in combination with ribavirin in 2001 common interferon- $\alpha$ three times weekly and ribavirin on a daily dosing was recommended. Sustained virological response rates were low and patients often stopped therapy due to side effects. Method: We started, before PEG-Interferons were available, an open-label study to investigate the efficacy and tolerability of high dose induction therapy with CIFN and ribavirin in treatment-naiive patients with chronic hepatitis C. 58 patients got enrolled receiving 18ug of CIFN daily for 8 weeks, followed by 9 ug daily for up to week 24 or 48 and $800 \mathrm{mg}$ of ribavirin daily. End point of the study was tolerability and eradication of the virus at week 48 and sustained virological response at week 72 . The study was approved by the local ethic committee and informed consent of patient was obtained prior to treatment. Results: More than $52 \%$ of patients did respond at week 48 showing a negative qualitative PCR (GT 1 fourteen patients (56\%), GT 2 two (50\%), GT 3 thirteen (87\%), GT 4 one (50\%)). Only five of the patients presenting negative PCR at EOT showed a relapse at week $72.48 \%$ of genotype 1 patients showed SVR six months after end of treatment. 13 patients did not respond to therapy. Six patients showed a relapse of i.v. drug use and one of them died due to an overdose. Four patients stopped treatment due to side effects (low WBC, epistaxis, depression). 4 patients 
did not show up for follow up visits during therapy. Conclusion: High dosing of CIFN on a daily basis was well tolerated and side effects like leuko- and thrombocytopenia were moderate. EOT rates were slightly lower compared to newer standard therapy with pegylated interferons. From our study CIFN on a daily basis might be a favourable therapy regimen for patients with subtype 1 and high viral load. Head to head studies to compare consensusinterferon and pegylated interferons are therefore warranted.

\section{S1928}

Real-Time, Acetylcholine-Induced Early Phasic Oscillatory Force Generation Analysis of 3D Bioengineered Colon Circular Smooth Muscle Constructs Using Dominant Negative RHOA Or Dominant Negative PKC $\alpha$

Saumya S. Vanderwyst, Suresh B. Patil, Robert R. Gilmont, Sita Somara, Khalil N. Bitar

Background: We have previously shown that bioengineered GI smooth muscle rings mimic the phasic physiological response of in vivo muscle tissue. Objective: To further understand the real-time, very early sequence of events in acetylcholine-induced force generation mediated by RhoA and PKC $\alpha$. Methods: Rabbit colon circular smooth muscle cells were harvested and cultured in vitro. A. Molecular biology: Cells were transfected with either dominant negative PKC $\alpha$ or dominant negative RhoA. B. Bioengineering: 3D rings were bioengineered from stably transfected dominant negative (-ve) constructs using a fibrin gel surrounding a circular mold. We have studied the response to acetylcholine $(0.1 \mathrm{uM})$ in these rings using an optical force transducer capable of micro-Newton sensitivity and a 0.1 second sampling rate. Results: 1) We were able to successfully create bioengineered rings from stably transfected cells. Also, we maintained the functional rings for up to 1 month post-ring formation. 2) Both bioengineered ring types responded to: a) 50\% stretch and b) $10 \mathrm{mM} \mathrm{KCl}$ addition. 3) The frequency of baseline oscillations were similar for both ring types; however, average baseline amplitudes were slightly lower for dominant negative RhoA (-ve PKC $\alpha=4.365 \pm$ $0.179 \mu \mathrm{N}$ vs. -ve RhoA $=3.929 \pm 0.002 \mu \mathrm{N}, \mathrm{p}<0.05)$. 4) Both bioengineered ring types responded equally to acetylcholine with maximum force generation. However, there was a significant difference in the early response pattern in reaching sustained force. The bioengineered rings expressing -ve PKC $\alpha$ (active RhoA pathway) showed a period of oscillation with $14 \pm 1$ peaks occurring within the first 30 seconds before reaching sustained force generation. The bioengineered rings expressing -ve RhoA (active PKC pathway) exhibited a sustained force generation in response to acetylcholine without any pre-oscillatory pattern. These data suggest a crucial role for RhoA in modulating very early (within first 10s) phasic oscillatory force generation response, in the absence of PKC $\alpha$. Summary: We have been able, for the first time, to successfully develop bioengineered rings from colonic smooth muscle cells transfected with either dominant negative PKC $\alpha$ or dominant negative RhoA. These rings show that RhoA modulates early phasic oscillatory force generation while PKC, in the absence of RhoA, is incapable of generating early oscillatory response. Conclusions: Using bioengineering and molecular biology tools, we have developed a technique to discern the molecular contribution of PKC and RhoA pathways, and the early sequence of events modulating phasic oscillatory force generation in response to acetylcholine in colonic smooth muscle

\section{S1929}

Colonic Electrical Stimulation with Trains of Short Pulses Not Long Pulses Enhances Colonic Transit in Awaken Rats

Shi Liu, Wenpo Li, Xiaohua Hou, Jiande Chen

Background: Our recent study has shown an acceleration of colonic transit with colonic electrical stimulation (CES) with trains of short pulses, this accelerative effect was prevented by nitric oxide inhibitor L-NNA. However, little is known about potential roles of CES with long pulses on colonic transit. Aims: To evaluate the effect of CES with long pulses on colonic transit in conscious rats and investigate whether cholinergic pathway was involved in accelerative effect of CES with trains of short pulses. Methods: Male rats implanted with one pair of stimulation electrodes on the proximal colon serosa were used for the study. A small catheter was inserted into the proximal colon and fixed with sutures. Three days after surgery, saline or muscarinic receptor antagonist atropine $(5 \mathrm{mg} / \mathrm{kg})$ were administered subcutaneously. $1.5 \mathrm{ml}$ solution of phenol red $(0.5 \mathrm{mg} / \mathrm{ml})$ was injected via the catheter as a marker of colonic transit. Colonic transit with or without CES was determined by calculating the output of phenol red from the anus every 10-min for 90-min. CES was performed during the first $40 \mathrm{~min}$. In saline group, the stimulus was composed of long pulses with a frequency of $20 \mathrm{cpm}$, a width of $200 \mathrm{~ms}$ and amplitude of $10 \mathrm{~mA}$. The parameters of stimulation in atropine group was trains of short pulses with an on-time of $2 \mathrm{~s}$ and off-time of $3 \mathrm{~s}$, a pulse frequency of $40 \mathrm{~Hz}$, a width of $4 \mathrm{~ms}$ and amplitude of $10 \mathrm{~mA}$. Results: 1) CES with long pulses had no effect on colonic transit at any times. There were no significant changes in the percentage of recovered phenol red between the group of CES with long pulses compared with the group without CES (at 90 -min: $46.4 \pm 5.9 \%$ vs. $49.5 \pm 5.3 \%$, $\mathrm{P}>0.05$ ). 2) Atropine delayed colonic transit compared with saline. The percentage of recovered phenol red at $90 \mathrm{~min}$ was decreased from $49.5 \pm 5.3 \%$ in the control session to $14.2 \pm 2.5 \%$ with atropine $(\mathrm{P}<0.001) .3)$ Atropine did not prevented accelerative effect of colonic transit caused by CES with trains of short pulses. At the presence of atropine, CES with trains of short pulses still accelerated colonic transit: the percentage of recovered phenol red was $14.2 \pm 2.5 \%$ in the control session (atropine alone) and $36.9 \pm 5.6 \%$ with CES session (atropine plus CES) $(\mathrm{P}<0.01)$. Conclusions: Colonic electrical stimulation with trains of short pulses not long pulses enhances colonic transit and this accelerative effect may not be mediated via cholinergic pathway.

\section{S1930}

Colonic and Gastric Muscle Contraction in the Obese (OB/OB) Mouse Ping Cong, Gyorgy Baffy, Piero Biancani, Jose Behar

The prevalence of gastrointestinal symptoms is higher in obese patients than in the genera population. However, the mechanisms responsible for this higher prevalence are not known Obese patients tend to have higher levels of serum cholesterol (Ch). We have previously shown that gallbladders with lithogenic bile with high $\mathrm{Ch}$ concentrations impair the muscle contraction and relaxation. Those muscle cells incorporate higher $\mathrm{Ch}$ levels in the caveolae and impair muscle contraction because of caveolar sequestration of receptors that results in decreased recycling back to the bulk plasma membrane (plasma membrane-caveolae) and lower ligand binding by membrane receptors. The aim of these studies therefore was to examine whether colonic and gastric muscle cell functions is affected by an abnormal incorporation of $\mathrm{Ch}$ in $\mathrm{OB} / \mathrm{OB}$ mice. We studied seven $\mathrm{OB} / \mathrm{OB}$ mice and six lean mice. The levels of serum $\mathrm{Ch}$ of $\mathrm{OB} / \mathrm{OB}$ mice was $10.9 \pm 2.0 \mathrm{mmol} / \mathrm{lt}$ significantly higher than the levels of lean mice of $3.1 \pm 0.6 \mathrm{mmol} / \mathrm{tt}(\mathrm{p}<0.00 \mathrm{l})$. The contraction of dissociated gastric and colonic muscle cells from $\mathrm{OB} / \mathrm{OB}$ mice in response to increasing concentrations of CCK-8 (10-11 to 10-8 M) were lower than those from lean mice (Anova, $\mathrm{p}<0.01$ ). In addition, caveola $\mathrm{Ch}$ levels in gastric and colonic muscle cells were higher in the $\mathrm{OB} / \mathrm{OB}$ mice than in lean mice $(p<0.001)$. Western blot studies using specific antibodies revealed higher concentrations of caveolin 3 proteins in the caveolae of muscle cells from $\mathrm{OB} / \mathrm{OB}$ mice than from lean mice. We also determined the distribution of CCK-1 receptors in the caveolae and bulk plasma membrane using specific antibodies. The optical density of the CCK-1 receptor band was significantly increased in the caveolae and decreased in the bulk plasma membrane in the OB/OB mice compare to those detected in the caveolae and bulk plasma membrane of muscle cells from lean mice $(\mathrm{p}<0.01)$. The results in this obese mouse model suggest that similar mechanisms may be responsible for the high prevalence of gastrointestinal symptoms in the obese population. That the gastrointestinal muscle cell response to agonists that ac on receptors that internalize through caveolae may be defective due to higher incorporation of caveolar $\mathrm{Ch}$ from high serum concentrations increasing the concentrations of caveolin 3 proteins and of receptors in the caveolae resulting in reduced recycling back to the plasma membrane

\section{S1931}

Biofeedback Is an Effective Treatment for Functional Constipation: A Randomized Controlled Study

Wayne H. Hu, Joanne H. Li, Annie O. Chan, Nina Y. Wong, Stephen H. Wong, Wai M. Hui

Introduction: Constipation affects up to $20 \%$ of the general population. Biofeedback is a newer way to treat constipation but most studies are uncontrolled. We performed a controlled trial to evaluate biofeedback in patients with functional constipation. Methods: Patients with chronic constipation according to the Rome 2 criteria with symptoms lasting more than one year were randomized. Full colonoscopy was performed to rule out organic pathology Patients were asked to stop all laxative medication for two weeks and record daily bowel habits on a diary card, as well as complete a constipation symptom severity questionnaire. Use of rescue laxatives was recorded. Biofeedback consisted of teaching of proper defecation posture, abdominal muscle exercise, pelvic floor retraining with pressure and biofeedback and also training of balloon expulsion from the rectum. Each session lasted 30-60 minutes and was repeated every two weeks for six sessions. Control group consisted of lifestyle dietary and general advice regarding defecation only. Bowel symptoms, defecation frequency were assessed at baseline and after twelve weeks. Severity of bowel symptoms were assessed in a 5 point Likert scale. Changes in symptom severity from baseline were compared using Mann-Whitney U test. Results: Sixty patients were recruited (mean age 44, 95\% female) and randomized equally to treatment and control groups. After completion of treatment the number of complete spontaneous bowel movements increased similarly in both treatment and control groups ( 1.3 to 2.6 and 1.2 to 2.3 ). In the biofeedback group, there was decrease in bloating (from 2.6 to 0.8 ), incomplete evacuation (from 2.4 to 1.0), straining (from 2.8 to 1.3 ) and laxative use (1.9 to 0.4$)$ after treatment. The control group did not show improvement in symptoms and laxative use $(2.2$ to $2.4,2.1$ to $2.4,2.3$ to 2.3 and 1.9 to 1.4 respectively). Change from baseline were significant between treatment and control groups for the symptoms assessed. Conclusions: Biofeedback treatment is effective in improving the dynamics of defecation and decreasing symptoms in patients with idiopathic constipation when compared to controls. Acknowledgements: This study is supported by the Simon To fund for nutrition and functional gastrointestinal disorders.

\section{S1932}

Responses of Rat Proximal, MID and Distal Colonic Muscularis Mucosae to ATP and Bradykinin Are Mediated By Different Mechanisms William Percy

Bradykinin and ATP each contract rat colonic muscularis mucosae (MM) in vitro, with an oral to aboral decrease in efficacy. The aim of this study was to determine the pharmacologic basis of the effects of these agents on this muscle layer in three separate colonic regions. METHODS: Strips of MM $3 \mathrm{~cm} \mathrm{x} 4 \mathrm{~mm}$ from the proximal, mid and distal colon were placed in $2 \mathrm{ml}$ tissue baths under a $0.5 \mathrm{~g}(4.9 \mathrm{mN})$ load for isometric recording. Responses were expressed as a percentage of each tissue's maximum contraction to acetylcholine. RESULTS: Bradykinin (4x10-13-4x10-6M) elicited MM contractions that reached $461.7 \pm 110.0 \%$, $136.3 \pm 8.0 \%$ and $108.6 \pm 12.1 \%$ ( $\geq 5$ each) in the proximal, mid and distal regions. Proximal responses to bradykinin were reduced to $228.7 \pm 23.1 \%$ and $270.4 \pm 55.8 \%$ following pretreatment with either indomethacin $(10-6 \mathrm{M})$ or TTX $(10-6 \mathrm{M})$ respectively (both $\mathrm{p}<0.05 ; \mathrm{n} \geq 5$ ) Mid and distal responses to bradykinin were unaffected under the same conditions. The selective B1 agonist Des-Arg9-bradykinin (5.2x10-12-5.2x10-6M) elicited proximal MM responses of $\leq 45 \%$ of the bradykinin maximum; in the mid and distal region the corresponding responses were $\leq 20 \%$ of the bradykinin maximum. Proximal, mid and distal MM responses to ATP (10-9-10-3M) reached $171.7 \pm 23.9 \%, 95.1 \pm 17.6 \%$ and $62.7 \pm 5.3 \%(\mathrm{n} \geq 7$ 
each). Proximal ATP responses were unaffected by indomethacin whereas, mid and distal responses were reduced to $18.5 \pm 8.7 \%$ and $21.5 \pm 6.8 \%(\mathrm{p}<0.01 ; \mathrm{n} \geq 7)$ respectively. Only distal MM responses to ATP were attenuated by TTX, falling to $43.9 \pm 2.3 \%(\mathrm{p}<0.001 ; \mathrm{n}=$ 8). When compared to other purines on an equimolar basis, the order of efficacy in each region was, $\mathrm{ATP} \approx \mathrm{ADP}>\mathrm{AMP}=$ adenosine. CONCLUSIONS: 1 . Colonic MM responses to bradykinin are mediated by $\mathrm{B} 2$ and, to a lesser extent, $\mathrm{B} 1$ receptors; responses to ATP occur via $\mathrm{P} 2$ receptors. 2. Proximal MM responses to bradykinin involve prostaglandin production and an excitatory neural component; bradykinin contracts mid and distal colonic MM by a direct action. 3. Proximal MM responses to ATP are direct, mid MM responses are prostaglandin-mediated, but in the distal region both prostaglandins and an excitatory neural pathway are utilized. 4. The inhibitory innervation of the rat colonic MM is unaffected by ATP or bradykinin. 5. Although both the excitatory innervation and prostaglandin synthesis associated with the colonic MM are involved in this muscle's responses to bradykinin and ATP, their relative contributions to the contractile process are region-specific

\section{S1933}

The Prokineticin Receptor 1 Mediates Inhibition of Spontaneous Giant Contractions in the Murine Proximal Colon

Willemijntje A. Hoogerwerf

Background: Prokineticins are novel peptides with reported effects on gastrointestinal contractility. We previously showed prokineticin receptor 1 (PKRl) expression in the myenteric plexus of the mouse proximal colon. The aims of this study were (1) to characterize the cell types expressing PKRl (2) to determine the effect of prokineticin 1 on mouse proximal colon contractility and (3) to determine the effect of prokineticin 1 on nitric oxide release in vitro. Methods: Immunohistochemistry was used to characterize the cell types expressing PKR1 by using double-labeling immunofluorescence in longitudinal muscle myenteric plexus (LMMP) preparations with antibodies to $\mathrm{HuC/D}$ (neuronal marker) and neuronal nitric oxide synthase (marker for myenteric inhibitory neurons). Proximal colon rings from adult male C57BLK6/J mice were used for circular muscle recordings in organ baths. The effect of PKC on nitric oxide release was determined from LMMP cultures using a commercial nitric oxide assay kit. Results: Quantification analysis showed that $23.8 \pm 1.85 \%$ of all $\mathrm{HuC/D}$ immunoreactive neurons express PKRl (504 neurons, 3 mice). Of PKRl- immunoreactive neurons, $7.7 \pm 1.02 \%$ express nNOS-immunoreactivity (233 neurons, 3 mice). Conversely, $5.6 \pm 1.98 \%$ of nNOS-IR neurons expressed PKR1-IR (318 neurons, 3 mice). PKC suppressed giant contractions (GC) in circular muscle. PKC $1 \mathrm{mM}, 10 \mathrm{mM}$ and $100 \mathrm{mM}$, significantly decreased the frequency from $2.22 \pm 0.48 / 5$ at baseline to $0.89 \pm 0.20,0.56 \pm 0.30$ and $0.22 \pm 0.15 \mathrm{GC} / 5$ minutes, respectively $(\mathrm{n}=8, \mathrm{P}<0.05)$. $\mathrm{PKC} 1 \mathrm{mM}, 10 \mathrm{mM}$ and $100 \mathrm{mM}$ significantly decreased the amplitude from $5.12 \pm 1.05$ to $3.25 \pm 0.85,2.20 \pm 0.80$ and $1.43 \pm 0.40 \mathrm{mN}$, respectively $(\mathrm{n}=8, \mathrm{P}<0.05)$. PKC $100 \mathrm{mM}$ did not significantly alter the frequency or amplitude in the setting of L-NAME. Under non-adrenergic, non-cholinergic conditions, PKC $100 \mathrm{mM}$ significantly decreased the amplitude of the contractions from $3.54 \pm 1.13 \mathrm{mN}$ to $1.04 \pm 0.27 \mathrm{mN}(\mathrm{n}=5 ; \mathrm{P}<0.05)$. PKC stimulated nitric oxide release from LMMP cultures in a dose-dependent manner in vitro: PKC $1 \mathrm{mM}, 10 \mathrm{mM}$ and 100 $\mathrm{mM}$ produced $0.59 \pm 0.22 \mathrm{mMol} / 10 \mathrm{mg}$ tissue weight, $1.19 \pm 0.28 \mathrm{mMol} / 10 \mathrm{mg}$ tissue weight, and $1.55 \pm 0.39 \mathrm{mMol} / 10 \mathrm{mg}$ tissue weight, respectively. This effect was blocked by l-NAME. Conclusion: PKRl mediates an inhibitory effect on spontaneous giant contractions in the murine colon, most likely through direct or indirect stimulation of nitric oxide release. PKRl and its natural ligand, prokineticin 1, may be important for modulation of colonic motility

\section{S1934}

Genome-Wide Transcriptional Signals from Colonic Neuromuscular Tissue in Patients with Slow Transit Constipation

Lu Liu, Rohan B. Williams, Fei Shang, Denis W. King, Elizabeth Burcher

Introduction: Slow transit constipation (STC) is a serious motility disorder of unknown etiology. Surgery is often the only effective treatment. In this study, we have applied a novel approach by using expression microarrays (measuring mRNA) with integrated bioinformatics follow-up (Gene Ontology $(\mathrm{GO})$ ) to describe transcriptional levels of individual genes, functional gene clusters and putative molecular mechanisms underlying the pathophysiology of STC. Methods: Microarray profiling was performed in sigmoid colon muscle from 6 female patients (age range 23-65 years) undergoing colectomy for STC, in comparison with normal colon from 6 age-matched female patients (30-64 years) undergoing resection for carcinoma. Normal colon was taken $10-20 \mathrm{~cm}$ from the tumor. Total RNA (sourced from circular and longitudinal muscle, and myenteric plexus) was extracted, pooled from each group, reverse transcribed and fluorescent dye coupled prior to hybridization onto microarray slides (19,000 unique human transcripts, $\mathrm{n}=6$ replicates). Following normalization, differentially expressed genes were identified using a Bayesian linear model (log-odds $>4$ significance level). Results: Of the 272 differentially expressed transcripts, 230 were significantly downregulated in STC, whereas only 42 genes were up-regulated (e.g. DEEPEST, NUP98, 4E-T, SNAP23). We employed GO annotations when known (163/272 transcripts), clustered to identify any major functional groupings of differentially expressed genes. The two top ranking clusters comprised 25 genes associated with protein metabolism and 23 genes with signal transduction (e.g. S100 calcium binding protein, guanylate cyclase activator). Unexpectedly, there was a notable down-regulation of multiple genes encoding for mucus production (mucins 2,3,5,11 and 12, and trefoil factors 1 and 3). Another unexpected finding was the down-regulation of EMAP2 (echinoderm microtubule associated protein 2: annotated to mechanosensation' in GO): an intriguing finding because it highlights potential differences in integration of cytoskeletal signaling that might have implications for mechanical function of smooth muscle in STC. There was no obvious change in genes linked to the enteric nervous system. Neuronal tissue comprised only a small portion of the total tissue examined, minimizing the likelihood of observing any neuronal changes. Summary: This study has described alterations in gene transcripts in human STC, measured using high-throughput ranscriptomics. We have identified several novel transcripts, cellular subsystems and mechanistic leads that may have relevance in the pathogenesis of this disorder. Supported by NHMRC Australia.
S1935

Differential Role of A1 and A2a Purinergic Receptors in the Control of Colonic Neuromuscular Function in the Presence of Experimental Colitis Luca Antonioli, Matteo Fornai, Corrado Blandizzi, Rocchina Colucci, Narcisa Ghisu, Maria Cristina Breschi, Mario Del Tacca

Introduction. Adenosine regulates innate immunity and is implicated in the pathophysiology of intestinal inflammation. Adenosine receptors are expressed throughout the digestive tract, but their involvement in gut dysmotility associated with inflammatory bowel disease has not been described. This study examines the role of adenosine Al and A2a receptors in the control of colonic motility in experimental colitis. Methods. Colitis was induced in male Sprague-Dawley rats by intrarectal administration of 2,4-dinitrobenzenesulfonic acid (DNBS) Colonic longitudinal muscle strips were suspended in Krebs solution containing antagonists of neurokinin receptors and connected to isotonic transducers (load $=1 \mathrm{~g}$ ) to determine the effects of $\mathrm{Al}$ and $\mathrm{A} 2 \mathrm{a}$ receptors ligands, dipyridamole (DIP, adenosine reuptake inhibitor) and adenosine deaminase (ADA) on atropine-sensitive motor responses evoked by: a) transmural electrical stimulation (TES: $0.5 \mathrm{~ms}, 30 \mathrm{~mA}$ ), delivered as single (sTES: $10 \mathrm{~Hz}, 100$ pulses) or repeated trains (rTES: $5 \mathrm{~Hz}, 25$ pulses every $60 \mathrm{~s}$ ); b) carbachol in the presence of tetrodotoxin. RT-PCR was used to examine Al and A2a receptor mRNA expression. Results. In normal colonic preparations, sTES-induced contractions were enhanced by DPCPX (Al antagonist; $+32.6 \%$ ) and ZM 241385 (ZM, A2a antagonist; $+21 \%$ ). In inflamed tissues, the enhancing action of ZM was more pronounced $(+45 \%)$, whereas DPCPX had no effects. The stimulant effects of ZM, in normal or inflamed colon, were prevented by N $\omega$-propylL-arginine (nNOS inhibitor), whereas those of DPCPX in normal colon were insensitive to nNOS blockade. After incubation of colonic preparations with DIP plus ADA, to decrease extracellular adenosine, CGS 21680 (A2a agonist) decreased motor responses to rTES, being more effective in inflamed (EC50 $=16 \mathrm{nM}$; Emax $=-58 \%)$ than normal tissues $(E C 50=20$ $n M ;$ Emax $=-27 \%$ ). Under these conditions, CCPA (Al agonist) inhibited rTES-induced contractions with similar efficacy in normal (EC50 $=120 \mathrm{nM}$; Emax= $-74.3 \%$ ) and inflamed tissues $(\mathrm{EC} 50=130 \mathrm{nM}$; Emax $=-66.5 \%)$. Al or A2a ligands did not affect carbacholinduced contractions. RT-PCR showed an increase in $\mathrm{A} 2 \mathrm{a}$, but not $\mathrm{Al}$, receptor expression in neuromuscular layer of inflamed colon. Conclusions. 1) Adenosine is involved in the inhibitory control of colonic motility via activation of $\mathrm{Al}$ and $\mathrm{A} 2 \mathrm{a}$ receptors; 2) $\mathrm{Al}$ receptors appear to directly modulate cholinergic nerves, whereas A2a receptors act through the recruitment of inhibitory nitrergic pathways; 3) Experimental colitis modifies this regulatory pattern, leading to an enhanced inhibitory control operated preferentially by A2a receptors.

\section{S1936}

Tegaserod Improves Symptoms and Function in Patients with Chronic Constipation

Brian Lacy, Jeffrey Kralstein, Michael Dolker, Michael Shetzline

Purpose The efficacy of tegaserod in patients with Chronic constipation (CC) is well established based on improvement in complete spontaneous bowel movements (CSBM) over a defined period. However, patients and physicians frequently use spontaneous bowel movements (SBM) and subjective symptoms to assess bowel function. This study assessed the benefit of 12 weeks of tegaserod (T) versus placebo (P) on SBM assessments as well as satisfaction of bowel habits in CC. Methods Data from separate double-blind, randomized, controlled clinical trials in CC for $\mathrm{T}$ dosed at $6 \mathrm{mg}$ bid $(\mathrm{N}=882)$ and placebo $(\mathrm{N}=863)$ were pooled for analysis. A bowel movement was considered spontaneous if the patient had not used a laxative or enema within the preceding 24 hours. Straining and satisfaction of bowel habits were assessed using 3 and 5 point ordinal scales and stool consistency was assessed using the Bristol stool scale. Data were analyzed for the intent to treat population comparing $\mathrm{T}$ and $\mathrm{P}$ for change from baseline using van Elteren tests at each time point over the 12-week active treatment period. Results Tegaserod demonstrated a consistent benefit quantitatively in stool frequency compared to $\mathrm{P}$ as well as a significant improvement in stool form, consistency, straining and satisfaction with bowel habits during the 12 week trial period (compared to baseline; all results $\mathrm{p}<.05$ ). Conclusion Tegaserod dosed at $6 \mathrm{mg}$ bid provided a sustained response over placebo for 12 weeks in both quantitative and qualitative assessments of constipation in patients with $\mathrm{CC}$.

\begin{tabular}{|c|c|c|}
\hline Variable & Weeks 1 - 12 Mean (SD) & $\begin{array}{c}\text { Change from Baseline Mean } \\
\text { (SD) }\end{array}$ \\
\hline SBM Frequency & $\begin{array}{c}\mathrm{T}=5.2 \pm(3.53) \mathrm{P}=4.4 \pm \\
(3.19)\end{array}$ & $\begin{array}{c}\mathrm{T}=1.9 \pm(3.08) \mathrm{P}=0.9 \pm \\
(2.54)\end{array}$ \\
\hline Daily straining score of SBM* & $\begin{array}{c}\mathrm{T}=0.9 \pm(0.40) \mathrm{P}=1.0 \pm \\
(0.43)\end{array}$ & $\begin{array}{c}\mathrm{T}=-0.4 \pm(0.48) \mathrm{P}=-0.2 \pm \\
(0.48)\end{array}$ \\
\hline Daily stool form of SBM & $\begin{array}{c}\mathrm{T}=3.6 \pm(1.04) \mathrm{P}=3.1 \pm \\
(1.02)\end{array}$ & $\begin{array}{c}\mathrm{T}=0.9 \pm(1.14) \mathrm{P}=0.4 \pm \\
(1.09)\end{array}$ \\
\hline $\begin{array}{c}\text { Complete evacuation }(\% \text { of } \\
\text { CSBM/SBM) }\end{array}$ & $\begin{array}{c}\mathrm{T}=35.8 \pm(28.81) \mathrm{P}=29.4 \pm \\
(27.52)\end{array}$ & $\begin{array}{c}\mathrm{T}=18.0 \pm(27.97) \mathrm{P}=12.3 \pm \\
(26.56)\end{array}$ \\
\hline Satisfaction of bowel habits* & $\mathrm{T}=2.3 \pm(.89) \mathrm{P}=2.5 \pm(.89)$ & $\begin{array}{c}\mathrm{T}=-0.8 \pm(1.05) \mathrm{P}=-0.5 \pm \\
(1.00)\end{array}$ \\
\hline
\end{tabular}

* A negative change denotes improvement

S1937

Corticotropin-Releasing Factor (CRF) Potentiates Rat Inferior Splanchnic Nerve Afferent (ISA) Unit Responsiveness to Serotonin (5-HT) in Vitro Yuhua Wang, Mulugeta Million, Lixin Wang, David Adelson, Jen Yu Wei, Yvette Tache

Background: CRF plays a role in regulating brain serotoninergic neurotransmission. Activation of $\mathrm{CRF}_{1}$ and $\mathrm{CRF}_{2}$ receptors in the dorsal raphe nucleus (DRN) inhibits and increases 5 -HT activity, respectively (Neuroscience 129, 509,2004). Our previous in vitro studies in 
a rat ISA-colon preparation model indicated that $\sim 53 \%$ and $\sim 78 \%$ of ISA fibers responded to intra-arterial (ia) injection of $\mathrm{CRF}$ and tegaserod, a 5- $\mathrm{HT}_{4}$ agonist, respectively (Gastroenterology 2001;120:A717 and 2002;122:A317). Aim: Determine whether CRF ia injection modulates 5-HT-induced ISA fiber activity in a rat in vitro preparation. Methods: The colorectal-inferior splanchnic nerve preparation was isolated from fasted, isoflurane anesthetized male SD rats $(250-300 \mathrm{~g})$ and put in a chamber as detailed previously (Gut 2005; PMID: 15985561). Responses of ISA fibers to consecutive ia injections of vehicle (saline $0.1 \mathrm{ml}), 5$-HT $(40 \mathrm{ng}), \mathrm{CRF}(400 \mathrm{ng})$ and a $2^{\text {nd }}$ dose of 5-HT (40 ng) at 15 - 30-min intervals, and to local mechanical receptor field stimulation (RFs) were recorded. In the control group the CRF injection was replaced by saline. Response magnitudes were presented as a $Q=5$ min spike count after/before treatment. A total of 28 units from 14 preparations were analyzed, including 20 units with basal activity averaging $1.10+0.13$ spikes $/ \mathrm{s}$ and 8 silent units. Control group (5 rats) had 9 units with 2 silent units, while test group (9 rats) had 19 units with 6 silent units. Results: CRF ia, which had no effect on ISA activity at this dose, significantly enhanced subsequent 5-HT-induced ISA fiber discharge (Table). In addition, CRF increased the number of 5-HT-responsive units from 6/13 to 12/13 and that of silent units from $2 / 6$ to $4 / 6$. In the control group, responses to the $1^{\text {st }}$ and $2^{\text {nd }} 5$-HT injections were similar. All 28 units responded to RFs. Conclusions: These in vitro studies provide evidence that CRF acts peripherally to enhance responsiveness of ISA fibers to 5HT, suggesting a potentiating interaction between CRF and 5-HT that may have implications for stress-related induction of hypersensitivity to colorectal distention. Supported by NIH grants DK 33061 and P50 DK64539 (YT)

Peptides activated ISA fiber

\begin{tabular}{|c|c|c|c|c|c|}
\hline Group & Vehicle & 5-HT $\left(1^{\text {st }}\right)$ & Vehicle & CRF & 5-HT $\left(2^{\text {nd }}\right)$ \\
\hline Control & $1.19 \pm 0.09$ & $1.41 \pm 0.11$ & $1.17 \pm 0.15$ & -- & $1.40 \pm 0.08$ \\
\hline Test & $1.01 \pm 0.04$ & $1.18 \pm 0.05$ & --- & $1.17 \pm 0.06$ & $1.66 \pm 0.11^{*}$ \\
\hline
\end{tabular}

Values are mean \pm SEM. $[\mathrm{F}=1.43, \mathrm{P}=0.26$, control group $(\mathrm{n}=7)$, and $\mathrm{KW}=23.11, \mathrm{P}<0.0001$, test group $(\mathrm{n}=13)$, parametric or nonparametric ANOVA test], ${ }^{*} \mathrm{P}<0.05$ vs. 5 -HT $\left(\mathrm{l}^{\mathrm{st}}\right)$.

\section{$\mathrm{S} 1938$}

Effect of Lactobacillus Casei Shirota On Colonic Transit Time in Patients with Slow-Transit Constipation

Franka Schlieger, Heinz Krammer, Andreas Franke, Hermann Harder, Irmtrud Wagner, Manfred V. Singer

Background: Slow-transit constipation is based on a motility disorder of the colon which leads to delaved transit ( $>72$ hours). Recently, it could be shown that Lactobacillus casei Shirota improves constipation related symptoms (stool frequency and consistency). Aim: To determine the effect of Lactobacillus casei Shirota on the colonic transit time in patients with slow-transit constipation under randomized double blind placebo controlled conditions. Methods: Colonic transit time of all consecutive outpatients with chronic constipation was determined by the Hinton-Test using radioopaque markers. If the transit time was at least 72 hours, subjects have been included in our study. 24 patients were given daily for four weeks either a dairy drink containing 6,5x109 colony forming units (cfu) of Lactobacillus casei Shirota or a placebo. After four weeks of therapy, measurement of colonic transit time was repeated. Results: The intervention with Lactobacillus casei Shirota resulted in a significant acceleration of the total colonic transit time (before: 95,6 hours, after: 77 hours, respectively; $\mathrm{p}=0,05$ ). This effect was mainly due to a reduction of the transit time in the sigmoid and rectum $(\mathrm{p}<0,007)$, see table 1 . In the placebo group, the decrease of the total colonic transit time did not reach statistical significance ( 93,7 vs 87,1 hours, respectively; $\mathrm{p}=0,393$ ). Conclusion: Lactobacillus casei Shirota decreases delayed colonic transit times in patients with slow-transit constipation. This effect is mainly due to an acceleration in the sigmoid and rectum.

Transit times before and after intervention with Lactobacillus casei Shirota

\begin{tabular}{|c|c|c|c|c|c|c|c|c|}
\hline & $\begin{array}{c}\text { Before } \\
\text { intervention } \\
\text { (hours) }\end{array}$ & & & & $\begin{array}{c}\text { After } \\
\text { intervention } \\
\text { (hours) }\end{array}$ & & & \\
\hline & Total & $\begin{array}{l}\text { Right } \\
\text { colon }\end{array}$ & $\begin{array}{l}\text { Left } \\
\text { colon }\end{array}$ & $\begin{array}{c}\text { Sigmoid/ } \\
\text { Rectum }\end{array}$ & Total & $\begin{array}{l}\text { Right } \\
\text { colon }\end{array}$ & $\begin{array}{l}\text { Left } \\
\text { colon }\end{array}$ & $\begin{array}{l}\text { Sigmoid/ } \\
\text { Rectum }\end{array}$ \\
\hline Verum & 95,6 & 22,7 & 44,6 & 28,3 & 77 & 22,5 & 37,3 & 16,7 \\
\hline Placebo & 93,6 & 26,8 & 47,5 & 21,5 & 87,1 & 26,2 & 43,9 & 17 \\
\hline
\end{tabular}

\section{S1939}

Regulation of Intestinal Smooth Muscle Cell Growth and IGF-I Production By Tegaserod

John F. Kuemmerle, Jennifer G. Bowers, John R. Grider

We have previously shown that intestinal smooth muscle cells express both $5-\mathrm{HT}_{4}$ receptors mediating relaxation, and 5- $\mathrm{HT}_{2 \mathrm{~A}}$ receptors mediating contraction. 5-HT stimulates proliferation of intestinal smooth muscle cells. Endogenous Insulin-like Growth Factor-I (IGF-I) is a key mediator of intestinal smooth muscle cell growth. Upregulation of IGF-I in the chronically inflamed muscle of Crohn's Disease regulates hyperplasia of muscle and may contribute to stricture formation. Tegaserod, a partial agonist of $5-\mathrm{HT}_{4}$ receptors and in some cells an antagonist of $5-\mathrm{HT}_{2 \mathrm{~B}}$ receptors, is used widely for a variety of gastrointestinal motility disorders although its effects on smooth muscle proliferation and interplay with IGF-I has not been examined. Aims: The aims of the present study were to determine whether tegaserod regulates muscle cell growth and identify the receptors involved, and to determine whether activation of 5-HT receptors can modulate IGF-I production by muscle. Methods: Colonic smooth muscle cells of the mouse colon were isolated by enzymatic digestion and used to initiate primary cultures. Muscle cell growth was measured in response to tegaserod using $\left[{ }^{3} \mathrm{H}\right]$ thymidine incorporation. IGF-I production was measured using a murine specific ELISA. GR113808A was used as a 5-HT 4 antagonist and BW723C86 was used as a $5-\mathrm{HT}_{2 \mathrm{~B}}$ antagonist. Results: Incubation of quiescent muscle cells for $24 \mathrm{~h}$ with tegaserod $(10 \mathrm{nM}-1 \mu \mathrm{M})$ elicited concentration-dependent inhibition of basal $\left[{ }^{3} \mathrm{H}\right]$ thymidine incorporation ( $1 \mu \mathrm{M}: 28 \pm 2 \%)$. In the presence of the 5-HT 4 antagonist, GRl13808A (1 $\mu \mathrm{M})$, the effects of tegaserod were inhibited by $40-70 \%$. Unlike tegaserod, incubation of muscle cells with the 5- $\mathrm{HT}_{2 \mathrm{~B}}$ antagonist, BW723C86 $(1 \mu \mathrm{M})$, did not affect basal $\left[{ }^{3} \mathrm{H}\right]$ thymidine incorporation. Incubation of quiescent muscle cells for $24 \mathrm{~h}$ with tegaserod also elicited concentration-dependent inhibition of IGF-I production ( $1 \mathrm{uM} \cdot 44 \pm 7 \%$ inhibition). The effects of tegaserod on IGF-I production were not altered by the 5-HT 4 antagonist, GR113808A (1 $\mu \mathrm{M})$. In contrast to proliferation, incubation of muscle cells with the 5$\mathrm{HT}_{2 \mathrm{~B}}$ antagonist, BW723C86, significantly inhibited IGF-I production (1 $\left.\mu \mathrm{M}: 25 \pm 5 \%\right)$ Conclusions: Tegaserod inhibits colonic smooth muscle cell proliferation by activation of 5-HT 4 receptors and inhibits IGF-I production via its ability to inhibit 5- $\mathrm{HT}_{2 \mathrm{~B}}$ receptors on muscle cells. Both effects suggest tegaserod might be useful in reducing the muscle hyperplasia associated with disorders such as Crohn's disease or intestinal obstruction.

\section{S1940}

Peripheral Corticotropin Releasing Factor (CRF) 2 Receptor Activation Decreases the $\mathrm{CRF}_{1}$-Mediated Colonic Motor and Secretory Function Mulugeta Million, Pu-Qing Yuan, Pascale Plaisancie, Paul R. Saunders, Karina Pambukhchian, Armen Karapetyan, Yvette Tache

Background: $\mathrm{CRF}_{1}$ receptor activation by stress or $\mathrm{CRF}$ induces defecation and diarrhea in rats (Gastroenetrology, 119:1569-79, 2000). Despite the presence of $\mathrm{CRF}_{2}$ receptors in the colon, and selective $\mathrm{CRF}_{2}$ ligands such as urocortin 2 (Ucn 2) their role in colonic function is not well known. We showed that peripheral $\mathrm{CRF}_{2}$ receptor activation dampens hypersensitivity to colorectal distention (Gut 2005; PMID: 15985561). Aim: Determine 1) whether the $\mathrm{CRF}_{1} / \mathrm{CRF}_{2}$ agonists, $\mathrm{CRF}$ and urocortin 1 (Ucn 1 ) stimulatory actions on colonic motor function(fecal pellet output) and secretion (mucus) is the result of simultaneous activation of $\mathrm{CRF}_{1}$ exitatory and $\mathrm{CRF}_{2}$ inhibitory pathways and 2) the location of $\mathrm{CRF}_{2}$ on nitric oxide (NO) colonic enteric neurons. Methods: Adult male SD rats (280-320 g) were injected IP in 10 min interval, with either saline + saline, CRF $(3 \mu \mathrm{g} / \mathrm{kg})$ or Ucn $2(10 \mu \mathrm{g} / \mathrm{kg})$ or Ucn $2+\operatorname{CRF}(10+3 \mu \mathrm{g} / \mathrm{kg})$, and fecal pellet output (FPO) was monitored for 60 min. Rats were then euthanized and distal colonic longituidinal muscle myenteric plexus (LMMP) whole mount preparation used for $\mathrm{CRF}_{2}$ and nitric oxide synthase (NOS) expression analysis. Fixed and parafin embedded colonic tissues were processed for mucus detection by alcian blue/PAS/hematoxylin staining. $\mathrm{CRF}_{2}$ antagonist astressin $2-\mathrm{B}(100 \mu \mathrm{g} / \mathrm{kg})$ was injected 10 min before Ucn 1 , at 3 and $10 \mu \mathrm{g} / \mathrm{kg}$ ) and FPO as well as diarrhea monitored for $2 \mathrm{~h}$. Results: Ucn $2(10 \mu \mathrm{g} / \mathrm{kg})$ + saline IP did not affect FPO, whereas CRF at $3 \mu \mathrm{g} / \mathrm{kg}$ or Ucn at 3 and $10 \mu \mathrm{g} / \mathrm{kg}$ increased FPO $(0.0 \pm 0.0 \vee 4.5 \pm 0.5 / \mathrm{h} ; 5.8 \pm 0.6 / 2 \mathrm{~h}, 7.7 \pm 1.6 / 2 \mathrm{~h}, \mathrm{p}<0.05$ respectively) and induced diarrhea. The CRF effect was reduced by Ucn $2(2.0 \pm 0.3 \vee 4.5 \pm 0.5$ $\mathrm{p}<0.05$ ). Conversely, Astressin $2-\mathrm{B}+\mathrm{Ucn} \mathrm{l}$ at 3 or $10 \mu \mathrm{g} / \mathrm{kg} v$ saline $+\mathrm{Ucn} 1$, had higher diarrhea prevalence $\left(0 \% \vee 44 \%, \mathrm{p}<0.05 ; 40 \% \vee 80 \%, \mathrm{p}<0.05\right.$ respectively). $\mathrm{CRF}_{2}$ receptors were expressed and colocalized with NOS positive neurons in colonic LMMP. Rats treated with Ucn 2, compared to saline, had increased number of mucus stained cells/crypt $(18.3 \pm 1$.0 $v 15.2 \pm 0.5, \mathrm{p}<0.05)$ whereas $\mathrm{CRF}$ treated rats had a decreased number. The effect of CRF on mucus cells was blocked by pretreatment with Ucn $2(15.5 \pm 0.6 v 10.5 \pm 0.6, \mathrm{p}<0.05)$ Conclusions: Blockade of peripheral $\mathrm{CRF}_{2}$ enhances the $\mathrm{CRF}_{1} / \mathrm{CRF}_{2}$ receptor agonistsinduced $\mathrm{FPO}$ and diarrhea while $\mathrm{CRF}_{2}$ activation blunts $\mathrm{CRF}_{1}$-mediated colonic motor and mucus discharge. The data suggest that $\mathrm{CRF}_{2}$ receptor exerts an inhibitory effect on a stimulated colon, probably involving NOS activation. $\mathrm{CRF}_{2}$ inhibitory signaling may represent a stress coping pathway in the $\mathrm{CRF}_{1}$-mediated alterations of colonic function during stress. Supported by R01 DK57238(YT) \& R21DK068155-01A1(MM)

\section{S1941}

A Course of Tegaserod Treatment Modulates CNS Processing of Visceral Afferent Information

Lisa Kilpatrick, Jen Labus, Steve M. Berman, Brandall Suyenobu, Jean Stains, Bruce D. Naliboff, Emeran A. Mayer

Introduction: Tegaserod (Teg) is a partial 5HT-4 receptor agonist which improves symptoms in IBS patients with constipation predominant bowel habit (IBS-C). The neural mechanism underlying subjective symptom improvement are incompletely understood, but may include effects on peripheral visceral afferent pathways. Aims: 1.Do changes in brain responses to rectal distention (INF)correlate with IBS symptom changes? 2. Does a 3 week course of Teg affect brain circuits concerned with visceral perception and autonomic control? Design and Methods: Study protocol: Brain responses to controlled rectal INF $(5,25$ and $45 \mathrm{mmHg})$ were assessed in 10 women with IBS-C following 3 week treatment with Teg $(6 \mathrm{mg}$ bid) and placebo during a randomized cross-over clinical trial. fMRI analysis: Normalized contrast images for effects of moderate (45mmH)INF in each session were generated using statistica mapping software(SPM2). Covariate analyses assessed the relationship of within-subject fMRI response to INF to symptom outcomes in regions of interest pre-selected for association with IBS symptom ratings. Multivariate task partial least squares (PLS) tested for distributed patterns of brain activity associated with the three INF levels. A seed PLS was subsequently applied to test for, and identify common and treatment-specific functional networks operating during rectal INF. Results: Positive correlations (more activity - more symptoms, $\mathrm{p}<0.01$ ) were observed between 24-hr (as well as diary) symptom ratings and the INF-induced activation in several brain regions including amygdala and dorsal pons. Negative correlations included those for left anterior insula, ventral striatum and bilateral PAG. Task PLS revealed a significant pattern of regions ( $\mathrm{p}<.001)$ that maximally differentiated 5 and $45 \mathrm{mmHg}$ INF across placebo and Teg treatment, and included medial thalamus. Seed PLS revealed a network of regions associated with the medial thalamus that functioned differently during the two INF conditions (but not during $5 \mathrm{mmHg}$ ) following Teg vs placebo treatment, $\mathrm{p}<.001$. This treatment-dependent medial thalamic network included the insula, amygdala, midbrain, ventral and dorsal ACC and prefrontal cortices. Conclusions: 1. Brain responses to rectal INF correlate with IBS symptom changes. 2. Network analyses are consistent with Teg treatment-induced differences in the functioning of a brain network comprised of regions 
consistent with a known spinal-thalamic-cortico-limbic circuit operating during experience of noxious visceral stimuli. FMRI assessment of brain responses to drug treatment in a small sample of patients may be a useful technique to assess drug effectiveness in IBS patients.

\section{S1942}

The Effect of Mosapride Citrate On Colonic Motor Function in Guinea Pig: Does It Differ Between Proximal and Distal Colon?

Hyojin Park, Eun Ju Choi, Sang In Lee

Backgrounds/Aims: Mosapride citrate (mosapride), a substituted benzamide, is a novel 5 HT4 receptor agonist. The 5-HT4 receptor detected in the myenteric plexus may be located on the excitatory neurotransmitter, and participate in the stimulation of motility, whereas stimulation of the receptors located on the circular muscle induce the relaxation of the muscle. We previously demonstrated mosapride significantly shortened the colonic transit in guinea pig (Yonsei Med J 2003;44:653-64). However, there have been some debates in its effects depending on gut regions what they used. Therefore, we undertook this study to validate our previous study, evaluate the different effect of mosapride between proximal and distal colonic motor function in guinea pig and, and to demonstrate the distribution of 5-HT4 receptor both in proximal and distal colonic wall. Methods: Proximal (approximately $8 \mathrm{CM}$ from the ileocolic junction) and distal colon (approximately $8 \mathrm{CM}$ from the anus) was removed. For the measurement of colonic transit time, artificial feces were inserted into the oral side of lumen, and moved toward the anal side by intraluminal perfusion via a peristaltic pump. Total $6 \mathrm{CM}$ was observed and the time taken from moving each $2 \mathrm{CM}$ was measured as a colonic transit. Tissue bath study by using electrical stimulation was done to estimate the contractile activity of the circular muscle of colon. Immunohistochemistry of 5-HT4 receptor of colonic wall of both proximal and distal colon was done to to clarify the mechanism of different action of mosapride according to the site of colon. Results: Transit time of proximal colon was significantly delayed compared with distal colon. Mosapride (10-9-10-7M) significantly shortened transit time of proximal colon but not distal colon. Mosapride increased the amplitude of contraction both in proximal and distal colon, which is rather more on the proximal colon. 5-HT4 receptor is located more predominantly in the myenteric plexus of the proximal colon, whereas receptor is more in the circular muscle of the distal colon. Conclusions: Mosapride shortened colonic transit in guinea pig and this is primarily due to an accelerated transit in proximal colon through the increased amplitude of contraction, which is rather more on the proximal colon. This effect may be relevant with the different distribution of 5-HT4 receptor in the gut wall.

\section{S1943}

\section{Dendritic Cells Can Secrete Corticotropin Releasing Factor}

Mariko Hojo, Toshifumi Ohkusa, Akira Harada, Tomoyoshi Shibuya, Harumi Tomeoku, Kaoru Kushima, Akihito Nagahara, Nobuhiro Sato

Purpose: Corticotropin releasing factor (CRF) influencing gut motor function and visceral perception appears to be involved in the pathogenesis of IBS. CRF has been found to be secreted not only by the hypothalamus, but also by lymphocytes and macrophage, and the secreted CRF appears to participate in the inflammatory-immune system. Dedritic cells in the intestinal mucosa seem to be key players in the intestinal immune system, but to our knowledge, there is no report on the secretion of CRF from these cells. In this study, we investigated whether CRF secretion occurred in dendritic cells, and if so, whether or not intestinal mucosal bacteria could stimulate and increase its secretion from the cells. Methods: JAWSII cells (ATCC CRL-11904), a mouse dendritic cell line, were seeded into 24-well culture plates $\left(1 \times 10^{5}\right.$ cells / well) and grown for three days. Commensal bacterial strains of E.coli (JCM1649), C.clostridiforme (JCM1291), B.vulgatus (JCM5856) or F.varium (ATCC8501) ( $1 \times 10^{8}$ cells each) were added to the cells, and incubated for two hours. After incubation, the culture medium was collected and CRF was measured by means of an ELISA assay. Results: CRF $(29.6 \mathrm{pg} / \mathrm{mL})$ was detected in the medium of JAWSIl cells treated with no bacteria. When E.coli, C.clostridiforme, or F.varium was added to cells, a small increase in the level of CRF was detectable. However, an approximately 7 -fold increase in the level of CRF $(209.3 \mathrm{pg} / \mathrm{mL})$ was observed when cells were treated with B.vulgatus. Conclusions: These results suggest that dendritic cells are able to secrete CRF, and that B.vulgatus in the gut may induce the secretion of CRF from dendritic cells. From these observations, we suggest that dendritic cells stimulated by commensal bacteria in the gut may be implicated in the development of IBS.

\section{S1944}

Irritable Bowel Syndrome (IBS) Patients Show Hyper-Reactive Motility Responses to Intraluminal Distention Which Are Unrelated to Pain Hypersensitivity

Motoyori Kanazawa, Olafur S. Palsson, Syed Im. Thiwan, Marsha J. Turner, Miranda Al. van Tilburg, Michael D. Crowell, Shin Fukudo, Douglas A. Drossman, William E. Whitehead

Previous studies reported that IBS patients have exaggerated colonic motility responses to experimental stress, intraluminal distention, or eating, but reports have been inconsistent. Aims: (1) Compare IBS patients to healthy controls with respect to colon motility during sustained intraluminal distention and following a standard meal, (2) determine what proportion of IBS patients show a motility response that is outside the normal range, and (3) determine whether motility reactivity is correlated with increased pain sensitivity in IBS. Methods: 89 IBS patients and 23 healthy controls were studied. A manometric catheter containing a $10 \mathrm{~cm}$ latex balloon with 2 perfusion ports above and 2 below the balloon for recording intraluminal pressure, was positioned in the descending colon. Pain threshold in the colon was assessed using an electronic barostat by the ascending method of limit before the motility recording. After a $10 \mathrm{~min}$ fasting baseline, the balloon was inflated to $20 \mathrm{mmHg}$ above intraoperating pressure (IOP) for $10 \mathrm{~min}$, then deflated for a $15 \mathrm{~min}$ recovery period. Next a standardized 810-cal meal was consumed and postprandial motility was recorded for $30 \mathrm{~min}$. Motility index was manually computed as the sum of the areas of all contractions divided by recording time. Average barostat bag volume was recorded as a measure of smooth muscle tone. Results: Motility indices during the distension were significantly higher in IBS patients than in controls $(944+94$ vs $531+98$; mean + SEM, $\mathrm{p}<0.05)$. There were no differences in motility indices during the baseline $(290+22$ vs $244+25)$, recovery $(434+37$ vs $308+36)$, or postprandial $(459+30$ vs $426+56)$ periods. $54 \%$ of IBS patients had pain thresholds below the range of healthy controls ( $95 \% \mathrm{CI} 41-49 \mathrm{mmHg}$ ). Barostat bag volumes during the baseline $(41.3+3.2$ vs $58.9+9.9 \mathrm{~mL}$ ), distension $(191.5+7.2$ vs $230.3+14.1 \mathrm{~mL}$ ) and recovery $(38.6+3.1$ vs $58.7+9.7 \mathrm{~mL})$ periods was significantly lower in IBS patients compared with controls (all $\mathrm{p}<0.05)$ but not during the postprandial period $(19.8+1.6 \mathrm{vs}$ $20.4+2.7 \mathrm{~mL}, \mathrm{p}>0.1)$. IBS patients showed lower pain threshold to the colonic stimulation (38 [10-50] vs 50 [16-50]; median with range, $\mathrm{p}<0.01$ ), but there was no relationship between colonic motility and visceral sensitivity. Conclusions: $45 \%$ of IBS patients showed hypercontractivity in the colon in response to intraluminal distension. IBS patients also showed increased smooth muscle tone. However, hypercontractility was not associated with hypersensitivity for pain. [Supported by RO1 DK31369, R24 DK67674, and RR00046.]

\section{S1945}

Alterations in Bile Acid Metabolism in Constipation

Hasse R. Abrahamsson, Ann-Margret Lindqvist, Ralf Nilsson, Magnus Simren, Per-Goran Gillberg

Bile acids (BA) are derived from cholesterol and are potent physiological laxatives. However, little is known about the role of BA in patients (pts) with functional constipation (FC) and constipation predominant IBS (IBS-C). We hypothesized that BA synthesis may be altered in constipation. METHODS: Female pts (23 IBS-C, 4 FC, Rome II criteria) were studied and compared with non-constipated (non-C) subjects (17 IBS-D, 20 healthy women). BMI, blood lipids, colonic transit time by a radiological method (OATT; Reference $\leq 4.3$ days) and stool frequency were measured. C4 reflecting bile acid synthesis was measured before breakfast ( 8 a.m.) and after lunch (1 p.m.). Lanosterol and sitosterol as markers of cholesterol synthesis and cholesterol absorption, respectively, were assessed. RESULTS: When the whole groups of constipated and non-constipated subjects were compared only stool frequency and OATT differed between groups $(\mathrm{P}<0.001)$. When constipated pts were categorized according to OATT marked differences related to colonic transit time were observed (see TABLE). Absence of the ordinary C4 peak at lunchtime was noted in $82 \%$ of pts with delayed OATT compared with $17 \%$ in subjects with normal OATT $(\mathrm{P}<0.001)$. Symptom severity and frequency did not differ between pt groups. CONCLUSION: Patients with IBS$\mathrm{C}$ and $\mathrm{FC}$ have marked changes in $\mathrm{BA}$ synthesis related to colonic transit. The diurnal rhythm is altered in slow transit colon with lack of the $\mathrm{C} 4$ peak at lunchtime. C4 levels indicate decreased BA synthesis in IBS-C with normal OATT. Alterations in bile acid metabolism may be involved in the pathophysiology of constipation.

TABLE. Mean values. P-values: Difference between Constipation with slow transit vs. Constipation with normal transit

\begin{tabular}{|c|c|c|c|c|}
\hline & Non-Constip. & $\begin{array}{c}\text { Constipation: Slow } \\
\text { transit }\end{array}$ & $\begin{array}{c}\text { Constipation: Normal } \\
\text { transit }\end{array}$ & P-value \\
\hline Stools/6 days & 11.8 & 2.3 & 5.9 & $<.001$ \\
\hline OATT, days & 1.7 & 5.5 & 3.0 & $<.001$ \\
\hline C4, nM, 8 a.m. & 63.6 & 93.2 & 43.0 & 0.007 \\
\hline $\begin{array}{c}\text { C4 increase, 1 p.m, } \\
\%\end{array}$ & 46.1 & -9.1 & 90.7 & 0.011 \\
\hline $\begin{array}{c}\text { Cholesterol, mmol/l } \\
\text { Triglycerides, } \\
\text { mmol/l }\end{array}$ & 5.01 & 5.66 & 5.31 & 0.018 \\
\hline Lanosterol, nM & 320 & 1.57 & 1.11 & 0.09 \\
\hline Sitosterol, nM & 2467 & 392 & 408 & 0.55 \\
\hline
\end{tabular}

\section{S1946}

Effect of Yohimbine On Gastrointestinal Transit and Symptoms in Healthy Subjects

Christopher N. Andrews, Adil Bharucha, Michael Camilleri, Barbara M. Seide, Kari L. Baxter, Duane D. Burton, Alan R. Zinsmeister

Background-The sympathetic nervous system tonically inhibits gastrointestinal (GI), and particularly colonic motility under physiological circumstances predominantly via $\alpha_{2}$ adrenoreceptors $\left(\alpha_{2} \mathrm{AR}\right)$. Yohimbine $(\mathrm{Y})$, a $\alpha_{2} \mathrm{AR}$ antagonist, increased colonic tone and can induce colonic HAPCs in healthy subjects; however, the effect of Y on GI transit is unknown. AimsTo evaluate the effects of $\mathrm{Y}$ on gastric emptying (GE), small bowel transit (SBT) and colonic transit $(\mathrm{CT})$ and on bowel symptoms in 30 healthy volunteers $(25 \mathrm{~F}$; mean age $30 \pm 1 \mathrm{yr}$ ) randomly assigned to p.o. placebo or Y $16.2 \mathrm{mg}$ p.o. tid for 7 days in double-blind fashion. Methods-Transit was measured by scintigraphy from days 5-7 using egg labeled with ${ }^{99 \mathrm{~m}} \mathrm{Tc}$ sulfur colloid for GE and SBT, and a delayed-release capsule containing ${ }^{111}$ In-activated charcoal for CT. Food challenge was simulated by assessing volume of Ensure intake to maximum satiation and postprandial symptoms 30 min later (100mmVAS). Transit data were analyzed by ANOVA adjusted for gender (and BMI, age, and baseline values where appropriate). Results-29 subjects completed the study. Y tended to increase maximum tolerated volume (MTV). Y also increased stool frequency relative to placebo $(p=0.02)$, and this effect was greater in males than females $(\mathrm{p}=0.05)$. The $\mathrm{Y}$ dose tested also increased $\mathrm{BP}$, heart rate, and norepinephrine (NE) levels. Conclusion-Yohimbine (16.2 $\mathrm{mg}$ tid) increased stool frequency but did not significantly affect GI transit in healthy subjects. Therefore, this dose is appropriate to assess for hypersensitivity at $\alpha_{2} A R$ in disease. Supported NIH PO1 DK-068055. 


\begin{tabular}{|c|c|c|c|}
\hline Variable $($ mean \pm SEM) & Yohimbine $(\mathbf{n}=\mathbf{1 4})$ & Placebo $(\mathbf{n}=\mathbf{1 5})$ & $\mathbf{p ~ v a l u e ~}$ \\
\hline GE half-time $(\mathrm{min})$ & $127 \pm 10$ & $135 \pm 10$ & 0.54 \\
\hline SBT (colonic filling) at $6 \mathrm{~h}(\%)$ & $54 \pm 9$ & $40 \pm 9$ & 0.19 \\
\hline Ascending colon half-time $(\mathrm{h})$ & $12.3 \pm 2.0$ & $13.5 \pm 2.1$ & 0.62 \\
\hline GC24 & $3.01 \pm 0.30$ & $3.04 \pm 0.32$ & 0.93 \\
\hline MTV (ml) & $1157 \pm 107$ & $1015 \pm 73$ & 0.11 \\
\hline Stool frequency (number/day) & $1.56 \pm 0.11$ & $1.20 \pm 0.12$ & 0.02 \\
\hline Stool consistency (Bristol scale) & $2.90 \pm 0.23$ & $3.56 \pm 0.23$ & 0.03 \\
\hline Systolic BP (2h post med) & $123 \pm 4$ & $113 \pm 4$ & 0.02 \\
\hline Diastolic BP $(2 \mathrm{~h} \mathrm{post} \mathrm{med)}$ & $74 \pm 2$ & $65 \pm 2$ & 0.002 \\
\hline Heart rate $(2 \mathrm{~h}$ post med) & $72 \pm 2$ & $62 \pm 3$ & 0.01 \\
\hline Pre drug NE (pg/ml) & $259 \pm 35$ & $124 \pm 33$ & 0.01 \\
\hline Post drug NE (pg/ml) & $340 \pm 41$ & $118 \pm 39$ & $<0.001$ \\
\hline
\end{tabular}

\section{S1947}

Role of Tachykinin Nk1 Receptors On Novelty Stress-Induced Defecation in Mongolian Gerbils

Shiho Okano, Hideaki Nagaya, Nobuhiro Inatomi

Background and aim: It is known that stress affects gastrointestinal functions such as gastric emptying, intestinal transit and colonic motility. We have previously demonstrated that tachykinin NK1 receptor antagonists inhibit restraint stress induced colonic motility. In this study, we established a new stress-induced defecation model involving the placement of animals in a novel environment (novelty stress), and examined the effects of TAK-637, an NK1 receptor antagonist, and several drugs on novelty stress-induced fecal pellet output. Methods: The male Mongolian gerbils kept in groups (10 animals per cage) were placed individually in small cages. The fecal pellets of each animal were collected every hour. The animals were given drugs orally $0.5 \mathrm{~h}$ (alosetron, a 5-HT3 receptor antagonist) or $1 \mathrm{~h}$ (other drugs) before 2-h novelty stress. The plasma concentration of adrenocorticotropic hormone (ACTH) was measured using a radioimmunoassay. Upper intestinal transit was evaluated using a charcoal method. Results: Animals under novelty stress, displayed active mobility and exploratory behavior compared to the non-stressed group, and the concentration of plasma ACTH was moderately but significantly increased by the novelty stress. The fecal pellet output significantly increased during the first $2 \mathrm{~h}$. The upper intestinal transit was suppressed during restraint stress, but not changed during novelty stress. TAK-637, alosetron hydrochloride, trimebutine maleate (a peripheral opioid receptor agonist) and atropine sulfate (a muscarine receptor antagonist) exhibited dose-dependent and significant inhibitory effects on novelty stress-induced defecation, with ID30 values of $0.03,0.38,12$ and $0.29 \mathrm{mg} / \mathrm{kg}$, respectively. To clarify whether the effect of the test drugs may be ascribed to the inhibition of normal defecation, stress-stimulated defecation or both, the effects of the drugs on the 4-h spontaneous fecal output was examined. TAK-637 did not inhibit normal defecation, however, alosetron hydrochloride, trimebutine maleate and atropine sulfate markedly inhibited normal defecation, with ID30 values of $0.96,22$ and $0.56 \mathrm{mg} / \mathrm{kg}$, respectively. Conclusions: The present study indicated that novelty stress increases fecal pellet output without affecting the upper intestinal transit, and this model may be useful for evaluating the effects of drugs on stress-stimulated colonic motility. The findings that NK1 receptor antagonist inhibited stress-stimulated defecation without affecting normal defecation suggest its therapeutic potential for improving stress associated colonic dysfunctions, such as irritable bowel syndrome.

\section{S1948}

Expression of 5-HT3, 5-HT4 and 5-HT7 Receptors in the Guinea Pig Distal Colon and Their Role in Peristaltic Activity

Barbara Balestra, Elisabetta Cervio, Roberto De Giorgio, Giovanni Barbara, Fabrizio de Ponti, Vincenzo Stanghellini, Catia Sternini, Marcello Tonini

Background: In the guinea pig colon activation of 5-HT3 and 5-HT4 receptors enhances peristaltic activity, whereas in ileal segments 5 -HT7 receptors participate in the accommodation of the circular muscle during the preparatory phase of peristalsis. Aims: To determine the presence of 5-HT3, 5-HT4 and 5-HT7 receptor mRNAs in the guinea pig distal colon by using reverse transcription-polymerase chain reaction (RT-PCR), to evaluate their relative expression by quantitative real time RT-PCR and to define their role in the peristaltic activity in isolated colonic segments. Methods: Male albino guinea-pigs (350-500 g) were used. Total RNA was isolated from distal colon fragments with the Rneasy Protect Mini Kit (Qiagen). First-strand cDNA was synthesized from the total RNA using the SuperScript III Reverse Transcriptase kit (Invitrogen) for the RT-PCR, or the ThermoScript RT-PCR system (Invitrogen) for real time RT-PCR. PCRs were carried out using primers specific for 5-HT3, 5HT4 and 5-HT7 receptors. Real time RT-PCR experiments were performed using FastStart SYBR Green Master kit (Roche Applied Science) and myosin light chain kinase (MLCK) was the housekeeping gene used as internal standard. In functional studies, propulsion was elicited by intraluminal distension of a thin rubber balloon $(0.05-0.1 \mathrm{ml})$. The velocity of balloon propulsion was considered as the main parameter of peristaltic activity. Selective 5-HT3 (ondansetron: $1 \mu \mathrm{M}$ ), 5-HT4 (RS-39604: $1 \mu \mathrm{M}$ ), and 5-HT7 (SB-269970: $100 \mathrm{nM}$ ) antagonists were used to determine the involvement of the three receptors in peristaltic activity. Results: PCR products corresponding to 5-HT3, 5-HT4 and 5-HT7 mRNA were all present in the distal colon and real time RT-PCR showed differences in the 5-HT3, 5-HT4 and 5-HT7 receptors expression. The 5-HT3 and 5-HT7 receptor mRNAs were found in comparable amounts, but lower in density compared to the 5-HT4 mRNA. In functional studies, peristaltic activity was reduced by approximately $65 \%, 85 \%$ and $40 \%$ by 5 -HT3, 5-HT4 and 5-HT7 receptor blockade, respectively. Peristaltic activity was invariably blocked by the simultaneous administration the three antagonists. Conclusions: In the guinea pig distal colon, 5-HT3, 5-HT4 and 5-HT7 receptors are all expressed. 5-HT plays a key role in the modulation of peristalsis since simultaneous blockade of the three receptors suppresses peristaltic activity. Among receptor subtypes, 5-HT4 receptors seem to be the most abundant and functionally important for peristalsis

\section{S1949}

Motility of the Interposed Colon for Esophageal Replacement

Susumu Ohwada, Susumu Kawate, Yoshihiro Sato, Masaru Izumi, Kunihiro Hamada, Kentaro Hirai, Yutaka Sunose, Hiroyuki Tsutsumi, Takayuki Yamada, Yuichi Iino

Backgrounds: Besides the stomach, the colon is considered a well functioning and durable esophageal substitute. However, long term motility of the interposed colon has not been determined, and effects of prokinetic agents on the interposed colon have not been studied Aim: The aim of this study was to evaluate the motor activity of the interposed colon for esophageal replacement following radical surgery for esophageal carcinoma, and to examine the effects of different doses of erythromycin (EM) on the interposed colon. Methods: We studied six patients with seven procedures, within six months ( $n=3$, ranging 1 to 6 months) and over one year ( $\mathrm{n}=4$, ranging 1.4 to 5 years) after the isoperistaltic left colon interposition in the esophageal bed following radical esophagectomy for esophageal carcinoma. The age of the patients ranged from 47 to 60 years (median, 54 years). Two patients had almos whole stomach, and other four patients 30\% remnant stomach. All patients had no disease recurrence. After the patients had fasted for 16 hours, we used a catheter and three microtransducers with $5 \mathrm{~cm}$ interval connected to a Microdigitrapper portable data-logger. The tip of the catheter was placed below the diaphragma. EM was intravenously administered at $1 \mathrm{mg} / \mathrm{kg} / 15 \mathrm{~min}$ and $5 \mathrm{mg} / \mathrm{kg} / 60 \mathrm{~min}$ at least four-hour intervals. Results: All patients exhibited the 14-16 cpm base line component. Spontaneous migrated contractions were observed in all three patients having the colon interposition within 6 months and one patient at 1.4 year after the colon interposition. Spontaneous segmental contractions appeared in the patients having the colon interposition over 2 years. EM $1 \mathrm{mg} / \mathrm{kg} / 15 \mathrm{~min}$ induced migratory contractions in all patients having the colon interposition within 6 months, and two of four patients having the colon interposition over one year. EM $5 \mathrm{mg} / \mathrm{kg} / 60 \mathrm{~min}$ also induced migratory contractions in all patients. The induced migrating contractions were similar amplitude, duration, frequency, and motor index to spontaneous. Conclusions: The interposed colon may become quiescent time long after surgery. Erythromycin may have therapeutic potential for the treatment of interposed colonic motility.

\section{S1950}

Intestinal Gas Retention in Patients with Slow-Transit Idiopathic Constipation (STC)

Ana C. Hernando-Harder, Hermann Harder, Heinz-Juergen Krammer, Manfred V. Singer BACKGROUND: The healthy gut avoids symptomatic gas retention during a jejunal gas challenge; duodenal lipids inhibit whereas acute fasting hyperglycemia accelerates gas propulsion and evacuation. Whether dysfunction of intestinal motor activity and sensory function in patients with STC affect intestinal gas dynamics and tolerance was evaluated. METHODS On 3 separate days, jejunal gas infusion $(12 \mathrm{ml} / \mathrm{min}$ ) was performed in 10 healthy volunteers and 10 patients with STC in randomized order for $120 \mathrm{~min}$ during duodenal lipids (1 Kcal), intravenous glucose (hyperglycaemic clamp; $\sim 12 \mathrm{mmol} / \mathrm{l}$ ) or saline infusion. Rectal gas evacuation was continuously measured; perception and abdominal girth changes were separately evaluated. RESULTS: In contrast to healthy controls, patients with STC exhibited significant final intestinal gas retention during saline perfusion which was similar during duodenal lipids and fasting hyperglycemia without significant differences between the groups. The increase in abdominal perimeter correlated with volumes of trapped intestinal gas $(\mathrm{r}=$ $0,82 \mathrm{p}<0.001$; pooled data for all patients) but without significant abdominal symptoms (Table 1). CONCLUSIONS: Dysfunction of intestinal motor activity in patients with STC includes neurohormonal mechanisms for clearance of gaseous intestinal contents, but without hypersensitivity for the gaseous intestinal stimulus.

\begin{tabular}{|c|c|c|c|c|c|c|}
\hline & Healthy & & & STC & & \\
\hline & A & B & C & A & B & C \\
\hline gas retention (ml) & $52 \pm 49$ & $361 \pm 67$ & $32 \pm 67$ & $301 \pm 63^{*}$ & $351 \pm 81^{*}$ & $345 \pm 75^{*}$ \\
\hline abdominal perception (0-6 score) & $1.4 \pm 0.3$ & $1.6 \pm 0.5$ & $1.3 \pm 0.3$ & $1.6 \pm 0.3$ & $1.8 \pm 0.7$ & $1.2 \pm 0.4$ \\
\hline girth increment (mm) & $0.1 \pm 0.0$ & $0.3 \pm 0.1$ & $0.1 \pm 0.0$ & $0.5 \pm 0.4^{*}$ & $0.8 \pm 0.5^{*}$ & $0.6 \pm 0.3^{*}$ \\
\hline
\end{tabular}

Table 1: Final gas retention, abdominal sensation and perimeter changes after 2-hour jejunal gas challenge in 10 healthy volunteers and 10 patients with STC during the studies with duodenal saline (A), duodenal lipids (B) and intravenous glucose (C) $\left({ }^{*} \mathrm{p}<0.01\right.$ vs. healthy)

\section{S1951}

The Effect of Naloxone-3-Glucuronide (NX3G) On Gastro-Coecal Transit Time in Healthy Volunteers Under Morphine Medication

Peter Netzer, Michael P. Wissmeyer, Alexander Sendensky, Catarina Batista, Stefan Baumeler, Rudolf Brenneisen, Thomas Krause, Peter Reber, Juergen M. Gschossmann, Ulrich Scheurer

Background: Constipation is one of the most troublesome side effects of opioid therapy While naloxone counteracts this unwanted side effect, it also reduces the analgesic potential of opioids. Recently, we could demonstrate in an animal model that naloxone-3-glucuronide (NX3G) antagonizes the motility lowering effect of morphine in the rat colon but withou absorption by the colonic wall (1). In healthy volunteers, we showed a partial inhibition of the morphine-induced delay of colonic transit time by NX3G without absorption into the intestinal wall (2). Aim: In the current study, our aim was to determine whether a) morphine 
also delays the gastro-coecal transit time and b) orally administered NX3G reduces this morphine-induced delay in healthy volunteers. Methods: Fourteen male volunteers were included into this double-blind, randomized 3-times crossover study. Gastro-coecal transit time was measured by scintigraphy. At the start of the experiment, NX3G $(0.16 \mathrm{mg} / \mathrm{kg} \mathrm{b}$.wt.) or placebo (crossover) was given orally together with the radio-nucleotide capsule. Thereafter, NX3G or placebo was administered 5 times every 4 hours. Morphine $(0.05 \mathrm{mg} / \mathrm{kg}$ b.wt.) or placebo (crossover) was injected subcutaneously 2 hours after start and thereafter 3 times every 6 hours. Finger pain tests were performed at start and 1 hour after morphine injection. Standardized meals were given during the day after the first medication. Results: In these 14 volunteers the slowest transit time was measured during the morphine phase (6.21hrs), which was significantly $(\mathrm{p}<0.05)$ longer than in the morphine/NX3G phase $(4.57 \mathrm{hrs})$ and the placebo phase ( $3.32 \mathrm{hrs}$ ). However, there was no statistically significant difference between the morphine/NX3G phase and the placebo phase ( $>>0.1)$. Pain perception was not significantly influenced by NX3G. Conclusion: In healthy volunteers, morphine significantly reduces the gastro-coecal transit time. This effect could be reversed by NX3G. Thus, our data suggest that NX3G may be a helpful therapeutic tool for prevention and therapy of constipation in patients under morphine medication. References: 1)Reber P et al. The effect of naloxone3-glucuronide (NXG) and N-methylnaloxone (MNX) on the motility of the isolated, perfused rat colon after morphine stimulation. Gastroenterology 2004;126:A219. 2)Netzer P et al The effect of naloxone-3-glucuronide on colon transit time in healthy volunteers under morphine medication. Gastroenterology 2005;128:A275

\section{S1952}

Evidence for Alterations in Central Autonomic Activity During Sleep in Women with Diarrhea-Predominant Irritable Bowel Syndrome (IBS-D) Heng Y. Wong, Emeran A. Mayer, Bruce D. Naliboff, Britta Dickhaus, Teresa I. Olivas, Vanessa Z. Ameen, Joyce Reinholdt, Lin Chang

Background: Patients with irritable bowel syndrome (IBS) show evidence for alterations in waking autonomic nervous system (ANS) activity, which may contribute to IBS symptoms such as alterations in bowel habits. It is not known if these ANS alterations are due to enhanced ANS responsiveness to environmental stimuli, or to a tonically increased ANS activity. Aims: 1) To quantify nocturnal ANS activity (colonic motility, cardioautonomic tone, plasma norepinephrine (NE) levels) during sleep; and 2) To determine the relationships between this tonic ANS activity and subjective GI symptom ratings in female patients with IBS-D. Methods: 11 female Rome II positive IBS-D patients (mean age $38 \pm 2.5 \mathrm{yrs}$ ) and 13 female age-matched healthy controls (Ctrls, mean age $33.8 \pm 2.7 \mathrm{yrs}$ ) were admitted for a 2 night sleep study to the General Clinical Research Center (GCRC). Patients with psychiatric, sleep, other chronic pain, or cardiovascular disorders, or who were on drugs that interfered with the ANS or sleep were excluded. Current gastrointestinal (GI) symptoms were selfassessed from none to very severe. Left colonic motility (motility index, MI), heart rate variability (HRV) and plasma NE were measured simultaneously at 90 min intervals during the second night of sleep after 1 night of acclimatization. HRV measures included power of the high frequency band (HF) to assess vagal tone and ratio of low frequency (LF)/HF to assess sympathetic/parasympathetic balance. Results: Compared to Ctrls, IBS-D patients had higher plasma NE levels $(\mathrm{P}<0.05)$ and lower cardiovagal tone (HF; $\mathrm{P}=0.08)$ throughout the sleep period. In contrast, colonic MI only showed a significant difference during the initial sleep period. Nocturnal NE levels correlated positively with overall GI symptom severity $(r=0.55$ to $0.76, P<0.05)$. Overall, cardiovagal tone correlated negatively with colonic MI $(\beta=-9.7 ; P=0.006)$. However, there was a significant interaction between groups, i.e., the slope was steeper in Ctrls than IBS-D $(\mathrm{P}<0.01)$. Conclusions: Female IBS-D patients show evidence for altered central ANS activity during sleep. While colonic motor activity is only altered during early sleep, patients show enhanced sympathetic/vagal balance throughout the night, and this alteration correlates with the severity of GI symptoms in IBS-D. These findings implicate alterations in nocturnal ANS tone in the pathophysiology of IBS symptoms. The IBS-related differences in the relationship between cardiac and colonic autonomic activity suggests alterations in central autonomic pathways. Supported by NIH grants GCRC \#M01RR00865, P50 DK64539, DK 48351 and GlaxoSmithKline.

\section{S1953}

Mechanisms Underlying the Neurokinin A - Induced Contraction of GuineaPig Taenia Coli

Takanori Nakatsuji, Satoshi Ieiri, Junko Akiyoshi, Tomoaki Taguchi, Sachiyo Suita

Background and Aims:Neurokinin A (NKA) belongs to tachykinins which are a family of neuropeptides that also includes substance P (SP) and neurokinin B (NKB). Tachykinins have a wide range of physiological functions including smooth muscle contraction, particularly in the gastrointestinal tract. However, no detailed analysis in the effects of NKA on digestive smooth muscle using a direct measurement of the intracellular $\mathrm{Ca}^{2+}$ concentration $\left(\left[\mathrm{Ca}^{2+}\right] \mathrm{i}\right)$, has yet been reported before. The mechanisms for the contraction induced by neurokinin A (NKA) in the smooth muscle of guinea-pig taenia coli were investigated by means of simultaneous measurements of the $\left[\mathrm{Ca}^{2+}\right] \mathrm{i}$ and tension. Methods:Hartley guineapigs (male, 6 week, body weight $350-400 \mathrm{~g}$ ) were used throughout the experiments. The guinea-pig taenia coli strips were loaded with $\mathrm{Ca}^{2+}$ indicator dye, fura-2 in the form of acetoxymethyl ester (Fura-2/AM). The peak level of both the force development and the fluorescence ratio in response to agonists was expressed as a percentage, assigning the values of $5.9 \mathrm{mM} \mathrm{K}^{+}$PSS and the steady state contraction induced by $60 \mathrm{mM} \mathrm{K} \mathrm{K}^{+}$PSS depolarization to be $0 \%$ and $100 \%$, respectively. Results: NKA (100 pM-10 $\mu \mathrm{M})$ caused a concentrationdependent contraction. A maximum response was obtained at $l \mu \mathrm{M}$ and the $\mathrm{EC}_{50}$ value was $84.1 \pm 12.9 \mathrm{nM}$. The peak levels of the $\left[\mathrm{Ca}^{2+}\right]$ i elevations induced by $1 \mu \mathrm{M}$ NKA was $104 \pm 3.80 \%$, whereas those of force development induced by $1 \mu \mathrm{M}$ NKA was $153 \pm 22.8 \%$. The rise in $\left[\mathrm{Ca}^{2+}\right]$ i induced $1 \mu \mathrm{M}$ NKA was similar to the increase in $\left[\mathrm{Ca}^{2+}\right] \mathrm{i}$ induced by $60 \mathrm{mM} \mathrm{K}+$ PSS depolarization, but the developed force during the contraction induced by $1 \mu \mathrm{M}$ NKA was significantly larger than that of $60 \mathrm{mM} \mathrm{K}^{+}$PSS depolarization. Both the Ltype $\mathrm{Ca}^{2+}$ channel blocker, diltiazem and the NK2 receptor selective antagonist, NK2-ra significantly inhibited the NKA induced $\left[\mathrm{Ca}^{2+}\right]$ e elevation and contraction. The NK1 receptor selective antagonist, TAK637 had no effect on the NKA induced contraction.NKA induced only transient increases in $\left[\mathrm{Ca}^{2+}\right] \mathrm{i}$ and the force in a $\mathrm{Ca}^{2+}$ - free solution in smooth muscle of guinea-pig taenia coli.Rho kinase inhibitor, Y-27632, significantly inhibited the NKA induced force development, while it had no influence on the $\left[\mathrm{Ca}^{2+}\right]$ i elevation. Conclusion:NKA was found to induce the contraction of the guinea-pig taenia coli by elevating $\left[\mathrm{Ca}^{2+}\right] \mathrm{i}$, as well as increasing, the $\mathrm{Ca}^{2+}$ sensitivity of the contractile apparatus. NKA induced elevation of $\left[\mathrm{Ca}^{2+}\right]$ i is caused by not only the $\mathrm{Ca}^{2+}$ influx but also by the $\mathrm{Ca}^{2+}$ release from the sarcoplasmic reticulum. The contraction induced by NKA based on $\left[\mathrm{Ca}^{2+}\right]$ i elevation was enhanced through the rho kinase pathway.

\section{S1954}

Purinergic P2X Neuro-Neuronal Transmission Underlies the 5HT3 Receptor Mediated Inhibition of Rabbit Distal Colon Propulsion

Luciano Onori, Annalisa Aggio, Giovanni Latella, Giuseppe Frieri, Gennaro Taddei, Dina Melideo, Marcello Tonini

BACKGROUND:Purine P2X receptors(P2X r) and serotonin 5HT3 receptors(5HT3 r) may contribute to cholinergic nicotinic receptors in the generation of fast-excitatory transmission in the enteric nervous system. We reported that neurally located P2X $\mathrm{r}$ and 5HT3 $\mathrm{r}$ may mediate either enhancement or inhibition of rabbit distal colon velocity propulsion. The inhibitory role of P2X and 5HT3 $\mathrm{r}$ was inferred by the prokinetic effect induced by lower concentrations of their receptor antagonists, pyridoxal phosphate-6-azophenyl-2' 4'-disulphonic acid (PPADS)(from 1X10-8M to 1 X10-7M) and Ondansetron (OND)( from 1X10$9 \mathrm{M}$ to $1 \mathrm{X10}-8 \mathrm{M}$ ), respectively. These enhancing effects on propulsion where reverted in an inhibitory effect in the presence of Ng-nitro-L-arginine(L-NNA), a NO-synthase inhibitor.The latter finding indicated that the inhibitory role of P2X $\mathrm{r}$ and 5HT3 $\mathrm{r}$ was mediated by a common mechanism represented by the final activation of a nitrergic pathway. AIM:To assess whether P2X r and 5HT3 r activate the nitrergic inhibitory mechanism independently of each other (receptors located in parallel neural circuitries) or by interacting along a sole circuitry (receptors located in series). METHOD:The propulsion was studied in vitro and elicited by distending an intraluminal balloon with 0.3 and $1 \mathrm{~mL}$ of water. The corresponding velocity of oro-aboral balloon displacement was 2.3 and $2.7 \mathrm{~mm} / \mathrm{sec}$,respectively. The role of P2X receptors was investigated by PPADS, a compound that at relatively low concentration (less than $1 \times 10-5 \mathrm{M}$ ) has been used as antagonist for neural P2X receptors in the guinea pig ileum (Bian, J Physiol. 2000, Monro; Neurogastr. 2002). The drugs were applied to the bathing bulk solution and the effect was expressed as \% of the basal value or of the velocity induced by the former compound in the sequential administration. RESULTS: In the presence of OND, which (at $1 \times 10-8 \mathrm{M}$ ) induced the enhancing effect on propulsion by $38.7 \%$ and $25.3 \%$ at 0.3 and $1 \mathrm{~mL}$ of distention, PPADS ( $1 \times 10-7 \mathrm{M})$, further induced a pro-propulsive effect by $26.3 \%$ and $23.2 \%$, respectively. Conversely, in the presence of PPADS (1x10-7M), which induced an enhancing effect by $58.8 \%$ and $27.3 \%$,at 0,3 and $1 \mathrm{ml}$ of distention, OND (1x10-8M), induced an inhibitory effect by $40.1 \%$ and $44.3 \%$, respectively. CONCLUSIONS: Our results indicate that a neuro-neuronal purine P2X $\mathrm{r}$ transmission mediates the serotonin-5HT3 $\mathrm{r}$ inhibition of the rabbit distal colon propulsion, suggesting that P2X $\mathrm{r}$ and $5 \mathrm{HT} 3 \mathrm{r}$ are located in series. Furthemore, the blockade of P2X r unveils the 5HT3 r-mediated enhancement of propulsion which, therefore, appears to be independent of purinergic P2X receptor transmission

\section{S1955}

CB1 and TRPVI Receptors Mediate Protective Effects On Colonic Electrophysiological Properties in Mice A. Sibaev, F Massa, B Yuece, G Marsicano, H A. Lehr, B Lutz, B Goeke, H D. Allescher, Martin Storr

Background: $\mathrm{CBI}$ and TRPVI receptors modulate enteric neurotransmission and colonic inflammation. AIM: The aim of this study was to investigate early electrophysiological changes in distal colon of wild-type and receptor deficient mice following an inflammatory insult set by dinitrobenzene sulfonic acid (DNBS). Methods: Colitis was induced by DNBS in CB1-/- mice, TRPV1-/- mice and their respective wild-type littermates. Electrophysiological properties consisting of membrane potentials (MP) and electrically induced inhibitory junction potentials (IJP) were evaluated at different time points. Additionally a histological colitis severity score was evaluated. Results: Inflammation caused spontaneous atropine insensitive rhythmic action potentials in CBI-/- and TRPV1-/- mice but not in wild-type animals indicating that membrane stability is disturbed, indicating a lack of protective mechanisms. Focal electrical neuronal stimulation of the myenteric plexus induced IJP in the smooth muscle cells. 24 hours after initiation of inflammation the duration of the IJP is prolonged in all animals, indicating disturbances within neuromuscular interaction. Interestingly in CB1-/- mice duration of IJP was significantly extended as compared to $\mathrm{CB} 1+/+$ mice pointing towards missing protective mechanisms in the $\mathrm{CBI} /$ - mice. Conclusion: Inflammatory insults in the mouse colon induces reproducible changes in the electrophysiological properties and such changes correlate with duration of colitis. In mutants, these electrophysiological changes display different patterns suggesting the lack of protective properties for neuromuscular interactions and membrane stability.

\section{S1956}

Platelet Activating Factor (PAF) Affects Sigmoid Smooth Muscle Contraction in Ulcerative Colitis (UC)

Sharad Kunnath, Victor E. Pricolo, Karen M. Harnett, Neal S. Leleiko, Weibiao Cao

We have previously shown that sigmoid circular muscle cells from UC patients exhibit reduced contraction and $\mathrm{Ca}^{2+}$ signaling in response to the endogenous neurotransmitter neurokinin A (NKA) and that hydrogen peroxide $\left(\mathrm{H}_{2} \mathrm{O}_{2}\right)$ is elevated in the UC circular muscle layer. The $\mathrm{H}_{2} \mathrm{O}_{2}$ scavenger catalase restored the decreased $\mathrm{Ca}^{2+}$ signal and cell shortening to normal levels, suggesting that $\mathrm{H}_{2} \mathrm{O}_{2}$ contributes to the motor dysfunction of UC. It is known that PAF levels are elevated in colonic mucosa from patients with ulcerative colitis. In the present study we examined the role of PAF in motor dysfunction and $\mathrm{H}_{2} \mathrm{O}_{2}$ 
production in UC. PAF levels were significantly elevated in sigmoid circular muscle from patients with ulcerative colitis, when compared to normal controls. Treatment of normal sigmoid circular smooth muscle cells with PAF $\left(10^{-6} \mathrm{M}, 2\right.$ hours $)$ significantly decreased NKA-induced contraction. Treatment of UC sigmoid muscle strips with the PAF antagonist CV3988 (10-6 M) for 2 hours, however, did not improve the motor dysfunction, suggesting that other inflammatory mediators e.g. $\mathrm{H}_{2} \mathrm{O}_{2}$ and IL-1 $\beta$ may be present and affect muscle contraction, even after blockade of PAF. PAF $10^{-8} \mathrm{M}$ significantly increased $\mathrm{H}_{2} \mathrm{O}_{2}$ production in sigmoid smooth muscle cells in primary culture, and $\mathrm{H}_{2} \mathrm{O}_{2}$ production was abolished by the NADPH oxidase inhibitor apocynin, suggesting that NADPH oxidases mediate PAFinduced production of $\mathrm{H}_{2} \mathrm{O}_{2}$. PAF-induced $\mathrm{H}_{2} \mathrm{O}_{2}$ was significantly reduced by the MEKl inhibitor PD98059, the P38 MAP kinase inhibitor SB203580 and the cytosolic phopholipase A2 inhibitor AACOCF3, suggesting that $\mathrm{PAF}$-induced $\mathrm{H}_{2} \mathrm{O}_{2}$ production depends on activation of MAP kinases (ERKI/2, p38) and cytosolic phospholipase A2. We conclude that PAF may contribute to motor dysfunction of sigmoid colon in UC and that PAF-induced $\mathrm{H}_{2} \mathrm{O}_{2}$ production depends on activation of MAP kinases (ERK1/2, p38), cytosolic phospholipase A2 and NADPH oxidases. Supported by NIDDK R21 DK62775-01.

\section{S1957}

Power Dynamics of Colonic Electrical Activity in Patients with Constipation and Irritable Bowel Syndrome Assessed By Transcutaneous Electrodes Manuel A. Amaris, Melchor V. Demetria, Bashar M. Attar, Daniel C. Sadowski, Martin P. Mintchev

BACKGROUND: The cause of chronic constipation is poorly understood. Recent data suggest impaired colonic myoelectrical function that manifests itself in some patients as weak or near absent electrical control activity (ECA). AIM: To determine if any abnormality in the colonic ECA can be found in patients with constipation using transcutaneous electrodes. MATERIALS AND METHODS: Ten patients with chronic constipation (age 49 \pm 13 , male/ female $=2 / 8$, intestinal habit $1.7 \pm 0.4 /$ week $), 11$ patients with constipation predominant irritable bowel syndrome (CPIBS) (age 40 \pm 10 , male/female $=2 / 9$, intestinal habit $1.3 \pm 0.6 /$ week), and 20 normal volunteers (age $40 \pm 16$, male/female $=10 / 10$, Intestinal habit $7.3 \pm 3.2 /$ week) underwent 8-channel bipolar fasting recording of colonic ECA via transcutaneous electrodes. Both groups of patients were defined by the Rome II criteria. Eight active electrodes were attached to the skin along the abdominal projections of the longitudinal axes of the right, transverse and left colon. Bipolar electrode combinations of these electrodes formed 8 ECArecording channels. Colonic electrical activity was recorded and digitally analyzed using custom-designed software in a frequency band of 0.02 to $0.2 \mathrm{~Hz}$. RESULTS. Spectral analysis showed no significant differences in the mean frequencies of patients and normals. Periods of unstable and/or weak power of ECA were recorded in 15/21 of the patients and only in $6 / 20$ of the normal volunteers. There was a consistent pattern of unstable power dynamics in patients with CPIBS. Analysis of the power stability in isolated colonic channels showed statistically significant differences $(\mathrm{p}<0.025)$ (Table 1). CONCLUSION: Weak or unstable colonic ECA may be the cause of constipation in selected patients. This may represent an increased level of physiological fluctuations in the power of ECA in some patients with irritable bowel syndrome and constipation. Further studies with a larger number of patients are needed to confirm these findings.

Table 1. Power dynamics.

\begin{tabular}{|c|c|c|c|c|c|c|}
\hline & \multicolumn{2}{|c|}{ Right Colon } & \multicolumn{2}{c|}{ Transverse Colon } & \multicolumn{2}{c|}{ Left Colon } \\
\hline & Stable & Unstable & Stable & Unstable & Stable & Unstable \\
\hline Constipation. $\mathrm{n}=10$ & 5 & $5^{*}$ & 9 & 1 & 7 & 3 \\
\hline CPIBS. $\mathrm{n}=11$ & 2 & $9^{*}$ & 2 & $9^{*}$ & 2 & $9^{*}$ \\
\hline Normals. $\mathrm{n}=20$ & 18 & 2 & 17 & 3 & 16 & 4 \\
\hline
\end{tabular}

Unstable: Power change of more than $80 \%$ for more than 2 consecutive minutes. Statistically significant differences are denoted with an asterisk*.

\section{S1958}

Serine 16 Phosphorylation-Dependent Translocation of HSP20 in Colonic Smooth Muscle Cells May Modulate HSP27 Dependent Contraction Robert R. Gilmont, Khalil N. Bitar

Background: Hsp20, a member of the stress response family of proteins is able to protect cardiac muscle cells against oxidant insult and ischemia/reperfusion injury. Hsp20 is known to bind to hsp27, and may form mixed oligomers. The PKG and PKA phosphorylation of hsp20 on serine 16 (p-ser16), is associated with relaxation, while hsp27 phosphorylation is associated with contraction in smooth muscle. Hsp27 phosphorylation leads to a translocation from the cytosolic fraction to the particulate. Objective: To characterize the role of hsp20 in colonic smooth muscle cells. To determine the presence of hsp20 in colonic smooth muscle, its phosphorylation status and if phosphorylation determines its cellular localization. Methods: 1. Cultured rabbit colonic smooth muscle cell lysates were analyzed by SDS-PAGE and Western blotting, using a commercially available antibody directed against hsp20. 2 . For indirect determination of hsp20 phosphorylation, whole cell lysates from cultured cells treated with either sodium nitroprusside (SNP), 8-bromo cAMP or acetylcholine (Ach) were separated by isoelectric focusing and analyzed by Western blotting. 3. Hsp20 was cloned by PCR using specific primers and cDNA from rat heart, and then ligated into pcDNA3.1/ myc-His. The $16 \mathrm{~S} / \mathrm{D}$ and $16 \mathrm{~S} / \mathrm{A}$ conversions were produced by site-directed mutagenesis. 4. These constructs were transfected into rabbit colon smooth muscle cells and maintained in culture under G418 selection for greater than two weeks. Particulate and cytosolic fractions were separated by high speed centrifugation. Results: 1 . The presence of hsp20 in rabbit colonic smooth muscle cells was demonstrated. 2. Changes in phosphorylation status following treatment with SNP or 8-bromo cAMP occurred slowly with maximum phosphorylation at 30 minutes post-treatment in each case, while Ach treatment induced a rapid (30 sec- 4 min) phosphorylation. 3. Phospho-hsp20 was preferentially localized in the pellet in normal smooth muscle cells. 4. In cells transfected with the $16 \mathrm{~S} / \mathrm{D}$ form, the recombinant protein is preferentially compartmentalized in the particulate fraction. 5. In cells transfected with the $16 \mathrm{~S} / \mathrm{A}$ mutant there was more protein associated in the cytosolic fraction. Summary: Phospho-hsp20 and the mimic of ser-16 phospho-hsp20 partition preferentially to the particulate fraction of colonic smooth muscle. Conclusion: Hsp20 translocates to the partic ulate fraction of colonic smooth muscle upon phosphorylation of serine residue 16. Here it may interact with hsp27 to inhibit hsp27 action and induce relaxation.

\section{S1959}

Effects of Sibutramine On Gastric Emptying, Intestinal Motility and Rectal Tone in Conscious Dogs

Junying $\mathrm{Xu}$, Jiande Chen

Background \& Aims: Sibutramine is a serotonin- norepinephrine uptake inhibitor, used in the treatment of obesity. Serotonin is a major neurotransmitter in Gastrointestinal (GI) tract It appears to be very important in the control of GI function. It has never been reported whether sibutramine affects gastrointestinal motility and myoelectrical activity. The aim of this study was to evaluate the effects of sibutramine on gastric emptying, small bowel contractions, rectal tone and compliance and GI myoelectrical activity. Methods: The stud was performed in 14 healthy female dogs $(18-27 \mathrm{Kg})$; seven of the dogs were surgically prepared for a duodenal cannula and placement of serosal recording electrodes. The study was composed of 3 separate experiments: gastric emptying of liquids and gastric and intestinal slow waves; small bowel contractions; and rectal tone and compliance. Each experimen included two sessions: a control session and a treatment session with sibutramine $(5 \mathrm{mg} / \mathrm{Kg}$ p.o, Generic Meridia, United Pharmacies) administrated 2 hours before the recording. Seven dogs with the cannula underwent a gastric emptying study of liquids and a small bowel contraction study in the fed state. Seven dogs without cannula underwent a rectal barostat study. Results: (1). Sibutramine significantly accelerated liquid gastric emptying at $75 \mathrm{~min}$ and 90 min after the meal but did not alter gastric or intestinal slow waves. Gastric emptying was $61.42 \pm 19.34 \%$ at $75 \mathrm{~min}$ and $66.32 \pm 19.38 \%$ at $90 \mathrm{~min}$ in the control session, and increased to $71.27 \pm 13.5 \%$ at $75 \mathrm{~min}$ ( $\mathrm{p}=0.046$, vs. control) and $75.93 \pm 15.05 \%$ at $90 \mathrm{~min}$ $(p=0.036$, vs. control) in the session with sibutramine. (2). Sibutramine significantly inhibited postprandial small bowel contractions. The area under the curve (AUC) was $14.57 \pm$ 4.11 in the control session and decreased to $10.64 \pm 4.18(\mathrm{p}=0.014$, vs. control) in the session with sibutramine. The contractile frequency was $12.49 \pm 0.88$ cycles/min in the contro session and reduced to $8.8 \pm 1.08$ cycles $/ \mathrm{min}(\mathrm{p}=0.0004$, vs. control) with sibutramine. (3) Sibutramine did not alter the rectal tone $(\mathrm{P}>0.05)$, but significantly increased rectal compliance The rectal compliance expressed as the slope of the pressure-volume curve was significantly increased from $7.59 \pm 2.05 \mathrm{ml} / \mathrm{mmHg}$ at baseline to $9.97 \pm 3.25 \mathrm{ml} / \mathrm{mmHg}$ after sibutramine administration $(\mathrm{p}=0.003)$. Conclusions: Sibutramine accelerates gastric emptying of liquids but inhibits small bowel contractions. These findings suggest the peripheral mechanisms of sibutramine in reducing food intake and causing weight loss in obesity patients.

\section{S1960}

Partial Replacement of Dietary Long-Chain Triglycerides (LCT) By MediumChain Triglycerides (MCT) Fed Ad Libitum Is Hepatotoxic Whereas Total Replacement Or Dietary Restriction Is Beneficial

Charles S. Lieber, Qi Cao, Leonore M. Decarli, Maria A. Leo, Ki M. Mak, Anatoly Ponomarenko, Chaoling Ren, Xiaolei Wang

We have previously shown that rats develop hepatotoxicity when given for 3 weeks our high fat NASH diet (Lieber et al. Amer J Clin Nutr 2004;79:502-509). When supplemented with MCT (replacing $35 \%$ of the dietary LCT calories) this resulted in a significant increase in hepatic inflammation associated with a rise in the inflammatory cytokine TNF- 0 (131.5 \pm 15.4 vs. $220.3 \pm 13.1 \mathrm{pg} / \mathrm{mg}$ protein; $\mathrm{p}=0.0002$ ). There was also an increase in collagen type $1(1017 \pm 201$ vs. $1947 \pm 151 \mathrm{pg} / \mathrm{mg}$ protein; $\mathrm{p}<0.001)$ as well as collagen type $1 \mathrm{mRNA}$ $(3.1 \pm 0.2$ vs. $3.93 \pm 0.3$ units; $p<0.01$ ). Since a comparable amount of MCT is being given for the treatment of obesity, we wondered how one could continue the therapeutic use of MCT while minimizing its toxicity. We now found that in rats given the same MCT diet but restricted to $2 / 3$ of the amount they were consuming when fed ad libitum, there was a striking reduction of the adverse effects, including less liver steatosis with lower hepatic triglycerides $(36.3 \pm 10.1$ vs. $81.1 \pm 10.9 \mathrm{mg} / \mathrm{gm}$ liver; $\mathrm{p}<0.01)$ and a decrease in the hepatic levels of TNF- $\alpha$ (from $220.3 \pm 13.1$ to $113.1 \pm 21.4 \mathrm{pg} / \mathrm{mg}$ protein; $\mathrm{p}<0.001$ ). There was also a reduction of collagen type I (from $1947 \pm 151$ to $1207 \pm 84 \mathrm{pg} / \mathrm{mg}$ protein; $\mathrm{p}<0.01$ ) and collagen type I mRNA (from $3.93 \pm 0.3$ to $1.97 \pm 0.3$ units; $p<0.001$ ). Furthermore, the plasma triglycerides decreased (from $288.1 \pm 22.4$ to $59.8 \pm 17.2 \mathrm{mg} / \mathrm{dl}$; $\mathrm{p}<0.001$ ). When MCT ( $35 \%$ of calories) was given together with carbohydrates (instead of LCT) there was also a reduction in hepatic TNF- $\alpha$ (from $131.5 \pm 15.4$ to $25.2 \pm 4.2 \mathrm{pg} / \mathrm{mg}$ protein; $\mathrm{p}<0.001$ ). Moreover, when $71 \%$ of the fat calories were replaced by MCT with no LCT remaining in the diet, excep for $3 \%$ of calories as safflower oil, no steatosis developed and hepatic TNF- $\alpha$ was low $(23.5 \pm 4.0 \mathrm{pg} / \mathrm{mg}$ protein). Thus, even in large amounts (such as $71 \%$ of dietary calories), MCT is not hepatotoxic provided the diet contains no significant amount of LCT. According to these rat studies, $35-40 \%$ of calories as MCT (commonly given to obese patients) should not be detrimental if no substantial LCT are simultaneously consumed. The mechanism whereby the combination of LCT with MCT acquires hepatotoxicity is now being investigated. CONCLUSION: Total replacement of dietary LCT with MCT fed ad libitum is beneficial whereas partial replacement becomes hepatotoxic, unless the dietary intake is restricted. Thus, if the adverse interaction between MCT and LCT shown in rats is verified in patients, especially obese subjects, the clinical implication is that diets containing some LCT with $40 \%$ of calories as MCT should not be given ad libitum to control obesity. 
S1961

Effects of Optifast Very Low Calorie Diet On Liver Size and Regional Adiposity

James Toouli, Mark Lewis, Madeleine Phillips, Sara Wahlroos, John Slavotinek, Robin Valentine, Lilian Kow, Campbell Thompson

Background: Obesity leads to liver steatosis and large volume livers. We determined the effect of a very low calorie diet (Optifast VLCD) on liver volume and regional lipid deposition in obese, non-diabetic females awaiting laparoscopic gastric banding surgery (LAGB) Methods: 14 obese subjects (mean BMI $45 \pm 7 \mathrm{~kg} / \mathrm{m} 2$ ) were provided with a 6 -week supply of the Optifast meal replacement and were seen two weekly in order to monitor adherence to the diet plan. Anthropometry (weight, waist circumference) and fasting blood; free fatty acids (FFA), liver enzymes, insulin and glucose were performed at baseline and following 6 weeks of the diet. Insulin sensitivity was estimated using the homeostasis model assessment (HOMA). Abdominal lipid deposition subcutaneously (SAT), viscerally (VAT) and in the liver were determined before and after 6 weeks on the VLCD using previously validated magnetic resonance spectroscopy and imaging techniques. Results: Presented as mean +/SD. All subjects adhered to the 6 weeks VLCD intervention. Mean body weight reduced by $8.5 \pm 3.0 \mathrm{~kg}$ (pre VLCD $119.2 \pm 16.0$ vs $110.6 \pm 16.9 \mathrm{~kg}$ post VLCD; $\mathrm{p}<0.001$ ). Mean liver volume and lipid content at baseline were $2.2+/-0.4 \mathrm{~L}$ and $0.13+/-0.12 \mathrm{AU}$ (range 0.01 to 0.34 ). Liver fat content in $64 \%$ of subjects $(n=9)$ was $>0.05 \mathrm{AU}$, indicative of steatosis. Baseline VAT and SAT were $0.22+/-0.11 \mathrm{~L}$ and $0.68+/-0.2 \mathrm{~L}$, respectively. Significant reductions occurred in liver volume $(0.35+/-0.35 \mathrm{~L} ; 15 \pm 12 \%)$, VAT $(0.04+/-0.05 \mathrm{~L} ; 15$ $\pm 14.8 \%)$ and SAT $(0.05+/-0.06 \mathrm{~L} ; 8 \pm 8.6 \%)$ but not in insulin sensitivity. Liver fat content reduced significantly by $63 \pm 25 \%$ in those subjects with liver steatosis. The reduction in liver fat was significantly associated with the reduction in liver size $(r=0.758, p=0.003)$. Conclusion: Adherence to a 6-week VLCD produces significant reductions in liver size and lipid content as well as VAT and SAT depots. Weight loss and VAT reduction following 6 weeks of VLCD ( $7.4 \%$ and $15 \%$ respectively) was similar to that occurring 3 months following LAGB at our centre (9\% and 18\%). In contrast to 3 months following LAGB however, 6 weeks of Optifast VLCD produces half the reduction in SAT and almost twice the reduction in liver fat. The perceived perioperative reduction in liver size seen in patients who have undergone pre-LAGB treatment with Optifast VLCD, is likely due to the preferential reduction in liver fat.

\section{S1962}

Laparoscopic Roux-en-Y Gastric Bypass Is a Viable Option in the Treatment of Mega Obesity (BMI $>70 \mathrm{~kg} / \mathrm{M} 2)$

William Watson, Bradley Needleman, Vimal Narula, S. Scott Davis, Matthew Goldblatt, W. Scott Melvin, Dean Mikam

Purpose: Super-obese patients (body mass index (BMI) $>50 \mathrm{~kg} / \mathrm{m} 2$ ), were previously not considered candidates for laparoscopic Roux-en-y gastric bypass (LRYGB) surgery. As surgical experience and technology improves, LRYGB in the super obese has become more widely accepted. The aim of this study is to define a new classification of obesity (megaobesity, BMI $>70 \mathrm{~kg} / \mathrm{m} 2$ ), as well as to evaluate the outcome of LRYGB in this patient population. Methods: We conducted a retrospective review of patients with BMI $>70 \mathrm{~kg} / \mathrm{m} 2$ undergoing LRYGB at a single university-based comprehensive weight loss program from January 2003 November 2005. We reviewed preoperative demographics, operative techniques and postoperative course. Results: Nineteen patients were eligible for the study (13 female, 6 male), with an average age of $42.1 \pm 8.3$ years of age. Preoperative mean weight was $217 \mathrm{~kg}$ (range: 180 - $297 \mathrm{~kg}$ ). Preoperative mean BMI was $76.8 \mathrm{~kg} / \mathrm{m} 2(70.1-119.0 \mathrm{~kg} / \mathrm{m} 2)$. Patients were $261 \pm 56 \%$ above their ideal bodyweight. Patients had on average $5.0 \pm 2.5$ co-morbidities attributed to obesity, and averaged $6.5 \pm 3.6$ medications for those co-morbidities. Eighteen of 19 cases were completed laparoscopically. One surgery was converted to open secondary to adhesions from previous surgeries. No patients required re-operation. There were no mortalities or anastamotic leaks. Operating times were $121.1 \pm 43.4$ minutes. Peri-anastamotic drains were placed in 9 of 19 patients (47\%) and all were removed prior to discharge. There were 4 minor postoperative complications in 4 patients: ileus, hypoglycemia, asymptomatic elevated WBC without source and myoglobinuria treated with hydration and alkylinization of urine. Hospital stays was $3.3 \pm 1.7$ days. Percent excess weight loss (\%EWL) was 14.3 $\pm 2.9 \%$ at 2 months $(\mathrm{m})$ postoperative $(\mathrm{n}=14), 24.8 \pm 4.3 \%$ at $6 \mathrm{~m}$ postoperative $(\mathrm{n}=8)$ and $42 \pm 6.6 \%$ at $12 \mathrm{~m}(\mathrm{n}=-6)$. Five of 19 patients (26\%) developed superficial wound infections. Two patients (11\%) developed anastamotic strictures. Four of 9 patients (44\%) reported decreased insulin requirements, 4 of 9 patients (44\%) discontinued as least 1 oral hyperglycemic agent and 3 of 12 patients (25\%) stopped at least one antihypertensive drug by 2 months postoperatively. Summary: LRYGB is a technically feasible surgery in the megaobese. Operative times are similar to LRYGB patients with a BMI of $<70 \mathrm{~kg} / \mathrm{m} 2$. Superficial wound infections appear to be more prevalent in the megaobese. Percent EWL at 6 months are similar to those with a BMI of $<70 \mathrm{~kg} / \mathrm{m} 2$. Long term follow-up will provide more information on resolution of comorbidities. LRYGB is a viable treatment option in the treatment of megaobesity.

\section{S1963}

Sibutramine Significantly Delays Gastric Emptying Without Altering Volume Or Postprandial Symptoms in Obesity

Maria I. Vazquez-Roque, Debra Stephens, Duane Burton, Kari Baxter, Michael Ryks, Matthew Clark, Michael D. Jensen, Sarah Kalsy, Karen Graszer, Alan R. Zinsmeister, Michael Camilleri

Background: Sibutramine is associated with weight loss and acts as a noradrenergic (NE) and serotonergic (5-HT) reuptake inhibitor. NE and 5-HT alter gastric functions. It is unclear whether weight loss with sibutramine is associated with altered motor and sensory functions of the human stomach. Aim: To compare in a randomized, double-blinded, controlled study with concealed allocation the effects of placebo and sibutramine, $15 \mathrm{mg}$ daily for 12 weeks, on gastric volumes, emptying, maximum tolerated volumes (MTV) and post-challenge symptoms in obese volunteers who were otherwise healthy. Methods: We planned a study of 24 obese [body mass index (BMI) $>30 \mathrm{~kg} / \mathrm{m}^{2}$ ] participants and documented lean mass and \% fat by body composition (DEXA). All subjects underwent at baseline and post-treatment the following measurements using validated tests: gastric emptying for solids and liquids by scintigraphy (GE tl/2); gastric volumes by SPECT; maximum tolerated volume and 30 minute post-challenge symptoms by satiation test. All participants received the same behavioral modification classes using the LEARN manual Statistical analysis used ANCOVA with baseline observations, gender, and BMI as covariates. Results: To date, 20 people have completed all studies. One subject was withdrawn due to hypertension. The table summarizes the data baseline and post-treatment, mean \pm SEM. Changes in weight were $-0.9 \pm 1.2 \mathrm{~kg}$ for placebo and $-4.4 \pm 0.9 \mathrm{~kg}$ for sibutramine $(\mathrm{p}=0.034)$. Changes in BMI were $-0.1+0.4 \mathrm{~kg} / \mathrm{m}^{2}$ for placebo and $1.6 \pm 0.3 \mathrm{~kg} / \mathrm{m}^{2}$ for sibutramine $(\mathrm{p}=0.011)$; changes were somewhat more pronounced in females. No significant differences in lean mass or $\%$ fat were noted in the two treatment groups. There was significantly delayed gastric emptying of solids and liquids with sibutramine, but no treatment differences in gastric volumes or satiation. Conclusion: Delayed gastric emptying may contribute to the weight reduction in response to sibutramine in obese people.

\begin{tabular}{|c|c|c|c|c|}
\hline & $\begin{array}{c}\text { Placebo, } \mathrm{n}=10, \\
\mathrm{BL}\end{array}$ & $\begin{array}{c}\text { Placebo, } \\
\text { POST }\end{array}$ & $\begin{array}{c}\text { Sibutramine, } \mathrm{n}=10, \\
\mathrm{BL}\end{array}$ & $\begin{array}{c}\text { Sibutramine, } \\
\mathrm{n}=10\end{array}$ \\
\hline Age, $\mathrm{y}$ & $45 \pm 4$ & $45 \pm 4$ & $43 \pm 4$ & $42 \pm 4$ \\
\hline Lean mass x $10^{-3}$ & $50.9 \pm 4.6$ & $48.8 \pm 3.3$ & $48.7 \pm 3.6$ & $52.1 \pm 3.1$ \\
\hline Tissue fat, $\%$ & $49.2 \pm 3.4$ & $47.4 \pm 0.66$ & $47.6 \pm 2.3$ & $46.2 \pm 0.66$ \\
\hline Fasting gastric vol, $\mathrm{ml}$ & $215 \pm 15$ & $243 \pm 20$ & $257 \pm 20$ & $232 \pm 21$ \\
\hline$\Delta \mathrm{PP}$ vol, $\mathrm{ml}$ & $453 \pm 26$ & $446 \pm 22$ & $464 \pm 27$ & $435 \pm 22$ \\
\hline $\mathrm{MTV}, \mathrm{ml}$ & $1289 \pm 133$ & $1242 \pm 94$ & $1224 \pm 71$ & $1129 \pm 94$ \\
\hline $\begin{array}{c}\text { Aggregate symptom } \\
\text { score }\end{array}$ & $158 \pm 22$ & $178 \pm 21$ & $122 \pm 14$ & $174 \pm 21$ \\
\hline GE t1/2 solids, min* & $119 \pm 16$ & $108 \pm 6$ & $119 \pm 12$ & $129 \pm 6$ \\
\hline GE t1/2 liquids, min\# & $21 \pm 4$ & $15 \pm 4$ & $21 \pm 3$ & $30 \pm 4$ \\
\hline
\end{tabular}

$* \mathrm{p} \leq 0.05 ; \# \mathrm{p} \leq 0.01$

\section{S1964}

Pre-PYLORIC Neural Electrical Stimulation Produces Cholinergically-Mediated Retrograde Peristalsis in Acute Canine Model of Microprocessor-Invoked Gastric Motility for the Treatment of Obesity

Emil Neshev, Denis Onen, Ehsan Jalilian, Martin P. Mintchev

BACKGROUND. Gastric electrical stimulation (GES) has been suggested as an alternative minimally-invasive surgical procedure for the treatment of obesity. Among the three known GES methods, neurostimulation (NGES) provides direct control of contractility without utilizing the spontaneous gastric electrical activity as an intermediary. AIM. We explored the possibility of producing NGES-controlled retrograde peristalsis in acute canine models, utilizing single and dual electrode sets implanted in the pre-pyloric area. METHODS. Eight healthy mongrel dogs underwent laparotomy and subserosal implantation of one distal and one proximal set of $10-\mathrm{mm}$ temporary cardiac pacing electrode pairs located at the opposite sides of the imaginary circumference perpendicular to the gastric axis in the distal antrum. The distal set was placed $2-3 \mathrm{~cm}$ proximally from the pylorus, and the second set was implanted 3.5-4 cm proximally to the first. In four of the dogs the electrodes were implanted parallel to the gastric axis. In the remaining four dogs the electrode implantations were perpendicular to the gastric axis. In both groups gastric motility patterns were captured using three force transducers implanted $3.5-4 \mathrm{~cm}$ apart along the projection of the gastric axis on the anterior wall. Custom-designed implantable neurostimulator was utilized for delivering controlled, charge-balanced, bipolar, $50-\mathrm{Hz} 16-\mathrm{V}$ (peak-to-peak) 8-second rectangular voltage waveforms to the distal electrode set only, and subsequently to both electrode sets in a sequential and overlapping fashion, starting with the distal electrode set. Normalized motility indices were utilized to quantify the produced contractions, and their propagation velocities were assessed. At the end of each test $0.05 \mathrm{mg} / \mathrm{kg}$ atropine sulfate i.v. was administered and NGES was re-applied. RESULTS. Both single and dual electrode set NGES produced circumferential lumen-occluding contractions in the vicinity of the electrode sets. The invoked contractions propagated in a retrograde fashion. The scope of the propagated contractions was 10-12 cm for dual-set stimulation, and 5-8 cm for single-set stimulation. Propagation velocities of the invoked retrograde contractions using parallel electrode implantations were significantly higher $(\mathrm{p}<0.05)$. Motility indices dynamics reflected by the three force transducers followed similar pattern regardless of the electrode implantation technique utilized. Dual-set NGES resulted in higher motility indices. Atropine sulfate inhibited all NGESinvoked contractions. CONCLUSION. Pre-pyloric NGES can produce controlled cholinergically-mediated retrograde peristalsis with minimal surgical intervention.

S1965

A Multi-Component Model Can Predict Outcome of Restrictive Surgery for Obesity At Baseline and 3 Months

Naeem Aslam, Hani Rashed, Atul K. Madan, David S. Tichansky, Teresa Cutts, William D Johnson, Thomas L. Abel

Introduction: We previously reported that a multi-component model of autonomic and enteric factors may correlate with ultimate weight loss or gain after restrictive obesity surgery (NGM 2005; 17:472). Patients: We report on 39 patients, 4 male, 35 female, mean age 37.2 years, followed for up to 16 years post-operatively after vertical banded gastroplasty. Methods: Two autonomic measures (adrenergic: PAR and VC and cholinergic: RRI) and one enteric 
measure (electrogastrogram: EGG) were recorded at baseline as previously described (DDS 44: $74 \mathrm{~s}-78 \mathrm{~s}, 1999)$. We performed a discriminant function analysis to investigate whether a patient's EGG, PAR, RRI, and VC values could be used to classify that patient as a loser or gainer following weight control surgery. The patients were divided into two categories (10 gainers, 29 losers), depending on the latest weight compared to baseline; discriminant criterion derived from the patient's data was applied to each patient's autonomic and enteric values to determine whether these measurements separated the patients into their true weight category. Results: A discriminate model based on baseline measures successfully predicted ultimate weight gain in $8 / 10(80 \%)$ of patients who subsequently gained weight and weight loss in 24/29 (83\%) of patients who in subsequently lost weight for a total correct classification rate of 32/39 (82\%). The same model with data at 3 months post-operatively predicted weight gain in 9 of $10(90 \%)$ of patients and weight loss in 24 of $29(83 \%)$ of patients, for a total correct classification rate of $34 / 39$ pts (87\%) (see table). Conclusions: A multicomponent model demonstrates that baseline and 3 months post-operative measures can predict ultimate weight outcome from restrictive obesity surgery.

\begin{tabular}{|c|c|c|c|c|c|c|}
\hline Time & Predicted Baseline & Outcome Lose & Total & 3 Month Predicted & Outcome Lose & Total \\
\hline Result & Gain & Lose & Total & Gain & Lose & Total \\
\hline Gain & $8(80 \%)$ & 2 & 10 & $9(90 \%)$ & 1 & 10 \\
\hline Lose & 5 & $24(83 \%)$ & 29 & 5 & $24(83 \%)$ & 29 \\
\hline
\end{tabular}

Four Component Model

\begin{tabular}{|c|c|c|c|c|c|c|}
\hline Time & Predicted Baseline & Outcome Lose & Total & 3 Month Predicted Gain & Outcome Lose & Total \\
\hline Gain & $8(80 \%)$ & 2 & 10 & $9(90 \%)$ & 1 & 10 \\
\hline Lose & 5 & $24(83 \%)$ & 29 & 5 & $24(83 \%)$ & 29 \\
\hline
\end{tabular}

\section{S1966}

Effects of Duodenum/Ileum Electrical Stimulations On Duodenum DistentionResponse (DD-R) Neurons in the Vmh of Rats J.N. Zhang, J D Z Chen

Background and Aims: Intestinal electric stimulation (IES, especially Duodenum electrical stimulation) has been shown to produce an inhibitory effect on gastric motility/secretion, gastric emptying and food intake in rats, suggesting a therapeutic potential of IES for obesity. However, the small intestine is lengthy and it is unknown whether stimulation at the proximal intestinal has a similar effect with stimulation at the distal intestine. It is well established that the VMH in the hypothalamus is closely related to the regulation of feeding behavior and plays an important role in the mediation of satiety. The aims of this study were to 1 ). explore the hypothesis that the neurons in the $\mathrm{VMH}$ are involved in the central mechanisms of the IES treatment of obesity; 2). compare the effects of IES at the duodenum and at the ileum on neuronal activities of the VMH. Methods: Extracellular potentials of single neurons in the VMH were recorded in 18 anesthetized rats. After VMH neurons with duodenum distension $(0.5 \mathrm{ml}, 10-30 \mathrm{~s})$ were identified, IES at the duodenum or ileum with 3 sets of parameters were performed for one minute randomly via one of 2 pair of platinum electrodes sutured onto the serosal surface of the duodenum $2-4 \mathrm{~cm}$ below the pylorus or $3-4 \mathrm{~cm}$ above the ileocecal junction. IES1 (pulse trains: 4mA, 2s-on, 3s-off, 2ms, 20Hz), IES2 (long pulse: $6 \mathrm{~mA}, 20 \mathrm{cpm}, 100 \mathrm{~ms}$ ), IES3 (same as IES1 but $40 \mathrm{~Hz}$ ). Results: Fifty nine percent of 34 (59\%) neurons in the VMH were responsive to duodenum distension (DD, $0.5 \mathrm{ml}, 10 \sim 30 \mathrm{~s}$ ). IES1 at duodenum and ileum activated $70.6 \%$ and $73.3 \%$ of the DD-responsive neurons respectively $(\mathrm{P}=1.0)$; Similar percentages of the neurons were activated with IES3 at the duodenum or ileum $(70.6 \%$ vs. $66.7 \% \mathrm{P}=0.91)$. However, IES2 at these locations activated only $25 \%$ and $46.2 \%$ of these neurons. Conclusions: IES with different parameters can activate the VMH neurons responsive to DD (25-73.3\%) and the pattern of stimulus (pulse trains or long pulses) plays an important role. However, it seems that the location of electrical stimulation in the small intestine does not alter its effects on the neuronal activity in the $\mathrm{VMH}$ of rats.

\section{S1967}

Intragastric Balloon for Refractory Obesity: Poor Compliance with Dietary Education and Follow-Up Does Not Preclude Weight Loss

Erik Francois, Jean Marc Dumonceau, Marie Barea, Karine Buedts, Michael Piagnerelli, Jacques Deviere, Axel Hittelet

Background: the intragastric balloon (IB) has been proposed as an aid to weight loss for patients with moderate obesity. By increasing satiety, the IB helps patients learn and adhere to new eating habits, which must be maintained after balloon removal if weight is to be stabilized. Thus, dietary education and follow-up before, during, and after IB placement are strongly recommended. Methods: From July 1999 to June 2004, 80 patients (68 women; body mass index, or BMI, range 28.4 to $58.5 \mathrm{~kg} / \mathrm{m} 2$; mean \pm SD: $36.9 \pm 7.8$ ) were treated with the BioEnterics ${ }^{\circledR}$ Intragastric Balloon (Inamed corporation, Santa Barbara, California, USA), placed endoscopically under sedation with midazolam or anesthesia with propofol. Patients were screened by a dietician before placement, and monthly dietary follow-up was prescribed thereafter. Results: IB placement was successful and uneventful in all cases. Three patients were lost to follow up. One-year post-removal follow-up was obtained in 77 patients. One IB migrated spontaneously and 76 IB were removed endoscopically, 3 of which had spontaneously deflated while remaining in the stomach (spontaneous deflation rate: $5 \%$ ). Early removal ( $<3$ months) for intolerance was necessary in $14 \%$ of patients. The only other complication was one case of severe hypokalemia. Mean duration of IB placement was 180 days (range: 8 to 548 days). Mean weight loss at removal was $11.3 \mathrm{~kg}( \pm 8.5 \mathrm{~kg})$; mean $\%$ of excess weight lost (EWL) was $44.7 \%( \pm 39.2 \%)$. Twenty-six percent of the patients lost less than $20 \%$ of excess weight. Six months after IB removal, the mean EWL was $26.2 \%$
$( \pm 40.3 \%)$ and was maintained at $22.4 \%( \pm 58.5 \%)$ at one year. Weight loss was greater if BMI was $>40 \mathrm{~kg} / \mathrm{m} 2$ (mean: $16.9+/-13.2 \mathrm{~kg}$ ) than if BMI was $<35 \mathrm{~kg} / \mathrm{m} 2$ (mean: $10 \pm 6$. $\mathrm{kg})(\mathrm{p}=0.01)$. Only 10 out of 80 patients were fully compliant with the prescribed dietary follow-up during IB and after removal. There was a trend to greater weight loss in the compliant group, but statistical significance was not achieved. Overall, $43 \%$ of the patients were satisfied with the IB. Conclusions: The IB appears to be safe and moderately effective in achieving sustained weight loss 1 year after removal despite poor compliance to dietary follow-up in this series.

\section{S1968}

Rediscovered Therapeutic Option for Morbid Obesity: Multicenter Pilot Study with a New Pneumatic Stomach Balloon

Yogesh Shastri, Irina Blumenstein, Udo Martin, Stephan Haass, Jurgen Stein, Wolfgang Caspary, Nicolas Hoepffner

Background: Morbid obesity is a rapidly growing problem mainly of western society. Over $60 \%$ of the American population is either obese or overweight. Conventional treatmen with reduced caloric intake, increased physical activity and pharmacotherapy are minimally effective in inducing sufficient weight loss, however the loss weight is often regained once the treatment is stopped. Given the comorbidities in these obese high risk patients, experienced bariatric surgeons often advocate a less invasive first stage intervention. The endoscopic implantation of various balloons in 80's and 90's could not come into vogue because of its associated complications. Since 2004 a new pneumatic intra-gastric balloon has come into practice. Ours is the first study reporting the safety, effectiveness, and tolerance of this new gadget in clinical practice. Patients and Methods: A pneumatic intra-gastric balloon system (heliosphere $®$ bag, Helioscopie, France) was placed endoscopically 27 times in 24 patients in a multicenter pilot study since September 2004 till November 2005. They were followed up prospectively with data about symptoms, complications, loss of weight, BMI, etc. Results There were 12 male and 12 females (age range 24-60, median 43 yrs) with a BMI range of 30-57 (median 37) and a body weight of 71-194 kg (median $113 \mathrm{~kg}$ ). In 2 female and 1 male patients the procedure was repeated twice (after 5, 10 and 11 months). There were no procedure related complications. $16(59 \%)$ of patients had balloon induced immediate side effects like occasional nausea with vomiting lasting 3 days in 6 patients (22\%), 7 patients $(32 \%)$ had more severe nausea, vomiting and abdominal pain, whereas $3(11 \%)$ patients required admission and intravenous fluid administration for the same. In two patients we had to perform premature extraction of the balloons after 5 and 14 days of implantation because of intolerance and severe bleeding (Dieulafoy's ulcer) respectively. The remaining balloons were explanted 9 months (range 5 to 13 months) after implantation. Except for one patient all patients lost weight ranging from 1 to $29 \mathrm{~kg}$ with a fall in BMI ranging from 1 to 9. Conclusion: With this new found "old" technique the results are promising. It wa efficient in inducing weight loss in mild or moderate obesity and can also be very useful for preoperative weight reduction as a first stage, low risk and less invasive procedure in morbidly obese patients.

\section{S1969}

Post Adjustable Gastric Banding Syndrome. pH-Metric and Manometric Recordings in 19 Patients

Mohamed Merrouche, Jean Marc Sabate, Pauline Jouet, Simon Msika, Severine Ledoux, Benoit Coffin

Background: Adjustable gastric banding (GB) is one of the most common surgical procedure for the treatment of morbid obesity. However, GB frequently induces upper gastrointestinal symptoms. The consequences of $\mathrm{GB}$ on acid reflux and oesophageal motility have been poorly evaluated. The aim of this study was to describe $\mathrm{pH}$-metric and manometric abnormalities in patients complaining of upper gastrointestinal symptoms after adjustable GB for morbid obesity. Patients and Methods: a standardized questionnaire, 24-hrs pH-metric recording (\% of time with $\mathrm{pH}<4$ ) and esophageal manometry [lower esophageal sphincter pressure (LES), morphology, amplitude and propagations of oesophageal contraction waves] were performed in patients with GB addressed for upper gastro-intestinal symptoms and/or GB failure (insufficient weight loss) to a tertiary unit specialized in the treatment of morbid obesity. Results: (mean \pm SD) 19 patients (F: 17 ; age $44.0 \pm 10.2$ yrs) were included 24 to 36 months after GB. BMI was $45.8 \pm 6.9 \mathrm{~kg} / \mathrm{m} 2$ (range : $39.9-67 \mathrm{~kg} / \mathrm{m} 2$ ) before surgery and $37.8 \pm 9.2 \mathrm{~kg} / \mathrm{m} 2$ (range: $22.2-59.4 \mathrm{~kg} / \mathrm{m} 2$ ) at the time of the study. Mean weight loss was $20.8 \pm 14.4 \mathrm{~kg}$ (range : $0-50 \mathrm{~kg}$ ). None of these referred patients had had preoperative $\mathrm{pH}$-metry or manometric recordings. At the time of the study, 13 patients $(68.4 \%$ ) had one or more upper gastrointestinal symptoms: vomiting more than 3 times a week in 9 (47.3 $\%$ ), dysphagia in $6(31.6 \%)$, heartburn in $5(26.3 \%)$ and epigastric pain in $3(15.7 \%)$. pHmetry was abnormal in 6 out of 15 patients $(40 \%)$ with a percentage of time with $\mathrm{pH}<4$ of $29.5 \pm 24.8 \%$ (range: $8.5-71 \%$ ). Esophageal manometry was abnormal in 12 out of 19 patients with incomplete LES relaxation in $9(47.4 \%)$ associated with abnormal oesophageal contractions in morphology, amplitude and duration; presence of non peristaltic waves were also noted. Conclusion: the presence of major reflux disease and oesophageal motor disorders that could be defined as pseudo-achalasia are frequent in patients consulting for upper gastrointestinal symptoms and/or insufficient weight loss after adjustable gastric banding These preliminary results in a small cohort of symptomatic patients without preoperative data suggest that complete oesophageal investigations are mandatory before performing bariatric surgery with adjustable GB, as it has been recommended before antireflux surgery. 


\section{S1970}

Incidence of Stomal Stricture and Efficacy of Endoscopic Intervention: A Tertiary Center's Experience with Gastric Bypass Surgery

Michael A. Veliuona, Fedele J. Depalma, Robert N. Cooney, Abraham Mathew

Introduction: The Roux-en-Y gastric bypass is a highly effective surgical approach for morbid obesity but is not without associated complications. We report our institution's experience with gastric bypass surgery and endoscopic intervention in treating stomal strictures. Methods: Patients who had undergone gastric bypass surgery at our institution between January 2000 and August 2004 were identified. Medical records were reviewed to obtain pertinent patient demographics and peri-operative data and outcomes. Results: Eight hundred and eighty eight patients underwent gastric bypass surgery at our institution by one of five surgeons. Among these patients, 94 (10.6\%) underwent esophagogastroduodenoscopy (EGD) for evaluation of possible stomal stricture and $58(6.5 \%)$ were found to have one. Laparoscopic approach was associated with more strictures ( 43 or $74.1 \%, \mathrm{p}=0.0003$ ). When controlled for the laparoscopic procedures, stomal stricture rate was similar between high volume and low volume surgeons. A total of 125 dilations were performed with an average of 2.2 dilations per patient (range 1-7). There was a total of 4 perforations (3.2\%) related to endoscopic dilation and $3(75 \%)$ perforations occurred in patients who had undergone repeated EGD and dilations (2, 5, 7 dilations). The one patient in which a perforation occurred with a single dilation was dilated to $20 \mathrm{~mm}$. The other 3 perforations occurred with dilations to $10 \mathrm{~mm}$ and $12 \mathrm{~mm}$ (2). The average number of post-operative days for endoscopically confirmed strictures was 66.2 days (range 12-365). Of the 94 patients thought to have stomal strictures, 87 underwent either computed tomography (CT scan) or barium Xray prior to endoscopic evaluation. Abnormal radiographic findings were seen in 42 patients and $5(11.9 \%)$ of these patients had a normal EGD without evidence of stomal stricture. $19(42.2 \%)$ patients with normal radiologic studies were found to have stomal strictures requiring dilation. Using EGD as the gold standard, radiographic studies demonstrated a sensitivity of $66 \%$ (95\% CI: 52-77), specificity of $83 \%$ (95\% CI: 65-93), positive predictive value (PPV) of $88 \%$, and negative predictive value (NPV) of $57 \%$. Conclusion: Endoscopic dilation of stomal strictures at our institution is safe with a perforation rate at the low end of the published range. Radiographic findings can assist in diagnosing the presence or absence of stomal strictures, but there is not always a direct concordance between radiographic and endoscopic findings.

\section{S1971}

Botulinum a Toxin Injected Into the Gastric Wall for the Treatment of Class III Obesity: A Pilot Study

Aloisio Cardoso Jr., Paulo Roberto Savassi-Rocha, Luiz Gonzaga Vaz Coelho, Maria

Matilde Mello Sposito, Walton Albuquerque, Marco Tulio Costa Diniz

BACKGROUND - Obesity represents a main public health problem in western countries. Initial studies suggest that injection of botulinum A toxin (Btx-A) into the antropyloric region inhibits propulsive contractions of the antral pump, with delay in gastric emptying, early satiety and body weight loss. METHODS -After approval by University Ethics Committee, we prospectively evaluated 12 patients with class III obesity divided into four groups of three patients each. In groups I and II, $200 \mathrm{U}$ Btx-A were injected into the antropyloric region at 8 and 16 sites, respectively. Groups III and IV received $300 \mathrm{U}$ Btx-A into the antropyloric region at 16 and 24 sites, respectively. Body weight and gastric emptying time (GET) of solids and semi-solids using 13C-octanoic acid breath test and 13C-acetic acid breath test, respectively, were determined before and after injection over a period of 12 weeks. RESULTS - Pre- and post-treatment body weight or solid and semi-solid GET did not differ significantly between groups $(\mathrm{p}>0.05)$. All patients reported a feeling of early satiety. No adverse effects related to Btx-A or complications resulting from the endoscopic procedure were observed. CONCLUSION - The injection of different doses of Btx-A at different sites in the antropyloric region of patients with class III obesity did not interfere significantly with the solid and semi-solid GET or body weight of these individuals. However early satiety was reported by all patients, the procedure was safe and no side effects of the treatment were observed. Further controlled studies involving different methodologies regarding dosage of Btx-A and sites of injection are necessary.

\section{S1972}

Short Duration Vs Continuous Bouts of Excercise in Childhood Obesity Treatment Programs

Sari Acra, Megan Neumman, Andrea Klint, Kong Chen

Introduction: Exercise and increased physical activity (PA) are integral to life-style weight management programs in obese children and success hinges on compliance. Studies have shown that children appear to spontaneously perform leisure PA in short bursts. We examined whether exercise regimens tailored after this pattern (10 min tid) led to increased compliance and total daily PA compared to a single bout of $30 \mathrm{~min} /$ day by obese children as part of a 10-week obesity lifestyle management program (ShapeDown). Both exercise prescriptions were for greater than moderate intensity PA performed daily. Methods:Using a randomized, crossover design (investigator- blinded), this pilot study examined PA patterns in 9 boys and 9 girls, ages 9-11, BMI 85-98\%. Minute-by-minute PA during awake hrs was monitored using an RT3 tri-axial accelerometer continuously for 11 weeks, including 1 week prior to any intervention (baseline $1 \mathrm{PA}$ ). Participants then entered the program and were randomized to: (Group 1) 4 wks of 10 min tid of exercise (period 1) followed by 4 wks of 30 min c day (period 2), or to (Group 2) 30min q day x 4 wks (period 1) followed by 4 wks of 10 min t.i.d (period 2). A l-week washout period followed by a second l-week baseline (baseline 2 PA), during which ad-lib PA occurred, separated each exercise period. Results: Baseline characteristics were similar between Groups 1 and 2 (BMI, gender, race, baseline 1 and 2 PA). Furthermore, Groups $1 \& 2$ had similar changes in BMI ( $\mathrm{p}=0.98 ;-0.02+/-0.03$ vs $0.02+/-0.04$, respectively) and dietary intake by $24-\mathrm{hr}(0.4 \%$ vs. $1.8 \%, \mathrm{p}=0.42)$ over the study period. While self-reported compliance was significantly greater for $30 \mathrm{~min} q$ day vs. 10 min t.i.d, measured compliance using accelerometers was similar between the two exercise forms ( $31 \%$ vs $55 \%$, respectively; t-test, $\mathrm{p}=0.12$ ). The normalized average changes (from the respective baselines) in PA during interventions were $1.06+/-0.27$ for 10 min t.i.d and $1.11+/-0.31$ for $30 \mathrm{~min} q$ day $(\mathrm{p}=0.67)$. No carryover or period crossover effects were noted. Conclusion: Short bursts of prescribed PA did not lead to an overall greater daily PA compared to a single daily bout of exercise, an effect mediated at least in part by poor compliance with both forms of exercise in obese children undergoing a life-style modification weight management program. This highlights the challenges facing obesity intervention programs in children.

\section{S1973}

Long Term Follow-Up of Morbidly Obese Patients Undergoing Bioenterics Intragastric Balloon (BIB) Placement

Spyros Michopoulos, Georgios Stamatis, Stephanos Karagiannis, Fotis Dimopoulos, Helen Chrysanthopoulou, Chris Karatsoras, Emmanouel Archavlis, Michael Theodorakis, Athanassios - Meletios Dimopoulos

The obesity worldwide epidemic poses serious health hazards, particularly in Western societies. Current clinical evidence suggest that surgery offers significantly beneficial long term results to people with morbid obesity, compared to conventional methods, since even modest weight loss is associated with substantial reduction of co-morbidities. However, a large number of patients are unwilling to undergo surgical procedures. Alternatively, the BIB® System consists of a small size, silicone-made balloon, filled with sterile-saline water that can be inserted via endocscopy so that the stomach is partly filled. To our knowledge, reports of long term results with this procedure is scarce in the literature. The aim of our study was to follow-up and compare two groups of obese (BMI > $40 \mathrm{~kg} / \mathrm{m} 2$ ) patients: Group A $(\mathrm{N}=14)$ was comprised of those reluctant to surgery, who underwent the BIB procedure, whereas patients of the other group (Group B; N=13) were only put on appropriate low caloric diet. Group A patients were put on the same diet scheme as those in Group B through out the follow-up period. Results: All patients were hospitalized for 1-4 days after the procedure. Discomfort, nausea and vomiting occurred frequently but did not lead to BIB removal during the first 6 months. There were no statistical differences in age, gender ratio, duration of follow-up period and initial BMI between groups. However, the magnitude of BMI reduction was significantly higher at 6 months as well as at the end of the follow-up period in patients having undergone the BIB procedure, compared to those that underwent no intervention. Results are summarized in the table (Mean \pm SD). Conclusions: 1) The BIB ${ }^{\circ}$ System leads to significant short- as well as long-term weight reduction in patients with morbid obesity, mainly when patients concurrently adhere to a diet protocol 2) Unfortunately, most patients regain weight in the long term although significantly less so following the BIB procedure. The persistence of difference is mainly due to the initial (during the first 6 months) weight loss, as the regain after the 6th month is the same in both groups.

\begin{tabular}{|c|c|c|c|}
\hline & Group A & Group B & $\mathrm{p}$ \\
\hline Age (years) & $41.0 \pm 7.9$ & $40.7 \pm 12.3$ & NS \\
\hline Men/women & $4 / 10$ & $5 / 8$ & NS \\
\hline Follow-up (months) & $27.0 \pm 4.9$ & $27.2 \pm 7.5$ & NS \\
\hline BMI (at entry) & $46.1 \pm 4.4$ & $46.7 \pm 6.4$ & NS \\
\hline$\Delta$ BMI (6 months) & $-7.0 \pm 2.8$ & $-4.3 \pm 1.88$ & 0.008 \\
\hline$\Delta$ BMI (throughout follow up) & $-4.6 \pm 3.2$ & $-0.96 \pm 1.64$ & 0.001 \\
\hline$\Delta$ BMI 6th month to the end & $2.3 \pm 1.4$ & $3.0 \pm 2.1$ & NS \\
\hline
\end{tabular}

M1073

Effect of Chromoendoscopy with Alcian Blue On the Detection of Incomplete Intestinal Metaplasia in the Stomach Serguei V. Mouzyka, Mykhailo Zakharash, Alexandra Fedoseeva

Background/Aims: Incomplete intestinal metaplasia (IIM) is generally considered to be an early stage in the multi-step process leading to the development of gastric cancer or to be associated with an increased risk of gastric cancer. The intestinal metaplasia is categorized as type II if the intermediate cells secrete neutral mucins and acid sialomucins. Alcian blue is a basic histological stain that stains sialomucins. Apparently, it can be used with endoscopy to diagnose IIM. However, there are no works studying the effectiveness of chromoendoscopy with alcian blue. The aim of our prospective study was to evaluate the use of chromoendoscopy with alcian blue in the differentiation of gastric lesions. Methods: A total of 40 patients (male: female $=35: 5$, age 49.5 years) with different gastric abnormalities participated in the study. After obtaining permission, we used to spray reagents onto the gastric mucosa in the following order: $1 \%$ alcian blue solution and intensive water irrigation (after 1 minute). The volume of each reagent depended on the size of the abnormalities (from 2 to $5 \mathrm{ml}$ of alcian blue solution). The overall alcian blue staining pattern on the mucosa was classified into: group A: no appreciable staining, group B: patchy, group C: diffuse. Biopsy specimens were obtained from alcian-stained areas as well as alcian-unstained areas. All endoscopic procedures were performed by a single endoscopyst. Earlier diagnosed IIM patients were excluded. Results: In group A all 25 lesions were classified as inflammatory changes. In group B lesions were classified as inflammatory changes (2), low grade dysplasia (2), complete intestinal metaplasia (2), IIM with high grade dysplasia (1). In group C all 8 lesions were IIM. The sensitivity and specificity of chromoendoscopy with alcian blue for diffuse staining for detecting IIM were $88,9 \%$ and $100 \%$ respectively. A significant positive correlation (Pearson coefficient $0,84, p<0,001$ ) was found between grade of endoscopic alcian-stained mucosa and IIM. CONCLUSION: Chromoendoscopy with alcian blue is simple and effective method of detection of IIM in the stomach. 


\section{M1074}

Terminal Ileal Photography Or Biopsy: Does the Microscope Agree with the Endoscope?

Nick Powell, David Yeoh, Bu Hayee, David Rowbotham, Vishal Saxena, Alistair McNair

Background: Some authorities advocate routine acquisition of terminal ileum (TI) biopsies in all patients undergoing colonoscopy to document the extent of examination. However, the additional costs and uncertain diagnostic yield in patients whose TI appears macroscopically normal raises issues regarding the cost effectiveness of this approach. We sought to demonstrate that photographs of the TI illustrating the presence of villi are a reliable means of documenting the extent of colonoscopy and that routinely acquired TI biopsies have a minimal diagnostic yield. Methods: We conducted a prospective study of 211 unselected patients referred for colonoscopy. In all patients the macroscopic appearance of the TI was described and photographs taken following instillation of water to flood the TI and demonstrate villi. Biopsies were sampled from the same visual field. Photographs were independently evaluated by 3 gastroenterology specialists and categorised as "definitely", "probably" and "definitely not" depicting villi. Results: Histological assessment of TI biopsies confirmed small bowel mucosa in 202/211 (95.6\%) patients. In 9 cases the sample was insufficient, the tissue received unidentifiable or no sample was received by the laboratory. Photographs taken from the 202 patients in whom TI intubation was confirmed histologically were evaluated by the 3 reviewers. Villi were described as "definitely" present in $93.0 \%$, "probably" present in $6.7 \%$ and "definitely not" present in $0.3 \%$ of cases. There was good agreement between the 3 reviewers (Cohen's kappa value for concordance $=0.78$ ). Routine ileoscopy and TI biopsy was associated with a low rate of abnormal findings. The macroscopic appearance of the TI was described as "normal" by the endoscopist in $96 \%$ of cases. In these patients microscopic abnormalities of the TI were only identified in $4(2.1 \%)$ patients. In all instances there were microscopic features of IBD present and in all cases there were macroscopic features of IBD present in the colon. Conclusions: A photograph of the TI that clearly demonstrates villi is a reliable means of documenting that the TI has been reached and that the whole colon has been examined. Routine TI biopsy has a poor diagnostic yield and is not necessary unless there are macroscopic mucosal abnormalities or other features to suggest IBD.

\section{M1075}

Decision Analysis: An Aid to the Diagnosis of Whipple's Disease Martin Olmos, Edgardo G. Smecuol, Eduardo Maurino, Julio C. Bai

Background/aims: The diagnosis of Whipple's disease (WD), a rare systemic infection affecting predominantly the small bowel, is based on the identification of the bacterium Tropheryma whipplei. Our aim was to make explicit diagnostic uncertainties in WD through a decision analysis considering patients with either mainly intestinal involvement or those with a predominant focal neurological compromise at presentation. Methods: Using appropriate software, we constructed a decision tree to estimate the consequences after testing different strategies for diagnosis of WD Probabilities and outcomes to determine the optimum expected value were based on a MEDLINE search through July 2005. Results: In patients with predominant intestinal compromise, diagnostic strategies considering endoscopic intestinal biopsies for histology, including PAS and Ziehl-Nielsen (ZN) staining, and the PCR testing for bacterial DNA were similarly effective. The expected value for both was 17.03 survival years. In case of failure of one procedure the best sequential choice was a PCR analysis after a negative histology compared with histology after a negative PCR determination (expected values: 15.74 and 15.11 survival years, respectively). For cases with predominant neurological involvement, of the five strategies tested ("watchful waiting", intestinal biopsy for histology [including PAS and ZN staining], PCR determination of either intestinal biopsy samples, cerebrospinal fluid or stereotaxis cerebral biopsies), evidenced the highest expected value (14.92 survival years) for the cerebral biopsy. However, using quality-adjusted life-years as utility considering morbidity of different methods, intestinal biopsy for PCR determination was the best choice (14.00 survival years). Conclusions: In this decision model, intestinal biopsies for histology (PAS-ZN) should be indicated firstly for WD with predominant digestive compromise and if negative, a PCR determination should be the next option. In neurological WD, the molecular PCR assessment of cerebral biopsies had the highest diagnostic yield; however, its associated morbidity turns intestinal molecular assessment or histological testing as with the highest utilities.

\section{M1076}

Predictive Value of Alarm Symptoms for Gastric Cancer in Korea Yu Kyung Cho, Myung-Gyu Choi, Jae Myung Park, Jung Hwan Oh, Chang Nyol Paik, Joon Wook Lee, In Seok Lee, Sang Woo Kim, In-Sik Chung

Background: The diagnostic value of alarm symptoms for predicting gastric cancer has not been evaluated in Korea where gastric cancer is prevalent. Aim: We aimed to evaluate the prevalence and predictive value of alarm symptoms in dyspeptic patients. Method: Patients with dyspepsia were recruited consecutively from non-referral and referral hospitals June 2001 to May 2002. They were evaluated by dyspepsia questionnaire, endoscopy and Helicobacter testing Alarm symptoms were defined as anemia, weight loss, dysphagia, gastrointestinal bleeding, recurrent vomiting, anorexia, palpable abdominal mass and familial history of gastric cancer. Result: Of 3,177 patients enrolled, 1368 (43.1\%) didn't have any alarm symptom (uncomplicated dyspepsia group) and 1809 (56.9\%) were presented with one or more alarm symptoms (complicated dyspepsia group). Of 196 gastric cancer patients, 31 (15.8\%) were identified in the uncomplicated dyspepsia group and $165(84.8 \%)$ were identified in the complicated dyspepsia group. The ages of the patients with gastric cancer in the uncomplicated dyspepsia group were $<35$ in 1,35 to 40 in 2,40 to 45 in 3, 45 to 50 in 2,50 to 55 in 9 patients and $\geq 55$ in 14 patients. Patients with gastric cancer less than 45 years old in uncomplicated dyspepsia group represented 3\% of all the gastric cancers. Using multivariate analysis, age (Odds ratios 1.07 (95\% confidence interval 1.06-1.08)), male (OR 2.1 (95\% CI 1.4-3.2)) anemia (OR 18.1 (95\% CI 11.3-28.8)), weight loss (OR 3.8 (95\% CI 2.7-5.3)), dysphagia (OR 2.0 (95\% CI 1.1-3.6)) and smoking (OR 1.9 (95\%
CI 1.2-2.8)) were predictive of gastric cancer. However, for only early gastric cancer, age (OR 1.07 (95\% CI 1.04-1.09)) and smoking (OR 2.9 (95\% CI 1.6-5.2)) were predictive features. The positive predictive value of anemia, weight loss and dysphagia were $34 \%, 16 \%$ and $16 \%$, respectively. Conclusion: Some alarm symptoms had predictive value for gastric cancer but were not optimal for predicting early stage cancer. The high prevalence of alarm symptoms in patients with gastric cancer, also in young patient, suggests that age and alarm symptoms will be an effective approach for selecting patients who have the risk of gastric cancer in Korea.

\section{M1077}

Performance Improvement (PI) Is a Valid Mechanism to Introduce SedationRisk Free (Unsedated) Colonoscopy Into Routine Practice

Felix W. Leung, Steven Aharonian, Paul H. Guth, S K. Chu, B T. Nguyen, P Simpson

Background Sedation is standard for colonoscopy in the U.S. Serious cardiopulmonar complications occur in up to $1.6 \%$ of sedation procedures (Joint Commission J Quality Improvement $27 \cdot 28-41,2001), 6.2 \%$ to $7 \%$ of one expert colonoscopist's patients requested no sedation (J Clin Gastro 2005;39:516-9). Predictors are being male and educated professionals. 4 other U.S. studies reported up to $1 / 4$ of all patients accepted unsedated colonoscopy when the option was offered. Hypothesis We tested the hypothesis that PI is a valid mechanism to introduce sedation-risk free (unsedated) colonoscopy into routine practice. Method In 2002, VAGLAHS consolidated sedation procedures at WLAVA. Patients at Sepulveda ACC requested alternative(s) to the limited local access to colonoscopy. The unsedated option as reviewed in Table 1 was offered. Outcomes were tracked by PI questionnaires. Results From 2002 to 2005, 145 of 483 patients (30\%) accepted the unsedated option. Colonoscopy was performed by supervised trainees. Overall success rate of cecal intubation was $81 \%$. Only 1 LVN was needed for technical assistance. Transient vasovagal reaction occurred in 2 patients. Table $2.85 \%$ of patients with successful cecal intubation reported good experience, and $90 \%$ indicated they would likely accept unsedated colonoscopy in the future. Mean wait time has increased from $27 \pm 4$ to $46 \pm 5$ and $72 \pm 6$ days from the lst to the 2 nd and 3 rd year, for 31,50 and 64 patients during each of the three periods, respectively. This program placed 3rd in a VAGLAHS-wide PI poster competition. Conclusion Sedation-risk free (unsedated) colonoscopy can meet $1 / 4$ of the workload demand successfully with minimal requirement for technical support. Patients value the interaction with the endoscopist devoid of amnesic influence of sedative medication. The lengthening of wait-time confirms the steady increase in patient acceptance of this "non-standard" option. Winning placement reflects recognition by the administration.

\begin{tabular}{|c|c|c|}
\hline $\begin{array}{c}\text { Table 1: Comparison of sedated and unsedated } \\
\text { colonoscopy }\end{array}$ & Sedated & Unsedated \\
\hline Medication Risks of hypoxia, hypotension, arrhythmia & $\begin{array}{c}\text { Very small, up to } \\
1.6 \%\end{array}$ & None \\
\hline Escort & Mandatory & Not required \\
\hline Drive a car same day after colonoscopy & No & Yes \\
\hline Discomfort reduced by medication & Highly likely & No medication \\
\hline Remember discomfort & No & Yes \\
\hline Remember discussion during and after colonoscopy & No & Yes \\
\hline Need monitoring for 1 hour after colonoscopy & Yes & No \\
\hline
\end{tabular}

\begin{tabular}{|c|c|c|}
\hline Table 2: Reasons for selecting no sedation ( $\mathrm{N}=128$ completed questionnaires) & $\mathrm{N}$ & Percent \\
\hline Able to communicate during and immediately after procedure & 107 & $84 \%$ \\
\hline Short wait time & 102 & $80 \%$ \\
\hline Able to drive self to \& from procedure, or no need to arrange for escort & 98 & $77 \%$ \\
\hline Familiarity with doctors or Short distance to facility & 84 & $66 \%$ \\
\hline Able to return to work on same day & 41 & $32 \%$ \\
\hline
\end{tabular}

\section{M1078}

Stool Caprotectin Detection Accurately Excludes Organic Disease in Patients Presenting to Gastrointestinal Clinic

Ariella Gar-gil Shitrit, Dan Braverman, Julien Paz

Background: Calprotectin (MRP8/14) is a heterodimer calcium-binding protein present in the cytoplasm of neutrophils and is expressed in the membrane of monocytes. Upon neutophil/ monocyte activation, calprotectin is released and can be detected in serum and body fluids, including stool. Therefore, it can be a potentially useful clinical inflammatory and neoplastic marker. Recent studies showed elevation of fecal calprotectin levels in inflammatory bowel disease patient as well as chronic inflammatory and neoplastic disorders. Gastroenterologists are often hampered by the lack of a non-invasive index of colonic pathology. Stool calprotectin can be a promising and useful non-invasive tool in evaluating patients with suspected bowel pathology. Aim To evaluate the predictive value of fecal calprotectin in the diagnosis of colonic pathology. Methods: Seventy two consequent patients referred for colonoscopic examination in the department of gastroenterology, Shaare-Zedek Medical Center, Jerusalem, Israel were included. All supplied a stool specimen taken before colonoscopic preparation. The stool specimen was stored in a household freezer and was brought by the patient on the day of examination. Fecal calprotectin levels were determined in our gastroenterology laboratory. In addition, all patients have performed blood tests for CRP, ESR, CBC, and a general stool tests for WBC and occult blood. The calprotectin levels were compared to the clinical and laboratory parameters of the patients. Results: Mean calprotectin level in patients with colonic pathology $(\mathrm{n}=27$ ) was $232 \pm 114 \mathrm{mg} / \mathrm{kg}$ compared to patients without pathology $80 \pm 98 \mathrm{mg} / \mathrm{kg}(\mathrm{n}=45) ;(\mathrm{p}=0.001)$. Calprotectin levels were statistically significant with C reactive protein, elevated sedimentation rate, fibrinogen and hemoglobin $(r=0.51, p=0.002$ 
$\mathrm{r}=0.50, \mathrm{p}=0.002 ; \mathrm{r}=0.36, \mathrm{p}=0.04 ; \mathrm{r}=0.32, \mathrm{p}=0.02$, respectively). The sensitivity, specificity, negative and positive predictive value of calprotectin to predict colonic pathology were $75 \%$, $84 \%, 75 \%$ and $80 \%$, respectively Conclusions: Stool caprotectin level can be a useful tool to exclude organic disease in patients presenting to gastrointestinal clinic

\section{M1079}

Quality Assurance Committee: An Old Concept with New Significance S. D. Feldshon, Glenda Tims

Traditional quality assurance activities have been overshadowed in recent years by quality improvement initiatives. Minnesota GI, a 50+ MD single specialty practice, has had a standing quality assurance committee for many years. The committee reviews all complaints and complications reported by physicians, staff, and patients. Database was reviewed from $9 /$ 04-9/05 to assess the types and frequencies of complications and complaints. In that time 35,000 clinic visits and 68,000 procedures were performed. A total of 203 complications were reported by physicians on 179 patients. $41 \%$ were self reported by the treating MD and 59\% were reported by a physician partner The most common complications reported were: hemorrhage with and without transfusion, perforation, and pancreatitis. There were no behavioral issues reported by MD's. The reported rate of complicatons is compared to expected national rates below. Procedure Complication \% of total \% Natl av Colonoscopy Bleed 0.15 0 03-1.9 Colonoscopy Perforation 0.04 0.2-0.4 Upper Endo Bleed $0.05<0.15$ Upper Endo Perforation 0.05 0.02-0.2 ERCP Pancreatitis 1.6 1.9-5 ERCP Bleed 0.22 1-2 There were a total of 108 patient complaints. $83 \%$ of the complaints were physician behavior related. $17 \%$ were concerns related to real or perceived complications. The most common issues reported were: rude, uncaring or disrespectful (36\%), appeared rushed (23\%), discomfort during or after the procedure (22\%). Interestingly, the 5 physicians with the highest number of complaints did not have the highest number of complications. In summary,this study of traditional quality assurance activities suggests that physicians in a private practice setting DO report their complications and can be responsible for self monitoring their quality of care. Complications are reported at a near expected rate and used as opportunities for improvement. In addition, the patient complaints demonstrate patient dissatisfaction with physician behavior. This aspect of the patient physician interaction has previously been relatively unappreciated, yet behavioral issues are known to correlate with litigation. We intend to utilize this data to improve our patient experience as well as minimize complications where feasible.

\section{M1080}

Outcome of Cases with Acute Pancreatitis in a Single Center and a Comparison of Ranson's and Sofa Scoring System in Predicting the Outcome Ari Wiesen, Kostas Sideridis, Pashun Jalal, Baez Mishiev, Simmy Bank

Background: Acute pancreatitis is associated with considerable morbidity and mortality throughout the world. The mortality of acute pancreatitis has been reported in literature as $10-15 \%$, but it may have improved significantly over time with early recognition of its severity based on various scoring systems and institution of appropriate management. Aim: To evaluate the outcome of cases admitted with acute pancreatitis in our center over the past 3 years (2001-2004), and compare Ranson's and SOFA scoring systems in predicting the outcome. Methods: Data from consecutive patients admitted with acute pancreatitis were analyzed retrospectively. The primary outcomes were ICU admission and mortality. Secondary outcomes were development of pancreatic necrosis, pseudocyst, culture positive sepsis, interventions required such as ERCP and surgery, and hospital stay. Ranson's score at admission and at $48 \mathrm{hrs}$, and SOFA scoring system were used in patients when recorded to predict the outcome parameters. Results: Total Number of cases with the diagnosis of acute pancreatitis over the period was 109 (age range 21-96 yrs, median 54 yrs; 59 male, 50 female). Aetiology was identified as gallstone in $44(40 \%)$, alcohol in $32(29 \%)$, postERCP in 4(3.6\%), hypertriglycerdemia in 2 , and 27 cases were diagnosed as idiopathic. ICU admission was required in 23 patients (21\%) and four patients (3.6\%) died. Pancreatic necrosis was seen in 9 cases (8.2\%); pseudocyst also developed in 9 cases (two with necrosis). Culture positive sepsis was seen in 12 cases (11\%). ERCP was done in 21 , surgical intervention (necrosectomy/cholecystectomy) in 36. The range of hospital stay was 1 to 49 days (median 8 days). Total parenteral nutrition (for $>1$ week) was required in 6 patients (5.5\%). No difference was seen in predicting mortality by Ranson's score at admission and SOFA score, possibly due to small number of deaths. However. SOFA scores at day 1 and day 3 were superior to Ranson's score at admission and at 48 hrs for ICU admission $(\mathrm{p}<0.05)$. Ranson's score of 3 or more at 48 hrs was significant for predicting necrosis $(\mathrm{p}<0.001)$, but not the score at admission. Similarly, SOFA score at day 3 was significant in predicting necrosis $(\mathrm{p}<0.001)$, but comparable with Ranson's at 48 hrs ( $>>0.5)$. Conclusions: Acute pancreatitis still has a very high morbidity and complications, but mortality has improved. Ranson's and SOFA scores are comparable in predicting mortality and necrosis, but SOFA score is superior in predicting ICU admission.

\section{M1081}

Fecal Occult Blood Testing in Thrombolytic Therapy

Alex J. Gershenhorn, Mojdeh Momeni, Rana Khan, Sowmya Kanikkannan, Kell Julliard, Linus Yoe, Vinod Kurupath, Sushil Duddempudi, Sury Anand, Kiran Bhat

Purpose: The incidence and severity of gastrointestinal bleeding (GIB) in patients receiving thrombolytic therapy is not well known. There is little data on the risk stratification of these patients for GIB. The purpose of this retrospective analysis was to evaluate the practice of performing fecal occult blood testing (FOBT) in patients prior to administering thrombolytic therapy and to determine whether FOBT positive stools led to worse outcomes. Methods: A retrospective chart analysis was done to evaluate all patients who received full-dose thrombolytic therapy from July 2003 to March 2005. There were no exclusion criteria. Ninety-eight charts were reviewed from three medical institutions - The Brooklyn Hospital Center, New York Methodist Hospital, and Lutheran Medical Center. The indications for thrombolysis included myocardial infarction, cerebral vascular accident, deep venous thrombosis, pulmonary embolism, and graft occlusion. Prior history of GIB, FOBT results prethrombolytic therapy, overt GIB post-thrombolysis, and all deaths were recorded. The chisquare test or fisher's exact test was used to determine statistical significance. The alpha was set at 0.05 . Results: Ninety-eight patients were studied; 58 (59.2\%) male and $40(40.8 \%)$ female. Fifteen (15.3\%) were under 50 years of age, 31 (31.6\%) were 50-65, and $52(53.1 \%)$ were over 65 . When comparing pre-thrombolytic FOBT positive and negative groups, there was no statistical difference in GIB rates post-thrombolysis $(\mathrm{p}=0.769)$. There was, however, a statistically significant increase in GIB post-thrombolysis in the prior GIB history group vs. no prior history of bleed $(p=0.022)$. There were no deaths from GIB. Conclusions: The incidence of GIB post-thrombolysis is low (4.1\%). The value of performing a FOBT prior to thrombolytic therapy is questionable, and a positive FOBT may not be predictive of GIB post-thrombolysis. In our study, a history of prior GIB was associated with GIB postthrombolysis $(\mathrm{p}=0.022)$. A positive FOBT alone should not necessarily be used to withhold thrombolytic therapy when indicated. Reliable criteria need to be defined to risk stratify patients for GIB when given thrombolytics

\begin{tabular}{|c|c|c|c|}
\hline \multicolumn{2}{|c|}{} & \multicolumn{2}{|c|}{ GIB Post-Thrombolysis } \\
\cline { 3 - 4 } & Pos & No \\
\hline \multirow{3}{*}{ FOBT Pre-Thrombolysis } & Negative & 0 & 5 \\
\cline { 2 - 4 } & No Record & 4 & 71 \\
\cline { 2 - 4 } & & 0 & 18 \\
\hline
\end{tabular}

\begin{tabular}{|c|c|c|c|}
\hline \multicolumn{2}{|c|}{} & \multicolumn{2}{|c|}{ GIB Post-Thrombolysis } \\
\cline { 3 - 4 } \multicolumn{2}{|c|}{} & Yes & No \\
\hline \multirow{2}{*}{ History of GIB } & Yes & 3 & 16 \\
\cline { 2 - 4 } & No & 1 & 78 \\
\hline
\end{tabular}

\section{M1082}

Clinical Efficacy of Gabapentin in Persistent Hiccups

Francisco Huerta-Iga

BACKGROUND: Hiccups have many causes that are difficult to establish. In terms of its duration they can be categorized as follows: acute, when last up to 48 hours; persistent, longer than 48 hours and intractable when last more than two months. Treatment options include both pharmacologic and nonpharmacologic agents. We present our experience with the use of GABAPENTIN in the treatment of persistent hiccups (PH). MATERIAL AND MÉTHODS: This is a clinical observational prospective study held form March 1999 to February 2005 and include patients more than 18 years old with diagnosis of $\mathrm{PH}$. We investigated history of hiccups, GERD, concomitant diseases and cancer and recent use of medications related to singultus. A subjective scale for grade the intensity of Hiccups was used for the patients during the study: $1=$ No Hiccups, 2 = Occasional hiccups. Don't affect sleep or daily activities, $3=$ Frequent hiccups that affect daily activities but don't affect sleep, $4=$ Frequent hiccups that affect daily activities and sleep, 5= Persistent hiccups that don't allow the patient to eat or sleep and is accompanied of weight loss. RESULTS: A total of 9 patients were enrolled in a 72 months period. All were men, with a mean age of 50.7 years (28-78). Three had history of Diabetes and one of Hypertension. Carlsson-Dent questionnaire were positive for GERD in $77.7 \%$. All were on PPI and cisapride at conventional dose treatment for at least one week. Two patients were also on carbamazepine. Initial mean symptom score was 3.88. The PPI and cisapride were continued and adjusted for all the patients, we only added Gabapentin $300 \mathrm{mg}$ twice a day. The patients filled the score cards every day until the disappearance of hiccups and Gabapentin was suspended three days after this. The mean first response day was 1.88 and the mean number of days until hiccups disappear was 3.77. Follow up for up to 6 months demonstrate no recurrence of hiccups in any patient. We can only correlate the hiccups disappearance with the use of Gabapentin in 8 of the $9(88.8 \%)$ patients in terms of a rapid response. Sedation and dizziness were present in $33.3 \%$. Only one patient accepted the $\mathrm{pH}$ monitoring after the study and was negative for acid reflux. CONCLUSIONS: Gabapentin is a useful empirical treatment for persistent hiccups with an $89 \%$ rate response in addition to PPI and prokinetic therapy via the GABA-ergic nerves on the hiccup centre. We suggest using it as a first line therapy because of its great clinical response and the less common, less important adverse effects rate comparison with Baclofen.

\section{M1083}

Clinical Utility of Esophagogastroduodenoscopy in the Management of Recurrent or Intractable Vomiting in Pregnancy Abraham Debby, Abraham Golan, Marek Glezerman, Haim Shirin

OBJECTIVE: The indications to perform esophagogastroduodenoscopy (EGD) and the yield of this procedure during pregnancy are still in debate. AIM: To determine the impact of EGD on the clinical management of pregnant women with recurrent vomiting and their pregnancy outcome. METHODS: We retrospectively evaluated 65 pregnant women who underwent diagnostic EGD between January 1987 to October 2003. Computer analysis of medical records for diagnostic codes, procedures codes, endoscopic results and pregnancy outcome were obtained from the medical charts of the patients. RESULTS: Pregnant women were divided into two groups according the indications for EGD: groupl- intractable vomiting with or without epigastric pain $(n=54)$ and group 2- vomiting and gastrointestinal bleeding $(\mathrm{n}=11)$. The endoscopic findings found in both groups were: esophagitis (28/65), gastritis (10/65), normal EGD (19/65) and other (8/65). The diagnostic yield for EGD was 68\% for group 1 and $82 \%$ for group 2. EGD was helpful for clinical management when performed for suspected gastrointestinal bleeding rather than for other indications. Mean gestational age at delivery, fetal weight and mean Apgar score did not differ by groups. No fetal malformations were observed. CONCLUSIONS: Results of EGD are not helpful for the 
clinical management in pregnant women with intractable or recurrent vomiting except for those with upper gastrointestinal bleeding. Whenever indicated, EGD can be safely performed in pregnancy with no maternal or fetal complications.

\section{M1084}

The Utility of Immunohistochemistry in the Diagnosis of Viral Enteritis in Small Bowel Transplant Recipients

Ivan A. Klement, Galen R. Cortina

BACKGROUND: Viral enteritis is relatively common in the immunosuppressed small bowel transplant recipient, and must be distinguished from acute rejection to assure appropriate clinical management of the patient. The purpose of this study was to assess the utility of immunohistochemistry in the diagnosis of cytomegalovirus, adenovirus, and herpes simplex virus infection in small bowel transplant (SBT) recipients. METHODS: Over a period of two years, immunoperoxidase stains for cytomegalovirus (CMV), adenovirus (AV), and herpes simplex virus (HSV) were performed on all biopsies of small bowel from transplant recipients. In general, $\mathrm{H} \& \mathrm{E}$ stained slides were available to the pathologist the day after the biopsy procedure, while immunoperoxidase slides were available after an additional one to three days. The pathologist had the option of holding the case until immunoperoxidase slides were delivered, or of issuing a preliminary diagnosis based on H\&E stain, and then later issuing an addendum to report the immunoperoxidase results. For this study, the results of immunoperoxidase staining were compared to preliminary diagnoses based only on H\&E stain, and cases which were held for immunoperoxidase were compared to those in which a preliminary diagnosis was rendered based on H\&E stain. RESULTS: A total of 117 biopsies from 35 patients were processed over the two-year period. For 13 of these biopsies, a final diagnosis was issued based only on $H \& E$ stain, and immunoperoxidase results were not recorded. For 11 biopsies, the case was held for immunoperoxidase; of these 10 were negative for viral inclusions and one was positive for AV. For the remaining 87 biopsies, a preliminary diagnosis was issued based on $H \& E$ stain and a subsequent addendum reported the immunoperoxidase results. In this group, there was one discrepancy between $\mathrm{H} \& \mathrm{E}$ diagnosis (negative for viral inclusions) and immunoperoxidase diagnosis (positive for AV). However, overall, there were only 3 biopsies demonstrating AV infection, and no biopsies were positive for CMV or HSV infection. CONCLUSIONS: In this series of SBT biopsies collected over a two-year period, the utility of immunoperoxidase stains to detect viral infection was modest, mainly due to the low overall frequency of positive biopsies. However, the sensitivity of H\&E for detection of adenovirus was only $67 \%$, and furthermore, $9 \%$ of the H\&E diagnoses were deferred to immunoperoxidase. Thus, immunoperoxidase may a useful adjunct method in selected cases where the clinical suspicion is high or the histologic diagnosis is equivocal.

\section{M1085}

Prescribing of Acid Suppressive Therapy: Interactions Between Hospital and Primary Care

Aleksander Krag, Jesper Hallas, Lars Teglbjerg, Axel Malchow-Moller, Peter Bytzer

Background: Overuse of acid suppressive therapy (AST) in the hospital setting as well as in primary care is well documented. In Denmark the defined daily doses (DDD) of AST per 1000 inhabitants rose from 20,6 in 2000 to 28,6 in 2004. Data on the interaction between prescriptions in primary and secondary care are lacking. Aim: To describe interactions between prescriptions of AST in hospital and in primary care. Methods: In a 24-months period all patients who were treated with AST while in the medical department were identified. Details about the prescription were retrieved from electronic medical records. All prescriptions on AST (H2RA and PPI) redeemed by these patients 12 months before and after discharge were retrieved from a large prescription database, which has complete capture of all prescriptions in the region. Results: 549 of 4477 patients (12.3\%) were treated with AST while in hospital, but AST was prescribed de novo in only $192(35 \%)$ of these cases. 439 patients $(80 \%)$ were discharged with AST. Information about indication for AST and planned duration of therapy were given in the discharge letter in only $25 \%$ and $17 \%$ of the cases, respectively. Among patients treated with AST during admission, prescriptions on AST were redeemed by $67 \%$ in the year before admission and by $74 \%$ in the year after discharge. Among patients who had the AST discontinued during admission $(n=67), 48 \%$ resumed AST within the following 12 months. Of all AST treated subjects in the catchment area, $7.8 \%$ were seen in our department. Conclusions: Decisions about acid suppressive therapy prescribing in hospital does not influence prescription behaviour in primary care. Communication between hospitals and primary care concerning the background for AST prescribing is insufficient and hampers rational pharmacotherapy.

Patients characteristics

\begin{tabular}{|c|c|c|}
\hline & Non AST users (N=3928) & AST users (N=549) \\
\hline Age (mean \pm SD) & $60.6 \pm 20.6$ & $70 \pm 16$ \\
\hline Gender & $48.2 \%$ males & $42.6 \%$ Males \\
\hline Days of admission & $5.7 \pm 8.9$ & $9.0 \pm 10.8$ \\
\hline NSAIDS/ASA users year before & $39.9 \%$ & $54.3 \%$ \\
\hline NSAIDS/ASA users year after & $39.8 \%$ & $43.7 \%$ \\
\hline AST year before & $8 \%$ & $67 \%$ \\
\hline AST year after & $10 \%$ & $74 \%$ \\
\hline
\end{tabular}

De novo prescriptions compared with continued therapy

\begin{tabular}{|c|c|c|}
\hline & De novo prescribed AST(N=194) & Continued AST (N=355) \\
\hline Endoscopy ( $\mathrm{n}=131)$ & $53.6 \%$ & $7.7 \%$ \\
\hline Indication for AST in discharge letter & $54 \%$ & $11 \%$ \\
\hline Duration of AST in discharge letter & $37.5 \%$ & $15.5 \%$ \\
\hline
\end{tabular}

\section{M1086}

The Availability of Faecal Occult Blood Tests Should Be Restricted in Hospital Practice

Bernard Denis, Stephane Jux, Pascale Chiappa, Gilles Breysacher, Jacques Bottlaender, Anne Marie Weiss, Andre Peter

FOBTs (faecal occult blood tests) are colorectal cancer (CRC) screening tools which are sometimes used as diagnostic tests. Aim: to assess the appropriateness and diagnostic yield of the use of FOBTs in hospital practice. Methods: retrospective chart review of all patients hospitalized in medicine services at Pasteur Hospital (Colmar. France) who had a FOBT during a 5 months period. FOBTs were performed on spontaneously voided stool samples using immunochemical tests. Results: FOBTs were performed in 200 patients: 81 men and 119 women with a mean age of 71 years (range 20 - 98). FOBT was performed on a single stool specimen in 58 patients ( $29 \%), 2$ samples in 32 (16\%) and 3 samples in $104(52$ $\%)$. No FOBT was performed for CRC screening. All were performed for the assessment of anemia $(n=141)$, gastrointestinal symptoms $(n=30)$, weight loss $(n=44)$ or deep vein thrombosis $(n=7) .23$ patients had a doubtful FOBT result but were not retested. 65 had a negative FOBT: 15 had nevertheless endoscopic procedures ( 6 colonoscopies, 3 flexible sigmoidoscopies and 11 upper endoscopies) and 45 had no further gastrointestinal investigation despite symptoms. $112(56 \%)$ had a positive FOBT: the test was repeated in 9 ( 8 $\%) ; 46$ (41.1\%) had no further gastrointestinal investigation; 33 (29.5\%) had a colonoscopy and $13(11.6 \%)$ a flexible sigmoidoscopy; $50(44.6 \%)$ patients had an upper endoscopy even though immunochemical FOBTs fail to detect upper GI bleeding. The diagnostic yield was colorectal neoplasia in 10 patients (CRC in 4 patients, adenomatous polyps $>1 \mathrm{~cm}$ in 1 and $<1 \mathrm{~cm}$ in 5 ) (positive predictive value $=8.9 \%$ ). Other endoscopic diagnoses included 6 non adenomatous polyps, 1 Crohn's disease, 1 colonic vascular ectasia, 3 gastric cancers, 16 gastroduodenal ulcers, 1 reflux esophagitis and 1 portal hypertension. In the anemia group, serum ferritin level had not been measured in 64 patients (41.6\%) and was normal or increased in $75(48.7 \%) .15$ patients had an iron-deficiency anemia: 10 had a positive FOBT, 6 of them had a colonoscopy and 8 an upper endoscopy; 1 had a negative FOBT but had both upper and lower endoscopy and 4 had a doubtful FOBT result, 1 of them had a colonoscopy. Conclusion: the FOBT, which is validated only for CRC screening, is almost always performed inappropriately in hospitalized patients. Whatever the result of the FOBT, only a minority is followed by appropriate investigation. The rate of positive FOBT is very high and the diagnostic yield quite low. Considering the potentially serious medical consequences and the cost of this widespread misuse, the availability of FOBTs should be restricted in hospital practice.

\section{M1087}

Audit of Biochemical Monitoring of Nasogastrically Fed Hospital Inpatients Ranjan Sanyal, Sanjeev Kumar, Shankar Dhandapani, C Emm, K Mairs, D Barrett, W Lycett, C Charles, K Evans, S.D. Hearing

Introduction: Malnourished patients given nasogastric tube feeding (NGTF) are at risk of developing potentially fatal refeeding syndrome (RFS). British Society Gastroenterology (BSG) guidelines recommend biochemical monitoring of all NGTF patients to detect and treat RFS at an early stage. Aims and Methods: In Staffordshire General Hospital, UK, it is recommended that all patients have biochemical monitoring including potassium, magnesium and phosphate performed for the first 4 days after NGTF is commenced, (Day $1=1$ st day after NGTF commenced) as per BSG guidelines. We undertook a prospective audit of all adult hospital patients receiving NGTF between Jan 2004 to Jan 2005. Result Number of patients $=93$ Median age $(\mathrm{IQR})=71(64-80)$ yrs. $\mathrm{M}: \mathrm{F}$ ratio=1:1. Median duration of feeding $(\mathrm{IQR})=5$ (2-14) days, 86 patients $(92 \%)$ were malnourished at commencement and $42(45 \%)$ had poor oral intake in 2 weeks prior to NGTF. After NGTF, 28 (30\%) resumed oral diet 16 (17\%), $14(15 \%)$ had a PEG placed and $3(3 \%)$ received TPN. Overall $48(52 \%)$ patients died, $18(19 \%)$ were discharged home, $22(24 \%)$ to Nursing Home and $5(5 \%)$ to other hospitals. Indications for NGTF included CVA (41\%) dementia (16\%) and Parkinson's disease $(11 \%)$. Patients were admitted to ITU $(55 \%)$, medical $(31 \%)$ surgical $(11 \%)$ and rehabilitation wards (3\%). Electrolyte abnormalities were detected in $42(45 \%)$ patients These patients were older (median $76 \mathrm{yrs}$ ), all were malnourished and 32 (76\%) had inadequate intake prior to NGTF. Of this subgroup, $26(62 \%)$ died. Hypophosphataemia was detected in $21(22 \%)$ patients, requiring treatment $(<0.5 \mathrm{mmol} / \mathrm{L})$ in 5 cases ( 3 intravenous 2 oral). Hypomagnesaemia was detected in $32(35 \%)$ cases, requiring treatment $(<0.5 \mathrm{mmol} /$ L) in 6 cases (intravenous). Hypokalaemia requiring treatment was detected in 18 cases (19\%), all requiring treatment. Peak incidence of electrolyte abnormalities were hypomagnesaemia (day 1) hypokalaemia (day 2) and hypophosphataemia (day 3). Biochemical monitoring was incomplete and performed as follow: Day 1 Day 2 Day 3 Day 4 Phosphate 34\% $34 \% 17 \%$ 17\% Magnesium 48\% 43\% 27\% 27\% Potassium 73\% 60\% 34\% 32\% The above table demonstrates that monitoring was incomplete and in particular measurement of serum phosphate was low. Furthermore 10(11\%) patients had no biochemical monitoring. 1 had monitoring performed for all 4 days after NGTF was commenced. Conclusion These data confirm that patients receiving NGTF are at high risk of developing electrolyte abnormalities in the first 4 days of feeding. However monitoring remains incomplete and further education is required. 
M1088

Usefulness of Transabdominal Ultrasonography in Diagnosing Gastric Submucosal Tumor

Madoka Nakao, Noriaki Manabe, Ken Haruma, Jiro Hata, Shinji Tanaka, Kazuaki Chayama

OBJECTIVES: We have made reports about the usefulness of transabdominal ultrasonography (US) in diagnosing gastric submucosal tumors (G-SMT). In the present study, we evaluated the diagnostic ability of US in cases of G-SMT as well as the usefulness of the technique in the follow-up of the course of the growth of G-SMT. METHODS: The present study assessed the visualization rate and diagnostic ability of US in 206 subjects (104 males and 102 females; mean age: $56 \pm 15$ ) who had been given the diagnosis of G-SMT during the period from April 1995 to April 2004 and then underwent both endoscopic ultrasonography (EUS) and US. Moreover, in 21 of the 206 subjects, we followed up the course of tumor growth by US over the period of 38.6 months on average. RESULTS: In the 206 subjects, lesions could be visualized in US in $84.5 \%$ (174/206 cases) and US-based diagnoses agreed with EUS-based ones in 58.7\% (121/206 cases). In the follow-up of the course of tumor growth, no subject had a lesion which had been once visualized and given a specific diagnosis but later became unvisualizable or was given different diagnosis. CONCLSION: US is useful in diagnosing G-SMT and may be a useful examination technique for use in the follow-up of the course of the growth of G-SMT which has been once diagnosed based on EUS findings.

\section{M1089}

Unique Case of Muscularis Mucosae Leiomyomata Polyposis Christine M. Hobbs, Rehana Begum

Background: Leiomyomas of the muscularis mucosae (MM) are known to occur in the Gl tract. GI leiomyomas present as submucosal nodules and are usually incidental. Multiple leiomyomas of the MM in the GI tract have not been previously described. Case: A 55 yearold male was evaluated for heme occult positive stools, mild anemia and constipation. His family history included a father with lymphoma and a sister with an intra-abdomina malignancy of unknown primary site. Colonoscopy revealed numerous sessile polyps, 0.6$1.3 \mathrm{~cm}$, extending from the rectum to the ascending colon. Snare polypectomies were done on one polyp from the ascending colon and four from the transverse colon. Subsequent EGD showed multiple sessile polyps in the antrum and distal esophagus. Representative antral polyps were biopsied. The duodenum was grossly normal. Microscopy of the ascending and transverse colon polyps showed classic histologic features of benign leiomyomas of the MM, namely, circumscribed bundles of cytologically bland, mitotically inactive, smooth muscle fibers, moderate in cellularity, predominantly occupying the submucosa but contiguous with the MM. The overlying mucosa was unremarkable. Case materials for the colon polyps were sent to a GI pathology consultant who agreed with the original histologic impression and confirmed the diagnosis immunohistochemically. Immunostains for desmin, CD117, CD34 and S100 were performed on one of the colon polyps. The spindle cells were diffusely and strongly positive with desmin and negative for CD117, CD34 and S100, essentially excluding a GIST or neural tumor. Microscopy of the antral biopsies revealed mucosal tissue only, as is typical for intramural lesions. Discussion: We refer to this patient's multiple leiomyomas of the MM of the GI tract as "muscularis mucosae leiomyomata polyposis," since this condition differs from leiomyomatosis, which occur exclusively in the esophagus, the veins of the uterus, and the peritoneum (in fertile females). Management: Since multiple leiomyomas of the MM have not been previously described, this patient will continue to receive regular endoscopic follow-up with polypectomies of representative polyps in each area of the GI tract along with polypectomies of any unusual polyps or polyps that have enlarged. In the future, extramucosal excision of a few of the esophageal and gastric antral polyps will be done to confirm their diagnosis. Capsule endoscopy is being considered to evaluate the small intestine for polyps. No treatment other than polypectomies is expected unless the patient should develop extensive ulceration of polyps with significant bleeding.

\section{M1090}

150 Endoscopic Mucosal Resections Carried Out Without Morbidity Having High Adrenalin Submucous Infiltration in 4 Different Techniques in the Digestive Tract

Mario Rey

Objective: Comparing 4 endoscopic mucosectomy techniques (EMR) in treating carcinomas and premalign lesions in terms of complete resections, bleeding, perforations, recurrence and survival in the digestive tract. Materials and Methods: 150 mucous or submucous lesions were endoscopically analysed in 145 patients having an average age of 59.6 (23-83 years); 28 lesions were in the oesophagus, 79 were gastric, 4 duodenal and 39 colorectal having an average size of $19.3 \mathrm{mms}$ diameter which were resected using plastic cap (68 cases), snare ( 45 cases), overtube ( 10 cases) or IT knife ( 27 cases) over a lapse of 6 years, following submucousal infiltration with saline solution plus adrenalin at $1: 1000$. They had a 2.1 year endoscopic follow up on average (0.2-7.1). Oesophageal lesions were type Ila and IIb ranging from 5 to $20 \mathrm{mms}$, half were associated with Barrett's oesophagus $81.2 \%$ of those in the stomach had an antral localisation; most rectal lesions were localised in the rectum and sigmoid. Results: final histological diagnosis was 41 carcinomas, 13 carcinoids, 7 leiomyomas, 61 high and low dysplasias, 24 adenomas, 2 aberrant pancreas, an angiolipoma and a gist. Most type I and IIa more severe lesions were resected with snare and overtube and flat or depressed ones required cap and IT knife. There were no significant differences between the average size of high degree dysplasias and carcinomas ( $15 \mathrm{mms}$ of $19 \mathrm{mms}$ ) $\mathrm{p}=0.17$. Lesions could be resected in one fragment in $85 \%$ of cases, mainly being done with IT knife and endoscopic overtube. Resected lesions in several fragments were significantly associated with the cap technique ( $\mathrm{p}=0.02)$ and these with positive edges ( 3 cases). Recurrence was identified in 1 carcinoid tumour $(0.7 \%)$, extensive submucous infiltration in 2 lesions and indifferentiation in another case requiring surgical treatment. 2 cases (1.3\%) had metachronous lesions after 1 and 3.5 year's follow-up; these were initially resected with cap and then treated again with mucosectomy leading to complete cure. No bleeding was presented during the procedure or later, perforations or mortality with the 4 EMR techniques in the series No side effects were found in relation to the high adrenaline dose infiltrated Conclusions: Endoscopic resection of the mucous was seen to provide effective and safe treatment in intramucous carcinomas and dysplasias when using the 4 techniques with high adrenaline submucous infiltration. However, resection in fragments was shown to have greater risk of having positive edges and recurrence associated with using the cap.

\section{M1091}

Prospective Randomized Controlled Trial Assessing the Impact of Feedback on Small Bowel Biopsy Performance

Diahann L. Seaman, Gavin Harewood, Bret Petersen, Beverly Ott

Introduction: Endoscopy is an operator-dependent procedure that relies heavily on both the judgment of the performing physician and their compliance with requests of referring physicians. This study aimed a) to characterize small bowel biopsy (SBBx) practice among patients undergoing EGD in whom SBBx was requested by the referring clinician, and $\mathrm{b}$ ) to prospectively assess the impact of feedback on SBBx practice among a large cohort of endoscopists. Methods: Procedural data was recorded from all outpatient EGD procedures performed between 07/01/05 and 10/28/05 for which SBBx was requested. Endoscopists were randomized to receive feedback or no feedback on SBBx practice; randomization was stratified according to endoscopist experience level. SBBx performance was recorded during 07/05 (pre-feedback baseline); the feedback group then received their performance data by e-mail every three weeks for 12 weeks (total of four feedback e-mails) documenting the proportion of patients in whom SBBx was performed when requested. Results: 54 endoscopists were randomized to receive feedback (29) or no feedback (25, control group). There was no difference in experience level between both groups $(\mathrm{p}=0.9)$ with overall mean of 10.3 yrs. In the feedback group, SBBx increased by $4.9 \%$, from $87.1 \%$ (95\% CI, 79-93\%) to $92.0 \%$ (95\% CI, 90-95\%), while in the control group, SBBx declined by $1 \%, 96.7 \%$ (95\% CI, 93-99\%) to $95.7 \%$ (95\% CI, 93-98\%) (Figure1). Conclusions: Feedback by e-mail appears to improve compliance among endoscopists for SBBx practice. Further study would help to characterize the utility of e-mail feedback in enhancing uniformity in endoscopy practice

\section{Pre- and post-feedback SBBx performance}

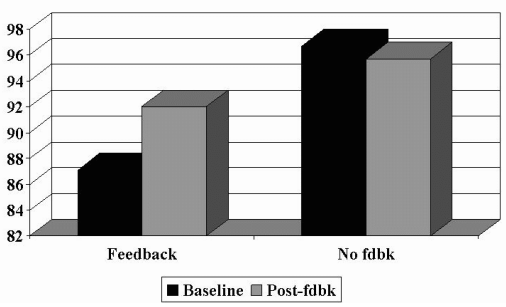

M1092

Use of Acid-Suppressive Therapy On the General Medical Ward Ifat Kamin, Tandon Manish, Julio C. Ayala

Medications to suppress gastric acid are among the most commonly prescribed drugs in the hospital and in the general practice. There is relatively little data regarding the overall use and misuse of acid suppressive medications in the general medical ward population. The purpose this study is to determine the frequency of appropriate or inappropriate use, and indications for prescribing acid suppressive medications to patients admitted to the general medical ward in a community teaching hospital. Chart review was undertaken of patients admitted to Mount Auburn Hospital, who were prescribed acid suppressive therapy upon admission or during their hospitalization. Data was gathered to determine the indication for prescribing acid suppressive therapy, type of medication prescribed and whether the medication was discontinued upon discharge. Note was also made if the patient was on therapy prior to admission or started subsequently in the hospital. A total of 194 charts were reviewed. Proton Pump Inhibitors were the most commonly prescribed (183 patients, 94\%). Overall 111 of 194 acid-suppressive prescriptions (57.2\%) were not indicated. Acidsuppressive therapy initiated in the hospital was not indicated in 67 of $83(80.7 \%)$ of the patients, whereas among patients receiving therapy prior to admission, the frequency of prescribing acid suppressive therapy without an indication was 44 of $111(39.6 \% ; \mathrm{p}<0.05)$ Of patients prescribed acid suppressive therapy in the hospital and subsequently discharged on therapy, 25 of $37(67.6 \%)$ of the prescriptions were not indicated. Among low risk patients started on acid suppressive therapy for stress ulcer prophylaxis, 31 of 72 (43\%) were discharged home with a prescription for the medication. In our logistic regression model we found that smokers were most likely to be put on acid-suppressive therapy for a non-indicated reason (OR - 3.119, CI 0.952-10.2). The odds ratio for anticoagulants was 1.851 (CI 0.868-3.950), steroids was $1.174(\mathrm{Cl} 0.482-2.860)$ and aspirin was $1.046(\mathrm{CI}$ $0.560-1.956)$. In conclusion, there is significant use of acid suppressive therapy in nonindicated cases on the general medical service in the hospital. Majority of inappropriate prescriptions are given for stress ulcer prophylaxis in low risk patients. A significant number of these patients are discharged with a prescription to continue the medication, inducing further inappropriate use of acid suppressive therapy. 
M1093

How Accurate Is a Clinical "Hunch" After the First Office Consultation in Gastroenterology Out-Patients?

Shital Amin, Niall van Someren, Kalpesh Besherdas, Steven Mann

Background: The United Kingdom National Health Service is under increasing pressure to cut costs and process patients as quickly as possible. Investigations focused on the initial working diagnosis are likely to be cost-effective if the clinical 'hunch' is accurate. Aim: To review the accuracy of our working diagnosis after first consultation in the office. Method: Consecutive new patients referred to Gastroenterology out-patient clinics (NVS, KB, SM) were given a working diagnosis (12 categories) following the consultation. Data was then re-analysed after appropriate investigations to determine the accuracy of the initial working diagnosis. Non-parametric analysis was used to evaluate any associations. Results: Data were available for 154 patients $(\mathrm{F}: \mathrm{n}=78[51 \%]) .95 \%$ of the initial diagnoses were the same as the final diagnosis following a diagnostic work-up $(\chi 2=16.93(n-1)<p 0.05=19.6)$. Conclusion: Our initial "hunch" in out-patients is accurate in more than $95 \%$ of cases. This is very reassuring and suggests that investigations can usually be targeted at confirming the diagnosis avoiding unnecessary follow-up appointments which may generate further investigations. We acknowledge that there will always be a few patients who are challenging and don't fit into an obvious diagnostic category.

\begin{tabular}{|c|c|c|c|c|c|}
\hline Diagnosis & Working Diagnosis & Final Diagnosis & Diagnosis & Working Diagnosis & Final Diagnosis \\
\hline 1 & 19 & 21 & 7 & 2 & 2 \\
\hline 2 & 17 & 23 & 8 & 9 & 8 \\
\hline 3 & 30 & 35 & 9 & 2 & 2 \\
\hline 4 & 3 & 1 & 10 & 14 & 12 \\
\hline 5 & 9 & 5 & 11 & 2 & 0 \\
\hline 6 & 14 & 23 & 12 & 32 & 22 \\
\hline
\end{tabular}

Legend

\begin{tabular}{|c|c|}
\hline $1=$ functional dyspepsia & $7=$ pancreatic disease \\
\hline $2=$ functional abdominal pain & $8=$ inflammatory bowel disease \\
\hline $3=$ functional colonic symptoms & $9=$ gallstones \\
\hline $4=$ upper GI neoplasm & $10=$ GORD \\
\hline $5=$ lower GI neoplasm & $11=$ coeliac \\
\hline $6=$ hepato-biliary pathology & $12=$ other \\
\hline
\end{tabular}

\section{M1094}

Need for ERCP Delays Surgery in Patinets with Acute Cholecystitis Christopher N. Dainiak, David Peretz, William Hale

Background: Until the 1970's, common practice was to "cool down" patients with acute cholecystitis and to subsequently perform open cholecystectomy several weeks later. In the early 1990s, with the advent of laparoscopic CCY, acute cholecystitis was regarded as a contra-indication to early operation. However, evidence from numerous retrospective and prospective studies have shown that laparoscopic cholecystectomy within 72 hours of admission decreases length of hospital stay and cost, while also decreasing patient complications and potentially mortality. Objectives: To describe our experience with acute cholecystectomy in a community-based hospital and to assess performance of our hospital in comparison with current recommendations. Methods: The authors performed a retrospective medical record review from January to December 2004 of 50 consecutive patients with acute cholecystitis at our institution. Data collected included age, sex, symptoms and physical findings at presentation, laboratory and radiological investigations performed (including ERCP), comorbidities, time from admission to surgery, length of stay, complications, and admission service (medical or surgical). Results: Mean age at presentation was 56 years old (range 3379). Median length of stay was 5 days (1-20). $12 / 50(24 \%)$ had a length of stay more than 6 days. Median time from admission to surgery was 24 hrs (1-204). 43/50 (86\%) were operated within 72 hrs of admission. 14/50 (28\%) were operated on the day of admission. Only $6 / 50$ (12\%) had surgery more than 72 hrs after admission. These patients with delayed surgery required ERCP more often ( $67 \%$ vs $2 \%$ ), had more underlying co-morbidities ( $83 \%$ vs $48 \%$ ), and had more post-operative complications ( $67 \%$ vs $15 \%$ ). Admission to the medical service vs the surgical service was associated with a prolonged time to operation ( 65 hrs vs 19 hrs), increased length of stay (6.0 days vs 3.0 days), a greater likelihood of a "cool down" period ( $18 \%$ vs 3\%), and a greater likelihood of ERCP (36\% vs $5 \%$ ). Conclusions: Early laparoscopic surgery for acute cholecystitis is being performed at this community hospital consistent with current recommendations. Patients admitted to the medical service are more likely to be older, sicker, and more often to require an ERCP. Patients requiring ERCP are more likely to have delayed operations and post-operative complications.

\section{M1095}

Association Between Proton Pump Inhibitors and Respiratory Infections: A Systematic Review and Meta-Analysis of Clinical Trials

Nabil Sultan, Jose Nazareno, Jamie Gregor

BACKGROUND: Proton pump inhibitors (PPI) have become the mainstay of treatment and prevention of many serious gastrointestinal diseases. Increase in gastric pH caused by PPIs may be linked to increased bacterial colonization of the stomach and possibly predispose to respiratory infections. AIM: To examine the association of proton-pump inhibitor treatment with respiratory infections. METHODS: A literature search was conducted in PubMed,
Medline and Cochrane databases of randomized placebo controlled trials evaluating the efficacy of proton pump inhibitors. Studies which listed and quantified the specific adverse events of 'respiratory infection' or 'upper respiratory infection' (or equivalent) and compared their rates between PPIs and placebo were included. Chi Square analysis was used to calculate significance of association in individual studies and a meta-analysis of the selected studies was performed. RESULTS: Out of 7457 studies initially identified and more than 60 relevant RCTs selected, 7 studies met the inclusion criteria. A total of 16 comparisons for Chi square analysis were possible given the multiple dosage arms used in several studies. PPIs included in the studies included esoprazole, rabeprazole, pantoprazole and omeprazole. More than half of the studies showed a trend towards association between PPI use and respiratory infections, although the majority of the studies failed to show significant correlation. A single study using high dose esoprazole $(40 \mathrm{mg}$ ) showed a significant association: $4.3 \%$ rate of respiratory infections in the active group compared to $0 \%$ in placebo $(\mathrm{p}<0.05)$. Meta-analysis showed a trend towards association of PPI and respiratory infections, although it failed to reach significance (odds ratio $=1.42,95 \% \mathrm{CI}: 0.86$ to $2.35, \mathrm{P}=0.17$ ). CONCLUSION: Although a trend was evident in both Chi Square analysis of individual studies as well as meta-analysis, this review and meta-analysis failed to show a conclusive association between PPIs and respiratory infections. Our study revealed that very few RCTs actively sought out respiratory infections which excluded the majority of RCTs identified. A well structured placebo controlled, prospective study would help determine if a true association between PPIs and respiratory infections exists.

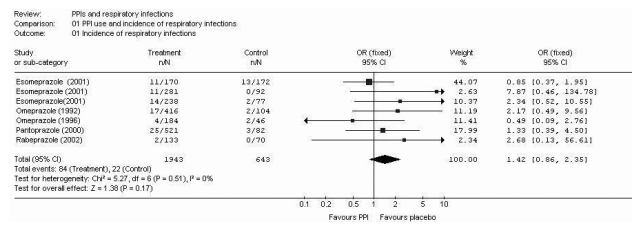

M1096

Misinterpretation of the Acid-Inhibitory Effect of PPIs in Western Populations Due to Cyp2c19 Genotype?

N G. Hunfeld, B H. Graatsma, E J. Kuipers, W P. Geus

The acid-inhibitory effect of PPIs is influenced by the CYP2C19 status of an individual. In Western populations $70 \%$ of the individuals are homozygous extensive metabolizer (homEMs), 25-30\% are heterozygous extensive metabolizers (hetEMs) and 2-5\% are poor metabolizers (PMs). In most studies with PPIs in Western populations the incidence of CYP2C19 genotype and the influence of genotype on the acid-inhibitory effect have not been assessed. Aim: To investigate the incidence of $\mathrm{CYP} 2 \mathrm{Cl} 9$ genotype and the influence of genotype on the acid-inhibitory effect of PPIs in studies performed in Western populations Methods: CYP2C19 genotype was assessed in Hp-negative Caucasian subjects who participated in 2 randomized two-way cross-over trials. In the first study the acid-inhibitory effect of lansoprazole $15 \mathrm{mg}$ (L15) was compared with omeprazole $10 \mathrm{mg}(\mathrm{O} 10)$. In the second study omeprazole $20 \mathrm{mg}$ (O20) was compared with pantoprazole $40 \mathrm{mg}$ (P40). In both studies intragastric $\mathrm{pH}$ was monitored continuously for $24 \mathrm{hr}$ at day 0 (baseline), day 1 and day 6 of dosing. Subjects' percentages of time above $\mathrm{pH} 4$ in 24-hr periods were calculated, corrected for baseline. Acid-inhibitory effects of hetEMS and PMs were compared to the effect in homEms. Results: Study L15 vs. O10: 11 subjects: 6 subjects were homEMs (55\%), 5 were hetEMs (45\%). Study P40 vs. O20: 16 subjects: 12 subjects were homEMs (75\%), 3 were hetEMs (19\%) and 1 was PM (6\%). At day 1 , responses of hetEMs and PM were significantly higher with $\mathrm{O} 10, \mathrm{O} 20$ and $\mathrm{P} 40$ compared to the homEMs (O10: $\mathrm{p}=0.04, \mathrm{O} 20 \mathrm{p}=$ $0.004, \mathrm{P} 40: \mathrm{p}=0.01$ ), with L15 there was a trend visible. At day 6 , a trend was seen towards increased acid-inhibition in hetEMs and PMs for all studied PPIs. Individual responses and median values are shown in Figure 1. Conclusions: In Western populations: 1.The acidinhibitory effect of PPIs is influenced by CYP2C19 status, especially at day 1 of administration. 2.Misinterpretation of the acid-inhibitory effect of PPIs is possible when (incidence of) CYP2C19 genotype is unknown.

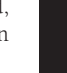




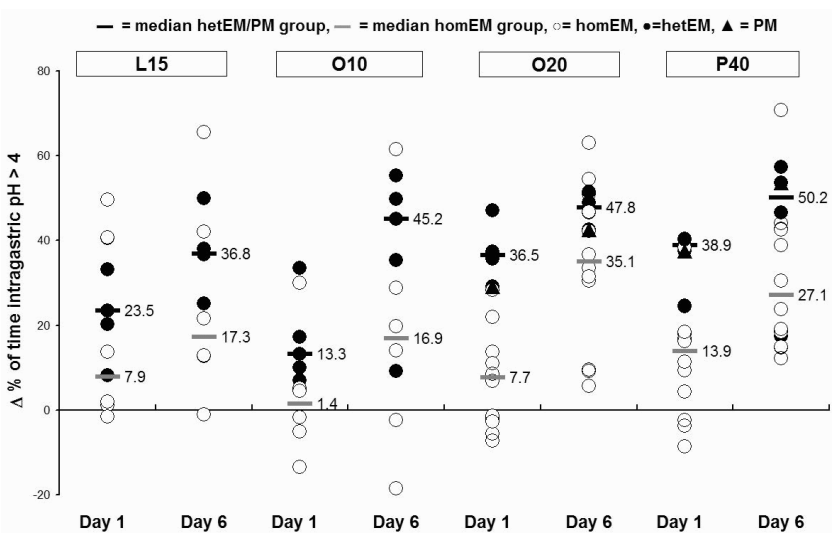

M1097

Endoscopic Surveillance After Gastrectomy: What Do We Accomplish? Mona Skoog, Stephen J. Sontag, Thomas G. Schnell, Jack Leya

Introduction: Gastric surgery (GS) is associated with a higher incidence of gastric adenocarcinoma (AdCa) and regular endoscopic surveillance is accepted as a means of early AdCa detection. There is little information on whether such surveillance actually improves the lives of those we seek to help. Nevertheless, large numbers of surveillance EGDs are performed in GS patients (Pts). Methods: From our large Hines VA GI Database, which contains the data of all procedures performed since January 1979, we identified all GS Pts who were referred for EGD between Jan 1990 and July 2003. The electronic and paper records of these GS Pts were reviewed in detail. The number of EGDs, EGD findings, Histology findings, H. Pylori status and outcomes were recorded. A control group comprised all Pts without GS (NGS) from the same period. The "Index EGD" was the first EGD performed at Hines VA. Only Pts without AdCa at Index EGD were included. To avoid missing Pts with AdCa, we used 3 identification methods: (1) the Hines VA GI Database, (2) the VA DHCP Pathology Data System, (3) review of individual records. No systematic EGD surveillance or biopsy protocol was followed. Results: Index EGD's were performed on 347 Pts with GS and 2751 Pts with NGS. In both groups, 98-99\% were males. The mean ages of the GS and NGS groups at Index EGD were similar (63.8 Vs 63.9), but the mean follow-up times were significantly different: 4.1 yrs Vs 5.3 yrs (Diff=1.2; CI: 0.7-1.7). The shorter follow-up time was due to a significantly shorter survival of GS Pts (median survival: 79.7 Vs 81.9: X2= 6.0; $\mathrm{P}=0.01$ )(Hazard Ratio: 0.80; CI: 0.65-0.95), but this shorter survival was independent of AdCa. During the 13-year period, 774 EGDs were performed to detect the 7 Ca's in the GS group. Incident $\mathrm{Ca}$ was detected in $7(2.0 \%)$ of the 347 GS Pts: $0.5 \%$ per year. The mean and median ages at diagnosis of $\mathrm{Ca}$ were 74 and 78 years, respectively, and the mean time from index EGD to Ca was 6 years. Each Ca pt had an average of 4.3 EGDs. Of the 7 Ca pts: - 3 died of non-Ca related causes. - 1 died from complications 2 months after total gastrectomy. - 3 died of metastatic disease from recurrent gastric AdCa. Conclusion: Our data do not support surveillance of GS patients in order to detect early curable AdCa.

\section{M1098}

A Study On the Clinicopathologic and Endoscopy Ultrasonographic Characteristics of Gastrointestinal Mesenchymal Tumor in 265 Cases Ji Feng, Jianwen Ning, Lijun Wang, Guoqiang Xu, Youming Li

Objective:to investigate the clinicopathologic characteristics of gastrointestinal mesenchymal tumor(GIMT) and combine with edoscopic ultrasonography(EUS) of pre-operation and immunohistochemistry of post-operation for exploring the value of EUS in the diagnosis Methods: the morphologic characteristics of GIMT in 265 cases was observed by light microscopy and the expression of CD117,CD34,SMA,S-100,KI-67 was detected by Envision immunohistochemical method, 32 of 265 cases performed EUS examination were retrospected to explore the value of EUS in the diagnosis and differentiate benign and malignant. Result:among 265 GIMT cases, there were 146 gastrointestinal stromal tumor (GIST) cases , 113 leiomyoma or leiomyosarcoma cases and 6 neurogenic tumour cases. CD117 showed diffuse strong expression in 135 of $146(92.5 \%)$ GIST cases and CD34 in 109 of $146(74.6 \%)$ cases SMA and S-100 were specially differential expressed in leiomyoma or leiomyosarcoma and neurogenic tumour respectively. 7 among 9 GIST(77.8\%) cases expressed ki-67 with more karyokinesis $(\geq 3 / 50 \mathrm{HPF})$ were malignant tumors. Most borderline and malignant tumors were found in male. The site specific positive predictive value of EUS for diagnosis of leiomyoma(leiomyosarcoma) and GIST was $96.9 \%$, qualitative diagnosis rate was $84.4 \%$ differentiating diagnosis rate of nonmalignant and malignant was $71.9 \%$. Conclusion:GIST is the most common group of GIMT but not leiomyoma and it express various differentiation and morphologies. In morphology it is difficult for GIST to be distinguished from leiomyomas and neurogenic tumour it is very necessary to distinguish them using a series of immunohistochemical markers such as CD117.CD34 SMA S-100. The expression of ki-67 combined with more karyokinesis supports diagnosis of malignant tumor . EUS is helpful in evaluating the gastrointestinal origin of GIMT, in differentiating between benign and malignant GIMT and in instructing clinical treatment and estimating prognosis.
M1099

Effect of Serum Gastrin Concentration On Insulin Resistance Yoshihisa Urita, Susumu Ishihara, Kenichiro Arai, Jun Kondo, Tastuo Akimoto, Hiroto Kato, Noriko Hara, Hideyuki Koshino, Yoshiko Honda, Yoko Nagai, Kazushige Nagai, Nagato Shimada, Motonobu Sugimoto, Kazumasa Miki

Background: Incretin hormones are gut-derived factors, which are secreted in response to the presence of nutrients in the intestinal lumen, and which act to potentiate meal-induced insulin secretion. Following oral ingestion the nutrients first come into contact with the Gand D-cells of the antrumand elicit the release of gastrin and somatostatin. It has been reported that gastrin-17 and its C-terminal tetrapeptideamide are able to stimulate insulin in man. This suggests a possible association between serum gastrin and insulin secretion. On the other hand, a serum gastrin level increases in patients with atrophic gastritis. We therefore investigate the insulin secretion in patients with atrophic gastritis. Patients and Methods: A standard $75 \mathrm{~g}$ oral glucose tolerance test (GTT) was performed in 42 subjects, 29 women and 13 men, aged 39-87 years. The patients received $75 \mathrm{~g}(225 \mathrm{ml})$ of glucose solution in the sitting position after an overnight fast. Venous blood samples were obtained before ingestion and at 30,60,90, 120 minutes and blood glucose and serum insulin concentrations were measured. A serum gastrin level was also measured before ingestion. Results: A high value of serum gastrin more than $200 \mathrm{pg} / \mathrm{mg}$ was detected in 14 patients who were classified into group $\mathrm{G}$. The remaining 28 were classified into the control group. HOMA-R was significantly higher in group $G$ than in control $(2.05+$ +/ 1.11 v.s. 1.44 +/$0.71, \mathrm{p}=0.036$ ). Fasting insulin concentration and a total of insulin were also higher in group $\mathrm{G}(6.3+/-2.4 \mu \mathrm{U} / \mathrm{ml}$ and $177+/-126 \mu \mathrm{U} / \mathrm{ml}$, respectively) but with no statistical significance, compared with the control group $(4.9+/-2.3 \mu \mathrm{U} / \mathrm{ml}$ and $162+/-92 \mu \mathrm{U} / \mathrm{ml}$, respectively). There was no difference in insulinogenic index, which was considered a parameter of initial insulin secretion, between two groups $(0.39+/-0.64$ v.s. $0.29+/-0.29)$. Conclusions: It is concluded that gastrin is a potential candidate to affect insulin resistance, which has a close association with metabolic syndrome, We should duly recognize the effect of serum gastrin concentration on insulin resistance when the patient receive a drug affecting serum gastrin level, such as proton pump inhibitors, or H2-receptor antagonists.

\section{M1100}

The Efficacy of High Dose Ondansetron for Reducing Motion Sickness in Highly Susceptible Subjects

Eric R. Muth, Amanda Elkins

Current drug remedies for motion sickness are not always effective and often induce undesirable side-effects such as drowsiness. Ondansetron is currently being explored as an alternative due to its prophylactic effect on post-operative nausea, the nausea and vomiting associated with chemotherapy, as well as its lack of side effects. There were 63 participants screened to have a history of frequent motion sickness and positive report of self treatment of motion sickness with over-the-counter medications selected from 750 student volunteers. Participants were divided into three condition groups: placebo, $100 \mathrm{mg}$ dimenhydrinate, or $24 \mathrm{mg}$ ondansetron. Participants were given 1 of the 3 treatments 1 hour before exposure to the rotating chair stimulus. Then participants were rotated in complete darkness for up to 20 minutes at $20 \mathrm{rpm}$ while making timed head movements. Electrogastrogram (EGG) data were collected prior to and during chair rotation. 60 participants were included in the overall data analysis while 48 were included in the EGG analysis. There were no statistically significant differences between dimenhydrinate, ondansetron, or placebo in the number of head movements tolerated, the amount of time tolerated in the rotating chair, or on subjective motion sickness symptoms as reported on questionnaires. All groups showed a marginally significant decrease in normal 3 cycle per minute activity $(\mathrm{F}[1,45]=3.04, \mathrm{p}<.10)$ and a significant increase in gastric tachyarrhythmia $(F[1,45]=9.71, p<.05)$, a pattern typically associated with motion sickness development. There were no significant differences between the groups and no significant interactions of time (baseline vs. rotation) found in the EGG data. This study revealed that ondansetron is not an effective remedy for motion sickness for highly susceptible individuals. Interestingly, the findings that ondansetron did not affect the gastrointestinal measures is in direct contrast with previous findings that suggest ondansetron may inhibit tachyarrhythmia (Levine et al., 2000). This study also provides evidence that dimenhydrinate may not be effective for reducing motion sickness in highly susceptible individuals. From both a subjective and objective perspective, all groups developed motion sickness. Interestingly, not even the "gold standard" dimenhydrinate treatment was effective. Therefore, it appears that there is a group of motion sickness sufferers who lack the adequate treatment that they need.

\section{M1101}

Autologous Haematopoietic Stem Cell Transplantation in Refractory Coeliac Disease with Aberrant T-Cells : A Pilot Study

Abdulbaqi Al-Toma, Otto Visser, Cindy van Roessel, B Blomberg, Gerrit Ossenkoppele, Peter Huijgens, Chris Mulder

Background: Autologous Haematopoietic Stem Cell Transplantation (ASCT) is now being investigated for patients with severe refractory autoimmune disorders unresponsive to conventional therapies. Refractory Coeliac Disease with monoclonal aberrant T cells (RCD-type II) is usually unresponsive to available therapeutic regimens and carries a high risk of transition into Enteropathy Associated T-cell Lymphoma (EATL). The objective of this pilot study is to assess the feasibility, safety and efficacy of high dose chemotherapy followed by ASCT in patients with RCD type II. Methods: Four patients [ ( 3 males, 1 female, mean age 64.2 years (range 61-69 years )] with RCD-II have been treated. Stem cells were collected by leucopheresis after pre-treatment with granulocyte colony-stimulating factor(G-CSF). After conditioning with fludarabine and melphalan, $\mathrm{ASCH}$ was performed. Patients were monitored for response of disease, adverse effects and haematopoietic reconstitution. Results: All 4 patients completed the mobilization and leucopheresis procedures successfully and subsequently received conditioning and transplantation. Engraftment occurred in all of these patients. No major non-haematological toxicity nor transplant related mortality was observed. 
Marked improvement of clinical condition was recorded in all patients (Follow-up 11.2 months, range 3-19 months). There was a significant reduction in the percentage of aberrant T-cells in duodenal biopsies associated with improvement in clinical wellbeing , and normalization of haematological and biochemical markers. Conclusions: High-dose chemotherapy followed by ASCT seems feasible and safe, and might result in long-term improvement of clinical condition in RCD patients with monoclonal aberrant $\mathrm{T}$ cells whose condition previously did not respond to the available drugs, and possibly prevents or delays the development of EATL. Long term follow up and a prospective randomized study will be needed to confirm the efficacy of this therapy.

\section{M1102}

Nature and Prevalence of Gastrointestinal Symptoms in Fabry Disease - A Retrospective Analysis from the Fabry Outcome Survey

Bjoern Hoffmann, Martin Schwarz, Atul Mehta, Satish Keshav

BACKGROUND: Fabry disease is a rare X-linked lysosomal storage disorder caused by deficiency of $\alpha$-galactosidase A. The condition typically manifests in childhood with acroparaesthesia in hands and feet. The disease is progressive, and causes premature death from cerebrovascular accident, cardiac failure, and renal insufficiency. In 2001 enzyme replacement therapy (ERT) has been approved. So far, no systematic analysis of gastrointestinal (GI) symptoms and their response to ERT has been documented. AIM: To describe the nature and prevalence of GI symptoms in a large cohort of patients with Fabry disease and to evaluate the possible response of these complaints to ERT with agalsidase alfa. METHODS: Retrospective analysis of data from the Fabry Outcome Survey (FOS), which is a Europewide registry of patients. Data from the medical history, physical examination, and clinical investigations are recorded on entry into FOS, and updated periodically. In addition, specific questionnaires are used to evaluate limb pain, and health-related quality of life. RESULTS: 752 patients from 11 countries in Europe had been enrolled in the database by October 2005. Evaluable clinical data on GI symptoms were available in 691 individuals ( 349 females and 342 males). Mean age at entry into the database was $32.8+15.8$ years, and $31.1 \%$ of the patients reported abdominal pain at that time ( $34 \% .0$ males, $28.0 \%$ females). Other complaints include diarrhea (overall $21 \%$; males $28.5 \%$ females $14.2 \%$ ), nausea ( $14.8 \%$; $16.0 \%$ vs. $13.6 \%)$, constipation $(13 \% ; 10.4 \%$ vs. $15.3 \%$ in females), and vomiting $(8.8 \%$; $10.4 \%$ vs. $7.4 \%$ ). Despite the high prevalence of these symptoms, no cases were initially diagnosed by GI physicians. CONCLUSION: GI symptoms in Fabry disease affect a large proportion of patients, and increased recognition of the condition may now allow the prompt initiation of a novel and effective treatment. Furthermore, quantification of these symptoms may allow the rapid evaluation of the effect of ERT.

\section{M1103}

Quality and Outcomes Assessment of Endoscopic Retrograde Cholangiopancreatography: A Prospective Audit of 500 Procedures Bernard Denis, Andre Peter, Gilles Breysacher, Pascale Chiappa, Anne Marie Weiss, Jacques Bottlaender

The American Society for Gastrointestinal Endoscopy (ASGE) recommended that some quality indicators should be routinely tracked on all patients undergoing endoscopic retrograde cholangiopancreatography (ERCP)(GIE 2002). Aim: to assess the quality of ERCPs performed in our hospital according to ASGE recommendations. Methods: prospective audit of 500 consecutive ERCPs performed by 2 operators between July 2002 and November 2005. 45 process and outcomes measures were assessed for each ERCP. Results: mean age was 69 years and $60 \%$ were women. American Society of Anesthesia Score was III in 28.3 $\%$ of patients. An endoscopic ultrasonography (EUS) was performed before $33 \%$ of ERCPs (93\% during the same endoscopic session). During the same period EUS performance avoided 110 unnecessary diagnostic ERCPs. The annual procedure volumes of both operators were respectively 69 and 87 ERCPs (62 and 80 therapeutic ERCPs). The main indications were bile duct stones (65.8\%) and malignant biliary obstruction (27\%). All were ASGE approved. The procedure intent was diagnostic in $9 \%$ of cases, therapeutic in $45.5 \%$ and mixed in $45.5 \%$. The procedure difficulty was grade 1 in $85.1 \%$ of cases, 2 in $5.6 \%$ and 3 in $9.3 \%$. Rates of complete technical success at first attempt were $92.7 \%$ for cholangiography, $94.5 \%$ for pancreatography, $91.1 \%$ for sphincterotomy, $87.9 \%$ for stone extraction and $80 \%$ for stent insertion. Rates of success of cholangiography differed significantly according to anatomy and indication: intradiverticular papilla (53.8 \%), Billroth II (75\%), choledocholithiasis $(95.6 \%)$, malignancies $(88.7 \%)(\mathrm{p}<0.01)$. A precut sphincterotomy was needed in $7.8 \%$ of ERCPs, successful in $87.1 \%$ of cases. Rates of clinical success by intent-to treat were $89.5 \%$ for diagnostic procedures and $81.3 \%$ for therapeutic procedures. The latter decreased significantly with difficulty grade: grade $1(83.8 \%), 2(71.4 \%)$ and 3 $(67.4 \%)(\mathrm{p}=0.01)$. In case of cannulation failure, the appeal to a backup endoscopist was successful in $72.2 \%$ of cases. Second attempts after prior failure were successful in $80 \%$ of cases. The rate of complications was $3.6 \%$ without difference according to operator, difficulty grade, indication, and use of precut sphincterotomy. Conclusion: Modern imaging techniques have lowered the rate of diagnostic ERCPs to less than $10 \%$. In the real world the rate of clinical success of therapeutic ERCPs is around $80 \%$. This audit enabled us to modify certain practices and to improve patient care: pancreatobiliary EUS prior to ERCP to lower the rate of diagnostic ERCPs; appeal in case of failure to a backup endoscopist either in the same session or in another.

\section{M1104}

Evaluation and Management of Patients Referred for Suspected Sphincter of Oddi Dysfunction Type III (SODIII)

Niraj D. Jani, Jennifer Dorfmeister, Arnold Wald, Adam Slivka

Introduction: In clinical practice, SODIII comprises a heterogeneous group of patients with abdominal pain and no objective markers of disease. Standard therapy, performing a sphincterotomy of the biliary and/or pancreatic segment if basal pressures are elevated, is associated with high risk, variable outcomes, and lacks validation. Aims: To prospectively characterize patients referred to a tertiary care center for evaluation and management of SODIII. Methods: Sequential patients referred to a single provider for suspected SODIII over a 3-year period were enrolled in an IRB-approved clinical registry. Patients with known structural biliary abnormalities or elevated liver/pancreas enzymes were excluded. In addition to routine labs/imaging, patients completed an SF-36 ${ }^{\mathrm{TM}}$ quality of life (QOL) questionnaire at enrollment and during subsequent office visits. Follow-up ranged from 2 months to 18 months. Physical and mental outcome scores were calculated to determine baseline and treatment response. Results: 38 patients with abdominal pain $(35 \mathrm{~F} / 3 \mathrm{M})$, mean age 45 years were enrolled. Common concurrent medical conditions included thyroid disease in $24 \%$, psychiatric illnesses in $22 \%$, and cholecystectomy in $58 \%$. Out of the 38 patients, only (10\%) met clinical criteria for SODIII according to Rome II consensus. Subsequent workup disclosed gastroparesis in 2 patients and breakthrough reflux in 1 . The remaining 31 patients were assigned a diagnosis of functional bowel disease. Baseline SF 36 scores demonstrated significant impairment in physical and mental well being for all patients. A total of 25 out of 38 patients including the 4 SODIII patients, were treated with visceral analgesics (tricyclics) and/or anti-spasmodic agent. None of the patients were subjected to manometry or sphincterotomy. For physical wellness, 17 (68\%) improved (including 3 of the 4 SODIII), 5 had no change and 3 were worse. For mental wellness, 18 (72\%) improved, 3 had no change and 4 were worse. Conclusions: The vast majority of patients referred for evaluation of SODIII does not meet defined clinical criteria, and should not be routinely subjected to high-risk endoscopic procedures. Significant impairment in QOL exists in these patients Improvement can be expected with pharmacological therapy for functional disease in the majority of patients, including those fulfilling criteria for SODIII.

\section{M110}

Major GI Events Among Elderly Chronic Users of Coxibs and Non-Selective NSAIDs, with/Without Aspirin

Jingshu Wang, C. Daniel Mullins, John Naradzay, Kimberly B. Howard

Objective: The gastrointestinal (GI) risks associated with the use of COX-2s versus traditional non-steroidal anti-inflammatory drugs (NSAIDs) were documented in clinical trials. The objective of this study was to estimate the rates of major GI events among elderly chronic users of COX-2s versus traditional NSAIDs, with and without aspirin (ASA), in routine clinical practice. Methods: This analysis utilized a retrospective cohort from the GE logician database (Centricity EMR), which contained the medical records of 3 million patients seen by 5,000 physicians across 27 states. Inclusion/exclusion criteria: chronic use (defined as 2 or more medication mentions of the same drug class within 60 days) of NSAIDs or COX2s between January 1, 1999 and June 30, 2003, age 65 or older on index date, no switch between COX-2s and NSAIDs during the follow-up period, which continued until the earlier of the end of one year or when a major GI event occurred. Major GI events were defined as GI hemorrhage including melena (ICD-9 codes: $578,578.0,578$ 1, and 578.9). Descriptive and multivariate logistic analyses were conducted to determine how the rate of major Gl events differed across chronic users of COX-2s (reference group), NSAIDs, COX-2s+ASA and NSAIDs+ASA. In order to account for channeling bias, we controlled for major and minor GI events in the year prior to the index date, and prior GI protective drug use. Other control variables included: gender, age, and pre or post-index GI harmful drug use. Results: Of the 12,729 patients in the study, 7,338 were on COX-2s alone (105 major GI events in the year prior to the index date and 127 in the year after), 3,826 were on NSAIDs alone (40 and 79), 963 were on COX-2s+ASA (13 and 17), and 602 were on NSAIDs+ASA (4 and 16). Compared to chronic COX-2s-alone users, NSAIDs-alone users had a statistically significantly higher rate of GI events (OR=1.35, 95\% CI: 1.01-1.80). Chronic users of NSAIDs+ASA also had a higher rate of GI events, and the effects approached statistical significance $(\mathrm{OR}=1.68,95 \% \mathrm{CI}: 0.99-2.86)$. COX-2s+ASA users had similar rates of Gl events as COX-2s-alone users ( $\mathrm{OR}=0.96,95 \% \mathrm{CI}$. 0.57-1.61). Conclusions: The risk of major GI events was highest among chronic users of NSAIDs+ASA, followed by those on NSAIDs alone. Only the chronic users of NSAIDs alone had a statistically significant higher risk than users of COX-2s alone. The addition of ASA did not significantly increase the risk of GI events among COX-2 users.

\section{M1106}

\section{Adverse Lower Gastrointestinal Effects of Non-Selective and COX-2 Selective} NSAIDs: A Systematic Review Loren Laine, Rebecca A. Smith, Kejia Min, Connie Chen, Robert W. Dubois

BACKGROUND: NSAIDs have been suggested to cause adverse effects on the lower G (LGI) tract, but no published systematic review exists. We reviewed the literature on LGI effects of non-selective (NS) and COX-2 selective (coxib) NSAIDs to determine if the evidence indicates that they increase the risk of LGI effects and to determine if coxibs decrease the risk compared to NS NSAIDs. METHODS: PubMed and Cochrane (CCTR) from 1950 to 2005 were searched. Inclusion criteria were studies of NSAID use reporting on LGI integrity (permeability, intestinal inflammation, microscopic lesions), visualization of LGI lesions at endoscopy or autopsy, and LGI clinical events (bleeding, perforation, diverticular disease) Two reviewers independently evaluated 930 published titles, identified 46 studies that me inclusion criteria, and performed independent data extraction. RESULTS: Study designs were RCTs $(\mathrm{N}=18)$, case-control $(\mathrm{N}=13)$, cohort $(\mathrm{N}=8)$, and before-after comparisons $(\mathrm{N}=$ 7). Methodologic quality was highly variable. NS NSAID vs No NSAID or Placebo: 11 studies assessed LGI bleeding: 2 underpowered RCTs had non-significant increases in bleeding with NS NSAID (RRs $1.6,0.5-5.0 ; 3.0,0.1-73.6) ; 7$ of 8 case-control studies had significant associations with NS NSAIDs (ORs 1.9-18.4); and an 8-patient before-after study showed no NSAID effect. The 2 LGI perforation studies (case-control) noted a significant association with NS NSAIDs: ORs 2.5 (1.2-5.5), 8.1 (2.5-26.6). Complicated/symptomatic diverticular disease was associated with NS NSAIDs in 5 case-control studies (ORs 1.8-11.2) and 1 cohort study (RR 1.5), but not in a 10-day RCT (RR 1.5, 0.1-36.5). Gross mucosal injury (e.g., breaks, ulcers) was increased with NS NSAIDs in 5 of 7 studies; a low-dose aspirin flexible sigmoidoscopy study $(\mathrm{N}=29)$ and a retrospective study of 96 colonoscopies showed no effect. NS NSAIDs caused significantly greater injury in 18 of 21 integrity studies. Coxib

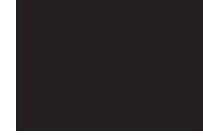


vs NS NSAID: In the 2 RCTs, coxibs had fewer LGI clinical events (RR 0.5, 0.2-0.9) and less hematochezia (RR $0.4,0.2-0.8$ ) than NS NSAIDs. In the 1 endoscopic study, coxibs had fewer mucosal breaks than NS NSAIDs (RR 0.3, 0.2-0.5) but more than placebo (RR $2.2,1.002-4.9$ ). Coxibs caused less injury than NS NSAIDs in 3 of 4 integrity studies, but no increase vs placebo in 2 of 2 studies. CONCLUSIONS: An increase in LGI injury and clinical events with NS NSAIDs appears relatively consistent across this heterogeneous collection of trials. Coxibs also appear to cause less LGI injury than NS NSAIDs. Additional high-quality trials are warranted to more precisely estimate the effects of NS NSAIDs and coxibs on the LGI tract.

\section{M1107}

Longterm Prevention of Esophageal Variceal Bleeding with rabeprazole Results of a 5 Year Study in Japan

Kuwayama Hajime, Nishiki Ryoichi

Our previous reported that the PPI treatment was effective in prevention of esophageal variceal bleeding and recurrent variceal bleeding in cirrhotic patients. We investigated the effect of rabeprazole (RPZ) in prevention of esophageal variceal bleeding in 5 years using a randomized controlled trial design. Subjects \& Methods: We enrolled 134 patients with confirmed cirrhosis and esophageal varices. 41 of these patients had at least one previous episode of confirmed variceal bleeding and had been treated with endoscopic ligation. The 134 patients were randomized to receive either PPI (oral rabeprazole 20mg/day, $\mathrm{N}=73$ ) or no anti-secretory treatment $(\mathrm{N}=61)$, and followed for up to 5 years. Patient demographics (age, gender, Child-Pugh score, variceal size, red color signs, and previous variceal bleeding) were not significantly difference in both groups. The evaluated association between vareices bleeding and patients risk factors, odds ratios (OR) and the corresponding 95\% CI were computed by means of logistic regression models. Results: The rate of variceal bleeding was significant lower in the PPI-treated group $(6.8 \%, 5 / 73)$ than the untreated group $(26.2 \%$, $16 / 61, P=0.0034)$. Five- year rate for occurrence of variceal bleeding, calculated by KaplanMayer analysis, was $14.0 \%$ in the PPI-treated group, which was significantly lower than the rate of $29.0 \%$ in the untreated group, $\mathrm{P}=0.0019$ by log rank test. Subanalysis of patients with pre-study variceal bleeding (secondary prevention) showed that variceal re-bleeding rate was significant lower in PPI-treated pts $(10.0 \%, 2 / 10)$ than untreated patients $(52.4 \%$, $11 / 21, P=0.0063$ ). Five-year rate for recurrence of variceal bleeding, calculated by KaplanMayer analysis, was $11.3 \%$ in PPI-treated patients, significantly lower than the $56.5 \%$ rate in untreated patients, $\mathrm{P}=0.0091$ by log rank test. Rabeprazole use was found to be significant factor for varices bleeding (OR: 0.207, 95\% CI:0.071-0.604, P=0.0004. Conclusion: Rabeprazole treatment was effective in prevention of esophageal variceal bleeding and recurrent variceal bleeding for 5 years.

\section{M1108}

Ethnicity and Gender Are Not Predictors of in-Hospital Mortality for Patient with Initial Esophageal Variceal Hemorrhage

Linda Diteodoro, Juan Carlos Munoz, Louis Lambiase, Kenneth Vega

Ethnicity and gender have been found to effect the outcome of a number of conditions such as heart failure, oral cancer, hepatitis C, post liver transplant infections, and survival after portosystemic shunting. Recent advances in the management of bleeding esophageal varices have lead to improvements in patient outcomes. Very little is known regarding the effect of ethnicity and gender on outcome in these patients. Previously we found that African American patients had a higher in-hospital mortality rate after their initial variceal hemorrhage. AIM:To examine a large sample of patients with variceal hemorrhage to determine if there was any difference in presentation or outcome when the patients were grouped by ethnicity or gender. Also, to determine whether ethnicity or gender were independently associated with differences in in-hospital mortality. Methods: A retrospective study was conducted. All patients undergoing endoscopy for their initial episode of esophageal variceal bleeding from April 1999 to October 2005 were identified via the Shands Jacksonville endoscopy database. These patient's charts were reviewed. Data collected included: demographics, self reported ethnicity and gender, etiology of liver disease, clinical presentation, laboratory data, Child-Pugh classification at presentation, variceal grade at endoscopy, initial therapy for bleeding control and hospitalization outcome. The data was analyzed for differences between race and gender using one-way ANOVA and Kruskal-Wallis test. Further analysis was performed via binary logistic regression using survival as the dependent variable. Results:200 patients were identified. There were 150 Non-Hispanic Caucasians and 50 African Americans. There were 154 men and 46 women. The in-hospital mortality was $11.5 \%$. African Americans were more likely to have a more prolonged prothrombin time, lower albumin, more advanced grade of varices at endoscopy, and more advanced Child classification at presentation $(\mathrm{p}<0.05)$. There was no difference in outcome between the groups. Women were more likely to have lower diastolic blood pressure and less likely to have alcoholic liver disease $(\mathrm{p}<0.05)$. There was no difference in outcome between men and women. Logistic regression identified only age $(\mathrm{p}=0.001)$, lower systolic blood pressure at presentation $(\mathrm{p}=0.004)$ and prolonged PTT $(\mathrm{p}=0.01)$ as predictors of outcome. Ethnicity $(\mathrm{p}=0.866)$ and Gender( $\mathrm{p}=0.256)$ did not influence survival. Conclusion: Although African Americans present somewhat differently than Non-Hispanic whites there does not appear to be any difference in outcome between the two groups. Likewise, gender also appears to not predict outcome.

\section{M1109}

Serum Markers of Prognosis Following Emergency Department Presentation for GI Bleed

Sara M. Echelmeyer, Latha G. Stead

Objective: To study mortality after presentation to the Emergency Department (ED) for GI bleed, and to determine whether any serum laboratory values were associated with a worse prognosis. Methods: All patients presenting to the ED of an academic medical center for acute GI bleed between 1/2002-6/2004 were included. Patients had standard laboratory evaluation. Time-to-event methodology was used for the analysis of patient survival. Duration of follow-up was calculated from the date of ED visit to the date of death or last followup, with all events censored at 1 year. Survival estimates were based on the Kaplan-Meier method. Cox proportional hazards models were fit to evaluate the association between each of the laboratory values and death. Associations were summarized with risk ratios (RR) and 95\% confidence intervals (CI). Laboratory values were evaluated univariately by fitting separate models, and then with a multivariate model using a combination of stepwise backward procedures and an inclusion criterion of $\mathrm{p}<0.05$. A total of 255 adults presented during the study period. Of these, 24 denied research authorization. The cohort was $55 \%$ male with a mean age of 64.2 years (SD, 18.0; median, 68; range, 18-100 years). A total of 50 patients died; 9 within 30 days, 11 within 31-90 days, 9 within 91-365 days, 17 within 1-2 years, and 4 after 2 years. Among the remaining 181 patients alive at last followup, the median duration of follow-up was 1.2 years (interquantile range, 0.5-2.0 years). The survival estimates at 6-months, 1-year, 18-months, and 2-years following ED presentation were $89.0 \%, 85.4 \%, 79.0 \%$ and $69.0 \%$, respectively. Univariate analysis identified the following variables as being significantly associated with poorer patient survival: older age, leukocytosis, elevated creatinine, elevated troponin, elevated AST, elevated lactate, low platelet count, low sodium, low bicarbonate, low chloride, high potassium, high glucose, high aPTT, and high bilirubin (all p<0.05). Multivariate analysis identified older age, elevated troponin, elevated creatinine, low sodium, and high aPTT as being jointly associated with poorer patient survival. Conclusion: Hyponatremia, renal insufficiency, an elevated troponin or aPTT are associated with increased risk of death at one year following ED presentation for gastrointestinal hemorrhage.

\begin{tabular}{|c|c|c|}
\hline Prognostic Factor & RR $(95 \% \mathrm{CI})$ & P-value \\
\hline Age & $1.4(1.1,1.8)$ & 0.005 \\
\hline Creatinine (high) & $4.2(1.1,16.1)$ & 0.037 \\
\hline Troponin (high) & $3.2(1.4,7.0)$ & 0.004 \\
\hline Sodium (low) & $3.1(1.6,6.3)$ & 0.001 \\
\hline aPTT (high) & $2.8(1.3,5.8)$ & 0.008 \\
\hline
\end{tabular}

\section{M1110}

Upper and Lower Gastrointestinal Findings in Patients with Anemia, But Without Evidence of Iron Deficiency

Nick Powell, Alistair McNair

Background: Occult blood loss in to the gastrointestinal (GI) tract is the most common cause of iron deficiency anemia (IDA). Evaluation of both the upper and lower GI-tract is warranted to identify culprit lesions. IDA is readily defined by hemoglobin levels below laboratory reference ranges in combination with microcytic/hypochromic red cell indices or reduced serum ferritin concentrations. Gastroenterologists frequently receive referrals for invasive investigation of patients with anemia in the absence of supporting evidence of iron deficiency. Methods: We conducted a retrospective study designed to evaluate whether significant GI-tract pathology exists in patients with a macrocytic or normocytic anemia and elevated, normal or undetermined serum ferritin concentrations. For comparison we studied a control group with documented IDA. We identified 130 patients referred to our unit for endoscopic evaluation with a macrocytic or normocytic anemia without evidence of iron deficiency. All 130 patients were investigated with upper GI endoscopy and 65 patients $(50.0 \%)$ were additionally investigated with colonoscopy or double contrast barium enema. The comparator group comprised 201 patients with documented IDA (microcytic/hypchromic anemia and reduced serum ferritin concentrations), all of whom were investigated with both upper GI endoscopy and lower GI tract investigations. Results: There were no lower GI-tract malignancies identified in the 65 patients with a macrocytic or normocytic anaemia who underwent either colonoscopy or barium enema, as compared to 23 colon cancers diagnosed in patients with documented IDA $(11.4 \%),(\mathrm{p}<0.01)$. Similarly, there were no upper GI-tract cancers found in the macrocytic/normocytic group as compared to $9(4.5 \%)$ upper GI tract cancers found in their iron deficient counterparts $(p<0.025)$. Other potential cause of occult blood loss were identified in the upper GI tract (ulceration in the esophagus, stomach or duodenum, celiac disease or vascular lesions) in $21.9 \%$ of patients with documented IDA as compared to only $7.7 \%$ of patients without evidence of IDA $(p<0.001)$. Conclusion: Evaluation of the GI tract in patients with macrocytic or normocytic anemia without evidence of iron deficiency has a very low diagnostic yield and is not warranted.

\section{M1111}

Association Between Referral Indications for Open Access Upper GI Endoscopy and Endoscopic Findings

Lieke A.S. V. Kerkhoven, Sally J. van Rijswijck, Leo G.M. van Rossum, Robert J.F. Laheij, Ellen M. Witteman, Adriaan C.I.T.L. Tan, Jan B.M.J. Jansen

Background: General practitioners refer patients with gastrointestinal symptoms for open access upper gastrointestinal endoscopy when symptoms possibly underlying a malignancy are present or when empirical treatment fails to relief symptoms. The aim of this study was to investigate the association between referral indications for upper gastrointestinal endoscopy, as given by general practitioners, and endoscopic outcomes. Method: Endocopy outcomes of consecutive patients who were referred to a primary care hospital for open access endoscopy between January 2002 and December 2004 were included. Their general practitioners were asked to specify the reason for referral on a specially designed form. Relevant organic disorders were defined as findings at endoscopy that might explain symptoms or influence treatment strategy, i.e. gastroesophageal carcinoma, peptic and duodenal ulcer, duodenitis, Barrett's oesophagus or reflux-oesophagitis. Results: A total of 1,298 subjects was included. A relevant organic disorder was found in 35\% of patients. Alarm symptoms had a positive predictive value (PPV) of $4 \%$ for carcinoma and 5\% for Barrett's 
oesophagus, while prevalences were $2 \%$ and $5 \%$, respectively. The PPV of treatment failure for carcinoma and Barrett's oesophagus are $1 \%$ and $5 \%$, respectively. The only indication with a slightly increased PPV was reflux-like symptoms; PPV was 33\% for reflux-oesophagitis, while prevalence was $22 \%$. Conclusion: None of the positive predictive values of different referral indications varies substantially from prevalences of organic endoscopic findings. Only reflux-like symptoms have a slightly increased PPV for reflux-oesophagitis, which is, however, of little clinical relevance since this finding rarely alters therapeutic strategy. In particular treatment failure and the presence of alarm symptoms, which are referral indications for open access endoscopy as described in the Dutch guideline for general practitioners, are not associated with any relevant organic finding. In conclusion, referral indications do not distinguish organic from functional diseases.

\section{M1112}

Comparative Study On the Efficacy of Famotidine and Rebamipide for Gastri Mucosal Lesions in Patients Receiving Long-Term Administration of NSAIDs Junichi Yamao, Hideyuki Kojima, Akira Mitro, Motoyuki Yoshida, Masayuki Yoshikawa, Eiryo Kikuchi, Masami Matsumoto, Masaki Nakayama, Tatsuichi Ann, Hiroshi Fukui

(Purpose) We used an endoscopy procedure to compare the effects of famotidine and rebamipide on gastric mucosal lesions (hemorrhage and/or erosion) in patients receiving non-steroidal anti-inflammatory drugs (NSAIDs) for a long period. (Subjects) The subjects were 261 patients who had been receiving NSAIDs (excluding aspirin) for 28 days or longer and were enrolled after providing written informed consent. (Methods) An upper gastrointestinal endoscopy was performed in all patients, with and without symptoms. Gastric mucosal lesions were evaluated using a modified LANZA scoring system. Patients with the presence of gastric hemorrhage and/or erosion (LANZA score 1-4) were divided into 2 groups, F and R, by dynamic storage allocation based on the presence of the anti-Helicobacter pylori antibody and LANZA scoring severity. Famotidine $(20 \mathrm{mg} /$ day $)$ was administrated to the patients in group $\mathrm{F}(\mathrm{n}=57$ ), while rebamipide ( $300 \mathrm{mg} /$ day) was given to those in group $\mathrm{R}(\mathrm{n}=55)$. Without stopping NSAID administration, an endoscopy was re-performed following the 4-week administration of famotidine and rebamipide. LANZA scores were evaluated by a endoscopist who belongs to another institute and had no knowledge of the drugs used or timing of the endoscopy. (Results) The screening endoscopic procedures revealed gastric hemorrhage and/or erosion in $137(52 \%)$ of the 261 patients, while gastric ulcers were seen in $20(8 \%)$, duodenal ulcers in $5(2 \%)$, and combined gastroduodenal ulcers in $2(1 \%)$. Ninety-six (37\%) of all patients had no gastric mucosal lesions. In addition, malignant lesions were found in $3(1 \%)$ patients (gastric cancer $n=2$, esophageal cancer $n=1$ ). The mean LANZA score for group F significantly $(\mathrm{p}<0.001)$ decreased from 2.4 to 1.3 following the 4 -week administration of famotidine, whereas that for group R did not show a significant change ( 2.4 to $2.2, p=0.478$ ). The efficacy ratios for group $\mathrm{F}$ and group $\mathrm{R}$ were $68 \%$ and $36 \%$, respectively. The difference in LANZA score between before and after medical treatment in group $\mathrm{F}(1.2)$ was significantly greater $(\mathrm{p}=0.002)$ than that in group $\mathrm{R}(0.2)$. (Conclusion) Gastric mucosal lesions were observed in $63 \%$ of patients who had been receiving NSAIDs for a long period. Famotidine demonstrated an excellent efficiency toward gastric mucosal lesions (hemorrhage and/or erosion) induced by NSAIDs and during their continued administration. We concluded that the effect of famotidine was significantly superior than that of rebamipide.

\section{M1113}

Is a short Infusion of $80 \mathrm{Mg}$ OMEPRAZOLE Once Daily the Optimal Dose for Stress Ulcer Prophylaxis in ICU Patients?

W P. Geus, W J. Hofste, J A. de Haas, N G. Hunfeld, E J. Kuipers

Key factor in the prophylaxis for stress ulcer bleeding is a postulated critical $\mathrm{pH}$ target of at least 4 . It is known that PPIs such as omeprazole can give a more predictable and sustained $\mathrm{pH}$ control during prolonged dosing than $\mathrm{H} 2$-receptor antagonists like ranitidine. However, the optimal dose and way of PPI administration for stress ulcer prophylaxis need to be established. Aim: To assess the acid-inhibitory effect of a short infusion of $80 \mathrm{mg}$ omeprazole given once daily in different ICU populations. Methods: Glass electrodes connected to data loggers were positioned in the gastric corpus to monitor intragastric $\mathrm{pH}$ continuously during day 0 (baseline) and day 1 and 2 of dosing in postoperative non-ventilated (NV) patients and during day 0 and day 1,2 and 3 in mechanically ventilated $(V)$ patients. Only patients whose intragastric pH fell below 4 for $70 \%$ of time on day 0 (baseline) were included. At day 1 all patients received a short infusion of $80 \mathrm{mg}$ omeprazole. At day 2 patients were randomized to placebo or continuation of omeprazole treatment. Severity of illness was estimated in both groups with APACHE II score. Results: 17 patients were included in the $\mathrm{NV}$ group and 10 in the $\mathrm{V}$ group. APACHE II score was significantly higher in the $\mathrm{V}$ patients ( 16 vs. $10, p=0.013$ ). At day 0 median $\%$ of time with intragastric $\mathrm{pH}<4$ did not significantly differ between the $\mathrm{V}$ and $\mathrm{NV}$ patients ( $98 \mathrm{vs.} 94 \%, \mathrm{p}=0.2$ ). During day 1 , following a single dose of omeprazole the $\%$ of time $<\mathrm{pH} 4$ was $9.7 \%$ in $\mathrm{V}$ patients and $48.8 \%$ in NV patients $(\mathrm{p}=0.024)$. During day 2 , NV patients had an intragastric $\mathrm{pH}<4$ for $3.9 \%$ of time when treated with omeprazole $(n=9)$, resp. $81 \%$ of time when given placebo $(n=8, p=0.01)$. For the $\mathrm{V}$ patients the corresponding figures were $8.6 \%$ after omeprazole $(\mathrm{n}=5)$ and $62 \%$ after placebo $(n=5, p=0.465)$. At day 3 the $\%$ of time $<\mathrm{pH} 4$ in the latter group increased to $21.7 \%$ during omeprazole treatment and to $100 \%$ during placebo $(\mathrm{p}=0.113)$. Conclusions: A once daily dose of $80 \mathrm{mg}$ omeprazole given as short infusion is not the optimal dose for stress ulcer prophylaxis in ICU patients: 1. In postoperative non-ventilated patients, intragastric $\mathrm{pH}$ remains below the target $\mathrm{pH}$ of 4 for $49 \%$ of the first 24 hours. 2 . In mechanically ventilated patients the acid-inhibitory effect of this dose omeprazole is still insufficient after 72 hours of treatment, with further increase of acid-output despite repeated treatment on consecutive days.

\section{M1114}

Disaster Medicine: Is Stress Ulcer Prophylaxis Needed for Crush Syndrome Patients After Earthquakes? Experience of a Referral Hospital,Turkey Hakan Akin, Zekaver Odabasi, Huseyin Atalay, Huseyin Kocak, Gulcin Kantarci, Nadi Bakirci, Cetin Ozener, Osman Ozdogan, Nurdan Tozun

Objective: To determine the frequency and the risk factors of stress ulcer related gastrointestinal (GIS) bleeding in patients with Crush Syndrome (CS) after Marmara Earthquake Disaster Turkey. Background: On 17 August 1999 a very destructive earthquake disaster has stroked the north-west region of Turkey which has resulted in 17480 deaths and 43953 injured victims. Marmara University Hospital was one of the nearest backup hospital to the disaster epicenter. Methods: After the Earthquake out of 476 hospital admission, 87 earthquake victims developed CS that mainly consist of acute renal failure secondary to the traumatic rhabdomyolysis. All CS patients were given i.v. Famotidine $20 \mathrm{mg}$ /day from the first day of the admission and observed for overt GIS bleeding related to stress ulcer. The possible risk factors are determined by univariate and multivariate analyzes. Results: Out of $87 \mathrm{CS}$ patients nine $(10.3 \%)$ had overt GIS bleeding which was shown to be related to stress ulcers by endoscopy. In 8 patients the bleeding was originated from upper GIS site whereas in one patient it was both upper and lower site. All the bleeding patients were male. Mean bleeding day was $7.7 \pm 3.4$ (between 3rd-14th days) after the rescue from the ruins. Mean age was $43.8 \pm 19.2$ years (range $24-71$ years).Seventy six CS patients were admitted within the first 3 days of the disaster but 11 patients were transferred to our hospital after the third day. Five patients out of $76(6.6 \%)$ who were admitted in the first 3 days of earthquake developed GI bleeding whereas 4 patients out of $11(36.4 \%)$ that were transferred to our hospital after the third day had GI bleeding (p: 0.012). All of the study patients had multiple extremity trauma and 41 of them had (47.1\%) fasciotomies. Intermittent hemodialysis was needed in 58 of the patients whereas 29 patients could be managed by conservative renal replacement therapy. In univaried analyzes; male sex (p: 0.01) and having no i.v. famotidine treatment in the first 3 days (p: 0.01 ) were found to be the significant risk factors for GI bleeding. In multivariate logistic regression analyze; initializion iv famotidine treatment in the first 3 days was the only independent factor for the protection of stress ulcer related G bleeding in CS patients (p:0.007, beta:-2.55, OR:0.077, \%95 CI 0.012-0.504). Discussion: This is the only CS series report in the literature that evaluates specifically overt GIS bleeding and its risk factors. In our series overt GIS bleeding is highly frequent in CS patients group and intializing i.v. famotidine treatment within the first 3 days seems be decreasing that significantly.

\section{M1115}

Use of Blatchford and Clinical Rockall Score in Screening Low Risk Patients with Acute Upper Gastro-Intestinal Haemorrhage - Which Score Is More Useful?

Asad R. Shah, Nadia A. Malik, David Sheridan, Athar Saeed

Introduction Acute Upper GI haemorrhage is one of the commonest medical emergencies.Reliable early identification of low risk patients can help guide decisions relating to early hospital discharge and define a group of patients who may not even require endoscopy The risk scores employed either use clinical parameters only or include endoscopic findings as well. Both Blatchford and Clinical Rockall Scores make use of only clinical parameters to screen low-risk patients (a value of 0 for both). Objective The purpose of this study was to assess the use of Blatchford and Clinical Rockall score in screening out patients at low risk of re-bleeding and mortality and to find out which score screens more patients and how effectively. Methods This study was conducted in two limbs. One limb was a retrospective case-review of patients undergoing OGD for acute upper GI haemorrhage between November 2003 and March 2004. The second limb was a prospective audit of patients presenting in the Accident and Emergency Department with Acute upper GI haemorrhage between March and May 2005. Both Blatchford and Clinical Rockall scores were determined for each patient as were the endoscopic findings, length of hospital stay, the incidence of re-bleeding, surgery mortality. Results There were 37 patients in the first group and 45 in the second. The mean ages were 57.5 years ( SD 19.9) and 61.1 years (SD 21.5) with a male predominance (57\% in both). The commonest presentation was haematemesis either alone or with melaena in both groups. $4(10 \%)$ and $8(18 \%)$ patients were screened out as low risk by the Blatchford score while the Clinical Rockall score was able to screen out $9(24 \%)$ and $11(24 \%)$ patients none of them had recurrent bleeding or died (100\% negative predictive value). The Mean age of the low-risk group was 39.2(SD 12.4, Range 19-57)years. The median length of hospital stay was 1 day (range $1-10$ days). 16 of the 20 (80\%) low-risk patients had endoscopy; none had any stigmata of recent haemorrhage. The range of endoscopic diagnoses was: 4 normal, 4 erosive esophagites, 1 gastritis, 1 Barrett's esophagitis, 2 gastric erosions 1 pyloric and 1 duodenal ulcer. The difference in performance of both scores was found to be statistically significant $(\mathrm{p}=0.05)$. Conclusion Our study has validated both Blatchford and Clinical Rockall scores to have equal negative predictive value for re-bleeding and mortality. The Clinical Rockall score screens out more patients and therefore is more useful than Blatchford score at identifying low risk patients. Furthermore a case can be made for early discharge of the low-risk patients without having endoscopy

\section{M1116}

How Did Rofecoxib Withdrawal Impact on NSAID and PPI Prescribing? Kathleen Bennett, Mary Teeling, John Feely, Colm O'Morain, Humphrey O'Connor In September 2004 the selective COX-2 inhibitor rofecoxib (vioxx $®$ ) was withdrawn globally from the market due to concerns about increased risk of cardiovascular mortality and morbidity with its use. AIMS: The aim of this study was to examine the switching patterns of patients prescribed rofecoxib after withdrawal in September 2004 to alternative nonsteroidal anti-inflammatory drugs (NSAIDs), and the impact this had on proton pump inhibitor (PPI) prescribing. METHODS: A national prescribing database was used to identify study subjects in the Eastern Health Region, the largest in Ireland. Medicines are coded according to WHO ATC. Adults (>16 years) prescribed rofecoxib for 3 or more months 
(chronic use) between Jan-Sept 2004 were identified. A longitudinal monthly prescribing history was used to determine the switching pattern to other COX-2 inhibitors, non-selective NS) NSAIDs or nimesulide (marketed as a COX-2 inhibitor) from Oct 2004-April 2005. Chi-square tests and logistic regression was used to determine the likelihood of switching to an alternative COX-2 inhibitor versus a NS NSAID, and the effects on coprescription of PPIs. Adjusted odds ratios (for age and gender, ORadj) and 95\% confidence intervals are presented. RESULTS: A total of 1,954 patients were identified as chronic users of rofecoxib prior to its withdrawal. Of these, 620 (31.7\%) did not receive a prescription for any NSAID after September 2005. Of the remainder, only $363(18.6 \%)$ were switched to an alternative COX-2 inhibitor; $404(20.7 \%)$ to a non-selective NSAID, 207 (10.6\%) to nimesulide, and $360(18.4 \%)$ to a combination of NSAIDs. Of those who had received rofecoxib, $37.8 \%$ were concurrently prescribed aspirin, compared with $27.6 \%$ of those prescribed NS NSAIDs $(n=13,677)$, between Jan-Sept $2004(p<0.0001)$, and $44.2 \%$ were concurrently prescribed a PPI compared with $29.4 \%$ on NS NSAID, between Jan-Sept 2004 (p<0.0001). The likelihood of switching to an alternative COX-2 inhibitor versus a NS NSAID was related to aspirin being previously prescribed or not (ORadj $=0.66,95 \%$ CI $0.49,0.90)$, and male gender (ORadj=1.39, 95\% CI 1.01, 1.90). Chronic users of rofecoxib, who were previously on PPI therapy, were most likely to continue on PPI therapy after rofecoxib withdrawal (ORadj= $27.6,95 \%$ CI 21.4, 35.7). CONCLUSIONS: Withdrawal of rofecoxib usually led to either withdrawal of NSAID therapy altogether or a switch to a NS NSAID. Rofecoxib withdrawal had no obvious impact on coprescription of PPIs and patients continued with PPI therapy after withdrawal. These prescribing trends also call into question the need for NSAID therapy in the first place in a substantial proportion of patients.

\section{M1117}

Prevalence and Predictive Factors of Upper Gastrointestinal Symptoms in Patients Treated with NSAIDs: COMPLAINS study Gerard Thiefin, Rene-Marc Flipo, Thierry Schaeverbeke, Philippe Barthelemy, Christine Soufflet

Introduction-Aim Ten to $40 \%$ of all patients treated with NSAIDs complain of upper gastrointestinal symptoms. The pattern of these symptoms and the factors influencing their occurrence are not well known. The aim of this study is to evaluate the prevalence and type of upper gastrointestinal symptoms in patients treated with NSAID as well as the predictive factors of occurrence. Method This transverse epidemiological study was carried out on a representative sample of hospital and private practice rheumatologists. The data were collected using a standardized questionnaire from all patients over 18 years old, treated with NSAID for at least 3 days and seen in consultation over a period of one week. Results Six hundred and thirty rheumatologists included 8269 patients (4878 women; mean age : 57 \pm 15 years) treated by non selective NSAID $(82.8 \%)$ or by coxibs $(17.2 \%)$. Half of these patients received their treatment for at least 2 weeks and $61.7 \%$ received a gastro-protection (PPI in $97 \%$ of cases). The prevalence of the upper gastrointestinal symptoms was $34.7 \%$ (2799 patients): $7.6 \%$ of the patients suffered from heartburn, $8.8 \%$ acid regurgitation, 17.3 $\%$ epigastric burning, $14.3 \%$ epigastric pain/discomfort, $4.8 \%$ nausea and $4.3 \%$ bloating. According to the definition used, the prevalence of gastroesophageal reflux (GER) symptoms was: heartburn and/or acid regurgitation, $12.8 \%$ and pyrosis and/or acid regurgitation and/ or epigastric burn $24.5 \%$. The prevalence of dyspeptic symptoms (epigastric discomfort/ pain and/or nausea and/or bloating) was $18.2 \%$. In multivariate analysis, the predictive factors of GER disease were a history of GER symptoms (OR : 2.4) or upper gastrointestinal symptoms (OR : 1.4). The predictive factors for dyspeptic symptoms excluding those associated with GER disease were a history of upper gastrointestinal symptoms (OR :3.5), UGD $(\mathrm{OR}: 2)$ and GER disease (OR : 1.8). Age and sex did not have a significant effect. The difference was not significant between patients treated with non selective NSAID and those treated with coxibs. Conclusion One patient out of 3 treated with NSAIDs suffers from upper gastrointestinal symptoms. Among those, symptoms of GER are most common. History of upper gastrointestinal symptoms or GER or peptic ulcer significantly increase the risk of upper gastrointestinal symptoms in patients treated with NSAID.

\section{M1118}

Identification and Distribution of Distinct Patterns of Symptoms in NSAID Induced Dyspepsia: Results from a Survey in 3500 Patients

Dominique Lamarque, Philippe Goupille, Christine Soufflet, Pierre Clerson, Pierre-Louis Prost, Philippe Barthelemy

Dyspepsia affects 10 to $40 \%$ of NSAID users. Dyspeptic symptoms are usually classified using the Rome criteria. However these criteria have been established in non NSAID users The aim of this survey was to differentiate among the different patterns of dyspeptic symptoms associated with NSAID. Methods: The study was conducted in a random sample of general practitioners (GPs) who included patients with upper abdominal symptoms occurring or worsening under NSAID treatment. Patients with history of abdominal surgery were excluded. The following symptoms were assessed: epigastric pain, heartburn, eructation, regurgitation, bloating, postprandial fullness, nausea and dysphagia. The intensity of each symptom was graded from mild to severe. Risk factors associated with NSAID were searched. A multivariate analysis was performed to isolate the independent patterns of symptoms. Distinct clinical presentations were clusterized around these patterns (proc fastclus SAS 8.2 SAS Institute, Cary, NC, USA). Results: From September 2004 to January 2005, 3500 patients entered the survey. The analysis incorporated $81 \%$ of patients with a fully completed questionnaire. The mean age $( \pm S D)$ was $50 \pm 14.58 \%$ were male. History of heartburn or dyspepsia was present in $29 \%$ and 19\%, respectively. Onset of symptoms occurred after initiation of NSAID in $78 \%$. Epigastric pain, heartburn, eructation, regurgitation, bloating, postprandial fullness, nausea and dysphagia was reported in $90 \%, 70 \%, 34 \%, 34 \%, 33 \%, 29 \%, 22 \%$ and $16 \%$ respectively. These symptoms were experienced daily on more than $60 \%$. Multivariate analysis isolated 14 patterns of symptoms and patients could be grouped within 3 clusters. The first cluster, which included $95 \%$ of the patients, was associated with mild to moderate epigastric pain, heartburn and regurgitation. The second cluster (3.6\%) included patients with nausea, dysphagia associated with severe heartburn and regurgitation. The third cluster concerning $1.4 \%$ was characterized by bloating and postprandial fullness associated with severe epigastric pain. No risk factor was significantly associated with a specific cluster. GPs stopped NSAID treatment in 54\% (cluster 2: 60\% vs other clusters : 54\%, p< 0.001 ) and referred $6 \%$ of the cohort to endoscopy (cluster 2 : $14 \%$ vs other clusters: $6 \%$, $\mathrm{p}<0.001$ ). Conclusion. Gastroesophagal reflux and epigastric pain are the main clinical complaints experienced under NSAID treatment. A cluster of patients with dysphagia and severe gastroesophagal reflux was identified. Those patients more frequently stopped NSAID and were referred to endoscopy.

\section{M1119}

Upper Gastrointestinal Symptoms in Patients Treated with NSAIDs : Evaluation of Physician-Patient Agreement and Impact On Compliance (COMPLAINS study)

Gerard Thiefin, Rene-Marc Flipo, Schaeverbeke Thierry, Philippe Barthelemy, Christine Soufflet

The aims of the study were to evaluate the physician-patient agreement in the assessment of upper gastrointestinal symptoms associated with NSAID intake, the impact on patients daily life and the impact on the NSAID treatment compliance. Method This transverse epidemiological study was carried out on a representative sample of rheumatologists. All the patients were over 18 years old, treated with an NSAID for at least 3 days and were seen in consultation over a one week period. The data were collected in a register by means of a standardized questionnaire. In the first 2 patients with upper gastrointestinal symptoms seen by each rheumatologist, 2 additional questionnaires were completed, one by the rheumatologist, the other by the patient, thereby enabling analysis of the physician-patient agreement in the evaluation of the symptoms. Results 630 rheumatologists participated in this study. 8269 patients were included in the register. Among them, 1213 patients presenting upper gastrointestinal symptoms were selected for the physician-patient questionnaires. The table summarizes the results of the questionnaires The physician-patient agreement was good for the epigastric pain, poor for the heartburn (under-estimation by the physician) and fair for the other symptoms. The fear of serious gastric complications was higher among patients than among physicians (67.3\% versus $38.3 \%$ ). Thirty-five $\%$ of the patients said that they were "fairly bothered » by the upper gastrointestinal symptoms and $16 \%$ « a great deal » or « enormously bothered » in daily life ( 5 graded Likert scale). The impact of NSAIDs on daily life was under-estimated by the physicians. The occurrence of upper gastrointestinal symptoms with NSAID resulted in the definitive withdrawal of the NSAID treatment by the patient $(10.8 \%)$, a temporary withdrawal $(17.2 \%)$ and a reduction in the dosage $(5.8 \%)$. Conclusion The evaluation of the physician-patient agreement regarding upper gastrointestinal symptoms associated with NSAID revealed : 1) an under-estimation of the diagnosis of heartburn by the physicians and 2) an under-estimation of the impact on daily life. Upper gastrointestinal symptoms also have a negative impact on NSAID treatment compliance in one patient out of three.

Type and distribution of the upper gastrointestinal symptoms reported by the questionnaires

\begin{tabular}{|c|c|c|c|c|c|c|}
\hline & Heartburn & $\begin{array}{c}\text { Acid } \\
\text { regurgitation }\end{array}$ & $\begin{array}{c}\text { Epigastric } \\
\text { burning }\end{array}$ & $\begin{array}{c}\text { Epigastric } \\
\text { pain/discomfort }\end{array}$ & Nausea & Bloating \\
\hline $\begin{array}{c}\text { Physician } \\
\text { questionnaire }\end{array}$ & $36.5 \%$ & $36.9 \%$ & $67.5 \%$ & $66.6 \%$ & $21.3 \%$ & $24.8 \%$ \\
\hline $\begin{array}{c}\text { Patient } \\
\text { questionnaire }\end{array}$ & $63.4 \%$ & $38.9 \%$ & $62.1 \%$ & $59.9 \%$ & $23.6 \%$ & $33.1 \%$ \\
\hline $\begin{array}{c}\text { Kappa } \\
\text { agreement }\end{array}$ & 0.43 & 0.67 & 0.58 & 0.56 & 0.81 & 0.73 \\
\hline
\end{tabular}

M1120

Concomitant Bisphosphonate Use Tends to Peptic Ulcer Formation in Rheumatoid Arthritis Patients Taking Long-Term Non-Steroidal AntiInflammatory Drugs

Kazumasa Miyake, Masafumi Kusunoki, Tomotaka Shindo, Tatsuhiko Hamamoto, Nobue Ueki, Kenji Suzuki, Yoko Shinji, Tetsuro Hiratsuka, Hitoshi Nishigaki, Seiji Futagami, Ken Wada, Katya Gudis, Taku Tsukui, Atsuo Nakajima, Choitsu Sakamoto

Objective: Non-steroidal anti-inflammatory drugs (NSAID) usage is aggressive factors for peptic ulcer disease. Although patients with rheumatoid arthritis (RA) require long-term therapy with NSAID, the pathogenesis of peptic ulcer in RA patients has been considered to be multifactorial. Although elderly age, prior gastric event and concurrent use of anticoagulant or prednisolone (PSL) have been identified as markers of risk for NSAID-related gastric event, high-risk NSAID users have yet to be characterized in Japanese population. Therefore, we estimated the potential risk or the protective factors for peptic ulcer occurrence in Japanese patients with RA taking long-term NSAID. Methods: Users of proton pump inhibitors, prostaglandin, and high-dose histamine-H2 receptor antagonists (H2RA), which have been demonstrated to have the high preventive effects for NSAID-induced peptic ulcers, were excluded. 162 patients with RA treated over a long-term with NSAID were enrolled. Their demographic data were recorded and laboratory data were measured. Endoscopy was performed to assess the prevalence of peptic ulcer. Ulcers were defined as mucosal loss over $3 \mathrm{~mm}$ or mucosal loss accompanied by fold convergence. Helicobacter(H.) pylori infection was diagnosed on the basis of serologic test and histological examination. The calculations were performed using SPSS software (for Windows). Results: Thirty-seven (22.8\%) of 162 RA patients had peptic ulcer disease, including 30 with gastric ulcer, 6 with duodenal ulcer and 1 with gastric plus duodenal ulcer. 52 patients (32\%) had used bisphosphonate (BP) and 76 patients (48\%) had H. pylori infection. The majority of peptic ulcer disease subjects showed the characteristics of age $>60$ years and BP users $(P>0.05)$. Multivariate analysis revealed that $\mathrm{BP}$ use [odds ratio $(\mathrm{OR}), 2.38$; $95 \%$ confidence intervals $(\mathrm{CI}), 1.01-5.59 ; \mathrm{P}=$ 0.047] was the predominant risk factor for the development of peptic ulcer among these patients, whereas regular or half-dose H2RA showed a tendency of protective role (OR, $0.38 ; 95 \% \mathrm{CI}, 0.14-1.05 ; \mathrm{P}=0.062$ ). In another population which was composed of $29 \mathrm{RA}$ 
patients without NSAID and anti-ulcer agent treatment, no peptic ulcer disease was found in BP users $(0 / 12,0 \%)$. Conclusions: Concomitant BP use tends to peptic ulcer formation in RA patients under long-term NSAID treatment. It should be used with caution in those patients

\section{M1122}

Upper Gastrointestinal Bleeding in General Population: Prelimininary Results of a French Prospective Study Including More Than 1000 Patients

Herve Hagege, Jean Paul Latrive, Bernard Nalet, Isabelle Rosa, Bruno Bour, Roger Faroux, Philippe Gower, Jean Pierre Arpurt, Jacques Denis, Jean Henrion, Alex Pariente, Lizzie Marek, Groupe Hemorragies Digestives Hautes Angh

Aims Upper gastrointestinal bleeding (UGIB) in general population represents a common emergency in clinical practice. The aim of this study was to describe current epidemiology, medical practices, morbidity and hospital mortality in a large, prospective series of UGIB. Patients and methods The inclusion period is scheduled to be one year, from March 2005 to February 2006. From March 1 to August 31, 2005, consecutive patients with UGIB were recruited in 53 French hospitals. Clinical, biological and endoscopic data were collected until the hospital discharge. Results 1682 patients were included during this period of the study. The current analysis includes the first 1080 patients: 729 men and 351 women, of mean age $64 \pm 17$ years. The mode of presentation of the haemorrhage was hematemesis with or without melena in $57 \%$ of the cases, melena only in 38\% and acute anaemia in $5 \%$. At admission, systolic blood pressure was $120 \pm 25 \mathrm{Hg}$ mm and heart rate was $94 \pm 20$ / min. Haemoglobin was $9 \pm 3 \mathrm{~g} / \mathrm{dl}$. An antecedent of UGIB was noted in $23 \%$ of the cases and of cirrhosis in 33\%. Before the UGIB episode, $11 \%$ of the patients had taken non steroidal anti-inflammatory drugs, $28 \%$ aspirin or anti platelet agents and $10 \%$ vitamin $\mathrm{K}$ antagonists. During endoscopy, signs of recent bleeding were present in $60 \%$ of the cases. The cause of the UGIB was attributed to: gastric ulcer in 129 cases, duodenal ulcer in 198, gastroduodenal erosions in 62, esophageal or gastric varices in 228 , portal hypertensive gastropathy in 57, esophagitis in 233, oesophageal cancer in 8, gastric cancer in 18 and other lesions in 73 . Mean Rockall score was: $5.11 \pm 2.34$. Hemostatic therapy was performed during the first endoscopy in $34 \%$ of the cases. Transfusion of $3.8 \pm 2.6$ units was realized for $63 \%$ of the patients. Rebleeding occurred among $10 \%$ of the patients. In multivariate analysis, predictive factors of rebleeding were: cirrhosis $(\mathrm{p}=0.01)$, anti platelet agents or anticoagulants $(\mathrm{p}=0.04)$, blood systolic pressure $(\mathrm{p}=0.03)$, haemoglobin rate $(\mathrm{p}=0.01)$, Rockall score $(p<0.0001)$ and transfusion $(p<0.0001)$. In-hospital mortality was $9 \%$. In multivariate analysis, predictive factors of death were: age $(\mathrm{p}=0.008)$, precedent UGIB episodes ( $\mathrm{p}=$ $0.003)$, systolic blood pressure $(\mathrm{p}=0.03)$ and Rockall score $(\mathrm{p}<0.0001)$. Conclusion During upper gastrointestinal bleeding, mortality remains important in spite of medical management progresses and particularly the use of endoscopic hemostasis. The continuation of this study will allow knowledge of predictive factors for rebleeding and of mortality and also will contribute to the improvement of medical management.

\section{M1123}

Modifying Physician Behavior On the Correct Use of IV Proton Pump Inhibitors in the ICU

Dennis Sinar, Mary Seymour, James Worden

Background: Proton pump inhibitors, with their high safety and effectiveness, are used in the ICU for a variety of clinical conditions. Purpose: to measure the effect of prescriber education on appropriate use of intravenous proton pump inhibitors (IV PPI) in an ICU setting. The study was conducted among two groups of tertiary medical center practioners: private physicians (caring for their own patients) and academic physicians (orders written by housestaff on monthly rotations through the ICU). For a 3 month baseline period followed by a 3 month intervention period, all patients admitted to the ICU were included. During each period, IV PPI use in each patient was assessed. Appropriate indications were defined as treatment of gastric/duodenal ulcer and/or UGI bleeding and/or erosive esophagitis in patients who are NPO (patients may have received oral PPI prior to admission). Appropriate dosing was defined as a single bolus dose of IV PPI q24 hrs. Appropriate switch time was when the patient resumed oral intake. In the academic/housestaff group the baseline period was followed by a required monthly lecture and dialog with a GI attending reviewing indications, dosing, and pharmacology of IV and oral PPI. Attendance at one or more of the lectures was $95 \%$. No informational lectures were given to private physicians. The proportion of patients that physicians prescribed the correct indications, dosing, and appropriate switch to oral PPI during the 3 month intervention was compared to those proportions at the baseline period for both groups. Results: 511 patients were included (total 372 academic, 139 private). Eighty five percent of ICU patients were on IV PPI during the baseline period and 70\% of ICU pts were on IV PPI during the test period. Unit acuity and occupancy were similar during both periods. The academic/housestaff group showed a significant improvement in correct dosing after lectures (37.3\% to 62.7\%, p $<0.001$ Pearson Chi Square); numeric improvements, but not significant changes were made in other measurements. For private physicians, no change in practice was observed. The most common incorrect doses were BID or infusion. Appropriate switches to oral medications or discontinuation of PPI were high ( $94 \%$ academic, $88 \%$ private physicians). Conclusions: ICU patients who are NPO have a high likelihood of receiving IV PPI. Instruction on indications, dosing, and pharmacology can improve the physician choice of an appropriate PPI dose. In this study population, there is a high appropriate switch schedule within $24 \mathrm{hrs}$ of the patient resuming oral intake to either receive oral PPI or discontinue PPI.

\section{M1124}

The Value of Baylor and Rockall Scores in Inpatients with Non Variceal Upper Gastrointestinal Bleeding

Jose A. Gonzalez-Gonzalez, Juan O. Gaytan-Torres, D.R. A. Garcia-Cantu, Hector J. Maldonado-Garza, Arnoldo Guerrero-Chapa

Introduction: Differences in etiologies have emerged in recent studies in both outpatients (OUTPTs) and inpatients (INPTs) with non variceal bleeding (NVB). Little data about the use of Baylor score and Rockall score in INPTs is available Objectives: To compare the clinical characteristics, bleeding etiologies, co-morbidities, Baylor score and Rockall score among OUTPTs and INPTs with NVB.Material and Methods: We prospectively studied al pts admitted to our institution with NVB (2001-2005) with information obtained from ou database. We studied age, gender, co-morbidities, alcohol, tobacco and NSAIDs use, history of previous GI bleeding and Hp. status. We compared bleeding manifestations, diagnoses, treatment, transfusions required, rebleeding, Baylor score and Rockall score, surgery and mortality. Statistical Analysis: The variables are expressed as mean, range. Chi-square and Fishers exact test were used for comparisons. Results. 653 pts: 85\% OUTPTs; M/F 360/ 197, mean age 58 vs 15\% INPT; M/F 61/36 mean age 57. Risk factors as DM, CRF, HTN pulmonary and liver disease, $\mathrm{OH}$, tobacco, NSAIDs use, Hp status and previous GI bleeding was not different among OUTPTs and INPTs, only cardiac disease 9\% (16\%) was significan in INPTs $(p=0.03)$. Presence of bright red blood hematemesis 36\% (19\%) and melen $64 \%(50 \%)$ was significant in OUTPTs $(p=0.001, p=0.01)$. The gastric and duodena ulcer, tumors and erosive duodenitis, was not different. Only erosive gastritis was significant in INPTs $23 \%(33 \%) \mathrm{p}=0.04$. The need for endoscopic treatment, units of blood requirement, rebleeding rate and surgery was not different, only the mortality rate was more frequent in the INPTs $(p=0.02)$. In the OUTPTs the Baylor score was useful to predict rebleeding and mortality $(p=0.0001, p=0.003)$ and the Rockall score was only significant for mortality $(p=0.0001)$. Both scores for INPTs are shown in Table 1 Conclusions: In our study, INPT with NVB had more coronary disease, erosive gastritis and higher mortality when compared to OUTPTs who usually presented with either melena or bright red blood hematemesis. In OUTPTs, the Baylor score was useful in predicting re-bleeding rates and mortality but was not useful in INPTs, whereas the Rockall score was helpful in predicting mortality in both groups.

\begin{tabular}{|c|c|c|c|}
\hline INPTs & $\mathrm{N}$ & Rebleeding & Death \\
\hline Rockall $\leq 2$ & 22 & 0 OR 1.28 IC 95\% (0.89-1.84) & 1 (5\%) OR 0.12 IC 95\% (0.01-0.96) \\
\hline Rockall > 2 & 75 & $7(9 \%)$ OR 0.77 IC95\% (0.54-1.11) & 21 (28\%) OR 8.1 IC 95\% (1.03-64.6 \\
\hline $\mathrm{p}$ & & 0.19 & 0.02 \\
\hline Baylor $\leq 10$ & 68 & $5(7 \%)$ OR 1.07 IC 95\%(0.19-5.86) & 12(18\%) OR 0.40 IC 95\%( 0.15-1.09) \\
\hline Baylor > 10 & 29 & 2 (7\%) OR 0.93 IC 95\%(0.17-5.11) & $10(34 \%)$ OR 2.45 IC 95\% (0.91-6.59) \\
\hline$p$ & & 0.99 & 0.11 \\
\hline
\end{tabular}

\section{M1125}

Deletion and G:C to A:T Transitions Are the Specific Mutation Patterns in the Inflamed Colon of the IL-10 Knockout Mouse

Yuichirou Sato, Seiichi Takahashi, Yoshitaka Kinouchi, Eiki Nomura, Kenichi Negoro, Sho Takagi, Hiroyuki Aihara, Tooru Shimosegawa

Background \& aims: Individuals with inflammatory bowel disease are at increased risk of developing gastrointestinal cancer. It has not been fully elucidated what kind of genomic mutations is critical for tumorigenesis. Recently, a new transgenic mouse line, gpt delta (gpt+), was established to facilitate the detection and analysis of mutations in vivo. The striking feature of gpt+ mice is their ability to reveal deletions and point mutations. To examine genotoxic effects of chronic inflammation of the colon, we used recombinan mice, gpt delta IL10-/-, which spontaneously develop intestinal inflammation and facilitates detection of genomic mutations. Then we characterized mutation spectrum of the inflamed colon. Methods: Genomic DNA was extracted from the colon. The 6-TG selection(detection of point mutation and small deletion) and the Spi- selection(detection of large deletion) were performed. The 6-TG mutant frequency and the Spi- mutant frequency were calculated Then DNA sequencing of the mutants were carried out. Results: The 6-TG mutant frequency of the inflamed colon in the gpt+IL-10-/- mice was about 5 times higher than that of the normal colon in the gpt+IL-10+/+ mice. The frequency of G:C to A:T transitions of the $40-$ weeks gpt+IL-10-/- mice was 4.1 times higher than that of the 40-weeks control mice, and 4.1 times higher than that of the 15-weeks gpt+IL-10-/- mice, and the small deletions and insertions strikingly increased in the gpt+IL-10-/- mice. The majority of the deletion or insertion mutations were observed in the monotonous base runs or adjacent repeats of short tandem sequences. The Spi- mutant frequency of the inflamed colon in the gpt+IL-10-/ mice didn't significantly increase compared with that of the normal colon in the gpt+IL$10+/+$ mice, and the pattern of the mutations was not significantly different. Conclusions In our data of the gpt+IL10-/- mice, several types of mutations increased, but in the mutations, short deletions or insertions in the monotonous base runs or adjacent repeats of short tandem sequences and G:C to A:T transitions were striking mutations.

\section{M1126}

Risk Factors for Ulcerative Colitis-Associated Colorectal Cancers in a Hungarian Cohort of Ulcerative Colitis Patients. Results of a Population-Based Study

Laszlo Lakatos, Zsuzsanna Erdelyi, Gyula David, Tunde Pandur, Mihaly Balogh, Simon Fischer, Peter Vargha, Peter L. Lakatos

Background: There is an increased risk of colorectal cancer (CRC) UC and prevalence of $\mathrm{UC}$-associated CRC is different in various geographical regions. The risk depends primarily on the duration and extent of disease. The aim of this study was to identify the risk factors 
for and the epidemiology of CRC in Hungarian UC patients. Patients and methods: We retrospectively evaluated the relevant epidemiological and clinical data of all UC patients in Veszprem province in our thirty-year IBD database ( 723 UC patients, m/f:380/343, familial disease: $5.2 \%$, non-CRC related colectomies: $3.7 \%$ ). Results: $C R C$ was diagnosed in 13 patients (male: 6, female: 7, 13/8564 person year duration) during follow-up. The median onset of UC in patients with UC associated CRC was 30 (13-61) years, 6 years younger compared o UC patients without CRC. Median age of UC-CRC patients at diagnosis of CRC was 51 $(27-70)$ years, almost 15 years younger than the average in sporadic CRC population in Hungary (duration of UC at the diagnosis of CRC: 17 (1-33) years). Eight patients are still alive (survival: 58.5 (10-163) months), five patients died because of CRC (survival: 6.5 months), one died due to unrelated cause after 10 years of the diagnosis of CRC. Longer disease duration, chronic continuous disease, more extensive colitis, the presence of iron deficiency or chronic anaemia, primary sclerosing cholangitis (PSC) and dysplasia were identified as risk factors for developing CRC in univariate analysis. In a logistic regression model longer disease duration, extensive colitis, PSC and dysplasia were associated with increased risk. The cumulative risk for developing CRC after a disease duration of 10 years was $0.6 \%$ (95\%CI:0.2-1.0\%), 20 years $5.4 \%$ (95\%CI:3.7-7.1\%) and 30 years $7.5 \%(95 \% \mathrm{CI}$ 4.8-10.2\%). CRC diagnosed at surveillance colonoscopy was associated with a tendency for longer survival $(\mathrm{p}=0.08)$. Conclusions: The cumulative risk of $\mathrm{CRC}$ was high in our UC patients; however it was lower compared to that reported in Western European and North American studies. CRC developed approximately fifteen years earlier compared to sporadic CRC patients in Hungary. Longer disease duration, extensive colitis, dysplasia and PSC were identified as important risk factor for developing CRC

\section{M1127}

Increased CPG Methylation Status of Low-Grade Dysplasia in Patients with Extensive Ulcerative Colitis

Niraj Jani, Yuan Yao, Adrian Gologan, Miguel Regueiro, Antonia R. Sepulveda

Introduction: With increasing duration of disease, patients with ulcerative colitis (UC) are at increased risk of colonic dysplasia and cancer. Currently, histologic examination of colonic biopsies is the only tool available to determine the presence and grade of dysplasia. CpG methylation of multiple gene loci has been reported in colorectal cancer, and studies in UCassociated neoplasia have focused on carcinomas and high-grade dysplasia. Little is known about the CpG island methylation status in low-grade dysplasia (LGD) arising in UC patients. Aims: To compare the promoter methylation status of pl6INK4a, MLH1, and MGMT in the background colitis-positive mucosa of UC patients and co-existing dysplastic lesions, and to correlate the clinical phenotype with histologic findings and molecular genotype. Methods: Cases were selected among UC patients identified with LGD from a prospectively clinically phenotyped IBD patient registry. Fifteen biopsy samples of LGD from nine UC patients and non-dysplastic mucosa from the same patients were studied. Normal colonic mucosa from 10 individuals without UC or dysplasia was selected as control. Paraffin embedded tissue sections were microdissected, DNA was extracted, and then treated with bisulfite to convert unmethylated cytosines to uracils. Methylation specific PCR (MSP) was performed with primers for pl6, MLHl, and MGMT CpG islands. The presence of a band on gel electrophoresis indicated methylation was present. Results: There were nine patients with LGD, (7 M/2F), mean age 54.6 years. Mean duration of UC was 13.3 years. All patients had extensive disease, moderate colitis activity and were on mesalamine. Positive CpG methylation status for p16, MLH1 and MGMT was identified in the following number of cases, respectively: $30 \%, 20 \%$ and $10 \%$ in normal colonic mucosa controls; $13 \%, 36 \%$, and $10 \%$ in the background colitis-positive colonic mucosa of patients with co-existing lowgrade dysplasia; $42 \%, 60 \%$, and $21 \%$ in dysplastic lesions. Although the differences were not statistically significant trends could be observed towards increased frequency of methylation in LGD at the three loci when the background colitis-positive mucosa and the dysplastic lesions of the same individuals were compared Conclusions: The frequency of methylation at each gene locus in LGD specimens was higher than in colitis specimens and the overall frequency of methylation was higher in dysplasia and colitis specimens compared to normal controls. Quantitative methylation analyses are needed to improve the diagnostic applications of CpG methylation status to distinguish non-neoplastic mucosa from dysplasia in UC patients.

\section{M1128}

Inflammatory Cells and Factors Modulate Intestinal and Mammary Carcinogenesis in $A P C^{\mathrm{Min} /+}$ Mice

Varada P. Rao, Theofilos Poutahidis, Zhongming Ge, Prashant R. Nambiar, Bruce H. Horwitz, James G. Fox, Susan E. Erdman

Cancers of bowel and breast are increasingly frequent in humans in recent years. Chronic inflammation is known to be a risk factor for these malignancies, yet cellular and molecular mechanisms linking inflammation and carcinogenesis remain poorly understood. Here we apply a widely used T-cell transfer paradigm, involving adoptive transfer of pro-inflammatory $\mathrm{CD}^{+} \mathrm{CD} 45 \mathrm{RB}^{\text {hi }}\left(\mathrm{T}_{\mathrm{E}}\right)$ cells and anti-inflammatory $\mathrm{CD} 4{ }^{+} \mathrm{CD} 45 \mathrm{RB}^{\text {lo }} \mathrm{CD} 25^{+}\left(\mathrm{T}_{\mathrm{p}}\right)$ cells in mice, to investigate roles of inflammation in the Apc ${ }^{\mathrm{Min} /+}$ mouse model of intestinal polyposis. We find that transfer of $T_{E}$ cells significantly increases intestinal and mammary tumor multiplicity, whereas cotransfer of anti-inflammatory $T_{R}$ cells prevent tumors. Although $T_{E}$ cells promote intestinal and mammary tumors in this model, cells of adaptive immunity are not required for tumors. Indeed, Rag-deficient $A p c^{\mathrm{Min} /+}$ mice lacking functional lymphocytes develop increased number of tumors in both intestine and breast when compared with $\mathrm{ApC}^{\mathrm{Min} / \mathrm{+}}$ mice. Both intestinal and mammary tumors are abolished by neutralization of key pro-inflammatory cytokine TNF- $\alpha$. These findings define a novel mouse model of inflammation-driven intestinal and mammary carcinoma and suggest that epithelial carcinogenesis is modulated by cells and cytokines integral in inflammatory bowel diseases in humans and mice.

\section{M1129}

Retrospective Analysis of Surveillance Colonoscopy for Patients with Longstanding Ulcerative Colitis

Kenichi Shindo, Masahiro Iizuka, Hiroaki Itou, Shiho Sagara, Yasuo Horie, Sumio Watanabe

[Background] The risk of colorectal cancer is critical for patients with longstanding ulcerative colitis (UC). Thus, surveillance colonoscopy for such patients has been conducted to detect colorectal cancer in early stage. However, it is controversial how we should perform the surveillance colonoscopy, namely, with random biopsies or with targeted biopsies. We retrospectively analyzed the results of surveillance colonoscopy for UC patients performed in our hospital and assessed the effectiveness of the methods of surveillance colonoscopy. [Methods] Surveillance colonoscopy was done 62 times for 23 patients with longstanding UC (male 5, female 18; average age: 49.2 yrs) between 1987 and 2005. Between 1987 and 1998 , surveillance colonoscopy was performed with 20 random biopsies and some targeted biopsies from dysplasia-like lesions using 0.03\% methylene blue. Between 1998 and 2005, surveillance colonoscopy was performed with only targeted biopsies using 33\% indigo carmine dye spraying. [Results] In 62 times of surveillance colonoscopies, 822 biopsies were taken. High- grade dysplasia was found in 3 patients (13\%) with total colitis. Low-grade dysplasia, or sporadic adenoma was found in $2(8.7 \%)$, or 3 patients $(13 \%)$, respectively All dysplastic lesions were detected by targeted biopsy from polypoid lesions, large polyp, or flat elevations. No dysplastic lesions were detected by random biopsies. [Conclusion] Our results suggest that the surveillance colonoscopy with targeted biopsies is more effective for detecting dysplasia in patients with longstanding ulcerative colitis compared to that with random biopsies.

\section{M1130}

Ordering the Activating Mutations of BRAF and KRAS Genes During Oncogenesis in Inflammatory Bowel Diseases

Celine Bossard, Marc G. Denis, Jean-Paul Galmiche, Magali Svrcek, Jean-Francois Flejou, Pierre Levillain, Anne-Sophie Thirouard, Claire Magois, Genevieve Aillet, Christian L. Laboisse, Jean-Francois Mosnier

The search for KRAS mutations and BRAF mutations have contributed to our understanding of sporadic colorectal oncogenesis. In fact, KRAS mutations are associated with the chromosomal instability pathway while BRAF mutations are associated with the microsatellite instability pathway. More than $80 \%$ of sporadic colorectal carcinomas (CRC) display either KRAS or BRAF mutations, that are mutually exclusive. In order to explore the oncogenetic pathway involved in inflammatory bowel disease (IBD)-associated oncogenesis, we studied BRAF and KRAS mutations during the progression of inflammatory mucosa to colorectal cancer in a series of 104 patients with IBD-related neoplasias [ulcerative colitis (UC) n=76, Crohn's disease $(C D) n=27$, and 1 indeterminate colitis]. Samples comprised adenocarcinoma ( $=$ 79), high-grade dysplasia $(n=17)$, low-grade dysplasia $(n=37)$, indefinite for dysplasia $(n=$ 7). We collected also samples from regenerative mucosa $(n=9)$ and matched inflammatory mucosa $(n=35)$. We selected 22 patients with IBD without neoplasia (UC, $n=20$ and $C D$, $\mathrm{n}=2$ ) who served as controls. We determined BRAF codon 600 (V600E) (1) as well as KRAS codon 12 and 13 mutations by a quantitative allele-specific PCR from genomic DNA extracted from histologically microdissected paraffin-embedded tissue sections. BRAF mutations were found in two lesions from 1 patient with UC, i.e. a serrated adenoma with low-grade dysplasia and a mucinous adenocarcinoma from the right colon. KRAS mutations were found in 21 samples from 10 patients ( $C D, n=9$ and $U C, n=1$ ), including 12/70 adenocarcinoma $(17 \%), 8 / 49$ dysplasia $(16 \%)$ and 1 inflammatory mucosa $(2.8 \%)$. One mutation was also found in the inflammatory controls. We observed a mutually exclusive relationship between BRAF and KRAS mutations. In conclusion, this study is the first to correlate the epithelial changes (regenerative, dysplastic, cancer) to the molecular alterations in BRAF and KRAS genes. Here we show that both BRAF and KRAS mutations occur early during the oncogenetic process. Finally, although BRAF and KRAS mutations are less frequent, their proportion is similar to that observed during sporadic CRC oncogenesis. (1) Jarry et al., Mol Cell Probes. 2004;18:349-352. Supported by grants "Projet Hospitalier Régional de Recherche Clinique" BRD04/6/P and BRD05/10-C

\section{M1131}

Are Crohn's Disease and Ulcerative Colitis More Common Among Patient with Digestive System Cancers in the U.S.? A Population-Based Case-Control Study

Aasma Shaukat, David Howard, Jonathan M. Liff

Background: Adenocarcinoma of the small bowel has been reported in patients with Crohn's Disease. However the association of Crohn's Disease and Ulcerative Colitis with esophageal, gastric and small bowel adenocarcinoma has not been well studied at the population level. Aims: To study the prevalence of Crohn's Disease or Ulcerative Colitis (CD/UC) among cases with adenocarcinoma of esophagus, stomach, small bowel and colorectum and compare it to the prevalence in controls never diagnosed with one of these tumors using a nationally representative database. Methods. We used the SEER-Medicare database, which consists of records from the SEER cancer registry linked with Medicare claims. Cases consisted of patients $65+$ diagnosed with cancer of the esophagus, small intestine, colon, rectum, and stomach diagnosed in 1994-1999. Controls consisted of Medicare beneficiaries never diagnosed with one of these cancers but residing in a SEER registry catchment area. We required that both cases and controls be enrolled in Medicare for at least two consecutive years. We identified CD/UC using the ICD-9 codes on Medicare outpatient, office, and inpatient claims during the two year window centered on the date of diagnosis for cases or a randomly selected date for controls. We assessed the statistical significance of differences between groups using logistic regression controlling for age (65-74 yrs, 75-84 yrs, 85+ yrs), sex, and year of diagnosis. Results: The final sample consisted of 62,097 cases and 99,485 controls. The prevalence of CD/UC, standardized by age and sex to the 2001 US population, among cases was $0.95 \%$ versus $0.40 \%$ for controls $(\mathrm{p}<0.001$ ). There was a significant association 
between CD/UC and adenocarcinoma of the stomach (OR 1.972; 95\% CI: 1.485, 2.617;pvalue $<0.001$ ), small bowel (OR 7.22; $95 \% \mathrm{CI} 4.97,10.629$; p-value <0.001) and colorectum (OR 2.76; 95\% CI 2.408, 3.179; p-value <0.001). Among cases, the age and sex-standardized prevalence of $\mathrm{CD} / \mathrm{UC}$ by tumor type was as follows: esophagus, $0.43 \%$; stomach, $0.71 \%$, small bowel, $2.84 \%$; colorectum, $1.10 \%$. Among cases, the prevalence of CD/UC was higher among patients diagnosed with a non-gastrointestinal cancer (for example, lung cancer), $0.92 \%$, versus cases never diagnosed with cancer of any type, $0.39 \%$, suggesting confounding by some other factor or a detection bias. Conclusions: These results suggest that the prevalence of esophageal, gastric, small bowel and colorectal adenocarcinoma is higher in patients with Crohn's Disease or Ulcerative Colitis. However, the higher prevalence of Crohn's Disease or Ulcerative Colitis among cases may be due in part to better detection.

\section{M1132}

Cytokeratin 7/20 and Mucin Core Protein Expression in Ulcerative ColitisAssociated Colorectal Neoplasms - A Distinct Histogenetic Pathway Showing Gastric Phenotype

Natsuko Tatsumi, Ryoji Kushima, Michael Vieth, Ken-Ichi Mukaisho, Hidetoshi Okabe, Rie Kakinoki, Franz Borchard, Manfred Stolte, Takeshi Okanoue, Takanori Hattori

INTRODUCTION: Different histogenetic pathways have been suggested between ulcerative colitis (UC)-associated neoplasia and sporadic colorectal neoplasia. Little is known about the cytokeratin (CK) and mucin expression in UC-associated neoplasms. CK7 has also been reported to be a possible marker of transient dedifferentiation in the sequence between gastritis, metaplasia, dysplasia and cancer of the stomach. AIM: In this study, we targeted the expression of CK7, CK20, MUC2, MUC5AC and MUC6 in UC-associated colorectal neoplasms as compared it to sporadic colon adenomas and adenocarcinomas, to further investigate colitic cancer carcinogenesis. MATERIAL AND METHODS: we examined the immunohistochemical expression of CK7, CK20, MUC2, MUC5AC and MUC6 in 90 colorectal neoplasms; including 22 UC-associated adenocarcinomas (colitic cancer; CC), 10 highgrade dysplasias (HGD) in UC, 9 low-grade dysplasias (LGD) in UC, 24 sporadic tubular adenomas (TA) and 25 adenocarcinomas (AC). The extent of positivity was scored according to the percentage of neoplastic cells stained: -, no positive cells or essentially none $(<5 \%)$; $1+$, some positive cells $(5-30 \%) ; 2+$, well-defined areas of positive cells $(30-60 \%) ; 3+$, extensive areas of positive cells ( $>60 \%)$. The chi-square test with Yates correction was used for statistical analysis. Differences were considered to be significant at $\mathrm{p}<0.05$. Fisher's test was applied whenever appropriate. RESULTS: CK7 was positive in most of UC-associated neoplasms: $59 \%$ of CC cases, $80 \%$ of HGD and $89 \%$ of LGD respectively, whereas in nonUC associated neoplasia $21 \%$ of TA and $12 \%$ of AC. None of sporadic adenoma-carcinoma group showed 3+, whereas 39\% (16 of 41) of UC-associated neoplasms expressed 3+. The frequency of MUC6 expression in UC-associated neoplasia was 32\% in CC, 30\% in HGD and $44 \%$ in LGD respectively, whereas in non-UC associated neoplasia: $4.2 \%$ in TA and $0 \%$ in AC MUC5AC expression in UC-associated neoplasia was detectable in $73 \%$ of CC, $90 \%$ of HGD and $89 \%$ of LGD respectively, in non-UC associated neoplasia $67 \%$ in AC and $20 \%$ in TA. There were obvious differences in the expression of CK7 and MUC6 between UC-associated neoplasms and sporadic tumors. The incidence of MUC5AC expression in UC-associated neoplasms was also higher than sporadic tumors. There was no significant difference in MUC2 and CK20 expression among all categories. CONCLUSION: These results suggest that UC-associated neoplasms develop and progress under induction of gastric differentiation. CK7 and gastric-type mucins may be useful in the differential diagnosis between UC-associated neoplasms and sporadic tubular adenomas and adenocarcinomas.

\section{M1133}

Hepatocyte Growth Factor May Not Accelerate Neoplastic Development in Two Experimental Models

Masatsugu Numata, Mayumi Kodama, Hirofumi Uto, Chihiro Nakanishi, Shuji Kanmura, Hiroo Abe, Tadashi Miike, Kazunori Kusumoto, Satoru Hasuike, Kenji Nagata, Katsuhiro Hayashi, Akio Ido, Hirohito Tsubouchi

[Background] Hepatocyte growth factor (HGF), originally purified from the plasma of patients with fulminant hepatic failure, is the primary agent that promotes hepatocyte proliferation. HGF also functions as a pleiotropic factor, acting as a mitogen, morphogen, and motogen for multiple subsets of epithelial cells, including gastrointestinal epithelial cells. Recently, we reported that HGF promoted colonic mucosal repair in animal models of experimental colitis; thus, HGF has the potential to be an important new treatment modality that promotes intestinal mucosal repair in patients with inflammatory bowel disease (IBD). However, we cannot exclude the possibility that the repeated administration of recombinant human HGF might accelerate carcinogenesis. This study sought to clarify if repeated administration of recombinant human HGF affects colonic carcinogenesis in two experimental models. [Methods] (A) F344 male rats were injected subcutaneously with 1,2-dimethylhydrazine (DMH, 20mg/kg body weight) once a week for four consecutive weeks. Four weeks later, recombinant human $\mathrm{HGF}(0.5 \mathrm{mg} / \mathrm{kg} /$ day $)$ was injected intravenously every day for four weeks. Control rats were injected with PBS. (B) F344 male rats were injected subcutaneously with $\mathrm{DMH}(40 \mathrm{mg} / \mathrm{kg}$ body weight) three times a week. The $1.0 \%$ dextran sodium sulfate (DSS) is administered to the rat concomitantly with the DMH, rats were also administered DSS for 2 weeks. Recombinant human HGF $(0.5 \mathrm{mg} / \mathrm{kg} /$ day $)$ was injected intravenously for 2 weeks during the administration. These rats were maintained until final sacrifice at week 26. Control rats were injected with PBS. [Results] Recombinant human HGF did not affect the size of DMH-induced colonic aberrant crypt foci (ACF) compared to PBS-treated rats. Furthermore, development of ACF or colorectal tumors induced by DMH/DSS was not significantly accelerated by administration of human HGF compared to control rats. The incidence of adenocarcinoma and multiplicities did not differ significantly between HGFand PBS-treated rats. [Conclusions] These findings suggest that HGF may not accelerate neoplastic development in the colon. However, because the possibility of HGF-associated carcinogenesis has not been completely excluded, complete informed consent is required.

\section{M1134}

High-Resolution Endoscopy and the Additional Value of Chromoendoscopy in the Evaluation of Duodenal Polyposis in FAP-Patients

Evelien Dekker, Jan Dees, Lisbeth Mathus-Vliegen, Jan Werner Poley, Johan Offerhaus, Joep Bartelsman, Ernst Kuipers, Paul Fockens

Introduction: Duodenal polyps occur in approximately $90 \%$ of all patients with familiar adenomatous polyposis (FAP). An estimated $5 \%$ of them develop duodenal cancer, nowadays being the leading cause of death in FAP-patients. Endoscopic surveillance of the duodenum has become standard care in these patients, making use of the Spigelman classification. This classification includes number, size and histology of duodenal polyps and correlates with the risk of developing duodenal cancer. Guidelines for endoscopic surveillance have been developed in which interval is dependent on the Spigelman-classification. Since the introduction of these guidelines, the quality of endoscopic imaging has dramatically improved and chromo-endoscopy has further enhanced our ability to detect small polyps. Aim: To investigate the use of high-resolution endoscopy and the additional value of chromoendoscopy in the evaluation of duodenal polyposis in FAP-patients. Methods: All consecutive FAP-patients scheduled for a surveillance endoscopy in 2 academic centers underwent gastroduodenoscopy with high-quality forward- and sideward-viewing endoscopes. After scoring the number and size of polyps in the duodenum (bulb, DI and DII seperately), indigocarmine $0.5 \%$ was sprayed onto the mucosa and the polyps were scored again. Biopsies were taken from the larger lesions and the papilla and were evaluated by an expert pathologist. Spigelman classifications were assessed for both procedures. Results: A total of 47 endoscopies were performed in 39 patients ( 19 men, mean age 48 yrs) were examined. Before the application of dye, in 37 patients (95\%) duodenal polyps were seen. Spigelman-classifications were: stage 0, 2 patients (5\%); stage I, 2 patients (5\%); stage II, 9 patients (23\%), stage III, 13 patients $(33 \%)$ and stage IV, 13 patients (33\%). The papilla was enlarged in 21 patients (54\%); biopsies revealed dyplasia in 18 (46\%). Chromoendoscopy detected more duodenal polyps in 13 procedures (mean \# of polyps 27 vs $30, p=0.03$ ) and maximum size of the polyps increased in 5 ( $15 \mathrm{vs} 16 \mathrm{~mm}$, NS). The total number of points for the Spigelmanclassification was increased in 7 procedures. However, this resulted in an increased Spigelman-classification in only $3(6.4 \%)$. Discussion: Compared to historic endoscopic studies evaluating duodenal polyposis in FAP-patients, the use of high-resolution endoscopes results in increased polyp detection and subsequently a higher Spigelman-score. Although chromoendoscopy detected significantly more polyps, this resulted in a higher Spigelman stage in only $6 \%$ of endoscopies and therefore its additional value seems to be limited compared to high-resolution endoscopy

\section{M1135}

Novel Fiberoptic Confocal Fluorescence Microendoscope

Shai Friedland, Roy Soetikno, Jonathan Liu, Peyman Sahbaie, Mandeep Singh, James Crawford, Christopher H. Contag, Thomas D. Wang

Background: Confocal fluorescence microscopy (CFM) provides high resolution images of tissue with optical sectioning, and has the potential to provide in vivo histopathology. Here we demonstrate the use of a novel catheter-based confocal imaging system (Cellvizio-GI Mauna Kea Technologies, France). Methods: Images were collected from 180 patients undergoing routine upper and lower endoscopy. The mucosa was prepared by spraying $5 \%$ acetic acid followed by $0.05 \%$ fluorescein. 0 - and 50 -micron working-distance probes were used to obtain fluorescence images at 12 frames per second using $488 \mathrm{~nm}$ excitation. Results: Architectural and cellular features of normal mucosa were readily appreciated. Significant differences in glandular architecture and fluorescein uptake between normal and neoplastic colonic mucosa were observed. Average lamina propria fluorescence intensity was $94 \pm 4$ in normal mucosa and $44 \pm 14$ in adenoma. Average intensity in the basolateral colonocyte border was $96 \pm 24$ in normal mucosa and $172 \pm 16$ in adenoma. The fast frame rate of the device allows for tracking of dynamic physiological events such as fluorescein uptake into tissue and cellular movement within mucosal capillaries. Conclusions: CFM images and movies can be collected in vivo in real time using a novel microscopy catheter compatible with conventional endoscopes, demonstrating the potential to perform real time histopathology and to study dynamic physiological events such as cellular uptake of fluorescent molecules.

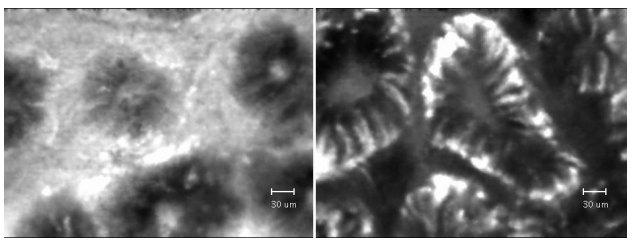

Normal Colonic Mucosa (Left) and Tubular Adenoma (Right)

\section{M1136}

Nuclear-Cytoplasmic Exchange of PKD3 in Human Pancreatic Cancer (PANC1) Cells: Tracking Photoactivable GFP-PKD3 By 2-Photon Activation and 1Photon Confocal Microscopy

Steven H. Young, Osvaldo Rey, Jennifer Lippincott-Schwartz, Enrique Rozengurt

Background: The development of photoactivatable GFP (PA-GFP) has increased the resolution of studies of the distribution of functioning proteins in living cells in real time. After exposure to a short flash of $405 \mathrm{~nm}$ light, PA-GFP photoconverts to new configuration that exhibits a 30 -fold increase in green emission intensity (at $520 \mathrm{~nm}$ ) when being scanned by blue (488 nm) light. In time, if these molecules move away from the activation region, they can be traced anywhere within the cell, as they irreversibly maintain increased green fluorescence. Methods: We have further increased the spatial resolution of photoactivation 
in single cells through the use of 2-photon confocal microscopy, which produces a very limited focal volume, in order to photoconvert the PA-GFP, and then using 1-photon confocal microscopy to track the movement of activated molecules. Protein kinase D3, a molecule present in both nucleus and cytoplasm is a member of a family of serine/threonine kinases which are implicated in the regulation of many cellular processes. We have expressed a PAGFP PKD3 fusion protein in PANC-1 cells, and have photoactivated this protein within narrow depth planar regions of the nucleus or the cytoplasm of single living cells. Results: We directly demonstrate that in time, PA-GFP PKD3 will translocate out of the nucleus and into the cytoplasm. Reciprocally, we show that activation of PA-GFP PKD3 in a restricted area of the cytoplasm results in a spread through the cytoplasm as well as into the nucleus, resulting in a dynamic distribution of PA-GFP PKD3 between nucleus and cytoplasm. Conclusion: The narrow depth of activation of 2-photon photoactivation provides for a far more precise region of activation than can be achieved by l-photon activation. We expect this technology to extend capabilities of spatial resolution in studies of the movement of molecules within and between compartments within living cells.

\section{M1137}

Detection and Classification of Adenomas Using High Resolution Endoscopy, Video Autofluorescence Imaging and Narrow Band Imaging Incorporated in One Colonoscope

Evelien Dekker, Mohammed A. Kara, Christine C. Cohen, James Hardwick, Johan Offerhaus, Bergman J. Jacques, Paul Fockens

INTRODUCTION: Video autofluorescence imaging (AFI) and narrow band imaging (NBI) are novel techniques that may improve the detection and classification of colonic polyps. In a recent prototype, high resolution endoscopy (HRE) has been combined with AFI and NBI (Olympus, Tokyo, Japan). AIMS \& METHODS: The aim was to evaluate the feasibility and efficacy of HRE, AFI and NBI for the detection and classification of adenomas. The prototype used in this study enables switching between HRE, AFI and NBI by using two knobs on the handle of the endoscope. During AFI, blue light is used for autofluorescence excitation and a narrow band of green light is used for reflectance. During NBI, narrowed red, green and blue light bands, with a higher intensity of blue light are used for excitation, enabling optimal visualization of the mucosal patterns without the use of stains. We enrolled patients attending for surveillance colonoscopy because of prior adenoma(s) or a family history for CRC. Segmental examination of the colon during withdrawal was performed using HRE and AFI in a randomized sequence. NBI was used to classify the pit-patterns of polyps according to the Kudo classification. Lesions were photographed using all three modalities prior to polypectomy or biopsy. Procedures were performed by 2 colonoscopists and the pathologists were blinded to the findings with AFI and NBI. RESULTS: Up to date, 41 patients were enrolled in this ongoing study ( 22 males, mean age 54 yrs SD 15). A total of 106 polyps ( 56 adenomas) were detected in 25 patients. In patients examined with HRE first ( $\mathrm{N}=21), 43$ polyps were detected with HRE (24 adenomas) and 12 additional polyps ( 7 adenomas) were detected with AFI; 17 of the 24 adenomas detected with HRE were also suspicious on AFI. In patients examined first with AFI, 31 polyps were detected with AFI including 15 adenomas and 20 additional polyps (10 adenomas) were detected with HRE The table below shows the diagnostic test values for AFI, NBI and the combination of both for the detection and classification of adenomas. CONCLUSION: The consecutive use of high resolution endoscopy (HRE), video autofluorescence imaging (AFI) and narrow band imaging (NBI) incorporated in one colonoscope is feasible. HRE and AFI increase the detection rate of adenomas and NBI can be used to classify polyps and diagnose adenomas without the need for staining solutions. The combination of AFI and NBI may improve the diagnostic accuracy for adenomas.

\begin{tabular}{|c|c|c|c|c|}
\hline Technique & Sensitivity & Specificity & $\begin{array}{c}\text { Positive predictive } \\
\text { value }\end{array}$ & $\begin{array}{c}\text { Negative predictive } \\
\text { value }\end{array}$ \\
\hline AFI (detection technique) & $76 \%$ & $33 \%$ & $47 \%$ & $84 \%$ \\
\hline $\begin{array}{c}\text { NBI (classification } \\
\text { technique) }\end{array}$ & $80 \%$ & $75 \%$ & $67 \%$ & $85 \%$ \\
\hline $\begin{array}{c}\text { AFI-NBI (detection/ } \\
\text { classification) }\end{array}$ & $76 \%$ & $81 \%$ & $73 \%$ & $84 \%$ \\
\hline
\end{tabular}

\section{M1138}

Detection of Residual Barrett's Mucosa After Photodynamic Therapy (PDT) Using High Definition Narrow Band Imaging (HD-NBI) Herbert Wolfsen, Lois L. Hemminger, Michael B. Wallace

Endoscopic ablation using porfimer sodium PDT significantly decreases the risk for progression to invasive carcinoma in Barrett's high grade dysplasia (BE+HGD) patients. After PDT, Barrett's mucosa persists in nearly $50 \%$ of patients and must be detected and destroyed to prevent metachronous carcinoma. Use of chromoendoscopy with Lugol's dilute iodine for residual disease detection is cumbersome and associated with risk of toxicity. The aim of this study was to compare detection of residual Barrett's mucosa after PDT with standard endoscopy, high definition (HD) endoscopy and HD-NBI. Methods: After IRB approval, consecutive patients undergoing treatment with Ps-PDT for BE+HGD were studied using standard definition white light endoscopy (Olympus GIF-Q180), high definition white light endoscopy (Olympus XGIF-H160Y2) and HD-NBI (Olympus XGIF-H160Y2, 415 and 540 $\mathrm{nm})$. Endoscopic imaging was performed at a single session under identical conditions with digital imaging documentation. Detection rates of residual areas of glandular mucosa were compared. Results: After Ps-PDT, 50 consecutive BE+HGD patients were studied (42 men, 8 women; mean age $68 \mathrm{yrs}$, range 51-83 yrs). The pre-Ps-PDT mean BE segment length was $4 \mathrm{~cm}$; range $1-12 \mathrm{~cm}$. The mean time interval after PDT was $20 \mathrm{mos}$; range 3-66 mos. Areas of persistent glandular mucosa (peninsulas or islands of glandular epithelium) were found in 22 pts using standard endoscopy, 25 pts using HD white light endoscopy and 38 pts using HD-NBI imaging. Conclusions: 1) The use of Narrow Band, high definition endoscopic imaging in patients treated with PDT for Barrett's high grade dysplasia detected additional areas of glandular dysplasia in more than 50\% of cases compared with standard conventional and high definition video endoscopy. 2) The use of NBI obviated the need for Lugol's chromoendoscopy. 3) Long term studies are underway to determine the impact of NBI on clinical outcomes in patients who have undergone endoscopic ablation therapy.

\section{M1139}

In Vivo Molecular and Subsurface Imaging By Confocal Real Time MiniMicroscopy

Martin Goetz, Christian Fottner, Esther Schirrmacher, Sebastian Gregor, Peter Delaney, Constantin Schneider, Dennis Strand, Manfred Relle, Andreas Schwarting Mathias M. Weber, Oliver Kempski, Martin Anlauf, Guenther Kloeppel, Michael Vieth, Peter R. Galle, Peter Bartenstein, Markus F. Neurath, Ralf Kiesslich

Introduction: Early and rapid diagnosis is essential for the successful management of diseases, but remains a technical challenge. We evaluated a newly developed miniaturized hand-held confocal laser mini-microscopy probe for in vivo molecular, functional and morphological fluorescence imaging in rodent models of human diseases. Methods: In the confocal laser probe, a single line laser delivers an excitation wavelength of $488 \mathrm{~nm}$. Optical slice thickness is $7 \mu \mathrm{m}$, lateral resolution $0.7 \mu \mathrm{m}$. The range of the z-axis is $0-250 \mu \mathrm{m}$ below tissue surface. Imaging was performed using different fluorescent staining protocols. Fluorescein-labeled octreotate was synthetized for molecular targeting of Somatostatin receptor (sstr) 2 . Results: Cellular and subcellular details could be readily visualised at high resolution ( $\mathrm{n}=30$ healthy mice). Sstr2 targeting allowed in vivo molecular staining of AR42-J induced carcinoma ( $\mathrm{n}=$ 2 mice) and pancreas islet cells ( $\mathrm{n}=2$ rats). Blood cell labeling visualised homing in small vessels and a spontaneous thrombosis formation in gerbils. Vessels in HEK-293-induced tumor ( $\mathrm{n}=2$ mice) could be easily differentiated from normal vessels, and glomerulonephritis was diagnosed ( $\mathrm{n}=2 \mathrm{MLR} / \mathrm{lpr}$ mice). In vivo imaging was confirmed by ex vivo (immuno-) histology. Discussion: Confocal mini-microscopy allows real-time subsurface in vivo molecular, functional and morphological imaging of inflammation, cancer and thrombosis. Since this probe is applicable to humans, it will be of fundamental impact on clinical and basic science.
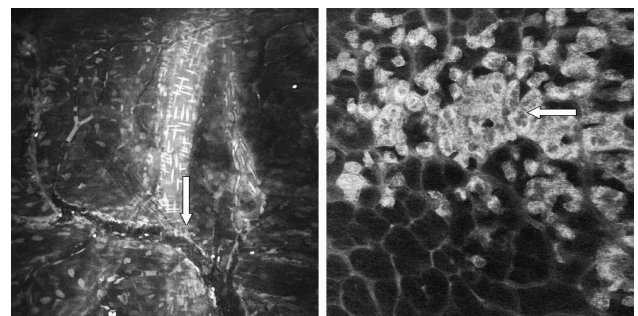

Left: Thrombosis of a brain venule (FITC-dextran, Acriflavine, FITC-labeled RBC) Right: Molecular targeting of sstr2, cytoplasmic islet cell fluorescence in rat pancreas

\section{M1140}

Fractal Analysis with Classification Tree for Classification of Dysplasia in Barrett's Esophagus Using Multiple Endoscopic Optical Coherence Tomography Images

Xin Qi, Douglas Y. Rowland, Michael V. Sivak, Andrew M. Rollins

We have shown that endoscopic optical coherence tomography (EOCT) using a first prototype has a 70\% accuracy for the diagnosis of dysplasia in Barrett's Esophagus (BE) (GIE 2003; $57 \cdot A B 77$ ). Also, we showed that a computer-aided-diagnosis (CAD) algorithm, based on a standard texture analysis of digital EOCT images (center-symmetric auto-correlation with principal component analysis), achieved a sensitivity of $82 \%$, specificity $74 \%$ and accuracy 84\% (SPIE Proceedings, 2004; Vol.5316:33-40) in the diagnosis of dysplasia. This analysis yields parameters representing texture on a single spatial scale, but EOCT features that differentiate dysplastic and non-dysplastic tissue occur on various spatial scales. Also this analysis classified images based on a single parameter, rather than the several quantifiable parameters available. Finally, the CAD algorithm used only one EOCT image per site, although multiple images are usually available. HYPOTHESIS: fractal analysis with a classification tree using multiple EOCT images of a single tissue site is more accurate than our previous CAD algorithm in defining dysplasia in EOCT images of BE. METHOD: EOCT images were recorded at biopsy sites in patients undergoing $\mathrm{BE}$ surveillance. At each site, EOCT frames acquired before the forceps entered the EOCT field of view were used as our CAD data set. With our biopsy/EOCT system, there is an exact correlation between histology and corresponding EOCT images. 812 EOCT images (13 patients, 70 non-dysplastic biopsy sites [499 images] and 38 dysplastic sites [313 images]) were analyzed. Fractal analysis was used to compute a fractal signature of the region of interest which is a quantitative measure of texture and self-similarity at many spatial scales. The scores of the first 10 principal components of the fractal signature were input to the classification tree to differentiate images of normal and dysplasitc tissue. RESULT: Using only one frame for classification, sensitivity was $95 \%$ (95\% CI: 82\%, 100\%) and specificity 94\% (95\% CI: 86\%, 98\%). Using three frames per site (with a requirement that 2 be positive for the site to be classified as positive) sensitivity was $100 \%$ (95\% CI: 89\%, 100\%) and specificity 100\% (95\% CI: $94 \%, 100 \%$ ); $97 \%$ of the sites had at least three frames available. CONCLUSIONS: Versus our previous algorithm, fractal analysis with classification tree substantially improved in the classification of dysplasia in BE in EOCT images. Use of multiple images from a single biopsy site improved accuracy. With further improvements, CAD could potentially enable EOCT surveillance of large areas of Barrett's mucosa for dysplasia. 


\section{M1141}

Confocal Microscopy: A Novel Method to Assess Aberrant Crypt Foci in Apcmin+/- and Apcmin+/- (Pparo-/-) Mice

Shonde Anthony, Krish Ragunath, George K. Anagnostopoulos, D R. Bell, A J. Bennett, Christopher J. Hawkey

Background: Peroxisome proliferator-activated receptor $\alpha$ (PPAR $\alpha$ ) is expressed at low levels in colon tumours. APCmin+/- mice fed with methylclofenapate (a PPAR $\alpha$ ligand) have 50\% less tumour burden in the small intestine and colon than controll. Recent studies showed that aberrant crypt foci (ACF) are the earliest morphological lesion detectable in colorectal epithelium that may lead to cancer. However the relationship between PPAR $\alpha$ and ACF formation is not known. Dissection microscopy is the most common method used for quantifying ACF. However it is time consuming and requires a great deal of skill to obtain a good preparation. Aims: (1) To assess whether Confocal Microscopy is a viable method for identifying ACF in scientific practice. (2) Examine the role of PPAR $\alpha$ in colon carcinogenesis using APCmin+/- and APCmin+/-(PPAR $\alpha-/-)$ mice. Method: Once weaned APCmin+/mice and APCmin+/-(PPAR $\alpha-/-)$ mice were fed standard chow throughout the study. Mice were weighed weekly, until mice showed signs of anaemia or lost $>20 \%$ of weight at which time they were sacrificed. ACF were assessed using Cell vizio Confocal Microscopy probe (Mauna Kea technologies, Paris) made of 30,000 optical fibers following colonic staining with acryflavine. Result: High definition images with $2.5 \mu \mathrm{m}$ lateral resolution of normal crypts and ACF were clearly identified using Confocal Microscopy. APCmin+/-(PPAR $\alpha-/$-) mice have more ACF than APCmin+/-mice in the colon, but this failed to reach significance $(\mathrm{n}=9)$. The mean number of ACF was 5.0/ 10 fields (CI 2.10- 7.90) in APCmin+/-(PPAR $\alpha-/-$ ) mice and 2.75/ 10 fields (CI 1.22- 4.27) in APCmint/- mice. Conclusions: Confocal Microscopy using the Cell vizio probe is a simple and quick method of accessing ACF, with the potential of refining experiments so that mice can be studied serially. PPAR $\alpha$ role in colon carcinogenesis may be to inhibit the formation of ACF rather than their progression. Greater understanding of PPAR $\alpha$ dependant genes may enable the pathogenesis of colon cancer to be further understood. 1. Potential role for peroxisome proliferator activated receptor (PPAR) in preventing colon cancer. Jackson L, et al Gut. 2003 Sep;52(9):1317-22.

\section{M1142}

Clinical Utility of Narrow Band Imaging (NBI) Endoscopy in Patients with Gastroesophageal Reflux Disease (GERD)

Prateek Sharma, Amit Rastogi, Ajay Bansal, Srinivas R. Puli, Sharad Mathur

Background : NBI is a novel imaging technique that uses narrow band filters $(415,445$, $500 \mathrm{~nm}$ ) and higher intensity blue light (smaller wavelength-less penetration) to show details of esophageal mucosal and vascular patterns. Aim: To compare findings seen only on NBI in pts with GERD and controls and to calculate sensitivity and specificity of these findings for GERD diagnosis. Methods: Reflux pts and controls without GERD symptoms filled 2 validated questionnaires (GERQ and RDQ). Then, distal esophagus was examined by white light followed by using NBI endoscope(Olympus GIFQ240Z, and $115 \mathrm{X}$ ) by a single investigator. Features seen only by NBI were noted:number, dilation and tortuosity of intra-papillary capillary loops (IPCL), micro erosions (ME), increased vascularity at squamocolumnar junction $(\mathrm{SCJ})$, columnar islands $(\mathrm{CI})$ in distal esophagus $(\mathrm{CI})$ and ridge villous pattern (R/V) below squamocolumnar junction. NBI images were then evaluated by another blinded endoscopist. IPCLs were classified as increased, dilated or tortuous and other findings noted to be present or absent. Findings were compared using Fisher's exact test; sensitivity and specificity of findings individually and in combination for GERD diagnosis were calculated. Results:72 pts were prospectively evaluated (mean age 60 yrs,67 males)- 48 GERD and 24 controls. NBI revealed a striking contrast of squamous and columnar mucosa at SCJ. Increased number, dilation and tortuosity of IPCLs, presence of micro-erosions and increased vascularity at SCJ were significantly higher in GERD pts compared to controls (Table 1). Accuracy of their combinations are shown in Table 2. Conclusion: NBI shows details of mucosa and vascularity in distal esophagus - IPCLs, micro erosions and increased vascularity at SCJ being significantly higher in GERD pts vs. controls. Also, distinctive features like increased number, dilation or tortuosity of IPCLs have a high accuracy for GERD diagnosis. These findings support an emerging role of NBI in GERD diagnosis

Table 1

\begin{tabular}{|c|c|c|c|c|c|}
\hline & GERD & Controls & $\mathrm{p}$ & Sens. & Specificity \\
\hline ICPL Increased & $65.2 \%(30 / 46)$ & $15.3 \%(4 / 26)$ & 0.0005 & $65.2 \%$ & $84.6 \%$ \\
\hline ICPL tortuous & $83.7 \%(36 / 43)$ & $38.4 \%(10 / 26)$ & 0.001 & $83.7 \%$ & $61.5 \%$ \\
\hline ICPL Dilated & $81.4 \%(35 / 43)$ & $15.3 \%(4 / 26)$ & $<0.0001$ & $81.4 \%$ & $84.6 \%$ \\
\hline ME & $50 \%(23 / 46)$ & $0 \%(0 / 26)$ & $<0.0001$ & $50 \%$ & $100 \%$ \\
\hline SCJ & $43.4 \%(20 / 46)$ & $7.6 \%(2 / 26)$ & 0.006 & $43.4 \%$ & $92.3 \%$ \\
\hline R/V & $23.9 \%(11 / 46)$ & $11.5 \%(3 / 26)$ & 0.36 & $23.9 \%$ & $88.4 \%$ \\
\hline CI & $39.1 \%(18 / 46)$ & $34.6 \%(9 / 26)$ & 0.08 & $39.1 \%$ & $57.7 \%$ \\
\hline
\end{tabular}

Table 2

\begin{tabular}{|c|c|c|}
\hline & Sensitivity & Specificity \\
\hline Increased or Dilated or Tortuous IPCL & $88.7 \%$ & $61.5 \%$ \\
\hline Increased + Dilated + Tortuous IPCL & $60.5 \%$ & $92.3 \%$ \\
\hline Increased or Dilated or Tortuous IPCL or ME & $93 \%$ & $61.5 \%$ \\
\hline Increased + Dilated + Tortuous IPCL + ME & $32.6 \%$ & $100 \%$ \\
\hline
\end{tabular}

M1143

Novel Ultrahigh Resolution Endoscopic Optical Coherence Tomography Imaging of the Stomach

Saleem Desai, Hiroshi Mashimo, Aaron D. Aguirre, Yu Chen, Qin Huang, Shu-Wei Huang, Joseph Schmitt, James G. Fujimoto

BACKGROUND/AIMS: Optical coherence tomography (OCT) provides high resolution crosssectional images of tissue microstructure in real time. Images are obtained in a minimally invasive manner without tissue excision using a small catheter introduced through the biops channel of a standard endoscope. Most OCT investigations in the upper gastrointestinal tract have focused on the diagnosis of Barrett's and dysplastic esophagus. There are relatively few studies of stomach pathology. In contrast to standard OCT which has 10-15 um axia resolution, the recently developed ultrahigh resolution endoscopic OCT (UHR EOCT) is capable of imaging with 5 um axial resolution and allows enhanced discrimination of tissue features in vivo. The goal of this study was to evaluate UHR EOCT for imaging of normal stomach and of various lesions of the gastric mucosa. METHODS: UHR EOCT imaging was performed of the normal stomach and of gastric lesions in vivo using an ultrahigh resolution OCT system based on a Cr:Forsterite laser source. Images were acquired with a $1.8 \mathrm{~mm}$ diameter linear-scanning catheter introduced through the accessory port of a standard endoscope. Specimens removed by endoscopic mucosal resection (EMR) were also subsequently imaged with an OCT microscope, and three-dimensional (3D) reconstructions were made of polyp microstructure. Specimens were submitted for histopathology an correlated with OCT images. RESULTS: In normal stomach, UHR EOCT could clearly visualize the gastroesophageal junction as well as gastric pit architecture. UHR EOCT also discriminated mucosal subtypes in the cardia, corpus, and pylorus, although imaging depth was significantly reduced compared to images of the esophagus. Gastric polyps could be surveyed and various polyps including mucinous, hyperplastic, inflammatory, adenomatous and dysplastic were characterized. Ex vivo images of resected gastric polyps with UHR OCI showed similar features to in vivo images but allowed three-dimensional reconstructions and high-resolution visualization in any section plane. CONCLUSIONS: UHR EOCT provides visualization of subsurface tissue structure in the stomach. This technology may prove useful in characterizing gastric polyps and masses. Future work on high speed and 3D UHR OCT promises new visualization methods in vivo

\section{M1144}

Novel Technique in Understanding the Sequence of Biochemical Changes in Carcinogenesis of Esophagus

Geeta Shetty, Catherine Kendall, Neil Shephard, Nicholas Stone, Hugh Barr

Background: Most reports emphasise genetic changes in understanding the carcinogenesis sequence of esophageal adenocarcinoma. Biochemical changes occurring in carcinogenesis have not been evaluated to their full potential. We propose a technique, which helps to understand the biochemical events involved in the development of malignancy in Barrett's esophagus. Methods: 70,404 Raman spectra were measured on 24 snap frozen endoscopic biopsy samples. Contiguous sections were stained with $\mathrm{H} \& \mathrm{E}$ for analysis by a consultant

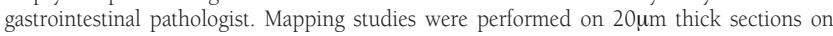
$\mathrm{CaF} 2$ slides with spectra measured at $100 \mu \mathrm{m}$ steps across the samples. Analysis of spectra was done in Matlab generating principal components and pseudocolour maps. Results: Using pseudocolour maps the lines of interest were selected representing the surface changes or understanding the depth profile of tissue (Figure 1). The spectra along the selected line were analysed for the relative concentration of different biochemical constituents using mathematical least fit square method. Marked variation in the distribution of oleic acid actin, triolene, glycogen and DNA were seen. Conclusion: Raman spectroscopy is an optical diagnostic tool, which can be used to demonstrate the biochemical changes in carcinogenesis of esophagus and identify early disease. Work is in progress to develop an in vivo endoscopic probe for real time analysis.

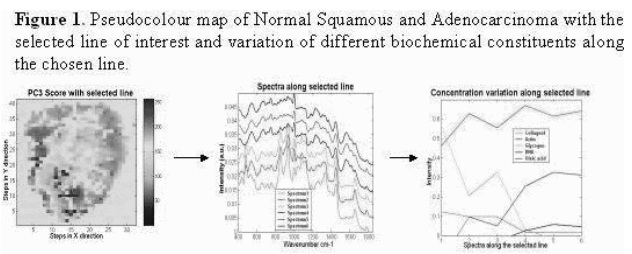

M1145

In Vivo Diagnosis of Microscopic Colitis By Confocal Laser Endomicroscopy Ralf Kiesslich, Arthur Hoffman, Martin Goetz, Katharina Lammersdorf, Stefan Biesterfeld, Michael Vieth, Peter R. Galle, Markus F. Neurath

Background: Microscopic colitis is subdivided into collagenous and lymphocytic colitis. The diagnosis of microscopic colitis relies on histopathologic examination of biopsy specimens. Aim of the current study was to investigate the value of confocal laser endomicroscopy enabling in vivo histology for immediate diagnosis of microscopic colitis during ongoing 
colonoscopy. Methods: Patients scheduled for colonoscopy with chronic diarrhea were examined with a newly developed Endomicroscope (Pentax, Tokyo, EC-3870CIFK). Fluorescein was applied intravenously to enable confocal endomicroscopy of colonic mucosa (0$250 \mu \mathrm{m})$. Confocal images from macroscopic normal apparent mucosa were obtained from the right, transverse colon and rectum. Confocal image data were collected at a scan rate of $1.6 \mathrm{sec} / \mathrm{frames}$ (1024x1024 pixels) using an optical slice thickness of $7 \mu \mathrm{m}$ (lateral resolution $\leq 1 \mu \mathrm{m})$. The presence of collagenous bands or lymphocytes within the lamina propria was graded and targeted biopsies were applied. Results: In 4 out of 57 patients with normal apparent mucosa microscopic colitis ( 3 collagenous, 1 lymphocytic) could be diagnosed by confocal laser endomicroscopy which was proved by histology in all cases (sensitivity $100 \%$ ). Subepithelial, thickened collagenous bands or lymphocytes could be readily identified and quantified by performing endomicroscopy. In addition crypts and vasculature could be seen at high resolution. The patchy distribution of the affected mucosa with accentuation of the right colon could be predicted with high accuracy (97.5\%) within these patients. Conclusions: Confocal laser endomicroscopy allows localizing and measuring the amount of collagenous bands and lymphocytes in the mucosal layer. Thus, endomicroscopy offers the possibility to diagnose and targeted biopsy microscopic colitis during ongoing colonoscopy

\section{M1146}

Automated Virtual Microcopy of Specimens from Gastric Biopsies Can Accurately Diagnose Gastritis and Adenocarcinoma

Levente Ficsor, Viktor Varga, Lajos Berczi, Pal Miheller, Attila Tagscherer, Mark Li-Cheng Wu, Bela Molnar, Zsolt Tulassay

Background: Automated virtual microscopy of specimens from gastrointestinal biopsies is based on cytometric parameters of digitized histologic sections. Diagnoses rendered by automated virtual microscopy are potentially more reproducible and more efficient than diagnoses rendered by conventional optical microscopy. To our knowledge, cytometric parameters of gastritis and of adenocarcinoma have yet to be fully characterized. Our objective was to classify gastritis and adenocarcinoma based on cytometric parameters. We hypothesized that automated virtual microscopy using this novel classification can reliably diagnose gastritis and adenocarcinoma. Methods: Routinely processed hematoxylin-andeosin-stained histologic sections that showed normal mucosa ( 14 specimens), gastritis ( 25 including 6 non-atrophic, 17 atrophic, 12 intestinal metaplasia), and adenocarcinoma (30 specimens) were scanned and digitized at high resolution $(0.3 \mu \mathrm{m} / \mathrm{pixel})$ using the Zeiss Mirax Scan (Carl Zeiss Jena GmbH, Germany, Jena). Thirty-eight cytometric parameters based on density and morphometry were applied to glands, foveolae, and superficial epithelium. Twelve cytometric parameters based on cytologic detail were applied to individual cells. Statistical analysis was performed using Statistica 6.0 software (StatSoft Inc., USA). Results: Statistically significant differences in cytometric parameters for normal mucosa, gastritis, and adenocarcinoma were found $(\mathrm{p}<0.0 .5$,). The most discriminatory parameter was the ratio of the total number of cells to the number interstitial cells (normal mucosa $1.43 \pm 0.12$; gastritis $1.23 \pm 0.13$; adenocarcinoma $1.1 \pm 0.05$; $<<0.01$ ). These differences correctly classified normal and gastritis cases versus adenocarcinoma (100\%) with reasonable accuracy ( $86 \%$ overall). The different gastritis cases could be classified by $56 \%$ (atrophic gastritis), $86 \%$ intestinal metaplasia, $65 \%$ gastritis without atrophy and intestinal metaplasia , respectively. Conclusions: We describe a novel classification of gastric mucosa based on cytometric parameters. Automated virtual microscopy can be uses to diagnose normal mucosa, gastritis, and adenocarcinoma with reasonable accuracy. Downstream classification of gastritis groups needs further alteration specific paremeters to be included in the morphometric parameters.

\section{M1147}

Cell Membrane Fluidity Assays of Fluorescence Anisotropy and Excimer Formation in Indomethacin-Treated Gastric Epithelial Cell-Line RGM-1 Tsuyoshi Kaneko, Hirofumi Matsui, Osamu Shimokawa, Takashi Mamiya, Jyunpei Udo, Nagano Yumiko, Mutsumi Muramatsu, Ken-Ichi Hirano, Atsunori Tsuji, Yasushi Matsuzaki, Ichinosuke Hyoudou

[Bachgrounds \& Aims] Gastric complications of indomethacin, a non steroidal anti-inflammatory drugs (NSAIDs), involves reactive oxygen species (ROS) formation, which induces cell membrane lipid peroxidation and gastric musocal injuries. When peroxidized by ROS, the amounts of unsaturated fatty acids in cell membrane lipid bilayers are reportedly decreased, which affects cell membrane fluidity. We thus investigate the cell membrane fluidity change after indomethacin treatment both with fluorescence anisotropy and with excimer formation using fluorescence indicators decanoylaminofluorescein (DAF) and 1,3di (1-pyrenyl) propane (PC3P), respectively, in gastric epithelial RGM-1 cell line. [Methods] Indomethacin-pretreated RGM-l cells were treated with $\mathrm{DAF}$ and $\mathrm{PC} 3 \mathrm{P}$ and both their fluorescence anisotropies and fluorescence intensity ratios were measured and calculated with an image intensified CCD (IICCD)-mounted fluorescence microscope and an imageprocessor. Indomethacin-pretreated RGM-1 cells were also treated with another fluorescence indicator, diphenyl-1-pyrenylphosphine (DPPP), which reacts with membranous organic hydroperoxides and hydrogen peroxide to emit fluorescence, and their fluorescence intensities were measured with the previous system. [Results] After indomethacin treatment the fluorescence intensities of DPPP in RGM-1 were increased in a time- and dose-dependent manner, indicating cell membrane lipid peroxidation was induced. The fluorescence anisotropy values of DAF and the fluorescence intensity ratios of PC3P excimer to monomer were both increased, indicating that the fluidity of cell membrane was decreased by the indomethacin treatment. [Conclusion] We demonstrated indomethacin treatment decreased cell membrane fluidity in a gastric epithelial cell line. Together with the result of DPPP fluorescence observation, the fluidity change reflects compositional changes of membranous lipid bilayers induced by lipid peroxidation. We thus propose the fluorescence observation of the cell membrane fluidity can apply to the detection of cellular pathphysiological changes in NSAID-induced gastric lesions.

\section{M1148}

Antibody Aided Confocal Laser Endomicroscopy Allows Molecular Imaging of Colorectal Lesions in Humans

Ralf Kiesslich, Martin Goetz, Brigitte Bartsch, Katharina Lammersdorf, Arthur Hoffman, Constantin Schneider, Manfred Vieth, Manfred Stolte, Peter R. Galle, Markus F. Neurath

Introduction: Confocal laser endomicroscopy (CLE) enables in vivo histology of the mucosal laver with cellular and subcellular resolution during ongoing endoscopy. Aim of the current study was the evaluation of distinct fluorescein labeled antibodies for immediate molecular imaging and characterisation of different colonic lesions in humans. Methods: Patients with known colorectal adenomas and/or aberrant crypt foci were enrolled in the study and underwent total colonoscopy with CLE (Optiscan, Australia; Pentax, Japan; excitation of $488 \mathrm{~nm}$ argon ion laser; detection $>515 \mathrm{~nm}$; optical slice thickness $7 \mu \mathrm{m}$; lateral resolution 0.7 um; frame rate 0.8 or 1.6 frames/sec with $1024 \times 1024$ or $1024 \times 512$ pixels). After reaching the colonic lesions, a fluorescent contrast agent (fluorescein) for transient staining of vessels and crypts was administered intravenously and endomicroscopic evaluation was performed using the confocal pattern classification. Endoscopic resection was performed and the resected specimens were immediately incubated with FITC-labeled Anti-EGFR ( $\mathrm{n}=4)$, Anti-CD44v6 $(\mathrm{n}=2)$ or Anti-Actin $(\mathrm{n}=1)$ antibodies (dilution; 1:50) for 30 minutes. Lesions were re-assessed ex vivo with CLE-endoscope after a thorough washing step. Subsequently, conventional histology and ex vivo immuno-staining on resected specimens was performed to correlate histology with the CLE findings. Results: Antibody aided confocal laser endomicroscopy specifically highlighted the corresponding epitopes on the cell surface. Cellular and subcellular detail could be seen at high resolution. Aberrant crypt foci were readily visible and highlighted by staining with Anti-CD44v6 antibodies. EGFR receptors could be quantified at the cell membrane with CLE and were confirmed histologically by ex vivo immunostaining. Anti-Actin antibodies aimed at intracellular targets showed no contrast by CLE in the absence of cell permeability and served as a negative control. Conclusions: Confocal laser endomicroscopy enables molecular imaging in humans at high resolution. Resected colonic lesions can be immediately analysed and characterised for the presence of different receptors. Thus, antibody aided CLE may offer a rapid analysis of colorectal lesions with important implications for targeted tumour therapy.

\section{M1149}

Quantum Detection of Inflammatory Markers in Vivo in DSS Model of Colitis Amolkumar Karwa, Sreekant Murthy, Elisabeth Papazoglou, Kambiz Pourrezaei, Som Tyagi

The ability to sense and image the biomolecules with nanotools has significant effect in unraveling the disease pathogenesis as well as nanodelivery of drugs at the disease site. Quantum Dots (QDs) are novel nanometer sized fluorescent particles, with distinct advantages of high quantum yield and excellent photostability making them great tools for dynamic monitoring of disease progression. With these excellent properties of QDs it is possible to image and quantify biomarkers that are important indicators of inflammation. ILl $\alpha$, Myeloperoxidase (MPO), and TNF $\alpha$ are three different biomarkers that play an important role in Inflammatory Bowel Diseases. These markers show an increased presence in the acute and chronic phase of animal model of DSS induced colitis, which resembles human ulcerative colitis. We report here targeting of these biomarkers in vivo with QDs and their subsequent quantification in the DSS model of ulcerative colitis. To target these biomarkers in vivo, the antibodies for Myeloperoxidase and cytokines ILl $\alpha$ and TNF $\alpha$ were conjugated to different sizes of QDs with emission at different wavelength. Acute and chronic colitis was induced in Swiss Webster mice according to the established protocols with conjugates being tested in vivo on pre determined days. A colonic loop was prepared and colon was directly exposed to QD conjugates, washed, excised, and frozen. Histological slides (15 micron thickness) were prepared and imaged with a confocal microscope. The subsequent images obtained showed gradual increase in presence of QDAb conjugates with increase in inflammation. In the acute stage of the colitis starting from Day 3 to Day7 of DSS feed the fluorescent intensity of QDs gradually increased with the disease severity (correlation, R= 0.96). In the acute phase MPO intensity was observed to be highest followed by ILl $\alpha$ whereas TNF $\alpha$ intensity was the lowest. In the chronic phase MPO could be still detected, however TNF $\alpha$ showed increased presence and ILl $\alpha$ presence showed gradual decline. QDs were absent in control colon tissue devoid of inflammation. The observations made here is in accordance with the previous biochemical studies done in the same DSS model albeit the nanotechnology based method applied here is less cumbersome and highly reproducible. In summary our results showed that QDs can be successfully used to image the biomarkers presence and relate their presence to the disease severity. The technique developed here can be extended in future to identify potential biochemical targets using multiple biomarkers for different pathological events taking place in the progression of disease.

\section{M1150}

Probiotics May Reduce Inflammation By Enhancing PPAR $\gamma$ Activation in Intestinal Epithelial Cells

Chang Soo Eun, Dong Soo Han, Seung Hyun Lee, Yoon Kyoung Park, Byoung Kwan Son, Hyun Joo Jang, Sea Hyub Kae, Jin Lee, Yong Seok Kim, Ronald M. Evans

Background: The peroxisome proliferator-activated receptor gamma (PPAR $\gamma$ ) is a nuclear receptor highly expressed in the colon and playing an anti-inflammatory role through inhibition of NF-KB pathway. Probiotics, living organisms in food and dietary supplements, have been shown to exert beneficial effects on clinical trials of inflammatory bowel diseases treatment. However, the exact mechanism by which probiotics exerted protection against the intestinal inflammation is not well understood. The aims of this study were to evaluate attenuation of inflammation by probiotics in intestinal epithelial cells and to study the possible relation between probiotics and PPARy. Methods: HT-29 human epithelial cells were stimulated with E. coli LPS $(20 \mu \mathrm{g} / \mathrm{mL})$ and probiotics, Lactobacillus casei $(10 * 5-10 * 7$ $\mathrm{cfu} / \mathrm{mL})$, or with LPS $(20 \mu \mathrm{g} / \mathrm{mL})$ alone for 24 hours. L. casei were administered either 6 hours before LPS stimulation or concomitant with LPS stimulation. COX-2, IL-8, TLR-4 
and PPAR $\gamma$ mRNA expression were assessed by RT-PCR. IL- 8 protein secretion levels were analyzed by ELISA. To evaluate whether the action of L. casei was correlated with the activation of PPAR $\gamma$, HT-29 cells were transfected with tk promoter-luciferase plasmid containing a PPRE. After stimulation with L. casei or PPAR $\gamma$ agonist (15d-PGJ2, ciglitazone), luciferase activities were measured. Results: LPS stimulation on HT-29 cells increased COX2, IL-8, TLR-4 mRNA expression and IL-8 protein secretion. Costimulation with LPS and L. casei, in comparison to LPS stimulation alone, decreased COX-2, IL-8, TLR-4 mRNA expression and IL-8 protein secretion. Costimulation with LPS and PPARy ligand (15dPGJ2) decreased COX-2, IL-8, TLR-4 mRNA expression and IL-8 protein secretion compared to LPS stimulation alone. L. casei increased PPAR $\gamma$ mRNA expression in proportion to stimulation doses. L. casei activated PPRE in HT-29 cells transfected with PPRE3-tk-luciferase construct in a dose-dependent manner. Conclusions: Probiotics. L casei, suppress inflammation in intestinal epithelial cells. The anti-inflammatory action of L. casei may be partially related to PPAR $\gamma$ activation.

\section{M1151}

Systemic Exposure to E.Coli DNA and Microbial Antigens During the Neonatal Period Triggers a Loss of Tolerance to Gut Microflora in Later Life Phil Bach, Francis Cheung, Dave Thiel, Brendan Diederichs, Julia Ewaschuk, Claire Arrieta, Karen Madsen

Impairment of innate immunity and heightened T-cell responsiveness to enteric microflora are associated with inflammatory bowel disease (IBD). Failure of the innate immune system to clear microbes could result in increased exposure of the adaptive immune system and induce a loss of tolerance to gut microflora. Previous studies have shown that some $\mathrm{CpG}$ DNA can render protein antigens highly immunogenic. Bacterial DNA, which contains CpG motifs, elicits variable immune responses in a strain-dependent manner and can be found in the serum of patients with increased gut permeability. In children, the onset of IBD often follows intestinal infection, when gut barrier function may be compromised. Aim: The aim of this study was to determine if exposure of the neonatal adaptive immune system to microbial antigens and immunogenic bacterial DNA would trigger a loss of tolerance to enteric microflora. Methods: Neonatal (3 wks of age) and adult $129 \mathrm{~Sv} / \mathrm{Ev}$ mice were injected subcutaneously once only with Clostridium sordelli (CS; $\mathrm{n}=24)$ or Viridans group Streptococci (VGS; $\mathrm{n}=24$ ) protein sonicates \pm E. coli or Bifidobacterium DNA (50ug). After 4 wks, ileal and colonic tissue were removed for histological assessment and measurment of basal and microbe-induced secretion of TNF $\alpha$ (as an indicator of innate immunity) and IFN $\gamma$ (adaptive immunity) by organ culture. Splenocytes were isolated and cytokine secretion assessed. Results: 4 wks following systemic exposure, intestinal tissue from mice injected with E. coli DNA and CS responded to CS and other bacterial sonicates with a significantly increased IFN $\gamma$ and TNF $\alpha$ secretion compared with mice receiving CS alone $(\mathrm{p}<0.05)$. In contrast, intestinal tissue from mice injected with Bifidobacteria DNA and CS did not respond to CS sonicates, but did have increased basal TNF $\alpha$ secretion, suggesting an enhancement of innate immune function. This mucosal response was strain-dependent, as mice injected with VGS \pm bacterial DNA did not demonstrate any changes in gut cytokine secretion. Neonatal mice injected with CS or VGS sonicates in the absence of bacterial DNA did not exhibit any differences in gut cytokine secretion. Adult mice injected with $C S$ or VGS $\pm E$. coli DNA did not demonstrate any alterations in intestinal cytokine secretion, but did have a heightened systemic response to CS protein. Conclusions: Exposure of the neonatal systemic immune system to E. coli DNA and bacterial antigens results in a loss of mucosal tolerance to gut microflora which is maintained into later life. The presence of probiotic bacterial DNA can suppress these adaptive immune responses, while enhancing the innate immunity of the gut.

\section{M1152}

1.4-Dihydroxy-2-Naphthoic Acid (DHNA) Shows Anti-Inflammatory Effect On NSAID-Induced Colitis in IL-10-Knockout Mice Through Suppression of Inflammatory Cell Infiltration and Increased Number of Genus

\section{Bifidobacterium}

Yoshikiyo Okada, Ryota Hokari, Shingo Kato, Norikazu Mataki, Keisuke Okudaira, Koichi Takebayashi, Hisayuki Matsunaga, Yoshikazu Tsuzuki, Shunsuke Komoto, Chikako Watanabe, Atsushi Kawaguchi, Shigeaki Nagao, Kazuro Itoh, Soichiro Miura

BACKGROUND: 1.4-dihydroxy-2-naphthoic acid (DHNA), a component of Propionibacterium freudenreichii ET-3, is known to promote proliferation of Genus Bifidobacterium. Therefore this compound is expected to have a beneficial effect as a prebiotics for intestinal inflammation. We previously reported that administration of DHNA can ameliorate dextran sodium sulfate-induced colitis (Okada et al. Gut 2005). In this study, we examined whether DHNA attenuates inflammation in nonsteroidal anti-inflammatory drug (NSAID)-induced colonic injury in IL-10 knockout (K/O) mice. MATERIALS \& METHODS: DHNA (2.0 mg/ $\mathrm{kg}$ ) or vehicle (control) was administered for 14 days in free drinking water. Thereafter, for the induction of colitis, IL-10 K/O mice ( 5 weeks of age) was fed with rodent chow containing the NSAID (piroxicam, 200ppm). After seven-days as feeding period, mice were euthanized and the colon was removed for further experiments. The degree of colonic inflammation was determined using H\&E sections. CD4, CD8, B220, beta7-integrin, F4/80 and MAdCAM1 expressions were determined by immunohistochemistry. mRNA levels of proinflammatory cytokines (IL-1 beta, IL- 6 and TNF-alpha) were determined by quantitative real-time PCR. In addition, cecal contents were examined for bacterial flora. RESULTS: A rapidly progressed colitis was induced after piroxicam treatment in IL-10 K/O mice characterized by the infiltration of the colonic mucosa with lymphocytes and monocyte lineage cells. DHNApretreatment significantly attenuated the piroxicam-induced histological changes including cell infiltration in the colonic mucosa. The increased infiltration of beta7-integrin+ cells, CD4+ cells, F4/80 cells and increased vascular MAdCAM- 1 expressions in the colonic mucosa were also significantly attenuated by DHNA pre-treatment. The increased mRNA levels of TNF-alpha, IL-1 beta, and IL-6 and TNF-alpha in the colonic tissue induced by NSAID were also significantly suppressed by DHNA pretreatment. Furthermore, the increased number of Genus Bifidobacterium was observed in cecal contents after DHNA administration. CONCLUSIONS: NSAID treatment in IL-10 K/O mice induced significant colonic inflammation accompanied by inflammtory cell infiltration, up-regulation of vascular adhesion molecules, and the expression of proinflamatory cytokines. These changes were significantly ameliorated by DHNA treatment concominant with the recovery of Genus Bifidobacterium, suggesting the beneficial role of this component as prebiotics in NSAID-induced colonic injury.

\section{M1153}

Effects of the Probiotic E. Coli Strain M-17 On Chronic Dextran Sulfate Sodium-Induced Colitis in Mice

Leo R. Fitzpatrick, Jeffrey Small, Eileen Bostwick, Robert Hoerr, Walter A. Koltun

Probiotic bacteria are effective for attenuating colitis in animal models of inflammatory bowel disease. The mechanisms of anti-colitis action for probiotics include decreasing the production of pro-inflammatory cytokines and maintenance of the colonic mucosal barrier. Purpose: Our primary goal was to assess the efficacy of the probiotic E. coli strain M-17 (EC-M17) in a chronic dextran sulfate sodium (DSS) colitis model. A secondary goal was to determine the effects of this probiotic on pro-inflammatory cytokines, as well as two parameters (colonocyte proliferation and apoptosis) associated with colonic mucosa homeostasis. Methods: A chronic model of DSS-induced colitis was established in C57 BI 6 mice, by alternating 7-day cycles of 1\% DSS and water. On day 21 (end of the second DSS-cycle), mice ( $\mathrm{n}=19$ to 20 per group) were administered EC-M17 ( 6 x10^8 CFU) metronidazole $(40 \mathrm{mg} / \mathrm{kg}$ ), or $0.6 \%$ saline (vehicle). Disease Activity Indices (DAI's) were determined every other day and on day 35 . The colons were excised from mice on day 35 , and distal colonic samples were taken for histological evaluations ( 0 to 40 point scoring system). Pro-inflammatory cytokines (IFN- $\gamma$, IL-12, IL-1 $\beta$ ) and IL-10 were measured by ELISA, from an adjacent segment of colon. In a subset of mice, colonic epithelial cell proliferation was determined by the PCNA method, while epithelial cell apoptosis was determined by TUNEL histochemistry. Results: On day 35, the DAI's were: $0 \pm 0$ (vehicle/ Water), $1.6 \pm 0.2$ (vehicle/DSS), $0.5 \pm 0.2$ (EC-M17/DSS) and $0.8 \pm 0.2$ (metronidazole/ DSS). Mice treated with the probiotic, or antibiotic, had significantly less histological damage $(\mathrm{p}<0.01)$ than in vehicle treated mice. The colonic histology scores were: $5.4 \pm 0.5$ (vehicle/ Water), $15.0 \pm 1.5$ (vehicle/DSS), $9.8 \pm 0.8$ (EC-M17/DSS) and $10.7 \pm 0.5$ (metronidazole/ DSS). Levels of IFN- $\gamma$ and IL-1 $\beta$, but not IL-12, were reduced (38 to $46 \%$ ) in the colons of mice receiving EC-M17. IL-10 was not affected by the probiotic or antibiotic treatments. An increase in the number of PCNA positive cells was found only in the crypts of vehicle/ DSS treated mice. In mice treated with EC-M17, the mean number of TUNEL positive cells per 20 crypts $(73 \pm 14)$ was reduced, as compared to the number of positive cells in vehicle treated mice (112 \pm 19$)$. Summary: EC-M17 attenuated various parameters of chronic DSS induced colitis in mice, including pro-inflammatory cytokines and colonic epithelial cell apoptosis. EC-M17 demonstrated a similar efficacy profile to that of metronidazole. Conclusion: EC-M17 represents a novel probiotic therapy, which can potentially be used for the treatment of intestinal inflammation.

\section{M1154}

DSS Induced Colitis Is Prevented By Lactobacillus Reuteri Through Reduction in P-Selectin Expression in Rats

Joel Petersson, Olof Schreiber, Stefan Roos, Michael Perry, D. Neil Granger, Lena Holm

Background: Recent findings indicate that DSS induced colitis is associated with a prothrombogenic phenotype in the colonic microcirculation, with P-selectin playing a major role in recruitment of platelets. Since probiotics have been suggested to reduce the severity of colonic inflammation we wanted to investigate how pretreatment with Lactobacillus reuteri influence P-selectin expression and colitis severity in DSS treated rats. Methods: Rats were divided into four groups; I/Non treated, II/DSS treated, III/L. reuteri treated and IV/ L. reuteri and DSS treated. The L. reuteri treated groups were given four different strains of reuter (JCM 5869, R2LC, MM2-3 and ATCC55730) by gavage ( $10^{9}$ bacteria in $0.5 \mathrm{ml}$ saline) daily for 16 days. Colitis was induced by feeding rats $5 \%$ dextran sulphate sodium (DSS) in drinking water for 9 days. Group IV was given DSS from day 8 to day 16 of the L. reuter treatment. The rats were anesthetized with inactin $\left(120 \mathrm{mgxkg}^{-1} \mathrm{ip}\right)$ and the dual radiolabeled monoclonal antibody technique was used to quantify the expression of P-selectin in differen parts of the GI tract (Circ Res 1996;79:560-569). The colon was divided in half; proximal and distal colon. The superficial mucosa was scraped off one part of the distal colon (distal colon mucosa) leaving the deeper mucosa, submucosa and muscularis (distal colon subm+musc). Severity of colitis was assessed using clinical parameters (Lab Inves 1993;69:238-249). Results: DSS in the drinking water for 9 days induced a colitis with a disease activity index (DAI) of $2.18 \pm 0.18$. Pretreatment with $L$. reuteri resulted in substantial protection against DSS induced colonic inflammation (DAI 0.67 \pm 0.11 ). P-selectin expression increased significantly in ceacum and the entire colon after DSS treatment, but was significantly reduced with $L$. reuteri treatment during the DSS period (Table 1). Conclusion Lactobacillus reuteri protects against DSS induced colitis in rats. The protection is accompanied by a reduced P-selectin expression down to control levels. Table 1. P-selectin expression (ng P-selectin Ab/g tissue)

\begin{tabular}{|c|c|c|c|c|}
\hline & $\begin{array}{c}\text { Non } \\
\text { Treated }\end{array}$ & DSS Treated & $\begin{array}{c}\text { L. reuteri } \\
\text { Treated }\end{array}$ & $\begin{array}{c}\text { L. reuteri and DSS } \\
\text { Treated }\end{array}$ \\
\hline Ceacum & $1.66 \pm 0.29$ & $5.71 \pm 1.56^{*}$ & $1.86 \pm 0.62$ & $4.52 \pm 2.4$ \\
\hline Proximal colon & $0.77 \pm 0.15$ & $3.65 \pm 0.92^{*}$ & $0.96 \pm 0.85$ & $1.51 \pm 0.32 * \dagger$ \\
\hline Distal colon & $0.55 \pm 0.11$ & $4.58 \pm 0.68^{*}$ & $0.76 \pm 0.72$ & $1.43 \pm 0.47^{*} \dagger$ \\
\hline Distal colon mucosa & $0.41 \pm 0.18$ & $10.80 \pm 1.60^{*}$ & $0.00 \pm 0.00$ & $1.76 \pm 0.92 \dagger$ \\
\hline $\begin{array}{c}\text { Distal colon } \\
\text { subm+musc }\end{array}$ & $2.54 \pm 0.74$ & $12.99 \pm 2.04 *$ & $2.52 \pm 0.60$ & $4.39 \pm 1.07 \dagger$ \\
\hline
\end{tabular}

Values are mean \pm SEM. ${ }^{*} p<0.05$ vs non treated $\uparrow p<0.05$ vs DSS treated 


\section{M1155}

Bifidobacterium Infantis Modulates Human Intestinal Epithelial Cell Function by Attenuating the Secretion of $\mathrm{Ccl} 20$

Ann M. O'Hara, Jude Ryan, Aine Fanning, Liam O'Mahony, Fergus Shanahan

Background and aims: Enteropathogenic bacteria elicit mucosal immune responses that can lead to the secretion of pro-inflammatory mediators including CCL20. Bacterial flagellin is a key inducer of CCL20 secretion by intestinal epithelial cells. CCL20 is chemotactic for immature dendritic cells, and T and B lymphocytes. Previous studies have demonstrated that probiotic bacteria may limit intestinal inflammation by attenuating pathogen-induced interleukin (IL)-8 secretion. In this study, we investigated whether the probiotic bacterium Bifidobacterium infantis modulates CCL20 induction by flagellated pathogens. Methods: HT29 human intestinal epithelial cells were incubated with increasing doses of $B$. infantis, or with Clostridium difficile (ribotype 001), Salmonella typhimurium, or $0.5 \mathrm{mg} / \mathrm{ml}$ of its flagellin for varying times. In some studies, HT-29 cells were pre-treated with B. infantis for $2 \mathrm{~h}$ prior to infection or flagellin stimulation. CCL20 protein levels in cell culture supernatants were measured by ELISA. Results: Compared to untreated cells $(106 \pm 23 \mathrm{pg} / \mathrm{ml})$, C. difficile $(1044 \pm 233 \mathrm{pg} / \mathrm{ml})$, S. typhimurium $(6167 \pm 1700 \mathrm{pg} / \mathrm{ml})$, and flagellin $(2620 \pm 690 \mathrm{pg} / \mathrm{ml})$ stimulated a significant secretion of CCL20 by HT-29 cells. B. infantis did not augment CCL20 production $(123 \pm 35 \mathrm{pg} / \mathrm{ml})$. In contrast, B. infantis dose-dependently reduced the baseline secretion of CCL20, and at a concentration of $1 \times 10^{7} / \mathrm{ml}$ this inhibition was significant ( $22 \%$ reduction). In cells pre-treated with the probiotic bacteria, $C$. difficile-, S. typhimurium, and flagellin-induced CCL20 secretion were attenuated by $15 \%, 22 \%$, or $28 \%$, respectively. Conclusion: Flagellin and flagellated pathogens, but not B. infantis, stimulate CCL20 secretion by intestinal epithelial cells. This study is the first to demonstrate that a commensal strain can attenuate CCL20 secretion at baseline and in response to pro-inflammatory stimuli. Collectively, the study suggests that by mediating CCL20 secretion, B. infantis can exert immunomodulatory effects on intestinal epithelial cells that mediate host responses to flagellin and enteric pathogens.

\section{M1156}

Distinct Regulation of Cell Activation and Apoptosis in $\gamma / \delta$ T Cells By the Probiotic Bacterium E. Coli Nissle 1917 (EcN)

Claudia Guzy, Daniela Paclik, Uta Berndt, Daniel C. Baumgart, Bertram Wiedenmann, Axel Dignass, Andreas Sturm

BACKGROUND: $\gamma / \delta$ T cells directly recognize unprocessed or non-peptide antigens such as pathogen-associated molecular patterns (PAMPs), respond rapidly to infections, display an increased cytotoxicity and expression of TNF-a and IFN-g and localize to mucosal surfaces. PAMPs are recognized by corresponding receptors of the innate immune system, such as Toll-like receptors (TLRs). We previously demonstrated that EcN modulates T cell function through a TLR-2 dependent pathway, prompting us to investigate the effect of $\mathrm{EcN}$ on $\gamma / \delta$ T cells. METHODS: Peripheral blood T cells (PBT) from healthy volunteers were magnetically separated in $\alpha / \beta$ and $\gamma / \delta$ T cells. Cells were then stimulated with anti-CD3 and -CD28 mAb and cultured in the absence or presence of $25 \%(\mathrm{vol} / \mathrm{vol})$ sterile filtered EcN supernatants for $48 \mathrm{~h}$ after which cell activation (CD69), cell cycling (Cyclin Bl and PI), apoptosis (annexin V) and necrosis (PI) was determined by flow cytometric analysis. RESULTS: As determined by CD69 staining, cell free supernatants of $\mathrm{EcN}$ inhibited T cell activation in $\alpha / \beta$ T cells significantly more potent that in $\gamma / \delta$ T cells (reduction by $37 \pm 2.1 \%$ vs $21.0 \pm 1.8 \%$, respectively; $\mathrm{p}<0.05$ ). In addition, $\mathrm{EcN}$ inhibited cell cycle progression into the $\mathrm{S} / \mathrm{G} 2 / \mathrm{M}$ phase significant more potent in $\alpha / \beta$ (reduction by $60 \pm 4.8 \%$ ) than in $\gamma / \delta$ T cells (reduction by $32+3.1 \%)(\mathrm{p}<0.05)$. When apoptosis and necrosis was determined by annexin- $\mathrm{V}$ and propidium iodide staining, EcN did not induce apoptosis or necrosis of stimulated $\alpha / \beta$ PBT. In contrast, in $\gamma / \delta$ T cells, EcN increased the proportion of apoptotic cells by $24 \pm 4.8 \%$ and necrotic cells by $38 \pm 5.1 \%(\mathrm{p}<0.05)$ compared to $\gamma / \delta \mathrm{T}$ cells cultured in the absence of $\mathrm{EcN}$. CONCLUSION: EcN exclusively induces apoptosis and necrosis in $\gamma / \delta \mathrm{T}$ cells and thus may explain down-regulation of TNF-a and IFN-g secretion by EcN. The distinct regulation of $\alpha / \beta$ and $\gamma / \delta$ T cell activation, cycling, and apoptosis by EcN provide evidence that probiotic bacteria have the ability to directly influence the human immune system and may explain the beneficial effect of EcN in intestinal inflammation.

\section{M1157}

Survival of E. Coli Strain Nissle 1917 Given in Combination with Oral mesalamine to Healthy Volunteers

Thai Hoa Nguyen-Xuan, Gabriele Blum-Oehler, Barbara Plaschke, Joerg Hacker, Lars Joeres, Stefan Saar, Juergen Schulze, Wolfgang Kruis

Background: Mesalamine and the probiotic E. coli strain Nissle $1917(\mathrm{EcN})$ are both therapeutically effective agents for the treatment of ulcerative colitis. In the interest of even better efficacy the effects of a combined therapy are highly worth to look for. However, for some theoretical reasons, there may exist antimicrobial effects of Mesalamine against EcN. Methods: In this prospective, randomized, double-blind, placebo-controlled study healthy volunteers $(\mathrm{n}=48)$ were treated with either $\mathrm{EcN}$ ( 2 capsules Mutaflor ${ }^{\circledR}$ od; $2.5-25$ x 109 viable bacteria per capsule) plus Mesalamine (Salofalk®; $1500 \mathrm{mg}$ bid) or EcN plus placebo. After a runin period of 17 days with all volunteers taking only $\mathrm{ECN}$, volunteers detected positive for $\mathrm{ECN}$ received combination treatment for 7 days. Faecal samples were examined in 2- to 3days intervals for EcN by Multiplex-PCR assay and cultivation. Diaries were completed for tolerability of the treatment and compliance. Blood samples and urine samples were additionally taken for safety and compliance. Results: During the run-in period faecal samples with positive results of the EcN analysis increased and viable $\mathrm{ECN}$ was detected in $83 \%$ of the vols (40/48). 8 volunteers were not eligible for combination treatment as no $\mathrm{EcN}$ was detected during the run-in period. From day 1 to day 7 of combination treatment, the number of volunteers with a positive EcN detection varied between $70 \%(14 / 20)$ and $80 \%(16 / 20)$ in the Mesalamine group and between $85 \%$ (17/20) and $95 \%$ (19/20) in the placebo group. The differences between the groups were statistically not significant (normal approximation, day $3 p>0.15$, day $5 p>0.25$, day $7 p>0.076$ ). After one week of the follow up when treatments were stopped, the proportion of positive EcN detection amounted to 16/20 in the Mesalamine group and 15/20 in the placebo group and dropped down continuously up to week 12 after combination treatment. It decreased to $7 / 20$ and 4/20 in the Mesalamine and placebo group, respectively. No differences between both groups in tolerability and safety could be seen. Conclusions: EcN was detected in the intestine of $83 \%$ of the healthy subjects after the treatment with $\mathrm{EcN}$ for 17 days. Mesalamine shows no significant effects on the survival of $\mathrm{EcN}$ in the intestine of healthy subjects. Therefore a combination of both treatments seems to be possible, further studies are necessary to estimate a benefit of a combined therapy with EcN and Mesalamine.

\section{M1158}

Development of Strain-Specific Molecular Method for the Identification of Bifidobacteria Infantis 35624

Duane Charbonneau, Russ Poehner, Rebecca Donovan-Brand, Jun Xu, Diana Fawcett

Background \& Aim: Evidence from controlled clinical trials indicates that the benefits of probiotic supplementation are strain specific, and limited to specific clinical conditions. Bifidobacterium infantis 35624 is the only probiotic strain clinically proven to provide multiple symptom relief in IBS. The literature also shows that many commercial probiotic preparations lack necessary quality control, as often the probiotic preparations are nonviable, incorrectly identified or worse contain microorganisms not recognized as probiotics. Thus, there is a need for strain-specific methodologies to assure consumers of product quality. We examined a method for the strain-specific identification of B. infantis 35624. Methods: A library of 32 strains of Bifidobacteria were obtained from ATCC and used as references. Where appropriate, library strain identity was confirmed using species-specific PCR reactions. Library strain identity was further confirmed via $16 S$ gene sequencing. B. infantis 35624 was grown in pure culture, and examined in a freeze-dried powder preparation. The method of rep-PCR was examined as a means for strain differentiation. DNA was isolated using the Ultra Clean Microbial DNA Isolation Kit as modified by Diversilabs. DNA extracts were processed using the Diversilab Bacterial Bar-Code system. Results: The Diversilabs Bacterial Bar-code system was very reproducible and effective in strain-specific identification among Bifidobacteria. This assay distinguished B. infantis 35624 from the other Bifidobacteria regardless of whether the DNA was isolated from pure cultures or the freeze-dried preparation. Conclusions: Bacterial Bar-Code assay provided a molecular method for strain-specific identification of B. infantis 35624 . These results further confirm the uniqueness of this clinically effective probiotic Bifidobacterium infantis 35624

\section{M1159}

In Vitro Experimental Data Supporting the Zinc Use in the Treatment of Acute Gastroenteritis in Children

Roberto Berni Canani, Francesco Porcaro, Serena Ruotolo, Vittoria Buccigrossi, Annalisa Passariello, Maria Concetta Siani, Roberta Maiorino, Bracale Ileana, Francesco Maria Ruggeri, Silvia Lima, Giulio de Marco, Alfredo Guarino

Background: Acute diarrhea continues to carry a high morbility and mortality in the pediatric age worldwide. $\mathrm{Zn} 2$ has been proposed in the treatment and prevention of diarrheal disease, but the mechanisms and the specificity against common intestinal pathogens (i.e. Rotavirus, RV) of these effects are largely undefined. We have recently demonstrated that, through a direct interaction with the enterocytes $\mathrm{Zn} 2$ induces a pro-absorptive effect in basal condition and inhibits V. cholera toxin but not E.coli heat-stable enterotoxin secretive action (JID, 2005). However, the Zn2 effects against others mechanisms of diarrhea and RV are still unknown. Aims: To investigate whether $\mathrm{Zn} 2+$ is able: 1) to reduce intestinal ion secretion mediated by $\mathrm{Ca} 2+$ or by $\mathrm{NO}$ in an in vitro model of secretory diarrhea; 2) to inhibit the enterotoxic and cytotoxic activity of RV in human enterocytes. Methods: Ion transport was investigated by monitoring electrical parameters of a human intestinal epithelial cells monolayer (Caco-2 cell line) mounted in Ussing chambers and exposed to increasing doses of $\mathrm{Zn} 2+$ in the presence of an agonist of $\mathrm{Ca} 2+$ : carbachol (10-9M), or NO: Interferon- $\gamma$ (IFN- $\gamma, 300 \mathrm{U} / \mathrm{ml}$ ), or RV (strain SA-11, 5 PFU/cell). Intracellular concentration of Ca2+, as well as the end stable degradation products of $\mathrm{NO}$ (NO2-/NO3-) were also measured. Transepithelial electrical resistance (TEER) was monitored up to $96 \mathrm{~h}$ in Caco-2 cell monolayer exposed to RV, in the absence or in the presence of $\mathrm{Zn} 2+$. The viral load in cell culture supernatant was determined at the end of each experiment by immunocytochemistry. Results: $\mathrm{Zn} 2+$ addition to Caco-2 cells resulted in a significant reduction of ion secretion elicited by carbachol $(-68 \%)$ or by IFN- $\boldsymbol{\gamma}(-100 \%)$. Zn $2+$ was also able to significantly inhibit intracellular increase of $\mathrm{Ca} 2+$ and $\mathrm{NO}$, in response to carbachol and IFN- $\gamma$, respectively. $\mathrm{Zn} 2+$ was able to inhibit RV-induced ion secretion $(-86 \%, \mathrm{p}<0.01)$ and TEER decrease $(-70 \%, \mathrm{p}<0.01)$. These effects were associated with a significant reduction of viral load $(12000+/-120$ vs. $6000+/-490 \mathrm{pfu} / \mathrm{ml})$. Conclusions: Through a direct interaction with the enterocyte $\mathrm{Zn} 2+$ inhibits ion secretion elicited by activation of 3 out of 4 main intracellular signal transduction pathways of diarrhea. $\mathrm{Zn} 2+$ is able to reduce viral replication and to counteract RV-induced enterotoxic and cytotoxic activity in human enterocytes. These data support the $\mathrm{Zn} 2+$ use as adjunctive preventive/therapeutic strategy against diarrheal diseases induced by the majority of enteric pathogens, specially RV diarrhea.

\section{M1160}

Treatment of Travelers' Diarrhea: Rifaximin, Rifaximin Plus Loperamide Or Loperamide Alone

Herbert L. Dupont, Zhi-Dong Jiang, Jaime Belkind-Gerson, Pablo C. Okhuysen, Charles D. Ericsson, Shi Ke, David B. Huang, Margaret W. Dupont, Javier A. Adachi, F. Javier de la Cabada, Francisco M. Sandoval

Antimotility agents such as loperamide can be useful for rapid symptomatic relief of travelers diarrhea, but they do not eradicate causative bacteria. Antibacterial drugs can eradicate causative bacteria and effect a clinical cure. The combination of an antimotility agent and an antibiotic may confer better therapeutic benefit than treatment with either alone. This 
study was conducted to compare the efficacy of the combination of the nonabsorbed $(<0.4 \%)$ antibiotic rifaximin and loperamide with that of each agent administered alone. Consenting adults $(\mathrm{n}=319) \geq 18$ years of age from the U.S. studying in Mexico during the summers of 2004 and 2005 with acute diarrhea (passage of $\geq 3$ unformed stools in $24 \mathrm{~h}$ with $\geq 1 \mathrm{sign} /$ symptom of enteric infection) lasting $\leq 72$ hours were enrolled (1:1:1) in a double-blind study to receive one of three drug regimens: rifaximin $200 \mathrm{mg}$ TID for 3 days (R); loperamide $4 \mathrm{mg}$ initially followed by $2 \mathrm{mg}$ after each unformed stool, not to exceed $8 \mathrm{mg} /$ day for 48 $\mathrm{h}(\mathrm{L})$; or both drugs in the same dosage schedule (R/L). Placebos were used to blind the study. Patients completed a daily diary reviewed each day in the clinic for 5 days. The major cause of illness was enterotoxigenic Escherichia coli found in 112 patients (35\%). Over the 5-day study period, the median time from first dose of study drug until passage of last unformed stool (TLUS) was shorter with both rifaximin-containing regimens: $\mathrm{R} 23 \mathrm{~h}$; R/L $19.5 \mathrm{~h}$; than with loperamide alone: $41.5 \mathrm{~h}(P=0.01)$. The incidence of treatment failure (not achieving wellness in 5 days) was lower with the rifaximin-containing regimens: $\mathrm{R} 8$ / 107 (7.5\%); R/L 7/108 (6.5\%); vs. L 17/104 (16.3\%) ( $P=0.032)$. The median/mean numbers of unformed stools passed for the duration of illness were lower with R/L 2.5/3.99 than with either treatment alone $\mathrm{R} 4 / 6.23 ; \mathrm{L} 4 / 6.72(P=0.002 / 0.004)$. In the first $10 \mathrm{~h}$ after dosing, results for TLUS favored loperamide; however, after the first $10 \mathrm{~h}$, results favored rifaximincontaining regimens. An initial loperamide response was also observed for median number of stools during the first $0-24 \mathrm{~h}: \mathrm{R} 2 ; \mathrm{L} 1$; R/L 1 ( $P=0.002)$. 48 subjects had a TLUS of 0 h: R10; L 15; R/L 23 ( $P=0.049)$. While loperamide alone conferred rapid symptomatic improvement in travelers' diarrhea, the effect was transient, with a high rate of continuing diarrhea. Rifaximin treatment resulted in clinical cure. The combination of rifaximin and loperamide, which together confer rapid initial symptomatic improvement coupled with clinical cure, may be the optimal treatment for non-dysenteric travelers' diarrhea.

\section{M1161}

The Probiotic E. Coli Nissle 1917 Stops Acute Diarrhoea in Infants and Toddlers

Jobst R. Henker, Boris Blokhin, Jury Bolbot, Vitaly Maydannik, Lars Joeres, Corinna Wolff, Juergen Schulze

Background and Objective: In most cases acute diarrhoea will be self-limiting during the first few days after onset. For young children health risks may arise when diarrhoea lasts longer than 3 days. The purpose of the present trial was to normalize the stool frequency of infants and toddlers suffering from acute diarrhoea by administering the probiotic E. coli strain Nissle $1917(\mathrm{EcN})$ quicker than by treatment with placebo. Patients and Methods: In total, 113 children (aged 2 to 47 months) with acute diarrhoea (>3 watery or loose stools in 24 hours) were randomized to either verum (probiotic EcN suspension, $n=55$ ) or placebo $(\mathrm{n}=58)$ in a confirmative, double-blind clinical trial. Depending on the age of patients 1 $3 \mathrm{ml}$ per day of verum suspension (108 viable EcN per $\mathrm{ml}$ ) placebo were administered orally. Results: As a reason for the acute diarrhoea viral infection was stated in 31\% (35/ 113) of the cases, bacterial infection in $11.5 \%$ (13/113), and mainly unspecific infection in the rest of the children. Onset of the response to treatment (reduction of stool frequency to $\leq 3$ watery or loose stools in 24 hours over a period of at least 2 consecutive days) in the $\mathrm{EcN}$ group was more rapid (median of 2.5 days) than in the placebo group (median of 4.8 days). The time saved by EcN therapy in comparison to placebo was a significant 2.3 days $(\mathrm{p}=0.0007$, Kaplan-Meier analysis). The number of patients showing response was significantly higher $(\mathrm{p}=0.0003)$ in the $\mathrm{EcN}(94.5 \%)$ than in the placebo group $(67.2 \%)$. Conclusion: EcN showed significant superiority compared to placebo in the treatment of acute diarrhoea in infants and toddlers. This concerns the time to response as well as the response rate which were much more favorable than under self-limiting conditions. Keywords: acute diarrhoea, infants, probiotics, Escherichia coli Nissle

\section{M1162}

Efficacy of Partially Hydrolyzed Guar Gum (Benefiber) Added WHORecommended Oral Rehydration Solution in the Treatment of Cholera in Adult

Nur H. Alam, Hasan Ashraf, Shafique A. Sarker, Shahanaz Parvin, Maryam Olesen, Niklaus Gyr, Remy Meier

Introduction: In cholera, small intestinal function is affected involving stimulation of secretory process and reduction in absorption of water and electrolytes from small and large intestine in response to cholera toxin. That Leads to profuse watery diarrhea resulting in hypovolemic shock or death if immediate rehydration therapy is not instituted. The human colon has the capacity to absorb water and electrolyte and absorption is increased in the presence of short chain fatty acids (SCFAs). SCFAs also inhibit c-AMP mediated chloride secretion in the colon. Partially hydrolyzed guar gum (Benefiber) is a water-soluble fiber and if added to oral rehydration solution (ORS) undergoes fermentation in the colon liberating SCFAs. SCFAs stimulate water and sodium absorption in the colon thus reducing the severity of diarrhea. Aim: examine the effect of Benefiber added ORS in reducing the stool output and duration of diarrhea in the treatment of adult patients with cholera. Methods: In an open randomized controlled trial 130 male adult cholera patients were studied; a) 65 received ORS plus $25 \mathrm{~g}$ of Benefiber; b) 65 received ORS alone (control). All patients received doxycycline $300 \mathrm{mg}$ once immediately after randomization. The study was carried out at Dhaka Hospital of ICDDRB from August 2003 to July 2005. The study protocol was approved by the Ethics committee of the ICDDRB. Written informed consent was obtained from all the patients. Main outcome measures were stool weight and duration of diarrhea. Results: Baseline clinical characteristics were comparable between the groups. No significant differences were found in stool weight $(\mathrm{g})$ during lst $24 \mathrm{~h}$, mean $\pm \mathrm{SD}$ (Benefiber plus ORS group, $10206 \pm 5770$ vs. control group, $10231 \pm 3750, \mathrm{p}=0.977$ ); 2nd 24h (Benefiber plus ORS group, $2418 \pm 3472$ vs. control group $2172 \pm 3931 \mathrm{p}=0.708)$. Duration of diarrhea (h) after admission in hospital was also similar in both groups (Benefiber plus ORS group, 31.4 \pm 11.1 vs. control group, $32 \pm 12.5, \mathrm{p}=0.805$ ). However, in a subgroup analysis (excluding very high purging patients, stool weight 1 st $24 \mathrm{~h}>10$ kilogram), the stool weight was significantly reduced in 1 st $24 \mathrm{~h}$ in the Benefiber receiving group (Benefiber plus ORS group, $5940 \pm 2920$ vs. control group $7913 \pm 1515, p=0.001)$. Conclusion: Benefiber added to
WHO-recommended ORS is beneficial in reducing the stool weight in less severely purging adult choera patients.

\section{M1163}

Open-Label, Dose Escalation Phase I Study in Healthy Volunteers to Evaluate the Safety and Pharmacokinetics of Clostridium Difficile Toxin a Human Monoclonal Antibody (CDAl)

Claribel Taylor

Claribel P. Taylor, Sanjeev Tummala, Deborah C. Molrine, Richard J. Farrell, Anthony Lembo, Patricia L. Hibberd, Lisa Davidson, Israel Lowy, Ciaran P. Kelly Background: Clostridium difficile is the leading cause of nosocomial infectious diarrhea in industrialized countries, and $15-20 \%$ of patients develop recurrent diarrhea after treatment with metronidazole or vancomycin. Prior human studies correlate antibody responses to C. difficile toxin A and protection against symptomatic disease and recurrence. We examined the safety and pharmacokinetics of a novel neutralizing human monoclonal antibody against $C$. difficile toxin $A$ (CDAl) in healthy adult volunteers. CDAl was developed by the Massachusetts Biologic Laboratories in partnership with Medarex, Inc. Methods: Five cohorts with 6 subjects each received a single intravenous infusion of CDAl over at least 2 hours at escalating doses of $0.3,1,5,10$, and $20 \mathrm{mg} / \mathrm{kg}$. Subjects returned on days $1,2,3,7,14,28$, and 56 for safety evaluations. The immunogenicity of $\mathrm{CDAl}$ was determined by measuring serum human anti-human antibody (HAHA) titers by a bridging ELISA. Samples for pharmacokinetic (pk) analysis were obtained pre-infusion, and $0.5,1,2,4,6$, and 8 hours post-infusion, and a each study visit through day 56 . Serum CDAl antibody concentrations were measured by a standardized ELISA, and pk analysis performed using a noncompartmental model. Results: Thirty subjects were enrolled; the median age was 27.5 years, $73 \%$ were white, and 10 were men. All subjects completed the study. There were no serious adverse events (AE) assessed to be possibly, probably, or definitely related to $\mathrm{CDAl}$. Of the 48 non-serious $\mathrm{AE}$ reported during the study period, 21 were possibly related to CDAl, and included transient changes in blood pressure requiring no treatment, nasal congestion, headache, abdominal cramps, nausea, and diarrhea that occurred on days 0 to 2 post infusion. Mean CDAl concentrations increased with escalating doses; the Cmax was $6.82 \mathrm{mcg} / \mathrm{mL}, 26.3 \mathrm{mcg} / \mathrm{mL}, 109 \mathrm{mcg} / \mathrm{mL}$ $223 \mathrm{mcg} / \mathrm{mL}$, and $511 \mathrm{mcg} / \mathrm{mL}$ for the $0.3,1,5,10$ and $20 \mathrm{mg} / \mathrm{kg}$ cohorts respectively. The geometric mean values of the half-life of $\mathrm{CDAl}$ for the five cohorts ranged between 25.3 and 31.8 days, and the volume of distribution approximated that of serum. HAHA titers were not detected in any subject. Conclusion: CDAl was safe and well tolerated among healthy adults, and $\mathrm{Cmax}$ increased proportionally with escalating doses. A randomized study of CDAl in hospitalized patients with $\mathrm{C}$. difficile-associated diarrhea is now underway.

\section{M1164}

Successful Therapy of Unspecific Prolonged Diarrhoea in Infants and Toddlers with the Probiotic E. Coli Nissle 1917

Jobst R. Henker, Boris Blokhin, Jury Bolbot, Vitaly Maydannik, Lars Joeres, Corinna Wolff, Juergen Schulze

Background: Infants and toddlers with prolonged diarrhoea over several days are in danger of developing dehydration and an acute deterioration of their general state of health So far no effective causal therapy exists. Therefore in a confirmatory, randomized, double-blind clinical trial the efficacy of the probiotic bacterial strain E. coli Nissle 1917 (EcN) as compared to a placebo was tested. Patients and Methods: In total, 151 children aged 1 month to 47 months ( $\varnothing 25$ months) with unspecific prolonged diarrhoea (>3 watery or loose stools without blood in 24 hours of a diarrhoeal episode, which has been persisting for more than 4 consecutive days but not longer than 14 days) were randomized in a double-blind design to receive either the probiotic EcN suspension $(n=75)$ or placebo $(n=76)$. All children were dehydrated to a medium extent ( $5-10 \%$ loss of body weight) and received 1 to $3 \mathrm{ml}$ verum or placebo suspension orally per day depending on the age $(1 \mathrm{ml}$ suspension contained 108 viable EcN bacteria) for 21 days. At study commencement, rehydration (ORL according to $\mathrm{WHO}$ ) was performed once. Results: The number of patients showing a reduction o stool frequency to less than 3 watery or loose stools in 24 hours over a period of at least 4 consecutive days (response rate) was higher in the $\mathrm{EcN}$ group than in the placebo group already on day 7 (EcN $78.7 \%$, placebo $59.2 \%$ ) The response rate increased continuously as was measured on days $14(\mathrm{EcN} 93.3 \%$, placebo $65.8 \%)$ and $21(\mathrm{EcN} 98.7 \%$, placebo $71.1 \%)$. The two-sample test of rates following a group sequential test design showed statistically significant superiority for $\mathrm{EcN}_{\mathrm{C}}$ on both days $14(\mathrm{p}=0.0017)$ and $21(\mathrm{p}<0.0001)$. Conclusion: Prolonged diarrhoea can be successfully treated. Due to its excellent efficacy the probiotic EcN is a suitable remedy. Keywords: prolonged diarrhoea, infants, probiotic Escherichia coli Nissle 1917

\section{M1165}

Increasing Incidence of Clostridium Difficile Associated Diarrhea in African American and Hispanic Patients - Is the Use of Proton Pump Inhibitor a Culprit? Abbasi J. Akhtar, Magda A. Shaheen, Cindy Vo

Background: Clostridium difficile (C. difficile) associated diarrhea (CDAD) has been increasingly diagnosed in recent years especially in hospitalized patients. The number of prescriptions for proton pump inhibitors (PPI) has also been increased significantly during last few years. Prolonged and intense suppression of gastric acidity may adversely effect the protection against ingested microorganisms. Few studies have reported an association between CDAD and PPI use, however the results are inconclusive. Aims: to determine the relationship between CDAD and the use of antibiotics and PPI in African American and Hispanic patients. Methods: A retrospective review of medical records of 684 patients with CDAD over 9 years diagnosed by the presence of $C$ difficile toxin in the stools, was undertaken in our hospita caring for predominantly African Americans and Hispanics. We excluded 44 patients because of incomplete data, leaving 640 patients for study (age 19 -101 years). An age/sex matched 
650 patients with diarrhea (age 21-97 years) but absent C. difficile toxin in stool were used as controls. There was no significant difference between the two groups for age, gender, and race/ethnicity. Data were analyzed to assess the relationship between the CDAD and the use of antibiotics and PPI using the Chi Square test and calculate the Odds Ratio (OR) and $95 \%$ Confidence Interval (CI). Results: Of the 640 patients in CDAD group, 576 (90 $\%)$ received antibiotics during the preceding 3 months, and 32 (5\%) received chemotherapy within preceding 3 months. In comparison, of the 650 control patients with diarrhea but negative C. difficile, $540(83 \%)$ received antibiotics and $39(6 \%)$ received chemotherapy. The odds of experiencing CDAD for subjects treated with antibiotics or chemotherapy is about 2.3 times that of those who are not using antibiotics or chemotherapy $(\mathrm{OR}=2.3,95 \%$ $\mathrm{CI}=1.5-3.7)$. Of the $608 \mathrm{CDAD}$ patients receiving antibiotics or chemotherapy, 274 (45\%) also received PPI within the preceding 3 months. Of the 579 control patients who received antibiotics or chemotherapy, $169(26 \%)$ also received PPI within the preceding 3 months. The odds of experiencing CDAD for subjects treated with PPI is about 2 times that of those who are not using PPI $(\mathrm{OR}=2.0,95 \% \mathrm{CI}=1.6-2.6)$. Conclusions: PPI therapy may increase the risk of CDAD in hospitalized African American and Hispanic patients receiving antibiotics. Prospective studies are needed to determine whether PPI therapy is an independent risk factor for CDAD. In the interim, the clinician should discourage liberal use of PPI therapy in hospitalized patients needing antibiotics, unless strongly indicated.

\section{M1166}

Nitazoxanide in the Treatment of Clostridium Difficile Associated Disease Daniel M. Musher, Nancy Logan, Richard Hamill, Herbert L. Dupont, Arnold Lentnek, Arvind K. Gupta, Jean-Francois Rossignol

Clostridium difficile associated disease (CDAD) has emerged as a major nosocomial infection in the developed world, having increased in incidence and prevalence, as well as in severity. Failure of treatment with metronidazole has increasingly been documented. Concern over vancomycin-resistant organisms in the hospital environment has motivated the search for an alternative therapy. Methods. We carried out a prospective, randomized, double-blind study comparing metronidazole with nitazoxanide in treating hospitalized patients with CDAD. Inpatients from 7 hospitals in the United States were eligible for inclusion if they had diarrhea ( $\geq 3$ unformed stools in $24 \mathrm{hr}$ ), a positive EIA for C. difficile, and fever, abdominal pain or leukocytosis. Patients were excluded if they had other known causes of diarrhea, were currently taking intravenous metronidazole or had been treated with more than 2 doses of oral metronidazole or vancomycin within the preceding 7 days. Patients were randomized to receive metronidazole $250 \mathrm{mg} 4$ times daily for 10 days, nitazoxanide $500 \mathrm{mg}$ twice daily for 7 days or nitazoxanide twice daily for 10 days in double-blinded fashion. Response was evaluated after 7 days of treatment as either "well" (resolution of all symptoms of CDAD at baseline) or "failure". Secondary efficacy endpoints included sustained response at study day 31, time from first dose to passage of last unformed stool and time from first dose to resolution of symptoms. Results. After 7 days of treatment, 28 of 34 $(82.4 \%)$ patients had responded to metronidazole vs. 68 of $76(89.5 \%)$ who had received nitazoxanide (difference $=+7.1 \%, 95 \% \mathrm{CI}:-7.0 \%,+25.5 \%$ ). Thirty-one days after the beginning of treatment, sustained responses were observed in 19 of $33(57.6 \%), 25$ of 38 $(65.8 \%)$ and 26 of $35(74.3 \%)$ of patients who had received metronidazole for 10 days, nitazoxanide for 7 days and nitazoxanide for 10 days, respectively $(P=0.34$ for comparison of all groups, $\mathrm{P}=0.20$ for comparison of nitazoxanide 10 -day and metronidazole groups). The median time to last unformed stool and resolution of symptoms was 3 days for the nitazoxanide and metronidazole treatment groups. All isolates of $C$. difficile collected during the study were susceptible to metronidazole, nitazoxanide and its metabolite, tizoxanide. Median MICs $(\mu \mathrm{g} / \mathrm{mL}$ ) were 0.5 (range 0.25 to 2 ) for metronidazole, 0.5 (range 0.25 to 1 ) for both nitazoxanide and tizoxanide, and 4 (range 1 to 8 ) for vancomycin. Conclusions. Nitazoxanide is at least as effective as metronidazole in treating CDAD.

\section{M1167}

Symptoms, Course, and Complications of Abdominal Attacks in Hereditary Angioedema Due to C1-Inhibitor Deficiency

Konrad Bork, Petra Staubach, Eckardt J. Alexander, Jochen Hardt

OBJECTIVES: Recurrent abdominal attacks belong to the cardinal symptoms of hereditary angioedema (HAE) due to $\mathrm{Cl}$ inhibitor deficiency. They are characterized by crampy pain, but may include vomiting, diarrhea, and other features. Detailed clinical data about the symptoms and course of abdominal attacks have not been previously reported. METHODS: This observational study was divided into a prospective and a retrospective part. A total of 33,671 abdominal attacks were observed in 153 patients in the retrospective analysis (19732004). A subgroup of 23 patients was prospectively followed over a 2-year period (20022004). Symptoms, course, frequency of attacks, and complications were analyzed. RESULTS: The relation of mild, moderate and severe attacks was 1:1.4:5.6 in the prospective part. Our data suggests that a typical abdominal attack can be divided into 5 stages. One hundred percent of the prospectively reported abdominal attacks followed this clinical course(phase 14). Extra-abdominal symptoms preceded the abdominal symptoms (pre-phase/phase 0 ) in $70 \%$ of patients. The mean maximal pain score was 8.4 (range 1-10). Vomiting occurred in $73 \%(24,696)$ and diarrhea in $41 \%(13,682)$ of the attacks. Circulatory collapse accompanied $4.4 \%$ (1468) of the attacks, with loss of consciousness occurring in $2.2 \%$ (739). Nine patients could clearly distinguish 2 types of abdominal attacks, one with vomiting and one with diarrhea. Rare complications included tetany, hemorrhagic stools, and intussusception of the colon. In $28 \%$ (43) of the patients, recurrent abdominal attacks had started before the characteristic swelling of the skin had ever occurred. The average onset of the first abdominal pain attack occurred at $14.1+/$ - 10.1 years (range: 1 to 59 years). Most patients experienced their first abdominal pain-attack within the first two decades of life. CONCLUSIONS: This is the first systematic study of untreated abdominal attacks in HAE. Our data suggests that these attacks are more disabling and complex than previously assumed. The retrospective analysis showed that abdominal attacks usually begin during adolescence, and may predate the characteristic swelling of the skin. Based on prospective data, we propose that a typical attack can be divided into 5 distinct clinical phases, and prodromal symptoms may aid in early recognition of an impending attack.

\section{M1168}

Altered Bowel Morphology, Function and Bacterial Colonization in Patients with Chronic Heart Failure

Juergen Bauditz, Anja Sandek, Alexander Swidsinski, Sabine Buehner, Jutta Weber-Eibel, Mathias Rauchhaus, Stephan von Haehling, Tim Karhausen, Wolfram Doehner, Stefan D. Anker, Herbert Loch

Introduction: Chronic heart failure (CHF) causes edematous swelling and impairment of several organ systems. However, the effects of CHF on morphology and function of the small bowel and colon are unclear. A disturbed gut barrier function facilitates penetration of luminal bacteria, possibly contributing to the chronic inflammatory state in CHF. Aim: The aim of the study was to investigate whether chronic heart failure is associated with disturbances in intestinal morphology, function and bacterial colonization. Methods: A total of 42 patients were studied. Bowel investigations were performed in $21 \mathrm{CHF}$ patients (LVEF $31 \pm 2 \%$, NYHA class $2.3 \pm 0.1$, peak VO2 $15.6 \pm 1.0 \mathrm{~mL} / \mathrm{kg} / \mathrm{min}$, BMI $26.4 \pm 1.7 \mathrm{~kg} / \mathrm{m} 2)$, and 21 normal controls (LVEF $68 \pm 2 \%$, peak VO2 $25.2 \pm 1.4 \mathrm{~mL} / \mathrm{kg} / \mathrm{min}$, BMI $25.0 \pm 1.0$ $\mathrm{kg} / \mathrm{m} 2$ ). Bowel wall thickness was measured by high-resolution transcutaneus sonography, intestinal permeability was assessed by lactulose-mannitol test, passive carrier-mediated transport by D-xylose test. Mucosal bacterial biofilm was investigated by fluorescence in situ hybridisation (FISH) in sigmoidal biopsies. Results: Patients with CHF demonstrated edematous thickening of the entire colon and terminal ileum as compared to normal controls (terminal ileum: $1.5 \pm 0.1$ vs $0.9 \pm 0.1 \mathrm{~mm}$, ascending colon: $2.3 \pm 0.2$ vs $1.2 \pm 0.1 \mathrm{~mm}$, transverse colon: $2.2 \pm 0.2$ vs $1.2 \pm 0.1 \mathrm{~mm}$, descending colon: $2.5 \pm 0.2$ vs $1.3 \pm 0.1 \mathrm{~mm}$, sigmoid: $2.9 \pm 0.2$ vs $1.5 \pm 0.2 \mathrm{~mm}$, ( $\mathrm{p}<0.002$ for all segments). Corresponding to edematous thickening of the bowel wall, an increased intestinal permeability (lactulose/manitol ratio: $2.2 \pm 0.1 \%$ vs $1.8 \pm 0.1 \%, \mathrm{p}=0.023)$ and decreased $\mathrm{D}$-xylose absorption indicating bowel ischemia ( $25 \pm 3 \%$ vs $38 \pm 2 \%, \mathrm{p}=0.003)$ was found in CHF patients. FISH demonstrated a higher concentration of bacteria in the mucosal biofilm of CHF patients as compared to controls $(\mathrm{p}=0.046)$. Conclusion: CHF is associated with edematous thickening of the small intestine and colon, a disturbed intestinal barrier and an increased concentration of bacteria within the colonic biofilm. These observations further support the hypothesis that a disturbed gut barrier function is involved in chronic inflammation in CHF

\section{M1169}

Colonic Mucosal Lesions Associated with Long-Term and Short-Term Administration of Nonsteroidal Anti-Inflammatory Drugs

Tomoyoshi Shibuya, Toshifumi Ohkusa, Takeshi Terai, Satoshi Abe, Osamu Kobayashi, Kazuko Beppu, Naoto Sakamoto, Akihiko Kurosawa, Taro Osada, Mariko Hojo, Akihito Nagahara, Tatsuo Ogihara, Nobuhiro Sato

Background: The effects of long-term and short-term administration of non-steroidal antiinflammatory drugs (NSAIDs) on the colon have not been well characterized. There are no case-control studies in Japan on the prevalence of NSAID-induced colitis, so we investigated colonoscopic findings and the prevalence of adverse events following the long-term and short-term use of NSAIDs. Methods: The study included 2157 patients who underwent colonoscopy between June 2001 and October 2004. They were divided into the three groups; 258 patients treated for over six months with NSAIDs, 67 patients treated for under one week with NSAIDs, and 1832 patients not treated with NSAIDs as a control group. A casecontrol study was performed by medical record review for endoscopic findings, underlying disease, pre-endoscopic symptoms, category of NSAID used, and duration of use. Results: Colonic lesions were found in 16 of the 258 patients (6.2\%) with long-term use of NSAIDs. Among these, the duration of use ranged from 0.5 to 18 years (mean 4.4 years). The most frequently used NSAIDs were low-dose aspirin by nine patients (56\%), followed by loxoprofen, and diclofenac. The most frequent features of colitis were longitudinal linear ulcers and redness, seen in five patients (31\%). The rectum (6 patients), and either sigmoid colon ( 3 patients), descending colon ( 3 patients) or both were commonly involved. In the short-term NSAID group, 4 of the 67 patients $(6.0 \%)$ had colonic lesions. Three of the patients were taking a cold complex preparation, and one patient was taking loxoprofen. Of the 4 patients, two had rectal ulcers, one had an unshaped ulcer on the ileocecal valve, and one had redness in the descending to sigmoid colon. In the control group, 55 of the 1832 patients (2.8\%) had colonic ulcers or colitis. The group with long-term use of NSAIDs had a significantly higher incidence of colonic lesions than the control group with the chisquare test $(\mathrm{p}=0.0077)$. Conclusion: The prevalence of colonic lesions in long-term and short-term NSAID users was found to be about $6 \%$. It was shown that especially long-term usage of NSAIDs had a statistically significant increased incidence of colonic ulcers or colitis.

\section{M1170}

Delayed Gastric Emptying Accelerate Bacterial Overgrowth in Both the Stomach and the Intestine

Yoshihisa Urita, Susumu Ishihara, Kenichiro Arai, Jun Kondo, Tatsuo Akimoto, Hiroto Kato, Noriko Hara, Hideyuki Koshino, Yoshiko Honda, Yoko Nagai, Kazushige Nakanishi, Nagato Shimada, Motonobu Sugimoto, Kazumasa Miki

Background: Bacteria represent the sole source of gut hydrogen (H2), making this particular gas attractive for the identification of bacterial overgrowth states. Previous studies showed the close relationship between gastric emptying and intragastric fermentation, reflecting bacterial overgrowth in the stomach, in diabetic patients. Several neuroendocrine peptides, such as peptide YY (PYY) and glucagon-like peptide (GLP) l, have been proposed as ileocolic mediators of upper digestive tract inhibition, and fermentation in the upper digestive tract may affect the gastric motility. The aim of this study was to evaluate a relationship between gastric emptying and fermentation in the digestive tract. Patients and Methods: Studies were performed in 25 patients (mean age 63.1 years, male/female $=6 / 19$ ) undergoing esophagogastroscopy. At the time of endoscopic examination, we intubated the stomach and the duodenum without inflation by air, and $20 \mathrm{ml}$ of intraluminal gas samples of both sites was collected through the biopsy channel. Intraluminal hydrogen concentrations were measured by gaschromatography. Within a week, 13C-acetate breath test was performed in all patients 
to measure the gastric emptying. Breath tests were performed in the sitting position after an overnight fast. The patients received a liquid test meal (OKUNOS-A) containing 100mg of 13C-acetate in the sitting position. Breath samples were collected at baseline and at 5 , $10,15,20,30,40,50,60,75,90,105,120,135,150,165,180,210,240$ min after ingestion of a test meal. $13 \mathrm{C}$ was measured as the $13 \mathrm{CO} 2 / 12 \mathrm{CO} 2$ isotope ratio and was expressed as delta per mil (). The results of $13 \mathrm{C}$-acetate breath test, gastric emptying, were expressed as the time of peak excretion (Tmax). Delayed gastric emptying was defined when Tmax was more than 90 min. Results: Twelve patients had Tmax more than $90 \mathrm{~min}$ and their mean values of intragastric, intraduodenal, and breath $\mathrm{H} 2$ gas were $12.3+/-16.7(0$ 49), $58.0+/-141.5(0-502)$, and $11.1+/-21.9 \mathrm{ppm}(0-79)$, respectively. Those in patients with normal gastric emptying were $5.6+/-6.6(0-21), 4.5+/-3.9(0-12)$, and $0.8+/-0.9$ ppm (0-3), respectively. Patients with delayed gastric emptying had higher intraluminal and breath $\mathrm{H} 2$ concentrations, possibly reflecting bacterial overgrowth not only in the stomach but in the intstinal tract. Conclusions: In conclusion, there was a close correlation between the bacterial overgrowth and delayed gastric emptying.

\section{M1171}

Long-Term Efficacy of Lubiprostone for the Treatment of Chronic Constipation John F. Johanson, Raymond Panas, P. Christopher Holland, Ryuji Ueno

Constipation is a common and bothersome gastrointestinal condition. Current treatment options are often ineffective. Lubiprostone is a novel type- 2 chloride channel (ClC-2) activator (AJP Cell Physiol 287:C1173-C1183, 2004) that has been shown to be efficacious and well tolerated by subjects with chronic constipation in a number of well-controlled clinical trials of 3-4 weeks duration (Gastroenterology 122:A315, 2002; Gastroenterology 124:A38, 2003; Am J Gastroenterol 100:S324, S328 and S329, 2005). We describe results from three openlabel, long-term ( 6 to 12 months) trials where efficacy assessments were periodically captured (approximately every 6 weeks). Methods: Study 1 was a 24-week open-label extension to a 4-week double-blind pivotal trial that enrolled 308 subjects. Study 2 was a 48 -week openlabel trial in 248 subjects, 87 of whom were examined in a 7-week randomized withdrawal study period prior to the open-label portion. Study 3 was a 48-week open-label trial in 324 treatment-naive subjects. In each of the three trials, subjects assessed treatment effectiveness, their constipation severity, and abdominal symptoms of bloating and discomfort using a 5point scale at each study visit. Results: Improvements in assessments of constipation severity, abdominal bloating, and abdominal discomfort were statistically significant in all studies and at all visits $(p<0.0001)$. Across all three studies, constipation severity was improved by an average of 1.28 points at Weeks $4-6(\mathrm{~N}=828), 1.47$ points at Week $24(\mathrm{~N}=512), 1.38$ points at Week 48 (Study 2 and 3 only, $\mathrm{N}=281$ ), and 1.15 points for the last on-drug measurement $(\mathrm{N}=866)$. Abdominal bloating was improved by an average of 0.89 points at Weeks 4-6 $(\mathrm{N}=829), 0.98$ points at Week $24(\mathrm{~N}=512), 1.00$ points at Week 48 (Study 2 and 3 only, $\mathrm{N}=282$ ), and 0.79 points for the last on-drug measurement ( $\mathrm{N}=867)$. Abdominal discomfort was improved by an average of 0.74 points at Week $1(\mathrm{~N}=619), 0.91$ points at Week $24(\mathrm{~N}=512), 0.87$ points at Week $48(\mathrm{~N}=282)$, and 0.72 points for the last on-drug measurement $(\mathrm{N}=867)$. Conclusion: These results demonstrate that the effects shown in the short-term, double-blind trials continue for at least 24 to 48 weeks, as observed in these three long-term trials. Lubiprostone provides significant relief for a variety of constipation symptoms and efficacy is potent and sustained for up to 48 weeks of treatment.

\section{M1172}

Is Visceral Obesity Really a Risk Factor for Colorectal Neoplasm? Tae-Hoon Oh, Jeong-Sik Byeon, Seung-Jae Myung, Suk-Kyun Yang, Ja Young Kim, KwiSook Choi, Hyung Joon Lee, Jun-Won Chung, Kee Don Choi, Gin H. Lee, Hwoon-Yong Jung, Jin-Ho Kim, Jae H. Byun

Backgrounds/Aims: Although several risk factors for colorectal neoplasm (CRN) have been suggested, there has been controversy about obesity as a risk factor for CRN because of the methodological difference in the measurement of obesity between studies. We conducted a prospective cross sectional survey to evaluate obesity as a risk factor for CRN. We used abdominopelvic CT scan for more objective analysis of visceral and parietal obesity. Method: We consecutively enrolled asymptomatic average-risk subjects who underwent both colonoscopy and abdominopelvic CT scan for the routine health evaluation. The amount of visceral and parietal fat was calculated from abdominopelvic CT scan. Other indices of obesity such as body mass index (BMI) and waist circumference were also evaluated. Various blood markers for metabolic syndrome such as fasting blood glucose, $\mathrm{Hb} \mathrm{Alc}$, and serum lipid profile, and family history of CRN were investigated. The presence or absence of CRN was analyzed in relation to obesity and metabolic markers. Results: 200 subjects were enrolled. Of these, 53 subjects (26.5\%) had CRN. Male to female ratio was not different between those with CRN and those without ( $3.0 \cdot 1.0$ vs. $1.7: 1.0, \mathrm{p}=0.127$ ). Mean age was significantly higher in those with CRN than those without $(55.0 \pm 7.7$ vs. $49.5 \pm 8.3$ years, $\mathrm{p}<0.001)$. Visceral fat amount was significantly higher in those with CRN than those without $(124.8 \pm 49.8$ vs. $99.7 \pm 51.6 \mathrm{~cm} 2, \mathrm{p}=0.003)$. Waist circumference was also higher in those with CRN $(86.5 \pm 7.7$ vs. $83.1 \pm 9.0 \mathrm{~cm}, \mathrm{p}=0.018$ ), but BMI was not different between those with $\mathrm{CRN}$ and those without. Out of various metabolic markers, only fasting blood glucose was higher in those with CRN than those without $(103.9 \pm 26.9$ vs. $96.5 \pm 14.6 \mathrm{mg} / \mathrm{dL}, \mathrm{p}=0.021)$. In multivariate analysis for the risk of CRN with an adjustment of age, sex, and family history of CRN, odds ratio of those with visceral fat over $136.61 \mathrm{~cm} 2$ (quartile 4) to those with visceral fat under $67.23 \mathrm{~cm} 2$ (quartile 1) was 4.6 (95\% confidence interval 1.1-18.4). Visceral fat amount was the only independent risk factor for CRN. Waist circumference and various metabolic markers were not risk factors for CRN in multivariate analysis. In cases with CRN, visceral fat amount was not associated with the location, number, and degree of villous component or dsyplasia. However, visceral fat amount tended to increase as the size of CRN increased $(r=0.29, p=0.03)$. Conclusion: Our data shows that visceral fat is an independent risk factor for the presence of CRN and it may influence the progression of small CRN to large one. Further large scale study is necessary to clarify the role of visceral fat in the development of CRN.

\section{M1173}

Helicobacter Pylori Infection Is Associated with An Increased Risk of Coloni Adenomatous Polyp

Takashi Ando, Shigeto Mizuno, Yoshinori Morita, Toshio Inui, Akihiro Asakawa, Naohiko Ueno, Takeshi Ishikawa, Yasuyuki Nagao, Nobuyuki Seto, Akiko Komatsu, Tuguhiro Matsumoto, Haruki Kato, Toshikazu Yoshikawa, Akio Inui

Compelling evidence from epidemiological, histopathological and animal studies has linked Helicobacter pylori infection to the subsequent development of gastric cancer.2 Nevertheless, the currently available data regarding a possible link between Helicobacter pylori seropositivity and colorectal cancer risk are limited and inconclusive. We sought to determine whether Helicobacter pylori is associated with colon neoplasia in Japanese population. We examined 332 patients who underwent routine highresolution total colonoscopy and serologic testing for IgG antibodies agonist Helicobacter pylori. Subjects who received cyclooxygenase-2 inhibitors or previous eradication therapy and those with borderline titer levels were excluded from data analysis $(n=27)$. Seronegative control subjects were from the same study population to maximize the representativeness. There were no significant differences in age and sex between the two patient groups. A significant increase in the incidence of adenomatous polyps $(\mathrm{p}<0.0001)$ and decrease in normal colonoscopic findings $(\mathrm{p}<0.0005)$ were observed in seropositive patients than those seronegative. This study indicates an etiological link of Helicobacter pylori infection to colorectal neoplasia and the need of routine colonoscopy in seropositive patients.

Presence or absence of Helicobacter pylori and colonoscopic findings

\begin{tabular}{|c|c|c|c|}
\hline & Helicobacter Positive & Helicobacter Negative & \\
\hline Age-yr & $58.5 \pm 1.18$ & $62.7 \pm 1.07$ & \\
\hline Sex Male & 126 & 48 & \\
\hline Sex Female & 84 & 47 & \\
\hline Endoscopic diagnosis-no & 210 & 95 & \\
\hline Normal & $67(31.9 \%)$ & $52(54.7 \%)$ & $\mathrm{p}<0.0005$ \\
\hline Hyperplastic polyp & $26(12.3 \%)$ & $18(18.9 \%)$ & \\
\hline Tubular adenoma & $89(42.4 \%)$ & $18(18.9 \%)$ & $\mathrm{p}<0.0001$ \\
\hline Yubulovillous adenoma & $2(1 \%)$ & $0(0 \%)$ & \\
\hline Villous adenoma & $2(1 \%)$ & $0(0 \%)$ & \\
\hline Cancer & $24(11.4 \%)$ & $7(7.4 \%)$ & \\
\hline
\end{tabular}

Numbers in parentheses are percentages. Plus values are mean plus and minus SEM. Analysi of variance followed by Bonferronit test were used to assess the differences among groups $\mathrm{P}$ was considerd to be statistically significant.

\section{M1174}

A Dose-Finding Study of Rifaximin in the Treatment of Small Bowel Bacterial Overgrowth in Patients with Irritable Bowel Syndrome Ernesto Cristiano Lauritano, Andrea Lupascu, Emidio Scarpellini, Maurizio Gabrielli, Alessia Cazzato, Mario Pescatori, Angelo Santoliquido, Roberto Flore, Paolo Tondi, Giovan Battista Gasbarrini, Antonio Gasbarrini

Introduction: Small bacterial overgrowth (SIBO) is a condition characterized by abnormally high bacterial population level in the small intestine, exceeding 105 organisms $/ \mathrm{ml}$. SIBO often requires lifelong management based on sporadic or periodic oral antibiotic therapy Up to date, given the diversity of intestinal bacterial populations involved and the small number of clinical trials, the choice of antibiotic and the duration of the therapy are still uncertain. Recent findings suggest that rifaximin, a broad spectrum non-assorbable derivate of rifamycin, is effective in the treatment of SIBO. The aim of the present study was to investigate the efficacy and tolerability of two different doses of rifaximin in the treatmen of SIBO. Patients and methods: We enrolled 60 pts affected by SIBO among our IBS population. The diagnosis of IBS was based on the Roma II criteria. The presence of SIBO was based on the occurrence of a peak of $\mathrm{H} 2$ values more than $10 \mathrm{ppm}$ above the basal value after $50 \mathrm{gr}$ glucose ingestion. All pts were randomised in two 7 days treatment: rifaximin $1200 \mathrm{mg} /$ die group 1 and rifaximin $1600 \mathrm{mg} /$ die group 2. Glucose breath test was reassessed 1 month after the end of therapy. Results: Two drop-outs were observed in group 2 due to taste disturbance whereas no drop-outs were observed in group 1. Glucose breath test normalization rate was $60 \%$ in group 1 in both intention-to-treat and per protocol analyses whereas glucose breath test normalization was achieved in 63 and $68 \%$ in group 2 in intention-to-treat and per protocol analysis, respectively. Differences between groups were not statistically significant. Side-effects were significantly lower in group 1 than group 2. Conclusions: Seven days- $1200 \mathrm{mg}$ rifaximin therapy is a good option in terms of efficacy and tolerability for SIBO treatment. Higher dosage of rifaximin doesn't increase the therapeutic efficacy in SIBO eradication and is associated with major intensity of side effects.

\section{M1175}

Improved Outcomes and Potential Avoidance of Transplantation with Multidisciplinary Intestinal Rehabilitation for Intestinal Failure - Intent to Treat Analysis

Kishore Iyer, Timothy Sentongo, Valeria Cohran, Lisa Keys, Kim Kazmerski, Margaret Richard

Background Intestinal transplantation is the standard of care for patients with irreversible intestinal failure and life-threatening complications of parenteral nutrition (PN). A very high mortality rate on the national waiting list $(25-30 \%)$ has led us to search for rationa management strategies. We report our preliminary experience with a multi-disciplinary 
approach to managing pediatric intestinal failure in a transplant program. Methods Retrospective review identified all patients who were managed within the program over a 3-year period. The primary end-point was patient survival; secondary endpoints were impact on PN utilization and impact on liver dysfunction (PNALD). Even in the presence of established transplant indications at referral, transplantation was reserved for patients who fail all conservative strategies. Where PN dependence and PN failure appear irreversible, patients are listed for intestinal transplantation, often in parallel with application of conservative strategies. Results of 64 patients managed with this approach, 60 patients are alive for an overall patient survival of $93.8 \%$. Two of the 4 deaths occurred in patients who were listed elsewhere for combined liver-intestinal transplantation (LIT) and transferred to our care with worsening advanced PNALD. In both cases, death occurred within weeks from sepsis without the opportunity for transplant. One death occurred after isolated intestinal transplant (SBT) and 1 death occurred from complications of lung-disease of prematurity. 49 of the $60(81.7 \%)$ survivors achieved full enteral autonomy from PN and $8(13.3 \%)$ achieved partial autonomy. Of 28 patients who met criteria for PNALD, 25 patients are alive. 24 of the 25 patients have normalized biochemical tests of liver dysfunction. 2 patients underwent SBT for PNALD, and 1 patient underwent isolated liver transplantation after achieving enteral autonomy from PN. Of 6 SBT performed since September 2004, 5 patients are alive, 4 with functioning grafts. Discussion Our preliminary results suggest that multidisciplinary intestinal rehabilitation may help address the significant mortality on the waitlist in infants awaiting combined LIT and allow overall improved patient survival. Majority of these patients achieve enteral autonomy from PN even in the setting of complicated intestinal failure and can achieve biochemical liver recovery often without need for transplant. Two of the 4 deaths in our series emphasize the need for rapid access to transplantation for success with this approach.

\section{M1176}

Racial Differences in the Distribution of Initial and Recurrent Colonic Adenomatous Polyps Among Veterans

Nadeem Ullah, Sunita Duttala, Martin Tobi, Adhip Majumdar

OBJECTIVES: Previous small studies have suggested that African Americans have predominantly proximal distribution of colonic adenomas as compared to general population but whether this distribution persists on follow up after polypectomy is not known. The purpose of current study was to determine the differences in the distribution of colonic adenomas in African American (AA) Veterans as compared to Caucasian Veterans and to see if this difference persists on follow up colonoscopy. METHODS: We retrospectively analyzed the data of 151 patients who were found to have at least one adenoma on clinically indicated colonoscopy and had follow up colonoscopies. Based on polyps distribution the patients were divided into three groups; 1- Only left-sided polyp, if the patient had all polyps distal to splenic flexure. 2- Only right-sided polyp, if the patient had all the polyps proximal to splenic flexure. 3- Right and left-sided polyps, if the patient had synchronous polyps proximal and distal to splenic flexure. Eighty AA subjects were matched with 71 Caucasians for age, BMI, nutritional assessment, serum B12, folate and calcium levels. RESULTS: All 151 subjects except 8 were male. On initial colonoscopy AA had statistically significant increased incidence of only right sided adenomas as compared to Caucasians $(\mathrm{p}<0.002)$ as shown in the table. On follow up (average 3 years) although more AA patients showed recurrence of adenomas ( $70 \%$ AA vs $55 \%$ C) but this difference was not statistically significant. The distribution of adenomas on follow up colonoscopy was not statistically significant different between these two groups (table). CONCLUSION: AA Veterans have increased incidence of only right sided colonic adenomas but once these adenomas are removed the distribution of recurrence of colonic adenomas assumes the same distribution as in Caucasians. Therefore, the surveillance strategies should be tailored accordingly.

Distribution of Colonic Adenomatous Polyps on Initial and Follow up Colonoscopy

\begin{tabular}{|c|c|c|c|}
\hline Parameter & $\begin{array}{c}\text { \% of Patients with } \\
\text { Left-sided Adenomas }\end{array}$ & $\begin{array}{c}\text { \% of Patients with } \\
\text { Right-sided Adenomas }\end{array}$ & $\begin{array}{c}\text { \% of Patients with Left \& } \\
\text { Right-sided Adenomas }\end{array}$ \\
\hline $\begin{array}{c}\text { Initial AA } \\
\text { Caucasian }\end{array}$ & 4356 & $30^{*} 10$ & 2734 \\
\hline $\begin{array}{c}\text { Follow up AA } \\
\text { Caucasian }\end{array}$ & 2634 & 3242 & 4224 \\
\hline
\end{tabular}

AA --> African American, ${ }^{*}$-- $>$ p $<0.002$, Initial --> Initial colonoscopy, Follow up --> Follow up colonoscopy.

\section{M1177}

Same Day Bi-Directional Endoscopy in Patients with Functional Gastrointestinal Disorders

Ivan Bottoli, Michael Shetzline, Nora Mattek, Jennifer Holub, David Lieberman, Glenn Eisen

Background: It is apparent that numerous patients with functional gastrointestinal disorders undergo endoscopic evaluation. The utility of these exams remains in question as patients tend to be younger and the yield of significant findings may be less than for patients with other indications such as bleeding and weight loss. Same day bi-directional endoscopy (panendoscopy) may be performed in this patient group but the overall yield is unknown. Our group utilized the CORI national endoscopic database to assess this question. Methods: All patients undergoing same day bi-directional endoscopy between 2000-2004 were included in the dataset. Demographics, indications and significant findings were assessed. Functional GI disorders for EGD were defined as dyspepsia and/or abdominal pain/bloating excluding reflux symptoms, dysphagia and Barrett's surveillance. For colonoscopy, functional GI disorders were defined by abdominal pain/bloating, diarrhea, constipation and or change in bowel habits excluding surveillance of or established IBDm weight loss or bleeding (anemia, iron deficiency, positive FOBT, hematochezia or melena). Significant findings for EGD were any of the following: suspected malignancy, AVM, ulcer, Barrett's, stricture; for colonoscopy: suspected malignancy, polyp $>9 \mathrm{~mm}$, or AVM. Results: During the study period, 706,775 unique patients had EGD and/or colonoscopy. 66,265 (9.4\%) patients underwent same day bi-directional endoscopy. 23,360 (33.7\%) of these patients had a functional GI disorder. Of these $33.9 \%$ had a functional disorder as the indication for the EGD only, $41.7 \%$ for the colonoscopy only and $24.4 \%$ for both procedures. Patients who had functional indications for both procedures tended to be younger (mean age 55.9) and more often female $(68.3 \%)(\mathrm{p}<.0001)$ than the other 2 groups and the procedure was more likely to be performed in a community/HMO setting. Conclusions: A significant number of patients with functional GI disorders undergo same day bi-directional endoscopy according to our national endoscopic database. The yield of significant findings is very low. Patients with functional indications for both procedures have the lowest rate of findings.

\begin{tabular}{|c|c|c|c|c|}
\hline & $\begin{array}{c}\text { Functional GI } \\
\text { disorder on EGD } \\
\text { only N=7029 }\end{array}$ & $\begin{array}{c}\text { Functional GI } \\
\text { Disorder on COL only } \\
\mathrm{N}=10071\end{array}$ & $\begin{array}{c}\text { Functional GI Disorder } \\
\text { on both procedures } \\
\mathrm{N}=6015\end{array}$ & p-value \\
\hline EGD Findings & $830(10.94 \%)$ & $1654(17.75 \%)$ & $437(8.0 \%)$ & $<0.0001$ \\
\hline $\begin{array}{c}\text { Any significant } \\
\text { EGD finding }\end{array}$ & $619(8.1 \%)$ & $510(5.47 \%)$ & $245(4.49 \%)$ & $<0.0001$ \\
\hline COL Findings & $103(1.36 \%)$ & $104(1.12 \%)$ & $30(0.55 \%)$ & $<0.0001$ \\
\hline $\begin{array}{c}\text { Any significant } \\
\text { COL finding }\end{array}$ & & & & \\
\hline EGD \& COL & & & & \\
\hline
\end{tabular}

\section{M1178}

Systematic Evaluation of the Causes of Chronic Watery Diarrhea with Functional Characteristics

Fernando Fernandez-Banares, Maria Esteve, Montse Alsina, Carme Farre, Clarisa Gonzalez, Montse Buxeda, Montse Forne, Merce Rosinach, Jorge C. Espinos, Antonio Salas, Josep Maria Viver

Causes of chronic watery diarrhea are multiple. A diagnosis of either functional diarrhea or diarrhea-predominant IBS is performed when routine complementary explorations are normal and there are not alarm symptoms/signs. However, the same clinical picture may be caused by several entities which should be excluded by diagnostic testing. Although tests to rule out bile acid malabsorption (BAM) and sugar malabsorption (SM) are recommended, they are not routinely performed. Besides, it has been described that some of these patients have mild forms of gluten-sensitive enteropathy (GSE). Aim: To assess prospectively the presence of GSE, BAM and SM in consecutive patients with chronic watery diarrhea of origin previously unexplained fulfilling Rome II criteria of functional disease. Methods: 62 patients $(52.2 \pm 2$ yrs; $15 \mathrm{M}, 47 \mathrm{~W}$ ) with chronic watery diarrhea (24-h stool weight>200 g/d), and with normal physical exam and routine blood analysis (including $\mathrm{C}$ reactive protein, T4/TSH, celiac serology, IgA), negative stool ova \& parasites, and normal full colonoscopy with multiple biopsies were included. Median previous duration of diarrhea was $24 \mathrm{~m}$ (range, 7-72). In all cases the following tests were performed: 1- HLA-DQ2/DQ8 genotyping; 2- In case of DQ2/DQ8+, endoscopic biopsies from distal duodenum were obtained. The number of intraepithelial lymphocytes $(\mathrm{N}<25 \%)$ and villous architecture were assessed, and intestinal damage was classified according Marsh; 3- SeHCAT abdominal retention test $(\mathrm{N}>10 \%$ at 7th day); 4- Small bowel follow-through; and 5- Hydrogen breath test (lactose, fructose+sorbitol) if previous tests were normal. Gluten- or sugar-free diet, or cholestyramine were administered according to results. Functional disease was diagnosed if all tests performed were normal or if there was no response to specific therapy after a follow-up of 6-12 m Results: 12 GSE (11 Marsh I, 1 Marsh II), 37 BAM and 22 SM were found. BAM was considered to be the cause of diarrhea in $29(46.8 \%)$ patients, SM in $10(16.1 \%)$, GSE in $9(14.5 \%)$, and both BAM and SM in 2. Twelve (19.3\%) patients were diagnosed as functional diarrhea/IBS. Diarrhea stopped in the 50 patients with an specific diagnosis, decreasing the daily stool number from $5.4 \pm 0.3$ to $1.5 \pm 0.1(\mathrm{p}<0.0005)$, without relapse after follow-up (12 $\mathrm{m}$ in $79 \%$; $>6 \mathrm{~m}$ in $100 \%$ ). Conclusions: The diagnosis of functional disease in patients with chronic watery diarrhea should be performed with caution since in most cases there is an organic cause which justifies diarrhea. Mild GSE is an entity to be considered in the diagnostic work-up of chronic watery diarrhea. Sponsorized by Fundació Banc Sabadell.

\section{M1179}

Primary Bile Acid Malabsorption - A New Insight Into Prevalence Gautam Mehta, Mathena Vinayaga-Pavan, Neil Galletly, Benedict Ancock, Geoff V. Smith, Devinder Bansi, Andrew V. Thillainayagam

Background and Aims: Bile acid malabsorption (BAM) is increasingly recognised as a cause of chronic diarrhoea. Malabsorption of bile acids can occur secondary to gastrointestinal disease or due to a primary defect in the distal ileal sodium-dependent bile acid transporter. The prevalence of primary BAM is unclear. The aim of this study was to clearly establish the aetiology and prevalence of BAM in chronic diarrhoea. Methods: A retrospective review was conducted of 149 patients with chronic diarrhoea, investigated for BAM using SeHCAT imaging between 2001-2005. A positive result was defined as $<15 \%$ retention of SeHCAT at 7 days. All patients had previously undergone assessment of haemoglobin, C-reactive protein, colonoscopy, colon histology, barium small bowel imaging and either duodenal histology or anti-tissue transglutaminase antibodies. Patients with BAM were classified as (i) BAM1 - previous ileal resection, ileocolonic resection or ileal disease, (ii) BAM2 - idiopathic BAM, or (iii) BAM3 - previous cholecystectomy, peptic ulcer surgery, irritable bowel syndrome, coeliac disease, chronic pancreatitis, diabetes mellitus or bacterial overgrowth. Results: The mean age of the study population was 50 years ( 57 males, 92 females). Incomplete previous investigations excluded 23 patients. There was no significant difference in age between the groups with normal and abnormal SeHCAT retention (49.3yrs vs 53.0yrs) Normal SeHCAT retention was found in 78 patients. Impaired SeHCAT retention was found in 48 patients (38\%). Of these, 13 patients $(27 \%)$ were classified as BAMl, 19 patients 
(40\%) as BAM2, and 16 patients (33\%) as BAM3. Patients with primary BAM were significantly younger than those with secondary BAM (47.0yrs vs $57.0 \mathrm{yrs}, \mathrm{p}=0.02$ ). Discussion: This cohort is more comprehensive than any in the current published literature. Our study is in accordance with the largest study to date by Wildt and colleagues ${ }^{1}$. They diagnosed primary BAM in $33 \%$ of patients with abnormal SeHCAT retention, but did not exclude enteropathy assiduously by ileal imaging and coeliac disease testing in every patient. The results of our study validate and enhance previous work with more stringent exclusion criteria. Idiopathic BAM accounts for a considerably greater proportion of cases with abnormal bile acid absorption than previously recognised. Therefore this condition must be actively sought in patients with chronic diarrhoea, particularly those of a younger age. 1. Wildt S, Nørby Rasmussen S, Lysgard Madsen J, Rumessen JJ. Bile acid malabsorption in patients with chronic diarrhoea: clinical value of SeHCAT test. Scand J Gastroenterol 2003:38:826-830.

\section{M1180}

Serum 7alpha -Hydroxycholestenone in Patients with Idiopathic Bile Salt Malabsorption - A Detailed Comparison with Conventional SeHCAT Testing Simon Campbell, Nischay Chandra, Gladman Lisa, Mee Anthony

INTRODUCTION: Bile salt malabsorption(BAM) is a common cause of chronic diarrhoea Causes include Crohn's disease, SB resection, bacterial overgrowth, post infective, post cholecystectomy and idiopathic(IBAM). A diagnosis of IBAM is made by measuring bile salt turnover/excretion, usually by SeHCAT test. This measures \% retention of $75 \mathrm{Se}$ labelled bile acid 7 days after oral admin.; $<19 \%$ uptake indicates BAM. Another Ix is measuring serum 7alpha-hydroxycholestenone (7HC), a bile acid metabolite. This is raised in BAM pts(normal $<35 \mathrm{ng} / \mathrm{ml}$ ). $7 \mathrm{HC}$ is a quicker Ix than SeHCAT and avoids radiation exposure. Serum 7-HC is not widely used because the standard material was not commercially available until recently There are few data regarding cost-effectiveness of $7-\mathrm{HC}$, its correlation with symptoms in IBAM or sensitivity compared to SeHCAT. AIMS \& METHODS: To compare serum 7-HC in pts with IBAM, compare sensitivity and cost with SeHCAT. We collected data from 64 pts who had SeHCAT test. Pts with SeHCAT of $<19 \%$ were included and pts with other causes of BAM excluded. 40 pts had a diagnosis of IBAM and 11 healthy controls were included - all had serum 7HC measured. Other data included demographics, all clinical Ix, stool freq. pre/post Rx. Comparison was by log rank analysis and MWT. Statistical significance was taken as $\mathrm{p}=0.05$. RESULTS: $40 \mathrm{pts}(22 \mathrm{M}: 18 \mathrm{~F}$ median age 53 yrs, range $25-75)$ had IBSM, with a SeHCAT $<19 \%$ and normal clinical Ix (median SeHCAT $7.3 \%$, range0.3$17.6 \%$ ). All pts had serum $7 \mathrm{HC}$ measured. Median bowel freq. pre-Rx was $5 \mathrm{x} /$ day (range312 ) - all pts improved post $\mathrm{Rx}$ (median $3 \mathrm{x} /$ day, rangel-6). 11 healthy asymptomatic controls (6M:5F, median age 53 yrs, range26-60) also had serum $7 \mathrm{HC}$ measured. There was a strong correlation between $7 \mathrm{HC}$ vs. SeHCAT results $(\mathrm{r} 2=0.37, \mathrm{p}<0.0001)$ and $7 \mathrm{HC}$ vs. stool freq $(\mathrm{r} 2=0.33, \mathrm{P}<0.001) .7 \mathrm{HC}$ levels in IBAM pts (median $67.0 \mathrm{ng} / \mathrm{ml}$, range $14-280)$ were higher than controls (median 27.0ng/ml, range 10-40) and was significant(MWT, $\mathrm{p}<0.001$ ). Median cost of Ix each pt was $£ 587$ (rangef347-£1170). The cost of $7 \mathrm{HC}(£ 60 /$ test) was less than SeHCAT( $£ 257 /$ test $)$ comparing well with other Ix(eg EMA- $f 45 /$ test $)$. CONCLUSION: 7HC is an accurate method of diagnosing BAM in pts with IBAM ( $93 \%$ sensitivity). In IBAM serum $7 \mathrm{HC}$ correlated with degree of BAM and symptoms. We speculate if $7 \mathrm{HC}$ was widely used the convenience of the Ix would lead to earlier diagnosis and avoid other invasive Ixsaving more money. Serum 7HC is cheaper, safer and should replace SeHCAT.

\section{M1181}

The ${ }^{13} \mathrm{C}$-Xylose Breath Test Is Inferior to the $\mathrm{H}_{2}$-Glucose Breath Test for the Diagnosis of Intestinal Bacterial Overgrowth in Children with Cystic Fibrosis Arne R. Schneider, Stefanie Klueber, Hans-Georg Posselt, Benjamin Funk, Lydia Murzynski, Wolfgang F. Caspary, Juergen Stein

Introduction: The ${ }^{13} \mathrm{C}$-xylose breath test has been proposed as an alternative to the $\mathrm{H}_{2}$ glucose breath test for the diagnosis of intestinal bacterial overgrowth. However, data on the accuracy of the xylose breath test with ${ }^{13} \mathrm{C}$ labeled substrates are lacking. Since recent studies reported a high prevalence of bacterial overgrowth in cystic fibrosis $(\mathrm{CF})$, we aimed to compare both breath tests in this group of patients. Patients and methods: 40 children with CF ( 21 boys; median age 12,5 years (4-18)), 10 healthy children ( 7 boys; 10 years (6-15)) and 22 adult patients ( 10 men; 53 years (21-73)) underwent a combined $\mathrm{H}_{2}$-glucose/ ${ }^{13} \mathrm{C}$-xylose breath test. In children, $50 \mathrm{mg}\left[{ }^{13} \mathrm{C}\right]$-D-xylose and $\mathrm{lg} / \mathrm{kg}$ b.w. glucose were dissolved in water and orally applied. Adults received $100 \mathrm{mg}\left[{ }^{13} \mathrm{C}\right]$-D-xylose and $80 \mathrm{~g}$ glucose. Breath samples were collected every 30 minutes for 3 hours and analysed for $\mathrm{H}_{2}$ concentration [ppm] and ${ }^{13} \mathrm{CO}_{2}$-enrichment (DOB [\%o]). An $\mathrm{H}_{2}$-increase of $>20 \mathrm{ppm}$ above baseline was considered pathologic. Results: In 27/40 CF-children (68\%), already baseline breath hydrogen content exceeded 20ppm and remained above this threshold during the test. None of the remaining $13 \mathrm{CF}$-children developed pathologically increased breath hydrogen concentrations. ${ }^{13} \mathrm{CO}_{2}$-enrichment (DOB) did not differ between $\mathrm{CF}$-children with or without pathologic $\mathrm{H}_{2}$-breath test results $(\mathrm{p}>0,05)$. The $27 \mathrm{CF}$-children with increased baseline $\mathrm{H}_{2}$-concentrations had a significantly lower $\mathrm{DOB}$ compared with the 10 healthy children $(\mathrm{p}=0,04)$. DOB after $3 \mathrm{~h}$ correlated significantly with age if all 50 children of the study population were considered $(\mathrm{r}=-0,65, \mathrm{p}<0,0001)$. Among 22 adult patients, $\mathrm{H}_{2}$ concentration pathologically rose in $8(36 \%)$. This subgroup produced significantly higher DOBs compared with the remaining 14 adults $(\mathrm{p}=0,02)$. A DOB-cutoff of $6,8 \%$ yielded a sensitivity of $79 \%$ and a specificity of $63 \%$ in adults. Discussion: Bacterial overgrowth is frequently encountered in children with cystic fibrosis. Compared with the $\mathrm{H}_{2}$-glucose breath test, the ${ }^{13} \mathrm{C}$-xylose breath test lacks sensitivity and specificity, probably due to an agedependent decrease of ${ }^{13} \mathrm{CO}_{2}$-enrichment. Moreover, considerably higher costs for the labelled substrate and a long period of ${ }^{13} \mathrm{CO}_{2}$-breath sampling are major drawbacks of the ${ }^{13} \mathrm{C}$-xylose breath test, the latter particularly in young children.

\section{M1182}

13c-Sucrose Breath Test to Differentiate Congenital Sucrase-Isomaltase Deficiency from Pandisaccharidase Deficiency

Claudia Robayo, Antone R. Opekun, Roberto Quezada-Calvillo, Susan S. Baker, Buford L. Nichols

Introduction: A substrate-paired breath test using 13C-sucrose (S) and 13C-glucose (G) has been developed to assess congenital sucrase-isomaltase deficiency (CSID). The aim was to determine if CSID could be detected without duodenal enzyme assay. Methods: Two patients (1F:1M, aged $1 \& 15$ yrs.) with CSID (duodenal mucosa biopsy-proven), 3 patients with pandisaccharidase deficiency (P) (1F:2M, aged 10-15 yrs.) \& 5 normal patients (4F:1M aged 3-15 yrs.) were studied. All had mucosal enzyme activity determinations done. Uniformly-labeled 13C-substrates ( $\mathrm{G} \& \mathrm{~S} ; 20 \mathrm{mg}$. each, Isotec, Miamisberg, $\mathrm{OH}$ ) were given in $10 \mathrm{gm}$ unlabeled glucose oligomers $(10 \%)$ vehicle after an overnight fast and reference sampling on 2 separate days. Serial $13 \mathrm{CO} 2$ breath enrichments (every 15 min *6) were assayed using a $13 \mathrm{CO} 2$ infrared spectrophotometer (POCone $®$, Otsuka Electronics, Tokyo, Japan). A coefficient of glucose oxidation (CGO) was calculated for $\mathrm{S}$ breath enrichment to adjust for individual differences for each time-paired sample. Normal control patients were used to define CGO lower reference levels (LL) defined as mean-1 SD. Results: The CSID patients had S-CGO significantly below the LL and the P patients had relatively norma S-CGO results above the LL. Duodenal S enzyme activity levels and S-CGO results closel correlated $\left(R^{\wedge} 2\right.$ 0.72). Conclusions: The S/G substrate-paired breath test distinguished CSID patients from controls and differentiated CSID patients from the secondary forms of $S$ deficiency. It appears that patients with low S-CGO could benefit from mucosal biopsy to identify additional enzyme deficiencies.

SUCROSE / GLUCOSE CGO INDEX

IN NORMAL SUBJECTS AND DEFICIENT PATIENTS

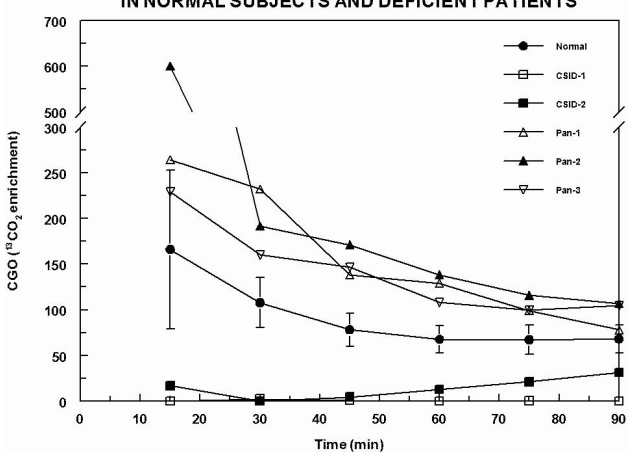

M1183

Variability in Gastrointestinal Permeability in Healthy Subjects

Paul Lundin, Jonas E. Pettersson, Karin Nelander, Lars Lofgren

BACKGROUND: Intestinal permeability has previously been assessed using different marker as a single test. Variations in repeated permeability tests over a period are not well known. Aim of the study was to investigate daily inter- and intraindividual variability of the intestinal permeability in healthy subjects. Furthermore we evaluated whether a fasting or non-fasting state alter the urine recovery of administered markers. METHODS: A combination of four sugar markers was used to reflect the gastroduodenal (sucrose), small intestinal (mannitol $\&$ lactulose) and distal gastrointestinal (sucralose) permeability. After overnight fasting, healthy subjects (five male, five female) ingested a solution of the markers, then urine was collected for $0-3,3-6,6-12$ and $12-24 \mathrm{~h}$. To reduce variability, all subjects had a standardized diet during the study days. This procedure was repeated in five consecutive weeks. On the fifth visit subjects did not fast prior to marker administration. The sugar markers in urine were analysed using gas chromatography - mass spectrometry (GC-MS). A statistical mode was designed to estimate inter- and intraindividual variability for each marker. RESULTS All subjects had a normal kidney function measured as glomerular filtration rate. The major part of the recovered sugar markers was found in the 0-6 h interval. For all sugar markers in fasting state, variations within and between subjects were of the same order. At the nonfasting day the sucrose recovery at the $0-3 \mathrm{~h}$ interval was significantly $(\mathrm{p}<0.05)$ lower than at the fasting visits. For sucralose, more than $20 \%$ of the dose recovered in urine was found in the 12-24 $\mathrm{h}$ interval, indicating an uptake in the distal GI-tract. CONCLUSIONS: In fasted healthy subjects, the inter- and intraindividual variability was of the same magnitude for each sugar marker and interval, respectively.

$\%$ of dose recovered in urine $0-12 \mathrm{~h}$, median (10th-90th percentiles)

\begin{tabular}{|l|c|c|c|c|c|}
\hline & Visit 1 & Visit 2 & Visit 3 & Visit 4 & Visit 5* \\
\hline Sucrose & $\begin{array}{c}0.057 \\
(0.027-0.066)\end{array}$ & $\begin{array}{c}0.058 \\
(0.039-0.077)\end{array}$ & $\begin{array}{c}0.063 \\
(0.048-0.071)\end{array}$ & $\begin{array}{c}0.058 \\
(0.043-0.064)\end{array}$ & $\begin{array}{c}0.039 \\
(0.032-0.055)\end{array}$ \\
\hline Mannitol & $19(16-21)$ & $18(15-22)$ & $19(15-23)$ & $18(14-20)$ & $21(15-30)$ \\
\hline Lactulose & $0.35(0.24-0.40)$ & $0.30(0.18-0.37)$ & $0.32(0.22-0.39)$ & $0.32(0.22-0.42)$ & $0.28(0.17-0.49)$ \\
\hline Sucralose & $1.9(1.7-2.0)$ & $1.9(1.6-2.3)$ & $1.9(1.5-2.1)$ & $1.7(1.7-2.1)$ & $2.2(1.3-3.0)$ \\
\hline
\end{tabular}

*Non-fasting subjects - 
M1184

Von Willebrand Disease - An Underestimated Cause of Gastrointestinal Bleeding from Angiodysplasia?

Bruno Neu, Sebastian Noe, Monther Bajbouj, Georg Moessmer, Alexander Meining, Christian Prinz, Holger Seidl, Wolfgang Schepp, Michael Anzinger, Wolfgang Schmitt, Roland M. Schmid

Background: Angiodysplasias (AD) are the most common reason of chronic bleeding from the gastrointestinal (GI) tract. However, asymptomatic $\mathrm{AD}$ are often seen during routine endoscopy. In this study we looked systematically, in a prospecitve manner for concomitant disorders of hemostasis, underlying diseases, and characteristics of AD which may be linked to bleeding from AD. Patients and Methods: 38 patients ( 22 women, mean age 71 years) with gastrointestinal bleeding from $\mathrm{AD}$ were included. All subjects were investigated by upper, lower, and capsule endoscopy. Platelet function was measured by closure time in a whole blood test, activated by collagen/epinephrine resp. collagen/ADP (platelet function analyser, PFA-100). Furthermore, PT, aPTT, thrombin time, factor XIII, factor VIII:C, ristocein cofactor activity and von Willebrand factor (vWF) Ag were determined. Results: Before study entry the patients suffered from bleeding for 2 years, needed 2 units of packed red cells, and had a minimal hemoglobin level of $7.4 \mathrm{mg} / \mathrm{dl}$ (median values). An average of 2.5 $\mathrm{AD}$ was found (1-10 $\mathrm{mm}$ in diameter), localised $5 \%$ in the stomach, $55 \%$ in the upper part, $6 \%$ in the middle part, $5 \%$ in the lower part of the small intestine, and $29 \%$ in the colon 25 of 38 patients had a disorder of primary hemostasis. 13/38 patients showed a pathological platelet function, measured by an increased closure time as a possible hint for a functiona vWF-defect. 3 of them had also low levels of vWF-Ag and therefore, were suspicious of a quantitative vWF-defect. 12 patient presented with increased closure times due to ASS medication or were under ASS treatment during the bleeding episode. Conclusion: Interestingly, most of the $\mathrm{AD}$ are localised in the upper third of the small intestine. Two-third of patients with bleeding angiodysplasia had a dysfunction in primary hemostasis. Half of them might suffer from a defect in the vWF, the other half had platelet-inhibiting medication.

\section{M1185}

Long-Acting Octreotide As Rescue Therapy in Preventing Rebleeding from Gastrointestinal Angiodysplasias

Giuseppe Scaglione, Lorena Pietrini, Pasquale Sarracco, Domenico Di Pietro, Mariarosaria Franco, Francesca Russo, Fernanda Mirco, Italo Sorrentini

BACKGROUND: angiodysplasias are the most common source of obscure gastrointestinal bleeding (GIB) in the elderly. Management of such vascular abnormalities is still an open clinical problem in patients not elegibile for surgical or endoscopic therapy. Octreotide has shown to have some benefit in this subgroup of subjects, but it has not been extensively studied. AIM: to investigate the efficacy of long-acting octreotide (LAR-OCT) in the prevention of rebleeding from gastrointestinal angiodysplasias in patients not candidate for surgery and resistant to endoscopic treatment. PATIENTS AND METHODS: ten patients (M/F 4/6, mean age+SD 77.6+8.1) with a long history of chronic occult or overt GIB due to multiple upper and lower angiodysplasias referred to our unit from 2000 to 2004 entered the study. In each patient diagnosis was made by upper and lower endoscopy, push enteroscopy and wireless video capsule endoscopy, when appropriate. After obtaining written informed consent, LAR-OCT was administered intramuscularly to all patients at a dosage of $10 \mathrm{mg}$ monthly for a minimum period of 1 year. Patients were followed up monthly and haemoglobin levels, number of blood transfusions, amount of iron parenteral supplementation, number of hospitalizations one year before and after starting LAR-OCT therapy were recordered to evaluate the efficacy of the treatment. RESULTS: follow-up ranged from 12 to 60 months, median 33. There was a significant difference in haemoglobin values (mean \pm SD $5.8 \pm 1$ vs $9.1 \pm 1.6, \mathrm{p}=0.002)$, iron parenterally administered $($ mean \pm SD $26.6 \pm 14$ vs $13.3 \pm 10, \mathrm{p}=0.05)$, blood units transfused (mean \pm SD $6.6 \pm 2.4$ vs $3.1 \pm 3.8, \mathrm{p}=0.01$ ), number of hospitalizations (mean \pm SD $3.6 \pm 1.2$ vs $1.6 \pm 1.5, \mathrm{p}=0.002$ ) between the year before and after LAR-OCT treatment. Six patients successfully stopped blood transfusions and iron supplementation during the follow up period. In one patient a partial improvement, requiring only iron supplementation, was observed, while no effect was found in the other three. No significant side-effect was registered in any patient. CONCLUSIONS: to our knowledge, this is the first nonanecdotical paper reporting on LAR-OCT treatment for preventing rebleeding from gastrointestinal angiodysplasias in patients who cannot undergo surgery due to old age and/or concomitant diseases. Our data demonstrate that chronic administration of octreotide may be useful as rescue therapy in this subgroup of patients.

\section{M1186}

Long-Term NSAID Use and Small Bowel Pathology: A Cross-Sectional Quantification By Capsule Enteroscopy

Laurence P. Maiden, A Seigal, Ingi Bjarnason, B Birgisson, Bjarni Thjodleifsson, Ingvar Bjarnason

Background \& Aims: Capsule enteroscopy has shown small bowel mucosal breaks (erosions and ulcers) in $40 \%$ of volunteers taking the NSAID diclofenac (with a proton pump inhibitor) for 2 weeks, however the full range of pathology in a large group of long-term users has not been assessed. We undertook a capsule enteroscopy in 117 patients on long-term NSAIDs to assess the range of small bowel damage caused by these agents. Methods: Patients with established osteoarthritis, rheumatoid arthritis or low back pain who had been regularly taking any one NSAID for 3 months or longer underwent a capsule enteroscopy. Patients with known or suspected Crohn's disease, spondylarthropathy, including seronegative athritides, and other small bowel pathologies were excluded as the gastrointestinal damage might not readily be distinguished from that of NSAIDs. Sixty healthy volunteers acted as controls. Results: The volunteers had no damage. 81 of the $117(69 \% ; \mathrm{p}<0.001)$ had evidence of intestinal pathology attributable to the drug on enteroscopy. Forty-one (35\%) had more than one lesion concurrently. The commonest lesion seen was the denuded lesion in 47 $(40 \% ; \mathrm{p}<0.001)$. Forty-one $(35 \% ; \mathrm{p}<0.001)$ had red spots. Thirty-one $(26 \%)$ demonstrated mucosal breaks, of which 1 was bleeding at the time of enteroscopy. Reddened folds were seen in 14 patients $(12 \% ; \mathrm{p}<0.05)$. Strictures without a mucosal break or bleeding were seen in 2 of the patients $(2 \% ; p>0.05)$ and a further 2 patients revealed evidence of bleeding without an obvious source. Conclusion: Capsule enteroscopy demonstrates small bowel lesions in $69 \%$ of patients on long-term NSAID. Clinically significant pathology (mucosal break, stricture and bleeding) is detected by capsule enteroscopy in 30\% of patients on long-term NSAIDs, which is greater than that seen in the stomach. This may explain why the serious outcomes (perforation, bleeds and obstruction) associated with NSAIDs from the upper and lower gastrointestinal tract are comparable.

\section{M1187}

Safety and Efficacy of $0.5 \mathrm{mg}$ Qd, $1 \mathrm{mg}$ Qd and $1 \mathrm{mg}$ Bid Alosetron Hydrochloride in Women with Chronic, Severe Diarrhea-Predominant IBS (DIBS)

Richard Krause, Vanessa Ameen, Susan Gordon, Marquita West, Amy Heath, Teresa Perschy, Eric Carter

The 5HT3 receptor antagonist alosetron is indicated for women with chronic, severe D-IBS who have not responded adequately to conventional therapy. Previous trials with alosetron lmgBID showed efficacy with an NNT $=7$, but $29 \%$ of pts reported constipation. Lower alosetron doses were assessed in severe D-IBS pts in a randomized, double-blind, placebocontrolled study (S3B30040) to determine efficacy and evaluate changes in the constipation rate. Methods: 705 women with chronic, severe D-IBS were equally randomized to 12 wks of placebo, $0.5 \mathrm{mgQD}, 1 \mathrm{mgQD}$, or $1 \mathrm{mgBID}$ alosetron. The primary endpoint was the proportion of responders on the Global Improvement Scale (GIS) at wk12, defined as pts with moderate or substantial improvement in IBS symptoms. Secondary endpoints were the average rate of Adequate Relief (AR) of IBS pain and discomfort, and improvements in bowel symptoms, (urgency, frequency, and consistency). Results: The proportion of GIS wk 12 responders was significantly higher for all doses of alosetron compared to placebo (treatment effects $\geq 11.9 \%, \mathrm{p} \leq 0.012$ ). Results were similar for the average adequate relief rate (treatment effect $\geq 12 \%, p \leq 0.038$ ). The $0.5 \mathrm{mgQD}$ and $1 \mathrm{mgQD}$ regimens were superior to placebo in improving all bowel symptoms. Alosetron $1 \mathrm{mgBID}$ was superior to placebo in reducing stool frequency and improving stool consistency. Constipation was the most common adverse event $(9 \%, 16 \%$, and $19 \%$ of pts in the 0.5mgQD, lmgQD, and $1 \mathrm{mgBID}$ groups, respectively). 27 serious adverse events occurred in alosetron treated pts, including levent of intestinal obstruction and 1 event of ischemic colitis in the $0.5 \mathrm{mgQD}$ group, and 1 event of fecal impaction in the $1 \mathrm{mgBID}$ group. The events were self-limited and resolved without sequelae. Conclusion: The $0.5 \mathrm{mgQD}$ and $1 \mathrm{mgQD}$ alosetron dosing regimens are effective in providing global improvement in IBS symptoms, adequate relief of IBS pain and discomfort, and improvement in bowel urgency, frequency, and consistency in women with chronic, severe D-IBS. These regimens also resulted in a decreased incidence of constipation relative to $\operatorname{lmgBID}$.

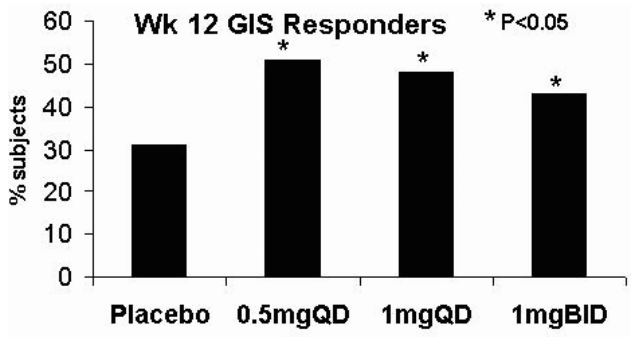

M1188

Risk Factors for Colonic Diverticular Bleeding

Atsuo Yamada, Hirotsugu Watabe, Makoto Okamoto, Yutaka Yamaji, Keiji Ogura, Shintaro Kondo, Miki Ohta, Takafumi Sugimoto, Takao Kawabe, Masao Omata

Background: Colonic diverticula are occasionally responsible for severe lower gastrointestinal bleeding. A majority of the cases are elderly and often have concomitant diseases. Nonsteroidal anti-inflammatory drugs (NSAIDs) have been previously reported as a risk factor for diverticular bleeding. However, little has been studied about concomitant conditions as risk factors for diverticular bleeding. We conducted the present study to elucidate their significance as a risk factor. Methods: From July 1988 to September 2005, patients with diverticular hemorrhage were enrolled in this study among 13320 colonoscopic examinations performed in the University of Tokyo Hospital and its affiliated hospitals. Their clinical features such as age, gender, site of bleeding diverticular, and outcome of the patients were reviewed. Control subjects $(n=74)$ were chosen from 2487 patients with diverticula who have no history of lower gastrointestinal bleeding, as age, sex and site of diverticulum were matched to each case. Effects of concomitant diseases including hypertension, hyperlipidemia, diabetes mellitus, alcohol consumption, smoking and NSAIDs use on diverticular bleeding were evaluated. Results: During the study period, 37 patients (mean age; $68 \pm 12$ years, male: female 30:7) had diverticular bleeding. Twenty-one patients (57\%) bled from right-sided diverticulum. Eleven patients were successfully treated by endoscopic procedures (clipping or local injection of epinephrine saline). Eleven patients needed blood transfusion because of severe anemia (2-14 units of packed red blood cells). The patients with diverticular bleeding had hypertension more frequently $(78 \%, \mathrm{P}=0.0012)$ than in the control $(45 \%)$ Thirteen patients (35\%) in the bleeding group have received NSAIDs while $11(15 \%)$ had in the control group $(\mathrm{P}=0.026)$. The estimated odds ratio of hypertension for bleeding risk was 3.6 (95\% CI: $1.32-10.04, \mathrm{p}=0.012$ ). The odd ratio of NSAIDs was 2.9 (95\% CI: 0.997$8.648, \mathrm{p}=0.051)$. Conclusion: The results of the present study suggest that hypertension is an independent risk factor of diverticular bleeding. Treatment of hypertension may lead to prevention of diverticular bleeding 
M1189

The Spectrum of Luminal Gastrointestinal Involvement in Amyloidosis Dustin G. James, Hanlin L. Wang, Gary R. Zuckerman, Chandra Prakash

Background: Amyloidosis is characterized by accumulation of insoluble proteins in multiple organ systems. Gastrointestinal involvement is likely underestimated, and characterization of endoscopic findings is sparse in the literature. We reviewed our institutional experience with amyloidosis, with the aim of describing gastrointestinal manifestations, endoscopic findings, and outcome. Methods: The pathology database was interrogated to identify patients with the finding of amyloid on tissue samples obtained during endoscopy over the past 15 years. Clinical presentation, endoscopic findings, patient course and outcome were then analyzed from review of inpatient and endoscopy records. Results: Amyloidosis was diagnosed on histopathology of endoscopic biopsies in 22 patients $(8 \mathrm{~F} / 14 \mathrm{M})$ during the study period. The mean age at diagnosis was $68 \pm 0.6$ years (range $46-90$ years), and $77.3 \%$ of patients were $\geq 60$ years at presentation. The most frequent clinical presentation was gastrointestinal bleeding seen in 11 patients (50\%), with overt bleeding in 9 and occult bleeding in 2 patients. Other presenting symptoms included change of bowels in $22.7 \%$, dyspepsia in $22.7 \%$, anorexia with weight loss in $13.6 \%$, abdominal pain and small bowel obstruction in $4.5 \%$ each; amyloid was incidentally found in a polypectomy specimen in one instance. On further evaluation, $45.5 \%$ had evidence of renal involvement, $50 \%$ had evidence of neural involvement (including at least 18\% with gut related symptoms), and $27.3 \%$ each had skin findings and cardiac involvement. Abnormal hematologic testing was noted in $63.6 \%$. Endoscopic abnormalities were seen in the colon in 50\%, small bowel in $31.8 \%$ and stomach in $18.2 \%$. Macroscopic findings included nodules in $36.4 \%$, and ulcers or erosions in $27.3 \%$. Nodules with a bluish appearance, visualized endoscopically in five patients $(22.7 \%)$, were significantly associated with overt gastrointestinal bleeding as a presenting manifestation $(\mathrm{p}<0.005)$. On histopathologic examination, amyloid deposition was most commonly seen in the submucosa and deeper layers in $40.9 \%$, with evidence of vascular involvement in $45.5 \%$. Amyloid type was known in 18 patients, and was as follows: AL type in 13, AA type in 2, and localized amyloid in 3. At least 18.2\% died within a month after diagnosis. Conclusions: Gastrointestinal manifestations of amyloidosis are varied, and awareness of the spectrum of gastrointestinal involvement is important for accurate diagnosis. Characteristic bluish nodules on endoscopy may represent a marker for overt gastrointestinal bleeding from amyloidosis. Multisystem involvement is frequent, even when presentation is predominantly gastrointestinal.

\section{M1190}

Testing for Lactose and Fructose Intolerance Should Be Standard Protocol for Patients with Chronic Abdominal Complaints

Charles W. Randall, Carlo M. Taboada, Gary Gossen, David Stump, Franz Zurita, Victor Ostrower, Russell Havranek, Susan Ryan, Janet Ford

Introduction Deficiencies in Lactase and Fructase are relatively common but often not considered in patients with chronic gastrointestinal (GI) complaints. The purpose of this study was to determine if these patients with chronic symptoms benefited from testing for disaccharidase deficiencies. Methods 191 patients ranging in age from 17 to 87 years of age presented to our clinic for evaluation. 46 were male and 145 were female. Appropriate labs, imaging studies and endoscopic procedures were performed when deemed appropriate. Each patient underwent hydrogen breath testing to determine presence of disaccharidase deficiency. Dietary management was initiated in all patients testing positive with appropriate follow-up to determine efficacy of therapy. Results Of the 191 patients, 42 were found to be lactose intolerant, 46 fructose intolerant and 16 were intolerant to both. Dietary management was initiated with a mean follow up time of 10.8 months and a median follow up time of 10 months. 97 patients experienced improved quality of life, while 7 had unsatisfactory results. Improvement was described as a return to a normal lifestyle and not requiring any additional therapy. Conclusions 1. - In this cohort of patients, $54.5 \%$ were found to have disaccharidase deficiencies. 2. - Dietary management allowed significant symptom relief in 93.3\% of patients. 3. - Determination of a patient's lactose and fructose tolerability clearly has a positive impact on patients with chronic GI symptoms and should be utilized in the initial evaluation.

\section{M1191}

Symptom Overlap and Irritable Bowel Syndrome (IBS-C) Comorbidity in Women with Chronic Constipation (CC)

Michael Crowell, V. Ann Schettler, Lucinda Harris, Ivan Bottoli, Jeffrey Kralstein

Background: Chronic constipation is a multi-symptom disorder thought to result from different pathophysiologic etiologies. However, the prevalence of overlapping symptoms between CC and IBS-C has not been well defined. Additionally, the differential impact of psychosocial function on constipation-related symptoms has not been established between patients with CC and IBS-C. Aim: To assess the prevalence of IBS in women presenting with a diagnosis of $\mathrm{CC}$ from their referring physicians and to evaluate symptom overlap and comorbidities between these patients. Methods: Consecutive patients clinically diagnosed with CC $(\mathrm{n}=136)$ without organic disease completed a validated GI symptom questionnaire and the SCL90-R. The proportion of patients reporting upper and lower GI symptoms were evaluated using Pearson Chi-square analyses and the Mantel-Haenszel Odds Ratios for categorical variables and the Student's t-test or the Mann-Whitney Test as indicated. Significance levels were set at $\mathrm{p}<0.05$. Results: In this tertiary female sample, the majority of patients met the Rome Criteria for IBS-C (70/136; $51.5 \%)$. Therefore, one group included CC patients who met criteria for IBS-C and the second CC patients who did not meet IBSC criteria. Both groups did not differ in age or other demographics. Stepwise logistic regression revealed significantly higher odds in the IBS-C group for pain frequency $(\mathrm{OR}=$ $1.22,95 \% \mathrm{CI}=1.06-1.40)$, bowel frequency $(\mathrm{OR}=1.09,95 \% \mathrm{CI}=1.01-1.17)$, and incomplete evacuation $(\mathrm{OR}=1.68,95 \% \mathrm{CI}=1.22-2.31)$. The number of pain sites was also increased in the IBS-C group $(\mathrm{OR}=1.40,95 \% \mathrm{Cl}=1.07-1.84)$. Somatization was slightly lower in CC patients compared to IBS-C $(\mathrm{OR}=0.94,95 \% \mathrm{CI}=0.90-0.98)$, otherwise psychosocial distress was not significantly different between the two groups. No differences were seen in Quality of Life measures between groups. Conclusions: A substantial percentage of patients with clinically diagnosed CC will be found to meet diagnostic criteria for IBS-C, and the chie distinction between the clinically diagnosed CC patients that meet criteria for IBS-C is the degree to which patients report abdominal pain, bowel frequency and incomplete evacuation. Psychosocial distress does not reliably differentiate patients with CC from IBS-C.

\section{M1192}

Complete Spontaneous Bowel Movement (CSBM) Correlates Better Than Spontaneous Bowel Movement (SBM) to Global Endpoints in Chronic Constipation (CC) Trials

Ivan Bottoli, Williams D. Chey, Cornelia Ddunger-Baldauf, Jeffrey Kralstein

Background: According to current guidelines ${ }^{1,2}$, chronic constipation (CC) is characterized by multiple symptoms (straining, hard stools, sensation of incomplete evacuation, insufficien frequency, need for manual maneuvers, sensation of anorectal obstruction). Yet most physicians focus on stool frequency when diagnosing and assessing the success of medical therapies for this condition. CSBM is an outcome that incorporates a qualitative component to the assessment of bowel habits and, therefore, may provide a better outcome measure for clinical trials than quantification of stool frequency alone (SBM). In a post-hoc analysis from two randomized, placebo-controlled, double-blind clinical trials, we determined how CSBM and SBM correlated with two global assessments (satisfaction with bowel habits and bothersomeness of constipation). Methods: The pooled data from two twelve week trials including CC patients on tegaserod 6mg BID $(n=882)$ or placebo $(n=863)$ were used for this analysis. Bowel habit parameters included frequency of spontaneous (not induced by laxatives or enemas) bowel movements with a sense of complete evacuation (CSBM) and of spontaneous bowel movements (SBM). The global assessments were satisfaction with bowel habits ("How satisfied were you with your bowel habits over the past week?"; 0 = a very great deal satisfied $4=$ not at all satisfied) and bothersomeness of constipation ("How bothersome was your constipation over the past week?"; $0=$ not at all, $4=$ a very great deal). Spearman correlations with bowel habit and bothersomeness of constipation averaged over weeks 1-12 are presented. Results: CSBM had a higher correlation than SBM to both global assessments (Table 1) Both global assessments showed almost identical correlations to each symptom, therefore showing equivalent global reach. The correlations were independent from the treatment. Conclusion: CSBM, with its additional qualitative component, better correlates to globa efficacy variables than SBM, supporting that frequency alone is likely not sufficient to assess therapeutic outcomes in CC patients. 1. Brandt et al, 2005 Gut;100(Suppl1) :S1-S4 2. Thompson et al, 1999Gut:45(Suppl II):II43-II47

CSBM had a Higher Correlation than SBM to both Global Assessments

\begin{tabular}{|c|c|c|c|c|}
\hline & \multicolumn{2}{|c|}{ Tegaserod 6mg BID } & \multicolumn{2}{c|}{ Placebo } \\
\hline $\begin{array}{c}\text { Satisfaction with } \\
\text { Bowel Habit }\end{array}$ & $\begin{array}{c}\text { Constipation } \\
\text { bothersomeness }\end{array}$ & $\begin{array}{c}\text { Satisfaction with } \\
\text { Bowel Habit }\end{array}$ & $\begin{array}{c}\text { Constipation } \\
\text { bothersomeness }\end{array}$ \\
\hline CSBM & -0.59 & -0.56 & -0.61 & -0.56 \\
\hline SBM & -0.43 & -0.40 & -0.45 & -0.39 \\
\hline
\end{tabular}

\section{M1193}

Gastrointestinal Transit Measurement May Simplify the Detection of Motility Disturbances in Clinical Practice

Riadh Sadik, Per Ove Stotzer, Magnus Simren, Hasse Abrahamsson

The management of patients with GI-symptoms may vary between different centers. The aim of this prospective study was to analyze the yield and utility of a GI-transit measuremen procedure in clinical practice. Methods: All patients referred by gastroenterologists to a tertiary centre for transit measurements were prospectively included. 243 patients were included. Smoking status and body mass index were recorded. The patients were categorized according to the main GI-symptom into five groups: diarrhea, constipation, nausea, an vomiting and abdominal pain. The patients recorded their bowel movements and three GIsymptoms daily for the week before the transit measurement. These symptoms were abdominal pain, gas and distention. The patients had to chose between four levels of symptoms: $0=$ no symptom, 1 =light symptoms, $2=$ pronounced symptoms, $3=$ =evere symptoms. All patient underwent a measurement of gastric emptying, small bowel residence and colonic transi using radiopaque markers (Sadik et al. Am J Gastroenterol.2004:99:711-8). The area under the colonic filling profile was utilized to calculate the colonic filling time. Percentile five and 95 of the transit values in 83 healthy subjects served as reference values. Results: 63\% of the patients had at least one transit abnormality. The transit abnormalities are shown in the figure. These abnormalities were more frequent in men compared with women, $\mathrm{p}=0.003$. Smoking women had more abnormalities compared with non-smoking women, $\mathrm{p}=0.01$. Patients with underweight had also more disturbances compared with other patients with normal weight and with overweight, $\mathrm{p}=0.03$. The reported severity of abdominal pain, gas and distention had no influence on the frequency of the detected transit abnormalities. Conclusion: Large-scale transit measurement is useful in a clinical prospective and may elucidate the relationship between some GI-symptoms and abnormal motility 


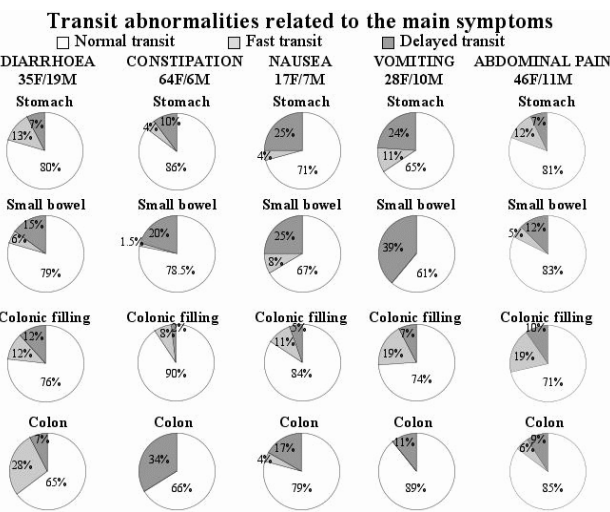

M1194

The Prevalence of Irritable Bowel Syndrome Among the General Population in Japan

Sen Shimaya, Masashi Matsuzaka, Makoto Yaegaki, Takao Oyama, Ippei Takahashi,

Takashi Umeda, Shigeyuki Nakaji, Daisuke Sasaki, Eiko Oka, Tadashi Shimoyama

Introduction: Irritable bowel syndrome (IBS) is a common condition among the general population worldwide, however, few studies have reported on the degrees of prevalence among the general population in Japan. Subject and methods: We surveyed bowel habits in a general population in northern Japan ( $\mathrm{n}=1067,407$ males and 660 females; 51 under 30 y.o., 92 in the 30-39 yrs age group, 142 from 40-49yrs, 262 from 50-59 yrs, 286 from 60-69 yrs, and 23470 yrs or over), and diagnosed IBS based on Rome II criteria. Result: The questionnaire was completed by 1067 individuals ( 407 males and 660 females, $100 \%$ response rate). The prevalence of IBS and constipation were as follows (see Table). 1. The number (prevalence) of IBS with constipation (IBS-C); 5 (1.2\%) in men, $23(3.5 \%)$ in women and $28(2.6 \%)$ in total. 2. The number (prevalence) of IBS with diarrhea (IBS-D); $8(2.0 \%)$ in men, $6(0.9 \%)$ in women and $14(1.3 \%)$ in total. 3 . The number (prevalence) of mixed IBS (IBS-M); 12 (2.9\%) in men, 20 (3.0\%) in women, and 32 (2.9\%) in total. 4. The number (prevalence) of total IBS (CIBS+DIBS+MIBS); $25(6.1 \%)$ in men, 49 (7.4\%) in women and $74(6.9 \%)$ in total. On the other hand, the prevalence of IBS in subjects in their 20's was $10.0 \%$ and for those in their 30 's was $9.8 \%$, the highest rates among all generations. By contrast, the prevalence of IBS for subjects of 70 yrs and over was $2.9 \%$, the lowest rate. As for prevalence by gender, the prevalence of IBS in men $(6.1 \%)$ was lower than in women (7.4\%). Discussion: The prevalence of IBD $(6.9 \%)$ in the current survey was lower than those previously reported in the Japanese and Western literature (over 10\% in most studies). This difference may be due to differences in lifestyle, physical characteristics and so on. The number of prevalence of IBS by age-group

\begin{tabular}{|c|c|c|c|c|c|}
\hline & & IBS-C & IBS-M & IBS-D & prevalence(\%) \\
\hline 10 's & 1 & 0 & 0 & 0 & 0 \\
\hline 20 's & 50 & 2 & 2 & 1 & 10.0 \\
\hline 30 's & 92 & 2 & 3 & 4 & 9.8 \\
\hline 40 's & 142 & 3 & 4 & 1 & 5.6 \\
\hline 50 's & 262 & 11 & 10 & 3 & 9.2 \\
\hline 60 's & 286 & 6 & 9 & 4 & 6.6 \\
\hline 70 's & 215 & 1 & 4 & 1 & 2.8 \\
\hline 80 's & 19 & 2 & 0 & 0 & 10.5 \\
\hline & 1067 & 28 & 32 & 14 & 6.9 \\
\hline
\end{tabular}

IBS-C: IBS with constipation, IBS-M: Mixed IBS, IBS-D: IBS with diarrhea The number of prevalence of IBS by gender

\begin{tabular}{|c|c|c|c|c|c|}
\hline & & IBS-C & IBS-M & IBS-D & prevalence(\%) \\
\hline Men & 407 & 5 & 12 & 8 & 6.1 \\
\hline Women & 660 & 23 & 20 & 6 & 7.4 \\
\hline
\end{tabular}

IBS-C: IBS with constipation, IBS-M: mixed IBS, IBS-D: IBS with diarrhea

\section{M1195}

Efficacy and Safety of Lubiprostone for the Treatment of Chronic Constipation in Male vs Female Subjects

Ryuji Ueno, Taryn R. Joswick, Aimee Wahle, Yan Zhu, P. Christopher Holland

Constipation is a common and bothersome gastrointestinal condition, affecting nearly 30 million individuals in North America. Lubiprostone is a novel type-2 chloride channel (ClC2) activator (AJP Cell Physiol 287:C1173-C1183, 2004) that has been shown to be efficacious and well tolerated in a number of well-controlled clinical trials of 3-4 weeks duration (Gastroenterology 122:A315, 2002; Gastroenterology 124:A38, 2003; Am J Gastroenterol $100:$ S324, S328 and S329, 2005). We examine pooled results from these trials so that subgroups of male and female subjects can be compared. Methods: In order to create an adequate pool of male subjects, data were combined from three well-controlled clinical trials. Spontaneous bowel movement (SBM) frequency rates, stool consistency ratings, and bowel straining assessments were compared between treatment groups (placebo and lubiprostone $48 \mu \mathrm{g} /$ day) via inferential statistics and used to assess efficacy. Mean improvements over placebo are compared between male and female subjects. Adverse event (AE) incidence rates were also compared between treatment groups and genders. Results: The pooled subgroup of males consisted of 27 placebo and 32 lubiprostone subjects. Male subjects taking lubiprostone experienced approximately twice as many SBMs per week as their placebo counterparts (5.69-6.05 SBMs/week vs 2.55-3.23 SBMs/week). Despite the small sample size, differences were statistically significant for the first 3 weeks $(\mathrm{p}<0.0489)$ and were nearly significant at Week $4(\mathrm{p}=0.0503)$. Compared to female lubiprostone subjects, these rates are slightly higher (rates among female lubiprostone subjects ranged from 4.99$5.75 \mathrm{SBMs} /$ week). With regard to safety, one-third (33.3\%) of male placebo subjects experienced at least one AE compared to $50.0 \%$ of male lubiprostone subjects. Conclusion: Lubiprostone is an efficacious and well-tolerated treatment for chronic constipation in male subjects.

\section{M1196}

Effect of Tamoxifen On Clinical Course of Sclerosing Mesenteritis Salma Akram, Darrell S. Pardi, Thomas C. Smyrk

BACKGROUND: Sclerosing mesenteritis (SM) is a rare, fibro-inflammatory disorder of unknown etiology that affects the small bowel mesentery. The rarity of cases and the heterogeneity of the natural history have limited the ability to study this disease and to evaluate its response to therapy. In 2002 we reported our institution's twenty years experience with this disorder. We have since identified 28 additional cases of SM or its variants, many of whom we have treated with tamoxifen. AIM: To describe clinical characteristics, therapy and outcome of all patients with SM or its variants evaluated at the Mayo Clinic, Rochester between 12/02 and 10/05. METHODS: Cases were identified through the diagnostic index and Department of Pathology database. Demographic, treatment, and clinical outcome data were abstracted from their records. Results: Among 28 patients, 23 (82\%) were male with median age at diagnosis of 64.5 yrs (range 42-82). Median follow-up was 10 month (mo) (range 1-60). Presenting symptoms were: abdominal pain (36\%), weight loss (29\%), diarrhea (25\%), nausea/vomiting (18\%), anorexia (7\%), fever (7\%). Prior history of abdominal surgery was present in $25 \%$. SM was an incidental finding in one pt (4\%). Concurrent intraabdominal pathology was noted in 5 (18\%): Non-Hodgkin's lymphoma 2 (7\%), sclerosing pancreatitis 1 (4\%), metanephric adenoma l (4\%), retroperitoneal fibrosis 1 (4\%). SM related complications included bowel obstruction (36\%), chylous ascites (18\%), need for long-term TPN (11\%), superior mesenteric vein thrombosis $(7 \%)$. Two (7\%) deaths were noted during the study period. Surgical resection of the mesenteric mass was done in $10(36 \%), 6(60 \%)$ of whom remained symptomatic. Overall, $19(68 \%)$ received pharmacotherapy, 4 improved after surgery alone, and 5 (18\%) received no treatment. Response was assessed by clinical symptoms and imaging studies. Tamoxifen ( $10 \mathrm{mg}$ BID) in combination with a prednisone taper (beginning at 20-40 mg daily) was used in 13/19 (68\%) for a median of 6 mo (1-33 mo) and $7 / 13(54 \%)$ improved within 4 to 6 weeks of treatment. Prednisone alone or combination with colchicine (0.6 mg BID, n=3) or azathioprine $(1-1.5 \mathrm{mg} / \mathrm{kg}, \mathrm{n}=2)$ was given to $5 / 19$ for a median of 4 mo (1-13 mo) and $1 / 5$ (20\%) improved. One pt received thalidomide $(200 \mathrm{mg} / \mathrm{d})$ for 5 mo without improvement. Serious side-effects include deep venous thrombosis on tamoxifen $(n=2,7 \%)$ and sepsis-related death on thalidomide in one patient. CONCLUSION: This uncontrolled data suggests that symptomatic SM patients may benefit from tamoxifen/prednisone combination treatment. Long-term follow-up is needed to substantiate the results.

\section{M1197}

Peppermint Oil (Mintoil $\left.{ }^{\circledR}\right)$ in the Treatment of Irritable Bowel Syndrome: A Prospective Double Blind Placebo Controlled Randomized Trial Giorgio Cappello, Mariangela Spezzaferro, Daniela Coraggio, Laurino Grossi, Leonardo Marzio

Introduction: Peppermint oil has been shown to relax gastrointestinal smooth muscle in vitro and to be useful in patients with irritable bowel syndrome (IBS) and bacterial overgrowth (BO). Aim: Aim of the study has been to test the efficacy of peppermint oil (Mintoil囚, Cadigroup, Rome, Italy) in patients with IBS without $\mathrm{BO}$ and lactose intolerance. Methods: Forty eight patients with IBS according to Rome II criteria with normal lactose and lactulose breath test were randomly treated with Mintoil ${ }^{\circledR}$ ( 2 enteric-coated capsules twice per day or placebo) for four weeks. Symptoms score was assessed before the beginning of therapy at the end of therapy (4 weeks). The symptoms evaluated were: diarrhea (Di), abdominal bloating (AB), constipation (Co), pain lower abdomen (PLA), pain on defecation (PoD), feeling of incomplete evacuation (FIE), difficulty on evacuation (DoE). For each symptom intensity and frequency graded 0 to 4 were scored. Intensity 0 : absent, 1: mild, 2 moderate, 3:severe, 4: intolerable, Frequency : 0 absent, 1 once a month, 2: once a week, 3 three times per week, 4 everyday. The mean value between intensity by frequency was calculated for comparison. Results: The results show statistical significant improvement in all symptoms evaluated after 4 week of Mintoil® (24 patients) (Table 1), while only diarrhea and abdominal bloating improved after placebo (26 patients) (Table 2). The improvement in symptoms score for $\mathrm{AB}$ is greater with peppermint oil than with placebo. Conclusion: Peppermint oil and placebo both improve abdominal symptoms in patients with irritable bowel syndrome. Peppermint oil however, improves more symptoms than placebo with a better score of improvement.

Table I

\begin{tabular}{|c|c|c|c|c|c|c|c|}
\hline & Di & Bl & Co & PLA & PoD & FIE & DoE \\
\hline Mintoil & 1.960 .23 & 2.920 .12 & 0.960 .11 & 2.920 .81 & 2.330 .87 & 1.580 .21 & 1.130 .21 \\
\hline Mintoil@ 4 weeks & 1.410 .11 & 1.580 .21 & 0.250 .04 & 1.500 .14 & 1.420 .12 & 0.960 .03 & 0.580 .11 \\
\hline P & 0.03 & 0.001 & 0.05 & 0.001 & 0.04 & 0.05 & 0.05 \\
\hline
\end{tabular}




\begin{tabular}{|c|c|c|c|c|c|c|c|}
\hline & $\mathrm{Di}$ & $\mathrm{Bl}$ & $\mathrm{Co}$ & $\mathrm{PLA}$ & PoD & FIE & DoE \\
\hline placebo & $1.88 \pm 0.28$ & $3.12 \pm 0.45$ & $1.42 \pm 0.23$ & $3.00 \pm 0.45$ & $2.08 \pm 0.45$ & $2.12 \pm 0.15$ & $1.00 \pm 0.12$ \\
\hline placebo 4 weeks & $1.12 \pm 0.15$ & $2.31 \pm 0.67$ & $0.96 \pm 0.24$ & $2.81 \pm 0.05$ & $2.42 \pm 0.89$ & $1.65 \pm 0.54$ & $0.88 \pm 0.34$ \\
\hline $\mathrm{P}$ & 0.03 & 0.03 & 0.17 & 0.3 & 0.025 & 0.09 & 0.36 \\
\hline
\end{tabular}

\section{M1198}

Development of a Hybrid Algorithm Based On Learning Classifiers That Improves Diagnosis of Inflammatory Bowel Disease and Differentiation Between Crohn's and Ulcerative Colitis in a Six-Marker System Augusto Lois, Frank Wadleigh, Bruce Neri

The use of combinations of serological markers has resulted in improved IBD diagnosis. Currently, marker cut-offs are commonly used to predict IBD. Generalized linear and nonlinear regression analyses have improved the prediction capability of these assays. However, significant advances have been made recently in the use of learning statistical classifiers like Neural Networks (NN), Support Vector Machines (SVM) and Classification and Regression Trees (C\&RT) to classify complex data, find underlying patterns and discover non-linear relations in data from multiple variables. These methods have been used successfully to predict the outcome and prognosis of cancer, glaucoma and other diseases. In this study, learning statistical classifiers were used to create an algorithm that predicts IBD and differentiates UC from CD with a six-marker system. C\&RT and NN were chosen because they can produce models with complementary analytical capabilities. Serological data was obtained from multiple markers using a cohort of 813 patients with $60 \%$ IBD prevalence. The markers comprised IgG antibodies to Cbirl, IgA to the E. coli-associated outer membrane protein C (OmpC), IgA and IgG anti-)Saccharomyces cerevisiae mannan antibodies (ASCA-IgA and ASCA-IgG), anti-neutrophil antibodies with a DNase-sensitive perinuclear staining. Multilayer Perceptron NN was trained with Back Propagation using results from 500 patients of the cohort. Multiple NN models were then tested using data from the remaining 313 patient samples. Similarly, standard and boosted C\&RT were trained and tested using different data sets. Both, NN and C\&RT produced models that improved the sensitivity and specificity of the assay in comparison to regression analysis ( $5 \%-15 \%$ increase in sensitivity and specificity). However, the best outcome was obtained with a hybrid algorithm that uses a C\&RT model in tandem with a NN model. The sensitivity and specificity of the hybrid algorithm for IBD prediction was $>90 \%$. Positive and Negative Predictive Values were $90 \%$ and $80 \%$ respectively. The sensitivities and specificities for CD and UC prediction were above $88 \%$. Conclusion: The use of a hybrid algorithm based on C\&RT and NN to analyze the results from six serological markers resulted in a significantly more accurate detection of IBD, CD and UC.

\section{M1199}

Multiple Adenomatous Polyposis (MAP) Patients in France: Prospective Characterization of This Population

Graber Ivan, Moussata Driffa, Gelas Dore Benedicte, Barthelemy Claude, Pelletier Michel, Phelip Jean-Marc, Lapalus Marie George, Pillon Didier, Nallet Bernrd, Leroux Dominique, Prieur Fabienne, Saurin Jean-Christophe

Aim: MAP is defined by the presence of 3-100 colorectal adenomas. The clinical and endoscopic characteristics of this population are poorly known. Retrospective series have suggested a role for APC/familial adenomatous polyposis (FAP) germline mutations in $8 \%$ of cases. Our first aim was to identify epidemiologic and endoscopic characteristic features of these patients. Patients and methods: Consecutive patients with 3-100 synchronous colorectal adenomas, from 9 hospitals of the French Rhône-Alpes region were included prospectively in this study after exclusion of known FAP and HNPCC cases. The study protocol included colonoscopy and duodenoscopy with indigo-carmin dye, a standardized epidemiological questionnaire and genetic counselling. Biological analysis of adenomas and analysis of germline APC and MYH mutations will be performed in a second part of this study. Results: From September 2003 to April 2005, 118 consecutive patients (90 males, $76.2 \%$, mean 61.2 years old, $95 \%$ CI $45-80$ ) were included. Colon ( $82 \mathrm{pts})$ : diffuse polyposis after dye spraying in the right colon $1.2 \%$, in the sigmoid colon $7.1 \%$. At least 1 adenoma per site: right colon $76.4 \%$, transverse colon $47.1 \%$, left colon $72.3 \%$, rectum $40 \%$.Adenoma maximal size $(\%$ pts): $<5 \mathrm{~mm}$ 22.6, 5-10mm: $37.8,>10 \mathrm{~mm}$ : 39.6. Histology (\%pts): highgrade dysplasia 22.7, intra-mucosal cancer: 2.7 , >or=sub -mucosal: 8.1 . Morphology(\%pts): pedunculated 36.5\%; plan or sessile $98.0 \%$. Stomach and duodenum (42pts): At least 1 duodenal adenoma 2 cases (4.76\%); fundic gland polyp 2 cases $(4.76 \%)$. Epidemiology (84pts): tobacco consumption $71.4 \%$ (mean duration 26.9 years, $95 \% \mathrm{CI}: 2.35-52.45,23.3 \%$ ongoing); alcohol $76.2 \%$ (wine, mean 17.9 glasses/week, mean duration 23.5 years). NSAIDs intake $22.7 \%$ (aspirin 16.7\%). Genetic counselling (52 pts) revealed the existence of a first degree relative with adenoma or colo rectal cancer in $50 \%$ and $40 \%$ of cases respectively. Criteria for HNPCC were unfrequent: one case of colorectal cancer before 50 years in the family in $9.4 \%$ and two first degree relatives with colorectal cancer in $9.4 \%$. Conclusion: We indentified unexpected epidemiologic characteristics of the MAP population: high male predominance with alcohol and tobacco consumption background. Less than $7 \%$ of MAP patients present endoscopic features compatible with attenuated FAP. Genetic counselling is not in favour of a high proportion of HNPCC cases.
Pancreas Evaluation Using the Endoscopic Ultrasound in Crohn's Disease Everson F. Malluta, Fauze Maluf-Filho, Carmem O. Agostinho, Ieda Nishitokukado, Andre A. Leite, Damiao O. Aderson, Sipahi Aytan

BACKGROUND Pancreas injury usually is not mentioned as an extra-intestinal manifestation of Crohn's disease, but the few available series suggest pancreatic injury in a significant proportion of these patients, ranging from $6 \%$ to $38 \%$ in this series. Endoscopic ultrasound (EUS) presents the advantage of having a much higher sensitivity than the abdomina ultrasound or computadorized tomography in evaluating pancreas abnormalities. Compared to the endoscopic retrograde cholangiopancreatography, EUS has at least an equal sensitivity, with a lower complication rate. AIMS To evaluate the incidence of pancreatic alterations by means of the EUS in Crohn's disease, correlating them with clinical, endoscopic and biochemical data, in order to determine possible predictive factors. METHODS Fifty patients with Crohn's disease, age between 18 and 60 years-old (mean $=38$ ), without previous history of pancreatic disease, diabetes mellitus or alcoholism, were submitted to EUS. Thirteen variables were analyzed, both in pancreatic parenchyma and ducts. The patients with 3 or more alterations were submitted to magnetic resonance. The control-group was formed by patients submitted to EUS with no previous history of pancreatic or Crohn's disease. RESULTS Of the 50 analyzed patients, $55 \%$ were female, with mean diagnosis time of seven years (125 ) and Crohn's Disease Activity Index (CDAI) of 102 (20-419). Two patients (4\%) presented 4 alterations in the EUS exam, $3(6 \%)$ presented 3 alterations, 10 (20\%) had 2 alteration and $13(26 \%)$ had 1 alteration in the EUS, which were statistically significant when compared to the control-group, in whom only $16 \%$ presented 1 exam alteration $(\mathrm{p}<0.001)$. In both groups, all the abnormalities were parenchymal, while ductal changes were not found. The patients with 3 or more alterations in the exam were submitted to magnetic resonance; however, pancreatic lesions were not detected. The only predictive factor that correlated with the number of alterations in EUS was ileal disease. CONCLUSIONS Patients with Crohn's disease had a higher incidence of pancreatic abnormalities (10\% with 3 or more alterations in endoscopic ultrasound) in comparison to the control-group. These alterations were found in the pancreatic parenchyma, which might explain the lack of correlation with the magnetic resonance, which is more prone to detect duct abnormalities. The only predictive factor to these alterations on EUS was ileal disease

\section{M1201}

Good Long-Term Results Are Possible with Anterior Anal Sphincter Repair Sushil K. Maslekar, Angela B. Gardiner, Graeme S. Duthie

Introduction Anterior anal sphincter repair (ASR) is standard treatment for faecal incontinence due to a structurally damaged anal sphincter. Long-term results of repair have generally been shown to be poor. This review of single surgeon series aimed to determine the longterm outcome of primary ASR for patients with faecal incontinence due to anatomical sphincter defects. Methods This study included patients undergoing ASR from 1995 to 1999. We perform standard ASR, but external and internal sphincters are repaired separately. The internal sphincter is repaired only if damaged. Telephonic interview was conducted with all the patients, following which questionnaires including SF-36 survey, faecal incontinence quality of life (FIQOL) questions and Wexner score-type questions were sent at 6 months and at median follow-up of 6 years. The questionnaire and telephonic interview also assessed bowel function and continence, restriction in activities related to bowel control and overall satisfaction. Demographic data and anorectal physiology was obtained from a review of clinical case notes. Results 64 of 72 patients returned questionnaires and operation was considered success in $75 \%$ of patients at median follow-up of 72 months. Six patients had undergone further procedure for incontinence and 58 patients were analysed. 10 patients reported complete continence to stool and flatus (18\%), which is best ever reported. Median continence scores had improved from Wexner score of 14 to 5 ( $\mathrm{p}<.0 .001) .75 \%$ of patients reported an improvement of continence, with $70 \%$ patients who improved at 6 months continuing to do so. $95 \%$ of patients were satisfied with their operation. There was an improvement in all aspects of FIQOL and in the mental health components of SF-36. None of the anorectal physiology variables including pudendal nerve terminal motor latency were of any value in predicting the outcome. Conclusion We have shown that good long term results can be achieved with anterior anal sphincter repair. The modified technique could explain the improved outcomes.

\section{M1202}

Association of Helicobacter Pylori Infection and Persistent Diarrhea in Malnourished Young Bangladeshi Children

Pradip K. Bardhan, Shafiqul A. Sarker, Zahid Hasan, Niklaus Gyr, Christoph Beglinger

Background: Gastric acid is an important nonimmune defence mechanism against gastrointestinal infections. In the developing countries, H. pylori (HP) infection occurs very early in the life, and the resulting gastritis may lead to hypochlorhydria and increased susceptibility to gastrointestinal infections, particularly in malnourished children. Studies examining the relationship between HP infection and diarrhea have reported conflicting results. Aim: To examine the association between HP infection and acute diarrhea (AD) and persistent diarrhea (PD) in young Bangladeshi children. Methods: This was a clinic-based case-control study with community controls. The cases were children aged 6-24 months suffering from $\mathrm{AD}$ (diarrhea $<3$ days) or PD (diarrhea $>15$ days, acute onset). The controls were asymptomatic age-matched children selected randomly from rural and peri-urban Bangladeshi communities. None received any antibiotic in the preceding 3 months. Status of HP infection was determined by ${ }^{13} \mathrm{C}$-urea breath test (UBT) and HP fecal antigen (FA) test. Only children with concordant results from both UBT and FA were included. Anthropometric, fecal microscopic and microbiological, nutritional, socioeconomic, and dietary data were also collected. Data were analyzed by $X^{2}$ test and logistic regression. Results: The study included 61, 51 and 64 children as $\mathrm{PD}, \mathrm{AD}$ and controls respectively. Age and socioeconomic status among the three groups were comparable. Malnutrition (wt./age $<75 \%$ of NCHS median) was noted in $41(67 \%)$ children with PD, $30(59 \%)$ with $\mathrm{AD}$, and $44(69 \%)$ in controls. The number 
of $\mathrm{HP}$ positive children was 34 (56\%) in $\mathrm{PD}, 23$ (45\%) in $\mathrm{AD}$, and 25 (39\%) in controls, without any significant difference. However, among the malnourished children, HP infection was significantly more often present in the PD group, but not in the AD group, when compared to the controls (Table). In logistic regression, HP infection appeared to be a significant risk factor for PD (Odds ratio 1.91, 95\% confidence interval 1.06-3.44, $\mathrm{p}=0.042$ ), but not for $\mathrm{AD}$. The covariates in the regression equation were age, gender, nutritional status, socioeconomic status, fecal pathogens, breast-feeding, immunization, mother's education, and place of residence. Conclusion: A significant association was noted between HP Infection and PD in malnourished Bangladeshi children. Intervention of HP infection may decrease the incidence of PD in this vulnerable population.

\begin{tabular}{|c|c|c|c|}
\hline & PD & AD & Controls \\
\hline HP (+) & 24 & 13 & 15 \\
\hline HP (-) & 17 & 17 & 29 \\
\hline
\end{tabular}

$X^{2}$ test, $\mathrm{p}=$ Overall 0.08; PD vs Control 0.04; $\mathrm{AD}$ vs Control 0.58

\section{M1203}

Gastrointestinal Inflammation Modulates Differentially the Expression of Cellular Prion Protein PRPC

Peter C. Konturek, Karolina Bazela, Vitalyy Kukharskyy, Detlef Schuppan

Background and aims: The main entry of infectious prions inducing transmissible spongiform encephalopaties (TSE) represents the gastrointestinal (GI) tract. Our previous studies demonstrated that mucosal epithelial cells in GI tract express PrPc which may represent a first target for infectious prions after oral inoculation. However, it is not known whether GI inflammatory states could influence PrPc expression and thus potentially modeluta the propagation of prions from $G$ tract. The analysis of PrPc expression at mRNA and protein level was analyzed in biopsy specimens from 3 different chronic human diseases of GI tract : H.pylori gastritis, sprue nad Crohn disease. Patients and methods: The biopsy material was obtained during endoscopy from patients with $\mathrm{Hp}$ gastritis before and after eradication therapy $(n=12)$, sprue under gluten free diet $(n=10)$ or without treatment $(n=10)$ and in patients with active Crohn disease $(\mathrm{CDAI}>150)$. The expression of $\mathrm{PrPc}$ was analyzed at mRNA and protein level by quantitative RT-PCR (Taqman) and Western blot, respectively. In addition, two different cell lines (gastric MKN 45 and colonic LoVo) were incubated with different mediators of inflammation such as prostaglandin PGE2 (10-100 $\mu \mathrm{M})$, IL-1 $\beta$ (1$10 \mathrm{ng} / \mathrm{ml}), \mathrm{TNF} \alpha(1-10 \mathrm{ng} / \mathrm{ml}), \mathrm{TGFb}(0.1-1 \mathrm{ng} / \mathrm{ml})$ and gastrin $(1-100 \mathrm{nM})$. Results: PrPc was upregulated in Hp gastritis and decreased after eradication to the level observed in the control (gastric mucosa without $\mathrm{Hp}$ ). In contrast, in the intestinal mucosa of sprue patients and colonic mucosa of Crohn patients a significant downregulation of PrPc as compared to normal intestinal mucosa was observed. The incubation of gastric epithelial cells with gastrin and PGE2 caused a significant dose-dependent upregulation of PrPc. Colonic cells (LoVo) incubated with PGE2 and TGF $\beta$ and ILI $\beta$ caused a significant downregulation of $\operatorname{PrPc}$ expression in these cells. Conclusion: Chronic inflammatory states can differentially modulate the expression of PrPc in the GI tract. PrPc is significantly upregulated in Hp gastritis and this upregulation may be linked to Hp-induced hypergastrinemia and increased PGE2 and ILl $\beta$ synthesis. In the intestinal mucosa of sprue patients and colonic mucosa of Crohn patients PrPc is downregulated. Ill $\beta$, TGF $\beta$ and PGE2 may mediate at least partly this downregulation.

\section{M1204}

Double-Balloon Enteroscopy Had Superior Diagnostic and Therapeutic Impact Than Push Enteroscopy for the Diagnosis and the Treatment of Lesions Identified By Videocapsule Endoscopy

Thibaut Maniere, Bruno Landi, Marion Simon, Joel Edery, Bernard Boboc, Raymond Jian, Christophe Cellier

Introduction Double balloon enteroscopy (DBE) is a new tool for the investigation of the small bowell. The aim of the study was to compare DBE and with classical push enteroscopy (PE) in patients with a lesion diagnosed by the videocapsule endoscopy (VCE) Patients and methods Seventy-eight DBE were performed between january 2004 and september 2005 in 67 patients $(37 \mathrm{~F}, 30 \mathrm{H}$, mean age 64 years [12-90] for an obscure gastro-intestinal bleeding (occult GI bleeding $30 \mathrm{pts}$, macroscopic bleeding $38 \mathrm{pts}$ ). A standardized data form was prospectively filled for all the patients. Sixty-two oral route and 16 anal route DBE were performed (double route DBE in 8 pts, 2 oral route DBE in 2 pts). All patients have had previously negative upper and lower GI endoscopy and at least one positive finding at VCE. The potential bleeding of the lesions visualized at CE were classified as follow: PO (low) in 14 cases, P1 (intermediate) in 32 cases, and P2 (high) in 21 cases. The results of DBE were compared with those obtained in a control group of 27 patients explored for obscure GI bleeding by PE after VCE in a previous prospective study of our group. Results DBE was considered as normal or unconclusive in 31 pts. The diagnostic yield was of $60 \%: 58 \%$ in occult GI bleeding, $62 \%$ in macroscopic bleeding. The diagnostic yield was related to the degree of bleeding potential of the lesion at VCE: 74\% in case of lesion P2, 45\% in case of lesion $\mathrm{Pl}, 44 \%$ in case of lesion P0 ( $\mathrm{p}=0.1)$. The diagnostic yield was higher by upper way ( $67 \%$ ) than by lower way ( $37 \%$; $\mathrm{p}=0.06)$. A treatment by argon plasma electrocoagulation was performed in 39 cases $(61 \%)$. The diagnostic yield was higher for DBE than PE $(60 \%$ vs $41 \% ; \mathrm{p}=0.1$ ), as well as the therapeutic yield ( $61 \%$ vs $22 \% ; \mathrm{p}=0.003)$. Conclusion: In patients explored for GI bleeding with a lesion diagnosed at CE, DBE is superior to PE in terms of diagnotic and therapeutic yield.

\section{M1205}

Routine Ascitic Fluid Analysis in Ambulatory Outpatients with Ascites Is Not Necessary

Karin Cesario, David Barnes, William Carey, Robert O'Shea, Nizar Zein

Background/Aims: AASLD practice guidelines (2004) recommend ascitic fluid analysis with outpatient therapeutic paracentesis. Aims were to determine the incidence and significance of neutrocytic ascites in an ambulatory population and to evaluate if routine cell counts are warranted. Methods: All patients who had therapeutic paracentesis between November 2004 and May 2005 were identified. Those hospitalized, on antibiotics [other than spontaneous bacterial peritonitis (SBP) prophylaxis] and/or complaining of fevers, chills or abdominal pain at the time of the procedure were excluded. Demographic and laboratory parameters were examined; neutrocytic ascites was defined as a polymorphonuclear (PMN) count of $>$ 250/mm3. Patients with neutrocytic ascites underwent repeat paracentesis with ascitic fluid analysis/cultures within 24 hours and prior to initiation of antibiotic therapy. Results: 361 therapeutic paracenteses in 95 patients were performed. Subject mean age and MELD scores were $58.4+9.6$ years and $15.0+6.7$, respectively. The majority of subjects were male $(64 \%)$, Caucasian $(78 \%)$ and had cirrhotic ascites secondary to alcohol (25\%), chronic hepatitis C virus infection $(13 \%)$ or a combination of both $(11 \%)$. Four cases of neutrocytic ascites were identified; a $1.1 \%$ incidence. There were no significant differences in age, serum albumin or sodium between subjects with and without neutrocytic ascites (all p-values $>0.05$ using unpaired t-test). Likewise, there were no statistical differences in race, gender or ascites etiology (all p-values $>0.05$ using Fisher's exact test). Upon repeat paracentesis and prior to antibiotic therapy, two of the four subjects no longer had elevated PMNs and had negative ascites cultures. The third again had elevated PMNs and was treated with a course of intravenous antibiotics. The fourth refused repeat paracentesis and was treated with a 5day course of oral antibiotics. Paracentesis after completion showed resolution of neutrocytic ascites and negative ascites cultures. Conclusions: In asymptomatic outpatients, neutrocytic ascites is (1) uncommon (2) may be transient and (3) is not well correlated with positive cultures or clinical infection. Our data suggests that routine ascitic fluid analysis in an ambulatory outpatient population may not be cost-effective or justified. This issue should be readdressed in future AASLD practice guidelines.

\section{M1206}

Predictive Factors of Survival After TIPS Insertion for Refractory Ascites Sylvie Evrard, Frederik Nevens, Abbas Mroue, Michael Adler, Jacques Deviere, Olivier Le Moine

Background: TIPS may improve the control of refractory ascites (RA) in patients with cirrhosis. However, it may induce severe liver failure and therefore jeopardise their chance to benefit from liver transplantation. Currently, no criteria exist to adequately select patients for TIPS and assess their short-term prognosis after the procedure. Aim: To identify preprocedural clinical and biological variables associated with survival after TIPS placement in a large cohort of patients with RA. Methods: 117 consecutive patients, from 2 belgian referral centers, who underwent non covered TIPS for RA between January 1992 and August 2004 were retrospectively analysed. Univariate and multivariate analysis (Cox regression) were performed to find the independent variables associated with 1 and 6 months survival. Results: The population included $75 \%$ of alcoholic cirrhosis and $62 \%$ of Child B patients. No procedure related deaths were encountered and survival was $85 \%, 66 \%$ and $58 \%$ at 1 , 6 and 12 months, respectively. Ascites control was observed in $87 \%$ and $91 \%$ of surviving patients after 1 and 12 months, respectively. Thirty eight \% of patients developed encephalopathy after TIPS. Alcoholic etiology (ETIO), pre-TIPS low bilirubin level (BILI) and high PTT were independent predictors of 1-month survival (OR: 2,5; 1,08 and 0,96 respectively). A prognostic index (pi) could be calculated as following: pi=exp $\{(0,085 \times B I L I)+(-$ $0,038 \mathrm{xPTT})+(0,903 \mathrm{xETIO})\}$ where BILI is expressed in $\mathrm{mg} / \mathrm{dl}$, PTT in $\%$ and ETIO as 1 if alcoholic or 2 non-alcoholic. By plotting pi on a Youden curve, the best cut-off value was 1,2 . Values of pi $<1,2$ predicted $96 \%$ of the patients who will survive 1 month after TIPS (specificity) whereas values $>1,2$ were less sensitive to predict death (44\%). When analysing 6 months survival, ETIO and PTT were still independently predicting outcome. Conclusions: Alcoholic cirrhosis and preserved liver function are predictive of survival after TIPS for RA. The prognostic index (pi) may help select the patients who will benefit the most of the procedure without jeopardising further liver transplantation. Prospective validation of this index is now mandatory.

\section{M1207}

Cost Comparison of Transjugular Intrahepatic Portosystemic Shunt Versus Paracentesis in the Treatment of Refractory Ascites Jason Taylor, Layla Hajjafar, Anthony Post

Background: Ascites is a common complication of cirrhosis, refractory to medical treatment in about $10 \%$ of cases. Current treatment modalities include repeated large volume paracentesis (LVP) or transjugular intrahepatic portosystemic shunts (TIPS). Neither treatment has been shown to be superior with respect to mortality. We performed a cost comparative study of TIPS vs. repeat LVP with albumin infusion for cirrhotic patients with refractory ascites. Methods: We created a decision model using data from 5 previous randomized controlled trials. We determined the one and two-year rates of treatment failure, complications, and mortality for both TIPS and LVP. In our analysis, we assumed that when patients failed the treatment in which they were randomized, they would then receive the alternative treatment. The costs of procedures and complications were calculated for each treatment group using Medicare reimbursement rates for the corresponding DRG or CPT codes on an intention-to-treat basis. The software program Data 3.5 was used (TreeAge, Williamstown,MA). Results: As illustrated in the table below, only at a frequency of $1.5 \mathrm{LVPs}$ per month over one year of treatment did we find the cost per patient treated to be less for the LVP group than for the TIPS groups. Otherwise, the cost of treating with TIPS was lower for all other frequencies and time periods. In addition, TIPS has lower mortality rates than 
LVP at both one and two years of treatment. Conclusion: TIPS is more cost-effective than LVP for the treatment of refractory ascites.

\begin{tabular}{|c|c|c|c|c|}
\hline \multirow{2}{*}{} & \multicolumn{2}{|c|}{ Cost per patient treated } & \multicolumn{2}{c|}{ Mortality } \\
\cline { 2 - 5 } & LVP & TIPS & LVP & TIPS \\
\hline 1 YEAR 1.5 LVPs/mo 2 LVPs/mo & $\$ 22,532 \$ 26,343$ & $\$ 24,849 \$ 25,165$ & $43.5 \%$ & $38.3 \%$ \\
\hline 2 YEAR 1.5 LVPs/mo 2 LVPs/mo & $\$ 35,436 \$ 42,315$ & $\$ 32,890 \$ 33,591$ & $57.2 \%$ & $50.0 \%$ \\
\hline
\end{tabular}

\section{M1208}

The Hemodynamic Role of Albumin in the Therapy of Type II Hepatorenal Syndrome

Carmen Fierbinteanu-Braticevici, Laura Tribus, Lucian Negreanu, Andreea Bengus, Radu Usvat, Dan Andronescu

Background: Type II hepatorenal syndrome(HRS) is a major complication of cirrhosis associated with gradual reduction of renal function and with a median survival time of 6 months,which recommends liver transplantation Aim: to assess the role of albumin in the therapy with terlipressin in type II HRS cirrhotic patients. Methods: 38 cirrhotic patients with type II HRS, were hemodynamicaly studied at diagnosis and after 14 days of treatment with terlipressin : 19 patients with albumin supplement (group I) and 19 patients without albumin (group II. The groups were matched for age, etiology and severity of cirrhosis. The patients underwent: creatinine clearance, urinary metanephrines, plasma renin activity (PRA), serum aldosteron and sodium homeostasis. Systemic, renal and hepatic hemodinamics were evaluated by mean arterial pressure (MAP), cardiac index $(\mathrm{CI})$, resistance index (RI) of renal artery, portal flow volume and velocity. Paired - $t$ test was used to compare the values among the groups. Results: Albumin treatment caused a significant improvement of the circulatory function as compared with group II indicated by an increse in the CI $(4,6$ vs 3,2 - $p<0,05)$, MAP (85 vs71 - $<<0,03$ ) as well as in portal flow velocity ( 15 vs $11 \mathrm{~cm} / \mathrm{sec}-\mathrm{p}<0,04$ ). Albumin substitution therapy also improved renal function consisting of a decrease in serum creatinine more than $30 \%$ from baseline values, normalisation of RI, increased urine volume and urinary sodium excretion. These changes were associated with a significant decrease of PRA (3,6 vs $7 \mathrm{ng} / \mathrm{ml} / \mathrm{h}-\mathrm{p}<0,001)$ and aldosterone ( $8 \mathrm{vs} 14 \mathrm{ng} / \mathrm{dl}-\mathrm{p}<0,05)$. Conclusions: Due to the beneficial circulatory effectiveness, the combination of terlipressin and albumin therapy restored renal function in cirrhotic patients with type II HRS, and this may be a solution for increasing the survival after liver transplantation.

\section{M1209}

Prevalence and Predictors of Venous Thrombosis in Hospitalized Patients with Cirrhosis

Kuntal M. Thaker, Raman Battish, Pradnya Mitroo, Kuldip S. Banwait, Madhusudhan R. Sanaka, Victor Navarro, Steven K. Herrine

Background: Cirrhosis results in impaired regulation of coagulation due to an imbalance of procoagulants, anticoagulants, and fibrinolytics. Despite the coagulopathy commonly seen in patients with cirrhosis, vascular thromboses have been described. Previous studies have shown an incidence of deep vein thrombosis (DVT) in the general hospital population of $0.1-3.17 \%$. However, the incidence of DVT in patients with cirrhosis has not been determined. Aim: To investigate the incidence and predictors of venous thrombosis in hospitalized patients with cirrhosis. Methods: A retrospective review of patients discharged from a tertiary care institution from 1/1/1985 to 3/31/2005 utilizing an electronic billing database was conducted. Diagnoses of venous thrombosis, DVT, pulmonary embolism (PE), and cirrhosis were searched. Charts were manually reviewed and only those admissions with documented acute venous thrombosis were included. Results: There were a total of 3547 patients ( 1254 females, 2293 males) with cirrhosis with mean age of $55 \pm 15$ years. There were 16 cases of PE in patients with cirrhosis $(16 / 3547=0.4 \%)$. DVT occurred in total of $103(2.9 \%)$ patients. Patients with cirrhosis and DVT (group 1) were compared to patients with cirrhosis and without DVT (group 2). Compared to group 2, patients in group 1 were older and had higher rate of mechanical ventilation, and previous history of DVT. There was no difference between the groups in respect to rate of malignancy, use of central lines, and coagulation profile (See Table 1). Conclusions: 1) Prevalence of DVT in patients with cirrhosis is similar to that of a general hospital population. 2) DVT and PE occur in patients with cirrhosis even in the setting of coagulopathy. 3) Older age, previous history of DVT and use of mechanical ventilation may serve as risk factors for DVT in patients with cirrhosis. 4) Future studies are warranted to establish guidelines for DVT prophylaxis in hospitalized patients with cirrhosis.

Table 1

\begin{tabular}{|c|c|c|c|}
\hline & Cirrhosis and DVT & Cirrhosis without DVT & p-value \\
\hline Age (years) & $61 \pm 14$ & $55 \pm 14$ & $<0.0001$ \\
\hline Mechanical ventilation (\%) & 34 & 10 & $<0.0001$ \\
\hline History of DVT (\%) & 8 & 0.41 & $<0.0001$ \\
\hline Central lines (\%) & 7 & 9 & NS \\
\hline Malignancy (\%) & 21 & 23 & NS \\
\hline PT (sec) & $17 \pm 5.4$ & $18 \pm 4.2$ & NS \\
\hline PTT (sec) & $37 \pm 16$ & $38 \pm 11.3$ & NS \\
\hline INR & $1.56 \pm 0.63$ & $1.45 \pm 0.48$ & NS \\
\hline Platelets (B/L) & $137 \pm 89$ & $130 \pm 89$ & NS \\
\hline
\end{tabular}

\section{M1210}

Diabetes Mellitus Worsens the Clinical Outcome of Acute Variceal Bleed in Cirrhotic Patients

Hasnain A. Shah, Shahid Majid, Mohammad Salih, Fasail W. Ismail, Khalid Mumtaz, Saeed S. Hamid, Wasim Jafri

Introduction: Association of Diabetes Mellitus (DM) and hepatic cirrhosis is well recognized and hyperglycemia induces splanchnic hyperemia with increase in portal pressures. There is scanty data regarding the clinical outcome in cirrhotic diabetics following variceal bleed. Aim: To compare the clinical outcome of gastro-esophageal variceal (GOV) bleed in cirrhotic diabetic patients with those without diabetes. Patients and Method: We reviewed the case notes of patients with GOV bleed admitted from June 2000-2005, under gastroenterology service at Aga Khan University Hospital. Diagnosis of cirrhosis was made on clinical, laborat ory, radiological findings. Liver biopsy was done in selected patients. Acute Variceal bleeding failure to control bleed and rebleeding were defined according to Baveno III consensus report. Results: A total of 839 admissions with variceal bleed occurred in 382 patients. Diabetes was present in $148(39 \%)$ patients.Comparison between diabetic and non diabetic cirrhotics with variceal bleed is presented in Table 1. Conclusion: The presence of Diabetes mellitus in patients with cirrhosis signifies a worse prognosis for control of acute gastroesophageal bleed and incidence of rebleed in hospital. The risk of rebleed following discharge is also increased in diabetic cirrhotics as compared to non diabetics.

Comparison between diabetic and non diabetic cirrhotics with variceal bleed is presented in Table 1.

\begin{tabular}{|c|c|c|c|}
\hline Variable & Diabetics n (\%) & Non diabetics n (\%) & P value \\
\hline 1) Age (yrs.) 2) Gender Male & $53.4 \pm 10.295(64 \%)$ & $52.2 \pm 13.4145(62 \%) 89$ & \\
Female 3) Child Pugh class. A B C & $53(36 \%) 7(4.7)$ & $(38 \%) 11(4.7) 73(31.2)$ & NS NS NS \\
4) Bilirubin <2 2-3 3 3) Ascites & $64(43.2) 77(52)$ & $150(64.1) 116(49.6)$ & 0.01 NS NS \\
6) No. of admissions with GI bleed & $101(68.2) 26(17.6)$ & $43(18.4) 75(35.1)$ & NS 0.001 0.001 \\
7) Failure to control bleed 8) & $21(14.2) 88(59.5)$ & $138(59) 2.03 \pm 1.0$ & 0.050 .02 \\
Rebleeding during hospital stay & $2.49 \pm 1.317(11.5)$ & $13(5.6) 17(7.3)$ & \\
\hline
\end{tabular}

\section{M1211}

Proinflammatory Cytokines Are Not Associated with Malnutrition in Liver Cirrhosis But with Altered Electric Tissue Properties

Kristina Norman, Nina Babel, Herbert Lochs, Petra Reinke, Matthias Pirlich

The inflammatory state has been hypothesized to trigger cachexia in liver cirrhosis and impair outcome. In addition low capacitance and phase angle as measured by bioimpedance (BIA) have been shown to be predictors of poor clinical outcome in liver cirrhosis and liver transplantation. Aims: We therefore investigated correlations between proinflammatory cytokines, the nutritional status and impedance parameters in 65 patients with liver cirrhosis Methods: Nutritional status was assessed with the Subjective Global Assessment, arm muscle area, arm fat area were measured anthropometrically. Bioelectrical impedance analysis (BIA$2000 \mathrm{M}$, Data Input Germany) was performed in order to obtain the impedance parameters capacitance and phase angle. Cytokines, tumour necrosis factor (TNF-alpha) and interleukin 6 (IL-6), were measured with ELISA. Albumin and CRP were measured according to standard methods. Results: Sixty-five patients with liver cirrhosis (age $56,6 \pm 12,4$ years, 35 male and 30 female Child A: 26 B: 22 and C: 17 patients; albumin 3,4 $40.62 \mathrm{~g} / \mathrm{dl}$ ) were recruited. 35 patients were classified well (SGA A) vs. 30 patients malnourished (SGA B or C). The capacitance and the phase angle were $72,2 \pm 12,8 \mathrm{Ohm}$ and $4,9 \pm 1,0^{\circ}$ in well vs. $42,1 \pm 14,0$ Ohm $4,1 \pm 0,9^{\circ}$ in malnourished patients. No correlations were found between TNF-alpha, IL-6 and nutritional parameters such as arm muscle area or arm fat area and cytokines were not higher in the patients classified malnourished by the SGA. (TNF alpha $12,1 \pm 10 \mathrm{pg} / \mathrm{mL}$ IL-6 9,6 $\pm 6,1 \mathrm{pg} / \mathrm{mL}, \mathrm{CRP} 0,27 \pm 1,1 \mathrm{mg} / \mathrm{dl}$ in well nourished patients vs. TNF alpha $11,4 \pm 4,4 \mathrm{pg} /$ mL, IL-6 14,0 $\pm 8,8$ pg/mL, CRP 2,33 $\pm 2,4 \mathrm{mg} / \mathrm{dl}$ in malnourished patients, all n.s.). However IL- 6 was shown to correlate closely with capacitance $(r=-0.420, p=0.001)$ and with the phase angle $(\mathrm{r}=-0.440, \mathrm{p}=0.01)$, whereas TNF-alpha did not correlate at all. Capacitance was also correlated to CRP $(r=-0.297, p=0.04)$ and albumin $(r=0.411, p=0.001)$. No correlations were obtained between cytokines, impedance parameters and disease severity as determined by the Child Pugh score. Conclusion: Inflammatory state does not seem to be associated with nutritional state as determined by the SGA or anthropometry. However IL6 and CRP are associated with altered electric tissue properties indicating impaired cell membrane integrity 
M1212

Cardiovascular Disease in Liver Cirrhosis. Correlations of Nutritional Status and Insulin Resistance with Serum Cholesterol Levels and Arterial Blood Pressure

Evangelos Kalaitzakis, Katrin Rabie, Einar Bjornsson

The prevalence of cardiovascular disease (CVD) in patients with liver cirrhosis(LC) has been reported to be low despite the frequent presence of diabetes(Marchesini et al, Am J Gastro 99). Malnutrition, low cholesterol and low arterial blood pressure(BP) are common in cirrhotics and have been proposed to be involved in this "protective" effect of LC. However data on the possible relation of CVD with nutritional status, cholesterol and BP in cirrhotics are scarce. The effects of nutritional status and insulin resistance on cholesterol and BP in LC are unclear. Methods:128 consecutive cirrhotics (mean(SD) age 57(11); Child-pugh 8.6(2.3); MELD 13.2(5.6);50F:55 alcoholic etiology) were prospectively evaluated for a history of CVD(stable angina, previous myocardial infarction or unstable angina), diabetes and hypertension. BP was measured and nutritional status assessed by anthropometry. In a subgroup of 95 patients fasting serum low- and high-density lipoprotein(LDL,HDL), glucose and insulin were measured. Insulin resistance was expressed as homeostasis model assessment index(HOMA). 59 healthy subjects(age 48(11),37F) served as controls. Results:20 cirrhotics $(16 \%)$ had CVD(8 stable angina, 9 previous infarction, 7 previous unstable angina). $33(26 \%)$ patients had diabetes, $32(25 \%)$ had hypertension and $38(30 \%)$ were malnourished. Patients with vs without CVD were older(66yr(10) vs $56 \mathrm{yr}(11) \cdot \mathrm{p}<0.01)$, more often hypertensive $(60 \%$ vs. $20 \%$; $<<0.01)$ or diabetic $(50 \%$ vs $22 \%$; $<0.01)$ and had lower $\operatorname{LDL}(1.8 \mathrm{mmol} /$ $\mathrm{l}(0.9)$ vs $2.5 \mathrm{mmol} /(1.4) ; \mathrm{p}<0.05)$ but did not differ in nutritional status, etiology or severity of LC. Patients with vs without malnutrition had lower diastolic $\mathrm{BP}(70 \mathrm{mmHg}(10)$ vs $75 \mathrm{mmHg}(11) ; \mathrm{p}<0.05)$. The correlations of age, BMI and insulin resistance with cholesterol and BP were found to be altered in LC compared to controls(table). Cirrhosis severity indices were negatively related to cholesterol(table). Conclusion:In cirrhosis, cardiovascular disease is related to age, hypertension and diabetes but not to etiology or severity of liver disease, malnutrition or cholesterol. Malnutrition is related to a lower diastolic blood pressure in cirrhotics. The effects of age, BMI and insulin resistance on cholesterol and blood pressure seem to be altered in cirrhosis.

\begin{tabular}{|c|c|c|c|c|c|c|c|c|}
\hline \multirow{2}{*}{} & \multicolumn{2}{|c|}{$\begin{array}{c}\text { Low-density } \\
\text { lipoprotein }\end{array}$} & \multicolumn{2}{c|}{$\begin{array}{c}\text { High-density } \\
\text { lipoprotein }\end{array}$} & \multicolumn{2}{c|}{$\begin{array}{c}\text { Systolic blood } \\
\text { pressure }\end{array}$} & \multicolumn{2}{c|}{$\begin{array}{c}\text { Diastolic blood } \\
\text { pressure }\end{array}$} \\
\cline { 2 - 9 } & Controls & Cirrhotics & Controls & Cirrhotics & Controls & Cirrhotics & Controls & Cirrhotics \\
\hline Age & $0.54^{*}$ & $\mathrm{~ns}$ & $\mathrm{~ns}$ & $0.22^{*}$ & $0.3^{*}$ & $\mathrm{~ns}$ & $0.29^{*}$ & $\mathrm{~ns}$ \\
\hline BMI & $\mathrm{ns}$ & $\mathrm{ns}$ & $-0.37^{*}$ & $\mathrm{~ns}$ & $0.29^{*}$ & $0.26^{*}$ & $0.46^{*}$ & $0.25^{*}$ \\
\hline HOMA & $0.29^{*}$ & $\mathrm{~ns}$ & $-0.33^{*}$ & $\mathrm{~ns}$ & $\mathrm{~ns}$ & $\mathrm{~ns}$ & $0.39^{*}$ & $\mathrm{~ns}$ \\
\hline Child & & $-0.29^{*}$ & & $-0.3^{*}$ & & $\mathrm{~ns}$ & & $\mathrm{~ns}$ \\
\hline MELD & & $-0.22^{*}$ & & $-0.21^{*}$ & & $\mathrm{~ns}$ & & $\mathrm{~ns}$ \\
\hline
\end{tabular}

* Pearson correlation coefficient for significant results $(\mathrm{p}<0.05)$

\section{M1213}

Tube Feeding Does Not Affect Subclinical Hepatic Encephalopathy in Patients with Liver Cirrhosis

Tatjana Schuetz, Kristina Norman, Ute Friedrich-Pagels, Johann Ockenga, Tam N. Luu, Herbert Lochs, Matthias Pirlich

Background: Protein-energy malnutrition is frequent in patients with liver cirrhosis (LC) However, nutritional supplementation with protein rich diets is considered contraindicated due to the risk of hepatic encephalopathy (HE). The aim of this study was, therefore, to investigate the effect of tube feeding with a high energy and protein intake on HE in a randomized controlled trial. Methods: Twenty-two hospitalized patients with LC (16 male; 6 female; age $59.9 \pm 10.4$ years) were randomly assigned to receive tube feeding in addition to normal food (TF-group, $\mathrm{n}=11 ; 3$ Child B, 8 Child $\mathrm{C}$ ) or normal food alone (C-group, n=11; 6 Child B, 5 Child C) for two weeks. The severity of HE was analyzed by 6 usually applied psychometric paper-pencil tests (number connection tests A and B, digit symbol test, pointing test, line tracing test time and line tracing test mistakes). In addition a newly established neurophysiologic tool (Gernot Schuhfried Inc., Austria) measuring visual discrimination ability (critical flicker frequency: CFF fusion frequency: FF) was used to identify patients with subclinical HE. Results: TF-patients received $2098 \pm 823 \mathrm{kcal} / \mathrm{d}$ and $80.8 \pm 42.2 \mathrm{~g}$ protein $/ \mathrm{d}$ compared to C-patients with $1409 \pm 527 \mathrm{kcal} / \mathrm{d}$ and $52.9 \pm 19.0 \mathrm{~g}$ protein/d. Before intervention both groups had a similar performance in both psychometric (TF: $-8.2+4.0$ points; $C:-7.6 \pm 5.0$ points) and neurophysiologic tests (TF: $F F=31.7 \pm 2.8 \mathrm{~Hz}$ $C F F=32.0 \pm 3.0 \mathrm{~Hz} ; \mathrm{C}: \mathrm{FF}=31.4 \pm 4.5 \mathrm{~Hz} ; \mathrm{CFF}=32.3 \pm 3.9 \mathrm{~Hz}$ ). Additional protein-rich tube feeding did not cause a deterioration in HE neither in psychometric ( $-8.5 \pm 3.7$ points) nor in the neurophysiological tests (FF: $31.4 \pm 4.5 \mathrm{~Hz} ; \mathrm{CFF}: 32.3 \pm 3.9 \mathrm{~Hz}$ ) after two weeks. Conclusion: Tube-fed LCI patients showed no deterioration in HE despite a high protein intake. Therefore high protein diets can be recommended in patients with LCI to meet their increased protein requirements.

M1214

Nonabsorbed (<0.4\%) Antibiotic Rifaximin Improves Hepatic Encephalopathy Symptoms in Patients with Cirrhosis Due to Hepatitis $C$

Melissa Palmer

Background: Lactulose, administered for the treatment of hepatic encephalopathy (HE), is often poorly tolerated. Data suggest antibiotics may be a useful treatment option for HE. Oral rifaximin (Xifaxan ${ }^{\circledR}$, Salix Pharmaceuticals, Inc., Morrisville, NC) is a gut-selective, nonabsorbed $(<0.4 \%)$ antibiotic with broad-spectrum activity in vitro. We prospectively investigated whether rifaximin in an outpatient setting would be effective and well tolerated for the treatment of stage $1 \mathrm{HE}$ in patients with cirrhosis due to hepatitis $\mathrm{C}$ virus (HCV) Methods: Consecutive patients with cirrhosis due to HCV diagnosed with stage $1 \mathrm{HE}$ (determined by West Haven criteria) were treated with rifaximin $400 \mathrm{mg} 3$ times daily for 14 days. All patients were assessed 24 hours prior to the start of therapy and 14 days after completion of therapy for multiple parameters, including ability to perform mental tasks, asterixis, and a quality of life (QoL) composite score (i.e., altered sleep patterns, personality change, short-term memory loss, attention span, reaction time, slow/slurred speech, and general QoL). Results: A total of 37 consecutive patients were enrolled and no patient was receiving therapy for $\mathrm{HE}$ at study entry. Twenty-three patients were receiving pegylated interferon (IFN) plus ribavirin for chronic $\mathrm{HCV}$, and 17 of these 23 patients were receiving a selective serotonin reuptake inhibitor for mild IFN-induced depression. Type 2 diabetes mellitus (DM) was reported in 12 patients. Rifaximin treatment lowered serum ammonia to normal levels in all patients, and overall, HE symptoms improved. Rifaximin was also well tolerated, with all patients completing treatment. One patient missed 2 rifaximin doses on day 9 due to complaints of flatulence and abdominal bloating, which resolved without medical intervention. There was a low incidence of adverse events (AEs): 3 patients (8\%) reported mild bloating, and there was 1 report each of mild abdominal pain, diarrhea, nausea, and headache. No hypo- or hyperglycemic episodes were reported during the study. Conclusion: This pilot study indicated that rifaximin is well tolerated and effective in improving symptoms of stage $1 \mathrm{HE}$ in patients with cirrhosis due to $\mathrm{HCV}$. In addition, no glycemic episodes were reported in patients with DM. Given that neomycin is associated with a risk for nephrotoxicity and lactulose administration in patients with DM is not ideal, rifaximin may be the more appropriate treatment for patients with DM. In addition, symptoms of HE can be mistaken for pegylated interferon and/or ribavirin AEs, and patients treated with these medications should be periodically evaluated for HE.

\section{M1215}

Disrupted Diurnal Rhythm of Autonomic Balance in Patients with NonAlcholic Liver Cirrhosis

Chung-Wang Ko, Chi-Sen Chang, Gran-Hum Chen, Hong-Zen Yeh

AIM: To evaluate the impairment of autonomic system in cirrhotic patients. METHOD:18 non-alcoholic cirrhotic patients and 17 normal subjects were enrolled. The signals of ECG for analysis of heart rate variability were measured by ambulatory 24-hr ECG.Power spectrum analyses of heart rate variability were performed. The low-frequency band (LF,0.04 0.15 $\mathrm{Hz})$ and high-frequency band $(\mathrm{HF}, 0.15 \sim 0.50 \mathrm{~Hz}$ ) were computed by estimating the power or variance of the beat-to-beat fluctuations in the heart period that fall into slow and fast rhythm. Parameters include power of HF, LF, and ratio of LF-to-HF (LF/HF) which represents parasympathetic, sympathetic modulation, and point of autonomic balance respectively. Each of them was evaluated in daytime(9AM 8PM)and nighttime(11PM $\sim 5 \mathrm{AM})$ periods. To evaluate the diurnal rhythm, the nighttime to daytime ratio of $\mathrm{HF}, \mathrm{LF}$, and LF/HF were calculated. Mann-Whitney U test was applied for statistic analysis. RESULT: Comparing to normal subjects, the daytime HF, LF, and LF/HF in cirrhotic group are $65.8 \pm 79.9$ vs $89.9 \pm 35.6 \mathrm{msec} 2(\mathrm{p}<0.05) ; 134.7 \pm 185.3$ vs $261.7 \pm 147.6 \mathrm{msec} 2(\mathrm{p}<0.05)$; and $2.17 \pm 1.40$ vs $3.15 \pm 1.75(\mathrm{p}<0.05)$. The nighttime $\mathrm{HF}, \mathrm{LF}$, and $\mathrm{LF} / \mathrm{HF}$ are $126.0 \pm 134.0 \mathrm{vs}$ $225.0 \pm 182.1 \mathrm{msec} 2(\mathrm{p}<0.05) ; 201.1 \pm 167.5$ vs $368.4 \pm 393.1 \mathrm{msec} 2(\mathrm{p}>0.05) ; 2.27 \pm 1.86$ vs $1.75 \pm 1.08(\mathrm{p}>0.05)$. The ratios of nighttime to daytime parameters are $2.68 \pm 2.01$ vs $2.89 \pm 2.97$ in $H F(p>0.05) ; 2.49 \pm 2.46$ vs $1.46 \pm 1.25$ in $L F(p>0.05) ; 1.05 \pm 0.71$ vs $0.61 \pm 0.29$ in $\mathrm{LF} / \mathrm{HF}(\mathrm{p}<0.05$, figure).CONCLUSION: Our unique finding is the disruption of diurnal variation in autonomic balance. Other abnormalities in autonomic regulations include suppressed parasympathetic modulation in both daytime and nighttime periods and suppressed sympathetic modulation in the daytime. (Supported by Taichung Veterans General Hospital Research Program-TCVGH93).

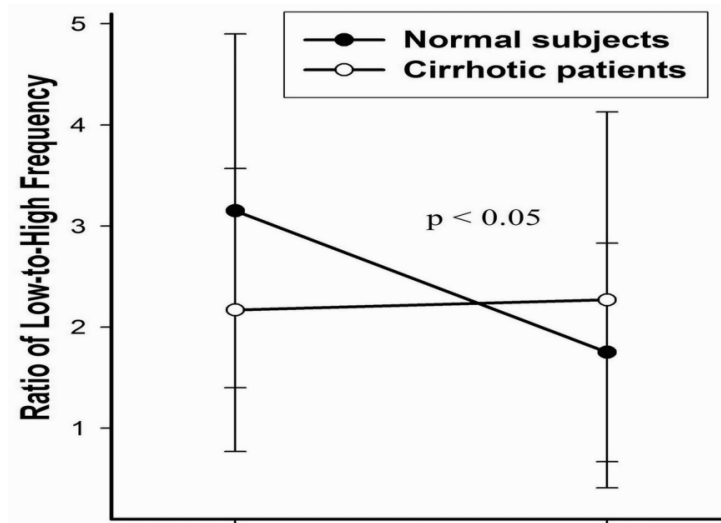

daytime

nighttime

Figure. Disruption of diurnal variation in autonomic balance

\section{M1216}

Autoimmune Pancreatitis: Response to Therapy in a UK Series Nicholas I. Church, Stephen P. Pereira, Adrian R. Hatfield, George J. Webster

Background Autoimmune pancreatitis (AIP) is a rare condition which usually presents with a pancreatic mass, biliary and pancreatic duct stricturing, jaundice and a raised serum IgG4 A response to steroids is a diagnostic criterion, although historical data from our unit suggest that spontaneous improvement may occur. This is the first UK series of AIP patients in 
which objective responses to active treatment are reported. Methods All 9 patients treated for AIP at our centre in 2004-5 were included. Intrahepatic/hilar stricturing was present in 8 at diagnosis, and plastic stents for jaundice/dominant CBD/hilar strictures were sited at ERCP in 5 (although 4 patients remained jaundiced despite apparently optimal stenting). Oral prednisolone $30 \mathrm{mg}$ OD was commenced in all patients, with gradual dose reduction over 4 weeks. In cases of disease relapse following steroid reduction, azathioprine was commenced. Response was assessed in terms of pre- and post-steroid symptoms, liver biochemistry and radiological appearances. Results Within 4 weeks of starting steroids, all patients reported significant symptomatic improvement. After 8 weeks, median bilirubin levels had fallen from $38 \mu \mathrm{mol} / \mathrm{l}$ (range 8-232) to $9 \mu \mathrm{mol} / /(5-27),(\mathrm{p}=0.004$ by Wilcoxon matched pairs test); median ALT from $88 \mathrm{IU} / \mathrm{(22-276)}$ to $57 \mathrm{IU} / \mathrm{l}(26-76),(\mathrm{p}=0.008)$, and ALP from $496 \mathrm{IU} / \mathrm{l}(149-978)$ to $193 \mathrm{IU} / \mathrm{(64-424)}(\mathrm{p}=0.004)$. Repeat ERCP (7 patients) and MRCP (2 patients) after 3-6 months showed improvement in pancreatic and extrahepatic/intra-hepatic biliary stricturing in all cases, with almost complete resolution in 4 . Biliary stents were removed in all cases, with no recurrence of jaundice (median follow-up 4, range 3-12 months). CT/MRI showed resolution of mass lesions in 3, and significant improvement in the rest. Eight patients remain on low dose steroids (range 3-12 months). Two patients relapsed on steroid dose reduction, with improvement on adding azathioprine. One patient discontinued steroids after nine months and has remained well. Conclusion Patients with AIP appear to show a good objective response to steroids. Disease relapse may occur following steroid reduction, but introduction of azathioprine may be of benefit. Further work is required to define the clinical course of this unusual condition, its aetiopathogenesis, and the optimal treatment regimen.

\section{M1217}

Clinicopathological Features of Autoimmune Pancreatitis in Relation to Elevation of Serum IgG4

Terumi Kamisawa, Naoto Egawa, Hitoshi Nakajima, Kouji Tsuruta, Atsutake Okamoto

Background and Aim: Autoimmune pancreatitis (AIP) is characterized serologically by elevation of serum IgG4 concentration. Incidence of serum IgG4 elevation in patients with AIP was reported to be from $68 \%$ to $95 \%$. In AIP, the characteristic histological finding of diffuse lymphoplasmacytic infiltration with fibrosis is observed in the peripancreatic retroperitoneal tissue, the biliary duct, and the salivary glands as well as in the pancreas. We have previously reported that infiltrations of abundant IgG4-positive plasma cells were detected in various organs in AIP patients but were not detected in other diseases, and suggested that AIP represented a pancreatic lesion of an IgG4-related systemic disease. This study aimed to examine the clinicopathological differences between patients with AIP in relation to presence or absence of elevated serum IgG4 concentrations. Patients and Methods: We divided 21 patients with AIP into two groups on the basis of serum IgG4 concentration measured before steroid therapy or surgical resection; elevated (>135 mg/dL) and low. Number of IgG4immunoreactive plasma cells in the pancreas $(n=9)$, bile duct $(n=8)$, gallbladder $(n=8)$, gastric mucosa $(n=15)$, and abdominal lymph nodes $(n=6)$ resected or biopsied from these patients was examined. Results: Serum IgG4 concentrations were elevated in 14 patients $(150-1890 \mathrm{mg} / \mathrm{dL}$ ) and ranged from 11 to $128 \mathrm{mg} / \mathrm{dl}$ in the other 7 patients. No significant differences in age (68.0 yr vs. 77.0) and sex (male/female; 10/4 vs. 5/2), frequencies of pancreatic swelling (14/14 vs. $7 / 7$ ), biliary stenosis (13/14 vs. 6/7), presence of autoantibodies (7/14 vs. $2 / 7)$, and salivary glands enlargement (5/14 vs. $0 / 7)$ were identified between the two groups. Abdominal lymphadenopathy was detected at laparotomy more frequently in patients with high serum IgG4 (5/5 vs. 0/3, p<0.05). Although, no difference in the number of IgG4-positive plasma cells infiltrating the pancreas (median 60.5/high power field (HPF) vs. $36.0 / \mathrm{HPF}$ ) and gallbladder (32.0/HPF vs. 8.0/HPF) was identified between the 2 groups, numbers of IgG4-positive plasma cells infiltrating the wall of the bile duct (56.0/HPF vs. $30 / \mathrm{HPF}, \mathrm{p}<0.1)$ and the abdominal lymph nodes $(55.0 / \mathrm{HPF}$ vs. $26.0 / \mathrm{HPF}, \mathrm{p}<0.1)$ tended to be greater in the group with elevated IgG4. The number of IgG4-positive plasma cells infiltrating the gastric mucosa was also significantly higher in this group (10.0/HPF vs. 1.5/ HPF, $\mathrm{p}<0.01$ ). Conclusion: IgG4-related phenomena occurred in various organs of patients with AIP associated with elevated IgG4, while these phenomena tended to be rather confined to the pancreas in patients with low serum IgG4.

\section{M1218}

The Japanese Diagnostic Criteria for Autoimmune Chronic Pancreatitis: Is It Completely Satisfactory?

Eun Kwang Choi, Myung-Hwan Kim, Jong Cheol Kim, Jimin Han, Dong Wan Seo, Sang Soo Lee, Sung Koo Lee

Objectives: Autoimmune chronic pancreatitis (AIP) is a very attractive disease to clinicians in terms of its dramatic response to the oral steroid therapy in contrast to ordinary chronic pancreatitis. In the year 2002, the Japan Pancreas Society published the diagnostic criteria of AIP and many clinicians around the world use this criteria for its diagnosis. The purpose of this study was to evaluate whether Japanese criteria for the diagnosis of AIP is adequate or not. Methods: We retrospectively analyzed the clinical, radiologic, laboratory and histologic features of 31 patients with AIP who have been successfully treated with oral corticosteroid. All the enrolled patients showed normalization or marked improvement in symptoms, laboratory and imaging findings after steroid treatment. Results: The mean patients' age was 56 years (range, 32-78 years) and were comprised of 25 males and 6 females. Seven patients who responded to the steroid did not satisfy the Japanese imaging criterion because the extent of irregular narrowing was less than one third of the entire length of main pancreatic duct. Among these seven patients, one patient did not meet the laboratory and histopathologic criteria as well. Another two patients fulfilled Japanese imaging criterion only and showed normal $\operatorname{Ig} G$ level, negative results of autoantibody measurements and nondiagnostic pancreatic histopathology. Taken together, nine $(29 \%)$ of 31 patients did not meet the Japanese diagnostic criteria for AIP, yet responded to the steroid. Conclusions: Clinicians may miss a substantial portion of AIP patients who may benefit from steroid therapy when the diagnosis is confined to those who satisfy the criteria proposed by the Japan Pancreas Society. It is necessary to convene a worldwide consensus to develop an improved diagnostic criteria for AIP. Key words: autoimmune pancreatitis, diagnosis, criteria

\section{M1219}

Pancreatic and Biliary Abnormalities in Patients with Autoimmune Pancreatitis (AIP) At MRI and Endoscopic Ultrasound (EUS)

Dermot O'Toole, Marie-Pierre Vullierme, Vinciens Rebours, Laurent Palazzo, Alain Aubert, Philippe Ponsot, Karine Nahon-Uzan, Anne Couvelard, Philippe Levy, Valerie Vilgrain, Philippe Ruszniewski, Pascal Hammel

Introduction: Although AIP is an increasingly recognised entity, in the absence of histology or a well-defined associated autoimmune disease $(\sim 40 \%$ [1]), the diagnosis remains difficult. Certain parenchymal and/or ductal abnormalities have been suggested to occur at both MRI and EUS but data are limited [2]. Aims: Examine for pancreatic and biliary signs of AIP at MRI and EUS in a cohort of patients (pts) with AIP followed in a single centre. Methods: Prospective study of 24 pts ( 15 men) with AIP (diagnosis based on either histology, or pancreatitis without a definable cause associated with another autoimmune disease and/or a favourable response to steroids) undergoing pancreatic MRI (axial sequences at Tl and T2, with MR-cholagio-pancreatography) and pancreatic and biliary EUS. Results: 1) MRI changes on Tl included: a hypointense zone $(n=14)$, a pseudotumour $(n=5)$; on T2: hyperintense ring $(n=11)$; parynchemyal pseudocyst $(P C)(n=5)$. Ductal changes at MRpancreatography were - main pancreatic duct (MPD): not visualised $(n=15,(63 \%)$; which was segmental in nature, $n=13)$ or a thin irregular MPD $(n=6)$; dilated segments $(n=5$ $>10 \mathrm{~mm}$ in $1 \mathrm{pt})$; - secondary ducts: moderate dilatation $(\mathrm{n}=5)$. Stenosis of the intra-pancreatic $\mathrm{CBD}$ was observed in 8 pts with dilatation of the intra-hepatic bile ducts in 6 ( 4 of whom were irregular). 2) EUS - the pancreatic parenchyma was diffusely hypertrophic in 8 pts. The echo pattern was heterogeneous or hypoechoic in 6 and 1 pts, respectively. Calcifications were observed in 1 pt. A focal hypoechoic mass was noted in 13 pts (54\%) (head/uncinate process: 9 , isthmus: 2 and body/tail: 2 ). Abnormalities of the MPD included a stenosis in 15 pts (63\% : diffuse, $n=13)$ with hyperechoic walls in 11 pts (disuse in 10). Segmentary or diffuse dilatation of the MPD was observed in 4 and 1 pts, respectively. A peri-pancreatic inflammatory reaction was noted in 4 pts including 2 cases of vascular encasement and 4 intra-pancreatic PCs. Stenosis of the intra-pancreatic CBD was found in 13 pts (54\%) with signs of cholangitis in 8 of these. Lymph nodes $>10 \mathrm{~mm}$ were visualised in $6 \mathrm{pts}$. Conclusion: 1) Abnormalities of the MPD appear constant in AIP observed at MRI (88\%) or EUS (75\%): including non-visibility or a thin irregular duct, and these changes are rare in pancreatitis from other causes. 2) Other frequent changes at MRI and EUS supporting the diagnosis of AIP include a pseudotumoral aspect and cholangitis. [1] Nahon-Uzan et al. Is idiopathic chronic pancreatitis an autoimmune disease? Clin Gastroenterol Hepatol 2005;3:903-9. [2] Sahani et al. Autoimmune pancreatitis: imaging features. Radiology. 2004;233:345-52.

\section{M1220}

Sclerosing Cholecystitis Associated with Autoimmune Pancreatitis Terumi Kamisawa, Naoto Egawa, Hitoshi Nakajima, Kouji Tsuruta, Atsutake Okamoto

Background and Aim: Patients with autoimmune pancreatitis (AIP) frequently have stenosis of the bile duct system. Fibrosis and dense lymphoplasmacytic infiltration with abundant IgG4-positive plasma cells is detected in the bile duct as well as in the pancreas of AIP These findings suggest a close relationship between AIP and sclerosing cholangitis, bu there is only one study that has evaluated the gallbladder in AIP. This study evaluated histopathological and radiological findings of the gallbladder in AIP patients. Patients and Methods: Radiological findings of the gallbladder of 19 AIP patients were reviewed. Stenosis of the extrahepatic bile duct occurred in 16 patients. At least 3 sections of the resected gallbladder of the 8 AIP patients were histologically examined and immunostained by antiIgG4 antibody. Pattern of gallbladder inflammation was classified into 2 types: transmura in which inflammatory infiltrates extended through the gallbladder wall and included involvement and focal destruction of the muscularis; and mucosal-based, in which the inflammatory infiltrates were prominently detected within the lamina propria. Controls consisted of gallbladders resected for symptomatic gallstones $(n=10)$ and those removed during pancreatoduodenectomy for pancreatic carcinoma $(\mathrm{n}=10)$, as well as extrahepatic bile ducts and pancreases removed by pancreatoduodenectomy for pancreatic carcinoma $(n=10)$. Results Thickening of the gallbladder wall was detected by US and/or CT in 10 patients with AIP (3 severe: thickness $\geq 8 \mathrm{~mm}$, and 7 moderate: thickness from $\geq 4 \mathrm{~mm}$ to $<8 \mathrm{~mm}$ ); in these patients severe stenosis of the extrahepatic bile duct was also noted. Histologically, thickening of the gallbladder was detected in 6 of 8 patients with AIP; 4 cases had transmural lymphoplasmacytic infiltration with fibrosis, and 2 cases had mucosal-based lymphoplasmacytic infiltration. Considerable transmural thickening of the extrahepatic bile duct wall with dense fibrosis and diffuse lymphoplasmacytic infiltration was detected in 7 patients. Immunohistochemically, infiltration of abundant IgG4-positive plasma cells (>10/high power field) was detected in the gallbladder, bile duct, and pancreas of all 8 patients, but was not detected in controls. Conclusion: Thickening of the gallbladder wall is one of the extrapancreatic lesions that are frequently detected in patients with AIP. It is characterized by transmural wall thickening with fibrosis and abundant infiltration of IgG4-positive plasma cells. We propose the use of a new term, sclerosing cholecystitis, for these cases that are induced by the same mechanism as sclerosing pancreatitis or sclerosing cholangitis in AIP.

\section{M1221}

High Prevalence of Nonalcoholic Steatohepatitis in Autoimmune Pancreatitis Takayoshi Nishino, Etsuko Hashimoto, Fumitake Toki, Hiroyasu Oyama, Itaru Oi, Makio Kobayashi, Keiko Shiratori

Background and Aim: Autoimmune pancreatitis (AIP) is known to frequently associate with type 2 diabetes mellitus (DM). DM is also a high risk factor for nonalcoholic steatohepatitis (NASH), however, there have been no studies about the prevalence of NASH in AIP. The present study was undertaken to identify characteristic features of liver biopsy and prevalence of NASH in patients with AIP. Methods: We retrospectively reviewed the clinical findings and outcomes of 30 AIP cases (M:F=20:10; age ranged from 45 to 83 yr, median age 65 yr). Liver biopsy was performed in 9 patients. The diagnosis of NASH was based on the following criteria: (1) steatohepatitis observed in the liver biopsy specimen, (2) daily ethanol

. 
intake less than $20 \mathrm{~g}$, and (3) exclusion of other liver diseases. A second liver biopsy was performed in 3 of the 9 AIP patients during predonisolone (PSL) therapy. Results: The profile of 9 biopsied-AIP patients (M:F=6:3; median age $72 \mathrm{yr}$ ) was as follows. Mean BMl was 22.2 (range 18.0 to 25.2). Insulin resistance was observed in $78 \%$ (7/9), hyperlipidemia was in $22 \%(2 / 9)$. The nine AIP patients in whom liver biopsy was performed reflected the characteristic profile of the 30 AIP patients. The characteristic liver pathological features were: (1) mild to moderate portal or periportal hepatitis manifested by minimal fibrosis. Inflammatory changes were consisting of lymphoplasmacytic infiltration with or without lobular hepatitis (9/9); Immunohistochemistry revealed many CD8-positive, CD4-positive lymphocytes and IgG4-positive plasma cells in the periportal area; (2) duct and ductuler proliferation with or without zone 3 cholestasis (9/9), and (3) steatohepatitis (8/9). Three patients had typical steatohepatitis (grade severe in three, stage 2 in two and 4 in one, Brunt's criteria) throughout the specimens. Cirrhotic NASH (stage 4) patient had 10 year history of overweight and DM before diagnosis of AIP. The other two patients with normal weight were diagnosed DM and AIP simultaneously. The other five patients were diagnosed with focal steatohepatitis (grade mild to moderate, stage 0-1), which located in zone 3 and consistent of $10 \%$ to $20 \%$ of the specimen. Examination of the second biopsy specimen revealed improvement of steatosis and ballooning degeneration in all three patients even during PSL treatment. Improvement of periportal and lobular inflammation with decrease number of IgG4-positive plasma cells was seen in two patients. Conclusions: This study revealed a high prevalence of NASH in AIP. This is a very important finding, and further study of the pathogenesis of AIP and NASH is needed.

\section{M1222}

Autoimmune Pancreato-Cholangitis - Which Parameters Help Diagnose the Disease? Results of a Multicenter Study Group

Annette Fritscher-Ravens, Menno Brink, Sjoerd D. van der Werf, Maarten J. Thomeer Maarten C. Meijssen, Henk R. van Buuren

Background: An autoimmune process causing pancreato-cholangitis (APC) has recently been proposed in Europe. The frequency of this entity, which presents with an enlarged pancreatic head, pancreatic and bile duct strictures and lympho-plasmocyticatic inflammation, fibrosis and good response to cortiosteroid therapy is yet unknown. As the awareness of this disease is not widespread in Europe correct diagnosis is rarely made at the onset of symptoms and many patients are subjected to unnecessary surgery. Immunosuppressive treatment is often only started some years after presentation, when the diagnosis is finally made. The aim of this study was to explore whether there are symptoms or labatory tests that may suggest this diagnosis and avoid unnecessary surgery prior to correct treatment. Materials and Methods: 40 cases with APC were collected in a multicenter approach and reviewed. The diagnostic criteria were evaluated retrospectively. Results: $37 / 40$ patients (92\%) presented with remarkable weight loss of $6-17 \mathrm{~kg}$ and nearly painless jaundice. $36 / 40(90 \%)$ patients had exocrine insufficiency. 10/40 (25\%) patients had gastro/duodenal ulcers with bleeding and BII/BI resection in 5. Lab investigations during follow up included antinuclear antibodies (ANA) in 37 patients, 32 of which were negative at diagnosis, but became positive within $1-5$ years after presentation in 6 cases. 5 patients had positive ANA at the time of diagnosis. All but one of these had or developed additional autoimmune diseases: Sjoegren, interstitial nephritis, lupus erythematosus, hypothyroidism or rheumatoid arthritis. Before treatment serum immunoglobulin (IGG) was available in 33 patients, $26(79 \%)$ of which were elevated (16-33 g/l: mean: 20.1). IGG4 subclass was measured before treatment in 24 patients, in $15(62 \%)$ it was elevated (2.1-30; mean: 8.4). If both of the tests (IGG and IGG4) were available prior to treatment, patients with APC had an elevated level for either IGG or IGG4 in $91 \%$ (21/23 patients). The CA19.9 was elevated at the time of diagnosis in $45 \%$ of cases. Conclusion: Patients with suspicion of pancreatic cancer, hilar cholangiocarcinoma or intrahepatic strictures of unknown origin with extreme weight loss, exocrine insufficiency, possible gastroduodenal ulcers, either elevated IGG and/or IGG4 have a strong likelihood of $>90 \%$ to have an autoimmune etiology of their disease.

\section{M1223}

Outcome of Autoimmune Pancreatitis Without Corticosteroid Treatment Kenji Hirano, Saburo Matsubara, Osamu Togawa, Toshihiko Arizumi, Takeshi Sasaki, Natsuyo Yamamoto, Yousuke Nakai, Takeshi Tsujino, Naoki Sasahira, Hiroyuki Isayama, Minoru Tada, Nobuo Toda, Takao Kawabe, Kazumi Tagawa, Tetsuo Katamoto, Ryo Nakata, Tateo Kawase, Masao Omata

Aim: Autoimmune pancreatitis (AIP) is a unique form of chronic pancreatitis characterized by irregular narrowing of pancreatic duct, swelling of parenchyma, lymphoplasmacytic infiltration and fibrosis, and a favorable response to corticosteroid treatment (CST). Since little is known about the outcome of AIP patients without CST, we attempted to clarify their clinical course. Patients and Methods: Diagnosis of AIP was based on imaging findings such as pancreas swelling and narrowing of main pancreatic duct and pathological features such as fibrosis with infiltration of lymphocytes and plasmacytes. Among 35 patients with AIP we experienced, 21 patients were followed up without CST at first because they were asymptomatic or became asymptomatic after biliary drainage. Their clinical features were retrospectively reviewed. Resoults: The patients without CST consisted of 17 males and 4 females and their average age was 67 years (range 41 to 80 ). Nine patients received biliary drainage before observation without CST. During follow-up of 54 months in mean (12 to 152 months), 12 patients developed symptoms including pancreatitis in 3 and bile duct involvement (sclerosing cholangitis) in 9. In analysis of risk factors for developing symptoms, severe bile duct stricture was identified. All the patients with late occurrence of sclerosing cholangitis had severe bile duct stricture at the diagnosis of AIP. Age, gender, serum IgG4 or abdominal pain was not related. The patients with developing symptoms were managed with CST and had favorable response. Conclusion: Patients with AIP tend to suffer from recrudescence without CST. Particularly in patients accompanied with severe bile duct stricture, CST is indispensable.

\section{M1224}

Utility of Total Immunoglobulin and IgG4 Levels in the Diagnosis and Management of Autoimmune Pancreatitis

Suresh T. Chari, Thomas C. Smyrk, Amaar H. Ghazale, Michael Levy, Mark Topazian, Naoki Takahashi, Jonathon Clain, Randall Pearson, Bret Petersen, Santhi Swaroop Vege, Michael Farnell

Background: Autoimmune pancreatitis (AIP) remains a diagnostic challenge. Presence of serologic abnormalities (elevation in titers of gammaglobulin and a variety of antibodies) are an important adjunct to diagnosis of AIP. Elevation of levels of the IgG4 subclass of IgG has been reported to be highly sensitive and specific for the diagnosis of AIP. Its usefulness in monitoring patients on steroid therapy remains unknown. Aims: Our aims were to a) assess the diagnostic accuracy of elevated levels of total IgG and IgG4 for AIP and b) determine the response of serum IgG4 levels to steroid therapy in AIP and its correlation with activity of the disease assessed radiologically. Methods: Total IgG levels (normal 600$1500 \mathrm{mg} / \mathrm{dl}$ ) and IgG4 (normal 8.0-140 mg/dl) levels were measured in 154 patients including 28 with AIP, 44 with pancreatic cancer, 36 with acute/chronic pancreatitis and 32 without pancreatic disease. Follow-up IgG4 levels were noted in the 15 AIP patients that had IgG4 measurement during and/or after completion of steroid treatment. Results: For diagnosis of AIP, elevation of IgG4 levels had better sensitivity compared to total IgG (23/28 (82\%) vs $16 / 28(57 \%), p=0.04$ ). All 16 AIP patients who had elevated total IgG also had IgG4 elevation. Of the 5 patients with AIP and normal IgG4 levels, one had the measurement after steroid therapy and another after pancreatic resection. Frequency of total IgG and IgG4 elevation was similar in patients that did not meet criteria for AIP $(9 / 126$ vs $14 / 126, p=0.1)$. Of the 14 patients with IgG4 elevation (range $146-366 \mathrm{mg} / \mathrm{dl}$ ) that did not meet criteria for AIP, 4 had biopsy-proven pancreatic cancer (range $168-330 \mathrm{mg} / \mathrm{dl}$ ), 3 had idiopathic pancreatitis, one had type $1 \mathrm{~b}$ diabetes and pancreatic steatorrhea, 3 had idiopathic pancreatic steatorrhea and 3 had no detectable pancreatic abnormality or any known extra-pancreatic manifestation of AIP. During steroid therapy IgG4 levels decreased or normalized in all patients studied. IgG4 were normal in all patients studied after completion of therapy. However, IgG4 levels did not correlate with intra-abdominal radiologic abnormalities. Conclusions: IgG4 elevation is more sensitive for diagnosis of AIP than total IgG. However, IgG4 elevation is characteristic but not diagnostic of AIP. In AIP patients on steroid therapy IgG4 levels normalize with treatment, but IgG4 levels do not appear to correlate with radiologic evidence of disease activity.

\section{M1225}

IgG4 Immunostaining in Autoimmune Pancreatitis and in Extra-Pancreatic Disease

Maesha G. Deheragoda, Nicholas I. Church, Manuel Rodriguez-Justo, Edward Seward, Marco R. Novelli, Steve Pereira, Adrian R. Hatfield, George J. Webster

Aims: Patients with autoimmune pancreatitis (AIP) usually present with a pancreatic mass, jaundice, and pancreatic and bile duct stricturing. Recognition of this entity is vital to avoid unnecessary resection of suspected malignancy. Originally thought to be restricted to Japan, we have recently reported a series of AIP patients from the UK, with a high frequency of extra-pancreatic involvement. Definitive diagnosis is difficult, relying on a combination of clinical, radiological and biochemical features including raised serum IgG4. The aim of this study was to investigate the utility of IgG4 immunostaining in the diagnosis of autoimmune pancreatitis and associated extra-pancreatic disease in biopsy specimens. Methods: All patients seen in our centre from 2004 to 2005, in whom the diagnosis of AIP was made on the basis of the typical characteristics were studied. Biopsy specimens from patients and controls were immunostained with human monoclonal IgG4 antibody. Numbers of $\operatorname{IgG4}$ positive plasma cells were assessed in 10 high power fields (HPF). Correlation was made with serum IgG4 levels.Results: Results IgG4 positive plasma cell numbers were consistently elevated in pancreatic and extra-pancreatic biopsy tissues even in cases with normal serum IgG4. The majority of AIP patients had $>10 \mathrm{IgG} 4$ positive plasma cells per HPF. Control tissues showed $\leq 2$ IgG4 positive plasma cells per HPF. The percentage of total plasma cells which expressed IgG4 was $>80 \%$ in AIP patients compared with $<10 \%$ in controls. Conclusions An IgG4 positive plasma cell infiltrate appears to be specific to AIP when compared with other inflammatory disorders. IgG4 immunostaining may be of use in the routine investigation of intra- and extra-pancreatic disease in clinically suspected cases of autoimmune pancreatitis.

\begin{tabular}{|c|c|c|c|c|}
\hline $\begin{array}{c}\text { Patient } \\
\text { ID }\end{array}$ & $\begin{array}{c}\text { Serum } \\
\text { IgG4 } \\
\text { N<1.5g/1 }\end{array}$ & $\begin{array}{c}\text { Biopsies from } \\
\text { clinically involved } \\
\text { organs }\end{array}$ & $\begin{array}{c}\text { Numbers if IgG4 } \\
\text { positive plasma cells per } \\
\text { High Power Field: } \\
\text { Patient samples }\end{array}$ & $\begin{array}{c}\text { Numbers of IgG4 positive } \\
\text { plasma cells per High } \\
\text { Power Field: Control } \\
\text { samples** }\end{array}$ \\
\hline 1 & Raised & Liver & 20 & 1 \\
\hline 2 & Raised & $\begin{array}{c}\text { Kidney Pancreas } \\
\text { Liver }\end{array}$ & 732021 & 021 \\
\hline 3 & Raised & $\begin{array}{c}\text { Bone Marrow } \\
\text { Submandibular gland } \\
\text { duodenum }\end{array}$ & 2510651 & 020 \\
\hline 4 & Normal & $\begin{array}{c}\text { Liver Pancreas } \\
\text { Gallbladder }\end{array}$ & $8^{*} 2261$ & 122 \\
\hline 5 & Normal & Pancreas & $2 * * * *$ & 11 \\
\hline 6 & Normal & Liver & 15 & 1 \\
\hline 7 & N/A & Liver Colon & 820 & 1 \\
\hline 8 & Normal & Liver & 18 & 11 \\
\hline 9 & Raised & Liver & 84 & \\
\hline
\end{tabular}

*insufficient portal tract tissue **Control tissues (10 per organ system): Liver (chronic hepatitis C), Pancreas (chronic alcoholic pancreatitis), Gall bladder (chronic cholecystitis), 
Kidney (tubulointerstitial nephritis), Salivary gland (chronic sialadenitis), Duodenum (chronic duodenitis), Bone marrow (normal bone marrow) *** Severe fibrosis

\section{M1226}

Novel HLA Allele Associated with Autoimmune Pancreatitis in the Korean Population

Do Hyun Park, Myung-Hwan Kim, Heung-Bum Oh, Sang Soo Lee, Dong Wan Seo, Sung Koo Lee, Sun-Joo Kim

Backgtorund/Aims: Autoimmune chronic pancreatitis (AIP) is associated with hypergammaglobulinemia, histologic evidence of lymphoplasmacytic infiltration, coexistence of other autoimmune diseases and a favorable response to steroid therapy, which are clinical evidence of autoimmunity. However, definite pathogenesis of AIP has been unclear. There was only one HLA study using low resolution typing in patients with AIP to determine close association between AIP and particular alleles of major histocompatibility complex genes. Sequencebased typing (SBT) for HLA has become the method of choice for HLA genotyping because of its ability to provide the highest resolution as well as to identify any new alleles. To determine whether certain alleles or haplotypes of major histocompatibility complex gene are associated with AIP in Korean population, we undertook this study with high-resolution typing (sequence-based typing) for HLA. Methods: Between October 2002 and August 2005, we obtained samples from consecutively enrolled 25 patients with AIP. there were 18 men and 7 women aged 34-74 years. HLA class I and II allelic genotypes from 153 healthy subjects were obtained for this study as a control. All patients with AIP met the diagnostic criteria of Japanese pancreas society. Results: In patients with AIP compared with healthy subjects, among class II antigen, DQ2 was significantly associated with AIP compared with health subjects ( $40 \%$ vs. $16 \%$, corrected $\mathrm{p}=0.01$ ), Among the DQ 2 subtype, we found a significant association between DQB1*0202 allele and AIP ( $28 \%$ vs. $11 \%$, odd ratio $=3.11$, $\mathrm{p}=0.022$ ). No other HLA class I and II antigen and DRBI or DQBI were significantly associated with AIP compared with healthy subjects. Conclusions: Using high-resolution typing for HLA, HLA DQ1*0202 may be associated with AIP in the Korean population. Staitistical analysis of DR and DQ subtype among patients with autoimmune pancreatitis, and healthy subject

\begin{tabular}{|c|c|c|c|c|}
\hline HLA & $\begin{array}{c}\text { Patients with } \\
\text { autoimmunepancreatitis } \\
(\mathrm{n}=25)\end{array}$ & $\begin{array}{c}\text { Healthy } \\
\text { subjects } \\
(\mathrm{n}=153)\end{array}$ & $\begin{array}{c}\text { Autoimmunepancreatitis } \\
\text { vs.healthy subjects (odds } \\
\text { ratio })\end{array}$ & $\begin{array}{c}\text { Corrected } \\
\mathrm{p} \text { value }\end{array}$ \\
\hline DRB1*0405 & 12 & $\begin{array}{c}\text { Frequency } \\
(\%)\end{array}$ & Standard p value & $\begin{array}{c}\text { Corrected } \\
\mathrm{p} \text { value }\end{array}$ \\
\hline DRB1*0406 & 24 & 13 & 1.000 & \\
\hline DQB1*0202 & 28 & 11 & 0.097 & 0.048 \\
\hline DQB1 $* 0302$ & 32 & 23 & $0.022(3.11)$ & \\
\hline DQB1*0401 & 12 & 12 & 0.323 & \\
\hline
\end{tabular}

\section{M1227}

Corticosteroids Are Efficacious in Patients with Lymphoplasmacystic Sclerosing Pancreatitis (LSP) But Relapses Are Frequent

Pascal Hammel, Vinciens Rebours, Dermot O'Toole, Marie-Pierre Vullierme, Karine Nahon-Uzan, Frederique Maire, Philippe Ponsot, Alain Aubert, Annie Sibert, Laurent Palazzo, Philippe Levy, Philippe Ruszniewski

Clinical, biological and morphological features of LSP (either "autoimmune": AIP or eosinophilic [EP]) are currently better characterized; however the effects of medical treatment to date has been poorly evaluated. Aim: To assess therapeutic management (indications, treatment modalities and results) of patients with LSP in a monocentric series. Patients (pts) and methods: Prospective inclusion of all pts with LSP between 01-2002 and 08-2005. The diagnosis of LSP was made in view of clinical biochemical and imaging according to Yoshida's criteria (1). Symptomatic pts received oral corticosteroids in the absence of untreated biliary stenosis or sepsis. Results: 29 pts (21 M, 8 F, median age 39 years (17-73), 25 with LSP, 4 with EP) were included with a median follow-up of 14 months (1-55). Median age at symptom onset was 39 years (16-69). The first symptom was abdominal pain, acute pancreatitis, weight loss, and jaundice in $35 \%, 24 \%, 21 \%$ and $17 \%$ of pts, respectively (fortuitous diagnosis in one symptom-free pt). Associated auto-immune or inflammatory bowel diseases were present in $6(21 \%)$ and $4(14 \%)$ pts, respectively. Pathological data obtained from EUS-guided biopsy were available in $12(41 \%)$ pts, showing typical features of LSP in 11 and fibrosis alone in 1 . Prednisolone $(1 \mathrm{mg} / \mathrm{kg}$ day for 1 month with progressive tapering over 3 months) was given to the 13 pts $(45 \%)$ with pain or jaundice. Biliary drainage was necessary in 3. Symptoms improved in 12 of the 13 pts (92\%), and radiological improvement was observed in $58 \%$ of cases. In one patient, septicaemia led to treatment withdrawal. Pancreatic $(n=3)$ or biliary $(n=5)$ symptoms relapsed in 8 pts $(67 \%)$ at time of treatment withdrawal, 3 of whom had $\geq 2$ symptomatic relapses. A second course of prednisolone was given in 7 pts ( 2 of whom also required biliary stenting), and biliary stenting alone was performed in $1 \mathrm{pt}$. Azathioprine or imatinib were prescribed in 2 and $1 \mathrm{pt}$ experiencing at least two relapses, respectively, with no further recurrence after 9-11 months. Conclusion: Corticosteroid treatment of pancreatitis was necessary in $45 \%$ of pts, occasionally associated with biliary drainage. Treatment efficacy concerning symptoms was high (>90\%) but relapses were frequent ( 2 out of 3 pts). Immunosuppressive therapy can be envisaged for steroid-dependent pts.

\section{M1228}

Serum IgG4 Levels in Pancreatic and Biliary Diseases

Kenji Hirano, Saburo Matsubara, Toshihiko Arizumi, Takashi Sasaki, Natsuyo Yamamoto, Yousuke Nakai, Takeshi Tsujino, Naoki Sasahira, Hiroyuki Isayama, Minoru Tada, Takao Kawabe, Nobuo Toda, Kazumi Tagawa, Tetsuo Katamoto, Ryo Nakata, Osamu Togawa, Tateo Kawase, Masao Omata

Purpose: Recently, it has been reported that the serum level of IgG4, a minor componen of IgG subclasses, is elevated in autoimmune pancreatitis. About IgG4 levels in other pancreatic or biliary diseases, however, little has been studies. Thus, this study was conducted to resolve this issue. Methods: Serum IgG4 was measured in 116 patients with pancreatic or biliary diseases ( 35 autoimmune panceatitis, 24 chronic pancreatitis except autoimmune pancreatitis, 11 primary sclerosing cholangitis, 23 pancreatic cancer, 3 islet cell tumor, 2 papilla cancer, 15 bile duct cancer, and 3 gallbladder cancer patients). We employed 135 $\mathrm{mg} / \mathrm{dl}$ as a cut-off level of IgG4. Results: Elevation of serum IgG4 was observed in 33 of 35 patients with autoimmune pancreatitis, 0 of 24 with chronic pancreatitis, 4 of 11 with primary sclerosing cholangitis, 0 of 23 with pancreatic cancer, 0 of 3 with islet cell tumor, 0 of 2 with duodenal papilla cancer, 0 of 15 with bile duct cancer and 0 of 3 with gallbladder cancer patients. Conclusions: Elevation of serum IgG4 was observed in autoimmune pancreatitis. In other pancreatic or biliary diseases except primary sclerosing cholangitis, it remain within normal limits.

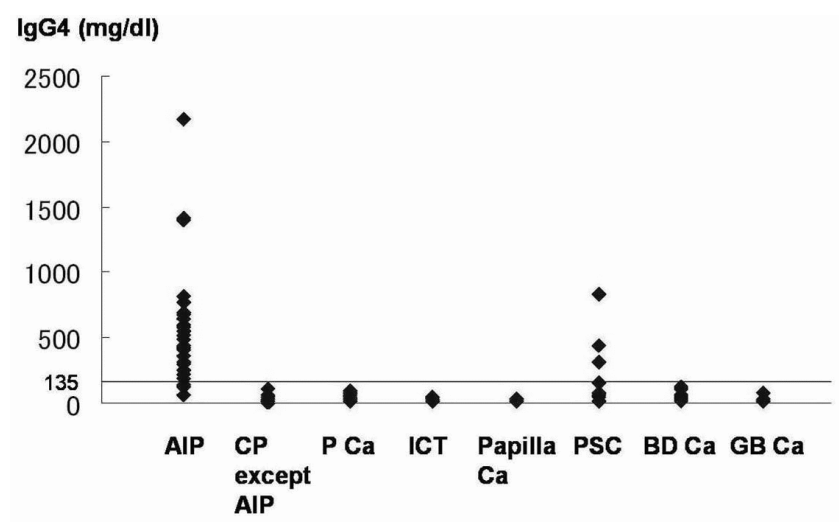

M1229

Clinical Aspect of Extra-Pancreatic Complications with Autoimmune Pancreatitis

Kazushige Uchida, Tsukasa Ikeura, Syoichiro Takamido, Masaaki Shimatani, Mitsunobu Matsushita, Makoto Takaoka, Kazuichi Okazaki

Background \& Aim: Autoimmune pancreatitis (AIP) is a new clinical entity of pancreatitis Recently several investigators reported that AIP has often complicated with extra-pancreatic disease. For example, sclerosing cholangitis with AIP (SC-AIP) is showing similar cholangiogram with classical primary sclerosing cholangitis (PSC). But SC-AIP is thought that pathogenesis is different from PSC. Because clinical coarse is quiet different. AIP sometime complicated with sialoadenitis also. This sialoadenitis might be different from Sjogren syndrome. To clarify these problems, we analyzed complications with autoimmune pancreatitis immunologically. Methods: Fifty patients with AIP ( 31 men and 19 women; average age 59.1 years old) were recruited for this study. Forty five patients were fulfilled the diagnosis criteria for AIP as Japan pancreas society's criteria. Five patients were diagnosed histrogically. These patients were measured serum $\operatorname{Ig} G, \operatorname{IgG} 4$ and autoantibodies (rheumatoid factor (RF), anti-nuclear, lactoferrin (LF), carbonic anhydrase II (CA-II), mitochondria (AMA), SS-A/SS-B, and GAD antibodies). All patients were screened extra-pancreatic complications Diabetes mellitus was complicated in $42 \%$ of the patients, sclerosing cholangitis $26 \%$, sialoadenitis $14 \%$, arthritis $12 \%$, retroperitoneal fibrosis $10 \%$, interstitial nephritis and thyroiditis $8 \%$, and hepatic pseudo-tumor $2 \%$. We also studied infiltrating cells by Immunohistochemistry. Results: High level of serum IgG was detected in $80 \%$ of the patients. IgG4 was $70 \%$. Anti-nuclear or LF antibody was detected in $75 \%$ of the patients. Anti CA-II antibody was detected in $55 \%$ of the patients. RF $25 \%$, SS-A/SS-B 3\%. AMA is not detected in any patients. Anti-LF or CA-II antibody was detected in $90 \%$ of the patients. High level of IgG4 was correlated with sclerosing cholangitis (PSC like cholangiogram, $\mathrm{p}<0.05$ ). Anti-LF antibody had a tendency with the showing of abnormal cholangiogram $(\mathrm{p}=0.08)$. In the immunohistochemical study, IgG4 positive plasma cells infiltrated in extra pancreatic lesions (salivary glands, retroperitoneum and liver). Two third patients with diabetes mellitus were improved $\mathrm{HbAlc}$ after steroid therapy. On the other hand, one-third patients were getting worse. Conclusions: To measure anti-LF and CA-II antibodies is useful for the diagnosis of AIP.

\section{M1594}

Commensal Microflora Attenuate Adaptive Growth in the Colon, But Not the Small Intestine, Following Ileo-Cecal Resection

Rachael J. Rigby, Christopher M. Dekaney, Susan J. Henning, P. Kay Lund, Michael A. Helmrath

Background: Surgical resection of ileum and proximal right colon is common in Crohn's disease and necrotizing enterocolitis. Adaptive growth of remnant bowel is beneficial in restoring normal nutrient, fluid, vitamin, bile acid and mineral absorption. Recent studies in models of intestinal inflammation indicate that commensal microflora impact on crypt homeostasis and promote mucosal healing. However, the role of bacteria in adaptive growth of the colon is not well defined. This study developed a mouse model of ileo-cecal resection

\section{.}


(ICR) in germ free (GF), conventionally-raised (CON), and conventionally-raised and antibiotic treated (CON-A) mice, to test the hypothesis that commensal microflora alter adaptive growth of the small intestine or colon. Methods: ICR or control transection (T) was performed on 8-12 week old GF or CON C57BL6 mice. CON-A mice were orally fed broad-spectrum antibiotics in liquid diet for 3 days before and 7 days after surgery. Mice were killed 7 days after surgery and jejunum and colon harvested. Crypt depth and BrdU incorporation were assayed. Adaptive growth after ICR in each group was assessed as percentage change in ICR versus T controls. Results: In T controls, crypt depth was significantly lower in small intestine of GF $(100 \pm 1)$ than CON $(124 \pm 14)$ mice, and CON-A mice had intermediate values $(114 \pm 7)$. After ICR, adaptive increases in crypt depth in small intestine were virtually identical in all groups GF ( $31 \pm 4 \%$ increase), CON ( $32 \pm 7 \%$ increase), and CON-A ( $35 \pm 4 \%$ increase). In both small intestine and colon, data for BrdU incorporation was consistent with crypt depth data. In colon, crypt depth did not differ significantly in T control groups. However, after ICR, colon of GF mice showed a dramatic increase in crypt depth $(95 \pm 2 \%$; $\leq 0.001)$, this response was absent in CON mice $(8 \pm 4 \% \mathrm{p}=0.19)$ and evident, but attenuated, in CON-A mice $(25 \pm 10 \% ; \mathrm{p} \leq 0.09)$. Conclusions: Commensal microflora do not significantly affect adaptive growth of the small intestine following ICR. In contrast, commensal microflora impair colonic adaptation after ICR. Significant adaptive growth of colon in GF, but not CON mice and moderate growth in the colon of antibiotic-treated CON mice, supports a concept that clinical therapies to modify commensal microflora may augment adaptive growth of colon and benefit patients after ICR

\section{M1595}

Effect of Ghrelin Administration On the Healing of Chronic Duodenal Ulcers in Rats. Role of Growth Hormone and Insulin-Like Growth Factor-1 Artur Dembinski, Zygmunt Warzecha, Ryszard Sendur, Piotr Ceranowicz, Jakub Cieszkowski, Marcin Dembinski, Wieslaw W. Pawlik, Peter C. Konturek, Atsukazu Kuwahara, Ikuo Kato

Background: Ghrelin, a 28-residue peptide, was primary isolated from the human and rat stomach. It is a natural ligand of growth hormone secretagogue receptor and apart from the stimulation of growth hormone, ghrelin exerts several biological activities including the stimulation of appetite, intestinal motility and protection of the heart, stomach and pancreas against damage evoked by different noxious agents. Study performed on rats has shown that induction of duodenal ulcers by cysteamine enhances plasma level of ghrelin, but the role of ghrelin in the maintenance of duodenal mucosal integrity has not been studied. The aim of this study was to examine the influence of ghrelin administration on the healing of chronic duodenal ulcers in animals with intact or removed pituitary. Methods: Two weeks after sham-operation or hypohysectomy, chronic duodenal ulcers were induced by $75 \%$ acetic acid applied to the serosal surface of duodenal wall. Animals were treated intraperitoneally twice a day with saline or ghrelin at the doses: 4,8 or $16 \mathrm{nmol} / \mathrm{kg} / \mathrm{dose}$. At 6 or 10 days after induction of ulcers, rats were anesthetized; the duodenum was exposed for measurement of duodenal blood flow and the duodenal ulcer area. Blood was taken for determination of serum level of growth hormone $(\mathrm{GH})$ and insulin-like growth factor-1 (IGF-1). Biopsy samples from the duodenal mucosa were taken for determination of mucosal DNA synthesis, as an index of mucosal cell proliferation. Results: In animals with intact pituitary, treatment with ghrelin increased serum level of ghrelin, GH and IGF-1. These effects were accompanied by the increase in mucosal cell proliferation, duodenal blood flow and acceleration of healing rate of duodenal ulceration. Ghrelin at the dose $8 \mathrm{nmol} / \mathrm{kg}$ exhibited maximal beneficial effect. After hypophysectomy, the healing of duodenal ulcers was delayed. This was accompanied by a significant increase in serum level of endogenous ghrelin and decrease in serum concentration of GH and IGF-1. Also, duodenal blood flow and mucosal DNA synthesis were reduced. In hypophysectomized rats, administration of exogenous ghrelin increased serum level of this peptide, but it was without effect on serum level of GH and IGF-1. Also, in hypophysectomized rats, treatment with ghrelin was without effect on healing rate of chronic duodenal ulcers, duodenal blood flow or mucosal cell proliferation. Conclusion: Treatment with ghrelin accelerates healing rate of chronic duodenal ulcers and this effect is dependent on the release of endogenous GH and IGF-1.

\section{M1596}

Induced Trpcl Expression Sensitizes Intestinal Epithelial Cells to Apoptosis By Inhibiting NF-KB Signaling Pathway Through a Process Involving $\mathrm{Ca}^{2+}$ Influx

Bernard S. Marasa, Jaladanki N. Rao, Tongtong Zou, Lan Liu, Kaspar M. Keledjian, AiHong Zhang, Jose Greenspon, Lan Xiao, Jie Chen, Jian-Ying Wang

Apoptosis occurs within crypts and at the luminal surface of gut and plays a critical role in mucosal homeostasis. We have recently demonstrated that TRPCl (canonical transient receptor potential-1) is expressed in intestinal epithelial cells (IECs) and functions as a $\mathrm{Ca}^{2+}$ permeable channel mediating capacitative calcium entry (CCE). TRPCl regulates intestinal epithelial cell motility by altering levels of cytosolic free $\mathrm{Ca}^{2+}\left(\left[\mathrm{Ca}^{2+}\right]_{\mathrm{cyt}}\right)$ through $\mathrm{CCE}$, but its involvement in modulation of apoptosis in IECs remains unknown. The current study determined if increased TRPCl expression sensitizes intestinal epithelial cells to apoptosis by inactivating NF-אB activity. Methods: Studies were conducted in IEC-6 cells, derived from rat intestinal crypts. Apoptosis was induced by tumor necrosis factor- $\alpha$ (TNF $\alpha$ ) and cycloheximide (CHX), while $\left(\left[\mathrm{Ca}^{2+}\right]_{\mathrm{cyt}}\right)$ was measured by fluorescence digital imaging analysis. Functions of TRPCl were investigated by stable expression of the TRPCl gene and transfection with small interfering RNA targeting TRPCl (siTRPCl). NF-kB activity was examined by measuring its sequence specific DNA-binding and NF- $\mathrm{KB}$ transcriptional activity. Results: Stable TRPCl-transfected IEC-6 cells (IEC-TRPCl) highly expressed TRPCl mRNA and protein. Levels of TRPCl mRNA and protein were 5 -folds the value of parental IEC6 cells. Although resting $\left[\mathrm{Ca}^{2+}\right]_{\mathrm{cyt}}$ and transient $\mathrm{Ca}^{2+}$ release from the store depletion were unchanged, sustained $\left.\mathrm{Ca}^{2+}\right]$ influx through CCE after $\mathrm{Ca}^{2+}$ store depletion was increased by $\sim 2$-folds in IEC-TRPCl cells. Susceptibility of IEC-TRPCl cells to TNF $\alpha / C H X$-induced apoptosis increased dramatically as measured by changes in morphological features, DNA fragmentation, and caspase-3 activity. Apoptotic cells were increased from $\sim 38 \%$ in parental
IEC-6 cells to $\sim 79 \%$ in IEC-TRPCl cells $4 \mathrm{~h}$ after exposure to TNF $\alpha / C H X$. Stable IECTRPCl cells also exhibited decreased NF- $\mathrm{KB}$ transactivation as indicated by decreases in levels of nuclear NF- $\mathrm{KB}$ proteins (including p65, p52, and p50), its DNA-binding activity, and NF- $\mathrm{KB}$-dependent promoter activity. Inhibition of TRPCl expression by siTRPCl in stable IEC-TRPCI cells and Cdx2-IEC cells (highly expressing endogenous TRPC1) not only increased NF-KB transactivation but also prevented the increased susceptibility to TNF- $\alpha$ / CHX-induced apoptosis. Similarly, exposure of IEC-TRPCl cells to the $\mathrm{Ca}^{2+}$ free medium also promoted NF- $\mathrm{KB}$ activity and blocked increased sensitivity to apoptosis. Conclusions: These results indicate that induced TRPCl expression sensitizes intestinal epithelial cells to TNF- $\alpha / C H X$-induced apoptosis by inhibiting NF- $\mathrm{KB}$ activity through a process involving $\mathrm{Ca}^{2+}$ influx

\section{M1597}

Deletion of the Mtgrl Transcriptional Co-Repressor Sensitizes the Colonic Epithelium to DSS-Induced Colitis

Jose A. Martinez, Joseph Amman, Isabel Moreno-Miralles, Kay Washington, Scott Hiebert

Myeloid Translocation Gene Related-1 (MTGR-1) is a member of the Myeloid Translocation Gene (MTG) family of transcriptional co-repressors. Two members of this gene family are disrupted by chromosomal translocations that are associated with acute myeloid leukemia. Chromosomal translocations affect master regulators of cell proliferation, differentiation and survival. Biochemical analysis of the fusion proteins created by these translocations suggest that all three MTG family member act as transcriptional co-repressors that associate with histone deacetylases and that are recruited to regulate gene expression. Genetic studies have now been undertaken to uncover the physiological role of this gene family. An Mtgrl knockout mouse model was developed and mice lacking this transcriptional co-repressor display impaired secretory cell lineage development in the small intestine, but no histological changes in the colon. However, there was an increase in proliferation in the crypts of both the small and large intestine. Despite this increase in proliferating cells, the colons of Mtgrlnull mice have a normal cell lineage distribution and normal architecture. The disruption of homeostasis between proliferation and apoptosis in the colonic epithelium contributes to the pathogenesis of human ulcerative colitis. To uncover colonic phenotypes in Mtgrl-/mice, we challenged the Mtgrl-null mice with low molecular weight Dextran Sodium Sulfate (DSS), which is a well-studied model of murine ulcerative colitis. Treatment with DSS uncovered a dramatic sensitization of the Mtgrl-/- colonic epithelium to apoptosis, which led to a severe colitis. Transplantation of wild type and Mtgrl-null bone marrow into irradiated wild type mice, demonstrated that the severe DSS-induced ulceration seen in Mtgrl-null mice was due to a colonic, rather than a hematological, defect. Analysis of crypt proliferation and apoptosis both before and after challenge indicated that Mtgrl-/- mice have higher levels of apoptosis when compared to wild type mice, which indicates an increased colonic cell turnover rate. Importantly, the epithelium of DSS-treated Mtgrl-null mice failed to completely regenerate, showing changes consistent with chronic colitis, even 10 weeks after a single DSS treatment. These findings suggest that Mtgrl has an important role in crypt survival and regeneration after colonic epithelial ulceration, which may indicate that Mtgrl functions at the level of the colonic stem cell or progenitor cells.

\section{M1598}

Smad3 Regulates Both Colonic Epithelial Cell Proliferation and Cell Positioning in the Crypt Epithelium

Kiyoshi Furukawa, Toru Sato, Tatsuro Katsuno, Kazuki Hatakeyama, Atsushi Isono, Atsuko Tokumasa, Yasuo Suzuki, Kotaro Yokote, Yasushi Saito

Background: We reported previously that deficiency of Smad3, an intracellular mediator of TGF- $\beta$, significantly accelerates re-epithelialization of the colonic mucosa (Tokumasa A. et al. BBRC 2004). However, little is known about how Smad3 affects proliferation of colonic epithelial cells. Aim: This study was performed to address the molecular mechanisms by which Smad3 controls colonic epithelial cell proliferation and colonic crypt formation. Methods: Smad3 ${ }^{\mathrm{ex} 8 / \mathrm{ex} 8} \mathrm{C} 57 \mathrm{BL} / 6$ mice (a gift from Dr. Anita B. Roberts, NCI) and wild-type littermates as controls were used in this study. Cell proliferation was assessed by monitoring Ki-67 staining and BrdU uptake. Protein levels of p21, p27, and $\beta$-actin were evaluated by Western blotting. Results: Hematoxylin \& eosin staining demonstrated that the colonic crypts of Smad3-/- mice were similar in structure to those of Smad3+/+ littermates. However, Ki-67 staining of the colonic mucosa indicated that number of proliferating cells in the crypts of Smad3-/- mice was twice as much as that of Smad3+/+ littermates. Analysis of BrdU uptake in isolated colonic epithelial cells also demonstrated a significant increase (205 $\pm 27 \%$ ) in number of cells in cell cycle in the colonic epithelium of Smad3-/- mice, even when treated with $10 \mathrm{ng} / \mathrm{ml} \mathrm{TGF}-\beta(288 \pm 83 \%)$. Protein levels of the cell-cycle inhibitors p21 and p27 were significantly decreased in the colonic epithelium of Smad3-/- mice. These results indicated acceleration of the cell cycle in the crypts of Smad3-/- mice due to downregulated p21 and p27 expression. Intriguingly, Ki-67 staining of the colonic mucosa also indicated that proliferating cells were scattered from the bottom to the top of the crypts in Smad3-/- mice, whereas they were restricted to the bottom of the crypts in Smad3+/+ littermates. This finding prompted us to examine whether the EphB/ephrin-B system, which is critical for maintenance of proliferating cells at the bottom of the crypts, was disturbed in the colonic crypts of Smad3-/- mice. In Smad3+/+ littermates, EphB3 protein expression was restricted to the bottom of the crypts, coinciding with the localization of Ki-67-positive cells. However, the level of EphB3 protein expression at the bottom of the crypts was significantly reduced in Smad3-/- mice. These observations suggest that Smad3 deficiency leads to mispositioning of the proliferating cells in the crypts by disturbing the EphB/ephrinB system. Conclusions: Smad3 not only negatively regulates proliferation of colonic crypt epithelial cells by up-regulating p21 and p27 expression, but also plays an essential role in maintaining the position of proliferating cells in the crypt epithelium. 
M1599

Implication of Ilk in the Control of Extracellular Matrix/Cytoskeletal Organization in Human Intestinal Epithelial Cells

David Gagne, Pierre H. Vachon, Jean-Francois Beaulieu

Interactions between cells and their surrounding matrix control many different functions of the cell. Integrin linked kinase (ILK) is a serine-threonine kinase found in focal adhesion points that has been found to be involved in the process of integrin signaling in many different tissues and its de-regulation leads to several pathologies including cancer. However, the role of ILK in normal intestinal cells has not yet been established. The objective of this study was to characterize the involvement of ILK in the proliferation, differentiation, migration and survival of human intestinal epithelial cells. Immunolocalization studies performed on cryosections of the intestinal crypt/villus axis showed a diminishing expression of ILK from the base of the crypt to the tip of the villus. Analysis of the expression of ILK in the HIEC and Caco-2/15 cell models revealed a strong expression of the ILK transcript and protein in proliferating cells. The forced decrease of ILK expression in the HIEC cell line using a synthetic siRNA led to an $80 \%$ reduction in the protein level without causing a toxic interferon response. This decreased ILK level did not affect cell survival however it did lead to a pronounced decrease in the rate of proliferation of the cells. We have also observed a marked change in cell morphology and actin cytoskeleton organization, associated with an inhibition of fibronectin deposition as well as a decrease in the rates of cell spreading and migration. Preliminary studies of actin cytoskeleton organization suggest the involvement of ILK in the modulation of the Rho-family GTPase protein. In summary, we have observed a differential expression of ILK along the intestinal crypt/villus axis and our data suggests an important function for ILK in the control of extracellular matrix/cytoskeletal organization in human intestinal epithelial cells.

\section{M1600}

Kinase Suppressor of RAS Promotes Raf-1 Kinase Activation in TNFR-Induced Cell Survival

Karen L. Edelblum, D. Brent Polk

Although inflammatory bowel disease is associated with increased production of apoptosisinducing cytokines such as tumor necrosis factor (TNF), activation of TNF receptor-1 (TNFR) promotes anti-apoptotic signaling during intestinal homeostasis. During inflammation KSR is required for the intestinal epithelial cell survival through the activation of anti-apoptotic signaling pathways including ERK/MAP kinase (MAPK). This study addresses the antiapoptotic role of Raf- 1 , a serine/threonine kinase that regulates ERK/MAPK and is the only known substrate of KSR. Methods: The impact of Raf kinase activity on cell survival was determined with apoptosis assays performed in young adult mouse colon (YAMC) cells pretreated with pharmacological Raf-1 kinase or MEK inhibitors prior to TNF exposure. To determine if TNFR-mediated Raf activation required Ras activation, whole cell lysates of TNF- or EGF-treated YAMC cells were subjected to an activated Ras pull-down assay. YAMC cells expressing dominant-negative Ras were also treated with TNF or EGF. Activation and membrane translocation of Raf was analyzed in TNF-treated YAMC and KSR-null colon epithelial cells by subcellular fractionation and immunofluorescence with phospho-specific and total Raf antibodies. Results: Loss of Raf kinase activity, but not MEK inhibition, increased YAMC cell sensitivity to TNF-induced apoptosis. To address the mechanism by which TNFR mediates Raf activation, we showed that while TNF and EGF treatment of YAMC cells activated Raf/MAPK signaling, only EGF stimulated Ras activation. Furthermore, expression of dominant-negative Ras completely blocked EGF-, but not TNF-stimulated MAPK activation. Activated Raf was localized to the plasma membrane following TNF treatment of YAMC cells, as was determined by immunostaining with phospho-specific antibodies for several Raf activation sites, including the proposed site of KSR activation, Raf T269. Phosphorylated Raf was localized to the plasma membrane following stimulation in YAMC cells but not in the absence of KSR, suggesting that both KSR kinase activity and scaffolding function is required for Raf activation in response to TNF. Conclusion: Our recent report citing the role of KSR in protecting the intestinal epithelium from cytokine-induced apoptosis may be attributed to KSR promotion of Raf activitation at the plasma membrane through a novel Rasindependent pathway. Furthermore, Raf kinase activation mediates TNF-induced intestinal epithelial cell survival in a MEK-independent manner, implicating this as an important regulatory kinase in gastrointestinal response to injury and repair mechanisms.

\section{M1601}

The Motogenic Effects of Cyclic Strain On Intestinal Epithelial Monolayer Wound Closure Are Matrix-Dependent

Jianhu Zhang, Cheri R. Owen, Jerrold R. Turner, Marc D. Basson

Complex deformation during peristalsis or villous motility may be trophic for the intestinal mucosa. Cyclic strain induces matrix-dependent intestinal epithelial proliferation on collagen (COL), an effect inhibited by fibronectin (FN). Since inflammation causes tissue FN deposition, we sought to evaluate the effects of strain and FN on intestinal epithelial wound healing. We made $1 \mathrm{~mm}$ circular holes in confluent monolayers on flexible membranes coated with COL I or tissue FN that were then subjected to static conditions or repetitive average $10 \%$ strain at 10 cycles/min. We used human Caco-2 and non-transformed rat IEC-6 intestinal epithelial cells to assess strain effects on wound closure, and Caco-2 cells to delineate the responsible mechanism. We measured wound closure morphometrically, and ERK and MLCK activity by blotting with antibodies for phospho-ERK (pERK) and phosphoMLC (pMLC). pERK and pMLC localization were evaluated by confocal microscopy. Although strain is mitogenic for intestinal epithelial cells on COL but not FN, strain inhibited wound closure on COL, but enhanced it on FN. Strain was motogenic, promoting motility and wound closure, as early as $6 \mathrm{hrs}$ on FN, too soon for proliferation. This persisted for 24$72 \mathrm{hr}$. Ablating proliferation with mitomycin $\mathrm{C}$ did not prevent the motogenic effect of strain. Strain activated MLCK on FN. Inhibiting MLCK by H-7, ML-7, or PIK, a peptide inhibitor of MLCK, prevented the motogenic effect. Strain activated ERK on both FN and COL. MEK inhibition by PD98059 blocked pERK and prevented motility on FN. Confocal microscopy revealed nuclear relocalization of pERK on COL and FN substrates following strain, but pERK redistribution to the lamellipodial edge only on FN. MEK inhibition did not prevent pMLC but disrupted its subcellular reorganization to the migrating edge in response to strain on FN Conversely, MLCK inhibition did not affect pERK activation or reorganization. These results suggest that repetitive strain acts in conjunction with the matrix to regulate intestinal epithelial biology. FN deposition induced by inflammation may trigger a shift from a mitogenic to a motogenic strain effect. Strain activation of ERK appears central to both the mitogenic effect of strain on COL and the motogenic effect of strain on FN, with specificity determined by matrix-dependent subcellular relocalization of pERK in response to strain. In particular, the motogenic effect of strain likely requires lamellipodial ERK activation, as well as MLCK activation and downstream pMLC at the migrating edge. Loss of straininduced signaling during ileus or sepsis may be an important target to promote mucosal healing

\section{M1602}

Epidermal Growth Factor Receptor (EGFR) Tyrosine Kinase Activity Attenuates TNF-Stimulated Intestinal Cell Apoptosis

Toshimitsu Yamaoka, Rebecca S. Dise, Wei Tong, D. Brent Polk

Background. EGFR tyrosine kinase activity, through cytokine and growth factor stimulation, is upregulated in ulcerative colitis and colon cancer suggesting that the activation of EGFR is a response to these diseases in humans. In the present study, we tested the hypothesi that EGFR tyrosine kinase regulates cytokine-mediated colon epithelial cell survival. Method Young adult mouse colon (YAMC) cells, colon cancer HT-29 cells, EGFR-null mouse colon epithelial (EGFR $\left.{ }^{-/} \mathrm{MCE}\right)$ cells, stable clonal cell lines expressing either wild-type (wt) or kinase-inactive (ki) EGFR and controls consisting of vector (v), ADAMl7-deficient mouse colon epithelial (ADAM17 $\left.7^{-1} \mathrm{MCE}\right)$ cells, retroviral expression of wild-type ADAM17 and catalytically-inactive (E406A) ADAM17 mutant MCE cells were used for these studies. Cells were treated with cytokines only, which include TNF $(100 \mathrm{ng} / \mathrm{ml})$, IL-1 $(10 \mathrm{ng} / \mathrm{ml})$ and IFN- $\gamma(100 \mathrm{ng} / \mathrm{ml})$, or in the presence of either a PI 3-kinase inhibitor (wortmannin 100 $\mathrm{nM}$ ), MAP kinase inhibitor (U0126 $10 \mu \mathrm{M}$ ) or EGFR tyrosine kinase inhibitor (AG1478 $150 \mathrm{nM}$ ). Apoptosis and signal transduction pathway activation were determined by TUNEL staining and Western blot analysis, respectively. Results. In EGFR ${ }^{-1} \mathrm{MCE}$, ki and v cells, TNF induced apoptosis 3-, 5- fold compared to YAMC or wt cells. Furthermore treatment of YAMC or wt cells with TNF in the presence of EGFR tyrosine kinase inhibitor increased apoptosis 10-fold. Similarly, treatment of YAMC or wt cells with TNF failed to increase apoptosis except in the presence of PI 3-kinase inhibitor, which increased the apoptotic response 10-fold. In HT-29 cells, TNF enhanced apoptosis 10-fold in the presence of EGFR tyrosine kinase inhibitor, but not other cytokines, IL-1 and IFN- $\gamma$. Treatment of YAMC and wt cells with TNF in the presence of MAP kinase inhibitor did not increase apoptosis. TNF stimulated AKT/PKB activation via EGFR tyrosine kinase activation in wt cells but not in ki or v cells and TNF enhanced apoptosis in ADAM17-- MCE and E406A ADAM17 mutant cells, but not in wild-type ADAM17 expressing MCE cells, suggesting TNF-stimulated EGFR transactivation via ADAM17 cleavage abrogates TNF-induced intestinal cell apoptosis. Conclusion. Loss of EGFR tyrosine kinase activity significantly increases TNF-initiated colon epithelial cell apoptosis and conversely, EGFR tyrosine kinase activation blocks TNF-induced apoptosis. These effects may be mediated through activation of PI 3-kinase/AKT pathway. This novel observation has significant implications for understanding the role of EGFR regulation in maintaining intestinal epithelial cell homeostasis in an environment of chronic inflammation, injury and repair.

\section{M1603}

Regulation of Intestinal Epithelial Cell Proliferation By the Nuclear Receptor Corepressor (N-CoR) Genevieve Doyon, Claude Asselin, Francois Boudreau

INTRODUCTION: The intestinal epithelium is a tissue that undergoes constant renewal where proliferation and differentiation processes are tightly regulated. However, the transcriptional mechanisms implicated in these processes remain to be ascertained. We have previously shown that $\mathrm{N}-\mathrm{CoR}$ protein is located in the bottom part of the intestinal crypts. Moreover expression of $\mathrm{N}-\mathrm{CoR}$ was associated with the proliferation state in the IEC-6/Cdx2 and Caco-2 cell models that are capable to differentiate in culture. AIM: To investigate the putative role of N-CoR in the control of proliferation and differentiation of intestinal epithelial cells. METHODS AND RESULTS. The profile of N-CoR protein expression was first evaluated in the IEC-6 intestinal epithelial cell line that do not differentiate in culture. A stable level of $\mathrm{N}$-CoR expression was maintained along with proliferation of the cells. An RNA interference based on the generation of shRNA was then used to knockdown N-CoR in IEC-6. Among three different shRNA constructs tested, one led to a drastic reduction of N-CoR protein expression as determined by Western Blot analysis. The decrease in N-CoR protein was highly specific since a related nuclear corepressor, the Silencing mediator for retinoid and thyroid receptors (SMRT), was not affected by the shRNA construct. The putative relationship between N-CoR expression and cell proliferation was then investigated. Kinetics over period of 10 days of cell proliferation assessments were done using the stable cell line expressing the shRNA against $\mathrm{N}-\mathrm{CoR}$. The downregulation of $\mathrm{N}-\mathrm{CoR}$ resulted in a decreased in cell growth rate of $50 \%$ as compared to IEC- 6 shRNA control cells without any significant change in the cell death rate. CONCLUSION: Our results suggest that N-CoR is important to maintain a proliferative state in intestinal epithelial cells. The identification of the molecular targets of $\mathrm{N}-\mathrm{CoR}$ involved in the regulation of cellular proliferation is currently under investigation. (This work was supported by CIHR, NSERC, CFI, FRSQ and CRS) 
M1604

Characterization of Molecular Targets of the Thyroid Hormone Signalling Pathway in Intestine Epithelium Progenitor Cells

Elsa Kress, Jacques Samarut, Michela Plateroti

Hypothyroid newborn mice show a strongly altered development of the small intestine with a deep reduction in the number of proliferating epithelial progenitor cells within the intestinal crypts (1). Inactivating either the $\alpha$ or the $\beta$ isotype of thyroid hormone receptors (TRs) in mice, showed that the TR $\alpha$ l receptor is the major actor mediating T3 signalling in these cells $(2,3)$. In order to analyse the mechanisms responsible, we studied the expression of genes belonging to and/or activated by the $\mathrm{Wnt} / \beta$-catenin pathway, a major actor in the control of physiological and pathological epithelial proliferation in the intestine (4). We show that T3-TR $\alpha$ l controls the transcription of the $\beta$-catenin gene in an epithelial cellautonomous way. This is parallel to a positive regulation of proliferation-controlling genes such as type D cyclins and c-myc, known targets of the Wnt/ $\beta$-catenin. In addition, we show that the regulation of the $\beta$-catenin gene is direct as TR binds in vitro and in chromatin in vivo to specific Thyroid Hormone Responsive Element present in the intron 1 of this gene (Plateroti et al, under revision). As Wnt/ $\beta$-catenin plays a crucial role in intestinal tumorigenesis, our observation opens a new perspective on the study of TRs in intestinal pathology. To further elucidate and to analyse in detail the molecular mechanisms which govern the thyroid hormone-dependent response of intestine epithelial cell progenitors, we are currently using two different experimental approaches: 1 . Analysis of intestine epithelium regeneration after gamma-ray treatment of WT and TR mutant mice. This will enable to clarify whether the TH signalling pathway plays a role in this function and which are the molecular mechanisms. 2. Gene profiling of crypt cells isolated by laser microdissection from WT and TR KO intestines. Affimetrix microarray technology has been used to compare the pattern of expression of genes increased or decreased in TR mutants compared to the WT. Bioinformatic analysis is going to be performed. Bibliography l.Flamant, et al. 2002. Congenital hypothyroid Pax8(-/-) mutant mice can be rescued by inactivating the TRalpha gene. Mol Endocrinol, 16: 24-32. 2.Plateroti et al. 1999. Involvement of T3Ralpha- and beta-receptor subtypes in mediation of T3 functions during postnatal murine intestinal development. Gastroenterology, 116: 1367-1378. 3.Plateroti et al. 2001. Functional interference between thyroid hormone receptor alpha (TRalpha) and natural truncated TRDeltaalpha isoforms in the control of intestine development. Mol Cell Biol, 21: 4761-4772. 4.Moon et al. 2004. WNT and beta-catenin signalling: diseases and therapies. Nat Rev Genet, 5: 691-701

\section{M1605}

Influence of Cell-Cell Contacts On Survival of Primary Human Colonic Epithelial Cells and Regulation of Bcl-2 Family Proteins

Claudia Hofmann, Monika Artinger, Johannes Grossmann, Werner Falk, Juergen

Schoelmerich, Gerhard Rogler, Florian Obermeier

Background: Intestinal epithelial cell homeostasis is crucial for the maintenance of the barrier function and prevention of cancer formation. Colonic epithelial cells (CEC) are highly anchorage-dependent. They undergo detachment-induced apoptosis (anoikis) as soon as they lose contacts. Cell-matrix anchorage and cell-cell anchorage are of equal importance for survival of primary human CEC as previous studies revealed, that the induction of anoikis can completely be blocked by maintenance of CEC cell-cell contacts despite the loss of cell-matrix anchorage. The signaling pathways mediating this anti-apoptotic effect, however, are unknown. Methods: CEC anoikis was modulated by incubation of ex-vivo isolated CEC in the presence of an E-cadherin-Fc construct. To maintain cell-cell contacts, intact CEC crypts were isolated and gently centrifuged to form a cell aggregate ("pellet"; PCEC) before disruption of CEC cell-cell contacts could occur. Induction of apoptosis in CEC was assessed by Western Blot (Caspase-3), a colorimetric assay (DEVD-pNA), and DAPI staining. Regulation of pro- and anti-apoptotic members of the Bcl-2 family (Bcl-2, Mcl-1, Bcl-xL, Bax, Bak, Bad, Bid, Puma, Bim) was investigated by Western Blot analysis. Results: After simulation of cell-cell contacts by soluble E-cadherin-Fc caspase-3-activation and consecutively anoikis was significantly reduced by $30 \%(\mathrm{p}=0.027)$. In contrast, a complete prevention of anoikis was achieved when existing cell-cell contacts were preserved as demonstrated by lack of caspase-3 activation and DAPI staining of P-CEC $(\mathrm{p}=0.0002)$. On the other hand, anoikis could still be induced in P-CEC after several hours in pellet culture by disrupting cell-cell contacts. P-CEC displayed increased levels of the anti-apoptotic proteins Bcl-2 and Mcl-1, whereas no significant changes in for Bcl-xL, Bax, Bak, Bad, Bid Puma and Bim were detected. In contrast, a degradation of Mcl-1 as well as a cleavage of Bid was observed in CEC undergoing anoikis. Conclusions: CEC anoikis can be partially blocked by simulation of E-cadherin-induced signaling and can completely be blocked by the preservation of cell-cell contacts. Anti-apoptotic members of the Bcl-2 family are involved in the cell-cell contact-mediated CEC survival. Cell aggregation in the pellet model does not change principal anoikis-mechanisms CEC as anoikis could still be induced after several hours of pellet culture. In summary, E-cadherin mediated cell-cell signaling serves as a cooperative mechanism providing survival signals capable of compensating the loss of cellmatrix contacts.

\section{M1606}

Adherens Junction-Dependent PI3K/Akt Activation Decreases Sensitivity to DNA Damage-Induced Apoptosis in Differentiated Colonic Epithelial Cells Tae Il Kim, Boah Chae, Kyoung Min Yang, Byung Chang Kim, Sung Jae Shin, Won Ho Kim

Background: The balance between proliferation and apoptosis is important for homeostasis during differentiation in crypt-villus axis of intestinal epithelium. In addition, cellular responses to diverse stimuli also vary by the degree of cellular differentiation. However, their mechanisms remain largely unknown. Aim: We investigated the differences in apoptotic sensitivities and its mechanism in genotoxin-induced apoptosis, based on the degree of differentiation of epithelial cells. Methods: Differentiation was induced by post-confluence culture in Caco-2 cells. MMS (methyl methanesulfonate), which is a direct-acting DNA alkylating agent, was used for apoptosis induction. The apoptotic cell death was measured by cytochrome $\mathrm{c}$ staining and MTT assay, and the expressional changes of differentiationand cell survival-associated molecules (NF-kB, p2l, $\beta$-catenin, E-cadherin, PI3K/Akt pathway) were evaluated by western blot, immunofluorescent study and immunohistochemical staining. EGTA and LY294002 were used for inhibition of cell-cell adhesion and PI3K, respectively. Results: Compared to the subconfluent Caco- 2 cells, 7 days post-confluent cells showed resistance to MMS-induced apoptosis. In our differentiation model, NF-kB activation or cytoplasmic p21 expressions, which are known to be important in differentiation-induced resistance to apoptotic stimuli in other cell systems, were not observed. The expression of nuclear and cytoplasmic E-cadherin and $\beta$-catenin decreased in 7 days post-confluent Caco2 cells, while their adherens junction components increased. E-cadherin and p-Akt expression increased in 7 days post-confluent Caco-2 cells, and, in human intestinal tissue, the expression of E-cadherin and p-Akt also increased in the upper portion of villi, compared to crypt. Inhibition of cell-cell adhesion using EGTA decreased Akt phosphorylation, which was reversed by calcium restoration. Akt phosphorylation by calcium-mediated cell-cell adhesion was more prominent in differentiated cells. In addition, LY294002 treatment inhibited Akt phosphorylation by calcium-mediated cell-cell adhesion. The difference in MMS-induced apoptotic sensitivity between subconfluent and 7 days post-confluent Caco- 2 cells was eliminated by inhibiting cell-cell adhesion or PI3K. Conclusion: Our data demonstrate that apoptosis-resistant phenotype of differentiated epithelial cells in DNA damage-induced apoptosis is mediated by activation of cell adhesion-mediated PI3K/Akt pathway.

\section{M1607}

Translocation of BMF During Anoikis in Primary Intestinal Epithelial Cells Martin Hausmann, Claudia Hofmann, Stephan Kiessling, Werner Falk, Juergen Schoelmerich, Hans Herfarth, Gerhard Rogler

BACKGROUND: Apoptosis is a conserved genetic program for the removal of malfunctioning or potentially dangerous cells. Anoikis is a specific form of apoptosis induced by loss of cell attachment. Loss of cell-anchorage specifically triggers the release of a BH3-only protein, $\mathrm{Bcl}-2$ modifying factor (Bmf)-2, from the actin cytoskeleton. Bmf is a sentinel placed to register damage at the cytoskeleton and then to convey the death signal to $\mathrm{Bcl}-2$. Bcl-2, intrinsic promoting cell survival, is neutralised by Bmf and cytochrome c release from the mitochondrium is initiated. As this mechanism might be of great importance during colonic epithelial cell (CEC) anoikis we investigated it in our primary CEC culture model. METHODS: Primary human CEC were isolated ex-vivo from surgical specimens. To induce anoikis a single cell suspension of epithelial cells was shaken for 2 hours in medium at $37^{\circ} \mathrm{C}$. Sub-cellular localization of Bmf in CEC was analysed by immunofluorescence (rat antiBmf, Alexis). Mitochondria were localized by a mitochondria selective probe (MitoTracker, Molecular Probes). Mitochondria were isolated from epithelial cells by digitonin-based subcellular fractioning. Bmf and cytochrome c was analysed by Western Blot. RESULTS: DAPI staining revealed that $>90 \%$ of primary human CEC exhibited a fractionated nucleus within 2 hours after ex-vivo isolation. Bmf protein could not be co-localized with mitochondria in freshly isolated CEC. However, immunofluorescence revealed a significant increase in Bmf protein associated with mitochondria after induction of anoikis by loss of cell attachment. Translocation of Bmf to the mitochondria was confirmed by Western Blot applying subcellular fractions. Initiation of cytochrome c release after translocation of Bmf was demonstrated by Western Blot. Re-constitution of cell-matrix or cell-cell contacts prevented Bmf translocation and subsequently induction of anoikis. CONCLUSION: This study shows for the first time the translocation of Bmf in primary CEC induced by the loss of cell attachment. This mechanism is likely to play an important role in the regulation of CEC survival, both in inflammation and carcinogenesis.

\section{M1608}

Cyclic-AMP Mediated Activation of NF- $\mathrm{B}$ in Intestinal Epithelial Cells: Implications for Cellular Survival

Jeffrey A. Rudolph, Julia Pratt, Kris A. Steinbrecher, Mitchell B. Cohen

Background: The ability of an intestinal cell to withstand a potentially lethal stress is in part governed by active cell survival signaling mechanisms. Two well characterized survival pathways include the extracellular signal regulated kinase (ERK1/2) cascade and nuclear factor $\kappa \mathrm{B}(\mathrm{NF}-\mathrm{\kappa B})$ signal transduction. We have previously shown cAMP to mediate cell survival in T84 cells, an intestinal epithelial cell line, via activation of the ERK1/2 signaling cascade. In some non-intestinal cell lines, the major effector protein of cAMP, protein kinase A, regulates transcriptional activity of NF- $\mathrm{KB}$ through direct serine phosphorylation. We hypothesized that in T84 cells, cAMP also mediates phosphorylation of NF-KB and nuclear translocation of its p65 subunit to activate anti-apoptotic NF- $\mathrm{KB}$ transcription. Methods: Confluent T84 cells were incubated with media alone, Tumor Necrosis Factor- $\alpha$ (TNF $\alpha$ positive control), or cholera toxin (CT) to increase intracellular cAMP. Protein was harvested from whole cell extracts for immunoblot analysis and separated into nuclear and cytoplasmic fractions for nuclear localization experiments. In a second set of experiments, RNA was obtained for real time RT-PCR analysis of two anti-apoptotic proteins, cIAP-2 and Mcl-1, based upon their known regulation by NF- $\mathrm{KB}$ transcription and a preliminary microarray analysis. Results: Nuclear localization experiments demonstrated that CT incubation led to a time dependent accumulation of $\mathrm{p} 65$ in nuclear extracts. Accumulation started in as little as 6 hours, peaked at 18 hours and subsequently decreased thereafter. Analysis of whole cell extracts for IkB $\alpha$ degradation and phosphorylation of p65 at serine 536, two markers of TNF $\alpha$ mediated NF- $\mathrm{KB}$ activation, were not present in CT treated samples. RT-PCR analysis of both cIAP-2 and Mcl-1 showed increased expression after a 24 hour exposure to CT, a time consistent with NF-אB transcriptional regulation. Conclusions: Cyclic-AMP accumulation in $\mathrm{T} 84$ cells leads to time-dependent activation of NF-KB signaling as evidenced by nuclear accumulation of p65. NF-KB does not appear to be activated by phosphorylation/ degradation of I $\mathrm{K} \mathrm{B} \alpha$ or phosphorylation of $\mathrm{p} 65$ at serine 536 , suggesting an alternative pathway of induction similar to that seen in some non-cytokine mediated NF-KB activation mechanisms. Expression of the anti-apoptotic proteins cIAP-2 and Mcl-1 is consistent with 
NF-KB transcriptional regulation and increased cellular survival. We speculate that the antiapoptotic effect of cAMP on T84 cells is more complex than ERKl/2 signaling alone and is

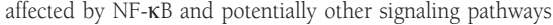

\section{M1609}

Reciprocal Targeting of Hathl and $\beta$-Catenin By Wnt-Gsk3 in Colonocyte Differentiation and Proliferation

Kiichiro Tsuchiya, Tstsuya Nakamura, Ryuichi Okamoto, Takanori Kanai, Mamoru Watanabe

Background \& Aims: The canonical Wnt pathway plays a crucial role in the cell fate decision of intestinal epithelial cells (IECs); however, the influence of the Wnt pathway on cellular differentiation remains unclear. Recently, it has been shown that a member of bHLH transcription factors, Math-1 and its human homolog; Hathl, acts as a critical factor for terminal differentiation of IECs, although Mathl- and Hathl- mediated regulation to balance between differentiation and proliferation remain poorly understood. In this study, we tested the possibility that Wnt pathway might directly target the Hath-1 function, especially focusing on the proteolytic mechanism of Math-1 in IECs. Methods: An expression plasmid encoding Hath-1 gene was transfected into 293T cells or colonic carcinoma-derived SW480 cells, and Western blotting was performed to analyze the expression levels of Hath-1 protein. The effects of various protease inhibitors were tested to assess the involvement of proteolysis in the regulation for Hath-1 protein. The molecular domain responsible for the degradation was analyzed by using various mutants of Hath-1 proteins. The role of Wnt signaling in the Hath-1 degradation was examined by cotransfecting the wild-type (wt) APC into SW480 cells, or the Wntl into 293T cells, respectively. Results: The Hath-1 protein was actively degraded in SW480 cells where the Wnt signal is constitutively activated. In contrast, the breakdown of Hath-1 did not occur in 293T cells in which the Wnt signal remains inactivated. The degradation of Hath-1 in SW480 cells was inhibited by the treatment with MG132, an inhibitor for the proteasome, and the accumulated Hath-l was shown to be ubiquitinated, indicating a role of ubiquitin-proteasome pathway in this process. Mutational analysis of Hath-1 showed that the critical residues regulating the proteolysis in SW480 cells are S54 and S58 that correspond to the consensus sequence for GSK-3 substrates. Indeed, pharmacological GSK-3 inhibitors blocked the Hath-1 degradation in SW480 cells. Importantly, the degradation of Hath-1 in SW480 cells was also inhibited by cotransfection of wtAPC, which reciprocally enhanced the degradation of $\beta$-catenin. Conversely, coexpression of Wnt 1 in 293T cells resulted in the proteolysis of Hath-1, while it facilitated the accumulation of $\beta$-catenin. Conclusion: We showed a novel branching of the Wnt-GSK-3 axis of signaling pathway, suggesting its important role in balancing the cellular differentiation and proliferation via the inverse regulation for the stability of Hath- 1 and $\beta$-catenin proteins.

\section{M1610}

Polyamine Depletion Stabilizes P53 and Nucleophosmin (NPM) By Regulating Cytoplasmic Translocation of RNA-Binding Protein Hur in Intestinal Epithelial Cells

Tongtong Zou, Lan Liu, Jaladanki N. Rao, Bernard S. Marasa, Kaspar M. Keledjian, Lan Xiao, Myriam Gorospe, Jian-Ying Wang

Our previous studies have demonstrated that polyamines are required for normal intestinal mucosal growth and that decreasing cellular polyamines inhibits intestinal epithelial cell renewal primarily by stabilizing p53 and NPM mRNAs. However, the exact mechanism by which polyamines modulate RNA stability remains unknown. The RNA-binding protein $\mathrm{HuR}$ is a critical regulator of the post-transcriptional fate of target transcripts and has shown to regulate the stability of labile mRNAs. Inactivated HuR is localized in the nucleus, but it can be activated after translocation to the cytoplasm. The present study tests the hypothesis that polyamines modulate stability of p53 and NPM mRNAs by regulating HuR in normal intestinal epithelial cells (IEC-6 line). Method: Cytoplasmic, nuclear and total HuR proteins were examined by Western blot analysis. HuR binding activity was identified by RNA pulldown technique and HuR-immunoprecipitation (IP). Stability of p53 and NPM mRNAs was examined by measurement of their mRNA half-lives with real-time RT-PCR analysis. Functions of HuR were investigated by using siRNA that specifically targets HuR (siHuR). Results: Depletion of cellular polyamines by DFMO, the specific inhibitor of polyamine synthesis, dramatically increased HuR translocation to the cytoplasm, along with a significant decrease in nuclear HuR, although it did not alter levels of total cellular HuR. The level of cytoplasmic HuR protein was increased by $\sim 4$-5-folds the control value on day 6 after DFMO treatment, while nuclear HuR was decreased by $>50 \%$, which were completely prevented by exogenous polyamine spermidine. Induced HuR in the cytoplasm following polyamine depletion directly bound to the 3'-untranslated regions (3'-UTR) of p53 or NPM mRNA as measured by RNA pull-down assays. HuR-IP study further showed the presence of endogenous p53 and NPM mRNAs in the materials immunoprecipitated by the specific anti-HuR antibody but not in those precipitated by nonspecific antibody (IgG1). Inhibition of HuR expression by transfection with siHuR in polyamine-deficient cells not only destabilized p53 and NPM as indicated by decreases in their mRNA half-lives but also prevented the increased levels of p53 and NPM proteins. Conclusions: These results indicate that 1 ) decreasing cellular polyamines results in the cytoplasmic translocation of $\mathrm{HuR}$ and 2) increased HuR in the cytoplasm binds to the 3'-UTRs of p53 and NPM mRNAs and plays a critical role in stabilization of NPM and $\mathrm{p} 53$ following polyamine depletion, thus involving in the negative control of intestinal epithelial cell proliferation.

\section{M1611}

BMP Signaling Regulates Proper Terminal Differentiation of Intestinal Goblet Cells in Mice

Yannick D. Benoit, Benoit A. Auclair, Yuji Mishina, Deborah L. Gumucio, Nathalie Perreault

BACKGROUND: Bone morphogenetic proteins (BMPs) are morphogens known to regulate numerous cell processes in various tissues. In the intestine, BMPs are primarily produced by the mesenchymal compartment. These factors can activate signaling pathways following a paracrine or autocrine route. Recent publications have shown links between BMP signaling and colorectal carcinogenesis. However, the potential effects of this cascade on other important cell functions in the gastrointestinal tract have not yet been explored. AIM: To investigate the paracrine role of BMP signaling during intestinal epithelial cell fate. METHODS AND RESULTS: With the use of the Cre/loxP system, we have generated a mouse with the BMPRIA receptor deleted exclusively in the gut epithelium by crossing floxed BMPRIA mice with the Villin-Cre line In order to characterize goblet cells in the jejunum of Villin-Cre/BMPRlAloxP/loxP mice, we performed Alcian Blue staining. We have observed that the number of goblet cells is not significantly changed in the mutants as compared to their wild-type littermates. However, these cells are consistently smaller in the mutant mice suggesting lack of maturity of goblet cells. Electron microscopy analysis exposed smaller mucigen granules and confirmed the reduction in size of the goblet cells in the mutant animals. We then analyzed, by quantitative PCR, the levels of expression of the goblet cell-specific marke Muc2 in both mutant and wild-type animals. We have confirmed a 2.2 fold decrease in Muc2 expression in the mutant compared to the wild-type animals. The zinc-finger transcription factor Klf4 is known to play a crucial role in goblet cell terminal differentiation in the colon. Comparative analysis of Klf4 expression between mutant and wild-type littermates has revealed a dramatic decrease of both protein and mRNA levels in the mutant animals. CONCLUSION: Altogether, these results demontrate that paracrine BMP signaling plays an important role in the terminal differentiation of intestinal goblet cell type through regulation of the zinc-finger transcription factor Klf4. Further analysis is currently underway in order to determine if other cell types from the secretory lineage are regulated by BMP signaling as well.

\section{M1612}

PDX-1 Regulates Gene Expression and Spatial Pattern Formation in the Small Intestine

Chin Chen, Rixun Fang, Ying Hao, Anson W. Lowe, Charalambos Maravelias, Philip L. Chou, Eric Sibley

Background: Transcription factor pancreatic duodenal homeobox-1 (PDX-1) plays a key role in pancreatic development and maintaining pancreatic islet function. However, the role of PDX-1 in intestinal development and gene expression is less well defined. Objective: We aimed to characterize PDX-1 regulation of spatial patterning of gene expression in small intestinal epithelial cells and to identify PDX-1 intestinal target genes. Methods: To characterize PDX-1 regulation of intestinal pattern formation and gene expression in vivo, conditional knockout $(\mathrm{KO})$ mice with PDX-1 inactivation restricted to the intestine were generated. Total RNA isolated from the small intestine segments of control and conditional KO mice was analyzed by real-time RT-PCR. To identify novel target genes regulated by PDX-1, microarray analysis and real-time RT-PCR were performed with total RNA isolated from transfected human intestinal Caco-2 cells stably expressing mouse PDX-1 or the contro empty vector. Results: Conditional inactivation of PDX-1 in the small intestine results in altered expression of candidate target genes along the anterior-posterior gut axis. The expression of adenosine deaminase (ADA) was reduced significantly in the proximal small intestine of the $\mathrm{KO}$ mouse, consistent with published reports that PDX-1 activates ADA. Sucraseisomaltase and lactase gene expression was increased in the proximal small intestine of the $\mathrm{KO}$ mouse, in agreement with reports that PDX-1 is a repressor for both genes. In Caco-2 cells stably expressing mouse PDX-1, 88 genes were activated while 49 genes were repressed ( $>4$-fold) by PDX-1 overexpression, when compared to the non-PDX-1 expressing Caco-2 control cells. The effect of PDX-1 on selected genes was confirmed by real-time RT-PCR Genes that were regulated by PDX-1 overexpression include transcription factors, growth factors, kinases, digestive glycosidases, nutrient transporters, nutrient binding proteins, and structural components. Conclusions: PDX-1 gene expression is necessary for appropriate spatial patterning of small intestinal target gene expression. The PDX-1 conditional KO mouse represents a useful tool for investigating in vivo the role of PDX-1 during gut development. The broad spectrum of genes regulated by PDX-1, as detected by microarray analysis, further supports an important role for PDX-1 in intestinal gene regulation.

\section{M1613}

Generation of a Functional in Vitro Model for Differentiation of Pluripotent Cells Into Multiple Intestinal Epithelial Cell Lineages

Carine R. Lussier, Benoit A. Auclair, Nathalie Perreault, Francois Boudreau

INTRODUCTION: As an ongoing renewal system in which proliferation, differentiation, migration and apoptosis are tightly regulated, intestinal epithelium is an excellent model to study these cellular processes at the molecular level. However, normal intestinal epithelial cells able to recapitulate these processes in culture is not yet available. The aim of this work was to produce a functional model for intestinal epithelial cell differentiation. METHODS AND RESULTS: An IEC-6 cell line that stably expressed Cdx2 was seeded on a monolayer of human fetal intestinal mesenchymal cells. Co-cultures were maintained for 12 days. At day 5 after seeding, up-elevated structures appeared and multiplied through day 12 . Electron microscopy revealed that these structures consisted in a monolayer of polarized epithelia cells presenting brush border and cell-cell junctions. These cells looked very similar to newly differentiated intestinal epithelial cells when compared to cells located at the base of the villus in the rat ileum. Western analysis showed a stable expression of HNF-lbeta and HNF3beta, an up-regulation of $\mathrm{Cdx} 2$ and GATA-4 along with the co-culture kinetic, and a drastic

. 
induction of HNF-lalpha and HNF-4alpha protein expression at day 6 after seeding when upelevated structures started to expend. This observation confirmed that all known transcription factors involved in the regulation of specific genes related to intestinal epithelial differentiation are present in this model. Consequently, it was possible to detect induction of three specific epithelial cell type markers by RT-PCR analysis. Indeed, the sucrase-isomaltase (enterocytes), trefoil factor 3 (goblet cells) and cholecystokinin (enteroendocrine cells) genes were induced after 8 days following the deposition of IEC6-Cdx2 cells on primary mesenchymal cells. Finally, cells were harvested after 3,5 and 7 days after seeding and submitted to a gene expression analysis (affymetrix) to identify novel molecular determinants for intestinal epithelial cell differentiation. CONCLUSION: Our results suggest that IEC-6 cells expressing exogenous $\mathrm{Cdx} 2$ are able to rapidly differentiate in a co-culture system with human intestinal mesenchymal cells, giving rise to at least three different intestinal epithelial cell types. Therefore, our co-culture system is the first in vitro model to reproduce normal pluripotent intestinal epithelial cell differentiation, generating a new opportunity to investigate molecular mechanisms involved in this process. (This work was supported by CIHR, NSERC, CFI CRS and FRSQ)

\section{M1614}

Crypt-Restricted Expression of the Integrin $\alpha 6 a$ Subunit in the Human Intestinal Epithelium: Implications in Proliferation But Not Differentiation Anders B. Dydensborg, Joelle Auclair, Elizabeht Herring, Inga C. Teller, Daniel Menard, Jean-Francois Beaulieu

Integrins are heterodimeric adhesion receptors that function as platforms for intracellular signalization of importance for cellular growth, differentiation and survival. The integrin $\alpha 6$ transcript undergoes alternative splicing leading to the A and B variants, which differ only in their short cytoplasmic domains. While no specific function has yet been ascribed to either variant in epithelial cells, distinct signalization capacities for the two variants exist such as the potentially exclusive activation of the Ras-MAPK pathway by the A variant. In this study, we have investigated the involvement of the $\alpha 6 \mathrm{~A}$ and $\mathrm{B}$ variants in human intestinal cells. Using indirect immunofluoresence we have evaluated the expression pattern of the two variants in the adult human intestine and have found that the A variant is predominantly expressed in the crypt while the B-variant is distributed evenly all along the crypt-villus axis. RT-PCR and western blotting showed the A variant to be primarily expressed at both the transcript and protein level in the normal undifferentiated human crypt cell line HIEC, while fully differentiated primary human epithelial cells were found to predominately express the B variant. Furthermore, two human intestinal epithelial cell lines capable of undergoing differentiation (Caco-2/15 and HT-29) were observed to down-regulate the expression of the A variant upon initiation of the differentiation process. To investigate whether these distinct expression patterns are of importance to cellular processes associated with proliferation and/or differentiation, we analyzed the capacity of the two variants to stimulate the transcriptional activity of reporter plasmids responsive to $\mathrm{c}$-Myc and $\beta$-catenin/ TCF (proliferation) as well as their capacity to stimulate lactase-phlorozin hydrolase and sucrase-isomaltase promoter activity (differentiation). Both reporter plasmids, responding to $\mathrm{c}$-Myc and $\beta$-catenin/TCF, were significantly stimulated by the over-expression of the $\mathrm{A}$ compared to the B variant, while the promoters of the two differentiation markers were not differentially stimulated by the two variants. We conclude that even though predominant in differentiated cells, the $\alpha 6 \mathrm{~B}$ variant does not appear to play a significant role in the differentiation process while the $\alpha 6 \mathrm{~A}$ subunit, which is predominant in less differentiated cells, promotes proliferation under a mechanism that appears to involve the $\beta$-catenin pathway

\section{M1615}

Diverse Cellular Responses to Glucocorticoid in the Developing Small Intestine

Murat B. Yaylaoglu, Barbara M. Agbemafle, Thomas J. Oesterreicher, Christina Thaller, Susan J. Henning

Glucocorticoids (GC) are known to exert both physiological and pharmacological effects on the gastrointestinal tract. Amongst the physiologic effects are an ability to elicit functional maturation. Although the latter has been widely studied in rodent models, to date the molecular mechanism of GC action on the developing intestine have not been elucidated. Our previous microarray studies identified 66 transcripts as being rapidly induced in the jejunum following dexamethasone (DEX) administration to suckling mice. In the current work we explored the hypothesis that these primary response genes would display cell specific patterns of expression. A secondary hypothesis was that robotic-based in situ hybridization (ISH) would allow quantitation of mRNA at the cellular level. Methods: Mouse pups at P8 received DEX (0.4 $\mu \mathrm{g}$ per $\mathrm{g}$ BW) or vehicle injections. Samples of jejunum were collected $3-4 \mathrm{hr}$ later for ISH and Northern blotting. Robotic-based ISH was performed with digoxygenin-labeled riboprobes using tyramide signal amplification. Results: A subset of 5 of the 66 DEX induced transcripts were studied: three known genes (Ndrg1, Sgk and c-fos) and two unknown genes (G9 and G36). As predicted, ISH revealed marked diversity of cellular expression. Four of the five transcripts were localized to the epithelium and of these: Sgk mRNA was found in all epithelial cells; c-fos mRNA was confined to the villus tip and Ndrg1 and G36 mRNAs were localized to the upper crypt and villus base. The remaining transcript (G9) was expressed in the lamina propria and the muscle layers. Quantitation of ISH signals showed that the average fold-increase in tissues from DEXtreated mice as compared with vehicle-treated mice was very similar to that measured by Northern blotting. Pseudo-color images allowed visualization of the location of strong, medium and weak ISH signals. In the case of G9, this approach showed that the magnitude of the DEX induction was much greater in the muscle layers than in the lamina propria Conclusions: Although this survey included a modest number of transcripts it demonstrated that, at the cellular level there is a great diversity in the immediate early genes of GC action on the developing intestine. Furthermore, the ISH methods used here represent a significant technical advance, being to our knowledge, the first report of quantitative ISH with G tissues. This work was supported by NIH grants R01 HD14094 and P30 DK56338.

\section{M1616}

Effect of Lactobacillus Plantarum 299v Administration On the Gut Function in Suckling Rats

Frida Fak, Siv Ahrne, Ann Linderoth, Goran Molin, Bengt Jeppsson, Bjorn Westrom

After birth, the gastrointestinal tract undergoes structural and functional maturation processes, eventually preparing it for the change in food at weaning. In the present study, we have studied whether these maturational changes can be affected by exposure to a probiotic Lactobacillus-strain, using a suckling rat model. Methods: Lactobacillus plantarum 299v (Lp299v, DSM9843) (Probi AB, Lund, Sweden) was administered (3.0 x 106 CFU/g body wt per day) for 7 days to Sprague Dawley rats pups aged 3, 7 or 14 days old, after which gut organ growth, intestinal disaccaridase pattern, intestinal permeability and pancreas proteins were analyzed. Results: Exposure to Lp299v resulted in different effects depending on the age of the animals. In the youngest age group, fed with Lp299v from 3 to 10 days of age, the intestinal mucosal protein content increased and a reduction in intestinal permeability, indicated by a decreased uptake of the gavaged marker, bovine $\operatorname{IgG}$, was observed as compared to the control littermates. This appeared to correlate with a ten-fold numerical increase in ceacal lactobacilli. The rat pups treated from 7 to 14 days of age, showed a decrease in pancreas weight and protein content, whereas the pups in the weaning group (14 to 21 days of age) showed little effect from the Lp299v treatment. Conclusions: Exposure to Lp299v during the early neonatal period stimulated the small intestine, leading to increased intestinal barrier properties. Administering Lp299v later during the suckling period resulted instead in decreased pancreas enzyme content, suggesting that an increase in gut lactobacilli could lead to decreased demand for pancreatic enzymes.

Effects of Lp299v on the mucosal protein content (mg/g body wt), the plasma level of BIgG $(\mu \mathrm{g} / \mathrm{ml}) 3 \mathrm{~h}$ after gavage and the pancreatic protein content (mg/g body wt) for 10,14 and 21 day-old rats treated during one week with either Lp299v or NaCl.

\begin{tabular}{|c|c|c|c|c|c|c|}
\hline Age & \multicolumn{2}{|c|}{3 to 10 days } & \multicolumn{2}{c|}{7 to 14 days } & \multicolumn{2}{c|}{$14-21$ days } \\
\hline Treatment & $\mathrm{NaCl}$ & Lp299v & $\mathrm{NaCl}$ & Lp299v & $\mathrm{NaCl}$ & Lp299v \\
\hline Mucosal protein & $102(52)$ & $151(45)^{* * *}$ & $131(31)$ & $131(27)$ & $275(51)$ & $272(54)$ \\
\hline Plasma BIgG & $233(78)$ & $174(74)^{* * *}$ & $194(34)$ & $187(22)$ & $0(0)$ & $0(0)$ \\
\hline Pancreas protein & $191(32)$ & $207(49)$ & $214(17)$ & $184(21)^{* *}$ & $398(80)$ & $412(37)$ \\
\hline
\end{tabular}

Values are mean (SD) and differences between Lp299v-fed and $\mathrm{NaCl}$ are considered significant when $\mathrm{p}<0.005(* *), \mathrm{p}<0.001(* *)$.

\section{M1617}

Molecular Mechanisms of Lactobacillus Rhamnosus GG (LGG) Regulation of Mouse Intestinal Functional Maturation

Fang Yan, Hanwei Cao, Robert Whitehead, D. Brent Polk

Background. Intestinal functional maturation occurring during weaning is coincident with the transition of aerobic to anaerobic bacteria in the gastrointestinal tract. Studies indicate that establishing the host-bacterial relationship plays critical roles in functional maturation of the intestine during early postnatal stage, including regulation of gastrointestinal maturationregulated gene expression. We reported that LGG regulates intestinal cell homeostasis, in part, through secreted proteins. To this end, we have isolated two LGG soluble proteins, p75 and p40, which promote cell growth and inhibit cytokine-induced apoptosis. The purpose of this study is to determine the mechanisms of LGG and its protein products regulating mouse intestinal functional development. Methods. Fetal C57BL/6 mouse midgut explants isolated from E20 embryos were cultured on top of transwell inserts in DMEM medium containing $0.5 \%$ FBS. After culture with LGG or 440 for 24 hours, total RNA was isolated from tissue for mouse MEEBO $38.5 \mathrm{~K}$ oligonucleotide microarray to detect intestinal transcriptional responses. Midgut and hindgut isolated from E20 embryos or small intestine and colon from P2 newborns were treated with LGG, LGG-conditioned medium (LGGCM) $\mathrm{p} 40$ or $\mathrm{p} 75$ for 24 hours, and paraffin-embedded tissue sections were studied by immunohistochemistry to detect epithelial proliferation by ki67 staining, differentiation by PAS staining, and epithelial glycosylation pattern by lectin staining. Results. LGG and soluble protein, p40, up-regulate expression of genes (greater than 2 -fold) involved in several important functions, including intestinal functional maturation (for example, Adenosine deaminase, Carbonic anhydrase, GATA, TGFb, and Fucosyltransferase), nutrient absorption (Colipase), barrier fortification (Integrin, Trefoil factor), cellular function ( $\mathrm{Na} / \mathrm{K}$ transporting ATPase), intestinal neuro-development (Glutamate transporter). Furthermore, LGG, LGG$\mathrm{CM}, \mathrm{p} 40$ and p75 all promote midgut and small intestinal epithelial proliferation and mature fucosylation, consistent with gene microarray results. Proliferation and PAS positive cells in hindgut and colon are also increased by LGG, LGG-CM, p40 and p75. Conclusion. These studies suggest that LGG modulates mouse intestinal development through secretory proteins, which enhance host transcriptional responses as one of the mechanisms of bacterial-epithelial interaction regulating intestinal maturation. Furthermore, this responsiveness to LGG and its secretory proteins suggests they may have preventive or therapeutic applications for the premature intestine in intestinal developmental diseases, such as necrotizing enterocolitis.

\section{M1618}

Role for L-Type Ca2+ Channels in Developmental Regulation of the ENS Phenotype Julien Chevalier, Philippe Naveilhan, Jean-Paul Galmiche, Michel Neunlist

Introduction: The developmental regulation of the phenotype of the ENS remains largely unknown. Therefore, the role of this study was to characterize in vitro the differentiation of enteric neurons and glial cells in an model of primary enteric cells culture and to study the effects of $\mathrm{KCl}$ upon the neurochemical phenotype. Methods: Small intestine of rat embryos (E15) were removed and finely diced. Tissue fragments were digested with trypsin and DNAse I and the cell suspension was seeded in gelatin coated 12 well plates. Following 
culture with conditioned medium in absence or presence of $\mathrm{KCl}(40 \mathrm{mM})$, cells were processed for immunohistochemistry or quantitative PCR after 3, 7 and 13 days (D). The effects of $\mathrm{KCl}$ were studied after D13 of culture. Immunohistochemical studies were performed using antibodies against Nestin, GFAP, PGP9.5, Tuj, VIP, NOS and TH and neurochemical coding was evaluated on at least 20 ganglia per preparation and per marker. Pharmacological studies with the L-type Ca2+ blocker nifedipine $(1 \mu \mathrm{M})$ were performed. Results: At day 0 (D0), $65 \%$ of the cells were Nestin-immunoreactive (IR). At D3, GFAP-IR cells appeared and localized within ganglionic structure. These structures also contained neurons that were Tuj-IR and PGP 9.5-IR. The number of neurons/ganglia increased over time and ranged from $3 \pm 1$ at $D 1$ to $35 \pm 6$ at $D 13(n=7)$. Over the 13 days of culture, there was a significant decrease in the proportion of VIP-IR and nNOS-IR neurons. At D3, $78 \pm 5 \%$ and $87 \pm 6 \%$ of neurons (\% of PGP 9.5; $n=5$ and $n=4$ respectively) were VIP and NOS-IR respectively. At D13, these proportions reached $30 \pm 8 \%$ and $33 \pm 7 \%$ respectively. Following 13 days of culture with $\mathrm{KCl}$, major changes in the phenotype were observed and characterized by a significant increase in the proportion of VIP $(+43 \%, p=0.007 ; n=4)$ but no change in the proportion of nNOS neurons. This increase in VIP was associated to an increase in VIP mRNA expression. $\mathrm{KCl}$ treatment induced also a massive induction of $\mathrm{TH}$ expression $(2 \pm 1 \%$ TH-IR cells in control vs $61 \pm 2 \%$ with $\mathrm{KCl}, \mathrm{p}<0.001 ; \mathrm{n}=7$ ) which was significantly blocked by nifedipine $(3 \pm 1 \%$ TH-IR cells, $\mathrm{p}=0.004 ; \mathrm{n}=3)$. Conclusion: In conclusion, this study suggests that $\mathrm{KCl}$ can modulate the developmental differentiation of the ENS, in part via L-type $\mathrm{Ca} 2+$ channels. The mechanisms and factors involved in this regulation remain to be identified.

\section{M1619}

Structure-Function Relationships in HLX Homeobox Transcription Factor Regulation of Intestinal Mesenchymal Promoters

Michael Bates, Angela Mendell

The Hlx homeobox transcription factor gene is expressed in developing intestinal and hepatic mesenchyme. Hlx is required for normal embryonic growth of intestine and liver as well as development of the enteric nervous system. Further, Hlx is required for differentiation of enteric smooth muscle, likely through direct regulation of enteric muscle-specific gene expression. However, the relation between Hlx protein structure and function is unknown. We addressed this question using deletions and point mutations (including reported nonsynonymous single nucleotide polymorphisms, or SNPs) of the 488 amino acid human HLX ( $\mathrm{hHLX}$ ) protein to define the importance of protein segments and specific amino acid residues of the HLX protein in regulation of promoters for $\alpha$-smooth muscle actin ( $\alpha$ SMA) and laminin $\alpha 4$ (lama4). The hHLX cDNA was subcloned into the bicistronic expression vector pMSCV-IRES-GFP. Point mutations in the hHLX cDNA corresponding to known SNPs or at other codons of interest were prepared using the Quik-Change system (Stratagene). Deletions were prepared by restriction digests resulting in in-frame deletions either using native restriction sites or sites engineered by silent point mutations. All mutations and deletions were confirmed by restriction digests and DNA sequencing. The gene regulatory activities of hHLX constructs (and control vector without HLX sequence) were tested by co-transfection with luciferase reporter constructs (pGL3, Promega) containing aSMA or lama4 promoter fragments into mesenchymal cell lines prepared from $\mathrm{Hl}^{-{ }^{--}}$embryos. We found that there was no effect on the regulation of either promoter by deletion of aa residues 78-164, which includes a proline-glutamine rich region that is conserved among mammals. A larger deletion (residues 27-221) still retained partial activity. Surprisingly, deletion of residues 216-290, which includes the N-terminal 15 aa of the 60 aa homeodomain (the DNA binding domain of the protein), retained significant gene regulatory activity. None of the non-synonymous SNPs had discernable effects on function. However, the mutation of a conserved tyrosine in the homeodomain that is within a tyrosine phosphorylation consensus sequence (Y299F) resulted in decreased HLX regulatory activity on both promoters, consistent with the idea that HLX activity may be regulated post-translationally by tyrosine kinases. These structure-function studies will help to identify mechanisms of gene regulation by the HLX transcription factor that are required for digestive system development. SUPPORT: NIH DK61219 and DK02791.

\section{M1620}

Melanin Concentrating Hormone (MCH) Stimulates Proinflammatory Responses in Human Colonic Epithelial Cells

Alan C. Moss, Kara Gross, Iordanes Karagiannides, Daniel Torres, Charlabos Pothoulakis, Efi Kokkotou

Background \& Objectives: Melanin-concentrating hormone (MCH) is a hypothalamic neuropeptide known to regulate appetite and body weight. We have previously shown that $\mathrm{MCH}$ deficient mice have reduced intestinal inflammation. Our studies also demonstrated that expression of $\mathrm{MCH}$, and its MCHRl receptor, is increased in the colonic mucosa during experimental inflammation in mice and human intestinal xenografts grown in SCID mice. Our aim was to delineate the inflammatory signaling pathways associated with the MCHRl receptor in human colonocytes. Methods: NCM460 cells were transfected with a MCHRIcarrying vector to increase the expression of this receptor in colonocytes. MCHRl overexpression was confirmed by real-time RT-PCR and western blotting in the transfected cells. NCM460/MCHRl cells were stimulated with MCH (10-6) and forskolin (10-6) for $30 \mathrm{~min}$ and intracellular cAMP levels were measured. Cells were exposed to MCH (10-6) for 0-60 minutes and cell lysates were examined by western blot for MAPkinase activation and IkappaB degradation. MCH-induced IL-8 mRNA levels and protein release at different time points was measured by real-time RT-PCR. Results: $\mathrm{MCH}$ significantly inhibited cAMP induction by forskolin in human colonocytes by $>50 \%$ ( $\mathrm{p}<0.001)$. It also induced erk $1 / 2$ phosphorylation as early as 2 min post-treatment; both findings are consistent with MCHRl signaling in other cell types. Stimulation of NCM460/MCHRl cells with $\mathrm{MCH}$ produced a time-dependent increase in IL-8 expression that was 2-fold greater than baseline at peak (4 hours) ( $\mathrm{p}<0.01$ ). Western blot analysis revealed that $\mathrm{MCH}$ exposure resulted in IkappaB degradation which was evident 20 minutes after treatment, suggesting activation of the NFkappaB pathway. Conclusions: This is the first study demonstrating a direct proinflammatory role for $\mathrm{MCH}$ in colonic epithelial cells. The current findings also provide a molecular mechanism that explains, at least in part, the relative resistance of the $\mathrm{MCH}$-deficient mice to intestinal inflammation. Grant support: supporter by a grant from Harvard Clinical Nutrition Research Center (P30-DK040561)

\section{M1621}

Adoptive Transfer of Th2 Polarized Cd4+ T Cells Up-Regulates 5-HT Production in Gut

Waliul Khan, Rami T. El-Sharkawy, Huaqing Wang, Monica Verma-Gandhu, Daniel Cohen-Lyons, Richard K. Grencis, Yasuaki Motomura

BACKGROUND/AIM: 5-hydroxytryptamine (5-HT) has been implicated in the pathophysiology of a number of functional and inflammatory bowel disorders but the mechanisms regulating the 5-HT production in the gut are unknown. Enterochromaffin (EC) cells are the main source of 5-HT in the gut and we have shown EC cell hyperplasia in nematode infection in mice. Here we studied whether the EC cell hyperplasia is driven by lymphocytes by determining $\mathrm{EC}$ cells and $5 \mathrm{HT}$ production are attenuated in nematode-infected SCID mice, and whether transfer of Th2 polarized CD4+ T cells from nematode-infected mice into naive recipient SCID mice induces changes in these parameters. METHODS: SCID mice on C57BL/6 background and control (C57BL/6) were infected with nematode, Trichuris muris and sacrificed to study EC cells and colonic 5-HT. To study the role of CD4+ T cells on EC cells and 5-HT, SCID mice were reconstituted with purified CD4+ T cells $\left(2.5 \times 10^{6}\right.$ cells/mouse,ip) from non-infected and infected C57BL/6 mice. Cytokine release was measured from Con A stimulated splenocytes. Immunohistochemical studies evaluated CD3+ T cells in colon. RESULTS: The number of EC cells was significantly higher in T. muris infected C57BL/6 mice. This infection-induced increase in EC cells was not evident in SCID mice $[9.9 \pm 2.5$ cells/10crypt unit (CU) vs. $2.9 \pm 0.5$ cells/10CU in C $57 \mathrm{BL} / 6$ vs. SCID mice on day 14 post-infection (pi)]. Colonic 5-HT amount was also significantly lower in infected SCID mice $(132 \pm 18.1 \mathrm{ng} / \mathrm{ml}$ vs. $93.2 \pm 11.9 \mathrm{ng} / \mathrm{ml}$ in C57BL/6 vs.SCID on day $14 \mathrm{pi})$. The number of EC cells significantly increased following reconstitution of SCID mice with CD4+ T cells from infected mice $(4.5 \pm 0.1$ cells/10CU vs. $2.4 \pm 0.6$ cells/10CU in SCID reconstituted with infected cells vs. SCID control on day 14 post-reconstitution). Colonic 5-HT amount was also markedly increased by $34 \%$ and $31 \%$ in SCID mice reconstituted with cells from infected mice on days 7 and 14 post-reconstitution. The increase in EC cells and 5-HT in reconstituted SCID mice was correlated with an up-regulation of colonic CD3+ T cells and IL-13 production (IL-13:319.7 $\pm 99.4 \mathrm{pg} / \mathrm{ml}$ vs. $22.5 \pm 1.9 \mathrm{pg} / \mathrm{ml}$ in SCID mice reconstituted with infected cells vs. SCID control). Number of EC cells or 5-HT amount was not significantly altered in SCID mice reconstituted with cells from non-infected mice. CONCLUSION: These results provide evidence for a critical role for Th2 polarized CD4+ T cells in 5-HT production in gut. Considering the importance of 5-HT in gut physiology, these results provide new insights into the mechanisms of gut function which may ultimately lead to improved therapeutic strategies in gut functional and inflammatory disorders.

\section{M1622}

Characterization of Pkce Translocation in Submucosal Neurons: Interaction with Cortical Actin and Altered Distribution During Inflammation Daniel P. Poole, Trung V. Nguyen, Shane Reeve, Ian Smith, Christopher J. Fowler, Craig B. Neylon, John B. Furness

Background: Protein kinase $\mathrm{C}(\mathrm{PKC})$ activation in myenteric intrinsic primary afferent neurons leads to hyperexcitability of these cells. Similarly increased neuronal excitability is exhibited in models of inflammatory bowel disease and these changes may be partially mediated through PKC. One consequence of inflammation is hypersecretion, suggesting that there may be altered secretomotor function. Purpose: 1) To characterize PKCE translocation in submucosal neurons; 2) To identify which neurons respond to inflammatory mediators 3 ) To identify PKC $\mathrm{P}$-interacting proteins; 3) To examine PKC $\varepsilon$ translocation in an experimental model of ileitis; 4) To identify molecular targets of PKC $\varepsilon$ activity. Methods: All experiments were performed using the guinea-pig ileum. PKC $\varepsilon$ translocation was analyzed immunohistochemically in submucosal neurons and by Western blotting. PKC $\varepsilon$ interacting proteins were identified by immunoprecipitation from submucosal lysates and mass spectrometry. Effects of PKC activation were examined electrophysiologically using intracellular microelectrodes Ileitis was induced by TNBS injection into the ileum, 6-7 days before tissue was taken Results: PKCE translocated from the cytoplasm to the plasma membrane in response to phorbol esters and to the protease activated receptor 2 (PAR2) activators trypsin and SLIGRL$\mathrm{NH} 2$. PAR2-evoked translocation was restricted to VIP-immunoreactive secretomotor neurons. Translocation was significantly reduced by PKCE-specific inhibition. Cortical actin was identified as a major PKC $\varepsilon$ interacting protein and confirmed by co-immunoprecipitation, f-actin disruption and immunohistochemistry. PKCE translocation was significantly increased 6-7 days post TNBS and was reduced by indomethacin treatment. Specific activation of novel PKCs resulted in an approximately 2-fold increase in input resistance of submucosa neurons. Conclusions: PKCE translocates to the actin cortex of submucosal neurons in response to activators and a similar response occurs during inflammation of the gut. We hypothesize that activated PKCE translocates to the actin cortex and is anchored close to $\mathrm{K}+$ ion channels in the plasma membrane. Phosphorylation of these channels results in channel closure and an increase in the excitability of the neurons. Based on our results, modulation of PKCE activity by pharmacological agents may be of therapeutic value in controlling the neuronal excitability changes associated with IBD and in limiting hypersecretion. 
M1623

Protease Activated Receptor 2 (PAR2) Sensitization of TRPV1 Currents Is Mediated By Protein Kinase $\mathrm{C} \varepsilon$ and Protein Kinase A

Francisco Bautista, Silvia Amadesi, Rustrum Karanjia, Carlos Barajas-Lopez, Nigel Bunnett, Stephen Vanner

Mast cell proteases cleave PAR2 on spinal dorsal root ganglia (DRG) neurons that innervate the intestine causing visceral hyperalgesia. Studies of the underlying mechanism demonstrate that PAR2 agonists sensitize TRPVl currents in DRG neurons and HEK cells expressing TRPVl by activating PKC (Amadesi et al. 2004). However, the PKC isozyme that mediates this sensitization of TRPVl currents and the contribution of PKA in this important signaling pathway remains to be determined. Therefore, we examined the role of PKCE and PKA using whole cell voltage clamp recordings in HEK cells naturally expressing PAR2 and stably transfected with TRPV1, a model system of PAR2-TRPV1 signaling in DRG neurons. Fast flow application of capsaicin $(300 \mathrm{nM})$ induced inward currents and repeat applications at 5 min intervals elicited reproducible responses in control cells $(n=5)$. Following application of the PAR2 agonist SLIGRL-NH2 (100uM) for 2 min currents elicited by re-application of capsaicin 30s later were increased 3 fold $(n=10)$, indicative of TRPVl sensitization. Repeated applications at $3 \mathrm{~min}$ and then $5 \mathrm{~min}$ intervals up to $18 \mathrm{~min}$ demonstrated this sensitization persisted during this interval. Peak responses ( 4.4 fold increase; $p<0.01$ ) were observed at $3 \mathrm{~min}$ after PAR2 application. When the PKCE inhibitor PKCEI $(200 \mu \mathrm{M})$ was included in the pipette, PAR2 sensitization of the capsaicin-evoked TRPVl currents was markedly inhibited (mean inhibition $=60 \%$ at 3 min; $p<0.05)$. Similarly, when the PKA antagonist H89 $(3 \mu \mathrm{M})$ was included in the pipette this sensitization was also markedly inhibited (mean inhibition = $70 \% \mathrm{p}<0.05)$. To test the selectivity of $\mathrm{H} 89$, we examined the effects of the PKC activator phorbol dibutyrate $(10 \mathrm{uM})$ combined with H89. Phorbol dibutyrate alone caused a 3 fold increase in the TRPV1 currents at $3 \mathrm{~min}$ applied as described for SLIGRL-NH2. When H89 was added to the pipette, this sensitization was not inhibited. To demonstrate an effect of AR2 activation on TRPV1 currents in mouse DRG neurons we used the same protocol in whole cell recordings from acutely dissociated mouse DRG neurons. SLIGRL-NH2 (100uM) elicited sensitization of TRPVl currents in a subpopulation of the neurons studied. These studies demonstrate that PAR2 sensitizes TRPV1 currents by a PKC $\varepsilon$ and PKA-dependent mechanism. Further studies are needed to determine if PKC $\varepsilon$ and PKA regulate TRPVI in an additive or synergistic fashion, or whether one pathway is upstream of the other.

\section{M1624}

Dual Role of Vanilloid Sensitive Neurons in the Concanavalin a Induced Model of Hepatic Tissue Injury

Jaroslaw Biernat, Ryszard Sendur, Rafal Obuchowicz, Michal Pawlik, Artur Dembinski, Wieslaw W. Pawlik

Vanilloid sensitive neurons endings have been described to have a close contact with the hepatic stellate cells. A dual role has been attributed to the neuropeptides released from the vanilloid sensory neurons in the liver. Substance $\mathrm{P}$ and other tachykinins are known to stimulate neurogenic inflammation by releasing TNF- $\alpha$ and IFN- $\gamma$ in a variety of experimental models of hepatic injury. However in our previous experiments we proved that CGRPmediated vascular mechanism has a protective effect in acetaminophen-induced liver injury. We performed the experiments using a Concanavalin A - induced liver injury to explore the role of both tachykinins and CGRP in the mediation of hepatic injury. Experiments were performed on Wistar rats weighing 200-220 under pentobarbital anesthesia. Mean arterial blood pressures (AP), portal blood flow (PBF) and microcirculatory hepatic blood flow $(\mathrm{HBF})$ using laser-Doppler flowmetry were measured. At the end of each experiment the venous blood samples were taken to establish the levels of hepatic tissue injury markers (ALT, AST). Experimental groups: control group (placebo pre-treated), ConA pre-treated animals, ConA + CGRP receptor antagonist (CGRP 8-37), ConA + NK-1, Con A + NK-2, Con A + NK-3 and ConA + NK-1, NK-2 and NK-3 receptor antagonist. In the additional series of experiments we used capsaicin-denervated and vanilloid receptor-blocked (ruthenium red) rats. In ConA alone pre-treated animals ALT and AST serum levels raised to $4233 \pm 1540$ and $2578 \pm 980 \mathrm{U} / \mathrm{L}$ whilst HBF were reduced by $12 \%$ (NS) in comparison to the values in the control group. In CGRP receptor- blocked rats both ALT and AST serum levels were raised by $35 \%$ and $21 \%$ and $\mathrm{HBF}$ was reduced by $27 \%$ in comparison to ConA alone pretreated rats. In capsaicin-denervated and vanilloid receptor-blocked (ruthenium red) ALT levels were increased by $42 \%$ and $36 \%$ respectively while HBF values were reduced by 32 and 29\% respectively (NS versus ConA alone pre-treated rats) In contrast NK-1 receptor blockade decreased ALT and AST serum levels to $3267+789$ and $2436 \pm 652 \mathrm{U} / \mathrm{L}$ without affecting the HBF values in this group. Both NK-1 and NK-3 receptor antagonists failed to influence the circulatory and enzymatic parameters in the Con-A induced model of hepatic injury. Vanilloid sensitive neurons play dual role in the Con-A induced model of hepatic injury. Tachykinins released from sensory endings exacerbate hepatic tissue injury acting via stimulation of NK-1 receptors whilst CGRP has a potent protective effect in this experimental model. The protective effect of vanilloid sensitive neurons is strictly correlated with vasodilatatory action of CGRP upon hepatic circulation.

\section{M1625}

Effects of TNBS-Induced Ileitis On Secretory Function in the Non-Inflamed Guinea Pig Colon

Jennifer R. O'Hara, Alan E. Lomax, Gary M. Mawe, Keith A. Sharkey

Altered gut motility and secretion are features of inflammatory bowel disease. Disturbances in intestinal function occur locally, but also at distant, non-inflamed sites of the gut. We therefore investigated whether animals with an inflamed small intestine had altered secretomotor function in the non-inflamed distal colon. Ileitis was induced by intraluminal administration of TNBS into the distal ileum of guinea pigs and experiments were conducted on day 3. The extent of inflammation was assessed macroscopically and by measurement of myeloperoxidase (MPO) activity. To assess secretory function, distal colon was excised and the external muscle layers were removed by blunt dissection. The submucosa/mucosa preparations were mounted in Ussing chambers, voltage clamped at $0 \mathrm{mV}$ and maintained at $37^{\circ} \mathrm{C}$ in oxygenated Krebs solution. Following an equilibration period, veratridine $(30 \mu \mathrm{M})$, bethanechol $(10 \mu \mathrm{M})$ or forskolin $(10 \mu \mathrm{M})$, in the presence or absence of the neuronal blocker tetrodotoxin (TTX), were added to the serosal compartment and the change in short circuit current (Isc) was recorded as a measure of electrogenic ion transport. Three days after induction of ileitis, the colon appeared macroscopically normal and the MPO activity was not different in the colon of animals with ileitis when compared to controls. Variations in basal Isc occurred such that $58 \%$ of the control group had a positive basal Isc; whereas $91 \%$ of the TNBS- treated animals had a positive basal Isc. When pooled, the basal Isc in the colon of animals with ileitis was significantly greater than the average basal Isc in the colon of controls (Control: $11 \pm 9 \mu \mathrm{A} / \mathrm{cm}^{2}$; TNBS: $91 \pm 18 \mu \mathrm{A} / \mathrm{cm}^{2}$ ). Mucosal resistance was also significantly increased in the colon of animals with ileitis when compared to controls (Control: $116 \pm 10 \Omega / \mathrm{cm}^{2}$; TNBS: $150 \pm 13 \Omega / \mathrm{cm}^{2}$ ). Addition of bethanechol or forskolin to the serosal bath evoked an increase in Isc that reached average peak responses of $180 \pm$ $38 \mu \mathrm{A} / \mathrm{cm}^{2}$ and $232 \pm 68 \mu \mathrm{A} / \mathrm{cm}^{2}$, respectively. The increase in Isc elicited by bethanechol and forskolin were significantly attenuated in the presence of TTX; however, there was no difference in the response to either drug in the colon of animals with TNBS-induced ileitis vs. controls. In contrast, the Isc response to veratridine was significantly attenuated in the colon of animals with TNBS-induced ileitis when compared to controls (Control: $494 \pm$ $40 \mu \mathrm{A} / \mathrm{cm}^{2} ;$ TNBS: $313 \pm 50 \mu \mathrm{A} / \mathrm{cm}^{2}$ ). The altered basal electrical parameters and hyporesponsiveness to veratridine in the colon of animals with ileitis suggest that inflammation at one site leads to functional consequences in secretomotor regulation at a distant, noninflamed site.

\section{M1626}

Targeted Disruption of the 5-Ht4 Receptor (5-HT4R) Gene in Mice Did Not Affect Gastric Emptying (GE) and Restraint Stress-Exaggerated Defecation But Paradoxically Increased Basal Defecation Rate

Robert Mader, Weber Eckhard, Bruno Tigani, Hans-Ulrich Gremlich, Stefan Marcaletti, Hans-Juergen Pfannkuche, Moriko Ito

BACKGROUND. In the gastrointestinal (GI) tract and in the central nervous system (CNS), 5-HT4Rs play multiple roles. Activation of peripheral 5-HT4Rs with agonists like tegaserod has been shown to accelerate GE and improve symptoms of constipation in animals and men. Our studies aimed to elucidate whether mice with targeted disruption of the 5-HT4R gene display a GI phenotype, e.g. delayed GE and/or reduced defecation. METHODS. Studies were performed in adult 5-HT4 knock-out mice $(\mathrm{KO})$ on C57BL/6 background $(7 \mathrm{f} / 7 \mathrm{~m}$, $22.4 \pm 0.7 \mathrm{~g})$ and corresponding wildtypes (WT) $(7 \mathrm{f} / 7 \mathrm{~m}, 22.0 \pm 0.9 \mathrm{~g})$. Mice were treated intraperitoneally or orally with tegaserod $(0.1 \mathrm{mg} / \mathrm{kg})$ or vehicle $30 \mathrm{~min}$ prior to experiments. Gastric emptying: Mice were fasted for $6 \mathrm{hrs}$; subsequently, $0.2 \mathrm{ml}$ of a viscous nutrient meal containing fluorescent beads was administered via gavage (meal: Novasource, $1 \mathrm{kcal} / \mathrm{ml}, 7.5 \%$ methylcellulose; beads: $50 \mathrm{mg} / \mathrm{ml}$ TentaGel $1 \%$ Cy5.5 diameter $0.1 \mathrm{~mm}$ ). GE was examined $10 \mathrm{~min}$ after the meal. The ratio of fluorescence activity (non-invasive near-infrared fluorescence imaging) measured in the stomach and, respectively, small intestine reflected the GE rate. Defecation: Following adaptation to the experimental conditions, non-fasted animals were isolated in metabolic cages, and basal defecation or exaggerated defecation during restraint stress was monitored for $1 \mathrm{hr}$ (number of fecal pellets/hour: FP/hr). RESULTS. The disruption of the gene encoding 5-HT4R by the insertion of lacZ was confirmed by negative RT-PCR and positive LacZ staining in gut and brain. GE of a viscous caloric meal was similar in $\mathrm{KO}$ and WT mice $(\mathrm{KO}: 39.5 \pm 7.9 \%$, WT: $36.0 \pm 2.4 \%$, mean \pm SEM, $\mathrm{P}=0.696$, ANOVA, $\mathrm{n}=$ 8). Tegaserod significantly accelerated GE in WT but not in KO mice (KO: $36.8 \pm 5.7 \%$, WT: $51.4 \pm 3.4 \%, P=0.046, n=8)$. Basal defecation was significantly higher in $\mathrm{KO}$ than in WT mice (KO: $3.8 \pm 0.6 \mathrm{FP} / \mathrm{hr}$, WT: $1.7 \pm 0.3 \mathrm{FP} / \mathrm{hr}, \mathrm{P}=0.007, \mathrm{n}=14$ ). Tegaserod significantly increased defecation in WT but not in $\mathrm{KO}$ mice $(\mathrm{KO}: 3.4 \pm 0.7 \mathrm{FP} / \mathrm{hr}, \mathrm{P}=0.446, \mathrm{n}=14$; WT: $4.4 \pm 0.5 \mathrm{FP} / \mathrm{hr}, \mathrm{P}<0.001, \mathrm{n}=14$ ). Restraint stress significantly increased defecation in both $\mathrm{KO}$ and WT mice (KO: $8.1 \pm 0.6 \mathrm{FP} / \mathrm{hr}, \mathrm{P}=0.013) \mathrm{WT}: 7.8 \pm 0.9 \mathrm{FP} / \mathrm{hr}, \mathrm{P}<0.001, \mathrm{n}=14)$. The difference observed between KO and WT mice under basal conditions was not apparent during stress $(\mathrm{P}=0.742, \mathrm{n}=14)$. CONCLUSIONS. 5-HT4R knock-out mice do not display an apparent phenotype in GE and stress-exaggerated defecation. The paradoxical finding of an increased defecation rate in $\mathrm{KO}$ mice under basal conditions could be due to an impaired adaptation of these animals to mild stressors (new environment stress, separation stress) and might reflect a CNS rather than a GI phenotype

\section{M1627}

Importance of Corticotropin-Releasing Factor (CRF) in the Development of Colonic Sensitivity Using $\mathrm{CRF}_{1}$ Receptor Deficient Mice

Anthony C. Johnson, Nancy Trimble, Alan C. Foster, Beverley Greenwood-van Meerveld

Symptoms of irritable bowel syndrome (IBS) are worsened in response to episodes of stress and anxiety. Corticotropin-releasing factor (CRF) is an important mediator in the stress response. Our previous studies in rodent models demonstrated that stress-induced colonic hypersensitivity was attenuated by a $C R F_{1}$ receptor antagonist. Since $C R F_{1} R$ deficient mice have an impaired stress-response our goal was to further explore the importance of $C_{R} F_{1} R$ in the development of colonic sensitivity. Methods: Using conscious $C_{R F} R(+/+), C R F_{1} R$ $(+/-)$ and $\mathrm{CRF}_{1} \mathrm{R}(-/-)$ mice, colonic sensitivity was assessed via a visceromotor behavioral response (VMR) recorded using an EMG electrode positioned on the external oblique abdominal musculature and quantified as the number of spikes $>300 \mathrm{mV}$ per $10 \mathrm{~s}$, to graded colorectal distension (CRD, 0-60 mmHg). Results: See Table for summary data (mean \pm $\mathrm{SEM})$. In the $\mathrm{CRF}_{1} \mathrm{R}(+/+)$ mice there was a pressure-dependent increase in the VMR to $\mathrm{CRD}$ that was attenuated in the $\mathrm{CRF}_{1} \mathrm{R}(+/-)$ mice. However in the $\mathrm{CRF}_{1} \mathrm{R}(-/-)$ mice a VMR to $C R D$ was only observed at the highest distension pressure $(60 \mathrm{mmHg}) . A C R F_{1} R$ antagonist, NBI 30775 (30 mg/kg i.p.) significantly decreased the VMR to CRD in all $C F_{1} R+/+$ mice. An identical inhibitory effect of NBI 30775 was observed in $43 \%$ of the $\mathrm{CRF}_{1}+/$ - mice, however in the remaining $\mathrm{CRF}_{1}+$ /- mice no inhibitory effect was seen. Conclusion: This study provides pharmacological and genetic evidence for the importance of $C_{R F} R$ in colonic sensitivity and substantiates a link between stress and visceral perception. VMR to CRD in the Various $C_{1} F_{1} R$ Genotypes (\# of spikes $>300 \mathrm{mV} / 10 \mathrm{~s}$ ) 


\begin{tabular}{|c|c|c|c|c|}
\hline Vehicle & $15 \mathrm{mmHg}$ & $30 \mathrm{mmHg}$ & $45 \mathrm{mmHg}$ & $60 \mathrm{mmHg}$ \\
\hline$+/+(\mathrm{n}=8)$ & $28.3 \pm 9.9$ & $45.3 \pm 6.7$ & $97.5 \pm 20.3$ & $131.6 \pm 14.6$ \\
\hline$+/-(\mathrm{n}=6)$ & $4.9 \pm 2.1$ & $16.3 \pm 4.6^{* *}$ & $33.6 \pm 10.3^{*}$ & $85.4 \pm 15.9^{*}$ \\
\hline$-/(\mathrm{n}=6)$ & $4.3 \pm 1.5^{*}$ & $4.9 \pm 1.7^{* *}$ & $12.7 \pm 4.9^{* * *}$ & $19.9 \pm 5.3^{* * *} \dagger$ \\
\hline NBI 30775 & $15 \mathrm{mmHg}$ & $30 \mathrm{mmHg}$ & $45 \mathrm{mmHg}$ & $60 \mathrm{mmHg}$ \\
\hline$+/+(\mathrm{n}=6)$ & $4.7 \pm 1.7$ & $6.8 \pm 3.4^{* * *}$ & $15.3 \pm 5.5^{* * *}$ & $21.9 \pm 7.3^{* * *}$ \\
\hline$+/-($ resp, $\mathrm{n}=3)$ & $0.4 \pm 0.2$ & $2.0 \pm 0.6$ & $7.3 \pm 2.9$ & $16.8 \pm 1.7 \uparrow$ \\
\hline+ +/ (no resp, $\mathrm{n}=4)$ & $6.4 \pm 3.5$ & $23.6 \pm 10.3$ & $33.4 \pm 12.8$ & $72.9 \pm 11.3$ \\
\hline
\end{tabular}

$* \mathrm{p}<0.05 .{ }^{* *} \mathrm{p}<0.01$ compared to $+/+, \dagger \mathrm{p}<0.05$ compared to $+/-$. resp $=$ responders, no resp $=$ non-responders.

\section{M1628}

Targeted Knock Down of NMDA Receptors in Dorsal Root Ganglia Neurons James A. McRoberts, Helena S. Ennes, Juan Carlos Marvizon, Bryce Vissel, Michael Fanselow, Emeran A. Mayer

BACKGROUND: NMDA receptors (NMDARs) expressed on the central and peripheral terminals of spinal afferent neurons are thought to play an important role in nociceptive transmission and possibly neurogenic inflammation, however their exact role remains controversial. The cell bodies of these nerves are located in the dorsal root ganglia (DRG), most of which express peripherin during development. AIM: To develop a genetic approach to this problem by targeting the knockout of the critical NRI subunit of the NMDAR to DRG neurons using transgenic mice expressing Cre recombinase under the control of the peripherin promoter. METHODS: Mice engineered with loxP sites flanking exons 11-22 of the NR1 subunit gene (floxed-NR1) were crossed with mice engineered to express Cre recombinase under the control of the peripherin promotor (Prph-Cre). At 100 days of age, adult male mice were sacrificed and various tissues isolated and assayed for expression of NRI using semiquantitative RT-PCR, Western blotting and immunocytochemistry. RESULTS: Homozygous floxed-NR1, Prph-Cre+ mice were viable and fertile. At 100 days of age, there was a $~ 43 \%$ reduction of NRl mRNA extracted from DRG, with no effect on NRI mRNA expression in the spinal cord, cortex, or cerebellum. Western blot analysis demonstrated a 59\% reduction in NRI protein expression in DRG neurons with no effect on expression in spinal cord or cortex. Immunocytochemistry with antibodies to NRI in sections of DRG, revealed less NRI immunoreactivity (-ir) next to the nucleus in the mutant mice with most of the remaining NR1-ir is next to the cell surface. Scion Image was used to quantitatively measure the intensity of the NRl-ir in confocal images from 3 sections per mouse. The integrated optical intensity (in arbitrary units) decreased from $9.5 \pm 2.8$ in phenotypically wild type littermates to $3.1 \pm 0.7$ (67\% decrease) in the targeted knock out mice $(\mathrm{p}<0.05$, t-test). The decrease in staining was observed in DRG neurons of all sizes. CONCLUSIONS: Targeted knock down of NMDARs using Cre expression driven by a peripherin promoter produced a $43-$ $67 \%$ decrease in NRl expression in DRG neurons with no change in NRl expression in the spinal cord or the CNS. These mice are likely to be useful in dissecting the role of NMDARs located on peripheral and central terminals of DRG neurons in nociception, sensitization and neurogenic inflammation. Supported by NIH grants DK 58173 and 1 P5O DK64539and R24 AT002681

\section{M1629}

Elucidating the Role of the Glucagon-Like Peptide 2 Receptor in the Enteric Nervous System

Sean C. Mcdonagh, Jenny Lee, Patricia L. Brubaker

Glucagon-like peptide 2 (GLP-2) is a proglucagon-derived peptide secreted from the enteroendocrine $\mathrm{L}$ cell of the distal gut in response to enteral nutrition. Administration of GLP-2 results in expansion of the small intestinal mucosa and improved nutrient absorption, primarily due to increased epithelial proliferation. GLP-2 also decreases gut motility and enhances intestinal blood flow. The mechanisms through which these effects of GLP-2 are achieved are poorly understood, but are likely indirect, as the G-protein coupled GLP-2 receptor (GLP-2R) is expressed in the enteric nervous system (ENS), enteroendocrine cells and the lamina propria, but not in proliferating enterocytes. To examine the role of the GLP-2R in the ENS, we have therefore studied the effect of GLP-2 administration in a mouse model of ENS deficit, the glial cell line-derived neurotrophic factor family receptor alpha 2 (GFR 22$)$ knockout mouse. As a result of defective mitotic and trophic support of neural crest cells as they migrate to colonize the gut during development, these mice exhibit a pronounced deficit in enteric cholinergic innervation. This deficit results in a demonstrable impairment in gut motility, as charcoal transit studies revealed a $39 \pm 9 \%$ decrease in the rate of transit of gut contents in knockout mice as compared to wild-type littermates $(\mathrm{P}<0.05$, $\mathrm{n}=5$ ). Furthermore, acute administration of GLP-2 decreased gut motility in wild-type (75 $\pm 7 \%$ of controls, $P<0.05)$ but not in GFR $22(124 \pm 23 \%$ of controls) mice $(n=4)$. In contrast, analysis of mucosal growth parameters (small intestinal wet weight, villus height, crypt depth and Ki67 staining of proliferating crypt cells) indicated no difference between GFR 22 knockout and wild-type animals ( $\mathrm{n}=5-10)$. Furthermore, the effect of chronic GLP-2 administration to increase small intestinal wet weight (by $44 \pm 6 \%, \mathrm{P}<0.001$ ), villus height (by 42 $\pm 4 \%, \mathrm{P}<0.001$ ) and crypt depth (by $16 \pm 5 \%, \mathrm{P}<0.001$ ) was not different between $\mathrm{GFR} \alpha 2$ and wild-type mice $(\mathrm{P}>0.05 ; \mathrm{n}=5-10)$. Intriguingly, GFR $\alpha 2$ knockout mice exhibited higher proglucagon $(314 \pm 133 \% ; \mathrm{P}<0.01) \mathrm{mRNA}$ transcript levels relative to wild-type littermates, as determined by quantitative RT-PCR ( $\mathrm{n}=5-7)$, suggesting the presence of adaptive mechanisms. These findings indicate that the ENS GLP-2R is important in the regulation of gut motility, but do not demonstrate a primary role in the tropic effects of GLP-2 on mucosal growth.

\section{M1630}

Haplotype Insufficiency for Socs2 Enhances Intestinal Growth and Promotes Polyp Formation in GH-Transgenic Mice

Carmen Z. Michaylira, Nicole Ramocki, James G. Simmons, Kirk K. McNaughton, John T. Woosley, Christopher J. Greenhalgh, Rachael Rigby, P. Kay Lund

Growth hormone $(\mathrm{GH})$ may improve intestinal growth or function in patients following intestinal resection or on total parenteral nutrition for short bowel syndrome. Excessive trophic effects of GH or its downstream target, insulin-like growth factor I (IGF-I), however, may contribute to neoplastic growth. Patients with acromegaly have increased risk of colorectal cancer. Identification of mechanisms that limit the tumorigenic potential of GH and IGFI is desirable. Suppressor of cytokine signaling-2 (SOCS2) limits GH action on body and organ growth, but its role in $\mathrm{GH}$ action on intestine is unknown. These studies tested the hypothesis that SOCS2 limits GH-induced intestinal growth or neoplasia in vivo. GHtransgenic (GH-TG) mice were crossed with SOCS2 null mice in attempt to generate transgenic (TG) or wild-type (WT) mice with 0 (HO-WT and HO-TG), 1 (HT-WT and HT-TG or 2 (WT-WT and WT-TG) copies of SOCS2. No HO-TG mice were derived from crossbreeding. WT-WT, HT-WT, WT-TG and HT-TG were therefore compared for body weight, small intestine and colon weight, length and BrdU incorporation as a measure of crypt proliferation. Levels of IGF-I and sucrase-isomaltase mRNAs were measured in jejunum. Colon was analyzed for the presence of abnormal lesions. HT-WT did not differ from WT-WT in any parameter. Compared with WT-TG, HT-TG had significantly greater $(\mathrm{P}<0.05) \mathrm{GH}$ transgeneinduced increases in body weight, small intestinal growth, crypt proliferation, and local IGF-I expression, and showed decreases in sucrase-isomaltase expression. In colon, HT-TG but not WT-TG developed multiple, histologically-confirmed, hyperplastic and lymphoid polyps which were positive for BrdU staining and composed primarily of B cells. Polyps had prominent nuclear staining for phosphorylated STAT3. In conclusion, haplotype insufficiency for SOCS2 promotes the trophic actions of GH in small intestine and, in association with GH excess, promotes pre-neoplastic changes in colon of young adult mice. Small variations in the levels of SOCS2 may have a major impact on the effects of therapeutic GH on small intestinal growth and on susceptibility of patients with acromegaly to the development of colonic polyps.

\section{M1631}

Use of Growth Hormone Releasing Peptide-6 for the Prevention of Hepatic and Multi Organ Failure

D Cibrian, H Ajamieh, J Berlanga, O Leon, J Alba, Mjt Kim, T Marchbank, J Boyle, E Ntatsaki, F Freyre, D Garcia del Barco, P Lopez-Saura, G Guillen, S Ghosh, R Goodlad, Raymond Playford

Background/Aims: Novel therapies for the treatment of multiple organ failure are required. Methods: We examined the effect of synthetic growth hormone releasing peptide-6 (GHRP6) on cell migration and proliferation using rat intestinal epithelial (IEC-6) and human colonic cancer (HT29) cells as in vitro models of injury. In addition, we examined its efficacy when given alone and in combination with the potent protective factor epidermal growth factor (EGF) in an in vivo model of multi-organ failure (using two hepatic vessel ischemiareperfusion protocols; $45 \mathrm{~min}$ ischaemia-45 min reperfusion and $90 \mathrm{~min}$ ischaemia- $120 \mathrm{~min}$ reperfusion). Results: In vitro studies showed GHRP-6 directly influenced gut epithelial function as its addition caused a three-fold increase in the rate of cell migration of IEC- 6 and HT29 cells $(\mathrm{P}<0.01)$ but did not increase proliferation $([3 \mathrm{H}]$-thymidine incorporation) In vivo studies showed that, compared to baseline values, ischemia/reperfusion caused marked hepatic and intestinal damage (histological scoring), neutrophilic infiltration (MPO assay, 5-fold increase) and lipid peroxidation (MDA assay, 4-fold increase). Pre-treatment with GHRP-6 (120 ug/kg sc.) alone truncated these effects by $50-85 \%$ (all $\mathrm{P}<0.05)$ and additional benefit was seen when GHRP-6 was used in combination with EGF (1 mg/ $\mathrm{kg} \mathrm{sc}$.). Lung and renal injuries were also reduced by these pre-treatments. Conclusion Administration of GHRP-6, given alone or in combination with EGF to enhance effects, may provide a novel, simple approach for the prevention and treatment of multi-organ failure and other injuries of the gastrointestinal tract. Further studies appear justified.

\section{M1632}

Upregulation of Proangiogenic Growth Factors By Leptin During Gastric Ulcer Healing

Tetsuya Tanigawa, Toshio Watanabe, E.J. Sasaki, Masatsugu Shiba, Kazunari Tominaga, Yasuhiro Fujiwara, Nobuhide Oshitani, Kazuhide Higuchi, Testsuo Arakawa

Background \& Aim; Leptin, an important regulator of food intake and energy metabolism, has been demonstrated to be involved in cutaneous wound healing. Leptin is produced in gastric tissue, but its role in gastric ulcer healing is unclear. In this study we investigated the role of leptin in healing of acetic acid-induced gastric ulcer using leptin-deficient mice. Methods; C57BL/6J wild-type and leptin-deficient mice (ob/ob mice) were used in this study Gastric ulcers were produced by focal serosal application of $60 \%$ acetic acid for $30 \mathrm{sec}$. Mouse recombinant leptin $(0.5 \mu \mathrm{g} / \mathrm{g} \mathrm{BW})$ or vehicle (normal saline) was given intraperitoneally twice daily to ob/ob mice from 3 days after ulceration for up to 7 days. Stomach was removed and ulcer size was measured by day 7 of treatment. Expression of mRNA for leptin, leptin receptor, vascular endothelial growth factor (VEGF) and basic-fibroblast growth factor (bFGF) in ulcerous tissue and normal mucosa were assessed by real-time RT-PCR, while localization of leptin, leptin receptor, and VEGF was evaluated by immunohistochemistry. Angiogenesis of ulcerous tissue was evaluated by counting the number of microvessels in the ulcer bed identified by immunohistochemical staining of CD31 (an endothelial cell marker). Results Genetic disruption of leptin resulted in delay of ulcer healing (ulcer index: $3.43 \pm 1.02 \mathrm{~mm}$ in wild-type mice vs. $5.36 \pm 1.45 \mathrm{~mm}^{2}$ in $o b / o b$ mice). In wild-type mice, ulceration increased mRNAs for leptin receptor, VEGF, and bFGF by 25.6-fold, 1.5-fold and 5.1-fold, respectively, whereas it did not change mRNA level of leptin. The levels of mRNAs for VEGF and bFGF in ulcerous tissue were lower in $o b / o b$ mice than in wild-type mice. In $o b / o b$ mice, restoration

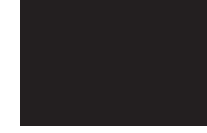


of leptin reversed the delay of gastric ulcer healing (ulcer index: $5.36 \pm 1.45 \mathrm{~mm}^{2}$ in vehicletreated group vs $1.60 \pm 0.84 \mathrm{~mm}^{2}$ in leptin-treated group), accompanied by upregulation of mRNA expression of VEGF and bFGF. The number of microvessels in the ulcer bed was smaller in $o b / o b$ mice than that in wild-type mice. Restoration of leptin also reversed the inhibition of angiogenesis in ob/ob mice. In epithelial cells at ulcer margins, leptin colocalized with VEGF. Conclusion; These results suggest that leptin contributes to gastric ulcer healing via upreguration of proangiogenic growth factors.

\section{M1633}

Characterisation of the Nk2 Antagonist Slv332 in Vitro and in Vivo Holger Sann, Daniel Jasserand, Reinhard Brueckner, Michael Firnges, Natasja Mj de Jong, Eric Ronken, Ulf Preuschoff, Jochen Antel

In preclinical models, blockade of neurokinin 2 (NK2) receptors reduce visceral hypersensitivity, suggesting that NK2 antagonists might be useful in the treatment of irritable bowel syndrome (IBS). Here we report the in vitro and in vivo activity of the newly developed NK2 antagonist SLV332. SLV332 exhibits a high binding affinity for the human (h) NK2 receptor (pKi=9.1) but was less active on the hNKl and hNK3 receptor (pKi NKl: 7.5, pKi NK3: 6.5). A receptor binding profile for SLV332 at $1 \mu \mathrm{M}$ using 72 other receptors did not reveal any displacement of more than 50\%, suggesting a high specificity for the NK2 receptor In functional assays, SLV332 concentration-dependently inhibited the neurokinin A (NKA)induced mobilization of intracellular calcium and phosphorylation of mitogen-activated protein kinase in $\mathrm{CHO}$ cells expressing the hNK2 receptor with a pA2 of 9.7 and 9.2, respectively. It was less active in functional $\mathrm{NK1}(\mathrm{pA} 2=7.6)$ and $\mathrm{NK} 3(\mathrm{pA} 2=6.6)$ assays. Furthermore, SLV332 concentration-dependently inhibited the (NKA)-induced contraction in the guinea-pig gall bladder preparation with a pEC50 of 10.1. To investigate the NK2 antagonistic potency of SLV332 in vivo, its ability to antagonise NKA induced changes in blood pressure, bronchoconstriction and colonic motility was investigated in anaesthetised guinea-pigs. The ED50 after i.v. administration was $9 \mathrm{nmol} / \mathrm{kg}$ for colonic motility. After oral administration, SLV332 dose-dependently inhibited the NK2 responses with an ED50 (determined using mean data from 1-180 minutes) of $1.1 \mu \mathrm{mol} / \mathrm{kg}$ for the colonic motility. SLV332 (30 and $100 \mu \mathrm{mol} / \mathrm{kg}$ ) was also tested in a rat model of visceral hypersensitivity by oral and subcutaneous administration. After sensitisation of colonic afferents by previous instillation of diluted acetic acid, colonic distension induced nociceptive behavioural responses such as abdominal cramps and writhing. SLV 332 inhibited significantly and long lasting ( $>2 \mathrm{~h}$ ) the visceral hypersensitivity by up to $80.3 \%$ after oral administration of the highest dose $(100 \mu \mathrm{mol} / \mathrm{kg})$. The data demonstrate that SLV332 is a potent and orally active NK2 antagonist. The ability of the drug to reduce visceral hypersensitivity suggests that SLV332 might be beneficial in IBS and potentially other GI disorders showing up with abdominal pain.

\section{M1634}

Differential Modulation of Colonic Mucus Secretion By Peripheral $\mathrm{CRF}_{1}$ and $\mathrm{CRF}_{2}$ Receptors in Conscious Rats

Mulugeta Million, Pascale Plaisancie, Paul R. Saunders, Yvette Tache

Background: Peripheral $\mathrm{CRF}$ acting on $\mathrm{CRF}_{1}$ receptors stimulates colonic myenteric neurons and motility in responses to stress and may have a role in stress-related manifestation of irritable bowel syndrome (IBS) (Br J Pharmacol 2004;141:1321-). In contrast, $\mathrm{CRF}_{2}$ activation dampens $\mathrm{CRF}_{1}$-mediated endocrine (Endocrinology 2001;144:2396-403) and colonic motor responses (Gut 2005; PMID: 15985561). Passage of mucus is part of symptoms supporting diagnosis of IBS (Gastroenterology 2002;123:2108-). Intraperitoneal (IP) injection of CRF causes colonic mucin release and a $\mathrm{CRF}_{1} / \mathrm{CRF}_{2}$ antagonist prevented restraint stress-induced colonic mucin release in rats (AJP 1996;271:G884). Aims: To investigate the influence of IP CRF (preferential $\mathrm{CRF}_{1}$ agonist) and urocortin 2 (Ucn 2, selective $\mathrm{CRF}_{2}$ ) on colonic mucus and processes of mucus discharge. Methods: Non fasted male SD rats (280-320 g) were injected IP twice at a $10 \mathrm{~min}$ interval with either saline + saline, CRF $(3 \mu \mathrm{g} / \mathrm{kg})$ or Ucn $2(10 \mu \mathrm{g} / \mathrm{kg})$ or Ucn $2+\mathrm{CRF}(10+3 \mu \mathrm{g} / \mathrm{kg})$, and fecal pellet output (FPO) was monitored. Sixty min later, distal colon was processed for mucus detection by alcian blue/ PAS/hematoxylin. Results: CRF, compared to saline, increased the percentage of cavitated mucus cells $(57.6 \pm 4.0 v 45.3 \pm 2.1 \%)$. The number of stained mucus cells per crypt section was also significantly reduced: CRF $10.5 \pm 0.6, v 15.2 \pm 0.5$ in saline. Pretreatment with Ucn 2 abolished the CRF-induced decrease in number of stained mucus cells $(15.5 \pm 0.6 v 10.5 \pm 0.6$ for $\mathrm{CRF}$ alone, $\mathrm{p}<0.05)$ and the percentage of cavitated mucus cells $(35.1+3.6 v 57.6 \pm 4.0 \%$ for $\mathrm{CRF}$ alone, $\mathrm{p}<0.05$ ). Interestingly, in Ucn 2 alone treated rats, the colonic epithelium exhibited an increase in the number of stained mucus cells compared with control preparations $(18.3 \pm 1.0 v 15.2 \pm 0.5, \mathrm{p}<0.05)$. Ucn 2 also showed a tendency to decrease the percentage of cavitated mucus cells: $35.1+3.1$ y $45.3 \pm 2.1$ for controls. Ucn 2 did not increase FPO $(0.0 \pm 0.0 \vee 0.5 \pm 0.5 \mathrm{FPO} / \mathrm{h}$ for saline), but blunted the stimulatory action of CRF $(1.8 \pm 0.5$ $v 5.4 \pm 1.0 \mathrm{FPO} / \mathrm{h}$ for $\mathrm{CRF}$ ). Conclusions: These data indicate that 1 ) $\mathrm{CRF}$ induces mucus secretion by a dual mechanism implicating both compound exocytosis, as shown by the cavitation of the apical membrane, and by the non cavitation process; 2) CRF action is $\mathrm{CRF}_{1}$ mediated because, Ucn 2 does not reproduce the effect of a preferential $\mathrm{CRF}_{1}$ agonist, $\mathrm{CRF}$ and 3) Ucn 2 prevents mucus discharge in the colon. These data demonstrate differential regulation of colonic mucus secretion by CRF receptor subtypes that may have relevance to the action of stress on colonic mucus secretion. Supported by ROl DK57238(YT) and R21 DK068155-01Al(MM)

\section{M1635}

Comparison of Selective Neurokinin Receptor Antagonists (NK1, NK2, NK3) and Mixed Profile Antagonists $(\mathrm{NK1} / 2, \mathrm{NKl} / 2 / 3)$ in a Model of Visceral Hypersensitivity in Rats

Dania Reiche, Daniel Jasserand, Holger Sann, Ulf Preuschoff, Jochen Antel

There is good evidence that the neurokinin (NK) family (substance P, NKA, NKB) may be present in many parts of the nociceptive system. The transmission of visceral nociception has been shown to be mediated by these neuropeptides acting on NK1, NK2 and NK3 receptors. In a preclinical model we evaluated the efficacy of selective NKI, -2 and -3 receptor antagonists as well as the effect of dual NK1/2 receptor antagonists and a triple $\mathrm{NK} 1 / 2 / 3$ receptor antagonist. Rats were fasted for 24 hours prior to the experiments with free access to water. Acetic acid $(0.6 \%)$ was injected into the colon. Colorectal distension (CRD) was performed by inserting a rubber balloon rectally and setting the balloon pressure to 100 mbar for 10 minutes. During this time the number of abdominal constrictions was monitored by visual inspection. The first CRD served as a control. Subsequently the animals received compound or vehicle and the CRD was repeated at 30,60,90 and 120 minutes post application. The number of abdominal constrictions was compared to prevalues by paired two sided t-tests. The selective NKI receptor antagonists SLV317 and vofopitant significantly reduced the visceral sensitivity by up to $70.3 \%$ and $78.2 \%$, respectively, after subcutaneous (sc) administration of $100 \mu \mathrm{mol} / \mathrm{kg}$. The selective NK2 receptor antagonists SLV321, SLV332 and saredutant reduced the visceral sensitivity by up to $28.6 \%, 21.3 \%$ and $52.1 \%$, respectively, after sc administration of $30 \mu \mathrm{mol} / \mathrm{kg}$. However, the oral administration of $100 \mu \mathrm{mol} / \mathrm{kg}$ showed a very prominent inhibition of the visceral sensitivity for SLV321 (80.1\%) and SLV332 (80.3\%), but not for saredutant. The selective NK3 receptor antagonist osanetant showed with a dose of $10 \mu \mathrm{mol} / \mathrm{kg}$ sc a significant reduction of the visceral sensitivity of up to $47.0 \%$. The dual NK1/2 receptor antagonists SLV336 showed a potent inhibition of the visceral sensitivity. After sc administration of $10 \mu \mathrm{mol} / \mathrm{kg}$ SLV336 inhibited the visceral sensitivity by up to $57.1 \%$. Another NK1/2 receptor antagonist ZD6021 (racemate) was less active $(10 \mu \mathrm{mol} / \mathrm{kg} \mathrm{sc}, 28.2 \%$ maximal inhibition). Similarily, the triple $\mathrm{NKl} / 2 / 3$ receptor antagonist SCH206272 (racemate) showed a reduction of up 52.5\% of the visceral sensitivity $(10 \mu \mathrm{mol} / \mathrm{kg} \mathrm{sc})$. The present results show that selective NK1, -2 and -3 receptor antagonists and antagonists with mixed profiles potently reduce the visceral sensitivity. We hypothesize, that the combined blockade of different NK receptors (NKl/2 and NKl/2/3) might show synergistic effects. In conclusion NK receptor antagonists might be beneficial in the treatment of patients with irritable bowel syndrome or other disorders associated with visceral pain.

\section{M1636}

Measurement of Ca2+ Signaling Dynamics in Exocrine Cells with Total Internal Reflection Microscopy

David I. Yule, Jong Hak Won

In non-excitable cells, such as exocrine cells from the pancreas and parotid, agonist-stimulated $\mathrm{Ca} 2+$ signals consist of both $\mathrm{Ca} 2+$ release and $\mathrm{Ca} 2+$ influx. We have investigated the contribution of these processes to near membrane $\mathrm{Ca} 2+$ signals in pancreatic and parotid acinar cells using Total Internal Reflection Fluorescence (TIRF) microscopy which allows imaging with unsurpassed resolution in the " $\mathrm{Z}$ " dimension. These near membrane $\mathrm{Ca2}+$ signals were compared to those obtained with conventional wide-field microscopy (WFM). Physiological agonist stimulation resulted in $\mathrm{Ca} 2+$ oscillations in both pancreas and parotid when measured an initial peak followed by a substantial maintained plateau phase. The contribution of $\mathrm{Ca} 2+$ influx and $\mathrm{Ca} 2+$ release in isolation to near plasma membrane $\mathrm{Ca} 2+$ signals was investigated. In the absence of extracellular $\mathrm{Ca} 2+$, the profile and magnitude of the response following stimulation with maximal concentrations of agonist was similar to that obtained with WFM. In contrast, when Ca2+ influx was isolated using a $\mathrm{Ca} 2+$ re-addition protocol, either following maximal agonist stimulation or after depletion of intracellular pools with SERCA pump inhibitor, the Ca2+ signals evoked were more rapid and robust than measured with WFM and in parotid acinar cells often resulted in the saturation of fluo-4, but not of the low affinity dye fluo-4 FF (kd=9700nM). Interestingly, Ca2+ influx as measured by this protocol in parotid acinar cells was substantially greater than that initiated in pancreatic acinar cells. Indeed, robust $\mathrm{Ca} 2+$ influx was observed in parotid acinar cells even at low, physiological concentrations of agonist. These data indicate that TIRFM is a useful tool to monitor agonist-stimulated near membrane $\mathrm{Ca} 2+$ signals mediated by $\mathrm{Ca} 2+$ influx but not $\mathrm{Ca} 2+$ release in exocrine acinar cells. In addition, TIRFM reveals that the extent of $\mathrm{Ca} 2+$ influx in parotid acinar cells is greater than pancreatic acinar cells when compared using identical methodologies.

\section{M1637}

Stimulatory Effects On the Exocrine Pancreas, Via Two CCK-Receptor Subtypes

Sofia A. Rengman, Bjorn R. Westrom, Stefan G. Pierzynowski

Introduction: In pigs, the secretion of digestive enzymes from the exocrine pancreas is stimulated by cholecystokinin, CCK, via CCK2-receptors, CCK2R, located in the duodenum and antrum, in doses mimicking postprandial levels $(1,2)$ while CCKl-receptors, CCKIR, are not believed to be involved (3). The main aim of the study was to establish a doseresponse curve and to examine the exocrine pancreatic response to increasing doses of CCK33 infused directly to the duodenum/antrum region or via the jugular vein, in order to determine how the response differs and whether more than one CCK-receptor subtype is affected. Methods: Five anaesthetised piglets were used in acute experiments. The piglets were surgically fitted with catheters in the gastric artery, the jugular vein and the pancreatic duct. CCK-33 was infused via the gastric artery in increasing concentrations (factor 2) from 4 to $128 \mathrm{pmol} / \mathrm{kg} / 10 \mathrm{~min}$. The pancreatic juice was collected every ten minutes. The piglets were given a 30 minute rest period, then the procedure was repeated but the increasing doses of CCK-33 was infused via the jugular vein instead. The pancreatic juice was analysed for protein and trypsin and the blood plasma was analysed for CCK. Results: During the arterial infusion, the pancreatic secretion increased (from 1 to $3 \mathrm{U} / \mathrm{kg} / \mathrm{h}$ for trypsin and $\mathrm{l}$ 
to $5 \mathrm{mg} / \mathrm{kg} / \mathrm{h}$ for protein) starting at the infusion levels of $4 \mathrm{pmol} / \mathrm{kg} / 10 \mathrm{~min}$ and reached a plateau at $32 \mathrm{pmol} / \mathrm{kg} / 10 \mathrm{~min}$. From 64 to $128 \mathrm{pmol} / \mathrm{kg} / 10 \mathrm{~min}$, a second phase of stimulation of pancreatic secretion was observed (up to $10 \mathrm{U} / \mathrm{kg} / \mathrm{h}$ for trypsin, and $15 \mathrm{mg} /$ $\mathrm{kg} / \mathrm{h}$ for protein). The infusion of CCK-33 via the jugular vein had to exceed $64 \mathrm{pmol} / \mathrm{kg} /$ 10 min to initiate any response. This dose is identical to the dose observed in the second phase of secretion after local, arterial CCK-33 infusions. Conclusions: The exocrine pancreatic response appeared to be elicited via two different receptor subtypes after local infusions to the duodenum/antrum region of CCK-33. Low doses of CCK-33, mimicking the postprandial plasma levels, stimulate the secretion in a dose-dependent manner. CCK-33 infused via the jugular vein can only reproduce the second phase of the response which does not involve CCK2R and demands high doses of CCK-33. The second phase, elicited by the high CCK33 doses, independent of infusion route, was twice as high as the response elicited via CCK2R in the duodenum-antrum region but is considered to be pharmacological since the plasma level of CCK-33 by far exceeds postprandial levels observed in pigs.

\section{M1638}

Interruption of the PDGF and EGF Signal Pathways By Curcumin Contributes to the Induction of Gene Expression of PPAR $\gamma$ in Activated Hepatic Stellate Cells in Vitro

Yajun Zhou, Anping Chen

BACKGROUND: A dramatic feature during the pathogenesis of liver fibrosis is enhanced cell growth of hepatic stellate cells (HSC), the major effector during hepatic fibrogenesis, triggered by PDGF and EGF. This process is coupled with the sequential up-expression of PDGF-beta receptor (PDGF- $\beta R$ ) and EGF receptor (EGFR), as well as a dramatic reduction in the expression of the peroxisome proliferator-activated receptor-gamma (PPAR $\gamma$ ). Signal pathways of PDGF and EGF, including the PI3K/Akt pathway and the MAPK pathways, are implicated in stimulating HSC proliferation. PPAR $\gamma$ has inhibitory effects on HSC growth. However, the relationship between the activation of the signaling for PDGF- $\beta$ and EGF and the expression of PPAR $y$ in activated HSC remains completely obscure. Curcumin, the yellow pigment in curry, was previously shown to inhibit cell growth of activated HSC and induce the expression of PPARy in activated HSC in vitro [(2003) Am J Physiol. 285, G20; (2004) Biochem J, 384, 149]. The underlying mechanisms are largely unknown. AIM AND HYPOTHESIS: This study is to elucidate the mechanisms of curcumin in the induction of PPAR $\gamma$ gene expression in activated HSC, focusing on the effects of the PDGF and EGF signaling on the expression of PPAR $\gamma$. We hypothesize that interruption of the PDGF and EGF signal pathways by curcumin contributes, at least partially, to the induction of gene expression of PPAR $\gamma$ in activated HSC. RESULTS. Studies herein indicate that exogenous PDGF or EGF inhibits the expression and trans-activation activity of PPAR $\gamma$ in passaged HSC. Further experiments show that curcumin interrupts the PDGF and EGF signaling in activated HSC by inhibiting the phosphorylation of PI3K/Akt, Erk and JNK and by suppressing the expression of PDGF- $\beta R$ and EGFR. Inhibiting the activity of PI3K/Akt, Erk or JNK respectively by specific inhibitors Ly294002, PD98059 or SP600125 significantly increases the expression of PPAR $\gamma$ in activated HSC. CONCLUSION: Our studies demonstrate that activation of PDGF and EGF signaling suppresses the expression of PPARy in activated HSC. The interruption of the PDGF and EGF signal pathways by curcumin contributes, at least partially, to the induction of gene expression of PPAR $\gamma$ in activated HSC. Our results provide novel insights into the relationship between the activation of PDGF and EGF signaling and the expression of PPARy during HSC activation. In addition, these results provide additional evidence and elucidation of the mechanisms of curcumin in inhibition of HSC activation. [The work was supported by the grant DK 47995 from NIH/NIDDK to A. Chen and by the Bridging Award to A. Chen from LSUHSC-S].

\section{M1639}

ACF Formation and Colon Cancer Growth Is Reduced in Growth Hormone Deficient Rats By Alteration in Proliferative Pathways and Receptor Signaling Robert Carroll, Robert Goodlad, Jai Marchandani, Heliodoro Medina, Young Choi, Sean Lee, Kevin Leung, Gregory Nemtsov, Tatiana Kouznetsova, Alexandra Ivanovic, Steven M. Swanson, R Brooks Robey, Terry G. Unterman

Background: The Spontaneous Dwarf rat (SDR) possesses a nonsense mutation in the growth hormone $(\mathrm{GH})$ gene that results in absent serum $\mathrm{GH}$ and reduced levels of insulin-like growth factor I (IGF-I). To evaluate the GH-IGF axis in colon carcinogenesis, we examined the formation of aberrant crypt foci (ACF) and tumor development in wild type (WT) and SDR littermates exposed to the carcinogen azoxymethane (AOM). Subsequently, we evaluated apoptosis and proliferation in response to carcinogen as well as the expression of hexokinase activity, IGF-I receptor (IGF-IR), epidermal growth factor receptor (EGF-R) and p27kip/cip protein expression in control and AOM treated WT and SDR animals. Methods: Eight week male Sprague-Dawley and SDR littermates received $15 \mathrm{mg} / \mathrm{kg}$ of AOM subcutaneously (s.c.) twice 7 days apart (total dose $30 \mathrm{mg} / \mathrm{kg}$ ). Rats were weighed weekly and euthanized by CO2 asphyxiation at 10,25 , and 28 weeks. ACF were quantified by stereomicroscopy and tumor number and weights were recorded for each animal. Cell proliferation was measured by vincristine metaphase arrest, flow cytometry and bromodeoxyuridine (BrdU) incorporation. Apoptosis was measured by Tunel staining and cleaved caspase 3 IHC. IGF-I was measured by RIA and northern blotting. Hexokinase activity was measured by spectrophotometric assay. PARP cleavage, and IGF-IR, EGF-R and p27kip/cip expression were measured by western blotting. Results ACF formation is markedly reduced ( $85 \%)$ in AOM treated SDR rats at $10 \mathrm{wks}, 25 \mathrm{wks}$, and $28 \mathrm{wks}$ compared to WT and tumor incidence. number and weight were reduced by $\sim 50 \%$ in SDR vs WT rats. Apoptosis rates are similar in AOM treated WT and SDR rats and correlate inversely with hexokinase activity in the colon. Though, baseline cell proliferation is increased in the proximal (1.5X) and distal (2.25X) colon of SDR rats compared to WT rats, it selectively decreases cell proliferation in the distal colon in response to $\mathrm{AOM} \sim-40 \%$, and these changes correspond to reduced ACF and tumor formation whereas AOM treatment significantly increases proliferation in WT rats as early as $72 \mathrm{hrs}$ post AOM. These changes correspond with reduction in serum IGF-I and colonic IGF message in SDR rats but significantly increased IGF-IR, EGF-R, and p27kip/ cip protein levels in the distal colon suggesting that IGFR/EGFR signaling and/or action may contribute to reduced proliferative response to AOM and decreased ACF formation in SDR vs WT rats. Conclusion: Early neoplastic lesions (ACF) are reduced in GH deficient animals. The SDR rat is a promising model for studies regarding the role of GH/IGF system in the initiation and promotion of colon cancer.

\section{M1641}

Role of Cholesterol $(\mathrm{Ch})$ and Caveolin 3 Proteins (CAV3) in the Regulation of CCK-1 Receptor Recycling in Human Gallbladder (GB) Muscle Ping Cong, Piero Biancani, Jose Behar

The contraction of muscle cells from $\mathrm{GB}$ with $\mathrm{Ch}$ stones is impaired in response to $\mathrm{G}$ protein coupled receptors such such as CCK-8 that act on CCK-1 receptors. This impaired response is associated with high caveolar Ch, CAV3, and CCK-1 receptors levels and a 50\% reduction of 125I-CCK-8 binding. These abnormalities are corrected when cells are incubated with $\mathrm{Ch}$-free liposomes that remove the excess $\mathrm{Ch}$ from the caveolae. The mechanism whereby high levels of $\mathrm{Ch}$ cause these abnormalities has not been elucidated. The present studies were performed in muscle cells from $\mathrm{GB}$ with $\mathrm{Ch}$ stones that have high caveolae $\mathrm{Ch}$ and from pigment stones (controls). We first examine the effect of CCK-8 on CAV3 phosphorylation (P) using antibodies against total and phosphorylated CAV3. In normal muscle cells CCK-8 increased the phosphorylated CAV3 band compared with basal levels ( $<<0.01)$. This increased phosphorylation of CAV3 was absent in muscle cells from GB with Ch stones or was blocked after incubation with Ch-rich liposomes. This inhibition of CAV3 phosphorylation was associated with increased caveolar levels of $\mathrm{Ch}, \mathrm{CAV} 3$, and CCK-1 receptors. Pretreatmen of normal muscle cells with PP2 (a Src tyrosine kinase inhibitor) blocked CCK-8 induced CAV3 phosphorylation and increased the concentrations of CCK-1 receptors in the caveolae. Sucrose gradients and co-immunoprecipitation studies using antibodies against EEAl (a marker of early endosomes) and Rab 11 (a marker of late recycling endosomes) show that in normal muscle cells CCK-8 increases the transfer of CCK-1 receptors to early endosomes at 5 and $10 \mathrm{~min}$, to late endosomes at 15 and $20 \mathrm{~min}$ and back to normal levels in the plasma membrane at $30 \mathrm{~min}$. In contrast, high caveolar $\mathrm{Ch}$ inhibit the transfer of receptors to the endosomes and decrease CCK-1 receptor band in the bulk plasma membrane. It is concluded that receptors that internalized through caveolae phosphorylate CAV3 that facilitate their transfer to recycling endosomes suggesting that acts as chaperone in recepto recycling. Excess $\mathrm{Ch}$ inhibits receptor trafficking by blocking CCK-1 dependent CAV3 phosphorylation.

\section{M1642}

Functional Epithelial Cells Proteomics Identified Novel Disease Targets for the Colitogenic Mechanisms of Enterococcus Faecalis in IL-10 Gene Deficient

Mice

Anna Shkoda, Pedro A. Ruiz, Sandy C. Kim, Hanelore Daniel, Balfour R. Sartor, D.R Haller

Background \& Aim. Colitogenic Enterococcus faecalis initiate and perpetuate experimental colitis in Interleukin 10 gene deficient mice (IL-10-/-). To identify novel disease mechanism for experimental colitis, we used 2D-gel SDS-PAGE and MALDI-TOF mass spectrometry to characterize altered protein expression profiles in primary intestinal epithelial cell (IEC) from E. faecalis-monoassociated wild type and IL-10-/- mice. Functional and molecular analysis revealed novel disease mechanisms for the glucose regulated protein (grp)-78 and galectin-3. Methods. Germ-free wild type and IL-10-/- mice were monoassociated with E. faecalis for 14 weeks. Primary IEC from cecal and colonic tissue were purified to perform proteome analysis. Molecular mechanisms of significantly altered proteins were characterized in the Mode-K cell culture model. Results. Intestinal epithelial cell proteomics identified 14 differentially regulated proteins in IEC from severely inflamed IL-10-/- mice (14 weeks) including creatine kinase, serpin clade B, dnaK type molecular chaperon grp-78 and galectin3. Immunoblot analysis linked the induction of grp-78 expression in IL-10-/- IEC with an

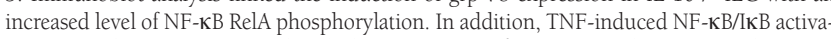
tion in IEC lines was associated with the recruitment of grp-78 into the IKK complex. Mos importantly for the understanding of molecular mechanisms, the siRNA-mediated knockdown of grp-78 inhibited TNF-induced RelA phosphorylation, suggesting a role for grp-78 in maintaining RelA phosphorylation. Interestingly, IL-10-mediated p38 signaling decreased grp-78 expression in primary wild type IEC and susceptible IL-10 receptor reconstituted IEC, suggesting protective mechanisms of IL-10 at the epithelial cell level. In addition to grp-78, the siRNA-mediated knock-down of galectin-3 in IEC lines triggered caspase- 3 cleavage. In vivo validation confirmed the increased expression levels of cleaved caspase-3 in IL-10-/- IEC. In addition, the decreased expression of galectin-3 was associated with decreased expression of the junctional protein E-cadherin in primary IEC from inflamed colonic tissue, suggesting a mechanistic role for galectin-3 in maintaining barrier integrity. Conclusion. Functional epithelial cell proteomics assigned novel disease mechanisms for grp-78 and galectin-3 expression in the epithelium under the pathological conditions of progressive chronic inflammation targeting the NF- $\mathrm{KB}$ signaling pathway and barrier function.

\section{M1643}

Flagellin-Dependent and Independent Responses to Attaching and Effacing Bacterial Pathogens in Intestinal Epithelial Cells

Mohammed A. Khan, Caixia Ma, Bruce A. Vallance

Introduction: Enteropathogenic Escherichia coli (EPEC) is an attaching and effacing (A/E) bacterial pathogen that causes diarrhea and intestinal inflammation in children. EPEC directly infects human intestinal epithelial cells which release pro-inflammatory interleukin-8 (IL8). EPEC Flagellin, a product of the fliC gene, is a major factor that triggers IL-8 release from epithelial cells. Presently, it is unclear if other inflammatory mediators are released from epithelial cells in EPEC infection, and whether these responses are fliC dependent or independent. Moreover, little is known about negative regulation of innate epithelia responses to bacterial pathogens. Studies suggest that Single Immunoglobulin IL-1R-related 
(SIGIRR) protein negatively regulates Toll-like receptor responses in epithelial cells; however, its role in innate response to $\mathrm{A} / \mathrm{E}$ pathogens has not been tested. Objectives: Our aims were a) to examine the mechanism of flagellin-dependent and independent IL-8 secretion and other pro-inflammatory mediators in $\mathrm{A} / \mathrm{E}$ infection; b) to compare temporal expression of these responses with SIGIRR expression in infected intestinal epithelial cells. Results: We infected Caco-2 colonic epithelial cells with wild type (WT) and $\Delta$ fliC EPEC and observed, in addition to IL-8 release, an up-regulated expression of several pro-inflammatory and antimicrobial genes such as MIP- $3 \alpha$, MCP-1, COX-2 and $\beta$-defensin, predominantly in a fliC dependent manner. These fliC dependent responses required PI-3K pathway and $\mathrm{p} 38 \mathrm{MAPK}$ activation, with involvement of key innate proteins e.g. MyD88 and IRAK. Interestingly, some of these genes were activated at later time points in epithelial cells infected with $\Delta$ fliC EPEC, but in a NF-kB dependent manner. Similarly, infection by Citrobacter rodentium $(\mathrm{Cr})$, a related $\mathrm{A} / \mathrm{E}$ pathogen, induced pro-inflammatory responses in the mouse colon independently of fliC, during $\mathrm{Cr}$ colitis. Preliminary data suggests that WT EPEC suppresses SIGIRR expression in a fliC dependent manner which temporally correlates with release of IL-8 from epithelial cells. Conclusion: The above findings indicate that A/E pathogens trigger an array of pro-inflammatory innate responses from infected intestinal epithelial cells through both fliC dependent and fliC independent mechanisms. Future studies will assess if innate epithelial response to A/E pathogens requires suppression of SIGIRR. These observations increase our understanding of how intestinal epithelial cells respond to bacterial pathogens, and may have important implications for inflammatory conditions triggered by enteric bacteria, including infectious colitis and inflammatory bowel disease.

\section{M1644}

IBD Mucosal E. Coli Isolates Mediate IL-8 Release from Colon Epithelial Cells By Shed Flagellin Acting Via Mapkinase Pathways

Sreedhar Subramanian, Jonathan M. Rhodes, Anthony C. Hart, Benjamin A. Tam, Lugang Yu, Helen M. Martin, Barry J. Campbell

Background \& Aims: Increased mucosa-associated E. coli are found in IBD and colon cancer. They commonly adhere to colon epithelial cells and cause IL-8 release(Gastroenterology 2004;127:80-93). We have now investigated the mechanisms for this IL-8 release. Methods: Confluent HT29 cells were incubated in triplicate wells for 4h with 33 E. coli mucosal isolates (14 colon cancer, 7 Crohn's disease (CD), 6 ulcerative colitis (UC) and 6 control (IBS/polyps) at a bacterium:cell ratio of 10:1 and IL-8 measured in the supernatant by ELISA. The effect on the IL-8 response of pre-treatment with U0126, SB203580, or SP600125 (60$90 \mathrm{~min}$, at $10 \mu \mathrm{M}$ ), specific inhibitors of the MAPkinases: ERK1/2, p38 and JNK, respectively, was studied. Pooled supernatants were also generated from 24 mucosal $E$ coli isolates, 6 from each of the 4 disease groups, passed through a $0.2 \mu \mathrm{m}$ filter and assessed for their ability to release IL-8 from HT29 cells. IL-8 response to whole bacteria was assessed in TLR5 transfected HEK293 cells or control LacZ transfected cells. Results: All 33 E. coli isolates induced an IL-8 response $(408 \pm 370 \mathrm{pg} / \mathrm{ml}, \mathrm{mean} \pm \mathrm{SD})$. This was significantly higher with haemagglutinin-positive bacteria $(\mathrm{n}=16 ; 548 \pm 427 \mathrm{pg}$ IL-8/ml) than for haemagglutininnegative bacteria $(\mathrm{n}=17 ; 281 \pm 252 \mathrm{pg} / \mathrm{ml})(\mathrm{p}<0.0001)$. Inhibition of ERK and $\mathrm{p} 38$ MAPK reduced average IL- 8 response ( $\mathrm{n}=33$ ) by $77 \pm 14 \%$ and $84 \pm 18 \%$ respectively (both $\mathrm{p}<0.001$ ANOVA) in contrast to JNK inhibition $(36 \pm 28 \%)$. The IL- 8 response to whole bacteria was TLR5 (flagellin receptor)-dependent in 14/33 E. coli of which 6/14 were haemagglutininpositive. IL-8 response was $389 \pm 249 \mathrm{pg} / \mathrm{ml}$ in TLR5 transfected cells versus $36 \pm 50 \mathrm{pg} / \mathrm{ml}$ in LacZ transfected control cells. Inhibition of LPS by polymixin B, $10 \mathrm{ug} / \mathrm{ml}$ caused no significant reduction $(7 \pm 4 \%)$ in IL- 8 response to whole bacteria. The IL-8 response to filtered supernatants (mean $659 \pm 239 \mathrm{pg} / \mathrm{ml}$ ) was approximately half that produced by whole bacteria $(1393 \pm 478 \mathrm{pg} / \mathrm{ml})$. Following ultracentrifugation of the pooled supernatant from 6 UC E. coli, $99 \pm 5 \%$ of the IL- 8 stimulating activity sedimented with outer membrane vesicles. Digestion of flagellin in the with neutrophil elastase inhibited IL- 8 release by $68 \pm 30 \%$ $(\mathrm{p}<0.001)$ or pre-treatment with anti-H6 flagellin antibody inhibited IL- 8 release by $50+19 \%$ $(\mathrm{p}=0.008)$. Conclusion: Mucosal E. coli cause IL-8 release from colon epithelial cells through ERK and p38 MAPkinase pathways, largely triggered by interaction between flagellin/TLR5. Much of the IL-8 triggering activity is present in a shed microvesicle preparation. The IL8 response is only partially related to bacterial adhesion and may be as relevant to UC as to Crohn's disease.

\section{M1645}

Further Characterisation of Mucosa-Associated Escherichia coli Isolates from Crohn's Disease and Colon Cancer Patients

Helen Martin, Barry Campbell, Sree Subramanian, Anthony Hart, Jonathan Rhodes

INTRODUCTION: We previously reported that E. coli isolated from biopsies taken from Crohn's disease and colon cancer patients displayed enhanced adherence to erythrocytes and intestinal cell lines whilst lacking the conventional markers of bacterial pathogenicity (Gastroenterology 2004; 127:80-93). Here we describe further phylogenetic and phenotypic characterisation of these strains. METHODS: For phylogenetic analysis, 21 strains of CD and colon cancer-associated E. coli (14 adherent and 7 non-adherent strains) were screened for the presence of the chuA, and yjaA genes, and the TSPE4.C2 DNA fragment (Applied and Environmental Microbiology 2000; 66:4555-4558). Haemagglutination (HA) and HEp2 cell adhesion assays were performed using all adherent E. coli strains. RESULTS: All adherent isolates and 2/7 non-adherent isolates were classed into E. coli phylogenetic groups D1 or D2, characteristic of virulent extra-intestinal E. coli. This grouping was performed on the basis of the presence of the chuA gene required for heme transport in enterohaemorrhagic O157:H7 E. coli. Five out of seven non-adherent isolates were classed into E. coli phylogenetic groups $\mathrm{Al}$ or $\mathrm{Bl}$, characteristic of commensal E. coli. All haemagglutinating isolates displayed adherence to HEp-2 cells in a pattern characteristic of diffusely adherent E. coli (DAEC). All adherent strains displayed chloramphenicol resistant HA indicative of specificity to cell surface receptors other than DAF (CD55), possibly CEACAMs. Strong haemagglutinating activity was found in some bacterial supernatants suggesting secretion of a bacterial adhesion factor, possibly on shed microvesicles. CONCLUSIONS: Adherent mucosa-associated E coli strains isolated from $\mathrm{CD}$ and colon cancer display attributes characteristic of virulent extraintestinal E. coli, and probably represent typical or atypical DAEC

\section{M1646}

Intestinal Alkaline Phosphatase, a Gut Defense Factor?

Xiaobo Zhang, Madhu S. Malo, Richard A. Hodin

INTRODUCTION: Intestinal alkaline phosphatase (IAP) is a brush border enzyme that is highly expressed in the mammalian small intestine and is thought to function as an inhibitor of dietary fat absorption. Previous investigations have reported the ability of the IAP enzyme to detoxify lipopolysaccharide (LPS), but the physiological significance of this finding has not been determined. We therefore sought to investigate the cellular functions of IAP in intestinal epithelia using an in vitro model system. METHODS: We first established a stable clone of T84 intestinal cells that over-expresses the IAP protein. The human $1.6 \mathrm{~kb}$ IAP cDNA was subcloned into the pIRES2-EGFP vector and transfected into T84 cells. Stable lines were purified through G418 selection, and confirmed by RT-PCR and IAP enzyme assay. Transient transfections were performed with an NF-kB-luciferase reporter plasmid along with expression plasmids for TLR4 and MD2, essential components of the LPS signaling pathway. Luciferase activity was determined as a function of LPS treatment. RESULTS: IAP mRNA was absent in T84 wild type (T84-WT) and T84 vector alone (T84-vector) cell lines, but was highly expressed in the IAP expressing cells (T84-IAP). IAP enzyme activity was

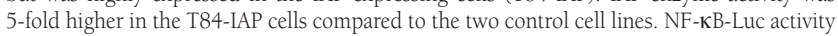
was induced in a dose-dependent manner by LPS (4-fold activation at $100 \mathrm{ng} / \mathrm{ml}$ ) in T84WT cells and T84-vector cells, but only when both TLR4 and MD2 were co-transfected. The magnitude of NF-KB-Luc activation by LPS in the T84-IAP cells was dramatically inhibited (approximately 40\% inhibition) compared to T84-WT and T84-vector cells. CONCLUSION Intestinal alkaline phosphatase is able to inhibit the LPS effects on intestinal cells in vitro suggesting that IAP represents a previously unrecognized component of the gut mucosal defense system. These findings could have important implications for our understanding of how the host is protected against gut-derived bacteria and toxins

\section{M1647}

Surface Expression of TLR9 Is Upregulated in Response to Pathogenic Bacterial DNA

Julia B. Ewaschuk, Jody L. Backer, Karen Madsen

Bacterial DNA elicits both pro- and anti-inflammatory responses in epithelial and immune cells in a strain-dependent fashion. In addition. DNA from probiotic bacterial strains can modulate and suppress epithelial cell cytokine secretion in response to pathogenic bacteria DNA. To date, the only known receptor that recognizes bacterial DNA is toll-like receptor 9 (TLR9). TLR9 appears to be localized in intracellular compartments in immune cells; however, the location of TLR9 in epithelial cells has not been investigated. Intestinal epithelial cells (IEC) are constantly exposed to high levels of bacterial DNA, and must recognize and respond appropriately to the presence of pathogenic bacteria. Objective: The aim of this study was to investigate TLR9 expression and localization in colonic epithelial cells under basal conditions and in response to stimulatory and inhibitory bacterial DNA. Methods: HT-29 epithelial cells were exposed to purified DNA from Bifidobacteria breve (Bb; probiotic) or Salmonella dublin (Sd; pathogenic) in varying concentrations (10-50 $\mu \mathrm{g}$ ). A time course of TLR9 mRNA expression was determined using real-time RT-PCR. IL-8 secretion as a response to bacterial DNA was measured by ELISA. Localization of TLR9 was determined by flow cytometry and confocal microscopy. Chloroquine and ethidium monoazide were used to block endosomal TLR9 interactions and label bacterial DNA, respectively. Results: Immunofluorescence and flow cytometric analyses demonstrated both intracellular and surface expression of TLR9 in HT-29 cells under basal conditions. Ethidium monoazidelabelled DNA from both Bb and Sd appeared at the cell surface of HT-29 cells. However, exposure of HT-29 cells to Sd DNA, but not to Bb DNA, resulted in a significant increase $(\mathrm{p}<0.05)$ in IL-8 secretion after 6 hrs. This was associated with a greater than 20 -fold increase in TLR9 mRNA within $30 \mathrm{~min}$, and an increased surface expression of TLR9, as assessed by flow cytometry. Chloroquine did not block IL- 8 secretion in response to Sd DNA, suggesting that TLR9 can signal from the cell surface. There was no change in mRNA levels or cellular localization of TLR9 in response to Bb DNA. Conclusion: These data indicate that intestinal epithelial cells can recognize pathogenic bacterial DNA and respond by increasing surface localization and expression of TLR9. This suggests that the inflammatory response to Salmonella DNA is mediated at least in part by this increase in TLR9 expression.

\section{M1648}

Helicobacter Pylori Strain Specific Induction of Cyclin D1 But Not BetaCatenin Regulates Gastric Epithelial Cell Proliferation

Dharmalingam Subramaniam, Vineet Ahuja, Douglas E. Berg, Courtney W. Houchen, Brian K. Dieckgraefe, Shrikant Anant

Helicobacter pylori $(\mathrm{Hp})$ is a genetically diverse species whose members vary markedly in vigor of inflammatory and other responses they elicit. Hp uses a cag pathogenicity island (PAI)-encoded type IV secretion system to inject CagA protein into gastric epithelial cells and induce proinflammatory interleukin-8 (IL-8) secretion. Recent studies have indicated that synthesis of $\leq$-catenin, which regulates numerous cancer-related genes, including cyclin Dl and cyclooxygenase-2 (COX-2), is induced by carcinogenic Hp strains in a CagAdependent manner. Here, we determined the importance of Hp strain diversity on induced beta-catenin and cyclin D1 expression in AGS gastric epithelial cells by western blot, and further, analyzed IL-8, and COX-2 expression by Real Time RT-PCR. Four different strains of Hp, based on their cag-PAI and VacA status were utilized in the study: SS1 (cag-PAI+, VacA s2m2), $\mathrm{CHl}$ and $\mathrm{CH} 8$ (both cagPAI+, VacAslml), and $\mathrm{CH} 5$ (cag negative, VacAslml) The levels of CagA protein following SS1 infection was 50\% less than that observed following $\mathrm{CHl}$ and $\mathrm{CH} 8$ infection. However, all four $\mathrm{Hp}$ strains increased beta-catenin protein levels to similar levels, $2 \mathrm{~h}$ following infection. At $6 \mathrm{~h}$ following infection, the beta-catenin levels were significantly reduced to levels below that observed in uninfected cells, suggesting that a transient increase in beta-catenin levels may be sufficient for Hp-mediated activity. However, nuclear beta-catenin levels were lower than that of controls at both 2 and $6 \mathrm{~h}$ following infection. This localization is similar to that observed in primary gastric cancers and suggests 
beta-catenin activity to be through cytoplasmic signaling and not at the level of transcription. We next determined the effects of Hp infection on the expression of cyclin Dl, an important mediator of cell cycle. Strains $\mathrm{CH1}$ and $\mathrm{CH} 8$ significantly inhibited, while strains SS1 and $\mathrm{CH} 5$ induced cyclin $\mathrm{Dl}$ expression. In contrast, while strains $\mathrm{CHl}$ and $\mathrm{CH} 8$ were robust inducers of proinflammatory mediators IL-8 and COX-2, SS1 and CH5 were weak inducers. In addition, while $\mathrm{CHI}$ induced apoptosis of AGS cells, SS1 induced proliferation. Together, these data suggest that Hp strains that induce a robust host gene response induce cellular apoptosis, while those that induce a weak host gene response enhance cell proliferation, probably through increased cyclin Dl expression

\section{M1649}

Hydrocortisone Modulates An Age-Dependent Cholera Toxin-Response By Regulating the Developmental Endocytosis in Immature Human Enterocytes Lei Lu, W Allan Walke

We have reported that cholera toxin (CT) elicited an excessive secretory response in immature enterocytes. Recently, we have also reported that this difference in response to CT is due, at least in part, to an enhanced endocytosis of the CT-GMl complex in immature enterocytes. In this study, we have tested the hypothesis that trophic factors in breast milk can modulate the CT-response in immature enterocytes by regulating the development of endocytosis. A human fetal small intestinal line (H4) and an adult crypt-like colonic carcinoma cell line (T84) were used in this study. Endocytic inhibitors and hydrocortisone, a known human breast milk trophic factor, were used to test our hypothesis. H4 cells were incubated with hydrocortisone $(1 \mathrm{mM})$ or media alone for 7 days before exposure to CT. CT-uptake and cAMP accumulation in the cells were monitored. In $\mathrm{H} 4$ cells, only chlorpromazine (CPZ, a clathrin-pathway inhibitor) significantly inhibited CT-uptake and the cAMP response, whereas in T84 cells only methy- $\beta$-cyclodextrin $(\mathrm{M} \beta \mathrm{CD}$, a caveolar pathway inhibitor) blocked CT-uptake and cAMP response. Hydrocortisone decreased CT-uptake and the cAMP response without affecting CT-GMl binding in $\mathrm{H} 4$ cells. Moreover, hydrocortisone significantly attenuated the inhibitory effect of $\mathrm{CPZ}$ on clathrin-endocytosis and the CT-response. In contrast, these cells were more sensitive to $\mathrm{M} \beta \mathrm{CD}$. Immunogold labeling and electron microscopy was used to confirm our biochemical observation that the predominant endocytosis of CT is via clathrin-coated pits in immature enterocytes and is down-regulated during enterocyte maturation. In $\mathrm{H} 4$ cells, a co-localization of the $\mathrm{CT}-\mathrm{B}$ subunit with clathrin was present initially but is absent in $\mathrm{H} 4$ cells pretreated with hydrocortisone. In contrast, there is a significantly increased co-localization of CT-B with caveolin-1/caveolae in hydrocortisone treated cells similar to that seen in T84 cells. To further investigate the role of clathrincoated pits in CT intracellular trafficking, we used RNA interference (RNAi) of clathrin with siRNA in $\mathrm{H} 4$ cells. There was a dose-dependent decrease in clathrin levels in $\mathrm{H} 4$ cells transfected with clathrin siRNAs and a diminished CT-uptake and cAMP elevation respectively. In contrast, though there was a similar level of clathrin knockdown in $\mathrm{H} 4$ cells pretreated with hydrocortisone, there was no inhibition of CT-uptake and decrease in cAMP elevation. Our studies suggest that the enhanced response to CT is partially due to an excessive uptake of CT by a developmentally regulated clathrin-endocytic pathway and that hydrocortisone can modulate CT-uptake by the induction of endocytosis in immature human enterocytes.

\section{M1650}

Transepithelial Transport of Cholera Toxin (CT) By Ganglioside Gml Sorts Strictly Away from the Golgi Apparatus: A Test of the Indirect Transcytosis Hypothesis

Joy Wan, Eli Kern, Anne Wolf, David Saslowsky, Randy Holmes, Wayne Lencer

Microbial products affect intestinal development and biology. The way this cross-talk occurs is highly diverse and not completely understood. Studies on microbial pathogens have been highly informative. Cholera toxin (CT) typifies the structure and function of the bacterial AB5-subunit toxins (including the E. coli and Shilgella toxins) that enter host cells by moving retrograde from the cell surface through the Golgi apparatus into the endoplasmic reticulum (ER). Over a decade ago, we discovered a pathway by which CT breeches the intestinal barrier by moving from the luminal surface all the way across the epithelial cell to the basolateral membrane via transcytosis. The toxin appeared to move across the cell by first binding its receptor, ganglioside GMl and entering the Golgi (and perhaps ER). This pathway is unlike the classic transcytotic pathway described for the polymeric Ig receptor pIgR and Fc-receptor FcRn, and we proposed the idea of "Indirect Transcytosis": a pathway from apical to basolateral membrane in mucosal epithelial cells that requires cargo movement through the Golgi and perhaps other compartments of the secretory pathway. Aim: We test this idea using CT that moves retrograde from PM to ER, the E. coli toxin LTIIb that sorts strictly away from the Golgi apparatus and ER, and a mutant of CT containing sulfation motifs (CT-GS). Methods: Selective cell surface biotinylation of intestinal T84 cell monolayers is used to measure transcytosis; and toxin-sufation to measure transport into the Golgi (this reaction is specific to the Golgi apparatus). Results: Only CT moves into the transcytotic pathway and can be detected on the basolateral membrane of T84 monolayers. This result is consistent with a requirement to enter the Golgi apparatus before moving to the basolateral membrane (indirect transcytosis). However, two independent initial studies show that the fraction of $\mathrm{CT}$ on the basolateral membrane is not sulfated, while the fraction of $\mathrm{CT}$ that moved into the retrograde pathway of the same cells is strongly sulfated. Conclusion: Ganglioside GMl sorts CT from the apical PM to ER and to the basolateral membrane by separate pathways. Sorting CT by GMl across mucosal barriers is specific to GMl as the related ganglioside GDla that binds LTIIb cannot do this.

\section{M1651}

Mesalazine (5-ASA), But Not Hydrocortisone, Inhibits E. Coli-Induced IL-8 Release from Colonic Epithelial Cells At Therapeutic Concentrations Sreedhar Subramanian, Barry J. Campbell, Helen M. Martin, Anthony C. Hart, Lugang Yu, Jonathan M. Rhodes

Background \& aims: Inflammatory bowel disease colonic mucosal E. coli isolates release interleukin-8 (IL-8) from colonic epithelial cells (Gastroenterology 2004:127:80-93). We have now investigated the effect of 5-ASA and hydrocortisone on this IL-8 response. Materials $\&$ Methods: Preliminary experiments showed that $0.2 \mu \mathrm{m}$-filtered supernatant, pooled from 6 E. coli mucosal isolates from 5 UC patients, induced a substantial IL-8 response from HT29 colon epithelial cells (pooled supernatant, $1262 \pm 110 \mathrm{pg}$ IL-8/ml; pooled whole bacteria, $1901 \pm 67 \mathrm{pg} \mathrm{IL}-8 / \mathrm{ml}$ add basal plus p value). HT29 cell monolayers were therefore pretreated for $1 \mathrm{~h}$ with $2.5-20 \mathrm{mM}$-ASA or 10-100 $\mathrm{MM}$ hydrocortisone followed by addition of the pooled supernatant. After $4 \mathrm{~h}$ treatment, IL-8 release was measured by ELISA. In paralle experiments, IL-8 release was assessed in the presence and absence of BAY11-7082, an NFKB inhibitor. Results: 5-ASA dose-dependently inhibited the IL-8 response to a pooled E coli supernatant, with reductions of $22+15 \%$ at $2.5 \mathrm{mM}, 45 \pm 17 \%(5 \mathrm{mM}), 51+11 \%(10 \mathrm{mM})$ and $97 \pm 1 \%$ at $20 \mathrm{mM}$ (all $\mathrm{p}<0.01 ;$ ANOVA). Hydrocortisone only caused significant inhibition of the IL-8 response $(49 \pm 13 \%, \mathrm{p}=0.01)$ at a supra-therapeutic concentration $(100 \mu \mathrm{M})$. This is in keeping with the fact that inhibition of NFKB, a principal target for corticosteroids, only had a modest effect on IL-8 release (mean reduction $29+23 \%, \mathrm{n}=33$ ). A parallel study showed that the IL-8 response was largely MAPkinase-mediated (submitted). In a separate experiment, 5-ASA $(5 \mathrm{mM})$ and hydrocortisone $(100 \mu \mathrm{M})$ in combination inhibited IL- 8 response by $82 \pm 7 \%$, compared with $38 \pm 8 \%$ for 5 -ASA alone and $51 \pm 6 \%$ for hydrocortisone alone $(\mathrm{p}<0.001)$. Conclusions: 5 -ASA, at therapeutic concentrations, markedly inhibits the release of IL-8 by colon epithelial cells in response to mucosal E. coli whereas hydrocortisone only has a significant inhibitory effect at concentrations almost 20 -fold greater than are likely to be seen therapeutically. This confirms the epithelial cell as the target for mesalazine (JEM 2005 201:1205-15) but implies that other cell-types probably represent therapeutic targets for corticosteroids in IBD.

\section{M1652}

Over-Expression of a 72-Kda Heat Shock Protein Protects Rat Gastric Mucosal Cells from H2O2-Induced Necrosis and Apoptosis Tamotsu Matsuhashi, Michiro Otaka, Sumio Watanabe

Background; We have demonstrated the importance of a 72-kDa heat shock protein (HSP72) for gastric and colonic mucosal protection in vitro and iv vivo experiments. However, the problem of our previous studies was that it was impossible to exclude a possible contribution of other cytoprotective factors other than HSPs, since systemic stress including heat shock (hyperthermia), chemicals or stress-related hormones had to be applied to induced HSP in those experimental systems. Therefore, in order to solve this problem, we have established HSP72-overexpressing gastric epithelial cells by transfecting full length of human hsp 72 CDNA, and we successfully cloned HSP72-highly expressed gastric epithelial cells that expressed approximately $350 \%$ of HSP72 (in protein level) compared with vector-contro cells. We examined the cytoprotective function of these cells against $\mathrm{H} 2 \mathrm{O} 2$-induced necrosis and apoptotic cell death. Methods; The RGM-1 cells were transfected with the pBK-CMV vector alone (pBK-CMV12) or transfected with the vector containing full length of cDNA of human hsp72 (7018-RGM1). The cationic liposomal-mediated method (lipofection method) was employed for transfection and the resistance to geneticine (G418) was used for selection of successfully transfected cells. To examine anti-necrotic ability, these cells were challanged with high concentration of $\mathrm{H} 2 \mathrm{O} 2(0-50 \mathrm{mM})$ and the cell viability was measured by WST-1 assay. To examine anti-apoptotic ability, these cells were treated with low concentration of $\mathrm{H} 2 \mathrm{O} 2(0-1 \mathrm{mM})$ for 24 hours and the cell viability was measured in the same manner. Also, to determine apoptotic cell damage by morphological changes, fluorescence staining for DNA was performed using Hoechst 33342 to quantify histonecomplexed DNA fragments. Further, we assessed the levels of Poly-ADP-Ribose-Polymerase (PARP) using western blot. Results; $\mathrm{H} 2 \mathrm{O} 2$ induced necrotic and apoptotic cell injury to pBK-CMV12 in a concentration-dependent manner. This phenomenon was significantly diminished in 7018-RGMl compared to pBK-CMV12. The numbers of apoptotic cells were reduced in 7018-RGMl morphologically and suppressed significantly genomic DNA fragmentation. The detection of a Western blot of PARP cleavage fragments was suppressed in 7018-RGM-1 compared to pBK-CMV12. Conclusion; These results suggest that HSP72 plays an important role in protecting gastric epithelial cells against oxidant stress-induced cellular apoptosis and necrosis

\section{M1653}

Flagellin Is the Essential Factor of E. Coli Nissle Mediated Human $\beta$-Defensin2 Expression in Caco-2 Cells

Miriam Schlee, Sabine Nuding, Artur Altenhoefer, Tobias A. Oelschlaeger, Jan Wehkamp, Eduard F. Stange, Klaus Fellermann

Background: The probiotic strain E. coli Nissle is established in maintaining remission in patients with ulcerative colitis as an alternative to 5-ASA. However, the mechanisms of action are still enigmatic. Recently, we have reported its capacity to stimulate the intestina innate defence by inducing the antimicrobial peptide hBD-2 in epithelial cells (Wehkamp et al., Infect Immun 2004). The aim of the present investigation was to identify the bacterial factor responsible for hBD-2 induction. Methods: Different E. coli Nissle deletion mutants encompassing known fitness factors (e.g. microcin $(\Delta S K 22 D)$, fimbriae ( $\Delta$ fim $\Delta$ foc), siderophore $(\Delta \mathrm{HPI})$, flagellin $(\Delta \mathrm{fliC})$, sigma factor $(\Delta \mathrm{fli} \mathrm{A})$, hook $(\Delta \mathrm{flgE}))$ were constructed. Bacteria were grown to mid-log phase and heat inactivated. $\mathrm{CaCo}-2$ cells were incubated with bacteria culture supernatants for $4.5 \mathrm{~h}$ or $6 \mathrm{~h}$, respectively and the hBD-2 induction determined by reporter gene assay or RT-PCR. Flagellin was isolated from different strains including E. coli Nissle, Salmonella enteritidis and the uropathogenic strain CFT073 (same flagellin sequence as E. coli Nissle). In addition, the flagellin mutant strains were complemented 
with the deleted genes to verify the flagellin dependent hBD-2 induction. Furthermore, the effect of an Hl-flagellin antiserum on hBD-2 inducing capacity of E. coli Nissle flagellin and supernatant was investigated. Results: E. coli Nissle supernatant was more potent to induce hBD-2 expression in $\mathrm{CaCo}-2$ cells than the bacterial pellet. Therefore, we searched possible secreted or shedded factors originating from E. coli Nissle. Of several mutants tested, only those with a deficient flagellin, hook or sigma factor expression, all necessary for proper flagellin synthesis, were unable to stimulate hBD-2 in CaCo-2 cells. Complemented mutants regained their inducing capacity comparable to wild-type E. coli Nissle. Isolated flagellins from E. coli Nissle, Salmonella enteritidis and CFT073 were potent inducers of hBD-2 mRNA expression. Coincubation experiments with Hl-flagellin antiserum reduced the hBD-2 induction of E. coli Nissle supernatant or flagellin by $70 \%$ or $50 \%$, respectively. Conclusion: E. coli Nissle strains ( $\Delta \mathrm{fliC}, \Delta \mathrm{fli} A$ and $\Delta \mathrm{flgE}$ ) with a loss of flagellin expression fail to induce hBD-2. This is in accordance with the finding that isolated flagellin is capable of inducing hBD-2 and identifies flagellin as the sole factor responsible for the E. coli Nissle mediated hBD-2 gene expression in Caco-2 cells. Flagellin with its immunomodulatory effects may be the mechanistic principle for remission maintenance in ulcerative colitis.

\section{M1654}

Helicobacter Pylori Induction of Matrix Metalloproteinase-7

Seth R. Ogden, Aime T. Franco, Uma S. Krishna, Mary K. Washington, Dawn A. Israel, Barbara Fingleton, Lynn M. Matrisian, Howard C. Crawford, Richard M. Peek

Helicobacter pylori infection is the strongest identified risk factor for distal gastric cancer. Strains that possess the cag pathogenicity island $\left(\mathrm{Cag}_{+}\right)$are known to augment this risk. Matrix metalloproteinase-7 (MMP-7), an epithelial cell-derived MMP that is induced by bacterial contact, is overexpressed within human gastric adenocarcinoma specimens and enhances tumor formation in rodents. We have shown that H. pylori cag+ strains are associated with MMP-7 protein expression in vivo and induce expression during co-culture with gastric epithelial cells in vitro. This induction is dependent on activation of ERK $1 / 2$ by specific components within the cag island. In addition, aberrant activation of beta-catenin, a multifunctional host molecule that plays an important role in regulating intercellular adhesion, has been implicated in transcriptional activation of target genes involved in carcinogenesis. Beta-catenin activation leads to its nuclear accumulation and the formation of heterodimers with the transcription factor lymphocyte enhancer factor/T-cell factor (LEF/TCF). Binding sites for this complex have been identified in the promoter region of MMP-7, implicating a role for MMP-7 as a beta-catenin target gene. To discern the level at which H. pylori regulates MMP-7, we determined whether induction of MMP-7 expression by $H$. pylori was transcriptionally regulated. Further, we examined the response of beta-catenin within gastric epithelial cells to co-culture with H. pylori. Methods: Following co-culture of AGS cells with the highly carcinogenic $H$. pylori cag+ strain 7.13 production of MMP-7 RNA transcripts were quantified by real time RT-PCR. In addition, immunofluorescence was used to determine the cellular localization of beta-catenin in response to co-culture with 7.13. Results: Transcription of MMP-7 was induced within two hours of bacterial-AGS cell contact and remained elevated for as long as 24 hours $(\mathrm{p}<0.005)$, with an increase as high as 4.5 -fold at 6 hours ( $p<0.003$ ). Nuclear localization of beta-catenin was observed in $45 \%$ of AGS cells co-cultured with 7.13 by 6 hours post infection, compared to $8 \%$ of untreated cells $(\mathrm{p}<0.05)$ Conclusions: The H. pylori cag+ strain 7.13 activates transcription of MMP-7, and this induction correlates with movement of beta-catenin to the nucleus, which may explain in part the increased gastric cancer risk associated with $c a g+H$. pylori

\section{M1655}

Carcinogenetic Potential of the WSR Caga Region of Helicobacter Pylor Carlo-Federico Zambon, Daniela Basso, Alessia Stranges, Alessandra Falda, Michela Fasolo, Laura Del Giudice, Graziella Guariso, Claudio Belluco, Francesco Di Mario, Filippo Navaglia, Eliana Greco, Paola Fogar, Massimo Rugge, Giuseppe Basso, Mario Plebani

Within the $H$. pylori virulence factor CagA a different number of repeats of the EPIYA, the tyrosine phosphorylation site of CagA, might be found in different strains. A higher phosphorylated CagA interferes much more with intracellular signalling and it is suggested to worse the outcome of $H$. pylori CagA positive infected patients. The aim of this study was to ascertain whether a different number of the first repeat region (FR) or of the second repeat region (WSR) repeats of the cagA gene, which comprise the sequence encoding for the EPIYAs, correlates with different inflammation patterns and outcomes of $H$. pylori cagA positive infected patients. We studied Italian patients with antral $(n=18)$ or diffuse $(n=16)$ gastritis, with duodenal ulcer $(n=16)$ and with histologically proven non-cardia gastric cancer $(n=25)$. All patients were infected by cagA positive $H$. pylori strains, the diagnosis being made on the basis of histology, culture and genetic analysis (ureA, cagA, $s$ and $\mathrm{m}$ vacA) of H. pylori isolates. FR and WSR regions were PCR analysed using isolates from the antrum; the number of repeats was evaluated on the basis of amplicons dimension (agarose gel electrophoresis). In a subset of 28 isolates, 14 from NCGC, the whole FR and WSR region was sequenced. In all patients the FR region contained one repeat. The WSR repeats ranged from 0 to 3. Some patients $(\mathrm{n}=22)$ were co-infected by strains with a different number of WSR repeats. H. pylori strains with less than 2 WSR repeats had more frequently the $\mathrm{ml}$ vacA allele $(X 2=5.88, p<0.05)$ and the infection was correlated with more severe corpus activity $(X 2=13.74, p<0.005)$. NCGC was associated with infections caused by $H$. pylori strains with two WSR repeats or with co-infecting H. pylori strains exerting two or more WRS cagA repeats, while those with less than two repeats were correlated with duodenal ulcer $(X 2=12.45, \mathrm{p}<0.05)$. No significant association was found between nucleotide sequences and disease diagnosis and degree of inflammation. In conclusion the FR region of cagA in Italian isolates does not vary. The variations of cagA WSR region are probably involved in causing different disease outcomes in patients infected by apparently similar H. pylori strains: those with less than two repeats mainly causing duodenal ulcer, those with two or more repeats probably being more carcinogenetic.

\section{M1656}

Gut Mucosal Immunity Against Core Types of LPS of Escherichia Coli- a Novel Strategy for Oral Vaccine

Syed S. Hoque, Ian R. Poxton

Background: Gut mucosa is an interface and acts as a barrier against infectious organisms or their products, gaining access to the systemic circulation. In sepsis syndrome,the source of LPS/Endotoxin is debated but still gut considered as one of the prime source. LPS is an essential component of the outer membrane of all Gram-negative bacteria including E.coli . Structurally, most types of LPS are composed of three distinct region: the lipid A, the core oligosaccharide and the O-polysaccharide.The LPS core regions are conserved, with a little variability in sugar molecules in outer core, it can be classified in 4 chemo types (R1-R4). Studies show that the antibodies to lipid A or core oligosaccharide should be more broadly cross-protective than antibodies directed against the more variable outer core and the Oantigen portion of LPS.It is essential to examine the mucosal antibody response against various core types of LPS of E.coli. These information would be highly relevant in oral vaccines against E.coli infection. Methods: 11 healthy male control from Dhaka, Bangladesh, median age 24(18-32 years and 12 healthy control (4 male, 8 female ) median age 39 (2348) from Edinburgh, Scotland were studied. All controls underwent Whole gut lavage using polyethylene glycol electrolytes-based solution drank at a standard rate of one litre/hour. The first clear effluent was collected. An ELISA technique was developed to measure IgA antibody against various core types LPS of E.coli. Results: There were IgA antibody responses against the whole range of available core types (R1,R2,R3,\&R4). In Edinburgh groups the antibody responses were in descending order of magnitude (Rl>R2>R3>R4). In Dhaka groups the order was $(\mathrm{R} 1>\mathrm{R} 3>\mathrm{R} 2>\mathrm{R} 4)$. There was a generalised increase in IgA response against whole range of core types in Dhaka control groups as compared to Edinburgh. However, significant differences were observed in R1, R3, \& R4 $(\mathrm{P}<0.021, \mathrm{P}<0.032$ \& $\mathrm{P}<0.015$ respectively. Conclusion:This study shows for the first time that there is mucosal IgA antibody response present against the specific LPS core types of E.coli. As several gram negative-enteric pathogen share closely related LPS core [ eg. E.coli O157:H7 and Shigella flexneri (R3), Shigella sonnei (R1)] and there was some constant association between $\mathrm{O}$ serotypes and core types, it is thus possible to develop orally administered mucosal vaccine targeting these conserved core types of LPS. This vaccine would be more broadly crossprotective against a wide range of gram-negative pathogens. However, further study will be needed to know the mechanisms that evokes immune response.

\section{M1657}

Therapeutic Effect of the Immunomodulator Glatiramer Acetate On Dextran Sulfate Sodium-Induced Experimental Colitis

Rina Aharoni, Basak Kayhan, Ruth Arnon

glatiramer acetate (GA, Copaxone, Copolymer 1), an approved drug for the treatment of multiple sclerosis, acts by induction of Th2 cells and a Th1 to Th2 cytokine shift. Recently we demonstrated that GA ameliorates the clinical signs in an animal model of Crohn's disease - trinitrobenzene sulfonic acid (TNBS) induced colitis. In this study, we have tested the efficacy of GA in another model - dextran sulfate sodium (DSS)-induced colitis. GA treatment significantly suppressed the various manifestations of DSS induced colitis, in the highly susceptible strain C57BL/6, exposed to either one or three cycles of various DSS concentrations and durations. This was manifested by significantly lower weight loss, reduced rectal bleeding, prevention of decrease in colon length, marked reduction of histological damage and improved survival. Combined results of 3 experiments demonstrate in the untreated group a loss of $33 \%$ of the original body weight with full mortality by day 12 , whereas in the GA-treated group (daily injections, $2 \mathrm{mg} / \mathrm{mouse}$ ), only slight weight loss with subsequently regain and full survival. The effect of GA on the immune system was manifested by suppression of the proliferation of local mesenteric lymphocytes in response to syngeneic colon extract and by diminished secretion of the detrimental cytokine tumor necrosis factor alpha. On the other hand, GA induced an elevated secretion of the beneficial cytokine transforming growth factor beta. In conclusion, the innunomodulatory drug Glatiramer Acetate is effective in abrogating DSS induced colitis. 


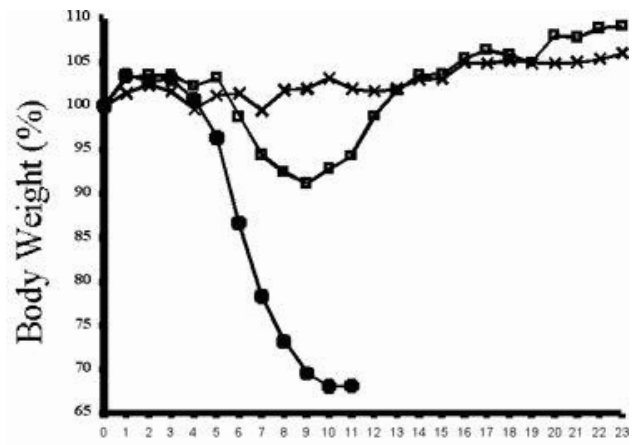

Time (day)

$\longrightarrow$ Naìve $\rightarrow$ Colitis $\rightarrow$ Colitis + GA

\section{M1658}

Protection Against Experimental Colitis By Filamentous Hemagglutinine a of Bordetella Pertussis Is Not Exclusively Mediated By IL-10 Inge L. Huibregtse, Henri Braat, Peter McGuirk, Kingston Mills, Sander J. van Deventer Introduction: Filamentous haemagglutinin A (FHA), a membrane antigen of the pathogenic B. pertussis causes immune evasion of this bacterium by inducing regulatory immune responses that include induction of IL-10. Our previous experiments revealed that FHA is able to ameliorate disease activity in a chronic T-cell mediated model of colitis by the induction of regulatory immune responses. We have now further explored whether IL-10 critically mediates the protective effects of FHA in experimental colitis. Materials and methods: The CD4+CD45RBhigh transfer model of colitis was adopted by using IL10 KO CD4+CD45RBhigh T-cells to induce colitis. Three groups of six mice were injected with CD4+CD45RBhigh T cells; the first group was also injected with CD4+CD45RBlow T cells. The second and third group were injected with respectively 10 ug FHA and $0.9 \% \mathrm{NaCl}$, subcutaneous, every two weeks. Group four was injected with IL-10 KO CD4+CD45RBhigh $\mathrm{T}$ cells and treated with FHA as previously described. After eight weeks, mice were sacrificed and the colon was taken out for histological assessment using routing H\&E staining. Results: Mice treated with FHA showed less weight loss compared to control mice during the entire course of the experiment $(\mathrm{p}<0.01)$, the colon weight was significantly lower $(140+/-1 \mathrm{mg}$. vs. $343+/-2 \mathrm{mg}, \mathrm{p}<0.01)$, the colon length significantly longer $(10.1+/-0.6 \mathrm{~cm}$. vs. 7.9 $+/-0.3 \mathrm{~cm} ., \mathrm{p}<0.01)$ and histological comparison showed a reduced inflammatory score $(1.7+/-0.3$ vs. $3+/-0.5, \mathrm{p}<0.01)$ after treatment with FHA. There was no significant difference between the wt and IL10 KO CD4+CD45RBhigh treated with FHA. Both were protected from the development of wasting disease. Conclusion: FHA of pathogenic B. pertussis is able to ameliorate disease activity in a chronic, $\mathrm{T}$ cell mediated model of colitis. The observation that also IL-10 KO T cells are able to prevent the development of colitis after injection of FHA indicate that IL-10 is dispensable for the induction of immune regulation by FHA.

\section{M1659}

Sirna Therapy of Inflammatory Bowel Disease (IBD)

Patricia Cristofaro, Amana Nasir, Tamako Konkin, Virginia Hovanesian, Paul R. Monfils, Murray B. Resnick, Bharat Ramratnam, Steven F. Moss

RNA interference (RNAi) mediated by single- or double-stranded RNA can silence specific genes through post-translational and post-transcriptional mechanisms. The therapeutic potential of this technology is currently being explored. Increasing understanding of the molecular basis of IBD affords opportunity for highly targeted therapy - especially for luminally delivered agents. Aim: To examine in detail the gene silencing durability and specificity of mucosal RNAi and its therapeutic potential in an animal model of IBD. Methods: Acute colitis was induced in C57BL/6 mice with dextran sodium sulfate (DSS) on days 0 through 5. siRNA targeting murine TNF- $\alpha$ or lamin A/C (expressed constitutively in epithelial cells) or control siRNA's (irrelevant, scrambled or mismatched) were administered intrarectally on days 0 and 2. Mice were sacrificed at days 0-10. Colitis severity was assessed histologically (Murano, Clin Exp Immunol 2000;120:51) and mucosal transcript levels evaluated by real-time PCR and Northern blot. Fluorescein-tagged siRNA was localized by confocal microscopy. Non-specific systemic effects were explored with a commercial mouse interferon response microarray (SuperArray). Results: Naked siRNA administration had no effect on mucosal gene expression or disease amelioration. In normal mice (no DSS treatment) $20 \mathrm{nmol}$ lamin A/C siRNA as a liposomal preparation [Lipofectamine 2000 (Invitrogen) with OptiMEM (Invitrogen)] at $4 \mathrm{nmol}$ siRNA/mouse produced lamin A/C knockdown persisting for 7 days, with maximal effect (70-85\% knockdown) on days 2 and 4, and recovery by day 10. Confocal microscopy showed fluorescein-tagged siRNA was taken up throughout the mucosa with penetration into the submucosa. In mice receiving DSS, colonic TNF- $\alpha$ mRNA levels were 58-fold increased compared with normal controls but only 5.5-fold increased in mice who received liposomal TNF- $\alpha$ siRNA intrarectally $(p<0.01$ ). Although severe colonic inflammation (grade 4) was evident in all mice treated with DSS, those treated with DSS and siRNA had a mean grade of 1.9 in the left colon $(\mathrm{p}<0.003)$. siRNA treatment had no appreciable effect on levels of $>100$ interferon-related genes in mucosal tissue compared to liposome-only treated animals. Conclusion: Intrarectal administration of siRNA produces a highly specific, potent and transient reduction in mucosal gene expression. Rectal TNF $\alpha$ siRNA markedly improves left colonic histology without promoting nonspecific interferon responses. These results provide proof of concept for the development of mucosally-delivered siRNA preparations to interrupt/inhibit the inflammatory cascade responsible for IBD.

\section{M1660}

Saccharomyces Boulardii Attenuates Mucosal Inflammation and Maintains Colonic Barrier Function in Citrobacter Rodentium-Induced Colitis in Mice Xiujuan Wu, Lee Boyer, Bruce A. Vallance, John Walker, Karen L. Madsen, Alison M. Buchan, Kevan Jacobson

Saccharomyces boulardii, a non-pathogenic yeast, has received increasing attention in prevention and treatment of infectious and inflammatory bowel diseases. While studies have begu to explore mechanisms of action of Saccharomyces boulardii further studies are required to elucidate its action. The aim of the present study was to examine the ameliorating effects of Saccharomyces boulardii on mouse colitis induced by Citrobacter rodentium in vivo and to further explore the relationship in vitro. Methods: C57BL/6 mice received $2.5 \times 10^{8} \mathrm{C}$ rodentium by gavage on day 0 , followed by $\mathrm{S}$. boulardii $\left(25 \mathrm{mg} / \mathrm{ml} ; 5 \times 10^{8}\right.$ live cells gavaged twice daily from day 2 to day 9 . Animals weights were monitored every second day and all animals were sacrificed on day 10 . The colons were removed and assessed for epithelial barrier function, histology and myeloperoxidase activity. For epithelial barrier function, tissues were mounted in Ussing chambers for measurement of permeability as assessed by mannitol movement and cAMP-dependent chloride secretion in response to forskolin. The antimicrobial effects of S. boulardii were assessed both in vitro and in vivo. Results: When tested in vitro, S. boulardii had significant bacteriocidal effect. Moreover, S. boulardii significantly reduced the number of adherent colonic C. rodentium by $82 \%$. C. rodentium infection was associated with mild diarrhea, significant decrease in body weight by day 4 post-infection, ( $31 \%$ decrease from baseline) with partial recovery by day 10 marked crypt hyperplasia, transmural infiltration on neutrophils and mononuclear cells and a disruption in epithelial barrier function. Treatment with S. boulardii ameliorated the disease associated with $\mathrm{C}$. rodentium infection. S. boulardii significantly attenuated the weight loss (13\% decrease from baseline at day 4) with full recovery by day 10, ameliorated the crypt cell hyperplasia $(234.7 \pm 7.2 \mu \mathrm{m}$ vs. $297.8 \pm 17.6 \mu \mathrm{m})$, the histological damage score $(0.5 \pm 0.2$ vs. $1.25 \pm 0.5)$ and elevated myeloperoxidase activity of infected mice $(2.1 \pm 0.4 \mathrm{U} / \mathrm{mg}$ vs. $4.7 \pm 0.9$ $\mathrm{U} / \mathrm{mg}$ ). Moreover, oral administration of $\mathrm{S}$. boulardii attenuated the increase in mannitol flux $\left(17.2 \pm 5.0 \mathrm{~nm} / \mathrm{cm}^{2} / \mathrm{hr}\right.$ vs. $31.2 \pm 8.2 \mathrm{~nm} / \mathrm{cm}^{2} / \mathrm{hr}$ ) and normalized the response to forskolin $\left(98.0 \pm 11.6 \mu \mathrm{A} / \mathrm{cm}^{2}\right.$ vs. $\left.63.2 \pm 8.3 \mu \mathrm{A} / \mathrm{cm}^{2}\right)$ in infected mice colons. Conclusion: This study demonstrated that $\mathrm{S}$. boulardii ameliorated the sequelae associated $\mathrm{C}$. rodentium infection through mechanisms that included inhibition of bacterial growth, reduction of mucosa inflammation and maintenance of colonic epithelial barrier integrity. S. boulardii may be beneficial in the treatment of infectious diarrheal illnesses and inflammatory bowel diseases.

\section{M1661}

22-Ene-25-Oxa-Vitamin D: A New Vitamin D Analogue with Profound Immunosuppressive Capacities in TNBS-Colitis

Carolin Daniel, Heinfried H. Radeke, Ulrich Zuegel, Juergen M. Stein

Background \& Aims: In addition to its well-established role as a regulator of calcium and bone metabolism, calcitriol has been established as a prominent regulator of immune cell functions. However, its clinical use as an immunosuppressant is limited because of the hypercalcemic toxicity occurring after systemic application. The aim of the following study was to investigate the in vivo immunomodulatory capacities of 22-ene-25-oxa-vitamin D (ZK156979), a new low calcemic vitamin D analogue, in TNBS-colitis in mice. Methods: A rectal enema of TNBS [100 mg/kg body weight (BW)] was applied to male Balb/c mice and calcitriol $[0.2 \mu \mathrm{g} / \mathrm{kg}]$ or ZK156979 [0.2 or $2.0 \mu \mathrm{g} / \mathrm{kg}]$ were administered intraperitoneally from day $0-3$ following the instillation of the haptenating agent. The study is conforming to the Guiding principles in the care and use of animals and was performed under approval of the ethical committee of Darmstadt/Germany (F134/03). Body mass and clinical activity score of colitis were determined daily. Cytokines (TNF- $\alpha$, IFN- $\gamma$, IL-10, IL-4) in colon homogenates were determined by immunoassay. Colonic myeloperoxidase (MPO)-activity was analyzed as a marker for neutrophil infiltration. Serum calcium was analyzed photometrically. Results \& Conclusion: Treatment with ZK156979 reduced the severity of TNBScolitis abrogating body weight loss, diarrhea, and macroscopic intestinal inflammation with similar potency compared to calcitriol. The therapeutic effects of ZK156979 were associated with a downregulation of TNF- $\alpha$ and MPO-activity, while IL-10 and IL-4 were induced. Thus the member of novel low calcemic vitamin D analogues ZK156979 may provide a considerable therapeutic potential in T-cell mediated diseases including inflammatory bowel disease.

Results 22-ene-25-oxa vitamin D (ZK156979) 


\begin{tabular}{|c|c|c|c|c|c|}
\hline \multirow{2}{*}{$\begin{array}{l}\text { Parameters } \\
\text { Treatments }\end{array}$} & KO & \multicolumn{4}{|c|}{ TNBS } \\
\hline & $\begin{array}{l}\text { untreated } \\
\text { control }\end{array}$ & untreated & $\begin{array}{l}0.2 \mu \mathrm{g} / \mathrm{kg} \\
\text { calcitriol }\end{array}$ & $\begin{array}{l}0.2 \mu \mathrm{g} / \mathrm{kg} \\
\mathrm{ZK} 156979\end{array}$ & $\begin{array}{c}2 \mu g / k g \\
\text { ZK156979 }\end{array}$ \\
\hline $\begin{array}{l}\text { Delta Body weight } \\
{[\% \text { of day } 0]}\end{array}$ & $+0.5 \pm 0.1$ & $-17.6 \pm 1.6$ & $-12.1 \pm 2.9 \mathrm{c}$ & $-13.4 \pm 1.7 \mathrm{c}$ & $-6.9 \pm 1.1 \mathrm{c}$ \\
\hline Clinical activity score & $0.2 \pm 0.1$ & $9.4 \pm 0.7$ & $7.3 \pm 2.0 \mathrm{c}$ & $7.4 \pm 0.7 \mathrm{c}$ & $4.3 \pm 0.4 \mathrm{c}$ \\
\hline $\begin{array}{c}\text { Colon weight day } 3 \\
{[\mathrm{mg}]}\end{array}$ & $150.3 \pm 7.4$ & $327.4 \pm 7.8$ & $258.4 \pm 17.9 \mathrm{c}$ & $271.6 \pm 12.1 \mathrm{c}$ & $210.1 \pm 9.1 \mathrm{c}$ \\
\hline $\begin{array}{l}\text { TNF- } \alpha[p g / m g \\
\text { protein] }\end{array}$ & $7.7 \pm 2.8$ & $100.2 \pm 17.3$ & $61.0 \pm 6.5 \mathrm{a}$ & $68.4 \pm 4.3 \mathrm{a}$ & $36.9 \pm 5.7 \mathrm{a}$ \\
\hline $\begin{array}{l}\text { IFN- } \gamma[\mathrm{pg} / \mathrm{mg} \\
\text { protein] }\end{array}$ & $11.3 \pm 2.7$ & $87.7 \pm 11.2$ & $34.7 \pm 6.5 \mathrm{a}$ & $42.8 \pm 6.5 \mathrm{~b}$ & $21.7 \pm 8.5 \mathrm{c}$ \\
\hline IL-10 [pg/mg protein] & $3.6 \pm 0.6$ & $3.3 \pm 0.2$ & $6.7 \pm 0.6 \mathrm{a}$ & $4.8 \pm 0.3 \mathrm{a}$ & $8.5 \pm 0.7 \mathrm{~b}$ \\
\hline IL-4 [pg/mg protein] & $3.1 \pm 0.6$ & $2.9 \pm 0.2$ & $5.9 \pm 0.4 \mathrm{~b}$ & $5.1 \pm 0.3 \mathrm{a}$ & $8.6 \pm 0.5 \mathrm{~b}$ \\
\hline $\begin{array}{c}\text { MPO-activity } \\
\text { [Units/g tissue] }\end{array}$ & $0.2 \pm 0.1$ & $11.6 \pm 1.5$ & $7.8 \pm 0.2 \mathrm{c}$ & $8.6 \pm 1.5 \mathrm{c}$ & $3.8 \pm 1.0 \mathrm{c}$ \\
\hline
\end{tabular}

$a=P<0.05 ; b=P<0.01 ; c=P<0.001$ vs. TNBS-treated mice.

\section{M1662}

Administration of Exogenous Interleukin-18 Prevents the Dextran Sodium Sulfate-Induced Colitis in Mice

Mana Mitamura, Hiroyuki Higashiyama, Lihua Shan, Toshihiko Kaise, Hideo Kikkawa Mine Kinoshita

BACKGROUND \& AIM Interleukin-18 (IL-18) is one of the key mediators that play important roles in host defense. IL-18 is a unique cytokine involved in both the destructive pathway through induction of interferon- $\gamma$ and the compensatory pathway through induction of Th2 cytokines and prostanoids via cycloxygenase-2 (COX-2). In inflammatory bowel disease (IBD) patients, IL-18 is highly expressed in the inflamed mucosa, however, its pathophysiological role in IBD has not been elucidated well. COX-2 is also highly expressed in the epithelium in IBD patients, and it has been reported to play a crucial role in the defense of the intestinal mucosa, and in the restitution and the repair of injured tissue via hepatocyte growth factor (HGF) production. In the present study, to elucidate the therapeutic potential of IL-18 in IBD, we examined the effect of exogenous IL-18 on clinical symptoms, histopathological features, and COX-2 and HGF mRNA expression in dextran sodium sulfate (DSS)induced colitis model in mice. METHODS Acute colitis was induced by ingesting 3\% DSS in drinking water from day 0 to 5 in BALB/c mice. Murine IL-18 (0.3 and $1 \mu \mathrm{g} / \mathrm{mouse} /$ day) was administered intraperitoneally from day 0 to 9 . The severity of colitis was evaluated by scoring the clinical symptoms, such as loss of body weight, stool consistency and occult blood in the stool, and the histopathological features in the colon. Gene expression of COX2 and HGF in the colon was analyzed by quantitative real-time PCR (TaqMan). RESULTS Severity of clinical symptoms induced by DSS was gradually increased and reached at peak on day 7. Exogenous administration of murine IL-18 ( $1 \mu \mathrm{g} /$ mouse/day) suppressed the development of clinical symptoms, especially body weight loss and occult blood in the stool in DSS-treated mice. Both COX-2 and HGF mRNA were up-regulated and reached at peak on day 7 , in well correlation with the development of clinical symptoms. In IL-18-treated mice, the up-regulation of COX-2 and HGF mRNA were accelerated in comparison with vehicle-treated mice: on day 5, the levels of COX-2 and HGF mRNA expression in the IL18-treated group were increased over 5.4 and 1.7 times of those in vehicle-treated group, respectively. Histopathological analysis on day 10 showed that the mucosal epithelial damage in the colon induced by DSS was suppressed by IL-18 treatment. CONCLUSION IL-18 attenuated the DSS-induced clinical symptoms and histopathological alteration in the colonic tissue. Up-regulation of COX-2 and HGF accelerated by IL-18 probably contribute to the maintenance of intestinal mucosal integrity, resulting in the prevention of DSS-induced colitis in mice.

\section{M1663}

Preventive Effect of Nimesulide On Colorectal Carcinogenesis in Experimental Murine Ulcerative Colitis

Takuya Inoue, Mitsuyuki Murano, Yosuke Abe, Eijiro Morita, Naoko Murano, Shingo Yasumoto, Ken Toshina, Takashi Nishikawa, Kentaro Maemura, Ichiro Hirata, Ken-Ich Katsu

Background: One of complications of ulcerative colitis (UC) is an increased risk for development of cancer of the colon and rectum, and the incidence of colorectal cancer increases as the region and duration of the disease increase. Cyclooxygenase- 2 (COX-2) inhibitor is known to suppress sporadic colorectal cancer, but preventive effect of selective COX-2 inhibitor in UC- associated neoplasia is still unknown. In the present study, we investigated preventive effect of a selective COX-2 inhibitor on colorectal carcinogenesis in experimental murine UC. Methods: Chronic colitis was induced in mice by four cycles of administration of dextran sulfate sodium (DSS) (i.e., 5\% DSS for 7 days and distilled water for the following 14 days), and the mice were sacrificed 120 days after the end of the fourth cycle. The mice were divided into the following five groups: Group A, fed only basal diet served as a disease control; Group B, received a diet mixed with $400 \mathrm{ppm}$ of nimesulide (NIM), a selective COX-2 inhibitor, during the whole period; Group C, received a diet mixed with 400ppm of NIM during the four cycles of DSS administration; Group D, received a diet with 400ppm of NIM for 120 days from the end of the fourth cycle; Group E, received no agents including DSS, served as a normal control. Results: The incidence of dysplasia and/or cancer was $28.0 \%, 15.0 \%, 11.8 \%, 6.7 \%$ and $0 \%$ in Groups A-E, respectively. In Group D, NIM significantly suppressed the occurrence of dysplasia and/or cancer $(\mathrm{P}<0.05)$. Tumor size in Groups A-D was $4480 \pm 469.9 u \mathrm{~m}, 2560 \pm 416.3 \mu \mathrm{m}, 4100 \pm 70.7 \mu \mathrm{m}$ and $2350 \pm 106.1 \mu \mathrm{m}$, respectively. Strong COX-2 expression was immunohistochemically found in cancer and dysplastic lesions in the colon of the disease control mice, but diffusely weak COX-2 expression was also found in the residual colon (i.e., lesion-free colon) of the disease control mice. Mucosal

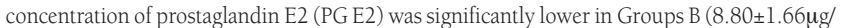
g protein) and D $(8.70 \pm 2.31 \mu \mathrm{g} / \mathrm{g}$ protein) than in Group A $(15.19 \pm 1.75 \mu \mathrm{g} / \mathrm{g}$ protein) Conclusion: NIM, a selective COX-2 inhibitor, showed suppressive effects on development of dysplasia and/or cancer in DSS mice colitis model, which had relevance to long standing $\mathrm{UC}$ in human.

Incidence of dysplasia and/or cancer

\begin{tabular}{|l|c|c|c|c|}
\hline & Negative and/or Indefinite & Dysplasia & Cancer & Dysplasia and/or Cancer \\
\hline Group A $(\mathrm{n}=25)$ & $18(72.0 \%)$ & $7(28.0 \%)$ & $5(20.0 \%)$ & $7(28.0 \%)$ \\
\hline Group B $(\mathrm{n}=20)$ & $17(87.5 \%)$ & $2(10.0 \%)$ & $3(15.0 \%)$ & $3(15.0 \%)$ \\
\hline Group C $(\mathrm{n}=17)$ & $15(88.2 \%)$ & $2(11.8 \%)$ & $2(11.8 \%)$ & $2(11.8 \%)$ \\
\hline Group D $(\mathrm{n}=30)$ & $28(93.3 \%)$ & $2(6.7 \%)$ & $2(6.7 \%)$ & $2(6.7 \%)^{*}$ \\
\hline Group E $(\mathrm{n}=10)$ & $10(100.0 \%)$ & $0(0.0 \%)$ & $0(0.0 \%)$ & $0(0.0 \%)$ \\
\hline
\end{tabular}

*p $<0.05$ vs Group A

\section{M1664}

Pegylated Arginine Deiminase (ADI-PEG) Protects Against DSS-Induced Mice Colitis By Decreasing Macrophage-Derived Nitric Oxide Jian Zhong, Marion Steiner, Sheldon Steiner, Helieh S. Oz, Munira Nasser, Willem J. de Villiers

Background \& Aims: The microbial enzyme arginine deiminase (ADI) metabolizes arginine into citrulline. Several tumor cell lines require arginine for growth and selective elimination of arginine from the circulation is a potential treatment modality. Previously we have shown that ADI covalently attached to polyethylene glycol (PEG) (ADI-PEG) is effective in attenuating intestinal inflammation in dextran sodium sulfate (DSS)-induced colitis in both IL-10 knockout (C57BL/6) and BALB/C mice. Here we further studied the potential mechanism of ADI-PEG treatment on the development of colitis in mice. Methods: Colitis was induced in BALB/c and IL-10-deficient mice by oral ingestion of 3\% for 7 days and assessed by serial weights, survival curves, colon weight and histological grading scores. ADI-PEG (5 IU i.p.) was administered on day 0 and day 5 . We measured levels of the acute phase protein, serum amyloid A (SAA). We also determined levels of Nitric Oxide (NO) and the pro-inflammatory cytokines, IL-6 and IL-12, in serum and supernatants from colonic explants. Furthermore, immunohistochemistry for CD68 (the macrophage specific marker) and iNOS in the colon tissues was performed to evaluate macrophage infiltration and activation. Results: DSS alone induced a severe colitis in BALB/C and IL-10-deficient mice demonstrated by increases in SAA levels and histological damage scores. ADI-PEG treated animals had significantly less severe colitis as manifested by improved survival, increased weights, decreased SAA, NO, and cytokine levels and decreased distal and proximal colonic histological damage scores. Colonic explants from ADI-PEG treated mice produced significant less cytokines compared with mice treated with DSS alone. Immunohistochemical study revealed that administration of DSS caused marked macrophage infiltration in the lamina propria of the colon. The expression of iNOS was up-regulated in the colons of DSS-treated mice but not in those of ADI-PEG treated mice. Conclusion: Macrophage infiltration/activation is one of the major potential mechanisms for the DSS model of colitis. We show that ADI-PEG treatment has a marked dampening and protective effect on the induction of colitis in two mouse models. The reduction in the intestinal inflammation is related to decreased macrophage-derived NO levels by ADI-PEG, as shown by decreased macrophage infiltration and iNOS expression. These data suggest ADI-PEG is of potential therapeutic use in IBD.

\section{M1665}

Angiotensin II Type 1 Receptor Blocker, Candesartan Attenuated Colonic Inflammation in Dextran-Sulfate Induced Colitis Via Inhibition of Madcam-1 Expression

Tsuneya Wada, Makoto Sasaki, Tadayuki Oshima, Naotaka Ogasawara, Satoshi Tanida, Hiromi Kataoka, Takashi Joh

Background: Mucosal cell adhesion molecule-1 (MAdCAM-1) is one of the most important adhesion molecules up-regulated in inflammatory bowel disease (IBD) and is thought to be associated with the onset and progression of IBD. We show in this study that angiotensin II receptor blocker, world-widely used for anti-hypertension treatment could prevent experimental colitis via inhibition of cytokine-induced MAdCAM-l expression. Method: The mRNA expression of angiotensin II type 1 receptor (ATl) was measured under stimulation of cytokine. The role of ATl in the expression of MAdCAM-1 on cytokine stimulated endothelial

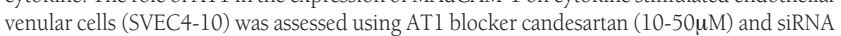
method. The effect of candesartan on p42/44 MAPK and NF- $\mathrm{kB}$ which are intracellular signaling of MAdCAM-1 expression by cytokine was examined. Furthermore, candesartan $(0.4 \mathrm{mg} / \mathrm{kg})$ was injected intraperitoneally in C57/BL6 mouse once a day during administration of $5 \%$ dextran sulfate sodium (DSS) orally for 5 days and colonic inflammatory scales (body weight (BW), disease activity index (DAI) including BW, stool characteristic, and fecal blood on a scale of 0 to 4 , myeloperoxidase (MPO) activity, and histology) were examined. Result: The mRNA expression of ATl on endothelial cell was up-regulated by TNF- $\alpha$ ( $50 \mathrm{ng} /$ $\mathrm{ml}$ ). TNF- $\alpha$ induced MAdCAM-1 expression was significantly prevented by ATl blocker, candesartan $(10-30 \mu \mathrm{M})$ dose-dependently. Furthermore, siRNA mediated knockdown of ATl blocked MAdCAM-1 induction by cytokine. Candesartan had no effects on p42/44 MAPK and NF-KB under TNF- $\alpha$ stimulation. Candesartan recovered BW loss (from 19\% to $13 \%, \mathrm{p}<0.05$ ) and colonic MPO activity (from 0.08 to $0.03, \mathrm{p}<0.05$ ). Control mice receiving DSS showed a significant increase in DAI, which was more attenuated by a treatment with candesartan $(\mathrm{p}=0.05)$. By H.E. staining, colon histology was altered by DSS and led to mucosal thickening, leukocyte infiltration, crypt damage, and loss of goblet cells; however candesartan protected against the DSS induced destruction of the colon and maintained 
epithelial integrity. Immunohistochemical staining of MAdCAM-1 was remarkably enhanced in the mucosa of DSS-treated mice. The increase in MAdCAM-1 staining was reduced but not eliminated by candesartan treatment. Conclusion: Angiotensin II type 1 receptor blocker, candesartan could be useful for the treatment of IBD, however further study is necessary about inhibition mechanism of MAdCAM-1.

\section{M1666}

Inhibition of Dipeptidyl Peptidase Activity in Dpiv Knock-Out Mice and Wild Type Mice Decreases Disease Activity in Experimental Colitis

Roger Yazbeck, Catherine A. Abbott, Gordon S. Howarth, Hans-Ulrich Demuth

Introduction: Glucagon-like peptide-2 (GLP-2(1-33)) is a potent intestinotrophic growth factor, whose activity is limited by dipeptidyl peptidase (DP)-mediated degradation. It has been established that the proteolytic enzyme, DPIV/CD26, cleaves GLP-2(1-33) to the biologically inactive GLP-2(3-33). Work in our lab has suggested that other DPs, including DP8 and DP9, may also cleave GLP-2(1-33). We hypothesised that DPIV knockout mice (DPIV-/-) treated with DP inhibitor, Isoleucine-thiazolidine (Ile-thia), would have an increased GLP-2(1-33) bioavailability, thus decreasing the severity of experimental colitis. Methods: Four groups of DPIV-/- $(n=52)$ and wild-type $(n=49)$ mice were treated twice daily by oral gavage with $0.9 \%$ saline, or a $10 \mathrm{mg} / \mathrm{kg}$ dose of Ile-thia in a 6,9 and 14 -day trial. Mice simultaneously consumed $2 \%$ dextran sulphate sodium for 6-days to induce colitis followed by a recovery period of 3 and 8-days for the 9 and 14-day time points. Inhibitor treatment continued throughout the entire experimental period. Disease severity was assessed daily using a disease activity index (DAI). Pair-wise comparisons with a Holmes post-hoc test between saline and treatment groups were made at each day with a familywise significance level of 0.05 . Results: Colon weight was greater in Ile-thia $(0.19 \pm 0.01 \mathrm{~g})$ treated wild-type mice compared to saline treatment $(0.16 \pm 0.01 \mathrm{~g})$ at day 6 , and this was consistent following correction for bodyweight. At day- 14 colon weight had increased from day-9 in the wild-type group treated with Ile-thia by $40 \%(0.15 \pm 0.02 \mathrm{~g}$ at day- 9 to $0.25 \pm 0.02 \mathrm{~g}$ at day-14, $\mathrm{p}<0.05)$ compared to saline treated wild-type mice, where no significant change was observed $(0.19 \pm 0.04 \mathrm{~g}$ at day- 9 to $0.21 \pm 002 \mathrm{~g}$ at day 9$)$. DPIV-/- and wild-type mice treated with Ile-thia had a significantly lower DAI $(\mathrm{p}<0.05)$ at 5 and 6 -days compared to saline treated DPIV-/- and wild-type mice for the 6 and 9-day trial. Conclusions: Inhibition of DP activity in DPIV-/- and wild-type mice partially ameliorates experimentally induced colitis, indicating that DPIV may not be the sole target of the inhibition. Although total recovery from experimental colitis was not observed, it may be that physiological levels of GLP-2(1-33) are insufficient to promote growth of a damaged intestine. Future work would thus look at mechanisms of elevating physiological GLP-2(1-33) alongside DP inhibitor treatment. DPIV/CD26 also has a role in immune modulation, and this may further explain the observed results. This study provides further evidence that DP inhibition has potential as a novel strategy for the treatment and control of IBD.

\section{M1667}

Photopheresis Induces the Generation of Regulatory T Cells Frank Strobl, Kim Campbell, Janine Huber, Amy Krutsick, Ulrich Thienel, David Peritt

Extracorporeal photopheresis (ECP) involves the clinical reinfusion of autologous apoptotic peripheral blood leukocytes that have been exposed ex vivo to 8-methoxypsoralen (8-MOP) and UVA light. ECP is approved for the palliative treatment of cutaneous T cell lymphoma and has exhibited promising activity in several autoimmune diseases including Crohn's disease (Aliment Pharm Ther 15:1313). The biological mechanism of action of ECP, however, remains unresolved. In animal models, delivery of apoptotic cells has been shown to regulate immune responses through the downregulation of antigen-presenting cell (APC) function, the modulation of cytokines, and the generation of regulatory $\mathrm{T}$ cells. We and others, have shown that activation of naive T cells in the presence of APCs that have engulfed ECPtreated apoptotic cells leads to the generation of a $\mathrm{T}$ cell population that can suppress syngeneic T cell proliferation and function. In the present study, we demonstrate that the direct interaction of ECP-treated peripheral blood mononuclear cells (PBMCs) with naive human $\mathrm{CD} 4+\mathrm{T}$ cells in vitro also promotes a $\mathrm{T}$ cell phenotype with regulatory activity. Transfer of ECP-derived regulatory T cells to a secondary mixed lymphocyte reaction results in a greater than $50 \%$ inhibition of syngeneic responder $\mathrm{T}$ cell proliferation and IFNg production at a Treg:responder ratio of 1:10. In addition, transwell assays demonstrate that inhibition of responder T cells by ECP-derived regulatory $\mathrm{T}$ cells is contact-dependent. The generation of human regulatory T cells by ECP is dependent on the presence of ECP-treated monocytes and can be reversed by the addition of IL-2. These regulatory T cells also proliferate in response to concanavalin-A, but do not express increased levels of Foxp3 or IL-10, nor do they secrete significant levels of the pro-inflammatory cytokines IL-2, IFNg, IL-4, and TNFa. Additional studies are underway to further characterize the phenotype of these regulatory cells and to determine their suppressive capacity in vivo. Regulatory T cells have shown protective effects in an animal model of colitis (J Immunol 170:3939). Two international open label phase II clinical trials in Crohn's patients who failed standard therapy including anti-TNFa are currently underway to determine the efficacy of photopheresis in these patients.

\section{M1668}

Differential Effects of Selective Or Complete PI3K Inhibition in a Murine Inflammatory Bowel Disease Model

Lindsey N. Jackson, Piotr G. Rychahou, Scott R. Silva, B. Mark Evers

Inflammatory bowel disease (IBD) affects the mucosal lining of the gastrointestinal tract; the etiology is unknown and treatment is directed at systemic immunosuppression. The phosphatidylinositol 3-kinase (PI3K) pathway, consisting of $\mathrm{p} 85 \alpha$ regulatory and $\mathrm{p} 110 \alpha$ catalytic subunits, regulates multiple cellular processes, including cell survival. Although PI3K plays an important role in inflammation, the mechanisms remain unclear. The purpose of this study was: (i) to define the role of PI3K and its subunits in the pathogenesis of IBD, and (ii) to determine if complete blockade of PI3K, using wortmannin, or selective inhibition, using siRNA directed to the p85 $\alpha$ subunit, will attenuate the inflammation associated with IBD. METHODS. (i) Female Swiss-Webster mice $(\mathrm{n}=40)$ were randomized into 8 groups of 5 mice to receive normal water or $5 \%$ dextran sulfate sodium (DSS) drinking water to induce colitis. Mice were treated with either vehicle $(5 \% \mathrm{EtOH})$ po or iv, or wortmannin $(0.75 \mathrm{mg} / \mathrm{kg}$ in $5 \% \mathrm{EtOH})$ po or iv every $12 \mathrm{~h}$ for 7 days. Disease activity index score (DAI) was determined daily. At sacrifice, tissues were harvested for H\&E, immunohistochemistry (IHC), and myeloperoxidase (MPO) assay. (ii) Female Swiss-Webster mice $(n=20)$ were randomized into 4 groups of 5 mice to receive normal or 5\% DSS drinking water. Mice were treated with either non-targeting control (NTC) siRNA or p85 $\alpha$ siRNA $(10 \mu \mathrm{g}$ ip, every $24 \mathrm{~h}$ starting day 3 after DSS exposure and continuing until the time of their death). DA was determined daily. RESULTS. (i) Wortmannin-treated mice had significantly more rectal bleeding and higher DAI scores when compared to the vehicle-treated group, with more colonic ulceration and less leukocytic infiltration in the affected colon, reflected by $\mathrm{H} \& \mathrm{E}$ staining and MPO assay, and decreased pAkt expression by IHC. (ii) p $85 \alpha$ siRNA-treated mice had lower DAI scores, improved stool consistency, and virtually no gross rectal bleeding throughout the course of treatment, with improved survival (12.8 days) when compared to NTC siRNA-treated mice ( 9.4 days). H\&E staining revealed increased mucosal regeneration and less ulceration in $\mathrm{p} 85 \alpha$ siRNA-treated mice. CONCLUSIONS. Total inhibition of PI3K with wortmannin decreased leukocyte infiltration but increased mucosal ulceration by inhibiting cell survival and proliferation. In contrast, targeted interference of $\mathrm{p} 85 \alpha$ resulted in increased mucosal cell regeneration, less ulceration, and increased survival in a murine IBD model, possibly by a paradoxical increase in PI3K activity. Selective targeting of specific PI3K pathway components by RNA interference may prove to be a novel and more effective treatment for IBD.

\section{M1669}

The Effect of Novel NF-kB Inhibitor, Dehydroxymethyllepoxyquinomichin in DSS Colitis Model Mice

Tadakazu Hisamatsu, Kumiko Arai, Nobuhiko Kamada, Hiroshi Chinen, Susumu

Okamoto, Kazuo Umezawa, Toshifumi Hibi

Background and Aims: NF-kB is a transcription factor that is rapidly activated in response to pro-inflammatory stimuli, infections and physical and chemical stressors. NF-kB participates in the induction of numerous pro-inflammatory cytokines, chemokines, and adhesion molecules that are important in pathogenesis of inflammatory bowel disease (IBD). In this study, DHME-Q (Dehydroxymethyllepoxyquinomichin), novel NF-kB inhibitor that prevents the nuclear translocation of $\mathrm{p} 65$ specifically, was used to elucidate the role of NF-kB in colitis. Materials and Methods: 1) In vitro, mice CD1lb+ bone marrow derived monocytes were stimulated by LPS with or without DHME-Q. TNF-a in culture supernatant was measured by cytkokine beads array. 2) The effect of DHME-Q on DSS colitis model mice in acute phase. To induce colitis, C57BL/6 mice were given 1.5\% DSS in their drinking

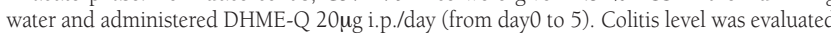
by body weight loss, DAI score, and histological score. 3) The effect of DHME-Q on DSS colitis model mice in recovery phase. To induce colitis, C57BL/6 mice were given $1.5 \%$ DSS in their drinking water from day 0 to 5 . On day 5 , they were switched to regular drinking water and started the administration of DHME-Q 20 $\mu \mathrm{g}$ i.p./day from day 5 to 12 Colitis level was evaluated by body weight loss,DAI score,and histological score. 4) The effect of DHME-Q on rat intestinal epithelial cell (IEC-6) restitution was studied by migration assay. Results: 1) DHME-Q significantly suppressed LPS induced TNF-a production from mice monocyte dose-dependently. 2) In acute phase, body weight loss at day4 $(\mathrm{p}<0.05)$ and DAI score at day5 $(\mathrm{p}<0.05)$ was significantly improved in DHME-Q treated mice. 3) Administration of DHME-Q in recovery phase significantly inclined and delayed the recovery of body weight(control 98\% vs DHME-Q 92.2\% of initial BW at dayl1, p<0.05). 4) DHMEQ inhibited IEC-6 cell restitution in dose dependent manner. Conclusion: 065 specific NF$\mathrm{kB}$ inhibitor, DHME-Q can inhibit pro-inflammatory cytokine production and may suppress inflammation in acute phase, however, in recovery phase, DHME-Q delays the restoration of body weight and inhibited intestinal epithelial cell restitution. Thus, because NF-kB has dual roles both in induction of inflammation and recovery of epithelial cells, careful consideration is necessary to use NF-kB inhibitor as therapeutic target of IBD

\section{M1670}

Selective Inhibition of IKK Prevents THI Triggered Colitis in Vivo Wataru Shibata, Shin Maeda, Keiji Ogura, Tomoya Ohmae, Ayako Yanai, Yoshihiro Hirata, Haruhiko Yoshida, Takao Kawabe, Masao Omata

Background \& Aims: IkappaB kinase (IKK) is a critical regulator for the activation NF-KB, which plays a key role in inflammation. In Crohn's disease (CD), clinical specimens display elevated NF- $\mathrm{\kappa B}$ activity and this may be involved in its pathogenesis. To determine a role of IKK/NF-KB activation in intestinal inflammation, we administered cell-permeable IKK complex inhibitor peptide NBD (NEMO Binding Domain) into TNBS-treated mice, a model of CD. Methods: TNBS-colitis was induced in C57BL/6 mice receiving intracolonic instillation of $200 \mu \mathrm{L}$ of TNBS in $50 \%$ ethanol, whereas control mice received $200 \mu \mathrm{L}$ of water or $50 \%$ ethanol alone. We injected mice intraperitoneally (i.p.) either with vehicle alone (DMSO) or with the wild-type or mutant NBD peptide at 4 hours after TNBS administration and again on day 1 and 2 . The mice were monitored for weight, mortality, colon length, colon histology, and cytokine mRNA expression in colon tissue 7 days after TNBS administration. Mice defected in interleukin- 6 , whose expression is tightly regulated by NF- $\kappa \mathrm{B}$, were also used. Results: Mice treated with wild-type NBD peptide had shown improvement of their weight loss and low mortality compared to those treated with mutant NBD peptide (mortality rate; wild-type vs. mutant, $10 \%$ vs. $60 \%, \mathrm{P}<0.05$ ). Wild-type improved intestinal injury and inflammation determined by microscopic grading 7 days after TNBS administration while mutant peptide did not (score; wild-type vs. mutant, $1.3+/-0.6$ vs. $2.3+/-0.6, p<0.05$ ). Th1 mediated NF-KB downstream genes mRNA expression of colonic homogenates significantly reduced in mice treated with wild-type but not with mutant NBD peptide (TNF- $\alpha$, IL1 $\beta$, IL-12, IFN- $\gamma$, ICAM-1, and IL-6). IL-6(-/-) mice showed less weight loss after TNBS administration, suggesting TNBS colitis partially depends on IL-6. Conclusions: Specific 
inhibition of IKK/NF-KB activation offers an effective therapeutic approach, in part through inhibition of Thl mediated cytokine expression, for treatment of human IBD.

\section{M1671}

Selective Inhibition of Leukotriene A4 Hydrolase Attenuates Tissue Injury, Cytokine Production and Inflammation in Rat Colitis

Paul J. Dunford, Csaba Vargas, Anne Fourie, Brendan J. Whittle

Leukotrienes (LTs), particularly LTB4, formed from arachidonic acid by the 5-lipoxygenase (5-LO) enzyme, are highly active pro-inflammatory lipid mediators, yet inhibition of 5-LO activity has not proved consistently effective in experimental models of colitis or in the therapeutic management of IBD. Moreover, 5-LO inhibitors may also prevent the production of endogenous anti-inflammatory factors such as the lipoxins that promote lesion resolution. Since leukotriene A4 hydrolase (LTA4H) is involved downstream of 5-LO in the biosynthesis of LTB4, we have now explored a more specific approach through selective inhibition of LTA4H and modulation of LTB4 production alone. Thus, we have evaluated the actions of the novel, highly selective LTA4H inhibitors, JNJ 10392980 and JNJ 26993135, that are active in the nanomolar range in vitro, in a hapten-provoked model of acute colitis. Colitis was induced in rats by the intracolonic instillation of trinitrobenzene sulfonic acid (TNBS) and the extent of colonic inflammation assessed after 3 days in terms of macroscopic injury and the colonic levels of the neutrophil marker, myleoperoxidase (MPO) and the inflammatory biomarker cytokines, tumor necrosis factor alpha (TNF $\alpha$ ) and interleukin-6 (IL-6). Oral administration of JNJ 10392980 (30 mg/kg b.i.d) commencing 24h prior to challenge, abolished the fall in body weight following TNBS challenge and significantly reduced the area of macroscopic injury by $57 \%(\mathrm{n}=15 ; \mathrm{P}<0.001)$ while attenuating the TNBS-provoked increase in the colonic levels of MPO, TNF $\alpha$ and IL- 6 by $59 \%, 67 \%$ and $82 \%$ respectively $(\mathrm{P}<0.001$ for each). Likewise, treatment with JNJ $26993135(5-30 \mathrm{mg} / \mathrm{kg}$, b.i.d., p.o.) significantly attenuated the fall in body weight and caused a dose-dependent reduction in all indices of colonic inflammation; thus, the highest dose of JNJ 26993135 which reduced colonic LTB4 levels by $79 \pm 5 \%$, reduced macroscopic injury, MPO, TNFo and IL-6 levels by $49 \%, 48 \%, 63 \%$ and $66 \%$ respectively ( $P<0.01$ for each). These findings with both JNJ 10392980 and JNJ 26993135 suggest that selective inhibition of LTA4H could provide a more specific approach to attenuating the biosynthesis of the chemotactic and pro-inflammatory lipid LTB4 in colitic tissue and invading cells. These novel pharmacological agents reduce the macroscopic, neutrophil and cytokine biomarker indices of experimental colitis and may thus point to a novel strategy for therapeutic intervention.

\section{M1672}

Disease Modifying Effects of Everolimus, Distinct from Immunosuppression, in Cd45rbhigh T Cell-Induced Murine Colitis

Henrietta D. Moore, Amy Martland, Martina Pfann, Marzieh Edjlalipour, Eva Kern, Isabell Buttron

BACKGROUND: We have shown that the mTOR inhibitor, everolimus (EVR) both protects against development of disease and can halt and reverse established severe inflammation in the SCID mouse model of IBD induced by transfer of naive T cells (Gut 2005,54(suppl.VII);A61) Calcineurin inhibition via FK506 protects against disease onset but only at doses with associated toxicity. AIM: To further differentiate the strong anti-inflammatory benefit of EVR in the intestine from its immunosuppressive properties we tested if EVR has prolonged effects after ceasing administration, as recently shown in a rat tumor model. (Cancer Res. 2004,64:252) METHODS: SCID mice were reconstituted with naive (CD4+CD45RbHi) T cells and treated with $1 \mathrm{mg} / \mathrm{kg} / \mathrm{d}$ EVR or $10 \mathrm{mg} / \mathrm{kg} / \mathrm{d}$ FK506 for 7 or 21 days. Mice were euthanized on day 29, serum haptoglobin measured by ELISA, colitis was evaluated histologically and lymphocyte numbers in blood, spleen and mesenteric lymph nodes (MLN) were measured by FACS analysis. Body weight (BW) was measured throughout and a score for diarrhea made at the end. RESULTS: In 2 similar studies with $\mathrm{n}=8$ mice per group placebotreated mice lost a mean \%BW of $23.1 \pm 2.6$ whilst EVR-treated mice lost only $8.8 \% \pm 5.3$ relative to non-transferred controls. In a third study, BW loss was the same in FK506 or placebo-treated mice. Colitis severity in the placebo-treated groups was 7 and 7.4 (out of 8) whilst EVR treatment for only the first $7 \mathrm{~d}$ resulted in reduced mean scores of 5.0 and 4.2 , respectively. EVR treatment for $21 \mathrm{~d}$ resulted in significantly $(\mathrm{p}<0.01)$ reduced mean scores of 2.3 and 0.1 , respectively. In stark contrast, no FK506-treated mice were protected against colitis. Serum haptoglobin in the 7d EVR-treated mice was reduced by 72 and $56 \%$, respectively whilst FK506 treatment, even for 2ld, failed to reduce this acute phase protein. However, a significant reduction of $75.9 \% \pm 4.9$ was measured when this dose of FK506 was given continuously for the entire $28 \mathrm{~d}$. There was no significant reduction in lymphocyte numbers in blood, spleen or MLN after EVR treatment. Diarrhea scores were also lower in $7 \mathrm{~d}$ EVR-treated mice with scores of 4 and 4.8 (out of 8 ) whilst placebo-treated mice scored 5.9 and 6, respectively. CONCLUSIONS: Since the half-life of EVR in the mouse is measured in hours these data suggest that EVR is modifying the early events of disease progression without preventing $\mathrm{T}$ cell expansion. Calcineurin inhibition clearly needs to be kept constant for any protective benefit. Further studies are necessary to elucidate if the EVR effects are mediated via specific factors of the mTOR pathway in intestine, eg the translational activator ribosomal protein $\mathrm{S} 6$ kinase 1

\section{M1673}

Hypnotherapy Decreases Rectal Mucosal Release of Substance P, Histamine and IL-13 in Patients with Active Ulcerative Colitis Joel Mawdsley, David Jenkins, David Rampton

Introduction: Hypnotherapy is effective for functional GI disorders and has been claimed to improve UC. The mechanisms by which hypnotherapy may act are unknown but could involve reductions in the mucosal production of inflammatory neurotransmitters, mast cell mediators and inflammatory cytokines. Aim: To assess the effects of one session of hypnotherapy on the concentrations of substance P (SP), histamine, TNF- $\alpha$ and IL-13 in rectal perimucosal fluid in patients with active UC. Methods: For 50 mins patients with active UC (Simple Clinical Colitis Activity Index $>3$, Baron sigmoidoscopy score $>1$ ) underwent either (i) Hypnotherapy - stress reduction and gut-focused symptom management (ii) Control session - listening to relaxing music. The autonomic response was assessed by pulse and BP every 15 mins. A sample of peri-mucosal fluid was collected before and after each protocol by placing a $7 \times 30 \mathrm{~mm}$ strip of filter paper through a rigid sigmoidoscope in contact with rectal mucosa for 1 minute until wet. The filter paper was incubated in $1 \mathrm{ml}$ Bovine Serum Albumin $(0.3 \%)$, sodium azide $(0.01 \%)$ and Tween $20(0.002 \%)$ in phosphate buffered saline for 24 hours and SP, histamine, TNF- $\alpha$ and IL-13 levels in the buffer measured by ELISA.1 Results: The control protocol caused no changes in any variable (Table). Hypnotherapy reduced pulse rate by median $7 \mathrm{bpm}$, systolic BP by $2 \mathrm{mmHg}$, and concentrations of SP by $81 \%$, histamine by $35 \%$ and IL-13 by $54 \%$. Conclusion: Hypnotherapy reduces rectal mucosal release of substance P, histamine and IL-13, but not of TNF- $\alpha$, in patients with active UC. These changes could provide a basis for a beneficial effect of hypnotherapy in UC. References 1. E. Carty, M. De Brabander, R. M. Feakins, D. S. Rampton, Gut 46, 487$492(2000)$

\begin{tabular}{|c|c|c|c|c|}
\hline & Protocol & $\mathrm{N}$ & Before & After \\
\hline \multirow{2}{*}{ Pulse rate (bpm) } & Hypno & 17 & $76(70-86)$ & $69(63-83)^{*}$ \\
\cline { 2 - 5 } & Control & 7 & $68(67-77)$ & $72(62-76)$ \\
\hline \multirow{2}{*}{ Systolic Bp(mmHg) } & Hypno & 17 & $125(116-132)$ & $123(112-137)^{*}$ \\
\cline { 2 - 5 } & Control & 7 & $134(120-155)$ & $138(112-153)$ \\
\hline \multirow{2}{*}{ Substance P(ng/ml) } & Hypno & 16 & $54(16-621)$ & $10(5-20)^{*}$ \\
\cline { 2 - 5 } & Control & 7 & $72(12-958)$ & $99(12-387)$ \\
\hline \multirow{2}{*}{ Histamine (ng/ml) } & Hypno & 16 & $26(15-34)$ & $17(6-25)^{*}$ \\
\cline { 2 - 5 } & Control & 7 & $14(6-34)$ & $17(2-32)$ \\
\hline \multirow{2}{*}{ TNF-alpha (pg/ml) } & Hypno & 16 & $111(42-252)$ & $140(62-253)$ \\
\cline { 2 - 5 } & Control & 7 & $112(52-231)$ & $137(50-396)$ \\
\hline \multirow{2}{*}{ Il-13(ng/ml) } & Hypno & 16 & $18.7(5.1-99.1)$ & $8.6(5.1-27)^{*}$ \\
\cline { 2 - 5 } & Control & 7 & $18.5(10.9-20.7)$ & $20.3(8.7-24.2)$ \\
\hline
\end{tabular}

* $\mathrm{p}<0.05$ from pre-hypnotherapy value. Median and IQR shown.

M1674

The Timing of Administration of Ro26-2198, a Vitamin D3 Analog, Causes Opposing Effects On Dextran Sulphate Sodium (DSS)-Induced Colitis Alessandro Fichera, Nathaniel Little, Sujatha Jagadeeswaran, Urszula Dougherty, Reba Mustafi, Sonia Cerda, Yanliang Sun, Yan C. Li, Lucas Campbell, Loren Joseph, John Hart, Amy Noffsinger, Marc B. Bissonnette

Introduction. Dextran sulfate sodium (DSS) induces an inflammatory colitis and repeated cycles induce colitis associated colon cancer. Vitamin D analogues are chemopreventive in several experimental models of colon cancer, but limited by hypercalcemia. While vitamin D compounds are anti-inflammatory in other models of inflammation and inhibit proliferation of transformed colonocytes, their effects on DSS-induced colonic mucosal inflammation and normal colonocyte regeneration have not been examined. For these studies we investigated a fluorinated non-hypercalcemic analogue of vitamin D3, Ro26-2198 (Ro26). The aim of this study was to compare the effects of Ro26, administered during or after DSS treatment, on clinical colitis and crypt loss. Methods. Male A/J mice were divided in three groups: controls, receiving standard mouse diet alone; group 2, receiving Ro26 $(0.05 \mu \mathrm{g} / \mathrm{kg}$ body $\mathrm{wt} /$ day) orally for the entire study; and group 3, receiving Ro26, after the peak of DSSinduced inflammation (day 12). All groups received 2.5\% DSS $\times 7$ days in the drinking water, followed by two wks recovery. The Disease Activity Index (DAI) that integrates clinical scores for weight loss, stool consistency and bleeding was evaluated daily. Two animals/ group were sacrificed at 2-3 day intervals. Crypt Loss Score (CLS) was determined from H\&E sections and Ki67 stained to assess proliferation. Results. DAI: In the group receiving Ro26 during DSS treatment, peak clinical inflammation occurred significantly earlier (day 7 vs. day 10) compared to control. This group, however, also showed an accelerated recovery that was complete by day 12 . When administered after DSS, Ro26 caused an accelerated reduction in DAI, with clinical resolution by day 18 vs. day 20 in the control group. CLS: When administered during DSS treatment, Ro26 caused a significantly earlier and higher peak (day 7 vs. day 10) in CLS compared with control. Following DSS withdrawal, this same group, however, recovered earlier than the other groups. When Ro26 was started following DSS treatment, prompt reduction of the CLS and complete histological recovery were achieved earlier than the control group. Proliferation: In the groups receiving Ro26, complete mucosal regeneration, with inhibition of residual proliferation, was noted earlier than the control group. Conclusion. When administered during DSS injury and recovery, Ro26 accelerated inflammation and recovery. When given after DSS withdrawal, Ro26 hastened recovery. Further studies are in progress to elucidate differential effects induced by the timing of Ro26 administration on the innate immune system and epithelial cells in DSS colitis. 
The Immunomodulatory Activity of 1,25-Dihydroxyvitamin $\mathrm{D}_{3}$ and Dexamethasone in TNBS-Colitis Is Mediated Via the Transcription Factors TBet, Gata3 and Foxp3

Carolin Daniel, Heinfried H. Radeke, Juergen M. Stein

Background \& Aims: Previously, we have demonstrated that 1,25-dihydroxyvitamin $\mathrm{D}_{3}$ (calcitriol) revealed in vivo strong Thl-selective immunosuppressive and immunomodulatory capacities in combination with classical immunosuppressants like the corticosteroid dexamethasone. The objective of the present study was to elucidate the relevant transcription factors mediating the effects of 1,25-dihydroxyvitamin $\mathrm{D}_{3}$ in combination with dexamethasone in experimental Thl-colitis. Methods: A rectal enema of TNBS [100 mg/kg body weight (BW)] was applied to male Balb/c mice, and dexamethasone $[0.6 \mathrm{mg} / \mathrm{kg}]$ and $/$ or calcitriol $[0.2$ $\mu \mathrm{g} / \mathrm{kg}]$ were administered intraperitoneally from day 0-3 following the instillation of the haptenating agent. The study is conforming to the Guiding principles in the care and use of animals and was performed under approval of the ethical committee of Darmstadt/ Germany (F134/03). Colitis severity was evaluated macroscopically and microscopically. Western blot analysis of T-bet-, GATA3- and FoxP3-expression, as well as of IL-23p19. and IL-27 was performed from colon protein extracts. IL-10 and TGF $\beta$ were analysed by immunoassay. Serum calcium was analyzed photometrically. Results \& Conclusion: The combined application of 1,25-dihydroxyvitamin $\mathrm{D}_{3}$ and dexamethasone most prominently downregulated the Thl-transcription factor T-bet, as well as the recently discovered cytokines IL-23p19 and IL-27, in contrast the Th2-lineage commitment factor GATA3 was significantly induced. Moreover, the provided Th1/Th2-shift was accompanied by a significant induction of FoxP3, raising evidence for a distinct enhancement of regulatory $\mathrm{T}$ cell capacities (see Table). These findings underlie the combined application of dexamethasone and calcitriol as an auspicious new treatment regimen for Thl-mediated diseases offering distinct corticoid sparing properties.

Results Calcitriol and dexamethasone in TNBS-colitis

\begin{tabular}{|c|c|c|c|c|}
\hline \multirow{2}{*}{$\begin{array}{l}\text { Parameters } \\
\text { Treatments }\end{array}$} & \multicolumn{4}{|c|}{ TNBS } \\
\hline & untreated & $\begin{array}{l}0.2 \mu \mathrm{g} / \mathrm{kg} \\
\text { calcitriol }\end{array}$ & $\begin{array}{l}0.6 \mathrm{mg} / \mathrm{kg} \\
\quad \text { dex. }\end{array}$ & $\begin{array}{c}0.6 \mathrm{mg} / \mathrm{kg} \text { dex. }+0.2 \mu \mathrm{g} / \mathrm{kg} \\
\text { calcitriol }\end{array}$ \\
\hline T-bet [\% of TNBS] & $100.0 \pm 0.0$ & $51.4 \pm 1.3 \mathrm{~b}$ & $26.0 \pm 1.0 \mathrm{c}$ & $18.2 \pm 0.8 \mathrm{c}$ \\
\hline $\begin{array}{l}\text { IL-23p19 [\% of } \\
\text { TNBS] }\end{array}$ & $100.0 \pm 0.0$ & $55.6 \pm 3.2 \mathrm{c}$ & $76.7 \pm 7.2 \mathrm{a}$ & $40.4 \pm 6.8 \mathrm{c}$ \\
\hline IL-27 [\% of TNBS] & $100.0 \pm 0.0$ & $57.2 \pm 4.9 \mathrm{c}$ & $74.4 \pm 6.8 \mathrm{a}$ & $41.3 \pm 7.2 \mathrm{c}$ \\
\hline $\begin{array}{c}\text { GATA3 [\% of } \\
\text { TNBS] }\end{array}$ & $100.0 \pm 0.0$ & $198.1 \pm 28.8 \mathrm{~b}$ & $\begin{array}{c}100.6 \pm 14.5 \\
\text { n.s. }\end{array}$ & $524.3 \pm 48.8 \mathrm{c}$ \\
\hline $\begin{array}{l}\text { IL-10 [pg/mg } \\
\text { protein] }\end{array}$ & $4.9 \pm 0.6$ & $7.8 \pm 0.3 \mathrm{~b}$ & $6.1 \pm 0.4$ n.s. & $9.5 \pm 0.9 \mathrm{c}$ \\
\hline $\begin{array}{c}\text { TGF } \beta[\mathrm{pg} / \mathrm{mg} \\
\text { protein] }\end{array}$ & $166.4 \pm 7.0$ & $267.0 \pm 36.8 \mathrm{~b}$ & $\begin{array}{c}199.6 \pm 17.9 \\
\text { n.s. }\end{array}$ & $328.9 \pm 18.3 \mathrm{c}$ \\
\hline FoxP3 [\% of dex.] & $\begin{array}{c}\text { not } \\
\text { detected }\end{array}$ & not detected & $100.0 \pm 0.0$ & $318.9 \pm 15.8 \mathrm{c}$ \\
\hline Macroscopic score & $3.9 \pm 0.3$ & $2.1 \pm 0.3 \mathrm{c}$ & $1.8 \pm 0.3 \mathrm{c}$ & $0.8 \pm 0.3 \mathrm{c}$ \\
\hline Microscopic score & $3.8 \pm 0.2$ & $2.7 \pm 0.2 \mathrm{c}$ & $2.5 \pm 0.2 \mathrm{c}$ & $1.4 \pm 0.1 \mathrm{c}$ \\
\hline
\end{tabular}

$\mathrm{a}=\mathrm{P}<0.05 ; \mathrm{b}=\mathrm{P}<0.01 ; \mathrm{c}=\mathrm{P}<0.001 ; \mathrm{n} . \mathrm{s} .=$ non significant vs. TNBS-treated mice. dex. $=$ dexamethasone

\section{M1676}

Dipeptidyl Peptidases and Their Expression in a Murine Model of Experimental Colitis

Roger Yazbeck, Melanie L. Sulda, Gordon S. Howarth, Catherine A. Abbott

Introduction: Glucagon-like peptide-2 (GLP-2) is a potent intestinotrophic growth factor; however its actions are limited by dipeptidyl peptidase (DP) IV mediated degradation. Recent work has suggested that there may be other enzymes with DP activity in addition to DPIV that are also responsible for the in vivo degradation of GLP-2. Fibroblast activation protein (FAP), DP8 and DP9 all exhibit DP activity and share sequence identity with DPIV as they are all members of the S9b enzyme family. Another DP also exists, DPII but this belongs to a different enzyme class. We hypothesized that there was greater than one enzyme with DP activity present in the colon and that the expression of these enzymes would differ in normal compared to colitic colon samples. We hypothesized that if any of these enzymes were present in colitis, then they may also be targeted by DPIV inhibitors. Methods: Wildtype (WT) and DPIV knockout (DPIV-/-) mice consumed 2\% dextran sulphate sodium (DSS) for 6 days to induce colitis ( $\mathrm{n}=6$ for each group). Mice were sacrificed at days 0 and 6 . Messenger RNA (mRNA), protein and enzyme levels of all enzyme members of the DPIV gene family and DPII were measured in the colon for each group using real-time RT-PCR, quantitative western blotting, DP-like enzyme activity assays and immunostaining techniques. Results: All enzyme members of the DPIV gene family and DPII were present in the colon of both normal and colitic mice at the mRNA level. DP8 and DPII mRNA levels were significantly increased $(\mathrm{p}<0.05)$ in WT mice with colitis compared to normal mice. DP enzyme activity but not DPIV protein was significantly increased $(\mathrm{p}<0.05)$ in colitic WT mice compared to normal WT mice, and DPII activity was significantly increased $(\mathrm{p}<0.05)$ in colitic DPIV-/- mice compared to day 0 DPIV-/- mice. DPIV, DP9 and fibroblast activation protein (FAP) mRNA levels were considerably reduced in colitic samples in both WT and DPIV-/- (DP9 and FAP only) mice. Conclusion: This study is the first to demonstrate that enzyme members of the DPIV gene family and DPII are present in the colon and that their expression differs in colitic samples compared to normal samples. Thus these other dipeptidyl peptidases may also play a role in the induction and resolution of colitis. This study together with previous work in our group provides further indication that DP inhibition may be a novel strategy for the treatment and control of IBD.
Fibratide, a Synthetic Fgf Mimetic,Alleviates Experimental Ulcerative Colitis Xinhua Lin, Paul O. Zamora, Kazu Takahashi

Existing IBD treatments modulate the immune response. However, many patients are nonresponsive or develop significant side effects. Hence new therapies are needed. In animals, recombinant human fibroblast growth factors (rhFGFs) limit the mucosal tissue damage but their delivery is largely limited to parenteral administration. Synthetic peptide mimetics of FGF, that have the potential for being tailored to oral or rectal administration, would be attractive as IBD treatment options. Fibratide, a synthetic FGF mimetic peptide, was evaluated for its ability to protect mice from experimental colitis. Colitis was induced by providing C57BL/6 mice drinking water containing 3\% dextran sulfate (DSS). Starting at 3 days after DSS treatment, animals were left untreated or treated once a day for 5 consecutive days with Fibratide ( 1 and $5 \mathrm{mg} / \mathrm{kg}$ ) or $\mathrm{rhFGF2}$ ( $1 \mathrm{mg} / \mathrm{kg}$; positive control) by intraperitoneal injection. On day 11 the animals were euthanized, and the lengths and weights of colons determined. Specimens were then processed by standard histological methods. In colitis mice with no therapy, body weights decreased gradually from day 4 to day 9 and the loss of body weight was not regained at the end of the experiment. The colons in these animals were shortened and had clear signs of inflammation including thickening of the bowel and increased vascularisation. Histological evaluation showed a loss of epithelium and cryp morphology, depletion of goblet cells, necrosis, ulceration and transmural infiltration o granulocytes. In contrast, colitis animals treated with Fibratide had slower rates of weigh loss during the study. Moreover, Fibratide promoted the recovery of weight loss compared to untreated mice once DSS treatment was stopped. Colons of these animals exhibited milder signs of inflammation including less shortening and thickening. Histologically, colons of mice treated with Fibratide exhibited morphologies more reminiscent of normal tissue architecture with an intact epithelium and complete crypts. Inflammation, while present, was mild and considerably less extensive than in untreated animals. The return to more normal morphology was especially noticeable in animals receiving doses of $5 \mathrm{mg} / \mathrm{kg}$. Animals treated with hrFGF-2, the reference treatment, also had increased body weights and histologically were similar to those treated with Fibratide at $5 \mathrm{mg} / \mathrm{kg}$. Collectively, the results demonstrate therapeutic effects of Fibratide in experimental ulcerative colitis. Future studies are targeted to modification of Fibratide to be permissive of oral or rectal administration to affect a local/regional therapy and to minimize dose and spurious systemic effects.

\section{M1679}

Integrin $\alpha 2 \beta 1$ Regulates Neutrophil Recruitment and Inflammatory Activity in Experimental Colitis in Mice

Sofie Lundberg, Johan Lindholm, Lennart Lindbom, Per M. Hellstrom, Joachim Werr

Receptors of the $\beta 1$ integrin family mediate leukocyte adhesion to extracellular matrix (ECM) proteins and are believed to regulate leukocyte migration to sites of inflammation and tissue injury. Human inflammatory bowel disease (IBD), e.g. Crohn's disease and ulcerative colitis, is associated with leukocyte accumulation in the inflamed intestinal tissue and recent studies strongly suggest a role of $\beta 1$ integrin receptors in regulating tissue damage and disease symptoms related to IBD. In this study, we investigated the role of the collagen-binding $\alpha 2 \beta 1$ integrin (CD49b/CD29) in dextran sodium sulfate (DSS) -induced colitis in mice. Rectal administration of function-blocking antibodies directed against the murine $\alpha 2$ integrin subunit was found to significantly reduce mucosal neutrophil infiltration in DSS-treated mice as shown by a decrease from $47.2 \pm 10.0$ to $6.6 \pm 8.0$ neutrophils per counted area ( $\mathrm{P}<$ $0.05)$. Clinical signs of colitis such as weight loss was reduced from $14.7 \pm 3.9 \%$ to $2.2 \pm 0.2 \%$ and shortening of colon length was reduced from $5.8 \pm 0.8 \mathrm{~cm}$ to $7.4 \pm 0.8 \mathrm{~cm}$, (healthy control $9.1 \pm 1.0 \mathrm{~cm}$ ). Blockage of $\alpha 2 \beta 1$ integrin also down-regulated gene expression of metalloproteinases (MMPs) $-2,-7$ and -9 . These MMPs have been demonstrated to be elevated in colitis and are known to contribute to inflammatory tissue damage in IBD. For comparison, the protective effect against colitis seen after anti- $\alpha 2 \beta 1$ integrin treatment was found to be favorable to the effect seen after high dose oral betamethasone. In conclusion, we demonstrate an alleviating action of the collagen-binding $\alpha 2 \beta 1$ integrin in experimental colitis in mice and suggest this effect to be mediated via inhibition of neutrophil migration and activation Local administration of function-blocking antibodies against integrin $\alpha 2 \beta 1$ may provide novel avenues to treat inflammatory bowel disease.

\section{M1680}

Therapeutic Effect of Tv-5010 On Dextran Sulfate Sodium - Induced Experimental Colitis Rina Aharoni, Basak Kayhan, Ruth Arnon

TV-5010 is a higher molecular weight version of glatiramer acetate (GA), the active ingredient of Copaxone. GA, which exerts its effect by inducing a Thl to Th2 shift, has been shown to ameliorate both trinitrobenzene sulfonic acid (TNBS) and dextran sulfate sodium (DSS) induced experimental colitis in mice. TV-5010 induces a similar immunomodulatory activity as GA, albeit with less frequent administration. In this study, we investigated the efficacy of TV-5010 on DSS-induced colitis, and compared it to the beneficial effects demonstrated previously for GA. TV-5010 ameliorated colitis in the highly susceptible strain C57BL/6 induced by either one or three cycles of DSS. This was manifested by significantly lower weight loss, improved long-term survival, reduced rectal bleeding, as well as by prevention of decrease in colon length and reduction of histological damage, in TV-5010 treated mice compared to untreated mice. In contrast to GA, which was more effective when administered daily by injection of $2.0 \mathrm{mg} /$ mouse, TV-5010 was most effective when administered every $5-7$ days, $0.2-1.0 \mathrm{mg} / \mathrm{mouse}$, indicating that smaller doses and less frequent injections of TV-5010 are needed to obtain therapeutic effect. The weekly injections of TV-5010 were most effective when started from the day of disease induction, but a beneficial effect was observed even when treatment started 10 days after disease induction, when the mice reached maximal weight lost. The effect of TV-5010 (injected weekly) as well as that of GA (injected daily) on the immune system was studied using RT-PCR analysis of cytokines in colons of DSS-induced mice. In both TV-5010 and GA treated mice, mRNA expression levels of the

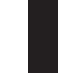

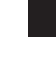


colitis associated Thl inflammatory cytokines interferon-gamma and tumor necrosis factor alpha were reduced. Moreover, the levels of the Th2/3 anti-inflammatory cytokines interleukin-10 (IL-10) and transforming growth factor beta were elevated, in comparison to untreated mice. In conclusion, TV-5010 was effective in the suppression of DSS induced colitis similarly to Glatiramer Acetate, with the advantage of less frequent administration.

\section{M1681}

\section{Improving the Objectivity and Sensitivity of Rodent IBD Models Using} Histometric Analyses and mRNA Profiling to Complement Traditional

\section{Readouts}

Catherine Booth, Gregory Tudor, Gerard Brady

Despite the wide range of rodent IBD models available the assays themselves remain notoriously poor indicators of eventual clinical efficacy. Several models are generally needed to provide sufficient supporting efficacy and mechanism of action data. Part of the problem is that the data is often not reproducible due to the insensitive or subjective scoring assessments used (animal weights, colon length, visual scores of inflammation) etc. We have developed a method to generate quantitative histometric data from simple $H \& E$ sections. Areas of lesions (overt ulcers and areas of intramural inflammation) are measured, along with indicators of mechanism of action such as crypt length, number of proliferative and apoptotic cells per crypt, changes in overall crypt cell turnover (including crypt density) and number of neutrophils and T cell phenotpyes per unit area of lesion or epithelium. An indicator panel of mRNA profiles is also generated from an adjacent area of the same treated tissue to demonstrate effects on key disease activity regulators such as IL-6, TNFa, IFNg etc. Dose and time dependent changes in the above parameters were measured in the mouse DSS IBD model, and time dependent changes were detected in the TNBS model. Significant suppression of some, but not all, parameters following treatment with clinical standards such as prednisalone, sulfasalasine and budesonide, were consistently observed in animals within and between studies. In IL10-/- mice the kinetics of development of crypt hyperplasia and subsequent ulceration was easily quantified, and were suppressed by IFNg inhibition. However, in all models the actual parameters most affected depended upon the drug and the model, indicating that careful consideration needs to be given to the MOA of any novel agent when determining the best model to screen suppression of disease severity. For example, some agents that reduced the intramural inflammation significantly had no statistical effect on the actual ulcer sizes, whereas some did both, and others the converse. The selected panel of parameters for each model will serve as useful biomarkers and may also be useful indicators in clinical trial biopsy material.

\section{M1682}

Targeting Epigenetics: Modifying Histone Acetylation As Novel Therapeutic Approach in Inflammation-Dependent Carcinogenesis Rainer Glauben, Arvind Batra, Inka Fedke, Hans A. Lehr, Paolo Mascagni, Martin Zeitz, Britta Siegmund

Histone deacetylase inhibitors (HDACi) are potent inducers of growth arrest and apoptotic cell death and are currently in clinical studies for solid tumors. In addition, an antiinflammatory potency was recently demonstrated by our group for HDACi from different classes in vitro as well as in vivo by using different models of experimental colitis in mice. Since IBD in humans is associated with an increased risk of developing colorectal cancer, a therapeutic approach including anti-inflammatory as well as anti-proliferative properties is highly intriguing. Thus with the present study HDACi from various classes (SAHA, trichostatin A, apicidine and valproic acid (VPA)) were first evaluated for their capacity to induce apoptosis and histone 3 (H3) acetylation in vitro. Here, for each HDACi class, a dose-dependent induction of apoptosis was observed in stimulated murine monocytes, CD4+ T-cells as well as lamina propria lymphocytes (LPL). These findings were associated with a parallel increase in $\mathrm{H} 3$ acetylation in the HDACi-treated cells. Second, in order to characterize the combined effect of the anti-inflammatory and anti-proliferative potency of HDACi in vivo, the AOM/DSS-model of inflammation-mediated colon carcinogenesis was applied. In this model the carcinogen AOM reveals its effect after the induction of a chronic DSScolitis within 40 days. The HDACi SAHA or VPA were administered throughout the entire experimental time course via oral gavage and tumor progression was monitored by lower endoscopy. HDACi treatment resulted in a delay of tumor development as evaluated by endoscopy and histology. In addition, the absolute number of adenomas was significantly reduced by $30 \%$ in the HDACi-treated group when compared to vehicle-treated animals as determined by histology. Subsequent immunoprecipitation experiments revealed, that this inhibition of tumor development went in parallel with a hyperacetylation of $\mathrm{p} 65$, a subunit of NF-KB, which was recently shown to act as a key molecule during the carcinogenesis in this model. In summary our results suggest that inhibition of HDAC exerts an anti-inflammatory as well as an anti-proliferative effect in vitro as well as in vivo. Therefore HDAC inhibitors could be of particular interest in the control of the hyperresponsiveness of mucosal immunity as seen in inflammatory bowel diseases, where chronic inflammation is linked to an increased risk for colon cancer.

\section{M1683}

Induction of Ho-1 Attenuates Trinitrobenzen Sulfonic Acid-Induced Colitis As Ho-1 Blocks Intracellular Calcium Mobilization and Inhibits TNF- $\alpha$-Induced Nf-kB Activity

Sang Wook Kim, In Hee Kim, Seung Hun Kim, Seung Ok Lee, Soo Teik Lee, Dae Ghon Kim, Woo Sung Moon, Geom Seog Seo, Suck Chei Choi, Yong Sung Kim, Chang Soo Choi, Jae Min Oh

Crohn's disease is characterized by a chronic relapsing inflammation of the bowel in which proinflammatory cytokines play an important perpetuating role. Heme oxygenage-1 (HO1 ) is the rate-limiting enzyme in heme catabolism, which leads to the generation of carbon monoxide (CO), biliverdin, and free iron. HO-1 has been known to show strong immunosup pressive properties although its mechanisms are not completely understood. In this study, it was therefore investigated whether HO-l has anti-inflammatory properties in vitro and in vivo In a mouse model, an $\mathrm{HO}-1$ inducer cobalt-protoporphyrin IX (CoPPIX) administration significantly improved the clinical and histopathologic symptoms of trinitrobenzene sulfonic acid (TNBS) colitis and also notably suppressed the expression of several inflammatory mediators such as TNF- $\alpha$, IL-1 $\beta$ and ICAM-1 induced by TNBS. The HO-l activity inhibitor, zinc protoporphyrin IX(ZnPPIX) reversed the protective effects of CoPPIX in TNBS colitis. Pre-treatment of human epithelial HT-29 cells with CoPPIX significantly blocked the I-kB $\alpha$ degradation by TNF- $\alpha$ or IL-1 $\beta$. Inhibition of HO-1 activity by ZnPPIX reversed the suppressive effects of CoPPIX on I-kB $\alpha$ degradation by TNF- $\alpha$ or IL-1 $\beta$ as the in vivo study shows. Calcium chelating agent BAPTA/AM suppressed I-kB $\alpha$ degradation by TNF- $\alpha$ in HT-29 cells. Like ZnPPIX, calcium ionophore A23187 also dose-dependently reversed the suppressive effects of CoPPIX on I-kB $\alpha$ degradation by TNF- $\alpha$. Calcium channel blockers such as Verapamil and Flunarizine blocked the effects of ZnPPIX on the suppressive effects of CoPPIX on I-kB $\alpha$ degradation by TNF- $\alpha$. Interestingly, the pre-treatment of ZnPPIX increased the intracellular calcium level in HT-29 cells. Collectively, these results suggest that HO-1 should exert anti-inflammatory effects by down-regulation of NF-kB activity via intracellular calcium regulation during the pathogenesis of TNBS-induced colitis.

\section{M1684}

Up-Regulation of Anandamide Levels As An Endogenous Mechanism and a Pharmacological Strategy to Limit Colon Inflammation

Giuseppe D'argenio, Marta Valenti, Giuseppe Scaglione, Vittorio Cosenza, Giovanna Mazzone, Ilenia Grandone, Lorena Pietrini, Valeria Dargenio, Italo Sorrentini, Vincenzo Di Marzo

Background. Direct stimulation of cannabinoid $\mathrm{CB} 1$ receptors exerts a protective function in animal model of inflammatory bowel diseases (IBDs). However, it is not known whether endocannabinoids are up-regulated during IBDs in animals or humans, nor whether pharmacological elevation of endocannabinoid levels can be exploited therapeutically in these disorders. Aim of this study was to addressed these questions. Methods. Colon inflammation was induced in mice and rats with 2,4-dinitrobenzene- and 2,4,6-trinitrobenzene sulfonic acids (DNBS and TNBS), respectively. DNBS-treated mice were treated chronically (for 3 or 7 days) with inhibitors of anandamide enzymatic hydrolysis ( $\mathrm{N}$-arachidonoyl-serotonin, AA-5-HT) or reuptake (VDM11), 10 or $5 \mathrm{mg} / \mathrm{kg}$, s.c., or with 5-amino-salicilic acid (5-ASA, $1.4 \mathrm{mg} / \mathrm{kg}$, i.r.). Endocannabinoids (anandamide and 2-arachidonoylglycerol, 2-AG) were quantified in mouse colon, or in rat colon mucosa and submucosa, and in bioptic samples from the colon of patients with untreated ulcerative colitis, by liquid chromatography-mass spectrometry. Results. A strong elevation of anandamide, but not 2-AG, levels was found in the colon of DNBS-treated mice, in the colon submucosa of TNBS-treated rats, and in the biopsies of patients with ulcerative colitis. VDM-11 significantly elevated anandamide levels in the colon of DNBS-treated mice and concomitantly abolished inflammation, whereas AA-5-HT did not affect endocannabinoid levels and was significantly less efficacious at attenuating colitis. 5-ASA also increased anandamide levels and abolished colitis. Conclusions. Anandamide is elevated in the inflamed colon of patients with ulcerative colitis, as well as in animal models of IBDs, to control inflammation, and elevation of its levels with inhibitors of its cellular reuptake might be used in the treatment of IBDs. Indeed, data from our laboratories indicate that also during celiac disease and diverticulosis, intestinal anandamide levels are increased, and that in the former case they are normalized during remission. Therefore, it is possible to hypothesise for this compound a general protective role against inflammatory conditions of both the upper and the lower tracts of the intestine.

\section{M1685}

Enhanced Anti-Inflammatory Activity of Hydrogen Sulfide Releasing Derivative of mesalamine in Rodent Models of Colitis Involves Direc Inhibition of Nf-kB

Stefano Fiorucci, Eleonora Distrutti, Andrea Mencarelli, Barbara Renga, Giuseppe Cirino, John L. Wallace

Background. Evolving research suggests that hydrogen sulfide $\left(\mathrm{H}_{2} \mathrm{~S}\right)$ may exert anti-inflam matory activities. We have previously shown that $\mathrm{H}_{2} \mathrm{~S}$ reduces neutrophils-endothelial interactions in acute models of mesenteric inflammation. ATB429 is an $\mathrm{H}_{2} \mathrm{~S}$-releasing derivative of mesalamine. Aim. To test the efficacy and immunomodulatory actions of ATB429 in TNBS and DSS-induced colitis in comparison with mesalamine. Methods. Colitis was induced by intrarectal administration of $1-2 \mathrm{mg} /$ mouse TNBS in $50 \%$ ethanol or $5 \%$ DSS in the drinking water for 7-10 days. Mice were administered 50 or $100 \mathrm{mg} / \mathrm{kg}$ mesalamine or 25 , $50,100 \mathrm{mg} / \mathrm{kg}$ ATB 429 by gavage. Mucosal cytokine content cytokines was assessed by quantitative (q)RT-PCR. Isolated lamina propria (LP) mononuclear cells composition was assessed by flow cytometry. Nuclear binding of NF-KB was assessed by electromobility shift analysis (EMSA). Results. At the dose of $100 \mathrm{mg} / \mathrm{kg}$ ATB429, but not mesalamine, protected against mortality induced by administering mice with $2 \mathrm{mg} /$ mouse TNBS. Mortality rate was: $30 \%$ in TNBS treated mice, $25 \%$ in mice administered mesalamine, while no mortality was observed in animals administered ATB429. When administered from day 1 to day 7 at the dose of 50 and $100 \mathrm{mg} / \mathrm{kg}$ ATB429 was effective in protecting against development of TNBS- and DSS-induced colitis by all disease endpoints measured: weight loss, inflammation, disease activity/diarrhoea scores and macroscopic/microscopic scores $(\mathrm{p}<0.05)$. Therapeutic administration (from day 4 to day 10) of equimolar doses of mesalamine (50 mg/kg) and ATB429 $(100 \mathrm{mg} / \mathrm{kg})$ resulted in a significant reduction $(50-60 \%)$ of colon inflammatory scores with ATB429 but not mesalamine ( $\mathrm{P}<0.05$ versus TNBS). In the treatment protocol ATB429, but not mesalamine, attenuated mucosal increases of COX-1, COX-2, iNOS IL-2, IL-6, IL-12, TNF- $\alpha$, IFN- $\gamma$ and RANTES mRNA expression as well as TNF- $\alpha$ protein induced by TNBS $(\mathrm{P}<0.05$ versus TNBS). Further, ATB429, but not mesalamine, reduced the accumulation of $\mathrm{CDllb} / \mathrm{Gr}$ l cells in the lamina propria as measured by flow cytometry. Using human peripheral blood-derived monocytes we found that ATB429 concentration dependently inhibits NF-kB translocation and TNF- $\alpha$ release induced by LPS. This effect was replicated by exposing cells to the $\mathrm{H}_{2} \mathrm{~S}$-releasing moiety of ATB429. Detectable amount 
of $\mathrm{H}_{2} \mathrm{~S}$ were found in supernatant of monocytes exposed to ATB429 but not to mesalamine. Conclusions .ATB429 prevents and treats DSS- and TNBS-induced colitis in mice. Protection by ATB429 in TNBS-colitis is associated with inhibition of NF-kB regulated genes.

\section{M1686}

Treatment of Murine IBD Via Non-Viral Delivery of NF-KappaB Decoy Christopher De Vry, Srinivasa Prasad, Laszlo Komuves, Carlos Lorenzana, Christi Parham, Tina Le, Sarvesh Adda, Jennifer Hoffman, Nicole Kahoud, Radhika Garlapati, Radha Shyamsundar, Wanda Williamson, Leslie M. McEvoy, Rolf O. Ehrhardt

Many of the currently available therapeutics for IBD, such as steroids, 5-ASA compounds, and Remicade work through interactions with the nuclear factor-kappa B (NF-kappaB) pathway. This key transcriptional regulator is utilized by a variety of immune cell types and signaling cascades involved in the inflammation process and represents a prime target for drug development. We have developed a fully phosphorothioated Decoy oligonucleotide with improved stability that specifically binds NF-kappaB and blocks key inflammatory mediators regulated by this transcription factor without the aid of viral-assisted delivery. In this preclinical study, we demonstrate that intracolonic administration of NF-kappaB Decoy results in the focal delivery of Decoy to inflammatory lesions in the colon which leads to amelioration of disease at the clinical, histological, and molecular levels in established acute and chronic murine colitis models. Specifically, in the Thl-driven TNBS-induced colitis model we find that mice receiving NF-kappaB Decoy treatment exhibit a dose-dependent reduction in disease severity and a more rapid recovery to normal body weight similar to a clinically-relevant dose of budesonide. This observed clinical efficacy is corroborated by significant reductions in both colitis pathology and the tissue levels of several pro-inflammatory markers. Rapid restoration of tissue homeostasis is observed in Decoy-treated animals as evidenced by the re-emergence of functional goblet cells and reduction in muscle cell proliferation. We also show that NF-kappaB Decoy mitigates disease activity in the Th2like oxazolone colitis and epithelial injury-related acute and chronic DSS colitis models indicating that locally administered NF-kappaB Decoy can effectively treat both Thl and Th2 mediated disease. This data supports the potential use of NF-kappaB Decoy as a crossfunctional therapeutic for the treatment of both Crohn's disease and Ulcerative colitis. Topical NF-kappaB Decoy is currently in Phase 1/2 clinical trials for atopic dermatitis.

\section{M1687}

The Nutrient and Octn Ligand L-Carnitine Induces Remission in Experimental Models of Crohn's Disease

Genevieve Fortin, Catherine Collette, Ekaterina Yurchenko, Alexandra-Chloe Villani, Ihsan Elimrani, Gary Wild, Alain Bitton, Ciriaco Piccirillo, Ernest Seidman, Denis Franchimont

L-Carnitine (L-C) is consumed in the diet and available as an over-the-counter nutritional supplement. This compound is a critical factor in the $\beta$-oxidation pathway of long chain fatty acids and is thus essential in mitochondrial metabolism. Additionally, the causative mutation at the IBD5 locus leading to an increased susceptibility to Crohn's disease (CD) was localized to the OCTN gene, which encodes the L-C transporter. We therefore chose to assess the potential impact of $\mathrm{L}-\mathrm{C}$ in mouse models of $\mathrm{CD}$ and in primary intestinal organ cultures. Methods: Animal study: TNBS $(n=12)$ and DSS $(n=10)$ colitis were induced in $\mathrm{Balb} / \mathrm{c}$ mice and treated IP with $150 \mathrm{mg} / \mathrm{kg}$ L-C per day. Drug efficacy was determined by weight loss, macroscopic score, serum cytokine levels by ELISA, and mucosal cytokine expression by quantitative real-time PCR. Cytokine secretion (ELISA) and apoptosis (PI/ Annexin V) were assessed in vitro in LPS- and anti-CD3-stimulated splenocytes and purified (cell sorted) CD4+ lymphocytes. Human study: Intestinal biopsies were taken from healthy subjects (HS) $(n=13)$ and patients with $C D(n=9)$. OCTN1 and 2 expression were determined by quantitative real-time PCR. Biopsies were also stimulated with LPS, treated with L-C, and cytokine production was measured by ELISA. Results: Expression of OCTN1 and 2 were confirmed in mouse and human intestinal mucosa, as well as in mouse CD4+ T cells. Mouse study There was a significant amelioration of TNBS colitis in L-C-treated mice as compared to untreated mice in all of the following parameters ( $\mathrm{p}<0.05)$ : body weight $(97.5+/$ $4.4 \%$ vs $85.2+/-5.5 \%$ of baseline), Wallace colitis severity score $(2.8+/-2.7$ vs $8.7+/-3.3)$, serum IL-1 $\beta$ (18.5+/-9.3 vs $37.5+/-7.5 \mathrm{pg} / \mathrm{ml})$ and IL-6 (53.5+/-26.8 vs $128.7+/-73.7 \mathrm{pg} /$ $\mathrm{ml})$, as well as colonic expression of IL-1 $\beta$ and IL-6 (2.7- and 4.8-fold decrease in treated mice, respectively). L-C was also beneficial in mice with DSS colitis, with a significant decrease in the expression of IL-1 $\beta$ and of IL- 6 by 2.3 - and 15.5 -fold, respectively. In vitro experiments demonstrated that L-C significantly inhibited pro-inflammatory cytokine secretion by LPS-induced mouse splenocytes. Similarly, L-C suppressed IFN $\gamma$ secretion by TCR-stimulated CD4+ lymphocytes without affecting cell survival or apoptosis. Human study: We confirmed the immunosuppressive action of L-C on human intestinal mucosa as L-C significantly reduced IL-6 secretion in LPS-stimulated intestinal explant cultures. Conclusion: L-C therapy is effective in treating experimental colitis and suppressing the production of pro-inflammatory cytokines in vitro. Therefore, L-C could represent a novel and safe alternative therapeutic strategy in $C D$.

\section{M1688}

TGF-Beta Induced Cd4+Foxp3+ Cells Prevent TH1 Colitis: Role of TGF-Beta and Exogenous IL-2

Massimo C. Fantini, Christoph Becker, Markus F. Neurath

Background: the imbalance between effectors cells and regulatory $\mathrm{T}$ cells contributes to the development of Inflammatory Bowel Diseases (IBDs). Thymus derived CD4+CD25+ regulatory $\mathrm{T}$ cells have been shown to mediate an important immunomodulatory effect in the intestinal mucosa. In addition to these cells, a novel class of regulatory cells characterized by the expression of the transcription factor FoxP3 has been recently shown to be induced by the TGF-beta from a population of naive cells. Aims: The aim of the present work was to evaluate the in vivo capacity of TGF-beta induced regulatory cells (Ti-Tregs) to suppress colitis and analyze the requirements for their function. Methods: In order to investigate the in vivo immunnoregulatory effect of Ti-Tregs, these cells were challenged in a model of Th colitis induced by the adoptive transfer of CD4CD62L+ T cells in SCID immunodeficien mice. Moreover we have analyzed the role played by IL-2 in the Ti-Tregs expansion and suppressive capacity. Results: We observed that Ti-Treg cells are able to suppress Thl colitis induced by the adaptive transfer of CD4+CD62L+ cells in SCID mice, as determined by macroscopic, histologic and endoscopic criteria. These cells were able to control the inflammatory process acting both on the acquired and innate immunity by preventing CD4+ and $\mathrm{CDll} c+$ cell accumulation in the lamina propria. Furthermore, we found that neutralization of IL-2 abrogates the generation of Ti-reg cells and that exogenous IL-2 is able to enhance survival of these cells. Consistent with the pivotal role of IL-2 in the peripheral generation of Ti-Treg cells we were able to induce Ti-Tregs cells from a population of CD4+CD25- T cells isolated from IL-2 deficient mice (IL-2-/-) upon activation with TGF-beta and exogenous IL-2. Conclusions: We propose a model in which TGF-beta induced regulatory T cells can be generated at the site of inflammation by both TGF-beta and exogenous IL-2, the latter provided by ongoing inflammatory immune responses. Once the pathologic immune reaction has been turned off, such regulatory cells release the immune system from their control by loosing their regulatory function due to lack of exogenous IL-2. Furthermore, such TGFbeta induced regulatory $\mathrm{T}$ cells emerge as a novel therapy for Thl mediated chronic intestinal inflammation.

\section{M1689}

Suppression of DSS-Induced Ulcerative Colitus in Mice By Orally-Available Inhibitors of Sphingosine Kinase

Lynn W. Maines, Leo Fitzpatrick, Charles D. Smith

We have developed novel, specific, small molecule inhibitors, A2 and A7, of human sphingosine kinase (SK) for the treatment of intestinal inflammation. Sphingosine-1-phosphate (SIP) produced by SK, is proving to be a critical component in inflammation, making SK an excellent, novel target for anti-inflammatory drugs. Purpose: Our goals were to investigate the mechanism of action of SK inhibitors in vitro and evaluate them in acute and chronic DSS-induced mouse colitis models. Methods: Cellular studies were conducted in rat IEC6 and human endothelial cells. SK activity was determined by mass spectroscopy of S1P levels. An NFKB reporter cell line (cat no. E1500, Promega) was used. Western analysis looked at TNF $\alpha$-induced expression of ICAM, VCAM and E-selectin. ELISA was used to measure TNF $\alpha$-induced PGE2 secretion. Acute 6 day (2\% DSS) and chronic 35 day (alternating water and 1.5\% DSS 7 day cycles) were used with oral administration of $50 \mathrm{mg} / \mathrm{kg}$ A2, A7 and dipentum, carried as a positive control, on days 0-5 for acute and 28-34 for chronic studies. Colons were excised with the distal third taken for 0 to 40 point scoring histology. MPO activity, cytokine profile (Cytokine Core Labs, Baltimore, MD) and S1P levels (mass spectroscopy) were evaluated in the middle third. Results: Cellular SK activity was present and markedly inhibited by the A2 and A7. NFKB activation by TNF $\alpha$ was suppressed by SK inhibitors (approximate 40 fold induction of activity reduced to near baseline). TNFa treatment resulted in marked increased ICAM, VCAM and E-selectin expression that was clearly attenuated by A2 and A7. TNF $\alpha$-induced Cox- 2 activity, as measured as PGE2 level, was strongly suppressed by both A2 and A7 (from 30-100\%). Both animal models revealed similar and excellent protection by all 3 compounds as compared to DSS controls. The three drug-treated groups in both models (representative acute data shown, day 6, W: water only control) showed significantly reduced DAI's (W:0.15, DSS:2.80, A2:1.1, A7:1.0, DP:1.4) histology scores (W:5.7, DSS:24.3, A2:19.0, A7:15.7,DP:13.86), MPO levels (W:12, DSS: 87, A2:41, A7:34, DP: 57 units/g tissue), as well as longer colons (W: 7.25, DSS:5.4, A2:6.5, A7:6.3, DP:6.30 cm) and a more favorable cytokine panel as measured by TNF $\alpha$, Il-1 $\beta$, IFN- $\gamma$, IL-6 and IL-10. Finally, colonic S1P levels were also shown to be reduced (W: 2.4 DSS:5.3, A2:3.2, A7:1.0, DP:0.7 pM) by the SK inhibitors in the in vivo models, providing an excellent pharmacodynamic marker for drug activity. Summary/conclusion: These studies lend evidence that our selective sphingosine kinase inhibitors may prove an effective treatment in intestinal inflammation.

\section{M1690}

Photodynamic Therapy As a Novel Therapeutic Approach for the Treatment of Crohn's Disease

Laurent Favre, Dominique Velin, Francois Borle, Daniel Bachmann, Stefan Schreiber, Hanifa Bouzourene, Hubert van den Bergh, Georges Wagnieres, Christian Felley, Gian Dorta, Pierre Michetti, Maria-Anna E. Ortner

Background: Photodynamic therapy (PDT) may modify the mucosal immune response and thus serve as a therapy for Crohn's disease (CD). Aim: Safety (1) was evaluated in BALB/C mice. The effect (2) of «low dose» PDT was investigated in a SCID mouse colitis model. Methods: 1) Delta-aminolevulinic acid (D-ALA $15 \mathrm{mg} / \mathrm{kg}$ bodyweight; Medac, Hamburg Germany) was orally administered 3 hours before illumination $[635 \mathrm{~nm}$; argon dye lase (Coherent Inc. Santa Clara, USA); cylindrical diffuser (length $20 \mathrm{~mm}$, diameter $0.95 \mathrm{~mm}$ Medlight, Ecublens, Switzerland); energy dose 5 or 10 joules (J)/cm2]. At 74 hours, body weight, overall wellness, histology, and cytokine expression index (normalized number of mRNA copies of each manipulated mouse/normalized number of mRNA copies of the unmanipulated mice) were measured. 2) A single «low dose» PDT (15mg/kg ( $\delta$-ALA; 10J/ $\mathrm{cm} 2$ ) was performed in 3 independent experiments. An endoscopic index of colitis (EIC consisting of ulcers, vascular pattern, granularity, erythema, length involved) was developed, Mice with moderate $(\mathrm{EIC}>7)$ or marked colitis (EIC>10) were randomized to «low dose» PDT (group A) or no treatment (group B). EIC, cytokine expression indices, weight and length of the colon and histology were evaluated. Results: 1) «Low dose» PDT neither induced inflammation, nor mucosal damage, nor changes in body weight or overall wellness in BALB/c mice. 2) EIC (mean/SEM) improved 1 week after PDT in moderate colitis (group A: $8.2+0.7$ vs. $5.2+0.7$; group B $7.4+0.4$ vs. $9.0+0.7 ; p=0.007$ ). Healing occurred already 3 days after PDT in marked colitis (group A: $11+0.6$ vs. $6.3+1.1$; group $B: 10.3+0.2$ vs $10.9+1.3, \mathrm{p}=0.01$ ). EIC correlated with standard indices of inflammation like reduction of colon length (correlation coefficient $\mathrm{R}=0.58 ; \mathrm{p}<0.0001)$, increase in colon weight $(\mathrm{R}=0.55$, $\mathrm{p}=0.0002$ ) and histopathologic severity index (Kappa coefficient 0.77 ). The expression 
indices of IFN- $\gamma(p<0.05)$, TNF- $\alpha(p<0.05)$ and IL-10 $(p<0.01)$ were lower than in the disease control group. EIC correlated with cytokine expression indices of IFN- $\gamma(\mathrm{R}=0.77$; $\mathrm{p}<0.0001)$, TNF- $\alpha(\mathrm{R}=0.62 ; \mathrm{p}=0.0033)$ and IL-10 $(\mathrm{R}=0.79 ; \mathrm{p}<0.001)$. Conclusion: «Low dose» PDT is a promising novel therapeutic approach for the treatment of CD. In a mouse model it downregulated the proinflammatory immune response and improved colitis. PDT is the first local treatment that excludes toxic systemic side effects and can therefore easily be repeated at any time point, if indicated. In our opinion, it is now expedient to evaluate the effect of «low dose» PDT in patients with $\mathrm{CD}$

\section{M1691}

Ethyl Pyruvate Ameliorates T-Helper-1 (TH1)-Mediated Murine Colitis Shaival H. Dave, Fengling Li, Richard A. Demarco, Donna B. Stolz, Antonia R. Sepulveda, Mitchell P. Fink, Michael T. Lotze, Scott E. Plevy

BACKGROUND: Degraded matrix and substances released by necrotic cells, or "damage associated molecular pattern" (DAMP) molecules, can initiate or perpetuate inflammation. A prototypical DAMP, high mobility group box 1 (HMGB1), activates innate immune responses and is elevated in murine chronic colitis. Ethyl pyruvate (EP) has been shown to inhibit secretion of HMGBI and other cytokines by immunostimulated macrophages and improve survival in murine models of endotoxemia, sepsis, and hemorrhagic shock. We reasoned that EP may be protective in chronic colitis, where pathogenesis also depends on activation of inflammatory cytokine responses. OBJECTIVES: The current study investigates the immunomodulatory effects of EP in cultured macrophages and in a murine model of chronic colitis. METHODS: Murine bone-marrow derived and cell line macrophages were incubated with EP for 1 hour before stimulating with LPS and IFN-y. Expression of IL-12 p40 was detected by RT-PCR ( 4 h) and ELISA ( $24 \mathrm{~h}$ ). Nitric oxide (NO) was assessed using the Greiss reagent. IL-10 deficient (-/) mice at 10 weeks of age were injected i.p. with EP ( $40 \mathrm{mg} / \mathrm{kg}$ every other day) for two weeks and compared to a cohort injected with vehicle. Histological colitis was scored by a pathologist blinded to treatment group. Spontaneous cytokine production in intestinal explants was assessed by ELISA (mean \pm SD). Colonic fecal samples were collected in PBS and analyzed for HMGBI via a specific ELISA. Values were normalized to total protein. Expression of HMGBl and its receptor, RAGE, was determined in murine intestinal sections by immunohistochemistry. RESULTS: In a concentrationdependent fashion, EP decreased expression of IL-12 p40 and NO production in cultured macrophages. IL-10-/- mice treated with EP $(n=4)$ demonstrate amelioration of colitis compared to IL-10-/- mice treated with vehicle $(\mathrm{n}=6$ ) (colitis score $0.7 \pm 0.5$ vs. $6.2 \pm 2.1$ ). Intestinal explants of EP-treated mice ( $\mathrm{n}=4)$ express less IL-12 $\mathrm{p} 40$ than explants from vehicle-treated controls $(n=4)(201+106$ vs. $621+142 \mathrm{pg} / \mathrm{ml})$. Furthermore, fecal HMGB1 levels were lower in EP-treated mice $(n=4)$ than vehicle-treated controls $(n=4)(18 \pm 28$ vs. $122 \pm 70 \mathrm{ng} / \mathrm{mg})$. IL-10-/- mice demonstrate increased HMGBl and decreased RAGE immunoreactivity in intestinal epithelial cells (IEC). EP-treated IL-10-/- mice demonstrate decreased HMGBl and increased RAGE immunostaining CONCLUSIONS: Anti-inflammatory effects of EP are mediated in part through inhibiting HMGB1 release and decreased proinflammatory cytokine expression in macrophages. EP ameliorates established chronic colitis in IL-10-/- mice and therefore may have therapeutic potential in inflammatory bowel diseas

\section{M1692}

A High Sucrose Diet Exacerbates Immune-Mediated Colitis in IL-10 Deficient Mice

Kang Moon Lee, Byung Ik Jang, R Balfour Sartor

Background: Environmental factors play a significant role in the pathogenesis of inflammatory bowel disease (IBD). Diet is an important environmental factor that provides luminal antigens, serves as substrate for bacterial metabolism, and alters commensal microflora. Many epidemiological studies found that increased sugar consumption is associated with IBD. The effect of fiber intake on disease activity is controversial, but some studies show increased growth of commensal probiotic bacterial species and production of protective butyrate. This study evaluated the effect of clinically relevant diets on Thl-mediated experimental colitis. Methods: Purified control (5\% fat from vegetable oils, 3\% cellulose, 15\% sucrose), high fiber (5\% fat, $20 \%$ cellulose, $15 \%$ sucrose), and high sucrose (5\% fat, $3 \%$ cellulose, $66 \%$ sucrose) isocaloric diet were given to specific pathogen free (SPF) IL-10-/- mice (susceptible 129SvEv background, 6/group). Feeding was started at the time of weaning (age $\sim 21 \mathrm{~d}$ ) and necropsy was performed at age 12 weeks. Inflammation was quantified by blinded histology scores $(0-4+)$, spontaneous IL-12p40 secretion by cultured colonic strips, and IFN $\gamma$ secretion from mesenteric lymph node cells stimulated with cecal content lysates from SPF mice. Results: Table 1 and 2 show colonic inflammatory scores and cytokine secretion. Mice on the high sucrose diet had more severe colitis and higher IL-12p40 and INF $\gamma$ production compared to the control diet group. There were no differences between the control and high fiber diet group. Conclusion: A high sucrose diet enhanced colonic inflammation in IL-10-/- mice. These results confirm the importance of dietary factors in IBD and suggest an opportunity for therapeutic manipulation.

Table 1. Histology scores (0-4). Mean (SEM)

\begin{tabular}{|c|c|c|c|}
\hline & Cotrol & High Sucrose & High Fiber \\
\hline Cecal Histology & $1.1(0.2)$ & $2.8(0.1)^{*}$ & $1.8(0.8)$ \\
\hline Rt. Colon Histology & $1.1(0.2)$ & $2.7(0.4)^{*}$ & $1.0(0.3)$ \\
\hline Lt. Colon Histology & $1.3(0.2)$ & $3.0(0.9)^{*}$ & $1.0(0.3)$ \\
\hline
\end{tabular}

$*<0.05$ vs. control

Table 2. IL-12p40 (pg/0.05 gm colonic tissue) and INF $\gamma$ production (pg/ml). Mean (SEM).

\begin{tabular}{|c|c|c|c|}
\hline & Control & High Sucrose & High Fiber \\
\hline IL-12p40 & $631(365)$ & $1,357(194)^{*}$ & $589(159)$ \\
\hline INF $\gamma$ & & & \\
\hline media & $0(0)$ & $1,147(1,049)^{*}$ & $8(12)$ \\
\hline CL $10 \mu \mathrm{g} / \mathrm{ml}$ & $2,973(2,744)$ & $26,854(4,521)^{*}$ & $7,556(2,591)$ \\
\hline CL $50 \mu \mathrm{g} / \mathrm{ml}$ & $8,108(4,081)$ & $21,097(5,637)^{*}$ & $7,340(3,931)$ \\
\hline
\end{tabular}

* $<0.05$ vs. control, CL: cecal content lysates

M1693

R-Spondin1, a Novel Intestinotrophic Mitogen, Reduces Acute and Chronic Colitis in Mice

Jingsong Zhao, Eric Beck, Servando Palencia, Josephine de Vera, Kyung-Ah Kim, Yi Liu, Pauline Shinkawa, Peter Emtage, Walter Funk

R-Spondinl (Rspol) is a novel epithelial mitogen that stimulates the growth of the small and large intestine. As an intestinotrophic protein, we investigated the therapeutic potential of Rspol in ameliorating dextran sulfate sodium (DSS) and trinitrobenzene sulfonic acid (TNBS)-induced colitis in mice. Therapeutic administration of Rspol reduced body weight loss, occurrence of diarrhea, and rectal bleeding in a mouse model of DSS-induced colitis. Histological evaluation revealed that Rspol improved mucosal integrity in both villus and/ or crypt compartments in the small intestine and colon in DSS-treated mice. Rspol overcame DSS-induced suppression of crypt epithelial cell proliferation in both the small intestine and colon in mice, suggesting that Rspol attenuates intestinal injury by stimulating crypt cell growth and mucosal regeneration in DSS-treated mice. Rspol significantly reduced DSSinduced myeloperoxidase (MPO) activity and overproduction of pro-inflammatory cytokines, including tumor necrosis factor (TNF)- $\alpha$, interferon- $\gamma$, interleukin (IL)-10, and IL-12, in mouse intestinal tissue, indicating that Rspol may down-regulate DSS-induced inflammation in mouse bowel by preserving the mucosal integrity. Moreover, in a chronic DSS-induced colitis model in mice comprising multiple cycles of colitis attack and remission, Rspol showed a regenerative effect in reducing colitis. Likewise, Rspol therapy also alleviated TNBS-induced interstitial inflammation and mucosal erosion in mouse colon. In addition, Rspol protein was localized to villus epithelium and crypt Paneth cells in mouse small intestine. Our results demonstrate that Rspol may be clinically useful in the therapeutic treatment of inflammatory bowel disease by stimulating crypt cell growth, accelerating mucosal regeneration, and restoring intestinal architecture.

\section{M1694}

A PPAR $\alpha$ Ligand Delays Whereas a PPAR $\delta$ Ligand Accelerates the Onset of Inflammatory Bowel Disease in Interleukin 10 Deficient Mice Jimmy W. Lee, Poonam J. Bajwa, Christian Lytle, Daniel S. Straus

Background and Aims: We demonstrated recently that rosiglitazone, a ligand for peroxisome proliferator-activated receptor- $\gamma$ (PPAR $\gamma$ ), delayed the onset of spontaneous colitis in the IL10 knockout model of inflammatory bowel disease (IBD) [Lytle et al. (2005) Inflam. Bowel Dis. 11:231-243]. The purpose of the present project was to test the possible therapeutic activity of the PPAR $\alpha$ ligand fenofibrate, and the PPAR ligand GW0742, in IL-10 knockout mice, and to determine the cellular distribution of PPAR in the colon. Methods: The effect of long-term treatment with fenofibrate or GW0742 on progression of spontaneous colitis in IL-10 knockout mice was evaluated. The cellular distribution of PPAR $\alpha$ in the colon was determined by confocal immunofluorescence microscopy. Results: Treatment with fenofibrate 1) delayed the onset of disease activity, 2) decreased the colonic histopathology score, and 3) decreased the colonic expression of genes encoding the inflammatory cytokines interferon $\gamma$ and interleukin 17 , in IL-10 knockout mice. The target for fenofibrate, PPAR $\alpha$, was expressed in crypt and surface epithelial cells of the colon, and treatment of mice with fenofibrate resulted in decreased electrogenic chloride secretion by isolated colonic epithelium. In contrast to the beneficial effect of fenofibrate, treatment of IL-10 knockout mice with the PPARS ligand GW0742 accelerated the onset of IBD in IL-10 knockout mice. GW0742 inhibited the catalytic activity of cyclooxygenase-1 (COX-1) and was shown by molecular modeling to fit into the substrate-binding pocket of COX-1. Action as a COX inhibitor may account for the adverse effect of GW0742 in IL-10 knockout mice. Conclusions: PPAR $\alpha$ is expressed in mucosal epithelial cells, and the PPAR $\alpha$ ligand fenofibrate has preventative activity toward IBD in IL-10 knockout mice. Conversely, the PPAR ligand GW0742 has an adverse effect in IL-10 knockout mice, possibly due to its ability to inhibit cyclooxygenase.

\section{M1695}

Pathophysiological Role of the Cholinergic Anti-Inflammatory and Immune Pathway in Mouse Oxazolone-Induced Ulcerative Colitis Model

Takeshi Yamamoto, Toshihisa Kodama, Naho Utsunomiya, Hirofumi Kuramoto, Makoto Kadowaki

It is well known that T helper cell type 1 (Thl) immune responses may play an important role in Crohn's disease (CD), whereas Th2 responses may be involved in ulcerative colitis (UC). However, there has been a fewer studies focused on UC than CD, because of the lack of suitable Th2-mediated experimental colitis models. Epidemiologic reports suggest that smoking and nicotine may improve the symptoms of UC. The purpose of the present study was to modify mouse oxazolone (OXZ)-induced colitis model which has been reported as Th2-mediated colitis model and investigate pathophysiological role of the cholinergic antiinflammatory and immune pathway via nicotinic receptors in the modified OXZ colitis. METHODS: To enhance the immune response to the colonic instillation of the haptenating agent, OXZ, mouse $(\mathrm{BALB} / \mathrm{c})$ was first primed with painting the low concentration of 
OXZ on the skin of abdomen on days 0 followed by intrarectal administration of the low concentration of OXZ on day 7. OXZ colitis was assessed with the disease activity score (DAS) on weight loss, diarrhea and hemorrhage until day 10. Macroscopic examination of the colitis with colonic damage score (CDS) was performed, and then the colon was used for myeloperoxidase (MPO) activity analysis. RESULT: Exposure of the primed mice to intrarectal OXZ challenge developed a colitis marked by weight loss, hemorrhagic diarrhea, ulceration, erosion etc. associated with about 13-fold increased MPO activities in the colon. However, OXZ failed to induce the colitis in C57BL/6 mice which are used for Th1mediated disease models. In addition, pretreatment with calcineurin inhibitor to suppress Th1 responses deteriorated OXZ colitis. Therefore, oxazolone colitis is Th2-mediated and has similar histologic features to UC. Although oral administration of $5-\mathrm{ASA}(100 \mathrm{mg} / \mathrm{kg})$ had no significant therapeutic effect on OXZ colitis, prednisolone ( $10 \mathrm{mg} / \mathrm{kg} \mathrm{p}$.O.) significantly alleviate the disease state. Noteably, the central stimulation of vagus nerves with 2-deoxyd-glucose $(200 \mathrm{mg} / \mathrm{kg}$ i.p. $)$ significantly $(\mathrm{P}<0.05)$ improved OXZ colitis [DAS: $5.9 \pm 1.8$ vs $2.3 \pm 0.8$; CDS: $4.4 \pm 1.3$ vs $1.5 \pm 0.3$; MPO: $3562.5 \pm 1034.2$ vs $1399.0 \pm 353.4$ units/g wwt (OXZ colitis control vs mice pretreated with 2-deoxy-d-glucose)]. Furthermore, subcutaneous administration of nicotine $(3.2 \mathrm{mg} / \mathrm{kg})$ significantly $(\mathrm{P}<0.05)$ suppressed OXZ colitis [DAS: $10.5 \pm 1.6$ vs $5.1 \pm 1.8$; CDS: $7.6 \pm 1.3$ vs $3.7 \pm 1.3$; MPO: $5258.6 \pm 1466.9$ vs $2585.7 \pm 234.7$ units/g wwt (OXZ colitis control vs mice pretreated with nicotine)]. CONCLUSION: Nicotinic acetylcholine receptors work on the cholinergic anti-inflammatory and immune pathway to alleviate Th2-mediated OXZ colitis.

\section{M1696}

Sp304, An Analog of Uroguanylin, Ameliorates Inflammation in a Model of Experimental Colitis

Refaat Hegazi, Fengling Li, Gabriel Calilao, Antonia Sepulveda, Kunwar Shailubhai, Scott Plevy

Introduction: Guanylyl cyclase C (GC-C) agonists (uroguanylin (UG) and guanylin), regulate water and ion homeostasis in a variety of tissues and organs, including the gastrointestinal (GI) tract, via cyclic GMP (cGMP). GC-C and its agonists are expressed by intestinal epithelial cells (IEC). The cGMP pathway mediates anti-inflammatory effects of cellular molecules such as nitric oxide and heme oxygenase-1, and therapies that induce cGMP (phosphodiesterase-4 inhibitors) demonstrate efficacy in murine models of IBD. Accordingly, we reasoned that agonists of GC-C, when orally administered may demonstrate anti-inflammatory effects in murine IBD. Aim: The present study investigates the immunomodulatory effects of a GCC agonist, SP304 (Callisto Pharmaceuticals, Inc., NY) in TNBS-induced murine colitis. Methods: GC-C, guanylin and UG mRNA expression was studied by RT-PCR in intestinal tissue from 12-week old IL-10 deficient (-/) mice. To induce colitis, $0.1 \mathrm{ml}$ of $2.5 \%$ trinitro benzene sulfonic acid (TNBS) in 50\% ethanol was administered via catheter into the colonic lumen of 8 week old female in BALB-c mice (day 0). Mice were administered the GC-C agonist SP304 at $10(n=6), 50 \mu \mathrm{g} /$ day $(n=5)$ or vehicle PBS for 7 days $(n=8$ each $)$ by oral gavage starting at day 0 . On day 7 , mice were sacrificed. H\&E stained colonic tissue sections were assessed for colitis by a pathologist blinded to treatment group. Intestinal explants were cultured for 24 hours and levels of IL-12 p40, IL-12 p70, IL-23 and TNF protein secretion were measured in culture supernatants by ELISA. Results: GC-C, guanylin and UG mRNA are expressed in intestinal tissue of IL-10-/- mice. Intestinal guanylin and UG mRNA expression is induced during interventions in IL-10-/- mice that ameliorate colitis. In TNBS-induced colitis, histological assessment of colonic tissue demonstrated significant improvement of the colitis in the SP304-treated mice compared with the vehicle treated mice (Table). Intestinal explant cultures from SP304 treated mice express less IL-12 p40, IL-12 p70, IL-23 and TNF than the vehicle treated control group. Conclusions: Histological improvement in IL-10-/- mice correlates with upregulation of UG and guanylin mRNA. Treatment with SP304 exhibited anti-inflammatory effects in TNBS-induced colitis in mice. Importantly, amelioration of colitis was associated with downregulation of proinflammatory cytokines such as TNF.

\begin{tabular}{|c|c|c|c|}
\hline & PBS $(n=8)$ & SP304 $(10 \mu \mathrm{g} / \mathrm{d})(\mathrm{n}=6)$ & $\mathrm{SP} 304(50 \mu \mathrm{g} / \mathrm{d})(\mathrm{n}=5)$ \\
\hline Average colitis score & 14.13 & $6.67^{*}$ & $0.8^{*}$ \\
\hline SD & 4.39 & 3.27 & 1.79 \\
\hline
\end{tabular}

$* \mathrm{P}<0.05$

\section{M1697}

Lansoprazole, a Proton Pump Inhibitor, Suppresses Induction of TNF- $\alpha$ in Experimental Colitis in Rats-New Molecular Implication for Therapy for Intestinal Mucosal Inflammation Beyond Acid Suppression

Tetsuya Tanigawa, Toshio Watanabe, E.J. Sasaki, Masatsugu Shiba, Kazunari Tominaga, Yasuhiro Fujiwara, Nobuhide Oshitani, Masahiko Nakamura, Kazuhide Higuchi, Tetsuo Arakawa

Background and Aim: Enteric bacteria play a crucial role in the pathogenesis of inflammatory bowel diseases which are characterized by leukocytic infiltration and overexpression of cytokines in intestinal mucosa. Lansoprazole (LAN), a proton pump inhibitor, has been shown to exert anti-inflammatory effect other than inhibitory effect on acid secretion. We have previously demonstrated that LAN inhibits development of dextran sodium sulfate (DSS)-induced experimental colitis in rats. In this study we investigated molecular mechanisms underlying preventive effect of LAN on colitis. Methods: Experiment (1) Experimental colitis was induced in male Wistar rats by administration of $3 \%$ DSS solution for 3 days. The rats were also orally given LAN $(30 \mathrm{mg} / \mathrm{kg}$ BW) or vehicle from day 1 of experiment for 3 days. Colonic tissue was subjected to measurement of myeloperoxidase (MPO) activity (a marker of neutrophil infiltration), assay of mRNA level of tumor necrosis factor- $\alpha$ (TNF $\alpha$ ) by real-time RT-PCR, and double-immunostaining with antibodies against TNF $\alpha$ and monocytes/macrophages. To clarify the uptake sites of LAN in the colonic tissues, 3H-labeled LAN $(0.5 \mathrm{mCi} / 100 \mathrm{~g} \mathrm{BW})$ was given through intraaortic catheter and localization of $3 \mathrm{H}-\mathrm{LAN}$ was visualized by autoradiography. Experiment (2) Human promonocytic THP-1 cells were pretreated with LAN (1 100 $\mu \mathrm{M})$ and then incubated with lipopolysaccharide (LPS). Produc tion of TNF $\alpha$ was determined by ELISA and phosphorylation and degradation of IкB $\alpha$ and phosphorylation of ERK were evaluated by Western blotting. Results: (1) Administration of DSS caused inflammation and damage in the colonic mucosa and increased MPO activity and expression of TNF $\alpha$ mRNA. Treatment with LAN inhibited increase in MPO activit and overexpression of TNF $\alpha$ mRNA induced by DSS by 70\% and $49 \%$, respectively. Doubleimmunostaining demonstrated that monocytes/macrophages were main source of TNFo The uptake site of 3H-LAN in the normal colon mucosa were few, while in inflamed colonic mucosa, 3H-LAN was extensively accumulated in inflammatory cells including macrophages and polymorphonuclear cells. (2) LPS induced production of TNF $\alpha$, which was inhibited by pretreatment with LAN. LPS induced phosphorylation and degradation of IкB $\alpha$ and phosphorylation of ERK within $60 \mathrm{~min}$. LAN inhibited phosphorylation and degradation of I $\mathrm{K} \alpha$ and phosphorylation of ERK induced by LPS. Conclusion: These results suggest that LAN suppresses colonic mucosal inflammation induced by DSS via reduction of TNFo expression in inflammatory cells and this reduction by LAN is due to inhibition of activation of NFKB and ERK signaling pathways.

\section{M1698}

Anti-Inflammation and Repair Induced By Bone Marrow-Derived Mesenchymal Stem Cells for Dextran Sulfate Sodium-Induced Colitis in Rats

Fumio Tanaka, Kazunari Tominaga, Masahiro Ochi, Tetsuya Tanigawa, E.J. Sasaki, Masatsugu Shiba, Toshio Watanabe, Yasuhiro Fujiwara, Nobuhide Oshitani, Kazuhide Higuchi, Tetsuo Arakawa

Background: Bone marrow-derived cells including a small amount of mesenchymal stem cells (MSCs) had therapeutic effects for clinical human and experimental animal colitis. Its detailed mechanism(s) may be partly mediated by mucosal regeneration, since MSCs have potential for differentiation to several parts of cells. But MSCs were thought to have other functions such as anti-inflammation as well as mucosal regeneration, because anti-inflammatory system is involved in the repair of colitis. We examined the therapeutic efficacy and anti-inflammatory effects of bone marrow-derived MSCs for dextran sulfate sodium (DSS)induced acute colitis in rats. Materials \& Methods: Experimental colitis was induced by orally administration of $0,1,2$, or $4 \%$ DSS in drinking water for 7 days in inbred male Lewis rats. Bone marrow was extruded from tibias and femurs. Then, its mononuclear cells were isolated and cultured in low-glucose DMEM containing $10 \%$ fetal calf serum for MSCs outgrowth. On 0,2 , and 4 days after the administration of DSS, MSCs $\left(5 \times 10^{6}\right.$ cells $)$ were injected via tail vein. We checked the volumes of food and water intake, stool condition, and body weight everyday. On day 7 , total colon was excised and each colonic mRNA expression of inflammatory cytokines such as TNF- $\alpha$, IL-1 $\beta$, IL-10, and COX2 was measured by real time RT-PCR method. Results: We confirmed the MSC's characterization by both the immunostaining for vimentin and $\alpha$-smooth muscle actin and the cell surface markers such as CD90, the bone marrow progenitor cell marker, but not CD45, HLA-DR, CD11b nor CD31 using flow cytometric technique. Optimal dose of DSS for the rats used was confirmed at $4 \%$ by the assessing for loss of body weight and appetite, bloody fluid stool, and the shortening of colon length. MSC treatment improved the bloody stool and body weight loss, and significantly inhibited the shortening of colon length. At the rectum of MSC-treated rats, expressions of local inflammatory cytokines such as TNF- $\alpha$ and IL-1 $\beta$ were markedly decreased to about 40 and $15 \%$. Local COX2 expression was also suppressed to $15 \%$. IL-10, an anti-inflammatory cytokine, expression was also decreased to $25 \%$. A the distal colon site (slightly oral side of rectum), similar tendency was observed about the expressions of cytokines in the MSC-treated colons. Conclusion: These findings suggested that MSC could have the therapeutic efficacy for the experimental colitis via anti-inflammatory functions.

\section{M1699}

Induction of Regulatory T Cell Capacities By the Sphingosine-1-Phosphate Analogue Fty 720 in TNBS-Colitis Carolin Daniel, Nico Sartory, Heinfried H. Radeke, Gerd G. Geisslinger, Juergen M. Stein Background \& Aims: The sphingosine-1-phopshate analogue FTY720 is known to alter migration and homing of lymphocytes via sphingosine-1-phosphate receptor interactions. We studied the effect of FTY720 in acute and established trinitrobenzene sulfonic acid (TNBS)-colitis focused on the induction of regulatory T cell capacities. Methods: A rectal enema of TNBS [100 mg/kg body weight (BW)] was applied to male Balb/c mice, and FTY720 [1 or $3 \mathrm{mg} / \mathrm{kg}$ ] was administered intraperitoneally from day 0-3 or from day 3-5 following the instillation of the haptenating agent. The study is conforming to the Guiding principles in the care and use of animals and was performed under approval of the ethical committee of Darmstadt/Germany (F134/03). Colon tissue was analyzed macroscopically and microscopically, and IL-10, transforming growth factor $\beta$ (TGF $\beta$ ) and FoxP3 expression were determined in colon protein extracts. Results: Treatment with FTY720 reduced the histopathologic severity of TNBS-colitis abrogating macroscopic and microscopic intestinal inflammation. Additionally, treatment with FTY720 resulted in a significant induction of IL-10, TGF $\beta$ and FoxP3 (see Table). Conclusion: FTY720 exhibits beneficial prophylactic as well as therapeutic effects in TNBS-colitis. Moreover, the induction of IL-10, TGF $\beta$ and FoxP3 raised evidence for a tolerance inducing activity also contributing to the beneficial capacities of FTY720 offering new auspicious therapeutic strategies for the treatment of inflammatory bowel disease Results FTY720 TNBS-colitis 


\begin{tabular}{|c|c|c|c|c|c|}
\hline \multirow{2}{*}{ Paramters Treatments } & KO & \multicolumn{4}{|c|}{ TNBS } \\
\cline { 2 - 6 } & $\begin{array}{c}\text { untreated } \\
\text { control }\end{array}$ & untreated & $\begin{array}{c}0.9 \mathrm{mg} / \mathrm{kg} \\
\text { dex. }\end{array}$ & $\begin{array}{c}1 \mathrm{mg} / \mathrm{kg} \\
\text { FTY720 }\end{array}$ & $\begin{array}{c}3 \mathrm{mg} / \mathrm{kg} \\
\text { FTY720 }\end{array}$ \\
\hline Macroscopic score day 3 & $0.2 \pm 0.1$ & $3.9 \pm 0.3$ & $1.8 \pm 0.2 \mathrm{c}$ & $1.8 \pm 0.4 \mathrm{c}$ & $1.0 \pm 0.4 \mathrm{c}$ \\
\hline Microscopic score day 3 & $0.3 \pm 0.1$ & $3.8 \pm 0.2$ & $2.4 \pm 0.3 \mathrm{c}$ & $2.5 \pm 0.2 \mathrm{c}$ & $1.4 \pm 0.2 \mathrm{c}$ \\
\hline IL-10 [pg/mg protein] & $3.3 \pm 0.5$ & $3.3 \pm 0.1$ & $4.1 \pm 0.7 \mathrm{n} . \mathrm{s}$. & $4.8 \pm 0.4 \mathrm{~b}$ & $8.4 \pm 0.5 \mathrm{c}$ \\
\hline TGFß [pg/mg protein] & $229.6 \pm 41.5$ & $226.3 \pm 35.9$ & $\begin{array}{c}199.6 \pm 18.0 \\
\text { n.s. }\end{array}$ & $372.9 \pm 32.3 \mathrm{a}$ & $453.7 \pm 75.9 \mathrm{a}$ \\
\hline FoxP3 [\% of TNBS] & not detected & $100.0 \pm 0.0$ & not detected & $259.3 \pm 32.1 \mathrm{~b}$ & $422.4 \pm 10.4 \mathrm{c}$ \\
\hline $\begin{array}{c}\text { Delta Body weight day } \\
\text { 5 [\% of day 0] }\end{array}$ & $0.0 \pm 0.6$ & $-22.1 \pm 1.6$ & $-13.8 \pm 2.5 \mathrm{a}$ & $-14.9 \pm 2.5 \mathrm{~b}$ & $-11.9 \pm 1.4 \mathrm{a}$ \\
\hline $\begin{array}{c}\text { Clinical activity score } \\
\text { day 5 }\end{array}$ & $0.4 \pm 0.2$ & $11.0 \pm 0.2$ & $7.3 \pm 0.7 \mathrm{c}$ & $6.2 \pm 0.5 \mathrm{c}$ & $3.8 \pm 0.9 \mathrm{c}$ \\
\hline $\begin{array}{c}\text { Colon weight day 5 } \\
\text { [mg] }\end{array}$ & $163.8 \pm 6.9$ & $402.2 \pm 12.8$ & $308.0 \pm 7.2 \mathrm{c}$ & $306.7 \pm 7.2 \mathrm{c}$ & $248.4 \pm 14.5 \mathrm{c}$ \\
\hline
\end{tabular}

$\mathrm{a}=\mathrm{P}<0.05 ; \mathrm{b}=\mathrm{P}<0.01 ; \mathrm{c}=\mathrm{P}<0.001 ;$ n.s. $=$ non significant vs. TNBS-treated mice. dex. $=$ dexamethasone

\section{M1700}

Therapeutic Efficacy of a Recombinant Soluble Interleukin-1 Type II Receptor (sIL-1RII) in Experimental Crohn's Disease: A Preclinical Study Genevieve Fortin, Catherine Collette, Thierry Gustot, Alexandra-Chloe Villani, Andre van Gossum, Jacques Deviere, Edouard Louis, Alain Bitton, Gary Wild, Denis Franchimont

Auto-inflammation is the fundamental underlying process of chronic relapsing inflammatory diseases such as Crohn's disease (CD) and involves an impairment in Interleukin-1 $\beta$ (IL$1 \beta$ ) processing. NOD2 knock-in mice for $C D$ mutations demonstrate an altered processing of IL-1 $\beta$, leading to an overwhelming mucosal production of this pro-inflammatory cytokine. Therefore, the neutralization of IL-1 $\beta$ is reemerging as a potential therapeutic modality in $\mathrm{CD}$. We have recently identified SILI-RII as a top corticosteroid-inducible gene with potent anti-inflammatory actions. In this study, we examined the regulation of sIL-1RII in CD, and the therapeutic potential of a recombinant sIL-1RII Fc fusion protein (sIL-1RII/Fc) in trinitrobenzene sulfonic acid (TNBS) colitis in mice. Methods: Serum and intestinal biopsies were taken from $C D$ patients $(n=50)$ and healthy subjects $(H S)(n=15)$. sIL-1RII levels were quantified in serum and biopsy supernatants by ELISA. TNBS colitis was induced in Balb/ $\mathrm{c}$ mice and treated with either $2 \mathrm{mg} / \mathrm{kg}$ sIL-1RIL/Fc $(\mathrm{n}=12)$ or the Fc fragment alone (Fc) $(n=9)$. Drug efficacy was assessed by mortality, the Wallace criteria, myeloperoxidase (MPO) activity and by measuring cytokine production. Results: Phase 1: Circulating levels of sILIRII were significantly decreased in CD patients in remission ( $\mathrm{rCD}$ ) as compared to those of healthy subjects (HS) (16007 [9855-25507] vs. 20073 [14537-28505] pg/ml; $\mathrm{p}<0.05)$. This decrease was even more pronounced in active CD $(12516$ [6270-23996] pg/ml) as compared to $\mathrm{rCD}(\mathrm{p}<0.05)$ and HS $(\mathrm{p}<0.01)$. sIL-1RII secretion was also lower in CD noninflamed (CDnon-inf) $(\mathrm{p}<0.05)$ and $\mathrm{CD}$ inflamed $(\mathrm{CDinf})(\mathrm{p}<0.01)$ mucosa than in healthy mucosa (HCM). Interestingly, this decrease in sIL-1RII was associated with higher IL-1 $\beta$ levels in CDinf than in HCM and CDn-inf ( $p<0.01$ and $p<0.01$ respectively). Phase 2: 5 days after TNBS instillation, sIL-1RII/Fc-treated mice displayed significant $(\mathrm{p}<0.05)$ improvements in all criteria, including: a greater percent body weight than Fc-treated (control) mice $(87.4+/-4.4$ vs $78.5+/-2.5)$, fewer mortalities ( $8 \%$ vs $34 \%)$, a lower Wallace score $(6.3+/-$ 4.1 vs $12.2+/-1.4)$, MPO activity index $(1.2+/-0.6$ vs $2.7+/-0.5)$, serum IL- 6 concentration $(43.9+/-15.3$ vs $123.3+/-63.5)$, and mucosal expression of IL- $1 \beta$ and IL-6 (3.6-fold and 8.4fold decrease in treated mice versus controls, respectively). Conclusion: sIL-1RII/Fc appears to be an effective therapy in experimental $C D$ that could compensate for the relative deficiency of endogenous sIL-1RII in CD patients.

\section{M1701}

Acylcarnitine Reduces the Severity of Murine DSS Colitis Pascal Muller, Ijaz Qureshi, Ihsan El Imrani, Catherine Collette, Ginette Fortin, Justine Dassa, Lynda Abed, Luc Oligny, Emile Levy, Serge Dionne, Denis Franchimont, Ernest G Seidman

INTRODUCTION: Carnitine is critical to cellular metabolism, including the $\beta$-oxidation of long chain fatty acids in mitochondria. Polymorphisms of the organic cation/carnitine transporters OCTN1 \& 2 (IBD5 locus 5q31) confer susceptibility to IBD. We have previously demonstrated the presence of OCTN 1 \& 2 in intestinal epithelium and shown that acetylcarnitine (AC) deficiency aggravates experimental colitis. We thus hypothesized that AC could improve the severity of colitis. METHODS: We utilized the dextran sulphate sodium (DSS) colitis model. BALB/c mice were divided into 4 groups of $8: 2$ groups were injected (IP) with AC (100 or $500 \mathrm{mg} / \mathrm{kg}$ ), and 2 groups with saline. Colitis was induced 1 week later using DSS 4\% in the drinking water (1 week) in 3 groups; a 4th control saline group was given plain water. The murine colitis disease activity index (DAI) was employed to compare outcomes (comprising \% body weight, severity of diarrhea and fecal blood loss). Mice were then sacrificed and their colons examined macro- and microscopically by pathologists unaware of treatments. Colonic IL-lb \& IL- 6 gene expression was determined by quantitative real time PCR. Short chain acylcarnitine and free radical levels (malondialdehyde, MDA) were also measured. RESULTS: The only deaths due to DSS colitis occurred in unsupplemented mice ( 3 of 8 ). Weight loss was reduced in the AC supplemented mice with colitis (16\% \& 4\% losses in DSS/carnitine $100 \mathrm{mg} / \mathrm{kg}$ and $500 \mathrm{mg} / \mathrm{kg}$ groups respectively, vs $24 \%$ in DSS/saline group; $\mathrm{p}<0.05 \& \mathrm{p}<0.01$, respectively) Overall DAI scores were also significantly lower in the 2 AC supplemented DSS colitis groups vs the DSS/saline group (total score 9.6 and 7.1 vs. 11.2 respectively; $\mathrm{p}<0.05$ and $\mathrm{p}<0.01$ ). Mean total histological damage scores were lowest for the $500 \mathrm{mg} / \mathrm{kg}$ AC group $(14.0+1-0.9 \mathrm{SD})$ compared to the DSS/saline treated mice $(22.8+/-0.5 ; \mathrm{p}<0.01)$. MDA levels were significantly higher in the unsupplemented DSS colitis animals $(\mathrm{p}<0.01)$, whereas tissue AC levels were highest in both AC supplemented groups. Colonic expression of IL-1b \& IL-6 were reduced in AC supplemented animals $(500 \mathrm{mg} / \mathrm{kg}$ ) compared to the non-supplemented DSS colitis group (4.4 and 3.8 fold, respectively). DISCUSSION: AC supplementation effectively reduced the severity of DSS induced colitis, suggesting a potential novel therapeutic intervention for IBD patients.

\section{M1702}

Novel Orally Active $\alpha 4$ Integrin Antagonist, T-728, Attenuates Dextran Sodium Sulfate-Induced Chronic Colitis in Mice

Ken-Ichi Hayashi, Hiroyuki Meguro, Sunao Hara, Rie Sasaki, Yoko Koga, Meiko Takeshita, Naoyoshi Yamamoto, Hiroe Hirokawa, Mie Kainoh

BACKGROUND AND AIM: Recruitment of circulating leukocytes into colonic tissue is a key feature of intestinal inflammation. Alpha4 integrin is expressed in leukocytes and plays an important role in adhesive interactions between leukocytes and endothelial cells. A humanized anti- $\alpha 4$ integrin $\mathrm{mAb}$ was recently reported to be effective in patients with Crohn's disease, and thus orally active $\alpha 4$ integrin antagonists have received much attention as a potential approach for the treatment of inflammatory bowel disease. In the present study, we investigated the effects of T-728, a novel orally active $\alpha 4$ integrin antagonist, on chronic colitis in mice. METHODS: Chronic colitis was induced in female BALB/c mice after three cycles of dextran sodium sulfate (DSS) application. T-728 (0.1-150 mg/kg) was orally administered once or twice daily over a 5-day period after the final dose of DSS. The effects of T-728 on disease activity index (DAI) and histological inflammation score were compared with those of anti-mouse $\alpha 4$ integrin $\mathrm{mAb}(10 \mathrm{mg} / \mathrm{kg}$, i.p.). The DAI includes weight loss, stool consistency and rectal bleeding, and the histological inflammation score considers crypt damage, intensity of inflammation and extent of inflammation. The effects of T-728 on lymphocyte homing to Peyer's patches were evaluated 75 min after oral administration in the chronic colitis model. RESULTS: T-728 at a dose of $0.1 \mathrm{mg} / \mathrm{kg}$ or higher improved DAI and/or histological inflammation score in DSS-induced chronic colitis. The efficacy of T-728 was comparable to that of anti- $\alpha 4$ integrin mAb. Moreover, T-728 at doses of 0.4 , 2 and $10 \mathrm{mg} / \mathrm{kg}$ prevented lymphocyte homing. CONCLUSIONS: Oral administration of T-728 or anti- $\alpha 4$ integrin mAb inhibited chronic colitis in mice. The novel orally active $\alpha 4$ integrin antagonist T-728 may be beneficial for the treatment of patients with inflammatory bowel disease.

\section{M1703}

Attenuation of Mouse DSS Colitis By Naked Hepatocyte Growth Factor Gene Transfer Into the Liver

Yusuke Kawauchi, Kenji Suzuki, Masaaki Takamura, Satoshi Yamagiwa, Hiroyuki Yoneyama, Gi Dong Han, Hiroshi Kawachi, Fujio Shimizu, Hitoshi Asakura, Jun-Ichi Miyazaki, Hiroki Maruyama, Yutaka Aoyagi

Background: Hepatocyte growth factor (HGF) has multiple biological effects on a wide variety of cells. It modulates intestinal epithelial proliferation and migration, and critically regulates intestinal wound healing. Aims: To investigate the therapeutic effect of the HGF gene transfer, we introduced HGF gene into the liver of mice with acute colitis. Methods: The rat HGF expression plasmid vector, pCAGGS-HGF was injected via the tail vein into C57BL/6 mice, followed by dosing with sodium dextran sulfate in distilled water. Firstly, the HGF gene was injected once on day 0 . Secondly, the HGF gene was injected on day 0 and again on day 2 . In addition we performed the ex vivo organ culture experiments, which could reveal the direct effect of HGF on immune response in the colon. We analyzed the expression of mRNA of IFN- $\gamma$ and IL-10 in the colonic explants of mice with DSS colitis Results: Injection of the HGF gene ameliorated colitis with inhibition of both loss of body weight and shortening of colon length. It protected the colon from epithelial erosions and cellular infiltration. Expression of mRNAs for IFN- $\gamma$, IL18, and TNF- $\alpha$ was reduced in the colon. In contrast, expression of mRNA for IL-10 was increased. The numbers of BrdUpositive intestinal epithelial cells were increased, and the numbers of TUNEL-positive apoptotic cells were decreased. Furthermore, a second injection prolonged the elevation of serum HGF levels, and ameliorated the symptoms better than a single injection. The empty pCAGGS plasmid did not ameliorate the acute colitis. The expression of mRNA for IFN- $\gamma$ was clearly reduced by the treatment with HGF on explants, whereas expression of mRNA of IL-10 was increased markedly in the ex vivo colon tissue cultured with HGF . Conclusions: HGF gene transfer attenuated acute colitis by facilitating intestinal wound repair as well as inhibiting the inflammation. HGF directly regulates the transcription of pro-inflammatory and antiinflammatory cytokine in the diseased colonic tissue. These results suggest that HGF gene therapy could be new therapeutic approach to inflammatory bowel disease

\section{M1704}

Stat5b Promotes Mucosal Tolerance in Crohn's Disease and Murine Colitis Xiaonan Han, Bankole Osuntokun, Nancy Benight, Kimberly Loesch, Stuart J. Frank, Lee A Denson

Background: Current data suggest that Crohn's disease (CD) is due to an exaggerated adaptive immune response to the enteric flora, driven by primary defects in the epithelial barrier and innate immune system. A recent analysis of the global pattern of gene expression in $C D$ and Ulcerative Colitis colonic biopsies has identified down regulation of the STAT5b.We have reported that growth hormone $(\mathrm{GH})$ dependent STAT5b activation is reduced in affected $\mathrm{CD}$ colon at diagnosis. However, the mechanism by which a local reduction in STAT5b activation and expression might promote chronic gut inflammation was not known.We hypothesized that STAT5b controls susceptibility to colitis by regulating genes involved in maintaining both the mucosal barrier function and the normal tolerant response to the enteric flora. Methods: STAT5b, PPARg, NFkB activation and mucosal barrier function were determined in normal and CD colon, in STAT5b deficient mice with TNBS-induced colitis, and in mice with colitis due to IL-10 deficiency. The effect of chronic GH administration on STAT5b activation and disease severity was determined in the two murine models of 
colitis. RNA interference was used to knock down STAT5b expression in the T84 colon epithelial cells (CEC) line and the effect upon TNFa dependent NFkB activation was determined. Results: GH dependent STAT5b activation and PPARg expression were reduced in affected $\mathrm{CD}$ colon, in both $\mathrm{CEC}$ and lamina propria macrophages. Chronic GH administration activated STAT5b and up regulated PPARg in both normal and inflamed IL-10 deficient mouse colon, leading to reduced NFkB activation in the inflamed colon. STAT5b deficient mice exhibited reduced colonic expression of the tight junction proteins (TJPs) ZO-1, ZO2 , and ZO-3, as well as reduced PPARg nuclear abundance. Occludin serine phosphorylation was also reduced. This was associated with mucosal edema and increased NFkB activation, and then more severe weight loss and proximal colitis following TNBS administration. Chronic GH administration following induction of TNBS colitis enhanced weight gain in wild type but not STAT5b deficient mice, relative to PBS treated controls. Knock down of STAT5b expression in T84 cells led to a significant increase in NFkB activation induced by TNFa. Conclusions: STAT5b activation is impaired in affected colon in CD, and STAT5b deficiency increases susceptibility to experimental colitis, through down regulation of TJPs which regulate the mucosal barrier, and PPARg which regulates the response to the enteric flora. STAT5b activation may therefore represent a novel therapeutic target for restoring both barrier function and mucosal tolerance in IBD

\section{M1705}

Dependence of Intestinal Granuloma Formation On a Unique F4/80+ Dendritic Cell Subset

Ken Sugimoto, Atsuhiro Ogawa, Yasuyo Shimomura, Takashi Nagaishi, Emiko Mizoguchi, Richard S. Blumberg, Atsushi Mizoguchi, Atul K. Bhan

BACKGROUND \& AIMS: Granulomas represent localized inflammatory reactions that are characteristically observed in several inflammatory conditions. In IBD, granulomatous inflammation specifically develops in CD but not UC. However, the mechanisms leading to granuloma formation have not been fully defined. Interestingly, the UC-like colitis in TCR $\alpha$ knockout (TCR $\alpha \mathrm{KO}$ ) mice changes to a distinct type of colitis characterized by granuloma formation in the absence of both IL-4 and B cells in TCR $\alpha$ KO mice ( $\alpha \mu 4$ triple KO mice). Therefore, this study was designed to define the mechanism of granuloma formation using $\alpha \mu 4$ TKO mice. METHODS: Cells from areas of granulomatous inflammation in $\alpha \mu 4 T K O$ mice were functionally and phenotypically analyzed and also subjected to local cell transfer studies. RESULTS: Flow cytometric and immunohistochemical analyses found that approximately $20 \%$ of cells within granulomas of $\alpha \mu 4 \mathrm{TKO}$ mice show immature myeloid dendritic cell phenotype as indicated by the surface profile $\left(\mathrm{CD} 1 \mathrm{cc}^{+} \mathrm{CD} 1 \mathrm{bb}^{+} \mathrm{CD} 86^{\text {low }} 22 \mathrm{~B}^{-} \mathrm{Grl}^{-} \mathrm{CD} 8 \alpha^{-}\right.$ ). Interestingly, these cells also expressed a macrophage marker, F4/80, but not another macrophage marker, MOMAl. Functionally, the unique $\mathrm{F} 4 / 80^{+}$immature myeloid dendritic (IMD)-like cells were characterized by the presence of phagocytic ability as judged by uptake of FITC-dextran and by the absence of antigen-presenting ability as judged by mixed lymphocyte reaction. Of note, the purified $\mathrm{F} 4 / 80^{+} \mathrm{CDll} \mathrm{c}^{+}$cells exhibited fine dendrites, a specific feature for dendritic cells. The IMD-like cells produced large amounts of IL-23 that was further upregulated in the presence of ligands of TLR9 ( $\mathrm{CPG}$ ) and TLR7 (loxoribine). In contrast, this IL-23 production was efficiently inhibited by the presence of IL-4 as well as intact IgG but not its $\mathrm{F}(\mathrm{ab})^{2}$ fragment. Importantly, local transfer of granuloma-derived $\mathrm{CD}_{11 \mathrm{c}^{+}}$cells induced granuloma formation in the ileocecal junction, liver and kidney of recipient TCR $\alpha$ KO mice in the absence, but not presence, of IL-4 and B cells. In addition, continuous administration IL-4 through implanted osmotic pump or repeated i.p. injection of IgG suppressed the IMD-like cell-mediated granuloma formation in the recipient mice. CONCLUSION: We have identified a unique $\mathrm{CDIlc}^{+}$dendritic subset that co-expresses the macrophage marker (F4/80) and induces granuloma formation under intestinal inflammatory conditions.

\section{M1707}

Transforming Growth Factor- $\beta$ Signaling and Matrix Metalloproteinase Pattern in Intestinal Strictures in Crohn's Disease

Antonio Di Sabatino, Mark Buckley, Karen M. Pickard, Raffaele Morera, Paolo Cazzola

Gino R. Corazza, Tom T. MacDonald, Sylvia L. Pender

"Background \& Aims": In addition to its crucial role in dampening tissue-damaging immune responses in the gut, transforming growth factor (TGF)- $\beta$ has a potent profibrogenic action that it exerts by inducing fibroblast proliferation, by down-regulating matrix metalloproteinase (MMP) expression and by enhancing tissue inhibitor of matrix metalloproteinases (TIMP) expression. To elucidate the mechanisms which may underlie intestinal fibrogenesis and stricture formation in Crohn's disease (CD), we explored intracellular proteins, whose phosphorylation is involved in activating ( $\mathrm{pSmad} 2 / 3$ ) or inhibiting (Smad7) TGF- $\beta$ signal transduction, and MMP patterns in CD patients. "Methods": Endoscopic ileal and colonic biopsy specimens were collected from strictured and non-strictured mucosa of 12 patients affected by fibrostenosing $C D$. Biopsies from inflamed mucosa of nine non-fibrostenosing CD patients and from 11 control subjects were also studied. pSmad2/3, Smad7, MMP-3, MMP-12 and TIMP-1 were determined by Western Blotting of homogenized tissue samples. Each blot was stripped and analyzed for Smad3 or $\beta$-Actin as an internal loading control. TGF- $\beta$ transcripts were analysed by RT-PCR. "Results": A lower expression of Smad7 was found in strictured than in non-strictured mucosa in CD patients. As expected, Smad7 was strongly upregulated in CD inflamed mucosa. pSmad2/3 was significantly higher in strictured than in non-strictured mucosa. Strictures expressed greater number of TGF- $\beta$ transcripts than non-stricures. MMP-3 and MMP-12 were significantly decreased in strictured in comparison to non-strictured mucosa. High expression of MMP-3 and MMP-12 was found in CD inflamed lesions. TIMP-1 was significantly higher in strictured that in non-strictured areas. "Conclusions": Our findings of decreased Smad7 and increased pSmad2/3 expression in strictures support the profibrogenic role of TGF- $\beta$ in CD. The reduced expression of MMP3 and MMP-12 together with the increased expression of TIMP-1 in strictured mucosa suggest that TGF- $\beta$ may induce intestinal fibrogenesis by changing the balance between MMP and TIMP expression in CD.

\section{M1708}

Cd4+ T Cells Are Regulators in An Hapten Specific Cd8+ T Cell Mediated Relapsing Colitis in Normal Mice

Stephane Nancey, Sebastien Holvoet, Ivan Graber, Bernard Flourie, Dominique Kaiserlian

Background : We have demonstrated that CD8+, but not CD4+ T cells, were pathogenic in an antigen-specific model of relapsing colitis in normal mice (Gastroenterology 2005, 128, T1576 abstract). In this model, mice progressively recover from colitis and a second hapten challenge is responsible for a relapse of colitis, mimicking the course of $C D$. Our aim was to study the role of CD4+ T cells in this colitis model. Materials and Methods: BALB/c mice previously sensitized by the hapten DNBS developed a relapsing colitis upon each hapten administration in the colon (challenge) using non inflammatory doses of the hapten. The role of $\mathrm{CD} 4+\mathrm{T}$ cells was investigated in $\mathrm{BALB} / \mathrm{c}$ and $\mathrm{C} 57 \mathrm{Bl} / 6$ mice, the former strain being sensible and the latter resistant to the usual load of the hapten. CD4+ T cell depletion using i.p. injections of CD4 mAb were performed. CD8+ T cells were isolated from mesenteric LN (MLN) and spleen and their proliferation and release of IFNg production (ELISA) after DNBS stimulation were studied. Results :Firstly, using BALB/c mice, we found that in vivo CD4+ T cell depletion did not affect significantly the severity of colitis after the first hapten challenge but enhanced its severity after the second challenge with DNBS (increased of body weight loss and histologic lesions). Secondly, a challenge dose of DNBS unable by itself to induce colitis in previously sensitized mice resulted in the development of evident signs of colitis only in CD4+ T cell depleted mice. Thirdly, CD4+ T cells regulated in vitro hapten specific CD8+ T cell priming in MLN but not in spleen (higher levels of hapten specific CD8+ T cell proliferation and IFNg production after hapten stimulation in CD4+ T cell depleted mice). Fourthly, whereas C57Bl/6 strain mice did not develop colitis, the resistance to colitis was abrogated in Abeta deficient syngeneic mice (MHC class II deficient) that are characterized by the absence of CD4+ T cells. Conclusion: Altogether these data strongly suggest that class II restricted CD4+ T cells behave as regulatory cells in the antigen-specific $\mathrm{CD} 8+\mathrm{T}$ cell mediated model of relapsing colitis. Their phenotype and the mechanism of regulation warrant further studies.

\section{M1709}

Btnl2, a Butyrophilin/B7-Like Molecule, Is a Negative Costimulatory Molecule Modulated in Intestinal Inflammation

Heather A. Arnett, Sabine S. Escobar, Eva Gonzalez-Suarez, Alison L. Budelsky, Lori A. Steffen, Norman Boiani, Ming Zhang, Gerald Siu, Avery W. Brewer, Jo L. Viney

Butyrophilin-like 2 (BTNL2) is a butyrophilin family member with homology to the B7 costimulatory molecules, polymorphisms of which have been recently associated through genetic analyses to sporadic inclusion body myositis and sarcoidosis. We have characterized the full structure, expression, and function of BTNL2. Structural analysis of BTNL2 shows a molecule with an extracellular region containing 2-4 Ig domains, a transmembrane region and a previously unreported cytoplasmic tail. Unlike other butyrophilin members, BTNL2 lacks the prototypical B30.2 ring domain. Taqman and Northern analysis indicate BTNL2 is predominantly expressed in digestive tract tissues, in particular small intestine and Peyer's patches. Immunohistochemistry with BTNL2-specific antibodies further localizes BTNL2 to epithelia and dendritic cells within these tissues. Despite its homology to the B7 family, BTNL2 does not bind any of the known B7 family receptors such as CD28, CTLA4, PD1 or ICOS. Because of its localization to the gut and potential role in the immune system BTNL2 expression was analyzed in a mouse model of inflammatory bowel disease. BTNL2 is overexpressed during both the asymptomatic and symptomatic phase of the Mdrla model of spontaneous colitis. In functional assays, soluble BTNL2-Fc protein inhibits the proliferation of murine CD4+ T cells from the spleen, mesenteric lymph node, and Peyer's patch, suggesting a role for BTNL2 as a negative costimulatory molecule.

\section{M1710}

Unfolded Structure of Paneth Cell $\alpha$-Defensin Disrupts the Microbicidal Function in Patients with Crohn's Disease

Atsuo Maemoto, Hiroki Tanabe, Toshifumi Ashida, Yutaka Kohgo, Tokiyoshi Ayabe

Background\&Aim: Paneth cells contribute to intestinal innate immunity by sensing bacteria and secreting microbicidal $\alpha$-defensins, such as HD5 in human. HD5 is stored as precursor; pro-HD5 in Paneth cells and cleaved by trypsin at secretion. In Crohn's disease (CD), the microbe-epithelia interaction has been suggested to contribute to the pathology. To clarify the involvement of Paneth cell $\alpha$-defensins in CD, we investigated processing of $\alpha$-defensins and their function in Paneth cells of patients with CD. Methods: Ileal mucosa were obtained from surgical specimens resected from patients with $C D$, and normal ileum from patients with colon cancer under informed consent. Immunohistochemical analyses on tissue specimens were conducted using anti-HD5 antibody. Crude peptides extracted from non-inflamed ileal mucosa from patients with CD were subjected to MALDI-TOF/MS for pro-HD5. Products cleaved by trypsin from crude peptides were analyzed by AU-PAGE western blotting against HD5. Isolated normal crypts or crypts from CD patients were co-cultured ex vivo at $37 \mathrm{C}$ for $30 \mathrm{~min}$ with S. typhimurium or E. coli 1,000 CFU/crypt, and supernatants are assayed for bactericidal activities against defensin-sensitive S. typhimurium. Results: HD5 co-localized in Paneth cell granules was detected in the ileum of CD patients. Western blot analyses showed that pro-HD5 peptides from CD patients cleaved by trypsin was degraded, meanwhile, those from normal controls donated mature HD5. MALDI-TOF/MS confirmed that the structure of pro-HD5 from CD patients was linear, i.e. tridisulfide array of pro-HD5 was disrupted, otherwise from controls was folded. We confirmed that the microbicidal activity of the degraded, linear HD5 was significantly low relative to those of folded. Bactericidal activities released from isolated crypts from $C D$ patients in response to S. typhimurium or E. coli were significantly diminished relative to normal controls on a per crypt basis. Conclusion: In Crohn's disease, the tertiary structure of Paneth cell $\alpha$-defensin was not folded and degraded during precursor processing so that those $\alpha$-defensins failed to kill bacteria effectively. The impaired innate defense mechanisms in Paneth cells might contribute to the pathology of $C D$

\section{(1)}




\section{M1711}

Negative Regulation of Toll-Like Receptor By C-Type Lectin in Murine TNBS Colitis

Hideki Iijima, Masahiko Tsujii, Satoshi Egawa, Shinichiro Shinzaki, Shuji Ishii, Takanobu Irie, Tsutomu Nishida, Masakazu Yasumaru, Yoshimi Kakiuchi, Hiroaki Murata, Tatsuya Kanto, Shingo Tsuji, Norio Hayashi

Background and Aims Toll-like receptors (TLRs) expressed on the intestinal innate immune cells play important roles for the initiation of inflammation against luminal pathogens. Inappropriate regulation of TLRs is suggested to have a role in the pathogenesis of inflammatory bowel disease (IBD). Recently, C-type lectins, e.g., mannose receptor (MR) and Dendritic Cell-Specific Intercellular Adhesion Molecule-3-Grabbing Nonintegrin (DC-SIGN), have been shown to downregulate inflammatory cytokines and to induce IL-10 in macrophages and dendritic cells under TLR4 stimulation. In the present study, we investigated the role of Ctype lectins in the mouse model of IBD. Methods Peritoneal macrophages from C57BL/6 mice were cultured in vitro with lipopolysaccharide (LPS; the lidgand for TLR4) for 24h and were further cultured with lipoarabinomannan (LAM: the ligand for MR and DC-SIGN) for 24h. Culture supernatants were collected and were analyzed for TNF- $\alpha$ and IL-10 production. TNBS colitis was induced in C57BL/6 mice (8-10 wks) and expression of MR and DC-SIGN after the induction of TNBS colitis was analyzed by flow cytometry. These mice were treated by intraperitoneal administration of LAM every other day started from one day before TNBS administration. Production of TNF- $\alpha$ and IL-10 from peritoneal macrophages was analyzed. Mononuclear cells from spleen and colonic lamina propria were stimulated in vitro with anti-CD3 and anti-CD28 mAb and culture supernatants were analyzed for IFN- $\gamma$ and IL-10 production. Results LPS induced TNF- $\alpha$ production from peritoneal macrophages in vitro and LAM induced significant suppression of TNF- $\alpha$ production and enhancement of IL-10 production in a dose dependent manner. Expression of DC-SIGN but not MR, on macrophages in the peritoneum and colonic lamina propria was upregulated by the administration of TNBS when compared with the PBS-treated mice. In the TNBS colitis model, administration of LAM induced IL-10 production from peritoneal macrophages and splenic mononuclear cells. IFN- $\gamma$ productions from spleen and colonic lamina propria were not affected by the treatment of LAM in the TNBS colitis model. Conclusions Ligation of C-type lectin suppressed the pathway of TLR4 to induce inflammatory TNF- $\alpha$ by LPS. On the other hand, regulatory IL-10 was induced by the treatment of LAM. Modulation of TLR4 pathway by C-type lectin can be a new therapeutic candidate of inflammatory bowel disease.

\section{M1712}

Phenotypic and Functional Characterization of Ccr9+ T Lymphocytes in Small Intestinal Crohn's Disease (CD)

Qi T. Yu, Masayuki Saruta, Stephan R. Targan, Konstantinos A. Papadakis

Background and Aim: C-C chemokine receptor (CCR)-9 expression characterizes a subset of T lymphocytes with homing potential to the small intestine (SI). CCR9+ T cells are increased in the circulation of patients with SI Crohn's disease (CD) and celiac disease. The aim of the current study was to analyze the phenotype and cytokine profile of CCR9+ T cells in mucosal lymphoid tissues in patients with CD. Methods: The phenotype of CCR9+ $\mathrm{T}$ cells in mucosal lymphoid tissues, lamina propria (LP) and mesenteric lymph nodes (MLN) was analyzed by flow cytometry. Analysis of IFN-gamma and IL-10-producing cells was performed by intracellular cytokine (IC) staining and IFN-gamma was measured by ELISA in culture supernatants. Results: Phenotypic analysis of T lymphocytes isolated from MLN draining inflamed $C D$ SB revealed that $C C R 9+T$ lymphocytes express higher levels of activation makers (CD25, CD69, HLD-DR), co-stimulatory molecules (OX-40, CD40L, CTLA-4), and chemokines receptors CXCR3 and CCR5, compared to CCR9- T lymphocytes; indicating that CCR9 expression is acquired on activated T cells in draining MLN in SB CD. Compared to CCR9+ T cells obtained from MLN draining normal SB, those from SB CD express higher levels of OX40, CD40L, and HLA-DR, but not CD25 or CD69. Similar phenotypic differences were observed in CCR9+ T cells isolated from $\mathrm{PB}$ of $\mathrm{SB} C \mathrm{CD}$ patients compared to normal donors. Interestingly, no major phenotypic difference among CCR9+ T cells was observed in LP between normal and inflamed CD SB. Most CCR9+ T cells from both normal and inflamed CD SB produced IFN-gamma and a low percentage of them were IL-10-producing cells as assessed by ic staining. However,IFN-gamma production was significantly higher among CCR9+ T cells isolated from inflamed compared to normal SB following CD3 or CD2 activation. In addition, priming of CCR9+ T cells with IL-12/IL-18 led to significantly higher level of IFN-gamma production in inflamed compared to normal SB mucosa. Moreover, the addition of TLIA markedly enhanced ( 4 fold) IFN-gamma production from CCR9+ T cells isolated from inflamed compared to normal SB. Conclusions: CCR9+ T cells represent effector Thl cells in SB CD since they produce large amount of IFN-gamma following anti-CD3 or -CD2 as well as IL-12/IL-18 and TL1A stimulation. Therefore, the use of CCR9 antagonists represents a rational novel treatment for human SB Crohn's disease. Supported by a grant from the Broad Medical Research Foundation

\section{M1713}

The Frequency of Mucosal $\mathrm{Cd} 4^{+} \mathrm{Cd} 25^{\text {High }}$ Regulatory T Cells Increases with Colonic and Systemic Disease Activity in Ulcerative Colitis; Presence of Functional Colonic Treg Cells

Nathalie Holmen, Anna Lundgren, Samuel Lundin, Ann-Marie Bergin, Anna Rudin, Henrik Sjovall, Lena Ohman

AIM: The aim of this study was to characterise regional differences in the colonic lamina propria $\mathrm{CD}^{4} \mathrm{CD} 25^{\text {high }} \mathrm{T}$ cell (Treg) population in ulcerative colitis (UC), the relationship of colonic Treg cell frequency and phenotype to the colonic and systemic disease score of UC patients, and to investigate the functional properties and the T cell receptor repertoire of colonic Treg cells. METHOD: Patients with UC in either remission or with active disease $(\mathrm{n}=25)$ were compared to controls $(\mathrm{n}=11)$ without gastrointestinal symptoms. Biopsy samples were taken during colonoscopy and intestinal tissue was also obtained from surgical resections of UC patients. The frequency of mucosal and peripheral blood Treg cells was analysed by flow cytometry. The suppressive effects and cytokine production of mucosal and peripheral blood Treg cells was compared and determined by co-culture proliferation assays and cytometric bead array, respectively. Furthermore, the diversity of the T cell receptor V $\beta$ repertoire of Treg cells was determined by flow cytometry. RESULTS: The frequency of Treg cells among $\mathrm{CD} 4^{+} \mathrm{T}$ cells was found to be significantly increased in the ascending colon $\mathrm{LP}$ (lamina propria) as compared to the sigmoid colon LP in healthy control subjects $(\mathrm{p}<0.05)$. This regional difference of colonic Treg cells was however not present in UC patients in either exacerbation or remission and both UC groups had comparable levels of Treg cells in right- and left regions of the colon. The frequency of colonic LP Treg cells was positively correlated to both colonic disease activity score $(\mathrm{p}<0.03)$ and the systemic acute phase protein CRP $(\mathrm{p}<0.05)$ in UC patients in exacerbation. Functional studies revealed that the colonic and peripheral Treg populations from UC patients were equally suppressive, according to the ability in reducing the proliferation of activated $\mathrm{CD}^{+} \mathrm{T}$ cells. Co-culture of $\mathrm{LP}$ Treg cells with activated LP T cells also resulted in decreased secretion of IFN- $\gamma$ and IL-2. Furthermore, analysis of the T cell receptor V $\beta$ repertoire showed that LP Treg cells in major reflected peripheral Treg cell receptor use, although the $\mathrm{V} \beta 2 \mathrm{~T}$ cell receptor was less frequent on Treg cells in the colonic mucosa than in the periphery. CONCLUSIONS: Inflammation in the intestinal mucosa of patients with ulcerative colitis is probably not due to a functionally impaired or decreased numbers of Treg cells failing to suppress $\mathrm{CD}^{+} \mathrm{T}$ cell activity. On the contrary, the LP Treg cell frequency increases with colitis severity.

\section{M1714}

The Ectonucleotidase CD39 Is a Selective Surface Marker of Regulatory T Cells and An Immunosuppressive Catalyst

Karen M. Dwyer, Silvia Deaglio, David Friedman, Beat M. Kunzli, Keiichi Enjoji, Terry B. Strom, Simon C. Robson

Introduction: Multiple mechanisms are involved in regulation of immune responses in transplanted organs, the liver and intestinal tract. These include active suppression by regulatory CD4+CD25+ T cells (Treg). Despite several recent advances, there are still no reliable, specific surface markers of Treg and minimal understanding exists of their cellular function. Extracellular nucleotides are released at inflammatory sites, bind specific purinergictype 2 receptors (P2-R) and trigger activation responses in multiple cell types. Ectonucleotidases hydrolyze these mediators to nucleoside derivatives and the balance of extracellular nucleotides to nucleosides appears to modulate immune reactions. Aim: To study expression of CD39/NTPDasel, an ectonucleotidase expressed on immune and vascular cells, on Treg and determine functional sequelae of this. Methods and Results: We demonstrate that functional CD39 is expressed by resting and activated Treg and efficiently distinguishes this functional subset from other T cells. CD39 serves as an integral functional component of Treg, modulating extra- and pericellular levels of adenosine through the phosphohydrolysis of nucleotides such as ATP and ADP. The co-ordinated expression of CD39 on Treg and of the adenosine A2A receptor on activated effector $\mathrm{T}$ cells generates an immunosuppressive loop. Importantly, Cd39 null Treg cells are unable to prevent skin allograft rejection in vivo. Furthermore, Cd39 null mice spontaneously develop autoimmune manifestations and exhibit exacerbation of experimental colitis secondary to deviated Thl responses. Conclusions: CD39 is a key molecule expressed by Treg and has functional relevance for cellular immunoregulation.

\section{M1715}

Analysis of NOD2-Mediated Proteome Response to Muramyl-Dipeptide in HEK293 Cells: Implications for Crohn's Disease

Philip C. Rosenstiel, Dieter Weichart, Johan Gobom, Gustavsson Niklas, Susanne Billmann, D.R. Seegert, Stefan Schreiber

Background \& Aims: NOD2, a cytosolic receptor for the bacterial proteoglycan fragment muramyl-dipeptide (MDP), plays an important role in the recognition of intracellular pathogens. Variants in the bacterial sensor domain of NOD2 are genetically associated with an increased risk for the development of Crohn disease, a human chronic inflammatory bowel disease. To understand the proteomic signature pattern associated with NOD2 dysfunction in Crohn disease, global protein expression changes after MDP-stimulation were analysed. Methods: Two-dimensional polyacrylamide gel electrophoresis of total protein extracts of human cultured 293 cells stably transfected with expression constructs encoding for wildtype NOD2 (NOD2 ${ }^{\mathrm{wt}}$ ) or the disease-associated NOD2 L1007fsinsC (NOD2 ${ }^{\mathrm{SNP} 13}$ ) variant. Differentially regulated proteins were identified by MALDI-TOF MS peptide mass fingerprinting and MALDI MS/MS. Results were verified in myelomonocytic cells. Gene specific knockdown to assess the impact of up-regulated genes on NOD2 signalling was performed using siRNA technology. Results: A complex pro-inflammatory program regulated by NOD2 ${ }^{\mathrm{wt}}$ that encompasses a regulation of key genes involved in protein folding, DNA-repair, cellular redox homeostasis and metabolism was observed both under normal growth conditions and after stimulation with MDP. The upregulation of Y-Box Factor 1 and Peroxiredoxin4 was specific for the NOD2 ${ }^{\text {wt }}$ response and was absent in NOD2 ${ }^{\text {SNP13 }}$ cells. Using a myelomonocytic cellular model, it is demonstrated that Peroxiredoxin 4 is part of a negative feed-back loop on NOD2-induced NF-KB activation. Conclusions: The comparison of NOD2 ${ }^{\text {wt }}$ and disease-associated NOD2 ${ }^{\mathrm{SN} 13}$ variant identified a proteomic signature pattern that may further our understanding of the influence of genetic variations in the NOD2 gene in the pathophysiology of chronic inflammatory bowel disease. The failure of NOD2 ${ }^{\mathrm{SNP} 13}$ to induce Peroxiredoxin 4 , a negative regulator of the NF- $\mathrm{KB}$ pathway, may contribute to the chronic intestinal inflammation observed in Crohn disease. 
M1716

A Novel Enterohepatic Helicobacter Species Induces Ulcerative Colitis-Like Intestinal Inflammation with Intraepithelial Neoplasia in Interleukin-10 Knockout Mice

Ulrich R. Bohr, Doerthe Kuester, Steffen Backert, Thomas Wex, Manfred Rohde, Wolfgang Koenig, Albert Roessner, Peter Malfertheiner

Background \& Aims: Interleukin-10 knockout (IL-10 KO) mice are known to develop a spontaneous intestinal inflammation that resembles Crohn's disease. However, several studies showed that initiatiation and perpetuation of intestinal inflammation depends on the presence of intestinal bacteria. In this study, we investigated if a novel enterohepatic Helicobacter species (HM-007) is capable to induce intestinal inflammation in IL-10 KO mice. Methods: C57BL/6J IL-10 KO mice and the corresponding wildtype strain C57BL/6J (WT) were bred under specific pathogen-free conditions. At the beginning of the experiment, all mice were tested free of Helicobacter spp. by fecal group-specific PCR. At the age of 12 months, WT and IL-10 KO mice were exposed to HM-007. These animals as well as uninfected control WT and IL-10 KO mice were monitored weekly for weight loss and diarrhea, and were sacrificed 60 days after exposure. The entire gastrointestinal tract was routinely processed for histopathology, and intestinal inflammation was scored by an established IBD-score. Results: $83.3 \%$ of the Helicobacter-exposed IL-10 KO mice developed colitis compared to $12.5 \%$ of the unexposed IL-10 KO mice ( $<<0.001)$. None of the unexposed and exposed WT animals developed IBD $(\mathrm{p}<0.001)$. In diseased IL-10 KO mice, intestinal inflammation was mainly found in the colorectum and was limited to the mucosa. It was characterized by severe acute and chronic inflammation through widespread infiltration with inflammatory cells, crypt hyperplasia, distortion of architecture and the presence of ulcers and crypt abscesses similar to human ulcerative colitis. Furthermore, focal colitis-associated intraepithelial neoplasia was frequently observed in the inflamed distal colorectum. Conclusions: Colonization of IL-10 KO mice with HM-007, a novel enterohepatic Helicobacter species, resulted in a unique model of colitis, whose distribution and histological features have characteristics resembling human ulcerative colitis and that may progress to intraepithelial neoplasia. Moreover, this study provides strong evidence that the composition of the intestinal flora decides (I) whether or not a susceptible host develops IBD and (II) whether the inflammation type is rather Crohn's disease or ulcerative colitis-like.

\section{M1717}

Defective TGF- $\beta$ and IL-10 Mediated Regulation of Interleukin 12-Stimulated Cytokine Responses in HLA-B27 Transgenic Rats

Bi-Feng Qian, Susan L. Tonkonogy, Balfour R. Sartor

We have reported that commensal luminal bacterial lysates induce an active in vitro IFN$\beta$ response in mesenteric lymph node (MLN) cells from HLA-B27 transgenic (TG) rats with chronic colitis but not from non-diseased specific pathogen free (SPF) non-TG, germ free (GF) TG or non-TG rats. However, equivalent amounts of IL-10 and TGF- $\beta$ are produced by the TG and non-TG cell types that make these two cytokines. In this study we examined IL-12 stimulation of cytokine responses to luminal bacteria and its regulation by suppressive cytokines in cells from TG and non-TG rats. Methods: Non-TG and TG MLN cells were collected from SPF or GF rats. Antigen presenting cells (APC) and T lymphocytes were enriched by negative selection. Cells were stimulated by cecal bacterial lysate (CBL), with or without exogenous recombinant rat IL-12, in the presence or absence of added TGF- $\beta$ or IL-10 for 3 days. IFN- $\gamma$ and IL-10 in supernatants were quantified by ELISA. The expression of IL-12p40 mRNA in cells after CBL stimulation for 18 hrs was analyzed by RT-PCR. Results: CBL induced much higher secretion of IFN- $\gamma$ in TG than in non-TG MLN cells, but IL-12 mRNA expression was equal in the two cell types. Addition of exogenous IL-12 elicited a significant increase in the CBL-induced IFN- $\gamma$ response in SPF TG cells. CBL and IL-12 synergistically induced IFN- $\gamma$ from baseline low response in non-TG cells (from both SPF and GF rats) and in GF TG cells. However, the interactions between proinflammatory and suppressive cytokines were different in non-TG vs. TG cells. TGF- $\beta$ fully counteracted the effect of IL-12 on non-TG cells, almost completely inhibiting IFN- $\gamma$ production. In contrast, TG cells were less susceptible to TGF- $\beta$-mediated down regulation with a substantial residual IL-12 induced IFN- $\gamma$ response. Cell mixing experiments further demonstrated that response to TGF- $\beta$ was defective in both TG APC and T cells. Addition of exogenous IL-10 partially inhibited IFN- $\gamma$ production by non-TG cells but did not affect TG cells. Conversely, exogenous IL-12 suppressed CBL-induced IL-10 production to a greater degree in TG compared to non-TG cells. Conclusion: IL-12 enhanced IFN- $\gamma$ production by MLN cells from both HLA-B27 TG and non-TG rats in response to in vitro stimulation with intestinal bacteria. The cells from TG rats that produce IFN- $\gamma$ in response to IL-12 are defectively regulated by suppressive IL-10 and TGF- $\beta$ with a concomitant inhibition of IL-10 production by IL-12. These results suggest that a self-perpetuating cycle of exacerbated induction and lack of regulation of $\mathrm{T}$ cell responses to commensal bacteria leads to uncontrolled chronic colonic inflammation in HLA-B27 TG rats.

\section{M1718}

Weakening of the Intestinal Epithelial Barrier with NSAIDs Can Lead to IBD Following Mono-Association with Non-IBD Causing Bacteria

Beate C. Sydora, Sarah M. Martin, Andrea L. Dmytrash, Thomas A. Churchill, Jason Doyle, Richard N. Fedorak

Background: In the IL-10 gene-deficient mouse development of intestinal inflammation occurs in conjunction with a defect in epithelial barrier integrity that permits passage of bacteria, or their antigens, into the lamina propria and thereby initiating a systemic immune and mucosal inflammatory response. Our mono-association experiments with endogenous Bacteroides vulgates (B. vulgatus) have shown that this bacterium, or its antigens, can initiate a systemic immune response but not an intestinal mucosal inflammatory (IBD) response. Objective: We thus investigated whether pre-weakening of epithelial barrier integrity was a prerequisite for non-IBD-causing bacteria, such as $B$. vulgatus, to cause a mucosal inflammatory response. Methods: A sub-optimal dose of indomethacin $(2 \mathrm{mg} / \mathrm{kg} / \mathrm{d})$ causes intestinal epithelial barrier disruption without any inflammatory response. Axenic IL-10 gene-deficient mice were mono-associated by oral gavage with B. vulgatus and were concomitantly fed suboptimal doses of indomethacin in the drinking water for $1 \mathrm{wk}$. At the end of the week epithelial barrier integrity was determined by mannitol transmural flux and systemic immune and mucosal inflammatory responses assessed by cytokine profile and histologic injury score. Results: Treatment with sub-optimal doses of indomethacin $(2 \mathrm{mg} / \mathrm{kg} / \mathrm{d}$ for 7 days $)$ alone caused a disruption of epithelial barrier integrity (mannitol flux of $28+3 \mathrm{~nm} / \mathrm{cm}^{2} / \mathrm{h}$ versus $19 \pm 1 \mathrm{~nm} / \mathrm{cm}^{2} / \mathrm{h}$ in untreated controls, respectively, $\mathrm{p}=0.002$ ) but did not cause a systemic nor mucosal inflammatory response (no significant increase in IFN $\gamma$ release in supernatants of intestinal explant or spleen cell cultures compared with those of untreated controls) Monoassociation with $B$. vulgatus alone did not cause epithelial barrier disruption nor a mucosal inflammatory response despite a systemic immune response. In contrast, monoassociation with $B$. vulgates into mice with an indomethacin-induced disruption of epithelial barrier integrity resulted in an intense mucosal inflammatory (7-fold increase in IFN $\gamma$ release from intestinal explants of indomethacin-treated, mono-associated mice compared with those from untreated control mice) and systemic immune (50-fold increase in IFN $\gamma$ release from spleen cells stimulated with B. vulgatus antigens compared to B. vulgatus antigen-stimulated spleen cells from untreated control mice) response. Conclusion: This study demonstrates for the first time, that non-IBD-causing endogenous bacteria are able to cause a marked intestinal inflammatory response provided that an initial weakening of the epithelial barrier function has occurred.

\section{M1719}

Fractalkine and Its Receptor CX3CR1 Are Upregulated in Inflammatory Bowel Disease

Taku Kobayashi, Susumu Okamoto, Yuko Iwakami, Atsushi Nakazawa, Tadakazu Hisamatsu, Toshio Imai, Toshifumi Hibi

Background: Fractalkine is a unique chemokine that mediates not only leukocyte migration but also adhesion. It has been reported that the interactions of fractalkine and its receptor CX3CRl might contribute to the pathogenesis of some inflammatory diseases mainly by recruiting monocytes to the inflamed sites. We investigated the pathogenic contribution of fractalkine/CX3CR1 in patients with inflammatory bowel disease(IBD). Methods: CX3CR expression on peripheral blood mononuclear cells was examined using flow cytometric analysis. About $C D 4+C X 3 C R 1+$ cells, expressions of surface antigens were assessed by flow cytometry. Productions of cytokines and cytotoxic granules were determined by flow cytometry and enzyme linked immunosorbent assay(ELISA). Fractalkine expressions in inflamed and non-inflamed tissue were analysed by immunohistochemistry and quantitative reverse transcription-polymerase chain reaction. Chemotaxis assay was examined using Transwell system. Additionally, Some patients having expanded CX3CR1+CD4+ cells were followed up to determine whether the expression of CX3CRI on CD4+ cells changes in the course of therapeutic interventions such as granulocyte apheresis, cyclosporine A, or surgery. Results: CX3CR1-expressing CD4+ and CD8+ cells were expanded in patients with IBD. Especially, CX3CRl+CD4+ cells were found only in IBD patients, and its percentage was correlated with disease activity. These unusual CD4 cells expressed natural killer cell markers such as CD11b, CD56, and CD161. More interestingly, they lacked co-stimulate molecule CD28, while CX3CRl-negative CD4+ cells always expressed CD28. CD4+CX3CRl+ cells produced more IFN- $\gamma$ and TNF- $\alpha$ but less IL- 4 and IL- 2 compared with CD4+CX3CRIcells. It was also confirmed that CD4+CX3CRl+ cells contained and released cytotoxic granules such as granzyme. Immunohistochemistry revealed that fractalkine was strongly expressed mainly on epithelial cells in inflamed tissue. Consistently, mRNA expression was about 4 folds higher in inflamed mucosa of ulcerative colitis(UC) and 6 folds in Crohn's disease $(C D)$ than control. Chemotaxis assay showed that fractalkine could work as a chemoattractant for CD4+ cells which express CX3CRl. Furthermore, in some patients, CX3CRI expression on CD4+ cells slightly decreased after treatment but had never reached to norma range. Conclusion: These data suggest that fractalkine/CX3CRl plays some roles in IBD not only through monocytes but also unique CD4+ cells found only in IBD patients.

\section{M1720}

IgG Plasma Cells in Inflammatory Bowel Disease Express Large Quantities of Stromelysin (MMP-3)

John N. Gordon, Karen M. Pickard, Patrick M. Goggin, Thomas T. MacDonald

Introduction: In both ulcerative colitis and Crohn's disease there is a marked increase in IgG plasma cells in the lamina propria which may be of primary pathogenic significance. In addition, gut plasma cells can secrete functionally active immune mediators capable of driving inflammation in the gut. Using a novel method for isolating gut plasma cells we have previously shown that long-lived IgG plasma cells persist in IBD and may contribute to tissue injury. In this study we have investigated the production of MMP-3 by gut plasma cells from normal subjects and patients with IBD. Methods: Biopsy and resection tissue specimens were obtained from patients with active IBD and from normal controls. Lamina propria mononuclear cells were isolated and immunomagnetic selection used to positively select plasma cell populations. MMP-3 expression was investigated using western blotting, and Taqman PCR. The types of plasma cell making MMP3 were studied by confocal microscopy. Results: Plasma cells from patients with CD and UC expressed significantly higher levels of MMP- 3 protein and transcripts $(100,000$ fold increase) than controls $(\mathrm{p}<0.001)$ Levels of TIMP-1 were not significantly different from controls. In patients with IBD, 92\% of IgG+ve plasma cells expressed MMP- 3 compared with $20 \%$ of IgA+ve cells. In contrast very few plasma cells from controls expressed any MMP-3. Conclusion: We have demonstrated that plasma cells from subjects with IBD express large amounts of MMP-3. Furthermore the primary source of MMP-3 is IgG+ve plasma cells. These results identify a new pathway by which IgG plasma cells can damage the gut. 


\section{M1721}

Activated Lymphocytes, Which Are Arrested from Proliferation By 6Mercaptopurine, Still Maintain Their Viability and Their Pro-Inflammatory Effector Functions: A Novel Mechanism for the Delayed Effect of Anti-Purines in IBD

Shomron . Ben-Horin, Itamar Goldstein, Orit Picard, Zohar Yerushalmi, Ella Fudim, Yifat Goldschmid, Alon Lang, Simon Bar Meir, Yehuda Chowers

Background: The reason for the slow onset of effect of purine analogues is still unclear, as the pharmacokinetic based explanation has been challenged by conflicting evidence. The aim of this study was to explore immune mechanisms possibly responsible for the delayed onset of effect of these drugs in IBD. Methods: Peripheral blood (PB) lymphocytes from IBD patients and healthy controls were triggered by anti-CD3 with or without 6-Mercpatopurine (6-MP) and 6-Thioguanine (6-TG). Proliferation of CD4+ and CD8+ T-cells was determined by CFSE dilution, and apoptosis induction was examined by Annexin-V/PI assays. Cytokine secretion was assessed by FACS read-outs of intra-cellular staining and by ELISA assays. Finally, expression of activation and adhesion molecules was examined by flowcytometry, and cell-mediated killing of K562 target cells was assessed. Results: 6-MP and 6-TG at pharmacologically-relevant concentrations abrogated the proliferation of PB CD4+ and CD8+ T-cells by $80 \%$ in 3-7 day cultures in controls $(n=8)$ and IBD patients $(n=7)$. However, the percentage of apoptotic cells was not different between lymphocytes triggered by anti-CD3 in the presence or the absence of $6-\mathrm{TG}(10.3 \pm 8.3 \%$ versus $10 \pm 6.4 \%$, respectively), and the viability of resting lymphocytes was also unaffected. T-cells, arrested from division by 6 TG, were still equally capable of secreting IFN-gamma and TNF-alpha, as were cells triggered via the T-cell receptor (TCR) in the absence of the drugs. Anti-CD3 stimulation resulted in upregulation of activation and adhesion molecules on CD4+ T-cells, which was only minimally inhibited by 6-TG and 6-MP, and adhesion to collagen IV and fibronectin was not reduced. Finally, T-NK cell-mediated killing of target cells was not inhibited by pre-treatment of effector cells with 6-TG. In contrast, Prednisolone rapidly induced marked apoptosis of Tcells and effectively inhibited the above lymphocyte effector functions. Conclusions: These data suggest that T-cells stimulated via the TCR are effectively arrested from expansion by anti-purine metabolites, but undergo little apoptosis and maintain their immune effector functions. These cell-cycle arrested lymphocytes, which nevertheless continue to exert their pro-inflammatory functions and perpetuate tissue inflammation, may constitute a novel mechanism accounting for the delayed onset of effect of 6-MP and Azathioprine in IBD.

\section{M1722}

Atypical Colonic Paneth Cell Metaplasia in Crohn's Disease with NOD2 Mutations

Jan Wehkamp, Hiu Chu, Bo Shen, Michael Weichenthal, Elke Schaeffeler, Matthias Schwab, Klaus Fellermann, Eduard F. Stange, Charles L. Bevins

Introduction: Paneth cells (PCs) are epithelial cells located in the crypts of the small intestinal mucosa and secrete antimicrobial peptides including the $\alpha$-defensins HD5 and HD6, lysozyme, and sPLA2. PCs also express NOD2, a receptor for muramyl dipeptide and an important susceptibility gene for Crohn's disease (CD). In CD of the small intestine, there is a specific reduction of PC $\alpha$-defensin expression, and this reduction is more pronounced in specimens harboring a L1007fs mutation (snp 13) in NOD2. In the presence of inflammation, PCs can be frequently found outside the small intestine, a process termed PC metaplasia. PC metaplasia in the colon is common in inflammatory bowel disease (IBD), but further details especially the impact of NOD2 on this process remain to be elucidated. Methods: Using real-time PCR and northern blot analysis, the mRNA expression of the 4 aforementioned PC antimicrobial peptides, anion trypsin as another PC product, and G3PDH were measured in two cohorts of colonic samples of a total of 65 patients with IBD, and nonIBD controls from both the colon and small intestine. To assess protein levels, we also performed immunohistochemistry and western blot analysis of selected products. Inflammation was assessed by histology and quantitation of IL-8 mRNA levels. All patients were screened for three common NOD2 mutations (SNP8, 12 and 13). Results: In metaplastic Paneth cells of the colon, the levels of HD5 and HD6 were about a factor of 100-1000 lower than in the small intestine. As compared to controls, PCs were significantly more often observed in the colon of IBD patients, especially in areas of inflammation. As compared to ulcerative colitis (UC) samples and CD specimens with wildtype NOD2, colonic specimens from CD patients with a NOD2 mutation showed a significant decrease of PC defensins HD5 $(p=0.001)$ and HD6 $(p=0.001)$ mRNA, and HD5 protein levels. Strikingly, in the colonic specimens with a NOD2 mutation, there were significant decreases of the other PC products anionic trypsin $(p=0.002)$ and sPLA2 ( $=0.02)$ mRNA and protein levels. Lysozyme was not altered by NOD2 status (mRNA and protein levels), but immunohostochemistry revealed that lysozyme was also found in many inflammatory cells and therefore is not PC cell specific. Conclusions: PC metaplasia is a common feature of $C D$ and UC. In patients with $C D$ and a mutation in the NOD2 gene, the colonic expression of a variety of PC products is altered, indicating that these metaplastic PCs are atypical. This suggests that NOD2 and bacterial recognition may be an important factor in PC metaplasia.

\section{M1723}

Adoptive Transfer of Mature Dendritic Cell Worsens Dextran Sulphate Sodium (DSS)-Induced Colitis

John Y. Kao, Brad Berndt, Min Zhang, Juanita L. Merchant

Background: Individuals with active inflammatory bowel diseases (IBD) have been shown to have more activated dendritic cells (DCs). Since antigen presenting cells such as dendritic cells are important for induction of host immunity against microbial pathogens, we hypothesize that an increased number of mature DCs may worsen colitis severity in IBD. Aims: To demonstrate the effect of adoptive transfer of mature dendritic cells on DSS-induced colitis. Methods: Optiprep-enriched, day 6, bone marrow-derived DCs from C57BL/6 mice were plated in culture with GM-CSF and IL-4 for $3 \mathrm{~h}$ and non-adherent DCs were isolated. FACS analyses for surface MHC class II, CD40, CD80 and CD86 were performed to show expression of these co-stimulatory markers on DCs. DCs were stimulated with live H. pylori SSI for $18 \mathrm{~h}$ and IL-12 release was measured by ELISA (BD PharMingen). A mixed leukocyte assay was performed with naïve syngeneic splenocytes and proliferation was quantified by the ViaLight Cell Proliferation Kit (Cambrex). For in vivo studies, mice were injected i.p. with 1 x106 mature DCs on day -1 and given $5 \%$ DSS water from days 0 to 7 . Daily stool hemoccult tests were performed and mice were sacrificed on day 7 and paraffin blocks of colon prepared in swiss-rolls for $\mathrm{H} \& \mathrm{E}$ staining and histological scoring (Rachmilewitz et al. 2002). Results: Non-adherent DCs expressed higher levels of MHC class II CD40, CD80 and CD86 compared to adherent DCs. There non-adherent cells are referred to as mature DCs. The production of IL-12 and the priming of naive splenocyte proliferation were higher by H. pylori-stimulated mature DCs than adherent (e.g., immature) DCs (IL-12: 366.4 pg/ $\mathrm{mL}$ vs. $180.3 \mathrm{pg} / \mathrm{mL}$ and splenocyte proliferation: 11460 vs. 2100 RLUs, respectively). Adoptive transfer of mature DCs prior to 5\% DSS treatment was associated with a significantly higher bleeding score (on day 1 post DSS-treatment: $0.63 \pm 0.18$ vs. $0.13+0.13, n=11$, $\mathrm{P}<0.05)$, and an increased colitis index $(30.4 \pm 6.6$ vs. $19.8 \pm 2.3, \mathrm{P}<0.05)$ compared to mice receiving PBS prior to 5\% DSS treatment. A higher mortality was observed in the mature DC group than the PBS controls ( $3 / 10$ or $33 \%$ vs. $0 / 11$ or $0 \%$, respectively). Conclusions: Adoptive transfer of mature DCs prior to initiation of DSS treatment worsened colitis disease activity. Our study provides further support that mature DCs play an important role in the pathogenesis of IBD and therapies that target these immune cells may be highly efficacious in the treatment of IBD.

\section{M1724}

Microsomal Triglyceride Transfer Protein Regulates Endogenous and Exogenous Antigen Presentation By Group 1 Cdl Molecules

Arthur Kaser, David L. Hava, Masaru Yoshida, Timothy T. Kuo, Takashi Nagaishi, Stephanie K. Dougan, Bryan Vander Lugt, Wael Haddad, Michael B. Brenner, Richard S. Blumberg

Background. Lipid antigens are presented to T cells by the non-polymorphic MHC class Irelated $\mathrm{CDI}$ molecules and play an important role in a variety of diseases. This laboratory recently reported that microsomal triglyceride transfer protein (MTP), which has until then been known for lipidating apolipoproteins B48 and B100 and generating chylomicrons and VLDL particles, regulates CDld function and hence natural killer T (NKT) cell biology. While CDld is expressed in humans and rodents, the type I CDl genes CDla, CDlb, and $\mathrm{CDlc}$ are only present in humans but not rodents and therefore not accessible for study in murine in vivo disease models. Aim. We therefore asked whether MTP regulates class I CDI $(\mathrm{CDla}, \mathrm{CDlb}, \mathrm{CDlc})$ antigen presentation, which primarily present microbial lipid antigens, in particular mycobacterial lipid antigens and are key restriction elements for the host immune response. Methods \& Results. Here we report that MTP regulates the function of type $1 \mathrm{CD} 1$ molecules CDla, CD lb, and CD lc. Pharmacological inhibition of MTP in human monocyte-derived dendritic cells (DC) and the lymphoblastoid B cell line ClR transfected with type $1 \mathrm{CDl}$ molecules resulted in a substantial decrease in endogenous antigen presenting function to several CDl-restricted T cell lines. Silencing MTP expression in CDlctransfected Hela cells similarly resulted in decreased antigen presentation. Inhibition of endoplasmic reticulum (ER)-resident MTP decreased presentation of exogenous mycobacterial lipid antigens to CDla- and CDlc-restricted T cells. Impaired presentation by MTP inhibition was associated with decreased cell surface staining for type $1 \mathrm{CD} 1$ molecules on DCs, but not C1R transfectants. The stability of CDlc heavy chain and beta-2-microglobulin was decreased in inhibitor treated ClR transfectants, suggesting that MTP-mediated lipid transfer in the ER is essential for proper folding and function of $\mathrm{CDl}$ molecules on the cell surface and/or that in the absence of initial lipid association in the ER, endosomal lipid exchange by saposins and GM2 activator proteins might be impaired. Conclusion. Our data implicate that the immune system has co-opted a key protein of lipid metabolism, MTP, for its antigen presentation pathways, and via MTP's regulation of $\mathrm{CDla}, \mathrm{b}$, and c function, this protein has a key role in the host immune response toward microbial pathogens. This might have implications for mucosal inflammation in IBD, as CDla, CDlb, and CDlc molecules are expressed on dendritic cells in the intestinal mucosa (unpublished observation, Per Brandtzaeg \& RSB). (AK and DLH contributed equally)

\section{M1725}

The Constitution and Distribution of Intestinal Bacterial Flora in Patients with Inflammatory Bowel Disease: Genus Or Species Specific Analysis Based On 16s rRNA Gene Sequences of Intestinal Bacterial Flora in Human Feces and Colonic Mucosa

Atsushi Nakazawa, Toshihiko Takada, Takahiro Matsuki, Shoichi Kado, Takashi Asahara, Koji Nomoto, Ryuichiro Tanaka, Atsushi Sakuraba, Tomoharu Yajima, Nagamu Inoue, Haruhiko Ogata, Yasushi Iwao, Hiromasa Takaishi, Toshifumi Hibi

Background \& Aims: Intestinal flora is thought to play a critical role in the inflammatory process at the intestinal mucosa of inflammatory bowel disease (IBD). As previously reported, Bacteroides fragilis group is assumed as one of the cause of IBD. The analysis of intestinal flora is important for elucidating the pathogenesis of chronic inflammation in IBD, but only $50 \%$ of bacteria consisting of intestinal flora can be cultivable by conventional cultivation techniques. To examine the intestinal flora constitution and distribution in the colon in IBD patients, we analyzed intestinal flora by fluorescence in situ hybridization (FISH) and quantitative PCR using 165 rRNA-gene-targeted group specific probes and primers. Methods: Fecal samples were collected from IBD patients and healthy volunteers. In order to detect the predominant intestinal bacteria (Bacteroides fragilis group, Bifidobacterium, Clostridium coccoides group, Clostridium leptum subgroup and Atopobium group) and to analyze the distribution of these flora in the colon, we newly established multi-color FISH system. FISH was conducted using $16 \mathrm{~S}$ rRNA-gene-targeted group-specific probes for the predominant intestinal bacteria in the feces or on the colonic mucosa. The ratio (\%) of each bacterial cluster shown in the multi-color FISH photographic images were calculated by setting the DAPI stained bacteria at 100 . To confirm the data through the novel FISH system, we performed previously established quantitative PCR. Results: Using our novel FISH system, Bacteroides fragilis group was predominantly detected in feces of ulcerative colitis (UC) 
patients, while hardly demonstrated on the colonic mucosa. In contrast C. coccoides group and Bifidobacterium were predominantly demonstrated on the colonic mucosa of UC patients. Bifidobacterium was mainly located on sigmoid colon $(27.0 \%$ on sigmoid colon; $6.4 \%$ on ascending colon) and $\mathrm{C}$. coccoides group was predominantly located on ascending colon ( $33.8 \%$ on ascending colon; $24.3 \%$ on sigmoid colon). By quantitative PCR, Bacteroides fragilis group, C. coccoides group, C. leptum subgroup and Atopobium cluster were decreased in feces of IBD patients compare to healthy volunteers, similar to the data from FISH. Conclusion: We established FISH system using group-specific probes based on 165 rRNA gene sequence. Unexpectedly Bacteroides fragilis group was rarely detected on the colonic mucosa of UC patients and this data suggested that Bacteroides fragilis group might not contribute to the pathogenesis of UC. The pathogenesis of IBD could be involved in the disorder of the intestinal flora constitution, not in specific intestinal bacteria cluster or species.

\section{M1726}

Increase Expression of Corticotropin Releasing Factor (CRF) Receptor Type 1(CRF1) On Macrophages in Colonic Mucosa of Patients with Ulcerative Colitis (UC)

Pu-Qing Yuan, Million Mulugeta, Peter A. Anton, Julie Elliott, Ekaterini Chatzaki, Dimitri Grigoriadis, Yvette Tache

Background: UC is characterized by a long-standing chronic inflammation of the bowel with intermittent periods of exacerbation and remission. Its pathogenesis and acute exacerbation appear to be modulated by various stress (Am J Gastroenterol 2000, 95:1213). CRF and its related peptide urocortin 1 (Ucn 1 ) acting on $C R F 1$ and CRF2 receptors are key mediators of stress response and CRF expression is elevated in the colonic mucosa of UC patients (Gut 1995,7:544; J Clin Endocrinol Metab 2004 89:5352). The actions of CRF and Ucn 1 on colonic function in experimental animals are mediated by CRFl receptor (Br J Pharmacol 2004,8:1321). Aims: To examine the local expression and cellular location of CRFl in colonic mucosa of healthy subjects (control) and in UC patients in active (UCact) and remission (UCrem) phases. Methods: Sigmoid colonic biopsies were obtained by colonoscopy from 6 control subjects, 6 UCrem and 4 UCact patients. The diagnosis of UC was based on history, clinical examination, endoscopic examination, and pathological findings. CRFl immunohistochemistry and double immunostaining of CRF1 and ED2(CD163), a marker for macrophages, were performed on 6-um paraffin-embedded sections. The number of CRFl immunoreactive (IR) positive cells and double labelled cells in lamina propria were counted in 5 fields from each specimen. The total RNA was extracted from 3 biopsy samples in each group and cDNA was synthesized and processed for CRFl real-time quantitative polymerase chain reaction (PCR) by ABI TaqMan. Data were quantitated using comparative cycle threshold $(\mathrm{Ct})$ method. Results: CRF1 immunoreactivity was mainly found in scattered cells of lamina propria and in the cell base of epithelia and crypts of the colonic mucosa. Compared to the control, the intensity of CRFl immunoreactivity and the number of CRFl IR positive cells in the lamina propria were significantly increased in UCact $(26 \pm 7$ vs $7 \pm 3$ / field in control, $\mathrm{p}<0.05)$, particularly in UCrem $(35 \pm 11$ vs $7 \pm 3$ field in control, $\mathrm{p}<0.05)$. Macrophages labeled by CDI63 are dramatically increased in UCact and UCrem and more than $90 \%$ of them were double-labeled by CRFl and CDI63. Real time PCR showed that CRFl mRNA level was remarkably increased by $150 \%$ in UCrem but not in UCact compared to the controls. Conclusion: CRFl is locally synthesized in both lamina propria macrophages and epithelial cells and significantly increased along with the increased population of macrophages in patients with UC, especially in those in remission periods. These findings indicate that $\mathrm{CRFl}$ receptor may be involve in the mediation of the immune-inflammatory reactions in colonic mucosa of UC patients. Supported by NIH P50 DK64539.

\section{M1727}

Bone Marrow Latently Retaining Colitogenic $\mathrm{Cd} 4^{+} \mathrm{T}$ Cells May Be a Pathogenic Reservoir for Lifelong Chronic Colitis

Yasuhiro Nemoto, Takanori Kanai, Shin Makita, Rei Fujii, Ryuichi Okamoto, Teruji Totsuka, Mamoru Watanabe

Background \& Aims: Crohn's disease (CD) is characterized by chronic intestinal inflammation. Surgery does not cure $C D$, and recurrence following surgery is the rule rather than the exception. There is no correlation between recurrence of the disease and the dissection of regional mesenteric lymph nodes and spleen. The evidence suggests that other sites might play a critical role in the recurrence of diseases as reservoirs of colitogenic memory T cells. Bone marrow (BM) is known to harbor memory $\mathrm{T}$ cells, suggesting that this compartment is a preferential site for migration and/or selective retention of memory $\mathrm{T}$ cells. However, linking $\mathrm{BM} \mathrm{CD} 4^{+}$memory $\mathrm{T}$ cells with a pathological role in chronic colitis is yet to be reported. Methods: To investigate the role of BM in consecutive immunopathology in chronic colitis, we assessed the latent BM CD4 ${ }^{+} \mathrm{T}$ cells in colitic SCID mice induced by the adoptive transfer of $\mathrm{CD}^{+} \mathrm{CD} 45 \mathrm{RB}^{\text {high }} \mathrm{T}$ cells. Results: We found that a high number of $\mathrm{CD} 4{ }^{+} \mathrm{CD} 44^{\text {high }} \mathrm{CD} 62 \mathrm{~L}-\mathrm{IL}-7 \mathrm{R}^{\text {high }}$ effector-memory $\mathrm{T}\left(\mathrm{T}_{\mathrm{EM}}\right)$ cells resided in the $\mathrm{BM}$ of the colitic SCID mice induced by the adoptive transfer of $\mathrm{CD}^{+} \mathrm{CD}^{+} 5 \mathrm{RB}^{\text {high }} \mathrm{T}$ cells. Colitic BM CD4 ${ }^{+}$ $\mathrm{T}_{\mathrm{EM}}$ cells divided to a greater extent of colitic lamina propria (LP) $C D 4^{+} \mathrm{T}_{\mathrm{EM}}$ cells, and produced large amount of Thl cytokines. Furthermore, a substantial part of the colitic BM $\mathrm{CD}^{+} \mathrm{T}_{\mathrm{EM}}$ cells expressed integrin a4b7 gut-homing receptors. Importantly, SCID mice transferred with the colitic BM T $\mathrm{F}_{\mathrm{FM}}$ cells induced colitis to a similar extent of mice transferred with colitic LP CD4 ${ }^{+} \mathrm{T}$ cells. Furthermore, these colitogenic BM CD4 ${ }^{+} \mathrm{T}_{\mathrm{EM}}$ cells were preferentially attached to IL-7-producing BM stromal cells, and the accumulation of BM $\mathrm{CD}^{+} \mathrm{T}_{\mathrm{EM}}$ cells was significantly decreased in IL-7-/- x Rag-1-/- recipients reconstituted with the colitogenic LP CD4 ${ }^{+} \mathrm{T}_{\mathrm{EM}}$ cells as compared with IL-7+/+ $\mathrm{x}$ Rag-1-/- recipients. The BM CD4+ $\mathrm{T}$ cells in IL-7-/- $\mathrm{x}$ Rag-1-/- mice expressed lower levels of Bcl-2 and CD69 as compared with those of IL-7+/+ x Rag-1-/- mice. Conclusions: Collectively, these findings suggest that the BM latently retaining IL-7-dependent colitogenic $\mathrm{CD} 4^{+} \mathrm{T}_{\mathrm{EM}}$ cells plays a critical role as a reservoir for persisting lifelong colitis.

\section{M1728}

Acute Gastroenteritis Is Followed By An Increased Risk of Inflammatory Bowel Disease

Julian Panes, Ana Ruigomez, Luis Alberto Garcia Rodriguez

Bacterial intestinal infections have been implicated as a possible cause of exacerbation of inflammatory bowel disease (IBD). We explored the relationship between infectious gastroenteritis (GE) and the occurrence of IBD using data from the General Practice Research Database. Methods: A cohort of patients aged 20 to 74 who had a first ever episode of acute gastroenteritis ( $\mathrm{n}=38,187)$ was identified. From the same source population an age, sex and calendar time-matched control group free of GE was sampled $(n=50,000)$. Both cohorts were followed-up during a mean duration of 3.5 years. Results: The estimated incidence rate of IBD in patients after an episode of GE was 70.9 per 100,000 person-years and 30.3 in the control cohort. The hazard ratio (HR) of IBD was 2.4 (95\% CI: 1.7-3.4) in the GE cohort compared to the control cohort and the excess risk was greater during the first year after the infective intestinal episode with a HR of 4.0 (95\% CI: 2.2-7.3). In patients with positive stool culture, the pathogens most commonly isolated were Campylobacter, Salmonella and Shigella species. The relative risk of developing Crohn's disease in the GE cohort was slightly greater than the one of ulcerative colitis, especially during the first year after the infective episode ( $\mathrm{HR}=6.0 ; 95 \% \mathrm{CI}: 1.7-20.7)$. A nested case-control analysis after adjusting for a number of risk factors, including age, sex, calendar time, smoking habits, appendectomy, and anxiety, produced similar results: The OR of IBD in the GE cohor compared to the control cohort was 1.9 (95\% CI: 1.3-2.7); the OR of UC was 1.8 (95\% CI: 1.1-2.9); and the OR of CD was 2.4 (95\% CI: 1.2-4.6). Treatment with antibiotics in the two weeks after the episode of GE was associated with a higher risk of IBD: HR=2.7 (95\% CI: 1.6-4.5). Conclusions: Our results are compatible with the hypothesis that infectious agents causing an episode of infectious gastroenteritis could play a role in the initiation and or exacerbation of inflammatory bowel disease.

\section{M1729}

Mixed IBD Families: A Distinct Entity Within IBD

Marie Pierik, Marie Joossens, Liesbet Henckaerts, Sofie Joossens, Karolien Claes, Paul Rutgeerts, Severine Vermeire

BACKGROUND \& AIM: Twenty percent of inflammatory bowel disease (IBD) patients report a positive familial history. Although there is high concordance for disease type, location and behavior within families, in some families both ulcerative colitis (UC) and Crohn's disease (CD) are present (=mixed families). We were interested in differences in genetic background serologic response and phenotypes between patients belonging to mixed families and other IBD patients. We also studied environmental, genetic and serologic factors that modulated the development of CD or UC in these families. MATERIALS \& METHODS We confirmed the diagnosis of $\mathrm{CD}$ and $\mathrm{UC}$ in 33 mixed families. Demographic and clinical characteristics in these patients were compared to 929 independent IBD patients and 209 controls. All subjects were genotyped for TLR4 D299G and the 3 CD associated CARD15 variants. Patients with IBD show antibodies in their serum directed against microbial antigens. ASCA are directed against the Saccharomyces cerevisiae strain Sul. Anti-Omp against Eschericia col outer membrane porin. gASCA and anti-Omp were assessed with ELISA (Glycominds Ltd and Inova diagnositics inc respectively). RESULTS: CD patients belonging to mixed families more often had pure colonic disease ( $27.3 \%$ versus $13 \% \mathrm{p}=0.03)$, and TLR4 variants $(28.6 \%$ versus $11.5 \%, \mathrm{p}=0.01$ ) than other familial CD patients. UC patients of mixed families more often carried mutations in CARD15 compared to sporadic UC patients and compared to controls $(51.7 \%, 19.3 \%$ and $20.6 \%$ both $\mathrm{p}<0.01)$. More UC patients of mixed families were female $(72.7 \%)$ compared to sporadic UC patients $(49.7 \%$; $<<0.01)$. Within the mixed families, active smoking and a positive ASCA were more often associated with a CD phenotype $(\mathrm{p}=0.02$ and $\mathrm{p}<0.01$ respectively). CONCLUSION: IBD patients belonging to mixed families behave differently and $\mathrm{CD}$ patients from these families more often present with pure colonic disease whereas UC patients more often carry CARD 15 variants. The reason for the divergent phenotypes in these families is not clear but smoking and ASCA appear important modulating factors. Furthermore female gender seems to play a role and future studies focusing on disease modulating genes on the X-chromosome might be interesting.

\section{M1730}

Proteomic Analysis Distinctively Separates Crohn's Disease (CD) and Ulcerative Colitis (UC)

Uta Berndt, Lars Philipsen, Sebastian Bartsch, Bertram Wiedenmann, Axel Dignass, Andreas Sturm

BACKGROUND: Although CD and UC share several clinical features, the mechanisms of tissue injury may differ in each condition. Up to now, most studies have been performed by analyzing protein lysates or RNA extracted from CD or UC tissue. However, since cellular function depends upon the protein network environment as a whole, we aimed to explore changes in the expression, distribution, and association of proteins to predict key protein involved in the disease pathways. METHODS: Endoscopic biopsies were taken during colonscopy from $\mathrm{CD}$, UC, and non-inflamed tissue. After fixation, Multi-Epitope-LigandKartographie (MELK) immunofluorescence microscopy with 30 different antibodies was performed. MELK is a novel, highly multiplexed robotic whole-cell imaging technology which stains the tissue subsequently with more than 30 different FITC-conjugated antibodies and bleach between every staining step. This technique allows integrating cell biology and biomathematical tools to simultaneously visualize dozens of proteins in a structurally intact cell or tissue with the ability to correlate cellular localization of proteins with function RESULTS: Performing MelTec'datamining procedure with the significance level of $\mathrm{p}<0.001$ (Student's t-test) and the search depth of combinations of 5 out of the 30 antibodies, controls and CD can be differentiated by 899, controls and UC by 1054 and CU from CD by 740 distinct protein combinations including extracellular, cell surface and intracellular target structures. For example, the number of CD45RA+, Bcl-2+,Caspase3-,Caspase8- and CD45RA+,CD1la-,Bax+,Caspase8+,p53- cells is significantly higher in CD than in UC or 
control tissue, whereas the amount of CD45RA-,Bcl-2+, Caspase3-,Caspase-8- cells is comparably in controls, $\mathrm{CD}$ and UC. Concerning regulatory T cells, the number of cells colocalising $\mathrm{CD} 4+\mathrm{CD} 25+$ - Caspase $3+$, Caspase $8+$ are decreased in $\mathrm{CD}$ compared to controls and $\mathrm{UC}$ explaining a higher expression of $\mathrm{CD} 4+, \mathrm{CD} 25+$ cells in $\mathrm{CD}$ than $\mathrm{UC}$ or controls tissue. In contrast, CD4+,CD25-,Caspase3+,Caspase8+ cells are increased in UC compared to control and CD tissue. Furthermore, CD29+,CD1la+ cells are, compared to controls, comparably lower in both, $C D$ and UC, indicating a down-regulation of integrin- $\alpha$ and $-\beta$ bearing cells in both forms of IBD. CONCLUSION: Analysis of key proteins involved in the pathogenesis of IBD and in-situ detection of their modification demonstrated for the first time that in $\mathrm{CD}$ and UC tissue apoptosis is executed distinctively within specific T cell populations. Thus, topological proteomic analysis may help to reveal the pathogenesis of IBD and understand the markedly different pathogenic events in CD and UC.

\section{M1731}

CD2 Deficiency Ameliorates Immunpathology and Improves Immune Defence in Murine T. Gondii Infection

Nina Pawlowski, Daniela Struck, Katja Grollich, Anja A. Kuehl, Martin Zeitz, Oliver

Liesenfeld, Joerg C. Hoffmann

Introduction: Infection of mice with Toxoplasma gondii ( $\mathrm{T}$. gondii) leads to a Thl mediated immunopathology of the small intestine. The aim of this study was to characterise the relevance of $\mathrm{CD} 2$ in $\mathrm{T}$. gondii infection with regard to parasite control and relevance for Thl mediated immunopathology. Material/Methods: WT $(\mathrm{n}=10)$ and CD2 deficient (CD2-/) $(\mathrm{n}=16)$ mice were infected with 100 cysts of $\mathrm{T}$. gondii (ME49) by gavage. On day 7 after infection 18 mice were killed (10 WT and 8 CD2-/- mice). Necrosis and the number of parasites per $\mathrm{cm}$ ileum were determined. Furthermore, cytokine levels in supernatants of CD3/CD28 or toxoplasmalysat antigen (TLA) stimulated cells as well as sera were evaluated. Survival of the 8 remaining CD2 $/$ - mice were analysed using Kaplan-Meier analysis; histologic scores, parasite load and cytokine levels were compared by Mann-Whitney Utest.Results: After oral infection with 100 cysts of T. gondii survival was significantly increased in CD2 deficient mice $(n=8)$ as compared to WT mice $(n=7 ; 23.5$ vs. $7.1, p=0.001)$. Histologic examinations 7 days after T. gondii infection showed less loss of weight and decreased inflammation of the ileum (histologic score: 2.3 vs. $4.3, \mathrm{p}=0.02$ ) of CD2-/ compared to WT mice. Furthermore, in the ileum of $\mathrm{CD} 2-/$ - mice the parasite load was significantly lower than in WT mice $(88 \pm 12$ vs. $349 \pm 58, p<0.01)$. The decrease in parasite load was paralleled by less production of IFN-g from TLA-stimulated lymphocytes of $\mathrm{mLN}(\mathrm{p}=0,039)$ and lower IFN-g levels in the serum $(\mathrm{p}=0,009)$. Surprisingly, TLAstimulated CD2-/- splenocytes induced higher levels of IFN-g $(p=0,021)$. On the other hand, IL-6 production by CD3/CD28 or TLA stimulated lymphocytes of mLN of CD2-/mice was decreased compared to WT cells $(\mathrm{p}=0,011)$. Discussion: In summary, CD2 deficiency decreases parasite load as well as intestinal immunopathology leading to increased survival. This is the first example with improved immune surveillance and decreased Thlmediated inflammation. Two possible mechanisms are the decreased IFN-g- and IL-6 production of mesenteric lymphnode lymphocytes. Further in vitro and in vivo studies are required in order to investigate the mechanisms behind these findings.

\section{M1732}

Constitutively Activated Notch Signaling Suppresses Goblet Cell Phenotype in Human Intestinal Epithelial Cells

Ryuichi Okamoto, Tetsuya Nakamura, Shigeru Oshima, Kiichiro Tsuchiya, Takanori Kanai, Mamoru Watanabe

Background \& Aims: The intestinal epithelia are a unique tissue in the human body that continues rapid renewal throughout life. Continuous generation of functional, differentiated intestinal epithelial cells (IECs) is maintained by intestinal stem cells residing in the lower part of the crypt. However, precise molecular mechanism how undifferentiated status of stem and progenitor cells is maintained remains largely unknown. Notch signaling is one of the signaling pathways that are known to have indispensable role in mammalian development. Recent studies have shown that Notch signaling also regulates cell fate decision of stem or progenitor cells in adult tissues such as blood, lung and skin. To determine whether Notch signaling also regulates cell differentiation within the human intestinal epithelia, we performed both immunohistochemical and cell-based functional assays and analyzed the functional role of Notch signaling in human IECs. Methods: Immunohistochemical analysis using surgically ressected human intestinal tissues was performed to determine expression and localization of cleaved Notchl and Hesl, a known target gene of Notch signaling. Cellbased functional assays were performed using human IEC-derived cells in which forced expression of constitutively activated Notchl could be induced by Tet-on system. Results: Immunohistochemical examination of human intestinal tissues showed nuclear localization of cleaved Notchl and its target gene Hesl protein in epithelial cells of the crypt region. Immunohistochemical analysis of Ki-67 expression suggested that Notchl is activated in the stem/progenitor cell population in the human intestinal epithelia. Surprisingly, Notchl was also broadly activated in distorted crypts observed in inflamed regions of ulcerative colitis patients, where goblet cells are depleted. Induction of constitutively activated Notchl in LS174T cells significantly up-regulated Hesl transcription, but reciprocally down-regulated E-box dependent transcription. More over, continuous expression of constitutively activated Notchl down-regulated both mRNA and protein expression of MUC2 gene, a gene specifically expressed in goblet cells, and subsequently reduced mucin production in LS174T cells. Conclusions: These results not only provide physiological distribution and function of Notchl activation in the human intestinal epithelia, but also suggests that constitutively activated Notchl in IECs might be involved in the pathogenesis of inflammatory bowel diseases.

\section{M1733}

Central Regulatory Role of the Transcription Factor NFATC2 in Cyclosporine a Treatment in Ulcerative Colitis

Benno Weigmann, Raja Atreya, Jonas Mudter, Hans A. Lehr, Peter R. Galle, L H Glimcher, Markus F. Neurath

Cyclosporine A (CsA) is widely used as a immunosuppressive drug in the treatment of inflammatory diseases. Various studies showed a therapeutic effect of CsA in the treatment of steroid-refractory ulcerative colitis (UC). After treatment with CsA an anti-inflammatory effect was observed, resulting in the induction of rapid remission. However this result could not be observed in patients with crohn<'>s disease. The aim of our study was to identify the molecular mechanism through which CsA exerts its therapeutic effect. Biopsies from patients suffering of UC and Crohn's disease (CD) were taken and fluorescence-stained with Anti-Nuclear factor of activated T cells (NFAT) c2 antibodies. Surprisingly a higher expression of NFATC2 was found in UC and CD tissue compared to control specimen. Furthermore isolated Lamina propria mononuclear cells (LPMC) from intestinal biopsies of patients with inflammatory bowel disease were examined regarding their rate of apoptosis induction upon treatment with CsA for $48 \mathrm{~h}$. Cytometric analysis of annexin V/propidiumiodide staining revealed a high number of apoptotic cells in UC compared to LPMC's from CD and control patients. ELISA of supernatants from cultivated LPMC revealed no significant difference regarding the production of IL-2, 4,5 . Significant reduction of the viability of cultivated CD4+ T cells from NFATc2 deficient mice in comparison to T cells from control mice could be observed. Due to downregulation of caspase 3 and 9 , the induction of apoptosis was elevated in control mice compared to NFATc2 deficient mice. Moreover, CD4+CD62L+ T cells from NFATc2 deficient mice and controls were isolated and transferred to RAG-2 deficient mice. In contrast to NFATc2 deficient mice, controls showed a significant loss of weight. Furthermore histological score and miniendoscopy revealed a significant lower intestinal inflammation in NFATc2 deficient mice. Our data points out the central regulatory role of NFATc2 in the pathogenesis of UC. This NFATc2 induced apoptotic pathway is responsible for the clinical efficacy of CsA in the treatment of acute steroid-refractory ulcerative colitis.

\section{M1734}

Altered Barrier Function Is Associated with Increased Serum Levels of Lipopolysaccharide and TNF-Alpha in Crohn's Disease

Vincenza Di Leo, Francesca Lamboglia, Daniela Pizzuti, Renata D'Inca, Sergio Varotto,

Maria G. Vettorato, Giacomo C. Sturniolo

BACKGROUND: Increased intestinal permeability and impaired response to bacterial products are both involved in the pathogenesis of Crohn's disease (CD). Binding of lipopolysaccharides (LPS), a component of Gram-negative bacteria, to its specific receptor CD14, leads to the production and release of proinflammatory cytokines, such as TNF-alpha. Serum levels of soluble CD14 (sCD14) reflect CD14 activation by LPS. We hypothesized that the inflammatory response associated with increased intestinal permeability may derive from abnormal passage of LPS through the gut wall. PATIENTS AND METHODS: Eight-two CD patients (39 F, 58 in remission) and thirty three healthy volunteers (HV) were studied. We analysed intestinal barrier function by lactulose/mannitol (L/M) test and in Ussing Chambers, serum levels of sCD14 and TNF-alpha by ELISA and LPS by LAL chromogenic assay. Statistical analysis was performed by non parametric tests. RESULTS: Median (25th-75th percentiles) L/M test was significantly increased in $C D$ patients compared to $H V(0,024$ $(0,013-0,038)$ vs $0,010(0,070-0,080) ; \mathrm{p}<0,001)$. Increased levels of $\mathrm{sCD} 14(3799 \mathrm{pg} / \mathrm{ml}$ (3163-4833) vs $2929 \mathrm{pg} / \mathrm{ml}(2603-3640) ; \mathrm{p}<0,05)$, TNF-alpha ( $7 \mathrm{pg} / \mathrm{ml}(4-10) \mathrm{vs} 3 \mathrm{pg} / \mathrm{ml}$ $(2-4) ; \mathrm{p}<0,001)$ and LPS $(0,28 \mathrm{EU} / \mathrm{ml}(0,15-0,68)$ vs $0,06 \mathrm{EU} / \mathrm{ml}(0,03-0,09) ; \mathrm{p}<0,001)$ were found in $C D$ patients vs HV. Altered intestinal barrier function in $C D$ patients was found in the Ussing chambers study as well. L/M test and LPS levels were significantly increased in patients with active disease compared to those in remission (L/M 0,037 $(0,020$ $0,050)$ vs $0,021(0,012-0,029 ; \mathrm{p}<0.05)$ (LPS $0,67 \mathrm{EU} / \mathrm{ml}(0,20-0,90)$ vs $0,23(0,14-0,46)$; $\mathrm{p}<0,05)$ with a significant correlation between the two parameters $(r=0,4, p<0,05)$ in $C D$ patients. No significant difference was demonstrated between sCD14 and TNF-alpha levels in patients with active disease vs patients in remission. CONCLUSION: A primary defect in barrier function, which allows increased passage of bacterial products into the intestinal wall, causes SCD 14 activation in CD. Active disease, albeit associated with increasing intestinal permeability and LPS absorption, does not result in further immunological stimulation.

\section{M1735}

Clinical Response to Visilizumab in Intravenous Steroid Refractory Ulcerative Colitis (IVSR-UC) Is Associated with Changes in Cd8+ T Cells Jacky Woo, Tanny Tsao, Lili Zhang, James Sheridan, Debbie Law, James Lowder, Vladimir Vexler

Background: Visilizumab (Nuvion $\left.{ }^{\circledR}\right)$, a humanized low FcR-binding IgG2 anti-CD3 monoclonal antibody, is currently being tested for the treatment of IVSR-UC. Visilizumab induces rapid and transient disappearance of blood T cells. CD8 counts recovered faster than CD4+ counts with CD4/CD8 ratio being inverted post-treatment. In clinical study to date $\sim 60 \%$ of IVSR-UC subjects treated with visilizumab had a clinical response. Clinical responses are durable and persist long after visilizumab is cleared from the circulation. Clinical response to visilizumab is hypothesized to be associated with the effect on CD8+ T cells. Aims: To compare the effects of visilizumab on (1) peripheral and mucosal CD8+ T cells among clinical responders and non-responders and (2) phenotype and function of CD8+ T cells in vitro. Methods: In phase I/II dose range-finding study of in IVSR-UC, IV bolus infusions of visilizumab on day 1 and day 2 were administered at $5,7.5$, 10 , or $12.5 \mathrm{mg} / \mathrm{kg}$ dose. Treated subjects with a MTWSI score that dropped below 10 , with at least a 3 points decline, were classified as responders. Absolute numbers of $\mathrm{T}$ cells were measured by flow cytometry (TruCOUNT). In 14 subjects colonic biopsies were collected at baseline and Day 30 and T cell composition was analysed by immunohistochenistry. Effects of visilizumab in vitro on the phenotype and function of CD8+ T cells was evaluated in PBMC isolated from healthy 
subjects. Results: Of 76 treated subjects evaluated, 51 responded to visilizumab. In responders $\mathrm{CD} 3+\mathrm{CD} 8+$ (but not $\mathrm{CD} 3+\mathrm{CD} 4+$ ) counts on Day 30 were significantly higher in the responder group compared to non-responders. Histological improvement was associated with a reduction in number of mucosal CD3+ cells and a relative increase in the percentage of CD8+ T cells. In vitro, visilizumab preferentially expanded CD8+ T cells and resulted in an inverted CD4/CD8 ratio. Visilizumab-treated CD8+ cells expressed CTLA-4+, a phenotype attributed to regulatory $\mathrm{T}$ cells. Regulatory activity of these cells was confirmed by inhibition of proliferation and CD25 upregulation of autologous PBMC. Conclusion: In visilizumabtreated IVSR-UC subjects, clinical response is associated with changes in peripheral and mucosal CD8+ T cells and visilizumab can induce CD8+ regulatory T cells in vitro. The induction of regulatory CD8+ T cells may contribute to visilizumab's observed clinical activity.

\section{M1736}

Endothelial Expression of PSGL-1 in Murine Colitis and Sepsis Thorsten Vowinkel, Katherine C. Wood, Christoph Anthoni, Norbert Senninger, Paul Kubes, D. Neil Granger, Christian F. Krieglstein

Introduction: P-selectin and P-selectin glycoprotein ligand-1 (PSGL-1) are vascular adhesion molecules that play an important role in leukocyte-endothelial, leukocyte-platelet and platelet-endothelial interactions during inflammatory responses. In contrast to P-selectin, little is known about the endothelial expression of PSGL-1. The aims of the present study were 1) to quantify endothelial PSGL-1 expression in different regional vascular beds and 2) to determine whether constitutive endothelial PSGL-l expression is altered in response to inflammatory stimuli. Methods: The dual radiolabeled monoclonal antibody (mAb) technique was used to quantify the expression of endothelial PSGL-l in different vascular beds of C57Bl/6J mice after 1) intraperitoneal injection of lipopolysaccharide (LPS) or 2) dextran sulphate sodium (DSS; $3 \%$ in drinking water for 7 days) induced colitis. Healthy wild-type (WT) mice, PSGL-1 knockout (KO) mice and WT mice transplanted with bone marrow (BM) from PSGL-1 KO mice (deficient in PSGL-1 on circulating blood cells) all served as controls. Results: Endothelial PSGL-1 expression was detectable in various tissues of untreated mice and was significantly reduced in almost every organ of PSGL-1 KO mice. Systemic administration of LPS increased PSGL-1 expression in the vasculatures of the intestines (small and large), kidneys, and brain as early as four hours. The LPS-induced increase in PSGL-1 was not associated with neutrophil accumulation since pretreatment with antineutrophil serum did not reduce PSGL-1 expression. However, PSGL-1 expression levels in BM chimeric mice deficient in PSGL-1 on circulating blood cells were significantly reduced, suggesting that platelets contribute to some of the PSGL-1 expression detected. Mice with DSS-induced colitis also exhibited significantly increased PSGL-1 expression in the large bowel, liver, lung and brain, compared to untreated controls. Conclusions: These findings suggest that PSGL-1 is constitutively expressed on vascular endothelium, where its expression is significantly increased in response to inflammatory stimuli such as sepsis and colitis. (DFG VO 998/1-1, DK65649)

\section{M1737}

Reversible Defect of Mitochondrial Thiolase Activity in Ulcerative Colitis Srikanth Santhanam, Aparna Venkatraman, Balakrishnan S. Ramakrishna

Background: Colonocyte oxidation of butyrate is defective in ulcerative colitis (UC). The cause of this defect has not been established. Aims: This study aimed to determine whether the activity of specific colonocyte enzymes involved in butyrate oxidation was defective in UC. Methods: Rectosigmoid mucosal biopsies were obtained at colonoscopy from 30 patients with UC and 41 control subjects (colon cancer and irritable bowel syndrome) and stored at -20 degrees $C$ until analysis. Biopsies were homogenized and the activities of the following enzymes estimated - thiolase, crotonase, and beta-hydroxyacyl CoA-dehydrogenase. Results: Mitochondrial thiolase activity was markedly reduced in biopsies from patients with UC compared to control (Table). This was reversible by addition of mercaptoethanol. In contrast, activity of crotonase and of beta-hydroxyacyl CoA dehydrogenase were not reduced. Further studies of mitochondrial thiolase in control biopsies showed that addition of $0.2 \mathrm{mM}$ hydrogen peroxide reduced enzyme activity to $60(8) \mathrm{nmol} / \mathrm{mg}$ protein $(\mathrm{P}<0.01)$, while addition of $300 \mu \mathrm{M}$ mercaptoethanol increased enzyme activity to 91 (15) $\mathrm{nmol} / \mathrm{mg}$ protein. Conclusions: There is a defect of mitochondrial thiolase activity in UC. Reversal of this defect by addition of mercaptoethanol suggests that the mechanism may be catalytic site inactivation by cysteine sulfhydryl oxidation or disulfide formation, a conclusion further strengthened by the demonstration of reduction in enzyme activity induced by hydrogen peroxide. Oxygen free radicals generated in mitochondria may be responsible for this defective enzyme activity which may in turn contribute to impaired butyrate oxidation by colonocytes in UC.

Activities of enzymes in biopsy homogenates.

\begin{tabular}{|c|c|c|}
\hline & Control & UC \\
\hline Mitochondrial thiolase & $113(16)$ & $47(05)^{*}$ \\
\hline$+300 \mu \mathrm{M}$ mercaptoethanol & $102(10)$ & $91(15)$ \\
\hline Crotonase & $391(60)$ & $331(40)$ \\
\hline Beta-hydroxyacyl CoA dehydrogenase & $283(43)$ & $261(10)$ \\
\hline
\end{tabular}

Values are mean (SEM) expressed as nmoles/mg protein. ${ }^{*} \mathrm{P}<0.01$ compared to control (t-test).

\section{M1738}

Unstable Fecal Microbiota in Ulcerative Colitis During Remission Cristina Martinez, Maria Antolin, Javier Santos, Antonio Torrejon, Francesc Casellas, Natalia Borruel, Francisco Guarner, Juan-Ramon Malagelada

Several studies have identified abnormal characteristics of the fecal microbiota in patients with clinically active IBD, but whether the changes are causal or secondary to inflammation remains uncertain. AIM: To investigate the composition and dynamics of the fecal microbiota in patients with ulcerative colitis (UC) in remission by using a molecular ecology approach (denaturant gradient gel electrophoresis, DGGE). METHODS: A group of 34 patients with $\mathrm{UC}$ in clinical remission $(\mathrm{CAI}<4)$ and on maintenance 5-ASA therapy were recruited. Fecal samples were collected over a period of one year, at entry (w0) and at months $1,3,6$, 9 and 12 (ml, m3, m6, m9, and m12). Eight healthy volunteers served as controls. Sixteen patients who remained in remission during the study period were included in the analysis. Total DNA was isolated from the fecal samples and used as a template to amplify by PCR the V6 to V8 variable regions of fecal bacterial 16S rRNA gene. The PCR amplicons were then separated by DGGE. The gel profiles were compared using the BioNumerics software package and similarity indices were calculated from densitometric curves using the Pearson product moment correlation coefficient. RESULTS: The DGGE profiles showed that each patient harbours a unique microbiota with a low similarity index (SI) between patient $(15 \pm 14 \%$, mean $\pm S D)$. The diversity of the microbiota estimated by the number of bands was $17 \pm 4$ in UC, and $28 \pm 5$ in healthy controls $(\mathrm{p}<0.05)$. Moreover, in UC, but not in healthy controls, the composition of the microbiota varied notably over time. Thus, similarity indexes versus $\mathrm{w} 0$ were $58 \pm 21 \%$ at $\mathrm{ml}, 42 \pm 24 \%$ at $\mathrm{m} 3,35 \pm 20 \%$ at $\mathrm{m} 6,26 \pm 20 \%$ at $\mathrm{m} 9$ and $23 \pm 19 \%$ at $\mathrm{m} 12$ (all $\mathrm{p}<0.05$ vs w0). In healthy subjects, however, the SI was $77 \pm 11 \%$ at 4 months. The SI of the same sample analyzed several times was $97 \%$. CONCLUSIONS: Molecular analysis of fecal microbiota in patients with inactive UC as compared with healthy individuals show low bio-diversity and temporal instability. These findings suggest an abnormal susceptibility of the intestinal ecosystem to environmental factors in UC.

\section{M1739}

Macrophage Migration Inhibitory Factor (MIF) -173 G/C Polymorphism Influences Upper Gastrointestinal Tract Involvement in Crohn's Disease Julia Dambacher, Zeljka Sisic, Tanja Staudinger, Simone Pfennig, Fabian Schnitzler, Katrin Hofbauer, Jan-Michel Otte, Julia Seiderer, Cornelia Tillack, Burkhard Goeke, Peter Lohse, Thomas Ochsenkuehn, Stephan Brand

Background: Macrophage migration inhibitory factor (MIF) is a proinflammatory cytokine which has been shown to be involved in the development of chronic murine colitis. A C nucleotide at position -173 in the 5'-flanking region of the MIF gene creates a binding site for the transcription factor activator protein-4 (AP-4). Aims: We analyzed the role of the $173 \mathrm{G} / \mathrm{C}$ single nucleotide polymorphism (SNP) in Crohn's disease (CD) and its influence on the $\mathrm{CD}$ phenotype. Methods: Using restriction fragment length polymorphism (RFLP) analysis, genomic DNA from 198 patients with CD and from 159 unrelated controls was analyzed for the $-173 \mathrm{G} / \mathrm{C}$ SNP in the MIF gene. Genotype distribution between CD patients and controls and the association of patients' genotypes with clinical parameters were statistic ally evaluated using Student's t-test for continuous data and Fisher's exact test for categorical data $C D$ phenotypes were classified according to the Vienna classification of $C D$ (Inflamm Bowel Dis 2000; 6:8-15). The diagnosis of upper gastrointestinal tract involvement was based on endoscopic and histologic findings. Results: No difference in the frequency of the $173 \mathrm{G} / \mathrm{C}$ genotypes was observed in $\mathrm{CD}$ patients compared to the control group. In the $\mathrm{CD}$ group, $74.2 \%$ were $\mathrm{G} / \mathrm{G}$ wildtype, $22.2 \%$ were $\mathrm{G} / \mathrm{C}$ heterozygous, and $3.5 \%$ were $\mathrm{C} / \mathrm{C}$ homozygous compared to $67.9 \%, 28.3 \%$, and $3.7 \%$, respectively, in the control group Thirty-one of the 147 wildtype carriers (21.1\%), but only one of the $44 \mathrm{G} / \mathrm{C}$ heterozygotes $(2.1 \%)$ were diagnosed of upper gastrointestinal tract involvement $(\mathrm{p}=0.002)$. In contrast there was no statistical significant difference between the different genotypes regarding other disease characteristics such as incidence of stenoses, fistulas, abscesses, surgical procedures and the use of immunosuppressive agents. Interestingly, $56.8 \%$ of the $\mathrm{G} / \mathrm{C}$ heterozygote were also carriers of at least one common CARD15 mutant (R702W, G908, or $1007 \mathrm{fs}$ ) compared to only $37.4 \%$ in the wildtype group $(\mathrm{p}=0.02)$. However, the observed negative association of the $-173 \mathrm{G} / \mathrm{C}$ genotype with upper gastrointestinal tract involvement was independent of the CARD15 genotype. Conclusions: The frequency of the -173 G/C MIF polymorphism in patients with $\mathrm{CD}$ is similar to that observed in the normal population Nevertheless, this polymorphism appears to be a factor contributing to a particular CD phenotype characterized by the lack of upper gastrointestinal tract involvement.

\section{M1740}

Low Human $\beta$-Defensin 2 Gene Copy Number in Crohn's Colitis Hartmut Schmalzl, Elke Schaeffeler, Matthias Schwab, Klaus Fellermann, Daniel Stange, Bo Shen, Jan Wehkamp, Walter Reinisch, Eduard F. Stange

Background: Defensins are small cationic peptides that are crucial components of innate immunity possessing a broad spectrum of antimicrobial activity. The human defensin gene cluster including HBD-2 as well as other beta defensins is one of few with a gene copy number polymorphism. HBD-2 copy number is known to correlate with HBD-2 expression in leukocyte cell lines. Beta defensins have been shown to be significantly induced in macroscopically inflamed colonic biopsy specimens of ulcerative colitis (UC) but less in Crohn's disease (CD), suggesting a defective antimicrobial barrier in CD. Aim: We hypothesized that this defective HBD-2 and 3 induction in colonic CD was related to a low HBD-2 gene copy number. Methods: We adopted a TaqMan protocol for the determination of HBD2 gene copy number from whole blood. Real-time PCR was performed and quantitative HBD-2 amplification data were normalized to albumin as an internal reference gene which was coamplified simultaneously in a single-tube biplex assay. The assay was validated with a selection of previously genotyped DNA samples containing three, four, five and seven HBD-2 gene copies.Mucosal HBD-2 mRNA expression was determined and correlated to gene copy number. Intestinal HBD-2 expression was measured by quantitative PCR. The 
phenotype of $\mathrm{CD}$ patients was categorized according to the Vienna classification. Statistical comparisons were performed with the Mann-Whitney test (two-sided). Results: The four independent cohorts consisted of 54 CD/38 UC (I), 111 CD/37 UC (II), 85 CD (III, surgical cohort) and 103 controls (IV). The gene copy number ranged from 2-10 copies per genome and the median HBD-2 gene copy number was 4 in the control group. Both ulcerative colitis cohorts (I and II) and ileal CD (I, II and III) had a distribution similar to the control groups with a median of 4 whereas colonic $C D$ had a median of only 3 copies per genome (colonic $C D$ vs, control $\mathrm{p}<0.031$ ). Median for gene copy number was 4 in case of ileal vs. 3 for colonic $C D(p=0.032)$. The differences between ileal and colonic $C D$ was most pronounced in the severe surgical cohort III $(\mathrm{p}<0.008)$. We confirmed our earlier findings of an enhanced HBD-2 mRNA expression in active UC. HBD-2 gene copy number was positively related to mucosal mRNA expression in inflamed specimen.Conclusion: The cohorts showed a significantly lower HBD-2 gene copy number in colonic CD compared to ileal CD, UC or controls. This affects mucosal HBD-2 expression as gene copy number translates into corresponding mRNA. Thus, we postulate that a low HBD-2 gene copy number predisposes to colonic $\mathrm{CD}$ through diminished beta defensins.

\section{M1741}

Cytoprotective Role of Multidrug Resistance Associated Protein 1 (MRP1) in Intestinal Inflammation: Involvement of the Cysteinyl Leukotriene Pathway Axel Van Steenpaal, Hans Blokzijl, Sara Vander Borght, Lisette I.H. Bok, Louis Libbrecht, Mariska Geuken, Tania A.D. Roskams, Gerard Dijkstra, Han Moshage, Peter L.M. Jansen, Klaas Nico Faber

Background: Multidrug Resistance-associated Proteins (MRPs) are known to cause multidrug resistance in cancer cells. Additionally, they may protect non-malignant cells against inflammation-induced cell damage. Here, we studied the regulation of MRP expression during intestinal inflammation and the role of MRPI in protecting the intestinal epithelium (IE) against cytokine and/or $\alpha$-Fas-induced cell death. Methods: Inflamed and non-inflamed colon tissue was obtained from patients with Crohn's disease (CD) $(n=20)$ or ulcerative colitis (UC) ( $\mathrm{n}=15)$. Quantitative RT-PCR and immunohistochemistry were used to analyse expression of MRP1-6 and MDR1. DLD-1 colon carcinoma cells and stable derivatives expressing MRP1-GFP were treated with $\alpha$-Fas or TNF- $\alpha$. MRP function and leukotriene C4 synthesis (LTC4; MRP1 substrate) were inhibited by MK571 and AA861, respectively. Apoptosis was assessed by caspase-3 activity assays and PARP-cleavage Western blotting. Results: MRP1, but not MRP2-6 or MDR1, was upregulated in inflamed IE from UC and CD patients, specifically in the crypts. $\alpha$-Fas- and TNF- $\alpha$-induced caspase- 3 activity and PARP cleavage in DLD-1 cells was strongly increased in the presence of MK571. Overexpression of MRP1-GFP resulted in reduced $\alpha$-Fas and TNF- $\alpha$-induced caspase- 3 activity and/or PARP cleavage in DLD-1 cells. MK571/ $\alpha$-Fas-induced caspase-3 activity was reduced by AA861 to levels comparable to AA861/ $\alpha$-Fas treatment. Conclusion: The leukotriene transporter $\mathrm{MRPl}$ appears to be involved in the cytoprotection of intestinal crypt cells during inflammation. Most likely, it exports proapoptotic compounds from the cysteinyl leukotriene biosynthesis pathway since 1) Inhibition of the leukotriene pathway decreases apoptosis and 2) LTC4 is a high affinity substrate for MRP1.

\section{$\mathrm{M} 1742$}

Mdp Induces Apoptosis in Immunocompetent Cells: A Possible Link Between NOD2/CARD15 Mutations and Crohn's Disease

Vanessa E. Beynon, Juergen Glas, Alexander Faussner, Rudolf Gruber, Kerstin Maier, Laurian Tonenchi, Ilona Endres, Matthias Folwaczny, Christian Folwaczny, Helga-Paul Toeroek

Background: The underlying pathogenetic mechanisms linking CD to mutations in NOD2 have not yet been elucidated. NOD2 mediates NF- $\mathrm{KB}$ activation in response to muramyl dipeptide (MDP), an effect impaired in presence of $C D$ associated mutations. For several pathogen-associated molecular patterns pro- and also anti-apoptotic effects have been described. Moreover, members of the NOD family have been shown to induce apoptosis. To determine the role of NOD2 in apoptosis, herein peripheral blood mononuclear cells (PBMC) and intestinal biopsies were stimulated with MDP, NOD2 variants were overexpressed in transfected cell lines and the effect on apoptosis has been studied. Methods: PBMC from 5 healthy controls (NOD2 wt) were isolated by gradient centrifugation. Lymphocytes and monocytes were further isolated from 4 healthy controls (NOD2 wt) by MACS. Intestinal biopsies from 2 healthy controls were collected during surveillance endoscopy. Cells or biopsies were cultured for 8 and $24 \mathrm{~h}$ in presence of $100 \mathrm{\mu g} / \mathrm{ml}$ MDP or medium alone. The Flp-In T-REx HEK 293 cell line has been stably transfected with wt-NOD2 and the variants L1007fs, R702W and G908R. NOD2 expression was induced by tetracycline for $48 \mathrm{~h}$. Apoptosis was determined by flow cytometry analysis of annexin $\mathrm{V}$ binding populations and immunohistochemical staining of biopsies for CytoDEATH and Bax. Results: The percentage of apoptotic PBMC significantly increased in presence of MDP after $8 \mathrm{~h}(13 \pm 5 \%$ vs. $17 \pm 7 \% ; \mathrm{p}<0.05)$. A similar effect was observed in isolated monocytes after $24 \mathrm{~h}(50 \pm 12 \%$ vs. $58 \pm 12 \% ; \mathrm{p}<0.05)$. In contrast, lymphocytes did not show an increase of apoptosis. Shortterm culture of intestinal biopsies in presence of MDP resulted in increased CytoDEATH and Bax positive epithelial cells. Overexpression of wt-NOD2 protein resulted in significant increase of apoptotic cells compared to parental HEK 293 cells $(17 \pm 10 \%$ vs. $31 \pm 17 \%$; $<<0.05)$. In contrast, expression of mutant L1007fs NOD2 protein did not result in increased apoptosis. For the R702W and G908R variants an intermediate response has been observed. Conclusion: Herein a proapoptotic role of MDP in human PBMC has been demonstrated for the first time. This effect was absent in isolated lymphocyte populations which suggests a mediation through monocytic cells. A proapoptotic effect of MDP has also been observed in intestinal epithelial cells ex vivo. These observations and the described proapoptotic effects of NOD2 overexpression in transfected cells are compatible with an implication of NOD2 protein in apoptosis. An impairment of this process caused by CD associated variants could represent an important pathogenetic link between NOD2 mutations and CD.

\section{M1743}

Increased Levels of $\beta 7$ Integrin On T Cells Activated By Colonic Dendritic Cells (DC) from Ulcerative Colitis Patients: Altered DC Control of T Cell Homing in Inflammatory Bowel Disease?

Rachael J. Rigby, Ailsa L. Hart, Neil McCarthy, Sabita Islam, Hafid Omar al-Hassi, Stella C. Knight, Michael A. Kamm, Michael Burke, John Lambert, Andrew J. Stagg

Introduction: Mucosal immune responses are compartmentalized. Studies in the mouse indicate that gut DC, but not those from non-intestinal sites, condition T cells that they activate to traffic selectively back to intestinal tissue by upregulating homing markers including $\alpha 4 \beta 7$ integrin. Although lymphocyte homing to intestinal tissue is central to the pathogenesis of human inflammatory bowel disease (IBD), it is not known whether DC condition gut-homing lymphocytes in Man or whether such imprinting is altered by intestinal disease. Therefore, we examined the expression of homing markers on human T cells activated by DC from colon (from healthy controls and ulcerative colitis (UC) patients), blood, skin and respiratory tract. Methods: Colonic and skin DC were enriched, using metrizamide gradients, from cells migrating from tissue during overnight culture. Blood DC were similarly enriched after overnight culture of mononuclear cells. Cells present in induced sputum were the source of respiratory tract DC. Naive CD4+ allogeneic peripheral blood T-cells were labelled with CFSE, and stimulated with DC (at $2 \%$ of T cells) for 4 days. Flow-cytometry was used to measure expression of gut homing ( $\beta 7$ integrin, CCR9) and skin homing (CLA, CCR4) makers on proliferating (CFSElo) cells. Results: Enriched DC from all tissues were potent stimulators of allogeneic CD4+ T cells $(\mathrm{p}<0.001$ to $\mathrm{p}<0.1$ ). Dividing cells expressing $\beta 7$ integrin, CLA, CCR4 and low levels of CCR9 were detected irrespective of the source of stimulating DC. A proportion of $\beta 7+$ dividing cells co-expressed the skin homing markers CCR4 or CLA; again this pattern of expression was observed for all DC. When compared with $\mathrm{T}$ cells activated by blood DC assessed in parallel, the level of $\beta 7$ integrin was higher on $T$ cells activated by DC from healthy colonic tissue $(p<0.02)$. Moreover the level of $\beta 7$ integrin on T cells activated by DC from non-inflamed colonic tissue of UC patients was greater than on cells activated by control colonic $(p<0.01)$ or blood $(p<0.005)$ DC. DC from UC tissue also generated a greater proportion of $\beta$ 7hi cells ( $p<0.05$ versus blood DC). Conclusions: Human tissue DC induce a broader range of homing markers than do their murine counterparts suggesting that other factors may contribute to compartmentalization of mucosal responses in Man. Nonetheless, colonic DC induced higher levels of the intestinal homing integrin $\beta 7$ than did blood DC. Increased induction of $\beta 7$ by colonic DC in UC supports a role for dysregulated control of homing by DC in IBD.

\section{M1744}

Characterization oF Reactive Oxygen Species Production (ROS) and Activity of Antioxidant Enzymes in Peripheral White Mononuclear Cells (PWMC) from Patients with Crohn's Disease (CD) At Onset

Belen Beltran, Pilar Nos, Francisco Dasi, Marisa Iborra, Guillermo Bastida, Marcial Martinez, Mariam Aguas, Vicente Ortiz, Julio Ponce

Background: CD patients have an increased ROS peripheral leukocyte damage and decreased plasma antioxidants. However, the ROS directly implicated, their place of production and the state of enzymatic antioxidant defenses have not been characterized. Mitochondria are organelles with high capacity of ROS production. Aims: To characterize at onset of $C D$ disease: 1) The basal and stimulated production of superoxide (O2-), hydrogen peroxide (H2O2) and nitric oxide (NO) in PWMC. 2) The activity of cellular antioxidant enzymes (Superoxide dismutase (SOD) and Catalase (CAT)). 3) Their mitochondrial activity. Methods: Blood samples from healthy subjects $(n=15$, mean age $32 \pm 8 y)$ and from patients at first flare of $C D(n=17$, mean age $36.9 \pm 9.2$ y) were obtained. PWBC were isolated by FicollHistopaque sedimentation. $\mathrm{O} 2-, \mathrm{H} 2 \mathrm{O} 2$ and $\mathrm{NO}$ production were measured by flow cytometry after $1 \mathrm{hr}$ incubation with hydroethidine $(5 \mu \mathrm{M}), \mathrm{H} 2 \mathrm{DCFDA}(20 \mu \mathrm{M})$ and DAF $(5 \mathrm{mM})$ respectively. Production of $\mathrm{O} 2$ - and $\mathrm{H} 2 \mathrm{O} 2$ were stimulated by adding plumbagin $(\mathrm{Pb})(2.5$ $\mathrm{mM})$ and t-butyl-hydroperoxyde $(\mathrm{Tb})(10 \mathrm{mM})$ respectively. SOD and CAT activity were measured spectrophotometrically by enzymatic assays. Mitochondrial activity was measured by searching the mitochondrial membrane potential (ym) after incubation (lh) of cells with TMRM $(0.5 \mathrm{mM})$. Results: C Reactive Protein $(\mathrm{CRP})$ levels were: controls $(2.7 \pm 0.7)$ and $\mathrm{CD}(63.67 \pm 21)$. Basal and stimulated $\mathrm{O} 2$ - and $\mathrm{H} 2 \mathrm{O} 2$ production are shown in the table. $\mathrm{NO}$ production was lower in $\mathrm{CD}(113 \pm 22 \mathrm{FU})$ than in controls $(167 \pm 20 \mathrm{FU}, \mathrm{p}=0.02)$. SOD activity was significantly increased in $C D(2.1 \pm 0.3 \mathrm{U} / \mathrm{mg}$ vs $0.97 \pm 0.1 \mathrm{U} / \mathrm{mg}$ in controls; $\mathrm{p}<0.001)$ whereas CAT activity was significantly inhibited $(14.9 \pm 4 \mathrm{U} / \mathrm{mg}$ in $\mathrm{CD}$ vs $35.5 \pm 4 \mathrm{U} / \mathrm{mg}$ in controls, $\mathrm{p}<0.01)$. ym was inhibited in $\mathrm{CD}$ patients $(25.4 \pm 1.4$ vs 14 \pm 1.1 in controls, $\mathrm{p}<0.001$ ). There was a significant negative correlation between ym inhibition and CRP (Rho Spearman -0.778). Conclusions: PWMC from CD patients have an increased production of $\mathrm{H} 2 \mathrm{O} 2$. The increased SOD activity prevents $\mathrm{O} 2$ - damage, however its $\mathrm{H} 2 \mathrm{O} 2$ yield cannot be completely cleared. CAT inhibition facilitates oxidative stress. The inhibition of ym shows impaired mitochondrial activity in CD which usually facilitates O2production. This inhibition does not depend on NO blocking the respiratory chain, as NO is diminished in PWMC of CD patients.

Reactive Oxygen Species (RFU +/- SEM)

\begin{tabular}{|c|c|c|c|c|c|c|}
\hline PWMC & {$[\mathrm{O} 2]$} & {$[\mathrm{O} 2+\mathrm{Pb}]$} & p-value* & {$[\mathrm{H} 2 \mathrm{O} 2]$} & {$[\mathrm{H} 2 \mathrm{O} 2+\mathrm{Tb}]$} & $\mathrm{p}$-value $\dagger$ \\
\hline Control $(\mathrm{C})$ & $1.2 \pm 0.2$ & $9.7 \pm 3.2$ & 0.001 & $0.9 \pm 0.2$ & $42.9 \pm 12$ & $<0.001$ \\
\hline $\mathrm{CD}$ & $1.2 \pm 0.3$ & $2.6 \pm 0.4$ & 0.004 & $2.9 \pm 0.8$ & $87.7 \pm 23$ & 0.01 \\
\hline p-value (C vs CD & 0.959 & 0.020 & & 0.03 & 0.06 & \\
\hline
\end{tabular}

(* p-value: [O2-] vs [O2- + $\mathrm{Pb}] \uparrow$ p-value: [H2O2] vs [H2O2+ Tb]) 


\section{M1745}

Increased Serum Levels of Anti-Tissue Transglutaminase Antibodies in Patients with Ulcerative Colitis

Helga P. Torok, Laurian Tonenchi, Juergen Glas, Rudolf Gruber, Molla T. Yirsaw, Daniel Schneider, Vanessa Beynon, Sebastian Cotofana, Christian Folwaczny

BACKGROUND: Several observations reported an increased prevalence of celiac disease among patients with inflammatory bowel disease. Tissue transglutaminases (tTG) represents the key autoantigens in celiac disease. Recent studies suggest that in the damaged colon transglutaminases are needed for the response to chronic injury and play an important role in the mucosal healing process. In the present study antibodies against ITG have been assessed in patients with inflammatory bowel disease. The levels of the antibodies were compared with those observed in healthy controls. SUBJECTS AND METHODS: The study population comprised 187 patients with Crohn's disease (CD), 138 patients with ulcerative colitis (UC) and 253 healthy blood donors. The levels of serum tTG antibodies have been assessed employing commercially available ELISA. Patients with CD and UC were characterised regarding disease phenotype, disease extension, age at diagnosis and disease duration. Statistical analysis was performed using the Mann-Whitney-U test and Pearson correlation. RESULTS: The levels of anti-tissue transglutaminase antibodies was significantly increased in the group of patients with UC when compared to controls ( $6.06 \pm 2.67$ vs. $4.57 \pm 2.03$ IU/ $\mathrm{ml}, \mathrm{p}<0.001)$ or patients with $\mathrm{CD}(4.54 \pm 2.26 \mathrm{IU} / \mathrm{ml})$. No significant correlation with disease extension (proctitis, left sided colitis or pancolitis) has been noted. A weak correlation of antibody levels with age (Pearson 0.263, $\mathrm{p}=0.002$ ) has been observed in UC. There was no significant increase in number of patients with CD or UC displaying sprue-significantly $(>25$ $\mathrm{IU} / \mathrm{ml}$ ) or border suggestive ( $>20 \mathrm{IU} / \mathrm{ml}$ ) levels of $\mathrm{tTG}$ antibodies. Only 1 patient with CD, 1 patient with UC and 4 control persons displayed antibody levels higher than $20 \mathrm{IU} / \mathrm{ml}$. DISCUSSION: In patients with ulcerative colitis a significant increase of the levels of antibodies against tissue transglutaminases has been found. This is not related to an increased prevalence of celiac disease in these patients, as highly positive, sprue specific levels have not been more frequently identified compared to controls. Rather, these slightly increased levels might suggest implication of $\mathrm{tTG}$ in the constructive mucosal healing processes taken place in the colon after ulcerative colitis related mucosal damages.

\section{M1746}

Inflammatory Bowel Disease After Liver Transplantation: Risk Factors for Recurrence and De Novo Disease

Robert C. Verdonk, Gerard Dijkstra, Elizabeth B. Haagsma, Valerie K. Shostrom, Aad P. van den Berg, Jan H. Kleibeuker, Alan N. Langnas, Debra L. Sudan

INTRODUCTION: Inflammatory bowel disease (IBD) is associated with primary sclerosing cholangitis (PSC) and autoimmune hepatitis (AIH) and can recur or develop de novo after liver transplantation (OLT). AIM: To investigate the incidence and severity of inflammatory bowel disease after liver transplantation and to perform an analysis for possible risk factors. PATIENTS AND METHODS: Ninety-one patients transplanted for PSC or AIH, without prior colectomy, were included. Sixty patients were transplanted for PSC, 31 for AIH. Inflammatory bowel disease activity before and after OLT were studied, and possible risk factors for recurrence or de novo disease analyzed in a multivariate model. RESULTS AND DISCUSSION: Forty-nine patients (54\%) had IBD before OLT, mainly ulcerative colitis. Forty patients (44\%) had active IBD after transplantation: recurrence in 32 and de novo in 8 patients. Cumulative risk for IBD after OLT was 15,39 and $54 \%$ after 1,5 and 10 years, respectively. In $59 \%$ of the patients with IBD prior to OLT the disease was more active after transplantation. Risk factors for recurrent disease were: symptoms at time of OLT, short interval between diagnosis of IBD and time of OLT and use of tacrolimus after transplantation. 5-aminosalicylates were protective. A cytomegalovirus (CMV) positive donor/negative recipient combination increased the risk for de novo IBD. Possible explanations for the increase in IBD seen in patients using tacrolimus are a decrease in regulatory T-cells, diminished apoptosis of T-cells, and an increase in intestinal permeability in patients using this drug. CMV infection could contribute to IBD-pathogenesis by influencing endothelial VCAM-1 expression, mucosal interleukin-6 production, and increasing intestinal permeability. CONCLUSIONS: 1. Recurrent and de novo IBD are common in patients transplanted for PSC or AIH 2. In patients with previous IBD the disease runs an active course compared to pretransplantation 3. Risk factors for recurrence are: symptoms at time of OLT, short interval between diagnosis of IBD and time of OLT and use of tacrolimus. 4. Risk factor for de novo disease is: a cytomegalovirus (CMV) positive donor/negative recipient combination

\section{M1748}

Non-T Cell-Derived Galectin-3 Is Immunoregulatory and Is Reduced in the Inflamed Intestinal Epithelium in IBD

Stefan Mueller, Beatrice Flogerzi, Thomas Schaffer, Andrew Fleetwood, Alain Schoepfer, Frank Seibold

Background and aims: Galectins represent a family of animal lectins and may play important roles at different stages in inflammation. Galectin-3, although mostly described as a proinflammatory molecule, can induce apoptosis in T cells. The present study aims at the determination of galectin-3 expression in the normal and inflamed gut and to define its role in T cell activation. Methods: Galectin-3 was detected by quantitative PCR with total RNA from endoscopic biopsies and by immunohistochemistry. Selected biopsies and PBMC samples were stimulated in vitro, and the latter were used to assess the functional consequences of inhibition or exogenous addition of galectin-3. Results: Galectin-3 mRNA was constitutively expressed at high levels in controls and IBD. In the normal mucosa galectin-3 protein was mainly observed in differentiated intestinal epithelial cells, preferentially at the basolateral side. Galectin-3 was significantly downregulated in inflamed biopsies from patients with IBD. In vitro stimulation of biopsies with TNF lead to similar galectin-3 mRNA downregulation as in vivo. No intrinsic differences in the regulation of galectin-3 mRNA expression could be detected between IBD and controls. Inhibition of galectin-3 mRNA during stimulation of PBMC resulted in increased proliferation and decreased activation-induced death of $\mathrm{T}$ cells, while addition of exogenous galectin-3 reduced proliferation. Conclusions: Downregulation of galectin-3 in the inflamed mucosa may reflect a normal immunological consequence an can be mimicked in vitro. Increased $\mathrm{T}$ cell activation after inhibition of galectin-3 mRNA in PBMC may indicate a regulatory role for galectin-3 when expressed by non- $\mathrm{T}$ cells. The constitutive expression of epithelial galectin-3 may, thus, help to prevent inappropriate immune responses against commensal bacteria or food compounds. Therefore, galectin-3 may proof valuable for manipulating disease activity.

\section{M1749}

Is Infection By Mycobacterium Avium Subspecies Paratuberculosis a Risk Factor for Crohn's Disease?

Antonio Lozano-Leon, Manuel Barreiro-Acosta, Enrique Dominguez-Munoz

Background: Etiopathogenesis of Crohn's disease (CD) remains unknown and the influence of inflammatory, infectious and genetic factors have been proposed. Mycobacterium avium subspecies paratuberculosis (MAP) is the causative agent of Johne's disease, a chronic granulomatous inflammatory bowel disease of the cattle, similar to human $C D$. The presence of MAP has been recently described in CD patients. We hypothesize that MAP infection could be associated to the risk of developing CD. Aim: To evaluate the potential association between $\mathrm{CD}$ and the presence of MAP infection. Methods: A case-control study was designed, in which patients with $C D$ and healthy unrelated controls (1:1), matched by ethnic, sex and age were included. Patients and controls were from Galicia, a region in the North-West of Spain. Diagnosis of CD was made by clinical, radiological, endoscopic and histologica criteria. All patients were classified according to the Vienna Classification. A venous blood sample ( $4 \mathrm{ml}$ ) was obtained from all cases and controls in EDTA tubes. Samples were immediately processed and the whole blood tube was transferred into a separate sterile tube $(1,5 \mathrm{~mL})$. Genomic DNA was extracted by Puregene DNA purification system and the genotyping was performed by polymerase chain reaction. The Oligonucleotide primers were derived from the DNA insertion sequence IS900, with is a unique to MAP. The primers P90 and P91 were used to amplify a unique 294 bp fragment of IS900 gene. The association of MAP with CD should be expressed as OR and $95 \%$ confidence interval. Results: A total of $73 \mathrm{CD}$ patients ( 40 female, 33 male, mean age 38 years, range $21-67$ years) and 73 matched controls were included. According to the Vienna classification, 21 patients suffered from a non stenotic-non penetrating disease (B1), 22 had a stenotic disease (B2), and 30 patients presented with a penetrating $\mathrm{CD}$ (B3). Ileal location (L1) was found in 34 patients, whereas 13 and 25 patients had colonic (L2) and ileo-colonic (L3) involvement, respectively. One patient presented with disease in the upper gastrointestinal tract (L4). Despite the analysis of an important number of patients, presenting with all different forms of $C D, P C R$ blood analysis for the presence of MAP was consistently negative in all cases and controls. Conclusion: MAP infection is not present in our patients with Crohn's disease. Therefore MAP is unlikely to be involved in the etiopathogenesis of the disease.

\section{M1750}

Detection of Helicobacter Species in Archival Human UC Tissue By Fluorescent in-Situ Hybridisation (FISH)

John Thomson, Ian Cooper, Georgina Hold, Nikki Fyfe, Malcolm Smith, Ashley Mowat, Zeli Shen, James G. Fox, Emad El-Omar

Background The role of Helicobacter species in human inflammatory bowel disease (IBD) is controversial. This in part is due to differences in techniques used and to lack of definition of disease phenotypes. Aim To investigate archival paraffin embedded tissue from patients with confirmed ulcerative colitis (UC) for the presence of Helicobacter species. Subjects and Methods 100 UC patients (65 relapsing, 35 de novo) and 39 controls with a norma colonoscopy, had archival biopsies investigated. The biopsies were taken from throughout the colon. FISH assays were designed to differentiate H. pylori from non-pylori Helicobacter species. The sections were analysed in triplicate. Results The Helicobacter genus was presen in 33 of the 100 patients with UC. Of these, 31 were non-pylori and 2 were pylori species However in the control group $23(60 \%)$ were positive for the Helicobacter genus but only $9(23 \%)$ were non-pylori Helicobacter and $14(35 \%)$ were H. pylori. The only statistically significant difference between the UC cases and controls was the lower prevalence of $H$ pylori in the colitis group $(\mathrm{p}<0.01)$. There was no difference in prevalence of non-pylor Helicobacters between de novo presenting and relapsing colitis patients. The distribution of Helicobacter species was predominately in the left side of the colon in both the colitis and control patients. The Helicobacter species were detected on the mucosa and in colonic crypts, in both inflamed and non-inflamed biopsies. Conclusions Non-pylori Helicobacter species are commonly detected in UC patients and subjects with normal colons. The role of these species in the pathogenesis of UC, if any, remains to be established.

\section{M1751}

Corticosteroid Usage in Patients with Crohn's Disease Is Associated with Decreased Peripheral T Regulatory Cells

Jeanne Tung, Phyllis A. Svingen, William A. Faubion

Background: Functional studies of human CD4+ T cells identified cells highly expressing CD25 (CD25++) with regulatory potential. CD25 positivity may reflect activated T cells thus contaminating functional analyses of CD25++ T cells. The transcription factor FOXP3 is a more reliable marker of these regulatory $\mathrm{T}$ cells ( $\mathrm{T}$ regs), particularly in patients with inflammatory diseases. However, intracellular staining for FOXP3 precludes functional analyses. Thus, we aimed to quantify coexpression of FOXP3 and CD25 in patients with Crohn's disease (CD). Methods: CD4+ T cells were isolated from the peripheral blood of 15 patients with $\mathrm{CD}$ and 4 controls by magnetic cell sorting and analyzed by flow cytometry. Data are expressed as percentage of $\mathrm{CD} 4+\mathrm{T}$ cells (median, range). Results: No difference exists in the percentage of CD25++ T cells [0.3 (0.1-1.4) vs. $0.3(0.2-2.2)$ ] or FOXP3+ T cells [3.4 $(1.8-6.3)$ vs. $3.8(2.6-7.2)]$ between patients with $\mathrm{CD}$ and controls. Subanalysis of patients with $\mathrm{CD}$ showed that those on corticosteroids $(C S, n=5)$ were deficient in FOXP3+ T cells 
[1.9 (1.8-3.1) vs. 4.4 (3.0-3.6)]. Furthermore, CD25++ T cells from patients on CS do not frequently express FOXP3, as compared to steroid-free patients and controls [38.5 (24.460.7 vs. $78.2(63.2-87.3)$ vs. 76.5 (52.1-85.6), respectively] (figure). CDAI did not differ between the two groups of patients. Expression of CD25 or FOXP3 did not correlate with disease activity, thiopurine use, or anti-TNF therapy. Conclusions: Patients with $C D$ do not differ from controls in the number of CD25++ or FOXP3+CD4+ T cells in the periphery, and the majority of CD25++ T cells express FOXP3. An important exception is those patients on CS, in whom fewer $\mathrm{T}$ regs exist and are not enriched in the CD25++ population. Isolates of $\mathrm{CD} 25++$ cells from $\mathrm{CD}$ patients may be used in future studies of $\mathrm{T}$ reg cell function, but one should avoid patients on corticosteroids.

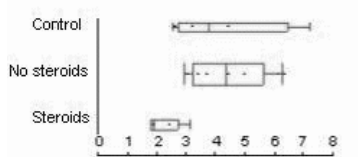

$\%$ FOXP3 gated on $\mathrm{CD} 4+$

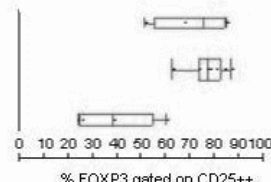

\% FOXP3 gated on $\mathrm{CD} 25++$

\section{M1752}

Sera from Active Ulcerative Colitis (UC) Contain IgG Antibodies Against Human Tropomyosin Isoform 5 (hTM5) That Kills Normal Colonic Epithelial Cells Through Antibody and Complement Lysis

Ellen C. Ebert, Xin Geng, Jim Lin, Kiron M. Das

Introduction: Patients with UC, but not Crohn's disease (CD) have IgGl antibodies in their serum and colon against hTM5, a cytoskeletal protein expressed both intracellularly and on the surface of colonic epithelial cells. Whether this is an epiphenomenon or related to the disease process is unknown. Since serum can gain access to the epithelium, we hypothesized that cytolytic IgG antibodies recognize and destroy epithelial cells in UC by antibody and complement lysis. Methods: Sera were obtained from UC patients $(n=60), C D$ patients $(n=$ 28), and normal individuals $(\mathrm{Nl})(\mathrm{n}=13)$ and were preincubated for 10 min with recombinant hTM5 or bovine serum albumin. Then, they were cultured for $4 \mathrm{hrs}$ with a ${ }^{51} \mathrm{Cr}$-labelled colonic adenocarcinoma cell line (LS180 cells) with up to $40 \%$ of the cells expressing surface hTM5. In some experiments, the IgG fraction was obtained by a protein $G$ column from pooled samples of UC or CD sera. Cytotoxicity was determined by the ${ }^{51} \mathrm{Cr}$-release assay. Results: To evaluate the role of hTM5, recombinant protein was added to the sera before addition of radiolabelled LS180 cells. There was a $20+3 \%$ reduction in cytotoxicity with addition of pure hTM5 to UC sera but not to CD or $\bar{N} l$ sera (both $p<0.001$ compared to UC). When evaluating the site of disease, those UC patients with left-sided or pan-colitis had significant declines in cytotoxicity with hTM5, while those with proctitis did not, suggesting that proctitis has a different pathogenesis. Disease activity and medications did not affect the results. However, increasing age correlated with the most inhibition by hTM5 $(\mathrm{p}<0.01)$, while increasing duration of disease had a similar, but nonsignificant, trend. Using $10 \%$ inhibition by hTM 5 as the cut-off, those values greater than $10 \%$ were from UC patients with a $15 \%$ false negative rate and a $2.5 \%$ false positive rate. To prove that IgG was involved, $\mathrm{UC}$ and $\mathrm{CD}$ sera were fractionated by a Protein G column. The IgG fractions from UC but not CD sera demonstrated hTM5-induced cytotoxicity. To determine whether complement was involved, sera were heat inactivated $\left(56^{\circ} \mathrm{C}\right.$ for $\left.45 \mathrm{~min}\right)$ or mixed with $\mathrm{Fc}$ fragments. Both maneuvers markedly reduced cytolytic activity by UC sera $(\mathrm{p}<0.05$ and $\mathrm{p}<0.01$ ), suggesting complement-mediated injury. Conclusions: This study shows that hTM5-specific IgG from UC, but not CD or Nl sera, kills LS180 cells by complement-mediated lysis. This provides a direct pathogenic role of anti-hTM5 antibodies in the disease process and leads the way to novel diagnostic and therapeutic possibilities.

\section{M1753}

Antibodies to Self and Bacterial Antigens in Patients with Crohn's Disease and Ulcerative Colitis: A Co-Twin Case-Control Study

Martina E. Spehlmann, Arno Kromminga

Abstract Introduction: Antibodies against self and bacterial antigens have been discussed to be associated with inflammatory bowel diseases (IBD). Methods: To evaluate these findings we have analyzed antibodies against the recombinant outer-membrane porin $\mathrm{C}(\mathrm{OmpC})$ from Escherichia coli and recombinant Pseudomonas fluorescens-associated I2 antigen as well as to saccharomyces cerevisiae antigens and tested perinuclear antineutrophil cytoplasmic antibodies in sera of monocygotic twin pairs discordant for Crohn's disease $(n=6)$ and ulcerative colitis $(\mathrm{n}=12)$. Results: Anti-OmpC-antibodies were exclusively found in twins with UC (5/12 of affected (41.7\%) and 4/12 of the corresponding non-affected co-twin (33.3\%)). Anti-I2-antibodies were measured in both UC (2/12 of the affected twins and $2 /$ 12 of the non-affected siblings $(16.7 \% ;))$ and $C D(1 / 6$ affected and $1 / 6$ of the non-affected siblings(16.7\%)). Surprisingly, ASCA were found in both UC (25.0\%) and CD (50.0\%) patients. P-ANCA were detected only in UC twins (9/12 in the affected (75.0\%) and 1/12 in the non-affected sibling (8.3\%)). Discussion: These findings confirm the specificity of pANCA for UC, whereas the detection of antibodies to OmpC, I2 and ASCA neither discriminated between $\mathrm{CD}$ and $\mathrm{UC}$ nor indicated a manifestation and severity of the disease. Due to the co-existence of anti-OmpC- and I2-antibodies in affected as well as non-affected UC patients we assume that the occurrence of these antibodies is a result of a genetic predisposition.

\section{M1754}

Invasive E. Coli Strains Are Increased in Crohn's Disease

Joel A. Alpern, Maiko Sasaki, Shanti V. Sitaraman, Robin E. Rutherford, Henry C. Olejeme, Qiang Cai, Jan-Michael A. Klapproth

Background: Bacteria and their products have been implicated in the pathogenesis of inflammatory bowel disease. Recent studies from Europe have described a new class of Adherent Invasive E. coli (AIEC) strains in patients with Crohn's disease (CD), but not ulcerative colitis (UC) or healthy controls. Representative AIEC strain LF82 was found to efficiently invade epithelial cell cultures and proliferate without inducing apoptosis. Hypothesis: Invasive E. coli strains are present in American patients with CD. Methods: Twenty-eight patients were enrolled (CD 11, UC 4, pouchitits 3, normal controls 10). Macroscopically normal and inflamed appearing endoscopic mucosal biopsies were subjected to DMEM/Gentamicin treatment for one hour, lysed, and plated on McKonkey agar. A total of 933 lactose fermenting bacterial strains were analyzed by invasion assay. Antibiotic-free, confluent Caco-2 monolayers were infected in triplicate with $5 \mu \mathrm{l}$ of an overnight culture of individual clones. Following three hours of infection, cells were treated with DMEM/Gentamicin. Dilutions of Triton-X-100 cell lysates and bacterial cultures were plated on LB agar. Colonies were enumerated and invasion indices expressed as percentage of initial inoculum. Invasion indices $\geq 1.0 \%$ were considered significant comparing pathogenic (LF82, EPEC, EIEC, EHEC) to non-pathogenic commensal E. coli strains. Invasive strains were further analyzed using Api20E bacterial identification kit. Results: Significant invasion was determined for $19.8 \%$ of all examined strains (CD $25.9 \%$, UC $18 \%$, pouchitis $20.4 \%$, control $13.5 \%$ ). As determined by biochemical analysis, the most common invasive isolate in all biopsies was E. coli (64.1\%). Invasive E. coli strains were more commonly identified in CD (88/98-89.8\%) and pouchitis (16/21-76.2\%) in comparison to UC (6/20-30\%) and normal controls (1/46$2.2 \%)$. Invasion indices for all $E$. coli isolates from patients with $\mathrm{CD}(8.8 \%, \mathrm{p}<0.05)$ were significantly higher in comparison to UC $(4.16 \%)$ or pouchitis (1.6\%). The highest invasion indices for E. coli strains were observed in inflamed mucosa from patients with CD (11.7\%, $\mathrm{p}<0.05)$ vs. non-inflamed mucosa $(8.8 \%)$, healthy or inflamed pouch mucosa $(1.27 \%, 1.63 \%$, respectively). Not a single invasive E. coli strain was isolated from inflamed UC mucosa. Summary and conclusion: $C D$ patients harbor a significantly increased number of $E$. coli strains in comparison to UC, pouchitis and normal controls. In comparison to normal appearing tissue, invasive E. coli strains are especially prevalent in inflamed CD mucosa. Invasive $E$. coli strains are a feature of and might contribute to the pathogenesis of $C D$.

\section{M1755}

Native, Circulating Plasmacytoid and Myeloid Dendritic Cells from Patients with Inflammatory Bowel Disease Secrete Increased Amounts of Inflammatory Cytokines and Drive a Pro-Inflammatory T-Cell Proliferation and Polarization Daniel C. Baumgart, Ingo Przesdzing, Christiane Bielecki, Diana Metzke, Saskia Thomas, Andreas Sturm, Bertram Wiedenmann, Axel U. Dignass

BACKGROUND: Breakdown of tolerance against the commensal microflora is believed to be a major factor in the pathogenesis of inflammatory bowel disease (IBD). The key role of dendritic cells (DC) in this process has been demonstrated in various animal models, while data on native human DC is still limited. PATIENTS AND METHODS: Plasmacytoid (PDC) and myeloid (MDC) dendritic cells and naive T-cells (TC) were isolated with magnetic cell separation from peripheral blood of 32 patients (Crohn's disease (CD) $n=18$, ulcerative colitis (UC) $n=14)$ and healthy controls $(n=22)$. DC cytokine production and secretion were evaluated following incubation with microbial surrogate stimuli such as CpG motifs, staphylococcal enterotoxin B (SEB) or lipopolysaccharide (LPS). PDC and MDC were also co-cultured with naive T-cells. FACS analysis was used to identify, enumerate, and phenotype cells. Proliferation was measured after CFSE staining. RESULTS: Upon culture and stimulation with microbial surrogate stimuli, PDC and MDC from IBD patients secrete more TNFa, IL8 and IL-6 than healthy controls (Table 1). DCs from IBD patients appear to induce a Thl type polarization and proliferation in naive T-cells. CONCLUSION: An aberrant response of DC to microbial surrogate stimuli suggests a disturbed interaction with commensals. The effect of DCs on T-cell proliferation and polarization underscore their critical role in the pathogenesis of human IBD Table 1: Cytokine Secretion

\begin{tabular}{|c|c|c|c|}
\hline & Mean TNF $\alpha[\mathrm{pg} / \mathrm{ml}]$ & Mean IL-8 [pg/ml] & Mean IL-6 [pg/ml] \\
\hline PDC-Control & 58 & 68 & 71 \\
\hline PDC-Crohn's disease & 2763 & 2200 & 2345 \\
\hline PDC-Ulcerative colitis & 7800 & 6112 & 5133 \\
\hline MDC-Control & 115 & 134 & 111 \\
\hline MDC-Crohn's disease & 4433 & 4511 & 3262 \\
\hline MDC-Ulcerative colitis & 5800 & 8305 & 3800 \\
\hline
\end{tabular}

\section{M1756}

Bacterial-Aluminum Interactions. The Role of Aluminum in Bacterial-Induced Colitis in IL-10 Deficient Mice

Aaron Lerner, Swathi Eluri, Daniel Perl, Sharon Moalem, David Sachar, Susan Tonkonogy Ryan B. Sartor

Aluminum (Al) is a common environmental element with immune adjuvant activity and the ability to induce granulomatous inflammation. Al exposure through food additives, municipal water supplies and air pollution is ubiquitous in Western cultures. Since loss of tolerance to commensal intestinal bacteria appears to be involved in the pathogenesis of $\mathrm{IBD}$, we postulated that dietary Al could increase luminal bacterial virulence and/or enhance mucosal immune responses to enteric bacteria. To explore the effects of $\mathrm{Al}$ on experimental colitis, we monitored its effects on bacterial growth, on the capacity of lyates from Al- 
preloaded murine E. coli to enhance immune responses in vitro, and the in vivo effect of dietary Al on immune-mediated murine colitis. Methods: Growth of murine intestinal bacteria in the presence of increasing $\mathrm{Al}$ concentrations $(0-662 \mathrm{uM})$ was assessed by spectrophotometry. Immune responses to bacterial lysates were measured in vitro by $[\mathrm{H}] 3$ thymidine incorporation and by IFN $\gamma$ secretion by IL-10 KO colitic mouse splenocytes. To assess the in vivo effects of dietary Al, IL-10 KO germ free mice were colonized with SPF enteric microbiota, fed a low Al, low iron diet, and exposed to 3 different Al concentrations: low $(0 \mathrm{uM})$, middle $(5 \mathrm{uM})$ and high $(500 \mathrm{uM}) \mathrm{Al}$ L-lactate added to their drinking water $(\mathrm{N}=5 /$ group). Iron (II) sulfate heptahydrate (luM) was added to the drinking water of all groups. Colitis was measured by histologic scores ( $0-4+)$ and spontaneous IL- 12 p 40 secretion by cultured colonic fragments. Results: Bacterial growth was suppressed slightly by high Al concentrations in the media. E. coli grown in low Al concentrations (150-200uM) stimulated, while those grown in higher concentrations inhibited in vitro T cell proliferation and IFNy secretion by splenocytes. In vivo Al feeding worsened colitis, with increased cecal histological scores accompanying progressively higher $\mathrm{Al}$ intake $(2.0+0.2$ low vs.3.8+0.2 high, $\mathrm{p}<0.05)$. IL-12 secretion by colonic strip cultures also increased with higher Al intake $(3.5+1.6$ vs. $4.9+0.4 \mathrm{ng} / \mathrm{ml}$ ). Dietary Al altered luminal bacterial metabolism by inducing pink E.coli colonies on MacConkey agar plates. Conclusions: Environmental Al stimulates immune responses to enteric bacteria in vitro and enhances bacterial-induced experimental colitis in IL-10 KO mice. Exposure to environmental Al may in part explain the increased incidence of IBD in countries adopting Western diets, industrial and public health practices.

\section{M1757}

Gene Expression Profile in Crohn's Disease Related NOD2 Variants Zuzana Zelinkova, Astrid J. van Beelen, Floor de Kort, Emiel Ver Loren van Themaat, Anje A. Te Velde, Esther C. de Jong, Sander J. van Deventer, Daniel W. Hommes

Background: NOD2 variants are associated with Crohn's disease (CD) but the underlying mechanism is not completely understood. Recent studies in CD patients showed a defective proinflammatory cytokines production upon TLR and NOD2 stimulation leading to hypothesis of impaired pathogen elimination that would result in a chronic inflammatory condition. However, our previously reported observations suggest that NOD2 deficiency may lead to a specific stimulation of interleukin-12 (IL-12) production in antigen presenting cells together with deficient IL-10 production upon NOD2 stimulation. In order to study the possible mechanism of $C D$ resulting from NOD2 mutations, we used this cell type to analyse differences in gene expression profile upon NOD2 stimulation in CD patients. Patients and Methods: Monocyte-derived dendritic cells (DCs) from CD carriers of double-dose NOD2 mutations, wild type $C D$ patients and wild type healthy volunteers were stimulated for 6 hours with NOD2 ligand muramyl dipeptide (MDP). Agilent Human Whole Genome Oligonucleotide arrays were used to assess the differential gene expression in the three studied groups. The statistical analysis was performed by ANOVA test using Rosetta Resolver v 50 Expression Data Analysis System. For pathway analysis gene ontology mapping online software has been used. The expression of selected significantly up-regulated genes was tested by confirmatory PCR. Results: The three groups showed substantially different gene expression profiles upon MDP stimulation with only 3 genes overlapping between all three groups. In NOD2 mutants group 301 genes were significantly changed among which different costimulatory molecules, chemokines and cytokines. Pathway analysis showed a clustering of these genes in pathways of negative regulation of apoptosis, regulation of DNA recombination and chromatin modification. In the group of wild type CD patients 694 genes were significantly changed, mostly genes clustering in pathways of response to external stimuli. Surprisingly, in the wild type healthy volunteers groups only 52 genes significantly changed, belonging to the response to pathogen pathway. Conclusion: Our observations support the hypothesis of NOD2 variants leading to Crohn`s disease through inappropriate non-specific inflammatory reaction.

\section{M1758}

Enhancement of Substance P mRNA Expression in Non-Inflamed Small Bowel of Crohn's Disease Patients

Christoph Michalski, Frank Autschbach, Federico Selvaggi, Michael Muller, Pierluigi Di Sebastiano, Markus W. Buchler, Thomas Giese, Helmut Friess

Background: Substance P (SP) as a mediator of neurogenic inflammation plays an important role in inflammatory disorders. In different bowel sections from patients with ulcerative colitis (UC), Crohn's disease (CD) or from normal control donors, we simultaneously analyzed the Neurokinin Receptor-1 (NK-1R) isoforms NK-1R-Long and NK-1R-Short, its ligand Substance P encoded by Preprotachykinin-A (PPT-A) and the SP degradation enzyme Neutral Endopeptidase (NEP). Methods: CD and UC tissues were obtained from patients undergoing intestinal resection due to complications of the disease. Normal human intestinal tissue samples were obtained from patients undergoing surgical bowel resection for other reasons than inflammatory bowel disease. Real-time quantitative reverse transcription polymerase chain reaction (RT-PCR) was used to simultaneously determine the expression of NK1R-L, NK-1R-S and PPT-A. Protein expression of NK-1R and NEP was determined by immunohistochemistry and immunoblot analysis. Results: In non-inflamed ileal samples of CD patients, PPT-A mRNA expression was significantly increased compared with normal controls. There was no difference between inflamed or non-inflamed UC tissues and normal intestinal tissue samples. Subgroups of diverse intestinal segments with various levels of inflammation revealed no differences in NK-1R-L or NK-1R-S expression. Immunoblot analysis revealed an upregulation of NK-1R protein levels in IBD with a more pronounced enhancement in $C D(p<0.001)$ than in UC $(p<0.003)$. Immunostaining confirmed the immunoblot findings and demonstrated stronger NK-1R immunoreactivity in the chronically inflamed tissues while the overall staining was more increased in CD than in UC samples. For NEP there was no difference in either immunohistochemistry or immunoblot analysis in normal, $C D$ and UC intestinal tissues. Conclusions: These observations suggest a contribution of substance $\mathrm{P}$ and its receptor NK-1R in the local inflammatory reaction in IBD and particularly in Crohn's disease Moreover, the significant upregulation of PPT-A mRNA in the non-inflamed ileum of patients with $\mathrm{CD}$ may constitute a precursor lesion of the local inflammation and therefore allow to predict the extent of inflammation of yet unaffected small bowel.

\section{M1759}

Acute Psychological Stress Increases Rectal Mucosal and LPS-Stimulated Whole Blood Release of TNF-Alpha in Patients with Inactive Ulcerative Colitis Joel Mawdsley, David Rampton

Introduction: Psychological stress is reported to increase the likelihood of relapse in ulcerative colitis (UC) but the mechanism is unclear. We hypothesized that stress might act by stimulating colorectal mucosal and lipopolysaccharide (LPS)-stimulated whole blood production o TNF- $\alpha$, an important cytokine in the pathogenesis of IBD. Aim: To assess the effects of acute psychological stress on the concentration of TNF- $\alpha$ in rectal peri-mucosal fluid, and on the production of TNF- $\alpha$ by LPS-stimulated whole blood in patients with inactive UC. Methods: For 50 mins, patients with inactive UC (Simple Clinical Colitis Activity Index $<4$ Baron sigmoidoscopy score <2) underwent either (i) Stress - modified dichotomous listening test (IQ test whilst listening to different types of music in either ear), or (ii) Control - relaxin music. The autonomic stress response was assessed by pulse and BP every 15 mins. Perimucosal fluid was collected before and after each protocol by placing a 7 × $30 \mathrm{~mm}$ strip o filter paper through a rigid sigmoidoscope in contact with rectal mucosa for 1 min until wet. The filter paper was then incubated in $1 \mathrm{ml}$ Bovine Serum Albumin (0.3\%), sodium azide $(0.01 \%)$ and Tween $20(0.002 \%)$ in phosphate-buffered saline for 24 hrs and TNF$\alpha$ concentrations in buffer subsequently measured by ELISA.(1) In blood taken before and after each protocol, LPS-stimulated TNF- $\alpha$ production $(1 \mathrm{ml}$ whole blood incubated with $25 \mathrm{mcg} / \mathrm{ml}$ LPS at $37 \mathrm{C}$ in $95 \% \mathrm{O} 2 / 5 \% \mathrm{CO} 2$ for 24 hours) was measured by ELISA. Results: The control protocol did not change any variable (Table). Stress increased pulse rate by median $7 \mathrm{bpm}$, systolic BP by $12 \mathrm{mmHg}$, TNF- $\alpha$ concentrations in rectal peri-mucosal fluid by $102 \%$ and LPS-stimulated TNF- $\alpha$ production by whole blood by $54 \%$. Conclusion: Acute psychological stress increases the release of TNF- $\alpha$ by rectal mucosa and by LPS-stimulated whole blood. Both mechanisms could contribute to the pathogenic effects of psychological stress in UC. Reference: 1. Carty E, De Brabander M, Feakins RM, Rampton DS, Gut 46, 487-492 (2000)

\begin{tabular}{|c|c|c|c|c|}
\hline & Protocol & N & Before & After \\
\hline \multirow{2}{*}{ Pulse rate (bpm) } & Stress & 25 & $70(65-74)$ & $77(70-85)^{* *}$ \\
\cline { 2 - 5 } & Control & 10 & $67(55-82)$ & $66(56-79)$ \\
\hline \multirow{2}{*}{ Systolic BP (mmHg) } & Stress & 25 & $118(113-133)$ & $130(119-148)^{* *}$ \\
\cline { 2 - 5 } & Control & 10 & $139(111-158)$ & $130(108-150)$ \\
\hline \multirow{2}{*}{ TNF-alpha in rectal perimucosal fluid (pg/ml) } & Stress & 25 & $\begin{array}{c}12.8(8.6 \\
-20.2)\end{array}$ & $\begin{array}{c}25.8(12.7- \\
41.5)^{*}\end{array}$ \\
\cline { 2 - 5 } & Control & 10 & $8.9(0-15.5)$ & $5.8(0.6-12.2)$ \\
\hline \multirow{2}{*}{$\begin{array}{c}\text { TNF-alpha production by LPS-stimulated blood } \\
\text { (ng/ml) }\end{array}$} & Stress & 25 & $28(17-57)$ & $43(20-62)^{*}$ \\
\cline { 2 - 5 } & Control & 10 & $18(13-59)$ & $20(16-72)$ \\
\hline
\end{tabular}

${ }^{*} \mathrm{p}<0.05$ from pre-stress value. ${ }^{* *} \mathrm{p}<0.01$ from pre-stress value. Median and IQR shown.

\section{M1760}

Exotoxin a from Pseudomonas Aeruginosa Promotes Leukocyte Adhesion to Gut Mucosal Smooth Muscle Cells Alana Majors, Melissa Jedlicka, Scott A. Strong

OBJECTIVE: Mounting evidence suggests that both luminal bacteria and the mucosal extracellular matrix play pivotal roles in the pathogenesis of inflammatory bowel disease (IBD) through mechanisms that have yet to be precisely identified. Pseudomonas aeruginosa is a normal component of the intestinal flora, but is also a pathogen whose virulence is primarily mediated by Pseudomonas exotoxin A (PEA) that inhibits protein synthesis and may elicit additional affects. In IBD, alterations in the hyaluronan (HA) of the extracellular matrix likely contribute to an enhanced binding interaction between immune cells and mesenchymal cells of the mucosa, but it is not unclear how bacterial products may affect the extracellular matrix. Accordingly, we attempted to determine whether PEA alters the extracellular matrix and influences mononuclear leukocyte binding to mucosal smooth muscle cells (M-SMCs) METHODS: Human M-SMC cultures were derived from fresh surgical specimens of patients undergoing colonic resection. Confluent cultures were treated both with and without PEA (optimal conditions: $100 \mathrm{ng} / \mathrm{ml} ; 23$ hours), incubated with non-stimulated mononuclear leukocytes, and subjected to morphologic as well as functional studies. RESULTS: Affinity histochemistry and confocal fluorescent microscopy demonstrated that HA was deposited in cable-like structures in PEA-treated cultures, but not in untreated, control M-SMC cultures Furthermore, quantitative leukocyte adhesion assays demonstrated that the PEA-treated cultures were significantly more adhesive for U937 cells as well as Jurkat and MOLT-4 Tcells than the untreated M-SMCs. Visualization of the bound leukocytes by fluorescent microscopy revealed that the leukocytes were bound to the HA-containing cables. DNA quantification confirmed that the PEA was not toxic under the experimental conditions. CONCLUSIONS: These results suggest that bacterial exotoxins may play an integral role in the pathogenesis of inflammatory bowel disease by altering the mucosal extracellular matrix and increasing its adhesiveness for leukocytes.

. 
Receptor Activator of Nfk-B Ligand (RANKL), Rank and Osteoprotegerin in Inflammatory Bowel Disease: Implication in Chronic Inflammation and Bone Loss

Catherine D. Reenaers, Nathalie Franchimont, Cecile Oury, Cecile Lambert, Jacques Belaiche, Michel Malaise, Philippe Delvenne, Edouard Louis

Crohn's disease (CD) and ulcerative colitis (UC) are inflammatory bowel diseases (IBD) associated with osteopenia. RANK (Receptor activator of NF-KB) is expressed by osteoclasts and by antigen presenting cells (APC). With its ligand RANKL, expressed by osteoblasts and T lymphocytes, RANK plays a critical role in bone resorption but also in DC-T cells interaction. Osteoprotegerin (OPG), a soluble decoy receptor for RANKL, inhibits the RANKRANKL interaction. The aim of our work was to study the expression of RANK, RANKI and OPG in IBD. Material and methods: Total RNA was extracted from normal colon samples. RANK, RANKL and OPG mRNA expression was studied by RT-PCR $(n=5)$. The level of soluble RANKL (sRANKL) and OPG in the gut was analysed by specific ELISA on supernatant of cultured colonic biopsies (15 CD patients, 7 controls patients). Fixed colonic samples from $14 \mathrm{CD}$ patients, $10 \mathrm{UC}$ patients, 10 diverticulitis patients and 5 controls were studied by immunostaining and immunofluorescence. Serum levels of sRANKL and OPG were also assessed by specific ELISA $(C D=18, U C=14$, inflammatory controls $=11$, controls=14). We studied the cells expressing RANK and RANKL in the serum by flow cytometry on PBMC isolated from CD, UC, inflammatory controls and healthy controls. Results: Transcripts for RANK, RANKL and OPG were confirmed in the human colon by RT-PCR. We observed by immunostaining and immunofluorescence an important number of RANK+ cells in areas of chronic inflammation in $\mathrm{CD}, \mathrm{UC}$ or diverticulitis. Double staining suggested that theses cells corresponded to CD68 and S100 cells. Levels of sRANKL and OPG were significantly increased in cultured colonic biopsies from $C D$, particularly in inflamed areas. Only OPG was correlated to pro and anti-inflammatory cytokines. Important levels of sRANKL and OPG were also detected in the serum of active CD. The study of PBMC in flow cytometry demonstrated that RANK and RANKL positive cells corresponded to 5 to $10 \%$ of PBMC All these cells were CD4+ and CDla+. Conclusion: There is an expression of RANKL, RANK and OPG in the human colon. The level of RANK in the gut is increased in chronic inflammation in different pathologies (CD, UC, diverticulitis). Moreover, we observed important levels of RANKL and OPG in the supernatant of cultured colonic biopsies and in the serum, mainly in active CD. The cells expressing RANKL and RANK in the colon and in the blood have characteristics of APC. These data suggest that RANK, RANKL and OPG system could be involved in immunology processes taking place in the gut in several inflammatory diseases. These proteins could also have a role in the associated bone loss, particularly in $\mathrm{CD}$.

\section{M1762}

Immunophenotype and Apoptosis of Colonic Mononuclear Cells in Active Steroid-Responsive Ulcerative Colitis

Rebeca Santaolalla, Maria Esteve, Josep Manye, Elisabet Pedrosa, Antonio Salas, Fernando Fernandez-Banares, Merce Rosinach, Montserrat Forne, Jorge Espinos, Eugeni Domenech, Miriam Manosa, Miquel Angel Gassull, Josep Maria Viver

BACKGROUND: Apoptosis is a programmed cell death that plays an important role in Tcells proliferation control. It has been suggested that corticosteroids may induce lymphocyte apoptosis by poorly known mechanisms. AIMS: To assess if steroid treatment may induce apoptosis and changes in the percentage of lamina propria (LP) colonic mononuclear cells in steroid-responsive ulcerative colitis (UC). METHODS: 10 patients with moderate or severe UC [ 5 men, 5 women, age: $44.9 \pm 4.8$ years] and 6 healthy controls [ 2 men, 4 women, age: $57.8 \pm 6.5$ years] were included. Disease activity was assessed by clinical (Truelove Index), endoscopic (Gomes Index) and histopathological (Gomes Index) criteria. Eigth endoscopic biopsies were taken just before starting steroid therapy and also 7-10 days after it, from the same afected area. Immunopheynotype and apoptosis were analized by flow citometry using a double staining with anti-CD3 (T lymphocytes), anti-CD38 (plasma cells), anti-CD19 (B lymphocytes), anti-CD14 (monocytes, macrophages) and annexin-V. Data are shown as mean \pm SEM of \% phenotyped population / LP total cells, and \% apoptotic phenotyped population / same type total cells. Analysis of the results were made by t Student test (paired and unpaired data). RESULTS: In the basal determination, an increase of CD 38+CD19- cells $(8.9 \pm 1.6$ vs. $4.5 \pm 1.1 ; \mathrm{p}=0.04)$ and CD38+CD19- apoptotic cells $(18.1 \pm 2.4$ vs. $8.8 \pm 1.6 ; \mathrm{p}=0.009)$ was found in UC patients compared to healthy controls After 7-10 days of treatment, a decrease in the clinical and endoscopic activity $(\mathrm{p}=0.005$ and $p<0.0001$ respectively) was found. Meanwhile there was only a tendency to a decrease in the histopathological activity index $(\mathrm{p}=0.152)$ and in the $\%$ of CD19+ cells $(\mathrm{p}=0.084)$. No significant changes neither in other cellular markers nor in the \% of apoptotic cells were detected. CONCLUSIONS: 1- The beneficial effect of steroids in UC is not related to an increase of LP apoptotic mononuclear cells in vivo, suggesting that this mechanism is not involved in its therapeutic effect. 2- The contrast between rapid clinical and endoscopical improvement observed in steroid sensitive patients and the relative resistance to histopathological and LP mononuclear cells restitution, suggests that the abnormalities observed in immunoregulatory mechanisms (increase of CD38+ and CD38+ apoptotic cells) are not primary phenomena but epiphenomena related to inflammation in ulcerative colitis. This study has received financial support from the "Fundacio La Marato de TV3" (Id.031730) of Catalonia (Spain).
Increased Expression of Lipocalin-Type-Prostaglandin D Synthase (L-PGDS) in Colonic Mucosa of Ulcerative Colitis Patients: Its Regulatory Role Against TH1 Response

Ryota Hokari, Keisuke Okudaira, Yoshikiyo Okada, Chie Kurihara, Hisayuki Matsunaga, Koichi Takebayashi, Yoshikazu Tsuzuki, Masaaki Higashiyama, Norikazu Mataki, Shunsuke Komoto, Chikako Watanabe, Atsushi Kawaguchi, Shigeaki Nagao, Kazuro Ito, Soichiro Miura

Background: The pathogenesis of ulcerative colitis (UC) is unclear, but abnormal infiltration of $\mathrm{T}$ lymphocytes and proinflammatory cytokines (cytokine imbalance) in the colonic mucosa has been implicated for colonic injury. Recently enhanced biosynthesis of prostaglandin (PG) $\mathrm{D}(2)$ has been suggested to contribute to resolution of inflammation in kidney or venular wall. PGD2 affects Th1/2 balance by stimulating Th2 cells via CRTH2 (chemoattractant receptor-homologues molecule specifically expressed on Th2 cells) and suppressing Thl cells via DP receptor in vitro. PGD2 is coverted from PGH2 by two kinds of enzymes, lipocalin-type-prostaglandin D synthase (L-PGDS) and hematopoietic-type-prostaglandin D synthase (H-PGDS). However, the change of these enzymes in colonic mucosa has not been studied at all. In this study we investigated how expression of enzymes for PGD2 synthesis altered its relation to the activity of colonic inflammation in UC. Methods: Tissue samples were obtained by colonic biopsies from patients with UC or colonic polyps with informed consent. Thl/2 cell infiltration was studied immunohistochemically using antibody against CC chemokine receptor 5 (CCR5) as Thl marker and CRTH2 as Th2 marker. Th1 related chemokine, MIP1-a and Th2 related enzymes, L-PGDS and H-PGDS were studied by mRNA expression using quantitative RT-PCR. mRNA expressions of IFN-gamma, IL-4 and Fox-P3 were also measured by quantitative PCR. Result: CCR5 positive cells significantly increased in the inflamed mucosa and their number pararelled with the disease activity of UC. Mipl alpha expression well correlated with the infiltration of CCR5 positive cells. CRTH2-positive cells was predominantly observed in the mildly inflamed or the margin of inflamed mucosa, but their number decreased in severely active UC patients. Both L-PGDS and H-PGDS mRNA expressions were observed in the colonic mucosa and the L-PGDS expression was increased in UC patients. On the contrary, H-PGDS expression in UC did not differ from control mucosa. L-PGDS expression pararelled with the disease activity Expression of FoxP3 was also increased as activity of inflammation advanced. IL-4 was not detected in the UC mucosa. Conclusion: We first reported the presence of two types of PGD synthase in the colonic mucosa and that only lipocalin-type PGDS increased with active mucosa of UC patients. From the absence of Th2 related cytokines and the decreased CRTH2 cells in severe colitis we speculate that L-PGDS-derived PGD2 may play important antagonizing roles through DP receptor against Thl response to ameliorate colonic inflammation in severe colitis.

\section{M1764}

Decreased Hyaluronidase 2 (Hyal2) Expression By Colon Smooth Muscle Cells (SMC) May Contribute to Pro-Inflammatory Hyaluronan (HA) Accumulation in Inflammatory Bowel Disease (IBD)

Hyunjin K. Rho, Carol A. de la Mott, Csaba Fulop, Scott A. Strong

Background: Increased hyalruronan (HA) levels in colonic mucosa involved in active inflammatory bowel disease (IBD), as compared to non-inflamed tissue, has been shown by our laboratory. HA is frequently muscularis associated. Additionally, we have shown the generation of leukocyte adhesive hyaluronan structures in vitro from colonic mucosal smooth muscle cells (M-SMC) stimulated with a virus or the viral mimetic, poly I:C (synthetic double stranded RNA). Conceivably, HA accumulation in tissue or on cells may be achieved either by increasing synthesis or inhibiting catabolism. Therefore investigation into the regulation of the three HA-synthase (HAS1, HAS 2, HAS3) and two active somatic hyaluronidase (HYAL1, HYAL2) enzymes by poly I:C treated M-SMC from Crohn's disease (CD), ulcerative colitis (UC) and non-IBD colon samples was initiated. Methods: Human M-SMCs were isolated from colon tissue obtained after surgical resection, grown in culture and assayed in the first sub-culture. M-SMC cultures from 25 patients were treated with or without poly I:C for 6 hours. RNA was extracted by a phenol-chloroform method, and comparative RTPCR was performed using primers of human HAS 1,2 and 3, and also Hyall and 2. The data was analyzed quantitatively after normalization to $\beta$-actin expression. HA production by M-SMCs was also monitored by microscopy and its function by measuring the binding of radiolabeled monocytic cells. Results and Discussion: The change in the HA synthetic enzymes HAS2 and HAS3 mRNA levels upon poly I.C treatment of M-SMC was variable among the patient samples, while HAS 1 was not detected in any. Likewise the HA degrading enzyme HYAL 1 was not detected in any M-SMC sample. Strikingly, however, HYAL 2 mRNA expression was dramatically decreased in poly I:C treated UC patients. Among the UC patients, $78 \%$ showed a $>75 \%$ decrease, while the rest were reduced 20 to $65 \%$. The response by $C D$ patient M-SMC $s$ was variable, with $50 \%$ showing an increase in HYAL2 expression upon poly I:C stimulation. Hyaluronan mediated leukocyte adhesion was also higher in poly I:C stimulated UC-derived M-SMCs than CD M-SMCs. This suggests disease specific differences in regulation of HA accumulation and function. Significance: We have reported inflammation-associated HA accumulation in IBD colon tissue and provided evidence that HA participates in the inflammatory process. The balance of synthesis and catabolism likely regulates the amount of HA sequestered in tissue. Here we provide novel evidence that controlling the break down, ie inhibiting clearance, of HA from M-SMC surface likely favors the formation of the HA pro-inflammatory environment in UC patients. 


\section{M1765}

Peripheral Natural Killer T-Cell Numbers and Serum IL-13 Concentrations Are Raised in Active Ulcerative Colitis and Correlate with Disease Activity Joel Mawdsley, Marion Macey, David Rampton

Introduction: The pathogenesis of ulcerative colitis (UC) is incompletely understood. It has been reported recently that mucosal biopsies from patients with UC contain a population of non-classical Natural Killer T (NKT) cells which may be important in disease pathogenesis (1). These NKT cells have been shown to produce IL-13, a cytokine which impairs the barrier function of colonic epithelial cells in vitro.(2) However, peripheral NKT cells numbers and serum IL-13 concentrations in patients with UC have not been studied. Aim: To measure the numbers of non-classical NKT cells and serum IL-13 concentrations in peripheral blood in patients with UC and to correlate this data with disease activity. Methods: To measure NKT cell numbers, blood samples were collected from 30 patients with UC, anti-coagulated with CTAD, stained with fluorescent monoclonal antibodies and analysed by flow cytometry. Non-classical NKT cells were identified as CD3+, CD4+ and CD161+ cells. Serum IL-13 levels were measured in a different but overlapping group of 50 patients with UC by ELISA. Disease activity was assessed by the Simple Colitis Activity Index (SSCAI).(3) Active UC was defined as SSCAI $>4$. Results: NKT cell numbers and serum Il-13 concentrations were higher in patients with active than with inactive $U C(p=0.03$ and $p=0.003$, respectively). SSCAI was directly related to both NKT cell numbers $(R=+0.39, p=0.04)$ and serum IL-13 concentrations $(\mathrm{R}=+0.58, \mathrm{p}<0.0001)$. However, in the 10 patients who had both variables measured, there was no correlation between NKT cell numbers and serum IL-13. Conclusion: The number of non-classical NKT cells and IL-13 concentrations in peripheral blood are increased in patients with active UC and correlate with disease activity. These findings are consistent with the possibility that IL-13-producing NKT cells contribute to the pathogenesis of UC. References (1) Fuss et al. Journ Clin Invest. 2004;113(10):1490-7 (2) Heller et al. Gastroenterology. 2005;175(5):3207-13 (3) Walmsley et al. Gut. 1998;43(1):29-32 NKT cell numbers and serum IL-13 concentrations in active and inactive UC

\begin{tabular}{|c|c|c|c|}
\hline \multirow{2}{*}{ NKT cells } & & Active UC & Inactive UC \\
\cline { 2 - 4 } & cells/ml & 15 & 15 \\
\hline \multirow{2}{*}{ IL-13 } & $\mathrm{N}$ & $3667(2095-6133)^{*}$ & $1195(526-3039)$ \\
\cline { 2 - 4 } & $\mathrm{pg} / \mathrm{ml}$ & 23 & 27 \\
\hline
\end{tabular}

Median and IQR are shown. ${ }^{*} \mathrm{P}<0.05$ from values for patients with inactive UC.

\section{M1766}

Dietary Microparticles Impair Macrophage Phagocytosis and Act As Adjuvants in the Presence of a Microbial Stimulus - Potential Relevance for Pathogenesis of Crohn's Disease

Matt Butler, J Boyle, R Playford, R Chaudhary, Jj Powell, Subrata Ghosh

Background and aims: Western diets regularly expose the gastrointestinal tract to a large quantity of man-made, submicron-sized, particles ( $>1012 /$ day) derived from food additives. These are taken up by $\mathrm{M}$ cells, accumulate in gut macrophages, and are resistant to degradation. Microparticles also have charged surfaces that readily adsorb luminal biomolecules such as bacterial lipopolysaccharide (LPS). Binding of luminal antigens or toxins to microparticles and delivery to intestinal macrophages in a particulate form could potentially overcome tolerogenic processes in susceptible individuals and lead to inflammatory bowel diseases such as Crohn's disease. We aimed to determine the effect of microparticles on human macrophage function. Methods: We have investigated the effect of two types of common dietary microparticle, (aluminium silicates and titanium dioxide) on human macrophage function in vitro. Monocyte-derived macrophages from active Crohn's patients or healthy controls were incubated with microparticles for 24 hours before being assayed for cytokine production (IL-8, TNF $\alpha$, IL-10, and TGF $\beta$ ), phagocytic activity and T cell stimulatory capacity. Results: Microparticles had little effect on macrophage function unless given at very high (toxic) doses $(>10 \mu \mathrm{g} / \mathrm{ml})$. However, in the presence of LPS, microparticles acted as adjuvants - improving cytokine responses by $\sim 10 \%$. This was shown to result from adsorption of LPS to the surface of microparticles in the presence of calcium $(\mathrm{Ca} 2+)$ in the culture medium. Microparticles also reduced the levels of phagocytosis, inhibited accumulation of engulfed latex beads and partly inhibited macrophage T cell stimulatory capacity. Significantly, although the responses were generally larger, cells from Crohn's disease patients were no more susceptible to the presence of microparticles than cells from healthy controls. Conclusions: Although they are immunologically 'inert' on their own, adsorption of luminal biomolecules to the surfaces of dietary microparticles could turn them into potent stimuli which might exacerbate established inflammation. Dietary microparticles also affect phagocytosis and T cell stimulatory capacity. In addition, since microparticles are selectively targeted to phagocytic macrophages, we believe they could potentially be used as delivery systems to enhance the efficacy of therapeutic agents. Our observations provide potential mechanisms by which dietary microparticles may alter immune regulation at the level of macrophages. Funded by: Broad Medical Research Program

\section{M1767}

NOD2 (CARD15) Mutations Are Associated with Mycobacterium Avium Subspecies Paratuberculosis (MAP) DNA in Intestinal Tissue But Not with Viable MAP in the Blood of Crohn's Disease (CD) Patients John F. Valentine, Xiao-Hong Song, Jerome Elam, Saleh Naser

BACKGROUND: MAP is an intracellular pathogen that causes Johne's disease, a chronic granulomatous enteritis in animals. The role of MAP as an etiologic agent or as an environmental cofactor for $\mathrm{CD}$ is controversial. Recent studies have been more consistent in the identification of MAP in patients with Crohn's disease. NOD2 is an intracellular pattern recognition receptor recognizing muramyl dipeptide, a component of bacterial cell wall peptidoglycans and is expressed in monocytes, macrophages, dendritic cells, paneth cells and intestinal epithelial cells. Mutations in NOD2 have been associated with impaired clearance of intracellular pathogens in the experimental setting. We determined the relationship of the presence of CD associated NOD2 mutations in patients that had blood cultured for MAP or had presence of MAP IS900 DNA evaluated in intestinal tissue. METHODS: DNA was available on 23 of $28 \mathrm{CD}$ patients that had previously had blood cultured for MAP (group 1). DNA was available for all 21 additional CD patients had previously had surgically resected intestinal tissue evaluated for the presence of MAP IS900 DNA (group 2). CD associated NOD2 mutations (R702W, G908R, 1007fsinsC) were determined using a TaqMan based allelic discrimination assay. The results were analyzed with respect to MAP culture data and MAP IS900 DNA data. RESULTS: For group 1, 12 of the $23(52 \%)$ had (+) MAP blood cultures, 6 of the $23(26 \%)$ had NOD2 mutations (1 heterozygous R702W 1 heterozygous G908R, 3 heterozygous 1007 fsinsC and 1 homozygous 1007fsinsC). There were no compound heterozygotes. 2 of the $6(33 \%)$ with NOD2 mutations also had positive MAP cultures (NS). For group 2, 17 of 21 (81\%) were MAP IS900 DNA (+), 8 of 21 (38\%) had NOD2 mutations (2 heterozygous R702W, 4 heterozygous 1007insC, and 2 compound heterozygotes R702W and 1007insC). 7 of the 8 (87.5\%) with NOD2 mutations also had MAP DNA detected in intestinal tissue $(\mathrm{P}<0.02)$. For groups 1 and 2 combined, 14 of 44 $(32 \%)$ carried NOD2 mutations. CONCLUSIONS: NOD2 mutations are associated with the identification of MAP DNA in the intestinal tissue of patients with CD but not with those with MAP bacteremia. The association of NOD2 mutations with MAP in intestinal tissue may be related to a decreased bacterial clearance related to a defect in NOD2 dependan intracellular killing or an increased viability due to NOD2 dependant defects in the epithelial barrier such as production of defensins.

\section{M1768}

Increased Expression of Tlrs' Is Associated with An Upregulation of Signalling Molecules Irak-1 and NfאB in Colonic Biopsies of Patients with Inflammatory Bowel Disease (IBD)

Anushka De Costa, Neil Rayment, Maria Mylonaki, Barry N. Hudspith, Liljana Petrovska, Jonathan Brostoff, Carlo Nunes, Gareth Parkes, Jeremy Sanderson, David Rampton

Background: Toll like receptors are innate cellular pattern recognition receptors (PRR's) which recognise specific bacterial antigens and are, in part, responsible for the immunological unresponsiveness towards normal commensal flora. We have previously reported changes in mucosal associated bacterial flora in IBD patients and the presence of E.coli within macrophages in the lamina propria. We have now investigated the activation status of both epithelial cells and lamina proprial macrophages and compared co-expression of TLRs' 2 4 and 5 with signalling molecules IRAK-1 and NFKB. Methods: Snap-frozen rectal biopsies were taken at routine colonoscopy from patients with ulcerative colitis (UC, $n=8$ ), Crohn's $(\mathrm{CD}, \mathrm{n}=8$ ) and controls with normal colorectal mucosa $(\mathrm{n}=8)$. Toll like receptor 2, 4 and 5 expression, signalling molecules IRAK-1 and NFKB and macrophages (CD68)were determined using indirect immunofluorescence. Epithelial cells were identified by thei histological appearance and co-localisation confirmed using confocal microscopy. Results Moderate epithelial expression of TLRs' 2 and 4 with little or no epithelial expression of TLR 5 was seen in controls and correlated with absence of signalling molecules IRAK-1 or NFKB. IBD biopsies showed increased epithelial expression of TLRs' 2 and 4 (not 5) and was associated with increased IRAK-1 and NFKB levels. A similar pattern of TLR expression was seen in lamina proprial macrophages with greater expression of TLRs' 2 and 4 in IBD patients compared to controls. Modest amounts of TLR-5 were also seen in this group but none in control samples. In IBD biopsies, co-localisation of TLR's with IRAK-1 and NFKB in lamina proprial macrophages showed a significant increase in expression compared to that seen in epithelial cells. Conclusion: Increased expression of TLRs' and signalling molecules IRAK-1 and NFKB in IBD is consistant with a heightened immunological response to enteric microflora and may contribute to the pathogenesis of the disease

\section{M1769}

Dose Dependent Interaction of NOD2 with TLR2 in Human Monocytes Michelle Borm, Adriaan A. van Bodegraven, Chris J. Mulder, Georg Kraal, Gerd Bouma

Background and aims: The mechanism by which mutations in NOD2 predispose to Crohn's disease (CD) is poorly understood. Nod2 is an intracellular receptor for the bacterial produc muramyl dipeptide (MDP). Previous studies in mice have shown that Nod2 signalling inhibits Toll-Like Receptor (TLR)-2 responses. So far, this effect could not be reproduced humans in fact, several groups have shown that simultaneous stimulation of Nod2 with MDP and different TLRs with their appropriate ligands may lead to a stronger inflammatory response. Here we show that the effect of Nod2 signaling on TLR responses is dose-dependent. In the current study we intend to further explore this discrepancy. Methods: Monocytes were isolated from venous blood from healthy volunteers. After stimulation with different doses of MDP (Nod2 ligand) together with synthetic TLR2 and TLR7 ligands, and lipopolysaccharide (LPS, TLR4 ligand), culture supernatants, nuclear extracts and RNA were isolated. NF-KB activation and production of pro-inflammatory cytokines were measured with ELISAs, and mRNA levels of the cytokines were measured with real time RT-PCR. Results: Monocytes respond to Nod2 stimulation in a dose dependent fashion, although this response is weak compared to TLR stimulation. Simultaneous addition of TLR ligand and low doses of MDP to the culture medium resulted in a significant increase in the NF- $\mathrm{kB}$ activation as well as cytokine production, (as compared to TLR stimulation alone) both on mRNA level and secreted protein. Importantly however, addition of higher doses of MDP abolished this effect specifically in TLR2 signaling, whereby the cytokine responses returned to the baseline production of cytokines with TLR2 stimulation alone. Conclusions: Contrary to the resent findings in mice, in which a clear reduction of cytokine responses was detected when mononuclear cells are co-incubated with Nod2 and TLR2 ligands, we did not observe such an inhibition in human monocytes in this pilot experiment. In fact, we show for the first time that monocytes from healthy Nod2 wild-type donors respond to Nod2 stimulation in a biphasic fashion. First, at low doses, Nod2 stimulation enhances the TLR2 response, then, at higher doses, this initial potentiating effect was lost. These results are indicative for a

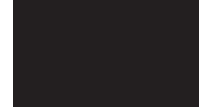


dose-dependent mode of interaction of Nod2 and TLR2 activation. Whether or not there is true negative regulation of TLR2 responses as is seen in mice, remains to be determined in humans. Studies with monocytes from CD patients in the CARD15/NOD2 are underway.

\section{M1770}

Critical Role of Cxcl12-Cxcr4 Interaction in the Pathophysiology of Inflammatory Bowel Disease

Sakae Mikami, Hiroshi Nakase, Takashi Nagasawa, Tsutomu Chiba

Background: Chemokines have been widely studied owing to their important role in regulating the trafficking of leukocytes under normal conditions and to sites of inflammation CXCR4 is a constitutive chemokine receptor, which is widely expressed on leukocytes and is involved in the recirculation of naive lymphocytes through high endothelial venules into lymphoid tissue. Moreover, recent studies indicate that CXCL12-CXCR4 chemotactic axis are involved in several inflammatory conditions. However, it remains unclear whether this interaction is associated with the pathophysiology of inflammatory bowel disease (IBD). Aim: The aim of our study is to examine the role of CXCL12-CXCR4 chemokine axis in the pathophysiology of IBD. Methods: (1) We evaluated the expression of CXCR4 in peripheral T cells and CXCL12 in colonic tissue of dextran-sulfate sodium (DSS)-induced colitis. C57/ B6 mice were fed 3\% DSS in their drinking water for up to 5 days. Infiltration of inflammatory cells into the mucosa and the expression of CXCL12 were analyzed by immunohistochemistry. Expression of CXCR4 in peripheral blood cells was analyzed by FACS. (2) We also evaluated the change of CXCR4 expression in peripheral T cells from 4 patients with ulcerative colitis (UC) during the leukocytapheresis (G-CAP or L-CAP). Result: (1) In animal study, FACS analysis revealed that CXCR4 expression in peripheral $\mathrm{T}$ cells was approximately five-fold in DSS-induced colitis mice compared to normal mice. Moreover, the expression of CXCL12 in inflamed colonic tissue was increased in colitis mice compared to control mice. (2) In human study, FACS analysis revealed that CXCR4 expression in peripheral T cells from patients with active UC was significantly higher than normal controls. Interestingly, after starting leukocytapheresis for these patients, CXCR4 expression was gradually decreased in all patients. Moreover, when these patients got into clinical remission, CXCR4 expression became normal. These results suggested that the intensity of CXCR4 expression in peripheral T cells was correlated with the activity of patients with UC. Conclusion: CXCR4-mediated lymphocytes recruitment may be critically involved in colonic inflammation. We firstly found the increased CXCR4 expression in T cells of DSS-induced colitis mice. Our results raise the possibility that the enhancement of CXCR4 expression in T cells contributes to the perpetuation of colonic inflammation. Therefore, the inhibition of CXCL12-CXCR4 interaction in peripheral $\mathrm{T}$ cells may be one of the promising therapies for patients with IBD in the future.

\section{M1771}

\section{Alternative Mechanisms of Action of Tacrolimus in IBD: Inhibition of} Intestinal Epithelial Cell- and NKT Cell- Activation

Jolanda M. Van Dieren, Janneke van der Woude, M E. Lambers, Ernst J. Kuipers, Edward E. Nieuwenhuis

Background: Tacrolimus is a calcineurin inhibitor, which has been shown to be effective in the treatment of IBD. Inhibition of T cell activation has been suggested as the major mechanism of action of tacrolimus function. Other mucosal cells such as the intestinal epithelial cells (IEC) are known to contribute to the pathogenesis of IBD through the production of cytokines and chemokines. Furthermore, CDld restricted T-cells have recently been found to be a major source of enhanced IL-13 production in human ulcerative colitis. The aim of this study was to determine whether tacrolimus exerts its effects through inhibition of activation of either CDld restricted T-cells and/or IECs. Methods: A CDld restricted Natural Killer (NK) - T cell-line (DN32.D3) was stimulated by overnight incubation with a CDld transfected intestinal epithelial cell-line (T84-d) that was pulsed with aGalactosylceramide (aGalCer). aGalCer is a glycolipid antigen that is known to activate NKT cells in a CDld restricted fashion. Another intestinal epithelial cell-line (Caco-2) was stimulated by overnight incubation with peptidoglycan at $10 \mu \mathrm{g} / \mathrm{ml}$. Both in vitro assays were performed in the absence or presence of different concentrations of tacrolimus. NKT cell activation was determined by measurement of IL-2 production and Caco- 2 stimulation by IL- 8 production by means of ELISA. Results: Pre-treatment of NKT cells with tacrolimus caused a dose-dependent reduction of IL-2 production upon stimulation with an exogenous antigen (aGalCer) as presented by T84-d cells, an IEC line. Interestingly, the same dose dependent reduction of NKT cell activation was observed when the antigen presenting T84-d cells were pre-treated with tacrolimus. Caco-2 cell activation by peptidoglycan could also be inhibited by tacrolimus, albeit at higher dosages. Conclusion: These data show that the inhibitory functions of tacrolimus can be extended to CDId restricted NKT cells as well as IECs, which may explain the effectiveness of tacrolimus in IBD.

\section{M1773}

Infliximab Induces a Genotype-Dependent Mucosa Protective Matrix Metalloproteinase Phenotype in Inflammatory Bowel Diseas

Martin J. Meijer, Marij A. Mieremet-Ooms, Wim van Duijn, Annie M. van der Zon,

Roeland Hanemaaijer, Jan H. Verheijen, Ruud A. van Hogezand, Cornelis B. Lamers, Hein W. Verspaget

Previous studies have shown an upregulation of matrix metalloproteinases (MMPs) in intestinal tissue of patients with inflammatory bowel disease (IBD) and a significant clinical and endoscopical improvement of the patients after administration of the anti-TNF- $\alpha$ antibody infliximab. We determined the expression and secretion of MMP-1, - 2, -3, -9 and their inhibitors TIMP- $1,-2$ by IBD vs control intestinal mucosa ex vivo and assessed the regulatory capacity by infliximab of the proteolytic phenotype. Intestinal mucosal explants from 20 IBD and 15 control patients, were cultured in medium with or without infliximab and/or the T-cell activator pokeweed mitogen (PWM). Explants and culture supernatants were analysed for MMPs, TIMPs and TNF- $\alpha$ protein, activity and/or mRNA levels by ELISA, BIA and semi-quantitative RT-PCR. All patients were genotyped for functional TNF- $\alpha$, MMP and TIMP single nucleotide polymorphism (SNP) loci by RFLP or tetra primer ARMS PCR. The expression of MMP and TIMP protein/activity in basal medium was higher for IBD vs control explants, and for the MMPs also within the explants. Infliximab downregulated genotypedependently MMP-1, MMP-3 and MMP-9 relative to TIMP-1 and -2, and also decreased MMP-1 and MMP-3 activities, while PWM enhanced these levels, partly counteracted again by infliximab. The expression of MMP-2 relative to TIMP did not change by treatment with infliximab and/or PWM. Conclusions: The high expression of MMPs in patients with IBD suggests a role for these proteinases in the pathogenesis of the disease. Infliximab induces a genotype-associated matrix protective phenotype by downregulating MMP-1, -3 and -9 relative to TIMP, which may contribute to the observed therapeutic efficacy of this drug in IBD, particularly at the mucosal surface.

\section{M1774}

The Peripheral Immune Cell Population Reflects the Local Disease Activity and Colonic Extension At the Onset of Ulcerative Colitis

Lena Ohman, Stefan Isaksson, Evangelos Kalaitzakis, P-O Stotzer, Magnus Simren, Hans Strid

AIM: The colonic localization of ulcerative colitis (UC) is highly individual and the understanding of underlying factors resulting in the individual variation of the disease severity and extension of the mucosal inflammation in UC is currently limited. The aim of this study was to investigate if peripheral immune activity is correlated to the local disease extension and/or disease activity at the onset of UC. METHOD: Blood samples were taken from 25 untreated patients ( 11 women, age $37.3 \pm 16.3$ ) within the first week of UC onset. The colonic disease extension was established during colonoscopy. 13 patients had an extensive colitis, 7 patients had a left sided dominated colitis, whereas 4 patients had proctitis. The disease activity was determined by using the well validated Mayo score. The frequency of peripheral blood T cells was analysed by flow cytometry and ANCA specific ELISA analysis was performed from serum samples. RESULTS: There was no correlation between the frequency of peripheral $\mathrm{CD}^{+} \mathrm{T}$ cells or $\mathrm{CD} 8^{+} \mathrm{T}$ cells and the disease activity or the colonic disease extension. However, the frequency of peripheral $\mathrm{CD}^{+}{ }^{+} \mathrm{T}$ cells expressing an activated phenotype, defined as $\mathrm{CD}_{62 \mathrm{~L}^{-} \mathrm{CD} 45 \mathrm{RA}}$, was negatively correlated to the disease activity $(\mathrm{p}=0.048)(\mathrm{R}=0.38)$. There was no correlation between the frequency of peripheral activated $\mathrm{CD} 8^{+} \mathrm{T}$ cells and the disease activity or the extension of the disease. There was no correlation between $\mathrm{CD}^{+}$or $\mathrm{CD}^{+} \mathrm{T}$ cells expressing integrin $\beta 7$ and the disease activity, although patients with a left sided colitis tended to have an increased frequency of $\operatorname{Int} \beta 7^{+} \mathrm{CD} 8^{+} \mathrm{T}$ cells $(56.7 \pm 12.8$ vs $46.6 \pm 17.0, \mathrm{p}=0.15)$ as compared to patients with an extensive colitis. The frequency of $\mathrm{CD} 19^{+} \mathrm{B}$ cells in blood was not associated to the disease activity or the colonic disease extension, although the frequency of activated CD19+ B cells, defined as $\mathrm{CD} 38^{\text {high }}$, was positively correlated to the disease activity $(\mathrm{p}=0.02)(\mathrm{R}=0.44)$. The frequency of peripheral natural killer cells (NK cells) was found to be negatively correlated to the disease activity $(\mathrm{p}=0.045)(\mathrm{R}=0.39)$, and tended to be increased in patients with left sided colitis/proctitis $(8.7 \pm 5.0)$ as compared to patients with extensive colitis $(6.0 \pm 3.3)(\mathrm{p}=0.08)$. The levels of ANCA in serum did not correlate to the disease activity or the colonic disease extension. CONCLUSIONS: The results from this limited number of patients indicate that the disease activity and the colonic extension of ulcerative colitis are reflected by the peripheral immune cell population. This might be a useful tool as part of the evaluation of disease activity and extension in patients with ulcerative colitis.

\section{M1775}

Clostridium Difficile Toxin a Increases Focal Adhesion Kinase (FAK) and Paxillin Dephosphorylation Through Inactivation of Src Kinase Ho Kim, Charalabos Pothoulakis, John T. Lamont

C. difficile toxin A glucosylates Rho, Rac, and cdc42 in cultured cells, inactivating these proteins and eventually causing actin filament disaggregation and cell rounding. We previously showed that toxin A impaired tight junction formation colonocytes. We investigated the roles of toxin A on FAK and paxillin involving focal contact formation. we assessed effects of toxin A on the Src kinase dependent FAK/paxillin activation in non-transformed human colonocytes (NCM460). The dephosphorylation of FAK and paxilline in response to toxin A was measured by western blotting. The changes of Src kinase activity induced by toxin A were studied by in vitro kinase assays. Toxin A increased dephosphorylation of paxillin at tyrosine 118 in colonocytes within 1 hour. In colonocytes exposed to toxin A, dephosphorylation of FAK, which has been known to phosphorylate paxillin at tyrosine 118 also strongly detected and which completely correlated with the time pattern of paxillin dephosphorylation. Blockage of glucosyltransferase activity of toxin A or toxin A induced production of reactive oxygen species by using several chemical inhibitors did not affect toxin A induced dephosphorylation of FAK and paxillin. However, Src kinase inhibition by PP2 or SU6656 increased dephosphorylation of FAK/paxillin similar to effect of toxin in colonocytes. We also found that EGF-induced phosphorylation of Src at tyrosine 416 (its active phosphorylation site) were markedly decreased in colonocytes exposed to toxin A. In vitro kinase assay also showed that addition of toxin A suppressed the kinase activity of Src. By analyzing immunoprecipitation, we demonstrated that toxin A added to whole colonocyte extracts could bind to intracellular Src protein in a dose dependent manner. Toxin A suppression of the Src kinase-mediated activation of FAK/paxillin did not require glucosyltransferase activity of toxin A. These results suggest that direct binding of toxin A to c-Src kinae, without involvement of protein tyrosine phosphatase, could cause inhibition of Src kinase and subsequent FAK/paxillin activation, which may result in failure of focal contact formation of colonocytes. These results provide insight into the profound disturbance in intestinal tight junction formation observed in C. dfficile infection. 
Identification of a Role for Shiga Toxin (STX1) in Enterohemorrhagic Colitis Includes Increased TNF $\alpha$ and Downregulation of Galectin-3

Marina Laiko, C. Zu, Okana Gustal, Edward Boedeker, Robert Cole, Mark Donowitz, Olga Kovbasnjuk

The rabbit cecum model of enterohemorrhagic colitis (EHEC), which most closely resembles the intestinal pathology seen in human EHEC, was used to test the hypothesis that Shiga toxinl (Stxl) contributes to the intestinal pathophysiology. In this model RDEC-1, an E. coli strain that adheres to rabbit mucosa and causes an attaching and effacing lesion, and RDEC-H19A, a strain engineered to express recombinant Stxl, were fed to New Zealand White male rabbits. 7 days later, experimental animals ( $\mathrm{n}=3$ ) infected with RDEC-H19A strain developed hemorrhagic diarrhea, while control RDEC-1 infected animals ( $\mathrm{n}=3$ ) developed mild watery diarrhea. Animals were sacrificed and cecal tissue was obtained for biochemical, immunofluorescence, and Mass Spectroscopy studies. The production of Stxl in rabbits infected with RDEC-H19A was confirmed in cecal lysates by Western blot analysis. Mass spectroscopy with iTRAQ analysis of protein expression profiles showed significant downregulation of galectin-3 (Gal-3) in rabbits with Stxl compared to the bacteria alone. Immunoblotting validated a significant decrease in Gal-3 expression from $0.28 \pm 0.03$ units in control to $0.14 \pm 0.01,(\mathrm{p}=0.02)$ in Stxl-exposed animals. TNF- $\alpha$ levels were increased $2-3 x$ in Stxl exposed cecum compared to bacteria controls. The downregulation of Gal-3 by Stxl in intestinal epithelial cells was also confirmed using a T84 cell model, in which incubation of cells for $72 \mathrm{~h}$ with holotoxin or B-subunit of Stxl (StxlB) both significantly decreased the Gal-3 amount compared to control non-treated cells. Gal-3 is a galactose binding protein, which is predominantly expressed in the upper crypt region of human colon and is involved in regulation of cell inflammation, maintenance of epithelial barrier function, and apoptosis. Gal-3 is downregulated in intestine in patients with Crohn's disease and this is due to elevation of TNF- $\alpha$. We conclude: 1) An animal model of EHEC showed that the presence of Stxl is associated with TNF- $\alpha$ upregulation and a downregulation in Gal-3 2) It is likely that the changes in TNF- $\alpha$ contribute to the changes in Gal-3. This is the first report of Gal-3 involvement in Stxl-induced intestinal disease. These results identify a potential role for Stxl in EHEC diarrhea, specifically that Stxl may trigger inflammatory mechanisms similar to those that occur in Crohn's disease.

\section{M1777}

Transgenic Epithelial Cell Toll-Like Receptor (TLR) Signaling Reduces Inflammation in DSS Colitis

Rachel S. Levi, Laurens Kruidenier, Lisa Meijer, Xiao Ping Lu, Meera Sebastian, Sven Pettersson, Ian R. Sanderson

Commensal bacterial may protect against intestinal inflammation through TLR signaling (Rakoff-Nahoum et al, Cell 2004,118:229). However, it is not yet clear which intestinal cell type transduces the beneficial bacterial signals. We hypothesized that TLR signaling by the epithelium would inhibit intestinal inflammation. Methods: We developed a transgenic mouse using a constitutively active human TLR4 cytoplasmic domain linked to the extracellular domain of $\mathrm{CD} 4$, under the control of the intestinal epithelial cell-specific fatty acid binding promoter (Fabpi). Colitis was induced in transgenic and wild-type male littermates with $1 \%$ dextran sodium sulfate (DSS). Weight loss and fecal consistency was monitored daily and after 1 week inflammation was assessed by histology. Results: Transgenic mice expressed CD4-TLR4 mRNA in the small and proximal large intestine, which appeared normal without any signs of spontaneous intestinal inflammation. However, their response to DSS-induced colitis differed significantly. Typical symptoms of colitis, such as loose stools, rectal blood loss, and loss of body weight, were observed in $7 / 8$ (88\%) of the wild type mice, but only in $7 / 37$ (19\%) of the TLR transgenic littermates. As a consequence, cumulative weight loss (Figure) was significantly more severe in the normal mice $(20.1 \pm 3.7 \%$ at day 7$)$ than in the transgenics $(5.5 \pm 1.1 \%, \mathrm{P}<0.01)$. Furthermore, histopathological assessment showed fewer neutrophils and lymphocytes and less mucosal damage and remodeling in the transgenic mice than in wild-type littermates. Conclusions: TLR signaling through intestinal epithelial cells is sufficient to reduce intestinal inflammation. Thus, the epithelial cell may transduce the effect of commensal bacteria in inhibiting IBD. against DSS colitis

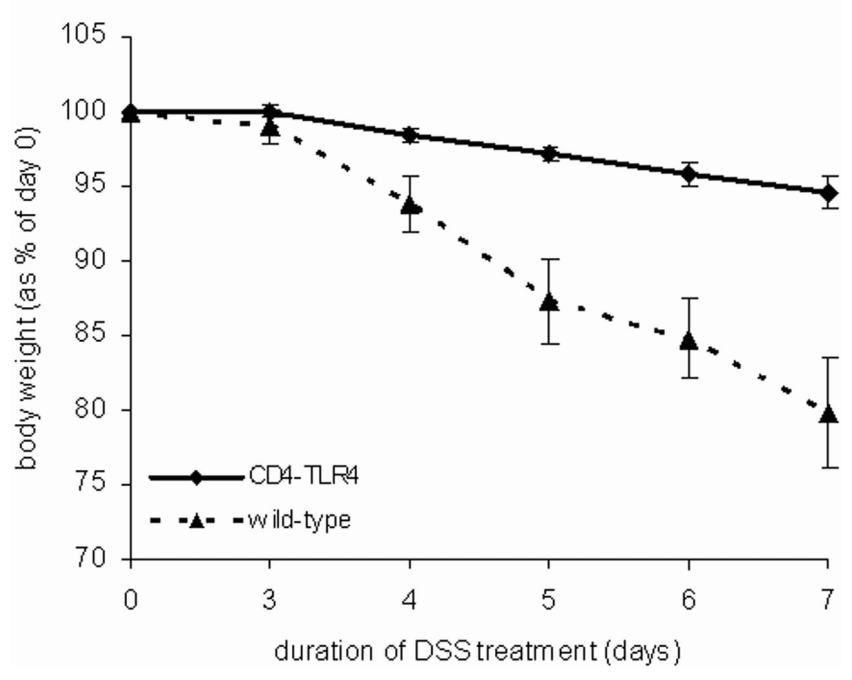

M1778

Helicobacter Pylori Caga Dependent Activation of the Signal Transducer and Activator of Transcription 3 (STAT3) Signaling Pathway Dana Bronte-Tinkew, Nicola Jones

Infection with CagA positive Helicobacter pylori strains enhances the risk for the development of gastric cancer. However the underlying mechanism(s) whereby this bacterium causes gastric cancer remain unknown. Recent evidence indicates that aberrant activation of the STAT3 signaling pathway may play a role in the development of gastric cancer. Therefore, we hypothesized that $H$. pylori infection modulates STAT3 signaling. To investigate possible alterations in STAT3 signaling, HEp-2 epithelial cells were infected with wildtype H. pylor strains (60190, G27 or 251) or the corresponding isogenic mutants: cagA, cagA EPISA with a mutation in the tyrosine phosphorylation domain (EPIYA), cagE, and cagM mutant strains at a multiplicity of infection (MOI) of 100:1 for up to 4hrs. As a positive control the known STAT3 activator IL-6 was employed. To determine STAT3 activation, expression of phosphorylated STAT3 was detected by Western blotting. In addition, fluorescence-recovery after photobleaching (FRAP) was performed on cells transfected with STAT3-GFP constructs To delineate potential mechanisms for STAT3 activation, cells were incubated with IL-6/ gp-130 receptor antagonists gp $130 \mathrm{mAb}$ or sant 7 . Wildtype H. pylori strains induced activation of STAT3 in HEp-2 epithelial cells, which was independent of changes in IL-6. In contras isogenic mutants of the type IV secretion system did not induce STAT3 activation. Furthermore, the cagA mutant failed to activate the STAT3 pathway. However, the H. pylori EPISA mutant strain activated the STAT3 pathway in a similar manner to its parental wildtype strain G27 indicating that tyrosine phosphorylation of cagA was not required. Inhibition of IL-6 receptor activation blocked the H. pylori-mediated increase in STAT3 phosphorylation Taken together these results indicate that $H$. pylori activates the STAT3 signaling pathway in epithelial cells in a CagA dependent manner, however tyrosine phosphorylation of the injected CagA protein is not required. Activation appears to occur early in the signaling pathway at the receptor level and is not due to a direct increase in IL-6. These findings provide a potential mechanism by which chronic $H$. pylori infection promotes the development of gastric cancer.

\section{M1779}

Dramatic Increase of Mucosal But Not Peripheral Regulatory T Cells in Untreated HIV Infection, Normalization By Suppressive Haart Hans-Jorg Epple, Christoph Loddenkemper, Desiree Kunkel, Hanno Troeger, Jochen Maul, Verena Moss, Reiner Ullrich, Jorg-Dieter Schulzke, Harald Stein, Rainer Duchmann, Martin Zeitz, Thomas Schneider

Background: Recent evidence indicates that regulatory T cells (Treg) play an important role in the pathogenesis of HIV infection. However, although the gastrointestinal mucosa is a key compartment in HIV disease, no data on mucosal Treg in HIV infection are available Aim: In this study, we compared the frequency of Treg in the duodenal mucosa and peripheral blood of treatment-naive and suppressively treated HIV-infected patients with those of normal controls. Methods: Mucosal Treg cells were determined in duodenal biopsies of 11 treatment-naive and 9 treated HIV-infected patients, 6 patients with norovirus infection and 12 patients without upper GI pathology (healthy controls) by immunhistochemistry with double immunoenzymatic (CD3+FOXP3+ or CD25+FOXP3+) detection of Treg. The specificity of the immunhistochemistry was confirmed by demonstration of co-expression of FOXP3 with cell surface markers for Treg by FACS (CD4+CD25high), immunhistochemical or confocal analysis (FOXP3+CD25high, FOXP3+GITR+). The frequency of Treg in peripheral blood was determined by FACS (CD3+CD4+FOX3+ and CD3+CD4+CD25high) and by immuncytochemistry (CD3+ FOXP3+). Results: Mucosal Treg were highly increased in untreated ( $40 \%$ of mucosal CD4+ T cells versus $1.2 \%$ in healthy controls, $\mathrm{p}<0.001$ ) but normal $(1.8 \%)$ in treated patients. In contrast, no significant differences in the frequencies of peripheral blood Treg were observed. In patients investigated serially, the frequency of

(1)

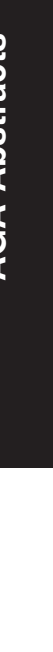


mucosal Treg returned to normal after suppression of HIV replication by HAART. Furthermore, in Norovirus infection, the frequency of mucosal Treg in the CD4+ T cell subset was only marginally elevated compared to normal controls. Therefore, the high increase in the frequency of mucosal Treg seems to be a characteristic feature of untreated HIV infection and is linked to active viral replication. Conclusions: The accumulation of Treg in a major site of HIV replication suggests a significant contribution of Treg to the pathogenesis of HIV disease. Their role, however, may be ambivalent, attenuating HIV-induced T cell hyperactivation while suppressing the immune response to HIV and opportunistic pathogens.

\section{M1780}

Differential Pattern of CD62l Expression On CDld-Unrestricted and CD1dRestricted T Cells Following Listeria Infection Is Regulated By IFN-Gamma Hyun Kim, Victor Arrunategui-Correa

BACKGROUND: CD62L, a member of the selectin adhesion family, is required for lymphocyte homing to peripheral lymph nodes (PLN) and to sites of inflammation. Unlike other leukocyte adhesion molecules, CD62L is rapidly down-regulated from the cell surface upon cell activation. CD62L expression is observed in a subset of CD4+ regulatory T-cell populations (CD25+). Previously, we have demonstrated distinct trafficking patterns of NKT cells in PLN, mesenteric lymph nodes (MLN), and non-lymphoid tissues isolated from Listeria monocytogenes (Lm)-infected WT, invariant NKT (iNKT) cell deficient J $\alpha 18 \mathrm{KO}$ and CDld KO mice. AIM: We have therefore investigated the role of CD62L and CD122 (IL-2/IL-15R) expression in PLN and MLN in these 3 strains of mice to determine if CD62L was an important determinant in the migration pattern we have observed. METHODS: WT, CDld $\mathrm{KO}$, and iNKT Ja18 KO mice were infected orally with $5 \times 108 \mathrm{Lm}$ and CFUs were measured. PLN and MLN were then prepared by standard methods and suspended in PBS/0.2\% BSA for flow cytometric analysis using CD62L and CD122 mAb. Production of cytokines from cultured supernatants of activated MLN was measured using an ELISA kit. RESULTS: Lm colonies were detected in MLN at day 1 , and reached the maximal CFU by day 3 postinfection in all three strains, with CDld KO mice displaying the highest CFU of viable Lm. Flow cytometric analysis at day 1 revealed that CD62L+ cells were decreased in both PLN and MLN isolated from iNKT Jal8 KO and CDld KO when compared with WT mice. In contrast, CD122+ cells were inversely increased in PLN and MLN of iNKT and CDld KO mice. The increased CD122+ cell population was most pronounced in MLN of CDld KO mice $(30 \%)$, while PLN from Jal8 KO and WT mice showed a modest increase (8\% and $2 \%$, respectively). Thus, CD122 is preferentially expressed in CD1d-unrestricted T lymphocyte subset, whereas $\mathrm{CD} 62 \mathrm{~L}$ is predominantly expressed in CDld-restricted $\mathrm{T}$ cell subset. Double CD62L+CD122+ cells were also increased only in the MLN of CDld KO mice, but not in PLN where the level was similar to Ja18 KO and WT mice. Cytokine production in the MLN culture supernatants showed a strong IFN-gamma production in CDld KO mice when compared with J $\alpha 18 \mathrm{KO}$ and WT mice, suggesting a Thl-dependent pathway in the regulation of CD62L and CD122 expression. CONCLUSION: CDld-restricted and CDld-unrestricted T lymphocytes show opposite CD62L and CD122 expression patterns following oral Lm infection. CD122-expressing CDld-unrestricted T cells were activated early after infection to produce IFN-gamma and display a unique trafficking pattern distinct from CDld-restricted T cells.

\section{M1781}

Helicobacter Pylori Infection Induces a T Regulatory Response Mediated By TGF-B and IL-10 in Children, in Contrast to the THI Response Characterized By IFN-G Observed in Adults

Lesley Smythies, Paul Harris, Ronald H. Clements, Lisa M. Mosteller-Barnum, Guillermo Perez-Perez, Phillip D. Smith

Background: In developing countries, such as Chile, $>60 \%$ of children are infected with $H$. pylori, but rarely develop ulcerative disease, whereas infected adults frequently develop ulceration. To determine if $\mathrm{T}$ cell responses are involved in this down-regulated inflammatory response to $H$. pylori in children, we characterized gastric $\mathrm{T}$ cell responses in infected children and adults and blood T regulatory (CD4+/CD25+) cell responses in adults. Methods: Gastric mucosal TGF-b, IL-10, IFN-g, IL-13 and IL-4 were measured in supernatants of homogenized biopsies from Chilean children ( $<12$ years; $n=36,50 \%$ infected) and adults ( $>18$ years; $n=$ $44,75 \%$ infected). Purified CD4+/CD25+ and CD4+/CD25- T cells from $H$. pylori-infected and uninfected patients were stained for FOXP3, LAG 3 and CTLA-4 (regulatory cell profile) and cultured alone or with homologous monocyte-derived dendritic cells pre-stimulated with H. pylori urease or Cag A, and culture supernatants were assayed for IL-2, IL-10, TGFb and IFN-g. Results: $11 \%$ of $H$. pylori-infected children had gastric ulceration compared to $40 \%$ of adults. Gastric mucosa from $H$. pylori-infected children had significantly higher levels of T regulatory cell cytokines IL-10 and TGF-b compared to infected adults. In contrast, gastric mucosa from infected adults had significantly higher levels of the Thl cytokine IFNg compared to infected children. Among uninfected children and adults, gastric levels of TGF-b were higher in children. Th2 cytokines IL-13 and IL-4 were similar among the groups and unchanged by infection. Unsorted T cells (and CD4+/CD25- cells) from infected persons produced IFN-g and IL-2 in responses to $H$. pylori antigens, and these responses were strongly inhibited by CD4+/CD25+ cells in association with increased IL-10 and TGF-b. Conclusions: H. pylori-infected children have significantly less gastric ulceration than infected adults. Increased levels of gastric TGF-b and IL-10 in infected children, but increased levels of IFN-g in infected adults, suggests that $H$. pylori introduces a gastric $T$ regulatory response in children and a Thl response in adults. Higher levels of TGF-b in gastric tissue from uninfected children may predispose children to mucosal $\mathrm{T}$ regulatory cell responses. The CD4+/CD25+ T cell-mediated down-regulation of IFN-g and IL-2 released from circulating $\mathrm{T}$ cells of $\mathrm{H}$. pylori-infected persons suggests the presence of a $\mathrm{T}$ regulatory cell population. We speculate that CD4+/CD25+ T cells down-regulate host responses to $H$. pylori infection in children and possibly adults without ulcer disease.

\section{M1782}

Bacterial Antigens Alone Can Break Epithelial Barrier Integrity While Live Bacteria Are Required to Initiate Intestinal Inflammation

Beate C. Sydora, Sarah M. Martin, Maryla Lupicki, John W. Walker, Levinus A. Dieleman, Thomas A. Churchill, Jason Doyle, Richard N. Fedorak

Background: It is well established that the intestinal microflora plays a critical role in the initiation and perpetuation of inflammatory bowel diseases (IBD) and that bacteria are involved in driving the intestinal and systemic immune responses. We have recently demonstrated that fecal slurry containing both live bacteria and bacterial antigens can rapidly (within 7days) lead to a disruption of epithelial barrier integrity and sustained intestinal inflammation when fed to axenic IL-10 gene-deficient mice. Objective: To further understand the mechanism of this inflammatory process we separated the role of live bacteria and bacterial antigens on the alteration of epithelial barrier integrity and the systemic and intestinal inflammatory response. Methods: Axenic (confirmed luminally sterile) IL-10 gene-deficient mice at $12 \mathrm{wk}$ of age were administered either fresh fecal slurry with live bacteria and bacterial antigens or a sterile fecal slurry with bacterial antigens only, devoid of live bacteria through lysation and filtration. Intestinal and systemic immune and inflammatory responses (intestinal and splenic cytokine profiles) and epithelial barrier integrity (mannitol transmural flux) were assessed lwk later. Results: The fecal slurry with live bacteria and bacterial antigens resulted in both a disruption of epithelial barrier integrity (mannitol flux: $22 \pm 3 \mathrm{~nm} / \mathrm{cm}^{2} / \mathrm{h}$ vs $13 \pm 1$ $\mathrm{nm} / \mathrm{cm}^{2} / \mathrm{h}$ in fecal slurry-treated vs axenic controls, respectively; $\left.\mathrm{p}=0.005\right)$ and increase in intestinal (5- and 10-fold increase in colonic and cecal IFNy release compared to axenic controls, respectively) and increase in systemic (up to 20 -fold increase in splenic IFN $\gamma$ release following stimulation with various bacterial antigens compared to axenic controls) inflammatory response. In contrast, the identical sterile fecal slurry, devoid of live bacteria and with bacterial antigens only, disrupted the epithelial barrier integrity (mannitol flux: $19 \pm 2$ $\mathrm{nm} / \mathrm{cm}^{2} / \mathrm{h}$ vs $13 \pm 1 \mathrm{~nm} / \mathrm{cm}^{2} / \mathrm{h}$ in sterile fecal slurry-treated vs axenic controls, respectively $\mathrm{p}=$ 0.01 ) but did not induce a systemic or intestinal inflammatory response (zero increase in IFN $\gamma$ release in cultures from intestinal or splenic tissue compared to cultures from axenic controls, respectively). Conclusion: We have demonstrated, for the first time, the discordance between the ability of bacterial antigens to disrupt the epithelial barrier integrity but not cause sustained intestinal or systemic inflammatory responses until live bacteria are present.

\section{M1783}

Intestinal Microflora Modulates Mucosal Expression of Macrophage ColonyStimulating Factor (M-CSF) and Granulocyte-Macrophage Colony-Stimulating Factor (GM-CSF)

Koichi Takebayashi, Ryota Hokari, Yoshikiyo Okada, Keisuke Okudaira, Chie Kurihara Hisayuki Matsunaga, Norikazu Mataki, Syunsuke Komoto, Chikako Watanabe, Atsushi Kawaguchi, Shigeaki Nagao, Kazuro Itoh, Yoshikazu Tsuzuki, Soichiro Miura

BACKGROUND \& AIMS: Enterobacteria have been implicated in the pathogenesis of inflammatory bowel diseases (IBD), and reported to be essential for the initiation of experimental murine colitis. Mucosal macrophages ( $\mathrm{mM} \Phi$ ) are also related to the pathogenesis of IBD by secreting both inflammatory and inhibitory cytokines in response to enterobacteria. Because mM $\Phi$ differentiates into two different phenotypes under macrophage colony-stimulating factor (M-CSF) or granulocyte-macrophage colony-stimulating factor (GM-CSF), we speculate enterobacteria may modulate intestinal inflammation through different CSF induction in the intestinal mucosa. However, there is no report how changes of bacterial flora affects CSF expression in vivo. In this study, we compared CSF expression in the intestine under germ free $(\mathrm{GF})$ or specific pathogen free (SPF) condition. We also examined effects of specific enterobacterial colonization on CSF expression in GF mice. MATERIALS \& METHODS: We used male IQI mice bred under GF or SPF conditions. Jejunal, ileal and colonic mucosa were removed. Messenger RNA of M-CSF, GM-CSF, M-CSF receptor, GM-CSF receptor and TNF- $\alpha$ were measured by quantitative RT-PCR. The expression of adhesion molecules in the ileal mucosa were evaluated by immunohistochemistry. In some GF mice, Bifidobacterium bifidum or Bacteroides vulugatus was inoculated and changes of CSF expression were evaluated after 21 days. RESULTS: In colonic mucosa, GM-CSF expression significantly decreased and GM-CSF receptor expression decreased slightly in GF mice compared with SPF mice. Interestingly, on the other hand M-CSF expression increased slightly in GF mice. TNF- $\alpha$ expression was significantly suppressed in GF mice, comparable with the deviation to MCSF dominant environment in GF condition. Immunohistochemical study revealed the significant increase in infiltration of CD4+ and $\beta 7$-integrin + cells and expression of MAdCAM$1+$ vessels in SPF mice than GF mice, Even after inoculation of enterobacteria in GF mice, however, there was no sign of inflammation and both bacteria significantly decreased GMCSF receptor expression, while M-CSF receptor expression increased slightly, maintaining the enhanced M-CSF dominance. In the jejunal or ileal mucosa, effects of enterobacteria to the M-CSF deviation was less dominant than in the colonic mucosa. CONCLUSIONS: Results suggest that intestinal microflora regulates cytokine production and adhesion molecule expression via modulation of M-CSF and GM-CSF expression. The M-CSF dominant environment in GF mice may be related to the decreased inflammatory cytokine response, thus giving us a suggestion of CSF as one of therapeutic targets for IBD.

\section{M1784}

Identification of Antigens Encoded By Enterococcus Faecalis That Display Serum Reactivity and Stimulate T Cell Responses in E. Faecalis Monoassociated IL-10-/- Knockout Mice

Sandra C. Kim, James P. Moran, Michael J. Lodes, Robert Hershberg, Susan L. Tonkonogy, R. Balfour Sartor

Background/aims: Germ-free (GF) IL-10 knockout (-/-) mice develop immune-mediated distal-predominant colitis when monoassociated with a non-pathogenic E. faecalis (Ef) strain. An oligoclonal TCR expression pattern in the colonic tissue and mesenteric lymph node cells (MLN) suggests that restricted T cell expansion in this model is due to activation by a limited profile of bacterial antigens. We sought to identify antigenic components of $E f$ 
that induce these T cell responses. Materials and Methods: A genomic random shear lambda expression library was generated from our published $E f$ strain and screened with sera from colitic Ef - monoassociated IL-10-/- mice. Three of 20 gene products identified by anti - Ef antibody binding were selected to be cloned and expressed as recombinant proteins (EF20, EF-33 and EF-60) and used for further analysis of T cell reactivity. GF IL-10-/- and wild-type (WT) control mice were monoassociated with Ef and studied 16-30 wks after inoculation; $E f$ - monoassociated IL-10 -/- animals develop severe colitis at this time point. CD4+ MLN were stimulated with antigen presenting cells (APC) that were pulsed overnight with a control protein $(\mathrm{KLH})$ or the recombinant Ef proteins. IFNy was measured in coculture supernatants as an indicator of $\mathrm{T}$ cell reactivity. To determine non-specific $\mathrm{T}$ cell activation, we tested each stimulant in unfractionated splenocyte cultures in the presence of an anti-IL-12 neutralizing antibody or an isotype matched control antibody. Results: EF60 stimulated IFN $\gamma$ secretion from IL-10 -/- CD4+ MLN/APC co-cultures that was significantly greater than the response to KLH. Responses to EF-20 and EF-33 were minimal (KLH: $0.2 \pm 0.1 \mathrm{ng} / \mathrm{ml}, \mathrm{EF}-20: 0.4 \pm 0.03$, EF-33: 0.5 \pm 0.1 , EF-60: 4.6 $\pm 1.3 ; \mathrm{p}<0.006)$. Anti-IL-12 neutralizing antibody reduced the IFN $\gamma$ response to EF-60 by nearly $80 \%$ in splenocyte cultures, but it had little effect on the IFN $\gamma$ levels in splenocyte cultures stimulated with EF-20 or EF-33 (Table). The same antibody concentration completely blocked IFN $\gamma$ production in cultures stimulated with $20 \mathrm{ng} / \mathrm{ml}$ of LPS. Conclusions: Colitic IL-10 -/- mice monoassociated with $\mathrm{Ef}$ develop immune responses that display both antibody and $\mathrm{T}$ cell reactivity to $E f$ - encoded antigens. Much of the T cell response to EF-60 was suppressed by IL-12 neutralization. Further studies are required to determine whether EF-60 contains adjuvant activity in addition to a $\mathrm{T}$ cell epitope.

Table 1. IFN $\gamma$ measurements in IL-10 -/- splenocyte (SPL) culture supernatants (ng/ml).

\begin{tabular}{|c|c|c|c|c|c|}
\hline & EF-20 & EF-33 & EF-60 & No Antigen & LPS \\
\hline IFN $\gamma$ SPL+anti-IL-12 & $0.6 \pm 0.4$ & $1.4 \pm 0.03$ & $3.2 \pm 0.6$ & Not detectable & $0.02 \pm 0.01$ \\
\hline IFN $\gamma$ SPL+ISO & $0.4 \pm 0.2$ & $1.1 \pm 0.3$ & $14.9 \pm 0.1$ & Not detectable & $5.0 \pm 1.2$ \\
\hline
\end{tabular}

\section{M1785}

Enteropathogenic E. Coli Promotes the Transactivation of Epidermal Growth Factor Receptor

Jennifer L. Roxas, Athanasia Koutsouris, V.K. Viswanathan

Enteropathogenic Escherichia coli (EPEC), a diarrheagenic pathogen, is responsible for significant infant mortality and morbidity, particularly in developing countries. While the precise mechanism by which EPEC causes diarrhea remains to be delineated, infectiondependent alterations in intestinal epithelial barrier function and the induction of proinflammatory cytokines contribute to the process. EPEC transfers specific virulence factors into host cells via a type III secretion system. EPEC, by an unknown mechanism, activates the PI-3 kinase and MAP kinase signaling pathway in host cells. EPEC activation of the MAP kinases ERK1/2 contributes to the production of the pro-inflammatory cytokine, interleukin 8. The aim of this study was to explore the effect of EPEC on the epidermal growth factor receptor, a known upstream activator of both the PI-3 kinase and MAP kinase pathways. Caco-2 BBE monolayers were infected with WT EPEC or a type III secretion mutant for specific time points, washed, and the extracts immunoblotted against EGFR, EGFR pY1068, ERKl/2, phospo-ERKl/2, Akt or pAkt. EPEC induced a progressive increase in EGFR autophosphorylation (pY1068) in intestinal epithelial cells, and this was blocked by the EGFR tyrosine kinase inhibitor, tyrphostin. A type III secretion mutant $(\Delta \operatorname{escN})$ also activated EGFR, ruling out the involvement of secreted effector molecules in EGFR autophosphorylation. Moreover, filter-sterilized EPEC growth medium promoted EGFR phosphorylation, suggesting that bacterial attachment is not required for this process. EPEC stimulated the phosphorylation of Akt, a PI-3 kinase substrate, and this was significantly attenuated by tyrphostin. Tyrphostin significantly inhibited EPEC-induced phosphorylation of ERK1/2, and attenuated interleukin 8 production in infected epithelial cells. The effect of tyrphostin was specific, since it did not impair attachment of bacteria to host cells, the recruitment of actin, or EPEC-induced barrier function alteration. Taken together, this suggests that EGFR transactivation is an early signaling event associated with EPEC infection that contributes to the innate immune response of host intestinal epithelial cells.

\section{M1786}

Important Role of Cytolethal Distending Toxin in Campylobacter JejuniInduced Host Proinflammatory Cytokine Responses and Loss of Transepithelial Electrical Resistance in Colonic Epithelial Cells Ming L. Chen, Zhongming Ge, James G. Fox, David B. Schauer

BACKGROUNDS AND AIMS: Campylobacter jejuni $(\mathrm{Cj})$ is the leading cause of human foodborne enteritis, however, microbial virulence factor(s) responsible for host inflammation have yet to be elucidated. Cytolethal distending toxin (CDT), a secreted tripartite toxin composed of A, B and C subunits, has been identified in several bacterial pathogens including $\mathrm{Cj}$. CDT has been shown to induce proinflammatory cytokine secretion and cell cycle arrest; the latter event could be partially attributed to the DNase-like activity of CDTB. We previously generated an isogenic $c d t B$ mutant by inactivating the gene in Cj strain 81-176 and found that this mutant lacks the capacity to induce gastroenteritis in NF-kB-deficient mice (Infect. Immun. 72: 1116). The aim of this study was to further investigate the mechanisms by which CDT contributes to gastroenteritis. METHODS: T84 cells were grown on collagencoated Transwell inserts ( $0.4 \mu \mathrm{m}$ pore-size) and reached $1500-2000 \mathrm{ohm}^{*} \mathrm{~cm} 2$ weeks after seeding. Polarized monolayer was apically infected with $\mathrm{Cj}$ or the $\mathrm{cdtB}$ mutant at a bacterial:cell ratio of 100:1. IL-8 secretion and transepithelial electrical resistance (TER) were determined by ELISA and with a voltohmmeter, respectively. RESULTS: Basolateral IL- 8 secretion was elevated from $159+/-19 \mathrm{pg} / \mathrm{ml}(\mathrm{n}=6)$ in mock infected cells to $328+/-29 \mathrm{pg} / \mathrm{ml}(\mathrm{n}=6)$ in Cj-infected cells 48 hrs post-infection. The amount of IL- 8 secreted by T84 cells infected with the $c d t B$ mutant $(231+/-62 \mathrm{pg} / \mathrm{ml}, \mathrm{n}=6)$ was reduced compared to the wild type. TER is a measurement of tight junction (TJ) barrier function. $\mathrm{Cj}$ infection caused a significant loss in TER $(22.2+/-12.8 \%$ of control, $n=4)$; while a more modest decrease in TER (43.4
$+/-12.3 \%$ of control, $\mathrm{n}=4$ ) was found in $c d t B$ mutant-infected cells. CONCLUSIONS Increased proinflammatory cytokines and disruption of $\mathrm{T} J$ barrier function are hallmarks o microbe-induced gastroenteritis. Our data demonstrate that $\mathrm{Cj}$ induces proinflammatory cytokine responses and disrupts TJ barrier function in polarized T84 cells. Furthermore, CDT plays an important role in mediating pathophysiological alterations in $\mathrm{Cj}$ infection. Future studies are aimed at elucidating the host intracellular pathways modulated by $\mathrm{C}$ leading to these changes. (This work was supported by NIH grant DK52413)

\section{M1787}

Upregulation of the THI Immune Response By Inducing Colitis Through TNBS Or Citrobacter Infection Results in Stereotypic Changes in Colonic Smooth Muscle and Epithelial Function

Thomas L. Sutton, Aiping Zhao, Justin E. Elfrey, Kathleen B. Madden, Carolyn A.

Sullivan, Allen Smith, Terez Shea-Donohue

IBD results from a complex interaction among genetics, bacteria, and the environmen resulting in a Thl dominant inflammation. Murine Citrobacter rodentium (CR) infection of the colon is an emerging model of IBD with notable similarities to clinical disease. The impact of $C R$ on colonic smooth muscle and epithelial cell function, however, is unknown Aim: To determine 1) the functional changes in smooth muscle and epithelium in response to $C R$ colitis and 2) compare these results with the more established model of TNBS induced colitis. Methods: BALB/c mice ( $\mathrm{n}>9 /$ group) received intrarectal TNBS $(2 \mathrm{mg} /$ mouse in $40 \%$ $\mathrm{ETOH}$ ) or saline and studied 4 days later. C57/BL/6 mice ( $\geq 3$ /group) were infected orally with live $C R\left(5 \times 10^{10} \mathrm{cfu}\right)$ or vehicle and studied 12 days later. Microscopic damage was assessed blindly. Stripped colonic mucosae were mounted in Ussing chambers to measure mucosal resistance $(\mathrm{R})$ and changes in short circuit current upon serosal addition of acetylcholine (ACH), 5-HT, or histamine (HIST). Colonic muscle segments were suspended in organ baths and responses to ACH ( $1 \mathrm{mM})$, HIST ( $1 \mathrm{mM})$ and electrical field stimulation $(\mathrm{EFS})(5 \mathrm{~Hz})$ were determined. Real-Time PCR determined the colonic expression of IFN-g, TNF-a, IL-4, and HIST 1 and 4 receptor (HIR; H4R). Results: CR and TNBS induced a colitis featuring significant $(\mathrm{p}<0.05)$ mucosal damage and upregulation of IFN-g $(51.3-\&$ 2.4-fold) and TNF-a (4.2- \& 2.1-fold) with no change in IL-4 (1 \& 1.4-fold). Despite evidence of mucosal damage, $C R$ improved mucosal resistance, likely due to marked mucosal hyperplasia. Smooth muscle and epithelial cell responses to $\mathrm{ACH}$ were unchanged. In contrast, both models inhibited contractility to EFS and HIST and reduced secretory responses to HIST and 5-HT. Inhibited responses to HIST were associated with a $>50 \%$ reduction in H1R expression. The $>3.5$-fold increase in the expression of the proinflammatory H4R reflects the increase in the number of hematopoeitic cells. Conclusions: These data demonstrate that 1) greater expression of Thl cytokines in $C R$ versus TNBS does not lead to a corresponding greater effect on gut function; and 2) upregulation of Thl cytokines induces a stereotypic hyposecretion and hypocontractility of the colon that is independent of the etiology.

\begin{tabular}{|c|c|c|c|c|}
\hline & VEH & TNBS & VEH & CR \\
\hline Smooth Muscle $\left(\mathrm{N} / \mathrm{cm}^{2}\right)$ & \multicolumn{3}{|l|}{} \\
\hline HIST & $6 \pm 1$ & $3 \pm 1^{*}$ & $7 \pm 1$ & $2 \pm 0.5^{*}$ \\
\hline EFS & $4 \pm 1$ & $1 \pm 1^{*}$ & $4 \pm 0.5$ & $1 \pm 0.5^{*}$ \\
\hline Epithelial Cell $\left(\mu \mathrm{A} / \mathrm{cm}^{2}\right)$ & \multicolumn{5}{|l}{} \\
\hline HIST & $42 \pm 10$ & $17 \pm 4^{*}$ & $\mathbf{5 0} \pm 19$ & $17 \pm 1^{*}$ \\
\hline SHT & $90 \pm 1$ & $34 \pm 10^{*}$ & $147 \pm 47$ & $38 \pm 9^{*}$ \\
\hline R $(\Omega \times \mathrm{cm}$ & $18 \pm 1$ & $24 \pm 3$ & $22 \pm 0.5$ & $35 \pm 5^{*}$ \\
\hline
\end{tabular}

$* \mathrm{p}<0.05 \mathrm{vsVEH}$

\section{M1788}

Downregulation of TNF- $\alpha$ Gene Expression in Human Intestinal Mucosa By Non-Adherent Probiotic Bacteria Marta Llopis, Maria Antolin, Francisco Guarner, Siv Ahrne, Goran Molin, Juan R. Malagelada

Introduction: The mechanisms by which certain bacteria modulate cytokine secretion by intestinal mucosa have not been clarified. The probiotic bacteria Lactobacillus plantarum $299 \mathrm{v}$ has the ability to adhere in vitro to human intestinal epithelial HT29 cells, but its spontaneous mutant strain (A-) lacks this property. Aim: To determine the potential antiinflammatory effect (TNF- $\alpha$ inhibition) of three known Lactobacillus strains and to investigate their mechanism of action. Methods: Macroscopically normal explants $(n=6)$ of colonic mucosa were obtained from patients undergoing colectomy for colonic neoplasia. Mucosal explants were stimulated ex vivo with PMA/lonomycin and incubated during 4 hours with 10E6 CFU/mL of test probiotic bacteria: Lactobacillus plantarum 299v (Lp),Lactobacillus plantarum 299vA- (LpA-), Lactobacillus casei ATCC334 (Lc) or no bacteria (blank). Release of TNF- $\alpha$ (ELISA), pH, and LDH enzyme (viability) were assessed in supernatant and relative quantification of the TNF- $\alpha$ mRNA was determined in the tissue. Mucosal colonisation by the probiotic bacteria was assessed by microbiological culture using the following formula (CFU in the tissue/ CFU in supernatant + tissue) $x$ 1000. The in vitro capacity of the probiotics to degrade TNF- $\alpha$ was tested in parallel by incubation with standard TNF- $\alpha$ in the absence of tissue. Results: All three strains decreased TNF- $\alpha$ release by the mucosa (\% inhibition versus blank, mean \pm sem: Lp $67 \pm 4.1 \%$ * LpA- $54 \pm 10.0 \%$, Lc $63 \pm 16.0 \%$ * ${ }^{*} p<0.001$ vs. blank). The three strains showed TNF- $\alpha$ degradation activity in vitro (\% degradation versus blank: Lp $68 \% \pm 13 *$, LpA- $66 \% \pm 3 *$, Lc $58 \% \pm 2 *,{ }^{*} p<0.001$ vs. blank) Both L.plantarum strains downregulated TNF- $\alpha$ gene expression, but Lc had no effect (relative TNF- $\alpha$ mRNA expressed as ratio test/blank, Lp $0.67 \pm 0.23$, LpA- $0.49 \pm 0.05^{*}$, Lc $0.89 \pm 0.22$ ${ }^{*} \mathrm{p}<0.05$ vs. blank). Mucosal colonization by the non-adherent strain LpA- was negligible (index of 0.7) and much lower than colonization by Lp (2.4) or Lc (35.7). Conclusion L.plantarum $299 \mathrm{v}$ (A-) shows a strain-specific regulatory effect on TNF- $\alpha$ gene expression. 
This effect is unrelated with epithelial adherence or mucosal colonization, which suggests mediation by a soluble anti-inflammatory substance of bacterial origin.

\section{M1789}

Cytomegalovirus Reverses the Normal Inflammation-Suppressing Effect of Intestinal Stroma On Blood Monocytes

Akhil Maheshwari, Lesley E. Smythies, Ronnie Clements, Yolanda Hartman, Meg Mosteller-Barnum, William J. Britt, Phillip D. Smith

Background: Cytomegalovirus (CMV)-mediated gastrointestinal disease is an important cause of morbidity and mortality in immunocompromised patients. CMV mucosal disease is characterized by prominent inflammatory cell infiltration despite the presence of relatively few CMV-infected cells. Since the absence of inflammation in normal intestinal mucosa is due, in part, to extra-cellular matrix (stromal) down-regulation of the pro-inflammatory function of monocytes newly recruited to the mucosa (JCI 2005; 115: 66), we hypothesize that CMV-induced mucosal inflammation is initiated when CMV infection of monocytes reverses the normal stroma-induced downregulation of chemokine and cytokine production. Reversal of the normal stroma-induced downregulation of IL-8 would enhance neutrophil recruitment to mucosal sites of inflammation. Methods: Blood monocytes were isolated by magnetic bead (MACS) separation or counterflow centrifugal elutriation. Lamina propria stromal tissue $(1 \mathrm{gm} / \mathrm{mL})$ was cultured in RPMI for 24 hrs to harvest stroma-conditioned media (S-CM). Monocytes were infected with a clinical (TR) or laboratory (AD169) isolate of CMV $(1 \mathrm{pfu} / \mathrm{mL})$. Cultures were treated with S-CM $(5-500 \mu \mathrm{g} / \mathrm{mL})$ for 2 hrs and then stimulated with LPS $(1 \mu \mathrm{g} / \mathrm{mL})$ for $1 \mathrm{hr}$. Monocyte IL-8 production was measured in supernatants after 24 hrs by ELISA (R\&D). Control CMV was UV-irradiated (to kill), heated (to inactivate), heparin-treated (to prevent viral binding to cell membrane heparan sulfate), or treated with CMV-treated serum (to inactivate). Results: Cultures of monocytes exposed to the TR and AD169 strains of CMV expressed CMV IE-1 gene product in $29 \%$ and $35 \%$ of the monocytes, respectively after $48 \mathrm{hrs}$ after infection. LPS increased monocyte IL-8 production ten-fold. Exposure to S-CM completely inhibited LPS-stimulated IL-8 production. However, CMV-infected monocytes, but not control CMV-infected monocytes, produced increased level of IL-8 (6-fold) following S-CM treatment and LPS stimulation. Conclusion: Our findings indicate that CMV-infection reverses the normal S-CM downregulation of monocyte chemokine production leading to an increase in inducible IL-8 production. Reversal of the normal stroma-induced downregulation of IL-8 provides a mechanism for enhanced neutrophil recruitment to mucosal sites of CMV infection.

\section{M1790}

Colonization with Helicobacter Bilis Increases Susceptibility to DSS-Induced Colitis in Gnotobiotic Mice

Abigail L. Henderson, Andrea Dorn, Zhiping Liu, Jennifer H. Wilson-Welder, Mark R. Ackermann, Albert E. Jergens, Michael J. Wannemuehler

Background: The etiology of inflammatory bowel diseases (IBD) including ulcerative colitis and Crohn's disease is unknown. In addition to genetic predisposition to IBD, the development of aberrant immune responses to the intestinal flora have been implicated in the pathogenesis of IBD. It is hypothesized that colonization of the intestinal tract with an environmental provocateur (e.g., Helicobacter bilis) may induce T cell responses to members of the normal flora that enhance susceptibility to colitis. Infection with Helicobacter has been associated with the development of IBD-like lesions in immunodeficient mice. The effect of H.bilis colonization on the development of host mucosal immune responses is poorly understood. Aim: To evaluate the mechanisms by which H.bilis colonization enhances susceptibility of DF mice to DSS-induced colitis. Methods: Groups of 6-8 week old, gnotobiotic C3H/ HeN:tac mice bearing the altered Schaedler flora (ASF) were colonized with H.bilis. Three to 20 weeks postinfection, mice were treated with low dose of DSS (1.5\%) in the drinking water for 5 days. Mice were euthanized 6 days after cessation of DSS treatment and evaluated for gross/histologic lesions and cell-mediated immune responses. Comparisons were made between DSS treated, H.bilis colonized, DSS/H.bilis (dual) treatment mice and non-treated, non-colonized (control) mice. Results: Gross cecal scores were significantly increased ( $\mathrm{p}<$ 0.05 ) in dual treated mice compared to mice in all other groups at all times post-colonization with H.bilis. Colon length of dual treated mice was significantly decreased $(\mathrm{p}<0.05)$ relative to control or H.bilis colonized mice. Cecal histopathology revealed increased mucosal cellularity, hyperplasia of lymphoid follicles and crypts, and moderate to severe epithelial erosions in the dual treated groups compared to all other groups. Mice colonized with H.bilis developed ASF antigen-specific T cell proliferative responses following in vitro stimulation. Sensitivity to DSS and the severity of lesions induced by DSS was correlated to the magnitude of the host response to the ASF antigens. Relative to control mice, cytokine analysis of cell culture supernatants showed increased secretion of IFN-Y and TNF- $\alpha$ for all 3 experimental groups. Additionally, increased antigen-specific secretion of IL-12 and IL-10 were observed in the dual group versus all other groups. Conclusion: These studies demonstrate that colonization of DF mice with H.bilis increased their susceptibility to DSS-induced colitis. The increased sensitivity to low dose DSS correlated with host immune responses as demonstrated by development of antigen-specific proliferation.

\section{M1791}

Antibiotic Therapy Temporaryly Suppresses Mucosal Bacterial Biofilms in IBD and Enhances Them Upon Cessation of Therapy

Alexander Swidsinski, Yvonne Doerffel, Vera Loening-Baucke, Laura P. Hale, Franz Theissig, Hartwig Andree, Herbert Lochs

Background: Antibiotics are commonly used in IBD for treatment of septic complications, abscesses, and wound infections. Little is known about the influence of antibiotics on the mucosal flora. Aims: To compare the concentrations and spatial organisation of mucosal bacteria in IBD patients treated with metronidazole and ciprofloxacin for different length of time with those who have not received antibiotics. Patients and methods: Six groups were investigated, including 20 patients each with ulcerative colitis or indeterminate colitis Groups were selected with regard to duration of/interval to antibiotic therapy: Group l= day of therapy; Group II $=7$ to 14 days of therapy; Groups III to V included patients 1 to 4,12 to 18 , and 26 to 36 weeks after the end of antibiotic therapy, respectively. The control group VI included patients without documented antibiotic therapy for $>2$ years. Biopsies from ileum, ascending colon and sigmoid colon were investigated using 40 different fluorescent in situ hybridization (FISH) probes that represented the diversity of the human intestinal flora. Results: No biofilms could be detected by rRNA-directed FISH in any of the biopsies obtained from patients during active treatment with antibiotics. However, adherent bacteria could be found in 12 patients of group I and two patients of group II using a DAPI stain that detects DNA. Prolific adherent biofilms could be observed after cessation of antibiotic therapy, with mean concentrations of mucosal bacteria of $13.2 \pm 4.3(\mathrm{p}<0.001) ; 5.8 \pm 2(\mathrm{p}<0.001) ; 1.1 \pm 0.8$ (ns); $0.5 \pm 0.4$ ( $\times 10^{10} /$ per milliliter) in groups III to VI, respectively. The composition of the biofilm differed significantly between these groups. For example, the Eubacterium rectale Clostridium coccoides bacterial group was the highest in the patients in group III, while Fusobacterium prausnitzii dominated in group IV. The concentration of both bacterial groups decreased in patient of groups V and VI. The concentration and occurrence of Enterobacteriaceae did not differ between patients in groups III to V, but was significantly higher $(\mathrm{p}<0.001)$ in these groups than in group VI with no exposure to antibiotics. Conclusions: Antibiotic treatment suppresses the viability of mucosal bacteria, however their concentrations rapidly increase upon antibiotic cessation to a level that is at least 10-fold higher than in patients not treated with antibiotics. Statistically significant changes in composition of mucosal bacteria can be found up to 8 month after the cessation of antibiotic therapy.

\section{M1792}

Alteration of TM7 Bacterial Phylogenies in Inflammatory Bowel Disease Tanja Kuehbacher, Ateequr Rehman, Ulrich R. Foelsch, Stephan J. Ott, Stefan Schreiber

Introduction: TM7 is a recently described subgroup of gram-positive unculturable bacteria originally found in natural environmental habitats. Recent studies have shown an association of TM7 bacterial division with inflammatory mucosal diseases, especially periodontitis and subgingival plaques. The TM7 family of bacteria is suggested to play an important role in the early stages of inflammatory mucosal processes, probably by modifying growth conditions for competing bacterial populations. Aims: To investigate the TM7 bacterial subgroup in inflammatory bowel diseases (IBD). Methods: Mucosal biopsy samples of patients with Crohn disease (CD) and ulcerative colitis (UC) were compared to healthy controls. The colonic mucosal TM7 consortia have been examined using molecular techniques based on the $16 \mathrm{~S}$ rDNA phylogeny, such as clone libraries, sequencing, and in-situ hybridization (ISH). Results: TM7 molecular 16S rDNA signatures could be cloned from mucosal samples of IBD patients and controls, but the composition of the clone libraries differed significantly $(\mathrm{p}<0.05)$ for both the $\mathrm{CD}$ and UC patients compared to healthy controls. Taxonomic analysis of the sequences revealed a higher diversity of TM7 subspecies in UC $(n=18)$ and CD $(n=16)$ compared to healthy individuals $(n=6)$. The clone libraries obtained from IBD patients showed a high number of novel sequences ( $n=27$ for $C D, n=10$ for $U C$ ) while no novel sequences were observed in healthy controls. A highly atypical base substitution for bacterial $16 \mathrm{~S}$ rRNAs associated with antibiotic resistance was detected in most of the TM7 sequences. In-situ hybridization of colon tissue section demonstrated the presence of TM7 group as filamentous bacteria. Conclusions: As previously demonstrated for oral inflammatory diseases, TM7 subgroup of bacteria might also play a pivotal role in inflammatory bowels diseases. The microbiotic shift of TM7 bacteria and the genetically determined antibiotic resistance of TM7 species in IBD could be a relevant part of a more general alteration of bacterial microbiota in IBD as recently found and could also be a basis for new therapeutic approaches

\section{M1793}

Changes in Mucosal Gene Expression Profiles Following Colonization of Gnotobiotic Mice with Helicobacter Bilis

Zhiping Liu, Andrea Dorn, Daniel S. Nettleton, Jennifer H. Wilson-Welder, Abigail L. Henderson, Albert E. Jergens, Michael J. Wannemuehler

Background: Dysregulation of host immune responses to gut microflora and genetic defects are considered as two important contributors to inflammatory bowel diseases. Helicobacter bilis is a gram-negative bacterium that has been shown to induce colitis in immunodeficient mice. Our data suggest that $H$. bilis infection can induce host immune responses to microflora without observable inflammation and increased susceptibility of mice to DSS-induced colitis. Aims: To define mucosal changes in gene expression in order to evaluate molecular mechanisms that may contribute to the increased susceptibility to colitis. Methods: Gnotobiotic $\mathrm{C} 3 \mathrm{H} / \mathrm{HeNTac}$ mice bearing the altered Schaedler flora were colonized with $\mathrm{H}$. bilis. As an acute inflammatory control, mice were treated with $2 \%$ dextran sulfate sodium (DSS) for 5 days. Mice were necropsied at 15,30 , or 45 days after $H$. bilis infection and 6 days after DSS treatment. Total RNA was isolated from mice ceca and converted into cRNA followed by submission to the Iowa State University Genechip Facility. The samples were evaluated for gene expression using Affymetrix Genechip Mouse Expression Set 4302.0 arrays. Data were analyzed with R software and Bioconductor package. Data were normalized by Robust Multi-array Average methods. Genes were selected by false discovery rate q value less than 0.1 and fold change more than 1.5 compared to negative controls. Results: 1) There were 3 gene expression patterns with the greatest changes in gene expression noted at 15 days postinfection (DPI). The gene expression pattern at $30 \mathrm{DPI}$ was similar to that at $45 \mathrm{DPI}$. 2) Innate immune response genes were notably upregulated (e.g., mast cell proteases and FcER) relative to other genes. 3) Series of interferon-regulated genes, including IFN-inducible GTPase P47 and P65 gene family, were significantly $(\mathrm{p}<0.05)$ upregulated at 15 DPI. Gene expression of IL-10, Stat 3 and Socs 3 were also significantly $(\mathrm{p}<0.05)$ at 15 DPI suggesting that there was no obvious Th1/Th2 bias in immune responses. 4) In contrast to DSS-induced colitis, gene expression induced by $\mathrm{H}$. bilis infection resulted in the upregulation of $\mathrm{B}$ cell differentiation and immunoglobulin genes. 5) Both DSS treatment and $\mathrm{H}$. bilis infection induced the downregulation of cytochrome P450 gene family. Conclusion: H. bilis infection induced upregulation of innate and acquired immune response genes in the absence of overt 
inflammation. Down-regulation of cytochrome P450 gene family may explain the increased susceptibility to DSS observed following H. bilis colonization.

\section{M1794}

Assessment of Immunological Ignorance Or Tolerance to Antigens-Derived from the Enteric Flora Prior to and After Infection with Helicobacter Bilis Jennifer H. Wilson-Welder, Albert E. Jergens, Charles Johnson, Jon Sullivan, Michael Drey, Andrea Dorn, Jesse M. Hostetter, Michael J. Wannemuehler

Background: The enteric flora is a critical co-factor in the pathogenesis of inflammatory bowel diseases. This is supported by increased serum antibodies to antigens derived from the flora (e.g., yeast antigens, $\mathrm{OmpC}$, flagellin) and that antibiotic treatment can ameloriate colitis. Data from our lab has previously shown that antigen-specific immune responses to the normal flora are increased following infection of gnotobiotic mice with Helicobacter bilis, a bacterial provocateur of colitis. It is unclear whether mechanism(s) governing the induction of host responses to the normal flora are related to a loss of mucosal tolerance or overcoming ignorance. Aim: These studies were designed to evaluate tolerance or ignorance to the host's enteric flora prior to microbial perturbations with $H$. bilis. We hypothesize that mucosal tolerance is broken following colonization with a novel bacterial species. This alteration induces local and systemic immune reactivity to resident bacterial antigens that contributes to the development of colitis. Methods: Bacterial antigens were prepared by acid gylcine extraction from each of the eight members of the altered Schaedler flora (ASF), H. bilis, or novel organisms (Escherichia coli, Staphylococcus epidermidis) isolated from healthy $\mathrm{C} 3 \mathrm{H} /$ HeOuJ mice. Immune responses (serum IgG, intestinal IgA, footpad delayed-type hypersensitivity (DTH) response and in vitro lymphocyte proliferation) to these antigens were evaluated in ASF flora-bearing naive, systemically primed, and $H$. bilis-infected mice. DTH responses were confirmed histologically. Results: Mice systemically primed with antigens derived from novel organisms exhibited a greater increase in serum antibodies and DTH response than naive mice or mice systemically primed with ASF antigens. Mice systemically primed with antigens from ASF 356, 360, 361, and 519 exhibited a very modest increase in serum antibody and little or no DTH response. ASF 500 and 492 induced both a significant increase in serum antibodies and DTH response in both naive and primed animals despite robust serum antibody response to 492 in the naive state. H. bilis colonization increased serum antibody, DTH responses, and lymphocyte proliferation to $\mathrm{H}$. bilis, normal flora, and novel antigens suggesting a heightened immune status in these mice. Conclusions: Gnotobiotic mice exhibit tolerance to their flora with heterogeneous levels of local and systemic reactivity. Heightened host immune responsiveness following selective colonization of gnotobiotic mice with $H$. bilis reflects dysregulation of tolerogenic mechanism(s) to the resident flora in a susceptible host.

\section{M1795}

Assessment of Microbial Diversity in Neoplastic Colonic Tissue Mairi E. Hope, Georgina L. Hold, Susan Berry, Mowat Ashley, Emad M. El-Omar

Background: The colonic microbiota is very diverse and is essential for promoting the host's health but imbalance of this microbiota has been implicated in the pathogenesis of colonic inflammation and colorectal cancer (CRC). Molecular techniques have recently shown that fecal microbial diversity does not accurately reflect mucosal populations, which are more relevant to colonic health and disease. Recent work shows that healthy individuals harbor their own unique microbial cohort that remains uniform throughout the healthy colonic mucosal surface. It is not known if this uniformity is disturbed in neoplastic lesions of the colon. Aims: To compare mucosal bacterial populations present in neoplastic (adenomatous polyp and CRC) tissue with immediately adjacent normal mucosa (less than $2 \mathrm{~cm}$ apart) using molecular techniques based on the 16S rRNA gene. Methods: Samples of paired mucosal biopsies from neoplastic and adjacent normal mucosa from the same individuals were obtained at colonoscopy (adenomatous polyps: $\mathrm{n}=72$ patients) and at surgical resection (CRC: $n=83$ patients). DNA was extracted from samples and subjected to PCR directed at the bacterial $16 \mathrm{~S}$ rRNA gene. Post-PCR analysis using denaturing gradient gel electrophoresis (DGGE) was used to generate microbial profiles for each paired sample set. Results: DGGE analysis showed the presence of bacterial DNA in both healthy and neoplastic tissues. Comparison of adjacent normal and neoplastic profiles showed that $40 \%$ of tubular adenomas and $70 \%$ of tubulovillous adenomas demonstrated altered microbial profiles. The more advanced polyps were more likely to harbor a different microbial profile from their paired normal tissue compared to the less advanced polyps $(\mathrm{p}<0.05$ ). Likewise, $60 \%$ of $\mathrm{CRC}$ samples had a different microbial profile compared to paired normal tissue, but the diversity did not correlate with Duke stage indicating that altered microbiota may play a role in progression of the earlier stages of neoplasia. Conclusions: To our knowledge this is the first demonstration of alterations in microbial diversity between paired normal and neoplastic colonic tissue. The perturbation of colonic microbial diversity in some neoplastic lesions may be a marker of disturbed homeostasis and may be relevant to the pathogenesis of CRC. The nature of the microbial diversity in normal and neoplastic tissue is currently under study.

\section{M1796}

Consumption of the Probiotic Bifidobacterium Infantis 35624 Influences Subsequent Immune Responses to in Vitro Challenge of Isolated Murine Peyer's Patch Cells with Bacterial Stimuli

Barbara Sheil, John MacSharry, Louise O'Callaghan, Avril O'Riordan, Alison Waters, John Morgan, John K. Collins, Liam O'Mahony, Fergus Shanahan

Background: The impact of Bifidobacterium infantis 35624 on proinflammatory cytokine production has been shown in models of enterocolitis. However, the influence of this probiotic in healthy animals and prior to onset of colitis has not been well investigated. Aims: To examine the effect of oral consumption of B. infantis 35624 on cytokine responses from isolated Peyer's patch cells following subsequent in vitro exposure to bacterial stimuli using wild-type (WT) mice and IL-10 knock-out mice prior to the onset of colitis. Methods:
Mice were fed B. infantis or placebo for 5 weeks. Peyer's patch cells were removed and cultured with and without Salmonella typhimurium. Cytokine production was assessed by ELISA. Results: Non-stimulated IFN- $\gamma$ secretion from Peyer's patch cells was significantly reduced for both WT and IL-10 KO mice following B. infantis 35624 consumption. However a striking difference between IL-10 KO and WT mice following probiotic consumption and in vitro stimulation with $S$. typhimurium was noted where IFN- $\gamma$ was significantly attenuated in IL-10KO mice but was significantly enhanced in WT mice. CCL2 was significantly reduced while CCL5 was significantly enhanced in Peyer's patch cells from IL-10 KO mice fed B. infantis 35624 following in vitro challenge. CCL5 and CCL2 levels did not change in WT mice following B. infantis 35624 consumption. Conclusions: Oral consumption of B. infantis 35624 can influence cytokine response in the Peyer's patch of healthy mice when subsequently exposed to a different organism. This response is not exclusively dependent on IL-10.

\section{M1797}

Increased Diversity of the Mucosa-Associated Fungal Microflora in Patients with Inflammatory Bowel Disease

Stephan J. Ott, Tanja Kuehbacher, Kenneth N. Timmis, Ulrich R. Foelsch, Stefan Schreiber

Introduction: An altered bacterial diversity on the intestinal mucosa may reflect the ne influence of life-style factors associated with the development of chronic inflammatory bowel diseases (IBD). While a reduced bacterial diversity as been reported in IBD, little is known about the fungal microflora, which is part of the fecal ecosystem. There is experimental and clinical evidence that fungi may be important trigger factors in the inflammatory pathophysiology. Aims: This study aimed at a systematic analysis of the intestinal fungal microflora in IBD. Patients and Methods: The mucosa-associated fungal microflora of 104 (colonic biopsy tissue of 47 controls and 57 IBD patients) was investigated using 185 rDNA based denaturant gradient gel electrophoresis (DGGE), clone libraries, sequencing, and fluorescence (FISH) or conventional in-situ hybridization. Results: Fungi-specific 18S rDNA signatures could be detected in all 104 patients. An overall fungal biodiversity of 43 different operational taxonomic units (OTUs) was found in the clone libraries. The qualitative composition of the fungal microflora was different between patients with IBD and controls. The DGGE profiles revealed a higher mean fungal diversity in the Crohn disease (CD) patients in comparison with controls $(10.8 \pm 3.1$ vs. $6.2 \pm 2.4$ for $C D, p=0.001)$. A separate analysis showed no association of fungal diversity with drug treatment or histological activity. Conclusions: A high number of fungal species is part of the normal enteric microflora, but diversity was increased and composition of the fungal communities varies in IBD. Further work is needed to investigate whether the alteration of the fungal flora in IBD is secondary to an imbalanced bacterial microflora or a independent etiologic factor.

\section{M1798}

\section{Role of the Reduced Folate Carrier (RFC) in Intestinal Folate Uptake} Krishnaswamy Balamurugan, Hamid M. Said

Background: Humans and other mammals cannot synthesize folate, and thus, they must obtain the vitamin from exogenous sources. We have previously investigated different aspects of intestinal folate uptake process and have cloned the RFC from human and mouse intestine and characterized its function in homologus and heterologus cellular systems (e.g., Gastroenterology. 112:783-91, 1997; J. Biol. Chem. 272:6226-31, 1997; J Biol. Chem. 277:3332533, 2002). To date, however, little is known about the contribution of the RFC system toward total carrier-mediated folate uptake by intestinal epithelial cells. Attempts to generate RFC knock out mice model to address such an issue have proven to be un-successful due embryonic lethality. Here we describe the use of the new approach of lentiviral- mediated short hairpin RNA (shRNA) to selectively silence the endogenous RFC and examined the effect of such silencing on intestinal carrier-mediated folate uptake. Methods: The rat-derived intestinal epithelial IEC-6 cells and RFC specific shRNA were used in this study. Levels of RFC mRNA and protein were determined by mean of Real-time PCR and Western blotting using RFC specific polyclonal antibodies. Results: Initial rate of uptake of $16 \mathrm{nM} 3[\mathrm{H}]$-folic acid in un-treated cells showed a marked increase as a function of lowering incubation buffer $\mathrm{pH}$ from 8.0 to 5.5. Addition of unlabelled folic acid to the incubation buffer lead to a severe inhibition in initial rate uptake of $3[\mathrm{H}]$-folic acid $(16 \mathrm{nM})$ at incubation buffer $\mathrm{pH} 5.5$ ( $95 \%$ ) but not at pH 7.4. Treating IEC-6 cells (for 72 hrs)with RFC specific shRNA lead to a severe reduction ( 80 and $78 \%$ ) in the endogenous levels of RFC mRNA and protein levels, respectively. Such a treatment also led to a severe inhibition $(\sim 90 \%)$ in carriermediated folate uptake at $\mathrm{pH} 5.5$ but not at $\mathrm{pH}$ 7.4. On the other hand, uptake of the unrelated biotin was not affected by treating the cells with RFC specific shRNA. Conclusions The results show that the carrier-mediated folate uptake system in intestinal epithelial cells is functional at acidic $\mathrm{pH}$ and that RFC is the major (if not the only) folate uptake system that is involved in intestinal folate absorption. [Supported by the grants from DVA and NIH (DK58057 and DK56061)]

\section{M1799}

Mechanism of Increased Intestinal $\mathrm{Na}^{+} / \mathrm{Glucose} \mathrm{Co}-$ Transporter (SGLT1) Expression in Human Type 2 Diabetes

J.N. Dyer, Anthony Ellis, Soraya P. Shirazi-Beechey

We have reported previously that there is a coordinated 4-fold increase in the expression of the luminal membrane monosaccharide transporters SGLT1 and GLUT5, and 3-fold increase in basolateral membrane GLUT2 in the small intestine of human type 2 diabetic patients. This increase is independent of blood glucose and insulin levels as low carbohydrate diet and the taking of sulfonyl urea or metformin drugs had no effect on reducing expression levels. In this work we set out to determine the mechanism(s) involved in this up-regulation. In order to perform nuclear run-on experiments we first had to modify current protocols for the isolation of intact nuclei to be applicable to duodenal biopsies (approximately $5 \mathrm{mg}$ wet weight). Furthermore we dramatically scaled down nuclear run-on assays to successfully 
produce strong, reproducible signals from minimal numbers of nuclei. Biopsies were removed, during routine endoscopy, from the second part of the duodenum of both diabetic and healthy individuals, with the permission of the Liverpool Ethics Committee and the written informed consent of the patients. Individual biopsies (both control and diabetic) yielded an average of $1.25 \times 10^{6}$ nuclei and nuclear run-on experiments with $3 \times 10^{6}$ nuclei produced around $10 \mathrm{kBq}$ of purified, newly synthesised [ $\left.\alpha^{32} \mathrm{P}\right] \mathrm{UTP}$ labelled, RNA. Three separate experiments, each utilising nuclei from two control and two diabetic patients, were carried out, and equal amounts of radiolabelled RNA were hybridised at $55^{\circ} \mathrm{C}$ for $20 \mathrm{~h}$ to $5 \mu \mathrm{g}$ of cDNAs for SGLT1, GLUT2, GLUT5, HNFl $\alpha$ (transcription factor involved in the glucose-regulation of SGLTl transcription) and $\beta$-actin (control) loaded onto nylon membrane using a slot blotter. Results show that the rate of SGLTl transcription is 4- to 4.5fold higher in nuclei isolated from diabetic duodenal biopsies than controls, indicating that the increase in the expression of SGLTl in the intestine of diabetic individuals is entirely due to increased SGLT1 transcription. However, the transcription rates for GLUT2 and GLUT5, along with HNFl $\alpha$, were essentially unchanged suggesting that post-transcriptional mechanisms are involved in changes seen in the expression of these genes.

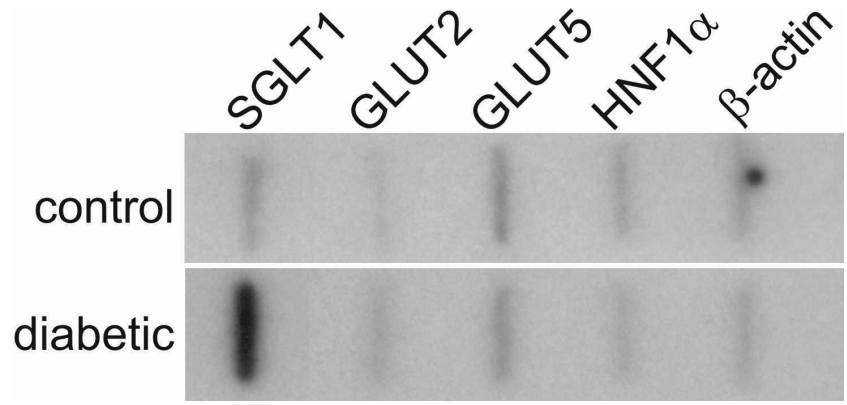

\section{M1800}

Calcium-Activated Potassium Channels in Human Colonic Crypts: A Target for the Treatment of Secretory Diarrhea?

John E. Linley, Arun Loganathan, Malcolm Hunter, Geoffrey I. Sandle

Background: In rat and human distal colon, $\mathrm{Ca}^{2+}$-activated $\mathrm{K}^{+}$channels are involved in $\mathrm{K}^{+}$and $\mathrm{Cl}^{-}$secretion. Apical large conductance (BK or KCNMAl) channels are a critical component of active $\mathrm{K}^{+}$secretion, whereas basolateral intermediate conductance (IK or KCNN4) channels contribute to maintaining the driving force for $\mathrm{Cl}^{-}$secretion, which is mediated by CFTR located in the apical membrane. In many examples of secretory diarrhea, an increase in intracellular $\mathrm{Ca}^{2+}$ concentration is the dominant factor involved in eliciting the $\mathrm{K}^{+}$and/or $\mathrm{Cl}^{-}$secretory responses, which depend on the relative magnitudes of the apical and basolateral $\mathrm{K}^{+}$conductances. Aims: To quantify the $\mathrm{Ca}^{2+}$-sensitive ion conductances of the apical and basolateral membranes of human colonic crypts. Methods: Human distal colonic biopsies were obtained with consent from patients undergoing routine colonoscopy as part of the investigation of altered bowel habit. In all cases, the colonic mucosa was normal macroscopically and histologically. Crypts were isolated by $\mathrm{Ca}^{2+}$ chelation (Bowley KA et al. Gut 52,854-860,2003). Whole-cell currents were measured from the middle third of crypts using the perforated patch-clamp technique $(240 \mu \mathrm{g} / \mathrm{ml}$ amphotericin in pipette). Results: Addition of the $\mathrm{Ca}^{2+}$ ionophore, ionomycin $(1 \mu \mathrm{M})$, increased whole-cell conductance from $1.9 \pm$ $0.4 \mathrm{nS}$ to $4.8 \pm 1.0 \mathrm{nS}(\mathrm{P}<0.0005 ; \mathrm{n}=17)$, which was accompanied by cell hyperpolarization from $-47 \pm 6 \mathrm{mV}$ to $-70 \pm 4 \mathrm{mV}$. The $\mathrm{Ca}^{2+}$-stimulated whole-cell current was inwardly rectifying with a reversal potential of $-70 \mathrm{mV}$, approaching the equilibrium potential for $\mathrm{K}^{+}$ $(-87 \mathrm{mV})$, indicating that it was predominantly $\mathrm{K}^{+}$selective. Addition of $100 \mathrm{nM}$ penitrem A a selective inhibitor of $\mathrm{BK}$ channels) in the presence of ionomycin reduced cell conductance from $5.3 \pm 0.5 \mathrm{nS}$ to $3.7 \pm 0.7 \mathrm{nS}(\mathrm{P}<0.05 ; \mathrm{n}=8)$. Subsequent addition of $10 \mathrm{uM}$ clotrimazole (an inhibitor of IK channels) abolished the penitrem A-insensitive conductance $(\mathrm{P}<0.01$; $\mathrm{n}=8$ ). Post-penitrem A and clotrimazole, cell conductance was $1.0 \pm 0.4 \mathrm{nS}$, with a reversal potential for the whole-cell current of $-39 \mathrm{mV}$, close to the equilibrium potential for $\mathrm{Cl}^{-}$($35 \mathrm{mV}$ ). Thus, the ionomycin-stimulated whole-cell conductance reflects activation of IK and BK channels, without any significant contribution from $\mathrm{Cl}^{-}$channels. Conclusions: In human colonic crypts, the dominant effect of increased intracellular $\mathrm{Ca}^{2+}$ concentration on ion channels is the activation of basolateral IK channels. This would hyperpolarize the cell, thereby increasing the driving force for $\mathrm{Cl}^{-}$secretion. IK channels are therefore a potential target for the treatment of secretory diarrheal diseases.

\section{M1801}

Prevention of Taurodeoxycholate (TDC) Cytotoxic Effect On Human Colon Mucosa By Sphingomyelin. Electrophysiological Studies in Ussing Chambers Piero Portincasa, Anna Diana, Laura Castellano, Antonio Moschetta, Helen H. Wang. David Q. Wang, Francesco Marino, Donato F. Altomare, Giuseppe Palasciano, Lucantonio Debellis

The secondary cytotoxic bile salt TDC has been shown to impair colonic ionic transport in animals and cell lines, but little is known in humans. Detrimental effects of TDC include prosecretory and detergent actions with relevance to clinical conditions such as bile salt malabsorption and colon cancer. Dietary sphingomyelin (SM) is resistant to intestinal hydrolysis and by reaching the distal colon may protect against bile salt-induced cytotoxic effects. Aims: We studied the human colon in Ussing chambers to assess if TDC-induced changes in human colonic epithelial function could be prevented by SM. Methods: The effects of TDC $(1,2,4$, and $8 \mathrm{mM})$ on electrophysiological parameters of human colon mucosa bathed in Krebs solution were studied in the absence or with the addition of $\mathrm{SM}$ at 0.1 to $0.3 \mathrm{SM}$ (SM+TDC) ratio. Transepithelial potential (Vt), resistance (Rt) and short circuit current (Isc) were measured by a computerized voltage clamp system in the Ussing chamber. Results: In
129 sigmoid colon specimens, basal Vt and Rt were $-3.5 \pm($ S.E. $) 0.2 \mathrm{mV}$ and $117 \pm 5 \Omega * \mathrm{~cm} 2$ respectively and Isc was $33.5 \pm 2.2 \mu \mathrm{A} / \mathrm{cm} 2$. With TDC $8 \mathrm{mM}(\mathrm{n}=11)$, Isc increased by $84.7 \pm 25.5 \%(\mathrm{p}<0.002)$ and Rt decreased by $46.9 \pm 5.25 \%(\mathrm{p}<0.001)$ while Vt remained virtually unchanged. Similar but less pronounced effects were observed with TDC 2 and 4 $\mathrm{mM}$, where Isc increased significantly by $18 \%(\mathrm{p}<0.02 \mathrm{n}=13)$ and $32 \%(\mathrm{p}<0.05 \mathrm{n}=11)$ respectively, while Rt was reduced by about $20 \%$; TDC $1 \mathrm{mM}$ was uneffective. SM prevented almost completely TDC 2 and $4 \mathrm{mM}$ changes of Isc and Rt. In the presence of the phospholipid, TDC $8 \mathrm{mM}$ increased Isc by only $27 \%$ and $5 \%$ at ratio $0.1 \quad(n=5)$ and $0.3(n=5)$ respectively. Incubations with SM alone $(4.28 \mathrm{mM})$ did not affect colonic epithelial transport function. Conclusions: In the human colon, the Ussing chamber model can accurately estimate prosecretory and detergent activities of secondary bile salts (e.g. TDC) within normal and supraphysiological concentrations. For the first time we show here that SM prevents TDC-induced epithelial transport alterations in human colon, possibly by decreasing free bile salt activity in the medium. Our findings may have implications for dietary manipulation during bile salt malabsorption or colon cancer prevention.

\section{M1802}

Acute Ileal Inflammtion Alters CFTR-Mediated Anion Secretion Sadasivan Vidyasagar, Hui Zhang, Nadia Ameen, Franca Golin-Bisello, James Melvin, Srisaila Basavappa

In vivo treatment of mice with anti-CD3 mAb is an established model of T-cell-mediated acute inflammation, characterized by increased pro-inflammatory cytokines, decreased absorption and acute diarrhea. In addition, increased fluid secretion by crypt cells during acute inflammation may also contribute to the diarrhea. As intestinal fluid secretion is driven by $\mathrm{CFTR} \mathrm{Cl}^{-}$conductane, we investigated the alterations in $\mathrm{Cl}^{-}$and $\mathrm{HCO}_{3}$ transport mediated by CFTR channels. For these studies, ileal tissues were removed three hours after intraperitoneal injection of anti-CD3 mAb or sham-injected mice. In Ussing chamber studies, the basal Isc was increased significantly by $260 \%$ in anti-CD3 injected mice. The subsequent exposure to forskolin significantly increased Isc in both sham- and anti-CD3-injected mice. However, the cAMP-mediated increase in Isc was significantly lower in anti-CD3 mAbinjected mice when compared to that of the control. Addition of $300 \mu \mathrm{M}$ glibenclamide abolished the cAMP-stimulated increase in Isc in both control and anti-CD3 mAb injected mice, suggesting CFTR mediated conductance. In contrast, in CFTR KO mice, the basal Isc was reduced to $5.9 \pm 0.9 \mu \mathrm{A} / \mathrm{cm} 2$ as compared to $11 \pm 1.2 \mu \mathrm{A} / \mathrm{cm} 2$ in WT mice. Treatment of CFTR KO mice with anti-CD3 did not alter the basal Isc. Furthermore, exposure to forskolin did not significantly increase Isc in either sham- or anti-CD3 injected CFTR KO mice. As CFTR is known to be permeable to $\mathrm{HCO}_{3}$, we investigated the effect of anti-CD3 treatment in CFTR KO mice using the $\mathrm{pH}$ stat method. In the presence of lumen $\mathrm{Cl}^{-}, \mathrm{HCO}_{3}$ secretion was $4.2 \pm 0.2$ and $4.6 \pm 0.2 \mu \mathrm{eq} / \mathrm{cm} 2 / \mathrm{h}$ in WT and CFTR KO mice respectively. Removal of lumen $\mathrm{Cl}^{-}$abolished $\mathrm{HCO}_{3}$ secretion in both WT and CFTR KO mice, suggesting ileal electroneutral $\mathrm{HCO}_{3}$ transport is not significantly altered in CFTR $\mathrm{KO}$ mice. Injection of anti-CD3 decreased $\mathrm{HCO}_{3}$ secretion by $\sim 31 \%$ and $8 \%$ in WT and CFTR $\mathrm{KO}$ mice, respectively, in the presence of luminal $\mathrm{Cl}^{-}$. The subsequent removal of luminal $\mathrm{Cl}^{-}$in WT mice did not abolish $\mathrm{HCO}_{3}$ secretion but decreased it by $~ 55 \%$, whereas as in CFTR KO mice $\mathrm{HCO}_{3}$ secretion was abolished. This suggests that CFTR-mediated $\mathrm{HCO}_{3}$ secretion is enhanced during acute inflammation. The role of CFTR playing a pivotal role during acute ileal inflammation was further confirmed as the protein expression of CFTR was significantly increased in vivo in anti-CD3 treated ileal crypts. In addition, cAMP levels as detected by immunoassay were elevated $\sim 3.7$ fold in anti-CD3 injected mice. Conclusion. The increased $\mathrm{Cl}^{-}$and $\mathrm{HCO}_{3}$ secretion via CFTR during acute ileal inflammation may contribute to the Tcell mediated acute diarrhea

\section{M1803}

Epidermal Growth Factor Chronically Enhances Colonic Epithelial Secretory Capacity By Upregulating NKCCl Expression

Fiona O'Mahony, Kim E. Barrett, Stephen J. Keely

Background: Although epidermal growth factor (EGF) is well known to promote intestinal epithelial barrier function, its role in regulating transport function is less well understood. Our previous data show that EGF chronically enhances epithelial responsiveness to both $\mathrm{Ca}^{2+}$ and cAMP-dependent secretagogues. Here we set out to examine the molecular mechanisms involved. Methods: $\mathrm{T}_{84}$ colonic epithelial cells were grown as polarized monolavers on permeable supports. Changes in $\mathrm{I}_{\mathrm{sc}}$, indicative of electrogenic ion transport, were measured by the Ussing chamber/voltage clamp technique. Protein expression was measured by Western blotting and real time PCR, respectively. Intracellular $\mathrm{Ca}^{2+}$ was measured by Fura- 2 fluorescence and protein kinase A (PKA) activity with a commercially available kit. Results: Acute treatment of $\mathrm{T}_{84}$ cells with $\mathrm{EGF}(100 \mathrm{ng} / \mathrm{ml} ; 15 \mathrm{~min})$ chronically enhanced subsequent $\mathrm{I}_{\mathrm{sc}}$ responses to the $\mathrm{Ca}^{2+}$ - dependent agonist, carbachol $(\mathrm{CCh}, 100 \mu \mathrm{M})$. This effect was only apparent after 3 hrs and was maximal by 6 hrs after stimulation with EGF at which time responses to CCh were $206.1 \pm 28.3 \%$ of those in control cells $(n=4 ; p<0.01)$. The potentiating effect of EGF was sustained for at least $24 \mathrm{hrs}$. The $\mathrm{Na} / \mathrm{K} / 2 \mathrm{Cl}$ cotransporter (NKCCl) inhibitor, bumetanide $(100 \mu \mathrm{M})$, abolished the effect of EGF, indicating $\mathrm{Cl}^{-}$ secretion to be involved. Neither basal nor agonist-stimulated levels of intracellular $\mathrm{Ca}^{2+}$ or PKA activity were altered by EGF pretreatment, implying the effects of the growth factor are not due to chronic alterations in levels of second messengers. Western blot analysis revealed that $\mathrm{EGF}$ increased the expression of $\mathrm{NKCCl}$ with a time course that closely paralleled its effects on $\mathrm{Cl}^{-}$secretion. This effect of EGF was maximal after $6 \mathrm{hrs}$, at which time NKCCl expression in EGF-treated cells was $199.9 \pm 21.9 \%$ of that in control cells $(\mathrm{n}=21 ; \mathrm{p}<0.0001$ ). EGF also increased the CFTR expression (by $3.9 \pm 1.1$ fold; $\mathrm{n}=5 ; \mathrm{p}$ $<0.01$ ) but not that of the Na/K ATPase pump. EGF-induced NKCCl expression was abolished by actinomycin D, implying EGF upregulates NKCCl expression at the level of gene transcription. Conclusions: These data reveal a novel role for EGF in chronically regulating epithelial secretion at the fundamental level of transport protein expression. EGF enhances epithelial secretory capacity by inducing $\mathrm{NKCCl}$ expression, an effect which in vivo would serve to promote epithelial hydration, thereby enhancing barrier function. These 
data support the idea that further elucidation of EGF-dependent signalling pathways will yield new targets for drug development in treatment of intestinal transport disorders.

\section{M1805}

The Intestinal Anion Exchanger DRA (Down Regulated in Adenoma) Is Inhibited By Intracellular Calcium By Not By PMA

Georg Lamprecht, Julia Schaefer, Michael Gregor

Introduction: In the intestine $\mathrm{NaCl}$ is absorbed by parallel $\mathrm{Na} / \mathrm{H}$ exchange and $\mathrm{Cl} / \mathrm{HCO}_{3}$ exchange. The respective proteins are NHE 3 and DRA (down regulated in adenoma = SLC26A3). In the native tissue and in Caco-2 cells NHE3 is inhibited by increased intracellular calcium. This mechanism is mediated by the interaction of NHE3 with the PDZ adapter protein E3KARP (NHE3 kinase A regulatory protein) involving $\alpha$-actinin- 4 and protein kinase $\mathrm{C}$. The interaction of NHE3 with the calcineurin homologous protein (CHP) may also mediate calcium inhibition. Aim: Is DRA also inhibited by intracellular calcium? Methods: DRA activity was studied in HEK cells stably expressing an EGFP-DRA construct. $\mathrm{Cl} / \mathrm{HCO}_{3}$ exchange was measured as transients of the intracellular $\mathrm{pH}\left(\mathrm{pH}_{\mathrm{i}}\right)$ upon $\mathrm{Cl}$ removal and $\mathrm{Cl}$ readdition in the presence of $5 \% \mathrm{CO}_{2} / \mathrm{HCO}_{3}$. Results: In these transfected cells exogenous DRA activity was 8-times higher than endogenous $\mathrm{Cl} / \mathrm{HCO}_{3}$ exchange. Raising the intracellular calcium by incubation with $10 \mu \mathrm{M} 4-\mathrm{Br}-\mathrm{A} 23187$ inhibited intracellular alkalinization by $45 \%$ upon $\mathrm{Cl}$ removal $(0.013 \pm 0.004 \mathrm{pH} / \mathrm{s}, \mathrm{n}=8$ vs. $0.007 \pm 0.003 \mathrm{pH} / \mathrm{s}, \mathrm{n}=9 ; \mathrm{p}<0.05)$. $10 \mu \mathrm{M} 4-\mathrm{Br}-\mathrm{A} 23187$ did not change basal intracellular $\mathrm{pH}_{\mathrm{i}}(7.28 \pm 0.12$ vs $7.32 \pm 0.06)$. But the maximal alkalinization was reduced from $7.89 \pm 0.09$ to $7.68 \pm 0.09(p<0.05)$. Similar effects were seen with $5 \mu \mathrm{M}$ ionomycin. Preincubation with $100 \mathrm{nM}$ phorbolesther (PMA) for 20 minutes did not inhibit DRA ( $\mathrm{n}=4-5$ for each group). In HEK cells, which do not express CFTR, DRA is also not influenced by stimulation of protein kinase $\mathrm{A}$ by forskolin. When a clonal cell line was measured that expresses an EGFP-DRA construct that lacks the C-terminal PDZ interaction motif (EGFP-DRA-ETKFminus) there was a tendency for stronger inhibition compared to the wild type construct. Conclusion: DRA is inhibited by increased intracellular calcium but the effect is not mimicked by direct activation of protein kinase C. This suggests a direct effect of calcium on DRA, that also does not require downstream activation of protein kinase $\mathrm{C}$. Preliminary data indicate that the effect of calcium does not require the interaction of DRA with the endogenous PDZ adapter proteins of HEK cells. This may be due to the relative overexpression of DRA compared to an interacting PDZ adapter protein. Alternatively other mechanisms may mediate the effect of calcium.

\section{M1806}

\section{SCFA-Dependent Colonic Transport Alters cAMP-Stimulated Ileal HCO3} Secretion in in Vivo Ileal and Colonic-Dual Loop Model

Sadasivan Vidyasagar, Srisaila Basavappa

In vitro $\mathrm{pH}$ stat studies of $\mathrm{HCO} 3$ secretion in stripped colonic sheets established short chain fatty acid (SCFA)-stimulated $\mathrm{HCO} 3$ secretion that is lumen $\mathrm{Cl}$ - independent. SCFA inhibited both $\mathrm{Cl}-\mathrm{HCO} 3$ exchange and cAMP-stimulated lumen $\mathrm{Cl}$-independent $\mathrm{HCO} 3$ secretion in rat distal colon (Am J Physiol Gastrointest Liver Physiol 288: G1217-G1226, 2005). Downregulation of $\mathrm{Cl}$-dependent and cAMP-induced $\mathrm{HCO} 3$ secretion was established to be secondary to the presence of SCFA and not due to one of its metabolite. However, exact mechanism by which SCFAs alter electrolyte transport is, however, not known. Ileal HCO3 secretion was measured in vivo using bi-burette $\mathrm{pH}$ stat technique in which lumen solution was continuously titrated and re-circulated using a peristaltic pump. Simultaneously ligated colonic loops were made which were then incubated with regular Ringer or with a SCFAcontaining solution. In the presence of lumen $\mathrm{Cl}$, there was significant $\mathrm{HCO} 3$ secretion (4.3 $\pm 0.13 \mu \mathrm{eq} / \mathrm{cm} 2 / \mathrm{h})$ in the ileum. Addition of $100 \mu \mathrm{M} \operatorname{DIDS}(0.25 \pm 0.03 \mu \mathrm{eq} / \mathrm{cm} 2 / \mathrm{h})$ or removal of lumen $\mathrm{Cl}$ abolished ileal $\mathrm{HCO} 3$ secretion $(0.12 \pm 0.03 \mu \mathrm{eq} / \mathrm{cm} 2 / \mathrm{h})$ indicating a basal $\mathrm{Cl}$-dependent $\mathrm{HCO} 3$ secretion. Addition of dibutryl cAMP to the colonic lumen solution resulted ileal lumen $\mathrm{Cl}$-independent $\mathrm{HCO} 3$ secretion $(3.7 \pm 0.11)$ that was inhibited by 10 $\mu \mathrm{M}$ NPPB $(0.01 \pm 0.01 \mu \mathrm{eq} / \mathrm{cm} 2 / \mathrm{h})$ and by $300 \mu \mathrm{M}$ glubenclamide $(0.05 \pm 0.01 \mu \mathrm{eq} / \mathrm{cm} 2 /$ h). In the presence of SCFA in colonic loop, glibenclamide had no effect on dibutryl cAMPstimulated $\mathrm{HCO} 3$ secretion in the ileal loop $(3.7 \pm 0.21$ vs $3.5 \pm 0.16 \mu \mathrm{eq} / \mathrm{cm} 2 / \mathrm{h})$ indicating that colonic SCFA had inhibited cAMP-stimulated HCO3 secretion in ileum. However, presence of SCFA in colonic loop did not alter lumen Cl-dependent, DIDS-sensitive HCO3 secretion in the ileal loop $(4.3 \pm 0.14$ and $4.7 \pm 0.11 \mu \mathrm{eq} / \mathrm{cm} 2 / \mathrm{h})$. Summary: 1$)$ presence of SCFA in a colonic loop inhibits cAMP-stimulated $\mathrm{HCO} 3$ secretion, but does not alter $\mathrm{Cl}$ dependent $\mathrm{HCO} 3$ secretion. We conclude that colonic SCFA are essential in regulating electrolyte transport at a site proximal to their production and play a more active role in a secretory state than under basal conditions.

\section{M1807}

Decreased Expression of Breast Cancer Resistance Protein (BCRP) in the Duodenum of Patients with Obstructive Cholestasis

Petr Hruz, Christian Zimmermann, Heike Gutmann, Luigi Terracciano, Lukas Degen, Ulrich Beuers, Christoph Beglinger, Juergen Drewe

Human breast cancer resistance protein (BCRP/ABCG2) is present on the apical membranes of the liver, in epithelial cells of the small and large intestine, and in kidney cells; the transporter mediates energy dependent efflux of drugs and potentially toxic compounds, and of various endogenous substrates such as bile acids. During obstructive cholestasis with a disrupted enterohepatic circulation, an adaptive regulation of several transporters for bile acids, bilirubin and cholesterol has been documented (Hepatology 2002;35:732f; Gastroenterology 2004;126:1044f; Gut 2006; in press). An adaptive regulation of human intestinal BCRP during obstructive cholestasis has, however, not been described before. Aim: The aim of this study was therefore to investigate the expression of $\mathrm{BCRP}$ in the duodenum of healthy controls and compare the results to patients with obstructive cholestasis. Methods: BCRP mRNA was quantified by real time PCR (Taqman) in duodenal biopsies of 14 control subjects and compared to the duodenal BCRP mRNA expression of 19 patients with obstructive cholestasis. In addition, duodenal BCRP mRNA expression was measured in 4 patients with obstructive cholestasis after reconstitution of bile flow. BCRP protein levels were determined in 6 cholestatic patients and in 6 healthy control subjects by immunohistochemistry. Finally, fasting bile acid and bilirubin concentrations were determined by specific assay systems. Results: Normalized duodenal BCRP mRNA expression (mean BCRP/Villin \pm SEM) was 0.51 $( \pm 0.03)$ in controls and $0.27( \pm 0.02)$ in cholestatic patients $(p<0.001)$. In a semiquantitative analysis of immunohistochemical protein assessment $(0=$ no expression, $1=$ low, $2=$ intermediate, 3 = high expression) mean BCRP protein expression (mean \pm SEM) was significantly reduced in 6 patients with obstructive cholestasis $(1.67 \pm 0.38)$ when compared to 6 healthy control subjects $2.91( \pm 0.08)(p<0.005)$. Bilirubin and bile acid plasma concentrations were inversely correlated with BCRP mRNA expression $(\mathrm{p}<0.05)$. After reconstitution of bile flow, bile acid and bilirubin plasma levels returned to normal and BCRP mRNA expression increased by 1.44-, 1.71-, 1.05-, and 1.90-fold, respectively ( $\mathrm{N}=4$ patients) Conclusion: $\mathrm{BCRP}$ is down-regulated in the human duodenum during obstructive cholestasis. We infer that a reduced intestinal BCRP expression during cholestasis influences the accumulation of bile acids, food-derived carcinogens, and the pharmacokinetics of various drugs that are transported by BCRP.

\section{M1808}

Short-Term Regulation of Intestinal Epithelial Type $2 \mathrm{Na}+\mathrm{K}+$ Exchanger By Serotonin

Fernando Magro, Patricio Soares-da-Silva

5-HT plays a critical role in the regulation of gastrointestinal motility, secretion, and sensation. 5-HT initiates peristaltic and secretory reflexes in gastrointestinal tract. The present study evaluated the effect of serotonin (5-HT) on intestinal $\mathrm{Na}+\mathrm{H}+$ exchange activity and the cellular signaling pathways involved in T-84 cells. T-84 cells express endogenous NHEl and NHE2 proteins, detected by immunoblotting. Eipa (IC50 $=519[465,579] \mathrm{nM})$ was more potent than cariporide (IC50 $=630[484,819] \mathrm{nM}$ ) and amiloride $(\mathrm{IC} 50=19[16,24], \mu \mathrm{M})$ on NHE activity inhibition. This different inhibitory sensitivity indicates that NHE 2 is a dominant isoform in T-84 cells. Short term exposure $(0.5 \mathrm{~h})$ of T-84 cells to 5-HT-induced an increase NHE activity in a concentration-dependent manner (vehicle: $0.0063 \pm 0.0005 \mathrm{Vs}$ 5-HT: $0.0090 \pm 0.0005 ; \mathrm{pH}$ units/s). This effect was not affected by cariporide $(300 \mathrm{nM})$ and S-3226 (300 nM). The next series of experiments were intended to explore the receptor and the signaling pathways from 5-HT stimulation. The stimulation induced by 5-HT was completely abolished by WAY $100135(300 \mathrm{nM})$ and Ketanserin (300nM), antagonists of 5 HTIA and 5-HT2A receptors, respectively. In the presence of 8-OH-DPAT and alpha-metil5-HT, agonists of 5-HT1A and 5-HT2A receptors, respectively, the NHE activity was increased (vehicle: $0.006330 \pm 0.0004$ Vs 8-OH-DPAT: 0.0093 \pm 0.0008 , alpha-metil- $5-\mathrm{HT}$ : $0.009 \pm 0.0003 ; \mathrm{pH}$ units/s). The coincubation with 5 -HT and pertussis toxin abolished the effects of 5-HT. Therefore the cells were treated with antibodies anti Gi, Gs and Gq/11. The effect induced by 5 -HT was blocked by both anti- $\mathrm{Gi}$ and $\mathrm{Gq} / \mathrm{ll}$. In contrast, the overnight treatment with antibodies anti- $\mathrm{Gi}$ and $\mathrm{Gq} / \mathrm{ll}$ only blocked the stimulatory effect of $8-\mathrm{OH}$ DPAT and alpha-metil-5-HT, respectively. Finally, the pretreatment with anti-bodies agains to alpha Gi -1,2 prevented the NHE stimulatory effect induced by 5-HT and 8-OH-DPAT Conclusions: Serotonin increases intestinal NHE isoform 2 activity by stimulation of 5-HTIA and 5-HT2A receptors,

\section{M1809}

\section{Leptin Inhibits $\mathrm{Na}+\mathrm{H}+$ Exchanger NHE3} Dongsheng Wang, Jennifer Johnson, Chris Yun

The epithelial brush border $\mathrm{Na}+\mathrm{H}+$ exchanger isoform-3 (NHE3) in the intestine and kidney is regulated by a wide variety of hormones and growth factors. Leptin is a hormone mainly secreted by the adipose tissue. Although the effects of leptin are mediated primarily via the central nervous system, studies have shown that nonadipose tissues, such as gastric and intestinal epithelia, can produce leptin, suggesting that leptin has effects on other organs. Previous studies have shown that leptin stimulates the intestinal absorption of peptide, butyrate, and cholesterol while it inhibits galatose absorption. In addition, leptin increases the activity of NHEl in erythrocytes. In the current study, we determined whether leptin affects the activity of NHE3. We utilized opossum kidney (OK) cells, which express NHE3 and lack other NHEs. The Na-dependent changes in intracellular $\mathrm{pH}$ by NHE3 was determined using the ratio-fluorometric, pH-sensitive dye BCECF-AM. Confluent OK cells were serum-starved and treated with $10 \mathrm{nM}$ leptin or carrier. An exposure of cells to leptin for less than 30 min showed no apparent effect on NHE3 activity. On the other hand, incubation of the cells with leptin for $16 \mathrm{~h}$ significantly decreased NHE3 activity in OK cells. The rate of Na-dependent recovery of intracellular $\mathrm{pH}$ was $0.0278+0.0013 \mathrm{\Delta pH} / \mathrm{sec}$ for control versus $0.0189+0.0013 \Delta \mathrm{pH} / \mathrm{sec}$ for cells exposed to leptin for $16 \mathrm{~h}$. Incubation of cells for $3 \mathrm{~h}$ with leptin exhibited a smaller effect compared with the $16 \mathrm{~h}$ exposure. The rate of Na-dependent pH recovery was $0.0250+0.0008 \mathrm{\Delta pH} / \mathrm{sec}$ for control versus $0.0214+$ $0.0017 \Delta \mathrm{pH} / \mathrm{sec}$. The mechanism underlying the leptin-mediated inhibition of NHE3 activity is not known. However, preincubation of cells with the protein kinase A blocker $\mathrm{H}-89$ completely blocked the effect of leptin at $3 \mathrm{~h}$, suggesting a potential involvement of protein kinase A. Our data show that leptin inhibits NHE3 activity but this inhitbition requires a at least $3 \mathrm{~h}$ of exposure to leptin.

\section{M1810}

Effects of Lubiprostone On Morphine-Induced Constipation and Analgesia Ryuji Ueno, Hiroyoshi Osama, Kory J. Engelke

Lubiprostone is a novel and selective activator of type-2 chloride channels (ClC-2) (AJP Cell Physiol 287:C1173-C1183, 2004) that is currently under development for the treatment of opioid-induced bowel dysfunction. In the present studies, the effects of lubiprostone on morphine-induced constipation and analgesia were examined using morphine-treated mice. ICR mice were initially administered $5 \mathrm{mg} / \mathrm{kg}$ morphine hydrochloride intraperitoneally, 
followed by an oral graphite marker. Immediately thereafter, vehicle or lubiprostone (0.1, 1,10 , or $100 \mu \mathrm{g} / \mathrm{kg}$ ) was administered orally. Control animals orally received the graphite marker and vehicle without morphine. All animals were sacrificed 150 minutes after administration of the marker. Animals were then scored based upon the presence of the marker found in the cecum. Lubiprostone significantly increased the intestinal transit of the marker in the morphine-treated animals when compared with that of the morphine-treated control group ( $<<0.01$ at $1 \mu \mathrm{g} / \mathrm{kg}$ or higher). In addition, the effect of lubiprostone on the analgesic effects of morphine was also evaluated. ICR mice received $5 \mathrm{mg} / \mathrm{kg}$ morphine hydrochloride intraperitoneally followed immediately by the oral administration of vehicle or $0.1,1,10$, or $100 \mu \mathrm{g} / \mathrm{kg}$ lubiprostone. An additional control group received vehicle without morphine. At $30,60,90,120$, and 150 minutes after administration of morphine, the base of the tail was pinched with forceps and the time required for a response was measured. A significant increase in response time was observed for the morphine-treated control group compared with the normal (no morphine) control group at 30 minutes after morphine injection. In addition, all animals treated with morphine and lubiprostone responded in a manner similar to that observed with the morphine-treated control group at all time points, even at doses 100 -fold higher than necessary to inhibit the morphine-induced constipation. These results indicate that lubiprostone significantly antagonizes the constipating effect of morphine without affecting the central analgesic effect of morphine.

\section{M1811}

Expression of Taste Receptors, Tlr Family Members, and the G-Protein, $\alpha$ Gustducin, Along the Crypt-Villus Axis of the Small Intestine Kieron S. Salmon, Soraya P. Shirazi-Beechey

We have shown previously, using real time PCR, that the taste receptors, T1R1, T1R2 and TIR3, and the $\alpha$-subunit of the taste - transduction G protein, gustducin, are expressed in the mouse intestine and in an enteroendocrine cell, STCl. Furthermore we have demonstrated, using western blotting, that the receptors along with Go-gustducin are expressed on, or associated with, the luminal aspect of the cells (1). The mammalian intestine is lined with a monolayer of epithelial cells that undergo rapid and continuous renewal. The stem cells located near the base of the crypts of Lieberkuhn give rise to daughter cells that differentiate into several different lineages; polarised absorptive enterocytes, mucous producing goblet cells, and enteroendocrine cells. In contrast to polarised absorptive enterocytes which constitute $90 \%$ of the villus enterocytes, enteroendocrine cells represent $<1 \%$ of the terminally differentiated cells of the intestinal mucosa. However, at least 15 different subpopulations of enteroendocrine cells have been defined (2). In this work we have determined the cell-specific expression of i) TlR family members, ii) $\alpha$ gustducin, iii) chromogranin A and iv) intestinal glucose transporter ( $\mathrm{Na}+$ /glucose cotransporter, SGLTl), along the crypt-villus axis of the mouse small intestine, using, in situ hybridisation and immunohistochemistry. We show that TIR2 and T1R3, the sweet taste receptors, are expressed in a subpopulation of the villus cells, with no expression in the crypt region. In contrast, SGLTl is expressed on the entire villus enterocytes, with negligible expression in the crypts. $\alpha$ gustducin is also expressed in same subpopulation of cells that manifest T1R2/R3 expression. Chromogranin A expression is depicted in a very few cells on the villus. We conclude that the sweet taste receptors along with the G-protein $\alpha$ gustducin are expressed in a subpopulation of the villus cells, likely to be enteroendocrine cells. References 1. Dyer, J., K.S.H., Salmon, L. Zibrik, and S.P. Shirazi-Beechey (2005) Biochem. Soc. Trans. 33(Pt 1): 302-305. 2. Lewin, K.J. (1986), Pathol. Annu, 1: 1-27.

\section{M1812}

New Insights On Genotype of Congenital Chloride Diarrhea Gianluca Terrin, Giuseppe Cardillo, Annalisa Passariello, Giuseppe Castaldo, Francesco Porcaro, Maria Concetta Siani, Cuono Puzone, Riccardo Troncone, Francesco Salvatore, Roberto Berni Canani

Background. Congenital Chloride Diarrhea (CLD) is an inherited disorder of intestinal electrolyte transport transmitted by autosomal recessive manner caused by a defect in SLC26A3/DRA gene that codifies for $\mathrm{Cl}-/ \mathrm{HCO} 3$ - exchanger. Patients affected by this disorder present clinically with intestinal $\mathrm{Cl}$ - malabsorption that induce severe life-threatening chronic diarrhea with neonatal onset. Early identification of this disorder is essential because an early introduction of appropriate therapy could be life-saving for these patients. Data on he prevalence and on molecular genetics of CLD are missing in many countries. Aims. To describe the genotype of children from different countries affected by CLD and to investigate genotype-phenotype correlations. Patients and Methods. Using automated direct sequencing (310 Abi Prism, PE, Transgenomic), we screened the whole coding region of SLC26A3 gene in 7 patients affected by CLD: 2 , unrelated, from Southern Italy, 3 ( 2 siblings) from Morocco, 1 from Zimbabwe and 1 from USA. Main clinical characteristics of each child were recorded (number of bowel movements per day; total fecal volume per day; stool consistency according the following score: 1: formed, 2: loose, 3: semiliquid; 4: liquid; urgency or incontinence; average doses of $\mathrm{Cl}$ - as $\mathrm{mmol} / \mathrm{kg}$ in the substitution therapy). Results. Molecular analysis revealed two novel missense mutations and a large homozygous novel deletion in patients from Southern Italy, a novel frameshift in one patient and a known Arabic homozygous mutation in siblings patients from Morocco, four novel homozygous mutations in patient from Zimbabwe and a missense and a point deletion, both novel, in the patient form USA Not clear correlation between genotype and phenotype was evidenced in this population. Conclusions. Molecular analysis can contribute to rapid and specific diagnosis of CLD, and can be used to investigate the disease prevalence in different ethnic groups. We confirm the strong genetic heterogeneity of CLD in ethnic groups where the disease is sporadic, and conflicting genotype-phenotype correlations in this condition. The complex genotype observed in patient from Zimbabwe, born to unrelated parents bearing to two different ethnic groups of Southern Africa, led to the hypothesis that such mutations could spread in such ethnic groups due to some yet unidentified protective effect.

\section{M1813}

Down-Regulated in Adenoma (DRA, Slc26a3) Is the Predominant $\mathrm{Cl}^{-} / \mathrm{HCO}_{3}$ Exchanger in the Lower Villous Epithelium of Murine Duodenum Janet E. Simpson, Nancy M. Walker, Clifford W. Schweinfest, Lane L. Clarke

Previous studies have shown that $\mathrm{Cl}^{-} / \mathrm{HCO}_{3}{ }^{-}$exchange contributes to duodenal mucosal $\mathrm{HCO}_{3}{ }^{-}$secretion and formation of the alkaline mucus barrier against gastric acid effluent. Three anion exchangers have been immunolocalized to the apical membrane in murine duodenum: DRA (Slc26a3), the putative anion transporter -1 (PAT-1, Slc26a6) and anion exchanger 4 (AE4, Slc4a9). We hypothesized that activity of each anion exchanger dominates in different regions along the crypt-villus axis. Using mice with gene-targeted deletion of these anion exchangers, we have recently shown that apical membrane $\mathrm{Cl}^{-} / \mathrm{HCO}_{3}{ }^{-}$exchange activity in the upper villous epithelium of the duodenum is dominated by PAT-1 (60-70\%) with a smaller contribution from DRA (20-30\%) and virtually no contribution from AE4. In the present study, we extended the investigation to evaluate the contribution of these anion exchangers to $\mathrm{Cl}^{-} / \mathrm{HCO}_{3}{ }^{-}$exchange in the lower villous epithelium. Using BCECF microfluorimetry, intracellular $\mathrm{pH}\left(\mathrm{pH}_{\mathrm{i}}\right)$ was measured in intact murine duodenal mucosa mounted in a horizontal Ussing-like chamber that allowed independent superfusion of the mucosal and basolateral surfaces. A short section of the mucosa was elevated to enable rapid solution changes and visualization of epithelial cells at the villous base. Epithelia of the villous base were defined by the presence of crypt openings in the microscopic field. In initial studies of wild-type (WT) duodenum, it was found that the baseline $\mathrm{pH}_{\mathrm{i}}$ was reduced, but that the rate of $\mathrm{Cl}^{-} / \mathrm{HCO}_{3}{ }^{-}$exchange in the lower villous epithelium was comparable in magnitude to the exchange rate in the upper villous epithelium. Investigation of PAT-1(-) and WT littermate duodena (gift of M. Soleimani, Univ. of Cincinnati) revealed that PATl, unlike its role in the upper villus, does not significantly contribute to $\mathrm{Cl}^{-} / \mathrm{HCO}_{3}{ }^{-}$exchange activity in the lower villous epithelium. In contrast, it was found that typical $\mathrm{Cl}^{-} / \mathrm{HCO}_{3}$ exchange activity was eliminated and, paradoxically, the $\mathrm{pH}_{\mathrm{i}}$ of the epithelial cells acidified then re-alkalized during luminal $\mathrm{Cl}^{-}$removal and exposure, respectively, in the DRA(-) lower villus. Preliminary studies of DRA(-)/CFTR(-) double knockout duodena $(\mathrm{n}=2)$ indicated that the paradoxical acidification in the absence of luminal $\mathrm{Cl}^{-}$was CFTR-dependent. We conclude from these studies that, in contrast to the upper villous epithelium, DRA is the dominant $\mathrm{Cl}^{-} / \mathrm{HCO}_{3}^{-}$-exchanger in the lower villous epithelium of the murine duodenum where it operates in a complex interaction with CFTR. Supported by NIH and CFF.

\section{M1814}

Ileal Bile Acid Transporter Expression in Patients with Chronic Diarrhea: Evidence for Differences in Transcriptional Regulation Julian R. Walters, Sara Balesaria, Konstantia-Maria Chavele, Umma Khair

Background: Primary idiopathic bile acid malabsorption is a frequent cause of unexplained chronic diarrhea and its causation is largely unknown. The aim of this study was to determine whether changes in expression or transcriptional regulation of ileal bile acid transporters could be identified. Methods: Ileal biopsies were obtained with informed consent at colonoscopy from two groups of patients: chronic diarrhea $(n=17)$ and controls without diarrhea $(n=21)$. No evidence of any ileal disease or of other causes of diarrhea was present. Duodenal biopsies were obtained from other patients for comparison of proximal and distal intestinal expression. RNA and cDNA were prepared using standard methodology. Transcript expression was measured by quantitative real-time RT-PCR (Taqman). Expression was normalised to that of the housekeeping gene GAPDH. Results: Expression of the apical membrane sodium-linked bile acid transporter (ASBT) and the cytoplasmic ileal bile acid binding protein (IBABP) was much higher in ileum than in duodenum. The putative basolateral organic solute transporters (OST) alpha and beta were found at similar levels. No significant differences in the mean or median values were found for transcripts of these transporters in the two patient groups. ASBT and IBABP were significantly correlated in those with diarrhea $(r=0.67, p<0.05)$ but not in controls. Expression of transcription factors that affect ASBT or IBABP expression was then determined. Farnesoid X receptor (FXR) and liver receptor homologue (LRH-1) were more abundant in ileum, unlike short heterodimer partner (SHP), c-Fos, or CDX2 Mean or median values for FXR, LRH-1, SHP, c-Fos or CDX2 were not significantly different between the two patient groups. The relationships of expression of these factors with ASBT and IBABP differed. Using multiple stepwise regression, ASBT was significantly related $(p<0.05)$ to LRH-1 and SHP in controls, and with SHP and CDX2, but not LRH-1, in diarrhea. IBABP was related to LRH-1, SHP and CDX2 in controls, but only to CDX2 in diarrhea. Fibroblast growth factor (FGF)-19, which is proposed to have a role in signalling between ileum and hepatic bile acid synthesis, was expressed much more abundantly in ileum than duodenum, but at similar values in both patient groups. Conclusion: Although differences between duodenum and ileum can be demonstrated, bile acid transporters and putative transcription factors were expressed at similar levels in ileal biopsy RNA from patients with chronic diarrhea and controls. Regression analysis suggests that ASBT and IBABP may be regulated differently in patients with chronic diarrhea.

\section{M1815}

Ontogenic Regulation of the Rat Heteromeric Organic Solute Transporter Ost $\alpha$-Ost $\beta$

Melissa Halpern, Nazzareno Ballatori, Jessica Clark, Sarah Doelle, Tara Saunders, Bohuslav Dvorak

Background Enterohepatic circulation of bile acids (BA) is regulated in the distal ileum through transport from the apical surface of enterocytes into the cell by the apical sodiumdependent bile acid transporter (Asbt). BA can then be bound to the ileal bile acid binding protein (Ibabp) for transport across the enterocyte. The recently described heteromeric organic solute transporter Ost $\alpha$-Ost $\beta$ may be a mechanism by which BA are then removed from the cell and transported into portal circulation. Aims To determine the ontogenic regulation of the Ost $\alpha$ and Ost $\beta$ genes in rats from birth to weaning. Methods Ileal tissue was taken from rats at day $0,1,3,7,13,21$ or 28 . To determine if introduction of BA would increase Ost $\alpha$ or Ost $\beta$ mRNA levels in the early neonatal period, newborn rats were 
given $288 \mathrm{mg} / \mathrm{kg}$ sodium deoxycholic acid 2X/day for 2 days (DOC). Control rats were gavaged with vehicle (PBS). Four day-old rats with Necrotizing Enterocolitis (NEC) have elevated ileal BA levels and mRNA were examined and compared to control pups without disease (Control). RNA was isolated from each sample and real-time-PCR assays were used to quantify steady-state mRNA levels for Ost $\alpha$ and Ost $\beta$. All mRNA levels were normalized according to the level of $18 \mathrm{~S}$ within each sample, which was used as an endogenous control. Results Under normal physiologic conditions, Ost $\alpha$ and Ost $\beta$ mRNA were very low in neonatal animals and increased to adult levels 28 days after birth (Table 1). Introduction of exogenous BA in the early neonatal period did not significantly increase expression of either Ost $\alpha$ or Ost $\beta$, even though Ibabp mRNA can be up-regulated in response to the same dose of BA. However, in neonatal animals with NEC, Ost $\beta$ expression was significantly increased (Table 2). Conclusions Like other ileal BA transporters, Osta and Ost $\beta$ are expressed at very low levels prior to the initiation of solid food diet. However, the Osta and Ost $\beta$ response to exogenous BA in the early neonatal period differs from Ibabp. Further, under pathological conditions when ileal BA levels are elevated, Ost $\beta$ mRNA is precociously up-regulated.

Table 1.

\begin{tabular}{|c|c|c|c|c|c|c|c|}
\hline & Day 0 & Day 1 & Day 3 & Day 7 & Day 13 & Day 21 & Day 28 \\
\hline Ost $\alpha$ & $1.0(0.3)$ & $0.9(0.3)$ & $1.7(0.6)$ & $1.0(0.4)$ & $0.9(0.3)$ & $3.0(0.9)^{*}$ & $4.8(1.3)^{*}$ \\
\hline Ost $\beta$ & $1.0(0.7)$ & $0.5(0.4)$ & $1.4(0.7)$ & $0.9(0.5)$ & $1.0(0.3)$ & $19.0(4.6)^{*}$ & $45.7(16.2)^{*}$ \\
\hline
\end{tabular}

All time points $\mathrm{n}=12$. Data are expressed as fold-change in mRNA (SD). $* \mathrm{p} \leqq 0.01$ vs day 0

Table 2

\begin{tabular}{|c|c|c|c|c|}
\hline & DOC $(\mathrm{n}=5)$ & PBS $(\mathrm{n}=5)$ & NEC $(\mathrm{n}=12)$ & Control $(\mathrm{n}=12)$ \\
\hline Ost $\alpha$ & $0.8(0.2)$ & $1.0(0.2)$ & $1.4(0.5)$ & $1.0(0.6)$ \\
\hline Ost $\beta$ & $0.9(0.6)$ & $1.0(0.6)$ & $2.2(0.6)^{*}$ & $1.0(0.3)$ \\
\hline Ibabp & $8.1(2.5) \#$ & $1.0(0.4)$ & $1.2(0.5)$ & $1.0(0.3)$ \\
\hline
\end{tabular}

Data are expressed as fold-change mRNA (SD). \# $\mathrm{p} \leqq 0.01$ vs PBS; * $\mathrm{p} \leqq 0.05$ vs control

M1816

The Relationship Between Bile Salt Malabsorption and Cholesterol 7-Alpha Hydroxylase Gene (CYP7A1) Promoter Polymorphism in Patients with Inflammatory Bowel Disease

Martin Lenicek, Milan Lukas, Lucie Budisova, Milan Jirsa, Jan Kovar, Libor Vitek

Background: Bile salt malabsorption (BSM) is a common complication of Crohn disease (CD) contributing to further clinical consequences such as increased prevalence of gallstone disease. In the present study the relationship between CYP7Al promoter polymorphism and serum levels of 7alpha-hydroxy-4-cholesten-3-one (cholesten), a novel marker of BSM, was investigated in patients with various clinical manifestation of inflammatory bowel disease. Materials and Methods: Serum levels of cholesten were determined by HPLC in a group of $C D$ patients with ileal resection $(n=31)$, ileal inflammation $(n=20)$, colonic involvement only $(n=12)$, ulcerative colitis (UC) patients $(n=12)$ and healthy controls $(n=40)$. Two common linked promoter polymorphisms of CYP7Al, $-203 \mathrm{~A} / \mathrm{C}$ and $-469 \mathrm{C} / \mathrm{T}$, were analyzed by PCRRFLP. Results: As compared to controls, serum cholesten levels corrected to serum cholesterol were increased in $C D$ patients with ileal resection and ileal inflammation and not affected in CD patients with colonic involvement only as well as in those with UC [median (min$\max ) ; 2.3(0.6-10.9)$ vs. $32.2(3.7-112.5) \mu \mathrm{g} / \mathrm{mM}, \mathrm{p}<0.001 ; 4.2(0.6-40.2) \mu \mathrm{g} / \mathrm{mM}, \mathrm{p}=0.009$; $2.4(0.6-60.2) \mu \mathrm{g} / \mathrm{mM}, \mathrm{p}=0.536$; and $2.9(1.2-22.7) \mu \mathrm{g} / \mathrm{mM}, \mathrm{p}=0.066$, respectively]. Patients with CD and UC homozygous for the alelles [203C; -469T] had lower serum cholesten/ cholesterol ratio $(\mathrm{p}<0.05)$ and higher serum cholesterol $(\mathrm{p}<0.025)$ than patients with 203A/A, -469C/C haplotype. Conclusions: 1) Serum cholesten level, in particular cholesten/ cholesterol ratio, is a useful marker of bile salt malabsorption and correlates well with the degree of ileal involvement. 2) CYP7Al gene promoter haplotype affects bile salt synthesis rate in patients with BSM

\section{M1817}

Sumo Modification of the $\mathrm{Na}^{+} / \mathrm{K}^{+} / 2 \mathrm{CL}^{-}$Cotransporter 1 in Colonic Secretory Epithelial Cells in Response to Epidermal Growth Factor Alfred E. Chappell, Kim E. Barrett

Background and Aim: Post-translational modification of proteins by the small ubiquitin-like modifier (SUMO) has been shown to regulate target protein activities through a number of different mechanisms including altered cellular localization, biochemical modifications, and protection from ubiquitin modification and subsequent targeting for proteosomal degradation. The epidermal growth factor receptor (EGFr) is an important regulator of chloride transport in intestinal epithelia and activation of EGFr has been shown to modulate activity of the $\mathrm{Na}^{+} / \mathrm{K}^{+} / 2 \mathrm{Cl}^{-}$cotransporter (NKCC), but the mechanisms were unknown. The aim of this study was to investigate the involvement of SUMO, if any, in regulating the activity of NKCC in secretory epithelia in response to epidermal growth factor (EGF). Methods: T84 human colonic epithelial cells were grown on transwell membranes. Cells were treated basolaterally with EGF for $1,3,5,10$, or 20 minutes or Ringers as a control at $37^{\circ} \mathrm{C}$. After treatment, cells were lysed at $2-8^{\circ} \mathrm{C}$ and lysates were immunoprecipitated using a commercially available Pan-SUMO antibody that is immunoreactive against all three SUMO isoforms. Precipitates were then analyzed by Western blot for NKCCl. Results: Treatment of T84 cells with EGF for 1 minute resulted in $66 \% \pm 13 \%$ more SUMO-modified NKCCl than in controls (mean $\pm \mathrm{SEM}, \mathrm{n}=6, \mathrm{p}<0.001$ ). However, levels of SUMO-modified NKCCl at later time points were not significantly different from those found in untreated cells. Conclusions: Basolateral exposure of T84 cells to EGF results in rapid modification of NKCC1 by one or more SUMO isoforms. Subsequent to this modification, NKCCl is likely rapidly de-SUMOylated. The dynamic regulation of SUMOylation of $\mathrm{NKCCl}$, and perhaps of othe transport proteins involved in chloride secretion, may account for the ability of EGF to regulate this important transport process. Our findings, therefore, have implications for disease states where chloride secretion is abnormal.

\section{M1818}

Altered Regulation of Lipogenic Genes in Mouse 3T3-L1 Fibroblasts and Adipocytes Co-Cultured with Human HEPG2 Hepatoma Cells Edwin Ouyang, David Cohen

Background: Adipocytes secrete adipokines, which are cytokine-like hormones that can influence lipid metabolism in the liver. Whether hepatocytes regulate lipid metabolism in extrahepatic cell types by endocrine mechanisms has not been well explored. Aim: To examine the influence of hepatocytes on expression of lipogenic genes in fibroblasts and adipocytes. Methods: Mouse 3T3-Ll fibroblasts were differentiated into adipocytes by a standardized procedure using dexamethasone, insulin and 3-isobutyl-1-methylxanthine. Fibroblasts or adipocytes were then cultured alone or together with an equal number of human HepG2 hepatoma cells for 48 hr in DMEM with 10\% FBS. Cellular RNA was purified and utilized to prepare cDNA. Quantitative real time PCR was performed using gene-specific primers for mouse sterol regulatory element binding protein-lc (SREBP-lc), a transcription factor that promotes lipogenesis, as well as the lipogenic genes stearoyl CoA desaturase 1 (SCD1) and fatty acid synthase (FAS). Gene amplification was quantified using SYBR green and normalized to beta-actin. Results: Compared with fibroblasts cultured alone, there was a marked 4.6-fold increase in SREBP-1c mRNA expression when fibroblasts were co-cultured with HepG2 cells. By contrast, SCD1 was decreased by $67 \%$ and FAS was unchanged. As expected, adipocytes alone displayed much higher expression of SREBP-lc, SCDI and FAS than fibroblasts by 30 -fold, 320 -fold and 18-fold, respectively. However, when adipocytes cells were co-cultured with HepG2 cells, mRNA expression levels of SREBP-lc, SCD1 and FAS in fibroblasts were reduced by $61 \%, 99 \%$ and $96 \%$, respectively. Similar changes in lipogenic gene expression in fibroblasts and adipocytes were observed when the percentage of HepG2 cells was increased from $50 \%$ to $90 \%$. Conclusions: HepG2 cells modulate expression of lipogenic genes in both fibroblasts and adipocytes. Because SCD1 and FAS are downstream target genes of SREBP-1c, the lack of coordinate upregulation of these genes in fibroblasts suggest that HepG2 cells influence lipogenesis by multiple mechanisms under co-culture conditions.

\section{M1819}

Impact of Ezetimibe and NPC1L1 On SRBI-Mediated HDL Selective Uptake and VLDL Secretion By Liver Cells

Philip Howles, Eric Labonte, Lisa Camarota, Dean Gilham, Yiannis Ioannou, Joanna Davies, David Y. Hui

Background: Ezetimibe potently blocks cholesterol absorption and is thought to be an inhibitor of NPClLl (Nieman Pick C type 1-like protein 1). Interestingly ezetimibe has also been shown to bind SRBI (scavenger receptor class B type I) and to decrease cholesterol uptake by SRBI transfected cells. The role of NPClLl and the effects of ezetimibe in the intestine have been extensively studied. However, NPC1L1, along with SRBI, is also abundant in human liver but the role of this protein in hepatic cholesterol metabolism is poorly understood. Thus, the purpose of these experiments was to determine the effects of ezetimibe and NPClLl on VLDL production and SRBI-mediated selective uptake of HDL. Methods: HepG2 cells were used as an in vitro model of human hepatocytes. Human $\mathrm{HDL}_{3}$ was radiolabeled with $\left[{ }^{3} \mathrm{H}\right]$ cholesteryl oleoyl ether or $\left[{ }^{3} \mathrm{H}\right]$ cholesteryl oleate. Selective uptake of HDL-CE (cholestery ester) by cells treated with $20 \mu \mathrm{M}$ ezetimibe or with SRBI blocking antibodies was measured and compared to untreated cells. After $4 \mathrm{hr}$, cells were harvested, homogenized and fractionated on iodixanol gradients. Selective uptake was determined on total homogenates and the distribution of radiolabel and protein was determined from gradient fractions. The amount and distribution of SRBI and ABCAl (ATP binding casette transport protein $\mathrm{Al}$ ) was determined by Western blot analysis. VLDL production by fasted control and NPC1Ll-null mice was determined by measuring plasma triglyceride increases after intravenous injection of triton WR1339. Results: Selective uptake of HDL-CE was reduced $25 \%$ by ezetimibe treatment as compared to $\sim 40 \%$ inhibition by SRBI blocking antibody. Acute ezetimibe treatmen reduced SRBI expression by $20 \%$, while overnight treatment increased SRBI expression by $40 \%$. ABCAl expression was similarly affected. Distribution of radiolabel in gradient fractions indicated that ezetimibe decreases the delivery of HDL-CE into intracellular cholesterol storage pools. The VLDL secretion rate was 35\% lower in NPC1L1-null mice as compared to normal mice. Conclusions: The data suggest that NPCILl is part of the SRBI-mediated selective uptake pathway in hepatocytes and that disruption of this pathway by ezetimibe results in futile overexpression of the SRBI protein. Reduced delivery of HDL-CE to the cellular pool observed in HepG2 cells treated with ezetimibe is consistent with reduced VLDL secretion by NPClL1-null mice.

\section{M1820}

The Molecular Structure That Allows FXR to Override the Stimulation of CYP7A1 By LXR

Quan Shang, Luxing Pan, Monica Saumoy, John Y L. Chiang, G Stephen Tint, Gerald Salen, Guorong Xu

We cloned the rabbit CYP7Al promoter and found that there is a 9 bp $\alpha$-fetoprotein transcription factor (FTF) binding site within the $16 \mathrm{bp}$ binding site for the liver $\mathrm{X}$ receptor (LXR). We hypothesized that this overlapping structure alters regulation of the CYP7Al promoter. Gel shift assays demonstrated that [35S]-FTF protein binds to the LXR binding site (LXRE). We further noted that FTF competes with LXR for binding to LXRE. In a ge shift assay of [35S]-LXR protein incubated with unlabeled LXRE, the addition of FTF reduced the intensity of the [35S]-LXR/RXR-LXRE band in a dose dependant manner. In another 
experiment, FTF reduced the binding of LXR/RXR to [32P]-LXRE. Although SHP (short heterodimer partner) alone was not able to bind to LXRE, adding SHP significantly enhanced the ability of FTF to compete with LXR for binding. This result was confirmed by our observation that, as more SHP was added, less amounts of [32P]-LXRE bound to LXR/RXR when a minimum amount of FTF was present. Furthermore, in HepG2 cells transfected with the rabbit CYP7Al promoter, adding SHP with FTF significantly enhanced the inhibitory effect of FTF and abolished the stimulating effect of LXR/RXR on the promoter. We conclude that FTF competes with LXR for binding to LXRE because its own binding site lies within the LXR binding motif. We propose that SHP enhances the ability of FTF to compete with LXR/RXR because it forms heterodimers with FTF which then bind more efficiently to the LXR binding site and block LXR/RXR to bind to LXRE. Activated FXR (farnesoid X receptor) is known to upregulate expression of its target gene SHP. Increased levels of SHP, together with FTF, will then prevent LXR/RXR from binding to LXRE and will abolish the stimulatory effect of LXR on the CYP7Al promoter.

\section{M1821}

Compensatory Hepatic Up-Regulation of Bile Acid Synthesis in Liver Fatty Acid Binding Protein (FABP1) Knockout Mice Provides Novel Insights Into the Physiological Role of FABP1

Mulugeta Seneshaw, Dietmar Klass, Newberry Elizabeth, Elke Preiss, Udo Seedorf, Guido Adler, Nicholas O. Davidson, Michael Fuchs

FABP1 is a cytosolic lipid binding protein expressed in hepatocytes and enterocytes. In vitro studies demonstrated that FABPI can bind bile acids in addition to fatty acids. Recently we and others demonstrated that bile acid secretion is decreased in Fabpl knockout mice. To explore the molecular mechanism responsible for bile acid hyposecretion, male Fabpl-/and wild-type mice were fed either a normal (ND) or cholic acid (CD) containing diet for 4 weeks. Hepatic bile was collected for 1 hour to measure bile flow and biliary lipids. Bile acid composition of hepatic bile was analyzed by gas chromatography mass spectrometry. Livers were harvested to study steady-state gene expression of bile acid synthetic enzymes employing TaqMan-PCR. Fabpl-/- mice had significantly reduced body (13\%) and liver weights $(23 \%)$ but food intake was unchanged compared with wild-type mice. Steady-state Cyp7al, Cyp27al and Hmgcr mRNA expression was increased by 2.3- $(\mathrm{p}<0.05), 1.7$ $(p<0.05)$ and 2 -fold $(p<0.001)$ in knockout mice, whereas Cyp8bl mRNA expression was slightly reduced by $43 \%(\mathrm{p}>0.05)$. The bile flow rate was $45 \%$ lower than in wild-type mice (1.15 vs. $0.63 \mu \mathrm{l} / \mathrm{min} / \mathrm{g}$ liver; $\mathrm{p}<0.05$ ). In addition, biliary secretion rates for cholesterol, phospholipids and bile acids were substantially reduced in Fabpl-/- mice (cholesterol: 0.6 vs. 1.3; phospholipids: 4.1 vs. 7.1 ; bile acids: 33 vs. $46 \mathrm{nmol} / \mathrm{min} / \mathrm{g}$ liver). The two major bile acid species present in hepatic bile of both groups of mice were cholic acid and betamuricholic acid, representing $70 \%$ of total bile acids. Alpha-muricholic acid (4\%) was only detectable in Fabpl-/- mice. This suggests that bile acid pool composition in mice is not critically dependent on proper FABPl expression. In response to the $\mathrm{CD}$, biliary lipid secretion rates increased significantly in all mice. However, bile acid secretion increased less pronounced in Fabpl -/- mice compared to wild-type mice ( 248 vs. $466 \mathrm{nmol} / \mathrm{min} / \mathrm{g}$ liver, $\mathrm{p}<0.01$ ). Based on our findings we propose that Fabpl gene ablation impairs intestinal bile acid absorption inducing increased fecal bile acid loss and compensatory hepatic up-regulation of bile acid synthesis.

\section{M1822}

Enteral Starvation Induces Enterohepatic Cycling of Bilirubin: A Putative Mechanism for Total Parenteral Nutrition (TPN)-Associated Liver Disease and Pigment Gallstone Formation

Scott A. Elisofon, Martin C. Carey

Aims: Liver disease and 'black' pigment sludge/gallstones are common in TPN patients. We asked whether enteral starvation with TPN infusion leads to induction of enterohepatic circulation (EHC) of bilirubin accompanied by conjugated "hyperbilirubinbilia". We hypothesized further that absence of secretin release might produce less alkaline bile via diminished cholangiocyte expression of CFTR. Both anomalies should provide the necessary conditions for increased hydrolysis of bilirubin glucuronides to unconjugated bilirubin (UCB) leading to bilirubinate injury to the hepatobiliary tree. Methods: Male Sprague Dawley rats received intravenous TPN/enteral starvation $(n=5)$ or sham surgery/regular chow diet $(n=5)$. At 7 days, hepatic bile was collected via acute biliary fistulae and assayed for $\mathrm{pH}$, bilirubin concentrations and molecular species, and common biliary lipids by standard methods. Hematocrit, haptoglobin, plasma indices of liver injury, and liver histology were examined. Stool outputs were weighed. Results: TPN rats displayed significantly lower 7-day stool weights (7.1 vs $59.3 \mathrm{~g}, \mathrm{p}<0.05)$. Bilirubin secretion rates were markedly higher in TPN rats than in controls $(51.5 \pm 6.7$ vs $25.3+1.2 \mathrm{nmol} / \mathrm{hr} / 100 \mathrm{~g} \mathrm{BW}, \mathrm{p}<0.05)$. Phospholipid (PL) secretion rates were significantly lower in TPN rats than in controls $(1.2 \pm 0.1$ vs $1.6 \pm 0.1$ $\mu \mathrm{mol} / \mathrm{h} / \mathrm{l} 00 \mathrm{~g}, \mathrm{p}<0.05)$, with a trend toward lower cholesterol $(\mathrm{CH})$ secretion rates $(0.14 \pm 0.02$ vs $0.21 \pm 0.04 \mu \mathrm{mol} / \mathrm{h} / 100 \mathrm{~g}, \mathrm{p}=0.09$ ). No significant differences in bile salt (BS) secretion rates or biliary $\mathrm{pH}$ occurred. In plasma, only total bilirubin levels were increased in the TPN group ( 0.20 vs $0.12 \mathrm{mg} / \mathrm{dL}, \mathrm{p}<0.05$ ). Over $50 \%$ of hepatocytes from TPN livers demonstrated microvesicular steatosis; control livers were healthy. Haptoglobin and hematocrit did not differ between the two groups. Conclusions: Seven days of enteral starvation/ TPN infusion in rats showed hepatic steatosis consistent with the human disease. Marked decrease in intestinal motility was evident by significant reductions in stool outputs, which allowed for more exposure time for EHC of UCB and hyperbilirubinbilia. In the absence of hemolysis or ineffective erythropoiesis, elevated serum total bilirubin in TPN rats is also consistent with induced EHC. In addition, decreased PL and $\mathrm{CH}$ secretion rates are consistent with lipid decoupling from BS secretion by increased endobiotic dianion excretion. Hyperbilirubinbilia as well as decreased biliary PL to BS ratios suggest that with prolonged TPN infusions, particularly in the setting of intestinal disease and/or resection, the biliary tree may become compromised by elevated UCB as well as increased detergency of bile acids.

\section{M1823}

No Evidence for Enterohepatic Cycling (EHC) of Bilirubin in the Slc10a2 (ASBT/IBAT)-/- Mouse

Folke U. Freudenberg, Bian B. Yu, Monika R. Leonard, Paul A. Dawson, Martin C. Carey

Introduction. Passive EHC of unconjugated bilirubin (UCB) is a well established contributor to neonatal hyperbilirubinemia, but there is inadequate proof that EHC of bilirubin occurs in adult life. Data from mouse and rat models with ileectomy or chemical inhibition of ASBT are consistent with EHC of bilirubin secondary to bile salt malabsorption. We hypothesize that, due to massive spillage of bile salts into the colon, there should be EHC of UCB in Slc10a2-/- mice. Methods. We collected hepatic bile from Slc10a2-/- mice within 30min of fistulation, and gallbladder (GB) bile after cholecystectomy. We assessed biliary pH by microelectrode, bilirubins by HPLC, and $72 \mathrm{hr}$ fecal bile acid excretion by enzymatic assay. Lipid analyses and total lipid concentrations were determined by standard methods. Results. In hepatic bile of Slc10a2-/- compared to wild type (WT) mice, conjugated bilirubins (CBR) are not elevated $(25.0 \pm 3.6$ vs $31.9 \pm 4.8 \mathrm{nmol} / \mathrm{hr} * 100 \mathrm{~g} \mathrm{BW})$ but UCB is slightly higher $(0.46 \pm 0.07$ vs $0.29 \pm 0.05 \mathrm{nmol} / \mathrm{hr} * 100 \mathrm{~g} \mathrm{BW})$. There is a modest difference in hepatic bile $\mathrm{pH}(8.7 \pm 0.06$ in Slc10a2-/- $\cdot 8.5 \pm 0.04$ in WT) GB size is sionificantly smaller in Slc10a2-/$(19.0 \pm 3.2 \mu l)$ than in WT $(47.9 \pm 5.8 \mu \mathrm{l})$ mice. Fecal bile acid excretion in Slc10a-/- mice is increased two- to threefold. Bile salts, phospholipids and cholesterol $(11.1 \pm 2.1 \mathrm{mM} ; 2.7 \pm 0.3$ $\mathrm{mM} ; 0.32 \pm 0.07 \mathrm{mM}$, respectively) are markedly decreased in hepatic bile of Slc10a2-/compared to WT mice $(26.5 \pm 7.4 \mathrm{mM}, 4.1+0.8 \mathrm{mM} \cdot 0.65 \pm 0.17 \mathrm{mM}$, respectively). In GB bile of Slc10a2-/- mice, bile salts are decreased fivefold ( $<<0.0001$ ), and $\mathrm{pH}$ is more alkaline $(8.1 \pm 0.1$ vs $7.7 \pm 0.1)$. Conclusions. There is no evidence for EHC of bilirubin in Slcl0a2-/mice. Massive bile acid loss leads to contraction of the bile acid pool, which reduces biliary lipid concentrations in hepatic and GB biles. Smaller GB sizes and higher pH values in Slc10a2-/- mice may be related to increased coprophagy and hyperphagia, resulting in more frequent GB emptying and more alkaline GB bile. Although increased bile acid losses have been shown to facilitate uptake of UCB from the colon in animal models and in patients with Crohn's disease, our study revealed no increases in CBR in hepatic bile. Flooding of the colon with bile salts most likely induces rapid colonic evacuation and insufficient time of exposure for UCB resorption. These results are consistent with the observation that congenital bile salt malabsorption secondary to ASBT null mutations does not pose a risk of pigment gallstone formation in humans.

\section{M1824}

An Ancillary Subunit Mutation Alters Activation of KCNQ1 Andrea E. Goldstein, Michael Duffey, Derek Beahm, Cynthia Dlugos, Michael Morales, Randall Rasmusson

In the liver, collecting ducts transport bile acid-rich bile to the duodenum, and secrete a $\mathrm{HCO}_{3}^{-}$-rich fluid. Basolateral membrane $\mathrm{K}^{+}$channels in duct epithelial cells play a critical role in secretion by establishing the negative cell interior membrane potential necessary for anion transport. In secretory epithelial cells, KCNQ1, a voltage-gated $\mathrm{K}^{+}$channel, associates with the KCNE3 subunit to form a constitutively active, voltage-insensitive channel. We hypothesize that KCNQ1+KCNE3 is the dominant $\mathrm{K}^{+}$channel in biliary duct cells. We demonstrated by RT-PCR the presence of KCNQ1 and KCNE3 mRNAs in the human liver secretory cell line, $\mathrm{Mz}-\mathrm{ChAl}$, and showed using immunofluorescence that $\mathrm{KCNQI}$ is expressed in rat liver secretory ducts. In the heart, KCNQ1 interacts with the KCNEl subunit to form a voltage-gated $\mathrm{K}^{+}$channel, mutations of which are responsible for long QT syndrome. To determine mechanisms of subunit interaction, we expressed KCNQ1 in Xenopus oocytes and determined the effects of the blocker $\mathrm{Ba}^{2+}$ on current-voltage relationships. Bath $\mathrm{Ba}^{2+}$ ( $5 \mathrm{mM}$ ) slowed activation and reduced (24\%) KCNQ1 currents. Ba ${ }^{2+}$ caused the constitutively active $\mathrm{K}^{+}$currents of KCNQ1 co-expressed with KCNE3 to become slowly-activating and dramatically reduced ( $42 \%)$. KCNQ1+KCNEl currents were very slowly activated and $\mathrm{Ba}^{2+}$ had little (16\%) effect. The dose-inhibition curve for $\mathrm{Ba}^{2+}$ on KCNQ1 showed two binding components with affinities at $10 \mu \mathrm{M}$ and $20 \mathrm{mM}$. The curve for KCNQ1+KCNEl showed only one component $(20 \mu \mathrm{M})$ and KCNQ1+KCNE3 showed one component $(100 \mu \mathrm{M})$. We determined the effects of a V72T mutation in KCNE3. This mutation altered a single amino acid within the "activation triplet" of the ancillary subunit KCNE3. KCNQ1+KCNE3(V72T) currents were slowly-activating, like KCNQl+KCNEl, and inhibition by $\mathrm{Ba}^{2+}(2 \mathrm{mM})$ was identical to that of KCNQ1+KCNE3. These results suggest that the site of regulation of KCNQ1 by KCNE3 or KCNE1 involves both the voltage-sensitive gating mechanism and extracellular pore region of KCNQ1. (NIH 62465)

\section{M1825}

Induction of $\mathrm{MLC}_{20}$ Gene Expression By Spontaneous Release of Acetylcholine At Neuroeffector Junction in Rat Colonic Circular Smooth Muscle Cells

Xuan-Zheng Shi, Sushil K. Sarna

During the course of normal gut motility function, acetylcholine (ACh) is released at the neuroeffector junction to contract circular smooth muscle cells. The aim of this study was to investigate whether spontaneous release of $\mathrm{ACh}$ also induces gene expression of specific signaling molecules in excitation-contraction coupling. The experiments were performed in fresh circular muscle strips and in conscious rats. Methods: Surgically implanted osmotic pumps infusing atropine intramuscularly blocked the effect of spontaneous release of ACh on colonic circular smooth muscle cells in intact conscious rats. The contractile activity of the colonic circular muscle was recorded in vivo by implanted strain gauge transducers and in vitro in muscle bath. Results: Osmotic pump infusion of atropine at 100, 200, and 300 $\mathrm{nmol} / \mathrm{kg} / \mathrm{h}$ for 24 or $48 \mathrm{~h}$ significantly inhibited the spontaneous contractile activity of the colon and it reduced the number of stool pellets from $16 \pm 3$ to $4 \pm 1,3 \pm 0.5$ and $3 \pm$ $0.4 /$ day respectively (all $\mathrm{p}<0.05$ ). The response of colonic circular muscle strips to $\mathrm{KCl}(30$ to $65 \mathrm{mM})$ and acetylcholine $\left(10^{-6}\right.$ to $\left.10^{-2} \mathrm{M}\right)$ was significantly suppressed in muscle strips from atropine-treated rats when compared to those treated with saline infusions. Atropine treatment did not alter the expression of $\alpha_{1 \mathrm{C}}$ and $\beta 3$ subunits of L-type $\mathrm{Ca}^{2+}$ channels and 
of smooth muscle $\alpha$-actin. However, it significantly reduced the expression of $\mathrm{MLC}_{20}$ to 42 $\pm 6 \%$ of saline control $(p<0.05)$. When naive colonic circular muscle strips were incubated with $1 \mathrm{mM}$ cholinergic muscarinic agonist methacholine, the expression of $\mathrm{MLC}_{20}$ was enhanced time-dependently. The expression of $\mathrm{MLC}_{20}$ began to increase at 6 hours, and the maximal increase at $24 \mathrm{~h}$ was $182 \pm 21 \%$ of control $(\mathrm{p}<0.05)$. Furthermore, the contractile response to $\mathrm{KCl}$ (30 to $65 \mathrm{mM}$ ) was significantly greater in the methacholine-treated muscle strips than in those cultured in control medium $(\mathrm{p}<0.05$. When the muscle strips were treated periodically, every $25 \mathrm{~min}$ for $5 \mathrm{~min}$ for a total of $6 \mathrm{~h}$, with $1 \mathrm{mM}$ acetylcholine, their response to $\mathrm{KCl}$ was also enhanced. The expression of $\mathrm{MLC}_{20}$ increased to $131 \pm 10 \%$ in ACh-treated strips compared with medium-treated strips. Conclusions: Our results demonstrate that spontaneous release of ACh at the neuromuscular junction not only causes colonic circular muscle contractions, but it also regulates gene expression of myosin light chain in colonic circular smooth muscle cells. This novel function of acetylcholine may help to maintain normal colonic smooth muscle contractility.

\section{M1826}

R-Type Calcium Channel Expression and Function At Nerve Terminals of Guinea Pig Ileum Myenteric Neurons

Xiaochun Bian, James Galligan

Introduction. Myenteric neurons in the guinea-pig ileum express N, L, P/Q and R-type calcium channels. Currents carried by R-type channels account for at least half of the total calcium current recorded from these neurons but the function of these channels is not known. Methods. We studied the localization and function of R-type calcium channels in guinea pig myenteric neurons maintained in primary culture using electrophysiological and immunohistochemical techniques. Calcium imaging and photometry with the calcium indicator dye, Fluo-4 $(2 \mu \mathrm{M})$, were used to assess calcium channel function in nerve cell bodies and nerve terminals. Results. Immunohistochemical studies revealed strong expression of R-type calcium channel immunoreactivity on most nerve cell bodies and nerve fibers. Patch clamp studies of individual neurons revealed that depolarizations from -70 to $0 \mathrm{mV}$ elicited inward currents in all neurons tested; these currents were blocked by $\mathrm{CdCl}_{2}$ (100 $\mu \mathrm{M})$, a non-selective calcium channel blocker indicating that the inward currents were calcium currents. SNX-482 $(0.1 \mu \mathrm{M})$ or $\mathrm{NiCl}_{2}(50 \mu \mathrm{M})$, selective R-type calcium channel blockers inhibited calcium currents by $50 \%$. R-type calcium currents recorded from the nerve cell bodies were inhibited in a pertussis toxin-sensitive manner by UK 14,304 (1 $\mu \mathrm{M})$, an $\alpha_{2}$-adrenergic receptor agonist. Pertussis toxin inhibits $\mathrm{Gi} / \mathrm{Go}$ G-proteins. $\mathrm{KCl}(60 \mathrm{mM})$ induced depolarization caused increases in nerve cell body and nerve terminal Fluo-4 fluorescence that were blocked by $\mathrm{CdCl}_{2}$. This result indicates that increases in Fluo-4 fluorescence were due to activation of voltage-gated calcium channels. UK 14,304 reduced $\mathrm{KCl}$-induced increases in Fluo-4 fluorescence by about $50 \%$ in nerve terminals and cell bodies. SNX-482 and $\mathrm{NiCl}_{2}$ also inhibited $\mathrm{KCl}$-induced fluorescence by about $50 \%$. UK 14,304 did not reduce Fluo-4 fluorescence in the presence of SNX-482 or $\mathrm{NiCl}_{2}$. Conclusions. R-type channels are expressed by most myenteric neurons and nerve fibers. These channels are modulated by $\alpha_{2}$-adrenergic receptors using a Gi/Go-dependent pathway. R-type calcium channels in nerve fibers are also the target for $\alpha_{2}$-adrenergic receptor modulation. Norepinephrine released from sympathetic nerves may inhibit transmitter release from myenteric neurons by acting at nerve terminal $\alpha_{2}$-adrenergic receptors to inhibit R-type calcium channels. (Supported by DK57039)

\section{M1827}

5-HT 4 Receptor Agonists Increase Transmitter Release Probability from Myenteric Nerve Terminals

Jim Ren, James Galligan

Background. 5- $\mathrm{HT}_{4}$ receptors are localized to nerve terminals in the myenteric plexus. Activation of $5-\mathrm{HT}_{4}$ receptors increases fast excitatory postsynaptic potentials (fEPSPS) mediated by acetylcholine (ACh) acting at nicotinic acetylcholine receptors (nAChRs). While it is known that $5-\mathrm{HT}_{4}$ receptors couple to the stimulatory G-protein Gs, the underlying mechanism(s) for $5-\mathrm{HT}_{4}$ receptor-mediated facilitation of fast synaptic transmission is unknown. Methods. This mechanism was investigated using acutely isolated longitudinal muscle-myenteric plexus (LMMP) preparations and myenteric neurons maintained in primary culture and intracellular and patch clamp electrophysiological recording techniques. Results. In LMMP preparations, a train of 20 electrical stimuli at $10 \mathrm{~Hz}$ evoked fEPSPs that declined in amplitude with a time constant of $0.6 \pm 0.1 \mathrm{~s}$ from an initial amplitude of $17 \pm 2 \mathrm{mV}$ to a steady state level of $0.7 \pm 0.3 \mathrm{mV}(\mathrm{n}=9)$. In the presence of the $5-\mathrm{HT}_{4}$ receptor agonist, renzapride $(0.1 \mu \mathrm{M})$, fEPSPs declined with a time constant of $0.6 \mathrm{~s} \pm 0.1 \mathrm{~s}$ but the amplitude of the first fEPSP was $28 \pm 3 \mathrm{mV}$ and this declined to a steady state level at the 20th stimulus of $6 \pm 2 \mathrm{mV}$ (both $\mathrm{P}<0.05 \mathrm{vs}$. control). Under control conditions, fEPSP amplitude recovered from run-down with a time constant of $7 \pm 2 \mathrm{~s}$ while in the presence of renzapride the recovery time constant was only $1.6 \pm 0.2 \mathrm{~s}(\mathrm{P}<0.05)$. Forskolin $(1 \mu \mathrm{M})$, an adenylyl cyclase activator, mimicked the action of renzapride. H-89 $(10 \mu \mathrm{M})$, a protein kinase A (PKA) inhibitor blocked renzapride's effects. We then tested the hypothesis that 5-HT receptormediated increases in fEPSP amplitude are due to an increase in release probability from single varicosities. This was accomplished using outside-out patches obtained from myenteric neurons containing nAChRs to detect ACh release from individual varicosities. Renzarpride increased release probability from $27 \%$ to $52 \%(\mathrm{P}<0.01)$. To confirm the $5-\mathrm{HT}_{4}$ receptor nature of the observed response, studies were also performed with the 5- $\mathrm{HT}_{4}$ receptor agonist, tegaserod $(0.1 \mu \mathrm{M})$. Tegaserod also increased release probability from $27 \%$ to $49 \%$ $(\mathrm{P}<0.01)$. Conclusions. These data suggest that an increase in release probability from single varicosities is a mechanism by which $5-\mathrm{HT}_{4}$ receptor agonists facilitate fEPSPs. This maintains synaptic transmission during periods of high frequency activity and accelerates recovery from transmitter depletion. Facilitation of fast synaptic transmission would contribute to the prokinetic actions of 5-HT 4 receptor agonists. (Supported by DK57039. Tegaserod was a generous gift from Novartis, E. Hanover, NJ)

\section{M1828}

Effects of Serotonin Transporter Inhibition On Gastrointestinal Motility and Colonic Sensitivity in the Mouse

Matthew D. Coates, Anthony C. Johnson, Beverley Greenwood-van Meerveld, Gary M. Mawe

Serotonin (5-HT) released from enterochromaffin cells is an important activator of intrinsic and extrinsic reflexes in the bowel. The serotonin-selective reuptake transporter (SERT) which is expressed on intestinal enterocytes, terminates the actions of 5-HT by removing it from the interstitial space. SERT expression is decreased in animal models of intestina inflammation and in individuals with inflammatory bowel disease (IBD) or irritable bowel syndrome (IBS). It is possible that resultant changes in serotonin signaling contribute to the manifestation of clinical features associated with these disorders. This investigation was conducted to test whether inhibition of SERT function leads to changes in gut motility and/ or sensitivity. METHODS: C57BL/6 mice (male; 5-8 weeks of age) underwent a 14 day treatment with the SERT inhibitor, paroxetine $(20 \mathrm{mg} / \mathrm{kg}$ ), or vehicle (50\% propylene glyco in $0.9 \% \mathrm{NaCl}$ ). In mice from both treatment groups, small intestinal transit was evaluated by dividing the distance the leading edge of a charcoal meal from the pylorus by the total length of the small intestines. Colonic motility was assessed by measurement of stool frequency. Electromyographic recording from the rectus abdominis muscle was used to assess sensitivity to colonic distension. RESULTS: Small intestinal transit was significantly decreased in mice treated with paroxetine (vehicle, $0.81 \pm 0.03, \mathrm{n}=10$; paroxetine, $0.67 \pm 0.03, \mathrm{n}$ $10 ; \mathrm{p}<0.05$ ). Stool pellet elimination was reduced in mice treated with paroxetine (vehicle $9.2 \pm 0.6$ pellets $/ 30 \mathrm{~min}, \mathrm{n}=12$; paroxetine, $5.8 \pm 0.7$ pellets $/ 30 \mathrm{~min}, \mathrm{n}=16 ; \mathrm{p}<0.05)$ Responsiveness to colorectal distension at a nociceptive level $(60 \mathrm{~mm} \mathrm{Hg}$ ) was attenuated in mice treated with paroxetine (control, $120.0 \pm 30.4$ spikes $/ 10 \mathrm{sec}, \mathrm{n}=9$; paroxetine, $50.1 \pm 19.9$ spikes $/ 10 \mathrm{sec}, \mathrm{n}=7 ; \mathrm{p}<0.05)$. SUMMARY AND CONCLUSIONS: These results demonstrate that pharmacological inhibition of SERT function significantly alters gastrointestinal motility and sensitivity in mice, and support the concept that altered SERT expression and function could contribute to symptoms associated with IBS and IBD. It is possible that the decrease in viscerosomatic sensitivity detected in the paroxetine group was due to an effect of the compound on serotonergic pain inhibitory pathways in the spinal cord. Supported by NIH grant DK62267, Novartis Pharmaceuticals and NIH P20 COBRE grant RR16435.

\section{M1829}

Role of Oxidative Stress and Prostaglandins On Colonic Muscle Cell Damage Induced By Exposure of Human Mucosa to a Pathogenic Lipopolysaccharide Michele Pier Luca Guarino, Carola Severi, Simone Carotti, Webiao Cao, Ivan Tattoli, Anna Maria Altomare, Rossana Alloni, Renato Caviglia, Sara Emerenziani, Michele Cicala

The suppression of intestinal muscle contractility induced by lipopolysaccharide (LPS) in animal models of septic ileus has been suggested to be mediated by acute activation of macrophages which primarily release inflammatory mediators and reactive oxygen species. Suppression of colonic smooth muscle cell (SMC) contractility induced by inflammation and oxidative stress seems to be mediated by the activation of the transcription factor NFkappa B. AIM: To define if human mucosal exposure to LPS is related to oxidative stress and whether this leads to suppression of muscle cell contractility via activated NF-kappa B and prostaglandin release. METHODS: From fresh specimens of human colon, the mucosa and submucosa were removed and sealed between two chambers, with the luminal side of mucosa facing upward and covered with $5 \mathrm{~mL}$ of Krebs solution, and the submucosal side facing downward into $20 \mathrm{~mL}$ of Krebs. Both chambers were oxygenated and kept at $37^{\circ} \mathrm{C}$ LPS (Sigma) obtained from the pathogenic strain of Escherichia coli 0111:B4 was added to the luminal side of the mucosa and kept for 30 minutes. Enzymatically isolated SMCs were exposed for 30 minutes to normal buffer or to buffer from the submucosal chamber of mucosa pre-exposed to LPS (LPS-buffer). SMCs were incubated for 15 minutes with LPSbuffer in the absence or presence of $\mathrm{H}(2) \mathrm{O}(2)$ scavenger catalase $(1,200 \mathrm{U} / \mathrm{mL})$ or indomethacin $(5 \mu \mathrm{M})$, a cyclooxygenase inhibitor. Furthermore SMCs were pre-incubated with MG132 $(0.1 \mu \mathrm{M}$, for $15 \mathrm{~min})$, an inhibitor of NF-kappa B activation, before direct LPS exposure $(50 \mathrm{ng} / \mathrm{mL})$. At the end of pre-incubation periods acetylcholine (ACh) (10-6M) contractionresponse was tested. RESULTS: SMCs incubated with normal buffer showed a maximal contraction of $26 \pm 2 \%$. SMCs exposed to LPS-buffer presented no change in the resting cell length $(5.5 \pm 2 \%$ decrease of unstimulated cell) but inhibition of maximal contraction o $75.3 \pm 6 \%$ compared to response in normal buffer. The inhibition was reversed by preincubation of SMCs with either indomethacin or catalase $(18.5+5 \%$ and $7.8 \pm 2 \%$ inhibition of maximal contraction, respectively). Inhibition of maximal contraction was also reversed in cells pretreated with MG132, before direct LPS exposure (11.2 $\pm 3 \%)$. CONCLUSIONS: The relevant impairment of SMC contractile response, observed after acute exposure of colonic mucosa to a pathogenic LPS, is reversed by the inhibition of cyclooxygenase expression and NF-kappa B activation and by removing $\mathrm{H}(2) \mathrm{O}(2)$. It is conceivable that excessive mucosal and/or muscular oxidative stress triggers an increased production of prostaglandins which leads to suppression of muscle cell contractility via NF-kappa B activation.

\section{M1830}

Heterogeneities in ICC Activity Within the Canine and Murine Large Intestine in Situ Are Revealed By Ca2+ Fluorescent Imaging

Peter Bayguinov, Hyun-Tai Lee, Kyu Joo Park, Grant W. Hennig, Nick J. Spencer, Sean M. Ward, Kenton M. Sanders, Terence K. Smith

Background: Both fast and slow electrical rhythms have been recorded across the muscularis of the large intestine in a variety of species including human, dog and mouse. A 20 cycles/ minute rhythm called myenteric potential oscillations (MPOs), has been observed in both the longitudinal muscle (LM) and myenteric circular muscle (CM) of the dog and human colon; this rhythm is unstable and waxes and wanes over time. In contrast, within the $\mathrm{CM}$ at the submucosal border a regular 5-7 cycles/minute rhythm is observed. Aims: We hypothesized pacemaker cells at the myenteric (ICC-MY) and submucosal (ICC-SM) borders 
would have different rhythms and firing patterns in the murine and canine large intestine. Methods: Segments of canine or murine proximal colon were opened, the mucosa removed, and pinned flat with either the LM uppermost (ICC-MY flap) or the submucosal surface uppermost (ICC-SM flap) in an organ bath and perfused with Krebs solution at $36^{\circ} \mathrm{C}$. In the ICC-MY flap, strips of LM were peeled away to reveal the ICC-MY network. Tissues were loaded with the $\mathrm{Ca2}+$ indicator (fluo-4) and activity monitored using an upright microscope and CCD camera. Results: In both species, individual ICC-SM cells within the submucosal network of ICC were observed to elicit rhythmic $\mathrm{Ca} 2+$ transients in a coordinated manner at a frequency between $5-8 / \mathrm{min}$. Activity in ICC-SM was associated with Ca2+ waves in CM followed by CM contraction. In dramatic contrast to ICC-SM, ICC-MY fired in a disorderly and uncoordinated fashion. Both intramuscular ICC (ICC-IM) aligned with the CM or LM and ICC-MY fired in a similar but irregular fashion ( 17/min). Conclusions: In both the murine and canine colon, ICC at the level of the myenteric plexus form a loosely coupled network of oscillators, whereas ICC on the submucosal surface of the CM are tightly coupled. These different activities of ICC may partially explain the differences in electrical activities generated on opposite sides of the CM. Supported by grants from NIH (NIDDK): RO1 DK45713 (TKS), DK41315 (KMS) and Core laboratory Cobre grant P2O RR-18751.

\section{M1831}

Control of Intestinal Epithelial Cell Proliferation By Enteric Glial Cells: Impact in Colorectal Cancer

Laurianne Van Landeghem, Tor Savidge, Philippe Aubert, Emmanuel Coron, Bernard Lardeux, Francois Paris, Jean-Paul Galmiche, Michel Neunlist

Introduction : Enteric glial cells (EGCs) are a major component of the enteric nervous system (ENS). Mucosal EGCs are organised in a dense cellular network that closely contacts intestinal epithelial cells (IEC). Recent in vitro studies suggest that EGCs greatly inhibit IEC proliferation. Nevertheless, it is not known whether EGCs control IEC proliferation in vivo and whether the EGC network is altered in colorectal cancer. Material et Methods : In vivo proliferation experiments were performed in transgenic mice expressing herpes simplex virus thymidine kinase under control of the GFAP promoter, a specific marker for EGCs in the intestine. Injection of ganciclovir in these animals induced a selective destruction of proliferating EGCs. After ganciclovir treatment, mice were injected i.p. with methyl $3 \mathrm{H}-$ thymidine (37kBq.g-l body weight) and intestinal tissues were processed for autoradiography studies. In addition, fragments of human colon (involved and margin) were obtained from patients undergoing surgery for colorectal cancer. These fragments were processed for immunohistochemistry and quantitative rtPCR studies of S100b, a marker of glial cells. In addition, the effects of soluble $\mathrm{S100 \textrm {b }}$ on human intestinal adenocarcinoma proliferation were characterized in vitro using Caco-2 cells seeded on Transwell filters for 6 days in the presence or absence of S100b. Results : EGC ablation in transgenic mice induced crypt cell hyperplasia with a significant increase in the number of 3H-thymidine labelled epithelial cells as compared to control nontransgenic littermates ( $28 \%+4 \%$ vs $19 \%+3 \% \cdot n=4 \cdot p<0,05)$. Immunohistochemisty performed on human colonic adenocarcinoma $(n=4)$ demonstrated a marked decrease in mucosal S100b-immunoreactivity when compared to marginal control tissues $(n=4)$. This was corroborated further by a significant $62 \%$ reduction in S100b mRNA expression in control tissues $(n=4)$. Finally, in vitro studies demonstrated that $\mathrm{S} 100 \mathrm{~b}(\mathrm{lnM})$ significantly reduced Caco-2 cell numbers as compared to controls $(19 \%, \mathrm{n}=6 ; \mathrm{p}<0,01)$. Discussion : Our results demonstrate a novel role for EGCs in inhibiting IEC proliferation in the gastrointestinal tract. The significant diminution of EGC found in the proximity of colonic tumours suggests that an alteration of the EGC network may favour the development of colorectal cancer.

\section{M1832}

Ca2+ Imaging of Pacemaker Activity in Interstitial Cells of Cajal in Human Jejunum in Situ

Hyun-Tai Lee, Grant W. Hennig, Neal Fleming, Sean M. Ward, Kathleen D. Keef, Nick J. Spencer, Kenton M. Sanders, Terence K. Smith

Background: Interstitial cells of Cajal (ICC) are present in human gastrointestinal tract between the longitudinal and circular muscle layers where they form a dense network (ICCMY), but we know very little about the function of these cells. Morphological studies of ICC in human small intestine suggest that they resemble ICC in both larger and smaller mammals. Aims: Therefore, we attempted to study whether ICC in the human jejunum have a similar pacemaker role as they do in other mammals. Methods: Segments of human jejunum were pinned flat with the longitudinal muscle uppermost in an organ bath perfused with Krebs solution at $36^{\circ} \mathrm{C}$. Strips of longitudinal muscle were peeled away to reveal the ICC-MY. The tissues were then loaded with the $\mathrm{Ca} 2+$ indicator (fluo-4 AM) and activity monitored using an upright microscope and CCD camera. Results: The structure of the ICCMY network in the human jejunum consisted of bands or bundles of multipolar cells that had long processes and were immunoreactive for Kit. Rhythmically oscillating Ca2+ transients were observed in these cells that were similar in waveform to slow waves. Activity within ICC-MY spread through the network of ICC at rates consistent with the propagation of electrical slow waves. Activity in ICC-MY was coupled to activity in muscle layers. Ca2+ signals in ICC-MY networks were not affected by nicardipine (1 3 $\mu \mathrm{M})$, TTX (1 $\mu \mathrm{M})$, atropine $(1 \mu \mathrm{M})$, or ryanodine $(10 \mu \mathrm{M})$, but their rhythmicity was reduced or abolished by CPA $(10 \mu \mathrm{M}), 2$-APB $(50 \mu \mathrm{M}), \mathrm{FCCP}(1 \mu \mathrm{M}), \mathrm{Ni} 2+(100 \sim 250 \mu \mathrm{M})$, or mibefradil (10 30 $\mu \mathrm{M})$. The gap junction blocker $\beta$-glycyrrhetinic acid $(30 \mu \mathrm{M})$ caused ICC to fire in an uncoordinated fashion. Conclusions: For the first time, we demonstrate that ICC-MY are pacemaker cells in human jejunum and that the spread of activity within the ICC-MY network can be readily studied using fluorescent $\mathrm{Ca} 2+$ imaging. Supported by NIH grants from NIDDK: RO1 DK45713 (TKS), PO1 DK41315 (KMS) and Cobre grant P2O RR-18751 which funded the imaging core.

\section{M1833}

Enteric Glia - Quantitative Comparison Between Human and Guinea Pig (GP) Enteric Nervous System (ENS)

Sebastian Hoff, Christian Pehl, Florian Zeller, Claus von Weyhern, Michael Wegner, Michael Schemann, Anne Ruhl

Enteric glia (EGC) are the most abundant cell type in the ENS, and loss of EGC has been shown to alter enteric neuronal phenotype and function. Because exact glial cell numbers have not yet been reported for the human ENS, we have used a recently identified monoclonal anti-Sox antibody as pan-glial marker in conjunction with a pan-neuronal marker to quantify EGC in the human vs. gp. Whole-mount preparations of the myenteric (MP) and the submucous plexus (SMP; 3 layers in man) from normal human and gp ileum, proximal and distal colon were immunostained with anti-Sox and anti-neuron specific enolase antibodies. Cell counts were performed semi-automatically and expressed as cells per ganglion to rule out stretch-dependent effects. In all human gut regions, EGC numbers per ganglion were 34.8 fold higher in the MP than in the SMP $(\mathrm{p}<0.01)$; in contrast, neuronal numbers were 9.5fold higher. This was mirrored by a 4fold higher glia:neuron ratio ("glia-index") in MP vs. SMP $(\mathrm{p}<0.05)$. Strikingly, neuronal numbers per ganglion were similar between man and gp MP, whereas glial cell numbers per ganglion differed significantly by a factor of 3.6 (cf. Table 1). This resulted in significantly different glia-indices, which are 5.9 for ileum, 6.5 for ascending and 6.1 for sigmoid colon while the respective gp regions have a gliaindex of 1.7 , indicating that the glia-index in the human ENS is consistently higher than in the corresponding gp tissues $(\mathrm{p}<0.01)$. It would further increase if extraganglionic EGC were included. Overall, our data constitute the first comprehensive data set on human EGC numbers and density and provide a basis for pathological assessment of glial proliferation or degeneration in the diseased human gut. Furthermore, we have revealed marked differences between the enteric glia indices of man and gp. The glia-index appears to be correlated with functional complexity and integrative potential of the respective neuronal structures. Supported by Broad Medical Research Program IBD-0105R

Glial cell numbers/ganglion in the human/gp ENS

\begin{tabular}{|c|c|c|c|c|c|c|}
\hline & \multicolumn{4}{|c|}{ human } & \multicolumn{2}{c|}{ guinea pig } \\
\hline & $\begin{array}{c}\text { inner } \\
\text { SMP }\end{array}$ & $\begin{array}{c}\text { intermediate } \\
\text { SMP }\end{array}$ & $\begin{array}{c}\text { external } \\
\text { SMP }\end{array}$ & MP & SMP & MP \\
\hline ileum & $8.0 \pm 2.5$ & $12.5 \pm 4.1$ & $12.8 \pm 2.2$ & $314.8 \pm 159.8^{* *}$ & $6.4 \pm 1.4$ & $109.5 \pm 29.4 * *$ \\
\hline $\begin{array}{c}\text { prox. } \\
\text { colon }\end{array}$ & $10.0 \pm 4.0$ & $21.6 \pm 8.2$ & $20.9 \pm 3.9^{*}$ & $440.9 \pm 33.8^{* *}$ & $15.8 \pm 4.2$ & $136.5 \pm 42.2^{* * *}$ \\
\hline dist. colon & $8.8 \pm 1.5$ & $13.5 \pm 1.2$ & $19.5 \pm 6.4$ & $489.3 \pm 191.1^{* *}$ & $15.5 \pm 2.7$ & $114.1 \pm 50.9 * *$ \\
\hline
\end{tabular}

Data are expressed as means \pm SD. *: significantly different from inner SMP; **: significantly different from all SMP and gp or human MP, respectively.

\section{M1834}

Regional Differences in Nitrergic Innervation to Mouse Lower Esophageal Sphincter (LES) Circular Smooth Muscle (CSM)

Yong Zhang, Hiroshi Mashimo, William G. Paterson

AIM: To characterize the inhibitory nitrergic innervation of mouse LES CSM and determine the relative contributions of nNOS and eNOS enzymes. METHODS: Nerve-mediated responses were studied using conventional intracellular recordings. Mouse LES, consisting of two " $\mathrm{C}$ "-like clasps (left and right), were studied separately. In the presence of atropine $(3 \mu \mathrm{M})$, nifedipine $(1 \mu \mathrm{M})$, guanethidine $(3 \mu \mathrm{M})$ and substance $\mathrm{P}(1 \mu \mathrm{M})$, resting membrane potential (MP) and response to nerve stimulation (NS; 4 square wave pulses, each $0.3 \mathrm{~ms}$ duration, $20 \mathrm{~Hz}$ ) were measured. RESULTS: In wild type mice, resting MP was -42 mV in both left and right LES. NS of left LES invariably induced a monophasic IJP with amplitude of $17.5 \pm 1.8 \mathrm{mV}$ and duration of $1091 \pm 108 \mathrm{~ms}(\mathrm{n}=4)$. However, NS of right LES evoked a biphasic IJP consisting of an initial faster IJP with amplitude of $29.4 \pm 1.5 \mathrm{mV}$ and duration of $720 \pm 45 \mathrm{~ms}$, followed by a slower IJP with amplitude of $9.5 \pm 1.8 \mathrm{mV}$ and duration of $14485 \pm 2097 \mathrm{~ms}(\mathrm{n}=5)$. Apamin $(300 \mathrm{nM})$ depolarized MP by $7.3 \pm 1.8 \mathrm{mV}$ over control and decreased IJP amplitude by $4.6 \pm 1.6 \mathrm{mV}(\mathrm{n}=6, \mathrm{p}<0.05)$ in left LES, while it depolarized MP by $6.5 \pm 1.2 \mathrm{mV}$ over control and decreased the fast and slow IJP amplitudes by 11.5 \pm 1.5 and $1.5 \pm 1.3 \mathrm{mV}$, respectively, in right LES $(\mathrm{n}=5, \mathrm{p}<0.05)$. L-NAME $(200 \mu \mathrm{M})$ markedly inhibited IJP amplitude from $19.5 \pm 3.5 \mathrm{mV}$ to $2.5 \pm 1.5 \mathrm{mV}(\mathrm{n}=3, \mathrm{p}<0.05)$ in left LES, and the fast and slow IJP amplitudes from $19.9 \pm 2.5 \mathrm{mV}$ and $8.3 \pm 1.4 \mathrm{mV}$, to $8.1 \pm 1.5 \mathrm{mV}$ and $0.3 \pm 0.2 \mathrm{mV}$, respectively, in right LES $(\mathrm{n}=5, \mathrm{p}<0.05)$. Moreover, nitrergic IJPs and biphasic IJPs were significantly inhibited by niflumic acid ( $200 \mathrm{nM})$, a $\mathrm{Ca}^{2+}$-activated $\mathrm{Cl}^{-}$channel blocker. To distinguish neuronal and myogenic contributions to the biphasic IJP in right LES, both nNOS- and eNOS-deficient mice were studied. In right LES of nNOSdeficient mice, only apamin-sensitive monophasic IJPs were recorded $(n=4)$. In eNOSdeficient mice $(n=4)$, the biphasic nitrergic IJPs recorded in right LES were identical to those recorded in control mice. Furthermore, sodium nitroprusside (SNP, $200 \mu \mathrm{M}$ ), a NO donor, hyperpolarized MP by $20.3 \pm 2.2 \mathrm{mV}(\mathrm{n}=3)$ and $22.4 \pm 1.5 \mathrm{mV}(\mathrm{n}=4)$ in wild type and eNOSdeficient mouse, respectively, and this hyperpolarization was unaffected by pre-application of L-NAME. However, SNP abolished the biphasic nitrergic IJPs and left apamin-sensitive purinergic IJPs intact. CONCLUSIONS: These data suggest nitrergic IJPs are regionally different in mouse LES (biphasic in the right and monophasic in the left). The mechanism(s) underlying the biphasic nitrergic IJP in right LES are unclear, but does not involve activation of myogenic NOS (eNOS) 
M1835

Interaction Between Enteric Glia and Neurons in a Primary Cell Culture Model Derived from Embryonic Mice

Priya Gomes, Werend Boesmans, Jozef Janssens, Jan Tack, Pieter Vanden Berghe

Disruption of enteric glial cells has detrimental effects on neuronal integrity in the enteric nervous system (Bush, 1998; Aubé, 2005). However, the role of glia in sustaining enteric neurons under non-pathological conditions has not been addressed. Our aim was to establish a cell culture model that allows studying glia-neuronal interactions. Therefore, intestinal cells were isolated using enzymatic dissociation of embryonic (E15) mouse intestines. Harvested cells were cultured in fetal bovine serum (10\%) medium containing and maintained for up to 14 days. Nerve growth factor (NGF), neuron (N2) or glia (G5) specific supplements (INVITROGEN) were used to modify neuronal and glial growth and maintenance. Expression of specific markers was checked by immunohistochemistry and intracellular $\mathrm{Ca}^{2+}$ changes were measured using $\mathrm{Ca}^{2+}$-sensitive dyes (Fluo-4). From day 7 onwards cells formed ganglialike structures, in which MAP-2/PGP9.5-positive neurons and GFAP/S100-positive glial cells were present. Within subsets of neurons we confirmed the expression of nitric oxide synthase (NOS), choline acetyltransferase (ChAT) and calretinin. Neurons established synaptic contacts as revealed by punctate expression of synaptophysin in the network. Growth and survival of neurons and glia could be directed by adding supplements. In the presence of N2, there was a 1.1-fold increase in number of neurons as compared to control. G5 supplemented cultures showed not only an increased amount of glia, but also an increased number of neurons (4.6-fold), which was further enhanced (14-fold) by N2. Combination of two neuronal supplements (N2 and NGF) was not able to yield as many neurons as G5 on its own. The viability of the neurons was tested by brief high $\mathrm{K}^{+}$exposure, to which they responded with a rise in intracellular $\mathrm{Ca}^{2+}\left(3.03 \pm 0.22, \mathrm{~T}_{500}: 15 \pm 1 \mathrm{~s}, \mathrm{n}=48, \mathrm{~N}=12\right.$ dishes). Neurons also responded $(2.08 \pm 0.07, \mathrm{n}=12, \mathrm{~N}=4)$ to electrical fiber stimulation $(1 \mathrm{~s}, 20 \mathrm{~Hz})$. None of the glial cells displayed $\mathrm{Ca}^{2+}$ transient caused by $\mathrm{K}^{+}$depolarization. We also used lipophosphatidic acid (LPA, $10 \mu \mathrm{M}$ ), an EDG2 receptor agonist and proposed glia activator. LPA induced a robust $\left[\mathrm{Ca}^{2+}\right]_{i}$ rise $\left(2.76 \pm 0.13, \mathrm{~T}_{50}: 20 \pm 2 \mathrm{~s}, \mathrm{n}=32, \mathrm{~N}=2\right)$ in the glial cells. Conclusion: We established a primary co-culture of enteric glia and neurons that allows studying interaction between these two cell types. Neurons and glia expressed the classical neuronal and glial markers respectively and displayed transient $\mathrm{Ca}^{2+}$ rises upon stimulation. The number of enteric neurons was highly dependent on glial cell supplements, which suggests an essential role for these cells in the growth and maintenance of enteric neurons.

\section{M1836}

Local Inhibitory Reflexes Evoked By Mucosally Applied Nutrients and Sensory Mediators in Isolated Guinea-Pig Jejunum

Rachel Gwynne, Joel Bornstein

Purpose. The motility of the gut depends on the chemicals contained in the lumen, but the stimuli that modify motility and their relationship to the enteric neural pathways are unclear. This study examined local inhibitory reflexes activated by various amino acids and putative sensory mediators (ATP, serotonin (5-HT), cholecystokinin (CCK)) applied to the mucosa to characterise effective physiological stimuli and the pathways they excite. Methods. Segments of jejunum were opened along the mesenteric border and dissected to allow access to the circular muscle on one half of the preparation. The mucosa was left intact on the circumferentially adjacent half. Circular muscle cells were impaled close to the intact mucosa and chemicals were transiently applied to the mucosa (duration $150 \mathrm{~ms}$ ) $1-2 \mathrm{~mm}$ circumferen-

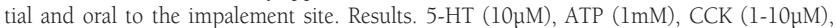
L-alanine, L-phenylalanine and L-tryptophan (all $1 \mathrm{mM}$ ) each elicited time-locked inhibitory junction potentials (IJPs) (latency $160-180 \mathrm{~ms}$, amplitude $3-15 \mathrm{mV}$, each $\mathrm{N}>6$ ) when applied to the mucosa. IJPs elicited by 5-HT were abolished by ondansetron and were resistant to 5-HT4, nicotinic, P2, NK1, AMPA or NMDA receptor antagonists (each $\mathrm{N} \geq 4$ ). ATP evoked IJPs were reduced by PPADS $(10 \mu \mathrm{M})$ but were unaffected by ondansetron or tropisetron $(10 \mu \mathrm{M})$. CCK evoked IJPs were reduced by the specific CCK B receptor antagonist L-365260 $(100 \mathrm{nM})$ or by a combination of 5-HT3 and 5-HT4 receptor antagonists. They were unaffected by the CCK A receptor antagonist devazepide (100nM), PPADS, or when the 5-HT3 or 5HT4 antagonists were added individually. The amino acids each evoked IJPs reduced by combined 5-HT3 and 5-HT4 receptor antagonism and also by PPADS. The CGRP antagonist alpha-CGRP 8-37 had no effect on responses evoked by CCK or L-alanine. In most preparations, a large component of the response remained even after addition of effective antagonists. Conclusion. Chemical stimulation of the mucosa activates distinct local reflex pathways whose pharmacologogy depends on the nature of the stimulus. 5-HT, CCK and ATP activate reflexes involving 5-HT3, CCK B and P2X receptors respectively. L-alanine, L-phenylalanine and L-tryptophan evoke reflexes that appear to involve P2X receptors and/or 5-HT3 and 4 receptors acting together. However, in most cases the responses were only partially reduced. Other transmitters involved in these reflexes remain to be identified, but do not appear to include acetylcholine acting at nicotinic receptors, CGRP, or glutamate acting at AMPA or NMDA receptors

\section{M1837}

Functional Metabotropic Glutamate Receptors Are Expressed On Enteric Glia and Their Expression Is Reduced During Colitis

Yasmin Nasser, Catherine M. Keenan, Adrienne C. Ma, Donna-Marie McCafferty, Keith A. Sharkey

Although glutamate is present in the enteric nervous system (ENS), its role in neurotransmission is not well understood. Glutamate is a neurotransmitter in the brain, but it also modulates glial function. Enteric glia are the most numerous cell in the ENS, but little is known about their role in gastrointestinal physiology or pathophysiology. We hypothesize that enteric glia are a target of neurotransmitters and play an active role in intestinal inflammation. We investigated whether enteric glia express functional metabotropic glutamate receptors, and whether receptor expression is altered during intestinal inflammation. In the myenteric plexus of the ileum and colon of mice, rats and guinea pigs, we observed widespread glial expression of the metabotropic glutamate receptor 5 (mGluR5). In vitro incubation of isolated guinea pig ileal segments with the specific mGluR5 agonist CHPG caused a dose-dependen increase in the expression of the immediate early gene product, Fos in enteric glia. We also observed that CHPG increased the expression of the phosphorylated form of the extracellular signal-regulated kinase (pERK1/2) in enteric glia. Preincubation of the tissues with the metabotropic glutamate receptor antagonist 4-CPG, had no effect on Fos expression alone but abolished the effects of CHPG. CHPG did not stimulate the expression of neuronal Fos or pERK1/2, suggesting that the effects of mGluR5 stimulation were specific to enteric glia In order to determine whether mGluR5 expression was altered by inflammation, we examined 10 week old IL-10 knockout mice, a model of spontaneous intestinal inflammation. Glia mGluR5 expression was significantly reduced in the colonic myenteric plexus when compared to controls, as assessed with real-time quantitative RT-PCR (relative expression of contro compared to GAPDH: $1.25 \pm 0.25, \mathrm{n}=4$; relative expression of IL- 10 knockout: $0.31=$ $0.22, n=7, p=0.02$ ). Substantial reductions in protein expression were also observed by immunohistochemistry and image analysis. Expression of the glial specific marker, glial fibrillary acidic protein, was unchanged in knockout mice, suggesting that inflammation did not cause widespread glial destruction. Our results suggest that enteric glia are a functional target for the glutamatergic neurotransmitter system and that changes in glutamatergic receptor expression may play a modulatory role in colitis.

\begin{tabular}{|c|c|c|c|c|c|}
\hline Treatment & $\begin{array}{c}\text { Incubation } \\
\text { Control }(\mathrm{n}=11)\end{array}$ & $\begin{array}{c}200 \mu \mathrm{M} \text { CHPG } \\
(\mathrm{n}=11)\end{array}$ & $\begin{array}{c}500 \mu \mathrm{M} \mathrm{CHPG} \\
(\mathrm{n}=5)\end{array}$ & $\begin{array}{c}200 \mu \mathrm{M} \text { CHPG }+ \\
300 \mu \mathrm{M} 4-\mathrm{CPG} \\
(\mathrm{n}=6)\end{array}$ & $\begin{array}{c}300 \mu \mathrm{M} \\
4-\mathrm{CPG} \\
\text { alone (n=6) }\end{array}$ \\
\hline $\begin{array}{c}\text { Fos positive } \\
\text { glia/ganglia }\end{array}$ & $19.97 \pm 1.73$ & $\begin{array}{c}38.31 \pm 7.54 \mathrm{P} \\
<0.001(\mathrm{vs} . \\
\text { control })\end{array}$ & $\begin{array}{c}29.34 \pm 7.65 \mathrm{P} \\
<0.01(\mathrm{vs} . \\
\text { control })\end{array}$ & $\begin{array}{c}25.92 \pm 11.14 \mathrm{P} \\
<0.05(\mathrm{vs} .200 \\
\mu \mathrm{M} \mathrm{CHPG})\end{array}$ & $18.37 \pm 9.09$ \\
\hline
\end{tabular}

\section{M1838}

Neuropeptide Y Promotes the Survival and Proliferation of nNOS-Containing Enteric Neurons

Mallappa Anitha, Simon Mwangi, Shanthi V. Sitaraman, Shanthi Srinivasan

Background: Alterations in NPY have been noted in diseases affecting the enteric nervous system such as diabetes. We investigated the role of NPY in the proliferation and suppression of apoptosis in a primary enteric neuronal culture as well as in an NPY knock out mouse model (NPY KO). Methods: Using a primary culture system of enteric neurons (EN)obtained by immunoselection from E14 rat embryonic intestines we examined the effect of NPY on enteric neuronal proliferation (BrdU incorporation) and apoptosis (Hoechst method and immunocytochemistry for cleaved caspase-3. nNOS and ChAT staining neurons were identified by immunocytochemistry. NPY-mediated phosphorylation of Akt was assessed by western blot analysis. For the in vivo studies segments of proximal colon obtained from WT and NPY KO mice were fixed and stained for peripherin, NADPH diaphorase and acetylcholine esterase to assess total number of myenteric neurons, nNOS containing neurons and excitatory neurons respectively. Electric field stimulation (EFS) of proximal colon strips was used to evaluate the inhibitory neuronal mediated relaxation of the colon. Results: Exposure of NPY $(1 \mu \mathrm{M}, 24 \mathrm{~h})$ significantly increased proliferation of $\mathrm{EN}(\%$ BrdU positive: Vehicle $37.6+4.3$; NPY: $60+2, n=4, p<0.006)$. NPY ( $1 \mu \mathrm{M}$, for $24 \mathrm{~h}$ ) also significantly reduced apoptosis in EN compared to controls assessed by the Hoechst method $(\mathrm{p}<0.002)$ and cleaved caspase 3 immunocytochemistry $(\mathrm{p}<0.0001)$. NPY induced a significant increase in nNOS+ EN compared to vehicle treated cells $(\mathrm{P}<0.01)$. No change in ChAT+ cells was noted with NPY for $24 \mathrm{~h}$. NPY significantly increased phosphorylation of Akt compared to vehicle and this was lost when treated with PI-3-Kinase inhibitor LY294002 ( $<<0.05$ vs. NPY, n= 3). We next examined the effects of NPY on enteric neurons in vivo. In NPY KO mice, there was a significant decrease in the number of NADPH staining neurons and large fibers in the colon compared to WT mice. (neurons:0.01; large fibers: $p=0.0009, n=6$ ). No change was noted in the number of acetylcholine esterase staining excitatory neurons or large fibers in the proximal colon of the NPY KO vs. WT mice ( $p=0.3760$, neurons; $p=0.2943$, large fibers; $n=6$ ). EFS evoked relaxation of the proximal colon was significantly impaired in NPY KO mice compared to WT mice. (\% relaxation at 48V: WT: $40.94+4.6$, NPY KO: 15.27 $+1.3, p=0.002, n=4)$. The neuronal staining as well as EFS data is consistent with a decrease in the inhibitory neurons in NPY KO Conclusions: Loss of NPY in the NPY KO mouse model is associated with a decrease in nNOS containing neurons demonstrated by neuronal staining and by EFS recording from muscle strips.

\section{M1839}

Characteristics of the Serotonergic (1A) Receptor Expressed By Mast Cells in Guinea-Pig Intestine

Xi-Yu Wang, Guo-Du Wang, Yun Xia, Sumei Liu, Na Gao, Gui-Jun Fei, Hong-Zhen Hu, Jackie D. Wood

Enteric mast cells sense the presence of threats in the intestine(food allergens and parasites) and signal their presence to the enteric nervous system (ENS) by releasing paracrine mediators. The ENS responds by programing secretory and motor behavior, which functions rapidly to eliminate the threat. Cramping abdominal pain,fecal urgency and explosive diarrhea are symptoms that reflect "running" of the mast cell/ENS program. In view of the large amounts of 5-HT known to be released from enterochromaffin cells in the mucosa, we tested the hypothesis that mast cells express 5-HT receptors, which influence release of mediators from mast cells to the ENS and other intramural sites (e.g., primary afferent nerve terminals). Immunofluorescence and ELISA assay were used to study expression and stimulation of 5-HT receptors expressed by mucosal mast cells (MMC) and connective tissue mast cells (CTMC) in 7 guinea-pigs. The results revealed immunoreactivity(IR)for the 5 $\mathrm{HT}_{1 \mathrm{~A}}$ receptor in $92.4 \%$ (2585/2798) of MMCs and 92.4\% (1521/1647) of CMCs in the small intestine. Nearly all tryptase-IR mast cells expressed 5-HT 1 -IR. No 5- $\mathrm{HT}_{1 \mathrm{~A}}$-IR cells expressed anti-S-100-IR, which is a glial cell marker, or anti-Hu-IR, which is a pan-neuronal marker. Immunoreactivity for neither the $5-\mathrm{HT}_{4}$ nor the $5-\mathrm{HT}_{2}$ receptor was expressed by the mast cells. Neurokinin-1 receptor-IR was expressed by 75 of 629 of $5-\mathrm{HT}_{1 \mathrm{~A}}$-IR mast 
cells and only by 3 of 246 that did not express $5-\mathrm{HT}_{4}-\mathrm{IR}$. Exposure to the $5-\mathrm{HT}_{1 \mathrm{~A}}$ agonist, BP554 $(10 \mu \mathrm{M})$ suppressed basal release of histamine from $720 \pm 56$ to $492 \pm 49 \mathrm{ng}$.gtissue This action of BP554 was suppressed when co-applied with the selective 5-HT 1 antagonist spiroxatrine $(20-50 \mu \mathrm{M})$. Exposure to $20-\mu \mathrm{M}$ 5-HT stimulated the release of histamine into the bathing medium. This stimulatory action of 5-HT was not reversed by any of the selective 5-HT receptor antagonists that included spiroxatrine, the 5- $\mathrm{HT}_{2}$ antgonist $\mathrm{LY}-53857$, the 5$\mathrm{HT}_{3}$ antagonist $\mathrm{Y}-25130$, the 5-HT $\mathrm{HT}_{4}$ antagonist GR1 13808, or the 5-HT antagonist SB269970 when applied at $30 \mu \mathrm{M}$ in 5 preparations. Tetrodotoxin $(1 \mu \mathrm{M})$ suppressed the stimulatory action of 5-HT. Application of 200-nM substance P elevated the concentration of histamine in the bathing medium by $25.37 \pm 2.3 \%$ of basal release. The results suggest that stimulation $5-\mathrm{HT}_{1 \mathrm{~A}}$ receptors on enteric mast cells suppresses the release of histamine and perhaps other mast cell mediators. Part of the 5-HT-evoked release of histamine in this study was secondary to stimulation of release of substance $\mathrm{P}$ from enteric neurons and activation of NK-1 receptors on the mast cells. (Supported by NIH ROI DK37238, ROI DK57075 and KO8 060468)

M1840

Nitric Oxide Enhances Inhibitory Synaptic Input to Non-Cholinergic Secretomotor Neurons of Guinea Pig Ileum, But Also Enhances Their Excitability

Joel Bornstein, Peipei J. Foong, Kathryn A. Marks, Zhihong Wang

Background and Aim: Nitric oxide synthase (NOS) is found in many nerve terminals within the submucous ganglia of the gut, but the role(s) of nitric oxide (NO) in enteric neural communication is unclear. The NO donor sodium nitroprusside (SNP) selectively enhances cyclic GMP production in non-cholinergic secretomotor neurons of guinea pig ileum, i.e. those immunoreactive for vasoactive intestinal peptide (VIP). This study aimed to determine whether NO modifies either synaptic or firing properties of these neurons. Methods: Guinea pigs were stunned and killed by severing their carotid arteries and spinal cords. Preparations of ileal submucosa were dissected using standard methods and pinned out for intracellular recording. Recording electrodes were often filled with biocytin dissolved in $1 \mathrm{M} \mathrm{KCl}$ to allow subsequent confirmation that impaled neurons contained VIP; but it was mostly assumed that neurons with inhibitory synaptic potentials (IPSPs) were VIP-neurons. Internodal strands were stimulated focally with $1,2,3,5$ and 10 pulses $(30 \mathrm{~Hz})$ to evoke slow excitatory synaptic potentials (EPSPs) and IPSPs in impaled neurons. All neurons exhibited fast EPSPs that were unaffected by the drugs used. Results: SNP $(100 \mu \mathrm{M})$ enhanced amplitudes of IPSPs in all VIP neurons studied $(\mathrm{n}=44)$ and for all stimulus protocols; e.g., IPSPs evoked by a single stimulus pulse increased from $4.7 \pm 0.9 \mathrm{mV}$ to $9.1 \pm 1.0 \mathrm{mV}(\mathrm{n}=29, \mathrm{P}<0.001)$ and those evoked by 5 pulses $(30 \mathrm{~Hz})$ increased from $21.8+1.3 \mathrm{mV}$ to $25.6 \pm 1.2 \mathrm{mV}(\mathrm{n}=34$ $\mathrm{P}<0.001$ ). SNP also depressed slow EPSPs evoked by $1,2,3,5$ and 10 pulse stimuli. The effects of SNP were blocked by an inhibitor of soluble guanylyl cyclase (ODQ, 10 $\mu \mathrm{M})$. The $\alpha 2$-receptor antagonist idazoxan $(2 \mu \mathrm{M})$ depressed IPSP amplitudes, without affecting slow EPSPs. In idazoxan, SNP enhanced IPSPs evoked by long stimulus trains ( 5,10 pulses), but not those evoked by short trains (1-3 pulses). VIP neurons typically fire action potentials only at the start of long depolarizing current pulses. SNP markedly enhanced firing in response to such depolarizations, often converting transient firing to sustained firing. In contrast, the NOS inhibitor NOLA $(100 \mu \mathrm{M})$ depressed IPSPs, enhanced slow EPSPs and depressed firing during depolarizing current pulses. Conclusions: NO enhances inhibitory transmission to VIP secretomotor neurons largely via an action on sympathetic inputs. Paradoxically, this increased inhibition is accompanied by enhanced excitability of these neurons indicating that NO differentially affects intrinsic and extrinsic neural pathways converging on the VIP neurons. Endogenous NO apparently has similar effects, suggesting new roles for enteric NOS neurons.

\section{M1841}

Stretch-Induced Relaxation of the Lower Esophageal Sphincter Ravinder K. Mittal, Ibrahim Dogan, Jianmin Liu, Valmik Bhargava

Swallow and esophageal-distension induced relaxation of the lower esophageal sphincter (LES) is associated with its cranial movement, of up to $20 \mathrm{~mm}$, due to a concurrent longitudinal muscle contraction of the esophagus. We hypothesized that longitudinal muscle contraction causes a cranially directed mechanical stretch of the LES, which in turn may be responsible for the LES relaxation. Methods: We studied 7 opossums. A silicon tube was placed via mouth into the esophagus and surgical laprotomy was performed. Two needles with silk sutures were passed, 90 degrees apart, through both esophageal walls and silicon tube 1-2 cm above the LES. Tube was withdrawn and one end of each of the 4 sutures was anchored to the esophageal wall and the other end exited through the mouth. The 4 sutures were used to exert graded cranial stretch on the LES using pulley and weights. LES pressure was monitored using a sleeve sensor anchored to LES. Effect of nitric oxide antagonist (LNNA $20 \mathrm{mg} / \mathrm{kg}$, I.V. bolus) on the stretch effect was also studied. Results: Cranial stretch caused LES relaxation with the recovery of LES pressure following cessation of stretch. Degree of LES relaxation induced by stretch increased with applied weight. A nearly complete LES relaxation was observed in all animals $78+12 \%($ mean + SD) before L-NNA (with a residual pressure of $11 \pm 6 \mathrm{mmHg}$ ); however, weight required to induce complete LES relaxation differed in animals $(714 \pm 348 \mathrm{~g})$. A cranial movement of $15-20 \mathrm{~mm}$ of the esophagus occurred with the weights used for the cranial stretch. Increased duration of stretch resulted in increased duration of relaxation. L-NNA, resulted in inhibition of the stretch induced LES relaxation ( $19 \pm 11 \%$ with a residual pressure of $46 \pm 14 \mathrm{mmHg}$ ). Conclusions: Our data support the presence of a stretch activated inhibitory neuron in the pathway to the LES. The role of stretch activated neuron in the mediation of swallow-induced LES relaxation requires further investigation.

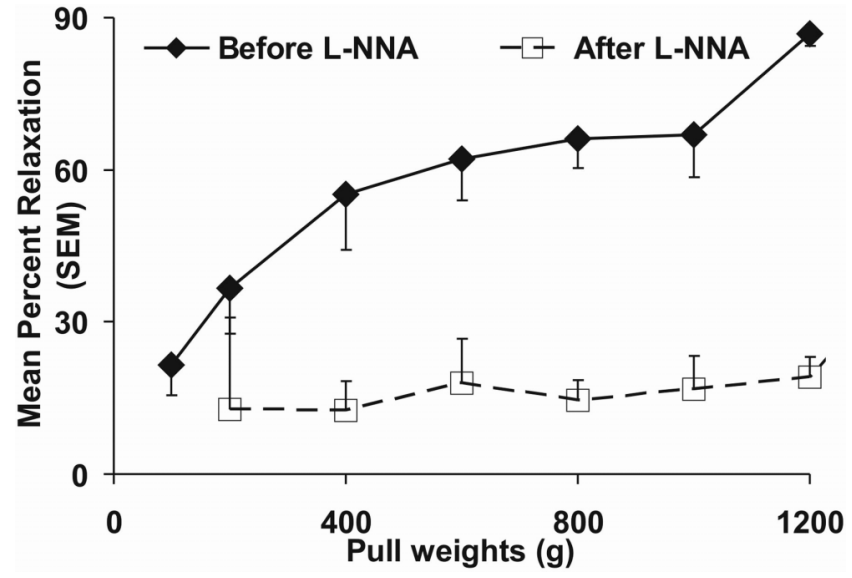

M1842

Neurogenic Mucosal Bicarbonate Secretion Mediated By the Purinergic P2yl Receptor in Guinea-Pig Duodenum

Gui-Jun Fei, Xiucai Fang, Xi-Yu Wang, Guo-Du Wang, Sumei Liu, Na Gao, Hong-Zhen Hu, Yun Xia, Jackie D. Wood

We reported earlier that ATP is released as a neurotransmitter in the guinea-pig submucosal plexus where it becomes a ligand for the $\mathrm{P} 2 \mathrm{Y}_{1}$ receptor subtype expressed by secretomotor neurons (J Physiol 2003.550:493-504) and that ATP, applied in Ussing flux chambers, acts at $\mathrm{P}_{2} \mathrm{Y}_{1}$ receptors on secretomotor neurons to increase short-circuit current as a reflection of stimulation of mucosal chloride secretion (Gastroenterology 2004;126:A148.). The present study investigated involvement of ATP and the neuronal $\mathrm{P}_{2} \mathrm{Y}_{1}$ receptor in stimulation of mucosal secretion of bicarbonate $\left(\mathrm{HCO}^{-}\right)$in guinea-pig duodenum. Muscle-stripped preparations were micro-dissected from the duodenum and mounted between the halves of Ussing flux chambers. Under continuous short-circuited conditions, the actions of exogenously applied ATP on mucosal $\mathrm{HCO}^{-}$secretion were investigated with the $\mathrm{pH}$-stat technique for measurement of the concentration of $\mathrm{HCO}^{-}$- in the bathing medium. Application of ATP to the submucosal side of the preparations increased $\mathrm{HCO}^{-}$in a concentration-dependent manner over a range of $10^{-7}$ to $10^{-5} \mathrm{~mol} / \mathrm{L}$ with an $\mathrm{EC}_{50}$ of $0.16 \mu \mathrm{M} / \mathrm{L}(\mathrm{P}<0.0001, \mathrm{~N}=6$ preparations). The maximal response to ATP occurred at $3 \times 10^{-6}$ mol/L (net peak $\mathrm{HCO} 3$ output $\left.0.49 \pm 0.06 \mu \mathrm{mol} . \mathrm{cm}^{2} \mathrm{~h}^{-1}\right)$. MRS2179 (10-5 mol/L), a selective P2Y purinergic receptor antagonist, significantly suppressed ATP-evoked $\mathrm{HCO}^{-}$- secretion by $47 \%$ $(\mathrm{P}<0.0001)$ and short circuit current $(\mathrm{Isc})$ by $63 \%(\mathrm{P}<0.002)$. Pretreatment with tetrodotoxin $\left(0.5 \times 10^{-6} \mathrm{~mol} / \mathrm{L}\right)$ or a selective $\mathrm{VIP}_{1}$ receptor antagonist $\left(10^{-6} \mathrm{~mol} / \mathrm{L}\right)$ suppressed ATPevoked $\mathrm{HCO}^{-}$secretion by $61 \%$ and $41 \%$ respectively $(\mathrm{P}<0.001)$ and $\mathrm{Isc}$ by $97 \%$ and $70 \%$ respectively $(\mathrm{P}<0.0001$ and $\mathrm{P}<0.001)$. Pretreatment with the muscarinic antagonist, scopolamine $\left(10^{-5} \mathrm{~mol} / \mathrm{L}\right)$, or the alpha 2 noradrenergic antagonist, idazoxan $\left(10^{-5} \mathrm{~mol} / \mathrm{L}\right)$, did not alter ATP-evoked $\mathrm{HCO}^{-}$secretion or Isc. The results suggest that ATP stimulates duodenal $\mathrm{HCO}^{-}$secretion mostly through an excitatory action at purinergic $\mathrm{P}_{2} \mathrm{Y}_{1}$ receptors on neurons in the submucosal division of the enteric nervous system. Stimulation of VIPergic secretomotor neurons accounts for a significant component of the ATP-evoked secretory response. (Supported by NIH R01 DK 068258)

\section{M1843}

Brain-Derived Neurotrophic Factor (BDNF) and Serotonin Are Co-Stored in a Population of Mucosal Enteroendocrine Cells of Intestine and Colon of Rat and Guinea Pig

John R. Grider, Melissa Gulick, Liya Qiao

Previously, we have shown in isolated segments of rat colon, that Brain-Derived Neurotrophic Factor (BDNF) is released during the peristaltic reflex and that BDNF enhances the release of serotonin (5-HT) and calcitonin gene-related peptide (CGRP) elicited by mucosal stimulation thereby leading to an augmentation of the peristaltic reflex. We have also shown that BDNF and CGRP are co-stored in neurons of myenteric ganglia cultured from guinea pig intestine indicating a neural source of BDNF, and suggesting that the augmentation of the peristaltic reflex occurs at the level of the intrinsic sensory neuron. Aim: to determine if BDNF is also present in the mucosa and if there is a relationship between BDNF-containing and 5-HTcontaining cells. Methods: Segments of colon and intestine from guinea pig and rat were fixed in $4 \%$ paraformaldehyde and transverse sections cut at a thickness of 10 micrometers. The sections were co-stained for 5-HT and BDNF. BDNF-immunoreactive mucosal cells were counted in each cross-section; the number of cells that were co-stained for 5-HT was determined and expressed as a percentage of total BDNF-immunoreactive cells. Results: In the rat colon, neurons of the submucosal and myenteric plexus, and cells in the mucosa were immunostained for BDNF. In the myenteric and submucosal plexus, both cell bodies and fibers were stained; BDNF-positive fibers were also present in circular smooth muscle. In the mucosa of the rat colon, BDNF-immunoreactive cells had a morphology typical of enteroendocrine cells and were more frequent in the basal area of the colonic crypts; there were $58 \pm 4$ BDNF-immunoreactive mucosal cells per transverse section. Dual staining with 5 -HT antibody indicated that $27.8 \pm 5.4 \%$ of these BDNF-immunoreactive mucosal cells were co-stained for 5-HT. Examination of the rat small intestine, and guinea pig small intestine and colon produced similar patterns of staining. In the rat small intestine, $30.1 \pm 4.8 \%$ of BDNF-immunoreactive mucosal cells were co-stained for 5-HT; in the guinea pig colon, $32.8 \pm 5.2 \%$ of the BDNF-immunoreactive mucosal cells were co-stained for 5-HT; and in the guinea pig intestine, $36.1 \pm 6.4 \%$ of the BDNF-immunoreactive mucosal cells were 
co-stained for 5-HT. Conclusion: BDNF is present in enteric neurons and in mucosal enteroendocrine cells of rat and guinea pig intestine and colon. A significant percentage of mucosal BDNF cells also contain 5-HT suggesting that the augmentation of the peristaltic reflex by BDNF might be mediated at the level of the mucosal sensory apparatus in addition to the augmentation at the level of the intrinsic sensory neurons

\section{M1844}

Enhanced Jejunal Secretory Function in Patients with Irritable Bowel Syndrome (IBS)

Magnus Simren, Jenny Gustavsson, Evan A. Thomas, Joel C. Bornstein, Henrik Sjovall

Background: The pathophysiology of IBS is complex and incompletely understood. A few small studies exists suggesting abnormal secretory function in the small intestine in IBS. Potential difference (PD) measurement in the small intestine reflects electrogenic chloride secretion, which depends on mechanosensitive submucosal neurons. Phase III of the migrating motor complex (MMC) represents a sustained mechanical stimulation of these neurons. Secretory events, measured by PD, before, during and after phase III therefore reflect submucosal neuronal function. We aimed to evaluate small intestinal secretory function, using PD, in a large sample of IBS patients. Methods: We included 82 patients with IBS according to the Rome II criteria (59 females; mean age 37 (19-67) years) and 25 healthy controls (13 females; mean age 39 (21-71) years). Submucous plexus function in the jejunum, and its linkage with motor activity, was studied by modified multilumen manometry, using two manometry channels as flowing electrodes. Motor activity and transmucosal PD were measured during 3 hours in the fasting state, and one hour in the fed state. Secretory events associated with the different phases of the MMC were evaluated using an automated system and compared between the groups. Results: Mean fasted PD did not differ between the groups $(-0.61 \pm 1.6$ vs. $-0.53 \pm 2.3 \mathrm{mV}$; NS), and there were also similar PD values during phase I and phase II in IBS patients and healthy controls. The maximal PD during phase III was higher (=more lumen negative) in the IBS group compared with controls $(-12.7 \pm 6.0$ vs. $-9.5 \pm 5.0 \mathrm{mV} ; \mathrm{p}=0.03)$, but the slope of the rise at onset of phase III did not differ between groups (NS). The PD during the fed state was also higher in the patient group ($4.9 \pm 2.6$ vs. $-3.8 \pm 2.6 \mathrm{mV} ; \mathrm{p}<0.05)$. As a measure of the responsiveness of the system the PD signal was corrected for pressure and this measure was also higher in the IBS patients than in the controls $(-1.0 \pm 0.6 \mathrm{vs} .-0.2 \pm 0.4 \mathrm{mV} / \mathrm{mmHg} ; \mathrm{p}<0.0001)$. There were no significant differences in PD between IBS subgroups based on the predominant bowel habit. Conclusion: Patients with IBS demonstrate enhanced jejunal secretory function, suggesting upregulated motility-secretion linkage in these patients. This probably reflects disturbed submucosal neuronal function in IBS patients. However, this seems to be unrelated to the predominant bowel pattern of the patient.

\section{M1845}

\section{Targets of 5-HT Innervation in Guinea Pig Myenteric Plexus} Kath B. Neal, Joel C. Bornstein

Serotonin (5-HT)-immunoreactive (-IR) interneurons in the myenteric plexus form long anally-directed chains in the small intestine and make connections to other uniaxonal cell types which have not been identified. We used confocal microscopy to survey 5-HT-IR appositions to other myenteric neurons. Methods: 10 guinea-pigs were stunned and their carotid arteries severed. Segments of jejunum $(4-6 \mathrm{~cm})$ were removed, cleaned and placed in an organ bath at $37^{\circ} \mathrm{C}$, containing $500 \mathrm{nM} 5$-HT in physiological saline bubbled with $95 \% \mathrm{O}_{2}$ and $5 \% \mathrm{CO}_{2}$, for 4 hours. This selectively loads intrinsic 5-HT interneurons and not sympathetic terminals. Tissue was pinned flat and fixed in Zamboni's fixative at $4^{\circ} \mathrm{C}$ for 12 hours. Myenteric plexus was dissected, washed and incubated at $4^{\circ} \mathrm{C}$ for 36 hours in antisera against 5-HT and one of calbindin, calretinin, nitric oxide synthase (NOS) or neuropeptide Y (NPY). Preparations were washed in phosphate buffered saline, incubated for 3 hours in cocktails of secondary antisera and mounted in fluorescence mounting medium. Stacked images of stained cells were collected with Biorad MRC 1024 confocal software using a 100x objective at a centre-to-centre step of 0.2-0.6um. Appositions between 5-HTIR terminals and other neurons were defined when no intervening pixel $(0.2 \mu \mathrm{m})$ was seen. Results: Each stain was analysed in tissue from 3-4 animals. $\mathrm{n}$ is the number of cells examined. $76 \%$ of calbindin-IR neurons $(\mathrm{n}=49)$ received 5-HT appositions; these cells received $5.3 \pm 0.5$ appositions. Calretinin cells were divided into inter- and longitudinal muscle motor neurons according to morphology and position. Interneurons $(n=22)$ received $28.9 \pm 1.7$ appositions which were distributed trimodally $(x<6 n=7 ; 16<x<36 n=9 ; x>53$ $\mathrm{n}=6) .63 .2 \%$ of calretinin motor neurons $(\mathrm{n}=23)$ received appositions; none received more than 11. NOS-IR neurons $(\mathrm{n}=19)$ received $30.7 \pm 1.8$ appositions which were bimodally distributed $(x<36 n=15 ; x>54 n=4)$. All NPY cells $(n=22)$ received large numbers of 5-HT appositions (81.5 1.0). Conclusion: Myenteric neurons targeted by 5 -HT comprise several cell types. Subgroups of calretinin-IR interneurons and NOS-IR neurons which are not currently distinguishable by other criteria receive large numbers of 5-HT appositions. Although some myenteric calbindin-IR cells receive serotonergic slow excitatory postsynaptic potentials, these cells receive very small numbers of appositions, suggesting that even small numbers of contacts can have physiological roles. Myenteric NPY-IR cholinergic secretomotor neurons are highly targeted by 5-HT, consistent with a role for 5-HT in cholinergic secretomotor pathways.

\section{M1846}

Expression of the Transient Receptor Potential Vanilloid 4 (TRPV4) Channel in the Enteric Nervous System of the Rat

$\mathrm{Na}$ Gao, Hong-Zhen Hu, Xiucai Fang, Sumei Liu, Gui-Jun Fei, Xiyu Wang, Guo-Du Wang, Yun Xia, Jackie D. Wood

Transient receptor potential vanilloid (TRPV4) belongs to the vanilloid subfamily of the transient receptor potential (TRP) channels. The expression of TRPV4 is found in a broad range of tissues, including brain, smooth muscle, liver, lung, spleen, kidney, fat, and vascular endothelium. In mammals, the physiological roles for TRPV4 are highly divergent because TRPV4 is activated by a variety of stimuli including thermal stress, fatty acid metabolites and hypotonicity. TRPV4 has been proposed to a mechanosensor and osmosensor. We used indirect immunofluorescence techniques to study the expression pattern for TRPV4 in the rat enteric nervous system. TRPV4 immunoreactivity (IR) was found in 46.0\% (1253/2721) of the population of submucosal neurons and $61.7 \%$ (1236/2002) of myenteric neurons by co-labeling with anti-Hu antibody. Neuronal localization of chemical codes, including calretinin, calbindin, neuropeptide Y (NPY), vasoactive intestinal peptide (VIP) and nitric oxide synthase (NOS) were used to study the chemical coding for TRPV4. In the myenteric plexus, $86.6 \%$ (1017/1174) of TRPV4-IR neurons expressed calretinin-IR and 80.7\% (1017) 1260) of calretinin-IR neurons expressed TRPV4-IR. All 486 NPY-IR neurons expressed TRPV4-IR and about $38.5 \%$ (486/1264) of TRPV4-immunoreactive neurons expressed NPYIR. About $40.1 \%$ (73/182) of calbindin-IR neurons expressed TRPV4-IR while only $4.4 \%$ (73/1677) of TRPV4-IR neurons expressed calbindin-IR. None of 1090 NOS -IR neurons had overlapping images with 1524 TRPV4-IR neurons in the myenteric plexus. In the submucosal plexus, TRPV4-IR was found in $71.4 \%$ (1088/1522) of calbindin-IR neurons and 56.5\% (1088/1925) of TRPV4-IR neurons expressed calbindin. All 1496 TRPV4-immunoreactive neurons expressed calretinin-IR and 84.1\% (1496/1779) of calretinin-IR neurons expressed TRPV4-IR. About $96.8 \%$ (655/677) of NPY-IR neurons expressed TRPV4-IR and $42.4 \%(655 / 1546)$ of TRPV4-IR neurons had NPY-IR. All 665 VIP-IR neurons expressed TRPV4-IR and 48.9\% (665/1360) of TRPV4-IR neurons expressed VIP-IR. The results sugges that TRPV4 is expressed abundantly in rat enteric nervous system and that the chemical coding for TRPV4-IR differs between the myenteric and submucosal plexuses. Expression of TRPV4 channels might be involved in sensing functions of neurons in the enteric nervous system. (Supported by NIH ROI DK37238 and DK57075)

\section{M1847}

Temporal and Regional Differences in the Gastrointestinal Motor Response to Infection with Schistosoma Manson Faiza B. Abdu, Osama Tayeb, David Grundy

Background: Mouse models of schistosomiasis have been used to investigate inflammation induced changes in intestinal motor function. Previously we described intestinal motor dysfunction during acute Schistosoma mansoni (S. mansoni) infection (Abdu \& Grundy, Gastroenterology Volume: 128, Suppl 2, page A-76, 2005). Our aim in this study was to investigate the role of inflammatory mediators in the mechanisms underlying post-inflammatory changes during chronic inflammation. Methods: Experiments were performed in Swiss mice 8- and 16-wk following infection with S. mansoni compared to untreated controls. Jejunal and colonic motility were assessed using a Trendelenburg type preparation to study aboral directed motor complexes (MC) as described previously (Abdu et al., AJP. Am J Physiol Gastrointest Liver Physiol. G624-G633). Data were expressed as mean \pm SEM (n = 4-17) and were analysed by paired or un-paired t tests as appropriate. Results: Infection had profound effects on jejunal and colonic motility where there was a significant increase in MC amplitude and an increase in frequency reflected by a decease in the interval between MC. Naproxen $(10 \mu \mathrm{M})$ caused a significant increase in amplitude and decrease in interval in the jejunum of control $(5.29$ versus $9.86 \pm 1 \mathrm{cmH} 2 \mathrm{O}, \mathrm{P}<0.002 ; 39.58 \pm 5$ versus 24.10 $\pm 2 \mathrm{~s}, \mathrm{P}<0.02$ respectively) and 8 -wk infected animals only $(17.93 \pm 1$ versus $24.73 \pm 0.3$ $\mathrm{cmH} 2 \mathrm{O}, \mathrm{P}<0.008 ; 49.06 \pm 3$ versus $19.01 \pm 0.6 \mathrm{~s}, \mathrm{P}<0.004$ respectively). In the colon Naproxen caused a decrease in amplitude in control animals and throughout the postinfection period $(\mathrm{P}<0.02)$ but increased the $\mathrm{MC}$ interval in control $(81.09 \pm 14$ versus 105.31 $\pm 14 \mathrm{~s}, \mathrm{P}<0.02)$ and early periods of infection only $(137.29 \pm 37$ versus $245.27 \pm 49 \mathrm{~s}, \mathrm{P}$ $<0.01)$. The 5-HT3 antagonist $\mathrm{Y}-25130(1 \mu \mathrm{M})$ had no effect on MC interval in control and infected animals $(\mathrm{P}>0.05)$ but in both jejunum and colon it caused a significant decrease in amplitude in control $(75.87 \pm 9$ versus $42.77 \pm 5 \mathrm{cmH} 2 \mathrm{O}, \mathrm{P}<0.008$ and 8 -wk $(79.45$ \pm 9 versus $41.10 \pm 5 \mathrm{cmH} 2 \mathrm{O}, \mathrm{P}<0.005)$ infected animals but not at 12 - and 16 -wk Conclusions: These data demonstrate temporal differences in the sensitivity of jejunal and colonic motor function following infection with S mansoni. Prostanoids and 5-HT3 play an important role in the regulation of motor function in both control and infected animals but altered sensitivity in the early and late post-infection period may point to plasticity in the mechanisms responsible for intestinal dysmotility.

\section{M1848}

Choline Transporter: An Additional Marker of Cholinergic Nerves in the Enteric Nervous System

Andrea M. Harrington, Margaret Lee, Sim-Yee Ong, Eric Yong, Pam Farmer, John M. Hutson, Bridget R. Southwell

Purpose: The cholinergic circuitry of the enteric nervous system (ENS) is labelled using antibodies against molecules involved in acetylcholine (ACh) synthesis and release. A new component of ACh synthesis, the high affinity choline transporter (CHT), which is essentia in the uptake of choline into cholinergic nerves, has been isolated and cloned. Recently, antiserum against CHT has been used in the central nervous system to label cholinergic nerves, but has not been located in the ENS. The aim of this study was to localise CHT immunoreactivity (IR) within rat and human intestine and determine if CHT colocalised with other cholinergic markers in the ENS. Methods: Segments of rat $(n=3)$ ileum and colon and human paediatric $(n=3)$ colon were fixed, prepared for sections and wholemounts and incubated with antisera against the full length human CHT sequence, followed by a fluorescent secondary antibody. Tissue was double labelled using antibodies to neuronal nitric oxide synthase (nNOS), common choline acetyltransferase (cChAT), substance P (SP) and vesicular acetylcholine transporter (VAChT). Colocalisation was quantitated in 3 confocal images from each segment. Results: In human and rat intestine, CHT-IR was present in nerve fibres in the circular muscle and myenteric ganglia and in myenteric nerve cell bodies. In human colon, CHT-IR was present in almost all VAChT-IR cholinergic nerve fibres in the circular muscle and myenteric ganglia. In contrast, in the rat only $56 \%$ of circular muscle VAChT-IR nerve fibres were CHT-IR, with this accounting for $79 \%$ of CHT-IR nerve fibres. In the myenteric ganglia, $48 \%$ of VAChT-IR nerve fibres were CHT-IR, accounting for $50 \%$ of CHT-IR nerve fibres. Surprisingly, no CHT-IR nerve cell bodies were CChAT-IR. Yet, 
$40 \%$ of CHT-IR nerve cell bodies were SP-IR (tachykinergic), accounting for 12\% of SP-IR nerve cell bodies. In both species, there was little localisation of CHT-IR in nitrergic NOSIR inhibitory nerve fibres. Conclusions: In human paediatric colon, there was almost complete colocalisation of CHT-IR and VAChT-IR in nerve fibres in the circular muscle and myenteric ganglia. Therefore the CHT antisera would be useful for studying the enteric cholinergic circuitry in human intestine and complimentary to currently used labels. In rat, CHT-IR identified VAChT-IR positive, VAChT-IR negative nerve fibres and cChAT-IR negative cell bodies. This result suggests the antibody does not bind to all the cholinergic circuitry identified by cChAT-IR or VAChT-IR. Further studies are needed to determine if the CHT antibody is specifically labelling cholinergic nerves in the rat.

\section{M1849}

Localization of 5-HT $\mathrm{HT}_{3}$ Receptors to Subsets of Myenteric Neurons in the Mouse Small Intestine

Dima Alkawwas, James Galligan

Introduction. 5-hydroxytryptamine (5-HT) is an important neurotransmitter and paracrine signaling molecule in the gastrointestinal tract. Enterochromaffin cells and neurons release 5-HT which acts on myenteric neurons to stimulate acetylcholine release causing contractions of the muscle layers. The present studies were designed to characterize 5-HT receptors on myenteric neurons in the mouse small intestine and to determine if $5-\mathrm{HT}_{3}$ receptors were localized to functional subsets of myenteric neuron. Methods. The aims of this studies were accomplished by using pharmacological methods. Isometric force transducers were used to measure longitudinal muscle contractions of segments of mouse ileum maintained in vitro. Results. 5-HT caused concentration-dependent (0.1-30 uM) contractions that were not inhibited by individual treatment with methysergide ( $1 \mu \mathrm{M}, 5-\mathrm{HT}_{1} / 5-\mathrm{HT}_{2}$ receptor antagonist), ketanserin ( $1 \mu \mathrm{M}, 5-\mathrm{HT}_{2 \mathrm{~A}}$ receptor antagonist), scopolamine (1 $\mu \mathrm{M}$, muscarinic receptor antagonist), tetrodotoxin (TTX, $0.3 \mu \mathrm{M}$, axonal sodium channel antagonist), or Y25130 (10 $\mathrm{MM}, 5-\mathrm{HT}_{3}$ receptor antagonist). However, combined application of methysergide and scopolamine or methysergide and TTX inhibited 5-HT induced contractions. Similarly, combined application of methysergide and Y25130 or ketanserin and Y25130 also inhibited 5-HT induced contractions. 5-HT-induced contractions were not inhibited by spantide (10 $\mu \mathrm{M})$ a NK1/NK2 receptor antagonist. The 5- $\mathrm{HT}_{3}$ receptor agonists 2-Me-5-HT $(0.3-30 \mu \mathrm{M})$ and m-chlorophenylbiguanide $(\mathrm{CPBG})(0.1-30 \mu \mathrm{M})$ mimicked 5-HT induced contractions. In addition, combined application of apamin and nitro-L-arginine to block inhibitory nerves, potentiated CPBG-induced contractions. All responses caused by CBPG were blocked by the $5-\mathrm{HT}_{3}$ receptor antagonist, Y25130 $(10 \mu \mathrm{M})$. Conclusions. These data indicate that 5$\mathrm{HT}_{3}$ receptors are localized to cholinergic excitatory neurons and to inhibitory neurons, but not to neurons that release tachykinin peptides supplying the longitudinal muscle. In addition, an excitatory, methysergide and ketanserin-sensitive 5-HT receptor is localized to the longitudinal muscle in the mouse small intestine. (Supported by DK57039)

\section{M1850}

Distribution of R-Type Calcium Channels in the Enteric Nervous System of the Guinea Pig

Vinogran Naidoo, Xiaochun Bian, James Galligan

Introduction. Previous electrophysiological studies have shown that many myenteric neurons in the guinea pig ileum express R-type calcium channels. However, the specific neuronal subtypes expressing R-type channels have not been established. The purpose of these studies was to characterize the distribution of R-type calcium channels in the enteric nervous system of the guinea pig gastrointestinal tract. Methods. R-type calcium channels were localized using an antibody against the $\alpha \mathrm{lE}$ calcium channel subunit which encodes R-type calcium channels. Neuronal subtypes were identified using antibodies against markers for specific neuronal classes. All studies were done in whole mount preparations of adult guinea pig gastrointestinal tract. Results. $\alpha$ lE-immunoreactivity (ir) was found in myenteric nerve cell bodies in the stomach and colon but not the small intestine. $\alpha$ lE-ir co-localized with substance $\mathrm{P}(\mathrm{SP})$ in myenteric neurons in the colon and with nerve fibers in the myenteric plexus and muscle layers throughout the gut. $\alpha$ lE-ir also co-localized with vasoactive intestinal peptide (VIP) and nitric oxide synthase (NOS) in nerve fibers supplying the muscle layers throughout the gut. $\alpha$ lE-VIP neurons were detected in the colon. Gastric myenteric neurons exhibited $\alpha 1 \mathrm{lE}$-nNOS staining. $\alpha 1 \mathrm{lE}$-ir was not co-localized with 5-HT in the myenteric plexus. $\alpha 1 \mathrm{lE}$-ir was also co-localized with tyrosine hydroxylase containing nerve fibers in the myenteric plexus throughout the gut. $\alpha$ lE-ir was not detected in the submucosal layer of any gut region. Conclusions. These data indicate that R-type calcium channels are present in SP-containing excitatory motorneurons, ascending interneurons and/or intrinsic primary afferent neurons. $\alpha$ lE-ir is also found in inhibitory (VIP, NOS) neurons supplying the muscle layers $\alpha$ lE-ir is not found in 5-HT-containing descending interneurons or submucosal neurons. Finally, $\alpha$ lE-ir is localized to the endings of sympathetic nerve fibers (tyrosine hydroxylase) supplying the myenteric plexus. Drugs targeting R-type calcium channels might be useful in facilitating or inhibiting functionally distinct subsets of myenteric neurons. (supported by DK57039)

\section{M1851}

Synaptic Transmission Between the Submucous Plexus and Myenteric Plexus of the Guinea Pig Ileum

Rebecca L. Monro, Paul P. Bertrand, Joel C. Bornstein

Background and Aim: In the ileum, there is a clear correlation between motility and secretion or blood flow. Vanner et al has shown that stimulation of the myenteric plexus results in activation of submucous neurons and dilatation of arterioles ${ }^{1,2}$. The present study aimed to examine the converse, whether myenteric neurons receive synaptic input from the submucous plexus. Methods: Guinea pigs were stunned then killed by severing the carotid arteries and spinal cord. A preparation (modified from Moore \& Vanner ${ }^{2}$ ) containing exposed myenteric ganglia in one half and submucous plexus in the other, was tightly pinned mucosa up in a bath of warmed physiological saline. Intracellular recordings were made from myenteric neurons while the submucous plexus was electrically stimulated. Submucous ganglia (up to $1.3 \mathrm{~mm}$ away) were stimulated using a 7 um carbon fiber bipolar stimulating electrode with either a single pulse or a train of 10 pulses at $100 \mathrm{~Hz}$, typically $0.075 \mathrm{~mA}$ and 0.5 ms duration. Fast excitatory post-synaptic potentials (EPSPs) were recorded at membrane potentials around $70 \mathrm{mV}$ while slow EPSPs were recorded near resting membrane potential. Muscarinic EPSPs were not blocked. In every $S$ neuron, the stimulating electrode was first tested on a myenteric fiber tract to ensure both that the neuron had synaptic input and that the stimulus strength was adequate to evoke a synaptic potential, but not so great as to stimulate myenteric ganglia through the submucous plexus. Results: Of the 29 neurons studied (13 S and 16 AH neurons), stimulation of the submucous plexus evoked a synaptic potential in only 7 cases, or $24 \%$ of neurons. When the stimulated submucous plexus was situated orally with respect to the myenteric plexus, 4 of 13 neurons (31\%) had synaptic input. When it was situated circumferentially, 2 of $8(25 \%)$ had input, and when it was situated anally 1 of $8(13 \%)$ had input. In terms of neuronal type, 5 of 13 S neurons (38\%) responded with fast EPSPs, 1 of which also had a slow EPSP, while 2 of $16 \mathrm{AH}$ neurons (13\%) responded with slow EPSPs. Conclusion: This study has shown that, contrary to the situation explored by Moore \& Vanner where many submucous neurons received input from the myenteric plexus, the synaptic input to myenteric neurons from the submucous plexus appears to be sparse. These data suggest that local input from the submucous plexus less important for the coordination of reflexes by the myenteric plexus. Supported by the NH\&MRC (Australia). 1. Vanner (2000) AJP 279(2); G380-7. 2. Moore \& Vanner (2000) AJP 278(2):G273-80

\section{M1852}

Sodium Hydrosulfide Changes Amplitude and Kinetics of Inward Sodium Current in Human Jejunal Circular Smooth Muscle Cells Robert Kraichely, Peter Strege, Michael Kendrick, Michael Sarr, Joseph Szurszewski, Gianrico Farrugia

Background: Gaseous molecules such as nitric oxide and carbon monoxide are known to play an important role in gastrointestinal physiology. $\mathrm{H}_{2} \mathrm{~S}$ is another gaseous monoxide that is endogenously produced and is found at micromolar concentrations in the serum and in tissues, including the gastrointestinal tract. $\mathrm{H}_{2} \mathrm{~S}$ has recently been shown to influence the activity of vascular smooth muscle, intestinal afferent fibers and voltage gated channels. Aims: The aim of the study was to determine the effect of $\mathrm{NaHS}$, a source of $\mathrm{H}_{2} \mathrm{~S}$, on the SCN5A-encoded $\mathrm{Na}_{\mathrm{V}} 1.5$ sodium channel expressed in human jejunal circular smooth muscle cells. Methods: NaHS (0.1 mM, l mM, $10 \mathrm{mM}$ ) was dissolved in Ringers solution buffered with HEPES. This was $\mathrm{pH}$ balanced to physiologic range $(7.35-7.45)$. At this $\mathrm{pH}$ one third of $\mathrm{NaHS}$ forms $\mathrm{H}_{2} \mathrm{~S}$ gas, soluble in aqueous solution. Inward currents from dissociated human jejunal smooth muscle cells were recorded by the amphotericin perforated-patch technique in HEPES-buffered Ringers solution using a $\mathrm{Cs}^{+}$based intracellular solution to block outward $\mathrm{K}+$ currents. NaHS was applied via pipette directly into the bath to avoid mechanoactivation of the currents. Results: NaHS increased peak inward $\mathrm{Na}^{+}$current $(0.1$ $\mathrm{mM} 18 \pm 6 \%$ increase, $\mathrm{P}>0.05, \mathrm{n}=3 ; 1 \mathrm{mM} 18 \pm 6 \%$ increase, $\mathrm{P}<0.05, \mathrm{n}=9 ; 10 \mathrm{mM} 34 \pm 11 \%$ increase, $\mathrm{P}<0.05, \mathrm{n}=9)$. At the highest concentration tested $(10 \mathrm{mM})$, a large $(20 \pm 2.9 \mathrm{mV})$ rightward shift in the activation voltage and voltage at maximum inward current was observed. This effect was largely reversed with washout of the NaHS with HEPES-buffered Ringers solution. Conclusion: A product of $\mathrm{NaHS}$ in aqueous solution, likely $\mathrm{H}_{2} \mathrm{~S}$, modulates $\mathrm{Na}^{+}$ current density and kinetics in human jejunal smooth muscle cells suggesting a potential role for a third gaseous molecule in regulating gastrointestinal smooth muscle function. Supported by NIH grants DK52766, DK57061 and DK 17238

\begin{tabular}{|c|c|c|c|c|c|}
\hline & $\begin{array}{c}0 \mathrm{mM} \\
\mathrm{n}=9\end{array}$ & $\begin{array}{c}0.1 \mathrm{mM} \\
\mathrm{n}=3\end{array}$ & $\begin{array}{c}1 \mathrm{mM} \\
\mathrm{n}=9\end{array}$ & $\begin{array}{c}10 \mathrm{mM} \\
\mathrm{n}=9\end{array}$ & $\begin{array}{c}\text { Washout } \\
\mathrm{n}=7\end{array}$ \\
\hline $\begin{array}{c}\text { Increase } \mathrm{I}_{\mathrm{Na}} \text { Amplitude }(\%, \\
\text { mean } \pm \mathrm{SEM})\end{array}$ & -- & $18 \pm 6 \%$ & $* 18 \pm 6 \%$ & $* 34 \pm 11 \%$ & $4 \pm 11 \%$ \\
\hline Activation $\mathrm{V}_{1 / 2}(\mathrm{mV})$ & -48 & -44 & -47 & -26 & -42 \\
\hline
\end{tabular}

$*=\mathrm{P}<0.05$

\section{M1853}

Exon 10 in the CFTR Gene Is Critical for the Anti-Apoptotic Pancreatic Phenotype in Mice: Lessons Learned from Separate CF Mouse Models Matthew J. Dimagno, Sae-Hong Lee, Yibai Hao, Chung Owyang

BACKGROUND AND AIMS: Multiple CFTR genotypes, including the DF508 mutation in exon 10 of the CFTR gene, are associated with idiopathic recurrent acute pancreatitis (AP). Recently, we showed that UNC cftr "null" mutant mice, which have an exon 10 replacement, develop more severe experimental AP compared to WT mice that was associated with an anti-apoptotic acinar cell response and exuberant pancreatic inflammation. We hypothesized that exon 10 in the CFTR gene is of critical importance to the anti-apoptotic pancreatic phenotype and aberrant apoptosis execution steps in CF models. To test this hypothesis we performed experimental AP studies in a more clinically relevant but less well characterized mouse model of CF having the DF508 CFTR gene mutation in exon 10 (the most common in humans). METHODS: DF508 cftr (+/-) mice were bred \& weaned offspring were fed Peptamen. In vivo AP was induced by 12 hourly IP caerulein injections $(50 \mathrm{ug} / \mathrm{kg})$. Histological severity of AP was characterized by assessing three sequential execution steps of apoptosis: caspase-3 catalysis, PARP cleavage and DNA fragmentation. RESULTS: During caeruleininduced AP, DF508 cftr mice had exaggerated neutrophil infiltration and tissue edema by blinded histological examination $(\mathrm{n}=3)$. Also, mirroring observations observed in the UNC cftr (-/-) mouse pancreas, during AP, DF508 cftr pancreas had reduced apoptosis, based on undetectable DNA fragmentation (laddering) in mutant mice ( $\&$ strong laddering in WT group) \& $50 \%$ less cleavage of PARP, a caspase-3 downstream target. Unexpectedly, after induction of AP, the upstream apoptosis execution step of capase- 3 catalysis was markedly 
enhanced in DF508 cftr vs WT groups ( $\mathrm{n}=3$ ); active caspase- 3 was immunohistochemically detected in the majority (95\%) of DF508 cftr pancreatic acinar cells vs $<5 \%$ of WT acinar cells $(n=3)$. CONCLUSIONS: This study supports the clinical observation that CFTR gene mutations may predispose to pancreatitis. Further, within exon 10 of the CFTR gene, both a "null" gene mutation and the DF508 mutation (arising from amino acid substitutions) contribute to the same anti-apoptotic pancreatic phenotype and more severe AP, but by distinctly different effects on apoptosis execution steps. This data raises the possibility that different amino acid substitutions in the exon 10 region may differentially modulate apoptotic pathways. Moreover, in the DF508 mouse CF model, the unexpected inhibition of apoptosis signal transduction from execution caspase- 3 to downstream PARP may be biologically relevant in the pathogenesis of AP associated with CFTR mutations and serve as a novel target for potential therapies.

\section{M1854}

Experimental Pancreatitis in Cathepsin B-Overexpressing Mice Matthias Beier, Barbara Brandt-Nedelev, Manuel Ruthenbuerger, Iveta Jaroscakova, Norbert Schachke, Thomas Reinheckel, Julia Mayerle, Walter Halangk, Markus Lerch

Background: Premature activation of pancreatic zymogens is believed to play a critical role in the initiation of acute pancreatitis. The lysosomal proteinase cathepsin B (CTSB) is thought to be involved in this process by directly activating trypsinogen within acinar cells. In experimental pancreatitis of CTSB-deficient mice we found trypsinogen activation to be reduced by $90 \%$. In the present series of experiments we investigated whether pancreatic overexpression of human CTSB in the mouse pancreas would increase the severity of pancreatitis. Materials and methods: Pancreatitis was induced in FVB wild type (WT) and FVB CTSB overexpressing mice (CTSB +++) by seven intraperitoneal injections of caerulein $(50 \mu \mathrm{g} / \mathrm{kg}$ body weight) at hourly intervals and mice were sacrificed after 8 hours. Using immunocytochemistry we determined the intraacinar localization of trypsin (polyclonal rabbit anti trypsin) and human CTSB (monoclonal mouse anti human cathepsin B). Acini were incubated with $100 \mathrm{nM}$ cholecystokinin (CCK) to measure time- and CCK-dependent intraacinar trypsin activity. Trypsin and trypsinogen activities (after enteropeptidase incubation) were measured fluorometrically. Trypsinogen and CTSB content was measured in zymogen granule, lysosomal and cytosolic subcellular fractions after density gradient centrifugation. Total CTSB was measured fluorometrically after incubation with dithioerythrol. To distinguish between total and active CTSB we probed subcellular fractions with NS-196, a high specific biotinylated CTSB inhibitor and Western blotting. Results: In spite of a 4 fold increase in pancreatic CTSB expression in transgenic animals we found no difference in trypsin activity or disease severity during caerulein-induced pancreatitis in comparison to WT animals. Immunocytochemistry of pancreatic sections and acini revealed the expected intracellular sorting of the transgenic human CTSB into the lysosomal and secretory compartment and this was confirmed in subcellular fractions. Trypsin and human CTSB were clearly colocalized. The 4 fold increase in total CTSB in CTSB +++ animals, however, did not result in an increase in total CTSB activity in the NS-196 biotinylated inhibitor studies. Conclusion: The transgenic overexpression of human CTSB does not result in increased trypsinogen activation or more severe pancreatitis. Whether this is caused by a failure of human CTSB to participate the intraacinar protease activation events, by a difference in intracellular processing of human and murine CTSB, or by the fact that maximal CTSB-induced trypsinogen activation cannot be increased by adding available CTSB, needs to be elucidated.

\section{M1855}

Pathogenic Role of Endothelial Nitric Oxide Synthase On Caerulein-Induced Acute Pancreatitis in Rats

Yusuke Sugiyama, Shinichi Kato, Shoji Mitsufuji, Takeshi Okanoue, Koji Takeuchi

Background/Aim: Nitric oxide (NO) is a potent pancreatic vasodilator, yet the pathogenic role of NO in acute pancreatitis still remains controversial. NO is generated from L-arginine by NO synthase (NOS), classified into three isozymes; neuronal (nNOS), inducible (iNOS) and endothelial NOS (eNOS). In the present study, we investigated the role of NO/NOS isozymes in the pathogenesis of caerulein-induced acute pancreatitis in rats. Methods: Acute pancreatitis was induced in male Wistar rats by two SC injections of caerulein $(20 \mu \mathrm{g} / \mathrm{kg})$. $\mathrm{N}^{\mathrm{G}}$-nitro-L-arginine methyl ester (L-NAME: a nonselective NOS inhibitor) or aminoguanidine (a relatively selective iNOS inhibitor) was given $\mathrm{PO}$, while tetrahydrobiopterin (BH4), a critical cofactor for NOS, was administered IP 30 min before the first caerulein injection. The animals were killed $6 \mathrm{~h}$ after the first caerulein injection, and then pancreas weight, serum amylase, myeloperoxidase (MPO) activity and thiobarbituric acid reactants (TBARS) were evaluated. The microvascular permeability was determined by Evans blue extravasation into pancreatic tissue $3 \mathrm{~h}$ after the first caerulein injection, while the levels of $\mathrm{NO}$ metabolites in the pancreas were determined by Griess method. The expression of total eNOS was examined by conventional Western blot, while dimeric eNOS was evaluated by Western blot after low-temperature SDS-PAGE. Results: Caerulein given repeatedly twice produced acute pancreatitis with concomitant increases in the serum amylase level, pancreas weight, MPO activity, TBARS and microvascular permeability. Prior administration of L-NAME, but not aminoguanidine, significantly prevented these changes, in a dose-dependent manner, and this effect was antagonized by the co-administration of L-arginine. The total eNOS was clearly expressed in the pancreas of normal rats and the expression was not affected by caerulein injection. However, the expression of dimetric eNOS in the pancreas was markedly suppressed by caerulein injections, together with a decrease in NO production, but the response was partly reversed by the prior administration of $\mathrm{BH} 4$. The increases in the serum amylase level and pancreas weight as well as TBARS in response to caerulein were significantly attenuated by the administration of $\mathrm{BH} 4$. Conclusion: These results suggest that the uncoupled eNOS, probably caused by the decrease in endogenous BH4 availability, plays a deleterious role in the pathogenesis of caerulein-induced acute pancreatitis. It may be assumed that the stabilization of eNOS by the supplementation of $\mathrm{BH} 4$ is a useful and novel approach for the treatment of acute pancreatitis.

\section{M1856}

Treatment with Anandamide, a Cannabinoid 1 Receptor Agonist, Increases the Severity of Edematous Acute Pancreatitis

Artur Dembinski, Zygmunt Warzecha, Jakub Cieszkowski, Wieslaw W. Pawlik, Ryszard Sendur, Romana Tomaszewska, Beata Kusnierz-Cabala, Jerzy W. Naskalski, Peter C Konturek

Background: Marijuana is well known herbal drug that has for centuries been use in native medicine for treatment of wide array of health concerns, acting on cannabinoid 1 (CBl) and cannabinoid 2 (CB2) receptor. $\mathrm{CB} 1$ receptor activation by exogenous and endogenous cannabinoids has been shown, between others, to reduce of ischemic myocardial necrosis area, to inhibit gastric and intestinal secretion, and gastrointestinal transit, and to protect gastric mucosa against stress-induced ulcers. In the pancreas, usage of $\mathrm{CBl}$ receptor antagonis has prolonged the survival of rats with taurocholate-induced pancreatitis. Aim of the presen study was to check whether the administration of $\mathrm{CBl}$ receptor agonist or antagonist affects the development of edematous pancreatitis. Methods: Acute pancreatitis was induced in Wistar rats by i.p. administration of cerulein $(50 \mathrm{mcg} / \mathrm{kg})$ five times with $1 \mathrm{~h}$ intervals Anandamide, a natural ligand for $\mathrm{CBl}$ receptor $(\mathrm{Ki}=61 \mathrm{nM})$, was administrated twice (30 min prior to cerulein or saline injection and after next $3 \mathrm{~h}$ ) at the dose: $0.3,1.5 \mathrm{or} 3 \mathrm{mcmol} /$ kg i.p. AM 251 (ALEXIS ${ }^{\circledR}$ Biochemicals), a synthetic CBl receptor antagonist $(\mathrm{Ki}=7.49$ $\mathrm{nM}$ ), was administrated i.p. at the dose $4 \mathrm{mcmol} / \mathrm{kg}$ alone or in combination with anandamide (at the dose $1.5 \mathrm{mcmol} / \mathrm{kg} /$ dose) twice ( $40 \mathrm{~min}$ prior to cerulein and after next $3 \mathrm{~h}$ ). After last injection of cerulein, the study was terminated. Results: Administration of anandamide or AM 251 did not affect pancreatic condition in animals without acute pancreatitis. In animals treated with cerulein, administration of anandamide increased dose-dependently the severity of acute pancreatitis. In histological examination, we observed an increase in pancreatic edema and inflammatory infiltration. Also, anandamide administration augmented the pancreatitis-induced increase in serum level of lipase, amylase, poly- $C$ ribonuclease, and pro-inflammatory interleukin-lbeta (IL-1), but was without effect on the pancreatitis-evoked fall of pancreatic blood flow. Treatment with AM 251 reduced histological signs of pancreatic damage, serum activity of pancreatic digestive enzymes and serum concentration of proinflammatory IL-1 in animals with acute pancreatitis. Also, administration of AM 251 reversed the anandamide-evoked aggravation of cerulein-induced acute pancreatitis. Conclusions (1) Activation of $\mathrm{CBl}$ receptors by anandamide increases the severity of edematous acute pancreatitis; (2) Blockade of $\mathrm{CBl}$ receptor by AM 251 attenuates pancreatic damage in acute pancreatitis.

\section{M1857}

Extracellular Acidification Sensitizes the Rat Pancreatic Acinar Cell to Cerulein-Induced Protease Activation

Thomas Jung, Thomas Kolodecik, Christine Shugrue, Fred Gorelick

Pathologic protease activation in pancreatic acinar cells initiates acute pancreatitis. Supraphysiologic concentrations [10-7M] of cerulein cause protease activation and pancreatitis. Clinical studies have shown a correlation between decreased extracellular $\mathrm{pH}(\mathrm{pHe})$ and AP. Work from our laboratory suggests that protease activation requires an acidic intracellular compartment that is vacuolar ATPase (vATPase)-dependent. This study examined the hypothesis that $\mathrm{pHe}$ acidification or inhibition of $\mathrm{Na}-\mathrm{H}$ exchange (NHE) could sensitize acinar cells to cerulein-induced trypsinogen/ chymotrypsinogen activation. Medium acidification (pHe 7.6 to 6.8) dramatically sensitized cells to cerulein [10-7M]-induced protease activation, bu did not affect basal protease activation or the secretory response to cerulein. The sensitizing effects of acidic pHe were observed with a range of cerulein [10-10 to 10-7 M]; the sensitizing effects were reversible. Although the low-pH sensitization was also observed with muscarinic agonist, carbachol (1 mM), it was less dramatic than cerulein. NHEs regulate proton extrusion from the acinar cell. PCR analysis confirmed the presence of NHEl and NHE4 in acinar cells. Addition of HOE-694, a selective inhibitor of NHEl that causes cytosolic acidification, enhanced cerulein-induced protease activation. Finally, the vATPase inhibitors bafilomycin and concanamycin $(100 \mathrm{nM})$ blocked the effects of an acidic pHe on cerulein-induced protease activation. These studies suggest that perturbations which potentially acidify the acinar cell cytosol sensitize it to ligand-induced protease activation. The mechanism of this effect may be through increasing the availability of protons for the vATPase. The effects of acidosis on acinar cell protease activation may be relevant to the pathogenesis of acute pancreatitis.

\section{M1858}

Dual Effect of $\mathrm{Ca} 2+$ On Cytochrome C Release from Pancreatic Mitochondria: Role of Reactive Oxygen Species

Irina V. Odinokova, Olga A. Mareninova, Kai Feng Sung, Yuri V. Evtodienko, Anna S. Gukovskaya

Background and Aims: We previously showed that mitochondrial dysfunction, resulting in cytochrome $\mathrm{c}$ release and depolarization, mediates death responses of pancreatitis. Cytochrome c release mediates apoptosis while loss of mitochondrial membrane potential (DYm) leads to necrosis. Excessive increases in $\mathrm{Ca} 2+$ and reactive oxygen species (ROS) are associated with pancreatitis; however, the regulation of pancreatic mitochondria by $\mathrm{Ca} 2+$ and ROS has not been studied. In this study, we determined the effects of $\mathrm{Ca} 2+$ and ROS on cytochrome c release and DYm of pancreatic mitochondria. Methods: Mitochondria were isolated from rat pancreas by differential centrifugation. We measured respiration with Clark electrode. DYm with TPP+electrode or fluorescent probe TMRM: ROS levels, with AmplexRed; and cytochrome c release, by Western blot. Free Ca2+ concentration in the medium ([Ca2+]e) was clamped at "zero" to $40 \mu \mathrm{M}$ using Ca2+/EGTA buffers. Results: Ca2+ at micromolar concentrations induced depolarization and cytochrome $\mathrm{c}$ release from isolated pancreatic mitochondria. Both effects were blocked by the permeability transition pore (PTP) inhibitor cyclosporin A, indicating that they are mediated by PTP opening. Depolarization only increased with $[\mathrm{Ca} 2+]$ (or duration of mitochondria exposure to $\mathrm{Ca} 2+$ ). By contrast, we found a non-monotonous dependence of cytochrome $\mathrm{c}$ release on $\mathrm{Ca} 2+$, with a maximum at $1.3 \mu \mathrm{M}[\mathrm{Ca} 2+]$ e. Blockade of cytochrome $\mathrm{c}$ release at higher $[\mathrm{Ca} 2+]$ e was associated with

. 
DYm loss. We hypothesized that depolarization blocks cytochrome c release by inhibiting mitochondrial ROS. We measured that $\mathrm{Ca2}$-induced depolarization, indeed, decreased mitochondrial ROS. We next measured that cytochrome c release was blocked by the mitochondrial uncoupler FCCP; the inhibitors of complex I, rotenone and diphenyl-iodonium; and by the lipid peroxidation inhibitor, butylene hydroxytoluene. The inhibitors (except FCCP) did not depolarize mitochondria, but all of them drastically decreased mitochondrial ROS. Conclusions: Ca2+ depolarizes pancreatic mitochondria and has a dual effect on cytochrome $\mathrm{c}$ release. It stimulates cytochrome $\mathrm{c}$ release via PTP and inhibits it through inhibition of ROS. These findings have important implications for the mechanism of pancreatitis. Excessive (or sustained) increases in Ca2+ could both stimulate necrosis through loss of DYm and inhibit apoptosis by blocking its key mediator, cytochrome c release. Thus, the intensity and/or duration of $\mathrm{Ca} 2+$ signals may determine the pattern of death responses in pancreatitis

\section{M1859}

\section{Expressions of TLR2 and TLR4 in the Intestinal Mucosa from Rats with} Severe Acute Pancreatitis

Hidehiro Sawa, Takashi Ueda, Yoshifumi Takeyama, Takeo Yasuda, Naoki Matsumura, Takahiro Nakajima, Yoshikazu Kuroda

Background and Aim. Infectious complication in the late phase is contributor to mortality in severe acute pancreatitis (SAP). This complication is thought to be a result of the bacterial translocation (BT) from the gastrointestinal tract, and breakdown of intestinal integrity is considered to be implicated in BT. We recently demonstrated that accelerated apoptosis of intestinal mucosa may be participated in BT. On the other hand, it is reported that toll-like receptors (TLRs) are involved in not only the innate immune response but also acquired immune activation, and that TLRs make important roles for the protection against infection. TLR2 recognizes lipoteicoic acid of Gram-positive bacteria, and TLR4 recognizes lipopolysaccharide of Gram-negative bacilli. We have recently reported that expression of TLR2 and TLR4 on alveolar macrophage was diminished in SAP. This study aimed to investigate the expressions of TLRs in the intestinal mucosa in SAP. Methods. Male Wistar rats weighing 250-300 g were used. Severe hemorrhagic necrotizing pancreatitis was induced by retrograde injection of 3\% sodium deoxycholate into the biliopancreatic duct. Ileum was removed 2 , 6,12 , and 18 hours after induction of SAP, respectively. The expressions of TLR2 and TLR4 proteins in intestinal mucosa were assayed by western blotting. Localization of TLR2 and TLR4 in intestinal mucosa was also evaluated by immunohistochemical staining. Results. The expression of TLR2 and TLR4 proteins were detected in intestinal mucosa from control rats. Two and 6 hours after induction of pancreatitis, both expressions were increased in comparison with control level. Thereafter, 12 and 18 hours after induction of pancreatitis, the expressions were decreased in comparison with control level. Immunoreactivities for TLR2 and TLR4 were detected at the top of villi and crypt in intestinal mucosa from control rats. They were increased at the top of villi and crypt (especially Paneth cells) 2 hours after induction of pancreatitis in comparison with control. They were diminished 12 hours after induction of pancreatitis accompanied with the breakdown of intestinal mucosa. Conclusions. In rats with SAP, expressions of TLR2 and TLR4 were increased in the early phase, and thereafter they were decreased to lower level as compared with control. These results suggest that enhancement of the mucosal immune response in the early phase may be related with the protection against infection, and that suppression of the mucosal immune response in the late phase may be implicated in the mechanism of bacterial translocation.

\section{M1860}

Ethanol Stimulates Akt Phosphorylation in the Pancreas Through PTEN Oxidation: A Predisposing Factor in Alcoholic Pancreatitis?

Olga A. Mareninova, Aurelia Lugea, Irina V. Odinokova, Hidekazu Tsukamoto, Ilya Gukovsky, Stephen J. Pandol, Anna S. Gukovskaya

Background and Aims: Although alcohol abuse is a major cause of pancreatitis, the mechanisms of ethanol toxicity to pancreas are poorly understood. We and others recently showed that the phosphatidylinositol 3-kinase (PI3K) pathway mediates key pathologic responses of cerulein (CR) pancreatitis, including intrapancreatic activation of trypsinogen and the transcription factor NF-KB. Most of PI3K effects are mediated through downstream Akt/ PKB kinase, which is negatively regulated by the phosphatase PTEN. It is not known how alcohol affects this key signaling pathway in pancreas. In this study, we determined the effects of ethanol feeding and CR pancreatitis on pancreatic PI3K/Akt/PTEN system. Methods: Rats were fed for 6 weeks control and ethanol-containing diet (either intragastrically or as liquid Lieber-deCarli diet). Pancreatitis was induced by 4 hourly i.p. injections of $50 \mu \mathrm{g} / \mathrm{kg}$ CR. In pancreatic tissue, we measured Akt phosphorylation by Western blot; PI3K activity with in vitro kinase assay; and PTEN oxidation and activity, with electromobility shift and enzymatic assays. Results: We found that CR time dependently stimulated pancreatic Akt phosphorylation in control-fed rats. The increase was evident at $30 \mathrm{~min}$ and reached 8-fold at $4 \mathrm{~h}$ after the lst CR injection. PI3K activity was not increased at these time points. PTEN oxidation was dramatically stimulated during $C R$ pancreatitis in control-fed rats, which resulted in inhibition of PTEN activity. Time-dependencies of PTEN oxidation and Akt phosphorylation superimposed suggesting PTEN oxidation as a mechanism for Akt activation in pancreatitis. Ethanol feeding by itself stimulated both Akt phosphorylation and PTEN oxidation in pancreas. In particular, 6 weeks of intragastric ethanol feeding increased Akt phosphorylation $\sim 9$-fold. In rats fed ethanol, CR stimulated Akt phosphorylation to a greater extent $(\sim 16$-fold) than in control-fed rats ( $\sim 8$-fold versus saline). Conclusions: The results indicate that ethanol feeding and CR pancreatitis both activate Akt. One mechanism of Akt activation is through oxidation and thus inhibition of PTEN. Persistent Akt activation could be a factor predisposing pancreas to alcoholic pancreatitis.

\section{M1861}

Pancreatitis Associated Protein (PAP) Modulates Cytokine Expression in Rat Macrophages

Domenico Viterbo, Yin Yao Lin, Cathy M. Mueller, Michael E. Zenilman, Martin Bluth

Background: Pancreatitis-associated proteins (PAP) are minimally expressed in normal pancreas but strongly induced in acute pancreatitis. We have previously demonstrated that expression of PAP may modulate the severity of acute pancreatitis, and that serum obtained from pancreatitic rats was able to induce phenotypic changes in macrophages. Since pancreatitis is associated with immune activation, we investigated the effect of PAP on macrophages Methods: Rat macrophages (NR8383 alveolar cell line) were cultured, with and without LPS (1.0ug/ml), in concentrations of $2.5-15 \mathrm{ug} / \mathrm{ml}$ of recombinant PAP for $24 \mathrm{hr}$ and then evaluated for cytokine (IL-1 $\alpha$, IL-1 $\beta$, TNF $\alpha$, IL-6) expression (RT-PCR) and morphologic changes (agglutination, adhesion, granule content) by light microscopy. Controls consisted of cells cultured with medium, vehicle alone or neutralizing PAP antibody. Significance between groups was set at $\mathrm{p}<0.05$ (Student's t-test). Results: Macrophages cultured in the presence of PAP resulted in increased expression of IL- $1 \alpha$, IL-1 $\beta$, TNF $\alpha$, and IL- 6 when compared with controls $(156,178,210$ and 242 fold, respectively at $5 \mathrm{ug} / \mathrm{ml} ; \mathrm{p}<0.05)$, which were reversed (IL-1 $\alpha$, IL-1 $\beta$ ) with neutralizing anti-PAP antibody. When macrophages were cultured in the presence LPS, similar increases in cytokine expression were observed. However, when PAP was co-cultured with LPS-stimulated cells, inflammatory cytokine expression was reduced (up to $50 \%$ ), with the observed reduction occurring at half the concentration of the stimulatory effect observed with PAP alone $(7.5 \mathrm{ug} / \mathrm{ml}$ vs $15 \mathrm{ug} / \mathrm{ml})(\mathrm{p}<0.05)$. When macrophages were cultured with PAP, phenotypic changes including increased agglutination/ adhesion, and granule content were observed and were dose dependent $(85 \%$ with $10 \mathrm{ug} / \mathrm{ml}$; $\mathrm{p}<0.05)$. These changes were not observed in any control groups. Conclusions: Pancreatitis is an immune phenomenon in which PAP is increased. PAP alone induces macrophage cytokine expression in a dose related fashion. However, PAP can modulate inflammatory cytokine expression by co-stimulants such as LPS, and induce morphologic changes in cultured macrophages. This dual effect may explain the modulatory role of PAP in acute pancreatitis.

\section{M1862}

Intragastric Ethanol Depresses Sphincter of Oddi Function in the Anaesthetized Australian Brush-Tailed Possum Yukio Sonoda, Masahiko Kawamoto, Charmaine M. Woods, Ann C. Schloithe, James Toouli, Gino Saccone

Background: The role of sphincter of Oddi (SO) function in alcoholic acute pancreatitis is unclear. Data from previous animal studies have been conflicting, however these studies have not used a technique that directly measures SO function (i.e. trans-sphincteric flow; TSF). Aim: To examine the effect of intragastric ethanol on sphincter of Oddi TSF and investigate possible mediation via neural mechanisms using prior acute vagotomy, pretreatment with atropine or nitric oxide synthase inhibition. Methods: Australian Brush-tailed possums were anaesthetized (sodium thiopentone) for the duration of the experiments. In TSF studies, ethanol $(1.0,2.0$ or $3.0 \mathrm{~g} / \mathrm{kg}$, in water) or water was instilled into the stomach $(n=20)$. In separate studies, possums were subject to bilateral cervical vagotomy, pretreatment with atropine $(360 \mathrm{ug} / \mathrm{kg} / \mathrm{h}$, continuous i.v. infusion) or with L-nitro arginine methyl ester (L-NAME; $15 \mathrm{mg} / \mathrm{kg}$ bolus followed by $6 \mathrm{mg} / \mathrm{kg} / \mathrm{h}$ continuous i.v. infusion) prior to administration of intragastric ethanol $(2 \mathrm{~g} / \mathrm{kg})$ or water $(\mathrm{n}=26)$. Blood ethanol concentrations were determined at $0.5 \mathrm{~h}$ intervals. Results: Intragastric ethanol decreased TSF whereas water had no effect. After $1.0 \mathrm{~g} / \mathrm{kg}$ ethanol, TSF decreased to $60 \%$ of baseline at $\mathrm{lh}$ and then recovered to $80 \%$ of baseline. The 2.0 and $3.0 \mathrm{~g} / \mathrm{kg}$ doses of ethanol gradually decreased TSF to 30 $40 \%$ of baseline at 3 h (both $\mathrm{P}<0.05$ vs water). Blood pressure was not significantly changed by ethanol administration (vs water). Instillation of $1.0,2.0$ and $3.0 \mathrm{~g} / \mathrm{kg}$ ethanol elevated blood ethanol levels peaking at $108 \pm 8(1.5 \mathrm{~h}), 221 \pm 12$ (3h) and $281 \pm 11 \mathrm{mg} / 100 \mathrm{ml}(3 \mathrm{~h})$ respectively. Acute vagotomy, pre-treatment with atropine or with L-NAME, reduced the blood ethanol level at $3 \mathrm{~h}$ to $131 \pm 17,178 \pm 26$ and $106 \pm 13 \mathrm{mg} / 100 \mathrm{ml}$ respectively. Following intragastric water, acute vagotomy did not alter the TSF rate over the $3 \mathrm{~h}$ period whereas pre-treatment with atropine or L-NAME reduced TSF to $77 \pm 4 \%$ and $47 \pm 5 \%$ of baseline at $3 \mathrm{~h}$ respectively. Acute vagotomy or pre-treatment with atropine or L-NAME delayed the increase in blood ethanol concentration following intragastric ethanol but the TSF rates under these conditions corresponded to those produced by the lower blood ethanol concentration. Conclusion: These data demonstrate that intragastric ethanol (and/or its metabolites) reduces sphincter of Oddi TSF. This effect is primarily due to the direct effect of blood ethanol. The depressed SO function would contribute to increased pancreatic duct pressure which could be one of the necessary events required for the onset of acute pancreatitis. Supported by the NH\&MRC of Australia (grant \# 229901) and the FMC Foundation.

\section{M1967}

Is Proton Pump Inhibitor (PPI) Therapy a Risk Factor for C. Difficile Infection?

Rohinee Beri, Michael P. Jones, Colin W. Howden

Background and Aims: PPIs suppress gastric acidity and may predispose to certain enteric infections. PPI use may increase the risk of C. difficile infection (J. Hosp Infect 2003; 54 : 243; CMAJ 2004; 171: 33). We performed a case-control study to examine this and assess whether PPI use increased the risk of $C$. difficile among patients with other known risk factors including antibiotic (ABx) use and cytotoxic chemotherapy (CC). Methods: 154 cases were identified from a hospital in-patient database. These were patients admitted between 2001 and 2005 for various reasons other than diarrhea or known C. difficile infection, but who had positive culture and toxin assay for $C$. difficile while in hospital. Median age was 62 years (range 23 to 92); 47.4\% were male. Controls, with no positive tests for $C$. difficile infection, were selected from a random list provided by the Medical Records department and matched individually to cases by age, sex, and month of hospital admission. Univariate and stepwise logistic regression analyses were performed to determine the association of drug classes with $C$. difficile infection. Results: Cases were significantly more likely to be using 
PPIs, ABx and CC than controls. OR (95\% confidence intervals (CI)) for PPI, ABx and CC use by cases were $3.06(1.87-5.01), 4.16(2.48-6.98)$ and 3.51 (1.82-6.77) respectively. Cases were not significantly more likely to be using $\mathrm{H}_{2}$-receptor antagonists $\left(\mathrm{H}_{2} \mathrm{RAs}\right.$; $\mathrm{OR}=$ $0.58 ; 95 \% \mathrm{CI}=0.29-1.16)$. Using stepwise logistic regression to predict $C$. difficile positive cases, a three step model was identified: $\mathrm{ABx}\left(\mathrm{R}^{2}=0.13\right) ; \mathrm{ABx}+\mathrm{PPI}\left(\mathrm{R}^{2}=0.194\right)$ and $A B x+P P I+C C\left(R^{2}=0.229\right)$. Conclusions: In this study, $A B x$ use was most highly associated with $C$. difficile infection. PPI but not $\mathrm{H}_{2} \mathrm{RA}$ use was also significantly associated with $\mathrm{C}$. difficile infection. In regression analysis, PPI use was more predictive of $C$. difficile infection than CC. Potent suppression of gastric acidity by PPIs likely impairs host defenses against C. difficile. $\mathrm{H}_{2} \mathrm{RAs}$, which cause less potent acid suppression, are not associated with an increased risk of $C$. difficile.

\section{M1968}

Acidic Media Increases the Expression of Dickkopf Homolog 1 (DKK-1) and 4 (DKK-4) in Esophageal Epithelial Het-1A Cells

Irshad Ali, Parvaneh Rafiee, Vicki Nelson, Aaron Rogaczewski, David G. Binion, Reza Shaker

INTRODUCTION: Dkk family of secretory proteins is inhibitors of the Wnt pathway, and activation of the Wnt pathway up-regulate Dkk-1 levels. Dkk-l over-expression increases apoptosis in stressed cells. $\mathrm{LiCl}$ activates the Wnt and the pro-survival phosphatidylinositol 3-kinase (PI3K) pathways. Recently studies have demonstrated increased Dkk-1 and Dkk4 expression in the non-injured distal squamous mucosa of esophagitis patients compared to the healthy controls and Barrett's patients suggesting that gastric acid exposure may upregulate their expression in this group. AIM: To determine whether low $\mathrm{pH}$ exposure of Het-1A cells leads to increased Dkk-1 and Dkk-4 expression and whether this mechanism involves the Wnt pathway. METHODS: Confluent Het-1A cells were incubated in basal media (free of growth factors) overnight. They were then treated with acidified ( $\mathrm{pH} 4.5$ ) media or $\mathrm{LiCl}$ for various times followed by incubation in fresh basal media for 3 hours. The effect of phosphatidylinositol 3-kinase (PI3K) inhibitor (wortmanin) was also determined. Dkk-1 and Dkk-4 mRNA expression was determined by real-time PCR and Dkk-1 protein levels in the culture media was determined by ELISA. RESULTS: Dkk-1 mRNA and protein expression were increased maximally after 10 minutes exposure to acidic media followed by $3 \mathrm{~h}$ incubation in normal basal media $(\mathrm{n}=4)$ whereas Dkk-4 mRNA expression was maximized at $30 \mathrm{~min}(\mathrm{n}=4)$. LiCl increased Dkk-1 and Dkk-4 mRNA expression in a time and concentration dependent manner. Dkk-1 protein was increased by LiCl $(40 \mathrm{mM}, \mathrm{n}=4)$. Wortmanin further increased the expression of Dkk-1 protein and mRNA by acidified media and $\mathrm{LiCl}(\mathrm{n}=4)$. Wortmanin decreased Dkk-4 mRNA expression by acidified media and did not affect Dkk-4 expression by LiCl $(n=4)$. CONCLUSION: Acidic media up-regulates Dkk1 and Dkk-4 expression in a temporally differential manner in esophageal epithelial HetlA cells suggesting a difference in sensitivity to low $\mathrm{pH}$ exposure for the activation of these genes. Increased expression of $\mathrm{Dkks}$ by $\mathrm{LiCl}$ suggests that the Wnt pathway may be involved and the enhanced expression of Dkk-1 by wortmanin suggests that the pro-survival PI3K pathway may also regulate its activity

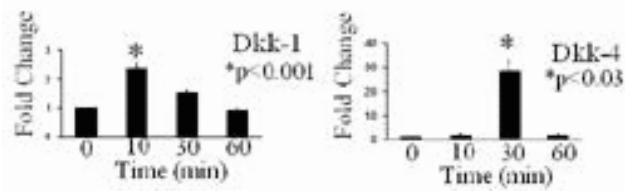

M1969

Ghrelin Attenuates the Experimental Induced Esophagitis

Peter C. Konturek, Tomasz Brzozowski, Grzegorz Burnat, Robert Pajdo, Eckhart G. Hahn, Konturek J. Stanislaw

Background and Aims: Ghrelin is a novel growth hormone (GH)-releasing and orexigenic peptide which was recently isolated from the fundic portion of the stomach. Recently antiinflammatory and gastroprotective effect of this hormone have been demonstrated, but the role of ghrelin in the protection of esophageal mucosa against injury has not been evaluated. The aim of the study was 1) to analyze the expression of ghrelin and its receptor in humans with esophagitis; 2) to assess the effect of exogenous ghrelin on experimentally induced esophagitis; 3) to study the effect of ghrelin on expression of COX-2 and proinflammatory cytokine IL-1 $\beta$ and inducible NO synthase (iNOS) in esophageal cell line (OE-19). Methods: 20 patients with reflux esophagitis were included in the study. The expression of ghrelin and its receptor was assessed by quantitative RT-PCR. In Wistar rats, the esophageal experimental ulcers were induced by serosal application of acetic acid on subdiaphragmatic portion of esophagus. Following experimental groups were included: 1) control (esophageal ulcers + placebo) and 2) esophageal ulcers + ghrelin $10 \mu \mathrm{g} / \mathrm{k} /$ day i.p. The ulcer area was assessed by planimetry and esophageal mucosal blood flow by $\mathrm{H} 2$ gas clearance method. In addition, in vitro experiments were performed in which OE-19 cells were incubated with ghrelin alone ( 150 and $450 \mathrm{nM})$ or ghrelin with addition of TNF- $\alpha(10 \mathrm{ng} / \mathrm{ml})$ to simulate inflammatory state. The expression of COX-2, iNOS and IL1 $\beta$ was assessed by quantitative RT-PCR. Results: In humans with reflux esophagitis expression of ghrelin and a significant upregulation of ghrelin receptor were recorded in the esophageal mucosa as compared to intact esophageal mucosa. In rats with esophageal ulcers, the treatment with ghrelin reduced significantly the esophageal injury and this effect was accompanied by a significant rise in esophageal mucosal blood flow. The "in vitro" experiments demonstrated expression of COX-2 in OE-19 cells which was significantly upregulated by TNF $\alpha$, but this effect was attenuated by ghrelin. Ghrelin caused also decrease in ILI $\beta$ expression and significant increase in inducible NO synthase. Conclusion: Ghrelin represents a novel ulcer healing and antiinflammatory factor for the esophageal mucosa. Its beneficial effects seems to be due to iNOS and increased generation of NO, contributing to anti-inflammatory effects of ghrelin.

\section{M1970}

Prevalence, Incidence and Disappearance Rate of Symptoms of Gastroesophageal Reflux During Pregnancy: A Prospective Longitudinal Study Enrique Rey, Puy Sanchez, Miguel Angel Herraiz, Angel Alvarez Sanchez, Fernando Rodriguez-Artalejo, Manuel Escudero, Manuel Diaz-Rubio

Gastroesophageal reflux symptoms (GERS) are frequent during pregnancy. Most studies on this issue have used a cross-sectional design and non-validated instruments, except one which showed a decreasing incidence of GERS. AIM: to examine the prevalence, incidence (new cases and worsening of pre-existing occasional symptoms), and disappearance rate of GERS during pregnancy. MATERIAL AND METHODS: We recruited 355 women with a pregnancy of 12 weeks or less. They completed three phone interviews at 12,24 and 36 weeks after the last menses, using the GERQ, adapted for a recall period of 12 weeks. In the first interview, information about GERS in the year before pregnancy was collected with GERQ. A control group of 350 women was interviewed once to compare basal information. GERS was defined as frequent (at least weekly) heartburn and/or acid regurgitation. A case was labelled as new case if she reported frequent GERS not previously reported. Disappearance rate was calculated as the percentage of GERS cases that reported no frequent symptoms in the subsequent interviews. Data were summarized as incidence (new cases) and disappearance rate in the 1st, 2nd and 3rd trimester of pregnancy. RESULTS: Data were analysed for the 272 pregnant women who provided complete information after discarding 34 abortions, 9 withdrawal of consent, 33 unreachable and 7 protocol violations. Prevalence of GERS a baseline was similar in pregnant $(22.8 \%$ with occasional and $7.0 \%$ with frequent GERS) and control women (28\% with occasional and $6.6 \%$ with frequent GERS). Prevalence o frequent GERS increased during pregnancy, with $27.6 \%, 32 \%$ and $40.8 \%$ of pregnant women suffering from them at 12,24 and 36 weeks of pregnancy, respectively. Incidence and disappearance rate, according to GERS status at baseline, is summarized in the table: CONCLUSION: During pregnancy, prevalence of GERS increased progressively, but incidence of new cases of GERS (about 25\% each trimester) and disappearance rate (about 30\% each trimester) remained stable. Occasional GERS before pregnancy was associated with a higher incidence and with a lower disappearance rate of GERS. Funded by FIS grant PI030718 (Instituto de Salud Carlos III, Ministry of Heath, Spain)

\begin{tabular}{|c|c|c|c|}
\hline Incidence & $1 \mathrm{st} \mathrm{T}$ & $2 \mathrm{nd} \mathrm{T}$ & $3 \mathrm{rd} \mathrm{T}$ \\
\hline No GERS before pregnancy & $20.4 \%$ & $23.0 \%$ & $22.2 \%$ \\
\hline Occasional GERS before pregnancy & $42.6 \%$ & $37.1 \%$ & $50.0 \%$ \\
\hline All & $25.8 \%$ & $25.7 \%$ & $26.6 \%$ \\
\hline Disappearance rate & $1 \mathrm{st} \mathrm{T}$ & $2 \mathrm{nd} \mathrm{T}$ & $3 \mathrm{rd} \mathrm{T}$ \\
\hline No GERS before pregnancy & & $38.5 \%$ & $37.1 \%$ \\
\hline Occasional GERS before pregnancy & & $15.4 \%$ & $23.1 \%$ \\
\hline Frequent GERS before pregnancy & $26.3 \%$ & $33.3 \%$ & $37.5 \%$ \\
\hline All & $26.3 \%$ & $29.7 \%$ & $33.9 \%$ \\
\hline
\end{tabular}

\section{M1971}

Long-Term Gastric pH Monitoring with Bravo Wireless System: Effect of Omeprazole On Acidic Environment in Patients with Endoscopically Resected Gastric Cancer

Jin Yonezawa, Mitsuru Kaise, Takuji Yamasaki, Yukinaga Yoshida, Mori Naoki, Kato Masayuki, Kenichi Gouda, Hisao Tajir

Background and aim: Superficial gastric cancers can be curably treated by endoscopic mucosa resection (EMR). Proton pump inhibitors (PPIs) are empirically administered to gastric ulcers artificially induced by EMR for preventing gastric hemorrhage. However, the effect of PPIs on an acidic environment in gastric cancer patients has not been well known. The study aims are (1) to evaluate the feasibility of long-term gastric $\mathrm{pH}$ monitoring with Bravo wireless system, (2) to demonstrate a gastric acidic environment of gastric cancer patients, and (3) to elucidate the effect of PPIs on an acidic environment in gastric cancer patients with EMR ulcer. Methods and subjects: Bravo capsule was detached from the delivery system and treaded with a suture string in advance. The capsule with a looped string was endoscopically attached with endoclips on the wall of the upper gastric corpus. Ambulatory pH monitoring with the system was performed in gastric cancer patients from one day before EMR (Prel) to 4 days after EMR (POD4). Acid inhibitory drugs were restricted from a week before EMR For two days from the day of EMR (POD1), omeprazole (20mg x 2 ) was intravenously administered to patients under nothing per os. From the day 3 after EMR (POD3), omeprazole $(20 \mathrm{mg} \times 2)$ was orally administered and ingestion was started. Eleven patients with mean age of 71 years old were enrolled. All of gastric cancers were intestinal types and limited in the mucosa. The percentage of time that the $\mathrm{pH}$ was less than $4(\%$ time $\mathrm{pH}<4)$ was calculated and used as a parameter of a gastric acidic environment with the system. Results: (1) All patients were successfully monitored with the system except for one patient, whose capsule were detached one day after attachment. (2) \% Time pH<4 on Prel were $30.2+/$ 33.4 (mean +/- SD). Gastric acidity environments of gastric cancer patients could be classified into hyper-acidic (\% time $\mathrm{pH}<4$ larger than $80, \mathrm{n}=3$ ), meso-acidic (\% time $\mathrm{pH}<4$ from 20 to $80, \mathrm{n}=5$ ) and hypo-acidic ( $\%$ time $\mathrm{pH}<4$ smaller than $20, \mathrm{n}=3$ ). (3) Average values of $\%$ time $\mathrm{pH}<4$ on Prel, POD1, POD2, POD3 and POD4 were $0,0,0,0,0$ and 31, 1, 0, 0, 0 in the hypo-acidic and meso-acidic groups, respectively. In contract, those were $92,88,86$, 68,57 in the hyper-acidic group. Conclusions: Long-term gastric $\mathrm{pH}$ monitoring was feasible with Bravo wireless system using novel methodology. Gastric acidic environments of gastric cancer patients can be classified into hyper, meso and hypo-acidic, and one third of gastric cancer patients were hyper-accidic. Omeprazole absolutely inhibited gastric acidic environment in hypo and meso-acidic patients, but poorly did in hyper-acidic ones.

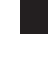


M1972

A Novel Technique for Prolonged Studies of Gastric Acid Secretion in Free Roaming Conscious Rats

Tobias Rudholm, Colin A. Campbell, Peter G. McLean, Per M. Hellstrom, Erik Naslund

Background: Evaluation of gastric acid secretion in rats has historically relied upon the use of gastric fistula or pylorus ligation techniques. The aim of this study was to assess the feasibility of measuring rat intragastric $\mathrm{pH}$ with the Bravo Capsule system (SynMed, Solna, Sweden) and compare these results with a gastric fistula model. Method: Male SpragueDawley rats (350 g) were used for this study. Using a midline incision, a small opening was made in the stomach along the greater curvature. The gastric contents were evacuated. A calibrated Bravo Capsule was placed inside the stomach with the $\mathrm{pH}$ sensor pointing distally. The capsule was anchored with a suture in the mucosa of the stomach. Studies commenced two days after surgery and the intragastric $\mathrm{pH}$ profile was studied during free feeding of standard chow for 5 consecutive days. The effects of an intravenous (IV) injection of esomeprazole $(1,3,5 \mathrm{mg} / \mathrm{kg})$, on 8-hour gastric $\mathrm{pH}$ was studied, as well as, the effect of IV esomeprazole $3 \mathrm{mg} / \mathrm{kg}$ on 2-hour gastric $\mathrm{pH}$. For the gastric fistula studies, a fistula was placed at the greater curvature of the stomach and passed through the skin slightly to the left of the midline. The fistula was capped/closed. During studies the rat was placed in a Bollman cage. Gastric fluid was collected at 30-min intervals for one hour before and 2 hours after IV esomeprazole $(3 \mathrm{mg} / \mathrm{kg})$ and acid output calculated. Data are mean \pm SEM Results: All rats rapidly recovered after surgery and placement of the Bravo capsule, and gained weight. The average 24 -hour $\mathrm{pH}$ with during free feeding was $1.6 \pm 0.2(\mathrm{n}=9)$. After treatment with esomeprazole at IV doses of 1,3 , and $5 \mathrm{mg} / \mathrm{kg}, 8$-hour $\mathrm{pH}$ was $3.2 \pm 0.5$ $(n=5), 3.6 \pm 0.5(n=8)$, and $4.0 \pm 0.5(n=5)$, respectively. $\mathrm{pH}$ one hour before and two hours after esomeprazole $(3 \mathrm{mg} / \mathrm{kg}$ ) was $1.7 \pm 0.3$ and $4.0 \pm 0.5$, respectively. Average gastric acid output, in the fistula model, one hour before IV esomeprazole ( $3 \mathrm{mg} / \mathrm{kg}$ ) was $78.2 \pm 7.4$ $\mathrm{mmol} / 30 \mathrm{~min}$ and $37.7 \pm 5.5 \mathrm{mmol} / 30 \mathrm{~min}$ for the two-hour period after esomeprazole. Conclusion: The Bravo Capsule system can be used for prolonged studies of gastric acid secretion in free roaming conscious rats over days and was well tolerated. The $\mathrm{pH}$ measurements with the Bravo Capsule system were stable and gave reliable recordings, comparable to those seen in the gastric fistula model. The attenuated variations in $\mathrm{pH}$ as seen after administration of a proton pump inhibitor indicate that true biological responses were recorded. The Bravo Capsule system optimizes our aim to record intragastric $\mathrm{pH}$ levels over prolonged periods in a closed environment and allows both short and long-term evaluation of drug effects.

\section{M1973}

Nighttime Acid Suppression with Proton Pomp Inhibitor (PPIs): Are All Nights the Same?

Jenny Liu, Yuhong Yuan, Richard H. Hunt

Background: Nighttime $\mathrm{pH}$ is an important outcome in pharmacodynamic studies, and several report early nighttime $\mathrm{pH}$ is lower than late nighttime. However, definition of nighttime varies widely between studies. This systematic review explores how differences in nighttime definitions impact on $\mathrm{pH}$ in PD studies of PPIs. Methods: Medline and EMBASE were searched for English-language RCTs from 1984-2005 investigating the effect of PPIs on intragastric $\mathrm{pH}$ in healthy volunteers on day 5-8. Keywords were: "intragastric $\mathrm{pH}$ ", "pH metry", "ambulatory pH", "proton pump inhibitor(s)", and PPI names. pH values (mean and/or $\%$ time $\mathrm{pH}>4$ /night) were obtained. Studies required a nighttime definition. Under the same PPI dose, unique nighttime periods were termed a group. When two or more arms under the same group reported the same $\mathrm{pH}$ parameter and included SD or 95\% CI, data was pooled. For the same PPI dose, pooled data for groups were compared using StatsDirect ${ }^{\circledR}$ version 2.4.6. Results: Ten relevant studies ( $25 \mathrm{arms}, \mathrm{n}=183$ ) provided intragastric nighttime $\mathrm{pH}$ values for healthy volunteers after 5-8 days PPI therapy [esomeprazole (ESO, 7 arms), lansoprazole (LAN, 9 arms), rabeprazole (RAB, 1 arm), pantoprazole (PAN, 3 arms), omeprazole (OME, 5 arms)]. Sample size ranged from 9 to 40 . All PPIs provided significant antisecretory effects at steady state compared with baseline. However, few studies reported all 2 parameters, while some did not report SD or CI. There were 17 groups, where nighttime varied $8-12 \mathrm{hrs}$. Of these, 13 groups were excluded because of single arms ( 7 studies). Only 6 groups ( 6 studies, 10 arms) had poolable values. For ESO 40mg om and OME 20mg bid, no comparison could be made since the complementary nighttime periods had only one arm, but mean \% time $\mathrm{pH}>4$ /night ranged widely from 46.8-57.9 and 71.7-91.1, respectively. LAN $30 \mathrm{mg}$ om was the only dose with two different nighttime definitions, each of which had a pooled mean \% time $\mathrm{pH}>4$ /night. For 22:00-06:00 and 23:00-08:00, the values were $26.27 \%(20.95-31.59)(2 \mathrm{arms}, \mathrm{n}=24)$ and $67.82 \%(45.35-90.29)$ ( 4 arms, $\mathrm{n}=38$ ), respectively. The $95 \% \mathrm{CIs}$ did not overlap, suggesting nighttime $\mathrm{pH}$ values between the two nighttime definitions were significantly different. Conclusion:In $24 \mathrm{hr} \mathrm{pH}$ studies, the definition of nighttime varies widely and ranges from $8-12 \mathrm{hrs}$, making comparisons between PPIs difficult. Simply pooling nighttime $\mathrm{pH}$ data may be inappropriate since the variability in nighttime definitions may influence the $\mathrm{pH}$ value. Standardized nighttime definitions are needed before comparing the role of antisecretory drugs in the management of nighttime reflux.

\section{M1974}

Validation of the Gastroesophageal Reflux Disease Impact Scale - A Patient Management Tool for Primary Care Roger Jones, Karin Coyne, Ingela Wiklund

To make appropriate management decisions, primary care physicians need to know how gastroesophageal reflux disease (GERD) affects their patients' everyday lives. Objectives: Our study aimed to develop and test a simple, one-page, patient-completed tool to communicate to the physician the frequency of reflux symptoms and their effect on patients' lives. Patients $\&$ Methods: A draft version of the GERD Impact Scale (GIS) was developed from focus groups of patients and primary care physicians, and a literature review. Items assessed the frequency of the following over the past week on 4-point scales: acid-related symptoms; chest pain; extra-esophageal symptoms; impact of symptoms on sleep, work, meals and social occasions; use of additional non-prescription medication. A validation study was conducted based on two consultations ( $16 \pm 2$ days apart) in primary care patients newly diagnosed with GERD or with an existing GERD diagnosis. Ancillary measures included the Quality of Life in Reflux and Dyspepsia (QOLRAD) and the Reflux Disease Questionnaire (RDQ). Results: A total of 100 newly diagnosed GERD patients and 105 patients with an existing GERD diagnosis from 9 primary care clinics participated in the study. Exploratory factor analysis reduced the number of items from 12 to 8 and identified 3 distinct factors: 'upper gastrointestinal symptoms', 'other acid-related symptoms', and 'impact of symptoms'. Psychometric validation demonstrated high internal consistency (Cronbach alpha: 0.8), reproducibility (intraclass correlation coefficient in stable patients with an existing GERD diagnosis: 0.75 ), and construct validity (Spearman correlations of the GIS with QOLRAD and RDQ: $0.5-0.8$ in both patient groups). Effect sizes in patients newly diagnosed with GERD and those with an existing GERD diagnosis were 1.5 and 0.4 , respectively. In $35 \%$ of patients ( $46 \%$ of newly diagnosed GERD patients versus $25 \%$ of patients with an existing GERD diagnosis), physicians reported altering their treatment decision based on information provided by the GIS. Overall, $76 \%$ of physicians found the GIS useful. Conclusion: The GIS demonstrated good psychometric properties, and was found to be useful by the majority of participating primary care physicians. By highlighting the impact of reflux symptoms on patients' lives, the GIS can help physicians identify newly diagnosed GERD patients in need of effective treatment and patients with an existing GERD diagnosis with uncontrolled symptoms in need of more effective treatment.

\section{M1975}

The Correlation Between Upper Gastrointestinal Symptoms and Plasma Ghrelin in Patients with Peptic Ulcer

Sun Young Yang, Oh Young Lee, Sung Hee Han, Dae Won Jun, Byoung Joo Roh, Chang Ok Yoon, Hang Lak Lee, Byung Chul Yoon, Ho Soon Choi, Jun Soo Hahm, Min Ho Lee, Dong Hoo Lee, Choon Suhk Kee

Background: Ghrelin is a peptide produced in the stomach, which influences appetitie, food intake, and gastric motility. Recently, Helicobacter pylori infection has been reported to be associated with a reduction in plasma and gastric ghrelin levels in patients with gastritis. However, the effect of $H$. pylori eradication on plasma ghrelin level remain controversial. The aims of this study were: (1) to compare the effects of plasma ghrelin on the course of healing of peptic ulcers; (2) to examine the effect of $H$. pylori positive peptic ulcer on plasma ghrelin expression; (3) to study the relationship of upper gastrointestinal symptoms with plasma ghrelin. Methods: Plasma ghrelin were measured via radioimmunoassay after overnight fast, before and after cure of gastric and duodenal ulcer in 20 patients (13 H. pyloripositive and $7 \mathrm{H}$. pylori-negative). 14 asymptomatic healthy volunteers who visited for health screening were enrolled as a control group. H. pylori status was assessed with rapid urease test. Patients with $H$. pylori-positive peptic ulcer received a triple regimen(esomeprazol, clarithromycin, and amoxicillin trihydrate) for 7 days, and esomeprazol following 3 weeks. Patients with $H$. pylori-negative peptic ulcer received only esomeprazol for 4 weeks. They were completed the questionnaires about 7 upper gastrointestinal symptoms (nausea, vomiting, epigastric pain, heartburn, acid reflux, early satiety, bloating) before and after treatment. They gave upper gastrointestinal symptom score from zero to ten before and after treatment. Results: Compared to the control group, plasma ghrelin was not significantly different in patients with peptic ulcer. The mean ghrelin level in patients with peptic ulcer seemed to increase (from $4.6 \pm 1.1 \mathrm{ng} / \mathrm{ml}$ to $4.9 \pm 0.7 \mathrm{ng} / \mathrm{ml}$ ) after treatment, but there was no significant difference $(\mathrm{p}=0.07)$. Only the ghrelin level in the $H$. pylori-positive peptic ulcer subjects was significantly increased (from $4.7 \pm 1.7 \mathrm{ng} / \mathrm{ml}$ to $5.3 \pm 1.9 \mathrm{ng} / \mathrm{ml}$ ) after treatment $(\mathrm{p}=0.02)$. Among 7 upper gastrointestinal symptoms, there was significant correlation between plasma ghrelin and symptom of early satiety $(\mathrm{r}=-0.57, \mathrm{p}<0.05)$ and bloating $(\mathrm{r}=-0.64, \mathrm{p}<0.001)$. Conclusions: The ghrelin level in the patients with $H$. pylori-positive peptic ulcer was significantly increased after treatment. There was significant correlation between plasma ghrelin and symptom of dysmotility

\section{M1976}

Is Abdominal Obesity Associated with Symptoms of Gastroesophageal Reflux in General Population? Enrique Rey, Cristina Moreno Elola Olaso, Fernando Rodriguez Artalejo, Manuel DiazRubio

Gastroesophageal reflux symptoms (GERS) are associated with excess weight. Increased in abdominal pressure due to higher content of abdominal fat in overweight subjects may be one of the main mechanisms. If so, abdominal obesity, which can be estimated by waist circumference, should be associated to GERS. AIM: To evaluate if waist circumference is associated to GERS in general population MATERIAL AND METHODS: 700 persons living in the 7th Health Area of Madrid were interview at home by trained professional interviewers. They were selected at random from the whole population and inclusion was stratified by age and sex. All of them answered the gastroesophageal reflux questionnaire; also we measured weight, height and waist circumference at their home. BMI was calculated and subjects were classified as normal (BMI lower than 25) or overweight. According to Lean et al (BMJ 1995), subjects were classified by waist circumference in normal ( $79 \mathrm{~cm}$ or lower for women and $93 \mathrm{~cm}$ or lower for men) and larger waist circumference (abdominal obesity). Typical GERS were assessed with GERQ and categorized as occasional (less than weekly) or frequent (at least weekly). We compared the annual prevalence of GERS by the existence of general overweight and abdominal obesity. Comparisons were done by chi squared test. RESULTS: We included 690 subjects who completed anthropometric measurements, but 97 subjects where excluded from the analysis to avoid any bias because they recognized to have taken any dose of PPI during the past year. Visceral obesity were significantly more frequent in overweight than non overweight subjects (79.5\% vs $30.5 \%$; $\mathrm{p}<0.0001$ ). Among those with normal BMI, prevalence of occasional symptoms was similar in subjects with and without abdominal obesity ( $17.3 \%$ vs $16.2 \%$; $=n s)$ but the prevalence of frequent symptoms was significantly higher in those with abdominal obesity compared to those without ( $11.1 \%$ vs $2.2 \%$; $=0.007$ ). Among those subjects with overweight, there were no 
differences between subjects with and without abdominal obesity in the prevalence of occasional ( $15.8 \%$ vs $10.4 \%$ ) and frequent GERS (5.8\% vs $4.5 \%$ ). CONCLUSION: Abdominal obesity is associated with frequent GER symptoms in non overweight subjects. This study was supported by an unrestricted grant from Fundacion Mutua Madrileña

\section{M1977}

Acid Output Control with Esomeprazole in Patients with Zollinger-Ellison Syndrome and Idiopathic Gastric Acid Hypersecretion: Final 12-Month Results David C. Metz, Joseph R. Pisegna, Philippe Ruszniewski, Christopher E. Forsmark, John Monyak, Mark B. Sostek

OBJECTIVE: To evaluate the effects of esomeprazole on basal acid output (AO) control in patients with documented Zollinger-Ellison syndrome (ZES) or idiopathic gastric acid hypersecretion (IGH) over 12 months. METHODS: This 12-month, open-label, multicenter study (D9612C00025) included 19 patients with ZES and 2 patients with IGH. Patients were switched from their previous PPI to esomeprazole $40 \mathrm{mg}$ twice daily $(\mathrm{n}=19)$ or 80 mg twice daily $(\mathrm{n}=2)$. At baseline and day 10 , AO was evaluated by nasogastric aspiration and $\mathrm{pH}$ titration. Adequate control of $\mathrm{AO}$ was defined as $\mathrm{AO}<5 \mathrm{mmol} / \mathrm{h}$ and $<10 \mathrm{mmol} / \mathrm{h}$ for patients who had and who had not had prior gastric-acid-reducing surgery, respectively. Patients with controlled $\mathrm{AO}$ were maintained on the same dose, and $\mathrm{AO}$ was measured again at 3,6 and 12 months. If $\mathrm{AO}$ was not controlled, doses were uptitrated (to a maximum dose of $240 \mathrm{mg} / \mathrm{d}$ ) until control was attained. At any time, doses could be increased at the investigator's discretion in patients reporting potentially acid-related symptoms or because of inadequate $\mathrm{AO}$ control. Endoscopies were performed at baseline and at 6 and 12 months. RESULTS: Of the 15 men and 6 women (mean age, 55 years), 3 had multiple endocrine neoplasia type- 1 syndrome, and 8 had metastatic ZE disease. AO was controlled in 19 patients at baseline. No patient withdrew from the study. At 12 months, AO was controlled with esomeprazole $40 \mathrm{mg}$ twice daily in 14 patients (67\%), with esomeprazole $80 \mathrm{mg}$ twice daily in 4 patients (19\%), and with $80 \mathrm{mg} 3$ times daily in 1 patient (5\%) and was not controlled with esomeprazole $40 \mathrm{mg}$ twice daily in 2 patients (10\%; see table). Of the 20 patients with controlled $\mathrm{AO}$ at day 10,18 had sustained $\mathrm{AO}$ control for the rest of the study. At 12 months, no patient had endoscopic evidence of erosive esophagitis or gastric or duodenal ulcers. Esomeprazole was generally well tolerated at all doses. Two patients had serious adverse events that the investigator attributed to treatment (hypomagnesemia and abdominal pain), and both resolved during continued treatment. CONCLUSIONS: Esomeprazole effectively maintains AO control in patients with ZES or IGH. These efficacy and safety results show esomeprazole is an effective and well-tolerated long-term medical therapy for these patients. Supported by AstraZeneca LP Basal Acid output (mmol/h)

\begin{tabular}{|c|c|c|c|c|}
\hline & Baseline visit & 10 Days & 6 Months & 12 Months \\
\hline Median (range) & $0.5(0-20.3)$ & $0.07(0-17.0)$ & $0.2(0-5.9)$ & $0.2(0-35.3)$ \\
\hline
\end{tabular}

\section{M1978}

Nitric Oxide Arising from the Lumen At Gastro-Esopahgeal Junction Could Have Cytotoxic Effect to the Adjacent Gastric Tissue in Human

Kiyotaka Asanuma, Katsunori Iijima, Nobuyuki Ara, Syuichi Ohara, Tetsuhiko Yoshimura, Tooru Shimosegawa

Back Ground The recent study reported high concentrations of nitric oxide (NO) were generated luminally at the gastro-esophageal junction (GEJ) through the entero-salivary recirculation of dietary nitrate in human. In our recent study, using a rat animal model in which luminal NO was generated mainly at GEJ, concentrations of NO in the gastric tissue of the rats were measured by using the NO trapping technique combined with EPR spectroscopy. It was revealed NO generated luminally was diffused into the adjacent tissues at a level comparable to that of iNOS derived-NO production. But the NO trapping reagents were not adapted to human because of cytotoxcity and it is unknown whether the NO arising from the lumen can actually affect the gastric tissues in human. Since an NO has a high affinity for Fe-S cluster proteins such as aconitase, which serves as an important catalyst of energy-yielding reactions, this protein can become one of an intracellular target of NO toxicity. Dinitrosyl dithiolato iron complex (DNIC) is considered to be generated as a product when the Fe-S cluster proteins interact with an NO, leading to inactivation of the enzyme activity. Aim To examine whether DNIC could be generated in the tissues as a consequence of the reaction of NO arising from the GE junctional lumen with $\mathrm{Fe}-\mathrm{S}$ cluster proteins in the adjacent tissues in human. Method At first, using the animal model, a DNIC and an aconitase activity in the gastric tissuewere measured. Secondly, Biopsy specimens of H.pylori negative volunteers were obtained from cardia, antrum after $10 \mathrm{mmol}$ sodium nitrate solution was administered and a DNIC of the tissue was measured. Results A DNIC was observed in the rats gastric tissue of the GEJ and was increased with time and dose-dependent manners, when administered nitrite, whereas no signal was observed at the distal stomach in same rats. An aconitase activity of the tissues at the GEJ was significantly lower in nitrite-administered rats compared to control rats $(22.6 \pm 2.2 \mathrm{mU} / \mathrm{mg}$ protein vs. $26.6 \pm 7.0 \mathrm{p}<0.05)$, while that in the distal stomach was similar between the two groups. in human, the DNIC was detected in the biopsy specimens from cardia, not antrum. Conclusion A DNIC formation and accompanying inhibition of aconitase activity at the GEJ was observed in the tissue which was exposed to abundant luminal NO. This suggests that the NO diffuses into the adjacent tissue and there interacts with Fe-S cluster proteins such as aconitase, leading to reduction of the enzyme activity. This mechanism may be involved in the high prevalence of inflammation and intestinal metaplasia at GEJ in human.

\section{M1979}

Angiotensin II Type 1A Receptor Blockade Attenuates Gastric Inflammation and Fibrosis in Experimental Ulcer Healing

Takahiro Suzuki, Masaaki Kuroda, Norimasa Yoshida, Tomohisa Takagi, Osamu Handa, Satoshi Kokura, Hiroshi Ichikawa, Yuji Naito, Takeshi Okanoue, Toshikazu Yoshikawa

Background \& Aims : We have reported that angiotensin II receptor blockade (ARB) has an anti-inflammatory effects via suppressing neutrophil-endothelial cell interactions. On the other hand, the recent report demonstrated that ARB could protect against progression of fibrosis in liver and pancreas or promote wound healing, however, the role of angiotensin II receptor in ulcer healing following gastrointestinal mucosal injury remains unknown, The aim of the present study was to determine the role of angiotensin II receptor in healing stage of chronic gastric ulcer model. Materials \& Methods: Expl. Seven weeks old male C57/BL6 mice and angiotensin II typel receptor (ATla) deficient mice were used in this study. Under anesthesia, mice underwent laparotomy via midline epigastric incision. After exposing the stomach, $40 \%$ acetic acid solution was focally applied to the anterior serosa surface of stomach for 30 seconds with a modified methods of Okabe et al. On day 14, we estimated the macroscopic and microscopic findings of gastric lesions. At the same time, total RNA was extracted from gastric mucosa and the gene expression related to inflammation (TNF, IL-1), cell growth (EGF, VEGF) and fibrosis (procollagen) was assessed by real time PCR. In addition, we determined myeloperoxydase (MPO) activity as index of neutrophil infiltration. Exp2. Seven weeks old male C57/BL6 mice were used in this study. In the same way as Expl, experimental gastric ulcers were made by acetic acid injection. On day 3 after operation, male C57/BL6 mice were divided randomly into the control group (physiologica saline) and ARB (candesartan at $0.1 \mathrm{mg} / \mathrm{kg} /$ day and at $1.0 \mathrm{mg} / \mathrm{kg} / \mathrm{day}$ ) groups. These agents were orally administered from 3 to 14 days after acetic acid injection. On day 14, we estimated various mediators in the same way as Expl. Results: Expl. On day 3, there were no significant differences in both the size of gastric lesion and MPO activity between ATla deficient mice and wild type mice. On day 14 , the size of gastric ulcer and MPO activity in ATl deficient mice were significantly decreased compared with wild type mice. The gene expression related to inflammation and fibrosis were decreased in ATl deficient mice. Exp2. In the ARB group, the gene expression related to inflammation and fibrosis was decreased but VEGF mRNA was significantly increased compared with control group without ARB Conclusions: These results indicate that inhibition of angiotensin II/ATla signal pathway is implicated in promotion of gastric ulcer healing. Its mechanisms may be partially mediated by inhibition of fibrosis and inflammation and progression of cell growth.

\section{M1980}

Endogenous Prostaglandins (PG) and Melatonin in Ulcer Healing Stanislaw Konturek, Tomasz Brzozowski, Peter C. Konturek

Background: (PG) and melatonin (MT) are generated by the gastric mucosa and exhibit gastroprotective activity but their role in mucosal repair and ulcer healing has not been established. Aims and Methods: This study carried out on rats with chronic acetic acid induced gastric ulcerations was designed to determine the involvement of endogenous PG and MT in ulcer healing process of gastric ulcerations in rats with intact and suppressed mucosal generation of PG by COX-1 and COX-2 inhibitors and blockers of MT receptors. Results: The ulcerated tissue and ulcer margin showed significant up-regulation of COX-2 and excessive generation of PGE2, but reduced formation of MT, when compared to intact mucosa. Misoprostol in gastroprotective dose $(10 \mu \mathrm{g} / \mathrm{kg}$ i.g) failed to affect the ulcer healing and COX-2-PGE2 system in the ulcerated mucosa. Larger, gastric acid inhibitory dose of misoprostol $(50 \mu \mathrm{g} / \mathrm{kg}$ i.g) accelerated ulcer healing that was significant after 1 and 2 wks of treatment and, paradoxically, was accompanied by the upregulation of COX-2 protein(without change in expression of COX-1) and increased PGE2 generation at the ulcer area. COX-2 (Celecoxib - $200 \mathrm{mg} / \mathrm{kg}$ ) and COX-1/COX-2 inhibition (indoemtahcin - 20 $\mathrm{mg}$ ) as well as blockade with Luzindole MT2 receptors, retarded dose-dependently ulcer healing and reduced ulcer tissue generation of PGE2, while increasing the expression of COX-2. Melatonin or its precursor L-tryprophan, also dose-dependently accelerated acetic acid ulcerations and this was accompanied by up-regulation of COX-2 and generation of PGE2 as well as expression of NO synthase (NOS) and increased formation and release of NO into the ulcerated tissue and gastric lumen. Omeprazole (proton pump inhibitor) in gastric acid inhibitory dose $(40 \mathrm{mg} / \mathrm{kg}$ ) accelerated ulcer healing, mainly due to its antisecretory activity but also through increasing the activity of COX-2-PGE2 system in ulcer tissue Conclusion: Endogenous PG are generated at the ulcer margin due to up-regulation of COX2 induced by melatonin

\section{M1981}

Intravenous Administrations of Proton Pump Inhibitor (Omeprazole) Before and After Meals Produce Similar Effects On Inhibitions of Gastric Acid Secretions

Pradermchai Kongkam, Varocha Mahachai, Sutep Gonlachanvit

Background: The inhibitory action of a proton pump inhibitor on gastric acid secretion is believed to depend on the administrative time related to a meal. Although it is recommended that PPIs should be administered before meals, the effects of PPIs on gastric acid secretions have not been well compared between before and after meal administrations. Aim: To stud the inhibitory action on gastric acid secretion of omeprazole when administered intravenously 30 minutes before meals compare to 2 hours after meals. Methods: 18 healthy volunteers $(10 \mathrm{M}, 8 \mathrm{~F}$; age $32 \pm 13$ years, mean $\pm \mathrm{SD}$ ) were randomized to receive either omeprazole (40 $\mathrm{mg}$ intravenously every 12 hours for 2 days) administered $30 \mathrm{~min}$ before meals or 2 hours after meals. All volunteers underwent 48 hour intragastric $\mathrm{pH}$ monitoring using Mark IIl Digitrapper with a pH catheter. Gastric $\mathrm{pH}$ sensor was positioned at $10 \mathrm{~cm}$ below the upper border of the LES localized by esophageal manometry. Results: All volunteers completed the studies. Mean basal gastric $\mathrm{pH}$ during $30 \mathrm{~min}$ fasting periods before iv omeprazole were $2.0 \pm 0.9$ for before meal and $1.2 \pm 0.4$ for after meal study arms, respectively $(p>0.05)$. The $\%$ time of gastric $\mathrm{pH}>4$ during the first 4, 8, 24, and 48 hour after iv omeprazole administered 
30 min before meals were $82 \pm 24 \%, 88 \pm 13 \%, 84 \pm 13 \%$, and $89 \pm 8 \%$, respectively. Whereas, the $\%$ time of gastric $\mathrm{pH}>4$ during the first $4,8,24$, and 48 hour after iv omeprazole administered 2 hour after meals were $80 \pm 19 \%, 84 \pm 20 \%, 86 \pm 18 \%$, and $90 \pm 14 \%$, respectively, not significantly different compared to the administration before meals $(p>0.05)$. Mean intragastric $\mathrm{pH}$ during the first $4,8,24$, and 48 hour after iv omeprazole given before meal were $5.7 \pm 1.4,5.9 \pm 0.9,5.7 \pm 0.9$ and $5.9 \pm 0.6$, respectively, not significantly different compared to after meal $(5.4 \pm 0.8,5.8 \pm 0.9,5.7 \pm 0.9$, and $6.0 \pm 0.8$, respectively, $\mathrm{p}>0.05)$. Conclusions: Omeprazole $40 \mathrm{mg}$ IV every 12 hours produces similar inhibitory effect on gastric acid secretion when given either 30 minutes before or 2 hours after meal. This result suggested that administration time related to meals did not significantly affect the inhibitory action on gastric acid secretion by IV omeprazole.

\section{M1982}

Effect of Naprosyn On Gastric Acid Secretion and Gastric pH Sheila Rodriguez-Stanley, Nancy Redinger, Philip B. Miner

Background: Non Steroidal Anti-Inflammatory Drugs (NSAIDs) are inflammatory to the stomach and associated with gastric ulcers and gastritis, however mechanisms for the inflammation are unclear. NSAIDs may change gastric secretory physiology. Aims: To determine if a commonly used NSAID, Naprosyn $500 \mathrm{mg}$ BID, alters pentagastrin-stimulated Maximal Acid Output (MAO), Basal acid output (BAO), and fasting gastrin in normal, $H$ pylori negative, asymptomatic subjects, aged 18-70. Methods: Informed consent and medical evaluations were performed during screening. Pre-NSAID BAO and MAO gastric aspirations were performed, followed by treatment with Naprosyn $500 \mathrm{mg}$ BID for 7 days. Then, post-NSAID BAO and MAO were performed on day 8 . BAO consisted of one hour of gastric fluid collection via nasogastric tube in 15-minute intervals ( 4 samples). At the end of the BAO period, pentagastrin (sc) was given at $6 \mathrm{mcg}$ per $\mathrm{kg}$ body weight, and gastric fluid was aspirated and collected for one hour in 15-minute intervals (4 samples) for MAO. Volume was measured in each sample and an aliquot was taken for determination of $\mathrm{pH}$ and $\mathrm{mEq}$ of acid by titration. Prior to gastric collections, blood was drawn for fasting gastrin levels. Significant differences between baseline $\mathrm{BAO}, \mathrm{MAO}$ and gastrin and post-NSAID BAO, MAO and gastrin were determined using ANOVA. Comparisons were made using paired T-tests $(\alpha=0.05)$. Results: Twenty-six subjects signed consent. Two subjects did not meet entry criteria. Twenty-four subjects were dispensed Naprosyn and completed the study ( 16 males; 8 females; age range $20-41$ years). Mean $\mathrm{pH}$ of the gastric fluid aspirate during the basal collection period was not altered by Naprosyn (3.34 vs 3.12; NS). Mean pH during the pentagastrin-stimulated maximal collection period was not altered by Naprosyn (2.70 vs 2.62 ; NS). Volume of gastric aspirate during the basal period was significantly decreased post-NSAIDS ( 83 vs 61 $\mathrm{ml} / \mathrm{hr} ; \mathrm{P}=0.012$ ), with no change in pentagastrin-stimulated gastric fluid volume post-NSAIDS (196 vs $188 \mathrm{ml} / \mathrm{hr}$; NS). Basal mean gastric acidity (meq/ml) was significantly increased post-NSAIDS ( 0.41 vs $0.49 \mathrm{meq} / \mathrm{ml} ; \mathrm{P}=0.03$ ), with no change in mean pentagastrin-stimulated maximal gastric acidity due to Naprosyn (0.95 vs 1.03. NS). Fasting gastrin level was not altered by Naprosyn (19.9 vs 17.45 ; NS). Conclusions: Naprosyn decreases basal gastric fluid volume in normal volunteers, which corresponds to the significant increase in basal gastric acid concentration. These data indicate another mechanism by which Naprosyn, and perhaps other NSAIDs contribute to gastric injury. This research was supported by an AstraZeneca ISS grant.

\section{M1983}

Gastrointestinal Complications in Post Renal Transplant Patients - A Single Center Experience

Nandini Nagaraj, Chukwuma I. Egwim, Douglas G. Adler

Background: The incidence of gastrointestinal (GI) disease following or related to renal transplantation has not been well described. The aim of this study was to examine the frequency and characteristics of post-transplantation GI complications. Methods: We retrospectively evaluated a database of renal transplant recipients at our institution since 1998 for severe gastrointestinal complications following renal transplantation. Results: There were 789 consecutive adult renal transplantations (190 living related donors and 599 cadaveric donors) performed on 781 patients during the period between January 1998 and November 2005 at our center. Of these, $87(11.1 \%)$ patients had severe or persistent GI problems requiring gastroenterology evaluation. Out of the 87 patients, 19 had combined renal pancreas transplant. There were 45 male and 42 female patients, with age range between 5 and 68 years (mean of 45 years). The median duration of follow up was 3 years (Range1 month to 88 months). The most common reason for evaluation was persistent, unexplained nausea/vomiting and abdominal pain (49\%). Thirty four of 87 (39\%) patients had severe post-transplantation GI complications including ulceration (16), hemorrhagic gastritis (2), severe opportunistic GI infections (9), severe colitis, diverticulitis with stricture or colon abscess requiring surgery (7), with $64 \%$ (22 patients) occurring in the first year of transplantation. Only 2 patients had Helicobacter pylori related gastritis. Six patients had biopsy proven cytomegalovirus infection of the gut, of these 4 occurred between 1998 and 2000 and only 2 occurred between 2001 and 2005 indicating a low incidence of post transplant opportunistic infections. The only tumor found was a gastric carcinoid, which occurred in one patient. Conclusion: Severe GI complications occur frequently following renal transplantation. Most complications occurred in the first year following transplantation. Prophylaxis with antisecretory medications seems warranted. The incidence of opportunistic GI infection appears to be low.

\section{M1984}

Is There Any Relationship Between Pernicious Anemia and Iron Deficiency? Stephanie Lagarde, Nicolas Jovenin, Marie-Daniele Diebold, Roland Jaussaud, Virginie Cahn, Eric Bertin, Damien Jolly, Gerard Thiefin, Guillaume Cadiot

Introduction : Previous studies have suggested that iron deficiency could be due to atrophic body gastritis. The aim of this study was to determine iron deficiency prevalence in patients with pernicious anemia and associated factors. Patients and methods: All patients with pernicious anemia diagnosed at our institution between January 1990 and February 2005 were included. Inclusion criteria were : 1- histological diagnosis of atrophic body gastritis and 2- autoimmunity criteria (antibody and/or autoimmune disease). A blind histological rereading of gastric biopsies was done. Iron deficiency was defined by serum ferritin level $<15 \mathrm{mg} / \mathrm{L}$ in women and $<40 \mathrm{mg} / \mathrm{L}$ in men. We estimated iron deficiency prevalence and associated factors. Results : 102 patients ( 73 women), mean age 60 years (range 23-90) were included. 20 patients (19.6\%) had normal hemogram; 14 patients $(13.7 \%)$ have microcytosis with or without anemia and 57 patients $(55.9 \%)$ have macrocytosis with or without anemia. Serum ferritin levels were measured in 64 patients, 17 (26.6\%) of whom had iron deficiency. These 17 patients were women, significantly younger ( 40.3 years) than patients without iron deficiency (61.7 years, $\mathrm{p}<0.0001) .76 .5 \%$ of women with iron deficiency were less than 50 years vs $28.6 \%$ of women without iron deficiency $(\mathrm{p}=0.002)$. The anemia frequency was similar in patients with or without iron deficiency (70.6\% and $70.2 \%)$. Microcytosis was more frequent in patients with iron deficiency $(47.1 \%$ vs $6.4 \%$ ) and macrocytosis more frequent in the other group (63.8\% vs $29.4 \%)$. Cobalamin deficiency frequency was similar in the 2 groups (56.2\% in group with iron deficiency vs $68.2 \%$ ). Serum gastrin levels did not differ between the 2 groups. The grade of atrophic body gastritis (graded from 1 to 3 ), the severity of the inflammatory infiltrate of the corpus and that of fundic endocrine cell hyperplasia were no significantly different between patients with or without iron deficiency. Multivariate analysis showed that iron deficiency was linked to female sex and age $<50$ years. Conclusion: Iron deficiency and microcytic anemia are not rare in patients with pernicious anemia and should not rule out the diagnosis. Iron deficiency does not appear to be related to the degree of atrophic body gastritis but is linked to female sex and young age, suggesting the role of menstrual blood loss. Whether decreased iron absorption due to acid hyposecretion favours the expression of gynaecological iron loss cannot be ascertained.

\section{M1985}

The Burden of Non-Heartburn Predominant Dyspepsia On Patients Lives. Results from the STARS II Study

Katarina Halling, Ingela Wiklund, Nigel Flook, Nimish Vakil, Sander Veldhuyzen van Zanten, Karsten Lauritsen, Tore Persson, Johan Brun, Lars-Erik Svedberg, Nicholas J. Talley

Objectives: To assess how dyspeptic symptoms affect patients' lives. Methods: Uninvestigated patients presenting in primary care, with predominant symptoms of pain or burning centered in the upper abdomen for more than 3 months, were included in this study (SD-NED0022). Prior to treatment patients completed the validated Reflux Disease Questionnaire (RDQ) rated 0-5 (worst score), the Gastrointestinal Symptom Rating Scale (GSRS) rated 17 (worst score), the Quality of Life in Reflux and Dyspepsia (QOLRAD) rated 1-7 (best score), SF-36 (0-100 best score) and the Hospital Anxiety and Depression (HAD) Scale (proportion of definite cases of anxiety and depression were calculated). The baseline values are presented for all questionnaires. Results: A total of 1250 patients (mean age 35.3, 59 $\%$ women) were analyzed. The most pronounced symptom burden was dyspepsia according to both GSRS and RDQ (mean GSRS abdominal pain 3.5 vs 2.9 reflux; mean RDQ dyspepsia 2.8 vs heartburn 1.5). A marked dysfunction was reported in the food/drink and vitality dimensions of QOLRAD (mean 4.5 in both) while the physical social functioning was least affected (mean 5.5). GSRS abdominal pain ( $\mathrm{r}=-.41$ food/drink problems, $\mathrm{r}=-.40$ physical/ social functioning, $\mathrm{r}=-.40$ vitality) and the RDQ dyspepsia dimension ( $\mathrm{r}=-.41 \mathrm{food} / \mathrm{drink}$ problems, $r=-.41$ vitality) correlated moderately with QOLRAD impairment $(\mathrm{p}<0.001)$. The SF-36 dimension scores were considerably lower than in a random reference population. The table shows greatest impairment in the bodily pain component and role physical (difference of 24.2 and 16.5 respectively). With the exception of physical function all dimensions differed by more than 5 score units which is considered clinically relevant. According to HAD, $24 \%$ were classified as definite anxiety cases and $5 \%$ as definite depression cases. Anxiety correlated moderately $(\mathrm{p}<0.001)$ with QOLRAD score impairment $(\mathrm{r}=-.48$ emotional distress, $\mathrm{r}=-.40$ vitality). Conclusion: Dyspeptic symptoms profoundly impact patients' lives. The most negative QOL influence was on bodily pain. 


\begin{tabular}{|c|c|c|c|}
\hline SF-36 Dimension & Patient Mean & US refs Mean & Difference \\
\hline Physical function & 85.4 & 89.6 & -4.2 \\
\hline Role physical & 70.4 & 86.9 & -16.5 \\
\hline Bodily pain & 53.9 & 78.1 & -24.2 \\
\hline General health & 62.5 & 75.4 & -12.9 \\
\hline Vitality & 52.8 & 62.0 & -9.2 \\
\hline Social function & 73.2 & 84.6 & -11.4 \\
\hline Role emotional & 70.7 & 82.9 & -12.2 \\
\hline Mental health & 65.3 & 74.6 & -9.3 \\
\hline
\end{tabular}

\section{M1988}

Chronic Urticaria Is Associated with Mast Cell Infiltration in the Gastroduodenal Mucosa

Francesca Minnei, Charlotte Wetzels, Gert De Hertogh, Peter van Eyken, Nadine Ectors, Rossano Ambu, Gavino Faa, Anne-Marie Kochuyt, Karel Geboes

Chronic urticaria (CU) is characterized by recurrent itching skin eruptions caused by mast cell degranulation. Relapses can be provoked by food intake. The aim of this study was to investigate if the mast cell number in the gastroduodenal mucosa is increased in CU patients, and whether mast cell counting by pathologists is clinically useful. We defined 2 study groups: 50 disease controls (16 Belgians and 34 Italians) and 43 Belgian CU patients. Mas cells were detected using immunohistochemistry for tryptase and CD117. The mast cell number in the disease controls was 20.2/HPF $(133.3 / \mathrm{mm} 2)$ in the stomach and $32.5 / \mathrm{HPF}$ (209.2/mm2) in the duodenum. There was no difference between Belgian and Italian controls, indicating that dietary habits have no influence on the normal gastroduodenal mast cell number. In CU patients mast cell numbers were significantly higher: $32.4 / \mathrm{HPF}(186.0 / \mathrm{mm} 2)$ in the stomach $(\mathrm{P}<0.0001)$ and $44.8 / \mathrm{HPF}(246.0 / \mathrm{mm} 2)$ in the duodenum $(\mathrm{P}=0.0002)$ Chronic urticaria is thus associated with mast cell infiltration in the gastroduodenal mucosa even if patients do not have gastrointestinal symptoms. Mast cell counting in gastroduodenal biopsies of $\mathrm{CU}$ patients can be useful to select patients who may respond to a therapy with intestinal mast cell stabilizing agents.

Acceptability and Tolerance of Lansop

Ruszniewski Philippe, Terpereau Arara

Introduction and objective: Lansoprazole is presently available in France as a capsule formulation. An orally disintegrating tablet has been developed as a therapeutic alternative to the capsule, allowing dosing without water at any time. The objective of this study was to evaluate the acceptability and tolerance of the new formulation of Lansoprazole oral dispersible tablet (LODT) $15 \mathrm{mg}$ in patients currently treated by lansoprazole $15 \mathrm{mg}$ capsule for at least 7 days . Patients and methods: A phase III, non comparative, multicentre, single arm, open label study was conducted in France. 30 general practitioners participated in this study. After a period of 7 days treatment with lansoprazole $15 \mathrm{mg} /$ day capsule, the LODT $15 \mathrm{mg}$ was substituted to all the patients. Each patient was seen at the inclusion visit and again at the end of the study. A patient questionnaire allowed an estimate of preference between the capsule and new formulation LODT. Acceptability concerning taste, ease of use, the speed of dissolution, mouth sensation and the possible presence of aftertaste, was collected by means of a visual analogue scale (VAS) going from 0 to 10 (0: completely unacceptable and 10 completely acceptable). Results: 105 patients were included, 103 analysed in the ITT analysis, with mean age 55.08 years $( \pm 14.01), 59.2 \%$ female. The patients were mainly treated for GERD (62.2\%). $53.4 \%$ of the patients preferred LODT, $21.4 \%$ preferred the capsule formulation and $24.3 \%$ were without preference between the 2 forms. On average on a scale of 10 , the ease of use was quoted as $9.17( \pm 1.7)$, convenience of use without water $8.9( \pm 2.19)$, rapidly dissolved in the mouth and pleasant taste was $8.64( \pm 2.2)$ and $7.9( \pm 2.92)$ respectively. Pleasant sensation in the mouth of the LODT was quoted 6.88 $( \pm 3.26)$ and the aftertaste in $6.8( \pm 3.41)$. These results are comparable when matched for sex, age and treated pathology. The lansoprazole orally disintegrated tablet (LODT) was well tolerated, with occurrence of three adverse events of which one was serious, all were considered not related to treatment. Conclusion: This study shows that in the patients requiring treatment with lansoprazole, the orally disintegrated tablet is well accepted. The majority of the patients preferred this new formulation, for its ease of use and the practicality of administration without water

\section{M1987}

Three Centuries of Stomach Symptoms in Scotland

J H. Baron, Fiona Watson, Amnon Sonnenberg

Aims: Stomach pain and discomfort have been reported since antiquity. The present study follows the long-term time trends of non-ulcer dyspepsia, gastric ulcer, duodenal ulcer, and benign oesophageal disease in Scotland to test when non-ulcer dyspepsia started to become a major clinical problem. Methods: The annual in- and outpatient records of the last three centuries from the Scottish Royal Infirmaries of Edinburgh, Aberdeen, Glasgow, and Dundee were analysed. In addition, dispensary attendances, clinicians' casebooks, students' notebooks and medical texts were scrutinised for historic statistics of upper gastrointestinal disease. Results: No hospital admissions for dyspepsia occurred before 1750. Non-ulcer dyspepsia was first recorded in the middle of the eighteenth century and subsequently increased markedly to levels ranging between 200 and 1000 per million living populations. The results of outpatients or public dispensaries during the first half of the nineteenth century show similarly high dyspepsia attendance with annual rates between 700 and 5100 per million living population, confirming the data of hospital admissions to the infirmaries at the same location and during the same time period. The high levels of hospitalization for dyspepsia persisted even after gastric and duodenal ulcers appeared in the late nineteenth century. Gastric ulcer started to occur only after 1850 mostly in females. Duodenal ulcer started to occur after only 1890 mostly in males. Within a few decades the hospitalization rates for gastric and duodenal ulcer rose from 0 to 500 per million living population. During the beginning of the 20th century hospitalization rates of gastric and duodenal ulcer came to surpass that of non-ulcer dyspepsia. In all four cities, hospital admissions for esophageal disease remained rare, never exceeding more than 50 per million with intermittent stretches of no admissions at all. Conclusion: The current twenty-first century commonest diagnosis of stomach pain, non-ulcer dyspepsia, dates from the mid-eighteenth century. Any explanations of its causation need to consider this peculiar time trend

\section{M1989}

Effect of Increasing Esomeprazole and Lansoprazole Dose On Day- and Nighttime Acid Control in Healthy Subjects

Clive Wilder-Smith, Tore Lind, Christina Lundin, Mia Svensson, Catharina NilssonPieschl, Kerstin Rohss

BACKGROUND: In clinical practice, doubling the standard dose of a proton pump inhibitor is often considered in response to problems such as nocturnal symptoms, which may occur as a result of inadequate 24-hour acid control. In this study, we compare the benefit to patient acid control of increasing doses of esomeprazole (ESO) and lansoprazole (LANSO) in terms of the amount of time with intragastric $\mathrm{pH}>4$ achieved during $0-12$ hours and 12-24 hours post-dose. METHODS: In an open-label, randomized, single center, 6 way crossover study, 40 healthy subjects (19 males; mean age: 31 yrs; mean weight: $69 \mathrm{~kg}$ ) received ESO 20, 40 and $80 \mathrm{mg}$ and LANSO 15,30 and $60 \mathrm{mg}$ orally once daily, 30 minutes before breakfast, for 5 days. Continuous 24-hour intragastric $\mathrm{pH}$ monitoring was performed following drug administration on day 5 of dosing using glass electrodes. Treatment periods were separated by a washout period of at least 13 days. RESULTS: See table below. ESO $40 \mathrm{mg}$ provided significantly greater acid control during both the day and night than ESO $20 \mathrm{mg}$, although no further statistically significant improvement was achieved when the dose was increased to $80 \mathrm{mg}$. LANSO $30 \mathrm{mg}$ provided significantly better daytime acid control than LANSO $15 \mathrm{mg}$, as did LANSO $60 \mathrm{mg}$ compared with LANSO $30 \mathrm{mg}$. ESO $40 \mathrm{mg}$ provided significantly better acid control than LANSO $30 \mathrm{mg}$ and $60 \mathrm{mg}$ for the full 24 hours after dosing, as did ESO $80 \mathrm{mg}$ versus LANSO $60 \mathrm{mg}$. ESO and LANSO were well tolerated throughout the study. CONCLUSIONS: In patients with inadequate acid control, either dose escalation from ESO $20 \mathrm{mg}$ to ESO $40 \mathrm{mg}$, or a change from LANSO to ESO may improve day and nighttime acid control.

Additional hours with gastric $\mathrm{pH}>4$ on day 5 of dosing with ESO and LANSO

\begin{tabular}{|c|c|c|c|c|}
\hline Dose comparison & $\mathrm{n}$ & \multicolumn{3}{|c|}{ Mean additional hours with gastric $\mathrm{pH}>4$ on day 5 (95\% confidence } \\
& & $0-12$ hours post-dose & $12-24$ hours post-dose & $0-24$ hours post-dose \\
\hline Eso $40 \mathrm{mg}$ vs Eso $20 \mathrm{mg}$ & 34 & $2.2(1.5 ; 2.8)^{* * * *}$ & $1.6(0.8 ; 2.3)^{* * *}$ & $3.7(2.8 ; 4.7)^{* * * *}$ \\
\hline Eso $80 \mathrm{mg}$ vs Eso $40 \mathrm{mg}$ & 35 & $0.4(-0.2 ; 1.1)$ & $0.6(-0.2 ; 1.5)$ & $1.1(-0.1 ; 2.3)$ \\
\hline $\begin{array}{c}\text { Lanso } 30 \mathrm{mg} \text { vs Lanso } 15 \\
\mathrm{mg}\end{array}$ & 36 & $1.8(1.1 ; 2.6)^{* * * * *}$ & $0.4(-0.5 ; 1.3)$ & $2.2(1.0 ; 3.5)^{* * *}$ \\
\hline $\begin{array}{c}\text { Lanso } 60 \mathrm{mg} \text { vs Lanso } 30 \\
\mathrm{mg}\end{array}$ & 35 & $1.1(0.4 ; 1.8)^{* * *}$ & $0.4(-0.3 ; 1.1)$ & $1.5(0.5 ; 2.4)^{* * *}$ \\
\hline $\begin{array}{c}\text { Eso } 40 \mathrm{mg} \text { vs Lanso } 30 \\
\mathrm{mg}\end{array}$ & 34 & $2.6(1.9 ; 3.2)^{* * * * *}$ & $1.1(0.3 ; 2.0)^{*}$ & $3.7(2.5 ; 4.9)^{* * * *}$ \\
\hline $\begin{array}{c}\text { Eso 40 mg vs Lanso } 60 \\
\mathrm{mg}\end{array}$ & 35 & $1.4(0.8 ; 2.1)^{* * * * *}$ & $0.8(0.2 ; 1.5)^{*}$ & $2.3(1.3 ; 3.2)^{* * * * *}$ \\
\hline $\begin{array}{c}\text { Eso 80 mg vs Lanso } 60 \\
\mathrm{mg}\end{array}$ & 35 & $1.9(1.2 ; 2.6)^{* * * * *}$ & $1.4(0.5 ; 2.4)^{* * *}$ & $3.4(2.0 ; 4.8)^{* * * * *}$ \\
\hline
\end{tabular}

${ }^{*} \mathrm{p}<0.05 ;{ }^{*} \mathrm{p}<0.01 ; * * * \mathrm{p}<0.001 ; * * * \mathrm{p} \leq 0.0001$

M1990

20mg Six Hourly Bolus Dosing of rabeprazole As Effective As Infusion: A Wireless Intragastric $\mathrm{pH}$ Assessment

Rupa Banerjee, Rajesh Gupta, Sandeep Lakhtakia, Arun Maseeh, Manu Tandan, Venkat G. Rao, Nageshwar D. Reddy

Introduction : Intravenous proton pump inhibitor (PPI) infusion is the mainstay of pharmacologic management of upper gastrointestinal bleeding where a high intragastric $\mathrm{pH}>6$ is required for adequate haemostasis. However data on the efficacy of these drugs in bolus doses are limited. Objectives of study: To assess and evaluate the effect of $20 \mathrm{mg}$ and 40 mg Rabeprazole in six and eight hourly doses as compared to bolus injection followed by infusion at $4 \mathrm{mg} / \mathrm{hr}$. Methods: Five subjects received Rabeprazole in a five -way crossover design study.48 hour intragastric $\mathrm{pH}$ assessment was done by the wireless $\mathrm{pH}$ capsule (Bravo,Medtronic) 1. The measured endpoints were the median time required to achieve a steady $\mathrm{pH}>6$, the percentage of time with $\mathrm{pH}>4$ and $\mathrm{pH}>6$. Safety was evaluated by incidence

\section{(1)}


and severity of adverse events. Results: $20 \mathrm{mg}$ and $40 \mathrm{mg}$ IV Rabeprazole achieved a steady $\mathrm{pH}>6$ at a median time of $10 \mathrm{~min}$ and $12 \mathrm{~min}$ respectively. The $\%$ time with $\mathrm{pH}>4$ and $\mathrm{pH}>6$ was $64 \%$ and $72 \%$ (mean) with eight hourly dosing. $20 \mathrm{mg}$ six hourly injections of IV Rabeprazole maintained a steady $\mathrm{pH}>6$ for the total duration of injections. This intragastric $\mathrm{pH}$ response was similar to $40 \mathrm{mg}$ IV bolus followed by infusion at $4 \mathrm{mg} / \mathrm{hr}$ (Graph).All doses were well tolerated. Graph: Intragastric $\mathrm{pH}$ tracing with infusion(a) and six hourly bolus dosing(b) Conclusions: Six hourly injections of Rabeprazole are as effective as infusion for maintaining a steady intragastric $\mathrm{pH}>6$. Referrence: 1.Banerjee R,Lakhtakia S,Reddy DN et al. Endocsopy assisted wireless intragastric pH measurement. Endoscopy 2005;37:922
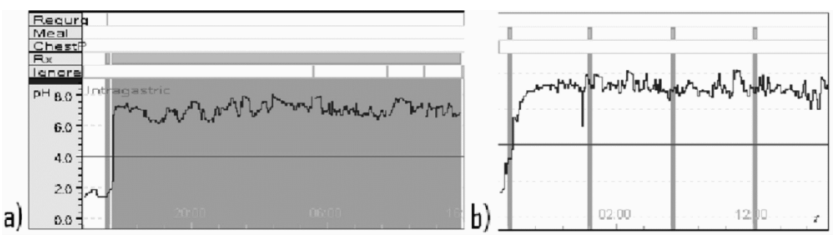

M1991

High Blood Pressure and Symptoms of Gastroesophageal Reflux: Impact On Health-Related Quality of Life

Enrique Rey, Cristina Moreno Elola Olaso, Fernando Rodriguez Artalejo, Manuel DiazRubio

Overweight is a risk factor of both high blood pressure (HBP) and gastroesophageal reflux symptoms (GERS). Association between HBP and GERS has not been evaluated, although an association between Barrett's esophagus and HBP was reported. Moreover, the relative impact of both HBP and GERS on health-related quality of life (HRQL), and the contribution of overweight to such relationship, is unknown. AIM: To examine: 1) the association of HBP with GERS; 2) the association of HBP and GERS with HRQL. MATERIAL AND METHODS: Study subjects were interviewed at home by trained professional interviewers. They were selected at random from the general population. Typical GERS (heartburn and acid regurgitation) was assessed with the GERQ. The SF-36 health questionnaire was used to ascertain HRQL. Lastly blood pressure (automatic device), weight and height were determined. Logistic regression was used to evaluate the predictors of GERS, including in the model HBP and other known risk factors. Differences in each of the dimensions of the SF36 by GERS, overweight, and HBP were analyzed with ANCOVA, including interactions. RESULTS: Study sample comprised 705 individuals (369 with HBP and 336 with normal pressure). There was no difference in the prevalence of GERS between both groups ( $24.9 \%$ in HBP and $24.1 \%$ in normal pressure). In logistic regression model, PSC score, obesity (BMI $>29.9 \mathrm{~kg} / \mathrm{m} 2$ ) and familiar history of GERS were independently associated with GERS, but HBP was not (adjusted OR 0.96 [0.6-1.4]). Results of SF-36 dimensions (mean and C195\%) according to the presence or not of GERS are shown in table In ANCOVA, GERS were statistically significantly associated with lower values of each dimension of SF-36 (except physical functioning). No association was observed between most dimensions of the SF-36 and HBP. BMI-GERS-HBP interaction was statistically significant for the social function dimension of the SF-36, and the BMI-GERS interaction attained significance for the body pain dimension. CONCLUSION: HBP and GERS are not associated in the general population. GERS is associated with lower HRQL, and this association is independent of overweight. Supported by a grant from Fundacion Mutua Madrileña

\begin{tabular}{|c|c|c|}
\hline SF36 dimension & No GERS (N=532) & GERS (N=173) \\
\hline Physical Functioning & $89.1(87.3-90.9)$ & $86.0(82.5-89.5)$ \\
\hline Role Physical* & $90.2(87.9-92.5)$ & $76.7(70.9-82.5)$ \\
\hline Bodily pain* & $83.9(81.9-85.7)$ & $66.2(61.5-70.8)$ \\
\hline General Health* & $74.8(73.2-76.3)$ & $66.7(63.5-69.9)$ \\
\hline Vitality* $^{*}$ & $73.1(71.7-74.6)$ & $60.9(57.8-64.1)$ \\
\hline Social functioning* & $93.0(91.6-94.4)$ & $86.4(83.0-89.7)$ \\
\hline Role Emotional* & $91.0(88.8-93.2)$ & $74.7(68.7-80.8)$ \\
\hline Mental Health* & $76.8(75.4-78.3)$ & $68.9(66.0-71.8)$ \\
\hline
\end{tabular}

${ }^{*} \mathrm{p}<0.001$ (ANOVA)

\section{M1992}

The Effect of Candida Albicans On Healing of Duodenal Ulcer Induced By Cysteamine in Rats

Longxue Jin, Masashi Yoshida, Tetsuya Nakamura, Hideki Ishikawa, Yoshiro Saikawa, Kaori Kameyama, Koichiro Kumai, Tetsuro Kubota, Yoshihide Otani, Go Wakabayashi, Minoru Tanabe, Shigeyuki Kawachi, Katsuko Sano, Masaki Kitajima

Background and purpose: It was known widely that Candida albicans (C. albicans) not only inhabits frequently the gastrointestinal tract of humans, but also was cultured in the ascites in the patients who were undergone the operation for gastroduodenal ulcer perforation. This experiment was performed to clarify the effect of $\mathrm{C}$.albicans infection to gastroduodenal ulcer in rats. Methods: [Experiment 1; Effects of Candida on incidence of duodenal ulcer perforation.] In this study, male Wistar rats $(220-250 \mathrm{~g})$ were separated into the Candida group and the saline group. After the rats were anesthetized with ether, the rats were administrated cysteamine $(310 \mathrm{mg} / \mathrm{kg}$ body weight) three times intragastrically every 4 hours; C. albicans was administrated to the Candida group rats at one hour before and 12,24 hours after the first dose of cysteamine. The control group rats were administrated saline. The rats were sacrificed after 72 hours after the first administration of cysteamine. The immunohistochemical stain was done with an anti-Sap2 (secretory aspartyl protease2) monocolonal antibody. ELISA was performed with anti-Sap2 monocolonal antibody. [Experiment 2; Effects of $\mathrm{C}$. albicans on healing of duodenal ulcer.] The rats in the Candida group were administrated with $\mathrm{C}$. albicans at 48 hours after the first dose of cysteamine administrated like above. Rats in the control group were administrated saline. The rats were sacrificed one week after the first administration of cysteamine. Result: [Experiment 1] The rate of duodenal ulcer perforation in the Candida group is $94.1 \%(16 / 17)$ that was higher than the control group $(26.7 \%, 4 / 15)$. The size of duodenal ulcer area in the Candida group $(40.89 \mathrm{~mm} 2)$ was larger than the control group $(16.53 \mathrm{~mm} 2)$; the survival rate of the Candida group was significantly lower than that of control group. In immunohistochemical analysis, the ulcer bed in the Candida group was positive, whereas the control group was negative. The Sap2 concentration in the duodenal ulcer bed in the Candida group was higher than that of the control group significantly. [Experiment 2] The incidence of duodenal ulcer in the Candida group $(63.6 \%, 14 / 22)$ was higher than that of the control group $(31.6 \%, 6 / 13)$. Conclusion: It was shown that the infection of $\mathrm{C}$. albicans not only promote the process of duodenal ulcer perforation but also delay the wound healing process of duodenal ulcer induced by cysteamine in rats

\section{M1993}

Severe Burn Injury Alters Gastric Slow Wave and Gastric Emptying in Rats Hanaa S. Sallam, Hermes M. Oliveira, Jiande Chen

Introduction: Severe burn injury is known to delay gastric emptying in both animals and humans; however no data are available on its effect on gastric myoelectrical activity (GMA) We aimed at studying the effect of burn injury on GMA as it might contribute to burninduced delayed gastric emptying. Methods: A pair of cardiac pacemaker wires was surgically implanted to the stomach serosa of 20 adult male Sprague-Dawley rats. Following recovery, GMA was recorded for $30 \mathrm{~min}$ in both fasting and fed conditions. The following day, rats received a sham or a 3rd degree scald burn injury under anesthesia. Proper fluid resuscitation and analgesia were given to all animals. Six hours after sham/burn injury, GMA was recorded for 30min again in both fasting and fed conditions. A methylcellulose meal was mixed with phenol red (marker), so that the percentage of gastric emptying could be calculated based on the amount of phenol red recovered from the stomach. GMA parameters were computed using previously validated spectral analysis software developed in our laboratory, including dominant frequency/power and percentages of normal slow waves, bradygastria, tachygastria and arrhythmia. The normal slow wave frequency range was 4-6 cycles per min (cpm) in rats, while $\leq 4 \mathrm{cpm}$ and $\geq 6 \mathrm{cpm}$ were considered bradygastria and tachygastria respectively. Arrhythmia was established if the slow wave frequency had no specific range. Results: 1) None of GMA parameters showed any statistical differences in the fasting condition after burn ( $p>0.05)$. 2) Significant changes in GMA with burn were only observed postprandially, including: a decrease in dominant frequency $(4.5 \pm 0.2$ vs. $5.1 \pm 0.2, p=0.002)$ and in the percentage of normal slow waves $(67.2 \pm 7.3$ vs. $84.5 \pm 4.1, p=0.02)$ and an increase in the percentage of bradygastria $(24.7 \pm 6.8$ vs. $9 \pm 3.9, p=0.02)$. 3) The percentage of gastric emptying was decreased after burn to $40.3 \pm 6.5$ vs. $77 \pm 3.2$ in previously obtained controls ( $\mathrm{p}=$ 0.0003 ), however, it did not correlate significantly to any of GMA parameters before or after burn, whether in fasting or in fed conditions ( $p>0.05)$. Conclusion: Severe burn injury impairs both GMA and gastric emptying in rats. However, these alterations are not correlated with each other, suggesting that gastric dysrhythmia is a manifestation of burn but not the cause of burn-induced delayed gastric emptying.

\section{M1994}

Pepsinogen I Correlates with Severe Esophagitis and Barrett's Esophagus Klaus Moenkemueller Ulrich Peitz, Thomas Wex, Daniel Jaspersen, Joachim Labenz, Wolfgang Meyer-Sabellek, Sn Willich, Marc Nocon, Manfred Stolte, Tore Lind, Peter Malfertheiner

Introduction: Gastrin and pepsinogens (pepsinogen I and II) reflect the functional state of the gastric mucosa. Preliminary data have indicated that circulating levels of gastrin are lower in pts with Barrett's esophagus than in healthy controls. Furthermore, the role of gastrin and pepsinogens in GERD is unclear. Aim: To evaluate whether serum levels of gastrin and pepsinogens correlate with the different forms of GERD [non-erosive (NERD), erosive reflux disease (ERD)] Patients and Methods: Four groups of 87 patients each (1: NERD; 2: ERD Los Angeles A and B, 3: ERD Los Angeles C and D; 4: BE) were selected from the prospective ProGERD study (multicenter open cohort study comprising an initial treatment phase and a 5-yr follow-up of 6,215 patients with various forms of GERD). The four groups were matched for age (median 56, range 29-79 years) and gender (male $\mathrm{n}=65$ ) All patients underwent clinical examination, responded to validated reflux questionnaires and underwent EGD (LA classification). The diagnosis of BE was based on endoscopic and histological criteria. Gastritis was classified according to the updated Sydney classification. None of the patients had taken any form of acid inhibition therapy for at least 4 weeks before endoscopy or giving the serum sample. Serum was analyzed for gastrin-17, pepsinogenI, pepsinogen-II und H. pylori using specific ElA tests (Biohit Plc, Laippatie, Helsinki, Finland). Statistical analysis: Mann Whitney. Results: Significant differences among groups were identified for pepsinogen-I, but not for pepsinogen-II or G-17 levels. Pepsinogen-I serum levels were lowest in NERD $(90.7 \mu \mathrm{g} / \mathrm{l})$ and highest in BE $(114.2 \mu \mathrm{l} / \mathrm{l}, \mathrm{P}=0.012)$ Pepsinogen-I serum levels were higher in BE (114.2 ug/l) compared to ERD Los Angeles $\mathrm{C}+\mathrm{D}=101.8 \mu \mathrm{g} / \mathrm{l}(\mathrm{p}=0.07)$. Pepsinogen levels were higher in $\mathrm{H}$. pylori- positive subjects, but this expected finding did not account for the differences of pepsinogen-I serum levels among various categories of GERD. Conclusions: The increased levels of pepsinogen I in more severe forms of GERD suggest that the mass of oxyntic glands may be a determinant among others for the severity of GERD. This finding correlates well with higher esophageal acid exposure previously reported in patients with more severe forms of GERD 
M1995

Polymorphisms of Exon 4 of P53 Gene in Gastric Intestinal Metaplasia in Hungary

Dominika Szoke, Bela Molnar, Zsolt Tulassay

Aims: Many studies have investigated the association of P53 polymorphisms with increased risk of different cancers, including gastric cancer. The literature of the polymorphisms in exon 4 in gastric cancer preceding intestinal metaplasia (IM) is not plentiful. Materials and methods: After informed consent, gastric biopsy samples were taken from the antrum of 51 consecutive patients with IM and 51 biopsy samples from histologically negative patients as control group. The genotype of the polymorphisms in exon 4 of P53 gene was determined by capillary sequencing. Results: We could not found any patient neither with codon 34 nor with codon 47 polymorphism. Codon 36 polymorphism was found in heterozygote form in three cases. The R72P polymorphism is the most common, the allele frequency for proline is 0.29 in 102 Caucasian individuals. The allele frequencies were significantly different with $\chi 2$-probe between H.pylori positive IM patients and the normal control group ( $\mathrm{p}=$ $0.0018)$, between H.pylori negative IM patients and the normal control group ( $\mathrm{p}=0.0005)$ and between the group of $H$ pylori positive and negative IM, male patients and the normal control group $(\mathrm{p}=0.0221)$. Discussion: There is a significant linear correlation between the frequency of R72P polymorphism and latitude. Our finding corresponds with the expected allele frequencies in our region. The control and IM patients group are not different statistically, however, significant differences can be found if we consider the H. pylory status and gender, too

\section{M1996}

Natural History of Dyspepsia in Primary Care: Incidence, Risk Factors, Comorbidity and Mortality

Mari-Ann Wallander, Saga Johansson, Ana Ruigomez, Luis A. Garcia Rodriguez

Upper gastrointestinal symptoms affect between one quarter and one half of the UK population at least once every six months. Many patients who consult primary care physicians with these symptoms do not receive a firm diagnosis of the underlying cause of their symptoms, and are classified as having dyspepsia. Objectives: To determine the natural history of upper gastrointestinal symptoms of unspecified origin presenting in primary care in the UK and identify risk factors and comorbidity associated with a diagnosis of dyspepsia. Methods: Data were extracted from the UK General Practice Research Database. Patients with a new diagnosis of dyspepsia in 1996 were identified $(n=6913)$ and were compared with an age- and sex-matched control cohort $(\mathrm{n}=11036)$. We determined the incidence of dyspepsia, potential risk factors and comorbidity, and the risk of mortality and of new onset morbidity in the year following the index date. Odds ratios (ORs) and 95\% confidence intervals (CIs) were calculated using unconditional logistic regression. Results: The incidence of dyspepsia was 15.3 per 1000 person-years (95\% CI: 15.0-15.6), and was greater in women than men. A slightly increased probability of a new dyspepsia diagnosis was associated with chest pain (OR: 2.4; 95\% CI: 2.1-2.7), general pain (OR: 1.8; 95\% CI: 1.7-1.9), sleep disorders (OR: 1.5; 95\% CI: 1.2-1.8), angina (OR: 1.5; 95\% CI: 1.2-1.8) and osteoarthritis/ rheumatoid arthritis (OR: 1.4; $95 \%$ CI: 1.2-1.6). The association of smoking (OR: 1.3; 95\% CI: $1.2-1.4$ ) and obesity (BMI $\geq 30 \mathrm{~kg} / \mathrm{m} 2$ ) (OR: $1.2 ; 95 \% \mathrm{CI}: 1.1-1.3$ ) with dyspepsia were of only borderline significance. Current use of non-steroidal anti-inflammatory drugs and aspirin was associated with a significant increase in the risk of dyspepsia (OR: 1.8; 95\% CI: $1.7-2.0$ and OR: $1.3 ; 95 \%$ CI: $1.2-1.5$, respectively). During the follow-up year, patients with dyspepsia were more than 60 times more likely to receive a diagnosis of gastroesophageal reflux disease (GERD) than patients without dyspepsia (OR: 62.8; 95\% CI: 31.1-127.0). They were also more likely to receive a subsequent diagnosis of angina (OR: $2.7 ; 95 \% \mathrm{CI}$ : 1.8-4.0) or chest pain (OR: 3.9; $95 \%$ CI: 3.7-4.2). There was an increased risk of death (RR: $1.6 ; 95 \%$ CI: 1.2-2.2) in the dyspepsia cohort compared with the control cohort during the follow-up year. Conclusions: Lifestyle changes appear unlikely to have a major role in the development of upper gastrointestinal symptoms. Many dyspepsia patients subsequently receive a GERD diagnosis, suggesting that GERD may be underdiagnosed in primary care.

\section{M1997}

Declining Incidence of Duodenal Ulcers Compared to Gastric Ulcers in a 10 Years Endoscopic Database

Marcel Groenen, Ernst J. Kuipers, Rob J.Th. Ouwendijk

Background and Study aims Over the past decades, important changes have occurred in the epidemiology of ulcer disease. Already more than forty years ago, a decline in the incidence of peptic ulcer was suggested on the basis of a cohort analysis. The discovery in the early eighties of $\mathrm{H}$. pylori as major cause of peptic ulcer disease had a further major impact on the incidence of ulcer disease. Our aim was to evaluate the further change of incidence in time of duodenal and gastric ulcer as diagnosed by endoscopy. Material and Methods With the help of Endobase ${ }^{\circledR}$, a computerized endoscopic database, the data of all patients who underwent upper endoscopy from 1996-2005 were analyzed. Data were collected in an open-access endoscopy unit of a district hospital. The incidence of duodenal and gastric ulcers was compared over time. SPSS was used to statistically evaluate the endoscopic data. Results Overall, 13081 upper endoscopies were performed. In 511 investigations $(3.9 \%)$ duodenal ulcers were diagnosed. Duodenal ulcers were found more frequent in men (56.6\%). 125 of the duodenal ulcers (24.5\%) had signs of recent bleeding. Twentyone of these duodenal ulcers (4.1\%) were classified as Forrest I, six of these as Forrest Ia. A visible vessel was diagnosed in 45 patients (8.8\%), Forrest IIb in 31 (6.1\%) and Forrest IIc in 28 patients (5.5\%). Gastric ulcers were found in 317 (2.4\%) endoscopies. 49 of the gastric ulcers (15.5\%) presented with signs of bleeding. Forrest I was diagnosed in 17 patients $(5.4 \%)$ and Forrest II in $32(10.1 \%)$. The incidence of gastric ulcers remained stable over time with about 30 cases per year. However, the incidence of duodenal ulcers declined from $4.1 \%$ to $2.8 \%$ with a linear regression coefficient of -.126 (p 0.087). There was a tendency of leveling incidence of gastric and duodenal ulcers in the last years of the investigated period. Conclusions In the past 10 years, the incidence of gastric ulcer disease has remained stable, whereas the incidence of duodenal ulcer disease decreases. With further treatment of $H$. pylori the incidence of gastric ulcers is expected soon to exceed the incidence of duodenal ulcer in the near future in our Western community

\section{M1998}

The Gastric Sling Fiber/Clasp Fiber Pressure Profile Is Significantly Decreased in Patients with GERD

Thomas McKenna, Annapurna Korimilli, Anil K. Vegesna, Parkman Henry, Brasseur James, Larry S. Mille

Purpose: To determine the contribution of the gastric sling fiber/clasp fiber complex to the antireflux barrier in normal control subjects and patients with GERD. Methods: A simultaneous ultrasound and manometry catheter was pulled through the proximal stomach and the gastroesophageal junction high-pressure zone at a constant velocity in ten normal contro subjects and 10 patients with GERD. Pressure was correlated to the ultrasound image during breath holding in expiration. The ultrasound images were used to locate the beginning of the right crural diaphragm. The area under the pressure curve was measured from the beginning of the pull-through to the beginning of the right crural diaphragm. Gastric pressure was used as a zero baseline. Any increase in pressure prior to the start of the crural diaphragm was assumed to be due to the gastric sling fiber/clasp fiber complex. Results: The area under the pressure curve in GERD patients, was only 18\% of the area under the pressure curve in normal control subjects. The pressure difference between the normal control subjects and the GERD patients was a mean of $19,15,13$ and $12 \mathrm{mmHg}$ at 5,10,15 and $20 \mathrm{~mm}$ before the beginning of the crural diaphragm. Conclusions: The gastric sling fibers form a $\mathrm{V}$ shape configuration on ultrasound imaging. This configuration acts as a pinchcock to prevent the reflux of gastric contents in normal subjects. It does this by providing a pressure barrier between the stomach and the crural diaphragm. This pressure barrier is significantly decreased in patients with GERD. We hypothesize that an underlying cause of GERD may be the absence of the pressure profile due to a defective gastric sling fiber/clasp fiber complex. Funded through an NIH grant R01 DK59500.

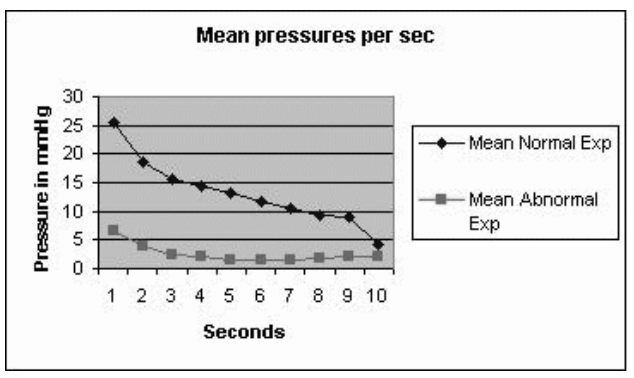

This graph shows the pressure profile of the gastric sling fiber clasp fiber complex in normal control subjects and patients with GERD. The beginning of the crural diaphragm is at $x=0$.

\section{M1999}

Changes in Compliance Following Laparoscopic Nissen Fundoplication Measured By a New Technique (FLIP)

Hanneke Beaumont, Barry P. McMahon, Willem Bemelman, Hans Gregersen, Guy Boeckxstaens

Background \& Aims: Increased distensibility or compliance of the esophagogastric junction (EGJ) was shown to play an important role in the pathogenesis of gastroesophageal reflux disease (GERD). However, to measure EGJ distensibility, radiographic imaging involving radiation is required limiting its application in research protocols. Therefore, a functional lumen imaging probe (FLIP) was constructed measuring eight cross sectional areas (CSA) at $4 \mathrm{~mm}$ intervals inside a saline-filled bag using impedance planimetry and containing two pressure side holes allowing assessment of EGJ compliance. The present study was designed to evaluate whether this technique could be used to detect changes in EGJ compliance between healthy volunteers, untreated GERD patients and patients who had a laparoscopic Nissen fundoplication (LNF) Methods: 5 GERD patients (57 (43-67), 3M), 4 post Nissen patients $(54$ (43-60), 3M) and 5 HV (40 (20-58), 3M) were evaluated. They underwent a compliance measurement using the FLIP, esophageal manometry and $24 \mathrm{hrs} \mathrm{pH}$ metry. The FLIP was positioned with the bag straddling the EGJ using manometry readings and point of respiratory inversion as a guide. The bag was filled with saline at a rate volume of 25 $\mathrm{ml} / \mathrm{min}$ to a maximum of $60 \mathrm{ml}$. Bag pressure and CSA were recorded. A pH below $4>$ $5.78 \%$ of total time was considered to be pathological. For statistical analysis of $\mathrm{pH}$ and manometry, unpaired Student's t-tests were used and data is presented as mean $\pm \mathrm{SE}$ Results: GERD patients have significant more pathological acid reflux $5 \mathrm{~cm}$ above the lower esophageal sphincter (LES) compared to LNF patients and healthy volunteers (GERD patients: $10.5 \pm 3.2 \%$, LNF: $0.7 \pm 0.4 \%, \mathrm{HV}: 2.3 \pm 0.7 \%(\mathrm{P}<0.031)$ ). The basal LES pressure did not differ between the three groups (GERD patients: $6 \pm 2 \mathrm{mmHg}$; LNF: $8 \pm 3 \mathrm{mmHg}$; HV: $9 \pm 3 \mathrm{mmHg}(\mathrm{NS}))$. At a bag pressure of $7 \mathrm{mmHg}$ during distension the average diameter of the EGJ in GERD patients and HV was $13 \mathrm{~mm}$ while the LNF average diameter was 7 $\mathrm{mm}$. However, at $13 \mathrm{mmHg}$, the diameter remained about $13 \mathrm{~mm}$ in $\mathrm{HV}$ and 8 in LNF patients whereas it increased to $22 \mathrm{~mm}$ in GERD patients. Conclusion: FLIP studies showed a greater distensibility in GERD patients compared to HV, accompanied by an increased esophageal acid exposure. Conversely, we showed that FLIP detects a significant decrease in compliance after LNF associated with a decrease in acid reflux. These results suggest that FLIP may offer a direct method to assess changes in compliance of the EG] 


\section{M2000}

The Effect of Food Composition (Energy Density and Fat Content) in Patients with Gastro-Oesophageal Reflux Disease

Mark Fox, Carole Barr, Suzanne Nolan, Miranda Lomer, Angela Anggiansah, Terry Wong INTRODUCTION \& AIM: Patients with symptoms of gastro-oesophageal reflux disease (GORD) are often told to avoid fatty food; however it is not certain whether reflux severity is associated with fat content or overall energy density of the diet. The catheter-free Bravo ${ }^{\mathrm{TM}}$ system provides prolonged oesophageal $\mathrm{pH}$ monitoring that is well tolerated by patients, providing an opportunity for repeated investigation of dietary interventions in GORD METHODS: Patients referred for investigation of reflux symptoms were recruited $(n=21)$. The Bravo ${ }^{\mathrm{TM}}$ capsule was placed by endoscopy. The study design allowed the effects of calorie density, fat content and food consistency on GORD to be assessed independently. High fat (50\%) vs. low fat (25\%) diet (calories controlled), and high calorie (1000kcal) vs. low calorie $(500 \mathrm{kcal})$ diet (fat controlled) were compared. The effects of food consistency on GORD were examined by comparing mixed solid/liquid vs. liquid diet. Meal volume was $800 \mathrm{ml}$ for all diets. All meals were supplied, providing $24 \mathrm{hr}$ in each 'dietary condition'. Patients returned after $48 \mathrm{~h}$ and $96 \mathrm{hr}$ to download data. Associations of dietary composition and consistency with esophageal acid exposure and reflux symptoms were analyzed by repeated measures ANOVA and pairwise comparisons. RESULTS: Complete data was available for $15 / 21$ patients (6M:9F, 48(26-70)yrs, BMI 26(21-35) kg/m2). Demographic variables and meal sequence had no effect on reflux parameters. ANOVA demonstrated significant effects of 'dietary condition' on esophageal acid exposure $(\mathrm{F}=7.4, \mathrm{p}<0.005)$ and reflux symptoms $(\mathrm{F}=24.2, \mathrm{p}<0.001)$. No effects of 'dietary consistency' on esophageal acid exposure or reflux symptoms were present. Esophageal acid exposure was less during the low calorie than high calorie diet (fat controlled); mean 5.2 \pm 1.4 vs. 8.6 $2.0 \%$ time@pH<4/24hr; $(\mathrm{p}<0.01)$. No difference was observed between low and high fat diets (calories controlled); mean $8.2 \pm 1.6$ vs. 8.6 $\pm 2.0 \%$ time@pH<4/24hr; ( $\mathrm{p}=\mathrm{ns}$ ). In contrast, the frequency of reflux symptoms was not affected by the calorie density of the diet (median 6 (2-12) vs. 8 (2-13) reports; $\mathrm{p}=\mathrm{ns})$ but was increased during the high fat diet (median 11 (5-18) vs. 6 (2-12) reports; $\mathrm{p}<0.05)$. Conclusion: This study provides clear evidence that calorie density rather than fat content of the diet determines the severity of acid reflux in GORD. However the fat content of the diet has important effects on the frequency of reflux symptoms. The effects of diet on GORD were clinically relevant with a $>30 \%$ decrease in esophageal acid exposure and $>40 \%$ decrease in reflux symptoms in low vs. high calorie and low vs. high fat diets respectively.

\section{M2001}

Does Gastric Leptin in the Refluxate Contribute to Healing of the Esophageal Mucosa?

Fritz Francois, Adam J. Goodman, Jatin Roper, Michelle Mourad, Asalia Z. Olivares de Perez, Guillermo I. Perez-Perez, Zhiheng Pei, Martin J. Blaser

BACKGROUND: Although heartburn is a hallmark of GERD, only a subset of affected individuals develop mucosal injury. Leptin, a hormone produced in the human stomach, stimulates cell growth in vitro and inhibits gastric ulcer formation in rats. We hypothesized that gastric leptin in the refluxate may contribute to the healing of the esophageal mucosa. Our aims were to evaluate whether leptin receptors are present in the esophagus and to determine whether regional gastric leptin levels correlate with esophageal pathology (esophagitis and/or Barrett's). METHODS: Patients referred for EGD were enrolled if they reported heartburn for $\geq 6$ months in the prior year. A detailed medical history, height, and weight were obtained, and BMI calculated. Serum was collected, and biopsies obtained from antrum, fundus, and esophagus were reviewed by a blinded GI pathologist. Based on endoscopic and histologic findings, patients were classified as having esophageal pathology (EP+) or not (EP-), and immunohistochemistry was performed on representative sections from both groups to determine density and localization of leptin receptors. Leptin levels in plasma and gastric biopsy samples were determined by specific ELISA, and gastric concentrations were normalized according to biopsy protein (pg/mg protein). RESULTS: Among 48 patients enrolled with heartburn, 27 were EP- and 21 were EP+. The two groups did not differ significantly in ethnicity, mean age, BMI, and gastric $\mathrm{pH}$, or in proportions using PPIs, or with H. pylori, or in plasma leptin. Receptors for leptin were highly expressed on esophageal epithelial cells, with similar density and staining pattern in the EP- and EP+ specimens. The EP- group had significantly higher antral leptin levels than the EP+ group (median 94 pg/

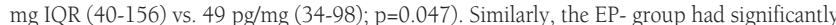
higher fundic leptin levels than the EP+ group (median $221 \mathrm{pg} / \mathrm{mg}$ IQR (93-357) vs. 103 $\mathrm{pg} / \mathrm{mg}$ (72-163); $\mathrm{p}=0.003$ ). Although the groups did not differ in symptom chronicity, the duration of heartburn was significantly correlated with fundus leptin levels in the EP- group $(r=0.59, p=0.005)$, but not the EP+ group. CONCLUSIONS: Extensive expression of the leptin receptor on esophageal epithelial cells suggests that they are susceptible to leptinmediated signal transduction. Differences in gastric leptin levels between EP+ and EP- persons could provide a mechanism for differential esophageal healing after reflux-induced injury. These data support the hypothesis that gastric leptin plays a protective role against esophageal pathology among patients with heartburn.

\section{M2002}

Patients with Non-Erosive Reflux Disease (NERD) Demonstrate Upregulation of TRPV1 Receptors in the Distal Esophagus As Compared to the Other GERD Groups

Ram Dickman, Pankaj J. Pasricha, John Winston, Mohan Shenoy, Diley Hernandez, Ronnie Fass

Background: There is no correlation between severity, frequency or intensity of symptoms and the presence or absence of esophageal mucosal injury. Visceral hypersensitivity and thus upregulation of TRPVl receptors has been suggested to play an important role in symptom generation of NERD patients. Aims: To compare the extent of distal esophageal expression and proximal distribution of TRPVI receptors among the different GERD groups and normal controls. Methods: Five normal healthy controls, 5 patients with NERD, 5 with erosive esophagitis (EE) and 5 with Barrett's esophagus (BE) were recruited into the study All participants underwent an upper endoscopy to ascertain the diagnosis and to obtain 2 biopsies from normal looking squamous mucosa, $6 \mathrm{~cm}$ and $15 \mathrm{~cm}$ above the esophagogastric junction. Patients with GERD symptoms and normal endoscopy underwent $\mathrm{pH}$ testing. Only those with an abnormal pH test $(>4.2 \%)$ were included in the study as NERD patients. The biopsies were evaluated for TRPVl expression using Western blots. Results: The mean age and $\mathrm{M} / \mathrm{F}$ ratio was $49.8 \pm 8.9 \mathrm{yrs}, 3 / 2$, for healthy controls. $47.8 \pm 4.8 \mathrm{yrs}, 4 / 1$, for NERD; $52.0 \pm 3.5,3 / 2$, for EE; and $62 \pm 4.3,5 / 0$, for BE. The mean length of the Barrett's mucosa was $5.9 \pm 0.5 \mathrm{~cm}(4.5-7.0 \mathrm{~cm})$. The distribution of erosive esophagitis was Grade A - 1, Grade B - 3 and Grade C - 1. The mean \% total time $\mathrm{pH}<4$ in the NERD group was $15.4 \%$ +3.7. Patients with NERD had the highest distal expression of TRPVl $=0.6572$ among the GERD groups, followed by EE $=0.4167$ and $\mathrm{BE}=0.0377(\mathrm{p}<0.05)$. TRPVl expression in normal controls was $0.5151(\mathrm{p}=\mathrm{NS})$. All participating groups demonstrated an increase in TRPVl expression in the proximal esophagus: $\mathrm{NERD}=0.7226, \mathrm{EE}=0.5074, \mathrm{BE}=0.6679$ and control $=0.5451$. Healthy controls demonstrated the least increase $(5.8 \%)$ in proximal distribution of TRPVl receptor as compared to the GERD groups (NERD $=10 \%, \mathrm{EE}=22 \%$ and $\mathrm{BE}=77 \%, \mathrm{p}<0.05)$. Conclusions: NERD patients demonstrate upregulation of TRPV1 receptors in the distal esophagus as compared to EE and BE. All GERD groups demonstrate increase in proximal distribution of TRPVl receptors, which may explain why proximal migration of an acid reflux event is associated with higher likelihood of symptom generation.

\section{M2003}

Influence of Intra-Esophageal Capsaicin Instillation On Heartburn Induction and Desensitisation in Man

Sebastien Kindt, Rita Vos, Daniel Sifrim, Jozef Janssens, Jan Tack

Heartburn is the most typical symptom in gastro-esophageal reflux disease (GERD). It has been suggested that heartburn sensation is mediated by the transient receptor potential vanilloid receptor 1 (TRPV1), a cation channel expressed by sensory neurons and activated by heat, acid pH, capsaicin, lipoxygenase products and anandamide. Exposure of the TRPV1 receptor to the agonist capsaicin, the pungent ingredient of chilli peppers, is characterized by activation, followed by receptor desensitization. The aim of the present study was to investigate the effect of intra-esophageal capsaicin instillation on esophageal symptom perception (activation) and on sensitivity to esophageal acid perfusion (desensitization). Methods: Ten healthy volunteers $(4 \mathrm{~m}$, mean age $27 \pm 1$ ) were studied on three separate occasions, at least one week apart. During standard esophageal manometry, a $10 \mathrm{ml}$ solution of saline containing 0 (placebo), 0.5 (low dose) or 3 (high dose) $\mathrm{ml}$ of a capsaicin solution $(0.17$ $\mathrm{mg} / \mathrm{ml}$ ) were instilled in the mid-esophagus. Thirty minutes later, $0.1 \mathrm{~N}$ hydrochloric acid was infused in the middle third of the esophagus at a rate of $6 \mathrm{ml} / \mathrm{min}$ for $30 \mathrm{~min}$. At 5minute intervals, throughout the study, the intensity of 10 symptoms (discomfort, pain, retrosternal burning epigastric burning, fullness, bloating nausea, belching, satiety, cramps) was assessed on $10 \mathrm{~cm}$ visual analog (VAS) scales. Areas under the curve (AUC) for symptom intensities under different conditions were recorded and compared by paired t-test and ANOVA. Results: Instillation of the high dose of capsaicin induced significantly higher symptoms of retrosternal burning and epigastric burning (AUC $203 \pm 61$ and $155 \pm 52 \mathrm{~mm} * \mathrm{~min}$ respectively) compared to the low dose $(18 \pm 11$ and $35 \pm 24 \mathrm{~mm} * \mathrm{~min}, \mathrm{p}<0.05)$ and to placebo $(65 \pm 40$ and $53 \pm 43 \mathrm{~mm} * \mathrm{~min}, \mathrm{p}<0.05)$. High dose of capsaicin also induced more pain (AUC $55 \pm 29$ vs. $15 \pm 15, \mathrm{p}<0.05$ ) and discomfort (AUC $138 \pm 63$ vs. $33 \pm 30, \mathrm{p}=0.05$ ) compared to placebo. During acid perfusion, regardless of the time interval of assessment, capsaicin pretreatment did not significantly alter perception scores for retrosternal burning (AUC $1015 \pm 254,1113 \pm 217$ and $890 \pm 243$ respectively for placebo, low dose and high dose, NS), epigastric burning (AUC $740 \pm 265,390 \pm 239$ and $408 \pm 207$ respectively for placebo, low dose and high dose, NS), or any other symptom. Conclusion: Instillation of the TRPV1 receptor agonist capsaicin in the esophagus induces symptoms of heartburn and epigastric burning in a dose-dependent fashion. However, as the doses used do not desensitize the esophagus to acid perfusion, identification of the receptor involved in esophageal acid sensitivity awaits other approaches.

\section{M2004}

High Resolution Manometry (HRM) of the EGJ in Normal Subjects and Symptomatic Patients; a Selective Analysis of the Crural Diaphragm (CD) Component

John E. Pandolfino, Qing Zhang, Sudip K. Ghosh, John Rice, John P. Norvell, Peter J. Kahrilas

Aim: HRM provides a spatially enhanced, dynamic representation of the EGJ high-pressure zone. This study utilized HRM to compare the $\mathrm{CD}$ contribution to EGJ pressure profile in normal subjects and symptomatic patients. Methods: 75 asymptomatic controls and 178 consecutive patients being evaluated for GERD symptoms underwent HRM with a 36 sensor solid state manometric assembly $(1 \mathrm{~cm}$ spacing) positioned to record from the hypopharynx to the stomach. The EGJ axial pressure profile was analyzed with spatial pressure variation plots over 5 to 6 respiratory cycles to quantify the position and contractile vigor of the $C D$ relative to the LES. EGJ and CD pressures were measured at end inspiration; LES pressure at expiration. In instances of a double pressure peak, the distal peak was taken to be the CD. Results: HRM can dynamically isolate the pressure signal of the $C D$ from that of the LES once they are spatially separated by $>2 \mathrm{~cm}$, a finding exhibited by $1 / 75$ normal subjects and $46 / 178(26 \%)$ of the patients ( $p<0.01$ ). There was no significant difference in expiratory LES pressure between normal subjects and patients (Table). In contrast, inspiratory EGJ pressure was significantly lower in patients with $\geq 2 \mathrm{~cm}$ LES/CD separation than in either patients with $<2 \mathrm{~cm}$ separation or controls (ANOVA $\mathrm{p}<0.01$ ). There was no difference in inspiratory EGJ pressure between normal subjects and patients with $<2 \mathrm{~cm} C D / L E S$ separation. There was an inverse correlation between LES/CD separation and inspiratory augmentation of $E G J$ pressure $(R=0.53, P<0.0001)$. In addition, it appeared that once axial separation was $\geq 3 \mathrm{~cm}$ LES pressure would decrease during inspiration and increase during expiration. Conclusions: $C D$ augmentation of the EGJ during inspiration becomes progressively attenuated once these EGJ elements are spatially separated by $\geq 2 \mathrm{~cm}$. Thus, this HRM parameter 
may be used as a surrogate marker for CD dysfunction and hiatus hernia. Although it appeared that inspiratory augmentation was a better discriminator between patients and normal subjects, future studies will need to determine if $C D$ dysfunction parallels GERD severity.

\begin{tabular}{|c|c|c|c|}
\hline & LES expiration & EGJ inspiration & Insp-Exp \\
\hline Normals (75) & $17.6(0.9)$ & $34.2(1.3)^{*}$ & $16.6(0.9) *$ \\
\hline All patients (178) & $16.6(0.8)$ & $25.9(1.2) \dagger$ & $9.3(0.8) \dagger$ \\
\hline Pts <2cm separation (132) & $17.7(1.0)$ & $30.4(1.2)^{*}$ & $12.6(0.8) * \dagger$ \\
\hline Pts $\geq 2$ cm separation (46) & $13.4(1.4)$ & $13.1(2.1) \dagger$ & $-0.3(1.4) \dagger$ \\
\hline
\end{tabular}

$\dagger \mathrm{P}<0.05$ vs. NL, ${ }^{*} \mathrm{P}<0.05$ vs. Patients with separation

\section{M2005}

Effect of Body Position Changes On Postprandial Gastroesophageal Reflux and Gastric Emptying in the Premature Neonate

Michiel P. Van Wijk, Nathalie Rommel, Ros Lontis, Louise Goodchild, Ross Haslam, Marc A. Benninga, John Dent, Geoff P. Davidson, Taher Omari

BACKGROUND: In infants, left side positioning decreases triggering of transient lower esophageal sphincter relaxations (TLESR) and gastroesophageal reflux (GER) but paradoxically delays gastric emptying (GE). ${ }^{1}$ The aim of our study was to establish a positioning regimen that would both promote GE and reduce GER by changing body position one hour after feeding. METHODS: Six healthy preterm infants (4 male, post menstrual age: 36 (3438) weeks, weight $2390 \pm 120 \mathrm{~g}$ ) were studied using combined esophageal impedance and manometry technique. After catheter placement, infants were positioned on the left or right side and then gavage fed via an infusion port. After one hour, the infant's position was changed to the opposite side. All infants were studied on two occasions such that position was changed from right to left (protocol 1) and from left to right (protocol 2) in a crossover fashion ( 3 patients protocol 1 first, 3 patients protocol 2 first). GE rate was determined with a 13C-Na octanoate breath test. Manometry and impedance tracings were blinded for analysis. RESULTS: Right positioning overall resulted in triggering of more TLESR and liquid GER than left positioning (mean: $3.3 \pm 2.2$ vs $1.9 \pm 1.5(p=0.004)$ and $4.8 \pm 3.4$ vs $0.8 \pm 1.3$ ( $p=$ $0.003)$ per hour respectively), but less gas GER $(0.3 \pm 0.7$ vs $1.3 \pm 1.05, p=0.04)$. Data before and after position change are presented in the table ( $\mathrm{p}$ values for right position (RP) vs left position(LP) shown in parentheses). The total number of all GER episodes did not differ between the two protocols however protocol 1 significantly reduced TLESR and liquid GER in the second hour after feeding. GE was faster during protocol 1 than during protocol 2 (table). The GE rate for protocol 1 was similar to premature infants previously studied in the right position only $(\mathrm{p}=0.89)$ and GE rate for protocol 2 was faster $(\mathrm{p}<0.001)$ than infants previously studied in the left position only. ${ }^{1}$ CONCLUSIONS: A postural strategy of right positioning for the first postprandial hour with a position change to left afterwards promotes GE while at the same time reduces liquid GER in the late post-prandial period when liquid reflux of $\mathrm{pH}<4$ occurs. Infants with GERD have increased acid GER in association with TLESR and right to left positioning may be a useful and simple therapeutic strategy for promoting GE and reducing acid GER. 1. Omari TI et al. J.Pediatr. 2004;145:194 - 200.

\begin{tabular}{|c|c|c|}
\hline & Protocol 1 & Protocol 2 \\
\hline TLESR & RP $3.7 \pm 2.2 /$ LP $1.7 \pm 1.7(<0.001)$ & LP $2.2 \pm 1.5 /$ RP $3.0 \pm 2.4(0.289)$ \\
\hline Liquid GER & RP $6.0 \pm 3.7 /$ LP $0.2 \pm 0.4(0.013)$ & LP1.5 $\pm 1.5 /$ RP $3.5 \pm 2.7(0.067)$ \\
\hline Gas GER & RP 0.3 \pm 0.8 / LP $1.8 \pm 1.0(0.060)$ & LP0.7 \pm 0.8 / RP $0.3 \pm 0.5(0.465)$ \\
\hline $\mathrm{GEt}_{1 / 2}$ & $34.1 \pm 11.1$ & $52.9 \pm 7.7$ (protocol 1 vs $2: \mathrm{p}=0.026)$ \\
\hline
\end{tabular}

\section{M2006}

Effects of Ecabet Sodium On Chronic Acid Reflux Esophagitis in Rats Daisuke Asaoka, Akihito Nagahara, Yuko Izumi, Akimitsu Ohkawa, Akihiko Kurosawa Masato Kawabe, Mariko Hojo, Toshoku Minoo, Ryuichi Ohkusa, Toshifumi Ohkusa, Hiroto Miwa, Nobuhiro Sato

Background: Ecabet sodium (ES) is a new anti-peptic ulcer drug, which is derived from a constituent of pine resin. ES exerts its effects on gastric ulcer by binding to gastric mucosal lesions directly in rats and humans. Further, protective effects of ES on ulcerative colitis have been also observed. Purpose: To investigate protective effects of ES on esophageal mucosa by observing esophageal mucosal damages macroscopically and histologically in rat chronic acid reflux esophagitis model. Methods: Chronic Acid Reflux Esophagitis in rats was developed by modified Omura's method. That is, using 10 week-old Wistar male rats, duodenum near the pylorus ring was wrapped with 18 Fr Nelaton catheter (width: $3 \mathrm{~mm}$ ) . Further, border between forestomach and glandular portion was ligated with 2-0 silk thread. ES $(65 \mathrm{mg} / \mathrm{kg})$ was only once intragastrically administered immediately after the surgery, and drinking water including ES $(21.39 \pm 2.74 \mathrm{mg} / \mathrm{kg} /$ day $)$ was taken from the next day to day 7 . Seven days after the surgery, esophageal damages were macroscopically and histologically examined in the upper (approx. $5 \mathrm{~mm}$ under crico-pharyngeus), the middle (midpoint between crico-pharyngeus and esophago-columnar junction, EC junction) and the lower (approx. $0.5 \mathrm{~mm}$ upper EC junction) areas. Results: In control group, esophageal damages of the lower area were the most severe among three areas (mucosal thickness of the lower, the middle and the upper area was $210.8,204.2$ and $47.5 \mu \mathrm{m}$, respectively). In ES group, mucosal thickness in the lower area was significantly decreased $(97.5 \mu \mathrm{m}, \mathrm{p}=$ 0.0112 ) compared with that of control. Furthermore, ES had the tendency to decrease areas and the number of ulcers in whole esophagus and to decrease the number of leukocytes infiltrated in the lower area of esophagus. Conclusions: Ecabet protected esophageal mucosa from chronic reflux gastric juice. This suggests its usefulness in the treatment for gastroesophageal reflux disease.
M2007

Pattern of Acid Reflux During Transient Lower Esophageal Sphincter Relaxations (TLESRs) Differ Among Patients with Mild and Severe Reflux Esophagitis and Healthy Subjects

Katsuhiko Iwakiri, Yoshinori Hayashi, Makoto Kotoyori, Noriyuki Kawami, Yuriko Tanaka, Akihiko Kawakami, Choitsu Sakamoto, Richard H. Holloway

Background: We have reported that there is no difference in the rate of TLESRs and the rate of acid reflux during TLESRs at $2 \mathrm{~cm}$ above the LES between patients with severe reflux esophagitis (RE) of LA grade $C$ and healthy subjects, but the groups differ with respect to whether or not refluxed gastric acid comes up to $7 \mathrm{~cm}$ above the LES. It is possible the proximal extent of reflux differs among healthy subjects, mild and severe RE patients, however, acid reflux patterns in patients with mild RE are unclear. In this study, we investigated whether or not there are any differences in acid reflux patterns during TLESRs among healthy subjects, mild and severe RE patients. Method: 13 patients with RE of LAgrade C, 13 patients with LA-grade A or B and 13 healthy subjects underwent esophageal manometry with a 21-lumen perfused assembly that monitored pressure in the pharynx, the upper esophageal sphincter, the esophageal body, LES and the proximal stomach Esophageal $\mathrm{pH}$ was monitored at 2 and $7 \mathrm{~cm}$ above the LES. Recordings were made in the sitting position for 3 hours after a meal ( $692 \mathrm{kcal}, 33$ \% fat). Results: There was no difference in the rate of TLESRs or the rate of acid reflux during TLESRs (a pH drop of at least $1 \mathrm{pH}$ unit) at $2 \mathrm{~cm}$ above the LES among the 3 groups. However, the incidence of acid reflux a $7 \mathrm{~cm}$ above the LES during TLESRs in severe RE patients (50.9\% (28.3-72.5), median (interquartile range)) was significantly higher than that in mild RE patients $(27.7 \%(14.2$ 45.9) $)(\mathrm{p}=0.0056)$ and healthy subjects $(5.7 \%(3.8-6.2))(\mathrm{p}<0.0001)$. In addition, the rate of acid reflux during TLESRs in mild RE patients was significantly $(p=0.0009)$ higher than that in healthy subjects. Conclusions: The rate of acid reflux during TLESRs does not differ in reflux esophagitis from that in healthy controls. However, reflux disease is characterized by a more proximal extent of the refluxate in patients with reflux disease and this extent is related to the severity of esophagitis.

\section{M2008}

What Is the Transsphincteric Pressure Gradient Before a TLESR Takes Place? R Frankhuisen, Ma van Herwaarden, Rch Scheffer, Hg Gooszen, Gs Hebbard, M Samsom Background: Increased pressure gradients across the esophagogastric junction $(\Delta \mathrm{EGJp})$ are pivotal for the occurrence of reflux during TLESRs. The question has arisen whether these observations are related specifically to that point in time, during sphincter relaxation, or whether comparable pressure differences are present before the sphincter pressure drops, leading to a TLESR. Aim: To compare $\triangle$ EGJp during a 3-min period before TLESR and during TLESR in patients with gastroesophageal reflux disease (GERD) and healthy controls (HC). Patients and methods: 17 GERD patients (10 men, mean age 50 (29-69)) with pathologic esophageal reflux $(\mathrm{pH}<4$ or $>6 \%)$ and or a symptom association probability index $>95 \%$ were enrolled along with $18 \mathrm{HC}$ (10 men, mean age 28 (18-53)). One hour before and $2 \mathrm{hr}$ following a liquid meal $(500 \mathrm{ml} / 300 \mathrm{kcal})$ combined esophageal $\mathrm{pH}$ and high-resolution manometry was performed using an assembly including 11 side holes spaced by $1 \mathrm{~cm}$ positioned across the EGJ. The 2 side holes proximal to the upper and distal to the lower border of the EGJ were used to calculate intrathoracic - and intragastric pressures at maximal inspiration. $\Delta \mathrm{EGJp}$ was defined as mean intragastric pressure minus mean intrathoracic pressure and was analyzed 180,60 and 10 seconds before TLESRs and during TLESRs. Statistics: One-way ANOVA was used to compare different time points in each group. If $\mathrm{p}<0.05$, a Bunnet post hoc test was performed. Results: The proportion of TLESRs associated with acid reflux was $49 \%$ in controls and $62 \%$ in GERD patients $(\mathrm{p}=0.05)$. In HC the $\Delta$ EGJp for TLESRs with acid reflux at $\mathrm{t}=-180 \mathrm{sec}(7.6 \pm 0.7 \mathrm{mmHg}), \mathrm{t}=-60 \mathrm{sec}(7.2 \pm 0.8$ $\mathrm{mmHg})$ and $\mathrm{t}=-10 \mathrm{sec}(6.9 \pm 1.1 \mathrm{mmHg})$ is significantly lower than at $\mathrm{t}=0(10.5 \pm 0.7 \mathrm{mmHg})$ (all $\mathrm{p}<0.05)$. In GERD, this difference is only observed between $\mathrm{t}=-10 \mathrm{sec}(8.0 \pm 0.6 \mathrm{mmHg})$ and $\mathrm{t}=0(11.8 \pm 0.9 \mathrm{mmHg})(\mathrm{p}<0.05)$. The $\Delta \mathrm{EGJp}$ for TLESRs without acid reflux was comparable during all time periods in both GERD patients as HC. The intragastric pressure for TLESRs with acid reflux in both GERD patients and HC increases between $\mathrm{t}=-10$ ( 3.9 and $2.1 \mathrm{mmHg}$ ) and $\mathrm{t}=0$ ( 6.6 and $3.6 \mathrm{mmHg}$ ) (all $\mathrm{p}<0.05)$, while intrathoracic pressure did not change. Conclusion: The transsphincteric pressure gradient observed during TLESR associated with acid reflux is not present before TLESR commences and is a consequence of an increase in intragastric pressure in both GERD patients and healthy controls.

\section{M2009}

One to One Relationship Between Esophageal Contractions, Bolus Transit and Perception of Dysphagia in Healthy Subjects and Patients with GERD George Karamanolis, Tony Lerut, Jiry Silny, Jan Tack, Daniel Sifrim

Abnormal clearance of swallowed food could lead to generation of esophageal symptoms Evaluation of patients with esophageal symptoms is usually performed using esophageal manometry. Recently, esophageal impedance has been added to assess bolus transport and combined manometry-impedance is considered an advanced tool for evaluating esophageal function. However, the relationship between the patient's perception of the esophageal passage of a bolus and the objective measurement of esophageal pressures and bolus transit during individual swallows is not clear. The aim of our study was to assess simultaneously perception, pressures and transit of viscous boluses in healthy subjects (HS) and patients with gastro-esophageal reflux disease (GERD). Methods: Simultaneous stationary esophageal manometry-impedance was performed in $10 \mathrm{HS}$ ( 7 men; mean age: $27 \mathrm{yrs})$ and 10 GERD patients ( 6 men; mean age: 48 yrs). Ten viscous swallows $(5 \mathrm{ml})$ were tested in each subjec in the supine position. Perception of each swallow was evaluated using a standardized 1-5 scoring system ( 1 =fluid passage and $5=$ complete blockade). Manometry was considered abnormal if the amplitude of contractions in the distal esophagus was $<30 \mathrm{mmHg}$ or if the contractions were simultaneous; bolus transit by impedance was abnormal if bolus exit was not identified at one or more of the measuring sites or if total bolus transit time was $>12.5$ min; and perception was increased if score was $>1$. Results: The pooled data analysis of 
200 viscous swallows showed 132 swallows with normal esophageal function and normal perception (score 1). 12 with abnormal function (ineffective peristalsis or incomplete bolus clearance) and increased perception ( 1 in HS vs. 11 in GERD, $p=0.003$ ), 45 with normal esophageal function (effective peristalsis and complete bolus clearance) and abnormal perception (13 in HS vs. 32 in GERD, p<0.001), and 10 with abnormal function and normal perception ( 5 in HS and 5 in GERD). The per-subject analysis showed concordance between manometry and impedance in $87 \pm 7 \%$ in $\mathrm{HS}$ and $88 \pm 7 \%$ in GERD. The concordance between bolus transit measured by impedance and perception scores was of $83 \%+6$ in HS and $60 \% \pm 12$ in GERD. Conclusion: The correlation between objective measurements of esophageal function and perception of bolus passage is good but still incomplete, particularly in patients with GERD. In healthy subjects 70-80\% of swallows showed concordance between objective measurements and perception. In contrast, patients with GERD showed a less accurate correlation with increased perception after swallows with both normal and abnormal function.

\section{M2010}

The Definition of GERD: A Global, Evidence-Based Consensus

Nimish Vakil, Sander Vanzanten, Dent John, Peter Kahrilas, Roger Jones

The aim of this study was to develop a globally acceptable definition and classification of reflux related diseases. Methods: A new definition was developed using a Delphi process over a period of 18 months. The Consensus Group of 45 experts from 19 countries, including a non-voting chairman, represented a diversity of views and expertise in GERD(Barrett's esophagus, surgery, and primary care). Systematic literature reviews, with defined inclusion and exclusion criteria, were conducted to identify the evidence to support each statement. The group voted anonymously on four iterations of the statements, twice electronically and at two Consensus Group workshops. Agreement with a statement by two thirds (i.e. $>67 \%$ ) of the group was classed as consensus. Results: The level of agreement strengthened through the Delphi process with consensus on all statements, for each iteration, accordingly $86 \%$, $88 \%, 94 \%$, and $100 \%$. Over $90 \%(n=39)$ of the group strongly agreed with $48(94 \%)$ of the 51 final statements. GERD was defined as a condition which develops when the reflux of stomach contents causes troublesome symptoms and/or complications. A classification system was developed based on symptoms and evidence of esophageal damage for the esophageal syndromes and the extra-esophageal syndromes of GERD. This classification makes it possible to diagnose GERD based on symptoms alone and also based on tests such as endoscopy. The consensus group concluded that the term Barrett's esophagus is variably interpreted and lacks the clarity needed for clinical and scientific communication about columnar metaplasia of the esophageal mucosa. Endoscopically Suspected Esophageal Metaplasia (ESEM) is a term proposed by the consensus group that describes endoscopic findings consistent with Barrett's esophagus that await histological evaluation. The group concluded that when biopsies of ESEM show columnar epithelium it should be called Barrett's esophagus and the presence or absence of intestinal metaplasia specified. The group concluded that chronic cough, chronic laryngitis, and asthma are usually multi-factorial disease processes and gastroesophageal reflux can be an aggravating co-factor, though it was rarely the sole cause. Conclusions: 1. A consensus of expert gastroenterologists from around the world is possible using structured consensus development techniques. 2. Critical reviews of evidence strengthen the level of consensus even when controversial subjects such as Barrett's esophagus are considered.3 The global definition and classification should help provide a common terminology that bridges cultures and countries. supported by Astra-Zeneca

\section{M2011}

Effect of Proton Pump Inhibitor Therapy On Inflammatory Changes in the Gastric Cardia (Carditis)

Shailender Singh, Sharad Mathur, Amit Rastogi, Srinivas R. Puli, Ajay Bansal, Prateek Sharma

INTRODUCTION: Inflammation and intestinal metaplasia in the gastric cardia have assumed importance given the rising incidence of adenocarcinoma in this location. The etiology of inflammation of the gastric cardia i.e. carditis is controversial with both GERD and H. pylori infection being proposed as possible factors. It has been shown that $H$. pylori eradication leads to a decrease in the degree of carditis, however, the effect of acid suppressive therapy on carditis has not been evaluated. AIM: To investigate the effect of proton pump inhibitor (PPI) therapy on the carditis score. METHODS: In GERD pts undergoing upper endoscopy, 4 quadrant biopsies were obtained from the gastric cardia (within $1 \mathrm{~cm}$ of the anatomic gastroesophageal junction) at baseline and after PPI therapy. All cardia biopsies were stained with $H \& E$ and reviewed by a single pathologist in a blinded fashion. The updated Sydney Classification was used to score the biopsies from 0 (absent) to 3 (marked) for the following: mononuclear cells, neutrophils, intestinal metaplasia, and atrophy. Carditis scores (pre and post PPI therapy) were compared using the paired $t$ test and Wilcoxon signed ranks test. RESULTS: 31 pts with a mean age of 61 years (range 47-82 years) were evaluated including 30 Caucasians and 30 males. Results from 5 pts were excluded from the analysis because at least one set of biopsies did not document the presence of cardiac mucosa. The mean duration of PPI therapy was 30 months (range:2-58 months). There was no significant change in the carditis scores after PPI therapy; the mean carditis scores before and after PPI therapy with their corresponding $p$ values (paired $t$ test) are shown in the table. Also, using the Wilcoxon signed ranks test, there were no statistically significant trends in the scores after PPI therapy. Only 4 pts had H. pylori infection and a sub-analysis showed that the presence of $H$ pylori did not affect the overall results. CONCLUSIONS: Long term acid suppression therapy with PPI's (mean duration of 30 months) does not lead to a significant reduction in the carditis scores. These results suggest that GERD does not play a major role in the pathogenesis of inflammation in the gastric cardia. These findings need to be confirmed by future prospective trials with larger number of patients.

Mean gastric carditis scores before and after PPI therapy

\begin{tabular}{|c|c|c|c|}
\hline & \multicolumn{2}{|c|}{ Mean Scores } & \\
\hline & Pre PPI & Post PPI & $\mathrm{p}$ \\
\hline Mononuclear cells & 1.23 & 1.73 & 0.07 \\
\hline Neutrophils & 0.35 & 0.62 & 0.17 \\
\hline Intestinal metaplasia & 0.46 & 0.31 & 0.38 \\
\hline Atrophy & 0.50 & 0.35 & 0.36 \\
\hline
\end{tabular}

\section{M2012}

Mast Cells Promote Neutrophil Infiltration in a Mouse Model of Reflux Esophagitis

Jeffrey Morganstern, Ming-Yu Wang, Barry K. Wershil

Background: Mast cells play a role in allergic and parasitic diseases of the gastrointestinal tract, but recent evidence suggest that mast cells may be involved in a variety of non-allergic conditions, including reflux esophagitis. However, the precise contribution of the mast cell to the pathogenesis of reflux esophagitis is not known. The goal of this study was to define the role of mast cells in acid reflux esophagitis. Methods: Mast cell-deficient Kitw/Kitw-v mice $(\mathrm{n}=14)$ and normal $(+/+)$ mice $(\mathrm{n}=15)$ were examined in a mouse model of reflux esophagitis described by Fujino, et al (DDW, 2004). Briefly, mice were fasted overnight in a metabolic cage and then underwent a surgical procedure to ligate the proximal duodenum and a region between the forestomach and the glandular portion of the stomach. The mice were sacrificed four hours after surgery and the distal third of the esophagus removed and processed for morphometric quantification of neutrophil infiltration in 1 micron, Giesmastained sections. As reported by Fujino, et al. this procedure induced a reproducible acute inflammatory response that was inhibited by acid suppression. The number of neutrophils in the esophageal tissue was determined and expressed as neutrophils per $\mathrm{mm} 2$ tissue. Results: The number of neutrophils in the esophageal mucosa and submucosa was significantly greater in the normal $(+/+)$ mice compare with the Kitw/Kitw-v mice ( $30 \pm 25$ vs. $9 \pm 7$, respectively $\mathrm{p}<0.01$ ). Neutrophil infiltration in the submucosa of $+/+$ mice was statistically greater than Kitw/Kitw-v mice ( $42 \pm 32$ vs. $17 \pm 16, p<0.05)$. Similarly, the neutrophil accumulation in the epithelium was greater in the $+/+$ mice, but did not reach statistical significant (18 \pm 16 vs. $3 \pm 2$, respectively, $p<0.1$ ). Conclusion: Mast cells contribute significantly to the neutrophil accumulation associated with a model of reflux esophagitis in mice.

\section{M2013}

Gene Expression of IL-8 and IL-1 $\beta$ : Quantitative Biomarkers of Esophageal Squamous Injury

Koji Tanaka, Daniel S. Oh, Steven R. Demeester, Jeffrey A. Hagen, Dong Yun Yang Hidekazu Kuramochi, Kathleen D. Danenberg, Peter V. Danenberg, Cedric G. Bremner, John Lipham, Tom R. Demeester

Background: Endoscopic and histologic criteria are used to define injury of esophageal squamous mucosa in patients with GERD. These endpoints are subjective, particularly when assessing response to therapy. Alternatively, measurement of the expression of inflammationrelated genes has potential as an objective and quantitative method of assessing injury. We hypothesized that injury in the setting of GERD would be associated with upregulation of the inflammatory genes Cox-2, IL-8, IL-1 $\beta$, and iNOS. Methods: 93 patients were evaluated for GERD with $\mathrm{pH} / \mathrm{Bilitec} \circledast$ montoring. Upper endoscopy was performed and squamous mucosal biopsies were taken within $3 \mathrm{~cm}$ of the squamocolumnar junction. Mucosal injury was defined as either endoscopic or histologic esophagitis. Controls were those with a normal

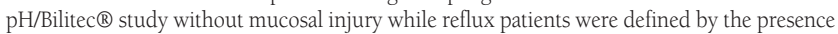
of abnormal acid and/or bile exposure in the distal esophagus. All patients were off acid suppression therapy and none had previous foregut surgery. Paraffin embedded biopsy blocks were sectioned and microdissected. RNA isolation and reverse transcription was followed by quantitative real-time PCR to measure gene expression relative to the reference gene $\beta$-Actin. Expression in controls was compared to that found in reflux patients with injury. The maximal chi-square method was used to determine the threshold value to best predict injury. Results: Expression of IL- 8 and IL- $1 \beta$ was significantly higher in reflux patients with injury compared to controls (Table). The optimal cutpoint value to predict injury in reflux patients was 0.48 for IL- 8 and 0.28 for IL-1 $\beta$. Cox- 2 expression did not differ in controls compared to reflux patients with injury ( $\mathrm{p}=0.33)$. There was no detectable iNos expression in any squamous biopsy. Conclusion: Gene expression of IL-8 and IL-1 $\beta$ is increased in injured squamous mucosa of GERD patients. Measuring the expression of these injury-related genes has potential usefulness as quantitative endpoints when assessing the effectiveness of antireflux therapy.

\begin{tabular}{|c|c|c|c|c|}
\hline & $\begin{array}{c}\text { No Reflux No } \\
\text { Injury (n=20) }\end{array}$ & $\begin{array}{c}\text { Abnormal Reflux } \\
\text { Histologic Injury (n=12) }\end{array}$ & $\begin{array}{c}\text { Abnormal Reflux } \\
\text { Endoscopic Injury } \\
(\mathrm{n}=25)\end{array}$ & $\begin{array}{c}\text { Kruskal-Wallis test } \\
\text {-value }\end{array}$ \\
\hline IL-8 & $0.01(0-0.15)$ & $0.26(0-0.68)$ & $0.26(0.07-0.82)$ & 0.01 \\
\hline IL-1 $\beta$ & $0.31(0.2-0.46)$ & $0.29(0.06-0.50)$ & $0.72(0.3-1.07)$ & 0.02 \\
\hline
\end{tabular}

Median values (IQR) based on gene x100/ $\beta$-Actin mRNA expression 
Excessive Post-Prandial Transient Lower Esophageal Sphincter Relaxation (TLESR) Causes GERD in Obesity. A Study of Esophageal Motility in Patients with Moderate and Severe Obesity

Justin Wu, Lik Man Mui, Carrian Cheung, Philip Chiu, Joseph Sung

BACKGROUND: Obesity has been associated with GERD and its complication but the underlying mechanism is unclear. We hypothesize that obesity leads to impairment of esophageal motility function and predisposes to development of GERD. AIM: To evaluate the esophageal motility function in patients with obesity. METHODS: We prospectively recruited consecutive patients referred for weight reduction endoscopic therapy or surgery because of moderate to severe obesity (BMI $>/=35$ ). Exclusion criteria included endoscopic erosive esophagitis, weekly attacks of heartburn and acid regurgitation, need of proton pump inhibitor therapy for upper gastrointestinal symptom, sliding hiatus hernia $>2 \mathrm{~cm}$ as diagnosed by endoscopy, and diabetes mellitus with established microvascular complication. All recruited patients underwent stationary esophageal manometry (EM), 2-hour postprandial EM and pH-metry after a standard 400 kcal test meal; and ambulatory 24-hour pH metry. Age-and-sex matched asymptomatic non-obese (BMI: 20-23) subjects were recruited as controls. RESULTS: 12 obese patients and 12 controls (10 male subjects in each group) were studied. In stationary EM, both groups had comparable mean lower esophageal sphincter (LES) pressure (Obese Vs control: $15.8+/-6.9$ Vs $14.7+/-8.8 \mathrm{mmHg}, \mathrm{p}=0.33$ ), mean LES length (Obese Vs control: $3.6+/-0.8$ Vs $3.8+/-0.4 \mathrm{~cm}, \mathrm{p}=0.28$ ), mean $\%$ of primary peristalsis (Obese Vs control: $86.6+/-10.3$ Vs $84.5+/-9.2 \%, \mathrm{p}=0.87$ ) and secondary peristalsis (Obese Vs control: $63.9+/-22.3$ Vs $68.5+/-26.3 \%, \mathrm{p}=0.15)$. In 2-hour postprandial study, however, obese patients had significantly higher rate of transient LES relaxation (TLESR) with and without acidic reflux than controls. Total, postprandial and upright esophageal acid exposure was also significantly higher in obese patients (Table). CONCLUSION: Obesity is associated with excessive TLESR during postprandial period, which results in higher postprandial and upright esophageal acid exposure. This finding suggests that obesity leads to abnormal postprandial LES function, which predisposes to development of GERD in susceptible patients.

\begin{tabular}{|l|c|c|c|c|c|c|c|}
\hline & $\begin{array}{c}\text { Mean } \\
\text { age } \\
(\mathrm{SD})\end{array}$ & $\begin{array}{c}\text { Mean } \\
\text { BMI } \\
\text { (SD) }\end{array}$ & $\begin{array}{c}\text { Median no. } \\
\text { of TLESR } \\
\text { without } \\
\text { reflux } \\
\text { (range) }\end{array}$ & $\begin{array}{c}\text { Median no. } \\
\text { of TLESR } \\
\text { with acidic } \\
\text { reflux } \\
\text { (range) }\end{array}$ & $\begin{array}{c}\text { Mean } \\
\text { DeMeester } \\
\text { score (SD) }\end{array}$ & $\begin{array}{c}\text { Mean \% time } \\
\text { postprandial } \\
\text { pH<4 (SD) }\end{array}$ & $\begin{array}{c}\text { Mean } \% \\
\text { time } \\
\text { upright } \\
\text { pH<4 } \\
\text { (SD) }\end{array}$ \\
\hline Obesity & $\begin{array}{c}36.5 \\
(8.7)\end{array}$ & $\begin{array}{c}38.9 \\
(3.2)\end{array}$ & $6(2-12)$ & $5(0-14)$ & $8.6(6.9)$ & $6.5(5.5)$ & $2.8(1.9)$ \\
\hline Control & $\begin{array}{c}34.8 \\
(7.5)\end{array}$ & $\begin{array}{c}22.6 \\
(1.9)\end{array}$ & $3(1-5)$ & $1(0-2)$ & $1.2(2.3)$ & $2.2(3.6)$ & $0.8(0.9)$ \\
\hline P value & 0.16 & $<0.001$ & 0.02 & 0.01 & 0.01 & 0.02 & 0.01 \\
\hline
\end{tabular}

\section{M2015}

The Acid Pocket Rivisited with Wireless pH Monitoring

Roberto Penagini, Ausilia Grigolon, Paolo Cantu', Ivana Bravi

Background and aim Data obtained by pH electrode pull-through have recently suggested the existence of a 2-3 cm layer of highly acidic unbuffered gastric juice at the gastric cardia after meals. This so called "acid pocket" could be involved in the high prevalence of disease at this site. The new Bravo wireless pH monitoring system could be a technique suitable to confirm the presence and relevance of this acid pocket by using $\mathrm{pH}$ electrodes clipped to the mucosa for a prolonged period of time. Aim of our study was therefore to measure $\mathrm{pH}$ at the gastric cardia and in the stomach for $24 \mathrm{~h}$ in healthy subjects using $3 \mathrm{Bravo} \mathrm{pH}$ capsules. Methods Nine healthy subjects, (aged 24-51 yr, 4 men) underwent transoral placement of three Bravo $\mathrm{pH}$ capsules during upper gastrointestinal endoscopy, so that the electrodes were at the squamo-columnar junction (SCJ), $2 \mathrm{~cm}$ and $9 \mathrm{~cm}$ below respectively. Two Bravo receivers were used, $\mathrm{pH}$ recordings were synchronized and lasted $24 \mathrm{~h}$ during which all subjects performed their usual activities. Position of the $\mathrm{pH}$ capsules was checked by xRay at the beginning and at the end of the studies. A standardised mixed nutrient 2.1 $\mathrm{MJ}$ lunch was given to all subjects. Median $\mathrm{pH}$ of $1 \mathrm{~h}$ before the standardised meal (fasting), $1 \mathrm{~h}$ after the standardised meal (post-prandial) and of all $24 \mathrm{~h}$ was compared among the three electrodes. Results (median; interquartile range). Recordings of all 3 capsules were available for analysis in 8 subjects. $\mathrm{pH}$ data were captured by the receivers for $93 \%+2$ of time. At the SCJ 6/8 tracing had an esophageal pattern with a baseline of $\mathrm{pH}$ 6-6.5 and acid reflux episodes, whereas the remaining two showed wide fluctuations in $\mathrm{pH}$. All tracing 2 and $9 \mathrm{~cm}$ below the $\mathrm{SCJ}(\mathrm{SCJ}+2 \mathrm{~cm}$ and $\mathrm{SCJ}+9 \mathrm{~cm})$ had an intragastric pattern with a fasting baseline of $\mathrm{pH}$ 0.9-1.5. $\mathrm{pH}$ readings at $\mathrm{SCJ}$ and $\mathrm{SCJ}+2 \mathrm{~cm}$ (gastric cardia) were not lower than those at $\mathrm{SCJ}+9 \mathrm{~cm}$ (gastric body). See table. Conclusions a) prolonged $\mathrm{pH}$ monitoring using the Bravo wireless system in healthy subjects does not confirm the existence of a layer of acidic unbuffered gastric juice at the cardia, as measured by the electrodes at the $\mathrm{SCJ}$ and $\mathrm{SCJ}+2 \mathrm{~cm}$, either in the postprandial period or during $24 \mathrm{~h}$. b) The Bravo wireless system is a valuable tool in the study of intragastric and SCJ pH environment.

\begin{tabular}{|c|c|c|c|}
\hline & & Median $\mathrm{pH}$ & \\
\hline & $\mathrm{SCJ}$ & $\mathrm{SCJ}+2 \mathrm{~cm}$ & $\mathrm{SCJ}+9 \mathrm{~cm}$ \\
\hline Fasting & $6.1 ; 4.0-6.4 *$ & $1.3 ; 1.1-1.5$ & $1.1 ; 0.9-1.3$ \\
\hline Postprandial & $5.1 ; 4.1-6.0 *$ & $2.3 ; 1.6-3.2$ & $2.3 ; 1.8-2.5$ \\
\hline 24 hours & $5.8 ; 2.8-6.3 *$ & $1.6 ; 1.2-2.0$ & $1.3 ; 1.0-1.6$ \\
\hline
\end{tabular}

*higher than $\mathrm{SCJ}+2 \mathrm{~cm}$ and $\mathrm{SCJ}+9 \mathrm{~cm}, \mathrm{p}<0.001$ by ANOVA followed by Fisher test.
Transient Lower Esophageal Sphincter Relaxation (TLESR) Is Associated with Arousal, But Reflux Esophagitis (RE) Has Multiple Causes in Patients with Obstructive Sleep Apnea Syndrome (OSAS)

Shiko Kuribayashi, Motoyasu Kusano, Yasuyuki Shimoyama, Osamu Kawamura, Masaki Maeda, Tatsuya Higuchi, Atsuto Nagoshi, Hiroaki Zai, Kunio Dobashi, Masatomo Mori

Background: We have reported that gastroesophageal reflux (GER) mainly occurs during the sleeping period and is most often induced by TLESR in patients with OSAS. RE is common in patients with OSAS, but the precise pathophysiology involved and the effect of GER on further arousal are unknown. Subjects and Methods: Seventeen OSAS patient, who had an apnea-hypopnea index (AHI) $\geqq 5$, and 8 normal volunteers (mean AHI: 2.9 ) were enrolled. Polysomnography (CHEST, Japan) and gastroesophageal manometry were performed simultaneously. Gastroesophageal manometry was done by using an infused catheter system (Arndorfer, USA) to monitor the pressures in the esophageal body and the LES with a Dent sleeve sensor as well as the gastric body. Esophageal pH was also measured at $5 \mathrm{~cm}$ above the LES by using a micro-glass electrode. All subjects ate a standard meal (800 kcal) together with $500 \mathrm{ml}$ of water, and the subjects were requested to adopt a supine position for 10 hours until the next morning. Manometry and $\mathrm{pH}$ data were recorded simultaneously with the polysomnography data and stored in a personal computer. Helicobacter pylori (Hp) infection was assessed by measuring the serum titer of Hp IgG antibody To investigate the effect of GER on arousal, changes of the sleep stage on the electroencephalogram (EEG) at one minute after reflux associated with TLESR and changes of the EEG after TLESR without GER were compared. All subjects underwent upper GI endoscopy, and the findings were compared among patients with $\mathrm{RE}(\mathrm{RE}+, \mathrm{n}=8)$, patients without $\mathrm{RE}$ (RE-, $\mathrm{n}=$ 9), and healthy volunteers $(C, n=8)$. Results: There was a statistically positive correlation $(\mathrm{R}=0.54, \mathrm{p}<0.05)$ between the number of arousal and that of TLESRs in OSAS patients, but the mean AHI did not differ between the RE+ group $(42.0 \pm 25.9$, mean \pm SD) and the REgroup ( $35.7 \pm 18.3)$. The number of TLESRs in both OSAS groups was significantly higher than in the $C$ group and the number of TLESRs associated with GER was significantly higher in the RE+ group (24/104 TLESRs) than in the RE- group (7/101). BMI of the RE+ group $(31.0 \pm 5.8)$ was significantly greater than that of the RE- group $(24.8 \pm 2.0)$. The Hp infection rate of the RE+ group (20\%) was significantly lower than that of the RE- group (62\%). A change of sleep stage (further arousal) was observed after $19 \%$ of GER events with TLESR versus $20 \%$ of TLESR events without GER, and there was no significant difference. Conclusions: TLESR was associated with arousal in OSAS patients, while RE was dependent on GER event, and on factors like dietary habits or gastric acid secretion. GER did not induce further arousal in patients with OSAS.

\section{M2017}

Do We Finally Understand the Underlying Mechanism of Increased Reflux During TLESRs in GERD Patients?

R Frankhuisen, Ma van Herwaarden, Rch Scheffer, Hg Gooszen, Gs Hebbard, M Samsom

Background: Increased pressure gradients across the esophagogastric junction $(\Delta \mathrm{EGJp})$ are pivotal for the occurrence of reflux during TLESR. It remains unclear if $\Delta \mathrm{EGJp}$ differ between GERD patients and healthy controls (HC). Aim: To investigate $\Delta \mathrm{EGJp}$ (=intragastric (IGp) - intrathoracic (ITp) pressures), during and 3 min preceding a TLESR in HC and GERD patients. Patients and methods: $18 \mathrm{HC}$ (10 men, mean age 28 (18-53)) and 17 GERD patients (10 men, mean age 50 (29-69)) were enrolled. One hour before and $2 \mathrm{hr}$ after a liquid meal $(500 \mathrm{ml} / 300 \mathrm{kcal})$ combined esophageal $\mathrm{pH}$ and high-resolution manometry, using an assembly with 11 side holes spaced by $l \mathrm{~cm}$, positioned across the EGJ, was performed. The 2 side holes proximal to the upper and distal to the lower border of the EGJ were used to calculate mean ITp and IGp. $\triangle E$ EJp was analyzed during TLESRs and at 180, 60 and 10 seconds before. Statistics: repeated measures ANOVA. Results: $\Delta E G J p$ and IGp were increased in GERD compared to $\mathrm{HC}$ at all points in time for TLESRs irrespective of reflux and both for TLESRs with and without reflux ( $\Delta \mathrm{EGJp}$ : all $\mathrm{p}<0.05$, IGp: all $\mathrm{p}=$ 0.001 ). ITp was comparable in both groups at all intervals. Conclusion: An increased $\Delta \mathrm{EGJp}$ caused by a higher intragastric pressure is responsible for the increased prevalence of GER during TLESRs in GERD patients. Lowering intragastric pressure might be a new strategy for the treatment of GERD. 


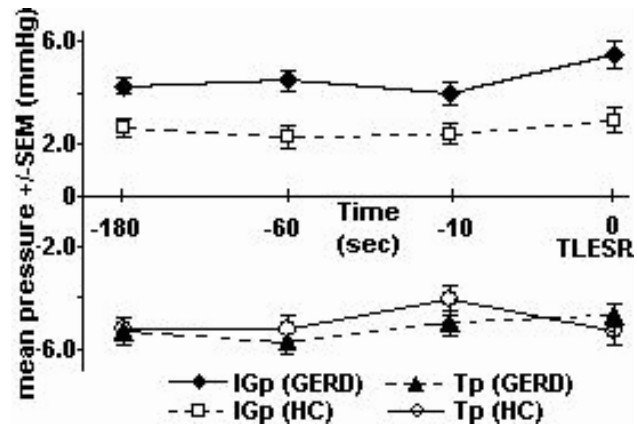

M2018

The Junctional Adehesion Molecules Occludin and Zonulin (ZO-1) Are Expressed in Squamous and Cardia Mucosa of Patients with Non-Erosive and Erosive Gastroesophageal Reflux Disease

Klaus Moenkemueller, Ulrich Peitz, Thomas Wex, Mike Beyer, Sven Kolfenbach, Peter Malfertheiner

Introduction: Erosive reflux disease (ERD) as well as non-erosive reflux disease (NERD) present with dilatation of the intercellular spaces of the esophageal mucosa. Junctional adhesion molecules may play a role in maintaining the integrity of the intercellular spaces. Little is known about the expression of these adhesion proteins in human gastroesophageal reflux disease (GERD). Aim: To determine the gene expression of Occludin and ZO-1 in cardia mucosa and distal esophagus in patients with NERD and ERD. Patients and Methods: We studied 73 GERD patients with typical esophageal symptoms, (ERD, n=35; NERD, n= 38). Fifty-five patients ( 25 NERD, 30 ERD were not taking any form of acid suppression therapy at the time of endoscopy. The presence of autoimmune gastritis, corpus-predominant H. pylori-gastritis and Barrett's mucosa were exclusion criteria. Endoscopic characterization of esophagitis was performed according to the Los Angeles classification. Histological classification of gastritis was based on the Sydney criteria. In addition to antrum and corpus biopsies for histology and rapid urease test, biopsies for molecular studies were taken from cardiac and squamous epithelium of each patient. The gene expression levels of occludin and Z0-1, as major components of tight junctions, were analyzed by quantitative RT-PCR and statistically evaluated by non-parametric Mann-Whitney U test. Results: In general, independent of underlying endoscopic appearance of GERD, Occludin and ZO-1 are expressed in the esophagus and cardia mucosa. Occludin transcript levels in cardia were 2 fold higher than those determined in distal esophagus [median: 0.096 vs. 0.045 artificial units (a.u.), $\mathrm{P}<0.0001]$. In contrast, $\mathrm{ZO}-1$ was found to be oppositely expressed, squamous epithelium revealed 1.6-times higher transcript levels than cardiac mucosa (median: 0.0082 vs. 0.005 la.u., $\mathrm{P}<0.0001$ ). Interestingly, no significant differences were found in the expression of both genes at both regions (cardia, esophagus) with respect to (I) the endoscopic grades of esophagitis and (II) the treatment with proton pump inhibitors (PPI) within the NERD and ERD groups. Conclusions: Both junctional adhesion molecules, Occludin and ZO-1, are expressed in both cardia and squamous mucosa of patients with ERD and NERD. There is no difference in expression of Occludin and ZO-1 between ERD and NERD. Ongoing studies are aimed to investigate the corresponding protein level of both genes and to characterize the subcellular localization of both, Occludin and ZO-1

\section{M2019}

Relationship Between Acidity of Gastroesophageal Reflux, Acidity of Gastric Content and Gastric Emptying. Effect of Erythromycin Daniel Sifrim, Kathleen Blondeau, Sara Emerenziani, Jan Tack, Jeremy Gale, Jozef Janssens, Jiry Silny

Acidity of gastroesophageal reflux determines patterns of symptoms and mucosal damage in GERD. The pH of the refluxate is not identical but correlates with gastric pH changes. After a meal, gastric $\mathrm{pH}$ follows a neutralization-re-acidification curve (gastric titration time $=$ time gastric $\mathrm{pH}>2$ ) which is shorter with increased postprandial acid secretion and/or faster gastric emptying. Erythromycin (ERY) accelerates gastric emptying and reduces gastric acid secretion. Aim: To further characterize the relationship between acidity of the refluxate, acidity of gastric contents and gastric emptying in healthy subjects. Methods: Concomitant esophageal impedance-pH and scintigraphic gastric emptying recordings were performed in 14 asymptomatic subjects [12 M, age 23 (19-60)]. The gastric emptying study used a caloric solid-liquid meal (700 Kcal, fat $60 \%$ ) and considered separately the emptying of total, proximal and subcardial stomach. Gastroesophageal reflux events (detected by impedance with acidity characterized by $\mathrm{pH}$ ) gastric $\mathrm{pH}$ (measured with a sensor located $10 \mathrm{~cm}$ distal to LES) and half emptying times and areas under the emptying curve for liquids and solids were measured during $3 \mathrm{hs}$ after the meal. During the meal, saline or ERY $3 \mathrm{mg} / \mathrm{kg}$ were infused IV (20 min) in a randomized, double blind, cross-over design. Linear mixed models were used to describe the evolution of $\mathrm{pH}$ of reflux as a function of gastric emptying and titration time and Wilcoxon test was used to assess the effect of ERY. Results: With saline infusion, the median gastric titration time was $50 \mathrm{~min}(27-72)$ and the longer the titration time, the higher the $\mathrm{pH}$ of reflux $(\mathrm{p}<0.05)$. The faster the proximal and subcardial gastric emptying of liquids, the shorter the titration time (r: 0.57) and the lower the $\mathrm{pH}$ of reflux ( $\mathrm{p}<0.05)$. There was no correlation between gastric emptying of solids and $\mathrm{pH}$ of reflux. ERY significantly prolonged gastric titration time to $90 \mathrm{~min}(49-140)$ and decreased the proportion of acid reflux events $(\mathrm{pH}<4)$ from $52 \%$ to $18 \%$ without reducing the total number of reflux events/3h [11(8-13) vs. 9.5 (7-10)]. ERY had no effect on gastric emptying of liquids but accelerated gastric emptying of solids ( $1 / 2$ time: $102 \pm 32 \mathrm{~min}$ vs. $67 \pm 30 \mathrm{~min}$, p< $0.05)$. Conclusion: The acidity of postprandial reflux is highly affected by the gastric titration time which depends on gastric secretion, buffering effect of the meals and gastric emptying.
ERY, in spite of accelerating gastric emptying of solids, prolongs gastric titration time and reduces acid reflux, probably by inhibiting postprandial acid secretion and enhancing the buffer capacity of the meal by a better mixing of acid and chyme.

\section{M2021}

Transient Lower Oesophageal Sphincter Relaxation and Mechanisms of Gastro-Oesophageal Reflux in Twins Discordant for Gord Symptoms Imtiyaz Mohammed, Angela Anggiansah, Roy Anggiansah, Lynn F. Cherkas, Tim D. Spector, Nigel J. Trudgill

Background: Transient lower oesophageal sphincter relaxation (TLOSR) is the principal mechanism associated with acid reflux episodes. We have therefore examined TLOSR in twin pairs who are discordant for GORD symptoms, i.e. only one of the pair has GORD symptoms, to establish whether differences in TLOSR may contribute to the aetiology of GORD. Methods: 4 monozygotic (MZ) and 12 dizygotic (DZ) twin pairs, who were repeatedly discordant for GORD symptoms over two years, were studied. LOS function using a sleeve sensor and oesophageal pH were monitored for 30 mins before and 60 mins after a $250 \mathrm{mls}$ (1200 Kcal) lipid meal. Results: MZ twins were aged mean 63 (range 53-75) years and DZ twins 57 (44-74) years. TLOSR frequency increased from median 2(0-4) to 3(1-8) per hour post-prandially $(\mathrm{p}=0.001)$ in the symptomatic twins and from $0(0-2)$ to $2.5(1-9)$ postprandially in the asymptomatic twins ( $\mathrm{p}=0.001$ ). Comparison between the groups revealed that pre-prandial TLOSR were more frequent in symptomatic twins $(\mathrm{p}=0.03)$ but there was no difference in post-prandial TLOSR frequency $(\mathrm{p}=0.6)$. However, only $6 \%$ of pre-prandial TLOSR were associated with acid reflux compared with $24 \%$ of post-prandial TLOSR. Acid reflux episodes increased post-prandially (symptomatic twins $0(0-2)$ to $1(0-9)$ per hour $(\mathrm{p}=$ $0.01)$, asymptomatic twins $0(0)$ to $1(0-8)(\mathrm{p}=0.01)$ ) but there was no difference between the groups in pre- $(\mathrm{p}=0.3)$ or post-prandial acid reflux episode frequency $(\mathrm{p}=0.9)$ or total post-prandial acid exposure (symptomatic twins median 11 (range 0-1051) s, asymptomatic twins $4(0-487) \mathrm{s}(\mathrm{p}=0.4))$. Conclusion: There was no difference in post-prandial TLOSR frequency in twins discordant for GORD symptoms. Although pre-prandial TLOSR were more common in twins with GORD symptoms this is unlikely to contribute to the aetiology of GORD, as they were rarely associated with acid reflux.

\section{M2022}

GERD, BMI, and Body Fat Distribution: A Large Cross-Sectional Study Douglas A. Corley, Ai Kubo, Wei Zhao

BACKGROUND: Almost no published data evaluate the association between GERD and body fat distribution; if present, such an association may help explain gender/ethnic differences in GERD complications such as esophageal adenocarcinoma. METHODS: We evaluated GERD risk factors in a large cross-sectional study. Participants were members of a large health maintenance organization undergoing a systematic health check-up. They completed detailed questionnaires, recorded symptoms in the last 6 months (including multiple heartburn questions), and underwent an examination that included height, weight, and anteriorposterior diameters of the abdomen and thigh. GERD was defined as burning abdominal pain that radiated into the chest. Fat distribution was the ratio of the abdomen/thigh diameters. Analyses utilized unconditional logistic regression and adjusted for age, smoking, alcohol use, and ethnicity. RESULTS: Questionnaire and BMI data were available for 189,447 unique participants; abdominal/thigh measurements were available for 111,398. There was statistical evidence of interaction between gender, fat distribution and GERD symptoms $(\mathrm{P}<0.01)$. A significant GERD:abdominal fat association (4th vs. 1st quartile) was present in white men (OR 1.2, 95\% CI 1.1, 1.3) but not in black men (OR 1.2, 95\% CI 0.9, 1.5). CONCLUSIONS: 1. GERD symptoms were independently associated with both an increased BMI and an increased abdomen/thigh ratio. 2. A substantial portion of the BMI-GERD association is mediated through an increased abdominal fat distribution pattern. 3. The GERD-abdominal fat association was strongest among those with a normal to moderately elevated BMI suggesting there may be a threshold BMI above which further increases in abdominal fat have less effect on GERD symptoms. 4. The strongest GERD-abdominal fat association was in males with a BMI between 22.9 and 24.2 (2nd quartile)

\begin{tabular}{|c|c|c|}
\hline GERD Risk Factors & Odds Ratio & $95 \% \mathrm{CI}$ \\
\hline BMI (4th vs. 1st quartiles) not adjusted for fat distribution & 1.57 & $1.50,1.64$ \\
\hline BMI (4th vs. 1st quartiles) adjusted for fat distribution waist/thigh ratio & 1.36 & $1.29,1.45$ \\
\hline Waist/thigh ratio (4th vs. 1st quartiles) stratified by quartile of BMI* & & \\
\hline Men, 1st quartile BMI & 1.22 & $0.97,1.55$ \\
\hline Men, 2nd quartile BMI & 1.69 & $1.25,2.27$ \\
\hline Men, 3rd quartile BMI & 1.35 & $1.11,1.63$ \\
\hline Men, 4th quartile BMI & 1.18 & $0.95,1.46$ \\
\hline Women, 1st quartile BMI & 1.37 & $1.19,1.57$ \\
\hline Women, 2nd quartile BMI & 1.41 & $1.10,1.81$ \\
\hline Women, 3rd quartile BMI & 1.18 & $0.97,1.44$ \\
\hline Women, 4th quartile BMI & 1.37 & $1.01,1.19$ \\
\hline
\end{tabular}

*All fully adjusted for BMI 
Don't Eat Before You Sleep, Or Your Nightmares May Come True Michael Piesman, Inku Hwang, Corinne Maydonovitch, Roy K. Wong

Background: Lifestyle modifications, including avoidance of late evening meals, are often prescribed to patients with gastroesophageal reflux disease (GERD) to improve symptoms of reflux. However, prospective evidence supporting the efficacy of this recommendation is lacking. The purpose of this study was to determine the difference in the degree of reflux in patients consuming early or late meals relative to bedtime on 2 consecutive nights. Methods: 32 adult patients with symptomatic GERD referred to our clinic were prospectively enrolled and acted as their own controls. Standard evening meal consisting of $1200 \mathrm{kCal}$ (35\% fat, $15 \%$ protein, and 50\% carbohydrates) was consumed either at 1700 or 2100 hours in a randomized fashion. The patients went to bed at 2300 hours on both nights and arose at 0600 . Acid exposure was measured for 48-hours using a Bravo wireless $\mathrm{pH}$ system. Reflux symptom score was recorded. Anti-secretory agents were discontinued for 10 days. Analysis was done using a paired T test. Results: 30 patients completed the study (19M/ $11 \mathrm{~F}, 70 \%$ Caucasian, mean age 46 (24-74), mean BMI 28 (18-40). EGD revealed esophagitis in $37 \%$ and hiatal hernia in $47 \%$ of patients. Average time from dinner to bedtime was 93 min for the late meal and $330 \mathrm{~min}$ for the early meal. There was a positive correlation between BMI and degree of reflux $\left(r^{2}=0.189\right)$. The $24 \mathrm{hr}$ JD score was significantly higher in patients following the late meal, as were the \% time total and supine reflux, and the longest episode of reflux. Following the late meal, patients had on average $228 \%$ greater supine reflux compared to the early meal. There was no significant difference in total symptom score between the late and early evening meal. (See Table). Conclusions: 1. GERD patients consuming a late-evening meal had significantly greater total JD score and supine reflux versus an early-evening meal. 2. GERD increased with increasing BMI. 3. There was no difference in individual and total symptom scores between the late and early-meal groups, suggesting that symptoms do not always correlate with acid reflux. 4. This study indicates that eating meals early before bedtime significantly decreases esophageal acid exposure. Reflux Parameters and Symptoms

\begin{tabular}{|c|c|c|c|}
\hline & Early Meal & Late Meal & P value \\
\hline JD 24 hr pH score & $27.2 \pm 3.7$ & $43.9 \pm 6.5$ & 0.001 \\
\hline \% Time Total Reflux & $6.5 \pm 0.8$ & $8.4 \pm 1.1$ & 0.017 \\
\hline \% Time Upright Reflux & $8.2 \pm 1.1$ & $8.8 \pm 1.3$ & 0.530 \\
\hline \% Time Supine Reflux & $2.3 \pm 0.8$ & $7.5 \pm 1.8$ & 0.003 \\
\hline \# Episodes >5 min & $3.5 \pm 0.6$ & $4.6 \pm 0.8$ & 0.144 \\
\hline Longest Episode & $15.6 \pm 3.4$ & $23.2 \pm 3.7$ & 0.031 \\
\hline Symptom Score & $7.7 \pm 1.2$ & $8.5 \pm 1.3$ & 0.352 \\
\hline
\end{tabular}

Data expressed as mean \pm SEM, JD=Johnson-Demeester

\section{M2024}

\section{Obstructive Sleep Apnoea (OSA) Is a Risk Factor for GERD in Morbidly}

\section{Obese Patients}

Mohamed Merrouche, Julien Pouzoulet, Dominique Maillard, Jean Marc Sabate, Pauline Jouet, Simon Msika, Severine Ledoux, Christine Clerici, Benoit Coffin

Obesity has been demonstrated to be a risk factor for GERD and to increase the risk of obstructive sleep apnoea (OSA). Previous studies have suggested that OSA could increase GERD because of the negative thoracic pressure produced by upper-airway collapse occurring during apnoea. The aim of this study was to evaluate in a group of patients with morbid obesity the prevalence of OSA and GERD and to look for a relationship between these two syndromes. Patients and methods: patients with morbid obesity [Body Mass Index (BMI) > $40 \mathrm{~kg} / \mathrm{m} 2$ or BMI $>35 \mathrm{~kg} / \mathrm{m} 2$ in association with comorbidities] selected for bariatric surgery were prospectively included in the study between April 2003 and January 2005. Every patient underwent a standard 24-hour ambulatory pH monitoring (GERD when \% of time with $\mathrm{pH}<4$ higher than $4.2 \%$ ), oesophageal manometry and nocturnal polysomnographic recording [OSA when apnoea-hypopnoea index $(\mathrm{AHI})>10$ ]. Results (mean \pm SD): 68 patients ( $59 \mathrm{~F}$, mean age $38.9 \pm 10.4$ yrs; BMI $46.5 \pm 6.4 \mathrm{~kg} / \mathrm{m} 2$ ) have been included. Thirty patients (44\%) had GERD (\% of time with $\mathrm{pH}<4: 7.5 \pm 2.7 \%$ ) and $55(80.9 \%$ ) had OSA. Severe OSA, defined by AHI>50, was present in $17.4 \%$. The lower esophageal sphincter pressure (LES pressure) was significantly lower in patients with GERD (11.6 \pm 3.4 vs $13.4 \pm 3.6 \mathrm{mmHg}$, respectively; $\mathrm{P}=0.039$ ). There was a significant relationship between AHI and BMI $(r=0.337 ; \mathrm{P}=0.005)$ in patients with $(\mathrm{P}=0.019)$ or without GERD $(\mathrm{P}=0.015)$. Patients with OSA were significantlly older $(40.5 \pm 10.9$ vs $33.5 \pm 10.4$ yrs; $\mathrm{P}=0.039)$. There was a trend for more frequent GERD in patients with OSA $(49.1 \%$ vs $23.1 \%$ respectively; $\mathrm{P}=0.089$ ). There was no significant relationship between $\mathrm{pH}$-metry criteria and AHI neither in the 24-h total recording time (\% of time with $\mathrm{pH}<4: \mathrm{r}=$ $0.173, \mathrm{P}=0.16$; number of acid reflux $\mathrm{r}=-0.179 ; \mathrm{P}=0.14)$, nor in the nocturnal recording time (\% of time with $\mathrm{pH}<4: \mathrm{r}=-0.088, \mathrm{P}=0,48$; number of acid reflux: $\mathrm{r}=-0,32, \mathrm{P}=$ $0,29)$. In multivariate analysis, GERD was significantly associated with a low LES pressure $(\mathrm{P}=0.031)$ and with OSA $(\mathrm{P}=0.045)$ but not with sexe, age and BMI. Conclusion: In this population of morbidly obese patients, OSA and GERD were extremely frequent and GERD was significantly associated with LES hypotonia and OSA. Motility disorders induced by OSA and their evolution after OSA specific treatment warrant further studies.
Esophagitis Is Associated with Enlarged Unbuffered Postprandial Acid Pocket Alan T. Clarke, Angela A. Wirz, Jonathan J. Manning, S Ballantyne, D Alcorn, Kenneth E. McColl

Background: Previous work has demonstrated the presence of an unbuffered pocket of highly acidic juice at the gastric cardia after a meal in healthy volunteers. Aims: To compare the postprandial acid pocket in healthy volunteers and patients with severe reflux esophagitis and to define it's position relative to the squamocolumnar junction and manometric landmarks. Methods: 12 healthy volunteers and 16 patients with grade III/IV reflux esophagitis or Barrett's esophagus were studied. At endoscopy radiopaque clips were placed at the most proximal margin of the gatric folds and, in the subjects with endoscopic evidence of hiatus hernia, at the diaphragmatic pinch. A barium meal was then performed to document the anatomy and confirm the position of the clips. While fasted, a pull-through study was performed using a combined dual $\mathrm{pH}$ and solid state manometry catheter, withdrawing at consecutive $1 \mathrm{~cm}$ intervals every minute. The position of the $\mathrm{pH} /$ manometry apparatus relative to the anatomical landmarks was obtained by identifying the radiopaque clips on latera chest $\mathrm{x}$-ray. The patients were then fed a standardized fatty meal and 15 minutes after the meal, the pull-through study, x-rays and barium meal repeated. Results: A hiatus hernia was identified endoscopically in 13 of the reflux esophagitis patients and it's median length was not significantly altered by the meal (fasting $=1.6 \mathrm{~cm}$, range $0.9 \mathrm{~cm}$ to $4.1 \mathrm{~cm}$ vs. postprandial $=2.3 \mathrm{~cm}$, range $0.6 \mathrm{~cm}$ to $4.7 \mathrm{~cm}$ ). Fasting and postprandial maximal mid-body intragastric $\mathrm{pH}$ were similar between the two groups studied. A region of unbuffered acid $(\mathrm{pH}<2)$ immediately distal to the proximal gastric folds was seen in $8 / 12$ healthy volunteers and 13/15 reflux esophagitis patients. This unbuffered "acid pocket" was a significantly longer in the esophagitis patients versus healthy volunteers (median length $2 \mathrm{~cm}$, range $0 \mathrm{~cm}$ to $15 \mathrm{~cm}$ vs. $0 \mathrm{~cm}$, range $0 \mathrm{~cm}$ to $4 \mathrm{~cm} ; \mathrm{p}=0.003$ ). It's location in the esophagitis patients corresponded with the hiatus hernia sac, the midpoint of which remained highly acidic (median $\mathrm{pH} 1.405$, range 0.78 to 7.54 ) after the meal. Postprandial barium studies showed no evidence of particulate food material within the hiatal sac. Conclusion: Severe esophagitis is associated with an enlarged unbuffered postprandial acid pocket. This may be partially due to the hiatus hernia providing a reservoir of highly acidic gastric juice isolated from food in the main stomach which may reflux into the esophagus.

\section{M2026}

Rilevance of Cell Proliferation in the Esophageal Mucosa Defence

Carlo Calabrese, Davide Trere, Anna Fabbri, Manuela Vici, Giovanna Cenacchi, Desiree Zahlane, Massimo Derenzini, Giulio Di Febo

Background: Erosive esophagitis (ERD) is a frequent macroscopic feature in patients with gastroesophageal reflux disease (GERD). However, most of patients with heartburn/regurgitation have a non-erosive reflux disease (NERD). The reason of this heterogeneous behavior of GERD is unknown to date. Aim: to value the cell proliferation of esophageal epithelium in healthy normal subjects and in patients with GERD with or without erosions. Material and methods: of the 60 subjects 9 were healthy controls with normal pH-testing and macroscopical, histological and ultrastructural pattern; 27 patients with ERD and 24 with NERD. Subjects underwent endoscopy and biopsies were taken from the distal esophagus Specimens were analyzed at histology and at transmission electron microscopy (TEM). Cell proliferation was evaluated by MIBI immunostaining and by measuring the nucleolar size after selectively silver staining. Results: at histology of the 27 patients affected by ERD,22 had normal mucosa and 5 showed a mild esophagitis. No patients with NERD showed signs of oesophagitis at histology. At TEM,all patients with GERD had an ultrastructural pattern of damage and all controls had a normal ultrastructural pattern. The mean $( \pm$ SD) MIBl-LI values of normal subjects and NERD and ERD patients were $62.2 \%( \pm 9.1), 30.3 \%( \pm 8.1)$ and $16.2 \%( \pm 5,6)$, respectively, and were significantly different among the three groups $(\mathrm{p}<0.001)$. On the other hand, the mean nucleolar areas did not differ significantly among normal,NERD and GERD patients. Conclusions: esophageal mucosa exposed to chronic acid and pepsin insult present a decreased proliferation status that could have a relevant role in determining ERD or NERD features in GERD patients.

\section{M2027}

A Polymorphism in PTPN11 and Not Interleukin-8 Is Associated with GERD Among Helicobacter Pylori Infected Japanese

Takafumi Ando, Yasuyuki Goto, Osamu Maeda, Kazuhiro Ishiguro, Osamu Watanabe, Naoki Ohmiya, Yasumasa Niwa, Nobuyuki Hamajima, Emad M. El-Omar, Hidemi Goto Background and aims: Gastro-esophageal reflux disease (GERD) patients have increased exposure to gastric acid and interleukin 8-mediated inflammation in the lower esophagus. A polymorphism of the PTPNll gene that encodes src homology 2 domain-containing protein tyrosine phosphatase-2 (SHP-2) is associated with gastric atrophy and might protect against GERD, while the IL-8 A-251T>A polymorphism affects IL-8 production. We aimed to study the association between GERD and the PTPN11 G>A and IL-8 A-251 T>A polymorphisms. Methods: Upper gastro-intestinal endoscopy was performed on 120 consecutive patients with GERD symptoms and 120 asymptomatic controls and biopsies were obtained from the squamo-columnar junction (SCJ) for histological examination and in vitro organ cultures for IL-8 measurement by ELISA. Polymorphisms in the PTPN 11 and IL-8 genes were genotyped by polymerase chain reaction with confronting two-pair primers. Results: The prevalence of $H$. pylori infection was significantly higher in the control (94/120,78\%) compared to the GERD group $(74 / 120,62 \%)(\mathrm{p}=0.005)$. Using the Los Angeles Classification, 74 patients (62\%) in the GERD group had reflux esophagitis (Grade A: 45, Grade B: 23 Grade C: 6 ), compared to 16 subjects (13\%) in the control group (Grade A: 14, Grade B 2) $(\mathrm{p}<0.001)$. The IL-8 T-251T >A polymorphism had no significant correlation with GERD symptoms, reflux esophagitis or IL-8 mucosal levels at the SCJ, despite the GERD patients having significantly higher IL- 8 mucosal levels than the controls (mean \pm SD $=107 \pm 59 \mathrm{ng} /$ $\mathrm{mg}$ protein compared to $59 \pm 32$ in the controls; $<<0.001$ ). In contrast, the PTPN11 G/A polymorphism had a significant effect on risk of GERD and reflux esophagitis. Compared 
to the common PTPNll G/G genotype, the A/A genotype (which protects against gastric atrophy) was associated with increased risk of GERD symptoms (odds $2.53,95 \%$ CI 1.06 6.03) and reflux esophagitis (odds ratio 5.23,95\% CI 2.09-13.07). Conclusions: The PTPN11 $\mathrm{G}>\mathrm{A}$ polymorphism appears to influence risk of GERD, most probably through its association with gastric atrophy while the IL-8 polymorphism is probably not relevant. This highlights the role of host genetic determinants of gastric acid in the pathogenesis of GERD.

\section{M2028}

Salivary Gland Scintigraphy in Gastro-Esophageal Reflux Disease

Yoshihisa Urita, Susumu Ishihara, Kenichiro Arai, Jun Kondo, Tatsuo Akimoto, Hiroto

Kato, Noriko Hara, Hideyuki Koshino, Yoshiko Honda, Yoko Nagai, Kazushige Nakanishi, Nagato Shimada, Motonobu Sugimoto, Masaaki Takano, Sanshin Hayashi, Kazumasa Miki

Background: Gastro-esophageal reflux disease (GERD) is associated with a decreased salivary flow as well as gastric acid production. Recent evidences prove the esophagoprotective ability of salivary secretions. For example, EGF is produced and secreted by salivary glands into the gastrointestinal tract for promoting regenerative functions. This study therefore aimed to investigate functional disorders of salivary glands in patients with GERD. Patients and Methods: Forty-four consecutive patients with GERD underwent salivary gland scintigraphy. GERD was diagnosed by endoscopy and gastro-esophageal reflux self-report questionnaires. Following intravenous injection of 180 to $200 \mathrm{Mbq} 99 \mathrm{mTc}$-pertechnetate, anterior sequential imaging was performed every minute for 40 minutes. At 20 minute4s after injection of radionuclide, a lemon candy was administrated intraorally to stimulate salivary secretion. Regions of Interests (ROI) were selected on the individual submandibular and parotid glands, oral cavity, and thyroid gland. Time activity curves were drawn for each of these. Washout ratio (peak count before lemon candy administration-lowest count after administration/ peak count before administration) was examined as a functional parameter. All of the patients were asked to refrain from drugs known to affect salivary secretion for at least one week prior to salivary scintigraphy. They were also asked to abstain from swallowing, chewing, sucking or any other mechanical stimulation of salivary flow. Results: The mean washout ratio was $55.8 \%$ in the right parotid gland, $59.8 \%$ in the left parotid gland, $61.6 \%$ in the right submandibular gland, and $65.3 \%$ in the left submandibular gland. If the washout ratio of less than $50 \%$ is defined as functional disorders of salivary glands, a decreased salivary secretion of four major salivary glands was found in $12(27 \%), 4(9 \%), 12(27 \%)$, and 8 cases $(18 \%)$, respectively. An earlier increase of oral cavity count, which may reflect spontaneous secretion from all four major salivaru glands, was found in 20 cases (47\%) with GERD. Overall, salivary function disorder of at least one major salivary glands was found in 36 patients $(82 \%$ ) with GERD . Conclusions: Salivary gland function was more frequently diminished than expected. We concluded that the presence of impaired salivary gland function was considered to be one of risk factors for developing RE.

M2029

Obesity Is Associated with Increased Esophageal Acid Exposure Hashem El-Serag, John Pandolfino, Stephanie Fitzgerald, Jennifer R. Kramer, Thomas Tran, Gulchin Ergun

Background: Obesity has been associated with GERD. The mechanism of this relation is unclear. The association between esophageal acid exposure and total body or abdominal anthropometric measures has not been examined. Methods: This is a prospective crosssectional study of consecutive patients undergoing 24 hour pH-metry at an open access Reflux Center. Standardized measurements of body weight, height, and waist and hip circumference were obtained. The association between several parameters of esophageal acid exposures and anthropometric measures were examined in univariate and multivariate analyses. Results: We enrolled 206 patients (63\% women) with a mean age of 51.4 years. The mean values for weight, height, and BMI were $76.4 \mathrm{Kg}, 168 \mathrm{~cm}$, and $27.0 \mathrm{Kg} / \mathrm{m} 2$, respectively. BMI>30 (but not BMI 25 to 29.9) was associated with a significant increase in the number of acid reflux episodes, the number of long reflux episodes ( $>5$ minutes), and time (and \% time) with $\mathrm{pH}<4$. This increase affected total, postprandial, upright and supine $\mathrm{pH}$ measurements at the distal probe (and less consistent associations at the proximal probe) Similar findings were seen for the waist circumference except for upright measurement where the correlation was not significant. However, when adjusted for waist circumference (included in the same model), the association between BMI>30 and measures of esophageal acid exposure became attenuated and no longer significant (Table) Conclusions: This indicates that obesity operates primarily through abdominal obesity to increase esophageal acid exposure and hence the risk of GERD.

Association between BMI $>30$ and several parameters of esophageal acid exposure at the distal probe

\begin{tabular}{|c|c|c|c|c|c|c|}
\hline & \multicolumn{3}{|c|}{ BMI $>30$ Unadjusted for Waist } & \multicolumn{3}{|c|}{ BMI $>30$ Adjusted for Waist } \\
\hline & estimate & Std. Err. & P- value & estimate & Std. Err. & P- value \\
\hline Number of Reflux Episodes & & & & & & \\
\hline Total & 46.54 & 19.62 & 0.019 & 20.41 & 31.11 & 0.512 \\
\hline Post Prandial & 33.17 & 10.13 & 0.001 & 19.77 & 16.04 & 0.219 \\
\hline Upright & 29.73 & 14.53 & 0.042 & 18.75 & 23.01 & 0.416 \\
\hline Supine & 16.03 & 8.14 & 0.050 & 1.48 & 12.83 & 0.908 \\
\hline Number of Long Reflux Episodes & & & & & & \\
\hline Total & 1.38 & 0.75 & 0.066 & 1.38 & 1.19 & 0.248 \\
\hline Post Prandial & 0.98 & 0.43 & 0.025 & 0.89 & 0.69 & 0.201 \\
\hline Upright & 0.52 & 0.50 & 0.297 & 0.34 & 0.80 & 0.671 \\
\hline Supine & 0.78 & 0.46 & 0.094 & 0.92 & 0.73 & 0.213 \\
\hline Time pH Below 4 & & & & & & \\
\hline Total & 40.78 & 19.75 & 0.040 & 19.86 & 31.41 & 0.528 \\
\hline Post Prandial & 25.58 & 9.76 & 0.009 & 18.02 & 15.52 & 0.247 \\
\hline Upright & 17.57 & 10.55 & 0.097 & 12.36 & 16.76 & 0.461 \\
\hline Supine & 22.79 & 12.80 & 0.077 & 7.43 & 20.40 & 0.716 \\
\hline
\end{tabular}

M2030

Characteristics of Gastroesophageal Reflux in Symptomatic Patients with and Without Excessive Distal Esophageal Acid Exposure

Albert J. Bredenoord, Bas L. Weusten, Robin Timmer, Andre J. Smout

Background and aim: In a subset of GERD patients a positive relationship between symptoms and reflux episodes can be demonstrated in the absence of excessive distal esophageal acid exposure. It is assumed that hypersensitivity of the esophagus to acid plays an important role in these patients. Besides acidity of the refluxate other factors such as its proximal extent may determine whether the episode is perceived or not. The aim of this study was therefore to investigate the profile of reflux episodes in GERD patients without excessive esophageal acid exposure. Methods: Combined 24-hr impedance and $\mathrm{pH}$ monitoring was performed in 14 GERD patients with excessive reflux (GERD pH+), 14 GERD patients with physiological reflux (GERD pH-) and 14 controls. All patients had a positive symptomreflux association during 24-hour monitoring. Results: The esophageal acid exposure time in GERD pH+ patients was $9.8 \%$ compared to $3.5 \%$ in the GERD pH- patients and $2.9 \%$ in the controls. The incidence of acid reflux episodes in GERD pH- patients $(25.5 \pm 4.9)$ and controls $(20.2 \pm 3.9)$ was comparable but significantly lower $(\mathrm{p}<0.05)$ than the incidence in GERD pH+ patients $(69.8 \pm 7.3)$. However, no differences in the number of weakly acidic reflux episodes were observed between GERD pH- patients, GERD pH+ patients and controls $(27.2 \pm 3.8$ vs $26.8 \pm 4.6$ and $21.0 \pm 3.7$, respectively). There was also no difference in the number of pure gas reflux episodes between GERD pH+ patients $(23.7 \pm 6.7)$, GERD pHpatients $(24.2 \pm 5.9)$ and controls $(20.8 \pm 2.9)$. The proportion of reflux episodes that reached the proximal esophagus was significantly higher in the GERD pH+ patients (19.6 $\%$ ) and GERD pH- patients ( $17.8 \%$ ) patients than in the controls $(5.7 \%, \mathrm{p}<0.05)$. Of the 158 symptomatic reflux episodes in the GERD pH+ patients 53 (33.5\%) were due to reflux episodes that reached the proximal esophagus. Of the 55 symptomatic reflux episodes in the GERD pH-patients, 21 (38.2\%) were due to reflux episodes that reached the proximal esophagus. Conclusions: Not only in GERD patients with excessive reflux but also in those with a normal distal esophageal acid exposure a higher proportion of reflux episodes reaches the proximal esophagus than in controls. These findings suggest that not only esophageal hypersensitivity but also proximal extent may play a role in symptom generation in GERD patients without excessive reflux.

\section{M2031}

Air Swallowing, Belching and Reflux in Patients with Gastroesophageal Reflux Disease

Albert J. Bredenoord, Bas L. Weusten, Robin Timmer, Andre Smou

Background and aim: It has been suggested that belching induces gastroesophageal reflux. The aim of this study was to investigate whether gastric air insufflation and spontaneous air swallowing and intragastric air promotes both belching and gastroesophageal reflux. Methods: Esophageal impedance, $\mathrm{pH}$ and pressure were measured during a 20-min recording period in 12 healthy controls and 12 patients with GERD before and after intragastric inflation of $600 \mathrm{~mL}$ of air in order to simulate an intragastric volume of swallowed air. This was repeated $90 \mathrm{~min}$ after ingestion of a meal. Subsequently, ambulatory 24-hr impedance$\mathrm{pH}$ measurement was performed and the incidence of air swallowing, belching and acidic and weakly acidic reflux was assessed. Results: After air infusion in the preprandial period the incidence of gas reflux episodes was increased in both patients $(1.00 \pm 0.35$ vs $3.33 \pm$ $0.65, \mathrm{p}<0.05)$ and controls $(0.42 \pm 0.15$ vs $5.67 \pm 1.14, \mathrm{p}<0.05)$. In contrast, the incidence of acid and weakly acidic reflux episodes was not increased after air infusion, neither in patients (acid: $0.25 \pm 0.13$ vs $0.25 \pm 0.13$, weakly acidic: $0.50 \pm 0.15$ vs $0.33 \pm 0.14$ ), nor in controls (acid: $0.08 \pm 0.08$ vs $0.0 \pm 0.0$, weakly acidic: $0.17 \pm 0.11$ vs $0.42 \pm 0.26$ ). Air infusion led to an increase in TLESRs in patients $(1.42 \pm 0.23$ vs $2.00 \pm 0.39, \mathrm{p}<0.05)$ and controls $(1.08 \pm 0.31$ vs $2.42 \pm 0.60, \mathrm{p}<0.05)$, but this was completely due to an increase in gas reflux-associated TLESRs. Similar results were obtained with air infusion after consumption of the meal. During the 24-hr impedance-pH study, patients showed a higher incidence of air swallows ( $287 \pm 45$ vs $176 \pm 24, \mathrm{p}<0.05)$, belches $(52.4 \pm 6.6$ vs $32.7, \mathrm{p}<0.01)$ and acid reflux $(42.3 \pm 7.6 \pm 19.1 \pm 2.8, \mathrm{p}<0.01)$ than controls. Patients did not show more weakly 
acidic reflux episodes $(21.0 \pm 5.3$ vs $14.5 \pm 1.1)$ and regular swallows $(782.1 \pm 89.0$ vs $818.0 \pm 57.3$ ) during the $24-\mathrm{hr}$ study. Conclusions: Patients with GERD swallow air more frequently and belch more frequently than healthy subjects. However, intragastric infusion of air does not result in an increase in acidic and weakly acidic reflux suggesting that air swallowing is not causing acidic and weakly acidic reflux

\section{M2032}

SERT Genotype in GERD Compared to Healthy Volunteers D.R. de Vries, Jose Ter Linde, Margot A. van Herwaarden, Melvin Samsom

Background: The insertion/deletion polymorphism in the promoter region of the serotonin transporter protein (SERT) is associated with many diseases. The polymorphism results in a short or long promoter region with a difference in length of $44 \mathrm{bp}$. The presence of a short promoter region results in lower SERT expression, less re-uptake and higher availability of serotonin (5HT). The short/short genotype (SS) is associated with diarrhoea predominant irritable bowel syndrome (IBS). In mice lacking SERT, gut motility increases. Moreover, the mice develop diarrhoea and intermittent periods of constipation. The aim of this study was to investigate whether the relation of the SERT polymorphism with gut motility results in an association with gastroesophageal reflux disease (GERD). Methods: GERD was defined using 24-hour esophageal $\mathrm{pH}$-monitoring and symptom association scores (total time $\mathrm{pH}<4$ $\geq 6 \%$ or $\mathrm{SI} \geq 50 \%$ or $\mathrm{SAP} \geq 95 \%$ ). Whole blood samples were taken from $260 \mathrm{GERD}$ patients (156 male, mean age 48,7, SD 13,4) and 138 age and sex matched healthy controls. DNA was extracted using QIAamp DNA Blood Mini Kit according to manufacturer's instructions. SERT genotype was determined by PCR amplification of the SERT promoter region and gel electrophoresis of the product. Allele frequencies were tested for Hardy-Weinberg equilibrium for both groups. Genotype frequencies were compared between groups using chi-squaretests. Results: In both the patient and the volunteer group, allele frequencies were in HardyWeinberg equilibrium. The genotype frequencies were similar for volunteers and patients, and are depicted in the figure. SERT genotype was not associated with GERD. Subsequently the phenotypic characteristics of the GERD patients were used to make subdivisions in the group. There was no association between SERT genotype and upright, supine or total esophageal acid exposure, nor was there an association with SI or SAP. Conclusion: There is no major association between SERT genotype and GERD in general, nor with characteristics of the reflux disease itself. This suggests that the pathophysiological mechanism which leads to GERD and GER symptoms could be located in another signalling pathway.

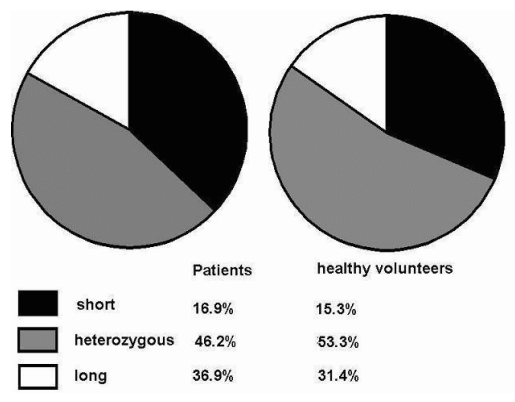

M2033

Prevalence of Nocturnal Heartburn and Night-Time Reflux in Patients with Non-Erosive Gastroesophageal Reflux Disease and Erosive Esophagitis Jose M. Remes-Troche, Maribal Gonzalez-Gutierrez, Mariel Mejia, Ana T. Abreu y Abreu, Jose A. Chavez-Barrera, Miguel A. Valdovinos

Background/Aim: Sixty to $70 \%$ of GERD patients report night-time symptoms. Nocturnal heartburn $(\mathrm{NH})$ interferes with sleep and impairs quality of life. Night-time reflux (NTR) has been associated with increased prevalence of GERD complications. The information regarding NH and NTR is limited, and there are no studies that address the frequency of these conditions in the different groups of GERD. Our aim was to evaluate the prevalence of NH and NTR according to the clinical variants of Non-Erosive GERD (NERD) and erosive esophagitis (EE). Material and Methods: Consecutive patients with symptomatic GERD were evaluated from January to June 2005. Esophageal mucosal injury was assessed by upper endoscopy. All patients underwent esophageal 24-h pH monitoring. $\mathrm{NH}$ was defined as presence of symptoms at night and in supine position. Abnormal $\mathrm{pH}$ was considered when total \% of time of $\mathrm{pH}<4$ was $>4.2 \%$. Abnormal NTR was defined as a $\%$ of time of $\mathrm{pH}<4$ $>1.2 \%$ at night. A symptom index $(\mathrm{SI})>50 \%$ was considered as positive $(+)$. Patients were classified in 4 groups: I) Erosive esophagitis, II) NERD with abnormal 24-h pH, III) NERD with normal 24-h pH,+SI, and IV) NERD with normal 24-h pH,-SI. Data were analyzed with Mann-Whitney U, Kruskal-Wallis and $\chi 2$ tests. Results: 83 patients, $66 \mathrm{~F}(81 \%)$ with a mean age of 45 years (range 20-75) were included. Distribution of patients according groups was: 26 (I), 14 (II), 7 (III) and 36 (IV). Overall prevalence for NH and NTR was $53 \%(n=44)$ and $54 \%(n=45)$, respectively. There was no correlation between NH and NTR ( $p>0.05$ ). Only 21 of 45 patients with NTR had NH. There was no difference in the prevalence of NH between groups $(p>0.05)$. However, prevalence of NTR, total reflux episodes, nocturnal reflux episodes, and \% of $\mathrm{pH}<4$ in the night were significantly higher in groups I and II (Table). Conclusions: Prevalence of NH is similar in the different groups of GERD patients. Despite this high prevalence (53\%), there was no correlation between symptom perception and abnormal acid esophageal exposure during the night. However, patients with EE and NERD with abnormal 24h-pH had an increased prevalence of NTR compared with the other groups. These findings suggest that other mechanisms besides acid reflux contribute to nocturnal symptoms in GERD.

\begin{tabular}{|c|c|c|c|c|}
\hline & Group I & Group II & Group III & Group IV \\
\hline Prevalence of NH $(\%)$ & 64 & 64 & 57 & 44 \\
\hline Prevalence of NTR $(\%)$ & $77^{*}$ & $64^{*}$ & 29 & 39 \\
\hline Total reflux episodes (Mean \pm SE) & $181 \pm 26^{*}$ & $180 \pm 58^{*}$ & $60 \pm 10$ & $44 \pm 4$ \\
\hline Night reflux episodes (Mean \pm SE) & $33 \pm 7 *$ & $56 \pm 21^{*}$ & $12 \pm 7$ & $9 \pm 2$ \\
\hline Total \% time $\mathrm{pH}<4$ & $15.4 \pm 2 *$ & $12 \pm 2 *$ & $2 \pm .4$ & $2 \pm .2$ \\
\hline Night \% time $\mathrm{pH}<4$ & $17.8 \pm 4 *$ & $12 \pm 4 *$ & $1.5 \pm .2$ & $1.1 \pm .1$ \\
\hline
\end{tabular}

$* \mathrm{p}<0.05$

M2034

Determinants of Proximal Extent of Gastroesophageal Reflux: Esophagitis, Acidity and Viscosity of the Refluxate

Sara Emerenziani, Michele Cicala, Xin Zhang, Mentore Ribolsi, Renato Caviglia, Michele Pier Luca Guarino, Daniel Sifrim

Proximal extent of reflux (PER) is a determinant of symptom elicitation in GERD patients The volume of the refluxate could affect PER, however, other factors i.e mucosal inflammation and/or physical characteristics of the refluxate might be involved. Weakly acidic reflux likely more viscous than acid reflux, is less perceived by patients. We evaluated proximal extent of reflux in the presence or absence of esophagitis both in patients with GERD and in an animal model of acute esophagitis and compared proximal extent of acid versus weakly acidic reflux in humans as well as more or less viscous refluxates in the animal model Methods: We measured proximal extent of reflux during 24-hr ambulatory esophageal pHimpedance monitoring in 17 patients with esophagitis (grade $\mathrm{I}=10$, grade $\mathrm{II}=7$ ), 27 patients with NERD and 10 asymptomatic controls. In 6 adult cats, reflux events were simulated by fast intraesophageal retrograde injection (via a percutaneous gastrostomy) of a radiopaque solution of increasing viscosity $[0,0.5$ and $1 \%$ of metilcellulose (MC)]. Proximal extent o simulated reflux was fluoroscopically assessed before and after acute esophagitis $(0.1 \mathrm{~N} \mathrm{HCl}$ perfusion, 80minutes). Results: Esophagitis was associated with higher proximal extent of reflux both in patients and cats. Compared to acid reflux, weakly acidic reflux showed a lower proximal extent in patients and controls (table). In cats, the most viscous solution $(1 \% \mathrm{MC})$ had the lowest proximal extent and reached the proximal esophagus significantly slower. Conclusions: Esophagitis, irrespective of refluxate volume, is associated with PER Weakly acidic and more viscous refluxates are characterized by lower proximal extent and slower migration.

\begin{tabular}{|c|c|c|c|c|c|c|}
\hline & \multicolumn{2}{|c|}{ CONTROLS } & \multicolumn{2}{c|}{ NERD } & \multicolumn{2}{c|}{ ESOPHAGITIS } \\
\hline LES -13 & \multicolumn{2}{|c|}{$27 \pm 2 \%$} & \multicolumn{2}{|c|}{$39 \pm 2 \% \%^{\circ}$} & \multicolumn{2}{c|}{$59 \pm 1 \% \%^{\circ}$} \\
\hline & Acid & Weakly acidic & Acid & Weakly acidic & Acid & Weakly acidic \\
\hline & $24 \pm 2 *$ & $17 \pm 2$ & $44 \pm 4 * 0$ & $27 \pm 5^{\circ}$ & $62 \pm 3^{* \circ} \#$ & $45 \pm 5^{\circ} \#$ \\
\hline LES - 20 & \multicolumn{2}{|c|}{$11 \pm 2 \%$} & \multicolumn{2}{|c|}{$22 \pm 1 \%^{\circ}$} & \multicolumn{2}{|c|}{$38 \pm 2 \%^{\circ} \#$} \\
\hline & $15 \pm 3^{*}$ & $7 \pm 2$ & $29 \pm 3 * 0$ & $16 \pm 4^{\circ}$ & $39 \pm 3 * \circ$ & $23 \pm 3^{\circ} \#$ \\
\hline & \multicolumn{3}{|c}{${ }^{\circ} \mathrm{p}<0.05$ vs controls, \# $\mathrm{p}<0.05$ vs NERD, ${ }^{*} \mathrm{p}<0.05$ vs weakly acidic } \\
\hline
\end{tabular}

\section{M2035}

Significant Enhancement of Salivary Protective Components in Males with Chronic Heartburn Symptomatology: A New Insight Into the Balance Between Aggressive Factors and Protective Mechanisms

Tomasz Jaworski, Marek Majewski, Irene Sarosiek, Stanley Edlavitch, Grzegorz Wallner Richard W. McCallum, Jerzy Sarosiek

Salivary glands elaborate a plethora of protective components that are instrumental in the maintenance of the integrity of the esophageal mucosa. Gender-related differences in the rate of gastric acid secretion are well established. Is the rate of secretion of salivary protective components in patients with chronic symptoms of heartburn also affected by gender, remains to be established? Aim: To measure inorganic and organic salivary protective components in basal conditions, during mastication as well as intraesophageal stimulation in patients with chronic GERD symptoms of both genders. Methods: The study was approved by HSC at KUMC and conducted in patients with chronic symptoms of heartburn (26F \& 12M mean age of 41,23-61 range). Subgroups of M \& F were comparable in terms of mean age and symptomatology. The samples of saliva were collected basally, during mastication as well as during the esophageal mechanical (tubing $\&$ balloons) and chemical (perfusion with $\mathrm{NaCl}$ followed by $\mathrm{HCl} /$ pepsin and final $\mathrm{NaCl}$ ) stimulation to mimic the natural gastroesophageal reflux scenario, using a specially designed esophageal perfusion catheter (WilsonCook Med.). In salivary samples their volume and the content of salivary buffers, protein mucin, EGF, TGF $\alpha$, and PGE2 were measured using commercial assays. Collected data were analyzed using SPSS statistical software. Results: The rate of salivary secretion among males was $79 \%(\mathrm{P}<0.01), 44 \%(\mathrm{P}<0.02)$, and $51 \%(\mathrm{P}<0.05)$ higher in basal conditions, mastication, and intraesophageal mechanical stimulation respectively. Salivary bicarbonate secretion among males was $191 \%$ higher $(\mathrm{P}<0.02)$ during basal conditions whereas salivary nonbicarbonate buffers were $71 \%$ higher $(\mathrm{P}<0.01)$ in basal conditions and $38 \%$ higher $(\mathrm{P}=0.05)$ during intraesophageal mechanical stimulation. The rate of salivary mucin secretion in males was $134 \%$ higher $(\mathrm{P}<0.01)$ in basal conditions and $62 \%$ higher $(\mathrm{P}<0.01)$ during mastication Salivary TGF $\alpha$ in males was also $66 \%$ higher $(\mathrm{P}<0.05)$ during mastication. Conclusions: 1 . Significantly higher rate of secretion of salivary inorganic and organic protective components among males may represent a gender driven phenomenon or compensatory mechanisms resulting from esophageal mucosal exposure to stronger acid from the gastric secretion. 2 The role of these protective components in prevention of injury and healing of mucosal changes resulting from gastroesophageal reflux warrants further exploration. 


\section{M2036}

Experience from the Progerd Study Suggests That GERD Is Not a Categorical Disease

Joachim Labenz, Michael Kulig, Marc Nocon, Stefan Willich, Daniel Jasperson, Andreas Leodolter, Tore Lind, Wolfgang Meyer-Sabellek, Michael Vieth, Manfred Stolte, Peter Malfertheiner

Background: There is controversy as to whether GERD exists as a spectrum of disease severity or as a categorical disease in three distinct groups: non-erosive (NERD) and erosive (ERD) reflux disease and Barrett's esophagus (BE). Aim: To assess whether there is progression or regression of GERD over 2 years in a large cohort of patients (ProGERD) under routine clinical care in Germany, Austria and Switzerland. Method: Patients with predominant heartburn with or without esophagitis were recruited and classified according to endoscopic status at baseline ie. NERD, ERD-LA grade A/B and -LA grade C/D and BE. After initial treatment with esomeprazole, they were followed, regardless of their response, and any medical therapy or endoscopy was instituted at the discretion of their primary care physician. At two years, endoscopy with biopsy was performed according to the protocol. Results: The proportions of patients (excluding those with confirmed BE at baseline) who progressed or regressed or remained the same in their GERD classification after 2 years were calculated (see table). At this timepoint, $22 \%$ of patients had been off medication for at least 3 months. Patients with ERD-LA grade C/D were at greatest risk of developing BE: $5.8 \%$ compared with $1.4 \%$ for $\mathrm{A} / \mathrm{B}$ and $0.5 \%$ for NERD. Conclusion: GERD does not seem to be a categorical disease. Progression and regression between grades was observed in this large cohort of patients under routine clinical care.

\begin{tabular}{|c|c|c|c|c|}
\hline Baseline status & No Change $\%$ & To NERD $\%$ & To LA A/B $\%$ & To LA C/D \% \\
\hline NERD $(n=1717)$ & 74.5 & N/A & 24.9 & 0.6 \\
\hline ERD A/B $(n=1512)$ & 37.1 & 61.3 & N/A & 1.6 \\
\hline ERD C/D $(n=278)$ & 7.8 & 50.4 & 41.8 & N/A \\
\hline
\end{tabular}

\section{M2037}

The Relevance of Helicobacter Pylori Eradication to Reflux Esophagitis Satoru Yagi, Hiroyuki Okada, Ryuta Takenaka, Yoshiro Kawahara, Masatsugu Miyoshi, Humiya Yoshinaga, Tatsuya Toyokawa, Jiro Miyaike, Atsushi Imagawa, Eiichiro Yumoto, Akiko Hujiwara, Yasushi Shiratori

Objectives: We analyzed the incidence of reflux esophagitis, and the change in the grade of pre-existing reflux esophagitis after Helicobacter pylori (H.pylori) eradication in correlation with the patient's factors.Methods: A total of 1030 Japanese patients, who were estimated the grade of reflux esophagitis according to the Los Angeles (LA) classification before and after H.pylori eradication therapy, were enrolled in this study Results: There was no esophagitis before H.pylori eradication therapy in 970 of the 1030 patients. H.pylori was eradicated in $806(83.1 \%)$ of them while $164(16.9 \%)$ patients had treatment failure. Reflux esophagitis developed after H.pylori eradication therapy in $67(8.2 \%$, LA classification $\mathrm{A} / \mathrm{B} / \mathrm{C} / \mathrm{D}=42 /$ $22 / 2 / 1)$ of the 806 patients who had successful therapy and 7 (4.3\%, LA classification A/ $\mathrm{B} / \mathrm{C} / \mathrm{D}=6 / 0 / 1 / 0)$ of the 164 patients with persistent infection. The incidence of reflux esophagitis tended to be higher among the patients cured of infection $(p=0.07)$. We also investigated the effects of sex, age, basal disease, the existence of hiatus hernia, and successful H.pylori eradication on the incidence of reflux esophagitis. In the Cox's proportional hazards model, the male sex and hiatus hernia were significant risk factors for the development of reflux esophagitis. Forty eight $(80.0 \%)$ of the 60 patients with pre-existing reflux esophagitis had successful H.pylori eradication therapy, and $12(20.0 \%)$ patients had treatment failure. The grade of reflux esophagitis worsened in $8(16.7 \%)$ of the 48 patients who had successful therapy and in $1(8.3 \%)$ of the 12 patients with persistent infection. Reflux esophagitis was improved in $22(45.8 \%)$ of the former 48 patients and $5(41.7 \%)$ of the latter 12 patients. There was no significant difference between the patients cured of infection and the patients with persistent infection in the change of the grade of pre-existing reflux esophagitis. In the Cox's proportional hazards model, none of the items mentioned above had a significant influence on the worsening or improvement of pre-existing reflux esophagitis.Conclusion The incidence of reflux esophagitis tended to be higher among the patients who had successfu H.pylori eradication therapy. The male sex and existence of hiatus hernia were significant risk factors for the development of reflux esophagitis.

\section{M2038}

Is Eradication of H. Pylori Infection Ever Followed By GERD? A Meta-Analysis Mohammad Yaghoobi, Yuhong Yuan, Richard H. Hun

Background: Some studies suggest that eradication of $H$. pylori (Hp) might cause reflux symptoms or erosive esophagitis (EE) in a proportion of patients. We aimed to investigate whether eradication of $\mathrm{Hp}$ infection is associated with the development of erosive esophagitis. Methods: A comprehensive English literature search was performed using PubMed from 1983 to August 2005 based on the keywords: H pylori, (o)esophagitis, reflux, heartburn, peptic ulcer, proton pump inhibitor (and individual commercial names of PPIs). 438 potential articles were reviewed. Only RCTs and prospective cohort studies comparing the prevalence of heartburn or erosive esophagitis (free from EE at baseline) in patients with Hp eradication versus those with persisted Hp were included. Only ITT data was used. Quality of studies was assessed by Jadad score. Meta-analysis of pooled odds ratio (fixed model) was performed using Review Manager 4.2. Results: Four RCTs $(n=1594)$ and four cohort studies $(n=1194)$ in patients with duodenal ulcer (DU) as well as 3 RCTs $(n=1405)$ and one cohort $(n=125)$ in dyspeptic patients using endoscopic diagnosis of $\mathrm{EE}$ as the endpoint were included. We also included 5 RCTs $(n=1422)$ in dyspeptic patients using development of heartburn as the endpoint. Test of heterogeneity was not significant for all following analyses. With metaanalysis of all RCTs, there was no association between Hp eradication and development of newly diagnosed GERD [OR=1.05 (CI: 0.89-1.23), P=0.57]. Similarly, there was no association in dyspeptic patients assessed either endoscopically [OR=0.96 (CI: 0.74-1.24), $\mathrm{P}=0.74$ or symptomatically [OR=1.03 (CI: 0.82-1.29), $\mathrm{P}=0.80]$. However, when analyzing studies in DU patients separately, the prevalence of EE in patients cured of $\mathrm{Hp}$ infection was consistently higher than in the control group in both cohort and RCT meta-analysis. There was no significant difference in length of follow-up between cohort studies and RCTs. In cohort studies, the average prevalence of new diagnosis of GERD (40.0\%) in patients with $\mathrm{Hp}$ eradication was significantly higher than in the control group $(16.4 \%)$ [OR=1.98 $(\mathrm{CI}$; $1.39-2.88), \mathrm{P}=0.0004]$. In RCTs, the rate was $6.3 \%(\mathrm{n}=55)$ in eradication group and numerically higher than the control group (3.4\%) [OR=1.58 (CI; 0.96-2.61), P=0.07]. Conclusion: There is no association between Hp eradication and development of new cases of GERD in the population of dyspeptic patients. However, in cohort studies, there appears to be a risk of development of EE by 1.5-2.0 folds in patients cured of DU. The effect in RCTs increased but non-significantly and this could be partly due to the small number of EE cases in study groups

\section{M2039}

Relevance of Liquid-Gas and of Weakly Acidic Reflux in Non Erosive Reflux Disease

Sara Emerenziani, Xin Zhang, Mentore Ribolsi, Renato Caviglia, Michele Pier Luca Guarino, Daniel Sifrim, Michele Cicala

The mechanism underlying symptoms in patients with GERD, particularly in non erosive reflux disease (NERD), is not completely known. Weakly acidic reflux and presence of gas in the refluxate could be relevant in the pathogenesis of NERD and it has been reported that balloon distension of the oesophagus may induce symptoms of heartburn and chest pain. AIM of this study was to assess the symptom association of weakly acidic, acid and mixed (liquid + gas) reflux, in NERD patients compared to a group of erosive reflux disease (ERD) patients. METHODS: Following a 3-week washout from PPI or H2 antagonists, 24hour ambulatory esophageal $\mathrm{pH}$-impedance monitoring was performed in $22 \mathrm{NERD}$ patients, without history of erosive disease, and in 20 ERD patients (grade I $n=12$, grade II $n=8$ ). Upper endoscopy was performed in all patients 3 to 11 days before pH-impedance. Reflux was classified as acid (pH drop below 4), weakly acidic (nadir pH between 4 and 7 ) and weakly alkaline (impedance drop without $\mathrm{pH}$ change below 7). Pure liquid reflux and mixed reflux were defined according to published criteria. Heartburn and acid regurgitation were considered in the analysis of symptoms. RESULTS: Weakly acidic refluxes were about $30 \%$ of all refluxes in patients and nearly 2/3 occurred in post-prandial periods. Gas was present in $40-50 \%$ of reflux in all study groups. 19 NERD patients reported 231 symptoms during the study day [mean 9 (range 1-23)], 13 ERD patients reported 72 symptoms [4 (1-16)] Out of the symptoms, heartburn was reported in nearly $70 \%$, regurgitation in nearly $30 \%$ of cases. In NERD patients, of all symptomatic refluxes ( $n=150), 17 \%$ were weakly acidic whereas $83 \%$ were acid (vs 6\% weakly, 94\% acid in ERD patients). Multivariate logistic analysis showed that the risk of reflux perception was very high when gas was present in the refluxate (odds ratio: $1.7 ; 95 \%$ confidence interval: $1.1-2.8, \mathrm{p}<0.01$ ), being maximal in patients ( $\mathrm{n}=7$ ) with normal acid exposure time (odds ratio: $2.5 ; 95 \%$ confidence interval: 1-6.1 vs 1.2; 95\% CI: $0.7-2$, p:ns in ERD patients). CONCLUSIONS: In all patients, the great majority of symptoms are related to acid reflux. In NERD patients, presence of gas in the refluxate significantly increases probability of reflux perception. NERD patients are more sensitive to less acidic reflux than ERD patients. These findings could play a role in the reduced response to PPI treatment, frequently observed in these patients.

\section{M2040}

Is the Postprandial Acid Pocket At the Gastro-Esophageal Junction Associated with a Bile Pocket?

Raf Bisschops, Daniel Sifrim, Rita Vos, Jozef Janssens, Jan Tack

Gastro-oesophageal reflux (GOR) typically occurs postprandially and is more pronounced in the distal oesophagus. Fletcher et al (2001) have previously established that a pocket of highly acidic gastric juice is present at the gastro-oesophageal junction (GOJ) after intake of a solid meal in healthy volunteers. Bilitec ${ }^{\circledR}$ studies have established that GOR often consists of a mixture of acid and bile, and that duodeno-gastric bile reflux is a physiological postprandial event. It is unclear how refluxed bile is distributed in the stomach after a meal. The aim of the current study was to investigate whether the postprandial acid pocket is associated with a bile pocket in healthy volunteers. Methods: 16 healthy volunteers underwent dual $\mathrm{pH}$ electrode and Bilitec $\AA$ probe stepwise pull-through $(\mathrm{lcm} / \mathrm{min}$, LES-10 to $+5 \mathrm{~cm}$ ) to measure regional difference in $\mathrm{pH}$ and bile absorbance before and 10, 30 and 50 min after a liquid meal $(200 \mathrm{ml}$ Nutridrink $®, 300 \mathrm{Kcal})$. LES position was determined preprandially with pull-through manometry. Results: Preprandial intragastric $\mathrm{pH}$ was significantly lower than the oesophageal $\mathrm{pH}(1.89 \pm 0.09$ vs $5.26 \pm 0.22, \mathrm{p}<0.0001)$. After the meal $\mathrm{pH}$ in the gastric body was increased to $5.18 \pm 0.39(\mathrm{p}<0.001)$. During pull-through, a significant $\mathrm{pH}$ drop of on average 1.8 units $(\mathrm{p}<0.05$ ), was present between LES-2 and $+2 \mathrm{~cm}, 10$ minutes after the meal. A similar pattern was found after 30 and 50 mins with a significantly lower $\mathrm{pH}$ at LES-lcm and at the level of the LES (pH drop 1.26 and 1.45 resp., $p<0.05)$. The averaged preprandial Bilitec $®$ pull-throughs $(n=14 ; 2$ failures $)$ did not show a significant variance in the proximal stomach. Postprandial gastric bile content was elevated compared to preprandially (absorbance $0.08 \pm 0.002$ vs. $0.11 \pm 0.003, \mathrm{p}<0.0001$ ), and Bilitec $®$ tracings displayed a small but non-significant absorbance drop at the level of the acid pocket. Analyses of individual tracings showed the presence of a "bile pocket" (segmentary rise in Bilitec $®$ absorbance $>0.14)$ on 11 of the $42(26 \%)$ successful Bilitec $®$ pull-throughs in 8 subjects (57\%).The majority of the "bile pockets" (7/11) were situated $2 \mathrm{~cm}$ distal to the acid pocket; $3 / 11$ coincided with the acid pocket and 1/11 was situated $2 \mathrm{~cm}$ proximal to the acid pocket. Conclusion: We confirmed the presence of a postprandial acid pocket at the GOJ in healthy volunteers. In half of the healthy subjects this was accompanied, at least transiently, by the presence of a "bile pocket". Studies in patients will be needed to confirm whether a synergistic action of acid and bile may contribute to the pathogenesis of GERD related distal oesophageal lesions. 
Impedance Monitoring (Imp) Shows That Body Position and Fasting Influence Reflux Composition

\section{Steven Shay}

Introduction: Imp is the only method that can determine if reflux composition is liquidonly, gas-only, or both liquid and gas. Aim: Assess with Imp the effect of posture and of a meal on reflux composition in different groups. Method: Nineteen patients with typical GERD symptoms either with $(\mathrm{n}=10)$ or without esophagitis $(\mathrm{n}=9)$ and 10 normal volunteers underwent Imp (6 sites: 3,5,7,9,15,17 cm above LES) simultaneous with manometry and $\mathrm{pH}(5 \mathrm{~cm}$ above LES). The 2-hr study consisted of three 40-min intervals, each 10-min left recumbent, 10-min right recumbent, and 20-min upright. One interval was fasting, and two intervals postprandial. Reflux events (RE) were defined by Imp as a retrograde fall (liquid) or rise (gas) in Imp $>50 \%$ from baseline in at least the two distal sites. Results: Reflux composition in the right recumbent posture was liquid-only near exclusively (93\%). In stark contrast, $85 \%$ of RE's in the left recumbent posture and $74 \%$ of RE's in the upright posture were gas-only or both liquid and gas (see figure, p<.001). There were more gasonly RE's fasting $(80 \%, \mathrm{p}<.01)$ vs postprandial as expected, though $26 / 30$ fasting RE's in the right recumbent posture were liquid-only. The proportion of RE's that were liquid-only, gas-only, and both liquid and gas was similar in the 3 study groups and the two postprandial intervals (all p>.25). Conclusions: 1) In contrast to the left and upright postures, RE's in the right recumbent posture are nearly all liquid-only even fasting 2) RE's fasting are more likely gas-only than postprandial RE's. Implication: Different posture and fasting times may partially explain variations in acid exposure during prolonged $\mathrm{pH}$ monitoring.

\section{Effect of Posture on Reflux Composition *}

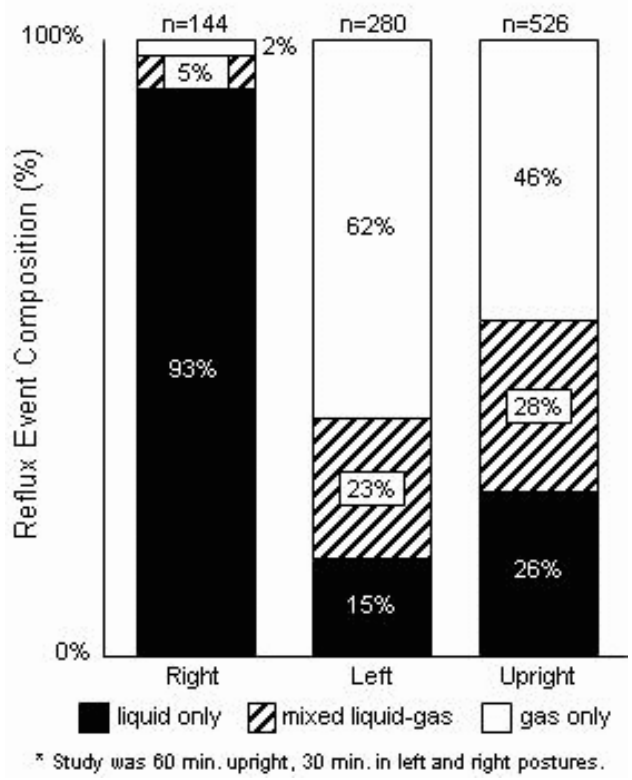

M2042

Facilitating Mechanisms for Gastroesophageal Reflux (GER): Evidences for Uneven Mixing of Gastric Juice and Food Content

Bermudez Jimena, Raul G. Yazigi, Pruzzo Rossana, Morales Barbara, Monica Pena

INTRODUCTION: GER is a frequent condition, not yet fully understood. Post prandial reflux may be an important symptom. There have been suggestions of an acid "pocket" in the cardial region and regional differences in postprandial acidity. Parietal cells exhibit Technetium (Tc-99m) uptake and later secrete it into the lumen. OBJECTIVES: To demonstrate that the mixture of swallowed food and gastric juice is not homogeneous and identify the area of higher concentration of postprandial acid. MATERIAL AND METHOD: After approval of the Ethics Committee a prospective SPECT study of 11 consenting healthy subjects ( 4 females, 7 males), mean age 27 years (19-35), using Tc-99 as a marker of parietal cell area and later of acid secretion. After an $8 \mathrm{hr}$. fast, Tc-99 was injected IV. Scans in the supine position were performed at 10,17 and 24 minutes later. (The scanner did not allow for scans in the upright position). The first scans define the gastric parietal cell area. Additional recordings register acid secretion in this same area. At the $17 \mathrm{th} \min .575 \mathrm{ml}$ of a liquid meal (ENSURETM Abbott Laboratories) was drank Two areas of interest were defined (proximal and mid third of the corpus of the stomach), quantifying the counts and pixels in each area. ANOVA and Student t test were used for statistical analysis. RESULTS: Both the anterior and posterior halves of the gastric proximal third in transaxial projections showed a statistically significant higher counts and pixels, with even higher activity in the anterior upper third; range $1.3-2.4(1.64 ; \mathrm{SD}=0.27)$. In the middle third no significant difference was found. The highest concentration of isotope (Tc-99m+acid secretion) was found in the lesser curvature. CONCLUSION: Uneven mixture of a liquid meal and an isotope in the supine position is demonstrated, confirming an acid pocket at the gastroesophageal junction. Solid meals and prone position should exaggerate the differences.
Shortening of the Abdominal Component of the Lower Oesophageal Sphincter Following a Meal

Jonathan J. Manning, Angela Wirz, Kenneth E. McColl

Introduction: There is a high prevalence of intestinal metaplasia and inflammation of the gastric cardia in $H$. pylori negative healthy subjects. This may represent relaxation of the distal (abdominal) portion of the lower oesophageal sphincter (LOS) and consequently, gastric juice damaging the most distal oesophageal squamous mucosa. The LOS is under most strain following a large meal. Aim: To study the effect of a large meal on the LOS of healthy volunteers. Method: 15 healthy volunteers ( 8 male), all Helicobacter Pylori negative by breath test, attended fasted on 3 separate occasions. Initially, a gastro-oesophageal pH-pullthrough profile was performed. Baseline slow motorised pull-through (SMPT) manometry was then performed, during quiet respiration, with a standard water-perfused manometry system. The subject was then given a battered fish and chip meal, with a glass of still water to drink The SMPT was repeated 5 minutes following the meal. Recordings were analysed from the 4 distal manometry channels. Pooled data was analysed $(n=45)$, each subject acting as their own control. Distances quoted are means and are measured from the nares. Results: Following the meal the total length of the LOS reduced from $4.94 \mathrm{~cm}$ to $3.48 \mathrm{~cm}(\mathrm{p}<0.0001)$. The upper border position did not change. The respiratory inversion point (RIP) elevated from $44.76 \mathrm{~cm}$ to $44.17 \mathrm{~cm}(\mathrm{p}<0.0001)$. The lower border of the LOS elevated from $47.63 \mathrm{~cm}$ to $45.98 \mathrm{~cm}(\mathrm{p}<0.0001)$. The thoracic portion of the LOS reduced from $2.1 \mathrm{~cm}$ to $1.7 \mathrm{~cm}$ $(\mathrm{p}<0.0001)$. The abdominal portion of the LOS reduced from $2.87 \mathrm{~cm}$ to $1.80 \mathrm{~cm}(\mathrm{p}<0.0001)$ The maximum end expiratory pressure reduced from $24.5 \mathrm{mmHg}$ to $21.2 \mathrm{mmHg}(\mathrm{p}<0.04)$ and the intragastric pressure increased from a baseline of $0 \mathrm{mmHg}$ to $4.9 \mathrm{mmHg}(\mathrm{p}<0.00001)$ Discussion: This study demonstrates shortening of the LOS after a meal due to shortening of the abdominal sphincter. There is also a small but significant shortening of the thoracic sphincter. As there is no movement of the upper border of the LOS, excluding shortening of the oesophagus, but there is movement of the RIP proximally, this probably reflects a mild elevation of the diaphragm caused by gastric distension. At only $6 \mathrm{~mm}$ this is likely to be missed if studied by $\mathrm{x}$-rays and radio-opaque clips. Conclusion: Shortening of the abdominal segment of the LOS following a large fatty meal will predispose to gastric acid reaching and damaging the squamocolumnar junction. This may explain the high prevalence of carditis and intestinal metaplasia at this anatomical site.

\section{M2044}

Gastroesophageal Reflux Disease, But Not Helicobacter Pylori Infection Leads to Major Changes of Lectin Staining Patterns in Chronically Inflamed Cardiac Mucosa

Helmut Neumann, Thomas Wex, Michael Vieth, Ulrich Peitz, Albert Roessner, Peter Malfertheiner

Background: Lectins are carbohydrate binding proteins which are ubiquitously expressed in nature. Commonly, plant and invertebrate lectins specifically bind defined carbohydrate structures, and therefore are commonly used as glycohistochemical tools for the analysis of normal and altered structures of glycans in histology and pathology. Aim: To investigate the staining patterns of the lectins UEA-I (binding to alpha-L-Fuc), DBA (alpha-D-GalNAc), HPA (D-GalNAc) and PNA (D-galactosyl-N-acetylgalactosamine) in cardiac and esophageal mucosa in context to the existence of gastroesophageal reflux disease (GERD) and Helicobacter pylori (HP) infection. Methods: In total, about 300 consecutive patients were initially investigated by endoscopy and characterized by histopathology and clinical aspects. The presence of autoimmune gastritis or corpus-predominant HP-gastritis were exclusion criteria. After complete clinical work-up and an unequivocal presence of HP-infection or GERD without Barrett's mucosa, 87 patients were stratified into four subgroups concerning both etiologies Furthermore, 33 unrelated GERD patients with histologically proven Barrett's esophagus (GERD+BE) were investigated. The staining pattern of lectins was assessed by immunohistochemistry in paraffin-embedded samples of cardiac and esophageal mucosa. Stainings were semiquantitatively evaluated using the Remmele score. Primary data were statistically analyzed by non-parametric Mann-Whitney U test. Results: UEA-I binding was increased in the stratum superficiale of GERD+BE compared to those with GERD $(\mathrm{P}<0.02)$, whereas the staining of all other lectins were unchanged in squamous epithelium among all subgroups. Major differences were identified for the binding of lectins to basal and superficial glands of cardiac mucosa in patients with GERD and GERD+BE compared to patients with HP or controls. HPA binding was increased in basal glands $(\mathrm{P}<0.02)$, whereas UEA-I and PNA staining of this compartment was decreased $(\mathrm{P}<0.001, \mathrm{P}<0.04$, respectively). In addition, superficial glands of cardiac mucosa revealed a decreased binding of DPA $(<0.001)$ and UEA-I $(\mathrm{P}<0.001)$. Conclusion: In general, GERD (with and without Barrett's mucosa) is associated with major changes in the lectin binding to cardiac mucosa implying molecular differences in glycosylation patterns or in the expression of glycoproteins. Since HP infection did not affect lectin staining in cardiac samples, the lectins PNA, DBA, UEA-I and HPA might be useful for differentiating histological changes in chronically inflamed carditis with respect to both etiological factors (HP-infection and GERD.

\section{M2045}

Role of Aracidonic Acid Metabolism in Rat Acid Reflux Esophagitis Tsuyoshi Hayakawa, Yasuhiro Fujiwara, Masaki Hamaguchi, Takashi Sugawa, Masatsugu Okuyama, E.J. Sasaki, Toshio Watanabe, Kazunari Tominaga, Nobuhide Oshitani, Kazuhide Higuchi, Tetsuo Arakawa

Background: Eicosanoids, products of aracidonic acid metabolism play roles in various inflammatory events. Their roles in pathogenesis of gastroesophageal reflux disease are unknown. Aims: We examined dynamics of cyclooxygenase (COX), prostaglandin E synthase (PGES), 5-Lypoxygenase (LOX), Leukotriene (LT) A4 hydrase and, synthetic activity of PGE2 and LTB4 to determine aracidonic acid's metabolism in rat acid reflux esophagitis. Methods Acid reflux esophagitis was induced in 9 week-old Wistar rats by ligating the transitional region between the forestomach and the grandular portion and wrapping the duodenum

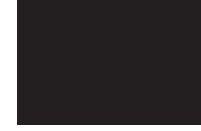


near the pylorus. Rats were killed on day 3 (acute phase) and day 21 (chronic phase) after induction of esophagitis. Esophageal proliferation was assessed by PCNA staining. Protein and mRNA expression by western blotting or immunohistochemistry and real-time RT-PCR. LTB4 and PGE2 synthesis were assessed by EIA. To determine role of COX-2 in esophagitis, rats were given subcutaneouly with celecoxib or nimesulide (10 mg/kg/day). Results: Expression of COX-2, microsoml PGES (mPGES)-1 was markedly increased in esophagitis, while modest changes in COX-1, cytosolic PGES (CPGES), mPGES-2 expression were observed. COX-2 and mPGES-1 were co-localized in epithelial cells of basal layer, and inflammatory and mesenchymal cells in lamina propria and submucosa. PGE2 synthesis of esophageal lesions was significantly increased. COX-2 inhibitors significantly reduced severity of chronic esophagitis but not affect acute esophageal lesions. COX-2 inhibitors significantly inhibited increase in PGE2 synthesis of esophageal lesions both on day 3 and day 21. Expression of 5-LOX and LTB4 synthesis were slightly increased in esophagitis. Epithelial proliferation on day 21 was significantly increased in the basal layer. Conclusion: Eicosanoid, especially PGE2 derived from COX-2 and mPGES-1, plays a significant role in pathogenesis of chronic acid reflux esophagitis.

\section{M2046}

A Study of Bile Exposure Immediately Above the Gastro-Oesophageal Junction in GERD Patients

Raf Bisschops, Daniel Sifrim, Toon de Greef, Rita Vos, Jozef Janssens, Jan Tack

Lesions in patients with gastro-oesophageal reflux disease (GERD) are thought to arise from a synergistic action of acid and duodeno-gastro-oesophageal reflux (DGER). Reflux is traditionally monitored $5 \mathrm{~cm}$ above the gastro-oesophageal junction (GOJ), but the majority of patients with erosive oesophagitis or Barrett's oesophagus display endoscopic lesions closer to the GOJ. Recently, an increased acid exposure and number of acid reflux events have been demonstrated $1 \mathrm{~cm}$ proximal to the GOJ in non-erosive GERD (Fletcher 2004). The aim of the current study was to investigate DGER exposure $1 \mathrm{~cm}$ proximal to the GOJ in suspected GERD. Methods: 18 consecutive patients with typical GERD symptoms underwent upper endoscopy followed by simultaneous Bilitec $₫$ and $\mathrm{pH}$ monitoring. The tip of the Bilitec probe was clipped $1 \mathrm{~cm}$ above the GOJ during endoscopy. A pH catheter was positioned conventionally $5 \mathrm{~cm}$ above the GOJ. Study patients were compared to an age-, sex- and endoscopy-matched control group who underwent conventional combined 24 hour $\mathrm{pH}$ and Bilitec monitoring at $5 \mathrm{~cm}$. Values are given as mean $\pm \mathrm{SEM}$ and compared with unpaired Student's t test or Mann-Whitney test. Results: In both groups, 3 patients had grade A oesophagitis and 1 had grade C (L.A.), and acid exposure time (3.4 4.0 vs. $6.0 \pm 2.3 \%$ of time, NS) and number of acid reflux episodes ( $48 \pm 13$ vs. $50 \pm 8$, NS) did not differ between study and control patients. For the entire patient group, DGER exposure time (absorbance $>0.14$ ) was similar at $1 \mathrm{~cm}$ or $5 \mathrm{~cm}$ above the GOJ (15.4 $\pm 5.8 \mathrm{vs} .15 .7 \pm 5.4 \%$ of time, NS), but the number of DGER episodes was significantly higher at $1 \mathrm{~cm}(53 \pm 14$ vs. $17 \pm 15, \mathrm{p}<0.05)$. Furthermore, in patients with pathological acid exposure at $5 \mathrm{~cm}$, a significantly higher number of DGER episodes was detected at $1 \mathrm{~cm}$ ( $87 \pm 36$ vs. $19 \pm 6.5$, $\mathrm{p}<0.05)$ and DGER exposure time tended to be higher $(28.2 \pm 13.8$ vs. $6.2 \pm 2.2 \%$ of time, $\mathrm{p}=0.07$ ). In patients without pathological acid exposure at $5 \mathrm{~cm}$, the difference in DGER episodes at 1 or $5 \mathrm{~cm}$ did not reach significance ( $40 \pm 13$ vs. $15 \pm 4$ respectively, NS). There was no significant difference in DGER exposure between erosive and non-erosive GERD. The difference in number of DGER episodes at $1 \mathrm{~cm}$ in patients with or without pathological acid exposure did not reach statistical significance $(87 \pm 36$ vs. $40 \pm 13$, NS). Conclusion: The distal oesophagus is exposed to significant high levels of bile reflux in patients with pathological acid exposure on conventional pH testing. The observations that both acid and bile reflux are more elevated close to the GOJ, is compatible with a synergistic action of acid and bile in the pathogenesis of GERD related distal oesophageal lesions.

\section{M2047}

Duodenogastro-Esophageal Reflux in Children with Refractory GastroEsophageal Reflux Disease

Ilse Hoffman, Toon de Greef, Nancy Haesendonck, Jan Tack

Duodenogastro-esophageal (DGE) reflux is involved in the pathogenesis of refractory reflux esophagitis in adults. The role DGE reflux plays in gastro-esophageal reflux disease (GERD) in children has not been addressed in depth. The aim of the study was to investigate acid and bile reflux in children with refractory GERD. Methods: Twenty two children (12 girls, mean age $13.2 \pm 0.4$ years) with GERD symptoms that persisted despite omeprazole (1 mg/ $\mathrm{kg}$ ) were investigated using upper gi endoscopy, $24 \mathrm{~h} \mathrm{pH}$ and bile reflux (Bilitec $($ ) monitoring, and a $13 \mathrm{C}$ octanoic acid gastric emptying breath test. Results: Patients presented mainly with epigastric pain (90\%), regurgitation (76\%) and nausea $(67 \%)$ followed by retrosternal pain (38\%), night-time pain (33\%) and anorexia (29\%). Pathological reflux was confirmed in $86 \%$ of patients. Endoscopy revealed persisting grade 1 esophagitis in 8 patients and grade 2 in 4 patients. Esophageal acid exposure and DGE reflux exposure were pathological in respectively 54 and $62 \%$ of the children. Acid exposure did not differ according to endoscopy findings $(4.5 \pm 0.8,7.4 \pm 3.7$ and $6.2 \pm 2.1 \%$ of time respectively in grade 0,1 and 2, NS). DGE reflux exposure increased with increasing severity of esophagitis $(9.6 \pm 1.9,9.1 \pm 5.3$ and $26.7 \pm 10.9 \%$ of time respectively in grade 0,1 and $2, p<0.05)$. Gastric emptying did not correlate with endoscopic or reflux monitoring results. Conclusions: Epigastric pain, nausea and regurgitation are the most common presenting symptoms of childhood with refractory GERD. DGE reflux seems to be an important contributor to the pathogenesis of refractory GERD and esophagitis in children.

\section{M2048}

Ecto-Enzymes Carbonic Anhydrase and Alkaline Phosphatase Coordinately Alter Rat Duodenal Bicarbonate Secretion

Yasutada Akiba, Misa Mizumori, Tetsu Takeuchi, Claudiu T. Supuran, Paul H. Guth, Eli Engel, Jonathan D. Kaunitz

Duodenal brush border membrane (BBM) has multiple highly-expressed ecto-enzymes, such as membrane carbonic anhydrase (CA) and intestinal alkaline phosphatase (IAP). The physiological function of these enzymes, despite their discovery decades ago, is incompletely understood. Since CA activity affects duodenal bicarbonate secretion (DBS), and since AP hydrolyzes organic phosphates at alkaline $\mathrm{pH}$, we hypothesized that these enzymes coregulate DBS. We examined the effect of $\mathrm{CA}$ and AP inhibitors on AP activity in rat BBM in situ and on DBS in vivo. BBM AP activity was measured in situ in duodenal frozen sections with the enzyme-linked fluorescent substrate ELF®-97 phosphate using fluorescence microscopy. Sections were reacted with ELF phosphate (0.1-0.5 mM) in pH 8.6 Tris buffer at $25^{\circ} \mathrm{C}$ for $5 \mathrm{~min}$. ELF fluorescence intensity was measured \pm IAP inhibitors L-cysteine, L-phenylalanine, or sodium tungstate (0.1-10 mM), or the tissue non-specific AP inhibitor levamisole (0.1-10 mM). We further examined the effects of high $\left(\mathrm{pH} 6.4, \mathrm{pCO}_{2}=206\right.$ $\mathrm{mmHg}$ ) or low ( $\mathrm{pH} 6.4$ Tris buffer, $\left.\mathrm{pCO}_{2} \approx 0\right) \mathrm{CO}_{2}$ solutions on AP activity \pm the cellpermeant $\mathrm{CA}$ inhibitor methazolamide $(1 \mathrm{mM})$ or novel cell-impermeant benzene sulfonamide CA inhibitors $(1 \mu \mathrm{M})$. DBS through duodenal loop was measured with a pH-stat or flow-through $\mathrm{pH}$ and $\mathrm{CO}_{2}$ electrodes. The loop was perfused with high $\mathrm{CO}_{2}$ solution $(\mathrm{pH}$ $6.4, \mathrm{pCO}_{2} 278 \mathrm{mmHg}$ ) or $\mathrm{pH} 2.2$ saline \pm cell-impermeant $\mathrm{CA}$ inhibitors $(0.1 \mu \mathrm{M})$ or $\mathrm{AP}$ inhibitors $(10 \mathrm{mM})$. ELF fluorescence appeared intensely on the BBM of villous cells, and was low intense in the mucus gel layer. Less activity was recognized in the crypt cells and no activity in Brunner's glands. IAP inhibitors dose-dependently inhibited ELF-based AP activity, whereas levamisole was less effective. Despite low $\mathrm{CO}_{2}$ solution decreased AP activity due to its $\mathrm{pH}$, the high $\mathrm{CO}_{2}$ solution enhanced BBM AP activity. Surprisingly, CA inhibitors inhibited the $\mathrm{CO}_{2}$-related augmentation of AP activity. Augmented DBS in response to perfusion of high $\mathrm{CO}_{2}$ or acid solution was reduced with methazolamide pretreatment, which inhibits cytosolic CA, whereas cell-impermeant CA inhibitors and AP inhibitors enhanced the DBS response. In vivo fluorescent microscopy revealed AP activity on the epithelial surfaces and in the mucus gel. ELF fluorescence enabled us to develop a simple in situ measurement assay of AP activity in the duodenal mucosa. Interaction between ectoCA and IAP suggests that these ecto-enzymes coordinately and negatively regulate DBS, which may affect juxtamucosal pH regulation. Regulation of DBS represents a novel and incompletely studied function of IAP.

\section{M2049}

Phosphodiesterase Isozymes Involved in Regulation of $\mathrm{HCO}_{3}{ }^{-}$Secretion in Mouse Duodenum in Vitro

Masamune Hayashi, Kazutomo Kita, Eitaro Aihara, Koji Takeuchi

Background and Aim: The secretion of $\mathrm{HCO}_{3}{ }^{-}$is one of the protective mechanisms in the duodenal mucosa against acid injury. Prostaglandins (PGs) and nitric oxide (NO) are known to stimulate duodenal $\mathrm{HCO}_{3}{ }^{-}$secretion both in vivo and in vitro, by increasing intracellular levels of cyclic nucleotides such as cAMP or cGMP, respectively. We reported previously that IBMX, the nonselective inhibitor of phosphodiesterase (PDE), potentiated the $\mathrm{HCO}_{3}$. response to $\mathrm{PGE}_{2}$, yet there is no information about the isozymes of PDE involved in this process. In the present study, we examined the effects of various isozyme-selective PDE inhibitors on $\mathrm{HCO}_{3}{ }^{-}$secretion in the mouse duodenum in vitro and investigated which type(s) of PDE isozymes are involved in the $\mathrm{HCO}_{3}{ }^{-}$response to $\mathrm{PGE}_{2}$ and NO. Methods: Male DDY mice were used. The duodenal mucosa was stripped of the muscle layers and mounted on an Ussing chamber. The tissue was bathed in saline gassed with $100 \% \mathrm{O}_{2}$ on the mucosal side and $\mathrm{HCO}_{3}{ }^{-}$Ringer's solution gassed with $95 \% \mathrm{O}_{2}-5 \% \mathrm{CO}_{2}$ on the serosal side. $\mathrm{HCO}_{3}{ }^{-}$secretion was measured at 7.0. using a pH-stat method and by adding $2 \mathrm{mM}$ $\mathrm{HCl}$. PGE $2\left(10^{-7} \sim 10^{-6} \mathrm{M}\right.$ ) or NOR3 (NO donor: $10^{-4} \sim 10^{-3} \mathrm{M}$ ) was added to the serosal solution. The EP4 antagonist (AE-3-208: $\left.10^{-6} \mathrm{M}\right)$ or methylene blue $\left(10^{-4} \mathrm{M}\right)$ was added to the serosal solution 10 min before $\mathrm{PGE}_{2}$ or 30 min before NOR3, respectively, while various PDE inhibitors $\left(10^{-7} \sim 10^{-4} \mathrm{M}\right)$ were applied to the serosal solution $30 \mathrm{~min}$ before. The expression of mRNAs of PDE isozymes was examined by RT-PCR. Results: Both PGE $\mathrm{P}_{2}$ and NOR3 increased the secretion of $\mathrm{HCO}_{3}{ }^{-}$in a dose-dependent manner, and the response to $\mathrm{PGE}_{2}$ or NOR3 was inhibited by the EP4 antagonist or methylene blue, respectively. IBMX, a nonselective PDE inhibitor, caused a significant increase of basal $\mathrm{HCO}_{3}{ }^{-}$secretion and potentiated the responses to both $\mathrm{PGE}_{2}$ and NOR3. Likewise, vinpocetine (PDE1 inhibitor) and cilostamide (PDE3 inhibitor), either alone at high doses, also stimulated the $\mathrm{HCO}_{3}$ secretion and significantly potentiated the response to $\mathrm{PGE}_{2}$ at the doses that had no effect by themselves on basal $\mathrm{HCO}_{3}{ }^{-}$secretion. The $\mathrm{HCO}_{3}{ }^{-}$stimulatory action of $\mathrm{NOR} 3$ was significantly potentiated by vinpocetine but not cilostamide. Other PDE inhibitors, including PDE2, PDE4 and PDE5, had no effect on the $\mathrm{HCO}_{3}{ }^{-}$secretion under both basal and stimulated conditions. RT-PCR analyses revealed the expression of PDEl and PDE3 mRNAs in the duodenal mucosa. Conclusion: These results suggest that both PDEl and PDE3 are involved in the regulation of duodenal $\mathrm{HCO}_{3}{ }^{-}$secretion and that the response to $\mathrm{PGE}_{2}$ is associated with both PDEl and PDE3 while the response to NO is mainly modulated by PDE1. 
M2050

Phosphodiesterase Isozymes Involved in Regulatory Mechanism of Gastric $\mathrm{HCO}_{3}{ }^{-}$Secretion

Yoko Nomura, Masamune Hayashi, Kazutomo Kita, Eitaro Aihara, Koji Takeuchi

Background and Aim: Gastric $\mathrm{HCO}_{3}{ }^{-}$secretion is stimulated by both prostaglandin $\mathrm{E}_{2}$

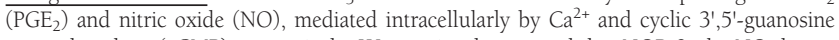
monophosphate (cGMP), respectively. We previously reported that NOR-3, the NO donor, increased $\mathrm{HCO}_{3}{ }^{-}$secretion in rat stomachs while forskolin the stimulator of adenylate cyclase or pituitary adenylate cyclase activating peptide did not, suggesting an involvement of cGMP in gastric $\mathrm{HCO}_{3}{ }^{-}$response. However, there is no information about the isozymes of phosphodiesterase (PDE) involved in this process. In the present study, we examined the effects of various isozyme-selective PDE inhibitors on the secretion of $\mathrm{HCO}_{3}{ }^{-}$in rat stomachs in vivo and mouse stomachs in vitro, and investigated which type(s) of PDE isozymes are involved in the $\mathrm{HCO}_{3}{ }^{-}$response to NO. Methods: Male SD rats and Male DDY mice were used. The rat stomach mounted on an ex-vivo chamber was perfused with saline, and $\mathrm{HCO}_{3}$ secretion was measured at pH 7.0 using a pH-stat method. NOR-3 (NO donor, $0.3 \sim 3 \mathrm{mg} /$ $\mathrm{ml}$ ) was given intraluminally while sildenafil $(1 \sim 10 \mathrm{mg} / \mathrm{kg}$, intraduodenally) the inhibitor of PDE5 was given 30 min before NOR-3. The mouse gastric mucosa was stripped of the muscle layers and mounted on a Ussing chamber, and the tissue was bathed in saline gassed with $100 \% \mathrm{O}_{2}$ on the mucosal side and $\mathrm{HCO}_{3}{ }^{-}$Ringer's solution gassed with $95 \% \mathrm{O}_{2}-5 \%$ $\mathrm{CO}_{2}$ on the serosal side. The $\mathrm{HCO}_{3}^{-}$secretion was measured at $\mathrm{pH} 7.0$ by a $\mathrm{pH}$-stat method using $2 \mathrm{mM} \mathrm{HCl}$. NOR-3 $\left(10^{-4} \sim 10^{-3} \mathrm{M}\right)$ was added to the serosal solution. Methylene blue $\left(10^{-4} \mathrm{M}\right)$ or various PDE inhibitors was added to the solution $30 \mathrm{~min}$ before NOR-3. The expression of mRNAs of PDE isozymes was examined by RT-PCR. Results: NOR-3 increased $\mathrm{HCO}_{3}{ }^{-}$secretion in a dose-dependent manner in both rat and mouse stomachs, and the response to NOR-3 was inhibited by methylene blue. Sildenafil, the PDE5 inhibitor, caused a significant increase of the secretion of $\mathrm{HCO}_{3}{ }^{-}$and potentiated the response to NOR-3 in rat stomachs. On the other hand, in the mouse stomach both vinpocetine (the PDEl inhibitor, $10^{-4} \mathrm{M}$ ) and zaprinast (the PDE5 inhibitor, $10^{-4} \mathrm{M}$ ), but not other PDE inhibitors including PDE2, PDE3 and PDE4, increased the $\mathrm{HCO}_{3}{ }^{-}$secretion and potentiated the response to NOR-3 at the dose that had no effect by itself on basal $\mathrm{HCO}_{3}{ }^{-}$secretion. RT-PCR analyses revealed the expression of PDEl and PDE5 mRNAs in the mouse gastric mucosa. Conclusion: These results confirmed that $\mathrm{NO}$ stimulates gastric $\mathrm{HCO}_{3}{ }^{-}$secretion mediated intracellularly by cGMP, and further suggested that this action is regulated by both PDEl and PDE5.

\section{M2051}

Hypoxia, Endothelial Dysfunction and Enhanced Apoptosis Are Key Mechanisms for Increased Susceptibility of Aging Gastric Mucosa to Ethanol Injury

Andrzej S. Tarnawski, Rama Pai, Tetsuya Tanigawa, Tomohiko Akahoshi, Jianyuan Chai, Aihua Li, Eric Chu

Aging gastric mucosa has increased susceptibility to injury and delayed injury healing. The molecular mechanisms of these phenomena are not fully explained. Methods: We examined abnormalities of aging (vs. young) gastric mucosa at baseline and following ethanol injury with focus on hypoxia, endothelial function and apoptosis-related factors. Studies: In 64 Fisher F344 rats, 3 months young and 24 months old (aging), non-treated (baseline) and 2, 8 and 24 hrs after intragastric ethanol administration ( $8 \mathrm{ml} / \mathrm{kg}$ of $50 \%)$ we studied: 1 ) mucosal histology and ultrastructure (TEM);2) blood flow with a laser-Doppler and mucosal hypoxia with specific hypoxy-probe; 3 ) apoptosis using TUNEL and apoptosis-related genes using Oligo-microarray; 4) expression of PTEN (negative regulator of PI 3-K/Akt pathway essential for cell survival), survivin (anti-apoptosis protein) and VEGF; 5) the extent of macroscopic and histologic mucosal injury and 6) angiogenesis following to injury by immunostaining and TEM. In in-vitro studies, we examined in human microvascular endothelial cells (HMVEC) derived from aging and young individuals, cells membrane permeability using LDH assay and cell ability to form tubes. We also examined expression of PTEN and survivin in gastric mucosa of patients older then 70 years and younger than 40 years. Results: Aging gastric mucosa at baseline exhibits reduced blood flow $(\sim 60 \%$; $<<0.002)$; prominent tissue hypoxia; increased Bax (2.9-fold), caspase-9 (3.6-fold), p53 (2-fold); decreased survivin (3-fold) and VEGF, and increased apoptosis (all $p<0.001$ vs. young rats). Ethanol administration increased gastric mucosal injury in aging rats by $\sim 3.5$-fold ( $p<0.001$ vs. young rats) and increased PTEN expression and apoptosis ( $\mathrm{p}<0.001)$. Injury of gastric microvascular endothelial cells preceded epithelial damage. At 24 hrs after ethanol injury in aging rats, angiogenesis in the mucosa bordering necrosis was reduced by 14 -fold; $p<0.001$ vs. young rats. Aged endothelial cells in vitro demonstrated increased LDH leakage and impaired tube formation. Gastric mucosa of patients $>70$ years of age exhibits a significant increase in PTEN and decrease in survivin expression vs. young patients. Conclusions: 1) Aging gastric mucosa exhibits at baseline significant hypoxia due to reduced blood flow, endothelial dysfunction and increased apoptosis; 2) HMVEC from aging patients exhibit membrane dysfunction, increased PTEN, reduced survivin and increased apoptosis; 3 ) In aging gastric mucosa alcohol-induced injury is increased by $350 \%$, endothelial cells of microvessels are the major targets of injury, and angiogenesis in response to injury is abolished.

\section{M2052}

Regulation of HCO3- Secretion in Esophageal Submucosal Glands Solange M. Abdulnour-Nakhoul, Nazih L. Nakhoul, Scott A. Wheeler, Roy C. Orlando

Introduction: The esophageal submucosal glands (SMG) secrete HCO3- and mucin into the esophageal lumen, which can contribute to esophageal protection against refluxed gastric acid. We have previously demonstrated that cholinergic receptor stimulation by carbachol almost doubles HCO3- secretion in these glands. This secretion is dependent on serosal $\mathrm{Cl}$ and is mediated by DIDS-sensitive transporters $\mathrm{Na}$ (HCO3-)n and Cl- - $\mathrm{HCO} 3-$, which we immunolocalized in the ducts and serous demi-lunes of the glands. This study was undertaken to further elucidate the pathways that govern $\mathrm{HCO} 3$ - secretion in this model. Methods: The experiments were done on pig esophagi stripped of their muscularis propria and bathed in HCO3- Ringer solution. HCO3- secretion was measured by cannulating the esophagus a both ends and recirculating unbuffered saline solution through the lumen to which a pH stat system was attached. Ion transporters were localized using specific antibodies and immunofluorescence technology. Results: Basal HCO3- secretion in these tissues ranged from 0 to $0.2 \mu \mathrm{Eq} / \mathrm{hr} . \mathrm{cm} 2$. Following serosal addition of forskolin, an adenylyl cyclase activator, and an activator of the luminal $\mathrm{Cl}$ - channel CFTR, HCO3- secretion increased from $0.03 \pm 0.007$ to $0.08 \pm 0.008 \mu \mathrm{Eq} / \mathrm{hr} . \mathrm{cm} 2(\mathrm{n}=6)$. Serosal addition of the phosphodiesterase inhibitor, 3-isobutyl-1-methylxanthine (IBMX), which stimulates both cAMP and CFTR also stimulated HCO3- secretion $(0.14 \pm 0.01$ to $0.22 \pm 0.01 \mu \mathrm{Eq} / \mathrm{hr} . \mathrm{cm} 2, \mathrm{n}=6)$. Genistein, an activator of CFTR, which does not increase intracellular cAMP, stimulated bicarbonate secretion from $0.016 \pm 0.001$ to $0.065 \pm 0.004 \mu \mathrm{Eq} /$ hour.cm2 ( $\mathrm{n}=7$ ). Addition of carbachol, following the use of forskolin, IBMX or genistein, resulted in no further stimulation of $\mathrm{HCO} 3-$ secretion. Immunofluorescence using an antibody to $\mathrm{Na}-\mathrm{K}-2 \mathrm{Cl}$ showed intense labeling of the basolateral membrane of mucous and duct cells. Conclusions: SMG HCO3secretion in pig esophagus is stimulated by elevation in intracellular cAMP. Na-K-2Cl, a likely mechanism for $\mathrm{Cl}$ - entry into the cell, is present at the basolateral membrane of mucous and duct cells. Compounds that activate CFTR and hence $\mathrm{Cl}$ - secretion cause similar effects on HCO3- secretion. Support: VA Merit grant

\section{M2053}

Phosphatidylinositol-3 Kinase Is Involved in Prostaglandin E2-Mediated Murine Duodenal Bicarbonate Secretion

Biguang Tuo, Ursula Seidler

Background \& Aims: Prostaglandin E2 (PGE2) plays an important role in regulation of duodenal mucosal bicarbonate (HCO3-) secrtetion. In the present study, we made investigation on signal pathways involved in PGE2-mediated duodenal HCO3- secretion. Methods Murine duodenal mucosal HCO3- secretion was examined in vitro in Ussing chambers by the $\mathrm{pH}$ stat in the presence of a variety of singal transduction modulators. Phosphatidylinositol-3 kinase (PI3K) activity was measured by immunoprecipitation of PI3K and ELISA. Results: PGE2 markedly stimulated duodenal HCO3- secretion. cAMP-dependent signaling pathway inhibitors MDL-12330A and KT5720 respectively reduced PGE2-stimulated HCO3- secretion by $22.99 \%$ and $19.54 \%$, and the Ca2+-influx inhibitor verapamil by $26 \%$, the calmodulin antagonist W-13 by $24 \%$, whereas the PI3K inhibitors wortmannin and LY294002 reduced PGE2-stimulated $\mathrm{HCO} 3$ - secretion by $51 \%$ and $47 \%$, respectively. Neither mitogen-activated protein kinase (MAPK) inhibitor PD98059 nor tyrosine kinase inhibitor genistein altered PGE2-stimulated HCO3- secretion. PGE2 application caused a rapid and concentrationdependent increase in duodenal mucosal PI3K activity. Conclusions: PGE2 stimulates duodenal mucosal HCO3- secretion via cAMP-, Ca2+-, and PI3K-dependent signaling pathways, and the PI3K pathway plays a major role in PGE2-mediated duodenal HCO3- secretion. This study has therefore revealed a novel signal transduction mechanism for the regulation of duodenal HCO3- secretion

\section{M2054}

Effect of Sparkling Water On $\mathrm{HCO}_{3}{ }^{-}$Secretion in Rat Stomach and Duodenum Yoko Sasaki, Eitaro Aihara, Yoko Nomura, Fumitake Ise, Koji Takeuchi

Background/Aim: The proximal duodenum is exposed to extreme elevation of $\mathrm{P}_{\mathrm{CO} 2}$ because of the continuous mixture of secreted $\mathrm{HCO}_{3}{ }^{-}$with gastric acid. Recent study showed that luminal $\mathrm{CO}_{2}$ diffused in the duodenal epithelial cells, followed by secretion of $\mathrm{HCO}_{3}^{-}$, the process being regulated by carbonic anhydrase and dependent on a basolateral $\mathrm{Na}^{+} / \mathrm{H}^{+}$ exchanger and endogenous prostaglandins (PGs). However, it remains unknown whether the same occurs in the stomach. In the present study, we examined the effect of sparkling water on $\mathrm{HCO}_{3}{ }^{-}$secretion in the rat stomach and duodenum and investigated factors involved in these responses. Methods: Male SD rats were used after $18 \mathrm{~h}$ fasting. Under urethane anesthesia, a chambered stomach or a duodenal loop was perfused with saline, and the $\mathrm{HCO}_{3}{ }^{-}$secretion was measured at $\mathrm{pH} 7.0$ using pH-stat method and by adding $2 \mathrm{mM} \mathrm{HCl}$ The mucosa was perfused with sparkling water (Suntory: pH. 4.5; 7.5 g/L CO 2 ) for $10 \mathrm{~min}$. Acetazolamide $(100 \mathrm{mg} / \mathrm{kg})$, indomethacin $(5 \mathrm{mg} / \mathrm{kg})$ or $\mathrm{N}^{\mathrm{G}}$-nitro-L-arginine methyl ester (L-NAME: 10 and $20 \mathrm{mg} / \mathrm{kg}$ ) was given SC $30 \mathrm{~min}$ or $3 \mathrm{~h}$ before the treatment while 5 (N,N-dimethyl)-amiloride (DMA:50 $\mu \mathrm{M}$ ) was add to the loop $40 \mathrm{~min}$ before. Ablation of capsaicin-sensitive afferent neurons was achieved by repeated SC injections of capsaicin (total dose of $100 \mathrm{mg} / \mathrm{kg}$ ) for 3 days. Effect of Coca-cola $\left(\mathrm{pH} 2.2 ; 7.1 \mathrm{~g} / \mathrm{L} \mathrm{CO}_{2}\right.$ ) on the secretion was also examined. Results: The mucosal perfusion with sparkling water markedly increased the secretion of $\mathrm{HCO}_{3}^{-}$in both the stomach and duodenum. The $\mathrm{HCO}_{3}{ }^{-}$response in the duodenum was partly inhibited by indomethacin, acetazolamide or sensory deafferentation, and totally abolished by co-administration of indomethacin and acetazolamide. In contrast, the $\mathrm{HCO}_{3}{ }^{-}$stimulatory action in the stomach was totally inhibited by acetazolamide but not by indomethacin or sensory deafferentation. DMA, an inhibitor of NHEl and NHE2, partially mitigated the $\mathrm{HCO}_{3}{ }^{-}$response to sparkling water in the duodenum but not the stomach. Similarly, Coca-cola ( $\mathrm{pH}$ 2.2, $\mathrm{g} / \mathrm{L} \mathrm{CO}_{2}$ ) also increased $\mathrm{HCO}_{3}{ }^{-}$secretion in both the stomach and duodenum, and the effects were significantly suppressed by indomethacin as well as acetazolamide. Conclusion: These results suggest that sparkling water induces $\mathrm{HCO}_{3}{ }^{-}$secretion in both the stomach and duodenum, but the mechanism differs between these two tissues; the response in the former is mainly due to the intracellular supply of $\mathrm{HCO}_{3}{ }^{-}$with the aid of carbonic anhydrase, while in the latter the response is mediated by both endogenous PGs and capsaicin-sensitive afferent neurons, probably related to the acidic $\mathrm{pH}$ of the solution, in addition to the intracellular supply of $\mathrm{HCO}_{3}$. 


\section{M2055}

Lafutidine, a Mucosal Protective $\mathrm{H}_{2}$ Receptor Antagonist, Prevents Acid-Pepsin Induced Interstitial Acidification in Rat Esophagus

Yasutada Akiba, Jonathan D. Kaunitz

Perfusion of acid in the rat esophageal lumen provokes a hyperemic response, implying that submucosal acid sensors are activated, although acid penetrates only superficially into the mucosa. Capsaicin-sensitive afferent nerve (CSAN) is involved in the acid-sensing mechanism in the duodenum and mediates capsaicin-induced hyperemia, one of important defenses in esophagus as well. Lafutidine is an $\mathrm{H}_{2}$ receptor antagonist possessing mucosal protective effects through sensitization of CSAN to acid, producing efferent responses that protect the mucosa from acid-induced injury in rat esophagus. Lafutidine, however, is not a direct agonist of acid-sensing TRPVl receptors. We thus hypothesized that lafutidine enhances the mucosal protective responses elicited by luminal acid in the esophagus by augmenting CSAN function. We examined the effects of lafutidine on the regulation of interstitial $\mathrm{pH}$ $\left(\mathrm{pH}_{\mathrm{int}}\right)$ and blood flow (BF) in response to luminal acid and on the acid-pepsin induced injury in rat esophagus using in vivo fluorescence microscopy. Under isoflurane anesthesia, the lower esophageal mucosa of rat was exposed and topically superfused with $\mathrm{pH} 7.0$ buffer, $\mathrm{pH} 1.0$ or $\mathrm{pH} 1.0$ pepsin $(1 \mathrm{mg} / \mathrm{ml})$ solution with or without lafutidine co-superfusion $(0.1-10 \mathrm{mM}) \cdot \mathrm{pH}_{\text {int }}$ was measured with 5,6-carboxyfluorescein $(5 \mathrm{mg} / \mathrm{kg})$ loaded into the interstitial space of the esophageal mucosa by bolus intravenous injection and BF with laser Doppler flowmeter. Lafutidine alone dose-dependently augmented $\mathrm{BF}$, and increased $\mathrm{pH}_{\text {int }}$. $\mathrm{pH} 1.0$ solution increased $\mathrm{BF}$, but did not change $\mathrm{pH}_{\mathrm{int}}$, whereas $\mathrm{pH} 1.0$ pepsin solution gradually lowered $\mathrm{pH}_{\text {int }}$, consistent with mucosal injury. During $\mathrm{pH} 1.0$ superfusion, $\mathrm{pH}_{\text {int }}$ was increased in the lafutidine-perfused rats, whereas sensory denervation using highdose capsaicin $(125 \mathrm{mg} / \mathrm{kg}, \mathrm{sc})$ pretreatment abolished the alkalinizing effect of lafutidine, acidifying the submucosa. Lafutidine also prevented interstitial acidification by $\mathrm{pH} 1.0$ pepsin solution and enhanced hyperemia in response to acid perfusion, the effect inhibited by sensory denervation. Lafutidine activated esophageal CSAN, producing hyperemia and interstitial alkalinization. The preventive effect of lafutidine on interstitial acidification by acid-pepsin solution through CSAN suggests that lafutidine enhances the defensive response via acid sensors on CSAN

\section{M2056}

Effects of Inhibition of Epithelial Ion Transport and Carbonic Anhydrase On the Regulation of Intracellular $\mathrm{pH}$ in Response to $\mathrm{CO}_{2}$ Perfusion in Mouse Duodenal Villous Cells

Tetsu Takeuchi, Yasutada Akiba, Misa Mizumori, Claudiu T. Supuran, Paul H. Guth, Eli Engel, Jonathan D. Kaunitz

Epithelial ion transporters and carbonic anhydrase (CA) participate in the regulation of intracellular $\mathrm{pH}\left(\mathrm{pH}_{\mathrm{i}}\right)$ in duodenal villous cells. Luminal acid or elevated luminal $\mathrm{pCO}_{2}$ $\left(\mathrm{CO}_{2}\right.$ stress) acidifies duodenal epithelial cells in rats. The $\mathrm{Na}^{+} / \mathrm{H}^{+}$exchange- 1 (NHE-1) inhibitor dimethyl amiloride (DMA) or the $\mathrm{Na}^{+}: \mathrm{HCO}_{3}{ }^{-}$cotransport (NBC) inhibitor DIDS enhances cellular acidification, whereas the CA inhibitors methazolamide (MTZ) or benzolamide (BNZ) abrogates acidification in rat duodenum. We examined the effects of these inhibitors on $\mathrm{pH}_{\mathrm{i}}$ regulation under $\mathrm{CO}_{2}$ stress in mouse duodenum. Mouse duodenal villous cells were exposed and loaded with a $\mathrm{pH}$-sensitive fluorescence dye $\mathrm{BCECF}$, and $\mathrm{pH}_{\mathrm{i}}$ was measured with in vivo fluorescent microscope. The duodenal mucosa was exposed to high $\mathrm{CO}_{2}$ solution (pH 6.4, $\mathrm{pCO}_{2} 260$ Torr) with or without DMA $(0.1 \mathrm{mM})$, DIDS $(0.5 \mathrm{mM})$, MTZ ( $1 \mathrm{mM}$ ), or novel cell-impermeant CA inhibitor positive-charged benzene sulfonamide compounds (1-10 uM). Furthermore, the expression of membrane-bound CAs (CA IV, IX, $\mathrm{XII}$ and XIV) was investigated in mouse and rat duodenum by immunofluorescence. Perfusion with the high $\mathrm{CO}_{2}$ solution $\left(\mathrm{CO}_{2}\right.$ stress $)$ rapidly acidified the cells, followed by $\mathrm{pH}_{\mathrm{i}}$ recovery over baseline (overshoot) after $\mathrm{CO}_{2}$ removal. DMA as well as DIDS enhanced $\mathrm{CO}_{2}$-induced $\mathrm{pH}_{\mathrm{i}}$ decrease, whereas DMA, not DIDS, delayed $\mathrm{pH}_{\mathrm{i}}$ recovery and abolished the overshoot. The cell-permeant CA inhibitor MTZ reduced the acidification during $\mathrm{CO}_{2}$ stress, but the cell-impermeant $\mathrm{CA}$ inhibitors had no effect on $\mathrm{pH}_{\mathrm{i}}$ response to $\mathrm{CO}_{2}$ stress. Immunofluorescence study revealed the basolateral expression of CA IX in mouse villous cells (mouse $=$ rat), and the apical, less expression of CA XIV (mouse $<<$ rat). Augmented acidification by DMA and DIDS confirms the involvement of the basolateral NHE-1 and NBC in the $\mathrm{pH}$ regulation in response to $\mathrm{CO}_{2}$ stress in mouse duodenal villous cells. The divergent actions of DMA and DIDS on $\mathrm{pH}_{\mathrm{i}}$ recovery and overshoot in mice, in comparison to their effects in the rat, suggest that $\mathrm{NHE}-\mathrm{l}$ is more important than is $\mathrm{NBC}$ for $\mathrm{pH}_{\mathrm{i}}$ regulation in response to $\mathrm{CO}_{2}$ stress in mice. The results that MTZ reduced, but cell-impermeant CA inhibitors had no effect on $\mathrm{pH}_{\mathrm{i}}$ response is consistent with the less expression of the apical $\mathrm{CA}$ in mouse villous cells. These data suggest a marked species difference between rat and mouse duodenum for $\mathrm{pH}_{\mathrm{i}}$ regulation by $\mathrm{CA}$ and by plasma membrane acid-base transporters.

\section{M2057}

Accumulation of the Anti-Apoptosis Protein, Survivin, in Gastric Epithelial Cells Following Exposure to Low-Dose Ethanol Is Mediated Via Increased Expression and Activation of Cyclin B1-P34 ${ }^{\mathrm{Cdc} 2}$ : Implications for Adaptive Cytoprotection Against Gastric Injury

Michael K. Jones, Oscar R. Padilla, Nicole A. Webb

BACKGROUND/AIMS: Survivin confers resistance to apoptosis and we have previously demonstrated a possible role for survivin in adaptive cytoprotection against ethanol (ETOH)induced gastric injury. It is postulated that survivin phosphorylation by the $\mathrm{p} 34^{\mathrm{cdc} 2}$ cell cycle-dependent kinase stabilizes it against degradation. This study was aimed to determine the mechanism by which mild irritant ETOH leads to increased survivin levels in the gastric epithelium and whether this is a key to adaptation against injury. METHODS: Rat gastric epithelial (RGMl) cells, pretreated with either vehicle alone, survivin antisense siRNA (to knockdown survivin protein levels), or control siRNA, were first incubated with serum-free medium alone (control) or serum-free medium containing $1 \% \mathrm{ETOH}$ and then incubated in medium containing $6 \%, 6.5 \%$ or $7 \%$ ETOH. STUDIES: 1) Total survivin, cyclin $\mathrm{Bl}$ and p34 cdc2 expression levels, and survivin and p34 cdc2 phosphorylation levels by Western blot; 2) apoptosis by TUNEL staining; 3) necrosis by MTT and LDH assay. RESULTS: Preincubation of RGMl cells in medium containing $1 \% \mathrm{ETOH}$ prior to incubation in medium containing 6-7\% ETOH resulted in increased expression levels of cyclin $\mathrm{Bl}$ and total p34cdc2 by $190 \%(P<0.05)$ and $85 \%(P<0.05)$, respectively, vs. controls. This treatment also resulted in reduced p34cdc2 phosphorylation (indicating an enhanced activation state) by $250 \%$ $(P<0.01)$, and increased survivin phosphorylation and total expression levels by $98 \%(P<0.05)$ and $170 \%(P<0.001)$, respectively, vs, controls. In contrast, these parameters were not significantly affected when cells were incubated in medium containing 6-7\% ETOH without prior exposure to $1 \%$ ETOH. Preincubation in medium containing $1 \%$ ETOH also reduced apoptosis and necrosis, in response to subsequent incubation in the presence of 6-7 \% ETOH by $83 \%(P<0.001)$ and $65 \%(P<0.01)$, respectively. The resistance to both apoptotic and necrotic cell death, as a result of $1 \%$ ETOH pretreatment, was significantly reduced $>73 \%(P<0.05)$ by survivin siRNA treatment. CONCLUSIONS: 1$)$ Pre-exposure of gastric epithelial cells to low-dose ETOH leads to activation of cyclin B1- p34 ${ }^{\text {cdc2 }}$ resulting in survivin accumulation via phosphorylation/stabilization. 2) Suppression of survivin attenuates the gastric adaptive cytoprotection provided by pre-exposure to low-dose ETOH against subsequent exposure to high-dose ETOH. 3) Activation of cyclin B1- p34 $4^{\text {cdc2 }}$ in response to low-dose ETOH likely mediates stabilization/accumulation of survivin resulting in increased resistance to apoptotic/necrotic cell death that underlies adaptive cytoprotection to the gastric epithelium. *NIH R01AAl4946

\section{M2058}

Ghrelin-Induced Gastric Secretory Activity and Gastroprotection Against Acute Gastric Lesions Involves Increased Expression of Histidine Decarboxylase (HDC) and Histamine Release

Tomasz Brzozowski, Peter C. Konturek, Zbigniew Sliwowski, Danuta Drozdowicz, Michal Pawlik, Greg Burnat, Stanislaw J. Konturek, Wieslaw W. Pawlik, Eckhart G. Hahn

Histamine and agonists of histamine $\mathrm{H}_{1}$ and $\mathrm{H}_{2}$ receptors were shown to afford gastroprotection against ethanol damage, predominantly due to enhancement in the protective mucus secretion and the formation of histodilutional barrier but whether histamine is implicated in gastroprotective action of ghrelin, an orexigenic peptide of gastric mucosa origin, remains unknown. We studied the effect of ghrelin applied intraperitoneally (i.p.) in a graded doses ranging from $2.5-40 \mu \mathrm{g} / \mathrm{kg}$ on gastric secretion in conscious rats equipped with gastric fistula (GF) and gastric mucosal lesions induced by $75 \%$ ethanol with or without: 1) inhibition of $\mathrm{H}_{2}$ receptors by ranitidine ( $30 \mathrm{mg} / \mathrm{kg}$ i.g.); 2) suppression of histidine decarboxylase (HDC) activity with $\alpha$-fluoromethylhistidine $(\alpha$-FMH), 3) subdiaphragmatic vagotomy, and 4 ) pretreatment with neurotoxic dose of capsaicin $(125 \mathrm{mg} / \mathrm{kg} \mathrm{s.c.}$ ) to induce inactivation of sensory nerves. The area of gastric lesions was measured by planimetry, the gastric blood flow $(\mathrm{GBF})$ was determined by $\mathrm{H}_{2}$-gas clearance technique and blood was withdrawn for the measurement of plasma ghrelin and histamine levels by specific radioimmunoassays (RIA) and the mucosal expression of ghrelin and HDC mRNA was evaluated by RT-PCR. Ghrelin dose-dependently increased gastric acid output in GF rats and this effect was completely abolished by ranitidine and significantly attenauted by $\alpha$-FMH, vagotomy and capsaicin denervation. Ethanol produced widespread mucosal lesions and this was accompanied by the gastric mucosal expression of mRNA for ghrelin and the increase in the plasma ghrelin levels. Pretreatment with exogenous ghrelin dose-dependently attenuated ethanolinduced gastric lesions; the dose reducing these lesions by $50 \%\left(\mathrm{ID}_{50}\right)$ was $20 \mu \mathrm{g} / \mathrm{kg}$. The protective effects of ghrelin was accompanied by the significant rise in the GBF (by 32\%) and the significant increase in plasma ghrelin and histamine levels. The ghrelin-induced protection and accompanying increase in the GBF were significantly reduced by pretreatment with ranitidine and $\alpha$-FMH and completely abolished by vagotomy and capsaicin-denervation. We conclude that 1 ) ghrelin increases gastric acid secretion via histamine release acting through $\mathrm{H}_{2}$ receptors and exhibits gastroprotective activity against acid-independent injury induced by strong irritant such as ethanol, and 2) this gastroprotective effect of ghrelin involves the enhancement in gastric microcirculation mediated by vagally-stimulated HDC activity and histamine release.

\section{M2059}

Profound Differences in the Rate of Secretion of Esophageal Protective Components Between Males and Females with Gastroesophageal Reflux Disease Could Be a Factor Contributing to Mucosal Injury and Complications Marek Majewski, Tomasz Jaworski, Irene Sarosiek, Kathy Roeser, Sandra Sostarich, Stanley Edlavitch, Grzegorz Wallner, Richard W. McCallum, Jerzy Sarosiek

The integrity of the esophageal mucosal depends upon equilibrium between aggressive factors and protective mechanisms (Sarosiek et al. J Pract Gastroenterol, 18:20J-202, 1994). Although males and females are equally affected by gastroesophageal reflux disease (GERD) symptomatology, females are less likely to develop its most severe complications such as Barrett's esophagus and esophageal adenocarcinoma. Little is know, however, if the rate of secretion of esophageal mucosal protective components, in response to aggressive factors is determined by gender. Purpose: To measure the esophageal mucosal secretory response in terms of protective components in GERD patients of both genders during the mucosal challenge mimicking the natural gastroesophageal reflux scenario. Methods: The study was approved by HSC at KUMC and conducted in 32 GERD patients (21F \& 11M; mean age of 42, 23-62 range). Subgroups of $M \& F$ were comparable in terms of mean age and GERD symptomatology. The esophageal secretions from submucosal mucous glands were collected, using a specially designed esophageal perfusion catheter (Wilson-Cook Med.), during mucosal exposure to initial $\mathrm{NaCl}$ followed by $\mathrm{HCl} /$ pepsin $(\mathrm{HClP})$ and final $\mathrm{NaCl}$ to mimic the natural gastroesophageal reflux scenario. In collected samples their volume and the content of bicarbonate and non-bicarbonate buffers, protein, mucin, EGF, TGF $\alpha$, and PGE2 were measured using commercial assays. Collected data were statistically analyzed using SPSS statistical software. Results: After the esophageal mucosal challenge with HClP, the rate of esophageal bicarbonate and non-bicarbonate secretion among females was $47 \%$ and $44 \%$ higher respectively $(\mathrm{P}=0.05)$ than in males. Esophageal mucin secretion among females was $150 \%$ higher and esophageal PGE2 secretion $257 \%$ higher $(\mathrm{P}<0.05)$ than in 
males. The rate of esophageal EGF secretion among males, however was 224\% higher $(\mathrm{P}<0.01)$ than in females. Conclusion: Profound differences in the rate of secretion of esophageal protective components after the mucosal challenge, mimicking the natural gastroesophageal reflux scenario, between males and females may at least partly explain the differences in the rate of development of mucosal injury and complications.

\section{M2060}

Tegaserod Stimulates Bicarbonate Secretion By Esophageal Submucosal Glands and Protects Against Acid-Pepsin Injury in a Pig Model

Solange M. Abdulnour-Nakhoul, Nelia Tobey, Ximena C. Vanegas, Scott A. Wheeler, Karen Brown, Roy C. Orlando

We assessed the effects of tegaserod, a 5-hydroxytryptamine-4(5HT4)agonist, on HCO3and mucin secretion by the esophageal submucosal glands (SMG). The experiments were done on pig esophagus whose muscularis propria was removed and which was bathed in HCO3- Ringer solution. The ends of the esophagus were cannulated for timed collections of instilled saline solution (mucin study) or for recirculation of an unbuffered saline solution attached to a pH-stat apparatus (HCO3- study). After baseline recordings, tegaserod, 0.25 $\mu \mathrm{M}-1 \mu \mathrm{M}$, or vehicle was added to the serosal solution and readings taken every $10 \mathrm{~min}$ for $1 \mathrm{hr}$. Following tegaserod, carbachol, $10 \mu \mathrm{M}$, was also added serosally and then atropine, 10uM. Results: Tegaserod increased HCO3-, but not mucin, secretion in a dose-dependent fashion, and at $1 \mu \mathrm{M}$, the increase in $\mathrm{HCO} 3-$ was $~ 33 \%$ greater than control $(0.15 \pm 0.05$ $\mu \mathrm{Eq} /$ hour.cm2 versus $0.21 \pm 0.05 \mu \mathrm{Eq} /$ hour.cm $2, \mathrm{n}=7, \mathrm{p}<0.005)$. Adding carbachol yielded no further increase in $\mathrm{HCO} 3$ - secretion while adding atropine abolished all secretion. Since serosal tegaserod stimulated SMG HCO3- secretion, it was also tested for a protective effect in esophagus against luminal acid-pepsin exposure ( $\mathrm{HCl}, \mathrm{pH} 2.5+$ pepsin $1 \mathrm{mg} / \mathrm{ml}$ ) for lhr. This was done in Ussing chambers using both SMG-bearing pig esophagus and, for comparison, SMG-free rabbit esophagus. Tegaserod had no effect on basal electrical data in either species; however following acid-pepsin washout, tegaserod treatment resulted in significantly higher resistances than controls for the pig, but not the rabbit, esophagus. Conclusions: Tegaserod stimulates HCO3-, but not mucin, secretion from esophageal SMGs, an effect mediated via a cholinergic pathway. Stimulation of SMG HCO3- secretion likely accounts for tegaserod's ability to protect SMG-bearing pig, but not SMG-free rabbit, esophagus from acid-pepsin injury. Supported in part by a grant from Novartis and by a VA Merit grant.

\section{M2061}

Lafutidine, a Mucosal Protective $\mathrm{H}_{2}$ Receptor Antagonist, Sensitizes the Mucosal Response to Luminal Acid in Rat Duodenum

Yasutada Akiba, Jonathan D. Kaunitz

Capsaicin-sensitive afferent nerves (CSAN) sense luminal acid and coordinate duodenal mucosal defense mechanisms, such as hyperemia and mucus secretion. Since lafutidine, an $\mathrm{H}_{2}$ receptor antagonist possessing CSAN-dependent mucosal protective effects, augments capsaicin-related calcitonin gene-related peptide release in rat stomach, we hypothesized that lafutidine upregulates mucosal defense responses to luminal acid in the duodenum. We thus examined the effects of lafutidine on the regulation of epithelial intracellular $\mathrm{pH}$ $\left(\mathrm{pH}_{\mathrm{i}}\right)$, blood flow $(\mathrm{BF})$ and mucus gel thickness $(\mathrm{MGT})$ in response to luminal acid in rat duodenum using in vivo fluorescence microscopy. Under isoflurane anesthesia, rat duodenal mucosa was exposed and topically superfused with $\mathrm{pH} 7.0,4.5,3.5$ or 2.2 solution with or without lafutidine co-superfusion $(1 \mathrm{mM}), \mathrm{pH}_{\mathrm{i}}$ was measured with pre-loaded BCECF, BF with laser Doppler flowmeter, and MGT with differential focal-plane measurements. Acid dose-dependently lowered $\mathrm{pH}_{\mathrm{i}}$, increased $\mathrm{BF}$ and MGT (Table). Lafutidine alone alkalinized the epithelial cells, augmenting BF and MGT increase. Co-perfusion of lafutidine with $\mathrm{pH}$ 2.2 solution reduced the acid-related decrease of $\mathrm{pH}_{\mathrm{i}}$, further increased $\mathrm{BF}$ and MGT. Deafferentation with high dose capsaicin (125 mg/kg, sc) abolished the lafutidine-related augmentation of these protective mechanisms, implicating the CSAN in lafutidine-mediated protection. Lafutidine reduced the intracellular acidification, and enhanced hyperemia and MGT increase induced by acid dose-dependently (Table). Lafutidine activated CSAN in duodenum, alkalinizing $\mathrm{pH}_{\mathrm{i}}$, a cellular signal preceding stimulated bicarbonate secretion and mucus secretion. Augmentation of protective mechanisms such as acid-induced hyperemia and mucus secretion suggests that lafutidine sensitizes CSAN to its endogenous ligand, $\mathrm{H}^{+}$.

\begin{tabular}{|c|c|c|c|c|c|}
\hline & Luminal $\mathrm{pH}$ & 7.0 & 4.5 & 3.5 & 2.2 \\
\hline \multirow{2}{*}{$\mathrm{pH}_{\mathrm{i}}$} & veh & $7.14 \pm 0.01$ & $6.95 \pm 0.05^{*}$ & $6.80 \pm 0.03^{*}$ & $6.57 \pm 0.05^{*}$ \\
\cline { 2 - 6 } & + laf & $7.24 \pm 0.02 \dagger$ & $7.23 \pm 0.07 \dagger$ & $7.04 \pm 0.06^{* \dagger}$ & $6.82 \pm 0.03^{*} \dagger$ \\
\hline \multirow{2}{*}{ BF (\%) basal) } & veh & $93.3 \pm 1.4$ & $95.5 \pm 4.8$ & $120.5 \pm 5.2^{*}$ & $136.4 \pm 9.3^{*}$ \\
\cline { 2 - 6 } & + laf & $118.2 \pm 2.9 \dagger$ & $123.8 \pm 9.9 \dagger$ & $138.4 \pm 8.3^{*} \dagger$ & $160.3 \pm 6.5^{*} \dagger$ \\
\hline \multirow{2}{*}{ MGT $(\mu \mathrm{m})$} & veh & $96.3 \pm 2.5$ & $98.0 \pm 5.1$ & $125.0 \pm 7.3 *$ & $177.8 \pm 16.3^{*}$ \\
\cline { 2 - 6 } & + laf & $139.9 \pm 6.4^{*}$ & $157.5 \pm 7.5^{*} \dagger$ & $171.5 \pm 6.7 * \dagger$ & $305.5 \pm 22.9 * \dagger$ \\
\hline
\end{tabular}

veh: vehicle, laf: lafutidine, ${ }^{*} \mathrm{p}<0.05$ vs $\mathrm{pH} 7.0$ group, $\uparrow \mathrm{p}<0.05$ vs veh group

\section{M2062}

Pathogenic Importance of Pepsin in Ischemia/Reperfusion-Induced Mucosal Injury in Rat Stomachs

Tohru Kotani, Atsushi Kobata, Kikuko Amagase, Koji Takeuchi

Background/Aim: Ischemia followed by reperfusion (I/R) leads to tissue injury. Although there is a substantial body of data characterizing the factors that promote gastric lesions under I/R-induced conditions, including superoxide radicals, the exact mechanism remains still unexplored. Gastric acid secretion is known to substantially decrease after I/R, yet the antisecretory agents reportedly had a protective effect on I/R-induced gastric lesions through the suppression of acid secretion. However, there is no information about the role of pepsin in the pathogenesis of I/R-induced gastric injury. In the present study, we focused on changes in pepsin secretion in rat stomachs during I/R-induced conditions and investigated the role of pepsin in the development of I/R-induced gastric injury. Methods: Male SD rats were used after $18 \mathrm{~h}$ fasting. Under urethane anesthesia, the pylorus was ligated, the celiac artery clamped, and $1 \mathrm{ml}$ of $100 \mathrm{mM} \mathrm{HCl}$ was instilled in the stomach. Reperfusion was established $15 \mathrm{~min}$ later by removal of the clamp, and after $2 \mathrm{~h}$ reperfusion period the stomach was examined for hemorrhagic lesions under a dissecting microscope. Pepstatin (a specific inhibitor of pepsin) or pepsin was given intraluminally after pylorus ligation. Cimetidine, omeprazole or atropine was given SC 30 min before ischemia. During I/R-induced conditions, both pepsin output and luminal acid loss were measured; the former was determined by the modified Anson method, while the latter was measured in presence of omeprazole. Results: $\mathrm{I} / \mathrm{R}$ produced hemorrhagic injury in the gastric mucosa in the presence of $\mathrm{HCl}$. The development of the I/R-induced gastric lesions was significantly prevented by atropine, yet neither omeprazole nor cimetidine had any effect. Intragastric administration of pepstatin also reduced the severity of I/R-induced gastric lesions in a dose-dependent manner, the effect being significant even at $0.3 \mathrm{mg} / \mathrm{kg}$, while that of pepsin markedly aggravated these lesions Of interest, the secretion of pepsin was significantly increased following $I / R$, and these changes were in parallel with the increase in luminal acid loss. In addition, the increased pepsin output during I/R-induced conditions was significantly inhibited by atropine but not cimetidine or omeprazole while pepstatin significantly inhibited the proteolytic activity of pepsin. Conclusion: These results suggest that 1 ) pepsin plays a pivotal role in the pathogenesis of $\mathrm{I} / \mathrm{R}$-induced gastric lesions, and 2) pepsin secretion is increased during I/R-induced conditions, the process being in association with acid back-diffusion and mediated through a cholinergic pathway.

\section{M2063}

Aggravation By Selective Serotonin RE-Uptake Inhibitors (SSRIs) of Antral Lesions Induced By Indomethacin in Rats: Effects of Antisecretory and Mucosal Protective Drugs

Akiko Tanaka, Yuka Takahira, Nahoko Izumi, Koji Takeuchi

Background/Aim: Recent clinical studies suggested a risk of gastric adverse reaction on the concomitant use of selective serotonin re-uptake inhibitors (SSRIs) with nonsteroidal antiinflammatory drugs (NSAIDs). In the present study, we used indomethacin as a conventional NSAID and investigated the adverse effect of SSRIs on gastric antral ulcers induced by indomethacin in rats and further investigated the effects of several antiulcer drugs on the occurrence of antral ulcers induced by NSAID and SSRI. Methods: Male SD rats were used. The animals fasted for $24 \mathrm{~h}$ were refed for $1 \mathrm{~h}$, and then administered indomethacin (30 $\mathrm{mg} / \mathrm{kg})$, dicrofenac $(30 \mathrm{mg} / \mathrm{kg})$ or flurbiprofen $(30 \mathrm{mg} / \mathrm{kg}) \mathrm{SC} 1 \mathrm{~h}$ after refeeding and killed $6 \mathrm{hr}$ later. Paroxetine $(1 \sim 10 \mathrm{mg} / \mathrm{kg})$ and other anti-depressants were given PO $30 \mathrm{~min}$ before indomethacin. Various serotonin (5-HT) receptor subtype-selective antagonists or severa antiulcer drugs were given SC or PO $1 \mathrm{hr}$ before indomethacin, respectively. Results: Indomethacin caused antral ulcers in re-fed rats. Paroxetine dose-dependently worsened the severity of antral ulcers in response to indomethacin, despite provoking by itself no damage the original ulcers induced by indomethacin were mostly non-hemorrhagic while they became hemorrhagic by co-administration of paroxetine. Similar results were obtained by proxetine when antral lesions were induced by other NSAIDs such as dicrofenac and flurbiprofen as well as SC-560 plus rofecoxib. This effect of paoxetine was mimicked by fluvoxsamine as well as milnacipran but not by imipramine and maprotiline. Exogenous $5-\mathrm{HT}(10 \mathrm{mg} / \mathrm{kg}$ SC) also worsened indomethacin-induced antral ulcers while the worsening effect of paroxetine was attenuated by ondansetron the selective 5-HT3 antagonist but not other subtype selective antagonists. The development of antral ulcers induced by indomethacin plus paroxetine was significantly prevented by omeprazole and atropine as well as pepstatin, although these agents, except pepstatin, had no effect on antral lesions induced by indomethacin alone. Likewise, the aggravating effect of paroxetine was significantly prevented by rebamipide and superoxide dismutase (SOD) but not by teprenon. Conclusion: These results suggest that SSRIs exert a harmful influence on the antral mucosa when given with NSAIDs, resulting in aggravation of antral ulcers, and 5-HT3 receptors may be involved in the aggravating action of SSRI. Rebamipide is useful for preventing such adverse effects in the antral mucosa caused by the combined administration of NSAID and SSRI, and this action may be partly accounted for by the scavenging action of superoxide radicals.

\section{M2064}

Bile Acids Induce a Shift Towards a TH2 Cytokine Profile in Barrett's Esophagus

L.M.G. Moons, P.D. Siersema, Z.M.A. Groothuismink, A.H.M. van Vliet, E.J. Kuipers, J.G. Kusters

Introduction: Barrett's esophagus (BE) is associated with a predominant humoral immunity (Th2). Analogous to other pro-inflammatory conditions predisposing for cancer, this development of a Th2 immune responses is associated with suppression of the cell-mediated immune response (Thl). Bile acids are known to suppress cell-mediated immunity in cholestatic liver disease and are thought to play an important role in the genesis of esophageal carcinogenesis. The latter specifically relates to deoxycholic acid (DCA) as this bile acid is present in increased concentrations in the bile pool of patients with BE. Aim: To determine whether DCA could suppress cell-mediated immunity in BE. Methods: Isolated human peripheral blood mononuclear cells (PBMCs) of 5 healthy controls were stimulated with $5 \mu \mathrm{g} / \mathrm{ml}$ Con A for 5 days in the presence of increasing concentrations of DCA $(0-100 \mu \mathrm{M})$. The effect on $\mathrm{T}$ cell responses was determined by measurement of proliferation (CFSE labelling), T cell activation (CD25/CD69), and IL-4, IFN- $\gamma$, IL-10, IL-2 cytokine production (ELISA \& RT-PCR). Induction of apoptosis was determined by FACS analyses of Caspase 3 and Annexin V. Cell viability was determined by 7-AAD. Results: $\mathrm{T}$ cell proliferation was decreased in a DCA concentration dependent manner, with a $1.4 \pm 0.1$ fold decrease at $100 \mu \mathrm{M}$ DCA as compared to untreated PBMCs $(\mathrm{p}<0.001)$. This reduced proliferation was not due to toxicity 
or induction of apoptosis, as there was no difference in cell viability (at all concentrations $>90 \%$ ), and Caspase 3 and Annexin V expression after 24 and 72 hours incubation between $100 \mu \mathrm{M}$ DCA and no DCA control. DCA was found to modulate activation of T cells, since T cells showed lower expression levels of the activation markers CD25 and CD69 (both $1.8 \pm 0.4$ fold $)$, lower levels of IL-2 mRNA ( $1.3 \pm 0.15$ fold; $\mathrm{p}=0.04)$ and IFN- $\gamma$ mRNA $(1.4 \pm 0.16$ fold; $\mathrm{p}=0.02)$, as well as lowered IL-2 protein levels $(5 \pm 1.2$ fold; $\mathrm{p}=0.03)$ at $100 \mu \mathrm{M}$ DCA versus untreated PBMCs. IL-4 mRNA levels were unaffected by treatment with DCA, whereas IL-4 protein levels were even increased at higher concentrations of DCA $(1.4 \pm 0.15$ fold $\mathrm{p}=$ 0.048 ) after 5 days of stimulation. IL-10 protein and mRNA levels were below detection limits. Conclusion: DCA exposure of PBMCs results in a concentration-dependent decrease of T cell proliferation, T cell activation, suppression of IL- 2 and IFN- $\gamma$ (Thl cytokines), and stimulation of IL-4 (Th2 cytokine) expression This indicates that DCA may be an important factor involved in suppression of cell-mediated immune responses in the esophagus, and may be involved in the observed shift towards a predominant humoral immune response in Barrett's esophagus.

\section{M2065}

Further Evidence for Involvement of Prostaglandin E Receptor Subtype 3 in Duodenal $\mathrm{HCO}_{3}^{-}$Secretion in Rats

Eitaro Aihara, Yoko Sasaki, Fumitaka Ise, Yoko Nomura, Koji Takeuchi

Background/Aim: Prostaglandins (PGs) play as a potent biological mediator in diverse physiological functions, including duodenal $\mathrm{HCO}_{3}^{-}$secretion. We previously reported that the $\mathrm{HCO}_{3}{ }^{-}$stimulatory action of $\mathrm{PGE}_{2}$ in the duodenum is mediated by the activation of both EP3 and EP4 receptors. However, these data were obtained only in EP3 receptor knockout mice and not confirmed pharmacologically. Thus, the involvement of EP3 receptors in duodenal $\mathrm{HCO}_{3}{ }^{-}$secretion remained pending. Recently, a selective EP3 receptor antagonist becomes available. In the present study, we examined using the specific EP3 agonist and antagonist the involvement of EP3 receptors in duodenal $\mathrm{HCO}_{3}{ }^{-}$secretion in rats and further confirmed a synergic phenomenon of the responses mediated by EP3 and EP4 receptors. Methods: Male SD rats were used after $18 \mathrm{~h}$ fasting. Under urethane anesthesia, a proximal duodenal loop was perfused with saline, and $\mathrm{HCO}_{3}{ }^{-}$secretion was measured at $\mathrm{pH} 7.0$ using a pH-stat method and by adding $5 \mathrm{mM} \mathrm{HCl}$. $\mathrm{PGE}_{2}(\mathrm{lmg} / \mathrm{kg})$, sulprostone (EPl+EP3 agonist, $1 \mathrm{mg} / \mathrm{kg}$ ) or AEl-329 (EP4 agonist, $3 \mu \mathrm{g} / \mathrm{kg}$ ) was given IV as a single injection. Mucosal acidification was performed by exposing the loop to $10 \mathrm{mM} \mathrm{HCl}$ for $10 \mathrm{~min}$. AE5599 (EP3 antagonist, 1 10 mg/kg, intraduodenally) or AE3-208 (EP4 antagonist, $1 \mathrm{mg} / \mathrm{kg}$, IV) was given $30 \mathrm{~min}$ or $5 \mathrm{~min}$, respectively, before the administration of $\mathrm{PGE}_{2}$ or Sul or the mucosal acidification. Results: Duodenal $\mathrm{HCO}_{3}{ }^{-}$secretion was stimulated by AEl-329 as well as sulprostone, the responses being equivalent to that induced by $\mathrm{PGE}_{2}$ at $1 \mathrm{mg} / \mathrm{kg}$ or mucosal acidification. The $\mathrm{HCO}_{3}{ }^{-}$stimulatory action of AE1-329 but not sulprostone was significantly attenuated by AE3-208. By contrast, AE5-599 dose-dependently inhibited the $\mathrm{HCO}_{3}{ }^{-}$response to sulprostone but not AEl-329. Both AE5-599 and AE3-208 partially but significantly mitigated the $\mathrm{HCO}_{3}{ }^{-}$responses to both $\mathrm{PGE}_{2}$ and mucosal acidification, and co-administration of these two agents completely blocked these responses. Duodenal damage caused by mucosal perfusion with $150 \mathrm{mM} \mathrm{HCl}$ for $4 \mathrm{~h}$ was worsened by pretreatment with the EP3 and EP4 antagonist as well as indomethacin and further aggravated by coadministration of these antagonists. In addition, neither the EP3 nor EP4 antagonist had any effect on gastric $\mathrm{HCO}_{3}{ }^{-}$response induced by $\mathrm{PGE}_{2}$ or mucosal acidification. Conclusion: These results suggest that 1) EP3 receptors are involved in the regulation of $\mathrm{HCO}_{3}{ }^{-}$secretion in rat duodenum, in addition to EP4 receptors, 2) the presence of both EP3 and EP4 receptors is essential for the full response of $\mathrm{HCO}_{3}{ }^{-}$secretion as well as the maintenance of mucosal integrity of the duodenum against acid injury.

\section{M2066}

Role of Glucocorticoid Hormones in Protective Influence of Preconditioning Mild Stress Against Stress-Induced Gastric Ulceration in Rats Ludmila Filaretova, Akiko Tanaka, Kozue Ishikawa, Tatiana Bagaeva, Koji Takeuchi

Background and Aim: It is known that preconditioning stress may attenuate stress-induced gastric injury and this effect is mediated by prostaglandings. Accordingly to our previous findings glucocorticoid hormones produced in a response to an acute stress are gastroprotective hormones, but not ulcerogenic ones as was generally accepted. This fact allows hypothesized the contribution of glucocorticoids to gastroprotective effect of preconditioning stress. The present study was designed to verify this hypothesis. Methods: Male SD rats weighing about $300 \mathrm{~g}$ were used after $18 \mathrm{~h}$ fasting. Rats were exposed to severe stress (cold-restraint stress at $10^{\circ} \mathrm{C}$ for $6 \mathrm{~h}$ ) with preconditioning mild stress (cold-restraint stress at $10^{\circ} \mathrm{C}$ for 30 min and restraint at room temperature for $60 \mathrm{~min}$ ) or without mild stress. The experiments were carried out: 1) in adrenalectomized rats (with or without corticosterone replacement) and in sham-adrenalectomized animals; 2) in medullectomized and sham-medullectomized animals; 3) in rats with occupation of glucocorticoid receptors by the antagonist RU-38486 and in control group. Adrenalectomy or medullectomy was performed one or three weeks, respectively, before stress. RU-38486 (20 mg/kg. PO) was administrated 30 min before mild stress. During experimental period, blood corticosterone and glucose levels as well as body temperature was monitored. At the end of each experiment, gastric erosions and myeloperoxidase (MPO) activity were measured. Results: Both mild and severe stress induced blood corticosterone and glucose rise and severe stress also produced gastric erosions. Mild stress decreased the gastric ulceration caused by severe stress and this effect was prevented by both adrenalectomy and occupation of glucocorticoid receptors but not medullectomy. An acute corticosterone replacement $(4 \mathrm{mg} / \mathrm{kg}, \mathrm{SC})$ mimicking the mild stress-induced corticosterone rise in adrenalectomized rats protected the gastric mucosa of these animals against the severe stress. Adrenalectomy resulted in dramatically decrease in body temperature and blood glucose levels during stress and the replacing corticosterone attenuated these effects. MPO activity was observed in gastric mucose of adrenalectomized rats. Conclusions: These results suggest that glucocorticoids released during preconditioning mild stress contribute to the protective effect of this stress on gastric mucosa against cold-restraint stressinduced gastric injury. The effect is functionally associated with prevention of lowering of glucose levels and body temperature. Manifestation of anti-inflammatory properties of glucocorticoids may contribute to their gastroprotective action

\section{M2067}

Mechanisms Underlying Compensatory Gastroprotective Action of Glucocorticoid Hormones During Desensitization of Capsaicin-Sensitive Sensory Neurons in Rats

Ludmila Filaretova, Peter Bobryshev, Tatiana Podvigina, Tatiana Bagaeva, Akiko Tanaka, Hanae Shiraishi, Koji Takeuch

Background and Aim: Capsaicin-sensitive sensory neurons (CSN) play a pivotal role in gastroprotection. Accordingly to our previous results glucocorticoid hormones also contribute to gastroprotection and may play a pivotal compensatory role in the maintenance of gastric mucosal integrity in the case of desensitization of CSN. The present study was designed to investigate mechanisms underlying compensatory gastroprotective action of glucocorticoids during desensitization of CSN. Methods: Male SD rats about $350 \mathrm{~g}$ were used after $18 \mathrm{~h}$ fasting. The effects of desensitization of CSN or adrenalectomy alone or their combination on indomethacin $(35 \mathrm{mg} / \mathrm{kg})$-induced gastric erosions, gastric microcirculation and mucus contents as well as blood glucose levels were investigated. Functional ablation of CSN was performed with neurotoxic dose of capsaicin (100 mg/kg SC, 3 days) two weeks before indomethacin administration. Adrenalectomy was created one week before indomethacin and followed by corticosterone replacement $(4 \mathrm{mg} / \mathrm{kg} \mathrm{SC}$ ). An in vivo microscopy technique for the direct visualization of gastric microcirculation and the analysis of red blood cell $(\mathrm{RBC})$ velocity was employed. For estimation of mucus production the hexose content was measured by the phenol-sulphuric acid method. Results: Indomethacin-induced gastric erosions were aggravated with similar extension by adrenalectomy or desensitization of CSN Desensitization of CSN profoundly aggravated the erosion formation in adrenalectomized rats without corticosterone replacement. Indomethacin slightly decreased RBC velocity and blood glucose levels in sham-adrenalectomized animals, but caused profound fall in both these parameters in adrenalectomized rats. Corticosterone replacement prevented these effects of adrenalectomy. Desensitization of CSN slightly potentiated the decrease in RBC velocity and did not significantly influence the blood glucose levels in sham-adrenalectomized rats. However, in adrenalectomized rats desensitization of CSN markedly potentiated indomethacin-induced falls in RBC velocity and in blood glucose levels. In adrenalectomized rats with corticosterone replacement the effects of desensitization of CSN on RBC velocity, blood glucose levels as well as gastric erosions were similar to those in sham-adrenalectomized rats. Conclusions: The results suggest that compensatory protective action of glucocorticoids against indomethacin-induced injury during desensitization of CSN is provided by their maintenance of gastric blood flow and glucose homeostasis. It is unlikely that gastric mucus production is involved in the mechanisms of this compensatory gastroprotective action.

\section{M2068}

Dual Influence of Portal Hypertension On Gastric Mucosal Defense Kazuhiro Fukushima, Takuya Endo, Yoshikazu Kubo, Shinichi Kato, Koji Takeuchi

Background/Aim: Portal hypertension, one of the most disastrous conditions related to chronic hepatic diseases, is known to be the major factor of pathogenesis of gastric abnormality. Indeed, the gastrointestinal bleeding is commonly observed in patients with liver cirrhosis. The purpose of this study was to investigate the change of gastric function in portal hypertensive rats. Methods: Male SD rats were used. Portal hypertension was produced by partial ligation of the portal vein, and 1 week later we carried out two experiments. First, animals were administered $\mathrm{HCl} /$ ethanol $\mathrm{PO}$, killed $1 \mathrm{~h}$ later, and the gastric ulcerogenic response was examined. Second, a rat stomach mounted in an ex vivo chamber was perfused with $100 \mathrm{mM} \mathrm{HCl}$ under urethane anesthesia, and transmucosal potential difference (PD) and gastric mucosal blood flow (GMBF) were measured before and after exposure of the mucosa to $20 \mathrm{mM}$ taurocholate (TC) for $30 \mathrm{~min}$. The expression of mRNA was examined by RTPCR. Levels of PGE 2 and NOx were measured by EIA and Griss reaction, respectively. Results: Portal hypertension by itself did not cause any damage in the stomach, and significantly decreased the mucosal susceptibility to $\mathrm{HCl} /$ ethanol-induced injury. Pre-treatment with L-NAME (a NOS inhibitor) reversed the protective effect of portal hypertension against $\mathrm{HCl} /$ ethanol. Aminoguanidine (a selective iNOS inhibitor) also mitigated the protective effect of portal hypertension, though the effect was much less as compared to L-NAME. Likewise, both indomethacin (a nonselective COX inhibitor) and rofecoxib (a selective COX-2 inhibitor) also restored the gastric ulcerogenic response to $\mathrm{HCl} /$ ethanol in portal hypertensive rats. On the other hand, mucosal application of TC caused a marked reduction in PD and an increase of GMBF. However, in portal hypertensive rats this treatment did not increase GMBF, despite causing a similar degree of PD reduction. Perfusion of TC-treated stomachs with $100 \mathrm{mM} \mathrm{HCl}$ caused only minimal damage in normal rats, yet produced gross lesions in portal hypertensive rats. Gastric $\mathrm{PGE}_{2}$ levels were significantly increased in portal hypertensive rats, in a rofecoxib inhibitable manner. Gastric NOx contents were also increased in portal hypertensive rats, in an aminoguanidine inhibitable manner. The expression of both COX-2 and iNOS mRNAs was up-regulated in the stomach of portal hypertensive rats. Conclusion: These results suggest that portal hypertension exerts a dual influence on the gastric mucosal defense, a protective effect mediated by PGs/COX-2 and NO/iNOS against $\mathrm{HC} / \mathrm{lethanol}$ damage and a deleterious effect on the damaged stomach through the impairment of GMBF response. 
M2069

Lansoprazole Protects Gastric Mucosa from H.pylori-Induced Oxidative Injury by Up-Regulating NRF2-Dependent Antioxidant Enzymes in Mice Akinori Yanaka, Ken Itoh, Hirofumi Matsui, Masafumi Tauchi, Hideo Suzuki, Takeshi Shibahara, Akira Nakahara, Ichinosuke Hyodo, Masayuki Yamamoto

Background: Lansoprazole (LPZ), a well known proton pump inhibitor, protects gastric mucosa from H.pylori (Hp)-induced gastric mucosal injury, presumably by its potent acid inhibitory action and/or by suppressing Hp activity. However, recent studies in ischemic colitis model have shown that LPZ protects intestinal mucosa from ischemia-reperfusion injury (World J Gastroenterol 2004;10:2814-7), suggesting that LPZ possesses an acidindependent antioxidant effect on intestinal mucosa. On the other hand, we have previously reported that nrf2 (NF-E2 p45-related factor-2), a transcription factor, which regulates cellular response against oxidative stress, plays an important role in activation of antioxidant enzymes in gastric mucosa during Hp infection (AGA meeting 2003, 2004). Based on these backgrounds, we hypothesized that, in addition to its acid inhibitory effects, LPZ up-regulates nrf2-dependent antioxidant enzymes, thereby affords gastric mucosal protection against oxidative stress during $\mathrm{Hp}$ infection. The present study was conducted to determine if LPZ stimulates antioxidant enzymes in Hp-infected gastric mucosa via nrf2-dependent mechanisms. Methods: Standardized mice model of $\mathrm{Hp}$ infection were set up by inoculating C57/ BL6 female mice (nrf2+/+ and nrf2-/-) with Hp Sydney Strain; SS1. Mice were maintained with high salt diet $(7.5 \% \mathrm{NaCl})$. Some mice were treated with $10 \mathrm{mg} / \mathrm{kg} /$ day LPZ. Mice were sacrificed at 4 wks later. Degree of gastritis was evaluated by updated Sydney system. DNA damage was estimated by measuring mucosal level of 8-OHdG. Expression of TNF$\alpha$, IL-1 $\beta$, COX-2 and HO- 1 (Heme Oxygenase-1) was analyzed by real time RT-PCR. Mucosal levels of PGE2, and 15-deoxy PGJ2 were measured using ELISA. Results: 1. In nrf2+/+ mice, treatment with $\mathrm{Hp}+$ high salt diet caused severe inflammation in corpus mucosa, accompanied by marked elevation of TNF- $\alpha$, IL- $1 \beta$, and $8-\mathrm{OHdG}$, while HO-1 expression was not prominent. 2. LPZ inhibited up-regulation of TNF- $\alpha$, IL-1 $\beta$, enhanced expression of HO-1, and mitigated corpus gastritis. 3. Treatment with NS-398 altered neither the protective effects of LPZ on corpus gastritis nor the expression of $\mathrm{HO}-1$, although it decreased expressions of COX-2, PGE2 and 15 dexy-PGJ2. 4. In nrf2-/- mice, stimulation of antioxidant enzymes by LPZ was completely abolished, and protective effect of LPZ on corpus mucosa against $\mathrm{Hp}+$ high salt was markedly attenuated. Conclusion: These results suggest that LPZ affords gastric mucosal protection against oxidative stress during Hp infection, at least in part by up-regulating nrf2-dependent, but not COX-2 dependent pathways.

\section{M2070}

Effect of Inflammatory Cytokines On the Expression of TFF1 (pS2) in Gastric Epithelial Cells

Tadahito Shimada, Takero Koike, Kyoko Tabei, Takashi Namatame, Akihiro Tajima, Masashi Yoneda, Hideyuki Hiraishi

Background and Aim: Trefoil factor family is a group of small peptides that play important roles in the defense of the gastrointestinal mucosa. Among TFF subtypes, TFFl (formerly pS2) is expressed at a high level in gastric epithelial cells. TFFl was originally discovered as an estrogen-inducible gene in MCF-7 breast cancer cells and TFFl expression is estrogendependent in MCF-7 and other hormone sensitive breast cancer cells. However. TFFl expression appears to be estrogen-independent in the stomach and the regulatory mechanisms of TFFl expression is not fully understood in gastric epithelial cells. In this study, we examined whether inflammatory cytokines (TNF-alpha and ILl-beta) affect TFFl expression in gastric epithelial cells. Methods: MKN45 and AGS, cell lines derived from human gastric cancers, were used. Endogenous TFFl mRNA expression was analyzed by real-time quantitative RT-PCR using ABI-PRISM 7700 sequence detection system. Beta-actin mRNA measurement was also performed for standardization. The promoter sequence of the human TFFl gene (-956 to +36) was cloned into pGL3-basic vector to make a TFFl reporter gene (TFFlLuc) and various mutant reporters were also made. In each reporter gene assay, phRL-TK vector was co-transfected for standardization. NF-kappaB activation was monitored by using a reporter vector that contained multiple copies of NF-kappaB responsive element upstream of the luciferase gene (NF-kappaB-Luc). Results: (1) TNF-alpha (10 ng/ml, 24 h-incubation) caused about 3-fold increase in the expression of endogenous TFFl mRNA in MKN45 and AGS cells. (2) Consistent with this, in reporter gene assays, TNF-alpha upregulated the transcription of TFFl-Luc in a dose-dependent manner. (3) IL-lbeta also showed similar stimulatory effect on endogenous TFFl mRNA expression and TFFl-Luc transcription. (4) TNF-alpha and IL-lbeta stimulated NF-kappaB activity in the cell types used in this study (5) TNF-alpha and IL-lbeta responsive element was mapped between -393 and -364 and between -256 and -216 from the transcription start site of the TFFl gene. Within the latter region, we found two potential NF-kappaB binding sites. Conclusion: These results suggest that inflammatory cytokines upregulate TFFl expression in gastric epithelial cells. NFkappaB pathway appears to be involved, at least in part. Upregulation of TFFl expression may benefit the protection of the gastric mucosa under inflammatory conditions.

\section{M2071}

Oral But Not Parenteral Aspirin Upregulates COX-2 Expression in Rat

Stomachs: A Causal Relationship Between COX-2 Expression and Prostaglandin Deficiency

Kaoru Nishikawa, Aya Yokota, Yuji Mashita, Masaki Taniguchi, Koji Takeuchi

Background/Aim: Nonsteroidal antiinflammatory drugs (NSAIDs) produce damage in the rat stomach, irrespective of the route of administration, yet aspirin (ASA) causes gastric lesions only when it is given orally. It is also known that NSAIDs up-regulate the expression of cyclooxygenase (COX)-2 in the stomach, and the inhibition of this enzyme is a key to their ulcerogenic action. In the present study, we compared the ulcerogenic effects of ASA and indomethacin in the rat gastric mucosa depending on the route of administration, together with the expression of COX-2, and investigated a causal relationship between COX2 expression and deficiency of prostaglandins (PGs). Methods: Male SD rats were used after
$18 \mathrm{~h}$ fasting. Animals were given ASA or indomethacin, either PO or SC, and the gastric mucosa was examined $4 \mathrm{~h}$ later. $\mathrm{PGE}_{2}$ contents were determined by EIA while COX mRNAs were examined by RT-PCR. Effects of these agents on gastric motility and transmucosa potential difference (PD) were also measured. Results: Indomethacin decreased the mucosal $\mathrm{PGE}_{2}$ level, increased gastric motility, and caused gastric lesions with the up-regulation of COX-2 expression, irrespective of the route of administration. ASA induced both damage and COX-2 expression in the stomach when given PO but not SC, despite decreasing the mucosal PGE 2 level similarly via either route of administration. Gastric motility was temporalily increased and gastric potential difference (PD) was markedly decreased by the administration of ASA PO. $\mathrm{PGE}_{2}$, although preventing ASA-induced gastric lesions as well as motility, affected neither the COX-2 expression nor PD reduction following the administration of ASA PO. By contrast, the COX-2 expression induced by indomethacin was prevented by both $\mathrm{PGE}_{2}$ and atropine at the dose that inhibited gastric hypermotility. In addition, SC 560 (a selective COX-1 inhibitor) but not rofecoxib (a selective COX-2 inhibitor) caused decrease in $\mathrm{PGE}_{2}$ content and an increase in gastric motility as well as COX-2 expression, without induction of any damage in the stomach. Conclusion: These results suggest that ASA given PO caused damage in the stomach, together with the up-regulation of COX-2 expression, and the up-regulated expression may be due to the topical irritative action (PD reduction), rather than being a result of PG deficiency. The expression of COX-2 after indomethacin treatment is associated with gastric hypermotility due to PG deficiency.

\section{M2072}

Involvement of Muscarinic Acetylcholine Receptors Subtype 4 in CarbacholInduced Acid and Pepsinogen Secretion in Mice

Takuya Endo, Takeshi Aihara, Kikuko Amagase, Minoru Matsui, Susumu Okabe, Koji Takeuchi

Background/Aim: Muscarinic acetylcholine receptors (mAChRs) consist of five subtypes ( $\mathrm{M}_{1}$

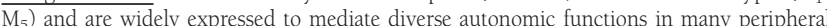
organs, including the gastrointestinal tract. It is considered that both $\mathrm{M}_{1}$ and $\mathrm{M}_{3}$ receptors play an important role in the regulation of gastric acid and pepsinogen secretion in response to acetylcholine. However, this idea is mainly derived from the data obtained by use of selective antagonist or those on their expressions in the tissue, and the precise role remains unclear. Especially, the data on pepsinogen secretion have mainly been obtained in-vitro but not in-vivo studies. In the present study, we investigated the roles of mAChRs subtypes in the cholinergic regulation of acid and pepsinogen secretion using muscarinic receptor knockout $(\mathrm{KO})$ mice and demonstrated the involvement of $\mathrm{M}_{4}$-receptors, in addition to $\mathrm{M}_{3-}$ and $\mathrm{M}_{5}$-receptors. Methods: C57BL/6J mice (12-16 wks old) of wild-type (WT) and $\mathrm{M}_{1-}$, $\mathrm{M}_{3^{-}}, \mathrm{M}_{4}-$ or $\mathrm{M}_{5}$-knockout $(\mathrm{KO})$ were used. Under urethane anesthesia, the abdomen was incised, the cardiac portion ligated, and an acute fistula prepared with a polyethylene tube was provided in the stomach through a pylorus. Then the stomach was instilled with saline $(0.4 \mathrm{ml})$ through the fistula, and the solution was changed every $20 \mathrm{~min}$. Acid secretion was measured by titrating the gastric content with $10 \mathrm{mM} \mathrm{NaOH}$. Carbachole $(30 \mu \mathrm{g} / \mathrm{kg}$ ) was given SC as a single injection. Atropine $(0.3 \mathrm{mg} / \mathrm{kg})$ was given SC $20 \mathrm{~min}$ before the administration of carbachol. Results: Carbachol caused an increase of both acid and pepsinogen secretions in WT mice, the effect being completely inhibited by prior administration of atropine. These acid and pepsinogen responses to carbachol were similarly observed in $\mathrm{M}_{1} \mathrm{KO}$ mice but significantly decreased in the animals lacking $\mathrm{M}_{3}$-receptors. Likewise, both the acid and pepsinogen secretions in response to carbachol were significantly impaired in $\mathrm{M}_{4} \mathrm{KO}$ mice as compared with WT mice; especially, the pepsinogen response was almost completely abolioshed. In $\mathrm{M}_{5} \mathrm{KO}$ mice, however, the pepsinogen response to carbachol was observed similar to WT mice, but the acid response was slightly reduced when compared to WT mice. Conclusion: These results suggest that under cholinergic stimulation the acid secretion is mediated mainly by the activation of both $\mathrm{M}_{3}$ - and $\mathrm{M}_{4}$-receptors and partly through $\mathrm{M}_{5}$-receptors but not $\mathrm{M}_{1}$-receptors, while the pepsinogen secretion is mediated mainly by the activation of $\mathrm{M}_{4}$-receptors and partly through $\mathrm{M}_{3}$-receptors but not $\mathrm{M}_{1}$-and $\mathrm{M}_{5}$-receptors.

\section{M2073}

Inhibitory Mechanism of Tenatoprazole in Vivo

Jai M. Shin, Michel Homerin, Florence Domagala, Herve Ficheux, George Sachs

Background: Tenatoprazole belongs to the class of proton pump inhibitors (PPIs). There is a surprisingly rapid rate of recovery of acid secretion after short or long term treatment with most of the PPIs except for pantoprazole and tenatoprazole. This has been ascribed to glutathione-dependent reversal of the inhibitory PPI-disulfide linkage in the luminal vestibule of the pump at cysteine 813 with the linkage of tenatoprazole at cysteine 822 being inaccessible and truly irreversible (Gastroenterology 123, 1588-97,2002 and Biochem Pharmacol, 2006 (in press)). These experiments were performed in vitro on the H,K-ATPase isolated from PPI inhibited rats and their relevance to the situation in the intact stomach needed to be established. Aims: To compare the binding sites and the stability of PPI binding to the gastric $\mathrm{H}, \mathrm{K}$ ATPase in vivo after stimulation of acid secretion in rats. Methods: Rats were injected with 14C-omeprazole or 14C-tenatoprazole after stimulation of acid secretion and the decay of radioactivity bound to the H,K-ATPase followed as a function of time after administration. Quantitative data reflecting stability of the PPI-pump complex were obtained using Western immunoblots of gradient-gel separated ATPase and determining specific radioactivity of the $100 \mathrm{kDa}$ band representing the target catalytic subunit. Results: The rate of loss of bound omeprazole from the pump in vivo was faster than expected from the 54 hr half-life of the protein whereas the rate of loss of tenatoprazole corresponded to the expected turnover of the ATPase. Conclusions: The results demonstrate that the PPI-cysteine 813 bond formed by omeprazole is accessible to glutathione in vivo and that reversal of inhibition by this mechanism plays a role in the rate of recovery of acid secretion in this species and probably also in people. In contrast the binding of tenatoprazole to cysteine 822 is not reversed in vivo as predicted from the ex vivo enzyme analyzed in vitro. These data would predict a longer duration of inhibition of acid secretion during tenatoprazole treatment as compared to omeprazole with also a major effect due to the long plasma halflife of tenatoprazole. 
Gastric Acid Secretion in Isolated Rat Gastric Glands Is Inhibited By Insulin Roman Schniepp, Philipp A. Lang, Philipp Kirchhoff, Basil Hirshowitz, John Geibel

Background Unregulated gastric acid secretion plays a major role in the pathophysiololgy of reflux diseases. Previous studies in diabetic patients demonstrated a reduced tendency to hypersecretory associated reflux disease, with in fact reduced basal and stimulated levels of acid secretion. However this is explained by loss of vagal function in these patients. In this study we chose to investigate the direct effects of insulin on secretagogue induced acid secretion. Methods We used a digital-imaging system to observe proton efflux from parietal cells. Gastric glands were isolated by a hand dissection technique, loaded with the $\mathrm{pH}$ sensitive dye BCECF, and acid extrusion was monitored in the absence of bicarbonate as intracellular alkalinization after removal of $\mathrm{Na}+$ and $\mathrm{K}+$ from the perfusate. Glands were stimulated with histamine $[100 \mathrm{\mu M}]$, carbachol $[100 \mathrm{\mu M}]$ or pentagastrin $[100 \mu \mathrm{M}]$ and rates of alkalinization were calculated following K+readdition to the perfusate $\pm(400 \mathrm{nM})$ insulin. Results We found that the potassium dependent recovery induced by histamine $(\mathrm{dpH} / \mathrm{dT}=$ $0.095 \pm 0.0075 \mathrm{pH} / \mathrm{min})$ or carbachol $(\mathrm{dpH} / \mathrm{dT} 0.101 \pm 0.0072 \mathrm{pH} / \mathrm{min})$, is decreased by insulin $(0.073 \pm 0.0078 \mathrm{pH} / \mathrm{min}),(0.068 \pm 0.0088 \mathrm{pH} / \mathrm{min})$ respectively, in rat gastric glands. There was no effect of insulin on the pentagastrin stimulated $\mathrm{K}+$ dependent alkalinization. (Arithmetic means \pm SEM). Conclusion This study demonstrates that insulin has a direct inhibitory effect on histamine or carbachol induced gastric acid secretion. However, insulin has no effect on pentagastrin stimulated acid secretion. These studies provide direct evidence that increased insulin levels, can reduce secretagogue dependent acid secretion. These observations may help to explain the reduced levels of acid related disease in diabetic patients and the reduction in both basal and secretagogue dependent acid secretion.

\section{M2075}

Identification of Rab27b in Gastric Parietal Cells Serhan Karvar, Lixin Zhu, James M. Crothers, John G. Forte

Acid secretion by parietal cells involves secretagogue-dependent recycling of the HK-ATPase. Rab proteins have been implicated as participants in membrane trafficking, docking, and fusing processes during the secretory cycle. Rabbit parietal cell tubulovesicle fractions were used to identify Rab proteins. Highly enriched HK-ATPase-containing tubulovesicle membranes were run on SDS-PAGE, followed by in-gel digestion and peptide analysis by mass spectrometry. Seven peptides were matched with Rab27b protein. We confirmed the Rab27b expression in highly purified tubulovesicle fractions by western blot. We also used cultured parietal cells to examine the cellular localization and stimulation-associated distribution dynamics of Rab27b. Parietal cells infected with adenoviral vectors containing Yellow Fluorescent Protein (YFP)-tagged wild type and mutant Rab27b were visualized by fluorescence microscopy. In resting parietal cells, YFP-Rab27b wild type and Rab27b Q78L constitutively active mutant were detected throughout the cytoplasm in a pattern that was similar to immunostaining for $\mathrm{H}, \mathrm{K}$-ATPase. After stimulation, YFP-Rab27b translocated to the apical plasma membrane. Dominant negative mutant Rab27b N133I was localized through the cytoplasm in resting and stimulated cells. Our results suggest that Rab27b is associated with tubulovesicle membranes in the parietal cell and Rab27b may play a role in stimulationassociated membrane recruitment and acid secretion.

\section{M2076}

Postprandial Gastric Acid Neutralization-Reacidification Measured with An Ingested pH Telemetry Capsule: Correlation with Emptying of Digestible and Indigestible Solids

William L. Hasler, Henry P. Parkman, Alan Hutson, William D. Chey, Kenneth L. Koch, Richard W. McCallum, John M. Wo, Braden Kuo, Michael Sitrin, Kevin Stevens, Beverly Landrigan, Jack R. Semler, Radoslav Coleski

Background: Gastric emptying profiles of digestible and indigestible solids exhibit distinct temporal characteristics which are proposed to relate to specific gastric motor patterns. Recently, an ingestible telemetry capsule was developed which continuously measures luminal $\mathrm{pH}$ and pressure (SmartPill Corp.). Using this method, indigestible solid emptying is measured by detecting the abrupt $\mathrm{pH}$ rise as the capsule passes into the duodenum. It is well known that gastric $\mathrm{pH}$ transiently neutralizes with solid meal ingestion and reacidifies with subsequent acid secretion and gastric emptying. Hypothesis: We hypothesized that intersubject differences in postprandial gastric $\mathrm{pH}$ profiles reflect differential rates of emptying of digestible solids. Methods: Data from 25 healthy volunteers and 45 patients with documented gastroparesis on prior scintigraphy from 7 centers were analyzed. Acid suppressants were discontinued for 1 week prior to study. Following capsule ingestion, subjects consumed standardized solid meals (120 g Egg Beater, $60 \mathrm{kcal}$; bread, $120 \mathrm{kcal}$; jam $74 \mathrm{kcal}$; and 120 $\mathrm{ml}$ water- $72 \%$ carbohydrate, $24 \%$ protein, $2 \%$ fat) with $1 \mathrm{mCi}{ }^{99} \mathrm{Tc}$-sulfur colloid tracers. Luminal pH measurements were obtained every $5 \mathrm{sec}$; scintiscans were acquired every 30 min for 4-6 h. Results: Meal ingestion elicited initial pH rises to a mean of $4.40 \pm 0.18$ followed by slow declines to stable $\mathrm{pH}$ nadirs of $0.93 \pm 0.12$ prior to capsule emptying. The time to empty $90 \%$ of the digestible radiolabelled meal on scintigraphy correlated modestly with the time needed to reduce gastric $\mathrm{pH}$ by $50 \%$ from the peak to the nadir values $(\mathrm{pHT} 50)(\mathrm{r}=0.47)$. $\mathrm{pHT} 50$ values correlated less well with duodenal passage of the indigestible capsule $(\mathrm{r}=0.30)$. In subjects with delayed scintigraphic emptying ( $<90 \%$ emptied at 240 $\mathrm{min})$, pHT50 values were significantly prolonged $(64.7 \pm 12.9 \mathrm{~min})$ vs. those with normal emptying ( $\geq 90 \%$ emptied at $240 \mathrm{~min})(25.8+2.2 \mathrm{~min})(\mathrm{P}=0.0001)$. The positive predictive value of a pHT50 value of $\geq 60 \mathrm{~min}$ for detection of delayed solid emptying defined scintigraphically was $89 \%$, whereas the negative predictive value was $78 \%$. Conclusions: Quantification of intragastric $\mathrm{pH}$ neutralization and subsequent reacidification recorded by an ingested telemetry capsule after consumption of a nutrient meal can provide a measure of gastric emptying of digestible solids which correlates with accepted scintigraphic methods and which complements the ability of the capsule to assess indigestible solid emptying. The capability to concurrently determine gastric emptying of both digestible and indigestible solids may be an important research and clinical tool in future investigations.
Arfgap-6 Is Essential for Acid Secretion in Gastric Parietal Cells Xia Ding, Zhen Guo, Zhiyou Fang, Fengsong Wang, Rihong Zhou, Xinwang Cao, John G. Forte, Xuebiao Yao

Stimulation of gastric acid secretion involves an activation of a cAMP-dependent protein kinase cascade that triggers the translocation and insertion of the proton pump enzyme, $\mathrm{H}, \mathrm{K}-\mathrm{ATPa} e$, into the apical plasma membrane of parietal cells. The stimulation-mediated relocation of the H,K-ATPase from the cytoplasmic membrane compartment to the apical plasma membrane is mediated by a small GTPases such as Rabll and ARF6. To further understand the role of ARF6 in parietal cell secretion, we searched for proteins in parietal cell extracts that bind the GTPase-deficient ARF6Q67L mutant but not wild-type ARF6. Mass spectrometric analysis of a $97 \mathrm{kDa}$ polypeptide specifically bound to ARF6Q67L led to the identification a 903 amino acid protein of unknown function. We named this protein as ARFGAP6 as our biochemical characterization demonstrated a PIP2-dependent GAP specific for ARF6. Immunocytochemical study revealed that ARFGAP-6 is mainly located to the apical membrane of parietal cell within the gastric glands. Our deletion analysis revealed that ARFGAP- 6 binds to ezrin via its N-terminus and is co-localized with ezrin to the apical canaliculi. The siRNA-mediated depletion of ARFGAP- 6 or a block of ARF6 GTP hydrolysis suppresses ARF6-dependent proton pump mobilization, suggesting that a continuous GTPase cycle is required for gastric acid secretion in parietal cells. Thus, ARFGAP6 may provide a novel link between signal transduction, vesicular trafficking and membrane cytoskeletal dynamics underlying parietal cell activation.

\section{M2078}

Dual Influence of Nitric Oxide On Gastric Hypersecretion Caused By Stomach Distension; Inhibition of Acid Secretion and Stimulation of Pepsin Secretion Ryo Matsuo, Yuuki Matsuzaka, Shinichi Kato, Koji Takeuchi

Background/Aim: It is known that distention of the stomach increases gastric secretion by both direct and indirect pathways such as neural reflex, although the detailed mechanism is still unclear. We previously showed that nitric oxide (NO) causes an inhibitory influence on the acid hypersecretion in response to stomach distension. However, the role of NO in pepsin hypersecretion in the distended stomach remains unknown. In the present study, we examined the effect of stomach distention on acid and pepsin secretion in rats and investigated the role of NO in these responses. Methods: Male SD rats were used after 18 $\mathrm{h}$ fasting. Under urethane anesthesia, the abdomen was incised, the cardiac portion ligated, and an acute fistula prepared with a polyethylene tube was provided in the stomach through a pylorus. Then the stomach was distended with instillation of saline $(6 \mathrm{ml})$ through the fistula, and the solution was changed every one hour. Acid secretion was measured by titrating the gastric content with $100 \mathrm{mM} \mathrm{NaOH}$ while pepsin concentration was determined by the Anson method. L-NAME (10 mg/kg), atropine ( $3 \mathrm{mg} / \mathrm{kg})$, cimetidine $(100 \mathrm{mg} / \mathrm{kg}$ ) or omeprazole $(30 \mathrm{mg} / \mathrm{kg})$ was given SC $1 \mathrm{~h}$ before the distention. In some cases, FK-409 ( 1 and $3 \mathrm{mg} / \mathrm{ml}$ ), the NO donor, was given intraluminally, and the acid and pepsin secretion was measured before and after the application. The amounts of NO in the luminal solution were determined by Griess method. Results: Both acid and pepsin secretions were markedly increased by distention of the stomach (the intraluminal pressure being about $20 \mathrm{cmH}_{2} \mathrm{O}$ ). The increased acid secretory response was completely inhibited by omeprazole, significantly mitigated by vagotomy, atropine and famotidine, while the response of pepsin was significantly inhibited only by vagotomy and atropine. By contrast, L-NAME markedly enhanced the acid hypersecretion in response to the distension while reduced the pepsin secretion induced by gastric distension, both in a L-arginine inhibitable manner. Distention of the stomach increased the luminal release of $\mathrm{NO}$, and the response was significantly suppressed by both vagotomy and L-NAME. Intragastric application of FK-409 dose-dependently stimulated pepsin secretion but reduced acid secretion in the stomach without distension. Conclusion: These results suggest that 1 ) the stomach distention increases both acid and pepsin secretions through vagal-cholinergic pathway, in addition to the luminal release of $\mathrm{NO}$, and 2) endogenous NO affects these responses in opposite directions, suppressing the acid response and enhancing the pepsin response.

\section{M2079}

Inihibition of COX-1, But Not COX-2, Enhances Proton Pump (PP) Expression in Rabbit Parietal Cells (PC)

Jyotirmoy Nandi, Natalie Pavelock, J. Michael Zinkievich, Robert A. Levine

We have previously shown that non-selective NSAIDs augment basal and histamine-stimulated gastric acid secretion in vivo (Gut 1984; 25:718) and in vitro (Gastroenterology 1991; 101:756). We reported that COX-1 is solely expressed in PC, and that COX-1, but not COX-2 inhibition augments basal and histamine-stimulated aminopyrine (AP) uptake in isolated rabbit PC with concomitant inhibition of $\mathrm{PGE}_{2}$ synthesis (Gastroenterology 2005; 128:A589). AIMS: To further investigate the precise mechanism of NSAIDs-induced augmentation of gastric acid secretion, we determined whether COX-1 inhibition enhances expression and/or activation of the PP. METHODS: Isolated rabbit PC were used (J Clin Invest 1990; 86.400) to assess $\left[{ }^{14} \mathrm{C}\right]$ AP uptake ratio and $\mathrm{PGE}_{2}$ synthesis, using a non-selective NSAID (indomethacin, INDO, 10-4 M) and selective COX-1 (SC-560, 10-5 M, Calbiochem) and COX2 (DFU, $10^{-5} \mathrm{M}$, Merck) inhibitors. Concentrations were selected based on $\mathrm{IC}_{50}$ values. PC $\left(5 \times 10^{6}\right.$ cells $\left./ \mathrm{ml}\right)$ were incubated in the presence and absence of INDO, SC-560, and DFU for $1 \mathrm{~h}$ at $37^{\circ} \mathrm{C}$. PP expression and activation were assessed by Western blot and pNPPase activity, respectively, in partially purified PC plasma membranes. RESULTS: Basal and histamine-stimulated AP uptakes were augmented by INDO and SC-560, but not by DFU. Augmentation of AP uptake was reduced to basal levels with lansoprazole $\left(10^{-5} \mathrm{M}\right)$, whereas ranitidine $\left(10^{-4} \mathrm{M}\right)$ failed to inhibit such augmentation, thereby implicating NSAIDs potentiation of acid secretion in PC to a locus in the PP. We found that INDO and SC-560 significantly enhanced PP expression by $28 \%$ and $29 \%$ and pNPPase activity by $65 \%$ and $61 \%$, respectively, compared to control PC, while DFU failed to enhance PP expression and pNPPase activity (Table). Concomitant inhibition of $\mathrm{PGE}_{2}$ synthesis was induced by INDO 
and SC-560, but not by DFU, indicating participation of COX-1 rather than COX-2 in modulating gastric acid secretion. CONCLUSION: Our data suggest that NSAIDs-induced gastric acid secretion in PC is due to enhancement of expression and activation of PP, presumably mediated by inhibition of COX-1, but not COX-2

NSAID Effect on PP Expression, pNPPase Activity, and PGE 2 Synthesis (\% of Control)

\begin{tabular}{|c|c|c|c|}
\hline & INDO $10^{-4} \mathrm{M}$ & SC-560 $10^{-5} \mathrm{M}$ & DFU $10^{-5} \mathrm{M}$ \\
\hline PP Expression & $128^{*}$ & $129^{*}$ & 100 \\
\hline pNPPase Activity & $165^{*}$ & $161^{*}$ & 115 \\
\hline $\mathrm{PGE}_{2}$ Synthesis & $10^{*}$ & $31^{*}$ & 83 \\
\hline
\end{tabular}

Control PP expression, pNPPase activity, and $\mathrm{PGE}_{2}$ synthesis were $0.75 \pm 0.06$ arbitrary units $/ 10^{5} \mathrm{PC}, 1.06 \pm 0.19 \mu \mathrm{mol} / \mathrm{h} / 10^{6} \mathrm{PC}$, and $461 \pm 23 \mathrm{pg} / 10^{6} \mathrm{PC}$, respectively $(\mathrm{n}=4)$. $* \mathrm{P}<0.05$ compared to control.

\section{M2080}

$\Delta$ F508 Is Necessary for CFTR-Modulated Secretagogue-Induced Acid Secretion Shafik M. Sidani, Philipp Kirchhoff, Elisa Ferreira, Marie Egan, John P. Geibel

Backround: The cystic fibrosis transmembrane conductance regulator (CFTR) is a multifunctional protein that plays a role not only in $\mathrm{Cl}^{-}$secretion, but also as a regulatory protein. The $\Delta$ F508 mutation is the most frequent CFTR defect causing the protein to be trapped and destroyed inside the endoplasmic reticulum before reaching the plasma membrane, resulting in cystic fibrosis. Acid secretion requires a complex interaction of transporters and channels at the apical pole of the parietal cell. Involved in this process is at least one $\mathrm{K}^{+}$ channel to recycle $\mathrm{K}^{+}$for the $\mathrm{H}, \mathrm{K}-\mathrm{ATP}$ ase, and a $\mathrm{Cl}^{-}$channel that secretes $\mathrm{Cl}^{-}$to combine with $\mathrm{H}^{+}$ions to generate $\mathrm{HCl}$ into the gland lumen. CFTR has previously been shown to interact with and modulate ROM-K channel activity when co-expressed in Xenopus oocytes, so that it is possible that CFTR could be acting as a regulatory protein for ROM-K and thereby modulate acid secretion. In the present study we measured secretagogue induced acid secretion from wild-type and $\Delta \mathrm{F} 508$ deficient mice in isolated gastric glands. Methods: We used a digital-imaging system to observe proton efflux from parietal cells in hand dissected gastric glands loaded with the $\mathrm{pH}$ sensitive dye BCECF. Individual glands were exposed to either: Histamine $(100 \mu \mathrm{mol})$, Pentagastrin $(100 \mu \mathrm{mol})$, or Carbachol $(100 \mu \mathrm{mol})$ and a $\mathrm{HCO} 3 / \mathrm{Na}^{+}$free perfusate to induce an acid load. We included 2 groups of mice: wild type and $\Delta \mathrm{F} 508$. To inhibit CFTR in wild type mice we used $100 \mu \mathrm{M}$ Glibenclamide and compared recovery rates to the transgenic mice. Results: $\Delta 508$ deficient mice had greatly reduced acid secretion. Glibenclamide effectively inhibited secretagogue induced acid secretion which was similar to the result obtained in the $\Delta \mathrm{F} 508$ deficient mice. Conclusion: Acid secretion induced by Histamine, Pentagastrin, and Carbachol in wild-type mice could be significantly reduced with an inhibitor of CFTR. In $\Delta 508$ deficient mice, Histamineinduced acid secretion was significantly less than wild-type mice due to absence of CFTR from the plasma membrane. These results demonstrate a substantial regulatory role for the CFTR protein in normal gastric acid secretion.

\section{M2081}

Partial Inhibition of Acid Secretion in Isolated Rat Gastric Glands with Low Concentrations of Etoh

Artur Vardanyan, John Geibel, Stanley Dudrick

Background: Multiple previous studies evaluating the effect of ethanol on gastric acid secretion demonstrated suppression of maximal acid output $(\mathrm{MAO})$ with high $(>10 \%)$ concentrations of $\mathrm{ETOH}$, which do not directly correlate with gastric mucosal injury caused by alcohol consumption. Additional human and animal studies investigating the effect of various alcoholic beverages produced by fermentation processes showed either marked stimulation or inhibition of acid secretion. The current study was designed to investigate the effect of low concentrations of pure ethanol on acid secretion by isolated rat gastric glands. Methods: To investigate the chronic vs. acute effects of ethanol on acid secretion 2 groups of animals were studied. In the first group male Sprague-Dawley rats were isolated and kept on regular food, with 5\% ETOH in the drinking water for 2 weeks (chronic). The second group were age/sex matched rats maintained on the same food and water minus ETOH (acute). Both groups were sacrificed and rates of $\mathrm{H}+$ efflux measured using the following protocol. We used a digital-imaging system and the $\mathrm{pH}$ sensitive dye BCECF to observe proton efflux from hand dissected gastric glands. Glands were stimulated with histamine (100umol) or Carbachol $(100 \mu \mathrm{M})$ and exposed to bicarbonate and $\mathrm{Na}+$ free $20 \mathrm{mM} \mathrm{NH} 4 \mathrm{Cl}$ perfusate to induce an acid load. $\mathrm{H}+$, K+-ATPase inhibition was determined by calculating pHi recovery $(\mathrm{dpH} / \mathrm{dT})$ rates. In a separate group $2 \% \mathrm{ETOH}$ was added to the bath perfusate. In a final series of experi-ments we used whole stomach $\mathrm{pH}$ measurements following Histamine $[100 \mu \mathrm{M}] \pm 5 \% \mathrm{ETOH}$ infusion with isotonic non-buffered saline. Serial luminal $\mathrm{pH}$ measurements were made for each group. Results: Our data shows: i) that addition of ETOH to the bath perfusate partially inhibits $\mathrm{H}+$ extrusion from parietal cells, and increases overall rate of alkalinization, ii) ingestion of 5\% ETOH for 2 weeks had no effect on acid secretion, iii) direct infusion of $5 \% \mathrm{ETOH}$ gave a modest reduction in the rate of histamine induced acid secretion in whole stomachs compared to saline controls. Conclusion: Acute exposure to low concentrations of ETOH has an inhibitory effect on neuro-endocrine mechanisms of gastric acid secretion. Chronic expo-sure to low concentrations of pure ethanol did not elicit any changes in $\mathrm{MAO}$, which suggests receptor/transporter remodelling to re-establish normal rates of acid secretion. Using low dose chronic and acute models of in vivo and in vitro acid secretion we have gained further insight into both alcohol-related malabsorption of food, and the pathogenesis of development of gastro-esophageal erosions secondary to excessive alcohol consumption.

\section{M2082}

Which Has Superior Acid-Suppressive Effect, Omeprazole $10 \mathrm{Mg}$ Once Daily Or Famotidine $20 \mathrm{Mg}$ Twice Daily? Effect of Single Or Repeated Administration of Omeprazole Or Famotidine in Helicobacter Pylori-Negative Cyp2c19 Extensive Metabolizers

Tomohiko Shimatani, Masaki Inoue, Tomoko Kuroiwa, Mutsuko Moriwaki, J.N. Xu, Masuo Nakamura, Hiroshi Mieno, Susumu Tazuma, Kazuro Ikawa, Norifumi Morikawa

Background: Low-dose omeprazole is superior to full-dose famotidine in maintenance therapy for gastroesophageal reflux disease; whereas, in 'on-demand' therapy, famotidine is more effective for relief of heartburn episodes than omeprazole. This discrepancy has no been fully explained. Aims: To compare acid-suppressive effects of single or repeated administration of omeprazole and famotidine considering of their respective pharmacokinetic/ pharmacodynamic characteristics. Methods: Study-1: In eight healthy Helicobacter pylorinegative cytochrome P450 (CYP) 2C19 extensive metabolizers, intragastric $\mathrm{pH}$ was measured for $24 \mathrm{~h}$ seven times; on the 1st, 8th, and 15th days of repeated administration of $10 \mathrm{mg}$ once daily omeprazole or $20 \mathrm{mg}$ twice daily famotidine, and before medication. Study-2: In nine healthy Helicobacter pylori-negative CYP2C19 extensive metabolizers, blood samples were collected in heparinized tubes before and at $1,2,3,4,6,8$ and $10 \mathrm{~h}$ after the lst, 8 th and 15 th dose of repeated administration of $10 \mathrm{mg}$ once daily omeprazole, and plasma concentrations were determined. Results were expressed as means \pm S.D. Results: Study-1 During repeated administration of omeprazole, the median value of intragastric $\mathrm{pH}$ and the percent of time that intragastric $\mathrm{pH}$ was above 4.0 for $24 \mathrm{~h}$ were significantly higher and became greater; $1.7 \pm 0.1$ and $6.5 \pm 3.3 \%$ before medication, $2.3 \pm 0.5 *$ and $19.1 \pm 11.9 \% *$ on the lst day, $3.3 \pm 1.4^{*} \#$ and $38.8 \pm 19.7 \% * \#$ on the 8 th day, and $4.3 \pm 1.3^{*} \#^{\dagger}$ and $53.2 \pm 23.6^{*}$ on the 15 th day, respectively $(* P<0.05$ vs. before medication, $\# P<0.05$ vs. the lst day, and $\uparrow P<0.05$ vs. the 8 th day). With famotidine, while these parameters were significantly higher the degrees became smaller; $3.9 \pm 0.6^{*}$ and $50.4 \pm 13.0 \% *$ on the 1 st day, $3.4 \pm 0.5^{* \#}$ and $36.2 \pm 8.7^{*} \#$ on the 8 th day, and $2.8 \pm 0.3^{*} \# \dagger$ and $22.9 \pm 8.2 \% * \# \dagger$ on the 15 th day. Con sequently, acid-suppressive potency was in the order; $20 \mathrm{mg}$ twice daily famotidine $>10$ $\mathrm{mg}$ once daily omeprazole on the lst day $(P<0.05)$, omeprazole $\approx$ famotidine on the 8 th day, and omeprazole $>$ famotidine on the 15 th day $(P<0.05)$. Study-2: Omeprazole increased its bioavailability following repeated administration: The area under the plasma concentration-time curve from 0 to $10 \mathrm{~h}$ for omeprazole also increased, with relative ratios of $1,1.77$ and 2.10 on the 1st, 8th and 15 th days, respectively. Conclusions: The effectiveness of full-dose famotidine in 'on-demand' therapy and that of low-dose omeprazole in maintenance therapy possibly result from the phenomenon of 'tolerance' to famotidine, and a 'potentiation of the acid-suppressive effect of omeprazole because of its increased bioavailability following repeated administration.

\section{M2083}

Somatostatin Receptor Type 2 Knockout Mice Display Inhibitory Effect of Galanin On ECL Cells But Enhanced Action of Gastrin On Parietal Cells Chun-Mei Zhao, Vicente Martinez, Laura Piqueras, Yvette Tache, Duan Chen

Background/Aim: Somatostatin receptor type 2 (SSTR2) and gastrin receptor (CCK2) are expressed in both parietal cells and histamine-producing ECL cells in the oxyntic mucosa of the stomach, whereas galanin Gall receptor is expressed mainly on the ECL cells. In the present study, we attempt to characterize the regulatory pathways that control acid secretion in SSTR2-deficient mice using a combined morphological and functional approach. Methods Gastric acid secretory responses to pentagastrin, histamine, somatostatin-14 and galanin were compared between SSTR2 knockout (KO) and wild-type (WT) mice that were anesthetized with urethane. Tissue samples were collected from the oxyntic mucosa in freely fed mice for quantitative analyses by immunocytochemistry and electron microscopy. Results The acid secretion induced by pentagastrin $(16 \mu \mathrm{g} / \mathrm{kg} / \mathrm{h}$, iv) was enhanced $(>15$-fold) in $\mathrm{KO}$ mice when compared to that in WT animals. On the other hand, histamine $(5 \mathrm{mg} / \mathrm{kg} / \mathrm{h}$, iv $)$ induced a similar acid response in both groups (WT: $11 \pm 2 \mu \mathrm{mol} / 2 \mathrm{~h}$ and KO: $13 \pm 1 \mu \mathrm{mol} /$ $2 \mathrm{~h})$. In KO mice, galanin $(10 \mu \mathrm{g} / \mathrm{kg} / \mathrm{h}$, iv) inhibited pentagastrin-induced acid secretion by $49 \%$ while somatostatin-14 $(20 \mu \mathrm{g} / \mathrm{kg} / \mathrm{h}$, iv) had no effect. Immunocytochemistry revealed that the expression of Gall receptor in the ECL cells was significantly increased ( $>7$-fold) whereas histidine decarboxylase and vesicle monoamine transport type 2 were significantly reduced (by 23-35\%) in KO mice. Further electron microscopic analysis showed an accumulation of the secretory granules ( $>2$-fold) in the ECL cells. The number and the proportion of secreting parietal cells containing secretory canaliculi were unchanged in $\mathrm{KO}$ mice. In addition, there were no differences between WT and KO mice in terms of the densities of immunoreactive cells stained for pancreastatin, ghrelin, or proliferating cell nuclear antigen Conclusion: Upon genetic removal of somatostatin SSTR2 inhibitory pathway, galanin inhibitory signalling may be up-regulated in the ECL cells, while the stimulatory action of gastrin is enhanced on the parietal cells.

\section{M2084}

Direct Visualization and Aspiration During Endoscopic Gastric Analysis Enhances Accuracy and Reproducibility of Acid Output Measurement in Zollinger-Ellison Syndrome

Hank S. Wang, David S. Oh, Gordon V. Ohning, Joseph R. Pisegna

Objectives: Gastric analysis (GA) has traditionally been considered the gold standard in the diagnosis of Zollinger-Ellison Syndrome (ZES). Patients with ZES initially require gastric analysis at 3 month intervals both to monitor disease progression and to assess therapeutic success. Conventional GA using a nasogastric tube is burdensome and complex, requiring more than an hour to complete and results in high levels of patient discomfort. In this study, we have validated endoscopic gastric analysis (EGA) as a more accurate and reproducible technique for measuring acid output in ZES and possibly in other hypersecretory conditions. Methods: In a prospective, crossover study, 12 patients with ZES underwen gastric analysis, first by GA and then by EGA performed on the same day by the same operator. Following an overnight fast, GA was performed in the standard way. The acid 
concentration of each 15 minute sample was determined by titration, the volume recorded and a 15 minute acid output level was calculated and added to give an hourly acid output. EGA was then performed with sedation, and acid output was determined in a similar manner. To assess reproducibility, 5 patients underwent repeat examination at least once including both GA and EGA at a mean follow-up of 7.2 months. Root mean square of the difference (RMSE) between EGA and GA was calculated to test for equivalence. Pearson's correlation coefficient was statistically significant when the calculated $\mathrm{p}$ for a two-tailed test was $<.05$. Results: No statistical equivalence was detected between volume (RMSE $13.4 \mathrm{ml}$ ), acid concentration (RMSE $.007 \mathrm{mEq} / \mathrm{ml}$ ) and acid output (RMSE $1.05 \mathrm{mEq} / \mathrm{hr}$ ). Moderate correlation was seen between EGA and GA regarding acid output $(r=.54, p<.02)$. In addition, EGA was highly reproducible $(r=.94, p<.01)$ while $G A$ was not $(r=.27, p>.05)$. Finally, measurements of acid concentration also had high correlation between EGA and GA ( $r=$ $.85, \mathrm{p}<.01)$, while volume did not $(\mathrm{r}=.059, \mathrm{p}>.05)$. Conclusions: We introduce a new, rapid, simple endoscopic technique to measure gastric acid output and report significant correlation between GA and EGA acid concentration and acid output. We derived a calibration equation in ZES patients showing that EGA can predict GA acid output values to within \pm $1.10 \mathrm{mEq} / \mathrm{hr}$ with a $95 \%$ confidence interval. While statistically nonequivalent, calculated differences between EGA and GA acid output values were of little clinical importance. Further evaluations of the accuracy and reproducibility of both GA and EGA in hypersecretory and non-hypersecretory conditions are required to determine which test is the true gold standard

\section{M2085}

Endogenous Cannabinoids (Endocannabinoids) Regulate Gastric Acid Secretion Via Cb1, Cb2, and VR1 Receptors

Robert Flynn, Prabhat Dwivedi, Shyamal Premaratne, Wei Hou, Robert McCuen, Mitchell Schuber

RATIONALE. We previously identified anandamide, an endocannabinoid, in rat gastric oxyntic mucosa by HPLC/gas chromatography/mass spectrometry. The activity of cannabinoids may be mediated via CB1, CB2, and VR1 receptors. Cannabinoids are inactivated by a two-step process that involves a transporter that promotes cellular uptake followed by intracellular hydrolysis. AIM. The aim of the present study was to determine the physiologic role and mechanism of action for gastric endocannabinoids in the regulation of gastric acid secretion. METHODS. CB1, CB2, and VR1 receptor expression were identified by RT-PCR and immunoblotting in rat oxyntic mucosa and isolated parietal cells. Acid secretion was measured by titration in isolated luminally perfused mouse stomach. RESULTS. CB1 and VR1 receptors were expressed in oxyntic mucosa; $C B 2$ receptors were expressed on isolated parietal cells. In isolated stomach, the anandamide membrane transport inhibitor, VDM1 1 $(1 \mu \mathrm{M})$, stimulated acid secretion $(18 \pm 2 \%$ above basal level; $\mathrm{P}<0.001)$, implying that gastric endocannabinoids are tonically active and contribute physiologically to basal acid secretion. Consistent with this notion, anandamide ( $1 \mathrm{pM}$ to $10 \mathrm{uM}$ ) caused a concentration-dependent increase in acid secretion (EC50, $70 \mathrm{pM}$; maximal, 33 $\pm 5 \%$ above basal level at $1 \mu \mathrm{M}$ $\mathrm{P}<0.01)$. The effect of $10 \mathrm{nM}$ anandamide $(23 \pm 1 \%$ above basal level; $\mathrm{P}<0.001)$ was augmented by the VRl antagonist, 5 '-iodoresiniferatoxin $(1 \mu \mathrm{M} ; 31 \pm 3 \%$ above basal level; $\mathrm{P}<0.01$ for the difference), implying that anandamide, acting via VRl receptors, inhibits acid secretion. Conversely, both the CB1 antagonist, SR141716 (0.1 $\mu \mathrm{M})$, and the CB2 antagonist, SR144528 $(0.1 \mu \mathrm{M})$, each inhibited basal acid secretion $(19 \pm 4 \%$ and $5 \pm 1 \%$ below basal level, respectively; $\mathrm{P}<0.01)$ and significantly attenuated anandamide-stimulated acid secretion, implying that activation of $\mathrm{CB} 1$ and $\mathrm{CB} 2$ receptors leads to stimulation of acid secretion. The axonal blocker, tetrodotoxin $(5 \mu \mathrm{M})$, partly inhibited the acid response to $10 \mathrm{nM}$ anandamide by $52 \%$; the residual response $(11 \pm 2 \%$ above basal level; $\mathrm{P}<0.01)$ was abolished by the $\mathrm{CB} 2$ antagonist but not the CBl or VRl antagonist. CONCLUSION. Endocannabinoids in gastric oxyntic mucosa physiologically regulate acid secretion. The dominant effect is stimulatory and mediated via $\mathrm{CB} 1$ receptors on neurons and $\mathrm{CB} 2$ receptors on parietal cells. A concurrent inhibitory effect is mediated via VRl receptors on neurons.

\section{M2086}

Expression of Prostaglandin E2 Receptor Subtypes in Barett's Esophagus and Esophageal Adenocarcinoma

Pilar Jimenez, Elena Piazuelo, Mark Strunk, Sonia Santander, Pilar Irun, Miguel Angel Simon, Asuncion Garcia, Ricardo Sainz, Angel Lanas

Background \& Aim: Accumulating evidence suggests that COX-2-derived prostaglandin E2 is involved in esophageal adenocarcinogenesis. PGE2 exerts its biological action through binding to specific receptors (EP1, EP2, EP3, EP4). Recent studies have shown that EP1, EP2 and EP4 are implicated in colon carcinogenesis. Since it is not known which receptors regulate PGE2 signals in esophageal adenocarcinoma (EAC), we investigated the expression pattern of PGE2 receptor subtypes as well as COX-1, COX-2, in tissues from normal squamous mucosa, Barrett's esophagus (BE) and EAC. Methods: m-RNA expression of COX1 , COX-2 and PGE2 receptor subtypes was determined by quantitative RT-PCR in esophageal biopsies taken from patients with BE and EAC. The housekeeping gene hu-TBP was used as reference gene for normalization the amount of sample ( $\Delta \mathrm{Ct}$ values). Relative quantification was expressed as fold-induction or decrease of the gene compared with the control (normal squamous epithelium) according to the formula $2^{-} \Delta \Delta \mathrm{Ct}$. The Mann-Whitney test was used to compared $\Delta \mathrm{Ct}$ values obtained among groups. Results: RT-PCR analysis showed that EP1, EP2, EP3 and EP4 are expressed in human esophageal mucosa. Levels of COX-2, EP2 and EP4 mRNA expression were increased in BE and EAC compared to normal esophageal mucosa. By contrast, COX-1 expression was decreased in both BE and EAC. The median relative values and range of the mRNA expression levels are listed on the table. Conclusions: PGE2 receptor subtype EP2 expression is markedly increased in Barrett's esophagus and adenocarcinoma, in clear contrast with the decrease observed for the COX-1 isoenzyme. In addition to COX-2, EP2 receptors could be a selective target in the prevention and/or reatment of esophageal adenocarcinoma associated with BE.

\begin{tabular}{|c|c|c|c|c|c|c|}
\hline & COX-1 & COX-2 & EP1 & EP2 & EP3 & EP4 \\
\hline BE Median & $0.21 *$ & $2.21^{*}$ & 2.33 & $63.11^{* *}$ & 1.42 & $4.59^{*}$ \\
\hline Range & $0.15-1.17$ & $0.22-8.5$ & $0.22-19.7$ & $8.6-116$ & $0.15-136$ & $0.17-7.63$ \\
\hline EAC Median & $0.064^{*}$ & $14.22 *$ & 3.6 & $21.55 *$ & 1.55 & 0.71 \\
\hline Range & $0.04-0.51$ & $4-95.6$ & $0.82-7.51$ & $17.6-27.28$ & $0.13-1.34$ & $0.4-1.05$ \\
\hline
\end{tabular}

(increase of expression: value $>1$ ) or (decrease of expression: value $<1$ ) vs (normal squamous mucosa, value $=1) .\left({ }^{*} \mathrm{p}<0.05,{ }^{* *} \mathrm{p}<0.01\right.$ vs normal squamous mucosa). $(\mathrm{n}=9-4)$

\section{M2087}

Endoscopic Mucosal Resection As a Diagnostic Modality: Interrater Diagnostic Correlation for Endoscopic Mucosal Resection Specimens Is Better Than Biopsy Specimens

Mari Mino-Kenudson, Mindy J. Hull, Ian Brown, Alona Muzikansky, Amitabh Srivastava, Joseph Misdraji, Do-Youn Park, Jonathan Glickman, Lawrence R. Zukerberg, Robert D. Odze, Gregory Y. Lauwers

Background: Endoscopic mucosal resection (EMR) is advocated for the treatment of superficial neoplasms arising in Barrett esophagus (BE). It represents a superior staging modality compared to EUS. EMR can also improve diagnoses compared to biopsies, and therefore facilitate optimal therapeutic decisions. However, the status of interobserver variation between pathologists reviewing EMR specimens has not been evaluated. Design: Nine pathologists from 3 academic centers reviewed 25 pairs of esophageal EMR specimens and peri-operative biopsy (either pre- or post- EMR) from the same lesion. Each cases were classified into the following diagnostic schema: 1) non-neoplastic; 2) low grade dysplasia (LGD); 3) high grade dysplasia (HGD); 4) intramucosal adenocarcinoma (IMC); and, 5) invasive adenocarcinoma (Inv Ca). The results were analyzed for concordance between raters, with the goal to evaluate if the observer agreement was the same for EMR specimens and biopsies. Analysis included intraclass correlation (ICC) and Kendall's coefficient (KC) of concordance (for ordinal data) to describe agreement of EMR \& biopsy specimens as individual groups. Significant difference between the rater classification of EMR versus biopsy specimens was assessed by finding the proportion of agreement of all possible permutations of paired raters, and seeing if this was higher for EMR than biopsy (unidirectional analysis). The proportion of agreement for any given lesion was evaluated for both exact classification (ex. lst and 2nd rater both diagnose LGD) and 1-grade discrepancies (ex. 1st rater diagnoses LGD, 2nd rater diagnoses HGD) using the one-sided Wilcoxon Signed-Rank test. Significance was considered to be $\mathrm{p}<0.05$. Results: The ICC and KC for the 25 esophageal biopsies was $0.938(95 \% \mathrm{CI}=$ $0.880-0.965)$ and 0.677 , respectively. The ICC and KC for the 25 EMR specimens was improved at $0.977(95 \% \mathrm{Cl}=0.957-0.987)$ and $0.831(\mathrm{p}=<0.0001)$. The proportion of agreement for EMR specimens was significantly better than that of biopsy specimens $(\mathrm{p}=0.015)$, indicating that pathologists are more likely to agree on a diagnosis on an EMR specimen than they are on a biopsy. Conclusion: The histopathologic assessment of dysplasia in BE, which remains the basis for therapeutic decision, is criticized for its lack of interobserver agreement. The causes for this limitation may be: biopsy sampling and artefacts, and lack of diagnostic confidence on small biopsy specimens. We demonstrate that the same group of pathologists has a better interobserver agreement on EMR specimens. It suggests that EMR, in addition to being a staging and therapeutic procedure, provides improved diagnostic consistency.

\section{M2088}

Reg I Expression in Untreated Endoscopic Biopsy Specimens Predicts LongTerm Survival Among T4 Thoracic Squamous Cell Esophageal Cancer Patients Treated with Chemoradiotherapy Followed By Esophagectomy

Satoru Motoyama, Toshihiro Sugiyama, Yasuharu Ueno, Hiroshi Okamoto, Shin

Takasawa, Hiroshi Nanjo, Kiyotomi Maruyama, Manabu Okuyama, Kenji Sasaki, Jun-Ichi Ogawa

Background and Aim: The prognosis of T4 (direct invasion to adjacent organs) thoracic esophageal cancer patients is extremely unfavorable, therefore we have been administering neoadjuvant chemoradiotherapy (CRT) followed by esophagectomy to these patients. The purpose of this study was to determine whether expression of REG I protein in untreated endoscopic biopsy specimens is predictive of their responsiveness to CRT and/or survival after treatment. Patients and Methods: Between 1992 and 2003, 50 patients with T4 thoracic esophageal cancer were administered neoadjuvant CRT followed by curative esophagectomy. REG expression was assessed in untreated endoscopic biopsy specimens and correlated with clinical and histological responses and survival in 37 patients. Results: Among the 37 cases that received CRT followed by surgery, the therapeutic response rate for neoadjuvant CRT was $68 \%$ and a complete histological response in resected specimens from the primary lesion was achieved in eight (22\%) patients. These clinical and histological responses to neoadjuvant CRT did not significantly correlate with survival, however. By contrast, nine patients were judged REG-positive based on analysis of their untreated endoscopic biopsy specimens, and their 5-year survival rate was significantly higher than that of the 28 REG-negative patients (Figure). Conclusions: T4 thoracic squamous esophageal cancer patients expressing REG in untreated endoscopic biopsy specimens achieved long-term survival when treated with neoadjuvant CRT followed by esophagectomy. 


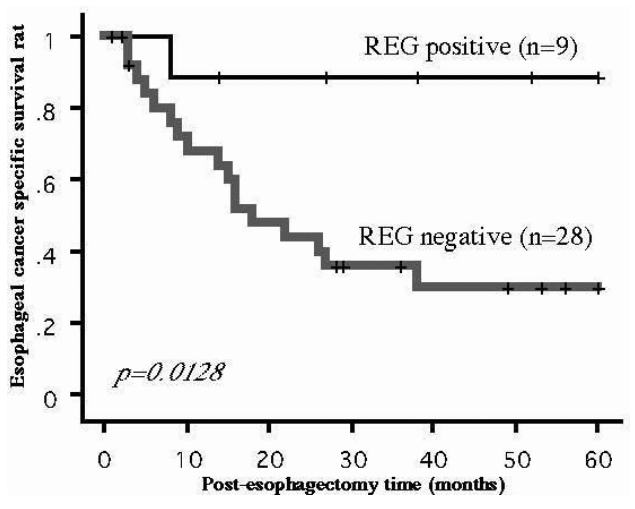

M2089

Value of Endoscopic Ultrasound (EUS) and Clinical/Endoscopic Findings As Predictors of Disease Extent and Regional Lymph Node Status in Esophageal Cancer (EC)

Gregory Zuccaro, Thomas W. Rice, John J. Vargo, John R. Goldblum, Lisa A. Rybicki, Rocio A. Lopez, Dumot A. John, David J. Adelstein, Patricia A. Trolli, Eugene H. Blackstone

Background. Either disease extent (defined as limited to wall: pTis-T2, pN0 vs. advanced beyond wall: $\mathrm{pT} 3-\mathrm{T} 4$, or $\mathrm{pNl}$ ) or lymph node status ( $\mathrm{pNO}$ vs. $\mathrm{pNl}$ ) may guide therapy in EC. EUS is commonly used to predict the presence or absence of advanced disease or $\mathrm{pNl}$; however, these predictions are more frequently erroneous than previously recognized (AJG 2005; 100:601-606). Purpose. Determine accuracy in predicting advanced disease and pN1 in EC using EUS alone, clinical/endoscopic information alone, and a combination of clinical/ endoscopic information with EUS. Methods. 314 pts with EC underwent clinical evaluation with endoscopy, and EUS, followed by esophagectomy. No pt received preoperative chemoradiation. Post-operative pathologic classification was the gold standard. Accuracy of prediction of both advanced disease and pNl was determined using: (1) EUS alone, (2) logistic regression using clinical/endoscopic (non-EUS) information alone, and (3) logistic regression combining clinical/endoscopic information with EUS result. The clinical/endoscopic factors examined were: age, gender, weight loss, dysphagia, tumor traversibility, length, location, and morphology, histopathologic type, and histologic grade. Receiver Operating Characteristic (ROC) curves were created, and area under each curve (AUC) calculated (AUC reflects accuracy of a diagnostic test and is used to compare two or more tests). Results. Pathologic classification at esophagectomy (gold standard): 138 (44\%) limited, 176 (56\%) advanced; 171 (55\%) No, $143(45 \%)$ N1. Risk factors for advanced disease and pNl were: dysphagia, weight loss, increased tumor length, poor histologic grade, and EUS prediction of advanced disease or pNl. AUC for each ROC curve is shown (table). Using all available information (clinical/ endoscopic plus EUS) was superior to EUS alone in predicting advanced disease and pNl. Clinical/endoscopic (non-EUS) information alone was equivalent to the combination of clinical/endoscopic and EUS information in predicting advanced disease and pN1. Conclusions. (1) While EUS alone is a good test for predicting advanced disease and $\mathrm{pNl}$, use of clinical/endoscopic information combined with EUS significantly improves prediction accuracy. (2) Clinical/endoscopic information without EUS is virtually identical to the combination of this information with EUS in predicting advanced disease and $\mathrm{pNl}$ in EC. AUC for Methods of Predicting Advanced Disease or pNl in EC

\begin{tabular}{|c|c|c|}
\hline & Advanced Disease & $\mathrm{pN} 1$ \\
\hline EUS alone & $0.85^{*}$ & $0.72 \#$ \\
\hline Clinical/Endoscopic Information Alone & 0.93 & 0.87 \\
\hline Combination of Clinical/Endoscopic and EUS & $0.94^{*}$ & $0.88 \#$ \\
\hline
\end{tabular}

* $\mathrm{P}<0.001 ; \# \mathrm{P}<0.0001$

M2090

Gastric Atrophy May Be Associated with the Development of Squamous Cell Carcinoma of the Esophagus in Japan

Katsunori Iijima, Tomoyuki Koike, Hitoshi Sekine, Akira Imatani, Shuichi Ohara, Tooru Shimosegawa

Background:Hypochlorhydric atrophic gastritis is a well-known premalignant condition of gastric cancer. Recent study in Swedish population demonstrated that gastric atrophy induced by $H$. pylori infection may also increase the risk of esophageal squamous-cell carcinoma (SCC) (W Ye, et al, JNCI 2004). However, this observation needs to be confirmed among different ethnic groups with high prevalence of $H$. pylori infection and accompanying gastric atrophy, like in Japanese population. The aim of this study is to investigate weather gastric atrophy was associated with the risk for esophageal SCC in case-control study. Methods: From April 2004 to March 2005, 64 consecutive subjects (60 men, mean age: 69 years) who had had early esophageal SCC and had received endoscopic mucosal resection before the entry were prospectively enrolled. For each case, age- and sex- matched asymptomatic control subjects were randomly selected from patients who visited our hospital during the same period. H. pylori status was determined by RUT, histology, and serum Ig-G antibody. Biopsy specimens from the gastric body were evaluated histologically regarding the prevalence of mucosal atrophy and intestinal metaplasia (IM). Gastric atrophy was also evaluated serologically by measuring serum pepsinogen (PG) concentration. A value of PG I/I ratio lower than 2.0 was considered as an indicator of gastric atrophy. Results: Prevalence of $\mathrm{H}$. pylori infection was similarly high in both groups (SCC group: $82 \%$, controls: $78 \%$ ). Mucosal atrophy and IM were more prevalent in SCC group compared with controls (atrophy: 56\% vs. $32 \%, p<0.05$; IM: $34 \%$ vs. $5 \%, p<0.01$ ). Serologically diagnosed gastric atrophy was also more prevalent in SCC group than in controls ( $53 \%$ vs. $33 \%, \mathrm{p}<0.05$ ). Proportion of current smokers were similar between SCC group and controls (37\% vs. 33\%). Conclusion Gastric atrophy concomitant with IM was more frequently observed in the stomach of SCC group compared with controls, suggesting its relevance to the pathogenesis of SCC of the esophagus. It is known that carcinogenic agent such as acetaldehyde or nitrosamine is produced by the microbial overgrowth in the hypochlorhydric atrophic stomach. Such carcinogens may reflux into the esophagus and be responsible for the development of SCC.

M2091

Evidence from Barrett's Carcinogenesis That CYP26Al Is Implicated in Cancer Chih-Long Chang, Pierre Lao-Sirieix, Rebecca C. Fitzgerald

Background Retinoic acid (RA) is an important signalling molecule for Anterior-Posterio (AP) patterning in embryogenesis. Tight control of intracellular RA levels results in an anterior shift in endoderm gene expression and induction of the caudal gene CdxA. CYP26A. is the major RA catabolising enzyme responsible for maintaining exquisite control over cellular RA concentrations. Hypothesis We hypothesised that RA signalling may have a role in determining the altered differentiation status in Barrett's carcinogenesis. Aims In this study we have investigated 1) the relationship between RA and CYP26Al on Barrett's metaplasia and associated dysplasia and adenocarcinoma. 2) The functional role of CYP26Al in vitro Methods and Results By using retinoic acid response element (RARE)- reporter cells we demonstrated that non-dysplastic Barrett's cell lines (BAR, QhTERT) and non-dysplastic Barrett's tissues had the highest levels of RA bioactivity, which decreased with dysplasia in cell lines (Chtert, Gihtert), and patient tissues ( $\mathrm{p}<0.001)$. The degree of RA bioactivity was inversely correlated with CYP26Al gene levels. Functionally, over-expression of CYP26Al in Gihtert cells increased proliferation as determined by their cytometric DNA profile and an MTT assay. An in vitro matrigel assay demonstrated that cells overexpressing CYP26Al were more invasive $(\mathrm{p}<0.001)$. The increased invasion is likely to be due to alterations in matrix metalloproteinase expression (up-regulation of MMP-3, down-regulation of TIMP-1 and TIMP-3 $\mathrm{p}<0.05$ ) which were demonstrated by quantitative RT-PCR. Using an organotypic culture system we also demonstrated that CYP26Al gene over-expressing cells are "dedifferentiated" as shown by co-localization with a stem-cell marker Oct-4. Pathway arrays showed induction of c-myc and EGFR expression in these CYP26Al over-expressing cells. Discussion Over-expression of CYP26Al causes intracellular RA depletion and drives the cell into a highly proliferative, undifferentiated and invasive state with induction of other known oncogenes. This data suggests a previously unknown role for this gene in cancer.

\section{M2092}

Expression of Stem Cell Markers CD34 and THY1 in Adenocarcinoma of Esophagogastric Junction and Their Prognostic Implications Kuniaki Aridome, Henning C. Fiegel, Bjoern-Christian Link, Dean Bogoevski, Jussuf T. Kaifi, Robin Wachowiak, Dietrich Kluth, Emre F. Yekebas, Takashi Aikou, Jakob R. Izbicki Background: The increase in cancer incidence associated with chronic injury supposed to be strongly correlated with stem cell renewal as a continuous state of repair. In addition bone marrow-derived cells, which are frequently recruited to site of chronic tissue injury and inflammation, are also suggested to represent a potential source of malignancy. Here, we focused on carcinoma of the esophagogastric junction as this type of carcinoma frequently arises after chronic inflammation in association with gastroesophageal reflux. Therefore, we examined the expression of CD34 class III and Thyl (CD90) as well established bone marrow stem cell markers in adenocarcinoma of the esophagogastric junction. Methods: Forty-nine patients with primary adenocarcinoma of the esophagogastric junction, who underwent operation with curative intention at the Department of General, Visceral and Thoracic Surgery at the University Medical Center of Hamburg-Eppendorf, Germany, were included in the study. Frozen sections were examined by immunohistochemical analysis using monoclonal antibodies against CD34 class III or Thyl epitopes. To assess correlation between immunohistochemical findings and clinicopathologic factors Fischer's exact test and Kaplan-Meier method with log-rank test was performed. Results: Expression of CD34 as a class III epitope was confirmed in $14(29 \%)$ out of 49 patients, as cluster of positive tumor cells. Thyl expression of tumor cells in the membrane and in the cytoplasm appeared in $35(71 \%)$ out of 49 patients. Thyl expression was significantly higher in tumors classified as Siewert's Type I/II compared to Type III tumors $(\mathrm{p}<0.05)$. Thyl positive patients had distant metastasis at significantly higher frequency than Thyl negative group $(\mathrm{P}<0.01)$. Patients with positive Thyl expression showed worse overall and disease free survival as compared to negative Thyl patients $(\mathrm{p}<0.05)$. On the other hand, we did not find any significant correlation between CD34 class III expression in primary tumors and clinicopathological features of carcinoma of esophagogastric junction. Conclusions: CD34 class III positive cell clusters were detected and may be regarded as evidence for cancer stem cells in adenocarcinoma of the esophagogastric junction. Expression of the stem cell marker Thyl has significant impact on overall and disease free survival.

\section{M2093}

Environmental Risk Factors for Cancer Development Are Not Different Between Patients with Esophageal and Gastric Cardia Adenocarcinoma Leonieke Wolters, Pieter-Jan F. de Jonge, Ernst J. Kuipers, Peter D. Siersema

Background: Over the past 3 decades, the major subtype of esophageal carcinomas has shifted from squamous cell carcinomas (SCC) to adenocarcinomas (EAC). This change in incidence is attributed to differences in etiology. However, it is largely unknown whether the same or different risk factors for cancer development are present in patients with EAC and gastric cardia adenocarcinoma (GCA). Aim: To compare environmental risk factors 
between patients with SCC and EAC on the one hand and patients with EAC and GCA on the other hand. Methods: Between January and November 2005, all patients who were evaluated in the Erasmus MC for esophageal or gastric cardia cancer by endoscopic ultrasonography were asked to fill out a questionnaire. This questionnaire comprised information on the subjects' history of diet habits, physical activity, gastro-esophageal reflux disease, history of proton-pump inhibitors (PPIs), H2-receptor antagonists (H2RAs) and NSAID/ aspirin use. Chi-square tests and t-tests were used for statistical analysis. $\mathrm{P}<0.05$ was considered statistically significant. Results: A total of 208 patients filled out the questionnaire of whom 112 patients were diagnosed with EAC (90\% males, mean age \pm SD: $62 \pm 10 \mathrm{yr}$ ), 42 patients with GCA ( $85 \%$ males, $65 \pm 11$ yr) and 54 patients with SCC $(65 \%$ males, $63 \pm$ $9 \mathrm{yr})$. Age, race and education were comparable between the three groups. More female patients were diagnosed with a SCC compared to EAC $(\mathrm{p}<0.001)$. Mean body mass index at age 20 years was significantly higher in EAC patients compared to SCC patients $(24 \pm 3$ vs. $22 \pm 3 \mathrm{~kg} / \mathrm{m} 2, \mathrm{p}<0.001)$ but did not differ between EAC and GCA $(24 \pm 5 \mathrm{~kg} / \mathrm{m} 2, \mathrm{p}=0.46)$. Symptoms of heartburn were more frequently present in EAC patients compared to SCC ( $50 \%$ vs. $23 \%, \mathrm{p}=0.001$ ) and GCA patients $(31 \%, \mathrm{p}=0.031)$. Use of PPIs was more common in EAC patients compared to SCC patients ( $48 \%$ vs. $30 \%, \mathrm{p}=0.04$ ), but did not differ between EAC patients and GCA patients ( $48 \%$ vs $38 \%, p=0.27$ ). As compared to GCA patients, EAC patients more often used NSAIDs or aspirin ( $47 \%$ vs $29 \%, \mathrm{p}=0.036 ; 51 \%$ vs $24 \%, p=0.003)$. No difference between EAC and SCC patients was observed in NSAID or aspirin use ( $47 \%$ vs $33 \%, p=0.078 ; 51 \%$ vs $39 \%, p=0.167$ ). Smoking and alcohol consumption was not different between the three groups. Conclusion: Patients with EAC and GCA do not differ grossly with regard to environmental risk factors for cancer development. In contrast, patients with EAC experienced more reflux symptoms than patients with SCC as could be expected from the etiologic sequence in development of EAC.

\section{M2094}

The Biological Role of Low Affinity Neurotrophin Receptor (p75NTR) in Esophageal Squamous Cell Carcinoma

Tomoyuki Okumura, Shigeru Tsunoda, Yukiko Mori, Tetsuo Ito, Keiji Kikuchi, Timothy C. Wang, Shigeru Yasumoto, Yutaka Shimada

Background: Recent observations using several malignancies have demonstrated the identification of a limited number of cells with a remarkable self renewal potential and extensive proliferation capacity. Most of these cells are isolated based on the expression of markers which characterize the stem cells of original normal tissues. The low affinity neurotrophin receptor (p75NTR) is expressed in a stem/progenitor cell fraction of human normal esophageal keratinocyte. However, the expression and biological role of p75NTR in esophageal squamous cell carcinoma (ESCC) have not yet been reported. Methods: Written informed consent was obtained from the patients for surgery and to use resected samples for research. First the expression of p75NTR in 187 resected ESCC specimens was immunohistochemically investigated. Next we investigated the expression of p75NTR in 30 ESCC cell lines (KYSEs) by RT-PCR, immunocytochemistry and flow-cytometry. We then isolated p75NTR-positive and negative cells from KYSE150 by magnetic beads to investigate their colony formation. The role of p75NTR in KYSEs was assessed by transient transfection of siRNA. Results: p75NTR was expressed in 92 of 187 (49.2\%) cancer specimens. In well differentiated tumors, positive staining was apparent in the first 1-2 layers from infiltrative margin of the tumors where most of the cells were actively proliferating. In moderately differentiated cases, p75NTR was expressed in more wide range from the margin of the tumors. The p75NTR was diffusely distributed in poorly differentiated tumors. p75NTR was expressed in all examined KYSEs and the mean proportion of the p75NTR-positive fraction was 30.1\% (ranged from $0.5 \%$ to $91.8 \%$ ). Our investigation using KYSE150 demonstrated that p75NTR was expressed in $118(60.8 \%)$ of 194 colonies. The mean diameter of p75NTR-positive and negative colonies were $969 \pm 513 u \mathrm{~m}$ and $414 \pm 182 u \mathrm{~m}$, respectively $(\mathrm{p}<0.0001)$. When the fractionated cells were plated and cultured, p75NTR was expressed in $178(82.0 \%)$ of 217 colonies and 65 $(30.1 \%)$ of 216 colonies derived from p75NTR-positive and -negative fraction, respectively $(\mathrm{p}<0.0001)$. Down regulation of p75NTR expression by SiRNA resulted in marked growth inhibition at 72 hours after siRNA transfection. The TUNEL assay demonstrated that the Apoptotic Index in the siRNA treated cells and control RNA treated cells were $15.5 \%$ and $3.0 \%$, respectively. Conclusion: Our findings suggest that p75NTR is expressed in the actively proliferating, undifferentiated cell component of ESCC and is necessary for survival and maintenance of the tumors, providing us with a potential target for novel therapies.

\section{M2095}

Socio-Economic Status Plays NO Role in Decision Making On Diagnosis and Treatment of Esophageal Cancer in the Netherlands

Evelyn P.M. van Vliet, Marinus J.C. Eijkemans, Ernst J. Kuipers, Ewout W. Steyerberg Ate van der Gaast, Peter D. Siersema

Background: The socio-economic status (SES) of patients with esophageal cancer in the United States correlates with tumor histology, staging approach and surgical treatment. Patients with lower SES have an excess incidence of esophageal squamous cell carcinoma, whereas those with higher SES more often develop adenocarcinoma. Lower SES patients more often do not undergo staging procedures and it is demonstrated that black patients less frequently undergo esophageal resection. It is unknown whether such correlations are also present in esophageal cancer patients in Western Europe. We therefore assessed whether a correlation existed between SES of esophageal cancer patients and tumor histology, staging approach and treatment in The Netherlands. Methods: Between 1994 and 2003, 888 esophageal cancer patients were included in a prospective database with findings on diagnosis and treatment of esophageal cancer in the Erasmus MC Rotterdam, The Netherlands. The SES of patients was defined as the mean yearly income, which is collected on a zip code level by the Central Office of Statistics in The Netherlands. Chi-Square testing (linear-bylinear association) and multivariate logistic regression analyses were used to determine a correlation between SES of patients with esophageal cancer and tumor histology, type of staging procedures and treatment modality. Results: Linear-by-linear association testing revealed that esophageal adenocarcinoma was more frequently observed in patients with a higher SES ( $p=0.02)$. Multivariate logistic regression analysis showed no correlation between
SES and the different staging procedures. The odds ratios (OR) for the correlations between SES and stent placement, and SES and undergoing chemotherapy were however statistically significant, with a negative correlation between SES and stent placement (OR: 0.79; 95\% C.I. 0.65-0.96) and a positive correlation between SES and chemotherapy (OR: 1.16;95\% C.I. 1.02-1.32). Finally, no correlation was found between SES and undergoing radiotherapy (OR: 1.05; 95\% C.I. 0.85-1.28), and SES and undergoing esophageal resection (OR: 1.15 95\% C.I. 0.99-1.32). Conclusions: Socio-economic factors do not play a role in the decision making on diagnosis and treatment of esophageal cancer in The Netherlands. The negative correlation between SES and stent placement, and the positive correlation between SES and chemotherapy probably suggests that patients in a higher income class are more eager to fight their disease.

\section{M2096}

The Changing Epidemiology of Esophageal Cancer in the Middle East Twenty Year Experience from Kuwait Iqbal Siddique, Saeed Akhtar, Anjum Memon, Fuad Hasan

Aim: The incidence of adenocarcinoma of the esophagus and gastric cardia has been reported to be increasing in a number of regions throughout the world, while the incidence of squamous cell carcinoma of the esophagus is mostly stable or decreasing. However, there are no reports of the incidence of these malignancies from the Middle East. The aim of this study is to determine the incidence rates of these malignancies in Kuwait over a 20 year period. Methods: A population-based Kuwait Cancer Registry was established in Kuwait in 1979. Notification of all cancers to the registry by all hospitals in the country is compulsory through a Ministerial Decree. The registry collects information on malignant neoplasms according to the recommendations of the International Agency for Research on Cancer (IARC). Kuwaiti nationals with adenocarcinoma of the esophagus and gastric cardia, as well as squamous cell carcinoma of the esophagus were identified in the registry for a 20 year period between 1980 and 1999. Age-standardized rates (ASR) were calculated for these malignancies using the World Health Organization (WHO) world standard population distribution as reference. Results: The ASR (per 100,000) for adenocarcinoma of the esophagus and gastric cardia and squamous cell carcinoma of the esophagus are shown in Table 1. The ASR for adenocarcinoma of the esophagus and gastric cardia has increased slightly from 4.6 in 1979-1984 to 6.0 in 1995-1999, while the ASR for squamous cell carcinoma has fallen from 31.2 in 1979-1984 to 7.9 in 1995-1999. Conclusions: The incidence of adenocarcinoma of the esophagus and gastric cardia has increased slightly, while the incidence of squamous cell carcinoma of the esophagus has decreased markedly in Kuwaiti citizens between 1980 and 1999. Further research into esophageal and gastric cardia adenocarcinoma is needed to clarify the risk factors and mechanisms responsible for these changing trends as well as geographical disparities in incidence.

Table 1. Age-standardized Rates for Adenocarcinoma of Esophagus and Gastric Cardia and Squamous Cell Carcinoma of Esophagus in Kuwait (1980-1999)

\begin{tabular}{|c|c|c|c|c|}
\hline \multirow{2}{*}{ Type of Malignancy } & \multicolumn{4}{|c|}{ Age-standardized rates (per 100,000) } \\
\cline { 2 - 5 } & $1980-1984$ & $1985-1989$ & $1990-1994$ & $1995-1999$ \\
\hline Adenocarcinoma of esophagus and gastric cardia & 4.6 & 6.3 & 6.5 & 6.0 \\
\hline Squamous cell carcinoma of esophagus & 31.2 & 14.6 & 17.8 & 7.9 \\
\hline
\end{tabular}

M2097

Clinical and Endoscopic Factors Predict Higher Pathologic Grades of Barrett's Esophagus

Sharmila Anandasabapathy, Marta Davila, Caimaio Wei, Jeffrey Morris, Robert Bresalier

Background: Barrett's esophagus is highly prevalent in the western world, however, only a minority of those who develop Barrett's progress to esophageal adenocarcinoma. While many studies have examined risk factors for development of Barrett's metaplasia, little data is available on risk factors for progression to neoplasia. Identifying simple, reliable, clinical and endoscopic predictors of risk for development of high-grade dysplasia and adenocarcinoma would be helpful for risk stratification in screening and surveillance programs. Methods: Clinical, endoscopic, and histologic data were retrospectively reviewed on patients with a new diagnosis of Barrett's esophagus seen at M.D. Anderson Cancer Center between the years 2002-2005. Patients were classified, by an expert GI pathologist, as having intestinal metaplasia alone, indefinite-for-dysplasia or low-grade dysplasia, high-grade dysplasia, and esophageal adenocarcinoma. Gender, age, race, hiatal hernia presence, hiatal hernia size, Barrett's length, H. pylori status, BMI, alcohol, smoking, PPI use and duration, and duration of reflux symptoms were evaluated by logistic regression analysis for their association with dysplasia severity. Results: 109 patients ( 26 women, 83 men, mean age: 58.8 ) were newly diagnosed with either Barrett's metaplasia $(n=39)$, indefinite/low-grade dysplasia $(n=35)$ and high grade dysplasia/esophageal adenocarcinoma $(n=35)$ over a three-year period. On logistic regression analysis, duration of reflux symptoms for $>20$ years (OR: 5.67, $\mathrm{P}=0.0090$ ), Barrett's segment length $\geq 6 \mathrm{~cm}(\mathrm{OR}: 8.37, \mathrm{P}<0.0001)$, hiatal hernia size $>4 \mathrm{~cm}(\mathrm{OR}: 10.63$, $\mathrm{P}=0.0270$ ), and male gender ( $\mathrm{OR}: 4.03, \mathrm{P}=0.0018)$ were associated with higher grades of disease. Duration of reflux symptoms, Barrett's length, and hernia size were significant as both discrete and continuous variables. Absence of $H$. pylori $(\mathrm{OR}: 2.73, \mathrm{P}=0.0607)$ approached significance in predicting dysplasia severity. In a bivariate model, gender and Barrett's length (OR: $2.52, \mathrm{P}=0.0490$ and $\mathrm{OR}: 1.30, \mathrm{P}<0.0001$,) were both significantly associated with grade when considered together, as were gender and hernia size (OR: 4.64, P=0.0049 and OR: 12.18, $\mathrm{P}=0.0197$ ). Conclusions: Male gender, longstanding GERD, hiatal hernia size, and length of Barrett's esophagus are strongly associated with higher grades of dysplasia at index diagnosis. These factors, as well as $H$. pylori status, warrant further prospective evaluation as predictors of risk for development of high-grade dysplasia and esophageal adenocarcinoma in Barrett's esophagus. 
M2098

Angiotensin Converting Enzyme Inhibitors Reduce the Incidence of Esophageal Cancer: A Study of Half a Million US Veterans

Vikas Khurana, Kondal Kyanam, Gloria Caldito

AIM: To investigate the effect of Angiotensin Converting Enzyme (ACE) Inhibitors use in reducing the incidence of esophageal cancer in the US veteran population. BACKGROUND: ACE Inhibitors are commonly used antihypertensive and nephroprotective agents. Vascular Endothelial Growth Factor (VEGF) is believed to play a major role in angiogenesis in human tumors. Blocking the VEGF inhibits angiogenesis and suppresses tumor growth. ACE inhibitors cause suppression of VEGF in experimental models, leading to their anticancer effect. ACE Inhibitors have been noted to suppress tumor growth by inhibiting tumor angiogenesis in several animal and experimental models. DESIGN: The VISN 16 database contains clinical and demographic information about all veterans ( $>1.4$ million patients) cared for in the South Central VA Health Care Network (eight states in south central United States). The data was queried from Oct 1998 to June 2004, using a retrospective case control design. Statistical analysis was performed using SAS software version 9.0 (Chicago, IL). Multiple logistic regression analysis was used with calculation of odds ratios and 95\% confidence intervals. The data was adjusted for age, race, gender, BMI, smoking, alcohol use, reflux, NSIAD use, and diabetes. Patients were placed in the ACE inhibitor user group if they were using ACE inhibitors prior to the diagnosis of esophageal cancer. RESULTS: A total of 483,733 patients were included in the analysis. $185,830(38.4 \%)$ of those were using ACE inhibitors. Esophageal cancer (ICD code of 150) was seen in $659(0.14 \%)$. ACE inhibitor users were 55\% less likely to develop esophageal cancer (Odds ratio 0.546; $95 \%$ $\mathrm{CI} 0.450-0.662, \mathrm{p}<0.01$ ). The protective effect of ACE inhibitors was independent of statin use. Other significant covariates include age (OR 1.021, 95\% CI for OR 1.015, 1,028, p $<0.01$ ); reflux (OR 2.20, 95\% CI for OR 1.849, 2.618, p <0.01); prior statin use (OR 0.452, $95 \%$ CI for OR 0.369, 0.553, p<0.01) and diabetes (OR 1.314, 95\% CI for OR 1.064, 1.622, p<0.01). CONCLUSION: ACE inhibitors are associated with a $55 \%$ reduced incidence of esophageal cancer after controlling for age, race, gender, BMI, smoking, alcohol use, reflux, NSIAD use, diabetes and statin use. The limitations of our data are the VA population, the database and the fact that this is a case control study. The dose, duration and type of ACE inhibitor used were not factored into the analysis

\section{M2099}

Loss of Klf4 Alters the Proliferation-Differentiation Equilibrium in Mouse Esophageal Epithelia Bree G. Goldstein, Yizeng Yang, Jonathan P. Katz

Background and aims: Klf4 (Krüppel-like factor 4, previously known as GKLF) is a DNAbinding transcriptional regulator highly expressed in skin and gastrointestinal epithelia, specifically in regions of differentiating cells. Homozygous null mice for Klf4 die shortly after birth from skin defects, and conditional ablation of Klf4 in stomach alters epithelial proliferation and differentiation. In esophageal epithelia, Klf4 transcriptionally regulates a number of genes vital to proliferation, differentiation, and transformation, including keratin 4 , keratin 19, and ED-L2. The aim of this study was to analyze the function of Klf4 in esophageal epithelial proliferation and differentiation in vivo. Methods: The ED-L2 promoter of Epstein-Barr virus, previously shown to target expression to the squamous epithelia of tongue, esophagus, and forestomach, was used to drive Cre recombinase in esophageal epithelia. ED-L2/Cre founder lines were mated to Gt(ROSA)26 ${ }^{\mathrm{tm} 1 \mathrm{Sor}}$ reporter mice to screen for Cre expression and to Klf4loxP/oxP mice to produce esophageal-specific gene ablation of Klf4. Mice were examined grossly and tissues were harvested at 1,2 and 6 months of age for histology, immunohistochemistry, and RNA analyses. Mice were injected with BrdU 1 hour prior to sacrifice. Results: Using Gt(ROSA) $26^{\text {tm1sor }}$ reporter mice, we demonstrated Cre-mediated recombination in esophageal epithelia of two independent ED-L2/Cre lines. ED-L2/Cre:Klf4loxPloxP mice were born at appropriate Mendelian ratios, appeared grossly normal, and survived into adulthood. Staining for Klf4 revealed expression in skin of both control and ED-L2/Cre; Klf4loxP/loxP mice. In contrast, Klf4 expression was absent from esophageal epithelia of $\mathrm{ED}-\mathrm{L} 2 / \mathrm{Cre} ; \mathrm{Kl}$ f $4^{\mathrm{loxP} / \mathrm{oxP}}$ mice. Esophageal epithelia of $\mathrm{ED}-\mathrm{L} 2 / \mathrm{Cre} ; \mathrm{Kl}$ f4 $4^{\text {loxP/oxp }}$ mice were hyperplastic and had evidence of proliferation in suprabasal cells, which normally have ceased dividing and begun the differentiation program. Moreover, Klf5, a KLF family member with pro-proliferative and transforming properties which is normally confined to proliferating basal cells, was expressed throughout the suprabasal layer of EDL2/Cre; Klf4 $4^{\text {loxP/loxP }}$ mice. Conclusions: ED-L2/Cre transgenic mice represent a valuable tool for studies of esophageal function of genes where ablation in skin leads to early lethality. Loss of Klf4 in esophagus causes epithelial hyperplasia, and Klf4 inhibits proliferation in differentiating esophageal epithelial cells and downregulates Klf5 during the switch from proliferation to differentiation in esophageal epithelia. Thus, Klf4 is a key regulator of esophageal epithelial proliferation and differentiation in vivo.

\section{M2100}

Her-2/Neu Amplification and Over Expression in Esophageal Adenocarcinoma Bernard S. Stacey, James D. Shutt, Ghasem Yadegarfar, Praful Patel, Adrian C. Bateman

Aims: To test the hypotheses that HER-2/neu amplification is a frequent genetic event in esophageal adenocarcinoma and that gene amplification is associated with HER-2/neu protein expression, histopathological tumour characteristics and clinical outcome. Methods: Fluorescence in situ hybridization (PathVysion; Vysis, Downers Grove, IL) and immunohistochemistry (Herceptest; Dako, Glostrup, Denmark) was performed on formalin-fixed and waxembedded sections of 100 archival specimens of esophageal adenocarcinoma with a minimum five year clinical follow up, to assess HER-2/neu gene amplification and protein over expression respectively. Both the Herceptest and Pathvysion kits have been approved by the US Food and Drug Administration for use in breast cancer for determining which patients might benefit from trastuzumab therapy. The relationship of gene amplification and protein over expression to histopathological tumour characteristics and survival was determined. Results: Amplification of HER-2/neu was identified in 12 cases (12\%) of esophageal adenocarcinomas and this correlated with HER-2/neu protein over expression (scoring $2+$ or $3+$ by the Herceptest protocol) in 4 of these cases $(p<0.001)$. Amplification of HER-2/neu and chromosome 17 polyploidy co-existed in 2 cases. Chromosome 17 polyploidy alone was present in 26 cases $(26 \%)$, with 2 of these cases over expressing HER-2/neu protein $(p=0.09)$. No HER-2/neu protein over expression was seen in the $62 \%$ of tumours that were diploid fo chromosome 17 and without HER-2/neu amplification. Cox regression analysis indicated that patients with HER-2/neu gene amplification (HR=1.95, 95\% CI: $0.94-4.01, \mathrm{p}=0.07$ ) and chromosome 17 polyploidy ( $\mathrm{HR}=1.73,95 \% \mathrm{CI}$. $1.04-2.88, \mathrm{p}=0.04$ ) had higher risk of death than patients with disomy. However, there was no association between HER-2/neu amplification or chromosome 17 polyploidy and histopathological features such as tumour grade, pathological stage and node metastasis. Conclusions: Amplification of HER-2/neu as determined by fluorescence in situ hybridization occurs in some oesophageal adenocarcinomas and is associated with a trend to impaired survival. It is possible that this gene amplification might indicate the potential response of these tumours to trastuzumab therapy

\section{M2101}

Prospective Comparison of Transthoracic (TTe) Versus Transhiatal (THe) Oesophagectomy Following Neoadjuvant for Oesophageal Cancer Matthew Morgan, Wyn Lewis, S Burrows, D Goricha, Neil Hopper, Michael Stephens, Ashley Roberts, Alison Brewster, Tom Crosby, Timothy Havard, Geoffrey Clark

Aims. Transthoracic esophagectomy has been championed as a better cancer operation than transhiatal esophagectomy because the approach facilitates meticulous wide tumour excision and lymphadenectomy. Neoadjuvant chemoradiotherapy (CRTS) and chemotherapy (CS) have recently been reported to improve outcomes, and we aimed to compare outcomes after neoadjuvant treatment related to operative approach. Methods. One hundred and nine consecutive patients managed by a regional upper GI multidisciplinary team ( 3 specialist surgeons) were studied prospectively. All patients were staged with both CT and EUS, and treatment decisions were stage and performance status, related with THe reserved for patients with impaired respiratory reserve. Results (table). Conclusion. Despite the fact that patients undergoing THe were less fit, operative time was significantly less, operative mortality was similar, and there was a modest survival benefit associated with the transhiatal approach in addition to neoadjuvant therapy. Although follow up is relatively short, these results support the role of neoadjuvant therapy followed by THe in patients with respiratory comorbidity. Results

\begin{tabular}{|c|c|c|}
\hline & TTe & THe \\
\hline Number & 79 & 30 \\
\hline Neo CRTS vs. CS & $33: 46$ & $14: 16$ \\
\hline Operative time mins & 390 & $210 *$ \\
\hline Operative mortality n (\%) & $2(3)$ & $2(7)$ \\
\hline Lymph node count & 12 & 14 \\
\hline Recurrence $n(\%)$ & $34(43)$ & $8(26)$ \\
\hline 5 yr survival $(\%)$ & 40 & 57 \\
\hline
\end{tabular}

Figures are median, $\mathrm{P}<0.0001$.

\section{M2102}

Over-Expression of mRNA and Protein of CDC25B and LAMC2 in Esophageal Squamous Cell Carcinomas from a High-Risk Population in China Philip Taylor, Jin-Zhong Shou, Nan Hu, Mark Roth, Laura Lee Johnson, Carol Giffen, Quan-Hong Wang, Chaoyu Wang, Yuan Wang, Hua Su, Li-Hui Kong, Michael EmmertBuck, Alisa Goldstein, Stephen Hewitt

Purpose: To evaluate $C D C 25 B$ and $L A M C 2 \mathrm{mRNA}$ and protein expression in esophageal squamous cell carcinoma (ESCC). Experimental design: (i) Seventy-three ESCC cases with frozen tumor and matched normal tissues were evaluated for CDC25B and LAMC2 mRNA expression using a real-time quantitative RT-PCR and the $2-\triangle \Lambda C T$ method; and (ii) 373 cores on a tissue microarray representing 294 ESCC cases were evaluated for CDC25B and LAMC2 protein expression using immunohistochemical analysis. Results: (i) CDC25B mRNA was over-expressed ( $\geq 2 \mathrm{~N}$-fold over-expression in tumor compared to normal) in $66 \%$ of the 73 cases evaluated, while LAMC2 mRNA was over-expressed in $89 \%$ of cases. (ii) CDC25B protein was detected by immunohistochemistry in $93 \%$ (240/259) of tumors on the tissue microarray compared with only $39 \%(9 / 23)$ of histologically normal esophageal tissue cores $(\mathrm{P}<0.05)$; LAMC2 was detected in $92 \%(263 / 296)$ of tumor cores as well as all cores of normal epithelium. LAMC2 protein expression was significantly associated with a number of favorable tumor characteristics (differentiation, non-metastatic disease, and lower tumor grade) and patients who expressed LAMC2 protein had longer median survival (24 versus 14 months, $\mathrm{P}=0.017)$. Conclusions: $C D C 25 B$ and $L A M C 2$ showed marked over-expression of both mRNA and protein in ESCC, suggesting that these two genes may play an important role in development of ESCC and are potential early detection and prognosis biomarkers for ESCC.

\section{M2103}

Comparison of DNA Ploidy Determination By Flow Cytometry (FC) and Image Cytometry (IC) in Esophageal Adenocarcinoma (EAC)

Qin Huang, Chenggong Yu, Raj K. Goyal

Introduction. Purpose of this study was to compare the sensitivity of detection of DNA aneuploidy in EAC by flow cytometry (FC) with DNA content analysis of the affected cells using image cytometry (IC). Methods and Materials. 2 lformalin fixed and paraffin embedded archived tissues from 12 EAC cases were investigated. For the FC analysis two $50 \mu \mathrm{m}$ section 
were dissociated to generate single cells suspension; cells were stained with propidium iodide and analyzed on the FACS Calibur instrument. IC was performed using two adjacent $7 \mu \mathrm{m}$ sections, one H\&E stained for identification of the affected areas and the adjacent one Feulgen-stained for IC on the Automated Cellular Imaging System (ACIS). In FC aneuploidy was diagnosed when there was a distinct aneuploid peak in addition to a diploid peak. In IC, DNA index (DI) of the primary peak was determined and cases with DI $\geq 1.3$ were considered to be aneuploid. Results. FC detected aneuploid peaks in 12 (57\%) and diploid peaks with a high S-phase fraction over $15 \%$ in additional 3 samples. In all, $15(71 \%)$ tissues were aneuploid and 6 (29\%) were diploid. The diagnosis of aneuploidy on FC was poorly reproducible. In contrast all (100\%) EAC tissues reproducibly showed aneuploidy on IC. IC also showed that $2 / 3 \mathrm{rd}$ of the samples also had elevated cell fractions $(>14 \%)$ outside the primary peak. These samples may represent cancers with unstable aneuploidy. Summary and Conclusion: Our preliminary data showed that: (1) 29\% of EAC tissues were diploid on flow cytometry but aneuploid on image cytomtry; (2) Aneuploidy determination by flow cytometry was poorly reproducible. (3) Aneuploidy detection by IC using ACIS is more sensitive and reproducible than that by FC.

\section{M2104}

Photodynamic Therapy Is of Benefit in Treatment of Early Oesophageal Carcinoma

Carol F. Craig, Mhari MacPherson, Heather Hodgson, Joanna Gray, Mark Zammit, Grant Fullarton

INTRODUCTION The current gold standard treatment of early oesophageal carcinoma is oesophagectomy. The primarily elderly population affected are often medically unfit for this radical intervention and less invasive curative options are required. AIM To relate our experience of photodynamic therapy (PDT) as an alternative to surgery in early oesophageal cancer patients. METHODS 30 oesophageal cancer patients $(\mathrm{M}=20, \mathrm{~F}=10$; median age 74 years; $80 \%$ adenocarcinoma, $20 \%$ squamous) over a six year period were found to have early stage disease based initially on endoscopy/CT assessment and latterly on CT/ Endoscopic ultrasound criteria. Although potentially suitable for major surgical resection these patients were judged to be medically unfit and were selected to have PDT. 48 hours prior to endoscopy patients received iv porfimer sodium (photofrin ${ }^{\mathrm{TM}}$ ), $2 \mathrm{mg} / \mathrm{kg}$ as a photosensitising agent. Red laser light activation of the drug was initiated at endoscopy using a $630 \mathrm{~nm}$ fibre delivering a light dose of $300 \mathrm{j} / \mathrm{cm}$. Patients were followed up endoscopically at 6-12 week intervals indefinitely. Routine biopsies of the treated area were obtained. Median follow up was 30 months (2-56 months). RESULTS Overall 20/30 patients had an initial complete endoscopic and histological response to therapy 8 weeks post procedure. 1 patient died before reassessment of unrelated disease. 9 patients had residual tumour at endoscopy and were considered treatment failures. $8 / 20$ complete responders remained disease free for a median follow up of 1103 days (249-2019). 12/20 developed recurrent local disease treated with further PDT with a median survival of 922 days (254-2049). Only the latter 14 patients assessed had EUS staging. EUS accurately predicted response as shown in table 1: All Tl N0 patients ( 9 / 14) has initial complete response to treatment although $5 / 9$ have required further PDT sessions. All remain disease free currently. No patients with T2/3 NO disease had complete response to treatment. The major complication of PDT encountered was stricture formation which occurred in $50 \%$ of cases and required a median of 5 dilations (Range 1-31). CONCLUSION PDT is a well tolerated and potentially curative treatment in selected patients with early oesophageal carcinoma. Accurate T staging with EUS is essential in selection of suitable patients

Table 1. Response to PDT.

\begin{tabular}{|c|c|c|c|c|c|c|}
\hline & & $\begin{array}{c}\text { Initial failed } \\
\text { treatment }\end{array}$ & $\begin{array}{c}\text { Initial } \\
\text { complete } \\
\text { response }\end{array}$ & $\begin{array}{c}\text { No } \\
\text { recurrence }\end{array}$ & $\begin{array}{c}\text { Recurrence } \\
\text { successfully } \\
\text { retreated }\end{array}$ & $\begin{array}{c}\text { Recurrence } \\
\text { palliated only }\end{array}$ \\
\hline EUS & T1N0 & 0 & 9 & 4 & 5 & 0 \\
\hline NO EUS & T2/3NO & 5 & 0 & - & - & - \\
\hline $\begin{array}{c}\text { ALL } \\
\text { PATIENTS* }\end{array}$ & & 9 & 11 & 4 & 5 & 2 \\
\hline
\end{tabular}

*1 patient died prior to reassessment

\section{M2105}

Prognostic Significance of Body Mass Indices (BMI) in Patients Undergoing Esophagectomy for Carcinoma

Matthew Morgan, Neil Hopper, Wyn Lewis, Xavier Escofet, Alison Brewster, Tom Crosby, Timothy Havard, Geoffrey Clark

Aims. Obesity is prevalent in Western society, even in patients with esophageal cancer, and apart from increased technical operative difficulty overweight patients are more likely suffer co-morbidity and post operative complications. The aim of this study was to determine the role of body mass index (BMI) in a Western population on outcomes after esophagectomy for esophageal cancer. Methods. Between June 1, 1998 and August 30, 2005, 203 consecutive patients underwent esophagectomy by three specialist consultant upper GI surgeons. Patients with a BMI of greater than $25 \mathrm{~kg} / \mathrm{m} 2$ were classified as overweight $[\mathrm{n}=111(55 \%)$, median age $62 \mathrm{yr}, 87 \mathrm{~m}$ ), and 29 patients ( $14 \%$ ) were classified as obese (BMI $>30 \mathrm{Kg} / \mathrm{m} 2$ ). Results (table). Conclusion. Thus $55 \%$ of patients were overweight, and although high BMIs were associated with higher anaesthetic risk, this did not translate into higher operative mortality. Recurrence rates were $16 \%$ higher and long-term survival 13\% worse after R0 esophagectomy in patients with high when compared with low or normal BMIs. Results

\begin{tabular}{|c|c|c|}
\hline & Normal BMI & High BMI \\
\hline Number & 92 & 111 \\
\hline Neoadjuvant therapy & 50 & 52 \\
\hline Operative time mins TTe vs THe & $343: 204$ & $390: 206 *$ \\
\hline Lymph node count & 14 & $12 *$ \\
\hline Operative mortality N (\%) & $8(9)$ & $3(3)$ \\
\hline Recurrence $\mathrm{n}(\%)$ & $25(27)$ & $41(37)$ \\
\hline 5 yr survival (\%) & 42 & 29 \\
\hline
\end{tabular}

Figures are median. $\mathrm{P}$ not significant. $\mathrm{TTe}=$ transthoracic esophagectomy. $\mathrm{THe}=$ transhiatal esophagectomy.

\section{M2106}

Prospective Cohort Comparison of Neoadjuvant Chemoradiotherapy Versus Chemotherapy for Patients Diagnosed with Oesophageal Cancer

Matthew Morgan, Sarah Burrows, Deenita Goricha, Neil Hopper, Xavier Escofet, Ashley Roberts, Alison Brewster, Tim Havard, Geoff Clark, Tom Crosby, Wyn Lewis

Aims. Despite neoadjuvant chemo radiotherapy's (CRTS) early promise, associated postoperative morbidity and the publication of the results of $\mathrm{OEO} 2$, have resulted in neoadjuvant chemotherapy (CS) being the current neoadjuvant treatment of choice for resectable T3 NO/ $1 \mathrm{M} 0$ oesophageal carcinoma. We aimed to study the effects of both therapies on outcomes for patients treated within the framework of a regional multi disciplinary team. Methods. Between January 1, 1998, and 2000, 88 patients with resectable oesophageal carcinoma of any cell type received neoadjuvant chemo radiotherapy [ 2 cycles cisplatin $(60 \mathrm{mg} / \mathrm{m} 2)$ with $225 \mathrm{mg} / \mathrm{m} 2$ per day of infusional 5FU, prior to $45 \mathrm{~Gy}$ in $25 \mathrm{~F}$ concurrent with 3 weekly cisplatin $(60 \mathrm{mg} / \mathrm{m} 2)$ and infusional $5 \mathrm{FU} 200 \mathrm{mg} / \mathrm{m} 2 /$ day]. After the publication of OEO2 in 2002, 97 patients received neoadjuvant chemotherapy (two 4-day cycles, 3 weeks apart of Cisplatin $80 \mathrm{mg} / \mathrm{m} 2$ plus fluorouracil $1000 \mathrm{mg} / \mathrm{m} 2$ ). Results (table). Conclusion. Patients receiving neoadjuvant chemo radiotherapy were almost twice as likely to survive long-term than patients receiving chemotherapy provided they did not suffer serious postoperative morbidity and mortality. Neoadjuvant chemoradiotherapy should arguably remain an option for oesophageal cancer patients of satisfactory performance status. Results.

\begin{tabular}{|c|c|c|}
\hline & Chemo radiotherapy & Chemotherapy \\
\hline Number & 88 & 97 \\
\hline Age & $56(31-75)$ & $60(36-74)^{*}$ \\
\hline No operation n (\%) & $17(19)$ & $12(12)$ \\
\hline Op. mortality n (\%) & $6(7)$ & $1(1)^{* * *}$ \\
\hline Lymph node +ve & $0(0-8)$ & $2(0-14)^{* * * *}$ \\
\hline Complete path response $\mathrm{n}(\%)$ & $6(7)$ & $0 * * * *$ \\
\hline 5 yr survival (\%) & 36 & 22 \\
\hline
\end{tabular}

Figures are median (range), $* \mathrm{P}=0.019 * * \mathrm{P}=0.04,{ }^{* * *} \mathrm{P}<0.00001$, $* * * * \mathrm{P}=0.049$, **** $\mathrm{P}=$ 0.009

\section{M2107}

Gadd45 and P27 mRNA Expression in Esophageal Cancer: Association with Histopathologic Response to Neoadjuvant Therapy and Prognosis Daniel Vallbohmer, Hiroshi Higashi, Shuichi Hokita, Ralf Metzger, Ute Warnecke-Eberz, Stephan E. Baldus, Jan Brabender, Martin Kocher, Rolf-Peter Mueller, Takashi Aikou, Arnulf H. Hoelscher, Paul M. Schneider

Background: The DNA damage-inducible gene GADD45 and the tumor suppressor gene p27 are key factors in cell cycle regulation and apoptosis. There is some evidence that both genes might play a role in chemo- or radiation sensitivity of solid tumors. Recently, we have demonstrated that histomorphologic regression is a significant prognostic parameter for patients with esophageal cancer receiving neoadjuvant radiochemotherapy followed by surgery. The aim of this study was to examine the association of quantitative Gadd45 and p27 mRNA expression with histopathologic response to neoadjuvant radiochemotherapy followed by transthoracic en bloc esophagectomy and prognosis of patients with locally-advanced esophageal cancer. Patients and methods: 46 patients with potentially resectable esophageal cancers (cT2-4, Nx, M0) received neoadjuvant radiochemotherapy (cisplatin, 5-FU, $36 \mathrm{~Gy}$ ) followed by transthoracic en bloc esophagectomy. Histomorphologic regression was defined as major response when resected specimens contained less than $10 \%$ of residual vital tumor cells and minor response when resected specimens contained more than $10 \%$ of residual vital tumor cells. RNA was isolated from endoscopic biopsies (paired tumor and normal tissue) prior to neoadjuvant treatment and quantitative real-time RT-PCR was performed to determine Gadd45 and p27 mRNA expression levels. RNA expression levels were standardized for $\beta$-actin. Results: The mRNA expression of Gadd45 and p27 in pretherapeutic biopsies was not significantly different between paired tumor and normal tissue specimens (Wilcoxon test: $\mathrm{p}=0.34, \mathrm{p}=0.32$ ). Neither absolute mRNA expression levels in tumors or normal tissues nor the RNA ratio between tumor and paired normal tissue was associated with $\mathrm{pT}$, pN, grading, or prognosis. Histopathologic regression following neadjuvant therapy itself was significantly associated with patients prognosis (log rank: $p=0.01$ ). Absolute or relative expression levels of Gadd45 or p27 in pretherapeutic biopsies however, did not indicate histopathologic response following neoadjuvant treatment. Conclusion: Expression patterns of Gadd45 and p27 appear not to be involved in the pathogenesis or progression of esophageal cancer. In addition, Gadd45 and p27 mRNA expression levels did not predict 
histopathologic regression to neoadjuvant therapy or prognosis in patients with esophageal cancer

M2108

What Is the Relationship Between H.pylori Infection and Esophageal Malignancy?

Changcheng Wang, Yuhong Yuan, Richard H. Hunt

Purpose: A possible role of H.pylori infection in esophageal malignancies is unclear. No systematic review has analyzed the relationship between H.pylori infection and different types of esophageal cancer. We explored the relationship between H.pylori infection and development of esophageal cancer. Methods: MEDLINE and EMBASE English language searches for human studies were performed using keywords "(o)esophageal cancer or (o)esophageal neoplasms or (o)esophageal carcinoma" and "Helicobacter pylori or Campylobacter pylori" published to Oct 2005. Searches generated 132 citations, and 10 met inclusion criteria: cohort or case-control studies with raw data on prevalence of H.pylori infection in esophageal cancer and controls; H.pylori detected by histology and/or serology; studies in adult populations. Exclusion criteria: studies without controls or raw data for retrieval and duplicate publications. Results: 1194 patients with esophageal cancer and 3497 controls were identified. Overall prevalence of H.pylori infection and cagA+ strains in esophageal cancer cases compared with controls was $32.6 \%(389 / 1194)$ vs $44.7 \%(1563 / 3497)$ $(\mathrm{p}<0.0001, \mathrm{OR}=0.60,95 \% \mathrm{CI} 0.48-0.76)$ and $25.9 \%(173 / 669)$ vs $33.5 \%(611 / 1824)$ $(\mathrm{p}<0.0001, \mathrm{OR}=0.66,95 \% \mathrm{CI} 0.54-0.81)$, respectively. The prevalence of H.pylori infection and cagA+ strains was significantly lower in esophageal adenocarcinoma than that in controls [34.3\% (184/536) vs $47.7 \%(1196 / 2506), \mathrm{p}<0.0001, \mathrm{OR}=0.55,95 \% \mathrm{CI} 0.41-0.73]$ and [18.9\% (79/417) vs 33.5\% (611/1824) ( $\mathrm{p}=0.0002, \mathrm{OR}=0.47,95 \% \mathrm{CI} 0.32-0.70)]$, respectively. In 3 small studies in squamous cell cancer $(n=91)$ there was marked heterogeneity (test for heterogeneity: $\mathrm{p}<0.0001$ ) and no significant difference between H.pylori prevalence in esophageal squamous cell cancer and controls [34.3\% (91/265) vs 40.5\% (356/880), p= $0.80, \mathrm{OR}=0.89,95 \% \mathrm{CI} 0.35-2.25]$. Conclusion: H.pylori infection and cagAt strains are inversely related to esophageal adenocarcinoma strongly suggesting that H.pylori infection has no aetiological role in esophageal adenocarcinoma. There is no clear relationship between esophageal squamous cell cancer and H.pylori infection.

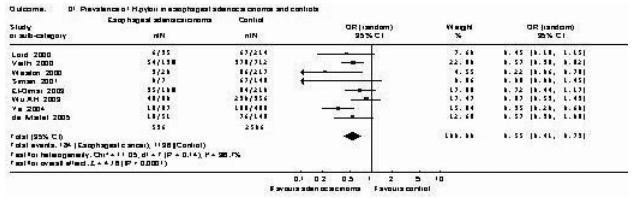

Fig. Prevalence of H.pylori infection in esophageal adenocarcinoma and controls

\section{M2109}

No Methylation Or Reduction in the Expression of TIMP3 in Squamous Cell Carcinoma of the Esophagus from a Region of High Incidence in China Eric Smith, Maria Caruso, Zi-Qing Tian, Jun-Feng Liu, John J. Kelly, Andrew R. Ruszkiewicz, Paul A. Drew

TIMP3 is an inhibitor of the proteolytic activity of matrix metalloproteinases and therefore may have the potential to reduce tumour invasion and metastasis. It has also been reported to induce apoptosis in cancer cells and suppress tumour growth and angiogenesis. It is downregulated in a number of cancers, and linked to a poor outcome. Loss of expression has been associated with methylation of the TIMP3 gene promoter in tumors, including esophageal adenocarcinoma. In a study of patients from Gunma, Japan, the only report of TIMP3 expression in esophageal squamous cell carcinoma, a reduction in its expression was seen in $67 \%$ of 90 tumours, which correlated with invasive activity and metastasis. Although methylation was suggested as a possible mechanism for the observed downregulation, it was not assessed in the study. The aim of this study was to measure methylation in the promoter of the TIMP3 gene in squamous cell carcinoma and relate this to any change of gene expression. Tumours and proximal resection margins were obtained from 51 patients operated on for esophageal squamous cell cancer at the 4th Hospital of Hebei Medical University, China. During the follow up period (mean 569 days) 9 patients had died (mean survival of 476 days after the operation), 27 were alive, and 15 were lost to follow up. There were $3 \mathrm{~T} 1$ tumours, $11 \mathrm{~T} 2,30 \mathrm{~T} 3$ and $4 \mathrm{~T} 4$, with node involvement recorded for $30 \%$, and for 3 no staging information was available. No methylation was detected in the TIMP3 gene promoter in any of the tumour or proximal margin tissues. Expression of TIMP3 was determined by immunohistochemistry. After microwave induced antigen retrieval the sections were stained using a monoclonal antibody to TIMP3 (Chemicon) and the MACH4TM universal probe HRP-polymer kit (Biocare Medical). All cases showed strong heterogeneous cytoplasmic staining in cancer cells. Strong heterogeneous cytoplasmic expression of TIMP3 was also observed in the normal proximal resection margin mucosa in 5 cases, but was limited to the basal layers of squamous epithelium. There was no evidence of reduced or partial staining. In this cohort of patients with esophageal squamous cell carcinoma of the esophagus there was no methylation of the TIMP3 gene promoter nor alteration in TIMP3 protein expression, regardless of the stage of or outcome from the disease. Further studies are required to determine if the conflicting results on TIMP 3 expression in this cancer are a result of methodological differences, or reflect a difference in aspects of the molecular biology of the disease between regions.

\section{M2110}

Late Toxicity After Definitive Chemoradiotherapy for Patients with Esophageal Squamous Cell Carcinoma

Yosuke Kumekawa, Kazuhiro Kaneko, Hiroaki Ito, Toshinori Kurahashi, Taikan Yamamoto, Meiko Kuwahara, Yotaro Kubota, Takashi Muramoto, Michio Imawari

Background/Aims: We retrospectively investigated the long-term toxicity after concurrent chemoradiotherapy (CRT) for patients with esophageal squamous cell carcinoma (ESCC) Patients and Methods: Concurrent chemoradiotherapy was performed in 110 patients with $\mathrm{Tl}$ to T4 disease containing Ml lymph node (LYM) disease. Our recruitment criteria were defined as follows: age $<75$ years, ECOG performance status 0 to 2, normal heart and pulmonary function, and clinical stage I to IVA. Chemotherapy consisted of protracted infusion of 5 -fluorouracil $400 \mathrm{mg} / \mathrm{m} 2 / 24$ hours on days 1 to 5 and 8 to 12 , combined with 2-hour infusion of cisplatin $40 \mathrm{mg} / \mathrm{m} 2$ on days 1 and 8 . Radiation treatment at a dose of 30 Gy in 15 fractions of the mediastinum was administered concomitantly with chemotherapy. A course schedule with 3 -week treatment and 2-week break was applied twice, with a tota radiation dose of $60 \mathrm{~Gy}$, followed by two or more courses of 5 -fluorouracil $800 \mathrm{mg} / \mathrm{m} 2 / 24$ hours on days 1 to 5 and cisplatin $80 \mathrm{mg} / \mathrm{m} 2$ on days 1 . For the assessment of toxicity, the Radiation Therapy Oncology Group/European Organization for Research and Treatment of Cancer late radiation morbidity scoring schema was adopted. Results: Of 110 patients who received definitive CRT, 29 patients who received palliative CRT and did not match the recruitment criteria were excluded from this study. A total of 81 patients were recruited, and their characteristics were as follows: median age, 63 years ranging from 42 to 75 years 74 men and 7 women. The clinical staging was as follows: stage I in 8 patients, stage II in 14 patients, stage III in 25 , and stage IV in 34 . There were 22 patients with T3 disease $(27 \%)$, and 44 with T4 disease (54\%). Of 81 patients, 33 had Ml LYM disease (41\%). The median survival time was 14 months, and the 3 -year survival rate was $22 \%$. Thirty-four patients ( $42 \%$ ) achieved complete response (CR). Of 34 patients with CR, one patient died as a result of acute myocardial infarction. Grade 2, 3, and 4 late toxicities occurred with the following incidences: pericarditis in 3 patients, 1 patient, and 2 patients, respectively heart failure in 0,0 , and 3 patients; pleural effusion in 2, 3, and 0 patients; and radiation pneumonitis in 0,0 , and 1 patient, respectively. In total, 4 patients died without cancer recurrence, and these causes of death may have been related to cardiopulmonary toxicity Conclusion: Definitive chemoradiotherapy for ESCC is effective with substantial toxicities. Further investigation is warranted to minimize the normal tissue toxicities.

\section{M2111}

The Effect of CDGP On Phosphorylation of Histone H2ax Induced By Irradiation in Esophageal Squamous Cancer Cells

Takahisa Furuta, Naohito Shirai, Mitsushige Sugimoto, Akiko Nakamura, Satoshi Osawa, Mutsuhiro Ikuma, Masayoshi Kajimura, Akira Hishida

Backgrounds/Aims: Chemoradiation therapy with irradiation (IR) and a platinum agent, such as CDDP (Cisplatin) or CDGP (Nedaplatin), yields high cure rate in esophageal cancer. However, the mechanisms by which the platinum agent enhances the cellular susceptibility to IR have not been fully elucidated. When irradiation induces DNA double strand breaks (DSBs) in cells, histone H2AX is immediately phosphorylated. The phosphorylated H2AX is called as $\mathrm{gH} 2 \mathrm{AX}$, which is the sensitive marker of DSBs. Then, we studied the effect of platinum agent, such as CDGP, on the IR-induced gH2AX. Methods: Esophageal squamous cancer cells (JCRB0190 [KYSE-70]) were exposed to 0 or 2 Gy of IR. At 15 min before IR CDGP or control medium was added to the culture medium (the final concentration of CDGP was $3 \mu \mathrm{M})$. The medium was changed with the fresh medium without CDGP at 3 hours after IR to remove the CDGP. Before and 1, 3, and 24 hours after IR, induction of gH2AX was determined by an immunofluorescence and westernblotting analyses. Tunnel staining was also performed. Results: 2 Gy of IR induced gH2AX, which reversed within 24. This reversal was inhibited by incubation with $3 \mu \mathrm{M}$ of CDGP for 3 hours. gH2AX induction by $3 \mu \mathrm{M}$ of CDGP alone was weak. At 24 hours after IR, tunnel positive cells were observed in cells treated with IR and CDGP. When cells were treated with 2 Gy of IR alone or $3 \mu \mathrm{M}$ of CDGP 3 hours alone, tunnel positive cells were very small throughou the study period ( 0 - 24hrs). Conclusion: The low dose of CDGP inhibits the repair of DSBs induced by IR, which would be related to the high cure rates of chemoradiation therapy for esophageal cancer with IR and CDGP.

\section{M2112}

Therapeutic Gene Transfer of a Glycosyltransferase, Which Is Exclusively Expressed in Normal Gastrointestinal Mucosa, Efficiently Prevents Gastric Cancer Metastasis

Yuki I. Kawamura, Yasuo Adachi, Rei Kawashima, Noriko Mizutani, Noriko ToyamaSorimachi, Norihiro Nishimoto, Taeko Dohi

Increased expression of sialyl Lewis $\mathrm{x}$ and sialyl Lewis a carbohydrate determinants correlates with clinical stages and formation of metastatic lesions for both gastric and colon cancer. Recently we reported that forced expression of a $\beta 1,4 \mathrm{~N}$-aceytylgalactosaminyltransferase synthesizing $S d^{a}$ carbohydrate antigen $\left(S^{a}{ }^{a}-\beta 1,4 G a l N A c T\right)$ in gastrointestinal (GI) cancer cells reduced their expression of sialyl Lewis x/a carbohydrates, ligands for E-selectin, and decreased the metastatic potential (Cancer Res., 65 , 6220-6227, 2005). Here, we aimed to control expression of sialyl Lewis $\mathrm{x} / \mathrm{a}$ carbohydrate determinants of cancer cells using $\mathrm{Sd}^{\mathrm{a}}$ $\beta 1,4 \mathrm{GalNAcT}$ gene in vivo, and eventually prevent metastasis. For this purpose, we used fiber-knob-modified adenoviral (Ad) vector, which contains the chimeric construct with a serotype 5 shaft and a serotype $3 \mathrm{knob}(\mathrm{Ad} 5 / 3)$. Using Ad5/3 vectors to introduce the $\mathrm{Sd}^{\mathrm{a}}$ $\beta 1,4$ GalNAcT gene into human gastric cancer cell line KATO III in vitro, we confirmed that almost all cells expressed $\mathrm{S} \mathrm{d}^{\mathrm{a}}$ along with the concomitant decrease of sialyl Lewis $\mathrm{x}$ antigen. Ad5/3-Sda $-\beta 1,4$ GalNAcT-infected KATO III cells showed significantly decreased adhesion to E-selectin-expressing CHO cells compared with Ad5/3-GFP-infected cells. We assessed the therapeutic effect of treatment with $\mathrm{Ad} 5 / 3-\mathrm{Sd} \mathrm{d}^{\mathrm{a}}-\beta 1,4 \mathrm{GalNAcT}$ vectors in vivo mouse peritoneal metastatic model using KATO III cells. Administration of Ad5/3-Sd ${ }^{a}-\beta 1,4$ GalNAcT 
vectors after inoculation of KATO III cells significantly reduced the incidence of metastasis compared with that of Ad5/3-GFP. These results indicate that $S d^{\mathrm{a}}-\beta 1,4 \mathrm{GalNAcT}$ gene transfer modified carbohydrate chains of cancer cells in vivo and has therapeutic interference with selectin-mediated tumor progression.

\section{M2113}

An Orthotopic Nude Mouse Model to Evaluate Novel Treatment Strategies for Gastric Cancer in Corpus and Cardia

Birgit Hotz, Hubert G. Hotz, Sarah Bhargava, Heinz J. Buhr

Background: Carcinoma of the stomach remains one of the most common causes of cancer death. In particular, the incidence for cancers of the gastro-esophageal junction (cardia) is rising in the western world. A clinically relevant animal model does not exist for this malignancy. The aim of the present study was to establish an orthotopic model for human gastric cancer of the corpus and the cardia in nude mice. Methods: 5 million cells of the human gastric cancer cell line MKN-45 (diffuse type) were injected subcutaneously into the flanks of nude mice. These donor tumors were harvested after 4 weeks and minced into small tumor fragments $(1 \mathrm{cmm})$. One donor tumor fragment was orthotopically implanted by microsurgery into the wall of either gastric corpus or cardia in 30 other mice. The animals were sacrificed 4,8 , and 12 weeks after tumor implantation. Volume of the primary tumor, local and systemic tumor spread (dissemination score) were determined at autopsy. $\mathrm{H} \& \mathrm{E}$ stained sections were analyzed from all resected organs in order to confirm the macroscopic findings. Results: The microsurgical implantation technique resulted in a tumor take rate of $100 \%$. An artificial dissemination of tumor cells into the abdominal cavity due to the procedure was not observed. The time course of local and systemic tumor development is summarized in the table (means $\pm \mathrm{SD}$ ). Conclusions: This study reports for the first time the development of a clinically relevant nude mouse model for human gastric cancer of the corpus and cardia. Primary tumors, local and systemic spread grow continuously during the observation period and mimic the human situation. This model may be suitable to evaluate novel treatment strategies for this still deadly malignancy.

\begin{tabular}{|c|c|c|c|c|c|}
\hline Corpus & $\begin{array}{l}\text { Tumor } \\
\text { volume } \\
(\mathrm{cmm})\end{array}$ & $\begin{array}{c}\text { Dissemination score } \\
\text { (points) }\end{array}$ & $\begin{array}{c}\text { Liver } \\
\text { metastasis ( } / \\
\text { n) }\end{array}$ & $\begin{array}{c}\text { Lung } \\
\text { metastasis (n / } \\
\text { n) }\end{array}$ & $\begin{array}{l}\text { Lymph node } \\
\text { metastasis }(n / n)\end{array}$ \\
\hline 4 weeks & $10 \pm 5$ & $1.0 \pm 1.2$ & $0 / 5$ & $0 / 5$ & $0 / 5$ \\
\hline 8 weeks & $270 \pm 240 *$ & $2.0 \pm 0.8$ & $0 / 5$ & $0 / 5$ & $0 / 5$ \\
\hline $\begin{array}{c}12 \\
\text { weeks }\end{array}$ & $560 \pm 477^{*}$ & $6.0 \pm 3.5^{*}$ & $0 / 5$ & $2 / 5$ & $1 / 5$ \\
\hline Cardia & $\begin{array}{l}\text { Tumor } \\
\text { volume } \\
(\mathrm{cmm})\end{array}$ & $\begin{array}{c}\text { Dissemination score } \\
\text { (points) }\end{array}$ & $\begin{array}{c}\text { Liver } \\
\text { metastasis ( } / \\
\text { n) }\end{array}$ & $\begin{array}{c}\text { Lung } \\
\text { metastasis (n / } \\
\text { n) }\end{array}$ & $\begin{array}{c}\text { Lymph node } \\
\text { metastasis }(n / n)\end{array}$ \\
\hline 4 weeks & $51 \pm 48$ & $1.8 \pm 0.8$ & $0 / 5$ & $0 / 5$ & $0 / 5$ \\
\hline 8 weeks & $171 \pm 134$ & $2.8 \pm 1.9$ & $0 / 5$ & $1 / 5$ & $0 / 5$ \\
\hline $\begin{array}{c}12 \\
\text { weeks }\end{array}$ & $306 \pm 300$ & $9.8 \pm 9.6$ & $1 / 5$ & $2 / 5$ & $1 / 5$ \\
\hline
\end{tabular}

(means $\pm \mathrm{SD} ; *=\mathrm{p}<0.05$ vs. 4 weeks)

\section{M2114}

Use of Angiogenesis Inhibitor Bevacizumab in Minimizing Peritoneal Dissemination of Stomach Cancer - A Randomized Control Trial in An Animal Model

Enders K. Ng, Angela M. Hui, Lydia K. Lung, Wai Keung Leung, Joseph J. Sung

Background: Transcelomic dissemination is a peculiar feature of gastric adenocarcinoma. Angiogenesis induced by free floating cancer cells may be an important mechanism for gastric cancer deposits to sustain and survive on the peritoneum. This study aims to determine whether Bevacizumab (Avastin), a humanized anti-VEGF monoclonal antibody, is effective in minimizing peritoneal spread of stomach cancer in an in vivo animal model. Methods: Fifteen female nude mice of age 4 to 6 weeks were inoculated intraperitoneally with a fixed dose (1 x 106) of MKN45 (gastric cancer cell line)suspension. They were immediately randomized into 3 groups. Group A and Group B were given once weekly intra-peritoneal Bevacizumab at low dose (5ug per g body weight) and high dose (10 ug per g body weight), respectively. Group $C$ was served as the control and administered with only normal saline. All nude mice were nursed in the same ambient environment and fed de liberal. Their body weight was monitored daily till abrogation on day-28. Results: By day-28, the overall weight loss was more prominent in the control group (14.5\%) when compared to that of Group A (5.9\%) and Group B (11.4\%). Upon laparotomy, significantly more peritoneal tumor deposit was found in the control group. In addition, the control group had significantly larger mean peritoneal tumor mass load than the treatment groups (Group $A=41 \mathrm{mg}$, Group B $=62 \mathrm{mg}$, Group C $=154 \mathrm{mg} ; \mathrm{p}=0.0001$, One-way ANOVA). When peritoneal tissues adjacent to the peritoneal tumour nodules were harvested for immunohistochemical stain, those treated with Bevacizumab had significantly lower microvascular density when compared to the control. Conclusion: Inhibition of VEGF reduces the extent and amount of peritoneal gastric cancer dissemination in a nude mice model. The low dose regimen appears to work better than the high dose regimen. Clinical studies are warranted to assess its potential role in preventing or controlling peritoneal dissemination related to stomach cancer.

\section{M2115}

Helicobacter Pylori and Upper Gastrointestinal Tract Cancers: The Results of a Long-Term Prospective Study

Farin Kamangar, You-Lin Qiao, Hormuzd Katki, Christian C. Abnet, Zhi-Wei Dong, Philip R. Taylor, Sanford M. Dawsey

Helicobacter pylori is an important risk factor for gastric non-cardia adenocarcinomas. However, its association with gastric cardia adenocarcinoma and esophageal squamous cell carcinoma is less clear. We conducted this long-term prospective case-cohort study to estimate the magnitude of the association of $H$. pylori seropositivity with risks of these upper gastrointestinal cancers, and to examine these associations by time from serum collection to cancer diagnosis, age, and CagA positivity. Cases and controls for this study were selected from a cohort of 29,584 healthy residents of Linxian, China, who participated in a nutrition intervention trial. The study participants were followed from 1985 to 2001. Loss to followup was $<1 \%$. We selected a random sample of esophageal squamous cell carcinomas $(N=300)$, gastric cardia adenocarcinomas $(N=600)$, all diagnosed gastric non-cardia adenocarcinomas $(N=363)$, and a random sample of the entire cohort $(N=1050)$. Ninetyfive percent of these subjects had adequate serum for $\mathrm{H}$. pylori assays and were enrolled in this study. Serum was evaluated for IgG antibodies to whole-cell and CagA H. pylori antigens by enzyme-linked immunosorbent assay (ELISA). Risks of both gastric cardia and non-cardia cancers were similarly increased in individuals exposed to H. pylori (Hazard ratio and 95\% $\mathrm{CIs}=1.64 ; 1.26-2.14$, and $1.60 ; 1.15-2.21$, respectively), whereas risk of esophageal squamous cell carcinoma was not associated with exposure to $H$. pylori $(1.17 ; 0.88-1.57)$. The strength of these associations was not modified by time from serum collection to cancer diagnosis or by CagA positivity. Consistent with previous studies, associations were stronger in younger individuals, but the interaction terms between age and $H$. pylori were not statistically significant. The associations between $H$. pylori exposure and gastric cardia and non-cardia adenocarcinoma development were equally strong, which is different from observations made in Western countries. This difference may be due to the absence of Barrett's esophagus and esophageal adenocarcinomas in Linxian, which makes all tumors classified as "cardia cancers" of gastric origin, rather than a mixture of gastric and esophageal tumors.

\section{M2116}

Comprehensive Analysis of Allelic Imbalance By Using High-Density Single Nucleotide Polymorphism Array in Gastric Cancer Cell Lines

Motohisa Tada, Fumihiko Kanai, Yasuo Tanaka, Lian-Jie Lin, Keisuke Tateishi, Osamu

Yokosuka, Masashi Sanada, Yasuhito Nannya, Seishi Ogawa, Masao Omata

(Background and Aim) Chromosomal copy number alterations can lead to activation of oncogenes and inactivation of tumor suppressor genes in human cancers and multiple genetic alterations, occurring sequentially in a cell lineage, underlie the carcinogenetic process in gastric cancer. Though comparative genomic hybridization $(\mathrm{CGH})$ was initially used to detect chromosomal copy number changes, it was difficult to identify key molecule for carcinogenesis because of low resolution (about $20 \mathrm{Mbp}$ ). In this study, we sought to identify the key molecule in gastric cancer pathogenesis by using high-density single nucleotide polymorphism array. (Materials and Methods) We analyzed 34 gastric cancer cell lines for areas of DNA copy number changes (high grade amplification and homozygous deletion) and loss of heterozygosity ( $\mathrm{LOH}$ ) through genome-wide analysis of 116, 204 SNPs distributed across all human chromosomes with Affymetrix GeneChip Human Mapping 100 K Set (mean intermarker distance 23.6 Kbp). Copy Number Analyzer for GeneChip (CNAG)(Nannya et al, Cancer Res 2004), our original analysis tool that automates the detection of copy number and regions of $\mathrm{LOH}$, was used for data analysis. The results from the array analysis were verified by PCR and quantitative real-time PCR. (Results) Thirty-four regions of high grade amplification ( $\mathrm{n} \geqq 5$ ) were found in at least two cell lines. The size of minimal common amplified region was $60 \mathrm{Kbp}$. Thirty regions of homozygous deletion were detected in at least one cell line. The size of minimal deletion was $11 \mathrm{Kbp}$. In addition to known genetic abnormality, such as amplification of K-ras gene and deletion of FHIT gene, high grade amplification of Chr.1lq21 region and homozygous deletion of Chr.16p13.3 region were newly identified. LOH at 3p, 4p, 4q, 5q, 7q, 8p, 8q, 9p, 12q, 17p, 18q was observed with high frequency. Moreover, this study detected allelic homozygosity (AH) at each chromosome. Among them, $\mathrm{AH}$ was detected at $4 \mathrm{q}, 16 \mathrm{q}, 17 \mathrm{p}$ and $18 \mathrm{q}$ with high frequency. (Conclusion) By using Affymetrix GeneChip Human Mapping $100 \mathrm{~K}$ Set and CNAG, we could detect minimal region of copy number alteration, which had never been able to detect by other methods. Moreover this study demonstrated that $\mathrm{AH}$ might be one of the mechanisms of gastric carcinogenesis. The novel amplified and deleted regions discovered in this study may be significant and useful for early diagnosis and novel therapy based on molecular targeting in gastric cancer.

\section{M2117}

Predictive Markers for Responsiveness to Chemo-Radiation Therapy in Patients with Esophageal Squamous Cell Carcinoma: Immunohistochemical Analysis of Pretherapeutic Biopsy Specimens Yukihiro Nakanishi, Yae Kanai, Setsuo Hirohashi

(Background) The responsiveness of esophageal squamous cell carcinoma (ESCC) to chemoradiation therapy (CRT) is difficult to evaluate before treatment. Therefore, it would be of considerable value to define parameters in pretherapeutic biopsy specimens that would identify patients likely to benefit from CRT for ESCC. (Methods) Nine previously reported predictive markers for responsiveness of ESCC to CRT (cyclin D1, p53, hypoxia-inducible factor, metallothionein, c-erbB-2, vascular endothelial growth factor, cyclooxygenase 2, MIB1, CD31 (microvessel density)) were investigated immunohistochemically in pretreatment biopsy samples from 75 consecutive patients staged at II and III who underwent definitive chemo-radiation therapy with 5FU+cisplatin+ radiation (60 Gy). We looked for correlations between these nine molecules and their associations with clinical response to CRT, and tried to determine the best combination of the molecules for predicting response to CRT. When more than $5 \%$ of the tumor cells were positively stained in pretreatment biopsy specimens, 
tumors were scored as positive. The percentage of MIB-1-positive tumor cells was obtained by counting positive tumor cell nuclei in the whole area of each biopsy specimen. Microvessel density was obtained by counting brown-stained vessels in the highest vascular density area within the tumor. In terms of clinical response to CRT, 27 (36\%) cases showed complete response (CR) without recurrence, 23 (31\%) cases showed recurrence after CR, and 25 (33\%) cases showed partial response, stable disease, or progressive disease (mean followup period: 40.8 months (range, 16.8-74.4 months)). (Results) Univariate analysis revealed significant associations between the responsiveness to CRT and negative expression of cyclin D1 and p53 ( $\mathrm{p}=0.03,0.01$, respectively). Multivariate analysis also revealed significant associations between the responsiveness to CRT and negative expression of cyclin Dl and $\mathrm{p} 53(\mathrm{p}=0.03,0.01$, respectively). Trend test revealed that a combination of cyclin Dl and p53 expression most accurately predicted the responsiveness to CRT $(p=0.001$ ). (Conclusion) Immunohistochemical examination of pretherapeutic tumor biopsy samples for expression of cyclin Dl and p53 could help to identify patients with ESCC who would benefit from CRT.

M2118

Inhibition of VEGFR Reduces Polyp Burden in a Mouse Model of Intestinal Cancer

Denis Alferez, Robert A. Goodlad, James Watkins, Anderson Ryan, Steve Wedge, Richard Poulsom, Ian Pyrah, Nikki Mandir, Robert Wilkinson

Introduction: AZD2171, a novel, orally active inhibitor of vascular endothelial growth factor receptor-2 (VEGFR-2) tyrosine kinase activity was used to study the effect of angiogenesis blockade in multiple intestinal neoplasia (ApcMin/+) mice. ApcMin/+ mice develop numerous polyps due to a mutation in the adenomatous polyposis coli (APC) gene, as in familial adenomatous polyposis (FAP) in humans. Methods: Two studies were performed in which $5 \mathrm{mg} / \mathrm{kg} /$ day of AZD2171 or vehicle was administered daily by oral gavage to 6-week old or to 10 week old ApcMin/+ mice for 28 days after which the number and size of polyps in the small and large intestines were scored. Results: AZD2171 reduced polyp number in the small bowel and colon by $46 \%$ and $62 \%$, respectively $\mathrm{P}<0.05$ in the first study. Polyp diameter was also reduced by $39 \%$ in the small bowel $(\mathrm{P}<0.001)$ but was unchanged in the colon. Mean tumour burden (the product of number and volume) in the small intestine was thus reduced $85 \%(\mathrm{P}<0.001)$. In the second study small bowel polyp number was not altered by AZD2171 but diameter was reduced by $24 \%$, in turn decreasing polyp burden by $46 \%(\mathrm{P}<0.059)$. AZD2171 had little effect on the nuclear localisation of $\beta$-catenin but reduced the number of cells expressing VEGFR-2 (from $7.4 \pm 0.6$ to $4.0 \pm 0.9$ per villus, $\mathrm{P}<0.022)$. Conclusion: AZD2171 significantly reduced the number and size of polyps, mainly in the earlier stages of polyp formation. VEGFR-2 signalling plays a key role in the development of intestinal adenomas.

\section{M2119}

The Disintegrin Metalloproteinase ADAM17 Is Increased in Gastric Cancer K Danjo, A Corvalan, Pa Robinson, Je Crabtree

Introduction: The ADAM (A disintegrin and metalloproteinase) family of enzymes are membrane-anchored cell surface glycoproteins which have a key role in ectodomain shedding of membrane bound receptors, growth factors and cytokines. Previous studies have shown increased ADAM17 transcripts in gastric tumour tissue, and that Helicobacter pylori upregulates ADAM17 transcript levels in gastric cancer cell lines in vitro. The aims of this study were to evaluate whether the differential expression of ADAM17 in gastric cancer tissue at the transcript level could be confirmed immunohistologically, and to evaluate the relation of ADAM17 to clinicopathological variables. Methods: Tissue and clinicopathological data were collected from 54 patients (mean age $58.3+14.9$ years) undergoing surgical resection for gastric cancer. Endoscopic gastric biopsies from seventeen patients without gastric cancer were used as non-cancer controls. ADAM17 was detected using a mouse monoclonal antibody M220 $(0.16 \mathrm{mg} / \mathrm{ml})$, biotinylated anti-mouse antibody and avidin-biotin-peroxidase. Primary antibody was omitted as a negative control. Cytoplasmic ADAM17 positivity in gastric cancer cells was scored on a scale of 0-3. Results: Forty four of fifty four (81.5\%) gastric cancer patients were positive for cytoplasmic ADAM17 with gastric tumour cells having immunoreactivity scores ranging from 1 to 3 . In contrast, in non-cancer controls, ADAMl7 was detected in lamina propria mononuclear cells, but cytoplasmic epithelial immunoreactivity was observed in only one of seventeen patients $(p<0.001)$. There was no significant difference between ADAM17 positivity and grading score between intestinal-type and diffusetype gastric cancers, or anatomic location of the tumour (cardia, body or antrum). Increased ADAM17 positivity was evident in gastric tumours greater than $5 \mathrm{~cm}(20 / 23 ; 87 \%)$ than tumours less than $5 \mathrm{~cm}(9 / 13 ; 69 \%)$. ADAM17 positivity was also increased in advanced (39/47; 83\%) cancers compared to early gastric cancers $(4 / 6 ; 67 \%)$. Conclusion: Higher ADAM17 transcript levels reported previously are matched by increased expression of ADAM17 in the cytoplasm of both intestinal and diffuse-type gastric cancer cells. Increased levels of ADAM17 expression in gastric cancer cells resulting in enhanced ectodomain shedding of EGF ligands may promote tumour progression

\section{M2120}

In Vitro Functional Assessment of CDHl Germline Missense Mutations and Implications for Clinical Management of Asymptomatic Mutation Carriers Gianpaolo Suriano, Fatima Carneiro, Carla Oliveira, Fernando Regateiro, David G. Huntsman, Sergio Castedo, Jose Carlos Machado, Raquel Seruca

Background: Hereditary diffuse gastric cancer (HDGC) is an autosomal dominantly inherited gastric cancer susceptibility syndrome caused by $\mathrm{CDHl}$ germline mutations. Carriers of these mutations have a $>70 \%$ lifetime risk of developing diffuse gastric cancer (DGC). Currently, total prophylactic gastrectomy is offered only to carriers of $\mathrm{CDHl}$ germline inactivating (truncating) mutations. Nevertheless, about $20 \%$ of the HDGC families harbor germline mutations of the missense type, and their clinical management remains controversial. We report here the first prophylactic gastrectomies performed on HDGC-carriers of CDHl germline mutations of the missense type, based upon in vitro assessment of their pathogenic nature. Methods: Mutation screening at the $\mathrm{CDHl}$ locus was performed by polymerase chain-reaction amplification and Single-Strand Conformational Polymorphism (PCR-SSCP) of the entire coding region of the $\mathrm{CDHl}$ gene, including all intron/exon boundaries and promoter region, followed by direct sequencing of the abnormal bands. Slow-aggregation and matrigel invasion assays were used to address the functional relevance of the missense changes. Total prophylactic gastrectomy was performed in two unrelated carriers of germline missense mutations, and the entire mucosa of the stomach sampled for histological analysis. Results: Both missense alterations impaired in vitro the ability of E-cadherin to mediate cellto cell adhesion and to suppress cell invasion, demonstrating their pathogenicity. Foci o early invasive diffuse carcinoma (signet ring cell type) were found in specimens from both individuals submitted to prophylactic gastrectomy. Conclusions: In vitro testing should be used as an adjunct in deciding on the potential pathogenic role of $\mathrm{CDHl}$ germline missense mutations.

\section{M2121}

An Important Role of Rhoc in the Metastasis Progress of Gastric Cancer Guoyun Zhang, Na Liu, Feng Bi, Yanglin Pan, Yulei Du, Daiming Fan

Objective: To study the effect of RhoC on the metastasis progress of gastric cancer cells. Methods: The expression of RhoC was examined by immunohistochemistry in gastric cance tissues and non-tumorous tissues. The vector PCEFL-GST- RhoC was transfected into gastric epithelial cells(GES-1).Under the assistance of computer, a group of RhoC-specific small interfering RNAs (siRNA) were designed and the related vectors were generated and transfected into human gastric cancer cell SGC7901 separately by the lipofectamine. The protein expression of RhoC in the transfectants was examined by Western blot. Cell growth rate was determined by counting viable cells with a Cell Counting Kit-8. MTT assay was used to evaluate the sensitivity of the transfectants to anticancer drugs. A wound-healing and a Transwell assays were used to examine the migration and invasion ability, respectively. Results: Only weak staining of RhoC was detected in 3 of 33 non-tumorous cases $(9.10 \%)$ The expression of RhoC was significantly higher in gastric cancer tissues $(23 / 42,54.76 \%$ ) than in non-tumorous tissues $(\mathrm{p}<0.01)$ and RhoC positively expressed in 18 out of 20 metastases $(90.0 \%)$, even higher than that in primary gastric cancer tissues. Western blot showed that RhoC was up-regulated in gastric cancer cell lines (AGS, XGC-9811,SGC7901 and KATOIII), but not expressed in SV40 transformed immortal GES-1. Overexpression of RhoC GTPase in GES-1 cells could produce a motile and invasive phenotype, but does no alter the monolayer growth rate $(p>0.05)$. Five RhoC-specific siRNAs were designed and the related vectors were generated. Western blot showed that RhoC-specific siRNA, RhoC s362, inhibited RhoC expression dramatically (62.57\%). Though down regulation of RhoC expression didn't affect the proliferation and chemo-sesitivity of the transfectants $(\mathrm{p}>0.05)$ it did restrain RhoC mediated cell migration and invasion(relative invasion index $0.263+0.01, p<0.05$ ). Conclusion: RhoC may play a very important role in the metastasis of gastric carcinoma. Therapeutic strategies targeting RhoC and RhoC-mediated pathways may be a novel approach for treating metastasis of gastric cancer.

\section{M2122}

Netl - A Novel Activator of RHOA and Gastric Cancer Cell Invasion David W. Murray, Jan Leyden, Gerry McEntee, Conor O'Keane, Padraic MacMathuna Peter Doran

Introduction Using computational informatics, we have identified a novel gastric cancer (GC) associated gene, NETl, a guanine nucleotide exchange factor. NETl activity has been reported in other cancers but its role in GC remains unclear. Aims To investigate the role of enhanced NETl expression GC by a) evaluating the effect of NETl knockdown on cancer invasion and proliferation and b) to determine whether the bio-activity of NETl in GC is promoted by LPA and is mediated by the GTPase RhoA. Methods Real time PCR was used to quantitate NETl expression levels in tumour and normal tissue. NETl knockdown using siRNA-mediated suppression was used to determine effects on the cell invasion and proliferation. The response of NETl expression to treatment with LPA, ILI- $\beta$ and TNFa was also monitored. Western blot analysis was used to determine levels of active RhoA. Results Netl expression was significantly enhanced by $34 \%$ in gastric tumour tissue samples as compared to healthy tissue $(\mathrm{P}<0.05)$. NETl siRNA transfection resulted in a dramatic decrease in RhoA activation as well as a $100 \%$ significant decrease in cell invasion and a $49 \%$ decrease in cell proliferation $(\mathrm{P}<0.05)$. NETl expression was increased 10 fold $(\mathrm{P}<0.05)$ in response to treatment with LPA but unaltered in response to ILI- $\beta$ and TNFa. Conclusions Using computational informatics, we have identified a novel gastric cancer (GC) associated gene, NETla and validated its function and enhanced expression in human GC tissue and cell lines. NETl is LPA responsive and it's bio-activity in GC proliferation and invasion is RhoA mediated. This data supports the involvement of NETl and RhoA in the progression of gastric cancer

\section{M2123}

Relative Benefit of Laparoscopy in Addition to CT in Staging Junctional Gastric Cancer

Jonathan Barry, Neil Hopper, Michael Stephens, Guy Blackshaw, Paul Edwards, Matthew Morgan, Gerald Thomas, Wyn Lewis

Aims. Junctional adenocarcinoma of the gastric cardia is believed to behave in a particularly aggressive fashion, and anatomical constraints limit the sensitivity and specificity of staging computed tomography (CT). The aim of this study was to assess the relative additiona staging yield of laparoscopy in patients diagnosed with adenocarcinoma of the gastric cardia when compared with helical CT. Methods. Eighty consecutive patients with Siewert type III adenocarcinomas of the oesophagogastric junction [median age ] were studied prospectively. All patients underwent a preoperative CT examination and laparoscopy with or without definitive surgery within 3 weeks. The strength of the agreement between the CT stage, the 
laparoscopic stage, and the final histopathological stage was determined by the weighted Kappa statistic (Kw, range -1 no agreement to +1 perfect agreement). Results. The strengths of agreement between the perceived preoperative stage as determined by CT compared with laparoscopy for T, Ml Liver and Ml peritoneum were 0.627 vs. $0.743,0.429$ vs. 0.719 and 0.108 vs. $0.964(\mathrm{P}<0.05)$ respectively. Unsuspected metastases that were not detected by $\mathrm{CT}$, were found in 15 patients at laparoscopy (4 Liver, 11 peritoneum) all of whom had T3 $(n=4)$ or T4 $(n=11)$ locally advanced tumours on CT criteria. Conclusion. Despite the potential difficulties of visualising primary junctional oesophagastric tumours at laparoscopy, this additional staging modality improved the detection of unsuspected distant metastases 9 fold and no fewer than $19 \%$ of patients were saved an open and close laparotomy. All patients diagnosed with an oesophagogastric type III junctional tumour of stage T3 and any $\mathrm{N}$ by CT criteria should undergo staging laparoscopy.

\section{M2124}

May Expression of Basic Fibroblast Growth Factor and Vascular Endothelial Growth Factor Combined with Epstein-Barr Virus Infection Influence On the Prognosis of Gastric Carcinoma?

Jeong-Seon Ji, Hiun-Suk Chae, Jin-Hyoung Kang, Kyoung-Mee Kim, Gyeong-Sin Park, Hyung-Keun Kim, Young-Seok Cho, Sung- Soo Kim, Kyu-Yong Choi, In-Sik Chung

Background: Angiogenesis has been shown to be necessary for tumor growth and metastasis. Vascular endothelial growth factor (VEGF) and basic fibroblast growth factor (bFGF) are important angiogenetic factors. And EBV (Epstein-Barr virus)-positive gastric carcinoma is $6-16 \%$ of total gastric carcinoma. Feature of pathology in EBV-positive gastric carcinoma is fibrosis. These lymphoepitheloid reaction is predominant in EBV-positive gastric carcinoma. So we hypothesized EBV-positive gastric carcinomas may be related with angio-fibrogenic factors, such as FGF and VEGF. We assessed VEGF and bFGF in the tissue of EBV-positive gastric carcinoma and also evaluated the relationship between this angiogenetic factors and other various clinicopathologic parameters. Methods: EBV In situ hybridization was performed at 621 patients who confirmed pathologically as gastric carcinoma after resection. Among them, 31 patients with EBV-positive gastric carcinoma and 69 patients with EBVnegative ones were randomly selected ( $72 \%$ male; mean age $58.0 \pm 1.2$ years). Gastric carcinoma tissues were stained with VEGF and bFGF antibody by immunohistochemical technique. Clinicopathologic parameters were morphologic and pathologic classification, location, stage, lymphatic invasion and survival data (mean follow up duration: $42.6 \pm 1.8$ months). Results: Male sex, positive VEGF, positive bFGF, upper location, tubular adenocarcinoma and early carcinoma were more common in EBV-positive gastric carcinomas than in EBV-negative ones ( $\mathrm{p}<0.05$ ). And positive EBV, upper location, lymphatic invasion and advanced carcinoma were more common in bFGF-positive gastric carcinomas than bFGF-negative ones ( $\mathrm{p}$ $<0.05$ ). And also positive EBV, lymphatic invasion, upper location, tubular adenocarcinoma and lymph node invasion were more common in VEGF-positive gastric carcinomas than VEGF-negative ones $(\mathrm{p}<0.05)$. However neither EBV or VEGF influenced to the survival rate of gastric carcinoma. And survival rate of bFGF-positive gastric carcinoma was statistically insignificant but lower than bFGF-negative one $(\mathrm{p}=0.128)$. Interestingly, survival rate of bFGF-positive gastric carcinoma was significantly lower than bFGF-negative one in patients with lymph node invasion. $(\mathrm{p}=0.036)$. Conclusions: As we expected, $\mathrm{bFGF}$ and VEGF expressed more in EBV-positive gastric carcinomas than EBV-negative ones. And interestingly, bFGF may be a marker of prognosis in the patients with gastric carcinoma, particularly in the case of lymph node invasion.

\section{M2125}

Possible Involovement of Ghrelin in Gastric Cancerogenesis

Peter C. Konturek, Kazimierz Rembiasz, Anna Zub, Grzegorz Burnat, Joanna Kania, Eckhart G. Hahn, Stanislaw J. Konturek

Background and aims: Ghrelin is brain-gut peptide with growth hormone-releasing and appetite-inducing activities. It is mainly secreted from the gastric mucosa. Recently, a possible involvement of ghrelin in cancerogenesis was postulated. The aim of the present study was 1) to compare the ghrelin expression in the gastric cancer with the ghrelin expression in the non-malignant gastric mucosa adjacent to the tumor; 3) to analyze the effect of ghrelin on cell proliferation and expression of COX-2 in gastric cancer cell line (KATO III). Methods: $10 \mathrm{H} . \mathrm{p}$. positive patients and $10 \mathrm{GC}$ patients were included in the study. In all patients was performed gastroscopy. The gastric biopsies were taken from the antrum, corpus and tumor. The expression of ghrelin and its receptor was assessed by quantitative RT-PCR. In addition, the effect of exogenous ghrelin on the cell proliferation (by BrdU) and the mRNA and protein expression of COX-2 was assessed by RT-PCR and Western blot, respectively. The plasma level of ghrelin was assessed by RIA Results: The expression of ghrelin in the antral gastric mucosa was significantly lower in than in corpus mucosa. In the gastric cancer, significant decrease in ghrelin expression was found, whereas in the adjacent corpus mucosa a significant overexpression of ghrelin was found. In contrast to negligible expression fo ghrelin in GC, ghrelin receptors were expressed in both GC tissue and adjacent gastric mucosa. Compared to $\mathrm{Hp}+\mathrm{ve}$ controls, the plasma level of ghrelin was significantly higher in GC patients. The in vitro analyzes showed a significant effect of ghrelin on cell proliferation after 64 hours of incubation (by 120\% of control). Ghrelin caused a significant increase in the expression of COX-2 in KATO III cells. Conclusion: Ghrelin is overexpressed in the gastric mucosa adjacent to gastric cancer and the ghrelin receptors are expressed by cancer what suggests a possible role of ghrelin in the gastric carcinogenesis. The possible mechanisms by which ghrelin promotes the gastric carcinogenesis involves an increased PG generation due to increased COX-2 expression.

\section{M2126}

Mixed Intestinal and Diffuse Type Histology Is a Risk Factor for Lymph Node Metastasis of Early Gastric Cancer

Naoko Tsuji, Singo Ishiguro, Masatoshi Kudo

Background: Technical progress in endoscopic procedures such as endoscopic submucosal dissection is expected to increase the number of node-negative early gastric cancers treated conservatively. Evaluation of lymph nodes status before any such procedure is very important. Methods: To clarify indications for conservative therapy, we investigated histologic characteristics of node-positive and node-negative early gastric carcinomas. Seven-hundred and forty cases of mucosal cancer (m-cancer) and 270 cases of submucosal invasive cancer (smcancer) treated surgically were analyzed clinicopathologically. Comparison was made between patients with node-positive ( $n=23$ in m-cancer and $n=33$ in sm-cancer) and node-negative cancer. Whole sections of surgical specimens were reviewed and reclassified as pure intestinaltype, pure diffuse-type and mixed intestinal and diffuse-type. The intramucosal and submucosal components of sm-cancer were also described histologically. Clinical outcome of all cases were also obtained. Results: The percentage of mixed intestinal and diffuse-type histology was significantly higher in the node-positive group than in the node-negative group (78\% vs. $33 \%$ in m-cancer, and $76 \%$ vs. $29 \%$ in sm-cancer, $\mathrm{P}<0.01$ ). The percentage of sm-cancer showing lymphatic permeation was significantly higher in the node-positive group (79\% vs. $36 \%, \mathrm{P}<0.01$ ). The percentage of mucosal components showing mixed-type histology was significantly higher in the node-positive sm-cancer group than in the node-negative group ( $58 \%$ vs. $33 \%, \mathrm{P}<0.01)$. Recurrence rates of the node-positive and the node-negative groups were $13 \%$ vs. $0.5 \%$ in m-cancer and $9.1 \%$ vs. $6.3 \%$ in sm-cancer respectively. Average disease free survival was 5.1 years, and there was no difference between node-positive and node-negative groups. Conclusions: Histologic heterogeneity is a risk factor for metastasis of early gastric cancer to lymph nodes. Heterogeneity of mucosal components of sm-cancer is also a significant risk factor and thus a good predictor of lymph node metastasis, potentially useful in distinguishing patients ineligible for conservative therapy. Long term follow up is necessary after conservative therapy, for disease free survival of early gastric cancer is long.

\section{M2127}

Regulation of HIF-1 Induced Multidrug Resistance By MGR1-Ag in Gastric Cancer Cells

Li Sun, Lili Liu, Yongquan Shi, Xiaoxuan Ning, Changcun Guo, Yanglin Pan, Tao Lin, Jie Liang, Shuang Han, Yu Chen, Kaichun Wu, Daiming Fan

Previous studies of our work reported that MGrl-Ag was a novel protein associated with multidrug-resistance (MDR) in gastric cancer cells. The aim of our study was to explore the role and the underlying mechanisms of MGrl-Ag in HIF-1 induced MDR of gastric cancer cells. Methods: Sense or siRNA vectors for MGrl-Ag and HIF-1 were introduced to achieve genetical up- or downregulation respectively. Western blot analysis or semiquantitative RTPCR were performed to characterize the expression level of MGrl-Ag, HIF-1, P-glycoprotein (p-gp), multidrug resistance associated protein (MRP), Bcl-2 and Bax. The sensitivity of gastric cancer cells to the chemotherapeutic drugs was detected by colony-forming assay and the evaluation of intracellular adriamycin accumulation and retention were conducted by flow cytometry. AnnexinV/PropidineIodide staining method was used to detect adriamycininduced apoptosis. Double immunofluorescence assay was recruited to characterize the coexpression of HIF-1 and MGrl-Ag. Results: The expression level of both HIF-1 and MGrl$\mathrm{Ag}$ in MDR gastric cancer cells were much higher than that in their parental cells. Drugsensitive gastric cancer cells showed resistance to 5-fluorouracil (2.74 \pm 0.86 fold IC50 increase) and vincristine (2.40 \pm 0.72 fold IC50 increase) under hypoxic condition. A significant increase of MGrl-Ag mRNA level was revealed under hypoxic condition than that of in normoxia. The coexpression level of HIF-1 and MGrl-Ag were increased concomitantly upon hypoxia pretreatment at indicated time via both Western blot and double immunofluorescence assay. This concomitance was also confirmed by the expression level of MGrl-Ag in exogenous up- or downregulated expression level of HIF-1 in gastric cancer transfected cells. Overexpression of exogenous MGrl-Ag could promote MDR phenotype and show

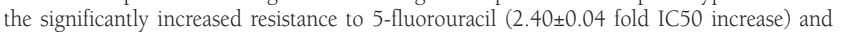
vincristine ( $2.65 \pm 0.33$ fold IC50 increase). Upregulation of MGrl-Ag could decrease intracellular adriamycin accumulation and retention ( $2.87 \pm 0.06$ fold releasing index increase) by downregulation of P-gp and MRP and also protect gastric cancer cells from adriamycininduced apoptosis $(0.43 \pm 0.19$ fold apoptosis index decrease) by downregulation of Bax and upregulation of Bcl-2, whereas downregulation of MGrl-Ag had the reverse effects. Conclusions: Under hypoxic condition, HIF-1 may promote MDR of gastric cancer cells by upregulating the expression level of MGrl-Ag via a decrease in intracellular drug accumulation and inhibition of drug-induced apoptosis.

\section{M2128}

Expression of Thrombospondin-1 and VEGF in Gastric Carcinoma Wan-Sik Lee, Chang-Hwan Park, Young-Eun Joo, Hyun-Soo Kim, Sung-Kyu Choi, JongSun Rew, Sei-Jong Kim, Chang-Soo Park

Backgrounds : Thrombosponding-1 (TSP-1) is an multifunctional platelet and extracellular matrix protein that is involved in angiogenesis on a variety of cancers. As to its role in angiogenesis, it has been proposed that TSP-1 functions as an angiogenic stimulator or inhibitor according to organ and histologic types. Vascular endothelial growth factor (VEGF) has a potent angiogenic activity and is well correlated with microvascular density. This study was to evaluate the expression of TSP -1 protein in gastric carcinomas and its relationship with VEGF expression and clinicopathological factors. Materials and methods: We conducted an immunohistochemical investigation of TSP-1 and VEGF in 108 tissue samples obtained from gastric cancer patients receiving curative surgical treatment. AJCC TNM classificaion of gastric cancer was used to analyze gastric cancer staging. Results : Positive staining for TSP-1 was seen in the cytoplasm of the cancer cells. Expression of TSP-1 protein was detected in $72 \%$ ( 77 of 108) of the cases, among which strong expression was encountered in $42 \%$. VEGF expression was significantly higher in tumors with strong TSP-1 protein 
expression than in those without expression or weak expression of TSP-1 ( $\mathrm{P}=0.01)$. Significant correlation was found between TSP- 1 expression and clinicopathological parameters of gastric carcinoma ( $\mathrm{T}$ and $\mathrm{N}$ factors). Conclusions: TSP-1 expression might be closely related to the development and progression of gastric cancer. Moreover, TSP-1 may contribute to the angiogenesis in the process of gastric carcinogenesis given the fact that it is closely correlated with VEGF expression

\section{M2129}

Expression of Nonsteroidal Anti-Inflmmatory Drug-Activated Gene in Gastric Adenoma and Carcinoma

Jeong Youp Park, Seungmin Bang, Myoung Hwan Kim, Kyung Hwa Park, Seung Woo Park, Jae Bock Chung, Sang Suk Koh, Si Young Song

NAG-1, one of TGF- $\beta$ superfamily gene, is reported to be responsible for NSAID-induced apoptosis. To find whether it can be also applied to gastric cancer which is one of the leading causes of cancer death worldwide, we tried to analyze its expression in gastric cancer according to cell differentiation and tumor stage. In addition, its expression in adenoma was also analyzed. Tissue specimens were obtained from individuals who underwent gastrectomy with curative purpose for gastric cancer, and with them tissue microarray was made. Immunostaining was performed using standard procedures with antibody to NAG-1. The intensity of immunostaining was evaluated using internal control. Tumor tissues with intensity weaker than normal tissue was graded as 0 (no expression) similar as + (weak expression) and stronger as ++ (strong expression). The level of NAG-1 expression was also evaluated using Western blot analysis. NAG-1 was stained in the cytoplasm of normal epithelial cells, adenoma and cancer cells. Among 15 cases of adenoma, 12 cases (80\%) showed strong expression. Western blotting confirmed strong expression in adenoma. Among 168 cases of gastric cancer, there were 69 cases with stage I, 25 with stage II, 43 with stage III, 31 with stage IV. According to stage by stage analysis, in stage I, 31 out of 69 cases (44.9\%) showed strong expression in tumor tissue. However, in stage IV, only three out of 31 cases $(9.7 \%)$ showed strong expression in tumor tissue and 18 cases (58.1\%) showed no expression. Western blotting showed NAG-1 expression was weaker than or similar to normal tissue. Significant correlations were noted between NAG-1 expression and the degree of cell differentiation, $\mathrm{T}$, and $\mathrm{N}$ stage. $(\mathrm{p}<0.05)$ Pearson correlation index for the degree of cell differentiation was -0.157 , for $\mathrm{T}$ stage -0.421 , and for $\mathrm{N}$ stage -0.397 . In summary, NAG-1 was expressed strongly in adenoma, and reversely correlated to tumor stages. This interesting finding may provide new targets for chemoprevention and future development of drugs.

\section{M2130}

C/EBP-Beta Is Over-Expressed in Gastric Carcinoma and Is Associated with the Expression of Cyclooxygenase-2

Goncalo Regalo, Paulo Canedo, Gianpaolo Suriano, Carlos Resende, Maria J. Oliveira, Ceu Figueiredo, Fatima Carneiro, Raquel Seruca, Jose C. Machado

The C/EBPbeta transcription factor has been associated with several cancer models. Recent studies suggest that the interleukin-6R/GP130 receptor signalling pathway, where C/EBPbeta plays a key role, is likely to be involved in gastric cancer (GC). In this study, expression of C/EBPbeta was analysed in a series of $90 \mathrm{GC}$. CEBPB gene mutations were screened for in a series of 35 primary GC. The functional activity of C/EBPbeta was analysed in GC cell lines. In normal gastric mucosa, C/EBPbeta expression was restricted to cells with a proliferative phenotype. In intestinal metaplasia, dysplasia, and GC of the intestinal and atypical subtypes, C/EBPbeta was overexpressed ( $\mathrm{P}=0.0005$, for the association with histological type). C/ EBPbeta and Ki67, a marker of cell proliferation, were also co-expressed in primary GC. In one GC we found a mutation of the CEBPB gene. Using GC cell lines we showed that transfected $\mathrm{C} / \mathrm{EBPb}$ ta is able to regulate the expression of endogenous cyclooxygenase-2 (COX-2). Back in the series of metaplasia, dysplasia and GC lesions we observed a high degree of overlap between expression of C/EBP-beta and COX-2. In GC cell lines we have also shown that transfected C/EBP-beta is able to modulate proliferation in these cells. These results suggest that $\mathrm{C} / \mathrm{EBPbeta}$ may both be a marker of neoplastic transformation and play an active role in gastric carcinogenesis through its ability to up-regulate COX-2 expression.

\section{M2131}

Usefulness of DAS-1 Monoclonal Antibody to Detect the Intestinal Metaplasia Associated with Gastric Cancer

Montse Forne, Clarisa Gonzalez, Jaume Casalots, Ferran Fernandez-Banares, Maria Esteve, Merce Rosinach, Jorge Espinos, Josep Maria Viver, Antonio Salas

Preventive strategies of gastric cancer should lead to diagnosis at an early stage. The role of endoscopic surveillance in patients with atrophic chronic gastritis and gastric intestinal metaplasia (IM) is not established. It has been suggested, recently, that Das-1 monoclonal antibody $(\mathrm{mAb})$ is useful in the identification of incomplete IM (IIM) -or colonic form of gastric IM- that is the specific subtype associated with an increased risk of intestinal type gastric adenocarcinoma in H. pylori (Hp) infected people (Mirza et al, Gut 2003). Aim: 1.To validate $\mathrm{mAb}$ Das-l in the diagnosis of IM associated with gastric cancer in our geographical area. 2. To identify the expression of Das-1 in the histological subtypes of IM and its possible relationship with the infection for Hp. 3.To assess the usefulness of mAb Das-1 in the detection of IIM as compared to routine histological techniques. Methods: The following patients were studied: Group -: $(\mathrm{n}=50)$ gastric cancer (paired samples of mucosa from both cancer areas and histological proved IM areas distant from cancer segment). Group 2: ( $\mathrm{n}=$ 25) gastro-duodenal ulcer, $\mathrm{Hp}(+)$, IM and chronic gastritis. Group 3: $(n=25)$ autoimmune atrophic chronic gastritis with IM and without Hp infection. Group 4- $(\mathrm{n}=25)$ chronic gastritis with and without $\mathrm{Hp}$ infection and without evidence of IM. Conventional histological study (H\&E) was carried out, and complete IM (CIM) and IIM were diagnosed. Immunohistochemical assay using mAb Das- 1 was performed, and the results were interpreted according to Mirza et al (Gut 2003). Results: Group 1: 22 patients had CIM, 25 IIM, and 3 both types of IM (Mixed). Das-1 was positive in 20 patients in cancer areas and in 13 in areas of IM
(CIM 5, IIM 8). In 7 patients Das-lwas positive in both areas studied. Group 2: 17 patients had CIM and 8 IIM. Expression of Das-1 was observed in 2 CIM. Group 3: 17 cases had CIM, 7 IIM and 1 mixed. No case was positive for Das-1. Group 4: No case was positive for Das-1. In summary expression of Das-1 was observed in 7 of 56 CIM and in 8 of 40 IIM (ns). On the other hand, there were differences between the expression of Das- 1 in the IM of group 1 as compared to IM of groups $2-3$ (13/50 vs 2/50; $<<0.01$ ). Conclusions: 1 . In our geographical area the described association of gastric cancer with IIM was not observed. 2. mAb Das-1 was not useful to differentiate between CIM and IIM. 3. The high expression of Das-1 in the group of gastric cancer, even in non neoplastic area, suggest that it might be a risk marker of neoplasia, and thus, patients with Das-1 expression would benefit from an strict follow-up. This possibility has to be further assessed.

\section{M2132}

Clinical Significance of CT-Defined Minimal Ascites: An Analysis Focusing On Survival Outcome of Gastric Cancer

Hyuk Lee, Dong Kyung Chang, Poong-Lyul Rhee, Jae J. Kim, Jong Chul Rhee

Backgrounds: The clinical significance of minimal ascites, which was only defined by dynamic $\mathrm{CT}$, is unknown. Furthermore, this CT-defined minimal ascites (CTMA) in preoperative staging for gastric cancer tends to force into a dilemma on therapeutic modality or outcome The objective of this study was to investigate the clinical significance of CTMA in patients with gastric cancer, in relationship to survival outcome. Methods: The medical records and the dynamic CT films of 5080 patients, who had been examined 6 years ago, were retrospectively reviewed. Asymptomatic 892 and symptomatic 2928 patients without history of prior trauma, abdominal surgery and chemoradiotherapy were selected. Experienced two radiologists calculated the volume of ascites in all patients presenting ascites. Results: The prevalence of minimal ascites $(<50 \mathrm{~mL})$ was $1.12 \%$ and $7.48 \%$ in asymptomatic and symptomatic group, respectively. The proportion of patients without accompanying other pathologic finding was $90.0 \%$ and $8.2 \%$ in both groups with CTMA. $57.1 \%$ of the symptomatic patients with CTMA were associated with malignant disease. Gastric cancer with CTMA was identified in $21.5 \%$ of CTMA in symptomatic group. Peritoneal carcinomatosis was $37.5 \%$ of CTMA of which the nature was surgically confirmed. CTMA without lymph node enlargement and peritoneal enhancement, which were $23.3 \%$ of CTMA with gastric cancer, was not associated with peritoneal carcinomatosis. No recurrence occurred in these patients, and they survived for 6 -year period. On the contrary, mean survival time of patients with CT finding accompanying lymph node enlargement was 63.4 months and that of patients with peritoneal enhancement was 46.9 months. The patients with both findings presented the mean survival time of 33.6 months. Conclusions: Gastric cancer with only CTMA tends not to be associated with peritoneal carcinomatosis if it does not accompany other CT finding. Therefore, curative resection should be attempted if CTMA is the only ambiguous sign.

\section{M2133}

Does Early Upper GI Cancer Cause Symptoms? Martin J. Maida, Rosie Crimmins, Jill Swift, Green John

INRODUCTION Current British Society of Gastroenterology guidelines recommend open access gastroscopy for all patients with new onset dyspepsia over the age of 55 years and in those with alarm symptoms at a younger age, to look for malignancy. We previously found that patients with upper gastrointestinal (GI) malignancy usually present with alarm symptoms and those without alarm symptoms often had advanced diseasel. AIMS AND METHODS In this study, we identified prospectively patients who over an eighteen month period from April 2004 to October 2005 had findings suggestive of malignancy at their index gastroscopy, and determined via a questionnaire their presenting symptoms, the duration of presenting features, and how often they had seen their general practitioner (GP) in the lead up to referral. RESULTS 31 patients were found to have upper GI malignancy (12 gastric 19 oesophageal) during the study period. The mean age was 72 years (44 - 91 years), 19 male patients and 12 female. The average duration of symptoms prior to gastroscopy was 5 months ( 2 weeks - 18 months). All 12 patients with gastric carcinoma presented with weight loss as the initial symptom, with associated dyspepsia in 6 patients (50\%), anaemia in $4(33 \%)$, nausea in $3(25 \%)$ and melaena in 2 patients. Two of the 19 patients with oesophageal carcinoma presented with a four week duration of reflux symptoms having previously been asymptomatic; one additional patient had anaemia and worsening of longstanding reflux symptoms, and the remaining 16 patients (84\%) were referred with dysphagia as their initial symptom. 13 out of 31 patients were on antacid treatment, which in all but one case had been commenced for their current symptoms, and only the one patient with longstanding reflux symptoms had seen their GP for dyspeptic symptoms prior to presenting with alarm symptoms. CONCLUSION Patients with upper GI cancer only had a short history of symptoms, and had not been to their GP or been on treatment that could mask symptoms prior to presenting with cancer. This implies that the cancer could not have been diagnosed any earlier, confirming that early upper GI cancer is asymptomatic. 1. GUT 2003;52(Suppl VI): A200

\section{M2134}

Early Gastric Carcinoma: Results of Endoscopic Mucosal Resection Michael Sackmann, Klaus-Dieter Schmidt, Hans Brehm, Hans-Martin Baumueller

BACKGROUND: According to first data mainly provided from Japanese groups, early gastric carcinoma may be treated by endoscopic mucosal resection (EMR) in selected patients. We attempted to prospectively evaluate the results of EMR and the early recurrence rate after successful therapy. METHODS: During a time period of 18 months, $n=22$ patients (age, 57 to 83 years; median, 78 years) were referred consecutively to our institution for evaluation of early gastric cancer. All patients underwent video endoscopy including application of zoom endoscopy (Fujinon) and chromo-endoscopy, endoscopic ultrasonography, mapping biopsy, and computed tomography. The options for treatment were then discussed by an interdisciplinary panel including gastroenterologists, oncologists, pathologists, and surgeons 
$73 \%$ ( $n=16 ; 6 f, 10 \mathrm{~m}$ ) of the 22 patients fulfilled the criteria suggested by the Japanese Society of Gastroenterology for endoscopic therapy of early gastric cancer, e.g., macroscopically type I or type II, histopathological grading G1 or G2, tumor restricted to the mucosal layer or to the first submucosal layer $(\mathrm{Sml})$, tumor size $<21 \mathrm{~mm}$. The ligation technique or the resection cap technique were used for EMR. Subsequently, the patients were followed up regularly at months $1,3,6,9,12,18,24$, and 36, respectively. RESULTS: Complete EMR (TlGlR0V0) was obtained in $13(81 \%)$ of the 16 patients. Three patients were operated due to tumor exceeding the lateral margins $(n=2)$, or due to invasion of lymph vessels $(n=$ 1). Two complications were handled non-surgically (small perforation by clip application and antibiotic therapy, bleeding seven days after EMR by endoscopic injection therapy). During follow-up (1-22 months; median, 8 months), recurrence of early gastric cancer was observed in three patients. The three patients were again treated by EMR (RO). By actuarial analysis, the probability of recurrence was $23 \%$ at two years. CONCLUSIONS: In selected patients with low-risk early gastric cancer, EMR is a suitable alternative to gastric surgery. Due to the risk of recurrence, regular follow-up of the patients is mandatory after EMR

\section{M2135}

Influence of Gender On Precancerous Changes Leading to Intestinal Type Upper Gastrointestinal Cancer

Mohammad H. Derakhshan, Reza Malekzadeh, Valerie Fyfe, Abbass Yazdanbod, Masoud Sotoudeh, Nasser Rakhshani, Abbass Kazemi, Kenneth E. McColl

Background and Aim: Intestinal type upper GI cancers are more prevalent in males than females. This type of cancer arises due to progression of chronic inflammation to atrophic gastritis to intestinal metaplasia and finally to adenocarcinoma. The histological stage in this pathway where gender acts is unclear. We have investigated the influence of gender on the frequency of the intestinal type cancer and its precancerous lesions. Method and Materials: The association between gender and histological subtypes was assessed in 124 cases with upper GI cancer in Ardabil province of Iran. In addition, in the general population (234 males, 274 females) with mean age of $54.6 \pm 11.3$ of the same region, we evaluated the relationship between gender and precancerous histological changes. Mean scores of active and chronic inflammation, as well as mucosal atrophy and intestinal metaplasia in 3 different biopsy sites were compared between male and female. Results: Male gender showed potent relationship with intestinal vs. diffuse type adenocarcinoma [OR: 5.7 (95\% CI: 2.5-13.1)] In the general population, the mean scores of active inflammation in all biopsy sites between both genders were similar. Chronic inflammation at antral site (incisura angularis) in males was more severe than females, but at other biopsy sites, there was no difference between sexes. Mean scores of non metaplastic atrophy at all biopsy sites were similar in both genders. Intestinal metaplasia showed significant relationship with male gender at each subsites (Table): Conclusion: In this population, male gender is associated with increased frequency of intestinal metaplasia as well as intestinal type adenocarcinoma.

Relationship of male gender with precancerous lesions of intestinal type cancer (OR, 95\% CI)

\begin{tabular}{|c|c|c|c|c|}
\hline Site & Active Inflammation & Chronic Inflammation & Atrophy & Intestinal Metaplasia \\
\hline Cardia & $1.1(0.8-1.6)$ & $0.8(0.6-1.2)$ & $12.0(0.7-1.4)$ & $3.1(1.2-8.1)$ \\
\hline Corpus & $0.8(0.8-1.2)$ & $0.9(0.7-1.3)$ & $0.7(0.5-1.1)$ & $7.4(1.6-33.3)$ \\
\hline Antrum & $0.8(0.5-1.1)$ & $1.5(1.0-2.2)$ & $0.8(0.6-1.2)$ & $2.1(1.1-4.0)$ \\
\hline
\end{tabular}

\section{M2136}

Clinical and Pathologic Characteristics of Gastric Cancer in Elderly Koreans Dong Ho Lee, Youn Joo Kim, Nayoung Kim, In Sung Song, Hyun Chae Jung, Young Soo Park, Suk Hyang Jung

Purpose:Gastric cancer is one of the most important cancers in Korea, considering its incidence and mortality rate. Since the number of elderly people is increasing in Korea, the proportion of elderly patients with gastric cancer will continue to increase. We have obtained preliminary data concerning the diagnosis and treatment of gastric cancer in elderly people by analyzing the clinical features, endoscopic findings, pathology, postoperative recovery time and operation-related complications. Method:We retrospectively analyzed 270 patients who were diagnosed with gastric cancer from May, 2003 to May, 2005, and who underwent surgical procedures in Bundang Seoul Nationall University Hospital. Patients were divided into two groups, age $<65(\mathrm{~N}=164)$ and age $\geq 65(\mathrm{~N}=106)$. We compared sex, age, symptoms, location, morphology, pathology, stage, ASA classification, postoperative recovery time and operation-related complications between the older and younger groups. Results:There was no statistically significant difference in sex, symptoms and family history between the elderly group and the younger group. There were no differences in the distribution of early gastric cancer and advanced gastric cancer, or stages. The younger group had a higher incidence of poorly differentiated carcinoma(38.3\%) and signet ring cell carcinoma(21.6\%), and the elderly group had more patients with moderately differentiated carcinoma $(33.0 \%)$, based on the WHO classification $(\mathrm{p}<0.001)$. Using the Lauren classification, the younger group had a higher incidence of diffuse type $(58 \%)$ and the elderly group intestinal type $(59.4 \%)(\mathrm{p}<0.001)$. The incidence of high frequency microsatellite instability(MSI-H) was higher in the elderly group $(17 \%$ vs $5.6 \%)(\mathrm{p}=0.01)$ and an intact hMLHl gene was higher in the younger group $(80.2 \%$ vs $68.9 \% \mathrm{p}=0.034)$. The incidence of $\mathrm{p} 53$ was similar between the two groups. Even though the younger group had more patients with lower risks, according to the ASA classification, there was no significant difference in postoperative recovery time $(\mathrm{p}=0.154)$ and the operation-related complication rates between the two groups. Conclusion: Gastric cancer in elderly patients demonstrated more pathologic types known to have a favorable outcome, when compared to the younger patient group. The elderly patients had a higher incidence of MSI-H, which is known to be associated with less frequent lymphatic metastasis. Since the older patients, when compared to the younger ones, did not have a longer postoperative recovery time or a higher incidence of operation-related complications, we should more frequently consider surgery in the elderly patient with operable gastric cancer

\section{M2137}

Protein Kinase C Theta $(\mathrm{Pkc} \theta)$ Differentiates Gastrointestinal Stromal Tumors (GISTs) from Other Spindle Cell Tumors Expressing Kit Linan Wang, Anette Duensing, Adrian Gologan, Shih-Fan Kuan

Background: Immunoperoxidase staining for the receptor tyrosine kinase KIT (CD117) is the key diagnostic marker to establish the diagnosis of GIST. However, KIT expression is not uncommon in other spindle cell tumors, thereby impeding the differential diagnostic process. PKC $\theta$ has recently been found to be expressed in the interstitial cells of Cajal (ICC) of the digestive tract and has shown promising features as a supplemental diagnostic tool in assisting with the diagnosis of GISTs. This study is to establish the prevalence of PKC $\theta$ expression in KIT-positive and -negative non-GIST spindle cell tumors in comparison to GIST. Methods: The study group comprised of 62 spindle cell tumors (both intra- and extraabdominal) from 62 unselected patients ( $F / M=29 / 33$, mean age: 60.7$)$ in which KIT immunoperoxidase staining was performed at UPMC from 2000 to 2005. The pathology reports and status of KIT immunostaining were reviewed to divide cases into KIT-positive and -negative groups. The KIT-positive group consisted of 30 histologically and immunohistochemically proven GISTs ( $F / M=12 / 18$, mean age: 62.9$)$ and 10 non-GISTs ( $F / M=5 / 5$, mean age: 52.8). The remaining 22 cases were KIT-negative non-GISTs ( $F / M=12 / 10$, mean age: 61.5). The non-GISTs included a wide spectrum of spindle cell tumors: schwannoma (4), leiomyosarcoma/leiomyoma (6), desmoid (2), glomus tumor (1), angiomyolipoma (1), reactive spindle cell proliferation (2), low-grade malignant spindle cell tumors (4), inflammatory fibroid polyp (1) and high-grade sarcoma, NOS (11). Immunoperoxidase staining was performed on formalin-fixed paraffin-embedded sections using a mouse monoclonal antibody to PKC (BD Biosciences; 1:50 dilution). Results: All 30 KIT-positive GISTs (100\%) also expressed PKC $\theta$ in a mostly diffuse pattern. By contrast, PKC $\theta$ expression was absent in $80 \%(8 / 10)$ of the KIT-positive spindle cell tumors, and only barely detectable in 2 cases. Moreover, PKCA was not expressed in 91\% (20/22) of the KIT-negative non-GISTs; the PKCO expression in 2 cases of this group was focal weaker than that in GISTs. Conclusion: Immunohistochemical expression of PKC $\theta$ was confirmed to be specific for GIST and can be a useful adjunct marker in separating GISTs from other KIT-positive spindle cell tumors.

\section{M2138}

The Natural History of Gastrointestinal Stromal Tumors and Leiomyomas: A Prospective Evaluation Via Serial Endoscopic Ultrasound Surveillance Vu Kwan, Ian D. Norton, David B. Jones

Introduction: The natural history of gastrointestinal stromal tumors (GIST) is not clearly defined. Recent consensus guidelines suggest that malignant transformation occurs invariably and that surgery is mandatory[1]. The majority of current literature has an oncological or surgical bias, with little data regarding small lesions found incidentally at endoscopy. Periodic surveillance with endoscopic ultrasound (EUS) may be another option for lesions of apparent low malignant potential, as EUS can accurately monitor size and internal echotexture. We aim to provide further insight into the natural history of small GISTs \& leiomyomas (GIST/ Ls) by evaluating outcomes of serial EUS surveillance. Methods: Consecutive patients with an EUS diagnosis of GIST/L were identified from an endoscopic database. Clinical progress $\&$ management were documented via mail-out to patients, contact with referring practitioners $\&$ review of surgical pathology. Patients managed non-surgically were prospectively recalled for repeat EUS. Growth velocity was determined as change in size(mm) over time(years). Results: Between 1997 and 2005, 125 patients received an EUS diagnosis of GIST/L. Median age $=65$ (range 31-92), males $=62$, median follow-up $=4.1$ years $(0.5-15)$, lesion location gastric $=87$, oesophageal $=35$, duodenal $=2$, rectal $=1$. Median lesion size was $17 \mathrm{~mm}(3-65)$. Surgery was performed in 41 patients based on EUS criteria for potential malignancy or patient preference. EUS diagnosis of GIST/L was confirmed in 33 (others: inflammatory = 4 , cyst $=1$, pancreatic cancer $=1$, pancreatic rest $=1$, hemangioma $=1$ ). None were of high malignant potential at histopathology. Serial EUS has been performed in 30 patients. Median follow-up since index EUS was 5 years (1-15yrs, some with initial EUS elsewhere). Median initial size was $15 \mathrm{~mm}$ (3-44). Median growth velocity was $0 \mathrm{~mm} / \mathrm{yr}(0-5)$. The majority $(\mathrm{n}=$ 24) have not changed in size. No EUS-related complications occurred. Of the remaining patients, 2 are deceased due to advanced GIST/L, 3 deceased from other causes, 8 lost to follow-up. Clinical and/or endoscopic follow-up is available for the remaining, with no cases of malignant transformation identified. Conclusion: The natural history of this subset of GIST/Ls appears to be characterised by slow growth, with the majority exhibiting no change in size over the follow-up period. Abrupt malignant transformation was not observed. Therefore, serial EUS surveillance of GIST/Ls may be a reasonable alternative to surgery for lesions with benign appearance at index examination. 1.Blay et al. Report of the GIST Consensus Conference of 20-21 March 2004, under the auspices of ESMO. Ann of Oncol,2005. 16, 566-578.

\section{M2139}

Significant Correlation Between Helicobacter Pylori Genotypes and Time to Clinical Remission After Eradication Therapy in Low-Grade Gastric MALT Lymphoma - First Results from a Prospective Multicenter Trial Renate Schmelz, Dana Grossmann, Christian Thiede, Monic Dawel, E Kuhlisch, Stephan Miehlke, Daniela Aust, Gerhard Ehninger, Manfred Stolte, Andrea Morgner-Miehlke

Background: Helicobacter pylori ( HP) may possess different factors of pathogenicity which can be associated with diseases like peptic ulcer or gastric adenocarcinoma. Prevalence of HP pathogenic factors in positive low-grade gastric MALT lymphoma have been determinded to some extend, however their potential influence on clinical outcome after eradication of HP is unknown. Aim \& Methods: The HP genotypes cagA, babA2, iceAl, vacAm, vacAs, JHP950, JHP1462, oipA and sabA were determined using formalin fixed paraffin wax embedded gastric tissue from patients with HP positive MALT lymphoma $(n=35)$ and Patients with gastritis $(n=33)$ only. The different genotypes of HP were correlated with time to complete remission (CR), age at first diagnosis, monoclonality, $\mathrm{t}[11 ; 18]$ and disease stage at first diagnosis. Results: HP from 35 patients (18 female, age range 30-78 years) with 
MALT lymphoma have been analyzed. 3 patients were lost of follow up (9\%) and another $3(9 \%)$ had to be irradiated because lack of complete remission after 12 months. First complete remission occured 3.6 months (mean) after HP eradication. $56 \%$ of patients showed monoclonality, $7 \%$ had the translocation $\mathrm{t}(11 ; 18)$. There was a significant correlation $(\mathrm{p}<0.01)$ between a positive status for JHP1462 and time to CR. Patients with positive JHP1462 ( $n=9)$ were in CR after a mean of 1.4 months compared to 3.4 months in JHP1462 negative patients $(\mathrm{n}=26)$. Patients with vacAml positive HP also showed a significant $(\mathrm{p}<0.05)$ shorter time to $\mathrm{CR}$ than VacAm2 positives ( 1.7 vs 3.7 months). Conclusion: Patients with low-grade gastric MALT lymphoma and positive JHP1462 status appear to achieve clinical remission faster after HP eradication than those with a negative JHP1462 status. The same trend has been seen for patients with vacAml positive HP. Larger patient numbers are needed to further confirm these findings.

\section{M2140}

Epidemiology of Gastrointestinal Stromal Tumors in a Defined Canadian Health Region: A Population Based Study

Brian Yan, Gil G. Kaplan, Stefan Urbanski, Carla L. Nash, Paul L. Beck

Background and Objectives: Gastrointestinal stromal tumors (GISTs) are the most common mesenchymal tumor of the GI tract. At present there is only one population based study on the epidemiology of GIST. The aim of the present study was to determine the incidence of GIST within a well defined Canadian Health Region. We also describe the demographics, clinical presentation, disease associations, risk factors, and tumor and pathological characteristics of GIST. The use of KIT staining (CD117) in the diagnosis of GIST was assessed. Methods: This was a population based retrospective analysis for all adult patients diagnosed with GIST in the Calgary Health Region from April 12000 to March 31, 2004. Cases were identified through the Calgary Lab Services pathological data base with the search terms "GIST" and "gastrointestinal stromal tumors". Charts of all included patients were reviewed for clinical history, pathology and immunohistochemical characteristics. All pathological specimens were stained for CD117, CD34, vimentin, smooth muscle actin, keratin, and protein $\mathrm{S} 100$ and reviewed by a single pathologist for specific mitotic rates, predominant tumor cell type and antigenic determinants. Category-specific risks for developing GIST were reported as relative risks (RR) with exact $95 \%$ confidence intervals (CI). Results: A total 22 cases were identified in the study giving a crude incidence rate of $0.68 / 100000$, and age and gender adjusted rate of $0.73 / 100000$. Of these, $59 \%$ were female $(\mathrm{p}=0.4)$ and the average age was 62.5 years. An increased incidence with advanced age was noted: 18$44(0.26 / 100000), 45-64(0.63 / 100000)$, and $\geq 65(2.84 / 100000)$, resulting in a RR4.6(p= $0.0006)$ for age $\geq 50.8$ cases were identified prior to routine CD117 staining of which only 3 were stained. 14 cases were identified post routine CD117 staining of which 11 were stained. On repeat staining, all cases were positive for CD117 and vimentin, 96\% for CD34, $64 \%$ for smooth muscle actin, and 45\% for protein S100. Gastrointestinal bleeding (59\%) and abdominal pain $(55 \%)$ were the most common symptoms. No specific diseases were associated with GIST in our series. Stomach (50\%) and small bowel (27\%) were the most common primary tumor sites. Metastatic disease at presentation was found in $19 \%$ of cases. $73 \%$ were spindle cell tumors. At presentation, $27 \%$ were very low risk, $36 \%$ low, $19 \%$ intermediate, and 19\% high. Conclusions: GISTs are rare tumors of the GI tract with an age and gender adjusted incidence of 0.73/100000. Those over age 50 are at increased risk. Cases are more readily identified with KIT staining. Most cases are low risk at presentation, but $37 \%$ will be intermediate to high risk.

\section{M2141}

Long-Term Outcome of Low-Grade Gastric MALT Lymphoma After Helicobacter Pylori Eradication: A Prospective Single Center Study You Sik Choi, Jae Hee Cheon, Jong-Yeol Lee, Byung Duk Ye, Sang Gyun Kim, Joo Sung Kim, Hyun Chae Jung, In Sung Song

Background/aims: Most cases of low-grade gastric MALT lymphoma are associated with Helicobacter pylori infection. Therefore, eradication of $H$. pylori has been accepted to be a main strategic target against localized gastric MALT lymphoma. However, long-term outcome of gastric MALT lymphoma after H. pylori eradication remain unknown. We aimed to evaluate prospectively the long-term outcome of gastric MALT lymphoma after $H$. pylori eradication in single center. Methods: Sixty-five patients with H. pylori-positive low-grade gastric MALT lymphoma (Ann Arbor stage IEl and IE2) were prospectively enrolled from January 1996 to October 2004. Diagnosis and staging were performed by endoscopic biopsy, chest Xray, abdominal $\mathrm{CT}$, bone marrow examination and endoscopic ultrasonography. After the eradication of $\mathrm{H}$. pylori, the patients were followed up with endoscopic and histologic assessment at every 2-3 months in first year, every 6 months in the second year and thereafter. Results: H. pylori eradication was confirmed in all of the 65 patients by first- and secondline therapy. Complete remission of low-grade gastric MALT lymphoma was achieved in 56 patients $(86.2 \%)$. Nine patients without remission of gastric MALT lymphoma underwent gastrectomy and one of them underwent radiation therapy because of incomplete resection. The median duration of follow-up of the 56 patients with complete remission after $\mathrm{H}$. pylori eradication was 28.7 months (range 6.1-106 months). During follow-up period, H. pylori reinfection occurred in 6 of 65 patients $(9.2 \%)$ with no evidence of disease relapse, and $H$. pylori was eradicated in all of them. Only one patient (1.5\%) with complete remission experienced disease relapse after 12 months without the evidence of $H$. pylori reinfection and underwent gastrectomy. Complete remission of gastric MALT lymphoma was not associated with the age, sex, endoscopic features, location or eradication regimens. Furthermore, there was no difference in remission rate between the lymphomas restricted to mucosa and those that invaded submucosa by endoscopic ultrasonography. Conclusions: Our study demonstrated that $H$. pylori eradication is an efficacious primary treatment option for lowgrade gastric MALT lymphoma because of high remission and very low recurrence rate of gastric MALT lymphoma by a long-term observation

\section{M2142}

Single Nucleotide Polymorphisms (SNPs) Associated with Sporadic Colorectal Cancer: A Case-Control Study

David Weinberg, Kathryn Irenze, Daniel Chasman, Kristin Ardlie

Introduction: Colorectal cancer (CRC) is a common, multi-factorial disease resulting from the variable contribution of lifestyle, dietary and genetic factors. The search for genetic susceptibility factors has been extensive with a particular recent emphasis on low penetrance genes or gene variants. Ultimately the comprehensive identification of the various genetic factors that contribute to CRC development will likely require whole genome scanning in large populations, with follow-up association studies of promising SNPs or gene candidates At present, most studies have employed a candidate gene approach where well-described variants of genes already implicated, or putatively implicated, in colorectal carcinogenesis are interrogated in an effort to identify those variants more frequently seen in patients with colorectal cancer compared to healthy controls. Methods: A panel of single nucleotide polymorphic (SNPs) variants of a range of genes associated with colorectal cancer was identified. Using a candidate gene approach, we genotyped multiple (5-10) SNPs from each of 21 genes on a sample of 750 patients with well phenotyped, sporadic CRC. In addition a similarly sized group of matched, healthy controls were also collected and genotyped. We then compared individual SNP frequencies in all cases and controls. Results: Of the 29 mos promising SNPs, 5 demonstrated significant association between genotype and case/control status: TCF4 (rs599550), MSH2 (rs2303428), DCC (rs2298606), TGFBl (rs 1800472), IGF2R (rs894817). Although associated odds ratios were relatively small (0.5 to 1.4), allele frequencies were in the range of $10-20 \%$, suggesting that the overall impact on attributable risk for a common disease like CRC may be substantial. Conclusions: Beyond potentially shedding light on the underlying biology of CRC, use of these SNPs or others may provide more accurate estimates of CRC risk in the future. Improved gene-based risk assessmen may ultimately allow for more tailored screening programs, where the "molecular" risk level determined by the assessment of a panel of relevant, but low penetrance markers informs the frequency or the type of screening

\section{M2143}

Gastric Cancer Susceptibility Is Not Linked to Pro- Or Anti-Inflammatory Cytokine Gene Polymorphisms in Caucasians: A Nation-Wide Multicenter Study

Maria Asuncion Garcia-Gonzalez, Mark Strunk, Rafael Benito, Angel Lanas, Enrique Quintero, David Nicolas, Santos Santolaria, Federico Sopena, Miguel Angel Simon, Cristina Pascual, Adolfo Parra-Blanco, Jesus Espinel, Rafael Campo, Marisa Manzano, Fernando Geijo, Maria Pellise, Ferran Gonzalez-Huix, Miguel Nieto, Jorge Espinos, Lluisa Tito, Luis Bujanda, Manuel on Behalf of the Spanish AEG Zaballa

Background \& aim: Recent studies have supported the relevance of host immunogenetic factors on gastric cancer (GC) susceptibility. The aim of our study was to analyze the influence of $H$. pylori infection and host genetic factors in a population of Spanish Caucasian GC patients by studying several functional polymorphisms localized in pro- and antiinflammatory genes. Methods: DNA from 404 unrelated Spanish patients with GC and 40 sex- and aged- ( \pm 5 years) matched healthy controls (HC) with no gastrointestinal symptoms was typed for several functional polymorphisms in the pro-inflammatory IL-1B $(-511,+3954)$ TNFA (-238, -308), LTA (LTA NcoI, LTA BsI), and IL-12p40 (+1188) genes, and the antiinflammatory IL-4 -590, IL-1RN (VNTR in intron 2), IL-10 (-597, -1087) and TGFBI (+869 +915) genes by PCR, RFLP and TaqMan assays. H. pylori infection and CagA/VacA antibody status were also determined by western blot in patients and controls. Results: $H$. pylon infection with cagA strains was associated with increased risk of GC (OR: 2.5; $95 \% \mathrm{CI}$ 1.85-3.37; $\mathrm{p}<0.0001)$. H. pylori prevalence was identical $(70.3 \%)$ in patients with cardia GC $(n=70)$ than in patients with non-cardia GC $(n=334)$ When considering the histological type of the tumor, H. pylori infection was significantly higher in patients with diffuse GC than in patients with the intestinal subtype (80\% vs $69 \%$; OR: 1.98 ; $95 \%$ CI: $1.11-3.52$ $\mathrm{p}=0.024$ ). Among the overall cytokine gene polymorphisms analyzed in this study, only TNF-A -238 SNP was weakly correlated with decreased risk of non-cardia GC. Thus, individuals homozygous for the rare allele TNFA-238A were significantly less frequent in patients with non-cardia GC compared to controls (8/334 in GC and 1/334 in HC, OR $0.12 ; 95 \% \mathrm{CI}: 0.01-0.98 ; \mathrm{p}=0.03$ ). In addition, some simultaneous combinations of proinflammatory genotypes reported to be associated with increased risk of GC were analyzed. Frequencies of IL-1B-511T/IL-1RN*2/*2 combination in patients and controls were $8.2 \%$ and $5.5 \%$ respectively. The haplotype IL-10 $-597 \mathrm{~A} / \mathrm{IL}-10-1087 \mathrm{~A}$ frequency was almost identical in GC patients (22.3\%) than in HC (19.4\%), and carriage frequency of IL-1B511 T/IL-1RN*2*2/TNFA-308A alleles was $6.6 \%$ in GC and $7.4 \%$ in HC. Conclusion: Our data show that IL-1B, IL-1RN, IL-12p40, LTA, IL-10, IL-4 and TGF-B1 gene polymorphisms are not involved in the susceptibility to GC in Caucasians. Studies to elucidate the real contribution of genetic host factors on GC susceptibility are warranted at different geographic areas and ethnic groups

\section{M2144}

Influence of Single Nucleotide Polymorphism in the Promoter Region of Interleukin-8 Gene On Progression and Metastasis of Gastric Cancer Ayumu Taguchi, Naoki Ohmiya, Nobuyuki Mabuchi, Takafumi Andoh, Osamu Maeda, Akihiro Itoh, Yoshiki Hirooka, Yasumasa Niwa, Hidemi Goto

Background: Host genetic susceptibility may influence gastric carcinogenesis caused by Helicobacter pylori infection. We previously reported that Interleukin (IL)-8 T-251A single nucleotide polymorphism (SNP) is associated with the elevated risk of atrophic gastritis and gastric cancer. In this study we aimed to investigate the influence of IL-8 T-25lA SNP on progression and metastasis of gastric cancer. We also investigated the effect of IL-8 polymorphism on IL-8 production and on histological degree of gastritis in gastric mucosa. Methods A total of 863 subjects were enrolled, including healthy controls $(\mathrm{n}=252)$, individuals with atrophic gastritis $(\mathrm{n}=215)$, and patients with gastric cancer $(\mathrm{n}=396)$. IL-8 T-251A SNP

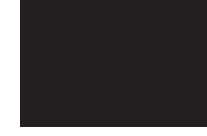


was genotyped by PCR-RFLP. The levels of IL-8 protein were measured by chemiluminescent immunoassay. Results: A/A genotype held a higher risk of overall gastric cancer (OR, 2.22; $95 \% \mathrm{CI}, 1.08-4.56)$ than $\mathrm{T} / \mathrm{T}$ genotype. We also found that $\mathrm{A} / \mathrm{A}$ genotype increased the risk of diffuse (OR, 2.79; 95\% CI, 1.21-6.39), poorly differentiated (OR, 2.70; 95\% CI, 1.146.38), lymph node (OR, 2.50; $95 \% \mathrm{CI}, 1.01-6.20)$ and liver metastasis (OR, 5.63; $95 \% \mathrm{CI}$, $1.06-30.04$ ), and p53-mutated (OR, 1.91; $95 \% \mathrm{CI}, 1.13-3.26$ ) subtype of gastric cancer. A/ $\mathrm{A}$ and $\mathrm{A} / \mathrm{T}$ genotypes were significantly associated with higher levels of IL-8 protein than T/T genotype. Neutrophil infiltration score was significantly higher in A/A genotype than in T/T genotype. Conclusion: We demonstrated that IL-8 T-251A SNP is associated with higher expression of IL-8 protein, severer neutrophil infiltration and increased the risk of gastric cancer, especially diffuse type, poorly differentiated adenocarcinoma, lymph node and liver metastasis, and p53 mutations. These findings suggest that IL-8 T-25lA SNP influence progression and metastasis of gastric cancer by autocrine and/or paracrine mechanism

\section{M2145}

Contribution of MYH Mutations to Colorectal Cancer in a British Asian Population

Sunil Dolwani, Geraint Williams, West Kevin, Joseph Newman, David Stock, Paul Griffiths, Jacqueline Best, Jeremy Cheadle, Julian Sampson

Bi-allelic mutations in the base excision repair gene MYH have recently been discovered to cause the multiple colorectal adenoma and carcinoma phenotype. Our previous study identified 25 such cases from 111 eligible families with non dominant inheritance out of 614 studied from 6 UK regional polyposis registers. An interesting observation in this study was that all 4 cases with homozygous E466X mutations and the case with a Y90X mutation were unrelated individuals of British Asian origin. We postulated that MYH mutations might contribute more significantly to colorectal cancer among British Asians than indigenous Northern Europeans. We therefore conducted a retrospective study to assess the contribution of MYH mutations to colorectal cancer a condition which is otherwise rare in this ethnic group. Subsequent to Multicenter ethics approval from the MREC 120 British Asian cases with CRC and 100 controls with inflammation without neoplasia from the same ethnic group were identified from pathology depts of the 5 participating centres. DNA was extracted and successfully analysed from paraffin blocks of normal tissue from these. All samples in the case group were subjected to histopathological re-examination to ensure analysis of only normal background tissue. PCR and restriction enzyme digestion was used for identification of the Asian mutations E466X (using ApoI ) and Y90X (using RsaI) and also to identify the common Northern European mutation G382D (using BglII). ARMS PCR was used for detection of the second common European mutation Y165C. Positive results were confirmed by bidirectional sequencing (ABI Prism 3100). One case (1/120) and one control (1/100) were found to be heterozygous for the E466X variant in MYH. Sequencing of all exons of $\mathrm{MYH}$ in these 2 individuals did not reveal any second mutations. We conclude that these four MYH mutations are unlikely to be a major contributor to colorectal cancer in British Asians. A much larger series would be required to quantify accurately the proportion of colorectal cancer attributable to MYH in this group. None the less, the frequency with which the E466X allele was observed is comparable to the frequency of Y165C and G382D in the general British population and there is a strong case for initial testing for this mutation in Asian individuals with adenomatous polyposis or colorectal cancer with apparent recessive transmission.Studies in other ethnic subgroups have also suggested that the identification and characterisation of population-specific ancestral variants such as 1395delGGA in Italians may be helpful for the provision of molecular diagnostics for MYH associated polyposis (MAP).

\section{M2146}

Possible Association of Polymorphisms in the COX-2 Gene with Risk of Adenoma Development in An African-American Case-Control Study Hassan Ashktorab, Lucile Adams-Campbell, Mamoon Elbedawi, Mohammad Daremipouran, John Kwagyan, Ahmad Akhgar, Akhter Shahina, Shirly Tsang, David J. Munroe, Iu Ali, Duane Smoot

Cyclooxygenase-2 (Cox-2), a key enzyme in the arachidonic acid signaling pathway is often over-expressed in colorectal adenoma. Recent evidence indicates that single nucleotide polymorphisms (SNPs) in the Cox-2 gene may modulate the risk of adenoma development. We explored this possibility of association between Cox-2 polymorphisms and risk of adenoma in an African American case-control study. We used an exhaustive approach of genotyping 13 haplotype-tagging SNPs (ht SNPs) in the Cox-2 gene in 72 cases of advanced adenomas and 146 polyp-free controls. All ht SNPs, except for one, were from the regulatory regions distributed over the entire gene (three in the promoter region at positions -466, 663, and -861; one in exon 1 at 2331; two in intron 5 at 5072 and 5229; one in intron 6 at 5625; one in intron 7 at 6064; five in the 3' untranslated region (UTR) of exon 10 at $8334,8494,10494,10848$, and 10935). Associations were analyzed with logistic regression models assuming a dominant effect for rare variants to increase statistical power. Our analysis showed an association of the intron 5-5229 G variant with increased risk of adenoma development. Compared to individuals with the wild type genotype GG at intron 5-5229, carriers of TG/TT genotypes had more than two-fold risk of adenoma development. (odds ratio (OR) 2.1 with $95 \%$ confidence interval (CI) $1.07-4.41, p=0.0044)$. Another trend of increased risk was observed at the 3'UTR- position 10935 (OR, 1.6: CI, 0.73 - 3.38). Interactions between COX2 genotype and use of nonsteroidal anti-inflammatory drugs, smoking, alcohol consumption, and risk of colorectal cancer were also explored. Our results, if confirmed in a larger African-American cohort, would be helpful in identifying individuals at increased risk for developing colorectal cancer

\section{M2147}

The Risk of Interleukin-lbeta Gene in Esophageal, Gastric and Colorectal Carcinomas

Hiroaki Ito, Kazuhiro Kaneko, Toshinori Kurahashi, Taikan Yamamoto, Yosuke Kumekawa, Meiko Kuwahara, Yutaro Kubota, Takashi Muramoto, Michio Imawari

[Background] Interleukin (IL)-1 gene polymorphisms are associated with development of gastric atrophy and with increased risk of gastric carcinoma. A -31 C-to-T base transition in the promoter region of this gene is involved in carcinogenic changes within the stomach, especially in Helicobacter pylori infected individuals. We examined association between 1L1 locus polymorphisms and risk of esophageal, gastric,colorectal carcinomas, and ulcerative colitis (UC) in Japanese patients with H. pylori infection. [Patients and Methods] Control subjects and all patients with esophageal, gastric, colorectal carcinoma cases, and UC cases were confirmed H.pylori infection by the use of 14C-urea breath test, 18 culture, and histopathological examination. IL-1B and ILl-RN polymorphisms were analyzed in 136 controls, 75 patients with esophageal carcinoma, 186 patients with gastric carcinoma, 69 patients with colorectal carcinoma, and 18 patients with UC. For IL-1B-511 and -31 polymorphisms were determined by fluorescence-based polymerase chain reaction-single-strand conformation polymorphism (PCR-SSCP) analysis. For IL-1 receptor antagonist gene (IL-1RN), pentaallelic variable number of tandem repeats (VNTR) was determined by PCR-standard agarose gel electrophoresis. [Result] For gastric carcinoma, IL-1B-511 heterozygotes (OR: 0.48, 95\% CI: 0.3-0.9 $\mathrm{p}=0.0115$ ) and T carriers (OR: $0.52,95 \% \mathrm{CI}: 0.3-1.0, \mathrm{p}=0.0185$ ) had a significantly reduced risk of carcinoma. For colorectal carcinoma, IL-1B-511 heterozygotes (OR: $0.34,95 \%$ CI: $0.2-0.7, p=0.0028$ ) and T carriers (OR: $0.43,95 \% \mathrm{CI}: 0.2-0.9, \mathrm{p}=0.0015$ ) had a significantly low risk of carcinoma. Genotype frequencies in patients with esophageal carcinomas and UC were similar to controls, showing no genotypic specific risk. No significant difference also was observed in the frequencies of IL-1B-31C/T and IL-1RN genotypes between controls and the esophageal carcinoma patients, and the UC patients. [Conclusion] Our results in Japanese subjects showed that IL-1B -31 and -511 genetic polymorphisms and IL-1RN polymorphisms are not associated with the risk of esophageal carcinoma. However, IL-1B -511 C/T and T carrier state may be less at risk for gastric and colorectal carcinoma in the Japanese population.

\section{M2148}

APC Polymorphism in Subjects At Average and Increased Risk for CRC Oleg Dolkart, Eliezer Liberman, Moshe Leshno, Miri Revivo, Nadir Arber

Background: Mutations in the APC gene are the major event in familial adenomatous polyposis and in the majority of sporadic CRC. I1307K APC gene variant occurs in about $6 \%$ of Ashkenazi Jews, and has been suggested to represent a genetic change leading to susceptibility for colorectal cancer (CRC) in this ethnic group. Nevertheless, recent publications have questioned the useful role of I1307K in determining the risk for CRC (OR 1.4-2.0). Much less is known about the E1317Q variant in the APC gene. Objective: To prospectively assess the clinical importance of these two polymorphisms in Israeli Jews at average and increased risk for CRC. Methods: 3650 consecutive attendees at the Integrated Cancer Prevention Center (between 12.97-12.05) were consented, including an average risk group (asymptomatic subjects without a personal or family history of colorectal neoplasia) and a high risk group (patients with a personal and/or family history of colorectal neoplasia). DNA from peripheral blood lymphocytes was amplified by real time PCR using primers that were specifically designed to detect, simultaneously, I1307K and E1317Q. E1317Q was evaluated in the last 1911 subjects (12.02-12.05). A life-style habits questionnaire, (cigarette smoking, alcohol consumption, BMI, physical activity, vitamins, and medications and anti-oxidants intake) was administered to all participants. Results: Overall I1307K was detected in 301 subjects (8.6\%) (9\% among Ashkenazi and 2.4\% among non-Ashkenazi Jews). The carrier rate was $10.1 \%$ and $7.7 \%$ in high and average risk subjects, respectively $(\mathrm{P}=0.78)$. In both average and high risk study groups, no significant differences were found among carriers and non-carriers in the incidence of colorectal neoplasia, their histological features and age of diagnosis. The odds ratio (OR) for CRC was 1.2. E1317Q was detected in only 19 (1.1\%) cases. However, 18 of these subjects (95\%) had colorectal neoplasia (9 CRC and 9 adenoma). The last E1317Q carrier is a young subject that was enrolled into a tight surveillance program. Both, I1307K and E1317Q variants, were not associated with any of the known epidemiological risk factors for CRC, or vitamins/supplements intake. Conclusions: E1317Q is not just a polymorphism, but a rare gene variant of APC that carries a significant high risk for CR neoplasia (OR-4.3). Meticulous surveillance program is recommended in this setting. $11307 \mathrm{~K}$, on the other hand, posses only very slight increases in CRC risk (OR-1.4) as we had previously shown (CEBP, 2002). Il307K carriers should be involved in CRC screening programs like the average risk population.

\section{M2149}

The Role of Alcohol Dehydrogenase in Alcohol-Associated Colorectal

\section{Carcinogenesis}

Michael Marks, Monika Benesova, Felix Stickel, Markus W. Buechler, Helmut K. Seitz

Background and Aims: Chronic alcohol consumption has an increased risk for colorectal cancer (Su and Arab, Nutr Cancer 50: 111, 2004). It has been shown that acetaldehyde (AA) causes DNA damage and contributes to cancer development. In the upper aerodigestive tract individuals homozygous for the ADHIC*1 allele which codes for an ADH enzyme with high AA producing capacity have an increased risk for cancer (Visapää et al, Gut 53: 871, 2004). ADH is also important for the oxidation of retinol to retinoic acid, an important factor for cell differentiation which is reduced following chronic alcohol consumption, at least in the liver. The aim of this study was to investigate whether the ADHIC*1 allele also plays a role in alcohol-associated colorectal cancer and whether the expression of the $\mathrm{ADH}$ phenotype changes during carcinogenesis. Methods: Genotype and allele frequency of $\mathrm{ADHIC}$ were studied in 101 patients with colorectal cancer and an alcohol intake of more than $20 \mathrm{~g} /$ day (average $70+45 \mathrm{~g}$ ), in 60 patients with colorectal cancer and an alcohol intake of less than $10 \mathrm{~g} /$ day, and in 204 healthy controls without cancer and an alcohol intake of 
$14+8 \mathrm{~g} /$ day. Genotyping of the ADHlC locus was performed using polymerase chain reaction based on restriction fragment length polymorphism methods on leucocyte DNA. In addition, starch gel electrophoresis for the characterization of ADH phenotype was performed using biopsies from normal colonic mucosa $(M)(n=17)$, polyps $(P)(n=8)$ and from carcinoma $(C)$ $(n=9)$. In addition, total ADH activity was measured. Results: The allele frequency of the ADHIC* 1 allele was found to be significantly increased in alcohol consumers with colorectal cancer as compared to lightly drinking cancer patients and healthy controls $(65.4 \%$ vs. $54.6 \%$ vs. $51 \%, \mathrm{p}<0.05)$. In addition, $\mathrm{ADHlC}^{*} 1 / 1$ homozygosity was also significantly different between cancer patients who consumed alcohol compared to the other groups ( $41 \%$ vs. $23 \%$ vs. $22 \%$, p $<0.05$ ). Furthermore, total mucosal ADH activity decreased from $2.6+0.34(\mathrm{M})$ to $0.94+0.34(\mathrm{P})$ to $0.44+0.27(\mathrm{C}) \mathrm{nmol} / \mathrm{mg} \mathrm{prot} / \mathrm{min}$. ( $\mathrm{p}<0.05)$ during carcinogenesis. This was associated with a decrease in the presence of $\gamma$ - $\mathrm{ADH}(94 \%$ vs. $50 \%$ vs. $33 \%)$ and $\chi$-ADH ( $100 \%$ vs. $88 \%$ vs. $88 \%$ ). However, $\sigma-A D H$ was neither detected in normal mucosa nor in cancer tissue, whereas in $38 \%$ of polyps $\sigma-\mathrm{ADH}$ was found. Conclusion:These data shown an increased risk for the development of colorectal cancer in alcohol consuming individuals with the $\mathrm{ADHlC} * 1$ allele. One explanation for the de novo expression of $\sigma-\mathrm{ADH}$ in polyps could be retinoic acid deficiency in a critical premalignant condition to guarantee increased generation of retinoic acid.

\section{M2150}

MDR1 Polymorphism Is Associated with Reduced Risk of Gastric Carcinoma in Japanese Population

Tomomitsu Tahara, Tomiyasu Arisawa, Tomoyuki Shibata, Mituo Nagasaka, Yoshio Kamiya, Hiroshi Fujita, Ichiro Hirata, Hiroshi Nakano

Background: Host genetic factors play a key role in gastric carcinogenesis, but all the mechanism is not clearly demonstrated. P-glycoprotein/Multidrug resistace 1(MDRl) mediates the active transport of various substrates including xenobiotics, and it thus has a protective function in various tissues and organs including gastrointestinal epithelial cells. Recently, it has been reported that P-glycoprotein/MDRl is associated with intestinal tumorgenesis in Apc(Min/+)mice. Further, MDRl gene C3435T polymorphism in exon26 influences P-glycoprotein expression and activity in gastrointestinal tract Aims: We investigated the effects of a MDRl gene polymorphism on the risk of gastric cancer. Subjects: The study was performed in 157 gastric cancer (GC) cases [mean age 64.9, $\mathrm{F}: \mathrm{M}=0.28$, intestinal type (58.2\%), diffuse type(37.0\%), mixed type(4.8\%)] and 107patients without evidence of GC [mean age 64.0, F:M=0.31, Peptic ulcer diseases (43.0\%), gastritis $(57.0 \%)$ ] as a control group. Methods: MDRl C3435T polymorphism was determined by PCR-RFLP in all the patients. Results: MDR-1 3435 TT genotype frequency was significantly higher in controls than GC cases ( $31.1 \%$ v $14.7 \%$, OR: 2.12, p<0.05). Furthermore, 3435 TT was more strongly associated with reduced risk of intestinal type histopathology (31.1\% v 12.2, OR: 2.55 , $\mathrm{p}<0.05)$. There was no significant difference in $\mathrm{CT}$ and $\mathrm{CC}$ genotype frequencies between GC and controls. Conclusion: Our data suggest that MDR1 3435T/T is associated with reduced risk of gastric cancer in Japanese population.

\section{M2151}

The Angiotensin I-Converting Enzyme Gene Insertion/Deletion Polymorphism Is Linked to Early Gastric Cancer

Matthias Ebert, Jutta Dierkes, Christian Folwaczny, Manfred Stolte, Christoph Roecken, Peter Malfertheiner

The insertion/deletion polymorphism of the angiotensin I-converting enzyme (ACE) gene has recently been linked to the pathogenesis and progression of human cancers. Using genomic DNA from 88 patients with early gastric cancer confined either to mucosa (pTla) or submucosa (pTlb) we assessed the insertion (I) and deletion (D) polymorphism by PCR analysis and compared it with a large non-cancer control population $(n=145)$. In the noncancer control group the II genotype was observed in 33 (23\%) individuals, whereas the ID and DD genotypes were found in $72(50 \%)$ and 40 (27\%) individuals, respectively. Interestingly, in the cancer group we found the II genotype in $6(7 \%)$ patients and the ID genotype in 46 (52\%) individuals, while the DD genotype was observed in 36 (41\%) individuals ( $\mathrm{p}=0.0034)$. Accordingly, the odds ratio (OR) for the II genotype was 0.20 ( $95 \%$ $\mathrm{CI} 0.08-0.54, \mathrm{p}=0.009$ ) and 0.55 for the ID/II genotype (95\% CI $0.31-0.96, \mathrm{p}=0.044)$ using the high-activity genotype DD as the reference category. No correlation was found between tumor type, tumor stage, the presence of Helicobacter pylori and the ACE genotype. Our study provides further evidence that the ACE insertion/deletion gene polymorphism may be linked to the development of early gastric cancer

\section{M2152}

Effect of Vitamin D Receptor Polymorphisms On Colorectal Cancer Progression

Damien Matusiak, Robert Carroll, Richard Benya

Activation of the vitamin D receptor (VDR) is thought to play an important role attenuating and modulating $C R C$ progression. However, the VDR gene is highly susceptible to mutation, with over 200 single nucleotide polymorphisms (SNP) having been identified. Of those altering function, Bsml, Taql and Fokl are among the best studied. Yet the clinical consequence of each SNP has only been considered in the context of altering CRC risk; while the studies to date have reported variable, conflicting and inconclusive effects. In contrast, no studies have been performed evaluating the effect of these SNPs on CRC behavior post malignant transformation. METHODS: 67 consecutively resected flash-frozen CRC from 3/ 04 to 5/05 were obtained from the NCI Cooperative Human Tissue Network (CHTN). Sections of adjacent non-malignant colon along with the primary tumor and all lymph node (LN) metastasis were evaluated for predominant histology. Regions that were well differentiated were scored 1 , moderately differentiated 2 , and poor 3 , and entire specimen scored using a weighted average. Relevant regions of the VDR genome were amplified by PCR, SNP status assessed, and wild type (ie, SNP not present) indicated as TT, BB, or FF, while mutations to 1 or both alleles are identified by small case. CHTN pathologists determined tumor size. Statistical comparisons were performed by ANOVA in all circumstances. RESULTS: There was no correlation between tumor differentiation and tumor size. Taq status had no effect on tumor size, differentiation, or number of LN metastases (Table). Fokl status significantly affected tumor size and number of LN metastases, but had no impact on overall differentiation. Finally, Bsml SNPs resulted in progressively smaller and better differentiated CRC that decreased tumor metastasis. CONCLUSIONS: VDR SNP status significantly alters $\mathrm{CRC}$ growth, differentiation, and propensity to metastasize. Although the mechanism whereby VDR SNPs alter tumor behavior has yet to be determined, assessing this status may identify patients benefiting from vitamin D chemoprevention.

\begin{tabular}{|c|c|c|c|}
\hline Genotype & Size $(\mathrm{cm} 3)$ & Differentiation & LN (\#) \\
\hline Taq1: TT & $73.0+/-9.2$ & $1.8+/-0.0$ & $4.7+/-2.8$ \\
\hline $\mathrm{Tt}$ & $70.9+/-3.4$ & $2.1+/-0.0$ & $4.1+/-0.2$ \\
\hline tt & $67.5+/-5.3$ & $2.2+/-0.1$ & $4.8+/-0.4$ \\
\hline Bsm1: BB & $86.5+/-8.8$ & $2.2+/-0.0$ & $5.7+/-0.5$ \\
\hline Bb & $76.9+/-3.0$ & $2.1+/-0.0$ & $4.1+/-0.2$ \\
\hline bb & $34.4+/-3.0$ & $1.8+/-0.2$ & $2.3+/-0.6$ \\
\hline Fok1: FF & $100.2+/-16.3$ & $2.1+/-0.1$ & $6.2+/-1.2$ \\
\hline Ff & $66.9+/-4.1$ & $2.1+/-0.0$ & $4.6+/-0.3$ \\
\hline ff & $60.2+/-2.2$ & $2.0+/-0.0$ & $3.1+/-0.4$ \\
\hline
\end{tabular}

Data expressed as means+/-SE.

\section{M2153}

Cost As a Barrier to Acceptance of a Pancreatic Cancer Surveillance Program in Familial High-Risk Individuals

Jennifer Simon, Sara Olson, Emmy Ludwig, Robert C. Kurtz

Familial pancreatic cancer (FPC) represents about 10\% of pancreatic cancers. Surveillance programs for healthy FPC family members use expensive imaging and endoscopic modalities. We have shown that the cost associated with such surveillance is a barrier to recruitment of FPC family members. Methods: In 2002, we began our FPC registry for FPC patients and their relatives. Our protocol includes both a family registry and a surveillance program that utilizes Magnetic Resonance Cholangiopancreatography and Endoscopic Ultrasonography. In 2005, a grant to help defray the cost of surveillance was obtained and applied to newly recruited FPC family members; those who had previously refused surveillance were re-contacted. We compare acceptance of our FPC surveillance program after the lst year of the study, and after obtaining grant support. Results: During the lst year of our program, 51 eligible FPC patients and relatives were asked to join the study. Eighteen FPC relatives ( 8 female) agreed to participate and 14 relatives ( 5 female) refused. The main reason for refusal was the cost of surveillance in 12 of the 14 (86\%) relatives. Eight of the $18(44 \%)$ relatives who accepted the registry also refused surveillance for the same reason. At 34 months 273 patients and relatives had been approached and 152 people agreed to participate. Of these 96 are healthy FPC relatives ( 60 female, 36 male, mean age 49.1 years). Only 13 of the $96(14 \%)$ eligible relatives refused surveillance due to cost. Thirty-one relatives (22 female) refused our registry. Seven of the $31(23 \%)$ relatives who refused gave the cost of surveillance as the reason. We re-contacted 19 relatives (10 female) who initially entered the program but refused surveillance due to cost; 8 agreed to participate in surveillance, 4 refused again, and 7 are pending. FPC family members who accepted surveillance are more likely to be self-referred, under 50 , female, have graduate-level education, and have 3 or more relatives with pancreatic cancer. Conclusions: Since obtaining grant support to cover surveillance costs, the percentage of relatives who rejected FPC surveillance has decreased from $44 \%$ to $14 \%$ for those who initially entered our FPC registry only. The overall rejection rate for both the registry and surveillance decreased from $86 \%$ to $23 \%$. While some of this decrease in rejection rate may be attributed to personnel changes and to our increasing experience with the program, we believe that the key factor is the ability to support the cost of FPC surveillance.

\section{M2154}

\section{Ubiquitin Specific Protease 14 Induced Pancreatic Cancer Cell Growth} Inhibition and Apoptosis

Toshiyuki Ishiwata, Kazumitsu Cho, Shunji Ishiwata, Taeko Suzuki, Kiyoshi Teduka, Yoko Kawamoto, Takenori Fujii, Kiyoko Kawahara, Nando Nakazawa, E.J. Uchida, Takashi Tajiri, Zenya Naito

Background: Ubiquitin specific protease 14 (USP 14), also known as the $60 \mathrm{kDa}$ subunit of tRNA-guanine transglycosylase (TGT $60 \mathrm{kD}$ ), belongs to the ubiquitin-specific processing protease (UBP) family. UBP family releases ubiquitins that are conjugated to various sizes of proteins by aNH-peptide bonds and an eNH-isopeptide bond. One of the members of the UBP family, herpesvirus associated ubiquitin-specific protease (HAUSP), stabilized p53 even in the presence of excess $\mathrm{Mdm} 2$ by which p53 was ubiquitinated and thereafter degraded. Furthermore, HAUSP induced the p53-dependent apoptosis. USP 14 expression was reported in leukemic and colorectal cancer cells, however, the role of USP 14 in cancer cells has not get been well elucidated. In the present study, we attempted to examine the expression and role of USP 14 in human pancreatic cancer cell lines and pancreatic cancer tissues. Methods: USP14 mRNA and protein expression were examined in Panc-1, MIA$\mathrm{PaCa}-2, \mathrm{PK}-45 \mathrm{H}$ and PK-8 pancreatic cell lines by real time-PCR and Western blot analysis. Immunohistochemical analysis using anti-USP 14 antibody was performed in 53 pancreatic cancer cases. Furthermore, in situ hybridization analysis was performed to examine the expression of USP 14 mRNA in pancreatic cancer. USP 14 cDNA was cloned into pIRES2 EGFP protein expression vector and transfected to Panc-1 cells. After selection by G418, 
USP 14 stable transfectants were obtained. Results: USP14 mRNA and its protein were detected in four pancreatic cancer cell lines. Immunohistochemically, USP 14 was weakly localized in the islets of control pancreatic tissues. In 19 of 53 pancreatic cancer patients (35.8\%), USP14 was strongly detected in the cytoplasm of cancer cells and USP-14 positive patients tended to show a better prognosis. USP14 stably transfected Panc-l cells decreased the growth rates as compared to mock cells. The USP 14 transfectants showed a balloonlike appearance and apoptosis-related proteins, Bax and cleaved Caspase-3, were markedly increased in the cells. Conclusion: USP 14 was expressed in some human pancreatic cancer cells and overexpression of USP 14 induced apoptosis and cell growth inhibition in pancreatic cancer. These findings indicate that USP 14 may be a novel target for pancreatic cancer therapy.

\section{M2155}

Impact of CIS-Hydroxyproline On JAK/STAT3 Pathway in Pancreatic Carcinoma Cells

Gisela Sparmann, Christoph Mueller, Robert Jaster, Stefan Liebe, Joerg Emmrich

Background and Aims: The proline analogue cis-hydroxyproline (CHP) has been shown to mediate endoplasmic reticulum (ER) stress resulting in growth inhibition and apoptosis induction of the rat pancreatic tumor cell line DSL6A (Mueller et al., Gastroenterology 2004:126:A548). The aim of this study was the characterization of biological and molecular effects exerted by CHP on human pancreatic tumor cells. Methods: The human cell lines Capan I, BxPc-3, MiaPaca-2 and rat pancreatic stellate cells (PSC) as a control were used. Cell proliferation was assessed by bromo-deoxy-uridine (BrdU) incorporation. Apoptosis was analyzed by detection of caspase- 3 activation, necrotic cell death was demonstrated through the intake of ethidium bromide using fluorescence microscopy. ER stress was investigated by RT-PCR and Western blot technique detecting the proteins glucose related protein (GRP)78 and growth arrest and DNA damage inducible factor (GADD)153. Activation of signalling pathway components (STAT3, ERK) was analyzed by immunoblotting using antibodies specific for the respective phosphorylated protein. Results: CHP inhibited the proliferation and induced cell death in all investigated cell lines. However, there were differences in the susceptibility of individual cell lines with respect to the required $\mathrm{CHP}$ concentrations and incubation time. CHP had no significant influence on the levels of ER stress marker proteins which were constitutively expressed in human pancreatic tumor cells. The constitutive activation of STAT3 in the cell lines has been completely abolished by CHP. We could exclude that the CHP-mediated dephosphorylation of STAT3 was only an unspecific toxic reaction since ERK activation remained unchanged or even increased after CHP incubation. Conclusion: We could show that the impairment of human pancreatic tumor cells by CHP has been associated with the disruption of the JAK/STAT3 pathway. The CHP-mediated 'knock-out' of constitutively activated signal cascades in tumor cells could provide the basis for a potent anticancer strategy.

\section{M2156}

Down Regulation of LET-7 Micro RNA in Human Pancreatic Adenocarcinoma Michele Bouisson, Barbara Bournet, Nicole Vaysse, Lucien Pradayrol, Jean Escourou, Louis Buscail, Pierre Cordelier

Background: pancreatic ductal carcinoma (PC) is the 5th leading cause of cancer related deaths in Western countries, with an increasing incidence and a poor prognosis. Thus, there is an urgent need for the development of new modalities to improve diagnosis and treatment of PC. Micro RNAs (miR) are small non-coding transcripts that regulate gene expression by promoting the degradation of transcribed messages or by inhibiting translation. Emerging studies suggest that many miR may participate in human disease, including oncogenesis. Recently, expression levels of the human let-7 gene have been shown to vary among various adult tissues. Down regulation of let- $7 \mathrm{miR}$ has been reported in lung tumors and hepatocarcinoma. The aim of the present study was to establish the pattern of expression of Let- $7 \mathrm{miR}$ in PC-derived samples. Methods: RNA was purified from PC-derived cell lines ( $n=7), P C$ tumors $(\mathrm{n}=5)$ and endoscopic ultrasound-guided, fine needle aspiration biopsies (FNAB) ( $\mathrm{n}=11$ ). RNA collected by FNAB was amplified using the Full Spectrum @ ${ }^{\circledR}$ kit(SBI). As controls, we used ready-made RNA (Ambion), and RNA purified from normal, adjacent pancreas to PC. Let- 7 miR al, a2 and a3 precursors were quantified using real time PCR followed by in situ hybridization. GAPDH RNA was used as a control. Results: using realtime RT-PCR, we demonstrated the down regulation of let- 7 al $(-79 \pm 5 \%, \mathrm{p}<0.05)$, a2 ($88 \pm 4 \%, \mathrm{p}<0.05)$ and a3 $(-89 \pm 8.5 \%, \mathrm{p}<0.05)$ in Capanl, AsPCl, Capan2, Pancl, Miapaca, BxPC3 and NP29 PC-derived cells. In contrast, S100P, which plays a major role in the aggressiveness of $\mathrm{PC}$, was found elevated in all of the cell lines tested $(+1743 \pm 511 \%, \mathrm{p}<0.05)$. In addition, let-7 miR levels were reduced $(-84.7 \pm 3.8 \%,-75.7 \pm 5.8 \%$ and $-75.8 \pm 5.5 \%$ for let-7al, a2 and a3 respectively, $\mathrm{p}<0.05)$ and S100P levels elevated $(+1736+612 \%, \mathrm{p}<0.05)$ in PC tumors compared to normal adjacent pancreatic tissue. Eventually, let-7 miR were found down expressed in FNAB $(-37.4 \pm 26.7 \%,-97.4 \pm 1.3 \%$ and $-70.6 \pm 17.6 \%$ for let-7al, a 2 and $\mathrm{a} 3$ respectively, $\mathrm{p}<0.05)$, whereas S100P levels were elevated $(+31050 \pm 1356, \mathrm{p}<0.005)$. Expression patterns of let-7 miR in PC tumors were confirmed by in situ hybridization. Conclusion: the present study represents the first report of reduced expression of let-7 in PC samples. Because (i) activating mutations of k-ras are frequently detected in PC, and (ii) the 3'UTRs of the human RAS genes contain multiple let-7 complementary sites, such alteration in let-7 expression might participate in PC induction or progression. Taken together, the present study could lead to the development of new molecular markers for either early diagnosis or targeted therapy for PC.

\section{M2157}

The Bioflavonoid Myricetin Induces Pancreatic Cancer Cell Death by a Mechanism Which Is Independent of HSP70

Phoebe Phillips, Vikas Dudeja, Rifat Sharif, Rajinder Dawra, Ashok Saluja

Pancreatic carcinoma is the fourth leading course of cancer deaths in the United States due to its poor response to chemotherapy and radiation treatments. One of the underlying mechanisms is their resistance to apoptosis. Studies suggest that foods rich in polyphenolic compounds contribute to reducing the risk of cancer and that these compounds can reduce tumor growth in vivo. We have previously shown that the bioflavonoid quercetin induces pancreatic cancer cell apoptosis via the inhibition of heat shock protein 70 (HSP70). The aim of the present study was to examine the effect of the flavonoid myricetin [structurally similar to quercetin, with an additional $\mathrm{OH}$ group on the 5' position of benzene ring $\mathrm{B}$ ] on i) pancreatic cancer cell viability compared to non-transformed pancreatic ductal cells; and ii) HSP70 levels in cancer cells. Methods - The human pancreatic cancer cells (PANC-1 or $\mathrm{MiaPaCa}-2)$ or normal pancreatic ductal cells were incubated with varying concentrations of myricetin $(5-100 \mu \mathrm{M})$ for 24 hours in serum free culture medium. Control cells were incubated with the vehicle alone $(<0.2 \%$ DMSO). Cell viability was studied using the tetrazolium salt WST-8 assay. HSP70 expression was assessed by real-time quantitative PCR and western blotting of cell lysates. Results - Incubation of both PANC-1 and MiaPaCa-2 cells with myricetin resulted in significantly reduced cell viability at concentrations greater than $25 \mu \mathrm{M}(40 \mu \mathrm{M}$ myricetin decreased cell viability by $50 \%$ and $100 \%$ in PANC-1 and MiaPaCa-2 cells respectively). In contrast, normal duct cell viability was unaffected by incubation with myricetin. Both of these pancreatic tumor cell lines had very high levels of endogenous expression of HSP70 levels compared to normal pancreatic duct cells, however, myricetin had no effect on HSP70 mRNA or protein levels. Conclusions - The presence of an additional hydroxyl group in the B ring of the flavonoid base skeleton of myricetin results in a greater inhibitory effect of this flavonol on pancreatic cancer cell viability as compared to our previous observations with quercetin. It also alters the mechanism of action, while quercetin's effect is mediated by its inhibition of HSP70 expression, myrecetin inhibits cell growth by a mechanism which is independent of HSP70 expression. These results suggest that myricetin may be a potential therapeutic agent to prevent the progression of pancreatic cancer.

\section{M2158}

Prognostic Factors for Survival in Pancreatic Cancer: A Population-Based Study

Mohamad Eloubeidi, Renee Desmond, Charles M. Wilcox, Reda Wilson, Pavan Manchikalapti, Mona Fouad, Isam Eltoum, Selwyn Vickers

Background: There is currently no population-based study from the Deep South of the United States (with $>25 \%$ African American residents) evaluating survival of patients with pancreatic cancer. Our aims were tol) analyze prognostic factors influencing pancreatic cancer survival using the population-based Alabama Statewide Cancer Registry (ASCR) 2) to determine whether race/ethnicity is an independent determinant of outcomes in patients with pancreatic cancer. Methods: Eligible cases included all persons diagnosed with pancreatic cancer from 1996-2000 and reported to the ASCR. Survival time was calculated from time of diagnosis to death for pancreatic cancer deaths or to date of last contact or death from other causes for censored cases. Risk factors associated with survival were assessed with the Kaplan-Meir survival method and the log-rank test. Demographic, tumor and treatment variables were assessed using Cox proportional hazards model. Results: Of 2,230 patients, the median age at diagnosis was 71 years and the male to female ratio was approximately 1:1. Seventy-three percent of patients were White, and $27 \%$ of patients were Black. The distribution by stage was $12.5 \%$ localized disease, $29.6 \%$ regional, $35.3 \%$ distant and $22.6 \%$ unstaged. The median survival for all cases was 0.39 (SD 0.01) years. Patients that underwent surgical treatment were less likely to die from pancreatic cancer (HR $0.4895 \% \mathrm{CI}, 0.41$ 0.56). Similarly, patients that underwent either chemotherapy or radiation therapy had improved survival (HR $0.6295 \% \mathrm{CI}, 0.53-0.73$ ). Across all stages, Black patients were significantly less likely to receive chemotherapy compared to White patients $(27.8 \%$ vs. $32.7 \%, p=0.03)$ and were significantly less likely to receive surgical intervention ( $15.2 \%$ vs. $18.9 \%, \mathrm{p}=0.049$ ). When examining patients who were offered the therapy of choice but refused, we found across all stages that a greater proportion of Black patients refused therapies vs. Whites ( $4.3 \%$ vs. $2.1 \%(p=0.02)$ for chemotherapy; $2.1 \%$ vs. $0.8 \%(p=0.05)$ for radiation and $3.3 \%$ vs. $1.4 \%$ ( $\mathrm{p}=0.01$ for surgery). Cox proportional hazard model showed no effect of race on overall survival while controlling for stage at presentation, type of therapy received, age at diagnosis and site of primary tumor. Conclusion: Survival in patients with pancreatic cancer remains dismal. Tumor characteristics and treatment factors are directly related to survival in patients with pancreatic cancer. Black patients were less likely to receive therapy but were also more likely to refuse indicated therapy. Factors leading to racial disparity in treatment of pancreatic cancer warrant further investigation.

\section{M2159}

Predictive Markers of Treatment Response to 5-FU and Gemcitabine in Pancreatic Cancer. Results from the RTOG 9704 Prospective Randomized Adjuvant Treatment Trial

James J. Farrell, Raymond Lai, Kathryn Winter, Marius van Rijnsoever, William Regine, Philip Schaefer, A Bowen Benson, John MacDonald, Chris Willett, Hany Elsaleh, John Mackey

Introduction: Thymidylate synthase (TS) thymidine phosphorylase (TP) and methylenetetrahydrofolate reductase (MTHFR) are key enzymes in the metabolism of folate and 5-fluorouracil. Human equilibrative nucleoside transporter (hENTl) is involved in gemcitabine transport into cells. Retrospective studies in pancreatic cancer suggest that these markers may have prognostic and predictive value. We aim to evaluate these markers in resected tumors from patients particpating in the RTOG 9704 prospective multicenter randomized adjuvant treatment trial which compared 5-FU with Gemcitabine. Methods: A Tissue Microarray was 
constructed using 3 separate cores from 225 resected pancreatic tumors. Immunohistochemistry was performed to define TS, TP and hENTl protein expression. TS promoter genotype ( 2 or 3 tandem repeats) and MTHFR genotype (C667T) was assessed using tumor DNA. Correlations between protein expression or genotype and prognostic or predictive factors were sought, by unconditional logistic regression analysis. Results: High TS nuclear expression, TP cytoplasmic expression, TP nuclear expression and TP stromal expression is seen in 30\%, $86 \%, 65 \%$ and $92 \%$ of cases. There are statistically significant associations between higher pathologic grade and high expression of TP nuclear $(\mathrm{p}=0.05)$ and TP cytoplasmic $(\mathrm{p}=0.02)$. There is a trend towards nodal status and high TP nuclear marker levels $(p=0.09)$. The frequencies of TS promoter tandem repeat $2 \mathrm{R} / 2 \mathrm{R}, 2 \mathrm{R} / 3 \mathrm{R}$ and $3 \mathrm{R} / 3 \mathrm{R}$ are $27 \%, 33 \%$, and $38 \%$. There is a non-significant trend towards 3R/3R TS genotype and high TS protein expression. $22 \%$ of cases had no expression of hENTl, compared with $38 \%$ with high expression. The frequencies of the MTHFR 667CC, 667CT, and 667TT genotypes are $48.8 \%$, $41.1 \%$, and $10.1 \%$, respectively. $27.6 \%$ of patients with either $667 \mathrm{CC}$ or $667 \mathrm{CT}$ vs. $8.3 \%$ of patients with $667 \mathrm{TT}$ are early $\mathrm{T}$ stage $(\mathrm{p}=0.12)$. Correlation analysis with treatment outcome and toxicity for this trial is currently underway. Conclusions: This is the first study to analyze prognostic and predictive markers in a prospective randomized pancreatic cancer clinical trial. There is limited association between TS or TP protein expression and tumor demographic factors. There is a trend towards correlation between TS genotype $3 \mathrm{R} / 3 \mathrm{R}$ and high TS protein expression in pancreatic cancer. MTHFR TT genotype was more likely to be associated with advanced $\mathrm{T}$ stage and may be associated with disease progression. The majority of tumors expressed hENTl. These markers may serve as useful predictors markers of response and toxicity in pancreatic cancer treatment.

\section{M2160}

TGF[Beta] Activates Calcium-Dependent PKC[Alpha] to Suppress PTEN Expression in Pancreatic Cancer Cells

Jimmy Y.C. Chow, Jennifer Cabral, Hui Dong, Sherry C. Huang, John M. Carethers

BACKGROUND: TGF[beta] switches to promote tumor growth at the later stages of tumor progression. TGF[beta] downregulates PTEN, a tumor suppressor gene that is mutated in the germline of some pancreatic cancer prone hamartomatous syndromes. PTEN, however, is rarely mutated in sporadic pancreatic cancers, and its regulation by TGF[beta] may mediate TGF[beta]'s growth suppression and/or oncogenic actions. Protein kinase CS (PKC) are widely expressed in cancer cells in various isoforms and have various physiological functions. Here, we examined if TGF[beta]-induced PTEN modulation was influenced by PKCs. METHODS: We used a SMAD4-null pancreatic cancer cell line (BxPC3), which expresses TGF[beta] surface receptors. Cells were treated with TGF[beta]l $(10 \mathrm{ng} / \mathrm{mL})$ that has been shown previously to reduce PTEN expression at both mRNA and protein levels, and observed up to 48 hours. The cells were lysed and separated into cytosolic and membrane fractions and analyzed by Western blotting using antibodies against various PKC isoforms (conventional: [alpha], [beta], [gamma], novel: [delta], [epsilon], [mu], [theta], and atypical: [iota]/[lambda], [zeta]). PTEN mRNA levels were assessed by reverse transcriptase PCR. A conventional PKC inhibitor, Gö6976 (a calcium-dependent PKC inhibitor) was used to block activation of PKC[alpha]. The levels of intracellular calcium was also determined using FURA-2/AM. RESULTS: TGF[betal was previously shown to reduce PTEN mRNA expression 24 hours and protein expression 48 hours after treatment. Most of the PKC isoforms tested except PKC[theta] were expressed in BxPC3 cells at the protein level. TGF[beta] treatment led to membrane translocation of PKC[alpha] and [iota]/[lambda] as early as 15 min and remained activated until 48 hours after treatment. Other isoforms were not activated by TGF[beta]. Furthermore, extracellular calcium influx was induced immediately after TGF[beta] treatment in BxPC3 cells. Using the conventional PKC inhibitor, Gö6976, TGF[beta]-induced PTEN suppression was reversed. Moreover, Gö6976 treatment suppressed TGF[beta]-induced cell growth in SMAD4-null cells, while Gö6976 treatment alone had no effect on PTEN expression and cell growth. CONCLUSIONS: Calcium-dependent PKClalphal appears to mediate TGF[beta]-induced transcriptional downregulation of the tumor suppressor PTEN in SMAD4null pancreatic cancer cells and induces cell growth. PKC[alpha] appears to constitute a TGF[beta]-induced pathway that promotes tumor growth in opposition to the classical SMAD pathway, which suppresses growth

\section{M2161}

Blocking Programmed Death 1 Ligands and PD 1 Pathway Induces Antitumor Effect of CC Chemokine CCL2 in Pancreatic Cancer Keisuke Okudaira, Ryota Hokari, Yoshikazu Tsuzuki, Yoshikiyo Okada, Chie Kurihara, Norikazu Mataki, Hisayuki Matsunaga, Koichi Takebayashi, Syunsuke Komoto, Chikako Watanabe, Atsushi Kawaguchi, Shigeaki Nagao, Kazuro Itoh, Ko Okumura, Miyuki Azuma, Hideo Yagita, Soichiro Miura

Background The negative signal provided by interactions of programmed death-1 (PDl) and its ligands, PDL1 (B7H1) and PDL2 (B7DC), has been suggested to play important roles in tumor evasion from host immunity. However, the role of PDl signals on pancreatic cancer development has not been reported. CCL2 positively correlates with tumor macrophage infiltration in pancreatic cancer, possibly induces recruitment of tumor infiltrating lymphocytes. In this study we investigated whether CCL2 immunotherapy is effective in murine pancreatic cancer model and if not, we examined how PDI/PDL systems are involved as negative signals in tumor immunity. Material \& Method C57BL6 mice and murine pancreatic cancer cells (pan02) were used. 3x105 tumor cells were orthotopically inoculated in mice pancreas. 4 weeks later, tumor sizes were measured. Recombinant CCL2 $(3 \mu \mathrm{g})$ was injected intraperitoneally once a week for 4 weeks. Tumor proliferation rates were calculated from their size after treatment. Effects of CCL2 on leukocytes migration to cancer vessels were observed by intravital microscope. Blocking study by antibodies against PD1, PDL1 or PDL2

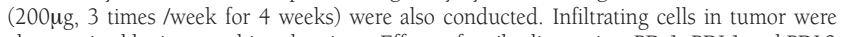
characterized by immunohistochemistry. Effects of antibodies against PD-1, PDL1 and PDL2 on cytokine expression in tumor were studied by quantitative RT PCR. Results Panc02 cells expressed PD1, PDLl and PDL2 at rest, but after interferon (IFN)gamma stimulation, only PDL1 was expressed in vitro. Panc02 cells developed tumor of $5.6 \mathrm{~mm}$ diameter on average.
Microscopic study showed increased leukocytes migration to tumor vessels by CCL2. Immunohistochemical study showed significant increase in CD4 and CD8 cells and PD1 positive infiltrating cells in tumor area after CCL2 treatment. PDL2 + cells were observed in peritumor area. PDl and PDL 2 mRNA expression significantly increased after CCL 2 treatment. Despite the significant infiltration of immune cells, long term treatment of CCL2 alone did not decrease tumor proliferation rates. However, antiPD1 or antiPDL2 treatment significantly decreased the tumor expansion. Cytokine study showed increased intratumoral expression of IL4 and IL10 after CCL2 treatment. This increase was ameliorated by antiPD1 or antiPDL2 Conclusion PD1 ligand/PD1 pathway upregulated by CCL2 appeared to induce tumor evasion from immunity in spite of the increase in tumor infiltrating immune cells in murine mode of pancreatic cancer. A successful inhibition of tumor growth by blockade of the PDl ligand/ PDl signalling pathway may implicate a new approach of immunotherapy for pancreatic cancer.

\section{M2162}

Inhibition of the Proto-Oncogene Slug Modulates Proliferation in Pancreatic Cancer (PC) Through Activation of $\beta$-Catenin Signaling

Jennifer L. Koetsier, Harrison Brand, Dhananjay Kunte, Randy Brand, Ramesh Wali, Hemant Roy

Background: $\beta$-catenin dysregulation is an important early event in the pathogenesis of $\mathrm{PC}$ however, the mechanisms are unclear. The transcriptional repressor SLUG modulates $\beta$ catenin signaling in several cancers. There are no previous reports on the role of SLUG in PC. Methods: Immunohistochemical analysis of Tissue sections (PC and control) were performed using standard methods. The human PC cell line Mia PaCa-2 was transfected with SLUG siRNA and subjected to mRNA and protein analysis by RT-PCR and Western blot analysis. A scramble siRNA was used as a control. $\beta$-catenin signaling was assessed using the Tcf TOPFLASH reporter plasmid. Results: SLUG was detectable in PC tissue sections and $3 / 3$ cell lines tested. In Mia PaCa- 2 cells, siRNA caused a reduction in proliferation as measured by PCNA (64.5\% of control) with a corresponding decrease in $\beta$-catenin transcriptional activity as assessed by Tcf TOPFLASH reporter assay (68.6\% of control). Furthermore, there was a corresponding decrease in cyclin Dl ( $85 \%$ of control), a proproliferative $\beta$-catenin-regulated protein. $\beta$-catenin protein, but not message, was decreased with SLUG siRNA $(63.4 \%$ of control, $\mathrm{p}<0.05)$ suggesting altered $\beta$-catenin stability. Intriguingly, Mia PaCa-2 cells do not express E-cadherin indicating a novel mechanism is involved. In this regard, we noted that SLUG siRNA altered Akt activity $(55.4 \%$ of control, $\mathrm{p}<0.05)$ implicating the Akt/GSK-3 $\beta$ axis. Furthermore, by pre-treatment with the PI-3 kinase inhibitor, LY294002, we were able to abrogate both the ability of SLUG siRNA to down regulate $\beta$-catenin and also to inhibit proliferation. Conclusion: We demonstrate, for the first time, that SLUG expression in PC is involved in the dysregulation of $\beta$-catenin signaling with concomitant hyperproliferation. While the mechanisms are unclear, our data indicates that this may be through regulation of the PI-3 kinase cascade.

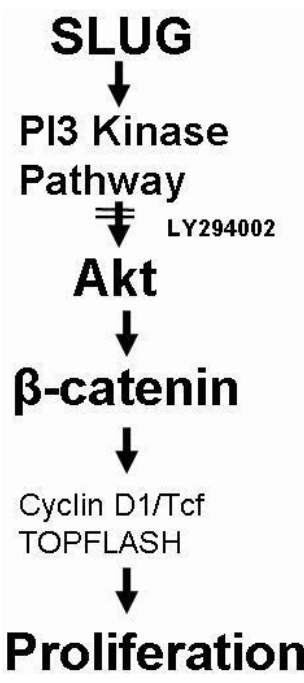

M2163

Angiotensin Converting Enzyme Inhibitors Reduce the Incidence of Pancreatic Cancer: A Study of Half a Million US Veterans Vikas Khurana, Ankur Sheth, Gloria Caldito, Jamie S. Barkin

AIM: To investigate the effect of Angiotensin Converting Enzyme (ACE) Inhibitors use in reducing the incidence of pancreatic cancer in the US veteran population. BACKGROUND ACE Inhibitors are commonly used antihypertensive and nephroprotective agents. Vascular Endothelial Growth Factor (VEGF) is believed to play a major role in angiogenesis in human tumors. Blocking the VEGF inhibits angiogenesis and suppresses tumor growth. ACE inhibitors cause suppression of VEGF in experimental models, leading to their anticancer effect. ACE Inhibitors have been noted to suppress tumor growth by inhibiting tumor angiogenesis in several animal and experimental models. DESIGN: The VISN 16 database contains clinical and demographic information about all veterans ( $>1.4$ million patients) cared for in the South Central VA Health Care Network (eight states in south central United States). The data was queried from Oct 1998 to June 2004, using a retrospective case control design. Statistical analysis was performed using SAS software version 9.0 (Chicago, IL). Multiple logistic regression analysis was used with calculation of odds ratios and $95 \%$ confidence intervals. The data was adjusted for age, race, gender, BMI, smoking, alcoho 
use, diabetes and statin use. Patients were placed in the ACE inhibitor user group if they were using ACE inhibitors prior to the diagnosis of pancreatic cancer. RESULTS: A total of 483,733 patients were included in the analysis. 185,852 (38.43\%) of those were using ACE inhibitors. Pancreatic cancer (ICD-9 code 157) was seen in 475 (0.1\%). ACE inhibitor users were $48 \%$ less likely to develop pancreatic cancer (Odds ratio 0.484 ; $95 \%$ CI 0.386-0.607, $\mathrm{p}<0.01$ ). The protective effect of ACE inhibitors was independent of statin use. Other significant covariates include age (OR 1.020,95\% CI for OR 1.012, 1,027, p <0.01); prior statin use (OR $0.458,95 \% \mathrm{CI}$ for OR $0.363,0.580, \mathrm{p}<0.01$ ) and diabetes (OR $3.837,95 \%$ CI for OR $3.089,4.767, \mathrm{p}<0.01)$. CONCLUSION: ACE inhibitors are associated with a $48 \%$ reduced incidence of pancreatic cancer after controlling for age, race, gender, BMI, smoking, alcohol use, diabetes and statin use. The limitations of our data are the VA population, the database and the fact that this is a case control study. The dose, duration and type of ACE inhibitor used were not factored into the analysis.

\section{M2164}

Proteosome Inhibitor Bortezomib Induces Lysosomal Disruption and a Caspase-2-Dependent Apoptosis in Human Pancreatic Carcinoma Cells Ho Yee Bonnie Yeung, Der Chen Huang, Frank A. Sinicrope

BACKGROUND. Bortezomib is a potent and reversible proteosome inhibitor that functions to degrade intracellular polyubiquitinated proteins. Bortezomib has shown broad in vitro antitumor activity and can induce apoptosis. We determined the effect of bortezomib on lysosomal and mitochondrial permeabilization, including the role of caspase-2 in apoptosis induction in human pancreatic carcinoma cells. METHODS. BxPC-3and CFPAC-l cells were incubated with bortezomib (Millenium Pharmaceuticals) at indicated concentrations and times. For measurement of ROS, cells $\left(3 \times 10^{6}\right)$ were incubated in dihydroethidium \pm tiron. Mitochondrial depolarization was analyzed using JC-1. Apoptosis was measured by annexin V-FITC labeling. Expression of caspase-2, -8, -9, cytochrome c, Smac, and Bcl-2 family members were analyzed by immunoblotting; $\mathrm{Bcl}-2, \mathrm{Bcl}-\mathrm{x}_{\mathrm{L}}$ mRNA by RT-PCR. Inhibitors of caspase-2 (z-VDVAD-fmk) and cathepsin B (R-3032: Celera Genomics) were utilized. BxPC 3 cells were stably transfected with an RNA interference (siRNA) construct against caspase-2. Cathepsin B redistribution was analyzed using fluorescence confocal microscopy. RESULTS. Bortezomib induced a dose-dependent apoptosis in association with reactive oxygen species (ROS) generation and cleavage of caspase- 2 to its $33 \mathrm{kD}$ and $14 \mathrm{kD}$ fragments. Bortezomib disrupted lysosomes with redistribution of cathepsin B to the cytosol that was blocked by the free radical scavenger tiron, but not by a caspase-2 inhibitor (z-VDVAD-fmk). Bortezomibinduced caspase-2 activation was attenuated by a selective cathepsin B inhibitor (R-3032), suggesting that cathepsin B release occurs upstream of caspase-2. Bortezomib-induced mitochondrial depolarization was attenuated by z-VDVAD-fmk, tiron and bongkrekic acid (inhibitor of mitochondrial permeability transition pore), and by caspase-2 siRNA compared to control cells, confirming that caspase-2 directly regulates mitochondrial permeabilization. Bortezomib-induced cytochrome $\mathrm{c}$ release and annexin $\mathrm{V}$ binding were attenuated by $\mathrm{z}$ VDVAD-fmk and partially by R-3032. In support of these data, bortezomib also activated caspase- 2 and induced apoptosis in CFPAC-1 pancreatic cancer cells that was attenuated by z-VDVAD-fmk, and to a lesser extent by R-3032 compared to BxPC-3 cells. Bortezomib activated the BH3-only proteins Bik and Bim and downregulated Bcl-2 and Bcl- $x_{\llcorner}$mRNA and proteins. CONCLUSION. Bortezomib induces lysosomal cathepsin B redistribution upstream of caspase-2. Caspase-2 activation regulates bortezomib-induced mitochondrial depolarization and apoptosis, suggesting that caspase- 2 may serve as a link between lysosomal and mitochondrial permeabilization.

\section{M2165}

Evaluation of the Combined Use of Cyclooxygenase-2-Inhibitors and Tumor Antigen-Loaded Dendritic Cells for the Immunotherapy of Pancreatic Carcinoma in a Murine Vaccination Model

Christian A. Bauer, Franz Bauernfeind, Max Schnurr, Rosi Kiefl, Walther Heldwein, Stefan Endres, Andreas Eigler, Marc Dauer

Background: Overexpression of cyclooxygenase-2 (COX-2) plays an important role in gastrointestinal carcinogenesis. Here, we investigated whether COX-2-inhibitors can increase the efficacy of dendritic cell (DC)-based vaccination therapy for pancreatic carcinoma. Material and methods: DCs were loaded with lysate from the pancreatic carcinoma cell line Panc-1 and cocultured with autologeous T cells. Restimulations with autologeous, tumor-lysate pulsed DCs were performed at weekly intervals. Lysis of Panc-l cells left untreated or pretreated with the COX-2-inhibitor NS398 was determined in a standard chromium release assay. In addition, apoptosis of the pancreatic carcinoma cells Panc-1, MiaPaca and BxPc3 treated with NS398 (20 or $100 \mu \mathrm{M})$ or left untreated was induced by CD95-activating antibody $\mathrm{CH}-11$ and measured by Annexin-V/PI-staining. The in vivo effect of the COX-2inhibitor Parecoxib on DC-based vaccination was investigated in a murine model of pancreatic carcinoma. DCs derived from the bone marrow of C57BL/6 mice were pulsed with syngeneic apoptotic Panc02-cells and matured with LPS/IFN-gamma. After establishment of subcutaneous Panc02-tumors, DCs were injected into the contralateral flank of C57BL/6 mice at weekly intervals. Parecoxib was administered i.p. daily; tumor size and weight loss were monitored. Results: Lysate-pulsed DCs primed tumor-specific, IFN-gamma-producing T cells in vitro. CTLs derived from cocultures with lysate-pulsed DCs specifically lysed Panc-1 cells Pre-treatment of Panc-1 cells with NS-398 prior to coculture with CTLs enhanced MHC class I-restricted, specific lysis. Blocking of CD95 on tumor cells prevented this increase in lysis. In vitro, COX-2-inhibitor NS398 sensitized Panc-1 cells to apoptosis (14 \% apoptotic cells after CD95-treatment in untreated cells versus $23 \%$ in NS398-treated cells). In the in vivo-model, DC vaccination and Parecoxib therapy were successful in preventing tumor growth and increased overall survival. However, combined therapy with Parecoxib was not more effective in preventing tumor progression than DC-based vaccination alone. Conclusions: Pancreatic carcinoma cells can be sensitized in vitro to CTL-mediated lysis by treatment with COX-2-inhibitors. Studies using CD95-blocking antibody as well as agonistic antiCD95 antibody indicate that COX-2-inhibitors specifically sensitize tumer cells to CD95mediated CTL killing. Currently, studies are performed to analyze whether the observed effects are COX-2-dependent and to characterize alternative downstream mechanisms involved in tumor cell sensitization to CTL-mediated killing

\section{M2166}

Sphingolipid Metabolite Levels Determine Pancreatic Cancer Cell Responsiveness to the Chemotherapeutic Gemcitabine Drug Julie Guillermet-Guibert, Lise Davenne, Dimitri Pchejetski, Nathalie Saint-Laurent, Olivier Cuvillier, Christiane Susini, Corinne Bousquet

Resistance to chemotherapy is the major reason for failure of clinical cancer treatment Pancreatic cancer is the fifth leading cause of death by cancer, and chemotherapy using gemcitabine has unfortunately not improve prognostic of this deadly disease. Compelling evidences suggest that aberrant sphingolipid metabolism results in chemotherapeutic drug resistance. Therefore, a correlation between pancreatic cancer cell resistance to gemcitabine and sphingolipid metabolism was here attempted. Two pancreatic cancer cell lines, BxPC3 and Panc-1, were chosen regarding their relative sensitivity and resistance to gemcitabine, respectively. When assessing endogenous levels of the tumor-suppressor ceramide versus tumor-promoting sphingosine-1-phosphate (SIP) lipids, which is a metabolic product of ceramide into sphingosine and then into SIP through ceramidase and sphingosine kinase (SK) activity, respectively, BxPC-3 showed higher level of ceramide as compared to Pancl cells. Conversely S1P levels were not different in both cell lines, which is consistent with a similar SK activity measured in these cells. As a consequence BxPC-3 cells displayed higher ceramide/S1P ratio as compared to Panc-1. Interestingly, cell-treatment with gemcitabine did not affect this ratio although gemcitabine unexpectedly up-regulated SK activity through increased SK mRNA expression. This was a consequence of gemcitabine-induced ceramide level as well. To investigate whether unbalancing the ceramide/S1P ratio might affect cellsensitivity to gemcitabine, BxPC-3 and Panc-1 cells were either treated with exogenous C2ceramide or processed to up- or down-regulate SK activity. Interestingly, C2-ceramide treatment decreased Panc-l cell survival in a greater extent than BxPC-3. Furthermore, ceramide sensitized Panc-1, but not BxPC-3, cells to gemcitabine cytotoxic action. Alternatively, overexpressing SK in BxPC-3 cells decreased the ceramide/S1P ratio by upregulating $\mathrm{SIP}$ production, and consequently rendered these cells more proliferative, invasive and resistant to gemcitabine cytotoxicity, as assessed in vitro and in a xenograft mouse model Conversely, inhibiting SK activity through Panc-1 cell-treatment with a SK-directed siRNA or with specific SK inhibitors increased the ceramide/S1P ratio, and consequently sensitized these cells to gemcitabine cytotoxicity. We therefore conclude that the ceramide/S1P ratio level is critical to predict pancreatic cancer cell-sensitivity to gemcitabine action. Balancing this ratio in gemcitabine-resistant cells using SK inhibitors might represent a promising approach to improve the clinical benefit of gemcitabine chemotherapy for pancreatic cancer.

\section{M2167}

Role of RAC1-Dependent NADPH Oxidase in the Growth of Pancreatic Cancer Jingru Liu, Larry W. Oberley, Joseph J. Cullen

Background: K-ras mutations occur in as high as $95 \%$ of patients with pancreatic cancer K-ras activates Racl-dependent NADPH oxidase a key source of superoxide. Our group has demonstrated that superoxide plays an important role in pancreatic cancer cell proliferation and that scavenging or decreasing the levels of superoxide inhibits pancreatic cancer cell growth both in vitro and in vivo (Cancer Res. 63:1297, 2003; Molecular Cancer Therapeutics $2: 361,2003)$. DNA microarray analysis and RT-PCR has demonstrated that Racl is also upregulated in pancreatic cancer (Oncogene 20:7437, 2001). The aim of this study was to determine if inhibiting Racl would alter pancreatic tumor cell behavior. Methods: Racl dependent effects were inhibited by using an adenoviral vector encoding a dominant-negative isoform of Racl (AdN17Racl). Human pancreatic cancer cells with mutant K-ras (MIA PaCa2) and wild-type K-ras (BxPC-3) were infected with AdN17Racl (25, 50, 100, and 200 MOI). Cells were harvested and analyzed via Western blot analysis for the HA-tag expressed by AdN17Racl. To determine pancreatic cancer cell behavior, cell growth and plating efficiency were determined following infection with the AdN17Racl. Results: In MIA PaCa2 human pancreatic cancer cells, AdN17Racl (25-200 MOI) increased Racl protein as demonstrated by immunoblots. AdN17Racl (25, 50, 100 and $200 \mathrm{MOI})$ significantly inhibited cell growth $(\mathrm{P}<0.01$ vs controls), while AdN17Racl (100 and $200 \mathrm{MOI})$ significantly decreased plating efficiency $(\mathrm{P}<0.01$ vs controls). In contrast, AdN17Racl had no effect in altering growth in the BxPC-3 human pancreatic cancer cell line. Conclusions: These results suggest that mutant K-ras activation of Rac-1-dependent NADPH oxidase leads to pancreatic cancer cell proliferation. In pancreatic cancer cells with mutant K-ras, inhibition of Racl may be a potential therapeutic target. Support: DK60618, CA66081

\section{M2168}

Promotor Polymorphisms of the UGT-Glucuronosyltransferase UGT1A4 Impact Activity and Inducibility of Human Hepatic Glucoronidation Thomas J. Erichsen, Ursula Ehmer, Tim O. Lankisch, Tordis M. Mueller, Tracey Gillman, Nicole Freiberg, Michael P. Manns, Christian P. Strassburg

Introduction: UDP-Glucuronosyltransferases (UGT) are a family of metabolizing enzymes which glucuronidate endogenous and exogenous substrates including the detoxification of carcinogens. We and others have described functional polymorphisms (SNPs) of individual members of the UGTIA gene family. Among these functional SNPs for UGTIAl and UGTIA7 have been associated with the glucoronidation of chemotherapeutics. Recently two functional SNPs of exonl of the human hepatic UGTIA4 gene were reported. The aim of this study was to identify and charactericize UGTIA4 SNPs outside the coding region with potential impact on UGT1A4 expression. Methods: Genomic DNA of 109 patients was analysed by polymerase chain reaction and re-sequencing. Promoter activity, point mutations and inducibility with TCDD and 3-MC were tested in cellculture by luciferase reporter gene assays using in Hep G2 cells. Human liver microsomes of genotyped samples were examined for specific UGT activity. Results: In the 5' UTR region of the UGTlA4 gene we identified 
two novel polymophisms (-163 G>A and $-219 \mathrm{C}>\mathrm{T}$ ) with an allelic frequency of 0.12 . A linkage disequilibrium was observed between $-163 \mathrm{G}>\mathrm{A},-219 \mathrm{C}>\mathrm{T}$ and the L48V SNP in exon 1 of the UGTIA4 gene. Reporter gene assays demonstrated a $40 \%$ reduction of the UGTlA4 transcription of the variant 5' UTR. TCDD and 3-MC were found to be able to induce UGTIA4 expression, which was significantly lower in the genetic variants. Patients with UGTIA4 SNPs showed a reduction of microsomal hepatic glucuronidation of steroids and cyproheptadines. Discussion: In our study we identified two novel functional SNPs of the UGTIA4 gene promotor region leading to a $40 \%$ reduction in transcriptional activity. The specific activity of the UGTIA4 protein with steroids, analgesics, anti-histaminics, anticonvulsive drugs and carcinogens suggests that genetic variations in the coding and promoter regions are the genetic basis for interindividual variations of metabolism. These data were functionally correlated and confirmed by glucuronidation activity determinations using human liver microsomes indicating that promoter and exon-based SNPs of the human hepatic UGTIA4 gene are biologically relevant factors for individual drug and possibly disease predisposition.

\section{M2169}

Gilbert's Disease and Drug Therapy: Lessons from Jaundice Under Atazanavir Treatment

Tim O. Lankisch, Ulrike Moebius, Georg Behrens, Michael Wehmeier, Reinhold E.

Schmidt, Michael P. Manns, Christian P. Strassburg

Introduction: Single nucleotide polymorphisms (SNP) of drug metabolizing enzymes such as uridine diphosphate glucuronosyltransferases (UGT) have been studied regarding their association with inheritable and mutagen-induced diseases, as well as risk factors for unwanted drug effects. Gilbert's disease is characterized by a TATA box polymorphism of the hepatic bilirubin-UGT (UGTlAl*28) leading to mild unconjugated non-hemolytic hyperbilirubinemia. Other SNPs at the 5'-UTR of the UGTIA gene locus such as UGTIA3$66 \mathrm{~T} / \mathrm{C}$ at the hepatic UGTlA3 gene and UGTlA7-57 T/G at the extrahepatic UGTlA7 gene have been identified and functionally lead to a reduction of steroid, carcinogen or drug glucuronidation. The protease inhibitor atazanavir represents a major drug for the treatment of patients with HIV infection and has been shown to lead to severe jaundice in individual patients. Based on the hypothesis of genetically determined metabolic risk factors we performed an UGT gene haplotype analysis in atazanavir treated HIV patients including UGTlAl*28, UGT1A3-66 T/C as well as UGTIA7-57 T/G to test the involvement of these UGT in atazanavir toxicity. Methods: SNP detection of UGTlAl*28, UGT1A3 -66 T/C, UGTIA7-57 T/G was performed by DNA sequencing and Taqman 5'-nuclease assays in 82 atazanavir-treated HIV-patients and 104 healthy blood donors. Patients were divided in 4 groups depending on their bilirubin serum level (group $0=$ normal value, group $4=5 \mathrm{x}$ upper limit) Results: We detected a significantly higher incidence of homozygous allelic variants and a higher allelic frequency of all three SNPs in the HIV patient group compared to healthy blood donors. Furthermore, most of all HIV patients with allelic variants were found in group 3 and 4 and all patients in group 4 carried the homozygous allelic variants of UGTIA1, UGT1A3, and UGT1A7. Statistical analysis revealed that the combination of all SNPs tested is the best predictor of atazanavir-induced hyperbilirubinemia. Discussion: Our findings show a higher frequency of hepatic and extrahepatic functional polymorphisms at the UGTlA gene locus in HIV patients. The combination of these SNPs of hepatic and extrahepatic UGT genes (haplotype) are a significant predictor of atazanavir-induced hyperbilirubinemia in HIV patients. In these patients Gilbert's associated jaundice is a phenotype which indicates an additional predisposition towards drug side effects based on the specificities of the other involved UGT genes. Therefore, the detection of variants at the UGTlA gene locus serve as a potential pharmacogenetic tool to predict drug associated toxicity and disease susceptibility.

\section{M2170}

Pathophysiology of Hepatobiliary Disease in Cystic Fibrosis (CF) Employing a $\Delta$ F508 Mouse Model

Folke U. Freudenberg, Jonathan N. Glickman, Bian B. Yu, Monika R. Leonard, Martin C. Carey

Introduction. Hepatobiliary complications of CF include cholestasis and focal biliary cirrhosis, but their pathobiology is not understood. We hypothesize that impaired CFTR function on large cholangiocytes prevents alkalinization of biliary $\mathrm{pH}$ and leads to a physical-chemical environment that facilitates precipitation of metal salts of unconjugated bilirubin (UCB) and bilirubin monoglucuronides (BMG) in the biliary tree and liver cells; moreover, hyperbilirubinbilia could aid this by enterohepatic cycling (EHC) of UCB secondary to bile acid spillage into the colon. Methods. Following genotyping, we studied homozygous $\Delta \mathrm{F} 508$ and wild type (WT) mice. We assessed liver histopathology and $\mathrm{Fe}$ and $\mathrm{Ca}$ accumulation by $\mathrm{H} \& \mathrm{E}$, Prussian blue and von Kossa staining. We measured hepatic and gallbladder (GB) bile pH and quantified bilirubin species by HPLC. We assessed gallbladder bile by light microscopy for mucin gel and gallstones and assessed GB sizes gravimetrically. Fecal bile acid excretion was quantified enzymatically. Results. Histologically, CF livers show mild cholangiopathy characterized by reactive changes in biliary epithelium, bile ductular proliferation and mild portal fibrosis. Those findings that were not present in WT mice. Multifocal Fe and $\mathrm{Ca}$ deposition was found in $17 \%$ and $33 \%$, respectively, of mutants compared to $6 \%$ in WT. Hepatic bile $\mathrm{pH}$ values were not significantly different, but gallbladder biles were more acidic in $\mathrm{CF}$ ( $\mathrm{pH} 7.2 \pm 0.2$ vs $7.9 \pm 0.1 ; \mathrm{p}<0.05)$. GB sizes were markedly larger in $\mathrm{CF}$ than in WT mice $(26.4 \pm 4.5$ vs $12.5 \pm 2.1 \mu \mathrm{l} ; \mathrm{p}<0.01)$, and contained mucin gel and significantly higher total bilirubin levels, but black stones occurred in only 1 of 7 mice. Paradoxically, conjugated bilirubin (CBR) levels were lower in hepatic bile of CF than WT mice $(56.3 \pm 6.7$ vs $80.3 \pm 5.0 \mu \mathrm{M} ; \mathrm{p}<0.05)$, due to marked decreases in BMG $(38.8 \pm 3.6$ vs $54.5 \pm 4.0 \mu \mathrm{M}$, $\mathrm{p}<0.05)$. Fecal bile acid excretion is elevated in all CF mice, but significantly ( $2-3$ fold $)$ in males only. Conclusions. Our study indicates that, although increased fecal bile acid losses should facilitate EHC of UCB, paradoxically hepatic hypobilirubinbilia was observed in $\mathrm{CF}$ mice. This is most likely caused by resorption of UCB and BMG by large cholangiocytes secondary to CFTR dysfunction. Taken together with increased Fe and Ca staining of the liver, presumably by UCB and BMG salts, our data suggest that chronic liver disease in CF may be triggered by both intrahepatic hydrolysis of CBR as well as deposition of insoluble metal salts of BMG and UCB in cholangiocytes and hepatocytes.

\section{M2171}

Identification of Multiple Interacting Quantitative Trait Loci (QTL) for Hepatic Fibrosis in Mice

Sonja Hillebrandt, Tilman Sauerbruch, Frank Lammert

Background: Recently we identified genetic loci that determine susceptibility to liver fibrosis in experimental crosses between fibrosis-susceptible and resistant inbred strains of mice (Hillebrandt et al. Nat Genet 2005;37:835-43). Despite the potential significance of genegene interactions (epistasis), their role in fibrosis susceptibility is unknown, since previous QTL analyses for liver fibrosis modeled the effects of single QTLs only. Our aim now was to identify interacting QTLs for liver fibrosis. Methods: We intercrossed $F_{1}$ hybrids of the fibrosis-susceptible strain $\mathrm{BALB} / \mathrm{cJ}$ and the resistant strain $\mathrm{FVB} / \mathrm{NJ}$ to obtain $350 \mathrm{~F}_{2}$ progeny. To induce liver fibrosis, we challenged the mice with $\mathrm{CCl}_{4}(2 \mathrm{mg} / \mathrm{kg} / \mathrm{wk}$ i.p. for 6 wks $)$. For phenotypic characterization, we determined the histological stage of liver fibrosis and measured hepatic collagen contents. We performed a genome scan with microsatellite markers (average distance $14 \mathrm{cM}$ ) in 100 mice with the largest deviations from the mean to the upper and lower ends of the distribution of each trait, followed by simultaneous genome scans of all possible pairwise interactions, as implemented in R/qtl. LOD thresholds were estimated by permutation tests. Results: In addition to the previously described fibrogenic QTLs (Hfib1 and Hfib2), we detected a new QTL on mouse chromosome 1 (Hfib3). According to standard nomenclature, this major QTL with significant effects on the progression of liver fibrosis (LOD 3.9) is named Hfib3. Furthermore, the two-dimensional scans identified a locus on chromosome 18 that affects fibrosis by interacting with loci on chromosomes 1 and 6 . In addition, we found interacting gene pairs between chromosomes 6 and 7, 9 and 13 as well as 3 and 16. Of note, the interacting loci display no significance on their own however, their interactions made notable effects on both the stage of fibrosis and hepatic collagen concentrations. Conclusions: Our findings demonstrate the importance of both single susceptibility loci and gene-gene interactions in liver fibrogenesis and provide the basic framework to investigate how systems of genes work together affecting this complex trait during chronic liver injury.

\section{M2172}

Increased Susceptibility to Estrogen-Induced Cholestasis in Mice Overexpressing the Hepatic $A B C B 11$ Gene

Helen H. Wang, Minoru Sakomoto, Anne Schmitz, Frank Lammert, David Q. Wang

Estrogen could be involved in intrahepatic cholestasis of pregnancy and oral contraceptiveinduced cholestasis, which represent a prominent feature of liver injury characterized by accumulation of bile salts (BS) and other toxic bile constituents within hepatocytes and blood. Because only a minority of patients develops cholestasis, we hypothesized that differences in estrogen metabolism could lead to differential inhibition of Abcbll-mediated BS transport in predisposed individuals. Methods: We investigated ovariectomized female wild-type (WT) and Abcb11 transgenic (tg) mice with or without disruption of the estrogen receptor $\alpha$ (Er $\alpha$ ) gene (i.e., Abcbll.tg and Abcbll.tg/EraKO), and treated the mice with $17 \beta$-estradiol (E2) at $3,6,12,24 \mu \mathrm{g} /$ day for 14 days. Biliary BS outputs were examined by HPLC and bile flow was determined gravimetrically. Expression levels of the genes encoding hepatic BS transporters and synthesis enzymes were analyzed by real-time PCR. Results There were dose-dependent 5 to 7 -fold increases in expression levels of the hepatic Er $\alpha$ Abcb11, Ntcp, Cyp7a1, and Cyp27 genes in WT mice, coupled to significant dose-dependent increases in bile flow and biliary BS output from $91 \pm 8$ to $136 \pm 15 \mu \mathrm{l} / \mathrm{min} / \mathrm{kg}$, and $215 \pm 5$ to $327 \pm 82 \mu \mathrm{mol} / \mathrm{h} / \mathrm{kg}$, respectively. The highest dose ( $24 \mu \mathrm{g} / \mathrm{day})$ of E2 significantly downregulated expression levels of the genes for hepatic BS transporters and synthesis and reduced bile flow and biliary BS outputs to $45 \pm 7 \mu \mathrm{l} / \mathrm{min} / \mathrm{kg}$ and $125 \pm 36 \mu \mathrm{mol} / \mathrm{h} / \mathrm{kg}$, respectively. In contrast, when doses of E2 were $\geq 6 \mu \mathrm{g} /$ day, Abcbll.tg mice displayed significant decreases in expression levels of the genes regulating hepatic BS transport and synthesis by 30 to $50 \%$, coupled to significant reductions in bile flow and biliary BS output to $45 \pm 7 \mu \mathrm{l} / \mathrm{min} /$ $\mathrm{kg}$ and $118 \pm 29 \mu \mathrm{mol} / \mathrm{h} / \mathrm{kg}$, respectively, all in a dose-dependent manner. Although being treated with various high ( 6 to $24 \mu \mathrm{g} /$ day) doses of E2, Abcbll.tg/EraKO mice showed essentially stable bile flow $(95 \pm 10 \mu \mathrm{l} / \mathrm{min} / \mathrm{kg})$ and biliary BS output $(238 \pm 64 \mu \mathrm{mol} / \mathrm{h} / \mathrm{kg})$ as well as constant mRNA expression levels of hepatic BS transporters and synthesis enzymes, all of which were comparable to those in WT mice treated with $3 \mu \mathrm{g}$ E2/day. Conclusions: Era plays a critical role in the biphasic (choleretic vs. cholestatic) effects of E2 on the regulation of hepatic BS transport and synthesis, as well as bile formation. The expression levels of Abcb11 partly account for variations in susceptibility to E2-induced cholestasis in mice. These studies point to a better design of individualized estrogen therapy in genetically susceptible patients and to improved prediction of the cholestatic potential of novel drugs.

\section{M2173}

Bcl-2,Bax and Bad mRNA Expression in Chronic Active Hepatitis, Cirrhosis, and Hepatocellular Carcinoma

Marina Bortolami, Andromachi Kotsafti, Chiara Carlotto, Romilda Cardin, Fabio Farinati

Background: Members of the $\mathrm{Bcl}-2$ family are important key apoptosis regulators, eithe promoting or inhibiting molecules. In HCV- and HBV-related chronic liver diseases, the apoptotic process seems to be the result of interaction between virus, hepatocyte and immune response. Failure of apoptosis is now known to contribute to the onset of human malignancies. Aims: To clarify the role of intracellular anti- and pro-apoptotic pathways, we studied the mRNA expression of Bcl-2, Bax and Bad in liver biopsies from patients with different degrees of liver damage, to determine whether HCV and HBV infection modulates their expression. Methods: We examined 87 liver samples from: 39 patients with chronic active hepatitis (CAH) (31 HCV-related and $8 \mathrm{HBV}$-related); 14 patients with cirrhosis (13 HCV-related and 1 HBV-related); 17 patients with HCC [tumorous and the corresponding non-tumorous 
tissues of 7 in virus-related liver cirrhosis (6 HCV-related and 1 HBV-related) and 5 in nonviral cirrhotic tissue]. Bcl-2, Bax and Bad mRNAs were quantified by absolute Real-Time PCR using beta-actin as the housekeeping gene. Results: Bcl-2 transcripts were significantly higher in cirrhotic tissues than in cases of $\mathrm{CAH}(\mathrm{p}=0.0008)$. CAH coincided with higher Bad levels than in cirrhotic or HCC tissues ( $\mathrm{p}=0.0007$ and $\mathrm{p}=0.00005$, respectively). These data were confirmed by the difference in Bcl-2/Bad ratio between CAH and cirrhosis ( $\mathrm{p}=$ $0.0002)$ and between $\mathrm{CAH}$ and HCC $(\mathrm{p}=0.011)$. No aetiology-related difference was found in Bax expression. In $\mathrm{CAH}$, only Bad mRNA expression was significantly different in HBVrelated infection versus HCV-related infection $(\mathrm{p}=0.018)$. Conclusion: The upregulation of the Bad-induced pro-apoptotic pathway in $\mathrm{CAH}$ seems to be more relevant in controlling apoptosis in liver tissues with $\mathrm{HBV}$ infection. Moreover, the Bcl-2 hyperexpression observed in cirrhotic tissues seems to play an important part in regulating cell survival in damaged liver cells, which may contribute to the persistence of HBV or HCV and thus activate cell proliferation and cell transformation.

\section{M2174}

Evaluation of Topo Iia and Topo I Gene Co-Expression in Hepatocellular Carcinoma: A Combined Immunohistochemistry and Chromogenic in Situ Hybridization Study Using Tissue Microarrays

Evangelos Tsiambas, Theodore Rokkas, George Vilaras, Andreas Karameris

Background: Hepatocellular Carcinoma (HCC) is one of the most frequent cancers worldwide and remains a highly chemoresistant neoplasm. Among chemotherapeutic agents, DNA Topoisomerase (Topo) I and II inhibitors, such as irinotecan-topotecan and etoposidedoxorubicin respectively, demonstrate a strong cytotoxic activity. But the selection of patients' sub groups, which may earn survival benefits and avoid treatment complications, remains unknown. Although Topo I and II immunohistochemical expression is observed in many cancers, only a small proportion is eligible for target chemotherapeutic response. Materials and Methods: Using Tissue Microarray Technology (TMArrayer 100, Chemicon), twenty $(\mathrm{n}=20)$ histologically confirmed primary hepatocellular carcinomas (Grade I 7, Grade II 8 and Grade III 5) were obtained and embedded into one-paraffin block (2 spots of each case with a core diameter of $1 \mathrm{~mm}$ ). Immunohistochemical stain for anti-Topo II and Topo IIa was performed using monoclonal antibodies in $2 \mu \mathrm{m}$ tissue sections. Chromogenic In Situ Hybridization (CISH-Zymed/In Vitrogen) for the detection of chromosome 17 instability and Topo Ila gene status was performed in $5 \mu \mathrm{m}$ serial sections. Finally using a semiautomated Computerized Image Analysis System we evaluated the immunohistochemical stain optical density of both proteins. Statistical analysis was performed by the application of SPSS version 11.0 software. Results: A significant proportion of the tumors showed over expression of the proteins. Topo I and Topo Ila overexpression were observed in 14/20 (70\%) and 16/20 (80\%), respectively. CISH application showed Topo Ila amplification in cell sub populations of 4/16 and gene deletion in 2/16 cases. Chromosome 17 instability (aneuploidy) was detected in 5/20 cases. Correlation between protein overexpression, chromosome instability 17 and biological behavior (Grade-Stage) of the tumors was statistically significant $(\mathrm{p}<0.05)$, especially in co-expression of Topo IIa and Topo I. But Topo Ila over expression was not significantly correlated with gene amplification $(\mathrm{p}>0.05)$. Interestingly, only Topo Ila overexpression was significantly correlated with history of the patients (HBV infection). Conclusions: The results indicate that accurate detection of Topo Ila amplification is a critical process because this genetic event correlates with response to chemotherapy (anthracyclines) in many cancers including hepatocellular carcinoma. Also chromosome 17 instability and Topo I-Topo Ila gene co-deregulation correlates with aggressive biological behavior

\section{M2175}

Hepatic Malignancies in Patients with Nonalcoholic Steatohepatitis Ken Sato, Hiroaki Hashizume, Akira Kojima, Naondo Sohara, Satoru Kakizaki, Hitoshi Takagi, Masatomo Mori

Background: Hepatic malignancies including HCC arising in nonalcoholic steatohepatitis (NASH), without hepatitis B or C virus infection have been a rare observation. Recently, NASH comes to be recognized as a cause of hepatocellular carcinoma (HCC). However, few studies have focused on a large series of studies of liver tumors with NASH. Methods: One thousand three hundred and ten patients with hepatic malignancies, diagnosed and observed from January 1992 to November 2005, were included in this study, and their clinical data were collected prospectively. Clinical and histological changes were required to diagnose NASH. Among them, thirty-four cases are arising in liver diseases without hepatitis B or C virus infection and autoimmune liver diseases. Of these patients, nine cases were diagnosed as NASH histologically. In addition, we comfirmed the presence of HCC by ultrasonographyguided tumor biopsy or surgery. Results: Hepatic malignancies include eight HCC and one cholangiocellular carcinoma (CCC). Nine patients comprise of six men and three women, aged 45-82 years. Of these patients, seven, five and four patients were associated with obesity, hyperlipidemia and hypertension, respectively. Hepatic malignancies measured 15 $70 \mathrm{~mm}$ in diameter and two cases of HCC were well differentiated. When hepatic malignancies were diagnosed, chronic hepatitis or liver cirrhosis was present in four or five cases, respectively. Homeostasis model assessment of insulin resistance values in all cases were high (3.536.2) except one case of CCC (1.76). Treatment of HCC consisted of surgery in five cases, radio frequency ablation in one case, transcathether arterial embolization in one case or infusion in the case of CCC and symptomatic therapy in one case. The median survival time is 35 months (25-38) after surgery and all cases after surgery have no recurrence even though the observation time is short. Conclusions: HCC arising from NASH is not always based on liver cirrhosis. The prognosis of HCC cases arising from NASH after surgery seems to be relatively good, suggesting that active treatment for HCC arising from NASH should be considered. Hepatic malignancies develop commonly to warrant regular screening for this complication in NASH patients.

\section{M2176}

Can Biliary Cytology Predict Prognosis of Biliary Tract Carcinoma? Its Relation to Mucin and Adhesion Molecules

Takeshi Tomokane, Naoki Muguruma, Tetsuo Kimura, Hiromi Yano, Yoshitaka Imoto, Tatsuzo Itagaki, Miyako Niki, Masako Kaji, Hiroshi Miyamoto, Seisuke Okamura, Susumu Ito

Purpose: Biliary cytology is feasible and noninvasive method following ERCP or drainage of obstructive jaundice to obtain definitive diagnosis of biliary tract lesions. However, its sensitivity is not sufficient. It is known that MUCl role as an anti-adhesion molecule and MUC2 may control cell proliferation. The aim of this study is to evaluate the correlation between mucin and cell adhesion protein expressions of surgical specimens and preoperative cytological positive rate, and to examine if cytology can predict postoperative prognosis. Methods: Sixteen patients with biliary tract carcinoma treated surgically in our hospital whose bile cytology was evaluated presurgically were included in this study. Immunohistochemical stainings were performed on paraffin-embedded tissue sections by $\mathrm{ABC}$ method. Biliary juice cytology was classified into 2 categories by Papanicolaou classification: negative and suspicious as Group-N and positive as Group-P. We analyze the relationship between positive rate of biliary cytology and expression of mucin, cadherin, catenins, and clinical stage, number of cytological examination, and evaluate cytological positivity. Evaluation of mucin immunoreactivity was based on the percentage of positively staining of cancer cells; $0 \%$ : none, $1-10 \%$ : mild, $11-50 \%$ : moderate, $50<$ : strong Reduced expression of cadherin and catenins was defined that positively stained neoplastic membrane is extended less than $80 \%$ comparing with normal glands. Results: There was no relationship between cytological positivity and clinical stage grouping, and also number of cytological examination. Preoperative cytology significantly proved positive in mucin group A (MUCl moderate-strong and MUC2 none), while it proved negative in mucin group B (MUCl none-mild or MUC2 mildstrong). Postoperative survival time of mucin group A was significantly poor than that of mucin group B. Postoperative survival time of Group-N tended to be better than that of Group-P (median survival time: $1243.1 \pm 671$.1davs vs, $693.7 \pm 853$.4days). There was no significance in expression of cadherin, catenins and cytological positivity. Conclusion: These results suggest that characteristics of mucin expression is highly associated with exfoliation of neoplastic cell from biliary tract to bile juice and cytological positivity. Considering MUCl/ MUC2 expression reflects various cancer prognosis, this study suggests mucin expression is relevant to prognosis of biliary tract carcinoma and cytological positiveness could be informative for predicting the courses and choosing postoperative adjuvant treatments in biliary tract carcinoma.

\section{M2177}

Caveolin-1 Expression in Patients with Biliary Tract Cancers

Hong-Ja Kim, Se Young Yoon, Bung Ha Kim, Seok Bae Kim, Jung Eun Shin, Il Han Song

Background; Caveolin-1 is the principal protein of caveolae, the vesicular invaginations of the plasma membrane. Several reports have suggested that caveolin-1 may have a role in intracellular signaling pathways and thus is associated with carcinogenesis. But previous studies of the expression and function of caveolin-1 in cancer have still shown controversial results. We studied the expression of caveolin-1 in normal and noncancerous bile duct epithelium and biliary tract cancer and evaluated its possible role in carcinogenesis. Method; Formalin-fixed, paraffin-embedded sections of 20 cases of bile duct carcinoma, 20 cases of bile duct from the benign gallstone diseases, and 5 cases of normal bile duct were stained immunohistochemically with anti-caveolin-1 antibodies. Caveolin-1 RNA expression was also analyzed by RT-PCR in normal biliary epithelium and biliary tract cancer cell line (TGBE2TKB). Results; Expression of caveolin-1 was mainly observed in the bile duct cancer cell. 12 of 20 cases of bile duct cancers (67\%) showed positive staining results. But none of normal bile duct epithelium and bile duct epithelium of benign disease showed staining $(\mathrm{p}<0.05)$. RT-PCR result showed increased expression in cancer cell line compared to no expression in normal bile duct epithelium. However, there was no significant statistical correlation between expression of caveolin- 1 and clinical status of biliary cancer patients such as age, sex and laboratory findings (SGOT, SGPT, bilirubin, CA19-9), differentiation status of tumor cells, tumor stage or LN metastasis. Conclusion; Caveolin-1 showed positive staining in $67 \%$ of patients with biliary tract cancers. There was no staining in bile duct epithelium of normal and benign disease. Increased expression of Caveolin-1 RNA was also observed at biliary tract cancer cell line. We will further evaluate the role of caveolin-1 expression in carcinogenesis of bile duct.

\section{M2178}

Clinical Characteristics of the Patients Initially Unsuspected But Finally Proven to Have Gallbladder Cancer Jae Seon Kim, Byung Gyu Kim, Ji Hyun Kim, Jin Yong Kim, Jong-Jae Park, Young-Tae Bak

Background/Aim: Gallbladder (GB) cancer is a malignancy with poor prognosis because of its rapid progression. Although early diagnosis is essential in the management, it is challenging even in the advanced stages. The aim of this study was to clarify the clinical characteristics of patients finally confirmed as GB cancer, initially unsuspected by radiological and/or other preoperative studies. Methods: We selected the cases diagnosed as GB cancer from 1997 to 2004 in a single tertiary hospital. After reviewing the medical records, we investigated the clinical characteristics including the radiologic findings (US, CT) of patients initially diagnosed as other than GB cancer. Results: Among 91 patients with confirmed GB cancer, 18 cases ( 7 men and 11; mean age 65.4 years) were diagnosed as other diseases initially (table) About $60 \%$ of the 18 cases were advanced stages (stage III and IV according to TNM classification). GB wall appearances of GB stone cases are as follows: diffuse thickening 8 cases, suspicious focal or irregular thickening 4 cases and unremarkable 4 cases. In cases of GB polyp, the size of the polyps ranged from $8 \mathrm{~mm}$ to $10 \mathrm{~mm}$; one case showed anomalous pancreaticobiliary ductal union and underwent operation (stage I), $10 \mathrm{~mm}$ polyp case also underwent operation (stage II). The patient with $8 \mathrm{~mm}$ sized polyp and the patient with 
adenomyomatosis were lost from follow-up and then showed up with stage IV state two years later. Mean serum CEA and CA 19-9 levels were $17.9(2.9-118.5) \mathrm{ng} / \mathrm{ml}$ and 153 (3.1-1983) U/ml respectively. Conclusion: There was no definite tool for early detection and differential diagnosis about GB cancer. We cannot overemphasize the intensive followup even with minimal suspicion of GB cancer and aggressive cholecystectomy case by case. Table. Initial Diagnoses of Unsuspected Cases

\begin{tabular}{|c|c|}
\hline Diagnosis & No. of patients (\%) \\
\hline GB stone Acute cholecystitis Pain only & $13(72.2) 9(50.0) 4(22.2)$ \\
\hline GB polyp $(<=10 \mathrm{~mm})$ & $3(16.6)$ \\
\hline Adenomyomatosis & $1(5.6)$ \\
\hline Klatskin's tumor & $1(5.6)$ \\
\hline Total & $18(100)$ \\
\hline
\end{tabular}

M2179

What Happens to Patients with Obstructive Jaundice?

Einar Bjornsson, Jonas Gustafsson, Jakob Borkman, Anders Kilander

Background: Patients with obstructive jaundice $(\mathrm{OJ})$ is a heterogeneous group with different etiologies and prognosis. Limited systematic data exists on the etiology and prognosis of unselected patients with OJ. We aimed to study the clinical features, etiology and prognosis of patients presenting with OJ. Methods: Over a two year period, 2003-2004, all adult patients with bilirubin $>100 \mu \mathrm{mol} / \mathrm{L}$ were identified by the clinical chemistry laboratory serving a university hospital in Sweden. Patients with an ultrasound evidence of obstructive jaundice with dilated biliary ducts were identified and relevant clinical information obtained. Results: A total of 749 patients with severe jaundice were identified (bilirubin $>100 \mu \mathrm{mol} /$ L) and out of these 243 (32\%) had obstructive jaundice (median age 72 years, 130 females), no patient was lost to follow-up. The biliary obstruction was due to malignancy in 151 (62\%) cases (median age 72 years, 83 females). Of malignancies, pancreatic cancer was the cause in $69(46 \%)$ cases, cholangiocancer (CC) in $41(27 \%)$, papilla vateri cancers in $6(4 \%)$ and other malignancies in 35 (23\%). Among the 92 cases with benign obstruction (median age 69 years, 46 females), choledocholithiasis (CDL) was present in 57 (62\%), biliary strictures in $14(15 \%)$, PSC in $5(5 \%)$ and other causes in $16(17 \%)$. Abdominal pain was present in $34 \%$ of patients with malignant obstruction vs. $68 \%$ of benign obstruction $(\mathrm{p}<0.05)$. Abdominal pain occurred among $38 \%$ in PC, $27 \%$ with CC, and no abdominal pain was present in $6(10 \%)$ out of 57 patients with CDL. The median bilirubin was higher in malignant vs. non-malignant cases (290 (210-420 IQR) vs. 155 (120-200); p<0.001) and ALP was also significantly higher in malignant vs. non-malignant cases, respectively. A total of 48 patients were operated on, 22 out of 151 with malignancy (15\%) whereas 26 out of the non-malignant cases $(28 \% ; \mathrm{p}<0.05)$ underwent surgery. At the end of the follow-up only $7(4.6 \%)$ out of 151 with a malignant obstruction were alive, whereas $70(76 \%)$ out of those with benign obstruction were alive. Of 57 with CDL, 11 (19\%) died, but in only one of these could death be attributed to gallstone disease. The median survival time of CC patients was 103 days $(27-217, \mathrm{IQR})$ and 74 days in PC (34-228). One and two years survival rates were $6 / 69(8.7 \%)$ and $1 / 69(1.4 \%)$ in $\mathrm{PC}$ and $3 / 41(7.3 \%)$ and $2 / 41(5 \%)$ in CC. Conclusion: Among patients with severe jaundice, one third was due to OJ. The majority of $\mathrm{OJ}$ was due to malignancy with high bilirubin levels indicating prolonged biliary obstruction. OJ due to malignancy with severe jaundice at diagnosis carried a very poor prognosis with a $95 \%$ two years mortality.

\section{M2180}

The Role of Ascitic Fibronectin and Cholesterol Concentrations in the Differential Diagnosis of Malignant Ascites

S Karatapanis, L Skorda, Philip Lisgos, A Spiropoulos, N Papantoniou, K Komnianides, S Afantenou, Th. Anagnostou, K Prodromidou, C Psellas, A Delaportas, A Zervos, A Koliadi, M Gini, S Manolitsis, V Artikis

Background: There have been few trials comparing the efficacy of determinations of ascitic fibronectin and cholesterol in the differential diagnosis of ascites. Aim : To evaluate the efficacy of ascitic cholesterol and fibronectin levels in the differential diagnosis of ascites in comparison with other known parameters. Patients and Methods : In our study we included 210 patients with ascites ( 102 with cirrhosis, 54 with extra-hepatic tumors, 41 with hepatocellular carcinoma-HCC and 13 with spontaneous bacterial peritonitis-SBP). Their ascitic fluid $\mathrm{pH}$, cholesterol, fibronectin, albumin, $\mathrm{LDH}$, and total proteins were analyzed to distinguish between different etiologies of ascites. The diagnosis of cirrhosis was based on histological findings and/or clinical, biochemical and endoscopic/imaging findings in those patients where liver biopsy was contraindicated. Results: Ascitic levels of fibronectin (81.5+-23.4 $\mu \mathrm{g} / \mathrm{ml}$ vs $28.5+-6.7 \mu \mathrm{g} / \mathrm{ml}$ ) and cholesterol (91.6+-23.8mg/dl vs $23.4+-9.8 \mathrm{mg} / \mathrm{dl}$ ) were significantly higher in patients with extrahepatic tumors compared to patients with cirrhosis $(\mathrm{P}<0.001$ in both parameters), while serum-ascites albumin gradient was significantly higher in patients with cirrhosis compared to the patients with extrahepatic tumors $(\mathrm{P}<0.001)$. We found no differences in these parameters between cirrhotic ascites and malignant ascites due to primary hepatocellular carcinoma.We found that ascitic fluid LDH and cholesterol were best at diagnosing malignant ascites, whereas ascitic fluid $\mathrm{pH}$ and $\mathrm{LDH}$ were best for diagnosing SBP. Fibronectin, cholesterol and serum-ascites albumin gradient at discrimination values of $52 \mu \mathrm{g} / \mathrm{ml}, 45 \mathrm{mg} \%$ and $1.1 \mathrm{~g} / \%$ respectively, separated patients with malignant ascites from patients with cirrhosis with an accuracy of $94 \%, 96 \%$ and $88 \%$ respectively. Conclusions : Ascitic fluid determinations of fibronectin and cholesterol offer an excellent method of discriminating between cirrhotic ascites and ascites due to peritoneal carcinomatosis,and are superior to conventional tests. These parameters don't help in differentiating patients with ascites due to cirrhosis and HCC.

\section{M2181}

MicroRNA-21 Regulation of Survival Signaling: A Novel Mechanism of Chemoresistance in Cholangiocarcinoma

Fanyin Meng, Roger Henson, Hania Wehbe, Jinmai Jiang, Janna M. Mize-Berge, Thomas Schmittgen, Tushar Patel

Background: Cholangiocarcinomas are highly treatment refractory cancers. microRNA (miRNA) are endogenous regulatory RNA molecules that modulate gene expression. Altered expression of miRNA has been shown in many cancers and could contribute to chemoresistance by altering the functional expression of critical genes involved in cell survival. Ou AIMS were to identify specific miRNA involved in the modulation of chemoresistance in human cholangiocarcinoma. Methods: miRNA expression in malignant $(\mathrm{KMCH}, \mathrm{MzChA}-1$ TFK) and non-malignant (H69) human cholangiocytes was assessed using a hybridization based microarray. Expression of selected miRNA and their precursors was evaluated by Northern blots and real-time PCR respectively. miRNA specific antisense oligonucleotides or precursor miRNAs were used to decrease or increase cellular expression respectively. In vivo miRNA expression was assessed in tumor cell xenografts in nude mice under basa condition or during treatment with systemic gemcitabine. Results: Compared to non-malignant cholangiocytes, the expression of most miRNA was decreased in cholangiocarcinoma cells. However, some miRNA such as miR-2l were highly over-expressed in several cholangiocarcinoma cell lines (MzChA-1 $2.7+0.1$-fold, KMCH $1.8+0.2$-fold, TFK $4.4+0.1$ fold). Inhibition of miR-2l in MzChA-l cells decreased sensitivity to gemcitabine in vitro. Furthermore gemcitabine treatment of MzChA-1 xenografts increased miR-2l by $4.1+0.3$ fold. Using a bioinformatics approach, the PTEN tumor suppressor gene was identified as a potential target gene for miR-21. PTEN is a PIP3 phosphatase which negatively regulates PI-3 kinase signaling which mediates cell survival. Antisense inhibition of miR-2l increased PTEN expression by $210 \pm 30 \%$ in MzChA-1 malignant cholangiocytes. Meanwhile, the expression of phosphorylated PI-3 kinase p85 subunit, a downstream target of PTEN was reduced to $49 \pm 2 \%$ of controls. Moreover, gemcitabine treatment of tumor cell xenografts decreased PTEN expression and increased activation of PI-3 kinase in vivo. Conclusions: We have identified a microRNA (miR-21) that is over-expressed in human cholangiocarcinoma, and report a previously unrecognized mechanism for chemoresistance involving this microRNA. Chemotherapeutic stress increases the expression of miR-2l which can activate survival mechanisms by modulation of the PI3 kinase-PTEN signaling pathway. Targeting miR-2l dependent signaling pathways involved in chemoresistance may provide new therapeutic approaches for the treatment of cholangiocarcinoma.

\section{M2182}

Lack of Effect of Elevated Plasma Cholecystokinin Concentration On Proximal Gastric Motor Activity in Critically Ill Patients

Laura Bryant, Carly Burgstad, Nam Q. Nguyen, Robert J. Fraser, Marianne Chapman, Richard H. Holloway, Michael Horowitz

Delayed gastric emptying is a major cause of feed intolerance in critical illness. In these patients, proximal gastric relaxation in response to duodenal nutrient is also impaired ${ }^{1}$. In patients intolerant of nasogastric feeding, Cholecystokinin concentrations [CCK] are elevated. Whether plasma [CCK] plays a role in proximal gastric dysmotility in the critically ill is unknown. Aim: To assess the relationship between [CCK] and proximal gastric motility in critically ill patients. Methods: Proximal gastric motility was measured (using a barostat) in 22 critically ill patients ( $18 \mathrm{M} ; 49 \pm 4$ years) during a continuous $60 \mathrm{~min}$ duodenal infusion of Ensure ( $2 \mathrm{kcal} / \mathrm{min}$ ). Plasma [CCK] was measured (using radioimmunoassay) at baseline and every 20 min during nutrient infusion. Proximal gastric volume and fundic wave activity (changes in volume $>30 \mathrm{ml}$ that revert to a volume within $50 \%$ of the previous level in less than $2 \mathrm{~min}$ ) were determined. Patient data were compared to 28 healthy subjects ( $21 \mathrm{M} ; 43$ \pm 2 years). Results: Fasting [CCK] was higher in critical illness than in health $(8.7 \pm 1.1 \mathrm{v}$ $4.7 \pm 0.4 \mathrm{pmol} / \mathrm{L} ; \mathrm{P}<0.05)$, with an augmented rise in $[\mathrm{CCK}]$ during nutrient infusion in patients $(\mathrm{P}<0.01)$. Baseline gastric volumes were similar in the two groups. Proximal gastric relaxation in response to nutrient infusion was less in critically ill patients (peak volume response: $252 \pm 25 \mathrm{v} 148 \pm 30 \mathrm{ml} ; \mathrm{P}=0.01$ ). There was a positive correlation between changes in $[C C K]$ and proximal gastric volume during nutrient infusion in healthy subjects $(r=0.525 ; \mathrm{P}<0.001)$, but not in critically ill patients. Fundic wave frequency during fasting and in response to nutrient infusion was reduced in patients compared to healthy subjects(fasting: $6.1 \pm 1.2$ v $11.7 \pm 1$ waves/10min; and nutrient infusion: $3.9 \pm 0.8$ v $7.7 \pm$ 0.8 waves $/ 10 \mathrm{~min} ; \mathrm{P}<0.01$ ). There was a negative correlation between peak $[\mathrm{CCK}]$ and fundic wave frequency during nutrient infusion in healthy subjects $(r=-0.51 \cdot \mathrm{P}<0.05)$, and a nonsignificant trend in patients $(r=-0.38 ; \mathrm{P}<0.1)$. Conclusion: Elevated [CCK] does not appear to directly influence proximal gastric motility in the critically ill, in contrast to healthy subjects where increased [CCK] may contribute to a slowing of gastric emptying in response to duodenal feedback. In patients, [CCK] may contribute to slow gastric emptying via actions on the distal stomach or the integration of proximal and distal gastric motor activity. Nguyen NQ, Chapman M, Fraser R, Bryant L, Holloway RH, Vozzo R, Feinle-Bisset C, Horowitz M. Impaired proximal gastric relaxation in response to small intestinal nutrients in critically ill patients. Gastroenterology 2005; 128 (4): A548.

\section{M2183}

Gastric Emptying (GE), Major Contributor to Postprandial Hyperglycemia in Patients with Type 1 Diabetes Mellitus (T1DM)

Juergen Woerle, Maximilian Albrecht, Rainer Linke, Mathias Nicolaus, Martin Storr, Joerg Limmer, Burkhard Goeke, Joerg Schirra

Postprandial (PP) hyperglycemia has been identified as an important risk factor for cardiovascular disease. The pancreatic hormone amylin is released postprandially and delays gastric emptying. TIDM are amylin deficient. Accordingly, we hypothesised that 1) in the absence of neuropathy, TIDM have accelerated GE, 2) accelerated GE may be an important regulator of PP glycemia, 3) amylin substitution should delay GE in TIDM and reduce PP glucose excursions. Methods: We studied 12 healthy subjects (HS) and 12 otherwise healthy subjects

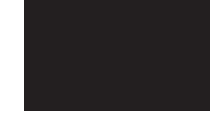


with TIDM on two occasions each, after ingestion of a mixed $472 \mathrm{kcal}$ meal with s.c. administration of either placebo (PBO) or the amylin analogue pramlintide $(30 \mathrm{mcg})$. T1DM were infused with IV insulin to establish a fasting euglycemia of $90 \mathrm{mg} / \mathrm{dl}$ and to prevent PP hyperglycemia $>180 \mathrm{mg} / \mathrm{dl}$ in order to avoid the effects of hyperglycemia on gastric emptying. Insulin was infused in an identical manner on both days. GE was determined by high-resolution scintigraphy (20 fr/min) over $330 \mathrm{~min}$. Results: PP glucose excursions in TIDM exceeded those of HS during the first 150 min after meal ingestion (AUC 416 \pm 64 vs $307 \pm 24 \mathrm{mg} / \mathrm{dl} / 150 \mathrm{~min}, \mathrm{p}<0.05$ ), and remained greater throughout, even though PP plasma insulin levels were 3-fold greater in TIDM. During this time period, GE was accelerated in TIDM $(55.7 \pm 4.7$ vs $41.7 \pm 4.6 \%$ emptied, $\mathrm{p}<0.05)$. GE was closely correlated with peak plasma glucose excursions $(\mathrm{r}=0.75, \mathrm{p}<0.001)$. Pramlintide delayed gastric emptying in HS ( $50 \%$ emptying at $225+16$ vs $177+10 \mathrm{~min})$ and TlDM $(217+10$ vs $149+10 \mathrm{~min}$; both $\mathrm{p}<0.001)$. It abolished the increase in glycemia during the first $150 \mathrm{~min}$ in TlDM $(-12 \pm 56$ vs $416 \pm 64 \mathrm{mg} / \mathrm{dl} / 150 \mathrm{~min}$ ) and markedly reduced overall PP glucose excursions in both HS and TIDM compared to PBO $(195 \pm 21$ vs $283 \pm 25$ and $119 \pm 73$ vs $389 \pm 75 \mathrm{mg} / \mathrm{dl} / 150$ min, both $\mathrm{p}<0.01$ ). Although GE was significantly accelerated between 150 and $330 \mathrm{~min}$ in TIDM receiving pramlintide, peak glucose levels did not exceed $138 \pm 8 \mathrm{mg} / \mathrm{dl}$ in T1DM compared to $\mathrm{PBO}(178 \pm 9 \mathrm{mg} / \mathrm{dl}, \mathrm{p}<0.01)$. None of the volunteers reported nausea or bloating. Discussion: Gastric emptying was increased rather than delayed in TIDM and that may be an important contributor to PP hyperglycemia. The amylin analogue pramlintide delayed gastric emptying and significantly reduced PP hyperglycemia in TIDM. Modulation of gastric emptying is a promising tool in regulating intestinal nutrient flow and reducing PP glycemia

\section{M2184}

Somatostatin Accelerates Gastric Emptying, But Does Not Improve Symptoms in Functional Dyspepsia

Jeoffrey J. Haans, Ingrid M. de Zwart, Albert de Roos, Ad A. Masclee

Background: It has been suggested that the somatostatin analog octreotide reduces symptoms in patients with functional dyspepsia (FD). Symptoms in patients with FD may result from several factors such as visceral hypersensitivity, impaired fundus relaxation or alterations in gastric emptying. Magnetic Resonance Imaging (MRI) is unique in that it allows simultaneous recording of motility, emptying and volume changes of the stomach. The present study was undertaken to investigate the effect of somatostatin on volume changes of the stomach, gastric emptying and motility in patients with FD. Methods: Eight patients with functional dyspepsia (6F; mean age $46 \mathrm{yrs}$; range 38-62 yrs) agreed to participate. MR images of the stomach and symptom scores in response to meal ingestion were obtained during two sessions in random order under continuous intravenous infusion of saline or somatostatin $(250 \mu \mathrm{g} / \mathrm{h})$. Gastric emptying and motility were evaluated using 3D-volume scans and 2Ddynamic scans. Measurements were obtained before consumption of a liquid meal and at regular intervals $(15 \mathrm{~min})$ thereafter. The liquid meal $(400 \mathrm{ml} ; 600 \mathrm{kCal})$ was labeled with the paramagnetic MRI contrast agent meglumine gadoterate. Symptoms scored included nausea, epigastric pain and postprandial fullness and tension (individual symptom score 010). Data obtained during somatostatin infusion were compared to data obtained during saline infusion. Results (mean $\pm \mathrm{SD}$ ): Initial total gastric volume did not differ between both experiments before ( $40 \pm 26 \mathrm{ml}$ vs. $42 \pm 24 \mathrm{ml}$, somatostatin vs. saline) and after meal ingestion ( $476 \pm 71$ vs. $493 \pm 108 \mathrm{ml}$ ), but reduced significantly $(\mathrm{p}<0.001)$ faster during somatostatin infusion ( $298 \pm 58$ vs. $432 \pm 128$ at $90 \mathrm{~min}$ ). During somatostatin infusion gastric emptying was significantly $(\mathrm{p}<0.001)$ faster than during saline infusion $(64 \pm 20 \mathrm{vs}$. $94 \pm 13 \%$ at $45 \mathrm{~min} ; 43 \pm 14$ vs. $73 \pm 14 \%$ at $90 \mathrm{~min}$ ). Total postprandial symptom score did not differ between both experiments neither before (1.1 \pm 3.2$)$ nor after meal ingestion $(9.5 \pm 9.1$ vs. $10.9 \pm 8.8$, somatostatin vs. saline). For epigastric pain the scores were even significantly higher during somatostatin infusion compared to saline infusion. Gastric motility (contraction frequency) did not differ significantly between both experiments. Conclusion: Administration of somatostatin accelerates gastric emptying. Administration of somatostatin does not result in postprandial symptom reduction in patients with FD. On the contrary postprandial symptoms tended to increase. This may be related to more rapid gastric emptying, as other motility parameters were not affected.

\section{M2185}

Gastroscopy Guided Implantation of Temporary Gastric Electrodes: A New Minimal Invasive Technique

Hasse R. Abrahamsson, Anders Elfvin, Stina Andersson, Anders Edebo, Magnus Simren, Hans Lonroth

Temporary (temp) leads have been used for gastric electrical stimulation (GES) to evaluate whether gastroparetic patients (GP pts) respond to GES (Abell et al 2002\&2005). Previous techniques required general anesthesia, or an oral or PEG route. We have elaborated a simple, minimal invasive principle for electrode implantation and tested the appropriateness of placement in animals and in pts by various recording and stimulation techniques. METHODS: Leads were constructed so that the tip of the lead could be anchored to the gastric submucosa. Part I: Acute 1-2 hr experiments (expts) were performed in anesthetized pigs. Under endoscopic control two leads were introduced and gastric EMG (G-EMG) recorded. Part II: Three pts (1 male, M; 2 females, F) with drug refractory nausea (N) and/ or vomiting $(V)$ with non-established indications for GES were evaluated with percutaneous temp leads, implanted at gastroscopy (slight sedation plus local skin anesthesia). Detailed studies of electrode function were done measuring distance between electrodes (fluoroscopy), impedance, G-EMG compared with manometry and the effect of temp GES for 7-9 days on symptoms (sympt) (stimulator: Enterra, Medtronic, $12 \mathrm{imp} / \mathrm{min}, 5 \mathrm{~mA}$ ). Diagnoses and main sympt were: chronic intestinal pseudoobstruction $(\mathrm{CIP} ; \mathrm{l} F, \mathrm{~V}+\mathrm{N})$, functional dyspepsia (FD) without GP (1 F, N; $1 \mathrm{M}, \mathrm{V})$. RESULTS: Part I: In the pigs a slow wave (SW) rhythm, $3-4 / \mathrm{min}$, was recorded throughout the expts with some decrease in frequency at the end of expt. SWs were accompanied by a second potential, suggestive of phasic contractions. Part II: In the pts implantation time from start of gastroscopy to end of electrode placement was $12-20 \mathrm{~min}$. Electrode distance varied from 12 to $45 \mathrm{~mm}$. Impedance was $1100-420$ ohm at gastroscopy, decreased after implant in 2 pts and was 510-629 on day 7-9. G-EMG showed a regular SW rhythm about $3 / \mathrm{min}$. Antral pressure waves had intervals being multiples of the SW to SW-time observed in each patient. With temporary GES for 7-9 days weekly frequency of main sympt decreased: CIP, F: N 20 to 1 hrs, V 7 to 1 . FD, F $\mathrm{N} 17$ to lhrs; M: V 5 to 3/w. The sympt decrease was independent of electrode distance. No complications from the leads or lead extraction were noted. Permanent implant and yr follow up showed very good results in the two pts with marked effect of temp GES (CIP; $\mathrm{FD}, \mathrm{F})$, the third $\mathrm{pt}(\mathrm{FD}, \mathrm{M})$ had moderate effect at $1 \mathrm{yr}$. CONCLUSIONS: Temporary percutaneous gastric leads can easily be implanted at endoscopy and may be used for testing of GES and study of gastric electrophysiology.

\section{M2186}

Disruption to Integration of Proximal and Distal Gastric Motor Activity in Critically Ill Patients

Nam Q. Nguyen, Robert J. Fraser, Marianne Chapman, Laura Bryant, Carly Burgstad, Richard H. Holloway

Normal gastric emptying is complex and involves coordination of the proximal and distal gastric motor activity in response to small intestinal nutrient feedback. Fundic waves (FWs) may play a role in transferring gastric contents more distally for antral trituration and emptying. Slow gastric emptying and subsequent intolerance of gastric feeds are common in critically ill patients. Although both proximal and distal gastric motility are abnormal in critically ill patients, the effect of critical illness on the coordination of 2 regions is unknown. Aim. To determine the association between proximal and distal gastric motility during fasting, and in response to duodenal nutrient stimulation in critical illness. Methods. Concurrent proximal and distal gastric motility was recorded in 10 critically ill patients and 10 healthy volunteers. Proximal gastric motility (barostat) and antro-pyloro-duodenal (APD) motility (perfused multi-lumen manometric assembly) were recorded during fasting, and two 60 -min duodenal infusions of Ensure ( $1 \mathrm{kcal} / \mathrm{min}$ and $2 \mathrm{kcal} / \mathrm{min}$ ) administered in random order separated by a $2 \mathrm{~h}$ "washout" period. Proximal gastric volume (PGV), FW activity (number and volume amplitude) and APD waves were determined. The association between these variables were also assessed. Results. During fasting, PGVs were similar (patients: $206 \pm 47 \mathrm{~mL}$ vs. healthy: $196 \pm 274 \mathrm{~mL}, \mathrm{P}=0.7$ ). Patients had fewer FWs and propagated antral waves (PAWs) than healthy subjects. In patients, duodenal nutrients was associated with less proximal gastric relaxation (at 10th min: $45+26$ vs. $196+29 \mathrm{~mL} ; \mathrm{P}<0.001$ ) and fewer FWs and PAWs than in healthy subjects (Table). During both fasting and in response to duodenal nutrients, the median proportion of FW that were followed by a distally PAW was lower in patients $(0 \%(0-8 \%))$ than in healthy subjects $(36 \%(11-44 \%) ; \mathrm{P}<0.001)$. The volume amplitude of $\mathrm{FW}$ in patients was smaller than that in healthy subjects $(87+8 \mathrm{~mL}$ vs $44+3 \mathrm{~mL} ; \mathrm{P}<0.001$ ). Conclusions. In critical illness, both proximal and distal gastric motility are impaired. Furthermore, the coordination between proximal and distal gastric motor activity is also disrupted, which may further contribute to delayed gastric emptying in these patients by interfering with transfer of gastric contents from proximal stomach to distal peristaltic pump.

\begin{tabular}{|c|c|c|}
\hline$* \mathrm{P}=0.003, * * \mathrm{P}<0.001$; vs. healthy subjects & Healthy controls & Critically ill \\
\hline $\begin{array}{c}\text { Fasting (mean } \pm \mathrm{SEM} \text { ) Fundic waves (No./10 min) } \\
\text { Propagated antral waves (No./10 min) }\end{array}$ & $15.3 \pm 1.58 .1 \pm 2.1$ & $\begin{array}{c}7.4 \pm 2.0 * 0.9 \pm 0.4 \\
* *\end{array}$ \\
\hline $\begin{array}{c}\text { Nutrient infusion (mean } \pm \text { SEM) Fundic waves (No./10 } \\
\text { min) Propagated antral waves (No./10 min) }\end{array}$ & $8.8 \pm 1.24 .5 \pm 1.8$ & $\begin{array}{c}4.3 \pm 1.2 * 0.4 \pm 0.1 \\
* *\end{array}$ \\
\hline $\begin{array}{c}\% \text { FWs followed by a PAW (median (IQR)) Fasting } \\
\text { Nutrient infusion }\end{array}$ & $\begin{array}{c}36 \%(13-44 \%) \\
(11-38 \%)\end{array}$ & $\begin{array}{c}0 \%(0-3 \%) * * 0 \% \\
(0-8 \%) * *\end{array}$ \\
\hline
\end{tabular}

\section{M2187}

Gastric Antro-Fundic Sensitivity and Helicobacter Pylori Infection in Idiopathic Dyspepsia

Maria Pia Caldarella, Flora Sacco, Angelo Milano, Chrysanti Balatsinou, Francesco Laterza, Elena Serio, Franco Cuccurullo, Matteo Neri

BACKGROUND: Recent data (Caldarella MP et al, Gastroenterology. 2003; 124:1220-9) have shown that the gastric antrum, in addition to the gastric fundus, may be the site of origin of symptoms in dyspeptic patients. Helicobacter pylori (H. pylori), has been advocated as a cause of dyspepsia, but this association is still unproven. AIM: To evaluate the role of H. pylori infection on gastric fundic and antral sensitivity in patients with idiopathic dyspepsia (ID). METHODS: $8 \mathrm{H}$. pylori positive patients with ID (mean age $36+6,6$ females and 2 males) and $8 \mathrm{H}$. pylori negative healthy subjects (mean age 26+6, 5 females, 3 males) were studied. H. pylori status and grade of gastritis (Sidney System) were assessed by 13C-UBT and histology. Gastric antral and corpus biopsies in patients were taken at endoscopy at baseline and six months after $H$. pylori eradication. In all participants, in two separate days a flaccid bag was located into the gastric antrum or into the fundus under fluoroscopy guide and connected to a tensostat; subsequently, isotonic distensions of the distal or proximal stomach were performed by 4 gr increments up to $64 \mathrm{gr}$ or discomfort. These studies were performed at entry and at least six months after $H$. pylori eradication. RESULTS: All patients with ID exhibited antral and fundic hypersensitivity in comparison to healthy subjects. At baseline, antral perception threshold was $13+3$ gr in patients vs. $32+6$ gr in health; and the discomfort threshold was $29+5$ gr in patients, while healthy subjects tolerated all distensions without discomfort. In the gastric fundus, the threshold of perception and discomfort were significantly lower in patients ( $8 \pm 2$ gr and $16 \pm 1$ gr respectively) than controls $(\mathrm{p}<0.05)$. In all patients the main symptom evoked at antral distension was cramp/colicky sensation $(58+8 \%)$. After eradication, fundic sensation thresholds did not change in comparison to baseline, while in the antrum a significant increase of the discomfort threshold was observed $(48+4$ gr, $p<0,05$ vs. baseline). At this time, an equal distribution of evoked symptoms was present (cramp/colicky $30+4 \%$ nausea $32+6$, fullness $28+6$, other $10+2 \%$ ). After eradication, activity of gastritis was significantly decreased ( 1,25 vs 0,4 score, $p<0.001)$. CONCLUSION Hp infection may modulate gastric antral hypersensitivity and symptoms pattern in patients with idiopathic dyspepsia, while having no effect on fundic sensitivity 
M2188

A Sham-Controlled Study of Intra-PYLORIC Injection of Botulinum Toxin in Gastroparesis

Joris Arts, Philippe Caenepeel, Lieselot Holvoet, Daniel Sifrim, Dominiek Dewulf, Verbeke Kristien, Jozef Janssens, Jan Tack

Recent uncontrolled studies in limited numbers of patients suggest a benefit of intrapyloric injection of botulinum toxin (botox) for the treatment of gastroparesis. Improvement seems to occur within the first 4 weeks, but controlled data are lacking. The aim of the present study was to investigate the effect of botox on symptoms and gastric emptying in gastroparesis in a double-blind randomised cross-over design. Methods: Consecutive gastroparesis patients underwent two upper gastrointestinal endoscopies with 4 weeks interval, during which intra-pyloric injection with saline or botox $4 \times 25 \mathrm{U}$ was performed in a randomised doubleblind fashion. Injections were prepared by a nurse who was otherwise not involved in the care of these patients. Before the start of the study and 4 weeks after each treatment, they underwent a solid and liquid 14C octanoic acid and 13C glycin gastric emptying breath test, and symptom severity was assessed using the Gastroparesis Cardinal Symptoms Index (GCSI). GCSI scores and gastric half emptying times (t1/2) were calculated and results $($ mean \pm SEM), both parallel and pooled data, were compared using Student's $t$ test. Results: 23 gastroparesis patients ( 5 men, mean age $45 \pm 3$ years, 21 idiopathic, 2 diabetic and 2 post-Nissen) were recruited; 12 received botox first and 11 saline first. After initial saline injection, significant improvement was seen for GCSI sore and solid and liquid $\mathrm{t} 1 / 2$ (respectively $32.1 \pm 1.7$ vs. $26.3 \pm 2.2 ; 121 \pm 14$ vs. $87 \pm 8 \mathrm{~min}$ and $107 \pm 11$ vs. $69 \pm 6 \mathrm{~min}$, all $\mathrm{p}<0.01$ ). After the second injection, with botox, GCSI improved further to $21.4 \pm 3.0(\mathrm{p}<0.05)$ while no significant changes were seen for solid and liquid tl/2 (respectively $101 \pm 17$ and $81 \pm 10$ min, NS). Initial botox injection was associated with similar significant improvement of GCSI score and solid t1/2 (respectively $21.3 \pm 2.8$ vs. $15.7 \pm 3.2$, and $111 \pm 7$ vs. $93 \pm 9 \mathrm{~min}$, both $\mathrm{p}<0.05$ ), while liquid $\mathrm{t} 1 / 2$ did not reach significance ( $104 \pm 30$ vs. $76 \pm 10 \mathrm{~min}$, NS). No significant further improvement occurred after the second injection, with salin (GCSI $13.4 \pm 3.3$, solid $\mathrm{tl} / 294 \pm 11 \mathrm{~min}$ and liquid $\mathrm{tl} / 274 \pm 8 \mathrm{~min}$, all NS). When data for both treatment periods were pooled, no significant difference was seen for improvements respectively after botox or saline injection for GCSI $(6.1 \pm 1.5$ vs. $3.8 \pm 1.5, \mathrm{NS})$, solid tl/2 $(3.4 \pm 7.4$ vs. $16.3 \pm 8.3, \mathrm{NS})$ and liquid $\mathrm{t} 1 / 2(8.2 \pm 13.7$ vs. $22.5 \pm 7.7, \mathrm{NS})$. CONCLUSION: In a cohort of gastroparesis patients of predominantly idiopathic origin, intra-pyloric injection of botulinum toxin does not seem superior to placebo in improving symptoms and solid or liquid gastric emptying rate.

\section{M2189}

Noninvasive Recordings of Electrical Response Activity Using a Squid Biomagnetometer

Andrei Irimia, William O. Richards, Leonard A. Bradshaw

Clinical efforts in electrogastrography (EGG) are typically focused on the evaluation of the gastric slow wave frequency and amplitude. The study of spiking potentials (electrical response activity or ERA, which is associated with smooth muscle contraction) may have significant clinical relevance, though no one has ever recorded ERA using cutaneous EGG. To record ERA noninvasively, we acquired magnetogastrogram (MGG) recordings from ten healthy New Zealand rabbits using a 37-channel Superconducting Quantum Interference Device (SQUID) biomagnetometer located in the Gastrointestinal SQUID Technology (GIST) Laboratory at Vanderbilt University. After a 30 minute baseline recording, the stomach of each animal was surgically divided and MGG data were acquired for an additional 30 minutes. We employed principal and independent component analysis (PCA and ICA, respectively) to isolate the gastric slow wave and ERA from MGG recordings. Whereas PCA is a dimensionality reduction technique for multivariate data, ICA is a powerful analysis method for extracting signal components from a set of multi-channel recordings. By using PCA and ICA, we ensured that extraneous artifacts such as motion, cardiac and intestinal activities, etc. were removed from the analyzed gastric signal. The slow wave frequency measured across subjects was $3.2 \pm 0.6 \mathrm{cpm}$ (cycles per minute, mean $\pm \mathrm{SEM}$ ) pre-division and $1.3 \pm 1.4 \mathrm{cpm}$ post-division. The mean percentage of slow waves exhibiting ERA spiking dropped from $52 \%$ pre-division to $23 \%$ post-division, a statistically significant decrease of $44 \%$ after stomach division $(\mathrm{p}<0.001)$. The ERA dominant frequency was $73.4 \pm 3.5 \mathrm{cpm}$ pre-division and $47.1 \pm 5.7 \mathrm{cpm}$ post-division, a significant post-division decrease of $64 \%$ $(\mathrm{p}<0.001)$. In all animal subjects, ERA frequencies as cited above were found to be significantly below those of the cardiac signal, whose dominating frequency was found to be $213.7+8.9 \mathrm{cpm}$ across rabbits. In conclusion, stomach division in rabbits is accompanied by significant changes in MGG-recorded ERA patterns which can be detected noninvasively using a SQUID. The capability of SQUIDs to noninvasively record ERA in addition to the frequency, amplitude and propagation velocity of the slow wave demonstrates the future diagnostic potential of noninvasive SQUID recordings in human subjects.

\section{M2190}

Continuous PYLORIC Manometry Determines the Effectiveness of Botulinum Toxin Injection in Patients with Gastroparesis

\section{Linda Anh B. Nguyen, Shelly Parker, Stephen Bunker, William J. Snape}

Background: Gastroparesis is a disorder of gastric motility that results in delayed gastric emptying in the absence of mechanical obstruction. Pylorospasm (tonic pyloric contractions $\geq 10 \mathrm{mmHg}$ ) has been implicated in gastroparesis(Mearin et al.Gastro 1986). Botulinum toxin A injection (80-200 U) into the pylorus has been shown in a number of studies to improve symptoms and gastric emptying. The success rate has been variable. Gupta and Rao(Gastrointest Endosc 2002) showed that botulinum toxin(BT) attenuated isolated pyloric pressure waves on ambulatory antropyloroduoenal manometry 3 weeks after injection with 200 units of BT. Our hypothesis is that the subset of patients with pylorospasm are the patients who would benefit from BT injection. Aims:1)Evaluate the feasibility of recording pyloric pressures using a Dent sleeve before and immediately after the injection of BT and 2)Correlate post-injection pyloric pressures with clinical response. Patients and Methods: 7 patients with gastroparesis ( 4 diabetics, $M=6$, age $35.1 \pm 2.5$, mean 4 hr gastric retention $23.5 \% \pm 6.9$ ) underwent upper endoscopy with pyloric pressure measurements using a Den sleeve (Mui Scientific). Positioning of the sleeve was confirmed flouroscopically. Basal and peak pyloric pressures were measured before and immediately after BT injection. The Den sleeve was left in position during BT injection. Patients with tonically elevated basal pyloric pressures received a total of 100 units of BT injection into the pylorus. 3 patients had a follow up gastric scintigraphy (1,3 and 18 days) after the injections. One patient underwen 2 treatment sessions after not responding to the first injection. Results: Basal pyloric pressures (PP) and peak PP decreased immediately after the injection of BT $(28.8 \mathrm{mmHg}+5.2 \mathrm{vs} .9 .9$ $\mathrm{mmHg} \pm 2.1, \mathrm{p}=0.002 ; 68.5 \pm 8.3$ vs. $34.8 \pm 5.4, \mathrm{p}=0.01) .3$ patients who were hospitalized had immediate improvement and were discharged from the hospital 2 days later. The 3 patients who improved, had their basal PP decrease from 12.8 to $0,33.0$ to 7.0 and 14.7 to $7.6 \mathrm{mmHg}$, respectively. Two patients who did not have symptomatic improvement had post BT basal PP the decreased but remained elevated at 18.7 and 17.0. The patient whose basal PP decreased from 38.6 to 18.7 had an improvement in gastric emptying but not symptoms. This patient had a second procedure 3 weeks later where the post BT pressure decreased to 10.9, at which point he symptomatic improvement. Conclusions: 1)Pyloric pressures decrease immediately following BT injection. 2)Since 100 units of BT did not reduce basal PP to $<10 \mathrm{mmHg}$ in all patients, this may indentify patients who need higher doses of BT

\section{M219}

Characteristics of Gastric Emptying and Neuropathy in Patients with Different Durations of Type-1 Diabetes

Tamas Varkonyi, Eva Borcsok, Robert Takacs, Richard Roka, Csaba Lengyel, Mate Lazar, Miklos Papos, Laszlo Pavics, Peter Kempler, Janos Lonovics

The roles of the disease duration and age in the pathogenesis of impaired gastric emptying in type-1 diabetes (DM) are still not clearly elucidated. The aim of this study was to determine the postprandial gastric emptying, and the autonomic and the sensory neuropathy in groups of DM patients (pts) with different disease durations and age. Patients, methods: 17 pts had a shorter, and 23 pts a longer history of type-1 DM (age: $30.7 \pm 2$ vs $44.6 \pm 2.6$ yrs, $p<0.001$ duration: $8 \pm 1.4$ vs $25.3 \pm 2.3$ yrs, $p<0.0001 ;$ mean \pm SE). The emptying of the stomach was evaluated by a scintigraphic gastric emptying procedure. Autonomic neuropathy (AN) was assessed by means of the five cardiovascular reflex tests. Sensory nerve function was studied with a Neurometer (Neurotron Inc. Baltimore, Md), using transcutaneous electrical stimulation. Results: There was a tendency to a longer half-time of gastric emptying (HTE) in the type-1 DM pts with longer duration, but the difference between the groups did not reach the level of significance (HTE: $93.5 \pm 10.1$ vs $67.8 \pm 6.7 \mathrm{~min}$., $p>0.05$; long-standing vs shortstanding type-1 DM). The overall severity of AN and the results of three reflex tests differed between the groups (AN score: $4.3 \pm 0.5$ vs $2.5 \pm 0.4, p<0.05$; heart rate response to breathing. $12.7 \pm 1.5$ vs $19.4+2.2$ beats $/ \mathrm{min}, \mathrm{p}<0.05$. Valsalva ratio: $1.3 \pm 0.05$ vs $1.75 \pm 0.1, \mathrm{p}<0,0001$ orthostatic hypotension: $23 \pm 3.4$ vs $11 \pm 3.7 \mathrm{~mm} \mathrm{Hg}, \mathrm{p}<0.05$ ). A positive correlation was observed between HTE and the AN score in long-standing DM $(r=0.57, p<0.01)$. The correlations between heart rate response to breathing and HTE $(r=-0.57, p<0.01)$ and between the Valsalva ratio and HTE $(\mathrm{r}=-0.43, \mathrm{p}<0.05)$ in this group were negative, while a positive association was found between the orthostatic hypotension and $\operatorname{HTE}(\mathrm{r}=0.51, \mathrm{p}<0.01)$. The current perception thresholds (CPT) detected at $2 \mathrm{kHz}$ frequency differed between the groups in the upper and lower limbs (CPT at the median nerve: $3.66 \pm 0.35$ vs $1.84 \pm 0.25$ $\mathrm{mA}, \mathrm{p}<0.001$; $\mathrm{CPT}$ at the peroneal nerve: $5.39 \pm 0.71$ vs $3.70 \pm 0.68 \mathrm{~mA}, \mathrm{p}<0.05)$. Conclusions: Impaired gastric emptying was found more frequently in pts with long-standing type-1 DM but this difference between the groups was not significant. Both the parasympathetic and the sympathetic systems and the sensory function of the large myelinated fibres were more seriously altered in long-standing DM. The severe autonomic dysfunction exhibited a correlation with the slower gastric emptying. These data support the hypothesis that the most progressive manifestations of diabetic neuropathy might be responsible for the development of gastroparesis.

\section{M2192}

Patients with Gastroparesis Have a Decreased Expression of the 5-Ht4(C) Splice Variant in the Duodenum

Niels Van Lelyveld, Jose Ter Linde, Marguerite Schipper, Melvin Samsom

Background \& Aims: Studies using 5-HT3 and 5-HT4 receptor agonists, selective serotonergic reuptake inhibitors (SSRI), and tryptophan depletion, have revealed that serotonergic signalling is involved in the regulation of gastric emptying rate and, potentially, in the generation of upper abdominal symptoms. To increase the understanding of the pathophysiologic basis of idiopathic gastroparesis at the molecular level, we compared the expression of genes encoding components of serotonergic signalling in the duodenum and stomach between patients with idiopathic gastroparesis and healthy controls. Methods: Mucosal biopsy specimens were collected from the duodenum, antrum, and fundus of 11 patients with idiopathic gastroparesis ( 2 male, mean age $46.5 \pm 4.0$ years) and 11 healthy controls ( 1 male, mean age $46.3 \pm 2.5$ years). Enteroendocrine (EE) cells (chromogranin immunoreactive) and enterochromaffin (EC) cells (serotonin immunoreactive) were counted and mRNA levels of TPH-1 (the rate-limiting enzyme of 5-HT biosynthesis), SERT (regulates 5-HT availability by high affinity uptake), 5-HT3(C) and 5-HT3(E) (subunits of the 5-HT3 receptor), the 5HT4 receptor, and three 5-HT4 splice variants (A, B, and C) were quantified by real time RT-PCR and compared between the two groups. Results: EE and EC cell number were comparable in the three regions between the two groups. In addition, no differences were found in the percentage of EE cells that were EC cells in any of the three regions. The expression of TPH-1 and SERT in all three regions was comparable between the groups ( $\mathrm{P}$ $>0.05$ ). In antrum and fundus, the expression of the 5-HT3 receptor subunits, the 5-HT4 receptor, and the 5-HT4 receptor splice variants was too low for accurate quantification. In the duodenum, the overall expression of the 5-HT4 receptor was comparable, however the 5-HT4(C) splice variant was expressed more abundantly in healthy controls compared to patients $(\mathrm{P}=0.015)$. No differences were found in 5-HT4(A) or 5-HT4(B) expression. Finally, the 5-HT3 receptor subunits $\mathrm{C}$ and $\mathrm{E}$ showed comparable expression in both groups 
Conclusion: These data suggest that delayed gastric emptying or the presence of upper abdominal symptoms does not result from altered 5-HT biosynthetic and uptake capacity or changed expression levels of the 5-HT3 and 5-HT4 receptor in the stomach and duodenum at the mucosal level. However, a different distribution of 5-HT4 splice variants, as shown by the lower expression level of the 5-HT4(C) variant, may cause a different functional response of 5-HT4 receptors upon stimulation and be of importance in the pathophysiology of gastroparesis.

\section{M2193}

Impaired Fundo-Antral Reflex in Diabetic Gastroparesis

Ashok Attaluri, Issam Nasr, Konrad Schulze, Satish Rao, Anjana Kumar

BACKGROUND \& AIM: Fundic balloon distension induces reflex antral pressure waves in normal subjects- the fundoantral reflex. This may play a role in trituration and transport of food. Here, we tested whether an altered fundo- antral reflex plays a role in the pathophysiology of diabetic gastroparesis. METHODS: We examined 13 diabetics $(\mathrm{m} / \mathrm{f}=5 / 8$; mean age 39 yrs) with gastroparesis and 15 healthy volunteers $(\mathrm{m} / \mathrm{f}=7 / 8$, mean age $32 \mathrm{yrs}$ ). Antral activity was assessed by positioning two antral pressure sensors $5 \mathrm{~cm}$ apart and fundic distension was accomplished by a $10 \mathrm{~cm}$ long, highly compliant balloon connected to a barostat. Phasic distensions were performed in increments of $3 \mathrm{~mm} \mathrm{Hg}$. The number and area under the curve (AUC) of antral pressure waves were analyzed. RESULTS: Both in controls and diabetics, the antral activity was higher during balloon inflation, compared to the deflation periods $(\mathrm{p}<0.05)$. All healthy controls showed reflex antral activity, compared to $62 \%$ of diabetics $(\mathrm{p}<0.05)$. The number and AUC of antral pressure waves were significantly lower $(\mathrm{p}<0.05)$ in diabetics (table; mean \pm SD). Also, in diabetics, antral pressure activity did not correlate with either gastric emptying time or symptoms such as postprandial fullness, nausea, bloating, abdominal pain, vomiting, and weight loss (Spearman correlation coefficient $r s=-0.24$ to $0.32 ; p>0.05$ ). CONCLUSIONS: Diabetic patients with gastroparesis demonstrate either an impaired or absent fundo- antral reflex and this may play a role in the pathophysiology of diabetic gastroparesis. However, there is poor correlation between reflex antral activity, gastric emptying and individual upper GI symptoms. This may either be due to multiple independent mechanisms that contribute to the pathogenesis of diabetic gastroenteropathy or a type II error. Supported by ACG reseach grant and R01 DK-57001-03.

\begin{tabular}{|c|l|l|l|r|}
\hline Balloon pressure & \multicolumn{2}{|c|}{ Number of waves/minute } & \multicolumn{2}{c|}{ Area under the curve $(\mathrm{mm} \mathrm{Hg})$} \\
\hline$(\mathrm{mm} \mathrm{Hg})$ & Controls & Diabetics & Controls & Diabetics \\
\hline 6 & $1.83 \pm 0.7$ & $1.15 \pm 0.5$ & $230.6 \pm 57$ & $151.1 \pm 23$ \\
\hline 9 & $2.23 \pm 1.1$ & $0.89 \pm 0.3$ & $275.3 \pm 71$ & $181.8 \pm 35$ \\
\hline 12 & $1.91 \pm 0.9$ & $0.89 \pm 0.3$ & $269.7 \pm 97$ & $173.4 \pm 53$ \\
\hline 15 & $2.08 \pm 1.2$ & $0.67 \pm 0.2$ & $308.8 \pm 85$ & $118.8 \pm 41$ \\
\hline 18 & $1.54 \pm 0.6$ & $0.63 \pm 0.3$ & $325.6 \pm 79$ & $145.1 \pm 61$ \\
\hline
\end{tabular}

\section{M2194}

Gastric Compliance By Barostat Does Not Correlate with Gastric Emptying Or Accommodation

Linda Anh B. Nguyen, Shelly Parker, Stephen Bunker, William J. Snape

Background: Proximal gastric compliance is a function of the physical elasticity of the stomach wall. Accommodation is a vagally mediated reflex that occurs after a meal. Patients with nonulcer dyspepsia and early satiety have been found to have impaired gastric accommodation compared to normals (Tack et al.Gastro 1998). There has been no difference in compliance between controls and patients (Mearin et al. Gastro 1991). Our hypothesis is that compliance correlates with accommodation and inversely with gastric emptying. Aims: 1)Correlate gastric compliance with gastric emptying and accommodation and 2)Determine if there is a physiologic difference in patients with impaired compliance compared to those with normal compliance. Patients and Methods: 36 patients ( $F=32$, age 38.3 \pm 11.1 ) with upper GI symptoms had gastric barostat testing to evaluate their symptoms. Minimal distention pressure (MDP), sensory threshold, compliance(stepwise distention) and accommodation(dV) were assessed. All patients had gastric scintigraphy(GET) to measure 2 and 4 hour gastric retention. The correlation coefficient of compliance with GET, sensory threshold and $\mathrm{dV}$ were calculated. In addition, the correlation of $\mathrm{dV}$ with GET was also assessed. Patients were then grouped by compliance (normal vs. abnormal $<20 \mathrm{ml} / \mathrm{mg}$ ). The 2 groups were compared with GET, dV and gastric volume change after sumatriptan (5HTl agonist). Results: $19.4 \%$ of patients had impaired compliance (mean $=13.1 \pm 2.3 \mathrm{mg} / \mathrm{mmHg}$ ). Gastric compliance did not correlated with $2 \mathrm{hr}$ GET $(\mathrm{r}=-0.05, \mathrm{p}=0.77)$, 4hr GET $(\mathrm{r}=0.17, \mathrm{p}=0.32)$, sensory threshold $(\mathrm{r}=0.09, \mathrm{p}=0.61)$ or $\mathrm{dV}(\mathrm{r}=-0.02, \mathrm{p}=0.93)$. There was also no correlation between $\mathrm{dV}$ and GET. When comparing patients with impaired compliance vs. normal compliance (mean $=43.5 \pm 3.5 \mathrm{ml} / \mathrm{mg}$ ), there was no differences in $\mathrm{dV}(76.7 \pm 28.7 \mathrm{ml}$ vs $57.1 \pm 24.5 \mathrm{ml}, \mathrm{p}=0.7), 2 \mathrm{hr}$ GET $(28.1 \pm 3.9 \%$ vs. $35.0 \pm 9.7 \%, \mathrm{p}=0.5)$, or $4 \mathrm{hr}$ GET $(9.1 \pm 3.1 \%$ vs. $7.3 \pm 2.7 \%, \mathrm{p}=0.8) .5$ patients in the impaired compliance group and 6 patients in the normal group received sumatriptan $6 \mathrm{mg}$ SQ after being found to have impaired accommodation. The post sumatriptan response was greater in the normal group $(252.8 \pm 115.1 \mathrm{~mL}$ vs. 11.4 $\pm 11.4 \mathrm{~mL}, \mathrm{p}=0.9$ ) but not statistically significant. Conclusions: 1) Gastric emptying cannot predict gastric compliance or accommodation as measured by gastric barostat. 2)Impaired gastric accommodation with normal compliance may indicate an abnormality in the neural reflex as seen by the trend toward increase response to sumatriptan. In comparison, patients with both abnormal dV and compliance may have problems with gastric elasticity which would not improve with a 5-HT agonist.

\section{M2195}

Effect of a Proton Pump Inhibitor On Postprandial Gastric Volume, Emptying and Symptoms

April B. Grudell, Duane D. Burton, Debra A. Stephens, Michael Camilleri

Background: Intravenous ${ }^{99 \mathrm{~m}} \mathrm{Tc}$-pertechnetate is taken up by gastric mucosa. This principle is used to image stomach volume in single photon emission computed tomography (SPECT) Proton pump inhibitors (PPIs) are beneficial in some patients with functional dyspepsia. The effects of PPIs on measurements of gastric volume and emptying are incompletely understood. Aim: To assess the effect of inhibition of gastric secretion on gastric volume and emptying measurements obtained with SPECT. Methods: In a double-blind, placebocontrolled trial, 13 participants were randomized to a PPI, rabeprazole, $20 \mathrm{mg}$ b.i.d., or placebo 2 days prior to the study visit. Medication allocation was concealed. During the study visit, fasting gastric volume was measured using SPECT- ${ }^{99} \mathrm{~m} T \mathrm{Tc}$-pertechnetate. After the last dose of study medication, an ${ }^{111}$ In-chloride charcoal-labeled egg meal (300kcal) was ingested, followed by SPECT measurements of postprandial gastric volume and gastric emptying (GE 2h, GE 4h) by a previously validated method (Am J Physiol 2005;289:G2616). Symptom ratings using a visual analog scale (fullness, nausea, bloating, abdominal pain) were obtained postprandially at 30 minutes. Group comparisons were performed using the Mann Whitney test. Results: Technical failure due to subcutaneous infiltration of ${ }^{99} \mathrm{mcO}_{4}$ led to exclusion of 1 participant's data. Gastric imaging was not compromised by the administration of the PPI. Patient demographics, fasting and postprandial gastric volumes, and gastric emptying percentages are shown in the table. There were no significant differences in the 2 groups. Rabeprazole resulted in reduced aggregate postprandial symptoms, particularly fullness, 30 minutes after the meal $(\mathrm{p}=0.01$ ). Post-hoc analysis showed that 30 participants per treatment group would be required to show a significant effect of rabeprazole on gastric emptying and 48 per group to show an effect on gastric volume. Conclusion: In healthy participants, rabeprazole $20 \mathrm{mg}$ b.i.d. did not significantly change gastric volume or emptying, but it reduced symptoms postprandially. The mechanism of reduced postprandial symptoms with a PPI requires further study.

\begin{tabular}{|c|c|c|}
\hline & Placebo $(\mathrm{n}=5)$ & Rabeprazole $(\mathrm{n}=7)$ \\
\hline Age (y), mean (SD) & $36(22-45)$ & $32(28-43)$ \\
\hline Gender (M/F) & $3 / 2$ & $3 / 4$ \\
\hline Fasting gastric vol (mL), median (IQR) & $222(171-307)$ & $290(221-316)$ \\
\hline Postprandial gastric vol (mL), median (IQR) & $810(761-875)$ & $909(877-944)$ \\
\hline Fullness (mm, VA scale) & $31(22-45)$ & $15(5-24)$ \\
\hline GE 2h (\%) median (IQR) & $34(30-36)$ & $35(21-40)$ \\
\hline GE 4h (\%) median (IQR) & $85(77-85)$ & $65(59-84)$ \\
\hline
\end{tabular}

Data show median (IQR)

M2196

Effect of Gastric Electrical Stimulation On Cerebral Blood Flow in Patients with Gastroparesis

Thomas V. Nowak, Gary Hutchins, Joel Hammond, Yang Wang, Lyn Ring, Kativa Leal

Gastric electrical stimulation (GES) of the stomach is an effective treatment of nausea and vomiting in patients with gastroparesis (Gastroenterology 125: 421-28, 2003). However, the mechanism is unclear, as many patients show resolution of nausea and vomiting yet continue to exhibit delayed gastric emptying. This suggests GES may relieve symptoms by activation of a mechanism involving the central nervous system. The purpose of this study was to determine whether GES produces changes in the brain evidenced by alterations in cerebral blood flow on positron emission tomography (PET). Eight patients ( 6 female, 2 male) ranging in age from 27 to $68 \mathrm{yr}$ were studied. Each patient had profound gastroparesis and had undergone implantation of a GES device from 6 to 57 mo (mean 25) prior. All patients studied had significant improvement in symptoms of nausea, vomiting, and early satiety after implantation of the GES device. PET scans were performed after an overnight fast and with the GES device in the ON mode. Each patient was then given an iv injection 30-40 $\mathrm{mCi}$ Ol5-water. After PET image data were obtained the GES device was then turned to the OFF mode for $30 \mathrm{~min}$. The O15-water injection and PET image scanning was then repeated. Respective PET images obtained with the GES device in the ON and OFF modes were then analyzed and compared. PET images were analyzed using SPM2 (Statistical Parametric Mapping), implemented using Matlab 6.5 Prior to statistical analysis all images were realigned to correct motion movements and spatially normalized into the MNI standard template to remove inter-subject anatomical variability. Spatially normalized images were smoothed using an isotropic Gaussian kernel with $16 \mathrm{~mm}$ FWHM to increase the signalto-noise ratio. Statistical comparisons between ON and OFF scans were performed on a voxel-by-voxel basis using paired t statistics. Significant voxels were superimposed on a high resolution MRI template provided by SPM2, thus allowing anatomical identification. Significant increases in blood flow during GES were noted in the middle, superior, and inferior gyri, in the middle occipital and middle temporal gyri, and in the amygdala and parahippocampal gyri $(\mathrm{P}<.05$ for each locus, respectively). Likewise, significant decreases in blood flow during GES were noted in the pre and post central gyri, in the anterior cingulate gyri, and in the caudate head and putamen ( $\mathrm{p}<.05$ for each locus, respectively). Conclusions: 1)GES produces significant alterations in cerebral blood flow in gastroparetic patients; 2)central nervous system mechanisms during GES may be responsible for the relief of nausea and vomiting in these patients. 
M2197

Influence of Helicobacter Pylori Infection On Gastrointestinal Motility of Dyspeptic Patients-Real-Time Ultrasonographic Study Hiroaki Kusunoki, Ken Haruma, Jiro Hata, Tomoari Kamada, Motonori Sato, Toshiaki Tanaka, Hideki Koga, Masaharu Takeda, Kenichi Tarumi, Keisuke Honda

Background: Helicobacter pylori infection has been proposed as one of the pathogenic mechanisms of functional dyspepsia (FD), but its effects on proximal gastric compliance and gastrointestinal motility are still unclear. The aim of the present study was to clarify these effects using ultrasonography. Methods: Fourteen FD patients with Helicobacter pylori infection (mean age, 59.8 years) and 14 uninfected FD patients (mean age, 43.2 years) were studied ultrasonographically. Ultrasonographic assessment of gastrointestinal motility was performed using previously reported methods (Scand J Gastroenterol 1994; 29 897-902, J Gastroenterol Hepatol 2000; 15 1022-1027, Gut 2002; 51 A188). For the assessment of gastric compliance, a cross-sectional area of the proximal stomach was measured after stepwise ingestion of a liquid meal (consomme soup: $3.25 \mathrm{kcal} / 100 \mathrm{ml}$ ), up to $400 \mathrm{ml}$. Then, gastric emptying, antral contractions and duodenogastric reflux (DGR) were observed in the sitting position. Result: There were no significant differences in \% gastric emptying, antral contractions and DGR between the FD patients with and without Helicobacter pylori infection (\% gastric emptying : 57.2 vs 57.8 , motility index : 8.90 vs 8.79 , DGR : 22 vs 26 ). Although the cross-sectional area of the proximal stomach tended to be smaller in the FD patients with Helicobacter pylori infection than in the patients without infection after the ingestion of 300 $\mathrm{ml}$ (30.3 vs $34.8, \mathrm{p}=0.166$ ) and $400 \mathrm{ml}$ (39.2 vs $43.3, \mathrm{p}=0.273$ ) of a liquid meal, there were no statistically significant differences. Conclusion: Impaired meal-induced gastric compliance was present in a higher proportion of FD patients with Helicobacter pylori infection than in patients without infection. There was little influence of Helicobacter pylori infection on gastrointestinal motility.

\section{M2198}

Artificially Sweetened, Compared to Regular Mixers, Accelerate Gastric Emptying and the Rate of Alcohol Absorption

Keng-Liang Wu, Reawika Chaikomin, Selena Doran, Karen L. Jones, Michael Horowitz, Chris Rayner

Mixed alcoholic drinks are increasingly being consumed in "diet" varieties, as opposed to "regular" versions containing sugar. Almost no consideration has been given to the potential for artificially sweetened drinks to empty more rapidly from the stomach, with an increased rate of alcohol absorption, when compared with the original formulations. Eight fasting male volunteers were studied twice, in randomized order. On each day, subjects consumed an orange-flavored vodka beverage ( $30 \mathrm{~g}$ ethanol in $600 \mathrm{~mL}$ ), made with either a "regular" mixer containing sucrose (total $478 \mathrm{kcal})$, or a "diet" mixer (225 kcal). Gastric emptying was calculated from ultrasound measurements of antral area. Blood was sampled at intervals for 180 minutes for measurement of blood ethanol concentration. Data are mean \pm standard deviation. The gastric half-emptying time was less for the "diet" than the "regular" vodka drink $(21.1 \pm 9.5$ vs $36.3 \pm 15.3$ minutes, $\mathrm{P}<0.01)$. Both the peak blood ethanol concentration $(0.053 \pm 0.006$ vs $0.034 \pm 0.008 \mathrm{~g} \%, \mathrm{P}<0.001)$ and the area under the blood ethanol concentration curve between $0-180$ minutes $(5.2 \pm 0.7$ vs $3.2 \pm 0.7$ units, $\mathrm{P}<0.001)$ were substantially greater with the "diet" drink. Substitution of artificial sweeteners for sucrose in mixed alcoholic beverages may have a marked effect on the rate of gastric emptying and the blood alcohol response.

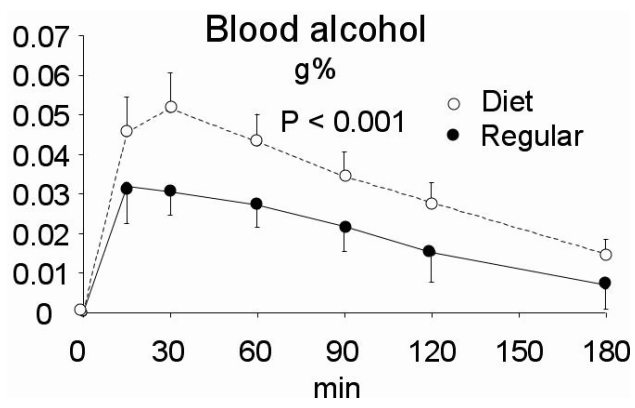

M2199

Which Time Interval Is Required Between Performance of Two 13c-Breath Tests?

Jutta Keller, Karola Schmidt, Christiane Fibbe, Peter Layer

Background: 13C-breath tests have become important gastroenterological diagnostic tools and several 13C-breath tests may be performed in one patient for varying indications. The 13C-octanoic acid breath test allows measurement of gastric emptying of solids. However, it has been demonstrated that $13 \mathrm{CO} 2$ continues to be excreted long after gastric emptying was shown to be complete by scintigraphy. This might be due to the fixation of the isotope in the bicarbonate pool of the body and its subsequent delayed release. Thus, it is of importance to know which time interval is required before performance of a second 13Cbreath test in order to avoid overlap. Methods: On day 1 a standardised 13C-octanoic acid breath test was performed in 60 patients with upper abdominal symptoms (450 kcal meal including scrambled egg, egg yolk doped with $91 \mathrm{mg}$ 13C-octanoic acid, breath sampling at $15 \mathrm{~min}$ intervals for $4 \mathrm{~h}$, analysis by isotope-selective infrared spectrometry). Data were evaluated using non-linear regression analysis (Ghoos et al Gastroenterology 1993). On day $2,24 \pm 1 \mathrm{~h}$ after collection of the first basal breath sample, another fasting breath sample was obtained from every patient. Results $($ mean \pm SEM): For all patients, mean half and lag time were $173 \pm 15$ and $98 \pm 11$ min, respectively, and $23 \pm 1 \%$ of the $13 \mathrm{C}$-dose administered were cumulatively exhaled within $4 \mathrm{~h} .18$ patients showed delayed gastric emptying (t $\mathrm{l} /$ $2: 327 \pm 23 \mathrm{~min}$ ) according to our laboratory's normal values $(\mathrm{t} 1 / 2<200 \mathrm{~min})$. These patient had significantly longer $t$ lag and lower cumulative $4 \mathrm{~h}-13 \mathrm{C}$-exhalation than patients with normal gastric emptying $(194 \pm 18$ vs. $58 \pm 7 \mathrm{~min}, \mathrm{p}<0.0001 ; 20 \pm 2$ vs. $24 \pm 1 \%$ of dose, $\mathrm{p}=$ 0.026 ). Basal $13 \mathrm{C}$-concentrations in breath were similar before and $24 \mathrm{~h}$ after application of 13C-octanoic acid for patients with normal and delayed gastric emptying and did not differ between the two groups (tab). Moreover, the delta between basal 13C-concentrations on day 1 and 2 did not correlate with half or lag time of gastric emptying or cumulative 13C-exhalation in either group. Conclusions: 24 hours after ingestion of a standard dose of 13C-octanoic acid fasting 13C-exhalation has returned to basal in patients with normal and prolonged gastric emptying. Thus, a second 13C-breath test may be performed on the next day even in patients with gastroparesis.

Fasting 13C-exhalation before and $24 \mathrm{~h}$ after performance of a 13C-octanoic acid breath test

\begin{tabular}{|l|c|c|c|}
\hline & All patients $(\mathrm{n}=60)$ & Patients with normal GE $(\mathrm{n}=42)$ & Patients with delayed GE $(\mathrm{n}=18)$ \\
\hline Day 1 & $-25.5+/-0.3$ & $-25.5+/-0.4$ & $-25.7+/-0.5$ \\
\hline Day 2 & $-25.4+/-0.4$ & $-25.4+/-0.5$ & $-25.4+/-0.3$ \\
\hline
\end{tabular}

Data are given as delta \%o, mean+/-SEM

\section{M2200}

Poor Correlation Between Electrogastrography and Antroduodenal Manometry in Patients with Motility Disorders

Shahab Abid, Greger Lindberg

Antroduodenal manometry (ADM) records lumen-occluding contractions of the gastric antrum, while electrogastrography (EGG) measures gastric myoelectric activity using cutaneous electrodes placed on the abdominal wall. Under physiological conditions there is a temporal correlation between myoelectric slow waves and contractile activity of the antrum. It is unclear if there is also a spatial correlation between electric and contractile activity. Aim of the study. To determine the relationships between electrical activity measured by EGG and antral contractile activity measured by ADM. Material and methods. This is a retrospective analysis of patients with various motility disorders of the gut subjected to simultaneous ADM and EGG recording. A single bipolar channel measured the EGG and ADM was done using either 6 or 8 channels. EGG parameters analysed were: (i) percentage of time with a dominant frequency (DF) in normogastric $(2-4 \mathrm{cpm})$, tachygastric $(4-10 \mathrm{cpm})$ and bradygastric $(0.5-2 \mathrm{cpm})$ frequency bands, (ii) EGG power attributed to the three frequency bands (normogastria, tachygastria and bradygastria), (iii) the ratio of postprandial to fasting power of the DF, (iv) the overall $\mathrm{DF}$, (v) variability of the dominant frequency (DFIC) and (vi) variability of the dominant power (DPIC). The ADM parameters measured were: mean frequency and amplitude of contractions and the motility index that was calculated by multiplying the amplitude ( $\mathrm{mm}$ ) by the frequency per minute. Data from one hour before and the first hour after a test meal were compared. Results. A total of 151 patients, $125(83 \%)$ females, mean age $45 \pm 14$ years, were analysed. There were 52 patients with irritable bowel syndrome, 26 patients with slow transit constipation, 22 patients with enteric dysmotility, 11 patients with chronic intestinal pseudo-obstruction and 13 patients with gastroparesis or functional dyspepsia, three patients with previous gastric surgery and 24 patients with other motility disorders of the gut. We found no relation between any of the 21 EGG parameters and 8 ADM parameters. Comparison between EGG response to meal ingestion (ratio between postprandial and fasting power) and ADM response to meal ingestion (ratio between postprandial and fasting motility index) revealed no correlation $(\mathrm{r}=0.06 \mathrm{p}=$ $0.44)$ Conclusions. No consistent relationship was observed between EGG and ADM in this series of patients with motility disorders. EGG and ADM give different kinds of information.

\section{M2201}

Postprandial Gastric Acid Neutralization and Reacidification in Diabetic Vs. Idiopathic Gastroparesis: Relation to Degree of Gastric Stasis

William L. Hasler, Radoslav Coleski, William D. Chey, Kenneth L. Koch, Richard W. McCallum, John M. Wo, Braden Kuo, Michael Sitrin, Kevin Stevens, Beverly Landrigan, Jack R. Semler, Henry P. Parkman

Background: Gastric acid-related complaints including GERD are prevalent in patients with gastroparesis, especially with diabetes. Demographic and clinical differences in diabetic and idiopathic gastroparesis suggest the two conditions have distinct pathophysiology. Though acid-associated symptoms have been related to autonomic neuropathy, the acid milieu of the stomach in gastroparesis is poorly understood. Further, the roles of different etiologies of gastroparesis and the magnitude of gastric stasis on gastric $\mathrm{pH}$ are unknown. Recently an ingestible telemetry capsule was developed to continuously measure luminal $\mathrm{pH}$ and pressure (SmartPill Corp.). Hypotheses: We hypothesized (i) severely delayed gastric emptying is associated with increased gastric acidity and (ii) postprandial $\mathrm{pH}$ profiles differ in diabetic vs. idiopathic gastroparesis suggesting dissimilar disease pathogenesis. Methods: Data from 44 patients with gastroparesis on prior scintigraphy (20 diabetic, 24 idiopathic) from 7 centers were analyzed. Acid suppressants were stopped x 1 week prior to study. After capsule ingestion, subjects consumed standard meals (120 g EggBeaters, bread, jam, and $120 \mathrm{ml}$ water; $255 \mathrm{kcal}$ ) with $1 \mathrm{mCi}{ }^{99} \mathrm{Tc}$-sulfur colloid. Luminal $\mathrm{pH}$ was measured every $5 \mathrm{sec}$; scintiscans were acquired every $30 \mathrm{~min}$ x 4-6 h. Results: Meals elicited initia $\mathrm{pH}$ rises that were greater in diabetics $(4.98 \pm 0.32)$ than idiopathics $(3.89 \pm 0.39)(\mathrm{P}=0.04)$. This declined to stable $\mathrm{pH}$ nadirs which were similar in diabetics $(1.50 \pm 0.23)$ and idiopathics $(0.93 \pm 0.31)(\mathrm{P}=0.16)$. Cumulative postprandial reacidification was quantified by measuring areas under $\mathrm{pH}$ curves (AUC) from meal ingestion to the time of nadir $\mathrm{pH}$. AUCs ( $\mathrm{pH} \times$ $\mathrm{hr})$ were greater in diabetics $(4.10 \pm 0.51)$ than idiopathics $(2.61 \pm 0.40)$ indicating less gastric acidity after eating in diabetics $(\mathrm{P}=0.02)$. Times for $90 \%$ gastric emptying (T90) were similar in diabetics $(249 \pm 15 \mathrm{~min})$ and idiopathics $(249 \pm 16 \mathrm{~min})$. Compared to those with mild gastroparesis (T90 $\leqq 300 \mathrm{~min}$ ), patients with profound stasis (T90>300 $\mathrm{min}$ ) had higher peak $\mathrm{pH}(5.30 \pm 0.50$ vs. $3.99 \pm 0.30)$, nadir $\mathrm{pH}(2.23 \pm 0.42$ vs. $0.74 \pm 0.17)$, and $\mathrm{AUC}(4.63 \pm 0.86$ vs 
2.79 \pm .29)(all $\mathrm{P}<0.03$ ). Conclusions: Diabetics with gastroparesis exhibit lesser degrees of postprandial gastric acidity than idiopathic gastroparetics that are not due to differences in gastric emptying rates. However, those with profound stasis exhibit higher rather than lower gastric $\mathrm{pH}$ regardless of etiology. These findings suggest that the pathophysiology of diabetic gastroparesis differs from idiopathic disease and indicate that factors other than low gastric $\mathrm{pH}$ may contribute to symptoms in diabetics with gastric retention.

\section{M2202}

Exaggerated Cephalic Gastric Relaxation and Gastric Accommodation in Nausea-Predominant Patients with Functional Dyspepsia

Tomotaka Shoji, Fukudo Shin, Yuka Endo, Kazuto Karahashi, Yashuhiro Sagami, Joe Morishita, Yuko Kimura, Atsushi Utsumi, Michio Hongo

(Back ground) Patients with functional dyspepsia (FD) are considered to have heterogeneous pathophysiology. Nausea-predominant patients may have specific gastric function. Therefore we tested whether fundic function was altered in nausea-predominant patients with functional dyspepsia (npFD). (Subject and method) Ten healthy subjects and seven npFD patients participated in this study after informed consent. The npFD patients satisfied the Rome II criteria and their dominant symptom was nausea. After 12 hours fasting, barostat bag was placed in the gastric funds. Gastric perception was measured by $1 \mathrm{mmHg} / \mathrm{min}$ step wise gastric distension and two first (FS) and discomfort (D) gastric sensation thresholds were determined. After the perception session, two (sham feeding and tube feeding) barostat sessions were performed with MDP (minimal distending pressure) $+2 \mathrm{mmHg}$ distending pressure. After the gastric volume measurement for 30 minutes, sham feeding was carried out by repeated taste and spit of Ensure liquid $₫$ for 10 minutes and then the measurement continued for 30 minutes. After 20 minutes interval, baseline was measured again. After the second baseline, Ensure liquid@ $(200 \mathrm{ml}, 200 \mathrm{kcal})$ was injected into gastric funds through a feeding tube and gastric volume was measured for 30 minutes. In both the perception session and barostat session, all subjects evaluated gastric sensation with visual analogue scale (VAS). All data was analyzed by Mann-Whitney's U test or ANOVA. (Result) Two gastric sensation thresholds ( $\mathrm{mmHg}$ ) were same in both healthy subjects and npFD patients (FS: $10.6+/-3.3$ vs. 10.8+/-4.4, D: $13.7+/-4.0$ vs. 12.3+/-3.9). In the barostat session, the baseline gastric volume ( $\mathrm{ml}$ ) was tended to be higher in $\mathrm{FD}$ than healthy subjects (193+/124 vs. $93+/-46, p=0.087$ ). After both sham and tube feeding, gastric volume significantly increased in npFD than controls (after sham: $309+/-204$ vs. $129+/-65$ and after tube: $476+/-$ 284 vs. $280+/-94 \mathrm{ml}$, ANOVA; $\mathrm{p}<0.05$ ). On the VAS, only nausea score was significantly higher in npFD after both sham and tube feeding (after sham: $23.6+/-15.7$ vs. $11.0+/-21.4$ and after tube: $47.1+/-38.7$ vs. $10.3+/ 17.0, \mathrm{p}<0.05$ ). (Conclusion) These data suggested that excessive cephalic gastric relaxation, excessive gastric accommodation and nausea were induced by nutrient stimulation in npFD patients. Nausea-predominant FD patients may have different fundic response to stimuli from the other form of FD patients.

\section{M2203}

Correlation of Plasma Ghrelin Levels and Delayed Gastric Emptying Time in FD Patients with Loss of Emptiness Feeling

Tetsuro Hiratsuka, Seiji Futagami, Tomotaka Shindo, Tatsuhiko Hamamoto, Nobue Ueki, Kenji Suzuki, Yoko Shinji, Masafumi Kusunoki, Ken Wada, Kazumasa Miyake, Katya Gudis, Taku Tsukui, Katsuhiko Iwakiri, Choitsu Sakamoto

Background/Aims: Since functional dyspepsia (FD) seems to be caused by multifactorials such as gastric motility, depression, and visceral hypersensitivity, its clinical symptoms are complex and resistant to cure. Gastric emptying has been reported to be delayed in some FD patients. However, the involvement of gut hormones and local cytokines in gastric emptying has yet to be elucidated in these FD patients. Thus, we measured gastric emptying time and plasma ghrelin levels, one of the motility factors released into the peripheral circulation from the stomach, and examined the relationship between these data and symptom patterns in FD patients. Methods: 47 patients with FD and 30 healthy controls were enrolled according to Rome II criteria after endoscopy for this study. 47 FD patients were separated into three groups: subjects with symptoms of dismotility-like dyspepsia (dismotility group), ulcer-like dyspepsia (ulcer-like group) and unspecified dyspepsia (unspecified group). Gastric motility was evaluated by measuring gastric emptying time using 13C-acetate breath test, and partly assessed by abdominal ultra-sonography. Upper abdominal symptoms including pain, nausea, fullness, discomfort, and emptiness were assessed by questionnaire scores. Scores from the Self-rating Questionnaire for Depression (SRQ-D) were also used to evaluate depression. Plasma ghrelin levels were measured by ELISA method in patients with FD. Results: Gastric emptying time in the dismotility group ( $33 \%$ of FD) was significantly delayed compared to the healthy control group ( $58.6 \pm 3.6$ vs. $46.2 \pm 0.65 \mathrm{~min}, \mathrm{p}<0.05$ ), while emptying time in the ulcer-like (14\% of FD) and unspecified (53\% of FD) groups did not differ significantly from healthy controls. In contrast, SRQ-D scores for the ulcer-like and unspecified groups $(11.7 \pm 2.5$ and $11.7 \pm 1.3$, respectively $\mathrm{p}<0.05)$ were significantly higher than for healthy controls, while scores for the dismotility group did not differ from that of the healthy control group. One of the symptoms of FD patients with delayed gastric emptying time was characterized as loss of emptiness feeling compared to patients with normal gastric emptying time $(3 \pm 1.5$ vs. $8.9 \pm 1.3, p<0.05)$. However, states of depression did not differ between FD patients with normal and delayed gastric emptying. Furthermore, levels of ghrelin phenotypes, acylated and desacylated ghrelin, decreased significantly in patients with delayed gastric emptying time compared with those in patients with normal gastric emptying time. Conclusion: Our results suggest that decreased plasma ghrelin levels might be involved in delayed gastric emptying time and loss of emptiness feeling in FD patients.

\section{M2204}

Symptoms in Functional Dyspepsia (FD) and Irritable Bowel Syndrome Patients with Dyspepsia (IBS+D) Are Correlated with Impaired Gastric Relaxation Identified By Magnetic Resonance Imaging (MRI)

Ivo R. van der Voort, Marco Schmidtmann, Peter Kobelt, Hassan Abou-Rebyeh, Bertram Wiedenmann, Hubert Monnikes

Impaired gastric relaxation has been proposed to be present in up to $40 \%$ of patients with FD using invasive barostat techniques. This technique itself might interfere with physiological gastric function. The aims of this study were to compare postprandial (pp) gastric relaxation and emptying in healthy volunteers, patients diagnosed with FD, and IBS plus dyspepsia using the non-invasive technique of MRI, and to assess if altered gastric function is correlated with dyspeptic symptoms. Material \& Methods: Fifteen healthy controls (HC; 7 female, mean age (MA) 36 yrs), 20 patients ( 10 female, MA 41 yrs) diagnosed with FD, and 20 patients suffering from IBS and dyspeptic symptoms (IBS+D; 11 female, MA 38 yrs) underwent MRI studies in supine position. Symptoms were assessed using the Nepean Dyspepsia Index (NDI) and a gastrointestinal symptom questionnaire (GSQ). MRI sequences covering the complete gastric region were performed before and after a liquid meal $(500 \mathrm{ml}, 500 \mathrm{kcal})$. Gastric and meal volumes were measured up to 90 minutes pp and changes in gastric and meal volumes calculated. Results: The NDI symptom scores in FD and IBS patients with dyspepsia were significantly higher compared to $\mathrm{HC}(\mathrm{p}<0.001)$, but were not different between patient groups. The quality of life $(\mathrm{QoL})$ score of the NDI was significantly higher in both patients groups compared to $\mathrm{HC}(\mathrm{p}<0.001)$, indicating a diminished quality of life. The cumulative increase of gastric volume in the early pp phase was significantly lower in FD and IBS+D compared to HC. Nine out of 20 patients ( $45 \%$ ) with FD and 6 out of 20 patients $(30 \%)$ with IBS+D showed no increase in pp gastric volume. In contrast, an increase in the pp gastric volume was found in all HC. Patients with impaired gastric relaxation had significantly higher dyspepsia scores in the GSQ (median: 78.5, range 68.0-94.0) and NDI (median: 106.5, range 94.0-117.0) compared to patients with normal gastric relaxation (median: GSI: 64.0, range 61.0-70.5, $\mathrm{p}=0.021$; NDI: 72.0 , range 59.0-91.5, $\mathrm{p}<0.001$ ). The increase in intragastric liquid volume in the early pp phase was significantly diminished in FD and IBS+D. Overall gastric emptying, calculated as area under the curve, showed a significantly delayed gastric emptying in FD compared to IBS+D and HC (ml/90min: FD= $213 \pm 7, \mathrm{IBS}+\mathrm{D}=198 \pm 6, \mathrm{HC}=174 \pm 7 ; \mathrm{p}<0.05)$. Conclusion: The technique of MRI allows to non-invasively identify alterations of gastric function in a subgroup of patients with otherwise unexplained dyspepsia. Impairment of pp gastric relaxation as measured by MRI is clearly accompanied with more severe dyspeptic symptoms FD and IBS plus dyspepsia.

\section{M2205}

Smartpill, a Novel Ambulatory Diagnostic Test for Measuring Gastric Emptying in Health and Disease

Braden Kuo, Richard W. McCallum, Kenneth Koch, William D. Chey, John M. Wo, Michael Sitrin, William Hasler, Jeffrey Lackner, Leonard Katz, Beverly Landrigan, Kathy Selover, Alan Hutson, David Barthel, Jack Semler, Henry P. Parkman

Introduction: Gastric emptying scintigraphy (GES) using a radiolabeled solid phase meal, is widely used to measure gastric emptying but the technique is non-ambulatory, involves radiation and is performed variably. Recently an ambulatory wireless capsule SmartPill (SP) which measures $\mathrm{pH}$ and pressure of the GI lumen when swallowed has been developed. The rapid luminal $\mathrm{pH}$ change from acidic to alkaline marks the transit of the ingested SP from the stomach into the duodenum. Aim: 1)To demonstrate the correlation between gastric residence time (GRT) as determined by the gastric emptying of the SP and the gastric emptying time of a radiolabeled meal in both healthy subjects and patients with documented gastroparesis, 2)To demonstrate that GRT can discriminate between healthy subjects and gastroparetics. Methods: In a multicenter study, simultaneous measurements of transit of SP and GES was performed in 86 healthy subjects and in 60 patients with previously diagnosed gastroparesis based on scintigraphy. SP was ingested after an overnight fast and then GES was performed in a standardized fashion with imaging taken up to 6 hours after ingestion of a 99mTc-SC radiolabeled meal (120 g Eggbeaters, 2 pieces of bread with jam; $255 \mathrm{kcal}, 2 \%$ fat) and $120 \mathrm{cc}$ water. Correlations between GRT and time to 50\% emptying (T-50\%), time to $90 \%$ emptying (T-90\%) were performed. Receiver operating characteristic (ROC) curves were used to summarize the sensitivity and specificity in differentiating the normal subjects from gastroparetic patients for the T-50, T-90\%, and GRT. Results: The correlation between GRT and T-90\% emptying was $0.85 \pm 0.06$ and for T-50\% emptying, $0.66 \pm 0.15$. The c statistic for the area under the ROC curve (AUC) assessing overall diagnostic accuracy for classifying normals and gastroparetics for GRT was 0.83 (95\% CI 0.74-0.87) and for T-90\%, 0.85 (95\% CI 0.80-0.90), not statistically different from each other. The AUC for T-50\% was 0.77 (95\% CI $0.70-0.83$ ). The cut off point that maximizes both sensitivity and specificity for T-90\%, $182 \mathrm{~min}$, gave a sens of $84.8 \%$ and spec of $77.9 \%$, whereas the cut off point for T-50\% was 102 , giving a sens of $71 \%$ and a spec of $74 \%$. The cutoff for GRT was $240 \mathrm{~min}$, giving $85 \%$ sens and $72 \%$ spec. Conclusion: The GRT of the SP has a high correlation with T-90\% of scintigraphy suggesting that the GRT more closely represents a time near the end of the emptying of a solid meal. The study demonstrates that GRT of the SP was able to differentiate normals from gastroparetics similar to scintigraphy This study suggests that the SmartPill can be used as a novel ambulatory method for assessing gastric emptying in health and disease 
M2206

Factors Associated with Gastric Dysrhythmias in Patients with Type 1 Diabetes and Without Dyspeptic Symptoms

Francesco De Giorgi, Clelia Verde, Lisa Faiella, Elisabetta Atteo, Riccardo Pascucci, Giovanni Sarnelli, Giovanni Annuzzi, Angela Rivellese, Rosario Cuomo

Impairment of gastric function in diabetes has been attributed to autonomic neuropathy (AN). Multichannel electrogastrography (EGG) is a non-invasive method to detect gastric electrical activity (GEA). Type 1 diabetes occurring at early age allows to study GEA abnormalities without interference of aging. Our aims are to study the presence of early GEA disturbances in patients with type I diabetes and to test the correlation between GEA and glycaemia levels, duration of disease and presence of AN. 37 patients with type-1 diabetes (17 m; 23$54 \mathrm{y}$ ), diagnosed from more than 5 years, without dyspeptic symptoms, 34 dyspeptics (15 $\mathrm{m}, 20-51 \mathrm{y})$ and 10 healthy volunteers $(7 \mathrm{~m}, 23-37 \mathrm{y})$ that served as positive and negative controls were studied. After an overnight fasting, all subjects underwent multichannel EGG study $30 \mathrm{~min}$ before and after a standard liquid meal $(300 \mathrm{kcal})$. Pre and postprandial percentage and power of normogastria $(2-4 \mathrm{cpm})$, bradygastria, tachygastria and arrhythmias were calculated by running spectral analysis. In diabetics AN was assessed by ECG beat-tobeat variation analysis and lying-to-standing blood pressure variations. Dyspeptic symptoms were assessed by a standardized questionnaire. Data were expressed as mean \pm SD. Diabetics had a significantly higher rate of gastric arrhythmias than dyspeptics and controls during the fasting phase $(27+16 \%, 18+11$ and $20+14, \mathrm{p}<0.05)$ especially at the pace-maker recording site. In a longer history of diabetes $(>20 \mathrm{y})$ percentage of pre-prandial normogastria was lower than in subjects with a history $<10$ y $(59 \pm 15$ vs $79 \pm 15, p<0,01)$. Fasting glycaemia $>200 \mathrm{mg} / \mathrm{dl}$ correlated with a higher percentage $(8,4 \pm 5,9$ vs $4,2 \pm 4,8, \mathrm{p}<0,05)$ and power $(44,5 \pm 15,8$ vs $33,7 \pm 15,3, \mathrm{p}<0,05)$ of postprandial bradygastria. Presence of AN correlated with lower fasting normogastria power $(22 \pm 8$ vs $33 \pm 17 \mathrm{p}<0,05)$ and postprandial bradygastria percentage $(4,1 \pm 4,8$ vs $10,9 \pm 6,5, p<0,001)$. We confirmed that gastric electric activity is altered in diabetics. Type I diabetics without dyspepsia had gastric arrhythmias and increased power of bradygastria. These events mainly occurred at the gastric pacemaker region and were associated with disease duration, severity of hyperglycaemia and AN. Gastric dysrhytmias precede dyspeptic symptoms appearance and probably represent an early gastric neuromuscolar injury.

\section{M2207}

Gastric Emptying Response to Temporary Gastric Electrical Stimulation Is Independent of Symptom Improvement But Dependent On Baseline Emptying Thomas L. Abell, Anil Minocha, Nighat Abidi

Introduction: Gastric electrical Stimulation (GES) is an accepted therapy for drug refractory gastroparesis, but its effect on gastric emptying is controversial. Patients: To examine the effect of GES on GET we examined 140 consecutive patients undergoing temporary endoscopic GES as previously described (GIE 2005: 61:455-461). Pts were $29 \mathrm{~m}, 11 \mathrm{l}$, mean age 41 yrs with diagnosis (DX): 44 diabetes (DM), 14 post-surgical (PS) and 82 idiopathic (ID). Methods: Symptom Assessment, Gastric emptying and EGG were evaluated at baseline and after temporary endoscopic GES (mean 3 days of therapy). Patients at baseline were stratified into 3 groups based on GET: Delayed, Normal and Rapid, as previously described (AJG 2004: 99: S4l-42). Symptoms (SX) of nausea (N), vomiting (V), and total SX (TSS), and GET were compared by paired t-tests and reported as mean values. Results: All patients had Symptoms improvement, irrespective of their DX or baseline GET:( N: 3.5 baseline to 0.9 after; V: 2.5 to 0.6 ; TSS: 15.2 to $5.1, \mathrm{p}<0.0001$ for all patients with similar changes in subgroups.) There were no significant changes in GET for the whole patient group. However, when stratified by baseline GET: the delayed group accelerated and the rapid group slowed (see table). Conclusion: Temporary GES performed on patients with the SX of gastroparesis shows an immediate symptom improvement, which is independent of baseline GET. The effect of temporary GES on gastric emptying is dependent on baseline emptying, with improvements seen in both delayed and rapid gastric emptying sub-groups. Investigations into the effect of electrical stimulation on gastric emptying need stratification for baseline gastric emptying.

\begin{tabular}{|c|c|c|c|c|c|c|}
\hline Group & B GET 1 & B GET 2 & B GET 4 & AFT GET 1 & AFT GET 2 & AFT GET 4 \\
\hline All $(\mathrm{n}=140)$ & 66.6 & 43.9 & 20.7 & 66.3 & 44.1 & 20.6 \\
\hline Delay $(\mathrm{n}=78)$ & 77.3 & 57.3 & 31.6 & 57.1 & 41.1 & 21.3 \\
\hline P. Value & --- & --- & --- & 0.04 & 0.08 & 0.18 \\
\hline Rapid (n=15) & 27.3 & 9.45 & 2.7 & 30.5 & 14.3 & 3.1 \\
\hline P value & --- & --- & --- & 0.08 & 0.04 & 0.4 \\
\hline
\end{tabular}

\section{M2208}

Effects of Erythromycin and Tegaserod On Upper Gut Motility - A Comparative Study Issam Nasr, Askok Attaluri, Konrad Schulze, Robert Summers, Satish Rao

BACKGROUND \& AIM: Both Erythromycin and Tegaserod are increasingly used for their prokinetic effects on the upper gut. To date, the effects of these agents on the stomach, duodenum and jejunum have not been directly compared. Our aim was to assess and compare the effects of Erythromycin and Tegaserod on upper gut motility in an open label study. METHODS: 22 patients $(\mathrm{M} / \mathrm{F}=4 / 18$; mean age $=37 \mathrm{yrs})$ with upper gut dysmotility underwent 24 hour manometry using a 6 sensor solid state probe, with 2 sensors located in each of the antral, duodenal and jejunal segments. On day 1 , in the fasted state, all patients received $125 \mathrm{mg}$ of intravenous Erythromycin Lactobionate, and on day 2, $12 \mathrm{mg}$ of oral Tegaserod. Baseline ( $30 \mathrm{~min}$ prior to drug) and post-drug motility changes were analyzed (Erythromycin for $90 \mathrm{~min}$, Tegaserod for $180 \mathrm{~min}$ ) by measuring the Area Under the Curve $(\mathrm{AUC})$ of pressure waves at each sensor and by examining motor patterns.
RESULTS: Both drugs increased motor activity in all 3 segments compared to baseline period (table), but after Erythromycin the motor response was significantly higher $(\mathrm{p}<0.05)$ in the antrum and occurred within the first $30 \mathrm{~min}$, whereas after Tegaserod it was higher $(\mathrm{p}<0.05)$ in the jejunum and occurred during the 2nd and 3rd hours. Following Erythromycin, large amplitude (> $100 \mathrm{mmHg}$ ) antral contractions at 3 cycles/min were seen whereas after Tegaserod a 'fed-response' like pattern was seen. Following Erythromycin and Tegaserod, Phase III MMCs were seen in 12(55\%) and 10(41\%) patients, respectively (p> 0.05 ). CONCLUSIONS: Both drugs increase upper gut motility, and induce MMC's, bu exert a differential response. Erythromycin predominantly increases antral motor activity but its effect is short lived, in part because the drug was given intravenously. In contrast, Tegaserod induces a more pronounced prokinetic effect in the jejunum and causes a more sustained increase in upper gut motor activity.

\begin{tabular}{|c|c|c|c|c|c|c|c|c|}
\hline \multicolumn{9}{|c|}{ AUC in $\mathrm{mm} \mathrm{Hg} \sec \times 10^{3}$ (\% } \\
\hline \multirow[t]{2}{*}{ Segment } & \multirow[b]{2}{*}{ Baseline } & \multicolumn{3}{|c|}{ Eryhtromycin } & \multirow[b]{2}{*}{ Baseline } & \multicolumn{3}{|c|}{ Tegaserod } \\
\hline & & 30 & 60 & 90 & & 60 & 120 & 180 \\
\hline Antrum & 22 & $33(53 \%)$ & $29(326)$ & 21(-4\%) & 24 & $28(144)$ & $28(14 \%)$ & $30(24 \%)$ \\
\hline Duadenum & 21 & $25(18 \%)$ & $28(3456)$ & $19(-9 \%)$ & 27 & $29\left(66^{\circ}\right)$ & $32\left(166_{5}\right)$ & $34\left(255_{0}\right)$ \\
\hline Jejunum & 18 & $2(189 \%)$ & $24\left(399^{8}\right)$ & $17(-5 \%)$ & 18 & $22(22 \%)$ & $25(3885)$ & $29(61 \%)$ \\
\hline
\end{tabular}

\section{M2209}

Characteristics of Symptoms and Functional Gastric Tests in Patients Referred for Electrogastrography (EGG)

Deborah M. Bethards, Lihua Xu, Kwangmi Ahn, Kenneth L. Koch, Ann Ouyang

The association between symptoms (Sx) and tests of gastric function remains unclear. The aims of this study were to determine whether specific clinical presentations and the histor of gastroesophageal reflux disease (GERD) were associated with gastric emptying (GET) or electrogastrogram (EGG) parameters. Charts of 163 patients (39 M,124 F) who underwent both GET and EGG were reviewed for the clinical Sx of nausea, pain, vomiting, bloating and altered bowel habits as well as weight and diabetes. Sx were scored as absent/mild or moderate/severe. The GET at $1 \mathrm{hr}$ and $2 \mathrm{hr}$, the EGG final diagnosis as well as the percent power in the frequencies $1-2.5 \mathrm{cpm}, 2.5-3.75 \mathrm{cpm}, 3.75-10 \mathrm{cpm}$ and $10-15 \mathrm{cpm}$ at baseline and volume of water tolerated during the water load test during the EGG were noted. Data is reported as median, 5-95 percentile. Comparisons were performed using the WilcoxonMann-Whitney and Chi-Squared tests. Results: Women(F) complained of pain more than men (M) and more men had altered bowel habits. Sx complaint data F:M: Pain 70\%:43\% ( $\mathrm{p}=0.003$ ), Nausea $89 \%: 85 \%$, Vomiting $58 \%: 54 \%$, Bloating $46 \%: 31 \%$, Normal bowel habits $43 \%: 23 \%(\mathrm{P}=0.02)$. Women weighed less than men and drank less during the water load test (480 vs $604 \mathrm{ml}, \mathrm{p}=0.001$ ). Gastric emptying was slower in women than men: at $2 \mathrm{hr}$ the $\%$ emptied was $63 \%, 7-97$, (median, 5-95 percentile) in women and $78 \%, 27-99$, in men, $\mathrm{p}<0.001$. There was no major difference in EGG diagnoses between women and men. When comparing diabetic patients vs nondiabetic, diabetic patients were older (52 vs 42 $\mathrm{p}<0.01$ ) and weighed more ( $187 \mathrm{lb}$ vs $146 \mathrm{lb}, \mathrm{p}<0.0001)$. Nondiabetics had more norma baseline EGG frequency spectra with a tendency towards baseline bradygastria in diabetics. Overall, the Sx of pain was associated with a lower volume of water tolerated on water load test, $450 \mathrm{ml}, 150-750$ vs $600 \mathrm{ml}, 250-750, \mathrm{p}=0.002$. The $\mathrm{Sx}$ of pain was associated with delayed gastric emptying at one hour but the relationship did not persist when adjusted for gender. No association between the other Sx and GET was noted. No Sx complaint was associated with any specific final EGG diagnosis. As expected, a complaint of GERD was associated with greater weight $(156 \mathrm{lb}$ vs $140 \mathrm{lb}, \mathrm{p}<0.05)$ but not with a greater delay in GET or any specific EGG characteristics. Conclusions: Gastric emptying was slower in women than in men. Pain was a more common presentation in women in the patients referred for study and the association of pain with a lower tolerated water load volume suggests that this symptom is related to an abnormal sensory process. These finding suggest a different therapeutic approach to patients in whom pain is a major complaint.

\section{M2210}

Pathophysiological Studies of Gastrointestinal Manifestations of Addison's Disease

Hubert Piessevaux, Dominique Maiter, Yves Horsmans

Background: Upper GI symptoms are frequent in Addison's disease. The mechanism leading to these symptoms is unknown. Aim: To study upper GI motor physiology in patients with Addison's disease before and after chronic glucocorticoid replacement therapy. Patients and methods: Three patients (A: F 42y; B: M 25 y; C: M 42 y) recently diagnosed with Addison's were studied in our motility lab. Esophageal manometry, 24-hour small bowel manometry, gastric emptying and gastric barostat studies were performed before and after initial replacement therapy as accepted by the patients. Results: Esophageal manometry $(n=2: A \& B)$ showed severely decreased wave amplitudes $(12+/-5 \mathrm{mmHg}$ ) when peristaltism was still presen (20\% of swallows). The LOS resting pressure was normal (17 and $12 \mathrm{mmHg}$ respectively) and relaxation was complete. After substitution both studies were completely normalized. Small-bowel manometry $(n=1: A)$ showed reversible antral hypomotility, with normal conversion to the fed pattern. Scintigraphic gastric emptying studies $(n=3)$ showed completely reversible severe gastroparesis (mean $\mathrm{tl} / 2$ for solids $243 \mathrm{~min}$ ). Gastric barostat (patient B\&C) showed normal sensitivity to distension and defective accommodation. No control study was performed. Conclusions: Myogenic motor abnormalities of the upper GI tract may be responsible for upper GI symptoms in Addison's disease. These findings are reversible after substitution 


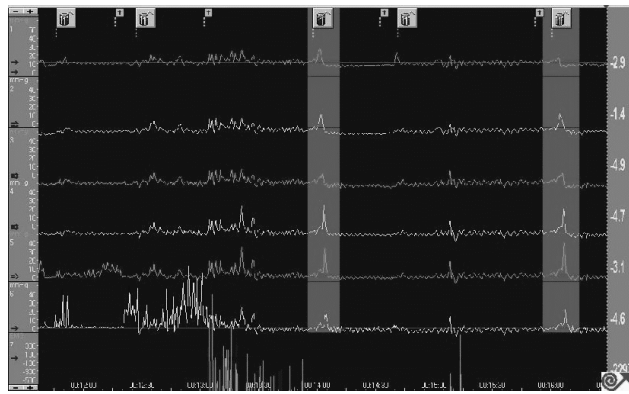

initial

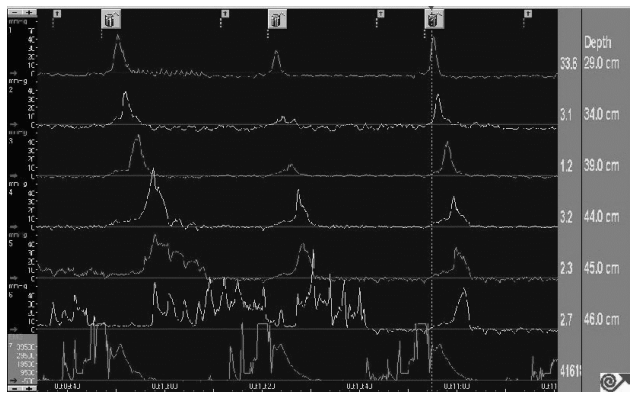

after R/

M2211

Electrogastrography Is of Limited Value in the Diagnosis of Motility Disorders Shahab Abid, Greger Lindberg

Electrogastrography (EGG) is a technique for recording gastric myoelectric activity. Conflicting results were obtained in previous studies when EGG was applied in motility disorders of the stomach associated with various systemic diseases. Aim of the study. To investigate whether the underlying diagnosis or the presence of symptoms could be predicted from EGG parameters. Material and methods. This is a retrospective analysis of patients with motility disorders who underwent EGG as part of their clinical work-up. A single bipolar channel measured the EGG. The ability of EGG to identify diagnostic groups and epigastric symptoms was tested by comparing EGG parameters for each diagnostic group or symptom against all other patients in the sample. Results. A total of 151 patients, 125 (83\%) females, mean age $45 \pm 14$ years were analysed. There were 52 patients with irritable bowel syndrome (IBS), 22 with enteric dysmotility, 26 with slow transit constipation (STC), 11 with chronic intestinal pseudo-obstruction (CIP), 13 with gastroparesis or functional dyspepsia, 3 postsurgical, and 24 patients with other diagnoses. In general, the discriminatory power of EGG for underlying motility disorder was low. Only those with STC showed a reasonable number of differences in EGG parameters compared to all other groups (Table). Patients with IBS had lower fasting and postprandial variability of the dominant frequency (DF) and a higher percentage of postprandial time with normogastric DF and those with CIP had higher power in normal and tachygastric ranges during fasting and a higher fasting period dominant power. Among the symptoms, vomiting was associated with a higher postprandial normal power $(4.2 \pm 0.6$ vs. $3.9 \pm 0.5)$, a higher postprandial period dominant power $(2.6 \pm 0.7$ vs. $2.3 \pm 0.5)$ and a lower postprandial percentage in bradygastric range. Conclusion. The ability of EGG to identify motility disorders as well as symptoms in general was poor. EGG findings in patients with STC require validation.

Significant EGG variables in slow transit constipation (STC) and other diagnoses

\begin{tabular}{|c|c|c|}
\hline EGG parameter & STC $\mathbf{n = 2 6}$ & Other diagnoses $\mathbf{n = 1 2 5}$ \\
\hline Postprandial normal power $^{\S}$ & $3.7 \pm 0.4$ & $4.0 \pm 0.5$ \\
\hline Postprandial tachygastria power $^{\S}$ & $3.5 \pm 0.4$ & $3.7 \pm 0.4$ \\
\hline${\text { Percentage of postprandial time with bradygastric } \mathrm{DF}^{\dagger}}^{\dagger}$ & $2(0-20)$ & $0(0-17)$ \\
\hline${\text { Percentage of postprandial time with normogastric } \mathrm{DF}^{\dagger}}^{\dagger}$ & $87(56-100)$ & $96(0-100)$ \\
\hline${\text { Percentage of postprandial time with tachygastric } \mathrm{DF}^{\dagger}}^{\dagger}$ & $9(0-33)$ & $3(0-100)$ \\
\hline Postprandial DFIC $^{\dagger}$ & $26(8-72)$ & $18(2-77)$ \\
\hline Postprandial PDP $^{\S}$ & $2.1 \pm 0.6$ & $2.4 \pm 0.5$ \\
\hline Power ratio $^{\S}$ & $0.2 \pm 0.5$ & $0.4 \pm 0.5$ \\
\hline
\end{tabular}

$\mathrm{DF}=$ dominant frequency; $\mathrm{DFIC}=$ dominant frequency instability coefficient $; \mathrm{PDP}=$ period dominant power; $\S=\log$ values; $\dagger=$ median (range)
M2212

The Role of Gastric Sensorimotor Dysfunction in Gastrointestinal Symptoms and Energy Intake in Liver Cirrhosis

Evangelos Kalaitzakis, Magnus Simren, Hasse Abrahamsson, Einar Bjornsson

Altered gastric sensorimotor function is related to the symptomatology in several gastrointestinal (GI) diseases. GI symptoms in liver cirrhosis may result in low energy intake contributing to malnutrition and increased morbidity. We aimed to investigate gastric accommodation to a meal and sensitivity to gastric distension with reference to energy intake, nutritional status and GI symptoms in cirrhosis. Methods: 16 cirrhotics (median age 56 (IQR 48-61); 3 female; 7 with alcoholic cirrhosis; 3 with mild ascites; Child-Pugh score 7(6-9); MELD score 9.5 (7.5-13.8) underwent a gastric barostat to assess gastric accommodation to a caloric $200 \mathrm{ml}$-drink. Gastric accommodation was expressed as the difference of the area under the time-volume curve between the post- and pre-prandial periods. Sensory thresholds for first perception and discomfort were evaluated. Patients underwent also a satiety drinking test, where a caloric drink $(1.5 \mathrm{kcal} / \mathrm{ml})$ was ingested at a constant rate, $15 \mathrm{ml} / \mathrm{min}$, until maximal satiety. A validated questionnaire was used to measure GI symptoms in cirrhotics. Energy intake was assessed by a dietary diary and nutritional status by anthropometry and estimation of weight loss in the previous 6 months. 15 healthy subjects (age 52 (49-62); 5 female) served as controls. Results: Cirrhotics compared to controls had increased gastric accommodation $(1985 \mathrm{ml} \times \min (1337-2776)$ vs. $1025 \mathrm{ml} \times \min (353-2137) ; \mathrm{p}<0.05)$ but lower daily energy intake $(21 \mathrm{kcal} / \mathrm{kg}(15-29)$ vs. $26.5 \mathrm{kcal} / \mathrm{kg}(24.5-37.5) ; \mathrm{p}<0.05)$. Patients with vs. without compromised nutritional status had increased gastric accommodation ( $2776 \mathrm{ml} \times \mathrm{min}$ (2197$3605)$ vs. $1813 \mathrm{ml} \times \min (692-2451) ; \mathrm{p}<0.05)$. Gastric accommodation was related to daily energy intake in controls $(r=0.67, p<0.05)$ but not in cirrhotics $(r=0.2 ; p>0.4)$. The endpoint of the satiety test was inversely related to gastric volumes before $(r=-0.7, p<0.005)$ and after the meal $(\mathrm{r}=-0.55, \mathrm{p}<0.05)$ in cirrhotics. Mean postmeal gastric balloon volumes were related to the Child-Pugh $(r=0.57, p=0.026)$ and MELD $(r=0.61, p=0.017)$ scores. Sensory threholds did not differ between patients and controls $(\mathrm{p}>0.05)$. In cirrhotics, discomfort thresholds were inversely related to GI symptom severity $(\mathrm{r}=-0.63, \mathrm{p}=0.01$ ), and the Child-Pugh ( $\mathrm{r}=-$ $0.64, \mathrm{p}<0.01)$ and MELD $(\mathrm{r}=-0.54, \mathrm{p}<0.05)$ scores. Conclusion: Gastric accommodation is increased in cirrhotics compared to controls and its relation to energy intake seems to be disturbed. The satiety drinking test is not a good surrogate marker of accommodation in cirrhotics. In cirrhosis gastric sensitivity is related to severity of GI symptoms and cirrhosis severity indices.

\section{M2213}

Management of Patients with Constipation Within a Physician Group Setting Allison Egger, Vickie Andros, Ken Wong, Annamaria Cerulli, Vipin Popat

Background: Constipation affects up to $27 \%$ of North Americans and has been shown to significantly reduce patient quality of life. The objectives of this study were to assess the characteristics of patients with constipation and examine the management of patients with this condition in a physician group setting. Methods: Retrospective claims data and medical record review of patients with constipation was conducted at 3 large physician group practices consisting mostly of primary care physicians. A random sample of 300 patients per group, between the ages of 18-79, were identified through outpatient billing records using International Classification of Diseases, Ninth Revision, Clinical Modification (ICD-9-CM) codes from June 2003 to July 2004. Subsequent medical record review was conducted; data collected included patient demographics, specialist referral, other gastrointestinal (GI)-related symptoms, GI-related procedures, and treatment. Patients were excluded from the review if they were deceased, had received treatment with tegaserod, or had a primary or secondary claim for intestinal tract infections, malignancies, inflammatory conditions, or history of malabsorption in the previous 12 months. Results: A total of 738 patients were identified with constipation and were eligible for medical record review. Mean patient age was 51.4 years; $67.8 \%$ of patients were female. Physician-confirmed diagnosis of constipation was noted in $74 \%$ of the medical records versus outpatient billing claims; however, 1 physician group had confirmed diagnosis in only $26.5 \%$ of medical records. Almost half of patients (42\%) were referred to a GI specialist; of these, $76.2 \%$ had constipation diagnosis confirmed in their chart. Patients reported having 2 other GI-related symptoms on average; abdominal pain was the most commonly reported GI-related symptom (48\%). Other symptoms included straining (26.2\%), gastroesophageal reflux disease-related (19.5\%), and bloating (19.2\%). Patients underwent 1 GI-related procedure on average; colonoscopy was the leading GIrelated procedure in $26.7 \%$ of patient records, followed by pelvic and/or abdominal radiology imaging $(19.5 \%)$ and computed tomography $(12.9 \%)$. Thirty-four percent $(n=251)$ of patients had at least 1 constipation-related office visit in the previous 12 months. No documentation of drug use was available in $64.8 \%$ of patients' records. Conclusions: Our study found physician documentation of constipation may vary by physician group. Patients reported multiple GI-related symptoms; $48 \%$ reported abdominal pain. The variety of GI-related symptoms reported indicates that treatment of constipation may require a multi-symptom approach

M2214

A Multi-Component Model Can Predict Gastric Emptying in Diabetic Gastroparesis

Naeem Aslam, Hani Rashed, William D. Johnson, Thomas L. Abell

Introduction: We have previously reported that a multi-component model of autonomic and enteric factors can help predict gastric emptying in diabetes mellitus. Patients: We studied 87 patients (38 males, 49 females; mean age 39 years) with Diabetes Mellitus presenting with signs and symptoms of Gastroparesis. Methods: We profiled the patients by autonomic nervous sytem testing including adrenergic tests by VC (vasoconstriction to cold) and PAR (postural ratio), and cholinergic tests by EKG R to R interval (RRI). The enteric nervous system measure (EGG) was measured by cutaneous electrogastrography. (Previously described: DDS, 1999; 44:74-78). These data were compared to results of standardized solid gastric emptying reported as T50. Results: Patients were distributed in 5 groups based on gastric emptying 
time (GET): $28 \%$ very delayed, $15 \%$ delayed, $22 \%$ normal, $17 \%$ rapid and $18 \%$ very rapid. There were statistically significant differences among the 5 group means for VC and RRI but not for EGG and PAR.(See table). Conclusions: A multi-component model demonstrates specific autonomic and enteric differences in subgroups of diabetic gastroparesis. Therapies for diabetic gastroparesis, including drugs and devices, may need to take into account these physiologic differences when looking at therapeutic outcomes.

Means

\begin{tabular}{|c|c|c|c|c|c|}
\hline GET & T50S & VC & RRI & EGG & PAR \\
\hline VERY DELAYED & 258.0 & 41.7 & 11.2 & 3.25 & 24.2 \\
\hline DELAYED & 202.6 & 60.8 & 9.2 & 3.46 & 33.7 \\
\hline NORMAL & 152.5 & 57.7 & 15.6 & 3.11 & 22.6 \\
\hline RAPID & 113.1 & 52.9 & 11.4 & 3.53 & 20.2 \\
\hline VERY RAPID & 67.6 & 75.3 & 25.1 & 3.38 & 27.9 \\
\hline P VALUES & --- & $<0.002$ & $<0.004$ & $>0.33$ & $>0.62$ \\
\hline
\end{tabular}

\section{M2215}

A Novel Method for the Assessment of Gastric Motility in Patients Using Wireless Capsule Endoscopy

Kishore Gaddipati, Henry Parkman, Benjamin Krevsky

Wireless capsule endoscopy (WCE), first FDA approved in August 2001 for diagnosis of obscure GI bleeding, is being increasingly used for the evaluation of esophageal, gastric, and small intestinal pathology. However, WCE has not been used previously to assess gastric motility. The aim of this study was to determine if WCE could be used as an investigative and perhaps clinical modality to simultaneously image and quantitate gastric motility. Methods: We analyzed gastric contractile activity on a retrospective basis by reviewing 25 WCE recordings performed between 2003-2005 (PillCam ${ }^{\text {TM }}$ SB, Given Imaging). No patients were known to have a gastric motility disorder. Periods of fasting gastric slow wave activity were identified by searching for repetitive contraction patterns of greater than 3 complete cycles in length. The length and frequency of the cycles, \% of gastric imaging time with recognized slow wave activity, and esophageal, gastric, and small bowel transit times were determined. A second observer reviewed several cases to determine interobserver variability. Results: 7 males and 18 females were evaluated; mean age $=63$ years. All subjects underwent WCE for the evaluation of occult GI bleeding. Two of the patients were diabetic. The capsule remained $>8$ hours in the stomach in 2 patients and did not reach the small intestine in 2 patients. For the remaining 21 patients the average gastric contractile activity was $3.1 \pm 0.1$ $($ mean $\pm S E M)$ cycles $/ \mathrm{min}(\mathrm{cpm})$, with a range of 2.4 to $5.5 \mathrm{cpm}$. The gastric contractile activity constituted $24.9 \pm 3.7 \%$ of the overall gastric transit phase. Average esophageal transit time was $141 \pm 60 \mathrm{sec}$, gastric transit time was $21.7 \pm 3.9 \mathrm{~min}$, and small bowel transit time was $3.9 \pm 0.3 \mathrm{hr}$. These transit times excluded patients with gastric (2) and small bowel (2) capsule retentions. No obvious gastric or small bowel MMC activity was identified. The average cycles/min determination for the 2 observers differed by less than $4 \%$ (NS). Conclusions: This is the first report of the assessment of gastric contractile activity using wireless capsule endoscopy. The average fasting periodic contractile activity in our subjects was 3.1 $\mathrm{cpm}$, which correlates well with established values of $2.7-4.2 \mathrm{cpm}$ in healthy individuals as determined by electrogastrography. Wireless capsule endoscopy may have a novel application as a complimentary test to scintigraphic emptying tests, manometry, and electrogastrography in the evaluation of gastric dysmotility. It permits a quantitative analysis of fasting contractile and emptying activity with simultaneous endoscopic imaging using a minimally invasive modality.

\section{M2216}

Acute Cold Stress Impairs Postprandial Gastric Accommodation in Healthy Humans

Maria Maluenda, Jordi Serra, Fernando Azpiroz, Juan R. Malagelada

Patients with functional dyspepsia often complain that postprandial symptoms are precipitated or worsened by stress. Previous studies have shown that either nutrients or stress alone induce reflex relaxation of the stomach, but the interaction of concomitant nutrients and stress is unknown. Thus, our present aim was to establish the effect of acute stress on nutrient-induced gastric relaxation. METHODS. In 8 healthy subjects ( 3 female, 5 men) gastric tone variations were quantified as isobaric volume changes using a barostat. Gastric tone was monitored: a) during a 15-min basal period; b) during a 30-min duodenal nutrient infusion mimicking normal gastric emptying (Ensure ${ }^{\circledR} 2 \mathrm{Kcal} / \mathrm{min}$ ) and c) during duodenal nutrient infusion plus two consecutive periods of either cold stress or sham stress, applied in random order (hand immersion in $4^{\circ} \mathrm{C}$ or $30^{\circ} \mathrm{C}$ water for $10 \mathrm{~min}$, respectively). RESULTS. During basal recording, intragastric volume showed minimal variations $(-10 \pm 7 \%$ volume change after $15 \mathrm{~min}$; NS). Nutrient infusion was followed by a progressive relaxation of the stomach, that reached its maximum after $20 \mathrm{~min}$ (volume increased by $200 \pm 57 \mathrm{ml} ; \mathrm{p}<0.05$ vs basal), and thereafter plateaued $(-10 \pm 15 \%$ volume change; NS). Application of cold stress during duodenal nutrient infusion produced an immediate contraction of the stomach that after 2 min had completely blocked the nutrient-induced gastric relaxation (volume reduced by $205 \pm 44 \mathrm{ml} ; \mathrm{p}<0.05$ vs nutrients alone), remaining steady thereafter $(10 \pm 35 \%$ volume variation; NS). Sham stress (hand immersion at $30^{\circ} \mathrm{C}$ ) induced no significant effects on gastric tone (volume reduced by $85 \pm 50 \mathrm{ml}$; NS vs nutrients alone). CONCLUSION. Acute cold stress antagonizes the response of the stomach to nutrients, resulting in defective gastric accommodation. These observations may have important pathogenetic implications in patients suffering from functional dyspepsia.

\section{M2217}

Influence of Naloxone On Gastric Sensitivity and On Gastric Response to a Meal in Health

Brecht Geeraerts, Lukas van Oudenhove, Rita Vos, George Karamanolis, Jan Tack

BACKGROUND: It has been suggested that failure of descending anti-nociceptive pathways (with opioids as the main candidate neurotransmitters) contributes to the pathogenesis of functional disorders, including functional dyspepsia (FD). Barostat studies of the proximal stomach have revealed hypersensitivity to gastric distension and impaired accommodation to ingestion of a meal in FD. The AIM of the present study is to investigate the effect of suppression of endogenous opioid action by naloxone on the sensorimotor function of the proximal stomach in normal volunteers. METHODS: Ten healthy subjects (mean age 29.0 1.7 years) participated in a placebo-controlled, double blind, and randomized gastric barostat study to assess sensitivity to distension and meal accommodation. Two stepwise distensions ( 2 mmHg steps at 2 minute intervals, until discomfort or pain) separated by 30 minutes of adaptation were followed by 90 minutes of gastric tone measurement. Thirty minutes after the second distension a standardized meal (Nutridrink $§, 200 \mathrm{ml}, 300 \mathrm{kcal}$ ) was ingested Continuous infusion of saline or naloxone $(20 \mu \mathrm{g} / \mathrm{kg} / \mathrm{h}$ after $0.4 \mathrm{mg}$ bolus) was started 15 minutes before the 2 nd distension.. Perception during distensions was scored on $0-6$ graded scales $(0=$ no sensation, $6=$ maximal) and VAS scales were used to quantify individual dyspeptic symptoms. RESULTS: Naloxone did not influence gastric compliance ( $43.5 \pm 5.3$ vs. $46.4 \pm 6.4$ $\mathrm{ml} / \mathrm{mmHg}$, NS) and sensitivity to gastric distension compared to saline. Consequently, the pressures needed to induce first perception (score $=1)(11.7 \pm 0.9$ vs. $12.4 \pm 1.0 \mathrm{mmHg}$, NS) or discomfort (score $\geq 5)(19.7 \pm 1.0$ vs. $19.2 \pm 0.9 \mathrm{mmHg}$, NS) and corresponding intraballoon volumes (respectively $218 \pm 46$ vs. $319 \pm 66$ and $565 \pm 74$ vs. $643 \pm 71 \mathrm{ml}$, NS) did not differ between saline and naloxone. Naloxone did alter VAS scores for pain or discomfort (AUC respectively $22.2 \pm 9.9$ vs. $24.5 \pm 9.2$ and $37.8 \pm 11.4$ vs. $43.1 \pm 11.0 \mathrm{~mm} * \mathrm{mmHg}$, NS) Naloxone did not alter preprandial gastric volumes $(202 \pm 20$ vs. $203 \pm 33 \mathrm{ml}$, NS), but gastric accommodation to a meal (volume increase after the meal) was significantly lower during naloxone compared to placebo (two-way ANOVA for repeated measures, $\mathrm{p}=0.001$ ). The intensity of epigastric symptoms after meal intake did not differ between placebo and saline. CONCLUSION: In healthy subjects, naloxone does not alter gastric compliance or sensitivity to distension, but inhibits gastric accommodation to a meal. These observations suggest that the endogenous opioid system is involved in control of gastric tone rather than sensitivity.

\section{M2218}

Multi-Channel Electrogastrography (MEGG) in Patients with Functional Dyspepsia

Weihong Sha, Nonko D Pehlivanov, P Jay Pasricha, Jiande Chen

The aim of this study is to assess differences in gastric slow waves measured by a newly approved multi-channel electrogastrogram (MEGG) device between patients with functional dyspepsia and healthy controls. Materials and Methods: 64 patients $(20 \mathrm{M}, 44 \mathrm{~F}$, mean age 46.1, Range 14-79) with functional dyspepsia were assessed for severity of upper gastrointestinal symptoms. Gastric myoelectrical activity of this group of patients and 16 health volunteers ( $8 \mathrm{M}, 8 \mathrm{~F}$, mean age 40.9 . range 20-68) was measured by surface multichannel electrogastrography for one hour in the fasting state and for two hours after a solid meal (Turkey sandwich-450 cals.). Computerized spectral analysis methods were utilized to compute the following EGG parameters in the fasting and fed state: dominant frequency (DF)/power (DP), \% of 2-4 cycles/min slow waves, \%of tachygastria, \% of bradygastria, \%o arrhythmia and slow wave coupling. Common gastrointestinal symptoms were assessed for symptom severity and graded. Results: 1) EGG was abnormal in 70.3\% of patients. Response to the test meal was abnormal (a decrease in dominant power) in $32.8 \%$ of patients. 2) Compare with the controls, the patients had a significant decrease in \% of normal slow waves and a significant increase in $\%$ of tachygastria both in fed state. No significant difference was recorded in any other EGG parameters. 3) The response to the test meal was similar between the controls and patients. That is, postprandially, there was a significant increase in DF and DP, but no significant changes in \% of normal slow waves, \%of dysrhythmias or slow wave coupling. 4) This group of patients had high symptom scores particularly to nausea, upper abdominal pain and bloating, with an average symptom score of 2.5/4 for nausea; $2.3 / 4$ for upper abdominal pain and 2.1/4 for bloating. The mean total score was $21 / 52$ for all symptoms. However, no significant correlation was noted between the EGG and the symptoms. Conclusions: EGG is abnormal in most FD patients. Postprandial percentage of normal slow waves and postprandial percentage of tachygastria are more sensitive than other parameters of EGG in distinguishing FD patients from healthy controls.

\section{M2219}

Measurement of Changes in Intragastric Pressure During Air Insufflation in Patients with Functional Dyspepsia - Evaluation of Gastric Function Using a Pharmacological Load

Tsuyoshi Suzuki, Masanori Hirano

(Aims)Visceral hypersensitivity and impaired gastric relaxation are thought to play a part in functional dyspepsia (FD). We previously reported that visceral hypersensitivity and impaired gastric relaxation were readily assessed through the measurement of changes in intragastric pressure (IP) during air insufflation as part of upper gastrointestinal tract endoscopy (UGITE) (JIMR 33(2),160-9,2005). In this study, we further examined the effects of the administration of antispastic agents to patients with FD. (Methods)The 30 FD patients and 18 healthy controls were enrolled. During UGITE, we induced gastric distension by air insufflation with epigastric symptoms, and measured IP using a pressure transducer introduced through the instrument channel. The parameters calculated were basal IP (mmHg), IP at onset of symptoms (threshold value: $\mathrm{mmHg}$ ), time to reach threshold value (sec) and rate of increase in IP ( $\mathrm{mmHg} / \mathrm{sec}):(0-5 \mathrm{mmHg}$ gradient and $5-10 \mathrm{mmHg}$ gradient). A pharmacological load was administered in the form of atropine sulfate $(0.5 \mathrm{mg})$ to 3 controls and $3 \mathrm{FD}$ patients, scopolamine hydrobromide $(20 \mathrm{mg})$ to 4 controls and 6 FD patients, and glucagon ( $1 \mathrm{mg}$ ) to 4 controls and $8 \mathrm{FD}$ patients. (Results) 1) Although no difference 
was detected between the control and FD in basal IP, pressure at threshold (21.5 vs 26.6 $\mathrm{mmHg}$ ) and time to threshold (45.0 vs $66.5 \mathrm{sec}, \mathrm{P}=0.002$ ) tended to be lower in the FD than in the control group. The rate of increase in IP tended to be higher in the FD than in control for both the $0-5 \mathrm{mmHg}$ and $5-10 \mathrm{mmHg}$ gradients $(0.52$ vs $0.33 \mathrm{mmHg} / \mathrm{sec}$ and 0.30 vs $0.19 \mathrm{mmHg} / \mathrm{sec}$ ). 2) Pharmacological load (i) The time to threshold tended to decrease in control, and increase in the FD, following the atropine load. (ii) The rate of increase in IP tended to be lower in both groups following the scopolamine load. The time to threshold was unchanged in control, but tended to be longer in the FD (from 45.8 to $52.6 \mathrm{sec}$ ). (iii) The rate of increase in IP tended to be lower in both groups following the glucagon load at the $0-5 \mathrm{mmHg}$ gradient. The time to threshold was unchanged in control, but tended to be longer in the FD (from 44.2 to $55.4 \mathrm{sec}$ ). (Conclusions) We attempted to evaluate of gastric function using a pharmacological load during air insufflation, finding different responses to antispastic agents in control and FD. Furthermore, the response to an atropine load was different to those to the other two agents in FD.

\section{M2220}

Evaluation of Gastrointestinal Motility in Vivo Using Fluoroscopy

Kent C. Williams, Robia G. Pautler, Norman Weisbrodt, Margaret E. Conner

Currently, evaluation of gastrointestinal motility often utilizes invasive or ex-vivo methodologies that are not feasible for many models and limit longitudinal analyses of motility. In light of this, we tested whether fluoroscopy was useful to measure gastrointestinal motility in vivo in control and rotavirus infected mice. Neonatal (12 day) and adult (6-8 week) CD1 mice were inoculated with murine ECwt rotavirus or media. Gastrointestinal motility was evaluated at 0,2 , and 4 (adult) or 0 and 2 (neonatal) days post inoculation. To study motility in vivo, mice were gavaged with barium, digital images of the gastrointestinal tract were obtained with a C-arm fluoroscopy unit, and rates of contraction and changes in stomach sizes were determined by image analysis. To measure rates of intestinal contraction in vitro, whole sections of intestine were mounted in organ baths using standard procedures and analyses. In adults, rates of contraction in the stomach and small intestine of rotavirus infected mice $(n=5)$ did not vary significantly from the controls $(n=4)$ at any time point with either method. However, the average rate of intestinal smooth muscle contractions measured in vivo $(40.5 \pm 3.9$ contractions/min. $)$ was slightly less than that recorded in vitro $(45.9 \pm 5.4$ contractions/minute; $n=8)$. In neonates, rates of intestinal contractions measured by either method were not significantly different between control and rotavirus infected mice, but similar to the adults the in vivo rates of intestinal contraction $(28.7 \pm 2.4$ contractions/min., $\mathrm{n}=8)$ were slightly less than measured in vitro $(35.9 \pm 2.0$ contractions $/ \mathrm{min}$., $\mathrm{n}=$ 10). Of note, the basal rates of contraction determined by both methods differed significantly between neonatal and adult mice. Gastric contractions were rarely observed in neonates but visible differences in the amount of barium leaving the stomach and entering the small intestine in control and rotavirus infected mice were noted. Therefore, gastric size was determined in fluoroscopic images as a measure of gastric emptying. A significant difference in initial gastric size $(64.7 \pm 14.5 \mathrm{~mm} 2$ vs $52.2 \pm 6.5 \mathrm{~mm} 2)(\mathrm{p}=0.009)$ and change in gastric sizes over 5 minutes $(2.6 \pm 1.6 \mathrm{~mm} 2$ vs $8.5 \pm 3.5 \mathrm{~mm} 2)(\mathrm{p}=0.0005)$ were observed in rotavirus infected $(n=10)$ compared to control neonates $(n=8)$. Rotavirus infection did not alter gastric contraction rates but did delay gastric emptying in neonates. Fluoroscopy and digital image analysis provided a reliable and accurate method to detect differences in gastrointestinal motility in vivo. This work was partially supported by the Texas Gulf Coast Digestive Disease Center.

\section{M2221}

Meal Related Dyspeptic Symptoms in Healthy Subjects: Association with Gastric Motor Function and Clustering of Symptoms

Guru Iyngkaran, Birgit Adam, Tobias Liebregts, F Dylan Bartholomeusz, Max Bellon, Jacob Bolwell, Suresh Sivanesan, Nicholas J. Talley, Gerald Holtmann

Introduction: Little is known about the association between symptoms and gastric motor function during the ingestion of a meal. We hypothesized that a) in healthy subjects meal related symptoms are associated with delayed gastric emptying and impaired gastric nutrient distribution (i.e. due to impaired fundic relaxation), b) factor analysis will separate meal induced dysmotility-type and ulcer-type symptoms. Methods: 14 healthy subjects ( 8 males, aged 21- 45 mean 27.3) without a history of relevant dyspeptic symptoms were recruited. After at least an $8 \mathrm{hrs}$ fast, they received every 5 minutes $200 \mathrm{ml}$ of a standardized 99mTclabelled enteral feeding solution (EnsureR) up to a cumulative volume of $800 \mathrm{~mL}$. After each $200 \mathrm{ml}$ drink, symptoms were assessed with the Adelaide-Dyspepsia-ToolR (ADT). This tool assesses 7 key symptoms (fullness, abdominal pain, retrosternal/abdominal burning, nausea, regurgitation, other) and determines peak and cumulated symptom response. The gastric nutrient distribution and emptying was assessed with a gamma camera. Data analysis: The cumulated symptom response during the 240 minute meal and post meal period was calculated separately for all symptoms. In addition, the time from the start of nutrient ingestion until the peak of symptoms was determined. Non parametric correlations were utilized to assess the association between symptoms and gastric emptying parameters. Factor analysis with varimax rotation was used for the categorization of symptoms. Results: The average peak of symptoms was different for the various symptoms ( 50 minutes for pain, 22 minutes for fullness, $18.1 \mathrm{~min}$ for nausea, $18.3 \mathrm{~min}$ for burning and $26.7 \mathrm{~min}$ for regurgitation). Maximal pain was significantly correlated to maximal fullness $(r=0.57, p=0.03)$ and cumulative fullness $(\mathrm{r}=0.60, \mathrm{p}=0.02)$ but not with gastric retention at 100 minutes. Cumulative fullness was linked to gastric retention at $100 \mathrm{~min}(\mathrm{r}=0.64, \mathrm{p}=0.01)$. Factor analysis addressing the maximum symptom intensity revealed a three factorial model. While pain, fullness and nausea loaded on one factor (35\% of variance), epigastria and retrosternal burning ( $21.6 \%$ of variance) and regurgitation ( $18.8 \%$ of variance) loaded on separate factors. Conclusions: Based upon these experiments in healthy subjects, meal related symptoms can be categorized into three categories: 1) pain, fullness and nausea, 2) retrosternal and epigastric burning and 3) regurgitation. Severity of symptoms appears inversely associated with the gastric emptying rate and lag time. The data suggest that the spectrum of meal related symptoms consists of distinct domains that are linked to different underlying mechanisms.

\section{M2222}

Comparison of Gastric Emptying Time and Plasma Ghrelin Levels in Patients with Functional Dyspepsia and Non-Erosive Reflux Disease

Tomotaka Shindo, Seiji Futagami, Tetsuro Hiratsuka, Tatsuhiko Hamamoto, Nobue Ueki, Yoko Shinji, Masafumi Kusunoki, Ken Wada, Kazumasa Miyake, Katya Gudis, Taku Tsukui, Katsuhiko Iwakiri, Choitsu Sakamoto

(Background/Aims) Reports have shown that NERD (Non-Erosive Reflux Disease) is not just a milder form of erosive GERD, but rather to have its own pathogenesis under the control of various independent mechanisms; including visceral hypersensitivity, prolonged contraction of the esophagus and distention of the upper stomach. However, there have been no clinical studies regarding the association between gastric motility and NERD. Therefore, we analyzed gastric emptying time and plasma ghrelin levels, a possible candidate factor for gastric motility in NERD patients, and compared data with that of patients with functional dyspepsia (FD). (Material and Methods) 30 patients with FD, 28 patients with NERD, and 30 healthy controls were consecutively enrolled according to Rome II criteria after upper gastrointestinal endoscopy. In this study, diagnosis of NERD was based on typical reflux symptoms of 12 weeks duration within a one year period, despite the lack of endoscopically detectable mucosal lesions. Gastric motility in the three groups was evaluated by gastric emptying time (T-max) using the 13C-acetate breath test, and partly assessed by abdominal ultra-sonography. We also evaluated upper abdominal symptoms, and depression based on SRQ-D scores. Plasma levels for both acylated and desacylated ghrelin were measured by ELISA method. (Results) Gastric emptying time was significantly delayed in FD patients versus healthy controls $(62.4 \pm 4.1$ vs. $46.2 \pm 0.65 \mathrm{~min}, \mathrm{p}<0.05)$. Although gastric emptying time in NERD patients $(51.46 \pm 4.7)$ was either delayed or shortened compared to healthy controls and FD patients, respectively, the differences were not significant. SRQ-D scores for FD and NERD patients $(10.7 \pm 2.5,12.3 \pm 2.3$, respectively $\mathrm{p}<0.05)$ were significantly higher than for healthy subjects. However, there was no significant difference in SRQ-D scores between FD and NERD patients. BMI for NERD patients $(22.3 \pm 2.1)$ was near the level of that of FD patients $(20.4 \pm 2.4) .51 \%$ of FD patients versus $14 \%$ of NERD patients had delayed gastric emptying time. Plasma acylated ghrelin levels in FD patients with delayed gastric emptying time was significantly decreased compared with that in FD patients with normal gastric emptying time. In contrast, acylated ghrelin levels in NERD patients were the same levels as in healthy subjects. Conclusions: Our results suggest that in a subset of NERD patients delayed gastric emptying might be associated with the occurrence of symptoms.

\section{M2223}

Anatomical Evidence of Region Specific Effects of Acupuncture On Rat Gastric Motor Function

Masahiro Iwa, Megumi Matsushima, Theodore N. Pappas, Mineko Fujimiya, Toku Takahashi

Background; Acupuncture has been used for gastric diseases, such as functional dyspepsia (FD) and diabetic gastroparesis. However, the mechanisms of acupuncture in affecting gastric function remains unknown. Our previous functional studies suggest that acupuncture at the lower limbs stimulates gastric motility via vagal cholinergic pathways (Am J Physiol 285, R862-72, 2003), while acupuncture at the abdomen inhibits gastric motility via sympathetic pathways in rats (Dig Dis Sci, 48, 59-68, 2003). To obtain the anatomical evidences of possible neural pathway in mediating acupuncture-induced gastric motor responses, we studied c-Fos immunohistochemistry of the brain stem in response to electroacupuncture (EA) in rats. Methods; Acupuncture needles were inserted at the bilateral acupoints of ST36 (lower limb) or ST-25 (abdomen) and needles were stimulated by electricity $(0.1 \mathrm{msec}$, $1 \mathrm{~mA}$ and $2 \mathrm{~Hz}$ ) for $30 \mathrm{~min}$ in conscious rats. Non EA-treated rats served as controls. Immediately after EA, rats were transcardially perfused with $0.01 \mathrm{M} \mathrm{PBS}$ and $4 \%$ paraformaldehyde for $10 \mathrm{~min}$. The brainstem was removed from the skull, immersed for 24 hours in the post fixative. The brainstem was cut into $20 \mu \mathrm{m}$ coronal sections by a cryostat. The sections were incubated in c-Fos antibody at $4{ }^{\circ} \mathrm{C}$ for 1 week. The total number of c-Fos immunoreactive cells was counted in the dorsal motor nucleus of the vagus (DMV), the nucleus tractus solitarius (NTS) and the rostral ventrolateral medulla (RVLM). Results; EA at ST-36, but not ST-25, significantly increased the number of c-Fos immunopositive cells at the DMV to $8.1+/-0.4$ cells/section, compared to that of controls $(1.8+/-0.3$ cells/ section) $(n=3, P<0.05)$. EA at ST-25, but not ST-36, significantly increased the number of c-Fos immunopositive cells at the RVLM to $9.9+/-0.7$ cells/section, compared to that of controls $(5.1+/-0.3$ cells/section $)(n=3, P<0.05)$. EA at ST-36 also increased the number of c-Fos immunopositive cells at the caudal NTS. On the other hand, EA at ST-25 increased the number of c-Fos immunopositive cells at the medio-caudal NTS. Conclusion; It is suggested that somatic afferents activated by EA at ST-36 is conveyed to the caudal NTS and stimulates the DMV neurons. In contrast, somatic afferents activated by EA at ST-25 is conveyed to the medio-caudal NTS and stimulates the RVLM neurons. The RVLM neurons are known as premotor sympatho-excitatory neurons that provide drive to the sympathetic preganglionic neurons in the intermediolateral nucleus of the spinal cord. Thus, EA at ST36 stimulates gastric motility via vagal efferents, while EA at ST-25 inhibits gastric motility via sympathetic efferents in rats.

\section{M2224}

TRP Channels in Murine Spinal and Vagal Visceral Afferents Pieter J. Peeters, Jeroen Aerssens, Ronald de Hoogt, Kirk Hillsley, Andrzej Stanisz, David Grundy, Ronald H. Stead, Bernard Coulie

Background: Mammalian Transient Receptor Potential (TRP) channels comprise six related protein families. All TRP channels are putative six-transmembrane proteins assembling as tetramers to form cation-permeable pores. TRPs mediate sensory transduction both at the whole organism and single cell level. The aim of this study was to evaluate murine TRP channel expression in dorsal root (DRG) and nodose ganglia (NG) projecting to the gut. Methods: Intraperitoneal injection of Cholera toxin B-488 in 11 Balb/c mice identified 
neurons projecting to the abdominal viscera. Fluorescent neurons in DRG (T10 to T13) and NG were isolated using laser-capture microdissection. RNA was hybridised to Affymetrix Mouse whole genome arrays. Differential expression was defined as at least 1.5 fold difference in expression and false discovery rates were calculated using significance analysis method for microarray data (SAM) and expressed as a q-values. Results: Arrays allowed detection of mRNA expression levels of 22 different TRP channels, of which 15 were reliably detected in DRG or NG. Of these 7 were equally expressed in DRG and NG, 4 had significantly $(\mathrm{q}<0.01)$ higher expression in DRG and 4 were higher in NG. As expected, mRNA for the capsaicin receptor Trpvl was present in both DRG and NG, with 3.8-fold higher levels in the former $(q<0.001)$. The membrane stretch sensitive Trpv2 is the only other Trpv channel family member detected in visceral afferents (more abundant in NG vs. DRG). The Trpal channel, gated by mechanical stimuli as well as by cold temperatures, mustard oil, and cannabinoids, showed high levels of expression in both NG and DRG, with 7-fold more abundance in the latter $(q<0.001)$. As such Trpal could confer mechanosensitivity on the peripheral endings of both spinal and vagal afferents. Trpm8, another cold activated channel was not detected in visceral afferents. Other Trpm family members that were detected include Trpm2, Trpm4, Trpm6 and Trpm7. The latter two can heteromerize and are important for $\mathrm{Mg}^{2+}$ homeostasis. Whereas Trpm7 was equally expressed in DRG and NG, Trpm6 was enriched in DRG (3-fold). With respect to Trpc channels, the putative store-operated $\mathrm{Ca}^{2+}$ channels $\operatorname{Trpcl}$, Trpc3 and Trpc5 were detected in visceral afferents. Trpc 3 is predominantly expressed in DRG, whereas Trpcl and Trpc5 are equally expressed in DRG and NG. Conclusions: These data provide an inventory of TRP channels present in NG and DRG neurons supplying the gut and demonstrate clear differences in TRP channel expression. These divergences may underlie the contrasting roles of NG and DRG, and contribute to differences in chemo-and mechanosensitivity of the gastrointestinal tract.

\section{M2225}

Anxiety-Induced Colonic Hypersensitivity: The Role of Glucocorticoid (GR) and Mineralocorticoid Receptors (MR) in the Amygdala

Brent Myers, Armstrong Isiaho, Beverley Greenwood-van Meerveld

In patients with IBS, abdominal pain is worsened during periods of anxiety. Although the amygdala is a key limbic structure involved in the regulation of anxiety, the importance of amygdaloid-mediated mechanisms in the regulation of colonic function is not completely understood. Our previous studies have shown that stimulation of the central amygdaloid nucleus (CeA) with corticosterone (CORT) increases indices of anxiety coupled with a hypersensitive colon. The goal of this study was to examine the relative importance of amygdaloid GR and MR-mediated mechanisms in the development of a hypersensitive colon as demonstrated by an exaggerated visceromotor response (VMR) to colorectal distention

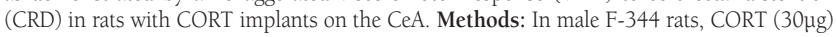
or inert cholesterol $(30 \mu \mathrm{g})$ micropellets were stereotaxically implanted bilaterally at the dorsal boundary of the CeA either alone or in combination with a GR antagonist, mifepristone $(30 \mu \mathrm{g})$ or a MR antagonist, spironolactone $(30 \mu \mathrm{g})$. After 5 days, anxiety was assessed on the elevated plus maze (EPM) and quantified by the percentage (\%) of time spent in the open arms. On day 7 , colonic sensitivity was assessed by measuring the VMR i.e. the number of abdominal muscle contractions in response to CRD $(0-60 \mathrm{mmHg})$. Results: In CORT implanted rats there was a significant reduction in the \% time spent in the open arms compared to cholesterol controls $(\mathrm{CORT}=28.3 \pm 7.2 \%$, cholesterol $=67.8 \pm 16.1 \% ; \mathrm{p}<0.05, \mathrm{n}=$ 5 ), indicative of anxiety. Furthermore, a higher number of abdominal contractions were observed in response to CRD when compared to rats with cholesterol implants (CORT: $20 \mathrm{mmHg}=20.0 \pm 3.9,40 \mathrm{mmHg}=25.8 \pm 5.1,60 \mathrm{mmHg}=34.4 \pm 4.5 ; \mathrm{p}<0.01, \mathrm{n}=5$; cholesterol: $20 \mathrm{mmHg}=6.5 \pm 0.9,40 \mathrm{mmHg}=14.3 \pm 1.7,60 \mathrm{mmHg}=20.2 \pm 2.8)$. In rats with CORT implants combined with the GR or the MR antagonist there was an inhibition of anxiety and a significant reduction in the VMR induced by CRD at all distention pressures (CORT/GR antagonist: $20 \mathrm{mmHg}=5.6 \pm 0.8,40 \mathrm{mmHg}=12.8 \pm 2.5,60 \mathrm{mmHg}=24.6 \pm 4.6 ; \mathrm{p}<0.01, \mathrm{n}=5$; CORT/MR antagonist: $20 \mathrm{mmHg}=7.0 \pm 1.6,40 \mathrm{mmHg}=11.8 \pm 1.7,60 \mathrm{mmHg}=16.0 \pm 2.6 ; \mathrm{p}<0.01$, $\mathrm{n}=5$ ). Summary: In rats, elevating amygdaloid CORT induces anxiety and colonic hypersensitivity. When combined with either a GR or MR antagonist, the CORT-induced responses were significantly inhibited. Conclusion: We have shown that at a concentration of CORT known to induce anxiety, both GR and MR in the amygdala play a role in colonic hypersensitivity, supporting the hypothesis that both amygdaloid GR and MR play key roles in the brain-gut axis.

\section{M2226}

The Vagus Nerves Contributed to LPS-Induced Increase of Colonic Paracellular Permeability By Central Release of IL-1 $\beta$

Raphael Moriez, Helene Eutamene, Vassilia Theodorou, Jean Fioramonti, Lionel Bueno

Subdiaphragmatic vagotomy (SDV) has been shown to inhibit behavioral effects of peripheral lipopolysaccharide (LPS), including anorexia and fever response. These behavioral effects implicate the central release of IL-1 $\beta$, abrogated by previous SDV. However, while it has been showed that IP administration of LPS increased the colonic paracellular permeability (CPP), no data report the involvement of vagus nerves in this process. The aim of this study was to evaluate the role of vagus nerves (afferent vs. efferent fibres) in the LPS-induced increase of colonic permeability. Methods: All experiments were carried out in male Wistar rats equipped with an intracolonic catheter. Firstly, a bilateral SDV was performed on 2 groups of 10 rats; a similar surgical approach was conduced on 2 other groups without section of the vagal trunks (SHAM group). Each of these 2 groups received either $\mathrm{NaCl}$ $0.9 \%(250 \mu \mathrm{L}, \mathrm{IP})$ or LPS from E.coli 0111:B4 $(250 \mu \mathrm{L}, 1 \mathrm{mg} / \mathrm{kg}$, IP). A second series of experiment was carried out on 5 groups of 10 rats prepared for intracerebroventricular (icv) injections using polyethylene catheters inserted into the lateral ventricles of the brain. These 5 groups received respectively $\mathrm{NaCl} 0.9 \%(5 \mu \mathrm{L}, \mathrm{icv})+\mathrm{NaCl}(250 \mu \mathrm{L}, \mathrm{IP}) / \mathrm{NaCl}$ (icv) + LPS $(250 \mu \mathrm{L}, 1 \mathrm{mg} / \mathrm{kg}, \mathrm{IP}) /$ recombinant rat IL-1 receptor antagonist (IL-1ra, $2 \times 20 \mu \mathrm{g}$ in $5 \mu \mathrm{L}$ at 30 min before and 3h after LPS, icv) + LPS (IP) / recombinant rat IL-1 $\beta$ (rrIL-1 $\beta$, l $\mu$ g in $5 u \mathrm{~L}, \mathrm{icv})+\mathrm{NaCl}$ (IP) with/without a previous SDV. In all these groups, animals recovered during 14 days and CPP was evaluated by intracolonic infusion of 51Cr-EDTA and expressed as percent of radioactivity collected in the urine for $6 \mathrm{~h}$ after LPS. Results: In SHAM rats,
LPS increased the CPP $(1.69 \pm 0.16 \%$ vs. $0.69 \pm 0.03 \%$ in controls; $\mathrm{P}<0.05)$. In SDV group administration of LPS failed to increase the CPP $(0.68 \pm 0.07 \%$ vs. $0.69 \pm 0.03 \%$ in SHAM control, $\mathrm{P}>0.05)$. SDV alone had no effect on $\mathrm{CPP}$ at $0-6 \mathrm{~h}$ period. In the second series of experiment, icv injections of IL-1 ra diminished the effects of LPS on the CPP $\left(0.87 \pm 0.19^{\circ}\right.$ vs. $1.87 \pm 0.22 \%, \mathrm{P}<0.05)$. In the same way, icv administration of rrIL- $1 \beta$ reproduced the effects of LPS characterized by an increase of the CPP $(1.53 \pm 0.20 \%$ vs. $0.55 \pm 0.09 \%$ for controls, $\mathrm{P}<0.05)$. A previous SDV abolished the effects of central administration of rrIL$1 \beta$ on the $\mathrm{CPP}(0.79 \pm 0.13 \%$ vs. $1.53 \pm 0.2 \%, \mathrm{P}<0.05)$. Conclusion: These data indicate a key role of vagus nerves in the alterations of gut permeability induced by IP administration of LPS. These effects involved central release of IL-1 $\beta$, activating in turn vagal efferents fibers to produce alterations of the colonic permeability.

\section{M2227}

Post-Infective Changes in Behavior: A Model of Functional Dyspepsia in the Mouse

Premysl Bercik, J.N. Foster, Patricia Blennerhassett, Elena F. Verdu, Stephen M. Collins

Background/Aim: The concept that previous infection can be a basis for the development of functional GI disease is now accepted. However, the lack of symptomatic improvemen following eradication of Helicobacter pylori (Hp) infection is construed as evidence agains the role of this organism in functional dyspepsia. Early post-prandial satiety is a dominan feature of functional dyspepsia. Here, we examine the hypothesis that $\mathrm{Hp}$ infection produces long lasting changes in feeding behavior reminiscent of functional dyspepsia. Methods Control and $\mathrm{Hp}$ infected Balb/c mice were studied before and two months after Hp eradication. Gastric emptying was assessed by videofluoroscopy image analysis. Frequency of eating bouts and amount of food was recorded during 24 hours by a computer-assisted method and IL- $1 \beta$ and TNF- $\alpha$ were detected in brain frozen sections by in situ hybridizations of 35S-labeled RNA probes. Results: Hp infected mice exhibited changes in feeding behavior characterized by the more frequent ingestion of smaller meals - analogous to early satiety and no weight loss. Uninfected mice had $14 \pm 4.1$ eating bouts/24 hours while Hp infected mice had $19.0 \pm 4.5$ bouts $/ 24$ hours $(p<0.05)$. Hp infected mice consumed $0.20 \pm 0.11 \mathrm{~g} /$ bout compared to $0.32 \pm 0.14 \mathrm{~g}$ /bout in controls $(\mathrm{p}<0.05)$. Infected mice exhibited a significant $42 \%$ delay in gastric emptying. Successful eradication of $\mathrm{Hp}$ resulted in normalization of gastric emptying but the altered feeding behavior persisted. This was accompanied by the selective up-regulation of TNF- $\alpha$ expression in the circumventricular median eminence whereas IL- $1 \beta$ expression was similar in both groups. Conclusions: Hp infection in mice is accompanied by a feeding pattern reminiscent of early satiety. While delayed gastric emptying may contribute to the altered feeding during active infection, it cannot explain its persistence after $\mathrm{Hp}$ eradication. The expression of TNF- $\alpha$ in the brain is a putative mechanism for altered feeding behavior in this model. We thus extend the current concept of post-infective gut dysfunction to include changes in the CNS which, in the context of functional dyspepsia, may explain the lack of symptom resolution post-eradication of $\mathrm{Hp}$.

\section{M2228}

Sensitization of the Entire Esophagus to Distension By Distal Esophageal Acid Exposure

Adeyemi Lawal, Mark Kern, Arthi Sanjeevi, Tanya Rittmann, Reza Shaker

Peripheral and central esophageal sensitization is believed to contributes to symptom development in esophageal chest pain and acid-related disorders. We have previously shown that esophageal central sensitization is manifested in both insula and cingulate gyrus. However, regional contribution within the esophagus to sensitization has not been systematically studied. AIM: To compare the effect of sensitization on processing of subliminal(neural activity) and liminal (neurocognitive activity) mechanical stimulation of the proximal and distal esophagus.METHODS: We studied 12 healthy adult subjects(age:18-35)during fMRI scanning of the insula $(n=6)$ or cingulate gyrus $(n=6)$ (eleven $2.5 \mathrm{~mm}$ thick sagittal slices, $96 \times 96$ pixel resolution, $24 \mathrm{~cm}$ FOV).Paradigm-driven, 2-minute fMRI scans were performed during randomly timed 15-second intervals of barostatically controlled proximal and distal esophageal distensions and rest before and after $30 \mathrm{~min}$ of distal esophageal acid perfusion $(0.1 \mathrm{~N} \mathrm{HCl}, 1 \mathrm{ml} / \mathrm{min}$ ). We tested two pressure levels: $2-6 \mathrm{mmHg}$ (unperceived/subliminal stimulating neural activity without perception) and barely perceived distension. FMRI volume elements(voxels) showing magnetic signal changes temporally related to the stimuli were tallied as a representation of the regional cortical activity associated with distal and proximal esophageal distension. RESULTS: The effect of distal esophageal acid exposure on regional cortical fMRI activity showed significant increases in the average number of activated voxels after acidification for both subliminal and liminal esophageal distension in the proximal and distal esophagus $(* \mathrm{p}<0.05)$. (Table) The amount of cortical activity associated with distal distension was significantly greater than that associated with proximal distension both before and after acid exposure $(\Delta \mathrm{p}<0.05)$ (Table). There were also significant differences in the amount of cortical activity during subliminal compared to liminal stimulation. (\# $\mathrm{p}<0.05$ ) (Table). CONCLUSIONS: 1) Distal esophageal acid exposure results in sensitization of the entire esophagus to distention. This sensitization is manifested at both neural and neurocognitive levels. 2) Registration of distal esophageal stimulation in the insula and cingulate seems to be more robust than in the proximal esophagus. 3) These findings may explain the prevalence of some common symptoms in GERD patients such as chest pain and belching. 


\begin{tabular}{|c|c|c|c|c|c|c|c|c|}
\hline \multirow{4}{*}{} & \multicolumn{4}{|c|}{ subliminal } & \multicolumn{4}{c|}{ liminal } \\
\cline { 2 - 9 } & \multicolumn{2}{|c|}{ pre-acid } & \multicolumn{2}{c|}{ post-acid } & \multicolumn{2}{c|}{ pre-acid } & \multicolumn{2}{c|}{ post-acid } \\
\cline { 2 - 9 } & proximal & distal & proximal & distal & proximal & distal & proximal & distal \\
\hline cingulate & $12 \pm 1$ & $37 \pm 9 \Delta$ & $25 \pm 5 *$ & $51 \pm 10 * \Delta$ & $19 \pm 4 \#$ & $80 \pm 25 \Delta \#$ & $38 \pm 6 * \#$ & $105 \pm 29 * \Delta \#$ \\
\hline insula & $9 \pm 3$ & $21 \pm 6 \Delta$ & $31 \pm 3 *$ & $36 \pm 3 *$ & $23 \pm 4 \#$ & $57 \pm 5 \Delta \#$ & $36 \pm 3 *$ & $85 \pm 12 * \Delta \#$ \\
\hline
\end{tabular}

\section{M2229}

Sex Differences in Brain Responses to Aversive Pelvic Visceral Stimulation in Healthy Subjects - An FMRI Study

Steven M. Berman, Bruce D. Naliboff, Brandall Suyenobu, Jennifer S. Labus, Joshua Bueller, Kim Ruby, Michael Minnema, Jean Stains, Emeran A. Mayer

Background: Women often have lower thresholds and tolerance for pain than men and a greater prevalence of a variety of functional pain disorders. Brain imaging studies using somatic and visceral stimuli have shown some sex based differences in brain activation. However, potentially sex-related deactivations have not been studied. Aim \& Methods: fMRI brain imaging (3T) of the BOLD signal was used to compare brain responses to aversive rectal distention and cued anticipation of distention between 6 healthy women (mean age $35 \pm 8$ ) and 7 age-matched men. Ten regions of interest (ROIs) were assessed for the presence of significantly activated or deactivated voxels $(\mathrm{p}<0.05)$ in response to moderate distention $(45 \mathrm{~mm} \mathrm{Hg})$, mild distention $(25 \mathrm{~mm} \mathrm{Hg})$ and sham distention $(5 \mathrm{~mm} \mathrm{Hg})$. Results: Rectal distention reliably increased the BOLD signal in typical pain regions (dorsal anterior cingulate, insula). Some subjects also showed significant effects in prefrontal cortex, and subcortical or limbic regions (thalamus, peri/infragenual cingulate, amygdala, ventral striatum, dorsal brainstem). Deactivations within all ROIs were also noted. Men activated more voxels than women in the majority of structures during moderate (10 of $10 \mathrm{ROIs})$, mild (9/10), and sham distention (10/10). However, women deactivated more voxels than men $(9 / 10$ in all 3 distention conditions). ANOVA indicated that the proportion of affected voxels that deactivated was significantly influenced by sex (Sex x ROI interaction $F=3.48, p<0.001$ ). Women showed a greater proportion of deactivations than men in brainstem, thalamus and ventral striatum $(\mathrm{p} s<0.05)$. Planned comparisons indicated no significant sex differences during sham inflation, but $\mathrm{p}<0.05$ effects in all three ROIs and the amygdala during mild inflation, and in thalamus and dorsal cingulate during moderate inflation. Conclusion: Sexbased differences in CNS response to aversive pelvic visceral stimulation consist of a greater proportion of deactivations in women in regions implicated in pain processing and modulation. These deactivations may reflect inhibition or differential connectivity with other modulatory brain regions. Supported by DK48351, R24 AT002681, P50DK64539 (EAM), NR04881 (BN).

\section{M2230}

Central Glucagon Like Peptide-1 Delays Solid Gastric Emptying Via a Central CRF and Peripheral Spinal-Celiac Pathway in Conscious Rats Yukiomi Nakade, Kiyoshi Tsukamoto, Constant Masere, Theodore N. Pappas, Toku Takahashi

Background: Glucagon like peptide-1 (GLP-1), one of the gastrointestinal (GI) regulatory peptide, is secreted from the alimentary canal in response to absorbed nutrients. A major role of GLP-1 is to stimulate insulin secretion on the islet beta cell to maintain glucose control. Besides the role of incretine, GLP-1 is also thought to be a brain neurotransmitter mediating GI function. Previous report has shown that central administration of GLP-1 inhibits liquid gastric emptying via vagal mediated pathways in rats (Am J Physiol 273, G920-927, 1997). This mimics the effect of central-injection of corticotropin releasing factor (CRF), which also delays liquid gastric emptying. However, it remains unknown how central GLP-1 delays solid gastric emptying. It also remains unknown how GLP-1 interacts with CRF within the brain in mediating the inhibitory effect of solid gastric emptying. Methods: Male Sprague-Dawley rats were fasted for 24 hours. Ten min after the feeding of solid rat chow (1.5 g), GLP-1 or saline was injected intracisternally (IC) or subcutaneously (SC). Ninety minutes after the peptide injection, rats were sacrificed. The gastric content was recovered from the stomach, dried and weighed for calculating solid gastric emptying. To examine the mechanism of GLP-1-induced delay of solid gastric emptying, atropine (50 $\mu \mathrm{g} /$ $\mathrm{kg}$, IP) or NG-nitro-L-arginine methyl ester (L-NAME) (10 mg/kg, IP) was applied 20 minutes before the feeding. Celiac ganglionectomy underwent 1 week before the gastric emptying study. CRF antagonist, astressin, was applied (IC) 20 minutes before the feeding. Results: Gastric emptying was $66.2+/-4.5 \%$ in vehicle-treated rats $(n=6)$. IC-injection of GLP-1 (0.03-3.0 nmol) dose-dependently delayed solid gastric emptying. Maximum effect was observe by GLP-1 ( $3.0 \mathrm{nmol}$ ), which significantly delayed gastric emptying to $20.3+/-4.5 \%$ $(\mathrm{n}=6)$. In contrast, SC-injection of GLP-1 ( $3 \mathrm{nmol})$ did not affect gastric emptying $(61.6+/$ $3.5 \%, \mathrm{n}=6)$. IC-injection of GLP-1 ( $3 \mathrm{nmol})$-induced delay of gastric emptying was partially inhibited by celiac ganglionectomy $(43+/-3.4 \%, \mathrm{n}=6, \mathrm{P}<0.05$, but not by atropine $(25.8$ $+/-3.6 \%, n=6)$, or L-NAME $(11.3+/-3.6 \%, n=6)$. IC- injection of astressin $(2.8 \mathrm{nmol})$ partially inhibited GLP-1-induced delay of solid gastric emptying $(41.3+/-2.5 \%, n=6$, $\mathrm{P}<0.05)$. Conclusions: These results indicate that central CRF and peripheral spinal-celiac pathway are, at least in part, involved in mediating central GLP-1-induced delay of solid gastric emptying in conscious rats.

\section{M2231}

Acupuncture Improves Restraint Stress-Induced Delay of Gastric Emptying Via Central Glutaminergic Pathways in Conscious Rats

Masahiro Iwa, Yukiomi Nakade, Theodore N. Pappas, Toku Takahashi

Background; Acupuncture has been used for treating functional gastrointestinal (GI) disorders, including irritable bowel syndrome (IBS) and functional dyspepsia (FD). Animal studies demonstrated that acupuncture improves various stress-induced physiological responses. We have recently showed that electroacupuncture (EA) at the lower limbs (ST36) significantly improved delayed gastric emptying induced by restraint stress in rats (Dig Dis Sci; in press). Glutamate and $\gamma$-Aminobutyric acid (GABA) are major excitatory and inhibitory neurotransmitters within the dorsal vagal complex (DVC) regulating GI motility. We studied whether glutamate and GABA receptors are involved in mediating the stimulatory effects of EA on delayed gastric emptying induced by restraint stress. Methods; Rats were given a solid pellet $(1.5 \mathrm{~g})$ for 10 minutes. Immediately after the feeding, the rats were placed on a stereotaxic apparatus under short isoflurane anesthesia. GABAA receptor antagonist (bicuculline methiodide; $5 \mathrm{nmol}$ in $5 \mu \mathrm{l}$ ), GABAB receptor antagonist (phaclofen; $100 \mathrm{nmol}$ in $5 \mu \mathrm{l}$ ), NMDA receptor antagonist (kynurenic acid; $0.1 \mu \mathrm{mol}$ in $5 \mu \mathrm{l})$ and saline $(5 \mu \mathrm{l})$ were injected intracisternally (IC). Then, the rats were submitted to the restraint stress for 90 minutes. Acupuncture needles were inserted to a depth of $5 \mathrm{~mm}$ into the skin and underlying muscles at ST-36 bilaterally. Needles were stimulated by electricity $(0.1 \mathrm{msec}$ and $10 \mathrm{~Hz}$ ). Electroacupuncture (EA) was performed for 90 minutes throughout the stress loading. Ninety minutes after the stress loading, rats were euthanized and the gastric content was recovered from the stomach, well dried, and weighed for calculation of solid gastric emptying. Results; Solid food gastric emptying in 90 min was significantly delayed by restraint stress $(27.3+/-2.1 \%, \mathrm{n}=8)$, compared to that of controls $(64.3+/-2.1 \%, \mathrm{n}=8)$. Restraint stress-induced delay of gastric emptying was significantly restored by the ICinjection of bicuculline methiodide $(46.5+/-3.1 \% ; n=6)$ and phaclofen $(48.3+/-3.3 \%$; $=$ $6)$. Delayed gastric emptying induced by restraint stress was significantly improved by EA at ST-36 $(49.7+/-1.4 \%, n=8)$. The stimulatory effect of EA on stress-induced delay of gastric emptying was prevented by pretreatment with IC-injection of kynurenic acid (30.1 $+/-2.1 \%, n=6$ ). Conclusion; Restraint stress-induced delay of gastric emptying is mediated via central GABAA and GABAB receptors. EA at ST-36 stimulates glutaminergic neurons in the brainstem resulting in improvement of stress-induced delay of gastric emptying. Acupuncture may be useful for the treatment of delayed gastric emptying associated with stress.

\section{M2232}

A Comparison of the Cortical Responses to Selectively Attended Visceral and Somatic Pain

Paul Dunckley, Qasim Aziz, Richard G. Wise, Jonathan Brooks, Irene Tracey, Lin Chang

Background/Introduction. Visceral and somatic pain differ in several key aspects - visceral pain is described as ill-defined and is often described as more unpleasant than somatic pain. These differences in perception must be reflected in differing cortical patterns of activation. We have used a paradigm of attentional modulation to study the differences in selectively attended visceral and somatic pain matched for both pain intensity and unpleasantness. Methods 12 healthy, pain-free, right-handed subjects were recruited for the study. Simultaneous electrical pulses and auditory tones lasting 6 seconds were delivered to the subjects during a whole-brain functional magnetic resonance imaging scan acquisition. The electrical catheter was placed into the rectum for the visceral scan and onto the lower abdomen for the somatic scan. Subjects were instructed to attend to and count either the auditory tones or electrical pulses. Electrical pulses and auditory tones were delivered at either 2, 3.5 or $4.3 \mathrm{~Hz}$ thereby varying the cognitive demand of the tasks. Pain intensity, unpleasantness and tone/pulse count were recorded after each stimulus. Anxiety levels were measured prior to the scan. Results. Pain intensity and unpleasantness were well matched for the 2 sensory modalities. Selective attention to both modalities resulted in activation in sensory-discriminative (secondary somatosensory cortex), affective (anterior insula, anterior mid cingulate cortex $(\mathrm{ACC})$ ) and cognitive (ACC) regions. Subtraction analysis identified greater activation during visceral pain in the right thalamus, ACC, bilateral cerebellum, the right anterior insula (during the $4.3 \mathrm{~Hz}$ condition alone) and right amygdala. There were no areas of greater activation during somatic pain. A separate focus of activation was observed in the anterior ACC during visceral pain at each frequency. Right anterior insula cortex activity correlated with anxiety rating for both somatic and visceral pain. Conclusions. Selectively attended visceral pain results in greater activation of affective (right anterior insula, right amygdala and anterior mid-cingulate) and attentive (ACC) regions compared with matched selectively attended somatic pain. Visceral pain is preferentially represented in the right anterior insula cortex. As unpleasantness and anxiety were both matched between sensory modalities, this suggests an added interoceptive role of this region during visceral pain. Greater representation of visceral pain in the affective regions of the cortex is consistent with the James-Lange theory of emotion whereby the physical sensation of emotions are dependent on visceral sensation.

\section{M2233}

Effects of Glucagon-Like Peptide-1 (GLP-1) On Identified Pancreas-Projecting Vagal Motoneurons

R. Alberto Travagli, Kirsteen N. Browning, Shuxia Wan

Glucagon-like peptide-1 has been shown to increase insulin secretion via a vagally-dependent mechanism. A high density of GLP-1 containing neurons is present in the vagal circuits within the brainstem. The dorsal motor nucleus of the vagus (DMV) comprises the neurons forming the preganglionic parasympathetic vagal motor output to abdominal viscera, including the pancreas. The aims of the present study were to investigate whether DMV neurons were responsive to exogenously applied GLP-1 and, if so, whether the responsive neurons could be distinguished from DMV neurons controlling other pancreatic functions. The hypothesis is that DMV neurons that control endocrine secretion (i.e. responsive to GLP1) are different from those that control exocrine secretion (i.e. responsive to pancreatic 
polypeptide, PP). Whole cell patch clamp recordings were made from pancreas-projecting DMV neurons identified by prior application of the retrograde tracer, DiI, to the body of the pancreas. Perfusion with GLP-1 induced a concentration-dependent excitation in approximately $50 \%$ of pancreas-projecting DMV neurons ( $\mathrm{N}=56$ of 114 cells tested). The threshold of the excitatory response was $10 \mathrm{nM}$, the EC50 was approximately $30 \mathrm{nM}$ and Emax was $300 \mathrm{nM}$. At $100 \mathrm{nM}$, GLP-1 induced a $5.8 \pm 0.4 \mathrm{mV}$ depolarization and increased the firing rate from $8.4 \pm 4.0$ to $35.9 \pm 5.3$ a.p. $/ \mathrm{min}(\mathrm{HP}=-60 \mathrm{mV})$. The GLP-1 mediated excitation was mimicked by Exendin- $4(100 \mathrm{nM})$, which induced a $5.5 \pm 0.8 \mathrm{mV}$ depolarization $(\mathrm{N}=$ 4), and was completely antagonized by Exendin 9-39 (100nM; N=3). The GLP-1 induced depolarization was reduced from $6 \pm 1.1$ to $2.2 \pm 1.4 \mathrm{mV}$ by TTX $(1 \mu \mathrm{M})$ suggesting that the effects were mediated both directly, via an effect on the DMV membrane, and indirectly, via an effect on local synaptic circuits. Experiments conducted in the voltage clamp configuration indicated that GLP-1 increased by $44 \pm 9.4 \%$ the amplitude of excitatory postsynaptic currents induced by stimulation of the nucleus tractus solitarius ( $\mathrm{N}=11$ of 15 cells). Cells that responded to GLP-1 with an excitation were, instead, unresponsive to PP (which reduces pancreatic exocrine secretion). Viceversa, cells that were responsive to PP were unresponsive to GLP-1 ( $\mathrm{N}=38$ cells tested). These data indicate that 1 ) GLP-1 may control pancreasendocrine secretion via an effect on pancreas-projecting DMV neurons; and 2) DMV neurons that control endocrine secretion (i.e. responsive to GLP-1) are different from those that control exocrine secretion (i.e. responsive to PP). Supported by NSF grant 0456291

\section{M2234}

Altered Expression of Trp, $\mathrm{Ca}^{2+}$ and $\mathrm{K}^{+}$Channels in Vagal Visceral Afferents in a Post-Infectious Model of Irritable Bowel Syndrome (IBS)

Jeroen Aerssens, Pieter J. Peeters, Ronald de Hoogt, Kirk Hillsley, Andrzej Stanisz, David Grundy, Ronald H. Stead, Bernard Coulie

Background: Extrinsic afferent neurons supplying the gut are prime targets for new treatments of chronic visceral pain disorders such as IBS. The pathogenesis of IBS is heterogeneous but at least in a subpopulation of patients emotional stress and enteric infection have been implicated. The aim of this study was to investigate long-term changes in gene expression of ion channels in vagal visceral afferents in a mouse model of IBS. The model consisted of a transient inflammation induced by the nematode Nippostrongylus brasiliensis ( $\mathrm{Nb}$ ) combined with exposure to stress. Methods: Balb/c mice housed in control / stress conditions were infected with $500 \mathrm{~L} 3 \mathrm{Nb}$ larvae. Four groups of animals were studied: sham/non-stressed (ShNS), infected/non-stressed (InfNS), sham/stressed (ShS) and infected/stressed (InfS). After acute inflammation had resolved (2l days post infection), vagal gut-specific neurons in nodose ganglia (NG), labeled by intraperitoneal injection of cholera toxin B-488, were isolated by laser-capture microdissection. RNA was hybridized to Affymetrix mouse whole genome 4302.0 arrays. Expression profiles were analyzed by two-way ANOVA for microarray data evaluating the effects of stress and infection with control of false discovery rate (using the Benjamini and Hochberg approach). Confirmation was done using quantitative PCR. Results: In NG visceral neurons, significantly different expression levels were found for 14 ion channel genes including a putative $\mathrm{Ca}^{2+}$ channel, 3 TRP channels and $3 \mathrm{~K}^{+}$channel subunits. Cacng2, also known as stargazing (which modulates AMPA receptor gating and trafficking), was expressed significantly lower in infected animals (2.3-fold). With respect to $\mathrm{K}^{+}$channels, significant changes were found for $\mathrm{Kcndl}\left(\mathrm{K}_{\mathrm{V}} 4.1\right), \mathrm{Kcnj10}\left(\mathrm{K}_{\mathrm{ir}} 4.1\right)$ and $\mathrm{Kcnabl}\left(\mathrm{K}_{\mathrm{v}} \beta 1\right)$. The increased expression of Kcnabl (1.5-fold), the auxiliary subunit of voltage-gated $\mathrm{K}^{+}$channels, was observed in both stress (ShS) and infection (InfNS and InfS) exposed animals. This could differentially affect the function of multiple $K_{V} l$ channels. The TRP channels Trpal (2.1-fold), Trpc4 (2-fold) and Trpvl (2.1-fold) were all upregulated after combined exposure to stress and infection (InfS and to a lesser extent in InfNS). All of these channels are thought to be involved in chemosensation. Conclusion: A plethora of ion channel genes are altered in visceral sensory NG neurons by $\mathrm{Nb}$ infection and stress, at a time point when the acute inflammation has subsided. Overall these changes point towards long-term alterations in synaptic transmission/plasticity and increased chemosensitivity. These findings may impact on future therapeutic strategies for IBS.

\section{M2235}

Cross-Talk Between Colon and Bladder Involves the Up-Regulation of Calcitonin Gene-Related Peptide (CGRP) and Receptor Tyrosine Kinase TRKB in Bladder and Colon/Bladder Convergent Afferent Neurons in DRG Following TNBS Colitis

Liya Qiao, John R. Grider

The concurrency of gut and bladder symptoms is a common occurrence in humans. Experimental animals with colitis induced by tri-nitrobenzene sulfonic acid (TNBS) showed overactive bladder. We hypothesized that primary afferent neurons located in dorsal root ganglia (DRG) may contribute to the cross-activation of bladder in colitis. AIM: to determine the effects of TNBS-induced colitis on rat bladder afferent neurons by examining the expression of CGRP and TrkB in these neurons. METHODS: Bladder and colon afferent neurons were labeled by injection of neuronal tracing dye Fast Blue into muscle wall of bladder or dicarbocyanine dye Dil into muscle wall of colon. Colitis was induced by colonic instillation of TNBS $(0.25 \mathrm{ml}$ of $60 \mathrm{mg} / \mathrm{ml}$ in $50 \%$ ethanol) and animals were killed on day 7 by intracardiac perfusion with 4\% paraformaldehyde. DRG from lumbosacral regions L1, L6 and S1 were removed, sectioned, and immunostained with specific CGRP or TrkB antibody. DRG from control rats were also isolated, dispersed and cultured in DMEM-10 plus Ara C (10 uM). The effects of $2 \mathrm{nM}$ of nerve growth factor (NGF), neurotrophin-3 (NT-3) or brain-derived neurotrophic factor (BDNF) on CGRP expression were determined in these cultures. RESULTS: Bladder afferent neurons from DRG of control rats constitutively expressed CGRP and TrkB. After TNBS colitis, the percentage of bladder afferents expressing CGRP increased in L1 (56.5 \pm 5.1 in colitis vs. $23.2 \pm 3.7$ in controls; $\mathrm{p}<0.05)$ but not in L6 or in S1. Colitis also caused a significant increase in the percentage of bladder afferents expressing TrkB in $\mathrm{Ll}(38.3 \pm 3.5$ in colitis vs $25.5 \pm 5.2$ in controls, $\mathrm{p}<0.05)$ and in L6 $(62.1 \pm 5.2$ in colitis vs $19.3 \pm 4.1$ in controls, $\mathrm{p}<0.01$ ), but not in S1. After colitis, the percentage of convergent neurons (dually projecting from colon and bladder) in DRG from Ll expressing CGRP was significantly increased 5-fold $(\mathrm{p}<0.01)$. Co-localization studies showed that there was also an increase in the subpopulation of DRG neurons from Ll expressing both CGRP and TrkB $(2.5$-fold, $\mathrm{p}<0.05)$. In cultured DRG neurons, all three Trk agonists increased CGR expression with the rank order NGF>NT-3>BDNF. CONCLUSION: TNBS-induced colitis results in up-regulation of CGRP in bladder and colon/bladder convergent afferent neurons in DRG. Colitis also increased TrkB expression in bladder afferent neurons. The increase in the subpopulation of DRG neurons co-expressing TrkB and CGRP in colitis and the ability of neurotrophins to increase CGRP in cultured DRG suggests a role for neurotrophins in the induction of CGRP expression during colitis.

\section{M2236}

Alterations in Behaviour, Hpa Axis Function and Cytokine Profiles Following Neonatal Maternal Separation: Evidence for Dysregulation of the Brain-Gut Axis

Paul Scully, S M. O Mahony, S Murphy, L O Mahony, E M. Quigley, T G. Dinan

Background \& Aims: Evidence suggests that early-life trauma and psychological stress can affect the course of intestinal disorders and increase the risk of irritable bowel syndrome (IBS) development. Studies have demonstrated that neonatal stress following maternal separation in the rat results in an altered adult phenotype, which includes development of cardinal IBS features such as visceral hyperalgesia and reduced somatic analgesia. Permanent functional alterations in the hypothalamic-pituitary-adrenal (HPA) axis have also been observed. Using the maternal deprivation model, the aim of this study was to assess the long-term impac of early life stress on brain-gut function in rats by assessment of alterations in behaviour, HPA axis function, systemic inflammatory response, and gut associated cytokine production. Methods: 22 Male Sprague-Dawley rats were randomly divided into two groups. From postnatal day 2 to 14 , neonates were separated daily from the dams for $180 \mathrm{mins}$. Control pups remained with their mothers. Behavioural tests were performed at days 50-70 and the rats were then sacrificed by decapitation. Following sacrifice, blood samples were obtained, stimulated with LPS and cytokine production measured after 48 hours. Plasma levels of cortisol, corticosterone and TNF- $\alpha$ were analysed by ELISA. Peyer's patches (PP) and mesenteric lymph node cells (MLNCs) cytokine levels of TNF- $\alpha$, IL-4, IL-6, IL-10 and IFN- $\gamma$ cytokine levels were measured by cytometric bead array. Results: Maternally deprived animals showed a significant increase in anxious behaviour in open field experiments $(\mathrm{P}<$ 0.05 ). Whole blood cultures for the maternally deprived group showed a significant increase in TNF- $\alpha$ following LPS stimulation when compared to controls $(\mathrm{P}<0.05)$. No significant changes in TNF- $\alpha$ were observed in plasma. Plasma levels of corticosterone were significantly increased in the maternally deprived animals $(\mathrm{P}<0.01)$. The levels of TNF- $\alpha$, IL-4, IL- 6 , IL-10 and IFN- $\gamma$ in the MLNCs were found to be consistently higher in the control animals compared to maternally deprived animals. No significant differences were observed in the level of cytokines in the PP. Conclusions: This study indicates that maternal separation results in alterations in behaviour, HPA axis function, mesenteric cytokine profiles and the systemic cytokine response to inflammatory stimuli. Such changes may be important in the pathophysiology of IBS.

\section{M2237}

Feasibility and Reproducibility of Functional Magnetic Resonance Imaging in Medetomidine Sedated Rodents

Venelin J. Kounev, James Hyde, Mark Kern, Jyoti Sengupta, Christopher Pawela, Douglas Prah, Eric Paulson, Reza Shaker

INTRODUCTION:Visceral sensory function in animals has traditionally been evaluated by assessing the visceromotor response to colonic distension. Although quantitative,this technique is an indirect measure of neurosensory response to visceral stimulation.Brain imaging techniques provide a direct representation of visceral sensation, but because of the use of anesthesia necessary for restraining the animals, their full potential remains unrealized. Our aim was to study the feasibility and reproducibility of direct quantification of cerebra cortical response to visceral stimulation using fMRI technique in a rat model sedated by Medetomidine.METHODS:We studied adult Sprague-Dawley rats(480-550g) under continuous infusion of Medetomidine $(100 \mathrm{mcg} / \mathrm{kg} / \mathrm{hr})$, a potent non-narcotic $\alpha$-2 adrenoreceptor agonist.Phasic barostat controlled air distensions $(20,40,60,80,100 \mathrm{mmHg})$ were applied to the hind gut of rats in a block paradigm fashion.All animals were imaged on a 9.4T MRI scanner(Bruker Biospin)utilizing BOLD contrast(gradient echo EPI, TR 2s, TE $21 \mathrm{~ms}$,FOV 4 $\mathrm{cm}$, matrix 96x96, slice thickness $1.5 \mathrm{~mm}$, voxel size $0.625 \mathrm{~mm} 3$ ). We used AFNI,deconvolution method for analyses. RESULTS:All tested animals exhibited cortical activity with distension pressures as low as $20 \mathrm{mmHg}$. The number of fMRI activated voxels in response to increasing distension pressures progressively increased $(\mathrm{P}<0.05)$ (Fig)in a dose-dependen manner $(\mathrm{R}=0.87)$. Moreover, these findings were reproduced in each experiment with different duration of stimulus presentation. The cerebral activity was spread throughout the brain with largest magnitude in MR signal change observed in voxels in the cingulate cortex. CONCLUSIONS:Functional imaging of the cerebral cortex by fMRI is feasible in rats under Medetomidine sedation and yields reproducible results.Increasing rectal distension pressure induces a dose-dependent increase in fMRI activity of the cortex in this model. 
Brain Activity

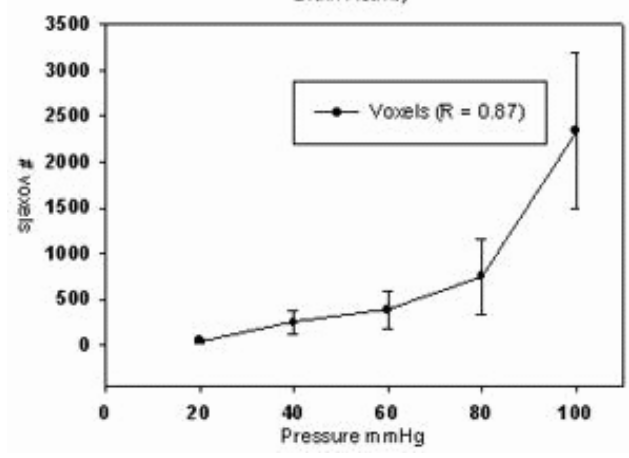

M2238

Ghrelin Stimulates Solid Gastric Emptying Via a Vagal Afferent Pathway in Conscious Rats

Yukiomi Nakade, Cindy Chen, Kiyoshi Tsukamoto, Theodore N. Pappas, Toku Takahashi

Background: Ghrelin is a newly discovered orexigenic peptide originating from the stomach and has been shown to regulate gastric motor activities. Previous reports showed that ghrelin accelerates liquid gastric emptying in mice (Regulatory peptide 2004120 23-32). We studied whether exogenous ghrelin accelerates solid gastric emptying and modify postprandial antral contractions in conscious rats. It has been demonstrated that ghrelin receptors are located at vagal afferent. We also studied whether ghrelin-induced acceleration of solid gastric emptying is mediated via vagal afferent pathway. Methods: Using male Sprague-Dawley rats, one strain gauge force transducer was sutured on the serosal surface of the antrum. One week after the operation, rats were fasted for $24 \mathrm{hrs}$. After recording of basal gastric motility for $120 \mathrm{~min}$, rats were fed of solid rat chow $(1.5 \mathrm{~g})$. Ten minutes after the feeding, ghrelin (4 microg/kg) was administered by intraperitoneal (IP)-injection and antral motility was monitored for $120 \mathrm{~min}$ thereafter. For gastric emptying study, rats were fasted for $24 \mathrm{hrs}$. Ten minutes after the ingestion of rat chow (1.5 g), ghrelin ( $0.3-8 \mathrm{microg} / \mathrm{kg})$ was administered by IP injection. Ninety minutes after the feeding, rats were sacrificed. The gastric content was recovered from the stomach, dried and weighed for calculating solid gastric emptying. To investigate whether ghrelin-induced acceleration of solid gastric emptying is mediated via vagal afferent pathway, capsaicin was applied on cervical vagus nerve bilaterally. Two weeks after the perivagal capsaicin treatment, the gastric emptying study was performed. Control rats received the vehicle-treatment on the vagus nerve. Results: Postprandial-injection of ghrelin induced phase III- like contractions of the antrum for over 30 minutes. Postprandialinjection of ghrelin (0.3-8 microg/kg) dose dependently increased solid gastric emptying. Maximum effect was obtained by ghrlin ( 4 microg $/ \mathrm{kg}$ ), which significantly accelerated gastric emptying to $76.6+/-3.6 \%(\mathrm{n}=6)$, compared to that of saline-injected rats $(58.3+/-3.6, \mathrm{n}=$ 6). Ghrlin (4 microg/kg)-induced acceleration of gastric emptying was attenuated in rats treated with perivagal capsaicin $(66.3+/-3.2 \%, \mathrm{n}=6, \mathrm{P}<0.05)$, compared to that of vehicle treated rats $(73.0+/-3.6 \%, n=6)$. Conclusions: These results indicate that postprandial ghrelin-injection changes postprandial gastric motor pattern to interdigestive motor pattern, resulting in acceleration of solid gastric emptying. The acceleration of solid gastric emptying induced by ghrlin is mediated via capsaicin-sensitive vagal afferent pathway.

\section{M2239}

The Gut Response to Stress Can Be Pharmacologically Modulated Amitriptyline Modifies Visceral Hypersensitivity in Irritable Bowel Syndrome (IBS)

Charles Murray, Wendy J. Winchester, Eric Tripoli, Maxton C. Pitcher, Michael A. Kamm, Anton V. Emmanuel

Introduction: Acute physical stress causes a measurable acute alteration of gut autonomic function and visceral hypersensitivity in patients with IBS (Murray et al Gastroenterology 2004). Low doses of amitriptyline are effective in over $50 \%$ of IBS patients, through mechanisms that are unclear. We present the first report of the effects of amitriptyline on the gut neural response to acute stress. Methods: 19 patients with constipation-predominant IBS (16 female, mean age 32, range 19-58) were given amitriptyline $25-50 \mathrm{mg}$ at night. Patients underwent stress assessment at baseline and after three months of treatment. Stress assessment comprised a physical (cold-pressor) and psychological (dichotomous listening) stress given in random order at least one day (median 1, range 1-4 days) apart. Physiological parameters measured included: perception of stress (visual analogue scale); systemic autonomic tone (heart rate and blood pressure); gut-specific autonomic innervation (laser Doppler flowmetry of rectal mucosal blood flow (RMBF)); and visceral sensitivity (rectal electrosensitivity). 13 patients underwent barostat assessment of rectal sensitivity. Results: 14 of $19(74 \%)$ patients were symptomatically improved after 3 months of amitriptyline (median dose $25 \mathrm{mg}$ ). Acute stress resulted in increased perception of stress and systemic autonomic tone, and reduced RMBF, which was similar in responders and non-responders ( $p>0.05$ for all). In contrast, all non-responders and only three out of 14 of responders, continued to exhibit stressinduced reduced pain threshold at 3 months (change from baseline $-31 \%$ vs $+2 \%, p<0.03$ respectively). Responders increased thresholds for pain from 21 (pre-) to $31 \mathrm{mmHg}$ (posttreatment) above distending pressure $(\mathrm{p}<0.03)$. No such change was seen in non-responders (from 23 to $24 \mathrm{mmHg}$, respectively). Discussion: Amitriptyline is effective in constipationpredominant IBS patients. This effect is primarily on stress-induced visceral hypersensitivity, independent of autonomic tone. The gut response to acute stress is a viable target to study drug efficacy in IBS.
M2240

Abnormal Vagal Regulatory Function of Brainstem Thyrotropin-Releasing Hormone (TRH) Is Involved in the Delayed Gastric Emptying (GE) in Type 2 Diabetic (T2D) Goto-Kakizaki (GK) Rats

Yan Ao, Natalie Toy, Moon K. Song, Viang W. Go, Mulugeta Million, Hong Yang

Background: Gastroparesis is common in diabetes, occurring in 30-50\% of diabetic patients. Caudal brainstem TRH-containing raphe-dorsal vagal complex (DVC) pathways play an important role in vagal regulation of gastric motility (Tache \& Yang, 1994). Neuropeptide $\mathrm{Y}$ (NPY) in the brainstem selectively innervates caudal raphe nuclei and the DVC and modulates TRH action in regulating vagal efferent function (Yang et al, Br J Pharmacol, 1998). We found altered brainstem TRH, NPY, and TRH receptor l (TRH Rl) gene expression in male T2D GK rats (Ao et al, Society for Neuroscience, 2005). Aim: To test the hypothesis that abnormal vagal regulatory function of brainstem TRH is responsible for the delayed GE in T2D. Methods: Brainstem NPY, TRH, and TRH Rl mRNA of normally fed adult female Wistar and GK rats (4-6/group) were measured by Northern blot analysis. Additional groups of female Wistar and GK rats were fasted overnight and intracisternally (ic) injected with saline or the stable TRH analog RX77368 (10 ng) under short isoflurane anesthesia; 20 min later, liquid GE was measured by the methylcellulose/phenol red method. Some rats were pretreated $(-20 \mathrm{~min})$ with atropine $(1 \mathrm{mg} / \mathrm{kg}, \mathrm{sc})$. Results: 1) Brainstem TRH gene expression is significantly reduced by $47 \%$ in female T2D GK rats while TRH Rl gene expression increased by 2-fold compared with Wistar rats; 2) liquid GE in $35 \mathrm{~min}$ is $32 \%$ slower in GK rats; 3) ic TRH analog-induced acceleration of GE is 61\% greater in GK than in Wistar rats; 4) the elevated GE response to ic RX 77368 in GK rats is reversed by atropine. Conclusion: 1) Altered vagal function resulting from reduced brainstem TRH gene expression may contribute to the abnormal gastric motility in T2D;2) T2D GK rats are extremely sensitive to exogenous ic TRH analog due to increased brainstem TRH Rl gene expression as a compensatory adaptation to reduced brainstem TRH synthesis.

Table 1. Brainstem TRH, NPY, and TRH Rl mRNA levels in female Wistar and GK rats

\begin{tabular}{|c|c|c|c|c|}
\hline & Blood glucose $(\mathrm{mg} \%)$ & TRH/GAPDH & NPY/GAPDH & TRH R1/GAPHDH \\
\hline Wistar rats & $92.6 \pm 6.5$ & $0.47 \pm 0.05$ & $0.81 \pm 0.06$ & $0.38 \pm 0.03$ \\
\hline GK rats & $151 \pm 8.3^{*}$ & $0.25 \pm 0.06^{*}$ & $1.31 \pm 0.27$ & $0.75 \pm 0.11^{*}$ \\
\hline
\end{tabular}

* $\mathrm{P}<0.05$ vs Wistar rats

Table 2. Effect of ic RX77368 on liquid GE (duration in min) in Wistar and GK rats (\%)

\begin{tabular}{|c|c|c|c|c|}
\hline Treatment (ic) & saline (35) & saline (20) & RX77368 (20) & RX77368+atropine (20) \\
\hline Wistar rats & $74 \pm 6$ & $34 \pm 3$ & $54 \pm 10$ & $51 \pm 15$ \\
\hline GK rats & $50 \pm 7 *$ & $34 \pm 7$ & $87 \pm 2 *$ & $52 \pm 10$ \\
\hline
\end{tabular}

* $\mathrm{P}<0.05$ vs Wistar rats

\section{M2241}

Corticotrophin Releasing Hormone (CRH) Acts On Mast Cells to Induced Intestinal Barrier Dysfunction

Ping-Chang Yang, Aaron A. Teitelbaum, Jennifer Jury, Mary H. Perdue

Ping-Chang Yang, Aaron A. Teitelbaum, Jennifer Jury, Mary H. Perdue Intestinal Disease Research Program, McMaster University, Hamilton, Canada The intestinal epithelial barrier plays a critical role in maintaining intestinal homeostasis. Several intestinal diseases such as IBD and food allergy are associated with intestinal barrier dysfunction. We and others have reported that psychological stress increases intestinal barrier permeability that is mast cell dependent; however, the mechanism of the stress-induced intestinal barrier dysfunction and its relation with mast cells remain to be further elucidated. Here we report that stress hormone $\mathrm{CRH}$, via a mechanism of receptor ligation and mast cell activation, plays a critical role in stress induced intestinal barrier dysfunction. Methods: Mast cell-deficient (Ws/Ws) rats and their +/+ normal littermates were implanted with minipumps containing CRH (50 $\mu \mathrm{g} / \mathrm{kg} / \mathrm{day}$ was delivered); minipumps containing saline were implanted in control rats. After 10 days of infusion, intestinal segments were excised and functional parameters were determined in Ussing chambers: ion secretion measured by short-circuit current (Isc); macromolecular permeability measured by flux of horseradish peroxidase (HRP). In vitro studies of epithelial barrier function were carried out in T84 epithelial cell monolayers co-cultured with CRH-stimulated mast cells (HMC-1 or RBL cells); barrier functions were assessed by measuring the transepithelial resistance (TER) and HRP flux. CRH receptor expression by mast cells was determined by RT-PCR and Western blot. Results: The $+/+$ rats treated with $\mathrm{CRH}$ showed significantly increased Isc $(48.4 \pm 3.2 \mu \mathrm{A} / \mathrm{cm} 2$, mean $\pm \mathrm{SE})$ compared with Ws/Ws rats $(13.2 \pm 1.5)$ and $+/+$ saline controls $(14.6 \pm 1.4)$. CRH-treated $+/+$ rats also showed significantly elevated HRP flux $(37.1 \pm 5.1 \mathrm{pmol} / \mathrm{hr} / \mathrm{cm} 2)$ vs Ws $/$ Ss rats $(7.1 \pm 1.2)$ and $+/+$ saline controls $(9.2 \pm 1.7)$. Mast cell degranulation was observed by electron microscopy: 1) in the intestine of the CRH treated rats, and 2) in cultured RBL mast cells after the addition of $\mathrm{CRH}(50 \mathrm{nM})$. The $\mathrm{CRH}$-activated mast cells significantly reduced the epithelial TER $(48.5 \pm 3.8 \%$ compared with control) and increased the HRP flux $(3.5 \pm 1.1$ fold) in T84 monolayers. Expression of $\mathrm{CRH}$ receptors were detected in the mast cells at mRNA and protein levels. Conclusions: Chronic administration of CRH mimics the effect of chronic stress in compromising intestinal barrier function. $\mathrm{CRH}$ activates mast cells; mast cell-derived mediators directly influence intestinal epithelial barrier function. 
Increased Colonic Sensitivity in Irritable Bowel Syndrome Is the Result of Increased Perceptual Response Bias Rather Than Increased Perceptual Sensitivity

Spencer D. Dorn, Olafur S. Palsson, Syed Im. Thiwan, Motoyori Kanazawa, W. Crawford Clark, Miranda Al. van Tilburg, Douglas A. Drossman, Yolanda Scarlett, Rona L. Levy, Yehuda Ringel, Michael D. Crowell, Kevin W. Olden, William E. Whitehead

Background: Patients with irritable bowel syndrome (IBS) report pain at lower pressures and volumes of intra-luminal balloon distension than subjects without IBS. These lower pain thresholds have been attributed to heightened visceral sensitivity and labeled a biological marker for IBS. However, because the methods used to ascertain pain thresholds are subject to perceptual response bias, differences in pain thresholds might reflect underlying psychological differences rather than biological differences [Gastroenterol 1998;115:1263-1271]. Aims: To determine and explain differences in pain and urge thresholds in IBS patients compared to controls. Methods: Sensory thresholds were tested in the descending colon using ascending methods of limits (AML) in 121 IBS subjects and 28 control subjects. In addition, potential psychological (response criterion bias: $1 / \beta$ ) and sensory (perceptual sensitivity: $p(a)$ ) determinants of pain and urge thresholds were determined using sensory decision theory [Anesthesiol 1974;40:272-287]. Psychological distress was assessed using the Brief Symptom Inventory (BSI) and Trauma Symptom Checklist (TSC-40). The data were analyzed using non-parametric statistical tests. Results: In response to painful stimuli IBS patients had lower AML pain thresholds (median: 36 vs. $50 \mathrm{mmHg}$; $<$.001) and a higher response bias ( .25 vs. $.19 ; \mathrm{p}<.001$ ) but similar perceptual sensitivity ( .50 vs. $.50 ; \mathrm{p}=.691)$. There was a strong inverse correlation between AML pain thresholds and response bias $(\mathrm{r}=-.75 ; \mathrm{p}<.001)$, and a weak correlation with perceptual sensitivity $(r=-.18 ; \mathrm{p}=.03)$. Response bias was weakly correlated with BSI global score $(r=.18 ; \mathrm{p}=.035)$, BSI somatization $(\mathrm{r}=.26 ; \mathrm{p}=.001)$ and TSC40 anxiety $(\mathrm{r}=.29 ; \mathrm{p}=.001)$. IBS patients scored higher than controls on each of these psychometric scales $(\mathrm{p}<.001)$. IBS patients also had lower AML thresholds for the nonpainful sensation of urge to defecate ( $26 \mathrm{vs.} 48 \mathrm{mmHg} ; \mathrm{p}=.002$ ) and a higher response bias ( .33 vs. $.24 ; \mathrm{p}=.006$ ) but similar perceptual sensitivity ( .55 vs. $.50 ; \mathrm{p}=.168$ ). There was a strong inverse correlation between AML urge thresholds and response bias $(\mathrm{r}=-.71 ; \mathrm{p}<.001)$ and a weak, non-significant correlation with perceptual sensitivity $(r=-.16 ; p=.052)$. Conclusions: Increased colonic pain sensitivity in IBS is largely explained by increased perceptual response bias rather than increased perceptual sensitivity. Thus, increased colonic sensitivity in IBS is determined more by psychological factors than by biological factors. [Supported by grants R24 DK67674, R01 DK31369 and RR00046]

\section{M2243}

Chronic Mild Stress Model in a Rat: A Real Model of the Visceral Hypersensitivity That Reflects a Sustained Stressful Situation of Irritable Bowel Syndrome in a Patient

Yong Sung Kim, Suck Chei Choi, Moon Young Lee, Young Woo Sohn, Yong Leol Oh, Tae Hyeon Kim, Geom Seog Seo, Eun Young Cho, Seoul Lee, Sang Wook Kim, Myung Gyu Choi, Yong-Ho Nah

Background/Aim: Psychological stress is believed to play a major role in irritable bowel syndrome(IBS). However, most of the studies about the effect of stress on the gastrointestinal(GI) tract used acute, short and intense stress tests such as cold water immersion, spinning, electrical shock or chemical injury. The effect of chronic stress on the GI tract needs to be investigated, but there were ethical limitations of human study and no valid animal model. We tried to find an appropriate animal model for chronic stress and adopt a chronic mild stress(CMS) model from psychiatric research field. In this study, we evaluate the impact of chronic mild stress exposure on GI tract and hypothalamic-pituitary-adrenal(HPA) activity using CMS model. Method: Adult Sprague-Dawley male(N=5) rat(200g) spent 6 weeks under CMS protocol. Control group( $\mathrm{N}=5)$ spent the same period under normal conditions. The stressors were: food $\&$ water deprivation, cage tilt at $45^{\circ} \&$ wet bedding, intruder sound, stroboscopic light, restriction in a small cage, replacement of sawdust with sand, strong illumination, white noise during dark phase. Each stressor lasted 8 18 hours each week. The body weight of each animal was measured once per week. After CMS protocol, we assessed EMG response of abdominal muscle to colorectal distension(CRD) using Fogarty embolectomy catheter and obtained blood sample for hormonal assay in both group. The adrenal glands were dissected for measurement of weight and colonic samples were collected to evaluate the change of mast cell using toluidine blue staining. Result: After following CMS protocol for 6 weeks, the weights of the CMS group were lower than the control group $(310.67 \pm 12.94 \mathrm{~g}$ vs $408.33 \pm 16.07 \mathrm{~g}, \mathrm{P}<0.01)$. The threshold to elicit a abdominal muscle contraction in response to $\mathrm{CRD}$ decreased from $0.58 \pm 0.09 \mathrm{~mL}$ in the control group to $0.39 \pm 0.09 \mathrm{~mL}$ in the CMS group $(\mathrm{P}<0.01)$. The plasma corticosterone level and absolute adrenal weight were slightly high in CMS group, but was not statistically significant. But the relative adrenal weight to body weight was significantly higher in the CMS group $(0.88 \pm 0.1 \mathrm{lmg} / \mathrm{g}$ vs $0.69 \pm 0.11 \mathrm{mg} / \mathrm{g}, \mathrm{P}<0.01)$. The number of mast cell increased more in the CMS group $(3.47 \pm 1.64 / \mathrm{mm} 2$ vs $0.55 \pm 0.39 / \mathrm{mm} 2, \mathrm{P}<0.05)$. Conclusion: These CRD response and mast cell changes are consistent with those of human studies and CMS model induce chronic change of HPA activity; therefore the CMS model of a rat seems to be an appropriate animal model to research the pathophysiology of IBS in terms of chronic stress. And chronic stress-induced change of HPA activity may contribute to the alteration of visceral sensation together with peripheral immune activation.
Gastric Electrical Stimulation Modulates Hypothalamic CRF Producing Neurons During Post Operative Ileus in Rat

Gourcerol Guillaume, Gallas Syrine, Mounien Lourdes, Leblanc Isabelle, Bizet Patrice, Leroi Anne Marie, Ducrotte Philippe, Vaudry Hubert, Jegou Sylvie

Introduction: Gastric electrical stimulation (GES), a new therapeutical approach in the treatment of medically refractory gastroparesis, causes symptomatic improvement despite modest acceleration of gastric emptying. We aimed to assess the effects of acute GES on cotricotropin-releasing factor (CRF) producing neurons in paraventericular nucleus o hypothalamus (PVN), which are involved in gastric ileus induction during laparotomy Method: Two electrodes were implanted in the rat gastric antrum during laparotomy, then stimulation (amplitude: $2 \mathrm{mV}$; duration $0,3 \mathrm{~ms}$; frequency: $2.1 \mathrm{~Hz} ; 1$ minute $\mathrm{ON} / 2$ minutes $\mathrm{OFF}$ ) or sham stimulation was applied. Using immunohistochemistry, the number of c-Fos protein-expressing neurons (FOS-IR) was quantified in PVN after 1 hour of stimulation ( $\mathrm{n}=$ 10) or sham stimulation $(\mathrm{n}=10)$. The number of neurons expressing simultaneously c-Fos protein and CRFmRNA was measured using in situ hybridization associated with immunocytochemistry, and silver grain density in PVN was calculated both in stimulated $(\mathrm{n}=6)$ and sham $(n=6)$ animals. Finally, $c$-fos and CRF mRNA levels were determined by quantitative PCR in the dorsal hypothalamus in sham $(n=8)$ and stimulated $(30 \mathrm{~min})$ animals $(\mathrm{n}=8)$ Results: Compared to sham group, FOS-IR in PVN decreased after 1 hour of stimulation (respectively $295+46$ vs $135+31 ; \mathrm{p}<0.05$ ), and was not significantly modified by sub-diaphragmatic vagotomy. In PVN, $86.4 \%$ and $72.4 \%$ CRF mRNA positive cells of sham and stimulated group were c-Fos-positive $(\mathrm{p}<0.05)$. Silver grain density throughout the PVN was 39\% reduced in stimulated group compared to sham group $(\mathrm{p}<0.05)$. Last, both $\mathrm{c}$ fos and CRF mRNA levels were respectively 56\% and 36\% decresed after stimulation ( $\mathrm{p}<$ 0.05). Conclusion: Acute GES modulates CRF producing neurons in PVN during postoperative gastric ileus and decrease CRF synthesis at this level.

\section{M2245}

Gastritis Causes Acid Hypersensitivity and Gender-Related Changes in Circulating Corticosterone and Anxiety-Related Behavior of Mice Peter Holzer, Evelin Painsipp, Thomas Wultsch, Anaid Shahbazian

Background and Aims: Gastrointestinal disease is associated with changes in gut function, abdominal pain and mood disturbances in a gender-dependent manner. In this study we investigated whether experimental gastritis would alter gastric acid sensitivity, circulating corticosterone and behavioral indices of anxiety and depression. Methods: Gastritis in female and male $\mathrm{OF} 1$ mice was induced by adding iodoacetamide $(0.1 \%)$ to the drinking water for 7 days; control mice received normal tap water. Inflammation was assessed by the myeloperoxidase (MPO) activity in the gastric wall, and the plasma levels of corticosterone were determined with an ELISA. The afferent signaling of a noxious stimulus, hydrochloric acid $(0.25 \mathrm{M} \mathrm{HCl})$, was visualized by immunohistochemistry for c-Fos in the nucleus tractus solitarii (NTS). Anxiety-related behavior was assessed on the elevated plus maze (EPM) and via stress-induced hyperthermia (SIHT), while depression-like behavior was estimated with the tail suspension test (TST). Results: Chronic addition of iodoacetamide to the drinking water induced gastritis as shown by an increase in MPO activity in the gastric wall. The increase in MPO activity in female mice was significantly larger than in male mice. The basal plasma levels of corticosterone in male mice remained unaltered by iodoacetamide but were reduced by $65 \%$ in female mice treated with iodoacetamide. Expression of c-Fos in the NTS evoked by gastric acid challenge was enhanced by $67 \%$ following treatment with iodoacetamide. The depression-related behavior in the TST was left unaffected by iodoacetamide. On the EPM, female mice treated with iodoacetamide exhibited fewer entries into the open arms than control mice, while iodoacetamide-treated male mice did not differ from their controls. The baseline rectal temperature of male mice treated with iodoacetamide was enhanced and SIHT was significantly reduced by iodoacetamide treatment. In contrast, the baseline rectal temperature of female mice was diminished and SIHT elevated following administration of iodoacetamide. Conclusions: These data indicate that iodoacetamideinduced gastritis is associated with hypersensitivity to gastric acid challenge. Female, but not male, mice also exhibit reduced levels of circulating corticosterone and behavioral signs of anxiety. It is hypothesized that inflammation of the stomach leads to gastric acid hypersensitivity and gender-dependent alterations in anxiety-related behavior and the hypothalamus-pituitary-adrenal axis. Supported by the Austrian Scientific Research Funds, the Jubilee Funds of the Austrian National Bank and the Zukunftsfonds Steiermark.

\section{M2246}

Trinitrobenzene Sulfonic Acid (TNBS) Colitis Activates ERK in Rat Lumbosacral Spinal Cord

Liya Qiao, Melisa Gulick, John R. Grider

Activation of extracellular signal-regulated kinase (ERK) in rat spinal cord and peripheral organs is reported to be essential for pain perception after inflammation or nerve injury Our previous study demonstrated activation of ERK5 but not ERK1/2 in lumbosacral dorsal root ganglia following TNBS colitis. The AIM of this study was to examine the changes in ERK (ERK1/2 and ERK5) activation in spinal cord following TNBS-induced colitis. METHODS Inflammation was induced in rats by colonic instillation of TNBS $(0.25 \mathrm{ml}$ of $60 \mathrm{mg} / \mathrm{ml}$ in $50 \%$ ethanol). After 7 days, rats were killed by intracardiac perfusion with $4 \%$ paraformaldehyde for immunohistochemistry or killed by $\mathrm{CO} 2$ inhalation and thoracotomy for western blot. Spinal cord segments from lumbosacral levels L1, L6 and S1 were collected for analysis. Specific primary antibody against phospho-ERKl/2 or phospho-ERK5 was used for immunohistochemical or western blot determination of phospho-ERK. Fluorchem 8800 software was used for densitometry analysis. RESULTS: Western blot analysis showed that TNBS colitis significantly induced phospho-ERKl/2 in Ll (3-fold, $\mathrm{p}<0.05$ ) and S1 (2-fold, $\mathrm{p}<0.05$ ) but not in L6 spinal cord levels. Immunostaining with the specific phospho-ERK1/2 antibody showed that the increase in phospho-ERK1/2 occurred at the superficial dorsal horn (DH) of Ll (1.5-fold increase, $\mathrm{p}<0.01)$ and $\mathrm{Sl}(1.2$-fold increase, $\mathrm{p}<0.05)$ spinal cord. TNBS colitis 
also caused an increase in the density and number of neurons expressing phospho-ERKl/ 2 in the region of the gray commisure of $\mathrm{Ll}$ spinal cord ( 3 -fold increase, $\mathrm{p}<0.01$ ). In $\mathrm{S} 1$ spinal cord, colitis caused a significant increase in the density of phospho-ERK1/2 in the region of the sacral parasympathetic nucleus (SPN, 1.7-fold, $\mathrm{p}<0.01$ ). After colitis, strong phospho-ERK1/2-stained nerve bundles appeared in S1 lamina I along the lateral edge of the DH and extended into the region of SPN. Western blot showed no changes in phosphoERK5 in any level of spinal cord examined. CONCLUSION: TNBS-induced colitis results in region-specific changes in phospho-ERK1/2, but not phospho-ERK5 in spinal cord. This is in stark contrast to our previous studies showing TNBS colitis-induced changes in phosphoERK5 but not phospho-ERK1/2 in dorsal root ganglia.

\section{M2247}

Abnormalities of Sleep Architecture and Colonic Motility Are Limited to Early Sleep in Diarrhea-Predominant Irritable Bowel Syndrome (IBS-D) Patients Heng Y. Wong, Emeran A. Mayer, Bruce D. Naliboff, Britta Dickhaus, Vanessa Z. Ameen, Joyce Reinholdt, Preetam J. Schramm, Jennifer S. Labus, Teresa I. Olivas, Lin Chang

Background: Subjective reports of poor sleep are a common extraintestinal manifestation of irritable bowel syndrome (IBS) and have been related to the severity of IBS symptoms. Even though some studies have reported objective evidence of abnormal sleep in IBS, the relationships between objective and subjective measures of sleep and colonic motility are not known. Aims: 1) To compare sleep EEG and colonic motility during consecutive REM sleep periods in IBS-D patients and healthy individuals, and 2) To assess the relationship between sleep EEG activity with subjective sleep and colonic motility. Methods: 11 female IBS-D patients (mean age $38 \pm 2.5 \mathrm{yrs}$ ) and 13 age-matched controls (Ctrls, mean age 33.8 \pm 2.7 yrs) were recruited for a 2-night sleep study in the General Clinical Research Center (GCRC). After 1 night of acclimatization, polysomnograms and left colonic motility index (MI) were measured simultaneously. Objective sleep measures included duration and number of REM periods, REM density and alpha wave intrusion during slow wave sleep (expressed as alpha/delta ratio). Quality of sleep was measured using the Medical Outcomes Study sleep questionnaire. Results: IBS-D patients showed several abnormal sleep EEG and colonic motility findings compared to Ctrls only during the first REM sleep period, but normalized during subsequent REM periods: 1$)$ longer REM duration $(27.4 \pm 5.4$ min vs $11.1 \pm 1.4$ min, $\mathrm{p}<0.05), 2)$ greater REM density $(4.0 \pm 0.5$ vs $2.7 \pm 0.3, \mathrm{p}<0.05)$, and 3$)$ greater colonic MI $(\mathrm{p}<0.05)$. In addition, alpha wave intrusion was greater during the first period of stage $3 / 4$ sleep in IBS-D patients vs Ctrls $(3.1 \pm 0.5$ vs $1.6 \pm 0.3, p=0.01)$. While there was a positive association between colonic MI and REM duration in all subjects ( $\beta=11.9$, $\mathrm{p}<0.05$ ), correlations between colonic MI and REM density were greater in IBS-D vs Ctrls $(\mathrm{p}<0.01)$. Subjective ratings of sleep adequacy were lower in IBS-D patients vs $\mathrm{Ctrl}(\mathrm{p}<0.005)$ and correlated negatively with duration of REM sleep $(r=-0.41, p=0.08)$ and REM density $(\mathrm{r}=-0.41, \mathrm{p}=0.08)$ during the first REM period. Conclusions: IBS-D patients have abnormal sleep EEG and colonic MI in the early part of sleep, but these abnormalities resolve with sleep progression suggestive of a restorative role of sleep. Increased alpha wave activity during non-REM sleep in IBS is a novel finding and has been associated with increased arousability and non-regenerative sleep. These findings are consistent with a compromised ability of the CNS to downregulate arousal mechanisms during the early phases of sleep. Supported by NIH grants GCRC \#M01-RR00865, P50 DK64539, and GlaxoSmithKline.

\section{M2248}

A Role of Stress and Stress-Related Neuropeptide of the Brain in the Progression of Non-Alcoholic Steatohepatitis (NASH)

Masashi Yoneda, Yukiomi Nakade, Kimihide Nakamura, Masaya Tamano, Tadahito Shimada, Akira Terano, Hideyuki Hiraishi

BACKGROUND: NASH is a distinct clinical entity characterized by steatosis with inflamation and fibrosis of the liver. Although the causes of NASH are not well defined, "two hit theory" is generally accepted but the second hit is still unclear. Epidemiological study has showed that mental stress is the risk factor for NASH, suggesting a crucial role of mental stress as the second hit in the progression of NASH. Several neuropeptides are overexpressed in the brain and play a role in the regulation of stress-related phenomenon. Corticotropin-releasing factor $(\mathrm{CRF})$ is synthesized in the hypothalamus under stress condition and acts as neurotransmitter to control hepatic functions. PURPOSE: To investigate an influence of stress and involvement of endogenous CRF of the brain in the progression of NASH from simple fatty liver. METHODS: Fatty liver was induced by subcutaneous injection of $\mathrm{CCl} 4(2 \mathrm{ml} / \mathrm{kg})$ Rats were subjected partial body restraint stress just after the CCl4 administration for $6 \mathrm{~h}$. Serum ALT and hepatic histological changes are assessed $24 \mathrm{~h}$ after CCl4 administration. In another experiment, Either CRF antagonist, astressin, (0.1-1 $\mu \mathrm{g})$ or saline vehicle was injected intracisternally just before and $6 \mathrm{~h}$ after $\mathrm{CCl} 4$ administration. 6-hydroxydopamine (6-OHDA, $100 \mathrm{mg} / \mathrm{kg}$ ip, -7 days and $80 \mathrm{mg} / \mathrm{kg}$ ip, -4 days), atropine methyl nitrate $(0.15$ $\mathrm{mg} / \mathrm{kg}$ ip, $-30 \mathrm{~min})$, hepatic sympathectomy ( -3 days), hepatic branch vagotomy ( -3 days), or respective vehicle treatment or sham operation was performed. RESULTS: CCl4 induced hepatic steatosis with an increase of serum ALT level (106 $\pm 15 \mathrm{IU} / \mathrm{L}) 24$ after the injection. Restraint stress induced hepatic necrotic change besides steatosis, and aggravated serum ALT level $(278 \pm 35 \mathrm{IU} / \mathrm{L})$. Intracisternal astressin dose-dependently lessened hepatic necrosis and elevated serum ALT level. The exacerbating effect of restraint stress on CCl4-indiced liver damage was abolished by sympathectomy or 6-OHDA pretreatment but not by hepatic branch vagotomy or atropine pretreatment. CONCLUSION: These findings provide a basical scientific evidence for a crucial role of stress and endogenous CRF of the brain in the progression of NASH.

\section{M2249}

Vagal Dependent Gastric Prostaglandin (PG) Release Induced By Intracisternal (IC) TRH Analog, Rx 77368, Dampens the Gastric Motility Response Through EP-1 Receptor in Anesthetized Rats

K.K. Kanamoto, Keishi Kawakubo, David W. Adelson, Yvette Tache

BACKGROUND: The stable TRH analog, RX77368 (RX) injected intracisternally (ic) at low doses (2-10 ng) induces a vagally-mediated, atropine-sensitive and dose-related increase in gastric PGE2 release that mediates gastric cytoprotection and blunts the acid response in rats (AJP 1998 274:G170-7). RX ic also induces vagal-dependent stimulation of gastric motor function (Endocrinology. 1998 139:3730-5). AIM: To assess whether endogenous gastric PGs also modulate the gastric motility response to ic RX via EP receptors (EPR). METHODS: Intragastric luminal pressure (ILP) was monitored via a catheter led into the corpus via a forestomach incision in urethane-anesthetized SD rats. Consecutive ic injections of RX (2.5, $10 \mathrm{ng}$ ) were done at a $60 \mathrm{~min}$ interval. Integrated (AR: $\left.\mathrm{cmH} 2 \mathrm{O}^{*} \mathrm{~min}\right)$ and peak responses (PR: $\mathrm{cmH2O}$ ) were calculated using computer software (DADiSP ver. 4.1). Basal ILP was $3.9 \pm 0.1 \mathrm{cmH} 2 \mathrm{O}$. Effects of pretreatment with indomethacin (IM, $5 \mathrm{mg} / \mathrm{kg}$, ip) or ONO8713 , a selective EP-1R antagonist (ONO, $10 \mathrm{mg} / \mathrm{kg}$, IP) were examined. RESULTS: Ic RX at 2.5 and $10 \mathrm{ng}$ induced a dose-related increase in ILP. In IM treated rats, the AR to ic RX at 2.5 and 10 ng was increased by $186 \%$ and $92 \%$, and PR by $220 \%$ and $67 \%$, respectively, compared with vehicle-pretreated group. Likewise, in ONO pretreated rats, the AR following ic RX was increased by $109 \%$ and $61 \%$, and PR by $292 \%$ and $45 \%$. IM and ONO had no effect by themselves. CONCLUSIONS: Blockade of PG synthesis or EP-1R potentiates the increased motility induced by ic RX at low doses. This indicates the existence of a PG/EP1 inhibitory mechanism recruited by low vagal stimulation that dampens vagally stimulated gastric motility.

Area of response (AR) and Peak Response (PR). Mean \pm SEM.

\begin{tabular}{|c|c|c|c|c|c|}
\hline Treatment & $\mathrm{n}$ & AR:RX2.5 & AR:RX10 & PR:RX2.5 & PR:RX10 \\
\hline RX ic+vehicle\#1 & 7 & $94.6 \pm 6.6$ & $251.7 \pm 18.4$ & $4.2 \pm 0.4$ & $15.2 \pm 3.2$ \\
\hline RX ic+IM & 7 & $270.2 \pm 36.6^{*}$ & $482.6 \pm 50.7^{*}$ & $13.1 \pm 2.8^{*}$ & $25.4 \pm 3.3^{*}$ \\
\hline Saline ic+IM & 5 & $24.1 \pm 8.7$ & $23.5 \pm 9.4$ & $1.2 \pm 0.3$ & $1.2 \pm 0.6$ \\
\hline RX ic+vehicle\#2 & 7 & $114.8 \pm 10.9$ & $279.1 \pm 14.5$ & $3.8 \pm 0.7$ & $14.5 \pm 1.3$ \\
\hline RX ic+ONO & 7 & $240.0 \pm 23.7 \dagger$ & $449.2 \pm 35.8^{\dagger}$ & $14.9 \pm 3.1 \dagger$ & $21.0 \pm 2.5 \dagger$ \\
\hline Saline ic+ONO & 5 & $5.8 \pm 4.2$ & $6.6 \pm 4.9$ & $0.3 \pm 0.2$ & $0.3 \pm 0.3$ \\
\hline
\end{tabular}

* $\mathrm{p} \leq 0.05$ vs. RX ic+vehicle\#l, $\uparrow \mathrm{p} \leq 0.05$ vs. RX ic+vehicle \#2 (ANOVA)

M2250

Neonatal Gastric Suctioning Results in Visceral and Somatic Hyperalgesia in Adult Rats

C.S. R. Smith, Jyoti N. Sengupta, Adrian Miranda

Objectives: Gastric suctioning of newborn infants is a common procedure in neonatal intensive care units. Recent evidence suggests that nasogastric suctioning in premature infants may play a role in the development of visceral hyperalgesia. Animal models have demonstrated a critical time during development in which the spinal cord is vulnerable to functional alterations in pain pathways. We hypothesize that repeated orogastric suctioning during the neonatal period results in chronic alterations in visceral and somatic sensation leading to hyperalgesia in adult rats. Methods: Neonatal male Long Evans rats $(n=13)$ received daily orogastric suctioning for 10 days starting at postnatal day two (P2). A separate group of control $(n=15)$ rats were handled similarly without orogastric suction. At 2 months of age, electrodes were implanted in the external oblique muscle in both groups for EMG recordings. The viscero-motor response (VMR) to graded colorectal distension (CRD) was used to assess visceral sensation $(10-80 \mathrm{mmHg}, 30 \mathrm{~s}$, 180s inter-stimulus intervals). Withdrawal thresholds using noxious heat applied to the hind paws were used to test alterations in cutaneous sensitivity. Results: Neonataly suctioned rats demonstrated a significant increase in the VMR to graded CRD when compared to non-suctioned controls $(\mathrm{p}<0.05)$. Cutaneous heat evoked paw withdrawal latencies of control rats (left hind: $13.8 \pm 0.7 \mathrm{~s}$, right hind: $14.2 \pm 0.7 \mathrm{~s}$ ) were significantly higher than orogastric suction rats (left hind: $10.0 \pm 0.7 \mathrm{~s}$, right hind: $9.4 \pm 0.4 \mathrm{~s}$, $\mathrm{p}=0.001$ ). Conclusions: Orogastric suctioning during the neonatal period results in global chronic somatic and visceral hyperalgesia in adult rats. These findings validate the existence of a critical period early in the development of the nervous system that can be associated with long-term neural plasticity.

\section{M2251}

The Esophagus Is Topographically Organized in the Brainstem According to Peripheral Location As Well As Receptor Type Ivan M. Lang, Bidyut K. Medda, Reza Shaker

Prior studies have found a topographical representation of the esophagus in the brainstem based on peripheral spatial distribution; but evidence suggests that other digestive tract organs, i.e. tongue, may also be organized in the brainstem according to receptor type. AIM: To determine whether the esophagus is organized in the brainstem according to receptor type as well as location. METHODS: Twenty anesthetized cats, 4 per group, were prepared to determine the brainstem projections of cervical or thoracic esophageal receptors sensitive to $\mathrm{H}^{+}$or distension; and the role of the vagus nerves. The cervical or thoracic esophagus was perfused $(1 \mathrm{ml} / \mathrm{min}$ ) with $0.1 \mathrm{~N} \mathrm{HCl}$ for 1.5 hours followed by $0.1 \mathrm{M} \mathrm{PBS}(\mathrm{pH} 7.3$ ) for 1.5 hours. A balloon was positioned in the cervical or thoracic esophagus and slowly inflated to $40-60 \mathrm{mmHg} 3$ times per minute for 3 hours. The cervical $\mathrm{H}^{+}$receptor protocol was performed after bilateral vagotomy. At the end of the experiments the brains were removed, sectioned, and stained for $c$-fos immunoreactivity. RESULTS: $\mathrm{HCl}$ perfusion but not distension of the cervical esophagus activated neurons in NTSis, NTScom and AP, but not in 
NTSce (Table). $\mathrm{HCl}$ perfusion or distension of the thoracic esophagus activated neurons in NTSce, but not in NTSis or AP (Table). Vagotomy blocked all responses to $\mathrm{HCl}$ perfusion of the cervical esophagus (Table). CONCLUSIONS: 1. Receptors of the cervical and thoracic esophagus project to different sets of brainstem sensory nuclei. 2. Mucosal $\mathrm{H}^{+}$-sensitive but not muscularis slowly adapting tension-sensitive receptors of cervical esophagus project to NTSis, NTScom and AP via the vagus nerves. 3. Muscularis slowly adapting tension and muscosal $\mathrm{H}^{+}$-sensitive receptors of the thoracic esophagus project to the NTSce. 4. Therefore, the esophagus is topographically organized in the brainstem according to periperal location as well as receptor type. Supported in part by DK-25731

Table. Number of $c-f o s$ positive neurons in brainstem nuclei activated under different conditions

\begin{tabular}{|c|c|c|c|c|c|c|}
\hline Experimental Groups & Comparisons & NTSce & NTSim & NTSis & NTScom & AP \\
\hline 1. Cerv-Eso-H ${ }^{+}$ & & $28 \pm 2$ & $21 \pm 2$ & $87 \pm 5$ & $64 \pm 4$ & $180 \pm 41$ \\
\hline 2. Cerv-Eso-Dist & vs 1 & $26 \pm 1$ & $16 \pm 3$ & $10 \pm 2 *$ & $8 \pm 1^{*}$ & $33 \pm 9^{*}$ \\
\hline Thor-Eso-Dist & vs 2 & $64 \pm 13^{*}$ & $7 \pm 1$ & $9 \pm 3$ & $8 \pm 1$ & $4 \pm 1^{*}$ \\
\hline Thor-Eso-H & vs 1 & $64 \pm 5^{*}$ & $8 \pm 2 *$ & $8 \pm 2 *$ & $33 \pm 5^{*}$ & $9 \pm 2 *$ \\
\hline Cerv-Eso-H $^{+}$-VX & vs 1 & $22 \pm 4$ & $13 \pm 2$ & $11 \pm 1^{*}$ & $7 \pm 1^{*}$ & $15 \pm 2 *$ \\
\hline
\end{tabular}

Values are mean $\pm \mathrm{SE}$ of number of $c$-fos positive neurons per section. Cerv, cervical; Thor, thoracic; Eso, esophagus; $\mathrm{H}^{+}, \mathrm{HCl}$ perfusion; Dist, distension; VX, vagotomy; NTS, nucleus tractus solitarius; CE, central; IM, intermediate; IS, interstitial; Com, commisural; AP, area postrema. *, $\mathrm{P}<0.05$ for a difference between groups.

\section{M2252}

Sex-Specific Alterations in Central Autonomic Function Among Patients with Irritable Bowel Syndrome

Kirsten Tillisch, Joshua Bueller, Steve Berman, Brandall Suyenobu, Emeran A. Mayer, Bruce Naliboff

Background: Sex related differences in autonomic nervous system (ANS) activity have been described in IBS patients. In general, male patients show greater sympathetic (SNS) activity compared to females. Skin conductance (SC) is a noninvasive measurement of SNS function highly sensitive to central arousal. Aims: To examine sex differences in central SNS response to rectal balloon inflation using PET imaging and SC. Methods: 36 patients (18 male) with non-constipated Rome+ IBS underwent $\mathrm{H}_{2}{ }^{15} \mathrm{O}$ PET imaging and simultaneous SC measurement during $0 \mathrm{mmHg}$ and $45 \mathrm{mmHg}$ rectal balloon inflations. Ratings of discomfort were obtained following the scans. Regression analysis (SPM 99) was used to identify voxels whose activation positively covaried with SC level across scans in males and females. A priori regions of interest including the amygdala, insula, midcingulate, and dorsolateral prefrontal (DLPFC) cortices were determined based on previously described ANS networks. Statistical threshold for these regions was based on small volume correction (significance set at $\mathrm{p}<0.05$ ). A whole brain analysis (significance set at $\mathrm{p}$ (uncorrected) $<0.001$ for apriori regions) was also performed. Results: Male IBS subjects showed SC positive covariance with the midcingulate cortex (left: $\mathrm{p}=0.004, \mathrm{t}=4.0$ and right: $\mathrm{p}=0.002, \mathrm{t}=5.9)$, right amygdala $(\mathrm{p}=$ $0.014, t=5.2)$, and left anterior and bilateral posterior insula $(\mathrm{p}=0.015, \mathrm{t}=3.6 ; \mathrm{p}<0.05, \mathrm{t}>3.5)$. Female IBS subjects showed a positive SC covariance with the DLPFC, left anterior insula $(\mathrm{p}=0.034, \mathrm{t}=3.5)$, and left amygdala $(\mathrm{p}=0.025, \mathrm{t}=6.2)$. As expected with the small samples size, the greater SC response seen in male patients did not reach statistical significance $(p>0.05)$. No sex differences were seen in discomfort ratings. Conclusions: 1 . Both male and female patients show a strong positive relationship between SC and limbic brain regions known to be involved in central autonomic control (amygdala, insula) 2. Sex related differences in laterality of the amygdala correlation are analogous to previously reported findings of laterality for memory processing of emotional material. 3. The strong correlation with MCC in male but not female patients is consistent with greater SNS responsiveness in men to a pelvic visceral stimulus found in previous studies. 4. The increased DLPFC - SC relationship found only in women may be related to sex-based differences in corticolimbic interactions. Supported in part by NIH grants P50 DK64539, DK 48351 and R24 AT002681

\section{M2253}

Chronic Restraint Stress Causes NO More Stimulation of Colonic Motor Function in Conscious Rats

Constant S. Masere, Cindy Chen, Yukiomi Nakade, Kiyoshi Tsukamoto, Christopher Mantyh, Kirk Ludwig, Theodore N. Pappas, Toku Takahashi

Background; Previous studies have shown that acute stress stimulates colonic motor function, at least in part, via a central corticotropin releasing factor (CRF) in rodents. However, little is known whether colonic motility is altered in chronic stress. We studied the changes of colonic motor function in response to chronic restraint stress or daily administration of CRF in rats. Methods; For colonic motility study, two force transducers were sutured on the proximal and distal colon. One week after the surgery, rats were subjected to wrapping restraint stress for 90 minutes every day for 5 consecutive days (chronic stress). Another group received intracisternal (ic)-injection of CRF $(1 \mu \mathrm{g})$ every day for five consecutive days. Colonic motility was monitored before, during and after the stress loading and CRF-injection. For colonic transit study, a catheter was inserted into the proximal colon. One week after the surgery, rats were subjected to chronic restraint stress for 5 consecutive days. On the 5 th day, $51 \mathrm{Cr}$ was injected via the catheter into the proximal colon before stress loading. Ninety minutes after the administration of $51 \mathrm{Cr}$, entire colon was removed and divided into 10 equal segments. The radioactivity of each segment was counted and the geometric center (GC) was calculated as a distribution of 51Cr. Results; At the 1st day of restraint stress, calculated motility index was significantly increased to $330+/-34 \%$ and $310+/-28 \%$ of basal in the proximal and distal colon, respectively $(n=6)$. Similar results were obtained at the 2 nd and 3rd day of restraint stress. In contrast, at the 5th day, restraint stress caused no more significant increase of colonic motility. IC-injection of CRF at the lst day increased colonic motility index to $231+/-38 \%$ and $271+/-42 \%$ of basal in the proximal and distal colon, respectively $(n=5)$. IC-injection of $\mathrm{CRF}$ at the $2 \mathrm{nd}$ and 5 th day showed similar increase of colonic motility in the proximal and distal colon. Colonic transit was significantly reduced following 5 consecutive days of restraint stress $(\mathrm{GC}=4.8+/-0.4 ; \mathrm{n}=6$ ), compared to that of acute restraint stress (GC; $7.3+/-0.5, \mathrm{n}=8, \mathrm{P}<0.01$ ). Conclusion; Our current study indicates that colonic motor function is no more stimulated following chronic restraint stress loading for 5 consecutive days. In contrast, colonic motor responses to central CRF are still observed following daily-treatment with CRF for 5 days. It is suggested that the decrease in colonic motor function in chronic stress is not be due to reduced sensitivity of central CRF receptors.

\section{M2254}

Clinical Characteristics of IBS Patients with Dysfunctional Endogenous Pain Modulation

Clive H. Wilder-Smith, Joan Yap-Robert

It has previously been demonstrated that a majority of IBS patients are hypersensitive towards tonic visceral and somatic stimuli, which coincides with dysfunctional endogenous pain inhibitory mechanisms (Wilder-Smith et al. Gastroenterology 2005;128(suppl 2):M1492). These mechanisms comprise a neuromatrix of brainstem, limbic and cortical pathways which filter and process noxious input and determine emotional, autonomic and motor responses to pain. In this study we compared characteristics of IBS patients with and without abnormal endogenous pain modulation. Endogenous pain modulation (diffuse noxious inhibitory controls, DNIC) was assessed by use of the validated heterotopic stimulation method in 40 female IBS patients (20 IBS-A/D and 20 IBS-C) and 20 matched female healthy controls. Heterotopic stimulation consisted of simultaneous painful rectal distensions (individual pain threshold pressure plus $10 \%$ for 120s) and cold pain on the left foot (immersion in water at $4^{\circ} \mathrm{C}$ for $\left.120 \mathrm{~s}\right)$. Rectal pain intensity was rated on a visual analogue scale $(0-100)$ during rectal distensions without and with the simultaneous cold foot pain. Correlation between functioning of DNIC and clinical characteristics was performed by multiple regression analysis. Mean rectal pain during heterotopic stimulation compared to rectal distension alone decreased in controls but increased in IBS patients ( $<<0.05), 63 \%$ of whom had pain inhibition values below normal range ( $<95 \% \mathrm{CI}$ of controls). Regression analysis showed IBS patients with abnormal DNIC were significantly more likely to have fibromyalgia, bloating, imperative urge, more frequent weekly bowel movements, belong to the IBS-A/D group $(\mathrm{p}<0.02)$ than those with normal DNIC. Abnormal DNIC was seen in $14 / 21(67 \%)$ of IBS patients with fibromyalgia, in $16 / 22$ (73\%) of IBS patients with imperative defecatory urge and in $14 / 20(70 \%)$ of IBS-A/D patients. DNIC malfunction correlated with pain ratings during separate rectal distension and foot cold pain stimulation $(R>0.45, \mathrm{p}<0.005)$. Abnormal endogenous pain modulation is evident in the majority of IBS patients and is reflected in clinical IBS characteristics, such as an increased risk of fibromyalgia and IBS-A/D status.

\section{M2255}

Gastric Symptom Threshold Requirements for Gastric Wall Electrical Stimulation in Different Body Mass Index (BMI) Classes Kathryn A. Stecco, Jacob A. Bashyam, Mohammed A. Habeeb, Chitoor M. Habibullah, Mir A. Imran

Background: Gastric wall electrical stimulation has been proposed as a way to treat obesity by altering the brain-gut-axis. Different electrical energy parameters elicit different gastric symptoms when applied to different locations within the stomach. There is currently no standard gastric electrical wall stimulation parameter set used to predict the effectiveness of adequate stimulation. It is also unknown if energy parameter sets will differ between patients of different body mass indices. Objective: The primary goal of this study was to electrically stimulate the gastric wall in order to determine the threshold necessary to produce gastric symptoms across body mass index class range. The secondary goal was to characterize the gastric symptoms for each regimen of stimulation used. Methods: 26 patients underwent endoscopic placement of temporary screw-in pacing electrodes in the distal stomach. Patients were stratified into BMI classes: 5 Normal, 2 Overweight, 7 Class-1, 7 Class- 2, and 5 Class3. Each patient received electrical stimulation in the microsecond (uS) range starting with a pulse width of $100 \mathrm{uS}$ that was increased by intervals of $100 \mathrm{uS}$ up to a maximum pulse width of 1000 uS. Next 22 out of the 26 patients received electrical stimulation in the millisecond $(\mathrm{mS})$ range starting with a pulse width of $100 \mathrm{mS}$ that was increased by intervals of $100 \mathrm{mS}$ up to a maximum pulse width of $500 \mathrm{mS}$. The pulse repetition frequency was fixed at 12 CPM and each regimen was repeated for amplitudes ranging from 3 milliamps (mA) to $20 \mathrm{~mA}$. Gastric symptom scores consisting of nausea, vomiting, satiety, fullness pain, bloating and other, were taken at baseline and after each regimen change. Results: The three most frequently reported gastric symptoms were abdominal pain (non-specific), crampy abdominal pain, and nausea across all electrical stimulation regimens. Pulses in the microsecond range were significantly more likely to produce symptoms of nausea than pulses in the millisecond range ( $34 \%$ vs. $0 \%$ ). Class- 3 obese patients required significantly higher pulse widths in the millisecond range to evoke gastric symptoms than people of smaller body masses. Conclusion: A certain minimum threshold of distal gastric wall electrical stimulation is necessary to elicit gastric symptoms in patients across all BMI classes (Normal through Class-3). Class-3 obese patients required statistically higher pulse widths to evoke gastric symptoms compared to patients in the lower BMI classes when millisecond pulse widths were used. The different gastric symptoms may serve as a marker to demonstrate that an adequate level of electrical stimulation has been attained. 


\section{M2256}

Central Neuronal Mechanism of Intestinal Electrical Stimulation: Effects On Duodenum Distention-Responsive (DD-R) Neurons in the VMH of Rats J.N. Zhang, J D Z Chen

Background and Aims: Intestinal electric stimulation (IES) has been shown to produce an inhibitory effect on gastric motility/secretion, gastric emptying, food intake and weight loss in rats, suggesting a therapeutic potential for obesity. However, possible central mechanism of this entero-gastric inhibitory effect induced by IES is unknown. It is well established that the VMH in the hypothalamus is closely related to the regulation of feeding behavior and plays an important role in the mediation of satiety. The aim of this study was to explore the hypothesis that the neurons in the VMH are involved in the central mechanisms of the IES. Methods: Extracellular potentials of single neurons in the VMH were recorded in 18 anesthetized, ventilated male adult rats. After VMH neurons with duodenum distension(DD, $0.5 \mathrm{ml}, 10 \sim 30 \mathrm{~s}$ ) were identified, IES with 3 sets of parameters was performed for one minute via a pair of platinum electrodes sutured onto the serosal surface of the duodenum $2-4 \mathrm{~cm}$ below the pylorus. IES1 (pulse trains: $4 \mathrm{~mA}, 2 \mathrm{~s}$-on, 3s-off, $2 \mathrm{~ms}, 20 \mathrm{~Hz}$ ), IES2 (long pulses: $6 \mathrm{~mA}, 20 \mathrm{cpm}, 100 \mathrm{~ms}$ ), IES3 (same as IESI but $40 \mathrm{~Hz}$ ). Results: Fifty nine percent of 34 (59\%) neurons in the VMH were responsive to duodenum distension. About Seventy percent $(12 / 17,70.6 \%)$ of the DD-responsive neurons with DD responded to IESI and IES3 (increased pulse frequency), which was significantly higher than that of IES2 (25\%, P=0.041). Conclusions: IES with different parameters activated $25-70.6 \%$ of the VMH neurons responsive to DD. Moreover, IES with trains of short-pulses seems more effective than IES with longpulses in activating the neuronal activity in the $\mathrm{VMH}$, but an increase in pulse frequency does not make a difference (IES1 vs. IES3, P>0.05). The modulatory effects of IES on the central neurons in the satiety center suggesting therapeutic potential of IES for obesity.

\section{M2257}

Effect of FMRI Signal Analysis Methods On Quantification of Brain Activity Associated with Esophageal Acid Exposure in GERD Patients and Healthy Controls

Mark Kern, Arthi Sanjeevi, Adeyemi Lawal, Venelin Kounev, Reza Shaker

Brain imaging has been frequently used in recent years to study the brain-gut axis. The effect of various signal analysis techniques on the absolute value of detected cortical activity volumes is not well known. Aim: To determine whether different analysis techniques are in concordance in quantifying brain activity during esophageal acid exposure. Methods: Seven controls (age: $20-44$ years, 4 female) and seven GERD patients (age: $20-62$ years, 4 female) were studied using a paradigm-driven fMRI experimental protocol to detect regions of brain activity associated with esophageal acid perfusion. Two 25-minute whole brain scans were performed. In the first scan, three randomly spaced, two-minute intervals of esophageal acid perfusion $(1 \mathrm{ml} / \mathrm{min})$ were alternated with saline perfusion. The second scan consisted of two 5-minute intervals of esophageal acid exposure alternated with saline perfusion. Measured parameters included the number of activated voxels in the fMRI rendered volumes from each type of scan for three methods of fMRI wave form analysis: 1) cross correlation analysis; 2) Deconvolution/linear regression analysis and 3) nonlinear regression analysis. For comparison purposes, the number of activated fMRI volume elements (voxels) shown to be associated with the acid perfusion stimuli detected by the three analysis techniques exceeding the probability threshold ( $<<0.05$, corrected for multiple comparisons) were evaluated. Results: Significant differences in the amount of cortical fMRI activity between 2 minute and 5 minute acid exposure scans were found in both GERD and control groups. $(* \mathrm{p}<0.05)$ These differences were independent of analysis method; however, the absolute number of activated voxels was highly dependent upon analysis technique. (table) Cortical region of interest Kappa analysis for the presence or absence of cortical activity showed that agreement of the three analysis techniques was moderate to very good with the percent agreement ranging from $71 \%$ to $100 \%$. Conclusions: Within- and between-group changes can be reliably detected irrespective of fMRI signal analysis technique. Absolute values of brain activity are analysis-technique-dependent and can be used only for comparative purposes. Comparative studies must be done using the same analysis technique. These findings need to be taken into account when using fMRI techniques for studies of the brain-gut axis.

\begin{tabular}{|c|c|c|c|c|c|c|}
\hline \multirow{2}{*}{} & \multicolumn{5}{|c|}{ \# of Activated Voxels } \\
\cline { 2 - 7 } & \multicolumn{3}{|c|}{2 min. acid } & \multicolumn{3}{c|}{5 min. acid } \\
\cline { 2 - 7 } & correlation & deconvolution & nonlinear & correlation & deconvolution & nonlinear \\
\hline GERD & $24 \pm 6$ & $30 \pm 4$ & $33 \pm 5$ & $46 \pm 9 *$ & $70 \pm 12 *$ & $80 \pm 15^{*}$ \\
\hline Control & $2 \pm 1$ & $4 \pm 1$ & $7 \pm 3$ & $14 \pm 3 *$ & $28 \pm 3 *$ & $40 \pm 5 *$ \\
\hline
\end{tabular}

\section{M2258}

Peripheral Injection of CCK-8s Induces FOS Expression in the Dorsomedial Hypothalamic Nucleus in Rats

Hubert Moennikes, Stephan Paulitsch, Marco Schmidtmann, Ivo R. van der Voort, Andrea Riedl, Burghard F. Klapp, Bertram Wiedenmann, Peter T. Kobelt

Cholecystokinin (CCK) plays a physiological role in the regulation of food intake. In the gastrointestinal tract, it is mainly synthesized in the upper small intestine and released postprandial. The dorsomedial hypothalamic nucleus (DMH) has been implicated in the brain regulation of food intake and satiety. The aim of this study was to determine if peripherally CCK affects neuronal activity in the DMH, as assessed by Fos expression. Methods. The study was performed in non-fasted Sprague-Dawley rats. At the end of the dark phase animals received intraperitoneally (ip) $2 \mu \mathrm{g}$ CCK-8S/kg body wt $(\mathrm{n}=6)$ or vehicle solution $(0.15 \mathrm{M} \mathrm{NaCl} ; \mathrm{n}=6)$. Brains were removed $90 \mathrm{~min}$ after injection. Fos immunohistology (FLI) and additional double staining with anti-thyrosine-hydroxylase (TH) were performed in free-floating technique. Density of Fos positive neurons was semi-quantitatively determined in the DMH, paraventricular nucleus of the hypothalamus (PVN), arcuate nucleus of the hypothalamus (ARC) and ventromedial hypothalamus (VMH) by use of a confocal laser microscope. Results. CCK-8S $(2 \mu \mathrm{g} / \mathrm{kg})$ injected ip increased Fos immunoreactivity in the DMH (mean \pm SEM: $108 \pm 10$ vs $54 \pm 6$ cells/section, $p<0.0009)$ and PVN $(120 \pm 12$ vs $20 \pm 3, p<0.0001)$ compared to the vehicle treated animals. CCK-8S had no effect on fos expression in the ARC $(13 \pm 2$ vs $12 \pm 1, p>0.75)$ and $\mathrm{VMH}(25 \pm 2$ vs $23 \pm 2$, p $>0.56)$ compared to the vehicle. The CCK-8S ip-induced FLI positive neurons, mainly localized in the ventral part of the DMH, were encircled in a network of TH-LI positive fibers. Conclusion. The present observation that peripherally CCK induces an increase of neuronal activity in the DMH suggests that the DMH is involved in the CNS regulation of satiety and food intake mediated via peripheral CCK. It has been shown that numerous afferents from the brainstem projecting to the DMH originate from the noradrenergic $\alpha 2$-cell group of the nucleus of the solitary tract (NTS); thus, one can speculate that activation of neurons in the DMH by peripheral CCK ensues from noradrenergic (i.e. TH-positive) cell groups in the NTS projecting to the $\mathrm{DMH}$.

\section{M2259}

Modulatory Effects and Afferent Pathway of Gastric Electric Stimulation

(GES) On Spinal Neurons Receiving Inputs from Stomach in Rats

J.N. Zhang, Robert D Foreman, J.D.Z Chen, Chao Qin

GES has been suggested as therapy for patients with obesity or gastric motility disorders. The aim of this study was to investigate the spinal mechanism of GES effects on gastric function. Extracellular potentials of single spinal (T9-T10) neurons were recorded in pentobarbital anesthetized, paralyzed, ventilated male rats $(\mathrm{n}=19)$. Gastric distension (GD) was produced by air inflation of a balloon. One pair of platinum electrodes $(1.0-1.5 \mathrm{~cm}$ apart) was sutured onto the serosal surface of the lesser curvature of the stomach. GES with four sets of parameters was applied for one minute: GES-A $(6 \mathrm{~mA}, 0.3 \mathrm{~ms}, 40 \mathrm{~Hz}, 2 \mathrm{~s}$ on, $3 \mathrm{~s}$ off), GES-B (6 mA, $0.3 \mathrm{~ms}, 14 \mathrm{~Hz}, 0.1 \mathrm{~s}$ on, $3 \mathrm{~s}$ off), GES-C ( $6 \mathrm{~mA}, 3 \mathrm{~ms}, 40 \mathrm{~Hz}, 2 \mathrm{~s}$ on, $3 \mathrm{~s}$ off), GES-D (6 mA, $200 \mathrm{~ms}, 12$ pulses/min). 61/157 (39\%) spinal neurons responded to GD (20 and/or $60 \mathrm{mmHg}$, 20s). Most GD-responsive neurons ( $\mathrm{n}=47$ ) were excitatory; the remainder was inhibitory $(n=10)$ or excitatory-inhibitory $(n=4)$. GES-A, $-B,-C$ and $-D$ affected activity of $32 \%, 16 \%, 78 \%$ and $35 \%$ of GD-responsive neurons, respectively. Bilateral cervical vagotomy did not significantly alter effects of GES on 6/6 neurons. Resiniferatoxin $(2.0 \mu \mathrm{g} /$ $\mathrm{kg}$, i.v.), an ultrapotent agonist of vanilloid receptor-1, abolished neuronal responses to GD and effect of GES in 4/4 rats. The results suggested that GES mainly had an excitatory effect on T9-T10 spinal neurons with gastric inputs; neuronal response to GES was strengthened with stimulation at an increased pulse width and/or number of pulses. The modulatory effect of GES involved thoracic spinal (sympathetic) afferent fibers containing vanilloid receptor-1. (Supported by a grant from OCAST)

\section{M2260}

Chemopreventive $\omega-3$ Fatty Acids Inhibit Colon Cancer Cell Proliferation By Pkc- $\delta$-Dependent Mechanisms

Sonia R. Cerda, Reba Mustafi, Merry J. G. Bolt, Anusara Chumsangsri, Holly Little, Marc Bissonnette

Introduction: Previously, we demonstrated PKC- $\delta$ inhibited cell cycle progression and enhanced apoptosis in Caco-2 colon cancer cells by mechanisms involving growth-inhibiting alterations in Gl cyclins and Bcl-2 family members. In preliminary studies chemopreventive fish oils ( $\omega-3$ fatty acids) increased PKC- $\delta$ expression, whereas tumor promoting $\omega-6$ fatty acids down-regulated the expression of this isoform in azoxymethane-induced colon tumors. We have recently shown in Caco- 2 cells that $\omega$ - 3 fatty acids up-regulate PKC- $\delta$ and antagonize its down-regulation by the physiological regulator diacylglycerol (DAG). In this study, we investigated the role of PKC- $\delta$ in the anti-proliferative actions of $\omega-3$ fatty acids in Caco-2, HT-29, and HCT-116 colon cancer cells. Methods: Colon tissues were obtained by endoscopic biopsy and immediately processed for protein analysis. Cells were cultured for 2-5 days in medium containing $2.5 \%$ serum, supplemented with albumin alone or with 100 $\mathrm{mM}$ indicated fatty acid complexed to albumin(3:1): $\omega-3$ [eicosapentaenoic acid (EPA), docosahexaenoic acid (DHA)] and $\omega-6$ [linoleic acid (LA)]. DAG molecular species were identified by reversed phase HPLC and confirmed by GLC analysis. PKC- $\delta$ expression was inhibited using PKC- $\delta$-specific siRNA oligonucleotides. Protein expression was assessed by quantitative Western blotting and cellular proliferation by WST-1 and BrdU assays. Results: In human colon, $\mathrm{PKC}-\delta$ was significantly decreased in adenomas and carcinomas by more than $60 \%$ and $80 \%$, respectively, compared to adjacent colonic mucosa $(n=8, p<0.05)$. Consistent with an anti-proliferative role for PKC- $\delta$ in colon cancer cells, PKC- $\delta$ knockdown with specific siRNA ( $>80 \%$ inhibition), significantly and dose dependently increased cell proliferation in Caco-2, HCT-116, and HT-29 cells $(p<0.05)$. Activating changes in PKC- $\delta$ phosphorylation were assessed in fatty acid treated cells, $\omega$ - 3 fatty acids (EPA,DHA) increased levels of phospho-Ser643 and phospho-Thr505 PKC- $\delta$. These free fatty acids also caused species-specific alterations in DAG fatty acid composition in Caco-2 cells. Furthermore, knock down of PKC- $\delta$ in these cells with specific siRNA blocked growth inhibition by $\omega$ - 3 fatty acids (EPA,DHA), supporting our hypothesis that the chemopreventive actions of fish oils are mediated in part by PKC- $\delta$ dependent mechanisms. Conclusion: We have identified novel DAG-dependent mechanisms regulating PKC- $\delta$ expression that contribute to the anti-proliferative actions of $\omega$-3 fatty acids. We postulate these PKC- $\delta$-dependent anti-proliferative pathways mediate the chemopreventive effects of fish oils in colonic carcinogenesis. 
M2261

Glutamine, Butyric Acid, and Antioxidants in Combination Inhibit Proliferation and Induce Differentiation in Colorectal Tumor Cell Lines Independent of Apoptosis

Torsten Steinbrunn, Theodor Kudlich, Juergen Schauber, Ralph Melcher, Hardi Luehrs, Andrea Gostner, Wolfgang Scheppach, Thomas Menzel

Rationale: Glutamine, butyric acid, and antioxidants are used as nutritional immune modulating compounds for critically ill patients. While glutamine serves as a major energy source for rapidly dividing cells it also promotes growth of neoplastic cells and inhibits apoptosis. Butyric acid stimulates growth of normal colonic cells in vivo but reduces proliferation of neoplastic cells in vitro. Antioxidants like ascorbic acid or selenium may selectively induce apoptosis in tumor cells while stimulating growth of normal cells. The combination of these substrates could lead to an immune and growth enhancing effect in normal cells without stimulating tumor cell growth. Methods: The colorectal carcinoma cell lines SW-620 and HT-29 and the adenoma cell line Geki-2 were incubated with an enteral immunonutrition supplement consisting of high doses of glutamine, butyric acid, and antioxidants (Intesta$\min \left({ }^{\circledR}\right)$ for $24 \mathrm{~h}, 48 \mathrm{~h}$, and $72 \mathrm{~h}$ in standard medium ranging from $1.25 \%$ to $10 \%$. Cell cycle distribution and apoptosis were measured with FACS using annexin V/propidium iodide staining. Cell proliferation and differentiation were determined by cell count and levels of alkaline phosphatase (AP), respectively. Expression of factors of cell cycle control and apoptosis (PCNA, P2 1, P53, Cdk-2, Bcl-2, Bax, Bak) was investigated by western blot and a cDNA-array testing 12000 genes was performed. Results: Proliferation was inhibited in all tested cell lines time and dose dependently. Compared to untreated controls, treated cells ( $5 \%$ vol.) showed a significant decrease in proliferation to $68 \pm 5 \%$ (HT-29, $24 \mathrm{~h}$ ), $74 \pm 7 \%$ (SW-620, 24h), and 33 \pm \% (Geki-2, 72h). AP levels significantly increased time and dose dependently from $3 \mathrm{U} / \mathrm{l}$ to $30 \mathrm{U} / \mathrm{l}$ in HT-29 (10\% vol., $72 \mathrm{~h}$ ) and from $28 \mathrm{U} / \mathrm{l}$ to $71 \mathrm{U} / \mathrm{l}$ in SW620 (2.5\% vol., $72 \mathrm{~h}$ ), indicating an increase in cellular differentiation. While P21 protein levels increased in SW-620 cells, the expression patterns of the other tested factors were not modulated. The cDNA-array showed all genes being regulated less than 1.5. Accordingly, cell cycle distribution and rate of apoptotic cells remained unchanged. Conclusion: The combination of glutamine, butyric acid, and antioxidants inhibits tumor cell proliferation and stimulates cell differentiation. Apoptosis is not inhibited and cell cycle remains stable. If clinically well tolerated, critically ill patients could benefit from the combined immune stimulation and energy provided by these substrates while growth of transformed and potentially malignant cells would not be promoted but selectively inhibited. Therefore, the combination might improve outcome also in cancer patients.

\section{M2262}

Omega-3-Fatty Acids Inhibit the Proliferation of Colorectal Cancer Cells

\section{Evidence for Ppary Independent Mechanisms}

Sandra Ulrich, Yasemin Turan, Loitsch Stefan, Juergen Stein

Introduction: Recent studies have shown that lipid emulsions, containing the $\omega$ - 3 polyunsaturated fatty acids ( $\omega-3-\mathrm{FA})$ eicosapentaenoic acid (EPA) and docosahexaenoic acid (DHA) exhibit potent antiinflammatory and antiproliferative properties. While the transcription factor PPAR $\gamma$ seems to mediate antiinflammatory effects, an involvement in $\omega$-3-FA-induced cell growth inhibition of cancer cells is discussed controversially. The aim of this study was to characterize anticarcinogenic effects of $\omega-3-\mathrm{FA}$ in colorectal cancer cells and to further specify a possible role of transcription factor PPAR $\gamma$. Materials and Methods: The colorectal cancer cell lines Caco-2 and HCT-116 were cultured under standard conditions and were treated with increasing concentrations of standardized lipid emulsions containing (1)-3- [10$100 \mu \mathrm{M}$ EPA and DHA] and $\omega-6-\mathrm{FA}$. Treatment with butyrate [2mM] was performed to induce PPAR $\gamma$ expression (BBRC, 2000) which is described to be a molecular target both of EPA and DHA. Cell growth was determined by MTT assay and crystal-violet staining. Caspase-3 induction was determined via an activity assay. PPAR $\gamma$, p27Kipl and Cyclin E protein was determined by Western blot analysis. A dominant-negative PPARgamma mutant was transfected in Caco-2 cells to suppress PPARgamma-mediated functions. Results: In contrast to $\omega$-6-FA, treatment with $\omega$-3-FA leads to a significant dose- and time-dependent reduction of cell counts and cell proliferation, whereas co-incubation with butyrate significantly enhances these effects [Means $\pm S E, * * * p<0.001$ at EPA $[50 \mu \mathrm{M}]$ ]. In contrast to $\omega$ 6 -FA, $\omega-3-F A$ further significantly induce caspase-3 activity Mean $\left.\pm \mathrm{SE},{ }^{*} \mathrm{p}<0,05\right)$. Incubation with $\omega-3$-FA on the one hand leads to an upregulation of $\mathrm{p} 27 \mathrm{Kipl}$ protein concentration and on the other hand to an decrease of Cyclin E expression. Althought PPARy mediated effects are suppressed, no differences in cell growth inhibitory actions could be observed in PPAR $\gamma$ mutant and empty vector cells after treatment with $\omega$-3-FA. Conclusion: The current results confirmed cell growth inhibitory effects of $\omega-3-\mathrm{FA}$ in a cell culture model of colorectal cancers, which seem to be mediated through an activation of cell cycle inhibitor p27Kipl as well as an inhibition of cyclin E. However in contrast to the involvement of PPAR $\gamma$ in antiinflammatory properties of $\omega-3-F A$, these effects are indicated to be mediated PPAR $\gamma$-independently.

\section{M2263}

Moderate Alcohol Consumption Protects Against Colorectal Adenomas Gregory Austin, Joseph Galanko, Christopher Martin, Robert Sandler

Background: Alcohol in moderation has been recommended to decrease the risk of cardiovascular disease. The effect of moderate alcohol consumption on other outcomes is not as well known. Previous studies have shown an association between excessive alcohol consumption and an increased risk of colon cancer. However, the effect of moderate alcohol consumption on the risk of colorectal adenomas and cancers has not been well-studied. Aim: To determine the effects of moderate alcohol consumption on colorectal adenomas. Methods: 725 eligible patients were included from the Diet and Health Study 4, a case-control study to evaluate risk factors for colorectal adenomas. There were 203 cases and 522 controls. All patients underwent a full colonoscopy. Alcohol consumption was obtained using the National Cancer Institute's Food Frequency Questionnaire. Patients were divided into five groups based on the average number of alcoholic drinks consumed per week: 1) 0 drinks/week; 2) $>0$ and $<2$ drinks/week; 3) 2-<7 drinks/week; 4) 7-<14 drinks/week; and 5) >=14 drinks/week Odds ratios were calculated using logistic regression, controlling for potential confounder sex, age, body mass index, use of non-steroidal anti-inflammatory medications, race, and smoking. Results: The groups consuming $>0$ and $<2$ and $2-<7$ drinks of alcohol/week had the lowest adjusted probability of having an adenoma. Using the group consuming $>0$ and $<2$ drinks/week as the reference group, non-drinkers had an adjusted OR of $1.41(95 \% \mathrm{CI}$ 0.83-2.39): patients consuming $7-<14$ drinks/week had an OR of 1.67 (95\% CI: 0.88-3.18); patients consuming $>=14$ drinks/week had and OR of 2.51 (95\% CI: 1.24-5.07). The patients consuming $>=14$ drinks/week were also at significantly increased odds of having an adenoma compared to the group consuming 2-<7 drinks/week (OR 2.34, 95\% CI: 1.144.83). Conclusions: Individuals who consume moderate amounts of alcohol are at lower risk of colorectal adenomas than nondrinkers and heavy drinkers. Moderate alcohol consumption may benefit the colon as well as the heart.

Adjusted Proportion with a Colorectal Aden oma by Alcohol Consumption.

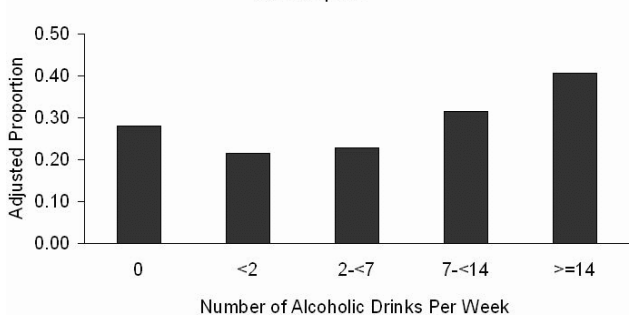

M2264

Antineoplastic Effects On Colorectal Cancer Cells of Plantago Ovata Husk After Fermentation with Human Gut Flora

Anna Giros, Xavier Llor, Elisenda Pons, Lurdes Fluvia, Rosa M Xicola, Anna Anguera, Miquel A Gassull

Introduction: Wide geographical differences in colorectal cancer (CRC) incidence and a rapid adaptation of migrant populations to the incidence of hosting regions suggest that environmental factors, among them nutrients, play a crucial role in CRC development. Data on the effects of dietary fiber on CRC incidence has often been inconclusive. At the experimental level, few studies have attempted to evaluate the effects of fiber on colorectal malignant cells under conditions that mimic the process that fiber undergoes inside the human gut. Objectives: To establish the capacity of products generated by plantago ovata husk fermentation in human feces to induce apoptosis and regulate proliferation in two colorectal adenocarcinoma cells lines, Caco-2 and HT-29. Methods: Fresh stool specimens were collected from healthy volunteers. Fecal homogenates (dilution 1:5 with sodium bicarbonate) were incubated with either plantago ovata husk powder $(20 \mathrm{mg} / \mathrm{ml})$ or with no fiber addition, under anaerobic conditions at $37^{\circ} \mathrm{C}$ for $48 \mathrm{~h}$. Bacterial fermentation was stopped by freezing at $-80^{\circ} \mathrm{C}$. Short chain fatty acids were determined by GLC using a semipolar column. Samples were then filtered with a $0.22 \mathrm{mc}$ filter for bacterial elimination and immediately frozen in small aliquots at $-80^{\circ} \mathrm{C}$. HT-29 and Caco-2 cells were supplemented with sera-free media plus: A)the products of fermentation with plantago ovata husk; B)control buffer; and C)pure butyrate at $1.5 \mathrm{mM}$. Apoptosis was assessed $48 \mathrm{~h}$ post supplementation by flow cytometry. Proliferation was evaluated through counting of [H3] thymidine incorporation $4 \mathrm{~h}$ after incubation at $24 \mathrm{~h}$ post supplementation. All experiments were repeated at least 6 times. Statistical analysis was performed using the Kruskal-Wallis one-way analysis of variance by rank and the MannWhitney U-test $(\mathrm{P}<0.0)$ Results: Apoptosis experiments: results are expressed as apoptosis index (AI) defined as percentage of apoptotic cells out of the total number of counted cells (10,000), SE+/-1: In HT-29 cells, mean AI was: cells supplemented with A: 44.3; supplement B: 11.24; and supplement C: 43.13. In Caco-2 cells, A supplement: 39.55; B: 9.04; and C: 42.11. In both cases there were no statistically significant differences between plantago ovata husk and butyrate and both were statistically different from control, $\mathrm{P}<0.005$. Thymidine incorporation with supplements A: 943; B: 4123; C: 4635 u. Plantago ovata husk was statistically different from control and butyrate $(\mathrm{P}<0.005)$. Conclusions: Products resulting from plantago ovata husk fermentation with human gut flora behave as antineoplastic agents through the induction of apoptosis and inhibition of cell proliferation.

\section{M2265}

Leptin and Leptin Receptor Expression in Colo-Rectal Cancer: Role of Microsatellite Instability

Nijez Aloulou, Marie-Therese Chaumette, Amel Sekour, Karen Leroy, Mohammad Abolhassani, Iradj Sobhani

Leptin regulates nutriment intake via specific hypothalamic receptor (ObRb). Colo-rectal cancer (CRC) is influenced by nutritional factors. Leptin stimulates colonocyte proliferation and invasiveness of CRC cells in vitro. The aim was to investigate leptin receptor (ObRb) expression in CRC in reference with serum leptin level and mismatch repair status. Patients $\&$ methods. Consecutive patients (73 F, 92M; 70+/-12 yrs) with rectal (33) or colon (132) adenocarcinoma underwent surgery. Microstallite instability (MSI) phenotype was characterized by immunohistochemistry (IHC) and PCR in tumours. ObRb was evaluated in tumours, homologous normal tissues, and culture cells (HT29, HCT116 and HCT116 with a transferred Ch-3) by both IHC and RT-PCR. Patients were ranged in two categories, with and without ObRb tumoral overexpression as compared to normal homologous tissue. The level of nuclear fraction of PPARgamma in normal colon tissue, in tumours ( $N=3$ ), and in HT29 culture cells before and $24 \mathrm{~h}$ after leptin $(0.1$ microM) treatment, was measured (ELISA, Kit). Exact Fisher, Anova t tests and multiple regression analysis were used for statistical analyses. Results. ObRb was expressed in normal and tumor tissues. In reference to normal tissue, ObRb was overexpressed in 118 tumours (71.5\%). This status was significantly associated 
with MSI-H phenotype ( $93.7 \%$ versus $62.5 \%$ in MSS; $\mathrm{P}=0,01$ ), right sided ( $85 \%$ vs $63 \%$, $\mathrm{P}<0,04)$, and individuals $>60$ yrs $(76 \%$ vs $57 \%$; $\mathrm{P}=0.04)$, when size-, pTNM- and differentiation of the tumors, were not significant related. ObRb was expressed more in HCT116 cells than in HCT116-Ch3. Serum leptin level, although was informative of BMI>30, failed to predict location, staging and ObRb expression in tumours or normal colo-rectal mucosa. The level of PPARgamma was significantly higher in tumour than in normal homologous tissue; in HT29 culture cells, it was three-five fold increase after treatment by leptin. Conclusion. Specific leptin receptor $\mathrm{ObRb}$ is overexpressed in CRC with microsatellite instability and may influence regulation of energy in tumours since it is accompanied with PPARgamma enhancement. This may be an adaptative pattern of some colon tumour cancer cells to energy uptake and expenditure.

\section{M2266}

Nutritional Status Is Predictive of Clinical Complete Response to Chemoradiotherapy in Locally Advanced Esophageal Cancer Stephane Lecleire, Frederic Di Fiore, Eric Lerebours, Pierre Michel

Introduction. Definitive chemoradiotherapy (CRT) is to date considered as an alternative curative option in patients with locally advanced esophageal cancer (LAEC). Severe undernutrition is found in approximately $60 \%$ of these patients. Impact of nutritional status on complete response to definitive CRT has not been demonstrated yet. The aim of the study was to assess the influence of ongoing undernutrition prior to CRT on the clinical complete response in patients with LAEC. Patients and Methods. Fifty-nine patients with LAEC treated by definitive CRT were retrospectively included in the study. CRT encompassed external beam radiation ( 50 to 60 Grays) and 5-Fluorouracile (5-Fu)-cisplatine chemotherapy courses. Clinical complete response was defined by the absence of tumor at endoscopy and the absence of distant metastases at CT scan two months after the end of CRT. Nutritional status of all patients was assessed prior to CRT by the serum albumin level and by the weight loss percentage as compared to the initial weight. Clinical and nutritional characteristics of responder patients to CRT were compared to those of non-responder patients using the chisquare and Student's tests. Results. Thirty-seven patients (63\%, group 1) were complete responders after CRT, and 22 (37\%, group 2) were non-responder patients. Mean age (63 vs 65 year-old), Tumor Node Metastasis Classification, Atkinson's dysphagia score (2.1 vs 2.4) and Who Performance Status (0.7 vs 1) were not different between the two patient groups. Prior to CRT, patients from group 2 presented with a poorer nutritional status as compared to patients from group 1. Patients from group 1 had a lower serum albumin level (33 vs $40 \mathrm{~g} / \mathrm{L} ; \mathrm{p}=0.025$ ) and a more important weight loss (10\% vs $5 \% ; \mathrm{p}=0.009$ ) than patients from group 2. More, esophageal dilation prior to the CRT was more often required in patients from group 2 ( $59 \%$ vs $19 \% ; \mathrm{p}=0.004)$. Conclusion. Patients with LAEC who do not respond to CRT appear to have significant undernutrition prior to CRT. Early nutritional intervention could increase the rate of complete response to CRT in these patients. These results suggest the value of nutritional status assessment prior to CRT in the management of patients with LAEC.

\section{M2267}

Impact of Bariatric Surgery On the Gastro-Esophageal Reflux Disease and Esophageal Motility in Morbid Obesity Patients

Vicente Ortiz, Ponce Marta, Marisa Iborra, Belen Beltran, Bastida Guillermo, Jose Luis Ponce, Garrigues Vicente, Julio Ponce

Esophageal motility disorders and gastro-esophageal reflux disease (GERD) are more prevalent in patients with morbid obesity (MO) than in the general population. However, the influence that bariatric surgery have on these variables is unknown. Aim: To evaluate the influence that bariatric surgery have on GERD and esophageal motility. Methods: Eightytwo consecutive patients with MO (body mass index (BMI) $\geq 40 \mathrm{~kg} / \mathrm{m} 2$ ) were prospectively evaluated before and six months after having bariatric surgery. In all cases the surgical technique used was a vertical gastroplasty plus a gastro-jejunal bypass. The parameters investigated were: 1) Esophageal symptoms registered through a structured clinical questionnaire. The consensus diagnostic criteria for GERD of having two or more episodes of heartburn in one week was used. 2) Esophageal exposure to acid which was achieved through a pH-metry (considered positive when time of exposure to $\mathrm{pH}<4$ was greater than $5 \%$ of the time). 3) Alterations of esophageal motility observed in a manometry. None of the patients was on anti-secretory therapies at least for 10 days previous to their evalutation. Results: The mean age of the patients was of $41.3+101$ years $(61$ women/21 men). The BMI previous to surgery was of $50.1 \pm 6.4$. Six months after the bariatric surgery their BMI was $37.5 \pm 5.4$ with a mean weight loss of $35.6 \pm 13.2 \mathrm{Kg}$. The main changes observed on symptoms and esophageal function are shown in table. The prevalence of GERD (symptoms) and the rate of pathological exposure to acid (positive pHmetry) were significantly reduced six months after the bariatric surgery. The percentage of time with $\mathrm{pH}<4(\mathrm{n}=82)$ was $8.1 \% \pm 11.8$ (females $5.9 \% /$ males $14.7 \%$ ) and after the surgery was $2.9 \% \pm 5.4$ (females $3.1 \%$ / males $2.6 \%)(p=0.005)$. The initial lower esophageal sphincter pressure was of $14.9 \pm 7.0$ $\mathrm{mmHg}$ and it was not modified after surgery. The manometry of the MO patients showed that they had an hyper-contractile oesophagus (nutcracker pattern which is defined by presenting peristaltic waves with a mean amplitude $>180 \mathrm{mmHg}$ ) in the $18.3 \%$ of the patients. This percentage was reduced after surgery to $6.1 \%$ of the patiens. No other changes in the esophageal motility was observed after surgery. Conclusion: The prevalence of GERD (symptoms and pHmetry) and their esophageal hypercontractility decrease after bariatric surgery in patients with MO.

\begin{tabular}{|c|c|c|c|}
\hline $\mathrm{n}=82$ & Basal & After Surgery & $\mathrm{p}$ \\
\hline Symptoms & $15(18.3 \%)$ & $2(2.4 \%)$ & 0.001 \\
\hline positive pH-metry & $40(48.8 \%)$ & $15(18.3 \%)$ & $<0.0001$ \\
\hline Nutcracker pattern & $15(18.3 \%)$ & $5(6.1 \%)$ & 0.021 \\
\hline
\end{tabular}

\section{M2268}

Effects of Retrograde Gastric Electrical Stimulation with Pulse Trains On Gastric Emptying of Solids and Plasma Hormones in Dogs Gengqing Song, Yan Sun, Xiaohua Hou, Bin Yang, Wei Qian, Jiande Chen

Retrograde Gastric Electrical Stimulation (RGES) is proposed as a novel therapy for obesity However, mechanisms of RGES involved in treating obese patients are not fully investigated. Aims: The aim of this study was to investigate the effects of RGES with trains of pulses on gastric slow waves, gastric emptying of solids and plasma concentrations of satiety-related peptides and glucose. Methods: Seven female beagle dogs implanted with four pairs of gastric electrodes on the gastric serosa were studied in two randomized sessions (control and RGES). Gastric emptying was measured by scintigraphy using a $99 \mathrm{mTc}$-labeled egg meal (255 kcal) for a period of 4 hours in each session. Blood samples were collected at 45 and $15 \mathrm{~min}$ before, 30,60 and $120 \mathrm{~min}$ after the meal. Plasma leptin insulin and glucagon were measured using radioimmunoassay method. Plasma glucose was assessed with a commercially available glucometer. In the RGES session, RGES was applied via the distal pair of electrodes $(2 \mathrm{~cm}$ above the pylorus) with trains of pulses (frequency: $40 \mathrm{~Hz}$; pulse width: $2 \mathrm{~ms}$; amplitude: $5 \mathrm{~mA}$; train on-time, $2 \mathrm{~s}$; off-time, $3 \mathrm{~s}$ ). RGES was initiated $30 \mathrm{~min}$ before the first blood sample and maintained for a period of 2.5 hours. Gastric slow waves and symptomatic response were also recorded in each session. Results: 1) Similar to long pulses, RGES with pulse trains (12 trains/min) was capable of turning regular gastric slow waves into tachygastria The percentage of tachygastria ( $>6$ cycles/min) in the fed state with RGES was $78.1 \pm 5.3 \%$ compared with $0.4 \pm 0.3 \%$ in the control session ( $\mathrm{p}<0.01)$. 2) RGES was able to delay gastric emptying of solids $(\mathrm{P}<0.05)$. The mean $\mathrm{T} 50$ of gastric emptying was significantly increased from $177.7 \pm 27.1 \mathrm{~min}$ without RGES to $254.6 \pm 71.7 \mathrm{~min}$ with RGES ( $\mathrm{P}=0.024)$. 3) Compared with the control session without RGES, the total AUC's of plasma insulin with RGES was significantly decreased in the fasting and postprandial periods $(\mathrm{p}<0.05)$. However, the total area under curves (AUC's) of plasma leptin, glucagon, and glucose were not significantly affected by RGES $(p>0.05)$. 4) This method of GES induced no noticeable symptoms. Conclusion: RGES with at a tachygastrial frequency decreases gastric emptying of solids and plasma insulin, but has no effect on plasma leptin, glucagons, and glucose. These findings are indicative of therapeutic potential for obesity.

\section{M2269}

Relationship Between Obesity, Nocturnal Heartburn and Night-Time Reflux Maribal Gonzalez-Gutierrez, Jose M. Remes-Troche, Mariel Mejia-Rivas, Ana T. Abreu y Abreu, Jose A. Chavez-Barrera, Miguel A. Valdovinos

Background/Aim: Night-time GERD symptoms are more troublesome than those that occur during the day. Several mechanisms have been related to night time reflux (NTR), including an impaired esophageal acid clearance, low production and flow of saliva, and reduced lower esophageal sphincter pressure. Although obesity has been associated with an increase risk for GERD symptoms and erosive esophagitis, the relationship with nocturnal heartburn $(\mathrm{NH})$ and NTR is not known. Our aim was compare the prevalence of NH and NTR in both obese and non-obese patients with GERD. Material and Methods: Consecutive patients with symptomatic GERD were evaluated from January to June 2005. Esophageal mucosal injury was assessed by upper endoscopy. All patients underwent esophageal 24-h pH monitoring. $\mathrm{NH}$ was defined as presence of symptoms at night and in supine position. Abnormal $\mathrm{pH}$ was considered when total $\%$ of time of $\mathrm{pH}<4$ was $>4.2 \%$. Abnormal NTR was defined as a $\%$ of time of $\mathrm{pH}<4>1.2 \%$ at night. According to body mass index (BMI) patients were classified in 2 groups: I) non-obese patients (BMI $\leq 30$ ), and II) obese patients (BMI $>30)$. Data were analyzed with Mann-Whitney U and $\chi 2$ tests. Results: 79 patients, $60 \mathrm{~F}$ (76\%) with a mean age of 45 years (range 20-75) were included. There were 50 patients in group $\mathrm{I}$ and 29 in group II. Overall prevalence for NH and NTR was $42 \%(\mathrm{n}=33)$ and $52 \%$ (n= 41), respectively. There was no correlation between NH and NTR. Prevalence of NH was similar in both groups ( $>0.05$, Table). In contrast, NTR prevalence was significantly higher in obese patients (OR 3.06, 95\% CI 1.18-7.9, $\mathrm{p}=0.04$ ). Total reflux episodes, nocturnal reflux episodes, and $\%$ of $\mathrm{pH}<4$ were also significantly higher in group II patients (Table). Conclusions: Obesity is a risk factor for abnormal acid esophageal exposure during the night. However, symptomatic perception during the night was not associated with a high BMI. These findings suggest that for nocturnal GERD symptoms perception, other mechanisms are more important than obesity

\begin{tabular}{|c|c|c|}
\hline & Group I (non-obese) $\mathrm{n}=50$ & Group II (obese) $\mathrm{n}=29$ \\
\hline Prevalence of NH $(\%)$ & 46 & 35 \\
\hline Prevalence of NTR (\%) & 42 & $68^{*}$ \\
\hline Total reflux episodes (Mean \pm SE) & $100 \pm 19$ & $158 \pm 24^{*}$ \\
\hline Night reflux episodes (Mean \pm SE) & $30 \pm 5$ & $49 \pm 7^{*}$ \\
\hline Total \% time $\mathrm{pH}<4$ & $9 \pm 1$ & $15 \pm 2^{*}$ \\
\hline Night $\%$ time $\mathrm{pH}<4$ & $12 \pm 2$ & $16 \pm 2^{*}$ \\
\hline
\end{tabular}


M2270

RELM $\beta$ Is an Intestinal Epithelial-Derived Hormone Responsible for Host Metabolic Adaptation to Chronic Parasitic Nematode Infections

Marie A. Hildebrandt, Qi Yong, Seana M. Thrasher, Laila M. McVay, Wolfgang Stehr, Brad W. Warner, Judith A. Appleton, Rexford S. Ahima, Gary D. Wu

We have shown that RELM $\beta$ is an intestinal goblet cell-specific protein that is constitutively expressed in the colon and can be strongly induced upon Th2 immune activation by intestinal parasitic nematode infections. Previously reported studies have demonstrated a role for a closely related protein, resistin, in the regulation of glucose homeostasis in mice. In order to elucidate the function of RELM $\beta$ in vivo, we have developed a RELM $\beta$ knock-out mouse and determined its metabolic response in a murine model of diet-induced type 2 diabetes mellitus and upon parasitic infection with Heligmosomoides polygyrus. Although RELM $\beta$ is secreted into the stool, it is also detectable in serum. Serum levels of this protein decrease dramatically after a subtotal colectomy in mice confirming the colon as the source of circulating RELM $\beta$. Through the use of glucose and insulin tolerance tests as well as hyperinsulinemic-euglycemic clamps, we show that compared to wild-type mice, RELM $\beta$ knockout mice exhibit increased insulin resistance through an increase in hepatic glucose production and a decrease in glucose uptake in both adipose tissue and skeletal muscle. These results demonstrate that RELM $\beta$ is a circulating hormone that functions to enhance insulin sensitization. Upon infection with the small intestinal nematode, $H$. polygyrus, there is a dramatic induction of RELM $\beta$ in the small intestine with a resultant 8 -fold increase in serum levels. Whereas wild-type mice are able to compensate well for the metabolic challenges imposed by parasitic infection, RELM $\beta$ knock-out mice demonstrate a significant loss in weight due to a decrease in adipose tissue mass. These results show that RELM $\beta$ enhances insulin-dependent effects on both glucose homeostasis and anabolic function in the same target tissues, thus protecting host nutritional status in the setting of a chronic parasitic infection. As a newly described gut hormone produced by goblet cells, RELM $\beta$ may provide novel insights into the pathogenesis and therapy of type 2 diabetes mellitus.

\section{M2271}

Gastric Electrical Activity Before and After Bionterics Intragastric Balloon (BIB) Placement

Gustavo A. Torres-Barrera, Francisco Bosques-Padilla, Hector Maldonado-Garza

BACKGROUND: Obesity is increasing worldwide. BIB has been used by some groups as an alternative to Surgery or in preparation to it. The mechanisms involved are partially studied. Recently the effect of BIB has been published, observing a reduction in Ghrelin levels as a factor in BIB effect. We hypothesized that the presence of the BIB can promote gastric dysrrhytmia, and early satiety.AIM:The aim of this study was to evaluate the effect of the presence of BIB on gastric electrical activity.METHODOLOGY: We included 15 overweight patients that meet the following criteria: 1) Body Mass Index (BMI) above 30, 2) Age above 18, 3) Informed consent. Exclusion criteria: 1) Previous gastric surgery, 2) Associated diseases with effect on gastric motility 3) Peptic ulcer or erosive esophagitis, 4) Hiatal hernia $>3 \mathrm{cms}$. METHODS. An electrogastrogram (EGG) was performed before and one month after BIB placement. We used a Medtronic polygraph and EGG software. The procedure was performed early in the morning, after an overnight fast and in the absence of medication with effect in motility. The recording included a preprandial and a postprandial period. We used a standard meal (250grs normal yogurt providing 204kcal) We evaluated the results in frequency in cpm in channels with the best recording of the electrical activity. A Wilcoxon test was used in statistical analysis. RESULTS. Patients were $13 \mathrm{~F} / 2 \mathrm{M}$, mean age $34 \pm 8$ range 20-51: mean BMI 36 4 range 30-43.6. In every patient gastric electrical activity before BIB placement was in the normal range $(2.5-3.5 \mathrm{cpm})$. Frequency was significantly reduced after BIB placement in all patients $\mathrm{p}<0.001$ and in $6 / 14$ reduction was below the normal range.Conclusion. BIB reduced gastric electrical activity in obese patients which can be one of the mechanism involved in its satiety effect

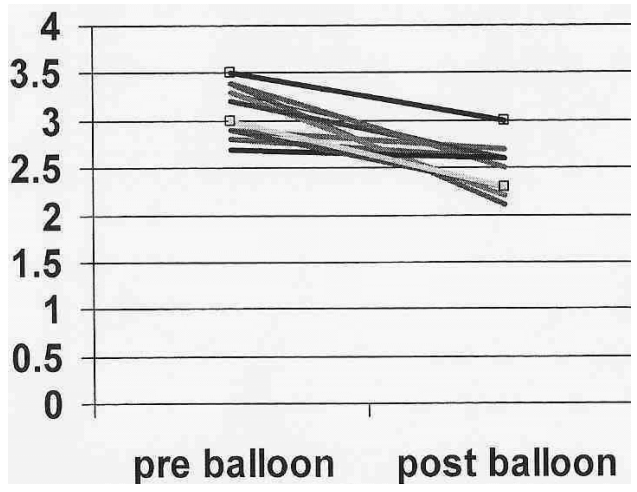

M2272

Gastric Motor and Sensory Functions in Normal Weight, Overweight and Obese People

Maria I. Vazquez-Roque, Debra Stephens, Duane Burton, Kari Baxter, Michael Ryks, Matthew Clark, Michael D. Jensen, Alan R. Zinsmeister, Michael Camilleri

Background: The literature on motor and sensory functions of the human stomach in relation to body mass presents contradictory information, in part because the functions have not been systematically studied in the same cohort of people. On the other hand, obesity is associated with several upper gut symptoms, and the mechanisms of symptom generation are unclear. In obesity, measured gastric volumes were normal except in the presence of bulimia (Physiol Behav 2004;81:735-40). Aim: To compare gastric volumes, emptying, maximum tolerated volumes (MTV) and post-challenge symptoms in normal, overweight, or obese volunteers who were otherwise healthy. Methods: We studied 68 of 72 participants in normal, overweight, or obese (WHO criteria) groups (all non-bulimic) and documented their lean mass and \% fat by body composition (DEXA). All underwent the following measurements using validated tests: gastric emptying for solids and liquids by scintigraphy (GE t1/2); gastric volumes by SPECT, a validated imaging method (Gut 2002:51:781-6); MTV and 30 min post-challenge symptoms by satiation test (Neurogastro Motil 2002;14:24953). Statistical analysis used one-way ANCOVA adjusted for gender. Results: The table summarizes the data, mean \pm SEM. There were significant differences in lean and fat mass and significantly lower fasting gastric volumes in the overweight and obese groups. We also observed borderline lower postprandial volume changes in obese and borderline faster gastric emptying of liquids. Conclusion: Being overweight or obese is associated with lower fasting gastric volumes, but there is no difference in food volume tolerated or symptom score after a challenge meal. The significance of lower fasting gastric volumes to upper gut symptoms in obesity requires further study.

\begin{tabular}{|c|c|c|c|c|}
\hline & $\begin{array}{c}\text { Normal weight } \\
(\mathrm{n}=24)\end{array}$ & $\begin{array}{c}\text { Overweight } \\
(\mathrm{n}=20)\end{array}$ & Obese $(\mathrm{n}=24)$ & $\begin{array}{c}\text { Overall } \mathrm{p} \\
\text { value }\end{array}$ \\
\hline Age, $\mathrm{y}$ & $37.4 \pm 3.0$ & $36.6 \pm 2.0$ & $44.3 \pm 2.6$ & 0.11 \\
\hline BMI, $\mathrm{kg} / \mathrm{m}^{2}$ & $22.9 \pm 0.3$ & $27.8 \pm 0.3$ & $34.8 \pm 0.7$ & \\
\hline Gender M:F & $8: 16$ & $6: 14$ & $11: 13$ & 0.05 \\
\hline Lean mass & $45593 \pm 1101$ & $48200 \pm 1216$ & $50607 \pm 1086$ & 0.007 \\
\hline Tissue fat, \% & $30.6 \pm 1.2$ & $39.9 \pm 1.3$ & $47.7 \pm 1.2$ & $<0.001$ \\
\hline Fasting gastric vol, ml & $258.7 \pm 13.1$ & $198 \pm 14.7^{*}$ & $236.0 \pm 13.0^{* * *}$ & 0.01 \\
\hline$\Delta$ PP vol, ml & $518.5 \pm 17.8$ & $489.1 \pm 20.0$ & $464.0 \pm 17.5 \$$ & 0.09 \\
\hline MTV, ml & $1230.6 \pm 71.7$ & $1197.7 \pm 79.1$ & $1260.9 \pm 69.1$ & 0.88 \\
\hline $\begin{array}{c}\text { Aggregate symptom } \\
\text { score }\end{array}$ & $145.3 \pm 14.5$ & $152.6 \pm 15.7$ & $140.1 \pm 14.0$ & 0.84 \\
\hline GE t1/2 solids, min & $119.8 \pm 6.3$ & $111.5 \pm 7.0$ & $116.9 \pm 6.1$ & 0.66 \\
\hline GE t1/2 liquids, min & $26.3 \pm 2.5$ & $19.4 \pm 2.9 \#$ & $20.4 \pm 2.4 \#$ & 0.13 \\
\hline
\end{tabular}

Compared to normals: * $\mathrm{p}=0.003 ; * * \mathrm{p}=0.055 ; \$ \mathrm{p}=0.03 ; \# \mathrm{p} \leq 0.10$

M2273

Organ-Specific Function of Ppara As Revealed By Gene Expression Profiling Meike Bunger, Heleen van den Bosch, Mechteld Grootte Bromhaar, Philip de Groot, Sander Kersten, Guido Hooiveld, Michael Muller

Introduction The peroxisome proliferator-activated receptor alpha (PPAR $\alpha)$ is a fatty acidactivated transcrintion factor expressed at high levels in small intestine and liver, two key organs in whole-body metabolism of (dietary) triglycerides (TGs). The importance of PPAR $\alpha$ in TG metabolism is illustrated by the fact that agonists targeting PPAR $\alpha$, such as polyunsaturated fatty acids (PUFAs) and fibrates, are commonly used for the treatment of dyslipidemia. Whether PPAR $\alpha$ functions differently in small intestine and liver is currently unknown. The aim of this study was to compare genome wide effects of PPAR $\alpha$ activation between small intestine and liver. Methods Wild-type and PPAR $\alpha$-null mice were fed a die supplemented with a synthetic PPAR $\alpha$ ligand (0.1\% WY14643) for 5 days. RNA was extracted from small intestine and liver and hybridized to Affymetrix MOE430 2.0 arrays. Data was analyzed applying the packages gcRMA for data processing, and LIMMA for statistical analyses. Differentially expressed transcripts were selected based on fold change $(>1.5)$ and false discovery rate $(<0.05)$. Data was functionally analyzed based on Gene Ontology using a score resampling method, metabolic and signal transduction routes using gene set enrichment analysis, and biological interaction networks using text-mining software. Results qRT-PCR analyses showed that PPAR $\alpha$ is expressed in small intestine and liver at comparable levels. Feeding studies identified 1918 respectively 4003 PPAR $\alpha$ target genes in SI and liver. These target genes could be divided into three sets, namely those regulated in both tissues (668 genes; corresponding to $35 \%$ respectively $17 \%$ of regulated genes in SI and liver), those regulated exclusively in small intestine (1250 genes) and those regulated exclusively in liver (3335 genes). Processes that were affected in both tissues include metabolism of lipids and energy-rich intermediates, organelle organization and biogenesis, and humoral immune response. Specific PPAR $\alpha$-dependently regulated processes in small intestine were antigen presentation via MHCII, B-lymphocyte proliferation, various cell signalling pathways, and bile acid metabolism. Liver specific processes were coagulation and wound healing, nonlymphocyte mediated immune response, non-lipid nutrient metabolism, and control of cell growth. Conclusion PPAR $\alpha$ in small intestine and liver controls the expression of a distinct set of genes, demonstrating that these organs use different strategies to adapt to high levels of agonists. We speculate that this differential regulation is an important mechanism explaining the well-known health-promoting effects of PUFAs and lipid-lowering drugs.

\section{M2274}

Comparison of Glycine and Leucine Kinetics in Obese Subjects Yoshihisa Urita, Susumu Ishihara, Kenichiro Arai, Jun Kondo, Tatsuo Akimoto, Hiroto Kato, Noriko Hara, Hideyuki Koshino, Yoshiko Honda, Yoko Nagai, Kazushige Nakanishi, Nagato Shimada, Motonobu Sugimoto, Kazumasa Miki

Background: Although it is well established that human obesity is accompanied by abnormal ities in glucose and lipid metabolism, it has been unclear whether protein metabolism also is disturbed. The aim of this study is to evaluate small intestinal absorption of nutrients, including glucose, amino acid, and short-chain fatty acid in obese subjects. Patients and methods: Consecutive 130 subjects who underwent upper gastrointestional endoscopy were recruited in this study. They were divided into three groups according to body mass index 
(BMI). The obesity group consisted of 21 patients with BMI of more than 26. At endoscopy, a tip of endoscope was placed to the second part of the duodenum and $20 \mathrm{ml}$ of water containing $100 \mathrm{mg}$ of $13 \mathrm{C}$-glycine $(\mathrm{n}=65)$ and $13 \mathrm{C}$-leucine $(\mathrm{n}=65)$ was sprayed into the second part of the duodenum. Breath samples were taken at baseline and at 5, 10, 20, 30, 40,50 , and $60 \mathrm{~min}$ after administration for analysis of the $13 \mathrm{C} / 12 \mathrm{C}$ ratio and were expressed as delta over baseline. Absorptive function was determined as cumulative \% dose at $60 \mathrm{~min}$. Results: Absorption values in 3C-glycine breath test (cumulative \% dose at $60 \mathrm{~min}$ ) in obese patients and non-obese patients were $13.6+-3.2 \%$ and $11.8+/-3.8 \%$, respectively. $13 \mathrm{CO} 2$ concentrations in the exhaled air were significantly $(\mathrm{p}<0.05)$ higher in the obesity group at 15,20 , and $30 \mathrm{~min}$, than in the non-obese groups. In contrast, for 13C-leucine breath test, breath $13 \mathrm{CO} 2$ concentrations were significantly $(\mathrm{p}<0.05)$ lower in the obesity group at 10 minutes and later. Absorptive function was not influenced by aging. Conclusions: Contrary to our expectations, absorption and oxidation of glycine was enhanced in obese subjects, whereas leucine metabolism was decreased. The decreased rate of leucine oxidation in the obesity may be a consequence of decreased substrate availability. It is concluded that changes in kinetics of amino acid may be one of causes of obesity.

\section{M2275}

Effects of Gastric Distension On Myoelectrical Activity of the Stomach in Obese People

Shukun Yao, Lili Chang, Ruixing Zhang

A number of studies have suggested that enlarged gastric accommodation and accelerated gastric emptying may play a role in obesity. Gastric motility is known to be regulated by gastric myoelectrical activities. For understanding the characteristics of myoelectrical activities in the obese people, this study was to observe the alterations of gastric myoelectrical activity induced by gastric distension between obese and non-obese healthy subjects. Methods: 31 obese (BMI $30.95 \pm 1.52 \mathrm{~kg} / \mathrm{m} 2$, age $30.95 \pm 1.52 \mathrm{yr}$ ) and 26 non-obese healthy volunteers (BMI $20.99 \pm 1.92 \mathrm{~kg} / \mathrm{m} 2$, age $22.73 \pm 6.93 \mathrm{yr}$ ) were studied with barostat and electrogastrography. Following an overnight fast, a polyethylene balloon attached at the end of a catheter was placed in the proximal stomach. By filling the gastric balloon with air, different visceral sensation (initial satiety and maximal tolerance) was induced and electrogastrogram (EGG) was recorded simultaneously. Results: (1)At baseline, initial satiety and maximal tolerance, the dominant power of the EGG was increased from $31.52 \pm 4.93$ to $33.15 \pm 4.85$ and $35.85 \pm 5.05$ in obese people, which were higher than those in non-obese group $(29.02 \pm 3.22,30.43 \pm 3.95$, $31.12 \pm 6.25, \mathrm{P}<0.05)$. The dominant frequency showed a tendency to decrease and no difference between the two groups. (2)A tendency was seen that the percentage of normal slow waves (N\%) decreased and tachygastria (T\%) increased as the volume of intragastric balloon was increased in obese group, and bradygastria (B\%) increased in non-obese group. (3)The dominant frequency instability coefficient (ICF), dominant frequency standard deviation (SDF) and power ratio (PR) in obese group were similar to the non-obese group $(P>0.05)$. The dominant power instability coefficient (ICP) and dominant power standard deviation (SDP) in obesity were higher than those in control subjects $(\mathrm{P}<0.05)$. Conclusion: Gastric distension results in an increase of DP, ICP and SDP. The increase of those in obese people was higher than those in non-obese people. These changes of gastric electrical activity may attribute to strengthened gastric motility and accelerated gastric emptying in obese people.

\section{M2276}

Effects of Gastric Electric Stimulation On the Expression of NPY, CCK and Growth Hormone Secretagogue Receptor mRNA in Rodent Brain Luo $\mathrm{Xu}$, Xiangrong Sun, Feng Zhong, J D Z Chen

Recently, gastric electrical stimulation (GES) has been introduced for treating obesity. However, the possible central mechanisms remain to be revealed Neusopeptide Y (NPY) (an orexigen), cholecystokinin (CCK) (a powerful anorexigen) and ghrelin have been shown to be involved in the regulation of food intake and energy metabolism. The purpose of this study was to explore the effect of GES on the contents of NPY immunoreaction (IR) and CCK-IR, and the expression of growth hormone secretagogue receptor (GHS-R) mRNA in rat brain. Methods: The contents of NPY-IR and CCK-IR in the brain, spinal cord and plasma were detected by radioimmunoassay (RIA) after GES (6mA, 3ms, $40 \mathrm{~Hz}, 2 \mathrm{~s}$-on, 3s-off) for $2 \mathrm{~h}$. The expressions of GHS-R mRNA in the brain and spinal cord were investigated using real-time PCR after GES for $2 \mathrm{~h}$. Results: The expressions of NPY-IR were decreased with GES in the hypothalamus, pituitary, solitary tract nucleus (NTS) and spinal cord $(\mathrm{P}<0.05)$, but increased with GES in the hippocampus $(\mathrm{P}<0.01)$. However no significant difference was observed for NPY-IR in plasma between GES and control groups. Conversely, the contents of CCK-IR were increased in hypothalamus $(\mathrm{P}<0.01)$, hippocampus $(\mathrm{P}<0.05)$, NTS $(\mathrm{P}<0.05)$ and plasma $(\mathrm{P}<0.05)$ after GES for $2 \mathrm{~h}$, but decreased in pituitary $(\mathrm{P}<0.05)$. The expression of GHS-R mRNA was not altered in the hypothalamus, pituitary, hippocampus or spinal cord with 2-h GES, compared with the control group $(\mathrm{P}>0.05)$. However, it was found that the expression of GHS-R mRNA was highest in pituitary ( $\triangle \mathrm{Ct} 5.877 \pm 0.34$ ), followed with the hypothalamus $(\triangle \mathrm{Ct} 7.605 \pm 0.45)$, the hippocampus $(\triangle \mathrm{Ct} 8.811 \pm 0.26)$ and the spinal cord $(\triangle \mathrm{Ct} 9.840 \pm 0.36)(\mathrm{P}<0.05 \sim 0.01)$. Conclusion: Gastric electrical stimulation increases the content of CCK-IR and decreases the expression of NPY-IR in rat brain, but does not alter the expression of GHS-R mRNA in brain. The expression of GHS-R mRNA is highest in the pituitary and lowest in the spinal cord.

\section{M2277}

Association of Variations in Candidate Genes Affecting Serotonergic and Adrenergic Mechanisms with Body Mass in Humans

Maria I. Vazquez Roque, Paula Carlson, Debra Stephens, Alan R. Zinsmeister, Michael Camilleri

Background: Genetic factors have a role in obesity. Previous studies have suggested that genetic variations in serotonergic, adrenergic, and several $G$ protein coupled receptors are associated with obesity, but this is still unclear. These are also candidate genes associated with gut dysfunction, as in irritable bowel syndrome (Gut 2004;53:829-37) and functional dyspepsia (Gastroenterology 2004;126:971-9). Obese people have high prevalence of gastrointestinal symptoms such as heartburn, postprandial bloating and diarrhea (Am J Gastroenterology 2004;99:1801-6). Aim: To characterize the association of polymorphisms of SERT-P (promoter for SLC6A4, the protein controlling 5-HT reuptake), $\alpha$-2 adrenoreceptor $(\alpha-2$ $\mathrm{MspI}$ ) and GNB3 (C825T) with body mass in normal, overweight, or obese individuals. Methods: Two hundred and six healthy, predominantly Caucasian volunteers from a Midwest U.S. population in the three body mass index $\left(\mathrm{BMI} \mathrm{kg} / \mathrm{m}^{2}\right.$ ) ranges using WHO criteria (normal $22.1 \pm 2.2$, overweight $27.0 \pm 1.1$, obese $34.7 \pm 4$ ) were recruited by public advertisement and letters. After informed consent, a venous blood sample was drawn for genetic analysis for SERT-P, $\alpha-2$ MspI and GN 33 C825T genotypes. Logistic regression and Chi-square analyses were performed to assess the associations of these genotypes and body mass. Results: The table summarizes the data, mean \pm SD and percent. No statistically significant associations were found between the genotypes and BMI; however there was a weak univariate association of $\alpha-2$ MspI with BMI group (likelihood ratio $x^{2}, p=0.05$ ). After adjusting for age, race, and gender, an increased odds for obesity was found for the polymorphic heterozygous $\alpha-2$ MspI polymorphism and obesity $(\mathrm{p}=0.04)$. However, this was not demonstrated for the overweight group. Conclusion: Our data suggest that genetic variation in $\alpha-2$ adrenoceptor function may be associated with obesity. The influence of this adrenoceptor genotype on development of gastrointestinal symptoms and responses to agents that affect noradrenergic reuptake, such as sibutramine, deserves further study.

\begin{tabular}{|c|c|c|c|c|}
\hline & Normal Weight $(\mathrm{n}=76)$ & Overweight $(\mathrm{n}=61)$ & Obese $(\mathrm{n}=64)$ & $\mathrm{p}$ value \\
\hline Age, $\mathrm{y}$ & $40.1 \pm 16.4$ & $39.8 \pm 12.4$ & $45.0 \pm 14.8$ & \\
\hline SERT-P (\%) LL & 32 & 37 & 31 & NS \\
\hline LS/SS & 41 & 26 & 33 & \\
\hline$\alpha-2 \mathrm{a}$ MSP (\%) CC & 41 & 35 & 24 & 0.05 \\
\hline CG & 35 & 27 & 39 & \\
\hline GG & 39 & 11 & 50 & \\
\hline GN33 (\%) CC & 37 & 31 & 32 & NS \\
\hline CT & 39 & 28 & 32 & \\
\hline TT & 33 & 24 & 43 & \\
\hline
\end{tabular}

\section{M2278}

Obesity Is An Independent Risk Factor for Chronic Gastrointestinal Symptoms in a Sample of Australian Adults Nicholas J. Talley, Stuart C. Howell

Introduction: Obesity has been identified as a potential risk factor for gastrointestinal (GI) complaints, although the data from community samples remains sparse. We assess the associations between GI symptoms and obesity in a population-based sample of Australian adults. Method: The prevalence of 26 gastrointestinal symptoms was determined by postal questionnaire which was sent to 5000 residents across western Sydney, Australia (60\% response rate). These were classified into five broader symptom groups according to the Rome II Criteria - abdominal pain, esophageal symptoms, dysmotility symptoms, diarrhea and constipation. Obesity was defined according to body mass index using self-reported height and weight. Socioeconomic characteristics and eating behaviors were also evaluated. Results: The prevalence of obesity (BMI $>=30 \mathrm{~kg} / \mathrm{m} 2$ ) was $25.1 \% ; 36.1 \%$ were overweight. There were significant and positive univariate associations between obesity and abdominal pain $(\mathrm{OR}=1.57 ; 95 \% \mathrm{CI}: 1.21-2.03 ; \mathrm{p}=0.001)$, esophageal symptoms $(\mathrm{OR}=2.21 ; 95 \% \mathrm{CI}$ : $1.65-2.95 ; \mathrm{p}<0.0001)$, dysmotility symptoms (OR=1.27; $95 \% \mathrm{CI}: 1.01-1.60 ; \mathrm{p}=0.05)$ and diarrhea $(\mathrm{OR}=2.11 ; 95 \% \mathrm{CI}: 1.69-2.65 ; \mathrm{p}<0.0001)$. The adjustment for socioeconomic characteristics and eating behaviors had minimal effect on the associations for abdominal pain $(O R=1.46 ; 95 \% C I$ : $1.10-1.95 ; p=0.01)$, esophageal symptoms $(O R=1.84 ; 95 \% \mathrm{CI}$ : $1.36-2.50 ; \mathrm{p}<0.0001)$ and diarrhea $(\mathrm{OR}=1.84 ; 95 \% \mathrm{CI}: 1.44-2.35 ; \mathrm{p}<0.0001)$; however, the association between obesity and dysmotility symptoms became non-significant $(\mathrm{OR}=1.26$; 95\% CI: 0.96-1.65; $\mathrm{p}=0.09$ ). Obesity was not related to constipation. Being overweight did not emerge as a significant risk factor for GI symptoms in this cohort. Conclusion: Obesity is an independent risk factor for abdominal pain, esophageal symptoms and diarrhea, but is not associated with dysmotility symptoms or constipation.

Table 1: Symptom prevalence for each BMI group 


\begin{tabular}{|c|c|c|c|c|}
\hline Symptom & Normal weight $(\mathrm{n}=952)$ & Overweight $(\mathrm{n}=929)$ & Obese $(\mathrm{n}=647)$ & $\mathrm{p}$-value \\
\hline Abdominal pain & 14.5 & 11.9 & 21.3 & $<0.0001$ \\
\hline Esophageal & 9.1 & 14.0 & 18.4 & $<0.0001$ \\
\hline Dysmotility & 21.1 & 17.1 & 25.6 & 0.001 \\
\hline Nausea/Vomiting & 5.9 & 4.4 & 6.8 & 0.09 \\
\hline Diarrhea & 19.5 & 21.6 & 34.6 & $<0.0001$ \\
\hline Constipation & 19.1 & 15.9 & 20.7 & 0.05 \\
\hline
\end{tabular}

intervals during the first 2 hours and at 30-minute intervals thereafter for 2 hours. Semisolid GET was measured by the (13)C-acetic acid breath test. The test meal consisted of 75 $\mathrm{ml}$ of milk mixed with $30 \mathrm{~g}$ of oat labeled with $150 \mathrm{mg}$ of 13C-acetic acid. Breath samples were collected before the meal (baseline) and at 5-minute intervals during the first 2 hour and at 10-minute intervals thereafter for 2 hours. The expired air samples were analysed using infrared isotope spectrometry (IRIS $\circledR_{\text {, }}$, Wagner Analysen Technik Vertriebs-GmbH, Bremen, Germany) and the data fitted to the established gastric emptying model. The lag times t(lag) and half excretion times $t(1 / 2)$ were calculated. The Student t-test was used to compare the mean data of the lean and class III obese groups. A p value $<0.05$ was considered to be significant in both analyses. RESULTS: The mean $t(1 / 2)$-values to the GET of solids (+/-standard error of the mean) were $203.6 \mathrm{~min}+/-76.0 \mathrm{~min}$ and $143.5 \mathrm{~min} \mathrm{+/-}$ $19.1 \mathrm{~min}$ for lean and obese, respectively $(\mathrm{p}=0.0010)$. The mean $\mathrm{t}(\mathrm{lag})$-values to the GET of solids were $127.3 \mathrm{~min}+/-42.7 \mathrm{~min}$ and $98.4 \mathrm{~min}+/-13.0 \mathrm{~min}$ for lean and obese, respectively $(\mathrm{p}=0.0044)$. There was no significant difference in the GET of semi-solids between the lean and class III obese groups. CONCLUSIONS: The present study demonstrated a significantly faster lag phase and a significantly enhanced gastric emptying in class III obese patients when compared to lean subjects. This findings are compatible with the hypothesis that gastric emptying, satiety, food intake and body weight are integrated parameters in subjects with class III obesity.

Man

Dan Lassman, Shane McKie, Simon Lal, Lloyd Gregory, Graham Dockray, Steve Williams, David Thompson

Ingested lipid signals via cholecystokinin (CCK) to limit food intake, but in man, brain responses to ingested lipid have not been shown. Methods: 5 fasted healthy volunteers underwent blood oxygenation level dependent (BOLD) $1.5 \mathrm{~T}$ functional magnetic resonance (fMRI) brain imaging before and after intraduodenal infusion of either saline or a CCKreleasing lipid on separate occasions. The average BOLD signal for each 2 minute time bin was compared to the pre-infusion 2 minute time bin. A random effects ANOVA was used to investigate significant differences over time between the two conditions. Visual analogue scores of satiety sensations were also recorded. Results: The comparison of lipid infusion to saline highlighted an increase of the BOLD response in the hypothalamic region (figure 1) and brainstem (figure 2) (mean $+/-95 \% \mathrm{CI} p<0.05$ ). At 10 minutes, infusion of lipid was associated with a $64 \%(4.9)$ increase in fullness scores compared to $10 \%(10)$ with saline. (Mean +/- SE, p0.007). Conclusion: These findings are consistent with a role of ingested lipid in the brainstem and hypothalamic control of eating in man.
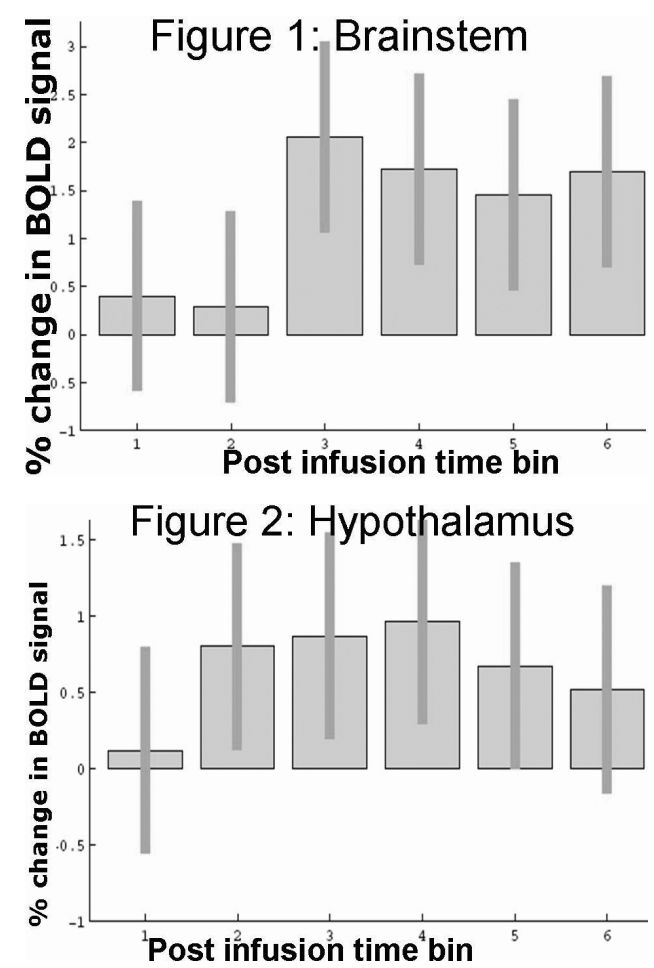

M2281

Gastric Emptying of Solids and Semi-Solids in Class III Obesity: An Assessment Using the Non-Invasive (13)C-Octanoic Acid and (13)C-Acetic Acid Breath Test

Aloisio Cardoso Jr., Luiz Gonzaga Vaz Coelho, Paulo Roberto Savassi-Rocha, Aline Miranda Almeida, Emanuela Eudes Dias, Gerival Vieira Jr., Mariana Moreira Castro, Yara Vieira Lemos

BACKGROUND: It has been suggested that obesity is associated with an altered rate of gastric emptying. An enhanced gastric emptying rate might reduce the satiating effect of food and thereby promote obesity. Gastric emptying rate has previously been compared between obese and lean subject with conflicting outcome. OBJECTIVE: The purpose of this study was to determine whether class III obese patients have different rates of solid and semi-solid gastric emptying compared to lean subjects. METHODS: Gastric emptying time (GET) of solid and semi-solid meals was measured using the (13)C-octanoic acid breath test and (13)C-acetic acid breath test, respectively, in 24 lean (healthy) and 14 class III obese individuals of both sexes. The age of the patients ranged from 17 to 77 years. Following an overnight fast, subjects were given a standard egg meal labelled with $100 \mathrm{mg}$ of (13)Coctanoic acid. Breath samples were collected before the meal (baseline) and at 15-minute

\section{$\mathrm{M} 2282$}

CNS Expression of Ghrelin and Cholecystokinin (CCK) with Gastric Electrical Stimulation in Rats

Suhuan Liu, Ming Tang, Shangmin Tao, Jiande Chen

Background and aim: Gastric electrical stimulation (GES) has recently been shown to be safe and effective in the treatment of obesity, in the peripheral tissue, it induces gastric distention, impairs gastric myoelectric activity, delays gastric emptying, but its effects in the central nervous system (CNS) are remained unclear, the aim of this study was to explore the possible central mechanisms involved in GES by investigation of the expression of food intake-related peptides. Methods: the experiment was designed in two parts: an acute experiment with $2 \mathrm{~h}$ GES and a long term experiment with 14- day continuous GES, each experiment contained two groups: stimulation group and Control group. After stimulation, the expression of orexigenic hormone - ghrelin in the hypothalamus and anorexigenic hormone -CCK in the hippocampus were detected by immunohistochemical method. GES was performed using pulse trains (train on-time of $2 \mathrm{~s}$, off-time of $3 \mathrm{~s}$, pulse amplitude of $6 \mathrm{~mA}$, width of $0.3 \mathrm{~ms}$ and frequency of $40 \mathrm{~Hz}$ ). Results: Compared with the control group, $2 \mathrm{~h}$ GES resulted in a decrease in the number of ghrelin immunoreactive neurons in the hypothalamic paraventricular nucleus (PVN, $34.8 \pm 1.86$ vs. $57.2 \pm 2.95, \mathrm{P}=0.02$ ) and in the supraoptic nucleus ( $\mathrm{SON}, 51.2 \pm 3.21$ vs. $82.8 \pm 3.08, \mathrm{P}=0.01$ ). The number of CCK immunoreactive neurons in the hippocampus was of no changes $(7.4 \pm 0.87$ vs. $6.2 \pm 0.58, P=0.29)$. After 14-day GES, the number of CCK immunoreactive neurons in the hippocampus was increased compared with that of the control group ( $4.0 \pm 0.32$ vs. $2.4 \pm 0.51, P=0.03)$. However there were no changes of the number of ghrelin immunoreactive neurons either in the PVN $(55.5 \pm 5.49$ vs. $61.6 \pm 3.53, \mathrm{P}=0.66)$ or in the $\mathrm{SON}(65.5 \pm 3.13$ vs. $80.0 \pm 3.39, \mathrm{P}=0.20)$. Conclusions: These results indicate that the expression of ghrelin and CCK can indeed be altered by GES. For the first time, we have demonstrated that GES may alter energy homeostasis by modulating the expression of food intake-related hormones in the central nervous system-reducing acutely the level of orexigenic ghrelin and increasing chronically the level of anorexigenic CCK.

M2283

Polyunsaturated Fatty Acids Derived from Korean Pine Nuts Affect Appetite Suppressing Hormones and Appetite Sensations in Overweight Women Wilrike J. Pasman, Jos Heimerikx, Carina M. Rubingh, Robin van den Berg, Marianne O Shea, Louisa Gambelli, Henk F. Hendriks, Alexandra W. Einerhand

Background: In the United States today almost two thirds of the entire adult population is either overweight or obese, roughly double the percentage twenty years ago. Western Europe is catching up fast and obesity is also on the rise in Asia and other parts of the world. In the face of this global epidemic there is an urgent need to find means to combat obesity. Controlling food intake by suppressing appetite might be one of the principal approaches to prevent overweight. The most widely investigated gut hormone in relation to appetite control is cholecystokinin (CCK). Previous experiments showed that polyunsaturated fatty acids (PUFAs) derived from Korean pine nuts (Pinus koraiensis) induced high amounts of CCK release by STC-1 enteroendocrine cells in comparison to other PUFAs and MUFAs Aim: This study investigates the effect of Korean pine nut PUFAs on appetite sensations and appetite-regulating hormones in humans. Methods: A randomized, cross-over, placebocontrolled double-blind study was carried out with 18 overweight post-menopausal women (BMI=25-30 kg/m2) receiving capsules with $3 \mathrm{~g}$ PUFAs derived from Korean pine nuts or olive oil (placebo) in combination with a light breakfast consisting of two slices of white bread and marmalade. At 0, 30, 60, 90, 120, 180 and 240 min following supplementation blood samples were taken for analyses of CCK and glucagon-like peptide-1 (GLP-1), two appetite suppressing hormones, and appetite sensations were evaluated by using visual analogue scales. Results: Korean pine nut PUFAs significantly induced CCK after 30 min and GLP-1 after 60 min relative to placebo. Over a period of 4 hours the total amount of plasma CCK and GLP-1 in response to pine nut PUFAs was $60 \%(\mathrm{P}<0.0001)$ and $25 \%$ $(\mathrm{P}<0.05)$ higher than in response to placebo, respectively. Korean pine nut PUFAs affected appetite sensations during the 4 hours after intake. Especially, at 30 minutes the "desire to eat" and the "prospective food intake" scores were, 29\% and 36\% lower relative to placebo, respectively. Similar results were obtained with Korean pine nut triglycerides. In conclusion Korean pine nut PUFAs induced CCK and GLP-1 levels significantly for up to 4 hours suggesting that Korean pine nut PUFAs suppress appetite and consequently may affect food intake. 


\section{M2284}

Comorbidities in Rural Obese Children

Mark Brunner, William Cochran, Michael Komar, Stacy Prall

Introduction The epidemic of childhood obesity is associated with multiple medical complications adversely affecting health in childhood and subsequently adulthood. Purpose To describe obesity-related comorbidities in children and assess for associations between insulin resistance or BMI with comorbidities. Methods Chart review of children seen in our obesity clinic from 1999-2005 to identify multiple comorbidities. BMI Z-score (BMIZ) was adjusted for gender and age. Hypertension was defined as 3 consecutive systolic pressures $>97$ th percentile for age, gender, and height. Insulin resistance was defined as fasting Homeostasis Model Assessment $>2.5$. Chi-square was used to correlate comorbidities with mean BMIZ and insulin resistance. Results 506 children were evaluated. 53\% were female, and 96\% were non-Hispanic white. Mean BMIZ for all patients was 2.61. Insulin resistance was present in $69 \%$. Table 1 summarizes BMIZ by comorbidity. Children with higher BMIZ were more likely to snore, have hypertension, and higher transaminases. Table 2 summarizes insulin resistance by comorbidity. Children with insulin resistance were more likely to have daytime somnolence, acanthosis, striae, and higher transaminases. Conclusions: Extremely obese children have obesity-related comorbidities. The presence of nocturnal snoring, daytime somnolence, and hypertension are associated with the degree of obesity. Insulin resistance is directly related to acanthosis, striae, dyslipidemia, and elevated transaminases. We sugges additional studies include normal and overweight children. This will allow more precise correlation of BMI with comorbidities, providing a target weight for children who cannot practicably reach ideal body weight.

Table 1: BMI Z-score for comorbidities

\begin{tabular}{|c|c|c|c|}
\hline Comorbidity (CM) & $\begin{array}{c}\text { CM present:N; mean } \\
\text { BMIZ }\end{array}$ & $\begin{array}{c}\text { CM absent:N; mean } \\
\text { BMIZ }\end{array}$ & $\begin{array}{c}\text { X association of CM and } \\
\text { BMIZ }\end{array}$ \\
\hline Nocturnal Snoring & $274(55.0 \%) ; 2.70$ & $224 ; 2.50$ & $\mathrm{p}<0.01$ \\
\hline $\begin{array}{c}\text { Daytime } \\
\text { Somnolence }\end{array}$ & $57(11.4 \%) ; 2.69$ & $442 ; 2.60$ & $\mathrm{p}=0.08$ \\
\hline T\& A & $136(27.0 \%) ; 2.60$ & $368 ; 2.61$ & $\mathrm{p}=0.85$ \\
\hline HTN & $235(46.8 \%) ; 2.75$ & $2.67 ; 2.47$ & $\mathrm{p}<0.01$ \\
\hline
\end{tabular}

$\mathrm{X}=$ Chi-Square testing. $\mathrm{T} \& \mathrm{~A}=$ tonsillectomy and adenoidectomy for obstructive symptoms Table 2: Insulin Resistance for Comorbidities

\begin{tabular}{|c|c|c|c|}
\hline Comorbidity (CM) & CM present: \% IR; $N$ & CM absent: \% IR; $N$ & X association of CM and IR \\
\hline Nocturnal Snoring & $78.2 \% ; 190$ & $77.4 \% ; 151$ & $\mathrm{p}=0.85$ \\
\hline Daytime Somnolence & $94.1 \% ; 48$ & $75.8 \% ; 294$ & $\mathrm{p}<0.01$ \\
\hline T\&A & $75.4 \% ; 92$ & $79.3 \% ; 257$ & $\mathrm{p}=0.37$ \\
\hline Acanthosis & $91.6 \% ; 152$ & $70.1 \% ; 195$ & $\mathrm{p}<0.01$ \\
\hline Striae & $88.1 \% ; 178$ & $63.2 \% ; 74$ & $\mathrm{p}<0.01$ \\
\hline Low HDL $(<40)$ & $86.1 \% ; 173$ & $71.8 \% ; 176$ & $\mathrm{p}<0.01$ \\
\hline High LDL $(>130)$ & $76.0 \% ; 57$ & $77.9 \% ; 275$ & $\mathrm{p}=0.72$ \\
\hline AST above median & $74.8 \% ; 154$ & $82.9 \% ; 160$ & $\mathrm{p}=0.05$ \\
\hline ALT above median & $85.1 \% ; 183$ & $70.8 \% ; 143$ & $\mathrm{p}<0.01$ \\
\hline
\end{tabular}

$\mathrm{IR}=$ insulin resistance.

M2285

Association of Ghrelin Receptor Gene Polymorphism (171T/C), Not of CCK-a Receptor $(-81 \mathrm{~A} / \mathrm{G},-128 \mathrm{G} / \mathrm{T}$ ) Or Beta3 Adrenalin Receptor (Try64Arg) Gene Polymorphism, with Bulimia Nervosa in a Japanese Population Kyoko Miyasaka, Hiroko Hosoya, Ayako Sekime, Minoru Ohta, Setsuko Kanai, Saeko Akimoto-Takano, Sachio Matsushita, Kenji Suzuki, Susumu Higuchi, Akihiro Funakoshi

Background: Ghrelin, an endogenous ligand for the growth hormone secretagogue receptor (GHSR), known to enhance appetite and increase food intake, and human ghrelin plasma levels are inversely correlated with body mass index (BMI). Extremely high levels of ghrelin were observed in patients with anorexia nervosa (AN). Aims: Eating disorders (EDs) have a highly heterogeneous etiology and multiple genetic factors might contribute to their pathogenesis. As no subject with ghrelin gene polymorphism (Leu72Met) has been detected in a Japanese population, we examined the 171T/C polymorphism of GHSR gene. In addition, beta3 adrenergic receptor (adr-R) gene polymorphism (Try64Arg) and cholecystokinin-A receptor (CCK-AR) gene polymorphism $(-81 \mathrm{~A} / \mathrm{G},-128 \mathrm{G} / \mathrm{T})$, which are both associated with obesity (Obes Res 2004), were investigated. Methods: The subjects consisted of 228 Japanese patients with EDs [96 AN, 116 bulimia nervosa (BN) and 16 not otherwise specified (NOS)]. AN patients were classified into two subtypes: the restricting type (ANR: $n=47$ ) and the binge-eating and/or purging type (ANP: $n=49$ ). BN patients were classified into the purging type (BNP: $n=91$ ) and the non-purging type (BNNP: $n=25$ ). The age- and gender-matched control group consisted of 284 unrelated Japanese subjects. Genomic DNA was extracted from peripheral leukocytes, and the171T/C in the coding region of the GHSRs were examined using a mismatch PCR-RFLP method (JCEM 2004). Beta3 adr-R and CCK-AR polymorphisms were determined according to the methods previously reported (Obes Res 2004). Results: In $\mathrm{BN}$ patients, the $\mathrm{CC}$ type occurred significantly more frequently than in control subjects (chi-square $=4.47, \mathrm{p}=0.035$, odds ratio $=2.05,95 \% \mathrm{CI}, 1.088-4.935$ ), while the frequency in AN patients did not differ from that in control subjects. The frequency of the CC type tended to be lower in the ANR group than in other subgroups (ANP + BNP + BNNP)(chisquare $=3.60, p=0.058)$. When the frequency of the CC type was compared between ED patients with binge-eating and/or purging (ANP + BNP) and those without purging (ANR
+ BNNP), there were no significant differences (chi-square $=1.83, \mathrm{p}=0.21$ ). The frequency of the beta3 adr-R gene polymorphism in AN patients tended to be lower than in control subjects (chi-square $=3.179, \mathrm{p}=0.075$ ). The difference between $\mathrm{AN}$ and $\mathrm{BN}$ subjects was also not significant (chi-square $=3.736, \mathrm{p}=0.053$ ). The distribution of CCK-AR gene polymorphism did not differ between ED and control subjects. Conclusions: The CC type of GHSR gene polymorphism (171T/C) is a risk factor for BN, but not for AN.

\section{$\mathrm{T} 1000$}

Comparison of GERD and Sleep-Related Medication Use Among Individuals with Daytime and Nighttime GERD

Robert W. Dubois, Ronnie Fass, Daniel Aguilar, Richard Lynn, Amitabh Singh, Michael A. Tedeschi

Objective:To compare GERD and sleep medication(med)use among individuals with daytime and nighttime GERD. Methods: An Internet-based survey was conducted among full-time working adults living in the US. A screening questionnaire that assessed frequency and severity of GERD symptoms (during past 12 months and past 7 days) was used to identify symptomatic GERD cases. GERD status was determined by using the validated GERD Symptom and Med Questionnaire (GERD-SMQ).Symptomatic GERD was defined as GERDSMQ score $>9$ and at least 1 episode of either heartburn or acid regurgitation during the past 7 days.Symptomatic nighttime GERD (NTG) was defined as at least 2 nights with GERD symptoms during the past 7 days. Questionnaire respondents were asked to report GERD symptoms as well as GERD and sleep related med use.Results:Of65,001 individuals invited to participate in the survey, 13,231 responded. Of these, 1,002 satisfied GERD study criteria and were defined as having symptomatic GERD $\{476$ NTG cases vs 526 daytime GERD (DTG) cases\}. Results are presented in the table below. Among NTG cases, 59\% of subjects took an OTC GERD med before going to bed to help prevent nighttime symptoms of GERD compared to $40 \%$ among DTG cases (at least 1 night/7days).70\% of NTG cases reported taking an OTC GERD med to reduce nighttime symptoms after waking vs $60 \%$ in the DTG group.GERD-related med use (in the past $6 \mathrm{M}$ ) was reported by a substantial number of GERD cases. Among cases with NTG and reporting the use of antacids, $28.3 \%$ reported the daily use of antacids vs $9.7 \%$ among DTG cases(p<.0001). Conclusions:A greater proportion of NTG patients depend on OTC and Rx GERD meds to help prevent GERD symptoms at night. NTG is associated with higher use of OTC\&Rx sleep meds to help subjects have a good night's sleep

Use of Sleep \& GERD Med to Prevent/Reduce Nighttime Symptoms of GERD Among NTG $(\mathrm{n}=476) \&$ DTG $(\mathrm{n}=526)$ Cases

\begin{tabular}{|c|c|c|c|}
\hline GERD Medication & $\begin{array}{l}\text { Study } \\
\text { Group }\end{array}$ & $\begin{array}{l}\text { At least } 1 \text { night } \\
\mathrm{n}(\%)\end{array}$ & P-Value \\
\hline \multirow{2}{*}{$\begin{array}{l}\text { OTC GERD med. before going to bed to help prevent } \\
\text { nighttime symptoms of GERD }\end{array}$} & NTG & $280(59.0)$ & \multirow{2}{*}{$<.0001$} \\
\hline & DTG & $210(40.0)$ & \\
\hline \multirow{2}{*}{$\begin{array}{l}\text { Rx GERD med. before bed to prevent nighttime symptoms } \\
\text { of GERD }\end{array}$} & NTG & $134(28.1)$ & \multirow{2}{*}{.0024} \\
\hline & DTG & $105(20.0)$ & \\
\hline \multirow{2}{*}{$\begin{array}{l}\text { OTC GERD med. to reduce nighttime symptoms (after } \\
\text { waking with symptoms) }\end{array}$} & NTG & $333(70.0)$ & \multirow{2}{*}{.0031} \\
\hline & DTG & $182(60.0)$ & \\
\hline \multirow{2}{*}{$\begin{array}{l}\text { Rx GERD med. to reduce nighttime symptoms (after } \\
\text { waking with symptoms) }\end{array}$} & NTG & $134(28.2)$ & \multirow{2}{*}{.0004} \\
\hline & DTG** & $52(17.1)$ & \\
\hline \multicolumn{4}{|l|}{ Sleep Medication } \\
\hline \multirow{2}{*}{ OTC sleep med. to help fall asleep } & NTG & $116(24.4)$ & \multirow{2}{*}{.0016} \\
\hline & DTG & $86(16.4)$ & \\
\hline \multirow{2}{*}{ Rx sleep med. to help fall asleep } & NTG & $64(13.5)$ & \multirow{2}{*}{.9491} \\
\hline & DTG & $70(13.3)$ & \\
\hline
\end{tabular}

** Only among respondents with DTG who reported 1 night with symptoms ( $\mathrm{n}=305)$

\section{T1001}

Risk Factors for Adulthood GERD in Persons with Childhood GERD Hashem El-Serag

Background GERD that develops during childhood in patients without underlying predisposing disorders may persist or predispose to GERD in early adulthood. However, the extent and determinants of adulthood GERD in these individuals are unclear. The predisposing factors for GERD in early adulthood are not well examined. Methods We identified a cohort of eligible individuals who were diagnosed with GERD in childhood between 1994 and 1996 in Texas Children's Hospital in Houston, TX. Patients with neurodevelopmental disorders, tracheoesophageal anomalies, or severe chronic pulmonary disorders were excluded. Computer assisted telephone interviews were conducted in 2004 and 2005 using a standard validated questionnaire for GERD symptoms and potential risk factors. We calculated the prevalence of GERD as defined by at least weakly heartburn or acid regurgitation. We examined potential determinants of GERD in unadjusted and adjusted logistic regression analyses. This cohort does not overlap with our previous pilot study (Am J Gastro, 2004). Results We have thus far recruited 95 subjects (participation rate: 67\%). The mean age at the time of the interview was 17.3 (SD 2.0), while the mean age at the time of childhood GERD diagnosis was 12.5 years. There were $59(62 \%)$ females. The racial distribution was $57 \%$ Caucasian, $2 \%$ black, $10 \%$ Hispanic, and rest $25 \%$ unreported. At least weakly heartburn or regurgitation was reported in 41/95 (43\%); most of whom (95\%) were taking PPI, H2RA, or antacids. An additional 18 subjects reported no symptoms but were using PPIs. Patients with adulthood GERD symptoms were more likely to have at least weakly dysphagia (32\% vs. $6 \% ; \mathrm{p}=0.003$ ) or chest pain ( $32 \%$ vs. $11 \% ; \mathrm{p}=0.013$ ) than those without GERD symptoms. GERD was significantly more frequent in females using OCP as compared to females not 
on OCP, or males (12/16 vs. 19/43 vs. 10/36, respectively; $\mathrm{p}=0.006$ ). In logistic regression, OCP use was associated with adulthood GERD independent of PPI use or recent weight gain; the adjusted odds ratio was 6.61 (95\% CI, 1.56 to 27.98; $\mathrm{p}=0.01)$. On the other hand, race, family history of GERD, education level, employment status, tobacco smoking, alcohol, or coffee drinking were not significant predictors of adulthood GERD. Conclusions Childhood GERD can persist and or predispose to GERD in early adulthood in approximately half of all cases. The use of oral contraceptives is a potential risk factor for GERD symptoms in early adulthood among persons of GERD in childhood.

\section{T1002}

\section{Temporal Trends in New and Recurrent Esophageal Strictures} Hashem El-Serag

Background There has been a significant recent increase in the prevalence of GERD (7-8), Barrett's esophagus, as well as esophageal adenocarcinoma. On the other hand, anecdotal experience suggests that gastroenterologists are encountering fewer patients with esophageal strictures. Conceivably the wide spread use of PPIs could have led to the decline in esophageal strictures, however, there has been no published data on the temporal trends of either new or recurrent esophageal strictures. Methods We examined the incidence rates of new esophageal strictures among patients presenting to VA facilities between 1998 and 2003 as a proportion of the annual frequency of all endoscopic procedures and/or imaging procedures. Recurrent strictures were defined as those recorded at least three months after the index date of a new stricture or the date of the preceding recurrent stricture. Stricture was defined only in the presence of an upper endoscopy or UGI contrast imaging within 30 days before or after the date of diagnosis. This algorithm was validated by manually reviewing the medical records of 180 patients; the positive predictive value was approximately $84 \%$. The risk of recurrent strictures was examined using Cox proportional hazards model for multiple failures using the PWP method. Results There were 19,157 patients identified between FY 1998 and FY 2003 with new esophageal strictures. The annual frequency of new esophageal strictures ranged between 3070 and 3425 with no clear temporal trend during 1998 - 2003. New strictures comprised a constant annual proportion of total EGD and UGI imaging studies. However, new strictures declined by $11.6 \%$ as a proportion of all upper endoscopy (not including UGI imaging). Among patients with new esophageal strictures, 15,930 (83.2\%) had no recurrent esophageal strictures through 10/2003, while the rest $(16.8 \%)$ had at least one episode of recurrence. The one-year incidence rate of recurrent strictures declined significantly $(-36.0 \%)$ from 13.9 per 100 person-years for patients diagnosed with new strictures in 1998 to 8.9 per 100 person-years for patients diagnosed with new strictures in 2003. In the full multivariable model, new strictures diagnoses during 2001-2003 were associated with $13 \%$ reduction in risk of having recurrent strictures independent of age, gender, race, geographic region, or the presence of BE. Conclusion The incidence of recurrent strictures has declined between 1998 and 2003 by approximately one third. On the other hand, there has been only a small decline in the incidence of new esophageal strictures. PPI use is likely to explain these trends.

\section{T1003}

The Prevalence of Heartburn in Elderly in Russia: Sub-Analysis from the Database of National Population-Based Study

Ekaterina S. Stavraki, Sergey V. Morozov, Vasily A. Isakov

The prevalence of heartburn is high in all age groups in developed countries. The data about prevalence of heartburn in Russia are scarce especially in different age groups. Aim: To evaluate the prevalence of heartburn and its impact on the quality of life in elderly in Russia. Methods: A questionnaire was designed for patients in the primary care setting. It included questions on the presence and frequency of heartburn, risk factors provoking heartburn, influence on the quality of life. Eight structured questions were organized in a self filled-in form. The questionnaire was validated in 115 GERD patients and 104 controls and found to have an acceptable sensitivity (89\%) and specificity (92\%). The mean time for completing the questionnaire was $5.4 \pm 1.2 \mathrm{~min}$. The study was performed in 14 of the largest cities in Russia. Results: Twenty thousand questionnaires were distributed, the response rate was $81.3 \%$ and 14,521 questionnaires were analyzed. The presence of heartburn was evaluated in 8037 women and 6486 men. Among them, 8661 subjects reported heartburn $59.7 \%$ of the respondents) and 1248 subjects $\geq 60$ years old. Thus, the heartburn was more prevalent in elderly then in other age groups: $61.9 \%$ vs $59.3 \%$; $<<0.03$. In total the frequency of heartburn was equal in women and men ( 59 . I v $60.4 \%, p=0$. 1). Elderly women experienced heartburn more often than men $(64.0 \%$ vs $58.2 \% \mathrm{p}=0.01)$. Frequent $(2-3$ times a week $)$ and daily symptoms were more prevalent in elderly in comparison to younger people $(30.6 \%$ vs $21.4 \% \mathrm{p}<0.001)$. The timing of heartburn in elderly was as follows: after a meal (47.7\%), bending $(15.6 \%)$, fasting $(10.5 \%)$, at night $(10 \%)$, during physical activity $(9.9 \%)$ or under the stress $(5.7 \%)$. The distribution of the frequencies of heartburn timing was similar in elderly and in younger people. However, heartburn appeared in elderly more often in bent position (15.6\% vs $12.0 \%$ ) in comparison to younger. Major differences in changes of the quality of life between elderly and others are shown in table. Conclusions. The prevalence of heartburn in elderly urban population in Russia is higher than in other age groups. Daily and frequent heartburn are more prevalent among the elderly. However, the quality of life is lower in younger patients who suffered from heartburn than in correspondent elderly population.

Quality of life changes in elderly and younger subjects

\begin{tabular}{|c|c|c|c|}
\hline & Elderly $(>60$ yo $)$ & Younger $(<60$ yo $)$ & $p$ \\
\hline General considerable discomfort & $37.4 \%$ & $56.8 \%$ & 0.001 \\
\hline Refuse of favorite dishesand beverages & $26.1 \%$ & $38 \%$ & 0.001 \\
\hline Limited physical activity & $11.3 \%$ & $26 \%$ & 0.001 \\
\hline Sleep disorders & $16.7 \%$ & $20.0 \%$ & 0.01 \\
\hline Low efficiency & $8.7 \%$ & $14.6 \%$ & 0.001 \\
\hline
\end{tabular}

\section{T1004}

The Efficacy of Several Over-the-Counter GERD Therapies: A Meta-Analysis Thomas Tran, Angus M. Lowry, Hashem B. El-Serag

Background: Over-the-counter (OTC) medications, including histamine-2 receptor antagonists (H2RAs) (half prescription dose), antacids, and alginate/antacids are commonly used for GERD. Many studies have evaluated the efficacy of individual agents. However, the overall efficacy of these agents has not been systematically reviewed. Methods: We performed a MEDLINE search up to December 2005 to evaluate randomized, placebo-controlled trials that examined the efficacy of H2RAs, antacids, and alginate/antacids. Study design and report qualities were measured by the Jadad scoring (from 0 to 5 ). Data on complete and adequate relief of GERD symptoms, subjective global improvement, and the use of rescue antacids were extracted and analyzed by using a random-effects model. Results H2RAs: A total of 12 randomized, double-blinded, parallel-group trials $(n=4075$, placebo $=3561$ mean Jadad score 3.5) compared H2RAs (4 different agents) versus placebo. Six studies examined adequate relief of GERD symptoms within 2 hours of drug ingestion that lasted for at least 3 hours; the combined effect favored H2RAs (OR 1.55, 95\% CI 1.41- 1.70, p $<0.0001)$. Three studies evaluated complete relief of GERD symptoms for at least 3 hours (OR 2.01, 1.59-2.66, p = 0.003). Five studies evaluated the \% of GERD episodes requiring rescue antacids; patients on $\mathrm{H} 2 \mathrm{RAs}$ were less likely use rescue antacids (OR $0.58,0.50$ to $0.67, \mathrm{p}<0.0001$ ). There were no significant differences between the 4 H2RA agents Antacids: Five randomized trials $(n=597$, placebo $=588$; mean Jadad score 3.8 ) compared the efficacy of antacid versus placebo. Four trials evaluated patients' symptomatic improvement after at least 2 weeks of therapy with a combined OR of $1.42(0.97-2.07, p=0.07)$ in favor of antacids. Two trials found patients on antacids less likely to have heartburn episodes requiring rescue antacids (OR 0.70, 0.58 to $0.85, \mathrm{p}<0.0001$ ). Alginate/antacid Three randomized trials $(n=108$, placebo $=108$; mean Jadad score 3.3$)$ compared alginate/ antacid combination versus placebo in improving heartburn with a combined OR of 3.86 in favor of alginate/antacid $(2.16$ to $6.87, \mathrm{p}<0.0001)$. Conclusions: OTC medications for GERD therapy are effective. Histamine-2 receptor antagonists are 50\% to $100 \%$ superior to placebo in providing adequate and complete symptom relief, improving patients' global rating, and reducing rescue antacids. Alginate/antacid combinations are also superior to placebo in improving symptom. There is a non-significant trend in symptomatic improvement in favor of antacid therapy versus placebo.

\section{$\mathrm{T} 1005$}

Objective Measurement of Hourly and Annual Productivity While At Work in Employees with Gastroesophageal Reflux Disease (GERD) Compared with Employees Without GERD

Peter Wahlqvist, Richard A. Brook, Sara Campbell, Mari-Ann Wallander, Ingela Wiklund, Nathan L. Kleinman, James E. Smeeding

OBJECTIVE: Results from studies using patient-reported data in employed population indicate that Gastroesophageal Reflux Disease (GERD) causes a reduction in productivity while at work of around $10 \%$. The aim of this study is to assess if a link between GERD and reduced productivity while at work can be established by using objective measurements of hourly and annual at-work productivity. METHODS: We examined the Human Capital Management Services Research Reference database of employees where units-of-production are captured on a per-employee-per-day basis. ICD-9 Codes were used to distinguish employees with GERD from the non-GERD cohort. The index date in the GERD cohort was defined as the date of first diagnosis during 2001 or later, while the average GERD index date was used in the non-GERD cohort. Hourly and annual productivity were measured during the year following each employee's index date. Regression modeling was used to measure the productivity differences between employees with GERD and employees without GERD while controlling for age, marital status, race, full-time/part-time status, location, and comorbidity (Charlson Comorbidity Index). RESULTS: Data were available for 27,316 employees: 541 employees with GERD and a control group of 26,775 employees withou GERD. The mean age of employees with GERD was 40 years, and $77 \%$ were full time employees. The analysis of at-work productivity (Table) show that GERD employees' average units processed were significantly lower by $4.4 \%$ hourly and $6.0 \%$ annually $(P<0.05)$ CONCLUSIONS: Even though quality aspects of work productivity are not captured, objective productivity measurements from real work-output data (the number of units of work performed by each employee on a daily basis) show that GERD has a significant impact on productivity while at work. Interventions focused on managing GERD could increase employees' ability to contribute to production 


\begin{tabular}{|c|c|c|}
\hline & Employees with GERD & Employees without GERD \\
\hline Productivity Output & $\mathrm{N}=541$ & $\mathrm{~N}=26,775$ \\
\hline Per Hour & & \\
\hline Mean Adjusted units processed per hour & 18.31 & 19.15 \\
\hline Standard error & 0.41 & 0.06 \\
\hline P-Value (GERD vs. Non-GERD, Hourly) & \multicolumn{2}{|c|}{0.0481} \\
\hline Per Year & \multicolumn{2}{|c|}{} \\
\hline Mean Adjusted units processed per year & 27,421 & 29,175 \\
\hline Standard error & 814 & 123 \\
\hline P-Value (GERD vs. Non-GERD, Annually) & \multicolumn{2}{|c|}{0.0391} \\
\hline
\end{tabular}

\section{T1006}

Long-Term GERD Symptom and Quality-of-Life Improvement in Subjects with Healed Erosive Esophagitis Treated for Up to 7 Years with Titrated-Dose Lansoprazole

Thomas O G Kovacs, Stuart Atkinson, James Spalding, Barbara Hunt

BACKGROUND: Gastroesophageal reflux (GERD), a chronic symptomatic condition, may be associated with erosive esophagitis (EE). Long-term GERD symptom prevention and patient quality of life (QoL) data are limited. Purpose: To determine the effect of maintenance lansoprazole (LAN) treatment on long-term GERD symptoms and QoL in subjects with healed EE. METHODS: All subjects had EE ( $\geq$ Grade 2 ) and had previously enrolled in an 8-week acute treatment followed by a double-blind treatment of up to 12 months. Subjects whose EE had healed started on LAN $15 \mathrm{mg}$ QD and the LAN dose was titrated up or down so that subjects received the minimum dose required to control their symptoms or EE. Questionnaires with disease-specific scales and validated QoL scales were self-administered annually. Scales were transformed to 0-100, with higher numbers indicating improvement. RESULTS: 195 patients (64 F/131 M; mean age, 50.8 years) received open-label LAN with a mean dosage of $24 \mathrm{mg} ; 120$ were exposed $\geq 5$ yrs. All disease-specific scales except sleep improved significantly $(\mathrm{p}<0.001)$ at each timepoint from open-label baseline. Of the global health scales, both general and comparative health had statistically significant improvements. CONCLUSIONS: Long-term LAN treatment in healed EE subjects resulted in sustained improvements from therapy start, not only in GERD symptoms and general health but also in QoL indicators, including activity, eating, drinking, and social functioning.

\begin{tabular}{|c|c|c|c|c|c|}
\hline & \multicolumn{4}{|c|}{ Open-Label Months Mean Change From Baseline } \\
\hline DISEASE-SPECIFIC SCALES & Baseline & 12 & 36 & 72 & 84 \\
\hline Heartburn Pain & & & & & \\
\hline Severity**** & 68.0 & +17.1 & +17.7 & +19.3 & +18.8 \\
\hline Bothersomeness Comparative**** & 47.9 & +17.8 & +12.6 & +11.0 & +10.9 \\
\hline Bothersomeness Degre* **** & 64.4 & +19.2 & +19.8 & +20.8 & +20.5 \\
\hline Related Problems & & & & & \\
\hline Activity Limits*** & 54.6 & +9.6 & +9.9 & +10.1 & +9.5 \\
\hline Eating/ Drinking*** & 59.2 & +8.6 & +8.1 & +8.5 & +8.3 \\
\hline Symptom Problem**** & 59.1 & +9.0 & +8.7 & +9.3 & +9.0 \\
\hline Social Function*** & 86.7 & +8.3 & +9.1 & +9.6 & +9.4 \\
\hline Sleep & 62.7 & +1.7 & +0.9 & +0.4 & +0.3 \\
\hline Treatment Satisfaction*** & 41.7 & +24.8 & +25.4 & +25.6 & +25.3 \\
\hline GLOBAL HEALTH SCALES & & & & \\
\hline General Health* & 54.2 & +4.3 & +4.3 & +3.6 & +3.5 \\
\hline Comparative Health** & 48.6 & +7.9 & +5.0 & +4.1 & +4.9 \\
\hline Health Distress (a) & 61.7 & +5.1 & +6.5 & +6.2 & +6.4 \\
\hline General Well-Being & 66.3 & +2.4 & +1.8 & +1.8 & +1.8 \\
\hline Energy and Vitality & 43.3 & +3.3 & +2.4 & +2.2 & +2.3 \\
\hline Mental Health & 65.6 & +0.9 & +1.3 & +0.8 & +0.4 \\
\hline
\end{tabular}

**** p $>0.05$ and 0.001 , respectively, all timepoints (a) p >0.05, all timepoints except month 60 (data not shown)

T1007

Health State Utilities and Health-Related Quality of Life (HRQOL) in Patients with Dysphagia

Nick Stern, Edel Doorley, Sanchoy Sarkar, Keith Bodger

BACKGROUND Swallowing disorders may impact on physical, psychological and social functioning. Clinical assessment often focuses on severity of dysphagia without capturing other aspects of HRQoL. Global valuation of health status is increasingly favoured in health technology appraisal but has not found a place in routine practice. Health state utility assessment produces a single cardinal measure of wellbeing grading health on a scale from zero (death) to one (perfect health). Techniques include direct measures of 'patient preference' (eg. Time Trade Off, TTO), visual analogue rating scale (VAS) and pre-scored multi-attribute health indices (eg. EQ-5D). There have been few studies of utilities in dysphagia. We aimed to measure utility weights in a range of dysphagic complaints by different methods and to correlate scores with simple clinical and endoscopic gradings and a multi-item, diseasespecific HRQoL instrument. METHODS Face-to-face interviews were conducted with dysphagic subjects prior to endoscopy, including oesophagogastric malignancy (Cancer), benign stricture (Benign) and non-obstructive dysphagia (Dysmotility). Clinical severity was graded by dysphagia scale (DysScale: $0=$ None; $1=$ Solids;2=Soft or Liquidised;3=Saliva) and luminal narrowing scale (EndoScale) for obstructive causes. Health utility weights were elicited by TTO, VAS and EQ5D. Patients completed the EORTC QLQ-C30 HRQoL instrument with oesophageal module OES18. Follow up interviews were performed after intervention to test reliability and sensitivity. RESULTS First 59 patients: mean age 62 yrs (25-85 yrs). 95\% completed the TTO question. Mean utilities by VAS (0.62) and EQ5D (0.63) were significantly lower than by TTO method (0.9), as reported in other diseases. Overall, Spearman correlation (rho) values for utility scores versus DysScale or EndoScale were weak (below 0.1). There were strong correlations for EQ5D (0.451) and VAS (0.77) utilities versus EORTC 'global health' domain $(\mathrm{p}<0.001)$. Sub-group analysis showed better correlation between utility scores versus DysScore for the benign group $(E Q 5 D=0.31, V A S=0.39, T T O=0.1)$ than for Cancer or Dysmotility. CONCLUSIONS Eliciting utility scores by different methods is feasible for dysphagic conditions but absolute values vary according to methodology. Utility scores and EORTC indicate significant impairment of well-being that is only weakly correlated with grading of dysphagia, particularly in the case of Cancer and Dysmotility. Diseasespecific instruments are time-consuming but measurement of utility scores is simple and may prove a useful adjunct to basic clinical assessment.

\section{T1008}

CDX-2 and P-27/CDK-2 Identify a New Pattern of Expression in Barrett Esophagus

Massimo Milione, Emilio Digiulio, Gabriele Capurso, Francesco Panzuto, Antonella Carnuccio, Giuseppe Montrone, Andrea Sbrozzi, Antonella Stoppacciaro, Emanuela Pilozzi, Andrea Vecchione, Cristina Colarossi, Ludovica Feigush, Luigi Ruco, Gianfranco Delle Fave

Background: Barrett's Esophagus (BE) is the single most important precursor lesion and risk factor for adenocarcinoma of the distal oesophagus. BE is defined as squamous epithelium replaced by specialized intestinal one. The histological diagnosis of BE involves the presence of goblet cells, but this finding may not be the first indicator of intestinal metaplasia. CDX2 , a transcription factors associated with intestinal differentiation may be engaged in $\mathrm{BE}$ development. CDX-2 is in relationship with cdk-2 and p27Kipl. cdk2 binds ciclinE and promotes cell progression into the $S$ phase 27 Kipl inhibits cyclinE/cdk2 complexes, and blocks cell in $\mathrm{Gl}$ phase. The targeted degradation of $\mathrm{CDX} 2$ following its phosphorylation by Cdk2 identifies a new mechanism through which CDX2 activity can be regulated in coordination with the cell cycle machinery. The possible link between CDX-2 and cdk-2/ p27 expression has not been specifically investigated in patients with ES and/or BE. Aim: To investigate the expression pattern of CDX-2, p27Kipl and cdk-2 in BE and in esophagitis. Material and Methods: Immunohistochemestry (IHC) with antiCDX-2, anti-p27Kipl and anti-cdk-2 monoclonal commercial antibodies was performed on paraffin embedded tissue sections of $12 \mathrm{BE}$, of 18 esophagitis (ES), and of 10 healthy gastro-esophageal junction (GEJ), from 30 patients. At least four biopsies for each patient were obtained during endoscopy. Results: CDX-2 was positive in all BE samples (12/12), negative in both $\mathrm{ES}(0 / 18)$ and in GEJ (0/10). p27Kipl staining was positive in $\mathrm{ES}(18 / 18)$ and in $\mathrm{BE}(12 / 12)$, negative in GEJ (0/10).cdk-2 was positive in all GEJ samples (10/10), negative in $\mathrm{BE}$ and in $\mathrm{ES}$ sections. Conclusions: The link between CDX-2, p27Kipl and cdk-2 seems a promising model to explain the pathogenesis of BES and ES. Evaluation of their expression may suggest the presence of three different expression pattern showed in table. Moreover the co expression of CDX-2 and p27 could be a predictive marker of BES, which comes first goblet cells. cdk2 may be useful to distinguish columnar gastric cells against intestinal ones. Table DIAGNOSIS CDX-2 + p27 + Cdk-2 + BE 12/12 12/12 0/12 ES 0/18 18/18 0/18 GEJ 0/10 0/10 10/10

\section{T1009}

The Effect of Definitive Chemoradiotherapy (ChemoRT) Against Esophagectomy On Pulmonary Function in Patients with Squamous

Esophageal Cancer - Results from a Prospective Randomized Trial By Chinese University Research Group for Esophageal Cancer (CURE)

Philip W. Chiu, Frances K. Cheung, Wilfred L. Mui, Man Yee Yung, Candice C. Lam, Angus C. Chan, Wing Tai Siu, Enders K. Ng

Background From our randomized study, ChemoRT achieved a similar early survival compared to esophagectomy for squamous esophageal cancer (J GI Surg 2005; 9:794-802). This study investigate the effect of ChemoRT compared to esophagectomy on the pulmonary function of patients with squamous esophageal cancer. Patients \& Method From 2000 to 2004, 80 patients with resectable carcinoma of mid or lower esophagus were randomly assigned to receive ChemoRT or esophagectomy. Patients treated with ChemoRT received continuous 5-FU infusion $(200 \mathrm{mg} / \mathrm{m} 2 / \mathrm{day})$ and cisplatin $(60 \mathrm{mg} / \mathrm{m} 2)$ on days 1 and 22 . The tumor was concomitantly irradiated to 50-60 Gy. The esophagectomy included a two or three-staged esophagectomy with two field dissection. Pulmonary function were assessed before and after treatment at 3 monthly intervals. Results 44 patients received standard esophagectomy, while 36 were treated with chemo-radiotherapy. No difference in the early cumulative survival was found between the two groups (RR $0.89,95 \%$ CI 0.37 to 2.17 ; Log rank test $\mathrm{p}=0.45$ ). The pretreatment FEVl and FVC were similar between the two groups, and there is a significant decline in FEVl and FVC after treatment for both groups at 3 months (Figure 1). The ChemoRT group showed a significant recovery of both FEVl and FVC as compared to esophagectomy group from 6 months after treatment till 24 months. From 30-question based survey, patients treated with esophagectomy had a significantly poorer dyspnoea on various activities than those treated with ChemoRT (56.1 vs. 33.6; $p=$ 0.05). Conclusions Both ChemoRT and esophagectomy induced a significant reduction in the pulmonary function in patients with squamous esophageal cancer. ChemoRT group showed a significantly better recovery in pulmonary function and less dyspnoea during daily activities when compared to esophagectomy group. 


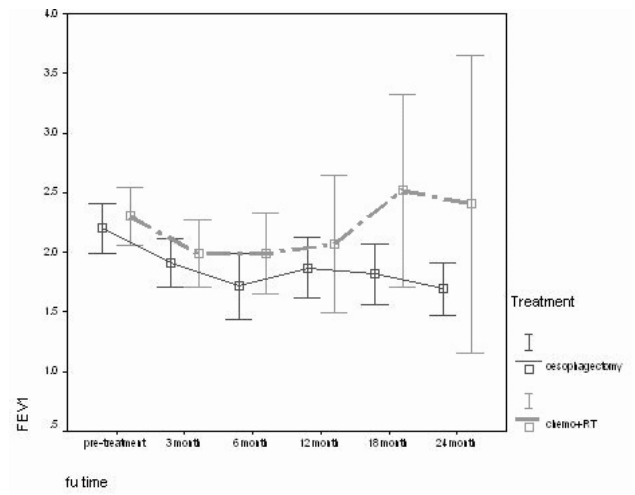

Figure 1

$\mathrm{T} 1010$

Treating Nighttime GERD Reduces Overall Costs More Substantially Than Treating Daytime GERD - A Population Based Economic Model

Stephen M. Lange, Quan Doan, Adam B. Elfant, Lynda S. Welage, Stephen Brunton, Richard B. Lynn, Robert W. Dubois

OBJECTIVE: To assess the economic impact of treating daytime (DG) and nighttime (NG) GERD with proton pump inhibitors (PPIs). METHODS: An economic model was constructed from the perspectives of employer and payer to compare the overall costs of treatment (Tx) with no treatment over 1 year among a population of 1 million (M) lives. Patients received daily PPI at standard doses. Patients with breakthrough symptoms or incomplete symptom resolution were given double doses of daily PPI for 4 weeks and then resumed standard daily doses. Parameter estimates were obtained from the literature and modified by experts as necessary to reflect generalizability to all GERD patients. GERD prevalence rate of $20 \%$ was assumed with half classified as DG and the rest NG. Fifty-six percent experienced complete symptom resolution after 4 weeks of standard dose PPI therapy; $78 \%$ in those requiring treatment of breakthrough symptoms. Without treatment, symptom relief of $24 \%$ after 4 weeks was assumed based on placebo response in clinical trials. Breakthrough symptoms at 6 months were $25 \%$ and $68 \%$ for PPI and no treatment, respectively. The published per patient per year costs of visits, hospitalizations, and procedures attributable to GERD was $\$ 240$. Applying utilization weights of $0.4,0.6$, and 2.4 to $\$ 240$, the costs for patients with mild, moderate, and severe symptoms were $\$ 97, \$ 146$, and $\$ 590$. Indirect costs represented the hours of productivity loss multiplied by the national average hourly earnings. The daily cost of regular dose PPI therapy was $\$ 2$. RESULTS: The burden of GERD was substantial $(\$ 663 \mathrm{M})$. Treatment resulted in an overall savings of $\$ 386 \mathrm{M}(\$ 1,930$ saved per treated patient per year) driven mostly by reduction in "indirect" costs compared with no treatment. Similar trends were observed in DG and NG, but treatment produced greater savings among NG patients. Savings associated with treating DG disappeared if the price of daily regular doses of PPI exceeded $\$ 3.80$. In contrast, results supporting treating NG were robust when varied from $\$ 1$ to $\$ 5$ per day for standard dosing of PPI. CONCLUSIONS: Treating GERD patients with PPI daily resulted in a substantial cost savings over a wide range of PPI costs, driven mostly by reducing indirect costs. Treating NG may yield even greater cost savings.

Economic impact (population=1M)

\begin{tabular}{|c|c|c|c|c|c|c|}
\hline & \multicolumn{2}{|c|}{ GERD $(\mathrm{n}=0.2 \mathrm{M})$} & \multicolumn{2}{c|}{$\mathrm{DG}(\mathrm{n}=0.1 \mathrm{M})$} & \multicolumn{2}{c|}{ NG (n=0.1M) } \\
\hline & Tx & No Tx & Tx & No Tx & Tx & No Tx \\
\hline Direct costs & $\$ 184 \mathrm{M}$ & $\$ 46 \mathrm{M}$ & $\$ 91 \mathrm{M}$ & $\$ 17 \mathrm{M}$ & $\$ 92 \mathrm{M}$ & $\$ 26 \mathrm{M}$ \\
\hline Indirect costs & $\$ 93 \mathrm{M}$ & $\$ 618 \mathrm{M}$ & $\$ 29 \mathrm{M}$ & $\$ 172 \mathrm{M}$ & $\$ 78 \mathrm{M}$ & $\$ 428 \mathrm{M}$ \\
\hline Total & $\$ 277 \mathrm{M}$ & $\$ 663 \mathrm{M}$ & $\$ 120 \mathrm{M}$ & $\$ 189 \mathrm{M}$ & $\$ 171 \mathrm{M}$ & $\$ 454 \mathrm{M}$ \\
\hline Net savings & $\$ 386 \mathrm{M}$ & & $\$ 69 \mathrm{M}$ & & $\$ 283 \mathrm{M}$ & \\
\hline Savings per treated patient per year & $\$ 1,930$ & & $\$ 689$ & & $\$ 2,834$ & \\
\hline
\end{tabular}

\section{T1011}

Incidence of Esophageal Adenocarcinoma After Endoscopic Ablative Therapy for Barrett's Esophagus: A Systematic Review and Meta Analysis Srinivas R. Puli, Brenda Westhoff, Nicholas J. Shaheen, J Wei, Sanjeev Slehria, Ajay Bansal, Amit Rastogi, Prateek Sharma

Purpose: The goal of ablative therapy in pts with Barrett's esophagus (BE) is to decrease the risk of developing cancer. The extent of any reduction of cancer incidence in $\mathrm{BE}$ pts following endoscopic ablation is not known. Our aim was to determine the cancer incidence after ablative therapy in pts with no dysplasia (ND BE), low grade dysplasia (LGD), and high grade dysplasia (HGD). Methods: We performed a MEDLINE search of the English language literature from 1980 to 2005. The search combined the keywords Barrett esophagus, esophageal adenocarcinoma, ablation, laser, argon, photodynamic, multipolar, and recurrence. Abstracts from DDW and ACG meetings from 2000 to 2005 were also included. All studies with mean/median follow-up duration of $<6$ months were excluded. Because most studies had no control arm, the rate of cancer development in the ablation studies was compared to historical data on BE pts not undergoing ablation The standardized incidence rate difference (IRD) was obtained using both the fixed and random effect models. Tests of heterogeneity were performed using the DerSimonian-Laird random effects analysis. Weighted mean event rate for a study population was utilized to obtain a summary standardized rate, which was expressed as the direct standardized incidence rate (DSR). Approximate confidence intervals for DSR were calculated by Chiang's normal approximation to Poisson rate sums. Results: 62 articles met the inclusion criteria and were included in the analysis. These studies included 1,153 pts with ND BE, 239 with LGD, and 611 with HGD. For pts undergoing ablative therapy, the DSR for cancer was 1.99/1000 pt-yrs (95\% Cl: -0.108 to 4.04$)$ for ND BE pts 1.58/1000 pt-yrs ( $95 \% \mathrm{Cl}$ : -0.66 to 3.84 ) for LGD, and $26.89 / 1000 \mathrm{pt}-\mathrm{yrs}(95 \% \mathrm{Cl}$ : 17.33 to 36.46$)$ for the HGD pts. Pooled data for controls obtained from 8 published articles showed a cancer incidence of 5.3/1000 pt-yrs in ND BE, 6.4/1000 pt-yrs in LGD, and 59.4/ 1000 pt-yrs in HGD. The Standardized IRD for ND BE, LGD and HGD were 5.29, 6.12 and 58.08, respectively $(\mathrm{p}<0.001)$. The number needed to treat to avoid an additional cancer in ND BE, LGD and HGD were 303, 208 and 31 respectively. Conclusions: Endoscopic ablation of $\mathrm{BE}$ is associated with a statistically significant reduction in cancer incidence rate compared to historical controls not undergoing ablation therapy; the higher the grade of dysplasia, the greater the incidence rate reduction following ablation. Although in ND BE and LGD pts undergoing ablation, the incidence of cancer was also reduced significantly, the NNT was prohibitive. The greatest benefit of ablative therapy appears to be in pts with HGD patient group.

\section{$\mathrm{T} 1012$}

Complete Symptom Resolution in Patients with Erosive Esophagitis and NonErosive Reflux Disease: A Meta-Analysis and Systematic Review

Srinivas R. Puli, Ajay Bansal, Amit Rastogi, Jiten Patel, Sandra Hall, Prateek Sharma

Purpose: To compare the efficacy of treatment with proton pump inhibitors (PPI), histamine 2 blockers (H2B), and placebos in completely resolving gastroesophageal reflux disease (GERD) symptoms in patients with erosive esophagitis (EE) and non-erosive reflux disease (NERD). Method: Study Selection Criteria: Only studies which were randomized, doubleblinded, and compared complete symptom resolution with two difference classes of drugs, were selected. The patient selection criteria included: age 18 to $80 \mathrm{yrs}$, GERD symptoms for at least 7 days before trial, no intake of acid suppressive medications 2 week prior to enrollment, and patients had endoscopy proven EE or NERD before start of treatment. Data collection and extraction: Articles were searched in Medline, Pubmed, Ovid journals, Cumulative index to nursing \& Allied health literature, International pharmaceutical abstracts, old Medline, Medline nonindexed citations, FDA database, and Cochrane control trial registry. Two reviewers independently extracted data. Areas of difference were resolved by mutual agreement. Statistical Method: A meta-analysis was conducted by combining data using both fixed and random effect models. Mantel-Haenszel and DerSimonian-Liard methods were used to analyze data using those models. Test of Heterogeneity was done using Mantel-Haenszel method. Results: Eleven studies for patients with EE (N=4227) and twelve studies for patients with NERD $(\mathrm{N}=3768)$ met study criteria (two studies had data for both the groups). At 4 weeks, the odds ratio (OR) of being symptom free on treatment with PPI compared to H2B was 6.0 (95\% CI: 4.81-7.49) in the EE group and 1.94 (95\% $\mathrm{Cl}$ : 1.05-3.62) in the NERD group (test of heterogeneity, $\mathrm{p}=0.6$ and 0.3 respectively). The OR of being symptom free at 4 weeks on treatment with PPI compared to placebo was $10.23(95 \% \mathrm{Cl}: 8.59-12.2)$ in the EE group and $4.76(95 \% \mathrm{Cl} ; 4.08-5.58)$ for the NERD patients (test of heterogeneity, $\mathrm{p}<0.001$ for both EE and NERD groups). Although test of heterogeneity was significant in PPI compared to placebo, on manual comparison, there were no significant heterogeneities between the studies. Conclusion: Complete symptom resolution at 4 weeks in EE patients is significantly higher in patients treated with PPI compared to either $\mathrm{H} 2 \mathrm{~B}$ or placebo. In the NERD subgroup, although treatment with PPI's is significantly better than $\mathrm{H} 2 \mathrm{~B}$ in complete symptom resolution, the benefit is marginal and much lower compared to EE patients. Future studied should attempt to improve symptom response in the NERD population as well as help define those NERD patients who will respond to acid suppressive therapy.

\section{$\mathrm{T} 1013$}

A Ten-Year Natural History of Gastro-Esophageal Reflux Disease Alexander C. Ford, Alastair G. Bailey, David Forman, Anthony T. Axon, Paul Moayyedi Introduction: Gastro-esophageal reflux disease (GERD) is common in the general population, with a reported incidence as high as 20 percent. However, there is little information regarding the long-term natural history of the disorder. Methods: The authors performed a 10-year follow-up cross-sectional survey of individuals recruited into a community screening and treatment program for Helicobacter pylori. All surviving, traceable participants were contacted, by validated postal dyspepsia questionnaire, which contained items relating to GERD symptoms. Baseline demographic data and GERD symptom data were stored on file from the original study. Results: Of 8407 individuals, 4003 (48\%) responded to the questionnaire. The mean age of responders was 55 years, and 2247 (56\%) were female. Males, those with dyspepsia at baseline, smokers, and those of lower social class were significantly less likely to respond $(\mathrm{p}<0.0001)$. There were $550(14 \%)$ subjects with GERD symptoms at a frequency of more than once a week at baseline. Of these, 240 (44\%) still had at least one dyspeptic symptom at a frequency of once a week or more at ten-year follow-up, and heartburn remained the predominant symptom in 167 (30\%), whilst 301 (55\%) had symptoms less than once a week or were entirely asymptomatic. Of the 3421 individuals who were asymptomatic at baseline, $629(18 \%)$ had heartburn at a frequency of once a month or more, 196 $(5.7 \%)$ once a week or more, and $39(1.1 \%)$ once a day or more. Heartburn was the predominant dyspeptic symptom in 540 (16\%) of these individuals at ten years. Conclusions: During ten years of follow-up approximately a third of individuals with GERD at baseline remained symptomatic, whilst in those without symptoms more than five percent had developed new onset GERD symptoms at a frequency of once a week or more. 
T1014

Prevalence of Heartburn in Russia: Preliminary Results of a National Population-Based Study

Vasily A. Isakov, Sergey V. Morozov, Ekaterina S. Stavraki

Introduction. Heartburn is a common symptom of gastroesophageal reflux disease (GERD). It has been shown that more than half of the adult population of Western Europe and the United States experience some heartburn, and a third of them suffer from heartburn daily or 2-3 times a week. The prevalence of heartburn in Russia has not yet been studied. Aim. To evaluate the prevalence of heartburn in Russia. Methods. A questionnaire was designed for patients in the primary care setting, which included questions on the presence and frequency of heartburn, risk factors provoking heartburn, influence on the quality of life. Eight structured questions were organized in a self filled-in form. The questionnaire was validated in 115 GERD patients and 104 controls and found to have an acceptable sensitivity $(89 \%)$ and specificity $(92 \%)$. The mean time for completing the questionnaire was $5.4 \pm 1.2$ min. The study was performed in 14 of the largest cities in Russia. Results. Twenty thousand questionnaires were distributed, the response rate was $81.3 \%$ and 14,521 questionnaires were analyzed. The presence of heartburn was evaluated in 8,037 women and 6,486 men. Among them, 8,661 subjects reported heartburn (59.7\% of the respondents). The heartburn prevalence was similar in women and men $(59.1 \%$ vs $60.4 \% ; \mathrm{p}=0.1)$. Amongst subjects with heartburn, $46.1 \%$ experienced infrequent heartburn, $16.4 \%$ weekly, $21.9 \%$ several times a week and $15,6 \%$ had daily symptoms. In total $22.7 \%$ of the respondents experienced frequent heartburn (daily or 2-3 times a week). The timing of heartburn was as follows: after a meal $(74.3 \%)$, bending $(19.1 \%)$, fasting $(16.1 \%)$, during physical activity $(13.5 \%)$, at night $(12 \%)$, or under stress $(10.4 \%)$. The presence of heartburn had an effect on the quality of life of those subjects: general considerable discomfort was noted by $56.8 \%, 38 \%$ avoided favorite dishes and beverages, $26 \%$ reported limited physical activity, sleep disorders were noted in $20 \%$, and low efficiency was reported by $14.6 \%$ of the respondents. Conclusion. This is the largest and the first population based study to report the prevalence of heartburn in more than 14,000 subjects in Russia. More than half of the studied Russian urban population suffers from heartburn, $75 \%$ of them have post-prandial symptoms. Every fifth respondent experiences heartburn frequently and this also has a major impact on their quality of life.

\section{T1015}

Epidemiology of Esophageal Carcinoma in a Statewide Cancer Registry Linda C. Cummings, Gregory S. Cooper

Background: Differences in etiologic factors and demographics associated with esophageal cancer have not been extensively studied at a population-level. Specifically, the impact of race, gender, age, and histology on the incidence and staging of esophageal cancer deserves further study. The primary aim of this study was to compare age-adjusted incidence rates of invasive esophageal adenocarcinoma (EAC) and squamous cell carcinoma (ESCC) by race and gender. Other aims were to evaluate the impact of race, age, gender, anatomical site, and histology on stage at presentation; and to assess tobacco use among patients with esophageal cancer. Methods: This is a population-based cross-sectional study using state cancer registry data. Cases of invasive esophageal cancer (ESCC, $n=937$ and EAC, $n=1,815$ ) diagnosed between 1998 and 2002 were included. Analysis of predictors of stage was restricted to incident staged cases of EAC or ESCC. Results: The incidence rate of ESCC was higher among African-Americans (5.0 cases/100,000/year) than whites (1.3 cases/ 100,000 /year). However, EAC incidence was higher among whites, with the highest rate in white males (6.4 cases/100,000/year). $80 \%$ of esophageal carcinoma, $85 \%$ of ESCC, and $77 \%$ of EAC cases for which tobacco history was reported occurred in past or current smokers. In univariate analyses, histology, race, age, gender, and primary site differed significantly by stage. $36 \%$ of males but only $26 \%$ of females had distant disease ( $\mathrm{p}=0.001$ ). $31 \%$ of patients $\geq$ age 65 presented with distant disease, compared with $37 \%$ of patients $<$ age 65 ( $\mathrm{p}<0.001) .32 \%$ of African-Americans presented with distant stage, versus $34 \%$ of whites $(\mathrm{p}=0.048)$. However, in a logistic regression model, male gender [OR 1.43, CI(1.10, $1.86)]$, and age $<65$ [OR 1.28, CI(1.05,1.57)], but not race, predicted distant stage. There was a trend towards EAC histology being a predictor of distant disease, but it did not reach statistical significance $[\mathrm{OR} 1.27, \mathrm{CI}(0.99,1.62)]$. Conclusions: EAC incidence rates are higher among whites, whereas African-Americans have higher ESCC rates. Most EAC cases were associated with smoking. To our knowledge, this is the first population-based study evaluating tobacco use in EAC. Unlike prior studies, we found that African-Americans are not more likely to present with distant stage. Further research is needed to elucidate racial differences in stage at presentation. Male gender and age $<65$ were independent predictors of distant stage, and although these differences could be due to socioeconomic factors, further studies are needed to explore this possibility.

\section{T1016}

Impact of BRAVO pH Telemetry On Ambulatory Esophageal pH Monitoring John M. Wo, Jennifer Koopman, Robert Cacchione, Steven Harrell

Bravo $\mathrm{pH}$ telemetry is a more tolerable method of ambulatory $\mathrm{pH}$ monitoring without a $\mathrm{pH}$ catheter through the nose. It is unclear if Bravo has altered the use of $\mathrm{pH}$ monitoring. AIM: To compare the clinical utilization and associated costs between transnasal and Bravo $\mathrm{pH}$ testing. METHODS: A comprehensive review was performed on physician ordering patterns for all $\mathrm{pH}$ tests during the past 2.5 years since Bravo became available at our institution. The following parameters were obtained: \# of pH tests (transnasal and Bravo), patient's chief complaints, ordering physician (community physician through open access or division faculty), test condition (on or off antireflux meds), \# of esophageal manometry studies (EMS) to locate lower esophageal sphincter for transnasal pH catheter placement, and \# of EGDs to locate gastroesophageal junction (GEJ) for Bravo capsule placement. Professional and facility fees for transnasal test, Bravo, EMS and EGD were determined using Medicare reimbursement for our state from 2003 to 2005 . New technology ambulatory payment category for Bravo was used for facility reimbursement after 4/1/2004. Cost per pH test, including EMS or EGD, was determined for each subject. Unpaired t-tests and $\chi 2$ tests were used. RESULTS: $642 \mathrm{pH}$ tests (51\% Bravo and $49 \%$ transnasal) were performed since Bravo was introduced. Results are shown in table. There were no significant differences in patient's age or gender between the two test methods. $70 \%$ of Bravo pH tests were performed on antireflux therapy compared to only $13 \%$ of transnasal $\mathrm{pH}$ tests ( $\mathrm{p}<0.001)$. Repeat $\mathrm{pH}$ testing occurred in $5 \%$ of Bravo patients compared to only $2 \%$ of transnasal subjects $(\mathrm{p}=0.03)$. Open access referral from community physician accounted for only $4 \%$ of Bravo tests compared to $83 \%$ of $\mathrm{pH}$ transnasal tests $(\mathrm{p}<0.001)$. Of the 252 subjects who underwent EGD to locate the GEJ for Bravo placement, $132(52 \%)$ already had an EGD in the past. CONCLUSIONS: A dramatic shift toward Bravo $\mathrm{pH}$ telemetry for esophageal $\mathrm{pH}$ monitoring has occurred in a short period of time despite a higher cost and similar indications. Costs may be minimized by recording GEJ location during all routine EGDs. More $\mathrm{pH}$ tests were performed on antireflux therapy using Bravo.

\begin{tabular}{|c|c|c|c|}
\hline \multicolumn{2}{|c|}{} & $\begin{array}{c}\text { Bravo pH test } \\
(\mathrm{n}=329)\end{array}$ & $\begin{array}{c}\text { Transnasal pH test } \\
(\mathrm{n}=313)\end{array}$ \\
\hline Cheif complaints & $\begin{array}{c}\text { Heartburn Chest pain } \\
\text { Supraesophageal symptoms }\end{array}$ & $\begin{array}{c}221(67 \%) 69(21 \%) \\
42(13 \%)\end{array}$ & $\begin{array}{c}223(71 \%) 86(27 \%) \\
76(24 \%)\end{array}$ \\
\hline $\begin{array}{c}\text { Additional } \\
\text { procedures }\end{array}$ & Esophageal Manometry EGD & $--252(77 \%)$ & $291(93 \%)---$ \\
\hline \multicolumn{2}{|c|}{$*$ Associated Costs per pH test } & $* * \$ 779 \pm 266$ & $* * \$ 460 \pm 62$ \\
\hline
\end{tabular}

$*$ Mean $\pm \mathrm{SD} ;{ }^{*} \mathrm{p}<0.001$

\section{$\mathrm{T} 1017$}

Objective Definition of Symptom Relief in Clinical Studies: Determination of International GERD Symptom Thresholds Based On Request ${ }^{\mathrm{TM}}$

Vincenzo Stanghellini, David Armstrong, Hubert Monnikes, Karna Dev Bardhan, Holger Schmitt, Iris Velten, Peter Berghofer, Ronnie Fass

PURPOSE: Symptom assessment as a primary endpoint is of increasing interest in GERD clinical trials. The validated ReQuest ${ }^{\mathrm{TM}}$ offers daily monitoring of treatment-induced symptom changes. Since individuals without evidence of GERD may experience mild symptoms commonly ascribed to GERD, ReQuest ${ }^{\mathrm{TM}}$-based GERD symptom thresholds were recently evaluated in a German population. Thresholds were established as objective criterion for symptom relief. Here, we aimed to verify the validity of the concept in an international setting. METHODS: 2,032 individuals from France, Italy, Spain, and USA were screened for evidence of GERD. Applying stringent exclusion criteria (e.g. history of gastrointestinal, cardiac, respiratory disorders/symptoms, consultation of a specialist, history of endoscopic examination, intake of GERD medication), 1,167 were eligible for analysis. Participants completed ReQuest ${ }^{\mathrm{TM}}$ on 4 consecutive days. Two subscales exist: ReQuest ${ }^{\mathrm{TM}}$-GI (acid complaints, upper abdominal/stomach complaints, lower abdominal/digestive complaints, nausea) and ReQuest ${ }^{\mathrm{TM}}$-WSO (general well-being, sleep disturbances, other complaints). Intensity and frequency of these dimensions (general well-being intensity only) were scored and weighted resulting in sum scores. The maximum sum scores are 46.28 (ReQuest $^{\mathrm{TM}}$ ), 30.77 (ReQuest ${ }^{\mathrm{TM}}-\mathrm{GI}$ ), and 15.51 (ReQuest ${ }^{\mathrm{TM}}-$ WSO). Based on the maximum values for each patient over the 4-day period, sum scores of ReQuest ${ }^{\mathrm{TM}}$ and its subscales corresponding to the $95 \%$-percentiles were calculated as international GERD symptom thresholds. The influence of demographics (age, weight, height, BMI, gender) and country-specific effects was determined by covariance analysis and Kruskal Wallis Test. RESULTS: The international GERD symptom thresholds were 4.12 (ReQuest $^{\mathrm{TM}}$ ), 1.60 (ReQuest $^{\mathrm{TM}}$-GI), and 2.87 (ReQuest $\left.{ }^{\mathrm{TM}}-\mathrm{WSO}\right)$. They correspond well with values recently obtained in the Germany study: $5.04,1.73$, and 3.68 , respectively. Demographics and country-specific effects on GERD symptom thresholds could be excluded (p-values $>0.05$ ). CONCLUSIONS: This study confirms that individuals without evidence of GERD experience low levels of symptoms commonly ascribed to GERD. These individuals do not seek medical help and take no GERD medication demonstrating that the impact of symptom burden is below a subjective threshold of impairment. It is therefore sufficient to treat GERD patients until symptom burden is below the GERD symptom threshold. Comparable GERD symptom thresholds assessed in the German and this international evaluation suggest this concept of GERD symptom thresholds is reliable and valid to prospectively define symptom relief in GERD therapeutic trials on a global level

\section{T1018}

Relationship Between Gastroesophageal Reflux Symptoms and Esophagitis in the General Population: Results from the Loiano-Monghidoro Study Rocco Maurizio Zagari, Paolo Pozzato, Silvia Damian, Maria Luisa Bianchi, Liza Ceroni, Lorenzo Fuccio, Maria Eugenia Minardi, Mari-Ann Wallander, Saga Johansson, Bahman Farahmand, Enrico Roda, Franco Bazzoli

Background \& Aims: Gastroesophageal reflux disease (GERD) is characterized by typical symptoms of heartburn and regurgitation, and/or endoscopically evident lesions such as esophagitis. The relationship between symptoms and endoscopic findings in the general population is unclear, as endoscopic data are usually obtained only in selected subgroups. Our study aimed to establish the relationship between endoscopic findings and reflux symptoms in a representative sample of the general population. Methods: We approached a representative sample of 1533 adults from two Italian villages (Loiano and Monghidoro) between 2000 and 2004 to undergo symptom assessment and gastrointestinal endoscopy. Endoscopic findings were assessed using the Savary-Miller classification. We recorded the frequency of heartburn and regurgitation during the previous 12 months using a validated questionnaire. We also collected data on smoking, alcohol and coffee consumption, history of peptic ulcer disease and medication use. Odds ratios and $95 \%$ confidence interval calculated by unconditional logistic regression models were used as measures of association between esophagitis and reflux symptoms. Results: Data were obtained from 1069 individuals representative of the general population (a response rate of $70 \%$ ). The overall prevalence of 
heartburn and/or regurgitation was $45.3 \% ; 21.1 \%$ of the study population had symptoms monthly, $21.5 \%$ weekly and $2.7 \%$ daily. Esophagitis was a risk factor for monthly (OR: 2.0 ; 95\% CI: 1.2-3.5) and at least weekly (OR: 3.2; $95 \%$ CI: 2.0-5.1) reflux symptoms. Others potential risk factors such as smoking, alcohol and coffee consumption, and the use of aspirin, nonsteroidal anti-inflammatory drugs and corticosteroids had no effect. The overall prevalence of esophagitis was $11.9 \%$. Females were less likely than males to have esophagitis. Hiatus hernia (OR: 4.4; 95\% CI: 2.8-6.8) and typical reflux symptoms increased the risk of esophagitis. Esophagitis was detected in $6.8 \%, 14.2 \%, 20.4 \%$ and $27.6 \%$ of subjects with no, monthly, weekly and daily symptoms, respectively. Compared with subjects with no symptoms, esophagitis was associated with monthly (OR: $2.1 ; 95 \%$ CI: 1.2-3.5), weekly (OR: 2.8; 95\% CI: 1.7-4.6) and daily (OR: 5.0; $95 \%$ CI: 1.9-13.5) reflux symptoms. Conclusion: We detected a linear relationship between the prevalence of esophagitis and the frequency of reflux symptoms in the general population. Our data show that subjects with even monthly typical reflux symptoms have a two fold increased risk of esophagitis.

\section{T1019}

Assessment of Satisfaction Following the Treatment of Gastro-Oesophageal Reflux By Proton Pump Inhibitors in Primary Care : The "Reflex " PhysicianPatient Agreement Study

Etienne Dorval, Jean-Francois Rey, Philippe Barthelemy, Agnes Caekaert, Christine Soufflet

The paim of this study is to evaluate the physician-patient agreement concerning satisfaction with the treatment of gastroesophageal reflux (GER) symptoms on a large population of adult patients treated with proton pump inhibitors (PPI) in primary care. Patients and Methods A sample of 2500 PCPs were selected by random draw with geographical stratification. Each PCP had to select the first 3 patients suffering from GER symptoms who were treated for at least one month by PPI prescribed daily or at least 14 days during the previous month. Two questionnaires were filled in for each patient included, one by the physician, the other by the patient, assessing the presence, frequency and intensity of the GER symptoms. The impact of GER on everyday life, overall satisfaction and expectations with respect to treatment (symptoms, quality of life, self-medication) were assessed trough a 5 grades Likert scale. Agreement between the physician and patient answers were analyzed by the kappa method. Results 5326 patients were included (57\% men ;mean age : $53+15$ years. $45 \%$ had an upper GI endoscopy within the 3 previous years showing oesophagitis in $77 \%$ of the cases. PPI was prescribed continuously in $69 \%$ of the patients, intermittent in $20 \%$ and on demand in $11 \%$. The analysis included 5174 physician-patient binomes. The agreement was good for the presence and frequency of GER symptoms (kappa >0.6) and also good for the impact on everyday life (kappa $=0.64$ ). However, it was only fair for the intensity of the symptoms (kappa 0.4 to 0.5 ) with an assessment in general lower on the physician questionnaire. While the overall treatment satisfaction was deemed good or excellent by the physicians and the patients in $72 \%$ of the cases, the agreement for satisfaction was only fair (kappa $=0.6)$ and fair or poor $(k a p p a<0.40)$ for expectations with regards to treatment. Conclusion The level of satisfaction associated with the treatment of GER by PPI as perceived by the physicians and the patients is high and exceeds $70 \%$. However, there are disagreements in the perception between patients' and physicians' assessment concerning the intensity of residual symptoms and their expectations with respect to the treatment.

\section{$\mathrm{T} 1020$}

Epidemiologic Risk Factors for Reflux Esophagitis in Healthy Population Su Youn Nam, Chan Gyoo Kim, Il Ju Choi, Woo Jin Lee, Kyung Woo Park, Ho June Song, Joo Hyuk Lee

Background/Aims: The prevalence of gastroesophageal reflux disease (GERD) have increased in western world over the 20 years but are not known in Korean population. We investigated the prevalence and epidemiologic risk factors for reflux esophagitis in general population. Methods: A total of 16580 healthy individuals who had undergone the endoscopy in center for cancer prevention and detection from May, 2001 to October, 2005 were enrolled in this study. GERD symptoms, dietary intake, education, economic status, housing environment, current job, smoking, alcohol consumption, age, and sex were interviewed by participants. Body mass index (BMI), hiatal hernia, and rapid urease test (CLO test) were evaluated. Male:female ratio was 1.28:1. Data analysis was performed using the Pearson $\chi 2$ test and logistic regression with the SPSS 12.0 for windows statistical package. Results: The prevalence of reflux esophagitis (RE) defined as LA classification was 4 03\% (667/16580). RE was more common in men than in women $(5.9 \%$ vs $1.7 \%, \mathrm{p}<0.001)$, current smoker or ex-smoker than in non-smoker $(6.8 \%, 5.2 \%$ vs $2.2 \%, \mathrm{p}<0.001)$, current drinker than in non-drinker $(5.1 \%$ vs $2.2 \%, p<0.001)$, persons with heartburn than in those without heartburn $(7.8 \%$ vs $4.5 \%, \mathrm{P}=0.001$ ) and $\mathrm{CLO}$ negative individuals than in $\mathrm{CLO}$ positive individuals $(4.57 \%$ vs $1.83 \%, \mathrm{p}<0.001)$. RE was more common in persons with hiatal hernia than in those without hiatal hernia $(18.8 \%$ vs $4 \%, \mathrm{P}<0.001)$. The prevalence of RE was increased according to BMI $(\mathrm{p}<0.001)$; BMI $<18.5(2.6 \%), 18.5<\mathrm{BMI}<23(2.7 \%), 23<\mathrm{BMI}<25(3.5 \%)$, $25<\mathrm{BMI}<29(5.3 \%), \mathrm{BMI}>29(7.4 \%)$. RE was more common. The prevalence of RE was showing decreasing tendency to increased age $(\mathrm{p}=0.06) ;<40$ year $(4.6 \%), 40-60$ year $(4.1 \%),>60$ year $(3.6 \%)$. Dietary intake, educational level, economic status, housing environment, and current job did not affect reflux esophagitis. Multivariate analysis revealed that hiatal hernia $(\mathrm{P}=0.004 / \mathrm{OR}=3.105 / 95 \%$ CI 1.430-6.742), heartburn $(0.001 / 1.823 /$ 1.279-2.596), male sex $(0.004 / 1.702 / 1.182-2.452)$, current smoker $(0.018 / 1.496 /$ $1.072-2.086)$, current drinker (0.046 / $1.370 / 1.005-1.867)$, increased BMI (0.001 / 1.587 / 1.321-1.920), and negative CLO test (0.001 / 2.631 / 1.866-3.703) were confirmative risk factors for reflux esophagitis. Conclusion: Male sex, smoking, alcohol consumption, absence of H. pylori, increased BMI, presence of heartburn and hiatal hernia are distinctive risk factors for RE in general population.
T1021

Gastroesophageal Reflux Disease (GERD) and Gastroesophageal Reflux Disease (GERD) Related Non-Cardiac Chest Pain (NCCP) in the Population: Prevalence, Risk Factors and Health Care Seeking Guy D. Eslick, Nicholas J. Talley

BACKGROUND: Whether the epidemiology of gastroesophageal reflux disease (GERD) in the general population is changing remains uncertain. Less is known about GERD related chest pain. We aimed to evaluate risk factors for both these conditions in the community, and health care seeking. METHODS: A random sample of 1,000 residents of Penrith were mailed the Chest Pain Questionnaire (CPQ), a validated self-report questionnaire. Measured were symptoms, risk factors, psychological distress, quality of life, and demographics. RESULTS: The response rate was $73 \%$ ( $n=672$; mean age 46 years; $52 \%$ female) (figure). A total of 78 (12\%) were classified as having GERD (at least weekly heartburn and/or acid regurgitation); only $14 \%$ had consulted a physician about GERD in the prior year. There were two independent cardiac risk factors for GERD, high cholesterol $(\mathrm{OR}=3.28,95 \% \mathrm{Cl}$ 1.42-7.57, $\mathrm{p}=0.005)$ and current smoking $(\mathrm{OR}=2.47,95 \% \mathrm{CI}$ : 1.07-5.70, $\mathrm{p}=0.03)$. GERDrelated non-cardiac chest pain (NCCP) severity and frequency were independent predictors of healthcare seeking for GERD (OR=9.36, 95\% CI: 3.05-28.70, $\mathrm{p}<0.001$; OR=3.48, 95\% CI: 1.07-11.33), respectively. None of the psychological conditions (anxiety, depression and neuroticism) were significantly associated with GERD in the community. CONCLUSION GERD is remarkably common in the general population. High cholesterol levels (possible indicating a high fat diet) and smoking are independently associated with GERD. GERDrelated NCCP severity and frequency were independent predictors of healthcare seeking for GERD.

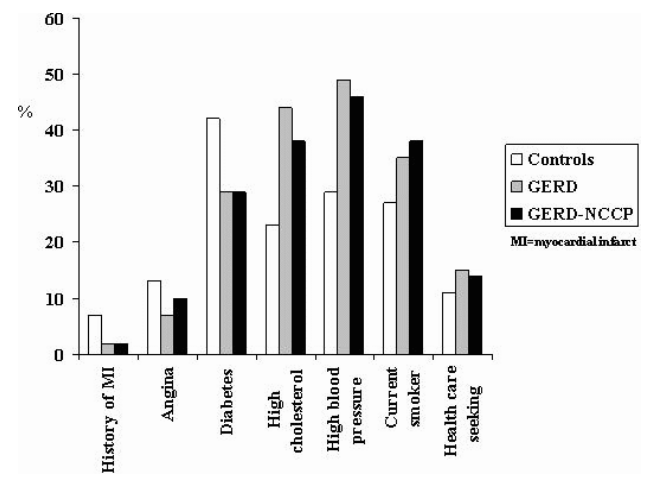

$\mathrm{T} 1022$

Increased Symptom Severity But Not Symptom Frequency Are Associated with a Worse Quality of Life (QOL) in Gastroesophageal Reflux Disease (GERD): A Population-Based Study Guy D. Eslick, Nicholas J. Talley

BACKGROUND: There is a dearth of population-based studies that determine the impac of gastroesophageal reflux symptoms on quality of life (QOL). We aimed to evaluate the magnitude of this problem in the community. METHODS: A random sample of 1,000 residents of Penrith were mailed the Chest Pain Questionnaire (CPQ), a validated selfreport questionnaire. Measured were GERD symptom severity and frequency, risk factors psychological distress, demographics and quality of life (using the SF-36). The symptom severity scale assessed mild, moderate, severe and very severe. The symptom frequency scale assessed $<1 /$ month, 1/month, 1/week, $>1 /$ week, and daily. RESULTS: The response rate among eligible subjects was $73 \%(n=672$; mean age 46 years; $52 \%$ female). GERD ever was reported by $52 \%$ of the population. The majority of QOL sub-scale scores were associated with the severity of both heartburn (Figure) and acid regurgitation symptoms. Those with mild, moderate, severe and very severe GERD symptoms had significantly poorer scores than those with no GERD ( $\mathrm{P}<0.05$ for sub-scales physical function, body pain, vitality, and social function). The frequency of heartburn and acid regurgitation (comparing $<1 /$ month and daily vs nil) were not associated with significantly reduced QOL sub-scale scores. CONCLUSION: Increasing GERD symptom severity is associated with worse QOL scores, whereas GERD symptom frequency $(<1 /$ month and daily) did not impact the QOL scores. We speculate this may be related to the development of tolerance for daily heartburn and acid regurgitation. 


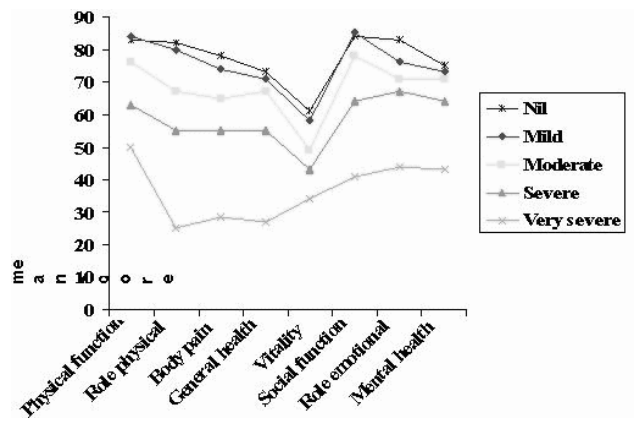

$\mathrm{T} 1023$

Gastroesophageal Reflux Disease (GERD) Associated Osteopenia

Stjepan Mise, Ante Tonkic, Ante Punda, Marina Titlic, Valdi Pesutic Pisac, Ivana Jukic, Sven Seiwerth, Predrag Sikiric

The pathophysiology of bone loss associated with gastrointestinal diseases has not been clearly defined. In addition to previous relation to inflammatory bowel disease or postgastrectomy status, we report an association with gastroesophageal reflux disease (GERD). In this study 131 subjects were randomly assigned, 62 with endoscopically determined GERD patients (35 female, 27 male), and 69 patients with normal endoscopy findings examined because of degenerative rheumatic disorders that need NSAIAs therapy (32 female, 37 male). Patients had not different ages GERD ( $\mathrm{min} / \mathrm{med} / \mathrm{max}: 34-65-84$ ), rheumatic patients ( $\mathrm{min} / \mathrm{med} / \mathrm{max}: 30-53-82)$, high GERD ( $\mathrm{min} / \mathrm{med} / \mathrm{max}: 146-166-186 \mathrm{~cm})$, rheumatic patients ( $\mathrm{min} / \mathrm{med} / \mathrm{max}: 151-165-190)$, weight GERD (min/med/max: 47-72-117 kg), rheumatic patients ( $\mathrm{min} / \mathrm{med} / \mathrm{max}:$ 48-76-107), menarche GERD ( $\mathrm{min} / \mathrm{med} / \mathrm{max}: 12-14-19$ years), rheumatic patients ( $\mathrm{min} / \mathrm{med} / \mathrm{max}: 11-14-18)$, menopause GERD ( $\mathrm{min} / \mathrm{med} / \mathrm{max}: 35-49-57$ years), rheumatic patients ( $\mathrm{min} / \mathrm{med} / \mathrm{max}: 38-49-55$ years). Lumbosacral spine and left hip were used for densitometry analysis ( $>-1=$ normal, $-1.0 /-2.5=$ osteopenia, $<-2.5=$ osteoporosis). The following values were obtained: lumbosacral spine: GERD ( $\mathrm{min} / \mathrm{med} / \mathrm{max}:-4.6 /-$ $1.3 / 2.1$ ), rheumatic patients ( $\mathrm{min} / \mathrm{med} / \mathrm{max}:-3.2 /-0.3 / 2.6)$ ( $\mathrm{P}<0.0002$ ), left hip: GERD (min/med/max: -2.9/-0.9/2.8) : rheumatic patients ( $\mathrm{min} / \mathrm{med} / \mathrm{max}:-2.5 /-0.2 / 2.2)(\mathrm{P}<0.003)$. Frequency of osteopenia: rheumatic patients: left hip $22 \%$, lumbosacral spine $10.1 \%$, GERD: left hip 43.5\% ( $\mathrm{P}<0.008)$, lumbosacral spine $22.6 \%(\mathrm{P}<0.05)$. Sex relation in GERD-patients: lumbosacral spine: male ( $\mathrm{min} / \mathrm{med} / \mathrm{max}:-2.7 /-0.6 / 1.4)$, female ( $\mathrm{min} / \mathrm{med} / \mathrm{max}:-4.6 /-1.4 / 2.1)$ ( $\mathrm{P}<0.014)$, left hip: male ( $\mathrm{min} / \mathrm{med} / \mathrm{max}:-1.5 /-0.4 / 2.8)$, female $(\mathrm{min} / \mathrm{med} / \mathrm{max}:-2.9 /-1.1 /$ 1.1) $(\mathrm{P}<0.028)$. No special difference was noted in nutrition, physical activity, alcohol consumption, as well as neither of patients received any therapy for osteoporosis. Conclusion. Thus, these data indicate an indicative association between GERD and osteopenia, and their relation remain to be further determined.

\section{$\mathrm{T} 1024$}

Can It Be Predicted Prior to Treatment the Necessity for Additional Prokinetics with PPI Using Frequency Scale for the Symptoms of GERD (FSSG), a GERD-Specific Questionnaire?

Miyamoto Masaki, Haruma Ken, Mizooka Masafumi, Kuwabara Masao

Background:PPI monotherapy cannot cure all GERD patients, and combination therapy with a prokinetics and PPI achieves symptomatic improvement for the patients. Few studies have been performed to predict the need for prokinetics before treatment for GERD commenced. Using the frequency scale for the symptoms of GERD (FSSG), a GERD-specific questionnaire developed in Japan (Kusano et al., J Gastroenterol 2004), we retrospectively investigated whether pre-treatment FSSG scores can be used to predict the need of prokinetics after 12 weeks. Patients and Methods:The 163 patients (64 male, mean age 53.1 16.6 years) with gastroesophageal reflux (GER) symptoms were evaluated using the FSSG and upper gastrointestinal endoscopy, and were administered rabeprazole $10 \mathrm{mg}$ daily. After 12 weeks, the patients were offered a choice of 4 treatment regimens according to their degree of satisfaction (satisfied group: 1)no need for further treatment, 2)opt for continued PPI treatment, 3)stepdown from PPI to H2RA; dissatisfied group: 4)dissatisfied with present treatment, so opt for combination treatment with prokinetics, mosapride citrate $5 \mathrm{mg} \mathrm{X} 3$ daily). The patients continued with the treatment of their choice for a further 12 weeks, and they were again offered the same 4 choices according to their degree of satisfaction. The evaluated the association predictor for choice of treatment after 12 weeks, odds ratio (OR) and corresponding $95 \% \mathrm{CI}$ were computed by means of logistic analysis models. Results: 1 . After 12 weeks, the satisfied groups were $79.1 \%<129 / 163,1) 21 \mathrm{pts}, 2) 98 \mathrm{pts}, 3) 10 \mathrm{pts}>$, and the dissatisfied group was $20.9 \%$ (34/163). After 24 weeks, $98.2 \%$ of subjects were in the satisfied group. 2. We compared 4 treatment regimens by baseline FSSG scores, the dissatisfied group $<4) 17.4 \pm 1.4>$ were significantly higher than the satisfied groups $<1) 12.3 \pm 1.3,2) 12.8 \pm 0.8$ and 3) $10.2 \pm 1.8)(P<0.05)$. 3. Male gender (OR 0.12: $0.02-0.56, P=0.007)$, the baseline FSSG score (OR 1.15: 1.03-1.28, $\mathrm{P}=0.017$ ), body mass index (OR 0.74: 0.60-0.91, $\mathrm{P}=0.005$ ), and symptomatic constipation (OR 21.6: 2.41-193.76, $\mathrm{P}=0.006$ ) were found to be significant predictors for additional prokinetics after 12 weeks. Conclusions As a result of selecting treatment regimens from request oneself, the patients satisfactory at 24 weeks in $98.2 \%$. The dissatisfaction by with continued PPI was useful for additional prokinetic The logistic regression analysis corrected for high baseline FSSG score and symptomatic symptomatic constipation show a significant predictor for the necessity for additional prokinetic.
T1025

Is Eosinophilic Esophagitis Underdiagnosed in Patients Presenting with Dysphagia Or Food Impaction? Rekha Attigere, Alison Schneider, Eric Miller, Richard Lee, Asyia Ahmad

Eosinophilic esophagitis is a disease of rising incidence and prevalence that should be considered in the differential of unexplained dysphagia and food impaction. Structural alterations may not be apparent during endoscopy and random esophageal biopsies are required to confirm the diagnosis. While the prevalence of eosinophilic esophagitis has increased, there are still no clear AGA guidelines for the diagnosis of this disorder. Our aim was to determine if the diagnosis of eosinophilic esophagitis is being considered by community and academic physicians during the evaluation of patients presenting with food impaction or dysphagia. METHODS: A survey was randomly sent to 700 gastroenterologists via electronic mail. Practitioners were asked about diagnostic and therapeutic interventions for dysphagia and food impaction. RESULTS: There were 117 responses ( 47 community gastroenterologists, 49 academic gastroenterologist, and 21 gastroenterology fellows). During the endoscopic evaluation of a patient presenting with a food impaction, $82 \%$ of academic, $79 \%$ of community gastroenterologists, and $81 \%$ of gastroenterology fellows stated they were likely to pursue further investigation. Surprisingly only $34 \%$ percent of academic physicians stated they would perform esophageal biopsies during the evaluation of a food impaction. However this was still significantly higher than both community gastroenterologists (14\%) and fellows $(17 \%)(p \leq 0.05)$. In addition, academic physicians were more likely to mention eosinophilic esophagitis as their reason for performing biopsies than both community and fellows (20.4\% academic vs $0 \%$ community vs $14 \%$ fellows; $p<0.01$ ). During the evaluation of unexplained dysphagia, all groups were likely to start with an EGD as their first test with community gastroenterologists performing this most commonly $(\mathrm{p}<0.05)$. Despite this, only $26 \%$ of academic physicians, $25 \%$ of community physicians, and $33 \%$ of fellows were likely to perform biopsies during upper endoscopy for unexplained dysphagia. There was no significant difference between these groups although there was a trend for academic physicians to mention eosinophilic esophagitis in their diagnosis more often. CONCLUSION: While eosinophilic esophagitis is an increasing cause of dysphagia and food impaction, the diagnosis is not being evaluated in practice. Academic gastroenterologists consider eosinophilic esophagitis more often than community physicians but neither group consistently includes eosinophilic esophagitis in their differential diagnosis or endoscopic plan. Further education is needed amongst all gastroenterologists to ensure that the diagnosis is not missed.

\section{T1026}

Usefulness of Rapid HP Star, a New Immunochromatographic, Monoclonal Antigen-Based Faecal Test, for Diagnosing Helicobacter Pylori Infection in Dyspeptic Patients

Mariela Quesada, Xavier Calvet, Victoria Calvet, Theresa Choat, Barbara Fallowfield, Isabel Sanfeliu, Ferran Segura

Objective: To evaluate the usefulness of two new monoclonal tests for detecting Helicobacter pylori antigens in dyspeptic patients' faeces: a rapid immunochromatographic test (RAPID Hp StAR, DakoCytomation, Cambridge, UK) and an ELISA (Amplified IDEIA Hp StAR, DakoCytomation, Cambridge, UK). Methods: H. pylori infection was determined in 68 dyspeptic patients who underwent endoscopy due to concordance of urease test and histology results. Faecal tests were performed according to the specifications of the manufacturer. Sensitivity, specificity, positive and negative predictive values (PPV and NPV respectively) were calculated for both RAPID Hp StAR and Amplified IDEIA Hp StAR. Concordance between different measurements was estimated by Kappa statistics. Results: The sensitivity of the RAPID Hp StAR ranged from $96-98 \%$ and its specificity was $67 \%$. PPV was $92 \%$ and PNV $80-90 \%$. Corresponding Amplified IDEIA Hp StAR values were $98 \%, 83 \%, 94 \%$ and 94\%. Conclusions: both RAPID Hp StAR and Amplified IDEIA Hp StAR were highly sensitive and easy-to-perform diagnostic tools for the diagnosis of $H$. pylori infection.

\section{$\mathrm{T} 1027$}

Evaluation of Four Different Faecal Tests for Determination of CURE After Helicobacter Pylori Treatment

Mariela Quesada, Xavier Calvet, Angelina Dosal, Victoria Calvet, Isabel Sanfeliu, Laura Ribera, Theresa Choat, Barbara Fallowfield, Antonia Montserrat, Valenti Puig, Ferran Segura

Background: Data evaluating the monoclonal tests for determination of cure after Helicobacter pylori treatment are scarce. Goals: This study was aimed to evaluate the usefulness of four stool tests - two new RAPID monoclonal immunochromatographic tests (RAPID Hp StAR ${ }^{\mathrm{TM}}$, DakoCytomation, Cambridge, UK and ImmunoCard STAT! HpSA, Meridian Diagnostics, Cincinnati, OH) a monoclonal EIA test (Amplified IDEIA ${ }^{\mathrm{TM}}$ Hp StAR, DakoCytomation, Cambridge, UK) and a polyclonal EIA test (Premier Platinum HpSA, Meridian Diagnostics, Cincinnati, $\mathrm{OH})$ - to confirm cure of $H$. pylori infection after eradication treatment. Study: Ninety-seven patients who underwent eradication treatment were included. Cure of H. pylori infection was determined using two consecutive reference tests. Faecal tests were performed according to the specifications of the manufacturer. Sensitivity, specificity, positive and negative predictive values (PPV and NPV) were calculated. Results: After H. pylori eradication, the RAPID Hp StARTM test has a sensitivity of $73 \%$ for detecting persistent infection, a specificity of $96-98 \%$, a PPV of $73-80 \%$ and a NPV of $96 \%$. For ImmunoCard STAT! HPSA the corresponding values were $91 \%, 97 \%, 77 \%$ and $99 \%$, for Amplified IDEIATM Hp StAR $73 \%, 97 \%, 73 \%$ and $97 \%$, and for Premier Platinum HpSA 91\%, 79\%, 35\% and $98 \%$. Conclusions: All tests except Premier Platinum HpSA were highly accurate confirming eradication after treatment. 


\section{T1028}

Serological Analysis of Helicobacter Hepaticus Infection in Japanese Patients with Chronic Hepatitis C Using New Monoclonal Antibodies

Tadashi Shimoyama, Ryoki Takahashi, Juichi Sakamoto, Takeo Ohmura, Shinsaku Fukuda, Akihiro Munakata

Background: Infection of Helicobacter hepaticus has been associated with the development of hepatocellular carcinoma in mice. It is generally known incidence of hepatocellular carcinoma in patients with chronic hepatitis $\mathrm{C}$ is high in Japan. However, prevalence of $H$. hepaticus infection has not been understood. We examined the prevalence of $H$. hepaticus infection serologically comparing with $H$. pylori, particularly in patients with chronic hepatitis C. Methods: Serum samples obtained from 182 patients with chronic hepatitis C (106 males and 76 females, mean age 47.4) and 142 control subjects who were not infected with hepatitis virus (69 males and 73 females, mean age 60.0). Seropositivity of $H$. pylori and $H$. hepaticus was tested by E-plate and antigen-capture ELISA using monoclonal antibody to $H$. hepaticus antigen, respectively. Results: H. hepaticus seropositivity was significantly higher in patients with chronic hepatitis C $(29.4 \%)$ comparing control subjects $(13.7 \%, \mathrm{p}<0.05)$ while $H$. pylori seropositivity was not different between groups $(67.0 \%$ and $77.4 \%$, respectively). Higher $H$. hepaticus seropositivity was observed in $H$. pylori-seropositive patients (32.1\%) than in H. pylori-seronegative patients $(15.3 \%, \mathrm{p}<0.01)$. Different seropositivity of $H$. hepaticus was observed according to birth year. H. hepaticus seropositivity was $37.3 \%$ in patients who were born before 1950 while it was only $12.7 \%$ in patients born after 1960 $(\mathrm{p}<0.01)$. H. pylori seropositivity was also showed the same trend in this series of patients. Conclusions: Present results suggest the possibility that prevalence of $H$. hepaticus is high in patients infected with hepatitis $C$ virus. Association of seropositivity may suggest the presence of similar transmission routes between $\mathrm{H}$. hepaticus and $\mathrm{H}$. pylori.

\section{T1029}

Helicobacter Pylori Infection Confers An Increase in Ten-Year Mortality from All Causes: Data from a Community Screening and Treatment Program Alexander C. Ford, Alastair G. Bailey, David Forman, Anthony T. Axon, Paul Moayyedi

Introduction: Population screening and treatment of Helicobacter pylori has been advocated as a means of reducing mortality from gastric cancer. Several screening programs have been conducted in the West, but follow-up has been limited to two years, therefore long-term mortality data are not yet available. Methods: The authors performed a ten-year follow-up study of individuals, currently aged 50 to 59 years, previously recruited into a community screening and treatment program for $\mathrm{H}$. pylori. Those testing positive were randomized to receive either eradication therapy, or placebo. All individuals originally involved were traced with a list cleaning service provided by the Office for National Statistics, and death certificates were obtained for deceased individuals. Results: Of 8407 original participants, 140 (1.7\%) were dead at ten years. $92(66 \%)$ were male. $64(46 \%)$ deaths were due to cancer, and 31 (22\%) to ischaemic heart disease (IHD). Mortality from all causes and IHD were significantly increased in $\mathrm{H}$. pylori positives compared to negatives (odds ratios (OR) 1.65; 95\% confidence interval (CI) 1.15 to 2.36 and 2.46 ; $95 \%$ CI 1.13 to 5.32 respectively), but there was no significant difference detected in cancer mortality (OR 1.37; $95 \%$ CI 0.78 to 2.36). Following logistic regression controlling for age at study entry, gender, tobacco and alcohol consumption, and socioeconomic status all-cause mortality remained significantly higher in $\mathrm{H}$. pylori positives (OR $1.46 ; 95 \% \mathrm{CI} 1.02$ to 2.09 ). There was a trend towards higher all-cause mortality and mortality from IHD in those receiving eradication therapy at ten years compared to placebo (relative risks $1.46 ; 95 \% \mathrm{CI} 0.85$ to 2.49 and $2.0 ; 95 \% \mathrm{CI} 0.69$ to 5.84 respectively), though neither were statistically significant. Two individuals died from upper gastrointestinal (GI) cancer, one gastric and one esophageal adenocarcinoma (both H. pylori negative), and a further died from upper GI hemorrhage from a duodenal ulcer (H. pylori negative). Conclusions: Infection with $H$. pylori is associated with an increased mortality from all causes, even when possible confounding factors are controlled for. The reasons for this are unclear. However, screening and treatment of positive individuals may confer an increased risk of death from IHD, which should be borne in mind when recommending the adoption of such a strategy.

\section{$\mathrm{T} 1030$}

Size of the Peptic Ulcer in H. Pylori Positive Patients: Association to the Clinical and Histological Characteristics

Perttu E. Arkkila, Arto Kokkola, Kari Seppala, Pentti Sipponen

BACKGROUND AND AIM: Based on the large trial of H. pylori (Hp) positive peptic ulcers, we clarified which are the symptoms and clinical characteristics to predict the size of peptic ulcer. We also studied if the size of the ulcer and other clinical and histological characteristics are independent factors for the healing of peptic ulcers. METHOD: A total of 333 consecutive patients with $\mathrm{Hp}$ infection and peptic ulcer were enrolled, the mean age of 54.8+/-12.7 years. Ulcer location was recorded by gastroscopy and the presence of $\mathrm{Hp}$ was assured by rapid urease test, histology and by serum $\mathrm{Hp} \operatorname{IgG}$ and IgA antibody measurement. The diameter of the peptic ulcer was measured by setting the opened biopsy forceps $(7 \mathrm{~mm})$ beside the peptic ulcer. Biopsy specimens were stained by haematoxylin and eosin, alcian blue-periodic acid Schiff and by modified Giemsa methods. Specimens were examined according to the Sydney system. RESULTS: Mean size of the peptic ulcer was in corpus $13.2+/-8.3$, in antrum $11.3+/-5.3$, in angulus $13.8+/-7.8$, in prepylorus $9.5+/-5.3$ and in duodenum $9.2+/-4.7 \mathrm{~mm}$ (duodenal vs, gastric type $\mathrm{p}<0.05$ ). The average size of ulcers was $9.4+/-5.3 \mathrm{~mm}$ in patients with Forrest III type of ulcer and 11.5+/-6.8 in other types of ulcers $(n=119) \quad(p<0.05)$. Patients, who were $\geq 50$ years old or currently smoking or who had corpus predominant chronic gastritis, atrophic gastritis or epigastric pain at day or night time, had larger ulcers than others. The size of index ulcer, the successful eradication of $\mathrm{Hp}$ and the presence of atrophic gastritis were independent factors for healing of the index ulcers. The odds ratio was 11.5 (95\% CI 3.3-40.5, p<0.01) for $\mathrm{H}$. pylori and 3.5 (95\% CI 1.1-11.2, $\mathrm{p}<0.05)$ for the size of index ulcer $(\leq 10 \mathrm{~mm}$ versus $>10 \mathrm{~mm})$ and for atrophic gastritis versus no atrophy 3.4 (95\% CI 1.2-9.8, p<0.05). CONCLUSIONS: A number of clinic and endoscopic variables (age, current smoking, corpus predominant gastritis, Forrest classification) associated with the size of peptic ulcer in $\mathrm{Hp}$ positive patients. The size of peptic ulcer, Hp infection and atrophic gastritis were indenpendent factors for the healing of peptic ulcers.

\section{T1031}

Evaluation of Endoscopy Outcomes a Decade After Nationwide Introduction of Protontonpump Inhibitors and Guidelines for Helicobacter Pylori Eradication Lieke A.S. Van Kerkhoven, Sally J. van Rijswijck, Leo G.M. van Rossum, Adriaan C.I.T.L. Tan, Robert J.F. Laheij, Jan B.M.J. Jansen

BACKGROUND: In several studies incidences of different gastrointestinal diseases have been investigated among patients referred for open access endoscopy. However, since the nationwide introduction of protonpump inhibitors and guidelines for $H$. pylori eradication over a decade ago, it is expected that incidences of endoscopic findings have changed. AIM: To compare the prevalences of different gastrointestinal diseases before and after nationwide introduction of protonpump inhibitors and guidelines for $H$. pylori eradication in a population referred for upper gastrointestinal endoscopy in an open access setting. METHODS: Dat about endoscopy outcomes of consecutive patients referred to the Canisius-Wilhelmina Hospital Nijmegen for open access endoscopy between January 2002 and December 2004 was collected from medical files. These were compared with data from three historical populations with similar characteristics publishes by Schaap et al, Numans et al. and Adang et al. a decade ago. Outcomes were compared using chi-square. RESULTS: Our curren study population consisted of 1298 subjects: 49\% were male and mean age (range) was 53.7(18-91). The historical population included a total of 3.062 subject, with a mean age of $50(\mathrm{SD}=15)$ and $57 \%$ was male. The prevalences of peptic ulcer disease and duodenitis statistically significant decreased from $18.2 \%$ to $5.7 \%$; a decrease of $12,5 \%(95 \% \mathrm{CI}: 10,2$ $14,9$ ) and from $11.8 \%$ to $5.4 \%$; a decrease of $6,4 \%$ (95\%CI: $4,4-8,4)$ respectively. The prevalences of reflux-oesophagitis, functional dyspepsia and Barrett's oesophagus statistically significant increased with $6,7 \%(95 \% \mathrm{CI}: 4,3-9,2), 10,5 \%(95 \% \mathrm{CI}: 7,3-13,7)$ and $2,0 \%$ ( $95 \%$ CI: 0,4-3,6), respectively. Gastroesophageal cancer is currently as frequent as it was in the historical population: $2.2 \%$ vs $1.8 \% ; \mathrm{p}=0.53$. CONCLUSIONS: Since the nationwide introduction of protonpump inhibitors and guidelines for $\mathrm{H}$. pylori eradication, prevalences of peptic ulcer disease and duodenitis have decreased significantly. The increase of oesophagitis at endoscopy supports the hypothesis that $H$. pylori eradication might contribute to the development of oesophagitis. 1 Schaap NPM et al. Ned Tijdschr Geneesk 1993;137(23):1142-6. 2 Numans ME et al. Scand J Gastroenterol 2001; 36(4):437-43. 3 Adang RP et al. Eur J Gastroenterol Hepatol 1994; 6:329-335.

\section{T1032}

The Relationship Between Previous Fluoroquinolone Use and Levofloxicin Resistance for Helicobacter Pylori Infections, 1998-2002 John J. Carothers, Michael G. Bruce, Thomas W. Hennessy, J Michael Bensler, Julie M. Morris, Alisa L. Reasonover, Debby A. Hurlburt, Alan J. Parkinson, John M. Coleman, Brian J. McMahon

Purpose: With increasing antimicrobial resistance among H. pylori to conventional therapies, interest in using fluoroquinolones as a viable second or third line therapy has increased. The relationship between prior fluoroquinolone use and levofloxacin resistance in $H$. pylor is unknown. Methods: We recruited adults from a clinical practice at a referral hospital in Alaska serving primarily Alaska Native/American Indians. One-hundred-twenty-five adults infected with $H$. pylori were enrolled. Medical records were reviewed for antimicrobial use in the 10 years prior to diagnosis with $H$. pylori infection. Fluoroquinolone susceptibility of $H$. pylori isolates obtained from endoscopic gastric biopsy was determined using the $\mathrm{E}$ Test method. The purpose of this study was to determine the relationship between prior fluoroquinolone use and levofloxacin resistance in $H$. pylori infected Alaska Native persons. Results: Among the 125 patients, 11 (8.8\%) were found to have H. pylori isolates resistan to levofloxacin. Resistance to levofloxacin was associated with any prior fluoroquinolone use ( 9 of 11 patients with levo-resistant isolates vs 19 of 114 patients with levo-sensitive isolates, $\mathrm{p}<0.001$ ). The odds of an isolate being resistant to levofloxacin increased in relation to the total number of courses of fluoroquinolones prescribed. Conclusion: Previous use of fluoroquinolones is associated with increased $H$. pylori resistance to levofloxacin. A history of fluoroquinolone use may be helpful in predicting $H$. pylori susceptibility to levofloxacin

\section{$\mathrm{T} 1033$}

Prevalence of Helicobacter Pylori Infection in Young Children: Non-Invasive Stool Antigen Testing

Michal Kori, Eva Goldstein, Esther Granot

OBJECTIVE: To noninvasively study the prevalence of Helicobacter pylori infection in young children attending day care facilities using an $H$. pylori stool antigen test (HpSA). METHODS: Children attending day care facilities aged 2 months and above were recruited. Stool samples were collected and tested for HpSA. Parents filled out a questionnaire regarding family size, residential crowding, parent's education, country of birth of the child and parent. RESULTS: 316 children (mean age $18.9 \pm 10.9$ months) were tested by HpSA. 78 (24.7\%) were positive and $236(76.3 \%)$ negative. $98(31 \%)$ of the children were younger than 12 months, 164 (52\%) were between 12 and 24 months and 54 (17\%) were older than 24 months. Seven (7.1\%) of children younger than 12 months tested positive for HpSA as compared to 52 (31\%) of the $12-24$ month old children and 19 (35\%) of the children older than 24 months $(\mathrm{P}<0.001) .158$ stool samples were collected and tested during the summer and an identical number were examined during the winter. 67 (42.4\%) of the samples tested during the summer were positive as opposed to $11(6.9 \%)$ tested during the winter $(\mathrm{P}<0.001)$. Mean age of the children tested during the different seasons was the same. CONCLUSIONS: By two years of age more than $30 \%$ of infants attending day care facilities in Israel are already infected with $\mathrm{H}$. pylori. The prevalence of infection during the first year of life is markedly 
lower (7\%). The observed increased incidence of HpSA positive tests during the summer as opposed to winter is intriguing and warrants further evaluation.

\section{$\mathrm{T} 1034$}

Effect of Sibling Number and Birth Order On Prevalence of Helicobacter Pylori: A Cross-Sectional Survey

Alexander C. Ford, Alastair G. Bailey, David Forman, Anthony T. Axon, Paul Moayyedi

Introduction: Social conditions influencing the acquisition of Helicobacter pylori in childhood are well recognized, but the effect of number of siblings, and birth order on prevalence of H. pylori has not been extensively reported. Methods: The authors performed a ten-year follow-up cross-sectional survey of individuals previously enrolled in a community screening and treatment program for $H$. pylori. Prevalence of $H$. pylori was assessed at baseline with 13carbon-urea breath testing. All involved individuals who were alive, and could be traced to a current address, were contacted by postal questionnaire, to obtain information on both total number of siblings, and their order of birth. Data concerning other social conditions in childhood were stored on file from the original study. Results: 3928 (47\%) of the 8407 original participants provided complete data. Prevalence of $H$. pylori infection increased steadily according to total number of siblings, from $20 \%$ in those with no siblings, to $63 \%$ with 8 or more. The odds of having $\mathrm{H}$. pylori infection was significantly increased when the individual had three siblings (odds ratio (OR) for infection 1.79. 95\% confidence interval (CI) 1.34 to 2.38), and a clear gradient of effect continued up to eight or more siblings (OR for infection 6.66; 95\% CI 3.94 to 11.44). In addition, odds of $H$. pylori infection was significantly higher in those individuals with two older siblings (OR for infection 1.32; $95 \%$ CI 1.04 to 1.68 ), and again increased steadily up to six (OR for infection 3.78; $95 \% \mathrm{CI}$ 1.51 to 9.74). Even amongst first-born individuals, prevalence of infection significantly increased with three or more siblings (OR 1.59; 95\% CI 1.02 to 2.44). When total number of siblings, and other social conditions in childhood, such as sharing a bed or bedroom with a sibling, lack of an inside toilet, and father's socioeconomic status were controlled for in a logistic regression model, the association between birth order and odds of H. pylor infection was no longer statistically significant. Conclusions: Number of siblings and birth order appear to influence prevalence of $H$. pylori infection, though the observed effect of birth order may be due to confounding by other factors in childhood.

\section{$\mathrm{T} 1035$}

Prevalence of Helicobacter Pylori Within Duodenal Ulcers in a District General Hospital

Shamaila K. Butt, Steve Mann, Niall van Someren, Kalpesh Besherdas

Background and Aims. The association between Helicobacter pylori and duodenal ulcers is well established, with infection being present in up to $95 \%$ of duodenal ulcer patients in published studies, the majority of the remainder being attributed to non steroidal antiinflammatory drugs (NSAIDS). One way of confirming presence of H.pylori infection at Endoscopy is by use of CLO test. In view of the high prevalence of H.pylori infection in duodenal ulcers, some advocate the empirical use of H.pylori eradication therapy in their management. The aim of this study was to assess the prevalence of H.pylori in duodenal ulcer patients from North London attending a district general hospital from the year 2000 . Methods. This was a retrospective analysis of patients (in a non-teaching hospital) with endoscopically diagnosed duodenal ulceration and considered to be infected with H.pylori if CLO test was positive. The data between July 2000 until July 2005 was gathered using the endoscopy Endoscribe reporting system along with CLO test records. Results. A total of 175 patients (age range was 20 to 101 years with a male to female ratio of 91:84) were identified as having duodenal ulceration on gastroscopy and were biopsied for H.pylori using the CLO test. The following table demonstrates the prevalence of H.pylori positive duodenal ulcers in our patients. Conclusion. We have shown that over five consecutive years the prevalence of H.pylori positive ulcers as measured by CLO test is in the range of $34 \%$ to $53 \%$, a much lower prevalence than proposed by previous studies. Although H.pylori infection is an important factor in the aetiology of duodenal ulceration, other factors (such as NSAIDS, and rarer causes like Crohn's disease, Coeliac disease, malignancy, hypergastrinaemia, gastrinoma and hyperparathyroidism) should be determined for as they may predominate. In addition, the empirical use of antibiotics in the management of patients with duodenal ulcers cannot be suggested by this study.

\begin{tabular}{|c|c|c|}
\hline Year & Number of duodenal ulcers & \% of duodenal ulcers CLO test positive \\
\hline 2000 & 15 & 53 \\
\hline 2001 & 30 & 43 \\
\hline 2002 & 56 & 39 \\
\hline 2003 & 51 & 41 \\
\hline 2004 & 32 & 34 \\
\hline 2005 & 23 & 48 \\
\hline
\end{tabular}

\section{$\mathrm{T} 1036$}

Calcium Channel Blockers May Radiosensitize Patients to Radiation Proctitis While Statins, NSAIDs May Radioprotect: A Case-Control Study

Bradley C. Irwin, Rasna Gupta, Kyung Kim, Sue Han, Edgar Ben-Josef, Bradley Axelrod, Martin Tobi

Background: We studied factors associated with the development of chronic radiation proctitis (RP) in prostate cancer $(\mathrm{PC})$ patients. We specifically targeted potential contributions of medications taken concomitantly with radiotherapy (RTx) since HMG-CoA reductase inhibitors (STATINS) and non-steroidal anti-inflammatory medication (NSAIDs) have been reported to enhance or reduce radiation effects in a number of organ systems. Methods:
From 1987-2002 there were 1,177 PC patients diagnosed at our center and 63 patients were found to have endoscopy-proven RP. 61 similarly diagnosed and irradiated PC patients with negative endoscopy served as controls. Demographics including body mass index were also compared. Results: Of the study population African Americans comprised 63\% of the sample. Average age was 67.7 years with median Gleason score of 7 . There were 61 controls and 63 patients with RP. Of the RP patients $60 \%$ were taking calcium channel blockers (CCB) versus $39 \%$ of controls; $19 \%$ of RP patients were taking STATINS versus $55 \%$ of controls; $15 \%$ of RP patients were taking NSAIDs versus $53 \%$ of controls. Logistic regression showed increased relative risk of RP in patients taking CCBs (OR 2.97, CI 1.29-6.87, p= 0.0107 ). A decreased relative risk was seen in patients taking Statins (OR 0.178, CI 0.069$.46, \mathrm{p}=0.0004$ ), and patients taking NSAIDs (OR $0.36, \mathrm{CI} .15-.82, \mathrm{p}=0.015$ ). Multivariate regression analysis revealed CCBs to be an increased independent risk factor for $\mathrm{RP}$ ( $\mathrm{p}=$ 0.023 ), whereas Statins and NSAIDs proved to be independent indicators for reduced risk $(\mathrm{p}=.0003$ and $\mathrm{p}=0.0158$, respectively). BMI, aspirin, folic acid, and multivitamin medications were also analyzed but no significant differences were detected between the groups. Conclusions: Medicinal intake of CCBs is a clinically significant and independent predictor for increased risk for RP. To the best of our knowledge this is a novel finding. Recent studies suggest that up-regulation of epidermal growth factor receptors (EGFR) plays a role in radioresistance in certain tumors. Calcium channel blockers interfere with the transactivation of endothelial EGFR and EGFR phosphorylation in vitro thus CCBs may Radiosensitize the rectal mucosa, thereby increasing the risk of RP. Conversely, STATINS and NSAIDs may be radioprotective by reducing RTX-induced chemokine and COX-2 expression, respectively. These findings may prove clinically useful to reduce the incidence of RP and prospective studies should be undertaken to determine their utility.

\section{T1037}

Does Colon Ischemia Have a Worse Prognosis When Isolated to the Right Side?

John Sotiriadis, Igal Khorshidi, Brandt J. Lawrence

The colon is the hollow viscus most commonly involved in gastrointestinal ischemia and colon ischemia (CI) accounts for approximately 1 in 1000 hospital admissions at our medical center. In general, $\mathrm{CI}$ has a very good prognosis but, because blood flow to the ascending colon and the small intestine are both from the superior mesenteric artery, it has been questioned whether the presentation of isolated right colon ischemia (IRCI) should be considered an emergency situation with a grave prognosis just as is ischemia acutely involving the small intestine. Indeed, we have seen several cases in which IRCI has been accompanied or followed by small bowel ischemia (acute mesenteric ischemia) and in which the mortality was higher than with the usual case of $\mathrm{CI}$. This study was designed to answer the following clinical questions: 1) does IRCI have a worse prognosis than $\mathrm{CI}$ involving other segments of colon? 2) does associated acute mesenteric ischemia account for any differences in observed morbidity or mortality and, if so 3) can emergency intervention alter the prognosis? A retrospective chart review was performed of all cases of biopsy-proven $\mathrm{CI}$ identified in the years 2000-2005. Age, gender, co-morbid conditions, anatomic location of the ischemia, pathology reports, radiologic imaging, colonoscopy reports and patient outcomes including hospital stay, need for surgery, morbidity and mortality were evaluated. 182 cases of CI were identified of which 46 (25.3\%) involved only the right side. In no case did acute mesenteric ischemia accompany or follow the index episode of IRCI. However, patients with IRCI had a worse outcome than those with $\mathrm{CI}$ involving other colon regions. 21 patients (45.7\%) with IRCI had a colectomy, of which $6(28.6 \%)$ died; another 4 died prior to surgery, yielding a total mortality rate of $54.3 \%$. The mortality rate for patients with nonright-sided $\mathrm{CI}$ was $4.4 \%$. In conclusion, IRCI is more common than previously reported, has a higher mortality rate than $\mathrm{CI}$ involving other regions of the colon and is not associated with or followed by acute mesenteric ischemia. Given these findings, it does not appear as if mesenteric angiography is appropriate in the evaluation of patients with IRCI, but the substantial mortality rate of this occurrence mandates its aggressive medical and surgical care.

\section{T1038}

The Spiraling Use of CT for Abdominal Pain: Is Colonoscopy Necessary If Colonic Thickening Is Reported?

Jordan H. Wolff, Jeffrey D. Potter, Waymon Lattimore, Murray B. Resnick, Brian L. Murphy, Steven F. Moss

Introduction: Computed tomography (CT) is often used in the investigation of acute abdominal pain. Not infrequently, colonic wall thickening is reported. Is a colonoscopy warranted to investigate this finding further? Only two small studies of a heterogeneous group totaling 73 patients have been published previously, with equivocal results. Given the lack of clinical guidelines regarding the management of such patients, our aim was to determine the utility of colonoscopy in the diagnosis and management of patients found to have colonic wall thickening on CT. Methods: All patients from July 2000 to April 2004 who were investigated by abdominal $\mathrm{CT}$ at our large university teaching hospital were considered. Cases were selected if any colonic wall thickening was reported. Patients were excluded if the indication for the CT did not include abdominal pain, if the patient had a history of a gastrointestinal disorder involving the colon such as a malignancy, any inflammatory bowel disease (IBD), infectious colitis or diverticulitis, or if they did not undergo colonoscopy within 30 days of the abnormal CT. Clinical, endoscopic and pathological data were extracted from the medical records of all eligible patients. Results: One hundred four cases were identified. Of these, $8(7.7 \%)$ were found to have adenocarcinoma of the colon or rectum. In 10 patients $(9.6 \%)$ a new diagnosis of IBD was made during the admission. Sixteen (15.4\%) had findings consistent with acute self-limited (infectious) colitis, $36(34.6 \%)$ had evidence of ischemic colitis, 27 (26\%) were found to have no abnormality that could explain the CT finding, and 7 patients (6.7\%) had other miscellaneous findings that may have been responsible for the "colonic thickening" - these were diverticulitis (3 cases), appendicitis (1), serosal abscess (1), acute proctitis (1), and mucosal friability related to thrombocytopenia (1). Notably, of those diagnosed with colorectal carcinoma or IBD, only 5 of the 18 patients (28\%) presented with evidence of gastrointestinal bleeding or anemia. Conclusions: Following colonoscopy a new diagnosis of colorectal carcinoma or IBD was made in $17.3 \%$ of these patients who 
had no known previous gastrointestinal disorder. Most of these patients had no other indication for diagnostic colonoscopy. Additionally, diagnoses of ischemic or infectious colitis were made in 50\% of patients overall. Based on this high frequency of significant findings, we recommend that patients with acute abdominal pain in whom CT demonstrates colonic thickening should undergo colonoscopy promptly.

\section{T1039}

The Cecum Is Inflamed in Health: The Danger of Considering a Diagnosis of Microscopic Colitis with Right Colon Biopsies

Shirley C. Paski, Robert Wightman, Marie Robert, Norman Pettigrew, Charles N.

Bernstein

BACKGROUND AND PURPOSE: Accurately diagnosing "normal colonic mucosa" is essential under many circumstances. An inherent degree of non-pathological mild inflammation, particularly in the cecum, has been informally described among gastrointestinal pathologists. This poorly described low-grade inflammation is often reported as "nonspecific colitis" which can be confusing to clinicians. The objective of this study was to characterize and quantify the low-grade inflammation present in the cecum and compare this to the rectum of healthy adults.METHODS: 85 healthy adult volunteers free of gastrointestinal symptoms and history of gastrointestinal disease consented to undergo colonoscopy + biopsy. Colonoscopy prep was with oral magnesium citrate and fleet phosphosoda. Biopsies were taken from the cecum and rectum. Biopsy slides were coded and mixed prior to scoring. Biopsy slides of cecum and rectum from 15 patients with ulcerative colitis were randomly distributed within our sample to limit observer bias. Histology scores 0 (none) to 3 (severe) were assigned for each of: lamina propria cellularity, subcryptal cellularity, crypt architecture, cryptitis/abscess, and epithelial injury. Activity = epithelial injury + cryptitis/abscess. Chronicity = lamina propria cellularity + subcryptal cellularity + crypt architecture changes. The total score for inflammation was measured as the sum of all histology scores for each slide.RESULTS: Histology scores for inflammation were significantly greater in the cecum vs rectum for all categories except there was no difference in subcryptal cellularity between cecum and rectum. See table. For persons with ulcerative colitis that did not reach the cecum, histology scores for cecal inflammation were no different compared with healthy individuals with respect to all measures of inflammation, activity, chronicity, and total scores. CONCLUSION: Increased microscopic inflammation of the cecum is present in healthy individuals free of gastrointestinal disease, compared to the rectum. Caution should be used when diagnosing "colitis" based on a cecal biopsy, particularly in the setting of low-grade inflammatory changes. Mean score (0-3)

\begin{tabular}{|c|c|c|}
\hline & Normal cecum & Normal rectum \\
\hline Lamina propria cellularity & 1.13 & 0.34 \\
\hline Crypt architecture changes & 0.25 & 0.09 \\
\hline Cryptitis & 0.40 & 0.10 \\
\hline Epithelial injury & 0.45 & 0.25 \\
\hline Activity score & 0.85 & 0.35 \\
\hline Chronicity score & 1.47 & 0.53 \\
\hline Total score & 2.32 & 0.89 \\
\hline
\end{tabular}

all comparisons significant at $\mathrm{p}<0.05$

\section{$\mathrm{T} 1040$}

\section{Treatment of Anal Ulcers in HIV-Infected Patients}

Claudia Gonzalez-Ruiz, Bill Briggs, Israelit Shlomo, Paul Selvidoss, Glenn Ault, Robert W. Beart, Andreas M. Kaiser, Petar Vukasin

Introduction: About 30\% of HIV-infected patients will seek attention for anorectal diseases at some point in their lives. The vast majority of their anorectal pathology is comprised of Human Papilloma Virus infections and anal ulcerations. The aim of this study was to review our experience in an HIV dedicated anorectal clinic within a large urban county system of the treatment of anal ulcers in HIV-Infected patients. Methods: The Los Angeles County University of Southern California HIV clinic, is an Institution solely dedicated to the care of HIV patients. A colorectal clinic was established within this environment in 1991, and has served as the exclusive provider for the care of anorectal pathology to these patients. A well established prospective database of patients treated at this clinic was reviewed for visits spanning Jan 2001 through Nov 2005. Included were patients with HIV-related anal ulcers. Excluded were patients with anal fissures anal pathology which did not include anal ulcers, and those with incomplete medical records. Data collected included general demographics, type of treatment and outcome. Treatment was considered successful if ulcer healing or resolution of anal symptoms was recorded. Results: A total of 307 HIV-infected patients were treated at our institution during the study period. Sixty patients $(20 \%)$ had the diagnosis of anal ulcers of which fifty one had adequate records for inclusion to the study. Nine patients were lost to follow-up. The Male: Female ratio was 57:3. Mean Age was 41 years (range 20-78). 50/60 patients (83\%) healed or became completely asymptomatic with a combination treatment of Acyclovir + metronidazol for 7 to 10 days. Ten patients (16\%) required two or three courses of antibiotics in order to heal completely. Only one patient required surgical intervention. Four patients presented with chronic ulcers that had burrowed in to perianal fistulas, all of which resolved with antibiotic treatment. Conclusion: Antibiotic treatment of HIV associated anal ulcer disease is highly effective in the resolution of symptoms and avoidance of surgical intervention.

\section{$\mathrm{T} 104$}

Profile of Patients Consulting for Ano-Rectal Symptoms and Having a Prescription of a Local Treatment in Primary Care Setting Marc Andre Bigard, Laurent Siproudhis, Francois Pigot, Ghislain Staumont

Background: Prescription of a local symptomatic treatment (ointment or suppository) is frequent by general practitioners but reasons of prescription are poorly understood. Methods : A survey was carried out in France by 161 general practitioners in a population of patients consulting for anal symptom. A questionnaire was filled out by physicians including questions about symptoms, history and prescription. Patients filled out a questionnaire with question about nature of treatment, symptomatic effect and tolerance. Results : A response was obtained for 885 patients (Female $=56,7 \%$ ). The mean age was 48,6 years (16-85 years) Patients described one symptom for $65,1 \%$, two symptoms for $25,1 \%$ and three symptoms or more for $9,8 \%$. The major symptoms were pain $(48,7 \%$ of patients), bleeding $(38,4 \%)$ swelling $(31,2 \%)$ and pruritus ani $(25,5 \%)$. Bleeding was more frequent in men $(42,2 \%$ vs $33,8 \%$ ) and pain was more frequent in women $(51,6 \%$ vs $42,7 \%)$. Reasons of medical advice were mainly intensity of symptoms $(61,5 \%)$ or anxiety $(32,5 \%)$. Medical prescription included 2 or 3 medications in $76,3 \%$. The most frequent prescription was an association of ointment, suppository and diosmine p.o (31,5\%). A combination of ointment and diosmine was made in $24,7 \%$. The duration of treatment was 10 days for ointment and suppositories and 30 days for oral diosmine. Conclusion : In case of anal symptoms (pain, bleeding, pruritus ani, swelling) attributed by general practitioners to hemorroids, symptomatic treatment with 2 or 3 medications is commonly prescribed in France. Satisfaction of patients is obtained in about $60 \%$ of the cases. In spite of lack of convincing randomized trials of these treatments, symptomatic effect seems correct in most of the cases.

\section{$\mathrm{T} 1042$}

Fecal Incontinence: Still Common and Underreported Susan McCormick

Background \& Aims: Fecal incontinence (FI) is a fairly common problem, though is is rarely discussed. There is no new medication to bring it to the limelight. Instead, it is an embarrassment. Patients rarely bring it up and doctors rarely ask, as the therapies are less than satisfactory. Though studies are sparse, the prevalence of FI has varied widely, from $1 \%$ (in an older study) to $18 \%$. Prevalence increases with age in most of the studies. This survey was undertaken to determine the prevalence and severity of FI in a young to middle aged group of health care professionals attending a gastroenterology lecture series. Methods 151 attendees of a Nurses and Associates Gastroenterology Update Conference were asked to fill out a questionnaire. Attendees included nurses, technicians, customer service representatives and secretaries. The questionnaire asked about demographics, obstetrical history, severity of FI (incontinence of mucous, liquid or solid stool; incontinence daily, weekly, 23 times a month or less than once a month; need for pads, restriction of activity) and whether FI had been discussed with their doctor. Results: 146 of 151 chose to answer the questionnaire (96.5\%). 5 respondents did not answer clearly, so 141 questionnaires were tallied (93\% of total). The mean age of the respondents was 45.2 (+/- SD 10). 136 of the respondents were women. 128 were caucasian, 6 were Asian, 4 were African American, 1 was Hispanic. 18 respondents had FI (13\%), mean age 49 (+/- SD 8). 8 were incontinent of liquid stool and 10 of solid stool. The frequency of FI was weekly in 3/18,2-3x a month in $4 / 18$, and $<1 /$ month in $11 / 18.3$ restricted their activities because of FI, 4 wore pads, and 2 had discussed the problem with their physician (1\%). 4 respondents had had primary caesarean section $(C / S)$. None of these had FI. 100 labored and then had either vaginal delivery or C/S. 15 of these women had FI (15\%), 32 of the women had no children. This was a younger group (mean age 38.4 vs. 47.5 in the group that had had children.) 3 of these had FI (10\%). Conclusion: In a survey of a relatively young group (mean age 45), Fl was still quite prevalent (13\%). More than half had FI of solid stool and 39\% had episodes weekly or 2-3x a month. Only $1 \%$ had discussed the symptoms with their doctor. FI remains common and underreported.

\section{$\mathrm{T} 1043$}

Joint Hypermobility and Rectal Prolapse in Children Sonia Michail, Adam Mezoff

The etiology of rectal prolapse is unknown, but it has been associated with long-standing constipation, cystic fibrosis, parasitic infection, chronic diarrhea, malnutrition, or neurological disease. A number of children with rectal prolpase do not display such associated disorders. An underlying connective tissue abnormality is a consideration in this subset of children but has not been previously described. The purpose of this study is to determine whether joint hypermobility is associated with rectal prolapse in the pediatric population Records of all children with rectal prolpase seen over a period of five years were evaluated. Children having any of the above associated disorders were excluded. Joint hypermobility as assessed by Beighton's criteria were evaluated in children with rectal prolapse as well as agematched controls. Nine children were identified with rectal prolpase without an associated disorder. Average age was 8.8 years (range: 3-17). Male to female ratio was five to four. Al children had a Beighton score more than 4 suggesting joint hypermobility $(5.6 \pm 0.53)$. All sixteen sex- and age-matched control children scored less than four. The average Beighton score for the control group was $1.6 \pm 0.67(\mathrm{p}=0.0018)$. The presence of joint hypermobility in children with rectal prolapse suggests an underlying connective tissue abnormality. This abnormality may contribute to a weakness in the rectal fixation and the subsequent prolapse of the rectum and rectal mucosa. 
T1044

Using Computed Tomography to Validate An Ultrasonography-Based Classification of Mild to Moderate Acute Colonic Diverticulitis

Akira Mizuki, Masayuki Tatemichi, Hiroshi Nagata, Satoshi Kaneda, Nobuhiro Tsukada, Toshifumi Hibi

Background \& Purpose: The purpose of this study was to validate an Ultrasonographybased (US-based) diagnosis and classification of mild to moderate acute colonic diverticulitis (ACD). We recently proposed a new US-based classification of ACD and indicated that patients with an inflamed diverticulum (with an abscess of $2 \mathrm{~cm}$ in diameter or smaller) diagnosed as US-grade I were treatable as outpatients (Aliment Pharmacol Ther. 21:889$97,2005)$. Since CT is considered the gold standard for ACD diagnosis, a study validating the US-based classification, using CT as a benchmark, was necessary. Material and Methods: From 1998 to 2005, both US and CT examinations were performed in 24 ACD patients (16 male and 8 female patients; (55.4 (16.5) years; mean (SD)). To compare US-based grade I severity with $\mathrm{CT}$, three sub-classifications were defined: Ia indicated an inflamed diverticulum, where the inflammation was limited to the diverticulum; Ib indicated an inflamed diverticulum with pericolitis (inflammation clearly extended to the pericolic tissue around the diverticulum); and Ic indicated an inflamed diverticulum with an abscess of 2 $\mathrm{cm}$ in diameter or smaller. Grade II indicated an inflamed diverticulum with an abscess greater than $2 \mathrm{~cm}$ in diameter, or with perforation from the colon into the abdominal cavity. All US and CT images were examined by three Radiologists. Results: Of 5 patients with USgrade Ia, 4 were diagnosed as grade Ia by CT, and one as grade Ib. Of 6 patients with USgrade $\mathrm{Ib}$, all cases were diagnosed as grade $\mathrm{Ib}$ by $\mathrm{CT}$, whereas of 6 patients with US-grade Ic, all cases were diagnosed as Ib by CT. In other words, a pericolic abscess with a maximum diameter of $2 \mathrm{~cm}$, as revealed by US, was not evident on the CT. Of 7 patients with USgrade II, 6 were classified as grade II by CT while one was diagnosed as grade Ib by CT (since the size of the abscess could not be determined by CT). According to our US-based findings, CT was unable to detect an abscess that was less than $3 \mathrm{~cm}$ in diameter. Conclusion: Our research shows that using CT to diagnose mild to moderate ACD can result in underestimating the severity of the ACD and, therefore, can result inappropriate treatment. Through our study we found that US-based diagnosis and classification is more accurate, which ultimately means that patients receive better treatment.

\section{$\mathrm{T} 1045$}

Impact of Alcohol Dependence On the Health Values of Patients Infected with $\mathrm{HCV}$

Muslim Atiq, Susan N. Sherman, Kenneth E. Sherman

Background: The CAGE questionnaire is a simple instrument used to assess alcohol dependence. The relationship between CAGE outcomes, health utilities and quality of life measures have not been previously assessed in HCV infected subjects. Materials and Methods: This cross-sectional study was carried out at the University of Cincinnati Medical Center. One hundred twenty-four patients with chronic $\mathrm{HCV}$ infection were administered a diseasespecific version of the SF-36 Health Survey, the Beck Depression Inventory, and 3 direct health value measures, including the Rating Scale, Time Trade-off (TTO), and Standard Gamble (SG). Correlation among measures and factor analysis was performed for the patients who were positive by CAGE criteria defined as $>2$ variables. Results: There were a total of 124 patients including $80(64 \%)$ males and $4 \overline{4}(36 \%)$ females. Mean age was $46.59+/-$ 8.78 years. Sixty patients $(48.4 \%)$ patients were found to have alcohol dependence by CAGE criteria. Alcohol dependence was associated with a history of depression $(\mathrm{p}=0.036)$ consumption of $>20$ grams/day of alcohol $(\mathrm{p}=0.01)$, history of IV drug abuse $(\mathrm{p}<0.001)$, nasal cocaine use $(\mathrm{p}=0.007)$ and tattoos $(\mathrm{p}=0.001)$; whereas it was independent of gender, ethnicity, marital status or sexual orientation. Alcohol dependence did not correlate with the presence of cirrhosis or decompensated liver disease. Mean RS, TTO and SG scores were not different between the two groups. Composite mental function QOL (MCS) scores were lower in patients with alcohol dependence $(\mathrm{P}<0.01)$. Social Functioning $(\mathrm{SF})$, Role emotional (RE) and Mental Health (MH) scores on the SF-36 questionnaire were lower amongst patients with alcohol dependence $(p<0.02 ; p<0.04$ and $p<0.08$ respectively). There was a strong correlation between RS and MCS $(\mathrm{r}-0.81 ; \mathrm{p}<0.01)$ in the subset of patients with alcohol dependence whereas $S G(r=0.44 ; p-0.02)$ and TTO $(r=0.56 ; p<0.01)$ were only weakly correlated with MCS. There was a very strong correlation between SG and TTO (R-value $0.77 ; \mathrm{p}$-value $<0.01$ ). The TTO and SG scores were significantly greater than the RS score $(\mathrm{P}<0.01)$ and did not differ from each other. Conclusion: Alcohol dependence significantly affects the QOL in HCV infected individuals; however the health utility indices are not affected. CAGE positive response is not associated with alteration of patient behavior related to medical decision making compared to those not meeting criteria for alcohol abuse. This study was funded by a grant from NIH AHRQ (grant number HS10366-01)

\section{$\mathrm{T} 1046$}

Outcome of Screening for Hepatitis C Virus Infection in the Veteran Population

Carol Mallette, Kittichai Promrat, Maura Flynn

Purpose: Screening for hepatitis C virus (HCV) infection in high risk individuals is currently recommended by most, but not all, health authorities. There is a lack of data on the outcomes of screening. This study identifies clinical characteristics and outcomes of patients diagnosed with HCV through a screening program targeting patients with increased risk. Methods: Veterans presenting for care in VA facilities are assessed for HCV risk factors by a selfadministered questionnaire. Those who identify a risk factor are offered an anti-HCV test. Review of the local HCV registry from October 1998 through May 2004 identified 25,701 patients who were assessed for risk factors. No risk factor was reported by 17,767 patients who were not offered anti-HCV testing unless clinically indicated. Risk factors for HCV were identified in 8471 patients, of which 5646 were tested for HCV antibody. Patients were considered to be diagnosed through the screening program if risk assessment led to anti$\mathrm{HCV}$ testing. Clinical characteristics and outcomes of these patients were prospectively obtained by chart review and medical evaluation. Candidacy for anti-HCV therapy was assessed using pre-determined criteria. Results: The prevalence of a positive HCV antibody in veterans who identified a risk factor was 7.3\%. Among 412 anti-HCV positive patients, 260 were diagnosed through the screening program. $98 \%$ of the patients were male with a mean age of $59.47 \%$ of patients were viremic, $34 \%$ were aviremic, and $19 \%$ had no confirmatory test. Among patients who had chronic HCV infection $(n=122), 34 \%$ had persistently normal ALT defined by ALT $<40$ on 3 separate occasions over 6 months. 39 patients (32\%) underwent liver biopsy, and two-thirds had minimal or no fibrosis (Scheuer stage 0 or 1). Advanced liver disease based on thrombocytopenia or abnormal synthetic function was evident in $10 \%$ of patients. After a mean follow up of 26 months, 22 patients (8\%) were deceased; 2 were liver-related deaths. Of patients with chronic HCV, 54 (44\%) were not immediate candidates for therapy secondary to medical and psychiatric co-morbidities or active substance abuse. Thus far, $16 \%$ of the screen-detected patients with chronic HCV infection have received anti-HCV therapy. Conclusion: The prevalence of a positive HCV antibody in veterans with risk factors is significantly higher than the general population. Approximately half of the patients are viremic but few have advanced disease. Approximately half are not immediate candidates for therapy based on contraindications or ongoing substance abuse. This data has major implications in designing an optimal screening strategy for hepatitis $\mathrm{C}$.

\section{$\mathrm{T} 1047$}

Initial HCV Response in Patients with End Stage Renal Disease Treated with Combination Pegylated Interferon $\alpha-2 a$ and Ribavarin

Wyel Hakim, Shehzad Sheikh, Irteza Inayat, Margaret Bia, Cary Caldwell, Dhanpat Jain, Douglas Smith, Fadi Lakkis, Amy Friedman, Marc Lorber, Richard Formica, Wajahat Mehal

Background. Treatment of hepatitis C virus (HCV) with pegylated interferon and ribavirin has been widely studied, but there is little data on combination treatment of patients with $\mathrm{HCV}$ and end-stage renal disease (ESRD). We designed a pilot study to determine the initial and sustained viral response of combination treatment of these patients. Methods. A nonrandomized, prospective observational study of combination therapy with low dose pegylated interferon $\alpha$-2a and ribavarin. 20 patients who met the criteria for inclusion (age over 18yrs, viremic for $\mathrm{HCV}$, any genotype, on dialysis) were enrolled for the study. Exclusion criteria were decompensated liver disease, immunosuppressive therapy, hepatocellular cancer, and use of antiviral therapy in the last 6 months. After undergoing liver biopsies, patients began PEG-IFN at 135 microgram/week sc, and 4 weeks later ribavarin was started at $200 \mathrm{mg} P O$ weekly, increasing gradually to three times a week for a total of 48 weeks. Results. 20 patients: M:F 18:2; mean age 52.4 yrs; African American 15, Caucasian 3, Hispanic 2; genotype 1: 18, non-1: 2. All patients underwent liver biopsy (stage I: 4; stage II: 9 ; stage III 7). Of the 20 patients, 4 withdrew from the study prior to starting treatment. 2 patients discontinued before three months of treatment ( 1 for moderate fatigue and 1 patient moved out of state). 11 patients have reached the three-month time point to determine initial viral response, while 3 have not. Of the 11,5 patients have had initial viral response, defined as at least a two log drop in their HCV viral count (45\%). Of the 3 patients that have not reached 3 months on treatment 1 has had a viral response. Side effects included 2 cases of anemia (Hgb less than 9.5 g/dl) requiring temporary withholding of ribavirin, and one patient with headaches not requiring stopping treatment. Conclusions. Low-dose pegylated interferon and ribavarin therapy of patients with HCV and ESRD in this study has not been associated with any serious adverse effects. The response rate in individuals taking three months of treatment is comparable to studies in non-ESRD patients. These results demonstrate that combination therapy with low dose pegylated interferon $\alpha-2 a$ and ribavirin is a safe therapeutic option in the ESRD population with HCV infection. Conclusive results will be obtained once all patients complete the treatment and the follow-up period for determining a sustained response. This study was supported by Roche Pharmaceuticals.

\section{T1048}

The Positive Impact of a Multi-Faceted Educational Intervention On Physician Knowledge and the Actual Practice of Primary Care Physicians Regarding Hepatitis C

Am Rebecca Daniel, Anna M. Jankowski, Naresh T. Gunaratnam, Thomas M. Shehab

Background: The majority of hepatitis $\mathrm{C}(\mathrm{HCV})$ patients remain undiagnosed. Multiple studies have shown knowledge and practice deficits among primary care physicians (PCPs). In addition, previous interventions have failed to improve patient care. Aim: To assess the impact of a multi-faceted educational intervention on HCV knowledge and practice of PCPs in a community based setting. Methods: Internal medicine faculty and residents were surveyed regarding knowledge of $\mathrm{HCV}$ risk factors, diagnosis and management. Charts were reviewed to determine physician practice. An intervention was then undertaken for 6 weeks. This intervention included lectures, e-mails, educational materials regarding HCV and chart cues prompting questions regarding risk factors. The physicians were resurveyed and a post intervention chart review was performed. Results: Over 800 pre intervention charts were reviewed (patients seen by 20 physicians). Patient Demographics: $58 \%$ female, $22 \%$ African American, mean age: $52 \mathrm{yr}$. At baseline, $0.4 \%$ of patients were asked about common HCV risk factors, $0.1 \%$ were identified as having a risk factor and $<0.1 \%$ were tested. There were no statistically significant differences between demographics of the pre and post intervention patient populations. There was notable improvement in all parameters of $\mathrm{HCV}$ management. There was a statistically significant difference in the proportion of patients asked about HCV risk factors ( 21.0 vs $0.4 \%, p<0.001)$, identified as having risk factors ( 3.1 vs $1.1 \%, p<0.001$ ) and undergoing testing ( 1.5 vs $0.08 \%, p=0.003$ ). The number of new HCV cases doubled post-intervention. Results were not impacted by race, gender or visit type. $72 / 79$ (91\%) physicians participated in the survey. The physicians reported low levels of confidence in caring for $\mathrm{HCV}$ patients. $62 \%$ had not made a new diagnosis in the last year. A significant proportion of physicians demonstrated a lack of knowledge with regard to HCV risk factors, testing, and effectiveness of therapy. Post intervention, there was a statistically significant improvement in risk factor identification, physician comfort in caring for HCV, and knowledge of treatment effectiveness $(\mathrm{p}<0.5)$. Conclusion: This multi-faceted intervention showed 
impact in virtually all areas of physician knowledge and a dramatic improvement in physician practice. The intervention led to increased screening and testing for HCV. This is one of the first studies to demonstrate this degree of impact on the identification of primary care patients at risk for HCV. Future studies should assess the durability of this change to determine the reproducibility in other settings.

\section{T1049}

Percutaneous Radio-Frequency Ablation Versus Surgical Resection in the Treatment of Small Hepatocellular Carcinoma

Shinichiro Nakamura, Yoshiyuki Kobayashi, Hironori Tanaka, Yasuhiro Miyake, Ryo Terada, Hideki Ohnishi, Bon Shoji, Shouta Iwadou, Kenji Miyoshi, Hirokazu Miyatake, Kazuhiro Nouso, Kohsaku Sakaguchi, Yasushi Shiratori

PURPOSE: The purpose of this study was to compare the effectiveness of percutaneous radio-frequency ablation (RFA) and that of surgical resection for the treatment of small hepatocellular carcinoma (HCC). STUDY DESIGN: Retrospective cohort study. METHODS: Between July 1997 and June 2005, consecutive 623 patients who were diagnosed with HCC for the first time were enrolled from our institute and Okayama Hepatocellular Carcinoma group. Two hundred and ninety-three patients received RFA (RFA group) and 330 patients received surgical resection (resection group). RFA was performed percutaneously using a 17-gauge internally cooled-tip electrode under real-time US guidance. All survival probabilities were estimated by means of the Kaplan-Meier method. Patients were stratified based on JIS score and CLIP score, and clinical outcomes in terms of survival and recurrence rates were compared. Furthermore, the multivariate analysis of factors affected to survival or recurrence rates were done using the Cox proportional hazards model. RESULTS: Clinical characteristics of patients were shown in Table 1. Four-year survival rates and recurrence rates were summarized in Table 2. Adjusted with age, gender, tumor number, Child-Pugh grade, tumor diameter, vascular invasion and serum level of alpha-fetoprotein, the relative risk of death and recurrence of RFA compared to resection was 1.58 (95\% C.I.: 0.79-3.18) and 1.26 (95\% C.I.: 0.87-1.82), respectively. CONCLUSION: No significant differences were found between the therapeutic outcome of RFA and surgical resection, even stratified according to JIS score or CLIP score.

Table 2. 4-year survival rates and 4-year recurrence rates

\begin{tabular}{|c|c|c|c|c|c|c|c|c|}
\hline & \multicolumn{2}{|c|}{ No. of patients } & \multicolumn{3}{|c|}{ 4-year survival rate (\%) } & \multicolumn{3}{|c|}{ 4-year recurrence rate (\%) } \\
\hline & RFA & Resection & RFA & Resection & P value & RFA & Resection & P value \\
\hline All patients & 293 & 330 & 85 & 82 & 0.74 & 48 & 45 & 0.49 \\
\hline JIS 0 & 105 & 64 & 98 & 91 & 0.28 & 42 & 38 & 0.07 \\
\hline JIS 1 & 117 & 158 & 84 & 82 & 0.62 & 33 & 43 & 0.24 \\
\hline JIS 2 & 54 & 86 & 71 & 82 & 0.07 & 55 & 48 & 0.09 \\
\hline JIS $\geq 3$ & 17 & 22 & 73 & 63 & 0.80 & 59 & 49 & 0.51 \\
\hline CLIP 0 & 146 & 184 & 98 & 88 & 0.08 & 39 & 44 & 0.79 \\
\hline CLIP 1 & 119 & 99 & 76 & 82 & 0.21 & 40 & 52 & 0.54 \\
\hline CLIP $\geq 2$ & 28 & 47 & 63 & 63 & 0.55 & 61 & 37 & 0.09 \\
\hline
\end{tabular}

Table 1. Clinical Characteristics of Patients

\begin{tabular}{|c|c|c|c|}
\hline Characteristics & RFA group & Resection group & P value \\
\hline Age, yr (median, range) & $69(35-87)$ & $65(24-83)$ & $<.0001$ \\
\hline Male: female & $2.1: 1$ & $3.5: 1$ & 0.0029 \\
\hline Anti-HCV Ab positive (\%) & 80 & 62 & $<.0001$ \\
\hline HBsAg positive (\%) & 11 & 22 & $<.0001$ \\
\hline Child-Pugh grade A/B/C (\%) & $72.2 / 27.4 / 0.4$ & $90.2 / 9.8 / 0$ & $<.0001$ \\
\hline Maximum tumor diameter, mm (median, range) & $15(5-47)$ & $36(10-150)$ & $<.0001$ \\
\hline Mean tumor number & 1.35 & 1.26 & 0.090 \\
\hline Follow-up period, Mo(median,range) & $29(8-57)$ & $54(7-98)$ & $<.0001$ \\
\hline
\end{tabular}

\section{$\mathrm{T} 1050$}

The Treatment of Malignant Ascites in Patient with Advanced Cancer: Peritoneovenous Shunt Versus Paracentesis

Masahiro Seike, Iruru Maetani, Takuro Endo, Hiroaki Shigoka, Tomoko Tada, Takeo Ukita, Yoshihiro Sakai

Background: Malignant ascites often appears in the end-stage patients with advanced cancer, and such patients must be admitted to the hospital due to refractory ascites. Malignant ascites is difficult to treat and effective palliation is often not achieved. Methods: We performed peritoneovenous shunt (PVS) placement in patients with malignant ascites, who were admitted to our institution between January 2000 and March 2005. The outcome of PVS placement was compared with the outcome in patients who underwent paracentesis to drain ascites during the same period. Comparison included changes in abdominal girth, hematocrit (Hct), blood urea nitrogen (BUN), and creatinine $(\mathrm{Ct})$ measurements before and up to seven days after PVS placement. The changes in performance score after the procedure, the median survival from the time of PVS placement and the first paracentesis, possibility of discharge, and complications were compared in the 2 groups. Furthermore, the median survival in PVS group was compared between patients who had malignant cells identified in their ascites cytology and those who did not have malignant cells in their ascites. Results: Twenty patients underwent PVS placement and 49 patients were subjected to paracentesis. After PVS placement, abdominal girth and Hct decreased significantly. BUN and $\mathrm{Cr}$ also tended to decrease. The postoperative performance score showed a significant improvement following PVS placement $(\mathrm{p}=0.0026)$. The median survival for PVS group was significantly longe than that for paracentesis group ( 42 days vs. 18 days; $p=0.003$ ). The rate of possible discharge was significantly higher in PVS group $(\mathrm{p}=0.0076)$. Severe complications associated with mortality were observed in 1 patient in the PVS group and in 7 patients in the paracentesis group. Patients with positive and negative ascites cytology evaluation for malignant cells had a median survival of 35 days and 54 days, respectively, and there was no statistically significant difference between them $(\mathrm{p}=0.9655)$. Conclusions: PVS placement decreased ascites in advanced cancer patients and improved circulating blood volume. The performance score, the median survival time, and possibility of discharge were better for the PVS group than for the paracentesis group. PVS was found to be a safe and effective treatment to improve the patient's quality of life.

\section{$\mathrm{T} 1051$}

What Is the Annualized Risk of Gastrointestinal Bleeding from Aspirin Use? The Results of Pooled RCT Trials Keith Tsoi, Yuhong Yuan, Richard H. Hunt

Background: Gastrointestinal injury due to aspirin is widely accepted. Although relative risk of aspirin-induced gastrointestinal injury has been reported, absolute risk data has only been shown in cohort studies. Recently the temporal profile of NSAID-induced gastrointestina bleeding has been shown to be linear. Using this assumption, we attempt to derive an annualized risk of aspirin-induced injury. Data Source: Medline, Embase, ACP Journal Club EBM databases from 1985 to 2004 Study Selection: Inclusion criteria for this study were as follows: 1) An RCT published in English which involves at least one arm with aspirin alone as the treatment group; 2) Upper gastrointestinal bleeding identified as a clinical outcome; 3) Clearly-defined follow-up period of at least 12 months; 4) Aspirin and NSAID groups must be analyzable separately; 5) Defined temporal period of aspirin use. Key word search strategies with Booleans algorithm were employed to the above databases. Results: A total of 5232 abstracts were identified; 333 full texts were further screened. Only $\overline{35 \text { were }}$ RCTs involving aspirin, and of these, 4 identified gastrointestinal bleeding as an outcome with well-defined temporal exposure of aspirin use and clearly delineated follow-up of at least 12 months. A total 12960 patients were involved in 4 trials. Aspirin dose ranged from $50 \mathrm{mg}$ to $1000 \mathrm{mg}$ per day. Average length of follow-up ranged from 12 to 38.2 months. A total of 26826.6 patient-years were observed, with 449 gastrointestinal bleeding events Limitations: Our study utilizes data in which gastrointestinal bleeding was not the primary endpoint, resulting in a population that carries confounding risk factors such as advanced age and use of SSRIs. Further analysis inclusive of retrospective and crossover studies may be beneficial for providing an estimate that is applicable outside the clinical study setting Conclusion: Assuming risk is linear for aspirin and gastrointestinal bleeding, the annualized risk in RCTs was 1.67\% per year.

\begin{tabular}{|c|c|c|c|c|}
\hline Study & $\mathrm{N}$ & $\mathrm{F} / \mathrm{U}(\mathrm{yr})$ & \# GI bleed & Rate/100 patient-yr \\
\hline Kurata 1990 & 2267 & 3.18 & 185 & 2.58 \\
\hline CAPRIE 1996 & 9586 & 1.91 & 255 & 1.39 \\
\hline Meade 1992 & 907 & 1.12 & 2 & 0.22 \\
\hline Silagy 1993 & 200 & 1.00 & 6 & 3.00 \\
\hline Total & 12960 & & 449 & 1.67 \\
\hline
\end{tabular}

$\mathrm{T} 1052$

Watermelon Stomach \& Portal Hypertensive Gastropathy in Patients with UGI Bleeding: Does Histopathology Help in Diagnosis?

Dennis M. Jensen, Wilfred M. Weinstein

For patients with chronic UGI bleeding (B), the diagnosis of Watermelon Stomach (WMS) or portal hypertension gastropathy (PHTG) is usually endoscopic. However, endoscopic biopsies (EndoBx) may help distinguish other non-vascular red antral lesions (MISC-RED) from WMS/PHTG \& may confirm endoscopic diagnoses. Purposes were: 1) to compare the histopathology (Histo) of WMS with PHTG \& MISC-RED lesions resembling WMS \& 2) to delineate whether EndoBx was safe \& helped distinguish WMS/PHTG from MISC-RED antral lesions. Methods: 22 consecutive patients with anemia from recurrent UGIB (10 WMS, 8 PHTG, 4 MISC-RED) gave informed consent for EndoBx \& endoscopic hemostasis. There were 15 females \& 7 males with mean age of 70 yrs (range 40 to 86). EndoBx were taken with large ("jumbo") forceps ( $3.2 \mathrm{~mm}$ diameter, $8 \mathrm{~mm}$ open) in pairs of mid-antral red stripes \& adjacent mucosa \& from the mid-body greater curvature (MBGC). Hemostasis was performed with MPEC or APC of WMS/PHTG \& biopsy sites with continued bleeding EndoBx's were fixed in formalin \& stained with $\mathrm{H} \& \mathrm{E} \&$ trichrome. All biopsies were read from coded sections by one investigator (WMW), without knowledge of the endoscopic subgroup diagnosis or clinical history. Results: Biopsy was safe \& often confirmed the clinical/endoscopic diagnosis of WMS or PHTG. Antral reactive gastropathy (including mucularis mucosae muscle splaying) was noted in all lesions. 90\% WMS \& 100\% PHTG (vs. $0 \%$ of MISC-RED) cases had vascular ectasia in deep mucosa with some penetrating the muscularis mucosae. For WMS or PHTG, oxyntic gland atrophy in MBGC was uncommon $(17 \%) \&$ inflammation was absent or mild. Edema of MBGC or antrum was always $(100 \%)$ seen with PHTG but rare in WMS (10\%) or MISC-RED (0\%). The previously reported focal fibrosis was seen in $30 \%$ of our WMS cases but not other lesions. Conclusion: 1) Endoscopic biopsy was safe $\&$ histology was clinically helpful in distinguishing WMS \& PHTG from MISC-RED antral lesions. 2) Reactive gastropathy was the most uniformly dominant \& frequent finding on antral biopsies for all lesions. 3) Edema without inflammation or atrophy in MBGC was always present in PHTG, but rare in WMS or MISC-RED lesions. 4) Vascular ectasia were seen in all cases of WMS \& PHTG, but not in MISC-RED antral lesions. 5) Although previous reports emphasized superficial location of vascular ectasia in WMS \& PHTG, we found that the most common location was deep in the mucosa \& some penetrated 
the muscularis mucosae. Identification was facilitated by trichrome stain. Partially supported by K24 NIH DK02650 \& CURE Human Studies Core NIH P30 DK41301.

\section{$\mathrm{T} 1053$}

\section{Stress Ulcer Syndrome: A Reappraisal}

Sonal Kumar, Gary R. Zuckerman, Scott T. Micek, Chandra Prakash

Although designated a dreaded complication of ICU stay, the incidence of upper gastrointestinal bleeding (UGIB) from stress ulcers (stress ulcer syndrome, SUS) is thought to be decreasing over the past decades. We reviewed our institutional experience with SUS in the current era of stress ulcer prophylaxis. Methods: The endoscopy database was interrogated to identify subjects with gastric ulcers or erosions with UGIB in five specialty ICUs. Subjects who developed UGIB from gastric erosions/ulcers after ICU admission and without prior peptic disease were extracted to form the SUS study group. Clinical characteristics were compared to ICU admissions with UGIB from non-SUS gastric ulcers/erosions during the same period. Results: Of 12,580 total ICU admissions over a 22 month period, 30 subjects $(0.24 \%, 11 \mathrm{~F} /$ $19 \mathrm{M}$, mean age $60 \pm 0.6 \mathrm{yr}$ ) fulfilled study inclusion criteria for SUS. In contrast, 93 subjects (39F/54M, mean age $65 \pm 1.4 \mathrm{yr}$ ), were admitted to the ICUs with non SUS gastric ulcer bleeding (incidence $0.74 \%, \mathrm{p}<0.001$ compared to SUS). Medical ICUs had the highest incidence of SUS (medical ICU: $0.53 \%$, cardiac ICU: $0.24 \%$, neuro ICU: $0.04 \%$, surgical ICU: $0.13 \%, \mathrm{p}=0.0004$ across groups). SUS prophylaxis was administered in 29 subjects (97\%) prior to the bleeding episode, and included enteral proton pump inhibitors (PPIs) in $80 \%$, IV PPIs in 3\%, enteral and IV histamine 2 receptor antagonists (H2RA) in 7\% each. Subjects had a mean of $3.3 \pm 0.1$ risk factors; $90 \%$ had $\geq 2$ risk factors. Mechanical ventilation was a risk factor in $80 \%$; others included shock $(73 \%)$, coagulopathy $(57 \%)$, sepsis $(53 \%)$, renal failure (47\%), cerebrovascular disease (20\%). Stigmata of bleeding were seen in $53 \%$; endoscopic therapy was performed in $40 \%$, and effective for hemostasis when attempted. There were 11 deaths (37\%), 9 within 30 days of SUS diagnosis. On regression analysis, only the presence of concurrent cerebrovascular lesions predicted 30-day mortality $(\mathrm{p}=0.04)$. Overall mortality rate was $50 \%$ in the medical ICU; other ICUs had lower mortality rates (cardiac ICU $25 \%$, surgical ICU $14 \%$, neuro ICU $0 \%, \mathrm{p}=\mathrm{ns}$ ). Ten subjects who died received enteral PPI prophylaxis, and one received IV H2RA. Mortality rate for non-SUS gastric ulcers was $3.2 \%$ ( $p<0.0001$ compared to SUS), all deaths occurring in the medical ICU. Conclusions: The incidence of SUS is low, if not rare in some specialty ICUs. Despite use of prophylactic PPIs, mortality from SUS is significantly higher than non-SUS gastric ulcers, especially in patients in medical ICUs. Presence of cerebrovascular lesions may predict a higher likelihood of 30-day mortality. Endoscopic therapy is highly successful for short term bleeding control.

\section{$\mathrm{T} 1054$}

Epidemiology of Acute GI Bleeding (AUGIB) Between 1983-85 and 2002-04: A Population-Based Study

Silvano Loperfido, Elena Piovesana, Ludovica Bellina, Katia Rossi, Marzia Groppo, Nadia Dal Bo, Helena Heras, Alessandro Caroli, Fabio Monica, Luisa Maifreni, Adelina Salvador, Luca Fabris, Lajos Okolicsany

Aim: To study in a population the incidence, causes and outcome of AUGIB during two different management eras. Methods: 539 hospitalized pts for AUGIB during 2002-04, were compared with 587 pts in 1983-85 prior to the introduction of endoscopic therapy (ET) in our hospital. Results: The overall incidence decreased from 112/100,000/year in 198385 to 90 in 2002-04: it decreased from 101 to 74 among emergency admissions, while it increased from 11 to 15 among in-hospital pts. The male/female ratio decreased from 2.7 in 1983-1985 to 1.8 in 2002-2004 and mean age increased from $61 \pm 14$ to $68.7 \pm 16 \mathrm{yr}$. The intake of NSAIDs ( $43 \%$ vs $46 \%$ ) and frequency of associated diseases (69\% vs $70 \%$ ) were similar; cirrhosis decreased from $29 \%$ to $20 \%$ ( $<<0.001$ ). Endoscopy was carried out in $96 \%$ in 1983-1985, while in $99 \%$ in 2002-2004. Diagnoses in 1983-1985 and 20022004 were: ulcer bleeding (UB) $59.1 \%$ vs $53.1 \%$, variceal bleeding (VB) $13.8 \%$ vs $11.5 \%$, miscellaneous $22.3 \%$ vs $30.4 \%$, and unknown $4.8 \%$ vs $5.0 \%$. The incidence of UB decreased from $66 / 100,000 / y r$ to 48 . In 2002-04, 208 pts (39\%) received ET, and in a quarter of them ET was repeated. Out of 57 pts with VB, 70\% underwent single techniques and 30\% combined or sequential techniques. Out of 151 pts with non-VB, 34\% underwent single techniques and in $66 \%$ combination techniques were performed. In 2002-04, no pts with VB were treated with vasoactive drugs; in $49 \%$ of pts with non-VB, PPI were used ( $80 \mathrm{mg} /$ $24 \mathrm{hr}$ in most cases) and others received H2-antagonists. Overall, from 1983-85 to 200204, further bleeding decreased from $32 \%$ to $7.4 \%$ ( $<<0.001$ ), surgery from $10 \%$ to $2 \%$ $(\mathrm{p}<0.001)$ and mortality from $15.5 \%$ to $9.1 \%(\mathrm{p}<0.05)$. The mortality decreased from $12.9 \%$ to $5.6 \%$ among emergency admissions ( $\mathrm{p}<0.001$ ), and from $37.7 \%$ to $26 \%$ among in-hospital pts (NS). The table shows the outcomes of VB and non-VB: there was a clear-cut decrease of further bleeding and surgery, while the difference in mortality achieved statistical significance for VB, but not for non-VB. Conclusions: 1) From 1983-85 to 2002-04, mean age increased from 61 to $69 \mathrm{yrs}$; 2) The male/female ratio decreased; 3) Annual incidence decreased overall by $20 \%$ and by $27 \%$ in UB; 4) ET dramatically reduced further bleeding and the need for surgery; 5) Mortality decreased both in pts with VB and non-VB, but the difference achieved statistical significance only for VB. Comparison of outcome between 1983-85 and 2002-04

\begin{tabular}{|c|c|c|c|c|c|c|c|c|c|}
\hline VB & $\mathrm{n}$ & FB & $\mathrm{S}$ & $\mathrm{M}$ & non-VB & $\mathrm{n}$ & FB & $\mathrm{S}$ & $\mathrm{M}$ \\
\hline $1983-85$ & 81 & $62 \%$ & $9.9 \%$ & $43 \%$ & $1983-85$ & 506 & $28 \%$ & $10.2 \%$ & $11 \%$ \\
\hline $2002-04$ & 62 & $15 \%$ & 0 & $18 \%$ & $2002-04$ & 477 & $6.5 \%$ & $2.3 \%$ & $8.0 \%$ \\
\hline p-value & & $<0.001$ & $<0.05$ & $<0.05$ & p-value & & $<0.001$ & $<0.001$ & $\mathrm{NS}$ \\
\hline
\end{tabular}

VB=Variceal Bleeding, FB=Further Bleeding, S=Surgery, M=Mortality

\section{T1055}

A Comparison of Intravenous (IV) Versus Oral Proton Pump Inhibitors (PPIs) for Patients with High-Risk Acute Nonvariceal Upper GI Bleeding (ANVUGIB) Laura E. Targownik, Leila Keyvani, Sanjay K. Murthy, Shanua Leeson

Background: The use of IV PPIs in patients with ANVUGIB following performance of endoscopic hemostasis is currently the standard of care. A 72 hour course of IV PPI following performance of endoscopic hemostasis for high-risk lesions has been shown to decrease the risk of rebleeding when compared to placebo. However, providing PPIs intravenously is associated with high medication costs and the need for a continuous intravenous infusion may prolong hospital stays. High-dose oral proton pump inhibitors have also been proven to be efficacious in reducing the risk of rebleeding for high-risk patients. There are no direct comparisons of IV versus oral PPIs for ANVUGIB patients following endoscopic hemostasis. Methods: We performed a retrospective review of all patients who presented to one of two tertiary care hospitals in Winnipeg, Manitoba from 1999-2004 with an admitting diagnosis of ANVUGIB who were found to have high-risk stigmata on diagnostic upper endoscopy and underwent therapeutic hemostasis. We separated the patients into one of two groups, based on whether they received intravenous PPIs following performance of endoscopy or they received oral PPIs only. Patients were excluded if the first dose of IV or po PPI was given more than $24 \mathrm{~h}$ after endoscopy. The primary outcome measure was the development of any adverse outcome (in-hospital rebleeding, surgery, in-hospital mortality, readmission within 30 days for ANVUGIB). Other outcomes included the time to endoscopy, transfusion requirements both prior to endoscopy and after $24 \mathrm{~h}$ following the initial endoscopy, and the length of hospital stay. Results: We identified 145 subjects who underwent therapeutic hemostasis of whom 59 received IV PPIs and 82 received oral PPI (4 received H2RAs only). There were no significant differences in baseline demographics, or severity of presentation by pre-endoscopic Rockall score. Patients receiving IV PPIs were non-significantly more likely have arterial bleeding or a non-bleeding visible vessel ( $68 \%$ vs. $56 \%, \mathrm{p}=0.17)$. There were no differences in the rates of adverse outcomes ( $22 \%$ IV PPI vs. $18 \%, p>0.2$ ), or likelihood of requiring a transfusion more than $24 \mathrm{~h}$ following initial endoscopy. (29\% vs. $26 \%, p>0.2)$. The overall length of hospital stay was comparable between the two groups. Conclusions. IV PPIs do not appear to provide any incremental benefit over oral PPIs for ANVUGIB patients undergoing endoscopic hemostasis for high risk lesions. Prospective research needs to be performed to determine whether provision of oral PPIs is an acceptable substitute for high risk ANVUGIB patients. Unrestricted Grant from Altana Pharma

\section{T1056}

Prospective Randomized Study Comparing the Effect of Oral Versus IV Pantoprazole On 30-Day Rebleeding Rates in Patients with Acute Non-Variceal GI Bleed: A Pilot Study

Kulwinder Dua, Jasmohan S. Bajaj, Kristin Hanson, Kenneth Presberg

Background: Previous studies have shown that IV proton pump inhibitors (PPI) reduce the rate of rebleeding in patients with acute non-variceal upper GI bleed (NVGIB). Studies have also shown that oral \& IV PPIs are equipotent in raising gastric $\mathrm{pH}$. Since majority of patients with NVGIB can take oral medications and IV PPIs are comparatively expensive and require dedicated IV lines for administration, the aim of the present study was to conduct a pilot study comparing the efficacy of oral vs IV pantoprazole on reducing rebleeding after NVGIB. Method: Consecutive patients with NVGIB were randomized to receive oral (PO) (80 mg BID for 3 days) or IV pantoprazole ( $80 \mathrm{mg}$ IV bolus and $8 \mathrm{mg} / \mathrm{hr}$ infusion for 3 days) followed by pantoprazole $40 \mathrm{mg}$ PO BID for 30 days. All enrolled patients underwent urgent upper GI endoscopy and if required, endoscopic intervention, within 24 hours of admission. Patients were followed for 30 days with the primary endpoint being rebleeding. Secondary endpoints were mortality, end-organ failure and blood transfusions. Results: Twelve patients were randomized to $\mathrm{PO}$ and 13 to IV pantoprazole. There were no significant differences in the age (IV 66.2 \pm 6 vs PO $59.5 \pm 19$ yrs), hematocrit (IV $25.7 \pm 8$ vs PO $24.9 \pm 9$ ), INR (IV $1.4 \pm 1$ vs PO $1.1 \pm 0.2$ ), Rockall scores (IV $5.3 \pm 2.5$ vs P0 $4.5 \pm 2.1$ ) and comorbidity scores (IV $3.3 \pm 2.6$ vs PO $2.2 \pm 1.9$ ) between the groups. Ulcer characteristics and therapeutic interventions were similar across groups (Table 1). Two patients in the IV arm rebled. Both these patients had adherent clot and underwent BICAP cautery and epinephrine injection on initial endoscopy and were retreated endoscopically after rebleeding. Another patient in the IV arm had reversible renal failure (Table 2). No patient in the PO arm had to be changed to IV pantoprazole. Conclusion: In this pilot study, the effect of oral pantoprazole on 30-day rebleeding rate in patients with NVGIB was similar to IV pantoprazole. Further trials with larger study populations are needed. Table 1

\begin{tabular}{|c|c|c|}
\hline ULCER STIGMATA & IV $(\mathrm{n}=13)$ & $\mathrm{PO}(\mathrm{n}=12)$ \\
\hline Active bleeding & 4 & 1 \\
\hline Visible vessel & 1 & 2 \\
\hline Adherent clot & 3 & 3 \\
\hline Flat spot & 0 & 2 \\
\hline Clean base & 5 & 5 \\
\hline THERAPEUTIC INTERVENTION & 7 & 5 \\
\hline
\end{tabular}

$\mathrm{p}=0.32$ Fisher exact test

Table 2

\begin{tabular}{|c|c|c|c|c|}
\hline Pantoprazole & Rebleeding & Organ Failure & Mortality & Blood Transfusion \\
\hline IV $(\mathrm{n}=13)$ & $2(15 \%)$ & $1(8 \%)$ & $0(0 \%)$ & $3.9 \pm 3.7$ \\
\hline Oral $(\mathrm{n}=12)$ & $0(0 \%)$ & $0(0 \%)$ & $0(0 \%)$ & $4.1 \pm 4.0$ \\
\hline
\end{tabular}

$\mathrm{p}=0.48$ Fisher exact test 
T1057

Eradication of Helicobacter Pylori for the Prevention of Ulcer Bleeding Recurrence

Javier P. Gisbert, R Garcia-Gravalos, D Olivares, X Calvet, F Feu, Jm Pique, A Cosme, F Bory, N Canete, S Santolaria, M Montoro, A Benages, P Almela, F Borda, R Aznarez, M Castro, A Perez-Aisa, N Fernandez

AIM: Eradication of $H$. pylori is associated with a very low rate of ulcer recurrence. Our purpose was to verify the effect of $H$. pylori eradication on ulcer bleeding recurrence secondary to peptic ulcer disease. METHODS: Patients with acute hemorrhage secondary to gastroduodenal ulcer were prospectively included. NSAID use was not considered an exclusion criteria. H. pylori infection was confirmed by rapid urease test, histology or 13C-urea breath test. Several therapies were used, mainly omeprazole or ranitidine-bismuth citrate based regimens. Afterwards, an $\mathrm{H} 2$-antagonist (ranitidine $150 \mathrm{mg}$ o.d.) was administered until eradication was confirmed by $13 \mathrm{C}$-urea breath test 8 weeks after completing eradication therapy. Patients with therapy failure received a second or third course of therapy. Patients with eradication success did not receive maintenance anti-ulcer therapy, and were controlled yearly up to 5 years with a 13C-urea breath test. NSAID use was not permitted during follow-up. RESULTS: Up to now, 238 patients have been followed up for at least 12 months, with a total of 410 patient-years of follow-up. Mean age was 59 years, $73 \%$ were males, and $39 \%$ were previous NSAID users. Seventy-one percent had duodenal ulcer, $22 \%$ gastric ulcer, and $8 \%$ pyloric ulcer. Recurrence of bleeding was demonstrated in 2 patients at 1 year (incidence: 0.49\% per patient-year of follow up), which occurred after NSAID use in both cases. CONCLUSION: Rebleeding does not occur in patients with complicated ulcers after $H$. pylori eradication. Maintenance anti-ulcer (antisecretory) therapy is not necessary if eradication is achieved. However, NSAID intake may cause rebleeding in $\mathrm{H}$. pylori-eradicated patients.

\section{$\mathrm{T} 1058$}

Coffee Ground Vomit - Can We Spot It?

Christian P. Selinger, Nicola M. Jones, Cornelle R. Parker

Introduction: It is assumed that healthcare professionals can identify coffee ground vomit as a sign of gastrointestinal (GI) bleeding. However, there is no published evidence to support this. Aims \& Methods: The aim of this study was to investigate the sensitivity and specificity of nurses' and physicians' assessment of coffee ground vomit. Photographs of nine samples of vomitus were obtained from medical inpatients. Two samples were classified as positive with signs of upper GI bleeding on endoscopy. Two negative samples were from patients suspected of bleeding with normal endoscopy. A further five negative samples were from patients suffering from other medical conditions with no clinical evidence (tachycardia, postural hypotension, drop in haemoglobin, rise in urea) of GI bleeding. One hundred and one healthcare professionals (56 nurses, 45 doctors) were asked to assess these pictures with the question: "Is this a coffee ground vomit from a significant GI bleed?" Results: The pictures of samples with confirmed bleeding were correctly identified in 197 cases and missed in five cases. Whereas the pictures of non coffee ground vomit were identified correctly in 419 cases and interpreted false positively in 288 cases. This results in a sensitivity of $97.5 \%$, specificity of $59.3 \%$, a positive predictive value of 0.41 and a negative predictive value of 0.99 . Subgroup analysis did not show a significant difference in assessment by nurses and physicians and by staff with different levels of experience. Conclusion: Coffee ground vomit is difficult to identify with certainty. High negative predictive value allows ruling out of a significant bleed. Low positive predictive value however does not allow the diagnosis of a GI bleed solely based on vomit appearance. Further clinical parameters and a risk factor profile should be taken into consideration when deciding on referral for upper GI endoscopy.

\section{T1059}

Cardiac Scoring Systems Predict Morbidity and Mortality in Patients with Acute Gastrointestinal Bleeding and Acute Coronary Artery Disease Praveen Rapolu, Tejal Shah, J.N. Preis, Jian Jun Li, Scott Tenner

The morbidity and mortality of patients with acute gastrointestinal bleeding (GI) is increased in the setting of acute coronary syndromes (CS), such as unstable angina and acute myocardial infarction. The management of patients with these disorders is further complicated by medications that increase the risk of bleeding while decreasing the risk of death from coronary artery disease. In order to better determine the factors that affect mortality in patients with Acute GI bleeding and Acute CS, we followed 100 consecutive patients who suffered from both gastrointestinal bleeding and acute coronary syndromes during the first week of hospitalization. One-hundred consecutive patients were studied. The mean age of the patients was $75.4+12.2,45$ female, 55 male. Twenty patients presented with acute gastrointestinal bleeding initially and developed acute coronary syndromes over the ensuing days. Eighty patients presented with acute coronary syndromes and subsequently developed gastrointestinal bleeding. There were 15 deaths within the hospitalization, all attributable to the underlying gastrointestinal bleeding and coronary artery disease. In order to determine independent variables that were associated with mortality, multiple regression analysis was performed. The risk of death was significantly associated with age $(\mathrm{p}=0.02)$, Goldman Criteria $(0.01)$, NYHA Congestive Heart Failure Class $(\mathrm{p}=0.02$ ). Eighty-six patients underwent upper endoscopy due to significant gastrointestinal bleeding. Clean based ulcers were found in $30 / 86$ patients $(34 \%$ ), varices in $3 / 86$ patients $(3.4 \%$ ). Stigmata or recent hemorrhage or active bleeding was found in 17/86 patients (19\%). Admission hematocrit, the presence of coagulopathy, and presenting vital signs were not associated with death. The time interval from admission to correcting the hematocrit, coagulopathy and vital signs did not correlate with mortality. Mortality was not associated with aspirin, clopidogrel, or anticoagulation with heparin, coumadin, thrombolytics, or IIb/llla inhibitors. We conclude that in the setting of acute gastrointestinal bleeding and acute coronary syndromes, mortality is related to the patients age, admission tropinin, the presence of congestive heart failure, renal failure and The Goldman Criteria. Gastroenterologists must become familiar with the Goldman Criteria and New York Heart Association Congestive Heart Failure Classification which appear to be most predictive of mortality in patients with acute gastrointestinal bleeding and acute coronary syndromes.

\section{$\mathrm{T} 1060$}

Success of Argon Plasma Coagulation for Management of Gastric Antral Vascular Ectasia

Kuldip S. Banwait, Madhusudhan R. Sanaka, Kuntal M. Thaker, Karl Kwok, Stephanie Moleski, David Assis, Thomas Kowalski, David Loren

Background: The gastric antral vascular ectasia (GAVE) syndrome is an uncommon cause of anemia and GI bleeding. A subset of patients with GAVE will require endoscopic therma ablation to manage bleeding complications and argon plasma coagulation (APC) has increasingly become the therapeutic modality of choice. We hypothesize that the success of thermal ablation may be affected by the coexistence of portal hypertensive gastropathy and present what we believe to be the largest reported experience using APC for the management of GAVE. Aims: The aims of this study were to evaluate the efficacy of APC in treating GAVE in a large patient cohort and to assess the impact of portal hypertensive gastropathy on the clinical success of ablation therapy. Methods: A retrospective review of all EGD reports from a single tertiary care hospital was performed between 2000 and 2005. All patients in whom GAVE was diagnosed based upon endoscopic findings were included in the analysis. The clinical presentation, number of treatment sessions with APC, response to therapy, and clinical recurrence were recorded. The chi square test and Students t-test were used where appropriate. Results: 24,349 upper endoscopies were performed during the study period o which $133(0.55 \%)$ patients were diagnosed with GAVE. The mean age of affected individuals was 65.2 yrs (range 34-99) compared to 57.7 yrs for the entire cohort. Women comprised $62.5 \%$ of affected individuals and represented $56.3 \%$ of all patients undergoing upper endoscopy. The most common presentation was GI bleeding (35.5\%) followed by anemia (30.9\%). Of patients with GAVE, 17.3\% (23/133) were diagnosed with coexistent portal hypertensive gastropathy. 69/133(51.9\%) of affected patients underwent ablation therapy with APC with a mean of 2.2 sessions per patient (median=2). 34/69 patients $(49.3 \%$ ) required only a single ablative session to manage bleeding or anemia. Of those requiring multiple sessions, the mean number was 3.5 (median=3, range=2-12). The mean interval between sessions was 6.3 months. When patients were stratified according to the coexistence of portal hypertensive gastropathy there was no significant difference in the percent of patients requiring therapy or the mean number of sessions required. No procedure related complications were observed. Conclusions: APC is a safe and effective treatment modality for the management of GAVE. The coexistence of portal hypertensive gastropathy does not appear to affect clinical outcomes of those requiring ablative therapy. Our results are similar to those of the previously reported smaller cohorts of patients with GAVE treated with APC

\section{T1061}

Accuracy of H. Pylori Diagnostic Tests in Patients with Bleeding Peptic Ulcer: A Systematic Review and Meta-Analysis

Javier P. Gisbert, Victor Abraira

OBJECTIVE: To perform a systematic review and a meta-analysis of diagnostic accuracy of the different tests aimed to detect $H$. pylori infection in patients with upper gastrointestinal bleeding (UGIB). METHODS: Selection of studies: assessing the accuracy of H. pylori diagnostic methods in patients with UGIB. Search strategy: electronic bibliographical searches Data extraction: independently done by two reviewers. Data synthesis: meta-analyses of the different tests were performed combining the sensitivities, specificities, and likelihood ratios (LRs) of the individual studies. RESULTS: Studies showed a high degree of heterogeneity. Pooled sensitivity, specificity, LR+ and LR- (95\% confidence interval) for the differen methods were: Rapid urease test (16 studies/1,417 patients): 0.67 (0.64-0.70), 0.93 (0.90$0.96), 9.6(5.1-18.1)$ and $0.31(0.22-0.44)$. Histology (10 studies/827 patients): 0.70 (0.66$0.74), 0.90(0.85-0.94), 6.7(2.5-18.4)$ and $0.23(0.12-0.46)$. When subanalysis depending on the biopsy site was performed, and only studies with samples obtained from both the antrum and corpus were considered, heterogeneity substantially decreased, and pooled sensitivity for rapid urease test and histology increased ( 0.78 and 0.83 , respectively). Culture (3 studies/314 patients): $0.45(0.39-0.51), 0.98(0.92-1.00), 19.6(4-96)$ and $0.31(0.05-$ 1.9). Urea breath test (8 studies/520 patients): $0.93(0.90-0.95), 0.92$ (0.87-0.96), 9.5 (3.9$23.3)$ and $0.11(0.07-0.16)$. Stool antigen test (6 studies/377 patients): $0.87(0.82-0.91)$ $0.70(0.62-0.78), 2.3(1.4-4)$ and $0.2(0.13-0.3)$. Serology (9 studies/803 patients): 0.88 $(0.85-0.90), 0.69(0.62-0.75), 2.5(1.6-4.1)$ and $0.25(0.19-0.33)$. CONCLUSION: Biopsybased methods, such as rapid urease test, histology and culture, have a low sensitivity, but a high specificity, in patients with UGIB. Biopsy samples from both the antrum and corpus may be recommendable, as with this strategy sensitivities of rapid urease test and histology seem to be higher. The accuracy of 13C-urea breath test remains very high in these patients. Stool antigen test is less accurate in UGIB. Although serology seems not to be influenced by UGIB, it cannot be recommended as the first diagnostic test for $H$. pylori infection in this setting.

\section{T1062}

The Mallory Weiss Tear

John Thomson, David Armour, Andrew Fraser, John Masson, Norman A. Mowat

Introduction Bleeding from Mallory Weiss Tear (MWT) is generally considered to be trivial, self-limiting and rarely requiring acute intervention. There is, however, only limited community based published data to support this benign perception. The 10 year prospectively collected data from the Aberdeen Gastrointestinal Bleeding Unit (AGIBU) has given us the opportunity to evaluate this further. Aim To evaluate the natural history of Mallory Weiss Tears in the stable at risk population $(458,536)$ of Grampian over a 10 year period. Method Prospectively collected data on all admissions with gastrointestinal bleeding to the AGIBU were stored on a Microsoft Access ${ }^{\circledR}$ database. Analyses were then performed on the data collected from all admissions with bleeding from Mallory Weiss Tears. Results From October

. 
1991 to October 2001, 9.3\% ( $n=658$ ) of admissions to the AGIBU, with suspected upper GI bleeding, bled from a MWT. A positive correlation $(\mathrm{p}<0.0001)$ was seen with young male patients, acute and chronic alcohol intake, smoking and a history of vomiting when compared to the other AGIBU admissions. Of those with haematemasis, $36 \%$ did not have a prior history of retching or vomiting. The bleeding episode was defined as significant (haemodynamic compromise, $\mathrm{Hb}<10 \mathrm{~g} / \mathrm{dl}$ ) in $36.7 \%$. The proportion with significant bleeding increased with age $(p<0.01)$ and was more common in females $(p<0.01)$. The majority had a short hospital admission with no complications. However $5 \%(n=28)$ rebleed within 30 days and $1.71 \%(n=9)$ died within 30days. All of the deaths were associated with significant comorbid disease. Conclusions Contrary to popular belief bleeding from MWT is not only common but is often significant. It often occurs without retching or prior vomiting and can be associated with rebleeding. There is a small but significant mortality.

\section{T1063}

Trends in the Incidence and Outcomes of Acute Non-Variceal Upper Gastrointestinal Bleeding (ANVUGIB) in Canada, 1994-2003 Laura E. Targownik, Alice Nabalamba

Background: ANVUGIB is a common reason for admission to hospital, and has been traditionally associated with a mortality rate of $5-10 \%$. There have been numerous innovations in the prevention and management of ANVUGIB in the last 10 years, though the magnitude of the effect of these innovations in unknown. Methods: We used the Canadian Institute of Health Information (CIHI) hospital database, which is contains data characterizing every inpatient hospital admission in Canada from April 1994 until March 2003. We identified admissions consistent with non-variceal upper gastrointestinal bleeding using a validated list of ICD-9 and ICD-10 codes. Data was extracted concerning patient demographics, length of hospital stay, incidence of surgery for complications of upper gastrointestinal bleeding, and overall mortality. Results: Between 1994 and 2003, the incidence of ANVUGIB decreased from 76.4 to 52.8 cases per $100,000 / \mathrm{yr}$. The decrease in incidence rates by age groups and year of admission are shown in Figure 1. The mean age of persons admitted with ANVUGIB rose from 62.06 to 66.77 years. The mean length of hospital stay decreased from 7.63 to 6.62 days, and the proportion of patients with hospital stays of less than 2 days increased from $22 \%$ to $33 \%$ of all admissions. The mortality rate remained constant at approximately 5\% between 1994 until 2003. Conclusion: The incidence of ANVUGIB appears to be steadily declining since 1994 in all groups, though there has been relatively little impact on mortality. The decreasing incidence of ANVUGIB may be due to a decreasing prevalence of Helicobacter pylori infection and increasing use of gastroprotective medications. The lack of effect on mortality suggests changes in management undertaken in this era have had little impact on clinical outcomes. Decreasing lengths in hospital stays may be reflective of adoption of early discharge guidelines for low-risk patients.

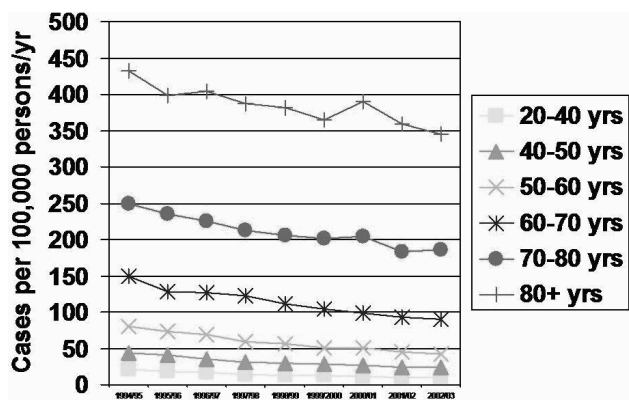

T1064

Are Patients with Acute Non-Variceal Upper GI Bleeding (ANVUGIB) Presenting Outside of Regular Hours Receiving Quality Care Laura E. Targownik, Leila Keyvani, Sanjay K. Murthy, Shanua Leeson

Background: ANVUGIB is among the most common reasons for presentation to a hospital's emergency room. The standard of care for patients presenting with signs and symptoms of ANVUGIB is to receive prompt resuscitation and early endoscopy to confirm the diagnosis and to provide endoscopic therapeutics. While endoscopists are generally on site during regular working hours, they generally provide "on call" services on evenings and weekends, only coming into hospital if endoscopy is required. Therefore, it is possible ANVUGIB patients presenting to hospital overnight or on the weekend may not receive the same level of care as those presenting when endoscopists are "in house". This disparity could result in poorer outcomes and longer hospital stays for patients presenting at night or on weekends. Therefore, we sought to determine if there are significant differences in the process and outcomes of care between ANVUGIB patients presenting during regular hours and those arriving while endoscopists are "on call" Methods: We performed a retrospective review of all patients presenting to one of two tertiary care hospitals in Winnipeg, Manitoba from 1999-2004 with a admitting diagnosis of ANVUGIB who underwent diagnostic upper endoscopy. We separated the patients into one of two groups, based on whether they presented to the emergency room during regular working hours (Mon-Fri 8am-5pm), or during "call" (all other times). The primary outcome measure was the development of any adverse outcome (in-hospital rebleeding, surgery, in-hospital mortality, readmission within 30 days for ANVUGIB). Other outcomes included the time to endoscopy, the need for endoscopic hemostasis, and the length of hospital stay. Results: We analyzed 500 subjects of whom 195 presenting during regular hours, and 305 presented while the endoscopist was on call. There were no significant differences in baseline demographics, severity of presentation by pre-endoscopic Rockall score, or likelihood of receiving endoscopy within $8 \mathrm{~h}$, or likelihood of undergoing endoscopic hemostasis. There were no differences in the incidence of adverse outcomes
( $19 \%$ vs. $20 \%, P>0.2$ ) or in the mean length of hospital stay. Patients presenting from $11 \mathrm{pm}$ $8 \mathrm{am}$ did not fare worse than those presenting from $5 \mathrm{pm}-1 \mathrm{lpm}$. Also, sicker patients (Preendoscopic Rockall Score >=3) fared equally well independent of time of presentation. Conclusions. ANVUGIB patients presenting outside regular hours do not suffer any compromise in the quality of care. It is reasonable for endoscopists to provide "on-call" services outside regular hours instead of remaining "in house."

\section{T1065}

The Effect of Various Doses of Pantoprazole On the Intragastric Acidity for Preventing Ulcer Rebleeding After Endoscopic Therapy in Korean Patients Myung-Gyu Choi, Jung-Hwan Oh, Jae-Myung Park, Chang-Nyol Paik, Joon-Wook Lee, Yu-Kyung Cho, In-Seok Lee, Sang-Woo Kim, Hae Won Han, Kyu Yong Choi, In-Sik Chung, Mi-Sook Dong

Background/Aim: Proton-pump inhibitor(PPI) therapy for ulcer bleeding has been more efficacious among Asian than non-Asian patients. This may be explained by an enhanced pharmacodynamic effect of PPIs in Asian patients related to lower parietal cell mass, ae well as high prevalence of both the slow metaboliser phenotype or of $H$. pylori infection. The aim of this study was to evaluate the effect of various doses of pantoprazole on the intragastric acidity for preventing ulcer rebleeding after endoscopic therapy in Korean patients. Methods: We enrolled 49 patients with bleeding pectic ulcers after successful endotherapy and 40 patients receiving endoscopic mucosal resection. Patients were randomly assigned to intravenously receive one of the 4 regimens: pantoprazole $40 \mathrm{mg}$ bolus qd, pantoprazole $40 \mathrm{mg}$ bolus bid, high dose of pantoprazole ( $80 \mathrm{mg}$ bolus then continuous $8 \mathrm{mg} / \mathrm{hr}$ ) and ranitidine $300 \mathrm{mg}$ continuous. Twenty-four hour intra-gastric $\mathrm{pH}$ monitoring was undertaken after second-look endoscopy. Genetic polymorphism of CYP2C19 and the presence of H. pylori were tested. Results: No patient in each group underwent surgery or died. Two patients had rebleeding after EMR. Pantoprazole groups, except pantoprazole $40 \mathrm{mg}$ qd, were superior acid inhibitions compared to ranitidine ( $\mathrm{p}<0.05$, table). A pantoprazole $40 \mathrm{mg}$ qd bolus provided worse intragastric acid control in the day's last half compared to the lst half ( $\mathrm{p}<$ 0.05 ), whereas there were no differences in acid control between the lst and 2 nd half of the day in the other regimens. The significant inter-individual variation of acid inhibition was observed in a low dose of pantoprasole, which was significantly dependent on the CYP2C19 genotype. There was no statistical difference in the medians of $24-\mathrm{h}$ pH values between pantoprazole $40 \mathrm{mg}$ bid and the high doses regardless of the status of $H$. pylori infection. Median intragastric $\mathrm{pH}<4$ was observed in only two patients of $40 \mathrm{mg}$ bid group $(\mathrm{N}=30)$, who were extensive metabolizer and $H$. pylori positive. Conclusion: Even a daily $40 \mathrm{mg}$ bid dose of pantoprazole sufficiently keeps the $\mathrm{pH}$ almost constantly $>6$ in Korean patients, except in a small number of patients with extensive metabolizer. The effect of various doses of pantoprazole on the intragastric acidity

\begin{tabular}{|c|c|c|c|c|}
\hline & $\begin{array}{c}\text { pantoprazole } 40 \mathrm{mg} \\
\mathrm{qd}(\mathrm{N}=25)\end{array}$ & $\begin{array}{c}\text { pantoprazole } 40 \mathrm{mg} \\
\text { bid (N=30) }\end{array}$ & $\begin{array}{c}80 \mathrm{mg}+8 \mathrm{mg} / \mathrm{hr} \\
\text { pantoprazole }(\mathrm{N}=13)\end{array}$ & $\begin{array}{c}\text { ranitidine 300 } \\
\mathrm{mg} / \mathrm{d}(\mathrm{N}=21)\end{array}$ \\
\hline $\begin{array}{c}\text { Median } \\
\mathrm{pH}\end{array}$ & $5.2 \pm 2.4$ & $6.4 \pm 1.3^{*}$ & $6.6 \pm 0.5^{*}$ & $4.4 \pm 2.4$ \\
\hline $\begin{array}{c}\% \text { time } \mathrm{pH} \\
>4\end{array}$ & $72.9 \pm 32.3$ & $89.5 \pm 21.3^{*}$ & $97.0 \pm 6.0^{*}$ & $59.7 \pm 33.9$ \\
\hline $\begin{array}{c}\% \text { time } \mathrm{pH} \\
>5\end{array}$ & $69.2 \pm 34.7$ & $86.6 \pm 23.3^{*}$ & $94.5 \pm 10.9^{*}$ & $55.7 \pm 35.2$ \\
\hline $\begin{array}{c}\% \text { time } \mathrm{pH} \\
>6\end{array}$ & $60.4 \pm 37.2$ & $75.8 \pm 26.7^{*}$ & $80.6 \pm 23.4^{*}$ & $46.0 \pm 35.8$ \\
\hline
\end{tabular}

mean $+\mathrm{SD}, *<0.05$ compared to ranitidine

T1066

Gastric Antral Vascular Ectasia: 75 Cases with Long Term Follow-Up Abdullah M. Al-Osaimi, Christopher W. Lawrence, Li H. Tri, Lawrence W. Comerford, Patrick G. Northup, Stephen H. Caldwell, Stephen J. Bickston

Background and Aims: Gastric antral vascular ectasia (GAVE) is an increasingly recognized entity involving mainly the gastric antrum that can be associated with significant gastrointestinal blood loss in patients with and without liver cirrhosis. Studies have been sparse on this entity. The aim of our study was to review the clinical features of this disease in a University based population including demographic features, presenting symptoms, associated diseases, endoscopic findings, methods of management, and the final outcomes when possible. Methods: A search of our institution's Clinical Data Repository between July 1997 and June 2002 using the ICD codes for nonbleeding and bleeding GAVE identified 278 patients. A review of the medical records distilled this to 75 patients whose records clearly substantiated the diagnosis. Results: 75 patients were followed for a mean of $32 \pm 27$ SD months. The majority of GAVE patients were females $77 \%$. The mean age was $69 \pm 10$ SD (range: 45-96) years, 47 (92\%) were Caucasian. Anemia was present in 63\%, while $21 \%$ had overt GI bleeding and $15 \%$ were discovered incidentally. Among symptomatic patients, the symptoms were present a mean of $12 \pm 12$ months before diagnosis. Cirrhosis was documented in $53 \%$ of patients; nonalcoholic steatohepatitis (NASH) in $48 \%$, cryptogenic cirrhosis in $30 \%$ and alcohol in $10 \%$ as the main etiologies. Endoscopic findings include watermelon type in $61 \%$, diffuse type in $28 \%$, and nodular type of GAVE in $11 \%$. Active bleeding was in $23 \%$, portal hypertensive gastropathy in $40 \%$, and esophageal varices in $37 \%$ of these patients. Patients were managed endoscopically in $57 \%$, of which 36 sessions were argon plasma coagulation, 15 bipolar electrocautery, and 4 Yttrium laser ablations. $43 \%$ of patients who underwent endoscopic therapy required multiple sessions with a mean of $4.2 \pm 3.3$ sessions. One patient underwent orthotopic liver transplantation for advanced liver disease with resolution of his GAVE. Conclusion: Because of the limitations of current therapy, GAVE remains a challenge to clinicians. Further analysis of this population may help identify patients at risk and to clarify which approaches to treatment are most promising. Regarding etiology, the proportion of patients with liver disease as an associated condition in our center 
is congruent with earlier reports. However, it is noteworthy that a substantial proportion of patients had either NASH, cryptogenic cirrhosis or alcohol-related liver disease -disorders united by abnormalities in fat metabolism. This suggests that the metabolic derangements caused by lipotoxicity with associated mitochondrial dysfunction may therefore underlie many cases of GAVE.

\section{$\mathrm{T} 1067$}

High-Dose Oral Proton Pump Inhibitor Is As Effective As Intravenous Administration in the Aspect of Increasing Intragastric $\mathrm{pH}$ and Reducing Rebleeding After Endoscopic Treatment of Bleeding Peptic Ulcers Jae-Young Jang, Seok Ho Dong, Ji Heon Jung, Myung Jong Chae, Nam Hoon Kim, Sang Kil Lee, Kwang Ro Joo, Hyo Jong Kim, Byung-Ho Kim, Young-Woon Chang, Joung Il Lee, Rin Chang

Badkground/Aims: The use of proton pump inhibitor (PPI) prevents rebleeding by elevating intragastric $\mathrm{pH}$ in patients with bleeding peptic ulcers after hemostasis has been achieved. We assessed that the high-dose oral pantoprazole is as effective as high-dose intravenous pantoprazole on 24-hour intragastric $\mathrm{pH}$ and determined their ability to prevent rebleeding after having achieved initial hemostasis in patients with active bleeding or nonbleeding visible vessels. Methods: Forty patients with bleeding peptic ulcers, who had obtained initial hemostasis, were enrolled in this randomized controlled trial. In the high-dose oral pantoprazole group $(n=20), 40 \mathrm{mg}$ of pantoprazole was given orally twice daily for 5 days. In the high-dose intravenous pantoprazole group $(n=20), 80 \mathrm{mg}$ intravenous bolus of pantoprazole was given, followed by $8 \mathrm{mg}$ /hour of continuous infusion daily for 3 days. Thereafter, $40 \mathrm{mg}$ of pantoprazole was given orally once daily for 8 weeks. A pH meter was inserted in each patients' fundus and then the pantoprazole was administered within 5 minutes (10 patients in oral group and intravenous group, respectively). Results: The two groups were similar with respect to all background variables. Rebleeding occurred in 2 patients $(10.0 \%)$ in the intravenous group and did not occur in the oral group by day 30 after enrollment $(\mathrm{p}=0.154)$. There was no significant difference in terms of therapeutic endoscopic sessions ( 1 vs. $1.18 \pm 0.53$ ), surgery ( $0 \%$ vs. $0 \%$ ), mortality ( $0 \%$ vs. $5.9 \%$ ), and mean number of units of blood transfused (1.9 vs. 1.9 pints). Pantoprazole maintained the mean gastric $\mathrm{pH}$ for 24 hours at the level of $4.8 \pm 1.4$ in the oral group, as compared with $5.7 \pm 0.3$ in the intravenous group $(\mathrm{p}<0.001)$. The duration of intragastric $\mathrm{pH}$ higher than 6.0 was longer in the intravenous group $(64.4 \% \pm 38.3 \%)$ than that of the oral group $(43.7 \% \pm 26.2 \%, p<0.005)$. But, after 7 hours of administration, there was no significant difference in the mean gastric $\mathrm{pH}$ (oral; $5.6 \pm 0.6$ vs. intravenous; $5.8 \pm 0.2, \mathrm{p}=0.554$ ). Conclusions: The high-dose oral pantoprazole is as effective as intravenous administration in increasing intragastric $\mathrm{pH}$ and reducing rebleeding episodes in patients with bleeding peptic ulcers after successful endoscopic therapy.

\section{T1069}

Prognosis Prediction of Stage II Colon Cancer By Gene Expression Profiling Alain Barrier, Antoinette Lemoine, Didier Brault, Sidney Houry, Francois Lacaine, Sandrine Dudoit, Antoine Flahault

Background and aims. The aims of the present study were: 1) to identify a prognosis signature (PS), based on microarray gene expression measures, in stage II colon cancer patients and to assess its accuracy with resampling techniques ; 2) to assess the accuracy, also with resampling techniques, of a previously proposed 23-gene PS. Material and methods. Colon tumor mRNA samples from 50 patients were profiled using the Affymetrix HGU133A GeneChip (22283 sequences). In a first part, the 50 patients were randomly divided into 2 groups (G1 and G2) of equal size that were considered alternately as training and validation sets. In a second part, the 50 patients were randomly divided into 1600 training (size=n) and validation (size $=50-n$ ) sets. Informative genes were selected on the training set by taking the 30 most differentially expressed genes between patients who recurred and those who remained disease-free; the accuracy of this PS was assessed by comparing the predicted prognosis (using a diagonal linear discriminant analysis (DLDA)) and the actual evolution for all the validation set patients. Using the same random splits, the accuracy of the 23gene PS was assessed with a DLDA that used learning set patients as reference samples. Results. The 30-gene PS that was identified from G1 (G2) patients yielded a $80 \%$ (84\%) prognosis prediction accuracy when applied on G2 (G1) patients. With resampling techniques, the prediction accuracy regularly increased with the learning set (LS) size: $65.5 \%$ (range $=52.5-75 \%$ ) with LS of size 10 , and $82.7 \%$ (range $=60-100 \%$ ) with LS of size 40 Comparisons of compositions of the $100 \mathrm{PS}$ for a given value of $\mathrm{n}$ suggested a high instability of informative genes; with LS of size 10, 7 genes were part of at least $10 \%$ of signatures; with LS of size 40, 7 genes were part of all the 100 signatures. The accuracy of the previously proposed 23-gene PS also increased with the learning set size. Conclusion. Microarray gene expression profiling represents a promising technique to predict the prognosis of stage II colon cancer patients. The present study also outlines the high instability of informative gene selection and suggests the usefulness of resampling techniques to obtain an honest assessment of prognosis prediction accuracy.

\section{$\mathrm{T} 1070$}

Prognostic Effects of Orotate Phosphoribosyl Transferase and Dihydropyrimidine Dehydrogenase in Colorectal Cancer After Surgery Yukihiko Tokunaga, Hirokazu Sasaki, Tohru Saito

Background and Objectives: Orotate phosphribosyl transferase (OPRT) is an essential enzyme for activation of 5-fluorouracil (5-FU) and its derivatives. Dihydropyrimidine dehydrogenase (DPD) is a rate-limiting enzyme for degradation of 5-FU. In colorectal cancer (CRC), few studies have evaluated the relationship between OPRT, DPD, and clinicopathological features, since monoclonal antibody sensitive for human OPRT and that for DPD have not been established. Now, new antibodies for human OPRT and DPD are available. Methods: The study included 150 patients whose CRCs were classified into stage II to IV, and resected surgically. OPRT and DPD expression were evaluated using immunohistochemistry with new antibodies. Relationships between their expressions and clinicopathological features Survival curves were calculated using Kaplan-Meier method, and differences were evaluated with log-rank test. Cox proportional hazards model was also used. Results: OPRT expression showed a negative correlation with advances in venous invasion $(\mathrm{p}=0.041)$, though DPD expression showed positive correlations with advances in venous invasion $(\mathrm{p}=0.0053)$, and cancer stage $(\mathrm{p}=0.0064)$. The patients survival rates were higher in those OPRT $(+)$ than in those OPRT $(-)(\mathrm{p}=0.004)$, and higher in those DPD $(-)$ than in those $\mathrm{DPD}(+)(\mathrm{p}=0.008)$. In regard to the combination of OPRT and DPD expression, the best survival curve was obtained for the OPRT(+) DPD(-)group followed by those for the OPRT(-) DPD(-), OPRT(+) DPD(+) and OPRT(-) DPD(+) groups in descending order. The estimated hazard ratio for patients death with OPRT and DPD expression were 2.43 and $6.55(\mathrm{p}=0.0047$ and 0.0096$)$ respectively. Conclusions: Using new sensitive antibodies, the present results indicated that OPRT expression was negatively associated with CRC progression and related with better prognosis in postoperative CRC patients, although DPD expression was positively correlated with CRC progression and related with poor prognosis. The overall patients survival rates were best in the patients OPRT(+)DPD(-), and worst in those OPRT(-)DPD (+) in treatment with fluoropyrimidine after surgery. The use of two determinants of response may identify a high percentage of responding patients.

\section{T1071}

Pathological Classification and Clinical Behavior of 63 Cases of Goblet Cell Carcinoids of the Appendix

Laura H. Tang, Jinru Shia, Douglas D. Wong, Philip Paty, Martin Weiser, Jose Guillem, Larissa Temple, Antonio Picon, Leslie Sobin, Klimstra D

Appendiceal tumors exhibiting neuroendocrine differentiation, mucin production and glandular differentiation are uncommon and have caused difficulty in pathological classification, prediction of prognosis, and the clinical management. They are variously designated as adenocarcinoid, goblet cell carcinoid (GCC), and mixed adenocarcinoma-carcinoid. We undertook a retrospective investigation of 63 such cases and classified them into low grade GCC, high-grade GCC, and mixed GCC-adenocarcinoma based primarily on their histological features. The clinical characteristics and prognosis were compared within the 3 subtypes and with the conventional appendiceal adenocarcinoma. The mean followup time was $49 \pm 5$ months. The stage IV-matched disease specific survival is demonstrated in Table 1. Our studies demonstrate that utilization of specific morphological criteria may predict the clinical behavior of GCCs. Both low grade and high grade GCCS share a similar immunoprofile, which includes preserved immunoreactivity for neuroendocrine markers, a normal intestinal type mucin glycoprotein profile (Table 2). The proliferative index (Ki67) is relatively low in this group of tumors, and is slightly increased from low grade to high grade tumors. The mixed GCC-adenocarcinoma demonstrated markedly increased proliferative activity and abnormal p53 immunoreactivity. In conclusion, the GCC is a distinct appendiceal neoplasm, which exhibits unique pathological features and clinical behavior. They display a spectrum of low and high-grade histology and possess a potential to differentiate or transform to an adenocarcinoma phenotype or frank adenocarcinoma. Thus careful evaluation of morphological features of the GCC at its primary site is crucial for the clinical management and may predict its outcome.

Table 1.Prognosis of Stage IV GCC Compared with Primary Adenocarcinoma of the Appendix.

\begin{tabular}{|c|c|c|c|c|}
\hline \multirow{2}{*}{ Tumor Type } & \multicolumn{4}{|c|}{ Stage IV } \\
\cline { 2 - 5 } & \# of Cases & DOD & 3-Year DSS & 5-Year DSS \\
\hline All GCC & $40 / 63(63 \%)$ & $14 / 40(35 \%)$ & $17 / 24(71 \%)$ & $8 / 19(42 \%)$ \\
\hline LG-GCC & $10 / 30(33 \%)$ & $1 / 10(10 \%)$ & $7 / 7(100 \%)$ & $5 / 5(100 \%)$ \\
\hline HG-GCC & $23 / 26(88 \%)$ & $7 / 23(30 \%)$ & $9 / 11(82 \%)$ & $3 / 8(38 \%)$ \\
\hline Mixed GCC-AdenoCa & $7 / 7(100 \%)$ & $6 / 7(86 \%)$ & $1 / 6(17 \%)$ & $0 / 6(0 \%)$ \\
\hline Adenocarcinoma & $19 / 28(68 \%)$ & $11 / 19(61 \%)$ & $4 / 13(31 \%)$ & $0 / 11(0 \%)$ \\
\hline
\end{tabular}

DOD = Died of Disease; DSS = Disease Specific Survival

Table 2. Immunoprofile of GCC and Adenocarcinoma of the Appendix.

\begin{tabular}{|c|c|c|c|c|c|c|c|c|c|}
\hline & Chromog & Synap & Ki67 & p53 & $\beta$-Cat & MUC1 & MUC2 & E-Cad & Rb \\
\hline LG GCC & $\begin{array}{c}100 \% \\
(1-2+)\end{array}$ & $\begin{array}{c}100 \% \\
(1-2+)\end{array}$ & $11 \pm 2 \%$ & Negative & Normal & Negative & $\begin{array}{c}100 \% \\
(4+)\end{array}$ & Normal & Normal \\
\hline HG GCC & $\begin{array}{c}100 \% \\
(1-2+)\end{array}$ & $\begin{array}{c}100 \% \\
(1-2+)\end{array}$ & $16 \pm 4 \%$ & Negative & Normal & Negative & $\begin{array}{c}100 \% \\
(4+)\end{array}$ & Normal & Normal \\
\hline $\begin{array}{c}\text { Mixed } \\
\text { GCC-Adenocarcinoma }\end{array}$ & $\begin{array}{c}100 \% \\
(1-2+)\end{array}$ & $\begin{array}{c}100 \% \\
(1-2+)\end{array}$ & $80 \pm 5 \%$ & $2 / 2(4+)$ & Normal & - & - & - & - \\
\hline Adenocarcinoma & Negative & Negative & $>80 \%$ & $\begin{array}{c}85 \% \\
(4+)\end{array}$ & - & $\begin{array}{c}89 \% \\
(4+)\end{array}$ & $\begin{array}{c}5 \% \\
(3+)\end{array}$ & - & - \\
\hline
\end{tabular}

"-" - immunostain not performed. + = Staining intensity (1-4)

\section{T1072}

Ultrasound or CT Scan for the Detection of Supraclavicular Lymph Nodes in Patients with Esophageal Or Gastric Cardia Carcinoma

Evelyn P.M. van Vliet, Aad van der Lugt, Ernst J. Kuipers, Hugo W. Tilanus, Ate van der Gaast, John J. Hermans, Peter D. Siersema

Background: Malignant supraclavicular lymph nodes in patients with esophageal or gastric cardia carcinoma are considered to be distant metastases. As the palliative treatment of these patients differs from the intent-to-cure treatment of patients with apparent non-metastatic disease, screening for malignant supraclavicular lymph nodes is thus of crucial importance in the work-up. Both ultrasound (US) and CT scan (CT) are used for this purpose. In some 
institutions, both methods are routinely combined, however others prefer to screen with $\mathrm{CT}$ alone and combining it with US with fine needle aspiration (FNA) if metastases are suspected. It is presently unknown whether CT scan alone is sensitive enough to investigate the supraclavicular region and, consequently, whether US with FNA should only be used in cases with suspected malignant lymph nodes on a CT scan. Aim: To retrospectively compare US, if indicated with FNA, with CT for the detection of supraclavicular lymph node metastases. Methods: From 1994 to 2004, 567 patients underwent US and CT of the supraclavicular region for staging of esophageal or gastric cardia carcinoma. The gold standard was a radiological result with $\geq 6$ months of follow-up $(\mathrm{N}=402)$, FNA $(\mathrm{N}=122)$ or postoperative detection of malignant lymph nodes in the resected specimen $(\mathrm{N}=43)$. Results: Based on the gold standard, $65 / 567$ patients (11\%) had malignant supraclavicular lymph nodes. Sensitivity of US with FNA was $72 \%(47 / 65)$, compared to $25 \%(16 / 65)$ for CT $(\mathrm{p}<0.001)$ Specificity was $100 \%(502 / 502)$ and $99 \%(500 / 502)$, resp. ( $\mathrm{p}=\mathrm{NS})$. Prior to performing FNA, US had classified lymph nodes as being malignant in 49 patients, resulting in a sensitivity of $75 \%(49 / 65)$ and specificity of $91 \%(458 / 502)$. In 4 patients with malignant nodes according to our gold standard, only CT classified lymph nodes as being malignant, whereas the initial US with FNA was negative. In 35 patients, US with FNA detected truepositive lymph node metastases where the CT scan was negative. Conclusions: Both US with FNA and CT of the supraclavicular region should probably be used in patients with esophageal or gastric cardia carcinoma, as both techniques are complementary for the detection of metastases. Nevertheless, US with FNA and CT scan alone were not able to detect malignant supraclavicular lymph nodes in as many patients as desirable. Therefore, a prospective, blinded, comparative study is needed to determine whether other preoperative investigations, such as PET scanning, alone or in combination with US with FNA or CT, are better able to determine the presence of malignant lymph nodes in the supraclavicular region.

\section{T1073}

Expression of Angiotensin II Receptor-Type 1 Is a Predictor of Nodal Spread in Gastric Cancer

Christoph Rocken, Stacy Carl-McGrath, Juliane Hoffmann, Peter Malfertheiner, Matthias Ebert

Aims: We studied the putative significance of angiotensin II-receptor type l (ATlR) and 2 (AT2R) on gastric cancer biology. Methods: Local expression of AT1R, AT2R and angiotensin converting enzyme (ACE) was investigated by immunohistochemistry in tumor and corresponding non-tumor specimens obtained from 100 patients with gastric cancer and compared with the ACE insertion/deletion gene polymorphism. Results: ATIR was expressed in tumor epithelial cells of $26(26 \%)$, and AT2R in tumor epithelial cells of $95(95 \%)$ patients. ATIR was found highly significantly more common in intestinal-type gastric cancer, where its expression correlated significantly with the $\mathrm{N}$-category $(\mathrm{p}=0.009)$ and the UICC tumor stage $\mathrm{p}=0.024)$. ATlR+ intestinal-type gastric cancers had a significantly larger number of lymph node metastases and a significantly higher UICC tumor stage than ATlR- tumors. Twentyone (21\%) of the 100 patients with gastric cancer had the ACE II-, 51 (51\%) the ACE IDand $28(28 \%)$ the ACE DD-genotype. Patients with ATIR-tumor cells and ACE II-genotype carried a low risk of developing lymph node metastases (OR 0.044; 95\%-CI: 0.004-0.453). In contrast, patients with ATlR+ tumor cells and an ACE ID- (OR 10 833. 95\%-CI: 12.00 97.798) or DD-genotype (OR 10.667; 95\%-CI: 0.551-206.36) carried a high risk of lymph node metastasis. Conclusions: We show that angiotensin II receptors are expressed in gastric cancer and that the combination of ATIR-expression and ACE I/D gene polymerphism allows the risk assessment of nodal spread in gastric cancer.

\section{T1074}

Clinical Significance of Prostaglandin E Synthase Expression in Colorectal Cancer

Tsuguhiko Seo, Atsushi Tatsuguchi, Ken Wada, Masaoki Yonezawa, Keigo Mitsui, Kazuhiro Nagata, Shu Tanaka, Shunji Fujimori, Katya Gudis, Seiichi Shinji, Tkashi Tajiri, Teruyuki Kishida, Choitsu Sakamoto

Background: Several lines of evidence suggest that the COX-2/PGE2 pathway is a significant contributor to the development and progression of cancer. In fact, elevated expression of cyclooxygenase-2 (COX-2) has been found in various types of human cancer, including colorectal cancer (CRC). Prostaglandin E Synthase (PGES) functions downstream of COX in the biosynthesis of PGE2. To date, at least three distinct isoforms of PGES have been cloned; one cytosolic PGES (cPGES), and two microsomal fractions, mPGES-1 and mPGES2. Although there have been reports concerning the expression of mPGES-1 in colorectal cancer, the clinical significance of each of the three different PGE synthases remains unclear. Aims: To investigate mPGES-1, mPGES-2, and cPGES expression in CRC tissue, and to examine the relationship of their expression to various clinicopathological factors and patient prognosis. Patients and Methods: A total of 99 CRC patients who had undergone surgery were examined. PGES mRNA and protein expression levels in cancer tissue were compared with those in normal tissue by real-time polymerase chain reaction (PCR) and Western blot analysis, respectively. mPGES-1 localization was analyzed immunohistochemically. Immunostaining results for mPGES-1 were compared with clinicopathological factors. Results: Realtime PCR and Western blot analysis of mPGES-1 mRNA and protein expression showed significantly higher expression levels in CRC tissue samples than in normal tissue counterparts. Western blot analysis showed cPGES and mPGES-2 protein expressed in all CRC and normal tissue samples, with no significant difference in their expression levels. Immunoreactivity for mPGES-1 was predominantly observed in the cytoplasm of cancer cells in 43 cases 43\%). Weak mPGES-1 expression was also seen in a few stromal cells such as fibroblasts and macrophages. mPGES-1 expression correlated with venous invasion $(\mathrm{P}=0.02)$, but with no other clinicopathological factors. mPGES-1 expression correlated with COX-2 expression. The prognosis was significantly worse in patients with mPGES-1-positive tumors than in those with mPGES-1-negative tumors. Conclusions: Among the three PGE synthases, only mPGES-1 immunoreactivity varied between colorectal cancer and normal tissue; CPGES and mPGES-2 were uniformly expressed in both tissue types. Thus, only mPGES-1 may play an important role in CRC progression in conjunction with COX-2.

\section{T1075}

Percutaneous Radio-Frequency Ablation Inproves the Prognosis of the Patients with Unresectable Hepatic Metastasis from Colorectal Cancer

Yukihiro Koike, Shuichiro Shiina, Haruka Kajiwara, Eriko Goto, Kazuhiko Ohira, Osamu Goto, Kazuhiro Watanabe, Masao Omata, Tateo Kawase

Background The liver is the most common site of metastases from colorectal cancer. At present, surgery is supposed to be the only therapy that offers the possibility of cure for patients with hepatic metastatic diseases. However, there are never many patients with colorectal liver metastases set as the candidates for surgical resection, while the remaining are treated with chemotherapy or best supportive care. This study was conducted to clarify the safety and efficacy of percutaneous radiofrequency ablation for unresectable liver meatstases of colorectal cancer. Methods; From Feb. 2002 to Dec. 2005, 67 patients with unresectable liver metastases from colorectal cancer were treated by percutaneous radiofrequency ablation, regardless of extrahepatic lesions. At the initial ablation, the mean number and size of tumor foci were $6.8 \pm 8.0($ mean $\pm S D$, range $1->30)$ and $50 \pm 36 \mathrm{~mm}($ mean $\pm S D$, range; $18-178 \mathrm{~mm})$ Patients were not considered for surgical metastatectomy due to extrahepatic metastases (26 patients), prior hepatic resection (11 patients), disease extent (more than 5 lesions, 22 patients, more than $50 \mathrm{~mm}, 22$ patients), age (over 75 y.o., 13 patients), and/or comorbidity. There were 29 patients with the previous history of anticancer agents administration. Complications and survival after radiofrequency ablation were calculated. Survival rates were compared with those of the 18 patients who did not receive either surgical resection or radiofrequency ablation for metastatic liver tumor of colorectal cancer developing from 1999 to 2000. Prognostic factors of the patients were evaluated by univariate Cox proportional hazard model. Results; The overall procedure mortality was $0 \%$. Two complications of live abscess were observed. 1- and 2-year survival rates of the patients who received radiofrequency ablation for liver metastases were $74 \%$ and $49 \%$, while those of the control were only $50 \%$ and $0 \%(\mathrm{P}<0.01, \log$ rank test). Prior administration of anticancer agents was the only significant prognostic factor. Conclusion; This study indicated that percutaneous radiofrequency ablation might safely prolong the prognosis of patients with unresectable liver metastases from colorectal cancer

\section{T1076}

Aspirin Use and Survival After Diagnosis of Colorectal Cancer Andrew T. Chan, Jeffrey A. Meyerhardt, Edward L. Giovannucci, Jennifer A. Chan, Eva S. Schernhammer, Charles S. Fuchs

Randomized trials and cohort studies have demonstrated that aspirin reduces risk of colorectal adenoma and cancer. In animal models, aspirin has been shown to inhibit tumor growth and metastasis. However, the influence of aspirin on survival in patients with established colorectal cancer is unclear. In an study of Stage III colon cancer patients enrolled in an adjuvant chemotherapy trial, aspirin users had a reduced risk of recurrent disease and death. Nonetheless, this study was not able to determine whether aspirin use before or after diagnosis was more influential for survival. Methods: We conducted a prospective study of aspirin use pre- and post-diagnosis of colorectal cancer among 724 women enrolled in the Nurses' Health Study. Since 1980, participants have provided aspirin data biennially. In the present study, we examined women diagnosed with Stage I, II, or III colorectal cancer between 1980 and 2002. We used Cox proportional hazards models with biennially updated aspirin data to assess the relation between aspirin use with colorectal cancer-specific and overall mortality. Results: Compared to non-users, women who used aspirin regularly after colorectal cancer diagnosis experienced a hazard ratio (HR) for colorectal cancer-specific mortality of 0.51 (95\% CI, 0.33-0.79) and overall mortality of 0.66 (CI, 0.47-0.92) after adjustment for age, age at diagnosis, date of diagnosis, stage, grade, tumor site, and aspirin use pre-diagnosis. In contrast, compared to non-users, women who used aspirin regularly before diagnosis did not have a survival benefit (HR 0.99; CI, 0.66-1.49 for colorectalcancer specific mortality and HR $0.84 ; \mathrm{CI}, 0.60-1.16$ for overall mortality). Notably, among 369 women who did not use aspirin before diagnosis, aspirin use initiated after diagnosis was associated with HRs for colorectal cancer-specific mortality of 0.42 (CI, 0.0.20-0.86) and overall mortality of 0.49 (CI, 0.28-0.85). Although low doses of aspirin were beneficial, the association between post-diagnosis aspirin use and survival appeared dose-dependent. Compared to non-users, the HRs were 0.53 (CI, 0.33-0.86) for women who used 0.5-7 standard aspirin tablets and $0.47(\mathrm{CI}, 0.23-0.96)$ for $>7$ tablets per week (Ptrend $=0.03$ ) Conclusions: Regular aspirin use after the diagnosis of colorectal cancer may reduce the risk of colorectal cancer-specific and overall mortality. In contrast, aspirin use before diagnosis did not influence survival. These results suggest that aspirin may favorably influence the biology of established colorectal tumors in addition to preventing their occurrence.

\section{T1077}

Evaluation of Prognostic Factors in Chemotherapy for Patients with Advanced Pancreatic Cancer

Ken Hirao, Hirofumi Kawamoto, Hironari Kato, Naoko Kurihara, Yuko Okamoto, Hirotoshi Fukatsu, Jun Kato, Hiroyuki Okada, Yasushi Shiratori

$<$ Purpose $>$ Pancreatic cancer is a disease of ominous prognosis. Therefore, the sooner the response to the chemotherapy is evaluated, the better management on these patients we can perform. As a reference index for early decision of prognosis, we evaluated prognostic factors of the patients with advanced pancreatic cancer <Methods>Between March 2003 and August 2005, forty-five consecutive patients with advanced pancreatic cancer were included in this study (Male: Female=27: 18, Median age 58.0 years old; range 38-80, Average observation period 218 days; range 11-638). All patients had histologically or cytologically proven stage IV pancreatic cancer. The performance status was evaluated using Karnofsky performance status (KPS) (KPS $50-60 \% \cdot 70-80 \% \cdot 90-100 \%=4 \cdot 24 \cdot 17$ ). The protocol of chemotherapy was as follows : gemcitabine alone $\left(1,000 \mathrm{mg} / \mathrm{m}^{2}\right.$ on days 1,8 and 15 of each 28 -day cycle; $\mathrm{n}=12)$, a combination of gemcitabine and carboplatin $\left(1,000 \mathrm{mg} / \mathrm{m}^{2}\right.$ gemcitabine and $10 \mathrm{mg} / \mathrm{m}^{2}$ carboplatin on days 1 and 8 of each 21 -day cycle; $\mathrm{n}=33$ ). To predict the prognosis of patients, we divided the patients into two categories according to the following 
parameters: age, gender, KPS, location of primary tumor, metastasis, protocol of chemotherapy, response to treatment, and decrease in two tumor markers level during initial one month after chemotherapy (carcinoembryonic antigen; CEA, carbohydrate antigen 19-9; CA19-9). The multivariate analysis was performed using the Cox proportional hazards model, respectively. <Results>The overall response rate was $15.5 \%$, and the overall survival time was 7.1 months. The independent prognostic factors identified by multivariate analysis were a KPS of $90-100 \%$ (RR: $4.3, \mathrm{CI}: 1.84-9.98$ ), a response to treatment of partial response (PR) or stable disease (SD) (RR: 33.5 CI: 9.34-120.0), and the decrease in both tumor makers level (RR: 5.3, CI: 2.07-13.57). Median survival time (MST) were as follows: KPS ( $\leqq 80 \%: \geqq 90 \%=6.3$ months: 11.5 months, $\mathrm{p}<0.01$ ), response to treatment $(\mathrm{PD}: \mathrm{PR}$ or $\mathrm{SD}=$ 1.6months: 10.3 months, $\mathrm{p}<0.001$ ), decrease in both tumor markers level (increase: decrease $=$ 2.6 months: 9.3 month, $\mathrm{p}<0.001)$. In patients with stable disease $(\mathrm{n}=25)$, combination of KPS $\geqq 90 \%$ and decrease in tumor markers level was also identified as a significant predictor of survival by univariate analysis (RR: 13.7, CI: 1.67-112.6). MST were as follows: KPS + both tumor makers level ( $\leqq 80 \%+$ increase: $\geqq 90 \%+$ decrease $=7.1$ months: 12.4 months, $\mathrm{p}<$ 0.05). < Conclusions>Good performance status and decrease in both tumor markers level during initial one month after chemotherapy may be helpful to predict the prognosis of patients with pancreatic cancer.

\section{T1078}

Colon Cancer in Young Patients: Have Worse Outcomes?

Hang Lak Lee, Oh Young Lee, Yong Chul Jeon, Dong Soo Han, Byung Chul Yoon, Ho Soon Choi, Seung Sam Paik, Jin Bae Kim

Background and Aims: Many articles have previously reported poor outcomes for young colon cancer patients relative to the older population. However, these studies tend to be small and have various biases. So we conducted a retrospective study concerning the clinical courses and survival rate of young colon cancer patient compared to old patients. Methods: All patients diagnosed with colorectal cancer between 1991 and 2001 were evaluated. Duke stage D patients were excluded and a total of 544 patients were included. All of 544 patients have been followed up regularly after surgery and/or chemotherapy. Patients were organized into two age categories: a young patient group ( $<50$ years) and an old age group ( $>50$ years). Cancer related death was only included. Results: (1) Among the total of 544 patients, young group included 123 patients, and older group contained 421 patients. (2) Cancer diameter was significantly large in young group $(6.06 \pm 2.19$ vs. $5.47 \pm 2.13 \mathrm{~cm}, \mathrm{p}=0.012)$ (3) There were no significant differences between two groups in stage at diagnosis, differentiation, degree of lymph node involvement, cancer location and gross finding. (4) Median cancer specific survival time was worse for the old group ( $>50$ years) compared with the young group ( $<50$ years)(3216.42 \pm 101.05 vs. $3696.99 \pm 160.35$ days)(Figure 1). We performed a subgroup analysis (age $<45$ years vs. age 55-60 years) to exclude effect of old age on survival time, and there were no significant differences between two groups in median cancer specific survival time. (5) Multivariate logistic regression analysis demonstrated that age ( $\mathrm{p}=$ $0.001)$, stage at diagnosis $(\mathrm{p}=0.002)$, lymph node involvement $(\mathrm{p}=0.041)$ are independent factors associated with survival rate. Conclusions: Contrary to prior reports, younger patients appear to have similar or better survival rate than older patients.

Survival Functions

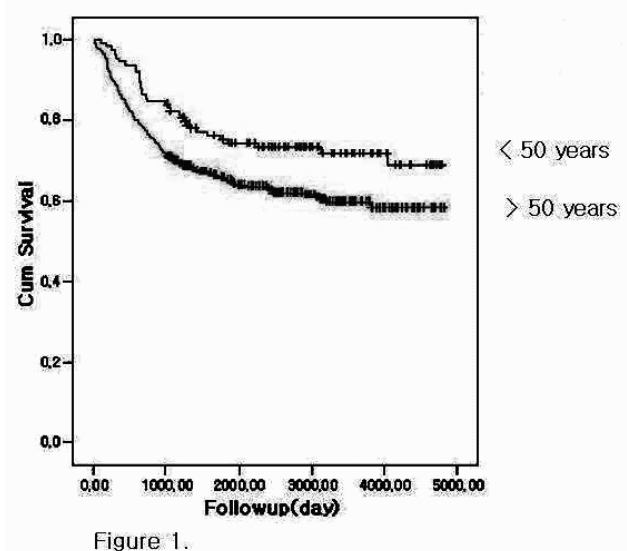

T1079

Ability of Surgery to CURE Patients with Entero-Pancreatic Endocrine Tumors Francesco Panzuto, Michela Di Fonzo, Cristina Nisita, Gabriele Capurso, Vito D. Corleto, Massimo Milione, Elsa Iannicelli, Massimo Falconi, Vincenzo David, Giovanbattista Grassi, Gennaro Nuzzo, Paolo Pederzoli, Vincenzo Ziparo, Gianfranco Delle Fave

Background. Surgery is the only treatment able to cure patients with entero-pancreatic endocrine tumors (EP ETs). However, data on the outcome of these patients during longterm follow-up are poor. Aim. To determine outcome and survival of EP ETs patients after surgery. Patients and methods. Among all consecutive patients with EP ETs evaluated at our Department, those who underwent surgical procedures were enrolled. Surgery was considered radical when all the visible tumor lesions were removed. Patients were considered cured when, after radical surgery, no disease recurrence occurred during follow-up. Coxproportional hazards regression and Kaplan-Meier method were used to calculate the risk factors for disease recurrence, and the survival. Results. Among the 168 EP ETs evaluated, 35 were excluded, due to the lack of follow-up data. 80 out of the 133 remaining patients (60.1\%; $42 \mathrm{M}$, median age $49 \mathrm{yrs)}$, underwent surgery, and were considered in the final analysis. Tumor originated from the pancreas (PETs) or the GI tract (GI carcinoids) in 38
(47.5\%) and 42 patients (52.5\%), respectively. At time of surgery, $41.2 \%$ of patients had primary tumor only, whereas $58.8 \%$ had metastases (nodes only $12.5 \%$, liver $46.3 \%$ ). Surgery was considered radical in 48/80 (60\%) patients [29/42 (69\%), and 19/38 (50\%) of patients with GI carcinoids and PETs, respectively $(\mathrm{p}=\mathrm{ns})]$. None received adjuvant treatment. Of them, 13 patients $(27.1 \% ; 7$ GI tumors and 6 PETs, $\mathrm{p}=\mathrm{ns})$ experienced disease recurrence at a median interval of 22 months (range 6-92) after radical surgery. Of these, before surgery, 6 had primary tumor only, whereas 7 had metastases ( 3 nodes only, 4 liver). The remaining 35 patients $(43.7 \%$ of the operated patients; $26.3 \%$ of the 133 enrolled patients) were considered cured after surgery, throughout a median 27 months follow-up. The presence of $\geq 2$ liver lesions was a significant risk factor for developing disease recurrence after radical surgery $(R R=4.85, p=.01)$. A total of 7 patients died of disease at a median interval of 15.5 months after non radical surgery, overall 5 -yr survival rate being $85.2 \%$. Conclusions. Surgery was considered radical in $60 \%$ of the operated patients. In this group, disease recurrence occurred in $27.1 \%$ of cases. The overall rate of curative surgery in EP ETs patient was $26.3 \%$. The presence of 2 or more liver metastases was the major risk factor for disease recurrence after radical surgery. Since recurrence occurred at a median interval of 22 months after surgery (range 6-92), patients receiving radical surgery should undergo long-term post-surgery follow-up. In these patients, the possible use of an adjuvant therapy should be considered.

\section{T1080}

Evaluation of An Inflammation-Based Prognostic Score (GPS) in Patients with Inoperable Gastro-Esophageal Cancer, Receiving Active Treatment Andrew Crumley, Donald C. McMillan, Margaret McKernan, Alexander C. McDonald, Robert C. Stuart

Background: There is increasing evidence, that the presence of an ongoing systemic inflammatory response, is associated with poor outcome in patients with advanced cancer. The aim of the present study, was to examine whether an inflammation-based prognostic score (GPS), was associated with survival, in patients with inoperable gastro-oesophageal cancer. Methods: Patients attending the Royal Infirmary, Glasgow between the lst January 2000 and the 31st December 2004 were included in the study. A blood sample was obtained for measurement of albumin and $\mathrm{C}$-reactive protein concentrations, at the time of diagnosis. Patients with an elevated C-reactive protein $(>10 \mathrm{mg} / \mathrm{l})$ and hypoalbuminaemia $(<35 \mathrm{~g} / \mathrm{l})$ were allocated a GPS score of 2 . Patients in whom only one of these biochemical abnormalities was present were allocated a GPS score of 1 and patients with a normal C-reactive protein and albumin were allocated a score of 0 . Results: Of the 195 patients studied $153(78 \%)$ patients died, 144 patients of their cancer and 9 of intercurrent disease in the follow-up period. On multivariate survival analysis, stage (HR 1.37, 95\%CI 1.15-1.63, p<0.001) and the GPS (HR $1.87,95 \%$ CI $1.45-2.42, \mathrm{p}<0.001$ ) were significant, independent predictors of cancer survival. Twelve month survival in patients with stage III/ IV disease was 57\%, 25\% and $12 \%$ for a GPS of 0,1 and 2 respectively. Conclusions: In the present study, the GPS predicted cancer specific survival, independent of stage and treatment received, in patients with inoperable gastro-oesophageal cancer. Moreover, the GPS may be used in combination with stage to improve the prediction of survival, in these patients.

\section{T1081}

Selection Criteria for Immediate Hepatectomy Without Neoadjuvant Chemotherapy in Patients with Multiple and Bilateral Liver Metastases Masami Minagawa, Masatoshi Makuuchi

Objective: To determine selection criteria for immediate hepatectomy without neoadjuvan chemotherapy in patients with multiple and bilateral liver metastases. Background Data Effectiveness of neoadjuvant chemotherapy followed by hepatectomy in patients with initially unresectable liver metastases has been shown. The indication of neoadjuvant chemotherapy is gradually extending, and the boundary of immediate resection and neoadjuvant chemotherapy followed by hepatectomy is becoming unclear. Methods: During the past 22 years, 369 patients underwent curative resection without neoadjuvant chemotherapy for liver metastasis from colorectal cancer; 156 had single, 76 had multiple and unilateral, 137 had multiple and bilateral metastases. Seventeen clinicopathological factors were analyzed in the last group. Results: The 5-year survival rate and the median survival time (95\% confidence interval) of 137 patients with multiple and bilateral metastasis were $28 \%$, and 2.3 year (2.1 2.8 years) respectively. There was no in-hospital death. Factors that significantly affected the prognosis were 4 or more lymph node metastases around the primary cancer $(\mathrm{P}<0.001)$ and carcinoembryonic antigen $>/=50 \mathrm{ng} / \mathrm{ml}(\mathrm{P}=0.003)$. Age, sex, number of liver nodules size of metastasis, synchronous or metachronous appearance of liver deposits, vascular of biliary invasion of metastasis, existence of extrahepatic metastasis or invasion, were not significant factors. The location, size, depth of wall invasion, lymphatic duct invasion, vascular invasion, and grade of differentiation of primary colorectal cancer were not significant. In patients with 3 or less lymph node metastases around the primary cancer, the 5-year survival rates of those with 2 3, and 4 or more liver metastases were $40 \%$ and $40 \%$ respectively, while these were $25 \%$ and $0 \%$ in patients with 4 or more lymph node metastases around the primary cancer. Conclusion: Resection of multiple and bilateral metastases was feasible and safe. Aggressive surgical approach may be supported if there are 3 or fewer lymph node 
metastases around the primary cancer. However, in patients with 4 or more colorectal lymph node metastases, biological selection by neoadjuvant chemotherapy may be more suitable.

$\mathrm{T} 1082$

The Expression of Sparc in Tumor Stroma May Facilitate Gastric Cancer Metastasis and Invasion

Christoph Rocken, Stacy Carl-McGrath, Uwe Lendeckel, Peter Malfertheiner, Matthias Ebert

Aims: Using cDNA microarray technology we searched for genes that might be involved in tumor spread in gastric cancers. Methods: cDNA microarray analysis of a primary gastric carcinoma and matched lymph node metastasis was carried out. Using quantitative real time RT-PCR, the expression levels of SPARC mRNA was confirmed in non-neoplastic, carcinoma, and metastasis tissues from 7 patients. Immunohistochemistry with anti-SPARC antibodies was performed on 40 cases of primary gastric carcinomas (20 diffuse, 20 intestinal) and the corresponding lymph node metastases. Results: SPARC was identified by a cDNA microarray as being up-regulated in the lymph node metastasis compared to the primary gastric carcinoma. The level of expression of SPARC mRNA in non-neoplastic tissues, carcinomas and metastases was validated by quantitative RT-PCR. Using immunohistochemistry, SPARC was found in intestinal metaplasia, as well as in fibroblasts, and occasionally in tumor cells. However, the level of immunoreactivity was extremely strong in stromal cells surrounding the tumor cells, and also frequently in metastatic carcinoma cells in lymph nodes. Conclusions: SPARC is a protein regulating cell-ECM interactions. The tumor-stroma interactions mediated by SPARC may contribute to the loss of cell-cell contacts that allows dissemination and metastasis of gastric cancer cells.

\section{T1083}

Arterial and Systemic Combination Chemotherapy of 5-Fluorouracil with Radiation in the Treatment of Advanced Gallbladder Cancer

Takashi Sasaki, Hiroyuki Isayama, Toshihiko Arizumi, Osamu Togawa, Saburou

Matsubara, Yousuke Nakai, Natsuyo Yamamoto, Naoki Sasahira, Kenji Hirano, Takeshi Tsujino, Nobuo Toda, Minoru Tada, Takao Kawabe, Masao Omata

BACKGROUND: The prognosis of patients with advanced gallbladder cancer is poor and existing chemotherapy is relatively ineffective. The aim of this study was to determine the efficacy of arterial and systemic combination chemotherapy of 5-Fluorouracil(5-FU) with radiation in the treatment of advanced gallbladder cancer. PATIENTS AND METHODS: The records of all patients who had histologically or cytologically confirmed gallbladder cancer were retrospectively reviewed. The regimen of arterial and systemic combination chemotherapy of 5-FU with radiation was as follows; arterial and systemic infusion of 5-FU(350mg m2) was given five consecutive days for four weeks with radiation therapy(Total 48Gy). Then 5-FU was administered biweekly at the same dose. Patients were evaluated for response rate, survival, toxicity and patency of metallic stent in case of malignant biliary obstruction. RESULTS: Of fourty-four patients with advanced gallbladder cancer, seven patients were treated by arterial and systemic combination chemotherapy of 5-FU with radiation. The response rate of this treatment was $71.4 \%$ (complete response rate of $14.3 \%$, and a partial response rate of $57.1 \%$ ) and a median survival period was 9.8 months (a mean survival period was $13.6 \pm 6.8$ months). The response rate of other treatment groups were $18.8 \%$ (Chemotherapy; $\mathrm{n}=16$ ) and 20\% (Radiation; $\mathrm{n}=5$ ). The median survival period of other treatment groups were 5.1 months(Chemotherapy), 3.8 months(Radiation), 2.4 months(Best supportive care; $\mathrm{n}=16$ ). As for patency of metallic stent, median stent patency were 9.7 months (Arterial and systemic combination chemotherapy of 5-FU with radiation; $\mathrm{n}=6$ ), 3.8 months (Radiation; $\mathrm{n}=4$ ) and 2.7 months (Chemotherapy; $\mathrm{n}=10$ ). The major toxicity of arterial and systemic combination chemotherapy of 5-FU with radiation was hematological (Grade 3 to 4 neutropenia occurred in $28.6 \%$ of patients without febrile neutropenia) CONCLUSION: Treatment with arterial and systemic combination chemotherapy of 5-FU with radiation resulted in clinically significant response rate, survival and local control in patients with advanced gallbladder cancer. Future clinical investigations for this treatment are warranted.

\section{$\mathrm{T} 1084$}

The Clinical Significance of Mucin Phenotype of Gastric Adenocarcinoma Ok-Jae Lee, Hyun-Jin Kim, Woo-Song Ha, Hidenobu Watanabe

Background/Aim: Histochemical and immunohistochemical studies for mucin contained in tumor allowed the mucous phenotypic classification of gastric adenocarcinomas. The clinical significance of mucin phenotype has not been clarified. We investigated the mucous phenotypic expression of gastric adenocarcinomas and its clinical significance as a prognostic factor. Methods: Histochemical and immunohistochemical stainings for paradoxical concanavalin A, MUC5AC, MUC-2 glycoprotein and CD10 of mucin were performed in 74 surgically obtained gastric adenocarcinomas. We determined mucin phenotype and analyzed its relationships with clinical and histopathological variables. Results: Among 74 gastric adenocarcinomas, $30(40.5 \%)$ expressed gastric phenotype (10 foveolar and 20 foveolo-glandular), 20 (27.0\%) intestinal phenotype ( 7 complete and 13 incomplete), 15 (20.3\%) mixed gastric-intestinal phenotype and 9 (12.2\%) undetermined type. The gastric and gastric predominant types were $54.1 \%(40 / 74)$, and intestinal and intestinal predominant types $31.1 \%(23 / 74)$. No relationship between mucous phenotypic expression and tumor location was found. Among 21 well differentiated adenocarcinomas, $12(57.1 \%)$ expressed predominantly intestinal phenotype and only 5 (23.8\%) gastric phenotype. Among 53 poorly differentiated adenocarcinomas, $35(66.0 \%)$ were gastric predominant and only 11 (20.8\%) were intestinal predominant phenotypes. Thirty-five out of $40(87.5 \%)$ gastric dominant cancers were poorly differentiated adenocarcinomas. Helicobacter pylori infection rates were $87.5 \%, 91.3 \%$, and $66.7 \%$ in gastric predominant, intestinal predominant and undetermined phenotypes, respectively. For about 6 years, 58 patients were followed up and the overall death rate was $39.7 \%$ with $62.5 \%$ for undetermined, $35.5 \%$ gastric predominant, $36.8 \%$ intestinal predominant phenotypes. Cumulative survival rate was lower in the undetermined than intestinal and gastric phenotypes. Conclusions: Gastric adenocarcinomas expressed predominantly gastric phenotype in Korea. Mucin phenotype may be associated with differentiation of gastric adenocarcinoma and Helicobacter pylori infection, and may have a role as a prognostic factor.

$\mathrm{T} 1085$

Prognostic Impact of DNA Ploidy Is Stronger Than and Independent of Microsatellite Instability in Human Colon Carcinoma Patients

Frank A. Sinicrope, Rafaela L. Rego, Kevin C. Halling, Nathan Foster, Daniel J. Sargent, Amy French, Megan Garrity, Richard M. Goldberg, John A. Laurie, Laurence J. Burgart, Stephen N. Thibodeau, Thomas E. Witzig

BACKGROUND. DNA ploidy and microsatellite instability (MSI) are highly correlated in human colon cancers and confer prognostic information. We determined whether MSI accounts for the prognostic impact of ploidy shown in prior studies. We studied the relationship between ploidy and MSI, allelic imbalance on chromosome arms 5q, 8p, 17p,18q, and p53 expression. MATERIALS \& METHODS. Dukes' stage B2 $(\mathrm{n}=158)$ and C $(\mathrm{n}=370)$ primary colon carcinomas from patients enrolled in six 5-fluorouracil-based adjuvant trials conducted by North Central Cancer Treatment Group. Tumors had been analyzed for DNA ploidy by flow cytometry. MSI and allelic imbalance were determined using 11 microsatellite markers [(instability at $\geq 30 \%$ of loci (MSI-H)]. Expression of mismatch repair proteins (hMLH1, hMSH2) and nuclear p53 were analyzed by immunostaining. Overall survival (censored at $8 \mathrm{yr}$ ) was analyzed in univariate and multivariate Cox models adjusting for stage, treatment, and stratifying by study. RESULTS. Diploid DNA content was detected in $223(42 \%)$ colon cancers. Ninety-five (18\%) tumors were MSI-H and 70 (74\%) of these were diploid. Of 58 MSH-H cases tested, 54 showed loss of hMLH1 $(n=50)$ or hMSH2 $(n=$ 4) proteins; all MSI-L $(n=30)$ and $240 / 241$ MSS cases tested showed intact expression. Female gender, proximal site, high histologic grade, infrequent p53, and diploidy were more likely to be MSI-H (vs. MSS/MSI-L; all p $\leq$ 0.0002). Patients with diploid tumors had a better overall survival (OS) compared to those with aneuploid/tetraploid tumors (HR= 0.64 , $\mathrm{p}=0.002)$ in all patients and in MSS/MSI-L cases $(\mathrm{p}=0.01)$. The prognostic impact of ploidy was similar within Dukes' B2 and C colon cancers (interaction p-value $=0.98$ ). Survival curves for diploid MSS/MSI-L and MSI-H patients overlapped ( $p=0.69)$. Neither MSI status $(\mathrm{p}=0.17)$ nor loss of mismatch repair proteins $(\mathrm{p}=0.13)$ were prognostic for OS. High grade histology $(\mathrm{p}=0.0002)$ and $8 \mathrm{p}$ allelic imbalance $(\mathrm{p}=0.034)$ were associated with poor OS and when stratified by ploidy, $8 \mathrm{p}$ status added additional prognostic information to diploid tumors $(\mathrm{p}=0.021)$. Allelic imbalance at $5 \mathrm{q}, 17 \mathrm{p}, 18 \mathrm{q}$ and $\mathrm{p} 53$ expression failed to predict outcome. In multivariate analysis, DNA ploidy $(\mathrm{p}=0.002)$ and histologic grade $(\mathrm{p}<$ $0.0001)$, but not MSI $(p=0.203)$, were stage-independent prognostic variables. CONCLUSION: DNA ploidy was the strongest stage-adjusted prognostic variable and was independent of MSI status. Diploid MSS/MSI-L patients had similar survival rates as did patients with MSI-H tumors. These data suggest that ploidy may be useful in therapeutic decision-making, including selection of patients for adjuvant therapy.

\section{T1086}

Frequency of Gastrointestinal Lesions in Iron Deficient Patients Without Anemia: A Prospective Endoscopic Study

Bani Chander, Edmund J. Bini

Background: Iron deficiency anemia occurs only after body stores of iron are completely exhausted and is, therefore, a late manifestation of the iron-depleted state. Although endoscopy is recommended for all patients with iron deficiency anemia, the need for an endoscopic evaluation in iron deficient patients without anemia is not known. The aims of this study were 1) to determine the frequency of gastrointestinal (GI) lesions in iron deficient patients with and without anemia and 2) to evaluate if colonoscopy can detect colorectal cancer at an earlier stage in iron deficient patients who have not yet developed anemia. Methods: Consecutive patients who were referred for the evaluation of iron deficiency anemia were prospectively enrolled at a single medical center. Patients were interviewed by a research assistant who obtained detailed demographic and clinical data, and all subjects had sameday EGD and colonoscopy. Iron deficiency was defined as a transferrin saturation of $<15 \%$ and a ferritin of $<20 \mathrm{ug} / \mathrm{L}$, whereas anemia was defined as a hemoglobin of $<13 \mathrm{~g} / \mathrm{dL}$ in men and $<12 \mathrm{~g} / \mathrm{dL}$ in women. Lesions were classified as clinically important according to standard predetermined criteria. Results: Of the 1,346 iron deficient subjects enrolled, 1,081 were anemic and 265 had normal hemoglobin levels. Clinically important lesions were identified in $60.7 \%$ of patients with anemia and $58.1 \%$ of those without anemia ( $\mathrm{P}=$ 0.44). The frequency of upper GI tract lesions ( $35.4 \%$ vs. $31.2 \%, P=0.20)$ and lower GI tract lesions $(40.5 \%$ vs. $35.1 \%, P=0.10)$ did not differ between anemic and non-anemic subjects. However, anemic patients were significantly more likely to have a clinically important colonic lesion identified proximal to the splenic flexure $(23.8 \%$ vs. $14.7 \%, \mathrm{P}=0.001)$ and were more likely to have clinically important lesions detected in both the upper and lower GI tract $(15.1 \%$ vs. $8.0 \%, \mathrm{P}=0.003)$. Although the prevalence of colonic adenomas $\geq 1 \mathrm{~cm}(24.1 \%$ vs. $23.4 \%, P=0.82)$ and colorectal cancer $(10.2 \%$ vs. $7.5 \%, P=0.19)$ was similar in anemic and non-anemic patients, non-anemic subjects with colorectal cancer were significantly more likely to have earlier stage cancers (Dukes' A \& B) than those with anemia $(50.0 \%$ vs. $19.1 \%, P=0.003)$. Conclusions: Iron deficient patients should undergo a thorough endoscopic evaluation of the GI tract, irrespective of the presence of anemia. Early endoscopy in iron deficient patients who have not yet developed anemia can detect colorectal cancer at a potentially curable stage. The effectiveness and cost-effectiveness of using ferritin to screen non-anemic patients for iron deficiency requires further evaluation. 
Home Fecal Occult Blood Testing in Average Risk Veterans Detects Only Advanced Colorectal Neoplasms

Christopher M. Mathews, David C. Whitehead, Christopher R. Marino

Background: Colorectal cancer (CRC) screening methods are designed to detect and remove pre-malignant lesions. Home fecal occult blood testing (FOBT) is used in many VA medical centers because it is easy to perform and has been shown by RCT to save lives. It is controversial, however, whether FOBT actually detects colorectal neoplasms or randomly targets individuals for interventions that subsequently identify these common lesions. We hypothesized that FOBT in average risk veterans does not select for individuals with colorectal neoplasia. Methods: The Clinical Outcomes Research Initiative (CORI) endoscopic database at the Memphis VAMC was retrospectively reviewed for male veterans age $\geq 50$ who underwent colonoscopy between 1999-2005 for a "positive home FOBT". Patients with anemia, personal or family history of colonic neoplasia, or symptoms such as abdominal pain, weight loss, change in bowel habits, or rectal bleeding were excluded by thorough review of medical records in the VA Computerized Patient Record System (CPRS). The prevalence of adenomatous polyps and CRC in veterans with a positive FOBT was compared to that in asymptomatic veterans who underwent screening colonoscopy using the same exclusion criteria (control group). Prevalence rates for colonic neoplasia in published autopsy studies were also compared. Results: The CORI database identified 663 veterans who underwent colonoscopy for positive screening FOBT and 301 who underwent screening colonoscopy without prior FOBT. CPRS review narrowed the FOBT cohort to 147 (45 black, 102 white) and the screening colonoscopy cohort to 138 ( 36 black, 102 white). Findings are summarized in the Table below. Published autopsy studies (Cancer 43:1847, 1979; Gut 23:835, 1983) report the prevalence of adenomatous polyps in males age $\geq 50$ to be $40-50 \%$ and CRC to be $3-4 \%$. Conclusions: The prevalence of adenomatous polyps in male veterans with positive screening FOBT does not differ from that in the general population. The lack of FOBT selectivity for adenomas results from the failure of FOBT to detect small $(<\mathrm{lm})$ adenomas present in a large percentage of individuals over age 50. FOBT positivity does, however, appear to select for the smaller subgroup of individuals with more advanced neoplasms (polyp size $\geq 1 \mathrm{~cm}$ or $\mathrm{CRC}$ ). Whether this sensitivity limitation enhances or detracts from the value of FOBT as a screening tool is subject to debate.

\begin{tabular}{|c|c|c|c|c|c|}
\hline Group & $\underline{\mathrm{N}}$ & *All Adenomas & $\underline{\text { Adenomas }<1 \mathrm{~cm}}$ & $\ddagger$ Adenomas $\geqq \underline{1 \mathrm{~cm}}$ & $\underline{\mathrm{CRC}}$ \\
\hline Control & 138 & $51(37 \%)$ & $41(30 \%)$ & $10(7 \%)$ & $1(1 \%)$ \\
\hline + FOBT & 147 & $65(44 \%)$ & $45(31 \%)$ & $20(14 \%)$ & $4(3 \%)$ \\
\hline
\end{tabular}

*p-value 0.21. †p-value 0.08. p-value 0.03 for advanced neoplasm (adenoma $\geq 1 \mathrm{~cm}$ or CRC)

\section{$\mathrm{T} 1088$}

RNA-Based Stool Assay Is Superior to a Single Immunochemical Fecal Occult Blood Test for Detecting Early Colorectal Cancer and Adenoma

Shigeru Kanaoka, Tetsunari Takai, Ken-Ichi Yoshida, Mutsuhiro Ikuma, Masayosh Kajimura, Naoyuki Miura, Haruhiko Sugimura, Akira Hishida

Background and Aims: It has been reported that fecal occult blood test (FOBT) is less sensitive for early colorectal cancer (CRC) and adenoma. We reported that Fecal COX-2 assay, one of RNA-based stool assays, is useful for detecting CRC (Gastroenterology 2004: 127: 422-427). It is reported that Snail is a repressor of both E-cadherin and vitamin D receptor gene expression, which is involved in colon carcinogenesis (Nature Cell Biol 2000; 2: 84-89, Nature Med 2004; 10: 917-919). We aimed to perform a study of whether RNAbased stool assay, combination of Snail and COX-2 as targets, allows us to improve further sensitivity for colorectal cancers and advanced adenomas compared the results with those of a single immunochemical FOBT (IFOBT). Methods: Standard histological techniques were used to classify adenoma or malignancy at I to IV stages according to TNM classification. We purified RNA from routinely collected stool samples and screened mRNA using COX2 and Snail specific nested RT-PCR as previously described, consequently compared the results with those of a single immunochemical FOBT (Magstream 1000/HemSp; FUJIREBIO, Inc., Tokyo, Japan) on same stool samples. Results: Stool samples from 65 patients with CRC, 13 patients with advanced adenoma, and 32 control patients without neoplastic disease were studied. The specificity of Fecal Snail assay as well as Fecal COX-2 assay was 100\% (95\% confidence interval [CI], 89.1-100\%), while that of IFOBT was $87.5 \%$ (95\% CI, 71.0$96.5 \%)$. The sensitivity of RNA-based stool assay was $87.7 \%$ (95\% CI, 76.6-94.5\%) for CRC, and $76.9 \%$ (95\% CI, 46.2-95.0\%) for advanced adenoma, while that of IFOBT was $73.0 \%(95 \% \mathrm{CI}, 60.2-83.5 \%)$ and $30.8 \%(95 \% \mathrm{CI}, 9.1-61.4 \%)$, respectively. COX-2 and/ or Snail mRNA was detected in $84.6 \%$ of stage I $(n=13), 93.5 \%$ of stage II $(n=31), 81.0 \%$ of stage III or IV $(n=21)$, while IFOBT was positive in $41.7 \%$ of stage I, $80.0 \%$ of stage II, $81.0 \%$ of stage III or IV. Snail mRNA was detected in 2 of 5 stool samples from patients with advanced adenoma who had negative Fecal COX-2 assay and IFOBT. Noteworthy, COX-2 and/or Snail mRNA was detected in 17 of 22 stool samples from patients with early cancer or advanced adenoma who had negative IFOBT. Finally, the sensitivity of this assay was $87.7 \%$ (95\% CI, 76.2-94.9\%) for advanced adenoma to stage II cancer, while that of IFOBT was $60.0 \%$ (95\% CI, 45.6-73.1\%). Conclusions: We could improve further sensitivity to add Snail as a target to RNA-based stool assay, which is superior to a single IFOBT in term of detecting curable colorectal cancer and advanced adenoma. These results strongly suggest that this assay would be useful for CRC screening.
Urgent Investigation of Alarm Symptoms of Suspected Upper Gastrointestinal Cancer Within Two Weeks Has a Low Yield for Diagnosis of Malignancy David Dewar, Manil Chouhan, David Reffitt, John O'Donohue

Background: In the UK, the 'two week wait' (TWW) referral initiative for suspected upper gastrointestinal cancer was introduced in July 2000. This standard allows general physicians to refer suspected cases of upper gastrointestinal cancer for urgent investigation which mus be performed within a 14 day period in an attempt to improve on the poor survival. Alarm symptoms include anemia, weight loss, dysphagia, new onset dyspepsia and jaundice. We wished to explore the outcome of investigating this group of patients urgently. Methods We identified all the urgent TWW referrals for the year 2004 and examined their medical records. This list was correlated with a database of all cases of diagnosed upper gastrointestinal cancers in our institution. The results were compared with a previous study at our institution which took place in 2000-2002, following the initial implementation of the two week wait. We included all diagnosed cases of upper GI malignancy. Results: In the year 2004, 32 patients (mean age $62.7 \mathrm{yrs}, 57 \%$ female) were referred using the TWW route, out of which 12 cases $(3.73 \%)$ were diagnosed with upper GI cancer. This constituted $16 \%(12 / 75)$ o all diagnosed cases of upper GI cancer in that year. In particular 288 endoscopies (11.4\% of total upper GI endoscopy activity) were performed of which 9 (3.1\%) showed cancer $50(17.4 \%)$ showed gastroduodenal pathology related to H.Pylori and $94(32.6 \%)$ were normal. Figures from 2001/2002 showed an annual TWW referral rate of 200.5 patients, with cancer being diagnosed in $5.7 \%$ of referrals. This constituted a similar proportion of the total upper GI cancers in this period (15.4\%). The incidence of diagnosis of upper G cancers remains similar with 65 cancers annually in 2001/2002 and 75 cases in 2004. It is noteworthy that only one of the urgently referred cancer was amenable to curative resection. Conclusion: The proportion of actual cancer cases amongst TWW referrals appears to have fallen, whilst the referral activity has risen. This may suggest that referring physicians have become less discerning in selecting patients for urgent investigation. The yield of cancers is low both in absolute terms $(3.7 \%)$ and in the proportion of total cancers (16\%) being diagnosed in this way. This reaffirms the fact that alarm symptoms correlate poorly with a diagnosis of cancer and that many cases of cancer present with more insidious symptoms which physicians may feel do not warrant urgent investigation. In the UK, the TWW route remains the preferred pathway for rapid cancer diagnosis but poses a significant workload for a small overall return.

\section{T1090}

Colorectal Cancer Screening By Primary Care Physicians: A Prospective Chart Audit

Bernard Denis, Guillaume Schon, Marcel Ruetsch, Jean Christian Grall, Michel Leveque, Jean Martin Meyer, Serge Moser, Jean Claude Tschiember, Philippe Perrin

Cancer screening requires 1) identification of individuals at high risk for cancer mainly through an adequate family history assessment and 2) documentation of dates and results of past screening examinations. Little data exist about the quality of charts in the field of colorectal cancer screening in primary care. Aim: to assess the completeness of the family history and past screening examinations interview in primary care. Methods: prospective chart audit of data on family cancer history and past screening examinations. Volunteer GPs self audited the completeness of their charts of 20 (10 men, 10 women) established patients aged 40-74 years for colorectal, breast and cervix cancer. Results: 37 GPs participated and 736 charts were analysed. Family history information on colorectal cancer was collected on $367(51.3 \%)$ charts. The individual rate of family history recording ranged from 5 to 100 $\%$ according to GP. Specific information regarding age at diagnosis and degree of kinship was present in 103 of the records. 134 charts $(20.5 \%)$ mentioned a past colon examination, but 85 (63.4\%) only mentioned the date of examination and its result. Among 490 50-74 years old average risk people, 251 charts (51.2\%) mentioned a past FOBT, $78.5 \%$ of them mentioned the date of the FOBT and $63.3 \%$ contained information about family history. Family history of breast cancer was documented in 139 (49.8\%) charts. 126 charts $(62.7$ $\%$ ) of women aged 50-69 years mentioned a past mammogram, but 52 charts only (25.9 $\%$ ) were complete and correct for breast cancer screening (family history, date and result of mammogram and $<2$ years mammogram). 99 charts (44.2\%) of women aged 40-65 mentioned a past pap smear but 53 charts only $(23.7 \%)$ were complete and correct for cervix cancer screening (date $<3$ years and result). Conclusion: quality of charts in primary care is globally poor but very heterogeneous, ranging from bad to excellent according to GP: family history of cancer is correctly documented in about a half of charts; about a quarter of charts are complete and correct for each cancer screening (date and result of past screening examinations documented and correct, family history collected). The collection of updated information on family history and past screening examinations must be improved in primary care. We designed paper tools to aid GPs in this task: posters and patientcompleted forms intended for GP's waiting rooms.

\section{T1091}

Reappraisal of Fecal DNA Testing (fDNA) in Light of Evolving Test Performance Characteristics and Increasing Colorectal Cancer (CRC) Treatment Costs

Murtaza Parekh, A. Mark Fendrick, Uri Ladabaum

BACKGROUND: CRC screening and treatment are dynamic fields. Some patients prefer non-invasive testing. As new tests evolve and CRC treatment costs rise with use of agents like bevacizumab and cetuximab, the cost-effectiveness of screening changes. Fecal occul blood testing (FOBT) decreases CRC incidence and mortality, but adherence with annual testing is poor. fDNA detects more CRCs and advanced neoplasia than FOBT (Imperiale NEJM 2004), it may have better patient acceptance, and fDNA test costs have decreased AIMS: To reappraise the cost-effectiveness of fDNA in people unwilling or unable to undergo colonoscopy, but amenable to non-invasive screening. METHODS: We used our published Markov model to compare life-yrs/pt and cost/pt (3\%/yr discount) for fDNA every 5 yrs, 
yearly FOBT or no screening (NS) from age 50-80. Colonoscopy was done after positive fDNA or FOBT, and every 5 yrs after polypectomy. Base case inputs included: fDNA sensitivity for CRC 0.52, large ( $\geq 1 \mathrm{~cm}$ ) polyp 0.18, specificity 0.94 , cost $\$ 300$ (Exact Sciences); FOBT sensitivity for CRC 0.4 , large polyp 0.1 , specificity 0.92 , cost $\$ 20$; CRC treatment costs $\$ 46,000 /$ localized, $\$ 91,000 /$ regional, $\$ 195,000 /$ disseminated CRC; test adherence $100 \%$. Sensitivity analyses focused on test performance characteristics and adherence. BASE CASE RESULTS: fDNA decreased CRC incidence by $24 \%$ and yielded 18.720 life-yrs/pt vs 18.686 life-yrs/pt with NS, costing $\$ 18,000 /$ life-yr gained (in contrast with our published estimate of $\$ 73,200 /$ /ife-yr gained assuming $\$ 695 /$ fDNA test and lower CRC treatment costs). With $100 \%$ adherence, FOBT was both more effective and less costly than either NS or fDNA CRC reduction 36\% vs NS; 18.742 life-yrs/pt). SENSITIVITY ANALYSES: A newer generation EDNA test with improved CRC sensitivity (0.65) would yield 18.725 life-yrs/pt at $\$ 14,200$ / life-yr gained vs NS. Further improved fDNA sensitivity (CRC 0.82, large polyp 0.2) at the expense of decreased specificity (0.82) would yield 18.733 life-yrs/pt at $\$ 13,000 /$ life-yr gained vs NS. fDNA with $100 \%$ adherence every 5 yrs and FOBT with $35 \% / y r$ adherence had comparable effectiveness, but FOBT remained cost-saving vs NS or fDNA. fDNA with $100 \%$ adherence cost $<\$ 100,00 /$ life-yr gained compared to FOBT when FOBT adherence was $\leq 24 \% / y r$. CONCLUSION: As CRC treatment costs increase and test costs decrease, fDNA may become a progressively more cost-effective CRC screening strategy in patients unwilling or unable to undergo colonoscopy. FOBT may be cost-saving given higher CRC treatment costs, but adherence with yearly testing is challenging. Present generation fDNA could be preferred if it achieved significantly higher adherence rates than FOBT.

T1092

Cancer Investigation At Peking University Using Fourier Transform Infrared (FT-IR) Spectroscopy. Immediate Spectroscopic Separation of Colitis from Cancer in Colon Biopsies

Q-B Li, X Li, Roger D. Soloway, Y-Z Xu, X-J Sun, G-J Zhang, Y-F Zhang, J-G Wu

Background: FT-IR spectroscopy can identify variability at the molecular level in biological specimens. This method is rapid and accurate and can be used intraoperatively to modify surgical procedures. Applications of newer statistical programs allow analysis of entire spectra simultaneously to utilize the maximum amount of information to characterize samples. Aim: To identify and separate cancer from colitis on endoscopic colon biopsies. Methods: After informed consent, one 1-3 mm pinch biopsy was obtained from each of 89 patients from the First Hospital of Xi'an Jiaotong University, China. 41 patients had colitis and 48 had cancer. Specimens were placed on a modified attenuated total reflectance (ATR) accessory linked to a WQD-500 FT-IR spectrometer with a MCT detector for greater stability and sensitivity. The spectral range was 4000 to $999 \mathrm{~cm}^{-1}$. Later, specimens were processed and read by pathologists without knowledge of the spectroscopic findings. Results: FT-IR spectral curves were smoothed by computer to reduce high-frequency noise. A Resampling HalfMean outlier statistical method was repeated 500 times and identified 4 samples as outliers ( 3 colitis, 1 cancer), thus 38 colitis and 47 cancer specimens were compared. Preprocessed spectra with first-order derivative after smoothing and normalization were used for discrimination analysis. A K-nearest neighbor model was constructed for diagnostic classification. A leave-one-out cross validation method was utilized to assess the discrimination results. 39 of 47 cancer specimens and 35 of 38 colitis specimens were correctly identified. This gave a sensitivity of $83 \%$ and specificity of $92 \%$ for cancer and a sensitivity of $92 \%$ and specificity of $83 \%$ for enteritis. This gave a $92.9 \%$ predictive value of a positive test for cancer and $81.4 \%$ predictive value for enteritis. Conclusions: 1 . Colon cancer can be distinguished from colitis with high accuracy using FT-IR spectroscopy and chemo metrics. 2. This should be extended to analysis of cancer vs normal colon or polyps. 3. This technique has promise for in vivo diagnosis with the development of endoscopic FT-IR miniprobes and is practical for immediate diagnosis at endoscopy. ***Project supported by State Key Project for Fundamental Research of MOST (2002CCA01900), National Natural Science Foundation of China (No. 30371604) and Doctoral Project of Education Ministry of China

T1093

Development of a Risk Assessment Tool for Patients Undergoing Colonoscopy to Identify Individuals At Risk for Hereditary Colorectal Cancer Syndromes Fay Kastrinos, David H. Stockwell, Radhika Venugopal, Elena M. Stoffel, Muthoka L. Mutinga, Earl F. Cook, Sapna Syngal

BACKGROUND: Complex criteria and time constraints are cited as reasons why clinicians fail to identify patients with hereditary colorectal cancer syndromes. Incomplete risk assessment can lead to inadequate surveillance for patients at highest risk for developing colorectal cancer (CRC). OBJECTIVE: To develop a practical and efficient risk assessment tool that will identify individuals at high risk for $\mathrm{CRC}$ and help gastroenterologists provide appropriate recommendations for surveillance intervals and referrals for genetic evaluation. METHODS: Patients undergoing outpatient colonoscopy at a tertiary-care center and a community hospital were asked to complete a 34-item self-administered questionnaire assessing CRC risk based on personal and family history of CRC, hereditary nonpolyposis colon cancer (HNPCC) related malignancies and colonic polyps. Subjects reporting any of the following criteria were defined as high risk: Personal history of CRC $<50$ years, polyps $<40$ years, endometrial cancer $<50$ years, $\geq 2$ HNPCC-associated cancers, $\geq 10$ cumulative polyps; Family history of first-degree relative (FDR) with $C R C<50$ years, FDR with polyps $<50$ years, $\geq 3$ relatives with CRC, FDR with HNPCC-associated cancer $<50$ years. HNPCC-associated cancers include genitourinary, gynecologic, gastric, small bowel, biliary, pancreatic or brain cancers. Recursive partitioning analysis was used to develop an algorithm of the fewest number of questions that successfully captured the largest proportion of individuals fulfilling high-risk criteria. RESULTS: Data were analyzed from 615 subjects. Of these, $18.9 \%$ of individuals fulfilled high-risk criteria. Recursive partitioning identified the following four questions as most informative: 1. "Do you have a FDR with an HNPCC-associated cancer at age $<50$ years?" 2. "Do you have three or more relatives with CRC?" 3. "Do you have a FDR with polyps at $<50$ years?" 4. "Do you have a personal history of polyps diagnosed before age 40?" When asked in succession, these questions captured $75.9 \%$ of high-risk individuals, with $100 \%$ specificity. CONCLUSION: We have developed a four-question Colorectal Cancer Risk
Assessment Tool that can efficiently identify high-risk individuals who may require more aggressive colonoscopic surveillance than average or moderate risk patients, and may benefit from referral for genetic evaluation. This tool can be easily incorporated into the routine pre-procedural evaluation for patients undergoing colonoscopy.

T1094

Oesophageal Cancer Survival According to Route of Diagnosis: Impact of the United Kingdom 'Two Week Rule' and Barrett's Surveillance in a Tertiary Cancer Centre

Nick Stern, Neil Kapoor, Richard Sturgess, Keith Bodger

BACKGROUND Oesophageal cancer (OCa) has a high mortality. Surveillance for patients with Barrett's metaplasia aims to detect early adenocarcinoma (AdCa). Most cases of O-Ca are diagnosed at a symptomatic stage. Delayed investigation is cited as a factor contributing to poor outcomes in the UK. The National Cancer Plan (2000) stipulated a 14-day waiting time standard for secondary care providers to investigate patients with alarm symptoms referred from primary care (the 'two week rule', TWR). This provided new fast-track services for patient with suspected cancer. AIMS (1) To compare survival for OCa before and after implementation of the TWR; (2) To examine survival according to route of diagnosis after implementation of the TWR, and to compare with outcomes for Barrett's surveillance; (3) To determine the proportion of all AdCa detected by surveillance. SETTING University Hospital serving a population of $>330,000$, implementing a Rapid Access Upper GI Cancer Service (RAUGICS) METHODS Using hospital IT systems, a cancer service database (Ref. 1) and casenote review, we audited outcome of all OCa diagnosed during two 2 -year periods, one before (Pre-) and the other after (Post-) service re-organisation. Data for tumour type, stage, operability and 2-year survival were obtained. For the post-TWR period, route of cancer diagnosis was designated as: 'RAUGICS' (fast-track route, $n=3008$ referrals to this service), 'Other' (includes non-urgent open access endoscopy, GI clinic and emergency room) and 'BarS' (Barrett's surveillance, $\mathrm{n}=420$ patients) RESULTS (1) Pre- versus Postperiods: Cases: 72 v 95; Mean age: 68 v 68 yrs; Surgery with curative intent: $38 \%$ v $26 \%$; 2-yr survival: $19.4 \%$ v $24.2 \%$ (AdCa: $22.5 \%$ v $25.4 \%$; Squamous Cell Ca: $16.1 \%$ v 25\%) All non-significant. (2) 2-year survival according to route of diagnosis (post-TWR period): RAUGICS: 18\%; Other routes: $30 \%$; and BarS 50\%. BarS detected $\mathrm{Ca}$ at a rate of $0.49 \%$ per annum ( $6.5 \%$ of all AdCa cases). Conclusions Fast-track service based on alarm symptoms selects patients with poor 2-year survival (18\%). It provides prompt palliation but doesn't improve survival. Patients who continued to access care via traditional routes had better survival (30\%), perhaps reflecting earlier stage disease, lack of alarm symptoms or "chance' diagnosis. Highest survival was achieved for surveillance detected $\mathrm{AdCa}(50 \%)$, though these represent only a minority of AdCa diagnosed in population (6.5\%). Screening for asymptomatic, early stage disease is required if outcomes are to improve for O-Ca. Ref 1 . Kapoor N et al. Gut 2005;54(1): 40-45.

\section{T1095}

Prospective Evaluation of Fecal Tumor Pyruvat Kinase Typ M2 (M2-PK) As a Screening Biomarker for Colorectal Neoplasia

Marc Naumann, Yogesh Shastri, Gerhard Oremek, Ernst Hanisch, Wolfgang Roesch Joachim Moessner, Juergen Stein

Introduction: Tumor formation is usually linked to an expansion of glycolytic phosphometabolite pools and aerobic glycolytic flux rates. To accomplish this, tumor cells generally overexpress a special glycolytic isoenzyme, termed fecal pyruvate kinase type 2 (M2PK) Here, we assessed fecal M2PK as a screening biomarker for colorectal neoplasia. Fecal M2PK was compared to fecal haemoglobin $(\mathrm{Hb})$ and colonoscopy which are currently the existing standards for stool screening and structural evaluation, respectively. Methods: A total of 347 consecutive patients referred for colonoscopy were included. Stool specimens were collected before purgation, processed appropriately, and quantitatively analyzed for M2PK A positive M2PK test was defined by a M2PK level $>4 \mathrm{U} / \mathrm{g}$. Experienced colonoscopist performing the procedure were blinded about the fecal M2PK results. Results: Based on the pre-established cut-point of $4 \mathrm{U} / \mathrm{g}$ of stool, the fecal M2PK assay had a sensitivity of $81.1 \%$, specificity of $71.1 \%$, PPV of $61.9 \%$, and NPV of $86.7 \%$ for subjects with any colorectal neoplasms. For the subset of subjects with clinically relevant disease, these performance estimates were $70.3 \%, 71.1 \%, 80.6 \%$, and $58.3 \%$, respectively. Fecal Hb concentrations of stool had a sensitivity of $36.5 \%$, specificity of $92.2 \%$, PPV of $72.9 \%$, and NPV of $71.5 \%$ for subjects with any colorectal neoplasms. For clinically relevant disease, these estimates were $38.4 \%, 92.2 \%, 89.4 \%$, and $46.6 \%$, respectively. Conclusions: Fecal M2PK was found to be a poor screening biomarker for colorectal neoplasia based on its prospective comparison against colonoscopy in a subject population at above average risk. We believe that the marginal performance characteristics demonstrated by the fecal M2PK assay do not warrant its application as a screening tool for colorectal neoplasia in this clinical setting.

\section{T1096}

Impact of a Public Educational Intervention On Colorectal Cancer Screening Behaviour

Antonio Z Gimeno, Adolfo Parra-Blanco, David Nicolas, Marta Garcia-Velazquez, Alejandro Jimenez, Enrique Quintero

Background: Low public awareness is an important barrier for increasing participation and compliance in colorectal cancer (CRC) screening campaigns. Aim: to evaluate the effectiveness of a public educational intervention for CRC screening in the average-risk population. Methods: during a CRC screening campaign, 158 subjects from 50 to 79 years were consecutively included. Participants were randomly assigned either to a control or an intervention group: the control group received written information about CRC and watched a 5 minutes non-related CRC video-tape, whereas the study group received additional written information about CRC prevention and watched a 5 minutes educational video-tape that specifically explained the prognosis of advanced and early CRC, the usefulness of performing a faecal 
occult blood test (FOBT) and showed how colonoscopy and polypectomy are performed under conscious sedation. Prior and after the provider's visit, every participant completed a 5 items of a previously validated questionnaire on the knowledge of CRC which included: age of risk for developing CRC, risk factors, warning symptoms, 5-year prognosis and incidence. Subjective risk perception for developing CRC barriers or benefits and intention of screening were also investigated. Finally, every subject received a FOBT and was requested to return it back in 2 weeks. Results: educational intervention significantly improved the the 5 items regarding public knowledge of $\mathrm{CRC}(\mathrm{p}<0.001)$. The intervention group returned significantly more FOBT than the control group (70\% vs $54 \%, \mathrm{p}<0.03$ ). In addition, barrier scores decreased significantly $(\mathrm{p}<0.05)$ only in the study group. Besides, educational intervention was the only independent predictor associated with compliance improvement (OR 1.91 [95\% IC, 1.0 to 3.7] $\mathrm{p}=0.05$ ). Conclusion: educational intervention highlighting the most important features of CRC significantly reduces the barriers to screening and improves public awareness and compliance in CRC screening campaigns.

\section{T1097}

Efficacy of a New Immunochemical Faecal Occult Blood Test for Colorectal Cancer Screening in a High-Risk Population

Antonio Z Gimeno, David Nicolas, Marta Garcia-Velazquez, Alejandro Jimenez, Manuel Hernandez-Guerra, Enrique Quintero

Background: Colonoscopy is empirically recommended as the first choice strategy for colorectal cancer (CRC) screening in the high-risk population, but its efficacy is limited by a low compliance. Aim: to determine the efficacy of a new faecal occult blood test (FOBT) for CRC screening in a family-risk population. Methods: 175 first-degree relatives of patients with CRC were prospectively assessed. On the whole, 38 were excluded because of a personal history of colorectal neoplasm, inflammatory bowel disease, coagulopathy, having recently been screened for CRC or age $<40$ years if the index case was older than 50 . All subjects were invited to receive colonoscopy and 2 FOBT: a qualitative inmunochemical latexagglutination test $(\mathrm{OC}$-Light () and a guayac-based test (Hemo-Fec () ). Results: 137 subjects were included: $106(77 \%)$ returned both FOBT tests and $86(81 \%)$ underwent colonoscopy. FOBT was positive in 19/106 (17\%): 18 to OC-Light ${ }^{\circledR}$ and 1 to both FOBT. Colonoscopy detected neoplasms in 43/86 (50\%): 33/43 (77\%) were non-advanced adenomas, and 10/ $43(23 \%)$ advanced adenomas ( $>1 \mathrm{~cm}$ in size, villous pattern or high grade dysplasia). None CRC was detected. OC-Light ${ }^{\circledR}$ detected $9 / 10(90 \%)$ of the advanced adenomas whereas Hemo-Fec ${ }^{\circledR}$ did not detect any. Considering colonoscopy as the gold standard test, OCLight ${ }^{\circledR}$ showed a sensitivity, specificity, positive and negative predictive values for advanced neoplasia detection of $90 \%, 88 \%, 50 \%$ and $98 \%$, respectively. One patient $(5 \%)$ with OCLight ${ }^{\circledR}(+)$ regret colonoscopy. Conclusión: In the family-risk population, FOBT screening with the qualitative immunochemical latex-agglutination test (OC-Light $₫)$ : 1) detects most colorectal advanced neoplasms; and 2) might improve adherence to screening, allowing for a better selection and saving of colonoscopies.

\section{T1098}

An Automated Developed, Quantitative, Immunochemical Fecal Occult Blood Test Has a Higher Positive Predictive Value for Significant Colonic Neoplasia Than a Sensitive Guaiac Test

Zohar Levi, Rachel Hazazi, Paul Rozen, Alex Wilkin, Amal Waked, Suzana Fishman, Yaron Niv

Background: The guaiac fecal occult blood test (G-FOBT) is the standard average-risk population screening test for colorectal cancer (CRC). An automated-developed, quantitative, immunochemical fecal occult blood (I-FOBT) is specific for human hemoglobin eliminates diet restriction and allows choosing a suitable hemoglobin threshold for follow-up colonoscopy. Aim: To compare the diagnostic yield of a sensitive G-FOBT with an I-FOBT. Methods: 101 asymptomatic patients with positive G-FOBT and 51 patients with negative G-FOBT underwent colonoscopy either because of the positive G-FOBT or their screening protocol. All patients underwent 3-day consecutive I-FOBTs prior to colonoscopy using the OCSensor automated instrument (Eiken, Japan) with hemoglobin threshold of $100 \mathrm{mg} / \mathrm{ml}$ to determine positivity; and 3-day G-FOBT with Hemoccult Sensa. The endoscopic end-point was "significant" neoplasia, CRC or advanced adenoma. Results: Significant neoplasia was identified in 10 patients; 8 patients had advanced adenoma and 2 patients had CRC. Sensitivity, specificity, negative and positive predictive values for significant neoplasia were $75.0 \%, 34.5 \%, 92.1 \%$, and $11.8 \%$, for G-FOBT and $75 \%, 94.8 \%, 96.9 \%$, and $63.1 \%$ for I-FOBT. The number colonoscopies needed to detect one significant neoplasia was 8.5 with the G-FOBT and 1.5 with the I-FOBT. Conclusions: A quantitative I-FOBT is more specific than G-FOBT of comparable sensitivity. Thereby, reducing the number of negative colonoscopies with their associated costs and morbidity.

\section{T1099}

Endoscopic Capacity and Manpower Planning in the Netherlands in the Era of Colorectal Cancer Screening

Jochim S. Terhaar Sive Droste, Mike E. Craanen, Jeroen J. Kolkman, Chris J. Mulder

Background: Currently, there is still debate on the necessity of population screening for colorectal cancer (CRC) in The Netherlands. One of the main arguments against an endoscopic screening program is the lack of adequate capacity and manpower. Before embarking on a nation-wide CRC screening program, it is mandatory to provide a clear insight in the Dutch endoscopic practice and manpower planning. This study is part of an interim analysis which will be conducted every five years in the Netherlands. In this way, trends in endoscopic production and manpower planning can be monitored. Aims: To determine endoscopic production and manpower planning in the Netherlands and to investigate the ability to meet the extra endoscopic demand associated with the intended implementation of a CRC screening program. Methods: A questionnaire was sent to all Dutch endoscopy units ( $\mathrm{N}=$
100) in the spring of 2005. Results: Ninety-eight percent of the units returned the questionnaire. The total number of endoscopies performed in 2004 was 408.982 . The total numbe of colonoscopies was 116.815 , corresponding with 719 colonoscopies per 100.000 inhabitants. The mean waiting time for routine gastroscopy and colonoscopy was 3,0 weeks (range 1-12 weeks) and 5, 1 weeks (range 1-15 weeks), respectively. The total number of endoscopists was 598. Fifty-two percent of the endoscopy units could not manage the projected increase in the number of colonoscopies related to a CRC screening program, resulting in 1334 to 1641 colonoscopies per 100,000 inhabitants. Conclusions: The quantitative and qualitative alterations in endoscopic practice will definitely hamper the implementation of a CRC screening program, due to the lack of adequate capacity and manpower. Although FOBT based screening may temporarily alleviate the capacity problems, it is a clearly inferior screening modality compared to colonoscopic screening. Hence, rigorous cost-effectiveness studies as well as adequate insights in the current and future endoscopic capacity and manpower will hopefully persuade the Dutch central government together with the healthcare insurance companies to create the opportunities and to provide the funding to realize the increase in colonoscopic capacity and manpower necessary for a successful, large-scale implementation of a nation-wide CRC screening program.

\section{$\mathrm{T} 1100$}

Primary Care Physicians Knowledge and Practices Regarding Colorectal Cancer Screening

Mohamad Eloubeidi, Michele Shipp, Renee Desmond, Tykeysha Boone, Norman Weissman, Marion Nadel, Mona Fouad

Background: Colorectal Rectal Cancer (CRCA) screening with Flexible sigmoidoscopy (FS) is associated with decreased mortality. Alabama Primary care physicians' (PCPs) knowledge and practices regarding CRCA screening are unknown. In particular, no data exist regarding training and practices of PCPs for FS to screen for CRCA. Objectives: 1) To gain understanding of Alabama PCPs knowledge and practices regarding CRCA screening, 2) to assess the need for PCPs to a) gain additional training in flexible sigmoidoscopy (FS) and b) to implement it in their offices. Methods: Self-administered questionnaires were mailed to 1916 PCPs in Alabama. Data were sought on personal demographics, practice characteristics, CRCA screening knowledge and practices as they pertained to fecal occult blood testing (FOBT), colonoscopy, double contrast barium enema (DCBE) and FS. The questionnaire also addressed issues of physicians' training in FS, the use of FS within practices, and physicians' willingness and ability to perform FS in their practices once trained. Results: Out of 1916, 660 (34\%) PCPs returned completed questionnaire. Sixty-one percent of the participants practiced in urban locations, while 39\% practiced in rural areas. Sixty-four percent recommended FOBT testing to their patients. In contrast, only $16 \%(95 \% \mathrm{CI}: 13.2,18.8)$ of the physicians recommended FS. Sixty-six percent recommended colonoscopy, while only $8 \%$ recommended DCBE. Sixty-three percent of PCPs received prior FS training and the majority of those trained received it while in residency training $(76 \%)$. Of those, only $21 \%$ performed more that 25 FS procedures during training. Only $17 \%(95 \%$ CI: $14.2,19.8)$ reported actually performing FS in their offices. Patients' non-compliance, cost to conduct the procedure, lack of practice time, cumbersome regulations related to endoscope disinfection, and difficulty in finding and training assistants were among the reported obstacles preventing PCPs from performing FS in their offices. Most of the physicians who did not perform FS reported that training is not available to them (49\%). Thirty-seven percent reported interest in pursuing training were it available. Eighty-seven percent of those interested in training reported that they would perform FS if adequately trained. Eleven physicians already enrolled in the hands-on-training program. Conclusions: Most survey respondents recommend adequate screening tests for CRCA. However, only a minority recommend FS as a screening procedure Very few PCPs perform FS currently in their office. Whether providing these PCPs with training opportunities in FS will increase FS utilization remains to be seen.

\section{T1101}

Cancer Investigation At Peking University Using Fourier Transform Infrared (FT-IR) Spectroscopy. FT-IR Separation of Gastric Cancer from Normal Stomach Using Chemometrics

Q-B Li, X Li, Y-Z Xu, Roger D. Soloway, X-F Ling, J-S Wang, G-J Zhang, X-S Zhou, J-S Shi, B-B Wang, Q-H Pan, J-G Wu

Background: The ability to accurately identify cancer during an operation can facilitate the proper surgical procedure at the time of the original surgery. We have previously reported the ability to utilize portions of the infrared spectra to separate gastric cancer from normal stomach. This required the involvement of an expert infrared spectroscopist. To simplify and automate this process, we have employed a series of computer based chemometric techniques to the spectra to make the separation more facile and more accurate. Aim: To improve FT-IR identification of gastric cancer from normal stomach by modifying spectra using chemometric techniques. Methods: Spectra were obtained using application of the biopsies from 37 cases of cancer and 26 normal gastric samples to an attenuated total reflectance (ATR) crystal attached to a Nicolet Magna $750 \mathrm{H}$ spectrometer. To use all of the spectral information, spectra were smoothed and then subjected to Resampling Half-Mean and standard normal variate methods. Samples were cross-validated using a KNN method. These programs were followed using Matlab 6.5 statistical package. Results: Among 37 samples from cancer patients, 36 were correctly diagnosed, one was judged to be benign. Among 23 samples from normal stomach 19 were correctly diagnosed while 4 were thought to be malignant. The sensitivity of cancer detection was $98 \%$ while the specificity was $83 \%$. Steps in the process will be presented. Conclusion: Applying chemometric statistical techniques to FT-IR spectra of biopsies improves and automates the spectral determination of cancer. The technique is highly sensitive but there were $17 \%$ false positive determinations. ***Project supported by State Key Project for Fundamental Research of MOST (2002CCAQ01900), National Natural Science Foundation of China (No. 30371604) and Doctoral Project of Education Ministry of China. 
$\mathrm{T} 1102$

Patient Experience and Preference of Conventional Colonoscopy Versus Stronger High Field Magnetic MR-Colonography (3-TESLA): A Feasability Study

Juergen M. Gschossmann, Bettina Saar, Corinna A. Messikommer, Wojtek Bielecki, Christoforos Stoupis, Peter Netzer

BACKGROUND: Overall, conventional colonoscopy (CC) is still the gold standard for coloreclancer screening. However, virtual colonoscopy has reached a technical level to become an increasingly attractive alternative for these questions given the non-invasive nature of the respective radiological imaging techniques. While comparative studies of CT colonography versus $C C$ suggest a patient preference for radiological examination, data on patient assessment of CC versus MR colonography (MRC) are missing. AIMS: to analyze and compare experiences of and preferences for CC versus MRC in patients scheduled for elective endoscopy of the lower gastrointestinal tract in a tertiary referral center. METHODS \& PATIENTS: In this feasibility study, $55 \mathrm{pts}$ (mean age $58 \mathrm{yrs}, 19-88 \mathrm{yrs}, 21$ females) were included. At he day of the scheduled endoscopy, all pts underwent first MRC with contrast enema, followed by CC. During CC, analgo-sedation (pethidin/midazolam) was only given upon patient request. To assess patient perception of both examinations, interviews were performed at two occasions: a) before discharge from the endoscopy unit (time point A) and b) 4-6 weeks after discharge (time point B). Main parameters of the assessment were "overal preference", "pain", "discomfort" and the question which technique they would prefer for re-examination of the colonic frame (only time point B). RESULTS: At time point A, 24/55 (44\%) pts preferred CC to MRC, the same number of patients preferred MRC, while 7 (12\%) had no preference. Twenty-nine of $55(53 \%)$ pts received analgo-sedation for CC. Fourteen of these pts were in favour of MRC while 13 pts preferred CC. The analysis of the parameter "pain" showed that $28(51 \%)$ pts experienced more pain during CC than during MRC while for 11 (20\%) pts MRC was more painful. Sixteen (29\%) pts did not notice any difference. As to "discomfort" associated with the examinations, 21 (38\%) pts regarded MRC more uncomfortable than CC whereas for 16 (29\%) pts CC was associated with more discomfort. Similarly, the intensity of pain during CC was higher compared to MRC while the intensity of discomfort was higher during MRC. At time point B, 28/55 (51 $\%$ ) of the pts preferred MRC to CC while $21 / 55(38 \%)$ chose CC as preferred examination technique. CONCLUSION: Directly after the examinations, patient satisfaction was similar for MRC and CC with analgo-sedation in case of painful examination. Whereas CC was more painful, MRC was more uncomfortable. In the follow-up interview, a tendency towards higher patience acceptance of MRC compared to CC was noted.

T1103

Oesophageal Cancer: How Reassuring Is a Negative Endoscopy in the Last 3 Years?

Benjamin Colleypriest, Peter Marden, Sarah Bailey, Aram Fard, John Linehan

Introduction Oesophageal cancer carries a grave prognosis with a 5-10\% 5 year survival. The incidence of oesophageal cancer has increased rapidly over the last 30 years. This corresponds to an increase of $8 \%$ per year and is thus the fastest growing cancer in the western world. Symptoms of early upper GI cancer are non-specific and many patients may be referred for endoscopy at this stage. The aim of this study was to look at the current frequency of normal investigations during a 3 year period prior to a diagnosis of oesophageal cancer in a hospital that serves a population of 500000 people. A similar study completed in Bath for colorectal cancer found that $4 \%$ of patients had had a previously norma investigation within the last 3 years. Methods All patients with a new diagnosis of oesophageal cancer, including tumours of the gastro-oesophageal junction, during a 3.5 year period (2001-2004) were identified. Records were examined to find patients who, during the 3 years prior to diagnosis, had had either a barium swallow or endoscopy. The results of all previous investigations during the three year period were recorded along with patient demographics. Results 180 patients, $67 \%$ males and 33\% females, were found with a new diagnosis of oesophageal cancer. The majority of tumours found were adenocarcinoma on histology. 17 patients had been investigated with a barium swallow. Of these tests 15 were abnormal and prompted an endoscopy. Both of the normal barium swallows were 21 months before the diagnosis. 33 patients were found to have had endoscopies in the 3 years prior to diagnosis. Of these 18 prompted repeat endoscopy, 6 were part of a Barrett's surveillance program and 7 were normal (6-36 months prior to diagnosis). Conclusion This study sought to answer the question, 'How reassuring is a normal investigation within the last three years?' We found a low but significant miss rate for upper gastrointestinal investigations in a three year period prior to diagnosis. $5 \%$ (3.9\% endoscopy $1.1 \%$ barium) of patients presenting with oesophageal cancer had been investigated. Oesophageal cancer is a rapidly growing tumour that often presents late with no previous symptoms. The rates of diagnosis of early cancer in the UK are lower than is other countries. Does this represent differences in techniques or the lack of a screening program for upper GI cancer? This low but significant miss rate suggests that patients who develop new symptoms need repeat investigation. Results

\begin{tabular}{|c|c|c|c|c|c|}
\hline Sex & $\begin{array}{c}\text { Number } \\
\text { Patients }\end{array}$ & $\begin{array}{c}\text { Average } \\
\text { Age }\end{array}$ & Adenocarcinoma & $\begin{array}{c}\text { Normal Barium } \\
\text { Studies }\end{array}$ & $\begin{array}{c}\text { Normal } \\
\text { Endoscopies }\end{array}$ \\
\hline Male & 121 & 68.6 & 82 & 1 & 3 \\
\hline Female & 59 & 75.3 & 47 & 1 & 4 \\
\hline
\end{tabular}

\section{$\mathrm{T} 1104$}

Characteristic of Colorectal Neoplasm in Symptomatic Asian Population: Prospective Survey of 4,681 Consecutive Patients Undergoing Colonoscopy Abdul Aziz Rani, Murdani Abdullah, Aru W. Sudoyo, Sung J. Yoseph, The Asia-Pacific Working Group on Colorectal Cance For

Background: The incidence of colorectal cancer is rising in some Asian countries. However, there is scarcity of data on prevalence and characteristics of colorectal neoplasia in Asian region. Aim: To determine the prevalence and characteristics of colorectal neoplasm in consecutive symptomatic subjects undergoing colonoscopic examinations in different Asian countries Method: From July to 2004 to September 2005, we conducted a prospective survey at 17 centers from 11 Asian cities. Participating centers entered data into a computerized registry from consecutive subjects who underwent colonoscopic examinations. The registry was designed based on CORI database of the ASGE. We included symptomatic subjects older than 16 . Those with a history of previous colorectal resection, with a history of colonoscopy within 5 years, with incomplete colonoscopy or indeterminate histological results were excluded. Those who underwent surveillance colonoscopy for inflammatory bowel disease were also excluded. Advanced neoplasm was defined as adenoma larger than $10 \mathrm{~mm}$, having $>25 \%$ villous features, or high grade dysplasia and invasive carcinoma. Result: A consecutive of 4,681 subjects (mean (SD) age 54.8+15.2 yrs; men 50.2\%) were studied. $132(2.8 \%)$ had a family history of colorectal cancer(defined by the first degree relatives being affected). The ethnicity was predominantly Chinese $(2373,50.7 \%)$ and Korean $(1012,21.6 \%)$. Indications for colonoscopy were abdominal pain $(20.0 \%)$, rectal bleeding $(15.0 \%)$, change in bowel habit $(13.5 \%)$, gastrointestinal bleeding $(8.1 \%)$, anemia $(8.1 \%)$, diarrhoea $(7.5 \%)$ and others. Predicting factors for prevalence of proximal advanced neoplasm in symptomatic and distal polyp-free patients were increasing age and Korean $(\mathrm{p}=0.004)$ and Japanese ( $\mathrm{p}=0.007$ )ethnic groups. Age groups $60-69$ years (OR 4.8,95\%CI:1.8-12.7), 7079 years (OR 6.7, 95\%CI:2.5-18.0), 80 year (OR 10.3,95\%CI:3.3-32.5) showed significant difference in prevalence of proximal advanced neoplasms. Prevalence of proximal advanced neoplasm according to the distal finding were $11.0 \%$ (RR3.8,95\% CI:2.4-5.9) after adjusting for age and sex Conclusion: There is an ethnic variation in prevalence and characteristics of colorectal neoplasms in the Asian population

Prevalence and location of neoplasm are listed in table 1.

\begin{tabular}{|c|c|c|c|c|c|c|}
\hline & \multicolumn{2}{|c|}{ All adenoma plus } & \multicolumn{2}{c|}{ Advanced neoplasm } & \multicolumn{2}{c|}{ Invasive cancer } \\
\hline & $\mathrm{N}$ & $\%$ & $\mathrm{~N}$ & $\%$ & $\mathrm{~N}$ & $\%$ \\
\hline Distal & 503 & 10.7 & 251 & 5.4 & 143 & 3.1 \\
\hline Proximal & 273 & 5.8 & 106 & 2.3 & 56 & 1.2 \\
\hline Synchronous & 209 & 4.5 & 31 & 0.7 & 2 & 0.0 \\
\hline Total & 985 & 21.0 & 388 & 8.3 & 201 & 4.3 \\
\hline
\end{tabular}

\section{T1105}

Acid Suppression and Upper Gastrointestinal Endoscopy Prior to Diagnosis in Patients with Adenocarcinoma of the Esophagus

Eva van Soest, Peter D. Siersema, Jeanne P. Dieleman, Miriam C. Sturkenboom, Ernst J. Kuipers

Background: The incidence of the adenocarcinoma of the esophagus (ACE) is rapidly increasing. The main risk factors for ACE are gastroduodenal reflux and Barrett's esophagus (BE) Gastroduodenal reflux symptoms are usually treated with $\mathrm{H}_{2}$-receptor antagonists $\left(\mathrm{H}_{2} \mathrm{RA}\right)$ or proton pump inhibitors (PPIs), and if persistent, further investigated by upper gastrointestinal (GI) endoscopy. There is limited knowledge how these resources are being used in patients at risk for ACE. Aim: To assess the use of medical resources in the medical history of ACE patients prior to diagnosis. Methods: We identified all ACE cases diagnosed between 1996 and 2003 within the Integrated Primary Care Information database, a general practice research database containing the complete longitudinal electronic medical records of more than 600000 persons. Rates of $\mathrm{H}_{2}$ RA use, PPI use and performed upper GI endoscopy in the 3 years prior to ACE diagnosis were examined. Results: In total, 51 incident ACE cases were identified with a mean follow-up of $4.1 \pm 1.8$ years prior to diagnosis. Thirty-nine (76\%) were males and mean age at diagnosis was $67.7 \pm 9.6$ years. In the 3 years prior to ACE diagnosis, 9 patients (26\%) with sufficient follow-up $(n=34)$ underwent diagnostic gastroscopy, which was in all but one performed in the last 12 months prior to ACE diagnosis. Only one of these patients was diagnosed with $\mathrm{BE}$, which was suspected for early malignancy. In the 3 years prior to ACE diagnosis, 18 (53\%) used acid suppressive drugs (22\% used $\mathrm{H}_{2} \mathrm{RAs}, 67 \%$ PPIs, $11 \%$ both). The proportion of patients using acid suppression was $38 \%$ in the $3^{\text {rd }}$ and $29 \%$ in the $2^{\text {nd }}$ year before ACE diagnosis, but increased to $57 \%$ in the $1^{\text {st }}$ year before ACE diagnosis. Conclusion: The use of medical resources is limited in patients at high risk for ACE. Only a small proportion is previously studied by upper GI endoscopy, and those who undergo endoscopy are often not diagnosed with BE. The majority of patients developing ACE have not used acid suppressive drugs in the $2^{\text {nd }}$ and $3^{\text {rd }}$ year before ACE diagnosis, which may be related to a relatively low frequency or severity of symptoms. The use of resources increases in the last year before ACE which is probably due to early symptoms of cancer development.

Number of ACE patients using medical resources prior to diagnosis 


\begin{tabular}{|c|c|c|c|c|}
\hline & $\begin{array}{c}1^{\text {st }} \text { year before } \\
\text { diagnosis } \mathrm{n}(\%) \\
\mathrm{N}=51\end{array}$ & $\begin{array}{c}2^{\text {nd }} \text { year before } \\
\text { diagnosis } \mathrm{n}(\%) \\
\mathrm{N}=42\end{array}$ & $\begin{array}{c}3^{\text {rd }} \text { year before } \\
\text { diagnosis } \mathrm{n}(\%) \\
\mathrm{N}=34\end{array}$ & $\begin{array}{c}\text { Total over 3 years } \\
\text { before diagnosis } \\
\mathrm{n}(\%) \mathrm{N}=34\end{array}$ \\
\hline $\begin{array}{c}\text { Use of acid } \\
\text { supression }\end{array}$ & $29(57)$ & $12(29)$ & $13(38)$ & $18(53)$ \\
\hline $\mathrm{H}_{2}$ RAs & $12(41)$ & $5(42)$ & $4(31)$ & $4(22)$ \\
\hline PPIs & $14(48)$ & $7(58)$ & $8(61)$ & $12(67)$ \\
\hline Both & $3(10)$ & 0 & $1(8)$ & $2(11)$ \\
\hline Gastroscopy & $13(25)$ & 0 & $1(3)$ & $9(26)$ \\
\hline Any resource & $36(71)$ & $12(29)$ & $13(38)$ & $23(68)$ \\
\hline
\end{tabular}

Performance of Capsule Endoscopy in Obscure Intestinal Bleeding - A MultiCenter Outcome Study

Joerg G. Albert, Mathias Dollinger, Leonore Hahn, Detlef Heinig, Konrad Schoppmeyer, Heiner Porst, Rainhard Lorenz, Mathias Plauth, Karel Caca, Wolfgang E. Fleig

Introduction: Capsule endoscopy (CE) has proven reliable in establishing bleeding sources in the small bowel in obscure intestinal bleeding. Its impact on therapeutic and long term outcome in defined patient groups is unknown. Methods: Patients in whom CE investigations were done for obscure intestinal bleeding (bi-directional endoscopy without findings) in five study centers between Dec 2001 and Dec 2004 have been included in this longitudinal prospective cohort study. Thereby, 299 investigations in 291 patients were identified and 293 investigations in 285 included. Results: A bleeding source was detected in 224 cases (76.5\%). CE was diagnostic in $177(79.0 \%)$ of these cases, and repeated or additional investigations resulted in findings in 47 cases (21.0\%), and therapeutic measures (endoscopic intervention, surgery, and medical) were initiated in 142 cases (48.5\%); (table 1). In 4 cases $(17.9 \%)$, patients were re-admitted due to digestive bleeding. Angiodysplasias, age older than 60 years, anticoagulants, and prior bleeding events were the main determinants to predict bleeding relapses during follow-up (table 2). Capsule retention occurred in four cases $(1.36 \%)$ and surgical removal of the capsule was necessary in three cases $(1.02 \%)$ Conclusion: Age, anticoagulants, treatment, and diagnosis determine the occurrence of bleeding relapses in obscure intestinal bleeding. Capsule endoscopy mainly contributes to the diagnosis, and might therefore be regarded as the first line diagnostic method in intestinal bleeding following negative bi-directional endoscopy

Ethnic Differences in the Prevalence of Advanced Colorectal Neoplasia in Asi Ida Hilmi, Kl Goh, Jjy Sung, Wk Leung, J Lau, Kg Yeoh, L Ho, Mh Chen, Cj Li, G Makharia, R Tandon, M Abdullah, R Fujita, Jy Kim, Hj Kim, Sk Yang, Wh Kim, Ti Kim, Js Byeon, Tan J, E Ong, J Sollano, Cw Wang, Dc Wu, P Kongkam

Introduction In the Asia Pacific region, colorectal cancer is the fastest emerging GI cancer. However, there are marked ethnic differences in Asia and in this multicenter collaborative study we sought to determine the prevalence of advanced neoplastic lesions (ANL) amongst the different races in the region. Methods An Asia-Pacific Working Group on Colorectal Cancer was formed in September 2003 to study the epidemiology of colorectal neoplasia in the region. Consecutive colonoscopy records from July 12004 to December 312004 were obtained from 17 centres in 11 cities (Bangkok, Delhi, Guangzhou, Hong Kong, Jakarta, Kuala Lumpur, Manila, Seoul, Singapore, Taipei, Tokyo). We used a common database modified from the CORI of American Society of Gastrointestinal Endoscopy. Data collected includes indication, clinical data, findings and histology. ANL was defined as adenomas $>1 \mathrm{~cm}$, severe dysplasia, $>75 \%$ villous feature or carcinoma. Results 7290 consecutive colonoscopies were carried out, of which 5474 with complete data were analyzed. Advanced colorectal neoplasms were found in 448 of cases $(7.8 \%)$. The prevalence in each ethnic groups was as follows: Japan 18\% (32/177) Korea 9.9\% (143/1441) Chinese** 7.2\% (203/ 2831) Thai 5.9\% (18/306) Malay* 5.7\% (17/297) Fillipino 5.1\% (19/371) and Indian 4.3\%** (11/257). Conclusions High prevalence races for ANL were Japanese, Korean and Chinese whereas the low prevalence races were Thai, Filipino, Malay and Indian. This suggests that there are host genetic or environmental factors specific to the racial group in the pathogenesis of ANL. Ethnic differences have major implications in screening strategies for CRC in Asia. * Malay and Indonesian were grouped together as they belong to the same racial stock. **Indian and Chinese patients from different Asian countries were grouped together

\begin{tabular}{|c|c|c|c|}
\hline Race & P value & Odds Ratio & $95 \% \mathrm{CI}$ \\
\hline Japanese & $\mathrm{p}<0.001$ & 3.86 & $1.99,7,52$ \\
\hline Korean & 0.016 & 1.92 & $1.12,3.35$ \\
\hline Chinese & 0.30 & 1.35 & $0.79,2.33$ \\
\hline Filipino & 0.94 & 1.09 & $0.53,2.27$ \\
\hline Filipino & 0.94 & 0.94 & $0.46,1.94$ \\
\hline Indian & 0.67 & 0.78 & $0.34,1.80$ \\
\hline
\end{tabular}

\begin{tabular}{|c|c|}
\hline Factor & Relative risk for relapse \\
\hline Diagnosis "Angiodysplasia" & $\mathrm{RR}=6.64 ; \mathrm{p}<0.01$ \\
\hline Age older than 60 yrs & $\mathrm{RR}=2.87 ; \mathrm{p}<0.01$ \\
\hline Anticoagulatory therapy & $\mathrm{RR}=2.65 ; \mathrm{p}<0.01$ \\
\hline Prior bleeding events & $\mathrm{RR}=2.90 ; \mathrm{p}<0.01$ \\
\hline No bleeding source found & $\mathrm{RR}=0.54 ; \mathrm{p}=$ n.s. \\
\hline
\end{tabular}

Table 2. Influence on recurrent bleeding events and subsequent hospital admission (RR relative risk; n.s. - not statistically significant)

\begin{tabular}{|c|c|c|c|}
\hline & Therapy & No therapy & \\
\hline Hemoglobin - mmol/l (mean \pm SD) & $5.59 \pm 1.46$ & $6.22 \pm 1.77$ & $\begin{array}{c}\mathrm{p}< \\
0.01\end{array}$ \\
\hline Transfusion requirements at hospital stay - units (mean \pm & $2.87 \pm 4.44$ & $1.86 \pm 3.48$ & $\begin{array}{c}\mathrm{p}< \\
0.01\end{array}$ \\
\hline SD) & $\begin{array}{c}61.01 \pm \\
17.78\end{array}$ & $\begin{array}{c}60.60 \pm \\
17.46\end{array}$ & $\mathrm{p}=$ n.s. \\
\hline Age - yrs. (mean \pm SD) & &
\end{tabular}

Table 1. Influence on therapeutic measures (RR - relative risk; n.s. - not statistically significant).

\section{T1109}

Biochromoendoscopy: Molecular Imaging with Capsule Endoscopy for Detection of Polypoid Lesions in the GI Tract Howard Zhang, Douglas Morgan, Gerald Cecil, P. Kay Lund

BACKGROUND: Capsule endoscopy (CE) is a minimally invasive technology designed to image the GI tract, primarily the small intestine. The ability of the current white-light CE imaging to discriminate different types of polyps is limited. Molecular imaging utilizing probes activated by neoplasm biomarkers, such as protease activated Near Infrared Fluorescent (NIRF) probes, is a potentially powerful tool to detect and distinguish premalignant and malignant lesions. In this study, we tested whether CE could be integrated with NIRF molecular probes to detect premalignant lesions. METHODS: A Prosense ${ }^{\mathrm{TM}} 680$ (VisEN Medical) probe activatable by proteases up regulated in polypoid lesions was used in the $\mathrm{ApC}^{\mathrm{Min} / \mathrm{+}}$ mouse model of polyposis. Apc ${ }^{\mathrm{Min} /+}$ mice ( 90 days of age) were injected intravenously with Prosense ${ }^{\mathrm{TM}} 680$ (2 nmol Cy 5.5 per animal). Animals were sacrificed 24 hours later and the entire intestine was dissected. In a custom built light-tight chamber, the dissected murine intestine was imaged to capture polypoid lesions with CE (Given Imaging, PillCam SB) under white light and near infrared light. For NIRF imaging, a near-infrared filtering device with excitation $(680 \mathrm{~nm})$ and emission $(700 \mathrm{~nm})$ filters was combined with CE. Resulting CE images were captured, transmitted, and recorded in usual fashion. This molecular imaging of the polypoid lesions was further verified by the standard NIRF detecting device, Xenogen (IVIS) Imaging System. In addition, positive and negative controls were imaged with $C E$ using Prosense ${ }^{\mathrm{TM}} 680$ samples added to buffer with and without trypsin on glass slides. RESULTS: NIRF images of polypoid lesions in the intestine of $\mathrm{ApC}^{\mathrm{Min} / \mathrm{+}}$ mice were readily visualized by CE molecular imaging. White-light images obtained by CE through the light-tight chamber were similar in quality to those from human applications. NIRF images captured by $\mathrm{CE}$ revealed discrete light spots on the control slides only in solutions with trypsin. CONCLUSION: Biochromoendoscopy proof of principle has been demonstrated. Polypoid lesions were detected with CE under near infrared light using a molecular probe specific for neoplastic intestinal lesions. Molecular imaging with capsule endoscopy may provide a novel approach to detect premalignant and malignant lesions in the GI tract. 64 without neoplasia using a newly developed bisulphite modification technique. RESULTS Promoter methylation was observed in all the gastrointestinal specimens and was a gradual increase in the number of genes methylated from normal to cancer, both in the large intestine stomach. As similar trend was observed in the stool samples, these findings provide proof of principal that gene methylation in stool can be used to detect gastrointestine methylation assay offered various cut-off values using six methylated genes to differentiate between the presence or absence of carcinoma, of carcinoma and advanced polyps, or of carcinoma, advanced and minor polyps. CONCLUSIONS The detection of early, asymptomatic gastrointestinal neoplasia can be facilitated by the analysis of fecal DNA methylation status 
T1110

An Integrated Approach to Iron Deficiency Anemia: Role of Videocapsule Endoscopy and CT-Enteroclysis

Angelo Milano, Chrysanthi Balatsinou, Francesco Laterza, Maria Pia Caldarella, Antonella Filippone, Nicola Genovesi, Flora Sacco, Franco Cuccurullo, Matteo Neri

Background: Iron deficiency anemia (IDA) is frequently encountered in clinical practice. Its main causes in developed countries is gynaecological in pre-menopausal women and gastroenterological in men and post-menopausal women. However $30 \%$ to $40 \%$ of patients usually remain undiagnosed even after extensive evaluation. Aims: i) to evaluate the efficacy of a systematic approach to IDA with a standardised diagnostic algorithm including ileoscopy and video capsule Endoscopy (VCE) in a large population of patients in whom extra-digestive causes of anemia have been excluded, and ii) to compare the diagnostic yield of VCE versus CT-enteroclysis. Materials and methods: Consecutive patients with IDA underwent upper endoscopy and colonoscopy plus ileoscopy. Endoscopy negative patients were further evaluated by small bowel follow- through and CT-enteroclysis and, if negative for stricture, by VCE. Out of 189 patients evaluated 144 were diagnosed at upper endoscopy (40 peptic ulcers, 20 erosive gastritis, 1 severe erosive esophagitis; 1 Cameron's lesions, 5 atero-venous malformations, 3 cancers, 5 celiac disease) and lower Endoscopy (4 diverticular disease, 12 angiodysplasia, 22 cancers, 4 IBD, 17 Haemorrhoids, 10 Bleeding polyps). In 45 patients (26 M, $19 \mathrm{~F}$; age 55, range 11-81) negative at gastrointestinal endoscopy, mean haemoglobin levels were $9.2 \mathrm{gr} / \mathrm{dl}$ (range 6-12) and ESR, CPR, fibrinogen and orosomucoid were negative. Overall the diagnostic rate by VCE was $77,8 \%$ versus $22,2 \%$ by $\mathrm{X}$-ray techniques $(\mathrm{p}<0,001)$. Interestingly, VCE was superior to entero-TC when mucosal lesions were found (angiodysplasia: $100 \%$ vs $0 \%$; small bowel Crohn's disease: $88 \%$ vs $50 \%$ respectively), while a comparable diagnostic accuracy was achieved on neoplastic diseases (both 85,7\%). Overall this diagnostic algorithm in 189 patients allowed a certain diagnosis in 96\%. Conclusions: This integrated diagnostic approach which includes endoscopy plus ileoscopy, VCE and CT-enteroclysis is highly efficient in the evaluation of patients with IDA of digestive origin. VCE is superior to CT-enteroclysis when angiodysplasia or Crohn's disease occur.

\section{T1111}

Double Balloon Enteroscopy - A Useful Diagnostic Tool? Analysis of a Large, Non-Selected Cohort of Patients Regarding Success and Complications of Double Balloon Endoscopy

Christian Maaser, Hansjoerg Ullerich, Karin Menzel, D.R. Domagk, Andreas Luegering, Wolfram Domschke, Torsten Kucharzik

Aim: Double balloon enteroscopy has become one of the most innovative diagnostic new tool for the examination of the small bowel also allowing therapeutic interventions. The aim of this analysis was the evaluation of the success on one hand and potential risks on the other hand in a large cohort of non selected patients of one endoscopic center. Methods: We evaluated all examinations performed in our center starting November 2004 until mid November 2005, which resulted in a total of 215 examinations. Endoscopies were performed with the double balloon enteroscope Fujinon EV EN-450F. The examinations were performed by a team of 4 experienced endoscopists. Results: Indications for double balloon endoscopy have been obscure/occult gastrointestinal bleeding in 94 patients, suspected Crohn s disease in 18 , strictures in 13 and suspected neoplasias in 14 patients. Overall we detected angiodysplasias in 32 cases, of which the most prominent ones were successfully treated with argonplasma coagulation in 17 patients. In 9 out of 18 cases suspected Crohn s disease was verified endoscopically and histologically. In 10 patients previously unknown neoplasias were detected. These included a tubulo-villus adenoma, a carcinoid tumor, three gastrointestinal stromal cell tumors (GIST), and intramural neurofibromatosis. Furthermore in 2 patients Peutz-Jeghers polyps were successfully resected during double balloon enteroscopy. In addition to the examination of the small bowel we used the double balloon technique to reach the Papilla vateri successfully in 5 patients that presented with a Y-Roux anastomosis and cholangitis. By using the double balloon technique we were able to successfully perform papillotomies and dilatations leading to successful drainages of the bile ducts. Regarding complications we recorded minor symptoms such as postinterventional abdominal pain or mildly, temporarily elevated temperatures in about $10 \%$ of all patients. However non of the double balloon enteroscopies led to any major complications, e.g. perforations, bleeding or pancreatitis, Conclusion: Double balloon enteroscopy in this large, non-selected cohort showed to be an effective and save procedure. The clear advantage of this new technique is the possibility for therapeutic interventions, e.g. coagulation of angiodysplasias, polypectomies and balloon dilatation of Crohn $\mathrm{s}$ disease strictures.

\section{$\mathrm{T} 1112$}

Clinical Outcome of Patients Submitted to Capsule Endoscopy for Suspect Crohn's Disease

Carlo M. Girelli, Paola Porta, Francesco Rocca

Capsule endoscopy (CE) has a diagnostic yield greater than radiology in the detection of subtle inflammatory changes of the small bowel mucosa, but their clinical significance is uncertain because of the lack of long term follow up studies. Aim of the study was to follow a cohort of patients with suspect Crohn's disease of the small bowel (SBCD) until a well established diagnosis in order to detect the clinical meanings of CE findings. From April 2002 to March 2005, we submitted to CE twenty-seven patients ( 11 males, $40 \pm 18$ yrs) with abdominal pain and diarrhea lasting more than three months, and at least one of the following features: anemia, weight loss, fever, extra-intestinal manifestation(s) of inflammatory bowel disease. All subjects underwent an unremarkable pan-endoscopy, serology for celiac disease and intestinal radiologic studies inconclusive for small bowel abnormalities. By CE findings, patients were assigned to three groups; group $\mathrm{A}$, severe stricturing lesions mandating surgery; group B, moderate inflammatory lesions further investigated by invasive means; group C, minimal inflammatory changes or normal findings (including lymphoid hyperplasia of the terminal ileum (LH)), clinically observed every three months until a final diagnosis was reached. Small bowel inflammatory lesions were found in 13 of 27 patients (diagnostic yield
48\%). Three patients underwent surgery (group A) and SBCD was confirmed in two; the remainder had ileal adenocarcinoma, but was considered a true positive anyway. Group B consisted of five patients and SBCD was histologically confirmed in 4 (one false positive; ulcer and erythema of the terminal ileum not confirmed at ileoscopy and final diagnosis of relapsing appendicitis). Finally, group C consisted of nineteen patients with a median follow up of 21 months (range 15-27); within those with positive findings at CE, SBCD was confirmed by clinical improvement to oral budesonide in seven of 8 ; the false positive one had jejunal erythema and a final diagnosis of splenic lymphoma. Only one patient with LH later developed overt ileal SBCD (one false negative). Sensitivity, specificity, positive and negative likelihood ratio were $93 \%, 84 \%, 5.8$ and 0.08 , respectively. Assuming a pre-test probability of disease of $40 \%, C E$ determined a post-test probability of $79 \%$. In our selected cohort, $\mathrm{CE}$ was a sensitive tool for the detection of small bowel abnormalities, doubling the pre-test probability of having a structural small bowel disease. Focal erythema and luminal debris may lower CE specificity. Patients with stricture are surgical candidates, whereas those with LH may later develop SBCD and a careful follow up is warranted.

\section{$\mathrm{T} 1113$}

Wireless Capsule for Esophageal Endoscopy: Comparison with Upper GI Conventional Endoscopy

Sylvie Sacher-Huvelin, Emmanuel Coron, Emmanuel Ben Soussan, Franck Cholet, Michel Antonietti, Michel Robaszkiewicz, Herve Lamouliatte, Marc Le Rhun, Jean Paul Galmiche

Wireless videocapsule endoscopy is a new technology that has been successfully applied for small bowel exploration. A new device (Pillcam ESO, Given Imaging) is currently in development for the investigation of esophagus. We report our preliminary experience of Esophageal Capsule Endoscopy (ECE) in patients referred for reflux symptoms or dyspepsia. The study is conducted prospectively, with conventional upper GI endoscopy as the "gold standard". Patients : 27 patients recruited in 4 academic centers have been prospectively enrolled after obtaining written informed consent. ECE was systematically swallowed before (from 30 minutes to 24 hours) upper GI endoscopy. Patients with severe dysphagia or symptoms suspect of bowel obstruction were excluded. The data recorded by ECE were downloaded on a computer and interpreted using a specific software (RAPID 3). Data were interpreted blindly by the investigator who red ECE and the gastroenterologist who performed upper GI endoscopy. The sensitivity analysis was done on an intention-to-diagnose basis (e.g inability to swallow ECE considered as diagnostic failure). Results : 27 patients were enrolled. Seventeen had reflux symptoms and 10 dyspepsia $(13$ men/ 14 women, mean age 53 years range 22-75). Only $50 \%$ of patients were on treatment, the remainders being "naive" subjects for endoscopy. All patients were able to swallow ECE, but in 3 cases imaging was incomplete regarding distal esophagus. In one case, a technical failure was responsible for loss of signal. Barrett'esophagus was detected by ECE in 6 out of 8 patients (comparison with upper GI endoscopy). The 2 false negative cases were mainly short segments. All 6 mild/moderate esophagitis were detected by ECE. In one patient with grade C esophagitis at upper GI endoscopy, ECE did not reach the distal esophagus. In 2 other patients, ECE detected mild erosions not confirmed by upper GI endoscopy. Hiatus hernia (present in 12 patients at upper GI endoscopy) was only found in 6 subjects by ECE. Gastric erosions present in 3 patients at upper GI endoscopy were also seen by ECE in 2 cases. No neoplasia was detected by both upper GI endoscopy and ECE. Conclusions: This preliminary study confirms the feasibility and tolerability of this ECE. Provided these results could be confirmed on a larger scale, ECE could represent a first-line investigation for the detection of Barrett'esophagus and esophagitis. However, the technique probably lacks sensitivity for short segment Barrett's and does not accurately detect the presence or absence of Hiatus hernia. The study is ongoing and results will be updated if abstract is selected for presentation.

\section{T1114}

Comparative Study of Capsule Endoscopy, Mesenteric Angiography and Double Balloon Enteroscopy in Obscur Gastrointestinal Bleeding: Preliminary Results

Begona Gonzalez-Suarez, Sara Galter, Jorge Carlos Espinos, Alfredo Mata, Carlos Castano, Jordi Ortiz, Candid Villanueva, Jordi Villalba, Adolfo Gallego, Edgar Ayala, Joaquim Balanzo

Introduction: Obscure gastrointestinal bleeding (OGIB) is cause of different studies, generating a high cost in the health system. That's why it would be useful to find out which is the most sensitive and efficacy test. Aims: 1. To compare the diagnostic yield of capsule endoscopy (CE) with mesenteric angiography (MA) in the diagnosis of the OGIB. 2. To compare the result of CE with double balloon enteroscopy (DBE). Patients and Methods: From March 2004 until November 2005, patients with OGIB (non-conclusive upper endoscopy and colonoscopy) were included. These patients were submitted to an emergency mesenteric angiography and a capsule endoscopy. Fron August 2005 until now mesenteric angiography has been substituted for a DBE. Results: 33 patients were included (17 men, 16 women), with a median of age of $70.8+/-12.1$ years. In 4 patients capsule endoscopy remained in the stomach and small bowel couldn't be visualized. Emergency mesenteric angiography wasn't performed in 6 patients because of technical problems. CE was diagnostic in 25/33 patients (75\%) observing small bowel lesions. 21 of the 27 mesenteric angiography done were described as normal and only in 4 patients we found lesions responsible of the bleeding $(14.8 \%)$, that were intestinal angiodisplasias in all the cases. Until now we have included 11 patients in the study with double balloon enteroscopy (5 men, 6 women). CE was diagnostic in $90 \%$ of patients ( 1 patient had CE retained in stomach during 8 hours). DBE diagnosed also $90 \%$ of patients, coinciding with CE and we were able to perform endoscopic therapy successfully in $81 \%$ of cases $(9 / 11$ patients). We have not had any complications for the moment. Conclusions: 1. Capsule endoscopy is superior to mesenteric angiography in the study of the OGIB ( $75 \%$ vs $14.8 \%, \mathrm{p}<0.001)$. 2. Double balloon enteroscopy is an emergent procedure with a high sensibility detecting lesions in small bowel (90\%) but we need more prospective and randomized studies to evaluate these findings. 


\section{T1115}

Minimally Invasive Measurement of Intestinal Edema Using An Impedance Analyzer Circuit

Ravi S. Radhakrishnan, Kunal Shah, Hasen Xue, Frederick A. Moore, Norman W. Weisbrodt, Brijesh S. Gill, Charles S. Cox

Acute intestinal edema has been shown to adversely affect intestinal transit, permeability, and contractility. Current resuscitation modalities, while effective in reversing shock, are associated with development of acute intestinal edema. Exact knowledge of levels of tissue edema would allow clinicians to monitor intestinal tissue water and may help prevent the detrimental effects of edema. While measurement of tissue edema is possible, there is no minimally invasive method to measure intestinal tissue water. We sought to develop a tissue impedance analyzer to quickly measure tissue edema without the need for invasive biopsy. Methods: A BK Precision Function Generator provided the oscillating voltage input, which was then fed into a voltage controlled current source (VCCS). Next, a Tektronix 2245100 $\mathrm{MHz}$ oscilloscope measured the voltage output across any load supplied by the VCCS and a resistor was added to the circuit to create a constant current. Rats (270g - 330g) were randomized to three groups: sham, mild edema (ME, $80 \mathrm{cc} / \mathrm{kg}$ of NS resuscitation), and severe edema (SE, $80 \mathrm{cc} / \mathrm{kg}$ of NS resuscitation with mesenteric venous hypertension). Intestinal edema was measured by wet to dry tissue weight ratio (W/D). Bowel impedance was measured and converted to capacitance using a standard curve. Results: Acute intestinal edema causes a significant increase in bowel capacitance. A linear correlation between tissue water and capacitance was established indicating that bowel capacitance can be used to predict tissue water concentration (Fig. 1). Conclusion: Using an impedance analyzer circuit, it is possible to measure intestinal edema reliably, quickly, and minimally invasively. This may prove to be a useful tool in the resuscitation of critically ill patients.

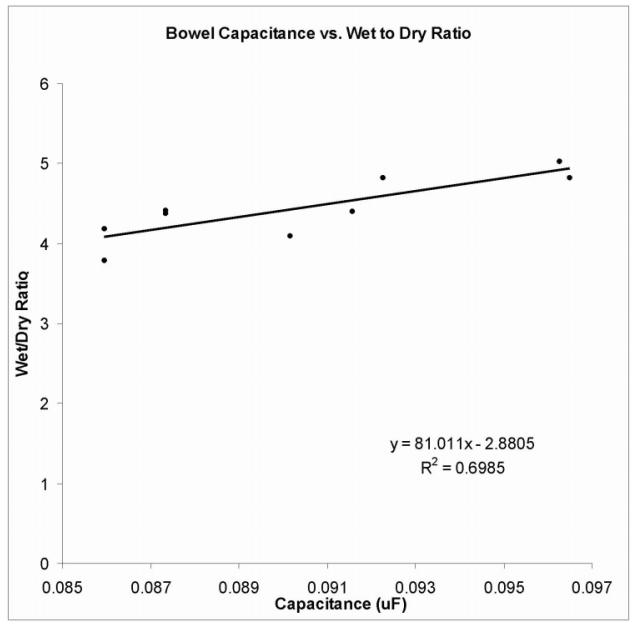

\section{T1116}

High-Resolution Sonography of the Bowel for Timely Diagnosis of Intestinal Acute Graft Versus Host Disease After Allogenic Haematopoietic Stem Cell Transplantation

Emma Calabrese, Massimiliano Postorino, Giovanna Del Vecchio Blanco, Luca

Franceschini, Raffaella Cerretti, Gottardo De Angelis, Carmelina Petruzziello, Micaela

Cretella, Livia Biancone, William Arcese, Francesco Pallone

Background. Acute Graft Versus Host Disease (aGVHD) is a major complication during the first 100 days after Allogenic Haematopoietic Stem Cell Transplantation (AHSCT), with 30$70 \%$ incidence. Target organs include the skin, the liver and the gastrointestinal (GI) tract. Intestinal aGVHD shows a progressive worsening of the symptoms, but the prognosis may be improved by an early diagnosis and timely treatment. Differential diagnoses include intestinal infections and chemo/radiotherapy side-effects. Conventional diagnostic procedures include the histologic assessment of the upper and lower GI tract, requiring invasive endoscopic procedures in severely ill patients. Aim Aim of the present study was to determine whether High Resolution Sonography (HRS) of the bowel may represent a useful non invasive procedure for the diagnosis and assessment of aGVHD. We also aimed at explaining whether HRS may be helpful for a timely treatment of aGVHD. Methods. From August 2004 to October 2005, 15 patients (11M, 42 yrs, range 18-58) underwent AHSCT (patients received: 11/15 peripheral blood stem cell transplantation and 4/15 bone marrow transplantation). Indication for AHSCT was: Acute Lymphocytic Leukemia in 2 patients, Acute Myeloid Leukemia in 5 patients, Chronic Myeloid Leukemia in 2 patients, Non Hodgkin's Lymphoma in 3 patients, Hodgkin's Lymphoma in 2 patients, Multiple Myeloma in 1 patient. GI symptoms were observed in 11/15 patients, including anorexia, nausea, vomiting, watery diarrhoea, intestinal bleeding. Symptoms onset began after a median of 48 days (range 7100) from AHSCT. Within 24 hours from the onset of GI symptoms, HRS was performed by using a $7 \mathrm{MHz}$ linear and a $3.5 \mathrm{MHz}$ convex array transducers. Results. Among the 11 patients with GI symptoms, HRS detected changes suggesting aGVHD in 6 patients: aGVHD was confirmed by clinical assessment in all 6 and by endoscopy in 3 patients. HRS changes included: increased bowel wall thickness, small bowel and/or colon dilation, double-lined inner echo. These 6 patients were treated with steroids and anti-TNFalpha MoAb. In the remaining 5 patients, HRS showed increased colonic thickness with normal echo pattern of the bowel layers. In 3/5 patients GI symptoms were related to chemo/radiotherapy toxicity, while in 2/5 patients a Campylobacter jejuni and a CMV infection was detected and successfully treated. Conclusion. HRS is a feasible and non-invasive diagnostic tool which may provide useful information for a prompt and appropriate treatment of patients with suspected
aGVHD. The possible role of HRS for predicting the development of aGVHD and for grading its severity warrants evaluation in prospective studies

\section{$\mathrm{T} 1117$}

Assessment of a New Simple Capsule Endoscopy Crohn's Disease Activity Index (CECDAI)

Eyal Gal, Alex Geller, Gerald Frase, Zohar Levi, Yaron Niv

Introduction: Capsule endoscopy (CE) (PillCam SB, Yoqneam, Israel) is a relatively new imaging tool for detection of small bowel pathology. It was found to be the most sensitive procedure for detection of small bowel Crohn's disease in several studies. Assessment of endoscopic severity of Crohn's disease by CE is not standardized and is limited by interobserver variation in interpreting findings and assessing their severity. Aim: To develop and validate a new, simple CECDAI in order to grade the severity of small bowel CE findings. Methods: The small bowel was divided into proximal and distal segments according to transit time, and each segment was given a score according to the worst mucosal lesion, extent of disease and presence of strictures (Table). The segmental score is calculated by multiplying the inflammation score by the extent score and adding the stricture score (A $x$ $\mathrm{B}+\mathrm{C})$. The final score is the sum of the 2 segmental scores so $\mathrm{CECDAI}=(\mathrm{Al} \times \mathrm{Bl}+\mathrm{Cl})$ $+(\mathrm{A} 2 \times \mathrm{B} 2+\mathrm{C} 2)$. Four experienced endoscopists (2 with experience in CE interpretation) reviewed 20 de identified, coded CE videos of Crohn's disease patients, and graded them according to CECDAI. Each examiner was blinded to the scores given by the others. The scores were compared by Spearman's correlation using SPSS for inter-observer variability Results: Total CECDAI score correlation was 0.867 (0.700-1.000 = strong degree association, WHO classification, $\mathrm{P}<0.0001$ ). CECDAI in 20 patients range was 0 to 26. All examiners agreed that the score was simple to learn and apply, even for endoscopists with no specific CE experience. Conclusions: The CECDAI score is a simple score for assessing small bowel Crohn's disease by CE, and shows a low inter-observer variability between examiners with extensive endoscopic experience. Table - CECDAI A. Inflammation score $0=$ none $1=$ Mild to moderate edema/hyperemia/denudation $2=$ Severe edema/hyperemia/denudation $3=$ bleed ing, exaudate, apthae, erosion, small ulcer (up to $0.5 \mathrm{~cm}) 4=$ moderate ulcer $(0.5-2 \mathrm{~cm}$ ) pseudopolyp $5=$ large ulcer $(>2 \mathrm{~cm})$ B. Extent score $0=$ none $1=$ Focal disease (single segment) $2=$ Patchy disease (multiple segments) $3=>$ Diffuse disease $C$. Narrowing score $0=$ none $1=$ single, passed 2= multiple, passed 3= obstruction Segmental score Total score

\section{T1118}

A Novel Diagnostic Approach in Patients with Indeterminate Colitis: Wireless Capsule Enteroscopy

Raffaele Bennato, Francesco Manguso, Agesilao D'Arienzo, Giovanni Lombardi, Gianfranco De Dominicis, Francesco P. D'Armiento, Oscar Nappi, Antonio Balzano

BACKGROUND/AIMS: the term indeterminate colitis was indicative of inflammatory bowel diseases in which there were difficulties in distinguishing between Crohn's disease and ulcerative colitis in colectomy specimens (10-15\% of cases). Today the significance of the term is more complex; it is linked not only to the histological findings but it is a clinicpathological definition with many aspects not yet well characterized. Wireless capsule enteroscopy (WCE) is able to directly visualize the entire small bowel mucosa and identify superficial lesions. The aim of our study was to evaluate small bowel mucosa by WCE in patients with indeterminate colitis at the onset. MATERIALS: from October 2004 to November 2005, 11 patients (M/F:5/6; mean age 38.9 yrs, range 26-58) consecutively admitted to our two Units with a first diagnosis of indeterminate colitis, were enrolled. The selection was made in a population of patients with symptoms suggesting colonic inflammatory bowel disease by a ileocolonoscopy. Mucosal biopsies were collected in terminal ileum and in all colonic segments (at least three specimens in the cecum, every $20 \mathrm{~cm}$ and in the mucosal areas judged to be the most severely inflamed). Histological assessment was made by two pathologists who were blinded to each other's evaluation, according to guidelines of the British Society of Gastroenterology. Discordances were resolved by consensus. A WCE was performed by two blinded observers in all patients. Patients had not been using 5-ASA, salazopyrin NSAIDs and corticosteroids for at least one month before the WCE. RESULTS: complete ileocolonoscopy was performed in all patients. In all cases there was not endoscopic and histological involvement of terminal ileum. Every patient well tolerated WCE. Outcome agreement between observers was $100 \%$. In one case multiple small aphthous ulcers were observed, in another patient two jejunal isolated aphthas were found, in two cases WCE showed proximal segmental oedema and hyperemia. The overall percentage of ileal inflammatory findings was $36,4 \%$. CONCLUSIONS: our study shows a small bowel inflammatory involvement, in particular of the bowel proximal to terminal ileum, in a high percentage o patients with indeterminate colitis, detected by wireless capsule enteroscopy. This novel safe diagnostic method seems to be able to find also signs of very mild ileal inflammation and could be used for a better classification of indeterminate colitis

\section{T1119}

Role of Wireless Capsule Endoscopy (WCE) in Geriatric Gastroenterology Adnan Muhammad, Yougandhar Akula, C.S. Pitchumoni

Introduction: The advent of WCE has provided a new diagnostic modality for diseases o small bowel. Its impact on patient care and usefulness in clinical practice has steadily been gaining ground. This new technology has been used in variety of clinical settings including localization of obscure GI bleeding, IBD ( Crohn's disease), suspected small bowel polyp or tumor, chronic diarrhea, unexplained abdominal pain and Celiac disease. Aim: The aim of this study is to identify WCE findings in patients who presented with different indications in Geriatric population and compare it with other age groups. Material and Method: Consecutive medical records of 333 patients presented for WCE at our Institution were recorded. Patients were divided into three age groups as listed in the table. The most common indications and findings were also recorded. Results: 1) The most common indication for WCE in Geriatric population is Anemia. 2) The most common finding in Geriatric population is Small Bowel 
Erosion. Conclusion: WCE provides a valuable tool in the visualization of Small Bowel pathalogy. WCE also plays a critical role in Geriatric population for the diagnosis of various small bowel diseases.

Indications for WCE in different age groups.

\begin{tabular}{|c|c|c|c|}
\hline Indications & Age $<50(\mathrm{~N}=95)$ & Age 50-64 (N = 78) & Age 65 and above (N = 160) \\
\hline Anemia & $17(18 \%)$ & $29(37 \%)$ & $80(50 \%)$ \\
\hline GI Bleeding & $14(15 \%)$ & $26(33 \%)$ & $63(39 \%)$ \\
\hline Abdominal pain & $27(28 \%)$ & $12(15 \%)$ & $8(5 \%)$ \\
\hline IBD (Unspecified) & $9(9 \%)$ & $2(3 \%)$ & 0 \\
\hline Crohn's disease & $17(18 \%)$ & $3(4 \%)$ & $2(1 \%)$ \\
\hline Chronic Diarrhea & $13(14 \%)$ & $4(5 \%)$ & $2(1 \%)$ \\
\hline Unexplained weight loss & 0 & 0 & $1(0.6 \%)$ \\
\hline R/o Small bowel polyps & $2(2 \%)$ & $2(3 \%)$ & 0 \\
\hline
\end{tabular}

Findings of WCE in different age groups

\begin{tabular}{|c|c|c|c|}
\hline Findings & $\begin{array}{c}\text { Age } \\
50\end{array}$ & $\begin{array}{c}\text { Age } \\
50-64\end{array}$ & $\begin{array}{c}\text { Age 65 and } \\
\text { above }\end{array}$ \\
\hline Gastric Ulceration & $5(5 \%)$ & $5(6 \%)$ & $16(10 \%)$ \\
\hline Gastric Erosion & $8(8 \%)$ & $12(15 \%)$ & $28(18 \%)$ \\
\hline Small Bowel Ulceration & $\begin{array}{c}22 \\
(23 \%)\end{array}$ & $21(27 \%)$ & $34(21 \%)$ \\
\hline Samll Bowel Erosion & $\begin{array}{c}24 \\
(25 \%)\end{array}$ & $23(29 \%)$ & $44(28 \%)$ \\
\hline Small Bowel Polyp & $6(6 \%)$ & $6(8 \%)$ & $12(8 \%)$ \\
\hline Celiac disease (Atrophy, Mosaicsm, Scalloping, Layering and & $5(5 \%)$ & $2(3 \%)$ & $5(3 \%)$ \\
\hline Intussusception) & $\begin{array}{c}16 \\
(17 \%)\end{array}$ & $13(17 \%)$ & $51(32 \%)$ \\
\hline Lymphangectasia & $4(4 \%)$ & $6(8 \%)$ & $10(6 \%)$ \\
\hline Small Bowel Nodularity & $4(4 \%)$ & $5(6 \%)$ & $17(17 \%)$ \\
\hline Active bleeding without identifiable lesion & 0 & $2(3 \%)$ & 0 \\
\hline Small Bowel mass & $4(4 \%)$ & $9(12 \%)$ & $21(13 \%)$ \\
\hline AVM & &
\end{tabular}

More than one WCE finding can be seen in one case.

T1120

Evaluating Mechanisms of Intestinal Intussusception Kristin E. Killoran, Robia Pautler, Norman Weisbrodt, Margaret E. Conne

Obstructing intussusception occurs in approximately 1:1,000 children in the US with a 2:1 male to female bias. Intussusception rates vary among locales suggesting the importance of genetics and/or environment. Intussusception is thought to be caused by dysregulated intestinal motility. E.coli lipopolysaccharide (LPS) affects intestinal motility, is a potent inducer of inflammation, and causes intussusception in a mouse model. In order to test our hypothesis that intussusception is impacted by multiple host factors and is associated with altered motility, adult mice are injected intraperitoneally with IPS and 6 hours later are examined for intussusception following laparotomy or magnetic resonance imaging (MRI). Intussusception rates differed significantly in male $(38.5 \%)$ and female $(55 \%)$ mice $(\mathrm{p}=$ 0.004 ) and among mouse strains (BALB/c $55 \%, C D-1,12 \%, C 57 B l / 60 \%)(\mathrm{p}<0.001)$. These data indicate a sexual and genetic dimorphism in the development of intussusception. In vivo imaging by MRI is being pursued to define for the first time the longitudinal kinetics of the development and resolution of intussusception and to assess intestinal contractile activity post-LPS injection in BALB/c and C57Bl/6 mice. Using MRI, intussusceptions from $2 \mathrm{~mm}$ to $3 \mathrm{~cm}$ in length have been visualized in the small intestine and colon. Despite use of anesthesia during MRI, motility of the intestine was observed. Examination of serial UFLARE scans showed a contraction-relaxation sequence in an intussusception. Additionally, we observed multiple intussusceptions that resolved during MRI. In one mouse that was imaged multiple times for 20 minutes, an intussusception was observed that subsequently resolved and then in a later series of scans a second intussusception formed in the same area of the intestine. Because detailed determination of the kinetics of intussusception in individual animals by MRI will require long-term anesthesia that may interfere with intestinal motility, we evaluated intestinal transit of FITC-dextran in BALB/c mice anesthetized with soflurane for 0,3 or 6 hours. In mice anesthetized for 3 and 6 hours, marker was transported to the terminal ileum in $8 / 10$ mice within 20 minutes. These data indicate that isoflurane anesthetized mice retain substantial intestinal motility. Taken together, our data indicate the feasibility of MRI for in vivo imaging of intussusceptions to obtain longitudinal acquisition of anatomical and dynamic physiological information from individual animals that will allow us to decipher the mechanisms of intussusception in vivo.
T1121

Video Capsule Endoscopy Findings in Cirrhosis with Portal Hypertension: A Prospective Study

Daniel Urbain, Danny de Looze, Pieter Hindryckx, Paul Ceulemans, Hendrik Reynaert Fazia Mana, Isabelle Colle

Background: Data concerning small bowel (SB) mucosal abnormalities in portal hypertension are scarce. Video Capsule Endoscopy (VCE) is a good tool to study the SB mucosa in a noninvasive way in these patients. Materials and methods: 24 patients with cirrhosis (mean age: $62 \mathrm{y}, 16$ males, 8 females) presenting endoscopic signs of portal hypertension (esophageal varices $>$ grade 2 and/or portal hypertensive gastropathy of mild or severe grade), but no clinical signs of bleeding, were evaluated by VCE in two university centers. Patients with former endoscopic therapy of the varices (mostly band ligation) were also included. Diagnosis of cirrhosis was based on biopsy and/or clinical and imaging evidence. VCE was performed within 1 month after oeso-gastro-duodenoscopy. Etiology of cirrhosis was: alcohol (16), $\mathrm{HCV}$ (4), NASH (3), haemochromatosis (1). Results: Abnormalities of the SB mucosa were observed in $87 \%$ of the patients ( 3 procedures were entirely normal). The lesions were described as limited vascular lesions (erythematous spots, angiodysplasia-like lesions, angioma) (62\% of the patients), edematous aspect of the mucosa and mosaic pattern (33\%), varices (25\%), ulcerations/erosions (25\%). In 3 cases (12\%), an active bleeding was seen without identification of the underlying lesion. Atypical vascular lesions and edematous aspect could be found as single lesions. Active bleeding and varices were always found in association with other abnormalities.. Conclusions: Mucosal abnormalities are found in a majority of patients with portal hypertension. The relevance of some findings (edema, limited vascular lesions, ulcerations/erosions) can be questioned, but the detection of SB varices and active bleeding in patients without clinical signs can be clinically important. A better definition of the endoscopic findings remains mandatory to further define the place of VCE in this field. Moreover, risk factors associated with the relevant findings like active bleeding and $\mathrm{SB}$ varices are needed.

\section{T1122}

Usefulness of the Capsule Endoscopy in the Study of the Inflammatory Bowel Disease: Preliminary Results

Sara Galter, Begona Gonzalez, David Monfort, Elena Ricart, Dolors Gonzalez, Edgar Ayala, Carlos A. Guarner, Ingrid Ordas, Joaquim Balanzo

Introduction: Capsule endoscopy (CE) has been proved to be a new and useful tool in the study of the inflammatory bowel disease, as Crohn's Disease (CD) of the small bowel has long presented diagnostic difficulties. Patients with colon CD or indeterminate colitis (IC) are the best candidates, because intestinal lesions may lead to a change in the diagnosis or the management of the patient. Aim: Assess the usefulness of the capsule endoscopy in detecting small bowel disease in patients with Colon $C D$, IC or suspected $C D$, after a normal small bowel follow through (SBFT). Patients and methods: Since January 2004 until November 2005 we included 22 patients ( 8 males, 14 females) divided in differents groups: established Colon CD (group 1, n=13), IC (group 2, n=7) and suspected CD (group 3, n= 2). 20 of the 22 patients had a previous normal SBFT. After an intestinal preparation and 8 hours of fasting, the exploration was performed. Results: CE was normal only in 6/22 patients $(27.2 \%)$ and pathological in $16 / 22(72.8 \%)$. In group 1 , the capsule showed lesions suggestive of $\mathrm{CD}$ in 7 patients (53\%); in group 2, 5 patients had intestinal lesions, leading to a diagnosis change $(70 \%)$ and in group $3, \mathrm{CD}$ was confirmed in 1 case $(50 \%)$. The cecum was reached in $90 \%$ of procedures and all capsules passed naturally. Conclusions: 1. Capsule endoscopy is effective in the study of Indeterminate Colitis, leading to a diagnosis of Crohn's Disease in $70 \%$. 2. The examination of the intestinal extent of Crohn's Disease shows lesions in 7 patients (53\%), although the clinical relevance needs to be assessed. 3. Capsule Endoscopy is superior to Small Bowel Follow Through in the detection of small bowel lesions.

\section{T1123}

Screening for Esophageal Varices with the PillCam ESO in Patients with Cirrhosis and Massive Ascitis

Tonantzin Matheus, Girish Anand, Neha Wadhwa, Philip O. Katz, Kenneth Rothstein, Santiago Munoz

Introduction: Esophageal varices are a major cause of morbidity and mortality in patients with cirrhosis. The benefits of primary and secondary prophylaxis of variceal hemorrhage have been established. The introduction of esophageal capsule endoscopy (ECE) for screening and surveillance of esophageal varices is currently under active evaluation. The presence of cirrhotic ascites is associated with increased intrabdominal pressure, esophageal vascular, anatomical and functional changes, which may affect variceal visualization by ECE. Aim: We evaluated the use of ECE using the PillCam ESO ${ }^{\mathrm{TM}}$ in patients with massive ascites due to end stage cirrhosis. Methods: Ten patients with end stage cirrhosis of varied etiology admitted for large volume paracentesis due to development of massive ascites. Patient demographics were recorded. Informed consent was obtained and patients were instructed about the procedure, which was performed prior to the paracentesis. The PillCam ESO capsule acquired video images from both ends of the device. Results were then interpreted using the following grading: F0: no varices present, Fl: small varices encompassing $<25 \%$ circumference of esophagus, F2: medium or large varices, involving $>25 \%$ circumference of esophagus. Results: All patients were male, mean age 52.8 yrs. Child Pugh class B: 6 pts, class C: 4 pts. Mean MELD score was 22 (13-40). Ethnicity was diverse. Average capsule transit time in esophagus was 3:58 min (range $20 \mathrm{sec}-7: 27 \mathrm{~min}$ ). 30\% patients had less than $1 \mathrm{~min}$. transit time, yet the ability to identify and grade the varices was not affected by short transit time. Varices were identified in 6 patients and $83 \%$ had medium/large F2 varices. Half of the patients had an EGD available for comparison within 1 year of the capsule and there was an $80 \%$ correlation between both tests. There were no severe adverse effects associated with ECE. Conclusions: Esophageal capsule endoscopy of esophageal varices appeared safe in advanced cirrhotics with massive ascites. The study demonstrated a spectrum of sizes of esophageal varices, even in the presence of rapid capsule transit time 
and severe ascites. $40 \%$ of patients had no esophageal varices in spite of having ESLD and massive ascites, confirming the need for endoscopy to determine the presence/absence of varices. These results indicate that further studies of ECE for esopagheal varices cirrhosis are warranted

\section{T1124}

Concomitant Immunosuppressive and Adalimumab Therapy in Patients with Crohn's Disease: 1-Year Results of the Classic II Study

R Panaccione, Stephen B. Hanauer, Richard Fedorak, Paul Rutgeerts, William J. Sandborn, Paul Pollack

BACKGROUND Adalimumab, a fully human anti-TNF monoclonal antibody, is approved for the treatment of rheumatoid arthritis and psoriatic arthritis. In CLASSIC I a 4-week randomized controlled study of adalimumab in the induction of remission in patients with active Crohn's disease (CD), immunosuppressant (IMM) —azathioprine, 6-MP, or methotrexate-use was permitted if patients entered the study on a stable IMM dose for 12 weeks prior to screening. IMM use did not influence the response to adalimumab in CLASSIC I. PURPOSE To assess the effect of concomitant IMM on the efficacy of adalimumab over 1 year in CLASSIC II. METHODS All patients in CLASSIC II completed CLASSIC I and received adalimumab $40 \mathrm{mg}$ sc at Weeks 0 (Week 4 of CLASSIC I) and 2. Patients with $\mathrm{CDAI} \geq 150$ at Weeks 0 and/or 4 received open-label (OL) adalimumab $40 \mathrm{mg}$ sc every other week (eow), weekly dosing was permitted for flare or persistent non-response. Patients with $\mathrm{CDAI}<150$ at both Weeks 0 and 4 were randomized to receive adalimumab, $40 \mathrm{mg}$ sc eow or weekly, or placebo for up to 1 year. CDAI was assessed at each study visit. Information on concomitant IMM use was collected at the start of the study through 56 weeks of treatment. The relationship of IMM and adalimumab on remission $(\mathrm{CDAI}<150)$ and $\triangle 100 \mathrm{CDAI}$ response (decrease in CDAI score $\geq 100$ points) and their potential interaction was assessed. RESULTS Of 276 patients enrolled in CLASSIC II, 30\% received concomitant IMM (IMM+). In the OL cohort $(\mathrm{N}=221), 46 \%$ of IMM+ patients achieved $\mathrm{CDAI}<150$ and $64 \%$ achieved $\Delta 100 \mathrm{CDAI}$, versus $40 \%$ and $60 \%$ of those not receiving IMM (IMM-), respectively. Results in the randomized cohort $(\mathrm{N}=55)$ are summarized in the table. IMM status did not notably influence the efficacy of adalimumab. CONCLUSION Adalimumab consistently improved CDAI outcomes with or without IMM use. Concomitant adalimumab and IMM will be evaluated in larger, long-term CD studies.

Week 56 Remission and Response: CLASSIC II Randomized Cohort

\begin{tabular}{|c|c|c|c|c|c|c|c|}
\hline \multirow{2}{*}{\multicolumn{2}{|c|}{ Therapy }} & \multicolumn{3}{|c|}{ CDAI $<150$} & \multicolumn{3}{|c|}{$\triangle 100 \mathrm{CDAI}$} \\
\hline & & \multirow{2}{*}{$\begin{array}{c}\text { Total } \\
8 / 18\end{array}$} & \multirow{2}{*}{$\begin{array}{c}\text { IMM+ } \\
1 / 3\end{array}$} & \multirow{2}{*}{$\begin{array}{c}\text { IMM- } \\
7 / 15\end{array}$} & \multirow{2}{*}{$\begin{array}{l}\text { Total } \\
10 / 18\end{array}$} & \multirow{2}{*}{$\begin{array}{c}\text { IMM+ } \\
1 / 3\end{array}$} & \multirow{2}{*}{$\begin{array}{r}\text { IMM- } \\
9 / 15\end{array}$} \\
\hline Dlo & $\mathbf{N}$ & & & & & & \\
\hline Hactoon & $\%$ & 44 & 33 & 47 & 56 & 33 & 60 \\
\hline \multirow{2}{*}{$40 \mathrm{mg}$ eow } & $\mathbf{N}$ & $14 / 19$ & $4 / 4$ & $10 / 15$ & $14 / 19$ & $4 / 4$ & $10 / 15$ \\
\hline & $\%$ & 74 & 100 & 67 & 74 & 100 & 67 \\
\hline \multirow{2}{*}{$40 \mathrm{mg}$ weekly } & $\mathbf{N}$ & $15 / 18$ & $4 / 5$ & $11 / 13$ & $16 / 18$ & $5 / 5$ & $11 / 13$ \\
\hline & $\%$ & 83 & 80 & 85 & 89 & 100 & 85 \\
\hline
\end{tabular}

LOCF

$\mathrm{T} 1125$

Adalimumab Maintains Improvement in Inflammatory Bowel Disease Questionnaire (IBDQ) Scores Over 1 Year Following the Initial Attainment of Remission in Patients with Moderately to Severely Active Crohn's Disease: Results of the Classic II Study

Paul J. Rutgeerts, Lisa E. Melilli, Ju Li, Paul F. Pollack

BACKGROUND Adalimumab, a fully human anti-TNF monoclonal antibody, is approved for the treatment of rheumatoid arthritis and psoriatic arthritis. In CLASSIC I, a 4-week randomized trial, the efficacy of adalimumab in the induction of remission in patients with Crohn's disease (CD) was demonstrated OBJECTIVE Assess the maintenance of improvement in physical function in subjects with active $C D$ who achieved remission $(\mathrm{CDAI}<150)$ when treated with adalimumab in CLASSIC I and maintained it at Week 4 of CLASSIC II, an extension trial. METHODS All subjects in CLASSIC II completed CLASSIC I and received adalimumab $40 \mathrm{mg}$ sc at Weeks 0 (Week 4 of CLASSIC I) and 2. Patients in remission at both Weeks 0 and 4 were randomized to receive adalimumab, $40 \mathrm{mg} \mathrm{sc}$ every other week (eow) or weekly, or placebo for up to 1 year. CDAI and IBDQ were assessed at each study visit. IBDQ measures disease-related functional changes in patients with IBD. A Total IBDQ $>170$ score has been correlated to clinical remission $(\mathrm{CDAI}<150)$. RESULTS The mean baseline CLASSIC I IBDQ score of 55 patients randomized was 137, consistent with active CD. The mean IBDQ score (186.4) at the start of CLASSIC II was consistent with remission. Remission was maintained with adalimumab treatment through Week 56 in $74 \%$ and $83 \%$ of patients treated with adalimumab $40 \mathrm{mg}$ eow and weekly, respectively, compared to $44 \%$ of placebo-treated patients (LOCF). IBDQ scores consistent with clinical remission were maintained through Week 56 in patients treated with adalimumab $40 \mathrm{mg}$ eow or weekly (figure), while IBDQ scores declined rapidly in patients receiving placebo. CONCLUSION In this randomized cohort, clinical remission and physical function were maintained with adalimumab therapy. Larger, long-term studies of adalimumab in patients with $C D$ are ongoing. ${ }^{1}$ Irvine EJ, Feagon B, Rochon J et al. Gastroenterology 1994;106:287-96

\section{Mean IBD Q Scores by Visit: Randomized Cohort of CLASSIC II}

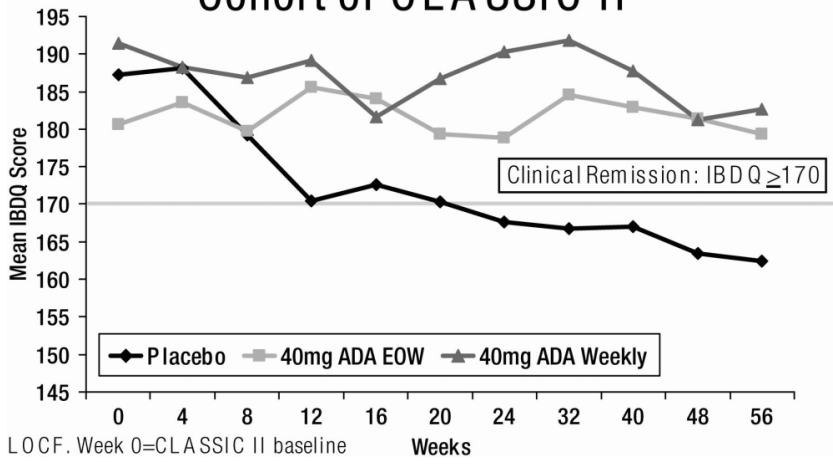

T1126

Safety and Tolerability of Subcutaneous (SC) Certolizumab Pegol in Active Crohn's Disease (CD): Results from Two Phase III Studies (PRECiSE program) Stefan Schreiber, Brian Feagan, Stephen B. Hanauer, Paul Rutgeerts, Juliet A. McColm, William J. Sandborn

Background: Tolerability and safety of certolizumab pegol were examined in 2 paralle Phase III trials (PRECiSE program) in active CD (CD Activity Index [CDAI] score 220-450) involving more than 1300 patients (pts). Methods: In PRECiSE 1, pts received sc certolizumab pegol 400mg ( $\mathrm{n}=331$ ) or placebo (PBO; $\mathrm{n}=329)$ at Weeks (Wks) 0, 2, 4, then 4-weekly up to Wk 24. In PRECiSE 2, 668 pts received open-label sc certolizumab pegol 400mg at Wks $0,2,4$. Pts who responded at Wk 6 received double-blind certolizumab pegol $400 \mathrm{mg}(\mathrm{n}=$ $216)$ or $\mathrm{PBO}(\mathrm{n}=212) 4$-weekly from Wks $8-24$. Adverse events (AEs) were monitored ( $\mathrm{n}=$ 1328). Results: Most AEs in both trials were mild or moderate. In the double-blind phases, the overall incidence of AEs was comparable for certolizumab pegol and PBO. For certolizumab pegol, the most common AEs included headache and nasopharyngitis (both studies), abdominal pain (PRECiSE 1), and cough (PRECiSE 2); the most frequently occurring infections included nasopharyngitis, urinary tract (UTI) and upper respiratory tract infections. In PRECiSE $1,10.3 \%$ of certolizumab pegol and $7.0 \%$ of PBO pts had serious AEs (SAEs) Corresponding data for the double-blind phase of PRECiSE 2 were $5.6 \%$ and $6.6 \%$, respectively. Non-CD-related infection SAEs were infrequent in the PRECiSE 2 double-blind phase (certolizumab pegol: 1 pulmonary tuberculosis [TB; responded well to anti-TB therapy], 1 pyelonephritis, 1 pneumonia; PBO: 1 bacteremia 6 days after the 1st $\mathrm{PBO}$ dose, 1 otitis media) and in PRECiSE 1 (certolizumab pegol: 3 abscesses, 1 UTI; PBO: 1 abscess). Incidence of reported malignancies in PRECiSE 1 was similar between certolizumab pegol (1 lung [10 months after withdrawal] and 1 rectal carcinoma) and PBO (1 non-Hodgkin's lymphoma and 1 cervical carcinoma [linked with human papillomavirus]) : none was reported in PRECiSE 2 . Injection reactions were less frequent for certolizumab pegol than PBO pts (PRECiSE 1 and 2: $11.8 \%$ vs $25.5 \%$ and $6.5 \%$ vs $19.8 \%$, respectively). One certolizumab pegol-treated pt in each study died (acute myocardial infarction/metastatic lung cancer (as noted above) and Fentanyl overdose); neither death was considered related to study drug. Proportions of pts negative for auto-antibodies at baseline, becoming positive at Wk 26/withdrawal were low (anti-nuclear: $\leq 8.3 \%$, and anti-double-stranded DNA: $\leq 1.4 \%$ ). No cases of lupus were reported. Conclusions: Experience in over 1300 pts with CD suggests that treatment with sc certolizumab pegol $400 \mathrm{mg}$ is safe and well tolerated. The low incidence of auto-antibodies observed following treatment with certolizumab pegol is strikingly different from that reported for other anti-tumor necrosis factor- $\alpha$ agents.

\section{T1127}

Endoscopically Measured Mucosal Healing of Delayed-Release Oral mesalamine 4.8 G/Day Versus 2.4 G/Day

Gary R. Lichtenstein, David Rubin, Gino Regalli, Rachelle Eusebio

Purpose: Determine the effect of delayed-release oral mesalamine $4.8 \mathrm{~g}$ /day (investigational $800 \mathrm{mg}$ tablet) vs $2.4 \mathrm{~g} /$ day (marketed $400 \mathrm{mg}$ tablet) on endoscopically measured mucosal healing in patients with moderately active ulcerative colitis (UC). Methods: Data from two Phase III, multi-center, randomized, double-blind, 6-week, controlled studies of similar design (ASCEND I\&II) were pooled and analyzed. Mucosal healing was defined as endoscopy subscore of 0 or 1 . Patients with moderately active UC (defined as a baseline Physician's Global Assessment score of 2) and baseline endoscopy subscore $>2$ were included in this analysis. Results: A total of 423 analyzable patients with moderate UC were randomized in the two studies, of which 391 patients met criteria for this analysis. The two treatment groups were balanced with regard to baseline and demographic characteristics, disease history, and baseline disease state characteristics. In the population of patients with moderately active UC and baseline endoscopy subscore $>2$, the percentage of patients achieving mucosal healing is shown in the table below: The $4.8 \mathrm{~g} /$ day dose of mesalamine was well tolerated, with adverse events comparable to $2.4 \mathrm{~g} /$ day. Conclusion: Regardless of dose and as early as 3 weeks, delayed-release oral mesalamine induces endoscopically measured mucosal healing in $>50 \%$ of patients. Compared to $2.4 \mathrm{~g} /$ day, initiating therapy with $4.8 \mathrm{~g}$ / day significantly improves endoscopic measured mucosal healing in patients with moderately active UC.

Mucosal Healing in Moderate UC Patients 


\begin{tabular}{|c|c|c|}
\hline & $2.4 \mathrm{~g} / \mathrm{day} \mathrm{n}=209$ & $4.8 \mathrm{~g} / \mathrm{day} \mathrm{n}=182$ \\
\hline Week 3 & $58 \%$ & $65 \%$ \\
\hline Week 6 & $68 \%$ & $80 \% *$ \\
\hline${ }^{*}<0.05$ & & \\
\hline
\end{tabular}

\section{$\mathrm{T} 1128$}

Efficacy of Weekend 5-ASA (mesalazine) Enema As a Maintenance Therapy in Patients with Ulcerative Colitis: A Randomized Controlled Study

Hiroshi Yokoyama, Sho Takagi, Shinichi Kuriyama, Shuichiro Takahashi, Hiroki

Takahashi, Masahiro Iwabuchi, Seiichi Takahashi, Yoshitaka Kinouchi, Ichiro Tsuji, Tooru Shimosegawa

Background and aims: Oral aminosalicylates are well established in the maintenance treatment of ulcerative colitis (UC). Mesalazine, both oral and topical (enemas and suppositories), has been shown to be effective either alone or in combination with oral or rectal administration for treating active UC. We report the results of a randomized controlled trial examining efficacy of $1 \mathrm{~g}$ mesalazine enema treatment twice a week at weekend regimen with $3 \mathrm{~g}$ oral mesalazine daily as a maintenance therapy for UC. This is the first randomized controlled study on treatment using mesalazine enema in Japanese UC patients. Methods: We scheduled a randomized controlled trial of weekend mesalazine enema as a maintenance treatment with 200 patients with UC. Patients with UC were considered as eligible for the study who had been just induced into a phase of clinical remission. They were randomly assigned to weekend enema group or oral alone group; the former received lg mesalazine enema twice a week at weekend (Saturday and Sunday) with $3 \mathrm{~g}$ oral mesalazine daily, the later did not use mesalazine enemas and received $3 \mathrm{~g}$ oral mesalazine daily. The clinical course was followed at regular intervals after randomization. The relapse of UC was defined as the primary endpoint of the study. We defined relapse as six and over of the clinical activity index (CAI) score and over three of the endoscopic index (EI) score. Recruitment commenced in January 2004 and is expected to be completed by January 2006 and follow-up will continue until December 2008. However, we stopped the study after 24 patients had been enrolled because an interim analysis showed the significant consequence. Results: Of 24 randomized patients, 11 were allocated to the weekend mesalazine enema group and 13 to the oral alone group. In the group receiving weekend enema, 2 patients (18.2\%) relapsed, as compared with $10(76.9 \%)$ in the group receiving oral mesalazine alone. The comparison was statistically significant $(P=0.037)$ and its hazard ratio was 0.19 . During the course of the study period, all patients did not have any adverse event related with the drugs. Conclusions: This study has demonstrated the beneficial effects of adding $1 \mathrm{~g}$ mesalazine enema twice a week at weekend with $3 \mathrm{~g}$ oral mesalazine for maintenance therapy of UC.

\section{T1129}

Comparison Between Intravenous Iron Gluconate Alone and Intravenous Iron Gluconate Plus Levofolinic Acid for the Treatment of Iron Deficiency Anaemia in Patients with Inflammatory Bowel Disease

Alfredo Papa, Silvio Danese, Simona Guglielmo, Italia Roberto, Marcello Covino, Antonino Grillo, Riccardo Urgesi, Stefano Semeraro, Michele Bonizzi, Franco Tagliaferri, Luisa Guidi, Italo de Vitis, Giuseppe Fedeli, Giovanni Gasbarrini, Antonio Gasbarrini

Background and aim: Anaemia is a common clinical feature in IBD patients, with rates between $30 \%$ and $50 \%$, leading to impaired quality of life. IBD-associated anaemia has a multifactorial aetiology but iron deficiency is the leading cause. Aim of this prospective open study was to compare the efficacy and safety of two different regimens for the treatment of iron deficiency anaemia (IDA) in IBD patients. Patients and methods: We enrolled 30 IBD patients (12 with Crohn's disease and 18 with ulcerative colitis) with IDA, defined as haemoglobin $(\mathrm{Hb})$ value $<10.5 \mathrm{~g} / \mathrm{dl}$ and hypochrome and microcytotic erythrocytes. None of the patients had hemolysis, macrocytic anaemia, or renal insufficiency. Patients were consecutively randomised in two groups comparable for disease type, activity and treatment. Fifteen patients were included in group A, receiving 10 daily infusions of $5 \mathrm{ml}$ of iron gluconate (each corresponding to $62.5 \mathrm{mg}$ of Fe+++) [Ferlixitâ, Aventis] and 15 patients were included in group B, receiving daily the same infusion of iron gluconate followed by the intravenous administration of $25 \mathrm{mg}$ of levofolinic acid [Levofoleneâ, Schering]. Clinical assessment of side effects and laboratory testing (blood cell counts, including iron, reticulocytes, mean corpuscolar volume, transferrin, ferritin, and C-reactive protein) were made at baseline and every five infusions. Results: At baseline, Hb levels, iron and ferritin concentrations were comparable between the two groups. In particular, the mean $\mathrm{Hb}$ was $9.4 \pm 1.4 \mathrm{~g} /$ $\mathrm{dl}$ in group A and $9.2 \pm 1.3 \mathrm{~g} / \mathrm{dl}$ in group B. At the end of the scheduled therapy, Hb levels increased to $10.2+1.5 \mathrm{~g} / \mathrm{dl}$ in group $\mathrm{A}(\mathrm{P}=0.142)$ and $11.1 \pm 1.8 \mathrm{~g} / \mathrm{dl}$ in group $\mathrm{B}(\mathrm{P}=0.025)$. Side-effects were generally mild and transient ( 2 in group A and 3 in group B); no serious adverse events were reported. Conclusions: The contemporary administration of intravenous iron gluconate and levofolinic acid showed a more consistent increase of the $\mathrm{Hb}$ concentration than iron gluconate alone in IBD patients with IDA. This results could be explained by the frequent finding of low levels of folates in IBD patients also in absence of overt macrocytic anaemia.
$\mathrm{T} 1130$

A Safety and Efficacy Study of a Novel Formulation of Prednisolone Metasulfobenzoate (PREDOCOL) in the Induction of Remission and Maintenance in Ulcerative Colitis

Jonathan M. Rhodes, Richard Robinson, Ian Beales, Stirling Pugh, Richard Dickinson, Michael Dronfield, Steven Wilkinson

Background/Aims Prednisolone metasulfobenzoate is effective topically in ulcerative colitis but is sparingly absorbed from the colon (McIntyre et al, Gut 1985). A modified-release oral formulation has been developed (Predocol) comprising a Eudragit L-coated capsule containing 200 controlled-release drug pellets. A pilot study showed very low systemic absorption. This study compares the safety and efficacy of Predocol with prednisolone in ulcerative colitis. Methods Patients were randomised to one of three treatment groups in a double-blinded study. Group A ( $\mathrm{n}=59)$ received Predocol $40 \mathrm{mgs} /$ day for 6 months plus placebo, Group B $(\mathrm{n}=61)$ received Predocol $60 \mathrm{mgs} /$ day for 6 months plus placebo, and group $C(n=61)$ received Prednisolone $40 \mathrm{mg} /$ day for 2 weeks which was then tapered to $30 \mathrm{mg} /$ day weeks 3 and $4,20 \mathrm{mg} /$ day weeks 5 and $6,10 \mathrm{mg} /$ day week $7,5 \mathrm{mg} /$ day week 8 then placebo only until 6 months. All drugs were given in twice daily divided doses. Steroidrelated side effects and efficacy were assessed by patients using a visual analogue scale (VAS) where $10 \mathrm{~cm}=$ symptom-free and $0=$ worst possible. Efficacy was also assessed by PowellTuck score, Physician global score and Baron endoscopic grading. The trial was designed to assess non-inferiority. Results Predocol at $40 \mathrm{mgs}$ per day but not at $60 \mathrm{mgs} / \mathrm{day}$ showed non-inferiority compared to prednisolone at 2 months and 6 months (Table). Physician's global assessment confirmed non-inferiority for Predocol $40 \mathrm{mg}$ vs prednisolone at month 2 (remission $36 \%$ and $43 \%$ respectively) and month 6 (remission $65 \%$ and $43 \%$ respectively). Similar efficacy was observed using Powell-Tuck and Baron grades. Patient's global assessment of steroid-related side-effects at 2 months was better for Predocol $40 \mathrm{mgs}$ or $60 \mathrm{mgs}$ ( $\mathrm{p}=$ 0.001 and 0.002 respectively) compared with prednisolone. Predocol was also significantly better than prednisolone on patient's scoring for moon face, hirsutes, flushing, mood changes and insomnia. Conclusion Predocol 40 mgs per day is non-inferior in efficacy compared with a conventional tapering prednisolone regimen and is associated with fewer steroidrelated side effects.

\begin{tabular}{|c|c|c|c|c|c|}
\hline \multicolumn{6}{|c|}{ Patient's Global Symptom VAS [cm, mean(sd)] ( $0=$ worst possible, $10=$ symptom-free) } \\
\hline & Entry & 2 weeks & 4 weeks & 2 months & 6 months \\
\hline Predocol 40mg & $3.99(2.06)$ & $6.26(2.36)$ & $7.09(2.68)$ & $8.20(1.78)$ & $8.20(2.80)$ \\
\hline Predocol $60 \mathrm{mg}$ & $2.97(1.76)$ & $5.38(2.46)$ & $6.82(2.01)$ & $7.47(2.28)$ & $6.84(2.84)$ \\
\hline Prednisolone & $3.95(2.12)$ & $7.61(2.11)$ & $7.98(2.39)$ & $8.03(2.16)$ & $7.94(2.40)$ \\
\hline \multicolumn{6}{|c|}{ Patient's Global Steroid Side-Effect VAS [cm, mean (sd)] ( $0=$ worst possible, $10=$ symptom-free $)$} \\
\hline & Entry & 2 weeks & 4 weeks & 2 months & 6 months \\
\hline Predocol $40 \mathrm{mg}$ & - & $8.38(2.20)$ & $8.29(9.50)$ & $8.14(2.59)$ & $9.04(2.12)$ \\
\hline Predocol $60 \mathrm{mg}$ & - & $8.18(2.61)$ & $8.39(2.14)$ & $8.07(2.59)$ & $7.48(2.73)$ \\
\hline Prednisolone & - & $6.55(2.45)$ & $6.44(2.65)$ & $6.74(2.77)$ & $9.16(1.58)$ \\
\hline
\end{tabular}

\section{T1131}

Double-Blind, Double Dummy, Randomized, Multicentre, Comparative Study On the Efficacy and Safety of Azathioprine (AZA) Versus mesalazine (5-ASA) for Prevention of Postoperative Endoscopic Recurrence in Crohn's Disease Hans Herfarth, Florian Obermeier, Christin Tjaden, Milan Lukas, Zuzana Serclova, Axel U. Dignass, Diether Ludwig, Ralph Mueller, And The Azt-1 Study Group Juergen Schoelmerich

Background: Postoperative recurrence of Crohns disease represents a considerable clinical problem. Two recently published studies addressing the therapy with 6-mercaptopurine (6MP) or azathioprine (AZA) postoperatively had several limitations (fixed low dose of $50 \mathrm{mg}$ 6-MP, statistical problems due to missing data or lack of blinding) and further clarification is needed. The aim of this study was to assess the efficacy of a postoperatively initiated therapy with AZA or 5-ASA for the prevention of endoscopic and clinical recurrence 12 months after resective surgery in patients (pat.) with Crohns disease. Methods: In a prospective double-blind, double dummy, randomized, multicentre study 279 patients were screened and 79 patients were randomized within 2 weeks postoperatively to $2.0-2.5 \mathrm{mg} / \mathrm{kg}$ body weight/day AZA (42 pat.) or $4 \mathrm{~g} 5$-ASA/day ( 37 pat.). The study was stopped prematurely after an interim-analysis due to a high therapy failure rate. 38 patients (AZA 18 pat.; 5-ASA 20 pat.) completed the study and could be evaluated regarding the primary endpoint therapy failure. The other pat. terminated the trial prematurely due to the study stop, but were also evaluated for adverse events (AE) and adverse drug reactions (ADR). Results: The overall failure rate was 19/38 pat. (50\%), equally distributed between both treatment groups (see table 1). In the AZA and 5-ASA groups, respectively, in $69 \%$ and $70 \%$ of the patients an $\mathrm{AE}$ and in $43 \%$ and $30 \%$ an ADR occurred. Overall the most frequent AE were headache and abdominal pain. Conclusion: In this prematurely stopped trial a superiority of AZA over 5-ASA could not be demonstrated due to a high percentage of therapy failures. No specific reason for therapy failure could be defined. The high percentage of therapy failures indicates the difficulties, which may arise in future clinical trials addressing the question of postoperative immunosuppressive therapy in Crohns disease. Supported by Dr. Falk Pharma GmbH, Freiburg, Germany

Table 1: Reasons for therapy failure in 19/38 patients 


\begin{tabular}{|c|c|c|c|}
\hline Reason (n=19) & AZA (n=18) & 5 -ASA (n=20) & Total (n=38) \\
\hline Completed (severe endoscopic recurrence) & $1(6 \%)$ & $2(10 \%)$ & $3(8 \%)$ \\
\hline Lack of efficacy & $1(6 \%)$ & $5(25 \%)$ & $6(16 \%)$ \\
\hline AE related to study drug & $7(39 \%)$ & $3(15 \%)$ & $10(26 \%)$ \\
\hline
\end{tabular}

\section{T1132}

Clinical Trial On the Efficacy and Tolerability of a Novel Low-Volume mesalamine Foam (2xlg 5-ASA/30ml) Vs mesalamine Foam (2xlg 5-ASA $60 \mathrm{ml})$ in Active Ulcerative Proctitis Or Proctosigmoiditis

Rami Eliakim, Zsolt Tulassay, Limas Kupcinskas, Kestutis Adamonis, Juris Pokrotnieks, Simon Bar-Meir, Ralph Mueller, Roland Greinwald, On Behalf of the International Foam Study Group Gross, Volker

AIM: To study the therapeutic efficacy and tolerability of a novel low-volume mesalamine foam $(2 \times 1$ g $5-A S A / 30 \mathrm{ml})$ vs mesalamine foam $(2 \times 1 g$-ASA $/ 60 \mathrm{ml})$ in patients with active ulcerative proctitis or proctosigmoiditis. METHODS: Patients with active ulcerative proctitis or proctosigmoiditis (Clinical Activity Index $[\mathrm{CAI}]>4$, Endoscopic Index $[\mathrm{EI}] \geq 4$ ) where elegible for this single-(investigator)-blinded, randomized, multicenter study. Patients received $2 \times 1 \mathrm{~g} / 30 \mathrm{ml}$ mesalamine foam $(\mathrm{n}=163)$ or $2 \mathrm{xlg} / 60 \mathrm{ml}$ mesalamine foam $(\mathrm{n}=167)$ for 42 days. Primary endpoint was clinical remission $(\mathrm{CAI} \leq 4)$ at the final/withdrawal visit (per protocol [PP] population). RESULTS: 330 patients were randomized, 330 were evaluable for safety and intention-to-treat (ITT), 290 for PP analysis. Efficacy See tables below. Safety Both formulations were safe and no drug-related serious adverse events were observed. The low-volume mesalamine foam was associated with a lower frequency of severe discomfort, pain, and retention problems as compared to the high-volume foam. CONCLUSION: Lowvolume mesalamine foam $(2 \times 1 \mathrm{~g} 5-\mathrm{ASA} / 30 \mathrm{ml})$ is as effective as high-volume mesalamine foam $(2 \mathrm{xlg} 5-\mathrm{ASA} / 60 \mathrm{ml})$ in the treatment of active ulcerative proctitis or proctosigmoiditis. Both mesalamine foams are safe. Supported by Dr. Falk Pharma GmbH, Freiburg, Germany Primary efficacy endpoint (final analysis):

\begin{tabular}{|c|c|c|c|}
\hline & \multicolumn{2}{|c|}{$\begin{array}{c}\text { Number (\%) of patients in clinical remission (CAI } \\
\text { 4) at Week 6 (LOCF) }\end{array}$} & $\begin{array}{c}\text { Shifted asymptotic } \chi^{2} \text { test for } \\
\text { comparing two rates* }\end{array}$ \\
\hline & 2x1g 5-ASA/30ml & 2x1g 5-ASA/60ml & p-value (one-sided) \\
\hline PP & $113 / 146(77.4 \%)$ & $111 / 144(77.1 \%)$ & 0.00002 (overall) \\
\hline ITT & $123 / 163(75.5 \%)$ & $121 / 167(72.5 \%)$ & $<0.00001$ (overall) \\
\hline
\end{tabular}

*Non-inferiority margin: 20\%; LOCF, Last observation carried forward Secondary efficacy endpoints:

\begin{tabular}{|c|c|c|c|}
\hline & & \multicolumn{3}{|c|}{ Number (\%) of patients } \\
\hline & & 2x1g 5-ASA/30ml & 2x1g 5-ASA/60ml \\
\hline Clinical remission (DAI <4) & PP & $99 / 144(68.8 \%)$ & $93 / 142(65.5 \%)$ \\
\hline & ITT & $105 / 157(66.9 \%)$ & $99 / 162(61.1 \%)$ \\
\hline Endoscopic remission (EI < 4) & PP & $87 / 146(59.6 \%)$ & $96 / 144(66.7 \%)$ \\
\hline & ITT & $96 / 163(58.9 \%)$ & $104 / 167(62.3 \%)$ \\
\hline Histological Remission & PP & $68 / 128(53.1 \%)$ & $65 / 126(51.6 \%)$ \\
\hline Therapeutic succes** (PGA) & ITT & $70 / 136(51.5 \%)$ & $65 / 140(46.4 \%)$ \\
\hline Therapeutic success** $($ PGA) & ITT & $107 / 146(73.3 \%)$ & $109 / 144(75.7 \%)$ \\
\hline & PP & $118 / 163(72.4 \%)$ & $117 / 167(70.1 \%)$ \\
\hline & ITT & $140 / 146(86.3 \%)$ & $131 / 144(91.0 \%)$ \\
\hline
\end{tabular}

*At least marked improvement; ** At least slight improvement DAI, Disease Activity Index (acc. to Sutherland); PGA, Physician's Global Assessment (acc. to Hanauer)

\section{T1133}

Pharmacokinetics of Adalimumab in a Long-Term Investigation of the Induction and Maintenance of Remission in Patients with Crohn's Disease (CLASSIC I and CLASSIC II)

Tushar S. Garimella, Joanna Z. Peng, Katrin Beck, Peter A. Noertersheuser, Kathleen G. Lomax, Susan K. Paulson, Paul F. Pollack

BACKGROUND Adalimumab, a fully human anti-TNF monoclonal antibody, is approved for the treatment of rheumatoid arthritis and psoriatic arthritis. In CLASSIC I, a 4-week randomized controlled study of induction of remission in patients with Crohn's disease (CD), serum adalimumab concentrations were dose-proportional. Additionally, adalimumab concentrations were sustained during the study period as a result of the initial loading dose (Paulson et al, DDW 2005). PURPOSE To assess the pharmacokinetics (PK) and immunogenicity of adalimumab following long-term administration in patients with $C D$. METHODS In CLASSIC II, an extension study of CLASSIC I, all patients received adalimumab $40 \mathrm{mg}$ subcutaneously (sc) at Week 0 (Week 4 of CLASSIC I) and Week 2. Patients in remission $(\mathrm{CDAl}<150)$ at both Weeks 0 and 4 of CLASSIC II were randomized to receive adalimumab, $40 \mathrm{mg}$ sc every other week (eow) or weekly, or placebo for up to 1 year. Patients not in remission at both Weeks 0 and 4 of CLASSIC II received open-label (OL) adalimumab, $40 \mathrm{mg} \mathrm{sc}$ eow. Dose escalation to $40 \mathrm{mg}$ /week was allowed for flare or persistent non-response. Trough serum samples for adalimumab and anti-adalimumab antibody (AAA) assays were obtained at Weeks 4,24 , and 56 to determine serum concentrations using validated ELISA methods. Population PK analyses were performed using the NONMEM software to estimate adalimumab apparent clearance (CL/F) combining the data from both CLASSIC I and II. A one-compartment model was used to describe adalimumab PK. RESULTS Of 276 patients entering CLASSIC II, 55 were randomized to receive adalimumab, $40 \mathrm{mg} \mathrm{sc}$ eow $(\mathrm{n}=19)$ or weekly $(\mathrm{n}=18)$, or placebo $(\mathrm{n}=18)$. The remaining 221 patient received OL adalimumab, $40 \mathrm{mg}$ sc eow. The median CL/F $(15.0 \mathrm{~mL} / \mathrm{hr})$ was consisten with that using CLASSIC I data only $(13.2 \mathrm{~mL} / \mathrm{hr})$, indicating relative time-independence. Concomitant therapy with either the immunosuppressant 6 mercaptopurine $(n=23)$ or azathioprine $(n=36)$ slightly lowered or had no impact on adalimumab CL/F. The effect of concomitant methotrexate alone on adalimumab $\mathrm{CL} / \mathrm{F}$ was inconclusive $(\mathrm{n}=6)$. The overall incidence of positive AAA in CLASSIC II was 3.0\% (8/269). Among 8 AAA+ patients, $3(37.5 \%)$ were in remission $(\mathrm{CDAl}<150)$ at Week 24 and $2(25 \%)$ were in remission at Week 56. CONCLUSIONS The pharmacokinetics of adalimumab in patients with CD remained constant over time. The overall incidence of AAA was low and adalimumab was well-tolerated.

\section{T1134}

Intravenous Clodronate for Prevention of Steroid-Induced Bone Loss in Patients Affected By Inflammatory Bowel Disease: A Prospective Randomized Double-Blind Placebo-Controlled Trial (Clastos study)

Vered Abitbol, Gabriel Baron, Philippe Seksik, Antoine Charachon, Yoram Bouhnik, Cecile Poupardin, Matthieu Allez, Dominique Lamarque, Christian Roux, Stanislas Chaussade

Background: Osteoporosis is common in patients affected by inflammatory bowel disease (IBD) with an increased risk of vertebral fractures (1). Corticosteroids induce a rapid and important bone loss (2). Clinical trials have shown oral bisphosphonates to effectively prevent steroid-induced bone loss. However, patients with IBD have been excluded from these studies by fear of digestive adverse events. Clodronate is a non-amine bisphosphonate (BP) available in intravenous form without expected digestive (oral BPs) or pro-inflammatory (amine BPs) side effects. Aim : to assess the efficacy of intravenous clodronate in preventing steroid-induced bone loss. Methods : 67 patients, 37 women, mean age 31 years, 52 Crohn's disease, 12 ulcerative colitis, 3 indeterminate colitis, at the start of a steroid therapy, were included. In this prospective, multicentric, double blind parallel and placebo controlled study, patients were randomized to receive during the steroid therapy, one infusion per 3 months of either intravenous clodronate ( $900 \mathrm{mg}, \mathrm{n}=33$ ) or placebo. Calcium (1g/day) and vitamin D (800 IU/day) were given to all of the patients during one year. Lumbar and femoral bone mineral density (BMD) were measured by dual energy X-ray absorptiometry at baseline, 6 and 12 months, with quality control procedures and analysis in a central facility, blind to allocation of the treatment. BMD changes from 0 to 12 months were tested to 0 ( $\triangle \mathrm{BMD}$ ) in each group (clodronate or placebo) through a Wilcoxon test and compared between the 2 groups. Results : Clinical and biochemical characteristics (age, gender, treatments, steroid intake and initial BMD) were not different in the 2 groups. T scores at baseline were $-1.3 \mathrm{SD}$ and $-1.2 \mathrm{SD}$ in the clodronate and placebo group, respectively. Tolerance to the treatment was good. After 1 year, BMD remained unchanged with clodronate whereas it decreased with placebo. There was a trend for a difference between groups at lumbar spine level $(\mathrm{P}=0.06)$ (Table 1). Conclusion: Quarterly intravenous clodronate may be useful for preventing vertebral bone loss when started early in a steroid therapy for IBD. (1) Abitbol $V$ et al. Gastroenterology 1995;108:417 (2) Roux C et al. Osteoporosis Int 1995; 5:156 Table 1. BMD changes after 1 year

\begin{tabular}{|c|c|c|c|}
\hline$\Delta$ BMD & Clodronate $(\mathrm{n}=33)$ & Placebo $(\mathrm{n}=34)$ & $\mathrm{P}$ \\
\hline Lumbar spine (L2-L4) & $-0.2 \%$ & $-2 \%$ & 0.06 \\
\hline Femoral total & $0.1 \%$ & $-0.8 \%$ & 0.28 \\
\hline Femoral neck & $2.3 \%$ & $-1.7 \%$ & 0.16 \\
\hline Trochanter & $0.1 \%$ & $-1.1 \%$ & 0.36 \\
\hline
\end{tabular}

\section{T1135}

Prospective Evaluation of Effects of NSAID Use On the Disease Course of the Ileal Pouch-Anal Anastomosis (IPAA)

Bo Shen, Feza Remzi, Bret Lashner, Ana Bennett, Aaron Brzezinski, Jean-Paul Achkar, Rocio Lopez, Ioannis Oikonomou, Kerry Sherman, Marlene Bambrick, Victor Fazio

The role of NSAID use in the exacerbation of IBD is controversial. There are no prospective studies. We hypothesized that pouch diseases in a subset of patients were exacerbated by chronic NSAID use and the cessation of NSAID use may help reducing disease activity and inducing remission. AIM: To prosectively study the effect of cessation of NSAID use on disease course of IPAA in patients with chronic daily use of the agents. METHODS: The 4wk study consisted of 2 consecutive cohorts of symptomatic patients seen in the Pouchitis Clinic who had ulcerative colitis, had colectomy and IPAA, and had chronic (> $6 \mathrm{mo}$ ) daily use of NSAID. The patients were treated either with avoiding NSAID (the Cessation Group, $\mathrm{N}=17$ ) or with avoiding NSAID plus being started with medical therapy with antibiotics, mesalamines, or corticosteriods (The Cessation + Therapy Group, N=12). The Pouchitis Disease Activity Index (PDAI) symptom scores, total PDAI scores, and Cleveland Global Quality of Life (CGQL, range 0 - 1.0), Irritable Bowel Disease Quality of Life (IBS-QOL, range 34-170), and short-form Inflammatory Bowel Disease Questionnaire (IBDQ, range 070) scores were measured before and after the 4 -wk trial. Compliance was assured. Appropriate statistical analyses were used. RESULTS: Demographic and clinical data were similar between the 2 groups. The Cessation Group consisted of 11 patients with chronic refractory pouchitis $(65 \%), 2$ acute pouchitis $(12 \%), 1$ cuffitis $(6 \%), 1$ cuffitis + chronic refractory pouchitis (6\%), 2 irritable pouch syndrome (12\%); and the Cessation + Therapy Group had 7 chronic refractory pouchitis (58\%), 2 acute pouchitis (17\%), 1 cuffitis + chronic refractory pouchitis $(8 \%)$, and 2 irritable pouch syndrome $(17 \%)(P>0.05)$. The cessation of NSAID use with or without concurrent medical therapy resulted in a significant reduction in symptom and PDAI scores and improvement in QOL scores (Table). There was no difference in 
treatment outcome between the 2 groups $(\mathrm{P}>0.05)$. CONCLUSIONS: Chronic daily NSAID use was associated with diseases of IPAA, particularly chronic refractory pouchitis. The majority of these patients benefited from complete cessation of NSAID use, suggesting that NSAID use can play an important role in the pathogenesis of pouch diseases, particularly chronic refractory pouchitis.

\begin{tabular}{|c|c|c|c|c|}
\hline & \multicolumn{2}{|c|}{ NSAID Cessation } & \multicolumn{2}{c|}{$\begin{array}{c}\text { NSAID Cessation + } \\
\text { Therapy }\end{array}$} \\
\hline Factor & Statistics & $\begin{array}{c}\text { P } \\
\text { value }\end{array}$ & Statistics & P value \\
\hline $\begin{array}{c}\text { Reduced Symptom Score, Median (25th, } \\
75 \text { th\%) }\end{array}$ & $\begin{array}{c}-1.0 \\
(-2.0,0.0)\end{array}$ & 0.001 & $-2.0(-2.0,-0.5)$ & 0.03 \\
\hline Reduced Total PDAI Score, Mean (SD) & $-3.6(3.0)$ & 0.0002 & $-3.0(3.7)$ & 0.02 \\
\hline Improved CGQL Score, Mean(SD) & $0.1(0.1)$ & 0.003 & $0.1(0.1)$ & 0.04 \\
\hline $\begin{array}{c}\text { Improved IBS-QOL Score, Mean(SD) } \\
\text { Improved IBDQ Score, Median (25th, 75th\% }\end{array}$ & $-9.3(21.1)$ & 0.09 & $-6.8(14.7)$ & 0.14 \\
\hline ) & $5(2.0,7.0)$ & 0.002 & $5(1.5,6.5)$ & 0.009 \\
\hline
\end{tabular}

T1136

Different Definitions of Remission for Ulcerative Colitis Result in Large Variations of Clinical Outcome Scores

Seymour Katz, Sunanda Kane, Peter Higgins, Rachelle Eusebio, Bruce Yacyshyn

Purpose: One of the treatment goals for ulcerative colitis (UC) is to induce remission. Currently, there is no standard/accepted definition of remission for UC. Thus, the definition of remission differs among clinical trials supporting various therapies for UC. The purpose of this analysis was to estimate remission rates using several different definitions of remission. Methods: Data from two Phase III, multicenter, randomized, double-blind, 6-week, controlled studies of similar design (ASCEND I and II) were pooled and analyzed using different definitions of remission. Definitions varied from complete resolution of clinical assessments to still having mild UC signs and symptoms. Remission rates of delayed-release oral mesalamine in patients with mildly to moderately active UC taking $2.4 \mathrm{~g}$ /day (marketed $400 \mathrm{mg}$ tablet) were examined using several different definitions utilized by various UC therapies. Results: A total of 687 patients with mildly to moderately active UC were included in the study, of which 349 received $2.4 \mathrm{~g} /$ day. Depending on the definition of remission used in the analyses (see table below), the percentage of patients in remission varied from $22-50 \%$. Conclusion: Remission rates vary widely depending on the definition of remission used. As there is no standard/accepted definition of remission for UC, physicians and other healthcare professionals should pay attention and be aware of the definition of remission being utilized when evaluating clinical efficacy.

\begin{tabular}{|c|c|}
\hline Definition & Rate \\
\hline $\begin{array}{c}\text { Complete Resolution (score=0) of stool frequency, rectal bleeding, sigmoidoscopy findings, } \\
\text { patient functional assessment adn physician's global assessment. }\end{array}$ & $22 \%$ \\
\hline $\begin{array}{c}\text { UCDAI score of \& } \# 8806 ; 1 \text { accompanied by a rectal bleeding and stool frequency subscore of } \\
\text { 0 or at least a 1 point decrease in sigmoidoscopy score from baseline. }\end{array}$ & $28 \%$ \\
\hline UCDAI score of \&\#8806;2, with no individual sbuscore $>1$. & $50 \%$ \\
\hline
\end{tabular}

$\mathrm{T} 1137$

Maintenance Therapy with Natalizumab Does Not Require Use of Concomitant Immunosuppressants for Sustained Efficacy in Patients with Active Crohn's Disease: Results from the ENACT-2 Study

William Sandborn, Jean-Frederic Colombel, Robert Enns, Brian Feagan, Stephen Hanauer, Ian Lawrance, Remo Panaccione, Paul Rutgeerts, Stefan Schreiber, Stephan Targan, Sander van Deventer

Introduction: In the ENACT-2 study, natalizumab treatment was associated with a clinically significant maintenance of sustained response and remission in patients who had previously responded to natalizumab induction therapy in ENACT-1. Many of the ENACT-1 patients had active Crohn's disease (CD) despite receiving an adequate course of immunosuppressants (IMM) at baseline of ENACT-1. Sustained response and remission rates in this subset of patients were compared with those achieved in patients who were not receiving IMM at ENACT-1 baseline. The protocol required that baseline IMM be continued as concomitant therapy throughout both trials. Methods: A total of 339 adult patients with $C D$ who achieved response ( $>70$-point reduction in baseline $\mathrm{CD}$ Activity Index [CDAI]) and/or remission (CDAI score $<150$ ) and had a CDAI score $<220$ after receiving 3 infusions of natalizumab in ENACT-1 were re-randomized in ENACT-2 to natalizumab $300 \mathrm{mg}(\mathrm{n}=168)$ or placebo $(\mathrm{n}=171)$ and received up to 12 monthly infusions. The primary endpoint was the proportion of patients that maintained response through 6 additional consecutive months of treatment in ENACT-2. Results: A total of 122 natalizumab-treated patients who were receiving concomitant IMM were in response and 94 were in remission at month 3 of ENACT-1, and were re-randomized in ENACT-2, compared with 216 patients who were in response and 156 who were in remission and not receiving concomitant IMM. Sustained response and remission rates following 12 monthly natalizumab infusions in ENACT-2 were similar between the 2 groups, and significantly greater than placebo in both cases (Table). The frequency and types of adverse events (AE) were also similar between the 2 groups. Persistent anti-natalizumab antibodies (Abs) occurred in $0 \%$ of patients on concomitant IMM and $7.5 \%$ of patients not receiving concomitant IMM. Hypersensitivity reactions occurred infrequently and at the same rate in both groups. Conclusions: Natalizumab was significantly better than placebo at maintaining sustained response and remission through 12 months, and the benefit was similar regardless of concomitant IMM use. The AE profiles between these subgroups were also similar. The rate of persistent anti-natalizumab Abs in patients not receiving concomitant IMM was relatively low and did not appear to significantly impact sustained efficacy at 1 year.

Proportion of Patients in Sustained Response or Remission Through 12 Months

\begin{tabular}{|c|c|c|c|c|c|c|}
\hline & \multicolumn{3}{|c|}{ Response } & \multicolumn{3}{c|}{ Remission } \\
\hline Baseline IMM & Placebo & Natalizumab & P-value & Placebo & Natalizumab & P-value \\
\hline Yes & $23 \%$ & $52 \%$ & 0.002 & $14 \%$ & $37 \%$ & 0.014 \\
\hline No & $18 \%$ & $55 \%$ & $<0.001$ & $16 \%$ & $41 \%$ & $<0.001$ \\
\hline
\end{tabular}

T1138

A Meta-Analysis of the Placebo Effect for the Induction of Clinical Remission/ Response in Mild to Moderate Active Ulcerative Colitis

Volker Gross, Martina Elze, Ralph Mueller, Roland Greinwald

AIM: The present meta-analysis aimed to evaluate the placebo effect in the treatment of mild to moderate active ulcerative colitis and to determine the effect of the route of drug administration (oral/rectal) and of the year of the study (up to 2000/2001 to date). METHODS: Published, randomised, controlled clinical trials, comparing the efficacy of active drugs versus placebo in the treatment of active ulcerative colitis were considered. The primary outcome measure was clinical remission. Secondary outcome measures were clinical improvement, endoscopic and histologic remission and/or improvement. RESULTS: 63 studies fullfilled the criteria for analysis. In 29/63 studies the investigational products were administered rectally, and in 27/63 studies orally. In 1/63, 4/63 and 2/63 studies intravenous, subcutaneous, or transdermal formulations were investigated, respectively. 51/63 studies were published until 2000; 12/63 from 2001 to date. The pooled clinical placebo remission rate across all studies was $13 \%(95 \% \mathrm{CI}, 10 \%-16 \% ; \mathrm{N}=38)$, across the studies with rectal administration it was $15 \%(10 \%-21 \% ; N=18)$, across the studies with oral administration it was $11 \%(8 \%-14 \% ; \mathrm{N}=14)$. The pooled clinical placebo improvement rate across all studies was $29 \%(95 \%$ CI, $25 \%-33 \%, N=54)$. Pooled rates of $19 \%(14 \%-24 \%$; N $=18)$, $30 \%(23 \% 38 \% ; \mathrm{N}=19), 9 \%(3 \%-14 \% ; \mathrm{N}=9)$, and $23 \%(18 \%-29 \% ; \mathrm{N}=13)$ were found for endoscopic remission, endoscopic improvement, histologic remission, and histologic improvement, respectively. The pooled rate of clinical remission in studies published up to 2000 was $12 \%(9 \%-16 \% ; \mathrm{N}=29)$, and in studies published from 2001 to date $14 \%(8 \%$ $20 \%$; N=9). CONCLUSION: The placebo remission rate of active ulcerative colitis is $13 \%(10 \%-16 \%)$. Similar clinical placebo remission rates were found for oral or rectal administration, as well as for studies published up to 2000 or from 2001 to date. Supported bei Dr. Falk Pharma GmbH, Freiburg, Germany

\section{T1139}

SPD476, a Novel, High-Strength 5-ASA Formulation Induces Remission of Active, Mild-to-Moderate Ulcerative Colitis in Subjects That Are Switched from Low-Dose Oral 5-ASA Therapy Or Are 5-ASA NaiVe: An Analysis of Pooled Data from Two Phase III Studies

William J. Sandborn, Michael A. Kamm, Gary R. Lichtenstein, Miguel Gassull, Stefan Schreiber, Lechoslaw Jackowski, Prabhakar Boddu, Natalya Gubergrits

Background: Reasons for patients switching from one therapy to another within a drug class may include improved convenience and/or clinical efficacy. During switching it is essential to maintain or even improve disease control. Here we report an analysis of subjects with ulcerative colitis (UC) who were switched from prior low-dose oral 5-ASA therapy to moderate or high-dose SPD476, a novel, high-strength (1.2g/tablet) oral formulation of 5ASA that uniquely combines a gastro-resistant film to delay drug release until the terminal ileum, with MMX Multi Matrix System ${ }^{\mathrm{TM}}$ technology to extend consistent release of the active drug throughout the entire colon. Methods: Data from two phase III studies (SPD476301 and SPD476-302) of subjects with active, mild-to-moderate UC who received SPD476 $2.4 \mathrm{~g}$ /day (given once or twice daily), SPD476 4.8g/day (given once daily) or placebo ( $\mathrm{Pb}$ ) were combined and further analyzed for subjects who were switched from prior low-dose oral 5-ASA therapy (discontinued oral 5-ASA therapy $[\leq 2 \mathrm{~g} /$ day $] \leq 5$ days prior to baseline) and subjects who were 5-ASA naive (no prior oral 5-ASA or had discontinued oral 5-ASA therapy $[\leq 2 \mathrm{~g} /$ day $]>5$ days prior to baseline). Remission (UC-disease activity index score of $\leq 1$ with rectal bleeding and stool frequency scores of 0 and $a \geq 1$ point reduction in sigmoidoscopy score from baseline) rates at week 8 were analyzed. Results: No difference in disease severity between treatment groups at baseline was observed. In the switched group, significantly more subjects receiving SPD $4764.8 \mathrm{~g}$ /day and numerically more subjects receiving SPD476 2.4g/day achieved remission compared with $\mathrm{Pb}$ (Table 1). In the 5-ASA naïve group, significantly more subjects achieved remission following either dose of SPD476 compared with $\mathrm{Pb}$ (Table 1). Conclusions: SPD476 was effective for the induction of remission of active, mild-to-moderate UC in subjects switched from recent oral 5-ASA therapies and subjects who were 5-ASA naive. These data show that subjects with active UC, despite prior oral 5-ASA therapy, can achieve remission following therapy with SPD476. The $4.8 \mathrm{~g}$ dose may give the best result in this setting. Switching to SPD476 may improve patient compliance and treatment success.

Table 1. Summary of remission rates

\begin{tabular}{|c|c|c|c|c|c|c|c|c|c|}
\hline & \multicolumn{3}{|c|}{$\mathbf{P b}$} & \multicolumn{3}{|c|}{ SPD476 2.4g/day } & \multicolumn{3}{|c|}{ SPD476 4.8g/day } \\
\hline Subjects & $n$ & $\begin{array}{c}\text { Remission n } \\
(\%)\end{array}$ & $\begin{array}{c}95 \% \\
\mathrm{CI}\end{array}$ & $\mathrm{n}$ & $\begin{array}{c}\text { Remission n } \\
(\%)\end{array}$ & $\begin{array}{c}95 \% \\
\text { CI }\end{array}$ & $\mathbf{n}$ & $\begin{array}{c}\text { Remission n } \\
(\%)\end{array}$ & $\begin{array}{r}95 \% \\
\text { CI }\end{array}$ \\
\hline Switched & 91 & $19(20.9)$ & $\begin{array}{l}13.3, \\
30.0\end{array}$ & 88 & $28(31.8)$ & $\begin{array}{l}23.0, \\
42.2\end{array}$ & 80 & $30(37.5)^{*}$ & $\begin{array}{l}27.1 \\
48.1\end{array}$ \\
\hline $\begin{array}{l}\text { 5-ASA } \\
\text { naïve }\end{array}$ & 80 & $11(13.8)$ & $\begin{array}{l}7.3, \\
22.6\end{array}$ & 84 & $36(42.9)^{\text {水水 }}$ & $\begin{array}{l}32.7, \\
53.7\end{array}$ & 94 & $31(33.0)^{* * *}$ & $\begin{array}{l}24.6 \\
43.8\end{array}$ \\
\hline
\end{tabular}

${ }^{*} \mathrm{p}<0.05 ;{ }^{*} \mathrm{p}<0.01 ; * * * \mathrm{p}<0.001$ vs $\mathrm{Pb} ; \mathrm{CI}=$ confidence interval 
$\mathrm{T} 1140$

How Effective Is Anti-TNF- $\alpha$ Therapy? A RE-Analysis of the Precise 2 Maintenance Trial of Monthly Subcutaneous Certolizumab Pegol in Active Crohn's Disease Using Remission to Redefine All Efficacy Measures Stefan Schreiber, Ian C. Lawrance, Juliet A. McColm, Ralph Bloomfield, William J. Sandborn

Background: In most clinical studies of biologics in Crohn's disease (CD), response defined as a drop of $\geq 70$ or $\geq 100$ points on the CD Activity Index (CDAI) - is used as an endpoint. Maintenance trials (eg ACCENT I, PRECiSE 2), where patients (pts) responding to open-label therapy are randomized to receive active agent or placebo (PBO) over 6-12 months, have evolved as a design which separates active treatment from PBO. However, 'remission' (CDAI $\leq 150)$ used as an efficacy measure throughout this clinical trial design may better reflect clinical need. Certolizumab pegol is a PEGylated Fab' fragment of a humanized monoclonal antibody that neutralizes tumor necrosis factor $\alpha$. In the PRECiSE 2 Phase III trial, 668 pts with active CD (CDAI 220-450) received open-label subcutaneous (sc) certolizumab pegol $400 \mathrm{mg}$ at Weeks (Wks) 0, 2, 4, followed by randomization of Wk 6 responding pts ( $\geq 100$-point decrease from baseline in CDAI) to every $4 \mathrm{Wk}$ certolizumab pegol $400 \mathrm{mg}(\mathrm{n}=216)$ or $\mathrm{PBO}(\mathrm{n}=212)$ up to $\mathrm{Wk} 26$. Remission $(\mathrm{CDAI} \leq 150)$ rates at Wk 26 were $47.9 \%$ for certolizumab pegol vs $28.6 \%$ for PBO $(\mathrm{p}<0.001)$ in the overall ITT population $(n=425)$. Methods: In a post-hoc analysis, remission after open-label induction at Wk 6 was used to select randomized pts for analysis. Remission rates at Wks $8,12,16$, 20, 24, and 26 were assessed in this population. In addition, absolute CDAI scores (last observation carried forward) for the overall PRECiSE 2 ITT population were analyzed. Results: At Wk 6, 278 pts (42\%) were in remission following induction; 132 pts were randomized to certolizumab pegol and 146 to $\mathrm{PBO}$. At Wk 26, 80 pts $(60.6 \%)$ in the certolizumab pegol arm vs 50 PBO pts (34.2\%) were in remission ( $\mathrm{p}<0.001$; Table). Median CDAI scores in the overall ITT population remained close to remission levels with certolizumab pegol (Wk 8: 142.0; Wk 26: 148.0), in contrast to PBO (Wk 8: 140.5; Wk 26: 195.4); $\mathrm{p}<0.001$ at Wk 26. Conclusions: Using maintenance of remission as an efficacy parameter demonstrates an efficacy signal for certolizumab pegol over $\mathrm{PBO}$ in a post-hoc analysis that may better reflect clinical need. Almost two-thirds of pts receiving monthly certolizumab pegol were in remission at Wk 26. Future induction/maintenance trials of biologics using the ACCENT I/PRECiSE 2 trial design could use remission instead of response as a clinically relevant endpoint.

\begin{tabular}{|c|c|c|}
\hline & \multicolumn{2}{|c|}{ Overall ITT population \% (No. of pts) } \\
\hline $\begin{array}{c}\text { Remission in pts who } \\
\text { were in remission at Wk }\end{array}$ & PBO n=146 & Certolizumab pegol 400 mg n=132 \\
6 & & \\
\hline Wk 6 Wk 8 Wk 12 Wk & $\begin{array}{c}100(146) 71.2(104) 56.2(82) \\
\text { 16 Wk 20 Wk 24 Wk 26 }\end{array}$ & $\begin{array}{c}100(132) 76.5(101) 72.7(96)^{* * *} 58.3 \\
(77)^{*} 59.1(78)^{* *} 56.8(75)^{* * * *} 60.6 \\
(80)^{* * * * *}\end{array}$ \\
\hline
\end{tabular}

$* \mathrm{p} \leq 0.05, * \mathrm{p} \leq 0.01, * * \mathrm{p} \leq 0.001$ vs $\mathrm{PBO}$

\section{T1141}

Moderate to Severe Crohn's Disease Induces High Productivity Losses: Work Productivity and Activity Impairment (WPAI) Baseline Data from the PRECiSE program

Brian Feagan, Stefan Schreiber, Bernard Selke, Margaret C. Reilly, William J. Sandborn

Background: The direct costs associated with Crohn's disease (CD) are substantial and are driven by hospitalizations and surgical procedures. In addition, $C D$ may lead to high indirect costs - as measured by productivity losses — owing to its early onset during economically productive years. However, limited information is available regarding work and activity impairment in these patients. Methods: We described the burden of $C D$ in terms of lost productivity (at work and in unpaid activities) using baseline data from 2 Phase III multicenter, randomized, placebo-controlled clinical trials (PRECiSE 1 and 2) of certolizumab pegol in moderate to severe CD (Crohn's Disease Activity Index [CDAI] score 220-450 points, inclusive). The Work Productivity and Activity Impairment (WPAI) questionnaire was used to collect data on impairment due to $\mathrm{CD}$ during the 7 days before the baseline visit. Four mean scores were calculated: absenteeism (\% of work time missed), presenteeism (\% of work time with impairment), overall work impairment (absenteeism plus presenteeism), and activity impairment. Higher scores indicated greater impairment and lower productivity. A univariate inferential analysis was used to identify possible factors associated with employment status. Results: At baseline, patients in the 2 studies of the PRECiSE program were similar regarding sociodemographic status, clinical characteristics and WPAI scores. Data from 1330 patients (mean duration of CD: 7.9 years) were analysed. In employed patients (62.4\% of the overall population), absenteeism was $19.5 \%$, presenteeism was $42.0 \%$, and overall work impairment was $47.5 \%$. Among all patients, the mean activity impairment was $53.3 \%$. Female gender, older age, and presence of prior bowel resection were significantly associated with being unemployed in patients with $\mathrm{CD}$ (Table) Conclusions: Moderate to severe $C D$ is associated with important loss of productivity. Assessments of the economic impact of $\mathrm{CD}$ treatments should therefore include indirect costs as measured by productivity loss.

\begin{tabular}{|c|c|}
\hline & Unemployed $(\%)(\mathrm{n}=1324)$ \\
\hline Gender $(45.4 \%$ male) $* * *$ & 31.442 .7 \\
\hline Male Female & \\
\hline Age (years, $\mathrm{n}=1324): * *$ & 43.731 .234 .044 .937 .6 \\
\hline$\leq 25>25$ to $\leq 35>35$ to $\leq 45>45$ All (mean age: 37.5$)$ & \\
\hline Prior resection (performed in $33.8 \%): *$ & 42.735 .0 \\
\hline Yes No & \\
\hline
\end{tabular}

** $\mathrm{p}<0.001 ;{ }^{*} \mathrm{p}=0.006 ; \chi^{2}$ test.

\section{$\mathrm{T} 1143$}

Maintenance Therapy with Tacrolimus in Patients with Crohn's Disease Refractory to Azathiopurine: 2 Years Trial

Hiroshi Nakase, Hiroyuki Tamaki, Minoru Matsuura, Satoko Inoue, Mitsunori Uza, Satoru Ueno, Hiroshi Kitamura, Katsuhiro Kasahara, Tsutomu Chiba

Background\&Aims: Azathiopurine (AZA) has been widely used in the treatment of inflammatory bowel disease (IBD), although many patients with IBD fail to respond to AZA or suspend it because of its side effect. Tacrolimus has been shown to be effective in the treatment of IBD. However, there are limited data about the effect of long-term use with tacrolimus on patients with Crohn's disease (CD) refractory to AZA. The aim of this trial was to compare the role of tacrolimus in the induction and maintenance of remission in CD patients with AZA. Patients and Methods: From 2003 to 2005, thirty seven patients with moderate to severe $\mathrm{CD}$ (8 ileum, 23 ileum and colon, 6 colon) were enrolled in a 2 year prospective controlled study. CD patients who had been refractory to AZA received tacrolimus therapy. 18 patients treated with tacrolimus were compared to 19 patients treated with AZA. Tacrolimus was administered orally to aim for serum trough level of 10 15ng/ $\mathrm{ml}$. After patients achieved their clinical remission, tacrolimus was tapered to the serum trough level $5 \sim 10 \mathrm{ng} / \mathrm{ml}$. AZA dose used in our institution is adjusted to aim for WBC count with less than 5000. Efficacy endopoints included clinical improvement (decrease in CDAl $>70$ ), and remission (CDAI $\leqq 150)$. Patients were also monitered for IBDQ. Results: No difference was present in mean CDAI, mean dose of prednisolone (PSL), and disease phenotype at study entry between the two groups. At 3 months after treatment, a significant clinical improvement was observed in both groups (tacrolimus vs AZA: complete remission $57 \%$ vs $63 \%$, clinical improvement $78.5 \%$ vs $79 \%$, p= n.s.). All patients in both treated groups could completely taper off PSL. At 12 months, a significant clinical improvement was observed in both groups (tacrolimus vs AZA: complete remission $72.2 \%$ vs $84 \%$, p= n.s.). IBDQ scores were also improved in both groups (tacrolimus vs AZA: $63 \%$ vs $53 \%$ ). At 24 months, 13 patients $(72.2 \%)$ were in remission in tacrolimus group, compared with 14 patients $(73.6 \%)$ in AZA group. $(\mathrm{p}=\mathrm{n} . \mathrm{s}$.). Patients had relapse in both groups, who were treated by additional infliximab therapy. In tacrolimus group, two patients experienced the increase of serum creatinine level, but this side effect was managed with dose reduction. No serious infection was observed during 2 years. In AZA group, no serious side effect was observed. Conclusion: Maintenance therapy with tacrolimus for 2 years resulted in complete withdrawal of steroid and keeping remission in patients with CD similar to AZA. Tacrolimus is a novel alternative option for patients with $C D$ who are not successfully managed on AZA

\section{$\mathrm{T} 1144$}

Treatment of Chronic Refractory Pouchitis - A Controlled Study Bo Shen, Feza Remzi, Bret Lashner, Ana Bennett, Aaron Brzezinski, Rocio Lopez, JeanPaul Achkar, Ioannis Oikonomou, Kerry Sherman, Marlene Bambrick, Victor Fazio

Management of chronic refractory pouchitis, one of the most common causes of pouch failure, is often challenging. AIM: to assess efficacy and safety of combined ciprofloxacin and tinidazole therapy in patients with chronic refractory pouchitis as compared with mesalamine therapy. METHODS. Two consecutive cohorts of ulcerative colitis patients with chronic refractory pouchitis (disease $>4$ wks and failure to respond to $\geq 4$ wks of singleantibiotic therapy) seen in the Pouchitis Clinic were assigned to receive a 4-wk course of ciprofloxacin $1 \mathrm{gm} / \mathrm{d}$ and tinidazole $15 \mathrm{mg} / \mathrm{kg} / \mathrm{d}(\mathrm{N}=16)$ or a 4 -wk course of oral $(4 \mathrm{gm} / \mathrm{d})$ enema $(8 \mathrm{gm} / \mathrm{d})$ or suppository $(1 \mathrm{gm} / \mathrm{d})$ forms of mesalamines $(\mathrm{N}=10)$. The Pouchitis Disease Activity Index (PDAI, range $0-18$ ) scores, clinical remission $(\mathrm{PDAI}<7$ ), clinical response (reduction in PDAI $\geq 3$ ), the Cleveland Global Quality of Life (CGQL, range 01), Irritable Bowel Syndrome-Quality of Life (IBS-QOL, range 34-170), and Short-Form Inflammatory Bowel Disease Questionnaires (SF-IBDQ, range 0-70) scores were calculated pre- and post- therapy and compared between the 2 treatment groups. Adverse effects were documented. RESULTS: The demographic and baseline clinical, baseline PDAI and QOL scores were similar between the 2 groups. The 4 -wk ciprofloxacin+tinidazole or mesalamine therapy resulted in significant reduction in PDAI scores, and the antibiotic therapy also led to a significant improvement in all QOL scores (Table). Patients in the antibiotic groups had a greater reduction of the PDAI score and a greater improvement in IBS-QOL and IBDQ scores than the mesalamine group $(\mathrm{P}<0.03)$. The clinical remission and response rates were $88 \%$ and $88 \%$ for the antibiotic group; $50 \%$ and $50 \%$ for the mesalamine group ( $\mathrm{P}=$ 0.07 ). Two patients in the antibiotic group developed adverse effects (peripheral neuropathy and dysgeusia)who were able to complete the trial, compared to none in the mesalamine group had adverse effects $(P=0.51)$. CONCLUSIONS: A combined ciprofloxacin and tinidazole therapy was generally well tolerated, was effective in treating patients with chronic refractory pouchitis by inducing remission, reducing disease activity scores and improving QOL scores, and appeared to be more efficacious than mesalamine therapy. The results support notion that chronic refractory pouchitis is of infectious etiology. 


\begin{tabular}{|c|c|c|c|c|}
\hline & \multicolumn{2}{|c|}{ Antibiotic Group } & \multicolumn{2}{c|}{ Mesalamine Group } \\
\hline Factors & Statistics & P value & Statistics & P value \\
\hline Reduced PDAI Score, mean (SD) & $-7.0(4.4)$ & $<0.0001$ & $-2.1(2.2)$ & 0.016 \\
\hline Improved CGQL Score, median (25th, 75th \%) & $0.2(0.1,0.3)$ & 0.0002 & $0.1(-0.1,0.2)$ & 0.12 \\
\hline Improved IBS-QOL Score, mean (SD) & $-28.9(30.5)$ & 0.002 & $-4.3(16.4)$ & 0.43 \\
\hline Improved SF-IBDQ Score, mean (SD) & $12.3(10.3)$ & 0.0003 & $4.4(6.6)$ & 0.064 \\
\hline
\end{tabular}

\section{T1145}

Efficacy of "Half Elemental Diet" As a Maintenance Therapy for Crohn's Disease: Crohn's Disease Half Elemental Diet Study in Sendai (CHESS): A Randomized Controlled Trial

Sho Takagi, Kyoko Utsunomiya, Shinichi Kurivama, Hiroshi Yokoyama, Shuichiro Takahashi, Masahiro Iwabuchi, Hiroki Takahashi, Seiichi Takahashi, Yoshitaka Kinouchi, Nobuo Hiwatashi, Yuji Funayama, Iwao Sasaki, Ichiro Tsuji, Tooru Shimosegawa

[Background \& aim] Crohn's disease (CD) is a chronic inflammatory bowel disease which is characterized by frequent relapses. In Japan, home enteral nutrition (HEN) has been used preferably for a maintenance therapy, which includes a way that the patients take the half of a daily consumption by elemental diet (ED) and the remaining half by food. We named this type of HEN therapy as "half ED". While there have been several reports on the efficacy of HEN, it is also argued to deteriorate the quality of life (QOL) of patients. The aim of this study was to investigate the efficacy of half ED as a maintenance therapy.[Methods] From December 2002 to June 2005, patients were recruited from two clinical centers in Sendai, Japan. Patients were eligible in the study, (1) when they had been diagnosed as CD clinically, endoscopically, radiologically and/or histologically, (2) when they had been just induced to remission stage and (3) when they had been guided properly in their eating by nutritionists or medical doctors once or more. 51 patients were randomly assigned to either a half ED group $(n=26)$ or a free diet group $(n=25)$. Participants belonging to the half ED group were required to take the half daily calorie by $\mathrm{ED}$ and the remaining half by normal meal. The dosage of half ED per day was set for 900-1200 kcal (3-4 packages) and was given through a self-inserted feeding tube and/or oral intake. Participants belonging to the free diet group were required to take all nutrition by normal unrestricted meal. The clinical course was followed and the trial was stopped for significant efficacy after 51 patients had been randomized. The primary end point was the incidence of relapse and the secondary end points included the QOL of participants and medical expenses. Remission and relapse were defined as the Crohn's disease activity index under 150 and over 200, respectively. [Results] A relapse rate in the half ED group is significantly lower $(34.6 \%$ vs $64.0 \%$; hazard ratio 0.36 $[95 \% \mathrm{CI} 0.15-0.83], P=0.016)$ than that in the free diet group. The compliance of the 2 groups was similar. No adverse events had occurred in all patients through the study. [Conclusion] This is the first randomized controlled trial showing the efficacy of half ED, and the results indicated that half $\mathrm{ED}$ is one of valuable candidates as a maintenance therapy for $\mathrm{CD}$.

\section{T1146}

Long-Term Safety Analysis of Budesonide in Crohn's Disease: A Pooled Analysis of Five 1-Year Studies

Gary Lichtenstein, Louise Hapten-White, William J. Sandborn

Aim: To evaluate the overall long-term safety profile of oral budesonide (BUD) for maintenance of remission in patients with mild to moderately active Crohn's disease (CD) of the ileum and/or ascending colon. Methods: Five 1-year, double-blind, placebo-controlled trials evaluating BUD 6mg QD as maintenance therapy in mild to moderate $\mathrm{CD}$ with identical protocols were analyzed. A total of 417 patients with $C D$ in remission $(C D A I \leq 150)$ were randomized to receive BUD 6mg (208 patients) or placebo (209 patients) QD for 12 months. The primary endpoint of the study was to analyze safety of BUD and to compare this with placebo. Results: Overall, reports of adverse events (AEs) by body system were similar between the 2 groups. There were no significant differences $(P \geq 0.30)$ in reports of $A E s$ in patients treated with BUD or placebo in the following body systems: gastrointestinal (39\% vs $44 \%$ ); general, whole body ( $21 \%$ vs $22 \%$ ); respiratory ( $19 \%$ vs $18 \%$ ); nervous system (14\% vs $11 \%)$; psychiatric (13\% vs $11 \%)$; or skin and appendages ( $13 \%$ vs $13 \%)$. The only significant differences in reported AEs by body system occurred in endocrine disorders (BUD $39 \%$, placebo $25 \% ; \mathrm{P}=0.0042$ ) and resistance mechanism disorders (BUD $11 \%$, placebo $5 \% ; \mathrm{P}=0.042$ ). The increased frequency in endocrine disorders was due to a higher, but not statistically different, incidence of acne (BUD $15 \%$, placebo $9 \% ; \mathrm{P}=0.07$ ) and moonface (BUD $11 \%$, placebo $6 \% ; \mathrm{P}=0.075$ ). There were no differences in other cutaneous corticosteroid (CS) symptoms such as swollen ankles (BUD 3\%, placebo $3 \%$; $\mathrm{P}=1.00$ ); bruising (BUD $11 \%$, placebo $7 \% ; \mathrm{P}=0.23$ ); hirsutism (BUD $6 \%$, placebo $2 \% ; \mathrm{P}=0.13$ ); or skin striae (BUD $<0.5 \%$, placebo $1 \% ; \mathrm{P}=1.00$ ). The majority of AEs were mild to moderate in intensity. Thirty-five BUD and 41 placebo patients reported serious AEs (SAEs). Risk of SAEs associated with CS use, such as adrenal insufficiency, cataracts, fractures, and osteoporosis were not increased with BUD treatment. The incidence of AEs was higher, although not statistically different, in female versus male patients in both the BUD group ( $82 \%$ vs $73 \%$, respectively; $\mathrm{P}=0.21$ ) and in the placebo group ( $82 \%$ vs $72 \%$, respectively; $\mathrm{P}=0.12$ ). The largest differences between female and male patients were seen in abdominal pain (17\% vs $10 \%, \mathrm{P}=0.20)$, vomiting ( $10 \%$ vs $2 \%, \mathrm{P}=0.052$ ) and nausea $(10 \%$ vs $3 \%, \mathrm{P}=0.12)$. Finally, discontinuation due to AEs occurred in $8 \%$ and $10 \%$ of the BUD and placebotreated patients, respectively $(\mathrm{P}=0.62)$. Conclusion: Long-term treatment with budesonide capsules is well-tolerated, with a safety profile comparable to placebo and with rare occurrences of SAEs causally-related to its treatment.

\section{$\mathrm{T} 1147$}

Once- and Twice-Daily Spd476, a Novel, High-Strength Formulation of 5-ASA, Induces Remission of Both Mild and Moderate Ulcerative Colitis: A Prespecified Analysis of Combined Data from Two Pivotal, Randomized, Placebo-Controlled, Phase III Studies

Gary R. Lichtenstein, Michael A. Kamm, William J. Sandborn, Miguel Gassull, Stefan Schreiber, Lechoslaw Jackowski, Prabhakar Boddu, Natalya Gubergrits

Background: Existing 5-ASA (mesalamine) formulations for the treatment of ulcerative colitis (UC) require multiple-daily dosing, which may lead to patient non-compliance and thus reduced treatment efficacy. SPD476 is a novel, high-strength (1.2g/tablet), once-daily formulation of 5-ASA that uniquely combines a gastro-resistant polymer film, to delay release of the active drug until the terminal ileum, and MMX Multi Matrix System ${ }^{\mathrm{TM}}$ (MMX) technology to extend consistent delivery of 5-ASA throughout the entire colon. In two pivotal, randomized, placebo-controlled, phase III studies (SPD476-301 [Study 301] and SPD476-302 [Study 302]), SPD476 was well tolerated and effective for the induction of remission of mild-tomoderate UC (a score of 4-10 on the UC disease activity index [UC-DAI]). To demonstrate the efficacy of SPD476 in subjects that experienced either mild (UC-DAI score 4 to $<6$ ) or moderate (UC-DAI score 6-10) disease, a prespecified analysis of combined data from these studies was performed. Methods: Data from these two phase III studies were analyzed for subjects with mild disease who received SPD476 2.4g/day (given once daily [QD; Study 302] or twice daily [BID; Study 301] combined $\mathrm{n}=67$ ), SPD476 4.8g/day (given QD [Studies 301 and 302] combined $n=70$ ) or placebo (combined $n=58$ ). Similarly, data were analyzed for subjects with moderate disease for each treatment regimen (combined $n=104,103$ and 112 , respectively). The proportion of subjects with either mild or moderate disease achieving remission (primary endpoint: UC-DAI score of $\leq 1$, with a rectal bleeding and stool frequency score of 0 and $\geq 1$ point reduction in sigmoidoscopy score from baseline) after 8 weeks treatment with SPD476 2.4g/day or 4.8g/day were compared with placebo. Results: At week 8, a significantly greater percentage of subjects with mild disease receiving SPD476 2.4g/ day achieved remission compared with placebo ( 44.8 vs $20.7 \%$; $\mathrm{p}=0.004$ ), while a numerically greater proportion of subjects with mild disease receiving SPD476 4.8g/day achieved remission ( 35.7 vs $20.7 \% ; \mathrm{p}=0.061$ ). Similarly, a significantly larger proportion of subjects with moderate disease achieved remission following treatment with SPD476 2.4g/day or $4.8 \mathrm{~g} /$ day compared with placebo $(32.7 \%$; $\mathrm{p}=0.004$ and $35.0 \%$; $=0.001$ vs $16.1 \%$, respectively) Disease severity was not a predictor of response to treatment. Conclusions: This unique 5ASA formulation, utilizing MMX technology, was effective for the induction of remission of active, mild and moderate UC at doses of $2.4 \mathrm{~g} / \mathrm{day}$ and $4.8 \mathrm{~g} / \mathrm{day}$. SPD476, by offering treatment of mild and moderate disease with a simple dosing regimen, may improve overall treatment success.

\section{T1148}

SPD476, a Novel Formulation of 5-ASA Given Once Or Twice Daily, Is Effective for the Induction of Remission of Left-Sided and Extensive Ulcerative Colitis: An Analysis of Combined Data from Two Pivotal, Randomized,

\section{Placebo-Controlled Phase UI Studies}

Michael A. Kamm, Gary R. Lichtenstein, William J. Sandborn, Miguel Gassull, Stefan Schreiber, Lechoslaw Jackowski, Prabhakar Boddu, Natalya Gubergrits

Background: Nearly $80 \%$ of patients diagnosed with ulcerative colitis (UC) have left-sided disease, affecting the colon below the splenic flexure. 5-ASA treatment regimens, the standard of care, often require inconvenient multiple-daily, oral dosing or enema administration in order to induce remission. Such regimens can lead to patient compliance issues, which may reduce drug efficacy. SPD476 is a novel, high-strength (1.2g/tablet), once-daily (QD) formulation of 5-ASA that uniquely combines a gastro-resistant film, to delay initial drug release until terminal ileum, and MMX Multi Matrix System ${ }^{\mathrm{TM}}$ (MMX) technology, designed to extend consistent delivery of the active drug beyond the splenic flexure. Two phase III studies (SPD476-301 and SPD476-302) have shown that SPD476 2.4g/day (administered $\mathrm{QD}$ or twice daily [BID]) or 4.8g/day (administered QD) is effective and well tolerated for the induction of remission of mild-to-moderate UC. Methods: In this analysis, data from Studies SPD476-301 and SPD476-302 were combined to compare treatment responses for subjects with left-sided disease and subjects with extensive UC (including pancolitis or involvement of the transverse colon). Subjects with left-sided disease received SPD476 2.4g/ day (given QD [SPD476-302] or BID [SPD476-301]; n=137), SPD476 4.8g/day (given QD; $\mathrm{n}=138$ ) or placebo $(\mathrm{n}=129)$. Similarly, subjects with extensive disease received the same treatment ( $\mathrm{n}=35,35$ and 42 for each treatment group, respectively). Subjects with proctitis only (inflammation $\leq 15 \mathrm{~cm}$ from the anus) were excluded from the original studies. The percentage of subjects with left-sided or extensive disease achieving remission (UC-disease activity index of $\leq 1$, with a rectal bleeding and stool frequency score of 0 and $\geq 1$ point reduction in sigmoidoscopy score from baseline) following 8 weeks treatment with SPD476 $2.4 \mathrm{~g} /$ day or $4.8 \mathrm{~g} /$ day was compared with placebo. Results: At week 8 , a statistically significantly greater proportion of subjects with left-sided UC receiving SPD $4762.4 \mathrm{~g} /$ day or $4.8 \mathrm{~g} /$ day achieved remission compared with placebo $(37.2 \%[\mathrm{p}<0.001]$ and $33.3 \%[\mathrm{p}=0.006]$ vs 18.6\%]). Similarly, statistically significantly more subjects with extensive UC receiving either dose of SPD476 achieved remission by week 8, compared with placebo (37.1\% [p=0.034] and $42.9 \%[\mathrm{p}=0.005]$ vs $14.3 \%$ ). No correlation between disease location and treatment activity was observed. Conclusions: SPD476, a unique, high-strength formulation of 5-ASA utilizing MMX technology, is effective for the induction of remission of left-sided and more extensive UC at doses of $2.4 \mathrm{~g} / \mathrm{day}$ and $4.8 \mathrm{~g} /$ day. Additional benefit should accrue from highstrength, once-daily dosing. 
Maintenance Treatment with Natalizumab Improves the Health Related Quality of Life (HRQOL) in Crohn's Disease Patients

Brian Feagan, Jean-Frederic Colombel, Robert Enns, Stephen Hanauer, Ian Lawrance, Remo Panaccione, Paul Rutgeerts, Stefan Schreiber, Stephan Targan, Sander van Deventer, William Sandborn

Purpose: Crohn's disease is associated with impaired HRQOL. This analysis investigated HRQOL outcomes during maintenance therapy with natalizumab (a humanized monoclonal $\operatorname{IgG}_{4}$ antibody to $\alpha 4$ integrin) in the ENACT-2 trial where higher rates of sustained response and remission were observed compared with placebo. Methods: Patients who responded to natalizumab induction therapy $(\mathrm{n}=339)$ were randomized to natalizumab $300 \mathrm{mg}(\mathrm{n}=$ 168) or placebo infusions ( $n=171$ ) given monthly for up to 12 months. HRQOL was measured by the Inflammatory Bowel Disease Questionnaire (IBDQ) and the Short Form-36 (SF-36) at months $0,3,6,9$, and 12 . Higher scores on each indicate better HRQOL. A Total IBDQ score $>170$ has been identified as indicating clinical remission. The Physical Component Summary (PCS) and the Mental Component Summary (MCS) of the SF-36 are computed such that a mean score of $50(\mathrm{SD}=10)$ corresponds to the US general population. Results: The mean total IBDQ scores of the natalizumab treated patients were significantly greater than those of the placebo group at months 3 through $12(\mathrm{p}<0.01)$ and their scores remained above 170 at each of these time points, while the placebo group fell below this threshold by the month 3 assessment. Among patients who were in remission, those receiving natalizumab had a higher quality of remission as measured by total IBDQ, with natalizumab patients reporting significantly greater scores at months 3 through $12(\mathrm{p}<0.01)$. HRQOL findings with the SF-36 paralleled those observed with the IBDQ, with natalizumab patient PCS and MCS scores significantly greater than those of placebo patients at all measurements post-randomization. With the exception of the MCS comparison at month 3 ( $p<0.011)$, all PCS and MCS comparisons were significant at $\mathrm{p}<0.01$. Mean month 12 scores of the two arms on each of the 8 scales of the SF-36 were compared to established norms of the US population. Natalizumab treated patients had significantly higher scores than the placebo arm on all scales $(\mathrm{p}<0.01)$. While placebo patients scored significantly lower than US norms, $(\mathrm{p}<0.011)$, natalizumab scores were not significantly different from US norms on 6 of the 8 scales (Physical Functioning, Role Physical, Bodily Pain, Social Functioning, Role Emotional, Mental Health). Conclusions: Maintenance therapy with natalizumab resulted in significantly improved HRQOL, as evidenced by both disease specific (IBDQ) and general (SF-36) measures. Patients receiving natalizumab were observed to achieve a higher quality of remission than those receiving placebo and, across the majority of scales of the SF-36, their quality of life was similar to that of the general US population.

\section{T1150}

Lysophosphatidic Acid (LPA) Stimulates Protein Kinase D2-Mediated Nf-кB Dependent Interleukin-8 Secretion in Nontransformed, Human Colonic Epithelial Cells- a Potential Signaling Pathway in Intestinal Repair Wai Yin Leung, Mary Moyer, Robert M. Strieter, Enrique Rozengurt, Terence Chiu

Background/Aims: LPA, which is released at injured epithelial surface (2-20 $\mu \mathrm{M}$ in serum), stimulates intestinal epithelial cell migration, proliferation and interleukin-8 (IL-8) secretion. We have shown that LPA stimulated Protein kinase D (PKD) activation in rat IEC-6 and IEC-18 cells. PKD and the newly identified isoforms, PKD2 and PKD3, constitute a novel family of serine kinases. Epithelial cell functions linked to PKD include migration, proliferation and polarized Golgi function. However, endogenous G-protein coupled receptor-mediated regulation and function of PKD2 had not been examined. PKD2 is the only detectable PKD isoform in nontransformed, human colonic epithelial NCM460 cells. We investigated the signaling pathways mediating LPA-stimulated PKD2 activation and the potential contribution of PKD2 in regulating LPA-induced IL-8 secretion in NCM460 cells. Results: Treatment of NCM460 cells with LPA (5uM) led to a rapid activation of PKD2, as measured by in vitro kinase assay and phosphorylation at the activation loop (Ser706/710) and autophosphorylation site (Ser876). Maximal effect was obtained after 1 min of LPA incubation. LPAinduced PKD activation was abrogated by preincubation with PKC inhibitors, GF-I and Ro 31-8220. Pertussis toxin partially inhibited LPA-induced PKD2 activation, suggesting involvement of other $G$ proteins in addition to Gi. RT-PCR yielded LPA receptor subtypes, $\mathrm{LPA}_{2}$ and $\mathrm{LPA}_{3}$ in NCM460 cells. Both receptors couple to $\mathrm{Gi}$ and $\mathrm{Gq}$ in multiple cell types, but $\mathrm{LPA}_{1}$ predominantly couples to Gi. To explore the significance of LPA-induced PKD2 activation, we tested whether LPA could stimulate IL-8 secretion. LPA induced a 20-fold increase in IL-8 production at 6 hours. Given the importance of NF-KB in IL-8 expression in human colonic epithelial cells, we examined whether PKD2 may mediate IL-8 production

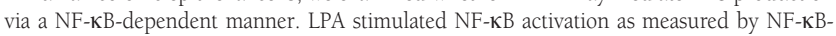

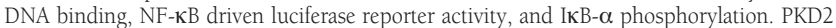
gene silencing utilizing siRNAs targeting distinct PKD2 sequences dramatically reduced LPAstimulated IL-8 secretion as well as NF-KB promoter activity. Conclusion: PKC-dependent PKD2 activation is a novel early event in the biological action of LPA and mediates LPAstimulated IL-8 secretion in NCM460 cells through a NF-אB-dependent pathway. We envisage that at sites of intestinal epithelial injury, high local concentrations of LPA can stimulate PKC/PKD2-mediated NF-KB activation, resulting in IL-8 secretion. This in turn may lead to neutrophil recruitment to counter potential bacterial invasion and stimulation of myofibroblasts, engaged in repair process.
Ginkgo Biloba Is a Potent Anti-Inflammatory Agent in Intestinal Epithelium: Therapeutic Potential for Suppression of NF-kB Activation and I-kb Degradation

Ali Banan, Lijuan Zhang, Philip Engen, Maliha Shaikh, Jeremy Z. Fields, Christopher B. Forsyth, Ashkan Farhadi, Ali Keshavarzian

$\mathrm{NF}-\mathrm{KB}$ activation is a crucial event in the development of inflammatory responses in a wide variety of GI disorders, including inflammatory bowel disease (IBD). To date, in spite of intensive investigation, an effective \& safe means of attenuating NF-kB activation in GI tract diseases is still lacking. Traditional Complementary \& Alternative Medicine (CAM) systems recommend putative anti-inflammatory herbs such as Ginkgo Biloba (GB). Despite claims, it has not been established whether this herb has anti-inflammatory effects \& is beneficial against intestinal inflammation $\&$ its mechanism of action is not known. Aims: We sough to determine whether NF-kB inactivation and cellular protection can be induced by GB under conditions of oxidative stress. Methods: Intestinal cells were pretreated with GB \&/ or NF-kB modulators prior to oxidants $(\mathrm{H} 2 \mathrm{O} 2$ or $\mathrm{HOCl})$. In other studies, cells were transfected with an IkB mutant to inhibit NF-kB. We measured monolayer integrity (LIVE/ DEAD assay, fluorometry), NF-kB p50/p65 subunit activities (EMSA, ELISA), NF-kB modulator, IkB- $\alpha$ levels/phosphorylation (PAGE), and structural protein integrity (laser confoca microscopy, SDS-PAGE), n=6/grp. Results: \{I\} Pretreatment with GB dose-dependently reduced IkB instability, decreased NF-kB nuclear translocation, and reduced NF-kB subunit activities while protecting barrier protein cytoarchitecture \& monolayer integrity. $\{\mathrm{II}\} \mathrm{IkB}$ stabilizers (MG-132, Lactacystin, etc) prevented oxidant-induced effects. Inhibitors of NF$\mathrm{kB}$ activation (e.g., PDTC) led to synergy induced protection in the presence of GB. Moreover selective potentiation of NF-kB activity by phosphoseryl/threonyl protein phosphatases [PAO, $\mathrm{PV}$ ] prevented GB protection during oxidative stress. \{III\} IkB stabilization by dominan mutant transfection also led to protection. Similar to blunting effects of the above pharmacological agents, mutant stabilization of IkB not only led to synergy induced protection with GB, but also with growth factor (EGF), and abolished NF-kB alterations. Conclusions: Our data indicate a unique anti-inflammatory affect for a traditional botanical dietary supplement Ginkgo Biloba. (1) Ginkgo Biloba appears to protect against oxidative injury through inactivation of the key proinflammatory factor, NF-kB. (2) The molecular mechanism underlying this potent anti-inflammatory affect of Ginkgo Biloba appears to depend on the stabilization of the endogenous inhibitor of NF-kB, namely IkB- $\alpha$. Accordingly, this botanical dietary supplement may be useful for development of novel therapies for a wide variety of inflammatory gut disorders where NF-kB is activated.

\section{T1152}

Tacrolimus (FK506), An Immunosuppressive Agent, Prevents IndomethacinInduced Small Intestinal Ulceration in Rats: Inhibition of iNOS Expression Through Suppressing Nf-Kappa B Activation Shinichi Kato, Kazuhiro Fukushima, Yoko Aoi, Koji Takeuchi

Background/Aim: Tacrolimus (FK506), an immunosuppressive drug, is widely used for organ transplantation and atopic dermatitis. Recent clinical studies have also demonstrated the beneficial effect in the treatment of rheumatoid arthritis (RA) and inflammatory bowe diseases. Nonsteroidal anti-inflammatory drugs (NSAIDs), which have been used for the treatment with RA, are known to cause damages in the small intestine as well as the stomach. Nitric oxide (NO) derived from inducible NO synthase (iNOS) plays a pivotal role in the pathogenesis of NSAID-induced small intestinal ulceration. Since tacrolimus has been also shown to suppress the expression of iNOS in various tissues and cells, this agent may have a beneficial effect on indomethacin-induced intestinal ulceration. In the present study, we examined the effect of tacrolimus on indomethacin-induced small intestinal ulceration in rats. Methods: Male SD rats were used. Indomethacin $(10 \mathrm{mg} / \mathrm{kg})$ was administered SC, killed $24 \mathrm{~h}$ later, and the area of lesions, myeloperoxidase (MPO) activity and thiobarbituric acid reactants (TBARS) were evaluated. Tacrorimus $(0.3 \sim 3 \mathrm{mg} / \mathrm{kg}$ ) was administered PO twice $30 \mathrm{~min}$ before and $6 \mathrm{~h}$ after indomethacin. The expression of iNOS mRNA was determined by a quantitative real-time TaqMan RT-PCR, while the activity of NF-kappa B DNA-binding was analyzed by electrophoresis mobility shift assays (EMSA) $6 \mathrm{~h}$ after indomethacin. Results: Indomethacin provoked severe hemorrhagic lesions in the small intestine, mainly the jejunum and ileum, accompanied with a marked increase in MPO activity and TBARS. Oral administration of tacrolimus significantly prevented indomethacin induced intestinal lesions in a dose-dependent manner a complete inhibition being observed at $3 \mathrm{mg} / \mathrm{kg}$. The increase in MPO activity and TBARS was also significantly attenuated by tacrolimus. The expression of iNOS mRNA was apparently enhanced by indomethacin at $6 \mathrm{~h}$ after the administration, and this response was almost totally attenuated by tacrolimus. Indomethacin also induced the NF-kappa B activation, in a tacrolimus-preventable manner Conclusion: Tacrolimus prevents indomethacin-induced small intestinal lesions. This effect may be due to inhibition of iNOS induction through suppressing the activation of NF-kappa B. It is thus assumed that tacrolimus is useful for not only the treatment of RA itself bu also for preventing the intestinal adverse effect of NSAIDs used in the treatment of RA.

\section{$\mathrm{T} 1153$}

Alterations of Expression and Function of the $\mathrm{Na}^{+} / \mathrm{H}^{+}$Exchanger NHE3 During Early Inflammation in IL-10 Knockout Murine Intestine

Tesfaye Tessema, Ayhan Cinar, Brigitte Riederer, Andre Bleich, Westendorf Astrid, Jan Buer, Michael P. Manns, Ursula Seidler

Introduction: Acute flares of Crohn's disease are often accompanied by watery diarrhea the pathophysiology of which is incompletely understood. In distal colon, inflammation strongly downregulates both mRNA levels and surface expression of the epithe-lial sodium channel $\mathrm{ENaC}$, but this channel is not expressed in the proximal colon and ileum. In this part of the intestine, electroneutral salt absorption predominantes, mediated by the $\mathrm{Na}+$ $\mathrm{H}+$ exchanger NHE3 and the anion exchanger SLC26A3. Aim: We investigated NHE3 mRNA and protein expression, localization and function in the terminal ileum and proximal colon 
of IL-10 deficent mice, which develop a proximally predominant inflamma-tion. Methods: IL-10 deficient mice on a C57/B6 backround were raised under SPF condi-tions, then in part transferred to a normal environment. Age and sex-matched IL-10 +/+ mice raised in identical environment were used as controls. mRNA expression levels of IF-gamma and ILlbeta, as well as NHE3 in the ileal and proximal colonic tissue of IL-10 deficient mice in the early stage of inflammation (8-12 weeks post exposure) were measured by quantitative PCR. NHE3 protein localization was studied by immunohistochemistry. Sodium absorption of isolated ileum and colon was measured in Ussing-chambers. To differentiate between distur-bances of NHE3 transport function and leaky tight junctions, NHE3 transport rates were as-sessed fluorometrically in the NHE3-expressing surface cells of BCECF-loaded IL$10+/+$ and $-/-$ colonic crypts. Total NHE exchange activity in the surface cell and cryptal region was also measured, as well as NHEl plus NHE2 activity in the crypts. Results: We found that in IL-10 deficient mice in normal but not in SPF conditions developed mild diarrhea and a virtual loss of active $\mathrm{Na}+$ absorption in inflamed tissue. IF-gamma and IL-lbeta mRNA were strongly upregulated, as expected. Histology showed mild crypt hyperplasia and leukocyte infiltration. Surprisingly, NHE3 mRNA was strongly upregulated, and the surface cell area which stained apically for NHE3 were found to penetrate more into the openings of the hyper-trophied crypts. On the other hand, NHE3 activity showed strongly decreased acid-activated transport activity as well as impaired regulation. This reduction in transport activity was seen only for NHE3, not for NHE in general. Conclusion: The data suggest that in the early stage of immune-mediated intestinal inflammation, mRNA expression of the $\mathrm{Na}+\mathrm{H}+$ ex-changer NHE3 is strongly upregulated and NHE3 protein is correctly located in the brush border membrane, but nevertheless transport activity is severely impaired due to as yet un-known intracellular events.

\section{$\mathrm{T} 1154$}

Effect of Interleukin-1 $\beta$ (IL-1 $\beta$ ) On Intestinal Epithelial Tight Junction Permeability Rana Al-Sadi, Thomas Y. Ma

IL-1 $\beta$ is a pro-inflammatory cytokine that plays a key role in intestinal inflammation. Recent studies have shown that pro-inflammatory cytokines TNF- $\alpha$ and IFN- $\gamma$ cause an increase in intestinal tight junction (TJ) permeability. It has been proposed that the cytokine-induced increase in intestinal TJ permeability is an important mechanism contributing to intestinal inflammation. Despite its central role in promoting intestinal inflammation, the role of IL$1 \beta$ on intestinal TJ barrier remains unclear. The aims of this study were to determine the role of IL-1 $\beta$ in the modulation of intestinal TJ barrier and to elucidate the intracellular pathway involved in the IL-1 $\beta$ modulation of intestinal TJ barrier. Methods: Filter-grown Caco-2 cells were used as an in vitro intestinal epithelial model system to study the TJ permeability. Results: IL-1 $\beta$ caused a concentration and time-dependent decrease in Caco2 transepithelial resistance (TER). Conversely, IL-1 $\beta(10 \mathrm{ng} / \mathrm{ml})$ caused a progressive timedependent increase in transepithelial permeability (20-fold increase) to paracellular marker inulin. IL-1 $\beta$ induced increase in Caco-2 TJ permeability was accompanied by a decrease in occludin expression and disruption in junctional localization. IL-1 $\beta$ treatment resulted in a sequential degradation of IKB- $\alpha$, cytoplasmic-to-nuclear translocation of NF-KB p65 and an increase in NF- $\mathrm{KB}$ binding to a known DNA binding site, indicating activation of

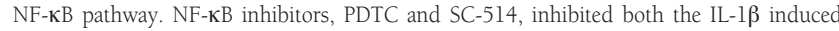
activation of NF-KB pathway and increase in Caco-2 TJ permeability. PDTC and SC-514 also prevented the IL-1 $\beta$ induced down-regulation of occludin protein expression and disturbance in junctional localization. To further validate the requirement of NF- $\mathrm{KB}$ in the IL-1 $\beta$ increase in Caco-2 TJ permeability, NF-KB p65 was depleted in filter-grown Caco-2 cells by siRNA transfection. NF-KB p65 siRNA transfection resulted in a marked knockdown of NF- $\mathrm{\kappa B}$ p 65 protein expression (95\% depletion). NF- $\mathrm{\kappa B}$ p 65 depletion completely inhibited the IL-1 $\beta$ induced increase in TJ permeability and IL-1 $\beta$ induced down-regulation of occludin protein expression. Conclusion: IL- $1 \beta$ caused an increase in Caco-2 TJ permeability and disturbance in occludin expression and junctional localization. The IL-1 $\beta$ induced increase in Caco-2 TJ permeability and alteration in occludin expression were mediated by the activation of NF- $\mathrm{kB}$ pathway.

\section{$\mathrm{T} 1155$}

The Opioid Antagonist Naltrexone Alleviates Chemically-Induced Colitis Gail L. Matters, John F. Harms, Leo R. Fitzpatrick, Anuj M. Parikh, Nicholas J. Nilo, Jill P. Smith

Background and Aims: Endogenous opioids have been shown to influence inflammatory responses through the modulation of cytokine production. Additionally, opioid antagonists promote tissue growth and repair. It was hypothesized that the opioid antagonist naltrexone could reduce inflammation and promote repair of the bowel in a chemically-induced mouse model of inflammatory bowel disease. Methods: Forty-eight C57BL/6J mice were divided into two groups: one received untreated drinking water and the other received $2 \%$ dextran sodium sulfate (DSS) for 5 days. On the third day after DSS was introduced, 8 animals in each group were injected subcutaneously with $0.1 \mathrm{ml}$ saline (control) or naltrexone (NTX, 0.1 or $10 \mathrm{mg} / \mathrm{kg}$ ) daily for 5 days. Disease activity index (DAI) scores, based on weight loss and stool hemoccult, were calculated daily. Mice were necropsied on day 9 and inflammation of the distal colons was analyzed histologically. Colonic RNA was evaluated by microarray and real-time RT-PCR. Results: By day 4, DSS-treated animals had significant weight loss $(p=0.006)$ and a higher DAI score $(p<0.001)$ compared to water controls. At necropsy, DSS-treated mice that received NTX $(10 \mathrm{mg} / \mathrm{kg})$ had less weight loss $(30.8 \%)$, lower DAI scores $(24.6 \%)$, and less inflammation $(25.0 \%)$ compared to DSS mice that received only saline. Real-time RT-PCR demonstrated an increased expression of the cytokines IL- 6 and IL-12 in DSS-treated mice. The increase in these pro-inflammatory cytokines was reversed by NTX treatment to levels near or equal to the expression in mice without colitis. Furthermore, the expression of the transcription factors STAT3 and STAT4, downstream effectors of cytokine signaling, was upregulated in the colons of DSS-treated mice and similarly reversed by NTX. Conclusions: NTX treatment reverses the disease manifestations and histologic evidence of inflammation in DSS-induced colitis. The mechanism by which NTX acts to reverse colitis is related in part to the decreased expression of pro-inflammatory cytokines (IL-6 and IL-12) and their downstream mediators (STAT3 and STAT4).

\section{T1156}

Stimulus Specific Role for Fer Tyrosine Kinase in Neutrophil Recruitment: Role of P38 MAPK and PI3 Kinase

Maitham A. Khajah, Graciela Andonegui, Donna-Marie McCafferty

Neutrophil recruitment and directional movement towards chemotactic stimuli are important processes in the innate immune response. This study examines if Fer PTK plays a role in neutrophil recruitment and chemotaxis to various stimuli in vivo and in vitro. Mice targeted with a kinase-inactivating mutation ( $\left.f e r^{\mathrm{DR} / \mathrm{DR}}\right)$ or wild type mice (WT) were used. In vivo time-lapse intravital microscopy was used to examine leukocyte recruitment and chemotaxis in response to keratinocyte-derived cytokine (KC; $5.2 \mu \mathrm{M})$ or chemotactic peptide WKYMVm $(0.1 \mu \mathrm{M})$. In WT and fer ${ }^{\mathrm{DR} / \mathrm{DR}}$ no difference in chemotaxis in response to $\mathrm{KC}$ was observed. However, in response to WKYMVm a two fold increase in leukocyte emigration was noted in $f e r^{\mathrm{DR} / \mathrm{DR}}$ mice $(\mathrm{p}<0.05)$. Bone marrow chimeras, where $\mathrm{fer}{ }^{\mathrm{DR} / \mathrm{DR}}$ received WT bone marrow, showed similar chemotaxis to WT in response to WKYMVm suggesting that endothelial Fer kinase was not playing a role in chemotaxis. In vitro neutrophil chemotaxis was assessed with bone marrow derived neutrophils using an under agarose gel assay. No difference in chemotaxis was noted between WT and fer ${ }^{\mathrm{DR} / \mathrm{DR}}$ mice in response to $\mathrm{KC}$ in vitro. However, a two fold increase in chemotaxis was noted in $f e r^{\text {DR/DR }}$ vs WT neutrophils in response to WKYMVm. This chemotactic response was inhibited by two p38 MAPK inhibitors (SKF86002 and SB203580) in WT mice but not in fer $r^{\mathrm{DR} / \mathrm{DR}}$ mice suggesting a regulatory role for Fer kinase on the p38 MAPK pathway. Interestingly, in fer ${ }^{\mathrm{DR} / \mathrm{DR}}$ mice a role for the PI3 kinase pathway in chemotaxis was revealed using a PI3 kinase inhibitor (LY294002). In addition, in fer $r^{\mathrm{DR} / \mathrm{DR}}$ neutrophils superoxide anion production $\left(\mathrm{O}_{2}\right.$ - $)$ started earlier than in WT neutrophils which suggests that activation of the PI3kinase pathway is responsible for this observation. These data suggest that Fer kinase regulates neutrophil chemotaxis in response to WKYMVm through the regulation of p38 MAPK signaling pathway. This work was funded by a Canadian Institutes of Health Research operating grant.

\section{T1157}

Proof of Gaps in Human Small and Large Intestinal Epithelium By in Vivo Histology with Confocal Laser Endoscopy

Alastair J. Watson, Ralf Kiesslich, Elizabeth M. Angus, Peter R. Galle, Constantin Schneider, Katharina Lammersdorf, Marshall H. Montrose, Markus F. Neurath

In vivo confocal microscopy has identified single-cell discontinuities in the small intestinal epithelium of mice (Gastro 2005;129:902). These gaps lack cellular material but are the diameter of epithelial cells and are created by the shedding of epithelial cells. Gaps are not visualized well in standard histology. Aim: To evaluate the presence gaps in human small and large intestinal epithelium using confocal laser endoscopy. Methods: Patients $(n=12)$ designated for IBD screening were enrolled with informed consent and underwent total colonoscopy with a confocal laser endomicroscope, enablingin vivo morphology of the mucosal layer to be imaged in real time (Optiscan, Australia; Pentax, Japan; 488nm excitation by argon ion laser; detection $>515 \mathrm{~nm}$ ) (Gastro 2004:127:706). Mucosal surface was analyzed for the presence of epithelial gaps at 2 or more focal depths into tissue. A Gap was defined as an area distinct from goblet cells which lacked nuclei and cytoplasm present in multiple focal planes despite their presence in neighboring cells. Results: Gaps (arrows) are present in both small and large intestine. At the colonic surface or villus tip their diameter varies from 2 to $10 \mu \mathrm{m}$. This is consistent with gaps being transient structures after single cell shedding. Gaps are not evenly distributed down the gut and appear to cluster on the colonic table or near the villus tip. $30 \pm 19 \%$ of analysable images contained gaps. Conclusion: The presence of frequent discontinuities in normal human intestinal epithelium is a new concept in gastrointestinal biology. The impact of these gaps on the pathogenesis of gastrointestinal diseases such as IBD and celiac disease must now be evaluated. 


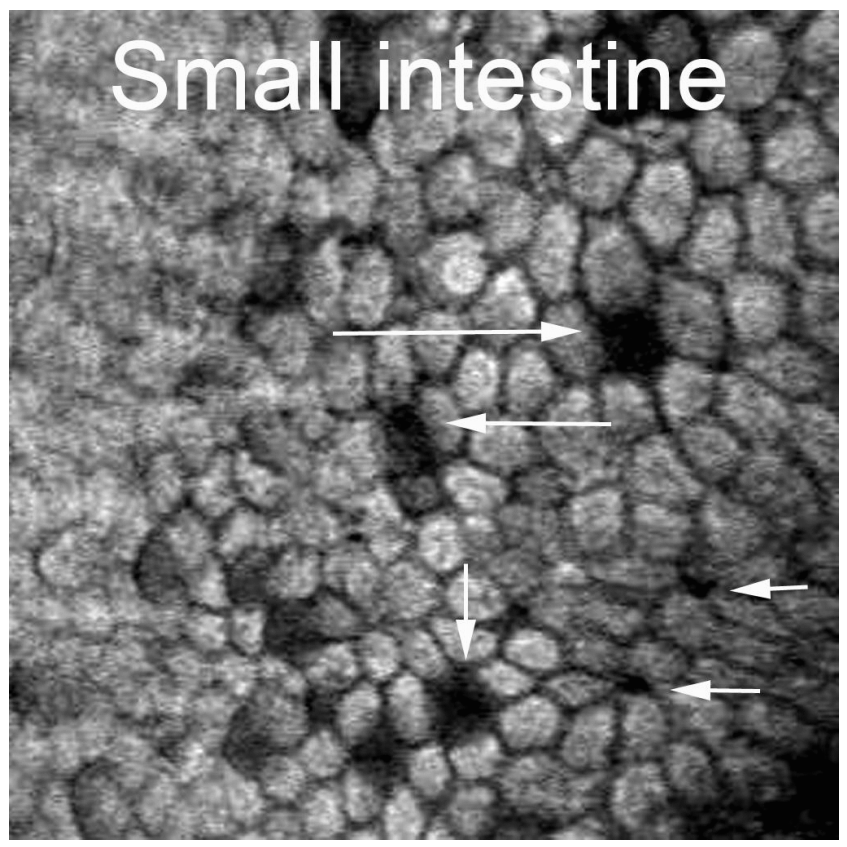

$\mathrm{T} 1158$

Prohibitin (PHB) Expression Is Decreased During Intestinal Inflammation and May Protect Against Inflammation-Induced Oxidative Damage

Arianne L. Theiss, Richard D. Idell, Lixin Wang, Dean P. Jones, Didier Merlin, Shanthi V. Sitaraman

INTRODUCTION: Prohibitin (PHB) is an evolutionarily conserved and ubiquitously expressed protein that is implicated in diverse cellular processes such as oxidative stress. The expression and function of PHB in the normal intestine and during intestinal inflammation are not known. Oxidative stress and its consequent effects on intestinal barrier dysfunction and wound healing have been shown to play an important role in the pathogenesis of chronic intestinal inflammation. We tested the hypotheses that the acute phase cytokine IL-6 increases PHB expression and that PHB modulates oxidative stress associated with intestinal inflammation. METHODS: PHB protein expression was assessed in mucosal biopsies from Crohn's disease (CD) and normal patients and in colitic changes from mice treated with dextran sodium sulfate (DSS) or from IL-10 knockout mice. PHB mRNA and protein expression was measured in Caco2-BBE intestinal epithelial cells treated with IL-6 (100 ng/ml) for various amounts of time. Localization of PHB was determined by confocal microscopy in Caco2-BBE cells treated with IL-6 or transfected with a His-tagged PHB expression vector. To determine the effect of oxidative stress on PHB expression, Caco2-BBE cells were treated with $0.5 \mathrm{mM} \mathrm{H} 2 \mathrm{O} 2$ for 1 hour to induce oxidative stress and assayed for PHB expression. Caco2-BBE cells treated with IL-6, H2O2, or pretreated with IL-6 to induce PHB and then treated with $\mathrm{H} 2 \mathrm{O} 2$ were assayed for glutathione (GSH) levels, an established marker of oxidative stress. RESULTS: PHB is down-regulated during active human CD, experimental colitis in vivo, and during oxidant stress in vitro. IL-6 potently induces PHB expression in Caco2-BBE cells. PHB is expressed in the cytoplasm and cell membrane of Caco2-BBE cells during basal conditions and translocates to the nucleus upon IL- 6 stimulation or when overexpressed. Finally, IL-6 pretreatment inhibits $\mathrm{H} 2 \mathrm{O} 2$-induced depletion of the antioxidant GSH in Caco2-BBE cells. CONCLUSIONS: These results demonstrate that PHB expression is decreased during oxidative stress and intestinal inflammation. Since IL-6-induced PHB expression is associated with reduced oxidative stress stimulated by $\mathrm{H} 2 \mathrm{O} 2$, perhaps $\mathrm{PHB}$ protects against oxidative stress associated with intestinal inflammation.

\section{T1159}

Prerequisite of Bile Acids for Expression of COX-2 and iNOS As Well As Lesion Formation in Rat Small Intestine Following Administration of Indomethacin

Yuka Takahira, Nahoko Izumi, Akiko Tanaka, Koji Takeuchi

Background/Aim: Nonsteroidal antiinflammatory drugs (NSAIDs) such as dicrofenac and indomethacin cause hemorrhagic lesions in the small intestine as the adverse reaction. Numbers of elements such as a deficiency of prostaglandins, bile acids, enterobacteria and inducible nitric oxide synthase (iNOS) are involved in the pathogenesis of NSAID-induced intestinal lesions. A recent study demonstrated the up-regulation of cyclooxygenase (COX)2 expression in this tissue following the administration of NSAIDs and sugrested that this event is a key to NSAID-induced intestinal lesions. However, the role of bile acids in these events remains unexplored. In the present study, we investigated the prerequisite of bile acids in the intestinal ulcerogenic response to indomethacin and its relation to the upregulation of COX-2 and iNOS expression. Methods: Male SD rats were used. The animals were given indomethacin (10 mg/kg) SC and killed various intervals after the administration. Bile duct ligation or cholestyramine treatment (bile acid sequestrant, 300 1000 mg/kg, PO) was performed $30 \mathrm{~min}$ before or $3 \mathrm{~h}$ after indomethacin. Enterobacterial count was determined $6 \mathrm{~h}$ after indomethacin treatment. The expression of COX-1, COX-2 and iNOS mRNAs was examined by RT-PCR. Results: Indomethacin caused hemorrhagic lesions in the small intestine $6 \mathrm{~h}$ later, and the severity increased with time up to $12 \mathrm{~h}$ later. Indomethacin increased bacterial count in the mucosa and up-regulated the expression of both COX-2 and iNOS in the small intestine without any change in COX-1 expression. Cholestyramine, given 30 min before, significantly prevented the development of these lesions in a dosedependent manner, whereas this agent, given $3 \mathrm{~h}$ after indomethacin, failed to affect the intestinal ulcerogenic response. Similar results were obtained by bile duct ligation; the ligation $30 \mathrm{~min}$ before completely prevented the occurrence of intestinal lesions while the ligation $3 \mathrm{~h}$ after indomethacin caused only a partial inhibition against these lesions. In addition, cholestyramine given $30 \mathrm{~min}$ before indomethacin treatment significantly prevented bacterial invasion in the mucosa and inhibited the up-regulation of COX-2 and iNOS expression after the administration of indomethacin. Conclusion: These results suggest tha 1) the presence of bile acids in the small intestine is essential for the occurrence of NSAIDinduced intestinal lesions, and 2) bile acids facilitate bacterial inversion in the mucosa and contribute to the up-regulation of both COX-2 and iNOS expression, confirming that the expression of these inducible enzymes is a key to NSAID-induced intestinal damage.

T1160

Roles of Endogenous Prostaglandins/Cyclooxygenases and EP4 Receptor Subtype in Healing of Indomethacin-Induced Small Intestinal Lesions in Rats Ryo Hatazawa, Ryoko Ohno, Mayu Tanigami, Akiko Tanaka, Koji Takeuchi

Background/Aim: Endogenous PGs play an important role in modulation of the mucosal defense in the stomach as well as the healing aspect of gastric ulcers, and it is believed that COX-1 or COX-2 is mainly involved in the former or the latter event, respectively. We recently reported that $\mathrm{PGs}$ produced by both COX-1 and COX-2 are important in maintaining the mucosal integrity of small intestine against NSAID-induced damage, yet the role of PGs in the healing of these lesions remains unknown. In the present study, the role of PGs/COX in the healing of indomethacin-induced intestinal lesions was examined in rats, using selective COX-1 and COX-2 inhibitors. Methods: Male SD rats were used. Intestinal lesions were induced by a single injection of indomethacin $(10 \mathrm{mg} / \mathrm{kg}, \mathrm{SC})$, and the rats were killed 1 , 4 and 7 days later. Indomethacin ( $2 \mathrm{mg} / \mathrm{kg}$ ), SC-560 (COX-1 inhibitor: $3 \mathrm{mg} / \mathrm{kg}$ ) and rofecoxib (COX-2 inhibitor: $3 \mathrm{mg} / \mathrm{kg}$ ) were given PO once daily for 6 days, during the first 3 or last 3 days of the experimental period. The effect of EP4 agonist and antagonist on the healing was also examined in the presence or absence of indomethacin. Vascular content was determined by the incorporation of Carmine. Expression of COX-2 mRNA in the mucosa was examined by RT-PCR, while PGE 2 content was measured by EIA. Results: All COX inhibitors given for 6 days significantly impaired the healing of indomethacin-induced small intestinal lesions. The healing was also impaired by rofecoxib given for the first 3 days or by SC- 560 given for the last 3 days. The expression of COX-2 mRNA in the intestine was up-regulated after ulceration, persisting for 3 days and dissipating thereafter. Levels of mucosal PGE 2 markedly decreased within $3 \mathrm{hr}$ after indomethacin was administered, recovered $24 \mathrm{hr}$ later, and increased above normal 1 3 days after ulceration. The $\mathrm{PGE}_{2}$ content on 4 days after ulceration was decreased by rofecoxib but not SC-560 while that on 7 days was suppressed by SC-560 but not rofecoxib. Vascular content in the ulcerated mucosa significantly decreased when the healing was impaired by COX inhibitors. The deleterious effect of indomethacin on the healing was mimicked by an EP4 antagonist and reversed by co-administration of $\mathrm{PGE}_{2}$ as well as an EP4 agonist. Conclusion: These results suggest that endogenous PGs play a role in the healing of intestinal ulcers, yet the COX isozyme responsible for the production of PGs differs depending on the stage of healing, COX-2 in the early stage and COX-1 in the late stage. In addition, the healing promoting action of PGE2 is associated with stimulation of the angiogenic response and mediated by the activation of EP4 receptors.

\section{T1161}

Bioactive Glass Stimulates Epithelial Wound Healing Hussila Keshaw, Alastair Forbes, Richard Day

Ulcer healing is a complex process requiring proliferation and migration of epithelial cells into the wound. A number of regulatory peptides mediate this process, many of which ar secreted from myofibroblasts, including basic fibroblast growth factor (bFGF) and vascular endothelial growth factor (VEGF). $45 \mathrm{~S} 5$ bioactive glass (BG) has previously been used to facilitate hard tissue regeneration. More recently it has been shown to stimulate angiogenesis and growth factor secretion from colonic myofibroblasts. Aim: To determine the effect o BG-induced growth regulatory peptides on epithelial wound healing. Methods: Conditioned culture medium (CM) was prepared by seeding CCD-18Co colonic myofibroblasts on surfaces coated with various concentrations $(0 \%, 0.01 \%, 0.1 \%, 1 \% \mathrm{w} / \mathrm{v})$ of BG. CM was collected after $72 \mathrm{~h}$. The amount of bFGF and VEGF present in the CM was measured by ELISA Confluent monolayers of human colonic epithelial cell line $\mathrm{CaCO}-2$ were wounded with a razor blade and cultured in $\mathrm{CM}$ for $24 \mathrm{~h}$. Triplicate plates containing four wounds were used to quantitate the $\%( \pm$ SEM $)$ changes in the area of cells across the wound border and the average distance of the leading edge of the repairing monolayer from the wound border using image analysis software. Results: CM collected from myofibroblasts cultured on BG contained increased amounts of bFGF compared with $0 \% \mathrm{w} / \mathrm{v}$ BG. The amount of VEGF in the $\mathrm{CM}$ was increased with $0.01 \% \mathrm{w} / \mathrm{v}$ and $0.1 \% \mathrm{w} / \mathrm{v} \mathrm{BG}$, but was reduced with $1 \% \mathrm{w} /$ $\mathrm{v}$ BG. Treatment of wounded monolayers with $0.01 \% \mathrm{w} / \mathrm{v}$ BG CM produced a significant increase in the area of cells across the wound border $(13 \% \pm 6 ; \mathrm{p}<0.05)$ compared with $0 \% \mathrm{w} / \mathrm{v}$ BG CM. The average distance of the leading edge was also significantly increased $(15 \% \pm 7 ; \mathrm{p}<0.05)$. Treatment of wounded monolayers with $0.1 \% \mathrm{w} / \mathrm{v} \mathrm{BG} \mathrm{CM} \mathrm{also} \mathrm{increased}$ the area of cells across the wound border $(10 \% \pm 7 ; \mathrm{p}>0.05)$ and average leading edge distance $(9 \% \pm 8 ; \mathrm{p}>0.05)$. Wounded monolayers treated with $1 \% \mathrm{w} / \mathrm{v} \mathrm{BG} \mathrm{CM}$ exhibited a significant reduction in the area of cells across the wound border $(54 \% \pm 15 ; \mathrm{p}<0.001)$ and the average leading edge distance $(54 \% \pm 17 ; \mathrm{p}<0.01)$ compared with $0 \% \mathrm{w} / \mathrm{v} \mathrm{BG}$. Conclusion This study demonstrates that myofibroblasts exposed to BG secrete regulatory peptides that can stimulate epithelial wound healing. It is likely that other factors in addition to those measured in the current study also contribute to the observed stimulatory effect. These findings, together with the known ability of BG to stimulate angiogenesis, could offer a novel therapeutic approach for ulcer healing and provide a rationale for further studies to identify the mechanism of BG-induced epithelial wound healing. 
T1162

Evidence of Monoclonality in Dysplasia in Ulcerative Colitis Simon Leedham, Lara Maia, Sean Preston, Macdonald Stuart, Oliver Sieber, Ian Tomlinson, Nicholas Wright

Aim: Ulcerative Colitis (UC) is a chronic inflammatory condition that increases the risk of developing colorectal cancer (CRC). UC associated colorectal cancers (UCACRC) often develop from areas of dysplasia, which can be widespread or multifocal. Loss of heterozygosity (LOH) of important tumour suppressor genes such as Adenomatous polyposis coli (APC), deleted in colon cancer (DCC) and SMAD4, can occur in UC associated dysplastic tissue LOH analysis can be used as a simple and reliable marker of clonality. The aim of this study was to use $\mathrm{LOH}$ at the above 3 loci for the assessment of clonality within dysplastic and inflamed tissue in UC to test the hypothesis that dysplastic lesions arise from a single mutated clone. Methods: Paraffin embedded tissue from colectomy specimens was obtained from St Marks Hospital pathology library. Tissue was categorised histologically into normal, acutely or chronically inflamed or dysplastic, based on $\mathrm{H} \& \mathrm{E}$ slides. Laser capture microdissection was used to isolate individual crypts. If no normal crypts were available from the tissue, regions of lamina propria or muscularis mucosae were taken to allow $\mathrm{LOH}$ analysis comparison. DNA was extracted and amplified for microsatellite markers close to the loci of APC (5q21.1), DCC (18q21) and SMAD4 (18q21.1). PCR product was analysed with ABI 3100 sequencer and genotyper software and abnormal tissue allelic areas were compared with normal tissue allelic areas as a ratio. Results: LOH for microsatellite markers was found in chronic inflammation in 1 patient at a low frequency (1 marker, $50 \%$ of crypts). $\mathrm{LOH}$ of the same microsatellite marker was seen in low grade dysplasia at a low frequency (44\%) but at a very high frequency across multiple high grade dysplastic patches in different patients (up to $100 \%$ ). LOH of multiple markers was seen in some patients. Conclusions: Lower $\mathrm{LOH}$ frequency in inflamed and low grade dysplasia compared to high grade dysplasia suggests which marker is lost first, and the earliest lost marker can be used a clonal marker. 'Across the patch' loss of the same allele in high grade dysplasia suggests monoclonal derivation. Increased crypt fission is likely to be responsible for mutation spread, and further work using mitochondrial DNA mutations to demonstrate shared mutations in neighbouring crypts in UC is underway.

\section{T1163}

15-Deoxy- $\Delta$ 12,14-Prostaglandin J2 Ameliorates Dextran Sodium SulfateInduced Colitis in Mice Via Induction of Heme Oxygenase-1

Tomohisa Takagi, Yuji Naito, Takahiro Suzuki, Hirokazu Kajikawa, Kouichi Terao, Hisato Tsuboi, Takeshi Ishii, Kazuhiro Kamada, Toshimitsu Okuda, Osamu Handa, Satoshi Kokura, Hiroshi Ichikawa, Norimasa Yoshida, Toshikazu Yoshikawa

Background: 15-deoxy-A12,14- Prostaglandin J2 (15d-PGJ2), a prostaglandin D2 metabolite, exerts anti-inflammatory activity as a negative feedback mechanism. Both peroxisome proliferator-activated receptor (PPAR)-dependent and -independent anti-inflammatory actions of 15d-PGJ2 have been identified. However, a definitive mechanism for 15d-PGJ2-induced reduction of intestinal inflammation has not been fully solved. In the present study, we found that enema treatment with 15d-PGJ2 induced heme oxygenase-1 (HO-1) expression and inhibited the development of murine colitis. Materials and methods: Acute colitis was induced with dextran sodium sulfate (DSS) in male BALB/c mice, 15d-PGI2 (5uM, $0.2 \mathrm{ml})$ was administrated everyday via rectal enema. Intestinal expression of HO-1 mRNA and protein were evaluated by real-time PCR and western blotting, and the activity of HO-1 was determined by luminal carbon monoxide $(\mathrm{CO})$ gas level. A disease activity index (DAI) was determined on a daily basis for each animal, and consists of a calculated score based on changes in body weight, stool consistency, and intestinal bleeding. Tissue-associated myeloperoxidase (MPO) activity was measured in intestinal mucosa as an index neutrophil infiltration. Moreover, we evaluated the effects by co-treatment of an HO-l inhibitor, zinc protoporphyrin IX (ZnPP 25mg/kg i.p., daily), or a specific PPAR-y antagonist GW9662. Results: Enema treatment with 15d-PGJ2 markedly induced HO-1 protein and mRNA expression in the colonic mucosa, and increased luminal $\mathrm{CO}$ gas. The induction of HO-1 by $15 d-P G J 2$ was not affected by co-treatment with GW9662. Increase in luminal CO gas level was significantly inhibited by the treatment with ZnPP. After DSS administration, DAl score were increased in a time-dependent manner, in addition to decreased colonic length and increased MPO activity. The treatment with 15d-PGJ2 ameliorated the increases in DAI score and MPO activity after DSS administration. The inhibition by 15d-PGJ2 against intestinal inflammation was cancelled by the co-treatment with ZnPP, but not by GW9662 Conclusion: These results demonstrate that 15d-PGJ2 down-regulates intestinal inflammation in mice by PPAR-independent and HO-1-dependent actions.

\section{T1164}

Alterations of Matrix Metalloproteinase-9, Hepatocyte Growth Factor Receptor and Insulin-Like Growth Factor Receptor-1 Expressions in Ulcerative Colitis Laszlo Herszenyi, Ferenc Sipos, Orsolya Galamb, Balazs Gyorffy, Istvan Hritz, Istvan Pregun, Bela Molnar, Zsolt Tulassay

Background: Epithelial cell growth regulation has been reported to be altered in inflammatory bowel disease. Matrix metalloproteinases play an important part in extracellular matrix remodelling. Hepatocyte growth factor receptor (c-met) and insulin-like growth factor receptor-1 (IGFR-1) modulate intestinal epithelial cell proliferation and migration, play a role in regenerative processes and intestinal wound healing. Aim: Determination of protein expression levels and mRNA of matrix metalloproteinase-9 (MMP-9), c-met and IGFR-1 in correlation with the severitiy of inflammation in ulcerative colitis (UC). Materials and methods: MMP-9, c-met and IGFR-l expressions were analyzed in 40 paraffin-embedded tissue samples by immunohistochemistry from patients with mild $(n=10)$, moderate $(n=10)$, severe $(n=10)$ $\mathrm{UC}$ and healthy control subjects $(\mathrm{n}=10)$. Immunostaining was determined semiquantitatively. The RNA expression profile from biopsy samples was evaluated using TaqMan® assay LowDensity Array to Gene Expression (Applied Biosystems). Statistical analysis with one-way ANOVA and LSD tests were performed. Results: MMP-9 expression was significantly lower in each inflammatory group compared with normal ( $p<0.05)$. IGFR-1 and c-met expressions were significantly higher in mild UC, but not in moderate and severe UC compared to normal $(p<0.005)$. MMP-9 and IGFR-1 mRNA expressions were significantly downregulated in UC compared to the normal tissue ( $\mathrm{p}<0.05$ ), while $\mathrm{c}$-met mRNA expression was slightly, but not significantly higher only in mild UC compared to normal. The immunohistochemical protein expression levels correlated to the mRNA expression array data. Conclusions: The downregulation of MMP-9, IGFR-1 and c-met expression correlated with the severity of inflammatory process in UC. The decrease of MMP-9 expression with the decreased IGFR1 and c-met expressions could contribute to the severity of epithelial damage in moderately and severely active UC

\section{T1165}

Aggravation of Indomethacin-Induced Intestinal Ulceration in Rats with Adjuvant-Induced Chronic Arthritis: Involvement of Increased Expression of Toll-Like Receptor (TLR)-4

Shinichi Kato, Kazuhiro Fukushima, Kikuko Amagase, Koji Takeuchi

Background/Aim: We previously reported that indomethacin-induced gastric lesions were markedly aggravated in adjuvant-induced arthritic (AA) rats. This phenomenon is accounted for by the overproduction of nitric oxide (NO) derived from inducible NO synthase (iNOS) On the other hand, it has been shown that the increased expression of iNOS induced by entrobacterial invasions in the intestinal mucosa plays a pathogenic role in the development of indomethacin-induced intestinal lesions. In the present study, we examined the intestinal ulcerogenic property of indomethacin in AA rats, in comparison with normal animals. Methods: Arthritis was induced in male DA rats by injection of Freund's complete adjuvant $\overline{(F C A)}$ into the right hind paw. Two weeks later, the animals were given indomethacin ( $3 \sim 10$ $\mathrm{mg} / \mathrm{kg}, \mathrm{PO}$ ), and the intestinal mucosa was examined for lesions 6 and $24 \mathrm{~h}$ later. The expression of iNOS and TLR-4, a receptor for bacterial lipopolysaccharide, was determined by Western blotting. Results: Oral administration of indomethacin produced multiple hemorrhagic lesions in the small intestine. The severity of these lesions was increased in AA rats as compared with normal rats, and the lesions were observed even at $3 \mathrm{mg} / \mathrm{kg}$, the lesion score at $6 \mathrm{mg} / \mathrm{kg}$ being significantly greater than that observed at $10 \mathrm{mg} / \mathrm{kg}$ in normal rats. $\mathrm{N}^{\mathrm{G}}$-nitro-L-arginine methyl ester (L-NAME) and aminoguanidine when given once 6 $\mathrm{h}$ after indomethacin significantly prevented these lesions in normal rats, but failed to suppress aggravation of these lesions in AA rats. However, aminoguanidine when given twice $30 \mathrm{~min}$ before and $6 \mathrm{~h}$ after indomethacin significantly prevented the severity of lesions in $\mathrm{AA}$ rats. Moreover, the onset of the lesions occurred much earlier in AA rats when compared to normal rats, the severe lesions being observed even $6 \mathrm{~h}$ after indomethacin. The development of these lesions in both normal and AA rats was potently prevented by dexamethasone and the antibiotic, ampicilline. Although the expression of iNOS and TLR4 was only slightly detected in the small intestine of normal rats, both of these expressions were clearly observed in the intestinal mucosa of AA rats without indomethacin treatment. Conclusion: These findings suggest that the ulcerogenic response to indomethacin in the small intestine is markedly aggravated in AA rats, similar to that in the stomach. Especially, the onset of the lesion formation is much earlier in AA rats than that in normal rats, and this may be partly due to the increased expression of TLR-4 in the small intestine during arthritis.

\section{$\mathrm{T} 1166$}

The Anti-Inflammatory Effects of Cv-11974,AT1 Receptor Antagonist, On Indomethacin-Induced Rat Enteritis

Toshimitsu Okuda, Norimasa Yoshida, Koichi Terao, Osamu Handa, Tomohisa Takagi, Satoshi Kokura, Hiroshi Ichikawa, Yuji Naito, Takeshi Okanoue, Toshikazu Yoshikawa

Background and Aims: Angiotensin II (ATII) goes through angiotensin II typel (AT1) and type2 (AT2) receptor on various cells including vascular endothelial cells and activates intracellular signal transduction mechanism. ATII plays an important role in blood pressure regulation, blood vessel remodeling. Recently, it has been proposed that ATII is implicated in the pathogenesis of oxidative stress-related vascular inflammation in various organs. The aim of the present study was to examine the effect of ATII typel receptor (ATIR) antagonist, CV-11974, on indomethacin-induced rat enteritis. Methods: Male Wistar rats weighting 190-210 grams were given indomethacin $(10 \mathrm{mg} / \mathrm{kg})$ subcutaneously and the intestinal mucosal damage was estimated. CV-11974 was given to the rats by intravenous injection $3 \mathrm{~h}$ before indomethacin injection. The area $(\mathrm{mm} 2)$ of macroscopic visible lesions, thiobarbituric acid-reactive substances (TBARS) as an index of lipid peroxidation, myeloperoxidase (MPO) activity as an index of neutrophils accumulation, and the content of cytokine-induced neutrophil chemoattractant-1 (CINC-1) in the intestinal mucosa were estimated $24 \mathrm{~h}$ after injection of indomethacin. ATIR in the intestinal mucosa was estimated using western blotting and immunostaining. Results: Single administration of indomethacin at $10 \mathrm{mg} / \mathrm{kg}$ provoked severe inflammatory lesions in the small intestine, mostly jejunum and ileum. Microscopic findings revealed multiple hemorrhagic erosions and edema with numerous inflammatory cells. The levels of TBARS, MPO activities and CINC-1 in the intestinal mucosa significantly increased prior to the occurrence of macroscopic injuries in indomethacintreated group compared with the sham-operated group. In addition, ATlR-positive cells in the mucosal layer increased in indomethacin-treated rats. The development of intestinal lesions in response to indomethacin was prevented by CV-11974 together with significant suppression of the increased level of TBARS, MPO activities and CINC-1 in the small intestine. Conclusions: These results indicate that CV-11974 protected against the small intestinal damage elicited by indomethacin, which suggesting that angiotensin II/ATIR interaction is involved in the pathogenesis of intestinal inflammation associated with oxidative stress. 


\section{T1167}

Evidence That a Novel Non-Chemical, Non-Electrical Intercellular Signaling System Causes Oxidative-Induced Changes in Cell Protein Content and Activation of Nf- $\mathrm{KB}$ in Distant Epithelial Cells

Ashkan Farhadi, Christopher Forsyth, Ali Banan, Maliha Shaikh, Phillip Engen, Jeremy Z Fields, Ali Keshavarzian

Background: Synchrony between physically separate biological units (e.g., synchronous illumination of whole populations of fireflies) is well known. We determined whether cells can induce synchronous behavior in physically separate neighboring cells and do so by a nonchemical, non-electrical (NCNE) mechanism. To investigate synchrony, we took advantage of the fact that cultured cells exposed to oxidants enter a state of emergency, decrease their protein content by half in a matter of minutes, and activate NF-KB as the key regulation point of cell stress signaling cascade. To determine whether information regarding this state can be transmitted to distant cells, we used cultures of a gastrointestinal cell line. Method: Caco-2 cell line cultures were divided into three groups: 1) Inducer cells were exposed to oxidant (H2O2); 2) Detector cells were not exposed to oxidant and were placed in a chemically isolated container in the proximity of inducer cells; 3) Control cells were also not exposed to $\mathrm{H} 2 \mathrm{O} 2$ and were kept in a separate room. Parts of the experiment were done using a newly designed container (Farhadi's cell container, patent pending) composed of two isolated chambers for inducer and detector cells that are side-by-side and share a thin, glass wall that precludes the passage of any chemical or electrical signals between the two containers. Total protein contents of cell samples were measured using spectrophotometry at $595 \mathrm{~nm}$. NF-KB activation was assessed using a quantitative ELISA of the nuclear extracts for the p65 sub unit. Results: Exposing inducer cells to hydrogen peroxide resulted in a significant reduction in their total protein content $(-50 \%)$ and increases in the level of nuclear NF- $\mathrm{\kappa B}$ p 65 subunits $(+38 \%)$. There were parallel and significant reductions in total protein content $(-48 \%)$ and increases in nuclear p65 subunit fractions of NF- $\mathrm{\kappa B}(+35 \%)$ in detector cells compared to controls ( $\mathrm{p}<0.0001$ and $\mathrm{p}=0.01$, respectively). Conclusions: Our data provide evidence for the existence of a NCNE signaling system among intestinal cells. This novel signaling system can be activated by oxidative stress and result in decrease in cell protein content and activation of NFKB pathway in distant cells. The mechanism underlying this unique signaling phenomenon is unknown, although electromagnetic signaling due to oscillating ions is a possible candidate. Intercellular NCNE signaling may play a pivotal role in the pathogenesis and possible therapeutic intervention of gastrointestinal disorders related to oxidative induced barrier injury such as IBD

\section{$\mathrm{T} 1168$}

Role of Thromboxane in Flow-Mediated Vasoconstriction in Inflammatory Bowel Disease (IBD)

Ossama A. Hatoum, David G. Binion, Hiroto Miura, Rebecca M. Selle, Shane A. Phillips, Doron Kipelman, David D. Gutterman

Background and Aims: IBD (Crohn's disease and ulcerative colitis) is characterized by areas of refractory inflammatory ulceration and ischemic damage to the gut which correlates with local microvascular dysfunction. Flow-induced vasodilation (FID) is a physiological mechanism for regulating mucosal perfusion. The mechanisms underlying impaired mucosal healing during IBD are not defined, therefore we hypothesize that flow-induced vasodilation is impaired in chronically inflamed gut microvessels in IBD patients. Methods: The diameter responses of freshly isolated intestinal submucosal arterioles $(75-200 \mu \mathrm{m})$, to flow were determined from IBD and non-IBD patients undergoing intestinal surgery and bowel resection. Microvessels were cannulated with glass micropipettes and pressurized to $60 \mathrm{mmHg}$ in a heated chamber and perfused with physiological salt solution. Responses of vessels to graded increases in flow (constant intraluminal pressure) were produced with intravascular pressure gradients $(0,20,40,60,80$ and $100 \mathrm{cmH} 2 \mathrm{O})$ between perfusion reservoirs. Diameters were recorded intermittently after 5 minutes of each pressure gradient (steady-state conditions) using videomicroscopy. Results: After constriction (30-50\%) with endothelin-1, FID was observed in non-IBD submucosal arterioles (max dilation (MD) $79.4+12 \%$, $\mathrm{n}=$ 7). Inhibition of NO synthase (L-NAME) reduced MD to $40.5 \pm 9.3 \%, P<0.05 n=5$. Flowinduced vasoconstriction was observed in chronically inflamed IBD arterioles $(-57.5+6 \%$, $\mathrm{n}=7, \mathrm{p}=<0.01$ vs. controls). Cyclooxygenase blockade with indomethacin (INDO, 10-5M) significantly attenuated the constriction $(-55.7 \pm 5 \%$ to $2.7 \pm 4 \%)$. Treatment of IBD microvessels with either the thromboxane receptor antagonist SQ-29548 (10-5M) or the thromboxane synthase inhibitor dazoxiben (10-5M) converted the vasoconstriction to vasodilation (MD: $81.9+8$ and 46.1+2, respectively). Immunohistochemical studies demonstrated a significant increase in the thromboxane staining in IBD arterioles when compared to vessels from patient without IBD. All responses to flow in both non-IBD and IBD submucosal arterioles were eliminated by endothelium denudation $(-3 \pm 5 \%$; $-4 \pm 2.2 \%$; respectively). Conclusions: Flow-induced endothelium-dependent vasoconstriction of gut microvessels during IBD may be related to increased synthesis of thromboxane. This microvascular dysfunction may contribute to the progression of reduced tissue perfusion and ischemia during IBD by limiting tissue perfusion, leading to ischemia and impaired mucosal healing which could enhance inflammation.

\section{T1169}

Tegaserod Has No Potential for Vasoconstriction or Impairment of Vasodilatation in Isolated Human and Non-Human Primate Mesenteric Arteries

Eckhard Weber, Christian T. Hamel, Mario Bernhard, Hans-Juergen Pfannkuche

BACKGROUND. Tegaserod (Zelmac®/Zelnorm $®)$, a potent 5-HT4 receptor agonist, is an effective and safe treatment of chronic constipation and irritable bowel syndrome with constipation. Since tegaserod has also affinity for human 5-HTlB receptors $(5-\mathrm{HTlBR}, \mathrm{Ki}=$ $60 \mathrm{nM}$ ) and agonists at 5-HTlBRs may cause mesenteric ischemia via local vasoconstriction, we conducted studies addressing the potential of tegaserod to trigger 5-HT1BR-mediated visceral vasomotor effects in isolated mesenteric arteries from human and non-human primates. METHODS. Peripheral mesenteric arteries were obtained from patients undergoin bowel resection for colon carcinoma ( $4 \mathrm{~m} / 4 \mathrm{f}, 44-92$ years) and from cynomolgus monkeys (8m/8f, 4-6 years). Artery rings (length: $3-5 \mathrm{~mm}$, diameter: $300-700 \mu \mathrm{m})$ were mounted in organ baths, contracted with $\mathrm{KCl}(100 \mathrm{mM})$, washed and examined for functional integrity of the epithelium. In a lst study, cumulative concentration-response curves were established for tegaserod $(1 \mathrm{nM}-10 \mu \mathrm{M})$ and the selective 5-HTlBR agonist, sumatriptan $(1 \mathrm{nM}-10 \mu \mathrm{M})$. In a 2nd study, tegaserod $(0.3 \mu \mathrm{M})$ and the selective 5-HTlBR antagonist, GR55562 (0.1 $\mu \mathrm{M})$, were investigated as to their inhibitory effects on sumatriptan-stimulated vasoconstriction A 3rd study was performed in monkey preparations only. Arteries were pre-constricted with phenylephrine $(10 \mu \mathrm{M})$ and relaxed with the endothelial and non-endothelial mediators of vasodilatation, acetylcholine, bradykinin and forskolin $(0.1 n \mathrm{M}-10 \mathrm{\mu M})$. These experiments were done in absence or presence of tegaserod $(1 \mu \mathrm{M})$. RESULTS. Tegaserod did not induce vasoconstriction in human $(n=4)$ or monkey mesenteric arteries $(n=6)$. In contrast, sumatrip tan concentration-dependently constricted both human $(\mathrm{H})$ and monkey $(\mathrm{M})$ artery preparations $(\mathrm{EC} 5 \mathrm{O}(\mathrm{H})=355 \pm 13 \mathrm{lnM}, \mathrm{Emax}(\mathrm{H})=16 \pm 12 \%$ of $100 \mathrm{mM} \mathrm{KCl}$ mean $\pm \mathrm{SEM}, \mathrm{n}=4$ EC50(M) $=214 \pm 137 \mathrm{nM}, \operatorname{Emax}(\mathrm{M})=23 \pm 12 \%, \mathrm{n}=8)$. Tegaserod $(\mathrm{T})$ and $\mathrm{GR} 55562(\mathrm{G})$ competitively inhibited sumatriptan-induced vasoconstriction $(\mathrm{IC} 50(\mathrm{TH})=145 \pm 107 \mathrm{nM}$, IC50(TM) $=$ $59 \pm 280 \mathrm{nM}, \mathrm{n}=4 ; \mathrm{IC} 50(\mathrm{GH})=8 \pm 30 \mathrm{nM}, \mathrm{IC} 50(\mathrm{GM})=36 \pm 77 \mathrm{nM}, \mathrm{n}=8)$. Tegaserod $(\mathrm{T})$, similar to vehicle (V), had no effect on acetylcholine (A), bradykinin (B) and forskolin (F)-triggered vasodilatation in phenylephrine-constricted monkey arteries (IC50(TA) $=129 \pm 28 \mathrm{nM}$ IC $50(\mathrm{VA})=143 \pm 7 \mathrm{lnM}, \mathrm{n}=5$; IC $50(\mathrm{~TB})=1.2 \pm 0.4 \mathrm{nM}$, IC $50(\mathrm{VB})=1.1 \pm 0.2 \mathrm{nM}, \mathrm{n}=6$; IC $50(\mathrm{TF})=$ $29 \pm 4 n M, I C 50(V F)=21 \pm 5 n M, n=6)$. CONCLUSIONS. The present findings in mesenteri arteries demonstrate that tegaserod can act as a 5-HTlBR antagonist and has no potential to cause visceral vasoconstriction or impair vasodilatation. Our mechanistic studies provide further evidence that tegaserod does not trigger visceral hemodynamic activities, which might lead to mesenteric ischemia.

\section{T1170}

Kayexalate (Sodium Polystyrene Sulfonate) in Sorbitol Is a Common and Frequently Unrecognized Cause of Intestinal Ischemia Susan C. Abraham, Alyssa M. Krasinskas

Background: Kayexalate in sorbitol for management of hyperkalemia has been associated both clinically and in animal studies with the development of bowel necrosis, potentially as a result of mucosal ischemia from the hypertonic sorbitol solution. It is estimated that gastrointestinal necrosis occurs in $\sim 1 \%$ of patients receiving Kayexalate in sorbitol. However, recognition of Kayexalate administration as an etiology of intestinal ischemia remains low, and the characteristic crystalline resin of Kayexalate is easily overlooked in histologic sections of intestinal biopsies or resections. Methods: We studied 115 surgical resections for acute ischemic bowel (41 colonic, 40 small bowel, and 21 large and small bowel ischemia) among 102 patients. Clinicopathologic etiology of the ischemia was evaluated, along with histologic evaluation for Kayexalate resin. Controls included 65 surgical resections for ileocolonic adenocarcinoma and 36 surgical resections of non-cancerous, non-ischemic bowel. Results Kayexalate administration was associated with colonic $(n=5)$ or ileocolonic $(n=3)$ ischemia in 8 of $102(7.8 \%)$ patients and 10 of $115(8.7 \%)$ surgical resection specimens. Patients with necrosis due to Kayexalate in sorbitol included 5 males and 3 females with a mean age of 59.4 years (range, 38-83 years). These demographics did not differ significantly from those of the entire population with ischemic bowel ( 45 males:57 females, mean age 60.5 years, range 18-91 years). In 5 patients, Kayexalate in sorbitol was the only identifiable etiology for bowel necrosis. The other 3 patients had alternative etiologies for ischemia that could have initiated or exacerbated bowel necrosis, including 81- and 83-year-old men with cholesterol emboli, and a 54 -year-old woman who experienced necrosis of a rectosigmoid anastomotic site after receiving Kayexalate 2 weeks following surgery for diverticular disease. Kayexalate administration had not been recognized as the etiology of ischemia pre-operatively in any of the 8 patients, and Kayexalate crystals were missed on initial histologic review of the specimens in 7 of 10 resections. None of the 101 control specimens of non-ischemi bowel contained Kayexalate crystals on histologic review. Conclusions: Administration of Kayexalate in sorbitol predisposes to acute intestinal necrosis in a sizable minority $(7.8 \%)$ of patients requiring surgical intervention for ischemic bowel, and continuing ischemia in some of these patients ( 3 of 8 in this series) necessitates additional bowel resection. Despite this, clinical and histologic recognition of the role of Kayexalate in intestinal ischemia remains low.

\section{$\mathrm{T} 1171$}

Enterocolic Lymphocytic Phlebitis: Its Prevalence and Role in Ischemic Bowel Alyssa M. Krasinskas, Susan C. Abraham

Background: Enterocolic lymphocytic phlebitis (ELP) is a form of vasculitis limited to the veins of the digestive tract. Histologically, ELP is characterized by mononuclear cell infiltrates within and around walls of small to medium-sized veins; venous thrombi and granulomas can be present. Since most cases of ELP reported in the literature are associated with intestinal ischemia, it has been assumed that ELP is a cause of ischemic bowel by promoting thrombosis on top of the phlebitic lesions. Although ELP appears to be uncommon, its prevalence is not known. This study examines the prevalence of ELP and its role in intestinal ischemia Methods: We studied 115 consecutive cases of surgically resected acute ischemic bowel (colon, $\mathrm{n}=46$; small bowel, $\mathrm{n}=52$; and both colon and small bowel, $\mathrm{n}=17$ ) from 102 patients. The control group included 101 surgical resection specimens ( 65 colonic adenocarcinomas and 36 non-malignant, non-ischemic bowel). Tissue sections were evaluated histologically for the presence and distribution of ELP. Results: Of the 115 cases of ischemic bowel, ELP was present in 6 specimens (5.2\%) from 6 patients (5.8\%). Patients with ELP had a mean age of 42 years (range 18-74 years), younger than the ischemic bowel patients without ELP who had a mean age of 61 years (range 18-91 years); $p=0.009$. There were 4 males and 2 females. The colon was involved in 4 and the small bowel in 2. The ELP was mild, with histologic evidence of perivenular lymphoplasmacytic inflammation within the submucosa through to the subserosa and mesentery; one case had venous thrombi. In 3 cases, the ELP extended outside the ischemic segment of bowel. Clinically, all patients had reasons other

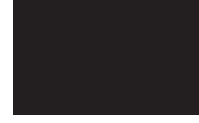


than ELP for their ischemia: nearby colon cancer, adhesions, incarcerated hernia, impacted stool with perforation, recent surgery for pancreatic cancer and recent surgery for a gunshot wound. Two of the cases had bowel resections 1 day and 10 days earlier and ELP was not present in those specimens. Interestingly, ELP was present in four of the control cases (4\%) and all were associated with colon cancer; ELP extend away from the tumor in only 1 case. Conclusions: ELP is uncommon, present in $5.2 \%$ of intestines resected for ischemia and $4 \%$ of non-ischemic resections. Although present in ischemic intestines, ELP does not appear causative. Based on our results, including known etiologies for the ischemia in cases with ELP, the presence of ELP in cancer and the absence of ELP in prior bowel resections in two patients, ELP likely represents a secondary response to an altered mucosal barrier, rather that a primary cause of ischemic enterocolitis.

\section{$\mathrm{T} 1172$}

The Chemokine Cxcl12 Regulates Intestinal Epithelial Restitution By Increasing Actin Rearrangement and Activity of the Rho Signaling Pathway Rebecca Moyer, Michael K. Wendt, Priscilla A. Johanesen, Michael B. Dwinell

The principal pathophysiologic features of inflammatory bowel disease and enterocolitis include epithelial ulceration, leukocyte infiltration, and mucosal remodeling and repair. Chemokines are essential regulatory factors that stimulate migration, proliferation and differentiation in chemokine receptor expressing target cells. Mucosal barrier homeostasis is dependent upon the tight physiologic balance between those processes suggesting chemokines may regulate the competency of the epithelial barrier to withstand injury from luminal stimuli and repair itself following damage. We have recently shown that binding of the chemokine CXCL12 to its cognate receptor CXCR4 stimulates epithelial restitution. The aim of the present study was to define the signaling mechanisms whereby chemokine receptors mediate epithelial cell migration. Normal, non-transformed IEC6 epithelial cells or differentiated polarized T84 epithelial monolayers were wounded and changes in F-actin assessed using fluorescence microscopy and flow cytometry. Rho activity was defined using a rhotekinGST assay in conjunction with immunofluorescence microscopy. CXCL12 stimulated the formation of F-actin, the accumulation of which was predominantly localized to the leading edge of CXCL12-treated wounded epithelial monolayers and was greater than unstimulated cells. In agreement with the increase in F-actin, CXCL12 binding to CXCR4 stimulated an increase in the levels of active RhoGTP in both unwounded and wounded IEC6 epithelial cells. Consistent with a role for Rho in chemokine mediated wound healing, inhibition of the downstream Rho-kinase significantly blocked IEC6 migration. Similarly, blockade of the myosin light chain kinase also prevented CXCL12 restitution. Our data demonstrate that CXCR4 and CXCL12 function as an autocrine and paracrine mucosal signaling network increasing activity of the canonical restitution mechanism. Our findings are consistent with the notion that chemokines, ubiquitous immune mediators produced within the gut mucosa, play a significant role in the restitutive repair processes of mucosal inflammation.

\section{T1173}

Increased Expression of Both Angiogenic VEGF, and Anti-Angiogenc Endostatin and Angiostatin in Ulcerative Colitis in Rats, Mice and Humans: An Explanation for Impaired and Delayed Healing Xiaoming Deng, Zsuzsa Sandor, Tetyana Khomenko, Longchuan Chen, Ximing Xiong, Andrzej Tarnawski, Sandor Szabo

Angiogenesis, which plays a key role in wound/ulcer repair, is governed by a balance between pro- and anti-angiogenic factors. We, therefore, determined the levels of potent angiogenic factor VEGF and angiogenesis inhibitors endostatin and angiostatin in colonic tissues of rats and mice with experimental ulcerative colitis, and human ulcerative colitis. Methods: In rats ulcerative colitis was induced by intracolonic administration of $6 \%$ iodoacetamide and the animals were euthanized at $0.5,1,2$, and $6 \mathrm{hr}$ after iodoacetamide. The mouse colonic tissues were obtained from an ulcerative colitis model induced by IL-10 knock-out, while the colonic biopsy specimens of human ulcerative colitis were obtained from surgical pathology archives. In the rat samples, levels of VEGF, endostatin and angiostatin were detected by Western blotting with respective specific antibodies (Santa Cruz Biotechnology). In human and mouse tissues, VEGF was detected by immunohistochemistry with a specific VEGF antibody. Results: Western blotting showed that the levels of $23 \mathrm{kDa}$ VEGF, $20 \mathrm{kDa}$ endostatin and $50 \mathrm{kDa}$ angiostatin in the rats with colitis were 3- to 5-fold higher than controls at al time points after iodoacetamide administration in rat colonic tissue. Immunohistochemistry demonstrated that VEGF was strongly expressed in endothelial cells of blood vessels and monocytes around the colonic lesion areas, while the control samples or the control negative staining of mouse and human colonic tissues showed no staining. Conclusions: Our studies demonstrated for the first time: 1) Both the angiogenic VEGF and anti-angiogenic endostatin and angiostatin were increased in early stages of experimental ulcerative colitis. 2) Increased VEGF was detected in endothelial cells of blood vessels and monocytes. 3) Active ulcerative colitis may be due, in part, to inhibition of angiogenesis by increased angiostatin and endostatin, even in the presence of markedly increased VEGF levels. 4) Thus, the enhanced expression of anti-angiogenesis factors may be a new explanation for impaired and delayed healing despite elevated VEGF.

\section{$\mathrm{T} 1174$}

PKC- $\beta 1$ Activation Is Required for Epidermal Growth Factor (EGF) Induced Cellular Migration and Cytoskeletal Remodeling in Enterocyte Monolayers: A Unique Wound Healing Mechanism in GI Epithelium

Ali Banan, Lijuan Zhang, Maliha Shaikh, Christopher B. Forsyth, Ashkan Farhadi, Jeremy Z. Fields, Ali Keshavarzian

Despite numerous studies of cell migration, the intracellular processes that orchestrate wound healing are unclear. Using monolayers of gut epithelial cells as a model of repair, we reported that oxidative stress injures monolayers by disrupting the cytoskeleton; EGF protects against such injury. But, the molecular events are unclear. Since the $\beta 1$ isoform of PKC is translocated to the cell membrane fraction by EGF in native gut cells, we hypothesized that PKC- $\beta 1$ activation is required for EGF-induced cell migration \& cytoskeletal remodeling in gut cells. Methods: Intestinal cells were transfected to stably over-express or under-express PKC- $\beta 1$. These innovative clones were preincubated with EGF (1-100 ng/mL) or PKC activator (DAG analog OAG, 0.01-1 uM) prior to exposure to oxidant (H2O2, 10-500 uM). Wild type (WT) cells were treated similarly. We monitored cell migration/monolayer repair \{2-D densitometry cell count, fluorometry\}, cytoskeletal integrity/oxidation \{laser confocal microscopy, SDSPAGE fractionation\}, PKC- $\beta 1$ subcellular activity/distribution \{in vitro kinase, immunofluorescence\}, oxidative stress (quenchable fluorescence), polymerized/monomeric actin (43 kDa subunits of actin, SDS-PAGE), n=6/grp. Results: \{I\} Pretreatment of WT cells with EGF or transfection of cells to overexpress (3.1 X) PKC- $\beta 1$ enhanced repair as indicated by native PKC- $\beta 1$ activation, oxidative stress suppression, cell migration enhancement, stable actin increases (F/G actin ratio) at the leading migration front, architectural remodeling of actin filaments at the leading front, $\&$ increased monolayer barrier integrity. The transfected clones exhibited enhanced cell migration $\&$ actin remodeling by low, previously nonrepair inducing doses of EGF or OAG. \{II $\}$ Enhancement of migration induced by PKC- $\beta 1$ overexpression was also potentiated by EGF or OAG. These effects were prevented by a PKC inhibitor, bisindolylmalemide. $\{$ III $\}$ Most of the overexpressed PKC- $\beta 1$ resided at the leading edge membrane/cytoskeletal fractions. \{IV\} In both transfected clones \& EGF treated WT cells, PKC- $\beta 1$ ( $78 \mathrm{kDa}$ ) was active $\&$ found with actin $(43 \mathrm{kDa})$, forming endogenous PKC- $\beta 1 /$ actin complexes. $\{\mathrm{V}\}$ Stably inhibiting expression of native PKC- $\beta 1$ substantially prevented all measures of EGF-induced repair. Conclusions: We show for the first time that: 1) EGF-induced cell migration requires the activation of the PKC- $\beta 1$ isoform in intestinal cells; 2 ) PKC- $\beta 1$ is perhaps a unique intracellular modulator of wound healing in epithelium; 3) The ability to promote normal dynamics of cell migration \& cytoskeletal remodeling is a newly discovered repair mechanism not previously attributed to this PKC isoform.

\section{T1175}

Development of a Collagen-Ceramic Composite for Fistula Repair Hussila Keshaw, Paul Sibbons, Alastair Forbes, Richard Day

Tissue adhesives, such as fibrin sealant, continue to be used for treatment of complex anal fistulae despite widely varying success rates. This is partly due to a lack of suitable alternatives and the desire for a relatively simple procedure with low morbidity. Permacol ${ }^{\mathrm{TM}}$, crosslinked porcine dermal collagen, has been used for treating small bowel fistulae and tissue bulking and is currently being investigated for perianal fistula repair. Growth factors regulate tissue healing and are likely to enhance fistula repair. Vascular endothelial growth factor (VEGF) is a regulator of angiogenesis. Stimulation of angiogenesis with exogenous VEGF dramatically accelerates healing of experimental ulcers. VEGF secretion from colonic fibroblasts is significantly increased by $45 \mathrm{~S} 5$ bioactive glass (BG). Aim: To modify Permacol ${ }^{\mathrm{TM}}$ with BG particles to enhance its bioactivity for use in fistula repair. Methods: $45 S 5$ BG particles $(0 \%-1 \% \mathrm{w} / \mathrm{v})$ were added to cryogenically milled Permacol ${ }^{\mathrm{TM}}$ (Permacol ${ }^{\mathrm{TM}}$ Injection) to produce a composite paste. Colonic myofibroblasts (CCD-18Co) were incorporated into the composite and cultured. Conditioned culture medium was collected after 24, 48 and $72 \mathrm{~h}$ and the amount of VEGF secreted was measured by ELISA. The amount of collagen-bound VEGF was similarly measured at $72 \mathrm{~h}$ after dissolving the composite with collagenase type 1A. Viability of the incorporated cells was determined at $72 \mathrm{~h}$ using an MTT assay. Results: At $48 \mathrm{~h}$, the amount of VEGF secreted from the composites containing $0.1 \% \mathrm{w} / \mathrm{v} 45 \mathrm{~S} 5 \mathrm{BG}$ was significantly increased $(2209 \mathrm{pg} \pm 26$ (SEM); $<<0.01)$ compared with $0 \% \mathrm{w} / \mathrm{v} 45 \mathrm{~S} 5 \mathrm{BG}$ $(1822 \mathrm{pg} \pm 50)$. The amount of VEGF secreted at $72 \mathrm{~h}$ was significantly increased from composites containing $0.01 \%(1720 \mathrm{pg} \pm 17 ; \mathrm{p}<0.001)$ and $0.1 \%(1804 \mathrm{pg} \pm 53 ; \mathrm{p}<0.01)$ w/v $45 S 5$ BG compared with $0 \% \mathrm{w} / \mathrm{v} 45 \mathrm{~S} 5 \mathrm{BG}(1602 \mathrm{pg} \pm 17)$. At $72 \mathrm{~h}$, the amount of VEGF retained in Permacol ${ }^{\mathrm{TM}}$ containing $0.1 \% \mathrm{w} / \mathrm{v} 45 \mathrm{~S} 5 \mathrm{BG}$ was significantly increased $(107 \mathrm{pg} \pm 18 ; \mathrm{p}<0.01)$ compared with $0 \% \mathrm{w} / \mathrm{v} 45 \mathrm{~S} 5 \mathrm{BG}(52 \pm 2)$. At $72 \mathrm{~h}$, there was no significant difference in cell number between composites containing $0 \%, 0.01 \%$ and $0.1 \%$ w/v 4555 BG indicating increases in VEGF secretion were independent of an increase in cell number. VEGF secretion was reduced with composites containing $1 \% \mathrm{w} / \mathrm{v} 45 \mathrm{~S} 5 \mathrm{BG}$ corresponding with a significant reduction in cell number at $72 \mathrm{~h}(72 \% \pm 10 ; \mathrm{p}<0.01)$. Conclusion: The current study indicates that Permacol ${ }^{\mathrm{TM}}$-45S5 BG composites might provide a safe filler material for repair of perianal fistulae. The increased secretion of VEGF from cells in contact with or infiltrating the material may accelerate the healing of fistulae treated with this composite.

\section{T1176}

PI3K-Dependent GSK3 $\beta$ Phosphorylation Is Implicated in the Intestinal Epithelial Cell Wound-Healing Response

Thomas Karrasch, Brigitte Allard, Christian Jobin

Introduction: Improper gastrointestinal restitution following various intestinal injuries may lead to acute and/or chronic inflammation through enhanced bacterial translocation and immune cell activation. The molecular mechanism involved in the wound-healing response in the intestine is poorly understood. The glycogen synthase kinase $3 \beta$ (GSK3 $\beta$ ) has been implicated in various biological processes such as cellular motility and spreading. Aim: To investigate the role of GSK3 $\beta$ in intestinal epithelial cell restitution. Methods: Rat IEC18 cells were serum-starved for 16-24h and wounded by multiple scraping. RelA(Ser536)-, GSK3ß(Ser9)-, $\beta$-catenin(Thr41,Ser45)-, Akt(Ser473)- and FAK(Tyr397)-phosphorylation were determined by Western blot using specific phospho-antibodies. The specific inhibitors AG1478 $(1 \mu \mathrm{M})$ and wortmannin $(100 \mathrm{nM})$ were used to block EGF-R autophosphorylation and PI3K-activation, respectively. $\beta$-catenin/LEF/TCF dependent transcription was determined by reporter gene assay (TOP/FOP system). C-jun gene expression was evaluated by reverse-transcriptase PCR. Results: Wounding induced focal adhesion kinase (FAK), Akt, GSK3 $\beta$ and $\beta$-catenin phosphorylation in IEC-18 cells. $\beta$-catenin phosphorylation/stabilization led to enhanced ( 2 fold) LEF-TCF transcriptional activity and c-jun mRNA accumulation in wounded cells. Blocking PI3K/Akt signaling with wortmannin prevented wound-induced GSK3 $\beta$ phosphorylation and significantly attenuated restitution. Additionally, wounding induced rapid and sustained NF-KB as well as FAK phosphorylation, which were inhibited by AG1478, but not by wortmannin. Conclusion: We conclude that PI3K mediated GSK3 $\beta$ 
activation is involved in the intestinal epithelial wound-healing response and operates independently of NF-KB and FAK signaling. Activation of GSK3 $\beta$ may be important for intestinal restitution by promoting cell motility, survival and proliferation.

\section{T1177}

Butyric Acid, a Physiologic Bacterial Fermentation Product, Represses Epithelial Inflammatory Signaling Via Changes in Cul-1 Neddylation Lauren S. Collier-Hyams, Shanthi Sitaraman, Andrew Neish

Background: The human enteric flora plays a significant role in intestinal health and disease, and administration of exogenous probiotic bacteria may modulate inflammation. We have shown that nonpathogenic enteric bacteria can inhibit the key proinflammatory NF-kappaB pathway by blockade of IkappaB-alpha ubiquitination, a process catalyzed by the E3-SCFbetaTrCP ubiquitin ligase complex. This enzyme is itself regulated via covalent modification of the Cullin-1 subunit by the ubiquitin-like protein NEDD8. We previously reported that interaction of a variety of commensal bacteria with epithelial cells resulted in a rapid deneddylation of Cullin- 1 and resultant repression of the NF-kappaB pathway. How bacteria mediate this process is not known, but conditioned media could recapitulate the inhibition. HPLC analysis of conditioned media detected large quantities of organic acids. Purpose: As short chain fatty acids have been previously shown to have immunomodulatory properties, we sought to determine if these naturally occurring small molecules could have inhibitory effects on IkappaB ubiquitination. Methods: T84, HeLa, and HT-29 epithelial cells, as well as human colonic biopsy specimens were incubated with physiological quantities of butyric acid $[0.5-5.0 \mathrm{mM}]$ and other short chain fatty acids. Cullin neddylation was assayed by immunoprecipitation and immunoblot. NF-kappaB responses were evaluated by immunofluorescence for $\mathrm{p} 65$ translocation and immunoblot analysis of IkappaB for phosphorylation, ubiquitination and degradation events. The inflammatory gene response was evaluated by ELISA for secreted chemokines. Results: We show that bacterial fermentation products including butyric acid and other short chain fatty acids could deneddylate Cullin-1 in cultured epithelial cells and intact human mucosa ex vivo in a dose dependent manner. Deneddylation occurred within 30-60 min and was reversible. Short chain fatty acids were able to inhibit IkappaB ubiquitination (but not phosphorylation), NF-kappaB translocation, and IL-8 secretion as efficiently as live bacteria, and with similar kinetics. Conclusions: As the normal flora produces significant quantities of organic acids, these observations may explain the ability of the intestinal bacterial communities to influence inflammatory regulation of the mammalian intestinal epithelia and may explain anti-inflammatory effects of probiotic microbes. As SCF ubiquitin ligases control many aspects of epithelial cell biology, these data also suggests how natural products of the normal intestinal flora could profoundly influence many epithelial signaling pathways

\section{$\mathrm{T} 1178$}

Remote Epithelial Dysfunction After Localized Intestinal Radiation Injury: Possible Role of IL-1 $\beta$ ?

Sandra Torres, Marc Benderitter, Jean-Pierre Caruelle, Agnes Francois

Motility disorders implicating unirradiated parts of the intestine have frequently been reported in patients irradiated for pelvic tumors, as a consequence of localized radiation injury to normal gut. However, less is known about intestinal transport capacity and epithelial barrier properties outside the irradiation field. We investigated here how a localized radiation exposure on the rat small intestine may induce intestinal dysfunction in shielded segments, and particularly far from the site of injury (duodenum). A possible role of ILI $\beta$ in this outof-field dysfunction was hypothesized. After laparotomy, 6 to $8 \mathrm{~cm}$ of rat small intestine was exteriorized and exposed to a single dose of $19 \mathrm{~Gy}$ (X-rays). Two weeks post-exposure, the functional capacity of the irradiated ileum (IR) and the non-irradiated segments (terminal ileum, jejunum 20 (Jej20) and $40 \mathrm{~cm}$ (Jej40) proximal to the IR and duodenum) were measured in Ussing Chambers. Basal parameters (short circuit current, potential difference, tissue conductance), as well as epithelial response to $35 \mathrm{~Hz}$ electrical field stimulation (EFS) and exogenously-added carbachol were measured. The structural integrity of the segments was studied by routine histology (morphometric measurements). Myeloperoxidase and enteric innervation density was followed by immunostaining. All basal electrophysiological parameters were diminished in IR as well as in shielded segments. Responses to a $35 \mathrm{~Hz}$ EFS were diminished in IR (-70\%) and Jej20 (-48\%). No difference was noticed in the others segments. Conversely, responses to carbachol were severely diminished all along the gut proximal to the injured area (IR: $-70 \%$, Jej20: $-49 \%$, Jej40: $-66 \%$ ), with the most important decrease observed in the duodenum $(-91 \%)$. The irradiated segments showed an important inflammatory infiltrate with areas of severe mucosal ulceration, and a global increase in both submucosal and myenteric ganglion numbers. Neither structural injury nor change in enteric innervation density was observed in shielded segments. Conversely a $4-$ fold increase of IL1 $\beta$ mRNA (RT-PCR) was observed in the duodenum of irradiated rats compared to those of control rats. Localized small intestinal irradiation in the rat induced severe intestinal dysfunction in the injured segment, due to severe epithelial disruption. The reason why intestinal transport capacity was impaired all along the small intestine, and more particularly in the duodenum, is less clear, but a possible explanation may be an increase in IL-1 $\beta$ expression. Ongoing studies investigate if this "remote effect" may be mediated by either systemic circulation or by neurogenic inflammation (enteric nervous system)

\section{T1179}

Cytostatic Drug-Induced Mucositis in Mucin 2 Deficient Mice Barbara de Koning, Maria Van der Sluis, Dicky Lindenbergh-Kortleve, Anna Velcich, Rob Pieters, Hans Buller, Alexandra Einerhand, Ingrid Renes

The structural component of the mucus layer, mucin Muc2, is up-regulated after chemotherapy. This suggests that Muc2 might be a potential target in mucositis prevention or reduction. Our aim was to determine the role of Muc2 during chemotherapy-induced mucositis. Mucositis was induced in Muc2 knockout $\left(\mathrm{Muc}^{-/-}\right)$and wild type $\left(\mathrm{Muc2}^{+/+}\right)$mice by injecting methotrexate (MTX). Animals were weighed and sacrificed on day 2-6 after MTX treatmen and jejunal segments were analyzed. Before MTX treatment, the small intestine of $\mathrm{Muc2}^{+/}$ and $\mathrm{Muc}^{-1}$ mice were similar with respect to epithelial morphology and proliferation Moreover, sucrase-isomaltase and trefoil factor-3 protein expression levels were comparable between $\mathrm{Muc}^{+/+}$and $\mathrm{Muc}^{-1-}$ mice. Up to day 3 , there were no differences in percentage weight-loss between $\mathrm{Muc2}^{+/+}$and Muc2 $2^{-/}$mice. Thereafter, Muc2 $2^{+/+}$mice showed a trenc towards regaining weight, whereas Muc $2^{-1-}$ mice continued to lose weight. Surprisingly, MTX-induced intestinal damage of $\mathrm{Muc}^{-1-}$ and $\mathrm{Muc}^{+/+}$mice was comparable. Prior to MTX treatment, interleukin-10 mRNA was significantly up-regulated in Muc2 ${ }^{-1-}$ mice, but not in $\mathrm{Muc2}^{+/+}$mice, probably due to continuous exposure of the intestine to luminal antigens CONCLUSION: Our data do not provide evidence that Muc2 deficiency leads to an increase in chemotherapy induced mucositis. This might be caused by the fact that Muc2 deficiency triggers the immune system to up-regulate the anti-inflammatory cytokine interleukin-10 prior to MTX-treatment, which limits mucositis after MTX treatment.

\section{$\mathrm{T} 1180$}

Rabbit Enteropathogenic E.Coli Strains Expressing Shiga Toxins 1 (Stx1) Or 2 (Stx2) Induce Renal Lesions of Hemolytic Uremic Syndrome (HUS) in Dutch Belted Rabbits

Edgar Boedeker, Chengru Zhu, Hernan Rios, Katherine Davis

Background: Shiga-toxin producing E. coli (STEC) strains, including those producing Stxl and/or Stx2, induce hemorrhagic colitis and hemolytic uremic syndrome (HUS) in humans. Although spontaneous occurrence of renal disease resembling HUS in Dutch Belted (DB) rabbits infected with an O153 E.coli strain producing Stxl has been reported, no reproducible animal challenge model for the induction of HUS following ingestion of STEC strains has been established. Aims: In order to develop orogastric challenge models for the induction of HUS by both Stxl and Stx2 strains, and to compare the virulence of the two toxins, we challenged DB rabbits with rabbit enteropathogenic E.coli (REPEC) strains, observed their clinical course and evaluated their kidneys histologically for lesions typical or characteristic of HUS. Design: $1 \mathrm{~kg}$ specific pathogen free DB rabbits were orgogastrically inoculated with saline(control), with the O153 Stxl REPEC strain from the natural infection, or with REPEC strains RDEC-H19A (O15 Stxl) and E22 Stx2 (O103 Stx2 which were selected in the laboratory for acquisition of bacteriophage encoding Stxl or Stx2. Animals were sacrificed at 7-10 days following infection and $H \& E$ stained sections of their kidneys evaluated for lesions typical or characteristic of HUS using a scoring system based on 4 quantifiable parameters: glomerular congestion, glomerular heterophils, fibrin like vascular deposits in medium vessels and cortical congestion. Results: no animals administered the WT O153 Stxl strain developed wt. loss, diarrhea or renal lesions. All animals administered RDECH19A-Stxl or E22-Stx2 developed wt. loss and diarrhea. Results for renal histology for REPEC strains selected for acquisition of Stx phages are tabulated below. Summary: WT STEC isolates did not cause reproducible disease in DB rabbits. This may have been because of previous exposure to the strain. REPEC strains which have laboratory-acquired Stx phages produce consistent clinical disease and histological lesions which are quantifiable and suggestive of HUS. Conclusions: Both Stxl- and STx2-producing REPEC strains induce reproducible and quantifiable renal lesions in DB rabbits following orogastric challenge. These challenge models should permit evaluation of therapies to prevent the onset of renal disease following STEC infection

\begin{tabular}{|c|c|c|c|}
\hline & Control & RDEC-H19A-Stx1 & E22-Stx2 \\
\hline Glomerular Heterophiles & $.85+/-.21$ & $3.04+/-0.9(\mathrm{p}=.02 \mathrm{vC})$ & $1.4+/-.36$ \\
\hline Glomerular Congestion & $2.4+/-1.2$ & $28.02+/-5.3(\mathrm{p}<.01 \mathrm{vC})$ & $15.3+/-6.6(\mathrm{p}<.05 \mathrm{vC})$ \\
\hline Fibrin-Like Vascular Deposits & $.13+/-.12$ & $19.75+/-3.1(\mathrm{p}<.01 \mathrm{vC})$ & $26.4+/-12.5(\mathrm{p}=.04 \mathrm{vC})$ \\
\hline Cortical Congestion & $.05+/-.05$ & $20+/-5.4(\mathrm{p}<.01 \mathrm{vC})$ & $3.4+/-2.7$ \\
\hline
\end{tabular}

\section{T1181}

Tumor Suppressor Foxo3a Is Involved in Regulation of Inflammation Induced By Enteric Pathogen Citrobacter Rodentium

Suzana Savkovic, Marinka Bulic, Amy Yang, Jameela Huq, Stanford L. Peng

Introduction: Enteric bacteria play an important role in initiating and maintaining chronic intestinal inflammation, which is closely associated with colon cancer development. Infection with Citrobacter rodentium (CR), a pathogen known to induce inflammation and promote tumorigenesis in the mouse colon is an accepted model to study bacteria-triggered mechanisms that contribute to these processes. It has been shown that the NF-KB pathway that controls intestinal inflammation is also involved in tumorigenesis. For example, the tumor suppressor Foxo3a is inactivated by and also regulates the NF-KB pathway in tumor cells Aim: The aim of this study was to determine the mechanism of regulation of Foxo3a in intestinal epithelial cells (IEC) and its contribution to CR-induced inflammation. Methods: Mouse IEC, CMT-93, were infected with CR and examined for Foxo3a inactivation by immunoblotting and immunofluorescent staining. IEC were transfected with retroviral vector pMX-Foxo3a-IRS-GFP, allowing over-expression of Foxo3a. pMX-IRS-GFP was used as a control vector, while independent expression of green fluorescent protein (GFP) was used as a internal control to assess the efficiency of retroviral transfection. These cells were analyzed for NF-KB activation by immunofluorescent staining of the p65 subunit of the NF$\kappa B$ complex and for expression of the cytokine macrophage inflammatory protein-2 (MIP2) by ELISA. Results: First we assessed whether infection with CR affects Foxo3a in mouse IEC. During infection of mouse IEC with CR, Foxo3a is phosphorylated, translocated from the nucleus into the cytosol, and degraded, suggesting its inactivation. Next, we examined whether Foxo3a plays a role in CR-induced NF- $\mathrm{KB}$ activation in mouse IEC by assessing the effect of over-expression of Foxo3a on CR-induced NF- $\mathrm{kB}$ translocation. CR-induced translocation of p 65 was attenuated by approximately $50 \%$ in the cells over-expressing the Foxo3a as compared to the control. To assess the effect of Foxo3a on cytokine expression, monolayers of mouse IEC over-expressing Foxo3a were treated with sterile supernatant (SN) 
from a CR culture known to induce expression of MIP-2 cytokine. Over-expression of Foxo3a attenuated CR-induced MIP-2 expression (pMX untreated $2 \pm 1$ pg/ml, CR-SN 4 h $97 \pm 8$ pg/ml; pMX-Foxo3a untreated $2 \pm 1$ pg/ml, CR-SN 4 h $60 \pm 7$ pg/ml, n=6, p=0.007 for SN treated pMX vs SN treated pMX-Foxo3a), suggests that Foxo3a plays a role in regulating CR-induced MIP-2 expression in mouse IEC. Summary: i) Infection of mouse IEC with CR induces phosphorylation, translocation, and degradation of Foxo3a; ii) Foxo3a regulates CR-induced NF-KB activation and CR-induced MIP-2 expression.

\section{$\mathrm{T} 1182$}

Lactobacillus Casei Strain Shirota Prevent NSAID-Induced Small Intestinal Injury By Suppressing Toll Like Receptor 4 Signaling Pathway

Hikaru Nishio, Toshio Watanabe, Tetsuya Tanigawa, E.J. Sasaki, Masatsugu Shiba

Kazunari Tominaga, Yasuhiro Fujiwara, Nobuhide Oshitani, Kazuhide Higuchi, Kato

Shinichi, Tetsuo Arakawa, Koji Takeuch

Background \& Aims: Experimental studies have shown that enteric bacteria play crucial roles in pathogenesis of NSAID-induced small intestinal injury. Probiotics have been used to treat a variety of gastrointestinal disorders, including inflammatory bowel disease, but effect on NSAID-induced small intestinal injury is unknown. In the present study, we examined effect of a probiotic Lactobacillus Casei strain Shirota (LcS) on indomethcin (IM)induced small intestinal injury in rats and mice. Methods: Animals were administered IM $\left(10 \mathrm{mg} / \mathrm{kg}\right.$ p.o.), and intestinal injury was evaluated $24 \mathrm{~h}$ later. Viable LcS $\left(10^{7}-10^{9} \mathrm{CFU}\right.$ p.o.) was administered once or for a week, while heat-killed LcS ( $10^{9} \mathrm{CFU}$ p.o.) was administered for a week. Ampicillin (active against both Gram-positive and Gram-negative bacteria, $800 \mathrm{mg} / \mathrm{kg}$ ), aztreonum (active against Gram-negative bacteria specific antibiotic, $250 \mathrm{mg} / \mathrm{kg}$ ) or vancomycin (active against Gram-positive bacteria, $50 \mathrm{mg} / \mathrm{kg}$ ) were given p.o. twice $24 \mathrm{~h}$ and $1 \mathrm{~h}$ before IM treatment. Result: IM induced hemorrhagic lesions in the small intestine of these animals. Both ampicillin and aztreonam suppressed the damage in rats, while vancomycin had no effect. One-week treatment with LcS prevented IM-induced intestinal injury with a 2.4-fold increase of the L-lactic acid (LA) concentration in small intestinal luminal contents, and also inhibited increases in myeloperoxidase activity (a marker for neutrophil infiltration) and expression of mRNA for tumor necrosis factor- $\alpha$ (TNF- $\alpha$ ) in small intestine by $24 \mathrm{~h}$ after administration of IM. Heat-killed LcS did not have the preventive effect on IM-induced small intestinal injury. In Toll-like receptor (TLR) 4-deficient mice, the intestinal damage was inhibited by $90 \%$ as compared with that in wild-type littermates. TLR4 was mainly expressed on inflammatory cells in the rat small intestine. In human monocytes cells (THP-1), both $10 \mathrm{mM} \mathrm{LA}$ and LcS culture supernatants containing $10 \mathrm{mM}$ LA inhibited increases in TNF- $\alpha$ mRNA expression and TNF- $\alpha$ protein secresion by lipopolysaccharide (LPS). Western blot analyses confirmed that LA and the LcS culture supernatants suppressed phosphorylation of IKB. Conclusion: These results suggest that TLR4 signaling pathway activated by LPS from Gram-negative bacteria plays a crucial role in IM-induced small intestinal damage, and LcS has a potential prophylactic effect on NSAIDinduced damage by suppressing TLR4 signaling pathway, which is mediated by LA

T1183

Metabolic Activity of Probiotics - Oxalate Degradation Caroline Murphy, Sharon Murphy, Sinead Jacob, Maurice O'Donoghue, Thomas Boileau, Gregory Sunvold, Gregory Reinhart, Barry Kiely, Fergus Shanahan, Liam O'Mahony

Background: Hyperoxaluria complicated by renal tract stones is an important clinical problem, particularly in patients with enteric hyperoxaluria secondary to conditions such as Crohn's disease. Aims: (a) To evaluate oxalate degradation by a range of lactic acid bacteria (LAB) in vitro; (b) To determine the impact of LAB consumption on oxalate excretion in vivo. Methods: Bacteria were grown in oxalate-containing media and their ability to degrade oxalate in vitro was determined using reverse-phased HPLC. Selected LAB, or placebo, were fed to rats (n=6/group) for 4 weeks. Urine was collected at weeks 0,2 and 4 and oxalate levels determined by HPLC. Results: In vitro oxalate degradation was detected for 11/18 of the Lactobacillus strains. In contrast, 0/13 Bifidobacterium strains degraded oxalate. Lactobacillus (L.) animalis 223C, L. murinus 1222, L. murinus 5323 and L. murinus 3133 were selected for investigation in the rat model. Urinary oxalate levels were significantly reduced $(p<0.05)$ in animals fed $L$. murinus 5323 ( $42 \%$ reduction) and $L$. animalis $223 \mathrm{C}$ ( $81 \%$ reduction) but were unaltered when fed with L. murinus 1222, L. murinus 3133 or placebo. Conclusion: Probiotic organisms vary widely in their capacity to degrade oxalate. In vitro degradation does not uniformly translate to an impact in vivo. The results have therapeutic implications and may influence the choice of probiotic, particularly in the setting of enteric hyperoxaluria.

\section{T1184}

Lactobacillus Farciminis Treatment Suppresses Stress-Induced Visceral Hypersensitivity Through Inhibition of Colonic Epithelial Cells Myosin Light Chain Kinase (MLCK)

Afifa Ait-Belgnaoui, Wei Han, Florence Lamine, Helene Eutamene, Jean Fioramonti, Lionel Bueno, Vasillia Theodorou

Background. Increase in gut sensitivity and intestinal permeability have been reported in irritable bowel syndrome. In rats, stress-induced increase in colonic paracellular permeability through epithelial cell MLCK activation is responsible for stress-induced colorectal hypersensitivity. Treatment with the probiotic Lactobacillus farciminis has been shown to prevent stressinduced increase in colonic paracellular permeability and colorectal sensitivity in rats, but the mechanism involved remains unknown. This study aimed to evaluate in rats (i) acute stress-induced changes in the levels of MLCK, myosin light chain (MLC) and its phosphorylated form (p-MLC) which is responsible for epithelial cells cytoskeleton contraction (ii) the influence of $L$. farciminis treatment on these alterations. Materiel and Methods. In a first series of experiments, 12 groups of 4 female Wistar rats were used for assessment of the kinetics of MLCK, MLC and p-MLC protein levels after 15, 30, 45, 60, 75 and $90 \mathrm{~min}$ of sham stress (groups 1-6) or partial restraint stress (PRS) (groups 7-12) proximal colonic mucosa was removed for determination of protein levels by Western immunoblotting. In a second series of experiments, 4 groups of 4 rats received orally during 15 days either saline (group 1,2 ) or $10^{11} \mathrm{cfu} /$ day of $L$. farciminis (groups 3,4 ). At the end of treatments p-MLC levels were determined after 45 min of sham stress (groups 1,3) or PRS (groups 2, 4). Results. Partial restraint stress did not modify MLCK levels. In contrast, p-MLC levels were significantly $(\mathrm{p}<0.05)$ increased from 15 to $45 \mathrm{~min}$ of starting PRS $(31.2 \%$ of increase at 45 min compared with sham stress). MLC levels were significantly increased at $60 \mathrm{~min}$ after the beginning of PRS. L. farciminis treatment significantly decreased p-MLC levels (- $26 \%$ compared to saline, $\mathrm{p}<0.05$ ) at $45 \mathrm{~min}$ of PRS. Conclusion. This study shows that a twoweek treatment by Lactobacillus farciminis reduces stress-induced MLC phosphorylation, suggesting that this probiotic strain prevents stress-induced increase of colonic paracellular permeability and colorectal sensitivity by inhibiting the contraction of colonic epithelial cells cytoskeleton and, the subsequent tight junction opening.

\section{T1185}

Lactulose Breath Testing Detects Small Intestinal Bacterial Overgrowth in Rats Justin H. Barad, Eileen O. Sullivan, Isella Nieto, Anna Hui, Henry C. Lin

Background: In rats, SIBO can be induced by feeding a diet containing raw red kidney beans (RKB) or its constituent lectin phytohemagglutinin as shown by direct culture and electron microscopy after euthanasia (Gastroenterology 1983;84:506). Recently, SIBO was found in $84 \%$ of patients with IBS as demonstrated by an abnormal lactulose breath test. The role of SIBO in IBS was supported by $75 \%$ improvement of symptoms with normalization of lactulose breath test with an antibiotic. A rat with SIBO would then be an appropriate animal model of IBS. Therapeutic strategies for IBS could be rapidly tested if lactulose breath testing were to be used in an animal model of SIBO to follow treatment response. The aim of this study was to test the hypothesis that hydrogen excretion following lactulose is greater in red kidney bean fed rats. Methods: Lactulose breath test was compared at time 0, week 1 and week 2 in two groups of rats fed either standard rat chow (control) or chow supplemented with $24 \%$ powderized red kidney beans (RKB). To perform the lactulose breath test, $0.5 \mathrm{ml}$ lactulose was delivered into the stomach by gavage at time 0 . The concentration of hydrogen in the exhaled breath was then measured every 15 min for 180 min while the rat was housed in a 3-L air-tight collection chamber that was equipped for timed air exchange. Breath hydrogen concentration was plotted against time over the 180-minute monitoring period. Data were analyzed by applying paired t-test against peak hydrogen concentration (mean \pm SE). Results: 1. At baseline, breath hydrogen profiles were similar between control and RKB. 2. At week 1 , while the peak concentration was $45 \pm 16.6 \mathrm{ppm}$ in the control group, the value was $186 \pm 123 \mathrm{ppm}$ in the RKB $(\mathrm{p}<.17)$. At week 2 , while there was a peak concentration value of $35 \pm 9.5 \mathrm{ppm}$ for the control, there was a peak concentration value of $107 \pm 24 \mathrm{ppm}$ for the RKB ( $\mathrm{p}<.05)$. Conclusion: Higher hydrogen excretion was detected in response to lactulose in the RKB fed rats to suggest SIBO after two weeks of RKB feeding. Lactulose breath test may be used to detect SIBO in rats. This study was supported (in part) by NIH P50 AAl1999.

\section{T1186}

Proteomic Analysis of Potential Mechanisms Involved in the Lactobacillus Paracasei -Mediated Restoration of Enteric Muscle Dysfunction Induced By Trichinella Spiralis Infection

Gabriela E. Bergonzelli, Elena F. Verdu, Luisa V. Lopes, Andreas Fuerholz, Laure MarvinGuy, Irene Corthesy-Theulaz, Stephen M. Collins

Background \& Aim: We have shown that L. paracasei NCC2461 or its spent culture medium (SCM) improved post-T. spiralis (Tsp) neuromuscular dysfunction in part through modulation of the immune response to infection. Here we investigate by proteomic analysis, other potential targets involved in the restoration of post-infective (PI) muscle dysfunction mediated by SCM. Methods: Tsp infected mice received $100 \mu$ l of growth media (MRS) or SCM from day 10 to 21 post infection. Uninfected mice received $100 \mu \mathrm{l}$ MRS. Protein extracts of myenteric plexus longitudinal muscle from jejunum were analyzed by 2D gel electrophoresis. Spots were quantified and excised for protein identification by MALDI-TOF-MS and NanoLCESI-MS/MS. Results: Tsp infection altered the expression of 39 proteins. SCM treatment resulted in total** or partial* normalization of $15 / 39$ proteins involved in different pathways. The table shows the most relevant functional clusters. SCM treatment restored Tsp-induced alterations in NO-associated pathways (reflected by creatin kinase and arginase 1 normalization), cytoskeleton organization, stress response and immune modulation but did not counteract the parasite effect at the respiratory chain level. Conclusions: Proteomic analysis suggests that the SCM-mediated normalization of PI muscle dysfunction is achieved through the modulation of multiple proteins involved in filament organization and other components of excitation-contraction coupling. This approach may be useful to identify novel therapeutic targets in PI-IBS 


\begin{tabular}{|c|c|c|}
\hline Protein names and function & $\begin{array}{c}\text { Tsp-MRS vs Uninfected } \\
\text { Fold changes }\end{array}$ & $\begin{array}{c}\text { Tsp-SCM vs Uninfected } \\
\text { Fold changes }\end{array}$ \\
\hline \multicolumn{2}{|c|}{ Cytoskeleton organization / Muscle function } \\
\hline Alpha-actin** & -2.66 & -1.09 \\
\hline Actin-like protein & 1.42 & 1.26 \\
\hline Annexin A4** & -5.24 & 1.05 \\
\hline Creatin kinase M chain** & 2.47 & 1.53 \\
\hline Gelsolin** & -3.77 & -1.42 \\
\hline Muskelin* & -3.64 & -1.90 \\
\hline Lamin A** & 1.72 & 1.02 \\
\hline \multicolumn{2}{|c|}{ Stress response / immune function } \\
\hline Heat Shock cognate prot 71 kDa** & 2.78 & 1.97 \\
\hline Heat shock prot 27 kDa & 2.33 & 2.03 \\
\hline BAG molecular chaperon regulator** & -3.76 & 1.76 \\
\hline Proteasome activator complex** & -2.45 & 1.35 \\
\hline \multicolumn{2}{|c|}{ Mitochondrial respiratory chain } \\
\hline NADH-ubiquinone oxidoreductase 30 \\
kDa
\end{tabular}

benefit from probiotics treatment. Aim: to assess the efficacy of Lactobacillus paracasei F19 in patients with SUDD. Materials and Methods: Multicentric randomized controlled study on 43 pts, aged 47-78 years ( $15 \mathrm{M}, 28 \mathrm{~F}$ ) with SUDD, diagnosed by double contras enema and biochemistry to exclude acute inflammation. SUDD was defined as: presence of abdominal symptoms (pain $<24 \mathrm{~h}$ and bloating) for at least 6 months before enrollment. All pts had a validated detailed questionnaire for abdominal symptoms and functional dyspepsia (FD) including a visual analogic scale (VAS). Exclusion criteria were: complicated diverticular disease, previous colon surgery, antibiotics or laxatives in the 30 days before enrollment, use of NSAIDs. In all pts a daily intake of $30 \mathrm{~g}$ of dietary fibre (vegetables and fruit) was recommended. Pts were randomly assigned to receive: 1 sachet b.i.d of L. paracasei F19 (containing 12.5x109 viable bacteria and $750 \mathrm{mg}$ of gluco-oligosaccharides ) for 14 days/ month for 6 months (Group A, n=14), only fibre diet (Group B, n=13) or 1 sachet once daily of L. paracasei F19 for 14 days/month for 6 months (Group C, n=16). Endpoint of the study was to evaluate the decrease of abdominal pain and bloating after 6 months of treatment by VAS. Results are expressed as mean \pm SD. Results: At baseline, 40 pts $(93 \%)$ had both abdominal pain and bloating, whereas 3 pts had only bloating. 34 patients (14 M, 20 F, 79\%) completed the study: 1 patient (Group A) was withdrawn for diarrhea and 8 patients (3 from Group A, 4 from Group B, 1 from Group C) were lost at follow-up. Symptoms related to FD were minimal and not affected by any treatment. Conclusion: Patients with SUDD benefit of treatment with L. paracasei F19 since it significantly decreases the intensity of abdominal pain and bloating. The efficacy of higher dosage of probiotics is similar to that of lower dosage.

\begin{tabular}{|c|c|c|c|}
\hline VAS of bloating & Group A & Group B & Group C \\
\hline Baseline & $5.1 \pm 2.7$ & $5.6 \pm 2.2$ & $4.9 \pm 2.3$ \\
\hline 6 mos treatment & $2.3 \pm 2.6^{*}$ & $3.8 \pm 2.9$ & $2.9 \pm 2.3^{*}$ \\
\hline VAS of pain<24 h & & & \\
\hline Baseline & $4.6 \pm 2.3$ & $4.1 \pm 1.4$ & $4.8 \pm 2.9$ \\
\hline 6 mos treatment & $1.9 \pm 2.4^{*}$ & $3.0 \pm 1.3$ & $2.1 \pm 2.6^{*}$ \\
\hline
\end{tabular}

$* \mathrm{p}<0.05$

T1189

The Probiotic Formulation Lacidofil $\AA /$ Entercine ${ }^{\circledR}$ Prevents Stress-Induced Increase in Colonic Permeability and Sensitivity to Distension in Rats Helene Eutamene, Chantal Chabo, Stephanie Guggisberg, Lionel Bueno, Jean Fioramonti, Henri Durand, Bernard Fabre, Vassilia Theodorou

$\mathrm{T} 1187$

Safety and Tolerability of the Probiotic Organism Bifidobacterium Infantis 35624: Clinical Experience and Molecular Basis

Eamonn M. Quigley, Peter J. Whorwell, Fergus Shanahan, Douwe Vansinderen, Jun Xu, Linda Altringer, Liam O'Mahony, Francisco Guarner, Progid Investigators

Background: Evidence for probiotic efficacy in a variety of gastrointestinal disorders accumulates. While probiotics are generally regarded as safe, there have been few large-scale investigations of short-term tolerance or long-term safety and theoretical concerns have been raised regarding short-term tolerability in functional disorders and risk of systemic infections among those with impaired barrier function. Aim: To evaluate the tolerability and safety of the probiotic organism Bifidobacterium infantis 35624. Methods: Data from two randomised, placebo-controlled, double-blind trials were used in this analysis. A four-week dose-ranging study, (Bifidobacterium $10^{6}$ vs. $10^{8}$ vs. $10^{10}$ ) in subjects with irritable bowel syndrome (IBS) and a one-year study among subjects with active Crohn's disease and ulcerative colitis, were reviewed for evidence of short-term tolerability and long-term safety, respectively. The genome of the organism was also evaluated for evidence of genetic features of pathogenicity. Results: In the IBS study, 270 subjects were randomised to one of the three doses of the organism and 92 to placebo; 330 completed the study, including 243 on active treatment. A total of 17 subjects withdrew due to adverse events (AE's), 9 from the placebo group and 8 from the three treatment groups combined. The majority were occasioned by worsening of IBS symptoms. The overall incidence of all AE's was similar in the four groups at $48 \%$, $37 \%, 52 \%$ and $43 \%$ for placebo, $10^{6}, 10^{8}$, and $10^{10}$, respectively with the majority $(29 \%$, $37 \%, 28 \%$ and $24 \%$ ) being IBS-type gastrointestinal symptoms. The incidence of severe AE's adjudged as treatment related was highest in the placebo group at $9 \%$; rates for the three treatment groups were $0 \%, 1 \%$ and $2 \%$, respectively. In the inflammatory bowel disease (IBD) study, 32 patients with Crohn's disease and 50 with ulcerative colitis completed the study. No instances of systemic or major organ sepsis were recorded. From genome analysis it was apparent that Bifidobacterium infantis 35624 did not contain DNA that was homologous to known pathogenicity islands or transferable antibiotic resistance markers. Conclusions: Bifidobacterium infantis 35624 is well tolerated in the short term by patients with IBS and is not associated, in long-term therapy, in a susceptible population (IBD), with any evidence of risk for systemic sepsis. These clinical findings are supported by genome analysis

\section{T1188}

6 Months-Treatment of Symptomatic Uncomplicated Diverticular Disease (SUDD) of the Colon with Lactobacillus Paracasei F19 Rosario Cuomo, Giovanni Maconi, Francesco De Giorgi, Edith Lahner, Giovanni Sarnelli, Bruno Annibale

Background: Abdominal pain and bloating are frequent symptoms in SUDD, which are generally accepted to be related to functional disorders. Albeit no specific standard treatment for SUDD is accepted, guidelines of the American College of Gastroenterology suggest an high-fibre diet in patients with SUDD. Some observations suggest a possible role of gut microflora in determining symptoms related to SUDD, thus these patients may potentially

Background. Acute stress-induced increase in colonic paracellular permeability is responsible for rectal hypersensitivity in rats (Pain 2005, 113: 141-7). In previous studies, the probiotic formulation Lacidofil ®/ Entercine ${ }^{\circledR}$ (Lactobacillus rhamnosus Rosell 11 and Lactobacillus acidophilus Rosell 52) has been effective in preventing and/or alleviating the symptoms of various gastrointestinal disorders. This study aims to evaluate the effect of the probiotic formulation Lacidofil $囚 /$ Entercine ${ }^{\circledR}$ on hyperalgesia to rectal distension (RD) and increased colonic paracellular permeability (CPP) induced by an acute stress. Methods. Four groups of eight female Wistar rats received orally during 15 days either saline or the probiotic formulation (8.4 $10^{9} \mathrm{CFU} /$ day). In order to measure abdominal cramps reflecting viscera pain, rats were chronically equipped with electrodes implanted in the abdominal striated muscle. At the end of treatments, rats were submitted to a partial restraint stress (PRS) during $2 \mathrm{~h}$. Four hours before and 20 min after PRS, a RD was performed using a Fogarty probe inflated from 0 to $1.2 \mathrm{ml}$. CPP was determined in rats fitted with an intracolonic (IC) catheter. At day 0,10 and 15 (20 min post PRS), rats received ${ }^{51} \mathrm{Cr}$-EDTA $(250 \mu \mathrm{L} ; 0.7$ $\mu \mathrm{Ci}$ IC) and $\mathrm{CPP}$ was assessed by ${ }^{51} \mathrm{Cr}$-EDTA recovery in urines collected for $24 \mathrm{~h}$. Results. In basal conditions, RD significantly $(\mathrm{p}<0.05)$ increased the number of abdominal cramps from $0.8 \mathrm{ml}$ of RD. A similar nociceptive response was observed in Lacidofil $\circledast /$ Entercine $®$ treated rats compared with vehicle. PRS significantly $(\mathrm{p}<0.05)$ increased the number of abdominal cramps for all RD volumes applied reflecting colonic hypersensitivity. The probiotic formulation Lacidofil $₫ /$ Entercine ${ }^{\circledR}$ significantly $(p<0.05)$ reduced the increase in the number of abdominal cramps induced by PRS, up to basal level, for all RD volumes applied. In absence of stress, at day 0 and day 10 similar values of CPP were observed in Lacidofilß/ Entercine ${ }^{\circledR}$ and vehicle group $(0.85 \pm 0.19$ vs $0.91 \pm 0.09 ; 0.96 \pm 0.23$ vs $0.87 \pm 0.11 \%$ respectively). PRS significantly $(p<0.001)$ increased CPP $(2.95 \pm 0.11$ vs $0.91 \pm 0.09 \%)$. Lacidofil $® /$ Entercine ${ }^{\circledR}$ treatment suppressed the PRS-induced increase of CPP $(1.06 \pm$ 0.05 vs $2.95 \pm 0.11 \%$ ). Conclusion. A two-week treatment with Lacidofil $₫ /$ Entercine $§$ suppresses the stress-induced visceral hypersensitivity and increase of colonic paracellular permeability in rats, suggesting a potential beneficial role of this probiotic formulation in functional gastrointestinal diseases treatment.

\section{T1190}

Acute Metabolic Stress Activates Intermediate Conductance Potassium (IK) Channels in the Basolateral Membrane of Human Colonic Crypt Cells Arun Loganathan, John E. Linley, Jeremy P. Lodge, Malcolm Hunter, Geoffrey I. Sandle

Background: Intestinal ischaemia is associated with increased mucosal permeability, bacteria translocation and sepsis. Acute metabolic stress produced by 2 , 4initrophenol (DNP) and deoxyglucose (DG) activates large conductance $\mathrm{K}^{+}$channels in cardiovascular tissues, and small conductance $\mathrm{K}^{+}$channels in liver and biliary cell lines. A possible link between $\mathrm{K}^{+}$ channel activity and intestinal epithelial permeability has come from studies using cultured monolayers of the $\mathrm{T}_{84}$ colonic adenocarcinoma cell line, which showed that an acute increase in the basolateral membrane $\mathrm{K}^{+}$conductance produced by mastoparan (an activator of $\mathrm{G}$ protein-coupled $\mathrm{K}^{+}$channels) caused a 4 -fold increase in paracellular permeability. Aims To determine whether acute metabolic stress leads to activation of basolateral $\mathrm{K}^{+}$channels in human colonic crypts. Methods: With ethics committee approval and informed consent, distal colonic biopsies were obtained from patients undergoing routine colonoscopy as part 
of the investigation of altered bowel habit. In all cases, the colonic mucosa was normal macroscopically and histologically. Intact crypts were isolated by $\mathrm{Ca}^{2+}$ chelation (Bowley KA et al. Gut 52,854-860,2003). Cells were exposed to $100 \mu \mathrm{M}$ DNP + 5mM DG to produce metabolic inhibition. Whole-cell $\mathrm{K}^{+}$currents were measured using the perforated patchclamp technique $(0.24 \mathrm{mg} / \mathrm{ml}$ amphotericin in pipette), and single channel activity in the basolateral membrane was studied in the cell-attached configuration. Results: $100 \mu \mathrm{M}$ DNP $+5 \mathrm{mM}$ DG activated whole-cell currents within 5 minutes. This response was associated with an increase in whole-cell conductance from $1.28 \pm 0.14 \mathrm{nS}$ to $2.60 \pm 0.17 \mathrm{nS}(\mathrm{P}<0.01$; $\mathrm{n}=12$ ), and hyperpolarization of the cell membrane voltage from $-72 \pm 3 \mathrm{mV}$ to $-83 \pm 3 \mathrm{mV}$ $(\mathrm{P}<0.025)$, consistent with activation of a $\mathrm{K}^{+}$current. In single channel recordings, $100 \mu \mathrm{M}$ $\mathrm{DNP}+5 \mathrm{mM}$ DG activated intermediate conductance (30pS, IK) $\mathrm{K}^{+}$channels, channel activity (expressed as N.Po, where $\mathrm{N}=$ number of channels in patch, and $\mathrm{Po}=$ single channel open probability) increasing from $0.58 \pm 0.2$ to $0.81 \pm 0.3(\mathrm{P}<0.05, \mathrm{n}=10)$. There was no evidence of activation of either large conductance (BK) or small conductance (SK) $\mathrm{K}^{+}$channels, both of which are normally present in the basolateral membrane of human colonic crypts. Conclusion: Metabolic stress activates basolateral $\mathrm{K}^{+}(\mathrm{IK})$ channels in human colonic crypts. If additional studies link increased $\mathrm{K}^{+}$channel activity with changes in paracellular permeability, specific $\mathrm{K}^{+}$channel blocking drugs may provide a way of preventing the increase in paracellular permeability, thereby decreasing the risk of sepsis in patients with intestinal ischaemia.

\section{$\mathrm{T} 1191$}

The Lambda ( $\lambda$ ) Isoform of PKC Is a Unique Modulator of Inducible NO Synthase (iNOS) Signaling and Is Critical to Monolayer Disruption By Oxidative Stress: A Novel Pro-Inflammatory Mechanism in Intestinal Epithelium

Ali Banan, Lijuan Zhang, Maliha Shaikh, Jeremy Z. Fields, Ashkan Farhadi, Christopher B. Forsyth, Ali Keshavarzian

iNOS upregulation \& gut barrier disruption are key to the pathogenesis of inflammatory GI disorders. Using monolayers of intestinal cells, we found that oxidants upregulate NO production (by activating iNOS) \& lead to disruption of cytoskeletal $\&$ barrier integrity. But, the mechanisms are unclear. Since the atypical $\lambda$ isoform of PKC is key to cell instability, we investigated whether PKC- $\lambda$ is crucial in oxidant-induced iNOS activation/NO overproduction, and cytoskeletal oxidation \& barrier disruption. Methods: We used innovative GI clones stably transfected to overexpress PKC- $\lambda$ or to inhibit its expression. Clones were incubated \pm oxidants $(\mathrm{H} 2 \mathrm{O} 2, \mathrm{HOCl}, \mathrm{ONOO})$ or PKC activators or inhibitors. Wild type (WT) cells were treated similarly. We monitored monolayer barrier integrity \{fluorometry\}, cytoskeletal integrity \& oxidation \{laser confocal microscopy, SDS-PAGE fractionation\}, PKC- $\lambda$ subcellular activity \{immunoprecipitation, in vitro kinase\}, iNOS activity \& expression \{scintillation, PAGE\}, NO levels \{chemiluminescence\}, oxidative stress (quenchable fluorescence $[\mathrm{QF}]$ ), polymerized \& monomeric actin (43 kDa subunits of actin, SDS-PAGE), $\mathrm{n}=6$ / grp. Results: $\{$ I $\}$ Relative to WT cells exposed to vehicle, transfected cells overexpressing (3.2 fold) PKC- $\lambda$ or WT cells incubated with oxidant exhibited oxidative damage as indicated by: PKC- $\lambda$ activation, iNOS upregulation, NO over-production, oxidative stress (increased $\mathrm{QF}$ ), actin nitration $\&$ oxidation, actin disassembly (decreased polymerization, increased disassembly), actin cytoarchitectural instability \& monolayer barrier disruption. \{II\} All measures of oxidative injury induced by $\mathrm{PKC}-\lambda$ overexpression were synergistic with oxidant effects. Most of the overexpressed PKC- $\lambda$ resided in membrane/cytoskeletal fractions ( $<8 \%$ in cytosolic fractions), indicating constitutive activity. In both transfected clones \& oxidanttreated WT cells, PKC- $\lambda(74 \mathrm{kDa})$ was active $\&$ found with iNOS (130 kDa), forming endogenous PKC- $\lambda$ iNOS complexes. \{III\} Dominant mutant transfection to inhibit native PKC- $\lambda$ expression $\&$ activity ( $99.9 \%)$ prevented all measures of oxidation $\&$ inflammation such as iNOS/NO \& monolayer instability. Conclusions: We report for the first time that: 1) Activation of PKC- $\lambda$ is necessary for cellular injury and oxidative stress of iNOS \& NO pathway activation; 2) PKC- $\lambda$ is a unique intracellular modulator of iNOS signaling in intestinal cells; 3) We have identified a novel proinflammatory mechanism - disruption of barrier \& cytoskeletal integrity through the upregulation of iNOS / NO signaling - among the PKC family of isoforms. \{Supported by NIDDK60511 \& NCCAM01581; NIAAA13745\}.

\section{T1192}

Role of Thrombin-Protease Activated Receptor 1 (PAR1) Pathway in Intestinal Ischemia-Reperfusion in Rats

Hisato Tsuboi, Yuji Naito, Takahiro Suzuki, Kouichi Terao, Hirokazu Kajikawa, Toshimitsu Okuda, Takeshi Hattori, Nami Nakabe, Tomohisa Takagi, Osamu Handa, Satoshi Kokura, Hiroshi Ichikawa, Norimasa Yoshida, Toshikazu Yoshikawa

Background: CXC chemokines including human interleukin-8 (IL-8) and rat cytokine induced neutrophil chemoattractant-1 (CINC-1) play a crucial role in the pathogenesis of intestinal injury induced by ischemia-reperfusion(I/R). Thrombin and its specific receptor, protease-activated receptor 1 (PAR1) act as important players in inflammation. However, the association between thrombin activation and chemokine production during $\mathrm{I} / \mathrm{R}$ is not well studied. Aims: We investigated whether thrombin and PARl might be involved in the pathophysiology of intestinal $\mathrm{I} / \mathrm{R}$, using in vitro and in vivo models. Materials and methods: (in vitro model) Human umbilical vein endothelial cells (HUVEC) were subjected to hypoxia in a Plexiglas chamber for $2 \mathrm{~h}$. Reoxygenation was initiated in an environment of normoxia for $8 \mathrm{~h}$. IL- 8 production was assayed by ELISA. The activation of nuclear factor- $\mathrm{KB}$ (NF$\kappa \mathrm{B})$ was determined by electrophoretic mobility shift assay. (in vivo model) Intestinal damage was induced by clamping the superior mesenteric artery for $30 \mathrm{~min}$ followed by reperfusion in male Wistar rats. ATIII was given to the rats intravenously just before the vascular clamping. Thrombin-antithrombin complex (TAT) was measured as indicator of thrombin activity. PARI was evaluated by real time-PCR and western blot. Tissue-associated myeloperoxidase (MPO) activity was measured in intestinal mucosa as index of neutrophil infiltration. The intestinal protein concentrations and mRNA levels of CINC-1 were determined by ELISA and real time-PCR. Results: Hypoxia-reoxygenation induced the production of IL-8 from HUVEC. Treatment with thrombin markedly enhanced the IL-8 production after reoxygenation, and ATIII blocked these enhancements by thrombin. Reperfusion after ischemia resulted in increase in luminal protein and hemoglobin concentrations. Reperfusion significantly increased the serum levels of TAT and enhanced the expression of PARl in the intestinal mucosa. Treatment with ATIII inhibited these increases after reperfusion in a dose-dependent manner $(1-30 \mathrm{U} / \mathrm{kg})$. The increase in MPO activity after reperfusion was significantly inhibited by treatment with ATIII. A profound expression of transcripts cording CINC-1 was detected in the course of reperfusion and ATIII significantly reduced protein and mRNA levels. After I/R, DNA binding activities of NF- $\mathrm{\kappa B}$ were markedly increased, whereas in ATIII treated intestines these effects were significantly reduced. Conclusion: These results suggest that thrombin-PARl pathway play an important role in the production of CINC-1 during $\mathrm{I} / \mathrm{R}$, and that ATIII has potent anti-inflammatory effects on this injury via inhibition of proinflammatory cytokines

\section{T1193}

Novel Action of Ptca Compared with Cysteine/GSH Prodrugs Against Colitis in Murine Model

Helieh S. Oz, Theresa Chen, Willem J. de Villiers, Jian Zhong, Herbert Nagasawa

Oxidant-mediated injury plays an important role in the pathophysiology of inflammatory bowel disease. Recently, we showed that antioxidants modulated colitis in mice. In this study, we further evaluated the protective effects of L-Cysteine and GSH prodrugs against progression of colitis in a murine model. Methods: Male ICR mice were fed above compounds incorporated into chow as follow: Group (A) received L-cysteine-glutathione mixed sulfide (CySSG), a ubiquitous GSH derivative present in mammalian cells and suggested to be a depot form of GSH. Group (B) was provided D-ribose-L-cysteine (RibCys) a cysteine prodrug that releases in vivo L-cysteine. Group (C) was given (RS)-n-propylthiazolidine-4(R)-carboxylic acid (PTCA) another cysteine prodrug involved in glutathione (GSH) biosynthesis. Group (D) was fed chow supplemented with the vehicle. After 3 days, animals were further provided with normal drinking water or water supplemented with $3.5 \%$ DSS, and the progression of colitis was assessed in each group. Results: Mice administered with DSS developed severe colitis with decreased hematocrit and weight loss. These parameters were improved with prodrugs therapy, PTCA being superior to the others. Colonic lesions (scored $0-4)$ significantly improved in treated animals with PTCA and RibCyc and to a less extend with CySSG therapy. Hepatic reduced GSH was depleted in colitis animals (control 6530+318 vs DSS $4814+298 \mathrm{nmol} / \mathrm{g} \mathrm{p}<0.001$ ) and normalized with prodrug therapies (control vs treatments $\mathrm{p}>0.05$ ). Protein expression of Serum amyloid A, inflammatory cytokines (TNF, IL-6 and IL-12) were significantly increased in colitis animals and improved with therapies. Immunohistochemistry showed significant upregulation of Cox-2, and CD68 expression (macrophage specific marker) suggesting macrophage activation and infiltration in the colonic lamina propria in colitis animals. These abnormalities were attenuated in prodrugs treated mice. Conclusions: PTCA, RibCyc and to a lesser extend CySSG provided protection against colitis in this murine model, further supporting a possible therapeutic application in IBD patients. These data strongly support our previous report on the novel action of PTCA against colitis

\section{T1194}

Metabonomic Investigation to Study Gut and Systemic Responses to Stress Doris M. Jacobs, Lionel Tornier, Ziad Ramadan, Sunil Kochhar, Carmen Alonso, Javier Santos, Mar Guilarte, Maria Vicario, Maria Antolin, Esteban Saperas, Juanramon Malagelada

Epidemiological, clinical and experimental evidence link life stress with the initiation, reactivation and perpetuation of gut mucosal inflammation although underlying biochemical events are still poorly understood. Aim \& Methods: To gain knowledge on the mechanisms involved in stress-induced inflammation in the human intestine, we have investigated metabolite changes in biofluids from 27 healthy volunteers ( 15 women and 12 men, average age of 23.3 \pm 2.5 ) during intestinal jejunal perfusion, in basal conditions and after the exposure to hand-cold pain stress for 15 minutes. Plasma and urine samples were collected at different time-points (Figure 1). One-dimensional 1H NMR spectra were recorded on a Bruker DRX600 NMR spectrometer operating at a proton NMR frequency of $600.13 \mathrm{MHz}$ and at a temperature of $300 \mathrm{~K}$. For plasma, Carr-Purcell-Meiboom-Gill (CPMG) spectra with the spin echo sequence (D[- $90^{\circ}$-(t-180 $\left.\left.-\mathrm{t}\right) \mathrm{n}-\mathrm{FID}\right)$ and the spin echo loop time of $128 \mathrm{~ms}$ were additionally acquired. Results: Cold stress pain induced time-dependent metabolic changes in plasma primarily in the glucose-alanine cycle and in anaerobic metabolism. The major metabolite changes in urine were citrate, formate, acetate, lactate, alanine suggesting a perturbed energy metabolism (Figure. 2). The accumulation of hypoxanthine in urine indicates oxygen depletion, which may be associated to an imbalance between oxidative stress and the defense mechanisms. Conclusion: Further in-depth analysis of the metabolite changes using metabonomics approach is needed to understand the underlying mechanism of stressinduced changes in gut functioning and its physiology.

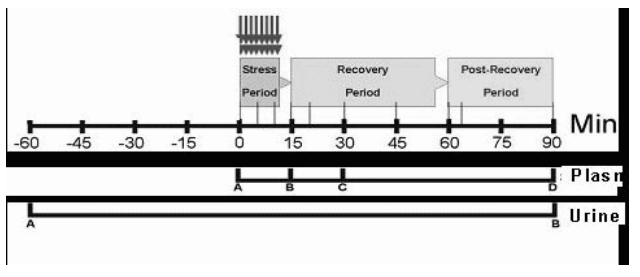

Experimental design 


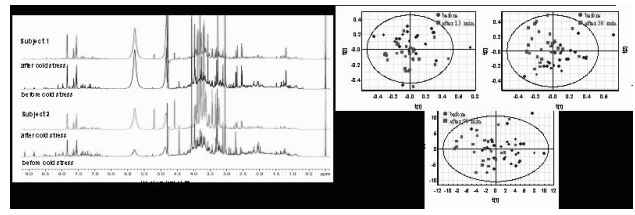

Analysis of plasma and urine samples before and after stress. Left panel, Urine 1H NMR fingerprints before and after stress from 2 randomly selected subjects. Right panel, pattern recognition by principal component analysis (PCA) of the plasma $1 \mathrm{H}$ NMR data

\section{T1195}

Calcium-Dependent Activation of Signaling Kinases Mediates the Inhibitory Effect of Hydrogen Peroxide On Colonic Epithelial Ion Transport Kim E. Barrett, Linh Pham, Declan F. Mccole

Background: Hydrogen peroxide $\left(\mathrm{H}_{2} \mathrm{O}_{2}\right)$ contributes to intestinal inflammation and dysfunction in inflammatory bowel disease (IBD). One feature of IBD is the decreased ability of the intestinal epithelium to transport ions. We have previously shown that $\mathrm{H}_{2} \mathrm{O}_{2}$ inhibits carbachol (CCh) stimulated, $\mathrm{Ca}^{2+}$-dependent $\mathrm{Cl}^{-}$secretion across $\mathrm{T}_{84}$ colonic epithelial cells, via a phosphatidylinositol-3 kinase (PI3-K) and extracellular signal-regulated kinase (ERK) dependent mechanism. As $\mathrm{H}_{2} \mathrm{O}_{2}$ has been shown to activate intracellular $\mathrm{Ca}^{2+}$ release, this study investigated the role of $\mathrm{Ca}^{2+}$ in the ability of $\mathrm{H}_{2} \mathrm{O}_{2}$ to activate signaling pathways that negatively regulate ion transport. Methods: Protein phosphorylation was measured in $\mathrm{T}_{84}$ colonic epithelial cell monolayers by Western blot, and ion transport responses were measured in Ussing chambers. Results: We have previously shown roles for ERK and the soluble tyrosine kinase, Src in the inhibitory effect of $\mathrm{H}_{2} \mathrm{O}_{2}$ on ion transport responses to $\mathrm{CCh}$. Pre-incubation of $\mathrm{T}_{84}$ cells with the intracellular $\mathrm{Ca}^{2+}$ chelator, BAPTA-AM $(20 \mu \mathrm{M})$, completely blocked $\mathrm{H}_{2} \mathrm{O}_{2}$-induced phosphorylation of ERK and $\operatorname{Src}(\mathrm{p}<0.001$, $\mathrm{p}<0.05$ respectively; $\mathrm{n}=5$ ). In addition, the $\mathrm{Ca}^{2+}$-dependent soluble kinase, Pyk-2, was also activated by $\mathrm{H}_{2} \mathrm{O}_{2}$ in a time-dependent manner, similar to the timecourse of Src activation $(\mathrm{p}<0.01 ; \mathrm{n}=$ 4). $\mathrm{H}_{2} \mathrm{O}_{2}$ activation of PI3-K signaling, as measured by phosphorylation of the downstream target, Akt1, was also significantly blocked by BAPTA-AM ( $<<0.05, \mathrm{n}=4)$. We previously observed that p38 MAPK is activated by $\mathrm{H}_{2} \mathrm{O}_{2}$. The p38 inhibitor, SB203580 $(10 \mu \mathrm{M})$, reversed $\mathrm{H}_{2} \mathrm{O}_{2}$ inhibition of $\mathrm{CCh}(100 \mu \mathrm{M})$-stimulated $\mathrm{Cl}^{-}$secretion $(\mathrm{p}<0.05, \mathrm{n}=5)$, and inhibited $\mathrm{H}_{2} \mathrm{O}_{2}$-induced Aktl phosphorylation, indicating that p38 lies upstream of PI3-K in the regulatory pathway $\left(\mathrm{p}<0.01 ; \mathrm{n}=3\right.$ ). Moreover, $\mathrm{H}_{2} \mathrm{O}_{2}$-induced $\mathrm{p} 38$ phosphorylation was partially reversed in the presence of BAPTA-AM $(p<0.01 ; n=6)$, indicating a prominent role for $\mathrm{Ca}^{2+}$ signaling in $\mathrm{p} 38$ activation by $\mathrm{H}_{2} \mathrm{O}_{2}$. Conclusions: Activation of $\mathrm{Ca}^{2+}$-dependent regulatory kinases by $\mathrm{H}_{2} \mathrm{O}_{2}$ reduces the ability of colonic epithelial cells to secrete chloride. These data increase our understanding of mechanisms involved in the suppression of colonic epithelial ion transport by inflammatory mediators associated with IBD.

\section{T1196}

Redox Mechanisms in the Pathogenesis of Cysteamine-Induced Duodenal Ulcers: Role of Iron in Organ-Specific Tissue Injury

Tetyana Khomenko, Joanna A. Bernstein, Xiaoming Deng, Klara Osapay, George Osapay, Gordon D. McLaren, Hamid M. Said, Sandor Szabo

Cysteamine (C), a reducing aminothiol, reacts with transition metals to generate $\mathrm{H} 2 \mathrm{O} 2$, which can oxidize reduced metals and produce hydroxyl radicals $(* \mathrm{OH})$ through the Fenton reaction. C specifically induces ulcers in rat proximal duodenum where most dietary iron absorption takes place. We hypothesized that iron may play a role in redox reactions involved in the pathogenesis of C-induced duodenal ulcers. Methods: Non-fasting rats were given either saline or $\mathrm{C}-\mathrm{HCl}(25 \mathrm{mg} / 100 \mathrm{~g})$ by gavage: once and euthanized 1, 2 hr later, twice and euthanized after $6 \mathrm{hr}$, or three times at $4 \mathrm{hr}$ intervals and euthanized 12, $24 \mathrm{hr}$ later. Duodenal mucosal oxygenation and redox status in rats at 2 and $12 \mathrm{hr}$ after $\mathrm{C}$ were measured using oxygen and redox electrodes. The production of hydroxyl radicals $(* \mathrm{OH})$ in duodenal mucosa was detected by fluorescence intensity of hydroxyphenyl fluorescein (HPF). The amount of $\mathrm{C}$ in duodenal, gastric, jejunal, colonic mucosa or liver was determined by HPLC. We also measured [C14]C uptake by isolated duodenal, gastric, jejunal and colonic enterocytes, and by IEC-6 and Caco-2 cells. Results: C administration reduced pO2 (vs. baseline) by $18-22 \%$ and significantly increased the redox status in the rat proximal duodenal mucosa. The production of $* \mathrm{OH}$ in duodenal mucosa of rats treated with $\mathrm{C}$ was increased from $9085 \pm 12$ in controls to $10069 \pm 68(\mathrm{p}=0.007)$ measured by fluorescence intensity. A detectable level of $\mathrm{C}$ was found by HPLC assay $2 \mathrm{hr}$ after $\mathrm{C}$ administration in duodenal mucosa $(0.018 \mathrm{ug} / \mathrm{mg}$ wet weight) and gastric mucosa $(0.004 \mathrm{ug} / \mathrm{mg})$, but not in jejunum, colon or liver. A similar amount of $C$ was detected in duodenal mucosa of rats given $C$ by gavage or subcutaneously. [C14]C uptake by isolated duodenal, gastric, jejunal and colonic enterocytes, and by IEC- 6 and Caco-2 cells increased linearly with time. [C14]C uptake by all cells studied was $\mathrm{pH}$ dependent, with lower uptake at $\mathrm{pH} 5.0$ and higher uptake in more alkaline buffer. Unlabeled C (1 mM) markedly inhibited [C14]C uptake by all cells. Overloading IEC-6 with iron (100 uM for $1 \mathrm{hr}$ ) accelerated uptake of [C14]C by $40 \%$, but incubation of cells with deferoxamine (100 or $200 \mathrm{uM}$ for $24 \mathrm{hr}$ ) inhibited C uptake by $30 \%$ and $61 \%$, respectively. Conclusions: 1) C induced local hypoxia, changed redox state and generated the production of hydroxyl radicals in rat proximal duodenum. 2) Absorption of $\mathrm{C}$ was mostly specific to the duodenal mucosa. 3) Absorption of $\mathrm{C}$ may be related to a carrier-mediated system of $\mathrm{C}$ uptake with high uptake in alkaline $\mathrm{pH}$ and dependence on iron. 4) Thus, iron-catalyzed redox reactions may play a key role in the pathogenesis of Cinduced organ-specific tissue injury
T1197

Alternative Method to Identify Susceptibility Genes for Crohn's Disease Through Transcriptome Analysis

Debby Laukens, Harald Peeters, Filip de Keyser, Eric Veys, Claude Cuvelier, D.R. Elewaut, Erik Remaut, Martine de Vos, Pieter Rottiers

Objective Crohn's Disease (CD) is a multifactorial disorder in which both genetic and environmental factors are involved. In general, molecular genetic approaches currently used to identify susceptibility factors for $\mathrm{CD}$ are hampered by variability in phenotype, genetic heterogeneity across populations, uncontrolled environmental influences and limited statistical power. Although the prospects for success have improved markedly, gene discovery in $\mathrm{CD}$ might require additional evidence. We integrated the growing knowledge of susceptibility loci to expression variation. Methods We compared basal gene expression levels in noninflamed colon biopsies from $16 \mathrm{CD}$ and 11 control patients using a focus microarray chip and looked for variation in baseline expression level of genes that are located near one of the known $C D$ linkage markers. We focused on a cluster of highly related genes, called metallothioneins (MT's), located near the IBD1 locus. Quantitative real-time PCR (QPCR) was used to specifically study the expression of different MT isoforms in an independent population of $\mathrm{CD}$ and controls. The induction of MTIK in peripheral blood mononuclear cells (PBMC's) isolated from CD and controls was compared using Zn, oxidative stress and several cytokines. The complete MTIK gene, and the promoter and coding regions of the main transcriptional regulator of metallothioneins, MTF-1, were screened for mutations. Results We identified 13 genes that differ in baseline expression and are located within CD loci, of which 5 have so far been verified by QPCR. Three isoforms of MT specifically show a decreased expression at basal level in colon biopsies of CD patients, as assessed by QPCR ( $<<0.01)$. This lowered expression level was also seen in PBMC's of CD patients, indicating a genetic aberration. Interestingly, the expression level greatly depends on disease location: the reduced MT expression observed in non-inflamed colon biopsies is more pronounced in $C D$ patients who have primarily a history of colitis. MTIK induction by Zn and cytokines was not impaired in $C D$, but the response to oxidative stress was significantly higher $(\mathrm{p}<0.005)$. No coding polymorphisms in MTIK were identified. Two missense mutations in MTF-1 were found in 7 of 95 patients, and we are currently evaluating the occurrence of a promoter polymorphism in $\mathrm{CD}$. Conclusions Microarray screening can be useful in identifying candidate susceptibility genes in $\mathrm{CD}$. We provide evidence that MT expression in $\mathrm{CD}$ is genetically determined, and we put forward potential candidate genes for mutation screening.

\section{T1198}

The Role of Amino Acid-Rich Preservation Solution in Alleviating Intestinal Ischemia-Reperfusion Injury in a Large Animal Model

Thomas Churchill, Payam Salehi, David Bigam

Introduction: Ischemia-reperfusion injury is a major determinant limiting the success of small bowel (SB) transplantation. Over the past few years, our lab has clearly revealed the effectiveness of an amino acid-based preservation solution (which is specifically tailored to the metabolic requirement of SB) in alleviating mucosal ischemia-reperfusion injury in ex vivo and in vivo rodent models. This study was designed to examine the role of this novel solution in ameliorating SB ischemia-reperfusion injury in a large animal model to bridge animal data to potential therapy protocols in the clinic. Methods: Under general anesthesia SB of Landrace-Yorkshire pigs (20-24 kg) (N=12) were randomly assigned for luminal pretreatment with $\sim 2$ liter of the following solutions: Group 1 - none (control); Group 2 University of Wisconsin (UW) solution; Groun 3 - an amino acid (AA) based solution. Total warm ischemia (WI) was induced via superior mesenteric artery occlusion using a vascular clamp, after $60 \mathrm{~min}$ the occlusive clamp was removed and reperfusion was monitored over $180 \mathrm{~min}$. Energetics, oxidative stress and histology were assessed at 0, 15, 30, 60, 90, 120 and 180 min reperfusion. Results: ATP and ADP were markedly better preserved in Group 3 throughout the entire reperfusion period ( $\mathrm{p}<0.05$, compared to control and Group 2). Group 3 also showed significantly greater ATP/AMP ratio compared to Groups 1 and 2 over the first $60 \mathrm{~min}$ of reperfusion ( $<<0.05$ ). After $60 \mathrm{~min}$ WI, Group 3 exhibited glutathione levels $\sim 40 \%$ greater than Groups 1 and 2 (13.1 vs 8.4 and $8.4 \mu \mathrm{mol} / \mathrm{g}$, respectively, $\mathrm{p}<0.05$ ). Group 3 glutathione (GSH) levels were up to 2.5-fold greater than corresponding values in Groups 1 and 2 after 60, 120 and 180 min reperfusion $(p<0.05)$. Malondialdehyde (index of lipid peroxidation) in all groups were inversely correlated with GSH values at all timepoints. After $60 \mathrm{~min} \mathrm{WI}$, Groups 1 and 2 showed $30 \%$ increase in tissue MDA contents; levels in Group 3 remained unchanged compared to freshly isolated tissues. Histology indicated superior preservation of mucosal architecture in amino acid treated tissues (Group 3) after ischemia and subsequent reperfusion. Conclusion: The strategy of administering a luminal flush with the amino acid-rich preservation solution improves overall SB quality when tested in a large animal model of ischemia-reperfusion. Applying an intra-luminal flush of small bowel with AA solution on the 'back table' after a common intravascular flush as part of multi-visceral procurement may be a practical way to minimize preservation injury

\section{T1199}

Stable Gastric Pentadecapeptide Bpc 157 Studied for Inflammatory Bowel Disease (PLD116, PL14736, Pliva) Inhibits Thromb Formation Following Abdominal Aorta Anastomosis in Rat

Masa Jasprica, Predrag Sikiric, Sven Seiwerth, Lovorka Batelja, Alenka Boban Blagaic, Miroslav Gjurasin, Leonardo Patrl

Abdominal aorta surgery is regularly complicated by blood cloth obstructed the blood flow Stable gastric pentadecapeptide BPC 157 (now studied for inflammatory bowel disease (PLD116, PL14736, Pliva)) protects endothelium, promotes angiogenesis and modulates NO-synthesis (Life Sci 54:PL63-68, 1994, European J Pharmacol 332, 23-33, 1997, J Physio $93,479-485,1999)$. Thereby, the focus was on injured abdominal aorta in rats, and we show that BPC 157 affects thrombosis and other vascular changes presented after terminoterminal abdominal aorta anastomosis. Methods. In deeply anaesthetized male Wistar rats

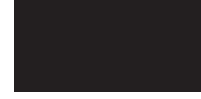


(400-500 g), aortal segment between the renal and common iliac arteries was clamped and cut, and a termino-terminal anastomosis performed using Prolene 9-0 sutures, and blood flow restored, ascertained by palpable pulse on distal aorta. Then, at the site of anastomosis, $\mathrm{l} \mathrm{ml}$ bath of gastric pentadecapeptide BPC 157 (dissolved in saline, 0/ml, $2 \mathrm{pg} / \mathrm{ml}, 2 \mathrm{ng}$ / $\mathrm{ml}, 2 \mu \mathrm{g} / \mathrm{ml}$ ) was given, and the assessment micro-scopically (program SFORM, VAMSTECHSoftware Company, Croatia) was 24 hours thereafter, and compared with the values obtained in corresponding healthy rats. Results. Regularly, following abdominal aorta anastomosis controls present with thromb formed at the anastomotic site, and almost no blood flow in blood vessel with apparently narrowed diameter. Contrasting with the findings in thrombotic controls, gastric pentadecapeptide BPC 157 shows a dose dependent effect, with a clear effectiveness in rats treated with $\mu$ g-regimen. As shown in Fig. 1, these pentadecapeptide BPC 157 rats present only a thrombotic ring at the site of the anastomosis, along with preserved blood flow. Also, blood vessel diameter in gastric pentadecapeptide BPC $157 \mu \mathrm{g}$ group being at the range of values noted in the healthy rats, is preserved, and much larger than in thrombotic controls. Conclusion. Together, an inhibition of all events related to abdominal aorta anastomosis is along with this pentadecapeptide BPC 157 as an agent known to protect mucosa, endothelium, and to modulate NO-system. Likewise, with respect to virtually no toxicity in clinical studies, these findings could be likely relevant for further therapy applications.

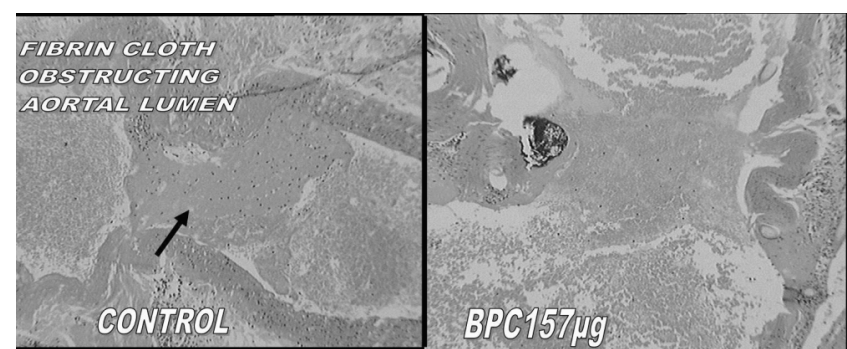

T1200

Stable Gastric Pentadecapeptide BPC 157 (BPC157) in Trials for Inflammatory Bowel Disease (PL-10, PLD-116, Pliva, Croatia). Heals Ileoileal-Anastomosis and Counteracts Corticosteroid-Negative Effect in Rat

Tihomir Vuksic, Predrag Sikiric, Sven Seiwerth, Bozo Radic, Robert Klicek, Luka Brcic

Gastric pentadecapeptide BPC 157 (BPC157) is an anti-ulcer agent is one of the promising agents in clinical trials for inflammatory bowel disease. More effective in ulcer and various wounds both locally and systemically (perorally, parenterally), unlike other peptides it is stable in human gastric juice, and used without carrier. Importantly, it competes corticosteroid-negative aspects. Thereby, it may be one of few pharmacological agents that could be also effective in inflammatory bowel disease complications, that follow small bowel resection and ileoileal anastomosis. Methods. Anastomotic healing was assessed in rats (at $1,2,3,4,5,6,7,14$ day after ileoileal anastomosis) by (i) ahddesions (0-7, (neighboring loops, stomach, liver "packed")), loop diameters (at $2 \mathrm{~cm}$ orally and aborally), anastomosis arcade vessels (between $10 \mathrm{~cm}$ orally and $10 \mathrm{~cm}$ aborally),(ii) the time (sec), the volume (ml)(infunded through syringe-perfusion pump system (Argus 600,Argus Medical A6) $\mathrm{lml} /$ $10 \mathrm{sec}$ ) and the pressure $(\mathrm{mmHg})$ to leak induction (catheter (BD Careflow 5Fr $200 \mathrm{~mm}$, Becton Dickinson, USA) connected with chamber (BD Gabarith PMSET IDT-XX, Becton Dickinson, USA) and monitor Sirecust 732 (Siemens, Germany) at $10 \mathrm{~cm}$ proximal to anastomosis, and (iii) microscopically. Treatment was once daily (first after surgery last at

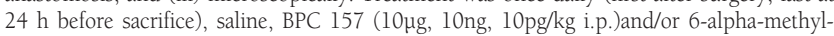
prednisolone (1 mg/kg i.p.).Results. BPC 157 significantly improves the all parameters of anastomotic wound healing. Besides, the low dose of pentadecapeptide BPC 157 that had no effect on those parameters by itself, became effective confronted with corticosteroids treatment that adversely affects healing of colonic anastomoses in the rat. Conclusion. the pentadecapeptide BPC 157 effect could be quite specific considering the all aspects of ongoing process in anastomosis healing.

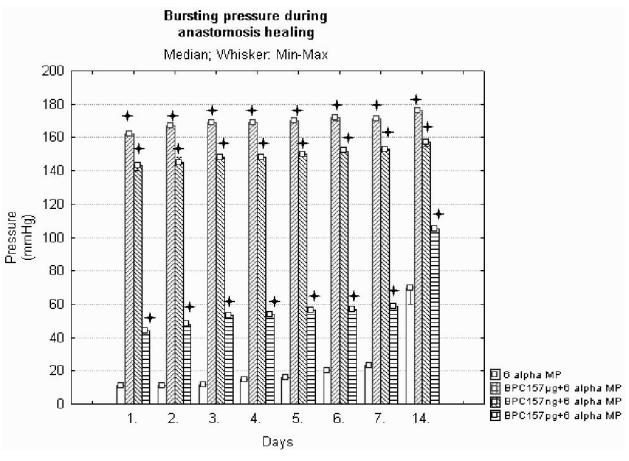

T1201

Hepatic and Mesenteric Steal Shown with Dynamic Angiography Relates to Ischemia in Non-Corresponding, Unaffected, Bowel Regions in Patients with Isolated CA or SMA Stenosis

Renzo Veenstra, Robert H. Geelkerken, Dick G. Gerrits, Ad B. Huisman, Jeroen J

Kolkman

Introduction Gastric exercise tonometry (GET) is the only clinically available method to detect GI ischemia. In patients with SMA stenoses, the GET may remain normal because of isolated small bowel ischemia. Moreover, mesenteric steal syndromes have been described which may lead to ischemia in the area of the unaffected vessel (f.e. gastric ischemia in SMA stenosis). In our cohort of chronic splanchnic syndrome (CSS) pts in whom gastric and jejunal tonometry was performed, we studied the relation between CA and SMA stenoses, and ischemia in stomach and small bowel, as well as dynamic angiography. Methods 220 pts were studied with gastric and jejunal tonometry, using dynamic angiography. In contrast to standard angiography, we studied the timing of appearance of contrast in the CA and SMA, and its collateral filling patterns. Results In 47 pts ( $25 \mathrm{M}, 22 \mathrm{~F})$ a single vessel stenosis was found, (43 CA, 4 SMA). Tonometry was false negative in 1 pt $(2 \%)$. Ischemia in the corresponding bowel region was seen in $83 \%(n=39)$, in $49 \%(n=23)$ in the corresponding area alone, in $34 \%(n=16)$ in both areas. In $15 \%(n=7)$ ischemia was only seen in the noncorresponding area. On angiography collaterals between CA and SMA were seen in 6/7 pts with non-corresponding ischemia and in $12 / 16$ pts with combined ischemia. Noncorresponding ischemia In all CA stenosis with small-bowel ischemia, an insufficient flow in the SMA was seen corresponding with rapid retrograde filling of the hepatic artery, suggesting 'hepatic steal'. In 1 of 2 pts with SMA stenosis and gastric ischemia, a similar defect in the CA was seen with rapid collateral filling of the SMA suggesting 'mesenteric steal'. Combined ischemia In 4 of 16 pts with combined ischemia, a pattern suggesting hepatic steal was seen. Conclusions This study shows that a single stenosis of either CA or SMA can lead to ischemia in non-corresponding bowel regions due to mesenteric and hepatic steal mechanisms. The latter is rather common and can be demonstrated using dynamic angiography with emphasis on the first phase of arterial filling and the distribution of contrast over both vessels. Future studies are needed to quantify the parameters in dynamic splanchnic angiography.

\section{$\mathrm{T} 1202$}

Survival Time of Hepatocellular Carcinoma Patients Stratified According to the Time-to-Recurrence

Kohsaku Sakaguchi, Yoshiyuki Kobayashi, Shin-Ichi Nakamura, Yasushi Shiratori

[Aim] The intrahepatic recurrence of hepatocellular carcinoma (HCC) is inevitable even after curative treatment. The period until recurrence is related to the prognosis of HCC patients However, in terms of the period until recurrence, the survival time of $\mathrm{HCC}$ patients has not been fully evaluated. The aim of this study is to clarify the survival time of HCC patients stratified according to the period until recurrence. [Patients and Methods] This study was designed as the retrospective cohort study including 402 HCC patients who underwent curative treatment (surgical resection, percutaneous ethanol injection therapy, or radio frequency ablation) for primary tumor between January 1991 and December 2004, and followed for tumor recurrence at our clinic. The time-to-recurrence is defined as the interval between the initial treatment and either recurrence of HCC or latest follow-up visit. We analyzed the survival time of HCC patients stratified according to the time-to-recurrence; Group I, 247 patients with time-to-recurrence less than 2 years, Group II, 82 patients with time-to-recurrence more than 2 and less than 4 years, and Group III, 46 patients with timeto-recurrence of 4 years and more. [Results] The 5- and 7-year probability of survival was $33.9 \%$ and $15.3 \%$ in Group I, $71.0 \%$ and $46.8 \%$ in Group II, and $95.1 \%$ and $89.4 \%$ in Group III, respectively. Median survival time was 3.8 (2.4-5.8) years in Group I, 6.8 (4.99.2) years in Group II, and 11.7 (8.4--) years in Group III ( $\mathrm{P}<0.001$, by log-rank test). The following analyses on the survival after the treatment for recurrent HCC were conducted solely among patients who had recurrence of HCC during follow-up period. One hundred fifty-nine patients in Group I, 60 patients in Group II, and 23 patients in Group III developed recurrence of HCC. The cumulative survival rates at 3-years after the curative treatment for recurrent HCC in Group I, Group II, and Group III were $76.8 \%, 67.5 \%$, and $63.5 \%$, and median survival time were 4.4 (3.1-6.9), 5.3 (2.4-8.6), and 3.6 (2.6- ) years, respectively $(\mathrm{P}=0.931$, by log-rank test). On the other hand, the 3-year probability of survival of patients who underwent non-curative treatment was $16.6 \%$ in Group I, $53.9 \%$ in group II, and $60.0 \%$ in group III. The median survival time after the non-curative treatment for recurrent HCC was 1.8 (0.8-2.5) years in group I, $3.2(2.0-5.1)$ years in group II, and 3.4 (2.1-4.8) years in group III, respectively $(\mathrm{P}=0.036$, by log-rank test). [Conclusion] The time-torecurrence significantly influences on the survival time of HCC patients, and the median survival time of patients with time-to-recurrence more than 4 years is more than 10 years. 


\section{T1203}

Prognostic Performance of the New Treatment Algorithm for Hepatocellular Carcinoma (HCC) in Japan: Compared with Prognostic Staging Systems Yoshiyuki Kobayashi, Shinichiro Nakamura, Yasuhiro Miyake, Kenji Miyoshi, Hirokazu Miyatake, Kazuhiro Nouso, Yoshiaki Iwasaki, Kohsaku Sakaguchi, Yasushi Shiratori

(OBJECTVE) Several prognostic staging systems and treatment strategies for hepatocellular carcinoma (HCC) that are linked with disease stage have been reported. Recently, a new HCC treatment algorithm by the study group of Japan that is based on liver damage by Liver Cancer Study Group of Japan and tumor factors (Japanese treatment algorithm) has been proposed. The purpose of this study is to assess the stratification ability of Japanese treatment algorithm compared with other prognostic staging systems. (SUBJECTS AND METHODS) A retrospective analysis of 2256 consecutive patients with HCC diagnosed between 1994 and 2004 was performed. Clinical characteristics of the patients was as follows; the mean tumor size and number were $26 \mathrm{~mm}$ and 1.6; Child-Pugh classification A was 1578 (70\%); tumor ablation, hepatic resection, and transarterial chemoembolization were performed in 901 patients (40\%), 425 patients (19\%), and 738 patients (32\%), respectively . We stratified HCC patients according to each 2 treatment strategies (Japanese treatment algorithm and BCLC staging classification) and 2 prognostic staging systems (CLIP score and JIS score). The goodness of fit of each prognostic system were assessed by the likelihood ratio (LR) test and Akaike information criterion (AIC) using Cox's proportional hazards model, and also the discriminatory ability was analyzed by linear trend test. (RESULTS) The proportion of all the HCC patients classified according to each staging system, CLIP score of $0,1,2,3,4,5$ and 6 was $33 \%, 34 \%, 17 \%, 9 \%, 5 \%, 2 \%$, and $0 \% ;$ JIS score of 0 , $1,2,3,4$ and 5 was $18 \%, 31 \%, 25 \%, 16 \%, 7 \%$, and $0 \%$; BCLC staging of $0, A, B, C$ and D was $6 \%, 62 \%, 16 \%, 10 \%$ and $6 \%$; Japanese treatment algorithm of A,B,C,D,E,F and G was $50 \%, 14 \%, 8 \%, 5 \%, 7 \%, 3 \%$, and $12 \%$, respectively. The cumulative 5 -year survival rates of the each best prognostic group, CLIP score 0, JIS score 0, BCLC stage 0 and Japanese treatment algorithm A were $64 \%, 67 \%, 75 \%$ and $58 \%$, respectively. In relation to homogeneity of survival within each stage, CLIP score have found to be the most fitting model for $\mathrm{HCC}$ prognosis ( $\mathrm{LR}=62.6, \mathrm{AIC}=13619, \mathrm{P}=.0000$ ), while Japanese treatment algorithm was more fitting model $(\mathrm{LR}=35.8, \mathrm{AIC}=13590, \mathrm{P}=.0000)$ than $\mathrm{BCLC}$ staging classification (LR= 18.5, $\mathrm{AIC}=13577, \mathrm{P}=.0009)$. Moreover, linear trend test showed that JIS score had the highest discriminatory ability $(\chi 2=984.9)$. (CONCLUSION) Prognostic staging systems have higher stratification ability with regard to prognosis in patients with HCC than treatment strategy, while Japanese treatment algorithm showed better prognostic performance than BCLC staging classification.

\section{T1204}

Repeated Recurrence Profiles of Hepatocellular Carcinoma Within Milan Criteria

Kenji Miyoshi, Yoshiyuki Kobayashi, Yoshiaki Iwasaki, Shinichirou Nakamura, Hironori Tanaka, Hideki Onishi, Shouta Iwado, Kohsaku Sakaguchi, Yasushi Shiratori

BACKGROUND Milan criteria has been considered in judging transplantability in patients with hepatocellular carcinoma (HCC). Most of HCC patients revealed tumor progression beyond the Milan criteria during follow-up. The purpose of this study was to clarify clinical profiles of $\mathrm{HCC}$ recurrence after treatment of $\mathrm{HCC}$ within Milan criteria. SUBJECTS AND METHODS One hundred and ninety-three curative HCC patients within Milan criteria who received hepatic resection $(n=57)$ or tumor ablation $(n=136)$ at Okayama University hospital between January 1999 and December 2004 were prospectively enrolled. The first, second, and third recurrence rate was analyzed by the Kaplan-Meier method. To identify risk factors for the recurrence of HCC beyond Milan criteria, 20 variables were analyzed with logistic regression model. RESULTS The group comprised 138 men and 55 women. Mean age was 68 years. Child-Pugh classification A was 149 (77\%). The mean tumor size and number were $21.4 \mathrm{~mm}$ and 1.3 . Of the 193 patients, $110(57 \%)$ presented the first recurrences of HCC and 75 (68 \%) of them met Milan criteria and 69 received further curative treatment. Of the 69 patients, $53(77 \%)$ patients presented second HCC recurrences and 39 (73\%) of them met Milan criteria and 18 received further curative therapy. Of the 18 patients, 9 (50\%) patients presented third HCC recurrences. In HCC patient within Milan criteria, the lst HCC recurrence rate at 1 and 2 years was $17 \%$ and $37 \%$, and the 2 nd HCC recurrence rate at 1 and 2 years increased to $46 \%$ and $75 \%$, respectively, and the 3rd recurrence rate was $43 \%$ and $71 \%$ respectively ( log-rank test $p=0.003$ ). The logistic regression analysis revealed that serum $\alpha$-fetoprotein $(\mathrm{AFP})$ level $(\mathrm{P}=0.03, \mathrm{RR}: 2.60, \mathrm{CI}: 1.06-6.15)$ and normalization of serum AFP level after the initial treatment $(\mathrm{P}=0.024$, RR: $0.36, \mathrm{CI}: 0.15$ 0.90 ) were related to the recurrence of HCC beyond milan criteria after initial cutative treatment. CONCLUSIONS The recurrence of HCC beyond Milan criteria after initial curative treatment is associated with the normalization of serum AFP level after the initial treatment, and the cumulative recurrence rate increased along with repeated recurrence of HCC.

\section{T1205}

Effect of Previous Interferon Treatment On the Recurrence of Hepatocellular Carcinoma in Patients with Hepatitis C

Hirokazu Miyatake, Yoshiyuki Kobayashi, Yoshiaki Iwasaki, Shinichirou Nakamura, Hironori Tanaka, Yasuhiro Miyake, Ryo Terada, Kenji Miyoshi, Hideki Ohnishi, Shouta Iwadou, Bon Shouji, Takeshi Kuwaki, Kohsaku Sakaguchi, Yasushi Shiratori

BACKGROUND; It has been reported that interferon (IFN) treatment for chronic hepatitis C could prevent the development of hepatocellular carcinoma (HCC). HCC are liable to recur frequently even after curative therapy mainly because of multicentric occurrence. The purpose of this study was to clarify the effect of previous IFN treatment before the development of HCC on the recurrence and survival of HCC patients. METHODS; One hundred and five out of 897 patients with HCC diagnosed at Okayama University Hospital between 1995 and 2005, had received with IFN therapy for chronic hepatitis C before the development of HCC. The first and second recurrence rate of HCC after curative treatment for HCC was statistically analyzed. The potential risk factors for HCC recurrence, including 27 variables at HCC development and recurrence, were analyzed by Cox proportional hazards model RESULTS; The group comprised 62 men and 43 women, and mean age at IFN therapy and development of HCC was 57 and 64 years old, respectively. Eighty nine out of 105 patients (85\%) were classified into Child-Pugh classification A. The mean tumor size and number were $21 \mathrm{~mm}$ and 1.4. Thirteen of them (12\%) showed a sustained virologic response (SVR) in previous IFN treatment. Of 105 patients $71(68 \%)$ developed the recurrences of HCC during mean follow-up period of 2.5 years. 44 (62\%) out of 71 patients with recurrence were successfully treated for the recurrent HCC. Of these 44 patients, $25(57 \%)$ patients developed 2nd HCC recurrences during mean follow-up period of 2.3 years. In univariate analysis, the age at the time of interferon therapy $(\mathrm{p}=.038)$ was the only factor related to lst HCC recurrence, while SVR $(\mathrm{p}=.014)$, tumor number $(\mathrm{p}=.021)$, serum AST levels ( $\mathrm{p}=$ .002), serum ALT levels ( $\mathrm{p}=.0008)$, prothrombin time $(\mathrm{p}=.039)$, and serum albumin at $1 \mathrm{~s}$ recurrence ( $\mathrm{p}=.045$ ) were related to 2 nd HCC recurrence. The multivariate analysis showed no significant factors of 2nd HCC recurrence. The period to 1st HCC recurrence after the curative treatment for primary tumor was not significantly different between SVR and nonSVR patients. However, the period to the 2nd HCC recurrence after the curative treatment for lst HCC recurrence in SVR patients was significantly longer than that in non-SVR patients. (1528 vs 667 days, (log-rank test; $\mathrm{p}=.026)$ ). Overall survival rate of SVR patients was higher than non-SVR patients $(\mathrm{p}=.007)$. CONCLUSIONS: Sustained virologic response to previous interferon treatment in patients with hepatitis $\mathrm{C}$ may reduce the risk for the second recurrence of HCC and improve survival.

\section{T1206}

Effects of Supplementation with a Branched-Chain Amino Acid-Enriched Preparation On Event-Free Survival and Quality of Life in Cirrhotic Patients with hepatocellular carcinoma: A Multicenter, Randomized Controlled Trial Haruhiko Kobashi, Youichi Morimoto, Toshio Ito, Toshinari Shimoe, Yasuhiro Makino, Yasuyuki Araki, Hiroshi Ikeda, Kouichi Takaguchi, Masaharu Ando, Masanobu Miyake, Yoshiaki Iwasaki, Kohsaku Sakaguchi, Yasushi Shiratori

Background \& Aims: Liver cirrhosis is often associated with protein energy malnutriotion $(\mathrm{PEM})$, and oral administration of branched-chain amino acids (BCAA) is known to correc PEM and improve health-related quality of life (QOL). However, the effect of BCAA in cirrhotic patients associated with hepatocellular carcinoma (HCC) is unclear. This study aimed to elucidate the effects of long-term BCAA supplementation on event-free survival and QOL in cirrhotic patients with HCC. Methods: A multicenter, randomized controlled trial for 3 years was conducted in 233 cirrhotic patients with HCC (159 men and 74 women, mean age of 69 ). The Child-Pugh stage was $\mathrm{A} / \mathrm{B} / \mathrm{C} /$ others in $58 / 162 / 10 / 3$ patients, and the TNM stage was $1 / 2 / 3 / 4 \mathrm{~A} / 4 \mathrm{~B} /$ others in $56 / 65 / 94 / 4 / 2 / 12$ patients. They were assigned randomly to the BCAA group $(n=123)$ and the control group $(n=110)$. Patients in the BCAA group were orally administered with $150 \mathrm{~g}$ per day of BCAA-enriched preparation, containing 40.5 $\mathrm{g}$ of protein including $18.3 \mathrm{~g}$ of $\mathrm{BCAA}$, and $630 \mathrm{kcal}$. Primary endpoint was event-free survival, that is, survival without development or deterioration of hepatic failure (jaundice, ascites, or encephalopathy), rupture of HCC, esophago-gastric bleeding, or liver-related death. Secondary endpoints were QOL estimated by the Short Form-36 questionnaires, and liver function tests such as serum albumin, serum ammonia, and BCAA/tyrosine ratio (BTR). The study conformed to the Declaration of Helsinki and was approved by the institutional ethical committee. All patients gave their informed consent. Results: (1) Before November 2005, when the mean observation time was 585 days, 107 patients died, 44 experienced development or deterioration of hepatic failure, 2 experienced esophago-gastric bleeding and 80 experienced no events. The mean event-free survival was 535 days in the BCAA group and 495 days in the control group (no significant difference by log-rank test). As for the patients with hypoalubuminemia at baseline (3.7g/dl or less), comprising 90 patient in the BCAA group and 68 in the control group, event-free survival was increased significantly in the BCAA group ( $\mathrm{p}=0.0374)$. (2) The "vitality", "role-emotional", and "social functioning" domains in the Short Form-36 measures were significantly improved ( $\mathrm{p}=0.0306,0.0347$, and 0.0262 , respectively) in the BCAA group. (3) Serum albumin increased significantly in the BCAA group $(\mathrm{p}=0.024)$. There was no significant change in serum ammonia and BTR Conclusions: Long-term BCAA supplementation in cirrhotic patients with HCC improves QOL and serum albumin level. It also improves event-free survival in cirrhotic patients with HCC and hypoalbuminemia.

\section{T1207}

Efficacy of Long Acting Release Octreotide in Patients with Advanced Hepatocellular Carcinoma: A Randomized Multicenter Phase III Study Gontran Verset, Chris Verslype, Hendrik Reynaert, Ivan Borbath, Philippe Langlet, Ann van den Brouck, Marc Peeters, Ghislain Houbiers, Peeter Michielsen, Marianna Arvanitakis, Jean-Luc van Laethem

Background/Aim: The role of somatostatin analogues in hepatocellular carcinoma (HCC) remains undetermined. We therefore designed a prospective randomized multicenter study to assess the efficacy of long acting release (LAR) octreotide for the treatment of advanced HCC. Primary study end point was patient survival. Patients and Methods: A total sample size of 90 patients was required to demonstrate with a power of $80 \%$ that octreotide LAR increases 1 year survival by $20 \%$ in patients with advanced HCC. 109 patients with HCC who were not amenable to curative resection, liver transplantation nor to local treatment, were included between March 2001 and March 2005 and were randomly assigned to the octreotide group $(n=56)$ or the control group $(n=53)$. Patients in both groups were allowed to receive best supportive care (BSC) and a daily dose of $20 \mathrm{mg}$ of tamoxifen. An intramuscular injection of $30 \mathrm{mg}$ octreotide LAR was administrated once monthly in the octreotide group. The clinical, biological and tumoral parameters were recorded every 3 months until death Groups were comparable with respect to age, gender, presence of cirrhosis, Child-Pugh score, etiology of hepatopathy, Okuda classification, KPS, $\alpha$-FP level, size of target lesion and previous treatement of HCC. Uni- and multivariate analyses were performed for assessment of specific prognostic factors. Results: On the last follow-up (median: 3 months, range: 0,131 months), all but 2 patients had died and the treated patients in the octreotide group had received a median number of 2 injections of octreotide (1-31 injections). The median 
survival was 3 months (C195\%: 1,4-4,6) for the ocreotide group and 6 months (C195\%:2$10)$ for the control group $(p=0,609)$. There was no difference in terms of $\alpha$-FP decrease, tumor regression or improvement of quality of life between the two groups. Variables associated with a better survival in the multivariable analysis were: presence of cirrhosis, $\alpha$-FP level< $400 \mathrm{ng} / \mathrm{ml}$ and Okuda stage I. Conclusion: In this study, octreotide LAR did not provide any benefit in terms of survival, tumor response or quality of life compared with BSC.

\section{$\mathrm{T} 1208$}

CT-Guided Brachytherapy of Hepatocellular Carcinoma in Liver Cirrhosis - A Novel Therapeutic Approach

Alexandra Bergk, Gero Wieners, Viola Weich, Bertram Wiedenmann, Thomas Berg, J.N. Ricke

Introduction: Unresectable hepatocellular carcinomas (HCCs) still are a major clinical chalenge due to poor prognosis and limited therapeutic options. This includes mainly HCCs exceeding a diameter of $5 \mathrm{~cm}$ or multifocal manifestations with more than 3 tumor nodes. Study: We treated 48 consecutive patients with unresectable HCC with a total of 63 single fraction, high dose rate brachytherapy procedures. A 192Iridium source was used to apply a minimal median dose of $20 \mathrm{~Gy}$ (15-25Gy) to the tumor tissue. Afterwards, patients were followed up closely until now or to their death ( 4 weeks - 4 years, median 16 month). Liver function was assessed by Child-Pugh criteria; 42 and 6 patients showing a Child A and B stadium, respectivley. The number of lesions treated simultaneously ranged from 1 to 5 with a median tumor size of $3.8 \mathrm{~cm}$ (range $1-9 \mathrm{~cm}$ ). Brachytherapy was generally well tolerated. Only one acute intervention related complication was seen (arterial bleeding, sufficiently treated by embolization), except for nausea $(n=1)$ and chills until 4 hours p.i. $(\mathrm{n}=1)$. Small perihepatic hematomas were seen in 4 patients 3 days after intervention, but no postinterventional liver function impairment was found. Results: After 6 month, 1 year and 2 year of follow up in $92 \%, 87 \%$ and $76 \%$, respectively no local recurrence of HCC could be detected by MRI in surviving patients. Nevertheless 8 patients (16\%) had an intrahepatic progression that could be treated with a second brachytherapy successfully. Overall survival after six month, one year and 2 years was $92 \%, 77 \%$ and $62 \%$, progression free survival $73 \%, 61 \%$ and $48 \%$ respectively. Observed survival was compared to the life expectancy according to the CLIP score by Kaplan-Meier analysis and showed a significant advantage for brachytherapy-treated patients. Conclusion: Brachytherapy is safe and efficous in the treatment of irresectable HCC. The direct comparison to other local ablative methods like TACE, PEI or RFTA concerning progression free and overall long-term survival needs to be examined.

\section{T1209}

Treatment Outcomes of Transcatheter Arterial Chemoinfusion (TACI) in Patients with Unresectable Hepatocellular Carcinoma (HCC) Bo Yoon Choi, Aijaz Ahmed, Mahmood Razavi, Daniel Sze, Joan Frisoli, Nicole Simpson, Ruel T. Garcia, Emmet B. Keeffe, Mindie H. Nguyen

PURPOSE: A common treatment for unresectable HCC is transcatheter arterial chemoembolzation (TACE), which carries a 5-16\% risk of serious complications. Compared to TACE, TACI without embolization may have similar efficacy and less side effects, but few studies have examined clinical outcomes of this modality. METHODS: We performed a retrospective study of 345 consecutive TACI cases in 165 patients between $1 / 98$ and 9/02 at single U.S medical center. We searched the National Death Index for death records of all patients. We examined tumor response (complete response [CR], partial response [PR], and no response/ progression of tumor [NR]) and survival rate (Kaplan-Meier curve). We used multivariate Cox's proportional hazards regression to identify independent predictors of survival function. RESULTS: Patient mean age was $59 \pm 12$ with most being male (79.4\%) and white $(29.7 \%)$ or Asian (59.4\%). Most had cirrhosis (75.8\%), and either chronic hepatitis C (45.5\%) or B $(35.2 \%)$. Mean MELD score was $9.2 \pm 2.7$ and mean CTP score was $6.0 \pm 1.1$. Tumor staging at diagnosis was: TNM I=4.9\%, $\mathrm{II}=31.5 \%, \mathrm{II}=21.8 \%$ and $\mathrm{IV}=41.8 \%$. Overall $\mathrm{CR}$ was $32.6 \%$, PR $33.3 \%$, and NR $34.1 \%$. There were statistically significant differences in tumor response by TNM staging. Only 7 patients were hospitalized for $>24$ hours (hemoperitoneum due to HCC prior to TACI, hepatic artery injury, and persistent nausea or fever or pain). Three patients (1.5\%) had worsening of liver function within 30 days following TACI. Overall survival rate is summarized in the Table. Survival was significantly poorer for patients with TNMIV compared to those with TNMI, II, and III (median survival=16 versus 33 months, $\mathrm{p}=0.004$ ). Following adjustment for age and etiology of liver disease, independent predictors of poor survival were high CTP $(\mathrm{HR}=1.51, \mathrm{p}<0.001)$ and TNMIV $(\mathrm{HR}=2.06, \mathrm{p}=$ 0.002 ). Independent predictors for higher survival rate were female $(H R=0.55, p=0.013$ ) and Asian ( $\mathrm{HR}=0.55, \mathrm{p}=0.01$ ) CONCLUSION: TACI is an effective and safe treatment for unresectable HCC with TNM stage I, II, and III. Rate of serious complications was only $2.7 \%$. In addition, most of our patients ( $98 \%$ ) required less than 24 hours of hospitalization, while patients undergoing TACE generally require longer hospitalization. Overall survival rates of TACI were comparable to those reported by Llovet et al and Lo et al in the only 2 individual RCT's that show survival benefits with TACE (Table).

\begin{tabular}{|c|c|c|c|c|}
\hline SURVIVAL RATES & 1-YEAR & 2-YEAR & 3-YEAR & 4-YEAR \\
\hline Current study & $69 \%$ & $46 \%$ & $39 \%$ & $30 \%$ \\
\hline Lo, Hepatol 2002 & $57 \%$ & $31 \%$ & $26 \%$ & -- \\
\hline Llovet, Lancet 2002 & $75-82 \%$ & $50-62 \%$ & $17-29 \%$ & -- \\
\hline
\end{tabular}

\section{$\mathrm{T} 1210$}

Thermo-Chemoembolization for Malignant Hepatic Tumors Is Clinically Useful

Mitsunori Yasuda, Motoharu Kondo, Satoshi Kokura, Yuji Naitoh, Norimasa Yoshida, Toshikazu Yoshikawa

Although surgical resection, chemotherapy, radiation, topical therapy, etc., are available for malignant hepatic tumors, treatment is often difficult in therapy-resistant cases or advanced ones. We recently used a combination of transarterial chemoembolization with Degradable Starch Microsphere (DSM) and hyperthermia (HT), i.e., thermo-chemoembolization (DSM/ HT), to treat such patients, and evaluated its clinical usefulness. Subjects and Methods: The subjects were 68 cases who underwent either HT alone or DSM-HT at our hospital between 2003 and 2005. HT group had 10 cases (5 primary, 5 metastatic). DSM/HT group was composed of 58 cases (20 primary, 38 metastatic liver cancers originating from the colorectum, stomach, pancreas, biliary, esophagus, breast, lung, etc). Response rate, survival, adverse reactions were compared between groups. DSM/HT was performed after a reservoir for hepatic-arterial infusion was inserted percutaneously. Immediately before each HT session, an appropriate amount of suspension containing DSM (an embolizing agent) and an anticancer agent (primarily MMC) was infused intraarterially under fluoroscopy to achieve temporary embolization of the entire liver. This was immediately followed by 50 -minute HT using a Thermotron RF-8 radiofrequency device (Yamamoto Vinita Co., Ltd.). Administration of DSM and anti-cancer agent just before HT was performed for the following reasons: (1) chemical effects of the agent on hepatic tumors, (2) anti-tumor effect of tumor ischemia induced by DSM, (3) anti-tumor efficacy by increase in temperature due to DSM-induced tumor ischemia, and (4) repeatable this treatment because DSM is degraded in about 1 hour by amylase, avoiding permanent damage of hepatic blood flow. This therapy was administered once a week for as long as possible at OPC. Results: No noteworthy adverse reactions other than temporary mild abdominal pain and nausea were noted in patients receiving DSM/HT therapy. Response rate, as determined by findings of diagnostic imaging and tumor markers, was about $40 \%$. Survival was significantly longer in the DSM/HT than in the HT group. In the DSM/HT group, survival period was positively correlated with frequency of treatment. When patients receiving DSM/HT were divided by number of treatment sessions into three groups ( 4 or less, 5 to 8 , and 9 or more sessions), mean survival period differed significantly between each groups, and increased with the frequency of treatment. Conclusion: Thermochemoembolization is relatively noninvasive, could be performed repeatedly in the outpatient clinic, and prolonged survival. It is thus a promising means of treating malignant hepatic tumors.

\section{T1211}

Incidence of Acute Hepatic Failure and Related Risk Factors After Transcatheter Arterial Chemoembolization of Hepatocellular Carcinoma Kyungsik Park, Byoungkuk Jang, Woojin Chung, Kwangbum Cho, Jaeseok Hwang, Sunghoon Ahn, Younghwan Kim, Jinsu Choi, Junyoung Hwang

Background/Aims : Although transcatheter arterial chemoembolization (TACE) is a major modality in treatment of unresectable hepatocellular carcinoma (HCC), acute hepatic failure after this procedure is not rare. But reports considering this important complication are not enough and results are various. So the purpose of this study is to evaluate the incidence and associated risk factors of acute hepatic failure after TACE. Methods: From year 2001 to 2004,632 procedures were done at 377 patients ( 294 men and 83 women). Mean age was $58.1 \pm 10.1$ years. Adriamycin mixed lipiodol solution and gelform were used for TACE. Various clinical and radiological data before and after the procedure were reviewed retrospectively. Univariate and multivariate analyses were done for evaluation of risk factors associated with development of acute hepatic failure. Results : Acute hepatic failure occurred in $76(12.0 \%)$ of the 632 sessions within 7 days. Univariate analysis revealed that ChildPugh class $(\mathrm{p}=0.005)$, history of previous TACE $(\mathrm{p}=0.004)$, total bilirubin level $(\mathrm{p}=0.005)$, number of involved segments $(\mathrm{p}=0.004)$, total size of tumor $(\mathrm{p}=0.002)$, presence of right portal vein thrombosis (PVT) $(\mathrm{p}=0.001)$ or main PVT $(\mathrm{p}<0.001)$, involvement of segment $1,5,6,7$ ( $p<0.05$ at each segment) were significantly different between the patients with or without hepatic failure after TACE. Of the variables, abscence of previous TACE $(p=$ $0.010, E(\beta)=2.096)$, elevated total bilirubin $(p=0.001, E(\beta)=1.334)$, large tumor size $(p=$ 0.032 , $E(\beta)=1.149)$, presence of right $(\mathrm{p}=0.013, \mathrm{E}(\beta)=2.421)$ or main $(\mathrm{p}=0.013, \mathrm{E}(\beta)=$ 4.014) PVT were independently associated factors in multivariate analysis. Conclusions The incidence of acute hepatic failure after TACE was $12.0 \%$ and as predictive variables, lst TACE, elevated bilirubin level, large tumor size, PVT can be considered.

\section{$\mathrm{T} 1212$}

Hepatic Hemodynamic Change After Percutaneous Radiofrequency Ablation of Hepatocellular Carcinoma - Long Term Follow-Up Observation Shinpei Sato, Hiroshi Miyakawa, Atsuko Takai, Eriko Kitazawa, Kentaro Kikuchi, Takayuki Kawakami

OBJECTIVE: Radiofrequency thermal ablation(RFA) is a minimally invasive treatment widely used for the treatment of liver neoplasms. The purpose of this study was to evaluate the changes in hepatic hemodynamics with long term follow-up observation after percutaneous RFA treatment of hepatocellular carcinoma using pulsed Doppler sonography. MATERIALS AND METHODS: Between April 2004 and April 2005, 50 subjects with hepatocellular 
carcinoma(1.2-4.3 cm in diameter, $1-3$ nodules) were treated by RFA. The RFA was performed percutaneously using a monopolar cooled-tip electrode needle under US guidance with local anesthesia. Hepatic hemodynamic change was examined in each patient two days before and after the RFA. In addition, we examined hepatic hemodynamic change in the outpatients in 3 and 6 months. The statistical analysis was performed on hemodynamic measurements recorded in the fasting state by pulsed Doppler sonography. The time averaged velocity and cross-sectional area of the portal vein, maximum velocity of hepatic artery were recorded. Portal vein blood flow was calculated. Hepatic venous flow was classified into normal or abnormal patterns. RESULTS: In the ablation zone, hepatic arterial velocity was significantly elevated after RFA, compared to pre-treatment level $(35.5 \mathrm{~cm} / \mathrm{s}$ vs $26.4 \mathrm{~cm} / \mathrm{s}), \mathrm{p}<0.05$. After 3 and 6 months, hepatic arterial velocity was reduced to pre-treatment level. while portal vein flow and hepatic venous flow were not significantly altered. In the non-ablated zone, there was no significant change in the hepatic hemodinamics before and after RFA. CONCLUSION: RFA treated zone had temporary elevated hepatic arterial velocity after RFA. But in the non-ablated zone, no significant change was observed in hemodynamics of artery, portal and hepatic veins, indicating that RFA is a safe and minimally invasive treatment, with no untoward effect on hepatic hemodynamics outside the locoregional area.

\section{$\mathrm{T} 1213$}

Long-Term Outcome in Patients with Advanced Klatskin-Tumors Undergoing Operative and/or Endoscopic Therapy

Andreas Weber, Sonja Landrock, Manfred Stangl, Roland Schmid, Christian Prinz

BACKGROUND: Tumors of the bile duct bifurcation (Klatskin tumors) are associated with a high mortality. Bile duct tumors are classified into 4 stages according to the Bismuth classification: stage I or II for tumors expanding up the hilus, type III A/B infiltrating left or right hepatic duct, and stage IV with infiltration of both hepatic ducts and subsegments. Because of the late presentation of symptoms, tumors are usually diagnosed in later stages and most therapy concept can not be curative. Survival rates reported so far describe a very limited life expectancy of $<3$ months if no treatment is offered. Possible treatment strategies include endoscopic retrograde cholangiopancreatography including stent therapy (ERCP), percutaneous transhepatic biliary drainage (PTBD) or surgery. METHOD: A long-term followup of patients with Klatskin-tumor undergoing an endoscopic and/or surgical therapy was performed by retrospective analysis. PATIENTS: 95 consecutive patients ( 57 male, 43 female; mean age 68 years) underwent treatment for malignant hilar bile duct tumors of Bismuth stages II-IV during a period of 8 years (1997-2004). RESULTS: 72/95 patients were treated only by endoscopic procedures using ERCP $(n=43)$ or PTBD $(n=26) .23 / 95$ patients could be treated by surgery initially and had no contraindications or lymph node metastasis. 4 / 23 operated patients had R0 operation with complete tumor removal in histology. 19 had recurrent obstruction and lateron required ERCP or PTBD. Overall mean survival was 372 days in the endoscopic group and 877 days in the group treated by surgery and subsequent endoscopic procedures. In the surgery group, mean survival was 2612 days in patients with BI/II stage $(n=4), 721$ days in B III $(n=5)$, and 511 days in B IV $(n=14)$ stages. In comparison, Patients in the endoscopic group had survival rates of 617,501 and 290 days in stage I/II $(\mathrm{n}=7)$, III $(\mathrm{n}=20)$ and IV $(\mathrm{n}=42)$, respectively. CONCLUSIONS. Surgery of bile duct tumors followed by endoscopic intervention significantly increases life expectancy; endoscopic stenting or PTBD alone is useful in patients with advanced disease (B IV; LN pos., contraindications towards surgery). Future therapies will aim at new strategies to improve the outcome.

\section{$\mathrm{T} 1214$}

Clinical Benefit of Radiation Therapy for Unresectable Extrahepatic Bile Duct Cancer

Osamu Togawa, Hiroyuki Isayama, Yousuke Nakai, Takashi Sasaki, Hiroshi Kogure, Natsuyo Yamamoto, Naoki Sasahira, Takeshi Tsujino, Minoru Tada, Yukiko Ito, Saburo Matsubara, Kenji Hirano, Toshihiko Arizumi, Nobuo Toda, Haruhiko Yoshida, Takao Kawabe, Masao Omata

Background: The patients with bile duct cancer presented obstructive jaundice. Both biliary drainage and anti-tumor treatment were important for those unarectable cases. The efficacy of self-expandable metallic stent (EMS) is being accepted. However, effective anti-tumor treatment for those patients did not established. We estimated radiation therapy for the patients with unrectable extrahepatic bile duct cancer. Methods: We treated 82 cases of unresectable extrahepatic bile duct cancer and analyzed 59 locally advanced cases (26 in Distal, 33 in Klatzkin tumor). Radiation strategies were total 60 Gy of external corporeal radiation and 24 Gy of intra-bileduct radiation with remote after loading system (RALS). We placed EMS for all cases, uncovered EMS (UMS) for hilar lesion (Klatzkin tumor) and both UMS or covered EMS (CMS) for distal stricture cases. Results: There were 31 (14 in distal, 17 in Klatzkin tumor) cases with both anti-tumor treatment and metallic stenting (Combination group), remaining cases received metallic stenting alone (Stenting Group). RALS was performed in 9 cases of Klatzkin tumor. Median survival period was 506 days in Combination group and 251 days in Stenting group. The cumulative survival period of Combinaton group was significantly longer than Stenting group $(\mathrm{p}=0.0014)$. In Combination group, 1-year survival rate was $53 \%$, 2-year $28 \%$ and 3-year $7 \%$. On the other hand, in Stenting group, 1 -year survival rate was $25 \%$ but 2 -year and more survival were $0 \%$. There was no significant difference in cumulative stent patency between Combination and Stenting groups. Conclusion: Radiation therapy was effective and contributes for prolongation of survival period for the locally advanced unresectable extrahepatic bile duct cancer.
T1215

Palliative Percutaneous Transhepatic Cholangiodrainage for Cholangiocarcinoma: Complications and Outcome

Dorothee H. Dorlars, Peter Fleischmann, Jenny Gebhardt, Juergen Pausch

Background: The primary treatment goal in patients with nonresectable cholangiocarcinomas (CC) and obstructive jaundice is to restore biliary flow via endostents. If ever, the placement of a percutaneous transhepatic cholangiodrainage (PTCD) is recommended in cases where the stenosis cannot be treated endoscopically. However, the PTCD treatment not only has comparatively few complications, it also increases the patients' survival time and quality of life. Patients and methods: From 12/2002 to 10/2004, we treated 26 patients with CC using PTCD (16 men, 10 women, mean age 71 [54-85] yrs; polyurethane PTCD, "Münchner Drainage", Pflugbeil \& Co., Germany). In all cases, a curative resection of the tumor was impossible, due either to the tumor itself or the patient's general condition. The CC stages (Bismuth classification) were as follows: Bismuth I $n=3$, Bismuth II $n=7$, and Bismuth IV $\mathrm{n}=16$. On average, 3.7 [2-7] sittings were required until the final PTCD was placed. The PTCD was placed in the left $(n=4)$, in the right $(n=12)$, or in both hepatic ducts $(n=10)$ The average drain caliber was 11 (8-16) F for the right duct and 10 (8-12) F for the left. Results: In 2 patients (7.7\%), restoration of biliary drainage was not successful. In all of the remaining cases, treatment was followed by a significant and sustained drop in elevated serum AST, ALT, AP and GGT. Prior to treatment, AST and ALT were elevated two-fold above the norm, AP and GGT even ten-fold. Bilirubin and inflammation parameters showed a moderate decrease immediately after treatment, but increased again post-treatment. Of 47 procedures performed, $14.9 \%$ had mild acute complications (bleeding), $85.1 \%$ had none During follow-up we found $56.9 \%$ mild complications (dislocation/obstruction of drain, leakage, cholangitis), and 3.8\% severe complications (liver abscess). The average time the drain remained in place was 185 days $(\min =30 \mathrm{~d}, \max =837 \mathrm{~d})$. The median survival time was 5.9 months. Conclusions: Compared to surgical intervention, PTCD is a safe and effective palliative treatment for $\mathrm{CC}$ with relatively few complications (3.8\% severe complications vs $29 \%$ severe surgical complications; $0 \% 30 \mathrm{~d}$-mortality vs. $15 \%$ surgical $30 \mathrm{~d}$-mortality). The average morbidity of our patient population was higher than is usual in surgical patients. Yet, in spite of these inauspicious conditions, the median life expectancy of our patients was 5.9 months, which compares favorably with the life expectancies reported in the literature (median 3-6 mo. with PTCD, 2 mo. with surgery).

\section{T1216}

Efficacy of Radiofrequency Ablation in Patients with Colorectal Hepatic Metastases

Kazuhiko Hosoda, Tomotaka Yasumura, Akiharu Yagawa, Ryota Sueki, Satoshi Kaji, Takanori Maruyama, Toshifusa Ozawa, Ryuichi Iida

PURPOSE: Radiofrequency ablation (RFA) is a new minimally invasive method that has been increasingly used in the treatment of hepatic metastases. The aim of this study was to examine the therapeutic efficacy of RFA in patients with colorectal liver metastases. PATIENTS AND METHODS: From April of 2000 until March 2005, 23 patients with colorectal liver metastases who were not candidates for hepatic resection underwent RFA. They were 16 males and 7 females with a mean age of 65 years (34-79). The mean number of metastases was 1.6 (1-4). The mean maximum diameter of tumor size was $1.96 \mathrm{~cm}$ (1.0-5.0). RFA was performed percutaneously with ultrasound guidance. All procedures were performed with a LeVeen needle electrode. Other 23 patients with colorectal liver metastases whose dominan lesion was less than $3 \mathrm{~cm}$ underwent hepatic resection between June of 1991 and December of 2004. The 5-year survival in the RFA group was compared with that in the hepatic resection group. RESULTS: The mean number of RFA treatment was 1.1 (1-2). The average hospital stay was 8 days. There was no treatment-related mortality. Major complications occurred in only one patient ( peritoneal dissemination ). Local recurrence was occurred in two patients $(9.5 \%)$. The cumulative 5-year survival rates for all patients were $31.8 \%$ after RFA. Patients with a carcinoembryonic antigen (CEA) less than $20 \mathrm{ng} / \mathrm{ml}$ had improved survival compared with those with a CEA more than $20 \mathrm{ng} / \mathrm{ml}(\mathrm{P}<0.05)$. Patients with the dominant lesion less than $3 \mathrm{~cm}$ in diameter had improved survival compared with those with the dominant lesion more than $3 \mathrm{~cm}(\mathrm{P}<0.005)$. The cumulative 5 -year survival rate after RFA and resection for patients with the dominant lesion less than $3 \mathrm{~cm}$ in diameter were $46.6 \%$ and $41.7 \%$, respectively (nonsignificant). The presence of extrahepatic metastases did not affect survival. CONCLUSION: RFA is a safe and effective treatment for colorecta liver metastases. Especially, for patients with the dominant lesion less than $3 \mathrm{~cm}$, survival is comparable with hepatic resection. A randomized prospective study comparing RFA with resection is necessary.

\section{T1217}

\section{Colonic Complications After Liver Transplant, An Under Appreciated} Significant Problem?

Antonio Sanchez, Hugo Bonatti, Rolland C. Dickson, Justin H. Nguyen, Walter C. Hellinger, Ronald A. Hinder, Heidi K. Chua, Jaime Aranda-Michel

BACKGROUND: Liver transplantation (LT) is the treatment of choice for end-stage liver disease. Liver diseases can be associated with diseases of the intestinal tract and post LT immunosuppressive agents might predispose to colonic complications. AIM: We retrospectively analyzed the incidence and spectrum of colonic complications in a cohort of $402 \mathrm{LT}$ patients. PATIENTS AND METHODS: A total of 467 consecutive LTs in 402 individuals were performed between 1998 and 2001 at the Mayo Clinic, Jacksonville, Florida. Standard immunosuppression consisted of Tacrolimus, Mycophenolic acid and steroids. RESULTS During a mean follow up period of three years 81 colonic complications developed in the 467 LT events. 4/19 patients transplanted for PSC and inflammatory bowel disease (ulcerative colitis $n=16$, Crohn's disease $n=3$ ) had persistent colitis; $8 / 19$ patients had colectomy prior to LT and 7/19 had no colonic symptoms during the follow up period. Combined colonic intervention and LT were performed simultaneously in 3 patients; one with colonic resection due to multiple colonic perforations during re-LT; one with resection for ischemic colitis 
associated with acute liver failure and the last case a preexisting transverse colostomy that had to be reinforced. Colonic polyps were endoscopically removed in seven patients and three patients were diagnosed with colorectal cancer (one cecal, two rectal cancers), which all were surgically treated. One patient developed a hemorrhage of the terminal ileum/cecal region in the course of intra-abdominal sepsis, and was treated by endovascular embolization of the ileocolic artery. Two patients developed hemorrhoids requiring surgical interventions, and two patients had perianal fistulas. Two patients developed sigmoid diverticulitis and one appendicitis requiring surgical intervention. There were 32 cases of Clostridium difficile associated enterocolitis. Nine patients developed CMV gastrointestinal complications with three cases of colitis, one leading to perforation, intra-abdominal sepsis and death. One patient developed a herpetic rectal ulcer and two perianal HSV associated lesions. Chronic diarrhea was seen in fifteen patients who had a negative evaluation that required withdrawal of mycophenolic acid. CONCLUSION: The frequency of colonic disorders in our series was higher than expected. Infection complications account for the majority of colonic complications seen in LT patients. The high incidence of clostridium colitis warrants improvement in screening and preventive measurements. Screening for polyps pre-transplant and post-transplant is highly recommended.

\section{$\mathrm{T} 1218$}

Incidence, Endoscopic Findings and Treatment of Biliary Tract Complications After Orthotopic Liver Transplantation - A Single Centre Experience Nicolas Hoepffner, Yogesh Shastri, Bora Akoglu, Christina Zapletal, Wolf O. Bechstein, Wolfgang Caspary, Dominik Faust

Background: Despite standardization for biliary reconstruction biliary complications after orthotopic liver transplantation (OLTx) occur in 7 to $51 \%$ of cases and are an important cause of early and late postoperative morbidity and mortality. The most common biliary complications are bile leaks and bile duct strictures. Early diagnosis and prompt treatment of biliary complications are necessary to reduce morbidity and mortality related to LTx. Here we have evaluated the role of endoscopic retrograde Cholangio Pancreatography (ERCP) in the diagnosis, treatment and outcome of post OLTx biliary complications. Patients and methods: This retrospective study included 162 patients (109 male, age $52 \pm 10$ yrs; 53 female, age $49 \pm 14$ yrs) who underwent OLTx between 1984 and 2004 and were regularly followed up at Frankfurt liver transplantation centre. The mean time since OLTx was 6 + 4 years. Endoscopic therapy was performed with balloon dilation and or plastic endoprosthesis. 154 patients underwent biliary continuity by choledocho-choledochostomy (end to side in 114 and side-to-side in 40) while 8 patients underwent Roux-en-Y choledochojejunostomy. Results: $40(24.6 \%)$ patients underwent ERCP for diagnosis and treatment of biliary complications. The median time between OLTx and first suspicion of biliary complications was 12 month (range 1 to 94 months). Overall 70 different biliary tract complications were identified. The most frequently diagnosed complication was isolated anastomotic strictures in $28(40 \%)$, followed by bile leakage in $17(24 \%)$ and non anastomotic strictures in $6(9 \%)$, whereas 19 patients (27\%) experienced miscellaneous complications like choledocholithiasis $7(10 \%)$, abscess $5(7 \%)$ or secondary cholangitis in $4(6 \%) .50 \%$ of the patients developed only 1 type of biliary complication after OLTx. Number of ERCPs performed per patient ranged from 2 to 52 . Sustained success was achieved in 26 (81\%), while endoscopic treatment failed in $6(19 \%)$. Out of this, 3 patients were re-operated. Till date, none of these were considered for re-transplantation. Conclusion: Biliary complications still remain an important problem in liver transplant patients. Endoscopic management is usually effective in majority of them but may require multiple procedures especially to treat strictures. Surgical intervention is required only in a few selected cases.

\section{T1219}

Endoscopic Treatment for Biliary Complications in Donors After Living-Donor Liver Transplantation

Seiji Shio, Shujiro Yazumi, Kazunori Hasegawa, Masaya Kida, Junichi Yamauchi, Shinsuke Tada, Hiroto Egawa, Shinji Uemoto, Tsutomu Chiba

Background and Aims. With the increased number of living-donor liver transplantation (LDLT), post-operative biliary complications of the donors have become one of the most significant problems of the LDLT. The aims of this study were to characterize the features of the biliary complications of the donors occurred after LDLT and to evaluate the feasibility of treating biliary complication endoscopically. Patients and Methods. A database of 613 consecutive donors (360 right lobes and 253 left robes) of LDLT from July 1999 through April 2005 in Kyoto University Hospital was reviewed. Biliary leakage and stricture were treated by endoscopic naso-biliary drainage (ENBD) and endoscopic biliary drainage (EBD), respectively. Results. The overall incidence of biliary complications was 4.1\% (25/613); the incidence in right-lobe donors $[6.1 \%(22 / 360)]$ was significantly higher than that in leftlobe donors $[1.2 \%(3 / 253)](\mathrm{p}>0.001)$. The complications were 23 biliary leakages and 7 biliary strictures; 5 of 7 biliary strictures occured after closure of biliary leakage. Out of 25 patients with biliary complications, 5 could not be treated endoscopically due to difficulty of cannulation $(\mathrm{n}=2)$, and large sized biliary leakage $(\mathrm{n}=3)$. They were all converted to surgical treatment. Biliary leakage $(n=18)$ and stricture $(n=7)$ were treated by ENBD and EBD, respectively. Finally, 20 of 25 patients $(80.0 \%)$ could be treated endoscopically. There were no significant ERCP-related complications. Conclusion. The right-lobe donors for LDLT have a significantly higher incidence of biliary complications than the left-lobe donors. The endoscopic treatment is useful for biliary complications of donors for LDLT and should be attempted before surgical conversion.

\section{T1220}

Evaluation of Transabdominal Ultrasonography for Biliary Complications After Liver Transplantation in Recipients with Choledochocholedochostomy Without T-Tube

Chad J. Long, Scott Biggins, Ruth B. Goldstein, Eugene Yen, Karen Bagatelos, Sandy Feng, James W. Ostroff

BACKGROUND: Biliary complications after liver transplantation (LT) are common, account for significant morbidity, and can be difficult to distinguish from graft rejection or disease recurrence. Transabdominal ultrasonography (TAUS) is a non-invasive initial diagnostic test for biliary complications after LT. Prior studies have predominantly evaluated TAUS in LT recipients with Roux-en Y biliary reconstruction or choledochocholedochostomy (CDCD) with a T-tube. AIM: To evaluate the diagnostic characteristics of TAUS for biliary complications after LT in patients with CDCD without T-tube METHODS: Records of patients who had LT with CDCD anastomosis without T-tube at our institution between 4/30/1998 and 5/30/2003 were reviewed for evidence of post-transplant endoscopic retrograde cholangiopancreatography (ERCP), percutaneous transhepatic cholangiography (PTC), or biliary surgery performed for the evaluation of a suspected biliary complication. Patients within this group having TAUS within 4 weeks prior to ERCP, PTC, or surgery were included in this study. TAUS reports were compared to ERCP, PTC, or surgical reports as the goldstandard. Biliary dilation, perihepatic fluid collections, or intraductal stones were considered positive sonographic findings. RESULTS: Of 459 LT recipients, 82 patients met inclusion criteria for this study. Mean age was 51 years (range 18-72) and 58\% were male. The most common reason for transplantation was hepatitis C. 5/82 patients received living donor transplants. The mean (range) time from TAUS to the gold-standard exam was $4.5(0-28)$ days. A biliary complication was confirmed in $71 / 82(87 \%)$ patients based on gold standard evaluation. The sensitivity of TAUS for detecting a biliary complication was 60/66 (91\%) with a specificity of $5 / 16(31 \%)$. The sensitivity for detecting anastomotic stricture, nonanastomotic stricture, and biliary leaks was $95 \%, 100 \%$, and $100 \%$ respectively. The positive predictive value and negative predictive value of TAUS in detecting any biliary complication in this study was 60/71 (85\%) and 5/11 (45\%), respectively. CONCLUSION: In LT recipients with CDCD biliary anastomosis, transabdominal ultrasonography is a sensitive screening modality for the evaluation of biliary tract complications. Transabdominal ultrasonography should be included in the initial evaluation of suspected post-tranplant biliary complications.

\section{$\mathrm{T} 1221$}

Predictive Factors for Graft and Patient Survival Following Liver Transplantation for Hepatitis C

Speranta Iacob, Susanne Beckebaum, Razvan Iacob, Vito Cicinnati, Christian Klein, Lian Gheorghe, Cristian Gheorghe, Irinel Popescu, Andrea Frilling, Massimo Malago, Guido Gerken, Christoph Broelsch

INTRODUCTION: Hepatitis $\mathrm{C}$ virus (HCV) associated liver disease is the most common indication for liver transplantation (LT). Short-term patient and graft survival for HCVinfected recipients have been reported to be similar to that of most other indications, but long-term survival rates are shorter. AIM \& METHODS: To assess the impact of different pre and post LT factors on graft survival. For this purpose we reviewed the records of 168 patients who underwent LT for HCV liver cirrhosis between January 1989 and October 2004. To identify potential predictors of graft and patient survival, univariate and multivariate Cox's proportional hazards regression model was used. RESULTS: One year overall graft and patient survival rates were $72 \%$ and $85 \%$ respectively and 5 year survival rates were $51 \%$ and $73 \%$. Median survival was 6.1 years for graft and 12 years for patient. In the univariate survival analysis the following parameters were identified as predictors of both graft failure and patient death: diagnosis of histologically proven recurrent hepatitis before 1 year after LT ( $<<0.0001$ and 0.001 ), presence of early (within the first 6 months) biliary complications $(\mathrm{p}=0.0007$ and 0.002$)$, administration of prednisone $<180$ days $(\mathrm{p}<0.0001$ and 0.01 ) and mycophenolate mofetil (MMF) $<180$ days ( $\mathrm{p}=0.0009$ and 0.02 ), induction without azathioprine $(\mathrm{p}=0.0002$ and 0.003$)$. Absence of post LT antiviral therapy was associated with a poorer graft survival $(\mathrm{p}=0.003)$ and severity of pre LT liver disease illustrated by Child-Pugh classification $C(p=0.009)$, MELD score $>18(\mathrm{p}=0.01)$, presence of hepatorenal syndrome $(\mathrm{p}=0.03)$ with patient mortality. Independent predictors of both graft failure and patient death in the multivariate survival analysis were: presence of early biliary complications $(p=0.0001$ and $p=0.004)$, administration of $M M F<180$ days $(p<0.0001$ and $p=0.02)$ and time to histologic recurrence less than 1 year $(\mathrm{p}<0.0001$ and $\mathrm{p}=0.001)$. Graft loss was independently influenced also by the recent year of LT after $2000(\mathrm{p}=0.0009)$, administration of prednisone $<180$ days $(\mathrm{p}=0.0004)$ and induction without azathioprine $(\mathrm{p}=0.0005)$. A higher MELD score $>18(\mathrm{p}=0.01)$ had a independent negative prognostic value for post LT patient survival. CONCLUSIONS: There are factors affecting both graft and patient survival, among which certain immunosuppressive strategies, the presence of early bile duct complications and the identification of the histologic HCV recurrence in the first posttransplant year seem to be of major importance.

\section{T1222}

Long-Term Outcome of Orthotopic Liver Transplantation in Cryptogenic Cirrhosis

Herve Boucard, Rajat Parikh, Arun Samanta, Baburao Koneru, Dorian Wilson, Adrian Fisher, Meelie Debroy, Andrew de la Torre, Kenneth Klein

Cryptogenic cirrhosis (CC) is one of the common indications for liver transplantation. Long term outcome in CC is not clearly defined with previous studies revealing conflicting results. It is being increasingly recognized that hepatitis following transplantation occurs in such patients with varying frequency (0-50\%). Aim: The present study compared the outcome of liver transplantation in CC with that in hepatitis $\mathrm{C}$ virus related cirrhosis (HCVC) by studying patient and graft survival. Method: This is a retrospective study involving 41 patients who underwent transplantation for CC between December 1994 and October 2003 
at our transplant center. The diagnosis of $\mathrm{CC}$ was made by negative serology for autoimmune liver diseases (ANA, Anti-smooth muscle Ab, AMA), negative viral hepatitis serology (anti-HCV, HCV-RNA by PCR, HBsAg, HBsAb, HBcAb), normal ceruloplasmin, $\alpha-1$ antitrypsin, transferrin saturation and confirmed by liver histology that was negative for viral or known metabolic liver diseases. Results: Mean follow up for the CC group (mean +/- SE) $5.76+/-0.44$ years and $4.14+/-0.31$ years for HCVC patients. For patients with CC the age, BMI and MELD score were $55.2+/-1.93$ years, $30.9+/-3.82 \mathrm{~m} 2 / \mathrm{kg}$, and $19.45+/$ 1.06 respectively compared to $52.04+/-1.15$ years, $27.58+/-0.68 \mathrm{~m} 2 / \mathrm{kg}, 19.87+/-0.97$ for HCVC patients. Diabetes mellitus was present in $55 \%$ of CC and $39 \%$ of HCVC (p= 0.17 ). Actuarial patient survival by Kaplan Meier analysis for CC was significantly better at 1 and 3 years being $90+/-7.5 \%$ and $77.5+/-6.6 \%$ compared to $84+/-4.6 \%$ and $62+/$ $6 \%$ for HCVC patients $(\mathrm{p}=0.0026)$. Actuarial graft survival for CC was also significantly better at 1 and 3 years being $87.8+/-5.1 \%$, and $78+/-6.5 \%$ compared to $77+/-4.96 \%$ and $54+/-5.9 \%$ for HCVC patients $(\mathrm{p}=0.0004)$. Conclusions: This study indicates that OLT for CC is associated with a favorable outcome for patient and graft survival compared to HCVC

\section{T1223}

Frequency of Development of Hepatocellular Carcinoma and the Utility of Surveillance Ultrasound Examination in Patients with END Stage Liver Disease (ESLD) from Hepatitis C Cirrhosis On the Liver Transplantation Waiting List

Anna Wieckowska, Nizar N. Zein

Background: Patients with hepatitis $\mathrm{C}$ virus (HCV) cirrhosis are at increased risk for the development of hepatocellular carcinoma (HCC). Periodic surveillance ultrasound examination for early detection for HCC has been recommended. Aims: 1) to determine the frequency of HCC in patients with ESLD due to HCV on the liver transplant (LT) waiting list, 2) to define the utility of surveillance ultrasound in identifying HCC in this population. Methods: Patients with HCV cirrhosis in the absence of HCC that were listed for LT at our institution between 1997 and 2004 were identified. Histologically proven HCC (by biopsy, explanted liver or on autopsy) was used to define HCC in this population. Patients who died prior to LT and had no autopsy examination were excluded. Patients who were still on the waiting list at the time of the analysis were excluded since histological examination was not available. All patients had at least one ultrasound examination while waiting for LT. A total of 211 ultrasound examinations were performed in 98 patients during the waiting time ( 1 to 8 ultrasounds per patients). The mean time from listing to transplantation was 10.7 months, Results: In total, 13 patients were found to have histologically proven HCC (13\%). Of 98 patients, $18(18.4 \%)$ had at least one positive ultrasound examination identifying a liver nodule during the waiting time on the list. Of these 18 patients, 9 were confirmed to have HCC by histology, while 9 appeared to have a false positive ultrasounds for HCC. Of the remaining 80 patients who had a negative ultrasound, $4(5 \%)$ were found to have $\mathrm{HCC}$ on histologic examination of explanted liver (2 of $4 \mathrm{HCC}$ were greater than $2 \mathrm{~cm}$ ). Overall, surveillance US had a specificity of $89.4 \%$, a sensitivity of $69.2 \%$ and a positive and negative predictive value of $50 \%$ and $95 \%$, respectively. Subjects with large nodules by ultrasound examination (over $2 \mathrm{~cm}$ ) were $60 \%$ more likely to have HCC than those with small nodules (OR $[95 \%$ CI]: $1.6[0.2,10.8]$ ). Conclusion: 1) Patients with ESLD due to HCV cirrhosis on the LT waiting list had a high frequency of HCC, supporting the need for surveillance measures with high sensitivity for early detection. 2) The sensitivity of surveillance ultrasound for early detection of HCC appears to be suboptimal, particularly in the current era where patients with HCC may receive a higher priority score for LT.

\section{$\mathrm{T} 1224$}

Hepatitis B, Tumor Treatment and Immunosuppression Influence Recurrence of Hepatocellular Carcinoma After Liver Transplantation

Sandra Ciesek, Thomas Becker, Tim Greten, Juergen Klempnauer, Michael P. Manns,

Christian P. Strassburg

Hepatocellular carcinoma (HCC) represents a considerable therapeutic challenge. Transarterial chemoembolization (TACE) and percutaneous ethanol instillation (PEI) are employed non-curing modalities. A potentially curative option is orthotopic liver transplantation (OLT). Aim of this study was to identify factors associated with HCC recurrence. Eighty-five patients with HCC transplanted between 1975-2004 at Hannover Medical School were analyzed retrospectively. Follow-up ranged from 13 to 349 months (mean 88 months \pm 75 .6). In 8/ $85(9.4 \%)$ HCC was established post OLT. Etiologies of chronic liver disease included HCV (39\%) and HBV (24\%) infection, and alcoholic cirrhosis (15\%). Cirrhosis was absent in 6/ $85(7 \%)$. Recurrent HCC occurred in $18.8 \%$ after a mean of 3.3 years following OLT. HCC stages at OLT were Tl (18\%), T2 (37\%), T3 (13\%) and T4 (12\%). Recurrence was associated with T4 ( $31 \%$ vs $7 \%, \mathrm{p}=0.018$, OR 5.82 ) but not with Tl-3, with $\mathrm{HBV}(60 \%$ vs $40 \%$, p= 0.02 OR 3.85), and with patients receiving tacrolimus ( $62.5 \%$ vs $37.5 \%, \mathrm{p}<0.0001$, OR 10$)$ and/or steroids ( $69 \%$ vs. $31 \%$, p=0.0006, OR 7.69) compared to cyclosporine or mycophenolate mofetil. After pre-operative TACE HCC recurrence $(62.5 \%)$ was more frequent compared to PEI (12.5\%) ( $<<0.0001$, OR 12.5), the PEI vs TACE group had 28\%/0\% Child C, 39\%/ $67 \%$ Child B, and 33\%/33\% Child A. Recurrence-free survival was achieved in $80 \%$ of patients undergoing OLT. Tumor stage, TACE before OLT, HBV infection and immunosuppression with tacrolimus and steroids were identified as risk factors for HCC recurrence. Preand post-operative management of HCC therefore influences the outcome in OLT in HCC.

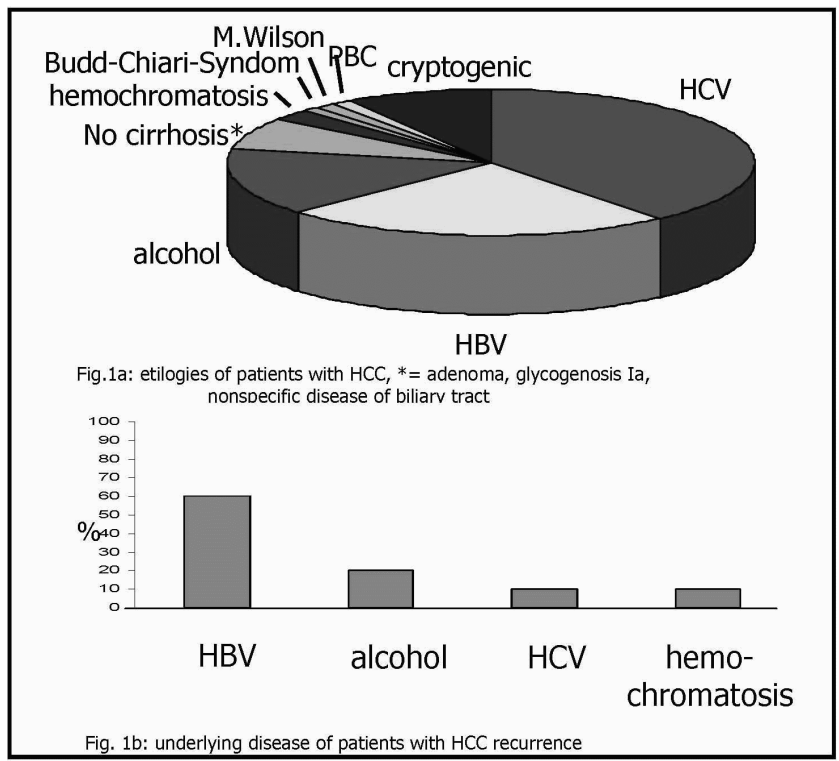

T1225

Effects of Tegaserod On Restraint Stress-Induced Visceral Hypersensitivity and C-FOS Expression On Spinal Cord in Rats

Yan Sun, Gengqing Song, Fengling Liu, Wei Qian, Xiaohua Hou

Aims: The aim of this study was to investigate the effects of Tegaserod on restraint stressinduced visceral hypersensitivity and c-fos expression on spinal cords in rats. Methods: The study was performed in two experiments. In the first experiment, male Sprague-Dawley rats were studied in control group without restraint stress, restraint stress groups with saline, Tegaserod at different doses $(0.3 \mathrm{mg} / \mathrm{kg}, 0.6 \mathrm{mg} / \mathrm{kg}, 1.2 \mathrm{mg} / \mathrm{kg}$, IP, respectively), or vehicle (1-methyl-2-pyrrolidone $10 \%$ ) at different doses $(0.3 \mathrm{ml} / \mathrm{kg}, 0.6 \mathrm{ml} / \mathrm{kg}, 1.2 \mathrm{ml} / \mathrm{kg}$, IP, respectively). The abdominal withdrawal reflex (AWR) score to colorectal distension (CRD) was assessed. The second experiment included a control group without restraint stress and three other groups with restraint stress. The experiment was conducted sequentially as follows restraint stress for $2 \mathrm{~h}$, administration of Tegaserod $(1.2 \mathrm{mg} / \mathrm{kg}$, IP), vehicle $(1.2 \mathrm{ml} / \mathrm{kg}$, IP) or saline $(1.2 \mathrm{ml} / \mathrm{kg}, \mathrm{IP})$ respectively, and repetitive CRD for $2 \mathrm{~h}$. The expression of $\mathrm{c}$-fos on the thoraxlumbar (T13-Ll) and lumbarsacral (L6-S1) spinal segments was detected by using immunohistochemistry method. Results: (1) The AWR scores in restraint stress groups with saline or vehicle were significantly higher than those in control group (Saline: $1.70 \pm 0.50$ vs. $0.86 \pm 0.32, \mathrm{P}<0.05$; Vehicle: $1.45 \pm 0.35$ vs. $0.86 \pm 0.32, \mathrm{P}<0.05$ ). (2) In comparison with the restraint stress groups with saline or vehicle, the AWR scores in restraint stress group with Tegaserod were significantly decreased in a dose-dependent manner at all distension pressures $(\mathrm{P}<0.05)$. (3) In contrast to the control group, $\mathrm{c}$-fos expression on L6-S1 and T13Ll spinal segments was significantly increased by repetitive CRD in restraint stress groups with vehicle $(45.5 \pm 3.9$ vs. $3.2 \pm 0.1$ and $40.1 \pm 5.6$ vs. $3.5 \pm 1.0, \mathrm{P}<0.05)$ or saline $(35.5 \pm 3.5$ vs. $3.5 \pm 1.0$ and $28.8 \pm 2.63$ vs. $3.8 \pm 1.1, P<0.05)$. The $c-$ fos immunoreactive positive neurons were mainly localized in laminae I-II, V-VII and X. (4) The c-fos expression in the restrain stress groups with Tegaserod was significantly lower than that in restraint stress groups with vehicle or saline ( $19.6 \pm 1.3$ and $15.7 \pm 1.8, \mathrm{P}<0.05)$. Conclusions: Tegaserod dose-dependently decreases restraint stress-induced visceral hypersensitivity and c-fos expression on L6-S1 and T13-Ll spinal segments. These findings suggest an antinociceptive property of Tegaserod under stress condition.

$\mathrm{T} 1226$

Medical Consultation for Children's Chronic Stomachaches Is Related to a Belief That the Symptoms Have a Physical Cause But Is Not Related to Anxiety About Serious Missed Diagnosis

Miranda Al. van Tilburg, Olafur S. Palsson, Denesh K. Chitkara, William E. Whitehead

Previous studies have shown that parental worries about their child's abdominal pain predict illness behaviors such as doctor visits and school absences (Gastroenterology 2004; 126(4), A372). Contrary to expectations, however, fear of an overlooked serious illness did not motivate consulting behavior. The aim of the present study was to confirm this observation using a more detailed evaluation of disease conviction. METHODS: We recruited 183 parents (97.8\% mothers) of children ages $1-15$ (M=8.1, 66.1\% female) through the UNC Cente for Functional GI disorders website. All parents filled out the Illness Attitude Scale (IAS) by Proxy. The IAS is a well validated measure of hypochondriac concerns with 2 subscales: Health Anxiety (HA) and Illness Behaviors (IB). Illness behavior refers to the way people perceive, evaluate and react to somatic symptoms and was measured by items such as number of doctor visits, number of treatments, school absences, and concentration difficulties. We revised the IAS to measure hypochondriacal concerns about one's child. Factor analysis yielded two similar scales for the IAS by proxy as for the original IAS, Cronbach $\alpha$ were .88 for HA and .80 for IB. RESULTS: 140 families consulted a doctor for their child's stomach aches $(76.5 \%)$. Consulting families $(\mathrm{CF})$ reported increased IB compared to nonconsulting families (NCF; $M=9.2$ vs $M=7.2, p<.01$ ). There were no significant differences in HA between CF and NCF. Additionally we tested 6 of the $11 \mathrm{HA}$ items that focus on disease conviction separately. CF were more likely to believe that their child was suffering

\section{(1)}


from a physical disease $(M=.72$ vs $M=1.72 ; \mathrm{p}<.01)$ but no significant differences were found for worrying about child's health, belief that pain is caused by a serious illness, or fear that child suffers from cancer, heart disease or another serious illness. Parents who suffered from chronic abdominal pain themselves $(78.7 \%$ ) did not report more HA or IB compared to non-sufferers. Duration of symptoms significantly correlated with IB $(\mathrm{r}=.23 ; \mathrm{p}<.01)$ but not with HA. CONCLUSIONS: Medical consultation for children's stomach aches is more likely if parents believe that a physical illness is causing the symptoms regardless of whether the illness is seen as serious or life threatening. Excluding major illnesses will most likely not be reassuring nor change parental illness behavior. Offering coping mechanisms might have more positive effects. [Supported by grant R24 DK67674]

\section{T1227}

Abdominal Pain and Co-Morbid Complaints from Childhood to Adulthood in a Population Based Birth Cohort

Denesh K. Chitkara, Nicholas J. Talley, Amy L. Weaver, Miranda van Tilburg, Slauvica K. Katusic, G. Richard Locke, Mary Jo Rucker, William E. Whitehead

Background: Abdominal pain (AP) of unknown origin is a common medical complaint and is associated with comorbid complaints (headache, limb pain, back pain, anxiety and depression). However, little is known about the medical presentation of AP with co-morbid complaints throughout the lifespan. Aim: To examine the prevalence and patterns of comorbidity in individuals who present for AP from childhood to adulthood. Methods: A birth cohort of all children born between 1976 and 1982 to mothers who were residents of Rochester, MN, and who remained in the community after age 5 years, was considered for this study. Medical visits for AP of unknown origin and co-morbid conditions (above) were identified by HICDA codes. Subjects were followed based on their diagnoses accumulated while $<21$ yo, and $80 \%$ of the population remained in the area until age 19. A group of age and gender matched control individuals without a diagnosis of AP before age 21 was used to compare co-morbid conditions. Results: Of the 5347 birth cohort members, 1358 $25 \%$ ) individuals had an incident medical visit for AP of unknown origin from 5 to $<21$ yo (single visit only $61 \%, 2$ visits $21 \%, 3$ visits $9 \%, 4+$ visits $9 \%$ ). Individuals with a medical visit for AP were more likely to present for headache (34\% vs. $16 \%$ ), back pain ( $18 \%$ vs. $8 \%$ ), limb pain ( $29 \%$ vs. $18 \%$ ), anxiety (34\% vs $16 \%$ ) and depression $(24 \%$ vs. $11 \%$ ) compared to those who did not present for AP. Individuals with multiple visits for AP had a progressively increased risk for presenting with a co-morbid diagnosis (Table; all $\mathrm{p}<0.001$ ). In individuals who had a visit for AP prior to being diagnosed with any of the co-morbid conditions (47\%), the average time between visits was 4.8 years (AP diagnosed at mean 10.4 years and co-morbid diagnoses at a mean of 15.2 years). For individuals with an $\mathrm{AP}$ diagnosis occurring after the co-morbid diagnosis (46\%) the average time between the diagnoses was 4.6 years. Conclusion: Individuals who present with 1 or more medical visit for AP are more likely to present with a co-morbid conditions throughout their lives. Comorbid diagnoses were no more likely to follow than to precede an AP visit. Supported by R24 DK67674 and an unrestricted research grant to the Dyspepsia Center from Novartis. The risk (O.R. 95\% C.I.) for comorbid conditions with 1 or more visit for AP compared to age/gender matched controls with no visit for $\mathrm{AP}$

\begin{tabular}{|c|c|c|c|c|c|}
\hline Group & Headache & Backpain & Limb pain & Anxiety & Depression \\
\hline AP, 1 visit & $2.0(1.6-2.4)$ & $1.6(1.2-2.2)$ & $1.8(1.5-2.3)$ & $2.1(1.7-2.6)$ & $2.0(1.6-2.5)$ \\
\hline AP, 2 visits & $3.5(2.6-4.6)$ & $2.8(2.0-3.9)$ & $2.5(1.9-3.4)$ & $2.8(2.1-3.7)$ & $2.9(2.1-4.0)$ \\
\hline AP, 3 visits & $4.7(3.2-6.9)$ & $4.1(2.6-6.4)$ & $3.0(2.0-4.4)$ & $4.9(3.3-7.2)$ & $4.0(2.6-6.1)$ \\
\hline AP, 4+ visits & $5.4(3.7-7.9)$ & $7.6(5.0-11.3)$ & $4.8(3.3-7.0)$ & $5.7(3.9-8.3)$ & $7.1(4.8-10.5)$ \\
\hline
\end{tabular}

T1228

Hypervigilance in Irritable Bowel Syndrome (IBS) Compared with Other Chronic Gastrointestinal Disorders Iris Posserud, Jan Svedlund, Jenny Wallin, Magnus Simren

Background: Selective attention (hypervigilance) to gastrointestinal (GI) sensations has been suggested as a contributing pathophysiological factor in IBS. IBS patients have previously been shown to selectively recall words describing GI sensations compared with healthy controls and patients with asthma (Gibbs-Gallagher et al Am J Gastroenterol 2001). However, it remains unclear whether there are differences between IBS and other chronic GI disorders. Aim: Evaluate the presence of hypervigilance towards the GI tract in IBS comparing these patients with patients with other chronic GI diseases. Method: We included 20 IBS patients (mean age 36 (22-55) years; 12 females) and 28 patients with organic GI disease (mean age 40 (20-60) years; 14 females) consisting of 15 patients with inflammatory bowel disease (IBD) and 13 with celiac disease. Patients with IBD and celiac disease were in clinical remission. They completed the Hospital Anxiety and Depression scale (HAD) and underwent three tests: 1) Word association - freely write down as many words as possible representing signs of disease during 60 seconds. 2) Word recall - memorize words representing $10 \mathrm{Gl}$ symptoms, 10 positive affects, 10 negative affects presented randomly (each word displayed for 3 seconds), then 15 minutes break before writing down remembered words. 3) Word recognition (tachistoscope) - 3 sets of 4 categories of words (positive affect, symptom of non-GI disease, GI symptom, negative affect) displayed (flashed) for increasing time (adding 5 milliseconds (msec) each step) until the subject identified the word. Results: For the word association test IBS patients wrote down on average 8.0 symptoms including $1.8 \mathrm{GI}$ symptoms compared with 7.6 symptoms including 1.3 GI symptoms in the organic group (NS). For the word recall test both groups remembered on average 10 words. The mean percentage of GI words among remembered words was $44 \%$ in the IBS group and $40 \%$ in the organic GI disease group. During the word recognition test the IBS patients were, compared with the organic GI disease group, significantly faster in recognizing words representing Gl symptoms ( 21 vs. 28 msec; $p=0.03$ ) and negative affects ( 25 vs. $36 \mathrm{msec} ; \mathrm{p}=0.04)$, but also tended to be faster in recognizing positive affects ( 25 vs. $32 \mathrm{msec} ; \mathrm{p}=0.06)$ and non-GI symptoms ( 22 vs. 30 msec; $p=0.09$ ). There were no differences in HAD score between the groups. Conclusion: Our results suggest that there might be a hypervigilance regarding GI sensations in IBS compared with patients with other chronic GI disorders. Despite no differences in anxiety and depression, IBS patients also seem to be hypervigilant regarding negative affects.

\section{T1229}

Effect of Autogenic Training in Patients with Irritable Bowel Syndrome: A Randomized Controlled Trial

Masae Shinozaki, Motoyori Kanazawa, Michiko Kano, Yuka Endo, Michio Hongo, Shin Fukudo

Background: Autogenic training (AT) is an useful and comprehensive relaxation technique However, there have been no studies that proved the effect of AT in irritable bowel syndrome (IBS). With the randomized controlled trial, we test the hypothesis that AT is effective on IBS symptoms. Subjects and Methods: Twenty-one IBS patients were randomly assigned to AT ( $\mathrm{n}=11,5$ male, 6 female, aged $33 \pm 3$ ) or control therapy ( $\mathrm{n}=10,5$ male, 5 female, aged $30 \pm 15$ ). IBS was diagnosed with Rome II criteria. AT patients were trained intensively by specialists eight sessions. The interval of each session was 2-4 weeks. Control therapy was discussion about their life styles with same frequency and interval. At each session, all patients completed to answer the adequate relief (AR) of IBS symptoms as a primary endpoint. Moreover, patients fulfilled the four questionnaires: Self-induced IBS Questionnaire (SIBSQ) for IBS severity, Self-reported Depression Scale (SDS) for depressive state, StateTrait Anxiety Inventory (STAI) for anxiety, and Medical Outcome Short Form 36 Health Survey (SF-36) for quality of life (QOL). Results: The proportion of AR in AT group was significantly higher than that in controls (ANOVA: group: $\mathrm{p}<0.0001$, interaction: $\mathrm{p}=0.017$, Figure 1). The two subscales of SF-36, SF and Bodily Pain, were significantly improved in AT group $(\mathrm{p}<0.05)$. Role Emotional $(\mathrm{p}=0.051)$ and General Health $(\mathrm{p}=0.068)$ showed trend improvement in AT group. SIBSQ, SDS, or STAI did not show the significant difference between AT group and controls. Conclusion: These results suggest that AT may be useful for IBS treatment, probably via improving impaired self-control feeling in patients with IBS Because AT is simple and comprehensive psychotherapy, further studies in IBS are warranted.

Figure 1

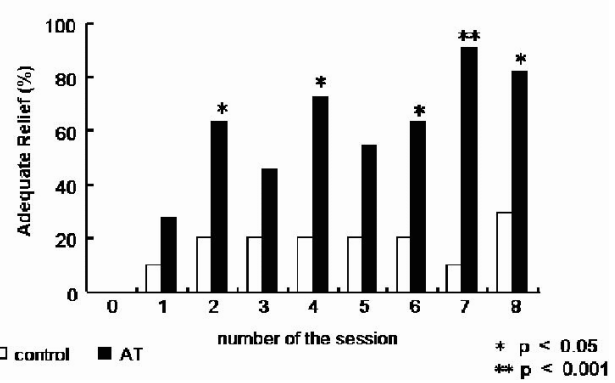

$\mathrm{T} 1230$

Minimally Important Difference (MID) for Health Related Quality of Life (HRQOL) Measures in Functional Bowel Disorders (FBD)

Douglas A. Drossman, Carolyn B. Morris, Yuming Hu, Brenda B. Toner, Nick Diamant,

William E. Whitehead, Christine B. Dalton, J.N. Leserman, Shrikant I. Bangdiwala,

Donald L. Patrick

Background. The study of HRQOL in FBD is of growing interest. What comprises a meaningful change in HRQOL for FBD patients that would impact interpretation of treatment benefit is unknown. Aim. To identify the MID using both anchor-based and statistical methods for condition specific (IBS-QOL) and generic (Sickness Impact Profile-SIP) measures among women with FBD undergoing a treatment trial. Methods. Females ( $n=402$ ) with FBD entering a 12-week treatment trial were studied at baseline and end of treatment with two HRQOL measures: 1) IBS-QOL (condition-specific), 2) SIP (generic-daily function). Change scores were compared using correlations and t-tests between the HRQOL measures and two more frequently used clinical anchors: a) change in Visual Analog Scale (VAS) pain averaged over 2-week periods prior and at end of treatment, and b) Treatment Efficacy Questionnaire (TEQ) obtained at end of treatment. Category definitions for pain and TEQ were defined as worsening $(<-25 \%$ drop in VAS; score $<20$ for TEQ), no change $(<-25 \%$ to $+50 \%$ for VAS, score $20-28$ for TEQ), and Improvement $(>+50 \%$ for VAS and $>28$ for TEQ - the definition of a responder). In addition, using the method of Norman (Medical Care, 2003). we calculated a statistically based MID using $1 \frac{12}{2}$ SD of the baseline values for the two HRQOL measures. Significance was $\mathrm{p}<0.05$. Results. 1) IBS-QOL more than SIP were moderately correlated with pain $(r=-0.22$. $p<0.0001$ for IBS-QOL; $r=0.17 ; p=0.003$ for SIP $)$ and Satisfaction ( $r=0.36 ; p<0.0001$ for IBS-QOL; $r=0.14 ; p=0.04$ for SIP); 2) The HRQOL values for clinical change categories are shown in Table 1, indicating MID for IBS-QOL and SIP of approximately 14 (13.8 for pain and 14.3 for Satisfaction) and 3.5 (4.77 for pain and 2.43 for Satisfaction); 3) 1/2 SD at baseline for IBS-QOL was 10.2 and for SIP was 3.5. Conclusion. We have estimated the MID in women with moderate to severe FBD is approximately 14 for the IBS-QOL and 3.0-4.0 for the SIP. Supported by NIH: RO1DK49334, R24 DK067674, and GCRC\#RR-000046 Table 1 


\begin{tabular}{|c|c|c|c|c|c|c|c|c|c|}
\hline \multicolumn{10}{|c|}{ QOL Scores by Pain Categories defined by \% improvement } \\
\hline & \multicolumn{3}{|c|}{ Worsening } & \multicolumn{3}{|c|}{ No Change } & \multicolumn{3}{|c|}{ Improvement } \\
\hline & \multicolumn{3}{|c|}{$<-25 \%$} & \multicolumn{3}{|c|}{-25 to $50 \%$} & \multicolumn{3}{|c|}{$>50 \%$} \\
\hline Label & $\mathrm{N}$ & Mean & SD & $\mathrm{N}$ & Mean & SD & $\mathrm{N}$ & Mean & SD \\
\hline QOL Change & 39 & 6.19 & 13.80 & 173 & 9.12 & 15.49 & 94 & 13.81 & 16.11 \\
\hline SIP Change & 39 & 2.38 & 5.16 & 173 & 1.47 & 4.91 & 94 & 3.06 & 4.77 \\
\hline \multicolumn{10}{|c|}{ QOL Scores by Satisfaction Categories defined by total score } \\
\hline & \multicolumn{3}{|c|}{ Worsening } & \multicolumn{3}{|c|}{ No Change } & \multicolumn{3}{|c|}{ Improvement } \\
\hline & \multicolumn{3}{|c|}{$8-<20$} & \multicolumn{3}{|c|}{$20-<28$} & \multicolumn{3}{|c|}{$28-40$} \\
\hline Label & $\mathrm{N}$ & Mean & SD & $\mathrm{N}$ & Mean & SD & $\mathrm{N}$ & Mean & SD \\
\hline QOL Change & 51 & 1.83 & 13.94 & 86 & 5.08 & 12.69 & 187 & 14.26 & 16.46 \\
\hline SIP Change & 51 & 1.23 & 3.99 & 86 & 1.21 & 4.34 & 187 & 2.43 & 5.35 \\
\hline
\end{tabular}

$\mathrm{T} 1231$

Somatization Trait and Somatization Disorder Have Different Impacts On IBS Miranda Al. van Tilburg, Olafur S. Palsson, William E. Whitehead

The psychological trait of Somatization and Somatization Disorders (SD) are both increased in Irritable Bowel Syndrome (IBS) and are related to symptom severity. However, somatization and SD are not completely overlapping concepts. Somatization refers to the tendency to report an excess of non-specific symptoms and is usually measured with questionnaires. Somatization Disorder is a psychiatric diagnosis characterized by a history of many clinically significant physical complaints that are not fully explained by a medical condition. It is usually diagnosed by a clinician according to the Diagnostic and Statistical Manual IV (DSMIV) criteria. We explored how useful both concepts were in explaining well-being and symptom status among IBS patients. METHODS: 156 IBS patients (age 18-73 $\mathrm{M}=35.2$; 83.3\% females) completed the Recent Physical Symptom Questionnaire (RPSQ; IBS specific somatization), the Brief Symptom Inventory-18 (BSI-18; psychological distress), IBSS (IBS Severity), IBS-QOL (Quality of Life) and the SD section of the Structural Clinical Interview for DSM-IV (SCID; Somatization Disorder). RESULTS: 22 Patients (14.7\%) met criteria for SD or undifferentiated SD. T-tests revealed significant differences between SD and non-SD patients in RPSQ $(\mathrm{M}=13.9$ vs $10.4 ; \mathrm{p}<.01)$ and IBSS $(\mathrm{M}=314.6$ vs $271.3 ; \mathrm{p}<.05)$, but not in BSI or IBS-QOL. Somatization as measured by the RPSQ correlated significantly with IBSS $(\mathrm{r}=.26 ; \mathrm{p}<.01)$, BSI-anxiety $(\mathrm{r}=.53, \mathrm{p}<.001)$; BSI-depression $(\mathrm{r}=.53, \mathrm{p}<.001)$ and IBS-QOL $(r=-.46, p<.001)$. In linear regression analyses with SD and RPSQ as independent variables; SD was only a significant predictor of BSI Anxiety $(\beta=-.16 ; \mathrm{p}<.05)$. RPSQ explained $6 \%$ of IBSS $(\beta=.18 ; p<.05) 20 \%$ of IBS-QOL $(\beta=-.44 ; p<.001) 19 \%$ of BSI Depression $(\beta=$ $.556 ; \mathrm{p}<.001)$ and together with SD, $32 \%$ of BSI anxiety $(\beta=.58 ; \mathrm{p}<.001)$. CONCLUSIONS: In this sample of IBS patients we found a high incidence of SD, similar to previous studies. However, SD was a poor predictor of IBS outcomes. The data indicates that somatization within the normal range affects IBS outcomes linearly. Somatization, as a psychological trait, is therefore a more sensitive measure than SD in determining impacts on IBS outcomes. As clinicians and researchers it is important to focus on the whole range of somatization rather than SD alone. [Supported by grants R24 DK67674, R01 DK31369 and RR00046]

\section{$\mathrm{T} 1232$}

Parental Concerns in Childhood Functional Abdominal Pain: A Comparison Study

Nader N. Youssef, Miranda van Tilburg, Annette Langseder, William E. Whitehead

BACKGROUND: Children with functional abdominal pain (FAP) have increased rates of school absenteeism and decreased participation in social activities as compared to children with abdominal pain of organic origin. One possible explanation is that FAP parents have a different set of concerns than parents of children with organic gastrointestinal disorders and may contribute to social withdrawl of these children. AIM: To compare concerns among groups of parents whose children have FAP or organic causes for their abdominal pain (ORG) to parents of healthy children without chronic abdominal pain (HC). SUBJECTS \& METHODS: From 04/ 2005 to 11/ 2005, 232 parents of children (mean age, 9.6 years, range 4 to 18 years, $55 \%$ female) presenting to a tertiary pediatric gastroenterology office completed a validated 25-item parent assessment instrument, Parental Worry of RAP Questionnaire (PWRQ), prior to their inital evaluation for abdominal pain. It consists of 4 subscales: (1) Fear to ignore symptoms-worries surrounding the willingness to ignore symptoms (6 items), (2) Desire for care- frustration with doctors (7 items), (3) Worry about copingunable to cope with symptoms ( 7 items), and (4) Exacerbating factors- recognizing the role of stress and family (5 items). Responses are scored on a 1 (not at all worried) to 5 (very much worried) scale. Scores on items were added to create a subscale score. A clinical diagnosis was assigned to each survey. A diagnosis of FAP was made utilizing the Pediatric Rome Criteria. Parents of children diagnosed with FAP (N=135) were compared to those with organic causes (gastroesophageal reflux, constipation, and inflammatory bowel, ORG $\mathrm{N}=65$ ) and a group of age $\&$ gender matched children without abdominal pain served as healthy controls ( $\mathrm{HC}, \mathrm{N}=36$ ). RESULTS: (Table) CONCLUSIONS: Parents of children with chronic abdominal pain have significant concerns when compared to those of healthy controls. There was no difference in specific parental worries between FAP and organic causes of pain. Therefore, decisions to limit school attendance and social activities do not arise from specific parental worries in FAP but may be a result of inappropriate parental responses to FAP and subsequent parental worries. Therapeutic interventions targeted at modifying parental response to FAP may serve as useful therapies for children with functional abdominal pain. (Grant R24DK67674).

PWRQ Scores

\begin{tabular}{|c|c|c|c|c|}
\hline & FAP & ORG & HC & SIG. \\
\hline Fear to Ignore Symptoms & 9.7 & 9.7 & 10.7 & ns \\
\hline Desire for Care & $16.1^{*}$ & $16.0^{*}$ & $11.5^{*}$ & $* \mathrm{p}<0.001$ vs. HC \\
\hline Worry about Coping & $22.7^{*}$ & $22.8^{*}$ & $13.0^{*}$ & $* \mathrm{p}<0.001$ vs. HC \\
\hline Exacerbating Factors & $13.1^{*}$ & $13.3^{*}$ & $8.8^{*}$ & ${ }^{*} \mathrm{p}<0.001$ vs. HC \\
\hline
\end{tabular}

\section{T1233}

Increase in Global Symptom Reporting Does Not Explain Increased Prevalence of Functional Bowel Disorders in First Degree Relatives of IBD Patients Lisbeth A. Selby, Freddy Caldera, Houssam Mardini, Willem de Villiers

There is mounting evidence that inflammatory bowel disease (IBD) and irritable bowel syndrome (IBS) may have a similar etiopathogenesis. In a recent study of 112 subjects we found that first degree relatives of IBD patients (FDR-IBD) meet Rome II criteria for functional bowel disorders (FBD) twice as often as general medical controls (GMC). Although this finding suggests that FBD, such as IBS, are part of the same spectrum as IBD biologically, there are numerous alternative explanations of these results. These include: increased symptom reporting in general, increased bowel symptom awareness by FDR-IBD, learned illness behavior, and response bias. Purpose: Evaluate the level of non-bowel symptom reporting in this group. High levels of non-bowel symptom reporting would suggest that the bowel symptoms endorsed by these FDR-IBD are part of a larger pattern of increased symptom reporting and do not necessarily reflect a true increase in FBD. Methods: A self-administered mail-back survey contained 16 Rome II bowel questions and 27 other questions on physica and psychological symptoms. Subjects were categorized as either FBD+ or FBD- based on Rome II criteria. Chi-square and Mann-Whitney U tests were used to assess the associations, estimate the odds ratios (OR) and the $95 \%$ confidence intervals of these associations. Results 112 surveys were evaluated, 55 from FDR-IBD and 57 from GMC. The mean age of FDRIBD was 39. The mean age of GMC was 48. In FDR-IBD with FBD mean age was 31 y. mean age of 46 in FDR-IBD without FBD (ns). FDR-IBD were $40 \%$ male; GMC were $32 \%$ male (ns). Aggregate non-bowel symptom reporting was similar among FDR-IBD and GMC (mean total no. symptoms $=5.88$ v. 5.09 , ns). Comparison of FDR-IBD who differed in FBD status also showed no significant differences in non-bowel symptoms. IBD relatives meeting FBD criteria did not have more non-bowel symptoms than GMC with FBD. Each of the 27 non-bowel symptoms was also analyzed separately. FDR-IBD with FBD significantly more often reported symptoms in only 3/27 categories: bruising - OR 5.5 (CI 1.3-23.8) urinary frequency - OR 6.7 (CI 1.2-36.6), and anxiety - OR 4.6 (CI 1.0 - 20.2). Notably musculoskeletal pain and fatigue, symptoms classically associated with FBD, were not more prevalent in FDR-IBD with FBD. Conclusion: FDR-IBD do not have globally increased somatic complaints compared to GMC. Therefore, our previous finding of an increased prevalence of FBD in this group seems to reflect a relatively isolated increase in bowe symptoms. The finding of increased anxiety in this group could suggest hypervigilance to bowel symptoms. Further study with validated instruments is needed.

\section{T1234}

Influence of Experimentally Induced Anxiety On Rectal Sensorimotor Function in Men

Brecht Geeraerts, Lukas van Oudenhove, Rita Vos, Jozef Janssens, Jan Tack

BACKGROUND: Various stressors, including emotions such as anxiety, may influence viscera perception and motor function, thereby eliciting or aggravating gastrointestinal (GI) symptoms. Experimentally induced anxiety was shown to affect gastric sensorimotor function, but it is unclear whether this also affects other parts of the GI tract. The AIM of the present study was to investigate the effect of experimentally induced anxiety on rectal sensorimoto function in health. METHODS: Eighteen healthy subjects (mean age $26.0 \pm 1.8$ years) participated in a rectal barostat study. To assess sensitivity to rectal distension and rectal compliance, stepwise isobaric distension ( $4 \mathrm{mmHg}$ steps at 1.5 minute intervals, until discomfort or pain) was performed during anxious and neutral emotional state. Both distension sequences were separated by a 30-minute adaptation period. Rectal compliance was calculated as the slope of the pressure-volume curve. Emotional context was altered using combined audiotape assisted recall of a neutral or anxious autobiographical experience and viewing of a set of validated neutral or fearful facial expressions. Emotional context was modulated for 10 minutes at the start of each distension sequence. Anxiety levels were assessed using visual analog scales (VAS) and the Spielberger State-Trait Anxiety Inventory (STAI). Lower abdominal sensation was scored on 0-6 graded scales ( $0=$ no sensation, $6=$ maximal) at the end of every distension step. RESULTS: Efficacy of anxiety induction was confirmed by VAS (AUC $0.6 \pm 0.3$ vs. $9.1 \pm 1.3 \mathrm{~mm} * \mathrm{mmHg} ; \mathrm{p}<0.00001)$ and STAI scores $(36.1 \pm 2.1$ vs. $45.6 \pm 2.5 ; \mathrm{p}=$ $0.005)$. Emotional context did not alter rectal compliance $(11.6 \pm 0.9 \mathrm{vs} .10 .6 \pm 1.0 \mathrm{ml} / \mathrm{mmHg}$ NS). Pressure needed to induce first perception $($ score $=1)(10.0 \pm 1.4$ vs $9.6 \pm 0.9 \mathrm{mmHg}$ NS) or discomfort (score $\geq 5)(29.3 \pm 1.4$ vs. $29.8 \pm 1.5 \mathrm{mmHg}$, NS), and corresponding intraballoon volumes (respectively $50.5 \pm 12.9$ vs. $40.6 \pm 5.4 \mathrm{ml}$, NS and $249.3 \pm 16.2$ vs. $231.4 \pm 21.2$ $\mathrm{ml}$, NS) were not significantly altered. CONCLUSION: Contrary to its influence on gastric sensorimotor function, experimentally induced anxiety does not affect rectal sensitivity or rectal compliance in healthy subjects. 
T1235

How Does Cognitive Behavioral Therapy for IBS Work? A Structural Equation Modeling Analysis of Results from a Randomized Controlled Trial Jeffrey M. Lackner, James Jaccard, Susan S. Krasner, Leonard A. Katz, Edward B. Blanchard

While evidence supports the efficacy of cognitive-behavioral therapy (CBT) for treating IBS, little is known about why it works or how to optimize CBT so that it is more potent, streamlined, and cost efficient. One hypothesis is that CBT achieves its therapeutic effects by reducing psychological distress (Aust NZ J Psychiatry, 39, 9, 807-815). Support for this hypothesis requires ruling out the possibility that changes in distress are a consequence, not cause, of symptom changes brought on by CBT. The relationship among symptom improvement, distress, and the important endpoint of quality of life (QOL) was formally evaluated using structural equation modeling (SEM) of data obtained prospectively from the acute treatment phase of an NIH funded clinical trial. 147 Rome II diagnosed IBS patients (81\% female) were randomized to 1 of 310 -wk conditions: (a) group-based CBT; (b) groupbased education (ED); or (c) wait list (WL). Primary endpoint was global ratings of IBS symptom improvement $(-100=$ substantially worse, $100=$ substantially improved $)$. Second ary endpoints were QOL (IBS-QOL) and psychological distress (BSI). SEM analyses yielded 3 major findings. First, CBT showed significantly greater symptom improvement than patients assigned to WL (mean post treatment difference $=33.83(95 \% \mathrm{CI}=16.26$ to 50.04 ). A comparison of CBT vs. ED (an active vs. passive control group) yielded statistically significant $(\mathrm{p}<0.01)$ relief of GI symptoms with CBT treated patients showing more symptom improvement than the ED patients (mean difference $=25.82(95 \% \mathrm{CI}=10.89$ to 39.45). Second, CBT reduced distress relative to $\mathrm{WL}(\mathrm{p}<0.05)$, but the effects were due to a complex chain of mediation. Specifically, data were consistent with a model where CBT tended to improve QOL and this, in turn, tended to lower distress (path coefficient $=-0.36,95 \% \mathrm{CI}=-0.57$ to $-0.10, \mathrm{p}<0.01$ ). Distress and QOL revealed a pattern consistent with a reciprocal causal relationship. Not only did QOL changes produce distress changes, but changes in distress produced changes in QOL (path coefficient $=-0.13,95 \% \mathrm{CI}=-.21$ to $-.06, \mathrm{p}<.01$ ). Third, 3 paths consistent with a model that CBT works by reducing distress did not approach statistical significance: (1) a direct path from global symptom improvement to posttest distress, (2) a direct path from posttest distress to global improvement or (3) a reciprocal influence between global improvement and posttest distress. Contrary to conventional wisdom, findings suggest that reducing psychological distress is not the primary mechanism through which CBT achieves global improvement of IBS symptoms. Supported by NIDDK grants $67878 \& 54211$.

\section{$\mathrm{T} 1236$}

Psychological Trait Dimensions in Functional Dyspepsia: Descriptive Data and Relationship with Gastric Pathophysiology

Lukas van Oudenhove, Joris Vandenberghe, Brecht Geeraerts, Rita Vos, Phillippe

Persoons, Sebastien Kindt, Koen Demyttenaere, Jan Tack

Background: Psychological trait dimensions including alexithymia and trait anxiety are believed to play a role in onset, course and outcome of functional dyspepsia (FD). This study aims to describe the prevalence of alexithymia and trait anxiety and their relationship with gastric pathophysiological mechanisms in FD. Methods: We studied gastric sensorimotor function with a barostat (sensitivity, compliance \& accommodation) and gastric emptying test in 139 consecutive tertiary care FD patients (102 women, mean age 39.5 512 ). Patients filled out the Toronto Alexithymia Scale (TAS-20) and the Spielberger State-Trait Anxiety Inventory (STAI) as a part of a larger set of questionnaires. Results: Pathophysiological mechanisms: 53 patients (38\%) were hypersensitive to gastric distension, accommodation was impaired in 52 patients (38\%) and 17 patients of the 92 for whom standardized emptying data were available (19\%) had delayed gastric emptying. Psychosocial dimensions: 1)TAS20 The median total TAS-score was 56 [19], which is significantly higher compared to healthy subject samples $(39-50, \mathrm{p}<.0001)$ and medical patient samples (42-49) and significantly higher than or similar to somatoform or psychiatric patient samples (49-55). Forty seven patients $(40 \%)$ scored low $(<51)$ on alexithymia, 34 patients $(29 \%)$ had an intermediate score (51-61) and 36 patients (31\%) scored high. The median scores on the TAS subscales "Difficulty Identifying Feelings", "Difficulty Describing Feelings" and "Externally Oriented Thinking" were 17 [10], 14[7] and 23[7], respectively. These scores are again higher compared to healthy subjects and medical patients and higher than or similar to from somatoform or psychiatric patients. No association was found between total or subscale TAS scores and gastric sensitivity status or accommodation. There was a higher total alexithymia score in the delayed emptying group (58 vs 51.7,p=.05). 2) Trait Anxiety The mean \pm SD STAI Trait score was $41.8 \pm 10.8$, which is significantly higher than the general population $(38.3, \mathrm{p}=$ .0004), similar to a medical patients sample $(40.6, p=.21)$ and significantly lower than a psychiatric patients sample $(51.8, \mathrm{p}<.0001)$. Trait anxiety did not differ relative to gastric sensitivity status, accommodation $\&$ gastric emptying. 3)Relationship alexithymia - trait anxiety TAS-20 total score and STAI Trait score were moderately correlated $(\rho=.35, p=.0002)$. Conclusion: Alexithymia and, to a lesser extent, trait anxiety scores are high in tertiary care FD patients. No association was found between psychological trait dimensions and gastric pathophysiological mechanisms, except for alexithymia and gastric emptying.

\section{T1237}

Increased Pre-Pulse Startle Inhibition in Irritable Bowel Syndrome (IBS): Evidence for Hypervigilance

Lisa A. Kilpatrick, Edward Ornitz, Hana Ibrahimovic, Mike Treanor, Michelle Craske, Maria Nazarian, Emeran A. Mayer, Bruce D. Naliboff

Background: The eye blink acoustic startle response (ASR) is a simple reflex elicited by a brief loud tone. Prepulse inhibition (PPI) of the ASR by an innocuous tone presented between 30-500 ms before the startle is a useful index for central inhibitory mechanisms regulating sensorimotor processes and is decreased in schizophrenia but is increased with attention to the prepulse or an increase in the threat value of the prepulse. Aims: To test IBS patients for either 1) decreased PPI and sensorimotor gating which would suggest deficient inhibition of interoceptive signals or 2) enhanced PPI which would suggest increased saliency of the pre-pulse and, therefore, general hypervigilance. Methods: ASRs were examined for 35 female ROME positive IBS patients (age $=35.2 \pm 12$ ) and 21 healthy female controls (age $=$ $30.7 \pm 8$ ) during a series of startle stimuli trials, some of which were preceded by $25 \mathrm{~ms}$ tones (75dB SPL, $1000 \mathrm{~Hz}, 4 \mathrm{~ms}$ rise and fall times). Startle stimuli consisted of a $50 \mathrm{~ms}$ burst of white noise at $104 \mathrm{~dB}$ SPL with a 0 ms rise time presented binaurally through earphones. ASRs were measured from the electromyographic response of the orbicularis oculi muscle. Peak startle amplitude was defined as the highest point within a window from $20 \mathrm{~ms}$ to $105 \mathrm{~ms}$ following startle stimulus onset. The amplitude was log-transformed to increase normality of the data. Results: Group and pre-pulse effects on ASR were examined using mixed model analysis of variance. ASR amplitudes during trials in which no pre-pulse stimulus was given did not significantly differ between IBS patients and healthy controls $(p=0.239)$. However, there was a significant group by trial type interaction $(p=0.004)$. This resulted from IBS patients showing smaller ASRs during the pre-pulse trails compared to controls, indicating greater PPI in IBS patients. Conclusions: The results demonstrate altered stimulus processing in IBS patients. Since the effect of the pre-pulse is dependent in part on its saliency, the increased PPI seen in IBS patients may reflect increased general vigilance. ASR responses may offer a simple and non-invasive approach to the study of neurocognitive mechanisms in functional disorders. Supported by NIH grants P50 DK64539, R24 AT002681, NR04881, and VA Medical Research.

\section{$\mathrm{T} 1238$}

Is a Structured Patient Educational Class An Efficient Tool in the Management of Patients with Irritable Bowel Syndrome (IBS)?

Gisela Ringstrom, Stine Storsrud, Sara Lundkvist, Berndt Westman, Hasse Abrahamsson, Magnus Simren

Background: IBS patients consume a lot of healthcare, but often feel that they are insufficiently informed about their condition. Medical treatment options are still limited. This is a report from a study evaluating the effect of a structured IBS patient educational class - an 'IBSSchool' - on symptom severity and the impact on daily life. Method: We included 62 patients (mean age 39 (23-67) years; 51 women) with IBS according to the Rome II criteria. The patients were referred from primary care physicians and gastroenterologists. The 'IBS-school' consisted of six two-hour lessons, once per week with eight to ten patients in each group. Each lesson was held by one out of five professionals: a nurse specialized in functional gastrointestinal (GI) disorders, a gastroenterologist, a dietician, a physiotherapist and a psychologist. All patients completed validated questionnaires before and three months after the start of the educational class to evaluate GI symptoms severity (Gastrointestinal Symptom Rating Scale-IBS, GSRS-IBS and IBS Severity Scoring System, IBS-SSS), GI symptom-specific anxiety (Visceral Sensitivity Index, VSI), quality of life (SF-36 and IBS Quality of Life, IBSQOL), psychological symptoms (Hospital Anxiety and Depression scale, HAD) and coping resources (Sense of Coherence index, SOC). The patients rated their perceived levels of IBS knowledge and satisfaction with the knowledge using a visual analogue scale. Results: The patients experienced increased knowledge about IBS at 3 months ( $42 \pm 23$ vs. $76 \pm 13 \mathrm{~mm}$ $($ mean $\pm S D) ; p=0.0001)$, and were more satisfied with their knowledge $(27 \pm 22$ vs. $77 \pm 17$; $\mathrm{p}=0.0001)$. Overall GI symptoms severity was reduced according to IBS-SSS $(322 \pm 87$ vs. $289 \pm 109 ; \mathrm{p}=0.003)$. Of the individual GI symptoms abdominal pain was less severe after the educational class (GSRS pain score $4.4 \pm 1.3$ vs. $3.9 \pm 1.5 ; \mathrm{p}=0.005$ ), whereas the bowel habits were unchanged. GI symptom-specific anxiety was reduced, as measured by VSI $(50 \pm 18$ vs. $56 \pm 16 ; p=0.006)$. The QOL improved after the educational class, especially for the mental component score on SF-36 ( $34 \pm 12$ vs. $39 \pm 14 ; \mathrm{p}=0.01)$, and the patients showed reduced levels of depression according to $\operatorname{HAD}(5.8 \pm 3.7$ vs. $5.0 \pm 4.4 p=0.03)$. Conclusion: A structured IBS patient educational class enhances the knowledge about IBS, which helps the patients to cope better with their symptoms. Patient education is efficient in order to reduce the impact of GI symptoms on daily life in IBS-patients, with improvements in quality of life. Performing patient education in a group setting probably enhances these positive effects.

\section{T1239}

Gastroenterologist Ratings of Patient Anxiety: Perception Or Attribution? Tiffany Havlicek, Michael P. Jones, Michael Williams, Robert Rahimi, Laurie Keefer Introduction: Numerous studies have shown that MD assessment of pt anxiety (ANX) and depression (DEP) is often poor. Further, studies suggest that MDs and other healthcare providers often harbor negative attitudes towards pts with functional GI disorders (FGID) This is problematic as accurate, unbiased assessments of psychosocial factors are needed to optimize outcomes. We assessed gastroenterologist (GE) ratings of pt ANX and DEP with respect to diagnosis. Methods: Subjs were pts making initial clinic visits at 2 university GI clinics and their GE. Pts were grouped by post-visit diagnosis as FGID, organic disorder (OrgDx; eg. IBD, PUD) or symptom-based diagnosis (SxDx; eg. abdominal pain, nausea) Pts completed HADS and the Visceral Sensitivity Index (VSI: a measure GI symptom specific ANX). GE rated ANX and DEP on 100mm VAS with anchors "not at all" and "severe." Results: 30 FGID, 29 OrgDx and 41 SxDx pts were evaluated by 26 GE. Groups did not differ by age or sex. No differences existed btwn groups for HADS ANX or DEP(Table). VSI scores were signif different btwn groups ( $F=4.254 ; p=0.019)$ with $S x D x$ but not FGID signif higher than $\operatorname{OrgDx}(\mathrm{p}=0.03$ and $\mathrm{p}=0.13$ by Bonferroni). GE ratings of ANX in FGID were higher than SxDx or OrgDx $(\mathrm{p}=0.11)$. GE ANX ratings correlated poorly with HADS-ANX $(\mathrm{r}=0.11 ; \mathrm{p}=0.26)$ and VSI. GE DEP ratings did not differ btwn groups $(\mathrm{p}=0.46) .(\mathrm{r}=0.17 ; \mathrm{p}=$ 0.18). Conclusions: MD ANX and DEP ratings do not correlate with pt self-report. No signif differences in DEP or generalized ANX exist btwn groups but VSI is higher in FGID and signif higher in SxDx. GE rate FGID higher in ANX and DEP while SxDx is rated similar to OrgDx, suggesting an attribution effect. Stigma assoc with FGID diagnosis may have influenced this outcome and warrants futher study. 


\begin{tabular}{|c|c|c|c|c|}
\hline & OrgDx & FGID & SxDx & $\mathrm{p}$ \\
\hline HADS(anxiety) & $6.44 \pm 0.68$ & $7.67 \pm 0.91$ & $6.87 \pm 0.70$ & 0.51 \\
\hline HADS (depression) & $4.44 \pm 0.54$ & $4.3 \pm 0.76$ & $4.16 \pm 0.51$ & 0.95 \\
\hline VSI & $21.73 \pm 2.80$ & $32.61 \pm 4.58^{* *}$ & $35.72 \pm 4.48^{*}$ & 0.02 \\
\hline MD VAS (anxiety) & $23.28 \pm 3.47$ & $36.40 \pm 5.69$ & $24.55 \pm 5.12$ & 0.11 \\
\hline MD VAS (depression) & $17.64 \pm 3.59$ & $22.63 \pm 4.84$ & $15.52 \pm 3.48$ & 0.46 \\
\hline
\end{tabular}

mean \pm SEM Bonferroni posttests comapred with OD: ** $(\mathrm{p}=0.13) *(\mathrm{p}=0.03)$

\section{T1240}

What Patients with Irritable Bowel Syndrome (IBS) Expect from Their Health Care Providers. National Survey On IBS Educational Needs and Expectations Albena Halpert, Christine Dalton, Olafur Palsson, Yuming Hu, Carolyn Morris, Shrikant Bangdiwala, Nancy Norton, Douglas Drossman

AIM: To identify patients' expectations from the health care providers related to IBS. METHODS: The study consisted of: 1) Questionnaire item generation using patient focus groups to develop the IBS-Patient Education Questionnaire (IBS-PEQ); 2) Cognitive item reduction of items considered important and relevant by IBS patients; and 3) Data acquisition. The IBS-PEQ was administered to a national sample of IBS via mail and on line. Expectations from the health care providers were assessed using a 7-point Likert scale questions. ANALYSIS: We report the six most desirable qualities of the health care providers based on \% of subjects agreeing or strongly agreeing with that quality. RESULTS: We report the results obtained from the first 495 subjects of a larger study. The mean age was $37.3 \pm 11.7$ years with an educational attainment of $15.1 \pm 2.6$ years, and $84 \%$ were female, $85 \%$ have seen an MD for IBS and $98 \%$ have previously used the web for health information. Illness duration was $6.7 \pm 4.0$ years. $50 \%$ of all subjects stated they have received some helpful information about IBS previously, but $100 \%$ wanted to know more about IBS. The most desired qualities of the health care providers and respective prior experiences are summarized in Table 1. Notably there is discrepancy between expectations and what patients report as their experience with the last MD they saw for IBS. CONCLUSION: 1) Patients with IBS feel they want more information about IBS and expect their health care provider to provide more in depth information, including additional learning sources and research options, to listen, and be supportive. 2) There is a discrepancy between the above expectations and patients' prior experiences with physicians who they have seen for their IBS. Better understanding of patients' needs and expectations is needed in order to improve our ability to effectively manage IBS. Supported by GIDH, Forest Laboratories and P\&G. Table 1

\begin{tabular}{|c|c|c|}
\hline $\mathrm{N}=495$ & $\begin{array}{c}\text { I expect my health care provider } \\
\text { to ...(\% of subject agreeing) }\end{array}$ & $\begin{array}{c}\text { The MD I saw about IBS } \\
\text { seemed to...\% of subject } \\
\text { agreeing) }\end{array}$ \\
\hline To provide in depth information & 94.3 & 20.3 \\
\hline Let me ask questions & 93.0 & 43.6 \\
\hline $\begin{array}{c}\text { Tell me where to get additional } \\
\text { IBS information }\end{array}$ & 90.9 & 19.7 \\
\hline To listen & 88.0 & 42.6 \\
\hline $\begin{array}{c}\text { Tell me about research } \\
\text { studies/medications }\end{array}$ & 87.0 & 20.8 \\
\hline To provide support & 75.8 & 28.8 \\
\hline
\end{tabular}

\section{T1241}

Family Physician Attitude Towards Irritable Bowel Syndrome Alexandra Ilnyckyj, Lesley Graffe, Lawrence Elliott, Gina Sunderland

Background: IBS is primarily managed by family physicians (FP). This study explored FP knowledge, clinical skill and personal satisfaction (attitude) in caring for patients with IBS and explored factors contributing to personal satisfaction. As well, FP attitudes were compared to those of other professionals. Aim: 1) Compare FP attitude towards IBS to other chronic conditions 2) Compare FP attitude towards IBS to that of other professionals 3) Identify factors contributing to FP satisfaction in managing IBS. Methods: Randomly selected FP, dieticians (D) and psychologists $(\mathrm{P})$ in a Canadian province were surveyed regarding their attitude with respect to IBS, depression, anxiety, chronic musculoskeletal pain, fibromyalgia, and obesity management. Comparison conditions were selected since education/ support is integral in treatment. Independent and paired sample $t$ tests were used, as appropriate, to compare FP attitudes regarding IBS to those of other professions, and to other conditions. Multiple regression analysis was used to explore the contribution of factors to FP satisfaction working with IBS. Results: 100 surveys were sent to each professional group, with responses from $46 \mathrm{FP}, 61 \mathrm{P}$, and $54 \mathrm{D}$. FP reported greater satisfaction treating the mental health conditions compared to IBS $(\mathrm{t}=-3.12, \mathrm{p}<.01)$, but lower satisfaction in treating those with chronic pain conditions compared to IBS $(\mathrm{t}=3.32, \mathrm{p}<.01)$. Similar results were found with respect to the dimensions of self-reported knowledge and clinical skill across these conditions. In comparison to other professionals, FP reported lower ratings than did D regarding their IBS knowledge $(\mathrm{t}=-2.06, \mathrm{p}=.04)$ and clinical skills $(\mathrm{t}=-2.45, \mathrm{p}=.02)$. There were no differences between $\mathrm{P}$ and FP. Similarly, FP satisfaction in working with IBS patients was lower than D satisfaction $(\mathrm{t}=-5.81, \mathrm{p}<.001)$. There was no difference between $\mathrm{P}$ and FP. A regression analysis assessing the effects of gender, knowledge, skills, time constraints and patient factors on FP satisfaction in working with IBS patients found that level of clinical skills was the strongest predictor $(\mathrm{F}=2.37, \mathrm{p}=.05)$, explaining $29 \%$ of the variance. Conclusion: Family physicians report their attitude to be greater towards the management of depression rather than IBS. However, their attitude towards IBS is greater than towards chronic pain conditions. Dieticians reported greater attitude than did family physicians towards managing IBS. Strong clinical skills are important in predicting family physician personal satisfaction in IBS management.

\section{T1242}

Interleukin-1 $\beta$ Stimulates Expression of NADPH Oxidases NOX1 and NOX4 in Colonic Smooth Muscle: Mediation By Nf- $\kappa \mathrm{B}$ and Differential Modulation By C-Jun Kinase and ERK1/2

Wenhui Hu, Fang Li, Karnam S. Murthy

Brief exposure $(<3 \mathrm{~h})$ of esophageal and colonic smooth muscle cells to IL-1 $\beta$ stimulates $\mathrm{H}_{2} \mathrm{O}_{2}$ production and inhibits smooth muscle contraction. Direct exposure to exogenous $\mathrm{H}_{2} \mathrm{O}_{2}$ also inhibits contraction raising the possibility that $\mathrm{H}_{2} \mathrm{O}_{2}$ is partly responsible for inhibition of muscle contraction after brief exposure to IL-1 $\beta$. The participation of $\mathrm{H}_{2} \mathrm{O}_{2}$ in inhibition of contraction after prolonged exposure depends on continuous generation of $\mathrm{H}_{2} \mathrm{O}_{2}$. An increase in NAPDH oxidase (NOX) expression or activity could provide a source for superoxide that can be readily dismuted to $\mathrm{H}_{2} \mathrm{O}_{2}$. NOXl and NOX4 are known to be expressed in colonic tissues and smooth muscle cells. Aim. To determine the effect and mechanism of action of IL-1 $\beta$ on NOX expression in colonic smooth muscle cells. Methods Cultured rabbit colonic smooth muscle cells in first passage were treated for 3 days with $\mathrm{IL}-1 \beta$ in the presence or absence of NF-KB and MAP kinase inhibitors. Expression levels of NOXl and NOX4 were determined by Western blot. Results. We have recently shown that IL-1 $\beta$ activates NF- $\mathrm{KB}$ and various MAP kinases, including ERK1/2, p38 MAP kinase, and c-Jun kinase (JNK). In this study, treatment of cultured muscle cells with IL-1 $\beta$ for 3 days induced 3- to 4-fold increase in NOXI and NOX4 protein expression. The increase in expression of both NOX1 and NOX4 was strongly inhibited by an IKK2 inhibitor (IKK2IV, $10 \mu \mathrm{M})$, suggesting that the increase in expression was mediated via a canonical NF-kB pathway (IKK2/IкB $\alpha / \mathrm{NF}-\kappa \mathrm{B})$. The increase in NOXl expression was selectively inhibited by the JNK inhibitor, SP600125 $(10 \mu \mathrm{M})$, whereas the increase in NOX4 expression was selectively inhibited by the MEK inhibitor, PD98059 $(20 \mu \mathrm{M})$. Expression of either enzyme was not affected by the p38 MAPK inhibitor, SB203580. Conclusion. IL-1 $\beta$ stimulates the expression of NADPH oxidases NOXI and NOX4 in colonic smooth muscle cells. The increase in expression is mediated by NF- $\mathrm{KB}$ and differentially modulated by ERK1/2 and JNK. The sustained increase in NOX expression provides a ready source for continuous generation of superoxide that can be readily dismuted to $\mathrm{H}_{2} \mathrm{O}_{2}$.

\section{T1243}

Affect and Perceived Stress in Functional Dyspepsia: Descriptive Data and Relationship with Gastric Pathophysiological Mechanisms

Lukas van Oudenhove, Joris Vandenberghe, Brecht Geeraerts, Rita Vos, Phillippe Persoons, Sebastien Kindt, Koen Demyttenaere, Jan Tack

Background: Psychosocial factors including affect and stress are believed to play an important role in the onset, course and outcome of functional dyspepsia (FD). The aim of this study is 1) to provide descriptive data on positive and negative affect and perceived stress in FD and 2) to describe the relationship of these psychological factors with gastric pathophysiological mechanisms. Methods: We studied gastric sensorimotor function with a barostat (sensitivity, compliance and accommodation) and gastric emptying test in 139 consecutive FD patients

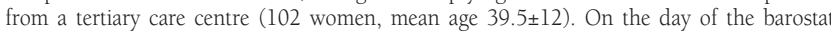
investigation, patients filled out the Positive And Negative Affect Schedule (PANAS, 20 items) and the Perceived Stress Questionnaire (PSQ, 30 items), as a part of a larger set of questionnaires. Results: Pathophysiological mechanisms: 53 patients (38.1\%) were hypersensitive to gastric distension, accommodation was impaired in 52 patients (37.7\%) and 17 patients of the 92 for whom standardized emptying data were available (19\%) had delayed gastric emptying. Psychosocial dimensions 1) PANAS: Patients rated their affect over the past two weeks. The mean \pm SD positive affect score was $28.1 \pm 7.4$ and the median [IQR] negative affect score was 17.8 [8]. The mean positive affect score was significantly lower compared to the normal population $(\mathrm{p}<.0001)$. PANAS scores did not differ relative to gastric sensitivity status, accommodation or gastric emptying. 2) PSQ: Patients rated their level of perceived stress over the past month. The mean PSQ index score was $0.33 \pm 0.13$, which is significantly lower than in healthy subjects $(0.37 ; \mathrm{p}=.005)$ and in a sample of GI out- and inpatients $(0.44 \& 0.49$ respectively; $\mathrm{p}<.0001)$. The PSQ scores did not differ relative to gastric sensitivity status, accommodation or gastric emptying. 3) Relationship between affect and perceived stress: perceived stress correlated negatively with positive affect $(\rho=-.53, p<.0001)$ and positively with negative affect $(\rho=.58, p<.0001)$. Conclusion: Tertiary care FD patients have lower positive affect rather than higher negative affect compared to healthy subjects. Surprisingly, perceived stress scores were lower than in both a sample of healthy subjects and of GI out- and inpatients. Positive and negative affect scores and perceived stress scores did not differ relative to gastric sensitivity, accommodation or emptying.

\section{T1244}

What Determines the Compromised Quality of Life in Patients with Irritable Bowel Syndrome (IBS)?

Magnus Simren, Gisela Ringstrom, Pia Agerforz, Per Jerndal, Martin Karpefors, Louis Akkermans, Robert Spaziani, Karen Miranda, Stephen Collins, Markad Kamath, Alfred Bayati

Background: Previous studies have demonstrated compromised quality of life (QOL) in patients with IBS compared with the general population. Factors of importance for this are incompletely known. Aim: To assess QOL in IBS and to evaluate the relative importance of demographic factors, gastrointestinal and psychological symptom severity for QOL in a large database study. Patients and methods: We included 88 healthy controls (58 females; mean age 33 years) and 290 patients fulfilling the Rome II criteria for IBS (208 females; mean age 42 years) at three centers. Demographic and disease-related factors were assessed using

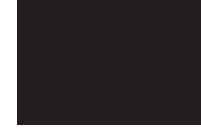


a structured interview. Patients completed two disease specific questionnaires, the Gastrointestinal Symptom Rating Scale - IBS (GSRS-IBS) and the Visceral Sensitivity Inventory (VSI) and two generic questionnaires, the Hospital Anxiety and Depression scale (HAD) and the Short Form-36 (SF-36). As the main outcome QOL variables we used the two summary scores on SF-36 - physical and mental component scores (PCS, MCS) Results: Compared with the controls patients with IBS had lower scores on all SF-36 domains (=lower QOL), including the two summary scores (PCS: $45 \pm 10$ vs. $51 \pm 11$ (mean \pm SD); $<<0.0001$; MCS: $43 \pm 13$ vs. $55 \pm 7 ; p<0.0001$ ). Using univariate analyses several domains on the questionnaires as well as demographic factors were associated with PCS and MCS, but no association was seen with IBS subgroup based on the predominant bowel pattern, duration of IBS, gender, family history of IBS or marital status. Regression analyses demonstrated independent associations between PCS and the overall GI symptom severity $(\mathrm{p}<0.0001)$ and frequency $(p<0.0001)$, GSRS Satiety Score $(p<0.0001)$, the presence of fibromyalgia $(p=0.002)$ and other functional GI than IBS $(\mathrm{p}=0.004)$, HAD depression score $(\mathrm{p}=0.01)$ and whether the patient was working or not $(\mathrm{p}=0.003)(\mathrm{R} 2=0.34)$. MCS was independently associated with HAD depression $(\mathrm{p}<0.0001)$ and anxiety scores $(\mathrm{p}<0.0001)$, age $(\mathrm{p}=0.003)$, recent psychological consultation $(\mathrm{p}=0.006)$ and VSI total score $(\mathrm{p}=0.03)(\mathrm{R} 2=0.64)$. Conclusion: $\mathrm{QOL}$ is severely compromised in patients with IBS. This is related to GI and psychological symptom severity, but also to some extent to demographic and social factors. The subgroup of IBS based on the predominant bowel habit does not seem to affect QOL. This study was supported by AstraZeneca.

\section{T1245}

Is There An Association Between Gn 33 C825t Genotype and Lower Functional Gastrointestinal Disorders?

Viola Andresen, Michael Camilleri, H. Jae Kim, Debra A. Stephens, Paula J. Carlson, Nicholas J. Talley, Yuri A. Saito Loftus, Raul Urrutia, Alan R. Zinsmeister

Background: GNB3 influences $G$ protein translation of a majority of ligand-receptor activations. It has been reported that functional dyspepsia (FD) is associated with homozygous genotypes of the C825T polymorphism in the GN $\beta 3$ gene. In a small group of 19 patients with FD overlapping with irritable bowel syndrome (IBS), borderline association with GN $\beta 3$ C825T was detected. It is unknown whether the GN 33 genotype is associated with lower functional gastrointestinal disorders (FGID). Aims: To compare the prevalence of the different GN33 C825T genotypes in patients with lower FGID and healthy controls, and to test associations of these genetic variations with subgroups of IBS grouped by bowel function, functional abdominal pain (FAP), and high somatic symptom scores. Methods: GNB3 C825T polymorphism was analyzed in DNA from blood samples of 233 patients with lower FGID and 152 healthy controls. Validated bowel and somatic symptom questionnaires characterized the FGID phenotype: 82 IBS-constipation, 94 IBS-diarrhea, 38 IBS-alternating bowel function, and 19 FAP. Logistic regression analyses assessed associations of the GN 33 genotypes with lower FGID as a group, subgroups of FGID and high somatic score. Results: GN33 C825T genotype distributions were similar between the 2 groups: $50.7 \%$ CC, $40.8 \%$ TC, and $8.6 \%$ TT in healthy controls and 51.5\% CC, 40.8\% TC and 7.7\% TT in patients with lower FGID. There were no significant associations of GN 33 C825T polymorphism with lower FGID overall or with the separate symptom subgroups including IBS and FAP (Fig 1), or with high somatic symptom scores. Conclusion: In contrast to the reported association with functional dyspepsia, GN 33 C825T polymorphism is not significantly associated with lower FGID or with different IBS or FAP phenotypes.

Figure 1:

Odds ratios with $95 \% \mathrm{CI}$ for IBS-D, IBS-C, IBS-A, or FAP of the GN $\beta 3$ C 825

CC genotype relative to the combined TC and TT genotypes.

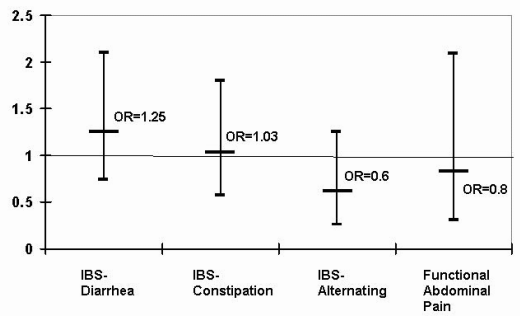

T1246

Subjective Bloating Is Not Correlated with Intestinal Gas Production But Is Weakly Correlated with Abdominal Distention

Syed I. Thiwan, Olafur S. Palsson, Marsha J. Turner, William E. Whitehead

Background: Volume of intra abdominal contents, abdominal muscle activity and altered perception are commonly proposed mechanisms to explain abdominal bloating. Bloating is one of the main symptoms in patients with lactose malabsorption and bacterial overgrowth, suggesting a possible role of intestinal gas production in bloating. Aims: To determine whether subjective bloating is due to 1 . volume of intestinal $\mathrm{H} 2$ and $\mathrm{Ch} 4$ produced by bacteria in response to oral sugar load or 2. objective abdominal distension. Methods: 80 subjects (10 males, 70 females, including 25 Healthy controls (H), 25 IBS, 23 Lactose Malabsorbers (LM) and 7 Slow Transit Constipation (C) underwent a hydrogen breath test with $50 \mathrm{~g}$ of lactose and $80 \mathrm{~g}$ of sucrose on an empty stomach in the morning. Breath samples for $\mathrm{H} 2$ and $\mathrm{Ch} 4$ were collected at baseline and every $15 \mathrm{~min}$ until $120 \mathrm{~min}$ and then every $30 \mathrm{~min}$ up to $180 \mathrm{~min}$. Abdominal girth was measured at the level of the umbilicus at endexpiration with subjects in a standing posture. Subjects marked the severity of bloating on a visual analog scale. Breath $\mathrm{H} 2$ and $\mathrm{Ch} 4$ were analyzed using a Quintron Microlyzer model SC. Analysis: Net gas production $(\mathrm{H} 2 \mathrm{CH} 4)$ was defined as area under the curve (AUC) of combined $\mathrm{H} 2+2 * \mathrm{Ch} 4$ - baseline $\mathrm{H} 2+2 * \mathrm{Ch} 4$. Net abdominal distention $(\mathrm{GM})$ was defined as AUC of girth measurement-baseline girth. Results: All 3 patient groups had significantly higher AUC bloating scores than controls $(\mathrm{p}<0.05)$. LM patients had significantly higher $\mathrm{H} 2$ production and greater increases in GM, while constipated patients had significantly greater increases in $\mathrm{CH} 4$ (see table). Net gas production ( $\mathrm{H} 2 \mathrm{CH} 4)$ was significantly increased in both LM and C. Regression analysis showed that $\mathrm{H} 2 \mathrm{Ch} 4$ and GM together accounted for $3 \%$ of the variance in subjective bloating and $6 \%$ of the variance in peak bloating, but only GM was significant $(\mathrm{p}<0.05)$. Conclusions: Increases in intra-intestinal gas volume in response to oral sugar load did not contribute significantly to bloating and the contribution of abdominal distension to the bloating sensation is marginal. We speculate that altered perception is the main mechanism of bloating. [Supported by Novartis Pharmaceuticals and grants RO1 DK31369, R24 DK67674, and RR00046].

\begin{tabular}{|c|c|c|c|c|c|c|c|c|}
\hline \multirow{2}{*}{} & \multicolumn{2}{|c|}{ H } & \multicolumn{2}{c|}{ LM } & \multicolumn{2}{c|}{ IBS } & \multicolumn{2}{c|}{ C } \\
\cline { 2 - 9 } & $\begin{array}{c}\text { Mean } \\
\text { AUC }\end{array}$ & $\begin{array}{c}\text { Mean } \\
\text { Peak }\end{array}$ & $\begin{array}{c}\text { Mean } \\
\text { AUC }\end{array}$ & $\begin{array}{c}\text { Mean } \\
\text { Peak }\end{array}$ & $\begin{array}{c}\text { Mean } \\
\text { AUC }\end{array}$ & $\begin{array}{c}\text { Mean } \\
\text { Peak }\end{array}$ & $\begin{array}{c}\text { Mean } \\
\text { AUC }\end{array}$ & $\begin{array}{c}\text { Mean } \\
\text { Peak }\end{array}$ \\
\hline H2 & 67.06 & 10.92 & $\mathbf{4 8 4 . 3 7}$ a & $\mathbf{1 1 0 . 0 9}$ a & 95.62 & 14.32 & 74.71 & 11.86 \\
\hline Ch4 & 57.27 & 6.32 & $\mathbf{1 0 8} \mathbf{b}$ & $\mathbf{1 1 . 8 3} \mathbf{b}$ & 6.08 & .96 & $\mathbf{2 2 3 . 2 9} \mathbf{c}$ & $\mathbf{2 4 . 8 6} \mathbf{c}$ \\
\hline GM & 4.72 & 2.48 & $\mathbf{2 8 . 2 6} \mathbf{c}$ & $\mathbf{4 . 0 2} \mathbf{d}$ & 14.4 & 3.1 & 4.89 & 3.64 \\
\hline VAS & 10.66 & 1.92 & $\mathbf{4 3 . 2 5} \mathbf{d}$ & $\mathbf{5 . 0 9} \mathbf{d}$ & $\mathbf{4 1 . 6 5} \mathbf{d}$ & $\mathbf{4 . 5 8} \mathbf{d}$ & $\mathbf{5 6 . 7 1} \mathbf{d}$ & $\mathbf{5 . 8 6} \mathbf{d}$ \\
\hline H2CH4 & 4.81 & 22.72 & $\mathbf{3 8 0 . 5 7} \mathbf{c}$ & $\mathbf{1 2 6 . 2 2} \mathbf{c}$ & -39.38 & 15.52 & 31.00 & $\mathbf{5 7 . 5 7} \mathbf{c}$ \\
\hline
\end{tabular}

( $a=v s . H, I B S, C ; b=v s . I B S ; c=v s . H, I B S ; d=v s . H \quad p<0.05$ or better)

\section{$\mathrm{T} 1247$}

Initial Approach and Pharmacotherapy for Functional Dyspepsia - A Large Clinical Trial in Japan

Michio Hongo

Though the functional dyspepsia (FD) is one of the common disorders in primary practice of gastroenterology, there are many controversies for initial approach and pharmacotherapy for FD. [AIM] This study has 3 main purposes; to clarify 1) the prevalence of organic disorders among the subjects presumed to be FD, 2) the effect of early endoscopy to the symptoms, and 3) the effect of mosapride in relieving the symptoms compared with teprenon which is indicated for gastritis. [SUBJECTS \& METHODS] Patients those who have dyspeptic symptoms for more than 4 days during the preceding 2 weeks, presumed to be functional origin, were asked to participate to the study after informed consent (IC). After IC, patients had endoscopy for evaluation of gastric mucosa. Symptoms were evaluated one week after endoscopy, and those who were still symptomatic were allocated to pharmacotherapy either mosapride $15 \mathrm{mg}$ tid or teprenon $150 \mathrm{mg}$ tid following the computer-controlled randomization. Symptom was evaluated in 2 domains, dysmotility-like symptom (feeling of stasis) and ulcer-like symptom (epigastric pain), with their degree of severity and frequency (both in 3 Likert scale). Sum of the scores were used as Symptom Score for both symptoms, at 1 and 2 weeks of medication. Patients' preference and SF-36 (v2 J acute form) were also obtained. [RESULTS] A total of 1,042 patients (57\% are from GPs) gave informed consent, and 1,025 patients had endoscopy. A total of 90 patients had organic lesions in their upper GI tract which could be responsible for the symptoms. Lesions frequently found were, reflux esophagitis (30\%), erosive gastritis (22\%), and gastric ulcer (22\%). Gastric cancers were found in 3\% of patients. 935 patients (M:F=1:1.3) (mean age 46 y.o.) were diagnosed as functional dyspepsia. One week after the endoscopy, dyspeptic symptoms were relieved in $30 \%$ of patients. 618 FDs were randomized to mosapride $(n=311)$ or teprenon. $(n=307)$. Two weeks after medication, stasis-symptoms and pain-symptoms improved with mosapride and stasis symptoms improved with teprenon. Improvement in symptom scores were higher in mosapride group in both symptoms. Subcales of SF-36 in bodily pain, general health, vigor and mental health were significantly higher in mosapride group. Proportion of patient's impression favoring medication was $91 \%$ with mosapride and 52\% with teprenon. [CONCLUSION] Presumed diagnosis of FD was correct in $90 \%$ of patients. Reflux esophagitis was the most common organic disorders among the presumed FDs. There were malignancies in 3\% of patients. Early endoscopy relieved symptoms in 30\% of patients. Mosapride showed better efficacy in relieving FD symptoms than teprenon.

\section{T1248}

Lactose Malabsorption, Small Bowel Bacterial Overgrowth and Celiac Disease in IBS Patients

Syed I. Thiwan, Olafur S. Palsson, Marsha J. Turner, William E. Whitehead

Background: Symptoms of small bowel bacterial overgrowth (SBBO), lactose malabsorption (LM) and Celiac disease (CD) are often similar to IBS and could be confused with IBS. LM is found in 23- $40 \%$ of IBS patients. Recent reports indicate high prevalence of SBBO as diagnosed by positive lactulose breath hydrogen test and Celiac disease in IBS patients, but this needs replication. Aims: To determine the prevalence of SBBO, LM and celiac disease in IBS patients. Methods: 197 subjects (158 IBS-mean age 35.2 yrs, 26 males, 132 females; and 39 healthy controls-mean age 36.54yrs, 8 males, 31 females) underwent hydrogen breath test with $50 \mathrm{~g}$ of lactose and $80 \mathrm{~g}$ of sucrose on an empty stomach in the morning. Breath samples for $\mathrm{H} 2$ and $\mathrm{Ch} 4$ were collected at baseline and every 15 min until 120 min and then every 30 min up to 180 min. Breath $\mathrm{H} 2$ and $\mathrm{Ch} 4$ were analyzed using a Quintron Microlyzer model SC. Patients completed the Rome II Modular Questionnaire and additional bowel habit questions. Anti-Gliadin and Anti-Endomysial antibodies were assayed using ELISA and qualitative indirect immunofluorescence assay respectively. Analysis: LM was defined as a rise of $20 \mathrm{ppm}$ of $\mathrm{H} 2$ over baseline after 120 minutes or a combined rise of $15 \mathrm{ppm}$ of $\mathrm{H} 2$ and Ch4 after 120 minutes. SBBO was defined as either a baseline H2 level of $>40 \mathrm{ppm}$ or $10 \mathrm{ppm}$ increase in $\mathrm{H} 2$ within 60 minutes of ingesting sucrose. Anti-Gliadin antibodies were interpreted as positive if the titer was > to $50 \mathrm{EU}$. Results: The prevalence of SBBO, LM, and positive antibody tests for celiac disease were similar in IBS patients and healthy controls (see table). IBS patients with and with out SBBO did not differ in predominant 
bowel symptoms nor in the frequency and severity of diarrhea or abdominal pain. There was only one patient with celiac disease confirmed by presence of Anti Endomysial antibody in the IBS group. Conclusions: Our findings do not support the previously reported increased prevalence of SBBO, LM, and Celiac disease in IBS patients. Failure to replicate an increased prevalence of SBBO in IBS may be due to our methodology: using sucrose is more accurate and specific than lactulose, and a 10 ppm rise in $\mathrm{H} 2$ is a more sensitive. However, SBBO in the distal small bowel may not have been detected with this method. [Supported by NIH grants RO1 DK31369, R24 DK67674, and RR00046].

\begin{tabular}{|c|c|c|c|c|c|c|c|c|}
\hline \multirow{2}{*}{} & \multicolumn{2}{|c|}{ BO } & \multicolumn{2}{c|}{ LM } & \multicolumn{2}{c|}{ AG IgA } & \multicolumn{2}{c|}{ AE IgA } \\
\cline { 2 - 9 } & $\#(\%)$ & Total\# & $\#(\%)$ & Total\# & $\#(\%)$ & $\#(\%)$ & $\#(\%)$ & Total\# \\
\hline H & $6(15.4 \%)$ & 39 & $5(18 \%)$ & 18 & $1(3.3 \%)$ & $1(3.3 \%)$ & 0 & 30 \\
\hline IBS & $32(20.3 \%)$ & 158 & $16(17.6 \%)$ & 91 & $5(3.5 \%)$ & $3(2.1 \%)$ & $1(0.7 \%)$ & 144 \\
\hline Total & $38(19.3 \%)$ & 197 & $21(19.3 \%)$ & 109 & $6(3.4 \%)$ & $4(2.3 \%)$ & $1(0.6 \%)$ & 174 \\
\hline
\end{tabular}

\section{T1249}

Optimizing Gastric Emptying Scintigraphy for the Detection of Gastroparesis Henry P. Parkman, Alan Hutson, Alan H. Maurer, Linda C. Knight, Braden Kuo, William L. Hasler, William D. Chey, Richard W. McCallum, John M. Wo, Kenneth L. Koch, Michael Sitrin, Jeffrey M. Lackner, Jack Semler

Gastric emptying scintigraphy (GES) using a radiolabeled solid-phase meal is currently considered the gold standard to detect gastroparesis. It is, however, performed in a variable manner at different institutions. A method to standardize gastric emptying using a low fat (EggBeaters) meal with imaging at 0,1,2 and 4 hours postprandially has been suggested (Tougas et al, Am J Gastro 2000;95:1456). Extending GES from 2 to 4 hours has also been advocated to detect more patients with gastroparesis (Guo et al, DDS 2001;46:24). The optimal duration of GES to maximize sensitivity and specificity for detecting gastroparesis has not been established. AIM: To determine the optimal duration of GES for detecting gastroparesis. METHODS: In a multicenter study evaluating the emptying of a $\mathrm{pH} /$ pressure recording capsule (SmartPill), GES was performed in 86 normal subjects and in 60 patients with previously diagnosed gastroparesis ( 37 idiopathic, 23 diabetic) based on scintigraphy within the past 2 years. GES was repeated in a standardized fashion with imaging at $0,0.5$, $1,1.5,2,2.5,3,3.5$, and 4 hours after ingestion of a 99mTc-sulfur colloid radiolabeled meal (120 g EggBeaters, 2 pieces of bread with strawberry jam; $255 \mathrm{kcal}, 2 \% \mathrm{fat}$ ) and 120 cc water. Receiver operating characteristic (ROC) curves were used to optimize sensitivity and specificity for differentiating gastroparetic patients from normal subjects for the $50 \%$ emptying time (T-50), 90\% emptying time (T-90), and the percent retention (\%R) at each imaging time. The concordance statistic (c-statistic) denotes the area under the ROC curve and is a global measure of diagnostic utility. RESULTS: The c-statistic was greater for \% $\mathrm{R}$ at 3 hours $(\mathrm{c}=0.843)$ than at 1 hour $(0.670), 2$ hours $(0.792)$, or 4 hours $(0.816)$. It was also greater than that for T-50 ( $c=0.766)$ and similar to that for T-90 ( $c=0.849)$. The cutoff point that maximizes both sensitivity and specificity for T-90, $182 \mathrm{~min}$, gives a sensitivity and specificity of $84.8 \%$ and $77.9 \%$, respectively, whereas the cutoff point for \%R at 3 hours, $9 \%$ retention, gives $85.0 \%$ and $75.6 \%$, and the cutoff point for $\% \mathrm{R}$ at 4 hours, $3 \%$ retention, gives $70.0 \%$ and $73.2 \%$. CONCLUSIONS: Using the previously validated EggBeaters meal, T-90 is better than T-50 to differentiate patients with previously diagnosed gastroparesis from normal subjects. The percent retention at 3 hours provides diagnostic utility similar to the $90 \%$ emptying time and avoids the need for data extrapolation. To optimally detect gastroparesis, assessment of gastric emptying at times greater than 2 hours is needed with the percent retention at 3 hours being the best individual time point.

\section{$\mathrm{T} 1250$}

Non-Diarrhea Irritable Bowel Syndrome Patients Exhibit Different Anorectal Symptoms and Physiology to Functional Constipation Patients Vid P. Suttor, Gillian Prott, Ross Hansen, John Kellow, Allison Malcolm

Background: Patients with functional constipation (FC) often manifest concomitant features of pelvic floor dyssynergia (PFD). Patients with non-diarrhea predominant irritable bowel syndrome (non-D IBS) can also present with symptoms of PFD similar to patients with FC [Talley, Rev Gastroenterol Disord 2005]. Aim: To compare the anorectal symptoms and sensorimotor function of patients with non-D IBS and PFD symptoms to FC with PFD symptoms. Methods: We studied 30 consecutive female patients referred with constipation and two or more features of PFD (straining, incomplete evacuation, blockage, digital evacuation, or anal pain). These patients comprised two groups: IBS ( $n=17$; mean age \pm SD $48 \pm 15$ yrs), and FC ( $\mathrm{n}=13 ; 51 \pm 9 \mathrm{yrs})$, according to ROME II criteria. Assessments included the Bowel Disease Questionnaire, the Hospital Anxiety and Depression score, a validated constipation questionnaire [Knowles, Dis Colon Rectum 2000], $10 \mathrm{~cm}$ visual analogue scales for patient satisfaction with bowel habit and impact on quality of life, as well as a comprehensive anorectal physiology study (balloon expulsion, anal sphincter pressures, pelvic floor descent, and rectal sensitivity). Results: Non-D IBS patients experienced a greater frequency of straining to defecate $(\mathrm{p}<0.01)$ and of incomplete evacuation $(\mathrm{p}<0.01)$ than FC patients. However there was no difference in the total number of PFD symptoms between the two groups. Overall, the patients with non-D IBS scored lower on satisfaction with bowel habits than the patients with FC ( $\mathrm{p}=0.05)$. Physiologically, the non-D IBS patients more often displayed impaired anorectal balloon expulsion $(\mathrm{p}=0.02)$. Non-D IBS patients however less often manifested paradoxical anal contraction with straining (6/17 IBS vs 10/13 FC; $\mathrm{p}=0.03$ ). There was no difference in the anxiety and depression scores for the two groups, and no difference in the impact of their symptoms on quality of life. Conclusion: While both FC and non-D IBS patients exhibit features of PFD, these findings show that non-D IBS patients with PFD can exhibit a different symptom profile (more straining and incomplete evacuation, and a lower satisfaction with bowel movements), and a different anorectal physiological profile (more abnormal bowel evacuation, and less paradoxical anal contraction with straining) compared to FC patients with PFD

\section{T1251}

Physical Activity Improves Quality of Life But Is Not Associated with Decreased Prevalence of Bloating

Ashok Tuteja, Nicholas J. Talley, Sandra K. Joos, Tolman G. Keith, David H. Hickam

Background: Abdominal bloating is reported frequently by otherwise healthy individuals and may be associated with prolonged intestinal transit. Because mild physical activity accelerates intestinal transit, it may prevent or relieve bloating. However, the effect of physical activity on the prevalence and severity of bloating has not been investigated. We conducted a cross-sectional study evaluating the association among physical activity, bloating and quality of life (QOL). Methods: 1069 employees (age range 24 to 77) of the Veterans Affairs Health Care System were mailed validated questionnaires inquiring about their symptoms including bloating (Bowel Disease Questionnaire), QOL (SF-36) and physical activity (modified Baecke questionnaire). Results: The questionnaire response rate was $72 \%$. Bloating was reported by $149(21 \%)$ subjects. More women than men reported bloating $(23.6 \%$ vs. $16.9 \%$; $\mathrm{p}=0.03$ ); but after controlling for age this difference was not significant. With increasing age, fewer subjects reported bloating $(\mathrm{OR}=0.98 ; 95 \% \mathrm{CI} 0.96-1.00 ; \mathrm{p}=0.03)$. Subjects with bloating reported lower QOL on all subscales of the SF 36 (all $\mathrm{p}<0.05$ ). There were no differences in any domain of physical activity scores (household, occupational, leisure-time sports or total activity) between subjects with and without bloating (Table). Among subjects' with bloating, higher levels of leisure-time, sports and total activity were associated with higher scores of the physical functioning and health perception domains of QOL ( $\mathrm{r}$ varied from 0.30 to 0.42 , all $p \leq 0.01$ ). Conclusions: The prevalence of bloating diminishes with older age and is not different between men and women. Individuals who experience bloating report significantly lower QOL. Levels of physical activity do not appear to reduce the risk of bloating. However, among individuals who experience chronic bloating, higher levels of physical activity are associated with better QOL. These results suggest that increased physical activity may reduce the impact of bloating but not the symptom itself. The Effect of Physical Activity Scores on Bloating

\begin{tabular}{|c|c|c|c|c|c|c|}
\hline & \multicolumn{3}{|c|}{ Men } & \multicolumn{3}{c|}{ Women } \\
\hline $\begin{array}{c}\text { Physical } \\
\text { Activity }\end{array}$ & $\begin{array}{c}\text { Bloat N=48 } \\
\text { Mean (SD) }\end{array}$ & $\begin{array}{c}\text { No-Bloat N=236 } \\
\text { Mean (SD) }\end{array}$ & P & $\begin{array}{c}\text { Bloat N=101 } \\
\text { Mean (SD) }\end{array}$ & $\begin{array}{c}\text { No-Bloat N=327 } \\
\text { Mean (SD) }\end{array}$ & P \\
\hline Leisure-time & $2.62(0.65)$ & $2.83(0.72)$ & 0.06 & $2.64(0.73)$ & $2.65(0.73)$ & 0.89 \\
\hline $\begin{array}{c}\text { Sports } \\
\text { Activity }\end{array}$ & $2.12(1.48)$ & $2.16(1.60)$ & 0.87 & $1.90(1.46)$ & $1.90(1.46)$ & 0.99 \\
\hline Total Activity & $2.21(0.53)$ & $2.32(0.58)$ & 0.22 & $2.28(0.59)$ & $2.28(0.57)$ & 0.96 \\
\hline
\end{tabular}

T1252

Effect of a Water Load Test On Gastric Myoelectrical Activity and Upper Gastrointestinal Symptoms in Patients with Nausea and Vomiting of Pregnancy

Kenneth L. Koch, T. Murphy Goodwin, Roberto J. Romero, Max E. Levine, Heather Tison

Nausea and vomiting of pregnancy (NVP) is a common syndrome in which symptoms predominate in the first trimester of pregnancy. Symptoms range from mild to severe and afflict up to $80 \%$ of pregnant women. The underlying mechanisms of NVP remain poorly understood. Provocative gastric function testing has not been performed in these patients AIMS of this study were to determine the effects of the water load test on gastric myoelectrical activity, nausea, and other upper gastrointestinal symptoms in pregnant women. METHOD. A sample of 46 women with NVP, ages $18-44$ yrs (mean age $=28.2 \mathrm{yrs}$ ), at an average of 11.5 weeks of pregnancy were studied prospectively. Gastric myoelectrical activity was recorded throughout the study with electrogastrogram (EGG) methods. A 15 min baseline period was followed by a water load test during which the women ingested water until full over a 5 min period. After the water load, an additional 30 min of EGG was recorded. EGG responses were categorized into four groups: normal (2.5-3.7 cycles/min), bradygastria (1.0-2.5 cycles/min), tachygastria (3.7-10.0 cycles/min), and mixed gastric dysrhythmia (combination of tachygastria and bradygastria). Nausea, bloating, stomach fullness, and abdominal discomfort were recorded before, and 10, 20, and 30 minutes after ingestion of water on $100 \mathrm{~mm}$ visual analog scales. RESULTS. In response to the water load test, 14\% of the women exhibited normal EGG responses, while significantly more (86\%) exhibited gastric dysrhythmias $(p<.01)$. Of the dysrhythmic EGG responses, 57\% were tachygastria, $11 \%$ were bradygastria, and $18 \%$ were mixed gastric dysrhythmia. Patients with tachygastria ingested less water $(338.0 \mathrm{ml})$ than patients with normal $(370.8 \mathrm{ml})$, bradygastria $(415.0 \mathrm{ml})$ or mixed gastric dysrhythmia $(393.8 \mathrm{ml})$ patterns, but the differences were not statistically significant. $46 \%$ of the women reported nausea at baseline; significantly more women (72\%) reported nausea after the water load test $(p<05)$. At 20 min after the water load test, a significant, direct relationship was observed between nausea severity and tachygastria $(p<.05)$. The other upper gastrointestinal symptoms were not significantly increased by the water load test. CONCLUSIONS. (1) $86 \%$ of the pregnant women in this sample developed gastric dysrhythmias in response to the water load test. (2) Tachygastria was the most common dysrhythmia, and increased tachygastria was correlated with increased nausea. Gastric dysrhythmias are evoked by the water load test in women with NVP, suggesting these dysrhythmias have a pathophysiological role in the mechanism of NVP, and are a target for future therapies. 
T1253

Cumulative Incidence of Chronic Constipation: A Population-Based Study 1988-2003

Rok Son Choung, Giles R. Locke, Cathy D. Schleck, Alan R. Zinsmeister, Nicholas J. Talley

Multiple studies have assessed the prevalence of chronic constipation (CC), but few have addressed its incidence. Recognition of incident cases is required to understand pathophysiology. Aim: To estimate the cumulative incidence of CC and evaluate potential risk factors. Methods: In past cross sectional studies beginning in 1988, random samples of Olmsted County, MN residents have been mailed valid GI symptoms surveys. A new, valid GI symptom survey was mailed in 2003 to all the remaining eligible (e.g. still alive) subjects that had been mailed to previously. CC was defined by at least 2 of 4 chronic symptoms (straining, hard or lumpy stools, incomplete evacuation, infrequent stools). An incident case of CC was defined as no constipation or IBS on the first survey but developed constipation on the second survey. Risk factors for incident constipation were assessed by logistic regression. Results: 5507 (79\%) subjects responded to a first survey and 2300 (55\%) subjects responded to a second survey in which chronic constipation could be defined. The median time between the two survey responses was 10.5 years. Those with new onset constipation had a mean age of $59( \pm 12)$ years and $54 \%$ were female. The incidence of the new onset constipation differed by gender in the young (under age 50) but not in the elderly (over 70 years) (see table). Previous GI symptoms, abdominal surgical history, history of GI infection, smoking, alcohol use, marital status and educational level were not significant predictors for new onset constipation. The subjects with abdominal pain but not constipation at baseline were at increased risk of developing CC. Conclusion: The cumulative incidence of chronic constipation over a decade is more than one in 10 but does not rise significantly in the elderly. Why young men have a low incidence remains unknown. Cumulative Incidence of Chronic Constipation (\%, 95\% CI)

\begin{tabular}{|c|c|c|c|}
\hline & Men & Women & Overall \\
\hline Age & & & \\
\hline Under 50 years & $3.8(0.8,10.7)$ & $15.2(8.1,25.0)$ & $9.5(5.4,15.2)$ \\
\hline $50-70$ years & $11.3(6.6,17.7)$ & $12.7(8.1,18.8)$ & $12.1(8.6,16.2)$ \\
\hline Over 70 years & $14.3(6.4,26.2)$ & $14.5(7.5,24.4)$ & $14.4(8.9,21.6)$ \\
\hline Overall & $9.8(6.5,13.9)$ & $13.8(10.2,18.0)$ & $11.9(9.4,14.8)$ \\
\hline
\end{tabular}

$\mathrm{T} 1254$

First Community-Based Study of Functional Gastrointestinal Disorders (FGID) in Mexico Using the Rome II Modular Questionnaire

Aurelio Lopez-Colombo, Dalia Bravo-Gonzales, Araceli Corona-Lopez, Maria Elena PerezLopez, Manuel Cervantes-Ocampo, Teresita Romero-Ogawa, Douglas Morgan, Max Schmulson

Aims: The prevalence of FGID in Mexico is unknown. We recently found an unexpectedly high frequency of IBS (35\%) in volunteers in Mexico City using the Rome II modular Questionnaire.(1) We sought to establish the prevalence of FGID in Mexico in a communitybased study. Methods: The State of Tlaxcala in central Mexico with a population of nearly 1 million inhabitants in 60 cities and villages, was chosen for the survey. We sampled 500 subjects $(0.05 \%)$ from 500 households based on the registry of the National Institute of Statistics and Geography (INEGI). Village population determined the number of households recruited locally. Two physicians used the validated Spanish-Mexico Rome II Modular Questionnaire with the first available adult of each sampled household. Additional questionnaire components included income, medical consultations, GI medications, and a depression scale (CES-D). The CES-D is a short regionally validated self-rating scale designed to rate depressive symptoms as absent, mild or severe. Results: Mean subject age was 40 years of which $61 \%$ were females. Reported occupations: 44\% Homemakers, 23\% Office Employees, 20\% Self Employed, 6\% Students, 4\% Laborers, 3\% Others. The table summarizes FGID prevalence. One or more FGID was found in $58 \%$. The FGID group reported higher frequencies of medical visits ( $35 \mathrm{vs}, 17 \%$ ) and GI medications (41 vs. $22 \%$ ), both $\mathrm{p}<0.001$. Also, mild ( 9 vs. $3 \%$ ) and severe (17 vs. $3 \%$ ) depression was more frequent in FGID subjects, $\mathrm{p}<0.001$. Female predominance was more frequent in IBS ( 77 vs. $58 \%, \mathrm{p}=0.001$ ), IBS-C ( 88 vs. $59 \%, p=0.001$ ) and in Fxnl Bloating ( 76 vs. $59 \%, p=0.017$ ), compared to those not fulfilling criteria for these FGID. In IBS the F:M ratio was 3.4:1. Older age was found among Fxnl Incontinence (48, $\mathrm{p}=0.005)$ and Levator Ani (55, $\mathrm{p}=0.016)$. Conclusions: This is the first community-based study of the prevalence of FGID in Mexico by Rome II criteria, showing similar frequencies to those reported in other global populations. These subjects report higher frequency of medical consultations, GI medication use and depression as well. Refs: 1. Schmulson M et al. Dig.Dis. in press.

FGID Prevalence in Mexico, Community-Based Survey. Expressed in percent (95\% C.I.)

\begin{tabular}{|c|c|c|c|c|c|c|c|}
\hline Globus & $1.8(0.8-3.4)$ & Dysmotility-like & $3.6(2.1-5.6)$ & Bloating & $10.8(8.2-13.9)$ & Incontinence & $4.6(2.9-6.8)$ \\
\hline Rumination & $0.8(0.2-2.0)$ & Aerophagia & $5.6(3.8-8.0)$ & $\begin{array}{c}\text { Fxnl } \\
\text { Constipation }\end{array}$ & $7.4(5.3-10.1)$ & Soiling & $2.8(1.5-4.7)$ \\
\hline Chest Pain & $3.0(1.7-4.9)$ & Fxnl Vomiting & $2.0(1.0-3.6)$ & $\begin{array}{c}\text { Fxnl } \\
\text { Diarrhea }\end{array}$ & $1.4(0.6-2.9)$ & Gross & $1.8(0.8-3.4)$ \\
\hline Heartburn & $19.6(16.2-23.4)$ & IBS & $16.0(12.9-19.5)$ & $\begin{array}{c}\text { Fxnl } \\
\text { Abdominal } \\
\text { Pain }\end{array}$ & $1.0(0.3-2.3)$ & Levator Ani & $1.4(0.6-2.9)$ \\
\hline Dysphagia & $1.8(0.8-3.4)$ & IBS-D & $2.4(1.2-4.2)$ & $\begin{array}{c}\text { Unspecified } \\
\text { Fxnl } \\
\text { Abdominal } \\
\text { Pain }\end{array}$ & $1.6(0.7-3.1)$ & $\begin{array}{c}\text { Proctalgia } \\
\text { Fugax }\end{array}$ & $6.2(4.3-8.7)$ \\
\hline Dyspepsia & $7.0(4.9-9.6)$ & IBS-C & $6.6(4.6-9.1)$ & $\begin{array}{c}\text { Gallbladder } \\
\text { Dysfunction }\end{array}$ & $1.2(0.4-2.6)$ & Dyssynergia & $2.0(1.0-3.6)$ \\
\hline Ulcer-like & $3.4(2.0-5.4)$ & Mixture & $7.0(4.9-9.6)$ & $\begin{array}{c}\text { Oddi } \\
\text { Dysfunction }\end{array}$ & $0.2(0-1.1)$ & & \\
\hline
\end{tabular}

Fxnl: Functional

\section{T1255}

The Bi Omnibus Study: An International Survey of Community Prevalence of Constipation and Laxative Use in Adults

Arnold Wald, Michael Kamm, Stefan Mueller-Lissner, Carmelo Scarpignato, Wolfgang Marx, Chris Schuijt

Reported prevalence of constipation range is $4-28 \%$, depending on definitions and methods used, making comparisons between countries difficult. To determine prevalence in a large community, self definition of constipation is important and use of a standard questionnaire is mandatory. AIM: To determine prevalence and demographics of constipation in 7 countries. METHODS: Representative samples of approximately 2000 persons in each of US, UK, Germany (GE), France (FR), Italy (IT), Brazil (BR), and South Korea (SK) were administered a standard questionnaire. Questions concerned occurrence, duration and frequency of constipation as well as treatment duration and frequency of laxative use by constipated persons. RESULTS: Of 13,958 persons interviewed, $12.4 \%$ reported constipation in previous year. This varied among countries, gender and age.

Table 1

\begin{tabular}{|c|c|c|c|c|c|c|c|}
\hline Constipation & US & UK & FR & GE & IT & BR & SK \\
\hline In females (\%) & 21 & 11 & 19 & 7 & 11 & 24 & 23 \\
\hline In males (\%) & 14 & 4 & 9 & 3 & 5 & 9 & 11 \\
\hline Age <30 (\%) & 14 & 6 & 12 & 2 & 6 & 14 & 18 \\
\hline Age 60+ yr (\%) & 20 & 9 & 22 & 9 & 11 & 19 & 14 \\
\hline All & 18 & 8 & 14 & 5 & 8 & 17 & 17 \\
\hline
\end{tabular}

In all countries, more women than men had constipation (OR2.43; CI 2.18-2.71) and except for SK, age $60+\mathrm{yr}$ had more constipation than $<30 \mathrm{yr}$ (OR 1.5; CI 1.25-1.73). Over $70 \%$ of constipated had constipation at least every 3 months; percentages of weekly constipation were highest in BR, SK, IT (36-59\%); those with daily constipation were highest in BR (19\%), lowest in US, SK, GE (5-7\%).

Table 2

\begin{tabular}{|c|c|c|c|c|c|c|c|}
\hline Laxative Use In Constipation Group & US & UK & FR & GE & IT & BR & SK \\
\hline In females (\%) & 38 & 35 & 35 & 33 & 29 & 34 & 16 \\
\hline In males (\%) & 43 & 38 & 27 & 23 & 46 & 17 & 17 \\
\hline Age $<30(\%)$ & 31 & 14 & 26 & 18 & 31 & 29 & 19 \\
\hline Age 60+ (\%) & 52 & 51 & 48 & 44 & 51 & 27 & 23 \\
\hline All & 40 & 36 & 33 & 31 & 34 & 30 & 16 \\
\hline
\end{tabular}

Laxative use ( $\mathrm{GE}=1$ ) $)$ was highest in US (OR 1 69. CI 1 04-2.74), lowest in SK (OR 0.56 ; CI 0.32-0.97); and similar in others. Use of laxatives increased with age $(60+\mathrm{vs}<30 \mathrm{yrs}=$ OR 1.63; CI 1.14-1.64). CONCLUSIONS: Prevalence of self defined constipation varies among countries and is related to gender and age in all but SK. Laxative use is highest in US, lowest in SK and is influenced by age but not gender, duration and frequency of constipation in all countries.

\section{T1256}

What Causes Functional Dyspepsia with Overlapping Symptoms? Zhiqiang Song, Meiyun Ke, Zhifeng Wang, Xiaohong Xu

BACKGROUND: Overlapping of gastrointestinal symptoms is very common in patients with functional dyspepsia (FD). It remains unknown what causes FD with overlapping symptoms (OS). Exploring whether other differences exist between FD patients with and without OS will facilitate us to understand the possible mechanisms. AIMS: To investigate the differences between the functional dyspeptics with and without OS at the aspects of demographic characteristics, symptomology, pathophysiology, quality of life, psychological and sleep status. METHODS: Eighty-two patients with FD (24M, 58F, mean age 45yr) surveyed with Rome II Modular Questionnaire are divided into two groups: the patients without OS (only dyspeptic symptoms) and with OS (esophageal, intestinal or anorectal symptoms). The data including demographic characteristics, dyspeptic symptoms subtypes (ulcer-like, dysmotilitylike, nonspecific) and severity scores (mild-1, moderate-2, severe-3), quality of life (SF-36), psychological status and sleep status (4-Likert scale) were recorded. The tests including liquid nutrient test (LNT), solid gastric emptying (GE, radiopaque makers and scintigraphic evaluation), multi-channels electrogastrography (MEGG) and autonomic nerve system (ANS) 
were performed. RESULTS: (1) The proportion of the patients with and without OS accounts for $63 \%$ and $37 \%$, respectively. (2) The two groups are matched in sex, age, BMI, occupation, education, marital status, economical status, working and living stress $(P>0.05)$. (3) No statistical differences are found in two groups at the aspects of symptom subtypes distribution and severity scores $(P>0.05)$. (4) No statistical differences exist in any index related to the tests of LNT, GE, MEGG and ANS (P>0.05). (5) However, the psychological status of the patients with OS is much worse (disturbed mildly $10 \%$, moderately $48 \%$, severely $15 \%$ ) than that of the patients without OS $(27 \%, 10 \%, 10 \%$ respectively, $\mathrm{P}<0.05)$. The same difference is also found in the sleep status (with OS: disturbed mildly $19 \%$, moderately $35 \%$, severely $29 \%$ vs. without OS: $30 \%, 17 \%, 13 \%$, respectively, $\mathrm{P}<0.05)$. The quality of life of the patients with OS is worse in the dimension of physical functioning, role limitations due to physical health and general health $(\mathrm{P}<0.05)$. CONCLUSIONS. The same features exit in demography, symptomology and pathophysiology between the functional dyspeptics with and without OS. The worse psychological and sleep status and quality of life in the patients with OS give us a hint that psychological factors are maybe involved in the mechanisms underlying OS and the treatment of psychological support and improving sleep is necessary.

\section{$\mathrm{T} 1257$}

Elevated Breath Methane in Constipation

Syed I. Thiwan, Olafur S. Palsson, Marsha J. Turner, William E. Whitehead

Background: Increased methane production was reported in patients with constipation or IBS-constipation. In animal studies, methane was shown to slow intestinal transit. Aims: To compare breath $\mathrm{Ch} 4$ and $\mathrm{H} 2$ at baseline (fasting) and following a carbohydrate meal in healthy controls, and patients with lactose malabsorption, IBS (without constipation) and slow transit constipation. Methods: 80 subjects (10 males, 70 females, 25 Healthy, 25 IBS, 23 lactose malabsorbers and 7 constipated) underwent hydrogen breath test with $50 \mathrm{~g}$ of lactose and $80 \mathrm{~g}$ of sucrose on an empty stomach in the morning. Breath samples for $\mathrm{H} 2$ and Ch4 were collected at baseline and every $15 \mathrm{~min}$ until $120 \mathrm{~min}$ and then every $30 \mathrm{~min}$ up to $180 \mathrm{~min}$. Breath $\mathrm{H} 2$ and $\mathrm{Ch} 4$ were analyzed using a Quintron Microlyzer model SC. Patients with constipation underwent Sitzmark study for assessment of colonic transit time. Analysis: One way ANOVA with Bonferreni correction was used to compare baseline as well as area under the curve (AUC) of $\mathrm{H} 2$ and $\mathrm{Ch} 4$. Results: Baseline $\mathrm{Ch} 4$ levels were significantly higher in constipation compared to IBS and was not significantly lower in IBS compared to healthy controls $(\mathrm{p}<0.05)$. Constipated patients also had significantly elevated $\mathrm{Ch} 4$ in response to the sugar load, compared to healthy controls and IBS $(\mathrm{p}<0.05)$. Mean colonic transit time among constipated subjects was $97.4 \mathrm{hrs}$ (77-115hrs). The transit time tended to correlate with AUC of $\mathrm{Ch} 4(\mathrm{r}=0.48)$, but this was not statistically significant in 7 subjects. Baseline $\mathrm{H} 2$ was not statistically different between the different groups but as expected, both $\mathrm{H} 2$ production and peak $\mathrm{H} 2$ level were significantly higher in subjects with lactose malabsorption compared to other groups $(\mathrm{p}<0.001)$. Conclusions: Increased Methane production is associated with constipation. Further experiments are needed to determine whether this association is due to methane inhibiting intestinal motility or whether delayed colonic transit facilitates the colonization of the colon with methanogenic bacteriae (i.e. whether elevated methane is a cause or consequence of constipation). [Supported by Novartis Pharmaceuticals and grants R24 DK67674 and RR00046.

\begin{tabular}{|c|c|c|c|c|c|c|}
\hline \multirow{2}{*}{ Group } & \multicolumn{3}{|c|}{$\mathrm{H} 2$} & \multicolumn{3}{c|}{ Ch4 } \\
\cline { 2 - 7 } & $\begin{array}{c}\text { Baseline } \\
\text { (Mean+SD) }\end{array}$ & $\begin{array}{c}\text { Peak } \\
\text { (Mean+SD) }\end{array}$ & $\begin{array}{c}\text { AUC } \\
\text { (Mean+SD) }\end{array}$ & $\begin{array}{c}\text { Baseline } \\
\text { (Mean+SD) }\end{array}$ & $\begin{array}{c}\text { Peak } \\
\text { (Mean+SD) }\end{array}$ & $\begin{array}{c}\text { AUC } \\
\text { (Mean+SD) }\end{array}$ \\
\hline Healthy & $5.44+6.246$ & $10.92+7.664$ & $67.06+59.83$ & $4.08+11.228$ & $6.32+15.884$ & $57.27+149.291$ \\
\hline $\begin{array}{c}\text { Lactose } \\
\text { Malabsorption }\end{array}$ & $6.09+8.586$ & $\begin{array}{c}\mathbf{1 1 0 . 0 9 + 7 9 . 0 5 0} \\
\mathbf{a}\end{array}$ & $\begin{array}{c}\mathbf{4 8 4 . 3 7 + 3 9 4 . 2 6 2} \\
\mathbf{a}\end{array}$ & $8.57+14.292$ & $11.83+16.353$ & $108.0+158.758$ \\
\hline IBS & $10.04+10.538$ & $14.32+10.877$ & $95.62+81.938$ & $0.64+1.868$ & $0.96+2.406$ & $6.08+18.866$ \\
\hline Constipation & $5.71+8.635$ & $11.86+10.792$ & $74.71+80.030$ & $\begin{array}{c}\mathbf{1 6 . 0 + 1 9 . 4 3 4} \\
\mathbf{b}\end{array}$ & $\begin{array}{c}\mathbf{5 1 . 4 3 + 3 3 . 3 8 1} \\
\mathbf{c}\end{array}$ & $\begin{array}{c}\mathbf{2 2 3 . 2 9 + 2 3 0 . 9 4 5} \\
\mathbf{c}\end{array}$ \\
\hline
\end{tabular}

( $a=$ vs. H, IBS, $C p<0.05 ; b=$ vs. IBS $p<0.05 ; c=$ vs. H, IBS $p<0.001$ )

\section{$\mathrm{T} 1258$}

Possible Role of Intraluminal Gas Production On Functional Gastrointestinal Disorders

Yoshihisa Urita, Susumu Ishihara, Kenichiro Arai, Jun Kondo, Tastuo Akimoto, Hiroto Kato, Noriko Hara, Hideyuki Koshino, Yoshiko Honda, Yoko Nagai, Kazushige Nakanishi, Nagato Shimada, Motonobu Sugimoto, Kazumasa Miki

Background: The presence of carbohydrates in the colonic lumen inhibits gastric and pancreatic secretions, and also influences lower esophageal sphincter function. Based on the fact that $2-20 \%$ of carbohydrates escape small intestinal absorption, colonic fermentation is considered to be present more frequency than expected and may result in various gastrointestinal symptoms. It is still unclear whether patients with functional gastrointestinal disorders have gastrointestinal fermentation more frequently. Because bacteria represent the sole source of gut hydrogen $(\mathrm{H} 2)$ and methane $(\mathrm{CH} 4)$, fasting breath $\mathrm{H} 2$ and $\mathrm{CH} 4$ gases have been used as markers of gastrointestinal fermentation. The aim of this study is to determine whether fermentation in the digestive tract may associate with developing gastrointestinal symptoms. Patients and Methods: A total of 53 patients (mean age 65 years, male/female= 9/44) diagnosed with functional dyspepsia according to the Rome II criteria were recruited in the study. At endoscopy, intragastric and intraduodenal gas samples were taken for measuring hydrogen and methane concentrations. Gastric emptying was measured with 13C-acetate breath test. After overnight fasting, the patient received a liquid test meal (200kcak, 200ml), containing $100 \mathrm{mg}$ of $13 \mathrm{C}$-acetate. Breath samples were collected at 10 min intervals of $120 \mathrm{~min}$ and gastric emptying were expressed as the time of peak $13 \mathrm{CO} 2$ excretion (Tmax). Results: Nineteen patients (36\%) had delayed gastric emptying (Tmax $>90$ $\mathrm{min}$ ). Of these 19 patients, a high hydrogen level (more than $10 \mathrm{ppm}$ ) of fasting breath, intragastric and ntraduodenal samples, was detected in 3.21. and $21 \%$, respectively. A high methane level (more than 10ppm) of three samples was found in 6,15 , and $6 \%$, respectively.
Among 34 patients with normal gastric emptying, 13 (25\%) had high hydrogen and/o methane levels of at least one sample. In 9 patients (17\%) with FGID, Tmax in 13C-acetate breath test and hydrogen and methane levels of all samples were normal. Conclusions: Delayed gastric emptying was found in only $36 \%$ of patients with FGID, whereas enhanced hydrogen or methane production in the digestive tract was detected in $42 \%$. This suggests the association between enhanced fermentation in the digestive tract and abdominal symptoms in patients with FGID

\section{T1259}

Familial Aggregation of IBS Is Specific to IBS Yuri Saito, Jamshid S. Kalantar, G. R. Locke, Alan R. Zinsmeister, Nicholas J. Talley

Purpose: IBS has been observed to aggregate in families-17\% in IBS relatives versus $7 \%$ of spouse control relatives (Gut 2003;52:1703-7). Whether other functional and non-functional gastrointestinal disorders cluster in families is unknown. Because of the overlap between IBS and other disorders, we hypothesized that there would be an increased frequency of other functional gastrointestinal disorders in first-degree relatives of IBS patients compared with relatives of controls (the patient's spouse). Methods: Patients attending an IBS educational program and residents of Olmsted County, Minnesota, who had been coded as IBS on a database and their spouses were mailed a validated self-report Bowel Disease Questionnaire (BDQ) including a somatic symptom checklist and a family information form (FIF) A BDQ was mailed to all first-degree relatives of subjects identified from the FIF. The prevalence of Gastroesophageal Reflux Disease (GERD), Functional Dyspepsia (FD), Functional Constipation (FC), Functional Diarrhea (Fdi), and Functional Bloating (FB), based on standard criteria, were calculated in patient relatives and spouse control relatives. Results: The BDQ was sent to a total of 303 eligible relatives, of which $73 \%$ responded. Relatives were comparable in mean age, sex distribution, and somatization score. The prevalence of the above disorders in IBS relatives and spouse control relatives are shown in the table below. Except for FB and FC, the prevalence of GERD, FD, and FDi were similar between the two relative groups. Conclusions: Familial aggregation of IBS had previously been shown in this dataset. Except for constipation, aggregation of other gastrointestinal disorders was not observed suggesting that aggregation of IBS symptoms is specific for IBS cases compared with controls. This suggests that IBS (and FC) may be a distinct entity separate from other functional disorders.

\begin{tabular}{|c|c|c|c|c|}
\hline & $\begin{array}{c}\text { Case Relatives } \\
(\%)\end{array}$ & $\begin{array}{c}\text { Control Relatives } \\
(\%)\end{array}$ & $\begin{array}{c}\text { Univariate LR* model } \\
\text { p-value }\end{array}$ & $\begin{array}{c}\text { Multivariate LR* }{ }^{*} \text { model } \\
\text { p-value }\end{array}$ \\
\hline GERD & 22.8 & 18.6 & 0.46 & 0.85 \\
\hline FD & 5.9 & 4.7 & 0.73 & 0.98 \\
\hline FC & 4.4 & 10.5 & 0.11 & 0.05 \\
\hline Fdi & 4.4 & 4.7 & 0.93 & 0.97 \\
\hline FB & 8.8 & 4.7 & 0.28 & 0.32 \\
\hline
\end{tabular}

*LR = Logistic Regression model accounting for correlation within families †adjusting for age, gender, and somatization

\section{$\mathrm{T} 1260$}

Anxiety and IBS: Influence On Severity of Symptoms, Consultation and Quality of Life

Marta Moreno Ortega, Monica Garcia Alonso, Enrique Rey, Manuel Diaz-Rubio

Anxiety is associated with IBS, although it is unclear its actual role. Anxiety is considered to be related to consultation behaviour. AIM: We aim to answer the following questions: is severity of IBS symptoms related to anxiety level? Is anxiety associated to consultation behaviour independently of severity of IBS symptoms? And how is the impact of anxiety and severity of symptoms on HRQL? MATERIAL\&METHODS. We targeted a population of IBS subjects representative of the full spectrum of the disease. For this purpose, symptomatic patients with the diagnosis of IBS were recruited from primary care and gastroenterologist office, and non consulters IBS subjects were recruited from the general population. All subjects (consulters and non consulters) must fulfil Rome II criteria for IBS to be included. All subjects completed a clinical questionnaire, including health resources use. Severity of IBS symptoms was evaluated using the functional disease severity index (FDSI); anxiety was evaluated as state anxiety and trait anxiety using the STAI questionnaire. HRQL was assessed with SF-36. Correlation was evaluated by Pearson coefficient. To evaluate factors associated with consultation, a logistic regression model was constructed including state anxiety, trait anxiety and FDSI as factors, adjusting by age and sex. Impact of anxiety (state and trait) and severity of symptoms on each subscale of SF36 was evaluated using a multiple lineal regression model, adjusting by age and sex RESULTS. We included 110 subjects, 52 IBS non consulters and 60 IBS consulters ( 85 women, mean age 40; range 18-73) IBS consulter suffered more severe symptoms ( 19 mild, 28 moderate and 13 severe symptoms) than non consulters (41 mild 11 moderate symptoms). There were no correlation between FDSI and state anxiety $(r=0.07)$ or trait anxiety $(r=0.15)$ even adjusting for consultation (partial correlation coefficients -0.07 and 0.08 , respectively). Consultation was independently associated with FDSI (adjusted OR 1.04 [1.02-1.06]) and state anxiety (1.03 [1.01-1.05]). In multiple linear regression model Physical Subscale of SF36 was associated with FDSI (Beta=0.41; $\mathrm{p}<0.0001$ ) and age (Beta $=-0.28 ; \mathrm{p}=0.001$ ); Mental Subscale of $S F 36$ was associated with trait anxiety (Beta $=-0.49 ; \mathrm{p}<0.0001)$ and state anxiety (Beta $=-0.28 ; \mathrm{p}=0.002)$. CONCLUSION Severity of IBS symptoms are not related to the degree of anxiety. Both severity of symptoms and anxiety are independently linked to consultation. Severity of symptoms and anxiety affect different aspects of HRQL. Supported by a Grant from FIS: PI03521 (Carlos III Institute. Ministry of Health, Spain) 
T1261

Additive Effect of Pathophysiological Abnormalities On Gastrointestinal Symptoms in Patients with Irritable Bowel Syndrome (IBS)

Magnus Simren, Hans Strid, Iris Posserud, Amanda Ersryd, Pia Agerforz, Anette Lindh, Henrik Sjovall, Hasse Abrahamsson

Background: Both central and peripheral factors are thought to contribute to the symptoms of IBS, including psychosocial factors, abnormal gastrointestinal (GI) motility and secretion, and visceral hypersensitivity. The relative importance of these pathophysiological factors for GI symptom pattern and severity is largely unknown. Methods: We included 79 IBS patients fulfilling the Rome II criteria for IBS (56 females; mean age 36 (19-62) years). They all underwent investigations to assess GI motility (stationary antroduodenojejunal manometry and oroanal transit time, OATT), secretion (jejunal mucosal potential difference) and visceral hypersensitivity (rectal sensitivity testing using an electronic barostat). Abnormal findings were defined based on normal values obtained at our lab and internationally accepted criteria (manometry).The patients also completed the Hospital Anxiety and Depression scale to evaluate the presence of clinically significant anxiety and depression (score $\geq 11$ ). Gastrointestinal Symptom Rating Scale (GSRS) was used to assess GI symptom severity. Results: All patients but 8 had at least one pathophysiological alteration - small intestinal dysmotility was seen in $51 \%$ of the patients (at least mild severity), abnormal jejunal secretion in $37 \%$, rectal hypersensitivity in 51\%, anxiety and/or depression in 32\%, accelerated OATT in 27\% and delayed OATT in 5\%. Patients with rectal hypersensitivity vs, normal sensitivity had more severe overall GI symptom severity (GSRS Total score: $3.6 \pm 0.8$ vs. $2.9 \pm 0.6($ mean $\pm S D)$; $\mathrm{p}<0.0001)$, and more severe abdominal pain $(\mathrm{p}<0.0001)$, indigestion $(\mathrm{p}=0.001)$, reflux $(\mathrm{p}=$ $0.04)$ and satiety $(p<0.0001)$. The presence of anxiety and/or depression was also related to more severe overall GI symptom severity $(3.7 \pm 0.8$ vs. $3.1 \pm 0.8 ; p=0.006)$, as well as more severe abdominal pain $(\mathrm{p}=0.04)$, constipation $(\mathrm{p}=0.01)$ and reflux $(\mathrm{p}=0.01)$. Patients with abnormal jejunal secretion tended to have more severe overall GI symptom severity ( $\mathrm{p}=$ $0.05)$ and more severe abdominal pain $(\mathrm{p}=0.04)$. Accelerated OATT was related to more severe diarrhea $(p=0.004)$ and delayed OATT with constipation $(p=0.02)$. Small intestinal dysmotility was unrelated to GI symptom severity and pattern. With increasing number of pathophysiological alterations a gradual increase in the overall GI symptom severity was found $(\mathrm{p}=0.009)$. Conclusion: Visceral hypersensitivity and psychological factors seem to be the strongest predictors for GI symptom severity in IBS. The pathophysiological factors in IBS seem to have an additive effect on GI symptom severity.

\section{$\mathrm{T} 1262$}

Idiopathic Bile Acid Malabsorption Is Prevalent in Patients with the Irritable Bowel Syndrome (IBS)

Antal Bajor, Hans Strid, Jenny Wallin, Kjell-Arne Ung, Hasse Abrahamsson, Magnus Simren

Background: Irritants within the gut may play a role in the pathophysiology of IBS. One class of such irritants may be bile acids not being reabsorbed in the terminal ileum, but leaking into the colon. In patients presenting with chronic diarrhea idiopathic bile acid malabsorption (IBAM) is present in up to $40 \%$, but the prevalence of IBAM in IBS is unknown. The SeHCAT retention test is a reliable indirect method for the diagnosis of bile acid malabsorption. Aims: To study the occurrence of IBAM in IBS patients and in the different IBS subgroups based on the predominant bowel habit. The secondary aim was to investigate whether symptoms are correlated to the SeHCAT values. Methods: IBS patients fulfilling the ROME II criteria referred to our centre has been consecutively included in the study, irrespective of the bowel habit of the patient. All patients completed the Bristol Stool Form scale daily during a week for registration of stool frequency and consistency. They also performed a SeHCAT test and a value of $\leq 10 \%$ on day 7 was considered abnormal Results: Forty-nine Rome II positive IBS patients were included (mean age 38 (20-72) years; 36 females). Of these, six patients had predominantly constipation (C-IBS), 22 diarrhea (DIBS) and 21 alternating bowel habits (A-IBS). A SeHCAT value $\leq 10 \%$ on day 7 , compatible with a diagnosis of IBAM, was found in 13 patients (27\%). In the IBS subgroups IBAM was demonstrated in 8 patients with D-IBS (37\%), in 5 patients with A-IBS (24\%) and in none the C-IBS group. The SeHCAT retention values differed significantly between the IBS subgroups, $\mathrm{p}=0.02$, with C-IBS patients having a median value of $54 \%$, D-IBS $15 \%$ and AIBS $19 \%$. There was no significant difference between the D- and A-IBS groups. Weak, but statistically significant correlations between SeHCAT values and the stool frequency ( $\mathrm{R}=$ $0,35, \mathrm{p}=0.02)$ and consistency $(\mathrm{R}=0.34, \mathrm{p}=0.02)$, respectively, were demonstrated. However, no clear "cut-off" level regarding stool consistency and frequency predicting an abnormal SeHCAT could be found. Conclusion: Idiopathic bile acid malabsorption is common in IBS patients with diarrhea predominance, but also in patients with alternating bowel habits. Stool consistency or frequency can not be used to reliably predict the presence of IBAM in IBS. Ongoing studies using bile acid binding agents to treat IBS patients with IBAM will further evaluate the clinical relevance of IBAM in IBS.

\section{T1263}

Performance Characteristics of a $\left[{ }^{13} \mathrm{C}\right]$-Spirulina Platensis Gastric Emptying Breath Test

Lawrence A. Szarka, Michael Camilleri, Duane D. Burton, Robert F Martin

Background: Previous clinical trials of $\left[{ }^{13} \mathrm{C}\right]$-Spirulina platensis gastric emptying breath test (GEBT) utilizing variable meal compositions have demonstrated accuracy similar to scintigraphy. The validation of a $\left[{ }^{13} \mathrm{C}\right]$-Spirulina platensis GEBT based upon a standardized meal preparation consisting of only shelf-stable components would be greatly desirable. Aim: To estimate the total variability of simultaneous scintigraphic and GEBT measurements in normal human subjects in order to appraise performance characteristics of the GEBT. Methods: Twenty healthy volunteers underwent assessment of gastric emptying in duplicate studies using a dual-labeled test meal consisting of $100 \mathrm{mg}\left[{ }^{13} \mathrm{C}\right]$-Spirulina platensis, $0.5 \mathrm{mCi}{ }^{99 \mathrm{~m}} \mathrm{Tc}$ sulfur colloid, $27 \mathrm{~g}$ freeze dried egg mix, 6 saltine crackers, and $6 \mathrm{oz}$ water. The test meal has a caloric content of $238 \mathrm{kcal}$. Breath samples and scintigraphic images were obtained at $45,90,120,150,180$, and 240 minutes after ingestion of the test meal. Tests were repeated within a median interval of 10 days. The primary transit parameter for breath test is the percent dose of carbon- 13 excreted (PCD) multiplied by 1000 to produce $\mathrm{kPCD}_{\mathrm{t}}$ at any time t. At respective times, the primary transit parameter for scintigraphy was percent emptied from the stomach using decay corrected, geometric mean data from anterior and posterior gamma-camera images. Coefficient of variation at each time point was calculated within and between individual participants for the primary transit parameters for the two tests. Results: The inter- and intra-subject coefficients of variation (\%) of the breath test and scintigraphy between 45 and 150 minutes were similar (table). At measurement times greater than 150 minutes, scintigraphic coefficients of variation are reduced (truncated) as a greater fraction of results reach the $100 \%$ emptied limit. Conclusions: The data suggest that gastric emptying results for $\left[{ }^{13} \mathrm{C}\right.$-Spirulina platensis breath test are generally as reproducible as those for scintigraphy and that imprecision within and between individuals reflects normal physiologic variation with both tests.

\begin{tabular}{|c|c|c|c|c|c|c|}
\hline & $45 \min$ & $90 \min$ & $120 \min$ & $150 \min$ & $180 \min$ & $240 \min$ \\
\hline GEBT & & & & & & \\
\hline Inter-subject & $23.5 \%$ & $19.5 \%$ & $14.5 \%$ & $13 \%$ & $12 \%$ & $16.5 \%$ \\
\hline Intra-subject & $20 \%$ & $15 \%$ & $12 \%$ & $8 \%$ & $8 \%$ & $7 \%$ \\
\hline Scintigraphy & & & & & & \\
\hline Inter-subject & $25.5 \%$ & $16 \%$ & $11 \%$ & $8.5 \%$ & $5 \%$ & $2 \%$ \\
\hline Intra-subject & $21 \%$ & $14 \%$ & $9 \%$ & $6 \%$ & $4 \%$ & $1 \%$ \\
\hline
\end{tabular}

\section{T1264}

Impaired Drinking Capacity in Patients with Functional Dyspepsia: Relationship with Intragastric Distribution

Bram D. Van den Elzen, Roel J. Bennink, Breg Braak, Guido N. Tytgat, Guy E. Boeckxstaens

Introduction: We previously demonstrated that the impaired drinking capacity in patients with functional dyspepsia (FD) could not be explained by altered proximal stomach function. To what extent abnormal distal stomach function or an altered intragastric distribution could explain the impaired drinking capacity in FD is unclear. Therefore, we determined gastric volumes after a drink test using SPECT imaging. Methods: After a baseline scan $20 \mathrm{HV}$ (13f) and $18 \mathrm{FD}(5 \mathrm{f})$ underwent a drink test $(100 \mathrm{ml} / \mathrm{min})$ followed by SPECT scanning. A MannWhitney $U$ test $(p<0.05)$ was used for statistical analyses. Results: Baseline gastric volumes measured by SPECT were significantly higher in FD compared to HV for total $(351 \pm 18$ $\mathrm{ml}$ vs. $225 \pm 18 \mathrm{ml} ; \mathrm{p}<0.001)$, proximal $(264 \pm 9 \mathrm{ml}$ vs. $184 \pm 12 \mathrm{ml} ; \mathrm{p}<0.001)$ and distal stomach $(87 \pm 12 \mathrm{ml}$ vs. $41 \pm 8 \mathrm{ml} ; \mathrm{p}<0.001)$. FD ingested significantly less water compared to $\mathrm{HV}(1148 \pm 143 \mathrm{ml}$ vs. $1818 \pm 153 \mathrm{ml} ; \mathrm{p}<0.001)$. The gastric volume increase induced by the drink test was significantly different between HV and FD for total $(2181 \pm 149 \mathrm{ml}$ vs. $1500 \pm 140 \mathrm{ml} ; \mathrm{p}=0.006)$, proximal $(1671 \pm 115 \mathrm{ml}$ vs. $1266 \pm 119 \mathrm{ml} ; \mathrm{p}=0.035)$ and distal stomach $(564 \pm 48 \mathrm{ml}$ vs. $234 \pm 33 \mathrm{ml} ; \mathrm{p}<0.001)$. There was a good correlation between the ingested amount of water and the increase in gastric volumes measured by SPECT (HV 0.61; $\mathrm{p}=0.004$ and FD 0.88; $<<0.001$ ). The ingested water was differently distributed with proportionally more ingested volume in the distal stomach of HV compared to FD (fig 1) and this resulted in a difference in proximal/distal stomach volume ratio for FD compared to HV $(6.4 \pm 1.3$ vs. $3.3 \pm 0.4 ; p=0.001)$. Conclusion: These data suggest that drinking capacity is mainly determined by antral volume, with a reduced antral filling in FD compared to HV at maximal ingested volume. Whether this is due to an altered antral compliance or an increased antral sensitivity for distension remains to be studied.

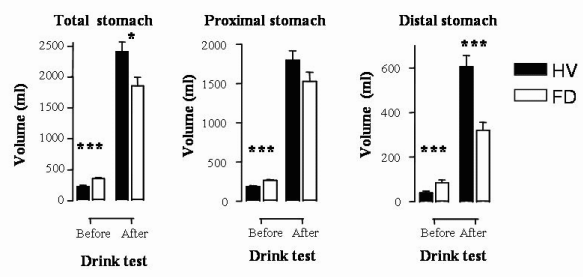

Fig. 1 Volumes measured by Gastric Volume Scintigraphy of total, proximal and distal stomach volume over time. (* $\mathrm{p}<0.05 ; * * \mathrm{p}<0.01$;

T1265

Distinguishing IBS Defined By the Manning Criteria from IBS Defined By the Rome II: Does Manning in the Absence of Rome Really Identify a Different Disorder?

Rok Son Choung, Nicholas J. Talley, Giles R. Locke, Yuri A. Saito, Cathy D. Schleck, Alan R. Zinsmeister

Irritable bowel syndrome (IBS) diagnosis is based on positive symptom criteria; however, more people meet the Manning criteria than the Rome criteria in population-based and clinical studies. Some have argued that those fulfilling Manning but not Rome criteria still have IBS, but the characteristics of this subpopulation have never been defined. Aim: To determine if those with Manning but not Rome II criteria differ from those with Rome II for IBS in the community. Methods: A population-based, cross-sectional survey was conducted by mailing a valid and reliable questionnaire to an age- and gender- stratified random sample of residents of Olmsted County, MN, aged 30 - 95 years (response rate 55\%). Manning was defined as abdominal pain plus 2 or more of 6 symptoms. IBS prevalence estimates were 
calculated for Rome II and Manning criteria, and subjects were categorized as 'Rome II (+) or Manning (+)/Rome II (-) based on their symptom status. A logistic regression model was used to estimate the odds ratios (OR $[95 \% \mathrm{CI}]$ ) for Rome II (-) vs (+) in subjects that met Manning criteria. Results: Among the 5119 subjects for which full data were available, 862 (17\%) met Manning criteria, while 620 (12\%) met Rome II criteria. Those subjects (N=242) with Rome II (-) and Manning (+) had a mean age of 60 years; $67 \%$ were female. Demographic characteristics were all similar in this group vs. Rome II subjects. Positive predictors for subjects with Rome II (-) and Manning (+) compared to Rome II $(+)$ were hard stools (OR 4.20 [3.06 - 5.76]), straining (OR 4.19 [3.01 - 5.83]), and less frequent stools (OR 2.06 $[1.15-3.69])$ (table). Negative predictors were loose or watery stools (OR $0.30[0.21$ $0.44]$ ) and urgency (OR $0.42[0.30-0.58])$. Severe and moderate pain severity were modest negative predictors for Rome II (-) and Manning (+) subjects. Conclusions: Discordant subjects with Rome II (-) but Manning (+) have similar demographic characteristics compared to those fulfilling Rome II (+) IBS, but they were more likely to report constipation type symptoms. Hence, Manning criteria may identify more people with the constipation subtype of IBS missed by Rome II. Supported in part by an unrestricted grant from Novartis. Individual symptoms that were independent predictors in different definitions of IBS

\begin{tabular}{|c|c|c|c|c|}
\hline & $\begin{array}{c}\text { Rome II (+) \% } \\
(\mathrm{n}=620)\end{array}$ & $\begin{array}{c}\text { Manning (+)/Rome II }(-) \% \\
(\mathrm{n}=242)\end{array}$ & OR & $95 \%$ CI \\
\hline Hard stools & 26.7 & 60.9 & 4.20 & $3.06-5.76$ \\
\hline Straining & 29.5 & 64.2 & 4.19 & $3.01-5.83$ \\
\hline $\begin{array}{c}\text { Watery or loose } \\
\text { stools }\end{array}$ & 42.6 & 18.0 & 0.30 & $0.21-0.44$ \\
\hline Urgency & 45.6 & 26.3 & 0.42 & $0.38-0.58$ \\
\hline
\end{tabular}

$\%$ is column percentage, OR calculated by row $\%$

\section{T1266}

Exploring Epidemiology of Irritable Bowel Syndrome to the Middle Teen-Ager Yuka Endo, Shin Fukudo, Yuko Kimura, Tomotaka Shoji, Kazuto Karahashi, Yasuhiro Sagami, Joe Morishita, Michio Hongo

BACKGROUND \& AIMS: Most of patients with irritable bowel syndrome (IBS) have IBS symptoms since their middle teen ages. However, the prevalence and the features of adolescent IBS were generally lacking. The objective of this research was to know the features of adolescent IBS in Japan. SUBJECTS \& METHODS: We randomly selected Junior high school students in Miyagi prefecture, Japan according to population of each area. The response rate was $76.9 \%, 833$ boys and 888 girls (age: 15 y.o.) participated in this study. They fulfilled self-reported questionnaires those include Rome-II Modular Questionnaire (RIIMQ), Self-reported IBS Questionnaire (SIBSQ) based on Rome-II criteria, Generalized Self-Efficacy Scale (GSES), SF-36 and other questions on their lives. RESULTS: One hundred and six boys (12.7\%) and 145 girls (16.3\%) were diagnosed as IBS by Rome-II Modular Questionnaire. IBS students had lower scores in GSES $(\mathrm{p}<0.0001)$ and every SF-36 subscales $(\mathrm{p}<0.0001$, respectively) than control students those have no abdominal symptoms (boys: $n=214$, girls: $n=148$ ). No IBS students consulted hospital frequently because of IBS symptoms in the present or in the past. Distribution of their resident area showed no significant difference between IBS students and controls. IBS students complained sleep disturbance more often than controls, especially initial insomnia $(\mathrm{p}=0.0007)$, difficulty in staying asleep $(\mathrm{p}=0.0005)$, lack of satisfaction with sleep $(\mathrm{p}=0.0036)$ and short sleeping time $(\mathrm{p}<0.0001)$. IBS students had more traumatic episodes and felt their lives more stressful than controls $(p<0.0001$, respectively). IBS girls had more traumatic episodes $(\mathrm{p}=0.0332)$ and felt more stressful in their lives $(\mathrm{p}=0.0407)$ than IBS boys. There were no significant differences in types of sleep disturbances except initial insomnia between IBS boys and IBS girls. In IBS symptoms of modular questionnaires, IBS girls had significantly severer symptoms in hard stool ( $\mathrm{p}=$ $0.0004)$, strain $(\mathrm{p}=0.0312)$ and abdominal distension $(\mathrm{p}=0.0023)$ than IBS boys and IBS boys had significantly severer symptoms in diarrhea $(\mathrm{p}=0.0116)$. Also, IBS girls showed severer soft stool $(\mathrm{p}=0.033)$, abdominal distension $(\mathrm{p}=0.0012)$, stress $(\mathrm{p}=0.0007)$ and exaggeration of symptoms under stress $(\mathrm{p}=0.0268)$ in a preceding week than IBS boys. In SF36 , IBS girls had higher PF scores $(\mathrm{p}=0.0367)$ and lower MH scores $(\mathrm{p}=0.0239)$ than IBS boys. CONCLUSION: Prevalence and some features of adolescent IBS have unique characters. Further analysis of adolescent IBS may contribute to prophylaxis of functional gastrointestinal disorders

\section{T1267}

Functional Disorders (FD): One Or Many? A Longitudinal, Observational Study of Patients (PTS) with FD in Binghamton, NY

Marcelo A. Barreiro, Gary D. James

Background: Functional Gastrointestinal Disorders (FGID) frequently coexist in pts with other FD. Whether FGID are the gastrointestinal manifestations of a more generalized syndrome that can produce symptoms referred to multiple organ systems, or whether multiple FD are all separate clinical conditions which may occur as "comorbidities" is not known. Objectives: To evaluate: 1 ) the characteristics of pts who present with multiple FD; 2) whether multiple FD present like a syndrome; and 3) whether there is a relationship between the number of coexisting FD and psychological symptoms, health related quality of life (HRQOL) and pain experience. Materials and Methods: The Disorders of Function Clinic was created to address the needs of patients with any FD, trying to avoid subspecialty bias. Pts were $>18$ years of age and without concurrent organic disease that could interfere with their evaluation. All pts signed informed consent. Published symptom-based diagnostic criteria were used to determine FD. Psychological symptoms were evaluated using the SCL90-R, and HRQOL was measured using the QOL Inventory (QOLI). Pain severity was evaluated using a $10 \mathrm{~cm}$ Visual Analog Scale and was characterized as visceral or somatic. Associations between the psychological symptom, QOL, pain and the number of FD were evaluated using ANOVA and correlations. Results: A total of 183 patients were evaluated,
167 of which presented with FD (mean age $47.0 \pm 13.0$ years). Of these, 143 were female $(78 \%)$ and $145(87 \%)$ presented with at least 3 FD (OR 6.57, CI $95 \%$ 4.45-11.35 $\mathrm{p}<.0001)$, far exceeding the possibility of association by chance. The most frequently observed syndromes (Psychiatric Disorder 76 \%, Chronic Fatigue Syndrome 74.3 \%, Fibromyalgi $73.1 \%$ and Irritable Bowel Syndrome $63.5 \%$ ) tended to group together and formed a cluster separate from other FD. In evaluating the SCL-90-R Global Severity Index, pts with 1-2 FD had a significantly lower score than those with 3 or more FD $(61.75$ vs $67.17, \mathrm{p}<.007)$. They also had a higher QOLI Raw Score ( 2.07 vs $0.85, \mathrm{p}<.013)$. Pain severity and type were not related to type or number of coexisting FD. Conclusions: In pts seeking care in a specialized clinic for FD: 1) The majority of pts are women. 2) Multiple FD coexist in the same patient with a frequency that far exceeds chance, suggesting a common pathogenesis and 3) The more FD that coexist in a pt the more severe the psychological symptoms and the poorer the QOL.

\section{T1268}

Is High-Frequency Gastric Electrical Stimulation Effective in Patients with Chronic Functional Nausea and Vomiting and a Normal Gastric Emptying? Richard W. McCallum, Zhiyue Lin, Irene Sarosiek, Jameson Forster

Previous reports have shown that long-term gastric electrical stimulation (GES) can improve symptoms and quality of life in up to $70 \%$ of patients with refractory gastroparesis. The aim of this study was to investigate the effectiveness of GES (Enterra ${ }^{\mathrm{TM}}$ Therapy) in patients with symptoms suggestive of gastroparesis but who had a normal gastric emptying and were given the diagnosis of functional nausea and vomiting not responding to standard approaches. Methods: We studied 15 such patients (3M, 12F, median age: 41 years, 5 diabetic and 10 idiopathic) who had a documented normal baseline 4-hour gastric emptying test (GET) (normal value at 4 hours $<10 \%$ gastric retention) and received GES therapy for at least 1 year. The GET, total symptom score (TSS) derived from 7 upper GI symptom sub-scores (0-4), quality of life (QOL) using SF-36 Health Status Survey questionnaire including physical composite score (PCS) and mental composite score (MCS) and nutritional status were evaluated at baseline and at 1 year of GES administrated according to our previous publications in gastroparesis (Am J Surgery 2001;182:676-81). Results: Of the 15 patients, 1 died at 4 months from complications of misdiagnosed anorexia nervosa and 1 had her device removed at 2 months due to infection in the pulse generator pocket. Results of 2 - and 4 hour gastric retention, nausea, vomiting scores, TSS and weight at baseline and 1 year for the remaining 13 patients are summarized in the table below. Overall there was no significan change in GET and approximately $67 \%$ patients continued to have a normal GET after 1 year. However, one third of patients did develop a slowing of GET (>10\% retention at 4 hours). Symptom severity of nausea, vomiting and TSS were significantly improved. $62 \%$ of patients had $\geqq 50 \%$ reduction in TSS and the median reduction of TSS was $51 \%$. Median PCS was significantly improved from 21.5 at baseline to 37.8 after 1 year but not MCS (43.2 vs 44.0 ). Weight remained stable. At implantation, 4 of the 13 patients required enteral feeding tubes for nutritional support and only one continued after 1 year for infrequent hydration needs. Conclusions: In patients with a normal GET and functional nausea and vomiting high-frequency GES is effective in improving upper GI symptoms, QOL and nutritional status with a good safety profile. These data extend the indications for GES therapy in patients with chronic nausea and vomiting

\begin{tabular}{|c|c|c|c|c|c|c|}
\hline $\begin{array}{c}\text { Median } \\
\text { (range) }\end{array}$ & $\begin{array}{c}\text { 2-hour gastric retention } \\
(\%)(\text { normal<60\%) }\end{array}$ & $\begin{array}{c}\text { 4-hour gastric } \\
\text { retention (\%) }\end{array}$ & $\begin{array}{c}\text { Vomiting } \\
\text { score (0-4) }\end{array}$ & $\begin{array}{c}\text { Nausea } \\
\text { score (0-4) }\end{array}$ & $\begin{array}{c}\text { TSS } \\
(0-28)\end{array}$ & $\begin{array}{c}\text { Weight } \\
(\mathrm{kg})\end{array}$ \\
\hline Baseline & $26(10-74)$ & $3(1-8)$ & $3(0-4)$ & $4(1-4)$ & $\begin{array}{c}18 \\
(9-26)\end{array}$ & $\begin{array}{c}59 \\
(43-82)\end{array}$ \\
\hline 1 year & $43(21-70)$ & $6(1-37)$ & $2(0-4)$ & $2(0-4)$ & $\begin{array}{c}8 \\
(0-19)\end{array}$ & $\begin{array}{c}60 \\
(49-84)\end{array}$ \\
\hline P value & 0.5 & 0.2 & 0.01 & 0.001 & 0.003 & 0.1 \\
\hline
\end{tabular}

\section{T1269}

Autonomic Nervous System Function During Sleep in Women with Irritable Bowel Syndrome

Monica E. Jarrett, Robert L. Burr, Kevin C. Cain, Anne P. Poppe, Margaret M. Heitkemper Objective: Autonomic nervous system (ANS) functioning may be linked to predominant bowel pattern in irritable bowel syndrome (IBS). In this study we examined the differences in ANS function during sleep between IBS and Controls, as well as among predominant stool patterns within the IBS group. Methods. 36 IBS and 40 Control participants slept for 3 nights in a research sleep laboratory. ECG-based R-R interval sequences were recorded in synchrony with a standard polysomnographic montage. Time and frequency domain HRV measures were computed to assess parasympathetic tone (lnRMSSD and lnHF) and sympathetic/parasympathetic balance (SDratio and sqrtLF/HF). Sleep was broken down into 4 sleep periods of successive NREM (non-rapid eye movement) and REM (rapid eye movement) across the night. The mean values across the 3 nights were computed. Rome II criteria were used to differentiate predominant bowel pattern (diarrhea $[n=13]$, constipation $[n=9]$, alternators $[\mathrm{n}=14]$ ). Results: There were no differences in parasympathetic tone or sympathetic/parasympathetic balance between the IBS and Control groups across the 4 sleep periods, or during NREM and REM sleep. However, within the IBS group, those who were diarrhea predominant had significantly higher levels of parasympathetic tone and lower levels of sympathetic/parasympathetic balance than those who were constipation predominant or alternators during both NREM and REM phases. Conclusions: These findings support a relationship between ANS functioning and predominant bowel pattern in IBS during sleep. Relationship of ANS Measures during REM Sleep to Predominant Bowel Pattern in IBS 


\begin{tabular}{|c|c|c|c|c|c|}
\hline & Diarrhea & Constipation & Alternators & P & $P^{*}$ \\
\hline Parasympathetic & & & & & \\
\hline InRMSSD & $3.81(0.59)$ & $3.25(0.47)$ & $3.17(0.44)$ & .006 & .009 \\
\hline InHF & $7.27(0.55)$ & $6.79(0.75)$ & $6.64(0.56)$ & .032 & .050 \\
\hline Sympathetic/parasympathetic & & & & & \\
\hline SDratio & $1.47(0.37)$ & $1.99(0.50)$ & $2.01(0.47)$ & .007 & .011 \\
\hline sqritLF/HF & $1.58(0.49)$ & $2.08(0.79)$ & $2.18(0.72)$ & .061 & .111 \\
\hline
\end{tabular}

P* controlling for age.

\section{T1270}

Irritable Bowel Syndrome in South Korea: Prevalence, Symptom Patterns and Risk Factors

Chang Hwan Choi, Sang Yon Hwang, Geun Jun Ko, Chang Woo Gham, Ki Joon Han,

Hyeon Geun Cho

BACKGROUND: Irritable bowel syndrome (IBS) is a common health problem affecting a substantial proportion of the population. The aim of this study was to evaluate the prevalence of IBS that satisfied ROME II criteria, and to determine the risk factors associated with this prevalence and probable risk groups in South Korea. METHODS: A total of 2345 subjects (1298 males and 1047 females) who have visited the Health Promotion Center of Kwandong University College of Medicine, Myongii hospital in South Korea from March 2005 through August 2005 were included in this study. They were interviewed by using a questionnaire comprising demographic features and ROME II criteria for the diagnosis of IBS. The prevalence of IBS was analyzed according to various factors such as demographic characteristics, body mass indexes (BMI), blood test, endoscopic and abdominal sonographic findings with multivariate regression analysis. RESULTS: The prevalence of IBS was $8.3 \%$ (7.9\% in male and $8.8 \%$ in female) according to ROME II criteria in our region. Of the IBS patients, $30.8 \%$ had characteristics of diarrhea-predominance, $23.6 \%$ constipation-predominance while $45.6 \%$ had alternating symptoms. The most frequent symptom was a sense of incomplete evacuation $(60.0 \%)$ followed by bloating or distention (54.9\%), straining (36.9\%), urgency (29.7\%), and mucus in stool (9.2\%). In univariate analysis, the prevalence of IBS was significantly higher in the younger age subjects (age<35) and in the under-weight subjects (BMI $<18.5$ ). In multivariate regression analysis, the younger age (odds ratio, 2.04: $95 \% \mathrm{CI}$ 1.33 to 3.12 ) and the under-weight (odds ratio, $2.7 ; 95 \% \mathrm{CI}, 1.37$ to 5.35 ) were the factors that independently affected the expression of IBS symptom. The IBS prevalence was not different in terms of gender, resident area, occupation, smoking, alcohol intake, diabetes and hypertension history, anemia, $\mathrm{ABO}$ blood groups, Helicobacter pylori infection, and presence of peptic ulcer or fatty liver disease. CONCLUSIONS: The prevalence of IBS according to ROME II criteria was $8.3 \%$ in general Korean population, and IBS with alternating bowel habit is the most common subtypes based on predominant bowel habit. IBS was more common in the younger age and in the under-weight people.

\section{T1271}

Determinants of Co-Existing Irritable Bowel Syndrome and Heartburn During Long-Term Follow-Up of Functional Dyspepsia

Sebastien Kindt, Philippe Caenepeel, Joris Arts, Jozef Janssens, Jan Tack

Background: Considerable overlap exists among different functional gastrointestinal disorders (FGID), especially between functional dyspepsia (FD), irritable bowel syndrome (IBS) and heartburn (HB). However very little is known about possible factors underlying this overlap. The aim of this study was to investigate the degree of overlap of IBS and HB with FD and to assess variables promoting this overlap. Methods: 253 tertiary care patients with newly diagnosed FD underwent a complete pathophysiological investigation with barostat, gastric emptying for solids and liquids, 24-hour ambulatory $\mathrm{pH}$-metry and $\mathrm{H}$. pylori testing, and were prospectively followed. After a follow-up of of $68+2$ months, FD, IBS and heartburn status were evaluated according to Rome II criteria by self-administered questionnaires. Anxiety and depression were assessed with STAI and HADS questionnaires. Quality of life was assessed with the Nepean Dyspepsia index (NDI). Differences between single and overlapping FGID were evaluated with Chi-square. Factors promoting IBS or heartburn were assessed with t-test, Wilcoxon test and logistic regression. Results: At follow-up, 24.9\% were asymptomatic, 66.0\% still had FD, 36.5\% had IBS and 26.5\% had HB. FD and IBS overlap were present in $29.5 \%$, FD and HB overlap in $23.7 \%$, and IBS and HB overlap in $14.9 \%$. Three overlapping FGID were present in $14.1 \%$. There was a significant positive association among the different FGID (all $\mathrm{p}<0.001)$. IBS overlap was associated with a greater weight loss at presentation, a lower MDP, higher STAI-trait, NDI and HADS-depression scores, female gender, use of FGID medication and diet. In logistic regression significant contributors were MDP, female gender and the interaction term gender*STAI-trait score. HB overlap was associated with a lower body length, a higher BMI, a greater weight loss at presentation, more chronic fatigue complaints, more dietary restrictions and use of FGID medication, and higher NDI, STAI and HADS scores. Body length was a significant contributor while STAItrait score and gastric emptying for solids showed a trend $(\mathrm{p}=0.058$ and 0.051 resp.). Conclusion: An important overlap among FGID is present in FD patients at follow-up. Anxiety and female sex are risk factors for IBS, while anxiety, shorter body length and delayed gastric emptying are risk factors for HB. FD patients with overlapping IBS or HB have a lower quality of life, and use more dietary and medical treatments.

\section{T1272}

Irritable Bowel Syndrome Aggregates Strongly in Families: A Family-Based Case-Control Study

Yuri Saito, Nicholas J. Talley, Janice M. Zimmerman, William S. Harmsen, Mariza De Andrade, Michael Camilleri, G. Richard Locke, Gloria M. Petersen

Background: Although environmental factors and learned illness behavior may contribute, twin and pharmacogenetic studies suggest a potential genetic basis for Irritable bowel syndrome (IBS). Positive family history has been shown to be predictive of IBS, but no detailed characterization of IBS pedigrees by direct data collection of entire families has been reported to date. Study aims: 1) To quantitate familial aggregation of IBS, and 2) To assess the accuracy of patient report of family history of IBS in cases and controls. Methods: The study design was a case-control study of 50 cases with IBS seen in GI clinics and 53 age-, genderand race-matched controls from General Medicine clinics at a major medical center in 2004-2005. Case and control probands completed a symptom questionnaire, and provided symptom and contact information for their first-degree relatives (FDRs). Questionnaires were then mailed to the FDRs for completion. Relatives were considered to have IBS if they met Rome criteria, reported a physician diagnosis of IBS, or reported constipation and/or diarrhea-type symptoms. Results: Case and control probands identified a total of 582 relatives in their family structure--286 among cases, 296 among controls. 75(13\%) were deceased, $48(8 \%)$ were minors, and $57(10 \%)$ were not allowed contact by the probands. $402(69 \%)$ surveys were mailed with $203(50 \%)$ responding with consent and symptom data. When comparing proband report with relative self-report data, case-probands reported $20 \%$ of their FDRs had IBS; however, relatives' self-reports showed 46\% had IBS. Control-probands reported $4 \%$ of their FDRs had IBS; in contrast, relatives' self-reports showed $25 \%$ had IBS. By chi-square analysis, both comparisons were statistically significant difference in $\%$ with IBS ( $<<0.05)$. Kappa statistics for agreement between proband-report and FDRs' self-report were poor for both case-FDRs and control-FDRs( 0.31 and 0.14 , respectively). When including proband information for non-participating relatives and unknowns were treated as a not having IBS, $24 \%$ of case-relatives were still affected, compared to $11 \%$ of control-relatives $(\mathrm{p}<0.001)$. Family comparisons showed that $70 \%$ of case-families have at least one affected family member, compared to $43 \%$ of control-families ( $p=0.01$ ). Conclusions: Cases and controls typically underestimated the frequency of IBS-type symptoms in their relatives, thus emphasizing the need for direct data collection from family members in any family study of IBS. IBS was observed to aggregate strongly in this study sample, compared to controlfamilies. The environmental and genetic reasons for this clustering merit further study.

\section{T1273}

Interleukin 10 Genotypes in IBS-Rome II Subjects in Mexico

Gabriela Gutierrez-Reyes, Rosalinda Martinez-Garcia, Norma Morales-Rochlin, Marina Gonzalez, Clarita Corona de Lau, Max Schmulson

Preliminary studies suggest that an inflammatory or genetic component may exist in some cases of IBS with lower production of IL-10. (Gut 2003:52:91) Therefore we sought to determine the frequencies of IL-10 and TNF genotype in a group of IBS subjects in Mexico. Methods: 36 volunteers fulfilling Rome II criteria for IBS were recruited. They were matched by age and gender with 73 non-IBS volunteers. Blood samples were obtained from each subject. Genomic DNA was extracted from peripheral blood leucocytes and genotyping was carried out by amplification refractory mutational system polymerase chain reaction methods (Transl.Immunol.1999;7:127), using allele specific primers to identify the high and low producer alleles of each biallelic polymorphic site of the IL-10 gene $(-1082 * \mathrm{G}$ and $-1082 * \mathrm{~A})$, respectively and the TNF-a gene $\left(-308^{*} \mathrm{G}\right.$ and $\left.-308^{*} \mathrm{~A}\right)$. Amplified DNA products were then analyzed using electrophoresis on $2 \%$ agarose gel and viewed under UV light. Allele frequencies were expressed in percentage. Genotypes for each subject were expressed as homozygous for the high and low producer and heterozygous or intermediate producer frequencies and compared by chi-sq. Results: $78 \%$ of IBS and $75 \%$ of controls were female. Age was similar in both groups: $35+/-2$ years. Allele frequency for IL-10 had a tendency to be lower for G in IBS compared to controls: 32 vs. $42 \%$, OR: 0.64 (95\% CI: 0.334-1.195), p=0.088. There was no difference in the overall genotype frequency in IBS vs. Controls (High, Low, Intermediate \%): $18,33,49$ vs. $8,45,47$ but according to gender, there was a tendency to a lower proportion of the low producer among females with IBS.(Table) Allele frequency for TNF-a was similar for the G and A in IBS: 83 and $17 \%$ vs. controls: 75 and $25 \%$. Nor was there any difference in the overall genotype frequency (High, Low, Intermediate \%): $66,0,34$ vs. $52,1,47$ or according to gender. Conclusions: These preliminary results suggest that in Mexico at least a subgroup of females may be genetically predisposed to developing IBS because of a lower production of the protective anti-inflammatory effect of IL-10. A larger study is warranted to determine if this hold true and if it is consistent among males.

IL-10 Genotypes in IBS and Controls.

\begin{tabular}{|c|c|c|c|c|}
\hline & High Producer & Low Producer & Intermediate Producer & P \\
\cline { 1 - 4 } IBS All n(\%) & $3(8)$ & $16(45)$ & $17(47)$ & \multirow{2}{*}{ NS } \\
\hline Controls n(\%) & $13(18)$ & $24(33)$ & $36(49)$ & \\
\hline Female-IBS & $1(4)$ & $15(53)$ & $12(43)$ & \multirow{2}{*}{0.081} \\
\hline Female-Controls & $8(14)$ & $17(31)$ & $30(54)$ & \multirow{2}{*}{ NS } \\
\hline Male-IBS & $2(25)$ & $1(12)$ & $5(63)$ & \\
\hline Male-Controls & $5(28)$ & $7(39)$ & $6(33)$ & \\
\hline
\end{tabular}

IBS: Female vs. Male, p=0.043 Controls: Female vs. Male, NS. 
$\mathrm{T} 1274$

Short-Allele Serotonin Transporter Gene Polymorphism Is Associated with Elevated Serotonin and Is Less Common in IBS with Constipation Compared to Healthy Controls

William E. Whitehead, Olafur S. Palsson, Syed Im. Thiwan, Temitope O. Keku, Motoyor Kanazawa, Shin Fukudo, Miranda Al. van Tilburg, Janie Peacock, Jeff Barnes, Marsha J. Turne

Single or double short allele ( $\mathrm{s} / \mathrm{l}$ or $\mathrm{s} / \mathrm{s}$ ) polymorphisms of the gene encoding the serotonin transporter protein (SERT) result in less effective serotonin reuptake at nerve synapses when compared to the long-long $(1 / 1)$ variant. This may affect bowel functioning. Association between short alleles and diarrhea-predominant IBS were reported in 2 previous studies. Short alleles were also associated with more severe psychological symptoms in some nonIBS studies. Aims: Examine SERT genotypes in relation to IBS subtypes, bowel symptoms, and psychological symptoms. Methods: 127 Rome II IBS patients and 27 healthy controls provided $10 \mathrm{ml}$ venous whole blood samples. DNA was extracted using a "Puregene" (Gentra Systems, Minneapolis, MN) kit according to manufacturer's protocol. After PCR amplification of the DNA, the product was denatured with Hi-Di formamide and subjected to capillary electrophoresis on the ABI 3100 Avant genetic analyzer. Alleles were determined with GeneMapper software. Subjects also completed the Rome II Modular Questionnaire, IBS Severity Scale, additional bowel habit questions and the Brief Symptom Inventory 18. Motility index scores and thresholds for first sensation and moderate urge and pain (ascending method of limits) were obtained with a balloon-tipped catheter placed in the sigmoid colon. Results: IBS-C patients had more $1 / 1$ and fewer $1 / \mathrm{s}$ alleles than controls $\left(X^{2}=5.845, \mathrm{p}=.054\right)$, while IBS-D were similar to controls (table). IBS patients with short alleles had elevated fasting whole blood serotonin (mean+SE: $186.9+7.0 \mathrm{ng} / \mathrm{mL}$ for $\mathrm{s} / \mathrm{l}$ and $\mathrm{s} / \mathrm{s}$ combined vs. $157.3+10.6 \mathrm{ng} / \mathrm{mL}$ for $\mathrm{l} / \mathrm{l} ; \mathrm{p}=.02$ ). IBS patients with $\mathrm{l} / \mathrm{l}$ had significantly more straining with defecation and tended to report more hard stools and fewer loose stools compared to $s / 1$ and $s / s$. There were no differences in motility index, sensory thresholds, or psychological symptoms. Conclusions: The SERT short allele was less common in IBS-C. It was associated with lower fasting levels of whole blood serotonin, and this was reflected in less straining and a tendency towards looser stools. However, we found no association between SERT polymorphisms and psychological symptoms. These data support the hypothesized association of SERT polymorphisms with IBS subtypes but require replication in larger samples. [Supported by Novartis Pharmaceuticals and grants R24 DK67674, R01 DK31369 and RR00046]

\begin{tabular}{|c|c|c|c|c|}
\hline & $1 / 1$ & $\mathrm{~s} / 1$ & $\mathrm{~s} / \mathrm{s}$ & Total \\
\hline Control & $8(29.6 \%)$ & $16(59.3 \%)$ & $3(11.1 \%)$ & 27 \\
\hline IBS-C & $16(50.0 \%)$ & $9(28.1 \%)$ & $7(21.9 \%)$ & 32 \\
\hline IBS-D & $10(29.4 \%)$ & $18(52.9 \%)$ & $6(17.6 \%)$ & 34 \\
\hline IBS-none & $8(40.0 \%)$ & $12(60.0 \%)$ & $0(0 \%)$ & 20 \\
\hline Total & $42(37.2 \%)$ & $55(48.7 \%)$ & $16(14.2 \%)$ & 113 \\
\hline
\end{tabular}

$\mathrm{T} 1275$

What Is the Correlation Between the Bloating Sensation and the AmountDistribution of Intestinal Gas in Patients with Functional Abdominal Bloating? Fermin Mearin, Antonia Perello, Monica Perona, Agustin Balboa, Mario Pages, Dian Hernandez, Antoni Castells

Many patients with functional abdominal bloating (FAB) relate their symptoms to excessive intestinal gas. However, this relationship has not been clearly established. AIM: To evaluate the amount and distribution of intestinal gas in patients with FAB in two different clinical situations: 1. Without abdominal bloating sensation; 2. With abdominal bloating sensation. METHODS: 9 patients diagnosed with FAB according to Rome II criteria were evaluated. The amount and distribution of intestinal gas was analyzed by using a high-resolution CT scan. Studies were conducted while patients experienced bloating sensation and while experiencing no bloating. In addition, results were correlated to changes in abdominal girth, measured multiple times using a diary. RESULTS: In "no bloating" conditions the mean total gastrointestinal gas was $261 \pm 44 \mathrm{ml}$ (stomach: $36 \pm 12 \mathrm{ml}$; colon: $182 \pm 26 \mathrm{ml}$ ), values being very scattered: total range from 66 to $507 \mathrm{ml}$, range in the stomach from 12 to $116 \mathrm{ml}$, and range in the colon from 42 to $312 \mathrm{ml}$. Volume change from "no bloating" to "bloating" conditions were: $158 \pm 129 \mathrm{ml}$ for total intestinal gas, $16 \pm 27 \mathrm{ml}$ in the stomach and $159 \pm 128 \mathrm{ml}$ in the colon, showing again scattered values: total range from 45 to $1172 \mathrm{ml}$, range in the stomach from -67 to $215 \mathrm{ml}$, and range in the colon from 58 to $1156 \mathrm{ml}$. No correlation was found between changes in abdominal girth during the day (going from 2.2 to $9.2 \mathrm{~cm}$; mean: $5.1 \mathrm{~cm}$ ) and changes in total gas amount. CONCLUSION: While studying patients with functional abdominal bloating no correlation between the bloating sensation experienced and the amount and distribution of intestinal gas was found. Other pathogenic mechanisms deserve to be considered.

\section{T1276}

Development and Validation of a Concise Point-of-Care Severity Index in IBS: The "B.E.S.T." Questionnaire

Brennan M. Spiegel, Bruce Naliboff, Emeran Mayer, Roger Bolus, Lin Chang

Background: Severity is a multidimensional outcome in IBS that combines intestinal and extraintestinal components. Although it is critical to accurately measure severity in IBS, data suggest that it is often underestimated in everyday practice. We sought to develop a concise, point-of-care index that accurately and rapidly measures IBS severity in the busy clinical setting. Methods: Based on a conceptual model of IBS severity (Lembo et al Clin Gas Hep 2005), coupled with previously identified predictors of quality of life in IBS (Spiegel et al Arc Int Med 2004), we identified 4 Likert-scaled items that balance brevity and scope. The resulting "B.E.S.T." questionnaire included: 1) how Bad are your bowel symptoms?, 2) can you still Enjoy the things you used to enjoy?, 3) do you feel like your bowel symptom mean there's something Seriously wrong?, and 4) do your bowel symptoms make you fee Tense? Scores were scaled on a $0-100$ range $(100=$ most severe). We prospectively measured the cross-sectional correlation of BEST with 6 severity measures in Rome-II IBS patients, including IBS-QOL, bowel symptom intensity [VAS] and duration, somatization [SCL-90], anxiety/depression [HAD], and coping [Likert]. Results: The validation group included 56 patients (mean age $=52 ;$ IBS-QOL $=55 ; 33 \%$ women). Mean completion+scoring of BEST was $<3 \mathrm{~min}$. Figure 1 shows a striking correlation of BEST with IBS-QOL $(\mathrm{r}=-0.83)$. There were moderate correlations between BEST and VAS $(r=0.49)$, symptom duration $(0.55)$ somatization (0.55), HAD (0.54), and coping $(r=-0.52)$. Conclusions: BEST is a concise, rapidly completed, point-of-care IBS severity index that demonstrates moderate-to-large correlations with disparate severity measures, and therefore has excellent cross-sectiona validity. Future work will measure the reliability and predictive ability of BEST, and evaluate its impact on clinical decision-making

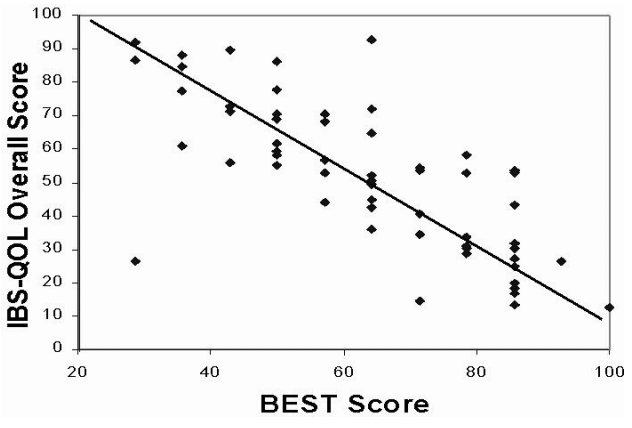

BEST vs IBS-QOL

\section{T1277}

Gastrointestinal Functional Disorders Are Common in Women Regardless of the Menstural Phase: A Rome II-Based Survey

Sun-Young Lee, Sang Kyun Yu, In-Kyung Sung, Hyung-Seok Park, Choon-Jo Jin, Won Hyeok Choe, So Young Kwon, Chang Hong Lee, Kyoo Wan Cho

Background and Aims: It is known that functional gastrointestinal disorders are more common in women in relation to the fluctuations of female sex hormones. We tried to detect the gender-related differences in the prevalence of functional dyspepsia (FD) and irritable bowel syndrome (IBS). In addition, we analyzed the symptoms according to their menstural phase in women. Methods: A total of 505 subjects (253 women before menopause and 252 men below age 50) who visited Digestive Disease Center of Konkuk University Hospital, from October to November 2006, were included in this prospective study. Subjects were examined by gastroenterologist after completing the questionnaire, and were diagnosed according to Rome-II criteria. Further examinations including blood test, endoscopic procedures, and imaging studies were done if needed. After the interview and examinations, subjects with malignant disease or acute infection, or those who have chronic disease requiring medications were excluded from the study. Women were subclassified into three groups according to their menstruation; menstrual phase (day 0-6), proliferative phase (day 7-14), and secretory phase (after day 15). Women under lactation or pregnancy, taking oral pills, or with irregular menstrual period more than 36 days were excluded. Finally, 179 men and 193 women (60 menstrual phase, 41 proliferative phase, and 92 secretory phase) were analyzed. Results: Women revealed higher prevalence of FD $(\mathrm{P}=0.03)$ and IBS $(\mathrm{P}=0.01)$ than men. In aspec of FD subtypes, reflux-dominant type was common in men while mixed or unclassified types were common in women $(\mathrm{P}=0.01)$. In aspect of IBD subtypes, diarrhea-dominant type was common in men while constipation-dominant or mixed types were common in women $(\mathrm{P}<0.001)$. Of 193 women, there was no significant difference in their gastrointestinal symptoms according to their menstrual phase. Conclusions: Regardless of the menstrual phase, functional gastrointestinal disorders are more frequent in women than in men. Physicians should consider gender-based approach in clinical practice since higher prevalence of FD and IBD in women may lead to reduced clinical reliability when accessing organic disease. In addition, different symptomatic manifestations between men and women should be considered when evaluating functional gastrointestinal disorders. Key Words: Functional gastrointestinal disorder, Women, Dyspepsia, Irritable bowel syndrome, Menstruation

\section{T1278}

Functional Gastrointestinal Disorders: Overlapping Symptom Or a Single Complex Syndrome? A Stratified Randomized Epidemiologic Study Using Cluster Sampling in Northern China

Xiucai Fang, Meiyun Ke, Guo-Zong Pan, Shaomei Han, Jiaming Qian, Zhaolu Ding, Sucai Lu

Functional gastrointestinal disorders (FGIDs) are commonly diagnosed in clinical practices in most regions of the world. Recent reports for community populations in the USA suggest that FIGD symptoms can occur in overlapping complexes. We analyzed stratified randomized epidemiologic data, obtained by cluster sampling in Northern China, and obtained an estimate of the prevalence of the major FGID symptoms and how they overlap in the general population. The study was designed for stratified and randomized selection in a cluster sampling of the inhabitant groups in the city of Beijing. A total of 2,486 subjectss (ages 18 - 70 years) were enrolled in the study. The subjects completed a questionnaire consisting of 165 items with the assistance of trained physicians or medical students during visits to their homes. Ten categories of symptoms were selected as representative of FGIDs. Chest pain of cardiac origin was excluded and abdominal pain was defined as occurring more 
than 6 times in the past year. Definitions for diarrhea, constipation and functional dyspepsia (FD), chronic constipation (CC) and the irritable bowel syndrome (IBS) were according to Rome II Criteria. Gastro-oesophageal reflux disease (GERD) was assesses by the intensity and frequency of heartburn, acid reflux and regurgitation ( $>6$ of 18). Acid reflux, heartburn, abdominal fullness, and pain and discomfort in the upper abdomen ranged of $14 \sim 22 \%$ in this general population. Chest pain, sensations of fullness and pain, constipation were most common in the women $(\mathrm{p}<0.05)$. FGIDs were reported in $21.9 \%$ of the population, and the prevalence of symptom complexes GERD, FD CC and IBS were $10.2 \%, 12.2 \%$, $6.3 \%$ and $1.0 \%$ respectively, with a higher prevalence of constipation in females than in males $(9.5 \%$ to $2.8 \%, \mathrm{p}<0.001)$. Two-complex overlap was present in $6.2 \%$, and threecomplex overlap was found for $1.1 \%$ of the population. Co-existence of upper FGIDs in with CC ranged from 12.9 to $13.8 \%$ while the range for IBS was 3.6 to $4.3 \%$. A complex of upper FGID symptoms co-existed with CC in approximately one-fourth of this CC group and in one-half of the IBS in the study. No significant relationship between the prevalence of overlap and age, BMI, occupation, education, marital status, smoking, alcohol abuse or emotional stress was found. The results suggest that FGIDs are present in the general population of Northern China in numbers reminiscent of findings of similar studies elsewhere. The significant overlap of symptoms between the upper and lower digestive tract suggests that a common pathogenesis might be responsible for the overlap in a given patient.

\section{$\mathrm{T} 1279$}

Pain and Health-Related Quality of Life in Irritable Bowel Syndrome (IBS) Clinicians' Perspectives Differ from Those of Patients Olivier Chassany, Martin Duracinsky, Marc Mathieu

Background. Although advances in psychometrics have enabled development of HealthRelated Quality of Life (QoL) questionnaires, from patient-to-GP information transmission remains informal. We sought to measure the degree of agreement of pain and QoL as reported by IBS patients, and that estimated by their general practitioners (GPs) during consultation. Methods. 307 IBS patients [64\% women; $58 \pm 16$ yrs] and their 131 GPs were included in a cross-sectional French study. Patients and GPs estimated patients' pain on a VAS, and QoL by the IBS-specific FDDQL questionnaire (patients) or 8 questions corresponding to the label of the 8 FDDQL dimensions (GPs). Before analysis [weighted Kappa coefficients $(\mathrm{Kw})$, ANOVA (p), regression (r)], VAS and QoL values were transformed to give values between 0 (no pain / bad QoL) and 100 (severe pain / good QoL). Results. Patient- and GP-rated pain correlated moderately $(\mathrm{Kw}=0.31)$ : the greater the pain according to the patient, the greater according to the GP. GPs, however, underestimated pain $(30.4 \pm 21.0$ versus

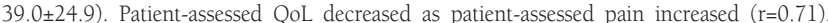
However, patient-assessed QoL scores varied drastically for a given pain intensity, suggesting that pain intensity only partially reflected QoL. Patients' and GPs' global QoL scores correlated poorly ( $\mathrm{Kw}=0.28$ ). On all dimensions (excepted $\mathrm{DA}$ and $\mathrm{AX}$ ), patients considered their QoL more adversely affected than did their GP (Tab). On all dimensions (excepted DA and AX), irrespective of GP ratings, patients' mean QoL scores were similar from those of patients rated 0 by their GP, suggesting that patients and GPs had discrepant perceptions of IBS impact. Conclusions. Patients' and GPs' perspectives, although partly overlapping, differed Moreover, although patient-assessments of pain and QoL overlap to some extent, they measure different concepts, i.e. QoL of IBS patients cannot be inferred from a pain score. Reliable patient perception measurement is essential to take account of all the aspects of IBS. Financial support: ALFIS (Association des Laboratoires et Firmes de Santé), Arcueil, France

\begin{tabular}{|c|c|c|c|}
\hline FDDQL questionnaire & Patients & GPs & Relationship between scores \\
\hline Number of data & 239 & 307 & 239 \\
\hline Global score & $57+/-15$ & $59+/-19$ & $\mathrm{Kw}=0.28$ \\
\hline Daily activities (DA) & $78+/-21$ & $58+/-25$ & $\mathrm{p}<0.01$ \\
\hline Anxiety (AX) & $63+/-22$ & $54+/-28$ & $\mathrm{p}<0.01$ \\
\hline Diet & $56+/-23$ & $60+/-26$ & $\mathrm{p}<0.01$ \\
\hline Sleep & $66+/-20$ & $72+/-26$ & $\mathrm{p}<0.01$ \\
\hline Discomfort & $51+/-18$ & $69+/-25$ & $\mathrm{p}<0.01$ \\
\hline Coping with disease & $55+/-20$ & $57+/-26$ & $\mathrm{p}<0.01$ \\
\hline Control of disease & $55+/-23$ & $59+/-26$ & $\mathrm{p}<0.01$ \\
\hline Stress & $32+/-25$ & $43+/-27$ & $\mathrm{p}<0.01$ \\
\hline
\end{tabular}

T1280

Methane Gas Is Associated with Constipation Predominant Symptoms in IBS Results from a Double-Blind Controlled Study Mark Pimentel, Sandy Park, Yuthana Kong, Kimberly Low, Soumya Chatterjee

Previous data suggests that methane gas on lactulose breath testing is associated with constipation among subjects with irritable bowel syndrome (IBS). In this study, we reevaluate the relationship between methane and constipation in a new cohort of IBS patients using both subjective and objective outcome measures. Methods: In a recent double-blind randomized controlled study evaluating the use of rifaximin (a non-absorbable antibiotic) for IBS, a nested study was conducted to evaluate the role of methane in constipation IBS. After consent, subjects filled out a stool diary as well as a daily Bristol Stool Score for 7 days. Subjects then returned for a qualitative evaluation of their IBS symptoms including diarrhea, constipation, bloating, urgency and abdominal pain on a VAS $(0-100 \mathrm{~mm})$ after which a lactulose breath test was conducted to determine the presence or absence of methane based on having methane on all samples through 180 minutes of breath samples. Results: 87 patients were included in the analysis. Of these, 15 subjects (17\%) were considered methane producers. Methane production was associated with a constipation severity of $78.2 \pm 23.7$ compared to $34.4 \pm 31.2$ for non-methane $(\mathrm{P}<0.000001)$. On the contrary, the diarrhea severity was lower in methane producers $(9.3 \pm 24.7)$ compared to non methane
$(45.1 \pm 34.9)(P<0.001)$. Bloating $(P<0.05)$ was more and urgency was less $(P<0.0001)$ in methane producers. Abdominal pain was not different. Based on objective scores, the stool frequency was $0.92 \pm 0.79$ per day among methane producers compared to $2.00 \pm 1.36$ in non-methane $(\mathrm{P}<0.01)$. The Bristol Stool Score was also lower in methane $(\mathrm{P}<0.05)$. Of methane producing IBS subjects, $93 \%$ were considered constipation-predominant compared to $31 \%$ for non-methane producers ( $\mathrm{OR}=18.5, \mathrm{Cl}=2.91-114.6, \mathrm{P}<0.001)$. Conclusions: Among IBS subjects, methane is significantly associated with constipation symptoms in both subjective and objective means of determining constipation

\section{T1281}

Overlapping Functional Gastrointestinal Disorders in Irritable Bowel Syndrome - Prevalence and Related Factors

Iris Posserud, Gunnar Midhagen, Hasse Abrahamsson, Magnus Simren

Background: Patients with irritable bowel syndrome (IBS) often complain of a wide range of symptoms, including symptoms from other parts of the gastrointestinal (GI) tract than the bowel. Overlap with other functional gastrointestinal disorders (FGIDs) has been reported but the actual prevalence and influencing factors are largely unknown. Aim: Evaluate the prevalence of other FGIDs and correlating factors in a large sample of IBS patients. Method: We included 308 patients attending our referral centre fulfilling the Rome II criteria for IBS (mean age 40 years, range 18-73; 226 females). Of these 117 had diarrhea predominant IBS (IBS-D), 60 constipation predominant (IBS-C), and 131 alternating diarrhea and constipation (IBS-A). They completed the Rome II Modular Questionnaire, and the presence of anxiety and depression was assessed with the Hospital Anxiety and Depression scale (HAD) using validated cut-off levels. Demographic data was also collected. Results: Rome II criteria for at least one additional FGID, was fulfilled in $76 \%$ of all IBS patients (mean number 1.3 FGID per patient, range 0-4). Functional anorectal disorders were most prevalent, and the single most common FGID besides IBS was proctalgia fugax, which was present in $40 \%$, whereas levator ani syndrome was observed in $11 \%$. Functional incontinence was found in $22 \%$, of which soiling accounted for $14 \%$ and gross incontinence for $8 \%$. The most frequent symptom, beside IBS symptoms, was dyspepsia, defined as pain or discomfort centered in the upper abdomen, reported by $48 \%$ (22\% ulcer like and $26 \%$ dysmotility like). However, only $6 \%$ fulfilled the Rome II criteria for functional dyspepsia since the majority reported a relation with bowel function and/or presence of reflux symptoms. Esophageal symptoms were also common, with $26 \%$ reporting reflux symptoms suggestive of functional heartburn and $8 \%$ reporting symptoms of functional chest pain. The number of simultaneous FGIDs in IBS patients was higher if the patients had anxiety ( $\mathrm{p}=0.003 \mathrm{vs}$. no anxiety) or depression ( $\mathrm{p}=0.002$ vs, no depression). Patients with IBS-A were more likely to have other FGIDs ( $\mathrm{p}=$ 0.04 vs. IBS-D and IBS-C) and patients with additional FGIDs tended to be older $(\mathrm{p}=0.05)$. There was no observed gender difference in the number of FGIDs. Conclusion: A majority of IBS patients also report symptoms compatible with other functional gastrointestinal disorders, implying a more widespread gastrointestinal disturbance. This is related to psychological factors, as well as subtype of IBS, but not with gender. Strict use of the present Rome II criteria probably underestimates the presence of some FGIDs, especially functional dyspepsia.

\section{T1282}

Women with Irritable Bowel Syndrome (IBS) in Pre- and Post-Menopausal Age Groups Report Similar Gastrointestinal (GI) Symptoms

Lin Chang, Claudia Alvarado, Emeran A. Mayer, Martijn G. van Oijen, Cathy Liu, Brennan Mr Spiegel

Background: Sex differences in symptoms, and physiologic and treatment responses have been reported in IBS (Gastro 2002;123:1686-1701). Animal and human studies support an influential role of female sex hormones on GI function. While the effect of menstrual cycle on IBS symptoms has been reported, the effect of menopause has not been well studied. Aims: We sought to compare the prevalence and severity of IBS symptoms in pre- vs. postmenopausal age groups in women with IBS, and to conduct similar comparisons in a control group of men with IBS. Methods: We analyzed data from 1634 Rome+ IBS patients aged 18-88 yr recruited from clinic and advertisement at a university-based functional GI disorders center. Subjects completed a bowel symptom questionnaire and validated psychological and quality of life surveys. We stratified the data by gender and age group ( $\leq 35 \mathrm{vs}$. $\geq 50$ ), and compared key IBS symptoms between groups using univariate chi-squared and $t$-tests. We then entered significant $(\mathrm{p}<0.05)$ variables into a series of regression analyses to determine whether menopausal status independently predicted any specific IBS symptoms, and performed similar analyses in the control group of men with IBS. Results: There were 459 women with IBS $\leq 35$ and $296 \geq 50$ years old, and 296 and 172 men with IBS, respectively. Univariate analyses in women revealed that only discomfort severity was significantly higher in pre- vs. post-menopausal age groups $(\mathrm{p}<0.05)$, although the effect size of this difference was not clinically significant $(0.16 \mathrm{SD})$. There were no important differences in men between age groups. In the regression analysis, the post-menopausal age group independently predicted a lower odds of having infrequent $(<3 / \mathrm{wk}$ ) bowel movements (OR $0.5,95 \% \mathrm{CI}=0.3$, $0.8)$ and mucus in the stool $(0.62 ; 0.4,0.97)$ and higher odds of having visible abdominal distension $(3.5 ; 0.3,0.8)$. These differences were not seen in men. There were no differences in overall symptom severity, abdominal pain, bloating, or other bowel habit symptoms. Conclusions: We found very few symptoms that were significantly different between prevs. post-menopausal women with IBS. The decreased stool frequency more often reported in younger women may be explained by the proposed inhibitory effect of female sex hormones on GI transit. However, taken together with the lack of effect of oral contraceptive agents and hormone replacement on IBS symptoms in past studies, these findings suggest that cyclical, physiologic changes in female sex hormones have only a minor effect on IBS symptoms. Future prospective studies are needed to confirm these findings. Supported by NIH grant \# P50 DK64539. 


\section{T1283}

Evaluation of a Fast, Low-Caloric Versus a Slow, High-Caloric Drink Test in Functional Dyspepsia

Trygve Hausken, Vernesa Dizdar, Ina Hjelland, Johan Lunding, Odd Helge Gilja, Arnold Berstad

Background: Drinking capacity is often reduced in functional dyspepsia. Drink tests may therefore have diagnostic potential. The aim was to find the best drink test in combination with ultrasonography to diagnose patients with functional dyspepsia (FD). Material and methods Sixteen patients with FD, age (median 33(18-57 yrs), BMI (mean (SD) 22 (4), male/female 6/9, and 15 healthy controls, age (median 25 (20-30 yrs), BMI mean (SD) 25 (3.6), male/female10/6 were included. In randomized order the subjects drank either meat soup ( $4 \mathrm{kcal} / 100 \mathrm{ml}$ with a velocity of $100 \mathrm{ml} / \mathrm{min}$ ) or Nutridrink $(150 \mathrm{kcal} / 100 \mathrm{ml}$ with a velocity of $15 \mathrm{ml} / \mathrm{min}$ ) until maximal drinking capacity. Intragastric volume at maximal drinking capacity was determined using 3-dimensional ultrasonography. Results: Drinking capacity $(p<0.003)$ and intragastric volume $(p<0.003)$ were significantly lower in patients than in the controls with the meat soup meal (696 (353) ml versus 1220(433) and 388 (190) $\mathrm{ml}$ versus $663(230) \mathrm{ml}$, respectively) and for the Nutridrink (drinking capacity 196 (124) $\mathrm{ml}$ versus 558 (254) ( $\mathrm{p}<0.0001)$, intragastric volume 164 (90) ml versus 386 (145) $(\mathrm{p}<0.0002)$. Receiver operating characteristic (ROC) analysis indicated that optimal discrimination between patients and controls was obtained by the combined test results of symptoms per intragastric volume using Nutridrink $15 \mathrm{ml} / \mathrm{min}$ as the test meal. Area under the curve 0.94 with a sensitivity of $93 \%$ and a specificity of $75 \%$. Conclusion Slow ingestion of a high-caloric Nutridrink is preferable compared to the rapid ingestion of a low-caloric meat soup to diagnose functional dyspepsia. Optimal discrimination between patients and controls was obtained by the combination of the drink test with ultrasonography.

\section{T1284}

Prevalence and Burden of Irritable Bowel Syndrome and Chronic Constipation in a Primary Care Sample of Healthcare Seekers Michael Warren, Daniel Belletti, Christopher Zacker, Annamaria Cerulli

BACKGROUND: Irritable bowel syndrome (IBS) and chronic constipation (CC) are common functional gastrointestinal (GI) disorders for which many patients never seek care or receive a diagnosis from a healthcare provider. AIMS: To establish the prevalence of IBS and CC in adult female patients among a primary care sample of healthcare seekers. The impact of IBS and CC on work productivity, daily activities, and over-the-counter (OTC) medication use was also evaluated. METHODS: Consecutive female patients $>18$ years, seeking care for any reason, from 32 primary care providers throughout PA, NJ and WV participated in the survey. A questionnaire including the Rome II criteria for IBS and CC, the Work Productivity and Activity Impairment index and questions on OTC drug use and alternative therapy in the past 4 weeks was used. Physicians completed a questionnaire for each patient assessing prior GI diagnoses and drug use. Comparisons were made between 3 groups: patients with IBS and patients with CC whose condition was confirmed by Rome II criteria or healthcare provider, and patients with neither condition (NC) using Chi Square and T-tests for categorical and continuous data, respectively. RESULTS: Out of 1,415 patients enrolled, $41.6 \%$ met the Rome II criteria or had a healthcare provider diagnosis for either IBS or CC (29.5\%, IBS and $12.1 \%, C C$ ) Of those patients meeting the Rome II criteria for IBS, only 100 $(28.7 \%)$ had a healthcare provider diagnosis of IBS, and of those meeting the CC Rome II criteria, only $35(18.9 \%)$ had their condition diagnosed by a healthcare professional. A total of $65.7 \%$ (174/265) of patients confirmed by Rome II criteria to have constipationpredominant IBS or CC had $\geq 1$ current GI-related symptom, and up to $42.6 \%(113 / 265)$ had 2 symptoms or more. Patients in both groups reported current symptoms of constipation (40.0\%), bloating (37.4\%), heartburn (26.0\%), abdominal pain (22.6\%), dyspepsia (18.9\%), and diarrhea $(4.9 \%)$. IBS and CC patients reported greater impact of their symptoms on overall work productivity compared to NE patients (19.3\% for IBS and $10 \%$ for CC vs. $7.3 \%$ for NE; p<.05 IBS vs. NE). Daily activity impairment due to GI problems was $21.4 \%$ for IBS, $13.6 \%$ for CC, and $5.5 \%$ for NE patients ( $\mathrm{p}<0.05$ for all). For patients taking OTC medication $(n=721 / 50.9 \%)$, the average number of medications taken was 2.1 (IBS) and 1.9 (CC) vs. 1.6 (NE; p<0.05). CONCLUSIONS: IBS and CC are common multiple symptom disorders among female patients presenting to primary care providers; however, many patients are never diagnosed despite the high symptom burden and adverse effect on patient work productivity and daily activities

\section{T1285}

Prevalence of Irritable Bowel Syndrome After Exclusion of Organic GI Disorders: Is Symptom Based-Diagnosis of IBS Precise Enough? Kyungsik Park, Byoungkuk Jang, Woojin Chung, Kwangbum Cho, Jaeseok Hwang, Sunghoon Ahn, Yuna Kang, Junyoung Hwang

Backgrounds/Aims; Irritable bowel syndrome (IBS) is a kind of common functional GI disorder. Recently, prevalence of this troublesome disease has been markedly increased in Asia. Prevalence of IBS has been reported variously maybe due to use of different diagnostic criteria. Because initial diagnosis of IBS is based on variable symptoms, the organic bowel or other GI diseases may be passed over. Therefore the aims of this study are first to find out precise prevalence of IBS by exclude organic components from patient group with IBS symptoms. Second, to determine the degree of agreement between different diagnostic criteria, and third, to analyze risk factors which were associated with presence of IBS. Methods; Health examinees who had visited health promoting center in Keimyung university, Dongsan medical center were requested to fill out questionnaire paper. Manning, Rome I, and Rome II criteria were used in all examinees and prevalence was calculated by each criteria. Degree of agreement between each criteria were analyzed by Cohen's kappa value. Risk factors were analyzed by comparing IBS and normal groups. Results; From January to November 2005, 2,793(58.1\%) out of 4,805 examinees, replied completely. Mean age was $45.4 \pm 10.3(16 \sim 82)$ years and male $(59.5 \%)$ was predominant. Prevalence of IBS by Manning, Rome I, and Rome II criteria were $22.4 \%, 14.9 \%$, and $17.5 \%$, respectively. Kappa value between Rome II and Manning criteria was 0.705 and between Rome II and Rome I was 0.474 . Of 490 IBS patients by Rome II criteria, $8(1.6 \%)$ patients showed abnormal fecal test. Colonoscopy was done at 390 persons and was abnormal at 34(8.7\%). Thus accurate prevalence by Rome II criteria was about $15.7 \%$. Other GI disorders which were observed at in IBS patients were peptic ulcer(1.2\%), esophagitis(12.9\%), GB stone $(6.5 \%)$ etc. Punch biopsy of intact-looking colonic mucosa was done at 71 informed IBS patients. Mucosal hyperplasia, lymphoid aggregates, lymphoid follicle, and increased eosinophil count were predominant pathologic finding. Gender( $\mathrm{M}: \mathrm{F}=15.4 \%: 20.7 \%, \mathrm{p}<0.001)$ ), occupation(no occupation, $\mathrm{p}<0.001)$, young age $(\mathrm{p}<0.001)$ were independent risk factors for presence of IBS in univariate and multivariate analysis. Conclusion; Exclusion of organic bowel problem is necessary to find out precise prevalence of IBS. Overall prevalence of IBS by Rome Il criteria 1 s $15.7 \%$ in Korea. Female gender, no occupation, and young age are independent risk factor associated with presence of IBS.

\section{T1286}

Clinical, Topographic and Pathophysiological Characteristics of Funcional Abdominal Bloating

Fermin Mearin, Antonia Perello, Monica Perona, Agustin Balboa, Diana Hernandez, Antoni Castells, Mario Pages

Abdominal bloating without an organic cause (functional abdominal bloating: FAB) is one of the most frequent digestive complaints but its clinical manifestations and causes are elusive. AIM: To evaluate clinical characteristics, some putative pathophysiological mechanisms and distribution of intestinal gas in FAB. METHODS: 34 patients with FAB (Rome II criteria) were evaluated to ascertain factors favoring distention and increase in abdominal girth (as recorded in a diary), presence of causative illnesses (celiac disease or hypothyroidism) carbohydrate intolerance (lactose and fructose-sorbitol breath tests), bacterial overgrowth (glucose breath test), visceral sensitivity (rectal barostat) as well as localization and amoun of intestinal gas ( $\mathrm{n}=17$; high resolution CT-scan with specific window). RESULTS: $85 \%$ of patients showed an increase in bloating through the day and $88 \%$ after meals; $61 \%$ complained of worsening with anxiety; $26 \%$ and $64 \%$ improved by belching or passing gas, respectively. Range of abdominal girth increased was 0.1 to 9.2 (mean: $3.7 \mathrm{~cm}$ ). Anti-tranglutaminase antibodies were negative in every case and in one case the diagnosis of hypothyroidism was made. Breath test for lactose, fructose-sorbitol and glucose were abnormal in 25\%,50\% and $5 \%$ of the cases, respectively. Visceral sensitivity was normal in $83 \%$ of the patients $12 \%$ showing an increase and $5 \%$ a decrease. In most of the cases gas was mainly located in the colon (63\%) (colon: $191 \pm 25 \mathrm{ml}$; stomach: $21 \pm 3 \mathrm{ml}$ ) while the remaining (37\%) showed mixed distribution in the stomach and colon (colon: $167 \pm 50 \mathrm{ml}$; stomach: 48 t $17 \mathrm{ml}$ ). Three out of 11 patients with gas mainly located in the colon had some carbohydrate malabsorption. In patients with mixed gas distribution some carbohydrate malabsorption was found in 5 out of 6 patients, and 1 had bacterial overgrowth. CONCLUSION: FAB is a homogeneous clinical syndrome but pathophysiologically heterogeneous with frequent carbohydrate malabsorption and normal visceral sensitivity. No clear correlation between pathogenic mechanisms and gas localization exists.

\section{T1287}

An Open Label Pilot Trial of High Dose Rifaximin in the Treatment of Patients with Constipation Predominant Irritable Bowel Syndrome James George, Michele Hunt, Asher Kornbluth, Peter Legnani

Introduction: A recent double-blind, placebo controlled trial (Pimentel M, et al ACG 2005) demonstrated efficacy with rifaximin, a nonabsorbable broad spectrum antibiotic, effective in the treatment of small intestinal bacterial overgrowth (SIBO) for patients with IBS Rifaximin was dosed at $200 \mathrm{mg}$. TID for 10 days and sustained improvement was noted in patients for up to 10 weeks in global improvement, bloating and diarrhea. Although symptoms of constipation were improved compared to placebo, this improvement did not reach statistical significance. Objective: The aim of this open label pilot study was to determine if higher doses of rifaximin would improve symptoms in patients with constipation-predominant IBS (IBS-C) who tested positive for bacterial overgrowth. Methods; Patients with IBS-C underwent lactulose breath testing for SIBO. Patients who had an elevation in $\mathrm{H} 2$ of at least $12 \mathrm{ppm}$ by 2 hours after ingesting $50 \mathrm{gm}$. lactulose were considered positive for SIBO. These patients were then treated with rifaximin $800-1200 \mathrm{mg} / \mathrm{d}$ in divided doses for 10 days. Patients were excluded if they had coexisting gastrointestinal diseases, recent antibiotic use or were on tegaserod Patient outcomes were recorded by office visit follow-up or telephone interview. Results: 14 patients with with IBS-C tested positive for SIBO with a mean increase of 37 ppm in $\mathrm{H} 2$ over 2 hours after oral ingestion of $50 \mathrm{gm}$ lactulose.. Seven patients were treated with rifaximin $400 \mathrm{mg}$ BID and 7 patients were treated with rifaximin $400 \mathrm{mg}$ TID. Six of 7 patients treated with rifaximin $800 \mathrm{mg} / \mathrm{d}$ had improvement in constipation and bloating and 3 of 7 patients treated with rifaximin $1200 \mathrm{mg} / \mathrm{d}$ had improvement in constipation and bloating. Symptomatic improvement occurred within the first week of treatment in al patients. 6 patients relapsed within 2 weeks of discontinuation of therapy and all 6 improved with reinstitution to rifaximin. The remaining patients remained in remission for at least 4 weeks after cessation of rifaximin. All patients tolerated rifaximin treatment without adverse events. Conclusion: Patients with IBS-C who test positive for SIBO have a $64 \%$ response rate for symptoms of bloating and constipation with doses of rifaximin of 800-1200 mg/ day, and these doses were well tolerated in all patients. Prospective placebo controlled trials of higher dose rifaximin with long term follow up are warranted to define its optimal dose and duration in the management of patients with IBS-C. 
T1288

Increased Risk of Ischemic Colitis Associated with Constipation and Irritable Bowel Syndrome

Mark Cziraky, Kristijan H. Kahler, Ralph Quimbo, Jeffrey Kralstein

Background: Recent reports have suggested an increased risk of ischemic colitis (IC) among patients with irritable bowel syndrome (IBS). More recently, it has been suggested that there is an increased risk of IC in patients with chronic constipation (CC). In this study, we attempt to evaluate the possibility that IBS and CC predispose patients to IC using a larger database than used in previous studies. Methods: The source of data was the HealthCore Managed Care Database which contains 12 million quality data lives, defined as those members with complete eligibility, medical, and pharmacy data. From this data source we identified two cohorts: patients newly diagnosed with IBS (ICD-9: 564.1) and patients newly diagnosed with CC (564.0). The IBS and CC cohorts were matched 1:1 to control cohorts using the propensity score method. Patients were identified from $1 / 1 / 2000$ to $2 / 28 / 2005$, and had to have 6 months eligibility prior and at least 3 months post the first diagnosis date. Patients were followed until the first occurrence of IC or the end of eligibility. Incidence rates (IR) were assessed using a Kaplan-Meier estimator, and relative risks (RR) were calculated using multivariate Cox proportional hazards models. Results: The IR for the development of IC among the 100,143 newly diagnosed IBS patients was 90.37 cases per 100,000 patientyears ( 167 cases) compared to 41.47 cases per 100,000 patient-years ( 77 cases) in the IBS controls. We also identified 81,399 newly diagnosed CC patients where the IR for IC was 80.44 cases per 100,000 patient-years (119 cases) compared to 43.03 cases per 100,000 patient-years ( 64 cases) among the CC controls. IRs for those with IBS and $\geq 65$ years old (152/100,000 patient-years) were about two-fold higher than the rates among those with IBS and $<65$ (76/100,000 patient-years). The RR for IC was 8.16 (95\% confidence interval [CI]: 4.85- 13.75) and 2.60 (95\% CI: 1.74-3.88) for IBS and CC, respectively. Conclusions: We found that patients with a diagnosis of IBS had an eight times greater risk of developing IC and patients with CC had a 2.6 fold increase in risk of IC relative to population controls. Given prior research in this area, and results of this current research using a much larger database than previous studies we conclude that IBS and CC are associated with the development of IC, and studies evaluating IBS and CC therapies must control for the background rate of IC among patients with these disorders.

\section{T1289}

Constipation-Predominant and Mixed/Alternating IBS Symptoms: Six-Month Results from the Irritable Bowel Syndrome Longitudinal Outcomes Study ILOS

G. Richard Locke, Kristijan H. Kahler, Nadia Leskinova, Robert Balshaw

Background: The different effects of therapies suggest that irritable bowel syndrome with diarrhea (IBS-D) and IBS with constipation (IBS-C) are not the same condition. The appropriate classification of IBS patients with an alternating bowel habit remains uncertain. Aim: To compare the outcomes of patients with IBS-C and mixed/alternating IBS (IBS-M/A) over 6 months in a community practice setting. Methods: ILOS was a 6-month, prospective, observational study which enrolled 380 patients across the US who had a physician diagnosis of IBS-C or IBS-M/A. Physician-reported clinical data were collected at baseline and patientreported symptom data were collected monthly for 6 months. Patients were classified as having IBS-C or IBS-M/A based upon the physician's assessment at baseline. Patients completed questionnaires to measure health-related quality of life (HRQOL), satisfaction with relief of abdominal pain or constipation, and work and activity impairment (WPAI). A total severity score (TSS) was calculated by summing the frequency, intensity and bothersomeness of constipation, gas, abdominal pain/discomfort and bloating. Chi-square and Wilcoxon rank sum tests were used for univariate comparisons between IBS-C and IBS-M/A subjects. Results: At baseline, $93 \%$ of subjects were female with a mean age of 42 years. The physicians characterized 224 (59\%) patients as having IBS-C and 141 (37\%) as having IBS-M/A. Sixmonth questionnaires were submitted by 231 subjects (61\%), with similar submission rates for IBS-C and IBS-M/A subjects ( $58 \%$ vs. $64 \%, \mathrm{p}=0.27$ ). Among 175 subjects with TSS data at baseline and 6 months, symptoms improved more for IBS-C subjects than IBS-M/A (change in mean TSS: IBS-C -6.7 vs. IBS-M/A $-2.8 ; \mathrm{p}=0.02$ ) with symptoms improving for $74 \%$ of IBS$\mathrm{C}$ subjects and $65 \%$ of IBS-M/A. Differences in symptom reduction were seen in constipation $(\mathrm{p}<0.01)$ and bloating $(\mathrm{p}<0.01)$, though not in gas $(0.48)$, abdominal pain $(\mathrm{p}=0.15)$, and diarrhea $(p=0.07)$. No significant differences were observed between IBS-C and IBS-M/A subjects with respect to change in HRQOL overall score ( $\mathrm{p}=0.611)$, or satisfaction with relief of abdominal pain $(\mathrm{p}=0.607)$ or constipation $(\mathrm{p}=0.227)$. Changes in WPAI overall impairment were similar for IBS-C and IBS-M/A subjects $(\mathrm{p}=0.179)$. Conclusion: In a community practice setting, patients with IBS-C reported greater symptom improvement over a 6-month period compared to patients with IBS-M/A. The availability of treatment for IBS-C and the lack of proven treatment for IBS-M/A may be a possible explanation; however, further research is necessary.

\section{$\mathrm{T} 1290$}

Efficacy of Oral Pancreatic Enzymes in Form of Enteric-Coated MiniMicrospheres for the Treatment of Maldigestion Secondary to Duodenopancreatectomy (DP)

Enrique Dominguez-Munoz, Maria Vilarino, Julio Iglesias-Garcia

Background: Maldigestion developing after gastroduodenal and pancreatic resection is a major therapeutic challenge. Enteric-coated pancreatic enzymes in mini-microspheres are the therapy of choice for maldigestion in chronic pancreatitis. The efficacy of this therapy in patients after pancreatic surgery is unknown. Aim: To evaluate the efficacy of this oral enzyme substitution therapy in patients with maldigestion after DP. Since the distal part of the stomach is also surgically removed, it was also evaluated whether this therapy can be improved by opening the enzyme capsules or by inhibiting gastric acid secretion. Methods: A prospective, randomized, open, comparative, crossover study was carried out in 18 patients (12 male, mean age 55 years, range 34-76 years) 32 months (range 3-164 months) after
DP. Patients with maldigestion were randomized to receive four capsules containing 10,000 $\mathrm{U}$ lipase each in form of enteric-coated mini-microspheres (Kreon®, Solvay Pharma, Germany) either closed or open during two consecutive 10-day periods. Esomeprazole $40 \mathrm{mg}$ od was added for further 10 days in patients with persisting maldigestion despite the enzyme therapy. Fat digestion before (basal) and on the last day of each one of the three treatment periods was evaluated by an optimized mixed 13C-triglycerides breath test. The cumulative recovery rate of $13 \mathrm{CO} 2$ was considered as the result of the test. Data are shown as mean [95\% CI] and compared by the Student-t test for related samples. Results: 15 patients (83\%) suffered from fat maldigestion and were included in the study. One of them was finally excluded because of protocol violation. Basal $13 \mathrm{CO} 2$ recovery rate was $35.9 \%$ [0-56.1\%]; (normal $>57.0 \%$ ). Therapy with oral pancreatic enzymes markedly improved fat digestion (13CO2 recovery $57.1 \%$ [15.8-84.9\%]; $<<0.01$ vs basal), independently of whether capsules were taken closed or open (13CO2 recovery $57.3 \%$ [0-94.1\%] and 56.9\% [31.7-95.2\%], respectively; n.s.). Eight patients (57\%) normalized fat digestion under enzyme therapy. The addition of esomeprazole in patients with persisting maldigestion increased significantly the efficacy of oral pancreatic enzyme substitution therapy (13CO2 recovery from $40.0 \%$ [15.8-55.0\%] to $52.2 \%$ [22.3-63.3]; $\mathrm{p}<0.01$ ), and 3 further patients were able to normalize fat digestion. Conclusion: Maldigestion develops in most patients after DP. Treatment with oral pancreatic enzymes in form of enteric-coated mini-microspheres is highly effective in this setting. Despite partial gastrectomy, opening enzyme capsules is not required. Inhibition of gastric acid secretion is of major help in patients with insufficient response to oral enzyme therapy.

\section{T1291}

How to Optimize Oral Pancreatic Enzyme Substitution Therapy in Patients with Chronic Pancreatitis to Assure a Normal Nutritional Status? Enrique Dominguez-Munoz, Maria Vilarino, Marta Iglesias-Rey, Julio Iglesias-Garcia

Background: We have previously shown that malnutrition persists in most patients with maldigestion secondary to chronic pancreatitis $(\mathrm{CP})$ despite an adequate clinical response to oral enzyme substitution therapy. As hypothesis, evaluation by objective methods of the digestive ability under therapy may allow optimizing the treatment and assuring a normal nutritional status in patients with CP. Aim: To evaluate the usefulness of the 13C-mixed triglyceride breath test (13C-MTG-BT) as a tool for optimizing the therapy of maldigestion related to $\mathrm{CP}$, and to analyze the impact of this therapeutic optimization on the patients' nutritional status. Methods: Prospective, interventionist, comparative and paired study. A total of 20 consecutive patients (17 male, mean age 55 years, range 43-77 years) with fat maldigestion secondary to alcoholic $\mathrm{CP}$, who had abnormally low circulating levels of retinolbinding protein (RBP) despite at least one year of adequate clinical control of maldigestion (absence of weight loss and diarrhea) by enzyme substitution therapy were included. In all of them, treatment of maldigestion was optimized by increasing the daily dose of oral pancreatic enzymes (enteric-coated mini-microspheres) as much as needed to obtain a normal 13C-MTG-BT result (13CO2 recovery rate $>57 \%$ ). A proton pump inhibitor was associated in case of insufficient response to oral enzymes. Serum levels of RBP (normal 3.0-6.0 mg/ $\mathrm{dL}$ ) and prealbumin (normal 21.0-41.0 mg/dL) were measured before and one year after optimization of the therapy. Results are shown as mean and $95 \%$ confidence interval, and compared by the Student-t test for paired samples. Results: Before optimization, patients were under treament with oral pancreatic enzymes at a median dose of 20,000 U lipase/ meal. A normal 13C-MTG-BT result was obtained in all patients by increasing the enzyme dose up to $40,000 \mathrm{U}(\mathrm{n}=18)$ and $60,000 \mathrm{U}(\mathrm{n}=2)$ lipase/meal. In addition, three patients $(15 \%)$ required the association of esomeprazole $40 \mathrm{mg}$ od to normalize the breath test result. By optimizing the therapy, serum levels of RBP increased from $2.4 \mathrm{mg} / \mathrm{dL}(2.1-2.7 \mathrm{mg} / \mathrm{dL})$ to $3.1 \mathrm{mg} / \mathrm{dL}(2.8-3.4 \mathrm{mg} / \mathrm{dL})(\mathrm{p}<0.001)$. Similarly, serum prealbumin levels increased from $20.7 \mathrm{mg} / \mathrm{dL}(18.8-22.6 \mathrm{mg} / \mathrm{dL})$ to $25.2 \mathrm{mg} / \mathrm{dL}(23.8-26.6 \mathrm{mg} / \mathrm{dL})(\mathrm{p}<0.001)$. Serum RBP and prealbumin levels reached normal values in $14(70 \%)$ and $20(100 \%)$ patients respectively. Conclusion: Therapy of CP-related maldigestion may be optimized by the 13C-MTG-BT This therapeutic optimization allows assuring a normal nutritional status in patients with CP.

\section{T1292}

Development of a Breath Test with Stable Isotopes for the Evaluation of Carbohydrate Digestion

Beatriz Cigarran, Maria Vilarino, Marta Iglesias-Rey, Enrique Dominguez-Munoz

Background: Diagnosis of carbohydrate digestion is becoming relevant in patients with pancreatic diseases due to the development of new enzyme preparations including lipase as the single enzyme. Carbohydrate maldigestion may also be an important aspect in diabetes mellitus-related exocrine pancreatic insufficiency. At present, methods to evaluate carbohydrate digestion are lacking. Aim: To develop a new breath test with stable isotopes for the evaluation of carbohydrate digestion. Methods: A prospective, open, crossover comparative study was performed. Eight healthy subjects (age rage 28-43 years, 6 male) were included. A test meal consisting on a muffin $(50 \mathrm{~g}$ corn flour, $14 \mathrm{~g}$ fat) and $300 \mathrm{ml}$ water was developed. After overnight fast, metoclopramide $10 \mathrm{mg}$ were orally given $20-30 \mathrm{~min}$ before test meal ingestion. Breath samples were collected in Exeteiner tubes before and in 30min intervals for 6 hours after test meal. The same test was repeated $7 \pm 2$ days thereafter, when acarbose $100 \mathrm{mg}$ was orally given just at the beginning of the test meal ingestion. The quotient $13 \mathrm{CO} 2 /$ $12 \mathrm{CO} 2$ was measured by mass spectrometry. The area under the curve (AUC) of the global $13 \mathrm{CO} 2$ exhaled over the 6 hour time, expressed as delta over baseline (DOB)/6h, was considered as the result of the test. Data are shown as median and range, and compared by the Wilcoxon test for related samples. Results: Administration of a corn-based test meal allowed us to obtain a $13 \mathrm{CO} 2$ exhalation curve concordant with the physiological process of carbohydrate digestion. $13 \mathrm{CO} 2$ elimination peak occurred at 180-210 minutes in all subjects and the AUC was 977 DOB/6h (range 632-1340 DOB/6h). Acarbose-mediated inhibition of glucosidase activity led to a marked reduction of $13 \mathrm{CO} 2$ elimination in breath (AUC $248 \mathrm{DOB} / 6 \mathrm{~h}$, range 72-503 DOB/6h) ( $\mathrm{p}<0.01$ ). Conclusion: A corn-based breath test allows carbohydrate digestion to be evaluated. In this test, glucosidase activity is the main factor determining the amount of $13 \mathrm{CO} 2$ exhaled. 


\section{T1293}

Are Monolithic Enteric-Coated Enzyme Preparations Effective in Pancreatic Exocrine Insufficiency? A Multicentre, Double Blind, Placebo Controlled Cross-Over Trial Jutta Keller, Peter Layer

Background: Due to dissociation between gastric emptying of meal nutrients and enzyme supplements, monolithic enteric-coated pancreatic enzyme preparations are assumed to be virtually ineffective in improving lipid digestion and absorption in severe pancreatic exocrine insufficiency. Instead, enteric-coated microgranule preparations are recommended as standard treatment. However, the effects of monolithic preparations on digestion and absorption have not been studied, yet. Aims: To test the effects of a monolithic enteric-coated pancreatin preparation on lipid digestion in patients with severe pancreatic exocrine insufficiency. Methods: 20 patients with documented chronic pancreatitis (19 males, 1 female, age: $52 \pm 2$ yrs, BMI: $23.1 \pm 0.7 \mathrm{~kg} / \mathrm{m} 2$, mean \pm SEM) and fecal elastase- 1 concentrations ranging between 15 and $105 \mu \mathrm{g} / \mathrm{g}(42.1 \pm 8.2 \mu \mathrm{g} / \mathrm{g})$ were included in 3 study centres. On two study days (separated by an interval of 3-14 days and each preceeded by at least 3 days without intake of enzyme supplements) patients received a monolithic enteric-coated pancreatin preparation containing $40,000 \mathrm{U}$ of lipase or placebo together with a standardised test meal containing $250 \mathrm{mg}$ of 13C-mixed triglycerides. Breath samples were collected at $30 \mathrm{~min}$ intervals for $8 \mathrm{~h}$ and analysed by isotope selective infrared spectrometry. The trial was performed according to a randomised, double blind, placebo controlled, cross-over study design. Differences between cumulative 13C-exhalation were evaluated as markers of lipid digestion during treatment with verum and placebo. Results (mean \pm SEM, statistics: ANOVA): One patient had to be excluded because of major protocol violations. Moreover, intestinal lipolysis was found to be normal during placebo treatment in 6 patients with decreased fecal elastase-1 concentrations. Thus, these patients were also excluded from the per protocol analysis. In the remaining 13 patients, mean cumulative 13C-exhalation over 6 and 8 hours was markedly higher following treatment with verum compared to placebo ( 6 h: $11 \pm 2 \%$ vs. $5 \pm 1 \%$ of dose, $\mathrm{p}=0.035 ; 8$ h: $18 \pm 3 \%$ vs. $9 \pm 1 \%$ of dose, $\mathrm{p}=0.015$ ). No treatment related adverse events occurred. Conclusions: In contrast to current belief, a monolithic enteric-coated pancreatic enzyme preparation containing 40,000 U of lipase significantly improves lipid digestion and absorption compared to placebo in patients with pancreatic exocrine insufficiency due to chronic pancreatitis. Thus, current treatment recommendations should be reconsidered.

\section{T1294}

Effect of Proton Pump Inhibition and/or Cigarette Use On the Results of An Endoscopic Secretin Stimulation Test

Neil Phelan, Wahid Wassef

Background: Recent data suggests that cigarette use may be an independent risk factor for pancreatic insufficiency or the progression of chronic pancreatitis. Endogenous secretin release is intimately linked to gastric acid production. A significant number of our patients with chronic pancreatitis are current or previous cigarette users, and are currently taking a proton pump inhibitor (PPI). Purpose: To determine the effect of cigarette use and/or PPI use on the results of an endoscopic secretin stimulation test (SST). Methods: A total of 44 patients with suspected chronic pancreatitis have undergone the combination of endoscopic ultrasound and an endoscopic SST at our institution. The SST involved measurement of [HCO3-] with duodenal aspiration at 15 minutes and 30 minutes after the administration of secretin. We retrospectively examined the effects of PPI use and tobacco use on the results of the SST. Tobacco use was subdivided into remote use, mild use ( $\leq 1$ PPD), and moderate use (> I PPD). The relationship between peak [HCO3-] and PPI use was evaluated with a T-test. The relationship between peak [HCO3-] and cigarette use was evaluated using linear regression analysis. The combination of the two variables was evaluated using multiple linear regression analysis. Cigarette use was also examined as a bivariate variable, using current smoking versus none/previous smoking. Results: The mean peak [HCO3-]'s of PPI users versus nonusers were 63.38 and 63.55 , respectively $(\mathrm{p}=0.98)$. The mean peak [HCO3-]'s of current cigarette users versus nonusers were 65.67 and 61.92 , respectively $(\mathrm{p}=0.58)$. There was not a statistically significant difference between any two groups when both variables were analyzed in combination. Conclusion: In our study, neither PPI use nor cigarette use, either alone or in combination, had a statistically significant effect on the results of the endoscopic secretin stimulation test. The results may be secondary to beta error, as the sample size within each group was small, but further study is ongoing to clarify this

\section{T1295}

Exocrine Pancreatic Function Testing in Pancreatic Sufficient Cystic Fibrosis Patients: A Correlation Study

Michael Wilschanski, Amir Weintraub, Huda Mussaffi, Hannah Blau, Eli Picard, Lea Bentur, Eitan Kerem

Background: Most patients with Cystic Fibrosis have pancreatic insufficiency (PI), however, $15 \%$ of the patients are pancreatic sufficient (PS). Many laboratory tests have been developed in order to examine exocrine pancreatic function to distinguish between PI and PS. The 'gold-standard' to determine pancreatic function apart from direct pancreatic stimulation test is the fecal fat excretion during $72 \mathrm{~h}$, expressed as the coeffiecient of fat absorption (CFA). Other tests include: fecal elastase and serum immuno-reactive trypsinogen (IRT). Aim: To test the correlation between elastase and IRT as compared to fecal fat excretion, thereby, to evaluate the best available test to determine exocrine pancreatic function in CF patients. Methods: 21 CF PS patients performed the 3 tests of fecal fat excretion, elastase and IRT. Correlation between the tests was evaluated by the kappa statistics test, sensitivity, specificity, and positive and negative predictive values. Results: The correlation between CFA and IRT was negative (kappa $=-0.154$ ), and between CFA and elastase was poor $(\mathrm{kappa}=0.213)$. The sensitivity, specificity, positive and negative predictive values of IRT vs. CFA were: $0 \%, 88 \%, 0 \%$, and $78 \%$, respectively, and for elastase were: $40 \%, 81 \%, 40 \%$, and $81 \%$ respectively. Conclusion: Poor correlation was found between IRT, elastase and
CFA, therefore, neither elastase in the stool nor IRT in the serum has the sensitivity or the specificity to replace the 'gold standard' test of fecal fat excretion during $72 \mathrm{~h}$. Thus, despite the technical difficulties and the inconvenient of collecting stool, there is a need to evaluate precisely the pancreatic function of the $\mathrm{CF}$ patients, at least at the time of diagnosis, and non-infrequently during the follow-up period, especially, when changes in clinical parameters occur.

\section{T1296}

Pancreatic Function Testing Is Best Determined By the One Hour Endoscopic Tube Assisted Technique Rather Than the Rapid 15 Minute Direct Endoscopic Aspiration Test

Picha Moolsintong, Frank R. Burton

BACKGROUND: Rapid 15 minute endoscopic secretin stimulated pancreatic function testing (ESPFT) has been proposed as an alternative to standard secretin stimulated duodenal tub pancreatic function testing. Various investigators have questioned the accuracy of ESPFT in determining peak $\mathrm{HCO} 3$ concentrations ( $\mathrm{PHC}$ ). Expanded collection periods with an oralduodenal drainage tube allow for additional collections to capture PHC after the initial 15 minute period without extending the EGD procedure time. AIM: To evaluate whether the one hour endoscopic secretin stimulated tube assisted technique is superior to the rapid 15 minute direct ESPFT in determining pancreatic exocrine function. METHODS: A retrospective study was done of patients with chronic abdominal pain and normal pancreatic imaging whom had ESPFT testing with the following modified endoscopic collection system. ESPF testing as described by Conwell et al was conducted. Synthetic secretin $(0.2 \mathrm{mcg} / \mathrm{kg})$ was given IV 15 minutes before endoscopic duodenal aspiration. The first 15 minute collection of duodenal secretions was obtained via direct endoscopic aspiration. A wire-guided Liguory nasal-biliary drainage tube with a distal pigtail multiport aspiration tip was placed in the 3rd portion of the duodenum under direct endoscopic visualization. The endoscope was withdrawn as the tube was brought out through the oropharynx and connected to continuous aspiration. Two additional 10 min-period collections were obtained via the drainage tube at 30 and $45 \mathrm{~min}$ after IV secretin. Patients were maintained in the left-lateral decubitus position during the procedure to prevent any gastric fluid contamination. All fluid collections were analyzed for bicarbonate $(\mathrm{HCO} 3)$ concentration and those $>80 \mathrm{mEq} / \mathrm{L}$ were considered normal. HCO3 concentrations at 15 minutes were compared to the 30 and 45 minute collections. RESULTS: Of the 28 patients studied, 9 were male and 19 were female, with a mean age of 47.9 years. PHC at 15 minutes occurred in 15 patients (54\%) and after 15 minutes in 13 patients (46\%). Normal concentrations of $\mathrm{HCO} 3$ were seen in 17 patients (60.7\%). Of the 17 patients with normal PHC, 9 had a PHC after 15 minutes. In 3 patients, the 15 minute PHC would have been inadequate to diagnose normal exocrine function. In the 11 patients who never achieved normal values, 4 required more than 15 minutes to reach PHC. CONCLUSIONS: The one hour secretin stimulated endoscopic tube assisted technique is superior to the rapid 15 minute direct ESPFT in pancreatic exocrine function testing. It appears that 1 hour collections were necessary in $3(18 \%)$ patients to determine normal pancreatic exocrine function.

\section{T1297}

Long-Term Outcome of Oral Anti-Oxidant Therapy in Patients with Painful Chronic Pancreatitis

Anil Bagul, Ajith K. Siriwardena

Introduction: The generation of short-lived oxygen free radicals(oxidative stress) has been implicated in the pathogenesis of chronic pancreatitis (CP). Anti-oxidants are endogenous inhibitors/scavengers of oxygen free radicals and their levels are depleted in patients with $\mathrm{CP}$. There is some evidence that supplementation with exogenous anti-oxidants modulates symptoms in CP. This study reports long-term outcome in a cohort of patients with CP given anti-oxidant therapy. Methods: Patients diagnosed as CP and having their index presentation to a regional hepatobiliary service in the calendar year 1993 were identified by computerised review of hospital discharge summaries using the search criterion of Internaional Classification of Disease 9:577-1. 39 patients fulfilled these criteria. These patients had undergone measurement of anti-oxidant levels and had then been commenced on antox (Pharmanord, UK) an anti-oxidant preparation comprising selenium, methionine and ascorbic acid. Their charts were retrieved and indepedently reviewed to confirm that they had either CT and/or ERCP evience of chronic pancreatitis. Chart review was then carried out with data recorded on baseline and subsequent annual levels of anti-oxidants, opiate use (expressed as a percentage of the study population and taking documented weekly prescription to signify "regular" opiate), endocrine and exocrine insufficiency, surgica intervention and mortality over the subsequent 10 years. Data are presented as median (range). Statistical comparisons are made using an assumption of non-parametric distribution of data with significance accepted at the $\mathrm{P}<0.05$ level. RESULTS: Serum ascorbic acid levels at recruitment were $14.7(1.9-28.3) \mathrm{mg} / \mathrm{l}$ and at 10 years were $15(1.9-27.1)$ [P=0.52;MannWhitney U-test]. Serum selenium at recruitment was $85(28-214)$ microgm/l compared to $143(86-266) \mathrm{microgm} / \mathrm{l}$ at 10 years $[\mathrm{P}<0.001$; Mann-Whitney $\mathrm{U}$ test]. The laboratory reference range for ascorbic acid was $4-20 \mathrm{mg} / \mathrm{l}$ and for selenium 83-152 microgm/l. At recruitment, 2 patients (5\%) were diabetic and by 10 years this had risen to $20(51 \%)$. Twenty six patients (78\%) presented with pain - and $25(75 \%)$ reported pain at 10 years. Eight $(38 \%)$ were on regular opiates at baseline compared to 21 ( $73 \%)$ at 10 years $[\mathrm{P}=0.01$; Fisher's 2 sided]. CONCLUSION: Although long-term anti-oxidant therapy appears to sustain selenium levels, there does not appear to be any sustained modulating effect on either the symptoms of chronic pancreatitis or the progression of this disease. 
Reoperation in Chronic Pancreatitis

H Ramesh, Sadiq S. Sikora

Aim: This retrospective analysis of prospective data aims at analyzing a) indications for reoperation, b) morbidity and mortality of late re-operation in chronic pancreatitis and c) to study the long-term outcome in these patients. Patients and Methods: During 1987 to 2004 623 patients underwent first surgery for chronic pancreatitis. During the same period, 57 patients underwent late re-operation (beyond 6 months of initial surgery). There were 3 groups: a) patients who had undergone other abdominal procedures for abdominal pain prior to referral to this center $(\mathrm{n}=16)$, b) who had undergone pancreatic procedures elsewhere $(n=22)$ and c) patients who had undergone re-operation in this unit earlier $(n=19)$. Results: The data is summarised in Table 1: The Indications for re-operation is shown in Table 2 Conclusions: Re-operation in chronic pancreatitis is often required when entire pancreatic duct system is not drained or if inflammatory mass involving head is not dealt with during the initial surgery. Re-operative surgery carries high morbidity and mortality even in a high volume center. Those who underwent re-operative surgery have good pain relief and enjoy good quality of life. The first surgical opportunity in chronic pancreatitis remains the best one. Table 1 :

\begin{tabular}{|c|c|c|c|c|}
\hline Parameters & Group 1 & Group 2 & Group 3 & P Value \\
\hline Age & 35 & 33 & 38 & NS \\
\hline Sex M:F & $11: 5$ & $15: 7$ & $13: 6$ & NS \\
\hline Diabetes & 6 & 6 & 9 & NS \\
\hline Presenting with complication & 3 & 14 & 15 & $<0.01$ \\
\hline Morbidity & 4 & 10 & 12 & $<0.01$ \\
\hline Mortality & 0 & 1 & 2 & NS \\
\hline
\end{tabular}

Table 2

\begin{tabular}{|c|c|c|}
\hline Indication & Group 2 & Group 3 \\
\hline Head masst & 5 & 8 \\
\hline head pseudocyst & 3 & 0 \\
\hline Hypersplenism & 1 & 0 \\
\hline Insufficient head drainage and stricture & 8 & 2 \\
\hline Tail abscess and stricture & 2 & 2 \\
\hline Cancer & 0 & 2 \\
\hline Biliary stricture & 4 & 6 \\
\hline
\end{tabular}

T1299

Differential Effect of Opioids in Patients with Chronic Pancreatitis: An Experimental Pain Stud

Thomas R. Sorensen, Camilla Staahl, Lars Arendt-Nielsen, Asbjorn M. Drewes

Background and aims: Chronic gastrointestinal pain can be difficult to treat sufficiently with classical opioids. This frequently results in a very poor quality of life for the patients. Animal experiments and clinical observations have indicated a different working profile of oxycodone compared to morphine, and we have previously shown that oxycodone attenuate visceral (but not skin and muscle) pain better than morphine. This study aimed at testing oxycodone and morphine on experimental pain in different tissues in patients with pain due to chronic pancreatitis. Methods: In this blinded cross-over study we included 10 patients suffering from painful chronic pancreatitis. Inclusion criteria were that the patients should fulfill the Marseille-Rome/Cambridge diagnostic criteria for chronic pancreatitis. They should have chronic abdominal pain typical for chronic pancreatitis despite the use of pain killers. The patients should not suffer from any other kinds of chronic pain and they should have no present alcohol abuse. The study was approved by The Ethics Committee of Northern Jutland and the Danish Medicines Agency. The analgesic effect of morphine (30 mg), oxycodone $(15 \mathrm{mg})$ and placebo was tested against multi-modal (mechanical, thermal and electrical) experimental pain in the skin, muscles and esophagus. Pain was assessed at baseline and 30,60 and 90 minutes after oral administration of the drugs. Results: In the skin and muscles oxycodone had better effects than placebo and morphine on mechanically (skin: $\mathrm{F}=12.4, \mathrm{P}<0.001$, muscle: $\mathrm{F}=11.0, \mathrm{P}<0.001$ ) and thermally (skin: $\mathrm{F}=8.5, \mathrm{P}<0.001$ ) evoked pain. In the esophagus oxycodone attenuated heat pain better than both morphine and placebo $(\mathrm{F}=9.5, \mathrm{P}<0.001)$. Morphine was equal to placebo in the above pain tests. Morphine and oxycodone both attenuated mechanical pain in the esophagus better than placebo $(\mathrm{F}=8.6, \mathrm{P}<0.001)$. After electrical stimulation no differences between the opioids and placebo in any tissue studied could be demonstrated. Conclusions: The findings indicate up-regulation of the opioid system in patients suffering from chronic pancreatitis. The model supports the clinical impression that oxycodone works well after opioid rotation in patients with persistent visceral pain
Is Pancreatic Insufficiency Significant in African American Patients with End Stage Renal Disease On Hemodialysis?

Kapil Gupta, Chekai Tsao, Reuven Yakubov, Safak Reka, Moro Salifu, Scott Tenner, Swaminath Iyer

BACKGROUND: Pancreatic exocrine insufficiency has been shown to be present in patients with end stage renal disease (ESRD) on hemodialysis (HD). This finding is based on previous autopsy studies, and evaluation of pancreatic exocrine function using standard tests like fecal fat and chymotrypsin. Recently studies have shown fecal elastase l (FEl) as an effective method to diagnose pancreatic insufficiency. The aim of this study was to determine the prevalence of pancreatic exocrine dysfunction in patients with end stage renal disease (ESRD) on hemodialysis using FEl. METHODS: This was a prospective cross-sectional study of 25 African Americans (14 women and 11 men), ranging in age from 29 to 71 years with mean $58 \pm 2.26( \pm$ SEM) with ESRD on HD 3 times a week. Patients with other causes of pancreatic exocrine dysfunction were excluded from the study, including significant alcohol use, history of acute or chronic pancreatitis, liver disease, HIV, and family history of pancreatic disease. Fresh stool samples were collected from all the patients. Samples were stored at $<-20$ degree $C$ until analysis. FEl was measured using an ELISA method (Joli Diagnostic, New York: Reference values: $200 \mathrm{to}>500 \mathrm{ug} / \mathrm{g}$ of stool $=$ normal; 100 to $200 \mathrm{ug} / \mathrm{g}$ of stool = moderate to slight insufficiency; $<100 \mathrm{ug} / \mathrm{g}$ stool = severe exocrine insufficiency). RESULTS: All patients had hypertension and the duration of hemodialysis varied from 1 yr to 20 yrs with mean $4.7 \pm 0.89$. There were 12 subjects with and 13 without diabetes. Twenty four of 25 patients (96\%) had normal levels of FEl. Eighteen $(72 \%)$ of the patients had levels $>500 \mathrm{ug} / \mathrm{g}$ of stool. Only 1/24 (4\%) patient had an FEl level of < 200 (175.9) ug/g stool. None of the patients had any clinical manifestation of pancreatic exocrine insufficiency. Mean serum albumin level was $3.75 \pm 0.11$ and serum albumin levels did not correlate with FEl levels $(\mathrm{p}=0.08)$. There was no evidence of malnutrition in any patient. The mean BMI was $27.95 \pm 1.33$. There was no correlation between BMI and FEl levels $(\mathrm{p}=0.2)$. Using the chi square test for independence there was no significant relationship between patients with diabetes and levels of elastase $(\mathrm{p}=0.5)$. Using the linear regression analysis the duration of dialysis also did not significantly affect FEl ( $\mathrm{p}=0.78)$. CONCLUSION: 1. In our pilot study, stool FEl levels did not indicate pancreatic exocrine insufficiency in subjects with ESRD on HD. 2. There was no clinical or laboratory evidence of pancreatic exocrine insufficiency in subjects with ESRD on HD. 3. There is no difference in presence of pancreatic exocrine insufficiency in patients with and without diabetes

\section{T1301}

Endoscopic Ultrasound (EUS)-Guided Fine Needle Biopsy (FNB) for the Histological Evaluation of Chronic Pancreatitis

Julio Iglesias-Garcia, Ihab Abdulkader, Jose Larino-Noia, Jeronimo Forteza, Enrique Dominguez-Munoz

Background: Histological diagnosis of chronic pancreatitis has been classically limited to the study of surgical specimens. Since pancreatic biopsies are rarely done in the context of chronic pancreatitis, histological characteristics at different stages of the disease are unknown. Aim: To evaluate the histological characteristics of chronic pancreatitis in samples obtained by pancreatic EUS-FNB according to EUS findings. Methods: Twelve consecutive patients (12 male, mean age 67 years, range 17-81 years) with known chronic pancreatitis, who underwent EUS-FNB for the study of pancreatic masses were included. EUS-FNB was performed under conscious sedation with a $22 \mathrm{G}$ needle guided by the lineal scanning Pentax FG-38UX echoendoscope. EUS criteria for the diagnosis of chronic pancreatitis were evaluated: parenchymal criteria included hyperechoic foci, hyperechoic strands, lobularity, cysts and calcifications; and ductal criteria included dilation, duct irregularity, hyperechoic duct margins, visible side-branches and intraductal calcifications. Following histological features were evaluated: presence of acini, ductal epithelium, fibrotic tissue (collagen) and inflammatory infiltration. Results: Adequate tissue sample for histological evaluation was obtained in all cases. Infiltration by inflammatory cells was observed in all tissue specimens. Samples included pancreatic acini in five cases (41.6\%), in number of 2 to 13 acini each. In the remaining 7 cases $(58.4 \%)$ the presence of ductal epithelium together with fibrotic tissue was observed. Biopsies including pancreatic acini were those obtained from patients with mild to moderate EUS changes of chronic pancreatitis (up to 5 EUS criteria). On the contrary, biopsy samples from more severe cases (8-10 EUS criteria) were those showing only ductal ephitelium with fibrotic component. Conclusion: EUS-guided pancreatic biopsy allows evaluating histological changes of all stages of chronic pancreatitis. This may be an important advance for a better knowledge of the disease.

\section{T1302}

Timed Secretin-Enhanced Magnetic Resonance Cholangiopancreatography As a New Non-Invasive Pancreatic Function Test - Results of a Pilot Study Darwin L. Conwell, Mansour A. Parsi, Gregory Zuccaro, Joseph Veniero

Background \& Aim: Secretin-enhanced magnetic resonance cholangiopancreatography (S$M R C P$ ) is a non-invasive imaging technique that allows the depiction of morphologic changes in pancreatic ducts and a qualitative assessment of exocrine function by monitoring duodenal filling after exocrine stimulation. In the present study we assessed the use of a timed SMRCP to assess pancreatic exocrine function during secretin stimulation and compared it to the already validated endoscopic pancreatic function test using secretin (S-ePFT). Methods: Sixteen patients with abdominal pain and suspicion of chronic pancreatitis underwent SMRCP and S-ePFT. After secretin stimulation during the MRCP, the timing of the duodenal filling and the volume of the pancreatic excretion were assessed. Patients were deemed as having pancreatic insufficiency if duodenal filling was not achieved or if the pancreatic secretion was significantly delayed. The S-ePFT was performed after injection of $0.2 \mathrm{mcg} /$ $\mathrm{kg}$ synthetic porcine secretin. Duodenal fluid was collected with an upper endoscope during endoscopy at 15, 30, 45 and 60 minutes after secretin injection and the peak bicarbonate concentration of the collected juice was determined. The peak bicarbonate concentration less 
than or equal to $80 \mathrm{mmol} / \mathrm{l}$ was defined as evidence of pancreatic insufficiency. Performance of the S-MRCP was compared to the S-ePFT as the gold standard. Results: Eight patients had a peak bicarbonate concentration of less than $80 \mathrm{mmol} / \mathrm{l}$ indicating presence of pancreatic insufficiency. The sensitivity, specificity, positive predictive value and negative predictive value of S-MRCP for detection of pancreatic insufficiency were all 63\%. Conclusions: This pilot study with a small number of patients suggests that S-MRCP may have clinical use as a non-invasive pancreatic function test. Further studies with larger number of patients are needed to determine the best cutt-off values for the time delay and volume of the pancreatic secretion that would indicate presence of pancreatic insufficiency.

\section{$\mathrm{T} 1568$}

Synergy of Helicobacter Pylori Vacuolating Cytotoxin and IL-4 in Eotaxin Expression in Human Gastric Epithelial Cells Through Stat6 Pathway Jung Mogg Kim, Joo Sung Kim, Hyun Chae Jung, In Sung Song

Helicobacter pylori-infected gastric mucosa from patients with active chronic inflammation is characterized by infiltration of various inflammatory cells such as neutrophils and eosinophils. Although several mechanisms for neutrophil infiltration are well known, there has been little known the role of eotaxin, which is a potent chemoattractant for eosinophils, on the inflammatory process of $H$. pylori infection. The present study was to investigate the mechanisms of eotaxin expression in gastric epithelial cells stimulated with $H$. pylori vacuolating cytotoxin (VacA). Stimulation with VacA purified from H. pylori slightly increased eotaxin expression in MKN-45 gastric epithelial cell lines and primary human gastric epithelial cells. In contrast, the combined stimulation with VacA and IL-4 synergistically increased the eotaxin expression, as assessed by quantitative RT-PCR and ELISA. In MKN-45 cells transfected with an eotaxin promoter-luciferase reporter plasmid, costimulation with VacA and IL-4 induced more luciferase activity than either VacA or IL-4 alone did. However, such up-regulation was significantly decreased in the cells transfected with luciferase reporter plasmid bearing an eotaxin promoter which has a mutation at STAT6 binding site. Tyrosine phosphorylation of STAT6 was definitely increased in both MKN-45 and primary gastric epithelial cells stimulated with VacA and IL-4, compared with VacA or IL-4 alone. Furthermore, eotaxin expression was significantly suppressed in MKN-45 cells transfected with STAT6 siRNA. These results suggest that up-regulation of eotaxin in VacA-stimulated gastric epithelial cells may be synergistically facilitated by IL-4 via a STAT6-dependent mechanism.

\section{T1569}

The Nikr Protein Mediates Nickel-Responsive Regulation of the Helicobacter Pylori Iron-Uptake Genes FECA3 and FRPB3

Florian D. Ernst, Arnoud H. van Vliet, Wannie M. Horrevoets, Jeroen Stoof, Johannes G. Kusters, Ernst J. Kuipers

Introduction: Intracellular homeostasis of iron is a necessity for most organisms, since both iron-deficiency and iron-overload will result in cell death. The gastric pathogen Helicobacter pylori has three copies of the fecA and frpB iron-uptake genes, of which the fecAl/2 and frpB1/2 genes are regulated by the Fur protein. Surprisingly, fecA3 and frpB3 are not Furregulated and thus were thought to be constitutively expressed. $H$. pylori expresses a second metal-regulatory protein, NikR, and this was previously suggested to be involved in regulation of the fecA3 and frpB3 genes, but the mechanims governing this regulation was not described. Aim: We therefore investigated whether the fecA3 and frpB3 genes are regulated by NikR, and characterized the molecular mechanism governing this regulation. Methods: H. pylori reference strain 26695 and isogenic mutants were grown in Brucella broth supplemented with $\mathrm{NiCl} 2$. Gene expression was monitored using Northern hybridization, primer extension and SDS-PAGE. Results: Expression of the fecA3 and frpB3 genes was nickel-repressed in wild-type $H$. pylori, but constitutively expressed in the nikR mutant. However, the genes fecAl/2 and $f r p B 1 / 2$ did not display any nickel or NikR-dependent regulation. On the translational level the nickel- and NikR-dependent regulation was confirmed for frpB3. Mutation of frpB3 had no effect on growth in nickel-supplemented medium, possibly due to the compensatory expression of the two other copies of the fecA and frpB genes. Direct regulation of fecA3 and frpB3 by NikR was confirmed by the specific binding of recombinant NikR to an operator sequence present in both the fecA3 and frpB3 promoters. Conclusion: The NikR and Fur proteins each regulate the expression of a subset of iron-transporter proteins, allowing differential expression of iron-uptake systems depending on the environmental conditions. This may help $H$. pylori to survive the acidic conditions in the gastric mucosa.

\section{$\mathrm{T} 1570$}

Iron-Responsive Repression of Urease Expression in Helicobacter Hepaticus Is Mediated by the Transcriptional Regulator Fur

Clara Belzer, Ernst J. Kuipers, Bart A. van Schendel, Johannes G. Kusters, Arnoud H. van Vliet

Introduction: The pathogen Helicobacter hepaticus colonizes the enteric and hepatobiliary tract of rodents, and causes inflammatory bowel lesions, hepatitis, and hepatic malignancies. Urease is an environmentally regulated key-virulence factor for gastric Helicobacter species, but little is known about its role or regulation in non-gastric Helicobacter species. Low iron is often used by pathogenic bacteria as a signal for entering the host. We have previously shown that urease expression in $H$. hepaticus is not nickel-responsive, unlike the $H$. pylori urease. In this study we have investigated the role of iron in regulation of $H$. hepaticus urease. Methods: H. hepaticus strain ATCC51449 and its isogenic fur mutant were grown both under low-iron and high-iron conditions. Gene expression was monitored by Northern hybridization, and protein expression was monitored by SDS-PAGE. Urease activity was measured via a colorimetric reaction representing production of ammonia. Results: Irondepletion of growth medium resulted in a three-fold increase in urease activity in wild type H. hepaticus strain ATCC 51449. Using Northern hybridization it was demonstrated that iron-repression of urease expression was mediated at the transcriptional level. Mutation of the fur gene abolished the effect of iron-depletion, suggesting a role for Fur in iron-repression of urease expression. This was confirmed using a gel-shift assay, as recombinant Fur displayed iron-dependent binding to the urease promoter region. Conclusion: Iron and Fur are likely to contribute to $H$. hepaticus virulence as they are both involved in the repression of expression of the urease virulence factor. This regulation strongly contrasts with the NikR- and nickelinduction of urease expression in $H$. pylori. Iron-mediated urease regulation has not been reported before and may be important in the colonization of the intestinal and hepatobilary tract by $H$. hepaticus.

\section{T1571}

Cautions Regarding Relationships Between Expression of Helicobacter Pylori Baba and Disease Outcome

Saori Fujimoto, Olabisi Ojo, Anna Arnqvist, Jeng Yih Wu, Stefan Odenbreit, Rainer Haas, Rita Reddy, David Y. Graham, Yoshio Yamaoka

Background: Gastric mucosal ABO blood group antigens are recognized by the blood group antigen binding adhesin BabA. The role of BabA in the outcome of $H$ pylori infections remains unclear. Proposed relationships between BabA status and clinical outcome have used PCR-based methods to distinguish functional babA2 genes from nonfunctional babA. genes. These methods are based on detection of a 10 base pair deletion in the signal peptide sequence of babA1. However, as expression of $b a b A$ may also be regulated at the level of transcription and by formation of chimeric $b a b A-b a b B$ gene it is not clear whether the PCR-based methods reflect BabA expression or whether the PCR-based methods provide misleading estimates of babA expression. Aims: To investigate the accuracy of PCR-based methods in providing an estimate of babA expression and to reassess the relation between BabA status and clinical outcome. Methods: We compared 16 published PCR-based methods for predicting BabA status with immunoblotting and Lewis b binding assays with Lewis B binding assays being the gold standard. We then used immunoblotting to examine the relationship between clinical presentation and BabA status. Results: Lewis binding assay and BabA immunoblot yielded identical results. In contrast, PCR-based methods had low sensitivity and specificity as the prevalence of the babA2-positive strains with different prime pairs ranged from $30 \%$ to $98 \%$ among Western strains and from $55 \%$ to $100 \%$ among Eas Asian strains. Sequence analyses showed among 137 strains deposited in GenBank and 18 strains examined here, only CCUG17875 carried the babA1 type of sequence. Of interest, the primer regions targeted were not located in the conserved region of the babA gene. We also examined $1,130 \mathrm{H}$. pylori isolates by immunoblot and found that BabA expression as determined by immunoblot was weakly, but significantly, related to the presence of both peptic ulcer and gastric cancer; peptic ulcer vs, gastritis (adjusted $\mathrm{OR}=1.9 \cdot 95 \% \mathrm{CI}=1.1$ $3.4)$, and gastric cancer vs. gastritis $(\mathrm{OR}=1.9 ; 95 \% \mathrm{CI}=1.1-3.3)$. However, BabA was expressed in the majority $(74 \%)$ of strains isolated from patients with simple gastritis. Conclusions: PCR-based methods correlate poorly with BabA expression status and can not be used to reliably possible examine BabA associations. Immunoblot is currently the most accurate and efficient method to assess BabA status. However, simple determination of BabA status appears not to be clinically useful. Supported in part by the Gulf Coast Digestive Disease Center.

\section{$\mathrm{T} 1572$}

Strain-Dependent and Independent Roles of the Alpab in Helicobacter Pylori Infection: In Vitro and in Vivo Evidences

Hong Lu, Ellen J. Beswick, Victor E. Reyes, David Graham, Yoshio Yamaoka

Background: Two adjacent homologous genes encoding the outer membrane proteins; adherence-associated lipoprotein ( $\operatorname{alp} A$ and $a l p B$ ) have been suggested to be associated with adhesion. alpAB isogenic mutants were reported not to induce Interleukin-8 (IL-8) from epithelial cells, but subsequent failed to confirm that finding. AlpAB were reported to be involved in colonization of the guinea pig; however the studies using other animals had never studied. Methods: We used $3 \mathrm{H}$. pylori cag pathogenicity island-positive strain (TN2GF4, JK51 and ATCC43504) and their precise whole alpA-alpB deleted mutants (alpAB KO). H. pylori were co-cultured with gastric epithelial cells (AGS, MKN28 or MKN45 cells). Adherence was evaluated by flow cytometry and IL-8 levels by ELISA. Binding of nuclear proteins to transcription factors was assessed by electrophoretic mobility shift assays and protein/DNA array. Colonization ability was examined in C57BL/6 mice after 8 weeks. Results: alpAB KO significantly decreased adherence (64-78\% reduction) irrespective of cell type or strain. However, the effect of alp $A B \mathrm{KO}$ on IL-8 induction from gastric epithelial cells was strain-dependent (e.g., a 52-91\% reduction compared to the parental strains with strains TN2GF4 and JK51 but no reduction with ATCC43504). The effect of AlpAB in activation of transcription factors located in IL-8 promoter (e.g., NF- $\mathrm{\kappa B}, \mathrm{AP}-1$ ) mirrored its effects on IL-8 induction. In contrast, the levels of the transcription factor cAMP response element-binding protein (CREB) were decreased with all alpAB KO strains. High IL-8 producing alpAB KO infected mice equally well as their parental strain. In contrast, low IL- 8 producing alpAB KO did not infect mice whereas the parental strain TN2GF4 did. Conclusion: Overall, our results confirm that $A l p A B$ is involved in adherence of $H$. pylori to gastric epithelial cells. AlpAB's effect on IL-8 induction was strain-dependent providing an explanation for prior conflicting results. The role of AlpAB as a colonization factor was independent of its effect on adherence but was related to the ability to induce IL-8. AlpAB appears to pay a complex role in $H$. pylori pathogenesis only part of which is related to a role in adherence. Supported in part by the Gulf Coast Digestive Disease Center. 
T1573

Adherence, Internalization and Persistence of Helicobacter Pylori in Human Hepatocytes

Kyoko Ito, Yoshio Yamaoka, Hala El-Zimaity, Hiroyoshi Ota, David Graham

Background: Helicobacter sp. including $H$. pylori have been identified in hepatocellular carcinoma and other liver diseases suggesting a possible association between Helicobacter infection and human liver disease. Aim: To explore the mechanisms of internalization and persistence of Helicobacter pylori in hepatocytes. Methods: H. pylori NCTCl1637 (CagA+, VacA+) or 401C (CagA-, VacA-) were co-cultured with Huh7 cells, a human hepatocellular carcinoma cell line. H. pylori adherence, internalization, and persistence were assessed by quantitative bacterial culture and gentamicin invasion assay as well as by histology using silver stains and electron microscopy. The mechanism for internalization was investigated using anti- $\beta 1$-integrin antibodies, chloroquine and cytochalasin $D$ to assess the requirements for attachment to $\beta 1$-integrin, receptor-mediated internalization and actin polymerization, respectively. Results: 1 hour after inoculation of 10(6) (to the 6th) CFU H. pylori, $5.2 \pm 1$ $\mathrm{X} 10$ (5) CFU (50\% of the inoculum) adhered to hepatocytes ( 7 CFU/cell) and $4.4 \pm 0.4$ X 10(3) CFU (1 CFU/ 10 cells) had invaded into the cells. By 6 hours the number of adherent $H$. pylori had increased to $8.3 \pm 0.5 \times 10(5) \mathrm{CFU}(\mathrm{P}<0.05)$ whereas as the number of intracellular $H$. pylori was essentially unchanged. After 48 hours the number of adherent and internalized $H$. pylori decreased slightly $(1.5 \pm 0.2 \times 10$ (5) CFU were adherent and $2.0 \pm 0.9 \times 10(3) \mathrm{CFU}$ were internalized $)(\mathrm{P}<0.05)$. Adherence and internalization were independent of the presence of CagA or VacA. Long term internalization assays revealed that H. pylori persisted in hepatocytes (confirmed by electron microscopy after 5 passages) for at least 13 passages ( 2 months). Cytochalasin D, chloroquine and anti- $\beta 1$-integrin antibody inhibited internalization of CagA/VacA-positive H. pylori. Internalization of CagA/ VacA-negative H. pylori was not inhibited by chloroquine, but was inhibited by cytochalasin $\mathrm{D}$ and anti- $\beta 1$-integrin antibody $(\mathrm{P}<0.05)$. Conclusion: $H$. pylori both adhered to and invaded hepatocytes. Further, H. pylori persisted within hepatocytes during subculture. Adherence and internalization were independent of $H$. pylori virulent factors. $\beta 1$-integrin is likely a receptor involved in internalization of $H$. pylori into hepatocytes and actin polymerization was required for internalization of H. pylori. CagA/VacA-positive strains appeared to utilize receptor mediated endocytosis. This is the first report of the adherence and internalization of Helicobacter into hepatocytes as well as the long-term persistence of Helicobacter inside human cells. Supported in part by the Gulf Coast Digestive Disease Center

\section{$\mathrm{T} 1574$}

Production of Reactive Oxygen Species in Peripheral Blood Is Increased in Individuals with Helicobacter Pylori Infection and Decreased After Its Eradication

Masayuki Mashimo, Manabu Nishikawa, Kazuhide Higuchi, E.J. Sasaki, Masatsugu Shiba, Kazunari Tominaga, Toshio Watanabe, Yasuhiro Fujiwara, Tetsuo Arakawa, Masayasu Inoue

Background: Helicobacter pylori (H. pylori) infection causes various diseases in the gastrointestinal tract. Some reports have already been reported that reactive oxygen species (ROS) production increased in local gastric mucosa. Recent studies have suggested that $H$. pylori infection may also cause other systemic diseases, including hematological disorders, skin and cardiovascular injury, by unknown mechanisms. We hypothesized that ROS contribute to the etiology of these systemic diseases other than gastrointestinal disorders in H. pylori infection. Methods: Production of ROS was determined in peripheral blood samples from 86 patients $(34 \mathrm{H}$. pylori-negative and $52 \mathrm{H}$. pylori-positive subjects) using a highly sensitive chemiluminescence probe [8-amino-5-chloro-7-phenylpyrido(3,4-d) pyridazine-1 and 4 $(2 \mathrm{H}, 3 \mathrm{H})$ dione] (L-012). Results: ROS production was significantly higher in individuals with $H$. pylori infection than in those without such infection (non-H. pylori-infected: 1.76 $\pm 1.26 \mathrm{kcpm} ; \mathrm{H}$. pylori-infected: $3.98 \pm 3.21 \mathrm{kcpm} ; \mathrm{P}<0.0005)$. Enhanced production of ROS was decreased significantly after eradication of $H$. pylori (Paired t-test: $\mathrm{P}=0.012$ ). No correlation was found between the extent of ROS production and sex, age, smoking status, alcohol ingestion, use of medications, or serum level of C-reactive protein. Conclusion: These findings suggest the possibility of involvement of enhanced ROS production in circulating blood in the etiology of various systemic diseases in patients with $\mathrm{H}$. pylori infection. Chemiluminescence analysis of blood samples using L-012 might permit evaluation of risk of systemic diseases in patients with $H$. pylori infection.

\section{$\mathrm{T} 1575$}

Caga Positive Strains of $H$. Pylori in Patients with Stable and Unstable Angina Pectoris

Francesco Franceschi, Antonio Gasbarrini, Giampaolo Niccoli, Alfonso Baldi, Marcello Candelli, Florinda Feroce, Maria Assunta Zocco, Micaela Conte, Davide Roccarina, Giovanni Gasbarrini, Filippo Crea, Nicolo Gentiloni Silveri

Background: Previous studies have reported an epidemiological association between CagApositive strains of $H$. pylori and ischemic heart disease (IHD). More recent studies, moreover, have shown that antibodies anti-CagA recognize antigens of both normal and atherosclerotic blood vessels, such as umbilical cord or atherosclerotic tibial arteries. In order to verify those findings we have designed a study aimed at determining the prevalence of CagApositive strains and the antibody titre in patients with stable (SA) and unstable angina pectoris (UA) and to verify whether anti-CagA antibodies cross-react with antigens of coronary atherosclerotic plaques collected from the same patients. Methods: 38 (30 males, mean age $64 \pm 11$ years) patients with angiographically documented UA, 25 patients with angiographically documented SA (21 males, mean age $62 \pm 10$ years) and 50 healthy volunteers (38 males, mean age $62 \pm 10$ years) were enrolled. The prevalence of $H$. pylori infection and CagA-positive strains and the antibody titres were evaluated in all subjects through ELISA (Radim, Italy). Fresh fragments of atherosclerotic plaques were obtained from all patients through directional coronary atherectomy, and prepared for immunohistochemistry using monoclonal antibodies anti-CagA (Austral Biologicals, USA). Results: Prevalence of H. pylori infection was significantly higher in patients with SA and UA compared to controls (60\% in SA, $61 \%$ in UA vs $38 \%$ of controls; $p<0.04$ ). Prevalence of CagA-positive strains was significantly higher in patients with SA and UA compared to controls (44\% in SA, 50\% in $\mathrm{UA}$ and $18 \%$ in controls; $\mathrm{p}<0.001$ ). Moreover, the titre of anti-CagA antibodies was significantly higher in patients with UA compared to those with SA $(161 \pm 120 \mathrm{RU} / \mathrm{ml}$ vs $78.7 \pm 63.1$ $\mathrm{RU} / \mathrm{ml} ; \mathrm{p}<0.03)$. Interestingly, anti-CagA antibodies recognized antigens localized in the cytoplasm of fibroblasts-like cells as well as lymphocyte-like cells inside atherosclerotic plaques. Conclusions: Anti-CagA antibody titre is significantly higher in patients with UA compared to those with SA. Interestingly, antibodies anti-CagA specifically recognize antigens localized inside the atherosclerotic plaques of the same patients. The binding of anti-CagA antibodies to those antigens could influence the progression of atherosclerosis. Antibody antiCagA titre may predict the destabilization of atherosclerotic lesions, in IHD infected patients.

\section{T1576}

Genotyping of the Cytotoxin Associated Gene Pathogenicity Island from Helicobacter Pylori Isolates in Alaska

Karen Miernyk, Michael Bruce, Brian McMahon, Thomas Hennessey, Frank Sacco, Gerry Sahagun, Paul Davis, Patrick Martinez, Michael Swenson, David Powers, Bane French, Helen Peters, Debra Hurlburt, Alan Parkinson

Introduction: Several studies have shown a relationship between the severity of gastric disease and the presence of the cag pathogenicity island (PAI). Presence or absence of the cag PAI tends to differ by geographic region. Additionally, in strains with the cag PAI, there are distinct differences in the 3' region of the cagA gene when comparing Asian strains with western strains. We studied Helicobacter pylori isolated from persons enrolled in a reinfection study to determine how common cag positive H. pylori strains are in Alaska. Methods: We enrolled American Indian (AI) and Alaska Native (AN) persons from three rural clinics in western Alaska and one urban medical center in southcentral Alaska and non-Native persons from two private urban clinics in southcentral Alaska. Study participants had an EGD and H. pylori was cultured from their tissue biopsy. We used real time PCR to identify the strain (either Asian or western) of cagA with primers amplifying portions of the cagA 3' repeat region. We used conventional PCR to identify the cag negative strains by amplifying the cag PAI empty site. Strains that could not be identified with either the strain typing PCR or the cag PAI empty site PCR were run with a second conventional PCR that detects the 5' end of the cagA PAI. Results: H. pylori was cultured and the isolate(s) genotyped from 257 persons. 201 (78\%) persons were infected with a cag positive strain of whom 35 (17\%) were co-infected with a cag negative strain. Fifty-six (22\%) persons were infected with a cag negative strain only. AI/AN persons were more likely to have a cag positive strain of $H$. pylori than non-Native persons ( $82 \%$ vs. $67 \% ; \mathrm{p}=0.01$ ). Of persons infected with a cag positive strain, $175(87 \%)$ were infected with a western strain of the cagA gene, 7 (3\%) with an Asian strain, $5(2 \%)$ with both western and Asian strains, and $14(7 \%)$ with a strain that could not be further identified. Conclusions: A high proportion of $H$. pylori strains isolated from persons residing in western and southcentral Alaska contain the cag PAI. Among cag positive strains, western cagA predominates. Further research is needed to determine how cag positivity relates to clinical symptoms and disease etiology.

\section{$\mathrm{T} 1577$}

Dramatic Effect of Changes in Standards of Living On the Acquisition of Helicobacter Pylori Infection in Childhood: A 10 Years Follow-Up Study in Russia

Hoda M. Malaty, Michael Tkachenko, Zhannat Z. Nurgalieva, Elena L. Blashenkova, Sergey V. Isachenko, David Y. Graham, Lev V. Erman

Background: The prevalence and rate of acquisition of $H$. pylori infection in children from developing countries is higher than in developed countries. This phenomenon has been related to differences in socioeconomic status, sanitation, and household hygiene. Russia is in the process transformation from an underdeveloped to a developed country. Aim: We examined the effect of recent improvement in standards of living on the prevalence of $H$. pylori in Russian children. Methods: We conducted 2 cross-sectional studies among children in St. Petersburg, Russia. The first study was conducted in 1995 and the second was conducted a decade later. H. pylori status was evaluated the same ELISA method for antiH. pylori IgG (HM-CAP). Demographic data were obtained from each individual; socioeconomic class was assessed by the education level of the mother and family income. Results: In 1995 the overall prevalence of $H$. pylori infection was 44\% and decreased to $12 \%$ ten years later. In both studies, the prevalence increased with age. In 1995 the prevalence was $30 \%$ among children younger than 5 years. A decade later the prevalence in the same age group is zero. The age specific patterns of $H$. pylori infection is shown in the table. The crude and the age-adjusted odds ratio risk of infection in children showed an inverse correlation between the mother's education level and $H$. pylori seropositivity $(\mathrm{OR}=1.8, \mathrm{CI}$ $95 \% 1$ to 3.2$),(p=0.05)$. No associations were found between prevalence of $H$. pylori and any factor tested including gender, type of dwelling, income or the number of persons living in the home. Conclusions: Improvements in standards of living in Russia have resulted in a marked reduction in H. pylori transmission. Different rates of acquisition H. pylori form the basis for the differences in prevalence of infection between and among populations. The changes in Russia are a dramatic example of how sensitive H. pylori acquisition is to improvement in standards of living.

table 


\begin{tabular}{|c|c|c|c|c|c|}
\hline & $<5$ years no (\%hp+) & $5-9$ no (\%hp+) & $10-14$ no (\%hp+) & $15-19$ no (\%hp+) & Total no (\%hp+) \\
\hline 1995 & $44(30 \%)$ & $102(39 \%)$ & $94(52 \%)$ & $67(48 \%)$ & $307(44 \%)$ \\
\hline 2005 & $21(0 \%)$ & $96(8 \%)$ & $45(13 \%)$ & $48(25 \%)$ & $210(12 \%)$ \\
\hline
\end{tabular}

\section{T1578}

Fur and Perr Mediate Iron-Responsive Regulation of Peroxide Stress Defense Genes in Helicobacter Hepaticus

Clara Belzer, Ernst J. Kuipers, Bart A. van Schendel, Theo Hoogenboezem, Peter W. Hermans, Arnoud H. van Vliet, Johannes G. Kusters

Introduction: Murine infection with Helicobacter hepaticus is associated with an active cellular immune response accompanied by the production of oxygen radicals. Iron also potentates the formation of reactive oxygen species. Therefore, pathogens are forced to maintain intracellular iron homeostasis and cope with oxidative stresses. The H. hepaticus genome sequence contains genes encoding homologs of bacterial oxidative stress defense proteins, and also homologs of the iron responsive regulatory proteins Fur and PerR, which mediates regulation of peroxide stress defense in several other bacteria. However, to our knowledge the function or of the regulation of expression of oxidative stress defense genes of $H$. hepaticus has not been described to date. Aim: We therefore investigated the expression and regulation of oxidative stress defense systems of H. hepaticus. Methods: H. hepaticus strain ATCC51449 and isogenic fur and perR mutants were grown both under low-iron and high-iron conditions. Gene expression was monitored by Northern hybridization, and protein expression was monitored by SDS-PAGE. Results: Growth of $H$. hepaticus in iron-restricted conditions resulted in altered expression levels of six proteins. Three of these proteins displayed ironrepressed expression, while three other proteins displayed iron-induced expression. Two of the iron-repressed proteins were identified as AhpC $(25 \mathrm{kDa})$ and $\mathrm{KatA}(55 \mathrm{kDa})$. Both proteins are involved in the degradation of peroxide compounds, and are known to contribute to the bacterial oxidative stress defense. Mutation of the fur and perR genes resulted in highlevel, iron-independent, expression of both AhpC and KatA. Conclusion: In H. hepaticus, iron metabolism and oxidative stress defense are intimately connected via the Fur and PerR regulatory proteins. This regulatory pattern resembles that seen in the enteric pathogen $C$. jejuni, but contrasts with the regulatory patterns observed in the human gastric pathogen H. pylori, where Fur regulates superoxide stress defense. Therefore, iron-dependent regulation of peroxide stress defense may be an adaptation advantageous for enteric colonization.

\section{$\mathrm{T} 1579$}

Helicobacter Pylori Antigens Operating At Early Infection

Hee-Shang Youn, Eun-A Kim, Jin-Su Jun, Ji-Hoe Park, Min-Ji Koo, Jae-Hee Kim, Ji-Hyun Seo, Gyung-Hyuk Ko, Hyung-Lyun Kang, Seung-Chul Baik, Woo-Kon Lee, Myung-Je

Cho, Kwang-Ho Rhee

OBJECTIVE: To identify the operating antigens of Helicobacter pylori during early infection, H. pylori proteomics and western blot analysis with sera of the H. pylori infected young infants were applied. METHODS: Two-dimensional large and small gel electrophoresis was performed with the H. pylori strain 51. Two-dimensional IgG, IgA, IgM immunoblotting using small gels were performed with the sera which were collected at the Gyeongsang National University Hospital from the infants who were confirmed $H$. pylori infected by urease test, histopathologic examination and pre-embedding immunoelectron microscopy. Spots with strong IgM and weak IgA immuno reactivities were considered early infection markers and those spots were compared to those of 2D large gel. Corresponding spots were applied to MALDI-TOF-MS. Obtained peptide finger printings were applied to NCBI database. RESULTS: Eight infant patients were confirmed to be $H$. pylori infected. One of 8 infants showed distinctive 14 spots on IgM 2D immunoblot without IgA immunoreactivities. These 14 spots were bounded on the gels by the pI range 5.0-6.5 and molecular weight range 30-80 kDA. Total 10 proteins were identified. These were ATP-dependent protease binding subunit, Elongation factor G, Putative hydantoin utilization, UreB subunit, Methyl-accepting chemotaxis protein, Flagella hook protein, Pyruvate ferredoxin oxidoreductase, Elongation factor TS, Putative proline peptidase, Fructose-1,6-bisphosphate aldolase. CONCLUSION: A total of 10 proteins operating early during acute $H$. pylori infection were identified by proteomics. These results might contribute to find the serological markers for the early $H$. pylori infection in infants.

\section{$\mathrm{T} 1580$}

Characterisation of the Proteome of Helicobacter Pylori Outer Membrane Vesicles

Erica M. Mullaney, Catherine H. Botting, Henry J. Windle, Ana M. Terres, Dermot P. Kelleher

INTRODUCTION: The effects of $H$. pylori infection may be mediated by direct interaction between the intact organism and the epithelium, and also, between released bacterial elements know as outer membrane vesicles (OMVs) and the gastric epithelium. H. pylori OMVs are small circular structures (Keenan et al, 2000) with an intact outer membrane that are constitutively shed from the surface of the organism. The mechanisms of OMV action are not clear but they may function as a vehicle for the delivery of virulence factors to the gastric mucosa. It is presumed that contact between OMV and host cells is necessary. AIM: To map the protein composition of $\mathrm{H}$. pylori OMVs in an attempt to gain a better understanding of their action in vivo. MATERIALS\&METHODS: OMVs were harvested from $H$. pylori by several rounds of ultracentrifugation. OMV samples were run on a $12.5 \% 1 \mathrm{D}$ mini gel, transferred to a PVDF membrane and blotted for H. pylori proteins. OMVs electrophoretically separated on a 1D mini gel were analysed by LC-MS/MS sequencing to determine the identity of the protein complement of the OMVs. RESULTS: Electron Microscopy (EM) confirmed the absence of whole cells and flagella in the OMV preparation and confirmatory Western blot analysis detected Helicobacter proteins in the OMV samples. The samples of OMVs separated on a ID SDS-PAGE gel were Coomassie and silver stained. Sequence analysis following mass spectrometry indicates that $H$. pylori OMV proteins contain many known outer membrane proteins including OMP 3, 5, 8, 11, 14, 15 and 20. Analysis has also revealed the presence of several virulence factors, namely CagA, VacA. NapA and urease. Interestingly, analysis also identified proteins from the $\mathrm{ABC}$ transporter system which is involved in the production of a catalytically active urease. Many iron regulated outer membrane proteins were also identified. CONCLUSIONS: This proteomic analysis of $H$. pylor OMV has demonstrated the presence of multiple OMP, including adhesions, in addition to recognised virulence factors. Given that OMV are constitutively shed by H. pylori in vivo, the possibility that OMVs are involved in OMV - epithelial cell interactions, leading to pathogenesis, both at the site of infection and at remote sites (e.g. duodenum) must now be considered.

\section{$\mathrm{T} 1581$}

Two-Dimensional Blue Native / SDS Gel Electrophoresis of Multiprotein Complexes from Helicobacter Pylori

Slovenie Pyndiah, Jean Paul Lasserre, Stephane Claverol, Armelle Menard, Valerie Prouzet-Mauleon, Francis Megraud, Frank Zerbib, Marc Bonneu

The study of protein interactions constitutes an important domain to understand the physiology and pathogenesis of microorganisms. A proteomic approach initially described for eucaryotic cells, including protein separation by two dimensional blue native/sodium dodecy sulfate polyacrylamide gel electrophoresis (2D BN/SDS-PAGE) and protein identification by mass spectrometry, was applied to strain $\mathrm{J} 99$ of the gastric pathogen Helicobacter pylori. It was possible to identify 131 proteins grouped in 91 multiprotein complexes: 66 from the cytoplasm, 22 from the membrane and 3 from both, including 43 complexes never reported before, as well as 36 multi-homooligomeric complexes. Beside complexes involved in $H$. pylori physiology, this method allowed to describe interactions involving known pathogenic factors such as urease with GroEL, or IlvC, and CagA with BabA, or GyrA, as well as to provide an insight on the partners of the adhesins. The 2D BN/SDS-PAGE combined with mass spectrometry could be applied to the study of the differences in complexes isolated in various situations and also to the study of the interactions between bacterial and eucaryotic cell proteins.

\section{$\mathrm{T} 1582$}

Analysis of the SHP-2 Binding Site of Helicobacter Pylori Caga Protein in Korean

Jun-Won Chung, Gin Hyug Lee, Hyung Joon Lee, Ja Young Kim, Kwi Sook Choi, Kee Don Choi, Hwoon-Yong Jung, Weon-Seon Hong, Jin-Ho Kim, Jin-Yong Jeong

Background/Aims: The incidence of gastric cancer is very high in Korea. Recently the pathological actions of CagA of H. pylori on gastric epithelial cells have been reported. There are two major CagA subtypes according to the amino acid sequence in the $3^{\prime}$ region of CagA; i) the East Asian type (A-B-D of EPIYA motifs) and ii) the Western type (A-B-C of EPIYA motifs). Repeated EPIYA motifs in the 3' region of CagA are involved in the interaction with SHP-2. The East Asian type conferred stronger SHP-2 binding activity than the Western type of CagA. Here we analyzed the amino acid sequences of the SHP-2 binding site of cagA gene in $H$. pylori, and investigated whether there is any relationship between the diversities of cagA and the disease outcome in Korea. We also examined the relationship between cagA and vacA alleles. Methods: The 62 clinical isolates of $H$. pylori from 14 patients with non-cardiac gastric cancer and 48 patients with chronic gastritis were studied. DNA sequencing of CagA SHP-2 binding site was performed by use of the dideoxynucleotide chain termination method. Then amino acid sequences were deduced from the results of DNA sequencing. To confirm vacA genotype, parts of the vacA s- and m-regions were amplified using appropriate primers. Results: Most of Korean H. pylori strains showed A-BD motifs (the East Asian type), and only one strain showed A-B-B-D motifs, which is also the East Asian type. All strains contained $\mathrm{sl}$ in the signal sequence region and $\mathrm{ml}$ in the middle region of vacA. The predominant vacA genotype in Korea was slc/mlb (43/62 $69 \%$ ). We also demonstrated that the predominant East Asia type CagA-positive strains had vacA slc/mlb genotype in Korea. Conclusions: In Korea, the incidence of atrophic gastritis and gastric cancer is significantly high compared with Western countries. The high frequency of the East Asian type CagA among Korean H. pylori strains would be involved in increasing the risk of gastric cancer in Korean populations.

\section{T1583}

The Cause for the Birth-Cohort Phenomenon of Peptic Ulcer Disease Amnon Sonnenberg

Background: The long-term time trends of gastric and duodenal ulcer have been shaped by a birth-cohort phenomenon with a rise in susceptibility to develop peptic ulcer among generations born during the 19th century and a subsequent decline among generations born during the 20th century. Infection of the upper gastrointestinal tract with $H$. pylori is the primary etiologic mechanism precipitating the occurrence of peptic ulcer. Since H. pylori infection was endemic among all human populations for millennia until the turn of the 19 th century, the cause of the rising ulcer epidemic during the second half of the 19th century has remained an enigma. The aim of the present study is to present a mathematical model of $H$. pylori epidemiology that explains the peculiar long-term trends of peptic ulcer disease. Methods: The epidemiology model is based on two simple and straightforward assumptions. First, the infection rate fell in the general population between 1800 and 2000 Second, gastric ulcer was caused by $H$. pylori infection contracted between the ages 5 and 15 years and duodenal ulcer by infection contracted after the age of 15 years. The timedependent fall of $H$. pylori infection is modeled by a logistic equation. The age-dependent rise in the fraction of subjects infected by $H$. pylori at increasingly older age is modeled by an exponential equation. The actual time trends of ulcer mortality from England \& Wales and their birth-cohort patterns are compared with the predictions of the mathematical model. 
Results: The birth-cohort phenomenon of peptic ulcer could be explained by the interaction of two opposing time trends, namely, declining infection rate and shifting of $H$. pylor acquisition towards older age. The superimposition of a declining and a rising trend resulted in a bell-shaped curve of ulcer occurrence among consecutive birth-cohorts. Similarly to the real data, the modeled cohort pattern of gastric ulcer preceded that of duodenal ulcer by 20 years. The time lag between the two curves of gastric and duodenal could be shortened by changing time intervals of infection. The time constants of the mode shifted the peak of ulcer occurrence along the time axis and determined the slopes of its rise and fall. Overall, the outcome of the model remained robust to most changes underlying its assumptions, and a very good resemblance was reached between the model and the actual epidemiologic data. Conclusion: The unifying concept of a receding $H$. pylori infection accompanied by a simultaneous shift in its age of acquisition secondary to increasing standards of hygiene suffices to explain the historic birth-cohort phenomenon of peptic ulcer disease.

\section{T1584}

Cag-a Positive H. Pylori Strains Are Highly Prevalent in Ischemic Cerebrovascular Disease: A Primary Care Multicenter Study

Rudy De Bastiani, Maurizio Gabrielli, Enzo Ubaldi, Edoardo Benedetto, Guido Sanna, Carmelo Cottone, Maria Assunta Zocco, Nathalie Saulnier, Marcello Candelli, Giovann Gasbarrini, Antonio Gasbarrin

Background. Previous studies suggested an association between ischemic cerebrovascular disease and H. pylori infection, in particular CagA-positive strains. Available data are conrasting and come from monocentric studies from referral tertiary centers. Moreover they often assessed $H$. pylori infection by serology, that is less accurate and does not distinguish between previous contact or active infection. Aim of the present study was to assess the prevalence of active H. pylori infection and its CagA-positive strains in patients with ischemic cerebrovascular infection with respect to controls without evidence of atherosclerotic-related diseases. Methods. A total of 106 consecutive patients (age 76,4 4 years; males 50\%) with well documented history of ischemic cerebrovascular disease and 97 sex-age (age 76,4 48 years; males $45 \%$ ) and social background-matched controls without relevant vascular diseases. Subjects come from 5 different regions of Italy ( 1 in the North, 1 in the Center and 3 in the South). Data concerning CT scanning and/or brain magnetic resonance imaging, duplex ultrasonography of the extracranial carotid arteries, history of atrial fibrillation were recorded in all patients. Risk factors for ischemic cerebrovascular disease (familiar history, arterial hypertension, smoke, diabetes mellitus, hypercholesterolemia, hypertrigliceridemia, obesity) were assessed in all subjects. Active H. pylori infection was assessed by 13C-urea breath test (Altana, Milano, Italy). In infected subjects, a serological assay for specific IgG against CagA was also performed (Radim, Pomezia, Italy). Results. The prevalence of active H. pylori infection higher in cases (63\%) with respect to controls $(54 \%)$, however without reaching statistical significance $(\mathrm{p}=0.2, \mathrm{OR} 1.21,95 \% \mathrm{CI} 0.85-2.61)$. On the other hand, a significant association was found between patients and controls as concerning Cag-A positivity ( $41.5 \%$ versus $17.5 \% ; \mathrm{p}<0.001, \mathrm{OR} 1.65,95 \% \mathrm{CI} 1.74-6.40)$. Conclusions. This is the first study assessing the prevalence of active $H$. pylori infection and CagA-positive strains in the setting of the general population. Our findings suggest that CagA-positive more cytotoxic strains of the bacterium are significantly associated to ischemic stroke. Future studies should aim to assess the effects of selected eradication of CagA-positive strains in patients with ischemic stroke to verify the causality of this epidemiological association.

\section{T1585}

H. Pylori Protease Activates Gastric Epithelial Cells to Produce IL-8 Through Protease-Activated Receptor 2

Norimasa Yoshida, Hirokazu Kajikawa, Kazuhiro Katada, Fumihiro Hirayama, Osamu Handa, Tomohisa Takagi, Satoshi Kokura, Hiroshi Ichikawa, Yuji Naito, Toshikazu Yoshikawa, Takeshi Okanoue

Background and Aims: IL-8 production by gastric epithelial cells infected with $H$. pylori has been implicated in the pathogenesis of gastric inflammation, however, its mechanism has not been fully understood. Recently, it has been demonstrated that proteases such as trypsin and thrombin activate many kinds of epithelial cells and endothelial cells to produce inflammatory mediators through protease-activated receptor (PAR). In the present study, we investigated whether PAR2 is involved in the IL-8 production by H. pylori-infected gastric epithelial cells. Materials and Methods: Expression of PAR-2 in human gastric epithelial cells, MKN45 cells, was assessed by reverse-transcriptase polymerase chain reaction (RT-PCR) method, western blotting analysis and immunocytochemistry. MKN-45 cells were stimulated with trypsin, agonist peptide (SLIGKV amide) for PAR-2 or H. pylori organism and expressions of IL-8 mRNA and proteins were measured by RT-PCR and enzyme-linked immunosorbent assay (ELISA). In addition, the effects of nafamostat mesilate and camostat msilate, serine protease inhibitors, on IL- 8 production from activated cells were determined. Finally, $H$. pylori-induced IL-8 production was estimated using antibody to PAR-2 and cells transfected with si RNA for PAR2. Results: PAR-2 mRNA and protein were constitutively expressed on unstimulated MKN-45 cells. Treatment of cells with $H$. pylori resulted in mild increase in PAR-2 expression. In addition, trypsin, agonist peptide for PAR-2 or H. pylori induced IL8 production from MKN- 45 cells in a dose- and time-dependent manner. Nafamostat mesilate and camostat mesilate protected against trypsin- and H. pylori-, but not agonist peptide-, mediated cytokine production from MKN-45 cells. Finally, H. pylori-induced IL-8 production was inhibited by antibody to PAR-2 and in PAR-2 deficient cells treated with siRNA Conclusions: These results indicate that $H$. pylori-derived protease activates gastric epithelial cells to produce inflammatory cytokines through PAR-2, suggesting an important role for PAR2 in the modulation of gastric inflammation associated with $\mathrm{H}$. pylori.

\section{T1586}

EGFR Inhibition Improves Helicobacter Pylori Induced Pathology and Epithelial Cellular Kinetics in Vivo

Aht Jeremy, K Danjo, Mf Dixon, K Bransfield, Pa Robinson, Je Crabtree

Introduction: H. pylori has been shown to transactivate the EGF receptor (EGFR) in gastric epithelial cells. H. pylori activation of the EGFR signalling pathway may be relevant to the epithelial hyperproliferation and increased risk of gastric carcinogenesis associated with infection. The aim of this study was to examine whether inhibition of the EGFR with the specific inhibitor EKB-569 would alter H. pylori induced pathology in the Mongolian gerbil model. Methods: Male Mongolian gerbils were infected with H. pylori (SS1 strain). 6 weeks post-infection, infected and uninfected controls were fed on either, control, or EKB-569 $(10 \mathrm{mg} / \mathrm{kg} / \mathrm{gerbil} / \mathrm{day})$ supplemented food. Gerbils were killed at 38 weeks post-infection. Gastric pathology was assessed and epithelial proliferation and apoptosis quantified respectively by anti-BrdU and activated caspase-3 histochemistry. Results: No difference in antral pathology was observed in treated and untreated $H$. pylori infected gerbils. EKB-569 treatment in $H$. pylori infected gerbils resulted in a significant reduction in corpus atrophy $(p<0.03)$ and mucous metaplasia $(\mathrm{p}<0.05)$ but chronic inflammation in the corpus was not significantly different from untreated gerbils. There was significant $(p<0.05)$ reduction in submucosal herniations in EKB-569 treated gerbils. Increased epithelial proliferation $(p<0.001)$ was observed in the antrum and corpus of both infected groups, whilst apoptosis was only significantly increased $(\mathrm{p}<0.001)$ in the EKB-treated group compared to uninfected control groups. There was a marked increase in apoptotic subepithelial mononuclear cells in the EKB-569 treated infected gerbils. In infected gerbils, the proliferation/apoptosis ratio in the untreated group was significantly greater $(\mathrm{p}<0.02)$ in both the antrum and corpus than in the EKB-569 treated group, where the ratio was comparable to uninfected controls. Conclusion: EGFR inhibition reduces H. pylori induced corpus pathology in vivo and promotes apoptosis in both epithelial and subepithelial mononuclear cells. This study was funded by Yorkshire Cancer Research

\section{T1587}

Restoration of RUNX3 Expression in H.pylori-Colonized Spasmolytic Polypeptide-Expressing Metaplasia (SPEM) After H.pylori Eradication Hidekazu Suzuki, Yuriko Minegishi, Kosei Ito, Toshihiro Nishizawa, Tatsuhiro Masaoka, Eisuke Iwasaki, Yoshiaki Ito, Nicholas A. Wright, Toshifumi Hibi

Chronic H. pylori infection induces spasmolytic polypeptide-expressing metaplasia (SPEM), which is positive for trefoil factor 2 (TFF2) (Gastroenterology 114:675, 1998). Recently, SPEM was reported to be a precancerous lesion, which induces gastric cancer formation with the recruitment of bone marrow-derived cells (Science 306:1568, 2004). RUNX3 (runt domain transcription factor 3), a tumor-suppressor gene involved in gastric carcinogenesis, has also been shown to be significantly downregulated in intestinal metaplasia (Cell 109:113, 2002). However, no studies have demonstrated RUNX3 expression in $H$. pylori-associated SPEM lesions or alterations after $H$. pylori eradication. The present study investigates RUNX3 expression in H. pylori-colonized SPEM lesions in Mongolian gerbils before and after bacterial eradication. Methods. Male Mongolian gerbils were inoculated with $H$. pylori $\left(10^{8} \mathrm{CFUs} /\right.$ $\mathrm{ml}$, ATCC43504) or with culture media alone and examined 12 or 24 weeks post-infection. Other groups of $\mathrm{H}$. pylori-inoculated gerbils in which infection was eradicated after 12 or 24 weeks of colonization, were examined 10 weeks after the eradication. RUNX3 mRNA expression was determined by quantitative RT-PCR and in-situ hybridization. RUNX3 and TFF2 protein expression was determined by immunohistochemistry. Results. Positive immunoreactivity for RUNX3 was observed in non-H. pylori-infected gerbils, predominantly in chief cells and to some extent in mucous neck cells and surface epithelial cells of the gastric fundic glands. RUNX3 mRNA expression was also detected in non-H. pylori-infected mice, predominantly in the nuclei of the chief and mucous neck cells. In gerbils examined 12 weeks after $H$. pylori inoculation, although the pyloric side of the gastric fundus showed the absence of RUNX3 immunoreactivity and mRNA expression and the presence of TFF2positive SPEM formation, RUNX3 expression was almost completely restored after eradication of H. pylori. In the cohort examined 24 weeks after H.pylori inoculation, SPEM was encountered from the pylorus to the proximal end of gastric fundus, with significant reduction of RUNX3 expression in these regions. In this cohort, RUNX3 expression was only partially restored in the proximal fundic mucosa after the eradication. Conclusions. Reduction of RUNX3 expression in regions showing SPEM in H. pylori-colonized stomach became more pronounced with increasing duration of colonization. Although early eradication almost completely restored the RUNX3 expression to normal levels with resolution of the SPEM lesions, longer-term colonization of H.pylori reduced the mucosal reversibility in terms of RUNX3 expression and SPEM formation

\section{T1588}

Persistence of Hypermethylation of Cell Cycle and DNA Repair Genes in Gastric Mucosa After $H$. Pylori Eradication Yuan Yao, Gabriel Calilao, Dong Il Park, Jae J. Kim, David Y. Graham, Antonia R. Sepulveda

Aims: To determine the methylation status of the gastric mucosa during Hp gastritis and the effect of $\mathrm{Hp}$ eradication on methylation in the mucosa, and to determine whether $\mathrm{Hp}$ organisms can induce $\mathrm{CpG}$ island methylation in epithelial cells in a co-culture system. Methods: Methylation specific PCR (MSP) for CpG islands in the promoters of MLHL, MGMT and pl6INK4a was performed with DNA from antral biopsies of 37 patients with Hp gastritis and at 6-8 weeks after Hp eradication (mean age $=46$ years, 9 women and 28 men) and 15 Hp-negative controls. Bisulfite sequence analysis was performed in MSP-positive cases Gastritis grade was determined with the updated Houston scale. Hp status was confirmed histologically and by PCR for the Hp $16 \mathrm{~S}$ rRNA and CagA gene. The methylation status of gastric AGS cells after co-culture with Hp (strain 43504) was determined by MSP in cells co-cultured with Hp bacteria or Hp water extracts. Results: P16 and MGMT CpG islands were more frequently methylated in Hp-positive gastritis as compared to control Hp-negative 
tissues. In contrast, MLHl was not (Table). The number of samples demonstrating MLHl and pl6 CpG island methylation was not different in pre- and after Hp eradication samples, while MGMT methylation was slightly reduced in the post-eradication group (Table). CagA status was not associated with differences in frequency of $\mathrm{CpG}$ methylation. The gastritis grades were not significantly different in methylated vs. unmethylated mucosal samples. Co-cultures of $\mathrm{Hp}$ or Hp water extracts and AGS cells, showed that Hp was able to induce methylation of the MLHI and MGMT promoter, but not of pl6. Conclusions: H. pylori gastritis leads to increased $\mathrm{CpG}$ island methylation of DNA repair and cell cycle genes. In vitro studies suggest that $\mathrm{Hp}$ bacteria may directly induce $\mathrm{CpG}$ island methylation. The increased $\mathrm{CpG}$ island methylation induced by $\mathrm{Hp}$ infection in the gastric mucosa persists after Hp eradication, suggesting that it is induced in progenitor cells with replicative potential.

\begin{tabular}{|c|c|c|c|}
\hline Samples & MLH1-methyl & MGMT-methyl & P16-methyl \\
\hline Before Eradication (B) & $10 / 37(27 \%)^{*}$ & $25 / 36(69 \%)^{* *}$ & $24 / 37(65 \%)^{* * *}$ \\
\hline After eradication (A) & $12 / 37(32 \%)^{*}$ & $18 / 36(50 \%)^{* *}$ & $26 / 37(70 \%)^{* * *}$ \\
\hline Control Hp-negative & $5 / 15(33 \%)$ & $4 / 15(27 \%)$ & $3 / 15(20 \%)$ \\
\hline Methylation (B vs. A) & $* \mathrm{P}=0.618$ & $* * \mathrm{P}=0.091$ & $* * * \mathrm{P}=0.619$ \\
\hline P value (B vs. control) & $\mathrm{P}=0.651$ & $\mathrm{P}=0.004$ & $\mathrm{P}=0.003$ \\
\hline
\end{tabular}

\section{T1589}

Effect of Helicobacter Pylori On Leptin and Ghrelin Levels in Plasma and Stomach

Jatin Roper, Fritz Francois, Michelle Mourad, Zhiheng Pei, Asalia Z. Olivares, Vivek M. Shinde, Chi-Hong Tseng, Guillermo I. Perez-Perez, Martin J. Blaser

Background : Helicobacter pylori (HP) colonization may affect production of ghrelin and leptin, possibly via the HP-induced inflammatory cytokine IL-1 $\beta$. Our aims were to evaluate circulating and gastric levels of leptin and ghrelin according to HP status and to correlate these levels with gastric IL-1 $\beta$. Methods: 217 patients referred for endoscopy were prospectively enrolled. BMI was measured, fasting serum collected, and antral / fundic biopsies obtained. Patients were categorized as HP+ if $\geq 2$ tests (urease, histology, culture, HP serology, and cagA serology) were positive, and HP- if all tests were negative. Levels of ghrelin, leptin and IL-1 $\beta$ in plasma and gastric biopsy samples (pg/mg protein) were determined by specific ELISAs, and results compared using non-parametric tests (Mann-Whitney U Test, Spearman Rank). Results: 64 patients were HP- and 100 were HP+. 53 patients of indeterminant status were not included in these analyses. Age and sex did not differ significantly between groups. Mean BMI was lower in the HP+ than HP- group ( $27.5 \pm 4.7$ vs. $29.0 \pm 5.2$; $\mathrm{p}=0.05)$. BMI was positively correlated with circulating, antral, and fundic leptin levels ( $R=$ $0.46, \mathrm{p}<0.0001 ; \mathrm{R}=0.19, \mathrm{p}=0.02 ; \mathrm{R}=0.29, \mathrm{p}<0.001$, respectively). After adjusting for BMI, leptin levels were lower in the HP+ group than the HP- group for both circulating (median $2166 \mathrm{pg} / \mathrm{ml}$, IQR (894-4057) vs. $4730 \mathrm{pg} / \mathrm{ml}(1831-9076) ; \mathrm{p}<0.001)$ and antral measurements (median $58 \mathrm{pg} / \mathrm{ml}(25-157)$ vs. $94 \mathrm{pg} / \mathrm{ml}(58-178) ; \mathrm{p}=0.04)$; circulating and gastric ghrelin levels did not significantly differ between HP groups. Regional gastric leptin and ghrelin levels were positively correlated in the HP+ group (antral leptin and antral ghrelin, $\mathrm{R}=0.52, \mathrm{p}=0.0001$; antral leptin and fundic ghrelin, $\mathrm{R}=0.38, \mathrm{p}=0.005$; fundic leptin and fundic ghrelin, $\mathrm{R}=0.47, \mathrm{p}=0.0001$ ), but not in the HP- group. Antral leptin levels were correlated with antral and fundic ILl $-\beta$ levels in the $\mathrm{HP}+$ group $(\mathrm{R}=0.34, \mathrm{p}=0.007 ; \mathrm{R}=0.26$, $\mathrm{p}=0.03$, respectively), but not in the HP- group. Antral ghrelin and ILl- $\beta$ levels were positively correlated in the $\mathrm{HP}+$ group $(\mathrm{R}=0.44, \mathrm{P}=0.002)$, but not in the HP- group. Conclusion: In this population, circulating and regional gastric leptin levels were lower in those who were HP+ than HP- independent of BMI. The positive correlation between gastric leptin and ghrelin in HP+ persons, associated with ILl- $\beta$ levels, suggests that HP colonization and induced inflammation affect gastric production of leptin and ghrelin.

\section{$\mathrm{T} 1590$}

Helicobacter Pylori CagA Induces Mitochondria-Dependent Production of Reactive Oxygen Species in Gastric Epithelial Cell

Osamu Handa, Yuji Naito, Takeshi Ishii, Hisato Tsuboi, Satoko Adachi, Tomohisa Takagi, Satoshi Kokura, Norimasa Yoshida, Hirofumi Matsui, Takeshi Azuma, Hai Ying Fu, Sunao Kawano, Toshikazu Yoshikawa

Background: Helicobacter pylori (H. pylori) has been defined as a definite carcinogen by IARC/ $\mathrm{WHO}$ in 1994. One of the candidate protein by which $\mathrm{H}$. pylori induce carcinogenesis is cytotoxin associated gene product A (CagA) injected into host gastric epithelial cell by type 4 secretion system of $H$. pylori. Although CagA positive $H$. pylori infection has been reported to produce excessive amount of reactive oxygen species (ROS) in human gastric mucosa, the mechanism by which CagA itself induce ROS production in gastric epithelial cell is not clear. Moreover, many in vitro studies have been done by using gastric "cancer" cell line. Aim: In the present study, we investigated the effect of CagA on ROS production by rat "normal" gastric mucosal epithelial cell (RGM-1). Materials and Methods: To this end, we used cagA gene- and a Tet-off system- transfected RGM-1, in which CagA expression could be controlled by tetracycline. CagA expression in the established cell line was detected by western blotting and laser scanning confocal microscopy (LSCM) with CagA specific antibody. ROS production in RGM-1 was assessed by redox sensitive fluorescent dye, RedoxSensor and mitochondria selective fluorescent dye, MitoTracker under ISCM and a fluorometer. Since nuclear factor kappa B (NFKB), a redox sensitive transcription factor, has been shown to play a key role in the pathogenesis of $H$. pylori- associated disease through the production of various cytokines, we also investigated the effect of CagA on NFKB nuclear translocation and subsequent production of cytokine by RGM-1. Results: As a result, we found that expressed CagA, after removal of tetracycline, induced significant ROS production by mitochondria, NFKB translocation into the nucleus and cytokine-induced neutrophil chemoattractant-1 (CINC-1) production by RGM-1. Conclusion: Taken together, CagA itself might have a pro-inflammatory effect on normal gastric epithelial cell through mitochondria- derived ROS-mediated mechanism and thus participate in the formation of vicious circle of inflammation.

\section{T1591}

Close Surveillance Is Necessary to Detect Gastric Neoplastic Lesions After Helicobacter Pylori Eradication in Japanese

Makoto Yonechi, Kazuhiko Ishida, Naotaka Fujita

Background The effect of $H$. pylori eradication on the prevention of gastric cancer development in chronic carriers is still unknown. Also remaining unclear is how often esophagogastroduodenoscopy (EGD) should be done to detect gastric neoplastic lesions in an early stage after successfulH. pylori eradication. The aim of this study was to determine whether frequent procedures of EGD were required or not after $\mathrm{H}$. pylori eradicaton to detect gastric neoplasia Patients and methods $H$. pylori was eradicated in a total of 1465 patients from December 1995 to September 2004. Of those patients, 929 (719 men and 210 women; mean age 55.9 years; range, $18-87$ years) who achieved successful eradication and were kept under surveillance with EGD for not less than 1 year, were included in this study. This group of patients was comprised of 524 cases of gastric ulcer, 233 cases of duodenal ulcer, 61 cases of gastroduodenal ulcer, 36 cases of early gastric carcinoma treated by endoscopic mucosa resection (EMR), 15 cases of gastric polyps, 27 cases of chronic gastritis, 7 cases of acute gastritis and 16 cases of MALT lymphoma. To detect neoplastic lesions in an early stage after eradication, the patients underwent EGD at two months, six months and twelve months after eradication. Thereafter, EGD was performed every 12 months. Results The mean follow-up period was 30 months (2-112) and the mean number of EGD examination was 4.5. During the follow-up, gastric cancer was newly detected in 26 cases (2.8\%). Background diseases in these 26 patients were gastric cancer treated by EMR in $10(38.5 \%)$ and gastric ulcer in 16 (61.5\%). Twenty-four cases $(92.0 \%)$ were intestinal-type carcinoma and the other two were diffuse type. All cancers but one were detected in an early stage. These early stage cancers were completely resected by surgery or EMR. The only patient diagnosed as gastric cancer of diffuse type in an advanced stage had undergone EGD until 24 months after eradication, but had then dropped out from the surveillance. Conclusions The chance of detecting gastric cancer increases after $H$. pylori eradication, probably due to facilitation of recognition of local changes following improvement of mucosal inflammation. As those patients who have had gastric ulcers or early cancer prior to $H$. pylori eradication have a high prevalence of gastric cancer development, such patients should be kept under close surveillance using EGD even after successful $H$. pylori eradication.

\section{T1592}

E-Cadherin Gene Promoter Hypermethylation in H.pylori-Induced Enlarged Fold Gastritis

Tamana Miyazaki, Yoko Murayama, Kenji Watabe, Shuusaku Tsutsui, Tatsuya Kiyohara, Shinji Tamura, Yasuhisa Shinomura, Norio Hayashi

Backgroud and Aim: We have previously reported that the odds ratio for gastric carcinoma and the prevalence of diffuse-type early gastric carcinoma in H.pylori-enlarged-fold gastriti increased with increasing fold width. However, the mechanism for the progression of gastric carcinoma in H pylori-associated enlarged fold gastritis is not clear. Recently, DNA methylation is reported as one of the regulatory mechanism associated with loss of function of tumour suppressor genes. In diffuse-type gastric carcinomas, E-cadherin expression is frequently reduced or lost. It is possible that hypermethylation of E-cadherin promoter is associated with this event. Thus, we investigated E-cadherin methylation in both gastric antral and body mucosa from H.pylori positive patients with enlarged fold gastritis, and compared with those of H.pylori positive and negative patients without enlarged folds Methods: 19 H.pylori-positive patients with enlarged folds, 22 H.pylori-positive and seven H.pylori-negative patients without enlarged folds were involved in the study. Genomic DNA extracted from the gastric antrum and body biopsy samples of all patients were treated with the sodium bisulfite. E-cadherin promoter methylation was studied using quantitative methylation specific polymerase chain reaction (PCR) and the methylation index (\%) was defined as the percentage of the fluorescence emission intensity values for the E-cadherin PCR products to those of the $\beta$ - actin PCR products. E-cadherin expression was studied by immunohistochemistry. Results: The average of the fold width were $8.0 \pm 0.27$, $4.6 \pm 0.15,3.4 \pm 0.22 \mathrm{~mm}$ in H.pylori-positive patients. with and without enlarged folds, H.pylorinegative patients. E-cadherin methylation index of the gastric antral mucosa in H.pylori positive patients with enlarged folds was much greater than that in both H.pylori positive and negative patients without enlarged folds $(20.2 \pm 2.0 v 11.9 \pm 1.4 v 1.4 \pm 0.48, \mathrm{p}<0.01)$. Ecadherin methylation index of the gastric body mucosa in H.pylori positive patients with enlarged folds was much greater than that in both H.pylori positive and negative patients without enlarged folds $(15.6 \pm 2.8 v 9.5 \pm 0.8 v 1.4 \pm 0.40, \mathrm{p}<0.05$ and $\mathrm{p}<0.01)$. After eradication of H.pylori in the patients with enlarged folds, E-cadherin methylation index of the gastric mucosa decreased significantly from $15.6 \pm 3.9$ to $8.8 \pm 2.2(\mathrm{p}<0.05)$. Expression of E-cadherin in H.pylori positive patients with enlarged folds was reduced. Conclusion: Our findings suggest that hypermethylation of the E-cadherin promoter might be involved in the process of gastric carcinomas in H.pylori-induced enlarged fold gastritis.

\section{T1593}

Effect of Caga Tyrosine Phosphorylation in Mmps Production By H. Pylori Infected Gastric Epithelial Cells

Eunju Ryu, Yong Chan Lee, Inohk Lee, Young-Hee Nam, Seung-Taek Lee

Backgound/Aims: Recently, it has been shown that H. pylori infection induces expression of matrix metalloproteases (MMPs), enzymes capable of degrading extracellular matrix components. cagA positive $H$. pylori is known to induce greater amount of MMPs induction and therefore, CagA, oncoprotein, could be implicated in a pivotal role in the release of MMPs in gastric epithelial cells. The aims of this study was to identify the mechanism of expression of MMP-9 and -7 in specific reference to CagA protein in human gastric epithelial cells 
Methods: MMP-9 and -7 production was measured by reverse transcription polymerase chain reaction and gelatin zymography or western blot. Inducible CagA expression cell line, W1804 (kind gift from Prof. Hatakeyama M. of Hokkaido Univ.) was used to evaluate the role of tyrosine phosphorylation of CagA protein. Also, a pair of naturally occurring cagA isogenic mutants $147 \mathrm{C}$ and $147 \mathrm{~A}$ ( different tyrosine phoshorylation activities) were used to confirm the role of CagA protein tyrosine phosphorylation in MMP-9 production in vitro. Results: CagA positive strains are able to induce greater magnitude of MMP-9 production. And MMP-9 production was well correlated with the status of tyrosine phosphorylation status of CagA protein. With the expression of CagA in AGS cells by inducible system, transient transfection and H.pylori strains including positive (147C) and negative (147A) EPIYA motif, gastric epithelial cells responded to $\mathrm{H}$. pylori infection with the increased production of MMP-9 in phosphorylation dependent manner. On the other hand, the expression of MMP-7 is increased by H. pylori infection which is CagA dependent but phosphorlation independent. Rather than phosphorylation of CagA, either presence of cagA and cagE or pathogenicity island may be important in increased level of MMP-7 by H. pylori infection. Conclusion: Taken together, these results suggest that tyrosine phosphorylation of CagA may influence the production of MMP-9 in gastric epithelial cells, whereas the phosphorylation status seems not to be important factor the expression of MMP-7 in gastric epithelial cells.

\section{T1594}

Fas Blockage in Gastric Epithelial Cells Treated with H. Pylori: Role of Reactive Oxygen Species and NFkB

Duane T. Smoot, Mohammad Daremipouran, Mamoon Elbedawi, Hassan Ashktorab

H. pylori (HP) infection is associated with an active chronic gastritis, peptic ulcer disease and gastric cancer. Adherence of this bacterium to gastric cells activates signal transduction pathways that lead to apoptosis. Studies have shown that $H$. pylori's cytotoxicity is mediated at least in part by the generation of reactive oxygen species (ROS) and inducing NFkB activity within gastric epithelial cells. The following study was conducted to determine whether $H$. pylori's activation of Fas contributes to the generation of ROS within gastric epithelial cells, and to determine whether or not this is associated with a reduction in active NFkB. AGS cells (a human gastric adenocarcinoma cell line) were transfected with siFas RNA and/or dominant negative-IKB (IkB-DN). The gastric cells were then exposed to a cagpositive H. pylori strain and treated with a non-fluorescent probe DCFDA that fluoresces after exposure to H2O2. ROS generation was measured using a flurometer. Cell death, proliferation and NFkB promoter activity were measured using trypan blue exclusion, tetrazolium assay and a luciferase reporter construct, respectively. SiFas reduced FAS levels by more than $90 \%$ in gastric cells. There was a rapid increase in ROS in gastric cells within 15 minutes of exposure to H. pylori, and this peaked (at a 3-fold increase) after 3 hours. siFas RNA resulted an increase in ROS generation in gastric cells exposed to $H$. pylori for up to 3 hours. Blocking FAS stimulated ROS production in gastric cells, and the combination of $H$. pylori and siFas RNA further increased both the ROS production and cell death in gastric cells above that seen by $\mathrm{H}$. pylori alone. Cell death did not increase in cells transfected with both siFas and IkB-DN; therefore, inhibiting NFkB activation protected cells from $\mathrm{H}$. pylori induced cell death. In addition, down-regulation of FAS prevented $H$. pylori induced increase in NFkB promoter activity gastric cells. In conclusion, inhibition of Fas induces more ROS in gastric epithelial cells treated with H. pylori. Down-regulation of FAS does not impair NFkB activation, which is important in $H$. pylori mediated cell death. The FAS signal transduction pathway appears to be important in mediating cytotoxicity from this bacterium.

\section{$\mathrm{T} 1595$}

Altered Bone Morphogenetic Protein Signaling in the Helicobacter Pylori Infected Stomach

Sylvia Pel-Bleuming, Ludmila L. Kodach, Maria J. Garcia Leon, Dick Richel, Maikel

Peppelenbosch, Pieter H. Reitsma, James C. Hardwick, Gijs R. van den Brink

Morphogens regulate epithelial cell fate decisions in the adult gastrointestinal tract. We hypothesized that influx of inflammatory cells into the lamina propria may disturb the normal expression gradients of morphogens (morphogenetic landscape) in gastrointestinal epithelia. We therefore examined changes in the activity of the Bone Morphogenetic Protein (BMP) pathway in the normal and Helicobacter (H.) pylori infected gastric mucosa. We show that BMP receptors, the activated (phosphorylated) form of the intracellular BMP signal transduction protein SMAD1 and BMP target ID2 all localize to gastric epithelial cells that are at the end of the axis of epithelial renewal in the normal mucosa. Colonization of the human gastric mucosa with $H$. pylori results in an increase of BMP2 expression caused by influx of inflammatory cells that produce BMP2. Furthermore whereas no BMP4 is detected in the normal antrum, Focal infiltrates of BMP4 expressing cells are found in the H.pylor infected stomach. This influx of BMP expressing cells is associated with an increase in epithelial BMP signaling. Interestingly, a shift of the activity of the BMP pathway is observed towards the precursor cell compartment (isthmus) of the gastric units. Thus, H. pylori infection results in an influx of inflammatory cells that disturb the normal activity gradient of a morphogenetic pathway with an established role in epithelial cell fate regulation. Our data suggest that morphological changes in epithelial histology may result from alterations in the morphogenetic landscape secondary to changes in the cellular composition of the lamina propria.

\section{T1596}

Secretory Leukocyte Protease Inhibitor (SLPI), a Target Gene of Helicobacter Pylori Infection, Regulates NF-kB Signaling in Gastric Tumor Cells Thomas Wex, Gerhard Treiber, Sabine Krueger, Michael Naumann, Peter Malfertheiner

Background: Recently, SLPI was identified as target gene in H.pylori (HP)infection. It is expressed by the gastric epithelium and exerts antiproteolytic activity towards serine proteases. In HP-infected patients, antral SLPI levels were strongly reduced independent of the associated disease (gastritis, ulcer disease or gastric cancer). Aim: To investigate functional implications as well as mechanisms regulating SLPI expression in HP-infection. Methods: Four gastric tumor cell lines (AGS, MKN-28, MKN-45, NCI-N87), co-incubated with HP strain $\mathrm{Pl}$ and recombinant SLPI, were investigated by quantitative RT-PCR and ELISA regarding their endogenous SLPI expression and NF-kB signaling. Furthermore, SLPI expression of antral biopsies was studied in context to the CagA status by immunoblot and neutrophil-derived elastase, a target protease of SLPI, by ELISA. Results: The co-incubation of all four cell lines with HP (MOI=50, up to 48 hours) resulted in a significant decrease $(-80 \%, \mathrm{P}<0.001)$ of the SLPI protein in AGS and MKN-28 cells and a similar trend for MKN-45 and NCI-N87, whereas corresponding transcript levels were induced up to 5-fold in all cell lines $(\mathrm{P}<0.01)$. These data together with previous data from clinical samples suggest a posttranslational mechanism like intracellular degradation as cause for reduced SLPI levels in HP-infected subjects. The co-incubation of cell lines with HP strain Pl and recombinant SLPI $(0-5 \mu \mathrm{g} / \mathrm{ml})$ for 24 hours lead to a dose-dependent inhibition of NF-kB signaling in all cell lines except MKN-28 implying an additional anti-inflammatory effect of SLPI. Antral biopsies of HP-infected subjects contained about 30-fold higher neutrophil-derived elastase activity compared to HP-negative persons $(\mathrm{P}<0.01$, ANOVA) proving the functional relevance of SLPI for inhibiting proteases, which are capable to degrade mucosal components. The presence of anti-CagA antibodies was strongly associated with a 50\% down-regulation of antral SLPI levels $(1914 \pm 455 \mathrm{pg} / 50 \mu \mathrm{g}$ protein, $\mathrm{P}=0.01)$, whereas HP-infected without anti-CagA antibodies and HP-negative subjects revealed similar SLPI levels of $3730 \pm 402$ and $3857 \pm 129 \mathrm{pg} / 50 \mu \mathrm{g}$ protein, respectively. Conclusions: The HP-induced reduction of antral SLPI levels is primarily regulated at the posttranslational level. The association between anti-CagA antibodies and reduced SLPI levels suggest a PAI-dependent mechanism for the SLPI loss in HP-infection. The increased elastase-derived activity in gastric biopsies with reduced SLPI levels and the inhibition of HP-induced NF-kB signaling by SLPI illustrate the functional relevance of SLPI for gastric mucosa.

\section{T1597}

Acidic Conditions Synergistically Enhance the Induction of Helicobacter Pylori Induced Gastric Mucosal Interleukin-8

Il-Ju Choi, Saori Fujimoto, Kazuyoshi Yamauchi, David Y. Graham, Yoshio Yamaoka

Background and Aims: Although acid and Helicobacter infection are two main causative agents for development of peptic ulcers, their interaction remains largely unstudied. Interleukin (IL)-8, a potent neutrophil chemotactic and activating peptide, is thought to play a major role in the pathogenesis of $\mathrm{H}$. pylori-associated diseases. We investigated the effect of low pH on IL-8 induction in H. pylori infected gastric epithelial cells. Methods: H. pylori (wild type strain TN2GF4 and its isogenic cag pathogenicity island [PAI] or oipA mutants) were co-cultured with gastric epithelial cells (AGS cells) at pH's ranging from 7.4 to 5.0. IL- 8 mRNA expression was evaluated by real time RT-PCR and IL- 8 protein production by ELISA. NF-KB binding activity was measured by electrophoretic mobility shift assay and phosphorylation of IKB- $\alpha$ by immunoblot. The regulatory sites in the IL-8 promoter were examined by luciferase reporter gene assay. Results: $\mathrm{pH}$ changes did not affect IL- 8 levels in uninfected control. Wild type H. pylori induced IL-8 mRNA in a pH dependent manner with maximal levels at pH 5.5 (30-fold compared with uninfected control at pH 5.5, and 4.2-fold compared with infected cells at $\mathrm{pH}$ 7.4). Changes in IL-8 protein levels paralleled mRNA levels (e.g., 2.9-fold increase at pH 5.5 compared with that at pH 7.4; 781 vs. 270 $\mathrm{pg} / \mathrm{mL}$, respectively). cag PAI or oipA mutants induced significantly lower amounts of IL-8 mRNA/protein than wild type strains; however both had similar $\mathrm{pH}$ dependent patterns.

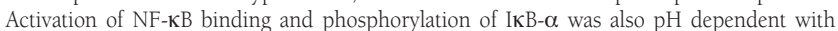
maximal levels at pH's 6.4-5.5. Luciferase activity using wild type IL- 8 promoter plasmid also increased in lower $\mathrm{pH}$ and was abolished when AGS cells were transfected with NF$\kappa \mathrm{B}$ mutated promoter plasmid. Conclusion: $H$. pylori and acidic conditions synergistically induced IL-8 via activation of NF- $\mathrm{KB}$. This provides a basis for reports that mucosal IL-8 levels are significantly higher in duodenal ulcer patients compared with gastritis only. The interaction of $H$. pylori with acidic gastric contents appears to enhance inflammation through increased IL-8 induction. Importantly, neither the cag PAI nor OipA were involved in pH dependent IL-8 production. Supported in part by the Gulf Coast Digestive Disease Center.

\section{$\mathrm{T} 1598$}

Different Risk Factors for Atrophic Gastritis and Intestinal Metaplasia in Korea

Nayoung Kim, Young Soo Park, Jin-Hyeok Hwang, Sook-Hyang Jung, Dong Ho Lee, Jung Mogg Kim, In Wook Kim, Yoo-Deok Won, Joo Sung Kim, Hyun Chae Jung, In Sung Song

Background/Aims: The prevalences of atrophic gastritis (AG) and intestinal metaplasia (IM) are very high in Korea. The possible risk factors for these precancerous lesions were comprehensively evaluated. Methods: The study subjects consisted of 825, that is 119 of control (87 Hp-positive), 234 patients of stomach cancer (202 Hp-positive), 253 of BGU (216 Hppositive), 162 of DU ( $155 \mathrm{Hp}$-positive) and 57 of dysplasia ( $52 \mathrm{Hp}$-positive). AG and IM were scored histologically by using the Sydney system classification on biopsy specimens taken from antrum and body of the stomach. Bacterial factors of $H$. pylori such as cagA, anti-CagA antibody, vacA $\mathrm{ml}, \mathrm{m} 2$, oipA were evaluated. Environmental factors such as smoking, alcohol drinking, drinking water, high salt intake, diet pattern such as vegetable and fruits, occupation, education and socioeconomic status were evaluated by questionnaires. Host factors such as genetic polymorphisms for IL-1B-511, IL-1RN, TNF-A, IL-10-592, IL10-819, IL-10-1082, GSTP1, p53, and methylation state of MGMT, p16, GSTP1 were evaluated. Risk factors were calculated by multiple logistic regression. Results: Risk factors for AG and IM were different. That is, H. pylori infection was risk factor for IM (OR, 2.0; $95 \% \mathrm{CI}, 1.3-3.1$ ) but not for AG. In addition, the effect of remaining factors on the AG and IM were different depending on $H$. pylori infected state. That is male gender, occupation (farmer and blue color labor), daily salty soup, and sharing the room by at least three children in the childhood were risk factors for AG and IM in the absence of H. pylori. In contrast, smoking and low education such as elementary graduation were risk factors for $\mathrm{AG}$ and IM in the presence of H. pylori. Low income was a common risk factor regardless 
of $\mathrm{H}$. pylori infection. Growing and living in the rural area was a risk factor for H. pyloripositive IM. Among the several genetic polymorphism variables only IL-10-1082 G/A type (OR, 5.2; 95\% CI, 1.7-16.5) and p53 pro/pro type (OR, 1.7; 95\% CI, 1.1-2.8) were risk factors for $H$. pylori-negative IM but methylated GSTPl was a risk factor for $H$. pylori-positive AG (OR, 5.0; $95 \% \mathrm{CI}, 1.1-22.9)$. Among bacterial factors only vac $\mathrm{ml}$ strain was a risk factor for both of $\mathrm{AG}(\mathrm{OR}, 3.6 ; 95 \% \mathrm{CI}, 2.1-6.2)$ and IM (OR, 3.0; $95 \% \mathrm{CI}, 2.0-4.5)$. Conclusions: These results that the risk factors are different for AG and IM, especially, depending on $H$. pylori infection suggest that intestinal metaplasia and atrophic gastritis might be caused by different mechanisms.

\section{T1599}

Regulation of Pathogenic Responses in Gastric Epithelial Cells By Helicobacter Pylori Sphingomyelinase

Robert E. Williams, Ramakrishnan Sitaraman, Uma S. Krishna, Dawn A. Israel, Daniel P. O'Brien, Seth R. Ogden, Aime T. Franco, Judith Romero-Gallo, Fang Yan, D. Brent Polk, Richard M. Peek

Helicobacter pylori activation of extracellular signal-regulated kinase (ERK) induces epithelial cell responses related to pathogenesis. ERK activation is dependent upon transactivation of the epidermal growth factor receptor (EGFR) but can also occur in response to $H$. pylori sphingomyelinase. Goals: To define the molecular pathways that mediate sphingomyelinase activation of ERK in the context of H. pylori infection. Methods: ERK activation was determined by Western immunoblotting. Sphingomyelinase activity was quantified using a specific sphingomyelin-N-( $\omega$-trinitrophenylaminolaurylsphingosylphosphorylcholine (TNPAL) assay. EGFR activity was blocked using the EGFR kinase chemical inhibitors AG 1478 and PD153035 (Compound 32). Results: H. pylori sphingomyelinase activity varied substantially among a population of clinical isolates $(\mathrm{n}=9)$ and H. pylori strain J166 exhibited the highest activity. Sphingomyelinase activity was significantly related to ERK activation in AGS gastric epithelial cells co-cultured with these same clinical isolate strains. H. pylori strain J166 reproducibly activated ERK in AGS cells; however, EGFR inhibition had no effect on ERK activation induced by this prototype isolate. Conclusion: The level of ERK activation in AGS cells was related to the level of sphingomyelinase expression by $H$. pylori, and this was not dependent upon transactivation of the EGFR. Implication: ERK activation by H. pylori likely occurs via both EGFR-dependent and independent pathways, with potential implications for pathogenesis of injury and inflammatory responses leading to tumorigenesis.

\section{$\mathrm{T} 1600$}

Catalytically Active Adam17 Is Required for TNF-Stimulated Transactivation of Erbb3

Stuart Hobbs, Robert Whitehead, Peter J. Dempsey, David-Brent Polk

Tumor Necrosis Factor (TNF) is a cytokine that regulates complex cellular responses during injury and repair such as apoptosis and survival through signaling by Tumor Necrosis Factor Receptor 1 (TNFR1) and Tumor Necrosis Factor Receptor 2 (TNFR2). As a result, deregulated signaling by TNF plays an important role in the pathogenesis of gastrointestinal diseases such as Inflammatory Bowel Disease (IBD) and Celiac disease in which there is an imbalance between cell survival and apoptosis. A number of observations suggest that TNF-stimulated signaling may additionally be important in the pathogenesis of gastritis and gastric cancer induced by the gram-negative bacteria $H$. pylori. For example, we have observed that $H$. pylori stimulates the expression of TNF in gastric epithelial cells. Furthermore, we have also observed that TNF is able to stimulate transactivation of members of the ErbB family of type I receptor tyrosine kinases. We have previously reported that H. pylori stimulates ErbB1 and ErbB3 transactivation in gastric epithelial cells. Therefore, we asked if TNF may play a role in $H$. pylori transactivation of ErbB receptors. Our lab has observed that $H$. pylori transactivation of the ErbB receptors ErbBl and ErbB3 requires the cleavage of membranebound ErbB ligands by the metalloprotease Tumor necrosis factor- $\alpha$ converting enzyme (TACE/ADAM17). Therefore, we hypothesize that TNF transactivation of ErbB receptors requires catalytically active ADAM17. Transactivation of ErbB receptors, such as ErbB3, that strongly couple to anti-apoptotic pathways may be critical for any TNF-stimulated cell survival and may contribute to the observed pathologies associated with gastric $H$. pylori infections. As a result, we monitored TNF stimulation of ErbB3 tyrosine phosphorylation in ADAM17 deficient Mouse Gastric Epithelial Cells (MGEC) in which wild-type ADAM17 (wt ADAM17) or a catalytically inactive E406A ADAM17 (E-A ADAM 17) mutant has been ectopically expressed. Here we report that TNF stimulates ErbB3 tyrosine phosphorylation in the ADAM17-/- MGEC wt ADAM17 cells. Moreover, TNF concentrations ranging from $0.1 \mathrm{ng} / \mathrm{mL}-100 \mathrm{ng} / \mathrm{mL}$ stimulate ErbB3 tyrosine phosphorylation. In contrast, TNF is unable to stimulate ErbB3 tyrosine phosphorylation in the ADAM17-/- MGEC E-A ADAM17 cells. In conclusion, TNF stimulates ErbB3 transactivation in gastric epithelial cells. Furthermore, TNF-stimulated ErbB3 transactivation requires catalytically active ADAM17. This suggests that TNF transactivation of ErbB3 may involve the cleavage of membrane-bound ErbB ligands by ADAM17.

\section{T1601}

Interleukin-1b Gene Polymorphisms and Gastric Mucosal Interleukin-1 $\beta$ in Benign Gastroduodenal Diseases with Helicobacter Pylori Infection Young W. Chang, Hyoung C. Oh, Jae Y. Jang, Ji H. Jung, Myung J. Chae, Sang K. Lee, Kwang R. Joo, Seok H. Dong, Hyo J. Kim, Byung H. Kim, Joung I. Lee, Rin Chang

Background \& Aims: Host genetic factor is suggested to be an important determinant that predispose to divergent clinical outcomes associated with Helicobacter pylori ( $H$. pylori) infection. The aims of this study were to examine Interleukin-1B (IL-1B) polymorphisms in benign gastroduodenal diseases with $H$. pylori infection, and to analyze the correlation between IL-1B genotypes and gastric mucosal inflammatory cytokines including IL-1 $\beta$, matrix metalloproteinase-3 (MMP-3), and IL-8. Methods: A total of $191 \mathrm{H}$. pylori-infected subjects included 70 non-atrophic gastritis (NAG), 47 atrophic gastritis (AG), 28 gastric ulcer (GU), and 46 duodenal ulcer (DU) group. PCR-RFLP was performed to analyze the genetic polymorphisms of IL-1B-31 and IL-1B-511. IL-1 $\beta$, MMP-3 and IL-8 level of gastric corpu mucosa were determined by using an ELISA. Serum pepsinogen I and II levels were measured by radioimmunoassay. Results: Since the alleles of IL-1B-511 were strongly linked with those of IL-1B-31, we examined linkage disequilibrium between the IL-1B-31 and IL-1B511 alleles. Estimation of observed haplotype frequencies and comparison with those of expected haplotype frequencies showed complete linkage disequilibrium coefficient in al subjects $\left(D^{\prime}=1.0, P<0.0001\right)$. Therefore, we examined the effect of IL-1B-31/-511 haplotype such as TT/CC, CT/CT, and CC/TT instead of IL-1B-31 or IL-1B-511 alleles each.on clinical manifestations. There were no significant differences in IL-1B-31/-511 haplotypes frequencies among the four groups. The haplotypes were not associated with the level of inflammatory markers. AG showed significantly higher basal IL-1 $\beta$ and IL-8 level but, basal IL-1 $\beta$ and MMP-3 were lower in DU compared to those of other groups. After H. pylori eradication IL-1 $\beta$, MMP-3, and IL-8 levels were significantly decreased $(\mathrm{P}<0.001)$ but, pepsinogen I/I ratio was significantly increased in all groups $(\mathrm{P}<0.001)$. Conclusions: Gastric mucosal levels of inflammatory markers including IL- $1 \beta$ were related to variable clinical outcomes associated with $H$. pylori infection. The genotypes of IL-1B polymorphisms do not affect the mucosal production of the inflammatory markers in benign gastroduodenal diseases.

\section{$\mathrm{T} 1602$}

Inhibitory Effect On Helicobacter Pylori and Anti-Oxidative Effect By Broccoli Sprout Extract Containing Sulforaphane

Jae-Young Jang, Young-Woon Chang, Ji Heon Jung, Myung Jong Chae, Sang Kil Lee, Kwang Ro Joo, Seok Ho Dong, Hyo Jong Kim, Byung-Ho Kim, Joung Il Lee, Rin Chang

Background/Aims: Isothiocyanate sulforaphane as the principal phase 2 inducer in broccoli extracts was a dose-related inhibitor of carcinogen-induced mammary tumorigenesis in rats and inhibited Helicobacter pylori (H. pylori) in vitro study. The aims of this study were to investigate 1) whether sulforaphane has the inhibitory effect on $H$. pylori infection, 2) whether sulforaphane has the preventive effect on lipid peroxidation and anti-oxidative effect on gastric mucosal damage in patients with nonulcer dyspepsia. Subjects and Methods: We designed a prospective, randomized controlled, single blind study. A total of 79 subjects included in $55 \mathrm{H}$. pylori-positive subjects and $24 \mathrm{H}$. pylori negative subjects. Fifty-five $\mathrm{H}$. pylori-positive subjects randomly assigned to receive a capsule containing sulforaphane 300 $\mu \mathrm{g}$ or placebo twice per day for 4 weeks. Tweenty-four $H$. pylori-negative subjects were also given sulforaphane twice per day for 4 weeks. All subjects were examined by C13-ure breath test and upper gastrointestinal endoscopy before and after suforaphane or placebo treatment. The concentration of ammonia was measured in gastric juice aspirate during gastroscopic examination. Malondialdehyde (MDA) and reduced glutathione (GSH) concentrations in gastric corpus mucosa were measured by ELISA. Results: H. pylori-positive sulforaphane group showed no significant changes in delta UBT values and gastric juice ammonia concentration before and after treatment. The mucosal MDA concentrations $(149.8 \pm 131.5 \mathrm{nmol} / \mathrm{g})$ were significantly reduced after sulforaphane treatment in $H$. pyloripositive subjects $(74.0 \pm 55.9 \mathrm{nmol} / \mathrm{g}, \mathrm{p}<0.01)$. The mucosal MDA concentrations $(120.3 \pm 62.0$ $\mathrm{nmol} / \mathrm{g}$ ) were also significantly reduced after sulforaphane treatment in $\mathrm{H}$. pylori-negative subjects $(72.3 \pm 49.0 \mathrm{nmol} / \mathrm{g}, \mathrm{p}<0.001)$. Difference in MDA concentration between before and after treatment was larger in sulforaphane groups irrespective of $H$. pylori infection than that of placebo group $(\mathrm{P}<0.05)$. There were no significant differences in basal reduced GSH concentrations in gastric mucosa between $H$. pylori-positive and negative subjects. Reduced GSH concentrations between before and after treatment were not differ significantly between sulforaphane group with or without $H$. pylori infection and placebo group. Conclusions: Sulforaphane has no effect on eradication or inhibition of $H$. pylori infection in human stomach. Sulforaphane may prevent lipid peroxidation in gastric mucosa and may play a role in the cytoprotection in $\mathrm{H}$. pylori-induced gastritis.

\section{$\mathrm{T} 1603$}

Low Serum Pepsinogen I/II Ratio Induces An Increase in Body Mass Index After Eradication of $\mathrm{H}$. Pylori

Hiroyuki Suto, Shinsuke Matsunaga, Ryuho Masaki, Tomoko Inagaki, Satoko Satomi Tomoyuki Okuda, Atsushi Muramatsu, Soshoku Lee, Yoshiyuki Ito, Yukinao Yamazaki, Masaru Kuriyama, Takeshi Azuma

Background and aims: Recently some reported that the eradication of $H$. pylori induces an increase in the body mass index (BMI). However the relationship between $H$. pylori infection and the BMI is still controversial. Here we investigated the relationship between the change in the BMI after the eradication of H. pylori and serum pepsinogen (PG) I/II ratio. Methods: One hundred and ninety five $H$. pylori positive patients with atrophic gastritis were chosen from subjects who visited multiphase health testing services and they underwent the eradication therapy for $H$. pylori. Cure of $H$. pylori infection was evaluated with $13 \mathrm{C}$-urea breath test 3 month after treatment. They were examined the relationship between the effect of infection cure in terms of the BMI and the serum PG I/II ratio before treatment. All these patients were investigated the BMI every year during 4 years after treatment. The BMI changes were calculated each year by subtracting the BMI before treatment from that after treatment. In addition, the serum leptin levels were also investigated before and one year after treatment. Results: The serum PG I/II ratio was classified into three groups; group A was $P G I / I I<2$, group $B$ was $2 \leqq P G ~ I / I I<3$ and group $C$ was $3 \leqq P G ~ I / I I$. The BMI changes $(\mathrm{kg} / \mathrm{m} 2)$ in 1 year after treatment in the groups $A, B$ and $C$ were $0.78 \pm 0.67,0.16 \pm 0.66$ and $0.04 \pm 0.71$, respectively. The mean BMI change in the group A was significantly higher than those in the groups B and $C(p<0.01)$. The mean BMI changes in 2 and 3 years after treatment in the group A were also significantly higher than those in the groups B and C $(p<0.01)$. The mean BMI change in 4 years after treatment in the group A was higher than those in the groups B and C, although it was not significant. The infection cure had a great effect on the BMI in the group A within 1 year after treatment and the influence of the weight gain remained during at least 4 years. We found the negative correlations between the serum PG I/II ratio and the BMI change in 1 and 2 years after treatment $(p<0.01)$ However we found no significant relationship between the change in the serum leptin levels 
and the serum PG I/II ratio. Conclusions: Low serum PG I/II ratio, especially PG I/II $<2$ induces an increase in body mass index after the eradication of $H$. pylori.

\section{$\mathrm{T} 1604$}

Helicobacter Pylori Itself and Interferon- $\gamma$ Suppresses the Expression of a Hmg Box Protein SOX2 Through Stat6-Mediated Interleukin-4 Signaling in Gastric Epithelial Cells

Sho Asonuma, Akira Imatani, Naoki Asano, Tomoyuki Oikawa, Shuichi Ohara, Tooru Shimosegawa

Background \& Aim: Helicobacter pylori $(\mathrm{Hp})$ is a major cause of the transdifferentiation to gastric atrophy and intestinal metaplasia which are risk factors of gastric cancer development. This progression is mediated by Thl-dominant host immune response to Hp infection. On the other hand, a SRY-related HMG box protein Sox2 is a transcription factor of organ development and differentiation in brain, lung and stomach. In stomach, Sox2 regulates pepsinogen and MUC5AC, but there has been no report of the relationship between $\mathrm{Hp}$ infection and Sox2. After evaluating Sox2 expression in Hp infected human gastric mucosa including gastric atrophy and intestinal meteplasia, we investigated the molecular mechanism responsible for the regulation of Sox2 by Hp itself and Hp-induced cytokines. Methods: We evaluated twenty cases of Hp infected human gastric mucosa for Sox 2 expression by immunohistochemistry. Human gastric cell lines AGS and MKN45 were employed as an in vitro model of human gastric epithelial cells. We stimulated them with CagA positive Hp (ATCC\#43504), IFN- $\gamma$, TNF- $\alpha$, and IL-4 for 24 hours independently, and also with IL-4 after the treatment with $\mathrm{Hp}$ or IFN- $\gamma$. We assessed the expression level of Sox 2 stimulated with $\mathrm{Hp}$ or those cytokines by western blotting respectively. RT-PCR analysis was performed to determine the mRNA levels of MUC2, MUC5AC and MUC6. We also assessed phosphoSTAT6/STAT6 activity by immunoblotting. Results: Expression of Sox2 protein in human oxyntic mucosa was observed in the nuclei of cells in the proliferative region, whereas in atrophic mucosa and intestinal meteplasia with $\mathrm{Hp}$ infection it was decreased. In vitro the expression level of Sox2 increased dose-dependently in both AGS and MKN45 after the phosphorylation of STAT6 mediated by IL-4, a Th2-associated cytokine. MUC5AC, a gastric specific mucin gene, was also up-regulated by IL-4 stimulation. Little changes of Sox 2 expression were observed after the treatment with Hp, IFN- $\gamma$ or TNF- $\alpha$, independently, but $\mathrm{Hp}$ itself and IFN- $\gamma$ inhibited dose-dependently the expression of both Sox 2 and MUC5AC induced by IL-4, and abrogated phosphorylation of STAT6. Conclusion: These results suggested that Hp itself and IFN- $\gamma$ suppressed the expression of Sox 2 through STAT-6 mediated IL-4 signaling, and down-regulation of Sox 2 may contribute to the progression of gastric atrophy and intestinal metaplasia. Hp itself and Thl-dominant host immune response may play an important role in the progression of them through the regulation of Sox2.

\section{$\mathrm{T} 1605$}

PCR for Immunoglobulin Gene Rearrangement Detects Residual MALT Lymphoma in Histologically Negative Gastric Biopsies

William Ahrens, Jeffrey Sklar, Dhanpat Jain, Vytas Patriub, Sue Lagarde, Marie Robert

Background: Eradication of $H$. pylori will cause regression of gastric marginal zone B-cell lymphoma (MALT) lymphoma in $60 \%$ to $90 \%$ of cases. However, monoclonal B cell populations can be detected by polymerase chain reaction (PCR) of immunoglobulin gene rearrangments (IGRs) in histologically negative follow up biopsies. The clinical significance of persistent clones in histologically negative biopsies is unclear. The value of surveillance, repeat PCR analysis, and the criteria for determining complete remission in relation to such clones has yet to be determined. Design: The pathology archives were searched for gastric biopsies that were positive for MALT lymphoma. Subsequent gastric biopsies on these patients were reviewed blindly by two pathologists and scored as being positive, negative or indefinite for involvement by lymphoma. PCR results of clonal IGRs were correlated with H\&E scores. PCR performed on sequential specimens were compared when possible. Result: 37/90 gastric lymphoma cases identified between 1992-2005 fit histologic/molecular criteria for low grade MALT lymphoma. 16/37 were $H$. pylori positive, and those patients were treated with antibiotic therapy. 15 patients had initial and at least one follow up PCR analysis to detect B-cell clonality. The correlation of histology and PCR on the same biopsy material is shown in Table 1. In 5 patients PCR positive on two biopsies, the clones detected were identical on both biopsies, although an additional subclone was found in one patient. In another of these five patients, PCR analysis on a third biopsy yielded products for IGRs different from those in the previous two analyses, indicating either instability of the IGRs in a single clone, or a new clone. $6 / 8$ total patients with persistent IGRs were negative for lymphoma at last follow up (range 4-89 months). Conclusion: Negative histology in follow up gastric mucosal biopsies is insufficient to exclude low grade MALT lymphoma after therapy. The addition of PCR for IGRs will detect small clonal populations that are frequently identical to the original clones, but unapparent histologically. In addition, lymphoma detected after treatment may have clonal IGRs that differ from those identified earlier. The clinical significance of persistent clones or new clones in the face of minimal histologic infiltrates remains to be determined.

Histology Score vs. PCR resul

\begin{tabular}{|l|c|c|}
\hline & Histology + & Histology - or Indefinite \\
\hline PCR + & 17 & 5 \\
\hline PCR - & 10 & 10 \\
\hline
\end{tabular}

T1606

Anti- Helicobacter Pylori Therapy Improves Iron Status and Gastric Acid Output in Young Bangladeshi Women with Helicobacter Pylori Associated Hypochlorhydria and Iron Deficiency Anemia

Shafiqul A. Sarker, Shamima Sultana, Shamima Sattar, Shafiqul Islam, George J. Fuchs, Christoph Beglinger, Niklaus Gyr

Bacground: Hypochlorhydria is a risk factor for iron deficiency anemia (IDA), which affects primarily infants and women of reproductive age. There are reports of $H$. pylori $(\mathrm{Hp})$ associated gastritis that may lead to hypochlorhydria. Aim: To determine if hypochlorhydria contributes to IDA and to evaluate whether IDA and hypochlorhydria can be managed by Hp eradication. Method: We studied 96 women, aged 20-40 years, infected with Hp based on a positive urea breath test. We measured hemoglobin ( $\mathrm{Hb})$, serum ferritin $(\mathrm{SF})$, and soluble transferrin receptor (sTfR) to assess iron status. Gastric acid output (in $\mathrm{mMol} / \mathrm{h}$ ) was measured during a 1-h basal period (BAO) and for an additional hour (1-hr stimulated period $=S A O)$ after stimulation with s.c. pentagastrin $(6 \mathrm{mg} / \mathrm{kg})$. Acid output and iron status were reevaluated in women who had exhibited both IDA $(\mathrm{Hb}<110 \mathrm{~g} / \mathrm{L}$, plus SF $<12 \mu \mathrm{g} /$ $\mathrm{L}$, and/or sTfR $>8.3 \mathrm{mg} / \mathrm{L}$ ) and hypochlorhydria ( $\mathrm{SAO}<14.0 \mathrm{mMol} / \mathrm{h}$ ), 60 days after completion of a supervised 2-weeks course of anti Hp therapy (amoxicillin, clarithromycin, and omeprazole.Results: Of the 96 infected women, 57 (59\%) had hypochlorhydria and 39 (41\%) had normochlorhydria (SAO> $14.0 \mathrm{mmol} / \mathrm{h}$ ). Compared to normochlorhydric women, the prevalence of IDA was significantly higher in hypochlorhydric women $(23 \%$ vs. $49 \%$, OR $0.31,95 \% \mathrm{CI} 0.16-0.6, \chi 2=14.6, \mathrm{p}=.0001$ ). In women who exhibited both hypochlorhydria and IDA ( $\mathrm{n}=28)$, anti-Hp therapy, which was $90 \%$ successful, improved both BAO (D-0 vs. D- $60 ; 1.8 \pm 1.6$ vs. $2.7 \pm 2.6, p=.024)$, and $\mathrm{SAO}(10.4 \pm 3.1$ vs. $13.7 \pm 4.4, \mathrm{p}=<.0001)$ and was accompanied by improvement of iron status; for $\mathrm{Hb}$ (mean $\pm \mathrm{SD}, 10.5 \pm 1.1$ vs. 10.8 $\pm 1.13, \mathrm{p}=0.13)$, for SF [(Geometric mean $(-1 \mathrm{SD},+1 \mathrm{SD}) 10.6(4.5,25.0)$ vs. $16.3(4.4,50.0$, $\mathrm{p}=.02]$, for sTfR [(Geometric mean $(-1 \mathrm{SD},+1 \mathrm{SD}) 10.2(5.6,18.2)$ vs. $6.5(3.9,10.8), \mathrm{p}=$ $<.0001]$. In women with hypochlorhydria, 15 (54\%) become normochlorhydric and $68 \%$ became non-anemic following anti-Hp therapy. Conclusion : Our findings indicate that hypochlorhydria and IDA is H-pylori associated in these women; both problems can be managed by appropriate eradication therapy.

\section{$\mathrm{T} 1607$}

Lipopolysaccharides from Helicobacter Pylori Nctc11637 Up-Regulate IL-8 and Vegf Via Toll-Like Receptor 2 in An Epithelial Cell System Sinead Smith, Anthony P. Moran, Dermot P. Kelleher

Helicobacter pylori is the causative agent of chronic gastritis and a predisposing factor for the development of both peptic ulcer disease and gastric cancer. Gastric epithelial cells provide the first point of contact between $H$. pylori and the host, responding to $H$. pylori infection by activating numerous signaling cascades. The best-characterised responses to date are those leading to the expression and activation of the transcription factors NF- $\mathrm{KB}$ and AP-1. One outcome of the activation of such transcription factors is the production of the inflammatory cytokine IL-8. Toll-like receptors (TLRs) have recently been suggested to mediate many of these cell signaling events. This study set out to investigate the role of TLRs in the response of epithelial cells to lipopolysaccharides (LPS) from $H$. pylori NCTC11637. Human embryonic kidney (HEK293) cells stably transfected with either TLR2 or TLR4 were treated with $10 \mu \mathrm{g} / \mathrm{ml} \mathrm{H}$. pylori LPS, and total RNA was isolated at intervals over 48 hours. IL-8 mRNA expression was monitored using a real-time PCR-based assay. It was observed that $H$. pylori LPS induced a transient up-regulation of IL-8 mRNA in HEK293 cells stably transfected with TLR2 (HEK-TLR2), with a maximum induction of approximately 18 -fold following 4 hours of stimulation. This up-regulation was not observed in HEK293 or HEK-TLR4 cells, suggesting that TLR2 was responsible for the LPS-mediated increase in IL-8 mRNA. The HEK-TLR4 cells were found to express functional TLR4, since treatment with the widely used TLR4 agonist, E. coli LPS, resulted in significant up-regulation of IL-8. A number of genes have been shown to be induced in response to H. pylori infection in vitro. The capacity of $H$. pylori LPS to stimulate a selection of these genes in HEK-TLR2 cells was next tested. VEGF was found to be significantly up-regulated by H. pylori LPS in HEK-TLR2 cells, when compared with HEK293 or HEK-TLR4 cells. However, other genes previously demonstrated to be induced by infection with live $H$. pylori, namely IL-1A, IL$1 B, A T F 3$ and C8FW, were not shown to be induced by H. pylori LPS in any of the HEK cell lines. It is likely that other $\mathrm{H}$. pylori-related virulence factors are involved in the induction of these genes. In conclusion, IL-8 mRNA was shown to be induced by LPS from H. pylori NCTC11637 via TLR2 in an epithelial cell system. In addition, VEGF was found to be upregulated during the TLR2-mediated response to LPS. Further studies are required to identify genes whose expression is mediated by TLR2 in response to H. pylori LPS, and to elucidate their role during $H$. pylori infection.

\section{$\mathrm{T} 1608$}

Effect of a Carcinogen On H. Pylori Infection: Expression of Cytokines and TGF $\beta$ Receptor 2 in Rhesus Monkeys Hui Liu, Cristina Semino-Mora, Steven Mog, Andre Dubois

Helicobacter pylori is recognized as a cause of gastric cancer, but only a fraction of infected subjects develop gastric cancer. Transforming Growth Factor $\beta$ (TGF $\beta$ ) is a tumor suppressor gene and mutations in TGF $\beta$ receptor II (TGF $\beta R 2$ ) are present in human gastric cancer. The present studies were undertaken to evaluate whether downregulation of TGF $\beta$ R2 does occur in rhesus monkeys infected with $H$. pylori and/or receiving the nitrosating carcinogen 
ethyl-nitro-nitrosoguanidine (ENNG). We also investigated whether gastric precancerous lesions and/or cancer emerge after ENNG administration, as reported in monkeys (J Natl Cancer Inst, 1986). Gastroscopies were performed in 23 monkeys naturally infected with H. pylori. Biopsies were fixed for histology, cultured, or flash frozen for gene expression analysis. The mRNA expression of H. pylori $16 S$ rRNA, IL-1 $\beta$, IL-8, and TGF $\beta R 2$ (copies/ 100 ng RNA) were determined using an absolute real-time quantitative RT-PCR (TaqMan) method, standard curves of cloned cRNA, and normalization with Rhesus monkey $18 \mathrm{~S}$ rRNA. After cure of the infection with quadruple therapy, H. pylori-negative monkeys were assigned to one of four groups: controls $(\mathrm{C}, \mathrm{n}=5)$; inoculation of a virulent $H$. pylori strain isolated from a patient with gastric cancer $(H, n=6)$; ENNG (E, $30 \mathrm{mg} /$ day, $\mathrm{n}=6)$; and $H$. pylori inoculation + ENNG (started 6 months post-inoculation; $\mathrm{H}+\mathrm{E}, \mathrm{n}=6$ ). Follow-up gastroscopies and biopsies were performed at regular intervals. Post inoculation, both $\mathrm{H}$ and $\mathrm{H}+\mathrm{E}$ groups had antral gastritis and $\mathrm{H}$. pylori were present in the gastric lumen, attached to, and inside, epithelial cells, and in the lamina propria. Starting at 21-month of ENNG administration, gastric body biopsies of $3 \mathrm{H}+\mathrm{E}$ animals demonstrated atrophy, inflammation with loss of parietal cells and conserved chief cells. At 31-month, gastric antrum biopsies of one $\mathrm{H}+\mathrm{E}$ animal showed intestinal metaplasia, small intestinal type, with marked epithelial hyperplasia and regeneration. IL-1 $\beta$ and IL-8 cytokine expression increased in $H$. pylori infected monkeys and TGF $\beta R 2$ increased only transiently in $\mathrm{H}$ and $\mathrm{H}+\mathrm{E}$ monkeys. Cytokine mRNA expression was directly and significantly correlated with $16 \mathrm{~S} r$ RNA expression both in $\mathrm{H}$ and $\mathrm{H}+\mathrm{E}(\mathrm{p}<0.05)$. TGF $\beta R 2$ expression was inversely correlated with $\mathrm{H}$. pylori $16 \mathrm{~S}$ rRNA during natural $H$. pylori infection and after experimental inoculation $(\mathrm{P}<0.01)$. This observation suggests that downregulation of TGF $\beta R 2$ may play a role in gastric carcinogenesis. Repeat endoscopies continue to be performed at 2-month intervals, the appearance of early gastric carcinoma is investigated, and the relation between TGF $\beta R 2$ and 16S rRNA expression is explored.

\section{T1609}

The Prevalence of a-251t Polymorphism of IL-8 and G-308a of TNF in H.pylori Positive and Negative Cases in Different Gastric Diseases

Dominika Szoke, Bela Molnar, Yvette Mandy, Zsolt Tulassay

Background: In vivo the H.pylori infection of the gastric mucosa induces the production of various cytokines, like interleukin- $1 \beta,-6,-8$ and TNF $\alpha$. Presently available evidences suggest that IL-8 may play a significant role in the pathogenesis of gastritis, which is closely associated with H.pylori infection. The A-251T of IL-8 is a functional polymorphism reported to be associated with the elevated expression level of IL8 and with pathogenesis of H.pylori related diseases. The G-308A polymorphism in the regulatory region of TNF $\alpha$ gene has been associated with susceptibility to a number of complex disorders, including infective diseases. We aimed to examine the prevalence of these two polymorphisms in H.pylori positive and negative cases in different gastric diseases. Materials and methods: Genomic DNA was extracted from biopsy samples of 136 consecutive patients with gastritis $(n=68)$, intestinal metaplasia (IM) $(n=28)$ and from histologically negative control patients $(n=40)$. The IL- 8 A-251T polymorphism was examined with using Amplification refractory mutation system method, the G-308A polymorphism of TNF $\alpha$ was determined with PCR-RFLP method, with Ncol restriction enzyme. H.pylori was determined in the samples by histology. The samples were sorted also by the endoscopic findings to erosion and normal groups. Results: In the case of the IL8 polymorphism the allele frequencies were significantly different with $\chi^{2}$ probe between H.pylori positive erosion group and the normal group $(\mathrm{p}=0.0441)$ and between H.pylori positive erosion and H.pylori negative erosion groups $(\mathrm{p}=0.0009)$. There were significant difference between the H.pylori negative and positive gastritis groups $(\mathrm{p}=6.63 \mathrm{E}-16)$ and significant difference were found between H.pylori positive IM and H.pylori positive gastritis groups ( $\mathrm{p}=1.04 \mathrm{E}-09)$. In the case of the TNF $\alpha$ polymorphism the allele frequencies were significantly different between the H.pylori positive IM and the negative control group ( $\mathrm{p}=$ $0.0087)$ and between H.pylori positive erosion and the normal group $(\mathrm{p}=0.0049)$. There were significant differences between the H.pylori negative and positive gastritis groups ( $\mathrm{p}=$ 2.73E-11) and between H.pylori positive IM and H.pylori positive gastritis groups ( $\mathrm{p}=1.04 \mathrm{E}$ 09). Discussion: Our findings suggest a relation between H.pylori infection and the presence of the heterozygous state of A-251T polymorphism of IL-8, and the presence of the wild type state of G-308A polymorphism of TNF $\alpha$. The prevalence of these two polymorphisms is significantly different between the different diseases groups independently from the H.pylori status.

\section{$\mathrm{T} 1610$}

The Role of Genetic Polymorphisms of Interleukin-1 $\beta$, Interleukin-Receptor Antagonist and Interleukin-6 in Helicobacter Pylori-Associated Diseases Gyu Jeong, Jae G. Kim, Sill M. Park

Background: Interleukin-1beta (IL-1 $\beta$ ), interleukin-1 receptor antagonist (IL-1RN) and interleukin-6 (IL-6) genetic polymorphisms are known to be associated with pathogenesis of the gastroduodenal disease infected with Helicobacter pylori. However, there has been debate in real significance of these polymorphisms. The ethnic difference as well as genetic heterogeneity should be taken into consideration to evaluate the significance of genetic polymorphisms in these cytokines. We investigated IL-1 $\beta$, IL-1RN and IL- 6 genetic polymorphisms in Korean gastroduodenal disease patients to define their significance. Methods: Gastric biopsy specimens were taken from $H$. pylori positive benign gastroduodenal disease patients (chronic gastritis, $n=23$; benign gastric ulcer, $n=59$; duodenal ulcer, $n=59$ : gastroduodenal ulcer, $n=13)$, H. pylori positive gastric carcinoma patients $(n=59)$ and $H$. pylori negative gastric carcinoma patients $(n=40)$. The DNA was extracted from gastric biopsy specimens. The IL- $1 \beta$ polymorphisms at position $-31,-511,+3954$ and IL- 6 polymorphisms at position $174,-572,-597$ were analyzed by PCR-restriction fragment length polymorphism. IL-1RN variable number of tandem repeat (VNTR) polymorphism was analyzed by PCR. Results: Among benign gastroduodenal diseases, the frequency of IL-1 $\beta-31 \mathrm{~T} / \mathrm{T}$ was significantly higher in benign gastric ulcer $(\mathrm{p}=0.013)$ and duodenal ulcer $(\mathrm{p}=0.025)$ compared to chronic gastritis. The frequency of $\mathrm{IL}-1 \beta-511 \mathrm{C} / \mathrm{C}$ was also significantly higher in benign gastric ulcer $(\mathrm{p}=0.010)$ and duodenal ulcer $(\mathrm{p}=0.015)$ compared to chronic gastritis. H. pylori positive gastric carcinoma patients had more IL-6-572* $\mathrm{G}$ allele compared to benign gastric ulcer and duodenal ulcer patients ( $\mathrm{p}=0.041$ ). Classifying of gastric carcinoma into intestinal and diffuse type, there was no significant difference in genetic polymorphisms of these thre cytokines. Between $H$. pylori positive and $H$. pylori negative gastric carcinoma patients there was also no significant difference. Conclusions: IL-1 $\beta$ polymorphisms and IL-1RN polymorphisms are not associated with gastric carcinogenesis in Korea. However, IL-6$572^{*} \mathrm{G}$ allele may be contribute to pathogenesis of $H$. pylori positive gastric carcinoma. In addition, IL-1 $\beta-31 \mathrm{~T} / \mathrm{T}$ and IL-1 $\beta-511 \mathrm{C} / \mathrm{C}$ genotypes may be associated with pathogenesis of peptic ulcer disease.

\section{T1611}

Use of Different Anti-Oxidant Strategies in Resisting Monochloramine-Induced Accumulation of Intracellular Ca2+ in Gastric Glands of the Rabbit Haley Naik, J. Matthew Dubach, David I. Soybel

AIM Monochloramine $(\mathrm{NH} 2 \mathrm{Cl})$, a thiol-directed oxidant which accumulates in $\mathrm{H}$. pylor gastritis, has been shown to induce $\mathrm{Ca} 2+$ increase in the cytoplasm of the gastric parietal cell due to intracellular store release and accelerated entry from extracellular spaces. This study aims to determine whether these effects are prevented and reversed by different antioxidant strategies, specifically thiol-reducing agent dithiothreitol (DTT) or oxidant scavenger Vitamin C (VitC). METHODS Glands were loaded with Ca2t-sensitive fluorescent dyes, fura-2 (to monitor cytoplasm, [Ca2+]i) or mag-fura-2 (to monitor intracellular stores, $[\mathrm{Ca} 2+] \mathrm{S})$. Fura-2-loaded glands were perfused with standard Ringer's solutions (Ca2+-free $+0.5 \mathrm{mM}$ EGTA or $1 \mathrm{mM} \mathrm{Ca2+)}$. Mag-fura-2-loaded glands were permeabilized with $190 \mathrm{nM}$ free-Ca2 + intracellular buffer and $10 \mu \mathrm{M}$ digitonin. Fluorescence excitation ratio $(340 \mathrm{~nm} /$ $380 \mathrm{~nm})$ was monitored in 6-8 parietal cells/gland. Heavy metal chelator, TPEN (20uM), was used to screen out contaminating heavy metal cation contributions. Results are expressed as mean +/- SEM and comparisons analyzed by t-test or ANOVA as appropriate. RESULTS In the presence of extracellular $\mathrm{Ca} 2+$, exposure of glands loaded with fura- 2 to $\mathrm{NH} 2 \mathrm{C}$ $(200 \mathrm{uM})$ led to sustained excitation ratio increases from $0.88+/-0.05$ to $1.40+/-0.09$, corresponding to $[\mathrm{Ca} 2+] \mathrm{i}$ increase of $300 \mathrm{nM}$ above baseline. In the absence of extracellular $\mathrm{Ca} 2+$ $\mathrm{NH} 2 \mathrm{Cl}$ exposure caused [Ca2+]i increases from $0.81+/-0.02$ to $1.01+/-0.13$, corresponding to $100 \mathrm{nM}$ increase above baseline. These effects were prevented by pretreatment of gland with DTT (100uM) or VitC ( $1 \mathrm{mM})$. Vitamin C introduction during ongoing $\mathrm{NH} 2 \mathrm{Cl}$ exposure arrested but did not reverse $[\mathrm{Ca} 2+] \mathrm{i}$ accumulation. These disturbances were arrested and partially reversed $1 \mathrm{~min}$ after exposure to DTT $(43.2 \%+/-6.5 \%$ decrease from max $)$, compared to a minimal reversal in the absence of DTT $(9.1 \%+/-1.9 \%$ decrease from max, $\mathrm{p}<0.01)$ In permeabilized glands loaded with mag-fura-2, $\mathrm{NH} 2 \mathrm{Cl}$ exposure irreversibly depleted intracellular stores. This was prevented by VitC, but not reversed by its addition during ongoing $\mathrm{NH} 2 \mathrm{Cl}$ exposure. DTT pretreatment prevented store-emptying, and its application during ongoing $\mathrm{NH} 2 \mathrm{Cl}$ exposure led to arrest of the depletion and partial refilling o intracellular stores. CONCLUSION NH2Cl causes intracellular store $\mathrm{Ca} 2+$ release and extracellular $\mathrm{Ca} 2+$ influx. $\mathrm{NH} 2 \mathrm{Cl}$-induced release of store $\mathrm{Ca} 2+$ is due to oxidation of intracellular thiol groups, with inhibition and recovery in the presence of thiol-reducing agent DTT Oxidant scavengers such as VitC may prevent or arrest such disturbances, but should not be expected to reverse ongoing injury due to $\mathrm{NH} 2 \mathrm{Cl}$.

\section{T1612}

Trichinella Spiralis Infection Alters Quality of Mucins in Previously H. Pylori (HP) Infected Gerbils

Mohamed Sagar, Waliul Khan, Richard Hunt

BACKGROUND: Mucin hypersecretion is an important component of the immune response to gastrointestinal nematode infection. Quantitative changes in mucus and qualitative changes in the terminal sugars of goblet cell mucins have also been observed during nematode infection. In contrast, H. pylori (HP) infection decreases gastric mucin synthesis via inhibition of galactosyl transferase. This effect may impair the gastric mucosal barrier and contribute to the mucosal injury induced by $H$. pylori infection. In this study we have investigated whether infection with the nematode, Trichinella spiralis can alter the quality of mucins in previously HP infected gerbils. METHODS: The gerbils were divided into 4 groups: control group, group infected with HP-Sydney strain (HP-SS1), group infected with Trichinell spiralis and group infected first with H. pylori and after 20 weeks with Trichinella spiralis. Gerbils were sacrificed to investigate the expressions of terminal sugars of mucins by lectin immunohistochemistry. Lectins used in this study were Griffonia Simplicifolia Lectin I (GSA I), Griffonia Simplicifolia Lectin II (GSL II) and Elderberry Bark Lectin (EBL) which specifically recognizes terminal N-acetylgalactosamine (GalNAc), N-acetylglucosamine (GlcNAc) and sialic acid, respectively. RESULTS: Subsequent infection by T. spiralis altered the expression of terminal sugars of mucins in HP infected gerbils. We observed a significant increase in the number of GalNAc and GlcNAc expressing goblet cells in HP infected gerbils which were also infected with T. spiralis (The number of GalNAc expressing cells/10 villi: 113 and 181 and the number of GlcNAc expressing cells/10 villi: 166 and 193 in HP infected and HP+TSP infected gerbils; respectively. There was no significant difference in sialic acid expressing goblet cells among the group. CONCLUSION: Our study demonstrated that $T$ spiralis infection altered the quality of mucins in gerbils previously infected with HP and suggests that helminth infection mediated changes in the composition of mucins may provide a protective effect in host defence in various GI disorders in which $H$. pylori infection is involved. 
$\mathrm{T} 1613$

Helicobacter Pylori Lipopolysaccharide Enhances the Production of iNOSDerived NO in Cooperation with the Induction of Toll-Like Receptor (TLR) 2 Through TLR4 in Gastric Epithelial Cells

Uno Kaname, Kato Katsuaki, Ohara Shuichi, Shimosegawa Tooru

[Background and Aims] Cell-surface Toll-like receptors (TLRs) play essential roles in the firstline of defense against virulent factors of Helicobacter pylori (HP). Microbial lipopolysaccharide (LPS) is a strong inducer of iNOS and its action is mediated through TLR4. Recent studies have suggested that HP-LPS may interact not only with TLR4 but also with TLR2. However, little has been known about HP-LPS induced signal transduction in the gastric epithelial cells. The present investigates the physiological roles of TLRs in HP-LPS induced iNOS in gastric epithelial cells. [Materials and Methods] Changes in expression of iNOS, TLR2 and TLR4 and downstream activation of MAPKs and NFKB were analyzed following stimulation of normal mouse gastric mucosal GSM06 cells with HP-LPS and IFN- $\gamma$ in the presence or absence of inhibitors for MAPKs and NFkB, as well as siRNA for TLR2 or TLR4. Localization of TLR2 in the human gastric mucosa was examined by immunohistochemistry. [Results] Incubation of GSM06 cells with HP-LPS increased iNOS and also enhanced TLR2 expression, but TLR4 expression remained. Increased expression of TLR2 in the human gastric mucosa with Helicobacter pylori infection was also demonstrated immunohistochemically. HP-LPSinitiated signal to induce iNOS and TLR2 expression was propagated through ERK phosphorylation and subsequent NFKB activation, independent on JNK and $\mathrm{p} 38$ pathways. The decline in TLR4 expression caused by specific siRNA transfection showed less response to TLR4-specific ligand, E.coli-LPS,i.e.; an increase in iNOS-induced NO production after E.coli-LPS treatment was inhibited in TLR4-diminished cells. Similar inhibition of induction of TLR2 and iNOS was observed in the cells treated with HP-LPS. TLR2-diminished cells lost the response to TLR2-specific ligand PAM3CSK4. Although TLR2-diminished cells retained iNOS induction following treatment with E.coli-LPS, the cells reduced the responsiveness to HP-LPS. These in vitro data indicated that HP-LPS might induce TLR2 mRNA through TLR4 signaling and that enhanced TLR2 might participate in iNOS-derived NO production by HP-LPS. In addition, HP-LPS may interact with TLR4 and inducible TLR2, resulting in facilitating HP-LPS stimulated iNOS induction. [Conclusions] TLR4 signaling initiated by HP-LPS and propagated by the activation of ERK and NFKB increased TLR2 expression and iNOS induction in gastric epithelial cells. This positive correlation between TLR2 and TLR4 may constitute a mechanism for stimulating the innate immune response against various bacterial components, including HP-LPS.

\section{$\mathrm{T} 1614$}

Proinflammatory Effects of Endothelial Nitric Oxide Synthase in Helicobacter Pylori-Induced Gastritis

Toshio Watanabe, Kazuhide Higuchi, Tetsuya Tanigawa, E.J. Sasaki, Masatsugu Shiba, Kazunari Tominaga, Yasuhiro Fujiwara, Nobuhide Oshitani, Tetsuo Arakawa

Background and Aim: Endothelial nitric oxide synthase (eNOS) is constitutively expressed on endothelial cells. The contribution of eNOS to inflammatory response has received less attention compared with inducible NOS, and the roles of eNOS in the pathogenesis of gastrointestinal inflammation have been conflicting and inconclusive. Infection with Helicobacter pylori (H. pylori) is associated with neutrophil infiltration and over-expression of inflammatory cytokines such as tumor necrosis factor- $\alpha$ (TNF- $\alpha$ ). In this study, we assessed the roles of eNOS in H. pylori-induced gastritis using eNOS-deficient mice. Methods: eNOSdeficient and wild-type mice were inoculated with $H$. pylori, and were sacrificed 6 months later. Expression of mRNAs for eNOS, TNF- $\alpha$, and intercellular adhesion molecule-l (ICAM1) in gastric tissue was determined by quantitative real-time RT-PCR. Measurement of myeloperoxidase (MPO) activity in gastric tissue, a marker of neutrophil infiltration, was assayed by the method of Bradley et al.. Expression of eNOS protein was evaluated by Western blotting, while localization of eNOS protein was examined immunohistochemically. Results: All mice inoculated with $H$. pylori were successfully infected, as assessed by bacterial culture. In wild-type mice, $H$. pylori infection increased mRNAs for TNF- $\alpha$ and ICAM-1 by 22.6-fold and 6.9-fold, respectively, comparing to un-infected mice. The MPO activity was also increased by 11.5 -fold in wild-type mice infected with $H$. pylori. H. pylori infection did not affect expression of both eNOS mRNA and eNOS protein. In wild-type mice, eNOS staining was limited to endothelial cells, and localization of eNOS expression did not change after induction of gastritis by H. pylori. Gastric inflammation induced by H. pylori was significantly inhibited in eNOS-deficient mice, the inhibition of TNF- $\alpha$ mRNA expression, ICAM-1 mRNA expression, and MPO activity being by $48 \%, 25 \%$, and $45 \%$, respectively. Conclusion: These results suggest that eNOS has proinflammatory effects on gastric inflammatory response to $H$. pylori.

\section{$\mathrm{T} 1615$}

A Functional Role of Interleukin-21 (IL-21) in Promoting Matrix Metalloproteinases Synthesis By Gastric Epithelial Cells During Helicobacter Pylori Infection

Roberta Caruso, Claudio Tosti, Daniele Fina, Ilaria Peluso, Valentina Gioia, Carmine Stolfi, Massimo C. Fantini, Thomas T. MacDonald, Francesco Pallone, Giovanni Monteleone

Background and Aim. Helicobacter pylori (Hp) infection causes a Thl cell-associated chronic inflammation which can lead to gastric ulceration. How Thl cell signalling promotes epithelial damage in Hp-infected subjects remains unknown. However, studies in other systems suggest that Thl cell-derived cytokines stimulate the local production of matrix metalloproteinases (MMPs), thereby promoting mucosal injury. Consistent with this, enhanced expression of MMP, such as MMP-2 and -9, has been seen in the Hp-infected gastric epithelium. Here we first examined whether in the Hp-infected gastric mucosa there is enhanced synthesis of IL-21, a T cell-derived cytokine, which associates with Thl cell response. Second, we examined whether gastric epithelial cells express IL-21 receptor (IL-21R) and whether these cells respond to IL-21 by increasing the production of MMPs. Methods. Gastric biopsies taken from patients with or without Hp infection were analysed for the content of IL-21 by Western blotting. IL-21R expression was examined in freshly isolated gastric epithelial cells and gastric epithelial cell lines by Western blotting. Induction of MMPs by IL-2l in gastric epithelial cells (AGS) was examined by Western blotting. To examine whether the IL-21 effect on MMPs relies on MAP kinases and/or NF-KB activity, AGS were treated with IL-21 for different time points and MAP kinase and NF-KB activation evaluated by Western blotting and EMSA respectively. The effect of TPCK, an inhibitor of NF-kB activation, on IL-21-mediated MMP production was also evaluated. Results. Constitutive production of IL-2l was seen in the gastric mucosa of controls and Hp-infected patients. However, a more pronounced expression of IL-21 was found in whole biopsies and isolated mucosal cells from Hp-infected patients in comparison to normal controls and patients with Hp-negative gastritis. Importantly, primary gastric epithelial cells and AGS expressed both the IL-21R and the common $\gamma$-chain, which are necessary for IL-21-driven signalling. Consistently, stimulation of AGS with IL-21 resulted in enhanced production of MMP-2 and MMP-9, but not MMP-7, whereas tissue inhibitors of MMPs remained unchanged. Time-course analysis also revealed that IL-21 enhanced NF-kB but not ERK1/2, p38 and JNK activation in AGS. Consistently, inhibition of NF-kB by TPCK inhibited the IL-21-induced MMP production. Conclusions. Data suggest that, in Hp-infected gastric mucosa, there is enhanced production of IL-21, and that this cytokine can contribute to the Hp-associated epithelial injury by promoting MMPs synthesis.

\section{T1616}

Ikk/Nf-kB Dependent Macrophage Activation Is Required for Helicobacter Pylori-Induced Chronic Gastritis

Ayako Yanai, Shin Maeda, Youko Hikiba, Tomoya Ohmae, Wataru Shibata, Keiji Ogura Masao Omata

Innate immune system is the first line of host defense against pathogens, and macrophages, main sources of pro-inflammatory cytokines, have been implicated. Helicobacter pylori ( $H$. pylori), one of the gram negative pathogens, induces chronic inflammation in stomach. To investigate the relationship between macrophage activation and gastric inflammation, we injected liposome-encapsulated clodronate for depletion of macrophages in H. pylori-infected Mongolian gerbils, which showed similar gastritis as human stomach. We found that gerbils treated with clodronate showed milder inflammation than ones without treatment. The NF$\kappa \mathrm{B}$ family of transcription factors plays an important role in regulation of inflammation through transcriptional regulation of pro-inflammatory cytokines in immune cells such as macrophages. To clarify a role of NF- $\mathrm{\kappa B}$ activation in $\mathrm{H}$. pylori-mediated gastritis, an inhibitor for IKK, which is a critical kinase for NF- $\mathrm{KB}$ activation, was administrated to $H$. pyloriinfected Mongolian gerbils every $24 \mathrm{~h}$ for two weeks. Inflammation levels were improved after the treatment of the inhibitor. Correspondingly, IL-1 $\beta$ and IFN- $\gamma$ levels of stomach of the gerbils were suppressed by the treatment of IKK inhibitor. Macrophage cells (J774A.1) were cultured with heat-killed $H$. pylori, it's culture supernatant, or $E$ coli lipopolysaccharide (LPS) for $24 \mathrm{~h}$, and the concentration of IL- 6 or TNF- $\alpha$, which is tightly regulated by NF$\kappa \mathrm{B}$ activation, was measured by ELISA. The culture supernatant, heat-killed $H$. pylori, or $E$. coli LPS induced IL-6 production. Cells treated with IKK inhibitor inhibited H. pylorimediated IL- 6 production compared to those without treatment. These results suggest that IKK/NF-кB dependent macrophage activation is required for development of H. pylorimediated gastritis. This mechanism may be one of the important factors for progression from chronic gastritis to gastric cancer.

\section{$\mathrm{T} 1617$}

The Neutrophil Activating Protein (HP-NAP) of Helicobacter Pylori Plays a Role in Adherence to Gastric Epithelial Cells and to Mucin Monique M. Gerrits, Ferry Namavar, Marion Sparrius, Arnoud H. van Vliet, Christina M. Vandenbroucke-Grauls, Johannes G. Kusters, Ernst J. Kuipers

Introduction: Adherence of Helicobacter pylori to the gastric epithelium is believed to be an important step in the induction of active inflammation of the mucosal laver. Several specific adhesins, like SabA, BabA2, AlpA and AlpB have already been identified, but other factors may well be involved. The H. pylori Neutrophil Activating Protein (HP-NAP) was previously identified as a protein involved in immune modulation and is considered to be a candidate for development of subunit vaccines against $H$. pylori. Aim: To assess the role of the Neutrophil Activating Protein (HP-NAP) in adherence of H. pylori to epithelial cells. Methods: The napA gene of H. pylori strain ATCC 43504 was disrupted by insertion of a kanamycin cassette. The wild-type ATCC43504 strain and its napA mutant were tested for their ability to adhere to Hela cells and gastric tissue sections. In addition, the binding of recombinant HP-NAP protein to mucin and sulfated oligosaccharides was studied by ELISA. Results: Wild-type H. pylori ATCC43504 displayed strong binding to Hela cells, but mutation of the napA gene resulted in a 10-fold reduction of this binding. The difference in adherence between the wild-type strain and the napA mutant was also observed when using gastric tissue sections. The wild-type strain primarily adhered to the mucus layer covering the epithelial cells. When compared to the wild-type strain, the napA-mutant displayed strongly reduced binding to purified mucin and sulfated oligosaccharides, suggesting that HP-NAP is indeed involved in binding to sulfated mucins. Conclusions: HP-NAP is a multifunctional protein that plays an important role in the persistence of $H$. pylori infection through binding to the gastric epithelium. The cell surface receptor involved in the interaction with HP-NAP is still unknown, but likely contains sulfated oligosaccharides. 


\section{T1618}

Helicobacter Pylori Infection Induces Accumulation of V $\delta 1$ T Cells Via CCR2 Upregulation

Seiji Futagami, Tetsuro Hiratsuka, Tomotaka Shindo, Tatsuhiko Hamamoto, Nobue Ueki, Kenji Suzuki, Masafumi Kusunoki, Ken Wada, Kazumasa Miyake, Katya Gudis, Hidemi Takahashi, Taku Tsukui, Choitsu Sakamoto

Background/Aims: Recent reports have shown that $\gamma \delta \mathrm{T}$ cells play an important role in mucosal immunity and possess anti-tumor effects. It has yet to be elucidated whether the response of the innate immune system, which includes $\gamma \delta \mathrm{T}$ cells, to antigenic stimulation such as by $H$. pylori component might contribute to the gastric mucosal defense system. Methods: Mucosal biopsies samples from $22 \mathrm{H}$. pylori-free and $75 \mathrm{H}$. pylori-infected patients were classified into grades I-III gastritis groups according to mononuclear cell infiltration based on the updated Sydney System. Phenotype analysis of peripheral T cells in H. pylori grade III gastritis were evaluated by FACScan. The number of $\gamma \delta$, V $\delta 1$, and V $\delta 2 \mathrm{~T}$ cells in gastric tissues was evaluated by immunostaining and then compared with counts in 17 patients after $H$. pylori eradication (Triple therapy consisting of omeprazole $(2 \times 30 \mathrm{mg} /$ day), clarithromycin $(2 \times 400 \mathrm{mg} /$ day $)$, and amoxicillin $(2 \times 750 \mathrm{mg} /$ day $)$ was prescribed for seven day). TGF- $\beta$ and IFN- $\gamma$ levels of peripheral V $\delta 1 \mathrm{~T}$ cells stimulated by H. pylori component (H. pylori urease, H. pylori-water extract protein (HPWEP) CagA+, HPWEP CagA-), IL-1 $\beta$, IL-7, and LPS were measured by ELISA method. CCR2 mRNA expression levels in stimulatedVס1 T cells were also evaluated by real-time PCR. Results: $\gamma \delta \mathrm{T}$ cell count was significantly higher in grade III gastritis patients, who exhibited strong IgA and IgG responses to $H$. pylori urease, than in other groups. Vס $1 \mathrm{~T}$ cells were dominantly resident in $H$. pylori-

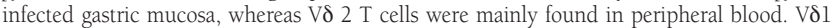
$\mathrm{T}$ cell count was significantly reduced after $H$. pylori eradication therapy. In vitro studies, H. pylori component stimulation significantly upregulated both TGF- $\beta$ and IFN- $\gamma$ production in supernatant from stimulated peripheral V $\delta 1 \mathrm{~T}$ cells. Moreover, CCR2 mRNA expression in Vठ1 T cells stimulated with $H$. pylori components was significantly increased. Conclusion: Vo $1 \mathrm{~T}$ cell accumulation of $\mathrm{H}$. pylori-infected gastric mucosa might occur through the upregulation of CCR2 expression.

\section{T1619}

Bone Morphogenetic Protein Signaling Regulates Fundic Gland Homeostasis and Suppresses Tumorigenesis At Gastric Epithelial Transition Zones in Mice Sylvia Pel-Bleuming, Xi He, Liudmilla Kodach, James Hardwick, Fiebo J. Ten Kate, Sander J. van Deventer, Maikel Peppelenbosch, Johan G. Offerhaus, Linheng Li, Gijs R. van den Brink

Background: Epithelial cells at the junction of two distinctive epithelial tissues are prone to receive conflicting information from the two different epithelial environments. This may make cells at epithelial junctions more vulnerable to tumorigenesis. Two important clinical examples of tumors associated with epithelial transition zones in humans are carcinomas that arise at the squamocolumnar junctions of the esophagogastric transition zone and the uterine cervix. Background \& aims: Morphogens regulate epithelial homeostasis in the gastrointestinal tract. Bone Morphogenetic Protein (BMP) signaling stimulates apoptosis and suppresses tumorigenesis in the small and large intestine. Here we examine the role of BMP signaling in the murine stomach. Methods: We examined the expression of the BMP signaling receptors Bmprla and Bmprlb and the localization of the phosphorylated (active) form of Smadl. We studied the role of the Bmprla in conditional Bmprla knock-out mouse. Results: The BMP signaling receptors are differentially expressed in the gastric epithelium. Bmprla is expressed in the gastric glands and by epithelial cells at the esophagogastric and gastrointestinal transition zones. Bmprlb is expressed by gastric pit (foveolar) cells and mesenchymal cells. Upon conditional inactivation of Bmprla mice develop fundic gland hyperplasia. Interestingly, these mice develop polyps that may develop intraepithelial neoplasia (dysplasia) specifically in the esophagogastric and gastrointestinal transition zones. We show that these transition zones are distinct areas of epithelium that lack some of the signals for differentiation of the adjacent gastric epithelium and show increased proliferation. Conclusions: Here we show that BMP signaling through Bmprla not only regulates fundic gland homeostasis but also specifically suppresses tumorigenesis at gastric epithelial transition zones in the mouse. This is the first example of a pathway that controls homeostasis at the junction between different epithelial layers. Our data may have important implications for the understanding of the genesis of esophagogastric tumors in humans

\section{$\mathrm{T} 1620$}

Gastric Mucosal Fas Proliferative Signaling Requires Raf-1 Recruitment to the Disc, Erk1/2 Activation and NF-kB Activity Brian Moquin, Sharif Zubair, Hanchen Li, J.N. Carlson, Jeanmarie Houghton

Fas mediated apoptosis is increased in the stomach of both Helicobacter infected humans and mice. This increase in Fas mediated apoptosis targets specialized cells within the oxyntic mucosa preferentially, resulting in the loss of parietal and chief cells, with resultant atrophy of the fundic mucosa. As cells become metaplastic and dysplastic however, they continue to express Fas Ag receptor, but become resistant to apoptotic signaling. In addition to apoptotic signaling, the Fas $\mathrm{Ag} / \mathrm{L}$ cascade has been shown to signal for proliferation. Our experiments suggest that Fas proliferative signaling may be important in driving gastric cancer growth. The pathway for proliferative signaling has not been well worked out, and the pathway used likely differs between cell types. Our experiments were designed to define Fas apoptotic and proliferative signaling pathways within gastric mucosal cells. Using a rat gastric mucosal cell line (RGM-1), we determined that gastric mucosal cells require a mitochondrial amplification loop (Type II signaling) for apoptotic signaling, but not for proliferative signaling. We manipulated the Fas Ag cascade at each known step of the Type II apoptotic path using a combination of dominant negative proteins (FADD-DN), chemical inhibitors (z-VAD-fmk, z-IEDT-fmk, UO126, ALLN) or naturally occurring inhibitory proteins (IkB, FLIP). We found that proliferative and apoptotic signaling bifurcate at the death inducing signal complex (DISC). Specifically, inhibition of FLICE with its known inhibitor-
Flice Like Inhibitory Protein (FLIP) abrogates apoptotic signaling, but leaves proliferative signaling intact. Increasing the strength of signal by increasing ligand availability or Fas Ag receptor abundance in the presence of FLIP shunts Fas signaling toward proliferation. To address the mechanism by which this switch occur, DISC complexes were immunoprecipitated under conditions favoring either apoptosis or proliferation and recruitment of adaptor proteins evaluated by Western blot. Raf-l is recruited early after receptor ligation and activates the MEK/Erk1/2 MAPK signal cascade. Erk1/2 activation of NF-kB has both proproliferative and anti-apoptotic effects through upregulation of the anti-apoptotic protein survivin. These studies demonstrate dual function for the Fas pathway within gastric mucosa cells. The switch from apoptotic to proliferative signaling may contribute to gastric cancer

\section{T1621}

High Expression of Metallopanstimulin-1 Reflects Tumor Progress and Stable Suppression of Its Expression Inhibits Tumorigenicity of Gastric Cancer in Vitro and in Vivo

Yunwei Wang, Ying Qu, Jianfang Li, Xuehua Chen, Bingya Liu, Qinlong Gu, Zhenggang Zhu

Metallopanstimulin-1 (MPS-1) belongs to the S27E family of ribosomal proteins, whose mRNA is highly expressed in a wide variety of actively proliferating cells. Convincing evidences have also shown that MPS-1 is overexpressed in many types of malignant tumors and maybe involved in progression toward malignancy. However, the expression of MPS $l$ in gastric cancer is still unknown and its potential role in cancer development and progression has not been explored. In this study, we produced the anti-MPS-1 polyclonal antibody and the expression pattern of MPS- 1 was determined in 90 primary gastric cancer specimens and 6 gastric cancer cell lines by immunohistochemistry and Western blot. To investigate the functional significance of MPS-1 expression, three recombinant plasmids producing hairpin small interfering RNA (siRNA) to target MPS-1 mRNA were constructed and transfected into gastric cancer cell line SGC7901, an adherent, moderately differentiated, human gastric cancer cell line, which we previously used to clone MPS-1 as a gastric cancerassociated antigen gene. The stable cell lines transfected with the recombinant plasmids containing the effective siRNA genes were selected and the biological features of the cells were examined. We found that MPS- 1 was overexpressed in $86 \%$ of the tumor samples and all gastric cancer cell lines when compared with normal gastric mucosa tissues and immortalized gastric mucosal epithelial cells. In addition, MPS-1 expression was even higher in tumors with late TNM clinical stage than those with early clinical stage $(\mathrm{p}=0.007)$, which implied that MPS-1 might also play an important role in malignant transformation of gastric cancer. The MPS-1-specific siRNA recombinant plasmids pRNAT-133 could significantly reduced the mRNA and protein expression levels of MPS-1 in pooled SGC7901/ pRNAT-133 cells. Moreover, Hoechst33342 staining and flow cytometry showed an increased spontaneous apoptosis rate of SGC7901/ pRNAT-133 transfectants. Furthermore, the stable transfectants also exhibited significantly decreased proliferation and tumorigenicity in compared with vector transfectants and parental cells in vitro and in vivo. These results provide the evidence that MPS-1 may play an important role in the control of cell proliferation and tumor development in gastric cancer and suggest MPS- 1 is a promising target for gastric cancer treatment.

\section{$\mathrm{T} 1622$}

5'-Untranslated RNA of the Human VEGF Transcript Facilitates Abnormal Cell Growth of Human Colon Cancer Cells

Kiyoshi Masuda, Shigetada Teshima-Kondo, Kensei Nishida, Hideyuki Sasaki, Kumiko Tominaga, Yuki Kuwano, Tomoko Kawai, Kyoko Morita, Atsuo Sekiyama, Keiya Nakamura, Kazuhito Rokutan

Non protein-coding RNA (ncRNA) accounts for up to $98 \%$ of the total transcripts from the human genome, and a large number of functional ncRNAs have been identified and demonstrated to play crucial roles in RNA-directed gene expression regulation. To explore a novel functional RNA, we have focused on the $5^{\prime}$ untranslated region (UTR) of human VEGF transcript. The 5' UTR is highly structured and unusually long (1,038 nucleotides) and contains sequences that are important in regulating gene expression, including mRNA stability and translational efficiency. During our investigation on specific roles of the 5 ' UTR in cap-independent translational regulation (FASEB. J. 2004), we found that VEGF 5' UTR could work as a trans-acting functional RNA molecule and regulate expression of distinct genes. We established HCT116/VEGF 5 ' stable transfectants that expressed the full length sequence of VEGF 5' UTR RNA. To identify genes specifically modulated by the 5' UTR, we performed cDNA microarray analysis. The CDNA microarray analysis showed that overexpression of VEGF 5' UTR specifically downregulated 7 genes including pro-apoptotic genes and upregulated 18 genes including cell cycle-promoting genes. Moreover, we demonstrated that HCT116/NEGF 5 ' cells showed an abnormal cell growth, which was demonstrated by their higher proliferation rate and cell cycle progression in low-serum conditions, and a higher degree of anchorage-independent growth. To identify the impact of VEGF 5' UTR RNA on malignant tumorigenicity, we injected HCT116/VEGF 5' or parental control cells subcutaneously into the flank fat pad of nude mice. At 28 days after injection, HCT116/ VEGF 5' cells formed larger tumors (2-fold increase) than parental control cells did. These suggest that the 5' UTR of VEGF messenger can function as a modifier of tumor cell development through modulating gene expression that are crucial for malignant transformation. 
$\mathrm{T} 1623$

Influence of P21 and P53 On HDAC-Mediated Apoptosis in HCT116 Colon Cancer Cells in Vitro and in Vivo

Mathias Ocker, Steffen Zopf, Daniel Neureiter, Marion Ganslmayer, Eckhart G. Hahn, Christoph Herold

Background: Inhibitors of histone deacetylases (HDAC-I) have been shown to exert antitumoral effects on different human cancers both in vitro and in vivo. We therefore investigated the effect of a novel HDAC-I, A-422378.0, on wild-type HCT116 colon cancer cells as well as derivatives lacking either $\mathrm{p} 21$ or $\mathrm{p} 53$. Methods: Cell lines were incubated with A-422378.0 at different concentrations $(0.001$ to $10 \mu \mathrm{M})$ for 3 to $120 \mathrm{~h}$. Cell viability, proliferation and apoptosis rates were determined quantitatively and verified qualitatively by western blotting detection of mitochondrial membrane potential breakdown, activation of caspases 3 and 8 and cytokeratin 18 cleavage.A subcutaneous xenograft model was established in NMRI mice. Animals were treated with daily intraperitoneal injections of $10 \mathrm{mg} / \mathrm{kg}$ for 14 days. Tumor size was measured, proliferation and apoptosis rate were determined immunohistochemically. Results: All three HCT116 cell lines responded to A-422378.0 treatment with a time- and dose-dependent induction of apoptosis with p21-/- cells being more sensitive than wildtype or p53-/- cells. Apoptosis induction was induced by breakdown of $\Delta \Psi \mathrm{m}$. Caspase 3 was activated in wild-type cells only. Xenografts showed a pronounced growth inhibition upon HDAC-I treatment, paralleled by down-regulation of PCNA and induction of apoptosis Conclusions: Treatment of wild-type or knock-out HCT116 cells with A-422378 0 exerts potent anti-proliferative and pro-apoptotic effects in vitro and in vivo. Loss of p53 leads to resistance to this treatment, while HCT116 cells deficient in p21 are sensitised to HDACinhibitor mediated apoptosis. A-422378.0 might therefore be a potent new therapeutic compound for the treatment of advanced colorectal cancers.

\section{T1624}

TGF- $\beta$ Modulates Ubiquitination of Tumor Suppressor, ELF, and SMAD3, by Praja, An E3 Ligase, That Control Hepatocellular Cancer Tapas Saha, Katuri Varalakshmi, Hye Beak, Sang S. Kim, Yi Tang, Jogunoori Wilma, Volpe A. Eugene, Bibhuti Mishra, Lopa Mishra

The TGF- $\beta$ superfamily plays essential roles in cell differentiation and liver development, as well as in both suppression and formation of hepatocellular carcinoma (HCC). TGF- $\beta$ is modulated through Smad activation and is dependent upon adaptor proteins such as ELF, a $\beta$-spectrin as well as modifying E3 ligases such as PRAJA. Interestingly nearly two thirds of elf-/- mutant mice develop hepatocellular carcinoma spontaneously at 12-15 months. PRAJA is expressed in abundance in HCC and is an E3 ligase regulated by TGF- $\beta$ and BMPs. Aims: To analyze molecular interactions between ELF, Smad3 and PRAJA in TGF- $\beta$ /BMP signaling pathway in both normal liver formation and in HCC. Results: 1. PRAJA is expressed in hepatocyte progenitor cells. Inhibition of PRAJA resulted in small livers, loss of liver architecture with loss of hepatocytes growth and apoptosis with lineage alteration to cartilage formation. 2. Confocal Microscopy and immunoprecipitation reactions demonstrate association of PRAJA with ELF in a TGF $\beta$-dependent manner. 3. PRAJA manifests substantial TGF- $\beta$ regulated ubiquitination of ELF and Smad3 via its C-terminal RING-H2 domain. Disruption of the RING finger domain of PRAJA (Delta-PRAJA) disrupts PRAJA ubiquitination of ELF. 4. ELF and Smad3 interact with the N-terminal fragment of PRAJA (Delta-150-395) in presence of TGF-beta. 5. PRAJA interacts with the Domain II of ELF (17 repeats region) that contains both ankyrin binding domain and NLS under TGF- $\beta$ stimulation. 6 . All three of the above interactions induce TGF-beta transcription through Luciferase reporter assays using 3TPLux and (SBE)4Lux reporter plasmids. 7. Phosphorylation of PRAJA induces association with ELF, however, this is not TGF- $\beta$ dependent. 8. Conclusions: PRAJA associates with ELF and PRAJA at independent sites from its RING finger domain. The RING finger domain is required for ELF and Smad3 for ubiquitination. Phosphorylation of PRAJA is not associated with the TGF- $\beta$ activity, however it induces PRAJA to associate with ELF. These studies reveal a mechanism for tumorigenesis in HCC whereby defects in adaptor proteins for Smads, such as ELF as well as Smad3, can undergo degradation by PRAJA, through the ubiquitin-mediated pathway and this RING E3 ligase serves as an important future therapeutic target in HCC.

\section{$\mathrm{T} 1625$}

Inhibition of Colon Tumor Progression and Angiogenesis By P16ink4a Amelie Boquoi, Tina Chen, Steven L. Gibson, Norman E. Sharpless, Colleen Brensinger, Greg H. Enders

The Ink4a/Arf tumor suppressor locus is widely inactivated in cancer but little is known about the tumor biology of its two products, p16Ink4a (p16) and Arf. Both the p16 and Arf promoters are methylated in a significant fraction of human colon carcinomas, implying a functional role for the locus in this tumor type. We have demonstrated previously that Ink4a/Arf-null colon tumors display increased growth and vascularity in C57Bl6 mice carrying the Min (multiple intestinal neoplasia) mutation. We present here an analysis of Min colon tumors $(\mathrm{N}=215)$ in mice with or without selective deficiencies in pl6 or Arf, respectively. Absence of Arf did not significantly alter tumor formation. In contrast, tumors in mice lacking p16 were moderately larger and redder. Histological analysis demonstrated that these tumors contained significantly more pockets of necrosis $(P=0.02)$, a marker of carcinoma in situ; less apoptosis $(P=0.02)$; and higher red blood cell density $(P=0.02$, 0.006 within vessels). Biochemical analyses demonstrated increased levels of vascular endothelial cell growth factor $(V E G F, P<0.01)$. Exogenous pl 6 expression in human colon tumor cells in vitro inhibited VEGF production. Microarray analyses of tumor mRNA expression have identified additional candidate mediators of increased angiogenic signaling in the pl6-null tumors. These results suggest that pl6 constrains colon tumor progression, in part through inhibiting angiogenic signaling.

\section{T1626}

Anoikis Induction By Galectin-1 in HCC Cells Requires Endocytosis of $\alpha 5 \beta 1$ Fibronectin Receptor

Hugo Sanchez Ruderisch, Martina Welzel, Katharina Detjen, Hans-Joachim Gabius, Bertram Wiedenmann, Stefan Rosewicz

Background: We have previously shown that Galectin-l (Gal-1) inhibits anchorage dependent growth of various human carcinoma cell lines via Gl cell cycle inhibition. In contrast, Gal1 activates proapoptotic signaling pathways when applied under anoikis conditions, i.e. to cells denied contact to an appropiate ECM. Gal-1 stimulated anoikis and cell cycle inhibition required the presence of the $\alpha 5 \beta 1$ fibronectin receptor (FNR) and relied on binding and functional interaction of Gal-1 with the FNR complex. Of the carcinoma cell lines studied, hepatocellular carcinoma (HCC) cell lines were consistently susceptible to Gal-1 stimulated anoikis, suggesting that Gal-1 activated proapoptotic pathways may be targeted for treatment of HCC. Here we used HCC to elucidate the mechanisms that specify underlie Gal-1 stimulated anoikis. Methods: Interaction between Gal-1 and integrins was analyzed using biotinylated Gal-1 and/or fluorescence labeled anti-integrin antibodies in flow cytometry and immunofluorescence microscopy. Apoptosis induction was determined based on the pre-Gl fraction in DNA-histograms using flow cytometry. Subcellular fractionation and subsequent immunoblotting were carried out to determine integrin or Gal-1 complement of the membrane compartment. Results: Gal-1 bound to the surface of Hep-G2 HCC cells and co-localized with the $\alpha 5 \beta 1 \mathrm{FNR}$. Under anoikis conditions, but not in adherent cultures, Gal-1 binding was rapidly followed by a reduction of FNRs on the cell surface, as determined by flow cytometry. This corresponded to a marked decrease of both $\alpha 5$ and $\beta 1$ subunits in membrane fractions of Gal-1 treated cells, confirming internalization of the FNR. Immunofluorescence studies further revealed a concordant redistribution of Gal-1 from the cell surface into the cytosol. In contrast to Gal-1, Galectin-3 was unable to stimulate internalization. Moreover, the amount of $\alpha 2, \alpha \mathrm{V}, \beta 4$ or $\beta 5$ integrin subunits on the cell surface was not influenced by Gal-1, suggesting that internalization was induced via a specific and selective interaction between Gal-1 and the FNR. Preatreatment of cells with filipin, a compound that inhibits endocytosis, prevented Gal-1 dependent internalization of the FNR and protected Gal-1 treated cells from anoikis. A functional requirement for FNR internalization was further supported by data from cell lines which despite Gal-1 binding failed to internalize $\alpha 5 \beta 1$ integrin, and were found resistant to Gal-1 stimulation of anoikis. Conclusions: Our data characterize the internalization of the FNR-Gal-1 complex as an integral functional step in the signal transduction process leading to apoptosis induction under anoikis conditions.

\section{$\mathrm{T} 1627$}

Fas AG Signaling in Colon Cancer: A Role in Proliferation and Metastasis Sharif Zubair, Hanchen Li, Brian Moquin, J.N. Carlson, Jeanmarie Houghton

Mechanisms to overcome apoptosis are crucial for cancer cells to acquire if they are to survive. The Fas Ag pathway of apoptosis is commonly dysregulated in cancer cells. Dysregulation occurs at several sites along the cascade, however complete loss of receptor is unusual. Thus retention of surface receptor suggests a pressure may exist to preserve the pathway for functions other than apoptosis. Colon cancer cells express Fas Ag but appear resistant to Fas mediated apoptosis. These cells also express Fas L on the surface which may function in immune counter attack. We hypothesized that Fas Ag is retained in colon cancer cells because it would give the cells a proliferative advantage. We also reasoned that Fas L expression would increase liver metastasis. MC38 cells are a colon cancer cell line derived from C57BL/6 mice. MC38 cells were transfected to express FLIP (FLICE -like inhibitory protein) +/- Fas L. Cell growth characteristics were assessed in vitro by Annexin V staining (apoptosis), cell counts, MIT assay and BrdU incorporation (proliferation). One million cells were injected subcutaneously or intravenously into C57BL/6 mice, and the incidence, size and invasive nature of local tumors, and the number of hepatic metastasis evaluated. MC38 cells and MC38 cells transfected with the empty vector express moderate amounts of Fas $\mathrm{Ag}$ and no detectable Fas L. Cells grow poorly in culture and undergo Fas L induced apoptosis. $30 \%$ of mice $(n=10)$ injected with these cells developed subcutaneous tumors. No hepatic tumors were identified $(\mathrm{n}=10)$ in mice receiving iv injections. Cells engineered to express FLIP (MC38/FLIP) were resistant to Fas mediated apoptosis, and increased proliferation with ligand. $100 \%$ of mice injected sq with MC38/FLIP cells developed tumors $(\mathrm{n}=10)$, and $30 \%$ of mice receiving iv injections $(\mathrm{n}=10)$ developed hepatic tumors. Cells expressing both FLIP and Fas L had high proliferation rates and were resistant to Fas L mediated apoptosis. Subcutaneous tumors developed in 100\% of mice. These tumors were large, vascular and invasive. Mice receiving iv injections developed numerous hepatic tumors, with 2 mice developing greater than 100 large lesions $(\mathrm{n}=10)$ within 2 weeks of injection. The ability to avoid Fas mediated apoptosis may be an early event in colon cancer. Blocking the apoptotic pathway while retaining proliferative signaling may offer cells a local growth advantage while the expression of ligand increases both proliferation (by bypassing the need for exogenous ligand for signaling) and metastasis. Agents targeted at this pathway may be suitable for colon cancer therapy.

\section{$\mathrm{T} 1628$}

Differentiation Regulator Musashi-1 Enhances Malignant Potentials in AGS Human Gastric Cancer Cells

Hiroaki Murata, Shingo Tsuji, Masahiko Tsujii, Masayo Matsuoka, Hiroshi Eguchi, Sunao Kawano, Norio Hayashi

[Background \& Aims] Musashi-1 (MSI-1) is an RNA-binding protein that regulates cell differentiation. We reported that MSI-1 was expressed in the stomach, in particular, in Helicobacter pylori-infected gastric mucosa. Previous reports showed that MSI-1 expression correlated with grades and stages of brain tumors, but MSI-1 expression in gastric cancer has poorly been investigated. Our preliminary investigation revealed that MSI-1 was expressed in human gastric cancer tissue. Here we aimed to explore the involvement of MSI-1 in the development of gastric cancer in vitro in terms of proliferation, apoptosis, and colony 
formation. [Materials and Methods] AGS human gastric cancer cells were transfected with Msi-1 cDNA (AGS-Msi-1 cells) and markers involved in proliferation and apoptosis were examined by RT-PCR and Western blotting. Cell growth was evaluated by WST-8 assay. After apoptosis was induced by actinomycin D $(0,0.01,0.1,1 \mu \mathrm{g} / \mathrm{ml})$ and $\mathrm{H} 2 \mathrm{O} 2(0,0.001$, $0.01,0.1 \%)$, cell numbers were counted by WST- 8 assay and apoptotic cells were visualized by Hoechst 33342 staining and quantified by flow cytometry with propidium iodide (PI) staining and double staining with annexin V. Cells were cultured in soft agar to examine anchorage-independent growth capacity. [Results] Compared with wild-type and mock AGS cells, AGS-Msi-1 cells grew significantly faster $(\mathrm{p}<0.05)$, which was supported by ERK activation. AGS-Msi-1 cells exhibited higher levels of anti-apoptotic Bcl-2 and lower levels of pro-apoptotic Bax and Bad. While actinomycin D and $\mathrm{H} 2 \mathrm{O} 2$ induced apoptosis in a dosedependent manner, AGS-Msi-l cells circumvented apoptosis and significantly more cells survived $(\mathrm{p}<0.05)$. Flow cytometry with PI staining and double staining with annexin $\mathrm{V}$ revealed a reduction in the sub-Gl cell population and the apoptotic cell fraction, respectively, in AGS-Msi-1 cells. After induction of apoptosis, ERK was activated while JNK activation was suppressed in AGS-Msi-1 cells. Soft agar assay revealed that AGS-Msi-1 cells formed markedly larger colonies. [Conclusions] MSI-1 expression induces pro-proliferative and antiapoptotic properties as well as large colony formation in AGS cells. MSI-1 enhances malignant potentials in gastric cancer cells

\section{T1629}

Role of MAT and SAME in Colon Cancer Pathogenesis

Hui Chen, Mark Lin, Meng Xia, Xiaopeng Ou, Heinz Josef-Lenz, Shelly C. Lu

Methionine adenosyltransferase (MAT) is a critical cellular enzyme that catalyzes the formation of S-adenosylmethionine (SAMe). In mammals, two different genes, MATIA and MAT2A, encode for two homologous MAT catalytic subunits, al (forms either a dimer MAT III, or tetramer MAT I) and a2 (MAT II); while a third gene MAT2b, encodes for a regulatory subunit b that regulates MAT II. MATlA is expressed mostly in liver whereas MAT2A is widely distributed. In liver, increased MAT2A and MAT2b expression is associated with lowering of cellular SAMe levels, increased growth and dedifferentiation. Interestingly, SAMe can inhibit liver cell growth and induce apoptosis in liver cancer cells while protecting normal hepatocytes against apoptosis. One small study found increased MAT II expression in resected colon cancer specimens as compared to normal colon, which correlated with stage of disease. The mechanism of MAT II up-regulation and whether this may play a role in the pathogenesis of colon cancer is unknown. Furthermore, whether SAMe also regulates growth and death response in colon cancer cells is also unknown. The current study examined these questions using both resected colon cancer specimens and normal colon counterparts from the same patients and colon cancer cell lines RKO and HT29. In six of seven randomly selected patients, the MAT2A mRNA levels were elevated 2-fold in colon cancer specimens as compared to normal colon controls. MAT2b mRNA levels were also higher (up to 5fold), particularly variant two (an alternatively spliced variant) in five of seven cancer specimens as compared to normal colon controls. EGF, IGFl and leptin, mitogens for colon cancer cells that have been implicated in the pathogenesis of colon cancer, up-regulated MAT2A (but not MAT2b) expression in RKO and HT29 cells. In RKO cells, transfection with RNAi against MAT2A lowered MAT2A expression by $80 \%$, inhibited cell growth by $15 \%$ and prevented the ability of these growth factors to induce MAT2A. Importantly, the mitogenic effect of these growth factors is abolished if MAT2A induction is blocked by RNAi. SAMe and its metabolite methylthioadenosine (MTA) lowered the baseline expression of MAT2A and blocked the growth factor-mediated increase in MAT2A expression in colon cancer cell lines. Furthermore, SAMe and MTA inhibited growth of colon cancer cells by inducing apoptosis. Collectively, these data suggest similar to the liver, up-regulation of MAT2A also provides a growth advantage and SAMe and its metabolite MTA regulate growth and death in colon cancer cells.

\section{$\mathrm{T} 1630$}

Unique Accumulation of Ser ${ }^{45}$-Phosphorylated $\beta$-Catenin Across Different Murine Models of Proliferation

Shahid Umar, Pomila Singh, Joseph H. Sellin

Background: Activation of $\beta$-catenin is recognized as a critical early factor in tumorigenesis. Several pathways of $\beta$-catenin activation have been delineated, e.g. APC mutations, Wnt signaling and gain-of-function mutations in $\beta$-catenin. We previously described a novel mechanism of $\beta$-catenin activation in vivo using the transmissible murine colonic hyperplasia (TMCH) model: GSK-3 $\beta$ inhibition with increases in $\operatorname{Ser}^{45}$-phosphorylated $\beta$-catenin ( $\beta$ $\mathrm{cat}^{45}$, DDW2005). It is unclear whether this is a unique pathway of $\beta$-catenin effects or whether other models share a similar series of molecular events. Aim: Examine alterations in the $\beta$-catenin pathway in independent animal models of proliferation. Methods: $\mathrm{TMCH}$ in Swiss-Webster outbred mice was induced by Citrobacter rodentium inoculation. Transgenic mice overexpressing progastrin (hGAS), and mdrla-deficient (-/-) mice that lack the membrane efflux pump p-glycoprotein and develop spontaneous colitis were used as models of proliferation. Crypt isolation and immunohistochemistry (IHC) studies were performed via standard techniques. Results: During TMCH, an 8-fold increase in colonocyte proliferation was accompanied by dramatic increases in abundance of cellular and nuclear $\beta$-cat 45 . IHC studies also exhibited significant increases in cytoplasmic and nuclear $\beta$-cat ${ }^{45}$ immunoreactivity and correlated with biochemical data. Ser33,37/Thr4l residues in $\beta$-catenin however, failed to show changes in phosphorylation despite in vivo blockade of proteasomal degradation via intra-peritoneal injections of proteasomal inhibitor PS-341. hGAS mice exhibited hyperproliferation and an elevated BrdU-labeling index of crypts predominantly in the proximal colon compared to wild type littermates. mdrla-/- mice develop spontaneous colitis at 12 weeks of age when housed in a conventional mouse colony. At 8 and 10 weeks of age however, these animals developed significant hyperplasia compared to wild type littermates, without detectable colitis. IHC/biochemical studies performed in proximal and distal colons of hGAS and mdrla-/- mice respectively, exhibited dramatic accumulation of cytoplasmic and nuclear $\beta$-cat ${ }^{45}$ without apparent increase in Ser33,37/Thr4l-phosphorylated $\beta$-catenin. Conclusions: A pattern of $\beta$-catenin related molecular changes found in TMCH is also found in other animal models of colonic hyperproliferation, demonstrating a common final pathway in seemingly diverse initiating events. Defining the specific mechanisms involved in GSK$3 \beta$ inhibition and increased $\beta$-cat ${ }^{45}$ accumulation may provide insight into the regulation of cell census in the colon and may help identify possible targets for early intervention/chemoprevention.

\section{T1631}

Reversal of Resistance to CPT-11 in Colorectal Cancer Following Inhibition of PI3K

Farnaz Taghizadeh, Isabella T. Tai

Treatment for metastatic colorectal cancers (CRC) now includes irinotecan (CPT), with RR of $\sim 35 \%$ when used in combination with 5 -fluorouracil (5-FU) compared to $21 \%$ with 5 FU alone. This still indicates that many patients have CPT refractory disease. To identify potential mechanisms contributing to CPT resistance, microarray results revealed a 2 -fold increase in PI3K in MIP/C in comparison to sensitive MIP101 (MIP) cells. Confirmation by RT-PCR led us to question whether PI3K can modulate therapy sensitivity in therapy refractory CRC AIM: To determine if modulation of PI3K mRNA and protein influences the sensitivity of CRC cells to chemotherapy. METHODS: In-vitro studies to determine if PI3K influences the sensitivity of cells to chemotherapy were performed by incubating MIPand MIP/C with a specific inhibitors of PI3K, LY294002 (LY), followed by exposure to $200 \mu \mathrm{M}$ CPT. To assess which subunit of PI3K was responsible for modulating chemotherapy sensitivity, MIP and MIP/C cells were transfected with siRNA targeting PIK3CA (p110a catalytic subunit) and PI3CG (p110y subunit), followed by exposure to CPT. Cell viability was determined by MTS assay, while the percentage of cells undergoing apoptosis were analyzed by TUNEL and FACS analysis. RESULTS: In-vitro results indicated that following inhibition of PI3K with LY, a significantly greater number of refractory MIP/C cells were undergoing apoptosis, from $0+0.04 \%$ in MIP/CPT cells exposed to CPT to $2.7+1.1 \%$ apoptotic cells when exposed to LY in combination with CPT. This increased sensitivity to CPT in the resistant cells following inhibition with LY was associated with a decrease in PAkt and P-Bad, and increased PARP by immunoblot in comparison to MIP/C cells exposed to CPT alone. Further evaluation revealed that inhibition of PIK3CA, and not PIKCG, by siRNA, had the greatest influence on cell survival in MIP/C, resulting in a decrease in cell viability from $5.9+3.9 \%$ with CPT alone to $36.8+6.1 \%$ following inhibition of PIK3CA and exposure to CPT. Interestingly, DNA sequencing of exon 9 and 20 of PIK3CA revealed a specific missense mutation in exon 20 (kinase domain) of MIP/C resistant cells that was not present in either the sensitive MIP CRC or normal CCD-112 CoN colon cell lines CONCLUSIONS: In-vitro studies revealed that resistance to CPT in MIP/C cells was associated with overexpression of PIK3CA, and that specific inhibition of the p110 $\alpha$ catalytic subunit re-sensitized therapy-refractory cells to undergo apoptosis following treatment with CPT Mutations in exon 20 that were specific to MIP/C may be responsible for enhancing PIK3CA activity, and promote CPT resistance.

\section{T1632}

XAF1 Mediates Inhibition of Extracellular Signal-Regulated Kinase 1/2 Induced Apoptosis in Human Colon Cancer Cells Lifen Yu, Jide Wang, Bing Zou, Yunlin Wu, Benjamin Cy Wong

Background/Aims: XIAP-associated factor 1 (XAF1) has been identified to negatively regulate the function of X-linked inhibitor of apoptosis (XIAP) which is a potent member of IAP family that exerts anti-apoptotic effects. Extracellular signal-regulated kinase (ERK) pathway is thought to increase cell proliferation and protect cells against apoptosis. However, the involvement of ERK in XAFl expression in colon cancer cells is not clear. Methods: Four human colon cancer cell lines were studied in present study, including HCT1116 and LoVo (wide-type p53), DLDl and SW1116 (mutant p53). Two LoVo stable transfectants, XAF sense and anti-sense were established. The effects of dominant-negative MEKl (DN-MEKl) and MEK-specific inhibitor U0126 on ERK signaling pathway were detected by Western blotting, so did the expression of XAF1 and XIAP protein. The transcription activity of core $\mathrm{XAFl}$ promotor was assessed by dual luciferase assay. The levels of XAFl and XIAP mRNA expression were detected by RT-PCR. Cell proliferation was measured by MTT assay. Apoptosis was determined by hoechest 33258 staining. Results: We found that U0126 (30uM) markedly induced XAFl protein expression in HCT1116 and LoVo cells at $48 \mathrm{~h}$ but XAF1 protein levels of DLDl and SW1116 were still very low. U0126 induced XAF protein expression in a dose- and time-dependent manner in LoVo cells. DN-MEKl also induced XAFl protein expression after transfection for $48 \mathrm{~h}$. Conversely, the XIAP protein expression was down-regulation by these treatments. MEKl/2 phosphorylation was inhibited similar to that of ERKI/2 inhibition. But expression of total ERK1/2 and MEK $1 / 2$ protein levels were not decreased in LoVo cells. The activities of XAFl promotors were also significantly increased by both U0126 treatment and DN-MEKl transfection. The over-expression of XAF1 mRNA induced by U0126 pretreatment was blocked by emetine. rhEGF-stimulated phosphorylation of ERK had little or no effect on XAFl expression, and was blocked by pretreatment with U0126. LoVo cells with stable transfectants of XAFl were more susceptible to U0126-induced apoptosis in a time-dependent manner. LoVo cells with stable transfectants of XAFl anti-sense rendered less sensitive to U0126 treatment in cells proliferation and lowered apoptotic cells levels compared with its parent cells. Conclusions: These findings suggested that inhibition of ERKI/2 pathway could induce XAFl expression which may correlated with different status of p53 in human colon cancer cells. XAF1 is necessary for U0126-induced apoptosis by transcriptional regulation. 
T1633

Comparative Promoter Analysis Suggests the Role of the NF Kappa B Transcription Factor in the Transition Between Adenoma and Carcinoma Balazs Gyorffy, Orsolya Galamb, Bela Molnar, Zsolt Tulassay

We have only scarce knowledge about the molecular mechanisms that cause the progression from adenoma to colon cancer (CRC). In our recent micorarray study we set up a gene list associated with the difference between adenoma and CRC. A major challenge in the genomic era is to decipher the regulatory networks responsible for the expression changes in a set of co-regulated transcripts. We aimed to identify transcription factors (TF) having overrepresented binding sites (TFBS) in the promoter regions of genes associated with the adenomacancer transition. We analyzed data obtained using Affymetrix HGU133A plus 2 gene chips. The genome-wide gene expression patterns of 15 colon cancer specimens and 15 adenoma specimens were compared. Using two independent normalization approaches, we identified the top 37 differentiating genes. Using the EZ-Retrieve web service proximal promoter sequences were available for 17 genes. The MotifScanner software, which searches the TRANSFAC database, was used to detect TFBS in this set of sequences. We have identified altogether 119 different TFBSs. The statistics tool of the Java program TOUCAN was applied to the data in combination with the appropriate background model of human expected frequencies (EPD - 0.1), thereby detecting over-represented TFBSs among the selected genes. Only one binding site was significantly over-represented: NFKappaB65 ( $\mathrm{p}=0.0339)$. In summary, using an in silico comparative promoter analysis we identified the NF kappa B65 transcription factor, which could represent a key element in a putative transcription regulatory network responsible for the transition from adenoma to colon cancer.

T1634

TRF2 Promotes Multidrug Resistance in Gastric Cancer Cells By Inhibiting DNA Damage Response

Zhiguo Liu, Hanbing Ning, Tingting Li, Lina Zhao, Daiming Fan

DNA double-strand breaks (DSBs) induced by anticancer drugs or irradiation increase TRF2 expression as an early response to DNA damage, which inhibits activation of the ATMdependent DNA damage response network. Therefore, TRF2 might probably be a general DNA-repair factor rather than just a telomere-binding factor. In this study, the possible involvement of TRF2 in anticancer drugs induced DNA damage response and drug resistance was investigated. Adriamycin (ADR) and etoposide are clinically important anticancer drugs which exert cytotoxic effect by inducing DSBs. Gastric cancer cell line SGC7901 and two multidrug-resistant (MDR) variants were treated with various concentrations of ADR or etoposide. It was found that telomerase activity and TRF2 were upregulated after treatment in all three cell lines, but not TRF1. More dramatic upregulation of TRF2 was found in MDR cells, which is accompanied by decreased ATM, H2AX, and p53 expression. Inhibition of TRF2 expression by RNA interference in MDR cells partially reversed its MDR phenotype and overexpression of TRF2 in SGC7901 promoted its MDR phenotype. Taken together, current results indicate that TRF2 plays an important role in DNA damage response, and is involved in drug resistance of cancer cell. Further study of the biological functions of TRF2 might be helpful to dissect the molecular mechanism of MDR and generate novel target to overcome MDR.

\section{$\mathrm{T} 1635$}

Involvement of P73 Protein in Gastrointestinal Tumorigenesis Katarina Tomkova, Wael El-Rifai, Alexander Zaika

p73, a new member of the p53 tumor suppressor family, is a transcription factor that is increasingly being recognized in cancer research as an important player in tumorigenesis, as well as in chemotherapeutic drug sensitivity. Recently, we reported that that p 73 isoforms have oncogenic properties and are frequently overexpressed in more than $60 \%$ primary adenocarcinomas of the stomach and esophagus. We have also demonstrated that this overexpression can lead to the upregulation of $\beta$-catenin and TCF/LEF-dependent transcription. Thus, despite substantial structural and functional similarities to $\mathrm{p} 53$, our results suggest that p53 and p73 may differently regulate its transcriptional targets and may have distinct roles in human tumorigenesis. Indeed, in contrast to $\mathrm{p} 53$, we found that $\mathrm{p} 73$ can upregulate the gastrin gene transcription. Our results show that $\mathrm{p} 73$, when overexpressed, can bind to the gastrin promoter in vivo. This leads to transcriptional upregulation of gastrin mRNA and an increase in progastrin level. Moreover, for the first time, we found that NF-Y transcription factor is a critical regulator of gastrin gene transcription can interact with p73 in promoter regulation. Taken together, our results demonstrate a novel mechanism for regulation of gastrin gene transcription and support a concept that p53 and p73 may have different biological roles in gastrointestinal tumorigenesis.

\section{T1636}

Capsaicin Induces Apoptosis in Gastric Cancer Cells - A Possible New "Hot" Cancer Treatment?

Justine Chow, Manith Norng, Jianyuan Chai

Capsaicin (C18H27NO3) is an organic compound found in "hot" chili peppers belonging to the genus Capsaicum. It causes insensitivity to painful stimuli through desensatation of its receptor VR-1, after prolonged contact. Currently capsaicin is used in pepper spray and pain medication. Studies have shown that capsaicin induces apoptosis in some cancer cells, but its effect on gastric cancer is not clear. The aims of this study were to examine the effect of capsaicin treatment on gastric cancer cells and mechanisms involved. METHODS: Human gastric cancer cells AGS were treated with capsaicin at $10 \mu \mathrm{M}, 50 \mu \mathrm{M}$, and $200 \mu \mathrm{M}$ for 2,6 and 24 hours. Apoptosis was determined by Annexin V and TUNEL staining. Expression of oncogene products was examined by cDNA array, Western blotting and Immunocytochemistry. Normal human gastric epithelial cells were used for comparison. RESULTS: 1) Capsaicin treatment at $50 \mu \mathrm{M}$ increased apoptosis in AGS cells by 5.5, 12 and 4.5 fold $(\mathrm{P}<0.001)$ at
2, 6 and 24 hours, respectively, compared to vehicle-treated control. Capsaicin treatment activated VR-1 expression and membrane localization at 2 and 6 hours, but the cellular sensitivity to the treatment was reduced by $80 \%$ after 6 hours. Normal cells responded to capsaicin treatment more slowly, compared to cancer cells. Apoptosis in normal cells came after 6-hour capsaicin treatment, 1.5 and 5.5 fold $(P<0.005)$ at 6 and 24 hours, respectively 2) After 6-hour treatment, the pro-apoptotic effect of capsaicin on AGS cells was increased as concentration, 4,12 and 14 fold $(\mathrm{P}<0.01)$ at $10 \mu \mathrm{M}, 50 \mu \mathrm{M}$, and $200 \mu \mathrm{M}$, respectively. In addition, 200 uM capsaicin caused severe cell death in both types of cells, indicating its toxicity. 3) In parallel to VR-1 activation, capsaicin increased Bax expression by $40 \%$ and $120 \%(\mathrm{P}<0.01)$ at 2 and 6 hours, respectively, compared to control, but did not alter $\mathrm{Bcl}-2$ expression significantly. Capsaicin also caused Bax translocation from cytoplasm to mitochondria and increased mitochondrial permeability to allow cytochrome c release. CONCLUSIONS: 1) Capsaicin induces apoptosis in both gastric cancer and normal cells, but cancer cells to a much greater extent; 2) Capsaicin induces apoptosis in a dose-dependent but not time-dependent manner, likely due to its desensatation property; and 3) Capsaicin induces apoptosis in gastric cancer cells through the mitochondrial pathway.

\section{$\mathrm{T} 1637$}

Effects of Dietary Fatty Acid Composition On Rat Colon Carcionogenesis Takehiro Fujise, Takashi Kakimoto, Ryosuke Shiraishi, Kanako Yamaguchi, Sadahiro Amemori, Yasuhisa Sakata, Seiji Tsunada, Ryuichi Iwakiri, Kazuma Fujimoto

Background and Aim Resentry, there is an upward trend in age-adjusted mortality rates for colon cancer in Japan. This upward trend in death rates due to colon cancer is mainly attributable to Westernization of Japanese food habits. Many nutritional epidemiologic studies have showed a positive association between dietary fat intake and colon cancer. Many studies demonstrated that the colon tumor-promoting effect of a high fat diet depends not only on the amount consumed but also on the fatty acid composition. Wnt signaling pathway and Oxidative stress was integrally associated with colon carcinogenesis. The aim of this study is to investigate an effect of different dietary fat on colon carcinogenesis in the rat pretreated with azoxymethane (AOM). Materials and Methods Male SD rats were given intraperitonial injections of AOM dissolved in $1 \mathrm{ml}$ physiological saline once a week for 2 weeks at a dose of $15 \mathrm{mg} / \mathrm{kg}$ body wt or saline and fed on a basal diet or supplemented with $10 \%$ corn, olive, beef, and fish oil for 44 weeks. Colonic aberrant crypt foci (ACF) were examined at 12 weeks and tumors, including adenomas and carcinomas, were examined at 44 weeks. Colonic proliferation were evaluated by BrdU uptake, and COX-2, Beta-catenin, cyclinD1, Wnt2 and Wnt5a were analyzed by Western blotting. Lipid peroxidation of colonic mucosa was evaluated by TBARS, and $8-\mathrm{OHdG}$, which indicated DNA damage due to Oxidative stress, were analyzed by immunohistochemistory. Results Long-term dietary olive and fish oil decreased ACF, considered biological precursors of colon cancer in rodents and humans, at 12 weeks and colon cancer incidence and multiplicity at 44 weeks even in AOM treated rats. In contrast, both corn and beef oil increased ACF in AOM-treated rats at 12 weeks and promoted colon cancer incidence and multiplicity. These two dietary oils increased BrdU uptake and expression of cytosolic beta-catenin, cyclin Dl in the colon mucosa. Wnt 2 and COX-2, in rats fed with dietary beef oil, and Wnt5a, in rats fed with dietary corn oil, expression increased in both AOM-untreated and AOM-treated rats. In rats fed with both dietary corn and beef oil, iNOS, expression and 8-OHdG positive cell in colon mucosa were increased. Conclusion In the AOM-induced rat colon cancer model, Long term feeding of dietary corn oil and beef oil increased cell proliferation via Wnt signaling pathway in the colon mucosa, leading to promotion of colon cancer in the AOM treated rats. Furthermore, increase of DNA damage due to oxidative stress and iNOS, COX-2 expression in the rat colon mucosa were participate in the effect of colon carcinogenesis among different dietary fatty acid composition.

\section{T1638}

Focal Adhesion Kinase (FAK) and C-SRC Protect Mammalian Cells from Hyperosmotic Stress-Stimulated Apoptosis: Evidence That FAK Signaling Lies Upstream of the Actin Cytoskeleton in IEC-18 Intestinal Epithelial Cells Responses to This Apoptotic Stimulus

J. Adrian Lunn, Enrique Rozengurt

Background and significance: High salt diet has long been touted as a risk factor for gastric cancer. Basic mechanisms underlying this phenomenon are lacking. Hyperosmotic stress(HS) induces acute activation of MAP kinase signaling pathways, concomitant cell shrinkage and F-actin rearrangement. Most studies show resultant apoptosis in response to chronic HS exposure. Studies on anti-apoptotic responses of cells to HS are lacking. FAK is a cytoplasmic tyrosine kinase critical to cell translocation as it regulates focal adhesion turnover. FAK also promotes tumor invasion, and protects epithelial cells from anoikis. As such, FAK is poised to play a central role in preventing HS-induced apoptosis. GPCR, RTK and integrin activation of FAK, all suggest that F-actin re-organization is a central requirement. In epithelial cells from rat intestinal IEC-18, human colorectal Caco2 and gastric NCI-N87 cancers, we examined specific FAK tyrosine residues phosphorylated in response to HS, and we tested the hypothesis that F-actin organization is necessary for this FAK activation. Methods and Results: Confluent, serum starved IEC-18/Caco2/NCI-N87 cells were exposed to media +/- 0.45M sorbitol for various times. Western blots using phospho-specific (anti-FAKpTyr-397, pTyr576, pTyr-861, and anti-total FAK antibodies, of PAGE-resolved cell lysates were performed. Basal levels of FAK pTyr-397 varied in the various cell lines, while levels of FAKpTyr-861 and FAKpTyr-576 were universally low. However, $15 \mathrm{~min}$ exposure to $0.45 \mathrm{M}$ sorbitol stimulated increases in FAKpTyr-576, and dramatic increases in FAKpTyr-861 in all cell lines. Using two distinct specific Src-family kinase inhibitors we demonstrated that HSstimulated increases in FAK pTyr-861 are Src-family-dependent. Pretreatment of confluent IEC-18 cells with either $2 \mu \mathrm{M}$ cytochalasin D, or $3 \mu \mathrm{M}$ latrunculin A, significantly decreased the high basal FAK pTyr-397 levels, but neither method of F-actin disruption inhibited HSinduced FAKpTyr-397, 576, or 861 stimulation. Conclusions: Results presented here are significant as they imply that FAK may be an important upstream regulator (rather than downstream effector) of F-actin reorganization in response to hyperosmotic stress; and that this FAK mediated reorganization of F-actin is critical to cell survival and anti-apoptosis 
signaling in response to that stress. We propose that FAK phosphorylation on tyrosine 861 by $\mathrm{Src}$, and subsequent reorganization of F-actin, initiate an important anti-apoptotic signaling pathway. This FAK/c-Src anti-apoptotic signaling pathway may explain the tumor promoting properties of high salt diet on gastric cancer.

\section{T1639}

Early Apoptosis and Cell Death By ATX-S10na(II)-Mediated Photodynamic Therapy Are BAX- and P53-Dependent in Human Colon Cancer Cells Makoto Mitsunaga, Akihito Tsubota, Kohichi Nariai, Makoto Sumi, Tetsuya Yoshikawa, Akira Torii, Hisao Tajiri, Kiyotaka Fujise

Background \& Aim: Photodynamic therapy (PDT) is a clinical approach that utilizes photosensitizers for cancer therapy, including esophagus, stomach and colon. Various studies showed that PDT kills tumor cells via apoptosis and/or necrosis in vivo and in vitro, that is induced by various mechanisms, such as intracellular generation of reactive oxygen species. Although the role of Bax and p53 in PDT has been investigated, it remains controversial. So we aimed to determine whether or not Bax and $\mathrm{p} 53$ protein functions contribute to photosensitivity of human colon cancer cells in PDT using a novel lysosomal photosensitizer ATX-S10Na(II). Methods: Using the mismatch repair-deficient HCT116 human colorectal cancer cell lines and Bax null or p53 null isogenic derivatives, we investigated contribution of Bax and $\mathrm{p} 53$ to the cytotoxic and apoptotic effects of ATX-S10Na(II)-PDT. Photosensitivity of these cells to ATX-S10Na(II)-PDT, irradiated with a diode laser using a wavelength of $670 \mathrm{~nm}$, was determined by MTT assays, cell cycle analysis, annexin V apoptosis assays, caspase activity assays and western blotting. Results: The extent of phototoxicity was intensity of laser irradiation- and concentration of ATX-S10Na(II)-dependent manner. Occurrence of cell death was significantly Bax- and p53-dependent in MTT assays. In Bax null and p53 null cells, induction of apoptosis was significantly inhibited as compared to wild-type HCTll6 cells in the sub- $G_{1}$ population in cell cycle analysis or flow cytometry with annexin $\mathrm{V}$. While activation of caspases 2,3 and 9 were significantly Bax- and p53-dependent, caspase 8 was activated little. A level of cytochrome $c$ release from mitochondria was reduced but not absent in Bax null cells. Meanwhile, downregulation (photodamage) of anti-apoptotic proteins Bcl-2 and Bcl-xl were Bax- and p53-independent. Conclusion: Induction of early apoptosis and cell death by ATX-S10Na(II)-PDT are Bax- and p53-dependent in human colon cancer cells. The intrinsic mitochondrial pathway of apoptosis through a p53-Bax network in addition to photodamage of Bcl-2 and Bcl-xl play a central role in the early apoptotic process by ATX-S10Na(II)-PDT.

\section{$\mathrm{T} 1640$}

Silencing of the Regenerating IV (Reg IV) Gene By Sirna Inhibits the Growth and Proliferation of Human Colon Adenocarcinomas

Qizhi Luo, Kumar S. Bishnupuri, Courtney Houchen, Shrikant Anant, Brian K

Dieckgraefe

The human Reg gene family encodes four small-secreted proteins expressed throughout the gastrointestinal (GI) mucosa. Individual Reg genes have characteristic regional expression profiles in the columnar epithelium of the GI mucosa. Ectopic or increased expression of Reg proteins has been considered as a potential marker of colorectal mucosa at risk for neoplasia. Reg IV is the dominant member of the Reg multigene family expressed in the distal GI tract including cecal and colonic mucosa. Reg IV expression is significantly upregulated in malignancies of the GI tract including colon carcinoma, indicating its possible role in the growth and development of human colon adenocarcinomas. The present study tests the hypothesis that silencing of Reg IV by using siRNA in colon adenocarcinoma cells may inhibit growth, proliferation and increase sensitivity to radiation-induced cell death. A panel of 21-nucleotide siRNAs were designed to target Reg IV gene expression. The effects were tested in HCT116 human colon adenocarcinoma cells. Reg IV protein levels were assessed by western blot analysis. Several siRNA constructs were identified that led to significant reductions in RegIV protein expression. Based on western blot analysis, the addition of Reg IV-siRNAs (100nM) to the HCT 116 cells led to a $\sim 65 \%$ reduction in Reg IV protein expression 48 hours after transfection. Furthermore, transfection of cells with Reg IV-siRNAs (50nM) led to a time dependent inhibition in cell proliferation when compared to cells transfected with negative control-siRNA (Percentage reduction in cell number: $24 \mathrm{hr}$ $\sim 8 \%, 48 \mathrm{hr} \sim 30 \%, 72 \mathrm{hr} \sim 46 \%$ and $96 \mathrm{hr} \sim 47 \%$ ). Increasing concentrations of siRNA led to increased inhibition of cell proliferation $(100 \mathrm{nM} ; 96 \mathrm{hr} \sim 64 \%)$. Next we determined the effect of siRNA on radiation-induced cell death. Gamma-irradiation treatment demonstrated a dose-dependent reduction in fractional cell survival. Furthermore, Reg IV-siRNA transfected HCT 116 cells demonstrated a significantly greater reduction in cell survival. Conclusion: Reduction in Reg IV protein expression by Reg IV-siRNA inhibits the growth and cell proliferation of human colon adenocarcinomas and increases the effectiveness of cell killing following radiation. Inhibition of endogenous Reg IV expression may be a new therapeutic strategy for human colon adenocarcinomas

\section{T1641}

Constitutive Activation of MEKl Signaling Suppresses Chemotherapeutic Agents Induced Apoptotic Cell Death in Rat Intestinal Epithelial Cells Via COX-2 Activation

Youhei Horikawa, Michiro Otaka, Koga Komatsu, Sumio Watanabe

[BACKGROUND] We previously reported that the constitutive activation of MEKl (CAMEK) signaling results in transformation of rat intestinal epithelial cells (RIE and IEC-6). These results may suggest that MEK-ERK signaling plays an important role in the development and maintenance of intestinal cancers. Resistance to the chemotherapeutic agents is one of the distinct features in cancer cells. Several reports have provided the evidence that MEK inhibitors sensitize cancer cells to some chemotherapeutic agents. However, the exact role and mechanism of MEK-ERK signaling in chemo-resistance has not been addressed. We focus current study to evaluate the role of activated MEK-ERK signaling in the sensitivity to chemotherapeutic agents using CAMEK transfected rat intestinal epithelial cells. [METHODS] To address the role of MEK-ERK signaling in chemo-sensitivity in intestina epithelial cells, two kinds of CAMEK permanently expressing rat IEC-6 cells(CAMEK1, CAMEK2) were used in this the study. Cell viability was determined by WST assay following treatment of various concentrations of chemotherapeutic agents (CDDP, 5-FU, VP-16, CPT 11). Drug sensitivity was evaluated by calculation of inhibitory concentration of $50 \%$ (IC50) To investigate the mechanisms of chemo-resistance in CAMEK cells, apoptosis induced by CPT11 were analyzed by DAPI staining, DNA fragmentation and Western blot analysis of the $\mathrm{Bcl}-2$ homolog family. Also we examined the regulation of COX-2 protein expression in CAMEK cells by Western blot analysis, and the effect of COX-2 selective inhibitor (NS398) combination with CPT-11 in CAMEK cell viability by WST assay. [RESULTS]CAMEK cells showed increased IC50 compared to each empty vector transfected cells. Apoptotic cells and DNA fragmentation was remarkably suppressed in CAMEK cells. Expression of antiapoptotic protein Bcl-2, Bcl-xL and Mcl-1 were increased in CAMEK cells. In contrast, expression o proapoptotic protein Bak was increased in empty vector transfected cells. And expression of COX-2 protein was markedly increased in CAMEK cells. CPT-11 combination with NS398 resulted in a significant dose dependent reduction in cell viability compared with eithe drug-treated alone in CAMEK cells. [CONCLUSIONS]MEK activation led intestinal epithelial cells to chemo-resistant variant. Therefore, the MEK-ERK signaling could be contributed to chemo-resistance in transformed cells by anti-apoptotic effect via COX-2 activation. These results also indicate that the MEK-ERK signaling pathway and inhibition of COX-2 may provide an important target for developing new cancer drugs.

\section{$\mathrm{T} 1643$}

LPA Protection of CACO-2 Cells from Etoposide-Induced Apoptosis Is Erk1/2 MAP Kinase Dependent

Raluca Rusovici, Chris Yun

Lysophosphatidic acid (LPA) is an important regulator of cell proliferation, differentiation and survival. The aim of this study was to decipher the signaling pathway involved in the anti-apoptotic effect of LPA in Caco-2 cells. Apoptosis was induced by treating cells with the chemotherapeutic drug etoposide. Apoptosis was determined by flow cytometry of cells stained with annexin V-fluorescein isothiocyanate antibody. Treatment with $175 u \mathrm{M}$ etoposide for $24 \mathrm{~h}$ resulted in a doubling of annexin V-positive cells compared to control. A significant decrease in an annexin V-positive cell population was observed in the presence of etoposide and $20 \mu \mathrm{M}$ LPA, suggesting that LPA has an anti-apoptotic effect in Caco-2 cells. Consistently, etoposide doubled caspase-3/7 activity in Caco-2 cells compared with untreated control, which was completely blocked by preincubation for $20 \mathrm{~min}$ with the general caspase inhibitor zVAD-fmk. Co-incubation with LPA significantly blocked the increase in caspase activity induced by etoposide. This was confirmed by an increase in the level of inactive procaspase 3 in cells treated with etoposide +LPA compared to etoposide alone. We have previously shown that LPA activates Erk1/2 MAP kinase and Akt in Caco-2 cells. To determine a potential contribution by Erk, cells were preincubated with the MAPK inhibitor U0126 $(25 \mu \mathrm{M})$ or the PLC $\beta$ blocker U73122 ( $5 \mu \mathrm{M})$. In the presence of U0126 or U73122, LPA did not protect the cells from etoposide-induced apoptosis indicating that the anti-apoptotic effect of LPA is dependent on Erk1/2. In contrast, pretreatment of Caco-2 cells with the PI3K inhibitor LY294002 $(50 \mu \mathrm{M})$ had a relatively smaller effect and LPA was able to protec cells from apoptosis even in the presence of LY294002. To confirm involvement of MAP kinase, phosphorylation of proapoptotic Bad was determined. Phosphorylation of Bad ( $\mathrm{p}$ Bad) at Serl12 and Serl36 is an ERK- and Akt-dependent process, respectively. We determined levels of $\mathrm{p}$-Bad after $13 \mathrm{~h}$ of treatment by western blot with antibodies against $\mathrm{p}$ Serl12 or p-Serl36 of Bad. The level of p-Serl12 was increased in LPA + etoposide treated cells compared to etoposide alone, whereas the level of p-Serl36 was not changed. The total amount of Bad remained constant. In summary, the anti-apoptotic effect of LPA in Caco-2 cells is mediated by an inhibition of caspase activity and this protection involves Erkl/2 MAP kinases.

\section{T1644}

Apoptosis and Cell Cycle Regulating Proteins in Gastroenteropancreatic Neuroendocrine Tumors: Role of P27

Patricia Grabowski, Joerg Schrader, Dieter Hoersch, Rudolf Arnold, Christian N. Arnold, Harald Stein, Martin Zeitz, Peter T. Daniel, Isrid Sturm

Background - Gastroenteropancreatic neuroendocrine tumors represent a heterogeneous tumor entity. The growth pattern ranges from very slowly to fast growing, aggressive types of tumors. In previous analysis, we established the prognostic relevance of alterations in apoptosis- and cell cycle regulation in various malignancies. Little is known about the role of apoptosis- and cell cycle regulating proteins in this tumor entity. Aim - Regulators of apoptosis (p53/Bax) and the G1-restriction point (p16, p21, Cyclin E, p27) were evaluated. Patients and Methods - Tumor specimens from 89 patients with a complete 5-year followup were studied immunohistochemically for BAX, p16, p21, p27 and cyclin E expression and for $\mathrm{p} 53$ mutations by SSCP-PCR. 29 patients with localized, well-differentiated gastroenteropancreatic neuroendocrine tumors (WDET, WHO class 1 ) had been curatively treated by surgical or endoscopic tumor resection. 50 patients had well-differentiated endocrine carcinomas (WDEC, WHO class 2), 10 patients were diagnosed with poorly differentiated neuroendocrine carcinomas (PDEC, WHO class 3). The functional relevance of p27 was evaluated in the human neuroendocrine cell line BON by the use of siRNA. Results - 26 29 WDETs showed a high expression of p27, whereas all 10 PDECs displayed a low expression of p27. In the 50 patients with metastatic WDECs, 20/50 (40\%) tumors had a low p27 expression. Those 20 patients had a statistically significant worse prognosis (median survival of 68 versus 113 months, $\mathrm{p}=0.037$ ). All other investigated proteins were not of prognostic significance in this subgroup of patients. Downregulation of the p27 protein in the human neuroendocrine cell line BON resulted in an increased phosphorylation of the RB protein as well as an increase of cells in the S-Phase and G2/M Phase of the cell cycle. Discussion - The loss of p27 seems to play a critical role in the progression of gastroenteropancreatic neuroendocrine tumors. The analysis of p27 expression identifies subgroups in metastatic disease with less favorable prognosis (p27 low expression). The 
underlying mechanism may be due to increased cell cycle progression in those tumors. We propose that the determination of $\mathrm{p} 27$ expression could be used to individualize therapeutic strategies in this tumor entity in the future.

\section{T1645}

FOXMl Transcription Factor Is Essential for Proliferation and Growth of Mouse Colorectal Cancer Induced by AOM/DSS Protocol

Yuichi Yoshida, Robert H. Costa

Colorectal cancer is one of the most common fatal malignancies in the world, with an incidence second only to lung cancer. The Forkhead Box Ml (FoxMl) transcription factor belongs to the Fox family proteins that share homology in the winged helix DNA binding domain. FoxMl is ubiquitously expressed in all proliferating mammalian cells and tumor derived cell lines where it regulates expression of cell cycle genes critical for DNA replication and mitosis. We previously showed that FoxMl deficient (-/-) mouse hepatocytes are resistant to developing carcinogen induced mouse hepatocelluar carcinoma. In this study, we demonstrated that human and mouse colon cancer cell lines and human colon cancer specimens express high levels of the FoxMl transcription factor. To investigate the role of FoxMl in colorectal cancer, we induced colorectal cancer in Rosa26-FoxMl transgenic (TG) mice that ubiquitously expressed FoxMl and mice conditionally deleted in the FoxMl gene with the Mx-Cre recombinase transgene using the carcinogen Azoxymethane (AOM) followed by tumor promotion with Dextran Sodium Sulfate (DSS) administration. Rosa26-FoxMl mice, Mx-Cre FoxMl -/- mice and wild type mice were subjected to a single IP injection of AOM (10 mg/kg body weight) followed by three cycles of one week of administrating $2.5 \%$ DSS in the drinking water, each cycle separated by a two week period. We also examined proliferation and transformation of human DLD1 and mouse CT26 colon cancer cell lines depleted in FoxMl levels by siRNA transfection. After 12 weeks of AOM/DSS exposure, Rosa26-FoxMl transgenic mice displayed an increase in the number and size of colorectal tumors, suggesting that increased expression of FoxMl promoted colon cancer growth Likewise, Mx-Cre FoxMl -/- mice exhibited a significant reduction in the number and size of colorectal tumors following AOM/DSS treatment, suggesting that FoxMl is essential for colon cancer progression. Moreover, transfection of siRNA against FoxMl into these cell lines caused a marked reduction of DNA replication and a significant inhibition of colony formation on soft agar. In conclusion, these results suggest that suppression of FoxMl function is a novel therapy to prevent proliferation and growth of colorectal cancer

\section{T1646}

Histone Acetyltransferase P300 Regulates KLF4-Mediated Transactivation Paul M. Evans, Chunming Liu

BACKGROUND/AIMS: Krüppel-like factor 4 (KLF4) is a transcription factor that controls the expression of several genes that are known markers of the terminal differentiation of colonic stem cells, such as intestinal alkaline phosphatase and mucin. Furthermore, KLF4 is a tumor suppressor in the gut, inhibiting the expression of genes controlling the cell cycle, such as Cyclin E and Cyclin D1, and simultaneously induces expression of the cell cycle inhibitor p21Cipl/WAF1. Since KLF4 interacts with p300 and recruitment of coactivators to the transcriptional apparatus is a common method transcription factors use to activate gene expression, we decided to study what effect KLF4/p300 interaction would have upon expression of known KLF4 target genes. RESULTS: Via GST-labeled pull-down assays, we confirmed that KLF4 and p300 do indeed interact. We then made several luciferase reporter constructs containing the full-length reporter sequence of various KLF4 target genes and tested the ability of p300 to synergize with KLF4 in transactivating these reporters. With some of these reporter constructs, p300 further increased KLF4-mediated transactivation, suggesting a synergistic interaction. To identify the KLF4 binding site, we made a panel of deletion mutants for each promoter. These binding sites were then confirmed using chromatin immunoprecipitation using a novel KLF4 antibody developed in our laboratory. Since KLF4 appears to recruit p300, a histone acetyltransferase, we decided to investigate the effect of KLF4 binding to its binding element on chromatin remodeling. We tested this using chromatin immunoprecipitation against acetylated histones. CONCLUSIONS: We confirmed that KLF4 and p300 interact and found that this interaction results in a synergistic activation of several known KLF4 target genes. Furthermore, we were able to identify the binding element for KLF4 on these target genes. Finally, we found that KLF4 plays a role in chromatin remodeling via recruiting p300 to the promoter, suggesting that acetylation is important for KLF4-mediated transactivation.

\section{T1647}

Characterization of a Human Neoplastic Enterochromaffin (EC) Cell Line, KRJ-1 and the Anti-Proliferative Effects of Lanreotide and Rapamycin Geeta N. Eick, Roswitha Pfragner, Mark Kidd, Manish C. Champaneria, John R. Murren, Irvin M. Modlin

Small intestinal carcinoid (SIC) is the most prevalent GI carcinoid and has increased in incidence by $740 \%$ over the last 3 decades (NCI SEER database, 2004). The proliferative and secretory regulation of its cell of origin - the enterochromaffin (EC) cell - is unknown and has hindered the development of rational therapeutic agents. The limited mechanistic and biological knowledge of SIC reflects the absence of a human EC carcinoid cell line. Other cell lines (BON, COLO320DM) are mixed cell (acinar-carcinoid/adenocarcinoid) and not of EC cell origin, and cannot be used to assess SIC pathobiology. We have characterized a neoplastic SIC cell line, KRJ-1, and evaluated its response to antisecretory and antiproliferative agents using serotonin (5-HT) EIA for secretion and MTT for proliferation. The KRJ-1 cell line was established from a malignant metastatic human SIC. Its doubling time is 1.8 days. EM demonstrated oval or irregular lobulated nuclei and irregular pleomorphic secretory granules that are Subst P and 5-HT-positive (immunohistochemistry (IHC)). Real time RTPCR detected transcripts for chromogranin A (CgA), VMATl, tryptophan hydroxylase (TPH) Subst P, guanylin and SERT (SLC6A4). All KRJ-l cells were positive (using IHC) for CgA,
SERT, VMATI and TPH, confirming a neuroendocrine, EC cell origin. Candidate secretory and proliferative receptors identified by transcriptome analysis (Affymetrix U133 Plus chip) included the somatostatin SSTR2/3, adrenergic $\alpha 1 C \& \beta 1$, dopamine D2, nicotinic-type cholinergic A5, A6, B1, muscarinic acetylcholine $\mathrm{M} 4$ and serotonin $2 \mathrm{~A}$ receptors. Incubation of KRJ-l cells ( $45 \mathrm{~min}$ ) with isoproterenol (a selective $\beta$-adrenergic receptor agonist) resulted in a dose-dependent increase in intracellular cAMP $(E C 50=3.4 \times 10-7 \mathrm{M})$, paralleled by a concentration-dependent increase in 5 -HT $(E C 50=8 \operatorname{lnM})$ that was inhibited by the cAMP antagonist 2',5'-dideoxyadenosine ( $1 \mathrm{mM})$ demonstrating that serotonin secretion in KRJ-1 cells is in part a cAMP-mediated event. Preincubation (10 min) with the SST receptor agonist, Lanreotide, inhibited isoproterenol-stimulated 5 -HT secretion $(\mathrm{IC} 50=4.3 \times 10-7 \mathrm{M})$. Secretory granules (by EM) increased in cells preincubated with Lanreotide (100nM). Lanreotide $(10 \mathrm{nM})$ and rapamycin (mTOR signaling, $50 \mathrm{nM})$ maximally inhibited KRJ-1 proliferation $(20 \pm 12 \%$ and $35 \pm 5 \%$ ), whereas Gefitinib (tyrosine kinase inhibitor, $1 n M-10 \mu \mathrm{M}$ ) had no effect. These data confirm the KJR-l cell line as a novel, neoplastic EC cell line and demonstrate its utility as an in vitro model of SIC to study tumor secretion and proliferation. Further transcriptome analysis will allow identification of novel therapeutic targets.

\section{$\mathrm{T} 1648$}

Regulation of Epithelial to Mesenchymal Transition By the Actin-Binding Protein Villin

Leon Chatman, Alok Tomar, Sudeep George, Narendra Kumar, Seema Khurana

Epithelial-mesenchymal transition (EMT) is an important process during development by which epithelial cells acquire mesenchymal properties and show reduced intercellular adhesion and increased motility. The mechanisms that govern EMT are only now being unraveled and many parallels have been found between these processes during embryonic development and in adult tissue maintenance such as wound-healing, organ remodeling during fibrogenesis as well as between mechanisms that propel invasive growth and metastases. Most recent studies point to a critical role of EMT during tumor progression and malignant transformation, bestowing the developing cancer cell with invasive and metastatic properties. EMT is always associated with cell scattering, defined by the loss of intercellular junctions, massive actin reorganization and acquisition of cell motility. In this study, we demonstrate the regulation of EMT by villin. Villin is a tissue specific actin-binding protein that is expressed in differentiated epithelial cells of the gastrointestinal tract and renal tubules including the absorptive cells of the small and large intestines and in the pancreatic and biliary duct cells. In addition, villin expression is maintained in tumor cells of intestinal origin. Previous studies from our laboratory have demonstrated that villin regulates actin reorganization, cell morphology and cell migration and that these functions of villin are regulated by tyrosine phosphorylation of villin and the ligand-binding properties of phospho-villin (F-actin, PIP2, PLC- $\gamma 1$ ). Using Madin-Darby canine kidney (MDCK) Tet-Off cells expressing wild-type villin or various phosphorylation site mutants of villin, we examined the role of villin in EMT during hepatocyte growth factor (HGF)-induced tubulogenesis. In a three-dimensional culture, the full-length villin, which was localized to the apical surface of MDCK cysts, enhanced HGFinduced tubulogenesis. In contrast, ectopic expression of the phosphorylation site mutant of villin, known to down-regulate PLC- $\gamma 1$ signaling, actin reorganization, cell morphology and cell migration, abolished the stimulatory effect on tubulogenesis. These findings, taken together, suggest that villin plays a role in enhancing induction of EMT and that this function of villin is regulated by its tyrosine phosphorylation and its ligand-binding properties.

\section{T1649}

AMP-Activated Protein Kinase Is a Positive Regulator of Poly(ADP-ribose) Polymerase-1

John Walker, Humberto Jijon, Karen Madsen

Peutz-Jeghers syndrome (PJS) is a dominantly conferred genetic disorder typified by multiple polyps in the intestine and the development of malignant intestinal tumours. Mutations of the LKB1 gene on chromosome 19p13.3 have been identified in the majority of PJS patients. The gene product (LKB1) has been identified as a serine-threonine kinase which functions as an upstream regulator of AMPK. AMPK acts as a cellular fuel gauge, and responds to decreased cellular energy status by inhibiting ATP-consuming pathways and increasing ATPsynthesis. We have previously shown a functional relationship between AMPK and poly(ADP ribose) polymerase (PARP), a nuclear enzyme involved in maintaining chromatin structure and DNA repair. It can be hypothesized that a mutation involving AMPK may affect PARP activity and contribute to tumorgenesis in PJS patients. Objective: The aim of this study was to examine the role of AMPK in modulating PARP activity. Methods: Constitutively active AMPK (Ad.CA-AMPK) was overexpressed in HT-29 epithelial cells and PARP activity assessed using biotinylated NAD as substrate for ADP-ribosylation. To evaluate whether the relationship between AMPK and PARP was mediated through direct interaction, HT-29 cells were treated with $\mathrm{H} 202(5 \mathrm{mM})$ for 0-60 min. PARP was immunoprecipitated and samples analyzed by Western Blot. Purified AMPK $(0-10 \mathrm{mU} / \mathrm{ml})$ was incubated with SAMS (synthetic AMPK substrate) or purified PARP \pm AMP $(300 \mu \mathrm{M})$ and phosphorylation assessed. To evalute the functional consequences of AMPK regulation. PARP was immobilized and the incorporation of bioNAD measured \pm AMPK and ATP $(125 \mu \mathrm{M})$. Results: Cells infected with Ad.CA-AMPK demonstrated a MOI-dependent increased PARP automodification and a significant $(\approx 2.8$ fold $)$ increase in bioNAD incorporation as compared with controls. AMPK and PARP co-immunoprecipitated under basal conditions and in response to H202, suggesting a physical interaction under both resting and stress-induced conditions. Incubation of PARP with purified AMPK resulted in the phosphorylation of PARP; further, the inclusion of AMP as an AMPK activator potentiated PARP phosphorylation. Finally, using immobilized PARP, the incorporation of bioNAD by PARP was dramatically increased following the addition of AMPK. Conclusion: These data suggest a novel role for AMPK in regulating PARP activity through a direct interaction involving phosphorylation. In the physiological setting of the intestine where genotoxic stressors are abundant, a dysregulation in LKBI as seen in Peutz-Jeghers syndrome could be translated into a deficiency in DNA repair through an inhibited AMPK signal to PARP. 


\section{$\mathrm{T} 1650$}

Constitutive Matrix Metalloproteinase-Mediated Release of An Epidermal Growth Factor (EGF) Receptor Ligand, HB-EGF, Regulates Basal Human Colon Cancer Cell Proliferation

Kunrong Cheng, Jean-Pierre Raufman

Previously, we showed that muscarinic ligands and conjugated secondary bile acids stimulate colon cancer cell proliferation by $\mathrm{M}_{3}$ muscarinic receptor-mediated transactivation of EGF receptors (EGFR) (Cancer Res. 2003:63:6744 and Biochem. Pharmacol. 2005;70:1035). In these studies, preliminary experiments with inhibitors of EGFR activation or EGFR-ligand release indicated that the rate of basal cell proliferation was reduced. The aim of the present study was to define the mechanism of basal EGFR-mediated cell proliferation in a wellcharacterized human cecal adenocarcinoma cell line (H508) that abundantly expresses muscarinic $\mathrm{M}_{3}$ and $\mathrm{EGF}$ receptors. We measured cell proliferation with the validated sulforhodamine B colorimetric assay and used several approaches to inactivate EGFR and block EGFR-ligand release. Increasing concentrations of PD168393 (0.1 nM - $1 \mu \mathrm{M})$, an inhibitor of EGFR activation, and LAl $(0.0001-5 \mu \mathrm{g} / \mathrm{ml})$, an antibody to the ligand-binding domain of EGFR, caused progressive reduction of $\mathrm{H} 508$ colon cancer cell proliferation. Maximal concentrations of the agents reduced basal cell proliferation by $70 \%$ ( $\mathrm{p}<0.005$ ). Incubation with GM6001 $(0.01-10 \mu \mathrm{M})$, a broad-spectrum matrix metalloproteinase (MMP) inhibitor, reduced basal cell proliferation by $59 \%(\mathrm{p}<0.005)$. Reduction of basal $\mathrm{H} 508$ cell proliferation was not observed when the cells were incubated with NC-GM6001, an inactive control analogue of GM6001. These results suggested that MMP-mediated release of an EGFR ligand is necessary to maintain basal cell proliferation. To identify the responsible EGFR ligand, we examined the effects of CRM197, a diphtheria toxin analogue that specifically blocks release of the EGFR ligand, HB-EGF. By a magnitude similar to that observed with maximal concentrations of PD168393, LAl and GM6001, CRM197 $\left(10^{-4}\right.$ to $\left.5 \times 10^{3} \mathrm{ng} / \mathrm{ml}\right)$ caused progressive reduction of basal $\mathrm{H} 508$ cell proliferation $(\mathrm{p}<0.005)$. Likewise, neutralizing antibody to HB-EGF ( 0.1 to $20 \mu \mathrm{g} / \mathrm{ml}$ ) reduced basal cell proliferation ( $\mathrm{p}<0.05$ ). To confirm that MMP-7-mediated release of HB-EGF can stimulate $\mathrm{H} 508$ colon cancer cell proliferation, we examined the actions of exogenous recombinant MMP-7 and HB-EGF. Increasing concentrations of both MMP-7 (l-100 ng/ml) and HB-EGF $(0.02-200 \mu \mathrm{g} / \mathrm{ml})$ stimulated a progressive increase in $\mathrm{H} 508$ cell proliferation $(\mathrm{p}<0.005)$. Collectively, these findings provide evidence that in the human H508 colon cancer cell line, basal cell proliferation is dependent on MMPmediated release of an EGFR ligand, HB-EGF, and autocrine activation of EGFR. MMPmediated release of HB-EGF may provide a therapeutic target for reducing both basal and stimulated colon cancer cell proliferation.

\section{T1651}

AKT-Dependent Mechanical Signaling Displays Isoform Specificity in Effector Outcome

David H. Craig, Vijayalakshmi Thamilselvan, Hiroe Shiratsuchi, Marc D. Basson

Akt is a serine-threonine protein kinase implicated in a diverse range of cell functions including cell migration, metabolism, growth and survival. Akt has three isoforms; Aktl and Akt2 are ubiquitously expressed, whereas Akt3 is predominately restricted to the heart, kidney, brain, lung, testes and skeletal muscle. Functional differences between Akt isoforms in mechanical signaling have not been described. Increased extracellular pressure activates colon cancer cell adhesion to matrix proteins, as well as stimulates phagocytosis by human monocytes and macrophage-like THP-1 cells. These effects are regulated by Akt-dependent signaling pathways and may both be prevented by global Akt inhibition. We hypothesized that these distinct effects of pressure might be mediated by different Akt isoforms. To study pressure-induced adhesion, human SW620 colon cancer cells were transfected with Akt1or Akt2-specific siRNA, subjected to $15 \mathrm{mmHg}$ increased pressure and allowed to adhere to collagen for $30 \mathrm{~min}$. Non-adherent cells were washed away and adherent cells counted. Aktl and Akt 2 expression were reduced by $50 \%$ and $60 \%$, respectively. Silencing Aktl, but not Akt2, completely prevented pressure-activated SW620 adhesion $(\mathrm{P}<0.001)$. Analysis of isoform-specific gene silencing effects on total Akt protein showed that SW620 cells express substantially more Aktl than Akt2 (approximately 9:1). We performed parallel studies in Caco-2 cells, which express more Akt2 (Aktl/Akt2 ratio approximately 3:7, similarly determined). As in SW620 cells, reducing Aktl by 80\% blocked pressure-stimulated Caco2 adhesion. An $80 \%$ Akt2 reduction did not alter the pressure effect $(\mathrm{P}<0.001)$. Thus, the isoform specificity of the pressure effect on adhesion is independent of the ratio of isoform expression. The same siRNAs were transfected into human PMA-differentiated (macrophagelike) THP-1 cells. Transfectants were incubated at $37^{\circ} \mathrm{C}$ with serum-opsonized fluorescencelabeled latex beads under ambient or $20 \mathrm{mmHg}$ increased pressure for $2 \mathrm{hrs}$, washed and fixed. Phagocytosed beads were counted under a fluorescence microscope. Pressurestimulated phagocytosis exhibited opposite isoform specificity to pressure-stimulated adhesion. Silencing of Aktl by $40 \%$ significantly reduced basal phagocytosis $(\mathrm{P}=0.03)$ without altering the pressure effect, but a $50 \%$ reduction of Akt2 inhibited pressure-stimulation of phagocytosis $(\mathrm{P}<0.05)$ without significantly affecting basal phagocytosis. Thus, despite an 81\% sequence homology, individual Akt isoforms, Aktl and Akt2, perform distinct roles in pressure-stimulated mechanotransduction with alternate effector outcomes.

\section{$\mathrm{T} 1652$}

FOXP3 Regulatory T Cells Regulate Cytolytic T Cell Function in Colorectal Cancer Via the T-Box Transcription Factor Eomesodermin Imke Atreya, Christoph Becker, Massimo Fantini, Christoph C. Schimanski, Martin R. Berger, Heike Dornhoff, Maximilian Waldner, Dennis Strand, Peter R. Galle, Markus F. Neurath

Introduction: An efficient cytolytic T cell function is essential for immune system mediated cancer rejection, whereas regulatory $\mathrm{T}$ cells are supposed to suppress the activity of such effector T cells. It has been shown, that the T-box transcription factor Eomesodermin is critically involved in the regulation of CTL differentiation. Methods: Expression of Eomesodermin and FoxP3 was analysed by RT-PCR and by Realtime PCR in colorectal tumors.
Results were compared with clinical data. Regulatory T cells were co-incubated with CD8 T cells and Eomesodermin expression was analysed in these cells. Results: The importance of Eomesodermin in the differentiation of cytolytic $\mathrm{T}$ cells was emphasized by showing that silencing of Eomesodermin in human CD8+ T cells by siRNA resulted in decreased perforin and IFN $\gamma$ levels, whereas Eomesodermin overexpressing cells showed increased expression of cytolytic genes. By analysing the expression of Eomesodermin in 88 colorectal tumors we found a significant negative correlation between the expression of Eomesodermin in these tumors and the infiltration of tumor cells into lymph nodes $(p<0,02)$. This inverse correlation was not dependent on varying numbers of tumor infiltrating CTLs. Furthermore Eomesodermin expression in tumors correlated with improved prognosis based on the UICC-classification $(p<0,02)$. No significant correlation was found between Eomesodermin expression and tumor grading, venous or lymph vessel invasion. Obviously, activation of tumor infiltrating $\mathrm{T}$ cells was able to prevent infiltration of tumor cells into lymph nodes Looking for factors, responsible for the regulation of Eomesodermin in tumors, we assumed that regulatory T cells might be critically involved. This hypothesis was underlined by reduced number of colorectal tumors in APCmin mice, depleteted for regulatory $\mathrm{T}$ cells. Furthermore, regulatory T cells prevented Eomesodermin expression in co-cultured CD8 $\mathrm{T}$ cells. To investigate the prognostic value of FoxP3 in colorectal cancer, the expression of FoxP3 in human tumors was analysed. In line with our model, high FoxP3 levels in tumors were associated with increased appearance of lymph node metastasis, implicating that high numbers of Eomesodermin expressing effector T cells and low numbers of regulatory $\mathrm{T}$ cells in tumors are beneficial for tumor patients. Conclusion: Based on our results, anti-tumor immunity is negatively regulated by tumor infiltrating FoxP3 regulatory $\mathrm{T}$ cells, which are able to suppress Eomesodermin induced activation of CTL mediated tumor defense.

\section{$\mathrm{T} 1653$}

Proteasome Inhibition and 5-FU Sensitises Hepatocellular and Colon Carcinoma Cells But Not Primary Human Hepatocytes for Trail-Induced Apoptosis By CFLIP Dependent and Independent Mechanisms At the Disc Tom Ganten, Ronald Koschny, Tobias L. Haas, Jaromir Sykora, Min Li-Weber, Kerstin Herzer, Wolfgang Stremmel, Henning Walczak

TNF-related apoptosis-inducing ligand (TRAIL) exhibits potent anti-tumor activity upon systemic administration in mice. Due to its apparent non-toxicity and proven in vivo efficacy TRAIL may serve as a novel antineoplastic drug for the treatment of human cancer. Pretreatment with the proteasome inhibitor MG132 or 5-FU rendered TRAIL-resistant hepatocellular and colon carcinoma cells but not primary human hepatocytes sensitive for TRAILinduced apoptosis. To elucidate the different mechanisms of sensitisation of proteasome inhibitiors and 5-FU for TRAIL-induced apoptosis, we investigated different levels of apoptotic signal transduction. Both chemotherapeutics revealed an up-regulation of TRAIL-R and $-\mathrm{R} 2$, and substantially increased caspase- 8 activation after sensitization. The increased activation of caspase- 8 could be explained by up-regulation of the death-inducing TRAIL receptors and an increase of the ratio of caspase- 8 to cFLIP within the DISC (Death inducing signalling complex). Although hepatoma cells pretreated with the proteasome inhibitor upregulated TRAIL-R1 and -R2 less efficiently than 5-FU pretreated cells, they where significantly more sensitive to TRAIL-induced apoptosis. Interestingly, at the same time cFLIPS was markedly up-regulated in the TRAIL-DISC by proteasome inhibitor pretreatment and downregulated when pretreated with 5-FU. To test a recent hypothesis that cFLIP under certain circumstance can reveal indeed a pro-apoptotic function we applied cFLIP siRNA in the presence of 5-FU and the proteasome inhibitior. The results suggest that TRAIL-mediated apoptosis in hepatocellular and colon carcinomas is controlled at the DISC level by a yet unidentified factor and cFLIP.

\section{T1654}

Overexpression of Wild-Type PKD2 Leads to Increased Proliferation and Invasion of BON Endocrine Cells

Lindsey N. Jackson, J.N. Li, L. Andy Chen, Courtney M. Townsend, B. Mark Evers

The BON endocrine cell line, established from a human pancreatic carcinoid tumor and characterized in our laboratory, represents a unique in vitro and in vivo carcinoid tumor model with production and secretion of serotonin, chromogranin A, and other peptides. Protein kinase D (PKD), a novel serine/threonine protein kinase, has been implicated in the regulation of transport processes in certain cell types. Recently, we have demonstrated an important role for PKD in stimulated peptide secretion from BON cells; however, the role of PKD isoforms, including PKD1 and PKD2, in the proliferation and invasion of carcinoid tumors remains unclear. The purpose of our study was to determine the role of the PKD2 isoform on BON cell proliferation and invasion. METHODS. BON cells were stably transfected with GST-tagged PKD2-wild type (WT), PKD2-kinase dead (KD), or empty vector (control) and selected in medium containing G418 (800 $\mu \mathrm{g} / \mathrm{ml})$; transfection was confirmed by assessment of GST expression. (i) To determine the effects of PKD2 on proliferation PKD2-WT, PKD2-KD, or control BON cell lines were plated in duplicate wells and MTT assays performed. (ii) Invasion was determined by plating cells in triplicate on BD Matrigel Invasion Chambers and counting cells invading the matrix after $24 \mathrm{~h}$. RESULTS. (i) PKD2WT BON cells exhibited a greater than 2 -fold increase $(\mathrm{p}<0.0001)$ in proliferation compared to either PKD2-KD or control (empty vector) BON cell clones. There was no significant difference in proliferation between PKD2-KD or control cells. (ii) PKD2-WT BON cells demonstrated significantly increased invasion of Matrigel ( $40 \pm 5.1$ cells/chamber) when compared with PKD2-KD BON cells $(8 \pm 1.5$ cells/chamber) or control cells transfected with empty vector $(13.3 \pm 1.2$ cells/chamber). CONCLUSIONS. PKD2 overexpression results in increased proliferation and invasion of the BON carcinoid cell line, suggesting an important role for PKD2 in carcinoid tumor progression. Targeted inhibition of PKD2 may prove to be a novel treatment option for patients with carcinoid tumors. 
$\mathrm{T} 1655$

Gastrin-Induced CCN2/CTGF Over-Expression in Mastomys Gastric Carcinoid May Be the Basis of ECL Transformation and Gastrin Autonomy

Mark Kidd, Irvin M. Modlin, Geeta N. Eick, Igor Latich, Michelle N. Zikusoka, Robert L. Camp, Shrikant M. Mane

Connective tissue growth factor or CCN2 (particularly in concert with EGF) has been shown to exert a mitogenic effect on a number of cell types including fibroblasts and stellate cells, but its presence and effects have not been evaluated in gastric neuroendocrine cells. In Mastomys, a rodent model of hypergastrinemia-induced gastric carcinoid neoplasia (enterochromaffin-like cell [ECL]) dCHIP analysis of Affymetrix microarray data revealed upregulation of CCN2 transcript $(+2.36$-fold, $\mathrm{p}<0.01)$. This gene is also upregulated in a transgenic mouse model (SV40 TAg under control of regulatory elements from the mouse Atp4b gene: PNAS 2004; 101:4471-6) of neuroendocrine cell carcinoma. We confirmed over-expression of CCN2 by Q RT-PCR in Mastomys tumor ECL cells, which exhibit elevated levels of transcript $(>20$-fold; $\mathrm{p}<0.001)$ compared to an enriched $(80 \%)$ elutriated population of normal ECL cells. To validate this observation as an ECL cell specific event, we developed a $>96 \%$ pure naive and tumor ECL cell fraction by FACS sorting histidine decarboxylase (HDC) positive-ECL cells. CCN2 transcript was significantly increased in pure tumor ECL cell fractions as opposed to normal ECL cells $(41.3$-fold $\pm 10.7, \mathrm{p}<0.005)$. Immunostaining of Mastomys gastric mucosa confirmed that while $\mathrm{CCN} 2$ protein was present in $\mathrm{ECL}$ tumors, it was not expressed in normal ECL cells. TGFbl, the primary upstream regulator of $\mathrm{CCN} 2$, elevated CCN2 transcript (3.5 \pm 0.6 -fold, p<0.01) in normal short-term cultured ECL cells $(E C 50=5 \mathrm{ng} / \mathrm{ml})$, whereas gastrin $(20 \mathrm{pg} / \mathrm{ml})$ had no effect. TGFbl had a similar effect on CCN2 transcript expression in tumor ECL cells in short-term culture (2.8 \pm 0.3 -fold, $\mathrm{p}<0.02$ ). Stimulation of tumor ECL cells with CCN2 induced proliferation (MTT assay) with an EC50 of $\sim 0.01 \mathrm{ng} / \mathrm{ml}$ and an ECmax of $0.1 \mathrm{ng} / \mathrm{ml}$, whereas normal ECL cells did not proliferate in response to CCN2. Addition of EGF significantly augmented the CCN2-induced proliferation of tumor ECL cells with an EC50 of $6 \mathrm{pg} / \mathrm{ml}$. No additive effect of CCN2 was noted on EGF-stimulated naive ECL cells suggesting that different downstream signaling pathways are active in naive and tumor ECL cells. In conclusion, CCN2 was absent in normal ECL cells and is present only in ECL cell tumor cells. Its expression was upregulated by gastrin and EGF, agents proven to induce ECL cell neoplastic transformation. Given its known mitogenic role in other cell systems, CCN2 may represent a proliferative regulator that drives ECL proliferation once cells become autonomous of gastrin regulation. Identification of CCN2 in carcinoid biopsies may be useful as an indicator not only of ECL cell transformation but also of gastrin-autonomy.

\section{$\mathrm{T} 1656$}

Functional Significance of Enhanced ETV4 and MYEOV Expression in Colorectal Neoplasia Using Sirna-Mediated Gene Knockdown Garrett Lowlor, Alan Moss, Donal Tighe, David W. Murray, Conor O'Keane, Peter Doran, Padraic MacMathuna

Introduction Bioinformatic approaches have the advantage of generating novel disease gene lists however; the functional significance of novel gene associations frequently remains unclear. We have previously reported a computational study to identify ETV4 and Myeov as novel genes with enhanced expression in colon cancer. However, the functional significance remained to be determined. Aims To assess ETV4 and Myeov expression in normal, adenoma and colon carcinoma tissue and to investigate the effect of gene knockdown on colon cancer cell invasion and proliferation. Methods Digital differential display (DDD) was used to identify differentially expressed colon cancer genes. Quantitative real time PCR was used to assess expression levels in ex vivo human tissue. The effect of siRNA-mediated gene knockdown on proliferation and invasion was evaluated in vitro using T84 cells. Results ETV4 expression was increased 4-fold in colon adenoma in comparison with normal, but unchanged in carcinoma tissue. Cell proliferation was decreased by $90 \%$ with ETV4 knockdown by $48 \%$ with Myeov suppression $(\mathrm{P}<0.05)$. Cell invasion was decreased by $60 \%$ with ETV4 knockdown. Myeov suppression resulted in $40 \%$ decreased cell invasion. Conclusions We have characterised two colon cancer related genes identified using DDD. ETV4 was shown to have enhanced expression in colon adenoma and to promote cell proliferation and invasion. Similarly, Myeov was shown to drive colon cancer cell proliferation and invasion. This data supports the importance of bioinformatic approaches in disease gene identification as well as the role of ETV4 and Myeov in colorectal neoplasia

\section{$\mathrm{T} 1657$}

Ying Yang 1 and NF-KappaB (p65) Are Markedly Increased in Metastatic Colon Cancer: Roles of Iaps and Fas in Cytotoxic Resistance to Apoptosis and Immune Privilege of Malignant Colon Cancer Cells, Respectively Sergio Huerta, Emily J. Goulet, Sara Huerta-Yepez, Mario I. Vega, Berenice Sanchez Arellano, Benjamin Bonavida, Edward H. Livingston

Background/Objectives: Cell lines SW480 and SW620 were established from the same patient at different stages of tumor progression: SW480 from a primary tumor (Duke's stage B) and SW620 from a lymph node metastasis. The SW620 cells are more resistant to apoptosis when induced both by chemotherapeutic agents (i.e. cisplatin induced apoptosis) and by treatment with anti-Fas monoclonal antibody, suggesting alteration of both the intrinsic and extrinsic pathways of apoptosis in this aggressive cell line. However, caspases 3, 8 and 9 are unaffected in both cell lines. The following study was undertaken to elucidate the mechanism rendering resistance to apoptosis in metastatic colon cancer cells. Methods: Cell lines SW480 and SW620 were obtained from the ATCC and grown under standard conditions. $1 \times 105$ cells were fixed in 4\% paraformaldehyde. Immunohistochemistry was then performed with the following antibodies: Fas (CD95), Bcl-xL, DR5, AIF, NFkappaB (p50 and P65), and YY1. Results: High levels of YYl and p65 were noted in the nucleus of the SW620 cell line and were almost undetectable in SW480 cells. Cytoplasmic p50 staining was low in SW620 cells and moderate in SW480 cells. Bcl-xL staining was very high in the cytoplasm of SW480 cells and moderate in SW620 cells. Fas levels were markedly high in
SW480 cells and low in SW620 cells. DR5 was absent in both cell lines. While there was a higher gradient of staining in the SW620 cells compared to the SW480 cells with AIF antibody, this staining was limited to the cytoplasm. Conclusion: The high levels of p65 in metastatic cells results in increased levels of survivin as well as TRAIL resistance to apoptosis (as previously described). The high nuclear presence of NFkappaB in metastatic colon cancer cells may explain the observed resistance to apoptosis by chemotherapeutic agents. The ubiquitous anti-apoptotic YY-1 correlates with down regulation of Fas, which may provide cancer cells immune privilege.

\section{T1658}

Side Populations of Human Gastrointestinal Cell Lines Julia Burkert, William R. Otto, Derek Davies, Nicholas A. Wright

A distinct population of human tumour cells, defined by the ability to efflux the nucleic acid staining dye Hoechst 33342, displays stem cell characteristics such as multipotential differentiation and self-renewal. These cancer stem cells (CSC), as defined by the side population (SP) phenotype, were first discovered in haematopoietic malignancies and have more recently also been shown in solid tumours of the breast, brain and lung. We are investigating the role of side populations in human tumour cell lines of the gastrointestinal tract and their verification as CSCS. 7 of 7 tumour cell lines ( 6 colorectal and 1 gastric, HGT101) show a distinct SP phenotype of between $0.3 \%$ and $10 \%$ of the whole population. SP cells retained stem cell properties in vitro (clonal self-renewal and differentiation) and their in vivo ability to generate tumours in NOD/SCID immunodeficient mice is under study. SP cells were analysed by multicolour FACS analysis for the surface markers CD34, CD44 and CD133, and confirmed by semiquantitative RT-PCR and immunofluorescence staining. SP cells from 4 lines showed two patterns of positivity: (Caco2: CD34- CD44+ CD133+; HT29: CD34- CD44+ CD133+/-) compared to (SW620: CD34- CD44- CD133-; HGT101: CD34- CD44- CD133-). Functional differences of the subpopulations with regards to stem cell characteristics are being further investigated, including possible stem cell markers such as musashi-1, c-kit, nanog. This may reveal useful information regarding the isolation and therapeutic targeting of the CSC population.

\section{T1659}

Comprehensive Analysis of Prostaglandin $\mathrm{E}_{2}$ Production in Pancreatic Cancer Cells

Hirozumi Sawai, Makoto Satake, Sascha Hasan, Howard A. Reber, Oscar J. Hines, Guido Eibl

Background and Aim: There is substantial evidence that prostaglandin $\mathrm{E}_{2}\left(\mathrm{PGE}_{2}\right)$ contributes to the malignant phenotype of several human cancers, including pancreatic cancer $(\mathrm{PaCa})$. Animal models have confirmed that $\mathrm{PGE}_{2}$ stimulates cancer cell growth, invasion, and tumor angiogenesis. Conversely, inhibition of $\mathrm{PGE}_{2}$ synthesis decreases tumor growth. In the past, studies focused almost exclusively on inhibition of the cyclooxygenase-2 (COX-2) isoform in an attempt to reduce $\mathrm{PGE}_{2}$ formation. However, recent concerns of serious cardiovascular side effects of selective COX-2 inhibitors necessitate a more comprehensive study of other components of the prostaglandin production pathway in order to identify other therapeutic targets. The aim of the present study therefore was to characterize $\mathrm{PGE}_{2}$ production in $\mathrm{PaCa}$ cells. Methods and Results: Liquid chromatography-mass spectroscopy analysis revealed comparable levels of the $n-6$ polyunsaturated fatty acid arachidonic acid (AA), the substrate of cyclooxygenases yielding prostaglandin $\mathrm{H}_{2}\left(\mathrm{PGH}_{2}\right)$, in six human PaCa cells: AsPC-1 (A), BxPC-3 (B), Capan-2 (C), HPAF-II (H), MIA PaCa-2 (M), and PANC-1 (P). Western blot analysis demonstrated expression of cytosolic phospholipase $\mathrm{A}_{2}\left(\mathrm{PLA}_{2}\right)$, which releases AA from membrane phospholipids, and COX-2 only in $\mathrm{B}, \mathrm{C}$, and $\mathrm{H}$. COX-1 was expressed in all six cell lines. The $\mathrm{COX}$ product $\mathrm{PGH}_{2}$ is converted to $\mathrm{PGE}_{2}$ by various $\mathrm{PGE}_{2}$ synthases (PGES). While cytosolic PGES and microsomal PGES-2 were detected in all six cell lines, microsomal PGES-1 expression was much more robust in B, C, and H. 15-deoxy-prostaglandin dehydrogenase (PGDH), which degrades $\mathrm{PGE}_{2}$, was similarly expressed in all six cell lines. $\mathrm{B}, \mathrm{C}$, and $\mathrm{H}$ produced significant amounts of $\mathrm{PGE}_{2}$, while $\mathrm{PGE}_{2}$ levels were virtually undetectable in $\mathrm{A}, \mathrm{M}$, and $\mathrm{P}$, as determined by ELISA. Baseline $\mathrm{PGE}_{2}$ production in $\mathrm{B}, \mathrm{C}$, and $\mathrm{H}$ was inhibited by selective COX-2, but not COX-1 inhibitors. Adding exogenous $\mathrm{AA}$ time- and dose-dependently increased $\mathrm{PGE}_{2}$ production in $\mathrm{B}, \mathrm{C}$, and $\mathrm{H}$, which was inhibited by a selective COX-2 inhibitor. AA only slightly increased $\mathrm{PGE}_{2}$ levels in $\mathrm{A}, \mathrm{M}$, and $\mathrm{P}$, which was inhibited by a selective COX-1 inhibitor. Conclusions: Taken together, our studies demonstrated for the first time that the components of the $\mathrm{PGE}_{2}$ production pathway are variably expressed in $\mathrm{PaCa}$ cells. $\mathrm{PGE}_{2}$ production correlated with the robust expression of cytoplasmic PLA 2 , COX-2, and microsomal PGES-1. These findings will help to identify novel targets for inhibiting $\mathrm{PGE}_{2}$ production in pancreatic cancer.

\section{$\mathrm{T} 1660$}

Intestine-Specific Transcription Factors and Interferon- $\gamma$ Cause Gastric CancerSpecific Expression of NADPH Oxidase 1 (NOX1)

Kazuhito Rokutan, Kumiko Tominaga, Yuki Kuwano, Kiyoshi Masuda, Kensei Nishida, Hideyuki Sasaki, Atsuo Sekiyama, Shigatada Teshima-Kondo, Keiya Nakamura

Epidemiologic data show a clear association between chronic inflammatory conditions and subsequent malignant transformation in the inflamed tissue. Gastric cancer is associated with Helicobacter pylori infection and is histologically classified into the intestinal and diffuse types. The carcinogenic pathway of intestinal-type gastric carcinomas involves well-characterized sequential stages; superficial gastritis, atrophic gastritis, intestinal metaplasia, and carcinomas. An intestine-specific CDX2 is one of the most likely candidates linked with the induction of intestinal metaplasia, while the underlying mechanism for displasia is still unkonwn. A novel NADPH oxidase 1 (Noxl) is implicated in the pathogenesis of inflammation and oxygen radical-dependent carcinogenesis. Northern hybridization and PCR of human stomach cDNA libraries showed that the Noxl mRNA was absent in the normal human stomach, while they were specifically expressed in intestinal-type and diffuse-type 
adenocarcinomas. Immunohistochemistry with use of polyclonal antiobody against human Noxl also demonstrated that Noxl protein was expressed in intestinal (15/17 cases), diffusetype carcinomas (7/7), and signet-ring cell carcinomas (10/10 cases), while it was absent in accompanying normal or chronic atrophic tissues with metaplasia. We cloned $5.0 \mathrm{kbp}$ of 5 '-frank of the human Noxl gene. The 500 bp-proximal promoter of the Noxl gene that contains binding sites for GATA6, CDX1/2, and HNF-l $\alpha$ factors appeared to be essential for the basal promoter activity. In fact, Noxl-expressing cancer cells possessed GATA6, CDXI/2 and HNF-l $\alpha$. Particularly, CDXl and HNF-l $\alpha$ were specifically colocalized in Noxl-expressing cancer cells. Furthermore, we found that a Thl cytokine (IFN- $\gamma$ ) was a potential activator for transcription of the Noxl gene. In this case, STATl and GAS motif located at -3960 to $-3645 \mathrm{bp}$ mediated the transcription stimulated by the Thl cytokine. These results suggest that inappropriate activation of specific developmental pathways (intestinal metaplasia) plus enhanced Thl cytokine production may cause dysregulated expression of the Noxl, which may be associated with Helicobacter pylori-associated, oxygen radicaldependent carcinogenesis in the stomach.

\section{T1661}

Establishment and Characterization of Hydroxycamptothecin-Resistant SW1116/HCPT of Human Colon Cancer Cell Line and Reversal of HCPT Resistant Phenotype

Zhi Hua Ran, Jian Zou, Shu Dong Xiao

Aims: To study multi-drug resistance mechanism of digestive tract tumor and reverse strategy. Methods: Stepwise concentration-increasing method was used to develop SW1116/HCPT which was hydroxycamptothecin-resistant cell line of human colon cancer. Cell growth curve, drug sensitive test, cell cycle, telomerase activity and karyotype analysis were detected. Human Cancer Drug Resistance \& Metabolism Gene Array was used to determine the expression profile of drug resistant genes. Expressions of 8 drug resistant genes were checked by RT-PCR. BCRP siRNA were designed and transfected into drug resistant cells. HCPT sensitiveness, cell cycle distribution and oncogenicity were detected in vitro. RT-PCR and western blot were used to check the efficacy of BCRP gene knock-out. Results: Compared with their parent cells, SW1116/HCPT was 120 times more resistant to cytotoxic action of HCTP. The number of SW1116/HCPT cells in G0/Gl phase increased and that of S phase decreased $(\mathrm{P}<0.01)$. Telomerase activity and karyotype of SW1116/HCPT cells had no apparent change. Karyotype is aneuploid 54-69. There were 30 drug resistant genes whose expression level were changed. Of them, 10 were down-regulated and 20 were up-regulated. The result of RT-PCR was in accordant with that of gene array. 2 genes were down-regulated, 4 were up-regulated and another 2 had no apparent change. BCRP siRNA were designed successfully, and the efficiency of liposome transfection was $42 \%$. Compared with drug resistant cells, sensitivity of HCPT was recovered and there was $62.5 \%$ decrease in IC50. The number of SWt cells in G0/Gl phase decreased and that of $\mathrm{S}$ phase increased $(\mathrm{P}<0.05)$. The oncogenicity of SWt cell were drop-down, and volum of clones were diminished. RTPCR and western blot shown that there was decrease in the expression of BRCP mRNA and protein in SWt cells. Conclusion SW1116/HCPT is an ideal model for the study of multidrug resistance in digestive tract tumor. Transfection of siRNA expressing plasmid is an effective reverse strategy of HCPT resistant phenotype in human colon cancer cells.

\section{T1662}

Nf-kB Activation Is Linked to Proximal Colon Tumor Induction in UCP2 Deficient Mice

Zoltan Derdak, Edmond Sabo, Rose Tavares, Murray Resnick, Jack R. Wands, Gyorgy Baffy

Background and aims. Uncoupling protein-2 (UCP2) has recently emerged as a negative regulator of mitochondrial oxidant production. When overexpressed, UCP2 protects cells from oxidative stress, while its absence may cause abundance of reactive oxygen species, release of pro-inflammatory cytokines, and persistent activation of $\mathrm{NF}-\kappa \mathrm{B}$, a pleiotropic transcription factor with an increasingly recognized role in cancer. We have recently found that mice deficient for UCP2 develop more aberrant crypt foci (ACF) and colon tumors when treated with azoxymethane (AOM), a chemical carcinogen typically targeting the distal colon (Gastroenterology 125(S1): A176, 2004). Surprisingly, increased susceptibility in $\mathrm{UCP2}-/$ - mice to AOM primarily occurred in the proximal colon in association with a disrupted balance between intestinal epithelial cell proliferation and apoptosis. To further study these events, we analyzed their association with oxidative stress and NF- $\mathrm{kB}$ activation. Methods. Proximal and distal colon tissue was obtained from 8-wk old male UCP2-/- mice $(\mathrm{n}=12)$ and wild type littermates $(\mathrm{n}=8)$ treated with $\mathrm{AOM}$ at a dose of $10 \mathrm{mg} / \mathrm{kg} / \mathrm{wk}$ i.p. for 6 wks and observed for an additional 24 wks. Oxidative stress was determined by immunohistochemical staining for malondialdehyde (MDA) and inducible nitric oxide synthase (iNOS). NF-kB activation was determined by Western blot analysis of phosphorylated IкB (pІкB) and by immunohistochemical detection for the nuclear presence of $\mathrm{p} 65$ subunit. Immunohistochemistry was evaluated by image analysis. Results. Increased MDA staining was found in the distal colon $(\mathrm{p}<0.05)$ regardless of the genotype, supporting the importance of regional differences. However, MDA staining was more intense in the proximal colon of UCP2-/- mice $(\mathrm{p}<0.05)$. Staining for iNOS resulted in a similar pattern. In wild type mice, less pIKB was found by Western blot analysis in the proximal colon, indicating diminished activation of NF- $\mathrm{KB}$ in this region $(\mathrm{p}<0.05)$. However, $\mathrm{pI \kappa B}$ was increasingly abundant in UCP2-/- mice with a loss of regional differences $(\mathrm{p}<0.05)$. Moreover, nuclear p65 staining was more intense in the proximal colon of $\mathrm{UCP} 2-/$ - mice $(\mathrm{p}<0.01)$ indicating excessive NF$\kappa \mathrm{B}$ translocation. Conclusion. Our findings indicate significant regional differences in mice for the degree of oxidative stress and NF-KB activation between proximal and distal colon. These differences are lost in UCP2 deficiency, suggesting a causal relationship to increased tumorigenesis in the proximal colon of UCP2-/- mice. Additional studies are warranted to assess the role of mitochondrial uncoupling in colon cancer development. Supported by NIH grant RR-17695.

\section{T1663}

Bone Marrow-Derived Stem Cells May Not Cause Fusion with Cancer Cells in Vivo

Shuji Ishii, Shingo Tsuji, Toshiyuki Yoshio, Shinichirou Shinzaki, Satoshi Egawa, Takanobu Irie, Tsutomu Nishida, Masakazu Yasumaru, Hideki Iijima, Hiroaki Murata, Yoshiyuki Kanazawa, Sunao Kawano, Norio Hayashi

[Background and Aims] There are hematopoietic stem cells and storomal stem cells in the bone marrow. On the other hand, a report suggests certain digestive cancers are bone marrow-derived. It is also reported that stem cells cause fusion with tumor cells in vitro. Another report suggests that the vascular endothelial cells in a tumor are a bone marrowderived. Therefore we investigated the fate of bone marrow-derived cells in colon cancer allografts in mice. [Methods] Whole bone marrow cells were collected from the femurs an tibias of GFP transgenic mice and were transplanted to C57BL/6 mice after whole body irradiation (10Gy). MC38, a mouse-derived colon cancer cell line, were transplanted subcutaneously to the mice. MC38 cells were also injected to the spleen of the animals to mak metastatic liver tumors. After tumors formed, mice were sacrificed and perfused with $4 \%$ paraformaldehyde/PBS. The tumors were removed immediately and frozen in cryo-enbedding compound. The frozen sections were immunohistologically analyzed for CD 45, CD 31, $\alpha$-SMA, vimentine and GFP using confocal microscopy. MC38 were transfected with pDsRed gene to label the cell with red fluorescence. The transfectant tumor cells (MC38pDsRed cells) were also transplanted into the back of the mice harboring GFP+ bone marrow. The tumor were also examined for the possible fusion of pDsRed+-tumor cells and bone marrow-derived GFP+-stem cells. [Results] Both the subcutaneous tumors and the metastatic liver tumors contained much GFP+ bone marrow-derived GFP+,CD45+ hematopoietic cells. Remaining several\% are bone marrow-derived myofibrobrasts and fibrobrasts (GFP+, $\alpha$-SMA+ and GFP+ vimentine+. The cell which differentiated to a endotherial cell with GFP + CD 31+ was not present. Furthermore, none of tumors did not contained GFP+ pDsRed+ cells, suggesting that fusion of a tumor cell and a bone marrow-derived stem cell may not occur in the tumor allografts. [Conclusions] Bone marrow is the source of tumor myofibrobrasts and fibrobrasts. Bone marrow-derived cells were also mobilized toward the tumor as hematopoietic cells. Fusion of bone marrow-derived stem cells and tumor cells may hardly occur in mice in vivo.

\section{T1664}

Transient Receptor Potential Ion Channel TRPM8 Is Expressed and Functionally Active in Neuroendocrine Tumor Cells Stefan Mergler, Mathias Strowski, Simone Kaiser, Thomas Plath, Ursula Ploeckinger, Bertram Wiedenmann, Carsten Groetzinger

BACKGROUND: TRPM8, a member of the melastatin-type transient receptor potential ion channel family, is activated by cold temperatures. TRPM 8 activation induces a rise in the concentration of intracellular free calcium $([\mathrm{Ca} 2+] \mathrm{i})$. This increase in $[\mathrm{Ca} 2+] \mathrm{i}$ occurs as a result of an influx of $\mathrm{Ca} 2+$ through the plasma mambrane via this non-selective cation channel. To date, neither the identity nor the functional meaning of this channel in neuroendocrine tumor (NET) cells is known. AIM: This study was undertaken to investigate electrophysiological properties of human NET primary cell cultures and permanent NET BON cells combined with the analysis of intracellular Ca2 + responses mediated by extracellular application of cooling compounds. METHODS: TRPM8 mRNA expression was detected by RT-PCR and protein expression was confirmed by immunofluorescence staining and microscopy. Ca2 + transients were measured by single cell fluorescence imaging. The wholecell patch-clamp technique was used to monitor icilin-mediated stimulation of TRPM8 activity. In addition, the effect of TRPM8 activation on neurotensin concentration was studied. RESULTS: Extracellular application of iclin $(300 \mathrm{nM})$ elicited non-selective cation channel currents in BON cells. In addition, [Ca2+]i increased from $104 \pm 8 \mathrm{nM}$ to a peak level of $185 \pm 14 \mathrm{nM}$ followed by a recovery to $120 \pm 7 \mathrm{nM}(\mathrm{n}=3)$. These results were reproducible if menthol $(100 \mu \mathrm{M})$ was used as stumlus. In addition, in three primary NET cell cultures icilin also led to an increase in [Ca2+]i (i.e from $104 \pm 3 \mathrm{nM}$ to $143 \pm 9 \mathrm{nM}$; recovery partially occurred to $130 \pm 16 \mathrm{nM} ; \mathrm{n}=4$ ). Furthermore, icilin increased neurotensin secretion in BON cells. CONCLUSION: For the first time, we could demonstrate by several methods that TRPM8 is expressed and functionally active in BON cells. Importantly, this TRPM isoform is associated with neurotensin secretion.

\section{T1665}

Gene Expression Profiling Reveals Different Regulated Pathways for Adenocarcinoma of the Esophagus and Cardia Sjoerd M. Lagarde, Emiel E. Ver Loren van Themaat, Perry D. Moerland, Lisa A. GilhuijsPederson, Fiebo J. Ten Kate, Pieter H. Reitsma, Antouine H. van Kampen, Koos H. Zwinderman, Frank Baas, Jan J. van Lanschot

Introduction: Adenocarcinomas of the distal esophagus and gastric cardia are two tumor that have many features in common. However, the etiology is different with gastroesophagea reflux disease playing a major role in adenocarcinoma of the distal esophagus, but not in that of the gastric cardia. The molecular analysis of differences between these tumors has mainly focused on single candidate genes. However, global gene expression profiling monitors changes in tens of thousands of genes. Extracting biological insight from these data remains a challenge. Gene Set Enrichment Analysis (GSEA) is used to detect modest but coordinate expression changes of groups of (functionally) related genes. The aim of this study was to identify differentially expressed genes and gene sets that are associated with tumor localization (esophagus versus cardia). Methods: Resection specimens of either adenocarcinoma of the esophagus $(\mathrm{N}=18)$ or cardia $(\mathrm{N}=16)$ were carefully selected. There was no difference in stage, differentiation grade and lymph node ratio between the two tumor types. Whole genome microarrays containing 44.000 probes were used to evaluate these 34 cancer specimens. After quality control and normalisation, differentially expressed genes were identified by Significance Analysis of Microarrays (SAM). Furthermore GSEA was applied to the

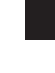


microarray data using 1325 biologically defined gene sets and 100 permutation tests. Results: SAM did not demonstrate differentially expressed genes between the two tumor types. However, GSEA found coordinate expressed gene sets that were related to the cell-cycle process and leptin pathway. Genes located on the chromosome loci $6 \mathrm{p} 22$ and 20q11 were differentially regulated between the two tumor types. Both chromosome loci have several interesting genes which are known to be associated with tumors in the digestive tract. Furthermore, both chromosomes contain a member of the E2F-gene family. The E2F family plays a crucial role in the control of the cell cycle, DNA replication and action of tumor suppressor proteins. Conclusions: Adenocarcinomas of the distal esophagus and gastric cardia have many features in common, but gene set enrichment reveals different regulated pathways for the two tumors. This suggests that different pathways underlie the oncogenesis of these tumor entities, which might necessitate distinct strategies for future targeted therapy.

\section{T1666}

Transcription Factor YY1 Is Overexpressed in Gastrointestinal Cancer Cell Dharmaraj Chinnappan, Dongmei Xiao, Anita Ratnasari, Thomas King, Chris Andry Horst C. Weber

The zinc finger transcription factor Yin Yang 1 (YYl) is a negative regulator of tumor suppressor gene $\mathrm{p} 53$, which is frequently mutated late in gastrointestinal (GI) carcinogenesis, and has functions in the control of cell growth, tumor suppression and differentiation. We recently identified YYl as a transcriptional activator of the GRP-receptor in colo-rectal cancer (CRC) cells, but the expression and regulation of YYl in CRC has not been investigated. Therefore, this study aimed to examine the expression of YYl in normal human tissues, CRC tissues and GI cancer cells in vitro. Methods: YYl-specific mRNA and protein expression was examined in human cancer cells by Northern and Western blot using radioactively labeled YY1 cDNA and a monoclonal anti-YYl antibody, respectively. Immunohistochemistry (IHC) was performed on CRC tissue arrays. Genomic DNA was used for Southern blot analysis, and FISH was performed with a human YYl-specific probe in human cancer cells. Results: Several distinct human YYl mRNA isoforms (1.0, 2.6, 4.6, and $7.5 \mathrm{~kb})$ were ubiquitously expressed in normal tissues, most abundantly in skeletal and heart muscle, but to a lesser extent also in colonic muscle and mucosa. In human GI cancer cells in vitro (Lovo, Caco-2, HT-29), two major YYl mRNA isoforms (7.5 and $2.6 \mathrm{~kb}$ ) were significantly over-expressed (2.6 kb: 3-6 fold: $7.5 \mathrm{~kb}: 2-20$ fold) compared with normal skeletal muscle. The YYl mRNA half lives in Lovo and Caco-2 CRC cells were 2 and 8 hours, respectively, and protein half lives were 6 hours (Lovo) and far exceeding 6 hours in Caco-2 cells. FISH mapped the YYl gene to chromosome $14 \mathrm{q} 32$ and showed various degree of aneuploidy in cancer cells without increased signal intensity. Only 5\% Lovo cells showed aneuploidy, whereas in 95\% of Caco-2 cells aneuploidy was noted (2n: 5\%, 3n:36\%, 4n:32\%, 5n and more: $27 \%$ ). YYl immunoreactivity of the colonic mucosa was confined to nuclear staining of epithelial cells both in normal and cancer samples. Some neutrophils, lymphocytes and muscle cells stained positive, whereas mitotic cells did not show YYl staining. When analyzed according to a standardized score (normalized intensity x \% nuclei staining), we detected a trend for increased score with increasing tumor grade. Conclusions: YYl is significantly over-expressed in cancer cells in vitro and some CRC tissue samples where it is confined to nuclear localization. In cancer cells in vitro, over-expression occurs in the absence of gene amplification and translocation and is regulated -at least in part- by mRNA and protein stability. YYl over-expression in CRC might be associated with cell dedifferentiation.

T1667

Novel Therapeutic Targets for Gastrointestinal Cancer

Eugene A. Volpe, Tapas Saha, Will J. Ferguson, Yi Tang, Varalakshmi Katuri, Wilma Jogunoori, Tiffany M. Blake, Bubhuti Mishra, Lopa Mishra, Stephen Rt Evans

The TGF- $\beta$ superfamily plays an important role in the formation of gastrointestinal carcinoma, cell differentiation, tumor suppression and liver development. The TGF- $\beta$ pathway is inactivated in most gastrointestinal cancers. TGF- $\beta$ is modulated through Smad activation and is dependent upon adaptor proteins such as ELF, a $\beta$-spectrin as well as modifying E3 ligases such as PRAJA. We have shown that disruption of the Smad4 adaptor protein ELF, a $\beta$ spectrin, disrupts TGF- $\beta$ signaling. Elf-/- mice die in midgestation from gastrointestinal, liver, neural and heart defects. Aim: To identify new therapeutic targets to control gastrointesinal cancer when the TGF- $\beta$ signaling pathway is inactivated. Methods and Results: 1 . We performed protein kinase assays and microarray analysis on ELF deficient mouse fibroblasts as well as in control mouse fibroblasts to dissect new pathways that could be inactivated when this key signaling pathway is inactivated. 2 . We have identified a number of TGF- $\beta$ regulated proteins important in early gut progenitor cell homeostasis. ELF heterozygotes develop gastrointestinal cancer. 3. Protein kinase assays demonstrate elevated expression of $\mathrm{Src}$ and ERKl in elf-/- fibroblasts when compare to elf $+/+$ when stimulated with TGF- $\beta .4$ Northern blot analysis and RTPCR confirms the above observations. 5. Microarray analysis using $44 \mathrm{~K}$ human array identified 77 genes that are up regulated and 65 genes that are down-regulated in ELF deficient mice with significant probability ( $\mathrm{p}$ value) when compared to the normal control mice. 6. SWI/SNF related, matrix associated, actin dependent regulator of chromatin (SMARCE1) protein has a 6 fold increase in expression in elf-/- mice. RING finger protein 10 (RNF10) is also up-regulated by 1.8 fold in ELF deficient mice. Interestingly, PRAJA, which is also a RING finger protein, is often found up regulated in gastric cancers and has been shown to ubiquitinate ELF and destroy it through 265 proteasome pathway. 7. Furthermore, G protein coupled receptor (GPR64; 7.84 fold), origin recognition complex subunit 6 (ORC6; 5.466 fold), transferrin (TF; 3.44 fold) and Jun B proto oncogene (JunB; 2.11 fold) are the most prominent proteins that are down-regulated in elf-/- mice. Conclusions: These studies reveal a new therapeutic approach for tumorigenesis in gastrointestinal cancers whereby defects in adaptor proteins for Smads, such as ELF, induce inactivation of the TGF- $\beta$ signaling pathway.

\section{$\mathrm{T} 1668$}

Induction of Enteric Neurons from Embryonic Stem Cells in Vitro and in Vivo Valentina Sasselli, Maria-Adelaide Micci, Pankaj Jay Pasricha

Introduction: In response to appropriate environmental signals, pluripotential embryonic stem (ES) cells can differentiate into a variety of cell types including central and peripheral neurons. However their potential for developing specifically into enteric neurons is unknown. As part of an ongoing project on stem cell replacement therapy for enteric neuronal disorders, this study examined the phenotypic response of ES cells to putative differentiating cues derived from the enteric nervous system and related tissues. Methods: Mouse ES cells were cultured as neurospheres for 7 days in serum free medium (NB27) supplemented with LIF EGF and FGF2. For in vitro experiments, neurospheres were co-cultured with a fresh preparation of mouse longitudinal muscle-myenteric plexus (LM-MP) placed inside transwells (no-contact) in NB27 medium without growth factors. After 10 days cells were processed for immunofluorescence staining, Western blot and real time PCR to assess neuronal and glial differentiation. For in vivo experiments, ES-derived neurospheres were transplanted at a density of 50,000 cells/ $\mu$ into the pylorus of C57BL/6J adult mice. Survival and differentiation of grafted cells was assessed 7 days after transplantation. Results: As compared with controls, exposure to LM-MP in vitro resulted in increased mRNA and protein expression of nestin, as well as $\beta$ III-tubulin, MAP 2 and nNOS (protein expression data showed a fold increase of 2.2 for nestin, 2.0 for $\beta$ III-tubulin, 1.9 for MAP2 and 1.9 for nNOS). Noticeably, expression of the glial marker GFAP was not detected in either group. In vivo studies revealed adequate survival of transplanted ES cells; however, these appeared undifferentiated and did not express any of the above markers. Conclusions: Our results indicate that enteric muscle and/or neurons secrete factors that can induce differentiation of ES cells to specific neuronal fates including the expression of nNOS, a key molecule for regulation of gut motility. On the other hand ES-derived neurospheres failed to adopt a neuronal phenotype in vivo in normal tissue at least, suggesting the presence of local inhibitory influences. We conclude that for an ES-based transplant strategy to successfully repopulate the ENS, the cells will either need pre-transplantation "nudging" or further manipulation of the posttransplant environment to acquire an enteric neuronal phenotype.

\section{T1669}

Turnover of Interstitial Cells of Cajal in the Murine Stomach: Regeneration from Local Progenitors and Limited Survival After Loss of Electrical Pacemaker Activity and Kit Expression

Doug Redelman, Andrea Lorincz, Viktor J. Horvath, Michael R. Bardsley, Hui Chen, Tamas Ordog

We have recently shown that reduced insulin/insulin-like growth factor-I (IGF-I) signaling and consequent decrease in stem cell factor (SCF) production by smooth muscle cells may be responsible for the depletion of interstitial cells of Cajal (ICC) in murine diabetic gastroparesis (Gastroenterology, in press). Preliminary results have also suggested a role for cell turnover in the maintenance of ICC. The goal of this study was to identify ICC precursors within the murine gastric tunica muscularis. We investigated the expression of CD34 and CD44, putative markers for ICC precursors, and receptors for SCF (Kit, the standard ICC marker), insulin, and IGF-I in gastric corpus+antrum muscles from adult and juvenile mice by immunohistochemistry and flow cytometry. Effects of long-term (32-85-day) treatments with SCF and IGF-I were studied in organotypic cultures. In freshly dissected tissues, CD34 was not detected in Kit ${ }^{\text {Bright }}$ ICC. In contrast, a small population ( $\leq 0.6 \%$ of all cells) of Kit ${ }^{\text {Low }}$ cells expressed high levels of CD34. These cells were oval-shaped, had no processes, and occurred in small clusters on the serosal and submucosal surfaces of the gastric musculature and in the myenteric region. Unlike mature ICC, they also expressed insulin and IGFI receptors. All Kit ${ }^{\text {Bright }}$ ICC and Kit ${ }^{\text {Low }}$ cells expressed CD44, a multifunctional glycoprotein found in mesenchymal stem cells. Long-term treatment of cultured gastric muscles with $\mathrm{SCF}$ caused the growth of the $\mathrm{Kit}^{\text {Low }} \mathrm{CD} 34^{+}$cells into densely packed clusters in the myenteric region or thick chords containing bipolar cells along the longitudinal axes of the nearby smooth muscle fibers. SCF stimulated Kit expression but reduced CD34 levels. In contrast, the IGF-I-treated cultures were dominated by normal, $\mathrm{Kit}^{+} \mathrm{CD} 34^{-}$ICC networks that also expressed CD44. The effects of IGF-I on ICC were blocked by inhibiting c-kit transcription with interferon- $\gamma$. Kit ${ }^{+}$ICC and electrical slow waves were lost in cultures maintained in the absence of growth factors for up to 85 days. However, partially disrupted networks of ICC-like cells could still be recognized by CD44 immunostaining. We conclude that maintenance of gastric ICC probably involves their insulin/IGF-I-dependent regeneration from Kit ${ }^{\text {Low }} \mathrm{CD} 34^{+}$precursors. While the development of these cells into ICC can be initiated with soluble SCF, their expansion and conversion into mature, CD34- ICC requires IGF-I or insulin, which can stimulate both soluble and membrane-bound SCF. CD44 expression is integral to the ICC phenotype and is maintained for some time after the loss of Kit expression and physiological function. Supported by NIH Grants DK58185 and RR16464.

\section{T1670}

A Conditionally Immortalized Colonic Epithelial Cell Line from a Genetically Engineered Mouse That Polarizes Spontaneously in Vitro Robert H. Whitehead, Angela L. Tyner, Jeffrey L. Franklin

Background. The colonic epithelium is a polarized epithelium, however the study of the mechanisms of polarization and transport has been limited by the lack of a normal epithelial cell line that will polarize in vitro. Because of this, studies have depended on the use of colon carcinoma cell lines that will polarize in vitro. Methods. Mice with disruption of the gene encoding the Brk tyrosine kinase were mated with an "Immortomouse" and the progeny carrying both genes mated to obtain homozygous knockout mice that carried the temperature sensitive SV40 large T gene. At 12 weeks of age the colons were removed and the crypts isolated using EDTA/DTT. These crypts were cultured in collagen-coated wells in LHC-9 medium. Results. A cell line was obtained that grew very slowly. The cells were sensitive to trypsin and had to be passaged using EDTA/EGTA. The cells were cultured on transwells that had been coated with Attachment Factor (Cell Systems Corporation). After 7 days 
culture at $330 \mathrm{C}$ the resistance of the monolayer was $>400$ milliOhms. Staining of the fixed monolayer demonstrated the polarization of these cells. The polarization was confirmed by fixing the filters with paraformaldehyde and staining with antibodies to E-cadherin, bcatenin, occludin and ZO-1. In all cases a polarized staining pattern was seen. Discussion. This is the first description of a polarizing conditionally immortalized murine colonic epithelial cell line. It is not yet known whether disruption of the brk gene or the fact that these cells were cultured in a serum-free medium was responsible for our success in deriving this cell line. Studies are underway to reintroduce Brk into the cells to determine if absence of functional Brk contributes to polarization

\section{T1671}

Gene Expression Profiles of Normal Proliferating and Differentiating Human Intestinal Epithelial Cells: A Comparison with the Caco-2 Cell Model Eric Tremblay, Joelle Auclair, Emile Levy, Daniel Menard, Nathalie Rivard, Ernest G. Seidman, Pierre H. Vachon, Jean-Francois Beaulieu

Background/Aim: cDNA microarray technology enables detailed analysis of gene expression throughout complex processes such as proliferation, differentiation and carcinogenesis. The aim of this study was to analyze the gene expression profile of normal human intestinal epithelial cells using cell models representing undifferentiated and mature intestinal epithelial cells in comparison to the widely used Caco-2 cell model. Methods: Four independent RNA preparations for each cell type were used on cDNA microarrays representing 19,200 human cDNA clones (University Health Network, Ontario, Canada). A hierarchical clustering algorithm was used to identify patterns of gene expression in the crypt-like proliferative HIEC and tsFHI cells, and isolated villus epithelial cells as well as Caco-2 cells at two distinct stages of differentiation (proliferative and twenty days postconfluence). Results: Unsupervised hierarchical clustering analysis of global gene expression among the cell lines identified two branches: one for the HIEC cells versus a second comprised of two sub-groups: a) the proliferative Caco-2 cells and b) the differentiated Caco- 2 cells and the closely related villus epithelial cells. At the gene level, supervised hierarchical clustering with 272 differentially expressed genes (one-way ANOVA with adjusted Bonferonni correction, $\mathrm{p}<0.05$ ) revealed distinct expression patterns specific to each cell phenotype. Among these expression patterns, we observed a striking difference in up-regulated genes involved in proliferation between the normal undifferentiated intestinal cells and proliferative Caco-2 cells suggesting a different molecular regulation of cancer cells. Conclusions: We identified several up-regulated genes that could lead to the identification of new regulatory pathways involved in cell differentiation and carcinogenesis. The combined use of microarray analysis and human intestinal cell models thus provides a powerful tool for establishing detailed gene expression profiles of proliferative to terminally differentiated intestinal cells. Furthermore, the molecular differences between the normal human intestinal cell models and Caco-2 cells clearly point out the strengths and limitations of this widely used experimental model for studying intestinal cell proliferation and differentiation.

\section{$\mathrm{T} 1672$}

Basal Promoter Activation of Rat Nhe2 Gene in Intestinal Epithelial Cells Ping Hua, Hua Xu, Jennifer K. Uno, Pawel R. Kiela, Fayez K. Ghishan

Introduction: $\mathrm{Na}+\mathrm{H}+$ exchanger isoform-2 plays important roles in intracellular $\mathrm{pH}$, cell volume regulation and ion transport in the kidney and intestine. Our previous studies have identified a minimal Spl-driven promoter fragment (nt -36/+116) directing high expression of NHE2 in mouse renal epithelial cells mIMCD-3. However, this minimal promoter region was not sufficient to promote active transcription in intestinal epithelial cells, suggesting the need for upstream regulatory elements to promote active transcription in the intestinal epithelium. In this study, we used non-transformed rat intestinal cells (RIE) as a model to identify the minimal promoter fragment and transcription factors necessary for the basal transcription of NHE2 gene in intestinal epithelial cells. Methods: A series of 5' deletion constructs $(-90,-85,-69$, and $-43 \mathrm{nt})$ were made by PCR and restriction enzyme digestion and subcloned into pGL3 basic reporter vector. All constructs had the same 3' end at nt +116 . These promoter constructs were transiently transfected into RIE cells, and assayed for reporter gene activity. Nuclear protein was extracted from RIE cells by standard methods, and electrophoretic mobility shift assay were performed to detect protein-DNA interactions. Results: Reporter gene assays demonstrated that promoter constructs $-90 /+116,-85 /+116$, and $-69 /+116$ were all functional in RIE cells, with construct $-69 /+116$ showing the highest promoter activity. Compared to this construct, $-43 /+116$ fragment had 40\% activity. EMSA detected DNA-protein complexes within the minimal promoter region spanning -73/-23 bp containing multiple and overlapping cis elements. Individual mutations at $-56 /-55 \mathrm{nt}$ or 52/-51 nt partially decreased protein binding affinity to a probe spanning $-67 /-43 \mathrm{nt}$ of NHE2 promoter, while in combination, these mutations completely abolished DNA-protein interactions in this promoter region. Mutation at $\mathrm{nt}-56 /-55$ also reduced the NHE-2 promoter activity by $\sim 40 \%$. Conclusion: The minimal promoter region for activating the rat NHE2 gene in intestine is located within -69/-36 nt upstream of transcription initiation site. The cis elements involved are distinct from those identified in the renal epithelial cells. Reporter gene assays indicated that $-56 /-55$ region representing a predicted core binding site of transcription factor MAZF is partially responsible for active transcription of NHE2 gene in RIE cells. Future work will focus on identifying transcription factors, their functional and physical interactions and their cognate cis elements participating in NHE2 gene transcription in the intestinal epithelium.

\section{T1673}

Monoclonal Conversion of Human Gastric Glands Gives Insights Into Stem Cell and Clonal Architecture

Stuart A. McDonald, Laura C. Greaves, Simon J. Leedham, Maesha Deheragoda, Robert W. Taylor, Sean L. Preston, Chung Yin-Lee, David Stoker, Marco R. Novelli, Janusz A. Jankowski, Douglass M. Turnbull, Nicholas A. Wright

Studies on the intestine of animals and man have confirmed, beyond reasonable doubt, that the structural units, the crypts, are clonally-derived. The unit found in the stomach, the gastric gland, especially in the human, remains an enigma. Some animal reports show clonalderivation, whereas others show that a minority of glands remain polyclonal, suggesting the possibility of multiple, possibly independent stem cells. In the human, results are also mixed. We have used mutations in the mitochondrial cytochrome c oxidase subunit 1 gene (COXsul), as a clonal marker (J. Clin. Invest. 2003:112(9)), detecting COXsul in conjunction with a nuclear-encoded oxidative enzyme, succinate dehydrogenase (SDH), by double enzyme histochemistry. We have observed body-type gastric glands which are partially filled with mutated cells, and others glands where all the cells in the gland are mutated (COXsul$\mathrm{SDH}+$ ). These suggest that a stem cell bearing this mutation expands to fill the entire gastric gland. Early observations suggest that all cell lineages in these mutated body-type glands are involved in this process. Moreover, the mutated gland then appears to spread in the mucosa by gland fission as shown by neighbouring glands having the same mitochondrial mutations. Several conclusions are made possible by these observations: 1.Gastric gland undergo a process of monoclonal conversion, where the gland becomes gradually replaced by mutated cells; 2 .Human gastric glands are maintained by a number of stem cells; 3 .Each body-type gland is derived ultimately from a single stem cell which gives rise to all the contained lineages, and are therefore multi-potential; 4.These mutated glands then appear to expand by gland fission, even in the adult stomach.

\section{T1674}

TGF-Beta Signaling Is Required for Ductal Progenitor Cell Differentiation and the Disruption of TGF-Beta Signaling May Be Involved in Development of Hepatocellular Carcinoma and Cholangiocarcinoma

Krit Kitisin, Natarajan Ganesan, Yi Tang, Varalakshmi Katuri, Jogunoori Wilma, Kirti Shetty, Lynt Johnson, Lopa Mishra

Human hepatic progenitor cells can most likely give rise to hepatocellular carcinoma as well as cholangiocarcinoma. In these models, a periportal population of small "primitive" oval epithelial cells proliferate either in association with or before hepatocyte multiplication. In mouse development, ventral floor of foregut endoderm produces cells destined to become hepatoblasts, bipotential cells committed to fetal hepatocytes and biliary epithelial cells. In adult human tissues, these hepatic progenitor cells (or mouse oval cells) are immature epithelial cells found residing in the canals of Hering. TGF-beta is important in modulation of biliary and liver epithelial cell morphogenesis. Both elf-/- and Smad2+///Smad3+/- mutant embryos display hepatic hypoplasia. In addition. TGF-beta signaling proteins such as TBRII, Smad4 and ELF are all implicated in suppressing HCC. Thus it is conceivable that cells expressing these proteins are progenitors that are susceptible to bile duct malformation and tumor development once tight TGF-beta control is lost. Aims: 1. To determine the role of TGF-beta signaling in liver progenitor cell proliferation and differentiation utilizing a mouse embryonic liver explant cultures. 2. To determine the localization of TGF-beta "progenitor cells" to a specific region of portal tracts, we analyzed human regenerating liver after donor transplant for TBRII, Smad4 and ELF expressions. Methods and Results: 1. Embryonic liver tissues isolated from normal, elf-/- and Smad2 $+/ / 3+/-$ mutants were cultured in TGF-beta growth factor to determine their growth properties. Explant cultures from Smad2 $+/-/ 3+/-$ mutants showed a lack of bile ductules 2. Addition of TGF-beta produced a rise in Smad2 and 3 activity, expression occurring at the rapid outgrowth of a limiting plate with bile duct formation. 3. Immunohistochemical labeling revealed a tight region around portal tracts where a 6-7 cell cluster specifically expressed TBRII, Smad4 and ELF. These clusters are more prominent in regenerating liver and in low abundance (one per low power view) Conclusion: Prominent expression of TGF-beta signaling proteins found in the periportal cluster of cells indicates that these cells are under tight TGF-beta control. The cluster of cells could represent the hepatic progenitor cell "niche" which is important in normal intrahepatic bile duct formation and in regenerating liver. Disruption of TGF-beta signaling has been implicated in numbers of human carcinogenesis. The exploration of these hepatic progenitor cell "niche" and the loss of TGF-beta signaling control could provide insights to the development of hepatocellular cancer and cholangiocarcinoma.

\section{T1675}

Failure of Vagal Sensory Axons to Innervate Aganglionic (Ret-deficient) Bowel: Relationship to Enteric Expression of Netrin-1 Elyanne M. Ratcliffe, Lena Fan, Tandi J. Mohammed, Michael D. Gershon

Vagal sensory axons are concentrated in myenteric ganglia, intramuscular arrays, and the lamina propria. This innervation develops during fetal life and results, in part, from the attraction by enteric netrins of vagal fibers, which express the receptor, deleted in colorectal cancer (DCC). We took advantage of the failure of crest-derived cells to form ganglia in the subdiaphragmatic bowel of Ret $\%$ mice to study the role ganglia play in establishing vaga sensory innervation. To label vagal sensory axons, Dil was applied bilaterally at E12 and E16 to the nodose ganglia of Ret $+/+,+/$ - and $-/$ - mice. DiI-labeled vagal sensory axons were identified in E12 Ret $+/+$ and $+/$ - fetuses in fasciculated paraesophageal bundles; extensions of these fibers were also seen in the gastric mesenchyme. In contrast, no labeled vagal axon could be detected distal to the esophagus at E12 in Ret -/- animals. By E16, in Ret +/+ and +/- mice, Dil-labeled vagal sensory fibers were well advanced into the small intestine; however, in Ret -/- mice Dil-labeled vagal fibers could not be visualized distal to the esophagus. Netrin-l immunoreactivity was located in the developing gut of Ret $+/+$ (E12 $\mathrm{P} 2$ ) and $-/$ - (E12 and E16) to test the hypothesis that the failure of the vagal sensory innervation to enter the subdiaphragmatic Ret -/- bowel was related to an abnormality of 
netrin-1 expression. Nerves and ganglia were simultaneously located with antibodies to PGP9.5. At E12 in Ret +/+ mice, netrin-1 immunoreactivity was concentrated in the outer gut mesenchyme and the mucosal epithelium of the presumptive esophagus, stomach and small intestine. Myenteric ganglia formed within the netrin-1 expressing mesenchyme and were themselves netrin-1-immunoreactive. In Ret -/- mice, at E12 and E16, little netrin-1 immunoreactivity was detected in the outer gut mesenchyme or mucosa. We suggest that the vagal sensory innervation of the gut depends on the formation of enteric neurons. The decrease in netrin immunoreactivity in the aganglionic gut is consistent with the idea that the vagal innervation fails in the Ret $-/$ - bowel because these axons are not attracted by netrins. Further studies are required to determine whether developing enteric neurons acquire netrin immunoreactivity because they synthesize or take up netrins. Additional studies of the interactions of developing ganglia with the enteric mesenchyme are also needed to determine why the absence of enteric ganglia causes mesenchymal expression of netrin to fail. Supported by K12-HD00850, AGA Research Scholar Award, NS12963 and NS15547.

\section{T1676}

Identification of Putative Transcriptional Regulatory Pathways Mediating the Genomic Response to Iron-Deficiency in Rat Duodenum

James F. Collins, Zihua Hu

Introduction/Aims: Our previous microarray studies identified differentially expressed genes (DEGs) in the duodenum of iron-deprived rats across different stages of postnatal development (sucklings through older adults). We demonstrated that many known iron-responsive genes were regulated in our rat model as expected, as well as many novel genes. We hypothesized that small groups of identified genes would likely be regulated by similar molecular mechanisms in response to iron-deprivation. Methods: To identify coordinately regulated groups of genes, we performed $8 \mathrm{H}$ Clustering Using Euclidean Distance and Complete Linkage, which has allowed us to identify several clusters of genes that are similarly regulated across the different age groups (and in the jejunum of adult iron-deficient rats). One of the clusters of strongly induced genes (\#7; containing 28 genes) was of the most interest, as it contained several iron transport genes, in addition to several novel genes. In order to identify transcriptional regulatory pathways that may coordinately control expression of some of the genes in this cluster, we utilized the Genomatix Suite software (Release 3.4.1) to extract promoter sequences of the 28 genes in this cluster and to search for complex transcription factor binding frameworks. Results: Interestingly, we found that several genes in cluster 7 including Divalent Metal Transporter 1 (DMT1), Transferrin Receptor 1, Menkes Copper ATPase (ATP7A) and a novel intestinal zinc transporter, contained putative binding sites for $\mathrm{p} 53$ and HOXF trans-acting factors (in close proximity to each other). We next searched the Genomatix rat promoter database with our p53/HOXF framework and found that the group of rat genes with similar binding sites was statistically enriched in iron transport-related genes. Importantly, Hephaestin (a copper binding ferroxidase important for intestinal iron transport) and some genes previously identified by the array studies were also found to contain this framework. Discussion: We have thus identified a potentially important regulatory pathway that may mediate the genomic response to iron-deficiency. The possible involvement of p53 is intriguing as this protein is known to mitigate stress responses and previous studies have demonstrated that p53 activity is increased by irondeprivation in various tissues and cell types. Moreover, iron-chelation is known to induce apoptosis in many cell types, a response that is partially mediated by p53. The possibility therefore exists that in addition to inducing apoptosis, p53 may play a role in iron homeostasis. Supported by NIH Grant 1R21 DK068349.

\section{T1677}

Human Colonic Crypt Self-Renewal Ex Vivo

Alyson Parris, Amy Reynolds, Theodore Spahos, Andrew Trim, Andrea Munsterberg, Richard Tighe, Michael Lewis, J.N. Cook, Alyson Prior, Chris Speakman, William Stebbings, James Hernon, Khalid Ellamin, Ian Beales, Mark Williams

Following on from classical animal studies and work on adenocarcinoma cell lines, recent gene targeted and transgenic mice studies have provided profound insights into the molecular determinants of epithelial renewal in the intestine. However, there is an increasing call for a complementary in vitro model of the intact human colonic epithelium that is amenable to real-time imaging and functional genomic analysis of tissue renewal. AIMS: To apply bioimaging and functional genomic approaches to the study of intestinal tissue renewal in cultured human colonic crypts. METHODS: Colonic crypts were isolated from tissue biopsies obtained at sigmoidoscopy from healthy-subjects (Ethical approval). Isolated crypts were attached to collagen-coated coverslips and cultured for 24 hours-7 days in serum-free DMEM $(5 \% \mathrm{CO} 2 / 37 \mathrm{oC})$. Validatory expression experiments were performed on cryosections of biopsy samples. Expression constructs and siRNA oligonucleotide duplexes were introduced to cells by low-voltage square wave electroporation. Protein location was visualised by immunofluorescence and confocal microscopy. A range of markers for cell polarity (e.g. NKCC1), cell proliferation (e.g. BrdU incorporation and Ki67) and viability (e.g. propidium iodide, calceinam) were utilised. Cell migration was assessed by monitoring Hoechst 33342 or $\alpha$ tubulinGFP labelled cells. RESULTS: Colonic crypts maintained their gross morphological flasklike appearance for at least 7 days in culture. Individual cells maintained the polarised expression of proteins (e.g. basolateral NKCCl and M3AChR) throughout the culture period. Expression of these proteins predominated at the crypt base and this topological expression gradient was preserved over the culture period. The proliferative hierarchy was also preserved in culture: Ki67 positive cells were located at the crypt base, as were BrdU positive cells $10 \mu \mathrm{M}, 4$ hour pulse). Accordingly, nuclear $\beta$-catenin labelled cells were confined to the crypt-base. Pulse chase experiments (4 hour pulse, 48 hour chase) demonstrated that BrdU labelled cells migrated to the upper crypt region (Ki67 negative). Tracking of Hoechst 33342 and GFP labelled cells confirmed a migration rate of approx. $5 \mu \mathrm{m} /$ hour. Cell shedding was visualised in real-time by propidium iodide/calcein status of detached cells. Cell viability was compromised in lower crypt compartments by siRNA knockdown of $\beta$-catenin. CONCLUSIONS: Isolated human colonic crypts are amenable to real-time imaging and functional genomic studies. We have demonstrated that many of the cellular processes associated with rapid renewal of the colonic epithelium are recapitulated ex-vivo.

\section{$\mathrm{T} 1678$}

Mapping Gene Expression Along the Anterior-Posterior Axis of the Adult Human Colon

Lawrence C. Lapointe, Robert Dunne, Daniel Worthley, Peter Molloy, David Wattchow, Graeme Young

Studies of colorectal adenocarcinomas suggest variable incidence, histopathology, and prognosis between proximal and distal tumors. While research has shown gene expression (GE) differences between the proximal and distal colon, there have been no studies exploring the nature of these expression gradients for such genes.AIM: To map individual gene variation and patterns of multi-gene expression longitudinally along the colon. METHOD: The data are from two sets of Affymetrix microarrays hybridized to mRNA isolated from non-diseased colorectal tissue specimens with an anatomically-identifiable site of resection by segment as one of cecum, ascending, transverse, descending, sigmoid or rectum. For discovery (hypothesis generation) we used a large commercial database of 184 microarrays. To validate our findings (hypothesis testing) we created a data set of 19 arrays from hospital tissue bank specimens. We analyzed anterior-posterior (A/P) GE using three techniques: single gene univariate diff. expression; linear models to compare a two-tier model of A/P GE change vs. a gradual, multi-segment model of change; and multivariate techniques to explore general GE patterns along the A/P axis. RESULTS: A total of 206 probesets, corresponding to approx. 154 known genes, were differentially expressed between the proximal $(\mathrm{n}=74)$ and distal $(n=110)$ specimens (Bonferroni corrected $p<0.05)$. Of these, $31(16.5 \%)$ were also differentially expressed in the validation data with a significant difference. $(\mathrm{p}<0.05)$. A subset of 115 probesets that were differentially expressed between the cecum and rectum were used for linear modeling. In $65(57 \%)$ of these transcripts, there was a two-tier A/P expression. The remaining $50(43 \%)$ transcripts were best modeled by a gradual, multi-segment colon model. Interestingly, 41 (82\%) of the 50 multi-segment models show a gradual GE increase along the colon. Multivariate methods, including principal components analysis (PCA) were also used to explore GE patterns along the A/P axis. These results suggest that there are two broad populations defined by the proximal vs. distal divide. CONCLUSION: We observe two forms of gene expression change in the colon. The first corresponds to a twotier, or proximal-distal, expression roughly patterned on the midgut vs. hindgut embryonic origins of the colon. A second set of genes exhibit a more gradual change from the cecum to the rectum. These results are consistent with univariate and multivariate analysis.

\section{T1679}

Induced C-Myc Regulates P21 Transcription Through Proximal Region of Its Promoter Following Increased Polyamines in Normal Intestinal Epithelial Cells

Lan Liu, Xin Guo, Jaladanki N. Rao, Tongtong Zou, Bernard S. Marasa, Jie Chen, Jose Greenspon, Robert A. Casero Jr, Jian-Ying Wang

Maintenance of intestinal mucosal epithelial integrity requires cellular polyamines that regulate expression of various genes involved in cell proliferation, growth arrest, and apoptosis. Our previous studies have shown that polyamines are essential for expression of the c-myc gene and that polyamine-induced c-Myc plays a critical role in stimulation of normal intestinal epithelial cell proliferation, but the exact down-stream targets of induced c-Myc are still unclear. The p2l is a major player in cell cycle control and it is primarily regulated at the transcriptional level. The current study went further to determine whether induced cMyc stimulates normal intestinal epithelial cell (IEC-6 line) proliferation by repressing p2l transcription following increased polyamines. Methods: c-Myc was increased by either increased cellular polyamines by stable transfection of the ornithine decarboxylase (ODC) gene (ODC-IEC cells) or transient infection with adenoviral expression vector containing $c$ myc cDNA. Expression of the $p 21$ gene was examined by measurements of its promoter mRNA, and protein. Deletion mutations and Sp-1 or Miz-1 point mutations were used to map the c-Myc-responsive region within the p21-promoter. Results: Increased levels of cellular polyamines by overexpression of the ODC gene induced c-Myc expression and inhibited 21 transcription as indicated by decreases in $\mathrm{p} 21$-promoter activity and its protein. Levels of c-Myc were increased by $~ 10$-folds in stable ODC-IEC cells, whereas levels of $\mathrm{p} 21$ promoter activity and its protein were inhibited by $\sim 80 \%$. In contrast, depletion of cellular polyamines by inhibiting ODC enzyme activity with $\alpha$-difluoromethylornithine decreased c-Myc but increased p2l transcription. Expression of c-Myc was decreased by $\sim 75 \%$ in polyamine-deficient cells, while p21 transcription was $>4$ times the control level. Ectopic expression of wild-type c-myc not only inhibited basal levels of p21 transcription in control cells but also prevented increased p21 in polyamine-deficient cells. Studies using different p21-promoter mutants showed that transcriptional repression of $\mathrm{p} 21$ by c-Myc was mediated through Miz-1- and Spl-binding sites within the proximal region of the p21-promoter in normal intestinal epithelial cells. Conclusions: These results indicate that p21 is one of the direct mediators of induced c-Myc following increased polyamines and that p21 repression by c-Myc is implicated in stimulation of normal intestinal epithelial cell proliferation.

\section{$\mathrm{T} 1680$}

Cooperative Interactions Among Intestinal Gata Factors in Activating the Rat Liver Fatty Acid Binding Protein Gene

Joyce K. Divine, Lora J. Staloch, Christopher W. Rowley, Hanna Haveri, Markku Heikinheimo, Theodore Simon

GATA-4, GATA-5, and GATA-6 are endodermal zinc-finger transcription factors that activate numerous enterocytic genes. GATA- 4 but not GATA- 5 or GATA- 6 are present in enterocytes of adult mice, and we now report the simultaneous presence of all three GATA factors in enterocytes of mice before weaning age. An immunohistochemical survey detected enterocytic GATA-4 and GATA- 6 at birth and one week of age, and GATA- 5 at one week but not birth. Interactions among GATA factors were explored utilizing a transgene constructed from the proximal promoter of the rat liver fatty acid binding protein gene (Fabpl). GATA-4 and GATA- 5 but not GATA- 6 activate the Fabpl transgene through a cognate binding site at 128. A dose-response assay revealed a maximum in transgene activation by both factors, 
where additional factor did not further increase transgene activity. At saturated levels of GATA-4, however, additional transgene activation was achieved by adding GATA-5 expression construct, and vice versa. Similar cooperativity occurred with GATA-5 and GATA-6. Identical interactions were observed with a synthetic target transgene consisting of a single GATA site upstream of a minimal promoter. Furthermore, GATA-4 and GATA-5 or GATA5 and GATA- 6 bound to each other in solution. These results are consistent with tethering of one GATA factor to the Fabpl promoter through interaction with a second GATA factor to produce increased target gene activation. Cooperative target gene activation was specific to an intestinal cell line and may represent a mechanism by which genes are activated in the intestinal epithelium during the period before weaning.

\section{T1681}

CNS-Derived Neural Stem Cells Can Form Neurons, Glia Or Smooth Muscle in Response to Putative Myogenic and/Or Neural Factors in the Enteric Nervous System

Maria-Adelaide Micci, Rochelle R. Simmons, Kristen M. Kahrig, Pankaj Jay Pasricha

Introduction: Recent studies have explored the potential of multipotent neural stem cells derived from the central nervous system (CNS-NSC) to repopulate the enteric nervous system (ENS). However, the spectrum of plasticity of these cells in response to various components of the ENS has not been characterized. The aim of this study was to investigate the effect of putative differentiating factors secreted by the muscle and/or enteric neurons on the differentiation of CNS-NSC in vitro. Methods: CNS-NSC were isolated from the subventricular zone (SVZ) of embryonic mice and propagated in culture in NB27 medium supplemented with EGF and FGF2. CNS-NSC were either (a) cultured for 10 days in the presence of conditioned media collected from primary cultures of intestinal mouse smooth muscle cells or (b) co-cultured with a fresh preparation of mouse longitudinal musclemyenteric plexus (LM-MP) placed inside transwells (no-contact) in NB27 medium without growth factors. Control cultures were kept in standard differentiating conditions (NB27 medium alone). After 10 days cells were processed for immunofluorescence staining and Western blotting to assess the expression of specific markers indicative of their phenotypical fate. Results: A strong induction of $\alpha$-smooth muscle actin was observed in CNS-NSC treated with smooth muscle-conditioned media. Moreover, as compared to controls, expression of the glial marker GFAP was increased (2.4 fold), while expression of the neuronal marker $\beta$ III-tubulin was markedly reduced (100 fold reduction). By contrast, exposure of CNSNSC to LM-MP resulted in increased protein expression of $\beta$ III-tubulin (2.24 fold increase compared with controls), as well as GFAP (2.04 fold increase). $\alpha$-smooth muscle actin expression, though more than controls (where it was undetectable), was markedly less than in CNS-NSC exposed to smooth muscle-conditioned media. Conclusions: Our data show that intestinal smooth muscle cells exert a strong myogenic and gliogenic effect on CNSNSC while inhibiting neuronal differentiation. In the presence of enteric neurons, however, this myogenic influence is overcome and there is a marked shift towards a neuronal phenotype at the expense of smooth muscle. Identification of these putative differentiating factors is in progress. Our results also show that under the appropriate circumstances, CNS-NSC can be induced to differentiate into muscle and thus suggest another potential therapeutic use for these cells in gastrointestinal disorders.

\section{$\mathrm{T} 1682$}

Microarry Analysis of Rat Duodenal Mucosa: Expression of Iron Related Genes Along the Crypt-Villus Axis Kwo-Yih Yeh, Mary Yeh, Jonathan Glass

Iron homeostasis is regulated at the level of intestinal absorption with dysregulation, e.g. in hemochromatosis, leading to iron excess with resultant tissue damage. The major proteins involved in iron absorption are divalent metal transporter l (DMTl), ferroportin l (FPN1), duodenal cytochrome b reductase (Dcytb) and hephaestin (Heph). How these proteins are regulated remains unclear. The transcriptional regulation of iron related genes was examined in rat duodenal villi by cryostat sectioning, isolating RNA from the top and bottom thirds of the axis, and examining global gene expression in the two poles with Affymetrix Rat Gene Chips comparing the iron deficient Belgrade $(\mathrm{b} / \mathrm{b})$ rats with wild-type $(+/+)$ rats and $\mathrm{b} / \mathrm{b}$ rats fed iron for 3 days $(b / b+F e)$. The location of the sections was confirmed by analysis of sucrase-isomaltase, a marker of differentiated mucosa, and cryptdin 5, a marker of Paneth cells in the crypts. Microarray data was sorted into genes that were either exclusively or preferentially expressed in the villus bottoms or tops. The genes involved in dietary iron absorption, Dcytb, Heph, DMTl and FPN, were expressed in the villlus tops and genes involved in systemic iron uptake, transferrin (Tf) and Tf receptor (Tfrc), were expressed in the villus bottoms. Differential expression was confirmed by real time RT-PCR and Northern and western blot analyses. In the b/b rats DMTl and Dcytb expression increased markedly and was reduced toward $+/+$ levels in the $b / b+F e$. Groups of genes were defined in both poles of the $\mathrm{C}-\mathrm{V}$ axis that were iron responsive either increasing or decreasing in the $\mathrm{b} / \mathrm{b}$ rats and returning to normal in bb+Fe rats. This analysis allowed us to define genes involved in iron transport because of differential spatial expression and changes in expression with changes in iron status. However, other genes, e.g. the heme basolateral transporter Abcg2, heme oxygenase 1 , the $\mathrm{Cu}++$ transporter ATP7a, solute carrier family 27 (fatty acid transporter) and cytochrome P450, 4al0 (Cyp4a10) had expression that was also spatially related on the $\mathrm{C}-\mathrm{V}$ axis and whose expression was altered in the $\mathrm{b} / \mathrm{b}$ rat, but not reversed with iron feeding. These findings, especially the persistence of gene expression after iron feeding for some genes and not others, strongly suggests that the $\mathrm{b} / \mathrm{b}$ rat mutation has effects on gene expression perhaps through epigenetic phenomena that occur as a result of iron deficiency. Supported by NIH DK-65101 (KY) and DK-41279 (JG)

\section{T1683}

Gata-6 and Hnf-4alpha Transcription Factors Regulate Murine Muc5ac Mucin Gene Expression During Stomach Development

Ingrid B Renes, Nicolas Jonckheere, Anita M Korteland-van Male, Marie-Paule Ducourouble, Isabelle van Seuningen

Introduction: During embryonic and foetal development of the gastrointestinal tract, the MUC5AC mucin gene has a spatio-temporal pattern of expression restricted to the stomach and the small intestine. In the adult, MUC5AC is only expressed in the surface epithelial cells of the stomach. Aim: To gain insight into the molecular mechanisms responsible for the restricted pattern of Muc5ac expression during mouse stomach development. Methods: Expression of Muc5ac mucin and of transcription factors known to be involved in cell differentiation programs during mouse gastric development was studied by immunohistochemistry. The transcriptional regulation of Muc5ac by HNF and GATA factors was performed in the murine CMT-93 cell line by co-transfection experiments. Cis-elements within the promoter were identified by electrophoretic mobility shift assays and chromatin immunoprec ipitation. Results: Expression studies indicate that GATA-4, HNF-lalpha, and HNF-3beta were already expressed in the pseudostratified epithelium of the hindstomach at embryonic day (ED) 14.5 and remained expressed at EDs 17.5/18.5, post natal day (PND) 1.5 and in adults. GATA- 6 and HNF-4alpha started to be expressed in the stomach as of ED17.5, concomitant with the outgrowth of the hindstomach into a glandular stomach. Their expression remained weak until ED18.5 but increased substantially after birth. As of ED17.5, al the transcription factors studied were expressed in epithelial cells from the bottom to the top of the glands. Interestingly, at PND1.5 we observed an increase in GATA-6 and HNF4alpha expression concomitant with the induction of Muc5ac. The implication of GATA-6 and HNF-4alpha in Muc5ac up-regulation during gastric development and differentiation was confirmed by in vitro studies in which we showed up-regulation of promoter activity by these two factors and binding of HNF-4alpha to two cis-elements in the promoter Conclusion: These results indicate that GATA-6 and HNF-4alpha play an important role in mediating the spatio-temporal pattern of expression of Muc5ac mucin in the stomach and may be responsible for its de novo ectopic expression in other tissues.

\section{$\mathrm{T} 1684$}

Colonic Stem Cells Are Unable to Selectively Segregate Their Genome At the Time of Mitosis: Replication Errors May Persist

Sean L. Preston, Nikki Mandir, Toby Hunt, Robert A. Goodlad, Mairi Brittan, Simon J. Leedham, Sabina Beg, Nicholas A. Wright, Malcolm R. Alison

Background: Compared to the large intestine, tumors of the small intestine (SI) are rare. No satisfactory explanation for this discrepancy has been identified. SI stem cells appear able to protect themselves from DNA replication errors by segregating their genome at the time of cell division; always retaining the old ('template') strand and passing on the newly synthesised DNA strand to their progeny (Potten et al. J Cell Sci 2002) - the Cairns hypothesis. Does the same process take place in the colon when new stem cells and new 'template' strands are being produced following radiation? Could the higher rate of tumors in the colon and the Min (APC +/-) be explained by an inability to selectively segregate thei genome at the time of division? Methods: $25 \mathrm{Min}$ and $25 \mathrm{C} 57 \mathrm{Bl} / 6 \mathrm{~J}$ mice underwent whole body irradiation at a dose of $8 \mathrm{~Gy}$, followed by 6 hourly ip injections of tritiated thymidine (3HTdR) over 48 hours - labelling the 'template' strand. The mice were then left for 8 days and 5 mice from each group were killed at this time. The remaining mice were injected with BrdU every 6 hours for 48 hours - labelling the newly synthesised strand, and killed from 40 minutes to 25 days following the final injection. Sections were immunostained for BrdU before autoradiography, and labelled cells mapped on a positional basis. Results: The distribution of the two markers in the SI was consistent with the Cairns hypothesis. However in the colon the results were different: after 8 days 1.9\% of colonic epithelial cells were labelled with 3HTdR, with no labelled cells seen after 20 days. Labelled cells were distributed equally throughout the length of the crypt and there were fewer labelled cells in the Min mice. Most crypt cells were BrdU labelled at 1 day, but labelling declined sharply 3-5 days after the last injection, with no labelled cells at 25 days. Cells containing both proliferative markers were rare, distributed throughout the crypt, occurring only in the immediate 24 hrs following labelling. Conclusions: Since the stem cell cycle time is reduced by irradiation the presence of 3HTdR labelling at 8 days is indicative of retention of part of the genome. In the colon the disappearance of the label over the following 12 days and the dilution of BrdU at the same rate suggests that the process is stochastic and there is no protective mechanism in the colon selectively retaining the 'template' strand. This may contribute to the higher incidence of tumors in the colon compared to the SI. The similarity in the distribution of the labels between the Min and the C57Bl/6J mice, suggests APC does not have a critical role in the process of segregation.

\section{$\mathrm{T} 1685$}

Differential Regulation of Intestinal Gene Expression By Hnfl $\alpha$ During the Weaning Transition in Mice

Tjalling Bosse, John J. Fialkovich, Eva Beuling, Christina M. Piaseckyj, Robert K. Montgomery, Stephen D. Krasinski

Lactase-phlorizin hydrolase (LPH) and liver fatty acid binding protein (Fabpl), both follow a developmentally coordinated pattern in which their expression declines during the weaning transition. Hepatocyte nuclear factor-l $\alpha(\mathrm{Hnfl} \alpha)$ is a transcription factor that is required for the expression of LPH and Fabpl in the adult jejunum as shown using hnfl $\alpha$ knockout mice (Bosse et al, Am J Physiol, In Press), but its function during postnatal development is currently unknown. Using this model, we investigated the importance of Hnfl $\alpha$ for the regulation of LPH and Fabpl during the weaning transition. Wild-type mice were analyzed for Hnfl $\alpha$ mRNA and protein levels in the jejunum 7, 14, 21 and 28 days after birth by real-time RT-PCR, Western analysis, and immunofluorescence. Although Hnfl $\alpha$ mRNA as measured by real-time RT-PCR was relatively constant throughout postnatal development, Hnfl $\alpha$ protein as determined by Western analysis was undetectable in the nuclear fraction 
of 7 day old mice and then increased incrementally to high levels in 28 day old mice, similar to that in adults. At 7 days of age, Hnfl $\alpha$ was localized to the cytoplasm of the absorptive enterocytes on villi, whereas at 28 days of age, $\mathrm{Hnfl} \alpha$ was restricted to the nucleus, as determined using co-immunofluorescent staining of Hnfl $\alpha$ with the DAPI nuclear stain. Co-staining with lectin HPA revealed that Hnfl $\alpha$ in the cytoplasm of the 7 day old mice was associated, in part, with the Golgi apparatus. Next, we investigated the function of Hnfl $\alpha$ in the regulation of LPH and Fabpl during postnatal development by comparing jejunal mRNA levels between wild-type and $\mathrm{Hnfl} \alpha$ null mice using real-time RT-PCR. LPH and Fabpl mRNA levels in the 7 day hnfl $\alpha$-/- mice were reduced $50 \%$ as compared to wild-type controls $(\mathrm{P}<0.05, \mathrm{n}=4)$, whereas LPH and Fabpl mRNA levels in 28 day old hnfl $\alpha$ null mice was nearly undetectable (95\% reduction). These data show that Hnfl $\alpha$ is indispensable for LPH and Fabpl gene expression in the intestine of 28 day old mice (similar to adults), but is only partially required for the expression of these genes before weaning. Conclusions: These data demonstrate (1) a previously unknown nuclear translocation process for $\operatorname{Hnfl} \alpha$ that is regulated during the weaning transition, and (b) a differential requirement for Hnfl $\alpha$ before and after weaning for LPH and Fabpl implicating differential regulatory pathways for intestinal gene expression during postnatal development.

\section{T1686}

Specific Antigen Expression and Novel Gene Expression in the Cells At the Bottom of the Colonic Crypt

Jeffrey L. Franklin, Paul Gregoli, Pamela S. Robinson, Walter Wakwe, Robert H. Whitehead

Background. Although the stem cells of the colonic epithelium have been extensively studied there are still no surface markers that would allow us to isolate these cells. The proliferative cells can be identified by BrDU but this does not allow for viable isolation of the cells. Methods. We initially bisected crypts and conducted comparative microarray studies using a cDNA chip. This identified CD24 as a marker for the bottom 5\% of the cells at the base of the crypt. We have labeled avidin-linked Dynabeads and biotinylated CD24 antibody to purify these cells and have undertaken further microarray studies using Affimetrix chips to identify genes that are either upregulated or specifically expressed in this cell population. We have repeated this study using CD44 antibody. Results. Both CD24 antibody and CD44 antibody have a very similar staining pattern on isolated crypts. Dynabead isolation with CD24 antibody yielded 4\% of the crypt cell population and CD44 yielded $6 \%$. Microarray studies showed that the CD24 + and CD44+ cell populations were more similar to each other than to their negative cell populations. Of 32050 gene probes that had similar expression in both CD24+ and CD44+ cells, 1650 were upregulated in the CD24+/CD44+ cells relative to CD24-/CD44- Cells. Discussion. CD24 and CD44 are the first 2 markers that allow for the viable isolation of the cells at the bottom of the colonic crypt. Using these markers we have been able to identify a panel of genes that are highly expressed in the 5\% of cells at the base of the crypt.

$\mathrm{T} 1687$

Imatinib Inhibits Kit Expression On Potential Interstitial Cells of Cajal in Human Intestinal Smooth Muscle Culture Cells Akiko Terauchi, Hiroshi Mashimo

Background/Aims: Imatinib inhibits KIT tyrosine kinase and can treat gastrointestinal stromal tumors thought to originate from the neoplastic transformation of interstitial cells of Cajal (ICC). Loss of ICC has been reported in various disorders of gastrointestinal motility. Efforts to identify factors influencing the population of ICC have been hindered by the lack of cell culture studies in humans. We reported that membrane-bound stem cell factor (mbSCF) a natural ligand for KIT, increases expression of KIT in human intestinal smooth muscle cells (HISM) and leads to unique morphological changes. In this study, we tested whether imatinib decreases the appearance of ICC-like round and uniquely dendritic cells in cultures of HISM when exposed to human mbSCF, and decreases the expression of KIT in HISM. Methods: HISM were co-cultured for 1, 5, and 8 days with either untransfected murine S1/ S14 fibroblasts (lacking murine SCF) or with Sl/Sl4 fibroblasts expressing transfected human mbSCF $(\mathrm{Sl} / \mathrm{Sl} 4+\mathrm{mbSCF})$ in the presence or absence of imatinib $(5 \mu \mathrm{M})$. The HISM were classified by shape (round, dendritic, spindle, or flat). HISM were distinguished from the fibroblasts using anti-human MHC-I antibody and stained with anti-human KIT antibody for detection of potential ICC-like cells. Results: Throughout the time span of culture, most of the round cells $(85.8+2.8 \%)$ and the dendritic cells $(94.6 \pm 3.2 \%)$ were KIT-positive. Compared to HISM co-cultured with Sl/SI4 alone, in vitro exposure to mbSCF increased ICC-like round cells (after 8 days, Table, $\mathrm{p}<0.05$ ). Uniquely dendritic cells also appeared with SCF exposure, not immediately, but after 8 days of mbSCF exposure (Table). This shift in the morphology of HISM correlated with increases in KIT expression from 1 day to 8 days of culture (after 8 days, Table). However, these increases in round and dendritic cells, as well as in KIT expression, were inhibited by imatinib (Table). Conclusions: KIT expression and morphological changes in HISM appear dynamic and responsive to membrane-bound SCF. Imatinib appears to block the effect of membrane-bound SCF, possibly via the tyrosine kinase activity of KIT, leading to decrease in KIT expression in HISM. This data allows further study of mechanisms of KIT expression, ICCs differentiation, and morphological changes driven by the KIT receptor and mbSCF interaction.

Round, dendritic and KIT-positive cells after 8 days culture

\begin{tabular}{|c|c|c|c|}
\hline Condition & Round cells & Dendritic cells & KIT-positive cells \\
\hline Sl/SI4 & $7.5 \pm 0.5 \%$ & $0.7 \pm 0.7 \%$ & $12.7 \pm 0.1 \%$ \\
\hline SI/SI4+imatinib & $4.0 \pm 1.1 \%$ & $0.3 \pm 0.3 \%$ & $6.9 \pm 4.2 \%$ \\
\hline SI/S14+mbSCF & $29.8 \pm 3.7 \%$ & $7.3 \pm 1.2 \%$ & $38.6 \pm 3.7 \%$ \\
\hline SI/SI4+mbSCF+imatinib & $5.1 \pm 0.7 \%$ & $3.0 \pm 0.3 \%$ & $7.0 \pm 0.2 \%$ \\
\hline
\end{tabular}

$\mathrm{T} 1688$

Expansion of Putative Colonic Stem Cells By Administration of Enteral Antibiotics Is Associated with Colonic Adaptation Following Ileocecal Resection in Mice

Christopher M. Dekaney, Susan J. Henning, Rachael J. Rigby, P. Kay Lund, Michael A. Helmrath

Introduction: Surgical resection of the ileum and proximal colon is commonly performed in patients with Crohn's disease. Mechanisms of adaptive growth in large bowel after such resections have not been well characterized. A murine model of ileocecal resection (ICR) was developed and data from our laboratories using this model demonstrate enhanced colonic adaptation in germ free mice. In addition, we have recently developed side population (SP) flow cytometry methods to measure putative colonic stem cells. In this study, we hypothesized that enteral antibiotics would expand the percentage of SP cells following ICR. Methods: Ileocecal resection (ICR) or sham surgery (S) was performed on 8-12 week old C57BL6 mice ( $n=4-6$ mice/group). Liquid diet with (AB) or without (CON) broad-spectrum antibiotics was provided 3 days before and for the duration of the experiment. Colonic tissue was harvested on postoperative day 3 and 7 for histology and fluorescent activated cell sorting (FACS) to evaluate changes in the percentage of CD45-negative SP cells. Results: ICR resulted in significant increases in colonic crypt depth in $A B$ vs. CON mice at both 3 $(78.8 \pm 5.6$ vs. $48.6 \pm 2.2 \mu \mathrm{m})$ and 7 days $(66.6 \pm 8.2$ vs. $52.9 \pm 1.6 \mu \mathrm{m})$ post resection. FACS analysis revealed that the addition of enteral antibiotics significantly increased the percentage of CD45-negative SP in sham operated mice at both $3(0.32 \pm 0.23$ vs. $0.79 \pm$ $0.38)$ and 7 days $(0.69 \pm 0.17$ vs. $0.58 \pm 0.15)$, despite no differences in crypt depth between groups. Significant increases in percent of SP following ICR in AB vs. CON were found at 3 days $(1.16 \pm 0.35$ vs. $0.14 \pm .07$ ), but not 7 days. Conclusion: Administration of enteral antibiotics increases the percentage of colonic CD45-negatvie SP cells. Following ICR, addition of enteral antibiotics results in early expansion of the percentage of SP cells and colonic adaptation. These data suggest that commensal microflora inhibit expansion of putative stem cells (SP) required for colonic adaptation following ICR. This work was supported by NIH grants K08 DK 067395 and P30 DK 56338.

\section{T1689}

Direct Effects of Nitric Oxide On Histamine Release from Rat Enterochromaffin-Like Cell

Naozumi Shibata, Hidetaka Matsui, Tomoyuki Yokota, Bunzo Matsuura, Kazutaka Maeyama, Morikazu Onji

Backgrounds: Although there is evidence that nitric oxide (NO) inhibits secretion of gastric acid, the contradictory results have also been reported. While enterochromaffin-like (ECL) cells have a central role in the regulation of gastric acid secretion, the direct effects of NO on these cells have not yet been demonstrated. In this study we investigated the direct effects of NO donors on histamine release from rat ECL cells. Methods: ECL cells were purified from the rats using an elutriator system, which can make the purity $80 \%$ or more. After $48 \mathrm{~h}$ of cultivation, the cells were incubated with test substance for $1 \mathrm{~h}$. Histamine released from ECL cells was measured by a high-performance liquid chromatography (HPLC)fluorometry technique. Intracellular Ca2+ levels were assayed in Fura-2/acetoxymethylester (AM) -loaded ECL cells. Intracellular cGMP content was measured by enzymeimmunoassay. Intracellular histamine content and histidine decarboxylase activity were measured after incubation with test substance for $8 \mathrm{~h}$. Results: The NO donor, NOR3 (10 $\mu \mathrm{M}$ and $100 \mu \mathrm{M})$ decreased gastrin-induced and PACAP (pituitary adenylate cyclase-activating polypeptide) induced histamine release significantly. In contrast, NOR3 over the concentration range 1 $u \mathrm{M}-100 \mathrm{uM}$ did not augment basal histamine release. The inhibitory effect of the NO donor appeared not to be caused by toxicity, as the cells were shown to be viable at the end of the experiments as judged by trypan blue dye exclusion and also showed continued responsiveness to gastrin after removal of the NO donor. $100 \mu \mathrm{M}$ NOR3 increased cGMP levels and reduced gastrin-induced calcium influx. ODQ, an inhibitor of guanylate cyclase, completely blocked NOR3-induced inhibition of histamine release. NOR4, an NO donor, did not affect intracellular histamine content and histidine decarboxylase activity. Conclusions: These results suggested that NO inhibits gastric acid secretion via suppression of gastrin-induced histamine release through a pathway in which $\mathrm{NO}$ activated soluble guanylate cyclase, in addition to increasing cGMP levels and reducing gastrin-induced calcium influx. The use of NO donor as a new type of gastric acid inhibitor that decreases histamine levels in the stomach would be beneficial as histamine that is increased by using an $\mathrm{H} 2$ blocker or proton pump inhibitor has various effects on tumors and immunological functions.

\section{T1690}

Down-Regulated Sonic Hedgehog Expression in the SPEM-Like Lesions in the Stomach of $\mathrm{H}_{2}$ Receptor-Null Mice

Yuriko Minegishi, Hidekazu Suzuki, Mamoru Arakawa, Yasushi Fukushima, Takashi Ishikawa, Nicholas A. Wright, Toshifumi Hibi

Spasmolytic polypeptide (trefoil factor family 2 ; TFF2)-expressing metaplasia (SPEM) has been know to be associated with the development of oxyntic atrophy and metaplastic cell lineages in response to H.felis infection (Lab. Invest. 79. 639, 1999: Gastroenterol. 127:582,2004), and mainly characterized as a loss of parietal cells. Sonic hedgehog (Shh) 
is an important signal protein which is expressed in the stomach and involved in the regulation of the homeostasis of fundic glands (Gut 51:28, 2002; Development 127:2763, 2000), and an in vitro study has showed that Shh expression is up-regulated under acidic conditions (Lab. Invest. 83;1829, 2003). The present study was designed to clarify the relation between gastric mucosal TFF2 and Shh under conditions of high $\mathrm{pH}$ in vivo, using histamine $\mathrm{H}_{2}$ receptor-null $\left(\mathrm{H}_{2} \mathrm{R}\right.$-null) mice as a model of impaired parietal cell phenotype and reduced gastric acid secretion. Methods. $\mathrm{H}_{2} \mathrm{R}$-null mice and wild-type (WT) mice aged 10 and 63 weeks were examined. The surface gastric mucosal pH was measured. Gastric Shh mRNA expression was examined by quantitative RT-PCR. In-situ hybridization (ISH) was performed to detect the localization of Shh mRNA in gastric mucosa. Immunohistochemistry against TFF2, Shh and gland-cell-specific markers was also performed. Results. Both the 10-week- and 63-week-old $\mathrm{H}_{2} \mathrm{R}$-null mice exhibited higher gastric $\mathrm{pH}$ than WT mice of corresponding age. The expression level of Shh mRNA was lower in the $\mathrm{H}_{2} \mathrm{R}$-null mice than in the WT mice ( 10 weeks: $0.8 \pm 0.06$ in the WT mice vs. $0.5 \pm 0.06$ in the $\mathrm{H}_{2} \mathrm{R}$-null mice; $\mathrm{p}<0.01 ; 63$ weeks: $0.9 \pm 0.09$ in the WT mice vs. $0.5 \pm 0.02$ in the $\mathrm{H}_{2} \mathrm{R}$-null mice; $\mathrm{p}<0.05$ ). ISH revealed strong Shh mRNA expression at the base of the fundic glands in the WT mice, mainly in chief cells, while only faint staining was detected in the $\mathrm{H}_{2} \mathrm{R}$-null mice. TFF2positive mucous cells were restricted to the mid-portion of the fundic glands in the WT mice, while they were distributed throughout the fundic glands in the $\mathrm{H}_{2} \mathrm{R}$-null mice. Mouse pepsinogen-positive cells, which represent chief cells and were distributed at the base of the fundic glands in WT mice, showed the shifted localization in the $\mathrm{H}_{2} \mathrm{R}$-null mice. Conclusion. The present study has shown for the first time that the gastric TFF2 expression pattern also extends in parallel with SPEM lineage in $\mathrm{H}_{2} \mathrm{R}$-null mice; while there is no loss of parietal cells, impaired parietal cell phenotype is noted even in the absence of inflammation. Taken together, down-regulation of Shh expression in vivo may lead the abnormal differentiation of parietal as well as mucous neck-chief cell linage and TFF2-positive metaplastic transformation in the gastric fundic gland.

\section{T1691}

The Antimicrobial Peptide Cathelicidin Promotes Gastric Ulcer Healing in Rats

William K. Wu, Ying H. Yang, Emily K. Tai, Helen P. Wong, Emily K. Lam, Vivian Y. Shin, Chi H. Cho

Cathelicidin, an antimicrobial peptide, has been shown to promote cutaneous wound repair and reaches high levels in the gastric mucosa during infection and inflammation. We therefore investigated whether this peptide contributes to gastric ulcer healing in rats. Ulcer induction increased cathelicidin expression in the gastric mucosa. Further increase in expression of this peptide by local injection of cathelicidin-encoding plasmid promoted ulcer healing by enhancing cell proliferation and angiogenesis. Cathelicidin directly stimulated proliferation of cultured rat gastric epithelial cells (RGM-1), which was abolished by inhibitors of matrix metalloproteinase (MMP), epidermal growth factor (EGFR), or MAPK/ERK kinase (MEK). Cathelicidin also increased EGFR and ERK1/2 phosphorylation via a MMP-dependent mechanism. Knockdown of transforming growth factor $\alpha$ (TGF $\alpha$ ), which is a ligand of EGFR, by small interfering RNA completely nullified the mitogenic signals evoked by cathelicidin in RGM-1 cells. These findings suggest that cathelicidin exhibits pro-healing activity in stomachs through TGF $\alpha$-dependent transactivation of EGFR and its related signaling pathway to induce proliferation of gastric epithelial cells.

\section{T1692}

Concomitant Activation of the Calcium-Sensing Receptor (CaSR) and the H2 Receptor Results in Inhibition of Acid Secretion in Isolated Rat Gastric Glands

Stephanie M. Busque, John P. Geibel

INTRODUCTION: Acid secretion in parietal cells occurs via a histamine dependent pathway. We have previously shown that an alternate acid secretory pathway exists in these cells by allosteric modification of the CaSR by L-amino acids and extracellular $\mathrm{Ca}^{2+}$. The subsequent increases in cAMP and intracellular $\mathrm{Ca}^{2+}$ during stimulation of these pathways results in acid secretion via $\mathrm{H}^{+}, \mathrm{K}^{+}$-ATPase activation. To determine the effect of simultaneous stimulation of both acid secretory pathways, we examined 1) the effect of L-phe or neomycin in the presence of $2 \mathrm{mM} \mathrm{Ca}^{2+} \pm$ histamine while monitoring cytosolic $\mathrm{pH}$ in isolated rat gastric glands; 2) the effect of the phosphodiesterase (PDE) inhibitor, IBMX, in histamine and CaSR activated glands on cytosolic pH. METHODS: Male Sprague-Dawley rats were fasted for 1824 hours, anesthetized, and individual gastric glands were hand-dissected from the excised stomach. Glands were loaded with the $\mathrm{pH}$ indicator dye BCECF $(10 \mu \mathrm{M})$, maintained at $37^{\circ} \mathrm{C}$, and perfused. Intracellular alkalinization rates $\left(\Delta \mathrm{pH}_{\mathrm{i}} / \mathrm{min}\right)$ were used as an index of $\mathrm{H}^{+}$extrusion or $\mathrm{H}^{+} \mathrm{K}^{+}$-ATPase activity. To induce an acid load, glands were exposed to a $20 \mathrm{mM} \mathrm{NH}_{4} \mathrm{Cl}, 0 \mathrm{mM} \mathrm{Na}^{+}$perfusate. The glands were then exposed to $2 \mathrm{mM} \mathrm{Ca}^{2+}, 0 \mathrm{mM} \mathrm{Na}^{+}$ solutions containing either $10 \mathrm{mM}$ L-phe or $400 \mu \mathrm{M}$ neomycin, both in the presence or

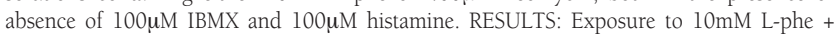
$2 \mathrm{mM} \mathrm{Ca}^{2+}$ resulted in a mean $\mathrm{H}^{+}$extrusion rate of $0.047 \pm 0.003 \Delta \mathrm{pH}_{\mathrm{i}} / \mathrm{min}$, while $400 \mu \mathrm{M}$ neomycin $+2 \mathrm{mM} \mathrm{Ca}^{2+}$ had a rate of $0.053 \pm 0.001 \Delta \mathrm{pH}_{\mathrm{i}} / \mathrm{min}$. When $100 \mu \mathrm{M}$ histamine was added to both experimental protocols, the rates were reduced to $0.023 \pm 0.001$ and $0.035 \pm 0.005 \Delta \mathrm{pH}_{\mathrm{i}} / \mathrm{min}$, respectively. In contrast, addition of $100 \mu \mathrm{M}$ IBMX in the continued presence of histamine, $2 \mathrm{mM} \mathrm{Ca}^{2+}$, and L-phe or neomycin led to enhanced $\mathrm{H}^{+}$extrusion rates of $0.036 \pm 0.007$ and $0.05 \pm 0.005 \Delta \mathrm{pH}_{\mathrm{i}} / \mathrm{min}$, respectively. CONCLUSIONS: These data suggest that: 1) the concomitant activation of the CaSR and histamine pathways result in a down-regulation of acid secretion. 2) down-regulation of acid secretion occurs via PDE activation. Our data demonstrate that activation of the CaSR in the continued presence of histamine results in a reduction in CAMP-associated acid secretion due to PDE activation.

\section{$\mathrm{T} 1693$}

Food Intake Dependent Expression of Mucosal Orexin Receptors Regulates Small Intestinal Secretion in Rats

Magnus Bengtsson, Kari Makela, Markus Sjoblom, Sanna Uotila, Karl Akerman, KarlHeinz Herzig, Gunnar Flemstrom

BACKGROUND \& AIM: The presence of orexins in intestinal enteroendocrine cells suggests a role of these orexogenic peptides as regulators of small intestinal secretion, and recen studies showed that low doses of orexin-A markedly increase mucosal electrolyte secretion. However, short (overnight) fasting abolishes the secretory response and stimulation only occurs in animals that have continuous access to food. Our aim was to further elucidate the intestinal secretory action of orexin-A. METHODS: Lewis x Dark Agouti rats were anesthetized and a $12-\mathrm{mm}$ segment of proximal duodenum with intact blood supply was cannulated in situ. Mucosal bicarbonate secretion (pH stat) was continuously recorded. Orexin-A was infused intra-arterially to the duodenum, administered to the central nervous system by intracerebroventricular infusion, or added to the luminal perfusate. Total RNA was extracted from duodenal mucosa specimens of fasted and fed animals and reverse transcripted to cDNA. Expression levels of prepro-orexin (PPO) and orexin receptors $(\mathrm{OX} 1)$ and $2(\mathrm{OX} 2)$ were measured by quantitative real-time PCR. RESULTS: Intra-arterially infused orexin-A (240-600 picomoles $/ \mathrm{kg}, \mathrm{h})$ caused a dose-dependent increase in duodenal bicarbonate secretion $(\mathrm{p}<0.01)$. The OXl-receptor antagonist SB-33487 (600-6000 picomoles/kg,h) had no effect on basal bicarbonate secretion, but abolished the orexin-induced secretory response. Intracerebroventricular infusion of orexin-A (2 - 20 nanomoles $/ \mathrm{kg}, \mathrm{h}$ ) did not affect the duodenal secretion, indicating a peripheral stimulatory action of the peptide. Orexin-A added to the luminal perfusate $(0.1-100 \mathrm{nmol} / \mathrm{L})$ did not elicit a secretory response, suggesting basolateral location of $\mathrm{OXI}$. Fasting significantly reduced expression levels of PPO $(\mathrm{p}<0.05)$ as well as OX1 $(\mathrm{p}<0.005)$ and OX2 $(\mathrm{p}<0.005)$. CONCLUSION The secretory response to orexin- $\mathrm{A}$ is mediated by OXl-receptors located in the duodena mucosa and expression of these receptors is markedly related to food intake. Supported by the Swedish Research Council and the Finnish Academy.

\section{T1694}

Hypophagia and Weight Loss Induced By Trichinella Spiralis Enteritis Are TNF $\alpha$-Independent John Worthington, Richard K. Grencis, John McLaughlin

Background: Enteric inflammation is closely coupled to reduced food intake but the linking molecular mechanisms are poorly understood, despite their potential clinical significance. We have recently demonstrated that increased CCK production driven by CD4+ T-lymphocytes and IL4/13 contribute to the marked hypophagic response caused by Trichinell spiralis, a nematode small intestinal infection (McDermott et al, Gut, in press). Food intake reaches a minimum $\sim 9$ days post infection (p.i.), then normalises as the gut expels the parasites. This model permits a mechanistic study of the mediators responsible for reduced food intake during gut inflammation. Aim: Since TNF $\alpha$ is pivotal in many gastrointestinal inflammatory responses, we explored its functional role in the hypophagic response in this model. Methods: Wild type C57BL/6 (WT) or TNF $\alpha$ receptor knockout (p55/75) adult male mice were infected by gavage with $300 \mathrm{~T}$. spiralis larvae. Food intake and body weight were monitored daily. The active role of TNF $\alpha$ in this process was assessed by quantifying worm expulsion, counting residual intestinal parasites at 20 days p.i. Results: WT mice displayed a significant fall in food intake: at day 9 p.i., infected mice consumed $1.77+1-0.24 \mathrm{~g} /$ mouse vs naive $2.94+/-0.04 \mathrm{~g} /$ mouse $(\mathrm{p}<0.05$ ). Body weight during infection fell to $90.3+/-0.8 \%$ of baseline, whilst rising to $108.5+/-1.7 \%$ in naive WT mice $(\mathrm{p}<0.001)$. However TNFa activity played no role in these effects: infected p55/75 KO mice consumed $1.75+/-0.15$ vs naïve $3.46+/-0.15 \mathrm{~g} /$ mouse $(\mathrm{p}<0.05)$. Body weight declined similarly in infected $\mathrm{p} 55 / 75 \mathrm{KO}$ mice, falling to $87.6+/-1.1 \%$ of baseline compared to $104.0+/-1.5 \%$ in naive $\mathrm{p} 55 / 75 \mathrm{KO}$ mice $(\mathrm{p}<0.001)$. Functional TNF $\alpha$ was however functionally important in the infection, as demonstrated by the host response to clear parasites. At day 20, WT mice retained 1.5+/1.0 larvae, whilst p55/75 KOs retained $18.1+/-4.5$ larvae $(\mathrm{p}<0.05)$. Conclusion: although TNF $\alpha$ contributes strongly to the host response to expel T.spiralis, this key cytokine is not implicated in the profound hypophagic response and rapid weight loss observed during small intestinal inflammation in this model.

\section{T1695}

Improvement of Anorexia By Rikkunshito Via Increased Ghrelin Secretion Hiroshi Takeda, Kanji Kato, Takehiko Katsurada, Tatsuya Ohkawara, Tomohisa Hattori, Masahiro Asaka

Abstract Body: Background/Aim : Rikkunshito (Chinese name Liu-Jun-Zi-Tang), one of the traditional Japanese medicines, is widely prescribed for patients with chronic hypofunction of the gastrointestinal tract, including gastric flatulence, anorexia, nausea, and vomiting However, the precise mechanism by which rikkunshito improves such dyspeptic symptoms remains to be determined. To clarify this, we investigated the effect of rikkunshito on an appetite-stimulating hormone, ghrelin,in CDDP-induced anorexic rats. Methods: To induce anorexia, male SD rats were intraperitoneally given $2 \mathrm{mg} / \mathrm{kg}$ of cisplatin(CDDP). Rikkunshito $(500$ or $1000 \mathrm{mg} / \mathrm{kg}$ ) was orally given once a day for up to 3 days. Changes in food intake and body weight were examined every day for 3 days. The plasma level of active ghrelin and desacyl-ghrelin were measured by ELISA. Results: Treatment of rats with CDDP significantly suppressed food intake from $17.3 \pm 0.9 \mathrm{~g} /$ day to $13.0 \pm 0.1 \mathrm{~g} /$ day $(\mathrm{p}<0.05)$. Oral administration of rikkunshito $(1000 \mathrm{mg} / \mathrm{kg})$ significantly improved the CDDP-induced reduction of food intake (from $13.0 \pm 0.1 \mathrm{~g} /$ day to $15.7 \pm 0.6 \mathrm{~g} /$ day, $\mathrm{p}<0.05$ ). In the CDDP-treated anorexic rats, plasma level of active and desacyl ghrelin was significantly decreased at 2 and 24 hours after the administration of CDDP. The ratio of active to desacyl ghrelin (A/D ratio) was also significantly reduced. After the oral administration of rikkunshito, the plasma level of active ghrelin and A/D ratio significantly increased. On the other hand, plasma desacyl ghrelin level did not changed. Conclusion:These results suggest that rikkunshito improve CDDPinduced anorexia via an increase in the plasma active ghrelin level.

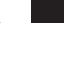




\begin{tabular}{|c|c|c|c|}
\hline & active ghrelin (fmol/mL) & desacyl ghrelin (fmol/mL) & A/D ratio \\
\hline Control & $32.1 \pm 4.1$ & $114.7 \pm 11.6$ & $0.29 \pm 0.04$ \\
\hline CDDP (2mg/kg) & $22.6 \pm 2.4 \#$ & $113.6 \pm 7.2$ & $0.20 \pm 0.01 \#$ \\
\hline + rikkunshito(500mg/kg) & $32.1 \pm 3.3^{*}$ & $122.7 \pm 10.8$ & $0.27 \pm 0.02$ \\
\hline + rikkunshito(1000mg/kg) & $31.9 \pm 2.8^{*}$ & $126.7 \pm 15.9$ & $0.29 \pm 0.04 *$ \\
\hline
\end{tabular}

\#: P $<0.05$ vs normal by t-test, $*: P<0.05$ vs control by Dunnett, The mean \pm S.E. of 10 12 rats, A/D ratio: plasma active /desacyl ghrelin ratio

\section{T1696}

GLP-1(7-36)Amid (GLP-1) Mediates the Ileal Brake in Human

Joerg Schirra, Samera Shakir, Mathias Nicolaus, Juergen Woerle, Burkhard Goeke

Nutrients appearing in the distal intestine induce a delay of upper gastrointestinal transit and inhibit gastropancreatic secretion. This 'ileal brake' is the primary inhibitory feedback mechanism which control transit and digestion of a meal. The factors mediating this response are unknown. GLP-1 is a gut hormone postprandially released from intestinal L-cells. The endocrine effects of GLP-1 are well described, it stimulates insulin and inhibits glucagon. However, the importance of the predominant presence of L-cells within the lower gut is not known. As GLP-1 is released by ileal nutrients, it is suggested as a candidate humoral mediator of the ileal brake. Accordingly, we examined the effects of ileal nutrients on pancreatic enzyme and gastric acid secretion with and without the specific GLP-1 receptor antagonist exendin(9-39) (Ex-9). Methods: In 12 healthy volunteers, an amino acid solution ( $3 \mathrm{ml} / \mathrm{min}, 0.6 \mathrm{kcal} / \mathrm{min}$ ) was continously perfused into the duodenum stimulating pancreatic enzyme and gastric acid secretion. After $40 \mathrm{~min}$, a mixed liquid meal $(90 \mathrm{ml}, 44 \mathrm{kcal}$, starch, maltose, sodium oleate, lipid) was perfused for $15 \mathrm{~min}$ into the ileum $(150 \mathrm{~cm}$ from the pylorus) approximating the amount of physiologically unabsorbed nutrients. Gastroduodenal secretion was sampled in 10-min intervals until $240 \mathrm{~min}$ after ileal perfusion and quantified using double-marker dilution technique. On 2 days in random order, Ex-9 $\left(300 \mathrm{pmol} / \mathrm{kg}^{-}\right.$ $1 / \mathrm{min}^{-1}$ ) or saline were IV infused. The output of gastric acid, amylase and trypsin was measured. Results: Duodenal amino acids significantly increased the output of gastric acid and pancreatic enzymes on both study days $(\mathrm{p}<0.05)$. Ileal nutrient perfusion induced the ileal brake by causing a pronounced reduction of endogenously stimulated gastropancreatic secretion. The latter was markedly inhibited by IV Ex-9 (see table). Conclusions: : Physiological amounts of nutrients within the ileum induce a prolonged inhibition of gastric acid and pancreatic enzyme secretion. As this 'ileal brake' is largely prevented by the GLP-1 receptor antagonist, we suggest GLP-1 as mediator of this intestino-gastropancreatic reflex. We conclude that beside its endocrine action, GLP-1 plays an important role as an enterogastrone. This may explain the very high densities of L-cells within the ileum and colon. (supported by a grant of Deutsche Forschungsgemeinschaft DFG)

\begin{tabular}{|c|c|c|c|c|}
\hline \multirow{2}{*}{ Inhibition of secretion by ileal nutrients } & \multicolumn{2}{|c|}{ Saline IV } & \multicolumn{2}{c|}{ Ex-9 IV } \\
\cline { 2 - 5 } & & \% reduction & & \% reduction \\
\hline Gastric acid $(\mathrm{mmol} / 90 \mathrm{~min})$ & $-17.7 \pm 1.9$ & $-61.7 \pm 3.5$ & $-11.9 \pm 2.7^{*}$ & $-31.6 \pm 5.1^{*}$ \\
\hline Trypsin $(\mathrm{kU} / 90 \mathrm{~min})$ & $-10.9 \pm 2.5$ & $-65.4 \pm 6.9$ & $-5.4 \pm 1.6^{*}$ & $-30.2 \pm 7.8^{*}$ \\
\hline Amylase $(\mathrm{kU} / 90 \mathrm{~min})$ & $-27.7 \pm 7.2$ & $-38.4 \pm 7.0$ & $-1.2 \pm 7.1^{*}$ & $-2.7 \pm 10.2^{*}$ \\
\hline
\end{tabular}

mean $\pm \mathrm{SEM} .{ }^{*}: \mathrm{P}<0.05$ vs saline IV

T1697

Curcumin Inhibits Neurotensin-Mediated Interleukin-8 Production and Migration in Hct116 Colon Cancer Cells

Xiaofu Wang, B. Mark Evers

Previously, we have shown that the GI hormone, neurotensin (NT), acting through its native high-affinity receptor (NTR1) and multiple downstream signaling pathways, selectively stimulates interleukin-8 (IL-8) gene expression and protein secretion in human colon cancer cells. IL-8, a chemokine regulated by NF-KB and AP-1 transcription factors, is increasingly recognized as an important factor in tumorigenesis. The purpose of this study was to determine the effect of curcumin, a natural dietary product, on NT-induced IL-8 production and cell migration in the human colon cancer cell line, HCT116, which possesses native NTRl. Methods. HCT116 cells were pretreated with curcumin $(5-25 \mu \mathrm{M})$ prior to NT (100 $\mathrm{nM})$ treatment. To determine the role of NF- $\mathrm{kB}$ activation on NT-mediated IL-8 induction, HCT116 cells were infected with an adenovirus encoding the super-repressor of IKB $\alpha$ (IKB$\mathrm{AA}$ ) or the control vector encoding GFP; IL-8 mRNA expression and protein secretion were determined by RNase protection assay and ELISA, respectively. The role of the MEK/ERK pathway on IL-8 induction was assessed using the MEK/ERK inhibitors, PD98050 and U0126. AP-1 and NF-KB binding activity was analyzed by gel shift assay. Intracellular $\mathrm{Ca}^{2+}$ mobilization was measured by fura-2 spectrofluorometry. $\mathrm{HCT} 116$ cell migration was assessed using collagen coated Costar Transwell plates. Results. (i) Curcumin significantly inhibited NT-induced IL-8 mRNA expression and protein secretion in a dose-dependent fashion. (ii) NT increased NF- $\mathrm{KB}$ and AP-1 DNA-binding activities, but not NFAT binding activity (another transcription factor that binds to the IL-8 promoter). Overexpression of the IKB $\alpha$ super-repressor significantly inhibited NT-mediated IL-8 mRNA induction. Both PD98059 and U0126 blocked NT-stimulated AP-1 binding activity and significantly suppressed IL-8 mRNA induction, but had no effect on NF- $\mathrm{KB}$ activation. Curcumin inhibited both ERK-dependent AP-1 and ERK-independent NF- $\mathrm{\kappa B}$ activation. (iii) Curcumin strongly inhibited NT-activated $\mathrm{Ca}^{2+}$ mobilization in a dose-dependent fashion. (iv) A low dosage of curcumin $(10 \mu \mathrm{M})$ blocked NT-induced HCT116 cell migration. Conclusions. NT stimulates growth of colon cancer cells with NTRl and increases expression and secretion of IL-8.
Curcumin inhibited NT-mediated IL-8 production through suppression of NT-induced AP1 and NF- $\mathrm{KB}$ activation as well as $\mathrm{Ca}^{2+}$ mobilization. Moreover, curcumin blocked NTinduced HCT116 cell migration. The overall inhibitory effects of curcumin on hormonemediated IL-8 induction suggests that this agent may be useful therapy for the treatment of certain colon cancers.

\section{T1698}

Different Expression of 5-Ht4 Receptor (5-HT4R) Transcripts Correlates with the Functional Responses of Tegaserod On Chloride/Water Secretion in the Human Ileum and Colon in Vitro

Moriko Ito, Eckhard Weber, Christian T. Hamel, Mario Bernhard, Stephan Marcaletti, Ursula Mueller, Frederic Bassilana, Hans-Juergen Pfannkuche

BACKGROUND. Tegaserod, a 5-HT4R agonist, has been shown to stimulate chloride secretion in the rat colon, suggesting a 5-HT4R-mediated modulation of intestinal fluid secretion by the drug in men. Our investigations aimed (i) to determine the expression profile of 5 HT4R transcripts and (ii) to demonstrate their function in 5-HT4R-mediated secretion in human ileum and colon preparations. METHODS. Specimens of ileum $(n=6)$ and colon $(n=$ 5) were obtained from patients undergoing bowel resection for colon carcinoma $(6 \mathrm{~m} / 5 \mathrm{f}$, 47-83 years). Mucosa/submucosa sheets, obtained by micro-dissection, were examined in parallel with respect to 5-HT4R mRNA expression and 5-HT4R-mediated secretion. Expression: RNA was extracted from tissues using standard methods and QRT-PCR was performed for universal 5-HT4R mRNA and 5-HT4R splice variant mRNAs (a) (b) (c). In addition, marker gene mRNAs specific for mucosa (CDXl) and muscle (desmin) were quantified. House keeping genes, ubiquitin $C(\mathrm{UBC})$ and $\beta$-2-microglobulin( $\beta$-2-MG) were used for normalization. Secretion: Mucosa/submucosa sheets were mounted in Ussing chambers and

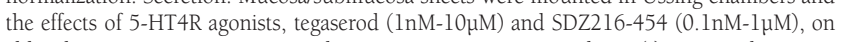
chloride secretion were investigated. RESULTS. Expression: In ileum (I), universal 5-HT4R mRNA was 3-fold more abundant than in colon (C) (I: $3.1 \pm 0.7, n=6 ; C: 0.9 \pm 0.2, n=5$, relative to $\beta-2-M G$, mean $\pm S E M, P=0.011, A N O V A)$. In both ileum and colon, splice variant (b) was the predominant splice variant, higher $(\mathrm{P}<0.001)$ in ileum than in colon (I: (a) 0.01 , (b) 0.06 , (c) $0.02, n=6$; C: (a) 0.004 , (b) 0.035 , (c) $0.012, n=5$, relative to UBC). The overall expression ratios of splice variants (a), (b) and (c) were similar in ileum and colon (10:70:20). Whereas CDXI was expressed in mucosa/submucosa preparations, desmin was not. Secretion: Tegaserod (T) and SDZ216-454 (S) stimulated chloride secretion in a concentration dependent manner in ileum sheets reflected by increases in short-circuit currents $(E C 50(T)=$ $92 \pm 27 \mathrm{nM}, \operatorname{Emax}(\mathrm{T})=41 \cdot 1 \pm 5.8 \mu \mathrm{A} / \mathrm{cm} 2 ; E C 50(\mathrm{~S})=19 \pm 11 \mathrm{nM}, \operatorname{Emax}(\mathrm{S})=85 \pm 31 \mu \mathrm{A} / \mathrm{cm} 2, \mathrm{n}=6)$. In contrast, both 5-HT4R agonists did not significantly alter baseline secretion in colon sheets $(\operatorname{Emax}(\mathrm{T})=6.2 \pm 5.1 \mu \mathrm{A} / \mathrm{cm} 2, \operatorname{Emax}(\mathrm{S})=2.0 \pm 1.1 \mu \mathrm{A} / \mathrm{cm} 2, \mathrm{n}=5)$. CONCLUSIONS. The study provides evidence for a higher 5-HT4R expression in the mucosa/submucosa of human ileum than colon. The differential expression profile may be responsible for the differences observed with respect to 5-HT4R-mediated chloride secretion. Our studies demonstrate that tegaserod can act as a stimulus of intestinal fluid secretion in the human intestine.

\section{T1699}

Pharmacological Characterization of Human and Guinea Pig Opiate Receptors M.C. O'Keefe, Elad Kaufman, Courtney Gee, Ngoc Mai, Jacqueline Smith, David Beattie, Ross G. Vickery

Management of chronic pain with morphine and other opiates is associated with adverse effects such as nausea, vomiting and debilitating bowel dysfunction (opioid-induced bowel dysfunction, $\mathrm{OBD}$ ) as a result of persistent activation of peripheral opioid receptors in the gastrointestinal tract. The peripherally-restricted opioid antagonists, methylnaltrexone (MNTX) and alvimopan, are currently undergoing clinical evaluation for the treatment of OBD and post-operative ileus. In the present study, a detailed comparison of the pharmacological properties of a panel of opioid antagonists, including MNTX, alvimopan and ADL 08-0011, the active metabolite of alvimopan, at human and guinea pig recombinant opioid receptors was undertaken using conventional [3H]-diprenorphine radioligand binding, guanine nucleotide exchange and adenylyl cyclase inhibition assays. The pKi values for MNTX for human $\mathrm{mu}(\mathrm{hMOP})$, delta (hDOP) and guinea pig kappa (gpKOP) opioid receptors were 8.0 \pm 0.1 , $6.2 \pm 0.1$ and $7.5 \pm 0.1$ (mean + sd; $n>11$ ), respectively. Alvimopan and ADL 08-0011 demonstrated up to 100 -fold higher affinity, with pKi values at hMOP, hDOP and gpKOP of $9.6 \pm 0.1,8.6 \pm 0.2$ and $8.3 \pm 0.1$ for alvimopan and $9.6 \pm 0.2,7.8 \pm 0.1$ and $7.5 \pm 0.1$ for ADL 080011 , respectively $(n>6)$. To evaluate mu opioid receptor activation, binding of europiumlabeled GTP to membranes expressing the hMOP receptor was monitored. Relative to the full agonist DAMGO, a range of intrinsic activity (IA) values were obtained for compounds reported to be antagonists, including nalorphine ( $59 \pm 9 \%)$, naloxone $(13 \pm 2 \%)$ and MNTX $(28 \pm 6 \%)$, whereas alvimopan and ADL 08-0011 demonstrated negative IA values under these assay conditions $(-7 \pm 9 \%$ and $-8 \pm 2 \%$, respectively) (mean $+s d, n>12)$. Nalorphine, naloxone and MNTX also behaved as partial agonists with respect to inhibition of forskolin $(3 \mu \mathrm{M})$-stimulated adenylyl cyclase, with IA values of $53 \%, 11 \%$ and $43 \%$, respectively. MNTX, alvimopan and ADL 08-0011 antagonized endomorphin-1-mediated reductions in electrical field stimulation (EFS)-evoked ileal contractions (pA2 values of 7.7, 9.6, and 9.4, respectively, $n=3$ ). For all compounds examined, there was good correlation between the $\mathrm{pKi}$ values for the hMOP opioid receptor and native gp ileal receptors $(\mathrm{r} 2=0.99)$. The clinical significance of the distinct pharmacological profiles of MNTX and alvimopan remains to be determined. 


\section{T1701}

Tegaserod Stimulates 5-Ht4 Receptor (5-HT4R)-Mediated Epithelial Chloride/ Water Secretion Through cAMP/PKA Activation But Not Through cAMP/Epac Or Ca2+-Dependent Signaling in the Rat Colon in Vitro Eckhard Weber, Mario Bernhard, Hans-Juergen Pfannkuche

BACKGROUND. Tegaserod, a 5-HT4R agonist, has been demonstrated to stimulate intestinal chloride/water secretion, presumably by coupling with Gs protein and thus activation of adenylate cyclase. The present study aimed at the elucidation of downstream signaling pathways potentially involved in epithelial electrolyte secretion, namely protein kinase A (PKA), EPAC (exchange protein directly activated by cAMP) and Ca2+-dependent processes. METHODS. Mucosa-submucosa preparations of rat colon (male Wistar rats, 230-470g) were mounted in Ussing chambers. Following equilibration, epithelial chloride secretion was triggered with tegaserod $(10 \mu \mathrm{M})$. Signaling pathways were studied by subsequent blockade

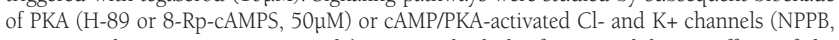
$100 \mu \mathrm{M}$, and 293B, $1 \mu \mathrm{M}$, respectively). Due to the lack of EPAC inhibitors, effects of the EPAC ligand, 8-pCPT-2'-O-Me-cAMP $(0.1-100 \mu \mathrm{M})$, were studied in the presence and absence of tegaserod $(10 \mu \mathrm{M}), \mathrm{H}-89$ and 8-Rp-cAMPS $(50 \mu \mathrm{M})$. The potential involvement of Ca2+dependent pathways was investigated following blockade of PKC (bisindolylmaleimide BIM, $0.1 \mu \mathrm{M}$ ) and large conductance Ca2+-activated K+-channels (charybdotoxin, $0.1 \mu \mathrm{M}$ ). RESULTS. Tegaserod induced sustained chloride secretion reflected by short-circuit currents of $48.2+18.5 \mu \mathrm{A} / \mathrm{cm} 2$ (mean $+\mathrm{SEM}, \mathrm{n}=9$ ). Inhibition of PKA using $\mathrm{H}-89$ and 8-Rp-cAMPS significantly reduced tegaserod-mediated secretion by $66.9 \pm 16.8 \%$ and $73.5 \pm 10.3 \%$, respectively ( $\mathrm{n}=4-8, \mathrm{P}<0.05$, ANOVA). Blockade of cAMP/PKA-activated $\mathrm{Cl}$ - and $\mathrm{K}+$ channels virtually abolished secretory responses to tegaserod (NPPB: $95.4 \pm 4.6 \% ; 293 \mathrm{~B}: 91.0 \pm 5.3 \%$ inhibition, $\mathrm{n}=6$ ). The selective EPAC ligand, 8-pCPT-2'-O-Me-cAMP, concentration-dependently increased chloride secretion $(E C 50=85 \pm 40 \mu \mathrm{M}, E \max =14.0 \pm 5.5 \mu \mathrm{A} / \mathrm{cm} 2, \mathrm{n}=5) . \mathrm{H}-89$ and 8-Rp-cAMPS alone or in combination with tegaserod did not alter, i.e. the latter did not cause leftward-shifts of the 8-pCPT-2'-O-Me-cAMP concentration-response curves (mean $\mathrm{EC} 50=75 \pm 30 \mu \mathrm{M}$, mean Emax $=16.5 \pm 4.1 \mu \mathrm{A} / \mathrm{cm} 2, \mathrm{n}=5$ ). Blockade of $\mathrm{PKC}$ and $\mathrm{Ca} 2+$-dependent Kt-channels did not alter tegaserod-induced chloride secretion either (BIM: $3.8 \pm 10.9 \%$; charybdotoxin: $4.2 \pm 7.5 \%$ difference to control, $n=4-6$ ). CONCLUSIONS. The data suggest that tegaserod stimulates intestinal chloride/water secretion via activation of cAMP/PKA signaling in naive epithelium of the rat colon. EPAC does not seem to be involved in tegaserod-mediated secretory effects. The complete blockade of tegaserod-induced secretion by $\mathrm{Cl}$ - and $\mathrm{K}+$ channel blockers, however, only partial inhibition by PKA blockers, suggests that additional signaling pathways are involved in 5-HT4R-mediated intestinal electrolyte secretion.

\section{T1702}

Tonic Inhibitory Influence of the Vagus in Chronic Experimental Colitis Jean-Eric Ghia, Patricia Blennerhassett, Hari K. Ondiveeran, Elena F. Verdu, Stephen M. Collins

Background/Aim: Previously we showed that the parasympathetic nervous system plays a counter inflammatory role in acute DSS colitis. As parasympathetic dysfunction has been identified in inflammatory bowel disease (IBD), we hypothesize that this may promote inflammation in this disease. To address this, we have examined whether parasympathetic nerves provide a tonic inhibitory influence in a chronic model of experimental colitis. Methods: C57BL/6 mice received 3, 2, 2 \% dextran sulfate sodium (DSS) in drinking water for 3 cycles during 5 days with 11 days of rest between each cycle. Control mice received normal drinking water. Bilateral truncal sub-diaphragmatic vagotomy plus pyloroplasty was performed 10 days before colitis induction. Sham vagotomized mice served as controls. After recovery, integrity of vagotomy was assessed by the sulfated cholecystokinin (CCK 8) satiety test. Disease severity index was evaluated daily after induction of colitis. At sacrifice, disease severity and inflammation were evaluated clinically and histologically. Myeloperoxidase activity (MPO) and colonic cytokine levels were determined. Results: CCK8 decreased food intake in sham operated but not in vagotomized mice, thus verifying the functional integrity of the vagotomy. After the lst DSS cycle (16 days), vagotomized mice exhibited an accelerated onset and increased severity of clinical disease as assessed by loose stools, weight loss and rectal bleeding. Vagotomy also increased the severity at the end of the 2nd cycle (32 days). After the third cycle ( 62 days), no macroscopic deterioration was seen in vagotomized mice. However, the histological score was significantly increased at 62 days following DSS administration compared to controls $(\mathrm{p}<0.05)$. In addition, MPO activity increased from $1.6 \pm 0.4$ in DSS-sham operated to $3.9 \pm 0.7 \mathrm{U} / \mathrm{mg} /$ tissue in DSS-vagotomized mice $(\mathrm{p}<0.05)$ and colonic tissue levels of IL-1b, IL-6, IL-18, INF-g and TNF-a,but not IL4 , were significantly increased in vagotomized mice compared to sham mice that received DSS. In control mice (without colitis) vagotomy per se did not affect any inflammatory marker. Conclusions: These results support the hypothesis that the vagus nerve modulates intestinal inflammation not only acutely but also in a chronic murine model of colitis. Identification of the molecular mechanism underlying the tonic protective role of parasympathetic nerves may lead to novel therapeutic targets in IBD.

\section{T1703}

Circulating Levels of Leptin, Adiponectin, Resistin and Ghrelin in Inflammatory Bowel Diseases

Konstantinos Karmiris, Ioannis Koutroubakis, Costas Xidakis, Maria Polychronaki, Theodora Voudouri, Elias A. Kouroumalis

Background: There is evidence that adipocytokines play an important role in metabolism and in inflammation. Since human metabolism dramatically changes in inflammatory bowel disease (IBD) and chronic inflammation is the hallmark of the disease, we studied serum levels of leptin, adiponectin, resistin and ghrelin in patients with ulcerative colitis (UC) and Crohn's disease (CD) in comparison with healthy controls (HC). Methods: Leptin, adiponectin, resistin and ghrelin serum levels were measured in 100 IBD patients (46 UC and 54 $\mathrm{CD}$ ) and in 60-matched HC using commercially available enzyme-linked immunosorbent assays (ELISA). The ghrelin ELISA detected the active form of the hormone. Leptin, adiponectin, resistin and ghrelin levels were correlated with disease activity, type, localization and treatment. Results: Mean serum leptin levels were $10.6 \pm 2.0 \mathrm{ng} / \mathrm{ml}$ in UC patients, $12.5 \pm 2.6$ $\mathrm{ng} / \mathrm{ml}$ in $\mathrm{CD}$ patients and $15.0 \pm 1.8 \mathrm{ng} / \mathrm{ml}$ in $\mathrm{HC}(\mathrm{P}=0.01)$. UC patients had significantly lower leptin levels than $\mathrm{HC}(\mathrm{P}<0.05)$. CD patients had lower but not significantly different leptin levels compared with the HC. Mean serum adiponectin levels were 9514.8 787.8 $\mathrm{ng} / \mathrm{ml}$ in UC patients, $7651.1 \pm 613 \mathrm{ng} / \mathrm{ml}$ in CD patients and $7270.6 \pm 559.4 \mathrm{ng} / \mathrm{ml}$ in $\mathrm{HC}$ $(\mathrm{P}=0.05)$. Only UC patients had significantly higher adiponectin levels than $\mathrm{HC}(\mathrm{P}=0.02)$ Mean serum resistin levels were $21.2 \pm 2.2 \mathrm{ng} / \mathrm{ml}$ in UC patients, $18.7 \pm 1.6 \mathrm{ng} / \mathrm{ml}$ in $\mathrm{CD}$ patients and $11.8 \pm 0.6 \mathrm{ng} / \mathrm{ml}$ in $\mathrm{HC}(\mathrm{P}=0.0002)$. Both $\mathrm{UC}$ and $\mathrm{CD}$ patients had significantly higher resistin levels than $\mathrm{HC}(\mathrm{P}<0.01)$. Mean serum ghrelin levels were $48.2 \pm 4.2 \mathrm{pg} / \mathrm{ml}$ in UC patients, $49.4 \pm 4.6 \mathrm{pg} / \mathrm{ml}$ in $\mathrm{CD}$ patients and $14.8 \pm 3.0 \mathrm{pg} / \mathrm{ml}$ in $\mathrm{HC}(\mathrm{P}<0.0001)$. Both $\mathrm{UC}$ and $\mathrm{CD}$ patients had significantly higher ghrelin levels than $\mathrm{HC}(\mathrm{P}<0.001)$. Mean serum level of ghrelin was significantly higher in male $(55.0 \mathrm{pg} / \mathrm{ml})$ compared to female IBD patients $(40.3 \mathrm{pg} / \mathrm{ml})(\mathrm{P}=0.02)$. No significant correlation was found with CRP levels, clinical indices of activity, disease localization, disease type (stenotic versus non stenotic), years of diagnosis (early versus late disease), smoking habits and the current use of medications. Only serum ghrelin was significantly higher in ileal compared to colonic $C D(P=0.04)$. Patients with BMI $<25$ had significantly lower serum leptin levels $(6.8 \mathrm{ng} / \mathrm{ml})$ compared to patients with BMl $\geqq 25(18.8 \mathrm{ng} / \mathrm{ml})(\mathrm{P}=0.004)$. Conclusions: Serum levels of adiponectin, resistin and ghrelin are increased whereas serum levels of leptin are decreased in patients with IBD. This observed dysregulation of protein secretion by adipose tissue may play an important role in the disease pathogenesis. Further studies are needed to elucidate the role of adipocytokines in IBD.

\section{$\mathrm{T} 1704$}

Down-Regulation of Granulocyte Colony-Stimulating Factor Receptors By the Condition of Iron Imbalance in Myelomonoblastic THP-1 Cells Sang Wook Kim, In Hee Kim, Seong Hun Kim, Seung Ok Lee, Soo Teik Lee, Dae Ghon Kim, Woo Sung Moon, Geom Seog Seo, Yong Sung Kim, Chang Soo Choi, Suck Chei Choi, Eun Young Choi, Chang Duk Jun

Granulocyte colony-stimulating factor receptor (G-CSFR) is primarily expressed in immature myeloid cells and mature neutrophils and transmits G-CSF signal for proliferation, differentiation, and survival of macrophages, neutrophils, and their precursors. G-CSFR, as well as G-CSF, expression is strictly controlled under steady-state and stress environments. In the present study, we report that iron chelator selectively down-regulates G-CSFR in both human myelomonoblastic THP-1 cells and peripheral blood mononuclear cells (PBMCs). Incubation of THP-1 cells with deferoxamine (DFO), an iron chelator, significantly down-regulated levels of G-CSFR mRNA and its protein and this was overcome by addition with ferric citrate (FC). Interestingly, FC alone also induced decrease of G-CSFR at even low concentrations, implying that conditions caused by either iron depletion or iron overload may influence expression of G-CSFR in monocytic cells. Treatment with DFO significantly decreased intracellular reduced glutathione (GSH) levels. However, restoration of GSH level by Nacetyl-cysteine (NAC), a glutathione precursor, showed little effect on G-CSFR expression, implying that down-regulation of G-CSFR expression by DFO is not likely due to oxidative stress. The signal transduction studies showed activation of ERK1/2 and p38 MAP kinases in response to DFO. Particularly, inhibition of ERK1/2 recovered the down-regulated GCSFR, suggesting that the DFO-activated ERK1/2 may contribute to the suppression of GCSFR expression. In conclusion, the present results demonstrate that conditions caused by iron imbalance may act as a regulator of G-CSFR expression in myeloid cells, thereby altering various cellular responses mediated by G-CSF.

\section{T1705}

$\mathrm{PGE}_{2}$ Stimulates Neurotensin Secretion and Gene Expression in the Human Endocrine Cell Line Bon

J.N. Li, Kathleen O'Connor, Xiaofu Wang, Courtney M. Townsend, B. Mark Evers

Neurotensin (NT), a gut peptide localized predominantly to the small bowel, plays an important role in gastrointestinal growth and colonic inflammation. Previously, we have shown that forskolin (FSK), an agent that increases cAMP levels, stimulated NT secretion in a novel human endocrine cell line BON, which produces and secretes NT peptide. Prostaglandin $\mathrm{E}_{2}\left(\mathrm{PGE}_{2}\right)$, a product of arachidonate metabolism and a potent mediator o inflammation, exerts its biological effects via cAMP/PKA and PLC/PKC pathways by binding to its receptors, EP2/4 and EPl, respectively. The purpose of this study was to define the role of $\mathrm{PGE}_{2}$ on NT secretion and gene expression. METHODS. (i) First, the presence of $\mathrm{PGE}_{2}$ receptors in $\mathrm{BON}$ cells was determined by RT-PCR. To delineate the role of $\mathrm{PGE}_{2}$ on NT secretion, cells were treated with varying doses of $\mathrm{PGE}_{2}$ for $1 \mathrm{~h}$ or $\mathrm{PGE}_{2}(1 \mu \mathrm{M})$ over a time course. NT release was measured by a NT EIA kit; (ii) to determine involvement of PKA or PKC, BON cells were pre-treated with PKA inhibitors, H89 and PKI, or transfected with siRNA to PKC- $\alpha,-\delta,-\varepsilon$ or $-\eta$; (iii) to determine if $\mathrm{PGE}_{2}$ regulates NT gene expression, BON cells were treated with $\mathrm{PGE}_{2}$ for $24 \mathrm{~h}$ and NT mRNA analyzed by real time PCR. In addition, human NT reporter plasmids containing varying lengths of the NT promoter or the empty vector (pXPl) were transfected into $\mathrm{BON}$ cells and treated with $\mathrm{PGE}_{2}$ or vehicle (control) for $24 \mathrm{~h}$; luciferase activity was measured. RESULTS. (i) EP1, EP2 and EP4 receptors are expressed in BON cells, and their agonists induced NT release. Furthermore, NT secretion from BON cells was significantly up-regulated by $\mathrm{PGE}_{2}$ in a dose- and timedependent fashion. (ii) $\mathrm{PGE}_{2}$-stimulated NT release was significantly suppressed by both H89 and PKI, suggesting the involvement of cAMP/PKA pathway downstream of EP2/4. In addition, PKC- $\varepsilon$ siRNA significantly decreased NT secretion from BON cells treated with $\mathrm{PGE}_{2}$, suggesting involvement of PLC/PKC pathway downstream of EP1. (iii) NT mRNA expression was increased $\sim 2$-fold by $\mathrm{PGE}_{2}$. Moreover, transfection of NT promoter constructs into BON cells, and treatment with $\mathrm{PGE}_{2}$ significantly induced NT promoter activity compared with vehicle treatment. CONCLUSIONS. We demonstrate that $\mathrm{PGE}_{2}$ regulates NT secretion and gene expression in BON cells. Furthermore, we showed that $\mathrm{PGE}_{2}$ regulates NT secretion by both cAMP/PKA and PLC/PKC-dependent pathways. Since both $\mathrm{PGE}_{2}$ and $\mathrm{NT}$ are crucial mediators of inflammation, our findings suggest that $\mathrm{PGE}_{2}$-regulated NT gene 
expression and protein release may play an important role in gut inflammation and may represent a target for the treatment of inflammatory diseases.

\section{T1706}

Prostaglandin E2 Induces IL-1 $\alpha$ Via Both Transcriptional and Posttranscriptional Mechanisms

Jinyi Shao, Hongmiao Sheng

Interleukin-1 (IL- $1 \alpha$ and IL-1 $\beta$ ) plays central roles in immune and inflammatory responses through stimulating the expression of large number of genes that participate in immune reactivity and inflammation. Cyclooxygenase- 2 (COX-2) is an IL-1 target gene and can be induced by IL-1 exposure. COX-2 derived prostaglandin E2 (PGE2) is thought to be an important mediator of IL-1 pro-inflammatory actions. In the present study, we demonstrate a positive feedback between the IL-1 signaling system and the PGE2 signaling pathway. PGE2 has been shown to modulate a number of signaling pathways and gene expression. Targeted microarray demonstrated that PGE2 specifically induced the expression of IL-l $\alpha$. Exposure to PGE2 rapidly increased the levels of IL- $1 \alpha$ mRNA and the production of IL$1 \alpha$ protein in colon cancer LS-174T cells. Transient transfection with the IL- $1 \alpha$ promoter construct showed that PGE2 treatment modestly increased IL-l $\alpha$ transcription. Therefore, we further determined whether PGE2 altered the stability of IL-1 $\alpha$ mRNA. LS-174T cells were treated with PGE2 and new transcription was blocked by addition of DRB (5, , dichlorobenzimidazole riboside). The decay rate of IL- $1 \alpha$ mRNA was evaluated by real time PCR. While the half-life of IL- $1 \alpha$ mRNA in untreated LS-174T cells was $\sim 2 \mathrm{~h}$, PGE2 treatment significantly stabilized IL-l $\alpha$ mRNA extending the half-life to $\sim 4.5 \mathrm{~h}$. To determine the role of the 3' untranslated region (3'UTR) of IL- $1 \alpha$ mRNA in PGE2 stabilization of IL- $l \alpha$ mRNA, we constructed a luciferase reporter vector (pCMV-Luc-3'UTR), in which luciferase cDNA was under the control of a CMV promoter and followed by the 3'UTR of IL-l $\alpha$ mRNA including class II AU-rich elements. pCMV-Luc-3'UTR was transiently introduced into LS$174 \mathrm{~T}$ cells, and the cells were treated with vehicle or PGE2. The presence of PGE2 increased the luciferase activity $\sim 3$-fold compared to the cells treated with vehicle, suggesting that PGE2 stabilized luciferase mRNA through the 3'UTR of IL- $1 \alpha$ mRNA. Our results provide additional insights to the understanding of intestinal inflammation and suggest that while IL- $1 \alpha$ exerts pro-inflammatory effects to the intestinal epithelium through induction of COX2 , which derived PGE2 may, in turn, further increase the expression of IL-l $\alpha$ through stabilizing IL-l $\alpha$ mRNA.

\section{T1707}

Gastrin Induces Leukocyte Adhesion to Human Endothelial Cells Through CCK-B/Gastrin Receptors

Sales Ibiza, Angeles Alvarez, Maria D. Barrachina, Carlos Hernandez, Irene Diez, Juan V. Esplugues, Sara Calatayud

Exogenous gastrin induces leukocyte / endothelial cell interactions in rat mesenteric venules through the CCK-B/gastrin receptor (Gastroenterology, 128: A591), and a recent study indicate that gastrin acts on human endotelial cells increasing the expression of leukocyte adhesion molecules and inducing angiogenesis (Clin Cancer Res 2004, 10:8250). The aim of the present study was to analyze whether gastrin acts on human endothelial cells to induce leukocyte / endothelial cell adhesion. Methods: The interaction between gastrin and human umbilical vein endothelial cells (HUVEC) was detected by measuring the binding of a fluorescently labeled gastrin derivative (rhodamine green heptagastrin, RG-7P). HUVEC were incubated with RG-7P (10-7M) for $30 \mathrm{~min}$ in the presence or absence of non-labeled gastrin (10-8-10-5M) or the CCK-B/gastrin receptor antagonist L-365,260 (10-9-10-6M). Adhesion of human monocytic cells (U-937) to HUVEC was analyzed in vitro in static conditions. HUVEC were incubated with vehicle or different concentrations of gastrin for $1 \mathrm{~h}$ and washed afterwards. A suspension of calcein-labeled U-937 cells was then dropped over these HUVEC monolayers and, 30 min later, the non-adherent cells were removed. The proportion of U-937 cells adhered was calculated by measuring the fluorescence emitted by these remaining cells. In some cases, HUVECs were pretreated with the CCK-B/gastrin receptor antagonist L-365,260 (10-7M). Results: RG-7P interacted with HUVEC through the CCK-B/gastrin receptors since the fluorescent signal emitted by the HUVEC-bound compound was significantly reduced by co-treatment with either non-labeled gastrin or the antagonist L-365,260 in a concentration-dependent manner. Treatment of HUVECs with gastrin $(10-11 \mathrm{M}, 10-10 \mathrm{M}$ and $10-9 \mathrm{M})$ induced significant increases in the adhesion of $\mathrm{U}$ 937 cells $(113 \pm 3 \% ; 125 \pm 3 \% *$, and $126 \pm 5 \% *$ of control response, respectively. * $\mathrm{P}<0.05$ vs control). This pro-adhesive effect of gastrin was partially prevented by pretreatment with L-365,260: $97 \pm 8 \%, 106 \pm 4 \% \#, 108 \pm 5 \% \#$ of control response, respectively. \# P<0.05 vs respective vehicle-treated group). Conclusions: Gastrin increases the adhesion of monocytic leukocytes to human endothelial cells through the activation of endothelial CCK-B/gastrin receptors. These results suggest that gastrin can exert a pro-inflammatory activity in humans.

\section{$\mathrm{T} 1708$}

Proliferative and Antiapoptotic Effects of Progastrin Peptides Are Attenuated On Down-Regulation of Nf- $\mathrm{KB}$ Expression in AR42J Cells William Rengifo-Cam, Sanjeev Choudhary, Shahid Umar, Pomila Singh

Background: Progastrin (PG) exerts proliferative and anti-apoptotic effects on pancreatic and colon cancer cells. We previously reported that $\mathrm{Src}$ was required for mediating proliferative effects while PI3K/Akt and p38/p44 MAP kinases were required for mediating anti-apoptotic effects of PG (DDW 2005). Aim: To examine the role of NF- $\mathrm{KB}$ in mediating the growth effects of PG on AR42J rat pancreatic cancer cells. Methods: Routine methods were used for all the assays in these studies. Results: AR42J cells exposed to 0.1nM PG resulted in I $\mathrm{B} \alpha$ phosphorylation and degradation followed by nuclear translocation of p65/Rel A. EMSA revealed an inducible band in response to PG which increased significantly in a biphasic manner peaking at $30 \mathrm{~min}$ and 6 hours. When super shift assays were performed with antibodies against Rel A/p65, p50 and c-Rel both Rel A/p65 and p50 antibodies exhibited super shifting of PG-inducible band while non-immune $\operatorname{IgG}$ and c-Rel antibodies failed to do so suggesting presence of $\mathrm{p} 50 / \mathrm{p} 65$ as predominant heterodimer after PG treatment. Activation of NF- $\mathrm{KB}$ was further confirmed by an ELISA-based -reporter assay. Significant inhibition of this activity in presence of NF-KB Essential Modulator (NEMO) peptide, established the specificity of the response. Mechanistically, 0.1nM PG increased phosphorylation of p65 subunit at Ser 536 by $\sim 5.0$ fold $(n=3)$. Thereafter, involvement of NF- $\mathrm{KB}$ activation in mediating the biological effects of PG was elucidated through p65/Rel A siRNA. Cells treated with randomly designed non-specific siRNA served as control. In control cells, PG significantly reduced the activation of caspase 3 in response to the pro-apoptotic agent, camptothecin; inhibition of NF-KB activation by p65 siRNA, resulted in the loss of antiapoptotic effects of $\mathrm{PG}$ and rescued the loss of activation of caspase 3 by $95 \%$. Interestingly, while the NEMO peptide decreased the proliferative effects of PG by only $20 \sim 30 \%$, p65 siRNA expression decreased this effect by almost $90 \%$, suggesting a possible involvement of IKK $\beta$ and IKK $\gamma$-independent pathways in PG-induced activation of NF-KB. Conclusions: 1. PG induces both phosphorylation/degradation of I $\mathrm{I} B \alpha$ and phosphorylation of $\mathrm{p} 65$ subunit which facilitates NF-KB's nuclear import. 2. NF-KB activation is mediating both the survival and proliferative effects of PG. 3. p65 siRNA significantly blocks PG-induced anti-apoptosis and cellular proliferation and may be a useful tool for treating pancreatic and colon cancers that are dependent on the growth effects of autocrine PG.

\section{$\mathrm{T} 1709$}

Morphogenic Protein Epimorphin Rescues Intestinal Epithelium from Oxidative Stress By the Activation of EGF Receptor-ERK,-Akt Signals Masahiro Iizuka, Kenji Sasaki, Yohei Hirai, Kenichi Shindo, Shiho Konno, Hiroaki Itou, Shigetoshi Ohshima, Yasuo Horie, Sumio Watanabe

[Background and Aim] Epimorphin is a mesenchymal protein that regulates morphogenesis of epithelial cells. Our preliminary study showed that epimorphin might modulate survival of intestinal epithelial cells (IEC). We conducted this study to elucidate whether epimorphin prolongs the life span of IEC and modulates cell survival and wound repair of IEC under oxidative stress condition. [Methods] (1) Rat intestinal epithelial cell line, IEC-6, was cultured in the absence or presence of epimorphin $(10,20 \mu \mathrm{g} / \mathrm{ml})$, and the life span of IEC was assessed. (2) Activation of the signaling proteins was assessed after treatment with epimorphin using Western Blot. (3) We assessed the function of epimorphin for protecting IEC (IEC6 cells and Caco- 2 cells) from severe oxidative stress conditions with hydrogen peroxide by counting dead cells including necrotic and apoptotic cells stained with DAPI, AnnexinV, Propidium iodide or by measuring lactate dehydrogenase (LDH) level in culture medium. (4) Finally, we assessed the effect of epimorphin for wound repair of IEC under oxidative stress condition using wound assays. [Results] (1) The mean life span of IEC-6 cells was prolonged 1.9 times $(\mathrm{p}<0.0006)$ by treatment with epimorphin. (2) We discovered that epimorphin phosphorylated EGF receptor, and activated both ERK1/2 MAPK and AKT signaling pathways, and phosphorylated 90RSK and Bad and induced Bcl-XL and survivin protein. (4) Hydrogen peroxide ( $1 \mathrm{mM}$ ) induced severe cell death $(78 \%)$ in IEC, but epimorphin decreased $67.3 \%$ or $79.3 \%$ of apoptosis or necrosis of IEC induced by hydrogen peroxide $(\mathrm{p}<0.0001)$. LDH level in culture medium of IEC increased 17.9 times by treatment with $100 \mathrm{mM}$ of hydrogen peroxide, but an increase in LDH level were dramatically $(86.3 \%$ ) suppressed by treatment with $20 \mu \mathrm{g} / \mathrm{ml}$ of epimorphin $(\mathrm{p}=0.0039)$. (4) We performed wound assays and found that the number of migrated cells in wounded areas decreased $65 \%$ by treatment with $30 \mu \mathrm{M}$ of hydrogen peroxide, but epimorphin 2.5 times increased the number of migrated cells in wounded areas $(p<0.0001)$. These functions of epimorphin were significantly suppressed by treating IEC with EGF receptor-neutralizing antibody or MEK inhibitor. [Conclusions] Our study indicates that morphogenic protein epimorphin also protects IEC from oxidative stress by the activation of EGF receptor-ERK1/2 MAPK and AKT signals, suggesting novel mesenchymal-epithelial relationship for protection of intestinal epithelia cells.

\section{$\mathrm{T} 1710$}

In Human Colon Cancer Cells, Bile Acid-Induced Selective Autophosphorylation of Epidermal Growth Factor Receptor (EGFR) Tyr992 Activates Post-Receptor Signaling

Kunrong Cheng, Jean-Pierre Raufman

In colon cancer cells conjugated secondary bile acids stimulate transactivation of EGFR, p44/42 MAP kinase (MAPK) activation and cell proliferation (Biochem. Pharmacol. 2005;70:1035). In H508 human colon cancer cells, inhibition of EGFR kinase activation (2 $\mu \mathrm{M}$ PD168393) or of p44/42 MAPK activation with a MEK inhibitor (25 $\mu$ M PD980959) inhibited both bile acid-induced p44/42 MAPK phosphorylation and cell proliferation $(\mathrm{p}<0.05)$. To determine the mechanism whereby bile acid-induced EGFR activation results in p44/42 MAPK activation, we examined EGFR autophosphorylation. Immunoprecipitation (IP) of H508 cell lysates with anti-EGFR antibodies was followed by immunoblotting (IB) with specific antibodies to 5 C-terminal EGFR tyrosine (Y) autophosphorylation sites (Y845, Y992, Y1045, Y1068, and Y1173). In H508 cells, incubation with deoxycholyltaurine (DCT; $100-300 \mu \mathrm{M})$ revealed selective phosphorylation of only Y992 whereas EGF $(10 \mu \mathrm{g} / \mathrm{ml})$ caused phosphorylation of all 5 EGFR tyrosine residues examined. Adding the EGFR kinase inhibitor (2 $\mu \mathrm{M}$ PD168393) abolished DCT- and EGF-induced Y992 autophosphorylation. EGFR Y992 is a high-affinity binding site for SHP2, a phosphatase whose activation is critical for sustained p44/42 MAPK phosphorylation, and phospholipase-C $\gamma$ (PLC $\gamma$ ), an enzyme that catalyzes phosphoinositol hydrolysis and increases cell $\left[\mathrm{Ca}^{2+}\right]$, thereby augmenting p44/42 MAPK activation. IB revealed that both DCT $(300 \mu \mathrm{M})$ and EGF $(10 \mathrm{ng} / \mathrm{ml})$ markedly increased phosphorylation of SHP2 Y542 (not SHP2 Y580). This provided evidence of bile acid-induced SHP2 activation. Incubation with DCT $(300 \mu \mathrm{M})$ followed by IP with antiEGFR antibodies and IB with antibodies to PLC $\gamma 1$, revealed a marked increase in the association of PLC $\gamma$ with EGFR. This action was mimicked by HB-EGF $(0.01 \mu \mathrm{g} / \mathrm{ml})$, an EGFR ligand, by matrix metalloproteinase-7 $(0.1 \mu \mathrm{g} / \mathrm{ml})$, an enzyme that releases HB-EGF, but not with EGF. To determine the importance of bile acid-induced PLC $\gamma$ activation and propagation of downstream $\mathrm{Ca}^{2+}$ signaling, intracellular $\mathrm{Ca}^{2+}$ was reduced using two strategies; chelation with BAPTA $(25 \mu \mathrm{M})$, and incubation with thapsagargin $(1 \mathrm{nM})$ to release 
$\mathrm{Ca}^{2+}$ from intracellular stores in the presence of $\mathrm{LaCl}_{3}(25 \mu \mathrm{M})$ to prevent $\mathrm{Ca}^{2+}$ re-entry Both strategies inhibited bile acid-induced p44/42 MAPK activation. Collectively, these findings provide evidence that bile acid-induced selective autophosphorylation of EGFR Y992 activates SHP2 and PLC $\gamma$. In H508 human colon cancer cells, EGFR kinase-mediated autophosphorylation of only Y992 in the C-terminal domain appears sufficient and necessary to propagate downstream signaling and to stimulate maximal bile acid-induced cell proliferation.

\section{T1711}

Cloning and Characterization of Rabbit Rgs4 Full-Length cDNA and Rgs4 Promoter

Wenhui Hu, Fang Li, Karnam S. Murthy

We have recently shown that the pro-inflammatory cytokine, interleukin-1 $\beta$ (IL-1 $\beta$ ) induces up-regulation of RGS4 mRNA and protein expression in rabbit colonic smooth muscle cells by activating NF- $\mathrm{KB}$. The increase in RGS4 expression is responsible for IL-1 $\beta$-induced inhibition of initial $\mathrm{Ca}^{2+}$-dependent contraction. In order to examine the mechanism of upregulation of RGS4 expression, it is essential to characterize the gene sequence of rabbit RGS4. Although the RGS4 cDNA sequence is available in other species, there are no reports of the cloning of the RGS4 promoter. Aim. To clone the full-length rabbit RGS4 cDNA and RGS4 promoter in rabbit colonic smooth muscle Methods. Degenerative RT-PCR and SMART CDNA 5'- and 3'-RACE were performed to obtain the full-length sequence of rabbit RGS4. A $1.4 \mathrm{~kb}$ sequence including the promoter region and a $50 \mathrm{bp} 5$ '-untranslated region (UTR) was cloned into pMlu renila luciferase reporter vector by PCR using genomic DNA from rabbit intestine as template. Results. Only one band of 5'-RACE PCR product was present, consistent with a single transcript of $2.8 \mathrm{~kb}$ in Northern blot analysis of rabbit colonic smooth muscle cells. There were 5 exons with long 3'-UTR. The 5'-UTR extended 120 bp nucleotides upstream of the RGS4 start codon with an in-frame stop codon, and shared 93\% homology with the 5'-UTR of the longest human RGS4 transcript. Analysis of the assembled genome of rabbit and comparison with human RGS4 genome identified the genomic organization of rabbit RGS4 gene. A putative promoter of $1.4 \mathrm{~kb}$ containing consensus binding sites for several potential transcriptional factors important in inflammatory responses was analyzed. Reporter gene assay showed that the cloned promoter region of RGS4 increased luciferase activity by 8-fold in rabbit smooth muscle cells, 50-fold in HEK293T cells, and 12-fold in Hela cells. Further analysis by mutagenesis suggested that deletion of the region containing NF- $\mathrm{KB}$ and AP-1 binding sites and core TATA box (P3: -1389 to -816) eliminated promoter activity, whereas deletion of the distal regions beyond the NF- $\mathrm{\kappa B}$ and AP-1 sites (P2 and P4) enhanced promoter activity. Electrophoretic mobility shift assay (EMSA) and chromatin immunoprecipitation (ChIP) assay confirmed the presence of NF$\kappa \mathrm{B}$ and $\mathrm{AP}-1$ binding sites within the proximal region $(-247)$. Conclusion. We report the first cloning of an RGS4 promoter and the cloning of RGS4 cDNA in the rabbit. The core promoter region contains binding sites for various transcription factors including NF- $\mathrm{KB}$ and AP-1, and possesses strong enhancer activity in regulating RGS4 transcription in rabbit colonic smooth muscle cells.

\section{$\mathrm{T} 1712$}

Glucocorticoids Inhibit T Cell Receptor Signaling via LCK and FYN - A New Mechanism for An Old Drug

Mark Lowenberg, Joyce Bilderbeek, Auke Verhaar, Jan van Marle, Frank Buttgereit, Maikel Peppelenbosch, Sander van Deventer, Daan Hommes

BACKGROUND: Glucocorticoids (GCs) form the basis of current IBD treatment and mediate well-defined genomic effects via the GC-receptor. There is increasing evidence for rapid nongenomic GC-effects on cellular function which can not be explained by the traditional mode of GC action. The aim of this study was to define rapid GC effects in CD4+ T lymphocytes. METHODS: Cells were pretreated for 10 minutes with the synthetic GC analogue dexamethasone and activated with anti-CD3 and anti-CD28 Abs for 15 minutes. Cell lysates were used for peptide arrays containing 1176 different kinase consensus substrates and results were validated with conventional techniques. RESULTS: Peptide array analysis revealed marked early differences in phosphorylation patterns between GC-treated and nonGC-treated cells. Suppressed phosphorylation of Lck/Fyn kinase consensus substrates was seen in lysates prepared from GC treated cells, indicating reduced Lck/Fyn enzymatic activities, and this was confirmed with in vitro kinase assays. Immunoprecipitation experiments demonstrated GC-induced inhibition of Lck and Fyn recruitment to the T cell receptor (TCR) complex, an essential step in Lck/Fyn-mediated TCR signaling. Western blot analysis showed suppressed phosphorylation of a series of downstream Lck/Fyn signaling targets, including PKB, PKC, ERK, JNK and p38 MAPKs due to short-term GC treatment. Experiments with a pharmacological GC-receptor ligand (RU486) revealed a GC-receptor dependent mechanism of reduced Lck/Fyn activity. Finally, parallel experiments conducted following the application of GCs in healthy individuals confirmed suppression of Lck and Fyn in T cells within $1 \mathrm{hr}$ in vivo. CONCLUSIONS: This study identifies a nongenomic GC-receptormediated inhibitory mechanism of Lck and Fyn, key-players in TCR signaling, providing a cellular substrate for rapid GC-induced immunosuppression. We hypothesize that agents selectively targeting Lck or Fyn could constitute a novel potent immunosuppressive therapy.

\section{$\mathrm{T} 1713$}

Evidence That Neither CCK1 Nor CCK2/Gastrin Receptors in the Duodenum/ Antrum Are Involved in the Physiological Regulation of the Exocrine Pancreas in Pigs

Stefan Pierzynowski, Sofia Rengman, Bjorn Westrom

Our recent results - including those presented at actual meetings - indicate the importance of CCK2 receptors, located in the duodenum and antrum after CCK-33 stimulation, to the physiological regulation of the exocrine pancreas via short local reflexes in pigs. Since CCK2 receptors were originally identified as gastrin receptors, the question arises whether gastrin, might stimulate the pancreas in the same way. Thus the aim of the study was to highlight and distinguish the role of gastrin from that of CCK in relation to the exocrine pancreas via the duodenal/antral CCK2 receptors. Materials and methods: Experiments were performed on 10 pigs $(12 \pm 3 \mathrm{~kg}$ b.wt. $)$ that were surgically fitted with catheters for the administration of CCK-33 and pentagastrin, in identical molar concentrations, to the local duodenal/antral arterial circulation and to the general (peripheral) circulation via the jugular vein and for pancreatic juice collection. During acute experiments in anaesthetised pigs after $2 \times 10 \mathrm{~min}$ baseline collections of juice, CCK-33 $(n=5)$ or pentagastrin $(n=5)$ was given as a bolus injection via the gastric artery at $13 \mathrm{pmol} / \mathrm{kg}$ and pancreatic juice was collected at $2 \times 5$ and $2 \times 10$ min intervals. The pigs were then given a 30 minute rest period before the procedure was repeated with injection of the hormones via the jugular vein to the genera circulation at $130 \mathrm{pmol} / \mathrm{kg}$. The pancreatic juice collections were analysed for trypsin activity. Results: Local injection of the low dose of CCK-33 stimulated the trypsin output from 1 to $20 \mathrm{U} / \mathrm{kg} / \mathrm{h}$ while injection of low dose pentagastrin had no effect on the trypsin output. Peripheral administration of the high doses of both hormones, stimulated both the trypsin outputs from 1 to $25 \mathrm{U} / \mathrm{kg} / \mathrm{h}$. In conclusion: pentagastrin, in contrast to CCK-33, did not stimulate the pancreas secretion via the local short reflex pathway originating in the duodenum/antrum. This indicates that the participation of CCK2 receptors sensitive to CCK33 in this reflex needs to be reconsidered. The existence of a third type of CCK receptor then $\mathrm{CCKl}$ and $\mathrm{CCK} 2 /$ gastrin receptors in the duodenum/antrum, and its participation in the physiological regulation of exocrine pancreatic regulation must be studied further.

\section{$\mathrm{T} 1714$}

Proteinase-Activated Receptor-2 (PAR-2) Increases Mucin Exocytosis Via Multiple Signal Transduction Pathways in Dog Pancreatic Duct Epithelial Cells (PDEC)

Mean-Hwan Kim, Bo-Hwa Choi, Kyong-Tai Kim, Thomas Sernka, Toan D. Nguyen, DukSu Koh

INTRODUCTION: Proteinase-activated receptor-2 (PAR-2) is activated through cleavage of its N-terminus by a protease (e.g. trypsin, tryptase) to expose a tethered ligand (activating peptide, AP). We reported that PAR-2 activation on $\operatorname{dog}$ PDEC causes elevation of intracellula $\mathrm{Ca}^{2+}\left(\left[\mathrm{Ca}^{2+}\right]_{\mathrm{i}}\right)$ to activate $\mathrm{Cl}^{-}$and $\mathrm{K}^{+}$channels (JCI, 1999, 103: 261-269). We now describe the effects of PAR-2 activation on exocytosis by PDEC and characterize the different signal transduction pathways responsible for this action. METHODS: Exocytosis of a single PDEC vesicle was monitored, using carbon fiber amperometry, as the exocytotic release of oxidizable dopamine, preloaded into intracellular vesicles. $\left[\mathrm{Ca}^{2+}\right]_{i}$ was measured with single-cell photometry using the $\mathrm{Ca}^{2+}$ sensitive dye, Indo- 1 , while mucin was measured as acetyl-D- $\left[{ }^{3} \mathrm{H}\right] \mathrm{gluco}-$ samine incorporated into TCA-precipitable protein. RESULTS: Trypsin $(1 \mu \mathrm{M})$ and AP (10 $\mu \mathrm{M})$ increased the average rate of exocytosis in PDEC $\sim 6$-fold in a biphasic manner: an early transient peak followed by a steady and sustained increase. Trypsin and AP also stimulated increases in $\left[\mathrm{Ca}^{2+}\right]_{i}$ with corresponding biphasic profiles. The early transient $\left[\mathrm{Ca}^{2+}\right]_{\mathrm{i}}$ increase was independent from extracellular $\mathrm{Ca}^{2+}(2 \mathrm{mM})$ and was resistant to 10 $\mu \mathrm{M} \mathrm{LaCl} l_{3}$, an inhibitor of the store-operated $\mathrm{Ca}^{2+}$ channels (SOC); this $\left[\mathrm{Ca}^{2+}\right]_{\mathrm{i}}$ increase most likely resulted from $\mathrm{Ca}^{2+}$ mobilization from internal stores. In contrast, the subsequen sustained $\left[\mathrm{Ca}^{2+}\right]_{i}$ increase was dependent on extracellular $\mathrm{Ca}^{2+}$ and inhibited by $\mathrm{LaCl}_{3}$, it likely reflected $\mathrm{Ca}^{2+}$ influx through SOC. When a $\mathrm{Ca}^{2+}$ chelator, BAPTA/AM $(20 \mu \mathrm{M})$, was used to abolish the $\left[\mathrm{Ca}^{2+}\right]_{\mathrm{i}}$ increase, exocytosis was markedly, but not totally, inhibited. This residual exocytosis was abolished with $100 \mathrm{nM}$ bis-indolylmaleimide I and $10 \mu \mathrm{M}$ calphostin $C$, specific inhibitors of protein kinase $C$ (PKC), but not with $500 \mathrm{nM}$ bisindolylmaleimide $\mathrm{V}$, the inactive analogue, or $1 \mathrm{mM} \mathrm{Rp-8-Br-cAMPS}$, an inhibitor of the cAMPactivated protein kinase. Accordingly, both trypsin and AP stimulated the enzymatic activities of the $\alpha$ and $\delta$ isoforms of PKC, as determined by histone Hl phosphorylation. AP $(1 \mu \mathrm{M})$ also stimulated mucin secretion. Consistent with previous localization of PAR-2 to the basolateral membrane of dog PDEC, luminal application of trypsin to the cell monolayer did not induce exocytosis. CONCLUSION: In PDEC, PAR-2 activation by trypsin and AP induced a biphasic $\left[\mathrm{Ca}^{2+}\right]_{\mathrm{i}}$ increase, through early mobilization of $\mathrm{Ca}^{2+}$ from intracellula stores followed by a sustained influx of $\mathrm{Ca}^{2+}$ through $\mathrm{SOC}$, to stimulate exocytosis. PAR-2 stimulated exocytosis is further partially mediated by PKC. Funded by NIH, VA, KRF, and POSTECH.

\section{$\mathrm{T} 1715$}

Attenuation of Proteinase-Activated Receptor-2 ( $\left.\mathrm{PAR}_{2}\right)$-Mediated Inflammation By Administration of Insulin

Eric Hyun, Steeve Houle, Nathalie Vergnolle

Trypsin, a serine protease, exerts various physiological roles in the body, including inflammatory process, mainly via activation of the G-protein coupled receptor proteinase-activated receptor-2 $\left(\mathrm{PAR}_{2}\right)$. In the intestine, $\mathrm{PAR}_{2}$ activation has been reported to lead to inflammation. Interestingly, trypsin has also been shown to regulate tissue functions, exerting "hormonelike" activity. Cleavage of insulin receptor (IR) by trypsin results in enhanced tyrosine kinase activity of the receptor that will no longer bind to insulin. On the other hand, activation of IR has been shown to decrease the activity of various GPCR proteins. We postulated here the existence of interaction between $\mathrm{PAR}_{2}$ and IR signaling pathways. To test this hypothesis we investigated whether or not administration of insulin would affect PAR $_{2}$-mediated inflammation in mouse paw and intestine. Basal paw thickness was measured using an electronic caliper, before, and then every hour for 6 hours after intraplantar injection of saline, SLIGRL$\mathrm{NH}_{2}$ (selective PAR 2 activating peptide) SLIGRL- $\mathrm{NH}_{2}+$ insulin, SLIGRL-NH $\mathrm{N}_{2}+$ inactivated insulin (boiled), LRGILS-NH 2 (control peptide), LRGILS-NH + insulin, carrageenan alone or carrageenan + insulin. The doses of compounds used for paw oedema experiments were $50 \mu \mathrm{g}$ for SLIGRL-NH $\mathrm{N}_{2} / \mathrm{LRGILS}_{-} \mathrm{NH}_{2}, 150 \mathrm{pmol}$ for insulin, and $200 \mu \mathrm{g}$ for carrageenan. Six hours after the intraplantar injections, mice were sacrificed and myeloperoxidase (MPO) activity was measured in paw tissues. Additionally, vehicle, SLIGRL-NH $(100 \mu \mathrm{g})$, SLIGRL$\mathrm{NH}_{2}+$ insulin $\left(100 \mu \mathrm{g}\right.$ and $150 \mathrm{pmol}$, respectively), or LRGILS- $\mathrm{NH}_{2}(100 \mu \mathrm{g})$ were injected intralumenally into mouse jejunum and intravital microscopy was performed to assess leukocyte rolling, adherence and recruitment on selected jejunal venules. Paw oedema and 
MPO activity in mice injected with SLIGRL-NH $\mathrm{N}_{2}+$ insulin were significantly reduced compared to mice injected with SLIGRL- $\mathrm{NH}_{2}$ alone. In contrast, insulin was not able to modulate the effects of control peptide. Injection of insulin was not able to reduce carrageenan induced paw oedema indicating that insulin does not exert general anti-inflammatory properties. Injection of SLIGRL-NH $\mathrm{N}_{2}+$ insulin resulted in significant reduction in both leukocyte rolling and adhesion along the venule of jejunum compared to mice injected with SLIGRL$\mathrm{NH}_{2}$ alone. The insulin-dependent attenuation of $\mathrm{PAR}_{2}$-mediated inflammation was not observed with inactivated insulin. Thus, this study shows for the first time in vivo that insulin can modulate $\mathrm{PAR}_{2}$-induced inflammation both in the jejunum and in paw tissues.

\section{T1716}

A Novel Mechanism for Inhibition of RHOA By $\mathrm{G}_{13}$-Coupled PAR2 Receptors in Smooth Muscle Involves PAR2-Mediated Activation of Nf- $\mathrm{KB}$ and Release of the Catalytic Subunit of PKA from Its Scaffolding Protein IKB $\alpha$ Wimolpak Sriwai, Sunila Mahavadi, Karnam S. Murthy

The protease-activated receptor PAR2 activates RhoA via $G_{13}$ in smooth muscle. In other

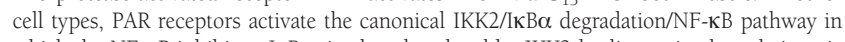

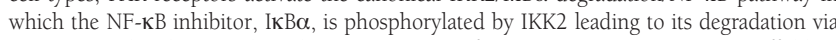

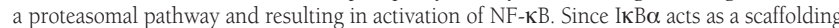
protein for PKA, and proteasomal degradation of I $\mathrm{KB} \alpha$ releases the catalytic subunit of PKA, we postulated that PAR-activated RhoA could be inhibited via feedback phosphorylation of Ser188 upon release of the catalytic subunits of PKA from its binding to IKB $\alpha$. We have previously shown that phosphorylation of Serl88 by PKA inhibits RhoA activity in smooth muscle. Aim. To characterize the link between activation of NF-KB by PAR2 and inhibitory phosphorylation of RhoA by cAMP-independent PKA. Methods. RhoA activation was determined from the increase in Rho kinase activity. The role of the IKK2/I $\mathrm{B} \alpha / \mathrm{NF}-\kappa \mathrm{B}$ pathway in feedback inhibition of RhoA was examined using the IKK2 inhibitor, IKKIV, and the proteasomal degradation inhibitor MG132, and in cells expressing dominant negative mutants

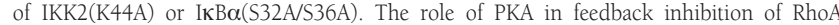
was examined using the PKA inhibitor (myristoylated PKI) and in cells expressing PKA phosphorylation-site deficient RhoA(S188A). Results. The PAR2 agonist, SLIGRL induced

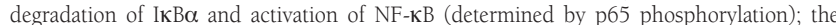
effects were abolished by the blockade of RhoA activity by Clostridium botulinum $\mathrm{C} 3$ exoenzyme suggesting that activation of NF- $\mathrm{KB}$ was downstream of RhoA. PAR2-stimulated Rho kinase activity was significantly augmented by PKI, IKKIV or MG132, and in cells expressing $\operatorname{IKK}(\mathrm{K} 44 \mathrm{~A}), \mathrm{I} \mathrm{KB} \alpha(\mathrm{S} 32 \mathrm{~A} / \mathrm{S} 36 \mathrm{~A})$ or RhoA(S188A). The results imply that RhoA was inhibited upon its phosphorylation by PKA at Serl88, and that the pathway involved activation of the canonical IKK2/IKB $\alpha / \mathrm{NF}-\mathrm{KB}$ pathway. Conclusion. We have identified a novel pathway for feedback inhibition of PAR2-stimulated RhoA. The pathway involves activation of the canonical NF- $\mathrm{KB}$ pathway by PAR2 leading to release of the catalytic subunit of PKA from its binding to I $\kappa \mathrm{B} \alpha$ and causing inhibitory phosphorylation of RhoA at Serl88.

\section{$\mathrm{T} 1717$}

Type-I-Interferon Mediates the Anti-Inflammatory Effects of Cpg Motifs On Colitic CD4+CD62l+ T-Cells in the SCID-Transfer Model of Colitis Florian Obermeier, Veit Philip, Ulrike G. Strauch, Nadja Dunger, Juergen Schoelmerich, Gerhard Rogler, Werner Falk

Introduction: Recently, we and others demonstrated a prophylactic effect of CpG motifs in experimental colitis. We could further demonstrate that $\mathrm{CpG}$ treatment of donor animals reverses the colitiogenic potential of $\mathrm{CD} 4+\mathrm{CD} 62 \mathrm{~L}+$ cells in the SCID model of colitis. A very recent report suggests that the protective $\mathrm{CpG}$-Oligodeoxynucleotide (ODN) effect in an acute model of chemically induced colitis is mediated by CpG-induced Interferon-alpha We tested whether IFN-alpha effects are involved in the potent T-cell dependent, protective effects of CpG-ODN in the SCID transfer model of colitis. Methods: Donor Balb/c mice were either left untreated or were treated with CpG-ODN i.p. (10 $\mu \mathrm{g} / \mathrm{d})$ for 5 days or CpG-

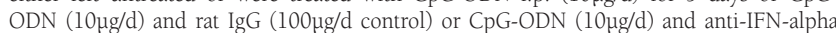
monoclonal antibodies $(\mathrm{mAb})(100 \mu \mathrm{g} / \mathrm{d})$. After treatment splenic CD4+CD62L+ cells were isolated and transferred to SCID recipients which were sacrificed 8 weeks after transfer. The body weight of the SCID recipients was monitored over 8 weeks and a standard histologic score (HS) (0-4) considering epithelial damage and the extent of inflammatory infiltration was used. Furthermore, cytokine secretion from isolated mesenteric lymph node cells (MLC) was measured by ELISA. Results: At the end of the experiment, SCID recipients which were not transferred increased their body weight averagely by $22 \%$. Mice which received cells from untreated donors showed no increase in body weight (untreated: average weight change: $-1 \%$ ) and developed colitis (HS \pm SEM: $3.3 \pm 0.2)$ in contrast to recipients transferred with cells from $\mathrm{CpG}-\mathrm{ODN}$ (average weight change: $+5 \%$; $\mathrm{HS} \pm$ sem: $0.7 \pm 0.2$ ) or $\mathrm{CpG}$-ODN $+\operatorname{IgG}$ (average weight change: $+6.5 \%$; $\mathrm{HS} \pm \mathrm{sem}: 0.5 \pm 0.3$ control) treated donors. In SCID recipients receiving cells from CpG-ODN + anti-IFN-alpha treated mice the CpGODN induced attenuation of colitis was significantly reversed (average weight change: $+1.8 \%$; $\mathrm{HS} \pm$ sem: $1.7 \pm 0.3, \mathrm{p}=0.03$ ). This was accompanied by a complete loss of the CpG-ODNinduced suppression of IL-6 (untreated: $800 \pm 30$ pg/ml, CpG-ODN + IgG: $66.0 \pm 5$ pg/ $\mathrm{ml}$; CpG-ODN + anti-IFN-alpha: $934 \pm 26 \mathrm{pg} / \mathrm{ml}$ ) and IFN-g secretion (untreated: $28 \pm 3.3$ $\mathrm{ng} / \mathrm{ml}, \mathrm{CpG}-\mathrm{ODN}+\mathrm{IgG}: 0.7 \mathrm{ng} \pm 0.12 \mathrm{ng} / \mathrm{ml} ; \mathrm{CpG}-\mathrm{ODN}+$ anti-IFN-alpha: $46 \pm 8.5 \mathrm{ng} /$ $\mathrm{ml}$ ) by MLCs isolated from SCID recipients. Conclusion: The prophylactic effect of CpGODN in experimental colitis is mediated by CpG-ODN induced IFN-alpha production, which effectively decreases the colitogenic potential of T-cells in the SCID transfer model of colitis.

\section{$\mathrm{T} 1718$}

Administration of TLR9L Modifies the Pro-Inflammatory Phenotype of Lp-Mdc to Acquire Anti-Inflammatory Properties

Kazumichi Abe, Jongdae Lee, Carol J. Shen, Ji-Hun Mo, Jose M. Gonzalez-Navajas, Sean Fine, Eyal Raz

The administration of TLR9L ameliorates DSS-induced colitis via the induction of type IFN. Dendritic cells (DC) are the major source of this cytokine. In this study we investigated how the administration of TLR9L modulates the composition and the properties of colonic lamina propria (LP) DC. The administration of TLR9L increased myeloid (M) DC (CD1lchigh CD1lb+), but not plasmacytoid DC (CD1lclow B220+) population in LP up to 14 days post-administration. This increase in MDC trafficking was not observed upon TLR2L, TLR3L, TLR4L or TLR7L administration. The administration of TLR9L enhanced the expression of CCL4 and CCL20 (DC chemoattractants) as well as GM-CSF (a growth factor of MDC) in the colonic tissue. LP-MDC in TLR9L-injected mice isolated one week post-injection differed from LP-MDC isolated one week post-DSS challenge. While TLR9L administration induced LP-MDC to express high levels of CD40, B7RP-1 and OX40L, LP-MDC isolated from DSSchallenged mice expressed low levels of CD80 and CD86. Administration of TLR9L to DSS treated mice resulted in the normalization of the expression levels of CD40, CD80 and CD86 but not of B7RP-1 and OX40L in LP MDC isolated from these mice. Finally, we investigated the physiological role of LP-MDC in DSS-induced colitis using the diphtheria toxin (DT) receptor (DTR)-tg mice. Injection of DT induced the depletion of MDC and attenuated the severity of colitis in DSS-challenged DTR-tg mice. In contrast, the injection of DT to TLR9L-treated, DSS challenged mice enhanced the severity of colitis. Collectively, our results indicate that LP-MDC play an important role in the induction or the inhibition of colonic inflammation and that the administration of TLR9L modifies the pro-inflammatory phenotype of LP-MDC to acquire anti-inflammatory properties.

\section{$\mathrm{T} 1719$}

Expression of Receptor Activator of Nuclear Factor- $\mathrm{KB}$ Ligand (RANKL) and Osteoprotegerin (OPG) in IL-10 \%- and Mdrla \%- Mouse Models of Colitis Silvia C. Resta-Lenert, Susan J. Lee

Background: Inflammatory bowel disease (IBD) is associated with osteoporosis and an increased risk of spontaneous bone fractures. Bone homeostasis is characterized by continuous remodeling through bone formation and resorption. The goal of the present study was to determine whether dysregulated bone homeostasis in two mouse models of colitis is attributable to alterations in bone formation, bone resorption, or both. Methods: 12 wk old colitic IL-10 -/- and mdrla -/- mice, and wild type (WT) counterparts matched for age and gender (8-10 mice/group), were tested for femoral length and bone mineral density (BMD), for serum OPG, RANKL and tartrate-resistant acid phosphatase (TRAP, a marker of osteoclast activity) levels, and colonic tissue OPG mRNA and protein levels, by dual energy X-ray densitometry, immunoassays, and Northern and Western blotting, respectively. Results: Compared to the respective WT, both IL-10- and mdrla-deficient mice had shorter femurs (in mm: $12 \pm 2$ vs $15 \pm 1$ and $13 \pm 1$ vs $16 \pm 2$, respectively; $p<0.05$ ), and lower BMD (in mg/ $\mathrm{cm} 2$ : $53.2 \pm 1.1$ vs $61.3 \pm 0.8$ and $49.8 \pm 1.5$ vs $65.7 \pm 0.5, p<0.05$ and $p<0.01$, respectively). However, serum OPG levels were significantly elevated and RANKL levels decreased only in mdrla -/- mice compared to WT counterparts and IL-10 -/- mice (OPG/RANKL ratio: 3.7 vs 1.0 vs 1.2 , respectively; $\mathrm{p}<0.05)$. OPG levels in supernatants from $24 \mathrm{~h}$ colonic tissue cultures was significantly elevated in mdrla $/-$, but not in IL-10 -/- tissues compared to the respective WT controls (in $\mathrm{ng} / \mathrm{ml}: 65.7 \pm 2.2$ vs $18.4 \pm 3.2, \mathrm{p}<0.001$, and $21.8 \pm 3.5$ vs $18.1+2.1$, respectively). OPG mRNA showed an increase only in mdrla -/- colonic tissue compared to WT (4.5-fold). Finally, serum TRAP levels were elevated significantly in mdrla -/- compared to WT and IL-10 -/- mice (in U/L: $10.5 \pm 1.1$ vs $5.4 \pm 0.5$ vs $6.5 \pm 1.0$, respectively; $\mathrm{p}<0.01$ and $\mathrm{p}<0.05)$. Conclusions: Our data suggest that the mechanisms underlying bone loss in colitis may correlate to disease pathogenesis, with prevalent alterations in either bone formation or resorption processes depending on the model examined. Greater insight into the mechanisms involved in bone homeostasis in the course of chronic inflammation may provide better guidelines for treatment and prevention of osteoporosis associated with IBD and other chronic diseases.

\section{T1720}

Aquaporin 4 Is Highly Upregulated in Experimental Colitis Jonathan J. Hansen, R Balfour Sartor

Inflammatory bowel diseases (IBD) are the result of dysregulated immune responses to commensal enteric bacteria in genetically susceptible hosts. The aberrant responses include up- or down-regulation of various effector and regulatory molecules on immune, epithelial, endothelial and mesenchymal cells in the gastrointestinal tract. These phenomena occur in both human IBD as well as the murine interleukin 10 knockout (IL10-/-) model of THImediated colitis. While expression levels of individual immunoregulatory molecules have been studied in IL10-/- mice, no published data exist on genome-wide surveys of differentially regulated inflammatory markers. Methods: Four wild-type (wt) and four IL10-/- mice born in germ free conditions were transferred to specific pathogen free conditions for four weeks. We isolated total RNA and protein from mesenteric lymph nodes (MLN) and various regions of the intestinal tract and harvested adjacent sections for histologic analysis and explant culture to measure interleukin 12 (IL-12) secretion. Total RNA from the ceca was analyzed on microarray of 33,000 murine genes and results were validated using real-time quantitative PCR, Western blotting, flow cytometry of MLN cells and/or immunofluorescence of frozen sections. Results: As previously reported, greater histologic inflammation scores were observed in ceca from the IL10-/- compared to wt mice ( $2.6 \pm 0.7$ vs. $0.8 \pm 0.21, p=0.006$ ) Similarly, IL-12 secretion from intestinal explants was greater in IL10-/- mice $(1424 \pm 331$ vs. $82 \pm 18 \mathrm{pg} / 50 \mathrm{mg}$ tissue, $\mathrm{p}<0.005)$. Microarray analysis demonstrated greatest induction of the following cell surface molecules in IL10-/- mice: aquaporin 4 (Aqp4), Sca-1, MHC class II, CD7, and CD38 with $24,14,12,9$, and 5 fold increase, respectively. We also observed down regulation of chloride calcium channel activated 3 and claudin 4 in IL10-/- 
mice with 5.3 and 4.9 fold decrease, respectively. These findings were validated using quantitative real-time PCR. In addition, we confirmed the mRNA results at the protein level with Western blots for Aqp 4 as well as immunofluorescence of frozen intestinal sections and flow cytometry of MLN cells for MHCII. Conclusion: While the majority of upregulated cell-surface markers in animals with experimental colitis are immune-related, interestingly Aqp 4, a membrane water channel gene not known to be associated with immune responses, was most highly upregulated. This may have implications not only for further elucidating the pathogenesis of chronic intestinal inflammation, but also for developing novel targeting strategies to diagnose, manage, and treat IBD and its clinical symptoms.

\section{T1721}

Osteopontin-Deficiency Protects Mice from DSS-Induced Colitis Jian Zhong, Erik R. Eckhardt, Helieh S. Oz, Dennis Bruemmer, Willem J. de Villiers

Background \& Aims: Osteopontin (OPN), a secreted glycoprotein that promotes Thl immune responses, is involved in several inflammatory conditions. Recently, OPN plasma levels have been demonstrated to be elevated in patients with Crohn's disease (CD). Based on this evidence we investigated in the present study whether OPN deficiency protects mice against dextran sodium sulfate (DSS)-induced colitis. Methods: Colitis was induced in OPN -/- mice and matched wildtype Black Swiss control mice by adding 3.5\% DSS to their drinking water. Disease progression was evaluated for 10 days by measuring body weight, stool consistency and rectal bleeding, colon lengths, histology, and immuno-histochemistry. Levels of the acute phase protein serum amyloid A (SAA), OPN, the pro-inflammatory cytokines IL-6 and IL-12, and the anti-inflammatory cytokine IL-10 were measured in the serum and, in case of IL-10 and IL-12, also in supernatants from colonic explants at the end of treatment. Results: Following DSS treatment, OPN -/- mice exhibited significantly decreased disease activity compared to wildtype mice, as evidenced by reduced rectal bleeding, weight loss, and histological intestinal injury $(\mathrm{P}<0.002)$. Furthermore, serum levels of SAA and IL-6 increased to a lesser extent $(\mathrm{P}<0.001)$, which was also the case for the release of IL-12 by colonic explants $(\mathrm{P}<0.01)$. The release of IL-10 by colonic explants, on the other hand, was increased $(\mathrm{P}<0.01)$. Serum levels of IL-10 and IL-12 were not affected by DSS treatment in both wildtype and OPN -/- mice. Macrophage infiltration into inflamed colonic tissue was also markedly attenuated in DSS-treated OPN -/- mice compared to wildtype mice. Conclusion: This study shows that OPN-deficiency significantly protected mice from colitis, by attenuating the Thl response and macrophage chemotaxis. OPN may represent a novel attractive target for pharmacological treatment of inflammatory bowel disease.

\section{T1722}

PECAM-1 Is a Key Mediator of Transendothelial Leukocyte Migration in Experimental Colitis

Emile J. Rijcken, Rudolf Mennigen, Sebastian Schaefer, Mike G. Laukoetter, Christoph Anthoni, Hans-Ullrich Spiegel, Norbert Senninger, Christian F. Krieglstein

Introduction: Recruitment of circulating leukocytes from the blood stream into the bowel wall plays a key role for development of the inflammatory infiltrate in inflammatory bowel disease. After initial adhesion to the vascular endothelium, transendothelial migration into the submucosa occurs. The cell adhesion molecule PECAM-1 (CD31) is expressed in the tight junction area of endothelial cells. The aim of this study was to determine the role of PECAM-1 in leukocyte transmigration and to show whether therapeutic blockade of PECAM1 has anti-inflammatory effects in experimental colitis. Methods: Chronic colitis was induced in female balb/c mice (20-22g) by cyclic oral administration of dextrane sodium sulfate (DSS) 3\% (wt/v). Three groups with each 10 animals were formed. The diseased control group A received the carrier PBS. Group B served as healthy controls. The treatment group $\mathrm{C}$ received $1 \mathrm{mg} / \mathrm{kg}$ anti-PECAM-1 (2H8) i.p. daily starting on day 26. On day 30 leukocyte adhesion and transendothelial cell migration was measured in the distal colon by intravital microscopy in N2O/isoflurane anaesthetised mice. Disease Activity Index (DAI) was scored and degree of inflammation was studied by histology. Statistics: Kruskal-Wallis or Wilcoxon test where appropriate $(\mathrm{P}<0.05)$. Results: Group A showed a marked increase in rolling $(112.9 \pm 18.2$ vs. $51.3 \pm 7.8 / \mathrm{mm} 2 / 30 \mathrm{~s})$, adherent $(27.1 \pm 11.2$ vs. $4.2 \pm 1.1 / \mathrm{mm} 2 / 30 \mathrm{~s})$ and transmigrated leukocytes $(404 \pm 40.8$ vs. $38.8 \pm 8.4 / \mathrm{mm} 2 / 30 \mathrm{~s})$ as compared to group B. The application of the $2 \mathrm{H} 8$ antibody reduced leukocyte transmigration $(65.8 \pm 6.8 / \mathrm{mm} 2 /$ $30 \mathrm{~s})$, rolling $(112.9 \pm 18.2 / \mathrm{mm} 2 / 30 \mathrm{~s})$ and sticking $(5.7 \pm 0.6 / \mathrm{mm} 2 / 30 \mathrm{~s})$ significantly. Treatment with anti-PECAM-1 diminished DAI $(2.8 \pm 0.3$ vs. $5 \pm 0.4$ pts. $)$ and histological inflammation score $(15.4 \pm 1.5$ vs. $23.9 \pm 1.2$ pts $)$ significantly compared to group A. Conclusions: Our results demonstrate that PECAM-1 plays a significant role for leukocyte extravasation through the endothelial barrier of colitic bowel. Blockade of PECAM-1 by specific antibodies inhibited leukocyte transmigration and resulted in attenuation of inflammation. Since $2 \mathrm{H} 8$ specifically inhibits leukocyte extravasation, the reduction of rolling and sticking leukocytes may be a secondary effect to a reduced activation of inflammatory cytokines. PECAM-1 should be considered as a novel target for anti-body based treatment in IBD.

\section{$\mathrm{T} 1723$}

\section{MUC2-IL-10 Knockout: Double Trouble}

Maria Van der Sluis, Janneke Bouma, Anna Velcich, Hans Buller, Alexandra Einerhand, Johannes van Goudoever, Isabelle van Seuningen, Ingrid Renes

Background \& Aims: Expression of mucin MUC2, the structural component of the colonic mucus layer, is lowered in inflammatory bowel disease. Furthermore, IL10 knockout (IL10KO) mice, which are known to develop colitis, have reduced Muc2 levels long before inflammatory infiltrates are present. Our aim was to obtain insight in the role of Muc2 and IL10 in epithelial protection. Methods: Thereto, Muc2-IL10 double knockout (DKO) mice were characterized and compared to Muc2 knockout (Muc2KO), IL10KO and their corresponding wild type (WT) littermates. We monitored clinical symptoms, intestinal morphology and differences in epithelial-specific protein and mRNA levels. Additionally, mRNA levels of the pro-inflammatory cytokine tumor necrosis factor- $\alpha$ (TNF- $\alpha$ ) in the colonic tissue were determined. Results: IL10KO mice were indistinguishable form WT mice throughou this experiment and showed no signs of clinical or microscopical signs of colitis. Muc2 IL10 DKO mice and Muc2KO mice showed significant growth retardation and clinical signs of colitis as of 4 and 5 weeks of age, respectively. Furthermore, Muc2-IL10 DKO mice had a high mortality rate [ $50 \%$ survival, 5 weeks of age] compared to all other types of mice, of which none died during the experiment. Microscopic analysis of the colon of Muc2KO showed mucosal thickening, increased proliferation and superficial erosions which was even more pronounced in Muc2-IL10 DKO mice. Colonic goblet cells in both the Muc2 KO and Muc2-IL10 DKO mice were negative for Muc2 but Tff3 was still detectable. Furthermore TNF- $\alpha$ mRNA was significantly up-regulated in the distal colon of Muc2KO and Muc2 IL10 DKO mice compared to IL10KO and WT mice. Additionally, TNF- $\alpha$ mRNA levels were the highest in the Muc2-IL10 DKO mice. Conclusions: Muc2KO mice develop severe colitis indicating that Muc2 and most likely the mucus layer is essential for mucosal protection and prevention of colonic inflammation. Muc2-IL10 DKO mice develop colitis which is more severe in every aspect compared to Muc2 KO and ILl0KO mice, indicating that in case of Muc2 deficiency, the anti-inflammatory cytokine IL10 can control epithelial damage, though to a limited extend.

\section{T1724}

B6.129p2-IL10 ${ }^{\text {Tm1cgn }}$ (IL-10 $0^{-/-}$) Mice Infected with Helicobacter Trogontum Develop Peracute Ulcerative Typhlocolitis While Concurrent Infection with $H$. Rodentium May Accelerate Preneoplasia of the Cecal-Colic Junction Mark Whary, Stephen J. Danon, Yan Feng, Zhongming Ge, Nataliya Sundina, Arlin B. Rogers, James G. Fox

Helicobacter trogontum is a urease-positive helicobacter first isolated from subclinically infected rats and is related to $H$. hepaticus and the ' $H$. rappini' taxon by $16 \mathrm{~s}$ rRNA analysis. $H$ rodentium, a urease-negative murine helicobacter, has only been associated with disease in SCID mice coinfected with $H$. bilis. In 3 experiments, $H$. trogontum infection was evaluated in B6.129P2-Ill $10^{\mathrm{tm} 1 \mathrm{Cgn}}$ mice (IL-10--) naturally infected with $H$. rodentium and in helicobacter-free IL10 $0^{-1}$ and C57BL/6 (B6) mice. IL-10 $0^{-/-}$mice naturally infected with H. rodentium and then dosed by gavage with $H$. trogontum at 6-8 weeks of age developed morbidity and severe typhlocolitis within 1 to 6 weeks post infection (PI). Dual infected IL-10-/- male mice developed severe disease with 10 of 14 mice developing accelerated dysplasia with features of gastrointestinal intraepithelial neoplasia (GIN) of the cecal-colic junction. Dual infected female IL-10 $\%$ mice remained either clinically normal (12 of 16) or developed morbidity at later time points $(\mathrm{n}=4)$ without as severe typhlocolitis or dysplasia. IL-10-1- mice infected with $H$. rodentium alone $(n=13)$ had subclinical disease with mild to moderate typhlocolitis. In a 2 nd experiment, male and female helicobacter-free IL $10^{-/}$and B6 mice ( $\mathrm{n}=5$ per group) were dosed with $\mathrm{H}$. trogontum and both genders of IL $10^{-/-}$mice developed severe typhlocolitis within 4 weeks but lesions did not develop to dysplasia consistent with GIN. B6 mice colonized with $H$. trogontum were resistant to disease. IL10 $0^{-/}$male and female mice $(n=10$ per group) infected with $H$. trogontum in a subsequent experiment became severely debilitated by 8 days PI. Clinical dehydration, weight loss and diarrhea were accompanied by severe ulcerative typhlocolitis characterized by crypt necrosis, crypt abscesses, ulceration of epithelium, submucosal edema and transmural inflammation progressing to serositis. A degenerative left shift in the hemogram of infected mice suggests sepsis. These results demonstrate that H. trogontum causes peracute ulcerative typhlocolitis in IL-10-/ mice while concurrent infection with $H$. rodentium promotes accelerated dysplasia of the cecal-colic junction, particularly in male IL-10-1 mice. H. rodentium infection may delay the course of disease attributable to H. trogontum sufficient to permit proliferation and dysregulation of intestinal epithelial cells resulting in development of GIN. The accelerated development of dysplasia at the cecalcolic junction in IL-10-1- mice coinfected with $H$. rodentium and $H$. trogontum provides a new rapid onset model for evaluation of acute severe inflammation and lower bowel tumorigenesis caused by bacterial pathogens.

\section{T1725}

VEGF-a Stimulation of Leukocyte Adhesion to Colonic Microvascular Endothelium: Implications for Inflammatory Bowel Disease

Steven Goebel, William C. Davis, Marilyn Jennings, Teruna J. Siahaan, Steven Alexander, Christopher G. Kevil

Inflammatory Bowel Disease (IBD) is a chronic inflammatory disorder characterized by increased leukocyte recruitment and subsequent tissue damage. An increase in the density of the microvasculature of the colon during IBD has been suggested, leading to the concep that angiogenesis may play a pathological role in IBD. Increased tissue and serum levels of the angiogenic cytokine VEGF-A have been reported in cases of active IBD. Here, we examine the hypothesis that VEGF-A exerts a pro-inflammatory effect on colon microvascular endothelium that contributes to colonic inflammation. Leukocyte adhesion to VEGF-A stimulated colon microvascular endothelial cells was examined using a parallel plate hydrodynamic flow chamber. ICAM-1 adhesion molecule expression on colonic microvascular endothelium was also determined in response to VEGF-A stimulation, along with characterization of leukocyte adhesion molecule expression. High dose VEGF-A ( $50 \mathrm{ng} / \mathrm{ml})$ stimulation increased neutrophil and T cell adhesion to and decreased rolling velocities on activated endothelium, whereas low dose VEGF-A (10 ng/ml) was without effect. Colonic endothelium constitutively expressed ICAM-1, which was significantly increased by treatment with $50 \mathrm{ng} / \mathrm{ml}$ VEGF-A or $10 \mathrm{ng} / \mathrm{ml}$ of TNF-alpha but not $10 \mathrm{ng} / \mathrm{ml}$ VEGF-A. T cells expressed CD18 and CD1la, with no expression of CD11b; whereas, neutrophils expressed CD18, CD1la, and CD1lb Lastly, VEGF-A dependent leukocyte adhesion was found to occur in a CD18 dependent manner. These results demonstrate that VEGF-A levels found in IBD exerts a pro-inflammatory effect similar to other inflammatory agents and suggests that this cytokine may serve as an intermediary between angiogenic stimulation and cell mediated immune responses. 
T1726

Role of Adiponectin in DSS and TNBS Induced Colitis in Mice Raja Fayad, Maria Pini, Joseph A. Sennello, Lawrence Chan, Giamila Fantuzzi

Role of adiponectin in DSS and TNBS induced colitis in mice Raja Fayad, Maria Pini, Joseph A. Sennello, Lawrence Chan, Giamila Fantuzzi Department of Human Nutrition, University of Illinois at Chicago, Chicago, IL; Department of Medicine, Baylor College of Medicine, Houston, TX. Adiponectin is a protein produced by adipocytes and is best known for its role in regulating insulin sensitivity. Adiponectin also displays prominent anti-inflammatory activities, particularly in the context of atherosclerosis. In Crohn's disease, adiponectin levels are elevated in fat wrapping that surrounds inflamed areas of the intestine. Our hypothesis is that adiponectin regulates intestinal inflammation and epithelial cell proliferation in models of inflammatory bowel disease (IBD). Methods: Adiponectin KO mice and their WT littermates in a C57BL6 background received 2\% DSS in drinking water for 5 days and regular drinking water for additional 5 days. A group of $\mathrm{KO}$ mice also received daily ip injection of 30 microgram of murine recombinant adiponectin. A clinical score including body weight, fecal haemoccult and diarrhea was assessed daily for each mouse. To evaluate proliferation of colonic epithelial cells, $1 \mathrm{mg}$ of BrdU was injected ip $2 \mathrm{~h}$ prior to sacrifice and incorporation analysed by immunohistochemistry. Colon cultures were performed to evaluate local cytokine production by ELISA. For trinitrobenzene sulfonic acid (TNBS) experiments, mice received $2 \mathrm{mg}$ of TNBS in $50 \%$ ethanol intrarectally on days 1 and 7. Parameters evaluated were the same as reported for the DSS model. Results: Compared to WT mice, adiponectin KO mice were protected from DSS-induced colitis, as evaluated by clinical and histologic findings. Increased proliferation of colonic epithelial cells was observed in KO compared to WT mice receiving DSS. Moreover, colonic MIP-2 and IL 6 levels were markedly reduced in KO compared to WT mice. Administration of adiponectin to $\mathrm{KO}$ mice restored their response to levels similar to those observed in WT mice, in terms of clinical score, histological findings, epithelial proliferation and cytokine production. Similar results were obtained using the TNBS model. Conclusions: Adiponectin KO mice were protected from DSS- and TNBSinduced colitis, suggesting that adiponectin might play a pro-inflammatory and anti-proliferative role in IBD. To our best knowledge this is the first report of a pro-inflammatory role for adiponectin. Supported by NIH grants DK61483 (to GF) and DK68037 and HL51586 (to LC).

\section{T1727}

\section{Fty720 Suppresses $\mathrm{CD} 4^{+} \mathrm{CD} 44^{\mathrm{High}} \mathrm{CD} 62 \mathrm{I}^{-}$Effector Memory T Cell-Mediated}

\section{Colitis}

Takanori Kanai, Rei Fujii, Yasuhiro Nemoto, Shin Makita, Teruji Totsuka, Mamoru Watanabe

Background: FTY720 (2-amino-2-(2-[4-octylphenyl]ethyl)-1,3-propanediol hydrochloride), a sphingosine-derived immunomodulator, causes immunosuppression via enhancement of lymphocyte sequestration into secondary lymphoid organs, thereby prevents their Ag-activated $\mathrm{T}$ cell egress to sites of inflammation. It has been suggested that the egress-blocking activity of FTY720 can be explained by its S1Pl down-modulating activity on lymphocytes. Although FTY720 is highly effective in inhibiting autoimmunity in various animal models in term of the prevention protocol, it is little known how FTY720 controls the migration property of memory T cells. Methods: We evaluate the effect of FTY720 on two types of colitis models, 1) our recently established memory T cell-mediated chronic colitis model induced by the adoptive transfer of colitogenic lamina propria (LP) CD4 ${ }^{+} \mathrm{CD} 44^{\text {high }} \mathrm{CD} 62 \mathrm{~L}^{-}$ effector-memory $\mathrm{T}\left(\mathrm{T}_{\mathrm{EM}}\right)$ cells into SCID mice, and 2) $\mathrm{CD} 4^{+} \mathrm{CD} 45 \mathrm{RB}^{\text {high }} \mathrm{T}$-cell-trans. We next evaluated expression pattern of S1P receptors in sorted normal splenic $\mathrm{CD}^{+}{ }^{+} \mathrm{CD}^{+}$ $\mathrm{CD}_{45 \mathrm{RB}^{\text {high }}}$, and colitic LP CD4+ $\mathrm{T}_{\mathrm{EM}}$ cells, using quantitative PCR analysis. Results: We demonstrate that FTY720 prevents the development of SCID colitis induced by the adoptive transfer of LP colitogenic CD4 ${ }^{+} \mathrm{T}_{\mathrm{EM}}$ cells and suppresses IFN- $\gamma$, IL-2, and TNF- $\alpha$ production by LP CD4 ${ }^{+} \mathrm{T}$ cells. The number of spleen, peripheral blood, mesenteric lymph nodes and LP CD4 $4^{+} \mathrm{T}$ cells in FTY720-treated mice was significantly reduced as compared with the control mice. Notably, LP CD4 $4^{+} \mathrm{T}_{\mathrm{EM}}$ cells as well as splenic $\mathrm{CD} 4^{+} \mathrm{CD} 45 \mathrm{RB}^{\text {high }} \mathrm{T}$ cells expressed several S1P receptors that are targets for FTY720. Furthermore, FTY720 also prevents the development of colitis induced by the adoptive transfer of splenic $\mathrm{CD}^{+} \mathrm{CD} 45 \mathrm{RB}^{\text {high }} \mathrm{T}$ cells into SCID mice. Conclusions: Collectively, the present data indicate that FTY720 treatment may control the colitogenic memory T-cell migration and suppress the memory T cell-mediated autoimmune diseases including inflammatory bowel diseases (IBDs). Although we have to pass many critical checkpoints, this study indicates that FTY720 offers a hope for the treatment, as well as the prevention, for human IBDs.

\section{$\mathrm{T} 1728$}

Impaired Production of Paneth Cell Defensin Precedes the Colonic Inflammation in IL-10 Deficient Mice Model of IBD Yuhei Inaba, Atsuo Maemoto, Toshifumi Ashida, Hiroki Tanabe, Jiro Watari, Tokiyoshi Ayabe, Andre J. Ouellette, Yutaka Kohgo

Backgrounds/Aims: There has been accumulating evidence that the distorted immunological response to intestinal bacteria is central for the pathogenesis of both human IBD and several mouse model of IBD. In IL-10 KO mouse model of IBD, that develop the colonic inflammatory response to the flora, abnormal Th- 1 type cytokine productions of $\mathrm{T}$ cells were wellinvestigated. However, the innate immune system is not fully understood in this model. Diminished production of intestinal alfa defensin has been found in patients with human Crohn's disease by our previous studies. We herein investigated the production of Paneth cell defensin in IL-10 KO mice, and revealed that the production of alpha defensin was similarly impaired during the development of the colonic inflammation. Materials/Methods: Five to 12-wk-old female wild type (WT) and IL-10 (-/-) C57BL/6 were used in this study. They were moved from SPF condition to the usual environment at the age of 5-wk-old, and given the normal chaw. Before and at 2, 4, and 6 weeks after settling to the contaminating condition, inflammations of small intestines and colons were evaluated. mRNA and protein expression of cryptdine-1 (cptd-1, mouse Paneth cell alpha defensin) expression in isolated intestinal crypts purified using the EDTA-disruption method, were examined by quantitative RT-PCR and AU-PAGE. Results: Average colon lengths of IL-10 (-/-) measured were significantly shorter, and the average tissue weight of colon of those were heavier than that of WT at the fourth and sixth week. Histological examination revealed the mild increase of inflammatory cells in lamina propria and the hyperplasia of colonic epithelia in IL-10 (-/-) at sixth week, suggesting the spontaneous colonic inflammation might require for more than a month of colonization period of intestinal flora from the environment. During in SPF condition, cptd-1mRNA was equally expressed in isolated intestinal crypt of both IL-10 (-/) and WT animals. However, cptd-lmRNA expression in intestinal crypts of IL-10 (-/-) was significantly suppressed during the observation period, to the average of $37 \%$ (at 2 week), $41.6 \%$ (at 4 week), and $42 \%$ (at 6 week) of the mRNA expression in WT animals. Protein expression of cptd-1 in IL-10 (-/-) crypts tested by AU-PAGE was also decreased. Conclusions: These results suggest that the secretion of cptd-1, innate antimicrobial peptide from intestinal Paneth cells in IL-10 (-/-) mice, is suppressed during the development, especially before originating the histological changes of colonic inflammation. Th-1-skewed cytokine environment in this model might influence to the suppression of cptd-1 expression in Paneth cells

\section{T1729}

\section{Targeted Disruption of TGF-Beta/SMAD3 Signaling Confers Resistance to} Intestinal Fibrosis

Giovanni Latella, Giuliana Zanninelli, Antonella Vetuschi, Roberta Sferra, Angela D'Angelo, Valentina Catitti, Maria Chiaramonte, Eugenio Gaudio, Renzo Caprilli

BACKGROUND \& AIM. Transforming growth factor-beta(TGF-beta)/Smad3 signalling plays an important regulatory role in the tissue fibrogenesis process acting as a potent stimulus of extracellular matrix (ECM) proteins accumulation. Since loss of Smad3 confers resistance to skin, kidney, lung and liver fibrosis, it could also be involved in intestinal fibrosis. The potential role of Smad3 in the pathogenesis of intestinal fibrogenesis was investigated in Smad3 knockout mice using a model of colonic fibrosis induced by intrarectal administration of trinitrobenzene sulfonic acid (TNBS). METHODS. Colonies of Smad3 wild-type, heterozygous and null mice were developed in our laboratory using pairs of Smad3 heterozygous mice kindly provided by A. Roberts (NCI, Bethesda, MD,USA). Genotype analysis was determined by the PCR method. Chronic colonic inflammation-associated fibrosis was induced by weekly intrarectal administration of TNBS for 6 weeks in 15 healthy adult Smad3 null and 13 wild-type mice. The colon was removed for macroscopic examination and histology (Haematoxilin \& eosin, Masson trichrome and silver staining), morphometry and immunohistochemistry (IHC) analysis. For IHC we used alpha-smooth muscle actin(alpha-SMA),vimentin desmin, TGF-betal, connective tissue growth factor(CTGF), Smad3, Smad7, collagen types I-III, and CD3 antibodies. RESULTS.At macroscopic examination the colon of Smad3 wild-type mice appeared significantly more indurated, thickened and shortened compared to the colon of the Smad3 null mice. 50\% of the wild-type mice showed strictures and adhesions of the distal colon associated with dilatation of the proximal colon. Histological and morphometric evaluation showed a significantly lower degree of colonic fibrosis and accumulation of collagen in Smad3 null mice compared to wild-type, whereas the degree of colonic inflammation did not differ between the two groups of mice. Immunohistochemistry evaluation showed a significant decrease in alpha-SMA, CTGF, and collagen I-III staining in the colon of Smad3 null mice compared to wild-type. TGF-betal and Smad7 staining was significantly increased in Smad3 null mice as compared to wild-type. CONCLUSIONS. The results indicate that Smad3 loss confers resistence to the development of TNBS-induced colonic fibrosis. Reduced fibrosis appears to be due to deregulated ECM-producing cell function, as the inflammatory response to TNBS was not significantly different between Smad3 null and wild-type mice. Smad3 could be a novel target for potential therapeutic treatment of intestinal fibrosis, especially in inflammatory bowel disease

\section{$\mathrm{T} 1730$}

A Critical Role for $\beta 7$-Integrins But Not CCR9 or CCL25 in the Pathogenesis of Experimental Ileitis

Maria Apostolaki, Konstantinos A. Papadakis, Menelaos Manoloukos, Marc-Andre Wurbel, Armine Avanesyan, Masayuki Saruta, Dimitris L. Kontoyiannis, Bernard Malissen, George Kollias

Background and Aims: The chemokine CCL25 and its receptor CCR9 have been reported to play an important role in small bowel immunity. The upregulated expression of CCL25 and the increased frequency of activated peripheral blood CCR9+ T cells in SB Crohn's disease (CD) implicate CCL25/CCR9 in the pathogenesis of the disease. The aim of our study was to determine the role of CCL25 and CCR9, as well as of the integrin $\beta 7$, in the pathogenesis of ileitis in the TNF $\triangle$ ARE model of Crohn's-like disease. Methods: CCL25 mRNA and protein expression in the small bowel (SB) of TNFAARE and wild-type mice were assessed by real-time PCR and ELISA, respectively. The effect of CCR9 and $\beta 7$ integrin in the course of ileitis was evaluated by backcrossing the TNFAARE mice with CCR9-/- and $\beta 7$ integrin-/- mice, whereas a neutralizing anti-CCL25 Ab was used to assess the effect of CCL25 in the course of experimental ileitis. Results: CCL25 was upregulated early (1 month) but not late (4 months) during the development of the disease in the TNF $\triangle$ ARE mice compared to wild-type mice. Administration of anti-CCL25 Ab at $5 \mathrm{mg} / \mathrm{kg}$ twice per week for 10 weeks, starting at 3 weeks of age, to TNF $\triangle$ ARE mice led to no significant improvement of the ileitis compared to isotype control-treated mice. Backcrossing of TNFAARE mice with CCR9-/- mice led to similar ileitis development compared to CCR9-sufficient mice. In marked contrast, backcrossing TNF $\triangle A R E$ mice with $\beta 7$ integrin-/- mice led to significant amelioration of the disease. Conclusion: $\beta 7$ integrin is critically involved in the pathogenesis of experimental ileitis, whereas CCL25 and CCR9 appear to play a dispensable role. CCL25 may be involved in the early, but not late stages of the disease. 


\section{T1733}

Modulation of Rat Colonic TNF- $\alpha$, iNOS and Acute Colitis Through DownRegulation of NF-kB By Glycogen Synthase Kinase-3 $\beta$ Inhibition

Brendan J. Whittle, Csaba Varga, Aniko Berko, Aniko Posa, Andor Molnar, Marika Collin, Christoph Thiemermann

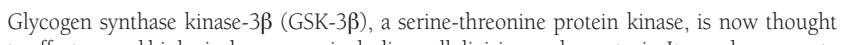
to affect several biological processes including cell division and apoptosis. It can also promote the the activity of the nuclear factor, NF-kB, a key transcription factor for the production of pro-inflammatory mediators found in colitis such as tumour necrosis factor a (TNF- $\alpha$ ) and the expression of inducible nitric oxide synthase (iNOS). Since GSK-3 $\beta$ may thus be involved in modulating the inflammatory process, the effects of two known selective inhibitors of GSK-3B, TDZD-8 and SB 415286 have now been investigated in a 3 day colitis model in the rat. Colitis was induced by the intracolonic instillation of trinitrobenzene sulfonic acid (TNBS) and the extent of colonic inflammation assessed after 3 days in terms of macroscopic injury and the colonic levels of the inflammatory biomarkers, myleoperoxidase (MPO), TNF- $\alpha$ and iNOS. Administration of TDZD-8 or SB $415286(0.1,0.33$ or $1.0 \mathrm{mg}$ $\mathrm{kg}-1$, s.c, b.i.d., for 3 days) caused a dose-dependent reduction in the area of macroscopic colonic injury (from $60 \pm 2 \%$ to $44 \pm 6 \%$ and $45 \pm 4 \%$ of the total area measured respectively, at the highest doses, $\mathrm{n}=8 ; \mathrm{P}<0.05)$ and also significantly reduced the macroscopic clinical score. The increase in colonic MPO as an index of neutrophil infiltration was also dosedependently inhibited by both TDZD-8 and SB 415286 (by 48\% and 59\% respectively at the highest doses, $\mathrm{P}<0.01$ ). Moreover, the increase in the levels of TNF- $\alpha$ in the inflamed colon was also significantly inhibited by either compound (by $46 \%$ and $51 \%, \mathrm{P}<0.01$ ) as was iNOS protein, determined by Western Blot densitometry (by $43 \%$ and $33 \%$ respectively). The elevated levels of the NF-kB subunit p65, also determined by Western blot densitometry, in the nuclear extracts from the TNBS-provoked inflamed colonic tissue, were reduced by TDZD-8 and SB 415286 treatment (by 56\% and $66 \%$ respectively at the highest dose, $\mathrm{P}<0.001$ ). These ovservations demonstrate that two chemically distinct selective GSK-3 $\beta$ inhibitors reduce the inflammatory biomarkers, iNOS, TNF- $\alpha$ and MPO and the tissue injury in a rat model of acute colitis. The mechanisms underlying their anti-inflammatory action is likely to be related to the observed down-regulation of NF-kB activity, involved in the generation of TNF- $\alpha$ and other pro-inflammatory mediators. Thus, the findings suggest a role for the protein kinase, GSK-3 $\beta$, in the promotion of the inflammatory response in colitis.

\section{T1734}

Mutant Major Histocompatibility Complex II Lacking Interactions with CD4 Selects for Immunoregulatory T Cells That Prevent Colitis Makoto Naganuma, Sanford H. Feldman, Peter B. Ernst

Background: Regulatory T cells suppress the ability of pathogenic T cells to induce autoimmune disease. Class II knock out (KO) mice lack Treg and develop colitis, although the percentage of peripheral $C D 8+C D 25+T$ cells from knock out mice is higher and these cells exhibit regulatory activity. The purpose of this study was to examine how class II MHC selects for regulartoy Th cells. Methods: Transgenic mice with a gene encoding mutant class II MHC molecules that no longer bind CD4 were generated and back-crossed onto the class II KO background (mutant mice). Mutant, class II KO and control mice were infected with Helicobacter hepaticus and infection was confirmed by PCR. All strains were monitored for colitis. The markers for Treg (CD25, CD45RBlo, CD62L, CD103, GITR and Foxp3) from thymocytes and peripheral lymphoid tissues were assessed by flow cytometry and Foxp3 RNA levels were measured by PCR. Cytokine production by Th subsets and their ability to inhibit Th cell proliferation in vitro was assessed. CD45RBhi T cells were transferred with Treg from control and mutant mice into SCID mice to assess Treg function. Results: When mice were infected with $\mathrm{H}$. Hepaticus, 9 (5 severe, 4 mild) of 11 class II KO developed colitis with mucosal prolapses, although no disease was observed in mutant and BL/6 mice. Histological analysis showed that goblet cells depletion, crypt abscess and transmural inflammation was found in the colonic mucosa from class II KO mice. Total CD4+ Th cells were decreased in thymocytes and the peripheral tissues of mutant mice but the relative percentage of CD4+CD25+, CD45RBlo, GITR+, CD103+ T cells were increased from these mice. However, neither CD4CD25+ nor CD8CD25+ T cells from class II KO expressed Foxp3, while mutant CD4+CD25+, CD45RBlo, GITR+ cells constitutively express Foxp3, suggesting that these Th subpopulation from mutant mice are Treg. IL-10 secretion and RNA levels of both CD25+ and CD25- T cells from mutant mice were significantly higher than those from control mice. In vitro, CD25+ cells from both control and mutant mice suppressed proliferation of effector cells as a ratio of $1: 2$ and partially suppressed at a ratio as low as 1:8. This inhibition was IL-10 independent but cell contact dependent. Co-transfer of Treg cells from mutant mice with CD45RBhi T cells of BL/6 mice blocked wasting disease and colitis in the SCID recipients. Conclusion: The expression of class II molecule is necessary for development of Treg to prevent colitis. However, recognition of the class II MHC-peptide complex can select for T reg that express FoxP3 in the thymus and peripheral tissues when signaling through CD4 is impaired.

\section{T1735}

A Comparative Genomic Perspective On Murine Inflammatory Bowel Disease Models

Trinidad Montero, Jose Maria Vieites, Xavier Llor, Olga Martinez, Fermin Sanchez de Medina, Antonio Suarez

Disease heterogeneity in IBD, reflecting differences in underlying environmental and genetic factors leading to the inflammatory mucosal phenotype, may be reflected in different intestinal gene expression profiles. The ability to analyze gene expression data may provide a basis for the knowledge of colitis development and for an improved classification and diagnosis of IBD. Over the last years, the use of animal models has had a significant impact on the understanding of the complex interplay of genes that ultimately result in mucosal inflammation. Although there is no a priori reason to assume the existence of common susceptibility genes between species, it is highly likely that key pathways may be conserved. We have performed a DNA microarray analysis of the gene expression profile in three colitis models (Dextran sulfate-induced colitis (DSS); Trinitrobenzene sulfonic acid-induced colitis (TNBS) IL10-/- knockout mice) to provide previously unachievable information about the pathogenesis of colitis and to perform a comparative genomic study. Experiments were performed as follows: DSS $5 \%$ was given orally to rats on days $1,3,5$ and 7 , continued with DSS $2 \%$ on days $9,11,13$ and 15 , and, finally, no DSS on days $17,19,21,23$. TNBS was given to rats and samples were collected in days 2, 5, 7 and 14. Samples from wild type C57BL/6 mice, germ-free and conventionally-grown IL10-/- mice were collected at 7,9 and 12 weeks. Colitis degree was assessed through biochemical, morphological and histological data. Total RNA from 3 representative animals at each time point was independently reverse-transcribed and hybridized on Affymetrix Rat Genome 2302.0 arrays for DSS and TNBS experiments and on Mouse Genome 4302.0 array for IL10-/- knockout mice. Gen chip data was analyzed and clustered with GeneSpring software. A set of comparisons for each individual experiment was performed: healthy controls versus injured animals; early versus late colitis; colitis versus recovery in DSS and TNBS experiments only. Probe set lists from each comparison were analyzed with GoMiner, Gene Ontology, Panther and Ingenuity software packages. Genes were associated with disease activity, and, categorized and grouped upon temporal expression pattern (k-means analysis), molecular function, pharmacological information or cellular marker. Results from these experiments are currently being cross-compared and show interesting concordances in pathophysiological processes. Chromosomal localization of differentially expressed genes partially matches with human chromosomal loci associated to IBD.

\section{T1736}

Cd30 Expression By Cd4 Cells At Inflammatory Sites in the Intestine Shinichi Somada, Hiromi Muta, Eckhard R. Podack, Kazuhiko Nakamura, Kuniomi Honda, Hajime Nawata, Kenzaburo Tani

Background: $\mathrm{CD} 30$ and its ligand $(\mathrm{CD} 30 \mathrm{~L})$ are members of the tumor necrosis factor receptor and tumor necrosis factor superfamily respectively. Although CD30 has long been recognized as an important marker on many lymphomas of diverse origin and as activation molecule on $\mathrm{B}$ and $\mathrm{T}$ cells, its primary function has remained obscure. We have shown previously that CD30-Ligand deficienct (CD30LKO) mice have attenuated intestinal disease after ant CD3 injection. Here we analyzed CD30 expressing cells in enteritis induced small intestine in mice by immunohistochemistory and also determined the level of soluble CD30 (sCD30) in the enteric mice and of patients with inflammatory bowel disease (IBD). Methods: $100 \mu \mathrm{g}$ of anti-CD3 antibody was injected intraperitoneally into BALB/c mice to induce inflammation of the small intestine. The $\mathrm{CD} 30$ positive cells were detected by immunohistochemistory in the paraffin embedded intestinal specimens obtained from mice 24 hours after the injection. $\mathrm{CD} 30$ positive cells were then analyzed by immunofluorescence staining to determine the cell type expressing CD30. The levels of SCD30 were measured by ELISA on the sample obtained from mie, IBD patients of ulcerative colitis (UC) and Crohn's disease (CD), and normal control Results: Anti-CD3 antibody injection induced small intestinal enteropathy CD30 positive cells in the small intestine obtained from anti-CD3 injected mice were detected by immunohistochemistory mainly in mucosal lesions. (Normal small intestine was negative for CD30 staining.) Immunofluorescence staining revealed CD30 expression by CD4+T cells The levels of SCD30 were increased in sera of the enteritis induced mice and patients with $\mathrm{UC}$ and $\mathrm{CD}$. The increased level of $\mathrm{sCD} 30$ in UC patients was correlated to the disease activity. Conclusion: We detected CD30 positive cells in the intestines of mice damaged by anti-CD3 antibody injection. Together with our previous report of CD30-Ligand knockou mice, these results support the hypothesis that CD30/CD30L signals activate T cells at inflammatory sites in the intestine. Although increased levels of sCD30 in UC patients has been reported before, our study revealed that the levels of $\mathrm{SCD} 30$ were increased not only in UC patients but also in CD patients. Furthermore, in UC patients, the level of SCD30 was correlated with the disease activity. As the level of serum SCD30 are thought to reflect the total number of $\mathrm{CD} 30$ positive cells in the body, CD30/CD30L signals appear to be augmented in IBD especially at the site of inflammation and may accelerate the disease activity. Thus, regulation of the CD30/CD30 ligand pathway could be an effective method for the treatment of IBD.

\section{T1737}

Both Experimental TH1 and TH2 Colitis and IBD Strongly Depend On IRF-4 Expression: Critical Role of IRF-4 in IL-6 Production and IL-6 Mediated Apoptosis Resistance

Jonas Mudter, Lioubov Amoussina, Benno Weigmann, Hans A. Lehr, Stefan Rose-John, Peter R. Galle, Michael Lohoff, Markus F. Neurath

Il-6 is known to be crucial in acute and chronic colitis in mice modulating T cell apoptosis and transmural inflammation. The interferon regulating factor-4 (IRF-4) is a central player in $T$ cell regulation and differentiation. Its function in IBD is still unknown. The aim of our work was to characterize the functional role of IRF-4 in the immunopathogenesis of experimental colitis and IBD. In our studies, we analyzed cryo sections from human colon specimen via immunofluorescence. Sections from both Crohn's disease and ulcerative colitis patients exhibited increased IRF-4 expression in the lamina propria (LP) compared to control patients. The majority of these cells were also positive for CD3. To perform in vivo colitis experiments, two murine models were evaluated in IRF-4 wild-type (WT) and knockou mice (IRF-4 -/-). Colitis was induced by the application of either TNBS (Th1) or Oxazolone (Th2). When exposed to TNBS, wild-type mice lost significantly more weight as compared to IRF-4 $-/$ - mice $(-25 \%$ vs $-11 \%)(n=12$ each group, three independent experiments). The protective effect of IRF-4 -/- was also mirrored by endoscopical and histological scores (wildtype+TNBS: $3.25+/-0.5$; IRF-4 -/- +TNBS: $0.7+/-0.4)$. Interestingly, the rectal application of oxazolone led also to significant weight loss in wild-type mice compared to IRF-4 -/- mice $(15 \%$ vs. $0 \%)$. This effect was also reflected by endoscopy and histology. Compared to TNBS treated IRF4 -/- mice and untreated WT mice, TNBS and oxazolone treated WT mice exhibited increased IL-6 m-RNA levels, whereas differences in the expression of the proinflammatory cytokine IL-18 were less significant in the late state of colitis. IL-6 Immunofluorescence staining of colon specimen showed similar results. In vivo, the protective effect of IRF-4 -/was abrogated by i. p. application of the designer cytokine hyper-IL-6. Ex vivo experiments 
exhibited enhanced apoptosis in splenic CD4+ T cells from IRF-4 -/- mice compared to those from WT as measured by AnnexinV/PI staining and flow cytometry. The increased rate of apoptosis was diminished by co-incubation with hyper-IL-6. Furthermore, TNBS treated IRF-4 -/- mice exhibited an increased number of apoptotic LP cells (TUNEL assay) compared to WT. Taken together, our data demonstrate a novel regulatory role for IRF-4 in experimental colitis and IBD. Both Thl and Th2 mediated colitis forms are critically dependent on IRF-4 that acts most likely as an upstream regulator of IL-6 production. IRF4 , thus emerges as an attractive target for treatment of Thl and Th2 dependent chronic intestinal inflammation.

\section{T1738}

Feeding Specific Ag to Prevent CD45RBhi T Cell-Induced Colitis Is Associated with Development of Foxp3 Expressing T Cells

Pengfei Zhou, Rajka Borojevic, Ken Croitoru

Antigen specific oral tolerance involves Ag induced T cell deletion or development of $\mathrm{T}$ cell anergy. Regulatory T cells (Treg) are thought to be involved in this process. We have shown that feeding OVA to SCID mice reconstituted with DO11.10 CD45RBhi T cells prevented the development of colitis in part due to the emergence of regulatory $\mathrm{T}$ cells expressing CD25. This was also associated with the development of tolerance of bacteria reactive I cells. Although regulatory $\mathrm{T}$ cells are thought to be involved in oral tolerance, specific induction of $\mathrm{T}$ cells expressing FoxP3 by oral Ag during the process of oral tolerance has not been previously demonstrated. METHODS: SCID mice reconstituted with DO11.10 CD45RBhi naïve T cells were fed OVA ( $5 \mathrm{mg} /$ day) or BSA ( $5 \mathrm{mg} /$ day). Mice were sacrificed after 10 weeks and intestinal tissues removed for histologic evaluation. Cells from spleen and MLN were stained for surface CD4, CD25, KJ1-26 and for intracellular FoxP3 expression and analyzed by flow cytometry. RESULTS: Less than 1\% of DO11.01 CD45RBhi donor cells transferred into the SCID mice expressed CD25+ or Foxp3+. OVA fed reconstituted mice showed a significant increase in CD25 and FoxP3 expressing spleen and MLN cells as compared to BSA fed controls. The percent of CD25+Foxp3+ cells increased from 0.83 to $5.21 \%$ in spleen and from 6.62 to $11.2 \%$ in MLN in OVA fed and BSA fed mice, respectively. CONCLUSION: These findings indicate that OVA feeding led to the differentiation of CD45RBhi naive T cells into cells that expressed CD25 and FoxP3, makers of Treg cells. Therefore, oral tolerance may well develop as a result of Ag specific induction of CD25+Foxp3+ Treg cells. Defining this process may lead to new startegies of preventing or treating intestinal inflammation. Funded by the CIHR and CCFC.

\section{T1739}

The Role of Mitogen-Activated Protein Kinase Phosphatase-1 in a Mouse Model of Inflammatory Bowel Disease

Tom K. Lin, John A. Barnard, Yusen Liu

Background/Aims: Mitogen-activated protein (MAP) kinases are important mediators of the innate immune response, integrally involved in the production of proinflammatory cytokines. MAP kinase phosphatase (Mkp)-l functions in a counterregulatory role in this inflammatory pathway. Research in our laboratory has demonstrated that Mkp-1 knock-out (null) mice have an augmented inflammatory response due to defective $\mathrm{p} 38$ and JNK inactivation in an endotoxic shock model. In the current study, we sought to determine the susceptibility of Mkp-1 null mice to inflammatory bowel disease (IBD) in the dextran sulfate sodium (DSS) model of IBD. Methods: Wild-type (WT) and Mkp-1 null mice were provided 2.5\% DSS (weight/volume) in drinking water for 10 days. Colitis development was monitored daily in a blinded fashion using a disease activity index scoring scale based on percentage weight loss, stool consistency and stool blood. Blinded scoring of histologic colon damage was performed using a modified, validated scoring scheme. Cytokine levels from colon homogenates were determined by ELISA analysis. Results: Both WT and Mkp-1 null mice developed weight loss and bloody diarrhea after DSS feeding. Surprisingly, WT mice developed more severe colitis than Mkp-1 null mice. The disease activity index was higher in WT mice versus Mkp- 1 null mice ( $\mathrm{P}=0.026$, by principal component analysis of the three variables); [percentage weight loss: WT $8.6 \% \pm 7 \%$ vs. KO $1.7 \% \pm 5 \%(\mathrm{P}=0.020)$; stool consistency: WT $3.3 \pm 0.6$ vs $\mathrm{KO} 2.6 \pm 0.7(\mathrm{P}=0.023)$; stool blood: WT $1.4 \pm 1.6$ vs $\mathrm{KO} 0.6 \pm 1.3(\mathrm{P}=0.227)]$. Histologically, colonic lesions in WT mice were also more severe [WT $6.2 \pm 2.8$ vs. KO $3.0 \pm 1.8(\mathrm{P}=0.01)]$. Tumor necrosis factor $\alpha(\mathrm{TNF}-\alpha)$ and interleukin (IL)-6 levels from colonic homogenates were elevated in WT mice relative to Mkp-1 null mice [TNF- $\alpha$ : WT $338 \pm 263 \mathrm{pg} / \mathrm{ml}$ vs. $\mathrm{KO} 106 \pm 100 \mathrm{pg} / \mathrm{ml}(\mathrm{P}=0.018) ; \mathrm{IL}-6$ : WT $335 \pm 219 \mathrm{pg} / \mathrm{ml}$ vs. KO $142 \pm 148$ $\mathrm{pg} / \mathrm{ml}(\mathrm{P}=0.033)]$, while levels of IL-10 and transforming growth factor (TGF) $-\beta$ were unchanged [IL-10: WT $40.9 \pm 9.4 \mathrm{pg} / \mathrm{ml}$ vs. $\mathrm{KO} 42.4 \pm 22.7 \mathrm{pg} / \mathrm{ml}(\mathrm{P}=0.854) ; \mathrm{TGF}-\beta$ : WT $3.10 \pm 1.23 \mathrm{pg} / \mathrm{ml}$ vs. KO $2.47 \pm 1.13 \mathrm{pg} / \mathrm{ml}(\mathrm{P}=0.289)]$. Conclusions: DSS colitis in Mkp1 null mice was significantly attenuated in clinical and histological severity and in the proinflammatory cytokine response. This effect was independent of IL-10 and TGF- $\beta$ secretion. These findings suggest an integral role for Mkp-l activity in the modulation of the inflammation found in IBD

\section{$\mathrm{T} 1740$}

Potential Role of Invariant Natural Killer T Cells On the Prevention of Acute Intestinal Inflammation Induced By Dextran Sulfate Sodium-Induced Colitis in Mice

Yoshitaka Ueno, Shinji Tanaka, Kyoko Yoshioka, Tsuyoshi Hatakeyama, Masaru Shimamoto, Yoshihiro Miyanaka, Masamoto Kanno, Kazuaki Chayama

BACKGROUND \&AIM: Invariant natural killer T (iNKT) cells recognize endogenous selfand exogenous microbial-lipid antigens presented by CDld on antigen presenting cells, promptly secrete cytokines, and contribute an innate-type immune response to provide the balance between tolerance and autoimmunity. However, the exact cellular mechanism of iNKT cells on the anti-inflammatory effect remains unclear. The aim of the present study was to investigate the role of iNKT cells in the early phase of intestinal inflammation by analyzing a murine colitis model induced by dextran sulfate sodium (DSS). METHODS: Colitis was induced in C57BL/6 (B6) and Jal8 NKT cell-deficient (KO) mice by the administration of various concentrations of DSS (1.5, 2.5, and 3.5\%) in drinking water. The survival rate, body weight loss, colon length, and histological score were investigated. Mononuclear cells were isolated from the liver and labeled with $\alpha$-galactosylceramide-loaded dimeric CDld:Ig fusion protein for the detection of iNKT cells. Activated CD4+T cells in the mesenteric lymph nodes were detected by staining with FITC-conjugated anti-CD69 or PEconjugated anti-CD25 on day $0,1,3$ and 7 after DSS administration. The populations of these cells were all analyzed by flow cytometry. RESULTS: KO mice exhibited increased susceptibility to DSS-induced colitis, as reflected by significantly higher lethality and higher clinical and histological scores, and more severe colonic shortening compared to B6 mice. Flow cytometric analysis revealed evidence of a significant increase in the percentage of mesenteric CD69+CD4+ and CD25+CD4+T cells in DSS-fed KO mice compared to DSSfed B6 mice on day 1 and 3. Interestingly, intake of DSS increased the percentage of hepatic iNKT cells from B6 mice. Moreover, the population of CD69+ iNKT cells but not CD69+ conventional T cells significantly increased in the B6 liver as early as day 1 after DSS administration. CONCLUSION: The present study demonstrated that liver iNKT cells are activated in the induction phase of intestinal inflammation and may exert an anti-inflammatory effect by reducing the population of the activated CD4+ T cells in the intestinal draining lymph nodes.

\section{T1741}

Influence of Carnitine On Butyrate Metabolism of Colonocytes in a Rat Model of Experimental Colitis

Giuseppe D'argenio, Menotti Calvani, Orsolina Petillo, Sabrina Margarucci, Monica Rienzo, Angela Torpedine, Giovanna Mazzone, Italo Sorrentini, Valeria Dargenio, Nicola Caporaso, Gianfranco Peluso

Background \&Aim.: It has been postulated that colitis may be the result of inability to oxidize butyrate, the main source of energy for colonocytes. Since carnitine plays a pivotal role in fatty acid metabolism, we evaluated carnitine transporter expression and carnitine uptake as well as butyrate metabolism in colonocytes isolated from TNBS-treated and control rats. Butyrate metabolism was analysed in cells before and after supplementation of liposomeencapsuled carnitine. Methods. Total, free, and acyl-carnitine fractions were determined by HPLC and tandem-mass-spectrometry. Butyrate metabolism was evaluated in isolated colonocytes incubated by 1-14C-labelled butyrate. Expression of carnitine transporters was analyzed by RT-PCR, real time RT-PCR and in-situ hybridization. Functional properties of carnitine transporters were investigated on the basis of substrate specificity. The inhibitory effect of several compaunds on L-carnitine transport was also determined. Results. The functioning of high-affinity (Octn2) and low-affinity carnitine transporters (Atb0+) was demonstrated in rat colonocytes. Expression of Octn2 and Atb0+ was also confirmed by real-time RT-PCR and in-situ hybridization. Experimental colitis induced a decrease in colonocyte carnitine uptake and a significant reduction of Octn2 and Atb0+ transcripts. The concomitant decrease in carnitine cell content paralleled with a substantial decrease in butyrate utilization by colonocytes. Carnitine supplementation in colonocytes by liposomes proved capable of restoring butyrate metabolism, while secondary carnitine deficiency in vivo increased colitis severity. Conclusions. In this study, we present evidence for the complex interactions between carnitine, carnitine transporters and butyrate metabolism. The altered colonocyte carnitine uptake in colitis leads to impaired butyrate oxidation and this, in turn, results in energy failure. Thus, it is not a decreased butyrate uptake but rather an altered utilization of this SCFA that produce toxic effects on the target cell. This may explain the contradictory therapeutic efficacy of butyrate in inflammatory bowel diseases, as proved by the last decades' clinical trials. Our results suggest that colitis is characterised by an energy deficiency, in which a lack of carnitine may lead to cell death and chronic inflammation by perturbing butyrate metabolism.

\section{T1742}

Downmodulation of STAT4 Expression Can Control Experimental Intestinal Inflammation Through Effects On Chemokine Expression and T Cell Trafficking

Ivan J. Fuss, Atsushi Kitani, Warren Strober

Background: Stats are important molecules involved in cytokine synthesis. With respect to Stat4, it has been shown that IL-12p70 signaling leads to phosphorylation of Stat 4 and IFNg production. It is important to recognize, however, that Stat 4 can be engaged in activities other that IFN-g production. This is evident from micro-array analysis of Stat $4-/$ - cells showing that while upregulation of the IFN-g is the earliest function of Stat4, the activation of other genes, such as chemokine receptors can be attributed to Stat 4 activation. Methods: Therefore, to further understand the function of Stat 4 in Thl development, we examined the latter in the murine model of Thl T cell-mediated inflammation, TNBS-colitis. In these studies we analyzed the effects of downregulation of Stat 4 in transgenic mice bearing a doxcycline-regulatable anti-sense oligonucleotide to Stat 4 under a CD2 promoter (AS-Stat 4 $\mathrm{Tg}$ mice). This inducible effect upon STAT4 lended itself to study the separate effects of Stat 4 on IFN-g expression from that on chemokine receptor expression. Results: In these studies, we demonstrate that during induction of TNBS-colitis whereas doxycline-induced up-regulation of AS-Stat4 in T cells led to inability to induce gut inflammation, this effect was not soley due to effects on IFN-g production at the local level. In further studies we observed a significant effect on chemokine expression and cell trafficking. This was exemplified by a negative influence on Thl T cell-associated chemokine synthesis (involving CCRl, CCR5 and CXCR3) in these Tg mice, which appeared to interfere with the trafficking of Thl cells to the site of inflammation. In this situation, Tg mice administered TNBS had Thl cells accumulated in the spleen and could not traffic to the lamina propria and thus did not cause TNBS-colitis in the latter site. In further studies, spleen CD4+ T cells obtained from AS-STAT4 TG mice when transferred to naive receipts (who receive TNBS re-challenge) did not traffic to the gut and did not transfer colitis to the recipient. Furthermore, T cells from the AS-STAT4 mice lacked migration responses in chemotaxis assays to MIP-la, MIP1b and RANTES. Finally, these findings correlated with the fact that in luciferase reporter gene 
studies specific Stat 4 binding sites were found within the CCR5 gene which are necessary for activation. Conclusion: These studies indicate that Stat 4 regulation is related to a range of Thl-related functional activities, such as chemokine expression and effects on cell trafficking, not just IFN-g production. The regulation of such may prove to be another useful avenue in the treatment of intestinal inflammation.

\section{T1743}

Dose Dependent Transfer of CD4+CD25+ T Cells Attenuates Hfutl Murine Colitis

Gregory T. Moore, Steven J. Brown, Mark Lust, Sally J. Bell, P R. Elliott, William R. Connell, Peter J. Cowan, Paul V. Desmond, Anthony J. D'Apice

Introduction: Human fucosyltransferase 1 (hFUT1) transgenic mice have thymic maturation defects and Thl colitis. Regulatory T cells (Treg) form in the thymus. hFUTl mice may be deficient in Tregs and transfer of normal Tregs may improve colitis. Methods: HFUTI Balb/ $\mathrm{c}$ and Balb/c thymocytes were assessed for number and phenotype of Tregs via FACS. hFUT1+ mice received $10^{\wedge} 5(\mathrm{n}=9)$ or $10^{\wedge} 6(\mathrm{n}=8) \mathrm{Balb} / \mathrm{c} \mathrm{CD} 4+\mathrm{CD} 25+\mathrm{T}$ cells, $10^{\wedge} 5 \mathrm{CD} 4+\mathrm{CD} 25-\mathrm{T}$ cells $(n=8)$ or $10^{\wedge} 5$ naive CD4+CD45RBhi T cells $(n=4)$ at 4 weeks of age. Control hFUT1 mice $(n=8)$ were similarly observed and euthanised eight weeks post transfer. Results: HFUT1 mice were deficient in mature $\mathrm{CD} 4+\left(1.92\right.$ vs $\left.55.6\left(\times 10^{\wedge} 6\right)\right)$ and $\mathrm{CD} 4+\mathrm{CD} 25+$ thymocytes (3.76 vs $\left.49.6\left(\mathrm{x1} 0^{\wedge} 4\right)\right)$. CD62L and GITR expression were reduced in hFUT1 Tregs. Foxp3 expression was similar. HFUTl mice receiving $10^{\wedge} 6$ and $10^{\wedge} 5$ CD4+CD25+ and $10^{\wedge} 5$ CD4+CD25- T cells had greater weight gain with mean weights (+/-SEM) of $21.1 \mathrm{lg}+/-0.58$, $(\mathrm{p}<0.05), 20.4 \mathrm{~g}+/-0.80,(\mathrm{p}=0.05)$ and $20.0 \mathrm{~g}+/-0.58(\mathrm{p}<0.05)$ versus hFUTl controls $17.7 \mathrm{~g}(+/-0.98)$. (CD4+CD45RBhi group $16.3 \mathrm{~g}+/-0.81$ ) Colitis improved in the $10^{\wedge} 6$ $\mathrm{CD} 4+\mathrm{CD} 25+$ group $(1.17+/-0.17, \mathrm{p}<0.01)$ and $10^{\wedge} 5 \mathrm{CD} 4+\mathrm{CD} 25$ - group $(1.40+/-0.50$, $\mathrm{p}<0.05)$ compared with the $10^{\wedge} 5 \mathrm{CD} 4+\mathrm{CD} 25+$ group $(2.67+/-0.41)$, CD $4+C D 45 \mathrm{RBhi}$ group $(3.75+/-0.25)$ and hFUTl group $(3.00+/-0.38)$. Conclusion: HFUTl mice are deficient in Treg number and transfer of normal CD4+CD25+ T cells improves hFUTl colitis dose dependently, along with CD4+CD25- T cells but not naive CD4+CD45RBhi T cells. HFUTl mice are deficient in CD4+CD25+ Treg and their colitis can be successfully treated with normal mouse Tregs.

\section{$\mathrm{T} 1744$}

Changes of Intestinal Leukocyte Migration After Exposure to Long Chain Fatty Acids: Interaction Between Platelets and Leukocytes

Masaaki Higashiyama, Ryota Hokari, Chikako Watanabe, Shunsuke Komoto, Yoshikiyo Okada, Chie Kurihara, Atsushi Kawaguchi, Shigeaki Nagao, Kazuro Itoh, Soichiro Miura

Background: Dietary fatty acids could affect immune functions in small intestine. We previously reported that fatty acids modulate cytokine production from intestinal epithelial cells and intraepithelial lymphocytes and enhance the migration of T lymphocytes to Peyer's patches. Recently it is reported that platelets contribute to the endothelial adherence of leukocyte. However, there is no report whether activation of platelets could modulate the leukocyte migration in the intestinal mucosa after fatty acid exposure through changes of platelet migration. The aim of this study was to assess the influence of dietary long chain fatty acid exposure on platelet adhesion to venular endothelium and interaction of leukocytes and platelets. Methods: Splenic lymphocytes were isolated from C57B6 mice and monocytes were isolated by MSP plate and labeled with rhodamine 6G. Blood of mice was collected and anticoagulated with $0.1 \mathrm{ml}$ acid citrate dextrose buffer and centrifuged at $600 \mathrm{~g}$. The platelet pellet was labeled with CFSE. Cell migration to intestinal microvessels was examined under an intravital fluorescence microscope after injection of splenic lymphocytes, monocytes and platelets labeled with CFSE. In some mice, platelets and leukocytes were simultaneously observed using 3 CCD camera. Oleic acid (main ingredient of olive oil) and linoleic acid were administered into the stomach 2 hours before examination. In addition, after treatment with antibody against PSGL-1 or P-selectin, changes of monocytes and platelet migration were also investigated. Changes of ICAM-1, VCAM-1 and MCP-1 expression were studied by quantitative RT-PCR. Results: In control, some platelets directly interacted with venular walls and a small number of leukocytes to which platelets are binding showed a rolling behavior. Intraluminal unsaturated fatty acid administration significantly enhanced the rolling and adhesion of both platelets and monocytes. Pretreatment with anti-P-selectin inhibited the increased platelet adhesion to venular walls and also significantly attenuated the monocyte adhesion or monocyte-platelet interaction induced by fatty acids. Pretreatment with anti PSGL-1 ameliorated only monocytes adhesion induced by fatty acids. RT-PCR study did not show significant changes in expression of ICAM-1, VCAM-1 and MCP-1. Conclusions: We successfully observed interaction of platelets and monocytes and demonstrated enhanced migration of platelets and monocytes by fatty acid exposure in intestinal microvessles. The rapid increase in monocyte migration was mediated by P-selectin-PSGL-1 interaction which may be largely influenced by platelet adhesion and leukocyte-platelet interactions.

\section{$\mathrm{T} 1745$}

Bone Marrow Transplantation Induces Remission in Crohn's Disease: Where Do the Cells Go?

Stefanie Schier, Natalie C. Direkze, Toby Hunt, Rosemary Jeffery, Thomas T. MacDonald, Malcolm R. Alison, Richard Poulsom, Nicholas A. Wright, Mairi Brittan

Introduction:Recent studies show that bone marrow (BM) transplantation induces remission in Crohn's disease. We used a mouse model of Crohn's colitis to elucidate the mechanism of action of BM transplantation. BM forms myofibroblasts in inflamed colons, which we now show can produce collagen. BM also forms multiple vascular lineages and contributes to both angiogenesis and neovasculogenesis. Methods: Female mice were lethally irradiated and rescued by a BM transplant from male donors. After 6 weeks, colitis was induced by injection of trinitrobenzene sulphonic acid (TNBS), and colons analysed 1-14 days later. In situ hybridisation for $\mathrm{Y}$ chromosome was combined with immunohistochemistry for specific antigens to determine cell phenotype. A novel triple staining method combined in situ hybridisation and immunohistochemistry with autoradiography for collagen mRNA. Results Cells derived from BM (Y chromosome-expressing) were abundant in inflamed colons and associated vasculature; vascular smooth muscle lining cells (VSMLCs) expressed alphasmooth muscle actin (SMA); endothelial cells expressed ICAMl and vWF; myofibroblasts expressed SMA and collagen mRNA. Inflammatory cell infiltration was ruled-out. BM contributed to $57 \%$ of myofibroblasts, which produced collagen. BM-derived endothelial cells, pericytes and VSMLCs were frequent in blood vessels, with 27\% of VSMLCs of BM origin in inflamed colons. BM contributes to both angiogenesis and neovasculogenesis, confirmed by vessels composed entirely of BM-derived cells. Discussion / Conclusion: This is the first observation of BM-mediated neovasculogenesis in colitis. We provide an insight into the regenerative function of BM by highlighting the capacity of BM to engraft within inflamed colons and form multiple, functional lineages.

\section{T1746}

Genetic Inactivation of JNK1 and JNK2 Aggravates the DSS Induced Colitis in Mice

Ansgar M. Chromik, Annette M. Muller, Julia Korner, Orlin Belyaev, Tim Holland-Letz, Frank Schmitz, Thomas Herdegen, Waldemar Uhl, Ulrich Mittelkoetter

Background: The c-Jun N-terminal kinases (JNKs) are considered as novel targets for therapy of IBD (inflammatory bowel diseases). However, the relevant isoforms have to be elucidated. Here, we have analysed the individual contribution of the JNK1 and JNK2 isoforms in Dextran Sulfate Sodium (DSS) model of experimental colitis. Material and Methods: JNK1 and JNK2 knockout mice (JNK1 ko, JNK2 ko) and their wildtype controls (WT1, WT2 received three cycles of DSS treatment, each consisting of $1.7 \%$ DSS for 5 days, followed by 5 days with water. Animals were daily evaluated by a disease activity index (DAI) comprising measurement of body weight, estimation of stool consistency and test for occult blood/gross rectal bleeding. After 30 days all animals were sacrificed, and the inflamed intestine was histologically evaluated by a crypt damage score. Results: Unexpectedly, neither JNK1 ko nor JNK2 ko prevented mice from developing a chronic colitis when compared to wildtype controls WT1 and WT2, respectively. In the very contrary, DAI and mortality were aggravated in JNK2 ko compared to WT2. DAI and mortality did not differ between JNKl ko and WTl, but the histological crypt damage score was significantly enhanced in the caecum of JNKL ko mice. Conclusion: Genetic deletion of JNK2 deteriorates the clinical outcome in an experimental model of murine colitis. We hypothesise that the functional deletion of the otherwise pro-apoptotic JNK2 prolongs the activity of pro-inflammatory immue cells with worsening of clinical course symptoms.

\section{$\mathrm{T} 1747$}

IL-12p35-Producing B Cells Regulate TH2-Mediated Chronic Colitis Ken Sugimoto, Atsuhiro Ogawa, Yasuyo Shimomura, Atsushi Mizoguchi, Atul K. Bhan

BACKGROUND \& AIMS: IL-10-porducing regulatory B cell subset appears in the mesenteric lymph mode (MLN) of T cell receptor knockout ( $\alpha \mathrm{KO})$ mice after the development of colitis and subsequently regulates the progression of this disease (Immunity 2002, 16:219) However, it is unclear whether the IL-10 produced by regulatory B cells directly or indirectly contributes to the regulation of this colitis. METHODS: Cytokine production by purified MLN B cells was examined by flow cytometric, ELISA, Q-PCR and RPA analyses. To investigate the functional role of $\mathrm{p} 35$ in the pathogenesis of colitis in $\alpha \mathrm{KO}$ mice, IL-12p35-deficien TCR $\alpha$ double knockout mice were generated. RESULTS: In the absence of B cells (B celldeficient TCR $\alpha$ double KO mice, $\alpha \mu \mathrm{DKO}$ ) or IL-10 (IL-10-deficient TCR $\alpha$ double KO mice, $\alpha$ IL10DKO), IL-12p35 expression was significantly downregulated in the MLN of $\alpha$ KO mice. Transfer of B cells from $\alpha$ KO but not $\alpha$ IL10DKO mice restored the IL-12p35 expression in the recipient $\alpha \mu D K O$ mice. Unexpectedly, studies using purified several MLN cell types (including B cells, T cells, macrophages, DCs, neutrophils, and NK cells) demonstrated that $\mathrm{B}$ cells per se are responsible for the production of IL-12p35 in the presence of IL-10. Flow cytometric and ELISA analyses revealed that the IL-12p35 production by purified MLN B cell from $\alpha \mathrm{KO}$ mice but not WT mice is further upregulated by the B cell receptor ligation in the presence of TLR9 ligand, $\mathrm{CpG}$. To test the functional role of $\mathrm{p} 35$ in the pathogenesis of colitis, IL-12p35-deficient TCR $\alpha$ double knockout ( $\alpha$ p35DKO) mice were generated. Of note, the $\alpha$ p35DKO mice spontaneously developed much more severe colitis compared to $\alpha K O$ mice. QPCR analysis showed that the deficiency of p35 enhances the IL-17 production of $\mathrm{CD}^{+} \mathrm{T}$ cells. To further confirm whether IL-12p35-producing B cells are actually involved in the regulation of colitis, $B$ cells from $\mathrm{KKO}$ or $\alpha \mathrm{p} 35 \mathrm{DKO}$ mice were transferred into $\alpha u \mathrm{DKO}$ mice. Transfer of $B$ cells from aKO mice but not $\alpha$ p35DKO mice efficiently inhibited the progression of colitis in the recipient $\alpha \mu D K O$ mice. In addition, the transfer of $\alpha p 35 D K O$ $\mathrm{B}$ cells enhanced the IL-17 production of $\mathrm{CD}^{+} \mathrm{T}$ cells. CONCLUSION: These results indicate that IL-12p35-producing B cells are induced by IL-10-producing B cells and are involved in the regulation of colitis. The deficiency of p 35 is associated with an increase in IL-17 producing CD4 ${ }^{+} \mathrm{T}$ cells (also called Th-17) in a model of Th2-mediated colitis.

\section{T1748}

Induction of Colitis in CDP/Cux Mutant Mice Is Associated with An Increase of Disease Severity

Mathieu Darsigny, Francois Boudreau

INTRODUCTION: The CCAAT displacement protein (CDP/Cux) is a transcription factor involved in the control of cell proliferation, differentiation and maintenance of the differentiated state. We have recently demonstrated that CDP/Cux can repress the intestinal specific gene sucrase-isomaltase. However, the intestinal physiological role of CDP/Cux remains to be ascertained. Recent observations from the literature have linked CDP/Cux functions to the development of the immune system as well as the regulation of inflammatory genes. AIM: To determine whether CDP/Cux could be involved in the regulation of the intestinal inflammatory response. METHODS AND RESULTS: The mouse knock-in model deficient for the homeodomain portion of $\mathrm{CDP} / \mathrm{Cux}$ was utilized. To test whether the CDP/Cux 
transcription factor was involved in the maintenance of the mucosal intestinal immunity, mice were challenged with a mild dextran sodium sulphate (DSS) induced-colitis protocol Three independent experiments were performed with groups of adult CDP/Cux mutant and wild type mice treated or not with $2.5 \%$ of DSS in drinking water ad libitum. All animals were sacrificed after 7 days and the disease severity was analysed among the individuals according to a disease activity index (stools aspect, rectal bleeding and weight loss), histological alterations, and cytokines profile in the distal intestine. CDP/Cux mutant mice showed severe colitis progression with the apparition of frequent fecal blood as well as rectal bleeding as compared to the wild-type animals. Histological examination of the colon of DSS-treated mutant mice with haematoxylin and eosin (H\&E) stained sections revealed a drastic destruction of the mucosa that was associated with an important recruitment of inflammatory cells. DSS-treated wild type mice displayed minor alterations of the mucosa with the formation of dispersed small inflammatory cells clusters. Proinflammatory cytokines were generally stable between groups with some mutants showing upregulated mRNA levels of TNF alpha and IL-1 beta as determined by a real-time PCR analysis. CONCLUSION Our results demonstrate that the loss of CDP/Cux transcription factor is associated with a significant increased disease severity of the colonic mucosa when subjected to a mild DSS treatment. Further investigations are ongoing to identify the molecular links between the transcriptional regulator CDP/Cux and intestinal inflammatory processes. (This work was supported by CIHR, NSERC, CRS, CFI and FRSQ).

\section{T1749}

The Role of the P1108 PI3-Kinase Isoform in Inflammatory Bowel Disease Klaartje Kok, Wayne P. Pearce, Maikel P. Peppelenbosch, Bart Vanhaesebroeck, Daan W. Hommes

Background IBD is thought to develop as a result of deregulated mucosal immune response to normal luminal flora but the molecular mechanisms remain poorly defined. Toll-like receptors (TLR) mediate innate immune responses and are present on immune cells such as macrophages. Increased cytokine production through these receptors is thought to play a role in the pathogenesis of IBD. Class IA PI3-kinases (PI3K) are lipid kinases that control many cellular responses. The pllod isoform of PI3K is mainly expressed in leukocytes. Inactivation of p110 (p1108D910A/D910A mice) leads to defects in B- and T-cell signaling and chronic focal colitis. This implies a role for pllod in the pathogenesis of IBD. Also the human p1108 gene PIK3CD maps to the IBD7 locus. Previous studies have shown that $\mathrm{PI} 3 \mathrm{~K}$ is a suppressor of cytokine production triggered by TLR signaling, preventing excessive innate immune responses. Here we investigated the role of normal luminal flora in pllos related colitis and of pl10 in bone marrow derived macrophages (BMDM) TLR signaling. Methods Colonic sections of p1108D910A/D910A mice derived from conventional and specific pathogen-free (SPF) environments were stained with $\mathrm{H} \& \mathrm{E}$ for histological analysis. Wild-type BMDM treated with or without D030 (p1108 inhibitor) or LY294002 (broadspectrum PI3K inhibitor) were stimulated with LPS $100 \mathrm{ng} / \mathrm{ml}$ and protein phosphorylation status was assessed. Results p1108D910A/D910A mice from a conventional environment spontaneously develop colitis at 16 weeks of age. Histological examination reveals mucosal hyperplasia, hyperplasic glands, elongated crypts, crypt abscesses, and leukocytic infiltration of the lamina propria and focal erosion of the mucosa. In contrast, SPF pl 108D910A/D910A mice show no inflammation or other pathology in any layer of the bowel. LPS induced Akt phosphorylation (indirect measure of PI3K activity) in BMDM is abrogated by LY294002 treatment and, to lesser extent, by D030 which is in contrast to phosphorylation of IкB $\alpha$, $\mathrm{pIKK} \alpha / \beta$, Erk and p38 which are unaffected by the inhibitors. Conclusion Mice lacking pl $10 \delta$ spontaneously develop IBD only in the presence of normal luminal flora. This finding emphasizes the role of p110 in the pathogenesis of IBD. Stimulation of BMDM with LPS leads to the activation of Akt/PI3K, MAP-kinase and NFKB pathways. PI3K inhibitors decrease Akt/PI3K activity but fail to inhibit the other pathways. This suggests that PI3K does not have a important role in LPS-induced cytokine production of BMDM and that other immune cells are more responsible for this pl108 IBD phenotype. Candidates are regulatory T-cells which are currently under investigation.

T1750

Role of Nitric Oxide (NO) and NO Synthases in Healing of Dextran Sulfate Sodium-Induced Rat Colitis

Yoko Aoi, Shyun Terashima, Hikaru Nishio, Shinichi Kato, Koji Takeuchi

Background/Aim: NO synthase (NOS) exists in two isoforms, constitutively expressed NOS

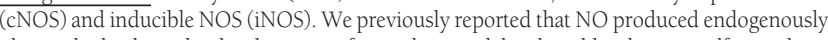
plays a dual role in the development of rat colitis model induced by dextran sulfate sodium (DSS), depending on the NOS isoform; a protective effect by constitutive cNOS/NO and a proulcerogenic effect of iNOS/NO. However, the roles of NO and NOS isozymes in the healing process of colitis remain unexplored. In the present study, we examined the effects of various NO inhibitors on the healing of DSS-induced rat colitis. Methods: Male Wistar rats were used. Experimental colitis was induced by feeding the rats for 6 days with $2.5 \%$ DSS in drinking water. After DSS treatment, the animals were fed normally with tap water thereafter and killed various days up to 7 days later. L-NAME (a nonselective NOS inhibitor, $5 \mathrm{mg} / \mathrm{kg}$ ) or aminoguanidine (a relatively selective iNOS inhibitor, $20 \mathrm{mg} / \mathrm{kg}$ ) was given PO twice daily for 6 days starting from the termination of DSS treatment. The ulcerated area, colon length, and myeloperoxidase (MPO) activity as well as thiobarbituric acid reactants (TBARS) were measured on day 7 after DSS treatment. Mucosal NO content was measured by Griess method. The expression of eNOS and iNOS mRNAs was examined by RT-PCR while that of basic fibroblast growth factor (bFGF) was determined by Western blotting. Results: DSS treatment caused severe lesions in the colon, accompanied by an increase in $\overline{\mathrm{MPO}}$ activity and TBARS as well as a decrease in body weight gain and colon length. The lesions healed gradually after discontinuation of DSS treatment, with a histological restoration and subsidence of inflammation. The healing of these lesions was significantly impaired by daily administration of L-NAME or aminoguanidine when examined both macro- and microscopically. The gene expression of eNOS was constantly observed in the mucosa while the expression of iNOS mRNA was up-regulated by DSS treatment, reaching a peak on day 1 , followed by a gradual decrease in later period, and these changes were associated with increase or decrease in NO content. The expression of bFGF was also up-regulated in the colonic mucosa following DSS treatment. Both L-NAME and aminoguanidine significantly suppressed the increase in NO content but had no effect on the expression of bFGF. Conclusion: These results suggest that endogenous NO produced by iNOS plays an important role in the healing of DSS-induced colonic lesions. Although the exact mechanism remains unknown, it is assumed that the healing promoting effect of NO is accounted for by actions other than modulation of the bFGF expression.

\section{T1751}

The PPARB Deficient Mice Are Highly Sensitive to the Oral Administration of Dextran Sulfate Sodium

Dominique Velin, Frederic Varnat, Daniel Bachmann, Christian Felley, Walter Wahli,

Pierre Michetti, Beatrice Desvergne

Background: The peroxisome proliferator-activated receptors (PPARs) are members of the nuclear receptor superfamily and are critical modulators of environmental and dietary stimuli. Ubiquitously expressed, PPARb mRNA expression is highest in the brain, adipose tissue, and epithelia. Aim: We studied the role of PPARb in the mouse model of distal colitis induced by oral administration of dextran sulfate sodium (DSS). Methods: Wild type or PPARb deficient mice received 2.5\% DSS in drinking water for 7 days. Body weight, overall wellness, colon histology, colon myeloperoxidase activity, and colon cytokine expression were followed at different time points. Results: In comparison to wild type mice, PPARb deficient mice lost significantly more body weight $(-4.2 \pm 3.2 \%$ of the initial body weight for PPARb deficient mice vs. $+2.2 \% \pm 2.8$ of the initial body weight for wild type mice; $\mathrm{p}<0.0001$; $n=14$ per group, Day 3 values), developed rectal bleeding more frequently (35\% vs $7 \%$; $\mathrm{p}<0.05 ; \mathrm{n}=14$ per group, Day 3 values) and died prematurely ( 2 out of 8 PPARb deficient mice died before Day 7 vs. none of 8 wild type mice). Histological analysis at Day 5 showed increased villi size in wild type mice, suggesting an ongoing regenerative process of the wild type colon epithelium. In contrast, the intestinal villi of PPARb deficient mice were smaller and a massive destruction of the colonic epithelium at Day 7 in PPARb deficient mice was further observed. At Day 7 myeloperoxidase activities measured in the colon of wild type mice ( $40 \pm 10$ unit $\mathrm{MPO} / \mathrm{mg}$ protein) were higher compared to PPARb deficient mice (20 \pm 7.5 unit $\mathrm{MPO} / \mathrm{mg}$ protein, $\mathrm{n}=7$ mice, $\mathrm{p}=0.03$ ). However, at Day 7 , no statistical differences were found in mRNA expression for TNFa, IFNg, MCP-1, IL-1b, IL-1Ra, and iNOS, in the colon of wild type or PPARb deficient mice. Conclusion: PPARb deficient mice are extremely sensitive to the DSS-induced colitis. The severe DSS-induced pathology in PPARb deficient mice is not due to a predominant inflammatory process. We are currently exploring how the lack of PPARb may interfere with the intestinal tissue repair capacity following DSS administration.

\section{$\mathrm{T} 1752$}

Sex Hormone Regulation of Colonic Macrophage Migration Inhibitory Factor Expression in Basal and Inflammatory Conditions in the Female Rat Eric Houdeau, Moriez Raphael, Leveque Mathilde, Waget Aurelie, Bueno Lionel, Fioramonti Jean

Background and aims: Clinical data highlighted the influence of sex steroids on gastrointestinal symptoms in female IBD patients. Macrophage migration inhibitory factor (MIF), a proinflammatory cytokine that promotes the release of tumor necrosis factor- $\alpha$ (TNF- $\alpha$ ) and interleukin-1 $\beta$ (IL-1 $\beta$ ), was shown as a target of sex steroids in inflammatory processes. This study aimed to determine the influence of estradiol (E2) and progesterone (P) on MIF expression and disease activity in a rat model of colitis. Methods: Basal concentrations of MIF, TNF- $\alpha$ and IL- $1 \beta$ were determined by ELISA at all stages of the ovarian cycle in female rats, or following ovariectomy (OVX) after a 8 day-treatment with subcutaneous capsules containing either estradiol (E2), progesterone (P), or saline (controls). Colitis was induced by intrarectal instillation of $80 \mathrm{mg} / \mathrm{kg}$ TNBS or vehicle in similar groups of rats. Twentyfour hours later, rats were sacrificed and colons removed for evaluation of macroscopic damage score (MDS), myeloperoxidase activity (MPO), and ELISA for MIF, TNF- $\alpha$ and IL$1 \beta$. Results: In cyclic rats, only MIF levels fluctuated during the cycle, with lowest values at estrus (endogenous E2 dominance) and highest in metestrus (endogenous P dominance) $(301 \pm 39 \mathrm{vs} 781 \pm 135 \mathrm{ng} / \mathrm{mg}$ of protein, respectively; $<<0,05)$. Similarly, E2 and P treatments in OVX rats did not affect basal levels in TNF- $\alpha$ and IL-1 $\beta$. In contrast, E2 decreased ($64 \%$ ) while P increased (+94\%) MIF expression ( $32.5 \pm 2.6$ and $173.1 \pm 11.5 \mathrm{ng} / \mathrm{mg}$ of protein, respectively) when compared to controls $(89.3 \pm 5.2 \mathrm{ng} / \mathrm{mg}$ of protein, $\mathrm{p}<0.001)$. In metestrus rats with TNBS colitis, an increase in MDS $(+78 \%, \mathrm{p}<0.001)$ and MPO activity was observed when compared with TNBS-induced colitis in estrus rats (1980 \pm 230 vs $882 \pm 100 \mathrm{U}$ MPO/ $\mathrm{g}$ of protein, respectively; $\mathrm{p}<0.01)$. In TNBS-treated OVX rats, MIF levels were reduced by E2 $(-56 \%, \mathrm{p}<0.001$ vs controls + TNBS $)$, and increased by $\mathrm{P}(+89 \%, \mathrm{p}<0.001)$. In the same animals, $P$ also induced a rise in TNF- $\alpha$ levels $(20.9 \pm 2.5 \mathrm{vs} 10.9 \pm 1.4 \mathrm{pg} / \mathrm{mg}$ of protein in controls), while E2 treatment was correlated to a diminution in IL-1 $\beta$ concentrations (4154 \pm 404 vs $6381 \pm 495 \mathrm{pg} / \mathrm{mg}$ of protein in controls). Conclusions: Our data show that E2 inhibits and P stimulates MIF expression in both normal and inflamed colon. The natural shift from E2 to P dominance during the ovarian cycle may promote alternatively resistance then susceptibility of the colon to inflammation, and modulates TNF- $\alpha$ and IL-1 $\beta$ production in colitis. Thus steroid hormone regulation during the cycle could modulate disease activity in women with IBD.

\section{T1753}

Constitutive Nitric Oxide Synthase Is Involved in Maintaining Colonic Mucosal Blood Flow in DSS and TNBS Treated Rats Olof Schreiber, Joel Petersson, Lena Holm

Background: The role of nitric oxide (NO) in inflammatory bowel disease is still controversial. Furthermore, the very few in vivo measurements of colonic blood flow in colitis models have not addressed the role of NO. The aim of this study was to investigate the involvement 
of NO in regulating colonic mucosal blood flow in two different colitis models. Methods: The rats were divided in four groups in respect to treatment: I/ saline and II/ trinitrobenzensulfonic acid (TNBS, $30 \mathrm{mg} / \mathrm{ml}$ in $50 \%$ ethanol) intrarectally 7 days prior to the experiments, III/ untreated and IV/ 5\% dextran sulphate sodium (DSS) in the drinking water 9 days prior to the experiments. On the experimental day the rats were anesthetized with Inactin ${ }^{\circledR}$ (120 mg.kg-lip) and the colonic mucosa exteriorized. Blood flow, measured with laser-Doppler flowmetry, and arterial blood pressure were continuously measured throughout the experiments (100 min) and vascular resistance calculated. All four groups were given the NOS inhibitor L-NNA (10 mg.kg-1 bolus followed by $3 \mathrm{mg} \cdot \mathrm{kg}-1 \mathrm{~h}-1$ iv) 20 min after start of the experiment. In a few experiments the iNOS inhibitor L-NIL was given in the same dose as L-NNA. Results: TNBS induced a severe colitis with penetrating ulcers, reduced colon length and thickening of the wall. DSS on the other hand induced a more diffuse mucosal inflammation covering the entire colon and with greater fecal blood content than the TNBS treated rats. Blood pressure before L-NNA was similar $(\approx 100 \mathrm{mmHg})$ in all groups and increased to $\approx 140 \mathrm{mmHg}$ in all groups except the TNBS treated rats in which blood pressure only increased to $121 \pm 7 \mathrm{mmHg}$. Colonic mucosal blood flow, calculated as perfusion units/ g tissue, was not significantly different in any of the four groups. The blood flow was not altered in the control groups (I and III) during L-NNA but substantially reduced in the TNBS and DSS treated rats $(67 \pm 4 \%$ and $67 \pm 7 \%$ of basal, respectively). Vascular resistance increased significantly more in the TNBS and DSS treated rats $(202 \pm 10 \%$ and $218 \pm 10 \%$ of basal, respectively) than in the control rats (169 $\pm 15 \%$ group I $145 \pm 15 \%$ group III of basal). L-NIL treatment did not significantly alter blood flow in any of the groups. Conclusions: The results clearly indicate that constitutively expressed NOS, probably eNOS since L-NNA most potently inhibits eNOS, is equally important in maintaining colonic mucosal blood flow in the TNBS and DSS colitis models.

\section{T1754}

Pivotal Role in Colonic Protection for the Mucin MUC2 in Unchallenged and Dextran Sulfate Sodium Challenged Mice

Maria Van der Sluis, Barbara de Koning, Adrianus De Bruijn, Anna Velcich, Johannes van Goudoever, Hans Buller, Jan Dekker, Isabelle van Seuningen, Ingrid. Renes, Alexandra Einerhand

Background \& Aims: Expression of mucin MUC2, the structural component of the colonic mucus layer, is lowered in inflammatory bowel disease. Our aim was to obtain insight in the role of Muc2 in epithelial protection. Methods: Muc2 knockout (Muc2 ${ }^{-/}$) and Muc2 heterozygous ( $\mathrm{Muc}^{+/-}$) mice were characterized and challenged by a colitis-inducing agent, dextran sulfate sodium (DSS). We monitored clinical symptoms, intestinal morphology and differences in intestine-specific protein and mRNA levels. Results: The Muc2 $2^{-1-}$ mice showed clinical signs of colitis (as of 5 weeks), aggravating as the mice aged. Microscopic analysis of the colon of Muc2 $2^{-1-}$ showed mucosal thickening, increased proliferation and superficial erosions. Colonic goblet cells in the Muc $2^{-1-}$ mice were negative for Muc 2 and Gob-5, but Tff3 was still detectable. In these mice, transient de novo expression of Muc6 mRNA was observed at 5 weeks, but expression declined as the mice matured. On day 2 of DSS treatment, the histological damage was more severe in $\mathrm{Muc2}^{+/-}$vs. Muc2 ${ }^{+/+}$mice, but the disease activity index (DAI) was not yet different. By day 7 , the DAI and histological score were elevated in $\mathrm{Muc}^{+/-}$vs. Muc2 $2^{+/+}$mice. The DAI of the Muc $2^{-/-}$mice was higher (vs. both $\mathrm{Muc2}^{+/+}$and $\mathrm{Muc2}^{+/-}$mice) throughout DSS treatment. The histological damage in the DSS-treated Muc2 $2^{-1-}$ mice was different vs. Muc2 ${ }^{+/+}$and Muc2 ${ }^{+/}$mice. Specifically, in Muc2 $2^{-1-}$ mice many crypt abscesses were seen, instead of mucosal ulcerations. Conclusions: This study shows that changes in the mucus composition (lack of Muc2) leads to inflammation of the colon, and contributes to the onset and perpetuation of experimental colitis.

\section{T1755}

Characterization of Ileal Dendritic Cell Distribution in a Rat Model of Acute and Chronic Inflammation

Manuel A. Silva-Munoz, Monica Porras, Jury Jennifer, Patri Vergara, Mary H. Perdue

Background: Crohn's disease (CD) is an inflammatory bowel disease characterized by an increased mucosal permeability and an imbalance in the distribution of dendritic cells (DC) subtypes. Enhanced antigen uptake may affect the DC response and render the intestine susceptible to immune activation and inflammation. Subcutaneous (sc) administration of indomethacin in rats leads to intestinal inflammation (Porras et al. Am. J. Physiol. 2004), with both acute and chronic stages having increased permeability (Porras et al Neurogastro Motility Meeting 2005, Toulouse, Fr). Here, our objective was to examine subpopulations of DC to determine their location and migration patterns. Methods: Sprawley-Dawley rats were treated with indomethacin $(7.5 \mathrm{mg} / \mathrm{kg} \mathrm{sc}, 2$ injections $48 \mathrm{~h}$ apart), and ileal segments were obtained in the acute stage at day $4(\mathrm{n}=4)$ and in the chronic stages at day 15 , and 30 after injection ( $n=6 /$ group). Control rats $(n=6)$ were injected with saline. Segments were processed for light microscopy; sections were examined for inflammation (score 0-7) and immunohistochemistry for CD103 and CDllb (rat DC markers), CD83 (DC marker of maturation), CDl63 (marker for macrophages), and double immune staining. Results: In controls, both immature DC sub-populations, CD103+CD11b+CD 163-CD83- and CD103+CD11b-CD163-CD83-, were observed in the lamina propria (LP), and the CD1lbpopulation was also present in the sub-epithelial dome of the Peyer's patches (PP). In the acute stage, areas with or without ulcers were observed; both had an increased $(\mathrm{p}<0.05)$ inflammation score compared to controls. CD103+ and CDIlb+ (CD83-) DC were absent from inflamed areas, reduced in non-inflamed tissue, but present in PP's. In chronic stages at day 15, CD103+ and CD1lb+ cells were located in inflamed and non-inflamed areas, and in PP. Also, CD83+ DC were detected in inflamed areas. At day 30, when we observed a complete microscopic resolution of inflammation, CD103+ and CD11b+DC were increased, and at day 30 there were CD83+ DC beneath the epithelial cells layer. Conclusions: In this model, at times of altered mucosal permeability, we documented changes in distribution of DC in the ileum. Acute and chronic stages of inflammation had different morphological patterns, DC distribution, and CD83 expression. Antigen uptake in acute stages may activate the resident immature DC, inducing their migration to lymphoid tissue and maturation, returning to the intestine in chronic stages to play a role in the local inflammatory response.

\section{T1756}

Caveolin-1 Regulation of Experimental Colitis

James Joshua M. Greer, John H. Chidlow, Pascal N. Bernatchez, William C. Sessa, Deept Shukla, Christopher G. Kevil

Inflammatory bowel disease and experimental models of colitis display microvascular dysfunction which contributes to colon tissue pathology. Recent studies demonstrate that caveolin-1 may be important in regulating microvascular function and inflammatory responses. However, the pathological role of caveolin-1 in experimental colitis is unknown. Here we subjected wild-type and caveolin-1-/- (Cav-1-/-) mice to the $3 \%$ Dextran Sulfate Sodium (DSS) model of experimental colitis. Analysis of weight change, stool consistency, occult blood, and evidence of gross bleeding showed a significant decrease in disease activity index (DAI) in Cav-1-/- mice compared to wild-type control mice. Moreover, examination of tissue histopathology showed significant protection in Cav-1-/- colons. In wild type colons, complete crypt dropout and transmural inflammation was observed with the infiltrate consisting primarily of neutrophils and the degree of inflammation ranging from severe to mild depending on disease severity. In contrast, DSS histopathology findings were significantly diminished in Cav-1-/- colons. DSS induced colitis was also evaluated in wild type mice treated with an $\mathrm{AP}-\mathrm{Cav}-\mathrm{AB}$ peptide $(3 \mathrm{mg} / \mathrm{kg}$ ) corresponding to the caveolin-1 scaffolding domain. Interestingly, the AP-Cav-AB peptide significantly attenuated DSS DAI and histopathology scores compared to the control peptide treated mice. These data identify a nove pathway whereby caveolin-1 plays an important pathophysiological role during experimental colitis.

\section{T1757}

Epithelial Contribution to Barrier Function and Carcinogenesis in the GI 22 Deficient Mouse Model of Inflammatory Bowel Disease

Robert A. Edwards, Jennifer Davis

The Gia2-/- mouse model of inflammatory bowel disease spontaneously develops colitis beginning at 6 weeks of age and is predisposed to developing non-polypoid, mucinous colon cancers. Lymphocytes, dendritic cells, and myofibroblasts all appear to contribute to disease in these mice, but the role of Gia2 signaling in the epithelium has not been investigated. Histologic sections from more than 80 Gia2-/- mouse colons reveal preneoplastic lesions present as early as 12 weeks of age, in the setting of mild colitis without ulceration. This suggests a direct effect of the loss of epithelial Gia2 on carcinogenesis, which occurs despite lower levels of mucosal PGE2. To test the hypothesis that Gia2 regulates the balance of colonic epithelial cell growth and apoptosis, we generated lines of human T84 colon cancer cells stably overexpressing the dominant negative human Gio2 mutant, G203T-Gia2 by Amaxa nucleofection. These cells express 20-fold higher levels of hGia2 mRNA and overexpress Gia2 protein by western analysis. G203T-Gia2 cells show enhanced basal proliferation rates in vitro, which correlate with increased BrdU incorporation in the colons of 5 week old, non-colitic Gio2-/- mice. Like Gia2-/- myofibroblasts, G203TGi 22 T84 cells are defective in releasing arachidonic acid. Since arachidonate is an important activator of sphingomyelinase that generates the pro-apoptotic factor ceramide, we tested whether G203T-Gia2 cells were resistant to camptothecin induced apoptosis. In contras to WT and vector-transfected T84 cells, 6 hours' treatment with $5 \mathrm{uM}$ camptothecin induced significantly less caspase 3 activity in G203T-Gi 2 cells, suggesting a link between altered arachidonate metabolism observed in multiple Gio2-/- cell types and the propensity towards early neoplastic transformation in the colonic mucosa of Gia2-/- mice

\section{T1758}

Course of Yersinia Enterocolitica Infection Is Dramatically Improved in CCR6 Deficient Mice - New in Vivo Model for M Cell Function?

Sabine Westphal, Andreas Luegering, Julia von Wedel, Ifor Williams, Christof von Eiff, Wolfram Domschke, Alexander Schmidt, Gerhard Heusipp, Hermann Herbst, Torsten Kucharzik

Introduction M cells are specialized cells within the follicle associated epithelium (FAE) of Peyer's patches (PP). We could recently demonstrate that CCR6-deficient mice lack M cells, the "gatekeepers" of the mucosal immune system. Yersinia enterocolitica exploit M cells to invade mucosal tissues and spread systemically. In our study, we therefore analyzed Y enterocolitica infection in CCR6 KO mice. Methods C57BL/6 wildtype mice (wt) and CCR6 deficient mice (KO) were orally infected with 2,5x108 CFU Y. enterocolitica. Intraperitonea (i.p.) injection was performed by $1 \times 106$ CFU. Invasion of PP by Y. enterocolitica was determined by histology. To evaluate kinetic of infection, bacterial excretion in stool and colonization in the PP and spleen was determined. Besides, we developed a scoring system to grade severity of infection with Y. enterocolitica. Cytokine mRNA expression was analyzed by real-time PCR. Results After oral application of Y. enterocolitica, CCR6-deficient mice showed no clinical symptoms of infection, whereas wt mice suffered from septic infection. Stomach and small intestine were extremely bloated by gas in wt mice. PP of wt mice were conspicuously swollen and highly inflamed. 7 days post infection, Y. enterocolitica could be found in stool and PP isolated from wt mice, whereas Y. enterocolitica was absent in PP of $\mathrm{KO}$ mice. No bacterial invasion could be observed in spleens neither in wt nor in $\mathrm{KO}$ mice. In addition, a significantly elevated level of TNF alpha RNA expression within PP of wt mice 7 days post infection could be evaluated using real-time PCR, whereas no change in TNF alpha level was assessed in $\mathrm{KO}$ mice. To bypass the routing of $\mathrm{Y}$. enterocolitica through PP mice were infected intraperitoneally (i.p.). In contrast to oral infection, no significant differences between wt and $\mathrm{KO}$ mice could be found in mice i.p. infected with Y. enterocolitica. Both groups showed severe symptoms of infection and revealed equal scores. Conclusions The interaction of CCR6 with its ligand Mip3 alpha is essential for the development of PP and M cells. Deletion of CCR6 negatively influences the lympho-epithelial interaction and causes inhibition of $\mathrm{M}$ cell differentiation within PP and consecutively decreased infectious potential of Y. enterocolitica. The CCR6 knockout mouse model represents a suitable instrument to characterize function of $\mathrm{M}$ cells in vivo.

\section{.}


T1759

Regulated Expression of Leukocyte Specific Transcript (LST)-1 in Human Intestinal Microvascular Endothelial Cells (HIMEC) - Evidence for Multiple Splice Variants

Jan Heidemann, Moritz Kebschull, Allon Canaan, Sherman M. Weissman, Kerstin Klimmek, Andreas Lugering, Matthias Bruwer, Wolfram Domschke, Torsten F. Kucharzik, Christian Maaser

Background: Leukocyte specific transcript (LST)-1, the human homologue of the murine B144 gene is encoded by a gene located in the major histocompatibility complex III. Located in close proximity of the TNF- $\alpha$ locus in humans and rodents, LST-1 is believed to possess immunomodulatory functions in the inhibition of mitogen-induced T cell proliferation, as well as functions required for dendritic cell differentiation and morphogenesis. The aim of this study was to determine expression of LST-1 in human microvascular endothelial cells (HIMEC). Material and Methods: Human intestinal microvascular endothelial cells were purified and cultured from normal human colonic tissue specimens. For regulation experiments, HIMEC were stimulated with TNF- $\alpha(20 \mathrm{ng} / \mathrm{ml})$, LPS $(1 \mu \mathrm{g} / \mathrm{ml})$, IFN- $\gamma(40 \mathrm{IU} / \mathrm{ml})$, or IL-1 $\beta(20 \mathrm{IU} / \mathrm{ml})$. Expression of LST-1 in human endothelial cells was assessed by Gene Array Analysis (Affymetrix HG-U 133), semi-quantitative RT-PCR, and sequence analysis of obtained PCR fragments using a capillary DNA sequencing system (ABI PRISM 3100 Genetic Analyzer). Protein expression in endothelial cells was assessed using polyclonal antisera by immunoblot analysis. Results: Gene array analysis revealed a strong and exclusive upregulation of LST-1 in HIMEC, but not the human reference microvascular endothelial cell line, HMEC-1, after stimulation with recombinant human TNF- $\alpha(20 \mathrm{ng} / \mathrm{ml})$ after $4 \mathrm{~h}$ and $24 \mathrm{~h}$, respectively. Semi-quantitative RT-PCR analysis of HIMEC stimulated with proinflammatory stimuli revealed a strong up-regulation of six LST-1 splice variants. Notably, of the stimuli tested, only IFN- $\gamma$ was unable to up-regulate LST-1 expression, which suggests that the mRNA expression of LST-1 in HIMEC is likely dependent on activation of the transcription factor, NF-KB. Sequencing analysis data obtained from purified RT-PCR fragments were suggestive of soluble and transmembrane splice variants of LST-1. Immunoblot analysis of whole cell lysates of HIMEC stimulated with TNF- $\alpha$ revealed multiple protein isoforms. Conclusions: This is the first report indicating regulated expression of LST-1 in human endothelial cells derived from human intestine. Given its potential functions in modulating the immune system and antigen-presentation, further research is warranted to closely define the role of soluble and membrane expressed LST-1 in the regulation of mucosal intestinal immunity and inflammation.

\section{$\mathrm{T} 1760$}

Cytoplasmic Domain of Intercellular Adhesion Molecule-1 Is Required for T Lymphocyte Adhesion and Migration: Implication for the Involvement of Actinin Binding Motif

Suck Chei Choi, Hyun Mee Oh, Ki Jung Yun, Geom Seog Seo, Young Woo Sohn, Tae Hyeon Kim, Yong Ho Nah, Chang Duk Jun

Intercellular adhesion molecule-1 (ICAM-1) has been known to enhance leukocyte adhesion, thereby inducing migration through blood endothelial cells. However, the molecular event during these processes is largely unknown. To identify whether ICAM-1 cytoplasmic domain participates in $\mathrm{T}$ lymphocyte adhesion and migration, following ligand-receptor binding event, we constructed several ICAM-1 cytoplasmic domain deletion mutants that were fused with green fluorescence protein (GFP). COS-7 cells expressing ICAM-1_GFP mutant without $\alpha$-actinin binding motif (ICAM-1_ $\triangle$ AT GFP) revealed no association with the actin cytoskeleton, while wild-type ICAM-1_GFP showed clear association with the actin. In addition, in contrast to the wild type ICAM-1_GFP, ICAM-1_AAT_GFP was not localized properly on microvilli and revealed no punctuate forms on the cell surface, suggesting that $\alpha$-actinin binding motif is important for the proper localization of ICAM-1 on the cell membrane. Membrane projection as well as T lymphocyte adhesion was decreased in the cells expressing ICAM- 1 without $\alpha$-actinin motif or entire cytoplasmic domain. We also found that $\alpha$-actinin biding motif deficient ICAM-1 resulted in significant decrease of transmigration as determined by transwell chamber assay. Collectively, these results suggest that $\alpha$-actinin binding motif in the cytoplasmic domain of ICAM-1 plays important role for T lymphocyte adhesion and transmigration, presumably through the regulation of actin dynamics

\section{T1761}

Novel Regulated Expression of Phosphatidylserine-Specific Phospholipase Al (PS-PLA1) in Human Intestinal Microvascular Endothelial Cells (HIMEC)Evidence for Secreted Enzyme Activity

Jan Heidemann, Christoph Ruther, Moritz Kebschull, Matthias Lehr, Matthias Bruwer, Wolfram Domschke, Torsten F. Kucharzik, Christian Maaser

Background: Phosphatidylserine (PS)-specific phospholipase A 1 (PS-PLAl) is a secreted enzyme that efficiently hydrolyzes PS exposed on the surface of apoptotic cells and activated platelets to produce 2-acyl-lysophosphatidylserine (lysoPS), which is a potent lipid mediator for mast cells, neural cells, and T cells. Effects of lysoPS on T cells include the inhibition of mitogen-induced proliferation, as well as activation of interleukin (IL)-2 production of $\mathrm{T}$ cells. Gene arrays performed to assess intestine-specific gene expression patterns of human endothelial cells after proinflammatory stimulation revealed a strong up-regulation of PSPLAl mRNA. We therefore aimed to assess the expression and enzymatic activity of human intestinal microvascular endothelial cells under proinflammatory conditions. Material and Methods: Human intestinal microvascular endothelial cells were purified and cultured from normal human colonic tissue specimens, and macrovascular endothelial cells from umbilical veins (HUVEC) were obtained according to standard protocols. For regulation experiments, endothelial cells were stimulated with TNF- $\alpha(20 \mathrm{ng} / \mathrm{ml})$, LPS $(1 \mu \mathrm{g} / \mathrm{ml})$, IFN- $\gamma(40 \mathrm{IU} / \mathrm{ml})$, or IL-1 $\beta$ (20 IU/ml). Expression of PS-PLAl was assessed by semi-quantitative RT-PCR and real time RT-PCR. Restriction fragment analysis was used to identify PS-PLAl splice variants in endothelial cells. A HPLC assay measuring oleic acid liberation from the PS-PLAl substrate
1-oleoyl-2-hydroxy-sn-glycero-3-[phospho-L-serine] was performed to detect PS-PLAl activity in cell culture supernatants and endothelial cell lysates. MG-132, an inhibitor of NF- $\mathrm{\kappa B}$ activation was used in stimulation experiments. Results: Gene array analysis revealed a marked up-regulation of PS-PLAl in HIMEC after stimulation with recombinant human TNF- $\alpha(20 \mathrm{ng} / \mathrm{ml})$ after $4 \mathrm{~h}$ and $24 \mathrm{~h}$. Likewise, endothelial PS-PLAl expression was markedly enhanced by proinflammatory stimuli utilizing the NF- $\mathrm{KB}$ pathway, including bacterial lipopolysaccharide (LPS) and IL-1 $\beta$. In turn, MG-132 inhibited the TNF- $\alpha$ induced stimulation of PS-PLAl expression in endothelial cells. Using RFLP analysis, HIMEC were shown to concomitantly express an active splice variant, PS-PLAl $\Delta \mathrm{C}$. Moreover, HIMEC cellular extracts and supernatants were shown to elicit regulated phospholipase Al activity. Conclusions: This is the first report describing the regulated expression of PS-PLAl in human endothelial cells. By secretion of PS-PLAl activity, human microvascular endothelial cells might mediate immediate and distant immune effects on $\mathrm{T}$ cells through generation of the lipid mediator, lysoPS.

\section{$\mathrm{T} 1762$}

Human Intestinal Intraepithelial Lymphocytes (IELs) Carry Out Fas Ligand (FL)-Mediated Spontaneous Cytotoxicity (sc) of Epithelial Cell (EC) Tumors Through Nkg2d

Ellen C. Eber

Introduction: Human IELs are TCR $\alpha \beta^{+} \mathrm{CD} 8^{+} \mathrm{T}$ cells located between ECs. Their SC activity specifically directed against EC tumors is likely to be an important host defense against colon cancer. The binding of NKG2D on NK and CD8 ${ }^{+} \mathrm{T}$ cells with its ligands (ULBP1, ULBP2, ULBP3, and MIC) on colon cancer is important for cytotoxicity. This study explores the mechanism of SC activity by IELs, particularly the role of NKG2D. Methods: IELs were co-cultured with ${ }^{51} \mathrm{Cr}$-labelled target cells, and the percentage lysis determined after 4- or 18-hrs. The involvement of NKG2D was studied using MIC-transfected CIR cells and chimeric NKG2D and ULBP proteins. Results: After 4 or 18 hrs, fresh IELs had little perforin-mediated cytotoxicity against anti-CD3-coated P815 cells. However, IELs carried out moderate TNF $\alpha$ and marked FL-mediated cytotoxicity against the WEHI cells and Jurkat cells, respectively, target cells that are particularly susceptible to each mechanism of lysis. For killing of HT29 cells, however, IELs utilized a combination of TNF $\alpha$ - and FL-mediated mechanisms. For this to occur, IELs needed 18 hrs of contact with HT-29 cells so that both TNF $\alpha$ and FL were up-regulated on the surface of the IELs. This is necessary because TNF receptors and Fas are minimally expressed on the surface of HT-29 cells. The involvement of the NKG2D pathway was demonstrated by a $75 \%$ reduction in cytotoxicity of HT-29 cells when NKG2D was blocked by specific antibodies or chimeric proteins. To determine whether the NKG2D ligands on EC tumors were necessary for lysis, the LOVO colonic adenocarcinoma cells were studied as they have low expression of the NKG2D ligands. The LOVO cells were as susceptible to lysis as HT-29 cells although blocking NKG2D reduced cytotoxicity by only $20-30 \%$ and the lysis was mainly TNF $\alpha$-mediated. This suggests that pathways not involving NKG2D can trigger TNF $\alpha$-induced lysis when NKG2D ligands are unavailable. Finally, IEL killing of MIC-transfectants was inhibited by blocking MIC or FL, but not TNF $\alpha$, suggesting that the NKG2D pathway induces FL-mediated lysis. Conclusion: This study shows that HT-29 cells are spontaneously lysed by IELs but only after an 18-hr co-culture during which TNF $\alpha$ and FL are up-regulated on the surface of IELs. NKG2D induces mainly FL-mediated cytotoxicity. When ligands to NKG2D are unavailable on the EC tumors, the lysis becomes mainly TNF $\alpha$-mediated

\section{$\mathrm{T} 1763$}

A Murine Model of Appendicitis and Its Impact On the Lymphocyte Constituents

Watson W. Ng, Andrew Llyod, Micheal C. Grimm

The appendix has long been regarded as an evolutionarily vestigial organ. Its role in gastrointestinal mucosal immunity has been highlighted by the demonstration that appendicectomy for appendicitis is protective against the development of ulcerative colitis. Although there are well established murine models of colitis, no murine model of appendicitis has been established. We therefore developed a murine model of appendicitis and examined its impact on the lymphocyte constituents of the murine appendix. Methods: SPF Balb/c mice were anaesthetised by intra-peritoneal injection of ketamine $(100 \mathrm{mg} / \mathrm{kg})$ and xylazine $(3.4 \mathrm{mg} /$ $\mathrm{kg}$ ). A $1 \mathrm{~cm}$ left-sided laparotomy was performed. The caecum was mobilised. The caecal patch (the murine equivalent of human appendix) was transformed into an appendix-like pouch by standardised continuous suction and a ligation rubber band was loaded around the neck of "appendiceal pouch". The caecum with obstructed "appendiceal pouch" was returned into the peritoneal cavity and sterile phosphate buffered saline was spread into the abdominal cavity. The peritoneum and the skin were closed in layers. Mice were then sacrificed at different time points and the "appendiceal pouches" were examined histologically for features of appendicitis. Appendiceal lymphocytes were examined by flow cytometry in particular for phenotypic markers of activiation and regulation. Results: All appendiceal pouches developed histological features resembling human appendicitis with mucosal ulceration, transmural inflammation with neutrophilic and lymphocytic infiltrates, with occasional eosinophils, and serositis. These changes were most evident between days 10 and 14 after induction of appendicitis. No ischaemic necrosis, appendiceal abscesses, significant peritonitis or perforations were observed. All mice remained well as determined by clinical scoring. Compared with normal murine appendiceal lymphocytes, there is an increase in CD3 CD4 T cells (from $17.5 \%$ to $30.5 \%$ ) and CD3 CD8 T cells (from 3.7\% to 10.2\%). In contrast, there is a reciprocal reduction in B cells (from $68.8 \%$ to $42.2 \%$ ). There is also an increase in CD25 Foxp3 T cells (from 1.07\% to 1.91\%). Conclusion: This is the first description of a murine model of appendicitis with features of mucosal ulceration, neutrophilic infiltration and serositis resembling human appendicitis. Preliminary data showed that there is marked shift of lymphocytes into a $\mathrm{T}$ cell predominant response associated with an increment in regulatory T cells. Further experiments are ongoing to examine the full impact of appendicitis on the lymphocyte constituents of the caecal patch and on the immunological outcome of colitis. 
$\mathrm{T} 1764$

PI3K Activates a Critical Step During Intestinal Inflammation-Induced Activation of Intestinal Stem Cell

Ramanarao . Dirisina, Navdha Mittal, Jennet Manjali, Yueming Tang, Goo Lee, Radhika Mutyala, Gery Grimm, Linheng Li, Xi C He, Gary Wu, Mei-Lun Wang, Samuel Ho, Xiao De Tan, Yaqin Zhu, Hans Clevers, Harry Begthel, Terrence A. Barrett

In the current study we have investigated if PI3K regulates stem cell activation. Data from human polyposis syndromes and gene knockout mouse models suggests that aberrant PI3K signaling promotes intestinal neoplasia via activation of intestinal stem cell (ISC). To examine regulation of ISC by local immune populations we utilized anti-CD3-induced T cell activation model of intestinal inflammation. Following $\mathrm{T}$ cell stimulation we see an increase in activated ISC as indicated by $>150 \%$ increase in nuclear beta-catenin staining in crypts. Analysis of isolated crypt cells revealed a rapid (3h) $125 \%$ increase in nuclear beta-catenin combined with elevated (4-12 fold) mRNA for a range of Wnt/B-catenin target genes (cMyc, cyclin D1, fra-1, MMP-7, cryptdin, Muc2, trefoil factor etc.). A robust proliferative response was shown by marked expansion of cells that dual-stained for cMyc and incorporated BrdU. Pluripotent effects on secretory lineage generation was determined by IHC, western blots and real-time RT-PCR for cryptdin, MMP-7 and lysozyme (paneth), trefoil factor and Muc 2 (goblet), chromogranin A (enteroendocrine) and cryptdin and trefoil factor (intermediate). Double staining of lineage marker with BrdU were used to determine accelerated generation of enterocytes as well as secretory linages. Role of PI3K and its downstream targets was assessed by western blot for pathway proteins (GSK3b, mTOR, BAD). Treatment of mice with PI3K inhibitor LY294002 (30mg/kg X 4 doses) drastically reduced the numbers of T cell induced activated ISC, cMyct/BrdU+ progenitors while abrogating generation of multipotent secretory progenitors. Taken together these data suggest that during T cell activation PI3K/ Akt signals led to nuclear beta-catenin accumulation and activation of ISC followed by enhanced production of enterocytic as well as secretory lineages. Similarly these pathways have increased production of goblet and Paneth cell factors to enhance barrier immunity as well.

\section{$\mathrm{T} 1765$}

Mucosal Delivery of Ovalbumin By the Genetically Modified L.Lactis Suppresses Systemic and Local Inflammatory T-Cell Responses in Dol1.10 Mice

Inge L. Huibregtse, Henri Braat, Veerle Snoeck, Koen van Laer, Anje A. Te Velde, Pieter Rottiers, Sander J. van Deventer

Background: Genetically modified Lactococcus lactis (Ll) can be used for local delivery of immunomodulatory proteins to the intestinal mucosa. We adopted this strategy to study the role of local and systemic immune responses after mucosal delivery of ovalbumin. For this purpose we genetically engineered ovalbumin (OVA) secreting $\mathrm{Ll}$ and we evaluated the immune response in OVA-TCR transgenic mice (DO11.10) after oral supplementation. Methods: A PCR amplified OVA cDNA fragment was ligated in the erythromycin resistant pTINX vector. Bacteria were grown overnight at $37 \mathrm{C}$ in erythromycin supplemented medium, diluted 1:50 and grown for another three hours and harvested at exponential growth phase (approximately 1x107 bacteria). Quantification of the secreted OVA was evaluated by ELISA and western blot. Mice were fed for 10 consecutive days with low dose OVA or lxl09 bacteria/day (Ll or LlOVA). Four days later mice were sensitized with 100ug OVA s.c. in the base of the tail. Another 12 days later, mice ears were injected with loug OVA, and ear thickness was measured after 24 hours. At day 27, the intestinal tract was isolated for histology. Bulk spleen and bulk MLN cells were used for FACS analysis on Tcell expression markers (CD4+OVATCR+ specific: CD25, foxp3, CD45RB, CD69, CD44 and $\mathrm{CD} 62 \mathrm{~L})$ and restimulated with OVA $(0.5 \mathrm{mg} / \mathrm{ml})$. Supernatants were used for cytokine measurements. Results: Sequence analysis confirmed the cloning strategy of the vector. In the exponential growth phase LlOVA produced $50 \mathrm{ng} / \mathrm{ml}$ OVA measured by ELISA. Daily intragastric administration of LlOVA led to a significant decrease of ear thickness compared to the sensitized Ll treated mice and the orally OVA fed mice (1x10-2mm vs $6.5 \times 10-2 \mathrm{~mm}$ vs $16.2 \times 10-2 \mathrm{~mm}$ respectively). A decrease in IFN-g production in bulk spleen cells and an increase in IL-10 production in MLN cells was seen in LlOVA compared to the Ll treated mice ( 67.8 vs $278.9 \mathrm{pg} / \mathrm{ml}$ and 41.4 vs $9.25 \mathrm{pg} / \mathrm{ml}$ ). Conclusion: The mucosal delivery of OVA by genetically modified Llactis induces suppression of local and systemic inflammatory OVA specific T-cell response in DO11.10 mice. These data strongly suggest that the mechanism of action is the induction of regulatory T-cells and that the mode of mucosal delivery of an antigen to the immune system critically determines immune activation.

\section{T1766}

Role of Trem-1 in PMN Transmigration

Titus A. Reaves, Lindsey M. Parent

Neutrophil (PMN) migration to sites of infection is the first line of defense serving as an essential component of the innate inflammatory response. When dysregulated, PMN migration underlies the basis of a number of inflammatory diseases in the intestine. Microbial products have been shown to have potent effects on the immune system and the intestinal lumen contains considerable amounts of such products. A recently discovered protein, a triggering receptor expressed on myeloid cells (TREM-1) has been implicated in PMN survival when PMN are exposed to specific microbes and/or microbial products. TREM-1 has also been shown to interact with toll-like receptors (TLR) and mediate the cell response to microbial products. We set out to further characterize and investigate the role of TREM-1 in PMN migration by exposing PMN to gram-positive and gram-negative bacterial products during fMLP directed migration across acellular filters. Following 120 minute incubation in the absence of antibodies, $50 \%$ of the applied load of PMN migrated. In contrast, when antibodies (mAb) to TREM-1 were used in transmigration $25 \%$ of the applied load of PMN migrated. Exposure of PMN to gram-positive bacterial products combined with mAb TREM1 produced a further reduction in migration. In particular, in the presence of lipoteichoic acid (LTA) 38\% of the applied load of PMN migrated. However, when LTA was combined with TREM-1, only $18 \%$ of the applied load of PMN migrated, representing a $65 \%$ decrease in migration. A similar transmigration response was observed when PMN were exposed to the gram-positive bacterial lipoprotein MALP-2. We also exposed PMN to gram-negative bacterial products such as lipopolysaccharide (LPS) and PAM3CSK4 (bacterial lipoprotein). Results show that PAM3CSK4 when combined with mAb TREM-1 reduced migration to $\sim 21 \%$ of the applied load of PMN. However, LPS when combined with mAb TREM-1 had minimal effects on PMN transmigration. Migration assays using antibodies to TLR2 combined with TREM-1 also had minimal effects on transmigration. Using Flow Cytometry, we confirmed that PMN expressed TREM- 1 and that this expression is up regulated when PMN are exposed to specific bacterial products. Furthermore, there appears to be immunofluorescence differences in TREM-l expression in PMN when PMN are exposed to gram-positive and gram-negative bacterial products. Taken together this data suggests that TREM-1 expression may affect PMN migration into the intestinal lumen and thus contributes to deregulated PMN migration.

\section{T1767}

Pathophysiological Role of the Cholinergic Anti-Inflammatory and Immune Pathway in a Murine Model of Food Allergy

Naho Utsunomiya, Takeshi Yamamoto, Toshihisa Kodama, Hirofumi Kuramoto, Makoto Kadowaki

The enteric immune system which works as a bioprotection builds up the enteric intranet with the enteric nervous system. It contributes to maintain enteric and whole body homeostasis as they interact each other. Although the number of food allergy patients has been dramatically increasing over the last several decades, the pathogenesis of food allergy has not been elucidated. Therefore, regarding to the disturbance of the enteric intranet, we focused on the cholinergic anti-inflammatory and immune pathway through vagus-nicotinic acetylcholine receptors in a murine model of food allergy. METHODS: A murine model of food allergy was developed using BALB/c mice. For induction of food allergy, a group of mice was primed with ovalbumin (OVA) and aluminum hydroxide gel as adjuvant by intraperitoneal injection, and the occurrence of diarrhea following repeated oral challenges with OVA in the primed mice was recorded. Next, the colon of the food allergy mice was removed and used for myeloperoxidase (MPO) activity analysis and for quantitative measurements of inflammatory cytokine mRNA. RESULTS: Exposure of the primed mice to repeated oral OVA challenges induced diarrhea $[85.4 \pm 8.5 \%$ (mean \pm S.E.) after six OVA challenges] associated with 2.4 fold increased MPO activities in the colon and with dramatically increased IgE levels in sera In addition, pretreatment with calcineurin inhibitor to suppress T helper cell type 1 (Thl) responses deteriorated the allergic diarrhea. The treatment with anti-allergic drug disodium cromoglycate, failed to significantly suppress the allergic diarrhea. Notably, the central stimulation of vagus nerves with 2-deoxy-d-glucose $(200 \mathrm{mg} / \mathrm{kg}$ i.p.) significantly improved the allergic states $(54.5 \%$ of the food allergy control, $\mathrm{P}<0.05)$. Furthermore, subcutaneous administration of nicotine $(3.2 \mathrm{mg} / \mathrm{kg}$ ) suppressed allergic diarrhea $(31.1 \%$ of the food allergy control, $\mathrm{P}<0.01$ ) and MPO in the colon $(57.6 \%$ of the food allergy control, $\mathrm{P}<0.01$ ), whereas nicotine exerted no inhibitory effect on IgE levels in sera $(121.2 \%$ of the food allergy control). On cytokine mRNA expression, there was a significant increase in IL-4, IL5 , and IL-10 (Th2 cytokines) in the food allergy mice, whereas IFN- $\gamma$ (Thl cytokine) was not detected even in the food allergy mice, suggesting that immune response is skewed toward Th2 in the food allergy mice. Those raised Th2 cytokines dropped back to the normal state by the treatment with nicotine. CONCLUSION: Nicotinic acetylcholine receptors work on the cholinergic anti-inflammatory and immune pathway to alleviate Th2-mediated food allergy.

\section{$\mathrm{T} 1768$}

Intestinal Anaphylactic Response to Allergen Challenge Is Impaired in Immunized Germ Free Mice

Robin M. Hopwood-Courville, Jody L. Gookin, Susan Tonkonogy, Bruce Hammerberg

The incidence and severity of food hypersensitivity is increasing. In sensitized individuals, oral allergen challenge triggers a series of events termed intestinal anaphylaxis. Allergenbound $\operatorname{IgE}$ interacts with low affinity $\operatorname{IgE}$ receptors (CD23) on the surface of intestinal epithelium and is transported transcellularly to the lamina propria. Subsequent recognition of allergen by mast cell bound IgE results in release of pro-secretory mediators that induce epithelial $\mathrm{Cl}$ - secretion. Intestinal microflora have been demonstrated to influence development of tolerance to intestinal allergens and clinical studies demonstrate differences in intestinal microflora between individuals that do or do not develop allergy. The objective of this study was to determine the influence of normal intestinal microflora on immunization and the intestinal anaphylactic response to allergen challenge. Specific pathogen free (SPF $\mathrm{n}=4$ ) and germ free ( $\mathrm{GF} \cdot \mathrm{n}=3) 129 \mathrm{~S} 6 / \mathrm{SvEv}$ mice were immunized by intraperitoneal injection of HRP (100 mg) in $0.2 \mathrm{ml}$ alum and $50 \mathrm{ng}$ pertussis toxin (days 0,7 and 14). Age and sex matched GF mice were immunized with adjuvant alone in PBS. On day 21-24 jejunal mucosa was mounted in Ussing chambers, acclimated for 30-min, and challenged luminally with $\operatorname{HRP}(2 \mathrm{mg} / \mathrm{ml})$ or unrelated antigen (ovalbumin, $2 \mathrm{mg} / \mathrm{ml})$ in the presence or absence of blocking anti-CD23 antibody $(0.1 \mathrm{mg} / \mathrm{ml})$. Short-circuit current (Isc; reflects $\mathrm{Cl}$ - secretion in this model) was recorded every 60 -sec for 15 -min. At 2-min after allergen challenge, sections were removed, processed, and examined for transepithelial HRP transport by TEM Epithelial expression of CD23 was examined by immunofluorescence, mucosal mast cells were enumerated, and HRP-specific serum IgE ELISA was performed. SPF mice demonstrated a secretory response to luminal allergen challenge $\left(\mu \mathrm{A} / \mathrm{cm}^{2}=19 \pm 3\right)$ while GF mice did not. Epithelial CD23 expression did not differ between SPF and GF mice. HRP-challenged GF mice demonstrated CD23-dependent transepithelial transport. Mast cell numbers did not differ between SPF and GF mice and both groups demonstrated equivalent Isc responses to the secretogogue aminophylline. HRP-specific $\operatorname{IgE}$ was significantly lower $(\mathrm{p}=0.03)$ in sera of immunized GF mice $(0.148 \pm 0.067)$ as compared to immunized SPF mice $(0.801$ \pm 0.178 ). Results of the present study suggest that GF mice have impaired allergen signaling post-transepithelial transport. In mice, elevated circulating IgE causes upregulation of highaffinity IgE receptors on the surface of mast cells. A relative deficiency of IgE in GF mice may be responsible for decreased sensitivity to luminal allergen challenge. 
T1769

Anti-TNF Agents Are Capable to Induce Apoptosis in Macrophages But Not in Dendritic Cells - Potential Role of Mtnf

Pia Lebiedz, Sabine Westphal, Stefan Koch, Wolfram Domschke, Andreas Lugering, Torsten Kucharzik

Introduction: Anti-TNF antibodies such as infliximab and adalimumab are established in the treatment of chronic inflammatory bowel diseases. Previous studies demonstrated that these antibodies may induce apoptosis in monocytes and T-lymphocytes. Furthermore, infliximab has been shown to act through the transmembrane form of TNF (mTNF). We herein investigated the effect of adalimumab and infliximab on human macrophages and dendritic cells (DC). Material and Methods: Macrophages and dendritic cells were in vitro differentiated from fresh human monocytes by stimulation with MCSF $(25 \mathrm{ng} / \mathrm{ml})$ or GMCSF (25ng/ml) plus IL-4 $(10 \mathrm{ng} / \mathrm{ml})$, respectively. Cells were stimulated with 5 and $50 \mathrm{\mu g} / \mathrm{ml}$ adalimumab and infliximab. Apoptosis was determined by flow cytometric analysis of annexin-V expression and immuno-blotting of cleaved PARP (poly [ADP-ribose] polymerase) The proteasome inhibitor Mgl32 was used as positive control for induction of apoptosis. Expression of mTNF on cell surfaces was also determined by flow cytometry. MAPK activation was analysed by immuno-blotting. Results: Infliximab and adalimumab were both able to induce apoptosis in macrophages but not in dendritic cells as shown by annexin-V staining and PARP cleavage. mTNF was found on the cell surface of human in vitro differentiated macrophages whereas dendritic cells were mTNF negative. No difference in MAPK activation could be determined between both cell populations. Discussion: We herein show that infliximab and adalimumab both are able to induce apoptosis in human macrophages but not in dendritic cells. As infliximab has been shown to induce apoptosis via reverse signalling by binding to mTNF, the missing induction of apoptosis in DCs is probably due to a lack of mTNF on this cell population. This observation further supposes the importance of mTNF as a target for pro-apoptotic anti-TNF signalling.

$\mathrm{T} 1770$

The Expression of Ccr6 Identifies Lymphoid Tissue Inducer Cells Within Cryptopatches

Andreas Luegering, Sabine Westphal, Christian Maaser, Jan Heidemann, Torsten Kucharzik

Introduction: The murine gut contains small clusters of lymphocytes expressing the chemokine receptor CCR6 known as cryptopatches (CP) that were recently identified as adult lymphoid tissue inducer cells and represent precursors of isolated lymphoid follicles (ILF) Although various studies documented the presence of ILF within the human intestine, CP were not detectable. The aim of this study was to characterize the significance of CCR6 expression by adult LTi cells in CP and to identify similar structures within the human intestine. Methods: Lymphoid tissue inducer cells were isolated from the lamina propria of the murine small and large intestine, and c-kit+ lineage-marker negative cells (=LTi-cells) analyzed by flow cytometry. Frozen tissue sections from the murine intestine were stained for CCR6 in parallel. To identify human CP-like structures sections from colonic biopsies of healthy controls as well as ulcerative colitis patients were stained with antibodies to ckit and CCR6. Results: Flow cytometric analysis of lin- c-kit+cells isolated from the complete lamina propria could demonstrate that $15-20 \%$ express CCR6 whereas $70-75 \%$ of CP cells express CCR6 in tissue sections suggesting that LTi cells are present inside and outside CP. The comparison of these two cell populations showed similar expression of IL-2R and IL$7 \mathrm{R}$, whereas other surface markers (CD4, CD8, CD45Rb) are differently expressed. In the human intestine, staining for c-kit could identify a limited number of positive cells in small lymphoid aggregates within crypts, whereas the majority of these cells express CCR6. A significant increase of CCR6 positive lymphoid aggregates was found under inflammatory conditions. Discussion: The chemokine receptor CCR6 is specifically expressed by LTi cells within CP and might play a pivotal role in the progression of CP to ILF. The presence of small lymphoid aggregates of CCR6 expressing cells within the human intestine suggests that CP-like structures are also present in the human gut. As CCR6 is known to drive the progression of CP to ILF this receptor may be a valuable target for intestinal inflammatory conditions, e.g. inflammatory bowel disease.

\section{T1771}

Regulation of Intestinal Inflammation By Lamina Propria Cd4+Cd25+/Bright $\mathrm{T}$ Cells

Shin Makita, Takanori Kanai, Yasuhiro Nemoto, Yumi Ito, Michio Onizawa, Teruji Totsuka, Mamoru Watanabe

Background \& Aims: It is well known that immune responses in the intestine remain in a state of controlled inflammation, suggesting that not only active suppression by regulatory $\mathrm{T}$ cells plays an important role in the normal intestinal homeostasis, but also its dysregulation leads to the development of inflammatory bowel disease. Here we assess the intestinal $\mathrm{CD} 4^{+} \mathrm{CD} 25^{+} \mathrm{T}$ cells both in humans and mice. Methods: 1) Human normal mucosal samples were obtained from unaffected areas in intestine resected from patients with colon cancer. Informed consent was obtained from all patients prior to the study. We isolated human LP $\mathrm{CD}^{+} \mathrm{T}$ cells and analyzed their surface molecules. We next sorted human LP CD4 ${ }^{+} \mathrm{T}$ cells into $\mathrm{CD} 4^{+} \mathrm{CD} 25^{-}$or $\mathrm{CD} 4^{+} \mathrm{CD} 25^{\text {bright }}$ subsets using FACS Vantage, and both subsets were examined expression of FOXP3. We further investigated whether $\mathrm{LP} C D 4^{+} \mathrm{CD} 25^{\text {bright }} \mathrm{T}$ cells possess suppressive function in vitro. 2) For animal experiments, C.B-17 SCID mice were injected i.p. with SP $C D 4^{+} C D 45 R B^{\text {high }}$ alone $\left(2 \times 10^{5}\right.$ per body), SP $C D 4^{+} C D 45 R B^{\text {high }}+\mathrm{SP}$ $\mathrm{CD} 4^{+} \mathrm{CD} 25^{+}$(1x10 $0^{5}$ per body), or SP CD4 ${ }^{+} \mathrm{CD} 45 \mathrm{RB}^{\text {high }}+\mathrm{LP} \mathrm{CD}^{+} \mathrm{CD} 25^{+}$( $1 \times 10^{5}$ per body) to assess the role of $\mathrm{LP} \mathrm{CD} 4^{+} \mathrm{CD} 25^{+} \mathrm{T}$ cells in intestinal homeostasis. The recipient mice after transfer were monitored the weight and clinical signs of illness. The histological score of inflammation in the colon and the cytokine production by LP CD4 $4^{+} \mathrm{T}$ cells were also examined. Results: 1) Human LP CD4 $4^{+} \mathrm{CD} 25^{\text {bright }} \mathrm{T}$ cells constitutively expressed CTLA-4, GITR and FOXP3. LP CD4 ${ }^{+} \mathrm{CD} 25^{\text {bright }} \mathrm{T}$ cells suppressed the proliferation and various cytokine productions of $\mathrm{CD} 4^{+} \mathrm{CD} 25^{-} \mathrm{T}$ cells. $\mathrm{LP} C D 4^{+} \mathrm{CD} 25^{\text {bright }} \mathrm{T}$ cells produced less amounts of IL-2, IFN- $\gamma$ and IL-10 as compared with LP CD4+CD25- T cells. 2) The mice transferred with the CD45RB high $\mathrm{T}$ cell population developed a wasting disease with severe colitis. In contrast, the co-transfer of $\mathrm{CD} 4^{+} \mathrm{CD} 45 \mathrm{RB}^{\text {high }}+\mathrm{SP}$ or $\mathrm{LPCD} 4^{+} \mathrm{CD} 25^{+}$cells prevented a wasting disease and leukocyte infiltration in the colon, and suppressed IFN- $\gamma$ and TNF$\alpha$ production by LP CD4 ${ }^{+} \mathrm{T}$ cells. Conclusion: These results suggest that LP is one of the critical sites to control intestinal homeostasis by LP CD $4^{+} \mathrm{CD} 25^{+ \text {bright }}$ regulatory T cells.

\section{$\mathrm{T} 1772$}

5asa Blocks Stem Cell Activation in Intestinal Epithelial Cells After T-Cell Activation:a Possible Explanation for the Chemo Preventive Effects of 5 asa Therapy On Ulcerative Colitis Patients

Jennet J. Manjali, Goo Lee, Gery R. Grimm, Navdha Mittal, Terrence A. Barrett

Background and Aims: Recent data in humans suggest 5ASA therapy reduces the risk for CRC in patients with IBD. We postulated that a potential mechanism for the chemopreventive effects of 5ASA involves 5ASA acting as a scavenger for oxygen radicals that activate intestinal stem cells thereby inhibiting proliferation during stages of intestinal inflammation. Methods and Results: To address this hypothesis, mice (C57BL/6 (B6) and TNFR-/- ) were given either low dose(LD,100mg/250g chow) or high dose(HD,300mg/250g chow) 5ASA(pellet chow) regime for 5 days followed by $\mathrm{T}$ cell activation (anti CD3 mAb,0.2mg i.p) and the epithelial response was studied. $\mathrm{T}$ cell activation increases proliferation of crypt progenitor cells studied by BrdU incorporation(2h label). Mice (B6 and TNFR-/- ) were sacrificed $18 \mathrm{~h}$ after anti-CD3, the proliferative responses seen are summarized in Table 1. Intestinal stem cell activation, as studied by immunohistogical staining of nuclear $\beta$-catenin increased on induction (Xi et al,Nature Genetics2004).Table2 summarises the nuclear $\beta$-catenin in mice sacrificed 3hours after induction with or without pretreatment with LD or HD 5ASA. Apoptosis as studied by TUNEL showed an increase from 0.072 to 1.8 TUNEL positive cells/colonic crypt in B6mice. HD 5ASA pretreatment showed a 16\%decrease in the TUNEL positive cells. Conclusion: Taken together these data suggest a)the chemopreventive effects of 5ASA is dose dependent. b)5ASA acts is more effective in blocking the stem cell activation in distal over the proximal colon. c)5ASA effects the stem cells via a TNF dependent pathway. Grant supported by Proctor and Gamble

Table 1 Shows effects of 5ASA treatment on proliferation in T-cell activated Colon( Numbers indicate BrdU positive cells/crypt)

\begin{tabular}{|c|c|c|c|c|}
\hline & Control & Anti-CD3induced & $\begin{array}{c}\text { LD 5ASA } \\
\text { +Anti-CD3 }\end{array}$ & $\begin{array}{c}\text { HD 5ASA } \\
\text { +Anti-CD3 }\end{array}$ \\
\hline Proximal Colon(B6 Mice) & $4\left(+/-1^{*}\right)$ & $10\left(+/-2^{*}\right)$ & $9\left(+/-1^{*}\right)$ & $10(+/-1 *)$ \\
\hline $\begin{array}{c}\text { Distal Colon(B6 Mice) } \\
\text { Proximal Colon(TNFR-/- } \\
\text { Mice) }\end{array}$ & $4\left(+/-1^{*}\right)$ & $10\left(+/-2^{*}\right)$ & $6\left(+/-1^{*}\right)$ & $4(+/-1 *)$ \\
\hline $\begin{array}{c}\text { Distal Colon(TNFR-/- } \\
\text { Mice) }\end{array}$ & $3(+/-1 *)$ & $6(+/-1 *)$ & $6\left(+/-1^{*}\right)$ & $6(+/-1 *)$ \\
\hline
\end{tabular}

*p value is less than or equals 0.05

Table 2 Shows the effects of 5ASA treatment on the expression of Nuclear Beta-Catenin in T-cell activated colon (Numbers indicate Beta-Catenin positive nuclei/1000 epithelial cells)

\begin{tabular}{|c|c|c|c|c|}
\hline & Control & $\begin{array}{c}\text { Anti-CD3 } \\
\text { induced }\end{array}$ & $\begin{array}{c}\text { LD } \\
\text { 5ASA+Anti-CD3 }\end{array}$ & $\begin{array}{c}\text { HD } \\
\text { 5ASA+Anti-CD3 }\end{array}$ \\
\hline Proximal Colon(B6 Mice) & 3.2 & 12.5 & 10.8 & 13.8 \\
\hline Distal Colon(B6 Mice) & 2.9 & 11.5 & 6.6 & 3.4 \\
\hline $\begin{array}{c}\text { Proximal Colon(TNFR-/- } \\
\text { Mice) }\end{array}$ & 2.3 & 7.5 & 6.2 & 5.8 \\
\hline Distal Colon(TNFR-/-Mice) & 2.1 & 6.8 & 4.8 & 5.5 \\
\hline
\end{tabular}

\section{$\mathrm{T} 1773$}

\section{Maternal Microchimerism in Crohn's Disease}

David Suskind, Melvin B. Heyman, Denice Kong, Baxter-Lowe Lee-Ann, Muench O. Meunch

Crohn's disease is a chronic disease characterized by inflammation of the gastrointestinal tract. The etiology is unknown. Crohn's disease shares many similarities with graft verses host disease (GVHD) seen after bone marrow transplantation. Disease location, histology and inflammatory responses are similar in both Crohn's and GVHD. Adaptive transfer models, using selective transfer of alloimmune cells, have been shown to cause transmural inflammation within the intestines of immunodeficient mice. This suggests engraftment of maternal cells during gestation could result in an immune response that leads to Crohn's disease. The aim of this study was to determine the presence and extent of maternal microchimerism (MM) in the tissue of patients with Crohn's disease. Methods: Eighteen patients were recruited from the Division of Pediatric Gastroenterology at Children's Hospital in Seattle, Washington. Blood samples were obtained from mothers and patients for HLA typing. Tissue samples from seven different regions of the gastrointestinal tract (stomach, esophagus, rectosigmoid, descending colon, transverse colon, cecum and terminal ileum) were obtained from patients during diagnostic endoscopy/colonoscopy. Using kinetic polymerase chain reaction (kPCR), patients' tissues were evaluated for MM. Results: Of the eighteen patients recruited, 7 were diagnosed with Crohn's disease; 10 patients did not have Crohn's disease. Using kPCR, MM was observed in the tissues of 4 of the 7 (57\%) patients with Crohn's disease and in 1 of the $11(9 \%)$ patients without Crohn's ( $\mathrm{p}=0.05)$. In patients with IBD, MM was identified in both inflamed and non-inflamed tissues ( $p=N S$ ). There was no association between MM and disease location, disease severity or granuloma formation. In individuals with IBD, tissue microchimerism was found with a range of 0.4 to 2.8 copies of maternal DNA per 25,000 copies of patients DNA. Conclusion: Maternal microchimerism 
is present in the gastrointestinal tissues of patients with Crohn's disease more frequently than in those individuals without Crohn's disease. Further investigation is required to help elucidate the role of MM in Crohn's disease.

\section{T1774}

Role of Appendix in Oral Tolerance Induction in Normal and Mice with Dextran Sulfate Sodium Induced Colitis

Li Youming, Yu Chaohui, Yue Min, Liu Yulan

Aims: Oral tolerance is a state of specific immunologic hyporesponsiveness by feeding antigens before challenging. Appendix, which was considered as a retrogressed organ, has been postulated to be involved in mucosal immune responses and the pathogenesis of dextran sulfate sodium induced colitis. We evaluated the presence of oral tolerance in appendectomized or sham operated mice and those mice with dextran sulfate sodium induced colitis. Materials and methods: Oral tolerance was induced via ingestion of ovalbumin with either single dose of $20 \mathrm{mg}$ or $250 \mathrm{ug}$ every other day in 10 days after 7 days of appendectomy or sham operation (BSA as control). DTH reaction was detected at 7,14,2ldays after intraperitoneal injection of ovalbumin in CFA. Feeding $5 \%$ dextran sulfate sodium in water was started 7 or 14 days after antigen challenging for 7 days to induce colitis. Clinical disease activity was assessed daily and colonic injury was graded by histology 7 days after the induction of colitis. Results: Feeding single dose of $20 \mathrm{mg}$ ovalbumin may cause oral tolerance after 14 days in sham operated mice with alleviated DTH reaction while not in appendectomized mice in 21 days after challenging. Repeated low dose of ovalbumin ingestion may induce oral tolerance after 7 days in sham operated mice but retarded to at least 14 days in appendectomized mice. No significant alleviation of DTH reaction was detected in both high dose ingested group in 21 days and repeated low dose ingested group in 14 days in appendectomized mice with DSS induced cilitis, while decreased DTH reactions were observed in both sham operated groups with colitis. No siginificant histological difference was observed except in appendectomized mice ingested with lower dose of ovalbumin repeatedly revealing significant lower damage scores in histology compared with BSA controlled appendectomized ones. Conclusions: Appendix is essential to oral tolerance induction and appendectomy may postpone the induction of oral tolerance. Oral tolerance still exits during the procedure of DSS induced colitis in normal mice but has been distorted in appendectomized mice with DSS induced colitis.

\section{$\mathrm{T} 1775$}

Absence of Cd1 Exaggerates Murine DSS-Induced Colitis: A Protective Role for NK T Cells

Jacob Rullo, Christina Hirota, Alison E. Fox-Robichaud, Derek McKay

Ulcerative colitis (UC) is a multi-factorial disease with unknown aetiology. At the cellular level, NK T cells have recently been illustrated as key immune regulators of mucosal inflammatory processes. Our objective was to characterize both the role NK T cells and dietary fat in murine dextran sodium sulphate (DSS) induced colitis which resembles human ulcerative colitis. Male C57Bl/6 mice or B6/CD1-/- (NK T cell deficient) were given $4 \%$ (w/ v) DSS for 5 days followed by 3 days normal drinking water. Mice were grouped into either a breeder (12\% fat) or low fat (4\%) diet. Mice were euthanized, a clinical score assigned based on macroscopic observation, and portions of the mid-distal colon removed for electrophysiological analysis in Ussing chambers and MPO activity. Normal mice on breeder diet, exposed to DSS, displayed typical signs of colitis: diminished responses to pro-secretary stimuli, namely electrical stimulation, carbachol and forskolin (all cause $\mathrm{Cl}$ - efflux from the epithelium) when compared to age, time and diet matched control. B6/CD1-/- mice on breeder diet responded to DSS with a $40 \%$ mortality rate, not seen in normal mice, in addition to diminished short circuit current, both baseline and stimulated, as well as macroscopic scores $(10.4 \pm 0.4$ vs. $2.3 \pm 0.3)$ when compared to normal mice, given DSS, on a breeder diet. It was also observed that a diet with reduced fat content increased the severity of DSS-induced colitis and ( $7.3 \pm 0.5$ vs. $2.3 \pm 0.3)$ decreased ion responses (Table). We have demonstrated that mice exposed to DSS-induced colitis have a greater degree of inflammation and altered ion-transport responsiveness when NK T cells are deficient. Diet also seems to be mediating the response to DSS, as C57Bl/6 mice on low-fat diet display more severe symptoms of colitis and comparatively diminished ion transport responses. This study may help in determining the underlying environmental and immune mechanisms involved in UC pathogenesis.

Ion Transport Responses and Inflammation Scores

\begin{tabular}{|c|c|c|c|c|}
\hline & $\begin{array}{l}\text { Electrical Stimulation } \\
(\mu \mathrm{A} / \mathrm{cm} 2)\end{array}$ & $\begin{array}{l}\text { Forskolin } \\
(\mu \mathrm{A} / \mathrm{cm} 2)\end{array}$ & $\begin{array}{l}\text { Carbachol } \\
(\mu \mathrm{A} / \mathrm{cm} 2)\end{array}$ & $\begin{array}{l}\text { MPO activity (g of } \\
\text { tissue) }\end{array}$ \\
\hline $\begin{array}{c}\text { C57Bl/6 Breeder } \\
\text { Diet }\end{array}$ & $20 \pm 3^{*}+\frac{1}{t}$ & $144 \pm 22^{*} \dagger$ & $58 \pm 12 *+\div$ & $6.4 \pm 0.2 *+$ \\
\hline $\begin{array}{c}\text { C57Bl/6 Low Fat } \\
\text { Diet }\end{array}$ & $7 \pm 3 *$ & $68 \pm 13 *$ & $11 \pm 6^{*}$ & $4.7 \pm 0.93^{*}+$ \\
\hline $\begin{array}{l}\text { C57B1/6 Breeder } \\
\text { Diet Control }\end{array}$ & $22 \pm 4$ & $326 \pm 9$ & $113 \pm 11$ & $0.2 \pm 0.1$ \\
\hline $\begin{array}{c}\text { B6CD1-/- Breeder } \\
\text { Diet }\end{array}$ & $4 \pm 2 *$ & $90 \pm 34^{*}$ & $11 \pm 7 * \%$ & $2.0 \pm 0.5+\$$ \\
\hline $\begin{array}{l}\text { B6CD1-/- Low Fat } \\
\text { Diet }\end{array}$ & $7 \pm 2 *$ & $126 \pm 17$ 米市 & $9 \pm 3 *$ & $6 \pm 1 *$ \\
\hline $\begin{array}{l}\text { B6CD1-/-Breeder } \\
\text { Diet Control }\end{array}$ & $13 \pm 2$ & $311 \pm 4$ & $38 \pm 11$ & $1.0 \pm 0.4$ \\
\hline
\end{tabular}

*statistically significant, compared to age, time, diet matched control, $\mathrm{P}<0.03 \uparrow$ itatistically significant, compared to same strain, DSS treated, opposite diet, $\mathrm{P}<0.03$ ₹statistically significant, compared to opposite strain, DSS treated, same diet, $\mathrm{P}<0.03$

\section{T1776}

The Transcription Factor Sox9 Contributes to the Defect in Ceacam1, 5, 6 Expression in CD

Giulia Roda, Stephanie Dahan, Ling Shao, Mee Sook Park, Franziska Roth-Walter, Okebugwn Kamalu, Xianyang Yio, Lloyd Mayer

AIM: We have previously documented a transcriptional defect in the expression of three of the CEA subfamily members expressed on intestinal epithelial cells (IECs), CEACAM6, CEACAM5 and CEACAMl in Crohn's disease (CD) but not in ulcerative colitis (UC). The consequence of the loss of expression is the lack of activation of CD8+ regulatory T cells. SOX9 is a transcription factor that regulates IEC differentiation through the Wnt and ? catenin pathway. CEA expression has been shown to be down-regulated by SOX9. The aim of this study was to define whether SOX9 was responsible for the defect in CEACAM expression. METHODS: SOX9 mRNA and protein was assessed by RT-PCR and Western Blot using a rabbit polyclonal antibody (Santa Cruz) in freshly isolated IECs from normal controls (diverticulitis, cancer) and IBD patients (inflamed and uninflamed tissues). Differential expression from crypt to surface was ascertained following sequential dispase treatments Paraffin sections were stained with the same antibody and counterstained with hematoxylin Regulation of SOX9 was assessed in fresh IECs and 2 IEC lines (T84 and HT29Cl.16E) using IL-6, TNF-? and IFN-? alone and in combination for 15 hours. RESULTS: SOX9 mRNA and protein expression was comparable in normal controls vs IBD IECs. Furthermore, there was no differential expression from crypt to surface. However upon immunohistochemical staining we noted that SOX9 protein was localized in the nucleus in CD IECs in contras to cytoplasmic localization in normal and UC IECs. ?-catenin expression paralleled SOX9 expression. SOX9 protein was decreased following treatment with IL-6, TNF-? and IFN-? while CEA expression was enhanced. CONCLUSIONS: These findings suggest that SOXO translocation to the nucleus in CD IECs suppresses transcription of CEA and that this is not related to the presence of inflammatory cytokines

\section{T1777}

Function of Intestinal Dendritic Cells Are Influenced By the Symphathetic Nervous System in the Gut That Modulates Acute and Chronic Colitis Ulrike G. Strauch, Florian Obermeier, Gisela Paul, Nicole Grunwald, Sonja Guerster, Juergen Schoelmerich, Rainer Straub

Background: Within the intestinal lamina propria a dense network of symphathetic nerves is found in close anatomical distance to intestinal immune cells such as lymphocytes and dendritic cells. During acute intestinal inflammation a decrease in the density of symphathetic nerves can be detected. The aim of our study was to investigate whether the sympathetic nervous system within the gut influences the function of intestinal dendritic cells (DC) and the course of colitis. Methods: Mice were sympathectomized by i.p. injection of 6-OHDA and mesenterial lymph node DC (MLN-DC) were isolated. Additionally, acute colitis was induced 5 days after sympathectomy by 3\% DSS within the drinking water for 7 days. Chronic DSS-colitis was induced by four cycles of 3\% DSS feeding and mice were sympathectomized directly after the last DSS-cycle. Weight loss was evaluated and colonic inflammation was quantified by a histological score. Cytokine secretion was measured within the supernatant of MLN-DC by ELISA. Immunohistochemistry for CDllc+ DC was performed on frozen sections. Results: After induction of acute DSS-colitis weight loss was reduced in sympathectomized animals (STX) as compared to control mice and colitis was significantly ameliorated as shown by histological score (control 7.6 \pm 0.5 , STX 4.2 $\pm 1.1, p=0.0079$ ) Immunohistochemistry was able to demonstrate a reduction in the number of activated DC within the lamina propria. On the other hand, in animals with established chronic colitis and symphathectomy intestinal inflammation was more severe (control 1.5 \pm 0.4 , STX $3.4 \pm 0.7$ $\mathrm{p}=0.0012$ ). MLN-DC from symphathectomized mice expressed more costimulatory molecules and higher levels of proinflammatory cytokines were secreted by these cells, representing an activated phenotype of antigen-presenting cells. Conclusion: During acute colitis the sympathetic nervous system plays a role within the induction and perpetuation of colitis as it directs immune cells e.g. dendritic cells to the inflamed tissue, whereas in chronic colitis it mediates protective effects as sympathetic stimuli inhibit activation of intestinal DC Therefore, the symphathetic nervous system influences gut inflammation by modulation of activation status and migration pattern of intestinal DC and could play a major role in the maintenance of tolerance within the healthy gut.

\section{$\mathrm{T} 1778$}

Iron Chelator-Induced IL-8 Production in Human Epithelial Cells Is Independent from Nf- $\mathrm{KB}$ But Involves AP-1 and CREB Activation

Eun Young Choi, Geom Seog Seo, Chang Soo Choi, Eun Young Cho, Young Woo Sohn, Ki Jung Yun, Tae Hyeon Kim, Suck Chei Choi, Yong Ho Nah, Chang Duk Jun

We have previously shown that a bacterial siderophore, deferoxamine (DFO), could trigger inflammatory signals in human intestinal epithelial cells by ERK1/2 and p38 pathways. In the present study, we determined transcription factors involved in DFO-induced IL 8 production. DFO markedly up-regulated the mRNA levels of essential components of transcription factor AP-1 in HT-29 cells. Although there was a slight expression of NF-kB subunit, pharmacological inhibitors that affect NF- $\mathrm{\kappa B}$ pathways showed little effect on the DFO-induced IL-8 production as well as the NF-kB transcriptional activity. In contrast, DFO significantly induced AP-1-dependent transcriptional activity and that transfection with dominant-negative (DN) c-Jun mutant markedly inhibited DFO-induced IL-8 promoter activity as well as AP-1-dependent transcriptional activity. Electrophoretic mobility shift assays confirmed that DFO increases the specific binding of AP- 1 but not NF-KB. Interestingly such AP-1 transcriptional and binding activities were blocked by pharmacological inhibitors of ERK1/2 and $\mathrm{p} 38$ kinase pathways, suggesting that MAPKs lie upstream of AP-1-dependent IL-8 production by iron chelator. DFO also induced phosphorylation of CREB and increased DNA-binding activity of CREB, both being affected by ERK1/2 and p38 kinase inhibitors.

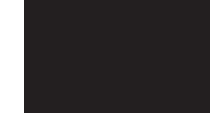


In summary, these results demonstrate that iron chelator-induced IL-8 production is independent from transcription factor NF- $\mathrm{KB}$ but involves AP-1 and CREB activation in human intestinal epithelial cells.

\section{T1779}

Membrane-Bound NAD(P)H Oxidase Repressess Cytotoxic Effects of Tumor Necrosis Factor (TNF)- $\alpha$ in Pancreatic Stellate Cells (PSCs) Via NF-kB Independent Mechanism

Eva C. Vaquero, Mariana Rickmann, Xavier Molero, Juan Ramon Malagelada

In many non-phagocytic cells $\mathrm{NAD}(\mathrm{P}) \mathrm{H}$ oxidase generates radical oxygen species (ROS) able to activate critical survival signals such as NF-kB. PSCs, the main cell type responsible for pancreatic fibrosis, are activated upon oxidative conditions. TNF- $\alpha$ is a redox-responsive molecule able to activate both survival and death pathways. In PSCS TNF- $\alpha$ induces prosurvival responses. AIM: To investigate the presence of a membrane-associated NAD(P)H oxidase as ROS source induced by TNF- $\alpha$ and its role in precluding TNF- $\alpha$ pro-apoptotic potential in PSCs. METHODS: Activated rat PSCs were preincubated for 30 min with the $\mathrm{NAD}(\mathrm{P}) \mathrm{H}$ oxidase inhibitor dyphenilene iodonium (DPI) and then stimulated with TNF- $\alpha$ for $30 \mathrm{~min}$ to $5 \mathrm{~h}$. Superoxide (O2.-) production by NAD(P)H oxidase was quantitated by lucigenin-enhanced chemiluminescence on membrane fractions. Cell death analysis was based on DNA fragmentation measured by ELISA; fluorescein diacetate (FDA)/propidium iodide (PI) staining by FACS; caspase- $8,-9$ and -3 activities by fluorometry; and mitochondrial membrane potential loss by FACS. mRNA expression of Nox subunits was analyzed by RTPCR. RESULTS: PSCs possess a functionally active NAD(P)H oxidase system as indicated by a basal production of O2.- and expression of Nox 1, Nox 2, Nox 4, p22, p47phox, and p67phox, key components of the enzymatic system. Exposure of cells to TNF- $\alpha$ for $30 \mathrm{~min}$ induced a 3 -fold increment in NAD(P)H oxidase activity. Both basal and TNF- $\alpha$ stimulated NAD(P)H oxidase activities were abolished by DPI. TNF- $\alpha$ by itself did not elicit cell death. However, when NAD(P)H oxidase activity was inhibited with DPI, TNF- $\alpha$ stimulated PSC death, as shown by DNA fragmentation (TNF- $\alpha+$ DPI: $8.6 \pm 2.2$ vs DPI: $6.7 \pm 2.5$ relative to control; $\mathrm{p}<0.05$ ), FDA-/PI- staining -apoptotic fraction- (TNF- $\alpha+$ DPI: $9.8 \pm 3.4$ vs DPI: $6.4 \pm 1.9 ; \mathrm{p}<0.05)$, FDA-/PI+ staining -aponecrotic fraction- (TNF- $\alpha+$ DPI: $18.5 \pm$ 1 vs DPI: $13.3 \pm 1.1 ; \mathrm{p}<0.05)$. The apoptotic effect of TNF- $\alpha$ upon NAD(P)H blockade was preceded by mitochondrial membrane depolarization and associated to large activation of caspase-8, -9 and -3 . Upstream caspase- 8 inhibition by IETD-fmk totally blocked DPIsensitization to TNF- $\alpha$ induced apoptosis, suggesting that DPI is uncovering the extrinsic caspase pathway of TNF- $\alpha$. TNF- $\alpha$ caused a rapid activation of NF-KB that was not prevented by DPI CONCLUSIONS: TNF- $\alpha$ stimulates ROS production in PSCs via NAD(P)H oxidase. This pro-oxidative effect silences the cytotoxic actions of TNF- $\alpha$ by an NF-kB independent mechanism. NAD(P)H oxidase can be a useful therapeutic target in the treatment of pancreatic fibrosis by facilitating PSC elimination.

\section{$\mathrm{T} 1780$}

Natural Vitamin E Tocotrienols, But Not a-Tocopherol, Induce Apoptosis and Autophagy in Pancreatic Stellate Cells (PSCs)

Mariana Rickmann, Eva C. Vaquero, Xavier Molero, Juan Ramon Malagelada

Natural vitamin E comprises two groups of compounds, tocotrienols and tocopherols. Although a-tocopherol is widely used in clinical practice, tocotrienols exert stronger antioxidant, cardioprotective, neuroprotective and tumor suppressive effects. Oxidative stress promotes activation and expansion of PSCs in chronic pancreatitis. In these settings, induction of PSC apoptosis might halt the progression of the disease. AIMS: To investigate the antioxidant and death promoting effects of both a-tocopherol and a palm oil derived tocotrienolrich fraction (TRF) in PSCs. METHODS: Activated rat PSCs were treated with TRF or atocopherol for 1 to $72 \mathrm{~h}$. Intracellular radical oxygen species (ROS) were measured by FACS using DCFH-DA; lipoperoxidation by fluorogenic assay in cells labeled with diphenyl-1pyrenylphosphine (DPPP); cell number by CyQUANT; apoptosis by DNA fragmentation, changes in mitochondrial membrane potential, and caspase-8, -9 and -3 activities; autophagy by detection of autophagic vacuoles stained with monodansyl cadaverine (MDC) and accumulation of LC3-II protein by immunoblot. RESULTS: Both TRF and a-tocopherol caused a mild decrease of ROS and a marked reduction ( $40 \%$ vs control) of lipoperoxidation. To exclude cell toxicity interference on ROS assessment, only viable cells (PI-excluding) were analyzed for DCFH-DA and DPPP labeling. Cell number decreased by 30\% after $24 \mathrm{~h}$ incubation with TRF but it was not affected by a-tocopherol treatment for up to $72 \mathrm{~h}$. In addition, TRF caused a significant increase in DNA fragmentation $(5.7 \pm 1.5$ fold vs untreated cells; $\mathrm{p}<0.0001$ ), which was associated with a large activation of caspase- $8,-9$, and -3 . Apoptotic changes were heralded by a pronounced mitochondrial membrane depolarization that was initiated during the first hour of TRF incubation. Interestingly, TRF did not induce apoptosis in quiescent (oil red positive) PSCs plated on basement membrane-like substrate (Matrigel). In contrast to TRF, a-tocopherol induced a weaker pro-apoptotic effect, shown by a modest increase in DNA fragmentation $(1.8 \pm 0.2$ fold vs untreated cells; $\mathrm{p}<0.0001)$ and by loss of mitochondrial membrane potential. Moreover, the apoptotic effect of a-tocopherol did not involve caspase activity. Cells exposed to TRF developed cytoplasmic autophagic vesicles as shown by MDC autofluorescence and accumulation of LC3-II. In contrast, atocopherol was unable to induce the above autophagic changes. CONCLUSIONS: Tocotrienols from palm oil, but not a-tocopherol, induce a robust cell death program (apoptosis and autophagy) in activated PSCs. Tocotrienols should be strongly considered as a potential therapeutic tool for chronic pancreatitis.

\section{T1781}

Overexpression of Thioredoxin-1 Prevents Cerulein-Induced Pancreatic Fibrosis in Mice

Shinya Ohashi, Akiyoshi Nishio, Hajime Nakamura, Junji Yodoi, Tsutomu Chiba

Background and Aims: Chronic pancreatitis (CP) is suggested to result from repetitive pancreatic injury, so-called necrosis-fibrosis sequence hypothesis. Various cytokines, growth factors, and chemokines released from injured tissues and inflammatory cells play an important role in the proliferation and activation of pancreatic stellate cells, which consequently induce pancreatic fibrosis. Considering the production of these mediators, oxidative stress is considered to play a crucial role. We previously reported thioredoxin-1 (TRX-1), an endogenous redox-regulating protein with an anti-oxidant effect, prevents experimental acute pancreatitis (AP) in mice. In this study, we aimed to clarify the protective role of TRX-1 on pancreatic fibrosis using $\mathrm{CP}$ model in transgenic mice overexpressing TRX-1 (TRX-1-TG mice). Methods: C57BL/6 wild type mice and TRX-1-TG mice of B6 origin were used for the experiment. At first, experimental AP was induced by intraperitoneal administration of cerulein and lipopolysaccharide injection. Histological changes and malondialdehyde levels in the pancreas were examined. Next, experimental $\mathrm{CP}$ was induced by three episodes of AP per week for 6 weeks. Pancreatic fibrosis was evaluated by histology and hydroxyproline content. Activation of pancreatic stellate cells was assessed by immunostaining and Western blotting for $\alpha$-smooth muscle actin ( $\alpha$-SMA). Moreover, pancreatic gene expression of transforming growth factor- $\beta$ (TGF- $\beta$ ), platelet-derived growth factor (PDGF), and monocyte chemoattractant protein-1 (MCP-1) was investigated using RT-PCR. Results: In AP model, overexpression of TRX-1 suppressed oxidative stress as determined by malondialdehyde levels, and attenuated acute pancreatic injury. In the pancreas of wild type mice in $\mathrm{CP}$ model, the acinar units were atrophied and some acinar units redifferentiated into tubular complexes. Moreover, thick bands of extracellular matrix accumulated around acinar units, and increased numbers of inflammatory cells were infiltrated within the matrix. However, pancreatic damages were obviously attenuated in TRX-1-TG mice. Pancreatic hydroxyproline contents and $\alpha$-SMA positive areas were significantly reduced in TRX-1-TG mice. Moreover, a gene expression of TGF- $\beta$, PDGF, and MCP- 1 in the pancreas was also significantly decreased in TRX-1-TG mice. Conclusions: TRX-1 suppressed oxidative stress in AP, which resulted in the attenuation of $\mathrm{CP}$. These results support the necrosis-fibrosis theory and oxidative stress is also supposed to be involved in the pathogenesis of $\mathrm{CP}$.

\section{T1782}

Suppression of IFN- $\gamma$ and Increase in IL-6 Is Associated with Ethanol-Induced Chronic Pancreatitis Responses in the CsA Model of Alcoholic Chronic Pancreatitis

Ilya Gukovsky, Mohammad Shahsahebi, Peggy P. Hong, Aurelia Lugea, Samuel W. French, Stephen J. Pandol

Background \& Aims: Alcohol abuse is a major cause of chronic pancreatitis; however, the pathogenesis of alcoholic chronic pancreatitis (ACP) remains unknown. A critical obstacle to understanding the mechanism of ACP is lack of animal models. We recently developed a model of alcohol mediated pancreatitis, termed "the CsA model of ACP", which for the first time reproduces all 3 responses characteristic of chronic human disease: loss of parenchyma, sustained inflammation, and fibrosis. These changes result from synergistic effect of ethanol diet, an episode of acute pancreatitis, and cyclosporin A (CsA) treatment. Our goal is to elucidate the mechanisms underlying these responses. Methods: Rats were pair-fed for 8 weeks Lieber-deCarli ethanol (E) or control (C) diet. For the last 2 weeks, they received CSA or vehicle. After 1 week on CsA, rats were subjected to 1 episode of cerulein (CR) pancreatitis, and killed at $3 \mathrm{~h}, 2,4$ or $7 \mathrm{~d}$ after CR treatment. Results: We found that ethanol feeding greatly inhibited pancreatic expression of IFN- $\gamma$ and up-regulated that of IL- 6 in our model. First, we compared the effect of all the treatments at 7 days after acute CR pancreatitis. Expression of both cytokines was detected by RT-PCR only with the combined treatment, i.e., $\mathrm{C}+\mathrm{Cs} \mathrm{A}+\mathrm{CR}$ and $\mathrm{E}+\mathrm{Cs} \mathrm{A}+\mathrm{CR}$, but not in other groups. In $\mathrm{E}+\mathrm{Cs} \mathrm{A}+\mathrm{CR}$ rats, IFN- $\gamma$ mRNA expression was several fold lower while that of IL- 6 was increased. These changes were associated with a much more severe pancreatic injury in the ethanol-fed rats, characterized by loss of up to $90 \%$ parenchyma, sustained inflammatory infiltration (9 times greater than in $\mathrm{C}+\mathrm{Cs} \mathrm{A}+\mathrm{CR}$ group), and widespread fibrosis. In time-course experiments on the two groups, IFN- $\gamma$ expression in pancreas remained undetectable acutely (i.e., at $3 \mathrm{~h}$ ) and dramatically increased at 2 days after the episode of acute CR pancreatitis. IFN- $\gamma$ mRNA levels then subsided, but in $\mathrm{E}+\mathrm{CsA}+\mathrm{CR}$ group they were several-fold less than in $\mathrm{C}+\mathrm{CsA}+\mathrm{CR}$ rats. By contrast, IL-6 mRNA expression was induced acutely, and it was significantly higher in $\mathrm{E}+\mathrm{Cs} \mathrm{A}+\mathrm{CR}$ rats than in their control-fed counterparts. Conclusions: Ethanol-induced suppression of IFN- $\gamma$, a key Thl mediator, together with up-regulation of IL-6 (that can be anti-inflammatory) may promote "immunoparalysis"-like condition, resulting in perpetuation of pancreatic injury. Because IFN- $\gamma$ is a major anti-fibrotic factor, its inhibition facilitates fibrosis induced by ethanol feeding in our model. These findings have important implications for the mechanism of ACP.

\section{$\mathrm{T} 1783$}

Sequence Variants of the CFTR Gene and Susceptibility to Idiopathic Chronic Pancreatitis

Bernhard Steiner, Jonas Rosendahl, Heiko Witt, Nils Teich, Volker Keim, Marie-Pierre Audrezet, Claude Ferec, Sabina Gallati, Macarena Gomez-Lira, Giorgio Cavallini, Kaspar Truninger

INTRODUCTION Compound heterozygous mutations in the CFTR gene are associated with idiopathic chronic pancreatitis (ICP), however, whether carriers of a single mutant CFTR allele have an increased ICP risk is unknown. We evaluated the role of common CFTR sequence variants, which we have recently shown to be associated with aberrantly spliced CFTR transcripts. METHODS 132 ICP patients and 394 healthy controls were genotyped for mutations and 5 sequence variants by SSCP-HD or dHPLC. We carried out a replication study involving 304 ICP patients and 309 healthy controls. RESULTS Mutations were 
identified in 43/132 (32.6\%) patients and in 22/394 (5.6\%) healthy controls ( $\mathrm{p}<0.001)$. No control subject, but nine patients $(7 \%)$ had a compound heterozygous genotype(OR 79.1, 95\% CI 4.6-1371.4; $\mathrm{p}<0.001)$. A single CFTR mutation increased ICP risk 5.6-fold (95\%CI 3.1-10.1; $<<0.001)$. None of the five sequence variants was associated with ICP, except the p.M470V variant. The $\mathrm{V}$ allele was more common among controls than ICP patients both in the first (53\% vs 47\%; p 0.08) and in the second population and ICP risk was increased in carriers of two V alleles (1. Popul OR 0.63, 95\% CI 0.36-1.08; p 0.09; 2. Popul OR 0.62,95\% CI 0.39-0.99\% $\mathrm{p}<0.05)$. The 5 sequence variants were used to infer haplotypes, of which one was associated with increased ICP risk (OR 1.91, 95\% CI 1.16$3.15 ; \mathrm{p} 0.02$ ) and one with decreased ICP risk (OR 0.68, 95\%CI 0.50-0.93; p 0.01). With the use of the UPGMA clustering method, haplotypes were grouped. Statistical analysis demonstrated one cluster to be associated with an almost 2-fold ICP risk increase (OR 1.92 , $95 \%$ CI 1.20-3.07; p 0.008), whereas another cluster showed a protective effect(OR 0.36, $95 \%$ CI 0.13-1.03; p 0.05). Patients and controls were assigned a CFTR cluster group combination depending on whether the haplotype group combination was associated with an adverse or protective effect on ICP susceptibility. The frequency of the homozygous risk genotype cluster was higher in patients (19\%) than controls (10\%)(OR 2.1, 95\%CI 1.1-4.0; $\mathrm{p}$ 0.02). Conversely, carriers of two protective genotype clusters were more common among controls ( $42 \%)$ than patients $(30 \%)(\mathrm{OR} 0.4,95 \% \mathrm{CI} 0.2-0.7 ; \mathrm{p} 0.002)$. These findings were confirmed in the second population both for the risk (OR 2.0,95\% CI 1.2-3.2; p 0.005) and the protective genotype cluster(OR $0.6,95 \% \mathrm{CI} 0.4-0.9 ; \mathrm{p} 0.02)$. Finally, in carriers of a single mutant CFTR allele, we found significantly more risk and protective clusters in patients and controls, respevtively. CONCLUSION Common CFTR sequence variants and their combinations modify ICP susceptibility, particularly in carriers of a single mutant allele.

\section{T1784}

Low-Dose Alcohol Redirects Postprandial Cholinergic-Stimulated Apical Exocytosis in Pancreatic Acinar Cells to the Basal Membrane, the Susceptibility Mechanism of Alcoholic Pancreatitis

Kai Yang, Patrick Lam, Herbert Y. Gaisano

BACKGROUND. We previously demonstrated that alcohol (ETOH) pretreatment of pancreatic acini caused the basal plasma membrane (BPM) to become receptive to aberrant BPM exocytosis upon stimulation with submaximal CCK-8. These actions of alcohol on the acinar cell were due to induction of PM-bound Muncl8c to dissociate into the cytosol, rendering the PM Syntaxin 4 to interact with PM-SNAP-23 and granule-VAMP to form a complex capable of mediating basolateral exocytosis into the interstitial space, which we had postulated to be a mechanism of alcoholic pancreatitis. Human pancreas however lack CCK receptors and instead responds to cholinergic stimulation. AIM: We therefore examined whether postprandial ETOH concentration $(20 \mathrm{mM})$ is able to alter postprandial cholinergic stimulation (luM carbachol) of rat pancreatic acinar cells. METHODS: Rat pancreatic acini were pretreated with non-stimulatory ETOH $(20 \mathrm{mM})$ followed by stimulation with postprandial carbachol (luM), a dose stimulating only half maximal amylase secretion. We then examined the effects on secretion by: (1) determination of amylase release, (2) real-time epifluorescent imaging of exocytosis using FMl-43; (3) confocal microscopy to examine the behavior of basolateral SNARE complex proteins; (4) cell subfractionation to quantify the SNARE protein levels on the PM. RESULTS: Whereas $20 \mathrm{mM}$ ETOH has no effect on amylase secretion, preincubation of acini with $20 \mathrm{mM}$ ETOH inhibited luM carbachol stimulated secretion. luM carbachol evokes apical exocytosis, but pretreatment with $20 \mathrm{mM}$ ETOH redirected exocytosis to the basal PM. While 20mM ETOH nor $1 \mathrm{uM}$ carbachol affected BPM-bound Munc18c, confocal microscopy and subcellular fractionation showed that $20 \mathrm{mM} \mathrm{ETOH}$ preincubation induced luM carbachol to displace Munc18c from the BPM into the cytosol. CONCLUSION: We conclude that ETOH renders acini PM-Munc 18c conducive for postprandial cholinergic stimulation to displace into the cytosol, which would promote basolateral exocytosis - which we propose to be the 'susceptibility' mechanism for human alcoholic pancreatitis. (Funded by: ABMRF and NIH).

\section{T1785}

Activation of PAK2 By CCK in Rat Pancreatic Acinar Cells Joo Weon Lim, John A. Williams

CCK activates small G proteins of the Rho family and both Rac and Rho play a role in regulating secretion, cell morphology and the actin cytoskeleton. However, little is known of their upstream regulators and downstream effectors. We used a GST-Racl fusion protein loaded with GDP or GTP $\gamma S$ as an affinity reagent to pull out interacting proteins from pancreatic lysate, which were then separated by ID gel electrophoresis. Two protein bands binding uniquely to Rac loaded with GTP $\gamma$ S were identified by MALDI TOF tandem mass spectrophotometry as $\mathrm{p} 21$ activated kinase (PAK) 2 and $\beta$ PIX. Both Western blotting and RT-PCR identified PAK2 as the major form of PAK present in acini. Using Western blotting and a pull-down assay with GST-PAK protein, we found two $\beta$ PIX isoforms, $\beta 1 P I X$ and $\beta 2$ PIX were present and that $\beta 2$ PIX was able to bind to PAK2. When PAK2 was immunoprecipitated and used in a kinase assay with myelin basic protein as substrate, CCK activated PAK activity, which was increased about 2 fold at 2 min and subsequently decreased but remained elevated to $20 \mathrm{~min}$. Using different concentrations of CCK, PAK activation showed a maximal increase at $100 \mathrm{pM}$. Both time course and dose response for PAK2 paralleled the activation of Rac as determined by a pull-down assay with GST-PAK-PBD domain. An important role for $\beta$ PIX was shown by use of a antisense oligonucleotide introduced into acini with a cationic liposome DOTAP which reduced $\beta$ PIX content by about $50 \%$ following overnight incubation. The antisense but not the sense oligonucleotide led to a reduction in activation of both PAK and Rac following stimulation with CCK. This result suggests that $\beta$ PIX is a regulator for both PAK and Rac activation and that Pak may mediate some of the actions of Rac in rat pancreatic acinar cells.

\section{T1786}

CCK-Induced Protein Kinase D Activation Is Regulated By Upstream PKC and Plays a Role in Pancreatic Acinar Cell Secretion

L. Andy Chen, J.N. Li, Hiroaki Watanabe, B. Mark Evers

Protein kinase D1 (PKDl) is a novel serine-threonine kinase which has been implicated in important intracellular functions such as cell survival and secretion. We have recently demonstrated that the secretagogue cholecystokinin (CCK) induces rapid translocation, phosphorylation, and activation of PKDl in pancreatic acinar cells; however, the mechanism and function of PKDl activation in these cells is not known. The purpose of our study was to delineate the signaling pathways upstream of PKDl activation and to determine whether PKDl inhibition affects CCK-stimulated secretion. METHODS: Pancreatic acinar cells wer isolated from female Swiss Webster mice (2 to 3-mo-old) by collagenase digestion. (i) CCKinduced PKDl activation in acinar cells was determined by Ser744/748 phosphorylation, kinase activity and translocation assays. A variety of pharmacological inhibitors were used to test whether members of the PKC, MAPK, and PI3K family are involved in CCK-induced PKDl activation. (ii) Isolated acinar cells were treated with either Gö-6976 (which inhibits PKC $\alpha$ and PKC $\beta 1$ as well as PKD) or Gö-6983 (which inhibits the $\alpha, \beta, \gamma, \delta$ PKC isoform but not PKD). Inhibition of PKD was confirmed by kinase activity assay, and CCK-stimulated secretion was assessed by amylase assay. RESULTS: (i) CCK-induced PKD1 phosphorylation in acinar cells was inhibited $>70 \%$ by pre-treatment with GF109203X $(5 \mu \mathrm{M})$, a broadspectrum PKC inhibitor and by rottlerin $(2 \mu \mathrm{M})$, a PKC- $\delta$ inhibitor; neither the Akt inhibitor $(5 \mu \mathrm{M})$ nor the PI3K inhibitors, LY294002 $(2 \mu \mathrm{M})$ or wortmannin (100 nM), significantly affected PKDl activation. (ii) CCK-induced amylase secretion was inhibited $\sim 20 \%$ by Gö$6976(1 \mu \mathrm{M})$, a PKC inhibitor effective against PKD, but not by Gö-6983 (1 $\mu \mathrm{M})$, a PKC inhibitor ineffective against PKD. CONCLUSIONS: Our results demonstrate PKC-dependent PKDl activation in pancreatic acinar cells following CCK stimulation. Importantly, our findings suggest a functional role for PKDl in pancreatic acinar cell enzyme secretion.

\section{T1787}

Calcium-Regulated Phosphorylation of Crhsp-28 At Serine 136 Mediates Its Translocation from a Perinuclear/Golgi Region to Plasma Membrane and Induces Acute Changes in Cho-Kl Cell Plasma Membrane Morphology Diana D. Thomas, Ning Weng, Christina L. Martin, Kala M. Kaspar, Megan D. Baumler, Guy E. Groblewski

CRHSP-28 is member of the Tumor Protein D52 family that is under normal conditions highly expressed in exocrine acinar cells, where it regulates $\mathrm{Ca}^{2+}$-dependent secretory activity. CRHSP-28 is also overexpressed in cancers of the breast, colon, lung, and prostate. Stimulation of acini induces a rapid translocation of CRHSP-28 from the trans-Golgi region to the apical plasma membrane and an early endosomal compartment and directly coincides with its acute serine phosphorylation. Ectopic expression of CRHSP-28 in CHO cells revealed that elevation of cellular $\mathrm{Ca}^{2+}$ by ionophore treatment induced a marked translocation of the protein from a perinuclear/Golgi region to the plasma membrane within $5 \mathrm{~min}$ and a pronounced increase in its phosphorylation. Mutation of multiple serine sites to alanine on CRHSP-28 revealed that ser 136 is the major phosphoacceptor site in both basal and stimulated conditions as indicated by immunoprecipitation following ${ }^{32} \mathrm{P}$-labeling and immunoblotting following isoelectric focusing. Although CRHSP-28 was previously shown as phosphorylated by CaM kinase II in vitro, serl36 is present within a casein kinase II consensus sequence. Mutation of the single CaM kinase II consensus site (ser100) was without effect. Likewise mutation of two additional casein kinase II consensus sites alone or in combination with serl36 did not further alter CRHSP-28 phosphorylation, confirming that serl36 is the primary site of phosphorylation in intact cells. Strikingly, the ser136/ala mutation failed to translocate to the plasma membrane following $\mathrm{Ca}^{2+}$-stimulation. Moreover, phosphomimetic mutation of serl36 to aspartate or glutamate resulted in a pronounced accumulation of CRHSP-28 along the plasma membrane. The CRHSP-28 serl36/asp and serl36/glut were localized within distinct plasma membrane invaginations resembling endocytic vesicles that appeared to be arrested during formation. Moreover, the plasma membrane of cells expressing the phosphomimetic mutants displayed multiple cytoplasmic projections suggestive of cell spreading/migration. These data demonstrate that CRHSP- $28 \mathrm{Ca}^{2+}$-mediated phosphorylation occurs at ser 136 and is the triggering mechanism for CRHSP- 28 translocation to the plasma membrane. Moreover, these data lend insight into the molecular nature of CRHSP-28's regulatory effects on acinar membrane trafficking and may have significant implications regarding its overexpression in epithelial cell cancers.

\section{$\mathrm{T} 1788$}

The Large Gtpase Dynamin Localizes to and Is Involved in Zymogen Secretion At the Pancreatic Acinar Cell Apical Membrane Vijay Singh, Barbara Schroeder, Mark A. McNiven

Introduction: Dynamin is a large GTPase involved in membrane dynamics required for endocytosis and secretion. In addition, dynamin provides a link between membranes an the actin cytoskeleton through a direct interaction with the actin-binding protein cortactin The actin cytoskeleton is known to be involved in zymogen secretion and we have recently shown that dynamin is involved in Golgi vesiculation in response to CCK in cultured pancreatic acinar cells. However, the role of cortactin in these processes in conjunction with dynamin is unknown. The GOAL of this study was to test the distribution and participation of dynamin and cortactin in CCK-stimulated zymogen secretion from isolated pancreatic acini. Materials and Methods: Male Sprague-Dawley rats weighing 100-150 grams were used. For in vivo studies, rats received $0.05 \mathrm{mcg} / \mathrm{kg}$ of CCK in $0.2 \mathrm{ml}$ saline or vehicle $(0.2 \mathrm{~m}$ saline) intraperitoneally and were sacrificed 3 hours later. Harvested pancreas was embedded in OCT and immunostained. For in vitro studies, acini were harvested in HEPES buffer and either used fresh or cultured overnight in DMEM with $0.5 \%$ BSA. Fresh acini were stimulated with CCK $(0.1 \mathrm{nM})$ for up to 3 hours before immunostaining. For secretion studies, cultured acini were transfected with $2 \times 106 / \mathrm{ml}$ PFU adenovirus to induce expression of either GFP wild type dynamin or a GTPase deficient dynamin (Dyn2K44A) and used 16-18 hours later.

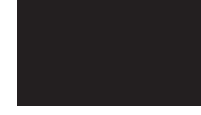


Untransfected acini were used as a control. Results: Stimulation of acini with physiologic doses of CCK resulted in a marked translocation of dynamin and cortactin to the apical domain both in vivo and in vitro. Amylase secretion in response to $0.1 \mathrm{nM} \mathrm{CCK}$ from cultured acini was not affected by expression of GFP or wild type dynamin compared to controls $(2.0+0.7 \%$ basal vs. $8.7+1.2 \%$ with $0.1 \mathrm{nM} \mathrm{CCK})$. In contrast, expression of the mutant dynamin, Dyn $2 \mathrm{~K} 44 \mathrm{~A}$, resulted in a $70 \%$ reduction in stimulated secretion $(2.5+0.3 \%$ basal vs. $5.3+0.7 \%$ with $0.1 \mathrm{nM} \mathrm{CCK}$ ). This reduced secretion was accompanied by a reduction in cortical actin at the apical domain, which was rich in untransfected, GFPand wild type dynamin-transfected acini. Conclusions: Dynamin and cortactin translocate apically, where they colocalize, in response to physiologic CCK stimulation. This apical translocation may be related to zymogen granule secretion as disruption of dynamin function resulted in the inhibition of CCK-stimulated secretion along with a loss in apical actin. Thus, dynamin may translocate with cortactin to the apical membrane and together they may aid in regulating CCK-stimulated zymogen secretion, possibly via a role in post-secretion membrane retrieval.

\section{T1789}

Role of Protein Kinase C Delta $(\mathrm{Pkc} \delta)$ in Cholecystokinin-8-Induced Amylase Secretion and Nuclear Factor Kappa-B (Nf- $\kappa \mathrm{B}$ ) Activation in Pancreatic Acini Jeffrey L. Wang, Akihiko Satoh, Lars S. Fischer, Stephen J. Pandol

Background and aims: Protein Kinase $\mathrm{C}$ represents a family of calcium and diacylglycerol activated kinases. It has multiple roles in the regulation of both the physiology and pathophysiology of the pancreas. Four specific isoforms of $\operatorname{PKC}(\alpha, \delta, \varepsilon$, and $\zeta)$ have been demonstrated to be present in pancreatic acinar cells. Recent studies provide evidence for roles of PKC in pancreatic enzyme secretion, and $\mathrm{PKC} \delta$ and $\varepsilon$ in activating inflammatory signal NF- $\mathrm{\kappa B}$. Experiments with pharmacologic inhibitors suggest that PKC $\delta$ plays a key role in acinar cells responses to cholecystokinin-8 (CCK). However, effects of genetic deletion of PKCX on acinar cells responses have not been studied. We investigated the role of PKC $\delta$ in both amylase secretion and NF- $\mathrm{KB}$ activation induced by CCK in pancreatic acinar cells obtained from PKCD deficient and wild type mice. Methods: $\mathrm{PKC \delta}^{-/-}$mice were generated in the laboratory of Dr. Nakayama from Kyushu University, Japan. PKCS deficiency was confirmed by genotyping. Pancreatic acinar cells were isolated from $\mathrm{PKC}^{-/}$and $\mathrm{PKC}^{+/+}$and incubated for 30 min with CCK concentrations ranging from $1 \rho \mathrm{M}$ to $0.1 \mu \mathrm{M}$. CCK-induced amylase secretion was measured using Phadebas kit; NF- $\mathrm{\kappa B}$ activation was measured using electromobility shift assay (EMSA). Results: CCK induced typical biphasic dose response curve for secretion of amylase in acinar cells isolated from both $\mathrm{PKC \delta}^{--}$and wild type mice with stimulation up to approximately $0.1 \mathrm{nM}$ and subsequent reduction of amylase release at supra-maximal doses of CCK. At both physiologic and supra-physiologic doses, CCK-induced amylase secretion was greater in acinar cells from $\mathrm{PKC}^{-/-}$versus that from wild type mice. For example, $10 \rho \mathrm{M}$ CCK stimulated amylase secretion by 20\% more so in the acinar cells from $\mathrm{PKC \delta}^{-1-}$ compared with the acinar cells from wild type mice. Our data suggest that PKC $\delta$ is a negative regulator of CCK-induced amylase secretion in acinar cells. On the other hand, CCK at supra-physiologic dose $(0.1 \mu \mathrm{M})$ stimulated NF-KB activation in acini isolated from both $\mathrm{PKC}^{-1-}$ and wild type mice. There was a $25 \%$ inhibition of NF-кB activation in the acini from $\mathrm{PKC}^{-/}$mice, suggesting that $\mathrm{PKC} \delta$ mediates $\mathrm{CCK}$ induced NF$\mathrm{KB}$ activation in pancreatic acinar cells. Conclusions: Experiments on pancreatic acinar cells from $\mathrm{PKC}^{-1-}$ mice indicate that $\mathrm{PKC} \delta$ negatively regulates CCK-induced amylase secretion and mediates CCK-induced NF-KB activation in pancreatic acinar cells. These effects of PKC may play a role in dysregulation of amylase secretion and inflammatory infiltration in pancreatitis.

\section{T1790}

Pancreatic Secretagogues But Not Growth Factors Activate Pkdl in Rat Pancreatic Acini By a Novel Mechanism

Marc J. Berna, Karl M. Hoffmann, Jose A. Tapia, Michelle Thill, Robert T. Jensen

Introduction: Protein kinase D (PKD) is a serine-threonine kinase that has been implicated in cell survival, proliferation, migration, invasion and protein trafficking. PKD activation is coupled to signaling by growth factors, G-protein coupled receptors and oxidative stress. There are no studies of the ability of pancreatic acinar cell stimulants to activate PKD or the possible processes involved. Methods: In dispersed rat pancreatic acini, PKD isoforms were studied by using specific PKD total and phospho-antibodies after SDS-PAGE and Western blotting. Immunocytochemistry was performed on cells after cytocentrifugation. Results: In quiescent acini both PKD1 and PKD2 were detected. After stimulation with CCK, a marked increase in serine-phosphorylated PKD1 (pPKD1) occurred both at the 916 and the 744/748 site. CCK, carbachol, bombesin and TPA induced pronounced PKDl phosphorylation, whereas stimulation with other secretagogues (VIP, secretin) and growth factors (HGF, EGF, insulin, IGFl) had no effect. CCK-stimulated pPKDl was studied in detail CCK-mediated PKDI phosphorylation was detectable within $30 \mathrm{sec}$, reaching a maximum after $10 \mathrm{~min}$ and lasting over $60 \mathrm{~min}$. CCK caused a dose-dependent increase in pPKD1 (EC50=0.1 nM). Stimulation with the high-affinity CCKA-receptor agonist and low-affinity antagonist JMV180 lead to a partial (20\% of maximal CCK) PKDl phosphorylation. Costimulation with CCK and JMV180 resulted in a dose-dependent inhibition of CCK-mediated PKD1 phosphorylation (IC50=600 nM JMV180). Inhibition of classical and novel PKC isoforms with GF109203X induced a total suppression of TPA-mediated, but only a partial (60\%) suppression of CCK-induced PKD1 phosphorylation. Calcium depletion with thapsigargin resulted in a nearly complete (90\%) inhibition of CCK-induced but had no effect on TPA-mediated PKD1 phosphorylation. The calcium ionophore A23187 did not induce PKD1 phosphorylation, nor did it potentiate TPA-mediated PKDl activation. After stimulation with CCK $(10 \mathrm{nM})$, a rapid but temporary translocation of PKDl from the cytosol to the membrane could be observed both in Western blots and in immunocytochemistry. Conclusions: A number of pancreatic secretagogues but not growth factors cause PKDl activation in rat pancreatic acini. CCK-induced PKDl activation is mediated by both the high $(20 \%)$ and low-affinity (80\%) CCKA-receptor state. In contrast to other cells, CCKmediated PKDl activation only partly relies on signaling through classical and novel PKC isoforms but is strongly dependent on calcium, suggesting pancreatic acini may possess novel signaling mechanisms of PKD activation.

\section{T1791}

ATP Release from Pancreatic Duct Epithelial Cells (PDEC): Effects of HypoOsmolarity and Ethanol

Toan D. Nguyen, Evelyn Tong, Thomas Sernka

INTRODUCTION: ATP may serve as an intercellular messenger by interacting with specific $\mathrm{P} 2$ receptors on cell membranes; it may also be released from epithelial cells to act in an autocrine fashion on the same cell, or in a paracrine fashion on neighboring cells. We previously demonstrated the expression of different P2Y and P2X receptors on PDEC; we now report the effect of hypo-osmolarity and ethanol on ATP release from these cells. METHODS: Two human PDEC cell lines were studied, PANC-1 and CFPAC cells; of note, CFPAC cells are derived from a patient with cystic fibrosis and do not express the CFTR $\mathrm{Cl}^{-}$channels. ATP released from these cells was monitored by measuring the luminescence generated when ATP reacts with luciferin through a reaction catalyzed by luciferase. RESULTS: When exposed to a hypo-osmolar medium, both PANC-1 and CFPAC cells release ATP. This release occurs at hypo-osmolar levels as low as 20\% and increases with increasing hypos-osmolar challenges, up to $60 \%$ (PANC-1: control: $12 \pm 4 ; 20 \%$ hypoosmolar: $22 \pm 5 ; 30 \%: 29 \pm 4,40 \%: 53 \pm 17,50 \%: 93 \pm 17,60 \%: 157 \pm 7.6 ;$ CFPAC control: $16 \pm 1,20 \%$ hypo-osmolar: $57 \pm 15,30 \%: 162 \pm 38,40 \%: 247 \pm 76,50 \%: 352$ $\pm 42,60 \%: 586 \pm 96$ [mean \pm SEM, in arbitrary luminometric units]). Ethanol at concentrations up to $1 \%$ did not cause ATP release. On the hand, when cells were preincubated with $1 \%$ ethanol, the response to a $50 \%$ hypo-omotic challenge was inhibited by $40 \%$ in PANC1 cells and 50\% in CFPAC cells. This effect may be partially mediated by the osmotic contribution of ethanol $(1 \%=172 \mathrm{mM})$ since cells pre-incubated with $172 \mathrm{mM}$ mannitol also showed a similar degree of inhibition. CONCLUSION: Hypo-osmolarity stimulates ATP release from PDEC in a dose dependent manner. The ability of CFPAC cells to release ATP suggests that the $\mathrm{CFTR} \mathrm{Cl}^{-}$channel, previously postulated to also serve as ATP conductance, does not mediate this release. If the released ATP interacts with $\mathrm{P} 2$ receptors to stimulate secretion, this may constitute a possible protection mechanism, in this case against hypoosmolarity-induced cell swelling. As ethanol impairs this reaction, this may be a mechanism whereby ethanol contributes to cellular injury. Funded by NIH RO-1 DK 55885 and VA Merit Review.

\section{T1792}

JNK Activation Is Required for CCK Induced Pancreatic Acinar Cell Growth in Culture

Lili Guo, Maria Dolors Sans, John A. Williams

Pancreatic growth occurs during fetal and early postnatal development and in the adult animal can be regulated by diet and gut hormones such as cholecystokinin (CCK). Our previous studies showed that endogenous CCK released by feeding trypsin inhibitor activated JNKs and ERKs as well as inducing expression of a variety of early response genes such as c-jun and c-fos. To more directly characterize the importance of the MAPK pathways and early response genes in CCK induced pancreatic growth, we used an in vitro cell culture model. Mouse pancreatic acinar cells were isolated with collagenase and cultured on type I collagen with $[3 \mathrm{H}]$ thymidine incorporation as a read out of DNA synthesis. CCK added after $24 \mathrm{~h}$ in culture enhanced DNA synthesis with a minimal effect at $30 \mathrm{pM}$ and a maximal effect of $96+2.1$ fold at $10 \mathrm{nM}$. EGF and FGF, the most potent growth factors, induced a maximal $7.8 \pm 1.2$ and $3.6 \pm 0.4$ fold increase of thymidine incorporation at $10 \mathrm{nM}$; much smaller effects on thymidine incorporation were seen with maximal concentrations of carbachol, bombesin, insulin and IGF-1. BrdU incorporation visualized by immunohistochemistry confirmed that stimulated DNA synthesis occurred in pancreatic acinar cells. JNK activity was increased $30 \mathrm{~min}$ after adding $\operatorname{lnM}$ CCK to the cultured acinar cells as shown by western blot with an activity state dependent antibody. CCK also induced early response genes expression at the mRNA level measured by quantitative RT-PCR including c-jun (17 fold), c-fos (44 fold) and ATF-3 (18 fold), while the expression of 18sRNA was not affected by CCK and GAPDH was slightly decreased. These increases were maximal at $1-2 \mathrm{~h}$ and decreased by $3 \mathrm{~h}$. C-jun protein induction was also observed after CCK stimulation with a maximal increase at $3 \mathrm{~h}$; this induction was 70\% blocked by the JNK inhibitor SP600125 at $30 \mu \mathrm{M}$. Specific chemical inhibitors of the JNK and ERK pathways were used to initiate studies of the importance of MAPKs to CCK induced acinar cell growth. SP600125 at 30 MM inhibited $80 \%$ of the thymidine incorporation. Two ERK pathway inhibitors U0126 and PD98095 blocked more than $80 \%$ of thymidine incorporation at $1 \mu \mathrm{M}$ and $5 \mu \mathrm{M}$ respectively, while the inactive analog U0124 had no effect. By contrast, Wortmanin, an inhibitor of PI$3 \mathrm{~K}$ had minimal inhibitory effect at $100 \mathrm{nM}$. Our results suggest that MAPK cascades and early response genes particularly c-jun participate in CCK induced pancreatic acinar cell growth.

\section{$\mathrm{T} 1863$}

The COX-2 -765c/-1195a Haplotype Predisposes for the Development of Esophageal Adenocarcinoma

L.M.G. Moons, J. G. Kusters, A. M. Rygiel, Z.M.A. Groothuismink, W.A. Bode, H. Geldof, K.K. Krishnadath, J.G.H.M. Bergman, A.H.M. van Vliet, P.D. Siersema, E.J. Kuipers

Introduction: The expression level of COX-2 is associated with an increased tendency for neoplastic progression in the esophagus, since COX-2 expression increases the metaplasiadysplasia-adenocarcinoma sequence. High expression levels are also associated with poor 5-year survival rates of esophageal adenocarcinoma. COX-2 is the rate-limiting enzyme for the production of prostaglandin E2 (PGE2), a compound associated with carcinogenesis in many inflammatory diseases, and recently, it was suggested that PGE2 levels were COX-2 promotor haplotype dependent. Aim: To determine the association between COX-2 haplotypes and the development of esophageal carcinogenesis. Methods: DNA was obtained from 140 Caucasian patients with an adenocarcinoma of the esophagus (mean age $63 \pm 11 ; 91 \%$ 
male), 250 Caucasian patients with histologically confirmed Barrett's esophagus (mean age $62 \pm 13 ; 68 \%$ male) with a mean Barrett-segment length of $4.2 \pm 2.3 \mathrm{~cm}$, and 247 Caucasian patients with endoscopically confirmed reflux esophagitis (mean age $56 \pm 14 ; 53 \%$ male). COX-2 haplotypes were determined by amplification of the promoter region by PCR, followed by determination of the polymorphisms at $-765 \mathrm{C} / \mathrm{G}$ and $-1195 \mathrm{~A} / \mathrm{G}$ by RFLP with restriction enzymes Acil and PvuII respectively. COX-2 haplotype dependent PGE2 levels were determined by EIA. Results: The tested population contained 171 (14\%) CA (-765C \& -1195A), 817 (66\%) GA and 258 (20\%)GG haplotypes, and none of the relatively rare GC-halotype. The haploptype distribution in patients with reflux esophagitis and Barrett's esophagus was similar (CA 12\%, GA, 68\%, GG 21\%), but differed significantly from patients with esophageal adenocarcinoma (Ca 21\%, GA 58\%, GG 20\%), in whom the CA-haplotype was significantly more common $(\mathrm{p}<0.0001)$. Furthermore, homozygosity of CA was only found in patients with Barrett's esophagus patients $(1.6 \%)$ and esophageal adenocarcinoma $(5.0 \%)(\mathrm{p}=0.002)$. $\mathrm{CA}$-carriership was associated with an increased risk for esophageal adenocarcinoma (OR 1.94; 95\%CI 1.23-3.08; $\mathrm{p}=0.005$ ), and homozygosity for the CA-allele was associated with an even greater risk (OR 3.83; 95\% CI 1.09- 13.84; $\mathrm{p}=0.036$ ). Conclusion: The COX-2 CAhaplotype is associated with an increased risk for the development of esophageal adenocarcinoma in Barrett's esophagus and reflux esophagitis patients. As the COX-2 CA-haplotype is associated with increased levels of PGE2, PGE2 seems to promote esophageal carcinogenesis in patients with a Barrett's esophagus and reflux esophagitis.

\section{T1864}

Expression of Tissue Inhibitors of Metalloproteinases and Target Proteases During Barrett's Carcinogenesis

Suhail Ahmed, A L Khan, L Pazmany, A Varro, K Bodger

INTRODUCTION A multi-step pathway for oesophageal adenocarcinoma (AdCa) proceeds from squamous epithelium (SqEp) via Barrett's metaplasia (BM) to dysplasia and cancer in the setting of reflux disease. These processes involve remodelling of tissue architecture and fundamental changes to extra-cellular matrix (ECM). Regulation of the ECM is under the influence of the matrix metalloproteinases (MMPs) and their natural tissue inhibitors (TIMPs), a system of molecules that have roles in ECM homeostasis, inflammation, angiogenesis and metastasis. METHODS The aim of the present study was to define expression profile of TIMPs (-1 to -4$)$ and selected MMPs $(-3$ and -8$)$ at different points in the cancer pathway. Oesophageal biopsies were obtained for mRNA extraction, histopathology and immunostaining. Real time PCR was performed on cDNA generated by reverse transcription of RNA extracts. Expression of transcripts was normalised for h-HPRT housekeeping gene. RESULTS Compared to $\mathrm{SqEp}$, BM was associated with a significant increase in mRNA abundance for TIMPs $-1,-2,-3$ and -4 and MMP-8 (but not MMP-3), whereas AdCa was characterised by increases in TIMP-4 (but not other TIMPs) and MMP-8 but a decrease in MMP-3. Compared to BM, there was reduced TIMP-3 and MMP-3 but increased MMP-8 in AdCa. Immunostaining confirmed cell-type specific changes in expression, with TIMP-4 in particular showing a progressive increase in epithelial and basal layer immunostaining from non-dysplastic BM through dysplastic BM to AdCa. CONCLUSION Neoplastic progression in the oesophagus is associated with altered expression of specific TIMPs and MMPs. Levels of TIMPs -1 to 3 mRNA are maximal at the stage of BM but decline at the stage of $\mathrm{AdCa}$. In BM, enhanced expression of protease inhibitors would reduce ECM degradation and favour fibrosis (eg. peptic stricture), whereas in AdCa the finding of elevated MMP-8 but falling TIMPs would suggest enhanced proteolyis favouring malignant infiltration and metastasis. TIMP-4 may have potential as a biomarker of progression.

Mean mRNA abundance (relative to HPRT)

\begin{tabular}{|c|c|c|c|}
\hline & Squamous SqEp, $\mathrm{n}=6$ & Barrett's BIM, $\mathrm{n}=9$ & Adenocarcinoma AdCa, $\mathrm{n}=8$ \\
\hline TIMP-1 & 0.0650 & $0.3995^{*}$ & 0.2728 \\
\hline TIMP-2 & 0.0130 & $0.0807^{*}$ & 0.0486 \\
\hline TIMP-3 & 0.0003 & $0.0956^{*}$ & 0.0006 \\
\hline TIMP-4 & 0.0218 & $0.0996 *$ & $0.1160 *$ \\
\hline MMP3 & 0.4108 & 0.4519 & $0.0419 *$ \\
\hline MMP8 & 0.0180 & $0.037^{*}$ & $0.3431 *$ \\
\hline
\end{tabular}

* $\mathrm{p}<0.05$ versus $\mathrm{SqEp}$

\section{$\mathrm{T} 1865$}

In Benign Barrett's Epithelial Cells, Acid Increases Reactive Oxygen Species That Activate the P38 MAPK Pathway

Linda A. Feagins, Lance S. Terada, Stuart J. Spechler, Rhonda F. Souza

Introduction: In extraesophageal tissues, reactive oxygen species (ROS) have been shown to activate signal transduction cascades, like the mitogen-activated protein kinase (MAPK) pathway, that regulate cell proliferation. In earlier studies, we showed that acid increases ROS production in benign Barrett's epithelial cells in vitro and that esophageal acid perfusion activates the MAPK pathway in Barrett's epithelium in vivo. Therefore, we sought to determine whether acid-induced ROS production causes activation of the MAPK pathway in Barrett's epithelial cells. Methods: Equally seeded wells of telomerase-immortalized, non-neoplastic Barrett's epithelial cells were exposed to acidic media ( $\mathrm{pH} 4.0$ ) for 3-minutes, followed by incubation in neutral pH media. The phosphorylation of ERK and p38 MAPKs (relative to total ERK and p38) were determined by Western blotting of cell lysates collected during and after the acid exposure in the presence and absence of TMPyP, a scavenger of ROS. To determine whether acid-induced ROS production was unique to benign Barrett's epithelial cells, we incubated a normal esophageal squamous cell line and 3 esophageal adenocarcinoma cell lines with H2DCF-DA, a fluorescent probe activated by ROS. The cell lines were then exposed to acidic media at $\mathrm{pH} 4$ for 3 minutes, followed by incubation in neutral pH media. Fluorescent intensity of the cells was used for quantification of ROS production. Results: Acid exposure increased the expression of phospho-p38 in Barrett's cells to levels 4-6 times those of control cells that were not exposed to acid. In contrast, acid exposure had no apparent effect on ERK phosphorylation. Incubation of Barrett's cells with TMPyP abolished the acid-induced p38 phosphorylation. In contrast to the benign Barrett's cells, acid exposure did not increase ROS production in the normal esophageal squamous cells or in any of the 3 esophageal adenocarcinoma cell lines. Conclusions: In benign, non-neoplastic Barrett's epithelial cells, acid induces the production of ROS which activate the p38, but not the ERK, MAPK pathway. In contrast, acid exposure does not induce ROS production in normal esophageal squamous cells or in esophageal adenocarcinoma cells. These findings highlight differences between benign and malignant Barrett's cells in their response to acid exposure, and indicate that acid-induced ROS production affects a key proliferation pathway.

\section{$\mathrm{T} 1866$}

Rho Kinase and ERK2 MAP Kinase Contribute to Acid-Induced Expression of NADPH Oxidase Nox5s (Short) in Barrett's Adenocarcinoma Cells Xiaoying Fu, Jose Behar, David Beer, Jack Wands, David Lambeth, Weibiao Cao

We have shown that NADPH oxidase NOX5S (short, i.e. without EF-hand motifs at its Nterminal) is overexpressed in SEGl esophageal adenocarcinoma cells associated with Barrett's esophagus (SEGl-EA). NOX5S mediates acid-induced hydrogen peroxide $\left(\mathrm{H}_{2} \mathrm{O}_{2}\right)$ production and contributes to increased cell proliferation and decreased apoptosis in SEGl-EA. In addition, acid-induced NOX5S expression and $\mathrm{H}_{2} \mathrm{O}_{2}$ production are mediated by increased intracellular calcium and activation of cyclic AMP response element binding (CREB) protein We now examine the role of Rho kinase and MAP kinases in acid-induced NOX5S expression and $\mathrm{H}_{2} \mathrm{O}_{2}$ production in SEGI-EA. NOX5 was sequenced by RT-PCR and 5'-RACE in SEGI EA cells. NOX5S was the only NOX5 isoform present in SEGl cells and its sequence was the same as in other tissues. SEGl-EA were exposed to $\mathrm{pH} 4.0$ for one hour, then washed and cultured at $\mathrm{pH} 7.2$ for 24 hours. Acid increased NOX5S expression and $\mathrm{H}_{2} \mathrm{O}_{2}$ production, that were significantly decreased by the MEKl inhibitor PD98059 and by the Rho kinase inhibitor Y27632, but not by the P38 MAP kinase inhibitor SB203580 nor by the protein kinase $\mathrm{C}$ inhibitor chelerythrine. These data suggest acid-induced, Rho kinase and ERKl/ ERK2 MAP kinases-dependent NOX5S expression and $\mathrm{H}_{2} \mathrm{O}_{2}$ production. We have shown that $\mathrm{CREB}$ is responsible for acid-induced NOX5S expression. To confirm the role of CREB a reporter plasmid of NOX5S was generated by ligating a NOX5S promoter fragment (1396 to - 6 from ATG) into the pGL3-basic vector. Overexpression of CREB caused 5.4-fold increase in luciferase activity, indicating CREB induced activation of NOX5S promoter. Acid treatment significantly increased CREB phosphorylation (serine 133), that was significantly inhibited by PD98059 and Y27632, but not by SB203580 nor by chelerythrine, indicating a role of ERK MAP kinases and of Rho kinase in acid-induced activation of CREB to overexpress NOX5S. To confirm the role of these kinases in NOX5S expression we examined the phosphorylation of the phosphatase catalytic subunit MYPTI and of ERK MAP kinases. Acid treatment significantly increased MYPTl and ERK2 MAP kinase phosphorylation. Acidinduced MYPTl phosphorylation on threonine 696 was blocked by Y27632, but not by PD98059. Conversely, acid-induced increase in ERK2 phosphorylation was blocked by Y27632, indicating Rho-dependent phosphoryation/inhibition of the phosphatase mediates ERK2 phosphorylation. We conclude that acid-induced NOX5S expression depends on sequential phosphorylation/activation of Rho kinase, ERK2 MAP kinase and CREB, that are potentiated by inactivation of phosphatases. Supported by NIH NCRR 1 P20 RR17695-01

\section{T1867}

Bile Salt Augments Caudal-Related Homeobox Gene CDX1 Expression in Esophageal Keratinocytes in Barrett's Epithelium

Hideaki Kazumori, Shunji Ishihara, Mohammad A. Rumi, Yoshikazu Kinoshita

Background \& Aims: The mechanism of transformation to intestinal metaplasia in Barrett's esophagus has not been clarified. $\mathrm{Cdxl}$ is the homeobox gene which appears to play major roles in the development of intestinal metaplasia in gastric epithelium. We investigated the direct effects of bile salt on the expression of $\mathrm{Cdxl}$ in cultured esophageal squamous epithelial cells. Further, we studied morphological and histochemical changes of squamous cells to intestinal epithelial cells in response to bile salt-induced expression of Cdxl. Methods: A rat model of Barrett's esophagus was produced by anastomosing the esophagus and jejunum and Cdxl expression was investigated by immunohistochemistry. The response of bile sal on Cdxl gene expression was studied in human colon and esophageal adenocarcinoma cel lines using a Cdxl promoter luciferase assay. Further, Cdxl gene expression was studied in primary esophageal squamous epithelial cells in response to bile salt. Results: Esophagojejunal anastomoses formed intestinal goblet cell metaplasia in rat esophagus specimens. Cdxl-positive cells with nuclear staining were mainly observed in the columnar cells in these areas and the adjoining squamous cells. These findings suggest the possibility that bile acid refluxate stimulates $\mathrm{Cdxl}$ expression in esophageal squamous epithelium, followed by the replacement with intestinal type columnar epithelium. When the effects of bile salt mixture on the expression of $\mathrm{Cdxl}$ were examined, bile salt mixture dose-dependently increased Cdxl promoter activity and Cdxl protein production in cultured esophageal keratinocytes. Conclusions: We showed that bile salt activates $\mathrm{Cdxl}$ promoter, and stimulate the production of $\mathrm{Cdxl}$ protein in esophageal keratinocytes.

\section{T1868}

Methylation, and Its Role in Intestinal Metaplasia of the Esophagus Xinyan Zhang, Xiaoxin L. Chen

Background \& Aims: As an intestinal transcription factor, caudal-related homeobox 2 (Cdx2) has been suggested as an early marker of intestinal metaplasia of the esophagus (Barrett's esophagus, BE). However, the mechanism of ectopic $\mathrm{Cdx} 2$ expression in the esophagus and its role in the development of BE remained unclear. Methods: RT-PCR, pyrosequencing and methylation-specific PCR were used to determine expression and promoter methylation of Cdx2 in human esophageal epithelial cells (HETlA, SEG1) after treatment with 5-aza-2' deoxycytidine (DAC), acid, bile acids and their combinations. HETlA cells with stable 
transfection of $\mathrm{Cdx} 2$ were characterized for morphology with light and transmission electron microscopes, and gene expression profiles with Affymetrix array. Results: DAC induced demethylation and expression of Cdx2 in HETlA and SEGl cells, and treatment with a DNA methylating agent counteracted the effect of DAC. Treatment of HETlA and SEGl cells with acid, bile acids or both also resulted in promoter demethylation and expression of Cdx2. HET1A cells with stable transfection of human Cdx2 formed crypt-like structures in vitro, and undertook electromicroscopic changes reminiscent of human $\mathrm{BE}$, such as increased microvilli, tight junctions, and lateral interdigitations. Microarray analysis and quantitative real-time PCR showed that stable transfection of Cdx2 up-regulated differentiation markers of intestinalized columnar epithelial cells and goblet cells in HET1A cells. Cdx2 might induce the goblet cell phenotype through the Notch signaling pathway. Conclusions: This study demonstrated that gastroesophageal reflux activated $\mathrm{Cdx} 2$ expression in human esophageal epithelial cells through promoter demethylation. Ectopic Cdx2 expression in esophageal squamous epithelial cells played a causative role in intestinal metaplasia of the esophagus.

\section{T1869}

Epithelial Cells Over-Express the Leptin Receptor During Malignant Progression in Barrett's Oesophagus

Suhail Ahmed, A L Khan, L Pazmany, K Bogder

INTRODUCTION Obesity is a recognised risk factor for oesophageal adenocarcinoma. The reason for a link between excessive weight and cancer is unclear. We hypothesised that leptin-receptor (OB-R) might be over-expressed in metaplastic or neoplastic oesophageal epithelium during carcinogenesis. This would provide a mechanism whereby obesity-related hyperleptinaemia might exert a direct growth-promoting effect on oesophageal mucosa, potentially favouring malignant progression. Leptin has been shown to stimulate proliferation of various epithelial cell lines expressing Ob-R. METHODS mRNA abundance of OB-Rb (main functional Ob-R isoform) was determined by quantitative PCR performed on cDNA generated by reverse transcription of RNA extracted from endoscopic biopsies. Values were normalised relative to h-HPRT house-keeping gene. Product was confirmed by agarose gel. Normal gastric fundus expresses $\mathrm{Ob}-\mathrm{Rb}$ and was used as positive control. Immunohistochemistry for Ob-R was performed on formalin fixed biopsies and western blots on lysates of oesophageal cancer cell line (OE33). RESULTS Ob-Rb is expressed at very low level in the normal squamous oesophagus but exhibits progressive expression during malignant progression at levels significantly in excess of the healthy stomach (See Table: Mean values (SD), $p<0.001$ ANOVA). Epithelial cell staining was confirmed by immunohistochemistry with marked upregulation of $\mathrm{Ob}-\mathrm{R}$ in surface epithelial cells noted at the stages of dysplasia and cancer. OE33 cells express Ob-R. CONCLUSION Over-expression of Ob-Rb occurs during malignant progression. This is consistent with our leptin-hypothesis. The effects of leptin on Barrett's epithelial cells require further study. Therapeutic manipulation of leptinreceptor signalling might provide a novel mechanism for the prevention or treatment of oesophageal adenocarcinoma

TABLE 1:Mean mRNA abundance(relative to HPRT)

\begin{tabular}{|c|c|c|c|c|}
\hline & $\begin{array}{c}\text { Squamous Epithelium } \\
(\mathrm{n}=5)\end{array}$ & $\begin{array}{c}\text { Gastric Body } \\
(\mathrm{n}=7)\end{array}$ & $\begin{array}{c}\text { Barrett'sMetaplasia } \\
(\mathrm{n}=8)\end{array}$ & $\begin{array}{c}\text { AdenoCA } \\
(\mathrm{n}=8)\end{array}$ \\
\hline $\begin{array}{c}\text { Ob-R } \\
\text { RNA }\end{array}$ & $0.028(0.015)$ & $0.103(0.075)$ & $0.693(0.203)$ & $0.899(0.121)$ \\
\hline
\end{tabular}

$\mathrm{T} 1870$

Lymphoid Aggregates Precede the Development of Barrett's Esophagus

Objective: Chronic inflammation of the alimentary tract predisposes to cancer. Isolated lymph follicles that develop in this inflammatory process play a role in the perpetuation of the inflammation. As a chronic inflammatory process is thought to play a role in the pathogenesis of Barrett's esophagus (BE), formation of lymph follicles may also develop in the progression of BE. Aim: To understand the function of a chronic inflammatory process in the induction of BE. Methods: Intestinal metaplasia (IM) was induced in 6 week old male Wistar rats by gastrectomy with esophagojejunostomy. Rats were sacrificed at 0,3 and 6 months. Mucosal thickness of the esophagus, area of lymphoid aggregates and distance to the anastomosis was determined with Nikon EclipseNet software in H\&E stained slides. In addition, tissue was immunohistochemically stained with antibodies specific for proliferation (Ki67), intestinal epithelium (CDX2), goblet cells (We9), B cells (OX33) and T cells (CD3) Results: At baseline and in controls ( $n=30)$, no inflammation was found in the rat esophagus, characterized by a mucosal thickness (MT) of $0.24 \pm 0.09,0.14 \pm 0.04,0.13 \pm 0.04$, and $0.11 \pm 0.03 \mathrm{~mm}$, at respectively $0,5,10$, and $20 \mathrm{~mm}$ from the anastomosis, and in the absence of lymphoid aggregates. At 3 months ( $n=3$ ), chronic inflammation was observed in the esophagus, which was associated with an increase of the MT to $0.78 \pm 0.17,0.75 \pm 0.48$, $0.52 \pm 0.10$ and $0.45 \pm 0.11 \mathrm{~mm}$, at $0,5,10$, and $20 \mathrm{~mm}(\mathrm{p}<0.001)$. Lymphoid aggregates were determined, with a surface area that increased equally with the MT $(r=0.67)$. At 6 months $(\mathrm{n}=21)$, the chronic inflammatory reaction was even stronger with a MT of $1.25 \pm 0.59$, $0.90 \pm 0.42,0.75 \pm 0.43$ and $0.59 \pm 0.30 \mathrm{~mm}$ at $0,5,10$, and $20 \mathrm{~mm}(\mathrm{p}<0.001)$. IM, which stained positive for CDX2 and We9, was observed in $21 / 21$ rats with a mean length of $3.94 \pm 1.48 \mathrm{~mm}$. The surface area of the lymphoid aggregates again correlated with the MT $(\mathrm{r}=0.68)$. Moreover, in $4 / 21$ rats significantly larger lymphoid aggregates were detected underneath the IM segment compared to the inflamed squamous epithelium (mean area $2.05 \pm 0.26$ vs. $0.04 \pm 0.02 \mathrm{~mm} 2$ ). The lymphoid aggregates in the IM tissue contained segregated B- and T cell areas and a high expression of Ki67 in the central B cell area, in contrast to those in the squamous epithelium. Conclusion: Esophageal lymphoid aggregates develop during the inflammatory process preceding $\mathrm{BE}$. These lymphoid aggregates contain central proliferation, suggesting an active local adaptive immune response. Local lymphoid aggregates may thus play a key role in the determination and maintenance of the chronic inflammation that is thought to be fundamental for the development of BE.
$\mathrm{T} 1871$

Morphological Transformation in Esophageal Submucosa By Bone Marrow Cells Esophageal Implantation Under External Esophageal Perfusion Yan Li, John M. Wo, Ruifeng R Su, Susan Ellis, Mukunda B. Ray, Whitney Jones, Robert C. Martin

Background: The bone marrow is an important resource of multipotential stem cells, and evidences indicate that stem cells from the bone marrow have the capacity to engraft into several organs including the esophagus in response to ongoing injury. Accumulating clinical and experimental studies indicate that Barrett's esophagus might arise through multipotential stem cells under the stress of gastroesophageal reflux. We have presented a novel external pump perfusion (EEP) rat model previously, and demonstrated that EEP with both acid and bile can induce severe esophagitis in 1 week with a similarly pathological change seen in humans. The aim of this study was to investigate the histological changes of esophagus after bone marrow cell engraftment with bile and acid EEP. Methods: The EEP procedure involved implantation of a micro-osmotic pump for esophageal perfusion. Bone marrow cells were obtained by flushing of the femur marrow, and the cell suspension was injected between the esophageal muscular and inner mucosa laver. Histological changes were determined after 4 weeks of perfusion. Proliferating cell nuclear antigen, 8-hydroxy-deoxyguanosine, manganese superoxide dismutase and apoptosis were measured by immunohistochemical staining and TUNEL assay, respectively. Results: Severe esophagitis were seen in both acid and bile EEP. Suspicious glandular structure in submucosa was observed after bone marrow esophageal implantation along with bile perfusion, but not with acid perfusion and controls. Alcin blue-PAS staining showed positive staining within the glandular structure area, no goblet cell was detected. Enhanced proliferation and apoptosis were observed, along with increased oxidative damage and suppression of antioxidation. Conclusions: 1) bone marrow implantation and EEP contribute the abnormal histological changes. 2) The engrafted bone marrow cells in esophageal submucosa could transform under the circumstance with bile perfusion. 3) Further studies are underway to explore the potential mechanism and induce Barrett's metaplasia.
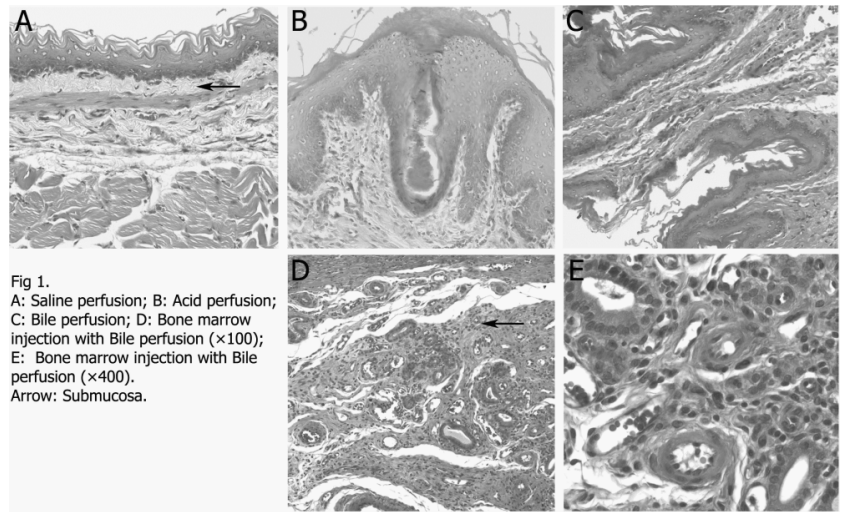

$\mathrm{T} 1872$

Constitutive Nf-кB Activation in Barrett's Epithelial Cells: Potential Role in Carcinogenesis

Cathrine Demars, Malini Madhavan, Sarah Papenfuss, Marlys Anderson, Lori Lutzke Ganapathy Prasad, Louis Wong Kee Song, Kenneth Wang, Navtej Buttar

BACKGROUND: Acid and bile salts in the reflux can promote pro-survival signaling such as activation of NF- $\mathrm{KB}$ and production of PGE2. Recent publications show that the clinical outcome of esophageal cancer is worse in patients that have increased NF- $\mathrm{KB}$ activation. Since many natural dietary products can inhibit NF- $\mathrm{KB}$ activation, the role of NF- $\mathrm{\kappa B}$ activation during carcinogenesis needs to be further explored. AIM: To determine the expression of activated NF- $\mathrm{KB}$ during carcinogenesis in Barrett's mucosa and to examine the functional relevance of NF- $\kappa B$ activation on proliferation and apoptosis of premalignant Barrett's epithelial cells. METHOD: Endoscopic mucosal resections from 12 patients were stained using an antibody that recognizes activated NF- $\mathrm{\kappa B}$. To determine the functional relevance of NF- $\mathrm{KB}$ activation, Barrett's epithelial cell cultures were treated with BAY 11-7082, which inhibits

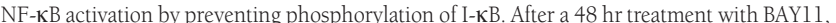
cells were assessed for proliferation (Ki67) and apoptosis (TUNEL). Experiments were repeated in presence or absence of PGE2, which is a downstream target of NF- $\mathrm{KB}$ to determine if the effects of NF- $\mathrm{KB}$ on cell survival would be altered. RESULTS: Activated NF-KB was localized to epithelial cells in dysplastic Barrett's, cancer and the deeper, rapidly dividing glandular cells. Normal squamous and non-dysplastic epithelium did not stain for activated

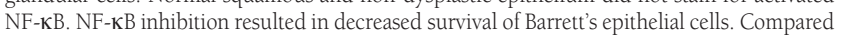
to vehicle only treated cells, 48 hour treatment with $1,2.5$, and $5 \mu \mathrm{M}$ BAYll decreased proliferation rates by $51 \%, 60 \%$, and $74 \%$, respectively $(\mathrm{p}<0.05)$. The apoptosis rates of cells treated for 48 hours with $1,2.5$, and $5 \mu \mathrm{M}$ of BAYll increased 2 fold, 2.4 fold, and 2.7 fold, respectively, compared to vehicle only treatment $(p<0.05)$. The addition of exogenous PGE2 reversed the effects of BAY11 on proliferation and apoptosis. In the presence of $2 \mathrm{ng} / \mathrm{ml} \mathrm{PGE2}$, BAY1l treatment had no significant effect on the proliferation or apoptosis of Barrett's cells. CONCLUSION: Constitutive NF- $\mathrm{KB}$ activation is noted in the epithelial cells during the progression of neoplasia in Barrett's esophagus. This activation appears to be functionally relevant since the loss of NF-KB activation can decrease proliferation and increase apoptosis in Barrett's epithelial cells. Exogenous PGE2 can reverse the effects of NF- $\mathrm{KB}$ inhibition on Barrett's epithelial cell survival, which raises the possibility that PGE2 (a downstream target of NF- $\mathrm{KB}$ activation) may have a role in the effect of constitutive NF$\kappa \mathrm{B}$ activation on the survival of Barrett's epithelial cells. 
T1873

Select Genomic Aberrations Occurring in Non-Dysplastic Barretts Esophagus (BE) Mucosa from Patients with Carcinoma Do Not Occur in Patients with Negative Long-Term Follow-Up: Array-Based Comparative Genomic Hybridization (A-CGH) Study

Marek Skacel, Daniel P. Gaile, Xiaotong Zhang, Jeffrey Conroy, Norma Nowak, Graham Casey, Thomas W. Rice, Mary P. Bronner, Gary W. Falk

Background: Genomic aberrations occur in long-standing inflammatory gastrointestinal disorders including BE. It is not known if the genomic profile of intestinal metaplasia in BE patients who have progressed to carcinoma is different from that encountered in patients who have not progressed to dysplasia. Aim: To compare the genomic profile, using A-CGH, of $\mathrm{BE}$ mucosa that is negative for dysplasia from patients who have progressed to esophageal carcinoma to that in patients who have not developed dysplasia over years of follow-up. Design: We studied 29 non-dysplastic biopsy- or esophagectomy-derived BE samples from patients without evidence of dysplasia ( $n=12$; in surveillance for a minimum of 3 years) and with BE-associated adenocarcinoma (at least intramucosal; $n=17$ ). Whole genomic DNA extracted from epithelial isolates from freshly frozen mucosa containing a minimum of $60 \%$ goblet cells was labeled by random priming using Cy3 (sample DNA) and Cy5 (normal human reference DNA). Labeled DNA was co-hybridized to microarrays containing triplicate spots of approximately 19,000 genomic RPCI-11 bacterial artificial chromosome (BAC) clones (Roswell Park Cancer Institute, Buffalo, NY). The fluorescence ratios and their logtransformed values were analyzed using DNAcopy as well as customized software. Results: The patients included 22 men and 7 women ages 53-74 years (mean: 62 years). Several recurring aberrations were detected in a subset of patients who progressed to cancer but never occurred in the nondysplastic epithelium of patients who had not progressed to carcinoma. These included genomic gains at 8q24.21 (two different BAC clones in this region), 3q24 and genomic loss at 12q31 (single clone in each of the latter two regions). Due to the sample size and testing multiplicity, these results are significant only in the context of an analysis with a liberal false discovery rate. Conclusion: Repetitive genomic changes occur in non-dysplastic intestinalized BE mucosa in patients with carcinoma. These changes were not detected in BE patients without dysplasia or cancer in this pilot cohort. Further study by fluorescence in situ hybridization will more accurately assess the precise distribution of these aberrations among BE patients.

\section{$\mathrm{T} 1874$}

Gene Promoter Methylation of ID4 and ARL4D in Barrett's Esophagus and Esophageal Adenocarcinoma

Eric Smith, Derek J. Nancarrow, Sandra Pavey, Nick K. Hayward, Neville J. de Young, John J. Kelly, David C. Whiteman, B M. Smithers, David C. Gotley, Paul A. Drew

Introduction: DNA methylation has been reported in the promoter region of a number of genes in oesophageal adenocarcinoma and its precursor lesion Barrett's esophagus, and may contribute to the progression to cancer. Analysis of gene methylation is important because there is a need for biomarkers in non-dysplastic Barrett's which might predict risk of progression to cancer, and it may provide insights into the pathogenesis of the disease. Aim: To determine the frequency of methylation in the promoter region of 10 genes in esophageal tissues and cell lines. Results: Methylation in the promoter region of 10 genes was measured in 3 oesophageal adenocarcinoma cell lines, single biopsies of Barrett's associated adenocarcinoma from 16 patients (AdCa), and multiple biopsies of metaplastic (BE) and single biopsies of proximal squamous epithelium from 18 patients with Barrett's esophagus. The percentage of patients or cell lines with methylation in each of the genes tested is shown in the table. In patients with Barrett's esophagus from whom multiple biopsies of BE were available, methylation often differed between the biopsies. Discussion: For the gene promoters examined there was less methylation in the squamous epithelium of the esophagus compared to the metaplastic or adenocarcinoma tissues. There was no consistent relationship between the methylation in the Barrett's and adenocarcinoma tissue. Two genes are of particular interest. ID4 is a member of the inhibitor of DNA-binding (ID) family, which inhibits DNA binding of basic helix-loop-helix transcription factors, and has been suggested to suppress tumour progression and metastasis. ARL4D is a member of the ARF family, and may play a role in membrane-associated intracellular trafficking. This is the first report of methylation of ID4 in Barrett's esophagus and esophageal adenocarcinoma, and of ARL4D in any cancer. This work is part of the Study of Digestive Health (Australia)

Percentage of patients or cell lines with methylation for each of the genes tested.

\begin{tabular}{|c|c|c|c|c|c|c|c|c|c|c|}
\hline & APC & MGMT & RBP1 & CDKN2A & TEMMF2 & RUNX3 & SFRP1 & TIMP3 & ID4 & ARL4D \\
\hline Squamous & 47 & 13 & 33 & 20 & 0 & 0 & 0 & 0 & 13 & 0 \\
\hline BE & 100 & 67 & 72 & 50 & 94 & 72 & 100 & 71 & 94 & 10 \\
\hline AdCa & 81 & 50 & 56 & 62 & 69 & 50 & 81 & 56 & 63 & 25 \\
\hline Cell lines & 33 & 33 & 100 & 0 & 100 & 67 & 100 & 33 & 67 & 33 \\
\hline
\end{tabular}

T1875

Hepatocyte Nuclear Factor-1-Alpha Is a Key Mediator of Muc4 Mucin Gene Regulation By Bile Acids

Guillaume Piessen, Nicolas Jonckheere, Brigitte Hemon, Christophe Mariette, Isabelle van Seuningen

Background: MUC4 is a membrane-bound mucin overexpressed in the early steps of esophageal carcinogenesis and associated with Barrett metaplasia and gastro-esophageal reflux. We previously showed that bile acids, a main component of reflux, upregulate MUC4 expression. Aim: To study the involvement of HNFl-alpha and HNF4-alpha, two transcription factors that mediate bile acids effects, in the bile-acid mediated upregulation of MUC4 in esophageal cells and tissues. Methods: MUC4, HNF-1 and HNF-4 expression was evaluated by immunohistochemistry in human esophageal tissues. MUC4 mRNA level, protein level and promoter regulation were studied in human esophageal adenocarcinomatous OE33 cells by RT-PCR, western-blot and transient transfection assays, respectively. HNFl and HNF cis-elements in the promoter of MUC4 were identified by electrophoretic mobility shift assay and chromatin immunoprecipitation and their functionality was assessed by site-directed mutagenesis. Results: MUC4 and HNFl-alpha are expressed by the same cells in esophageal tissues during the whole carcinogenetic sequence whereas HNF4-alpha is only expressed in adenocarcinomatous tissues. Studies on the promoter activity, at the mRNA level and at the protein level indicate that HNFl-alpha is an important mediator of MUC4 activation. We showed by gel retardation that HNFl-alpha mediates its effects by binding to two cognate elements, respectively located at $-3332 /-3327$ and $-3040 /-3028$, in the distal promoter. We confirmed their functionality by site-directed mutagenesis. Finally, we showed that HNF1alpha positively mediates taurodeoxycholic and taurochenodeoxycholic bile acid activation of MUC4 transcription whereas HNF4-alpha has no effect. These effects are dose-dependen and involve binding of HNFl-alpha to the -3332/-3327 and -3040/-3028 cis-elements previously identified. Conclusion: These results describe a new mechanism of regulation of MUC4 expression by bile acids that brings new insight in its up-regulation in oesophageal carcinoma associated with bile reflux.

\section{T1876}

Cigarette Smoking and Barrett's Esophagus: A Case-Control Study Nicholas J. Shaheen, Melissa B. Spacek, Joseph A. Galanko, Paris Heidt-Davis

Background: Several studies have assessed the role of cigarette smoking as a risk factor for adenocarcinoma of the esophagus. The association of cigarette smoking with the presence of Barrett's esophagus (BE) is less clear. Aim: To compare cigarette smoking habits between subjects with BE and subjects with reflux symptoms but no BE. Methods: We performed a case-control study comparing subjects with reflux symptoms who have BE to subjects with reflux symptoms but no BE. Cases were subjects with BE, defined as endoscopically visible tongues of any size in the tubular esophagus with histologic confirmation of specialized intestinalized epithelium. Controls were those presenting to GI clinic with a complaint of reflux symptoms, specifically heartburn or regurgitation. Cases and controls were between the ages of 18 and 80 . Subjects who had undergone endoscopic ablative therapy, those with previous esophageal resection, and those with carcinoma on the index endoscopy were excluded. All subjects underwent upper endoscopy to confirm their histology. They then completed a series of questionnaires, assessing demographics, GERD severity, and quality of life. Cigarette smoking was quantified both in magnitude and duration, as well as in proximity of cigarette use to the survey date. A summary statistic of pack-years was generated by multiplying the number of years smoked by the average number of packs smoked per day. Bivariate analysis was performed using Chi square and Student's t tests. Multivariate logistic regression was performed with case/control status as the outcome variable, and age sex, and measures of cigarette use as predictor variables. Results: Four hundred and seventyone subjects were enrolled ( 169 controls, 302 cases). Sixty-nine percent of cases were male, compared to $42 \%$ of controls ( $\mathrm{p}<0.0001$ ). The mean age of cases was 58.9 years (SD 11.9) and of controls 51.2 years (SD 13.1) $(\mathrm{p}<0.0001)$. In bivariate analysis, a past history o cigarette use was more common in subjects with $\mathrm{BE}$ than controls ( $58 \%$ vs. $47 \%, \mathrm{p}=0.04$ ) There was also a trend toward more pack-years in subjects with BE than controls (15.9 p$y$ vs. $13.4 \mathrm{p}-\mathrm{y}, \mathrm{p}=0.09)$. However, after controlling for age and sex in multivariate logistic regression, these differences disappeared $(\mathrm{p}=0.61)$. Conclusions: This large case-control study demonstrates that, after controlling for potential confounders, subjects with BE appear to be no more likely to have a history of cigarette smoking than subjects with reflux symptoms but no $\mathrm{BE}$. These data do not suggest that cigarette smoking is a risk factor for BE among those with GERD symptoms.

\section{T1877}

P53 Mutation Does Not Predict Malignant Progression in Biopsies of Patients with Barrett's Metaplasia (BM)

Mamoun Younes, Baomin Liu, Jiang Wang, Juan Lechago, Atilla Ertan, Gulchin Ergun, Margaret Bridges, Frank Meriano, Ray Verm, Karen Woods, Carl Schmulen, Craig Johnson, Alberto Barroso, John McKechnie, Jim Schwartz, Cecilia Fenoglio-Preiser

p53 gene mutation has been implicated by many authors in malignant progression in Barrett's metaplasia (BM). The significance of $\mathrm{p} 53$ mutations in BM has not been adequately addressed in prospective studies. We sequenced exons 2-10 of the p53 gene, utilizing microdissected samples from formalin-fixed and paraffin-embedded biopsies from 190 consecutive cases of BM. Morphological evaluation for dysplasia, was done by two gastrointestinal pathologists To date, 117 patients had one or more years of follow up (median 31 months), and these are included in this study. Of the 117 biopsies only 20 had p53 mutations, and 4 of these progressed to a higher grade of dysplasia (20\%). Ninety-seven biopsies did not have any p53 mutations, but 21 of these $(22 \%)$ progressed to a higher grade of dysplasia. The difference was not statistically significant $(\mathrm{p}=1.000)$. Additional studies and follow-up are in progress to determine whether specific p53 mutations are associated with malignan progression in BM. Supported by National Institutes of Health grant R01 CA81570-02, and in part by PriCara, Unit of Ortho-McNeil, Inc. and by Eisai Inc.

\section{T1878}

The Effect of Oral Administration of Ursodeoxycholic Acid and Proton Pump Inhibitors in Patients with Barrett's Esophagus

Andreas Bozikas, Willem A. Marsman, Wilda D. Rosmolen, Jantine W. van Baal, Wim Kulik, Fiebo J. Ten Kate, Sheila K. Krishnadath, Jacques J. Bergman

Background \& Aims Bile acids may play a role in the pathogenesis of Barrett's esophagus (BE). Bile composition can be influenced by oral administration of ursodeoxycholic acid (UDCA). We prospectively investigated the effect of proton pump inhibitors (PPI) supplemented with UDCA in vivo in patients with BE. Patients \& Methods Pts with no or lowgrade dysplasia who were clinically asymptomatic on PPI were eligible for the study. In 
order to exclude the effects of acid reflux, all pts were initially treated with $40 \mathrm{mg}$ esomeprazol (ESO) BID for six mo and continued on this dose till the end of the study ( $\mathrm{t}=12 \mathrm{mo}$ ). During a period of six months ( $\mathrm{t}=6 \mathrm{mo}-\mathrm{t}=12 \mathrm{mo}$ ) pts were treated with oral UDCA (600 mg BID). Pts underwent endoscopy at $\mathrm{t}=0 \mathrm{mo}, \mathrm{t}=6 \mathrm{mo} \& \mathrm{t}=12 \mathrm{mo}$ with multiple biopsies of the distal and proximal BE segment, normal squamous and gastric cardia. In addition, $\mathrm{pH}$ was measured at $\mathrm{t}=0 \mathrm{mo} \& \mathrm{t}=6 \mathrm{mo}$ using a BRAVO wireless $\mathrm{pH}$ capsule and bile was sampled at the beginning of the UDCA treatment and six mo later $(\mathrm{t}=6 \mathrm{mo} \& \mathrm{t}=12 \mathrm{mo})$. All biopsies were reviewed for the extent of metaplasia, dysplasia, and acute \& chronic inflammation. In addition proliferation (Ki67), differentiation (villin, cytokeratins $7 \& 20$ ) and inflammation (COX-2) were investigated by immunohistochemistry (IHC). Results Nine pts (mean age 60 years, median $\mathrm{BE}$ length $7 \mathrm{~cm}$ ) were included, of whom 3 had low-grade dysplasia. $\mathrm{pH}$ measurements revealed a normalisation of the intraesophageal $\mathrm{pH}$ at $\mathrm{t}=6 \mathrm{mo}$. In addition, bile composition analysis demonstrated the efficacy of UDCA. Combining the results of both phases of the study, no significant changes were seen in any of the histological \& IHC parameters. In 5 patients the amount of metaplasia and dysplasia was marginally increased, mostly pronounced distally. Differentiation and proliferation parameters showed no significant changes. Conclusion In this study, in BE pts who were clinically asymptomatic on PPI, increasing the PPI dose to the maximum for six mo followed by the addition of UDCA for six mo did not result in significant histological or IHC changes in their BE. This study was financially supported by Zambon Netherlands.

\begin{tabular}{|c|c|c|c|}
\hline Parameter (range) & $\mathrm{t}=0$ months & $\mathrm{t}=6$ months & $\mathrm{t}=12$ months \\
\hline Medication & $\begin{array}{l}\text { Standard } \\
\text { PPI }\end{array}$ & $\begin{array}{l}\text { ESO (40 mg } \\
\text { BID) }\end{array}$ & $\begin{array}{l}\text { ESO (40 mg BID) \& UDCA ( } 600 \mathrm{mg} \\
\text { BID) }\end{array}$ \\
\hline Fraction time $\mathrm{pH}<4(\%)$ & 4 & 2 & - \\
\hline UDCA in bile (\%) & - & 0.5 & 45 \\
\hline Extent of Metaplasia (1-3) & 2 & 2 & 3 \\
\hline Dysplasia $(0-4)$ & 0 & 0 & 1 \\
\hline $\begin{array}{l}\text { Chronic Inflammation } \\
\qquad(0-3)\end{array}$ & 2 & 2 & 2 \\
\hline Acute Inflammation (0-3) & 0 & 0 & 1 \\
\hline Proliferation (0-3) & 1 & 1 & 1 \\
\hline Differentiation (0-3) & 2 & 2 & 2 \\
\hline
\end{tabular}

T1879

Dual Roles for Nitric Oxide in the Development and Progression of Barrett's Adenocarcinoma

Nicholas Clemons, Ruwani Abeyratne, Rebecca C. Fitzgerald

Introduction: Oesophageal adenocarcinoma develops via a metaplsia-dyplasia sequence. Luminal components such as acid and bile are thought to contribute to the neoplastic progression. For example, pulsatile acid and bile exposure can alter proliferation and differentiation status in ex vivo and in vitro models and low $\mathrm{pH}$ can induce DNA damage in vitro. Recently, increased oesophageal concentrations of nitric oxide (NO) have been demonstrated in Barrett's oesophagus (BO) patients. NO and its derivates have been shown to induce DNA damage in colorectal carcinogenesis. In addition, NO has been implicated in the regulation of metastatic behaviours in cancer cell lines and in tissues. Aims: Using oesophageal cell lines we investigated whether NO could induce DNA damage by studying the phosphorylation of histone $\mathrm{H} 2 \mathrm{AX}(\gamma \mathrm{H} 2 \mathrm{AX}$ ), a specific and early marker of double strand breaks (DSBs) The ability of $\mathrm{NO}$ to regulate metastatic behaviour was also investigated via in vitro invasion and cellular aggregation assays. Methods: Adenocarcinoma cell lines BIC, SEG and FLO and Barrett's high-grade dysplasia cell lines ChTERT and GihTERT were used. Cells were treated for $45 \mathrm{~min}$ with $25-200 \mathrm{uM}$ of NO donor NOC-9 (Axxora) and $\gamma \mathrm{H} 2 \mathrm{AX}$ was assessed by flow cytometry and confocal microscopy. Long-term survival following NO exposure was assessed by clonogenic assay. For the in vitro invasion assay, FLO and GihTERT cells were added to Matrigel (BD) coated trans-well inserts (BD Falcon) and treated with 5-100 $\mu \mathrm{M} \mathrm{NO}$ incubated for $24 \mathrm{hr}$. The effect of $\mathrm{NO}$ on adhesive behaviour was examined by a cellular aggregation assay. NO-induced changes in the expression of genes implicated in metastasis were examined by SYBR-green real-time PCR. Results: NO induced $\gamma \mathrm{H} 2 \mathrm{AX}$ in a dose dependent manner ( $<<0.05$ at all doses). The ability of $\mathrm{NO}$ to induce $\gamma \mathrm{H} 2 \mathrm{AX}$ appears to occur preferentially in cells that are in S-phase. Long-term survival studies demonstrated that cells were able to continue to grow following this initial insult. NO enhanced the ability of GihTERT and FLO cells to invade through Matrigel ( $\mathrm{p}<0.05$ for $25 \mu \mathrm{M}$ and above). NO-induced metastatic behaviour correlated with the induction of osteopontin, COX-2, MMP2 and MMP 9. Maximum gene expression was observed $1-3 \mathrm{hr}$ after the addition of NO and had returned to basal levels after $22 \mathrm{hr}$. Conclusions: NO has the potential to generate DSBs in the oesophagus. If not repaired correctly these lesions could lead to gross genetic and chromosomal abnormalities that may aid in the progression of BE to adenocarcinoma. At later stages NO may promote metastasis by increasing invasive potential and regulating expression of genes nvolved in metastatic behaviour.

$\mathrm{T} 1880$

NADPH Oxidase Nox5s (short) Mediates Platelet-Activating Factor (PAF)Induced $\mathrm{H}_{2} \mathrm{O}_{2}$ Production in Barrett's Adenocarcinoma Cells Xiaoying Fu, Jose Behar, Ramy Eid, David Beer, Jack Wands, Weibiao Cao

A carcinogenic role of reactive oxygen species (ROS) has been demonstrated in several types of human cancer. Elevated ROS levels have been reported in Barrett's esophagus (BE) and in esophageal adenocarcinoma. We have previously shown that NADPH oxidase NOX5S (short, i.e. without EF-hand motifs at its N-terminal) is overexpressed in SEGl esophageal adenocarcinoma cells associated with Barrett's esophagus (SEGl-EA) and mediates acidinduced production of hydrogen peroxide $\left(\mathrm{H}_{2} \mathrm{O}_{2}\right)$. It has been reported that PAF levels increase in esophagitis and that PAF causes production of $\mathrm{H}_{2} \mathrm{O}_{2}$ in other cell types. In the present study we examined the role of $\mathrm{PAF}$ in $\mathrm{H}_{2} \mathrm{O}_{2}$ production in SEGl-EA. PAF dosedependently increased $\mathrm{H}_{2} \mathrm{O}_{2}$ in SEGl-EA cells. $\mathrm{PAF}\left(10^{-7} \mathrm{M}, 24\right.$ hours)-induced $\mathrm{H}_{2} \mathrm{O}_{2}$ production was abolished by the NADPH oxidase inhibitor apocynin, suggesting a role of NADPH oxidases in PAF-induced $\mathrm{H}_{2} \mathrm{O}_{2}$ production. The NADPH oxidase catalytic subunits NOXI and NOX5S are highly expressed in SEG1-EA cells and PAF significantly increased expression of NOX5S, but not of NOX1. Knockdown of NOX5S by NOX5S small interfering RNA significantly decreased PAF-induced $\mathrm{H}_{2} \mathrm{O}_{2}$ production, suggesting a role of NOX5S in PAF induced $\mathrm{H}_{2} \mathrm{O}_{2}$ production. PAF-induced $\mathrm{H}_{2} \mathrm{O}_{2}$ production was significantly reduced by MEK1 inhibitor PD98059, the P38 MAP kinase inhibitor SB203580 and by the cytosolic phopholipase $\mathrm{A} 2$ inhibitor $\mathrm{AACOCF} 3$, but not by the protein kinase $\mathrm{C}$ inhibitor chelerythrine, suggesting that PAF-induced $\mathrm{H}_{2} \mathrm{O}_{2}$ production depends on activation of MAP kinases (ERKL/ 2, p38) and cytosolic phospholipase A2. We conclude that PAF increases $\mathrm{H}_{2} \mathrm{O}_{2}$ production in SEGl-EA cells through activation of MAP kinases (ERK1/2, p38), cytosolic phospholipase A2 and NADPH oxidase NOX5S. Supported by NIH NCRR 1 P20 RR17695-01.

\section{T1881}

Oxidative Stress Is Induced By Bile Acids and Low pH in Barrett's Esophagus Biopsies and Esophageal Cell Lines

Katerina Dvorak, Melissa Chavarria, Lois Ramsey, Barbora Dvorakova, Ronnie Fass, Richard Sampliner, Claire M. Payne, Harris Bernstein, Carol Bernstein, Sylvan Green, Harinder Garewal

Background: Barrett's esophagus (BE) appears to result from chronic irritation by gastric acids and bile acids. Several studies implicate oxidative stress and induction of reactive oxygen species (ROS) in the development of BE and esophageal cancer. Nevertheless, studies evaluating the direct effect of low $\mathrm{pH}$ and bile acids on the production of ROS have been lacking. Our aim was to evaluate whether brief exposure to bile acids and $\mathrm{pH} 4$ induces oxidative stress in esophageal cells and human biopsies. Methods: Esophageal cell lines (normal squamous Het-1A cells, BE cells transfected with hTERT, Seg-l adenocarcinoma cells) were exposed to control medium, medium at $\mathrm{pH} 4$ and/or a $0.1 \mathrm{mM}$ bile acid cocktail (BA) for 10 minutes. BA consisted of glycodeoxycholic, glycocholic, taurocholic, glycochenodeoxycholic and deoxycholic acid. Oxidative stress was evaluated by staining with 5 uM MitoSOX Red, a compound that is oxidized by ROS to a fluorescent dye, using fluorescent microscopy or flow cytometry. Oxidative stress was also evaluated in BE biopsies with different grades of dysplasia (15 ND, 15 LGD, 15 HGD), duodenum and squamous epithelium by immunohistochemical staining for 8-hydroxy-deoxyguanosine (8-OH-dG), a marker of oxidative DNA damage. In addition, four separate biopsies from ten BE patients were evaluated for 8-OH-dG expression after ex vivo incubation with control medium and medium at $\mathrm{pH} 4$ and/or $0.5 \mathrm{mM}$ BA. Results: Exposure to $\mathrm{pH} 4$ and BA resulted in an increase in ROS in Het-1A, Seg-1 and BE cells, while no change in ROS was detected in these cells after treatment with $\mathrm{pH} 4$ alone or BA alone. Increased 8-OH-dG staining was detected in BE biopsies compared to squamous and duodenal epithelia. The strongest staining of 8-OH-dG was observed in the dysplastic glands, while a less intense signal was found in nondysplastic tissue. Statistical evaluation indicated that dysplastic BE had significantly greater $8-\mathrm{OH}-\mathrm{dG}$ levels than nondysplastic $\mathrm{BE}(\mathrm{p}=0.003)$. In the ex vivo experiment, six out of ten biopsies expressed elevated 8-OH-dG after $\mathrm{pH} 4$ and BA exposure compared with the biopsies exposed to control medium only. Interestingly, the majority of the biopsies (5/ 6) with increased 8-OH-dG were nondysplastic. In contrast, two out of four cases that did not show increased 8-OH-dG levels after $\mathrm{pH} 4$ and $\mathrm{BA}$ exposure were dysplastic and a strong 8-OH-dG signal was found in the biopsies exposed to control medium. Conclusion: Our data indicate that oxidative stress is induced by short exposures to $\mathrm{BA}$ and low $\mathrm{pH}$ in esophageal cell lines and human BE, especially in nondysplastic tissue. Such repetitive and chronic stress may lead to DNA damage and cancer progression.

\section{$\mathrm{T} 1882$}

Chromosome 17 Hyperploidy and Her2/Neu Oncogene Amplification Are Important Genetic Events in the Transformation of Barrett's Esophagus Into High-Grade Dysplasia

Agnieszka M. Rygiel, Franceska Milano, Jantine W. van Baal, Wilda D. Rosmolen, Pau Fockens, Febo J. Ten Kate, Jacques J. Bergman, Maikel P. Peppelenbosch, Kausilia K. Krishnadath

BACKGROUND: Barrett's esophagus (BE) is associated with an increased risk for developing esophageal adenocarcinoma (EAC). The progression of BE to cancer is a gradual process that is characterized by increasing dysplasia and the accumulation of several genetic abnormalities, for instance aneuploidy and amplification of the oncogene Her2/neu. The amplification of Her2/neu is generally considered as a late event in cancer progression correlating with poor survival. In BE however, studies examining the occurrence of this abnormality in patients with different stages of dysplasia are lacking. AIM: The purpose of this study was to investigate the frequency of Her2/neu (17q21) gene amplification and chromosome 17 aneusomy in a BE population with different stages of dysplasia. MATERIAL and METHODS: Multi-color FISH using a centromeric probe for chromosome 17 and a locus-specific Her2/ neu probe was applied on brush cytology specimens of $100 \mathrm{BE}$ patients. Brushes were taken separately from the $B E$ segment $(n=100)$ and from the adjacent normal squamous epithelium $(\mathrm{n}=20)$, the latter served as a controls. RESULTS: Of the $100 \mathrm{BE}$ samples, 73 had BE without dysplasia (ND), 8 had indefinite for dysplasia (IND), 10 had low grade dysplasia (LGD), and 9 high grade dysplasia (HGD). We observed chromosome 17 trisomy in: 3 of 73 (4\%) of ND cases, 1 of 8 (13\%) of IND cases, and in 5 of $9(56 \%)$ of the HGD cases. The Her2/ neu amplification was not seen in ND but was detected 1 of $10(10 \%)$ of LGD, and in 8 of $9(89 \%)$ of HGD cases. Interestingly, we observed that 5 out of 8 cases with HGD and Her2/neu amplification also had a trisomy of chromosome 17. CONCLUSIONS: Our results show that hyperploidy of chromosome 17 and/or Her2/neu gene amplification are important genetic events contributing in the progression of BE with ND into HGD. We postulate that this process starts with hyperploidy of chromosome 17 , subsequently followed by amplification of the Her2/neu gene. These genetic abnormalities are presently used as tumor markers for the surveillance of a BE cohort. 
Freqency of chromosome 17 trisomy and Her2/neu amplification in BE population

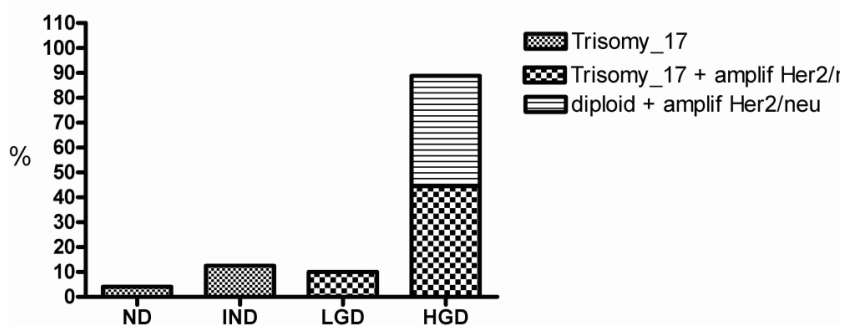

$\mathrm{T} 1883$

Conversion of Stratified Squamous to Columnar Epithelium By Bone MarrowDerived Cells -three-dimensional Reconstruction Using a Primary Culture of Epithelial Cells Isolated from Murine Esophagus and Forestomach -

Hidekazu Suzuki, Mamoru Arakawa, Yuriko Minegishi, Akifumi Ootani, Eisuke Iwasaki, Toshifumi Hibi

Barrett's esophagus is an acquired condition, in which squamous epithelium in the distal esophagus is replaced by specialized (metaplastic) columnar epithelium. It is considered to be mainly induced by chronic gastroesophageal reflux and to be a preneoplastic condition. Despite the great interest in the pathogenesis of this condition, little is known regarding the mechanism underlying the conversion of stratified squamous to columnar epithelium. Recently, the importance of bone marrow-derived cells during the development of gastric adenocarcinoma through the metaplastic and dysplastic stages was reported in a mouse model of Helicobacter infection (Science 306:1568, 2004). The present study was designed to investigate the involvement of bone marrow-derived cells in the conversion of stratified squamous to columnar epithelium, by establishing a three-dimensional culture system for highly differentiated murine esophageal epithelial cells. Methods. On postnatal dayl, tissues of the esophagus and forestomach of C57BL/6 mice were minced and digested in dispase I solution. To reconstruct the esophageal mucosa, type I collagen gel solution was mixed with fibroblasts isolated from the forestomach. As these fibroblast-containing collagen gel layers have been shown to be comparable with the esophageal lamina propria in vivo, isolated epithelial cells were cultured on the reconstructed lamina propria. Seven days later, the cultured epithelial cells were fixed with Carnoy's solution, vertically embedded in paraffin and sections were stained with periodic acid-Schiff (PAS). Immunohistochemistry for cytokeratin 14 (CKl4) was also performed to recognize cells of basal layers of stratified squamous epithelium. Samples of bone marrow cells obtained from the same strain of mice were incubated with the primary epithelial cell culture for 7 days. Results. A primary threedimensional culture system for epithelial cells isolated from the esophagus and forestomach was established. The isolated epithelial cells developed PAS-negative and CK14-positive stratified squamous cell layers, with keratinization of the surface layer. After the bone marrowderived cells were mixed into the culture, the isolated epithelial cells developed into a PASpositive and CKl4-negative columnar monolayer epithelium. Conclusions. A primary threedimensional cell culture system for epithelial cells of the esophagus and forestomach was established. Bone marrow-derived cells are considered to be involved in the genesis of Barrett's epithelium by promoting the transformation of stratified squamous epithelium into columnar epithelium.

\section{$\mathrm{T} 1884$}

Y Chromosome Abnormalities in Barrett's Esophagus: How Common Is It? Kenneth K. Wang, Ganapathy Prasad, Navtej S. Buttar, Michel Wongkeesong, Kevin Halling, Thomas Smyrk, Jason Lewis, Susan Abraham, Sheila Krishnadath, Marlys Anderson, Lori Lutzke, Catherine Demars, Sarah Papenfuss, Lynn Borkenhagen, Dawn Ferguson

Y chromosome abnormalities have been described to occur in 31-40 percent of Barrett's esophagus related cancer and dysplasia and has nearly always been reported to be loss of Y. However, these reports have been in small cohorts of patients. Due to the male predominance of Barrett's esophagus, there has been interest in delineating whether Y chromosome alterations are more common than initially reported. Aim: To determine the frequency of Y chromosome abnormalities in a cohort of patients with Barrett's esophagus and associated neoplasia. Methods: Patients were recruited from a specialized Barrett's Esophagus Unit where they underwent cytological brushing and four quadrant biopsies every centimeter of Barrett's esophagus. Y chromosome was assessed using fluorescent in situ hybridization (FISH) using a centrometric probe (pll.1-qll.1, Vysis, Des Plaines, Il). All histological interpretations were performed by two expert pathologists. FISH results were all interpreted by an experienced cytopathologist. Results: 166 males with Barrett's esophagus were entered into this study. 42 patients had non-dysplastic Barrett's mucosa, only $4(10 \%)$ had y chromosome abnormalities. 22 patients had low-grade dysplasia and only 5 or $23 \%$ had y chromosome abnormalities all being losses. 81 patients had high-grade dysplasia, 36 (44\%) had y chromosome abnormalities with 32 of these being deletions. 21 patients had adenocarcinoma with 13 (62\%) having y chromosome abnormalities with loss of y in 12 . . There was a statistically significant difference in Y chromosome abnormalities between those with highgrade dysplasia or cancer and those with low-grade dysplasia or no dysplasia $(\mathrm{p}<0.05)$. Conclusions: Y chromosome abnormalities are frequent in Barrett's esophagus and become significantly more common with increasing degrees of dysplasia. Y chromosome loss is the most frequent abnormality with gains found in $<5 \%$ of patients. This suggests that the $Y$ chromosome loss may play an important role in the pathogenesis of Barrett's esophagus.
$\mathrm{T} 1885$

CDX2 Overexpression Is a Marker of Intestinalization in GERD - Metaplasia Adenocarcinoma Sequence

Leandro A. Streher, Luise Meurer, Marcelo Binato, Carlos Renato A. Melo, Richard R. Gurski

Background: Barrett's esophagus develops as a result of severe esophageal mucosa injury from gastroesophageal reflux. It is a premalignant lesion and plays important role in the development of esophageal adenocarcinoma. The histologic diagnosis of BE requires the presence of globet cells, but this finding may not be the earliest indicator of intestina metaplasia (IM). The aim of the present study was to determine the expression of the Cdx2 in patients with GERD - metaplasia - adenocarcinoma sequence. Methods: We investigated 146 patients with gastroesophageal reflux disease assessing the imunoexpression of $\mathrm{Cdx} 2$ in biopsy specimens obtained by endoscopy, from patients with normal esophageal mucosa, esophagitis, columnar epithelium in distal esophagus and adenocarcinoma. The patient were distributed into five groups according to the histological diagnosis: Group 1: normal squamous epithelium (30), Group 2: chronic esophagitis (21), Group 3: columnar epithelium in distal esophagus without IM (24) Group 4: columnar epithelium in distal esophagus with IM (27), Group 4: adenocarcinoma (44). Results: Cdx2 immunoexpression was present in $0 \%$ in $\mathrm{G} 1,5 \%$ in $\mathrm{G} 2,0 \%$ in $\mathrm{G} 3,63 \%$ in $\mathrm{G} 4$ and $66 \%$ in $\mathrm{G} 5$ (p<0,001). We observed tha $\mathrm{Cd} 2 \mathrm{2}$ is a highly sensitive marker of intestinal metaplasia and its expression was related with presence of goblet cells. Conclusions: The $\mathrm{Cdx} 2$ protein expression may be one of the useful biomarkers for assessing the intestinal metaplasia in patients with Barrett's esophagus . It could be capable of detecting intestinal differentiation in Barrett's patients with missing of goblet cells.

\section{$\mathrm{T} 1886$}

Early Markers of Esophageal Intestinal Differentiation After Mucosal Ablation By Photodynamic Therapy

Gerda Spijkerboer, Leon M. Moons, Peter D. Siersema, Johannes G. Kusters, Jelle Haringsma, Ernst J. Kuipers

Objective: Barrett's esophagus (BE) is characterised by specialized intestinal metaplasia (SIM) that expresses CDX2. The time course of CDX2 expression in SIM development is largely unknown. Photodynamic therapy (PDT) results in ablation of the mucosal surface, followed by rapid re-epithelialisation. The new epithelium is thought to derive from stem cells in the esophagus. Aim: To determine the timing of CDX2 expression in the development of SIM in the esophageal mucosa. Methods: Biopsies taken from the esophagus of 12 patients (75\% male, mean age 68 years) with high grade dysplasia and a mean BE-segment length of 6 $\mathrm{cm}$ (range 2-12) treated with PDT, were used to determine the expression of CDX2, intestina differentiation markers (Villin and MUC2), and proliferation (KI-67) by immunohistochemistry. Paired biopsies were taken after 3 weeks and 16 weeks post treatment. The presence of SIM was determined in H\&E stained sections and confirmed by an Alcian Blue stain Results: Although there were characteristics of tissue regeneration based on a more central

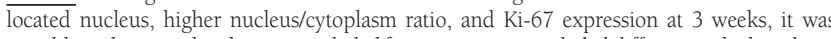
possible to distinguish columnar epithelial from squamous epithelial differention by histology The columnar epithelial cells were not homogeneous and were all in various stages of differentiation. At three weeks, well differentiated glandular epithelium could be detected, which concerned SIM (CDX2+, MUC2+ and Villin+), as well as cardiac type epithelium negative for these markers. In addition there was also epithelium with lacking clear signs of differentiation; i.e. it was CDX2+, but showed only faint cytoplasmic MUC2 and Villin expression. This epithelium also lacked the presence of goblet cells (H\&E). In 3 patients, several glands could be detected with large strong Ki-67+ nuclei, a very high nucleus/ cytoplasm ratio, and absence of cytoplasmic organization. Although most of these glands were not positive for CDX2, a few glands showed very weak nuclear CDX2 expression which was in $50 \%$ associated with perinuclear expression of MUC2. At 16 weeks only well differentiated epithelium was detected with either squamous-, IM- or gastric type epithelium. Conclusions: This study shows that the formation of glands in the esophagus precedes differentiation into gastric- or intestinal type epithelium, and that CDX2 was already expressec at very early stages of differentiation. Biopsies from early regenerating epithelium following PDT provides a good opportunity to study the process of intestinal differentiation of the human esophagus, and its underlying factors in a time dependent manner.

\section{$\mathrm{T} 1887$}

P63 Response to Bile and Acid Exposure in Esophageal Squamous Carcinoma Cell Lines

Sabine Roman, Amelie Thepot, Agnes Hautefeuille, Jean-Yves Scoazec, Francois Mion, Pierre Hainaut

Backgrounds and aim: The TP63 gene encodes for at least six different isoforms proteins with homology to the tumor suppressor protein p53. These isoforms are produced from a single gene using 2 promoters (TA and deltaN isoforms) and alternative splicing of the primary RNA transcript ( $\alpha, \beta$ and $\gamma$ isoforms). TA and deltaN isoforms are involved in keratinocyte stem cells differentiation and proliferation, respectively. P63 is present in esophageal squamous epithelium but not in Barrett's mucosa. Patients with Barrett's mucosa have an increased esophageal bile and acid exposure. Our hypothesis is that loss of deltaN p63 expression induced by bile and acid is necessary to the development of Barrett's metaplasia. The aim of this study was to determine p63 expression after bile and acid exposure in esophageal cell lines over expressing p63. Materials and methods: Two esophageal squamous carcinoma cell lines, TE-1 and TE-13, were exposed to bile and/or acid (pH5) Three bile salts, primary bile salt (chenodeoxycholate CDCA), secondary bile salt (deoxycholate DCA) and conjugated primary bile salt (taurochenodeoxycholate TCDCA) were applied at $200 \mu \mathrm{M}$ concentration during 5, 10, 20, 40 and 80 minutes. P63 expression was studied by western blot and RT-PCR at the end of bile and acid exposure. Actin was the reference protein and gene. Results: When cell lines were exposed to DCA at pH5, p63 protein expression decreased after 5 minutes exposure and disappeared after 20 minutes. P63 mRNA 
expression remained stable in all experimental conditions. No modification of $\mathrm{p} 63$ expression was detected after cell exposure to DCA or acid alone. The same results were obtained with CDCA whereas TCDCA exposure at acid or neutral pH had no effect on p63 expression. Results were similar in both cell lines. Conclusion: A brief exposure to unconjugated bile salt in acid medium induces a loss of p63 expression in esophageal squamous carcinoma cell lines. This synergistic effect of bile and acid occurs very soon after exposure and is probably linked to a post translation phenomenon. The potential role of bile and acid induced p63 loss of expression in the initiation of Barrett's metaplasia development will be tested in primary esophageal cell cultures and in experimental models of bile and acid reflux.

\section{$\mathrm{T} 1888$}

Identification of Novel Genes Modulated By Acid and Bile Exposure in a Barrett's Esophagus Cell Line and Associated Differential Expression in Esophagitis, Barrett's Esophagus and Esophageal Adenocarcinoma Clare Donnellan, Simon Everett, Chris Wild, Laura Hardie

Molecular changes linked to reflux exposure may serve as biomarkers to identify Barrett's esophagus (BE) patients at greatest risk of esophageal adenocarcinoma (EA) and improve current surveillance programmes. To identify genes central to the molecular response to reflux, we exposed the non-cancer BE cell line, qhTERT, to media containing acid, deoxycholic acid (DCA), or a mix of primary bile salts, all at $\mathrm{pH} 4.5$, for 15 minutes. RNA was extracted from treated and control cells, 2 and 6 hours later. Gene expression was assessed by hybridising cDNA to Affymetrix U133 Plus 2.0 microarrays. Following ethical approval, expression of selected genes was then assessed by Real Time (RT) PCR in matched biopsies from the mid and lower esophagus of patients with non-erosive reflux disease (NERD) (N= 31), esophagitis ( $\mathrm{N}=27), \mathrm{BE}(\mathrm{N}=20)$ and $\mathrm{EA}(\mathrm{N}=32)$. Compared to other conditions, treatment of the qhTERT cell line with DCA resulted in the greatest overall change in gene expression; 210 genes were up- and 65 down-regulated. Based on biological plausibility, 12 genes were selected for further study. For 11 of the 12 genes, changes in expression were confirmed by RT PCR in treated cells. Patient biopsy data revealed that none of the target genes were changed in NERD but four (including Anterior Gradient 2 homolog and COX-2) were increased in esophagitis tissue. In contrast, the levels of expression of 8 genes (including Manic Fringe and DNA-damage-inducible transcript 3) were increased and 2 (Ras-related associated with diabetes and heat shock protein-40) decreased in BE and EA compared to matched normal tissue. This research highlights novel genes associated with acid and bile exposure in BE and EA, which may have utility as biomarkers in the future

T1889

Insulin Resistance in Barrett's Esophagus Patients: Preliminary Results Kayode Olowe, Jennifer Kimble, Jason Taylor, Amitabh Chak

Background: Obesity is independently associated with Barrett's esophagus (BE) and esophageal adenocarcinoma (EAC). Consistent with the hyperinsulinemia hypothesis of obesity associated carcinogenesis, we propose that insulin resistance may contribute to the development of $\mathrm{BE}$ and its progression to EAC. Aim: To determine the prevalence of insulin resistance and measure body mass index (BMI) in subjects with BE. Methods: A fasting serum sample was taken from subjects with BE who provided informed consent. Serum was assayed for insulin and glucose levels. The lowest detectable insulin level was $3 \mu \mathrm{IU} / \mathrm{ml}$. Levels below this were treated as zero. Insulin resistance was calculated using the homeostasis model assessment of insulin resistance $(\mathrm{HOMA}-\mathrm{IR})$ equation $=$ [fasting serum insulin $(\mu \mathrm{IU} / \mathrm{ml}) \times$ fasting glucose $(\mathrm{mg} / \mathrm{dl})] / 405$. Insulin resistance was deined as HOMA-IR $\geq 3.80$. Measurements of weight and height were taken to determine the BMI $=\mathrm{kg} / \mathrm{m} 2$ with obesity defined as BMI $>30 \mathrm{~kg} / \mathrm{m} 2$. Results: Fasting serum samples from $21 \mathrm{BE}$ subjects were assayed. The mean age of the BE subjects was $64.1 \pm 11.7$ years. Eighteen of 21 subjects $(85.7 \%)$ were men. The median HOMA-IR value was 2.62 with a range of zero to 16.81 . Four of 21 (19\%) subjects had a HOMA-IR value $>3.80$, indicative of insulin resistance. Eight of $20(40 \%)$ subjects were obese with BMI $>30 \mathrm{~kg} / \mathrm{m} 2$. All four subjects with HOMA-IR $>3.80$ also had $\mathrm{BMI}>30 \mathrm{~kg} / \mathrm{m} 2$. Conclusion: Our preliminary data suggest that there is a significant degree of insulin resistance in obese subjects with Barrett's esophagus. This relationship between obesity, insulin resistance, and Barrett's esophagus needs to be further explored.

\section{T1890}

Antimicrobial Resistance for Primary and Secondary Helicobacter Pylor Strains Isolated from Korean Patient

Jung Mogg Kim, Joo Sung Kim, Nayoung Kim, Sang Gyun Kim, Hyun Chae Jung, In Sung Song

BACKGROUND and AIMS. The aim of this study was to assess MIC values and resistance rates of several antibiotics in primary and secondary $H$. pylori isolates from Korean patients. MATERIALS AND METHODS: Sixty-five strains of $H$. pylori were isolated from 65 patients that had not received antibiotics, PPI or nonsteroidal anti-inflammatory drugs during the preceding three months. Three hundred twenty-four strains of $H$. pylori were isolated from 67 patients who had taken PPI triple therapy consisting of amoxicillin and clarithromycin for one week. The MIC values of $H$. pylori isolates to the antibiotics were determined using the serial two-fold agar dilution method. The resistance breakpoints for amoxicillin, metronidazole, and tetracycline were defined as $>=0.5,>8$, and $>4 \mu \mathrm{g} / \mathrm{ml}$, respectively. The breakpoint for clarithromycin was set at $>1.0 \mu \mathrm{g} / \mathrm{ml}$, and those for azithromycin, ciprofloxacin, levofloxacin, and moxifloxacin were provisionally defined as $>1.0 \mu \mathrm{g} / \mathrm{ml}$. The mutation of penicillin-binding protein $1 \mathrm{~A}(\mathrm{pbplA})$ was determined by PCR amplification and nucleotide sequencing. RESULTS: The overall MIC values of secondary isolates showed a shift to high concentrations compared with those of primary isolates. Primary resistances to amoxicillin, clarithromycin, metronidazole, tetracycline, azithromycin, ciprofloxacin, levofloxacin and moxifloxacin were $18.5 \%, 13.8 \%, 66.2 \%, 12.8 \%, 32.3 \% 33.8 \%, 21.5 \%$ and $21.5 \%$, respectively. Secondary resistances to amoxicillin, clarithromycin, metronidazole, tetracycline, azithromycin, ciprofloxacin, levofloxacin and moxifloxacin were $31.3 \%, 85.1 \%$,
$70.1 \%, 0 \%, 89.6 \% 35.8 \%, 32.8 \%$ and $32.8 \%$, respectively. Sequence analysis of pbplA in H. pylori strains with more than $2 \mu \mathrm{g} / \mathrm{ml}$ of amoxicillin MIC revealed C206T (Asp69 to Val), Cl667G (Thr556 to Ser), Al684T (Asn562 to Tyr), Al777G (Thr593 to Ala), and C1798A (Pro600 to Thr). In contrast, the activity of $\beta$-lactamase was detected in neither the amoxicillin-resistant strains nor amoxicillin-susceptible strains. Eleven out of 67 treatment-failure patients (16.4\%) showed mixed infections with both antibiotic-susceptible and -resistant $H$. pylori. The most common multidrug resistance was to clarithromycin, metronidazole and azithromycin. CONCLUSIONS: These results indicate that MIC values of secondary isolates were higher than those of primary isolates and that resistance to amoxicillin is likely mediated through mutations in pbplA

\section{T1891}

Comparison Study On Effectiveness of Established Triple Therapy with Its Modified Therapies for Helicobacter Pylori Eradication Taewoon Park, Jae Eun Lee, Chang Woo Gham, Ja Sung Choi, Chang Hwan Choi, Ki Joon Han, Hyeon Geun Cho

Background: The rate of Helicobactor pylori infection as well as related morbidity are relatively high in Korea. H. pylori eradication is becoming an essential treatment not only for peptic ulcer patients but also for functional dyspepsia patients. However, the eradication rate by the established triple therapy (PPI + amoxicillin + clarithromycin, PAC) is still around $80 \%$. In this study, we compared the efficacies of the established triple therapy with its modified therapies for $H$. pylori eradication in order to obtain a better eradication rate Methods: This prospective study included a convenience sample of $355 \mathrm{H}$. pylori infected patients (mean age 48.4+12.4 years) with functional dyspepsia $(n=189)$ or peptic ulcer $(n=$ 166)(table 1). From March 2003 to August 2005, the patients were randomly assigned to PAC group (PPI: rabeprazole $10 \mathrm{mg}$, bid + amoxicillin $1 \mathrm{~g}$, bid + clarithromycin $500 \mathrm{mg}$, bid), PACR group (PAC + roxatidine $75 \mathrm{mg}$, bid at different timing from PPI), or DPAC group (doubling PPI dose in PAC). The patients in three groups underwent each eradication treatment for one week and the eradication was confirmed through follow-up endoscopy or urea breath test at least four weeks after the completion of treatment. Results: Tests for homogeneity demonstrated no significant differences between groups in age, gender, and diagnosis. In per protocol analysis, the eradication rates were $83.2 \%$ in PAC group, $92.6 \%$ in PACR group, and $81.6 \%$ in DPAC group. Significantly higher eradication rate was noted in PACR group than the other two groups $(\mathrm{p}=0.034 ; \mathrm{p}=0.019$, respectively). Intent-to-treat analysis also showed similar findings; $74.2 \%$ in PAC group, $84 \%$ in PACR group, and $69.0 \%$ in DPAC group ( $p=0.043 ; p=0.003$, respectively). In terms of drug tolerance, only seven patients out of 355 patients failed to complete the treatment, but there were no differences between groups. Conclusion: While doubling PPI dose in PAC did not change H. pylori eradication rate, adding H2RA(roxatidine) to PAC did significantly increase the eradication rate. Further studies with a large sample are recommended to support these results and drug interaction between PPI and H2RA needs to be examined. Tabel 1. Characteristics of Patients

\begin{tabular}{|c|c|c|c|c|}
\hline & PAC $(\mathrm{n}=120)$ & PACR $(\mathrm{n}=119)$ & DPAC $(\mathrm{n}=116)$ & Total $(\mathrm{n}=355)$ \\
\hline Sex $(\mathrm{M} / \mathrm{F})$ & $63 / 57$ & $62 / 57$ & $62 / 54$ & $187 / 168$ \\
\hline Age $($ Mean \pm SD $)$ & $50.3 \pm 11.5$ & $47.8 \pm 11.9$ & $47.0 \pm 13.8$ & $48.4 \pm 12.4$ \\
\hline PUD/FD & $65 / 55$ & $65 / 54$ & $59 / 57$ & $189 / 166$ \\
\hline
\end{tabular}

P: proton pump inhibitor (rabeprazole), A: amoxicillin, C: clarithromycin, R: roxatitidine, DP: double dose PPI PUD: peptic ulcer disease, FD: functional dyspepsia

\section{$\mathrm{T} 1892$}

Dose Atrophic Gastritis Affect the Accordance Between Stool Antigen Test and Serology to Determine Helicobacter Pylori Infection in the Mass Survey? Tadashi Shimoyama, Takao Oyama, Shinsaku Fukuda, Shigeyuki Nakaji, Akihiro Munakata

Background: In Japan, infection of Helicobacter pylori is often tested in the mass screening for gastric cancer along with the level of serum pepsinogen (PG) I and II. Although infection of $\mathrm{H}$. pylori is usually examined by serology, a stool antigen test (EIA) using monochronal antibody to catalase is now available. We examined $H$. pylori infection by both serology and this stool antigen test in a mass survey and studied whether age of subjects or atrophic gastritis affect the results of the two tests. Methods: 994 healthy adults who received mass survey in April 2005 were tested. There were 379 males and 615 females, and the mean age was $57.7 \pm 13.9$ years old. Stool samples were used to test the prevalence of $H$. pylori antigen by EIA using monochronal antibody to $H$ pylori catalase. Serum samples were tested for the prevalence of IgG antibody to H. pylori by ELISA. The level of PG I and II was also measured and atrophic gastritis was considered significant if both PG I less than $70 \mu \mathrm{g} / \mathrm{L}$ and PGI/II less than 3.0 were observed. Results: The prevalence of $H$. pylori infection was defined as $60.7 \%$ by serology and $55.4 \%$ by stool antigen test. There were 22 subjects who were positive only by stool antigen test and 75 subjects were positive only by serology. The concordance of both tests was not affected by age of the subjects ( $91.7 \%$ in $<50$ years old, $88.1 \%$ in 50 s, $92.1 \%$ in 60 s, and $91.2 \%$ over 70 years old). The concordance of these tests was not also different in subjects with atrophic gastritis $(91.1 \%, \mathrm{n}=418)$ and without atrophic gastritis $(90.1 \%, \mathrm{n}=576)$. Conclusions: Both age and atrophic gastritis did not have significant effect on the results of serology and stool antigen test. The discrepancy of the results seemed to depend on the sensitivity and specificity of individual tests. Since stool antigen test have been shown to have better sensitivity and specificity, it would be better non-invasive test to determine $H$. pylori infection in the mass survey. 
T1893

Significantly Different H.pylori Density and Genotypes in Erosive, Antral and Corpus Routine Gastric Biopsy Specimen Detected By PCR

Bela Molnar, Dominika Szoke, Agnes Ruzsovics, Zsolt Tulassay

Background and aim: Determination of local intensity and genotypic variations in H.pylori in gastric biopsy specimen could support the precise diagnosis and research of the H.pylori infection. The aim of this study was the development of real-time PCR based new techniques for this purpose and comparison with routine available techniques. Methods: Serial dilutions of H.pylori (0.016-16 mikrog/ $/ \mathrm{l})$ and control bacterial and human DNA samples were prepared. From 53 patients who underwent routine gastroscopy, fresh frozen antral and corpus gastric biopsy specimen were taken and DNA was isolated using a commercial assay. UreaseA quantitative determination using hybridisation probes with parallel evaluation of an internal human control gene (beta-globin) was performed on a real-time PCR system. CagA and vacA genotypic characterisation was done using intercalating dye too. Comparison of the data was made to UBT, histology, and Western blot analysis of human sera samples for H.pylori and its antigens (UreaA, B, VacA, CagA). Results: The presence of H.pylori could be detected by ureaseA-FRET(53\%), UBT(46\%) and Western blotting(43\%), histology(41\%) as compared to the gold standard (44\%). A significant correlation was found between the quantitative real-time ureaseA/beta-globin ratio based H.pylori frequency and the UBT results $(\mathrm{p}<0.01)$. Significantly increased bacterial density was found in the erosion as compared to the healthy part of the antrum and corpus $(\mathrm{p}<0.01)$. VacA genotyping was in significant correlation $(\mathrm{p}<0.01)$ between the Western blot and real-time PCR, but CagA not. The genomic profile $(\mathrm{VAC} / \mathrm{GAC})$ was different in $8,7 \%$ of the cases concerning three different locations in the stomach surface. Conclusions: Real-time PCR based quantitative and genotypic determination of H.pylori in biopsy specimen can yield important data for routine diagnosis and research. The density and genomic profile of gastric $H$. pylori infection shows quantitative and qualitative differences between the place of erosion, antrum and stomach.

\section{T1894}

Clinical Practice Patterns for the Management of Helicobacter Pylori Infection, Dyspepsia, and Gastroesophageal Reflux Disease

Steven W. Blume, Colin W. Howden, Gregory de Lissovoy

Background Since the recognition of Helicobacter pylori (H. pylori) as the major cause of peptic ulcer, clinical guidelines for the testing and treatment of $H$. pylori infection have been widely disseminated. One of the key recommendations has been that clinicians use noninvasive testing for $H$. pylori infection, reserving prompt endoscopy for older patients or those with alarm symptoms. Eradication therapy should be administered to all patients testing positive. Little is known about adherence to these recommendations and actual medical practice patterns. Methods Medical and pharmacy claims of a national sample of two million non-elderly individuals continuously enrolled in managed care plans from 2002 to 2004 were the basis of a longitudinal cohort study. We selected patients with claims for antisecretory medication, $H$. pylori tests, or endoscopies but having no disease-related claims in the first six months of the study period and having at least six months of follow-up. Patients were grouped by their initial clinical diagnosis: peptic ulcer disease (PUD) ( $\mathrm{n}=$ 3456), non-ulcer dyspepsia $(n=14,593)$, or GERD $(n=36,233)$. The proportions of patients treated contrary to guidelines, diagnostic procedures and medications received, and the sequencing of key procedures and medications were examined by initial diagnosis, age, and physician type. Results Two-thirds of the study population were enrolled in health maintenance organizations and almost half were located in the Midwest census region. Several practices contrary to guidelines were common. Twenty-five percent of suspected PUD patients were not checked for $H$. pylori infection, and only $25 \%$ were treated with eradication therapy. Of all patients aged $18-49,15 \%$ had an endoscopy as their first clinical event. A third of those receiving antibiotics for $H$. pylori infection had not been first tested for the infection. Serology was used for $87 \%$ of pre-treatment tests in 2004 and was improperly used by primary care practitioners for post-treatment testing. Nineteen percent of GERD patients were tested for $H$. pylori. Patterns of care varied little by type of medical plan or geographic region. Conclusions The study demonstrated substantial non-compliance with guidelines. A better understanding by physicians of the indications for $\mathrm{H}$. pylori testing and endoscopy would improve patient care and resource use.

\section{T1895}

Seroprevalence of Helicobacter Pylori in Two Asymptomatic Dutch Populations A. J. van Vuuren, R. A. de Man, H. F. van Driel, M. Ouwendijk, J. G. Kusters, E. J. Kuipers, J. H. Richardus, P. D. Siersema, M. Blankenstein

Background: Helicobacter pylori $(\mathrm{Hp})$ infection poses a potential risk for developing upper gastrointestinal disorders ranging from peptic ulcers to gastric malignancies. The Hp prevalence is thought to be declining in Western countries, probably reflecting a birth cohort effect resulting from improvements in hygiene. Aims: To examine the secular trends in the Hp seroprevalence in a native Dutch population and in non-Western immigrants by determining the age specific $\mathrm{Hp}$ seroprevalence in representative groups from both populations. Methods: Plasma samples were collected from 794 native Dutch blood donors living in the South-Western Netherlands, which includes Rotterdam. Information concerning age, gender, and geographic region was available, but no donor identity. Another 287 serum samples were collected from asymptomatic volunteers from an urban district of Rotterdam, predominantly populated by non-Western immigrants. All samples were tested for $\operatorname{Ig} G$ antibodies against $\mathrm{Hp}$ by a commercial ELISA (Orion Diagnostica), the results were divided into ten-year age groups. Results: are summarized in the Table. Hp seroprevalence was overall $32 \%$ in the native Dutch population: $34 \%$ in males, and 30\% in females. Logistic regression within donors reveals a higher age specific $\mathrm{Hp}$ prevalence compared to the younger age groups(p<0,001): odds ratios ( $95 \% \mathrm{CI})$ are respectively $2,1(1,22-3,57)$ group II, 2,4 (1,40$4,03)$ group III, $2,4(1,38-4,00)$ group IV, and 4,7 $(2,79-7,86)$ group V. In contrast, in the urban district $H p$ seroprevalence was far higher in all age groups, $72 \%$ in males, $66 \%$ in females, $69 \%$ overall $(\mathrm{p}<0.001 \mathrm{Chi}$-square test), without signs of an age specific increase.
No gender differences were observed in either population. Conclusions: The secular trend towards lower Hp infection rates was observed in the native Dutch population, but no in volunteers of the urban district, with many non-Western immigrants. However, both populations are living in the same region, $H p$ seroprevalence varies. These ethnical differences are of obvious importance to public health authorities planning interventions to lighten the burden of $\mathrm{Hp}$ associated disease.

\begin{tabular}{|c|c|c|c|c|c|c|}
\hline \multicolumn{4}{|c|}{ Donors } & \multicolumn{3}{|c|}{ Volunteers } \\
\hline $\begin{array}{c}\text { Age } \\
\text { Groups }\end{array}$ & $\begin{array}{c}H p \text { pos } \\
\text { male (\%) }\end{array}$ & $\begin{array}{c}H p \text { pos } \\
\text { female }(\%)\end{array}$ & $\begin{array}{l}H p \text { pos total } \\
(\%)\end{array}$ & $\begin{array}{c}H p \text { pos } \\
\text { male (\%) }\end{array}$ & $\begin{array}{c}H p \text { pos } \\
\text { female }(\%)\end{array}$ & $\begin{array}{c}\text { Hp pos total } \\
(\%)\end{array}$ \\
\hline $\begin{array}{c}\mathrm{I}=18-28 \\
\mathrm{y}\end{array}$ & $12 / 5(\mathbf{2 0} \%)$ & $\begin{array}{l}15 / 101 \\
(\mathbf{1 5 \%})\end{array}$ & 27/160(17\%) & $\begin{array}{c}14 / 19 \\
(74 \%)\end{array}$ & $21 / 38(\mathbf{5 5} \%)$ & $\begin{array}{l}35 / 57 \\
(61 \%)\end{array}$ \\
\hline $\begin{array}{c}\mathrm{II}= \\
\mathrm{y}\end{array}$ & $23 / 8(\mathbf{2 7} \%)$ & $24 / 73(33 \%)$ & $47 / 158(30 \%)$ & $\begin{array}{c}23 / 35 \\
(\mathbf{6 6 \%} \%)\end{array}$ & $36 / 47(77 \%)$ & $\begin{array}{c}59 / 82 \\
(72 \%)\end{array}$ \\
\hline $\begin{array}{c}\mathrm{III}= \\
39-48 \mathrm{y}\end{array}$ & $31 / 9(\mathbf{3 4 \%})$ & $21 / 69(\mathbf{3 0} \%)$ & $52 / 160(33 \%)$ & $\begin{array}{c}18 / 26 \\
(69 \%)\end{array}$ & $24 / 36(66 \%)$ & $\begin{array}{c}42 / 62 \\
(68 \%)\end{array}$ \\
\hline $\begin{array}{c}\mathrm{IV}= \\
49-58 \text { y }\end{array}$ & $\begin{array}{l}32 / 101 \\
(\mathbf{3 2 \%})\end{array}$ & 19/57 (33\%) & $51 / 158(\mathbf{3 2} \%)$ & $\begin{array}{c}23 / 28 \\
(\mathbf{8 2 \%})\end{array}$ & $22 / 33(66 \%)$ & $\begin{array}{l}45 / 61 \\
(74 \%)\end{array}$ \\
\hline $\begin{array}{c}V=59-70 \\
y\end{array}$ & $\begin{array}{l}49 / 101 \\
(49 \%)\end{array}$ & $28 / 57(49 \%)$ & $77 / 158(\mathbf{4 9 \%})$ & $\begin{array}{c}11 / 15 \\
(\mathbf{7 3} \%)\end{array}$ & $6 / 10(60 \%)$ & $\begin{array}{c}17 / 25 \\
(68 \%)\end{array}$ \\
\hline
\end{tabular}

\section{T1896}

Accuracy of Monoclonal Stool Antigen Test for the Diagnosis of H. Pylori Infection: A Systematic Review and Meta-Analysis Javier P. Gisbert, Felipe de la Morena, Victor Abraira

OBJECTIVE: To perform a systematic review and a meta-analysis of accuracy of monoclonal stool antigen test for the diagnosis of H. pylori infection. METHODS: Selection of studies: assessing the accuracy of monoclonal stool antigen test for the diagnosis of $H$. pylori infection. Search strategy: electronic and manual bibliographical searches. Data extraction: independently done by two reviewers. Data synthesis: meta-analyses of the different tests were performed combining the sensitivities, specificities, and likelihood ratios (LRs) of the individual studies. RESULTS: Pre-treatment studies: Twenty-two studies, including a total of 2,499 patients, evaluated the monoclonal stool antigen test for the diagnosis of $H$. pylori infection before therapy. Pooled sensitivity, specificity, LR+ and LR- were, respectively, 0.94 (95\% CI, 0.93-0.95), 0.97 (0.96-0.98), 24 (15-41) and 0.07 (0.04-0.12). The accuracy of both the monoclonal and the polyclonal stool antigen tests was evaluated together in 13 pretreatment studies, where a higher pooled sensitivity was demonstrated with the monoclonal technique ( 0.95 vs. 0.83 ). Post-treatment studies: Twelve studies, including a total of 957 patients, assessed the monoclonal stool antigen test to confirm eradication after therapy Pooled sensitivity, specificity, LR+ and LR- were 0.93 (95\% CI, 0.89-0.96), 0.96 (0.940.97), 17 (12-23) and $0.1(0.07-0.15)$. The accuracy of both the monoclonal and the polyclonal stool antigen tests was evaluated together in 8 post-treatment studies, where, again, a higher pooled sensitivity was demonstrated with the monoclonal technique $(0.91$ vs. 0.76). Although heterogeneity among studies was initially present, it disappeared when a single outlier study was excluded. When subanalysis depending on the reference method (gold standard based on only one diagnostic method vs. at least two reference methods) or on the study population (adults vs. children) was performed, the results of the meta-analysis were similar. CONCLUSION: Monoclonal stool antigen test is an accurate non-invasive method both for the initial diagnosis of $H$. pylori infection and for the confirmation of its eradication after treatment. The monoclonal technique has a higher sensitivity than the polyclonal one, especially in the post-treatment setting.

\section{T1897}

H.pylori RE-Infection in Insulin Dependent Diabetes Mellitus Patients: A Five Yaers Follow-Up

Veronica Ojetti, Dario Pitocco, Alessio Migneco, Michele Santoro, Ilir Leka, Giovanni Gasbarrini, Giovanni Ghirlanda, Antonio Gasbarrini

Objective: Previous data show that diabetic patients (pts) have a low H.pylori eradication and a high re-infection rate after 1 year of follow-up. The increased susceptibility of diabetics to infections are based probably on many mechanisms (reduced lymphocyte activity, neutrophil dysfunction, failure of chemiotaxis, frequent hospitalization and dental plaque as H.pylor reservoir). Aim of our study was to evaluate the H.pylori re-infection rate in insulin dependent diabetes mellitus (IDDM) pts and in dyspeptic controls five years after successful eradication, and the impact of H.pylori re-infection on glycaemic control. Methods: 40 pts (23males, 17females; mean age 48+/-9) affected by IDDM and 50 dyspeptic control matched for sex and age, previously treated for $H$. pylori infection and successfully eradicated, were submitted to $13 \mathrm{C}$ urea breath test (UBT) after five years of follow-up. Daily insulin requirement, glycosilated haemoglobin ( $\mathrm{HbAlc}$ ) expressions of glycaemic metabolic control and the development of organ damage were evaluated. Results: We found a significantly higher incidence of $H$. pylori re-infection in IDDM pts compared to controls. In particular, 11 of $40(27 \%)$ IDDM pts vs 2 of $50(4 \%)$ controls resulted re-infected after 5 years $(p<0.001)$. Among IDDM pts, re-infection occurrence was not affected by sex or age. At five years follow-up in the re-infected diabetic pts an increased levels of HbAlc compared to values at enrolmen ( 8.26 vs $7.48, \mathrm{p}<0.01$ ) was observed, while no significant increase in HbAlc was found in the negative group. Furthermore, the H.pylori positive group showed increased insulin requirements compared to negative pts $(0.69 / \mathrm{Kg}$ vs $0.61 / \mathrm{Kg})$. These data reached not statistical significance. Finally, we found statistical significant higher prevalence of diabetic organ damages in the re-infected group compared to negative pts: neuropathies: $42 \%$ vs $10 \%$ ( $<<0.01$ ); nephropathies: $42 \%$ vs $15 \%$ ( $p<0.01)$; retinopathies: $28.6 \%$ vs $5 \%(p<0.05)$, respectively. Conclusions: IDDM pts show higher H.pylori re-infection rates compared to dyspeptic controls. H.pylori re-infection in IDDM pts is associated with poorer glycaemic control and higher incidence of organ damage compared to not re-infected diabetic patients. 
UBT as follow-up screening in successfully eradicated IDDM pts seems therefore a useful tool in order to detect re-infected pts and optimize glycaemic control.

\section{$\mathrm{T} 1898$}

Lower Prevalence of Helicobacter Pylori Infection in Young Women: A 9-Year Observational Study

Sun-Young Lee, Sang Kyun Yu, In-Kyung Sung, Hyung-Seok Park, Choon-Jo Jin, Won Hyeok Choe, So Young Kwon, Chang Hong Lee, Kyoo Wan Choi

Background and Aim: Although many studies have reported on the trends of changes since Helicobacter pylori was first discovered in 1982, detailed information is still lacking. We surveyed the detailed information on the trends of changes in the prevalence of Helicobacter pylori infection relative to age and gender. Methods: A total of 8646 subjects aged 16 years or older (4099 men and 4547 women) who submitted to a rapid urease test during upper gastrointestinal endoscopy, from January 1997 to July 2005, were included in this study. We investigated how the prevalence of $H$. pylori infection varied with gender and age at the date of examination. Results: H. pylori infection was noted in 3747 cases $(43.3 \%)$ of all cases. The infection rate was $50.0 \%$ in 1997, but declined gradually down to $40.6 \%$ in $2005(\mathrm{P}<0.001)$. In addition, the prevalence was higher in men $(49.0 \%)$ than in women $(41.6 \%)$ before the age 50 years $(\mathrm{P}<0.001)$ in any of the years studied. In contrast, there was no difference in the prevalence of $H$. pylori infection between the men (39.2\%) and women $(41.1 \%)$ after the age 50 years $(\mathrm{P}=0.28)$. Conclusions: $H$. pylori infection has gradually decreased over the past decade, and there is a lower rate of infection among young women aged less than 50 years old when compared to men. Female sex hormone might be the cause of this gender-related difference in the prevalence of $H$. pylori infection, which explains indirectly the lower prevalence of intestinal-type gastric cancer among young women. Key Words: Helicobacter pylori, Prevalence, Young, Women

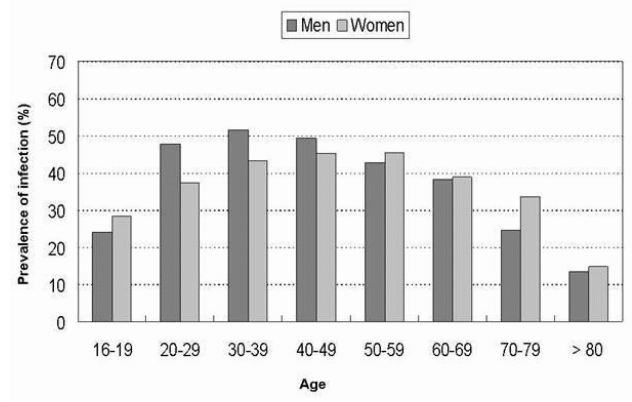

T1899

The Diagnostic Yield of Various Tests for Helicobacter Pylori Infection in Patients On Acid Reducing Drugs

Javed Yakoob, Wasim Jafri, Zaigham Abbas, Shahab Abid, Muhammad Islam, Saeed Hamid, Hasnain Alishah, Zubair Ahmed

Aim: The aim of this study was to compare the diagnostic yield of various tests for Helicobacter pylori (H. pylori) infection in patients on acid reducing drugs such as proton pump inhibitors (PPI) and histamine-2 receptor blocker (H-2RB). Methods: Eighty consecutive patients with dyspeptic symptoms on acid reducing drugs attending the endoscopy suite were enrolled. Gastric biopsy specimens were collected at endoscopy for the rapid urease test (Pronto Dry, Medical Instrument Corp, France), histopathology and polymerase chain reaction for $H$ pylori 165 ribosomal DNA (PCR $165 \mathrm{rDNA}$ ). Results: These are the preliminary results of an ongoing study. There were $57 \%$ (35/80) males and $43 \%$ (45/80) females with age range $17-80$ years and mean age of $44 \pm 14.8$ years. Fifty-three $(66 \%)$ patients were on PPI while $27(34 \%)$ on H2RB. PCR for H. pylori $16 \mathrm{~S}$ rDNA was positive in $65(81 \%)$ and negative in $15(19 \%)$. Of those positive $42(65 \%)$ were on PPI and $23(35 \%)$ on H2RB while those negative $11(73 \%)$ were on PPI and $4(27 \%)$ on $\mathrm{H} 2 \mathrm{RB} \mathrm{p}=0.52$. Pronto dry was positive in $23(29 \%)$ and negative in $57(71 \%)$. Of those positive, $10(43 \%)$ were on PPI and $13(57 \%)$ on $\mathrm{H} 2 \mathrm{RB}$ while those negative $43(75 \%)$ were on PPI and $14(25 \%)$ on $\mathrm{H} 2 \mathrm{RB} \mathrm{p}=0.009$. Histopathology showed $H$. pylori associated mild-moderate gastritis in $36(45 \%)$ and $H$. pylori negative mild non-specific gastritis in $44(55 \%)$. Of those with $H$. pylori associated gastritis $24(67 \%)$ were on PPI and $12(33 \%)$ on H2RB while of those with H. pylori negative non-specific gastritis $29(66 \%)$ were on PPI and $15(34 \%)$ on H2RB $p=0.63$. Conclusion This study shows that in patients on PPI with $H$. pylori infection, diagnostic yield of PCR on gastric tissue is higher than rapid urease test and histopathology. These patients on acid reducing drugs are likely to have a negative rapid urease test and a mild non-specific gastritis on histopathology

\section{$\mathrm{T} 1900$}

Rapid Stool Antigen Test for Diagnosis of H. Pylori Infection in Asymptomatic Childre

Victor M. Cardenas, Delfina C. Dominguez, Flor Puentes, Corinne C. Aragaki, Karen J Goodman, David Y. Graham, Yoshihiro Fukud

Background: Rapid immunochromatographic tests for H. pylori (HP) infection have been developed to allow testing in clinics and in field epidemiologic studies. Aims: To determine the diagnostic performance of the Testmate rapid H. pylori antigen detection test (Wakamoto Pharmaceuticals) in diagnosing HP infection in asymptomatic children. Methods: We used data and fecal specimens collected from children participating in the ongoing Pasitos Cohort Study that takes place in El Paso, Texas and Ciudad Juarez, Mexico on the US-Mexico border. All children in the study had an HP stool antigen EIAs test (HPSA) status (HpSA Premium, Meridian Bioscience) tested (positive $=O D>=0.16$ ). Children classified as Hpinfected had a positive urea breath test (UBT) (urea hydrolysis rate $=>10$ based on adjusted DOB readings from Wagner's IRIS) and a positive HPSA. Children classified as uninfected were negative on both tests and on serology (IgG concentration $<2.2$, HM-CAP IgG antibodies, Enteric Products). Children were classified as status uncertain if they were positive on either the UBT or HPSA test, but not both. For each subject, a dab of stored fecal specimen (frozen at $-70^{\circ} \mathrm{C}$ ) was thawed and tested with the Testmate rapid H. pylori antigen kit following the manufacturer's instructions. Results: We tested specimens from 52 children. Twenty-four were positive, 19 negative, and 9 had uncertain status. The sensitivity and specificity of the Testmate rapid H. pylori antigen were 100\% (24/24) (95\% mid-P binomial CI: 88.3-100.0) and 100\% (19/19) (95\% mid-P binomial CI: 85.4-100.0), respectively. There was $100 \%$ concordance between HPSA and Testmate for those with uncertain Hp status. The observed agreement between the EIA HPSA and this novel product was $96.1 \%$ with Kappa=92.4\% ( $95 \% \mathrm{CI}=82.3-100.0)$. Discussion: This rapid stool antigen test should prove useful for point of care testing and in epidemiologic field studies.

Results of Testmate rapid H. pylori testing in relation to H. pylori Infection Status of Asymptomatic Children in the US-Mexico Border

\begin{tabular}{|c|c|c|c|}
\hline Testmate Results & H. pylori infected & Uninfected & Total \\
\hline Positive & $24(100 \%)$ & 0 & 24 \\
\hline Negative & 0 & $19(100 \%)$ & 19 \\
\hline Total & 24 & 19 & 43 \\
\hline
\end{tabular}

Hp infection positive status: EIA Stool Antigen Test positive and UBT positive. Hp infection negative status: Negative to: 1) EIA Stool Antigen Test, 2) UBT and 3) HMCAP serology. Table excludes 9 children of uncertain H. pylori infection status.

\section{T1901}

Long-Term Follow-Up of 13c-Urea Breath Test Result After Helicobacter Pylori Eradication: Frequency and Significance of Borderline $813 \mathrm{co} 2$ Values

Javier P. Gisbert, David Olivares, Isabel Jimenez, Jose-Maria Pajares

AIM: To quantify the 13C-urea breath test (UBT) result for several years following $H$. pylori eradication, and to evaluate the frequency and the significance of borderline $\delta 13 \mathrm{CO} 2$ values. METHODS: Two-hundred H. pylori eradicated patients confirmed by 13C-UBT (100 mg of urea, citric acid), and having had repeated this test yearly up to 5 years, were studied. $\delta 13 \mathrm{CO} 2$ values between 2 and 5\%o were considered as borderline results. RESULTS: Eight H. pylori recurrences were observed during 406 patient-years of follow-up (1.97\% yearly). In $2 / 8$ reinfected patients, the reinfection was preceded by a negative $\delta 13 \mathrm{CO} 2$ value $>2 \%$. Borderline $813 \mathrm{CO} 2$ values were detected in $4 \%$ of the 606 UBTs performed, and in $25 \%$ when only patients in whom $H$. pylori recurrence was detected in subsequent UBTs were included $(\mathrm{p}<0.05)$. The negative predictive value of a post-treatment $\delta 13 \mathrm{CO} 2>2 \%$ or the diagnosis of $H$. pylori recurrence was $99 \%$. CONCLUSION: Positive and negative UBT results tend to cluster outside of the range between 2 and 5\%. Nevertheless, a borderline UBT $\delta$ value (e.g., very close to the selected cut-off point) should be cautiously interpreted, and the result should probably be confirmed either by repeating the UBT or by other diagnostic methods. On the contrary, a $\delta 13 \mathrm{CO} 2$ value $<2 \%$ very confidently confirms $\mathrm{H}$. pylori eradication

\section{$\mathrm{T} 1902$}

Helicobacter Pylori Infection As a Cause of Iron Deficiency Anaemia Nikitas Papantoniou, Stylianos Karatapanis, Philip Lisgos, Emmanuel Spanos, Ypakoi Papantoniou, Kostas Kladakis, Haridimos Tsibidakis, Kostas Komnianidis, Chrysostomos Psellas, Stylianos Manolitsis, George Manolakas, Ioannis Stouraitis

Background: It is estimated that $30 \%$ of patients presented with iron deficiency anemia remain undiagnosed despite complete upper and lower GI endoscopy and other tests. Helicobacter pylori (H.pylori) infection is the main cause o chronic gastritis, peptic ulcer disease and gastric cancer. H.pylori infection has a relation with iron metabolism but the exact mechanism is unknown. Aim: To investigate the role of H.pylori infection in patients with iron deficiency anemia of unknown aetiology. Patients and Methods : In the present study we investigated 115 consecutive patients presented with iron deficiency anemia during the period 2003-2004. We excluded patients with nutritional problems, with IBD, with a history of upper GI surgery, patients receiving NSAIDS or aspirine, pregnant women and patients with malignancy. Presence of H.pylori was assessed by histology (Giemsa), CLOtest and/or UBT. Iron deficiency anemia was diagnosed in the presence of low plasma ferritin and iron level and low transferrin saturation. Results : 54 out of the initial 115 patients excluded from further evaluation (due to medication 33, due to malignancy 6 , due to pregnancy 2 and 13 due to recent episode of GI bleeding). From the remaining patients 38/61 (62.29\%) had established Hp infection, while 23/61 (37.7\%) were negative to H.pylori. Mean haemoglobin levels were similar in both group of patients ( $8.9 \mathrm{gr} \%$ vs $8.7 \mathrm{gr} \%$ ). On the contrary the mean values of serum iron and ferritin levels and transferin saturation were significantly lower in those patients harboured H.pylori infection as compared with those negative to H.pylori testing. Conclusion: Chronic atrophic gastritis as a consequence of chronic H.pylori infection correlates with iron deficiency anemia of unknown aetiology. The interaction of H.pylori infection and iron metabolism remains obscure. 
T1903

Applicability of a Helicobacter Pylori Stool Antigen Test to Determine the Results of Eradication Therapy

Shinsaku Fukuda, Tadashi Shimoyama, Daisuke Nishiya, Akihiro Munakata

Background: ${ }^{13} \mathrm{C}$-urea breath test (UBT) is usually used to test the results of eradication therapy of Helicobacter pylori. However, in most hospitals, results can not be obtained immediately because this test requires particular equipments. H. pylori stool antigen tests are non-invasive and results are obtained within $10 \mathrm{~min}$. The aim of this study was to examine whether stool antigen test is applicable to determine the results of eradication therapy. Methods: 65 patients infected with $H$. pylori ( 35 males, 30 females, mean age 57.1) received eradication therapy consisted of lansoprazole, amoxicillin and one of clarithromycin or metronidazole. At least 5 weeks after finishing the treatment, results were evaluated by ${ }^{13} \mathrm{C}$-UBT. On the same day, Testmate rapid pylori antigen, which uses monochronal antibody to $H$. pylori native catalase, was also performed and the results were compared with ${ }^{13} \mathrm{C}$ UBT. Results: In all the 49 patients with negative ${ }^{13} \mathrm{C}$-UBT, Testmate rapid pylori antigen was also negative. In $14{ }^{13} \mathrm{C}$-UBT-positive patients, 12 were also positive by Testmate rapid pylori antigen. One patient could not be tested because his stool was watery. Two patients who had grey zone result by ${ }^{13} \mathrm{C}$-UBT was negative by Testmate rapid pylori antigen and they were tested by ${ }^{13} \mathrm{C}$-UBT again one month later and the results were negative. Overall accuracy of Testmate rapid pylori antigen to determine H. pylori eradication was $98.4 \%$ when compared with ${ }^{13} \mathrm{C}$-UBT. Conclusions: Testmate rapid pylori antigen is an immediate and accurate test to evaluate the results of $H$. pylori eradication therapy.

\section{T1904}

Detection of Helicobacter Pylori in Esophagus and Saliva in Human Luigina Cellini, Rossella Grande, Daniela Coraggio, Laurino Grossi, Leonardo Marzio

Introduction: Helicobacter pylori infection is the most common gastrointestinal bacterial disease worldwide. The microorganism has established its niche in the human gastric mucosa, but the presence of the germ has also been demonstrated in human dental plaque, in water distributions and in feces supporting the oro-oral and/or fecal-oral transmission. In a previous study we detected H.pylori in the esophagus of Balb/C mice 3 months after infection with human H.pylori, suggesting for this location a possible role of reservoir for the infection and transmission of the bacterium. Aim: to see if in humans the esophagus and saliva may be reservoir of H.pylori. Methods: Saliva samples, and mucosal biopsy from esophagus, gastric antrum and fundus were collected during upper g.i. endoscopy from 13 patients with positive Urea Breath Test (UBT). Gastric biopsies were cultured to isolate H.pylori, whereas saliva samples and esophagus biopsies were collected to investigate for H.pylori with a Nested-PCR targeting the $16 \mathrm{~S}$ rRNA gene using Hpl, Hp2 and Hp3 primers. Results H.pylori was isolated in gastric biopsies of 12 patients either in the antrum and/or in the gastric fundus. The analysis in Nested-PCR gave positive results in all saliva and esophageal samples studied. The amplified fragments of $109 \mathrm{bp}$ were sequenced and were found in accord with all the sequences present in the Data Base of the National Center for Biotechnology Informations (NCBI), confirming the identity of the microorganism. Conclusion: These data suggest that saliva and the esophagus may be considered reservoirs for $H$. pylori in humans. Identification of the transmission route of H.pylori is crucial in developing an effective plan of surveillance of the microorganism by finding new ways of managing the disease.

\section{T1905}

A Multiple-Step Polyclonal Versus a One-Step Monoclonal Enzyme Immunoassay to Detect Helicobacter Pylori Antigen in the Stools in Children Bruno Hauser, Ingrid Wybo, Gerard Tshibuabua, Denis Pierard, Yvan Vandenplas

Objective: Evaluation of a polyclonal and a monoclonal Helicobacter pylori stool antigen tests (HpSAT) for the detection of a Helicobacter pylori (HP) infection in children. Methods: 43 children (mean age 8.9 years, range 3.5-17.5 years) underwent a 13C-urea breath test (13CUBT) and 2 HpSATs for the detection of HP: the "Premier Platinum HpSA" test (PP-HpSAT) (multiple-step polyclonal enzyme immunoassay) and the "ImmunoCard STAT! HpSA" test (ICS-HpSAT) (one-step monoclonal enzyme immunoassay). A child was considered as HP positive if the 13C-UBT was positive. Results: Of the 43 children, 18 (41.9\%) were HP positive and $25(58.1 \%)$ were HP negative. The sensitivity and specificity of the PP-HpSAT was $94.4 \%$ and $100.0 \%$, respectively. Results were equivocal with the ICS-HpSAT in 5/ $42(11.9 \%)$ of the tests due to visual interpretation difficulties of the change in colour of the test-line. The sensitivity and specificity of the ICS-HpSAT was $100.0 \%$ and $76.0 \%$, respectively, when any change of colour of the test-line was considered as positive (according to the guidelines of the manufacturer). However, sensitivity and specificity of the ICS-HPSAT reached $100.0 \%$ and $96.0 \%$, respectively, when the test was considered positive in case a "significant change of colour" of the test-line occurred. Conclusions: Compared to the 13C UBT, the PP-HpSAT showed a comparable sensitivity and specificity and the ICSHPSAT had a comparable sensitivity but low specificity. However, sensitivity and specificity of the ICS-HPSAT became comparable when a weakly positive test was considered as negative. The ICS HpSAT is easy to perform with results available within 10 minutes, and is therefore of particular interest in ambulatory medicine.

\section{T1906}

Prevalence of Helicobacter Pylori Infection in Gastric MALT Lymphoma: A Systematic Review Javier P. Gisbert, Laura Martin-Asenjo

Objective: To perform a systematic review of the studies evaluating $H$. pylori prevalence in patients with gastric MALT lymphoma, and to analyze the factors influencing it. Methods: Bibliographic searches were conducted in MedLine, selecting the articles that studied $H$. pylori prevalence in patients with MALT lymphoma. Results: Thirty-eight studies were identified, including 1844 patients. The mean prevalence of $H$. pylori infection was $79 \%$.
In patients diagnosed of $H$. pylori infection with 2 or more methods, the prevalence was $85 \%$, whereas it was $77 \%$ when only one diagnostic method was performed $(p<0.0001)$ The $H$. pylori prevalence in patients diagnosed with histology was $75 \%$, but this figure increased up to $85 \%$ when serology was used $(\mathrm{p}<0.0001)$. The H. pylori prevalence in highgrade lymphomas was $60 \%$, and $79 \%$ in low-grade lymphomas $(\mathrm{p}<0.0001)$. H. pylori infection was detected in $74 \%$ of MALT lymphomas confined to the mucosa or submucosa (EII), bu in only $44 \%$ of those beyond submucosa $(\mathrm{p}<0.0001)$. Conclusions: The H. pylori prevalence in patients with MALT lymphoma is variable, which seems to depend, at least partly, on the number and the type of diagnostic methods used to detect the infection, on the histological grade and on the stage of tumoral invasion. If the adequate diagnostic methods are performed, and if only low-grade MALT lymphomas are considered, the H. pylori prevalence is remarkably high, nearly $90 \%$, which reinforces the role of this micro-organism in the pathogenesis of gastric MALT lymphoma.

\section{T1907}

Efficacy of Helicobacter Pylori Erradication Treatment in Gastric MALT Lymphoma: A Systematic Review

Javier P. Gisbert, Laura Martin-Asenjo

Objective: To perform a systematic review of studies evaluating the effect of $H$. pylor eradication treatment on the histological regression of gastric MALT lymphoma. Methods: Bibliographical searches were conducted in MedLine, and studies evaluating the effect of H. pylori eradication treatment on the histological regression of gastric MALT lymphoma were included. Results: Forty-one studies were identified, including a total of 1446 patients. After $H$. pylori eradication, complete remission was achieved in $74 \%$ of the cases, partial remission in $10 \%$, and no response in $16 \%$, during a mean follow-up of 23 months (range 12 to 35 months). After complete remission, tumoral relapse was detected in $6 \%$ of de cases; of these, $25 \%$ were associated with $H$. pylori reinfection, and in $15 \%$ a high grade MALT lymphoma component was identified. When only patients with MALT lymphoma stage EI (confined to stomach) and with purely low-grade histological type were included complete remission was achieved in $80 \%(95 \% \mathrm{CI}, 77-82 \%)$ of the cases. In stage EII (confined to mucosa or submucosa), the response to eradication treatment was higher than in stage EI2 (beyond submucosa): $84 \%$ vs. $31 \%$; p $<0.0001$. Conclusion: H. pylori eradication in patients with gastric low-grade MALT lymphoma and stage EI achieves complete remission in $80 \%$ of the cases. This figure increases up to $84 \%$ in EIl lymphomas, but is of only $31 \%$ in EI2 ones.

\section{T1908}

Levofloxacin-Based Rescue Regimens After Helicobacter Pylori Treatment Failure: Systematic Review and Meta-Analysis Javier P. Gisbert, Felipe de la Morena

OBJECTIVE: To systematically review the efficacy and tolerance of levofloxacin-based rescue regimens, and to conduct a meta-analysis of studies comparing these regimens with the quadruple therapy for $H$. pylori eradication failures. METHODS: Selection of studies: levofloxacin-based rescue regimens. For the meta-analysis, randomized controlled trials comparing levofloxacin-based and quadruple regimens were included. Search strategy: electronic and manual bibliographical searches. Assessment of study quality: independently by two reviewers. Data synthesis: "intention-to-treat" eradication rate. Meta-analysis combining the Odds Ratios (OR) of the individual studies. RESULTS: Mean eradication rate with levofloxacin-based regimens was $80 \%$. 10-day regimens were more effective than 7 -day combinations ( $81 \%$ vs. $73 \%$; $\mathrm{p}<0.01$ ). The meta-analysis showed better results with levofloxacin than with the quadruple combination ( $81 \%$ vs. $70 \%$; $\mathrm{OR}=1.80 ; 95 \% \mathrm{CI}=0.94-3.46)$. This difference reached statistical significance and heterogeneity markedly decreased when a single outlier study was excluded or when only high-quality studies were considered. Incidence of adverse effects in general, and severe adverse effects in particular, in levofloxacin treated patients was $18 \%$ and $3 \%$. The meta-analysis showed less adverse effects with levofloxacin than with quadruple regimen, both overall ( $19 \%$ vs. $44 \%$; $\mathrm{OR}=0.27 ; 95 \% \mathrm{CI}=0.16-0.46)$ and regarding severe adverse effects ( $0.8 \%$ vs. $8.4 \%$; $O R=0.20 ; 95 \% \mathrm{Cl}=0.06-0.67)$. CONCLUSION: After $H$. pylori eradication failure, levofloxacin-based rescue regimen is more effective and better tolerated that the generally recommended quadruple therapy. A 10-day combination of levofloxacin-amoxicillin-proton pump inhibitor constitutes an encouraging second-line alternative.

\section{T1909}

Third-Line Rescue Therapy with Levofloxacin After Two H. Pylori Treatment Failures

Javier P. Gisbert, Manuel Castro-Fernandez, Fernando Bermejo, Angeles Perez-Aisa, Julio Ducons, Miguel Fernandez-Bermejo, Felipe Bory, Angel Cosme, Luis-Miguel Benito, Laureano Lopez-Rivas, Eloisa Lamas, Manuel Pabon, David Olivares

AIM: Eradication therapy with proton pump inhibitor, clarithromycin and amoxicillin fails in a considerable number of cases. A rescue therapy still fails in more that $20 \%$ of the cases. Our aim was to evaluate the efficacy and tolerability of a third-line levofloxacin-based regimen in patients with two consecutive H. pylori eradication failures. METHODS: Design Prospective multicenter study. Patients: in whom a first treatment with omeprazole-clarithromycin-amoxicillin and a second with omeprazole-bismuth-tetracycline-metronidazole (or ranitidine bismuth citrate with these antibiotics) had failed. Intervention: A third eradication regimen with levofloxacin (500 $\mathrm{mg}$ b.i.d.), amoxicillin (1 g b.i.d.) and omeprazole ( $20 \mathrm{mg}$ b.i.d.) was prescribed for 10 days. Outcome: Eradication was confirmed with 13C-urea breath test 4-8 weeks after therapy. RESULTS: One-hundred patients were initially included, and 9 were lost for follow-up. All patients but 5 took all the medications correctly. Perprotocol and intention-to-treat eradication rates were 66\% (95\% CI=56-75\%) and 60\% (50$70 \%$ ). Adverse effects were reported in $25 \%$ of the patients, mainly including metallic taste $(8 \%)$, nausea (8\%), myalgia/arthralgia (5\%), and diarrhea (4\%); none of them were severe. 
CONCLUSION: Levofloxacin-based rescue therapy constitutes an encouraging empirical third-line strategy after multiple previous $H$. pylori eradication failures with key antibiotics such as amoxicillin, clarithromycin, metronidazole and tetracycline.

\section{T1910}

Gatifloxacin Resistance and Mutations in Gyra Aftre Unsuccessful Helicobacter Pylori Eradication in Japan

Toshihiro Nishizawa, Hidekazu Suzuki, Kumiko Kurabayashi, Tatsuhiro Masaoka, Hiroe Muraoka, Eisuke Iwasaki, Intetsu Kobayashi, Tshifumi Hibi

Background/Aims: Gatifloxacin (GFLX) has been shown to have excellent activity against H. pylori in vitro. Recently, higher eradication rates $(92 \%)$ were reported with the administration of a 7-day regimen of GFLX, amoxicillin and rabeprazole (Helicobacter 9:255, 2004). However, fluoroquinolones-resistant strains are more common in Japan than in Western countries. The quinolone resistance-determining region of gyrA gene is reported to play a critical role in ciprofloxacin resistance of $H$. pylori. The present study examines GFLX susceptibility and mutation patterns of the gyr A and gyrB genes in $H$. pylori strains obtained from Japanese patients after unsuccessful eradication therapy. Methods: H. pylori isolates were obtained from the stomach of 48 patients. The MICs of GFLX, clarithromycin (CAM) and metronidazole (MET) were determined by the agar dilution method. Twenty-three GFLX-resistant strains (defined by MIC $1 \mu \mathrm{g} / \mathrm{ml}$ ) and 25 GFLX-susceptible strains were examined by PCR amplification and sequencing of the quinolones-resistance-determinant regions of gyrA and gyrB. Four GFLX and CAM-susceptible strains were serially plated onto GFLX-containing agar $(0.12 \mu \mathrm{g} / \mathrm{ml})$ or CAM-containing agar $(0.015 \mu \mathrm{g} / \mathrm{ml})$ of increasing agar density. The GFLX and CAM susceptibility were examined by agar dilution method, and the quinolones-resistance-determinant regions of gyrA gene were examined by PCR amplification and sequencing. Results: The resistance rates to GFLX and CAM were $47.9 \%$ and $79.2 \%$. The GFLX resistance rate tended to be higher in the strains resistant to CAM than in those susceptible to CAM ( $\mathrm{p}=0.10)$. Twenty-two of the $23(95.7 \%)$ GFLX-resistant strains had point-mutations of gyrA at codon 87 Asn or 91 Asp. Only one of the 25 (4\%) susceptible strains had a mutation with substitution at amino acid 87 . Neither the susceptible nor the resistant strains showed mutations of gyrB. All four strains plated on GFLX- containing agar developed GFLX resistance, and three of the four strains had mutations of gyrA. In contrast, none of the strains plated on CAM-containing agar developed resistance, to either CAM or GFLX, until the 10th generation of repeated culture. Conclusion: Mutation of gyrA in H. pylori was significantly associated with higher MIC for GFLX. Although GFLX may be one of the drugs of choice for the treatment of $\mathrm{H}$. pylori infections, the selection of antibiotics ideally should be based on the results of drug susceptibility testing or gyrA gene analysis.

T1911

Mutations of 23s rRNA Gene of $\mathrm{H}$. Pylori Determined By a Selmap (Self Selective Multi Amplification Primers)-PCR Method

Takahisa Furuta, Naohito Shirai, Mitsushige Sugimoto, Akiko Nakamura, Mutsuhiro Ikuma, Yoshihiro Soya, Masayoshi Kajimura, Akira Hishida

Backgrounds and Aims. Susceptibility to clarithromycin (CAM) is one of the determinants of response to CAM-based therapy for $\mathrm{H}$. pylori ( $\mathrm{Hp}$ ) infection. Bacterial resistance to CAM is caused by single nucleotide polymorphisms (SNPs) of 23SrRNA gene. The SELMAP (Self Selective Multi Amplification Primers)-PCR can measure couples of SNPs easily in the single reaction tube within a short period by PCR amplification alone. Then, we intended to develop the SELMAP-PCR assay for determining SNPs at positions 2142 and 2143 of 235 rRNA gene of Hp. Methods. We designed the forward primer, FP3G, and the reverse primer, RP2G, which specifically anneal with the $2143 \mathrm{G}$ and $2142 \mathrm{G}$ mutations, respectively. We also designed the forward primer, FP-1, and reverse primer, RP-1, at the indicated sites in Figure 1. When the strain is wt, neither of FP3G nor RP2G anneals with the template and PCR amplification occurs between FP-1 and RP-1, resulting in the 320 bp amplicon. In the case of $2143 \mathrm{G}$ mutation, PCR amplification occurs mainly between FP3G and RP-1 rather than between FP-1 and RP-1, because PCR amplification preferentially occurs in the smaller size of amplicon, resulting in the $118 \mathrm{bp}$ amplicon. Similarly, in the case of $2142 \mathrm{G}$ mutation, PCR amplification occurs mainly between FP- 1 and RP2G, resulting in the 238 bp amplicon. Then, the SNP can be distinguished by the PCR amplicon sizes. DNAs extracted from 64 gastric tissue samples were subjected to the SELMAP PCR analysis of $23 \mathrm{~S}$ rRNA mutations. The results were compared with minimum inhibitory concentrations (MIC) for CAM. Results: 29 of 64 samples were wt and others had 2143G mutation. The median of MIC values for CAM in the wt strains was $<0.015 \mu \mathrm{g} / \mathrm{ml}$. That of $2143 \mathrm{G}$ strains was $2.0 \mu \mathrm{g} / \mathrm{ml}$. Conclusion: The SELMAP-PCR assay for $23 \mathrm{~S}$ rRNA mutation of $\mathrm{Hp}$ is a useful and easy method to detec CAM-resistant strains of $\mathrm{Hp}$, and could be a useful tool for the optimal antibiotic selection in Hp eradication therapy.

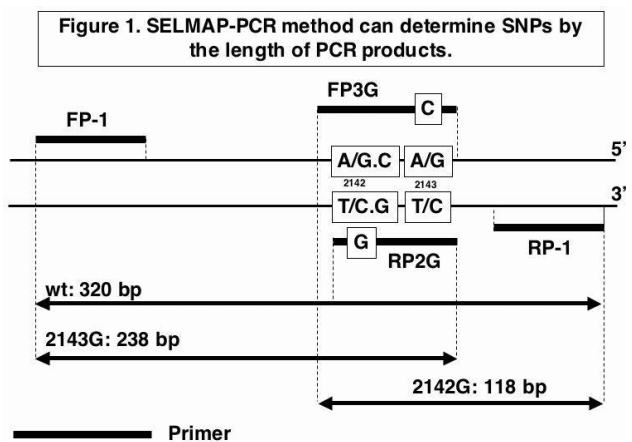

T1912

Recurrence of $\mathrm{H}$. Pylori Infection After Eradication: 5-Year Follow-Up Study of 1,000 Patients

Javier P. Gisbert, Marta Luna, Blas Gomez, Juan-Manuel Herrerias, Joan Mones, Manuel Castro-Fernandez, Pilar Sanchez-Pobre, Angel Cosme, David Olivares, Jose-Maria Pajares

AIM: To study the incidence of $H$. pylori recurrence, its chronological aspects, and the variables that might influence it. METHODS: 1,000 patients in whom $H$. pylori had been eradicated were prospectively studied. Therapies were classified as low and high efficacy regimens. Four to eight weeks after completion of therapy, 13C-urea breath test was performed, and it was repeated yearly up to 5 years. In some patients, endoscopy with biopsies was also performed to confirm $H$. pylori eradication. RESULTS: 1,000 patients were included, giving 2,744 patient-years of follow-up. Seventy-one H. pylori recurrences were observed (2.6\% per patient-year). Probability of being $H$. pylori-negative at 1 year was $94.7 \%$, and at 5 years $90.7 \%$. In the multivariate analysis, low age (OR: 1.84; $95 \%$ CI: 1.04-3.26) and low efficacy therapies (OR: $2.5 ; 1.23-5.04$ ) correlated with 1 year $H$. pylori recurrence. Differences were observed when Kaplan-Meier curves were compared depending on age and therapy regimen. CONCLUSION: Risk of post-eradication H. pylori recurrence is higher during the first year, which suggests that most recurrences during this period are recrudescences and not true reinfections. H. pylori recurrence is more frequent in younger patients and in those treated with low efficacy therapies, but is exceptional if high efficacy therapies are used, in which case post-therapy eradication can be safely confirmed at 4 weeks with 13C-urea breath test.

\section{T1913}

Where Does the Antimicrobial Resistance of Helicobacter Pylori Come from, Acquired After Birth Or Inherited from Parents? Different Mechanism Between Clarithromycin and Levofloxacin

Naomi Torio, Mutsuko Konno, Ikuya Miki, Daisuke Shirasaka, Yoshinori Morita, Yuko Matsumoto, Hideyuki Miyachi, Takao Tamura, Nobuo Aoyama

Background; Many reports revealed that most Helicobacter pylori (H. pylori) infection might be transmitted during childhood, mainly from their parents, and however, only a few reports clarified the different acquisition mechanism of antimicrobial resistance of $H$. pylori in childhood between antibiotics. Clarithromycin (CAM)-resistance is a major issue in standard anti-H. pylori both in children and adults, in which situation levofloxacin (LVFX) is considered one of alternative agents showing a good efficacy and tolerance. CAM can be used both for children and adults for many infectious diseases, while LVFX cannot for children. Aim; We revealed the mutation pattern and rate of $H$. pylori in the genes associated with CAM- and LVFX-resistance in Japanese children, and focusing on the difference of antibiotics used between children and adults, we clarified whether $H$. pylori antimicrobial resistance in children might depend on their usage of antibiotics after birth or on the inheritance from their parents. Methods; Forty H. pylori strains were isolated from 26 Japanese children and 14 parents using biopsy specimens obtained through endoscopy. In order to confirm CAMand LVFX-resistance, mutations were analyzed in the 23SrRNA gene (A2142G and A2143G) and the gyrA gene performing amplification by PCR and direct sequencing, and mutation patterns of $H$. pylori in children were compared to those in their parents. Results; Mutant strains in the 23SrRNA gene were found in 7 children (26.9\%), and none of their parents harbored mutants in this gene. Mutants in the gyrA gene were found in 3 children (11.5\%), and 2 of their parents harbored the same type pf mutation point in this gene (Asn to Lys at amino acid 87). Conclusion: CAM-resistance rate of $H$. pylori is very high in Japanese children compared to that in Japanese adults previously reported, which may depend mainly on its frequent use in childhood. In contrast, LVFX-resistance is often found although LVFX cannot be used for children, which suggests that LVFX-resistance may be originated from their parents. We have to realize the importance of proper usage of antibiotics in consideration of not only acquired but also inherited antimicrobial resistance of $H$. pylori.

\section{T1914}

Increased Helicobacter Pylori Clarithromycin Resistance Rates in Patients with Non-Ulcer Dyspepsia

Selahattin Unal, Meltem Yalinay Cirak, Sukru Dumlu, Tarkan Karakan, Doruk Engin, Sevgi Turet, Mehmet Cindoruk

Background: Recent studies suggest that the Helicobacter pylori eradication rate in patients with non-ulcer dyspepsia (NUD) is lower when compared to patients with peptic ulcer diseases. Clarithromycin resistance was reported to be higher in NUD patients compared to those with peptic ulcer. Aim: To determine and compare the clarithromycin resistance 
profiles in patients with NUD, endoscopic esophagitis and peptic ulcer diseases. Methods: A total of 120 consecutive patients referred for upper endoscopy were performed gastric biopsies at four quadrants. Presence of HP was diagnosed by pathology, rapid urease test and real time-PCR. Clarithromycin resistance was determined by real time-PCR. Endoscopic diagnosis was classified as: NUD, esophagitis and peptic ulcer disease. Results: HP was positive in 107 out of 120 patients (89.1\%). Clarithromycin resistance was present in 20 patients (19\%). Endoscopical diagnosis of patients was; $66.6 \%$ NUD, $15.8 \%$ esophagitis and $17.5 \%$ peptic ulcer disease. Clarithromycin resistance rate was higher in patients with NUD than in patients with esophagitis and peptic ulcer disease; $21.7 \%$ vs. $16.6 \%$ vs. $10 \%$, respectively $(\mathrm{p}<0.05)$. In patients with NUD, endoscopical biopsies from corpus anterior and posterior regions revealed significantly higher positive results for clarithromycin resistance. Conclusions: Clarithromycin resistance rate is higher in patients with NUD and probably this leads to sub-optimal treatment results in this patient population. The selective intragastric distribution of clarithromycin resistance species of HP in gastric corpus, which is a secondary finding in our study, might be related to the altered cell cycle kinetics of the bacteria. Further studies are needed on this issue.

\section{T1915}

Antibacterial Effect of Mupirocin On Helicobacter Pylori in Vitro Masaaki Minami, Takafumi Andoh, Osamu Maeda, Yasumasa Niwa, Naoki Ohmiya, Michio Ohta, Hidemi Goto

[Background] Mupirocin (MUP) is an effective antibiotic against methicillin-resistant Staphylococcus aureus.(MRSA). Its bactericidal effect is stable under acid condition and its mechanism is unique. The present study was conducted to investigate the effect of MUP on clarithromycin (CAM) or metronidazole (MNZ)-resistant and -susceptible strains of Helicobacter pylori ( $\mathrm{H}$. pylori), and the post-antibiotic effect (PAE) of H. pylori. [Methods] The susceptibility to MUP of $140 \mathrm{H}$. pylori including clinical strains, ATCC43504 (quality control strain), 26695 and J99 (genome sequenced standard strains) and the susceptibility of CAM and MNZ-resistant strains were measured by the agar dilution method. The clinical isolates were from lesion biopsy specimens obtained by endoscopy at Nagoya University Hospital and a regional health-care center. The time course of antibacterial effects was determined using strain ATCC43504. Bacteria cultured for 4 days were diluted into a final inoculum of 105 CFU/ $\mathrm{mL}$. Broth culture with MUP was incubated for 2 days at $37^{\circ} \mathrm{C}$. in a microaerophilic atmosphere. At $0,12,24,36$ and 48 hours, samples were removed and the number of colonies growing after 4 days' incubation was counted. We preformed post-antibiotic effect (PAE) test of MUP on ATCC43504. [Result] MIC90 and MBC of MUP on all 140 strains is less than $0.064 \mathrm{mg} / \mathrm{l}$ and $0.1 \mathrm{mg} / \mathrm{l}$, respectively. Ten of them were CAM-resistant strains and 3 were MNZ-resistant strains. There were no differences of MUP effect between susceptible and resistant strains either for CAM or MNZ. By adding MUP, time-kill curve showed that bacterial quantities decreased in dose and time-dependent manner and no viable colony was found after 12 hour culture with $0.1 \mathrm{mg} / \mathrm{l}$ MUP. The value of PAE is 12 and killing effect on $H$. pylori remained after cessation of MUP exposure. [Conclusion] MUP is a potential effective antibiotic for $H$. pylori even those for CAM/ MNZ-resistant strains.

\section{T1916}

The Effect of Mdrl C3435t Polymorphism On the Eradication Rates of $H$ Pylori By a Triple Therapy with Lansoprazole, Clarithromycin and Amoxicillin Takahisa Furuta, Naohito Shirai, Mitsushige Sugimoto, Akiko Nakamura, Mutsuhiro Ikuma, Masayoshi Kajimura, Akira Hishida

Backgrounds and Aims: Proton pump inhibitors (PPIs) are metabolized by CYP2C19 in the liver. We have reported that eradication rates of $H$. pylori by a PPI-based therapy depend on genotypic differences of CYP2C19. Recently, the polymorphism of MDRl gene (from C to $\mathrm{T}$ at the position 3435 ) has been made clear to be associated with the activity of the ATP-dependent efflux transporter, P-glycoprotein (P-gp). Individuals who were homozygous for 3435T (TT) had significantly decreased intestinal P-gp expression and increased bioavailability of some drugs after oral administration, such as PPIs. Then, we intended to examine whether eradication rates of a PPI-based therapy for $H$. pylori infection were associated with the MDRl polymorphism at the position 3435. Methods: Patients infected with $H$. pylori underwent the triple therapy with lansoprazole $30 \mathrm{mg}$ bid, clarithromycin $400 \mathrm{mg}$ bid and amoxicillin $750 \mathrm{mg}$ bid for 1 week. DNA was extracted from the blood or tissue sample of each patient. Polymorphisms of MDRl at the position 3435 and CYP2C19 genotype status wer measured by an Invader assay. The genotypes of MDRl was classified into the three groups: C/C, C/T, T/T. CYP2C19 genotype status was classified into rapid metabolizer (RM), intermediate metabolizer (IM) and poor metabolizer (PM) as previously reported (Clin Gastroenterol Hepatol 2005). Susceptibility of H. pylori to clarithromycin was also determined. Eradication of $H$ pylori was judged based on 13C-UBT. Results: Incidences of MDRl genotypes were C/C: $35.0 \%(n=41), C / T: 45.3 \%(n=53)$ and T/T: $19.7 \%(n=23)$. The total eradication rate was $79.5 \%$. The eradication rates in $\mathrm{C} / \mathrm{C}, \mathrm{C} / \mathrm{T}$ and $\mathrm{T} / \mathrm{T}$ groups were $79.5 \%, 88.7 \%$ and $69.6 \%$, respectively $(\mathrm{P}=0.0766)$. When patients were classified in the three $\mathrm{CYP} 2 \mathrm{Cl} 19$ genotype groups, eradication rates in $\mathrm{C} / \mathrm{C}, \mathrm{C} / \mathrm{T}$, and $\mathrm{T} / \mathrm{T}$ groups of MDR1 were $70.6 \%(12 / 17), 90.0 \%(9 / 10)$ and $66.7 \%(6 / 9)$-(N.S.) in RMs of CYP2C19, those in IMs of CYP2C19 were $76.1 \%(16 / 21), 83.3 \%(25 / 30)$ and $63.6 \%$ (7/11)-(N.S.), and those in PMs were $66.7 \%(2 / 3), 100.0 \%(13 / 13)$, and $100.0 \%(3 / 3)-(\mathrm{P}=0.0599)$. In PMs of CYP2C19 infected with clarithromycin-resistant strains of $\mathrm{H}$. pylori, eradication rates in $\mathrm{Cl}$ $\mathrm{C}, \mathrm{C} / \mathrm{T}$ and T/T groups of MDRl were $0.0 \%(0 / 1), 100.0 \%(5 / 5)$ and $100.0 \%(1 / 1)-(\mathrm{P}=$ 0.0302). Conclusion: The impact of MDRl polymorphism on the eradication rates of $H$. pylori by a triple therapy with lansoprazole, clarithromycin and amoxicillin was generally small but was significant in patients with PM genotype of CYP2C19 and infected with clarithromycin resistant strains of $H$. pylori.
T1917

A Cost-Effectiveness Analysis of Competing Strategies for Helicobacter Pylori Eradication in Relation to Regional U.S. Antibiotic Resistance Patterns Robert H. Harris, Miguel R. Arguedas

There are regional differences in Helicobacter pylori antibiotic resistance patterns in the United States, which may alter the optimal choice for antimicrobial eradication therapies. Growing antibiotic resistance to metronidazole and clarithromycin is often cited as a reason for the increase in $H$. pylori treatment failures. As resistance grows, the economic impact of empiric verses treatment guided by culture and antibiotic susceptibility testing will need to be further explored. Methods: We conducted a cost-effectiveness analysis comparing competing strategies for $\mathrm{H}$. pylori eradication. The competing strategies were compared in each of fou U.S. regions (South East, North East, Mid West and West) in regards to recently published regional antimicrobial resistance patterns. The strategies compared were: 1) quadruple therapy followed by triple therapy and then third-line therapy for failures (quadruple-first strategy) 2) triple therapy followed by quadruple therapy and then third-line therapy for failures (triple-first strategy) 3) antibiotic susceptibility testing prior to initial treatmen followed by third-line therapy for failures (susceptibility testing-first strategy). A decision analysis was created using Tree Age software. Probabilities and costs for the decision tree were obtained from a Medline search of published medical literature and from hospita pharmacy databases. Results: The cost per patient of using the quadruple-first strategy ranged from \$149.60-157.20 with $99-100 \%$ of patients achieving eradication. The cost per patien of using the triple-first strategy ranged from $\$ 274.30-\$ 287.10$ with with $99-100 \%$ of patients achieving eradication. The cost per patient of using the susceptibility testing-first strategy ranged from $\$ 570.80-584.50$ with $98-99 \%$ of patients achieving eradication. Even though the cost of the quadruple-first strategy is increased in regions where metronidazole resistance is higher it still dominated the other strategies tested. Conclusion: Based upon current regional antimicrobial resistance patterns the quadruple-first strategy is the most cost-effective method of H. pylori eradication across the U.S

\section{T1918}

Could Frameshift Mutations in the Frxa and Rdxa Genes of Helicobacter Pylori Be a Marker for Metronidazole Resistance?

Tatsuhiro Masaoka, Hidekazu Suzuki, Yohei Nomoto, Toshihiro Nishizawa, Toshifumi Hibi

Background We had previously reported that in order to increase the accuracy of clarithromycin-susceptibility testing and improve the eradication efficacy, dilution agar methods should be combined with PCR-RFLP analysis before second-line eradication therapy (Aliment Pharmacol Ther. Suppl 1:158, 2004). Although resistance of Helicobacter pylori (H.pylori) to metronidazole (MET) had previously been reported to be associated with mutations in the $r d x A$ or frxA gene, several recent studies have indicated that they may, in fact, contribute little to MET resistance. To clarify the roles of these two H.pylori genes in MET resistance, we examined them in strains that were not eradicated by first-line eradication therapy. Patients and Methods A total of 132 patients (92 males, 40 females, average age 53.8 years old)with H.pylori- positive peptic ulcer or chronic gastritis who underwent upper $G$ endoscopy after unsuccessful first-line 7-day triple therapy with lansoprazole, amoxicillin and clarithromycin were enrolled for this study. Endoscopically obtained biopsy specimens were used for examining the susceptibility of the H.pylori strains to MET using the agar dilution method. The H.pylori $r d x A$ and frxA genes were then sequenced. The cutoff value for resistance was $8 \mu \mathrm{g} / \mathrm{ml}$. Results In the MET-resistant strains, the sensitivity and specificity of detection of frameshift mutations in the $r d x A$ gene were $44.4 \%$ and $98.4 \%$, respectively. A significant association between the presence of frameshift mutations in the $r d x A$ gene and resistance to MET was observed ( $\mathrm{p}<0.001$, odds ratio 48.4). Among the strains with frameshif mutations in the $r d x$ A gene, none was successfully eradicated by the metronidazole-containing regimen. On the other hand, the sensitivity and specificity of detection of frameshift mutations in the frxA gene in the MET-resistant strains were $22.2 \%$ and $91.1 \%$, respectively and no significant association between frameshift mutations in the frxA gene and resistance to MET was observed. Among the strains with frameshift mutations in the frxA gene, $90.9 \%$ wer successfully eradicated by the metronidazole-containing regimen. Conclusion Mutation in the $r d x A$ gene was sufficient, but not necessary to confer resistance to MET in H.pylori. Mutation in the frxA gene was not necessary for resistance to MET in H.pylori.

\section{T1919}

The Efficacy of Levofloxacin Based Triple Therapy for Helicobacter Pylori Eradication

Sung Pyo Hong, Ji Hyun Lee, Chang Il Kwon, Lea Hyun Phyun, Byoung Sok Lee, Han Ul Song, Kwang Hyun Ko, Seong Gyu Hwang, Pil Won Park, Kyu Sung Rim

Backgroud/Aims: In first and second line therapies, the failure rate of Helicobacter pylon eradication therapy ranged from 15 to $20 \%$. This study aimed to compare the efficacy and safety of levofloxacin based triple therapy versus standard triple or quadruple therapy in Korea. Method: We enrolled two hundred and sixty seven patients who were documented Helicobacter pylori infection. One hundred and forty-one patients were treated with levofloxacin based triple therapy(LAP; levofloxacin, amoxicillin, PPI) to eradicate Helicobacter pylori, and one hundred twenty-six patients were treated with standard triple therapy. Those who failed in eradication were retreated with standard quadruple second-line therapy(MAPB metronidazole, amoxicillin, PPI, bismuth subcitrate) or levofloxacin based therapy(LAP or LCP; levofloxacin, clarithromycin, PPI). Result: In first line therapy, the eradication rate of levofloxacin based triple therapy and standard triple therapy were $69.8 \%$ and $74.0 \%$ respectively $(\mathrm{p}=0.52)$. In second-line therapy, the eradication rate of levofloxacin based triple therapy and standard quadruple therapy were $62.5 \%$ and $40.5 \%$ respectively( $\mathrm{p}=0.34)$. Conclusions: Levofloxacin based triple therapy was effective as standard regimen to eradicate Helicobacter pylori and could be useful as an alternative rescue therapy. Comparison of Helicobacter pylori Eradication Rates by Different Treatment Regimens

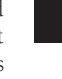




\begin{tabular}{|c|c|c|c|c|}
\hline Regimen & Eradication Rate, ITT(\%) & $\mathrm{p}$-value & Eradication Rate, PP(\%) & $\mathrm{p}$-value \\
\hline First Line Treatment & & & & \\
\hline LAP & 52.5 & 0.5266 & 69.8 & 0.5153 \\
\hline CAP & 56.3 & & 74.0 & \\
\hline Second Line Treatment & & & & \\
\hline First Line Therapy & CAP & & & \\
\hline LAP & 55.6 & 0.1956 & 62.5 & 0.3428 \\
\hline MAPB & 28.6 & & 40.5 & \\
\hline First Line Therapy & LAP & & & \\
\hline LCP & 60.0 & 0.4642 & 60.0 & 0.4902 \\
\hline MAPB & 40.0 & & 80.0 & \\
\hline
\end{tabular}

regardless of the MIC values, suggesting that molecular genetic approach appears to be a powerful indicator for treatment outcomes rather than the E test.

\section{T1922}

Levofloxacin- Versus Clarithromocyn-Based First Line Therapy for Helicobacter Pylori Infection

Antonio Rispo, Elena Di Girolamo, Antonio Cozzolino, Hamma Lamine Ali Taleb, Luigi Pasquale

line treatment of Helicobacter pylori $(\mathrm{Hp})$ infection. Several reports have shown the efficacy of levofloxacin for the rescue therapy of resistant Hp infection while its role as first line treatment is poorly investigated. AIM: To evaluate the efficacy and safety of levofloxacinbased triple therapy in the first line treatment of $\mathrm{Hp}$ infection when compared to the classical clarithromicyn-based protocol. PATIENTS AND METHODS: 60 consecutive out-patients with an histological diagnosis of $\mathrm{Hp}$ infection were prospectively randomised in two groups of weekly treatment: Group A (\#30) levofloxacin 250 b.i.d, amoxicillin 1 gr b.i.d., esomeprazole $20 \mathrm{mg}$ b.i.d: Group B (\# 30) clarithromycin $500 \mathrm{mg}$ b.i.d., amoxicillin 1 gr b.i.d., esomeprazole $20 \mathrm{mg}$ b.i.d. Cure of infection was determinated by urea breath-test. The cure rate was expressed as intention-to-treat. Statistics was calculated by using MedCalc software determining Pearson's $\chi 2$. RESULTS: Hp eradication was achieved in 27 patients in Group A and 26 patients in Group B (90\% vs $87 \%$; p=ns); 1 patient in Group B stopped therapy because of diarrhoea and abdominal pain. CONCLUSIONS: Levofloxacin-based triple therapy is effective and safe in first line treatment of Hp infection. Levofloxacin-based therapy could be suggested in first line treatment of Helicobacter pylori infection in the areas with high primary resistance to clarithromycin.

BACKGROUND/AIMS: The recent increase in resistant strains of Helicobacter pylori has become a serious problem. Ecabet is a novel anti-ulcer agent that acts directly on the gastric mucosa, has bactericidal activity, and inhibits adhesion of Helicobacter pylori to the gastric mucosa. We aimed to evaluate the efficacy of ecabet sodium combined salvage therapy for eradication of $H$. pylori in patients who had previously failed to eradication of $H$. pylori with peptic ulcer disease. METHODOLOGY: Forty-nine patients with peptic ulcer disease who had failed eradication of $H$. pylori by proton-pomp inhibiter (PPI) + amoxicillin (AMPC) +clarithromycin (CAM) therapy according to the Japanese guideline therapy were included in this prospective study. The inclusion criteria included one or more failed attempts at eradication and presence of $H$. pylori infection as evidenced by positivity of at least one of three tests: rapid urease test (RUT), 14C urea breath test (UBT), and histology. The subjects were treated with a 14-day regimen consisting of ecabet sodium $1000 \mathrm{mg}$ bid, OME $20 \mathrm{mg}$ bid, AMPC $750 \mathrm{mg}$ bid, and metronidazole(MTZ) $500 \mathrm{mg}$ bid. Four weeks or more duration after completion of therapy, $H$. pylori status was assessed by UBT. RESULTS: The study subjects included 34 males and 14 females with a median age of 53 years (range: 31-71 years). Successful H. pylori eradication as defined by negativity of UBT, was achieved in $98 \%(48 / 49)$ of patients by intention-to-treat and $100 \%$ (48/48) of patients by per-protocol analysis. Side effects were reported seven patients with diarrhea, 5 ; skin rash, 2; increased serum transaminase, 2 . There was no serious side effects were observed. DISCCUSION: We already reported the eradication therapy for $H$. pylori using same regime of OME+AMPC+MTZ without combined ecabet sodium as a first line therapy for the peptic ulcer patients previously no eradication treatment for H. Pylori ( J Gastroenterol Hepatol. 1998 Mar;13(3):301-4). The reported results of eradication ratio of $H$. pylori were $87 \%$ (20/23) by per-protocol analysis. These results suggested that OME+AMPC+MTZ combined with ecabet sodium is highly effective than without combined ecabet sodium. CONCLUSIONS: We conclude that ecabet sodium combined salvage therapy was successful regimen for eradication of $H$. pylor in patients who had failed to prior eradication of $H$. pylori by PPI+AMPC+CAM therapy.

\section{T1921}

Mutation Analysis in the Gyra Gene, Not the E Test, Can Be a Powerful Predictor for Treatment Outcomes of Helicobacter Pylori Infection with Levofloxacin-Based Therapy

kuya Miki, Daisuke Shirasaka, Hideyuki Miyachi, Yuko Matsumoto, Takao Tamura, Yoshinori Morita, Masanori Toyoda, Toshifumi Mitani, Naomi Torio, Nobuo Aoyama

Background: Levofloxacin (LVFX) is one of the most attractive agents for refractory Helicobacter pylori (H. pylori) infection, but LVFX-based rescue therapy cannot show a high eradication rate in Japan compared to in other countries. Therefore, it is an urgent issue how to predict treatment outcomes. Although antimicrobial susceptibility judged by culture method such as the E test may be useful, recent studies have revealed the disagreement among the results of susceptibility obtained by conventional culture method and molecular genetic method in the case of clarithromycin (CAM)-based therapy. As for LVFX-based therapy, no clinical studies are available concerning the clinical impact of molecular genetic approach on treatment outcomes in comparison with the E test. Aim: In order to confirm whether molecular genetic method can be a useful tool in LVFX-based rescue therapy, we evaluated the clinical impact of gyrA mutations on treatment outcomes. Methods: Of 102 patients who received 7-day-rescue therapy (lansoprazole, $30 \mathrm{mg}$ b.d., amoxicillin, $1000 \mathrm{mg}$ b.d., and LVFX, $400 \mathrm{mg}$ or $600 \mathrm{mg}$ b.d.), 52 patients whose first-line treatment failure were judged by positive culture were retrospectively analyzed. Susceptibility testing by the E test was performed using two biopsy samples from the greater curvatures of the corpus and the antrum after first-line therapy and before rescue therapy. Total DNA was extracted from the recovered H.pylori isolates in the frozen state, and the quinolone resistance-determining regions of $\mathrm{H}$. pylori gyrA gene were amplified by PCR and directly sequenced. The association between gyrA mutation and MIC values is analyzed according to treatment outcomes. Results: Twenty (39\%) of 52 strains had gyrA mutations. 11 and 8 strains respectively showed point mutations in the gyrA gene at amino acid (aa) 87 (Asn to Lys or Ile) and at aa 91 (Asp to Asn or Gly). Double mutations at aa 87 (Asn to Ile) and 91(Asp to Asn) were found in 1 strains. Nine out of 13 strains with MIC values to LVFX of $\geqq 1 \mathrm{ug} / \mathrm{mL}$, and 10 out of 39 strains with those of $<\mathrm{lug} / \mathrm{mL}$, were not successfully eradicated. Fourteen out of 19 refractory strains to rescue therapy showed the gyrA mutations. Twenty-five out of 32 strains without gyrA mutation were successfully eradicated. The existence of gyrA mutation showed a endency toward worse results regardless of the MIC values. Conclusion: In levofloxacinbased rescue therapy for H.pylori infection, mutants in the gyrA gene is hardly eradicated

\section{T1923}

Effects of Red Ginseng Supplementary Treatment After Eradication Regimen in Patients with Helicobacter Pylori-Associated Chronic Gastritis: A Prospective Randomized Double-Blind Study

Kee Myung Lee, Marie Yeo, Soo Jin Park, Mi Sun Kwak, Jeong A Lee, Young Bae Kim, Kwang Min Kim, Sung Won Cho, Ki Baik Hahm

The fact that mass eradication of Helicobacter pylori (H. pylori) can not be recommended as a cost-effective means to prevent the development of gastric cancer, in spite definition as class I carcinogen by IARC, drives us to search for better strategies for chemoprevention including either attenuation of bacteria colonizations or lessening gastric inflammations. Recent concept about the crossroads between inflammation and carcinogenesis stressed the importance of the intervention of anti-inflammatory strategy. Based on our previous study suggesting that red ginseng capsule could rescue gastric mucosa from $H$. pylori-associated cytotoxicity, a randomized controlled trial was done to document the efficacy and advantages of 10 weeks red ginseng capsules $(2.7 \mathrm{~g} / \mathrm{day})$ supplemented group compared with placebo supplemented group after the eradication-regimen with PPI based triple therapy. Total 84 patients were enrolled and 70 patients completed the trial (83.3\% per protocol analysis) Statistically, the eradication rates were significantly augmented in red ginseng supplemented group compared to placebo group $(79.4 \%$ in placebo group and $91.7 \%$ in red ginseng group, $\mathrm{p}<0.05)$. In respective analysis of gastritis based on Updated Sydney system, red ginseng group showed statistically significant improvement in neutrophil infiltrations, mononuclear cell infiltrations, gastric atrophy, and even intestinal metaplasia than placebo group $(\mathrm{p}<0.05)$. H. pylori infection was associated with significant generation and nuclear translocation of 8-OHdG but red ginseng supplemented group was excellent in lessening 8-OHdG generations in parallel with attenuated apoptosis and single cell nuclear damages (comet assay) than placebo group $(\mathrm{p}<0.05)$. Conclusively, red ginseng supplemented treatment after $H$. pylori eradication could achieve either quantitative advantages or qualitative improvement for $H$. pylori infection and the considerable gastroprotective properties or antiinflammatory activities of red ginseng might contribute to the improvement of $\mathrm{H}$. pyloriassociated chronic gastritis including the scope of chemoprevention with long-term administration

\section{T1924}

Emergence of Ciprofloxacin Resistance in Helicobacter Pylori Isolated from Korea: Mutational Changes in Gyra and Other Genetic Loci

Hyung Joon Lee, Gin Hyug Lee, Jun-Won Chung, Ja Young Kim, Kwi Sook Choi, Kee Don Choi, Hwoon-Yong Jung, Weon-Seon Hong, Jin-Ho Kim, Jin-Yong Jeong

Background/Aims: Failures of proton pump inhibitor-based triple therapies for eradication of $H$. pylori are frequently due to resistance to either clarithromycin or metronidazole. Thus, other antibacterial drugs, such as quinolones, will be needed for $H$. pylori therapy in the future. In this study, we selected ciprofloxacin-resistant $H$. pylori and studied the mechanisms of ciprofloxacin resistance. Methods: H. pylori strains were obtained from gastric biopsy specimens of 64 consecutive H. pylori-positive patients at Asan Medical Center (AMC) in Seoul, Korea. Genomic DNA of ciprofloxacin-resistant strains was extracted and used for PCR and DNA sequencing to determine the mutations in gyrA. To establish that the mutations in gyrA and gyrB caused ciprofloxacin resistance, the PCR products were used to transform a ciprofloxacin-susceptible strain of $H$. pylori to a resistant phenotype. Results: In one Korean Hospital (AMC) the rate of resistance to ciprofloxacin (MICs, $\leq 1 \mathrm{ug} / \mathrm{ml}$ ) in $H$. pylori was $10.9 \%$ (7 of 64 isolates) in 2004. High-level resistance (MICs, $\leq 4 \mathrm{ug} / \mathrm{ml}$ ) was mainly caused by mutations in gyrA. The isolates showing low-level resistance (MICs=1-2 ug/ml) have no mutation in gyrA and gyrB. There was a ciprofloxacin-resistant isolate (MIC=8 ug/ml) having no mutation in gyrA but double mutations (Asp481Glu and Arg484Lys) in gyrB. Transformation by the amplified fragment of gyrB of this isolate showed that the gyrB double mutations are not associated with ciprofloxacin resistance, suggesting that the mechanisms of ciprofloxacin resistance in $H$. pylori are not only mutational changes in gyrA but also in other genetic loci. Conclusions: The rate of ciprofloxacin-resistant $\mathrm{H}$. pylori was $10.9 \%$ at 
AMC, Seoul, Korea. The mechanisms of ciprofloxacin resistance in H. pylori are not only mutational changes in gyrA but also in other genetic loci, which is unknown so far

\section{T1925}

Novel Action of Gastric Proton Pump Inhibitor On the Suppression of Helicobacter Pylori-Induced Angiogenesis

Marie Yeo, Dong Kyu Kim, Sang Uk Han, Jong Eun Lee, Kee Myung Lee, Young Bae Kim, Yong Kwan Cho, Kwang Jae Lee, Jin Hong Kim, Sung Won Cho, Ki Baik Hahm

Though the activations of mitogen activated protein kinases (MAPKs) by Helicobacter pylori (H. pylori) infection, H. pylori infections are associated with induction of host angiogenesis, which might contribute to $H$. pylori-associated gastric carcinogenesis. However, the strategy for its prevention has not been identified yet. Since we previously reported a strong inhibitory action of gastric proton pump inhibitor (PPI) on MAPK ERKI/2 phosphorylation, we investigated whether PPI could suppress the H. pylori-induced angiogenesis via inhibiting MAPK ERK1/2 and resultant angiogenic growth factors. To address the relationship between $H$. pylori infection and angiogenesis, comparative analysis of density of CD 34+ blood vessel was performed in tissues obtained from $20 \mathrm{H}$. pylori-positive gastritis and $18 \mathrm{H}$. pylorinegative gastritis. Expression of HIF-1 and VEGF were tested by RT-PCR and secretion of IL-8 and VEGF was measured with ELISA. To evaluate the direct effect of $H$. pylori infection on the tubular formation of human endothelial HUVEC cells, in vitro angiogenesis assay was employed. Activation of MAPK and NF-KB was detected by immunoblotting. H. pyloripositive gastritis $(40.9 \pm 4.4)$ showed a higher density of CD34 blood vessel than $H$. pylorinegative gastritis $(7.2 \pm 0.8)$, which was well correlated with the expression of HIF-1. Conditioned media from $\mathrm{H}$. pylori-infected gastric epithelial cells directly induced a tubular formation of HUVEC cells and the increase of in vitro angiogenesis was suppressed by PPI treatment. Infection of $H$. pylori significantly up-regulated expression of HIF-l and VEGF in gastric epithelial cells and the expression of proangiogenic factors was mediated by MAPK activation and partially responsible for NF-kB activation. PPI effectively inhibited the phosphorylation of MAPK ERKl/2 that is a principal signal for $H$. pylori-induced angiogenesis. In conclusion, the fact that PPI could down regulate $H$. pylori-induced angiogenesis shed light on that anti-angiogenic treatment using PPI could be a promising protective therapeutic approach for $\mathrm{H}$. pylori-associated carcinogenesis.

\section{T1926}

Prevalence of Eosinophilia and Eosinophilic Esophagitis in Adults in the Community: A Random Population Based Study (Kalixanda) Jukka Ronkainen, Nicholas J. Talley, Pertti Aro, Tom Storskrubb, Elisabeth BollingSternevald, Tore Lind, Michael Vieth, Manfred Stolte, Marjorie M. Walker, Lars Agreus

Background and aims: Eosinophilic esophagitis (EE) is thought to be a very rare inflammatory condition in adults (estimated prevalence 1 in 10,000 ) but patient studies suggest the incidence may be increasing. However, the prevalence and incidence in the general population remain unknown, as endoscopy and biopsy are required in a random population sample. $\mathrm{EE}$ can be associated with allergic disorders and presents with food impaction or dysphagia. The aim of this study was to determine the presence of any eosinophilia in the distal esophagus and the prevalence of $\mathrm{EE}$ in a random sample of a Swedish population. Methods: A random sample $(n=3000)$ of the adult population $(n=21,610)$ in two Swedish municipalities was surveyed using a validated questionnaire assessing troublesome gastroesophageal symptoms (response rate 74\%). 1563 responders were randomly invited to an esophagogastroduodenoscopy (EGD). Esophageal biopsies were obtained $2 \mathrm{~cm}$ above the z-line and from areas of structural abnormality. An infiltration of the esophageal epithelium was defined as mild ( $<15$ eosinophils/hpf), moderate (eosinophils/hpf $\geq 15-<20$ ) or marked ( $\geq 20$ eosinophils/hpf). Symptomatic gastroesophageal reflux disease (GERD) was defined as the presence of heartburn and/or acid regurgitation during the last three months. Esophagitis was graded according to the Los Angeles classification. Results: EGD with biopsy was performed in 1000 subjects (mean age 53.5 years, $51 \%$ female). Eosinophils in the esophageal biopsies were found in $9(0.9 \%, 95 \%$ CI $0.3-1.5)$ : 5 with mild, 3 with moderate and one with marked infiltration. Esophagitis (LA-grade A) was found in one individual with mild and in one with moderate infiltration. No other endoscopic abnormalities were found in this group. GERD was found in $2 / 3$ with moderate and in the one with marked infiltration. Dysphagia and asthma only occurred in the individual with marked infiltration. GERD and esophagitis were not associated with eosinophilia but those with esophageal eosinophilia had significantly less H.pylori on histology or culture in the stomach compared with those without eosinophilia (0/9 vs. 323/942, $\mathrm{p}=0.032$ ) and were less likely to ever having smoked or used moist snuff ( $2 / 7$ vs. 538/942, p=0.045). Conclusions: This is the first population-based study to estimate the prevalence of eosinophilic esophagitis to our knowledge. Esophageal eosinophilia was found in nearly $1 \%$ of the general Swedish population: four $(0.4 \%)$ with $\geq 15$ eosinophils/hpf and one ( $0.1 \%$ ) with $\geq 20$ eosinophils/hpf. Asymptomatic eosinophilia $<20 / \mathrm{hpf}$ is not apparently explained by GERD, and is more common than has been appreciated in adults.

\section{T1927}

Psychological Factors Affect the Frequency of Belching in Patients with Aerophagia

Albert J. Bredenoord, Bas L. Weusten, Robin Timmer, Andre J. Smou

Background and aim: In patients with excessive belching (aerophagia) an organic cause is seldomly found and a psychogenic cause is often suspected. Aim of this study was to investigate the effects of attention and distraction on the frequency of belching and to investigate the mechanism of belching in these patients. Methods: In 10 patients with aerophagia combined esophageal manometry, $\mathrm{pH}$ and impedance monitoring was performed for 2 hours. These 2 hours consisted of 4 30-min recording periods. Period 1: patient under the impression that recording had not yet commenced. Period 2: patient informed of recording in progress. Period 3: patient being distracted by having him/her filling in questionnaires.
Period 4: patient not being distracted. Data are presented as median (iqr). The study was approved by the local IRB. Results: One patient stopped belching immediately after introduction of the catheters and was excluded from further analysis. In the remaining 9 patients, a total of 1258 belches was measured, 51 of which were the result of air that escaped from the stomach (gastric belches). The remaining 1207 belches (96\%) were events during which air was expelled in oral direction almost immediately after entering the esophagus, before reaching the stomach (supragastric belches). Gastric belches were distributed equally over the first $(1.5(0.5-2.0))$, second $(1.5(0.5-2.0))$, third $(1.0(0-2.0))$ and fourth $(1.0(0-2.0))$ recording period. In contrast, the incidence of supragastric belches increased significantly $(\mathrm{p}<0.05)$ from $0(0-32)$ in the first period to $30(18-60)$ in the second period, after patients were told that recording was started. During the questionnaires the incidence of supragastric belches decreased $(\mathrm{p}<0.05)$ to $14(4-30)$. In the fourth period the incidence of supragastric belches increased $(\mathrm{p}<0.05)$ to $21 \quad(10-49)$. Supragastric belches were initiated either by sucking air into the esophagus by a negative thoracic pressure ( 8 patients) or by injecting air into the esophagus by a pharyngeal contraction (2 patients) Conclusions: The vast majority of belches in patients with aerophagia is due to supragastric belching. When patients are unaware that they are being studied or when they are distracted the incidence of belching is significantly reduced. These findings confirm that a psychological factor is important in this disorder, which supports treatments such as behavioral therapy. The mechanisms the patients use to fill their esophagus with air during supragastric belching are similar to the techniques described for facilitation of esophageal speech in laryngectomized patients, which suggests that logopedic therapy might also be helpful.

\section{T1928}

Intra-Duodenal Capsaicin Infusion Induces Esophageal Hypersensitivity Which Is Dose Dependent

Barbara J. Unsworth, Qasim Aziz, Peter Holzer, John McLaughlin, Anthony Hobson

Background: Capsaicin activates TRPV-1 receptors on spinal and vagal afferents. Its infusion into the jejunum evokes burning and cramping sensations, the intensity of which are dose dependent. Herein, we hypothesized that capsaicin infusion into the proximal duodenum would result in increased excitability of spinal dorsal horn neurons (central sensitization) and the development of sensitization in a visceral region with convergent spinal innervation Methods: We recruited 15 subjects (11 Female). A catheter was positioned in the proximal duodenum with a 2 nd in the distal esophagus. Pain thresholds (PT) to electrical stimulation (ES) were assessed in the esophagus (500usec duration, $0.3 \mathrm{~Hz}$ frequency). Capsaicin was then infused into the duodenum ( $2 \mathrm{ml} / \mathrm{min}$ for 30-minutes). The concentrations of capsaicin used were 100 and $200 \mathrm{ug} / \mathrm{ml}$, or saline control. Subjects were studied on 4 occasions $(1 \times 100 \mu \mathrm{g} / \mathrm{ml}, 2 \times 200 \mu \mathrm{g} / \mathrm{ml}, 1 \times$ Saline $)$ in a double blind randomized order. Esophageal PT were recorded at 15 and 45 -minutes post infusion. Visual analogue scales (VAS) for pain unpleasantness, nausea and anxiety were recorded at 5-minute intervals during the infusion and a short McGill pain questionnaire was used to describe the psychophysical properties of the infusion. Results: Significant reductions in esophageal PT were seen on both occasions at $200 \mu \mathrm{g} / \mathrm{ml}$ (max group mean reduction in PT post infusion $=-9.2 \mathrm{~mA}$ and $-11 \mathrm{~mA}, \mathrm{p}=$ $0.004)$ but this was not significant at $100 \mu \mathrm{g} / \mathrm{ml}(-6.3 \mathrm{~mA}, \mathrm{p}=0.06)$ or with saline $(-0.7 \mathrm{~mA}$ $\mathrm{p}=0.77$. Baseline Esophageal PT was reproducible within subjects when compared for the $2 \times 200 \mu \mathrm{g} / \mathrm{ml}$ visits (Intra-class correlation coefficient $(\mathrm{ICC})=0.74$ ). Whilst group data for the magnitude of sensitization were similar for the $2 \times 200 \mu \mathrm{g} / \mathrm{ml}$ visits $(\mathrm{p}=0.35$ ), data within subjects were less reproducible (ICC at 15 mins post infusion $=0.431,45 \mathrm{mins}=0.421$ ) VAS scores for pain were higher than for other psychophysical measures and highest at the $200 \mu \mathrm{g} / \mathrm{ml}$ concentration. The most common verbal descriptors used to describe the capsaicin infusion were 'cramping', 'hot-burning' and 'aching'. Conclusion: Capsaicin infusion into the proximal duodenum induces sensitization in a visceral region known to have convergent afferent input at the spinal cord level. The magnitude of sensitization increases with increasing concentrations of capsaicin, as does the subjective awareness of the infusion. Whilst group data provide reproducible findings, there is some variability seen within subjects with regard to the development of sensitization. These data provide further evidence that central sensitization plays an important role in the development of visceral hypersensitivity.

\section{T1929}

Expression of Eotaxin-2 and Chemokine Receptor 3 in Eosinophilic Esophagitis Versus Reflux Esophagitis

James R. Carlsten, Murray B. Resnick, Patricia A. Meitner, Edmond Sabo, Adam Carter Sripathi Kethu, Baishali Bhattacharya

Background: Eosinophilic esophagitis (EE) is increasingly being recognized in biopsies from the esophagus and distinguishing it from gastroesophgeal reflux disease (GERD) is important given the differences in pathogenesis and treatment. Allergy is thought to play an important role in the etiology of EE also supported by the fact that many of the EE patients suffer from bronchial asthma and present with peripheral blood eosinophilia. Biopsies of EE cases show markedly increased intraepithelial eosinophils $>25 /$ high power field (HPF) in the esophageal squamous mucosa with luminal eosinophilic clusters and microabscesses. Recently there has been much interest in the role of Eotaxins which act through their receptor chemokine receptor 3 (CCR-3), in the recruitment and activation of eosinophils Much of the studies have been focused on bronchial asthma. But their role in EE has no yet been elucidated. Design: This was a retrospective study using formalin-fixed paraffin embedded blocks of biopsies taken from 12 cases of established EE, 22 cases of GERD and 17 normal esophagus controls. Comparison was made between eosinophil count and expression of Eotaxin-2 (CCL24)and Eotaxin receptor (chemokine receptor 3/CCR3) usin primers for mRNA. Result: All $12 \mathrm{EE}$ cases were males, age $2-49$ years, mean 17.25 years with mean eosinophilic count of $62.45 / \mathrm{HPF}$ (range 34.5-140.75). The 22 cases of GERD (13 males and 9 females)with a mean age 30.0 yrs had a mean eosinophil count of $3.6 /$ HPF. The 17 normal controls ( 8 males and 9 females)with a mean age 20.65 years had 0 eosinophil count. EE cases had symptoms of dysphagia (most common), food getting stuck, abdominal pain etc. Endoscopic appearance consisted of friable mucosa, furrows, ridging cracking, white spots resembling candida etc. The mean expression of Eotaxin- 2 among EE, GERD and normal controls consisted of $11.9+/-1.69 ; 4.05+/-0.98$; and $0.62+/-0.14 \mathrm{pg}$ / 
$\mathrm{ng}$, respectively. The differences were statistically significant between all groups ( $\mathrm{p}<0.0001$, one way ANOVA followed by Bonferroni test). For the CCR-3 receptor, a significant difference was found between EE $(1.80+/-1.05)$, and GERD $(0.15+/-0.12),(\mathrm{p}=0.016$, Kruskal Wallis ANOVA). A marginally significant difference was also found between EE and the normal controls $(\mathrm{p}=0.057$ ). Conclusion: Based on our study, Eotaxins and chemokine receptor-3 appear to play a significant role in the pathogenesis of EE and therapeutic agents targeting these molecules may have a promising role in the management and understanding of this disease.

\section{T1930}

The Circular Smooth Muscle of the Distal Esophagus Is Oriented At An Oblique Angle Leading to Axial Shortening of the Circular Smooth Muscle During Peristaltic Contraction

Keng-Yu Chuang, Anil Vegesna, Rina Raju, Wassim Asfari, Zeeshan Ramzan, Parkman Henry, Larry S. Miller

Background:The law of mass conservation implies that if the circular smooth muscle of the esophagus (CSM) is oriented perpendicular to the longitudinal smooth muscle (LSM), only the LSM should actively shorten in the axial (longitudinal) direction and any CSM shortening in the axial direction is as a consequence of LSM drag during peristaltic contraction. Yet, ultrasound studies demonstrate that the CSM actually shortens significantly more in the axial direction than does the LSM, especially in the distal esophagus. Purpose:We hypothesize that the CSM of the distal esophagus is oriented at an oblique angle to the perpendicular and that this angulation accounts for an increase in CSM shortening over LSM shorting in the axial direction. In addition we hypothesize that this angle is greatest in the distal esophagus accounting for greater distal esophageal shortening. Methods:Nine cadaveric esophagi underwent fine dissection to expose the LSM and CSM layers. The angles of the muscle fibers between the two smooth muscle layers were measured at every $\mathrm{cm}$, starting at the gastroesophageal junction (GEJ)using digital imaging. Results:A significant difference was found between the mean muscle angles of the CSM in the distal esophagus (at the GEJ) and the muscle angles of the CSM above the GEJ ( $p<0.001)$. (see figure). Conclusions:A significant difference was found between the muscle angles of the CSM at the GEJ and the muscle angles above the GEJ. We believe that this oblique angulation along with drag from the LSM leads to axial shortening of the CSM which is greater than the axial shortening due to the LSM alone. Furthermore we believe that this oblique angulation is partially responsible for the greater distal esophageal shortening of the esophagus during peristaltic contraction.

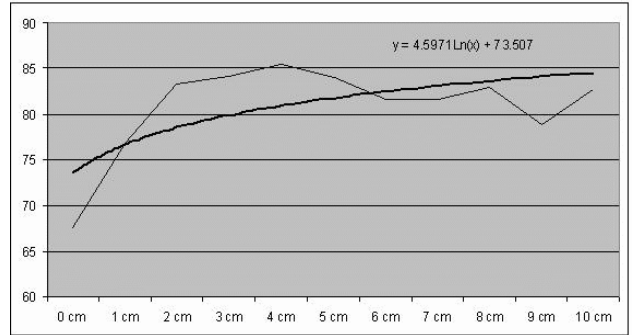

The angle of the muscle fibers is on the $\mathrm{y}$ axis. The distance from the GEJ is on the $\mathrm{x}$ axis.

\section{T1931}

The Tumor Suppressor Gene P53 As a Predictor for Development of Esophageal Cancer in Patients with Achalasia

Ivonne Leeuwenburgh, Astrid Capello, Monique M. Gerrits, Johannes G. Kusters, Peter D. Siersema, Ernst J. Kuipers

Introduction: Patients with longstanding achalasia, an esophageal motor disorder leading to unctional esophageal obstruction, have an up to $140 \mathrm{x}$-increased risk of developing esophageal cancer compared to the healthy population. Chronic food stasis (often present despite LES-lowering therapy) may lead to chronic inflammation, epithelial hyperplasia, multifocal dysplasia and squamous cell carcinoma (SCC). On the other hand, gastro-esophageal reflux, a complication of LES-lowering therapy may lead to Barrett's metaplasia and adenocarcinoma (AC). Surveillance-endoscopies are advocated, however optimal sampling and analysis methods as well as surveillance intervals remain to be determined. Aim: To investigate whether p53- (tumor suppressor gene) and Ki 67 (proliferation) expression, and changes in DNA-ploidy are early predictors for progression to malignancy. Methods: In our cohort of 414 achalasia patients, $16(4 \%)$ patients died of advanced esophageal cancer despite bi-annual endoscopic surveillance. Out of those 16 cancer cases, we selected 8 patients (5 males, mean age 45.5 yrs (range 30-66) at the start of achalasia symptoms) who developed esophageal cancer (6 SCC, 2 AC). Cancer cases had been under surveillance for a mean of 8 yrs (range 2-20). All biopsy sets obtained during surveillance were studied for p53 and Ki 67 expression using immunohistochemistry. Two independent researchers counted the samples. Samples were considered positive for either marker if more than 15\% of nuclei stained positive. Five patients ( 3 males, mean age 50 (31-68) yrs at start disease) with achalasia without esophageal cancer development during follow-up (mean 19 (18-20) yrs) served as controls. Results: 28 biopsy sets were obtained during surveillance in the cancer cases and compared with 25 sets from the controls. In 5/8 (62\%) cancer patients (4 SCC, 1AC), p53 was expressed in previous surveillance biopsies at a mean time of 6 (111) yrs prior to cancer development. In 1 SCC patient, p53 was negative, in I SCC patient the expression was $10 \%$ and in $1 \mathrm{AC}$ patient not enough material was available. In the controls, no p53 expression was detected. Ki 67 was expressed in all surveillance biopsy sets of all carcinoma and control patients. Flow cytometry of surveillance specimens did not reveal aneuploidy. Conclusions: p53- expression, but not proliferation and changes in
DNA-ploidy, appear useful to identify achalasia patients at increased risk of developing esophageal carcinoma. A further prospective follow-up study is needed to determine the effectiveness of intense surveillance of p53-positive patients for early detection of cancer.

\section{T1932}

Influence of Bolus Consistency and Position On Esophageal High Resolution Manometry Findings

Anita Bernhard, Daniel Pohl, Oliver Goetze, Heiko Fruehauf, Werner Schwizer, Michael Fried, Radu Tutuian

Background: Conventional manometry evaluating liquid swallows in recumbent position has been criticized as it measures pressure changes only at limited number of sites in the esophagus and it does not assess pressure changes during solid boluses in the physiologic upright position. High resolution manometry (HRM) allows a more comprehensive evaluation of the esophageal peristaltic and, using hydrostatic correction factors, allows evaluating esophageal peristalsis during bread swallowing in upright and recumbent position. Aim: To compare findings of esophageal motility abnormalities during water and bread swallows in upright and recumbent position. Methods: Patients referred for evaluation of dysphagia, chest pain and GERD symptoms (heartburn and regurgitation) underwent HRM testing using a 32-channel low compliance water-perfused system. After transnasal placement of the catheter patients received water swallows (10cc each) and bread swallows in upright and left lateral decubitus position. Swallows were considered normal if in the isocontur plot representation a peristaltic band $>30 \mathrm{mmHg}$ spanned over at least $5 \mathrm{~cm}$ in the distal esophagus, ineffective if the pressure band in the distal esophagus was less than $5 \mathrm{~cm}$ and simultaneous if the onset velocity of the pressure band $>30 \mathrm{mmHg}$ exceeded $8 \mathrm{~cm} / \mathrm{sec}$ in the distal esophagus. Abnormal esophageal manometry was defined as the presence of $30 \%$ or more ineffective or $20 \%$ or more simultaneous swallows. Patients with achalasia were excluded from the analysis. Results: From April 2003 to November 2005 data from 96 patients (64 F, mean age 50 years, range 19-79 years) who underwent HRM for dysphagia (56\%), chest pain $(22 \%)$ and GERD symptoms (22\%) were available for analysis. Change in position and bolus consistency increased the percentage of patients with abnormal manometry findings (Table). Conclusions: Manometry during bread swallows increases the sensitivity of finding abnormal motility patterns in patients with esophageal symptoms.

Percentage of patients with abnormal manometry

\begin{tabular}{|c|c|c|c|}
\hline & Dysphagia (N=54) & Chest pain (N=21) & GERD symptoms (N=21) \\
\hline water recumbent & $26 \%$ & $29 \%$ & $24 \%$ \\
\hline water upright & $33 \%$ & $48 \%$ & $52 \%$ \\
\hline bread recumbent & $36 \%$ & $62 \%$ & $38 \%$ \\
\hline bread upright & $37 \%$ & $71 \%$ & $67 \%$ \\
\hline
\end{tabular}

\section{T1933}

Relationship Between Abnormal Bolus Transport and Dysphagia Lilian R. Aprile, Ricardo B. de Oliveira, Roberto O. Dantas, Jiri Silny, Daniel Sifrim

The term dysphagia encompasses a number of sensations which are perceived as caused by abnormal bolus transit from mouth to stomach. The mechanisms responsible for nonobstructive dysphagia as well as its relations with altered esophageal motility and bolus transit remain unclear. Aim: To investigate the relations between sensation of dysphagia with esophageal motility and bolus tranport in normal volunteers whose esophagi were rendered transiently hypocontractile. Methods: Concurrent esophageal manometry and impedance were performed in 9 healthy volunteers. Subjects swallowed $10(5 \mathrm{ml})$ viscous and 10 bread boluses in sitting position. Distal esophageal contractility was impaired with sildenafil (50mg) ingested $10 \mathrm{~min}$ before the series of swallows. Perception of each swallow was evaluated using a standardized 1-5 scoring system (1 fluid passage and 5 complete blockade). Manometry was abnormal (A) if the amplitude of distal esophageal contractions was $<30 \mathrm{mmHg}$ or if they were simultaneous; bolus transit by impedance was abnormal (A) if bolus exit was not identified at one or more of the measuring sites or if total bolus transit time was $>12.5 \mathrm{~min}$; and perception was increased (I) if score was $>1$. Agreements between results were measured using Kappa statistic k. Results: Sildenafil provoked progressive esophageal peristaltic failure starting at $\pm 20 \mathrm{~min}$ after ingestion. At least one enhanced perception episode (I) was reported by eight of the nine subjects for bread swallows, and by 5 of them for gel swallows; 30 of the 86 bread swallows (34.8\%) and 15 of the 86 gel swallows (17.4\%) were perceived as (I). Agreement between manometry and impedance occurred for $88 \%$ of bread swallows $(\mathrm{k}=0.67 \cdot 95 \% \mathrm{CI} \cdot 0.48-0.87)$ and $86 \%$ of gel swallows $(\mathrm{k}=0.70 ; 95 \% \mathrm{CI}: 0.54-0.85)$. In contrast, agreement between impedance and perception occurred for only $52 \%$ of gel swallows $(\mathrm{k}=0.12 ; 95 \% \mathrm{CI}: 0.00-0.24)$ and $42 \%$ of bread swallows $(\mathrm{k}=0.04 ; 95 \% \mathrm{CI}:-0.09-0.17)$. Agreement between perception and manometry was even lower, observed in $36 \%$ of gel swallows $(\mathrm{k}=-0.04 \cdot 95 \% \mathrm{CI} \cdot-0.17-0.09)$ and $41 \%$ of bread swallows ( $\mathrm{k}=-0.02 ; 95 \% \mathrm{CI}:-0.16-0.09)$. Conclusions: In sitting healthy subjects, sildenafil-induced reduction of distal esophageal contractility is associated to impairment of esophageal bolus transit but not with significant dysphagia. The hypocontractility-induced abnormal bolus transport plays a minor role, if any, in the genesis of dysphagia. These results suggest that in patients with non-obstructive dysphagia, factors other than hypocontractility and abnormal bolus transport i.e hypersensitivity might be more relevant in dysphagia genesis. Support:CNPq,FAPES 
T1934

Intraluminal Impedance Detects Failure of Pharyngeal Bolus Clearance During Swallowing: A Validation Study in Adult Dysphagia Patients

Taher Omari, Michal Szczesniak, Nathalie Rommel, Phil G. Dinning, Sergio Fuentealba, lan J. Cook

Introduction: Multichannel intraluminal impedance (MII) has been shown to detect bolus flow through the pharynx and UES in healthy volunteers (1). The aim of this study was to determine if this technique can detect failure of pharyngeal bolus clearance in patients with dysphagia. Methods: Patterns of pharyngo-esophageal pressure and impedance were simultaneously recorded with videofluoroscopy (VF) in 5 adult patients (3M:2F, 57-78 yrs) with dysphagia due to a range of causes; post-myotomy cricopharyngeal stenosis, postradiotherapy myopathy, motor neuron disease and medullary infarction. A combined MIImanometry assembly with 6 impedance segments spaced at $1 \mathrm{~cm}$ intervals was positioned with 2-3 impedance segments located above the upper margin of the UES. Measurements were performed during bolus swallows of $2-10 \mathrm{ml}$ liquid and semi-solid and MII values were simultaneously recorded from bolus administration until bolus clearance. The VF determined presence/absence of bolus/bolus residue was compared with the MII determined bolus presence/absence (ie $\Omega \leqq 50 \%$ baseline = bolus present; $\Omega>50 \%$ of baseline = bolus absent) at intervals of $0.4 \mathrm{sec}$ from the onset of pharyngeal swallow. Agreement between VF and MII was then determined using Cohen's Kappa statistics. Results: A total of 39 bolus swallows were evaluated (15 liquid: 24 semisolid). Bolus residue following passage of the pharyngeal stripping wave was present on VF for 19 swallows this was also detected by MII on 16 occasions (84\%). The level of agreement between VF and MII in individual patients varied from slight agreement $(\kappa<2 ; 2$ patients) to fair to moderate agreement ( $\kappa 2-6 ; 3$ patients). Agreement was better for semisolid $(\kappa=0.47)$ than liquid boluses $(\kappa=0.43)$ and agreement improved with increasing bolus volume tested $(\kappa=0.41,0.43$ and 0.49 for $2 \mathrm{ml}, 5 \mathrm{ml}$ and $10 \mathrm{ml}$ respectively). Conclusion: These data indicate that pharyngeal impedance measurement can detect failed bolus clearance in patients with dysphagia. Severe pharyngeal dysfunction renders poorer agreement due to low baseline impedance values relative to nadir. 1. Omari TI et al Assessment of Intraluminal Impedance for the Detection of Pharyngeal Bolus Flow During Swallowing in Healthy Adults. Am J Physiol Gastrointest Liver Physiol. 2005

\section{T1935}

Topical Viscous Budesonide for Pediatric Eosinophilic Esophagitis Seema Aceves, John Bastian, Robert Newbury, Ranjan Dohil

Background: Eosinophilic esophagitis (EE) is a disease of increasing incidence in both adult and pediatric populations. Diagnosis depends on the presence of 20 eosinophils or more per high power field (hpf) at 400x microscopy. Although treatment with topical fluticasone appears to be effective, not all children can master the puff and swallow technique required for pan-esophageal particle deposition. This may lead to treatment failure. Methods: Children with upper gastrointestinal symptoms, including dysphagia and vomiting, who had endoscopic and histologic evidence of EE were treated with topical budesonide mixed with sucralose at doses of 1 to $2 \mathrm{mg}$ per day. Some patients had previously received fluticasone and/or elemental diet. All patients received concurrent acid-suppression therapy. At every UGI endoscopy, 2-3 biopsies were taken each from the distal, mid and proximal esophagus. Results: Fourteen children, 3 females and 11 males, with EE were treated with oral budesonide. The mean patient age was 5.7 years (range $3-14$ years). Three patients had previously failed topical fluticasone therapy. Upper gastrointestinal endoscopy with biopsy following 3 to 4 months of therapy demonstrated disease improvement or resolution in 12 of 14 children following topical esophageal therapy with budesonide. The highest number of eosinophils per hpf decreased from a mean of 78 (95\% CI: $56.3-99.4)$ to 19 (95\% CI: 4.9-33.4) $(\mathrm{p}=0.0007)$. Among the responders, the highest number of eosinophils per hpf decreased from a mean of $85(95 \%$ CI 61.9-107.3) to $11.5(95 \%$ CI 1.8-21.2) $(\mathrm{p}=0.00002)$ with 8 of 12 patients having less than 7 eosinophils per hpf following therapy. Histologic improvement was pan-esophageal. In addition, $67 \%$ of responders had histologic resolution of basal zone hyperplasia. None of the patients had a normal esophageal appearance on endoscopy prior to budesonide therapy and $57 \%$ percent of the patients had a completely normal esophagus at follow-up endoscopy. Of the responders $83 \%$ had improvement esophageal endoscopic appearance. One patient developed Candidal esophagitis. Patients typically had symptomatic improvement. None of the patients who were tested had suppression of their am cortisol level. Conclusions: Topical viscous budesonide is a once daily, safe, palatable, and effective therapy in children with EE and placebo controlled trials are warranted to evaluate the true disease modifying effect of therapy.

\section{T1936}

Does Endoscopic Ultrasound Help Predict Clinical Outcome of Pneumatic Dilation for Achalasia?

Woosuk Park, John J. Vargo, Edgar Achkar, Gary W. Falk, Michael F. Vaezi

INTRODUCTION: Pneumatic dilation (PD) is a widely accepted treatment option for idiopathic achalasia. Published studies on predictors of response to PD have not previously addressed morphology of the lower esophageal sphincter (LES). AIMS: To assess LES morphology using endoscopic ultrasound (EUS) and to determine clinical and physiologic predictors of PD outcome. METHODS: A prospective cohort study of all patients diagnosed with achalasia undergoing initial Rigiflex pneumatic dilation at the Cleveland Clinic Foundation between 2003 and 2005 was performed. Pre-dilation assessment included symptom score, EGD, EUS, timed barium swallow (TBS) and esophageal manometry (ESMO). All patients subsequently underwent Rigiflex pneumatic dilation using a $3.0 \mathrm{~cm}$ balloon. EUS of the LES was performed immediately before and after PD using Olympus UM DP-12 25R 12 $\mathrm{MHz}$ echoprobe by a single endoscopist. Subjects were then followed at $1,6,12$ and 18 months. At one month follow-up, all patients had repeat symptom score, TBS and ESMO. Treatment failure was defined as recurrence of symptoms and/or abnormal TBS requiring further therapy. RESULTS: 21 patients underwent $3.0 \mathrm{~cm}$ Rigiflex pneumatic dilation [Median age (IQR): 51 years $(37,56), 52 \%$ male]. The median (IQR) follow-up was 11 months $(6$,
12). A total of 10 patients ( $48 \%$ ) failed therapy: 8 failed at one month and 2 failed at one year. The treatment failure group $(n=10)$ and treatment success group $(n=11)$ showed no significant difference in age, gender, pre- and post-therapy symptom score, TBS, LES pressure, and LES inner circular muscle laver thickness. However, the treatment success group had significantly thinner post-therapy outer longitudinal muscle measurements compared to the treatment failure group [median thickness (IQR): $1.1 \mathrm{~mm}(1.0,1.2)$ and $1.6 \mathrm{~mm}(1.5,2.1$ respectively, $\mathrm{p}=0.02 \mathrm{l}$. ROC analysis showed that post-therapy outer longitudinal muscle thickness $\leq 1.5 \mathrm{~mm}$ was associated with $85.7 \%$ sensitivity and $99 \%$ specificity for treatment success using a $3.0 \mathrm{~cm}$ dilator. CONCLUSIONS: 1) There are no demographic, clinical, radiologic or manometric predictors of outcome with $3.0 \mathrm{~cm}$ PD for achalasia. 2) Posttherapy EUS assessing thickness of the longitudinal muscle may be useful in predicting clinical outcome in patients with achalasia undergoing PD.

\section{T1937}

Accuracy of Esophageal Impedance in the Evaluation of Esophageal Function in Patients with Systemic Sclerosis

Lilian R. Aprile, Luciana A. Bento, Roberto O. Dantas, Daniel Sifrim, Jiry Silni, Ricardo B. de Oliveira

Background: A number of studies show that esophageal impedance (EI), by allowing precise assessment of movements of intraluminal contents, may be useful in the evaluation of the esophageal function. Information about the accuracy of EI for detection of inneffective motor activity in specific conditions is lacking. Systemic sclerosis (SS) is an important cause of esophageal motor disorders. Aim: to determine the agreement between the data of conventional manometry and EI in SS patients. Methods: twenty five informed patients meeting the American College of Rheumatology criteria for SS (23 females, median age: 43 years, range 20-72 years) were submitted to both perfusion esophageal manometry and EI recording in separate days. During continuous EI recording, each subject was asked to give 6 swallows of a $5 \mathrm{ml}$-saline bolus, 3 in the supine and 3 in the sitting position. EI was considered abnormal if baseline impedance was lower than $1000 \mathrm{ohms}$ or if deglutitions had impedance nadir higher than $600 \mathrm{ohms}$ or the post-deglutition impedance failed to reach the previous baseline. Manometry was considered abnormal if severe distal esophageal body hypomotility, namely contractions $<30 \mathrm{mmHg}$ and very low lower esophageal basal pressure was found Agreements between results were measured using Kappa statistic $k$. Results: fifteen of the 25 SS subjects $(60 \%)$ had abnormal manometry. Agreement between manometry and basal impedance occurred in $84 \%$ of the subjects in the sitting position $(\mathrm{k}=0.67 ; 95 \% \mathrm{CI}: 0.37$ $0.96)$ and in $92 \%$ of them in the supine position $(\mathrm{k}=0.83 ; 95 \% \mathrm{CI} ; 0.60-1.00)$. Agreement between manometry and deglutitions data occured in $76 \%$ in the sitting position $(\mathrm{k}=0.46$ 95\% CI: $0.12-0.81$ ) and in $92 \%$ of them in the supine position ( $\mathrm{k}=0.83 ; 95 \% \mathrm{CI} ; 0.60$ 1.00). Taking manometry as "gold standard", calculated sensitivity was $100 \%$ and specificity was $80 \%$ for both supine EI parameters. Conclusions: the concordance between manometry and EI data performed in the supine, but not in the upright position is high in SS patients. The measurement of the baseline impedance is as accurate as the more complex analysis of the deglutition profile for the detection of abnormal esophageal motility in SS patients. EI may be a useful tool for detection of esophageal disorder in SS patients.

\section{T1938}

Impact of Age and Gender On Success of Pneumatic Dilation and Heller Myotomy in Achalasia

Sandra El-Hachem, A Rocio Lopez, Marcelo Vela, Thomas Rice, Michael Vaezi

Background: The current recommendation in patients with achalasia advocates a graded approach to pneumatic dilation (PD) starting with $3.0 \mathrm{~cm}$ balloon followed by 3.5 and 4.0 $\mathrm{cm}$ balloons in those who fail to respond. However, treatment response with this approach has been disappointing in young males (Clin Gastroenterol Hepatol 2004; 2: 389-94). There is controversy on whether Heller myotomy (HM) should be the initial treatment in this group. The aim of this longitudinal study was to compare age and gender dependent success of initial PD versus HM in previously untreated achalasia patients. Methods: Initial treatmen was PD with $3.0 \mathrm{~cm}$ balloon in 81 patients and HM (Heller Myotomy) in 53. Clinical symptoms and timed barium swallow were recorded pre- and post PD and HM. Follow up was 3 years and failure was defined as need for further treatment or a change in symptom assessment $<50 \%$. A Cox proportional hazards model was used to study the effect of therapy, age, and gender on outcome. Log-rank tests were used to compare success rates between PD and HM in these subgroups: men under 50 and over 50, women under 50 and over 50 years. Results: Adjusting for age and gender, subjects who had PD with a $3.0 \mathrm{~cm}$ balloon were 3.1 times more likely to fail than those who had HM (Figure, $\mathrm{P}=0.01$ ). Age and gender were not significantly associated with treatment failure, but a subgroup analysis showed higher success rates for HM compared to $\mathrm{PD}$ in men under 50 years $(\mathrm{P}=0.01)$ There was no age or gender difference in outcome for female patients. Conclusions: 1) Initial therapy with Heller myotomy has a higher long term success rate in males under the age of 50 than $3.0 \mathrm{~cm}$ PD 2) In this group graded approach to PD starting with $3.0 \mathrm{~cm}$ should not be the standard of care. 3) Future studies should assess the differential response between initiating with $3.5 \mathrm{~cm}$ PD versus HM in young men with achalasia. 


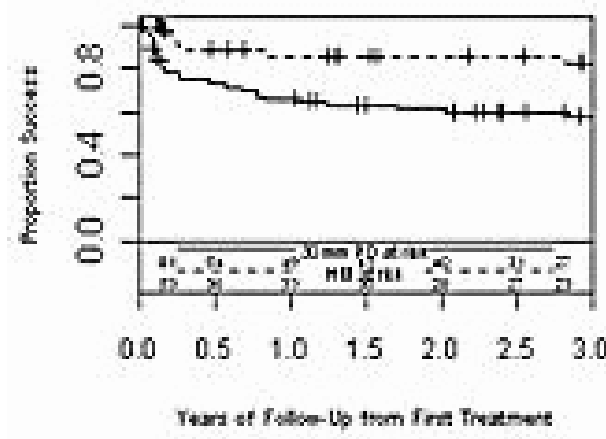

$\mathrm{P}=0.006$

\section{T1939}

Esophageal Testing and Diagnoses in Patients Discharged from the Hospital with Non-Cardiac Chest Pain: A Missed Opportunity?

G R. Locke, Ross A. Dierkhising, Peter A. Smars, Guy S. Reeder, Alan R. Zinsmeister, Nicholas J. Talley

Patients presenting to an emergency room (ER) with chest pain often do not have a cardiac cause for their pain. Gastroenterologists will investigate for esophageal diseases, but the degree to which esophageal diseases are considered in non-cardiac chest pain (NCCP) patients in the community is not clear. Aim: To estimate the frequency of esophageal testing, diagnoses and treatment in patients presenting to an ER and admitted with chest pain who were discharged with a diagnosis of NCCP. Methods: The resources of the Rochester Epidemiology Project were used to identify everyone who had presented to the ER in Olmsted County, MN with chest pain, admitted to the hospital and then dismissed without a cardiac diagnosis over an 8 year period. Their medical records were reviewed in detail to ascertain GI testing, GI diagnoses and GI medication use. Results: 2058 residents presented to an ER with CP; 1973 were admitted and 365 were not given a cardiac diagnosis ( 6 had to be excluded because of research authorization). Of these, 94 (26\%) received a digestive disease diagnosis in the hospital ( 82 were GI and 12 were biliary); their mean age was 62 years and $50 \%$ were male. 230 had no positive diagnosis given (NCCP group); there mean age was 56 years and $53 \%$ were male. Median follow up was 5.3 years (range $0-13.5$ yrs) for the GI group and 5.8 yrs (0-12.9) in the NCCP group. 23 (24\%) of the GI group died during follow up, (median time to death 4.1 years) 8 died of cardiac causes. 31 (14\%) of the NCCP group died, (median time to death 3.6 years) of which 15 died of cardiac causes. Among the 230 with an NCCP diagnosis, $22 \%$ had an EGD of which $65 \%$ were abnormal and $31 \%$ had an UGI of which $20 \%$ were abnormal yet none had a ambulatory pH study and just 5 had an esophageal manometry, of which 2 were abnormal. During follow up, $22 \%$ of the NCCP patients received a diagnosis of GERD. Conclusions: Although the GI literature focuses on GERD or esophageal dysmotility in NCCP; very few people who have sought care for NCCP get GI tests or a GI diagnosis. This highlights the referral bias in the literature regarding $\mathrm{pH}$ and manometry for NCCP. In addition, a GI or NCCP diagnosis does not protect a patient with chest pain from dying from cardiac disease. A better understanding of NCCP in the community is required. Supported by an unrestricted research grant from Tap Pharmaceuticals

\section{T1940}

Gastric Atrophy: A Major Determinant Factor Differentiating Non-Erosive Reflux Disease from Erosive Reflux Disease

Jin-Bae Kim, Yun-Jung Chang, Sung-Won Jung, Il-Hyun Baek, Myung-Seok Lee

BACKGROUND: It is still not known whether there are differences between erosive (ERD) and nonerosive GERD (NERD). Some large epidemiologic study has shown that male gender, overweight, and regular use of alcohol are independent predictors of ERD and Helicobacter status (HP) is associated with a lower risk of ERD. On the contrary, the presence of HP, HP gastritis, and female sex are reported to be more in relation with NERD. AIMS: The aim of this study was to evaluate any differences in demographic and clinical characteristics and in the degree of gastric atrophy between two forms of GERD. METHODS: We studied 114 consecutive patients with a clinical diagnosis of GERD (heartburn/acid regurgitation and endoscopy). Reflux esophagitis was classified according to the modified LA classification. HP status was determined with either rapid urease test or selology. Gastric atrophy was also defined serologically as present when a pepsinogen I/II ratio is less than 3 . The prevalence of ERD and NERD were analyzed in relation to age, gender, BMI, presence of hiatal hernia, HP status, alcohol intake, smoking, previous duodenal ulcer history or current showing ulcer scar, and serological atrophic parameters. RESULTS: One hundred and fourteen patients were diagnosed as having GERD based on either predominant symptoms of heart burn and acid regurgitation and/or findings of reflux esophagitis. Patients with concomittent gastrointestinal neoplasm (2), esophageal ulcer (1), clinical gastroduodenal ulcer (5), suspicious functional dyspepsia (2), IBS (1), and underlying psychiatric disorders (5) were excluded from the study. ERD was identified in $48(49 \%)$ patients (mean age: $52.1 \pm 13.2$ years) and NERD in $50(51 \%)$ patients (mean age: $46.5 \pm 13.2), \mathrm{p}=0.042$. HP infection was found in $30(76.9 \%)$ ERD patients, and 30 (88.2\%) NERD patients, $p=0.208$. Stepwise regression analysis identified the following independent predictors of ERD: male gender $(\mathrm{OR}, 11.6 ; \mathrm{p}=0.008)$, older age $(\mathrm{OR}, 1.1 ; \mathrm{p}=0.008)$. Serological atrophy were associated with an increased risk of NERD (OR, 2.4; $\mathrm{p}=0.004)$. CONCLUSION: Female sex, younger age, and gastric atrophy are associated with NERD. Chronic progressive gastritis may act as an important factor in patient with NERD and contribute to the esophageal hypersensitivity to acid. It remains to be seen whether HP eradication will be helpful or not in patient with NERD.
T1941

Characteristics of the Esophageal Pressure Trough in Patients with Esophageal Symptoms. Studies Using High Resolution Manometry (HRM) Daniel Pohl, Heiko Fruehauf, Oliver Goetze, Monika A. Kwiatek, Werner Schwizer, Michael Fried, Radu Tutuian

Introduction: Human esophageal motility studies have described a physiologic pressure trough in the transition zone from striated to smooth muscle. Combined fluoroscopy and high resolution manometry (HRM) studies have been used to investigate bolus and pressure dynamics in this region but there are limited data on the clinical relevance of the esophageal low-pressure zone (LPZ). Aims: To investigate the relationship between esophageal symptoms and the size of the LPZ, the hypothesis being that patients with esophageal symptoms may have a larger LPZ. Methods: Esophageal symptoms (dysphagia, chest pain and heartburn/ regurgitation) were quantified using a validated questionnaire (Eraflux) and stratified according to their main symptom. We analyzed 32-channel HRM data obtained during water swallows in left lateral decubitus performed in patients with esophageal symptoms and asymptomatic individuals. The analyzer was blinded to diagnosis and symptoms. Using a two-dimensional spatiotemporal colour plot we identified the distal border of the upper esophageal sphincter, the proximal border of the lower esophageal sphincter and the LPZ. The LPZ was measured extending from the point where the amplitude of the proximal contraction wave declined below $30 \mathrm{mmHg}$ to the point where the amplitude of the distal contraction wave first reached above $30 \mathrm{mmHg}$. Patients with achalasia were not included in the analysis. Results: Data from 101 patients (53f, mean age 48, range 16-80) were available for analysis. The average $( \pm S E M)$ length of the $L P Z$ measured $5.4 \pm 0.5 \mathrm{~cm}$ in asymptomatic individuals $(n=20)$ and did not differ $(\mathrm{p}>0.05)$ from the LPZ measured in patients $(\mathrm{n}=44)$ with dysphagia $(6.3 \pm 0.6 \mathrm{~cm})$, in patients $(n=17)$ with chest pain $(6.7 \pm 0.9$ $\mathrm{cm})$ and patients $(\mathrm{n}=20)$ with heartburn/regurgitation $(6.1 \pm 1.0 \mathrm{~cm})$. These results did not change even when the length of the LPZ was calculated as percentage of the total esophageal length. The time gap between the proximal and distal contraction waves (i.e. "time-width" of the LPZ) in asymptomatic individuals $(2.0 \pm 0.2 \mathrm{sec})$ did not differ $(\mathrm{p}>0.05)$ from that measured in patients with dysphagia $(2.4 \pm 0.3 \mathrm{sec})$, chest pain $(2.4 \pm 0.4 \mathrm{sec})$ or heartburn/ regurgitation $(2.4 \pm 0.5 \mathrm{sec})$. Conclusion: Our study provides no evidence to support a relationship between esophageal symptoms and the size of the physiologic esophageal low pressure zone.

\section{T1942}

Esophageal Clearance Patterns in Normal Older Adults As Documented with Videofluoroscopic Esophagram

Janice Jou, Jason Radowsky, Stephanie Kays, Jackie Hind, Eric Gaumnitz, Joanne Robbins

Objective: Normal esophageal bolus transport in asymptomatic older adults has not been well defined. This study assesses the radiographic esophageal motility patterns seen in normal older adults using videofluoroscopic recording. Esophageal clearance characteristics were evaluated, including intraesophageal stasis (IES) and intraesophageal reflux (IER). IES was operationally defined as any portion of the barium bolus failing to pass through the lower esophageal sphincter after completion of the initial swallow. IER occurred when the barium bolus traveled cephalad during the initial swallow before crossing the lower esophageal sphincter without regard of the effect of subsequent dry swallows. Methods: Twenty-four healthy asymptomatic adults, six males and six females in each of two age groups, 45-64 and older than 65 were enrolled. All subjects underwent videofluoroscopic esophagram consisting of three $10 \mathrm{~mL}$ thin liquid boluses, three $10 \mathrm{~mL}$ semi-solid boluses, and one $13 \mathrm{~mm}$ pill. All bolus types were administered in the upright and prone positions except the pill, which was administered upright only. Peristaltic bolus transport was noted for each patient. Inefficiencies in esophageal clearance were quantified by a three-point numerical scale signifying the degree of IES. The presence of IER was also recorded. Results: IES was more frequent with semi-solid as compared with liquid regardless of position, which was statistically significant $(\mathrm{p}<.0001)$. The rate of IES with liquid and semi-solid were $16 \%$ and $63-64 \%$ respectively of all swallows. The rate of IER with liquid was $12-15 \%$ and with semi-solid was $2-3 \%$ in all swallows. The prevalence of IER in males compared with females trended toward significance, with males demonstrating a higher rate. Furthermore, older males appeared to have an increased frequency of IER as compared to younger males. There were no other differences revealed in reference to position, bolus consistency, age, or gender. Conclusion: Semi-solid boluses elicit more stasis than liquid boluses, suggesting that a variety of barium product consistencies simulating food-like viscosities need to be used in addition to traditional fluids to fully evaluate esophageal transport with esophagram. Further studies are required to confirm these findings with larger numbers of normals and elucidate the observation of IER in older males. Our future efforts will provide a normative database for comparison with dysphagic patients.

\section{T1943}

High Prevalence of Esophageal Involvement in Lichen Planus: A Study Using Magnification Chromoendoscopy

Rutger Quispel, Ofke S. van Boxel, Marijke R. Canninga, Marguerite E. Schipper, Vigfus Sigurdsson, Andre J. Smout, Melvin Samsom, Matthijs P. Schwartz

Lichen planus (LP) is a mucocutaneous disease which can also affect the esophagus. The premalignant potential of oral LP has since long been established, but recently the first three cases of squamous cancer in esophageal LP have been described. Aims of this study were: 1. to screen for the prevalence of esophageal involvement in a cohort of patients with orocutaneous LP, 2. to assess the presence of esophageal dysplasia using magnification chromoendoscopy (MCE). Methods: Twenty-two patients (8M/14F; mean age 56 years) with a well-established history of LP were recruited from the dermatology outpatient clinic and evaluated by upper endoscopy. Validated questionnaires were used to assess upper GI symptoms. Mucosal abnormalities were described according to size, aspect and location. MCE using indigo carmine focussed on mucosal surface irregularities and vascular pattern. Biopsies were taken randomly at three levels in the esophagus and from all focal abnormalities. 
Results: In 95\% (21/22) of patients endoscopic findings compatible with, but not diagnostic of, esophageal LP were found, including: a friable, coarse appearance of the proximal mucosa in $41 \%(9 / 22)$, yellowish and whitish papules in $73 \%(16 / 22)$. In 9 of these 22 patients (41\%), histopathology confirmed the presence of lichen planus with characteristic features, such as a lymphocytic infiltrate, subepithelial cleavage and Civatte bodies (i.e. necrotic keratinocytes). Histopathology in the other patients showed no abnormalities in $27 \%$ (6/ 22) and aspecific chronic inflammation in 32\% (7/22). Endoscopy in the 9 patients with biopsy-confirmed LP showed coarse mucosa in 5 and papules in 7 . In the entire group other endoscopic focal abnormalities were: linear mucosal breaks typical for reflux disease in 55\% (12/22), cylindric epithelium above the squamo-columnar junction in $14 \%(3 / 22)$ and a proximal patch of cylindric epithelium in $4.5 \%(1 / 22)$. No lesions suspect for dysplasia were detected by MCE. In concordance with $\mathrm{MCE}$ results, dysplasia was not found in any of the biopsy samples. None of the 9 patients with documented esophageal LP reported dysphagia, 1 had mild reflux symptoms. Conclusions: Screening endoscopy detects a high prevalence of esophageal involvement in asymptomatic patients known with orocutaneous lichen planus. True prevalence might even be higher than now described, because biopsy sampling error cannot be ruled out. The potential benefit of surveillance endoscopy in patients with lichen planus remains to be established, because in this cohort of patients dysplasia was not found.

\section{T1944}

What Is the Prevalence of Motility Abnormalities in Patients with Non Acid Reflux On High Dose Proton Pump Inhibitor Therapy? Toan T. Nguyen, Matthew Gideon, Philip O. Katz

Background: The etiology of refractory GERD symptoms on antisecretory therapy is debated. Multichannel intraluminal impedance/pH (MII-pH) has been advocated as a means of determining if reflux (acid or non acid) is temporally associated with these continued symptoms. The prevalence of esophageal motility abnormalities in these patients and the potential association with symptoms is not clear. Aim: To determine the prevalence of motility abnormalities in GERD symptoms refractory to PPI and if these findings differ between patients with positive and negative symptom indices (SI) to reflux episodes determined by MII-pH. Patients and Methods: A retrospective analysis of patients referred for persistent reflux symptoms despite high dose PPI therapy between 2003-2005. Inclusion criteria: having had both ambulatory MII-pH and motility/manometry tests within a l-week period. MII-pH monitoring was performed with a 6-impedance electrode/2-pH electrode catheter (Sleuth; Sandhill Scientific). Reflux episodes categorized as acid ( $\mathrm{pH}<4$ ) or non-acid ( $\mathrm{pH}>4$ ). Positive SI defined as $\geq 50 \%$ positive correlation to reflux episode. Motility assessed using a combined manometry/impedance catheter (Sandhill Scientific). Liquid and viscous bolus transit was recorded. Motility abnormalities defined using standard manometric criteria. Results: 64 patients met inclusion criteria. $42 \%$ patients had positive SI. 58\% patients had negative SI. The following manometric findings were noted in order of prevalence: $28 \%$ Ineffective esophageal motility (IEM), 19\% incomplete LES relaxation, 16\% hypotensive LES, 14\% hypertensive UES, $9 \%$ nutcracker esophagus, $8 \%$ double high pressure zone, $5 \%$ hypertensive LES, 3\% incomplete UES relaxation, 3\% distal esophageal spasm, and 2\% hypotensive UES. There was a trend towards more IEM and impaired liquid bolus transit in patients with positive SI although statistical significance was not reached. See table. Conclusion: The pathogenesis of symptoms in patients with non-acid reflux does not appear related to abnormal motility or bolus transit. Further studies are needed in this population.

\begin{tabular}{|c|c|c|c|}
\hline Finding & + SI & -SI & P value \\
\hline IEM & $11(41 \%)$ & $7(19 \%)$ & 0.09 \\
\hline Abnormal viscous transit & $8(30 \%)$ & $9(24 \%)$ & 0.58 \\
\hline Abnormal liquid transit & $11(41 \%)$ & $7(19 \%)$ & 0.09 \\
\hline
\end{tabular}

\section{T1945}

Measurement of Esophageal Bolus Transit Is Affected By Bolus Size and Viscosity

Dawn Ferguson, Kenneth R. Devault, Ernest P. Bouras, Mark E. Stark, Sami R. Achem

Introduction: Multi-channel intraluminal impedance (MII) combined with esophageal manometry (EM) allows for simultaneous evaluation of esophageal pressure and bolus transit. Traditionally, $5 \mathrm{~mL}$ normal saline and viscous boluses have been used to assess transit. Varying volumes and viscosities of boluses have not been examined in regards to their performance in assessing esophageal dysfunction. Methods: We performed a chart review of patients undergoing combined EM and MII testing in our laboratory. Routine clinical procedure was followed for ten $5 \mathrm{~mL}$ normal saline swallows. An additional ten swallows of a viscous solution were given. The first five of these were $5 \mathrm{~mL}$ boluses followed by five $10 \mathrm{~mL}$ boluses. All swallows were given with the patient supine. The proportion of complete esophageal bolus transit was compared between the saline and viscous swallows. Esophageal bolus transit was considered abnormal if $30 \%$ or more of saline boluses had incomplete transit or if $40 \%$ of viscous swallows were incomplete. The total bolus transit time (TBTT) was compared between the saline boluses, the $5 \mathrm{~mL}$ viscous boluses and the $10 \mathrm{~mL}$ viscous boluses. The manometric findings were compared to the bolus transit results. Results: 40 patients underwent testing using this protocol. 93\% of the normal saline swallows resulted in complete transit compared to $83 \%$ of the viscous swallows $(\mathrm{p}<0.01)$. The $5 \mathrm{~mL}$ viscous bolus had the fastest mean transit time $(7.7 \mathrm{sec})$ compared to the $5 \mathrm{~mL}$ saline boluses $(8.4$ $\mathrm{sec}, \mathrm{p}<0.05)$ and the $10 \mathrm{~mL}$ viscous boluses $(8.9 \mathrm{sec}, \mathrm{p}<0.01)$. Table 1 outlines the comparisons of the EM and MII diagnoses. Conclusions: These results suggest that small volume $(5 \mathrm{~mL})$ viscous boluses transit the esophagus faster than similar volume saline boluses. The slowest transit is seen with larger $(10 \mathrm{~mL})$ viscous boluses. Abnormal viscous transit appears to be more predictive of a manometry disorder than normal saline transit.

Association with Bolus Characteristic and Esophageal Manometry

\begin{tabular}{|c|c|c|}
\hline EM Diagnosis & Abnormal Saline Bolus Transit & Abnormal Viscous Bolus Transit \\
\hline Normal $(\mathrm{n}=29)$ & $2 / 29(6.9 \%)^{*}$ & $4 / 29(13.8 \%)^{*}$ \\
\hline Ineffective Motility $(\mathrm{n}=5)$ & $0 / 5(0 \%)$ & $2 / 5(40 \%)$ \\
\hline Nutcracker Esophagus & $0 / 4(0 \%)$ & $1 / 4(25 \%)$ \\
\hline Diffuse Spasm & $0 / 2(0 \%)$ & $1 / 2(50 \%)$ \\
\hline
\end{tabular}

The viscous bolus transit was normal in all patients with abnormal saline bolus transit and the saline bolus transit was normal in all patients with abnormal viscous transit.

\section{T1946}

\section{Esophageal Pressurization: Clinical and Manometric Aspects}

Brendan J. Boland, Christian G. Peyre, Cedric G. Bremner, Colleen B. Gaughan, Jeffrey A. Hagen, Christian Rizzetto, Steven R. Demeester, John C. Lipham, Tom R. Demeester

Introduction: The baseline pressure of the intrathoracic esophagus is negative. In some pathologic states this baseline pressure is increased. This phenomenon occurs in achalasi and in other conditions and is thought to reflect outflow resistance. We measured esophageal pressure and correlated the degree of pressurization with symptoms and other manometric findings. Methods: A database of all motility studies from 2001 to 2005 was queried for the finding of esophageal pressurization. Corresponding patient tracings were reviewed. Esophageal pressurization was defined as a sustained elevation of esophageal pressure above the gastric baseline not associated with vascular or other artifacts, or esophageal body contractions. Esophageal pressure was measured by averaging the pressure in two channels within the esophageal body relative to the gastric baseline at mid respiration. Clinical and demographic information was collected. Results: There were 146 patients with esophageal pressurization, 94 (64\%) with achalasia and 52 (36\%) with other abnormalities (hypertensive lower esophageal sphincter 15 , other motility disorders 13 , and failed fundoplications 10) The most common primary symptom was dysphagia 115 (79\%) followed by chest pain 12 (8\%). The mean esophageal pressure was $8.2 \mathrm{~mm} \mathrm{Hg}$ and higher in achalasia than other diagnoses $[10.2$ v $4.6(\mathrm{p}<0.0001)]$. Esophageal pressure correlated directly with LES pressure $\mathrm{r}=0.5[\mathrm{CI} 0.36$ to $0.61,(\mathrm{p}<0.0001)]$ and LES residual pressure $\mathrm{r}=0.43[\mathrm{CI} 0.25$ to 0.58 $(\mathrm{p}<0.0001)]$. There was no association between esophageal pressure and findings on barium esophagogram. Conclusion: Patients with esophageal pressurization have disorders associated with outflow resistance such as achalasia, hypertensive LES, and failed fundoplication. This observation is confirmed by the correlation between esophageal pressure, LES residual pressure and LES pressure.

\section{T1947}

Distinct Allergic Predisposition of Children and Adults with Eosinophilic Esophagitis

Nirmala Gonsalves, Thuy Anh, Qing Zhang, Amir Kagalwalla, Anne Ditto, Ikuo Hirano

Background: Eosinophilic Esophagitis (EE) is increasingly recognized as an important entity in both children and adults. While the etiology of this illness is a topic of ongoing investigation, previous studies have suggested an association with both food and environmental allergens. The goal of this study was to compare allergic trends between children and adults with EE. Methods: We conducted a retrospective chart review of pediatric and adult patients (pts) with a confirmed histologic diagnosis of EE seen between 1999 and 2005. Demographic and allergy testing data were recorded. Results are presented as the mean \pm standard deviation. Results: Charts from 234 patients with EE were reviewed including 114 adults (age $39 \pm$ $13 \mathrm{yrs}$ ) and 120 children (age $4.7 \pm 4.5 \mathrm{yrs}$ ). The duration of symptoms prior to the diagnosis of EE was $6.7+6.2 \mathrm{yrs}$ in adults and $1.2+1.5 \mathrm{yrs}$ in children. There was a male predominance of $75 \%$ in both groups. 101 patients including 83 children and 18 adults underwent formal allergy testing with skin prick testing. Of those tested, $72 \%$ of children and $100 \%$ of adults were found to have documented allergies. The most common food allergens were milk, egg wheat, soy, peanut, and seafood. Other allergens were recorded if positive. Children were significantly more likely to have egg allergies $(\mathrm{p}<0.005)$ while adults were more likely to have nut allergies $(p<0.005)$. A significant difference was demonstrated in the following allergens based on age: milk $(p<0.005)$, egg $(p<0.001)$, and wheat $(p<0.005)$ with younger patients more likely to have these allergens. Having an allergy to one food allergen increased the likelihood of having additional food allergies. Environmental allergens were not routinely tested in children. The most common environmental allergens in adults were ragweed (81\%), tree $(75 \%)$, grass $(81 \%)$ and mite $(75 \%)$. Conclusions: (1) Allergies documented by skin prick testing were present in $72 \%$ of children and $100 \%$ of adults in our EE cohort (2) Younger patients were more likely to be allergic to milk, egg, and wheat, while adults were more likely to be allergic to nuts. (3) Environmental allergens were found in $83 \%$ of adults while food allergens were present in $61 \%$. (4) The role of formal allergy testing for patients with $\mathrm{EE}$ is still being defined.

\section{T1948}

Assessment of Esophageal Emptying in Achalasia Patients By Intraluminal Impedance Monitoring

Jose M. Conchillo, Mohamed Selimah, Albert J. Bredenoord, Melvin Samsom, Andre J. Smout

Background and aim: In achalasia patients, the timed barium esophagram is an objective test for the assessment of esophageal emptying but its use implies exposure to ionizing radiation. Intraluminal impedance monitoring can accurately determine the timing of esophageal filling and emptying in healthy subjects without radiation. Aim of this study is to evaluate the suitability of impedance monitoring for the assessment of esophageal emptying in achalasia patients. Methods: 10 achalasia patients ( 6 men, mean age 44 years, range 2064) underwent simultaneous fluoroscopy and impedance recording during the esophageal clearance of a 100-cc barium swallow. Impedance monitoring was recorded for $15 \mathrm{~min}$ and 
a fluoroscopic image was recorded every $20 \mathrm{~s}$. Fluoroscopic images were scored for bolus clearance time (BCT), defined as the time when the barium column passed the impedance channel at $1 \mathrm{~cm}$ above the LES. In addition, the height of the barium column level was analyzed at 1, 5, 10 and $15 \mathrm{~min}$. Impedance tracings were scored independently by 3 observers. Results: All patients showed a low baseline impedance level in the distal esophagus suggesting stasis of fluids. Three patients had at least one period of regurgitation of fluids and in 9 patients pathological air movement (air trapping) within the proximal esophagus was detected. On fluoroscopy, 8 patients showed a BCT longer than $15 \mathrm{~min}$. On impedance monitoring, the percentage of patients having a BCT longer than $15 \mathrm{~min}$ as scored by the 3 observers was 50,40 and $20 \%$, respectively (mean $37 \%$ ). Two observers scored BCT on impedance monitoring similarly $( \pm 1 \mathrm{~min}$ ) to the time on fluoroscopy in $70 \%$ of the patients while the third observer found a similar BCT in $40 \%$ of the patients. Correlations between height of barium on fluoroscopy and fluid level on impedance monitoring were poor at 1 and $5 \mathrm{~min}(\mathrm{r}=0.53, \mathrm{p}=0.11$ and $\mathrm{r}=0.42, \mathrm{p}=0.22)$ and moderate at 10 and $15 \mathrm{~min}(\mathrm{r}=0.65$, $\mathrm{p}=0.04$ and $\mathrm{r}=0.63, \mathrm{p}=0.05$ ). Kendall's coefficient of concordance between the 3 observers for assessment of fluid level on impedance monitoring was $0.31(\mathrm{p}=0.04)$ at 1 and $5 \mathrm{~min}$, $0.26(\mathrm{p}=0.08)$ at $10 \mathrm{~min}$ and $0.44(\mathrm{p}=0.01)$ at $15 \mathrm{~min}$. Conclusion: In achalasia patients pathological bolus transport patterns such as low baseline impedance and air entrapment in the proximal esophagus make impedance monitoring unsuitable for the assessment of esophageal emptying.

\section{T1949}

Validation of Platelet Count/ Spleen Diameter Ratio As a Noninvasive Method to Screen for Prescence of Varices in Cirrhotic Patients Trinh Meyer, Vidushi Golla, Helene N. Pena Sahdala, David J. Clain, Henry C Bodenheimer, Albert D. Mi

Esophageal varices are a common complication of cirrhosis. Cirrhotic patients are routinely screened for varices with upper endoscopy. A recent study reported $100 \%$ negative predictive value (NPV) of platelet count/ spleen diameter (PC/SD) ratio using a cut-off value of 909 for presence of esophageal varices (Gut 2003;52:1200). The aim of our study was to validate and asses PC/SD as a noninvasive predictor of varices in patients with cirrhosis. Method: Records of all patients age $\geq 18$ years with cirrhosis with and without esophageal and/or gastric varices on upper endoscopy between January 2003 and October 2005 at Beth Israel Medical Center, NY were reviewed. The data collected were age, gender, etiology of cirrhosis, spleen diameter on CT/MRI scan, INR, total bilirubin, platelet count, albumin, Child-Pugh score and endoscopic findings. Data were analyzed using t-test, chi-square and Fisher's exact test. Results: There were 137 patients, and 87 (63.5\%) were men. Seventy-six (55\%) patients had esophageal and/or gastric varices. The mean age, gender and etiology of cirrhosis were similar between the two groups (see Table). Patients with varices were more likely to have lower platelet count, splenomegaly, ascites, lower albumin, and higher Child-Pugh score. Using a PC/SD ratio with a cut-off value of 909, as previously reported, yielded a NPV of only $53.7 \%$. However, a PC/SD ratio with a cut-off of 200 yielded a NPV of $97 \%$. In contrast, using a platelet value of $>50,000$ alone, the NPV was $89 \%$, although the positive predictive value for presence of varices was only $11 \%$. In addition, using a spleen diameter value of $>14 \mathrm{~cm}$ alone, the NPV was 52\% for presence of varices. Conclusions: Lower platelet count, splenomegaly, ascites, lower albumin, and higher Child-Pugh score were positive predictors of varices. In our study, a platelet count/ spleen diameter ratio with a cut-off value of 909 as an independent variable had poor NPV for varices. Upper endoscopy remains the best method to screen for presence of varices in cirrhotic patients.

\begin{tabular}{|c|c|c|c|}
\hline Variables & NO VARICES $\mathrm{n}=61$ & VARICES $\mathrm{n}=76$ & $\mathrm{p}$ Value \\
\hline Mean age & $55(32-80)$ & $60(32-84)$ & 0.347 \\
\hline Male & $34(56 \%)$ & $53(70 \%)$ & 0.109 \\
\hline Ascites & $13(21 \%)$ & $44(58 \%)$ & $<0.0001$ \\
\hline Spleen Diameter $(\mathrm{mm})$ & $118(80-200)$ & $146(80-240)$ & $<0.0001$ \\
\hline Platelet & $138,442(1,000-43,500)$ & $103,605(35,000-430,000)$ & $<0.0001$ \\
\hline Albumin & $3.7(0-5)$ & $2.9(2-6)$ & $<0.0001$ \\
\hline Total Bilirubin & $2.4(1-30)$ & $3.9(1-49)$ & 0.135 \\
\hline
\end{tabular}

T1950

Endoscopic Findings in Eosinophilic Esophagitis (EE): A Morphologic Clasification According to Manometric and Histopathologic Relationship Alfredo J. Lucendo, Marta Navarro, Sonia Martin-Chavarri, Ramon Pajares, Juan Manuel Pascual, Emilio Burgos, Pilar Castillo, Javier Larrauri

Background: EE is an inflamatory disease with increasing incidence and immunoallergic etiology. It is defined by dense eosinophilic infiltration of the esophageal mucosa, descarting infiltration of another sections of the alimentary tract. Eosinophilic infiltration produces esophageal motility disturbances observed in manometry. Great variety of endoscopic findings have been described in EE, judging by this as a polimorfic disease. Objective: To analize endoscopic esophageal findings and to relate them with histopathologic and manometric findings. Define a EE clasiffication in endoscopic patterns. Patients \& Methods: Endoscopic appearance of esophagus was described and recorded in a prospective cohort of 25 patients with EE. Esophageal stationary manometry were achieved in all patients. Results: 8 patients showed normal esophageal diameter, 6 concentric stenosis. 11 had simultaneous contraction rings along the organ's lumen. Main mucosal findings: 4 patients showed multiple papules along the organ's wall (granular aspect). 8 presented longitudinal mucosal furrows in all the length of the organ 2 patients showed undulated smooth mucosa and 5 had white exudative lession of different size. 22 patients showed disturbances in esophageal manometry affecting the 2 lower thirds: 14 of them had a severe non-especific motor disorder with hypoperistalsis and low amplitude simultaneous waves. 8 presented great amplitude peristaltic waves. In 3 patients was normal. All histopathological exams showed the same type of lession characterized by epithelial edema with increasing of intercelular spaces, acanthosis, papillomatosis and basal cell hyperplasia, with outstanding inflamatory infiltration by eosinophils. Severity of this changes was correlated with different endoscopic patterns. Discussion: 1) Endoscopic appearance of EE is defined by two tipes of independent finding: Alterations in esophageal calibre, and lessions or disturbans in the organ's mucosa. The first is manifested like regular concentric estenosis preventting to observe distal lumen, or simultaneous contraction rings along the length of the organ. A normal esophageal diameter can also be observed, with independence of mucosal apearance. This alterations are variable for a same patien along the time in abscence of specific treatment, and don't respond to organic lessions. Mucosal lessions in our patients could be resumed in four well-deffined patterns: a) Granular type mucosa. b) Longitudinal forrows along organ length. c) Circular undulated mucosa D) Exudative (white spots or exudates). 2) We describe a correlation beetwen endoscopic mucosal pattern and severity of histopathologic changes.

\section{T1951}

An Essential Amino Acid, Isoleucine, Prevents Liver Metastases of Colon Cancer By Antiangiogenesis

Kazumoto Murata, Masami Moriyama

Background: In spite of recent advances in the treatment of colon cancer, multiple liver metastases are still difficult to treat. Antimicrobial peptides such as defensins play an important role in the innate immunity for all lives, including plants or insects that lack immune systems. $\beta$-defensins are strong chemotactic factors for memory $\mathrm{T}$ cells and dendritic cells, suggesting that they play an important role in the acquired immunity as well. On the other hand, $\beta$-defensins are inducible by an essential aminoacid, isoleucine. Therefore, we hypothesized that isoleucine may contribute to tumor immunity. Methods: Male BALB/c or nude mice $(8-10 \mathrm{w}$ ) were administered isoleucine, leucine ( $3 \mathrm{mg} /$ day) or PBS 1 week before implantation of colon 26 cells to the spleen until sacrifice on the 21 st POD. After checking liver metastasis, livers and spleens were taken for RT-PCR (VEGF: vascular endothelial growth factor), western blot ( $\beta$-defensin-3, p-4EBP-1, p-stat 3, HIFl- $\alpha$ ) and immunohistochemistry Blood samples were taken for serum albumin. Colon 26 cells were cultured with isoleucine, leucine or medium alone and subjected to RT-PCR or western blot and the culture medium was subjected to ELISA for VEGF. These cells were also used for MTT assay to check cytotoxicity by aminoacids. Results: Isoleucine $(\mathrm{n}=5)$ inhibited liver metastasis in a dose dependent manner whereas no inhibition was observed by leucine $(n=5)$ or PBS $(n=5)$. Serum albumin level was not impaired in the isoleucine-treated mice whereas significant reduction was observed in the leucine- and the PBS-treated mice. However, $\beta$-defensin in the liver was not induced even in the isoleucine-treated mice without any metastasis against our hypothesis. Furthermore, isoleucine completely inhibited liver metastasis even in nude mice $(n=5)$, suggesting immune responses did not contribute to this mechanism. VEGF in the culture medium of colon 26 cells was inhibited by isoleucine in a dose dependent manner without affecting cell viability. Isoleucine inhibited VEGF mRNA expression in both in vitro and in vivo experiment. In the liver of the isoleucine-treated mice, the expression of p-4EBP-1 and p-stat 3 were mildly down-regulated in comparison with the leucine-treated mice. The expression of HIFl- $\alpha$ was inversely correlated with expression levels of those. Immunohistologic CD31 staining in the isoleucine-treated mice showed a marked decrease in the number of blood vessels in comparison with the leucine-treated mice. Conclusion: Isoleucine inhibits angiogenecity by impairment of VEGF expression partially through mTOR pathway. Isoleucine could be a safe medicine for prophylactic use for liver metastasis of colon cancer.

\section{T1952}

A Novel Synthetic Diphenyl, Difluoroketone (L-2395) Compound with Potent in Vitro and in Vivo Anti-Cancer Activity

Dharmalingam Subramaniam, Sripathi M. Sureban, Robert George, Rama P. Ramanujam, Hideg Kalman, Periannan Kuppusamy, Courtney W. Houchen, Brian K. Dieckgraefe, Shrikant Anant

Curcumin is an anti cancer chemotherapeutic and chemopreventive agent. We have previously demonstrated that curcumin inhibits EGF mediated signaling and inhibits intestinal adenomas in $\mathrm{APC}^{\mathrm{min} /+}$ Mice. Using the structure of curcumin, we have now developed a new water-soluble molecule, diphenyl difluoroketone (L-2395, also known as EF24) and tested it against cancer cells. L-2395 demonstrated a dose-dependent inhibition of proliferation and induction of apoptosis of a variety of adenocarconoma cells. This activity was significantly higher and at lower doses than that observed with curcumin. In addition, L2395 induced G2/M cell cycle arrest, which was followed by the induction of apoptosis as evidenced by caspase- 3 activation and an increased number of cells with a sub-G1 DNA fraction. Significant inhibition in the number and size of colonies were also observed. On the other hand, the compound did not affect the proliferation of normal mouse embryo fibroblasts. L-2395 significantly suppressed epidermal growth factor mediated induction of AKT and ERK phosphorylation. Real Time PCR analyses were performed to determine the effect of L-2395 on gene expression. L-2395 significantly inhibited the expression of cyclooxygenase-2 (COX-2), interleukin-8 (IL-8) and vascular endothelial growth factor (VEGF). Microarray analyses demonstrated increased expression of TNF-receptor II coupled with induction of BAX, caspase- 6 and -9 at 24 h, while inducing BAX, caspase- 7 and -9 at $48 \mathrm{~h}$. In addition, there was significant induction of $\mathrm{p} 73$, a p53-related nuclear transcription factor within $24 \mathrm{~h}$. The effect of L-2395 on growth of tumors in vivo was determined using HCT-116 colon cancer tumor xenografts in nude mice. Xenograft tumors were established by injecting a single-cell suspension of $>95 \%$ viable HCT-116 cells implanted subcutaneously into the backs of Balb/c athymic nude mice. Formation and size of the tumors were monitored daily until day 30. L-2395 was injected intraperitoneally from day 7 onwards. L-2395 inhibited tumor growth in ectopic xenografts, with notably lower tumor volume and weight. The microvessel density was also significantly lower in the tumors following L-2395 treatment than in control tumors. Real Time PCR analyses demonstrated significant inhibition of COX2 and IL- 8 mRNAs in the tumors. Our results indicate that the antitumor effect of L-2395 in colon cancer cells is shown at least in part through TNF-receptor II-mediated activation of caspases and BAX genes, resulting in tumor growth inhibition with apoptosis induction. 


\section{T1953}

Inhibition of the Nuclear Transcription Factor PPAR $\gamma$ Prevents Growth of Colorectal Cancer By Directly Reducing Cellular Tubulin Concentration Katherine Schaefer, Hirokazu Takahashi, Victor Morales, Atsushi Nakajima, Lawrence J. Saubermann

Background: Colorectal cancer (CRC) is the third-most commonly diagnosed cancer worldwide, and adjuvant therapies are urgently needed for advanced disease. The nuclear transcription factor PPARy has been explored as a potential therapeutic target for a variety of cancers, and the thiazolidenedione PPAR $\gamma$ activating ligands have been shown to cause growth arrest and apoptosis in a number of cancer cell lines. However, despite these encouraging results, the effects of PPAR $\gamma$ activation in clinical trials have been extremely modest, with no beneficial effect seen in metastatic CRC. More recent results in animal models of CRC suggest that, although PPARy activation is important in preventing the development of CRC, it may in fact be harmful after CRC is established. We therefore investigated the effects of PPARy inhibition on CRC. Methods: The human CRC cell lines HT-29, Caco-2, and LoVo were treated with the PPAR $\overline{\text { r }}$ inhibitors T0070907, GW9662, and BADGE or PPAR $\gamma$-specific siRNA. The effects on apoptosis, cell cycle and tubulin function were investigated using flow cytometry, confocal microscopy, Western blot, and in vitro tubulin polymerization assays. The ability of T0070907 to inhibit tumor formation in a HT-29/SCID xenograft metastatic cancer model was also measured. Results: PPAR $\gamma$ inhibitors induce dual Gl and M cell cycle arrest and apoptosis in CRC cell lines, with an ED50 of $50 \mu \mathrm{M}$ for T0070907 and GW9662 and $100 \mu \mathrm{M}$ for BADGE. Before commitment to apoptosis and independent of cell cycle, PPAR $\gamma$ inhibitors disrupt epithelial morphology and microtubule networks. PPAR $\gamma$-specific siRNA has the same effect on the cytoskeleton as do the inhibitors. PPAR $\gamma$ inhibitors do not interfere with the polymerization of tubulin monomer into microtubules, but instead directly reduce the cellular concentration of both $\alpha$ and $\beta$ tubulin monomer proteins. This regulation occurs post-transcriptionally, as tubulin RNA levels are unaffected by PPAR $\gamma$ inhibitors. Consistent with these results, 1 and $5 \mathrm{mg} /$ $\mathrm{kg} /$ day T0070907 given daily starting the day after HT-29 cells were injected into the spleen reduced both the number of metastatic colonies in the liver (to 37 and 29\% of control, respectively), and the total tumor volume (to 23 and $12 \%$ of control, respectively). Conclusions: PPAR $\gamma$ inhibition can induce apoptosis of colorectal cancer cells through its effects on cytoskeletal regulation and reduce the number and volume of metastases in an animal model of CRC. These results suggest that PPARy inhibition should be considered as a therapeutic target in CRC.

\section{T1954}

Sulindac Independently Modulates ERK1/2 and PKG Pathways Stevany Peters, D. Rayburn Moore, K. Scott Beard, Dennis Ahnen, Pamela Rice

Colorectal cancer (CRC) is the 2nd leading cause of cancer mortality in the US. Substantial human and animal data support the ability of nonsteroidal anti-inflammatory drugs (NSAIDs) to cause regression of existing colon tumors, and prevent new tumor formation. Despite these chemoprotective effects, chronic use of NSAIDs is limited by GI toxicity. The mechanism by which the NSAID sulindac exerts its growth inhibitory effects is poorly understood, as sulindac modulates several growth-related pathways. We have described the ability of sulindac to inhibit the ERK1/2 mitogen-activated protein kinase cascade at times and doses consistent with the induction of apoptosis. Expression of a constitutively active MEKI gene prevents ERKl/2 inhibition and apoptosis induced by sulindac. Treatment with sulindac also leads to activation of protein kinase $\mathrm{G}(\mathrm{PKG}$ ) and phosphorylation of JNK. Expression of a dominant negative JNKl gene prevents apoptosis and JNK activation by sulindac. The purpose of this study was to determine if sulindac modulates ERK1/2 and PKG/JNK signaling pathways independently in CRC cells. Parental HT29 CRC cells, or stable HT29 clones expressing empty vector ( $\mathrm{PCEP}$ ) or constitutively active MEKl (R4F) were treated with sulindac metabolites, U0126 (MEKl/2 inhibitor), or YC-1 (activator of PKG). Sulindac treatment resulted in 1) inhibition of EGFR, HER2, and ERK1/2 phosphorylation, 2) activation of JNK and AKT phosphorylation, and 3) inhibition of beta-catenin protein expression. HT29-R4F clones were resistant to ERK1/2 inhibition and apoptosis induced by sulindac. However, inhibition of EGFR and HER2, activation of JNK and AKT, and inhibition of betacatenin expression occurred equivalently in drug treated HT29-pCEP and HT29-R4F clones. Inhibition of ERK1/2 with U0126 induces apoptosis and Gl arrest of CRC cells, but does not affect JNK activation. Activation of PKG with YC-1 leads to JNK phosphorylation, increases apoptosis, and causes S-phase arrest of CRC cells without modulating ERK1/2. Simultaneous treatment with U0126 and YC-1 produces synergistic apoptosis, and recapitulates the biochemical effects of sulindac treatment - namely inhibition of ERK1/2, activation of JNK and AKT, and decreased beta-catenin protein expression. These results indicate that sulindac modulates ERK1/2 and PKG pathways independently in CRC cells. Co-treatment with U0126 and YC-1 produces synergistic anti-tumor effects in vitro. Utilizing similar approaches in vivo may provide more effective, less toxic chemotherapeutic strategies against CRC. Such therapies could dramatically reduce the incidence and mortality from CRC

\section{T1955}

Violacein Synergistically Increases 5-Fluorouracil Cytotoxicity, Induces Apoptosis and Inhibits AKT-Mediated Signal Transduction in Human Colorectal Cancer Cells

Liudmila L. Kodach, Carina L. Bos, Nelson Duran, Maikel P. Peppelenbosch, Carmen V. Ferreira, James C. Hardwick

Despite recent additions to the armory of chemotherapeutic agents for colorectal cancer treatment, the results of chemotherapy remain unsatisfactory. 5-fluorouracil (5-FU) still represents the cornerstone of treatment and resistance to its actions is a major obstacle to successful chemotherapy. Therefore new active agents in colorectal cancer and agents that increase the chemosensitivity of cancer cells to 5-FU are still urgently required. Violacein, a pigment isolated from Chromobacterium violaceum in the Amazon river, has a diverse spectrum of biological activities, and represents a novel cytotoxic drug with known antileukemic properties. To assess the suitability of violacein as a chemotherapeutic agent in colorectal cancer its cytotoxic effects were evaluated both as a single agent and in combination with 5-FU. Its underlying mechanisms of action were further investigated by studying its effects on the cell cycle, apoptosis and cell survival pathways (PI3 kinase/Akt, p44/42 MAP kinase and nuclear factor kappa-B) in colon cancer cell lines. Violacein inhibits the growth of all four colon cancer cell lines tested. It induces apoptosis, and potentiates the cytotoxic effect of 5-FU in a poorly differentiated microsatellite unstable cell line (HCT116). Violacein causes cell cycle block at G1, upregulates p53, p27 and p21 levels and decreases the expression of cyclin-D1. Violacein leads to dephosphorylation of $\mathrm{Rb}$ and activation of caspase and a pancaspase inhibitor abrogates its biological activity. Our data provide evidence that violacein acts through the inhibition of Akt phosphorylation with subsequent activation of the apoptotic pathway and downregulation of NFkB signaling. This leads to the increase of chemosensitivity to 5-FU in HCT116 colon cancer cells. Taken together, our findings sugges that violacein will be active in the treatment of colorectal tumors and offers new prospects for overcoming 5-FU resistance.

\section{T1956}

Sulindac Inhibits Colon Tumor Cell Proliferation in Xenograft Mice Matthew Quallick, Matthew Sericati, Nathan Susnow, Linda Driggers, Dennis Ahnen, Pamela Rice

Regular use of nonsteroidal anti-inflammatory drugs (NSAIDs) reduces the risk of colorectal cancer (CRC) development. The NSAID, sulindac, prevents development of human adenomatous polyps and inhibits colon tumor formation in animal models. The mechanisms by which sulindac prevents colon tumor growth in vivo are complex and poorly understood. Inhibition of COX-1 and -2 , the classical targets of NSAIDs, does not adequately explain sulindac's growth inhibitory effects in vitro or in vivo. Several COX-independent mechanisms have been described for sulindac, including the extracellular signal-regulated kinase (ERK1/ 2) pathway. Sulindac metabolites inhibit ERKl/2 activation, and this inhibition is both necessary and sufficient for apoptosis of human colon cancer cells in vitro. The goal of this study was to determine if sulindac treatment inhibits ERK1/2 activity and stimulates apoptotic cell death in vivo, using the xenograft mouse model. Athymic mice were injected s.c. with HT29 human CRC cells and tumors allowed to grow to a palpable size. Mice were then placed on diets containing 0 or $320 \mathrm{ppm}$ sulindac for 15 days. Tumors were measured with calipers, harvested and prepared for immunohistochemistry using KI67 (a marker o proliferation), cleaved caspase-3 (a marker of apoptosis) and phospho-ERK1/2 antibodies Sulindac treatment inhibited tumor growth, and this inhibition was associated with a significant decrease in Ki67 staining. Sulindac-induced growth inhibition was also associated with increased cleaved caspase- 3 staining and decreased phospho-ERK1/2 staining, however these results were not statistically significant. Together these results suggest that inhibition of tumor cell proliferation - and not induction of apoptosis - is the biological mechanism by which sulindac prevents tumor formation in vivo.

\section{$\mathrm{T} 1957$}

Mechanism for Enhanced Anti-Tumor Effects of 5-Fluorouracil (5FU) By Celecoxib in An Advanced Colon Cancer Model: Angiogenesis, Or 5FU Metabolisms?

Takanobu Irie, Masahiko Tsujii, Shingo Tsuji, Shuji Ishii, Toshiyuki Yoshio, Shinichiro Shinzaki, Satoshi Egawa, Tsutomu Nishida, Yoshimi Kakiuchi, Masakazu Yasumaru, Hideki Iijima, Hiroaki Murata, Sunao Kawano, Norio Hayashi

Background \& Aims: Many reports have suggested that COX-2 inhibitors are effective chemopreventive agents against colorectal cancers. However, effect of COX-2 inhibito combined with chemotherapy for the treatment of advanced colon cancer remains obscure. Therefore, we examined efficacy of combination therapy of COX-2 inhibitor and chemotherapy for colon cancer cells, in vitro and in vivo, and whether COX-2 inhibitor influence metabolism of 5FU. Methods: (1) in vivo assay: Male BALB/c mice were inoculated subcutaneously with 1 x104 cells of colon 26 cells, a murine colon cancer cell line. When tumor allografts reached more than $5 \mathrm{~mm}$ in diameter, mice were randomized and given daily with either $5 \mathrm{FU}$ alone $(20 \mathrm{mg} / \mathrm{kg}$, i.p.), celecoxib alone $(3 \mathrm{mg} / \mathrm{kg}$, i.p.), or $5 \mathrm{FU}$ and celecoxib Tumor size was measured every 7 days. After 28 days, tumors were harvested and used for analyzing angiogenesis (expression of CD31) by immunohistochemistry and for measuring VEGF concentration by ELISA. (2) in vitro cytotoxicity: Colon 26, which is expressing COX2 , was used. The cells were cultured for 3 days with or without 5-FU, celecoxib and/or SN38. Cell survival was evaluated by WST assay. (3) Influence on 5FU metabolism: Colon 26 cells were cultured for 24 hours with or without $5 \mathrm{FU}$ and/or celecoxib. Cells were harvested and enzyme activity of thymidylate synthase (TS), intracellular 5FU and FdUMP (5-fluoro2'-deoxyuridine 5'-monophosphate) concentration and 5FU incorporate into RNA were measured. Expression of dihydropyrimidine dehydrogenase (DPD; catabolic enzyme of 5FU) mRNA was analyzed by RT-PCR. Results: (1) $5 F U(20 \mathrm{mg} / \mathrm{kg})$ and celecoxib $(3 \mathrm{mg} / \mathrm{kg})$ treatment significantly inhibited the growth of colon 26 allograft compared with control or other groups. 5FU/celecoxib treatment significantly reduced VEGF production and suppressed angiogenesis compared with $5 \mathrm{FU}$ alone. (2) Tumoricidal effect of $5 \mathrm{FU}(4 \mu \mathrm{M})$ combined with celecoxib $(10 \mu \mathrm{M})$ was significantly lower than $5 \mathrm{FU}$ alone, but it was recovered by adding SN-38. (3) Intracellular concentration of 5FU and FdUMP and incorporation of 5FU into RNA were lower, and TS activity and DPD mRNA expression were higher in 5FU/ celecoxib treatment group than $5 \mathrm{FU}$ alone. Conclusions: These results suggest that celecoxib enhances antitumor effect of 5FU via suppressing angiogenesis, not via enhancing intracellular metabolism of 5FU. Thus, COX-2 inhibitor, in combination with certain chemotherapeutic

agents, appears to have therapeutic value in patients with advanced colon cancer. 


\section{$\mathrm{T} 1958$}

The Nucleoside Analog ARC Induces Apoptosis in GI Tumor Cells Senthil K. Radhakrishnan, Andrei L. Gartel

Using a p21 promoter-lacZ reporter construct in LIM1215 colon cancer cells, we screened a library of structurally diverse compounds obtained from the National Cancer Institute for small molecule, transcriptional inhibitor of p21. We identified a nucleoside analog ARC (4amino-6-hydrazino-7 beta-D-ribofuranosyl-7H-Pyrrolo[2,3-d]-pyrimidine-5-carboxamide), which has properties of a general transcriptional inhibitor. Specifically, ARC inhibits phosphorylation of RNA polymerase II by PTEF-b (positive transcription elongation factor $\mathrm{b}$ ) leading to a block in transcriptional elongation. ARC was able to potently repress p53 targets p 21 and hdm2 protein levels, but dramatically increased p53 levels similar to other transcriptional inhibitors, including flavopiridol. This increase in p53 corresponded to the downregulation of short-lived protein hdm2, which is a negative regulator of p53. Furthermore, we found that ARC induces apoptosis in LIM1215 colon cancer, AGS gastric cancer and HepG2 liver cancer cells, where we detected cleavage of caspase-3 after ARC treatment. Interestingly, HepG2 cell line with stable knockdown of p53 (HepG2-p53si) also showed cleavage of caspase- 3 confirming that ARC-mediated apoptosis in these cells is $\mathrm{p} 53$-independent. Although ARC promoted accumulation of p53, ARC-induced apoptosis in tumor cells was p53-independent, suggesting that it may be useful for treatment of tumors with functionally inactive p53. In order to evaluate if ARC induces apoptosis in colon cancer cells comparable to that of the other known nucleoside analog DRB, we treated LIM1215 cells with either ARC or DRB for 48 hrs. We found that $5 \mu \mathrm{M}$ of ARC treatment resulted in $37.8 \%$ apoptosis, while a $50 \mu \mathrm{M}$ of DRB treatment only showed $23.4 \%$ apoptosis suggesting that ARC is much more efficient in eliciting apoptosis than DRB in LIM1215 colon cancer cells. Furthermore, cell death induced by ARC had a strong correlation with downregulation of anti-apoptotic gene survivin, which is often overexpressed in human tumors. To test how ARC may affect angiogenesis we used cord formation assay using human umbilical vein endothelial cells that were plated on a three dimensional layer of Matrigel, where they formed cord-like structures. They were treated with different concentrations of ARC for 24 hrs and cord formation was quantitated as a function of cord junctions and cord length. A dose dependent decrease was seen in response to ARC treatment in both junction and length with respective IC50 values of 344.87 and $382.3 \mathrm{nM}$, suggesting that ARC has an antiangiogenic activity. Taken together, our data suggest that ARC may be potentially useful drug against GI cancers.

\section{T1959}

The Tyrosine Kinase SRC Regulates Adhesion to the Substrate of Pancreatic Endocrine Cancer Cells

Alessia Di Florio, Gabriele Capurso, Massimo Milione, Francesco Panzuto, Raffaele Geremia, Claudio Sette, Gianfranco Delle Fave

In a recent PETs microarray screen we identified overexpression of the Src-like tyrosine kinase Lck. Src kinases belong to an oncogene family altered in several neoplastic cells and involved in the control of cell adhesion, proliferation and motility. The present study is aimed at testing the possibility that Src-like kinases are suitable therapeutic targets for PETs. We have tested the PET cell lines (CLs) CM and QGP1, for the expression of the Src-like kinases Lck, Fyn and Src by western blot (WB) and found Src to be the most aboundant isoform. Further WB with a phospho-Src ab revealed Src activation. We confirmed expression of Src in PETs in vivo by IHC on a series of 28 cases (18 primaries, 10 metastases) which were all positive. To test the role of Src, CLs were cultured in the presence or absence of the Src-kinase inhibitor PP2. Adhesion and initial spreading of cells on the culture plate was severely inhibited. WB analysis confirmed that Src activity was selectively inhibited in cells treated with PP2. Moreover, the effect of the inhibitor was specific for cell adhesion and initial spreading, while prolonged incubation showed that PP2 exerted no effect on cell cycle and/or apoptosis. In order to identify the molecular targets of Src in PET CLs, we examined the pattern of tyrosine phosphorylation during adhesion. We observed a transient increase of tyrosine phosphorylation in QGPl cells adhering to the plate, with a peak at 75 min after seeding, when approximately $75 \%$ of cells are attached. Treatment of cells with PP2 caused a dramatic reduction in the number of attached cells and abolished phosphorylation of candidate proteins with different molecular weight that we named pl80, p130, pl00, p70, $\mathrm{p} 55$ and $\mathrm{p} 40$. To determine if these proteins could be isolated from the cell extracts, we performed an immunoprecipitation experiment using an anti-phosphotyrosine antibody followed by WB analysis with the same antibody and demonstrated that all the above mentioned proteins could be immunoprecipitated from control but not from PP2-treated cells, indicating their phosphorylation is Src-dependent. A pull-down assay using a purified GST-SrcSH2 fusion protein showed that p180, p130, p70 and p55 can be specifically isolated by the GST-SrcSH2 pull-down but not by a control GST pull-down, indicating that they form a complex with Src upon phosphorylation. Identification of these Src substrates in QGPl cells by mass spectrometry and WB analysis of the immunoprecipitates is ongoing. Our results suggest that Src plays a specific role during adhesion in PET CLs and may be a therapeutic target able to limit their metastatic potential.

\section{T1960}

Final Results of a Randomized Comparison of Infusional 5-Fluorouracil/ Leucovorin Plus Oxaliplatin (FUFOX) Versus Capecitabine Plus Oxaliplatin (CAPOX) in Metastatic Colorectal Carcinoma (MCRC)

Rainer Porschen, S Kubicka, A Grothey, U Graeven, A Kretzschmar, R Greil, W Freier, I Seufferlein, Ht Arkenau, A Hinke, Hj Schmoll, W Schmiegel

Background: The combination of capecitabine and oxaliplatin has demonstrated good efficacy results in phase II studies. Therefore, we initiated a phase III trial comparing FUFOX and CAPOX as first line therapy in MCRC. Here, we present the final results of the efficacy and toxicity analysis. Patients and methods: 462 pts. were required in order to exclude noninferiority of the CAPOX regimen (i.e. HR $<1,29$ ). From 8/02 to 8/04 476 pts. (m:f = 299:177; median age 65 yrs) were randomized to receive either FUFOX (234 pts. arm A:
5-FU 2000mg/m2 24h inf., leucovorin 500mg/m2, oxaliplatin 50mg/m2 dl,8,15,22; q5 wks) or CAPOX (242 pts. arm B: capecitabine $1000 \mathrm{mg} / \mathrm{m} 2$ bid dl-14, oxaliplatin $70 \mathrm{mg}$ / $\mathrm{m} 2 \mathrm{dl}$ and $8 ; \mathrm{q} 3 \mathrm{wks}$ ). All pts. had measurable metastatic disease, ECOG performance status $0-2$, normal renal/hepatic/haematologic function. Results: To date 2635 treatment cycles (1073 FUFOX, 1562 CAPOX) were administered (median number of cycles per patient: FUFOX: 5, range 1-16; CAPOX: 6, range 1-24). Based on 371 events currently observed, median time to tumor progression (primary study end point) was 8.0 months in the FUFOX arm and 7.0 months in the CAPOX arm (HR: 1.19 (90\% CI: $1.0-1.41), p=$ 0.094). Median overall survival (OS) was 18.1 months in the FUFOX arm and 16.6 months in the CAPOX arm, respectively (HR: 1.23 (90\% C.I: 0.99-1.52), p=0.11). Response rates (intention to treat, (ITT)) were similar in both arms (table 1). Conclusions: Treatment with CAPOX and FUFOX induced similar response rates. There was no significant difference in median PFS. Today median OS data are too immature as only $51 \%$ events occurred. Ongoing studies will put our data into context and further define the role of the CAPOX regimen.

\begin{tabular}{|c|c|c|}
\hline Response rates (ITT), \% & CAPOX & FUFOX \\
\hline CR + PR & 47 & 50 \\
\hline SD & 27 & 23 \\
\hline PD & 26 & 27 \\
\hline
\end{tabular}

\section{T1961}

Antitumor Effect of Whole Body Hyperthermia Combined with Immunotherapy in Subcutaneous Tumor Model of Colon Cancer Takeshi Hattori, Satoshi Kokura, Tetsuya Okayama, Toshimitu Okuda, Satoko Adachi, Naoyuki Sakamoto, Tomohisa Takagi, Osamu Handa, Yuji Naito, Norimasa Yoshida, Toshikazu Yoshikawa

Aim: Whole body hyperthermia (WBH) has been applied as an adjunctive therapy with various established cancer treatments such as radio-therapy and chemotherapy. Recently, it has been reported that an activation of the immune system might contribute to the therapeutic effect of WBH. On the other hand, it has been established that alpha-galactosylceramide (alpha-GalCer), a glycolipid, is recognized by natural killer T (NKT) cells together with the monomorphic MHC-like antigen, CDld, in mice and humans. In this study, we investigated the antitumor effect of WBH combined with alpha-GalCer in mouse subcutaneous tumor model of colon cancer. Methods: Colon26 cells were inoculated subcutaneously in male $\mathrm{BALB} / \mathrm{c}$ mice to establish subcutaneous tumor. Colon26-bearing mice were treated with WBH by using far infrared rays 3 times a week. Rectal temperature was maintained for 60 min at 41 degrees Celsius. In some experimental groups, alpha-GalCer was intraperitoneally injected before WBH. We investigated the therapeutic effect of WBH, alpha-GalCer, and the combined therapy. Furthermore, we also investigated the mechanism by which alphaGalCer enhanced the antitumor effects of WBH. We monitored the serum level of IFNgamma, and we measured both NK activity and CTL activity in each group. Results: (1) Compared with control group, WBH alone showed a tendency to inhibit tumor growth and to prolong survival rate. (2) Alpha-GalCer did not show the inhibitory effect to tumor growth. (3) The combination of WBH and alpha-GalCer showed significant inhibitory effect of tumor growth and prolongation of survival rate. (4) Serum IFN-gamma increased after 3 hours and returned to its basal level after 24hours of alpha-GalCer administration. (5) Both NK activity and CTL activity were enhanced on the group of combination therapy with whole body hyperthermia and alpha-GalCer. Conclusion: (1) Whole body hyperthermia showed the antitumor effect on mouse subcutaneous tumor model of colon cancer. (2) The combination with alpha-GalCer increased the efficacy of whole body hyperthermia probably via the enhancement of both NK activity and CTL activity.

\section{T1962}

A Selective Cyclooxygenase-2 Inhibitor, Etodolac, Suppresses Stomach Carcinogenesis in Helicobacter Pylori-Infected Mongolian Gerbils Hirohito Magari, Yasuhito Shimizu, Ken-Ichi Inada, Izumi Inoue, Mkitaka Iguchi, Hideyuki Tamai, Kimihiko Yanaoka, Kenji Arii, Masashi Oka, Masae Tatematsu, Masao Ichinose

Purpose:Recent studies indicate that the selective cyclooxygenage-2 (COX-2) inhibitor reduces the risk of colorectal cancer. Expression of COX-2 in chronic atrophic gastritis $(C A G)$ and intestinal metaplasia is reported recently. CAG including intestinal metaplasia occurs in Helicobacter pylori (Hp)-infected Mongolian gerbils (MGs). Therefore, in the present study, we investigated the effect of a selective COX-2 inhibitor, etodolac, on Hp-associated stomach carcinogenesis in MGs. Methods:A total of $154 \mathrm{MGs}$ were divided into five groups (A-E). Animals were inoculated with $\mathrm{Hp}(3.0 \times 108 \mathrm{CFU} / \mathrm{ml}$, ATCC 43504) (groups A-D; $\mathrm{n}=$ $28,35,35$, and 40 , respectively) or vehicle alone (group $E ; n=16$ ). Groups A-D were given MNU in the drinking water at a concentration of $10 \mathrm{ppm}$ for 23 weeks. MNU was not administered to group E. In groups B-D, the animals were given the stock Etodolac diet from week 24 until week 53, whereas the animals in groups A and E received the control diet. The daily administered dosage of etodolac in groups A-D were $0,5,10,30 \mathrm{mg} / \mathrm{kg} / \mathrm{day}$ respectively. On the 53rd experimental week, all animals were sacrificed. Tissues sections were stained with $\mathrm{H \& E}$ and were also analyzed by immunohistochemistry for PCNA. Serum levels of anti-Hp antibodies and 8-OHdG were measured using ELISA. COX-2 m-RNA level in gastric mucosa was measured using RT-PCR. Results:In all the Hp-infected animals (groups A-D), marked infiltration was observed in the lamina propria and submucosa. The mucosal level of COX-2 mRNA tended to be dose-dependently reduced by etodolac treatment. Infection with Hp remarkably elevated the serum level of 8-OHdG but it was not significantly reduced by etodolac treatment. In contrast, the etodolac treatment dose-dependently reduced the serum level of anti-Hp antibodies. Infection with $\mathrm{Hp}$ greatly enhanced mucosal cell proliferation as revealed by immunohistochemistry using anti-PCNA antibodies. Etodolac treatment resulted in a step-wise and significant reduction in the proliferation of $\mathrm{Hp}$-infected mucosal cells. In addition, etodolac repressed the development of intestinal metaplasia. As a result of long-lasting infection with $\mathrm{Hp}, 14.8 \%$ of the animals in group A developed cancer. 
The incidence of cancer decreased in a step-wise manner with the dose of etodolac, and the development of the cancer was completely inhibited in group D Conclusion: Etodolac treatment dose-dependently inhibited the development of gastric cancer. These results suggest that COX-2 is a key molecule in inflammation-mediated stomach carcinogenesis and that chemoprevention of stomach cancer should be possible by controlling COX-2 expression or activity.

\section{T1963}

The Role of 99m-Technetium-Depreotide in the Management of Neuroendocrine Tumours

Tahir H. Shah, Ilona Kulakiene, John Buscombe, Ann-Marie Quigley, Christos

Toumpanakis, Martyn E. Caplin

Background: Neuroendocrine tumours (NETs) express somatostatin receptors which can be utilised for tumour imaging as well as for tumour targeted radiotherapy. 111Indiumpentetreotide (OctreoScan $囚$ ) is widely used for imaging and is associated with 67-95\% sensitivity. However, it mainly binds to somatostatin receptor subtype 2 (SSTR2) and will miss neuroendocrine tumours (NETs) lacking SSTR2 or NETs which lose SSTR2 upon alteration of tumour phenotype i.e. upon progression from low grade to high grade. Lack of strongly positive imaging with agents targeting SSTR2 normally rules out SSTR-targeted radiotherapy as an option $99 \mathrm{mT}$ c-depreotide is a new somatostatin analogue that binds SSTR2/3/5 with high affinity. Aim: To assess the diagnostic value of $99 \mathrm{mTc}$-depreotide scintigraphy in patients with negative or weakly positive 111 In-pentetreotide scans (Krenning scale $\leq 1$ [range 0-4]) and to determine its role in guiding therapy. Methods: 39 patients, with histologically proven NETs underwent paired $111 \mathrm{In}$-pentetreotide and $99 \mathrm{mTc}$-depreotide scans. The results were compared with CT and/or MRI imaging. Outcomes in patients subsequently receiving intravenously administered SSTR-targeted radiotherapy were analyzed. Results: Histologically $24 \%, 31 \%$, and $45 \%$ of tumours were of low, intermediate, and high grade respectively. The Krenning scores were: 0, 1, 2, and 3 in 36\%,32\%,25\%, and $7 \%$ of patients respectively when imaged with 111 In-pentetreotide; and $29 \%, 21 \%$ $29 \%$, and $21 \%$ respectively when imaged with $99 \mathrm{mTc}$-depreotide. Scores were equal for the 2 modalities in $29 \%$ of patients; $99 \mathrm{mTc}$-depreotide > $111 \mathrm{In}$-pentetreotide in $46 \%$; $111 \mathrm{In}$ pentetreotid $>99 \mathrm{mTc}$-depreotide in 14\%; and separate tumour sites were highlighted by the 2 agents in 11\%. 4 patients received SSTR targeted radiotherapy: 1 patient currently undergoing therapy; 2 patients had disease progression despite therapy; 1 patient had disease stabilisation for 6 months followed by disease progression. Conclusion: $99 \mathrm{mTc}$-depreotide is a significant new imaging modality in NET patients with intermediate / high-grade tumours which show complete absence or only limited uptake of 111 In-pentetreotide. It identifies patients amenable to radionucleotide therapy targeting SSTR subtypes 3 and 5 as well 2 . However initial results in patients identified for treatment using $99 \mathrm{mTc}$-depreotide are disappointing. This is due to the aggressive nature of NETs lacking SSTR2.

\section{T1964}

Inhibition of Nuclear Factor $\kappa \mathrm{B}$ Inhibits Cancer Cell Implantation in Vivo Brett W. Doxey, Phillip Gray, Scott K. Kuwada

The nuclear factor $\kappa \mathrm{B}(\mathrm{NF} \kappa \mathrm{B})$ family of transcription factors is commonly over expressed in malignant cells in which it promotes survival and angiogenesis. We previously showed that NF- $\mathrm{\kappa B}$ activity is transiently and strongly increased, and mediated cell survival, during readhesion of colon cancer (CCa) cells in suspension (Scaife et al. Cancer Res 2002). AIMS: To determine the vulnerability of digestive tract cancer cells to NF- $\mathrm{KB}$ inhibition during readhesion in vitro and in vivo. METHODS: The apoptotic effect of BAY 11-7085 was compared with vehicle on readhering human pancreatic cancer cells (Su.86, MIA PaCa-2) and CCa cells (DLD-1, HT-29, HCT-116) using Annexin V-FITC or cleaved PARP detection. Readhering HT-29, DLD-1, and HCT-116 cancer cells were treated with BAY 11-7085 with or without anti-TNF antibodies or soluble RANK (receptor activator of NF- $\mathrm{\kappa B}$ ) protein to determine the mechanism of NF- $\mathrm{kB}$ activation. 12 athymic mice were randomized to intraperitoneal (i.p.) BAY 11-7085 (5mg/kg) or vehicle (DMSO). The mice were treated with BAY 11-7085 or DMSO 24 hours prior to i.p. injection of 1 million Su.86 or MIA PaCa-2 cells, followed by i.p. treatment with BAY 11-7085 or DMSO every other day for 9 days. RESULTS: Treatment of readhering pancreatic cancer and CCa cells with BAY 11-7085 for 8 hours caused a large induction of apoptosis versus controls. Anti-TNF antibodies or soluble RANK inhibited readhesion-induced NF-KB activation in 0/3 and 3/3 CCa cell lines, respectively. While $67-100 \%$ of vehicle-treated mice demonstrated intraabominal seeding, only $33 \%$ of BAY $11-7085$-treated mice did $(\mathrm{p}<0.05)$. There was a $2.5-11$-fold reduction in the mean number of peritoneal implants in the treatment groups versus the control groups $(\mathrm{p}=0.045)$. CONCLUSIONS: Inhibition of NF- $\mathrm{KB}$ induced apoptosis and inhibited intraabdominal implantation of readhering digestive tract cancer cells. CCa cells utilize RANK but not TNF receptors to transiently activate NF-KB during readhesion. The strong dependence of digestive tract cancer cells on NF-KB and RANK activity for survival during readhesion provides a unique therapeutic opportunity to inhibit intraabdominal seeding and metastasis.

\section{T1965}

HSP70-Inducing Drug, Geranylgeranylacetone,, Enhances Anti-Tumor Effect of Whole Body Hyperthermia in Mice Subcutaneous Tumor Models of Colon Cancer

Satoko Adachi, Satoshi Kokura, Toshimitsu Okuda, Takeshi Hattori, Tomohisa Takagi, Osamu Handa, Yuji Naito, Norimasa Yoshida, Toshikazu Yoshikawa

Background and Aim: The transcription factor NF-kB is constitutively activated in many human cancers, and induces the expression of multiple genes including COX-2, VEGF, and IAP. Therefore, the blocking of NF-kB activity may be associated with the suppression of angiogenesis, and induction of apoptosis. Recently, we confirmed that heat pretreatment inhibited activation of NF-kB through the expression of heat shock proteins in a human colon cancer cell line. Thus, these facts raise a possible scenario that hyperthermia inhibits
NF-kB activation of a cancer cell, resulting in the inhibition of cancer growth by the induction of heat shock proteins. In this study, we investigated the effect of whole body hyperthermi combined with a heat shock proteins-inducing drug, geranylgeranylacetone (GGA, Hsp70 inducer) in mice subcutaneous tumor models of colon cancer. Methods: Colon26 cells were inoculated subcutaneously to establish subcutaneous tumor. Colon26-bearing mice were treated with whole body hyperthermia by using far infrared rays 3 times a week. Recta temperature was maintained for $60 \mathrm{~min}$ at 41 degrees Celsius. In some experimental groups, oral administration of GGA $(250 \mathrm{mg} / \mathrm{kg})$ was performed $2 \mathrm{hr}$ before whole body hyperthermia We investigated the therapeutic effects of whole body hyperthermia, single administration of GGA, and the combined therapy. Moreover, Hsp70 of tumor tissue in each treatmen group was detected by Western bloting. The activity of NF-kB in tumor tissue after each treatment was investigated by EMSA and ELISA. Results: 1) Whole body hyperthermia significantly inhibited the growth of subcutaneous tumor. 2) A single administration of GGA did not show the inhibitory effect of tumor growth. 3) The combination of whole body hyperthermia and GGA made further inhibitory effect of tumor growth. 4) GGA or whole body hyperthermia slightly induced Hsp70 in tumor tissue 24h after treatment. On the other hand, whole body hyperthermia combined with GGA administration increased the induction of Hsp70. 5) Whole body hyperthermia inhibited the NF-kB activation, which was enhanced by the combination with GGA. Conclusions: The development of subcutaneous tumor was inhibited by the treatment of whole body hyperthermia, and it might be suggested that Hsp70 induced by whole body hyperthermia play the important role of the inhibition of tumor growth because the effect of treatment was enhanced by using a Hsp70 inducer

\section{T1966}

A Phase I/II Study of a New Radiolabeled Somatostatin Analogue 90yttriumTyr3-Dota-Octreotate for the Treatment of Metastatic Neuroendocrine Tumours Christos Toumpanakis, Tahir Shah, Nick Chong, Neil Davies, John Buscombe, Martyn E. Caplin

Background: Radiolabeled somatostatin analogues can be used not only for tumor visualization but also for treatment of metastatic neuroendocrine tumours. Aim: To estimate efficacy and tolerability of a new radiolabeled somatostatin analogue 90Yttrium-Tyr3-DOTA-octreotate in patients with metastatic progressive neuroendocrine tumours. Patients \& Methods Sixteen patients $(8 \mathrm{~m} \& 8 \mathrm{f})$ with metastatic progressive neuroendocrine tumours were studied. Mean age was 60.4 years (range:34-83years). Patients received 1-3 cycles of the radionuclide, either intravenously (10/16) or intra-arterialy (6/16). In 4 patients of the latter group the intrarterial administration was combined with particle embolization of hepatic metastases Intravenous aminoacids were also administered for protection of renal function. Clinical and/or radiological response was assessed. Mean follow-up so far is 4.5 months (range: 2 9 months). Results: In all patients the post treatment scintigraphic scans demonstrated uptake and localization by the tumour. Clinical improvement was noted in 11/16 (68.75\% patients) after 1-3 treatments. Stabilization of tumor growth was achieved in all patients who had already completed the 3 cycles of treatment. Bone marrow suppression was noted in $4 / 16$, persisting at 3 months in one patient with significant bone metastases. Despite prophylactic aminoacids, one patient developed irreversible mild renal failure. Conclusion: 90Y-DOTA-octreotate seems to be a well-tolerated and safe treatment for patients with progressive neuroendocrine tumors, despite a risk for bone marrow toxicity in patients with large-volume bone metastases. Early results regarding efficacy are promising. Longer followup and more patients are required for better evaluation of tumor response.

\section{T1967}

Astragalus Saponins Induce Inhibition of Cell Proliferation in Association with COX-Independent Overexpression of NAG-1 in Colon Cancer Cells Joshua K. Ko, Mandy M. Tin, Chi-Hin Cho, Kelvin K. Chan

Background \& Aims: Active medicinal constituents of herbal origin may possess cytotoxic and/or growth inhibitory effects in various cancers. Our recent findings have indicated that the total saponin extract of radix Astragalus membranaceus causes significant growth inhibition in colon cancer cells and tumor xenograft on nude mice. In the present study, we aimed to examine the antineoplastic mechanism of extracted total astragalus saponins (AST) in HT29 colon adenocarcinoma cells and to identify the upstream molecular target. Methods: AST at various concentrations was incubated in HT-29 cell culture for 48 or 72 h. Cytotoxicity and cell proliferation were determined by MTT assay and BrdU ELISA, respectively. Flow cytometry was then performed to determine cell cycle arrest and apoptosis. Modulation of the apoptosis and growth-related proteins was assessed by Western analysis. The protein and gene expression of the transcriptional factors cyclooxygenase (COX)-2 and NSAIDactivated gene (NAG)-1 were also evaluated. Results: Cell growth inhibition was observed in HT-29 cells incubated with AST for $48 \mathrm{~h}$ (IC50=39.8 $\mu \mathrm{g} / \mathrm{ml})$ and $72 \mathrm{~h}(\mathrm{IC} 50=31.6 \mu \mathrm{g} /$ $\mathrm{ml}$ ). The cytotoxic effect of AST after $48 \mathrm{~h}$ incubation was accompanied by a dose-dependent reduction of cell proliferation $(30-100 \mu \mathrm{g} / \mathrm{ml})$. Data from flow cytometry had indicated tha AST ( $50 \mu \mathrm{g} / \mathrm{ml}$ ) caused G2-M phase arrest, with a slight increase in the amount of apoptotic cells $(\sim 10 \%)$ accumulated at the sub-Gl region of the DNA histogram. Western analysis had demonstrated that AST induced cleavage of procaspase- 3 and the caspase- 3 substrate PARP, markedly inhibited the expression of the anti-apoptotic protein $\mathrm{Bcl}-\mathrm{xL}$ and the growthrelated protooncogene c-myc, and caused overexpression of the cyclin-dependent kinase inhibitor p21 in HT-29 cells. In addition, a concurrent COX-independent NAG-1 protein overexpression was also revealed. By using quantitative real-time PCR, we had shown a time-dependent induction of NAG-1 mRNA by $3-6 \mathrm{~h}$ of AST incubation $(50 \mu \mathrm{g} / \mathrm{ml})$, of which the gene expression returned to control level at $24 \mathrm{~h}$. Conclusion: These findings envisage the cytotoxic effect and inhibition of cell proliferation induced by AST in HT-29 colon cancer cells. The mechanism of action involves cell cycle arrest by modulation of the growth-related proteins, with NAG-1 being identified as a vital molecular target. The upstream transcriptional factors (e.g. an early growth response factor EGR-1) that could be triggered by AST and subsequently facilitate NAG-1 induction are currently under investigation in our laboratory. 


\section{T1968}

Gene-Wide Haplotypic Analysis Demonstrates Novel Association Between ATP-Binding Cassete 3/Multidrug Resistance Protein 3 (ABCC3/MRP3) Gene and Inflammatory Bowel Disease

Gwo-Tzer Ho, Nicole Soranzo, Elaine Nimmo, Albert Tenesa, David Goldstein, Jack Satsangi

Background: Increasing evidence implicates the disruption of xenobiotic defence mechanisms in the pathogenesis of inflammatory bowel disease (IBD) 1,2. We have recently demonstrated association between variations of the ABCBI/MDRl gene and UC; microarray data now implicates a series of other genetic determinants involved in the maintenance of intestinal barrier function. Aim: We have analyzed the contribution of a panel of genes (derived from recent microarray data by Langmann et al.) consisting of the Pregnane-X receptor (PXR), ABCC2, ABCC3; and MEKKl genes using a gene-wide haplotype tagging approach. Methods/ Results: We have assessed the linkage disequilibrium pattern in these candidate genes by re-sequencing 24 CEPH Caucasian trios leading to the identification of 71 SNPs. A set of 22 haplotype tagging SNPs representing the common variations of these genes, both known and unknown were then selected using the multiple marker criterion of haplotype $r 2>0.8$ for all genotyped SNPs. These SNPs were then genotyped in the Scottish cohort consisting of $388 \mathrm{UC}, 328 \mathrm{CD}$ and $338 \mathrm{HC}$. Within the ABCC3 gene, 2 haplotype-tagging variants demonstrated significant associations with $\mathrm{CD}$ ( $\mathrm{rs} 739921 \mathrm{C} / \mathrm{G}, \mathrm{p}=0.005$, OR $1.41,95 \% \mathrm{Cl}$ 1.11-1.79) and UC(rs2277624 C/T SNP, $\mathrm{p}=0.003, \mathrm{OR} 1.52,95 \% \mathrm{CI} 1.15-2.00)$ respectively. In contrast, no associations were demonstrated at the allelic or haplotypic level (using loglikelihood ratio tests, $\mathrm{p}=0.9-0.1$ ) for the other genes studied. Conclusion: The present data provides the first evidence of the involvement of the $A B C C 3$ gene in determining susceptibility to IBD. ABCC3 gene encodes for multidrug resistance protein 3 (MRP3) which is expressed in the gut and liver. Further fine-mapping of associated interval of the implicated SNPs and replication in other datasets are in progress, together with functional and expression data. 1. Langmann, T. et al. Gastroenterology 127, 26-40 (2004). 2. Ho, G.T. et al. Gastroenterology $128,288-296$ (2005).

\section{T1969}

A Biological Pathway Approach to Disease Association Studies: Toll Receptor Pathway Alleles and Susceptibility to Inflammatory Bowel Disease Philip L. de Jager, Denis Franchimont, Alicja Waliszewska, Alain Bitton, Albert Cohen, Diane Langelier, Jacques Belaiche, Lisa Farwell, An Goris, Cecile Libioulle, Niraj Jani, Themistocles Dassopoulos, Gillian Bromfield, Benedicte Dubois, Judy H. Cho, Steven R. Brant, Richard Duerr, Huiying Yang, Jerome I. Rotter, Mark S. Silverberg, A. Hillary Steinhart, Mark J. Daly, Edouard Louis, David A. Hafler, John Rioux

The human gastrointestinal tract contains an abundant microbial flora that, along with pathogens, has long been thought to play a role either in initiating or in exacerbating inflammatory bowel disease (IBD). Host defenses such as the innate immune system are critical to the host/pathogen interaction and have been implicated in the pathophysiology of IBD. We therefore set out to characterize the role of genetic variation in a family of pattern recognition receptors, the Toll-like receptors (TLRs), and their signaling molecules in IBD. Our study implicates three of these molecules - IRAK2, TIRAP and TLR4 - in conferring susceptibility to IBD. Specifically, we examined a total of 1919 subjects with IBD and describe suggestive evidence that TIRAP (OR 1.16, 95\% CI 1.04-1.30; P=0.007) and IRAK2 (OR $1.15,95 \% \mathrm{CI} 1.00-1.31 ; \mathrm{P}=0.035)$ have modest effects on risk of IBD. In addition, our genotyping of 1539 cases of IBD and meta-analysis of 4805 cases of IBD demonstrate that one less common haplotype of the TLR4 gene is clearly associated with risk of IBD (OR $1.30,95 \% \mathrm{CI} 1.15-1.48 ; \mathrm{P}=0.00017$ ) and Crohn's disease (OR 1.33, 95\% CI 1.16-1.54; $\mathrm{P}=$ $0.000035)$, but not ulcerative colitis. This analysis of the TLR pathway in IBD is the first attempt towards a comprehensive evaluation of innate immunity in a chronic inflammatory disease and offers a road map for assessing other inflammatory diseases such as autoimmune thyroiditis, multiple sclerosis, rheumatoid arthritis, systemic lupus erythematosus, and type I diabetes that have been shown to share some susceptibility alleles.

\section{T1970}

The Nf-кB Regulator Card8/Tucan/Cardinal Is a Crohn's Disease Modifying Gene and Influeces Response to Microbial Antigens

Marie Pierik, Liesbet Henckaerts, Sofie Joossens, Isabelle Verstreken, Karolien Claes, Nele van Schuerbeek, Marc Ferrante, Paul Rutgeerts, Severine Vermeire

INTRODUCTION \& AIM Caspase recruitment domain (CARD) containing proteins are involved in apoptosis and NF-kB activation. The association between CARD15 variants and Crohn's disease (CD) confirmed the role of a disturbed innate immune response in inflammatory bowel disease (IBD). Recently an association between CD, especially ileal CD, and CARD 8 has been described. Given that patients with IBD show serum antibodies against microbial antigens we hypothesized that a genetically determined altered innate immune response may lead to an exaggerated adaptive immune response due to bacterial invasion. We therefore studied the relation between CARD8 and CARD15 variants and ASCA (antiSaccharomyces cereviciae) and anti-Omp (anti-Escheria Coli outer membrane porin). MATERIALS \& METHODS To study the association between CARD8 and IBD a cohort of 2016 individuals was genotyped for *10Cys with MALDITOFF mass spectrometry. CARD15 variants were genotyped with PCR RFLP. For 1190 subjects (760 CD, 193 UC and 237 controls) gASCA IgG and anti-Omp IgA were measured with ELISA (Glycominds and Inova).The clinical charts of these patients were reviewed for disease location and behavior, age at diagnosis, gender, disease duration, history of surgery and smoking at diagnosis. RESULTS: There was no significant distortion of transmission of the CARD8 T allele in IBD, CD or UC parent child trios. Multivariate analyses in $C D$ patients showed an association between the CARD8 $\mathrm{T}$ allele and stricturing disease ( $\mathrm{p}=0.02$ OR 1.73 95\% CI $\{1.09-2.76\}$ ) and the prevalence of anti-OmpC ( $\mathrm{p}=0.03 \mathrm{OR} 1.5195 \% \mathrm{CI}\{1.04-2.19\})$. The age at diagnosis was significantly lower in $C D$ patients with a CARD15 variant compared to $C D$ patients with no variants in CARD15 or CARD8 (24.59 and 27.64, p<0.01). However the mean age at diagnosis in patients with a concomitant CARD15 and CARD8 allele was not significantly lower compared to CD patients without any of the studied variants (24.79 and 24.59). After combining all subjects (IBD and controls) we found that Anti-Omp was more frequent and the titer was higher in subjects with a CARD8 T allele $(29.9 \%$ versus $24,1 \%, p<0.01$ and $\mathrm{p}<0.01)$. gASCA antibodies were more often present and the gASCA serum level was significantly higher in subject carrying at least one CARD15 variant $(54.0 \%$ versus $31.8 \%$, $\mathrm{p}<0.01$ and $\mathrm{p}<0.01$ ). DISCUSSION Our results show a higher prevalence of antibodies against microbial compounds (gASCA and anti-Omp) in patients carrying CARD15 and CARD8 variants. These data therefore give further evidence that a genetically determined altered immune response to microbial compounds plays a role in the molecular pathogenesis of IBD

\section{T1971}

Functional Polymorphisms of the Receptor for Advanced Glycation END Products (RAGE G82S and -374T/A) in Chronic Inflammatory Bowel Disease D.R. Foell, Frauke Friedrichs, Jochen Hampe, Torsten Kucharzik, Johannes Roth, Monika Stoll, Stefan Schreiber

Background and aims - An imbalance between innate and adaptive immune mechanisms may contribute to Crohn's disease (CD) and ulcerative colitis (UC). The receptor for advanced glycation end products (RAGE) is involved in innate immune mechanisms. Polymorphisms of RAGE have been described as a factor amplifying inflammation in susceptible patients, but the association of RAGE polymorphisms with inflammatory bowel disease (IBD) is not known. Patients and Methods - The most relevant functional RAGE polymorphisms, namely the promotor polymorphism -374T/A (rs1800624) and a coding polymorphism in exon 3 causing the amino acid exchange $\mathrm{G} \rightarrow \mathrm{A}$ (rs2070600), were studied in a German study sample consisting of $421 \mathrm{CD}$ patients, 211 UC patients and 549 controls. Results - Genetic epidemiological studies using logistic regression models revealed that the A allele of the RAGE promotor polymorphism at position -374 is negatively associated with $C D$ (odds ratio $(\mathrm{OR})=0.71,95 \%$ confidence interval $(\mathrm{CI})=0.53-0.93, \mathrm{p}=0.016$, additive inheritance model $)$. This result was confirmed by a transmission disequilibrium test in the families of the $C D$ patients that are part of the association study $(\chi 2=12.28, \mathrm{p}<0.001)$. The A allele was significantly associated to $C D$ when accounting for CARD 15 and IBD5 risk variants (adjusted $\mathrm{OR}=0.71,95 \% \mathrm{CI}=0.52-0.97, \mathrm{p}=0.031$ ). Genotype-phenotype analyses demonstrated that carrying the A allele reduces the chance to develop a stenosis $(\mathrm{OR}=0.627,95 \% \mathrm{CI}=0.41$ $0.98, \mathrm{p}=0.04)$. The $-374 \mathrm{~T} / \mathrm{A}$ variant was not associated with $\mathrm{UC}(\mathrm{OR}=0.99,95 \% \mathrm{CI}=0.71$ $1.38, \mathrm{p}=0.94)$. We were not able to demonstrate an association of rs 2070600 to $\mathrm{CD}$ or UC. However, the allele frequency of the rare variant is $3.3 \%$ in CD patients, $1.1 \%$ in UC patients and $2.6 \%$ in controls; thus larger study samples are needed to evaluate an association with IBD. Conclusions - We conclude that 374T/A variant leading to facilitated RAGE gene transcription may protect from developing $C D$ by augmenting innate immune mechanisms and increasing levels of soluble RAGE which neutralizes pro-inflammatory mediators. Further studies will confirm this effect in a secondary sample and confirm the impact on RAGE gene transcription in $C D$.

\section{T1972}

Novel Association Between the C3435t Polymorphism of the MDR1 Gene and Crohn's Disease

Simon Lal, Joanne M. Stempak, Elkadri A. Abdul, Law Christine, Thomas D. Walters, Anne M. Griffiths, Gordon R. Greenberg, Hillary Steinhart, Mark S. Silverberg

Background: Bacteria-host interactions are important in the pathogenesis of Crohn's disease (CD) and genes that affect these interactions are likely to be important factors in the susceptibility to developing disease. To further explore these pathways, we investigated the influence of important candidate genes that affect microbe-host interaction on disease susceptibility and phenotypic characteristics in a large Canadian cohort of patients with inflammatory bowel disease (IBD). Methods: 358 CD and 164 ulcerative colitis (UC) affected individuals and 809 unaffected family members from trio and tetrad families were evaluated. Genotyping was performed for the following SNPs: NODl/CARD4 (rs2075820), TLR4 (rs4986790) and MDRl (rs1045642 and rs2032582). Medical records were reviewed for all patients and phenotypic information compiled for disease type, behaviour and location according to the Montreal classification. Association with disease susceptibility and genotypephenotype relationships were analyzed using the family-based association test (FBAT). Results: Significant association between the C3435T (rs1045642) MDR1 polymorphism and $\mathrm{CD}(\mathrm{p}=0.02)$ was found. This result seemed to come primarily from Jewish $\mathrm{CD}$ patients $(\mathrm{n}=$ $86 ; \mathrm{p}=0.01$ ). The association was strongest among patients with both ileal and colonic involvement (vs. small bowel only or large bowel only) ( $\mathrm{p}=0.03$ ). There was no association between the C3435T polymorphism and UC, nor was there any association between the MDRl G2677T/A (rs2032582) polymorphism and either UC or CD.. The TLR4 Asp259Gly ( $r$ 4986790) polymorphism was not associated with $C D$ overall $(\mathrm{p}=0.06)$, however, there was a significant association with Jewish $C D$ patients $(p=0.03)$, those with inflammatory disease behaviour $(\mathrm{p}=0.02)$ as well as with ileocolonic location of disease $(\mathrm{p}=0.004)$. The TLR4 Asp259Gly (rs4986790) polymorphism was not associated with UC. There was no association between NOD1/CARD4 and either UC or CD. Conclusion: This is the first demonstration of an association between the C3435T MDRl variant and CD in a large cohort; this association arises primarily from Jewish individuals with $\mathrm{CD}$. We did not replicate the previously described association between this polymorphism and UC. The association of TLR4 (Asp259Gly) with CD primarily arose from Jewish individuals. These findings reinforce the importance of ethnicity when classifying patients for genetic studies. 
T1973

OCTN Variants Within Ibd5 Locus Contribute to Pediatric Crohn's Disease (CD) Susceptibility; Analysis of a Population-Based Pediatric Onset CD Cohort

Umesh Babusukumar, Tao Wang, Erin McGuire, Ulrich Broeckel, Subra Kugathasan

Susceptibility to CD by the IBD5 locus is now confirmed in adults. Recently, two polymorphisms (SNPs) in the OCTN gene cluster within IBD5 have been associated with CD. Higher LOD scores were associated with early age of onset $C D(<16$ yrs) by Rioux et al. We performed a comprehensive analysis of OCTN variants in an independent, exclusively pediatric-onset $\mathrm{CD}$ cohort and examined the genotype / phenotype correlations. We genotyped IBD5 variants (OCTN1\{SLC22A4\} \& 2 \{SLC22A5\} and 2 haplotype tagging SNPs (IGR2198, IGR2230)and analyzed them using family-based associations (TDT) and case control designs. DNA was genotyped from 264 Caucasian CD children (172 trios), 98 ulcerative colitis (UC) and from 527 Caucasian control children. Mean age of onset for $C D$ was 12 years. A significant association (TDT) was found with OCTN1 $(X 2=4.40, \mathrm{p}=0.036)$ and OCTN2 $(X 2=4.92$, $\mathrm{p}=0.027$ ) (table 1). All markers tested were in tight linkage disequilibrium with one another. Case control analysis (logistic regression) showed TC homozygosity genotype TC/ TC (SLC22A4/SLC22A5) (table 2) was significant but modest for CD risk (OR 1.66, 95\% $\mathrm{CI}(1.06-2.61), \mathrm{p}=0.041)$. No correlation is seen with regards to clinical phenotypes and the SLC22A4 / SLC22A5 diplotype. There was a trend towards colonic disease $(\mathrm{p}=0.09)$. There was no significant interaction between the SLC22A4 / SLC22A5 diplotype and the 3 CD-associated CARD15 SNPs. No associations were seen in UC. Conclusions: We confirm the association of IBD5 locus in pediatric onset $C D$ as seen in adult CD cohorts. However, compared with adults, a relatively weak association of the OCTN variants was observed. The contribution of OCTN1/2 variants is not independent of the IBD5 haplotype. The strong linkage disequilibrium in the IBD5 locus requires extended linkage disequilibrium analyses of OCTN variants before definitive conclusions can be drawn about these variants as causative genes in pediatric $C D$.

Table 1

\begin{tabular}{|c|c|c|c|c|}
\hline IBD5 Marker & Allele Frequency & Transmitted/ Untransmitted & TDT $\left(\mathrm{X}^{2}\right)$ & P value \\
\hline OCTN I (T) & 0.46 & $44 / 66$ & 4.40 & 0.036 \\
\hline OCTN 2 (C) & 0.49 & $76 / 51$ & 4.92 & 0.027 \\
\hline IGR2198 (C) & 0.51 & $49 / 60$ & 1.11 & 0.292 \\
\hline IGR2230 (A) & 0.42 & $51 / 75$ & 4.57 & 0.033 \\
\hline
\end{tabular}

Table 2

\begin{tabular}{|c|c|c|c|c|}
\hline OCTN1/OCTN2 diplotype & $\mathrm{CD}(\mathrm{n}=264)$ & Controls $(\mathrm{n}=527)$ & Odds ratio $(95 \% \mathrm{CI})$ & $\mathrm{P}$ value \\
\hline$(+/+)$ & $58(22.1 \%)$ & $86(16.4 \%)$ & $1.66(1.06-2.61)$ & 0.041 \\
\hline$(+/-)$ & $130(49.4 \%)$ & $258(48.9 \%)$ & $1.25(0.86-1.81)$ & 0.814 \\
\hline$(-/-)$ & $59(22.4 \%)$ & $144(27.3 \%)$ & 1.0 & - \\
\hline other diplotypes & $16(6.1 \%)$ & $39(7.4 \%)$ & $1.04(0.54-2.01)$ & 0.507 \\
\hline
\end{tabular}

$(+/+)$ refers to genotype TC/TC, (-/-) refers to genotype CG/CG, (+/-) includes the genotypes $\mathrm{TC} / \mathrm{TG}, \mathrm{TC} / \mathrm{CG}$ or TC/CC, and other diplotypes refers to genotypes TG/CG, TG/CC or CC/CC

\section{T1974}

Mutations in Innate Immune Receptors Modulate the Serologic Response to Microbial Antigens in Patients with Inflammatory Bowel Disease Liesbet Henckaerts, Marie Pierik, Karolien Claes, Marc Ferrante, Nele Vanschuerbeek, Nir Dotan, Gary Norman, Rom T. Altstock, Iris Dotan, Benjamin Schooler, Zakera Shums, Paul Rutgeerts, Severine Vermeire

Introduction: The pathogenesis of inflammatory bowel diseases (IBD) remains unknown, but a dysfunctional innate immune response in a genetic susceptible host seems important. Patients with IBD show various antibodies directed against microbial epitopes. Antibodies against Saccharomyces cerevisiae (ASCA) and the Outer Membrane Porin of bacteria (antiOmp) have been described in Crohn's disease (CD), although their pathophysiological role is unclear. Recently, three anti-glycan antibodies (Anti-Chitobioside-, Anti-Laminaribiosideand Anti-Mannobioside Carbohydrate Antibodies or ACCA, ALCA and AMCA) have also been associated with $\mathrm{CD}$. Aims: We hypothesised that mutations in innate immune receptors lead to altered serologic response in IBD. Methods: We genotyped a large cohort of 1225 patients with IBD (913 CD/272 UC/40 IC) for the main variants in CARD15 (R702W, G908R, 1007fs), Toll Like Receptor-4 (TLR4 D299G), OCTN (OCTN1 C1672T, OCTN2 207) and DLG5 (R30Q and _e26). Serum samples were analysed for gASCA IgG, ALCA IgG, ACCA IgA, AMCA IgG (Glycominds Ltd, Israel) and Omp IgA (Inova Diagnostics, San Diego) antibodies, using commercially available Elisa assays. Results: Compared with wild type, CD patients with 1 or more CARD 15 variants were more gASCA positive $(49.4 \%$ vs. $63.7 \%, \mathrm{p}<0.0001$ ), and had higher gASCA titres (61.9U vs. 83.7U, $\mathrm{p}<0.0001)$. A similar finding was observed for ALCA ( $32.7 \%$ vs. $45.9 \%, p<0.0001 ; 41 \mathrm{U}$ vs. $46.8 \mathrm{U}, \mathrm{p}=0.003$, respectively). Furthermore, the prevalence of gASCA and ALCA increased with the number of variants, suggesting a gene dosage effect: gASCA prevalence increased from $49.6 \%$ to $61.2 \%$ and $71 \%(\mathrm{p}<0.0001$ ), and ALCA prevalence from $32.7 \%$ to $45.3 \%$ and $47.5 \%$ ( $\mathrm{p}=$ 0.002), for 0, 1 and 2 CARD15 variants respectively. For TLR4 D299G, an opposite effect was seen, as $C D$ patients carrying a variant less frequently expressed ACCA $(22.5 \%)$ compared with wild type patients $(37.2 \%, \mathrm{p}=0.003)$. Similarly, UC patients with TLR4 variants were less frequently Omp positive (3.1\%) than UC patients without a variant $(28.5 \%, \mathrm{p}=0.001)$. Here also a gene dosage effect was observed: ACCA prevalence in CD decreased from $37.2 \%$ to $24.5 \%$ and $0 \%(\mathrm{p}=0.001)$ and Omp prevalence in UC from $28.5 \%$ to $3.3 \%$ and $0 \%$ ( $\mathrm{p}=$ 0.002 ) for 0,1 and 2 variants respectively. Conclusion: We found a significant association between variants in innate immunity genes and antibodies against microbial epitopes. Interestingly, an opposite effect of CARD15 and TLR4 variants was observed with respect to the antibody response. This finding merits further exploration, and may contribute to understanding the conflicting theories between loss-of-function and gain-of-function for the genetic variants.

T1975

Dose Dependent Genetic Interaction Between CARD15 Variants and a Promotor Polymorphism in Toll-Like Receptor (TLR)-9 in Crohn's Disease Helga P. Torok, Juergen Glas, Ilona Endres, Laurian Tonenchi, Peter Lohse, Thomas Ochsenkuehn, Matthias Folwaczny, Christian Folwaczny

BACKGROUND: Recognition of bacterial DNA through Toll-like receptor (TLR)-9 seems to be crucial for the maintenance of gut homeostasis and bowel inflammation. Recent data suggest functional interactions e.g. synergism between CARD15 and TLR9, but it is yet unknown whether this might be mirrored by genetic variations. We have previously described an association of Crohn's disease with the -1237 T/C promoter polymorphism in TLR9 (1) located in a susceptibility locus for IBD. Herein we assessed the role of the two polymorphisms in TLR9, which distinguish the four most common TLR9 haplotypes, in a second independen IBD population and tested for potential genotype-phenotype correlations and genetic interactions with Crohn's disease (CD) associated mutations in CARD 15. SUBJECTS AND METHODS: The polymorphisms - $1237 \mathrm{~T} / \mathrm{C}$ and $2848 \mathrm{~A} / \mathrm{G}$ in TLR9 and risk-associated CARD15 variants were assessed in a total of 457 patients with CD 247 patients with ulcerative colitis (UC), and 792 healthy controls. Association with disease susceptibility, clinical phenotypes defined by the Vienna classification, and possible genetic interactions with CARD15 variants in CD were tested by case-control analysis. RESULTS: A significant increase in the frequency of $-1237 \mathrm{C}$ carriers was observed in patients with $\mathrm{CD}$ bearing at least one disease associated variant in CARD 15 [overall increase of $10 \%, \mathrm{p}=0,008 \mathrm{vs}$. controls OR 1.62, $95 \%$ CI (1.13-2.32)]. Notably, a dose-effect was observed: the risk associated with $-1237 \mathrm{C}$ carrier status increased in patients with double-dose mutations in CARD15 $[\mathrm{p}=0.004$ vs. controls, OR 2.31 (1.28-4.16)]. In CD patients with wildtype CARD15 and in UC no significant differences were observed. No significant associations with $C D$ phenotypes were noted and allele and genotype frequencies of the $2848 \mathrm{~A} / \mathrm{G}$ polymorphism were comparable in patients and controls. CONCLUSIONS: Our results provide novel evidence for dose-dependent genetic interactions between CARD15 and TLR9 variants, increasing susceptibility to CD. Similar to CARD15, TLR9 is expressed close to secretory granules in Paneth cells, and is implicated in release of antimicrobial substances within mucosal crypts. Genetic variants in CARD15 and TLR9 could result in impaired interactions between these receptors and thus provide a first step in disturbance of mucosal homeostasis. Our observation sustains the hypothesis of an impaired cross-talk between different receptors of the innate immune system as a major first step in the pathogenesis of CD. 1. van Heel DA, et al. Gut 2005;54:1553-7. 2. Torok HP, et al. Gastroenterology 2004;127:365-6. 3. Rumio C, et al. Am J Pathos 2004;165:373-38

T1976

Case-Control Association Study of Tnfsf15 Polymorphisms in the Japanese Patients with Crohn's Disease and Ulcerative Colitis

Yoichi Kakuta, Yoshitaka Kinouchi, Kenichi Negoro, Masaki Matsuura, Hiroko Abe, Go Imai, Atsuhiro Mochida, Masaki Tosa, Eiki Nomura, Sho Takagi, Seiichi Takahasi, Tooru Shimosegawa

Background and Aims: Tumor necrosis factor superfamily (TNFSF) is considered to play an important role in the pathogenesis of inflammatory bowel disease (IBD). TNFSF15 (TL1A, V$E G I)$ is a novel member of TNFSF and its mRNA and protein expression is up-regulated in IBD particularly in involved tissues of Crohn's disease (CD). Recently, Yamazaki et al reported that the polymorphisms in TNFSF15 conferred susceptibility to Japanese and European CD (Human Molecular Genetics, 2005). To replicate the associations of TNFSF15 with CD and to investigate the genetic susceptibility to ulcerative colitis (UC), we genotyped single nucleotide polymorphisms (SNPs) of the TNFSF15 in the Japanese patients with CD, UC and healthy controls (HCs). Subjects: A total of 826 Japanese individuals, 286 patients with CD, 263 patients with UC and $277 \mathrm{HCs}$, were analyzed in this study. Methods: We first confirmed three SNPs, -640G/A (rs7848647) and -360C/T (rs6478109) in the 5'-flanking region and $+603 \mathrm{G} / \mathrm{A}(\mathrm{V} 201 \mathrm{~V}, \mathrm{rs} 3810936)$ in exon4, by direct sequencing of the whole gene using 24 $\mathrm{CD}$ patiens, but no other SNPs were found in $5^{\prime}$-flanking region and all exons. We then performed a case-control analysis of a total of six SNPs including additional three SNPs (TNFSF15 33, TNFSF15 31, TNFSF15 28 defined by Yamazaki) in introns which were previously reported to be associated with CD. Genotyping for SNPs was performed by polymerase chain reaction - restriction fragment length polymorphism analysis. We also analyzed clinical phenotypic statuses of affected individuals and TNFSF15 genotypes. Results: Minor allele frequencies of the $-640 \mathrm{G} / \mathrm{A},-360 \mathrm{C} / \mathrm{T},+603 \mathrm{G} / \mathrm{A}$, TNFSF15 33, TNFSF15 31, and TNFSF15_28 were $25.7 \%, 25.7 \%, 26.9 \%, 62.4 \%, 26.2 \%, 61.2 \%$ in CD, $36.3 \%, 36.3 \%$, $36.3 \%, 47.7 \%, 36.5 \%, 47.7 \%$ in UC, and $39.0 \%, 39.0 \%, 40.0 \%, 48.0 \%, 39.2 \%, 47.1 \%$ in $\mathrm{HCs}$, respectively. There was strong linkage disequilibrium between $-640 \mathrm{G} / \mathrm{A}$ and $-360 \mathrm{C} /$ T, consisting of GC/AT haplotype. All of 6 SNPs showed significant associations with susceptibility to $C D$ (the highest odds value was found in $-640 \mathrm{~A} / \mathrm{G}$ and $-360 \mathrm{C} / \mathrm{T}, \mathrm{p}=1.84 \mathrm{x}$ $10^{-6}$, odds ratio 1.85 ) but UC. No phenotypic statuses were related to $C D$ or UC genotype distribution. Conclusions: The association of CD with TNFSF15 previously reported was replicated in our Japanese case-control, however, no evidence for association of TNFSF15 with UC was observed. 
T1977

Analysis of the Utah Population Database Demonstrates Elevated Risk of IBD Among Family Members of IBD-Affected Individuals and Is a Source for Large Pedigrees in Which IBD Is Aggregating

Stephen Guthery, Richard A. Kerber, Richard Pimentel, Geraldine Mineau

Background: The Utah Population Database (UPDB) contains computerized genealogical records and family histories for over 6.4 million individuals dating back to the mid-18th century. UPDB is probabilistically linked to the University of Utah Health Sciences Center's administrative healthcare database. This allows for the ascertainment of individuals with diseases using ICD-9 codes and the aggregation of these diseases in UPDB pedigrees. We calculated the familial risk of IBD, Crohn's disease (CD), and Ulcerative colitis (UC) using this resource and identify large pedigrees in which IBD is aggregating. Methods: ICD-9 codes were used to ascertain individuals with Crohn's disease (555.x) and ulcerative colitis (556.x) dating from 1991 to 2005. Cases consist of all UPDB subjects having at least one of the indicated diagnoses who were also biologically related to at least one other UPDB individual. The controls were selected from a pool of UPDB individuals who had at least one hospital record and at least one relative in UPDB. Results: There were 1,093 cases of $\mathrm{CD}$ and 896 cases of UC identified within multigenerational pedigrees. The familial risk for first degree relatives of subjects with IBD is shown in the Table. The population attributable risk percent for familial CD was $10.5 \%$; for UC 5.3\%; and for IBD $12.1 \%$. Conclusions: IBD UC, and CD aggregate in families, and having a first degree relative with IBD is an important risk factor for IBD. The risk to lst degree relatives of probands with UC is higher than previously reported, although we cannot exclude referral bias as an explanation for this finding. Identification of specific Utah families in whom IBD aggregates is ongoing Extended pedigree studies in these families will likely complement other study designs delineating the genetic contribution to IBD. Relative Risks ( $95 \% \mathrm{CI}$ ) for first degree relatives.

\begin{tabular}{|c|c|c|}
\hline & Possible cases & Probable cases \\
\hline Crohn's disease & $4.5(1.9-10.8)$ & $4.6(1.1-18.2)$ \\
\hline Ulcerative colitis & $9.3(3.7-23.6)$ & $11.1(3.6-34.3)$ \\
\hline IBD & $4.9(3.0-8.2)$ & $6.8(2.9-16.0)$ \\
\hline
\end{tabular}

\section{T1978}

CARD15 Mutations Decrease the Mucosal Bacterial Flora in Crohn's Disease Carsten Buening, Thomas Fiedler, Enno Gentz, Janine Buettner, Hartmut Schmidt, Herbert Lochs, Alexander Swidsinski

Background and Aims: Crohn's disease (CD) patients carrying mutations within the CARD15 gene suffer from a more aggressive disease behaviour, but the mechanisms explaining these observations remain to be elucidated. CARD15 acts as an intracellular receptor in monocytes repsonsible for the recognition of bacterial wall components. We therefore hypothesized that CARD15 mutations have an impact on the composition of the mucosal bacterial flora in $C D$ patients. Methods: In total, 42 patients with CD (male: 17, female: 25; age: 21-57) were included in this study. For analysis of the composition of the mucosal bacterial flora, biopsy specimens were taken from the ileum, ascending and sigmoid colon and were analysed by fluorescence in situ hybridization (FISH). For FISH analysis rRNA-targeted oligonucleotide probes were synthesized and forty domain, group-, and species-specific probes were applied. In addition, genotyping for the three common CARD15 mutations (Arg702Trp, Gly908Arg, 1007 fins) was performed in all subjects. Furthermore, data were correlated to clinical characteristics of all CD patients (including age, disease localisation and behaviour, standard medical therapy). Results: Mucosal bacteria at concentrations greater than $10^{9} / \mathrm{ml}$ were overall found in $66.7 \%$ of $C D$ patients. Genoytyping revealed that $45.2 \%$ of patients carried at least one mutant allele within CARD15 (considered to be CARD15 positive, the remaining $54.8 \%$ considered to be CARD15 negative). We found that the presence of mucosal bacterial concentrations less than $10^{9} / \mathrm{ml}$ was significantly associated with CARD15 mutations ( $\mathrm{p}=$ 0.03 , Fishers exact test). We observed a similar composition of the mucosal flora. However, Enterobacteriaceae were elevated in the CARD15 group. We have previously reported that 5-ASA therapy dramatically reduces mucosal bacteria resulting in a complete disappearance of the mucosal flora in some $\mathrm{CD}$ patients. Interestingly, in this study we found that this was more pronounced in patients with CARD 15 mutations compared to CARD15 negative patients $(\mathrm{p}=0.003)$. Conclusion: Mucosal bacteria are found in significantly lower concentrations in CD patients with mutated CARD 15 compared to patients without mutated CARD15. 5-ASA therapy was associated with a complete disappereance of the mucosal flora predominantly in patients with CARD15 mutations. Further studies are warranted to investigate whether genotyping for CARD15 mutations might be used to identify CD patients with a more pronounced effect of 5-ASA therapy.

\section{T1979}

From Vienna to Montreal: The Impact of Changes in IBD Classification On CARD15 Genotype-Phenotype Relationships in a Population-Based Cohort Richard B. Gearry, Rebecca L. Roberts, Christopher M. Frampton, Michael J. Burt, Judith A. Collett, Bruce A. Chapman, Melanie D. Allington, Martin A. Kennedy, Murray L. Barclay

Introduction: The role of CARD15 mutations in the development of Crohn's disease (CD) at a population level is poorly understood. Recently, a new classification system aiming to integrate clinical phenotype and laboratory markers was proposed. Aim: To compare IBD phenotypes using the Vienna and Montreal systems, and to determine the CARD15 genotypephenotype relationship in a population-based IBD cohort. Methods: 1420 IBD cases (91\% of people with IBD) were recruited into the study from Canterbury, New Zealand (population 464,800). DNA and clinical information was obtained from the cases who were phenotyped according to both the Vienna and Montreal classification systems. Population-based control
(201) and cases were genotyped for the three major CARD15 mutations associated with (CD). Results: $C D$ phenotype distribution was similar to other population-based cohorts (Vienna system). The Montreal system led to fewer cases classified with penetrating disease. The frequencies for having any one of the three CARD15 mutations were 0.132 (CD), 0.07 (UC) and 0.06 (controls). The R702W ( $\mathrm{p}=0.002$ ) and Cinsl007fs ( $\mathrm{p}=0.003$ ) mutations, but not the G908R ( $p=0.091)$ mutation, were significantly associated with $C D$. The presence of a single mutation was associated with CD (odds ratio 2.3 [95\% CI 1.4-4.8]) as was the presence of two mutations $(9.2[1.2-68])$. The following significant phenotype-genotype associations were found using the Montreal system for carrying one or two mutations, respectively: ileal location: $(2.4$ [1.6-3.6], 3.5 [1.7-7.4]), colonic disease $(0.35$ [0.22-0.54], $0.27[0.10-0.72])$, inflammatory disease $(0.48[0.33-0.71], 0.17[0.04-0.42])$, stricturing disease (1.5 [0 98-2.2], $4.1[1.9-8.8]$ ) and penetrating disease (2.3 [1.3-3.9], 1.6 [0.614.4]). Other associations with one or two CARD15 mutations were previous bowel resection (2.3 [1.6-2.4], 3.2 [1.5-6.8]) extra-intestinal manifestations $(0.47$ [0.26-0.87], 1.3 [0.55$3.2])$ and first degree relative with IBD (2.8 [1.7-4.8], 4.8 [1.9-12]). Conclusions: Use of the Montreal system leads to fewer CD cases classified with penetrating disease. In turn, this strengthens the association between penetrating disease and carriage of one or two CARD15 mutations. While the association between CD and CARD15 mutations remains, it is weaker in this population-based cohort than in previous clinic-based cohorts.

\section{T1980}

Prognostic Significance of NOD2/CARD15 SNPs in Allogeneic Stem Cell Transplantation (SCT) from Unrelated Donors: Evidence for An Important Role of Macrophages

Julia Brenmoehl, Heike Bremm, Anne M. Dickinson, Hildegard Greinix, Gerard Socie, Daniel Wolff, Graham Jackson, Gerhard Fischer, Vanderson Rocha, Beate SteinerKrammer, Joachim Hahn, Gerhard Hildebrandt, Guenther Eissner, Joerg Marienhagen, Juergen Schoelmerich, Reinhard Andreesen, Ernst Holler, Gerhard Rogler

Introduction: NOD2/CARD15 has been associated with a significantly increased risk to develop Crohn's disease. Our previous studies demonstrated a major role of SNPs within the NOD2/CARD15 gene in stem cell transplantation (SCT) in HLA-identical sibling transplants. The presence of NOD2/CARD15 variants was followed by an increased risk to develop Graft versus host disease (GvHD) and an increased mortality. Our pilot data in unrelated donor (URD) transplantation were less clear, we now extended our analysis. Methods and patients: TaqMan PCR analysis for major SNPs 8,12, and 13 of the NOD2/CARD15 gene was performed in DNA samples from recipients (R) and their donors (D) of 341 URD transplants performed in 5 European centres. Results were compared with other known risk factors such as matching for class I and II, age, disease and stage at the time of HSCT, use of T cell depletion and reduced intensity conditioning (RIC) as well as stem cell source, and major outcome variables such as GvHD grade III/IV, 1 yr non-relapse related mortality (NRM) and overall survival were analysed in relation to these risk factors. Results: GvHD grade III/IV was $21 \%$ in transplants with wildtype NOD2 pairs or mutant NOD2 in R only, but increased to $34 \%$ in transplants with D variants and to $43 \%$ in a small group $(n=14)$ of simultaneously occurring NOD2 variants in R \& D. Further analysis revealed, if donors had a SNP13 variant $(\mathrm{n}=23)$ there was a higher risk for GvHD and NRM. GvHD grade III/ IV of these patients rose from 22 to 48\% (p 0.007) and 1 yr NRM from 27\% to 59\% (p 0.007 ). Multivariate cox regression analysis for $1 \mathrm{yr}$ NRM revealed a strongly increased risk of mortality in the presence of donor SNP13 variants (HR 3.7, 95\% CI $1.77-7.79, \mathrm{p} 0.001$ ) whereas other risk factors such as age, stage at the time of SCT and more important, mismatch at an allel- or even antigen-level did not reach significance. 1 yr NRM almost doubled in this high-group, but did not reach significance. Conlusion: Our data suggest a different but still relevant role of NOD2/CARD15 variants in URD transplants as compared to HLA identical sibling SCT. As NOD2/CARD15 SNP13 variants result in a stronger suppression of NOD2/CARD15 dependent function than other SNPs altered monocyte/macrophage functions in unrelated donors may contribute to these specific effects. Our data furthermore suggest for the first time that matching for a non-HLA gene could be more relevant than differentiated HLA-matching in URD-SCT and strongly support a role of intestinal bacterial translocation.

\section{T1981}

Influence of Gender and DLG5 R30q On Susceptibility for Pediatric Onset Crohn's Disease

Vincent F. Biank, Umesh Babusukumar, Frauke Freidrichs, Monika Stoll, Tao Wang, Erin McGuire, Ulrich Broechel, Subra Kugathasan

Using a positional cloning approach, a significant association has been found between the DLG5 variant (R30Q) and inflammatory bowel disease (IBD), as well as, Crohn's Disease (CD). These findings have subsequently been confirmed by Daly et al. in several independent adult IBD \& CD cohorts, but have yet to be investigated in pediatric onset IBD. In addition, there is growing evidence that gender significantly influences the effect of the DLG5 variant. Furthermore, a 2:1 male to female ratio has been observed in pediatric onset IBD. We, therefore, investigated the effect of gender and the association of the DLG5 variant in pediatric onset IBD. 281 Caucasian CD cases (181 trios), and 479 Caucasian population-based controls were genotyped for the DLG5 variant using Taqman assay. Logistic regression was performed between cases and controls. Overall allele frequency for R30Q was $8.5 \%$ in CD and 10.3 $\%$ in controls (Table 1 shows the allele frequencies separated by gender). While there was no significant difference in males, a significant lower allele frequency for the R30Q mutations was detected in females with $\mathrm{CD}(\mathrm{p}=0.0048)$. Logistic regression analysis showed no significant effect combining both genders (OR 0.81; 95\%CI 0.551-1.198, p=0.295). However, when stratified by gender a significant effect was detected in females only (OR 0.392; 95\%CI 0.2$0.765 ; \mathrm{p}=0.006$ ). Furthermore, analysis investigating gender, $\mathrm{R} 30 \mathrm{Q}$ and an interaction term of gender and $\mathrm{R} 30 \mathrm{Q}$, also demonstrates a significant interaction between gender and R30Q (OR $0.29,95 \% \mathrm{CI} 0.125-0.674, \mathrm{p}=0.004)$. Finally, adjusting for previously associated mutations in the CARD15 gene (3020InsC) as well as a marker in the IBD5 locus (Igr2198), we detect a significant effect of CARD 15 (OR 3.389, 95\%CI 2.394-4.799; $<<0.0001$ ) as well as significant interaction between R30Q and female gender $(\mathrm{p}<0.05)$. In conclusion, while 
we did not find a significant association with R30Q in the overall cohort, when stratified by gender, we show a significant negative association in females, therefore, suggesting DLG5 has a protective effect in female pediatric $C D$. Furthermore, we demonstrate a significant interaction between the DLG5 variant and gender. Although, for males, the allele frequency is higher in cases vs. controls, this did not reach statistical significance. Further studies are therefore needed to address the complex effects of gender, mutation frequency, and age of disease onset in pediatric IBD

Table 1: Allele Frequency of DLG5 in CD and Controls Stratified by Gender

\begin{tabular}{|c|c|c|c|c|c|c|}
\hline $\begin{array}{c}\text { DLG5 } \\
\text { marker }\end{array}$ & $\begin{array}{c}\text { CD Males } \\
(\mathrm{n}=164)\end{array}$ & $\begin{array}{c}\text { Control Males } \\
(\mathrm{n}=240)\end{array}$ & $\begin{array}{c}\mathrm{P} \\
\text { value }\end{array}$ & $\begin{array}{c}\text { CD Females } \\
(\mathrm{n}=117)\end{array}$ & $\begin{array}{c}\text { Control Females } \\
(\mathrm{n}=239)\end{array}$ & $\begin{array}{c}\mathrm{P} \\
\text { value }\end{array}$ \\
\hline $\mathrm{R} 30 \mathrm{Q}$ & $10.7 \%$ & $8.3 \%$ & 0.26 & $5.6 \%$ & $12.3 \%$ & 0.0048 \\
\hline
\end{tabular}

\section{T1982}

Array-Based Genetic Evaluation of Adenocarcinomas and Dysplastic Lesions (DALM) in Ulcerative Colitis

Jolanda M. Van Dieren, Janneke van der Woude, Kees J. Vissers, Ruud Schouten, Hans J. Tanke, Karoly Szuhai, Ernst J. Kuipers, Josiane C. Wink, Herman van Dekken

Background: Longstanding ulcerative colitis (UC) is associated with a high risk of developing UC-related colonic adenocarcinoma (UCC). These carcinomas originate from dysplastic regions referred to as Dysplasia Associated Lesion or Mass (DALM), whereas sporadic colon carcinoma (SCC) develops from adenomatous polyps. Methods: We evaluated the genomic spectra of 13 DALM/ UCC's by array comparative genomic hybridization (aCGH) with a 3500-element BAC-PAC array. Seven tumors comprised early TNM stages (2 stage 0 [c.i.s.], 5 stage I), six neoplasms were late stages ( 5 stage III, 1 stage IV). Results: Frequent losses of array clones ( $>20 \%$ of tumors) were detected at chromosome arms $4 \mathrm{p}, 5 \mathrm{q}$, and $18 \mathrm{q}$, frequent gains of array clones ( $>20 \%$ of tumors) were found at $1 \mathrm{q}, 5 \mathrm{p}, 6 \mathrm{p}, 7 \mathrm{p}, 7 \mathrm{q}, 8 \mathrm{p}, 8 \mathrm{q}$, $11 p, 11 q, 12 q, 14 q, 17 q, 19 q, 20 p$ and 20q. The pattern of alterations is dominated by gains on $5 p$ and $20 q$ with loss of $4 p$, all of which were already present in a patient with carcinoma in situ. However, gain of BAC clone RP11-150P21 at 8q22.3 was found more frequently in the late-stage sub-group $(\mathrm{P}=0.02)$. The distribution of gains and losses in DALM/ UCC is different from SCC, which is typically characterized by gains of whole chromosomes 7 and 13, gain on 20q, and loss on 17p and 18q. Conclusions: UCC's display profound genomic instability with multiple genetic abnormalities. Furthermore, the genomic changes underlying UCC differ from those seen in SCC supporting an alternative genetic pathway in the carcinogenesis of DALM and UC-associated adenocarcinomas.

\section{T1983}

CARD8: A Novel Association with Childhood-Onset Ulcerative Colitis (UC) Marla Dubinsky, Lirona Katzir, Iwona Wrobel, Antonio Quiros, Ling Mei, Yoana Picornell, Xiaowen Su, Ghassan Wahbeh, Gary Silber, Ron Bahar, Bruce Grill, Eric Vasiliauskas, Kent D. Taylor, Stephan R. Targan, Huiying Yang, Jerome R. Rotte

Background: CARD proteins play an important role in apoptosis and cytokine regulation, including NFKB, processes which are important in the pathogenesis of IBD. CARD15/NOD2 was the first novel gene reported to confer Crohn's disease (CD) susceptibility and influence disease phenotype. CARD4 has not been found to be associated with CD. McGovern et al reported a significant $\mathrm{CD}$ association with the CARD8/TUCAN/CARDINAL gene located at 19 q13.3 in adult patients. Aim: The Western Regional Pediatric IBD Research Alliance investigated the association of the CARD8-T10C polymorphism with susceptibility to UC and CD in children. Methods: DNA was collected from 342 subjects (75 CD trios, 39 UC trios). Both parents and the affected child were genotyped for 3 allelic variants of the CARD15 gene (R702W, G908R, 1007insC, also referred to as SNP 8, 12 and 13) as an association control and 1 variant of the CARD8 gene (T10C) using Taqman technology. The transmission disequilibrium test (TDT) was used to test association with either UC or CD using GENEHUNTER 2.0. Results: CARD8 allele T was present in $63 \%$ of CD patients and $77 \%$ of UC patients. CARD 15 frequency (any variant) was $25 \%$ and $11 \%$ in CD and in UC, respectively. Similar frequencies were observed for parents for both genes. As expected, transmission distortion was seen for all CARD15 variants in CD, but not in UC. No association was observed between CARD 8 and CD, however, in contrast, TDT showed a highly significant association with UC, with over transmission of the CARD8 common allele (Table). Conclusions: This is the first report of a CARD8 association with childhood-onset UC. The over transmission of the common allele in this analysis is similar to that which is seen with PPAR $\gamma$ in type 2 diabetes and the insulin gene polymorphism in type 1 diabetes. These findings are in contrast to the adult $\mathrm{CD}$ association suggesting different mechanisms for pediatric IBD. Larger genotype-phenotype association studies are underway to further explore this novel genetic association.

TDT analysis

\begin{tabular}{|c|c|c|c|c|c|c|}
\hline & \multicolumn{2}{|c|}{ CARD8 T allele } & pvalue & \multicolumn{2}{c|}{ CARD15 SNP 8,12,13 } & pvalue \\
\hline & TRANSMITTED & $\begin{array}{c}\text { NOT } \\
\text { TRANSMITTED }\end{array}$ & & TRANSMITTED & $\begin{array}{c}\text { NOT } \\
\text { TRANSMITTED }\end{array}$ & \\
\hline $\begin{array}{c}\text { CD } \\
(\mathrm{n}=75)\end{array}$ & 37 & 33 & NS & 30 & 11 & 0.003 \\
\hline $\begin{array}{c}\text { UC } \\
(\mathrm{n}=39)\end{array}$ & 23 & 8 & 0.007 & 4 & 7 & NS \\
\hline
\end{tabular}

T1984

Only Relapse Rate in the Ulcerative Colitis Patients Differs Between Sporadic and Familial Cases in a Prospective Population Based Norwegian Study May-Bente Bengtson, Camilla Solberg, Morten Vatn

Background and aims: Does familial aggregation of IBD change the clinical phenotype compared to sporadic cases? The aim of this study was to estimate the increasing prevalence of familial cases over a ten years period. We also wanted to compare the clinical phenotype between sporadic and familial cases both in Crohn (CD) disease and ulcerative colitis(UC) in a Norwegian population based follow-up study from onset of disease. Methods: This incidence study includes 415 patients with UC and 191 with CD enrolled 1990 to 1994, and followed up at one, five and ten years. Disease distribution and behaviour were confirmed throughou these years. At each follow up the patients were asked about 1 . degree relatives with IBD, which was later confirmed. Results: Affected first degree relatives were increased from 20 (10.5\%)at diagnosis to $33(14.1 \%)$ at ten years follow up in CD, and from $27(8.2 \%)$ to $52(12.5 \%)$ in UC. Assuming a population prevalence of $150 / 100000$ for CD, and 400 / 100000 for UC, the relative risks in siblings and parents increased with $69 \%$ and $17 \%$ in $\mathrm{CD}$, and $28 \%$ and $72 \%$ in UC respectively through ten years. Concordance for disease within families was $71,2 \%$ for UC, and $70.4 \%$ for CD patients. Cumulative relapse rate during ten years follow-up was significantly greater in familial cases compare to sporadic cases for UC (OR=3.6, 95 \% CI: 1.09-12,01) There were no statistical differences between the familial and sporadic cases regarding immune modulating therapy, bowel resection, colectomy rate, disease behaviour, location of disease or age at diagnosis. However we found a trend towards a lower age at diagnosis in familial cases in $\operatorname{CD}(\mathrm{p}=0,06)$. Conclusion: The prevalence of IBD increased in both parents and siblings with strong disease concordance. The marked trend of increased number of affected siblings compared to parents in $C D$, opposite to increased number in UC over ten year is a new observation. Only relapse rate in UC patients was significantly increased in familial cases compare to sporadic cases.

\section{T1985}

Association Between Ancestry Proportion and Inflammatory Bowel Disease in Puerto Ricans

Ling Mei, Yoana Picornell, Federico Gregory, Roberto Mera, Deb Dutridge, Jerome I. Rotter, Esther A. Torres, Huiying Yang

Mapping by admixture linkage disequilibrium (MALD) in an admixed population has advantages for mapping inflammatory bowel disease (IBD) genes compared to traditional LD methods in terms of the number of markers required. This is due to the fact that LD in a recently admixed population can extend over large chromosomal segments, so that substantially fewer markers, termed ancestry informative, can cover the entire genome. However the success of MALD is proposed to depend on the existence of different disease prevalence between ancestral populations. To investigate the potential feasibility of using a MALD approach for mapping susceptibility genes for IBD in the Puerto Rican (PR) population, we evaluated the association between ancestry and IBD in a PR IBD case-control sample. Methods: 204 IBD cases (128 Crohn's disease and 76 ulcerative colitis) and 110 controls were ascertained from Puerto Rico. 44 ancestry informative markers identified from NCBI SNP database and literature were genotyped. There were 31, 23, and 32 informative makers with allele frequency difference $>0.3$ between African and European ancestry, European and Native American (NA) ancestry, and African and NA ancestry, respectively. The genotype data from the HapMap Project, including 60 Yoruba, 60 European, and 45 Asian independen individuals, were used to determine specific ancestral representation within PR subjects. Individual and group admixture levels were calculated by using the program STRUCTURE. Results: There was a wide range of genetic ancestry at an individual admixture level. On average, IBD cases had a greater proportion of European ancestry ( $54 \%$ vs. $47 \%, \mathrm{p}<0.0001$ ) and lower proportion of African ancestry ( $7 \%$ vs. $14 \%, \mathrm{p}<0.0001$ ) compared to controls. There was no difference in NA proportion between cases and controls. Conclusions: The results suggest that the risk of IBD in PR population may be influenced by genetic factors that have different allele frequency in Europeans and Africans. Thus, for any candidate gene studies, population stratification should be taken into consideration in the analysis. Most important, MALD may be a viable approach to identify susceptibility genes for IBD in the PR population

\section{T1986}

Analysis of CCL20 Variants in Inflammatory Bowel Disease (IBD) Provides Further Evidence for Genetic Heterogeneity in Disease Susceptibility Charlie Lees, Elaine R. Nimmo, Richard K. Russell, Johan van Limbergen, Amanda Smith, Hazel E. Drummond, Leif Torkvist, Nagamu Inoue, Toshifumi Hibi, Nobuhide Oshitani, Ian D. Arnott, Jack Satsangi

Background. Expression of the chemokine CCL-20 is increased in colonic mucosa and peripheral blood mononuclear cells in IBD. In a S Korean population, the $1706 \mathrm{G} \rightarrow \mathrm{A}$ promoter polymorphism was strongly associated with susceptibility to UC $(\mathrm{p}<0.0001)^{1}$ These data have yet to be replicated. The CCL20 gene is located on chromosome 2q36.3 in a short haplotype block containing no other genes. Aims. To assess the contribution of CCL20 promoter polymorphisms to IBD susceptibility in Scottish, Scandinavian and Japanese 
populations. Methods. The Scottish population, 100\% white Caucasian, mean age at diagnosis 28.1 years, comprised 523 cases of ulcerative colitis (UC), 442 of Crohn's disease (CD), \& 351 healthy controls (HC). 292 Swedish UC cases \& 254 HC; 105 Japanese UC cases \& 84 Japanese HC were also studied. Direct sequencing and Taqman analysis were used for mutation screening of the CCL20 promoter and genotyping of variants. Results. Only two common variants $(1706 \mathrm{G} \rightarrow \mathrm{A} \& \mathrm{rs} 6749704 \mathrm{~T} \rightarrow \mathrm{C}$ ) were identified in these populations. There was no difference in variant allelic frequencies in Scottish \& Swedish HC ( $\mathrm{p}=$ $0.20)$, or between Japanese $\&$ S Korean HC $(p=0.53)$. However, $1706 \mathrm{G} \rightarrow$ A allelic frequencies were significantly different between N European (Scottish \& Swedish) and Eastern (Japanese \& S Korean) $\mathrm{HC}(13.3 \%$ vs $38.5 \%$, OR $4.09, \mathrm{p}<0.0001)$. The $1706 \mathrm{G} \rightarrow \mathrm{A}$ variant was significantly increased in Japanese UC (OR 1.59, p=0.035), but not in either N European population (see Table). In the $\mathrm{N}$ European population there was a significant protective effect with the rs6749704'CC' genotype in IBD \& UC compared with HC (6.6\% vs $10.6 \%$, OR $0.60, \mathrm{p}=0.0054)$. This variant was not associated with $\mathrm{UC}$ in the Japanese population, again mirroring the S Korean data ${ }^{1}$. Conclusions. These observations provide further evidence for genetic heterogeneity between Eastern and N European populations, paralleling the experience of NOD2/CARD15 in CD ${ }^{2}$, and HLA-DRB1*1502 in UC. The weak effect of $1706 \mathrm{G} \rightarrow \mathrm{A}$ in the Scottish and Scandinavian populations contrasts strongly with the strong effect in the S Korean and Japanese populations. The contribution of the rs6749704 variant in both $\mathrm{N}$ European datasets suggests that $1706 \mathrm{G} \rightarrow \mathrm{A}$ may not be the causal variant. Further detailed analysis of the CCL20 gene is warranted. References. 1.Choi SC et al Gastro 2005;128(4):A137. 2.Arnott ID et al. Genes Immun.2004 Aug;5(5):417-25.

\begin{tabular}{|c|c|c|c|c|c|c|c|c|c|c|}
\hline \multirow{3}{*}{$1706 \mathrm{G} \rightarrow \mathrm{A}$} & \multicolumn{6}{|c|}{ N EUROPEAN POPULATIONS } & \multicolumn{4}{|c|}{ EASTERN POPULATIONS } \\
\hline & \multicolumn{4}{|c|}{ SCOTTISH } & \multicolumn{2}{|c|}{ SWEDISH } & \multicolumn{2}{|c|}{ JAPANESE } & \multicolumn{2}{|c|}{ S KOREAN ${ }^{1}$} \\
\hline & $\mathrm{HC}$ & IBD & UC & CD & $\mathrm{HC}$ & $\mathbf{U C}$ & $\mathrm{HC}$ & UC & $H C$ & $U C$ \\
\hline $\begin{array}{l}\text { 'A' Allelic } \\
\text { freq. }\end{array}$ & $12.3 \%$ & $\begin{array}{l}13.7 \% \\
p=0.35\end{array}$ & $\begin{array}{l}14.0 \% \\
\mathrm{p}=0.31\end{array}$ & $\begin{array}{l}13.4 \% \\
p=0.56\end{array}$ & $14.8 \%$ & $\begin{array}{l}15.9 \% \\
p=0.66\end{array}$ & $36.3 \%$ & $\begin{array}{c}47.6 \% \\
p=0.035\end{array}$ & $39.1 \%$ & $\begin{array}{c}60.7 \% \\
p<0.0001\end{array}$ \\
\hline $\begin{array}{c}\text { 'A' } \\
\text { Carriage }\end{array}$ & $22.0 \%$ & $\begin{array}{l}25.7 \% \\
p=0.19\end{array}$ & $\begin{array}{l}26.2 \% \\
p=0.18\end{array}$ & $\begin{array}{l}25.0 \% \\
p=0.36\end{array}$ & $26.4 \%$ & $\begin{array}{l}30.1 \% \\
p=0.38\end{array}$ & $57.1 \%$ & $\begin{array}{l}68.8 \% \\
p=0.14\end{array}$ & $62.0 \%$ & $\begin{array}{c}82.9 \% \\
p<0.0001\end{array}$ \\
\hline 'AA' rates & $2.5 \%$ & $\begin{array}{c}1.7 \% \\
p=0.27\end{array}$ & $\begin{array}{c}1.8 \% \\
\mathrm{p}=\mathbf{0 . 3 7}\end{array}$ & $\begin{array}{c}1.7 \% \\
p=0.31\end{array}$ & $3.1 \%$ & $\begin{array}{c}1.7 \% \\
p=0.17\end{array}$ & $15.5 \%$ & $\begin{array}{c}26.7 \% \\
p=0.094\end{array}$ & $16.3 \%$ & $38.6 \%$ \\
\hline
\end{tabular}

T1987

NOD2/CARD15 Mutations in Crohn's Disease Decreases Endogenous Expression of Human Alpha 1 Defensin (DEFA1) After Salmonella Typhimurium Infection in Colonic Epithelial Cells Jesus K. Yamamoto-Furusho, Daniel K. Podolsky

Background: DEFAl is an antimicrobial peptide that is expressed predominantly in Paneth's cells of the ileum. NOD2 mutations include Arg702Trp (tryptophan substituted for arginine at codon 702), Gly908Arg (arginine substituted for glycine at codon 908), and leul007fsinsC (a frameshift mutation that truncates the 33 amino acids) may be involved in the defective response of intestinal epithelial cell lines against to bacteria. Aim: To study the effect of NOD2 mutants in the expression of DEFAl after Salmonella typhimurium infection. Material and Methods: SW480, HT-29, T84, Colo205, LS174 and HCT116 cells were obtained from he ATCC and used for experiments after they had reached $90 \%$ to $100 \%$ confluence. Cells were transfected with a cationic lipid (LipofectAMINE 2000, Invitrogen). An expression plasmid-encoding GFP-tagged NOD2 mammalian expression vector was made. All NOD2 mutants were generated by PCR using the QuickChange Site-Directed Mutagenesis Kit (Stratagene) and were sequenced. Real-time RT-PCR was performed in an ABI Prism 7000 Sequence Detector using SYBR Green JumpStartTM detection system. Total RNA of intestinal epithelial cell lines was extracted by Trizol. For reverse transcription, $2 \mu \mathrm{g}$ of total RNA was transcribed by SuperScript First-strand Synthesis System for reverse transcription polymerase chain reaction. Immunoblotting: Proteins were blotted onto polyvinylidene difluorid membranes and stained by affinity-purified biotinylated anti-DEFAl. Results: DEFAl is expressed in all the colonic epithelial cell lines studied. Expression of DEFAl was increased after infection with non-pathogenic bacteria (E.coli) and pathogenic (Salmonella typhimurium) for at least two hours in colonic epithelial cell lines. Transfection of the three most common NOD2-associated Crohn's disease mutants Arg702Trp, Gly908Arg and leul007fsinsC in several epithelial cell lines with NOD2 endogenous expression showed a significantly decreased of DEFAl in mRNA and protein levels after exposition with Salmonella typhimurium as compare to those cells without infection. Interestingly, DEFAl endogenous expression was not affected by NOD2 mutants after infection with Salmonella in cells which lack NOD2 endogenous expression. Conclusion: NOD2 Crohn's disease mutations are associated with decreased expression of DEFAl after infection with bacteria.

T1988

Defective MDP Signaling in a Subset of Crohn's Patients Not Explained By Common NOD2 Mutations

Vinay K. Katukuri, Phyllissa Schmiedlin-Ren, Raj Jagirdar, Mark Naddaf, Peter D.

Higgins, Kate Kleaveland, Tahira Khan, Ellen M. Zimmermann, Gabriel Nunez

Certain variants of the NOD2/CARD15 gene (C2104T, G2722C, 3020insC) are strongly associated with susceptibility to Crohn's disease (CD) in Caucasian populations. Current literature indicates that the major NOD2 mutations code for proteins that are unable to induce NF-kB activation and increased transcription of inflammatory cytokines in response to muramyl dipeptide (MurNAc-L-Ala-D-isoGln; MDP), a conserved structure of bacterial peptidoglycan. The aim of this study was to determine if NOD2 mutations are accompanied by differences in cytokine expression by peripheral white blood cells exposed to purified bacterial products in vitro. Methods: Heparinized blood was obtained from patients with $\mathrm{CD}$ and from healthy controls. White blood cells were exposed to MDP or Lipid A for 16 hours. Synthesis and release of IL-1, IL-6, IL-8, and TNF-ÿ was measured by ELISA. DNA was isolated from another portion of the blood. Genotyping for the three common NOD2 mutations was done by direct sequencing of PCR products. Results: Eleven CD patients were enrolled in the study. CD patients had mildly active disease with mean CRP 0.6 and mean ESR 12. Single NOD2 mutations were detected in $3 \mathrm{CD}$ and 1 control subject. Two mutations were detected in 2 of the CD patients. Notably, three patients were MDP nonresponders and did not have any of the three common NOD2 mutations. Viability and responsiveness of the peripheral white blood cells was confirmed by their robust elaboration of cytokines in response to Lipid A. IL-1, IL-6, IL-8, and TNF-ÿ production was lower in the cells from CD patients as compared to controls ( $<<0.008,0.019,0.072,0.015$ for IL1, IL-6, IL-8, and TNF-y , respectively); the difference was statistically significant for all but IL-8 whether or not the presence of NOD2 mutations was considered. There was a trend for all cytokines to be expressed at lower levels in response to MDP as the number of mutations increased from 0 to 1 to 2 , but statistical significance was not reached with our small sample size. Conclusion: Patients with $C D$ had decreased cytokine response of peripheral white blood cells to MDP. MDP non responders without common NOD2 mutations may have a novel defect in MDP signalling.

\section{T1989}

Analysis of Positional Candidate Gene HLA-B in Japanese Patients with Ulcerative Colitis

Eiki Nomura, Yoshitaka Kinouchi, Go Imai, Yoichi Kakuta, Hiroko Abe, Masaki Tosa, Atsuhiro Mochida, Kenichi Negoro, Sho Takagi, Seiichi Takahashi, Nobuo Hiwatashi, Tooru Shimosegawa

Background \& Aims: Evidence is accumulating that ulcerative colitis (UC) is a multifactorial disorder, with both genetic and environmental factors. We have previously reported the HLA region where a strong association was observed in UC, especially the centromeric region of class I and the telomeric region of class III by fine mapping using microsatellite markers. HLA-B gene is located in the center of this region. Previous study suggested the association between UC and HLA-B52 using serotyping. Thus, HLA-B is one of positional candidate genes for UC in Japan. The purpose of this study was to perform association study in HLA$B$ by genotyping and also elucidate the association between $H L A-B$ and disease phenotype in Japanese patients with UC. Methods: A total of 310 patients with UC and 284 healthy controls (HC) were included in this case-control study. All subjects were Japanese. Patients were classified according to disease extent, age at diagnosis, need for intravenous intensive steroid therapy and need for surgery. Genotyping of the HLA-B gene was performed by a polymerase chain reaction sequence-specific primer that can classify the gene into 23 allele groups. Allele frequencies were compared between UC and $\mathrm{HC}$ with chi-squared test using a $2 \times 2$ contingency table. $P$ values were corrected by the number of allele groups $(n=23)$ observed in the Japanese population or the number of clinical subgroups. Corrected $\mathrm{P}$ values (Pc) of less than 0.05 were regarded as statistically significant. The strength of association was estimated by the odds ratio (OR). The genotype relative risks (GRR) for homozygous and heterozygous for the risk allele $(B * 52)$ and the population attributable risk (PAR) were estimated. Results: HLA-B*52 and $B^{*} 4002$ allele were associated with UC compared with $\mathrm{HC}$, positively and negatively, respectively (28.5\% of UC vs. $9.7 \%$ of $\mathrm{HC}, \mathrm{Pc}=5.1 \times 10^{-14}$ $\mathrm{OR}=3.73)(7.9 \%$ vs. $15.0 \%, \mathrm{Pc}=0.0028, \mathrm{OR}=0.49)$. In the subgroup analysis according to phenotypes, none of the HLA-B alleles except $B * 52$ showed any disease phenotype-genotype associations. The allele frequency of $B * 52$ in patients diagnosed before $39 y 0$ was significantly higher than that in patients diagnosed after 40yo ( $\mathrm{Pc}=0.0045, \mathrm{OR}=2.04)$. The GRR for homozygous and heterozygous for $B * 52$ were 12.79 and 3.91 , respectively. The PAR regarding $B * 52$ was $38.3 \%$. Conclusions: High value of the PAR strongly suggest that HLA-B*52 itself determines susceptibility to UC in Japan and may affect earlier onset of the disease. HLA$B * 4002$ appears to be a disease resistance allele.

\section{T1990}

Age and Gender Dependent Distribution of the DLG5 R30q Variant in CD Patients and Healthy Controls

Liesbet Henckaerts, Robert Vlietinck, Isabelle Verstreken, Karolien Claes, Nele Vanschuerbeek, Steven Boonen, Paul Rutgeerts, Severine Vermeire

Introduction and aims: The inflammatory bowel diseases (IBD), Crohn's disease (CD) and ulcerative colitis (UC), are chronic gastrointestinal diseases of unknown etiology, but with a important genetic background. Recently, the R30Q variant of the disc large homolog 5 (DLG5) gene on 10q23 has been reported to be associated with CD in European patients, but we could not confirm these findings in a Flemish IBD population. Recent data suggest that age and gender effects could be responsible for the lack of replication of this gene. We therefore wanted to asses the influence of age and gender on the prevalence of the DLG5 R30Q variant in our cohort of CD patients, as well as in a large cohort of healthy controls. Methods: A total of $730 \mathrm{CD}$ patients (294 male; from 4 to $92 \mathrm{y}$ ) and 1101 healthy controls (618 male; from 1 to 98 y) were genotyped for the DLG5 R30Q variant. Results: In the CD group, no difference in allele frequency for DLG5 R30Q was observed between males (10.5\%) and females $(10.3 \%)$. When adding age as a covariate, we noted a higher allele frequency in men older than 40 years $(\mathrm{p}=0.028)$. Subsequently, we analysed the group of healthy controls, in function of both gender and age. Here, a higher allele frequency of DLG5 R30Q was observed in women $(12.4 \%)$ when compared with men $(9.6 \%, \mathrm{p}=0.036)$. An age effect was not seen in the healthy controls for either sex. Conclusion: There appears to be an age and gender effect on the frequency of the DLG5 R30Q variant in a large cohort of healthy controls. This may influence study results and might explain, in part, the divergent replication findings for the DLG5 gene. These findings furthermore stress the importance, in studying complex traits, of a large control cohort that is well matched, including for sex and age. The observed difference in allele frequencies between males en females could be due to gender dependent selection during development, but further research is needed in order to determine the role of the DLG5 R30Q variant in healthy subjects. Investigating the effect of the genetic variants on the function and expression of the gene might help to clarify its possible role in the development of IBD. 
T1991

Clinical and Molecular Characteristics of Isolated Colonic Crohn's Disease (CD)

Laura Hancock, Tariq Ahmad, John B. Beckly, Bryan F. Warren, Neil J. Mortensen, Derek P. Jewell

Background: Clinical, serological and molecular data support the existence of discrete subsets of $\mathrm{CD}$ defined by location of disease. Little is known about the epidemiology and natural history of isolated Crohn's disease of the colon (Montreal Classification L2) because most studies have not accurately distinguished L2 from L3 disease (ileo-caecal). Aims:1. To describe the clinical features and natural history of isolated colonic $C D$ in a rigorously characterised patient cohort. 2. To confirm the association with HLA-DRBI*0103, reported in smaller cohorts, and to investigate its role in predicting disease course and need for surgery. Methods: Patients with L2 disease were identified from a database of $1318 \mathrm{CD}$ patients. Only patients with a normal small bowel enema (70\%), ileoscopy alone (30\%) or both (20\%) were included. No patients had capsule endoscopy. HLA genotyping was performed using PCR-SSP. Results: 136 (10.3\%) patients were classified with L2 disease after a median follow-up of 10.8 years (range 1.4-39.8). The mean age at diagnosis was 30.7 years. L2 disease was more common in women $(74.3 \%$ vs. $61.0 \% ; P=0.001 ; R R=1.7)$ and in never smokers ( $52.2 \%$ vs. $41.3 \% ; P=0.007 ; R R=1.5) .29 .4 \%$ and $14.0 \%$ reported a family history (1st or 2nd degree) of CD and UC respectively. Stricturing colonic disease was noted in $10 \%$ of patients. $12 \%$ of the entire cohort received $\geq 1$ Infliximab infusion and $19 \%$ underwent colonic resection for severe disease (cumulative risk at $2 \mathrm{yrs}-10.6 \%$, 5yrs $17.1 \%, 10 \mathrm{yrs}-32.8 \%)$. We confirmed the association with HLA-DRB1*0103 (14.7\% cases vs. $2.7 \%$ controls; $P=5.5 \times 10-9 ; R R=3.2$ ) and report the novel association of this allele with time to first surgical event (Log Rank $P=0.002$ ) and time to first 'severity event' (resection/ diversion ileostomy/Infliximab) $(P=0.001)$. Conclusions: This study reports the clinical manifestations of isolated colonic CD. We confirm the association with HLA-DRBl*0103 and further demonstrate that this allele may predict disease course

\section{T1992}

CARD15 Susceptibility and Pediatric Onset Crohn's Disease (CD): Increased Risk in Male Gender

Subra Kugathasan, Umesh Babusukumar, Tao Wang, Manu Sood, Erin McGuire, Ulrich Broeckel

Gender difference in disease presentation and outcome is complex and needs thorough evaluation in $C D$. The overall incidence and prevalence of $C D$ among females exceeds that of males. The US, Canadian and UK studies involving children have reported nearly a 2:1 male-to-female ratio in the incidence of pediatric onset $\mathrm{CD}$, but not in ulcerative colitis (UC). Recent genetic progress documents that nearly $40 \%$ of Caucasian CD children have CARD15 mutations. We investigated the influence of gender on CARD15 by family based association and case control models in our population based, pediatric onset inception IBD cohort. Only Caucasian IBD was studied. 273 CD (172 trios), 98 UC (72 trios)\& 479 controls were genotyped for CARD15 variants (R702W, G908R, 3020InsC). There were 162 male and 111 female $C D$ children. The mean age of onset for males were 12.1 and females 12 yrs. The allele frequencies of CARD15 markers were not significantly different between males and females. TDT performed after stratified by gender of the probands, showed a significant association for all 3 CARD 15 markers with male CD, but not in female $\mathrm{CD}$ children (table 1). A significant association was seen in both male and female $\mathrm{CD}$ children compared with controls for CARD15 markers by case control analysis. However, a significant gender effect was seen for $\mathrm{CD}$ susceptibility in males when a logistic regression was performed adjusting for gender (table 2). In addition, a much stronger male gender effect for association was seen when all 3 CARD 15 markers were combined ( $p<0.0001$ ). Kaplan Meier analysis showed gender has no effect on the age of onset in children with CARD15. No differences in genotype-phenotype correlations were seen between males and females in CD. CARD15 was not associated in UC by TDT and case-control. Conclusions: A strong gender effect in the association of CARD15 and CD suggest that CD susceptibility to CARD15 is increased in male children. This novel finding is only seen in children and needs further investigation since several CARD15 studies involving adults did not report gender differences in CD. TDT Analysis of 172 Families stratified by gender of the proband

\begin{tabular}{|c|c|c|c|c|c|c|c|c|}
\hline \multirow{2}{*}{ Marker } & \multicolumn{2}{|c|}{ Allele Frequency } & \multicolumn{2}{|c|}{ Transmitted/ untrasmiitted } & \multicolumn{2}{|c|}{ TDT $\left(\mathrm{X}^{2)}\right.$} & \multicolumn{2}{|c|}{ P value } \\
\cline { 2 - 10 } & Male & Female & Male & Female & Male & Female & Male & Female \\
\hline R702W & 11.4 & 8.6 & $4 / 19$ & $6 / 7$ & 10.67 & 0.25 & 0.002 & 0.78 \\
\hline G908R & 6.2 & 5.7 & $4 / 12$ & $1 / 5$ & 4.76 & 3.57 & 0.04 & 0.1 \\
\hline 3020InsC & 10.4 & 11 & $6 / 20$ & $7 / 10$ & 7.54 & 2.25 & 0.006 & 0.47 \\
\hline
\end{tabular}

Gender effects on CARD15 mutation by logistic regression

\begin{tabular}{|c|c|c|c|}
\hline & Odds ratio-male gender on CARD15 & $95 \%$ CI & P value \\
\hline R702W & 1.97 & $1.28-3.5$ & 0.01 \\
\hline G908R & 4.2 & $2.3-7.9$ & 0.03 \\
\hline 3020InsC & 5.4 & $3.2-9.0$ & 0.03 \\
\hline
\end{tabular}

T1993

NOD2/CARD15 Variants Are a Risk Factor for Lung Complications After Stem Cell Transplantation (SCT)

Julia Brenmoehl, Gerhard Hildebrandt, Daniel Wolff, Bernd Hertenstein, Hildegard T.

Greinix, Christian Schulz, Anne M. Dickinson, Juergen Schoelmerich, Reinhard

Andreesen, Gerhard Rogler, Ernst Holler

Introduction: Recently our group has shown that NOD2/CARD15 variants (SNPs) not only are a risk factor to develop Crohn's disease but also play a major role for the outcome after SCT. As NOD2/CARD15 is involved in the recognition of bacterial translocation which also takes place in the lung we supposed that it could also play a role for lung diseases. Bronchiolitis obliterans syndrome (BOS) is a life-threatening complication after allogeneic SCT with high mortality. Risk factors and pathophysiology have yet to be defined. NOD2/CARD15 is expressed not only in monocytes/macrophages and intestinal epithelial cells IEC but also in bronchial epithelial cells. Methods and patients: The association of NOD2/CARD15 SNPs (SNP 8, 12, 13) with the occurrence of BOS was investigated 244 donor/recipient (D/R) pairs of patients receiving allogeneic SCT. Follow-up was performed for a mean of 1243 172 days (range: 327-2149). BOS was diagnosed by pathology or by functional airway obstruction ( $\mathrm{FEVl}</=80 \%$ of predicted). Results: 12 patients developed BOS with a mean time point of diagnosis at 678112 days after SCT (range: 186-1407). NOD2/CARD15 variants were observed at higher frequencies in D/R pairs developing BOS (donor: $60.0 \%$ vs. $12.9 \%, p=0.001$; recipient: $50.0 \%$ vs. $15.0 \% ; p=0.007) .50 \%$ of patients with BOS died between day +327 and day +1582 (mean: 844 195), whereas 6 patients are still alive (mean follow-up: 1642170 days). Incidence of BOS rose from 1.7\% (3/174) in D/R pairs without mutated SNPs to $10.0 \%(6 / 60)$ in pairs with mutated alleles in either donor or recipient $(\mathrm{p}=0.01)$ and to $30.0 \%(3 / 10)$ in pairs with mutated alleles in both donor and recipient $(\mathrm{p}=$ 0.009 ). Survival did not differ between patients with BOS in relation to the presence/ absence of NOD2/CARD 15 mutations. Finally, we analyzed whether other transplant-related parameters contributed to the incidence of BOS. Conditioning regimen intensity, stem cel source, donor type (matched unrelated donor vs. HLA-identical sibling), recipient age, donor or recipient gender, and acute GVHD were not associated with BOS development, whereas chronic GVHD was confirmed as a risk factor for BOS $(\mathrm{p}=0.01)$. Conclusion: Variations in the NOD2/CARD15 gene are not only associated with an increased risk to develop Crohn's disease and GvHD after SCT but also with the development of lung disorders such as BOS This indicates that either NOD2/CARD15 directly plays a role in the bronchial epithelial cells or bacterial translocation in the gut is relevant for lung disease.

\section{T1994}

The Single Nucleotide Polymorphism C3435t in the MDRl Gene Predicts the Efficacy of Steroid Tapering in Inflammatory Bowel Disease

Konstantinos Katsanos, Marc Ferrante, Liesbet Henckaerts, Marie Pierik, Karolien Claes, Nele van Schuerbeek, Marie Joossens, Sofie Joossens, Gert van Assche, Epameinondas V. Tsianos, Paul Rutgeerts, Severine Vermeire

BACKGROUND: Steroids are 'pumped out' of target cells by means of the P-glycoprotein pump (P-gp), the product of the multi-drug resistance (MDR) gene. A significant correlation between single nucleotide polymorphisms (SNPs) in the MDRl gene and P-gp expression and function has been reported. There are no studies investigating the effect of MDRl variants on the response to steroids in inflammatory bowel disease (IBD). AIM: To investigate the relationship between MDR1 variants and the ability of systemic steroid tapering in IBD patients. PATIENTS-METHODS: The prevalence of the MDR1 SNPs G2677T/A (Ala893Ser/ Thr), and C3435T(Ilel145Ile) was investigated using PCR-RFLP in 167 IBD patients (104 Crohn's disease, 63 ulcerative colitis) who were initiated on systemic steroids ( $1 \mathrm{mg} / \mathrm{kg})$ together with azathioprine. IBD patients were grouped as unable to taper steroids (group 1 ), able of partial steroid tapering [steroids remaining $>50 \%$ of the initial dose] (group 2), able of significant steroid tapering [maintenance steroids decreased to $<50 \%$ of the initial dose (group 3) and able of complete steroid tapering (group 4). RESULTS: Seventy-seven patients (45.9\%) were unable to taper steroids (group 1) while partial, significant and complete steroid tapering was recorded in $28(17 \%), 18(11.2 \%)$ and $44(25.9 \%)$ of patients respectively. The patients in groups 3 and 4 had a significantly lower prevalence of the MDRl-3435T mutated allele $[\mathrm{p}=0.027, \mathrm{OR} 0.58(95 \% \mathrm{CI} 0.35-0.94)]$ compared to patients in groups 1 and 2 (table). No significant differences in steroid tapering among the different groups were noticed for the G2677T/A SNP. CONCLUSIONS: In IBD patients the C3435T SNP in the MDRl gene correlates with steroid tapering. We found that patients carrying the MDRl 3435T allele were more resistant to steroid tapering, further underscoring that this variant contributes to the P-gp pump regulation. 


\begin{tabular}{|c|c|c|}
\hline IBD groups of steroid sparing & MDR1 3435 T allele \% & \% TT genotype \\
\hline Group 1 (unable to taper) & 61 & 32 \\
\hline Group 2 (partial tapering) & 57.1 & 32.1 \\
\hline Group 3 (significant tapering) & 46.9 & 18.7 \\
\hline Group 4 (complete tapering) & 46.1 & 18 \\
\hline
\end{tabular}

T1995

NO Association of Polymorphisms in the Pparg Gene Encoding for Peroxisome Proliferator-Activated Receptor- $\gamma$ (Ppar $\gamma$ ) with Inflammatory Bowel Disease Juergen Glas, Helga P. Torok, Laurian Tonenchi, Christian Markus, Martin Wetzke, Stephan Brand, Peter Lohse, Wolfram Klein, Joerg T. Epplen, Uwe Schiemann, Thomas Mussack, Thomas Ochsenkuehn, Matthias Folwaczny, Christian Folwaczny

Background: PPAR $\gamma$ is a member of the peroxisome proliferator-activated receptors (PPARs), a superfamily of nuclear hormone receptors. PPARy is considered to be anti-inflammatory limiting NF-KB activation similar to NOD2/CARD15. Recently Pparg was identified as a susceptibility gene in Crohn's disease (CD) in a mouse and in a cohort-based study investigating three polymorphisms in the human PPARG gene (SNPs 1-3) significantly associations with $\mathrm{CD}$ were detected. The PPARG gene maps to a IBD linkage region on chromosome $3 \mathrm{p} 25$. Herein SNPs 1-3 were assessed in an inflammatory bowel disease population and additionally tested for potential interactions with CD associated mutations in NOD2/CARD15. Aim: The present study sought to investigate whether polymorphisms of the PPARG gene are associated with inflammatory bowel disease. Methods: The study population comprised 620 patients with $C D, 368$ patients with ulcerative colitis (UC) and 791 healthy, unrelated controls. Genotyping of the three SNPs was performed by PCR and RFLP analysis. Statistical analysis was performed employing $\chi 2$-test. Results: The allele frequencies of all three polymorphisms showed no significant differences between Crohn's disease or ulcerative colitis when compared to the control group (table 1). Moreover, no significant associations could be found concerning genotype and phenotype frequencies. Moreover, no significant associations were detected with respect to NOD2/CARD15 status in CD. Conclusion: Herein, polymorphisms within the PPARG gene displayed no significant associations with inflammatory bowel disease an could not confirm the predescribed association with Crohn's disease. It has to be considered that the previously found association was observed in a relatively small group of $134 \mathrm{CD}$ patients. In conclusion, the present study could not give evidence for a role of the PPARG gene in inflammatory bowel disease and also no evidence for interactions between PPARG and NOD2/CARD15.

Allele frequencies of PPARG polymorphisms in Crohn's disease and ulcerative colitis

\begin{tabular}{|c|c|c|c|}
\hline SNP1 & Crohn's disease & Ulcerative colitis & Controls \\
\hline G & $950(76.7 \%)$ & $562(76.4 \%)$ & $1215(76.8 \%)$ \\
\hline A & $288(23.3 \%)$ & $174(23.6 \%)$ & $367(23.2 \%)$ \\
\hline$\Sigma$ & 1238 & 736 & 1582 \\
\hline SNP2 & & & $1409(89.7 \%$ \\
\hline G & $1101(90.0 \%)$ & $654(90.3 \%)$ & $163(10.3 \%)$ \\
\hline A & $123(10.0 \%)$ & $70(9.7 \%)$ & 1582 \\
\hline$\Sigma$ & 1224 & 724 & $1345(85.8 \%)$ \\
\hline SNP3 & & & $223(14.2 \%)$ \\
\hline C & $1082(87.3 \%)$ & $628(86.3 \%)$ & 1568 \\
\hline T & $158(12.7 \%)$ & $100(13.7 \%)$ & 728 \\
\hline$\Sigma$ & 1240 & & \\
\hline
\end{tabular}

\section{T1996}

The $1059 \mathrm{~g} / \mathrm{C}$ Polymorphism in the Crp Gene Is Associated with Involvement of the Terminal Ileum in Patients with Crohn's Disease

Dominik Thalmaier, Julia Dambacher, Astrid Konrad, Veronika Schachinger, Simone Pfennig, Fabian Schnitzler, Tanja Staudinger, Katrin Hofbauer, Jan-Michel Otte, Julia Seiderer, Cornelia Tillack, Burkhard Goeke, Thomas Ochsenkuehn, Peter Lohse, Stephan Brand

Background: The level of C-reactive protein (CRP) is used to evaluate inflammatory activity in Crohn's disease (CD). Recently, a single nucleotide polymorphism (SNP) in exon 2 of the CRP gene $(1059 \mathrm{G} / \mathrm{C})$ has been reported. Aims: Our aim was to analyze the influence of this CRP polymorphism on CD phenotype. Methods: Using restriction fragment length polymorphism analysis, genomic DNA from 223 patients with CD and from 199 unrelated controls was analyzed for the $1059 \mathrm{G} / \mathrm{C}$ substitution in the CRP gene. Disease phenotypes were classified according to the Vienna classification of CD (Inflamm Bowel Dis 2000;6:8 15). Results: No difference was observed in the genotype frequencies between $C D$ patients and the control group, although homozygous $\mathrm{C} / \mathrm{C}$ carriers were detected only among $\mathrm{CD}$ patients ( $p=0.13$ ). In the $C D$ group, $90.1 \%$ were wildtype $G / G, 8.1 \%$ heterozygous $G / C$, and $1.8 \%$ homozygous $\mathrm{C} / \mathrm{C}$. In the control population, no homozygous $\mathrm{C} / \mathrm{C}$ carriers were detected and $6.6 \%$ were $\mathrm{G} / \mathrm{C}$ heterozygotes, while $93.4 \%$ had two wildtype alleles. However, while only 117 of 201 patients with the wildtype G/G genotype (58.2\%) had an involvement of the terminal ileum, the terminal ileum was affected in 17 of $18(94.4 \%)$ of the heterozygous $\mathrm{G} / \mathrm{C}$ carriers $(\mathrm{p}=0.002)$. Similar genotype frequencies were observed for the $1007 \mathrm{fs}$ CARD15 mutation, for which we demonstrated ileal involvement, in wildtype (17.6\%) and heterozygous carriers (16.7\%) suggesting that the observed association between the $1059 \mathrm{G} / \mathrm{C}$ polymorphism and ileal disease location is independent of the CARD15 $1007 \mathrm{fs}$ mutant. Consistent with this finding was the observation, that none of the $\mathrm{G} / \mathrm{C}$ heterozygotes had disease involvement restricted to the colon only (L2 phenotype according to the Vienna classification), while the L2 phenotype was found in $21.4 \%$ of patients with two $G$ alleles $(p=0.027)$. Moreover, while only 2 of 22 heterozygous and homozygous carriers of the $C$ allele $(9.1 \%$ ) were diagnosed of a stricturing disease phenotype according to the Vienna classification, $68.2 \%$ of the hetero- and homozygotes had a penetrating phenotype as compared to $53.2 \%$ patients with a penetrating phenotype and $27.9 \%$ with a stricturing phenotype in the group with the $\mathrm{G} / \mathrm{G}$ genotype ( $\mathrm{p}=0.06$ ). Conclusions: Heterozygosity for the CRP 1059G/C polymorphism is associated with disease involvement of the terminal ileum and a tendency to develop the more severe penetrating disease phenotype. Homozygous $\mathrm{C} / \mathrm{C}$ carriers were found only in the $\mathrm{CD}$ group. Given the overall low frequency of the homozygous $\mathrm{C} / \mathrm{C}$ genotype, larger trials are necessary to evaluate if homozygosity for the $\mathrm{C}$ allele is associated with CD.

\section{T1997}

MDR1 As a Susceptibility Gene in Inflammatory Bowel Disease: Results of a Large Case-Control and Family-Based Analysis

Korinne Bradley, Alissa Walsh, Beth Fowler, Susan Mason, Douglas Lincoln, Rebecca I. Cooley, Graham L. Radford-Smith

Background: The MDRl gene located at $7 \mathrm{q} 22$ has been implicated as a susceptibility gene for ulcerative colitis (UC) and Crohn's disease (CD). Results of 5 studies (5 case-control, l pedigree) are conflicting. The gene codes for P-glycoprotein 170, an efflux transporter pump expressed on epithelial cells and lymphocytes. A murine knockout develops UC-like disease while overexpression has been associated with treatment resistance in IBD patientsl. At least two functional SNPs have been identified, C3435T and G2677T/A. Aims: To establish whether MDRI C3435T and G2677T/A contribute to IBD susceptibility using both casecontrol and family based approaches. To perform genotype-phenotype analysis if association is demonstrated. Methods: A population-based cohort of 1044 IBD patients were recruited for this study from the North Brisbane region. Population controls (405) were drawn from the same geographical region and matched for sex and ethnicity. Phenotyping was based upon original records of clinical history, histology, and radiology and confirmed by patients. Genotyping was performed by PCR-RFLP and results confirmed by sequencing in $10 \%$ of samples. Results: Analysis by genotype revealed significant differences in C3435T for UC $(\mathrm{P}<0.001)$ and $\mathrm{CD}(\mathrm{P}=0.04)$ compared to controls. An allele association analysis demonstrated significance for $\mathrm{CD}(\mathrm{p}=0.02)$ but not for UC. The results for $\mathrm{G} 2677 \mathrm{~T} / \mathrm{A}$ were not significant. A transmission disequilibrium test (TDT) using $89 \mathrm{UC}$ trios and $120 \mathrm{CD}$ trios for analysis of the C3425T SNP did not reach significance either for UC ( $p=0.26)$ or for CD ( $p=0.62)$. Phenotype-genotype analysis was based upon 398 UC patients and $450 \mathrm{CD}$ patients. The UC data demonstrated significant differences in C3435T genotype between patients suffering acute severe colitis, strictly based on the Truelove-Witts criteria, and those with no history of this complication $(\mathrm{p}=0.006$ ). This was based on a higher $\mathrm{CC}$ and lower TT carriage rate by those with severe disease. Colectomy for either acute, severe disease or chronic refractory disease did not show significant association with genotype $(\mathrm{p}=0.13)$. There were no significant findings for $\mathrm{CD}$. Conclusions: This large case-control study supports association between the C3435T MDRI SNP and UC but this is not confirmed by our TDT analysis. This may be due to lack of power in the latter. Phenotype-genotype analysis supports a possible role for increased MDRl expression in patients with severe disease. The issue of population stratification may have been underestimated for this gene, and a larger consortium-based investigation would be appropriate to address this. 1Farrell et al. Gastroenterol 2000 Feb;118(2):279-88

\section{T1998}

Is There Any Difference in Phenotypes Between Familial and Sporadic Cases of Inflammatory Bowel Disease? Results from a Population Based, Follow-Up Study

Magne Henriksen, Jorgen Jahnsen, Idar Lygren, Morten H. Vatn, Bjorn Moum

Background: The risk of inflammatory bowel disease (IBD) in family members of patients with ulcerative colitis (UC) and Crohn's disease (CD) is increased. If phenotypes in familial clustering differ from sporadic cases is not clear. However, there are few data from population based, follow-up studies. Aim: To compare phenotypes in familial IBD with sporadic IBD at diagnosis and after five years of follow-up. Methods: All patients $(n=843)$ diagnosed with IBD in four different counties in southern Norway in the period 1990 - 94 were followed prospectively (the IBSEN cohort). Diagnosis were re-evaluated after one and five years of follow-up. CD patients were retrospectively classified according to the Vienna classification at diagnosis and after five years. The extent of inflammation at diagnosis and the maximal extent of disease during the follow-up period was recorded for UC patients. Results: After five years of follow-up 454 patients with UC and 200 patients with CD were alive and had data sufficient for analysis. At diagnosis 7,5\% of the patients had a first degree relative with IBD increasing to $11,5 \%$ after five years ( $10,1 \%$ of UC patients and $14,5 \%$ of $\mathrm{CD}$ patients). $\mathrm{CD}$ patients with familial IBD were younger at diagnosis compared to sporadic cases (mean age 26,9 vs 34,1 median age 25,0 vs $29,0 \mathrm{p}=0.03$ ). This difference in mean age was only seen in patients with colonic CD. No difference in age was observed between UC patients with familial IBD compared to sporadic cases (mean age 41,4 vs 39,9 $p=0.6)$. In $C D$ patients no difference in localisation or behaviour was observed at diagnosis $(\mathrm{p}=0.1$ and $\mathrm{p}=0.7)$ or after five years of follow-up $(\mathrm{p}=0.3$ and $\mathrm{p}=0.4)$. In UC patients no difference in extent was observed at diagnosis or after five years $(\mathrm{p}=0.2$ and $\mathrm{p}=0.5)$. Conclusions: CD patients with a family history of IBD and colonic involvement was younger at diagnosis compared to sporadic cases. In $C D$ patients no difference in localisation or behaviour was observed between familial and sporadic cases. Furthermore, in UC patients no difference in extent was observed between familial and sporadic IBD. 
T1999

Na Transporters NHE3 and Na/K-ATPase, NHE3-Regulatory Factorl (NHERF1) and the Intracellular Chloride Channel Clc5 Are Down Regulated in IBD: Probable Contribution to IBD Diarrhea

Xuhang Li, Sean Sullivan, Mark Donowitz, Themos Dassopoulos, Steve Brant, Carmen Cuffari, Yueping Chen

Diarrhea is one of most common symptoms in IBD. Decreases in Na absorption appear to be the major electrolyte transport change in IBD based on mouse intestinal studies. To increase understanding of intestinal $\mathrm{Na}$ absorption in IBD, we tested the hypothesis that inhibition of $\mathrm{Na}$ absorption was due to changes in expression of intestinal $\mathrm{Na}$ transport proteins. The expression of multiple intestinal $\mathrm{Na}$ transporters were examined, including $\mathrm{Na} / \mathrm{H}$ exchanger 3 (NHE3) and $\mathrm{Na} / \mathrm{K}-\mathrm{ATPase}$, as well as the NHE3 regulatory protein $\mathrm{NHERF} 1$ and an intracellular $\mathrm{Cl}$ channel important for trafficking, $\mathrm{ClC} 5$. Studies examined 55 intestinal biopsies from 40 patients [ 43 sigmoid biopsies ( 12 normal, 13 UC active, 10 $\mathrm{CD}$ active, $5 \mathrm{UC}$ and $3 \mathrm{CD}$ inactive/unaffected) and 12 ileal biopsies (4 normal, 8 active CD)]. Analysis was done by SDS-PAGE and Western blotting, using GAPDH as a loading control. In $80 \%$ of biopsies from active UC and CD (sigmoid and ileal biopsies), NHE3 was down regulated. In addition, NHE3 was down-regulated, although to a less extent in $50 \%$ of sigmoid biopsies from inactive IBD. There was usually a coordinated down-regulation of multiple ion transporters, including NHE3, Na-K-ATPase, and $\mathrm{ClC} 5$ in biopsies from active $\mathrm{CD}$ or UC, although the change in Na-K-ATPase and ClC5 were less dramatic than that of NHE3. NHERF1, an NHE3 regulatory factor, was also down regulated in mucosa of most active CD or UC ( $>80 \%)$. In summary, (1) Na transport proteins, NHE3 and Na-K-ATPase as well as proteins involved in regulation of trafficking, $\mathrm{ClC} 5$, and in regulation of NHE3 function, NHERFl, are usually down-regulated in IBD. (2) The down regulation occurs more in the areas of active disease, but is also present in areas without active disease, suggesting systemic mechanisms. We conclude (1) The diarrhea of IBD is due to decreased $\mathrm{Na}$ absorption with the mechanisms including decreased expression of $\mathrm{Na}$ transport proteins, including at least NHE3 and Na-K-ATPase, and of the proteins that are involved in NHE3 regulation (NHERFl and $\mathrm{ClC}$ ). (2) In considering mechanisms for this coordinated regulation, common transcriptional elements or mechanisms of message stability that respond to inflammatory mediators of IBD should be considered.

\section{T2000}

Is There a Crosstalk Between DLG5 R30q and NOD2/CARD15 Or TLR4 Mutations in Hungarian Patients with Crohn's Disease? Phenotype-Genotype Correlations

Peter L. Lakatos, Karolien Claes, Tamas Molnar, Simon Fischer, Agota Kovacs, Istvan Altorjay, Claudia Willheim-Polli, Zsolt Tulassay, Ferenc Szalay, Janos Papp, Hungarian LBD Study Group, Paul Rutgeerts, Peter Ferenci, Severine Vermeire, Laszlo Lakatos

AIM: Mutations of NOD2/CARD15 gene predispose for Crohn disease (CD) and are associated with fibrostenosing behavior and recent data from Germany have suggested that specific haplotypic variants of the DLG5 gene on chromosome 10q23 may be associated with susceptibility to inflammatory bowel disease (IBD). In this study, we investigated possible associations between common NOD2/CARD15 mutations, D299G TLR4 polymorphism and the carriage of DLG R30Q risk allele in Hungarian CD patients. METHODS: 475 unrelated patients with $C D$ (m/f: $265 / 262$, age: $37.1 \pm$ D 12.6 years, duration: $8.4 \pm 7.0$ years) and 150 healthy subjects were included. DNA was screened for possible NOD2/CARD15 mutations (SNP8, SNP12 and SNP13) by denaturing-HPLC and if found mutations were confirmed by direct sequencing. TLR4 D299G and DLG5 R30Q were tested by PCR-RFLP. Clinical phenotypes - familial disease, age, age-at-onset, duration, location, disease behavior, perianal involvement, extraintestinal manifestations (arthritis, PSC, ocular and cutaneous), need for steroid or azathioprine, steroid resistance, need for surgery or multiple resections and smoking habits - were determined by questionnaire. Comparisons between groups were made by $\chi 2$ test using SPSS13.0 software. RESULTS: $20.8 \%$ of CD patients were carriers of the $113 \mathrm{~A}$ variant DLG5 allele compared to $28.0 \%$ of 150 healthy controls, associated with a tendency with decreased frequency for the $113 \mathrm{~A}$ allele (OR: $0.677,95 \% \mathrm{CI}$ : 0.44 1.03, $\mathrm{p}=0.07$ ). Similarly, TLR4 variant allele frequency was not different between patients and controls ( $10.1 \%$ vs. $12.6 \%)$ and only carriage of variant NOD2 allele was identified to confer risk (32.2\% vs. $12.6 \%$, OR: $3.3,95 \% \mathrm{CI}: 1.9-5.5$ ). There were no associations between DLG5, NOD2 and/or TLR4 in determining clinical phenotypes. In addition, solely carriage of variant NOD2 allele but not variant DLG5 or TLR4 allele were associated with disease location, behavior and increased risk for surgery in a logistic regression model. CONCLUSION: These results confirm in large cohorts of Hungarian CD patients the association of variant NOD2 alleles with disease susceptibility, ileal disease, stricturing disease behavior and risk for surgery. In contrast, presence of DLG5 R30Q or TLR4 D299G variant alleles was not different from controls as well as no associations were found between the carriage of these variants, NOD2 mutations and/or disease phenotype.

\section{T2001}

Mica and Micb Microsatellite Polymorphisms Are Associated with Ulcerative Colitis in Chinese Patients

Bing Xia, Min Lv, Jin Li, Mei Ye, Zhengguo Hu

Aims The human MHC class I chain-related gene A and B (MICA and MICB) play a role in regulating protective responses by intestinal epithelial $V \delta l \gamma \delta \mathrm{T}$ cells and the polymorphisms of MICA and MICB have shown to be related to several autoimmune diseases. The present study was aimed to investigate the association of the microsatellite polymorphisms in the exon 5 of MICA and in the intron 1 of MICB with the susceptibility to ulcerative colitis (UC) in Chinese population. Methods The microsatellite polymorphisms of the MICA and MICB were genotyped in unrelated 127 Chinese patients with UC and 193 ethnically matched healthy controls by a semiautomatic fluorenscently labeled PCR method. All the subjects were the Chinese Han origins. Results The frequency of MICA-A5.1 allele (29.0\% vs. $17.4 \%, \mathrm{P}=0.0010, \mathrm{Pc}=0.0050, \mathrm{OR}=1.936,95 \% \mathrm{CI}: 1.310-2.863)$, MICB-CAl 8 allele
(14.0\% vs. $5.8 \%, \mathrm{P}=0.0016, \mathrm{Pc}=0.024, \mathrm{OR}=2.637,95 \% \mathrm{CI}: 1.443-4.820$.) and MICA-A5.1 A5.1 homozygous genotype ( $18.5 \%$ vs. $7.0 \%, P=0.0032, \mathrm{Pc}=0.048, \mathrm{OR}=3.036,95 \% \mathrm{Cl}$ 1.447-6.372) were significantly increased in UC patients compared with healthy controls. And logistic regression analysis adjusted the effects of gender and age at onset confirmed our results. Moreover MICB-CA18 allele was significantly increased in frequency in the female UC patients compared with the female healthy controls ( $18.3 \%$ vs. $4.1 \%, \mathrm{P}=0.0006$ $\mathrm{Pc}=0.0080, \mathrm{OR}=5.224,95 \% \mathrm{CI}: 1.940 \sim 14.069)$. The comparison between MICA and MICB alleles and the subgroups of UC according to the extent of disease and extraintestina manifestations were not shown any association. MICB-CA18 was significantly in linkage disequilibrium with MICA-A5.1 although the MICA-A5.1/MICB-CA18 haplotype was no associated with UC after Bonferroni multiple correction. No MICA-MICB haplotypes were displayed significant differences between UC or UC subgroups and the healthy controls. Conclusions The MICA-A5.1 allele, MICA-A5.1 homozygous genotype and MICB-CAl8 allele were significantly associated with UC in Chinese population, but MICA and MICB haplotypes have not shown these significant associations.

\section{T2002}

Mdrl, Runx3 and TGF $\beta$ Rii Pathways Implicated in Human IBD Laura E. Harrell, Emily C. Baechler, Timothy W. Behrens, Sam M. Bracamonte, Betul Yilmaz, Judy H. Cho

"Background:" There are numerous experimental mouse models where induced deficiencies in host genes result in inflammatory bowel disease (IBD), but the relevance of corresponding pathways in human IBD is incompletely defined. It would be anticipated that pathogenic heterogeneity affecting different pathways would exist between individuals with IBD. Limited numbers of expression microarray studies from intestine have been reported implicating various impaired pathways in IBD, notably the pregnane X receptor (PXR) transcription factor pathway involved in xenobiotic responses and MDRl transcription. We sought to define the most differentially regulated transcripts from peripheral blood leukocytes (PBL) from individuals with active Crohn's disease (CD) compared to controls in order to identify transcripts also implicated in experimental mouse models. "Methods:" Nine patients with a history of $\mathrm{CD}$ and a Harvey-Bradshaw CDAI score of $\geq 5$ and 8 healthy controls were examined. RNA was extracted from whole blood and hybridized to Affymetrix U133 2.0+ GeneChips. Microarray data files were processed using GeneData Expressionist Refiner 1.0 Pro. Patients and controls were compared using the following criteria: i) p-value $<0.001$ from an unpaired student's t-test, ii) fold change $(\mathrm{FC})>1.5$ in expression between controlmean and patient mean, and iii) absolute difference in expression $>100$ between the means of the two groups. "Results:" Comparing CD to controls, 299 differentially expressed genes (200 down-regulated, 99 up-regulated) were identified. Among down-regulated transcripts, three genes were identified for which targeted deficiency in mice result in IBD; RUNX3 MDRl, TGF $\beta R I I$ transcripts demonstrated $-3.1,-2.1$ and -1.7 fold decreased expression, respectively. Some heterogeneity in down-regulation of these transcripts was observed, but overall, down-regulation of these transcripts was very consistently observed in all $C D$ patients. Down-regulation of PXR induced genes was not a general feature in $C D$, being observed only with MDRI and fatty acid synthase, among 14 expressed transcripts known to be induced by PXR. RUNX3 is an integral downstream component of TGF $\beta$ signaling. "Conclusion:" These data implicate the RUNX3, TGF $\beta$ RII and MDRl genes and pathways in the pathogenesis of human IBD, corroborating findings from murine models. We observe TGF $\beta$-RUNX3 signature in PBLs from patients with active CD. In addition, the present differences in PXR pathway regulation from previously reported intestinal microarray studies may reflect context-specific features, but further implicate MDR1 deficiency in human IBD

\section{T2003}

Detection of Novel CARD15 Mutations in IBD Patients with a Severe Disease Phenotype

Fabian Schnitzler, Stephan Brand, Tanja Staudinger, Simone Pfennig, Katrin Hofbauer, Julia Seiderer, Cornelia Tillack, Burkhard Goeke, Thomas Ochsenkuehn, Peter Lohse

Background: CARD15/NOD2 encodes a protein involved in the intracellular recognition of muramyldipeptide, a component of bacterial peptidoglycans. Three mutations in the CARD15 gene (c.2104C>T [p.R702W], c.2722G>C [p.G908R], and c.3019_3020insC [p.Leul007fsX1008]) have been found to be associated with Crohn's disease (CD). Aims: We performed a limited DNA sequence analysis in patients with inflammatory bowel disease (IBD) to identify additional mutations in the CARD15 gene. Methods: 89 patients with Crohn's disease (CD), 19 patients with ulcerative colitis (UC), and three patients with indeterminate colitis (IC), who were heterozygous carriers of one of the common CARD15 mutations (c.2104C>T [p.R702W], c.2722G>C [p.G908R], or c.3019_3020insC [p.Leul007fsX1008]), of a new amino acid substitution encoded by the 3'-end of exon 4 , or of the c. $2462+10 \mathrm{~A}>\mathrm{C}$ variant in intron 4 , were included in this study. In these patients, exons $4,5,6,8$, and 11 of the CARD15 gene were amplified by PCR and completely sequenced thereby theoretically covering $73.9 \%$ of the described CARD 15 variants and $96.6 \%$ of the mutated alleles. Results: Using this approach, eight novel amino acid substitutions (c.1171C > T [p.R391C], c.1387C > G [p.P463A], c.2138G>A [p.R713H], c.2278C>T [p.R760C], c.2368C >T [p.R790W], c.2371C >T [p.R791W], c.2475C > G [p.N825K], and c. $2546 \mathrm{C}>\mathrm{T}$ [p.A849V]) were detected in six CD, two IC, and one UC patient. Five of these amino acid replacements (p.R39lC, p.R713H, p.R760C, p.R790W, and p.R791W) are most likely true mutations considering Dayhoff's calculations (Atlas Protein Seq Struct 1979; 5:345-52) and the observed disease phenotype, while the p.P463A variant appears to be a polymorphism (frequency of exchange $29 \%$ ). The two remaining variants p.N825K and p.A849V with replacement frequencies of $14 \%$ and $10 \%$ have to be analyzed in functional tests in order to determine whether these substitutions are polymorphisms or true mutations. A severe disease phenotype was observed especially in those patients compound heterozygous for a common and a novel CARD 15 mutation. Conclusion: This study identified eight nove CARD15 variants. Compound heterozygosity for one of these variants in combination with one of the three common CARD15 mutations ([p.R702W], [p.G908R], and [p.Le $\mathrm{ul} 1007 \mathrm{fsX1008]}$ ) was associated with a severe disease phenotype. Complete sequence analysis especially of CARD15 exons 4 and 5 appears to be an efficient screening procedure to 
detect additional mutations in severely affected IBD patients with only one or none of the common mutations

\section{T2004}

Single Nucleotide Polymorphisms of the Mdrl Gene in Inflammatory Bowel Disease

Konstantinos Katsanos, Marc Ferrante, Karolien Claes, Nele van Schuerbeek, Marie Pierik, Liesbet Henckaerts, Marie Joossens, Sofie Joossens, Gert van Assche, Epameinondas V. Tsianos, Paul Rutgeerts, Severine Vermeire

BACKGROUND: There is conflicting data in the literature whether the multidrug resistance l (MDRl) gene by its product P-glycoprotein (P-gp) is implicated in susceptibility to inflammatory bowel disease (IBD). Current data suggest a role for the MDRI-C3435T variant in the susceptibility to ulcerative colitis (UC). We studied functional variants in MDR1 in a large cohort of IBD patients and healthy controls to look for association with the disease and with disease-phenotypes. METHODS: In total 837 IBD patients (716 CD, $121 \mathrm{UC}$ ) and 383 healthy subjects from the same tertiary referral center were genotyped for MDRlG2677T/A (Ala893Ser/Thr) and C3435T (lle1145Ile)] using PCR-RFLP. RESULTS: A significantly lower prevalence of the MDR1-3435 T allele was found in CD patients (51\%) comparing to controls (58\%) $[\mathrm{p}=0.004]$ while no association was observed in UC (54\%). Multivariate regression analysis in the $\mathrm{CD}$ patients showed that if the MDRl-3435T variant was present, this was associated with fistulising Crohn's disease (OR 1.84; $95 \%$ CI 0.98-3.5; $\mathrm{p}=0.058$ ) No significant differences between IBD, CD or UC patients and healthy subjects were noticed for the G2677T/A SNPs. CONCLUSIONS: In this large IBD cohort, we show for the first time an association of the MDRl-C3435T variant with susceptibility to CD. Although we could not confirm an association with UC, a modest effect of MDR variants cannot be excluded as our UC sample was relatively small. MDRl mutations may be associated with IBD susceptibility by disregulating the expression and functionality of the P-gp.

\section{T2005}

Multiple Genetic Testing to Explain Intolerance to Azathioprine Orazio Palmieri, Anna Latiano, Fabrizio Bossa, Maria Rosa Valvano, Maurizio Vecchi, Renata D'Inca', Renzo Caprilli, Francesco Tonelli, Salvatore Cucchiara, Angelo Andriulli, Vito Annese

Background: Adverse drug reactions (ADRs) to azathioprine (AZA) may lead to therapy interruption in up to one quarter of patients with inflammatory bowel disease (IBD). The TPMT gene polymorphisms explain only the minority of cases of bone marrow toxicity (BMT) and ADRs. Aim: The present study was undertaken to correlate the polymorphisms of TMPT but also ITPA gene with AZA toxicity in Italian patients with Crohn's disease (CD) and ulcerative colitis (UC). Methods: 424 patients and 245 healthy controls were included and genotyped for three polymorphisms (G460A/Alal54Thr and A719G/Tyr240Cys of the TMPT gene, and C94A of the ITPA gene), by sequencing (ABI 310) or RFLP-PCR, respectively. 249 patients had CD (134 males, mean age $35 \pm 14$ yrs), while 175 had UC (94 males, mean age $43.5 \pm 15$ yrs). 79 episodes of ADRs were reported in 69 patients: 20 cases of BMT, 12 of abnormal liver transaminases, 16 of pancreatitis, 4 of skin reactions, 9 of nausea vomiting 18 of fever/myaloias. Results: Main frequencies of risk haplotype (TPMT3*A, 3*B) and genotype (ITPA CA) are given in the table. The frequency of the TPMT risk haplotype (TPMT3*A, $3 *$ B) was similar in IBD patients $(5.6 \%$ ), and controls (3.2\%). In contrast, the risk haplotype was significantly increased in the whole group of patients with ADRs ( $13 \%$, $\mathrm{p}=0.001)$ and especially in patients with BMT $(30 \%, \mathrm{p}=0.00001)$. More specifically the risk haplotype was found in 9 out of 69 patients with ADRs and 6 out of 20 episodes of BMT. No significant association of the allele and genotype frequencies of C94A polymorphism of ITPA gene was found in the ADRs group (17.4\%) compared to patients without ADRs (13\%), or healthy controls (12\%). However, the ITPA risk genotype was found in 5 out of 20 episodes of BMT and 12 out of 69 patients with ADRs. Only one patient with severe BMT had both TPMT risk haplotype and ITPA risk allele. Conclusions: The combined genetic testing of TMPT and ITPA genes, enhanced the correlation between genotype and phenotype; 20/69 cases of ADRs, and more importantly 10/20 cases of BMT were explained. While the positive predictive value was only $23 \%$, the negative predictive value of both genetic tests reached the $85 \%$

\begin{tabular}{|c|c|c|c|c|c|c|}
\hline & $\begin{array}{c}\text { IBD pts } \\
(\mathrm{n}=424)\end{array}$ & $\begin{array}{c}\text { Controls } \\
(\mathrm{n}=245)\end{array}$ & $\begin{array}{c}\text { ADRs cases } \\
(\mathrm{n}=69)\end{array}$ & $\begin{array}{c}\text { No ADRs } \\
(\mathrm{n}=355)\end{array}$ & $\begin{array}{c}\text { MBT cases } \\
(\mathrm{n}=20)\end{array}$ & $\begin{array}{c}\text { Others ADRs } \\
(\mathrm{n}=49)\end{array}$ \\
\hline $\begin{array}{c}\text { TPMT } \\
\text { *A, 3*B }\end{array}$ & 5.6 & 3.2 & 13 & 4.5 & 30 & 4.1 \\
\hline ITPA CA & 13.5 & 12 & 17.4 & 13 & 25 & 14.3 \\
\hline
\end{tabular}

T2006

14 bp Deletion Polymorphism in the HLA-G Gene in Inflammatory Bowel Disease

Juergen Glas, Helga P. Torok, Laurian Tonenchi, Molla Yirsaw Teshome, Martin Wetzke, Wolfram Klein, Joerg T. Epplen, Uwe Schiemann, Thomas Mussack, Matthias Folwaczny, Christian Folwaczny

Background: HLA-G is a non-classical major compatibility complex (HLA) class I molecule highly expressed in cytotrophoblast. HLA-G supresses immune reactions mediated by T cells, natural killer cells and dendritic cells. A recent study found an increased expression of HLA-G in ulcerative colitis (UC), but not in Crohn's disease (CD) (1). Thus, HLA-G potentially is implicated in the pathogenesis of inflammatory bowel disease and is therefore a functional and also a positional candidate gene for inflammatory bowel disease, as it is localized in the linkage region IBD3. Within the HLA-G gene several polymorphisms have been described, in exon 8 encoding for the 3' untranslated region a 14 bp deletion polymorphism has been found (2). This polymorphism influences the mRNA stability und is of potential functional relevance (3). Herein, the 14 bp deletion polymorphism was assessed in a population of inflammatory bowel disease patients and additionally tested for potential interactions with CD associated mutations in NOD2/CARD15. Aim: The present study sought to investigate whether the 14 bp deletion polymorphism within the HLA-G gene is associated with inflammatory bowel disease. Methods: The study population comprised 359 patients with CD, 253 patients with ulcerative colitis (UC) and 731 healthy, unrelated controls. Genotyping was performed by PCR and agarose gel electrophoresis. Statistical analysis was performed employing $\chi 2$-test. Results: The allele frequency of the 14 bp deletion polymorphism (Del +) in the HLA-G gene displayed no significant differences between Crohn's disease or ulcerative colitis when compared to the control group. Moreover, no significant associations were found concerning genotype and phenotype frequencies and also no significant associations were assessed with respect to NOD2/CARD15 status. Conclusion: Herein, the 14 bp deletion polymorphism within the gene encoding for HLA-G displayed no significant associations with inflammatory bowel disease. The study could not give evidence for a role of the HLA-G gene in inflammatory bowel disease and also no evidence for interactions between HLA-G and NOD2/CARD15. However, as there are several partly functional polymorphisms within the promotor region potentially influencing expression of the HLA-G gene and the differences of HLA-G expression between UC and CD could be influenced by these polymorphisms.

Allele frequencies of the 14 bp deletion polymorphism in the HLA-G gene

\begin{tabular}{|c|c|c|c|}
\hline & Crohn's disease & Ulcerative colitis & Controls \\
\hline Del + & $464(64.6 \%)$ & $315(62.3 \%)$ & $931(63.7 \%)$ \\
\hline Del - & $254(35.4 \%)$ & $191(37.7 \%)$ & $531(36.3 \%)$ \\
\hline$\Sigma$ & 718 & 506 & 1462 \\
\hline
\end{tabular}

T2007

Association of CARD15 Variants with Clinical Subtypes of Crohn's Disease: A Study of 630 Cases

Clive Onnie, Sheila Fisher, Alastair Forbes, Jeremy Sanderson, Cathryn Lewis, Christopher Mathew

Introduction: The association of three common sequence variants in the CARD15 gene with susceptibility to Crohn's Disease (CD) is well established. Several studies have reported an association of these variants with ileal disease location and complicated disease behaviour We have examined the relationship of the CARD15 variants with CD in one of the largest cohorts of $\mathrm{CD}$ cases with detailed phenotype data collected to date. Methods: A total of 630 $\mathrm{CD}$ cases from two referral centers in London, UK were carefully phenotyped according to the Vienna Classification with some modifications recently suggested by the World Gastroenterology Congress. Details on drug history, histopathology, age at disease onset as well as a detailed surgical history were recorded. CARD15 genotyping was performed by TaqMan (ABI) (G908R) and pyrosequencing (R702W, L1007fs). Allele frequencies between cases and 314 healthy controls were compared using chi-squared tests. Cases were analysed using multinomial logistic regression, modeling CARD15 genotype on clinical factors of disease. Results: All three CARD15 variants were significantly associated with CD with a combined frequency of $21.3 \%$ in cases compared with $7.9 \%$ in controls ( $\mathrm{p}=8 \mathrm{x} 10-13) ; 33.8 \%$ of cases carried at least one CARD15 variant. CARD15 genotype was significantly associated with ileal disease $(\mathrm{p}=1.7 \times 10-6)$ and stricturing disease behaviour $(\mathrm{p}=0.001)$. However, the association with stricturing disease was not significant when adjusted for site of disease. A significant association of CARD15 variants was also observed with age at diagnosis ( $\mathrm{p}=0.001)$, surgery $(\mathrm{p}=0.003)$, recurrent small bowel resection $(\mathrm{p}=0.03)$ and granulomas $(\mathrm{p}=0.0006)$, which remained significant $(p=0.003)$ when adjusted for presence of resectional bowel surgery with full histopathology details. Conclusions: We have replicated the finding that CARD15 variants predispose to ileal disease and have demonstrated a novel association of CARD15 with the presence of granulomas. Carriage of CARD15 variants appears to predict aggressive disease, with not only the need for surgery but also recurrent small bowel resection. We propose that knowledge of CARD15 variant status in patients with small bowel CD could be helpful in disease management and may lead to a more aggressive clinical approach

\section{T2008}

Association Between NOD2/CARD15 Gene Variants and Response to Antibiotics in Perianal Fistulizing Crohn's Disease

Sieglinde Angelberger, Matthias Schwab, Clemens Dejaco, Thomas Waldhoer, Cornelia Lichtenberger, Marieluise Harrer, Elke Schaeffeler, Harald Vogelsang, Walter Reinisch

Background: NOD2/CARD15 gene variants have been associated with susceptibility to Crohn's disease (CD). NOD2/CARD15 is involved in the immune system's response to bacteria by recognizing a specific peptidoglycan motif, muramyl dipeptide, and subsequent activating NF-KB. Antibiotics are the first line strategy for perianal penetrating $\mathrm{CD}$ owing to their antibacterial activity. Aim: This study aimed to explore an association of NOD2/ CARD15 gene variants and clinical response of perianal fistulas to antibiotic treatment. Methods: 52 patients ( $\mathrm{f} / \mathrm{m}: 24 / 28$; median age:36) with draining perianal fistulas were treated with ciprofloxacin $(500-1000 \mathrm{mg} /$ day, $\mathrm{n}=49)$ or metronidazole $(1000-2000 \mathrm{mg} /$ day, $\mathrm{n}=3)$ for a median duration of 7 weeks. According to the Fistula Drainage Assessment complete response was defined as fistula closure or the absence of any draining fistulas despite gentle finger compression. Genotyping of the main coding mutations of CARD15/NOD2, denoted SNP 8, 12 and 13, was conducted by TaqMan analysis (Applied Biosystems, Foster City, CA, USA). Laboratory personnel were blinded to case status of the participants. Results: Ciprofloxacin was discontinued in 1 patient due to diarrhea after 2 weeks. 13 patients showed complete response to antibiotic therapy, whereas 39 were rated as non- or partial-responders At least one mutation in NOD2/CARD15 gene was detectable in none of the responders, but in $12 / 38(31.6 \%)$ of non- or partial responders $(p=0.01)$. Conclusion: Our preliminary data suggest a pharmacogenetic significance of NOD2/CARD15 in the treatment of CD for the first time. NOD2/CARD 15 wildtype seems to be relevant for efficacy of antibiotic treatment against bacterial flora in perianal fistulas. 
NOD2/CARD15 Genotype and Frequency of NOD2/CARD15 Allelic Variants

\begin{tabular}{|c|c|c|c|c|}
\hline & $\begin{array}{c}\text { Wildtype, } \\
\mathrm{n}=39\end{array}$ & $\begin{array}{c}\text { Heterozygous/Compound } \\
\text { heterozygous, } \mathrm{n}=10\end{array}$ & $\begin{array}{c}\text { Homozygous, } \\
\mathrm{n}=2\end{array}$ & \\
\hline $\begin{array}{c}\text { Non-/partial } \\
\text { responder }\end{array}$ & 26 & 10 & 2 & \\
\hline Responder & 13 & 0 & 0 & \\
\hline----- & ------ & ----- & ----- & $-\cdots$ \\
\hline & $\mathrm{SNP} 8$ & $\mathrm{SNP} 12$ & $\mathrm{SNP} 13$ & $\mathrm{SNP} 8+12+13$ \\
\hline All patients & $7.7 \%$ & $1.9 \%$ & $5.8 \%$ & $15.4 \%$ \\
\hline $\begin{array}{c}\text { Non-/partial } \\
\text { responder }\end{array}$ & $10.5 \% *$ & $2.6 \%$ & $6.6 \%$ & $19.7 \% * *$ \\
\hline Responder & $0 \%$ & $0 \%$ & $0 \%$ & $0 \%$ \\
\hline
\end{tabular}

Non-/partial responder vs. Responder $* \mathrm{p}=0.06,{ }^{*} \mathrm{p}=0.006$

\section{T2009}

Evaluation of Slc22a4 and Slc22a5 Ibd5 Locus Functional Variants in African American IBD Patients and Controls

Toshihiko Okazaki, Howard A. Kader, Kim L. Isaacs, Duane T. Smoot, James D. Lewis, Jerrold Canakis, Mary L. Harris, Themistocles Dassopoulos, Judy H. Cho, Yin Y. Shugart, Steven Brant, Niddk IBD Genetics Consortium

BACKGROUND: Inflammatory bowel disease (IBD) is a complex genetic disorder. Genetic associations have been observed for IBD, Crohn's disease (CD) and/or ulcerative colitis (UC) for CARD15 mutations (CD), HLA polymorphisms (CD and UC), and the chromosome $5 \mathrm{q}$ IBD5 risk haplotype (CD) in white populations. Although African American IBD prevalence is only modestly lower than white prevalence, IBD genetic research is only now being initiated in African American cohorts. METHODS: In collaboration with the NIDDK IBD Genetics Consortium, following informed consent, African American patients with confirmed IBD and matched healthy controls (HC) were recruited for genetic investigations. Additional similarly consented cases were previously recruited from University of Chicago and Duke University. We genotyped the SLC22A4 (OCTN1) L503F and SLC22A5 (OCTN2) G-207C polymorphisms (leading functional candidate variants for the IBD5 locus in white populations) by TaqMan for L503F and direct sequencing for G-207C. Allele and genotype frequencies were compared by ChiSquare analysis (STATA version 8.0). RESULTS: 165 CD, 43 UC and $56 \mathrm{HC}$ were genotyped. Similar to that observed in white populations, the $503 \mathrm{~F}$ and 207C alleles were increased in CD cases as compared to controls (Table). UC showed similar trends but was did not reach statistical significance. Carrier frequency of risk alleles in $\mathrm{CD}$ vs. HC did not reach significance (503F $19.6 \%$ vs. $9.3 \%, p=0.08 ;-207$ C $66.2 \%$ vs. $55.6 \%$, $\mathrm{p}=0.16$ ). Linkage disequilibrium between these two markers was markedly lower in African Americans as compared to whites (r2 African American controls, 0.11; r2 NIDDK Consortium white parental controls, 0.73). CONCLUSIONS: IBD5 functional variants of SLC22A4 and A5 show borderline association with $\mathrm{CD}$ in African Americans. The greatly reduced linkage disequilibrium in the African American population for these two markers suggests that additional genotyping of the IBD5 locus in this population may allow separation of causative risk variants from the background haplotype that is also associated. We are presently expanding our African American cohort of IBD cases and controls and will pursue genotyping of markers throughout the IBD5 candidate region.

\begin{tabular}{|c|c|c|c|c|}
\hline Variant & Genotype & HC $\%$ & CD $\%$ & UC $\%$ \\
\hline L503F & CC & 90.7 & 80.4 & 81.4 \\
\hline & CT & 9.3 & 17.2 & 16.3 \\
\hline & TT & 0.0 & 2.4 & 2.3 \\
\hline P value T allele & & & 0.048 & 0.119 \\
\hline G-207C & GG & 44.4 & 33.8 & 34.9 \\
\hline & GC & 50.0 & 47.8 & 44.2 \\
\hline P value C allele & CC & 5.6 & 18.5 & 20.9 \\
\hline
\end{tabular}

T2010

Mthfr, Mtr, Mtrr and Tcn Polymorphisms and Homocysteine in a French Population with Inflammatory Bowel Disease

Laurent Peyrin-Biroulet, Rosa-Maria Gueant-Rodriguez, Jean-Pierre Bronowicki, MarcAndre Bigard, Jean-Louis Gueant

Background: Mild hyperhomocysteinemia is commonly associated with inflammatory bowel disease (IBD). However, the association of 5,10-methylenetetrahydrofolate reductase polymorphisms (MTHFR C677T and A1298C) with either homocysteine level or IBD remains controversial. Furthermore, the influence of methionine synthase (MTR A2756G and MTRR $\mathrm{A} 66 \mathrm{G})$ and transcobalamin (TCN C776G) polymorphisms has never been evaluated in IBD. Methods: A study was carried out on a total of 121 IBD patients, including 111 cases of Crohn's disease and 10 cases of ulcerative colitis patients, compared to 100 healthy volunteers from a Center of Preventive Medicine. Results: The mean age of IBD patients was $35.2 \pm$ 14.8 years and the sex ratio 4:1 (F:M). Plasma homocysteine level (Hcys) was higher in IBD patients than in controls (median $11.8 \mathrm{vs} 10.4 \mu \mathrm{mol} / \mathrm{L} ; \mathrm{p}=0.0004$ ). The folate and vitamin B12 levels were significantly lower in IBD patients than in controls $(8.6 \mathrm{vs} 11.0 \mathrm{ng} / \mathrm{mL} ; \mathrm{p}=$ 0.0036 , and $207 \mathrm{vs} 255 \mathrm{pmol} / \mathrm{L} ; \mathrm{p}=0.0082$, respectively). In multiple linear regression analysis, age $(\mathrm{p}=0.0337)$, folate $(\mathrm{p}=0.0101)$ and vitamin $\mathrm{B} 12(\mathrm{p}=0.012)$ were independently associated with homocysteine in IBD patients. Hcys was higher in carriers of MTHFR 677TT genotype than in CC and CT genotypes ( $\mathrm{p}=0.0207)$ while a trend was observed when comparing Hcys of MTR 2756AA with that of AG and GG ( $\mathrm{p}=0.0759)$. The frequency of MTRR 66 A allele was higher in IBD, compared with controls (0.54 [95\% CI: 0 48-0.61] and $0.39[0.32-0.46]$, respectively, $\mathrm{P}=0.0009$ ), with an odds ratio for IBD risk estimated at 4.0 [1.7-9.2] after adjustment for sex and age. Conclusions: MTHFR 677TT homozygous genotype, folate and vitamin B12 levels are three determinants of homocysteinemia in IBD cases from a French population of European origin. Furthermore, we found an association of the MTRR A66G polymorphism with IBD which may be related to an increased cobalamin reduction associated with oxidant stress.

\section{T2011}

Candidate Gene Analysis of Runx1 and Padi4 in Japanese Patients with Inflammatory Bowel Disease

Masaki Tosa, Yoshitaka Kinouchi, Kenichi Negoro, Eiki Nomura, Yoichi Kakuta, Hiroko Abe, Go Imai, Atsuhiro Mochida, Sho Takagi, Seiichi Takahashi, Tooru Shimosegawa

Backgrounds: A substantial genetic component of susceptibility to inflammatory bowel disease has been demonstrated in epidemiological and linkage studies. It has been proposed that genetic susceptibility loci for Crohn's disease (CD) may be shared with other autoimmune/inflammatory diseases. Rheumatoid arthritis (RA) is also common chronic autoimmunopathy. CD and RA are characterized by chronic inflammation in which crucial roles for Thl-cell, macrophage, and TNF have been demonstrated. In addition, Infliximab is effective in the treatment of both $\mathrm{CD}$ and RA. Recently, three novel candidate genes associated with RA, SLC22A4 on chromosome 5 known as IBD5 and PAdi4on chromosome 1 known as IBD7 and RUNX1 on chromosome 21, were identified in Japan. We have already reported IBD5 including SLC22A4 is not a major component of the susceptibility for IBD in Japanese while Padi4 and RUNX1 have not studied in Japanese patients with IBD. Thus the aim of this study is to determine if the haplotype and SNP associated with RA in Japan were also associated with IBD in Japan. We analyzed a genetic variant in Padi4 (Exon3 JST083451), an intronic SNP in RUNX1 (intron6 JST022046G/C). Material and methods: A total of 758 Japanese individuals ( 241 patients with CD, 247 patients with ulcerative colitis (UC) and 270 healthy controls) were analyzed in this study. Genotyping for SNPs was determined by polymerase chain reaction-restriction fragment length polymorphism analysis. Case-control association analyses were performed using the chi-square test, and sub-stratification analyses according to disease phenotype were also performed. Results: No significant differences were observed between disease $(C D, U C, C D+U C)$ and controls in the two SNPs located in RUNX1 and Padi4. There were no significant association between clinical phenotype and haplotypes of the Padi4. On the other hand, in RUNX1, the very weak relationship was found in ileitis type of CD $(\mathrm{p}<0.05)$. Conclusion: We conclude that RUNX1, Padi4, does not influence susceptibility to both UC and CD in the Japanese.

\section{T2012}

Polymorphisms with Possible Functional Relevance in the Genes for Octnl and Octn2 in Ibd5 Locus in Inflammatory Bowel Disease

Helga P. Torok, Juergen Glas, Klaus Ott, Wolfram Klein, Peter Lohse, Thomas Ochsenkuehn, Matthias Folwaczny, Christian Folwaczny

BACKGROUND: Recently, functional relevant polymorphisms in the genes for the organic cation transporters OCTN1 and OCTN2 in IBD5 locus have been associated with Crohn's disease (CD). Based on statistical analysis and functional studies showing reduced function in OCTN genes activity in vitro, a causative role for the described variants (C1672T in SLC22A4 and -G207C in SLC22A5) has been suggested (1). However, due to the strong linkage disequilibrium among the IBD5 locus it is possible that other unassayed polymorphisms in these or nearby genes are responsible for disease susceptibility (2). The aim of the present study was to assess the frequencies of two other OCTN1 and OCTN2 polymorphisms, which may have effects on gene function, in inflammatory bowel disease SUBJECTS AND METHODS: The study population comprised 596 patients with CD, 355 patients with ulcerative colitis (UC) and 790 healthy unrelated controls. All study participants were Caucasians. The polymorphisms C917T resulting in the amino acid substitution T306I in OCTN1 and C1721T in the 3' untranslated region of the gene encoding for OCTN2 have been assessed by restriction fragment length polymorphism analysis. $\mathrm{CD}$ patients were stratified also with regard to presence or absence of major disease associated variants in CARD15 (L1007fs, R702W, G908R). RESULTS: No significant differences in the allele and genotype distributions between patients with $\mathrm{CD}, \mathrm{UC}$ and controls have been observed. The frequencies of the rarer 917T allele in OCTN1 were 0.373 in CD, 0.376 in UC and 0.378 in controls. The frequencies of the minor allele $1721 \mathrm{~T}$ in OCTN2 were 0.116 in $\mathrm{CD}$, 0.118 in UC and 0.135 in controls. A significant difference in the distribution of the two polymorphisms was also not obtained after stratification of $\mathrm{CD}$ patients with regard to the CARD15 genotype. $99.7 \%$ of individuals carrying the $917 \mathrm{~T}$ allele in OCTN1 and only 10.3 $\%$ of individuals carrying the $1721 \mathrm{~T}$ allele in OCTN2 displayed the homozygous riskassociated diplotype TT/CC concerning the polymorphisms OCTN1 C1672T and OCTN2 G207C (3). DISCUSSION: Herein two polymorphisms with possible functional relevance in the genes encoding for OCTN1 and OCTN2 have been studied in IBD for the first time. No independent association of these polymorphisms with CD or UC has been observed Due to the strong linkage disequilibrium in the IBD5 region, additional studies are required before definitive conclusion regarding the causative role of polymorphisms within these genes. 1. Peltekova VD, et al. Nat Genet 2004;36:471-5. 2. Urban TJ, et al. Inflamm Bowel Dis 2005;11:78-9. 3. Torok HP, et al. Gut. 2005;54:1421-7. 


\section{T2013}

The Functional Gene Polymorphisms for the Pathogenesis of Non-Alcoholic Steatohepatitis

Akira Hirose, Toshiji Saibara, Chikako Namikawa, Yasuko Nozaki, Masafumi Ono, Akemi Yoshioka, Masaya Takahashi, Naoaki Akisawa, Shinji Iwasaki, Saburo Onishi

BACKGROUND/AIMS: The pathogenesis of non-alcoholic steatohepatitis (NASH) is poorly understood. The aim of this study was to examine genetic influences on NASH pathogenesis in Japanese population. METHODS: Blood samples from 63 patients with biopsy-proven NASH and 150 healthy controls were analyzed by the polymerase chain reaction (PCR) and restriction fragment length polymorphism (RFLP). Four functional polymorphisms were studied: the $-493 \mathrm{G} / \mathrm{T}$ polymorphism in the promoter of microsomal triglyceride transfer protein (MTP), the 190 T/A polymorphism in $\beta 3$-adrenergic receptor, which results in Trp64Arg (W64R) amino acid replacement, the $1183 \mathrm{~T} / \mathrm{C}$ polymorphism in the mitochondrial targeting sequence of manganese superoxide dismutase (MnSOD), and the $-511 \mathrm{~T} / \mathrm{C}$ polymorphisms in promoter sequence of the interleukin-1 $\beta$. RESULTS: NASH patients had a much higher incidence of the MTP gene $G$ allele $(P=0.001)$ and of the $G / G$ genotype $(P=$ 0.002 ) compared to the controls. Fat occupied more area in liver lobules and the stage of NASH was advanced in patients with the G/G genotype than in patients with G/T genotype $(\mathrm{P}=0.04)$. $\beta 3$-adrenergic receptor $\mathrm{R}$ allele frequency $(\mathrm{P}=0.01)$ and the $\mathrm{R} / \mathrm{-}(\mathrm{W} / \mathrm{R}$ and $\mathrm{R} / \mathrm{R})$ genotype frequency $(\mathrm{P}=0.008)$ were significantly higher in NASH patients than those in control subjects. Obesity, hypertriglyceridemia and hyperinsulinemia were associated with NASH patients with the $\mathrm{R}$ - genotype $(\mathrm{P}<0.001)$, whereas a decrease in insulinogenic index was associated with NASH patients with the W/W genotype $(\mathrm{P}<0.01)$. NASH patients also had a higher incidence of the MnSOD T/T genotype $(\mathrm{P}=0.016)$. Interleukin- $1 \beta-511 \mathrm{~T}$ allele frequency $(\mathrm{P}=0.0001)$ and the $\mathrm{T} / \mathrm{T}$ genotype frequency $(\mathrm{P}=0.00001)$ were significantly higher in NASH patients than those in control subjects. CONCLUSION: The G allele in the MTP promoter leads to decreased MTP transcription, less export of triglyceride from hepatocytes, and greater intracellular triglyceride accumulation. The $\mathrm{R}$ allele in the $\beta 3$-adrenergic receptor leads to reduce energy expenditure. The T allele in MnSOD mitochondria targeting sequence leads to less transport of MnSOD to the mitochondria. The $\mathrm{T}$ allele in the interleukin- $1 \beta$ promoter leads to the development of inflammatory changes in the liver. Therefore, this study confirmed that the contribution of hepatic steatosis, obesity, glucose intolerance, hypertriglyceridemia and inflammation of the liver influenced by the functional gene polymorphisms for the development of NASH in the Japanese population.

\section{$\mathrm{T} 2014$}

The PPAR- $\alpha$ Agonist Wy-14643 Prevents Development of Nonalcoholic Fatty Liver Disease in Mice Heterozygous for a Mitochohdrial Trifunctional Protein Defect

Jamal A. Ibdah, Peter Perlegas, Hermina Borgerink, J. Mark Cline

Background and Aims: We reported previously that aging mice heterozygous for a mitochondrial trifunctional protein (MTP) defect develop insulin resistance and hepatic steatosis (Gastroenterology 128:1381-90, 2005). MTP is the enzyme complex that catalyzes the last 3 steps of long chain mitochondrial $\beta$-oxidation. The peroxisomal proliferator activated receptor (PPAR- $\alpha$ ) regulates the gene expression of mitochondrial fatty acid oxidation enzymes and is activated by the peroxisomal proliferator Wy-14643. We hypothesized that treatment of the heterozygous (MTP+/-) mice with Wy-14643 enhances mitochondrial fatty acid oxidation and prevents development of hepatic steatosis. Methods: A study group of 3-month old male mice (12 MTP $+/$ - and $12 \mathrm{MTP}+/+$ mice) were fed a diet containing the peroxisome proliferator $\mathrm{Wy}-14,643(005 \% \mathrm{w} / \mathrm{w}$ diet $)$ and a control group of 3-month old male mice (12 MTP+/- and $12 \mathrm{MTP}+/+$ mice) were fed a control chow diet. All mice were sacrificed after 12 months of dietary treatment and liver sections were evaluated using routine histology and Oil Red $O$ fat stain. Scores of 0 to 3 were assigned to hepatic steatosis. Serum insulin levels were also measured. Results: On the chow diet, MTP+/- mice developed mild but significant hepatic steatosis (score $1+$ steatosis) while MTP+/+ had no or minimal hepatic steatosis. There was no steatosis noted in the liver sections obtained from the MTP+/or MTP $+/+$ mice treated with the Wy-14,643 diet. However, the Wy-14643 diet caused development of hepatic tumors in both MTP+/- and MTP+/+ mice, a known effect of Wy14643 use in mice. Gross and histological examination revealed that $75 \%$ and $25 \%$ of the MTP+/- mice developed significant hepatic adenomatous and hepatocellular carcinomatous lesions, respectively, compared to $33 \%$ and $17 \%$ in the MTP $+/+$ mice. The MTP $+/-$ mice on the control chow diet had hyperinsulinemia $(2.31+/-0.45 \mathrm{ng} / \mathrm{ml}$, mean +/- SD) compared to MTP+/- on the Wy-14,643 diet $(1.1+/-0.8 \mathrm{ng} / \mathrm{ml}$, mean +/- SD, $\mathrm{P}<0.001)$, suggesting that the Wy-14,643 diet prevents development of hyperinsulinemia in the MTP heterozygous mice. Insulin levels in the MTP+/+ mice on the chow and Wy-14,643 diets were $0.95+/$ 0.63 and $1.3+/-0.6 \mathrm{ng} / \mathrm{ml}$, respectively. Conclusion: The PPAR- $\alpha$ agonist $\mathrm{Wy}-14643$ prevents development of hepatic steatosis in the MTP+/- mice suggesting that enhancement of mitochondrial fatty acid oxidation prevents development of NAFLD.

\section{T2015}

Effects of N-Acetylcysteine On Change of Oxidative Stress and Histopathology in Rats with Non-Alcoholic Steatohepatitis

Duangporn Thong-Ngam, Suchittra Samuhasaneeto, Onanong Kulaputana, Suthiluk Pathumraj, Piyawat Komolmitr, Naruemon Wisedopas

Background: Prevalence of non-alcoholic steatohepatitis (NASH) appears to be increasing. No therapy for NASH has been proven to be clearly effective. Aims: To determine the effects of N-acetylcysteine (NAC) on change of oxidative stress and histopathology in rats with NASH. Materials \& Methods: Thirty-nine male Sprague-Dawley rats, 220-250 gram, were divided into 4 groups. Group 1: control group, fed with 35\% fat standard diet for 6 wks $(\mathrm{N}=8)$. Group 2: NASH group, fed with $100 \%$ high-fat diet for 6 wks $(\mathrm{N}=8)$. Group 3: NASH with diet treatment group, fed with 100\% high-fat for 6 wks then 35\% fat standard diet for 4 wks $(\mathrm{N}=11)$. Group 4: NASH with NAC treatment group, fed with $100 \%$ highfat diet for 6 wks then NAC $20 \mathrm{mg} / \mathrm{kg}$ for 4 weeks $(\mathrm{N}=12)$. Whole blood total glutathione, hepatic malondialdehyde (MDA) concentration and liver histopathology were evaluated. Results: In rats fed with $100 \%$ high-fat diet for 6 wks, all livers developed moderate to severe macrovesicular steatosis and mild to moderate necro-inflammation. Whole blood total glutathione and hepatic MDA concentration were significantly reduced in NASH+diet and $\mathrm{NASH}+\mathrm{NAC}$ group compared to NASH group. Less histopathologic evidence of steatosis and necro-inflammation in NASH+diet and NASH+NAC group. More improvement of steatohepatitis was found in NAC than in control diet alone. Conclusions: NAC and dietary diet decreased oxidative stress via reducing total glutathione and hepatic MDA concentration and attenuated steatohepatitis in rat fed with $100 \%$ high-fat diet model of NASH.

\section{T2016}

[1-13c]-Acetate Breath Test Reveals Impaired Acetate Metabolism in Patients with Fatty Liver Diseases

Yoshihisa Urita, Susumu Ishihara, Kenichiro Arai, Jun Kondo, Tatsuo Akimoto, Hiroto Kato, Noriko Hara, Hideyuki Koshino, Yoshiko Honda, Yoko Nagai, Kazushige Nakanishi, Nagato Shimada, Motonobu Sugimoto, Kazumasa Miki

Background: [1-13C]-acetate breath test has been used to evaluate gastric emptying of liquid test meals. Acetate is absorbed rapidly from the small intesrine and reaches the liver where carbon dioxide (CO2) is produced and excretes in expired air. However, it has been unclear whether the rate of production of $13 \mathrm{CO} 2$ directly reflects the rate of substrate oxidation or gastric emptying. If acetate metabolism in the liver is changed, it is possible that $13 \mathrm{CO} 2$ excretion also changes regardless of gastric emptying. For example, it has been reported that acetate metabolism is impaired in diabetics. We therefore conducted [1-13C]-acetate breath test for the evaluation of acetate metabolism in patients with various liver diseases. Patients and Methods: [1-13C]-acetate breath test.was performed in 23 chronic liver diseases (mean age 67 years). The cause of liver disease was hepatitis $C$ virus (HCV) in 8 patients, hepatitis B virus (HBV) in 3, and primary biliary cirrhosis in 1, alcoholic liver disease in 4 , non-alcoholic fatty liver disease in 6 . The patients received $100 \mathrm{ml}$ of water containing $100 \mathrm{mg}$ of [1-13C]-acetate in the sitting position after an overnight fast. Breath samples were collected at baseline and at $5,10,15,20,30,40,50,60,70,80,90,100,110$, and 120min after ingestion to analyze $13 \mathrm{CO} 2.13 \mathrm{C}$ was measured as the $13 \mathrm{CO} 2 / 12 \mathrm{CO} 2$ isotope ratio and was expressed as delta over baseline per mil (\%o). Results: The $13 \mathrm{CO} 2$ concentrations increased from the beginning, and peak enrichment values were reached after $20 \mathrm{~min}$. A peak value of $13 \mathrm{CO} 2$ excreted was lowest in patients with alcoholic fatty liver (mean 19.1+/$8.6 \%$ ), followed by non-alcoholic fatty liver disease (mean $21.1+/-12.6 \%$ ), and viral hepatitis (mean 48,9+/-5.6\%o). There was a significant difference between alcoholic fatty liver group and viral hepatitis group Conclusions: In patients with chronic liver diseases, acetate metabolism differs variously. Especially, [1-13C]-acetate breath test is a useful non-invasive tool for distinguishing between patients with fatty liver diseases and those with viral hepatitis. When [1-13C]-acetate breath test is used to evaluate gastric emptying in the subject with fatty liver diseases, we should duly recognize the various metabolism of acetate.

\section{$\mathrm{T} 2017$}

Nonalcoholic Fatty Liver Disease in Japanese Patients with Type 2 Diabetes: Clinical Features and Risk Factors

Nobuyuki Toshikuni, Koji Nakashima, Akiyoshi Omoto, Mikio Mitsunaga

Aims: Type 2 diabetes is commonly associated with nonalcoholic fatty liver disease (NAFLD) An Italian study group has recently demonstrated that NAFLD is an independent risk factor for cardiovascular events in type 2 diabetic patients. Furthermore, type 2 diabetic patients with NAFLD have been reported to have a poor prognosis compared with nondiabetic patients with NAFLD. Thus, type 2 diabetes with NAFLD is a serious health problem. To date, there have been few studies investigating which factors play key roles in the development of NAFLD in type 2 diabetic patients. The aim of this study was to analyze clinical features of NAFLD and identify risk factors for NAFLD in Japanese patients with type 2 diabetes. Methods: Drug-naive patients with type 2 diabetes were enrolled. Exclusion criteria were as follows: hepatitis B surface antigen, antibody to hepatitis $C$ virus, alcohol consumption (more than $20 \mathrm{~g} / \mathrm{d}$ of ethanol). All patients underwent a clinical, anthropometric, and laboratory investigation. The insulin resistance (IR) index was calculated by the homeostasis model assessment (HOMA) method. The ratio of liver to spleen (LS ratio) for computed tomography numbers was used as an index of fatty infiltration of the liver; a L/S ratio $<1$ was considered to represent fatty liver. Abdominal fat distribution was estimated by computed tomography scans. Results: A total of 40 patients with type 2 diabetes $(25$ men, 15 women; a mean age of 62 years) were enrolled. L/S ratio was strongly correlated with HOMA-IR $(\mathrm{r}=-0.70, \mathrm{P}<0.0001)$. L/S ratio was also correlated with visceral fat area $(\mathrm{r}=-0.49, \mathrm{P}=$ $0.001)$, but was not correlated with subcutaneous fat area $(r=-0.24, P=0.13)$. The 40 patients were divided into two groups: the group with NAFLD (group A; $\mathrm{n}=15$ [1] men, 4 women]) and the group without NAFLD (group B; n=25 [14 men, 11 women]). HOMAIR in group A was significantly higher than that in group B $(6.1 \pm 4.3$ vs $2.1 \pm 0.8, \mathrm{P}<$ 0.0001 ), although fasting plasma glucose and $\mathrm{HbAlc}$ did not significantly differ. Furthermore, the incidence of the metabolic syndrome in group A $(80 \%[12 / 15])$ was higher than that in group $B(24 \%[6 / 25], P=0.0008)$. Multiple logistic regression analysis with stepwise variable selection showed that HOMA-IR $(O . R .10,95 \%$ C.I. 1.2-91, P = 0.03) and alanine aminotransferase (O.R. 22, 95\% C.I. 1.0-486, P = 0.04) were independent risk factors for NAFLD. Conclusions: This study suggests that insulin resistance may play a key role in the development of NAFLD in Japanese patients with type 2 diabetes. HOMA-IR and alanine aminotransferase may be serum markers of NAFLD in type 2 diabetes. 


\section{$\mathrm{T} 2018$}

Correlation of Ultrasonography and Histology in Patients with Steatosis Bashar M. Attar, Cory Halliburton, Erick Chinga-Alayo

Liver biopsy is currently the gold standard in the diagnosis of steatosis, particularly to exclude other diseases and to assess inflammation and fibrosis. It is common practice to assign a patient to a diagnosis of steatosis when it is positively identified on ultrasonography. Prior studies correlating histologic steatosis with ultrasound findings were smaller and did not reflect the actual clinical setting. Other studies used quantitative ultrasound attenuation which is not commonly used in clinical practice. Methods: To assess correlation of ultrasonography with biopsy proven cases of hepatic steatosis, we performed a retrospective study of 170 consecutive liver biopsies with complimentary ultrasound. Biopsies were performed for evaluation of non-alcoholic fatty liver disease or to aid in decisions regarding hepatitis C treatment. Cases were assessed for agreement between imaging and histology as the gold standard. Steatosis was defined on histology as: not significant $<5 \%$, mild $5-30 \%$, moderate $30-50 \%$, marked $50-80 \%$ and severe $>80 \%$. An ultrasound study was considered positive for steatosis if fatty liver was specified in the differential diagnosis. Results: Positive correlation between steatosis on liver biopsy and ultrasound was poor. Our study found that ultrasound had a sensitivity of $47 \%$, specificity of $63 \%$, positive predictive value of $11 \%$, and accuracy of $61 \%$ in diagnosing steatosis. However it does have good negative predictive value at $92 \%$ for any grade of steatosis, and the specificity improves to $96 \%$ with steatosis of $50 \%$ or greater. Interestingly the sensitivity, positive predictive value, or accuracy of ultrasound imaging for detecting fat did not significantly improve with increasing degrees of steatosis. Conclusion: Based on the very low positive predictive value of ultrasonography in identifying hepatic steatosis, the detection of fat by this imaging technique does not accurately reflect the presence of steatosis on histology. Our study demonstrates that ultrasonography should not substitute for liver biopsy when fatty liver is found, although we understand our limitation regarding the patchy distribution of fat in the liver. Ultrasonography is however a reasonable test for ruling out steatosis when clinical hepatitis is present.

\section{$\mathrm{T} 2019$}

Alteration in Fatty Acid Composition in Liver Biopsies of Patients with NonAlcoholic Fatty Liver Disease (NAFLD) Compared to Those with Reported Normal Liver Biopsy

Johane. P. Allard, Ellie Aghdassi, Ghazal Avand, Nita R. Prayitno, David W. Ma, Augustin Nguyen, Maitreyi Raman, Maha Guindi, Morris Sherman, Jenny E. Heathcote

Background: Insulin resistance and abnormal lipid metabolism contribute to the pathogenesis of NAFLD. In addition, alterations in the fatty acid (FA) composition in the liver may be associated with lipogenesis, lipid mobilization, mitochondrial B-oxidation or lipid peroxidation. Objectives: To compare the FA composition between liver biopsies of patients with NAFLD and those with reported normal liver biopsy with no fat (NF). Method:This is a prospective cohort study where 43 patients had liver biopsy for mildly elevated liver enzymes. Alcohol intake was $<20 \mathrm{~g} /$ day and all other causes of liver diseases were excluded. Thirtythree patients were diagnosed with NAFLD (14 steatosis, 19 steatohepatitis) while 10 had normal biopsies. In addition to histology, total lipid FA composition was determined by gas chromatography. Composition in \% for total lipid (TL) is reported. Unpaired t-test was used and $\mathrm{P}<0.05$ is considered significant. Results: Patients with NAFLD had a significantly higher: BMI $(30.01 \pm 0.85$ vs $22.50 \pm 1.28 \mathrm{~kg} / \mathrm{m} 2)$, ALT level $(68.09 \pm 6.35$ vs $47.85 \pm 6.61 \mathrm{u} / \mathrm{L})$, blood triglycerides $(2.14 \pm 0.23 \mathrm{vs} 1.13 \pm 0.20 \mathrm{mmol} / \mathrm{l})$, and a trend towards a higher glucose level $(6.14 \pm 0.33$ vs $5.10 \pm 0.40, \mathrm{p}=0.08 \mathrm{mmol} / \mathrm{l})$, total cholesterol $(5.46 \pm 0.21$ vs $4.27 \pm 0.34$, $\mathrm{p}=0.09 \mathrm{mmol} / \mathrm{l})$ and a lower $\operatorname{HDL}(1.27 \pm 0.05$ vs $1.56 \pm 0.12, \mathrm{p}=0.058)$ compared to those with NF. Other liver enzymes were similar. Compared to NF, NAFLD patients' total polyunsaturated FA (PUFA) $(8.3 \pm 0.5$ vs $17.8 \pm 2.8)$ was significantly decreased due to significant decreases in long chain n- 6 and n-3 FA. In addition, there was a significant increase in n6 to n-3 ratio ( $7.3 \pm 1.2$ vs $3.2 \pm 0.6)$ in NAFLD patients. Saturated fatty acids, palmitic $(30.1 \pm 0.5 \mathrm{vs} 23.9 \pm 3.2)$ and stearic $(23.3 \pm 1.0$ vs $18.4 \pm 2.7)$ were significantly higher in patients with NAFLD whereas C20:0 (2.4 \pm 0.4 vs $5.4 \pm 1.3), C 22: 0(0.7 \pm 0.1$ vs $2.7 \pm 0.8)$ and C24:0 $(0.3 \pm 0.09$ vs $1.1 \pm 0.3)$ were all significantly lower when compared to subjects with NF. The monounsaturated FA, oleic $(17.5 \pm 1.2$ vs $11.2 \pm 1.9)$ and the elaidic (trans fat, $9.4 \pm$ 0.7 vs $5.5 \pm 1.2$ ) were significantly higher in patients with NAFLD. The conjugated linoleic acids were significantly lower ( $1.6 \pm 0.3$ vs $4.5 \pm 1.6)$ in patients with NAFLD when compared to subjects with NF. Conclusion: patients with NAFLD have in their liver, a different fatty acid composition compared to NF. This includes a decrease of the PUFA particularly from the $n-6$ and $n-3$ series with a disproportionate increase in the n- 6 to $n-3$ ratio. This pattern of fatty acid composition may be the result of alteration in lipid metabolism favoring hepatic steatosis

\section{T2020}

Changes in Liver Histology After One Year of Treatment with Probucol in Nonalcoholic Steatohepatitis

Shahin Merat, Mohsen Aduli, Rozana Kazemi, Masoud Sotoudeh, Reza Malekzadeh

Background and Aims: Nonalcoholic steatohepatitis (NASH) is rapidly becoming a major health problem in many communities. No effective medical treatment has been yet approved for this entity. It appears that oxidative damage is an important step in the pathology of NASH. Probucol is a lipid lowering agent with significant antioxidant effects. We have previously shown its effectiveness in normalizing liver enzymes in NASH patients. In the current study, we report liver histology changes after using probucol for one year in 8 patients. Material and Methods: Eight cases of biopsy proven patients with NASH were included. Viral and autoimmune hepatitis and other liver disorders were ruled out. None of the patients used alcohol. The patients were given $500 \mathrm{mg}$ probucol daily for 1 year. Aminotransferases, lipid profile and weight changes were evaluated during the treatment. Liver biopsies were performed before treatment and after one year. The biopsies were staged using a modification of the system developed by Brunt et al. According to this modification, proposed by Sotoudeh et al. in DDW 2004, steatosis, hepatocyte ballooning, lobular Inflammation and portal inflammation were scored from zero through three. Fibrosis was staged from zero through four. The paired-samples T-test was used to compare variables before and after treatment. Results: The patients completed one year of treatment and underwen the second liver biopsy. At the end of therapy the mean alanine aminotransferase and alanine aminotransferase levels decreased from 94 and 55 to 41 and 26 respectively $(\mathrm{p}=0.012$ and 0.011 respectively). The mean weight of patients decreased by $2.9 \mathrm{~kg}$ and the mean body mass index by $0.67 \mathrm{~kg} / \mathrm{m} 2$, none reached statistical significance. The pathologic grade of liver histology decreased from 7.4 to $5.6(\mathrm{p}=0.021)$, the stage changed from 1.13 to 1.25 $(\mathrm{p}=0.76)$, and the total score decreased from 8.5 to $6.9(\mathrm{p}=0.048)$. No adverse drug effect were observed. Conclusion: Probucol is effective in normalizing aminotransferase levels in patients with NASH. It also significantly reduces the histologic grade of steatohepatitis afte one year of treatment. We failed to demonstrate statistically significant improvement in histologic stage.

\section{T2021}

Lovastatin Is Not Hepatotoxic to Patients with Pre-Existing Liver Disease Andrew L. Avins, Michelle M. Manos, Theodore R. Levin, Lynn M. Ackerson, Wei K. Zhao, Rosemary C. Murphy, Douglas J. Watson, Peggy May T. Hwang, Amy R. Replogle, Jeffrey G. Levine

BACKGROUND: Data in the literature addressing the risk of adverse hepatic outcomes associated with statin use among patients with pre-existing liver disease are limited. In addition, the effect of statins on lipid metabolism may have beneficial effects among patients with certain hepatic diseases. METHODS: This was a retrospective cohort study. Subjects were adult members of the Northern California Kaiser Permanente Medical Care Program from January 1994 through June 2004 who had at least two alanine aminotransferase (ALT) elevations 6-18 months apart, a medical-record diagnosis of liver disease, or chronic hepatitis $\mathrm{B}$ or hepatitis $\mathrm{C}$ infection. Patients were excluded if they used a statin medication within one year of study entry. The primary endpoint was defined as concurrent serum ALT>3 times the upper limit of normal, total bilirubin $>2$ times the upper limit of normal, and alkaline phosphatase $<1.5$ times the upper limit of normal (modified Hy's Rule). Secondary endpoints included any ALT $>3$ times the upper limit of normal, cirrhosis, and liver failure For univariate analyses, follow-up time was partitioned into lovastatin-exposed and unexposed time and rates of each endpoint were calculated and attributed to the appropriate exposure time. Multivariate analyses were conducted with Cox proportional hazards models. RESULTS: The study group consisted of 13,492 patients with lovastatin exposure and 79,628 patients without lovastatin exposure during the follow-up period. In univariate analyses, exposure to lovastatin was associated with strong and significant reductions in all primary and secondary endpoints, including Hy's Rule (Relative Rate (RR) $=0.29,95 \%$ Confidence Interval (CI): 0.14 to 0.60 ), elevated $\mathrm{ALT}(\mathrm{RR}=0.55,95 \% \mathrm{CI}: 0.47$ to 0.65 ), cirrhosis ( $\mathrm{RR}=$ $0.25,95 \%$ CI: 0.19 to 0.35 ), and liver failure ( $\mathrm{RR}=0.21,95 \% \mathrm{CI}: 0.14$ to 0.31 ). Results were similar when adjusted for age and gender in multivariate analyses. Rates of statin prescription were similar across subgroups defined by hierarchical categories of liver-disease certainty, suggesting that confounding by contraindication was not a likely explanation for these results. CONCLUSION: Exposure to lovastatin was associated with a large and significant decrease in adverse hepatic endpoints, possibly mediated through an effect on liver disease by lovastatin's alteration of lipid metabolism. These results are not consistent with an increased risk of lovastatin hepatotoxicity in patients with pre-existing liver abnormalities.

\section{T2022}

The Role of ADH3 and CYP2E1 Gene Polymorphism in Alcohol Liver Cirrhosis and Chronic Alcohol Pancreatitis Halina Lach, Krzysztof Celinski, Jadwiga Partycka, Maria Slomka

Genetic polymorphism of enzymes involved in alcohol metabolism plays a relevant role in etiopathogenesis of alcohol disease. The aim of the present study was to find in the Polish population the ADH3 and CYP2El genotypes, which are likely to be responsible for higher susceptibility to alcohol liver cirrhosis and chronic alcohol pancreatitis. The ADH3 and CYP2El genotype and alleles frequencies were examined in 198 patients: 57 with alcoholic liver cirrhosis, 44 with alcohol chronic pancreatitis, 43 abusing alcohol but without damage to gastrointestinal organs -"healthy drinkers" and 54 nondrinkers as a control group. Genotyping of the $\mathrm{ADH} 3$ and $\mathrm{CYP} 2 \mathrm{E} 1$ was performed using polymerase chain reaction -restriction fragment length polymorphism methods on white cell DNA. The genotype ADH3*1/ADH3*1 was found to be significantly more frequent in alcohol abusers compared to non-drinkers. The examinations of the group of alcohol abusers showed that the genotype ADH3*2/ADH3*2 occurred statistically significantly less frequently in patients with chronic pancreatitis than those without alimentary lesions and patients with cirrhosis. In the examined population the $c 2$ allele was present only in $1.5 \%$ of patients. It was found only in patients abusing alcohol. In the group of patients with alcoholic cirrhosis it was present in $3.5 \%$ of cases while in patients with chronic alcoholic pancreatitis in $2.3 \%$. The genotype $\mathrm{cl} / \mathrm{c} 2$ was present in $3 \%$ of subjects. The genotype $\mathrm{c} 2 / \mathrm{c} 2$ was not found in any patient. Heterozygotes $\mathrm{cl} / \mathrm{c} 2$ were present only in patients consuming excessive amounts of ethanol; in $7 \%$ of patients with alcoholic cirrhosis and in $4.5 \%$ of those with chronic alcoholic pancreatitis. Our studies suggest that allele $\mathrm{ADH}^{*}{ }^{*} 1$ may be a risk factor for the developing alcoholic liver diseases and allele $\mathrm{ADH} 3 * 2$ can protect against alcoholism and be the protective factor of chronic alcohol pancreatitis. The frequency of alleles $\mathrm{c} 2$ in Polish population is low, but their presence promotes alcoholic damage to alimentary organs amongst Poles. 
Central Role of Srebp Lipogenic Pathways in Alcohol-Induced Hepatic Steatosis

Cheng Ji, Christine Chan, Neil Kaplowitz

BACKGROUND: Alcohol induced fatty liver correlates with activation and induction of SREBPs, transcription factors which regulate expression of genes which promote fatty acid and triglyceride synthesis and inhibit $\beta$-oxidation. To determine the contribution of this mechanism of steatosis, the aim of the present study was to compare the effects of intragastric alcohol feeding on lipogenic pathways in wild type and SREBPlc null mice. METHODS: Wild type and SREBPlc null mice were fed alcohol alone or with betaine (1\% w/v) or control diet for 4 weeks. Alcohol-induced liver damage was assessed with H\&E and TUNEL staining. Changes in gene expression were analyzed with Real Time PCR, RT-PCR and immunoblotting. RESULTS: ALT, plasma homocysteine (Hcy), liver cholesterol (Chol), and hepatic apoptosis were increased in alcohol-fed mice as compared to control in both genotypes (Table 1). The alcohol induced liver cholesterol levels in SREBPlc null mice were higher than in wild type. Liver triglycerides (TG) were increased 4 times in alcohol-fed wild type but 1.8 times in alcohol-fed SREBPlc null as compared to the pair-fed controls. SREBP2 and HMG CoA reductase were increased slightly in wild type and significantly in knockouts in response to alcohol. Selective genes related to triglyceride synthesis were increased in wild type but not in SREBPlc null mice fed alcohol. Plasma adiponectin was reduced in both genotypes fed alcohol but no significant changes were found in AMPK $\alpha$ or activated AMPK $\alpha$. In addition, neither Scap nor S1P was changed in response to alcohol in both genotypes. Betaine reduced alcohol-induced changes in both genotypes. CONCLUSIONS: SREBPIc knockout protects against alcohol induced triglyceride accumulation. SREBP2 may provide compensatory pathways for lipid synthesis in the SREBPIc null mice fed alcohol and thus a complete protection against alcohol-induced fatty liver seems to require inactivation of both SREBPI and 2 regulated lipogenic pathways (supported by NIAAA R01AA014428-03 $\&$ NIDDK P30DK048522-11 to NK and CJ. chengji@usc.edu).

Table 1 Pathological features of wild type and SREBPlc (-/-) mice fed ethanol

\begin{tabular}{|c|c|c|c|c|c|c|}
\hline & \multicolumn{3}{|c|}{ Wild type } & \multicolumn{3}{|c|}{ SREBP1c KO } \\
\hline & Control & EtOH & EtOH/Betaine & Control & EtOH & EtOH/Betaine \\
\hline $\begin{array}{c}\text { Liver/Body (\%) ALT } \\
\text { (U/L) Hcy }(\mu \mathrm{M}) \\
\text { Steatosis score TG } \\
\text { (mg/g liver) Chol } \\
\text { (mg/g liver) } \\
\text { Necroinflammation } \\
\text { Apoptotic nuclei N }\end{array}$ & $\begin{array}{c}5.3 \pm 0.4 \\
13.1 \pm \\
4.22 .9 \pm \\
1.10 .3 \pm \\
0.322 .3 \\
\pm 3.12 .9 \\
\pm 0.40 .1 \\
\pm 0.20 .4 \\
\pm 0.36\end{array}$ & $\begin{array}{c}7.1 \pm \\
0.5^{*} \\
118.3 \\
\pm 35.5^{* *} \\
20.2 \pm \\
3.43 .2 \pm \\
0.5^{* *} \\
87.2 \pm \\
7.5^{* * 4} 4.3 \\
\pm 0.7^{*} \\
1.2 \pm \\
0.4^{*} 6.2 \\
\pm 4.3^{*} 6\end{array}$ & $\begin{array}{c}6.3 \pm 0.562 .3 \\
\pm 25.8^{*} 3.4 \pm \\
0.91 .5 \pm 0.4^{*} \\
38.2 \pm 4.2^{*} 3.5 \\
\pm 0.50 .4 \pm 0.3 \\
2.5 \pm 1.15\end{array}$ & $\begin{array}{c}4.5 \pm 0.5 \\
12.5 \\
\pm 3.61 .4 \\
\pm 0.60 .2 \\
\pm 0.1 \\
14.5 \pm \\
3.83 .5 \pm \\
0.50 .1 \pm \\
0.10 .5 \pm \\
0.55\end{array}$ & $\begin{array}{c}5.6 \pm 1.0 \\
79.7 \\
\pm 29.5^{* *} \\
12.4 \pm \\
6.5^{*} 0.9 \\
\pm 0.4^{*} \mathrm{a} \\
27.9 \pm \\
4.0^{*} \mathrm{a} 5.6 \\
\pm 1.0^{*} \\
0.4 \pm 0.3 \\
3.1 \pm 1.9 \\
6\end{array}$ & $\begin{array}{c}4.4 \pm 0.5 \mathrm{a} 49.4 \\
\pm 8.2^{*} 3.1 \pm \\
1.10 .3 \pm 0.2 \mathrm{a} \\
16.8 \pm 3.1 \mathrm{a} 3.6 \\
\pm 0.70 .1 \pm 0.1 \\
0.4 \pm 0.45\end{array}$ \\
\hline
\end{tabular}

$* \mathrm{P}<0.05 ; * * \mathrm{P}<0.01$ compared to control; a $\mathrm{P}<0.05$ compared to wild type.

\section{T2024}

N-Acetylcysteine and Ascorbic Acid Protect Against Oxidative Stress Related Toxicity Caused By Ecstasy and Ethanol

Kristina Norman, Conni Mueller, Manuela Jackstadt, Herbert Lochs, Tilman Grune. Matthias Pirlich

Introduction: Ecstasy (Methylendioxymethylamphetamine, MDMA) belongs to the most commonly used designer drugs and is often consumed together with alcohol. Both MDMA and ethanol are hepatotoxic and cause oxidative stress. They might be used as model to test anti oxidative strategies. Aim: We investigated the impact of MDMA in combination with ethanol on oxidative stress and cell viability in cultured hepatocytes and the effect of a coincubation with the antioxidants N-Acetylcysteine (NAC) and ascorbic acid. Methods: HepG2 cells were incubated with a) MDMA and b) MDMA and ethanol for 24 hours. Cell viability was measured with trypan blue exclusion and protein carbonyls were assessed as marker for protein oxidation. The tests were then repeated with a) NAC and b) ascorbic acid coincubation. Results: 24 hours incubation with $0.5 \mathrm{mM}$ MDMA increased protein oxidation $(+171 \%$, after $24 \mathrm{~h}, \mathrm{p}<0.01)$. When the HepG2 cells were incubated with MDMA as well as $200 \mathrm{mM}$ ethanol cell viability was further decreased (56.4\%, p=0.048). However, when NAC and ascorbic acid were simultaneously administered, cell viability was largely preserved $(72.9 \%$ with $0.05 \mathrm{mM}$ MDMA and $200 \mathrm{mM}$ ethanol with $3 \mathrm{mM} \mathrm{NAC} ; 63 \% 0.05 \mathrm{mM}$ MDMA and $200 \mathrm{mM}$ ethanol with $0.2 \mathrm{mM}$ ascorbic acid). Moreover protein oxidation ($30 \%$ with $0.05 \mathrm{mM}$ MDMA and $200 \mathrm{mM}$ ethanol and $0.2 \mathrm{mM}$ ascorbic acid) was also significantly reduced. Conclusion: MDMA increases oxidative stress and reduces viability of HepG2 cells. The effects on cell viability are accelerated when MDMA and ethanol are coincubated. However administration of the antioxidants ascorbic acid and NAC partially prevented these hepatotoxic effects. Studies should further evaluate these antioxidants in the therapy of oxidative liver damage.
Oxidative Stress and Lipid Peroxidation-Induced 1, N6-Ethenodeoxyadenosine DNA Adducts in CYP2E1 Overexpressing HEPG2 Cells Upon Ethanol Treatment Jagadeesan Nair, Dan Zili, Felix Stickel, Bartsch Helmut, Helmut K. Seitz Background and Aims: Alcohol abuse exerts detrimental oxidative stress and lipid peroxidation (LPO) in humans which is now generally accepted as one of the mechanisms for the induction of liver cancer in heavy drinkers. We have previously demonstrated that LPOinduced 1, N6-ethenodeoxyadenosine (edA) levels were elevated in hepatic DNA of alcohol liver disease patients (Frank et al., Carcinogenesis, 2004). We now investigated the role of CYP2E1, the primary enzyme responsible for ethanol metabolism on edA formation. Methods: HepG2 cell lines stably transfected with CYP2E1 (E47) and a vector mock transfected control (C34) [kindly provided by N.N. and A.I. Cederbaum, New York, NY, USA] were used for this study. $\varepsilon d A$-adducts were detected by immunohistochemistry using MAb EM-1-Al. Results: Ethanol treatment increased edA-staining in the nuclei of E47 compared to C34 cells. At 5, 10 and $25 \mathrm{mM}$ ethanol concentration the number of positively stained nuclei exhibited a dose response curve. Although all E47 cells were expected to show high CYP2El expression, an edA-increase was detected only in a fraction. Ethanol treatment reduced glutathione in E47 cells measured by monobromobimane, again only in a fraction of cells. It is likely that these cells are more prone to oxidative stress resulting in higher LPO-induced DNA damage. Conclusion: Our results indicate that formation of miscoding etheno-DNA adducts is an early response to CYP2El overexpression in ethanol-exposed liver cells. This suggests that the ensuing mutations may play a role in the ethiopathogenesis of benign and malignant alcohol liver diseases.

\section{T2026}

The Anti-Fibrogenic Effects of EGCG in Activated HSC in Vitro Are Mainly Derived from Its Antioxidant Property By De Novo Synthesis of Cellular Glutathione

Yumei Fu, Yajun Zhou, Shizhong Zheng, Anping Chen

BACKGROUND: Hepatic stellate cells (HSC) are the major players during hepatic fibrogenesis. Over-production of extracellular matrix (ECM) is a characteristic of activated HSC Transforming growth factor-beta (TGF- $\beta$ ) is the most potent fibrogenic cytokine while connective tissue growth factor (CTGF) mediates TGF- $\beta$-induced ECM production. HSC activation and hepatic fibrogenesis are stimulated by oxidative stress. Glutathione (GSH) is the most important intracellular antioxidant. We recently reported that (-)-epigallocatechin3-gallate (EGCG), a major and the most active component in green tea extracts, inhibited HSC activation by reducing cell growth and suppressing ECM gene expression [Chen at el. Biochem J (2002) 368:695-704]. The underlying mechanisms remain largely to be elucidated. AIM AND HYPOTHESIS: This study is to explore the mechanisms of EGCG in inhibition of ECM gene expression in activated HSC in vitro. It is hypothesized that EGCG inhibits ECM gene expression in activated HSC by interrupting TGF- $\beta$ signaling through attenuating oxidative stress. RESULTS: TGF- $\beta$ signaling is interrupted by EGCG in activated HSC by suppressing gene expression of type I \& II TGF- $\beta$ receptors. In addition, CTGF gene expression is inhibited by EGCG, leading to the reduction in the expression of ECM genes, including $f \backslash(\mathrm{I})$-procollagen. Exogenous CTGF dose-dependently eliminates the antifibrogenic effect of EGCG. To elucidate the underlying mechanisms, EGCG is found to attenuate oxidative stress in passaged HSC by scavenging basal cellular reactive oxygen species and reducing lipid peroxidation mainly by the de novo synthesis of GSH. Nacetylcysteine (NAC), a GSH analog, mimics the inhibitory effects of EGCG in passaged HSC. L-buthionine-sulfoximine (BSO) is a specific inhibitor of glutamate-cysteine ligase (GCL), a key rate-limiting enzyme in GSH synthesis. BSO eliminates the anti-fibrogenic effects of EGCG. CONCLUSIONS: This study elucidates the underlying mechanisms of EGCG in inhibition of ECM gene expression in activated HSC. The reduction of ECM production by EGCG is mainly facilitated by interrupting TGF- $\beta$ signaling and suppressing gene expression of TGF- $\beta$ receptors and CTGF. Our results, for the first time, demonstrate that the antioxidant property of EGCG plays a critical role in its anti-fibrogenic effects and is mainly derived from increasing intracellular GSH. These results provide novel insights into the mechanisms of EGCG as a possible anti-fibrogenic candidate in prevention and treatment of liver fibrosis. [The work was supported by the grant DK 47995 from NIH/ NIDDK to A. Chen, and by the Bridging Award to A. Chen from LSUHSC-S].

\section{T2027}

Tegaserod Enhances Gastric Accomodation By Antagonizing 5-HT2B Receptor Activity in the Gastric Myenteric Plexus and Smooth Muscle Ling Wang, Shi-Yi Zhou, Chung Owyang

Tegaserod (T) is a potent 5-HT4 receptor agonist with clinical efficacy in disorders associated with reduced GI motility and transit. T has been shown to enhance gastric accommodation in humans, but the underlying mechanism is unclear. In vitro studies indicate that $\mathrm{T}$ is not strictly selective for the 5-HT4 receptor, as it has similar affinity for the 5-HT2B receptor. We therefore investigated whether improved gastric accommodation evoked by $\mathrm{T}$ is mediated by 5 -HT4 or 5 -HT2B receptors. In vivo gastric accommodation studies were performed in anesthetized rats. A pressure transducer was inserted into the proximal stomach through the pylorus along with a catheter to infuse saline for gastric distension. Intragastric distension produced a stepwise increase in intragastric pressure (IGP). At 2 and $6 \mathrm{ml}$ of gastric distension IGP increased $7.25 \pm 0.8$ and $5.4 \pm 0.2 \mathrm{mmHg}$ respectively. $\mathrm{T}$ ( $1 \mathrm{mg} / \mathrm{kg}$ iv) significantly reduced this increase to $5.5 \pm 0.3$ and $3.4 \pm 0.2 \mathrm{mmHg}$. T's action was dose dependent and not affected by a specific 5 -HT4 antagonist (SDZ-205,557, $1 \mathrm{mg} / \mathrm{kg}$ ). Similarly, vagotomy did not affect T's action, suggesting T acts directly on 5-HT receptors other than 5-HT4 present in gastric myenteric plexus or smooth muscle. To investigate the effects of activation of 5-HT2B receptors on gastric motility, we showed that administration of a 5-HT2B agonist, $\alpha$-methyl$5 \mathrm{HT}(0.03 \mathrm{mg} / \mathrm{kg})$ increased IGP to $7.1 \pm 0.5 \mathrm{mmHg}$, which was dose dependently antagonized by T. Co-administration of SDZ-205,557 did not affect the antagonistic action of T suggesting 
$\mathrm{T}$ is a potent 5-HT2B receptor antagonist. This action likely contributes to T's ability to enhance gastric compliance. High levels of both mRNA and protein for 5-HT2B receptor were found in the gastric wall of rats by Rt-PCR and Western analysis. Immunocytochemistry showed 5-HT2B receptors were present predominantly in the longitudinal and circular smooth muscle layers and myenteric plexus. To investigate the mechanisms by which activation of 5-HT2B receptor increases gastric contractions, we showed that electrical field stimulation $(1-20 \mathrm{~Hz})$ of gastric muscle preparations resulted in a neuronally-mediated contractile response significantly potentiated by application of $\alpha$-methyl-5HT (10-6M) (30\% over control). Similarly $\alpha$-methyl-5HT enhanced carbacol (10-9-10-6M) induced gastric contractions by $50 \%$. This potentiation effect could be blocked by T (10-6M). Conclusion: $\mathrm{T}$ enhances gastric accommodation in rats. This action is not due to activation of 5-HT4 receptors but appears to be related to 5-HT2B receptor antagonist activity, which reduces the release of acetylcholine from the myenteric plexus and/or its actions on gastric muscle.

T2028

Mitemcinal (GM-611), Motilin Receptor Agonist Accelerates Both Upper GI Motility and Lower GI Motility in Dogs

Ken-Ichi Ozaki, Hirokazu Sudo, Hiroyasu Muramatsu, Tatsuo Yata, Kenshi Kamei, Reiko Sekimori, Zen Ito, Satoshi Omura

INTRODUCTION Mitemcinal is an erythromycin-derived macrolide that is a motilin receptor agonist to accelerate gastric emptying in dogs and humans. It remains unclear whether motility of other alimentary tracts is accelerated by mitemcinal. In this study, therefore, we investigated the actions of mitemcinal on lower gastrointestinal (GI) along with upper GI in dogs. METHOD Lower esophageal sphincter (LES) pressure of the anesthetized dog was measure by means of an infused catheter system. Proximal intragastric volume was measured with a barostat during the constant pressure procedure and changes in gastric motility were measured with force transducers sutured on the gastric antrum, simultaneously, in conscious dogs. The effects of mitemcinal on colonic motility by force transducers and defecation were examined in conscious dogs. RESULTS Mitemcinal (0.3-100 $\mu \mathrm{g} / \mathrm{kg}$, iv) increased the LES pressure in a dose-dependent manner (see Figure) with a potency 30 times that of erythromycin. This increase was inhibited in the presence of the motilin receptor antagonist GM-109 ( 1 and $10 \mathrm{mg} / \mathrm{kg} / \mathrm{h}$, iv infusion). Mitemcinal ( 3 and $10 \mu \mathrm{g} / \mathrm{kg} / \mathrm{h}$, iv infusion) decreased intragastric volume in a dose-dependent manner and increased gastric motility at the same time. The colonic motility was stimulated by mitemcinal (0.3-1 mg/kg, po) (see Figure) and abolished in the presence of GM-109 ( 1 and $10 \mathrm{mg} / \mathrm{kg} / \mathrm{h}$, iv infusion). The latency period of defecation was shortened without inducing diarrhea by mitemcinal ( $3 \mathrm{mg} / \mathrm{kg}$, po). CONCLUSION Mitemcinal accelerated both upper and lower GI motility as well as gastric emptying in dogs. Furthermore, it became clear that these actions by mitemcinal on upper and lower GI were demonstrated with the same doses which accelerate gastric emptying. This suggests that mitemcinal may be a promising agent for treatment of upper and lower GI motility disorders.

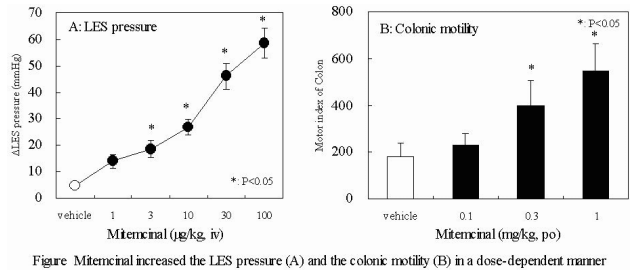

T2029

Effects of a Novel 5-HT4 Agonist, ATI-7505, On Gastrointestinal and Colonic Transit in Humans

Michael Camilleri, Duane Burton, Maria I. Vazquez-Roque, Thomas Ford, Sanna

McKinzie, Alan R. Zinsmeister

Background: ATI-7505 is a prokinetic 5-HT4 agonist shown to be devoid of QT prolongation effects and not metabolized by CYP 450. This study assessed the effect of ATI-7505 on gastrointestinal and colonic transit in healthy humans. Methods: This randomized, parallelgroup, double-blind, placebo-controlled study evaluated effects of ATI-7505, 3, 10 or 20 mg t.i.d., in healthy volunteers (12 per group). Validated scintigraphic methods were used to measure gastrointestinal and colonic transit with ${ }^{99} \mathrm{~m} \mathrm{Tc}$-egg meal and ${ }^{111} \mathrm{In}$-charcoal. Primary endpoints were gastric emptying (GE) tl/2, colonic geometric center (GC) at 24 hours and ascending colon (AC) emptying t1/2. Stool diaries were kept during the 9-day treatment period. An ANCOVA was used to assess overall treatment group differences, and post-hoc unadjusted pairwise comparisons were also examined. Results: There were borderline overall treatment effects (decrease) on $\mathrm{GE} \mathrm{tl} / 2(\mathrm{p}=0.154)$, with $20 \mathrm{mg}$ t.i.d. ATI7505 accelerating GE vs. placebo ( $\mathrm{p}=0.038$ ). ATI-7505 increased colonic transit (GC24, $\mathrm{p}=$ 0.031 , Fig.) with fastest transit at $10 \mathrm{mg}$ t.i.d. vs. placebo $(\mathrm{p}=0.065)$. ATI-7505 accelerated AC emptying $\mathrm{t} 1 / 2$ (overall $\mathrm{p}=0.075$, Fig.) with $10 \mathrm{mg}$ dose vs. placebo $(\mathrm{p}=0.042)$. There were no changes in stool frequency or ease of passage, but there was somewhat looser stool consistency (Bristol stool form scale, overall $\mathrm{p}=0.056$ ) with the 10 and $20 \mathrm{mg}$ t.i.d. doses. No safety issues were identified. Conclusions: The 5-HT4 agonist, ATI-7505, accelerates overall colonic transit, tends to accelerate GE and AC emptying and loosen stool consistency. The optimal doses in health appear to be $20 \mathrm{mg}$ and $10 \mathrm{mg}$ t.i.d. to accelerate GE and colonic transit, respectively.

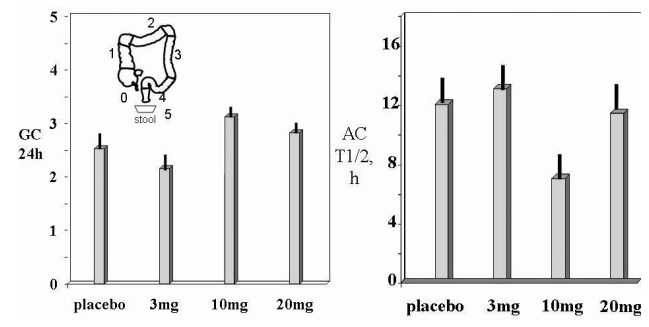

T2030

Dexloxiglumide, a CCK1-Antagonist, Improves Gas-Related Symptoms in Healthy Subjects

Beatriz Lobo, Jordi Serra, Fernando Azpiroz, Massimo D'Amato, Juan R. Malagelada

Patients with irritable bowel syndrome and functional dyspepsia, often complain of gasrelated symptoms, particularly after meals. Duodenal lipids impair intestinal gas transit and tolerance in healthy subjects, and this effect is exacerbated in patients with irritable bowe syndrome. Our aim was to test whether dexloxiglumide, a CCKl-antagonist, blocks the effect of lipids on intestinal gas transit and tolerance. METHODS. In 12 healthy subjects (6 female, 6 men) we used a validated model inducing gas retention in man (GE 2002; 123:700). Briefly, a mixture of gases mimicking physiological venous gas was infused into the jejunum at $12 \mathrm{ml} / \mathrm{min}$ for $3 \mathrm{~h}$ (total volume $2160 \mathrm{ml}$ ). Simultaneously, lipids were administered into the duodenum (Intralipid, $1 \mathrm{kcal} / \mathrm{min}$ ), and we quantified anal gas evacuation (via a barostat) and abdominal symptoms (by a 0-6 scale). Paired double-blind studies with iv infusion of either dexloxiglumide ( $2.5 \mathrm{mg} / \mathrm{Kg}$ bolus followed by $5 \mathrm{mg} / \mathrm{Kg} . \mathrm{h}$ continuous infusion) or saline (control) were performed on separate days in random order. RESULTS. As expected subjects developed significant abdominal symptoms $(2.6 \pm 0.6$ score $)$ and intestinal gas retention $(665 \pm 180 \mathrm{ml})$ in response to the gas plus lipids challenge test during i.v. saline. In contrast, i.v. infusion of dexloxiglumide reduced abdominal symptoms $(1.2 \pm 0.4$ score $\mathrm{p}<0.05$ vs saline) and evidenced a trend towards improving intestinal gas clearance $(589 \pm 197$ $\mathrm{ml}$ gas retention; NS vs saline). Interestingly, the effects of dexloxiglumide were much more pronounced in the women subgroup (symptoms improved by $56 \pm 16 \%$ and gas retention by $28 \pm 19 \%$; $<<0.05$ vs saline for both). CONCLUSION. CCK1-antagonist dexloxiglumide reduces gas-related symptoms and improves gas clearance, particularly in women.

\section{T2031}

Influence of Guanylate Cyclase C Binding Ligand Md-1100 On TNBS-Induced Visceral Hypersensitivity in Wt vs Ko Guanylate Cyclase C Deficient Mice Helene Eutamene, Vassilia Theodorou, Valerie Tondereau, Aurelie Waget, Jean Fioramonti, Alexander P. Bryant, Lionel Bueno

Background. MD-1100 acetate (MD-1100) is a novel therapeutic agent in clinical development for the treatment of irritable bowel syndrome with constipation (IBS-C) and chronic constipation. MD-1100 is an agonist of the receptor guanylate cyclase-C (GC-C). Ligand binding to GC-C stimulates cGMP production which initiates a signal transduction cascade that culminates in activation of the cystic fibrosis transmembrane conductance regulator (CFTR). In a previous study (DDW 2005) we showed that MD-1100 is active in a model of trinitrobenzene sulfonate (TNBS) colitis induced hypersensitivity in rats. The aim of this present study, using GC-C KO mice, was to elucidate the specific activity of MD1100 on GC-C in inflammation-induced visceral hyperalgesia. Methods. Two groups of 12 mice (C57Bl6), WT and GG-C KO (GCC, 22-25g) were used. To measure abdominal contractions reflecting acute visceral pain, animals were equipped with $\mathrm{NiCr}$ electrodes implanted in the abdominal striated muscle. Colorectal distension (CRD) was performed using a Fogarty probe $4 \mathrm{~F}$ inserted into the rectum at $1 \mathrm{~cm}$ from the anus. The balloon was gradually inflated with water by steps of $0.02 \mathrm{~mL}$ from 0 to $0.12 \mathrm{~mL}$ each step lasting $10 \mathrm{sec}$ at 5 min intervals. The electromyographic (EMG) recording system was composed of 8 channel-differential pre-amplifier associated with 4 amplifier analyser PowerLab/4SP connected to a computer Mice were submitted to control CRD performed before (basal condition) and 3 days after intra-rectal administration of TNBS $(20 \mathrm{mg} / \mathrm{kg}$ in $30 \%$ ethanol, $60 \mu \mathrm{L})$. Mice received MD$1100(0.01$ and $0.3 \mu \mathrm{g} / \mathrm{kg} \mathrm{PO})$ or its vehicle ( $\mathrm{NaCl} 0.9 \%), 1$ hour before distension in basal conditions and 3 days after colonic inflammation. Results. Under basal conditions, the sensitivity was different in WT vs KO mice. The EMG response thresholds were significant at 0.06 and $0.08 \mathrm{~mL}$ respectively and different between WT and $\mathrm{KO}$ mice. In these conditions, MD-1100 was effective to reduce the basal sensitivity at a dose of $0.3 \mu \mathrm{g} / \mathrm{kg}$ in WT but not in GC-C KO mice. TNBS instillation induced a similar hyperalgesia and allodynia in both WT and KO mice. After TNBS-induced colonic inflammation, MD-1100 administered at a dose of $0.01 \mu \mathrm{g} / \mathrm{kg}$ significantly reduced the hypersensitivity only in WT mice ( $48 \pm 7 \mathrm{vs}$ $20 \pm 5$ amplitude of contraction (mV/s) for $0.04 \mathrm{ml}$ of CRD), whereas no changes were observed in GC-C KO mice. Conclusion. In the basal condition, compared to WT, GC-C $\mathrm{KO}$ mice are less sensitive to $\mathrm{CRD}$, suggesting a key role of GC-C in the basal sensitivity of the gut. Moreover, MD-1100 reduces inflammation-induced hyperalgesia through GC-C binding, studies to elucidate the mechanism are underway.

T2032

Effect of Tegaserod On Meal-Related Symptoms Induced By the Nutrient Drink Test in Man

George Karamanolis, Sebastien Kindt, Rita Vos, Josef Janssens, Jan Tack

In the majority of functional dyspepsia (FD) patients, symptoms are triggered or aggravated by ingestion of a meal. Pathophysiological studies indicate that abnormalities of gastric sensorimotor may underlie the occurrence of meal-related symptoms in FD. Tegaserod, a 
5HT4 receptor agonist, enhances gastric emptying and gastric accommodation in man. The aim of the present study was to investigate the effect of tegaserod on the maximum tolerated volume of nutrient ingested and postprandial symptoms in man. Methods: Seventeen healthy volunteers $(7 \mathrm{~m}$, mean age $27 \pm 1$ ) were studied on two separate occasions, after 3 days pretreatment with placebo or tegaserod $6 \mathrm{mg}$ b.i.d. They underwent a slow satiety drinking test ( $1.5 \mathrm{kcal} / \mathrm{ml}$; $13 \%$ proteins, $48 \%$ carbohydrates, $39 \%$ lipids) until maximum satiety. At 5-minute intervals, the intensity of 9 symptoms (satiety, fullness, bloating, nausea, belching, pain, epigastric burning, heartburn, discomfort) was assessed on $10 \mathrm{~cm}$ visual analog (VAS) scales. Nutrient tolerance, area under the curve (AUC) for symptom intensities and symptom satiety scores were recorded and compared by paired t-test and ANOVA. Results: Pretreatment with tegaserod had no influence on nutrient tolerance (maximum intake $670 \pm 57$ vs. $671 \pm 65$ $\mathrm{ml}$, NS). After tegaserod, symptom intensity scores for fullness (AUC $1147 \pm 141$ vs. $1568+141$ mm*min, $\mathrm{p}<0.05$ ), bloating (AUC $825 \pm 165$ vs. $1352 \pm 227 \mathrm{~mm} * \mathrm{~min}, \mathrm{p}<0.05$ ), epigastric pain (AUC $66 \pm 42$ vs. $133 \pm 67 \mathrm{~mm} * \mathrm{~min}, \mathrm{p}=0.05$ ), and epigastric discomfort (AUC $701 \pm 50$ vs. $1158 \pm 203 \mathrm{~mm} * \min , \mathrm{p}=0.05$ ), were decreased (all ANOVA significant, $\mathrm{p}<0.05$ ), while scores for satiety, belching, heartburn, epigastric burning and nausea were not altered. Conclusion: The 5-HT4 receptor agonist tegaserod does not affect meal-induced satiety and nutrient volume tolerance, but tegaserod decreases the intensity of several meal-induced symptoms. These data support a potential benefit of tegaserod in the treatment of mealinduced FD symptoms

\section{T2033}

Newer Antiepileptic Drugs for Adults with Cyclic Vomiting Syndrome (CVS): A Novel Approach to Maintenance Therapy

Ray E. Clouse, Gregory S. Sayuk, Rajesh Shah, Patrick J. Lustman, Chandra Prakash

Management of CVS in adult patients is limited by the small number of effective agents either for abortive treatment of episodes or maintenance of the episode-free interval. Tricyclic antidepressants (TCAs) have been the most commonly used maintenance medications in adults, but their use often is limited by high side effect profiles. Newer antiepileptic drugs (AEDs), including zonisamide (Zonegran ${ }, \mathrm{ZN})$ and levetiracetam (Keppra $®$, KP), are well tolerated, are being used for off-label prophylaxis against migraine headache, but have not been tested in CVS. Methods: 18 adult patients with CVS (mean age $38.6 \pm 3.3 \mathrm{yr}$; range 19-71; 7 female/11 male) were treated with maintenance open-label AEDs after TCA failure (13 pts) or intolerance (5 pts). Each patient had typical Rome II features of CVS; 9 (50\%) also had a dominant abdominal pain component; and 5 subjects (28\%) had a CVS picture in face of diabetes mellitus without other satisfactory explanation for the stereotypical vomiting episodes. 2 (22\%) had personal or family history of migraine headache and $3(9 \%)$ had prodromal features. 4 subjects had used marijuana regularly but had no improvement on withdrawal. Duration of CVS averaged $5.4 \pm 1.4 \mathrm{yr}$ (range $0.2-21 \mathrm{yr}$ ), and episode rate over the 1 year pre-AED averaged $1.1 \pm 0.3$ /month. Each subject was followed for $\geq 3$ months on AEDs; response to treatment was evaluated on a 0-3 Likert scale (worse/no improvement to near complete or complete response) with comparison to pre-treatment course. Results: 15 subjects (83\%) were treated with ZN and 3 (17\%) with KP; median final daily dose (range) was $300 \mathrm{mg}(100-600 \mathrm{mg})$ for ZN and $1000 \mathrm{mg}(500-2000 \mathrm{mg})$ for KP. TCAs were discontinued in 10 subjects (75\%). Duration of follow-up on AEDs averaged $9.7 \pm 2.0 \mathrm{mo}$. Response $\geq 2$ on the Likert scale (at least moderate response) occurred in $13(72 \%)$ of subjects, while complete or near complete resolution of episodes was reported by 8 of these ( $44 \%$ of total). Episode rate reduced to $0.5 \pm 0.2 / \mathrm{mo}$ ( $\mathrm{p}<0.05$ compared with pre-AED value) for responders. Only 1 subject was intolerant (CNS side effects from ZN). Conclusions: Newer AEDs, specifically ZN and KP, are beneficial in open-label, maintenance use for reducing vomiting episodes in adults with CVS. Nearly three-fourths of subjects who were unsatisfactorily managed with TCAs benefited over this short follow-up period. Further investigation of these AEDs in CVS is indicated to better define efficacy, dosing and drug superiority. Considering their high tolerance and lower side effect profiles, they may prove superior to TCAs in primary maintenance strategies.

\section{T2034}

The Characterization of An Active Metabolite of MD-1100, a Therapeutic Agent in Development for the Treatment of IBS-C and Chronic Constipation Alexander P. Bryant, Robert W. Busby, Etchell A. Cordero, Marco M. Kessler, Christine M. Pierce, Jenny V. Tobin, Angelika Fretzen, Caroline Kurtz, Mark G. Currie

MD-1100 acetate (MD-1100) is currently in clinical trials for the treatment of irritable bowel syndrome with constipation (IBS-C), chronic idiopathic constipation and additional gastrointestinal (GI) disorders. MM-419447 is an in vivo metabolite of MD-1100 that was discovered in non-clinical pharmacokinetic studies. This metabolite was synthesized, characterized and found to be a potent agonist of the receptor guanylate cyclase-C (GC-C) sharing the pharmacological traits of the parent, MD-1100. Aim: To characterize the in vivo and in vitro pharmacology of MM-419447 in rats through in vitro activity assays, competitive radioligand binding assays, and in vivo efficacy experiments. Methods: MM-419447 was characterized in vitro by measuring the concentration-dependent production of cyclic GMP (cGMP) upon exposure to human T84 colon cancer cells. The binding of MM-419447 to cell surface GC-C receptors on T84 cells was characterized using a competitive radioligandbinding assay. The in vivo pharmacology was evaluated using widely accepted rodent models of GI transit and intestinal secretion. Results: MM-419447 stimulated cGMP production in vitro upon exposure to human T84 cells in a concentration-dependent manner over a range of concentrations (0.03 to $1000 \mathrm{nM}$ ). Direct administration of MM-419447 into ligated intestinal loops in the rat resulted in a statistically significant increase in lumenal fluid secretion and cGMP production. Both high and low affinity GC-C binding sites were identified for MM-419447. Finally, oral administration of MM-419447 resulted in a significant and dose-dependent increase in GI transit in rats when compared with vehicle-dosed animals. The lowest effective dose of MM-419447 in the GI transit study was $6.25 \mu \mathrm{g} / \mathrm{kg}$ compared to $5.0 \mu \mathrm{g} / \mathrm{kg}$ for MD-1100. Conclusion: MM-419447 is an active metabolite of MD-1100. Like the parent, this metabolite binds to the GC-C receptor, which results in elevated levels of cGMP, and increased fluid and electrolyte secretion. When orally administered, this GC$\mathrm{C}$ agonist accelerates intestinal transit in rats, with comparable potency to MD-1100. The
GC-C binding affinity of MM-419447 was similar to the parent compound and the in vitro activity of MM-419447 is not statistically different from MD-1100

\section{T2035}

Diabetic Gastroparesis: Effect of Mitemcinal By Subgroup Analysis in a 12Week, Randomized, Multi-Center, Double-Blind, Placebo-Controlled, Phase 2b Study

Richard W. McCallum, Barry J. Goldstein

INTRODUCTION: Mitemcinal, a macrolide derived motilin-receptor agonist can accelerate gastric emptying (GE) time in patients with idiopathic and diabetic gastroparesis. Obesity and poor glycemic control are thought to be independent risk factors for upper GI symptoms. We investigated how these factors affected placebo response and examined Mitemcinal efficacy in the subgroup using an eligibility baseline cut-off of $\mathrm{BMI}<35 \mathrm{~kg} / \mathrm{m}^{2}$ and $/$ or HbAlc $<10 \%$. METHODS: 392 insulin-requiring pts with type 1 or 2 DM were stratified by gastric emptying test results (solid meal scintigraphy, per site standard) and treated for 3 months with placebo (P), Mitemcinal $5 \mathrm{mg}$ or $10 \mathrm{mg}$ BID. Complete Response (CR) required positive monthly response (defined at least twice of "adequate symptom relief" in weekly global assessments of a month) for all 3 months. Overall Response (OR) required at least $75 \%$ of the weekly global assessments to be positive for the whole treatment period. 145 of 392 pts were excluded for: lack of baseline data ( 36 total: 9 BMI, 23 HbAlc, 4 both), BMI $>=35$ (89), $\mathrm{HbAl}>>=10 \%$ (18), both factors (7). Study subset (247 patients) included $74 \%$ type 1: $26 \%$ type 2; $62 \%$ female: $38 \%$ male; and $52 \%$ delayed: $48 \%$ non-delayed. RESULTS: Multivariate analysis in $\mathrm{P}$ demonstrated significant influences on placebo response rate by BMI (Odds Ratio 3.08, 95\% CI 1.00-9.46) and gender (2.73, 1.02-7.29). DM type, GE status, age and HbAlc affected PR rate without achieving statistical significance; DM history and symptom severity were not predictive. Multivariate analysis in ITT identified BMI<35 subgroup for CR of Mitemcinal $10 \mathrm{mg}$ vs. P (2.29 OR; 1.09-4.82, 95\% CI). The Weekly Response, $\mathrm{CR}$ and $\mathrm{OR}$ rates (\%) for pts with baseline $\mathrm{HbAl}<<10 \%$ and $\mathrm{BMI}<35$ are reported. CONCLUSIONS: 1) A significantly higher response rate was seen in mitemicinal treated patients; 2) BMI and gender were independent covariates for a greater placebo response; 3) Insulin requiring diabetics with symptomatic gastroparesis who have a BMI<35 and $\mathrm{HbAlc}<10 \%$ may represent a subset of study patients more likely to accurately reflect a clinically meaningful response with mitemcinal at $10 \mathrm{mg}$ bid; 4) Tachyphylaxis to Mitemcinal was not observed over the 3-month double-blind study period. 5) Additional trials with mitemcinal in diabetic gastroparesis and other gastropathies are warranted. [Research funded by Chugai Pharma USA, LLC]

\begin{tabular}{|c|c|c|c|c|c|c|c|c|c|}
\hline & \multicolumn{3}{|c|}{$\begin{array}{c}\text { Mean Weekly Response } \\
\text { Rate (\%) }\end{array}$} & \multicolumn{3}{c|}{ CR rate (\%) } & \multicolumn{3}{c|}{ OR rate (\%) } \\
\cline { 2 - 10 } $\begin{array}{c}\text { BMI }<35+ \\
\text { HbA1c<10\% } \\
(\mathrm{n}=247)\end{array}$ & $\begin{array}{c}\mathrm{P} \\
(\mathrm{n}=\end{array}$ & $\begin{array}{c}5 \mathrm{mg} \\
(\mathrm{n}=94)\end{array}$ & $\begin{array}{c}10 \mathrm{mg} \\
(\mathrm{n}=68)\end{array}$ & $\begin{array}{c}\mathrm{P} \\
(\mathrm{n}= \\
82)\end{array}$ & $\begin{array}{c}5 \mathrm{mg} \\
(\mathrm{n}=95)\end{array}$ & $\begin{array}{c}10 \mathrm{mg} \\
(\mathrm{n}=70)\end{array}$ & $\begin{array}{c}\mathrm{P} \\
(\mathrm{n}= \\
82)\end{array}$ & $\begin{array}{c}5 \mathrm{mg} \\
(\mathrm{n}=95)\end{array}$ & $\begin{array}{c}10 \mathrm{mg}(\mathrm{n}= \\
70)\end{array}$ \\
\cline { 2 - 10 } & $\begin{array}{c}28.4 \\
\pm\end{array}$ & $\begin{array}{c}34.9 \pm \\
31.6\end{array}$ & $\begin{array}{c}44.8 \pm \\
34.8\end{array}$ & 15.9 & $\begin{array}{c}24.2 \\
(0.168)\end{array}$ & $\begin{array}{c}34.3 \\
(0.008)^{*}\end{array}$ & $\begin{array}{c}9.8 \\
(0.17 .9\end{array}$ & $\begin{array}{c}32.9 \\
(0.121)\end{array}$ & $(0.0004)^{*}$ \\
\hline
\end{tabular}

Mean $\pm \mathrm{SD}(\mathrm{P}$ value $)$

T2036

Long-Term Quality of Life in Patients with Irritable Bowel Syndrome with Diarrhea Predominance (IBS-D) Receiving Cilansetron $2 \mathrm{Mg}$ tid: Results from a 52-Week Extension Trial Philip Miner, James S. Novick

INTRODUCTION: Patients with IBS-D have impaired quality of life (QOL). In various clinical trials, cilansetron, a 5HT3 antagonist has been shown to be effective in improving symptoms of abdominal pain and altered bowel habits as well as QOL in patients with IBSD. The purpose of this analysis was to determine whether the QOL improvements observed with cilansetron $2 \mathrm{mg}$ TID during a double-blind, placebo-controlled 3-month efficacy and safety study are maintained with long-term treatment. METHODS: Patients with Romedefined IBS-D who had completed the 3-month core efficacy study were eligible for enrollment in a 52-week extension study. All patients in this study received open-label cilansetron $2 \mathrm{mg}$ TID. OOL was evaluated as a secondary endpoint in both studies and was measured using the validated IBS QOL survey, a 34-item survey including 8 subscales. Patients completed the survey at Baseline which was defined as the final study visit in the core study, at Week 12, and at End of Treatment which was defined as the last post-baseline assessment on study drug. Higher scores indicate better QOL (maximum score is 100). RESULTS: In total, 543 subjects were enrolled in the extension study and 536 subjects were included in the safety population. The overall mean cilansetron exposure was 351 days for subjects who received cilansetron in both studies (C/C group) and 254 days for subjects who received placebo in the core study and cilansetron in the extension study (P/ C group). At the start of the extension study, the Baseline mean scores for all scales were higher for $\mathrm{C} / \mathrm{C}$ than for $\mathrm{P} / \mathrm{C}$ subjects (Table). An improvement from Baseline to End of Treatment was observed in all scales for the P/C group. At Endpoint, the mean IBS-QOL Overall scores were 70.7 in $\mathrm{C} / \mathrm{C}$ subjects and 70.9 in P/C subjects. The largest increases in IBS-QOL were observed in those subscales with the lowest baseline levels. CONCLUSION: The beneficial effect of cilansetron $2 \mathrm{mg}$ TID on QOL in patients with IBS-D observed during the 3-month core study was maintained over the 52-week extension study.

IBS-QOL overall and subscale scores 


\begin{tabular}{|c|c|c|c|c|}
\hline & \multicolumn{2}{|c|}{ Baseline Mean (SE) } & \multicolumn{2}{c|}{ End of Treatment Mean (SE) } \\
\hline & $\mathrm{C} / \mathrm{C}(\mathrm{n}=257)$ & $\mathrm{P} / \mathrm{C}(\mathrm{n}=279)$ & $\mathrm{C} / \mathrm{C}(\mathrm{n}=257)$ & $\mathrm{P} / \mathrm{C}(\mathrm{n}=271)$ \\
\hline Overall & $69.5(1.4)$ & $62.1(1.4)$ & $70.7(1.4)$ & $70.9(1.5)$ \\
\hline Interference with activity & $61.7(1.7)$ & $52.3(1.6)$ & $62.8(1.6)$ & $64.4(1.7)$ \\
\hline Body image & $75.3(1.5)$ & $66.5(1.6)$ & $75.0(1.5)$ & $74.1(1.6)$ \\
\hline Health worry & $77.8(1.1)$ & $73.3(1.2)$ & $77.2(1.1)$ & $77.1(1.2)$ \\
\hline Food avoidance & $58.4(2.0)$ & $47.6(1.8)$ & $59.5(1.8)$ & $59.1(1.8)$ \\
\hline Social reaction & $73.1(1.5)$ & $67.0(1.6)$ & $75.0(1.4)$ & $74.8(1.5)$ \\
\hline Sexual & $80.8(1.7)$ & $74.6(1.8)$ & $82.2(1.6)$ & $79.8(1.6)$ \\
\hline Relationship & $75.8(1.4)$ & $70.8(1.5)$ & $77.8(1.4)$ & $76.7(1.5)$ \\
\hline Dysphoria & $67.9(1.7)$ & $61.4(1.7)$ & $70.0(1.7)$ & $71.1(1.6)$ \\
\hline
\end{tabular}

T2039

Tegaserod Is Effective for Long-Term Use in Patients with Chronic Constipation

Lin Chang, Marielle Cohard-Radice, Cornelia Dunger-Baldauf, Jeffrey Kralstein, Michael A. Shetzline

BACKGROUND: Constipation is frequently chronic and presently only Tegaserod is approved for the treatment of this disorder. Although constipation may be treated acutely for symptom resolution, this approach does not address the underlying disease. In order to determine the clinical benefit of tegaserod in patients with chronic constipation, we explored dat accumulated in a long term safety study to query the benefit of long-term (13 month) tegaserod treatment in patients who respond to tegaserod $6 \mathrm{mg}$ bid after a 4 week period METHODS: In this blinded extension study, symptoms of constipation were assessed monthly for abdominal distension/bloating, abdominal pain/discomfort, bothersomeness of constipation and satisfaction with bowel habits (using a 5-point Likert scale). A responder in the core efficacy treatment period ( 12 weeks prior to the 13 month extension) was defined as having an average improvement of at least one complete spontaneous bowel movement per week during the first 4 weeks of therapy. Response at 12 weeks was defined similarly. Patients in the extension period continued on the same dose of tegaserod they were on at the completion of the core trial. A responder in the extension period was defined as a patien who had a reduction of the average symptom severity over month 1 to month 13 versus baseline of at least 0.5 points for at least $50 \%$ of reported symptom scores. Responders to tegaserod $6 \mathrm{mg}$ bid in the core trial who took study drug in the extension were included in this analysis. Average symptom scores were analyzed with paired t-tests against the withingroup baseline. RESULTS: 113 of the 278 patients who took $6 \mathrm{mg}$ bid in the extension, (41\%) were 4-week responders. 100/113(88\%) were responders at the end of the 12-week core efficacy period and over the 13 months extension, 91/107 (85\%) of the patients were responders (6 excluded due to unavailable data). Average severity reductions (mean(SD)) were $0.93(0.98)$ for abdominal distension/bloating, 0.88 (1.02) for abdominal pain/discomfort, 1.13 (1.04) for bothersomeness of constipation and 1.36 (0.93) for satisfaction with bowel habits. All improvements were statistically significant $(\mathrm{p}<0.0001)$ and clinically meaningful (at least 0.5 point improvement on a 5-point scale) CONCLUSION: $85 \%$ of patients who initially respond to tegaserod will continue to respond and this clinical response averaged one point improvement in a 5 point Likert scale over more than one year follow up. These results clearly demonstrate that the vast majority of patients who respond to tegaserod after 4 weeks of therapy will manifest long-term improvement in the multiple symptoms of chronic constipation. tion had no effect. The response was similar in small and large intestine and the data were therefore pooled. The increase was $9.7 \pm 2.9 \mu \mathrm{A} / \mathrm{cm} 2$ for $256 \mu \mathrm{g} / \mathrm{ml}, 22 \pm 7.9 \mu \mathrm{A} / \mathrm{cm} 2$ for $512 \mu \mathrm{g} /$ $\mathrm{ml}$ and $29 \pm 8.1 \mu \mathrm{A} / \mathrm{cm} 2$ for $1024 \mu \mathrm{g} / \mathrm{ml}$ ( $\mathrm{p}<0.05$ at all concentrations). The concentrations used are well below the dose used to treat patients with functional dyspepsia which is $3 \mathrm{x}$ $51 \mathrm{mg} / \mathrm{ml}$ daily. The STW5 evoked secretory effect was bumetanide $(100 \mu \mathrm{M})$ sensitive and therefore due to increased chloride secretion. Nerve blockade by tetrodotoxin $(1 \mu \mathrm{M})$ had no effect indicating a direct epithelial action of the drug. In addition the secretory response after electrical field stimulation of nerves remained unchanged in the presence of STW5. Our results indicate that STW5 has a powerful pro-secretory effect in the human intestine in vitro. It does not interfere with neurally mediated secretion but appears to stimulate chloride secretion at the level of the epithelial cell. Generally, patients with reduced secretion may profit from this drug. STW5 (Iberogast $®$ ) may represent a novel treatment option for IBS patients, in particular those with constipation predominant IBS

\section{$\mathrm{T} 2038$}

Protein and Ginger for the Treatment of Chemotherapy-Induced Delayed Nausea and Gastric Dysrhythmia

Max E. Levine, Marcum Gillis, Sara Yanchis, Anne C. Voss, Robert M. Stern, Kenneth L. Koch

Background: Delayed nausea occurs in many cancer patients during the three days following administration of a cytotoxic agent. Meals high in protein content decrease the nausea of motion sickness and pregnancy, possibly by enhancing normal gastric myoelectrical activity and/or by reducing gastric dysrhythmias. Ginger may also have anti-nausea properties. The aim of this study was to explore the use of a nutritional intervention consisting of high protein meals and ginger for the management of delayed nausea experienced by chemotherapy-naive cancer patients. Method: Twenty-eight cancer patients receiving emetogenic cytotoxic agents were assigned to one of three groups. During the three day study period following their first chemotherapy session, Control Group patients $(n=9)$ continued with their normal diet, Protein Group patients $(n=9)$ were provided with a protein drink (ProSure ${ }^{2}$; $15 \mathrm{~g}$ whey protein) and $1 \mathrm{~g}$ of dried ginger root to consume twice daily, and High Protein Group patients $(n=10)$ were provided with a protein drink with additional protein powder (ProSure ${ }^{\circledR}$ and ProMod $(31 \mathrm{~g}$ whey protein) and $1 \mathrm{~g}$ of dried ginger root to consume twice daily. All patients were asked to complete a symptom diary over the three days to assess the severity, frequency, and bothersomeness of the nausea they experienced, as well as their use of antiemetic medication. Gastric myoelectrical activity was assessed by electrogastrography as five patients ingested a protein meal with ginger on the first morning of the study. Results: Reports of nausea, of nausea being experienced often, and of nausea being bothersome were significantly less frequent in the High Protein Group than in the Control and Protein Groups $(p s<.05)$; the Control and Protein Groups were not significantly different from each other. Furthermore, significantly fewer patients in the High Protein Group elected to use antiemetic medication than patients in the other two groups $(p<.05)$. A significant increase in normal gastric myoelectrical activity ( 3 cycles/min), and a significant decrease in gastric tachyarrhythmia (3.7-10 cycles/min), the gastric dysrhythmia that frequently accompanies nausea, occurred with ingestion of the protein meals and ginger ( $p s<.05)$. Conclusions: (1) High protein meals with ginger reduced the delayed nausea of chemotherapy, and reduced the use of antiemetic medications. (2) Anti-nausea effects of high protein meals with ginger were associated with enhancement of normal gastric myoelectrical activity and decreased gastric dysrhythmias. High protein meals with ginger represent a novel, nutritionally-based treatment for the delayed nausea of chemotherapy.

\section{T2040}

Effect of a Novel Motilin Receptor Agonist, GSK326416, On Faecal Output in the Rabbit

William Unsworth, Julia E. Smith, Colin A. Campbell, Simon T. Bate, Kim L. Matthews, Alexander J. Stevens, Shaun McNulty, Kevin Lee, Susan M. Westaway, Sharon C. Stratton, Celestine T. O'Shaughnessy

Background: Previously, we have shown that the known motilin receptor agonist erythromycin $(10 \mathrm{mg} / \mathrm{kg}$ i.v.) increases faecal output in rabbits over a $2 \mathrm{~h}$ period (20th International Symposium on Neurogastroenterology and Motility, Toulouse, 2005-P096). In the present study the effect of GSK326416, a novel small molecule motilin receptor agonist, on faecal output in the rabbit was compared with that of erythromycin. The rabbit was chosen for these studies due to the apparent absence of a functional motilin receptor in rodent species, and the demonstrated expression of motilin receptors in the rabbit GI tract (Depoortere et al., 1991, Peptides 12:89-94). Methods: Male NZW rabbits (2.5-3.6kg; $\mathrm{n}=8$ ) received GSK326416 (1,3mg/kg), erythromycin $(10 \mathrm{mg} / \mathrm{kg}$ ) or vehicle (5\% glucose, $10 \% \mathrm{HPB}, 2 \%$ DMSO) on 4 separate occasions via i.v. bolus injection. Due to the pattern of faecal outpu in the rabbit following a clear circadian rhythm, dosing commenced at $4 \mathrm{pm}$ on each study day to coincide with a return to hard faecal pellet production. Each study day was separated by a period of at least $48 \mathrm{~h}$, with animals receiving treatments based on a randomised 4 period cross-over design. Faecal output was assessed following a $2 \mathrm{~h}$ period, during which the animals were left undisturbed. Data were analysed using a linear mixed model approach in SAS (Proc Mixed), with post hoc-planned comparisons between the predicted means. Results: When administered erythromycin, rabbits produced more faecal pellets $(67.9+/$ 7.5 vs $35.9+/-8.0$ pellets, $\mathrm{p}=0.0012)$ with an increased total faecal weight $(28.3+/-3.8 \mathrm{~g}$ vs $12.1+/-2.8 \mathrm{~g}, \mathrm{p}<0.0001)$ during the $2 \mathrm{~h}$ period, compared to vehicle. No increase in faecal output was observed when rabbits received $1 \mathrm{mg} / \mathrm{kg}$ GSK326416 (37.9+/-10.2 pellets and $13.5+/-4.4 \mathrm{~g})$. However, significant increases in both faecal pellet number $(61.5+/-7.8$ pellets $\mathrm{p}=0.0065)$ and total faecal weight $(23.8+/-4.0 \mathrm{~g}, \mathrm{p}=0.0014)$ were observed following dosing of $3 \mathrm{mg} / \mathrm{kg}$ GSK326416. Conclusion: We have demonstrated that the motilin receptor agonis GSK326416 possesses in vivo efficacy similar to that of erythromycin in a model of faecal output in the rabbit. GSK326416 therefore represents a novel class of motilin receptor agonist, distinct from erythromycin derivatives or motilides, which may prove useful in the treatment of gastrointestinal motility disorders including gastroparesis and functional dyspepsia.

\section{T2041}

Lactulose in the Treatment of Constipation in Pregnant Chinese Women: A Double-Blind, Randomized, Placebo-Controlled, Multicenter Study Daniel Becker, Daniele Compagnone, Lily Lu

Constipation is a common problem during pregnancy. The synthetic disaccharide lactulose is effective and safe in the treatment of constipation and, as it is not absorbed in the small intestine, presents no risk to the fetus. The aim of the study was to compare the efficacy, safety and tolerability of lactulose versus placebo in the treatment of constipation in pregnant Chinese women. Methods A randomized, double-blind, placebo-controlled, multicenter study was carried out in 63 pregnant Chinese women with constipation (Type 1, 2 or 3 on the Bristol Stool Form Scale (BSFS) in the week prior to study entry and in a one-week

. 
run-in phase, with at least one Type 1 or 2 stool in each period). The study was conducted according to ICH-GCP, and subjects provided written informed consent. After the run-in period, subjects were allocated by prespecified, concealed randomization list to oral lactulose solution ( $30 \mathrm{ml} /$ day during breakfast, adjusted to 15 or $45 \mathrm{ml} /$ day from day 3 if necessary) or placebo for 14 days and were asked to record all stools in a diary. Efficacy was assessed for the ITT sample in terms of stool consistency (BSFS), stool frequency and the subjects' and physicians' treatment satisfaction. The two primary efficacy variables, i.e. change in mean stool consistency from baseline to endpoint and responder rate (mean stool consistency of 3.5-5.5 or an increase of at least 1 in the last week), were tested using the Wilcoxon Mann Whitney rank sum test and the Fisher's exact test, respectively. Results A significantly greater change in mean stool consistency (BSFS) from baseline to endpoint occurred with lactulose than with placebo ( $1.3 \mathrm{vs} .0 .7 ; \mathrm{p}=0.0191$ ). Other clinically significant benefits were seen with lactulose versus placebo for the last week of treatment in terms of responder rate (61.3\% vs. $46.9 \%)$, proportion of subjects with a mean stool consistency of $3.5-5.5$ (32.3\% vs. $18.8 \%)$, and the percentage of subjects who were 'very satisfied'/fairly satisfied' (61.3\% vs. $45.2 \%$ ) and with 'marked improvement'/improvement' according to the investigator $(64.6 \%$ vs. $50 \%)$, although the differences were not statistically significant. Treatmentemergent adverse events were more common with lactulose than with placebo (48.4\% vs. $21.9 \%$ of subjects); all events in the lactulose group involved the gastrointestinal tract and were categorized as mild by the investigator. Lactulose had no notable effects on vital signs, physical findings or pregnancy outcome. Conclusion Lactulose was effective and safe in the treatment of constipation in pregnant Chinese women in this study.

\section{T2042}

Interruption of Daily Activities in Cilansetron-Treated Patients with Irritable Bowel Syndrome with Diarrhea Predominance (IBS-D): Results from a 16Week, Placebo-Controlled, Rerandomization Trial Carlos F. Francisconi, Douglas A. Drossman, Emeran A. Mayer, Steven Caras

Background: Cilansetron is a 5 HT3 antagonist currently under development for the treatment of IBS-D. In various efficacy trials, cilansetron has been shown to improve IBS-D symptoms (abdominal pain, bowel habits, and stool consistency) and IBS-related quality of life. AIM: To review the impact of cilansetron treatment on the interruption of daily activities (IDA) in patients with IBS-D. Methods: In a double-blind, placebo-controlled study, ROMEdefined IBS-D patients were randomized to cilansetron $2 \mathrm{mg}$ TID or placebo for 12 weeks, and then rerandomized to either treatment for an additional 4 weeks. IDA, a secondary endpoint, was determined by asking patients the following question at pretreatment and Months $1,2,3$, and 4 : Over the past 4 weeks, how have your IBS symptoms significantly interfered with your ability to effectively perform your activities? $0=$ not at all or rarely; $1=$ occasionally $(>10 \%$ of the time); $2=$ often $(>25 \%$ of the time); $3=$ very often $(>50 \%$ of the time), $4=$ almost always. IDA results were determined by intent-to-treat analysis. Results: At baseline, $>70 \%$ of all patients reported that their IBS symptoms "often," "very often," or "almost always" resulted in IDA and $\leq 8 \%$ reported symptoms that "not at all or rarely" resulted in IDA. By the 12 -week endpoint, $46 \%$ in the cilansetron vs $32 \%$ in the placebo group $(\mathrm{p}<0.001)$ claimed that their IBS symptoms "not at all or rarely" resulted in IDA (Table 1). Results for the proportion of patients whose symptoms "not at all or rarely" resulted in IDA during the rerandomization phase are presented in Table 2. Conclusions: In patients with IBS-D, an increase in IDA was observed following blinded withdrawal of cilansetron treatment while continued benefits were seen in patients maintained on cilansetron. Patients rerandomized from placebo to cilansetron demonstrated improvement in IDA while no relative changes were observed in patients maintained on placebo. Proportion of patients reporting IDA at baseline and week-12 endpoint

\begin{tabular}{|c|c|c|}
\hline Baseline & Cilasnetron n (\%) & Placebo n (\%) \\
\hline $\begin{array}{c}\text { Not at all or rarely Occasionally Often } \\
\text { Very often Almost always }\end{array}$ & $\begin{array}{c}29(8) 64(17) 129(34) 89 \\
(24) 66(18)\end{array}$ & $\begin{array}{c}21(6) 74(20) 120(33) 82 \\
(22) 71(19)\end{array}$ \\
\hline 12-Week Endpoint & 366 & 357 \\
\hline $\begin{array}{c}\text { Not at all or rarely Occasionally Often } \\
\text { Very often Almost always }\end{array}$ & $\begin{array}{c}168(46) 113(31) 44(12) \\
23(6) 18(5)\end{array}$ & $\begin{array}{c}116(32) 109(31) 54(15) \\
49(14) 29(8)\end{array}$ \\
\hline
\end{tabular}

Proportion of patients reporting IDA at rerandomization baseline and week-16 endpoint

\begin{tabular}{|c|c|c|c|c|}
\hline & $\mathbf{C / C} \mathbf{n}(\boldsymbol{\%})$ & $\mathbf{C} / \mathbf{P} \mathbf{n}(\%)$ & $\mathbf{P} / \mathbf{C} \mathbf{n}(\%)$ & $\mathbf{P} / \mathbf{P} \mathbf{n}(\%)$ \\
\hline Rerandomization Baseline & 149 & 139 & 135 & 146 \\
\hline Not at all or rarely & $78(52)$ & $70(50)$ & $54(40)$ & $44(30)$ \\
\hline 16-Week Endpoint & 149 & 137 & 134 & 145 \\
\hline Not at all or rarely & $81(54)$ & $51(37)$ & $67(50)$ & $53(37)$ \\
\hline
\end{tabular}

C, Cilansetron; P, Placebo

T2043

Effects of Lactobacillus Plantarum 299v On Symptoms and Rectal Sensitivity in Patients with Irritable Bowel Syndrome (IBS) - A Randomized, DoubleBlind Controlled Trial

Magnus Simren, Alma Syrous, Anette Lindh, Hasse Abrahamsson

The effects of probiotic bacteria on symptoms in IBS patients remain controversial. The majority of studies published so far are small and the effects of the different bacteria tested have varied. Lactobacillus Plantarum 299v (LP) has improved gastrointestinal (GI) symptoms in IBS patients in some (Nobaek et al 2000; Niedzielin et al 2001) but not all studies (Sen et al 2002). The mechanism of action is unclear, but recent animal studies have proposed a positive effect of probiotics on visceral sensitivity. The effects of probiotics on visceral sensitivity in humans are unknown. METHODS: We included 76 IBS patients fulfilling the Rome II criteria (48 females; mean age 40 years). After a screening period of two weeks eligible patients were randomized to receive six weeks of treatment with either $400 \mathrm{ml}$ per day of a rose-hip drink containing $5 \times 107 \mathrm{cfu} / \mathrm{ml}$ of LP or a plain rose-hip drink (control), comparable in colour, texture, and taste. Symptoms were assessed before and after the treatment period using the IBS severity scoring system (IBS-SSS) and the Gastrointestinal Symptom Rating Scale-IBS (GSRS-IBS) Rectal sensitivity testing was performed before and after the treatment period, both in the fasting state and after an $800 \mathrm{kcal}$ liquid meal. RESULTS: Twenty-nine patients in each group completed the trial, 10 patients were screening failures and 8 dropped out prematurely during the treatment phase, due to lack of effect of the treatment or to factors unrelated to the study. The overall GI symptom severity improved in both groups but the improvement did not differ statistically between the groups $(\mathrm{p}=0.17)$. The improvement in the control group was statistically significant (IBS-SSS total score: $305 \pm 73$ vs. $245 \pm 118($ mean \pm SD); $=0.0001)$ and a trend in the same direction was seen in the LP group $(295 \pm 95$ vs. $279 \pm 129 ; \mathrm{p}=0.08)$. The majority of individual GI symptoms assessed by the GSRS-IBS improved significantly in the control group, whereas only trends in the same direction was seen for the LP group. The change in the severity of the individual GI symptoms did not differ between the treatment groups. The rectal sensory thresholds were not affected in any significant way by the treatments, except for trends towards reduced pain thresholds after the treatment period in both groups, but with no difference between the groups. CONCLUSION: We could not detect a positive effect of Lactobacillus Plantarum $299 \mathrm{v}$ on GI symptoms and rectal sensitivity in IBS patients. The effectiveness of probiotics in IBS remains uncertain. This study was supported by an unrestricted grant from Probi AB.

\section{T2044}

Colonic Vascular Conductance Increased By Herbal Medicine Dai-Kenchu-to Via Calcitonin Gene-Related Peptide

Takashi Koseki, Toru Kono, Jun Iwamoto, Shinichi Kasai

Background \& Aims: Dai-Kencyu-To (DKT) is a traditional Chinese (Kampo) herbal medicine and has been used as the treatment of paralytic ileus DKT may increment gastrointestinal motility by an up-regulation of the calcitonin gene-related peptide (CGRP). CGRP is also the most powerful vasoactive substance. In the present study, we investigated whether DKT has any effect on the colonic blood flow $(\mathrm{CBF})$ in rats. Methods: Experiments were performed on 50 fasted anesthetized Wistar rats. Systemic mean arterial blood pressure (MAP) and heart rate (HR) were recorded. Red blood cell flux in CBF was measured using laser Doppler flowmetry, and colonic vascular conductance $(\mathrm{CVC})$ was calculated as the ratio of flux to MAP. We examined five key physiological mechanisms underlying the response using the blocker drugs, CGRP receptor blocker (CGRP8-37), nitric oxide synthase inhibitor (LNAME), vasoactive intestinal polypeptide (VIP) receptor blocker ([4-Cl-DPhe6, Leul7]-VIP), cyclooxygenase inhibitor (indomethacin), and substance P (SP) receptor blocker (spantide). After laparotomy, a cannula was inserted into the proximal colon to administer the DKT. Results: Intracolonal administration of DKT (100 and $300 \mathrm{mg} / \mathrm{kg}$ ) increased CVC (\% of basal CVC, $170 \%$ and $220 \%$, respectively) in a dose-dependent manner, whereas the MAP and HR were not affected. CGRP8-37 completely abolished the DKT-induced hyperemia, whereas [4-Cl-DPhe6, Leul7]-VIP, L-NAME and indomethacin partially attenuated the DKTinduced hyperemia. Spantide did not affect the hyperemia. Conclusions: The present study demonstrated that DKT increased CBF which was mainly mediated by CGRP. This suggests that DKT may be useful in the treatment of intestinal ischemia-related disease.

\section{T2045}

The Effect of Lipase Supplementation On Upper Gastrointestinal Symptoms and Gastric Myoelectrical Activity Induced By a High Fat Meal in Healthy Volunteers

Max E. Levine, Vikram D. Gopal, Sara Yanchis, Kenneth L. Koch

Background/Aims: Dyspepsia symptoms of abdominal discomfort, fullness, early satiety, and nausea occur after ingestion of meals in $20-30 \%$ of the population. Gastric dysrhythmias are exhibited by approximately $55 \%$ of dyspepsia patients. Currently there are limited therapies to reduce these symptoms. Gastric and pancreatic lipases are key enzymes in fat digestion, and hydrolyze fat into fatty acids and monoglycerides. The aims of this study were to characterize the effects of a high fat meal on upper gastrointestinal symptoms and gastric myoelectrical activity, and to evaluate the effect of acid-resistant lipase supplementation on the same outcomes. Method: Sixteen healthy volunteers ( 12 males; mean age $=31$ yrs) were enrolled in a double-blind, placebo controlled, cross-over trial. Volunteers were given a high fat meal (Pulmocare $®$ ) that was $55 \%$ fat, $28 \%$ carbohydrates, and $17 \%$ protein (237 ml; $355 \mathrm{Kcal}$ ). A capsule containing $280 \mathrm{mg}$ of acid-resistant lipase (Amano Enzyme USA) or placebo was administered immediately before ingestion of the meal. The order of conditions was counterbalanced, and visits were separated by at least one week. At each visit, individuals completed a Visual Analog Scale (VAS) concerning symptoms of nausea, stomach fullness, hunger, bloating, and abdominal discomfort. The VAS was completed at baseline, immediately after the meal, and at 10,20, 30, 45, and 60 minutes after the meal. Electrogastrograms (EGGs) were recorded throughout each visit to assess gastric myoelectrical activity. Results: Nausea, bloating, and stomach fullness were significantly increased 10 min after ingestion of the meal $(p s<.05)$, and hunger was significantly decreased $(p<.001)$; there was also a significant decrease in normal gastric myoelectrical activity ( 3 cycles $/ \mathrm{min}$ ), and a significant increase in tachygastria (3.7-10 cycles/min) at $10 \mathrm{~min}$ after the meal $(p s<.05)$. By $45 \mathrm{~min}$ after the meal, dyspepsia symptoms and tachygastria had decreased significantly from immediately after the meal, and normal gastric myoelectrical activity had increased significantly $(p s<.05)$. Stomach fullness was significantly lower with lipase supplementation than with placebo condition at 20 and $30 \mathrm{~min}$ after the meal $(p<.05)$; no effect of lipase supplementation on gastric myoelectrical activity was detected. Conclusions: 1) The high fat meal induced dyspepsia symptoms and gastric dysrhythmias, suggesting the meal may be a useful test for assessing gastric neuromuscular disorders; and 2) Acid-resistant lipase supplementation decreased stomach fullness after ingestion of the meal, and warrants further study in individuals with functional dyspepsia. 
T2046

A Novel Model to Study the Effect of Infused Gastric Content On Gastric Pressure and Content Distribution in Conscious Rats: Effect of Atropine, Molsidomine and $\mathrm{N}^{\mathrm{G}}$-Nitro-L-Arginine Methyl Ester Pieter Janssen, Maria Astin Nielsen, Per-Goran Gillberg, Leif Hultin

Introduction The stomach accommodates food without major intragastric pressure (IGP) increase. Impairment of gastric accommodation has been associated with abnormal gastric food distribution (overloading the antrum) and causes symptoms in functional dyspeptic patients. We present a novel model in which we can study the effect of increasing gastric content on IGP and gastric distribution in conscious rats. Atropine (muscarinic receptor antagonist; $1 \mathrm{mg} / \mathrm{kg}$ ), $\mathrm{N}^{\mathrm{G}}$-nitro-L-arginine methyl ester (L-NAME; nitric oxide synthase inhibitor; $30 \mathrm{mg} / \mathrm{kg}$ ) and molsidomine (MOL; nitric oxide donor; $20 \mathrm{mg} / \mathrm{kg}$ ), known to affect gastric muscle tone, were used to validate the model. Material \& Methods Experiments, performed in 5 female Wistar Kyoto rats, were run on a weekly basis alternating control (no drug) and drug treatment. After an overnight fast, a test-meal infusion system and a catheter to measure IGP were connected to a gastric fistula. Drugs were injected subcutaneously before start of the experiment. After a $20 \mathrm{~min}$ stabilisation period, the non-nutrient test-meal (containing $0.5 \mathrm{gr}$ barium sulphate/ml in a $3 \%$ hydroxypropyl methylcellulose solution) was infused at a preset speed of $0.25 \mathrm{ml} / \mathrm{min}$ for $2 \mathrm{~min}$, thereafter $2 \mathrm{ml} / \mathrm{min}$ until $4 \mathrm{ml}$ injected. Forestomach, corpus and antrum area, calculated as \% of the total stomach area, were evaluated from $X$-ray pictures continuously taken during the experiments. Results $($ mean \pm SEM) were compared using a paired $t$-test $(\mathrm{P}<0.05$ considered significant). Results IGP increased linearly with test-meal infusion. Atropine and MOL significantly reduced the maximum IGP from $13.3 \pm 0.4$ and $13.4 \pm 0.7$ to $12.0 \pm 0.3$ and $5.9 \pm 0.4 \mathrm{mmHg}$ respectively. L-NAME significantly increased the maximum IGP from $13.4 \pm 0.6$ to $15.9 \pm 1.3 \mathrm{mmHg}$. Atropine and MOL significantly reduced the antrum area to respectively $6.7 \pm 1.8$ and $4.0 \pm 1.6 \%$ compared to $13.9 \pm 1.6$ and $14.8 \pm 4.3 \%$ in control experiments. Atropine increased the forestomach area to $53.0 \pm 6.6 \%$ compared to $44.3 \pm 4.3 \%$ in control experiments whereas MOL significantly increased the corpus area to $52.9 \pm 2.5 \%$ compared to $41.3 \pm 1.7$ in control experiments. L-NAME, however, had no significant effect on content distribution between control and L-NAME treatment. IGP and content distribution did not significant differ between the control groups. Discussion \& Conclusion For same intragastric content, atropine and molsidomine reduced IGP and the gastric content was more proximally distributed. L-NAME increased IGP but did not have any effect on intragastric distribution. This novel rat model can be used to study the relationship between gastric content, gastric content distribution and intragastric pressure in a reproducible way.

\section{T2047}

Tegaserod Is Proven Safe in IBS-C Patients and Those with Mixed/Alternating Bowel Habits

Luis C. Sabbagh, William D. Chey, Gary Barton, Andrea Viegas, Gregory Ligozio, Michael A. Shetzline

BACKGROUND: Tegaserod (T), a promotility agent, has been shown to be safe and effective in the treatment of irritable bowel syndrome with constipation (IBS-C) and chronic idiopathic constipation. Recently, studies in patients with a mixed/alternating bowel pattern (IBS-M) have demonstrated efficacy for T. Since patients with a mixed/alternating bowel pattern are more likely to have softer stool consistency and increased stool frequency when compared with IBS-C patients, we studied safety and tolerability of $\mathrm{T}$ in this population. METHODS: A randomized, double-blind, placebo-controlled, multicenter study (North American, Latin American and United Kingdom centers) was performed in patients with IBS-C (Rome II) and IBS-M (those without IBS-C or IBS-D by Rome). Safety assessments consisted of reporting all adverse events, serious adverse events (SAEs), and pregnancies, as well as assessments of laboratory studies, vital signs, physical condition and body weight. RESULTS: 661 women were randomized; data were available for $329 \mathrm{~T}$ and 331 placebo (P) patients. Overall, $29.5 \%$ of patients experienced at least one adverse event during treatment with $\mathrm{T}$ and $25.7 \%$ for P. The most frequent adverse events were diarrhea (T 9.4\% and P $2.1 \%$ ), headache ( $\mathrm{T}$ $5.5 \%$ and P $6.6 \%$ ), abdominal pain (T $3.0 \%$ and P $2.1 \%$ ), and nausea (T $2.1 \%$ and P $3.3 \%$ ). Discontinuations due to adverse events were infrequent ( $1.8 \%$ vs P $3.0 \%$ ). Diarrhea led to study discontinuation in $1.5 \%$ receiving $\mathrm{T}$ vs $0.6 \%$ receiving $\mathrm{P}$. Diarrhea was reported more frequently in the IBS-M subset (T $12.1 \%$ and P $1.8 \%$ ) compared with the IBS-C subset ( $7.0 \%$ and P $2.4 \%$ ). However discontinuations due to diarrhea were low in both subsets ( $1.5 \%$ for IBS-M and $0.6 \%$ for IBS C). Diarrhea with T typically occurred soon after initiating therapy and lasted 2-3 days without recurrence. There were no cases of diarrhea with clinically significant consequences (i.e., hospitalization, need for IV fluids, abnormal lab values). No deaths occurred during the study. One patient receiving P experienced a SAE (appendicitis) and one T-receiving patient experienced chostochondritis. Neither SAE was suspected to be related to treatment. There were no reports of ischemic colitis. CONCLUSION: Diarrhea is reported more frequently in the IBS-M population, however diarrhea is well tolerated and infrequently results in discontinuation of medical therapy. T treatment of patients with IBS, including the subset of patients experiencing a mixed/alternating bowel pattern, is well tolerated and safe.

\section{$\mathrm{T} 2048$}

Quality of Life Improves in Both Male and Female Patients with Irritable Bowel Syndrome with Diarrhea Predominance (IBS-D) Following 12 Weeks of Cilansetron Treatment As Part of a Placebo-Controlled Rerandomization Trial Lucinda A. Harris, Braden Kuo

BACKGROUND: Cilansetron, a selective 5-HT3 receptor antagonist, is being evaluated for the treatment of irritable bowel syndrome with diarrhea predominance (IBS-D). In clinical trials, cilansetron treatment provided significant relief from IBS symptoms in both men and women. IBS has a negative impact on quality of life (QOL) in most patients and cilansetron has been demonstrated to increase IBS-QOL scores in 2 previous phase III cilansetron trials. AIM: To evaluate changes in QOL by gender in IBS-D patients treated with cilansetron.
METHODS: In this double-blind, placebo-controlled trial, 770 Rome II-defined IBS-D patients received either cilansetron $2 \mathrm{mg}$ TID or placebo for 12 weeks and were then rerandomized to either treatment for an additional 4 weeks. QOL data for the first 12 weeks were analyzed by gender. QOL was measured using the validated IBS-QOL survey, a 34 item survey comprising 8 domains: body image, dysphoria, food avoidance, health worry, interference with activity, relationship, sexual, and social reaction. Patients completed the survey at baseline and at Week 12 by responding to each of the 34 questions. Each response was associated with a QOL score; the total score was transformed to a 0-100 scale with higher scores reflecting better QOL. RESULTS: The IBS-QOL survey was completed by 744 patients ( 238 men; 506 women). There were no significant differences between treatmen groups in overall IBS-QOL score at baseline, although mean female scores (cilansetron: 49.6 placebo: 50.6) were numerically lower than mean male scores (cilansetron: 59.0 ; placebo: 59.2). At the end of treatment, the improvement in overall IBS-QOL survey scores was significantly larger in both men and women in the cilansetron group vs placebo (Table). In both men and women, statistically significant improvements were found in the cilansetron groups vs placebo for all 8 of the individual IBS-QOL subscales. CONCLUSION: Male and female IBS-D patients demonstrated significant improvements in overall IBS-QOL after 12 weeks of cilansetron $2 \mathrm{mg}$ TID treatment. Furthermore, significant improvements were observed in male and female cilansetron-treated patients in all 8 individual IBS-QOL subscales: body image, dysphoria, food avoidance, health worry, interference with activity, relationship, sexual, and social reaction. These data are similar to IBS-QOL results reported previously from 2 other placebo-controlled phase III trials of cilansetron.

Mean (SE) change from baseline to endpoint in QOL scores during the 12 week period

\begin{tabular}{|c|c|c|c|}
\hline & Cilansetron & Placebo & P-Value \\
\hline Males & $15.5(1.6)$ & $7.3(1.6)$ & $<0.001$ \\
\hline Females & $20.3(1.3)$ & $12.6(1.3)$ & $<0.001$ \\
\hline
\end{tabular}

\section{T2049}

Clinical Hallmarks of Cyclic Vomiting Syndrome (CVS) in Adults and Role of Long-Term Tricyclic Therapy

Farid Namin, Patel Jitan, Iman Joker, Zhiyue Lin, Reginald Dusing, Richard W. McCallum

Our goal was to prospectively investigate adult patients meeting Rome II criteria for CVS Methods: 24 patients (14M, 10F, mean age: 34 years, range 18-62) who were diagnosed over an 18-month period completed a clinical questionnaire as well as a Hamilton Rating Scale for Anxiety (HAM-A) and Zung Depression inventory. Gastric Emptying Time (GET) by a 4-hour scintigraphy of a solid meal was assessed and electrogastrogram (EGG) recorded between acute attacks. PET scan was performed in 5 patients during their attacks. 14 patients who were taking Amitriptyline at $1 \mathrm{mg} / \mathrm{kg}$ for minimum 6 months completed a follow up questionnaire including global assessment of quality of life using 1 to 10 visual analog scales Results: The mean age of onset of CVS was 29, range 14-53 years. All subjects reported that severe epigastric or diffuse abdominal pain accompanied the nausea and vomiting cycles. A typical attack, ranged from 1 to 14 days, majority of 4-6 days. Average \# of hospitalizations or ER visits was 42 per year. The HAM-A revealed that 21 (88\%) were suffering from anxiety disorder. Results of Zung Depression inventory indicated that $19(79 \%)$ suffered from mild to moderate depression. No history of migraine was reported, but $11(46 \%)$ had family history of migraine. GET was delayed ( $>10 \%$ isotope remaining at $4 \mathrm{hrs}$ ) in 2 patients, while $18(75 \%)$ had rapid GET ( $<30 \%$ retention at 2 hrs) and 4 were normal. The EGG revealed tachygastria in 4 of 10 subjects. PET scans showed similar patterns with decreased metabolism in the left inferior frontal cortex, thalamus and cingular gyrus in all. This was significant with 2.4 SD on a "Neuro Q" Program based test. Marijuana was used to relieve symptoms in $50 \%$ of patients and did not appear to explain cycles. For long-term prevention 14 were treated with Amitriptyline with a target dose of $1 \mathrm{mg} / \mathrm{kg}$ for minimum of 6 months. The average \# of ER visits and hospitalizations were significantly diminished from 32 to 14 when annually adjusted. Visual analog scale improved by average of 4 points. The major side effect of amitriptyline was hypersomnia and dizziness that caused one subject to stop the treatment. Conclusions: 1) Abdominal pain accompanies nausea and vomiting cycles; 2 There is a high prevalence of anxiety and depression; 3) Rapid gastric emptying and tachygastric EGG findings are present between cycles; 4) PET scan changes indicate central mechanisms are present during acute cycles. 5) Successful suppression of attacks can be achieved by long-term high dose amitriptyline therapy. This single center study extends the spectrum of CVS from pediatrics and emphasizes its increasing recognition in adults.

\section{$\mathrm{T} 2050$}

Growth Hormone Favorably Affects Bone Turnover and Bone Mineral Density in Patients with short Bowel Syndrome Undergoing Intestinal Rehabilitation Vin Tangpricha, Menghua Luo, Concepcion Fernandez-Estivariz, Niloofar Bazargan, Shanthi V. Sitaraman, Li H. Gu, Jan-Michael Klapproth, John R. Galloway, Lorraine M. Leader, Thomas R. Ziegler

Background: Patients with short bowel syndrome (SBS) have a high prevalence of metabolic bone disease due to nutrient malabsorntion and other factors. Human growth hormone (hGH) has been shown in some studies to have anabolic effects on bone, but hGH effects on bone in patients with SBS are unknown. Methods: Adults with PN-dependent SBS underwent a 7-day period of baseline studies while receiving usual oral diet and PN, then were placed on modified diets designed to improve nutrient absorption and daily oral calcium/vitamin D supplements (1500 mg elemental calcium and $600 \mathrm{IU}$ vitamin D3, respectively). Subjects received in a double-blind manner either sc hGH $(0.1 \mathrm{mg} / \mathrm{kg} / \mathrm{day}$ for 3 weeks, then on a $3 X$ weekly basis for 8 weeks; $n=14$ ) or saline placebo (control; $n=9$ ). Open-label hGH was given from weeks 13 to 24 in subjects who required PN at the end of week 12. Markers of bone turnover [serum osteocalcin (bone formation) and urinary Ntelopeptide (NTX; bone resorption)], and vitamin D nutriture [serum calcium, 25-hydroxyvitamin D (25-OH D) and parathyroid hormone (PTH) concentrations] were obtained a baseline and at week 4 and 12. Dual $\mathrm{x}$-ray absorptiometry of the hip and spine was performed 
to determine bone mineral density (BMD) at baseline and weeks 12 and 24. Results: The control and hGH-treated groups were similar in gender, age, residual small bowel length, $\%$ with residual colon in continuity, length of time on PN and daily PN requirements The majority of subjects in each group exhibited evidence of vitamin D deficiency at baseline (25-OH D levels $<30 \mathrm{ng} / \mathrm{mL} ; 78 \%$ of control subjects and $79 \%$ of hGH-treated subjects, respectively; mean values were control $22 \pm 3$ and $\mathrm{hGH} 27 \pm 3 \mathrm{ng} / \mathrm{mL}$; NS). Serum $25-\mathrm{OH}$ D levels at weeks 4 and 12 remained unchanged from baseline in each group. Subjects treated with hGH demonstrated a significant $62 \%$ increase from baseline in serum osteocalcin levels at 12 weeks (baseline $6.3 \pm 1.7$ vs week $1210.2 \pm 2.5 \mathrm{pg} / \mathrm{mL} ; \mathrm{p}<0.05$ ) and a $71 \%$ increase in urinary NTX levels at 4 weeks (baseline $51 \pm 20$ vs week $487 \pm 30 \mathrm{nM} / \mathrm{nM}$ creatinine; $\mathrm{p}<$ $0.05)$; osteocalcin and NTX remained unchanged over time in control subjects. BMD of the spine and total hip was unchanged from baseline in subjects treated with either placebo or hGH at 12 and 24 weeks. However, femoral neck BMD was slightly, but significantly, decreased in the placebo group at 24 weeks, but remained unchanged from baseline in the hGH-treated subjects. Conclusion: hGH therapy significantly increased markers of bone turnover during the initial 3 months of therapy and stabilized femoral neck bone mass over a 6-month period in patients with severe SBS undergoing intestinal rehabilitation.

\section{T2051}

Colostrum Supplementation Causes Hypertrophy in An Intestinal Smooth Muscle Cell Line (HISM) and in the Ileum of a Piglet Pre-Clinical Model of short Bowl Syndrome

Prue M. Pereira, Sarah L. Vaughan, Russell G. Taylor, Peter J. Fuller, Magdy Sourial, Eva S. Nagy, Julie E. Bines

Introduction:We have previously shown a trophic effect of colostrum protein concentrate (CPC) on intestinal adaptation following massive small bowel resection (MSBR). However no study has examined the effect of CPC on muscle following MSBR. Using a previously developed preclinical model of SBS, together with a human intestinal smooth muscle (HISM) cell line, the current study examines the impact of CPC supplementation on ileum muscle morphology. Methods: (A)4-wk-old piglets underwent a $75 \%$ MSBR or sham operation. Post-op, piglets received a polymeric infant formula diet (PIF; $n=3-6)$ or PIF supplemented with bovine $\mathrm{CPC}(\mathrm{PIF}+\mathrm{CPC} ; \mathrm{n}=6-9)$. At sacrifice ( 8 weeks post-op) serum IGF-1 levels were measured, and the ileum removed for histological assessment. Circular and longitudinal muscle area, and nuclei numbers were quantitated (5 random measurements/piglet). (B) $50 \%$ confluent HISM cells were treated with serum free media(SFM), 10\% protein in SFM, or $10 \%$ CPC in SFM ( $\mathrm{n}=3 /$ treatment) for 6 hours. The cell area was determined (40 cells/ coverslip), the number of cells in 4 fields of view/coverslip counted, and protein and DNA concentration calculated. Results: CPC supplementation resulted in increased circular and longitudinal muscle area in sham-operated piglets (Table 1) and moderate increases in MSBR piglets. Nuclei numbers were similar in all groups indicating a hypertrophic response. CPC treatment of HISM cells increased cell area and protein concentration compared with media alone treatment, but did not increase cell numbers (Table 2). Circulating unbound IGF-1 levels were significantly higher in CPC-fed, MSBR piglets compared with PIF alone MSBR piglets. Conclusion: Supplementation of an infant formula diet with bovine colostrum resulted in hypertrophy of ileum circular and longitudinal muscle. Hypertrophy was also observed following colostrum treatment of a human intestinal smooth muscle cell line. We have shown a significant increase in circulating levels of IGF-1 in CPC-fed, resected piglets and are currently investigating the mechanism behind this hypertrophic response. Table 2

\begin{tabular}{|c|c|c|c|c|}
\hline & Cell number & Cell area $\left(\mu \mathrm{m}^{2}\right)$ & DNA $(\mathrm{ng} / \mathrm{ml})$ & protein $(\mu \mathrm{g} / \mathrm{ml})$ \\
\hline SFM & $33 \pm 3$ & $4931 \pm 849$ & $77 \pm 4$ & $372 \pm 95$ \\
\hline $\mathrm{SFM}+10 \%$ protein & $54 \pm 5$ & $5045 \pm 676$ & $78 \pm 21$ & $620 \pm 107$ \\
\hline SFM+10\%CPC & $39 \pm 4$ & $5564 \pm 280$ & $63 \pm 4$ & $549 \pm 134$ \\
\hline
\end{tabular}

Table 1

\begin{tabular}{|c|c|c|c|c|}
\hline & PIF(sham) & PIF+CPC(sham) & PIF(MSBR) & PIF+CPC(MSBR) \\
\hline circular muscle area(mm ${ }^{2)}$ & $0.08 \pm 0.003$ & $0.11 \pm 0.008^{\mathrm{a}}$ & $0.09 \pm 0.009$ & $0.11 \pm 0.006^{\mathrm{c}}$ \\
\hline long.muscle area $\left(\mathrm{mm}^{2}\right)$ & $0.07 \pm 0.003$ & $0.09 \pm 0.01 \mathrm{~b}$ & $0.06 \pm 0.009$ & $0.08 \pm 0.012$ \\
\hline Free IGF-1 (ng/ml) & $351 \pm 38$ & $337 \pm 50$ & $89 \pm 13$ & $220 \pm 29 \mathrm{~d}$ \\
\hline
\end{tabular}

${ }^{a}$ sham vs sham, $\mathrm{p}<0.05,{ }^{\mathrm{b}}$ sham vs sham, $\mathrm{p}=0.06$, MSBR vs MSBR; ${ }^{\mathrm{d}} \mathrm{p}<0.05,{ }^{\mathrm{c}} \mathrm{p}=0.09$

\section{$\mathrm{T} 2052$}

Recombinant Human Growth Hormone (rhGH) Reduces Hpn Dependence (HPNd) of short Bowel Syndrome (SBS) Adult Patients: A Randomized, CrossOver, Placebo Controlled Study

Francisca Joly, Claude Baudoin, Abdul Monem Badran, Pascal Crenn, Bernard Messing

Background: following a positive placebo controlled 3 arms study, 4 weeks rhGH $(0.10 \mathrm{mg} /$ $\mathrm{Kg} / \mathrm{d}$ ), + a modified diet and glutamine continued 12 weeks, (Byrne TA, et al. JPEN 2003;27:S17) was recently FDA approved for HPN SBS patients weaning. This study was to confirm lower rhGH $(0.05 \mathrm{mg} / \mathrm{Kg} / \mathrm{d})$ alone efficacy. Methods: inclusion criteria were adult SBS HPNd patients (> 2 PN cycle/week), on sufficient oral intake, with no neoplasia, other organ failure, severe malnutrition, uncontrolled disease, steroids or octreotide. Patients received A and B sc daily cross over treatment with 1 PN cycle / wk decrease every 3-wk Ifor a maximum duration of 4(3-wk) treatment including no change in PN during run in and a 3-wk wash out between A \& B] until lean body mass (LBM) decline, evaluated on DEXA, was more than $5 \%$. It was expected at least 1 PN-decrease difference between A \& B with LBM change based on regression coefficient set up in each patient : a linear mixed model was used for statistical analysis of the cross over design (Chapman and Hall, London
1990). Results: 11 among 13 enrolled patients, completed the study (1 stop for personal convenience after the run in and 1 convulsion before treatment): $3 \mathrm{~F}, 8 \mathrm{M}, 44(22-60)$ (median, range) yr old, with a jejuno-colonic anastomosis, a small bowel remnant of $30(0-100) \mathrm{cm}$ and an initial PNd of 5(2-7) cycle / wk. PNd was -2(0-3) with A and -3(0-3) with B. LBM changes $(\mathrm{Kg} / \mathrm{wk}$, mean $\pm \mathrm{SD})$ were for $\mathrm{AB}$ sequence $(\mathrm{n}=6)-0.03 \pm 0.16 \&+0.12 \pm 0.21$, for $\mathrm{BA}$ sequence $(n=5):+0.22 \pm 0.10$ and $-0.21 \pm 0.10$ respectively. Difference between treatment $A$ and $B$ was better for $B:+0.29 \pm 0.08($ mean $\pm S E M)(p=0.007)$ with neither carry over $(p=$ 0.46 ) nor period effect $(\mathrm{p}=0.12)$. Conclusion : $\mathrm{rhGH}$ (treatment $\mathrm{B}$ ) reduced PNd better than placebo (treatmentA) with no LBM decline during rhGH treatment. The rhGH effect was not sustained during the placebo period treatment

\section{T2053}

The Effects of Glucagon-Like Peptide 2 (GLP-2) On Intestinal Mucosal BloodFlow Evaluated in the Stoma Nipple By Laser Doppler Flow in short Bowel Patients

Peter Hoeyerup, Peter T. Schmidt, Per Hellstrom, Per B. Mortensen, Palle B. Jeppesen

Background: GLP-2 is a hormone secreted from the endocrine L-cells of the intestine in response to enteral feeding. GLP-2 has been shown to be intestinotrophic in animal and human studies. In animal studies it has also been described that GLP-2 acutely stimulates proximal small intestinal blood-flow (Stephens-J et al. Am J Physiol Regul Comp Physiol sept. 2005). Short bowel jejunostomy patients describe hyperemia and enlargement in stoma nipple size in relation to GLP-2 treatment. Whether these observations are due to changes in mucosal blod flow is unknown. The aim of this study was to evaluate the acute effect of subcutaneous (s.c.) GLP-2 on stoma nipple mucosal blod flow (SNMBF) by Laser Doppler Technique (LDT). Methods: In 5 fasting short bowel IBD patients ( $1 \mathrm{~F}, 4 \mathrm{M} ; 56 \pm 6$ years; small bowel $204 \pm 52 \mathrm{~cm}$ ) a laser doppler flowmeter probe with 3 measure-points (site 1-3) was inserted approximately $5 \mathrm{~cm}$ into the stoma and fixated. A control probe was fixed to the skin in the maxillar region of the face (site 4) of the patients. SNMBF was measured employing a Periflux PF 4001 Master (Perimed AB, Järfälla, Sweden) in 2 minutes intervals at baseline and 14 and 59 minutes after injecting 800 microgram GLP-2 s.c. A wavelength of $780 \mathrm{~nm}$, a time constant of $0.2 \mathrm{sec}$, and a bandwidth of $20-25 \mathrm{kHZ}$ with a sampling frequency of $32 \mathrm{HZ}$ were used. The SNMBF was expressed in units of relative perfusion (perfusion units, PU). Results: The results are presented in the table. Conclusion: Although a numerical increase in perfusion units was detected in SNMBF after an s.c. GLP-2 injection, this did not reach statistical significance. Larger studies, more time intervals and possible longer observation periods are needed to answer whether GLP-2 increase SNMBF or not. Perfusion units (PU)

\begin{tabular}{|c|c|c|c|c|}
\hline $\mathrm{PU}(\mathrm{n}=5$, mean $\pm \mathrm{SD})$ & Baseline-2 to 0 minutes & $14-16$ minutes & $59-61$ minutes & ANOVA p-value \\
\hline Site 1 & $269 \pm 86$ & $322 \pm 94$ & $346 \pm 56$ & 0.39 \\
\hline Site 2 & $237 \pm 98$ & $334 \pm 114$ & $402 \pm 116$ & 0.07 \\
\hline Site 3 & $224 \pm 89$ & $291 \pm 88$ & $417 \pm 152$ & 0.12 \\
\hline Average & $243 \pm 83$ & $316 \pm 91$ & $388 \pm 99$ & 0.14 \\
\hline Site 4 (control) & $96 \pm 65$ & $97 \pm 62$ & $101 \pm 83$ & 0.95 \\
\hline
\end{tabular}

T2054

Increased Intestinal Absorption Rates By Segmental Reversal of the Small Bowel in Patients with short Bowel Syndrome

Francisca Joly, Sophie Rousseau, Xavier Dray, Kouroche Vahedi, Nathalie Kapel, Patrice Valleur, Yves Panis, Bernard Messing

Introduction : Segmental reversal of the distal remnant jejunum (SRJ) has been proposed as rehabilitative surgery to decrease parenteral nutrition dependence in adult patients with short bowel syndrome (SBS) type II (jejuno-colic anastomosis). However no solid data are available concerning change in absorption. Aim: To compare the intestinal absorption rates in SBS patients with and without SRJ. Methods: Intestinal absorption of total energy (bomb calorimetry), nitrogen (nitrogen elemental analysis), fat (Van de Kamer method) and carbohydrate (total - fat and protein) was analyzed during a 3-day period of (a) oral intake (no TCM) measured by expert dietetic enquiry and (b) stool collection on ice. All causes of SBS were included with the exclusion of SBS due to radiation enteritis. Seventeen SBS patients with SRJ (median age 52 years, range 21-80) were matched to 17 control SBS patients (median age 47 years, range 24-68) according to the post duodenal remnant small bowel length and remaining colon. All patients presented with a jejuno-colic anastomosis. Wilcoxon matched-pair signed-ranks test was used to compare continuous variables. Results: At the absorption study period, the 17 patients of the SRJ group and 13 patients of the control group were still on parenteral nutrition. The median remnant small bowel length was 35 $\mathrm{cm}$ (range 9-70) in the SRSB compared to $40 \mathrm{~cm}$ (range 0-80) in the control group ( $\mathrm{p}=$ 0.86). The median (range) delay between the reestablishment of the jejunocolic anastomosis and the absorption study was for SRJ 16(6-131) months and for SBS controls 47(5-227) months (NS). The median (range) length of the reversal segment was $10(8-15) \mathrm{cm}$. Oral intake and stool output were similar in both groups $(2572 \mathrm{kcal} / \mathrm{d}$ and $1577 \mathrm{ml} / \mathrm{d}$ in the SRJ group, $2666 \mathrm{kcal} / \mathrm{d}$ and $1676 \mathrm{ml} / \mathrm{d}$ in the control group, $\mathrm{p}=0.93$ and $\mathrm{p}=0.90$ respectively). Net caloric absorption was higher in the SRJ group compared to the control group (1809 vs $1508 \mathrm{kcal} / \mathrm{d}, \mathrm{p}=0.21$ ), as well as mean relative absorption rates of total calories (69\% vs $58 \%, \mathrm{p}=0.03$ ), nitrogen ( $63 \%$ vs $53 \%, \mathrm{p}=0.06$ ) and lipids ( $48 \%$ vs $33 \%, \mathrm{p}=0.05$ ), carbohydrate absorption being similar ( $91 \%$ vs $85 \%, \mathrm{p}=0.43$ ). Conclusion: SBS Patients with SRJ had a $10 \%$ to $15 \%$ improved intestinal caloric, fat and nitrogen absorption rates, which corresponded to $21 \%$ of their mean resting energy expenditure $(1442 \mathrm{kcal} / \mathrm{j})$. These results confirm SRJ as a tool to improve absorption in SBS. 
T2055

Effect of LGG On Gut Barrier Function and Breath Hydrogen Testing in Children with short Gut Syndrome: Results of a Randomized PlaceboControlled Clinical Trial

Timothy A. Sentongo, Xaiotan Zheng, Valeria Cohran, Simona Korff, Annie Xoomsai, Christine Sullivan, Kishore Iyer

BACKGROUND: The disproportionately high incidence of bacteremia illnesses with gram negative and enteric bacteria in children with short gut syndrome (SGS) is suspected from translocation of gut bacteria. There are currently limited data about gut barrier function (GBF) in patients with SGS. Lactobacillus casei (LGG) exerts an inhibitory effect on other enteric bacteria and also enhances GBF in patients with atopy. Therefore we examined GBF in children with SGS and explored the effects of probiotic therapy with LGG on GBF and indicators of bacterial overgrowth (SBBO). METHODS: 11-week prospective double-blind, placebo controlled cross-over study of LGG vs. placebo therapy in SGS. Children with SGS and healthy controls had GBF measured at baseline using Lactulose:Mannitol ratio adjusted for body surface area(LMR). Subjects with SGS then randomnly received either LGG or placebo for 4-weeks, followed by 3-weeks of no therapy (washout) then therapy crossedover for another 4-weeks so that those initially treated with LGG now received placebo and vice versa. LMR, hydrogen breath testing (HBT) for SBBO, and quantitative stool cultures for LGG (cfu/g of stool) were measured during therapy with LGG and placebo. Comparisons of LMR in SGS and controls at baseline and during therapy with LGG vs, placebo in SGS were examined using Wilcoxon's rank sum test. Associations between LMR and age were examined using Spearman correlation. Rates of increased HBT (SBBO) during therapy with LGG vs. placebo were examind using McNemar's test. Signficiance was $\mathrm{p}<0.05$. RESULTS: 21 subjects enrolled; SGS $(\mathrm{N}=9)$ median age 3.5 y (range $1.6-16.1$ y) and controls $(\mathrm{N}=$ 12) median age $10.7 \mathrm{y}$ (range $1.7-16.4 \mathrm{y}$ ). There was no difference in baseline LMR between SGS and controls $($ mean \pm SD) $0.15 \pm 0.12$ vs. $0.10 \pm 0.10(p=0.47)$. LMR was inversely correlated with age $(r=-0.82, p<0.0001)$ suggesting that permeability at tight junctions decreased with advancing age in all subjects. Therapy with LGG vs. placebo had no effects on $\operatorname{LMR}(0.33 \pm 0.35$ vs. $0.23 \pm 0.23, \mathrm{p}=0.58)$. Stool colonization with lactobacillus during therapy with LGG was (median)9.0x10(8) cfu/g, (range) $4.0 \times 10(5)$ to $4.0 \times 10(9) \mathrm{cfu} / \mathrm{g}$. The prevalence of increased HBT (SBBO) was $67 \%$ and did not change during therapy with LGG vs. placebo $(p=0.32)$. CONCLUSION: GBF in children with SGS and controls was similar and mainly influenced by age. Emperic therapy with LGG and colonization with lactobacillus had no measureable effects on GBF or prevalence of SBBO in this sample of children with SGS. These findings are important for defining the role of probiotics in patients with SGS. Supported by NIH Grant M01 RR-00048

\section{$\mathrm{T} 2056$}

Genetic Testing for Hypolactasia - Redefining Diagnostic Testing for Lactose Intolerance?

Daniel Pohl, Radu Tutuian, Oliver Goetze, Martin Hersberger, Arnold von Eckardstein Bernadette Stutz, Michael Fried

Introduction: Lactase deficiency (LD) is the most common cause of lactose intolerance, a highly prevalent condition and frequent cause of abdominal pain, bloating and diarrhea. The standard test to diagnose lactase deficiency is the hydrogen breath test (H2-BT). Recently the single-nucleotid-polymorphism C/T 13910 base pairs above the structural gene coding for the enzyme lactase has been associated with lactase deficiency with a reported sensitivity of $75 \%$ compared to lactose H2-BT. Aims: To evaluate the agreement between C/T13910 and lactose $\mathrm{H} 2-\mathrm{BT}$ as well as sensitivity, specificity and predictive values of gastrointestinal symptoms in response to lactose ingestion. Methods: 41 patients (32f; mean age 36, range 11-76 years) with clinical suspicion of lactose intolerance underwent a 3-hour H2-BT after intake of $50 \mathrm{~g}$ of lactose followed by genetic testing. During the H2-BT patients rated five symptoms (abdominal pain, borborygmi, diarrhea, bloating, nausea) every 15 minutes on a linear scale from 1-9. The H2-BT was considered positive with a rise of exhaled hydrogen $>20$ ppm over baseline and the test considered symptomatic after an increment of $>2$ scale units in at least two symptoms. Genotype CC13910 was considered indicative for lactase deficiency. Results: All but one patient (genotype CC, H2-BT negative, symptomatic) had concordant results in the genetic test and $\mathrm{H} 2-\mathrm{BT}$ indicating an almost perfect agreement (kappa $=0.95)$. Sensitivity and specificity of the genetic test when compared to H2-BT as gold standard were $100 \%$ and $96 \%$ respectively. All 17 patients with genotype CC were classified as symptomatic as did 39\%(7/18) of patients with genotype CT and 33\% (2/6) of patients with TT. Diarrhea had the highest positive predictive value $(67 \%)$ and bloating the highest negative predictive value (93\%) to identify patients with LD. Abdominal pain had the lowest diagnostic accuracy. Conclusions: Genetic testing has an excellent agreement with the standard lactose hydrogen breath test and could replace breath testing for the diagnosis of LD. Clinical evaluation of symptoms developed in response to the ingestion of $50 \mathrm{~g}$ lactose could be used as a simple screening test in patients suspected to have lactose deficiency: No symptoms in particular absence of bloating and borborygmi make the diagnosis of lactase deficiency unlikely.

\begin{tabular}{|c|c|c|c|c|}
\hline $\mathrm{n}=41$ & Sens. $(\%)$ & Spec. $(\%)$ & PPV $(\%)$ & NPV (\%) \\
\hline Bloating & 94 & 56 & 58 & 93 \\
\hline Borborygmi & 88 & 60 & 58 & 88 \\
\hline Nausea & 69 & 60 & 52 & 75 \\
\hline Abd. pain & 62 & 60 & 50 & 71 \\
\hline Diarrhea & 50 & 84 & 67 & 72 \\
\hline
\end{tabular}

\section{T2057}

Functional Alterations Following Small Bowel Ischemia-Reperfusion Injury As a Model of Infant Necrotizing Enterocolitis Brian M. Chung, Fatima Ahmed, Kelly Tappenden

Infant necrotizing enterocolitis (NEC) presents both clinical and experimental challenges. Clinicians are faced with the threat of bowel perforation, systemic multi-organ dysfunction syndrome and failure to thrive, while researchers are challenged with few models of spontaneous NEC. One proposed initiator of NEC injury is bowel ischemia coupled with overfeeding. We hypothesized that the presence of luminal nutrients in the face of ischemia-reperfusion (I/R) injury would alter ileal nutrient transport to induce NEC-like symptoms. Two-dayold neonatal piglets $(\mathrm{n}=20)$ were anesthetized and two $15-\mathrm{cm}$ loops of distal ileum were cannulated for the randomized luminal perfusion of saline or hydrolyzed infant formula $(20 \mathrm{ml} /$ hour $)$ for the duration of the study. The small intestinal branch of the mesenteric artery was then atraumatically clamped for 1 hour, followed by 1 hour of reperfusion injury. Perfused tissues were harvested for electrophysiological analysis of nutrient transport in modified Ussing chambers as well as gross morphology. Ileal tissues did not demonstrate alterations in basal short circuit current, resistance, or transmural potential difference, glycylsarcosine, proline, arginine or threonine transport, nor serotonin- or carbachol-induced secretion, regardless of perfusate. However, tissues perfused with formula demonstrated significantly $(\mathrm{P}<0.05)$ decreased glucose transport when compared to saline perfused loops (formula $2.76 \pm 1.67$ vs saline $9.66 \pm 1.67 \mathrm{uA} / \mathrm{cm} 2$ ), as well as decreased glutamine transport (formula $4.65 \pm 2.14$ vs saline $8.29 \pm 2.14, \mathrm{p}<0.05$ ). This was mirrored by fluid flux measurements using the inert marker sodium ferrocyanide, where formula perfusion stimulated net secretion $(97 \pm 2.87)$, whereas saline perfusion stimulated net absorption $(106.35 \pm 2.88 \%$ stock perfusate $\mathrm{p}<0.05)$. Villus height, crypt depth and mucosal wet weight were not altered but a significant increase $(p<0.05)$ in mucosal DNA in the formula perfused tissues (formula $1.87 \pm .15$ vs saline $1.32 \pm .15 \mathrm{mg} / \mathrm{mg}$ mucosa) was observed. $\mathrm{I} / \mathrm{R}$ in the presence of luminal nutrients appears to be a viable model for initiation of NEC, as supported by: the decrease in glucose and glutamine transport, coupled with net fluid secretion, and increase in mucosal DNA, without change in the villus/crypt length, thus indicating imminent intestinal failure associated with possible immune infiltration.

\section{$\mathrm{T} 2058$}

The Relationship Between Glucose Absorption and Gastric Emptying in Critical Illness

Marianne Chapman, Robert Fraser, Laura Bryant, Carly Burgstad, Francis Bartholomeusz, Stephanie Creed, Antonietta Russo, Karen Jones, Max Bellon, Barry Chatterton, Ross Butler, Geoffrey Davidson, Michael Horowitz

Delayed gastric emptying is common in critical illness, resulting in reduced tolerance to nasogastric feeding. In these patients, nutrient absorption is also impaired. As the small intestine is the major site of absorption, gastric stasis may be a limiting factor. Absorption may be further compromised by mucosal oedema, atrophy or reduced splanchnic blood flow. There are limited data on the factors reducing nutrient absorption in critical illness Aim: To assess the relationship between glucose absorption and gastric emptying in critically ill patients. Methods: Gastric emptying was measured (using scintigraphy) over $4 \mathrm{~h}$ in 15 mechanically ventilated, critically ill patients (12M; $60 \pm 4$ years; APACHE II score 18 (1230)) after the intragastric delivery of $100 \mathrm{ml}$ of Ensure labelled with ${ }^{99 \mathrm{~m}} \mathrm{Tc}$ sulphur colloid. Glucose absorption was assessed using 2g of 3-Ortho-Methyl-D-glucose (3-OMG) added to the test meal. The \% meal retention at 60, 120, 180 and $240 \mathrm{~min}$ was determined. Plasma 3-OMG concentrations were measured (using High Performance Exchange Chromatography) at baseline, every $15 \mathrm{~min}$ during the first hour, and every $30 \mathrm{~min}$ thereafter for $3 \mathrm{~h}$. The area under the curve (AUC) was calculated for the 3-OMG concentrations over time. Patient data were compared to 15 healthy subjects $(8 \mathrm{M} ; 35.2 \pm 4.7$ years) and are expressed as mean \pm SEM. Results: Meal retention at both 180 and 240 min was greater in critical illness than in health $(180 \mathrm{~min}: 32.1 \pm 9.2 \times 3 \pm 0.6 \%, \mathrm{P}<0.05$; and $240 \mathrm{~min}: 29.7 \pm 9.2 \times 1.7 \pm$ $0.4 \%, \mathrm{P}<0.05)$. All healthy subjects and 8 critically ill patients $(53 \%)$ had $<10 \%$ meal retention at $240 \mathrm{~min}$. The mean area under the $3-\mathrm{OMG}$ concentration curve was less in critically ill patients than healthy subjects (AUC: $32.9 \pm 4.8$ v $65.9 \pm 4.8, \mathrm{P}<0.0001$ ). There was a negative correlation between the \% meal retention (at all time-points) and 3-OMG absorption in critically ill patients $(r=-0.77$ to $-0.87 ; \mathrm{P}<0.001)$, but not in healthy subjects. Conclusion: Delayed gastric emptying has a negative effect on glucose absorption in critically ill patients, but not in healthy subjects, suggesting this may be the rate limiting step in patients. Prokinetic agents to stimulate gastric emptying should therefore enhance nutrient absorption in the critically ill. 
T2059

Development of a Simple Model of Orthotopic Small Bowel Transplantation in the Mouse

Fatima Javairiah, Scott G. Houghton, Michael G. Sarr

BACKGROUND: Small bowel transplantation (SBT), though a promising treatment for intestinal failure, is associated with multiple, still poorly understood functional problems of the transplanted gut. Ability to study SBT models in mice is hindered by the extreme technical difficulty of orthotopic SBT in the mouse. AIM: To develop an easily constructed preparation of orthotopic, extrinsic denervation of the entire jejunoileum in mice as a model of orthotopic SBT. HYPOTHESIS: This mouse model will allow more ready investigation of physiologic changes in gut function after extrinsic denervation without confounding factors of ischemia-reperfusion and/or immune phenomenon. METHODS: All neurolymphatic tissues accompanying the superior mesenteric artery and vein were transected at the base of the small bowel mesentery just distal to the middle colic vessels. The superior mesenteric artery and vein were stripped carefully of all adventitia, and the mesenteries up to the proximal jejunum and proximal colon were transected in radial fashion. The proximal jejunum and proximal colon were not transected and re-anastomosed. To confirm extrinsic denervation, 4 weeks later, still innervated duodenum and extrinsically denervated jejunum and ileum were harvested from 6 mice and stained for PGP9.5 (a non-specific neuronal marker) and tyrosine hydroxylase (a marker of extrinsic innervation because the only catecholaminergic nerves in the gut are extrinsic sympathetic nerves). Neurally intact mice provided control tissues. RESULTS: Immunohistochemistry showed complete lack of tyrosine hydroxylase activity in jejunum and ileum in denervated mice but preservation of activity in the duodenum. No obvious differences were noted in PGP9.5 staining. SUMMARY: This technique in the mouse accomplishes complete extrinsic denervation of the jejunoileum. CONCLUSIONS: This murine model of orthotopic SBT, by allowing the use of genetically modified (knockout mice), will allow study of molecular and genetic effects of selective extrinsic denervation on small bowel function.

\section{T2060}

Maltotriose, Product of $\alpha$-Amylase Starch Hydrolysis, Suppresses MaltaseGlucoamylase Activity and Slows Terminal Starch Digestion 44.5 Fold Roberto Quezada-Calvillo, Claudia C. Robayo-Torres, Susan S. Baker, Bruce Hamaker, Erwin E. Sterchi, Buford L. Nichols

Starches constitute the main caloric source in the average human diet. The digestion of starches is far more complex than sugars and requires six different enzyme activities to produce free glucose before absorption. Salivary and pancreatic $\alpha$-amylase activities initially hydrolyze internal 1-4 glucose bonds of starch to produce a limited pattern of soluble glucose oligomers including maltotriose. Terminally, mucosal enzymes maltase-glucoamylase (MGAM) and sucrase-isomaltase (SI) hydrolyze external linear bonds of starch oligosaccharides to free glucose. Our aim was to determine the relative roles of the two mucosal enzymes in terminal starch digestion. These experiments led to the novel discovery that rates of integrated mucosal starch digestion to glucose are regulated by lumenal maltotriose substrate inhibition, termed the maltotriose brake. Methods: Enterocytes were harvested from transplant donor jejunum by scraping. The membranes were solubilized with $10 \%$ Nonidet $\mathrm{P}$ 40. MGAM and SI activities were immunoprecipitated (IP) with monoclonal antibodies and incubated with dilutions of maltose, maltotriose and dextromaltose for $60 \mathrm{~min}$, Dextromaltose, partially hydrolyzed amylopectin, substrate was analyzed by Mass Spectrometry. Glucose production was measured by Sigma Infinity reagent. The protocol was approved by BCM IRB as H-1320. Results: Dextromaltose has a median polymer length of 7 glucoses. Enzyme IPs were shown pure and relative concentrations determined by Western blot. Concentration of SI is 20 times MGAM. Under clinical assay conditions, SI accounted for all mucosal maltotriase and $80 \%$ of IP maltase and dextromaltase activities. MGAM was inhibited (Ki $1.8 \mathrm{mM}$ ) by maltotriose. All activities followed Michaelian kinetics. Both enzymes hydrolyzed dextromaltose but $\mathrm{Km}$ was 1.3 for MGAM and 58 for SI. Conclusion: MGAM and SI accounted for $90 \%$ of all mucosal dextromaltase activity. MGAM is 44.5 times more active than SI. MGAM is substrate inhibited by maltotriose but SI is not. SI is a low activity, high capacity unregulated enzyme for terminal starch digestion. MGAM has higher activity (44.5 fold) and lower capacity (20 fold). MGAM provides rapid glucose production during early and late starch feeding or snacking but its high activity is suppressed by the maltotriose brake during heavy starch ingestion. MGAM matches Kms of amylase and the glucose transporter but when the brake is applied, slower SI becomes rate limiting for the digestion of starch to glucose. This work was supported in part by USDA/ARS/Children's Nutrition Research Center and Public Health Service grant DK56338, which funds the Texas Gulf Coast Digestive Diseases Center.

\section{T2062}

Comparison of a Genetic Test for Lactase Polymorphism with Two Standard Tests for Lactose Mal-Digestion in Adults

Andrew N. Szilagyi, Paula Malolepszy, Elise Hamard, Mary Ponniah, Xiaoqing Xue, Nir Hilzenrat, Elizabeth MacNamara, George Chong

Introduction: The breath hydrogen test (BHT) is the most common indirect test of lactose mal-digestion. A positive rise shows lactase non persistence (LNP). It agrees moderately with an earlier blood glucose test (BGT) where a positive rise shows lactase persistence (LP). The availability of a test for genetic polymorphism (GPT) in the LPH gene for LNP/LP status allows head on comparison among these 3 tests. GPT correlates with intestinal lactase. Participants: Previously established LNP/LP subjects, at a BH2 testing laboratory in a university hospital setting. Their LNP/LP status was determined according to BHT and/or ethnic background without IBS or other contraindications. Methods: Participants underwent a $50 \mathrm{~g}$ lactose challenge. The BHT ( $>10$ or $20 \mathrm{ppm}$ ) with q30 min scored symptoms was measured for $4.5 \mathrm{Hrs}$. by hand held electrochemical H2 sensor(Bedfont, UK). The BGT (>1.1 mmol/ L) was measured for $2 / 4.5 \mathrm{Hrs}$, by colorimeter (Roche Diagnostics). Blood drawn for GPT was assessed by real-time PCR fluorescent resonance energy transfer (FRET) on the Light
Cycler (Roche Diagnostics). Participants completed a validated lactose targeting 3 -day recall questionnaire to assess average pre- test daily consumption. Analysis: Kappa statistics were used to assess the agreement among the 3 tests. Wilcoxon signed rank test or chi-square test were also used for other comparisons. Results: Thirty subjects (12 male, 18 female) participated in the study. Among them, 17 are LNP and 13 are LP based on results from BHT. However, the results by GPT showed 15 CC (LNP), 12 CT (LP) and 3TT (LP). Of 14 symptomatic subjects, $11 / 15(73.3 \%)$ were LNP, and 3/15 (20\%) were LP (p-value $=0.01$ ). Symptomatic subjects including all Asians consumed significantly less lactose per day than asymptomatic subjects. Conclusions: Either BHT or BGT reflects GPT substantially. All 3 tests agree well also but BGT only moderately agrees with BHT. LNP subjects were significantly more symptomatic. Indirect tests like BHT or BGT may also reflect several physiological effects. Studies on the impact of dairy consumption in adults probably should use both GPT and another indirect test, i.e. BHT or BGT. Population prevalence studies could use GPT alone.

Distribution of test outcomes

\begin{tabular}{|c|c|c|}
\hline $\mathrm{GPT}(\mathrm{N})$ & $+\mathrm{BGT}(\%)$ & $+\mathrm{BHT}(\%)$ \\
\hline $\mathrm{CC}(15)$ & $1(6.7)$ & $14(93.3)$ \\
\hline $\mathrm{CT}(12)$ & $10(83.3)$ & $1(8.3)$ \\
\hline $\mathrm{TT}(3)$ & $3(100)$ & $2(66.7)$ \\
\hline
\end{tabular}

Kappa coefficients

\begin{tabular}{|c|c|c|c|}
\hline GPT/BHT/BGT & GPT/BGT & GPT/BHT & BGT/BHT \\
\hline $0.69(95 \% \mathrm{CI} 0.5-0.86)$ & $0.8(95 \% \mathrm{CI} 0.54-1)$ & $0.73(95 \mathrm{CI} 0.49-.97)$ & $0.53(95 \% \mathrm{Cl} 0.22-0.82)$ \\
\hline
\end{tabular}

\section{T2063}

High Prevalence of Vitamin D Deficiency in Patients with Inactive Crohn's

\section{Disease}

Treasa Nic Suibhne, Colm O'Morain, Maria O'Sullivan

Vitamin D is essential for bone and muscle metabolism. Crohn's disease (CD) patients are at increased risk of vitamin D deficiency due to low dietary intake, malabsorption and low exposure to sunlight, particularly during periods of high disease activity. AIM: To determine the prevalence of vitamin $D$ deficiency in inactive $C D$ and to evaluate vitamin $D$ and calcium (Ca) intakes in CD patients compared to healthy controls. METHODS: Nutritional and clinical assessments were conducted on $81 \mathrm{CD}$ patients and 81 healthy controls matched for age, sex and socio-economic status [CD patients, 60\% (49/81) female, $36.43 \pm 11.03$ years $v$ controls $60 \%(49 / 81)$ female, $37.03 \pm 10.87$ years]. Dietary vitamin D and Ca intakes were assessed in all subjects using a validated food frequency questionnaire. Serum 1,25dihydroxy vitamin D3 was measured by radioimmunoassay in $\mathrm{CD}$ patients. The majority of CD patients [68\% (55/81)] were in clinical remission (defined by Crohn's Disease Activity Index (CDAI) <150; median 92.09 ( 0 - 371.67), had normal C-reactive protein (CRP) levels [58\% (47/78); median 3.85 (2.9 - 53.3)], and were on corticosteroid [51\% (41/81)] or infliximab therapy [15\% (12/81)] during the previous 12 months. RESULTS: The majority $[63 \%(51 / 81)]$ of CD patients showed vitamin D deficiency $(<20 \mathrm{ng} / \mathrm{ml})$. Of these, $46 \%$ (37/ 81 ) had mild deficiency $(10-20 \mathrm{ng} / \mathrm{ml})$ and $17 \%(14 / 81)$ had moderate deficiency (5 $10 \mathrm{ng} / \mathrm{ml})$. Higher serum vitamin D was significantly associated with vitamin D intake from supplements $(r=0.31, P=0.00)$. There was no association with age $(36.86 \pm 10.91 \vee 35.70$ \pm 11.38 years; NS), gender [57\% (29/51) v 67\% (20/30) female; NS $\left.\chi^{2}\right]$, CRP levels [63\% $(30 / 48)$ v $57 \%(17 / 30)<5 \mathrm{mg} / \mathrm{L} ; \mathrm{NS} \chi^{2}$ ], serum tumour necrosis factor alpha (TNF $\left.\alpha\right)$ levels $(6.03 \pm 2.21$ v $6.14 \pm 1.88 ; \mathrm{NS})$, CDAI $\left[69 \%(35 / 51)\right.$ v $67 \%(20 / 30) ;$ NS $\left.\chi^{2}\right]$, seasonality $\left(P=0.52 \chi^{2}\right)$, disease site $\left(P=0.59 \chi^{2}\right)$ or previous surgery $\left(P=0.43 \chi^{2}\right)$. Dietary vitamin $D$ intakes were significantly lower in CD patients than controls $(159.87 \pm 13.96 \mathrm{IU} v 207.81$ $\pm 14.26 \mathrm{IU}, P=0.01)$, and only $22 \%(21 / 78)$ of patients met the Recommended Daily Allowance (RDA) of $200 \mathrm{IU}$ for vitamin D from diet alone. After addition of vitamin D intake from supplements, 53\% (41/78) of patients reached the RDA. Patients were more likely to be prescribed vitamin $\mathrm{D}$ supplements if they had received steroids within the past year $\left(r^{2}=\right.$ $0.3, P=0.01)$. Dietary Ca intakes were similar in both $C D$ and controls $(974.59 \pm 51.22 \mathrm{mg}$ $v 1062.92 \pm 57.16 \mathrm{mg}$; NS). CONCLUSION: Vitamin D deficiency is prevalent in quiescent $\mathrm{CD}$, a group not typically considered at risk.

\section{T2064}

13c-Glucose Hydrogen Breath Test to Evaluate Glucose Oxidation and Carbohydrate Malabsorption

Yoshihisa Urita, Susumu Ishihara, Kenichiro Arai, Jun Kondo, Tatsuo Akimoto, Hiroto Kato, Noriko Hara, Hideyuki Koshino, Yoshiko Honda, Yoko Nagai, Kazushige Nakanishi, Nagato Shimada, Motonobu Sugimoto, Kazumasa Miki

Background: Glucose is readily absorbed in the proximal small bowel. In patients with gastorectomized patients, it was reported that $100 \%$ of the glucose ingested was absorbed before reaching the colon. Therefore, the fact that any peak of breath hydrogen (H2) and methane $(\mathrm{CH} 4)$ excretion after ingestion of glucose is abnormal is the main advantage in terms of the interpretation of $\mathrm{H} 2$ breath test using glucose. It has been unknown whether carbohydrate malabsorption affect glucose metabolism. On the other hand, recent studies have targeted prediabetic individuals for early therapeutic intervention in the hopes of preventing progressikon to type 2 diabetes. Early intervention might be most appropriate at the stage of disease progression when insulin resistance is present but before glucose intolerance occurs. The aim of this study is to evaluate the association among glucose oxidation, insulin resistance, and carbohydrate malabsorption. Patients and Methods: A standard $75 \mathrm{~g}$ oral glucose tolerance test (GTT) was performed in 62 subjects, 32 women and 30 men, aged $39-87$ years. The patients received $75 \mathrm{~g}(225 \mathrm{ml})$ of glucose solution in the sitting position after an overnight fast. Breath samples were collected at baseline and at $5,10,15,20,30,40,50,60,70,80,90,100,110$, and $120 \mathrm{~min}$ after ingestion to analyze 
breath $\mathrm{H} 2$ and $\mathrm{CH} 4$ concentrations as well as $13 \mathrm{CO} 2$. An increase of at least $10 \mathrm{ppm}$ within a two-hour period is indicative of carbohydrate malabsorption. Venous blood samples were obtained before ingestion and at 30,60,90,120 min and blood glucose and serum insulin concentrations were measured. Results: $13 \mathrm{CO} 2$ excretion rate in expired breath increased up to $180 \mathrm{~min}$. Recovery rate of $13 \mathrm{CO} 2$ in expired breath is $1.7 \%$ and $6.1 \%$ at $60 \mathrm{~min}$ and $120 \mathrm{~min}$, respectively. Insulinogenic index (plasma insulin at $30 \mathrm{~min}$-baseline/plasma glucose at 30 min-baseline) significantly correlates with $13 \mathrm{CO} 2$ excretion at $60 \mathrm{~min}(\mathrm{R} 2=0.28)$. Carbohydrate malabsorption was found in 16 patients (26\%). These patients had slightly increased insulin resistance, compared to diabetic patients without carbohydrate malabsorption. There was no significant difference in insulinogenic index and $13 \mathrm{CO} 2$ excretion at any time point between two groups. Conclusions: It is concluded that carbohydrate malabsorption may have a role of keeping appropriate blood glucose concentration and progressing insulin resistance. There was no significant correlation among carbohydrate absorption, glucose oxidation, and insulin resistance.

T2065

Intestinal Absorption Capacity Is Normal in Recently Diagnosed Celiac Disease, But Not in Refractory Celiac Disease

Nicolette Wierdsma, Job H. Peters, Chris J. Mulder, Marian A. van Bokhorst- de van der Schueren, Adje A. van Bodegraven

Introduction Malnutrition due to malabsorption of nutrients is a common finding in celiac disease $(C D)$. It is not clear to what extent villous atrophy determines the level of malabsorption. To quantify intestinal absorption capacity may be helpful to distinguish patients that may benefit from nutritional support. This study compared absorptiometry in patients with de novo $C D$ prior to a gluten free diet, in patients with refractory celiac disease (RCD) and healthy controls (HC). Aims \& Methods The objective was to determine a difference in intestinal absorption capacity between the three groups. Absorptiometry was obtained by recording oral caloric intake and the extent of fecal calorie loss by using the gold standard, bomb calorimetry. A four days nutritional diary assessed oral intake and subsequent collection of feces was performed from day two to four. Intestinal absorption capacity was calculated as the difference in caloric intake and fecal loss expressed in percentage. Results A total of nineteen patients was included. Nine patients $(4 \mathrm{M}, 5 \mathrm{~F})$ in the de novo $C D$ group had a mean age of $50.1 \pm 11.8$ years, a BMI of $25.7 \pm 4.9 \mathrm{~kg} / \mathrm{m} 2$, a caloric intake of $2363 \pm 533$ $\mathrm{kcal} /$ day and a fecal caloric loss of $280 \pm 124 \mathrm{kcal} /$ day. Five patients $(2 \mathrm{M}, 3 \mathrm{~F})$ in the RCD group had a mean age of $62.8 \pm 1.8$ years $(\mathrm{p}=0.02$ compared to $\mathrm{CD}, \mathrm{p}=0.01$ compared to $\mathrm{HC}$ ), a BMI of $20.9 \pm 2.1 \mathrm{~kg} / \mathrm{m} 2$, a caloric intake of $3020 \pm 525 \mathrm{kcal} /$ day ( $\mathrm{p}=0.01 \mathrm{compared}$ to $\mathrm{HC}$ ) and a fecal caloric loss of $772 \pm 651 \mathrm{kcal} /$ day. Five patients $(5 \mathrm{~F})$ in the $\mathrm{HC}$ group had a mean age of $42.5 \pm 12.4$ years, a BMI of $23.7 \pm 3.3 \mathrm{~kg} / \mathrm{m} 2$, a caloric intake of 2021 $\pm 205 \mathrm{kcal} /$ day and a fecal caloric loss of $224 \pm 75 \mathrm{kcal} /$ day. Mean intestinal absorption capacity was $88.6 \pm 3.7,73.0 \pm 24.7,88.8 \pm 3.8 \%$ in $C D, R C D$ and $\mathrm{HC}$, respectively. Conclusion Intestinal absorption capacity in healthy controls is $90 \%$. In patients with de novo celiac disease, absorption capacity was equal to that of healthy controls. There was a trend towards a decreased intestinal absorption capacity in RCD compared to both de novo $\mathrm{CD}$ and $\mathrm{HC}$, possibly due to individual variability in the RCD group. In these patients nutritional support may be beneficial.

\section{T2066}

The Protein Absorption Capacity Is Intact in Human Neonates with An Enterostomy

Maaike W. Schaart, Adrianus C. De Bruijn, Dick Tibboel, Johannes B. van Goudoever, Ingrid $\mathrm{B}$. Renes

Background: The intestine plays a key role in the maintenance of an effective protein metabolism, which determines growth of human neonates. Bowel resection in the neonatal period leads to a drastic loss of absorptive and protective surface and consequently to malabsorption of nutrients. We therefore hypothesized that the net enteral protein absorption of the small intestine is reduced in the postoperative period of human neonates following bowel resection. Objective: To quantify the net absorption of dietary proteins in human neonates with an enterostomy following bowel resection during a 4-week period after surgery. Methods: Eighteen patients were included in the study [jejunostomy: $n=3$, ileostomy: $n=$ 15]. After introducing enteral feeding, the outflow fluid at the stoma exit was collected during 24-48 hours every week over a period of 4 weeks [wk 3 - wk 6] following bowel resection. Protein levels in the outflow fluid were determined by Bicinchoninic Acid (BCA) protein assay. The net absorption of dietary proteins was calculated by subtracting the protein amount excreted in the outflow product from the protein amount ingested and was expressed as the percentage of the total enteral protein intake. Results: The mean dietary protein intake of all patients was $2.64+1.28 \mathrm{gr} / \mathrm{kg} / \mathrm{d}$. The dietary protein absorptive capacity was constant over the 4 -week period for every individual patient. The mean net absorption of dietary proteins was $86.7 \% \pm 9.0 \%$ of the total enteral intake. The absorption capacity of patients with a jejunostomy tended to be lower compared to patients with an ileostomy [jejunum: $78.7 \% \pm 2.1 \%$, ileum: $88.3 \% \pm 9.0 \%, \mathrm{p}=0.066$ ]. Conclusions: In contrast to our hypothesis this study showed that the small intestine is capable to absorb dietary proteins from 3 weeks following intestinal surgery. Thus, the protein absorption capacity is intact in human neonates who underwent bowel resection in a very critical period of their life.

\section{T2067}

Determination of Residual Bowel Length Via Surgical, Radiological Or Historical Data in Patients with short Bowel Syndrome and Intestinal Failure Neha R. Parekh, Ezra Steiger, Douglas L. Seidner

Introduction: Treatment options for patients with short bowel syndrome (SBS) and intestinal failure (IF) vary directly with the length and condition of the residual bowel. An accurate assessment of remaining intestine is necessary to make optimal therapeutic decisions and to efficiently track the progress of SBS and IF patients. The most reliable way to assess intestinal length is by measuring along the antimesenteric border from the Ligament of Treitz at the time of surgery, although this is not often done. Radiologic techniques with use of an opisometer may give a quantitative estimate, however the results have not been entirely accurate. Our aim in this report is to describe the frequency with which surgical, radiological and historical data is used to assess intestinal length in patients referred to a tertiary center for intestinal rehabilitation. Methods: A total of 57 new patient referrals with SBS and IF were seen in the Intestinal Rehabilitation Program (IRP) from March 1, 2005 to November 30,2005. Remaining length of small bowel and colon was recorded in the IRP database at the initial visit. Method of determination of bowel lengths (surgical reports, radiology or patient history) and underlying diagnosis leading to SBS or IF were also recorded. Results: One-third (33.3\%) of new IRP patient referrals were diagnosed with Crohn's disease This was closely followed by ulcerative colitis $(21.1 \%)$, radiation enteritis $(17.5 \%)$ and mesenteric vascular infarction (12.3\%). Despite the presence of significantly more known vs. unknown anatomical information ( $\mathrm{p}<0.0001$ for colon and small bowel lengths), only $26(48.1 \%)$ of 54 known colonic measurements and $25(53.2 \%)$ of 47 known small bowe lengths were measured surgically. Conclusions: Although residual bowel length is important in determining appropriate IRP therapy, surgical measurements were taken in only half of SBS patients. The residual length of small bowel remains completely unknown in 15 to $20 \%$ of patients referred for IRP intervention. Additional (non-surgical) means of accuratel assessing intestinal length or enterocyte mass, such as plasma citrulline levels, are needed.

\begin{tabular}{|c|c|c|}
\hline & Colon & Small bowel \\
\hline Length & & $47 / 57(82.5 \%)$ \\
\hline Known & $54 / 57(94.7 \%)$ & $10 / 57(17.5 \%)$ \\
\hline Unknown & $3 / 57(5.3 \%)$ & \\
\hline Determination & & $25 / 47(53.2 \%)$ \\
\hline Surgery & $26 / 54(48.1 \%)$ & $3 / 47(6.4 \%)$ \\
\hline Radiology & $1 / 54(1.9 \%)$ & $19 / 47(40.4 \%)$ \\
\hline Patient History & $27 / 54(50.0 \%)$ & \\
\hline
\end{tabular}

T2068

Taste Receptor G-Protein $\alpha$-Gustducin Does Not Colocalise with Enteroendocrine Cell Markers in the Mouse Intestine Kate Sutherland, Nicole J. Cooper, Michael Horowitz, Robert F. Margolskee, L Ashley Blackshaw, Richard L. Young

A common complication of type 1 and type- 2 diabetes is disordered gastrointestinal motility which may reflect a hypersensitivity of the small intestine to nutrient-induced vagal reflexes The primary nutrient sensors of the intestinal tract are likely to be enterendocrine cells capable of detecting luminal stimuli and releasing paracrine and/or endocrine mediators, such as the incretin hormones glucagon-like peptide-1 (GLP-1) and glucose-dependent insulinotropic peptide (GIP) or 5-hydroxytryptamine (5-HT), to activate vagal afferent endings. However, the mechanism of intestinal glucose-sensing and signalling is currently unknown. Alpha-gustducin $(\alpha-G D)$ a G-protein subunit involved in lingual sweet taste transduction has recently been identified in the rat duodenum [1,2] and may be a key signalling molecule of the intestinal glucose-sensor. The aim of this study was to map the radial and longitudinal expression of $\alpha$-GD in the mouse intestine and identify potential enteroendocrine cells involved in signalling luminal glucose to vagal afferents. METHODS: Immunohistochemistry for $\alpha$-GD was performed in the tongue and intestine of 8 adult male C57BL mice and combined with dual-label immunohistochemistry for 5-HT, GLP-1, GIP and for nitric oxide synthase (NOS), a marker enriched in intestinal brush cells [1]. RESULTS We confirmed specific labelling of $\alpha$-GD in circumvallate and fungiform taste cells of the tongue. In the intestine a specific population of $\alpha$-GD labelled epithelial cells was identified showing strong immunoreactivity in cytoplasm and at the apical brush border membrane Individual $\alpha$-GD cells were scattered throughout the epithelium and expressed in a regional specific manner; rare in duodenum, frequent in mid-jejunum (5-10-fold more than in ileum) and less frequent toward the ileum. Radial $\alpha$-GD expression was commonly seen in the upper villus or villus tip, less frequently in the mid villus and was never seen in crypts. Labelling for $\alpha$-GD did not colocalise with 5-HT, GLP-1, GIP or NOS immunoreactivity in any region of the gastrointestinal epithelium. CONCLUSIONS: Identification of the taste molecule $\alpha$-GD in specific populations of intestinal epithelial cells associated with upper villi of the mid-jejunum suggests a potential role in chemosensory function. The transduction pathways and identity of effector signalling molecules of the $\alpha$-GD expressing cell type in the small intestinal epithelium are the subject of ongoing studies in our laboratory. 1. Hofer D et al Proc Nat Acad Sci USA 1996 93(13):6631-4 2. Gountounas N et al Gastroenterology 2005 128(4) suppl. 2:T1808 Supported by: University of Adelaide

\section{T2069}

Omega-3 Polyunsaturated Fatty Acids Change the Permeability Characteristics of Intestinal Monolayers By Remodeling the Tight Junctions Yasotha Duraisamy, Daniel Lambert, Catherine A. O'Neill, Philip Padfield

Aims: In this study we have examined the influence of eicosapentaenoic acid (EPA) and docosahexaenoic acid (DHA), the two main omega-3 polyunsaturated fatty acids (PUFas) found in fish oil, on the paracellular permeability characteristics of Caco-2 cell monolayers. Methods: Confluent, fully differentiated, monolayers of Caco-2 cells grown on Transwell filter inserts were treated with increasing concentrations of EPA and DHA for up to 8 days. Paracellular permeability was assessed by measuring transepithelial electrical resistance (TEER) and the transepithelial flux of fluorescently labelled $4 \mathrm{kDa}$ Dextran and Lucifer yellow. In addition, the expression of tight junction proteins was determined by densitometric analysis of Western Blots produced from extracts of control and treated Caco-2 cell monolayers. Results: EPA and DHA produced a time and concentration dependent decrease in transepithelial electrical resistance (TEER). By day 8, EPA and DHA (200uM) produced a 
$70 \%$ to $80 \%$ decrease in TEER. In contrast over the same time period there was no detectable increase in the paracellular permeability of either Dextran or Lucifer yellow. Next we examined whether EPA and DHA were influencing TEER by altering the expression of the main protein components of the tight junctions in Caco-2 cells, namely claudins 1, 3 and 4 and occludin. The PUFAs produced a significant increase in both claudins 3 and 4 , but a significant decrease in claudin 1. Maximally (on day 8) EPA and DHA both produced a 30\% to $35 \%$ rise in claudin 3, whereas claudin 4 increased by $64 \%$ (EPA) and 123\% (DHA). In contrast, EPA and DHA produced a $39 \%$ and $61 \%$ decrease in claudin 1, respectively. The PUFAs did produce a slight increase in occludin, however, the most profound influence was on the phosphorylation status of the protein. Both PUFAs stimulated a dramatic increase in the phosphorylation of occludin. These changes in the claudins and occludin were accompanied by small but significant alterations in the ion selectivity of the paracellular pathway. Specifically, EPA and DHA increased the relative permeability of divalent cations $(\mathrm{Mg}+)$ and monovalent anions ( $\mathrm{Cl}-$ ) but had no effect on monovalent cations $(\mathrm{K}+)$. Conclusions: EPA and DHA significantly changed the ionic but not the macromolecular permeability characteristics of the paracellular pathway in Caco- 2 cell monolayers through altering the relative protein composition and phosphorylation status of the tight junctions.

\section{$\mathrm{T} 2070$}

Expressions of TNF- $\alpha$, TNFR-1 and TNFR-11 in the Intestinal Mucosa of LPSInjected Rats Maintained On Low-Protein and High-Fat Diets Nilima Raina, Khursheed N. Jeejeebhoy

Background: Previously we showed that reduced energy and increased protein intake decreased the expression of TNF-alpha and TNFRs in organs and muscle. The effects of nutritional manipulations on TNF and TNFRs in intestinal mucosa are unknown. Objective: To determine the effect of nutritional manipulations on TNF and TNFRs in intestinal mucosa of endotoxic rats. Design: Thirty male wistar rats weighing $250 \mathrm{~g}$ were fed an isocaloric liquid defined-formula diet and randomly allocated to 1$) \operatorname{con} \operatorname{trols}(\mathrm{C} ; \mathrm{n}=6)$, receiving normal protein/ CHO/fat energy density of 49.25/263.7/52.72 kJ/60 mL + normal saline (NS); 2)lowprotein(LP; $n=3)$,receiving normal energy but a reduced protein energy density of $12.3 \mathrm{~kJ} /$ $60 \mathrm{~mL}+$ LPS; 3)high-fat (HF; N=3), receiving normal energy but increased fat energy density of $145.6 \mathrm{~kJ} / 60 \mathrm{~mL}$ + LPS; 4)refed(RF; $n=6)$, initially fed on low-protein/high-fat $\operatorname{diet}(10 \mathrm{~d})$ and then repleted on normal protein/fat $(10 \mathrm{~d})+$ LPS; 5)Pair-fed (P-F; $\mathrm{n}=12)$ rats were individually paired with LP, HF or RF rats + NS. After $10 \mathrm{~d}$ the rats were sacrificed and mRNA expressions of TNF, TNFR-1 and TNFR-11 in the mucosa were measured by RTPCR. Results: LP rats showed no significant difference in the mRNA expressions of TNF, TNFR- 1 and TNFR-11, but HF rats (Fig 1) showed significant increase in the mRNA expression of TNF and TNFR-11, compared with C and RF groups, respectively. Conclusions: High-fat but not protein deficiency in mucosa of endotoxic rats increases the expression of TNF and TNFR-11 which is suppressed by refeeding normal fat. This is clinically relevant in IBD, since the data suggests that a low fat diet may reduce the inflammatory mediators' expression in intestinal mucosa.

\section{FIG 1. HIGH-FAT DIET}

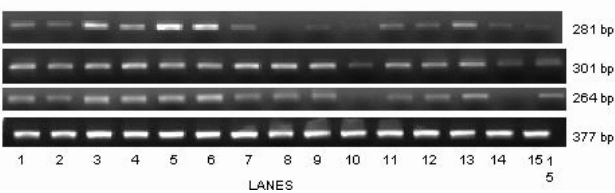

Fig 1: mRNA expression of TNF, TNFR-1 and TNFR-11. $281 \mathrm{bp}=$ TNF-alpha, $301 \mathrm{bp}=$ TNFR-1, $264 \mathrm{bp}=$ TNFR-11, $377 \mathrm{bp}=\mathrm{GAPDH}$. Lanes 1 to 3- C, lanes 4 to 6- HF, lanes 7 to 9 - P-F to HF, lanes 10 to $12-\mathrm{RF}$, lanes 13 to $15-\mathrm{P}-\mathrm{F}$ to RF.

T2071

Effect of Breakfasts with Different Glycemic Index and Glycemic Load On Metabolic and Hormonal Responses to the Breakfast and to the Following Meal

Luigi Benini, Fosca de Lorio, Laura Rigo, Anna Albiero, Furio Brighenti, Camilla Melegari, Roberto Ciati, John Blundell, Naika Tacchella, Elisa Nesta, Donatella Ieluzzi, Italo Vantini

BACKGROUND. Breakfast influences food intake; in the long term, subjects skipping breakfast gain weight and present a reduced productivity. Breakfasts rich in protein and fat are associated with an increase in BMI compared with carbohydrates rich ones. Aim of our study was to compare the effects of isocaloric breakfasts $(400 \mathrm{Kcal})$ with different glycemic index (GI) and glycemic load (GL=GI x carbohydrate content). MATERIAL AND METHODS Sixteen healthy volunteers ( 13 females, aged 23-30 years) were studied in 3 different days for 4 hours after $200 \mathrm{ml}$ of tea and breakfasts with high GI-high GL (HGI-HGL; rich in refined sugars: rusks $75 \mathrm{~g}$, jam $30 \mathrm{~g}$ ), high GI-low GL (HGI-LGL; higher fat content: rusks $45 \mathrm{~g}$, jam $30 \mathrm{~g}$, butter $22.5 \mathrm{~g}$ ), or low GI-low GL (LGI-LGL, higher fiber content: cereals biscuits $60 \mathrm{~g}$, jam $60 \mathrm{~g}$ ). On every occasion, they were studied also for 5 hours after the following, standard main meal (mixed solid meal, $800 \mathrm{Kcal}$ ). The following parameters were evaluated: gastric emptying and antral area (ultrasound), hunger, fullness and satiety (visual analogue scales), biochemical parameters (glucose, triglycerides, NEFA), hormones (insulin, CCK, ghrelin, leptin, PYY; Phoenix Pharmaceuticals). ANOVA for repeated measurements and, when significant, the paired data Student's $t$ test were used for statistical analysis. RESULTS. No differences were found in gastric emptying either of breakfasts or of the main meals. The HGI-HGL breakfast produced a greater satiety and fullness compared with the other ones after the breakfast, mirrored by a significantly greater antral distension during the early postprandial period. On the contrary, no differences in sensations were found after the following main meal. The HGI-HGL breakfast produced a higher peak both of glucose and of insulin compared with the other breakfasts during the morning $(\mathrm{p}<0.001)$. On the contrary, after the main meal, higher insulin levels were observed the day with fat rich breakfast (HGI- LGL; again, p<0.001). No clear differences were seen in triglycerids and NEFA levels. No differences were observed in ghrelin, leptin or CCK levels. On the contrary, PYY levels were slightly but significantly higher during the morning after the LGI-LGL breakfast. This suggests that some of the metabolic effects of slowly digestible fibers are due to their arrival to the distal intestinal tract and to PYY release. CONCLUSION. Isocaloric breakfasts with different glycemic index and glycemic load produce different metabolic responses in young healthy volunteers, possibly mediated by PYY release. The effect of these dietary manipulations in subjects at higher metabolic risk requires further studies.

\section{T2072}

Effect of Supplementing Different Macronutrients On Gastric Sensory and Motor Functions and Appetite in Normal Weight, Overweight, Or Obese

\section{Human}

Moo-In Park, Michael Camilleri, Helen O'Connor, Lavonne Oenning, Duane Burton, Debra Stephens, Alan R. Zinsmeiste

Background: The effects of supplementing different macronutrients on gastric sensory and motor functions are unclear. Aim: To compare effects of 2 weeks' supplementation of different classes of macronutrients on gastric function, satiation, and appetite in health and obesity. Methods: In a randomized, parallel-group, double-blind study, 52 healthy participants [14M, 38F, aged 18-64y, body mass index (BMI) $19-47 \mathrm{~kg} / \mathrm{m}^{2}, \mathrm{n}=13$ per diet group] were randomized to different isocaloric diets adjusted for BMI and activity level; standard diet was $20 \%$ protein (P), 30\% fat (F), and 50\% carbohydrate (C). High P, F, or C diet contained 500 additional kcal in the respective nutrient class. On 3 separate days, we measured gastric emptying of solids, gastric volumes, postprandial symptoms, appetite and food choice at a buffet meal with validated methods. Age, gender, BMI and baseline satiation were covariates in ANCOVA. Results: Fat supplementation was associated with increased maximum tolerated volume (MTV) in people with high baseline MTV $(\mathrm{P}<0.05)$, irrespective of BMI (figure shows MTV in each macronutrient-supplemented group). Gastric emptying and volumes, post-

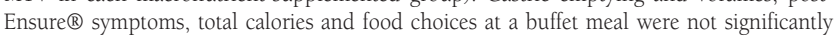
different following each dietary preload. Fasting gastric volumes tended to be higher with F-, than with C- or P-enriched diets $(\mathrm{P} \leq 0.1)$. Gastric emptying and volumes, and satiation were not different in the BMI categories $<30$ and $>30 \mathrm{~kg} / \mathrm{m}^{2}$. Conclusion: Supplementing $500 \mathrm{kcal}$ fat in excess of required calories for 2 weeks reduces satiation in normal weight or obese healthy persons without significant change in gastric functions. This suggests that calories from fat in excess of need may result in adaptation that leads to greater calorie intake, and may lead to obesity.

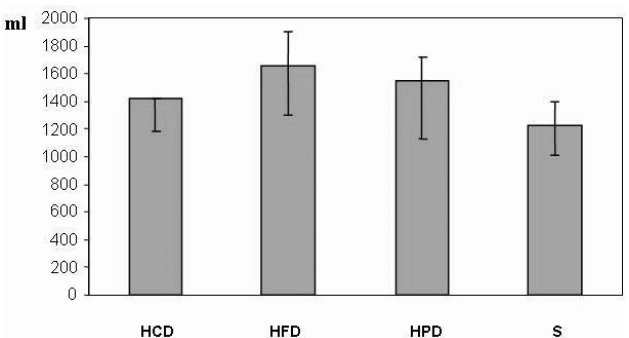

T2073

Gastric Distension Does Not Affect the Inhibitiory Effect of Pyy3-36 On Food Intake in Healthy Human Subjects

Lukas Degen, Philipp Cesena, Sibylle Oesch, Christoph Beglinger

Background: Intravenous (IV) administration of peptide YY (PYY3-36) dose-dependently inhibits food and calorie intake in humans (Gastroenterology 129.1430f-2005), but a physiologic role has not been established for the peptide. Also, gastric distension induced by the ingestion of a preload can alter food intake. The potential interaction between gastric distension and IV PYY3-36 on food intake has not been studied before. The aim of the present study was therefore to investigate whether a protein preload intensifies the inhibitory effect of IV PYY3-36 on food intake in order to define a potential physiologic role for the peptide. Methods: 16 healthy male subjects were studied in a randomized, double-blind, 4-period cross over design. On each study day, subjects ate a light breakfast before $8 \mathrm{a} . \mathrm{m}$. but no food was allowed afterwards until 12 noon, when subjects underwent one of the following treatments: a) water preload plus iv saline infusion; b) water preload plus iv PYY336 infusion $(0.4 \mathrm{pmol} / \mathrm{kg} / \mathrm{min}) ; \mathrm{c})$ protein preload plus iv saline infusion c) protein preload plus iv PYY3-36 infusion. The infusions were given throughout the experiments. A preload (400 ml water or protein drink) was given orally 20 min before presenting a standard test meal (ham sandwiches, orange juice, chocolate pudding, coffee). On each occasion, subjects were free to eat and drink ad libitum. Volunteers scored feelings for hunger and fullness on a standard visual analogue scale before and during food intake. The amount of food eaten and of fluid drunk were measured. Total intake of calories was calculated and the time to complete the meal was assessed. Plasma PYY and CCK were determined in regular intervals. Results:(Mean \pm SEM) Data were analysed using ANOVA followed by multiple paired t-test with Bonferroni's correction. Summary: 1) IV infusion of PYY3-36 at 0.4 pmol// $\mathrm{kg} / \mathrm{min}$ did reduce food and calorie intake, but the effect was not significant. Plasma PYY levels were above the physiological range. 2) The protein preload did not modulate the effects of PYY3-36. Conclusion: Whether PYY3-36 has a physiologic role in the regulation of satiety in humans remains questionable.

Effect of PYY with and without a preload on food parameters 


\begin{tabular}{|c|c|c|c|c|}
\hline Treatment & Food intake (g) & Fluid intake (ml) & Calories (kcal) & Eating time (min) \\
\hline a) water/saline & $715 \pm 43$ & $686 \pm 56$ & $1701 \pm 97$ & $37 \pm 3$ \\
\hline b) water/PYY3-36 & $648 \pm 36$ & $581 \pm 63$ & $1618 \pm 84$ & $37 \pm 2$ \\
\hline c) protein/PYY3-36 & $589 \pm 38$ & $618 \pm 58$ & $1578 \pm 78$ & $34 \pm 2$ \\
\hline d) protein/saline & $634 \pm 38$ & $687 \pm 57$ & $1594 \pm 97$ & $34 \pm 3$ \\
\hline
\end{tabular}

T2074

Peptide Yy (PYY) Release Is Increased in Critical Illness During Small Intestinal Nutrient Stimulation

Nam Q. Nguyen, Richard Holloway, Robert Fraser, Laura Bryant, Marianne Chapman, Carly Burgstad, Judith Wishart, Michael Horowitz

In critical illness, the entero-gastric feedback response to nutrient stimulation is enhanced and may contribute to slow gastric emptying. PYY, an enterogastrone released in response to small intestinal lipid, is known to slow gastric emptying. The potential role of PYY in disordered gastric emptying of critical illness has not been assessed. Aim. To investigate changes in PPY concentrations ([I) in response to duodenal nutrients in critically ill patients. Methods. 19 critically ill patients (12M; $48 \pm 7$ years) and 24 healthy volunteers (17M; $42 \pm 3$ years) received a $60 \mathrm{~min}$ duodenal infusion of Ensure, at either $1 \mathrm{Kcal} / \mathrm{min}$ (13 healthy vs. 9 patients) or $2 \mathrm{Kcal} / \mathrm{min}$ (11 healthy vs 10 patients). Blood samples for PYY and glucose concentrations were collected during fasting every 20 minutes during nutrient infusion and $2 \mathrm{~h}$ after the infusion. [PYY] were analysed by radioimmunoassay. Results. Fasting [PYY]s were similar in healthy subjects and patients prior both infusions. Both plasma and integrated $[P Y Y] s$ were higher in patients during duodenal nutrients stimulation(figure). There was a dose-dependent increase in [PYY]s in healthy subjects but not in patients. A greater elevation of [PYY]was observed in patients during $1 \mathrm{kcal} / \mathrm{min}$ but not $2 \mathrm{kcal} / \mathrm{min}$ infusion. In patients, there was a positive correlation between the integrated changes in [PYY]s and changes in blood glucose ( $r=0.6 ; \mathrm{P}<0.001)$, fasting [PYY] $(r=0.76, \mathrm{P}<0.0001)$, body weight $(\mathrm{r}=0.45, \mathrm{P}=$ 0.02 ) and length of ICU stay $(r=0.6, P=0.03)$. Conclusion. Plasma PYY concentrations are increased in critically ill patients. The greater elevation of PYY in response to $1 \mathrm{kcal} / \mathrm{min}$ but not to $2 \mathrm{kcal} / \mathrm{min}$ duodenal infusion suggests an increased small intestinal sensitivity to nutrients in triggering the "ileal brake" feedback. This abnormal response may contribute to delay gastric emptying in these patients.

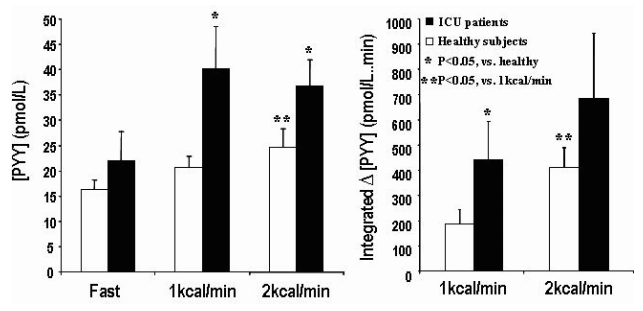

T2075

Protection Against Foodborne Bacterial Infections By Dietary Calcium: Role of Duration of Calcium Supplementation and Gut Microflora Composition in Rats

Ingeborg Bovee-Oudenhoven, Mischa Lettink-Wissink, Roelof van der Meer

Background: Even in industrialized countries $>20 \%$ of the population contracts a foodborne infection per year. We have shown earlier that calcium supplementation protects rats against intestinal colonization and translocation of Salmonella enteritidis. Calcium also prevents enterotoxigenic E. coli induced diarrhea in rats and humans. For now, the duration of calcium supplementation necessary to achieve protection is unknown as well as the mechanism involved. Aim: To determine the efficacy of acute versus continuous calcium supplementation on resistance of rats to salmonella and to address the role of the gut flora. Methods: SPF rats ( $\mathrm{n}=8$ /group) were fed a Western diet with a low (LCa) or a high (HCa) calcium content (20 vs $100 \mathrm{mmol} \mathrm{Ca} / \mathrm{kg}$ ). Two groups received LCa or HCa during the 20 study days. The 3rd group was mostly fed LCa but HCa 1 day before and 1 day after infection only. All rats were orally infected with $10^{9} \mathrm{CFU}$ S. enteritidis on day 10 . Salmonella colonization, and lactobacilli and enterobacteria were quantified by fecal culturing with time. Salmonella translocation was determined by urinary excretion of nitric oxide metabolites. Specific IgG was analyzed in sera obtained 10 days after infection. In a 2 nd study the effect of sterilized $\mathrm{LCa}$ and $\mathrm{HCa}$ (continuously supplied) was tested in SPF vs germfree (GF) rats housed in isolators. Salmonella was supplied as mentioned above, except that $10^{4} \mathrm{CFU}$ were given to GF rats. Gut mucosa, liver and spleen were isolated one day after infection for salmonella culturing. Results: Continuous calcium supplementation is superior in reducing salmonella colonization, but protection to a lesser extent was also observed in rats that received $\mathrm{HCa}$ for only 2 days. Additionally, salmonella translocation was inhibited by calcium supplementation for 2 days or longer. This corresponded with lower serum IgG levels against S. enteritidis in these groups. In contrast to the common thought that diet-induced changes in gut flora composition require weeks, lactobacilli increased and enterobacteria decreased already after 2 days of extra calcium. So, an intermediate role of the protective microflora could not be excluded. Therefore, the second infection experiment was performed. Calcium conferred clear protection against ileal and colonic mucosal colonization and translocation of salmonella to liver and spleen in SPF but not in GF rats, indicating that flora is involved in the protective mechanism. Conclusion: Only 2 days of calcium supplementation are already effective in reducing salmonella infection and this might be mediated by stimulation of lactobacilli and/ or inhibition of enterobacteria.

Carnosine and Histidine Suppress the Induction of Inflammatory Cytokine in Human Intestinal Epithelial CACO-2 and HT-29 Cells

Hideo Satsu, Dong Ok Son, Makoto Shimizu

Background/Aims: Carnosine, composed of beta-alanine and histidine, is one of antioxidative dipeptides and is contained mainly in meat and poultry. The physiological function of carnosine has been investigated in several tissues such as brain. However, little is known about the function of carnosine in the intestine, especially intestinal-immune system. We therefore investigated the effect of carnosine on the secretion of inflammatory cytokine, especially interleukin-8 (IL-8), using human colon-derived Caco-2 and HT-29 cells as a model of intestinal epithelial cells. The anti-inflammatory effect of histidine and beta-alanine was also examined in this study. Methods: The amount of IL-8 secreted from Caco-2 and HT-29 was assessed by sandwich-ELISA. The expression level of IL- 8 mRNA was determined by Real-time PCR analysis. The transcriptional activity of human IL-8 promoter was measured by reporter gene assay. The carnosine uptake into Caco-2 cells was measured using [3H]carnosine. Results: In both human intestinal epithelial-like Caco-2 and HT-29 cells, the hydrogen peroxide-induced IL-8 secretion was significantly reduced by the addition of carnosine. Carnosine also suppressed TNF-alpha-induced IL-8 secretion in a dose-dependent manner. Carnosine uptake into Caco-2 cells was also examined, suggesting that carnosine was transported into the cells via proton-coupled peptide transporter (PepTl). Then in the presence of Gly-Sar, typical substrate of PepTl, the intracellular content of carnosine was decreased and the inhibitory effect of carnosine on the induction of IL-8 was also weakened suggesting that intracellular carnosine suppressed the IL-8 induction. The expression level of IL-8 mRNA induced by hydrogen peroxide and TNF-alpha was not changed by carnosine, suggesting that carnosine acts at the post-transcriptional level. The increase of IL-8 secretion by hydrogen peroxide and TNF-alpha was also significantly suppressed by histidine, but not beta-alanine. Interestingly, increased expression level of IL-8 mRNA and increased transcriptional activity of IL-8 promoter by TNF-alpha were both significantly suppressed by histidine treatment. Further histidine suppressed TNF-alpha-induced NF-kappaB-dependent transcriptional activity, suggesting that histidine acts upstream of NF-kappaB pathway Conclusion: Both carnosine and histidine ameliorate the induction of IL-8 secretion in intestinal Caco-2 and HT-29 cells, whereas their suppressive effect was likely to be regulated in a different manner. Thus food-derived peptides and amino acids such as carnosine and histidine may improve intestinal inflammation.

\section{T2077}

Relationship Between Blood Glucose Control and Feed Intolerance in Critical Illness

Nam Q. Nguyen, Katrina Bland, Robert J. Fraser, Richard H. Holloway

Delayed gastric emptying and subsequent intolerance of naso-gastric feeding is common in critically ill patients. Many of these patients have stress-related hyperglycemia and receive insulin therapy. Hyperglycemia, even at physiological levels $(8-10 \mathrm{mmol} / \mathrm{L})$, disrupts antropyloro-duodenal motility and may slow gastric emptying. The contribution of physiological hyperglycemia to the high incidence of feed intolerance in critical illness is unclear, as is the role of tight glycemic control in preventing this complication. Aim. To assess the relationship between blood glucose concentrations (BSL) and feed intolerance in critical illness. Methods. Hourly BSLs and insulin requirements, over the first 10 admission days, were assessed prospectively in 87 consecutive mechanically-ventilated, feed-intolerant (6-h NG aspirate $+250 \mathrm{ml})$ patients $(57 \mathrm{M} ; 57 \pm 2 \mathrm{yrs})$ and 48 age-matched, feed-tolerant patients (32M; $56+2 \mathrm{yrs}$ ). Only patients fed for at least 5 days were studied. Results. The mean BSLs on admission, 24 hours prior to starting NG feeding and for the first 4 days of feeding, and the use of insulin therapy were similar between the 2 groups (Table). In comparison with feed-tolerant patients, feed-intolerant patients had slower rates of feeding $(46+2 \mathrm{ml} / \mathrm{h}$ vs $60+2 \mathrm{ml}, \mathrm{P}=0.01$ ), higher peak BSL and greater hourly variations in BSL both fasting and during enteral feeds. The variation increased significantly during the $24 \mathrm{hr}$ up to the development of feed intolerance. There was a weak but significantly negative correlation between the admission BSL and the time taken to develop feed intolerance $(r=0.2, P<0.0001)$ Conclusions. Feed intolerance in critical illness is associated with significantly higher peak and greater variation in BSL during both fasting and feeding. The contribution of these findings to the development of delayed gastric emptying and feed intolerance is unclear but they suggest more intensive insulin therapy may be required to minimize the variability in BSLs in feed intolerant patients.

\begin{tabular}{|c|c|c|}
\hline $\begin{array}{c}* \mathrm{P}<0.001 ; \\
\text { * } \mathrm{P}=0.02 ; * * * \mathrm{P}=0.04 ; \# \mathrm{P}=0.05 \text { Data } \\
\text { expressed mean } \pm \mathrm{SEM}\end{array}$ & $\begin{array}{c}\text { Feed-tolerant } \\
(\mathrm{n}=48)\end{array}$ & Feed-intolerant $(\mathrm{n}=87)$ \\
\hline Mean BSL - Admission - Pre-feed - During feed & $\begin{array}{c}8.0 \pm 0.27 .3 \pm 0.2 \\
7.4 \pm 0.2\end{array}$ & $8.6 \pm 0.37 .6 \pm 0.27 .6 \pm 0.3$ \\
\hline Peak BSL - Pre-feed - During feed & $8.1 \pm 0.28 .4 \pm 0.2$ & $9.3 \pm 0.3 * 9.6 \pm 0.3 *$ \\
\hline $\begin{array}{c}\text { Hourly variation in BSL - Pre-feed - During } \\
\text { feed - 24hr leading to intolerance }\end{array}$ & $2.4 \pm 0.22 .7 \pm 0.2$ & $\begin{array}{c}3.4 \pm 0.2 * * 3.6 \pm 0.2 * * * \\
4.0+0.2 *\end{array}$ \\
\hline
\end{tabular}

\section{T2078}

Membrane Targeting and Intracellular Trafficking of the Human Thiamine Transporter-2 (hTHTR-2) in Epithelial Cells

Veedamali S. Subramanian, Jonathan S. Marchant, Hamid M. Said

Background: The water-soluble vitamin thiamine (B1) is essential for normal growth and development. Humans cannot synthesize the vitamin and thus must obtain it from exogenous sources via absorption in the intestine. Studies from our laboratory have shown that thiamine absorption involves both the hTHTR-1 and hTHTR-2 transporters. In contrast to hTHTRl, nothing is currently known about targeting and intracellular trafficking of hTHTR-2 to the apical membrane of polarized epithelial cells. The aim of this study was therefore to identify the region(s) in the hTHTR-2 polypeptide that dictate its targeting to the apical membrane 
and also to study its intracellular trafficking. Methods: Live cell (Caco-2 and MDCK) confocal imaging was used to resolve the localization and trafficking of full-length hTHTR2 and truncated constructs fused to green fluorescent protein (GFP). Results: The fused full-length hTHTR-2 protein was found to be only localized at the apical membrane of filter grown polarized Caco-2 and MDCK cells and was functional. Complete or partial truncation of the cytoplasmic tail of hTHTR2 failed to prevent its targeting to the apical cell surface, although the level of expression of these $\mathrm{COOH}$ terminal truncations was reduced. In contrast, deletion of specific sequences within the polypeptide backbone completely abrogated cell surface expression of hTHTR2 and the truncated proteins were retained within the endoplasmic reticulum. Video-rate confocal imaging resolved rapid bi-directional movement of intracellular vesicles containing hTHTR2-GFP. Treatment of cells with microtubule disrupting agents lead to impairment in vesicles movement; disruption of the actin microfilament, however, was without an effect. Similarly overexpression of the dynactin subunit, dynamitin (p50) which inhibits cytoplasmic dynein led to impairment in apical expression of hTHTR2GFP. Conclusions: Our results shows for the first time that targeting of the hTHTR2 protein is dependent on specific sequence located in the transmembrane backbone of the polypeptide. In addition, apical delivery of hTHTR2 is depend on an intact microtubules and cytoplasmic dynein. [Supported by grants from the DVA and the NIH (DK 56061, DK 58057(HMS) and DK 63750 (VSS))]

\section{T2079}

Reduced Intestinal Cholesterol Absorption Lessens the Uptake of Long- and Medium-Chain Saturated Fatty Acids in Mice

Eric Labonte, Ronald J. Jandacek, Joanna P. Davies, Yiannis A. Ioannou, David Y. Hui, Philip N. Howles

Recent studies have documented the importance of Niemann Pick Cl-like 1 protein (NPClL1), a putative physiological target of the drug ezetimibe, in mediating intestinal cholesterol absorption. Mice deficient in NPClLl exhibit resistance to diet induced hypercholesterolemia similar to that observed with ezetimibe treated wild type mice. However, the effect of reduced cholesterol absorption and any direct or indirect role NPClLl in fat absorption has yet to be delineated. In this study, we explored the impact of reduced cholesterol absorption, by either ezetimibe treatment or NPClLl gene ablation, on fat absorption efficiency in mice. Methods: Both NPClL1+/+ and NPClL1-/- mice in C57BL/ 6 backgrounds, a strain utilized to study diet-induced obesity, were fed diabetogenic diets (58 kcal\% fat) consisted of a combination of soy bean oil and either or both lard and hydrogenated coconut oil in the presence or absence of ezetimibe. Lipid extracted from fecal pellets collected $24 \mathrm{hr}$ after feeding were analyzed by gas chromatography to detect dietary fat absorption utilizing the sucrose polybehenate method. Additionally, tissues were collected during necropsy for lipid analysis. Results: Ezetimibe treatment and NPClL1deficiency in mice both exhibited a similar reduction in the absorption of stearate (19\%), palmitate $(10 \%)$, and myristate $(9 \%)$. At the same time, the absorption of laurate, oleate, and linoleate absorption was unchanged in these animals. NPC1L1-/- mice treated with ezetimibe exhibited an exaggerated suppression of absorption for stearate $(28 \%)$, palmitate $(13 \%)$, and myristate (13\%). Gallbladder bile in NPClLl+/+ and NPClLl-/- mice in this study were found to have similar concentrations of bile acids, cholesterol, and phospholipids. Conclusion: Reducing intestinal cholesterol absorption in mice by NPClLl ablation or ezetimibe treatment hindered the absorption of long and medium chain saturated fatty acids. Simultaneously, the intestinal uptake of unsaturated fatty acids and of the shorter chain laurate was unaffected. These results demonstrate a link between cholesterol and fatty acid absorption in the gut and suggest that the metabolism of both types of lipids are linked in the enterocytes of the small intestine. The additive effect of both ezetimibe treatment and NPClL1 ablation on medium chain saturated fatty acid absorption further suggests that ezetimibe may have additional physiological targets.

\section{T2080}

Amino Acid Absorption in the Colon Is Evidenced By Means of a Colonic Instillation Study Using ${ }^{13} \mathrm{C}$-Leucine

Karen Geboes, Anja Luypaerts, Vicky de Preter, Paul Rutgeerts, Kristin Verbeke

Introduction : The colon plays an important role in fluid and electrolyte conservation, but presumably also in nutrient absorption, especially in pathological conditions. Under normal conditions, the absorption of protein digestion products almost entirely occurs in the small intestine. However, in patients with short bowel syndrome, functional adaptations such as increased colonic absorption of nutrients have been observed. Recently, an amino acid transporter capable of mediating the absorption of amino acids - including D-amino acids derived from bacterial metabolism - has been characterized in human colonocytes. Methods - Eleven healthy volunteers swallowed a narrow polyvinyl tube which progressed down the intestine overnight. On the following morning, radiographic screening ensured the position of the tube in the right colon. Baseline breath and serum samples were retrieved before bolus administration of a $40-\mathrm{ml} \mathrm{NaCl} 0.9 \%$ solution containing $200 \mathrm{mg}{ }^{13} \mathrm{C}$-leucine. After instillation, serum samples were obtained every 30 min during 6 hours. ${ }^{13} \mathrm{C}$-leucine serum concentrations $(\mu \mathrm{g} / \mathrm{ml})$ were calculated from the ${ }^{13} \mathrm{C}$-leucine enrichment and the leucine concentration which were both measured using gas-chromatography combustion isotope ratio mass spectrometry (GC-C-IRMS, Delta Plus, Thermo Electron). Four hours after the instillation, all volunteers received a sandwich with ham or cheese. Results : In all volunteers, the evolution of ${ }^{13} \mathrm{C}$-leucine concentration over $6 \mathrm{~h}$ was similar and characterized by two peaks. Within 30 minutes after instillation, a sharp increase in ${ }^{13} \mathrm{C}$-leucine serum concentration was observed $(0.39+/-0.16 \mu \mathrm{g} / \mathrm{ml}$ versus $0.21+/-0.04 \mu \mathrm{g} / \mathrm{ml}$ at baseline, means $+/$ $\mathrm{SD}, \mathrm{p}<0.01$ ). This initial peak however, may be due to absorption in the ileum through backwash. From $210 \mathrm{~min}$ after instillation on, a second peak in ${ }^{13} \mathrm{C}$-leucine concentrations was observed, $(0.22+/-0.06 \mu \mathrm{g} / \mathrm{ml}$ at $210 \mathrm{~min}, 0.32+/-0.08 \mu \mathrm{g} / \mathrm{ml}$ at $300 \mathrm{~min}$ (reaching statistically significant difference as compared to baseline, $\mathrm{p}<0.01$ ), means $+/$ - SD) suggesting absorption of ${ }^{13} \mathrm{C}$-leucine from the colon. This increase in ${ }^{13} \mathrm{C}$-leucine concentration could not be explained by consumption of the sandwich, since the rise in ${ }^{13} \mathrm{C}$-leucine concentration started before ingestion of the meal. Conclusion: Higher serum ${ }^{13} \mathrm{C}$-leucine levels were observed after direct colonic instillation of this labeled amino acid, demonstrating the possibility of colonic absorption of dietary amino acid, which subsequently can be used for human metabolism.

\section{T2081}

GIF 68a>G Genetic Polymorphism of Gastric Intrinsic Factor Is Not Associated with Low Vitamin B12 Nor with High Homocysteine Plasma Level in African and European Populations

Celine Chery, Cyrille Bessod, Rosa-Maria Gueant-Rodriguez, Emile Kousanta Amouzou, Nicodeme Chabi, Ambaliou Sanni, Paolo Bosco, Guido Anello, Renee Debard, Marc Andre Bigard, Jean-Louis Gueant

Background: Inherited GIF deficiency is a rare cause of pernicious anemia characterized by gastric intrinsic factor (GIF) impaired secretion, normal acid secretion and absence of atrophic gastritis and of anti-GIF auto-immunity. An association of a GIF $68 \mathrm{~A}>\mathrm{G}$ genetic polymorphism with inherited gastric intrinsic factor (GIF) deficiency has been reported recently in 5 patients (Yassin et al., Hum Mutation, 2004). Aim: to investigate the frequency of this polymorphism in populations from 3 world area and its association with blood vitamin B12 and homocysteine, a metabolic marker of B12 and folate deficiency. Methods: the GIF 68A $>\mathrm{G}$ was genotyped by Q-PCR in 172 young healthy adults from Sub-Saharian Africa, 230 from France and 123 from Italy. Frequencies were compared by Fisher test. Genotype associations with vitamin B12 and homocysteine were evaluated in univariate and multivariate analyses. Concentrations were given as median and inter-quartiles and compared by Mann Whitney test. Results: The frequency of allele GIF 68A was much higher in Africa than in France and Italy (0.192 [95\%CI: 0.154-0.237], 0.026 [0.014-0.045], 0.017 [0.006-0.042], respectively, $\mathrm{P}<0.0001$ ). $68 \mathrm{GG}$ homozygous genotype was reported in 5 cases from Africa but not in the population samples from France and Italy. It was not associated with low vitamine B12 deficiency, when compared with the 68AA+AG genotypes from the same African population ( 498 (452-529) vs 469 (321-687) pmol/L, $\mathrm{P}=0.8661)$, nor with homocysteine plasma level (9.9, 8.4-20.9 vs $12.7(9.0-18.0) \mu \mathrm{mol} / \mathrm{L}, \mathrm{P}=0.7052)$. There was no association of the GIF 68G allele with vitamin B12 nor with homocysteine and hemoglobin level in any of the 3 populations. Conclusion: The difference in GIF 68A allele frequency between African and Caucasian populations suggested either a founder effect and/or an environmental influence that contrasted with a lack of phenotypic effect of this polymorphism with vitamin B12 status and anemia. The previously reported association of this polymorphism with inherited GIF deficiency should be reconsidered in regard to our results

\section{T2082}

The Small Intestine Used Dietary and Systemic Threonine Simultaneously for Muc2 Synthesis in Human Neonates

Maaike W. Schaart, Adrianus C. De Bruijn, Henk Schierbeek, Johannes B. van Goudoever, Ingrid B. Renes

Background: The intestine utilizes $60-90 \%$ of the dietary threonine intake in neonatal pigs Mucin 2 (MUC2), the predominant intestinal mucin, is a large glycoprotein, rich in threonineproline-serine tandem repeats. The high visceral need of the intestine for threonine might reflect the high synthesis rate of MUC2. In this way threonine might be essential to maintain the protective mucus layer. We hypothesized that dietary more than systemic threonine is mainly incorporated into secreted proteins of the small intestine, especially MUC2. Objective: To determine the dietary and systemic threonine incorporation into MUC2 and to quantify the fractional synthetic rate (FSR) of MUC2 in human neonates with an enterostomy following bowel resection. Methods: Intragastric (IG, $\mathrm{n}=8$ ) and intravenous (IV, $\mathrm{n}=4$ ) infusion of $\mathrm{U}$ ${ }^{13} \mathrm{C}$ labeled threonine was used to determine the incorporation of threonine into secreted MUC2 in the outflow fluid at the enterostomy exit in human neonates. Small intestinal MUC2 was purified by $\mathrm{CsCl}$ equilibrium ultracentrifugation. Mucin containing fractions were identified by western-blot and Periodic Acid Schiff (PAS)-staining. Mucin containing fractions were pooled and the isotopic enrichment of threonine in MUC2 was measured by Gas Chromatography Isotopic Ratio Mass Spectrometry (GC-IRMS). Results: Dietary and systemic threonine were incorporated into MUC2 of the small intestine, which was subsequently secreted into the lumen of the small intestine. The FSR varied between $8 \%$ and $41 \%$ per day. The FSR tended to be higher during the intravenous threonine administration [IG: $18.5 \pm 9.9 \%$ vs. IV: $28.5 \pm 10.1 \%$, NS]. The maximal threonine enrichment of MUC2 during enteral threonine administration was not significantly different compared to the maximal threonine enrichment of MUC2 during intravenous tracer infusion [IG: $0.770 \pm$ 0.34 vs. IV: $0.714 \pm 0.36 \mathrm{MPE}$ ]. Systemically derived threonine accounted for $58 \%$ of the total amount of threonine used for MUC2 synthesis, whereas $42 \%$ was derived from the luminal site. Conclusions: Both dietary and systemic threonine are utilized for small intestinal MUC2 synthesis and secretion. The small intestine does not prefer dietary threonine above systemic threonine for MUC2 synthesis in human neonates receiving (some) enteral nutrition.

\section{T2083}

Influence of the Mitochondrial Taurine Transporter Expression On Hepatic Mitochondria Number in $\mathrm{CCL}_{4}$-Induced Liver Disease Teruo Miyazaki, Yasushi Matsuzaki, Akira Honda, Tadashi Ikegami, Yining Zhang, Bernard Bouscarel

Backgrounds/Aim Maintenance and enhancement of mitochondrial activity are key factors in the improvement of liver diseases (LD). Taurine is one of the most abundant amino acids and it is taken up by a specific transporter (TAUT). This cysteine derivative is suggested to accumulate in the mitochondria and to play a role in the regulation of oxidative stress and apoptosis. Therefore, the aim of the present study was to investigate the mitochondrial TAUT expression level as its expression and alteration in LD is still unclear. Methods SD rats were divided into four groups; normal, LD1, LD2 and LD treated with taurine (LDT) groups. The LDl and LDT groups received $\mathrm{CCl}_{4}$ for 5 weeks, and the LDT group was fed a $2 \%$ taurine diet along with, as well as one week prior to the $\mathrm{CCl}_{4}$ treatment. The LD2 groups received $\mathrm{CCl}_{4}$ for 15 weeks. The expression levels of TAUT, porin and cytochrome 
$\mathrm{C}$ (CytoC) were examined in the mitochondria, cytoplasm, and membrane fractions by Western blotting. Furthermore, the respective hepatic lobular and intracellular TAUT expression levels were studied by immunohistochemistry. Results The taurine concentration in hepatic tissue was significantly decreased in the LDl and was even further decreased in the LD2 group. Hepatic histological damage was observed mainly in the pericentral (PC) region, and was significantly aggravated along with the duration of $\mathrm{CCl}_{4}$ administration. Furthermore, the MitoTracker-positive mitochondrial numbers were markedly decreased in the liver of $\mathrm{CCl}_{4}$ administered groups. The taurine supplement led to improvements of the hepatic function, taurine concentration and histological parameters. The TAUT protein expression in the hepatic lobule was markedly and predominantly expressed in cells of the PC region. The TAUT was confirmed to be expressed in the mitochondria, cytoplasm and membrane by both confocal laser microscopy and Western blotting. The mitochondrial and cytoplasmic expressions of TAUT were significantly decreased in the LD1 and LD2 groups compared to that in the normal group. The mitochondrial expressions of both CytoC and porin were significantly reduced in the LD2 group, while these expression profiles were maintained in the normal and LDl groups. Taurine administration prevented the loss of TAUT in the cytoplasm but not in the mitochondria. Conclusions The present results show that hepatic damage with a reduction in mitochondria number in the $\mathrm{CCl}_{4}$-induced $\mathrm{LD}$ could be due to a decreased hepatocellular TAUT expression level. Therefore, these findings support that the maintenances of mitochondrial TAUT expression and taurine content are important factors for mitochondria and cellular viability in LD.

\section{$\mathrm{T} 2084$}

A Novel Type Hypertriglyceridemia Observed in Spontaneous Hepatic Steatosis Model FLS Mice

Masafumi Ono, Masaya Takahashi, Toshiji Saibara, Akira Hirose, Naoaki Akisawa, Shinji Iwasaki, Saburo Onishi

BACKGROUND/AIMS: The unique inborn hypertriglyceridemia and hepatic steatosis seen in FLS (fatty liver Shionogi) mice was relieved by the administration of purified apolipoprotein (apo) C-II. Lipoprotein lipase (LPL) and its cofactor, apoC-II, play a pivotal role in VLDL metabolism. Therefore, we investigated the genetic background involved in this hypertriglyceridemia. METHODS: Plasma levels of TG and total cholesterol as well as LPL activity were measured in male FLS mice and C57BL/6J mice. Agarose gel electrophoresis and fast protein liquid chromatography were used to analyze the lipoprotein profile. Male FLS mice were administered $0.1 \mathrm{ml}$ of C57BL/6J mice plasma, saline, or saline with purified human apoCII. A cross experiment was done to determine the genetic background of hypertriglyceridemia observed in FIS mice. cDNA sequences of apoC-II and apoC-III of FLS mice were determined. RESULTS: Prea-lipoprotein was the predominant lipoprotein class in FLS mice plasma. LPL activity remained in the range observed in C57BL/6J mice, and purified apoC-II transiently relieved FLS mice from hypertriglyceridemia. Intravenous administration of either a small amount of C57BL/6J plasma or purified human apoC-II restored LPL activity. Prea-lipoproteinemia was inherited in an autosomal recessive manner. ApoC-III appeared to be a causal factor for this unique hypertriglyceridemia. Microsatellite analysis, however, revealed that the responsible chromosome was not 7; rather, apoC-III mapped onto chromosome 9. CONCLUSION: We suggest apoC-III as a candidate causative factor for the hypertriglyceridemia observed in FLS mice because an excessive amount of apoC-III attenuates LPL activity in vivo and in vitro. Therefore, the FLS mice may be the good model for study of the abnormal hypertriglyceridemia such as apoC-II deficient patients.

\section{T2085}

Methionine Deficiency and Steatohepatitis in a Dietary Animal Model Helieh S. Oz, Willem J. de Villiers, Craig McClain

Methionine is an essential sulfur amino acid involved in DNA methylation, biosynthesis of cysteine and glutathione (GSH). Methionine (Meth) and choline deficient diets serve as animal models for nonalcoholic steatohepatitis (NASH), but the mechanism of action is not fully defined. We examined the effect of Meth on the development of steatohepatitis. Methods: Young rats were fed (ad libitum) amino acid-based total Methionine and choline deficient (MCD) or sufficient (MCS) diets. After 3 wks, MCD rats were daily gavaged with vehicle (MCD), or Meth administration (Meth/MCD). One group of MCS rats was fed deficient diet (MCS/MCD) for 2 additional wks. Result: Animals fed deficient diet for 2 wks (MCS/MCD) had a normal hematocrit value but lost $(29 \%)$ weight (MCS/MCD vs. MCS p<0.05). Rats fed a deficient diet for 5 wks (MCD) weighed only $1 / 3$ of those on sufficient diet (MCD vs MCS $p<0.001$ ) and developed anemia (hematocrit: MCD vs. MCS $p<0.01$ ), but otherwise appeared normal. Daily Meth administration ( $2 \mathrm{wks})$ normalized hematocrit and partially (19\%) improved weight (Meth/MCD vs MCD p<0.05). Liver transaminases elevated after only 2 wks (MCS/MCD vs MCS p<0.01) and further increased (AST: 6-fold; ALT: 3-fold MCD vs. MCS $\mathrm{p}<0.001)$ after 5 wks on deficient diet. Meth administration significantly suppressed these abnormal enzyme activities $(\mathrm{p}<0.01)$. Rats fed deficient diet developed severe liver lesions evidenced ( $2 \mathrm{wks}$ ) by fatty degeneration and inflammation (MCS/MCD score: $2.8+0.5)$ and progressed $(5 \mathrm{wks})$ to necrosis (MCD score: $3.6+0.5 \mathrm{p}<0.001$ ). Liver pathology was partially but significantly improved with Meth administration (2.4+0.2 Meth/ $M C D$ vs. MCD $p<0.01$ ). Blood and liver reduced GSH and SAM were significantly affected in rats fed deficient diet and improved with Meth administration. Deficient diet ( 2 wks) significantly upregulated genes responsible for inflammation and those involved in tissue remodeling and fibrosis; this upregulation was significantly ameliorated by Meth administration. Conclusion: Methionine deficiency resulted in markedly elevated liver enzymes, pathology and expression of inflammatory/ fibrogenic genes after only 2 wks and advanced further after 5 wks on a deficient diet. Meth administration partially but significantly improved these abnormalities. These data support a pivotal role for Methionine in the pathogenesis in this dietary model
T2086

Butyrate Up-Regulates the Bacterial Lipoprotein Stimulation of the NFkB Inhibitory Protein,A20, in Intestinal Epithelial Cells

Meiqian Weng, W.allan Walker, Ian Sanderson

Butyrate are produced in the intestine by bacterial fermentation of carbohydrate. Studies on the effect of butyrate on the expression of cytokines from intestinal epithelial cells have given differing results. Both up-regulation and down-regulation of IL-8 have been reported. Furthermore, butyrate enhances histone acetylation and render it more available to transcription factors. However, it is difficult to understand how such an opening of DNA downregulates gene expression. We hypothesized that these lack of understanding could be explained by butyrate up-regulating an inhibitor of NF-kB in a distinct time-dependent manner. We therefore examined the effect of A20 on bacterial lipoprotein (BLP) stimulation of IL-8, and how butyrate affected A20 -expression. Methods: Caco-2 cells were studied at 10 days after plating. A20-expression was inhibited by A20-specific SiRNA. Cells were stimulated with BLP to produce IL-8. Butyrate or trichostatin A (TSA), a histone deacetylase inhibitor, were given for 24 hours before stimulation with Toll-like receptor2 (TLR2) ligands. Results: BLP induced IL-8 collected over 24 hours in normal Caco-2 cells and in those transfected with non-specific RNA, but removing A20 -expression with specific SiRNA increased IL-8 expression about 4-fold (Figure). BLP enhanced A20-expression and this was increased by treating with butyrate or TSA. The effect of butyrate was dose dependent and time dependent, with A20 increasing within 2 hours, and decreasing beyond 18hours. Butyrate decreased BLP stimulated IL-8 expression at early time points, but greatly enhanced it later. Conclusions: A20 inhibits the BLP stimulation of IL-8. Butyrate enhances IL- 8 expression over 24 hours, but at early time points when $\mathrm{A} 20$ is increased butyrate inhibits IL-8. Thus the effect of butyrate on NF-kB dependent genes depends on the relative expression of the gene and its induced inhibition by $\mathrm{A} 20$.

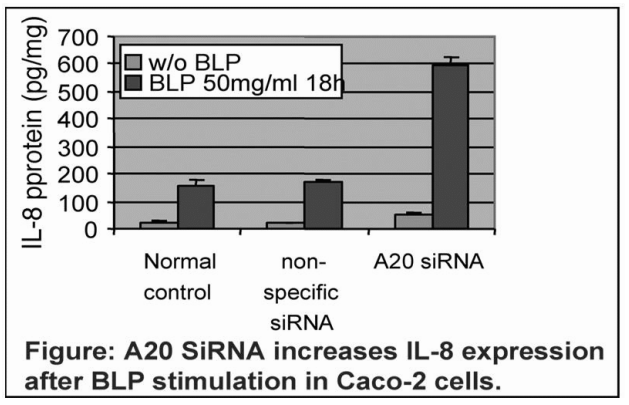

T2087

Gene Expression Profile of Stomach in Response to Food Intake in Mice Eli Helge, Chun-Mei Zhao, Turid Follestad, Paul van den Ijssel, Paul P. Eijk, Bauke Ylstra, Duan Chen

Background/Aim: The physiological role of stomach is generally believed to serve as a reservoir for ingested food and to secrete gastric juice containing $\mathrm{HCl}$. The aim of the presen study was to reveal the gene expression profile of the stomach in response to food intake in mice. Methods: Twenty mice (male, C57BL/6, 2-month old) were divided into fasted group (6 mice) and re-fed group (14 mice). The re-fed mice were fasted for $24 \mathrm{~h}$ and then given free access to the standard mouse food pellets for $4 \mathrm{~h}$. The intact stomachs were collected from each mouse and 6 stomachs in re-fed group were selected based on the weight of gastric contents. RNA was isolated from each mouse stomach using Trizol method and the quality was checked by spectrophotometer and electrophoresis. The gene expression profiling was performed by Affymetrix Mouse Gene Chips (39K), and verified by mouse oligo expression microarray $(21 \mathrm{~K})$, and qRT-PCR. Differentially expressed genes were annotated using the gene ontology tool eGOn v. 2.0 (www.genetools.microarray.ntnu.no). Results Affymetrix analysis revealed a total of 1842 genes to be changed significantly. Oligo microarray showed that 288 genes were changed significantly (false discovery rate $<0.05$ ), in which 151 genes were up-regulated ( $>2$-fold) and 121 down-regulated $(>50 \%), 4 \mathrm{~h}$ after starting re-feeding. Annotation analysis revealed that these genes modulate mainly the expression of transcripts that are involved in metabolism and catabolism $(92+11$ genes), cell growth and/or maintenance (57), molecular transport (50), cell communication (31), and morphogenesis and organogenesis $(14+12)$. Both expected and unexpected mRNA expressions were observed, such as ATPase, intracellular chloride channel protein, and mast cell protease 9 pre B-cell leukaemia transcription factor 1 . Moreover, pancreatic polypeptide (PP) mRNA expression was found in the stomach and was down-regulated by re-feeding after starvation Conclusions: In response to food intake, the stomach was almost entirely mobilized at the level of transcription. Gastric PP might play a role in the regulation of food intake.

\section{T2088}

Analysis of Small Intestinal Gene Expression in C57bl/6 Mice Revealed a Protective Effect of Calcium On Dietary Fat Induced Cell Proliferation Nicole de Wit, Hanneke Vermeulen, Lydia Afman, Mechteld Grootte Bromhaar, Michael Muller, Roelof van der Meer

Background and aim: Metabolic syndrome (MS) is characterized by obesity, insulin resistance and hypertension and is induced by chronic metabolic stress that can result from a disturbed balance between lipid metabolism and pro-inflammatory response. In this study, C57Bl/6 mice that develop characteristics of MS on a high fat diet were used to gain insight into molecular mechanisms underlying the aetiology of MS. Methods and results: Changes in gene expression were analyzed in small intestine of C57Bl/ $/ 6$ mice fed a high ( $45 \mathrm{kcal} \%$ ) or low (10 kcal\%) fat diet, using Affymetrix Mouse genome 4302.0 arrays. After 1, 2, 3, 4,

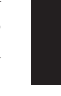


10 and 16 weeks of diet intervention, the small intestine of mice ( $\mathrm{n}=6$ per diet) was collected and divided in three equal parts. For microarray analysis, part I and II were pooled, while part III was analyzed separately. Already after one week of diet intervention gene expression changes could be detected. Moreover, a substantial number of genes showed a robust differential expression throughout diet intervention. Pathway analysis revealed differential expression of genes involved in biological processes, such as fatty acid metabolism and immune response. Interestingly, in part III of small intestine, cell cycle genes showed an increased expression on a high fat diet, especially in the first two weeks of diet intervention. This indicates regeneration of small intestinal cells to compensate damage that is caused by luminal free fatty acids and/or related metabolites. Additionally, this enterocyte damage might trigger a pro-inflammatory response associated with onset of MS. It is known that calcium precipitates luminal free fatty acids and can induce an antiproliferative effect in colon. To determine the effect of calcium on small intestinal gene expression profiles induced by a high fat diet, C57Bl/6 mice were fed a high fat diet with low $(50 \mathrm{mmol} / \mathrm{kg})$ or high $(150 \mathrm{mmol} / \mathrm{kg}$ ) calcium content. After 10 weeks of diet intervention, microarray analysis of part II and part III of small intestine showed a decreased expression of cell cycle genes on the high calcium diet. Additionally, performing immunohistochemistry for Ki67, small intestinal villi of mice fed the high calcium diet showed a lower labelling index compared to mice fed the low calcium diet. This implies that also in small intestine calcium can provide a cytoprotective and thus an antiproliferative effect. Conclusion: The results of the C57Bl/ 6 mice studies indicate that enterocyte damage and the accompanying proliferation is an early event in development of MS and that calcium can have a protective effect in this process.

T2089

The Enteroendocrine Fatty Acid Receptor GPR40 Retains Acyl Chain Length Response Specificity When Heterologously Expressed in PC12 Cells Andrew J. Higgins, Anthony D. Jackson, Craig P. Smith, John McLaughlin

BACKGROUND: Fatty acids (FA) cause secretion of cholecystokinin (CCK) from enteroendocrine cells (EEC), but the cellular mechanisms of FA detection remain unclear. The murine enteroendocrine cell line, STC-1, secretes CCK in response to FA via a rise in [Ca2+]i, in a chain length dependent manner. This requires a minimum of 12 carbons in the acyl chain, matching that observed in vivo in humans. The molecular sensing mechanism is unknown. The recent identification of the former orphan GPR40 as a FA receptor (expressed by pancreatic $\beta$ cells) has prompted this investigation into its potential role as a candidate lipid sensor in EEC. AIMS: To express mouse GPR40 in a heterologous system, and study the acyl chain length specificity of transferred fatty acid responsiveness. METHODS: mGPR40, obtained by RT-PCR from the STC-1 cell line, was subcloned into the mammalian expression vector $\mathrm{PEYFP}-\mathrm{Nl}$ in C-terminal fusion with the fluorescent protein EYFP, and stably expressed in the neuroendocrine cell line, PC12. Both wild type (WT) and mGPR40EYFP expressing $\mathrm{PC} 12$ cells were loaded with Fura- 2 to monitor $[\mathrm{Ca} 2+]$ i by fluorescence microscopy and analysis of the ratio of light emitted at 340 and $380 \mathrm{~nm}$. Cells were exposed to $500 \mathrm{uM} \mathrm{FA}$ of varying acyl chain lengths. RESULTS: FA of acyl chain length C4-C10 did not increase $[\mathrm{Ca} 2+] \mathrm{i}$ in either PC12-WT or mGPR40EYFP+ cells. However, mGPR40EYFP+ PC12 cells displayed significantly increased [Ca2+]i responsiveness to long chain FA compared to PC12WT cells. Dodecanoic acid $(\mathrm{C12}: 0)$ produced no increase in [Ca2+]i in PC12-WT, but a large response was elicited in mGPR40EYFP+ $\mathrm{PC} 12$ cells, with an average ratio increase of 0.5+/-0.05 units. Linoleic acid (C18:2) induced a small rise in [Ca2+]i in PC12-WT (0.1+/0.05 ratio units) which was amplified in mGPR40EYFP+ PC12 cells $(0.4+/-0.1$ ratio units; $\mathrm{p}<0.05)$. For comparison, the maximal [Ca2+]i response induced by $70 \mathrm{mM} \mathrm{KCl}$ was $0.7+1-$ 0.1 ratio units in PC12-WT and 0.6+/-0.1 in mGPR40EYFP+ PC12 cells. Responses were reversible and reproducible, data representing a minimum of 5 independent experiments CONCLUSIONS: mGPR40 of EEC origin functions as a FA receptor when heterologously expressed in the PC12 neuroendocrine cell line. The response to FA is chain length dependent, and remains entirely concordant with previous studies in both the STC-1 cell line and in humans, further supporting a functional role for GPR40 in FA-sensing by EEC. These data justify further detailed study into the signalling pathways and detailed molecular physiology of the GPR40 receptor in relation to EEC.

T2090

Expression of Metabolic and Signalling Genes Is Altered in Adipose Tissue of Obese Children

Antonio Suarez, Concepcion Aguilera, Carolina Gomez-Llorente, Ines Tofe, Mercedes Gil, Esther Farez, Jose Antonio Gomez-Capilla, Ramon Canete, Angel Gil

AIMS: To determine changes in gene expression profile of adipose tissue associated to obesity in prepubertal children. METHODS: We selected 10 children, 5 obese (BMI adjusted for age and sex $z$ score $>2$ ) and 6 control. During appendix surgery, about $400 \mathrm{mg}$ of adipose tissue was taken and immediately immersed in RNAlater solution; total mRNA was isolated and cRNA was hybridizated on the Human Genome U133 Plus 2.0 Arrays (Affymetrix $®$ ). To minimize array discrepancies all arrays were scaled up using the Affymetrix GCOS 1.1 software and the results of each array were compared with all the others. Expression gene profiles were classified using the the GeneSpring software. Transcripts were classified according to Gene Ontology and Panther softwares. RESULTS: 10976 sequences were expressed in obese and 11014 in control children. Bidimensional cluster analysis allowed to adequately separate samples by experimental condition (obese and control). 1588 genes at $\mathrm{P}<0.05,435$ at $\mathrm{P}<0.01$ and 57 genes at $\mathrm{P}<0.001$ were found to be differentially expressed. These transcripts were classified by biological process (30.5\% metabolism, $17.7 \%$ cellular physiological processes and $13,5 \%$ cell communication) molecular function $(14.5 \%$ nucleic acid binding, $10.4 \%$ protein binding and $8.5 \%$ metal ion binding), cellular components $31 \%$ intracellular, $14.6 \%$ membrane and $2.7 \%$ cell fraction) and signalling pathways $(2.3 \%$ Cytokine signalling, 2.3\% Wnt signalling and 2.2\% angiogenesis). CONCLUSIONS: Obesity in early childhood results in a marked alteration of gene expression. Metabolic genes are the most affected ones followed by membrane and intracellular signalling proteins.

\section{T2091}

Is Lys109lys in the Lepr Gene Associated with Protecting Obesity from Hyperglycemia?

Chen Shaohua, Yu Chaohui, Jiang Lingling, Li Youming

Background and aim: The leptin receptor plays a critical role in regulation of body weight by inhibiting food intake and stimulating energy expenditure. Mutations in LEPR gene have been shown to cause obesity. Whether lys109arg in leptin gene affect obesity is still unknown. The study analyse the association between lys109arg polymorphism in the lepr gene and obesity. Materials and methods A total of 180 subjects ( 90 cases with BMI $<25$ and 90 cases with $\mathrm{BMI} \geq 25$ ) who received physical examination were included in the study Genomic DNA samples were extracted from blood from subjects. Genotyping was performed using oligonucleotide microarray. Blood were used to detect total protein, albumin, ALT, triglyceride, cholesterol, HDL and fasting blood glucose. Results There are 144 (80\%) subjects with $G / G$ genotype(arg $109 \mathrm{arg}), 33(18.33 \%$ ) with $\mathrm{G} / \mathrm{A}$ genotype(lys109arg) and 3(1.67\%) with A/A genotype(lys109lys). All three cases (aged from 50 to 70 yrs) with lys109lys polymorphism in the lepr gene were female, short (with height from 152 to $158 \mathrm{~cm}$ ), with BMI26.31.The percentage of body fat and abdominal wall fat thickness is significantly higher in lys109lys $(37.73 \% \& 4.07 \pm 0.40 \mathrm{~cm})$ than that in $\arg 109 \arg (28.80 \% \& 2.72 \pm 0.74 \mathrm{~cm})$ and lys109arg $(28.95 \% \& 2.79 \pm 0.63 \mathrm{~cm})(\mathrm{p}<0.05)$. The level of triglyceride, cholesterol and HDL of the three cases is normal. Conclusion Lys109lys polymorphism in the LEPR gene may be involved in protecting obesity with high percentage of body fat and high abdominal wall fat thickness from hyperglycemia. And the distinct mechanism is still not known

$\mathrm{T} 2092$

Identification of the Acute Effects of Lactobacillus Plantarum WCFS1 On Transcriptional Responses in Human Intestinal Mucosa Freddy Troost, Michiel Kleerebezem, Willem M. de Vos, Robert-Jan M. Brummer

The impact of probiotics on gut health parameters in health and disease has drawn a lot of attention over the last years. However, the impact of probiotics on the regulation of human intestinal mucosa is largely unknown. This study aimed to identify the acute response of intestinal mucosa to Lactobacillus plantarum WCFS1 in vivo in healthy volunteers. Eight healthy subjects without a history of GI symptoms were recruited to participate in this randomized cross-over study. After an overnight fast, tissue samples from the horizontal part of the duodenum were obtained during duodenoscopy. Subsequently, a perfusion catheter was inserted into the proximal small intestine with an injection port located in the proximal descending duodenum. A $1 \%$ glucose solution was infused for three h at $10 \mathrm{ml} / \mathrm{min}$ and, after reaching steady state conditions, a solution containing either $10^{\wedge} 11$ Lactobacillus plantarum WCFS1 in $1 \%$ glucose, or placebo (1\% glucose) was infused for $60 \mathrm{~min}$. A second duodenoscopy was performed to obtain tissue samples after the perfusion experiments. Gene expression profiles in the tissue samples were measured using Affymetrix U133A microarray chips. A multivariate model was designed to correct the array data for interindividual dayto-day variability and effects of the procedure. Only gene reporters that were consistently regulated in all volunteers were considered for further analysis. A fold change $\geq 10 \%$ was considered as a differentially expressed gene reporter. All gene intensities were loaded in the Genmapp/Mappfinder public software programme (www genmapp.org) to extrapolate the gene results to functional biological processes. We observed significant changes in 689 gene reporters; 225 were upregulated, and 444 were downregulated. Twenty-three of the upregulated gene reporters were associated with ribosomal proteins, 15 with immunoglobulins, and many gene reporters could not clearly be allocated to a functional group of genes. Genmapp analysis showed that ribosomes, and specific subgroups of ribosomes, were importantly mediated by Lactobacillus plantarum WCFS1. Other pathways that were regulated were caveola, isomerase activity, translational initiation, ER to Golgi transport, GTPase activity, fatty acid beta-oxidation, ubiquitin conjugating enzyme activity, chymotrypsin and trypsin activity. This study shows that acute, 1-h exposure of intestinal mucosa to Lactobacillus plantarum WCFS1 triggers a consistent transcriptional response in small intestinal epithelium in vivo. Especially processes associated with protein synthesis, proteolysis, intracellular signal transduction, fatty acid metabolism, and immune function seem to be regulated.

\section{T2093}

Prokinetic Therapy for Feed Intolerance in Critical Illness: One Drug Or Two? Nam Q. Nguyen, Marianne Chapman, Robert Fraser J. Fraser, Laura Bryant, Richard H. Holloway

In critical illness, enteral feeding is the preferred method of nutrition. Initial therapy for intolerance of naso-gastric (NG) feeding is either IV metoclopramide (M) or erythromycin (E). Persistent or recurrent intolerance is managed with combination therapy and, if drug therapy fails, post-pyloric feeding. Data to support this practice are limited. Aims. (i) to compare metoclopramide and erythromycin in the treatment of feed intolerance in critical illness, and (ii) determine the success of 'rescue' combination treatment in patients who fail monotherapy. Methods. 90 feed-intolerant (6hly NG aspirate $>250 \mathrm{ml}$ ) critically ill patients received either $\mathbf{M}(\mathrm{n}=45 ; 10 \mathrm{mg}$ IV QID) or $\mathbf{E}(\mathrm{n}=45 ; 200 \mathrm{mg}$ IV $\overline{\mathrm{BD}})$ in a prospective, doubleblind randomized fashion. Age, sex and APACHE II scores were similar between the 2 groups. After the lst dose of prokinetic therapy, NG feeding was recommenced and 6hly aspirates were performed. If $\mathrm{NG}$ aspirate remained $>250 \mathrm{ml}$, combination therapy was given Patients were studied for 7 days. Results. The use of inotropes, opioids and sedations, and BSLs were similar in the 2 groups. Both $\mathbf{M}$ and $\mathbf{E}$ significantly reduced gastric residual volumes(GRV) and improved the \% of patients with successful feeding (M: $62 \%$ and $\mathbf{E}$ : $87 \%$ ) after $24 \mathrm{~h}$ of therapy. Treatment with $\mathrm{E}$ was more effective than $\mathrm{M}$ ( $\mathrm{P}<0.05$, figure). The $\%$ of patients who respond to either treatment on subsequent days declined rapidly. 'Rescue' combination therapy was highly effective and its effectiveness was maintained over $6 \mathrm{~d}$. Predictors of a poor response to single or combined prokinetic therapy were: older age, high admission APACHE II score, multiple trauma, high pre-treatment GRV, and impaired 
renal function. Conclusions. In critical illness, erythromycin is more effective than metoclopramide in treating feed intolerance. Rapid tolerance to prokinetic agents renders both treatments sub-optimal. Combination therapy in these patients is highly effective as 'rescue' therapy. Further study is required to examine the role of combined therapy as first line treatment.

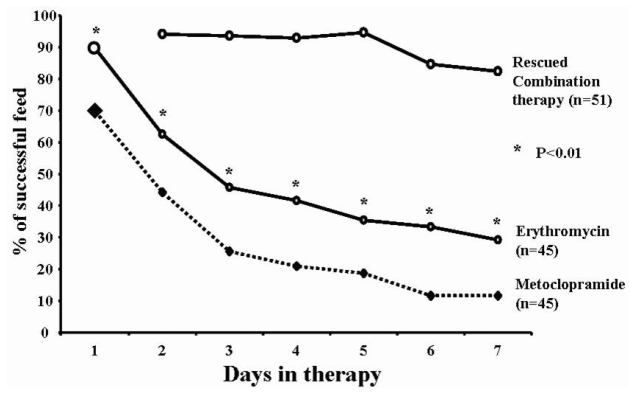

T2094

A Subjective Assessment of Skills and Social Support Required to Administer Home Parenteral Nutrition and Presence of a Psychiatric Diagnosis Can Predict the Development of Complications

Douglas Seidner, Lori Jeris de Burgoa, Cynthia Hamilton, Judy Stafford, Rocio Lopez, Ezra Steiger

Aim: A prospective cohort study was performed to help define the risk factors associated with complications of home parenteral nutrition (HPN). This report describes the impact that psychosocial and training issues have on patient outcome. Methods: All adult patients newly started on HPN and discharged to home from June 2003 through July 2005 were included. Major complications were recorded for the first $90 \mathrm{~d}$ after discharge. Data was collected prospectively and included reason for HPN, psychosocial assessment, severity of illness, and duration of training. A nurse and social worker skilled in assessing and training patients for HPN gave each patient/caregiver a subjective score based on their likelihood of success in performing the tasks of administering HPN. The grading was $A>B>C>D>F$. Complications were categorized by type, severity, cause, and whether they were preventable. Patients who had complications were compared with those who did not. Data is expressed as median (Q25,Q75) and was analyzed using the Chi-square and Wilcoxon rank sum tests. Results: 187 patients (age 51.4y $(41.6,61.5), 109 \mathrm{~F}, 78 \mathrm{M}$ ) were included in the study. The median duration of HPN was $90 \mathrm{~d}(41,90) .99(52.9 \%)$ had a psychiatric diagnosis, usually a mood disorder. There were 103 complications in 72 patients: 36 patients had one, 21 had two, and 5 had three complications. The analysis only included the first complication. The distribution of complications was infectious 52 (50.5\%), mechanical 24 (23.3\%), and metabolic 27 (26.2\%). Twenty three (22.7\%) of the complications may have been preventable. There was an $81 \%$ agreement in success grade between the nurse and social worker. This corresponds to a kappa value of 0.68 (95\% CI, 0.55-0.81). For every 1 grade worsening in the success score, the odds of having at least 1 complication increase $60 \%$ (OR [95\% CI]: $1.60[1.04,2.4])$. Subjects with a psychiatric diagnosis had an $88 \%$ higher odds of having at least 1 complication than those with no psychiatric diagnosis $(1.88[1.03,3.4])$. Conclusions: Patients with difficulty acquiring skills for HPN, a limited social support structure and an underlying psychiatric diagnosis are at greater risk for developing a complication of HPN. Identification of these factors is important and management may help decrease preventable morbidity associated with this therapy.

\begin{tabular}{|c|c|c|}
\hline Grade & RN & SW \\
\hline A & 102 & 84 \\
\hline B & 62 & 63 \\
\hline C & 21 & 19 \\
\hline D & 2 & 2 \\
\hline F & & 1 \\
\hline
\end{tabular}

\section{T2095}

Plasma Citrulline Concentration Is a Reliable Marker of Small Bowel Absorptive Capacity in Crohn's Disease and Is Independent of Intestinal Inflammation

Cinzia Papadia, Ra Sherwood, Tommy Kalantzis, Katharina Wallis, U Volta, E Fiorini, Alastair Forbes

Background \& aims: Post-absorptive plasma citrulline concentration has been proposed as a reliable marker of small bowel absorptive capacity in short bowel patients. Significant correlation between reduced citrulline and abnormal histology has also been shown in patients with intestinal damage, but the potentially confounding impact of intestinal inflammation has not been addressed. The present study aims to evaluate plasma citrulline as a surrogate of intestinal functional integrity in Crohn's disease (CD) and whether concentrations are influenced by inflammation. Methods: 55 patients were selected according to diagnosis, small bowel length and degree of bowel inflammation, to constitute 8 groups: 1) CD with massive small bowel resection: $\leq 50 \mathrm{~cm}$ remaining $(n=6) ; 2$ )resected $C D$ with $50-150 \mathrm{~cm}$ remaining small bowel (n=9); 3)CD with no resection but active inflammation (high CRP and Crohn's Disease Activity Index $(\mathrm{CDAI})>220)(\mathrm{n}=7) ; 4) \mathrm{CD}$ with no resection and no active inflammation (normal CRP and $\mathrm{CDAl}<150)(\mathrm{n}=6) ; 5)$ mesenteric infarction (MI) with massive small bowel resection with $\leq 50 \mathrm{~cm}$ remaining $(\mathrm{n}=6) ; 6)$ mesenteric infarction with resection leaving
50-150 cm small bowel; 7) active celiac disease $(n=6) ; 8$ ) healthy volunteers ( $n=6)$. Postabsorptive fasting plasma citrulline (in $\mu \mathrm{mol} / \mathrm{L}$ ) was measured using reverse-phase High Performance Liquid Chromatography (HPLC). All patients had standard laboratory marker performed and CD activity was scored according to the CDAI. Five hour urine collection were then carried out on each patient following sugar mix ingestion ( $5 \mathrm{~g}$ lactulose, $1 \mathrm{~g} \mathrm{~L}$ rhamnose, $0.5 \mathrm{~g}$ D-xylose and $0.2 \mathrm{~g}$ 3-O-methyl-D-glucose in $100 \mathrm{ml}$ of demineralised water) Results: The plasma citrulline concentration strongly correlated with small bowel length $(\mathrm{p}<0.0001)$ and carbohydrate absorption $(\mathrm{p}<0.001)$. No correlation was found between plasma citrulline concentration and small bowel permeability, and there was no correlation with CRP, sedimentation rate, white cell count, platelets or albumin. Plasma citrulline was significantly higher $(\mathrm{p}<0.0004)$ in $C D$ and MI patients with a remnant small bowel length of $50-150 \mathrm{~cm}$ (mean value $21.0 \mu \mathrm{mol} / \mathrm{L}$ ) than in those with $\mathrm{CD}$ and $\mathrm{MI}$ patients with a smal bowel length $\leq 50 \mathrm{~cm}$ (mean value $9.2 \mu \mathrm{mol} / \mathrm{L}$ ). No significant difference $(\mathrm{p}=0.734)$ was found between groups of patients with CRP $<10$ compared to those CRP $>10$, nor between active and inactive $C D$ patients in whom the intestinal length remained intact. Conclusion Citrulline plasma concentration is a simple, sensitive and reliable surrogate for small bowel absorptive capacity in Crohn's disease patients and is not influenced by intestinal inflammation.

\section{T2096}

Reduced Muscle Function and Muscle Stores Are Common in Quiescent Crohn's Disease

Treasa Nic Suibhne, Colm O'Morain, Maria O'Sullivan

AIM: To define the nature and prevalence of undernutrition in quiescent Crohn's Disease (CD). METHODS: Nutritional and clinical assessments were conducted on $100 \mathrm{CD}$ patients and 100 healthy controls (mean age $35.73 \pm 10.93 \vee 37.96 \pm 11.02 \mathrm{yrs} ;$ NS) matched for age, sex and socio-economic status. Protein stores were assessed by mid upper arm circumference (MUAC, $\mathrm{cm}$ ) and midarm muscle circumference $(\mathrm{MAMC}, \mathrm{cm}$ ), muscle function by grip strength (GS, $\mathrm{kPa}$ ) and fat stores by triceps skinfold thickness (TSF, mm). Muscle depletion was defined as MUAC or MAMC $<15$ th centile, reduced muscle function as GS $<$ population standard, and reduced fat stores by TSF $<15$ th centile. Serum Tumour Necrosis Factor alpha (TNF $\alpha$ ) was measured by ELISA. RESULTS: Reduced muscle function was significantly more common in CD patients than controls [72\% (72/100) v 43\% (43/100); $\left.P=0.00 \chi^{2}\right]$. Patients with reduced muscle function had significantly higher steroid usage in the past year [54\% (39/72) v $\left.32 \%(9 / 28) ; P=0.04 \chi^{2}\right]$ compared to those with normal muscle function. Importantly, physical activity [44\% (32/72) y 46\% (13/28) <30mins per week; NS $\left.\chi^{2}\right]$ was similar between groups. Other factors such as gender [61\% (44/72) v 57\% (16/28) female; NS $\left.\chi^{2}\right]$, age $[36.13 \pm 10.62$ v $34.67 \pm 11.81$ yrs; NS], Crohn's Disease Activity Index (CDAI) [68\% $(49 / 72)$ v $\left.64 \%(18 / 28)<150 ; \mathrm{NS}^{2}\right]$, C-reactive protein (CRP) [59\% (41/69) v 64\% (18/28) $\left.<5 \mathrm{mg} / \mathrm{L} ; \mathrm{NS} \chi^{2}\right]$ and disease duration $[8.55 \pm 6.50$ $7.07 \pm 6.39 \mathrm{yrs}$; NS] did not differ between groups. Screening using standard nutritional and clinical parameters showed the majority of patients with reduced muscle function were of normal BMI [88\% (63/72); mean $\left.25.29 \pm 6.07 \mathrm{~kg} / \mathrm{m}^{2}\right]$, were in clinical remission [68\% (49/72); median CDAI $95.24(0$ 422.76)], had normal CRP levels [59\% (41/69); median 3.90 (2.9 -38.6) mg/L], normal plasma total protein $[94 \%(66 / 70)$; mean $73.60 \pm 4.89 \mathrm{~g} / \mathrm{L}]$, normal serum albumin $[93 \%$ $(65 / 70)$; mean $42.62 \pm 4.36 \mathrm{~g} / \mathrm{L}$ ] and normal fat stores [94\% (68/72) with TSF $>15$ th centile], therefore, would remain undetected by standard measurements. Muscle depletion was similar in CD and controls $\left[42 \%(42 / 100)\right.$ v $41 \%(41 / 100)<15$ th centile; NS $\left.\chi^{2}\right]$. In CD, patient with muscle depletion had significantly higher serum TNF $\alpha$ levels $(6.85 \pm 1.96$ v $5.87 \pm$ $2.05 \mathrm{pg} / \mathrm{ml} ; P=0.02)$ than those without. CDAI $\left[62 \%(26 / 42) \vee 71 \%(41 / 58)<150 ; \mathrm{NS} \chi^{2}\right]$ CRP $\left[\left(60 \%(24 / 40) \vee 61 \%(35 / 57)<5 \mathrm{mg} / \mathrm{L} ; \mathrm{NS} \chi^{2}\right]\right.$, steroid usage [52\% (22/42) v 45\% (26/ $\left.58) ; \mathrm{NS} \chi^{2}\right]$ and physical activity $\left[40 \%(17 / 42)\right.$ v $48 \%(28 / 58)<30$ mins per week; NS $\chi^{2}$ did not differ between groups. CONCLUSION: Reduced muscle function is prevalent in $\mathrm{CD}$ patients with inactive disease and is associated with higher steroid usage.

\section{T2097}

The Effect of Distal Jejunal Feeding On in Vivo Trypsin Synthesis and Turnover

Wen Zhou, Justin Kiswardy, Neeraj Kaushik, Marie Pietraszewski, Stephen J. O'Keefe

Recent studies of ours showed that bypass of the cephalic phase had no appreciable effect on pancreatic secretion. However, converting the duodenal dietary infusion from polymeric to elemental form decreased secretion by $50 \%$, and delivery of the feed distal to $40 \mathrm{~cm}$ down the jejunum avoided pancreatic stimulation. In the present study, we extended our investigations to assess the effect of distal jejunal feeding on the synthesis of trypsin. Pancreatic trypsin synthesis and turnover was measured by the 6h IV infusion of 13C-labeled leucine and collection and isolation of trypsin from duodenal secretions, as described previously (AJPhysiol 2004), in 32 healthy volunteers studied during no nutrient (saline-placebo), IV duodenal or mid-distal jejunal nutrient infusion 20 or $120 \mathrm{~cm}$ past ligament of Treitz (LOT) given at $40 \mathrm{kcal} / \mathrm{kg}$ IBW/day and $1.5 \mathrm{~g}$ protein $/ \mathrm{kg}$ IBW/day. Table summarizes group mean (SE) results showing that duodenal feeding stimulated trypsin secretion and the rate of appearance of newly synthesized trypsin, while mid-distal jejunal feeding did not. Secondly, trypsin secretion and synthesis were significantly lower when the diet was changed from duodenal to distal jejunal feeding site. Amylase and lipase secretion followed a similar pattern. Although the response was exaggerated by infusion $60 \mathrm{~cm}$ beyond the LOT, the difference was not significant. Conclusion: Our results suggest that post-pyloric feeding with an extended tube placed $40-120 \mathrm{~cm}$ down the jejunum avoids the stimulation of pancreatic enzyme synthesis resulting in lower secretion. Further studies are needed to see whether ileal infusion can suppress basal enzyme synthesis for the management of pancreatic secretory disorders.

Trypsin Secretion \& Synthesis 


\begin{tabular}{|c|c|c|c|c|}
\hline & Placebo (n=6) & IV(n=5) & Duodenal (n=14) & Distal Jejunal (n=7) \\
\hline Trypsin secretion (BAEE u/h) & $134(22)$ & $171(33)$ & $408(51)^{* * *}$ & $119(16)+$ \\
\hline Trypsin Synthesis (minutes) & $91(5)$ & $89(10)$ & $71(4) * *$ & $137(26)+$ \\
\hline Trypsin turnover (\%/h) & $0.268(.017)$ & $0.269(.032)$ & $0.347(.037)$ & $0.335(.067)$ \\
\hline
\end{tabular}

Statistics: ${ }^{*} \mathrm{p}<0.05,{ }^{* *} \mathrm{p}<0.001$ Student's t test vs. placebo or baseline, $+\mathrm{p}<0.05$ vs. duodenal

\section{T2098}

Associations Between Growth and Dietary Intakes in Children After Liver Transplant

Timothy A. Sentongo, Simona Korff Korff, Roopa Seshadri, Ann Quinn, Meena Rammohan, Estella Alonso

BACKGROUND: Growth in children after liver transplant is improved but not normalized. There are limited data about dietary patterns and their associations with growth after transplant. Therefore this study examined relationships between dietary intakes and posttransplant growth. METHODS: Prospective study of pediatric liver transplant recipients. Weight $(\mathrm{kg})$ and height $(\mathrm{cm})$ were measured within three months (baseline) and at $\sim 24$ months (follow-up) post-transplant. Z-scores for weight (WAZ), height (HAZ), weight-forheight (WHZ) and body mass index (BMIZ) were computed. Average daily energy intake (EI, kcal/d) was determined from 3 randomn 24-hr diet recalls. EI was compared with NHANES III data and expressed as a multiple of estimated basal metabolic rate (EI:BMR) to assess level of intake above basal requirements. Calcium intake (mg/day) was expressed as a percentage of Adequate Intake (\%AI-Ca) and calcium-to-energy ratio (Ca:EI) to estimate intake per $1000 \mathrm{kcal}$. Catch-up growth (significant change in z-score) was examined using t-tests. Associations between growth and dietary intake were examined using correlations and non-parametric tests. Significance was $p<0.05$. RESULTS: 24 subjects ( 18 females) enrolled. The median age at transplant was $1.65 \mathrm{y}$ (range $0.40-14.8 \mathrm{y}$ ). The interval between baseline and follow-up was (Mean \pm SD) $18.4 \pm 5.3$ months. The baseline and follow-up growth status was: WAZ $-0.87 \pm 1.37$ vs. $-0.01 \pm 1.35 ; \mathrm{HAZ}-0.89 \pm 1.37$ vs. $-0.58 \pm 1.03$; WHZ $0.02 \pm 1.34$ vs. $1.03 \pm 1.29$; BMIZ $-0.16 \pm 1.30$ vs. $0.27 \pm 1.26$. Catch-up growth occurred in weight ( $\mathrm{p}=$ $0.021)$ and weight-for-height $(\mathrm{p}=0.006)$. The mean EI was $1464 \pm 475 \mathrm{kcal} / \mathrm{d}$ and did not differ from reference values $(p=0.21)$. The mean EI:BMR was $1.71 \pm 0.45$ and not dependent on age $(\mathrm{p}=0.16)$. EI:BMR was inversely correlated with change in WHZ $(r=-0.78, p=$ 0.002 ) suggesting that underweight children had relatively higher EI and also demonstrated more growth. The Ca:EI and \%AI-Ca were $646 \pm 268 \mathrm{mg} / \mathrm{kcal} / \mathrm{day}$ and $137 \pm 65 \%$ respectively. The Ca:EI and \%AI-Ca were higher in children aged $<3-y, p=0.048$ and $p<0.001$ respectively. There was no correlation between \%AI-Ca and total EI $(r=-0.33, p=0.16)$. CONCLUSION: Growth in the first 2-y after liver transplant was characterized by more rapid gains in weight than height. EI was influenced by nutritional status and correlated with rates of post-transplant growth, whereas adequacy of Calcium intake varied by age and was independent of total EI. Recognizing dietary trends and their association with age, growth and likelihood of adequacy will lead to optimized nutritional support in transplant recipients. Supported by NIH Grant: M01 RRR-00048

\section{$\mathrm{T} 2100$}

Dose Enteral Feeding Stimulate the Pancreas in Patients with Severe Acute Pancreatitis?

Stephen J. O'Keefe, Marie Pietraszewski, Neeraj Kaushik

Recent studies have shown that enteral feeding is superior to TPN in the managment of acute pancreatitis, despite tha fact that EN stimulates trypsin production and trypsin is the initiating factor in the disease. Previously we have shown that secretion is lower in AP patients than in healthy volunteers during enteral feeding, but we do not know whether secretion is even lower during bowel rest. Consequently, we studied 8 patients with acute severe necrotizing pancreatitis, first measuring secretion during bowel rest, and then again during enteral feeding. Results demonstrated that secretion rates of typsin, amylase and lipase were $>90 \%$ lower in necrotizing pancreatitis than healthy controls, and that enteral feeding had no significant effect on secretion ( 3 higher, 6 lower: Table) In conclusion, enteral feding has no appreciable effect on luminal secretion of pancreatic enzymes in necrotizing pancreatitis. However, further studies need to be performed to exclude the possibility that EN increases trypsin synthesis but that the secretions are lost into the inflammatory mass. Effect of Feeding on Secretion (mean(SE))

\begin{tabular}{|c|c|c|c|}
\hline & trypsin $(\mathrm{u} / \mathrm{h})$ & amylase $(\mathrm{u} / \mathrm{h})$ & lipase $(\mathrm{u} / \mathrm{h})$ \\
\hline Bowel Rest & $46(19)$ & $6499(3377)$ & $14617(11042)$ \\
\hline Enteral feeding & $19(4)$ & $4596(2320)$ & $50566(46812)$ \\
\hline
\end{tabular}

\section{$\mathrm{T} 2101$}

Survival Rate, Nutritional Independence, and Prognostic Factors in 100 Patients with Chronic Intestinal Failure

Italo Vantini, Silvia Montibeller, Luigi Benini, Giorgio Talamini, Elettra Marangoni, Fosca de Lorio, Ulrich Thalheimer, Franco Capra, Franco Benini

Chronic intestinal failure (CIF) affects nutritional status and life expectance. Even though recovery of nutritional independence is possible, about $50 \%$ of CIF need long-term artificial nutrition. Aim of this study was aimed at assessing what factors influence the long-term outcome of patients with CIF. PATIENTS AND METHODS. One hundred patients with CIF (90 with short bowel syndrome (SBS) with or without colon in continuity, 10 with intestinal pseudo-obstruction) were followed in our institution for a median of 36 months (range 6132). Twenty-two clinical and anatomical variables were considered. Statistical analysis was carried-out with Mann-Whitney U-test, Pearson Chi square, Spearman correlation test,
Kaplan-Meyer method and Cox's proportion hazards regression analysis. RESULTS. Twenty seven patients died, in $50 \%$ of the cases for catheter-related infections or for intestinal failure Survival rates were 94.8 and $79.4 \%$ at 1 and 5 years respectively. Survival was lower in patients with permanent CIF and with long term dependence on parenteral nutrition (5 year survival 91 and $71 \%$ for patients on oral supplements and on artificial nutrition respectively). Forty percent of CIF weaned from artificial nutrition, most of them within 3 months since hospital discharge. A 0.63 patient/year hospital readmisson rate was observed, mainly because of catheter-related problems or malnutrition. In univariate analysis, the variables which resulted associated with better outcomes were colon in continuity, length of residual bowel $>100 \mathrm{~cm}$, age $<45$ at onset and diagnosis of chronic idiopathic pseudoobstruction. Multivariate analysis showed that only the length of first hospital admission was as a negative prognostic factor (OR $1.016,95 \%$ CI 1.006-1.026). CONCLUSIONS. Chronic intestinal failure represents a transient condition in about $40 \%$ of patients; nutritional independence is achieved early in most cases, with a little gain thereafter. Age under 45, colon in continuity, length of remnant bowel are associated with better outcome and higher nutritional independence rate. The length of hospital stay as negative prognostic factor probably mirrors the severity of CIF and a more difficult stabilization to be achieved before discharging

\section{$\mathrm{T} 2102$}

Efficacy of Home Pamidronate Infusion in Patients with Metabolic Bone Disease On Home Total Parenteral Nutrition

Maitreyi Raman, Baun Mary, Ellie Aghdassi, Johane P. Allard

Background: Patients on long term home total parenteral nutrition (HTPN) are at increased risk of developing metabolic bone disease (MBD). Pamidronate is a bisphosphonate, proven to be effective at improving bone density in patients with osteoporosis. Pamidronate has been poorly studied in patients with MBD on long term HTPN. Objective: This study examined the change in bone density after receiving pamidronate in patients on long term HTPN with MBD. Methods: This is a prospective cohort study with 11 patients receiving intravenous pamidronate between 1998 and 2005. Bone mineral density (BMD) was performed prior to receiving pamidronate and after at least 18 months of treatment. Pamidronate was infused at home over 2 hours at a dose of $30 \mathrm{mg} \mathrm{q} 3$ months Paired t-test was used to compare t-score in the lumbar spine, femoral neck, and hip. Results are expressed as mean + standard deviation. Results: Eleven patients ( 7 female; 4 male) receiving HTPN for a mean of $10.6+6.3$ years were studied. At baseline, mean age was $59.9+14.2 \mathrm{yr}$ and body mass index was $24.3+3.7 \mathrm{~kg} / \mathrm{m} 2$. Indications for HTPN include: Short Bowel Syndrome $\mathrm{n}=7$, Crohn's Disease $\mathrm{n}=1$, malignancy $\mathrm{n}=1$, radiation enteritis $\mathrm{n}=1$, pseudo-obstruction $\mathrm{n}=$ 1. Two patients were receiving steroids. Mean duration of pamidronate treatment was 22.2 +5.4 months. There was a trend toward improvement in the mean t-score of the spine after receiving pamidronate (pre pamidronate: $-3.1+0.75$ vs. post pamidronate: $-2.9+0.69, p=$ 0.07 . Additionally, a trend toward improvement was noted in the BMD of the hip (pre pamidronate: $0.67+0.10 \mathrm{vs}$. post pamidronate: $0.73+0.09, \mathrm{p}=0.05$ ), and $\mathrm{t}-\mathrm{score}$ of the hip (pre pamidronate: $-2.6+0.9$ vs. post pamidronate: $-2.3+0.84, \mathrm{p}=0.09$ ). No significant change in bone density or t-score was noted in the femoral neck post pamidronate. After excluding the 2 patients on corticosteroids, there was significant improvement in the mean $\mathrm{t}$-score of the spine (pre pamidronate: $-3.4+0.57$ vs. post pamidronate: $-3.1+0.65, \mathrm{p}=$ 0.036). Conclusions: In this small sample size, patients on HTPN receiving intravenous pamidronate showed a trend toward improvement in BMD which became significant for the spine when those receiving steroids were excluded. This suggests that home infusion of pamidronate can be of benefit in this patient population. Further studies with longer treatment period and larger sample sizes are needed to evaluate the true efficacy of pamidronate.

\section{$\mathrm{T} 2103$}

Choline Deficiency Is Associated with Increased Risk for Venous Catheter

\section{Thrombosis}

Alan L. Buchman, Marvin E. Ament, Donald J. Jendon, Chul Ahn

Introduction Patients with intestinal failure that require long-term TPN develop catheter thrombosis as a complication. This patient group may also develop choline deficiency because of a defect in the hepatic transsulfuration pathway in the setting of malabsorption. This study was undertaken to determine whether choline deficiency is a risk factor for development of catheter thrombosis. Methods Plasma free and phospholipid-bound choline concentrations were measured in a group of patients that required long-term TPN. 22 had short bowel syndrome, 5 had pseudoobstruction, 3 unclassified sprue, and 11 had misc indications. Episodes of catheter thrombosis from onset of TPN to the time of blood testing were recorded. Results 41 subjects with 231 catheter years (one patient with a catheter for one year $=$ one catheter year) including 21 males (aged 34+/-26 yrs) that had received home TPN for 5.8+/5.0 yrs and 20 females (aged 55.1+/-18.1 yrs) that had received home TPN for 5.6+/-4.1 yrs were studied. 16 (39\%) patients developed catheter thrombosis and 5 had recurrent catheter thrombosis. Age, sex, TPN duration, and TPN indication were similar between patients that developed catheter thrombosis and those that did not. Plasma free choline was $7.7+/-2.7 \mathrm{nmol} / \mathrm{ml}$ in patients with no history of catheter thrombosis and $6.2+/-1.7 \mathrm{nmol} /$ $\mathrm{ml}$ in patients with previous catheter thrombosis ( $\mathrm{p}=0.076$ by Wilcoxin rank-sum test). The partial correlation between plasma free choline concentration and the frequency of clots after controlling for catheter duration was $\mathrm{r}=-0.33, \mathrm{p}=0.038$. Plasma phospholipid-bound choline concentration was $2191.7+/-679.0 \mathrm{nmol} / \mathrm{ml}$ in patients with previous catheter thrombosis and $2103.3+/-531.2 \mathrm{nmol} / \mathrm{ml}$ in patients without history of catheter thrombosis $(\mathrm{p}=\mathrm{NS})$. Relative risk for catheter thrombosis in patients with a plasma free choline concentration $<8 \mathrm{nmol} / \mathrm{ml}$ (normal: $10-12 \mathrm{nmol} / \mathrm{ml})=10.00,95 \%$ C.I. $=(1.134,88.167)$ Conclusion Choline deficiency is a significant risk factor for development of catheter thrombosis in patients with intestinal failure that require TPN. 
$\mathrm{T} 2104$

Nasojejunal Feeding in Severe Acute Pancreatitis: Comparison of Endoscopic and Self-Migration Tube Placement

Stephane Lecleire, Michel Antonietti, Eric Lerebours

Introduction. Nasojejunal feeding has become the first intention nutritional support in severe acute pancreatitis. While it is recommended to start enteral nutrition early after the onset of the disease, difficulties in tube placement in jejunum can delay the beginning of nasojejunal feeding. The aim of the study was to compare the delay between nasojejunal tube placement and the beginning of enteral nutrition regarding the feeding tube placement procedure, using endoscopy or self-migration tube. Patients and Methods. Thirty patients presenting with severe acute pancreatitis and enterally fed using nasojejunal tube were retrospectively included in the study. Ranson's score was $>3$ with a C-reactive protein (CRP) $>150 \mathrm{~m}$ and/or Balthazar CT scan assessment was scored D or E in the 72 first hours after the onset of epigastric pain. Thirteen patients underwent endoscopic nasojejunal tube placement using a nasofibroscope (group 1), and in 17 patients nasojejunal tube migration was stimulated by the intravenous injection of a prokinetic twice a day during 48 hours (group 2). Control of the jejunal site of the feeding tube was assessed by a plain radiograph at day 0 in patients from group 1 and day 2 in patients from group 2. Nasojejunal feeding delay after tube placement, duration of enteral feeding and length of stay were copared between the two groups. Results. Patients from group 1 and 2 did not differ for mean age (53 (32-79) vs 48 (36-74): ns): mean Ranson's score (3.6 (2-6) vs 3.4 (2-5); ns); mean Balthazar's score; initial CRP (243 (51-520) vs 262 (76-361); ns). Nasojejunal tube was placed in a median time of 5 days after admission in both groups (2-10 vs 2-12; ns). Nasojejunal tubes were all in jejunum at day 0 in groupl. In $18 \%$ of patients from group 2, the tube was not in jejunum at day 2, leading to an endoscopic correction of the tube position. Enteral feeding was started significantly earlier after admission in patients from group 1 as compared to patients from group 2 (5.6 days ( $2-11)$ vs 8.9 days $(4-15) ; \mathrm{p}<0.01)$. Length of stay was significantly shorter in patients from group 1 ( 17 days $(12-41)$ vs 22 days (15-45); $\mathrm{p}<0.05)$. Conclusion. Endoscopic placement of nasojejunal tube in patients with severe acute pancreatitis permits to start enteral nutrition earlier and to significantly reduce the hospital length of stay. These result should be confirmed by a large prospective study including a cost-analysis.

\section{W1000}

A Patient Navigator (PN) in An Open Access Endoscopy (OAE) Program Facilitates Completion and Satisfaction with Screening Colonoscopy (SC) Among Low-Income Underrepresented Minorities (URMs)

Stephanie A. Santos, Lina Jandorf, Steven Itzkowitz, Anabella G. Castillo, Jennifer Christie BACKGROUND: African-Americans and Hispanics have lower screening rates for colorectal cancer (CRC) than Caucasians. Organizational barriers such as lack of patient (pt) access to timely referrals, adequate follow-up and reminder systems have an impact on successful CRC screening. OAE is becoming more common in the United States. The advantages of OAE include shorter waiting times, better control by referring physicians over pt preventive services, and lower costs and inconvenience by eliminating an initial GI consultation. AIM: To assess pt satisfaction with the SC process in an OAE program. METHODS: Average risk, asymptomatic URMs from the primary care clinic at Mount Sinai Hospital were referred for SC through OAE. The majority of pts reside in East Harlem, a medically underserved community. The role of the PN included pt education, explanation of bowel preparation, scheduling appointments, assisting with transportation/escorts, and providing counseling and support to pts. After referral, the PN contacted each person to assess appropriateness for $\mathrm{OAE}$ and discuss the procedure and bowel prep. Two weeks after SC, pts were contacted by phone to conduct a 19-item patient satisfaction survey. RESULTS: From June-Oct 2005, 120 people completed their SC and 106 completed the pt satisfaction survey (response rate: 88\%). $85 \%$ were female. $56 \%$ were Hispanic, $29 \%$ African-American and $11 \%$ Caucasian. The mean age was $56.2(\mathrm{SD}=5.2) .84 .2 \%$ stated that their primary care provider $(\mathrm{PCP})$ adequately explained the reason for the SC. After contact with the PN, this rate increased to $92.1 \%$ ( $\mathrm{p}=.02$ ). $34.9 \%$ of pts were extremely satisfied with their PCP's explanation of the bowel prep in comparison to $58.5 \%$ following the PN explanation $(\mathrm{p}=.00) .83 \%$ were extremely satisfied or satisfied with PCP explanation of bowel prep versus $99.1 \%$ after PN explanation ( $\mathrm{p}=\mathrm{NS}$ ). $94 \%$ of pts were extremely satisfied/satisfied with the SC procedure itself, and $89 \%$ were extremely satisfied/satisfied with the sedation during the procedure. Only $2 \%$ of pts were dissatisfied with the quality of services received during the SC program. $10 \%$ of pts were extremely dissatisfied or dissatisfied with how the results were explained after the procedure. Importantly, $64 \%$ of participants said they would not have completed the $\mathrm{SC}$ without the assistance of the PN. CONCLUSIONS: PN, in an OAE program among low-income URMs, helps pts overcome barriers to SC. Nearly two-thirds of pts would not have undergone a SC without PN assistance. Overall, pts were highly satisfied with the SC program. A PN program may help reduce racial disparities in CRC screening.

\section{W1001}

Yield of Initial Colonoscopy Screening in Patients $<50$ Years of Age with a Family History of Colorectal Cancer

Brooks D. Cash, Cecilia H. Kim, Dolores Y. Rhodes, Mark H. Johnston, Peter W. Soballe, Philip S. Schoenfeld

Background: Current recommendations state that patients with a family history of colorectal cancer (CRC) should undergo screening colonoscopy at age 40 or 10 years prior to the age of the family member when diagnosed with CRC. These recommendations are largely based on expert opinion. This study characterizes the prevalence and location of adenomas any size and advanced neoplasia (adenomas $>10 \mathrm{~mm}$, villous adenomas, adenomas with highgrade dysplasia, or carcinoma) among 18-49 year-old patients with a family history of CRC undergoing index colonoscopy. Methods: Prospectively collected data from endoscopic databases at two tertiary medical centers was pooled. Inclusion criteria: asymptomatic men and women $<50$ years old with a family history of CRC in a first degree relative referred for initial colonoscopy. Exclusion criteria: family history of HNPCC, FAP, IBD or other inherited CRC syndromes, recent history of hematochezia or abdominal discomfort, or surveillance colonoscopy. Data was extracted on patient's age, polyp histology, size and location in the colon, prevalence of adenoma (any size) and advanced neoplasia. Results: From 1998-2005, 380 patients aged $18-49$ (mean age 41.9 years, $38.4 \%$ female) with a family history of CRC underwent screening colonoscopy. 46/380 (12.1\%) had at least one adenoma identified at index colonoscopy, but only $2.1 \%$ (8/380) had advanced neoplasia. When the distal colon is defined as colon distal to the splenic flexure (SF), 82.6\% (38/46) of patients with any adenomas and $50 \%(4 / 8)$ with advanced neoplasia had lesions proxima to the SF without accompanying distal colonic neoplasia. Among patients $<40$ years, $8.5 \%$ (8/94) had adenomas (any size) and 0\% (0/94) had advanced neoplasia. Among 40-49 year old patients, 10.5\% (30/286) had adenomas (any size) and 2.8\% (8/286) had advanced neoplasia. The prevalence of adenomas and advanced neoplasia was not significantly differen between these 2 age groups ( $\mathrm{p}=0.694$ and 0.208, Fisher's exact test). Conclusions: Asymptomatic individuals $<50$ years of age with a family history of CRC were usually found to have proximal colonic neoplasia, confirming the appropriateness of screening with colonoscopy. However, the prevalence of advanced neoplasia is relatively low. Additional data is needed to evaluate the appropriateness of current screening recommendations for patients with a family history of CRC

\section{W1002}

Can Artificial Neural Networks Predict Need for Colonoscopy in Patients Attending Colorectal Clinics?

Sushil K. Maslekar, Angela Gardiner, Graeme S. Duthie

Aims: Artificial neural networks (ANN) are computer programs used to identify complex relations within data that cannot be detected with conventional linear-statistical analysis. The routine clinical predictions of need for lower gastrointestinal endoscopy have been based on population statistics with little meaning for individual patient. This results in large number of unnecessary colonoscopies. We aimed to develop a neural network algorithm which can accurately predict presence of pathology in patients attending routine outpatient clinics. Methods: 300 patients undergoing lower gastrointestinal endoscopy prospectively completed a specifically developed questionnaire which included 40 variables based on clinical symptoms, signs, past and family history. Complete data sets of 50 percent of series were used to train the artificial neural network; the remaining 50 percent were used for internal validation. The primary output used was a positive finding on the colonoscopy, including polyps, cancer, diverticular disease or colitis. Results: The outcome and pathology reports of all the patients were obtained and assessed. Clear correlation between actual data value and artificial neural network value were found $(r=0.931: P=0.0001)$. The predictive accuracy of neural network was $95 \%$ in the training group and was $89 \%$ (95\% CI 84-96) in the validation set. This accuracy was significantly higher than the clinical accuracy (69\%) Conclusions: We have shown that ANN is more accurate than standard statistics when applied to prediction in individual patients of need for lower gastrointestinal endoscopy. These results have obvious implications, with at least $20 \%$ resultant decrease in need for unnecessary lower gastrointestinal endoscopy. The logistic and economic impact with this development is tremendous.

\section{W1003}

Should We Suspect Hereditary Non Polyposis Colorectal Cancer (HNPCC) in Patients with Colorectal Adenomas Before 40 ?

Driffa Moussata, Alexandra Glehen, Marie-Odile Joly, Beatrice Parmentier, Stephane Nancey, Clermont-Carre Nathalie, Jean-Yves Scoazec, Jean-Christophe Saurin

Detection of a colonic adenoma before 40 years is part of the Bethesda's criteria for suspicion of HNPCC. The frequency of MSI adenomas and of clinical criteria of HNPCC in thi population is however poorly known. We aimed at evaluating the biological and genetic status of these patients. Patients and methods: This was a retrospective study in 2 university hospitals, based on pathological register. We identified 90 patients with at least one adenoma diagnosed before the age of 40 between January 1999 and October 2004. Twenty-five cases were excluded because of known HNPCC or PAF. Patients were interviewed by phone about their familial history. The characteristics of the polyps were obtained from endoscopic reports. Immunohistochemical studies were performed on available material (MSH2 and MLH1, MSH6) and microsatellite instability (MSI) was tested. Results: Preliminary results were obtained in 31 patients ( 12 women, mean age $39.3 \pm 6.5$ years (range 17-45)). The mean age at diagnosis of adenoma was $36 \pm 5.7$ years (range $16-40$ years). Colonoscopy was scheduled because of family history of colorectal cancer or adenoma in 15 cases (48.5 $\%$ ), of digestive symptoms in 16 cases (haemorrage ( $32.3 \%$ ), abnormal transit (9.6\%), both $(9.6 \%)$ ). The mean number of adenomas was $1.4 \pm 0.73$ (range 1-4) per patient, the mean size was $10+15.2 \mathrm{~mm}$ (range $3-70$ ), there was 8 flat $(31 \%), 14$ sessile $(36.8 \%)$ and 16 pedunculated adenomas (32.2\%). The localisations were: left colon for 22 polyps $(57.9 \%$ ), rectal $8(21 \%)$, right colon for 8 polyps $(21 \%)$. Histology was of low grade dysplasia in 33 cases $(86.4 \%)$ and high grade dysplasia in 5 cases $(13.6 \%)$. Family history of colonic neoplasia : at least one first- or second- degree relative with adenomatous polyp in 2 cases $(6.6 \%)$, of colorectal cancer in 11 cases $(35.5 \%)$. Amsterdam criteria was observed in 1 case. Loss of MMR proteins expression was observed in 4/31 cases (12.9\%): 3 MSH6 and $1 \mathrm{MSH} 2$; microsatellite instability was tested on available material of $28 \mathrm{pts}$, and on Bat 26 mononucleotide was present in $1 / 30$ cases with loss of MSH6 protein expression and MSI. He had a familial history of colorectal cancer, compatible with HNPCC. Conclusion Systematic biological testing of adenoma developed before 40 years reveals biological abnormalities in $13 \%$ cases. It was associated in 1 case with clinical criteria compatible with Amsterdam criteria. That's why we think that biological testing of adenomas in young patients should be recommended for the identification of possible HNPCC patients. This suspicion is to be confirmed by the identification of MMR deleterious mutations. 
W1004

Both Body Mass Index and Familial History of Cancer Are Risk Factors of Colorectal Neoplasms

Takafumi Sugimoto, Yutaka Yamaji, Atsuo Yamada, Shintaro Kondo, Miki Ohta, Hirotsugu Watabe, Keiji Ogura, Makoto Okamoto, Takao Kawabe, Masao Omata

(OBJECTIVE)In recent years, colorectal cancers have increased in Japan. It has been debated that the westernized lifestyle of Japanese people is responsible for this increase. Indeed, many studies have suggested that lifestyle is related to colorectal cancer. However, few data are available about effects of lifestyle on colorectal polyps, that is considered precursor of cancer. Thus, we conducted the present study to elucidate it. (METHODS)We investigated the relationship between colorectal neoplasms and patient's status taken by questionnaires, including BMI(body mass index) as a parameter for lifestyle, and a familial history of cancer as a heredity factor. We prospectively investigated 1735 patients (male:female 1042:693, average age 61 yrs) who underwent total colonoscopy for their first examination of lower intestine in the University of Tokyo hospital from January, 2003 to September, 2005. We analysed the relationship of sex, age, BMI, and a familial history of cancer with the presence of colorectal neoplasms (adenomata or cancers) by using chi-square test for univariate analysis and logistic regression analysis for multivariate analysis. Age was classified by 10 years old interval, and BMI was classified to BMI $<18.5,18.5<\mathrm{BMI}<25,25<\mathrm{BMI}<30$ s, $30<\mathrm{BMI}$ according to a standard of Japanese obesity society. (RESULTS) We found colorectal neoplasms in $890(51.2 \%)$ of 1735 patients and invasive cancers in 24 (1.3\%). By univariate analysis, the presence of colorectal neoplasms was associated with familial history of cancer male gender, advanced age and high BMI. By multivariate analysis, it was significantly associated with male (odd ratio [OR], 2.78; 95\% confidence interval [CI], 2.26-3.41), advanced age (OR, 1.42; 95\%CI 1.30-1.55), familial history of cancer (OR, 1.24; $95 \% \mathrm{CI}$, 1.01-1.51) and BMI (OR, 1.24; 95\% CI, 1.05-1.46). The presence of invasive cancer was associated with only advanced age and familial history of cancer by univariate analysis. By multivariate analysis, it was significantly associated with advanced age $(\mathrm{OR}, 1.47 ; 95 \% \mathrm{CI}$, $1.01-2.15)$ and familial history of cancer (OR, 2.37; $95 \% \mathrm{CI}, 1.02-5.48)$, but not with male (OR, 2.01; 95\%CI, 0.79-5.14) and BMI (OR, 1.15; 95\%CI, 0.59-2.26). (CONCLUSION)The present study shows that colorectal neoplasms are associated with obesity and familial history of cancer as well as with gender and age. As for invasive colorectal cancers, however, it is suggested that the advanced age and familial history of cancer have greater association with the diseases.

\section{W1005}

How Much Does Polypectomy Actually Reduce Colorectal Cancer (CRC) Risk? Estimated Impact of Initial Colonoscopy/Polypectomy and Surveillance Murtaza Parekh, Uri Ladabaum

BACKGROUND: Screening reduces CRC incidence and mortality, but the actual impact of polypectomy is intensely debated. The National Polyp Study reported a post-polypectomy CRC standardized incidence ratio (SIR) of 0.24 vs SEER (general population). In contrast, data from 3 adenoma chemoprevention trials suggest a post-polypectomy CRC SIR of 0.98 vs SEER (Robertson Gastro 2005). Patients with adenomas are at elevated CRC risk. Thus, even a post-polypectomy CRC risk similar to that of SEER may represent a substantial CRC risk reduction. AIMS. To generate a quantitative estimate of the CRC risk reduction attributable to polypectomy, and to compare the relative impact of initial screening/polypectomy and post-polypectomy surveillance. METHODS: We constructed a Markov model of postpolypectomy adenoma recurrence and CRC development, calibrated to data from placebo arms of chemoprevention trials. We assumed that $90 \%$ of CRC arise from adenoma and accounted for missed adenomas and CRC at colonoscopy. We used the derived rates of adenoma formation, growth, and malignant transformation to project CRC incidence if 50 year-old patients with adenomas did not undergo screening/polypectomy (Natural History; $\mathrm{NH}$ ), and compared this with one-time colonoscopy/polypectomy at 50 (SCRN) or screening+surveillance for age 50-80 (SCRN+SURV). RESULTS: While SCRN was calibrated to trial data for only 4 yrs, SCRN continued to follow SEER CRC incidence closely through age 80 (Figure). We estimated a CRC risk 2.9-fold higher without polypectomy (NH) than with SCRN, representing a $66 \%$ CRC risk reduction attributable to initial polypectomy. SCRN+SURV resulted in $92 \%$ risk reduction compared with NH. Approximately $71 \%$ of the total risk reduction with SCRN+SURV was attributable to the initial polypectomy. CONCLUSIONS: Initial polypectomy and post-polypectomy surveillance are both likely to significantly reduce CRC risk. Even if post-polypectomy CRC risk is similar to that of the general population, this may represent a dramatic $66 \%$ risk reduction compared with no polypectomy.

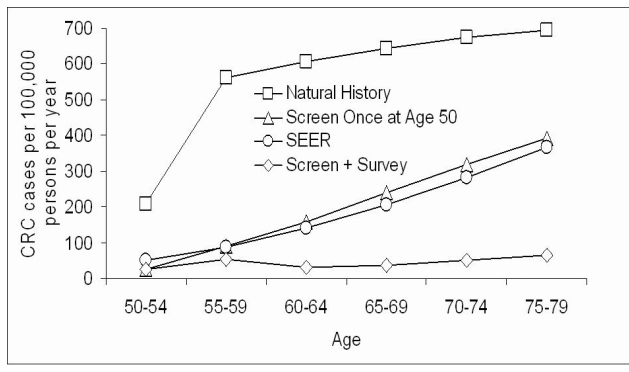

W1006

Age, Smoking Status and the ACE-27 Co-Morbidity Score Are Accurate and Independent Predictors of Survival from Colorectal Cancer in the Elderly Parita Patel, Muhammad Ayub, Samuel N. Forjuoh, Tushar Patel

Background: The incidence of colorectal cancer increases with age. There is a need for accurate prediction of survival in the elderly who may have other competing causes of death Although aging is associated with an increased prevalence of co-morbid conditions, the impact of these on survival from colorectal cancer are poorly understood. Thus, our aims were to (1) identify factors that can predict survival, and (2) quantitate the impact of comorbidities on survival after a diagnosis of colorectal cancer in the elderly. Methods: Patients aged 70 years or older diagnosed with colorectal cancer between 1997 and 1999 were identified. The main outcome measures were the duration of survival. Follow-up was assessed using a hospital-based cancer registry in which all patients were enrolled at the time of diagnosis and prospectively followed-up. Chart reviews were performed to assess co-morbidity which was described using the Adult Co-morbidity Evaluation 27 (ACE-27) Index. Univariate and multivariate analysis were performed to determine the impact of several clinical, demographic and laboratory variables on survival. Results: 243 patients were identified; age 70-74 yrs $(n=77), 75-79$ yrs $(n=77), 80-84$ yrs $(n=42)$ and age 85 and above $(n=$ 47). Follow-up data was available for all patients. The overall 1,3 and 5 -year survival rates were $81.9 \%, 60.9 \%$ and $51.4 \%$ respectively. The overall ACE- 27 co-morbidity index was mild, moderate or severe for $28.4 \%, 27.6 \%$ and $30.5 \%$ of patients respectively. Survival at one year was significantly associated with age group ( $<<0.0001)$, smoking status $(\mathrm{p}=.042)$, presence of metastases ( $p=.001)$, use of an NSAID ( $p=.017)$, and the ACE-27 index $(p<.0001)$. Current smoking and the presence of a metastatic solid tumor were associated with lower survival, whereas the use of an NSAID was associated with a higher survival. Multivariate analysis showed that age group was a significant predictor of survival with an Odds ratio (OR) of 16.0 (95\% CI 4.0-64.0) for ages 80-84 and 5.6 (95\% CI 1.4-22.0) for ages 85 and over. The ACE- 27 index and smoking status were also significant independent predictors with an OR 3.8 (95\% CI 1.3-11.3) for severe co-morbidity, and 3.7 (95\%CI 1.3-12.8) for active smoking status respectively. Summary and Conclusions: Elderly patients with severe co-morbidity, active smoking and increased age have a worse survival after a diagnosis of colorectal cancer. These parameters may be useful to develop a predictive model for survival and may help facilitate clinical discussions regarding screening or management of colorectal cancer in the elderly.

\section{W1007}

Evaluating Quality Improvement of Initial Colonoscopy and Polypectomy in Patients with Precancerous Polyps and Cancers: A Three Year Followup Study Harvey W. Olsen

Purpose: Prior studies of colonoscopy suggest a significant miss rate for advanced polyps and colon cancer. In our private practice, we also have been concerned and are striving to improve the effectiveness of the original colonoscopy and encourage compliance with followup colonoscopies. This 30 year study identifies reasons for quality improvement in colonoscopy and polypectomy by evaluating patient results from a three year follow-up period. Method: All patients with polyps identified at colonoscopy since 1973 have been continuously monitored. Four physicians in an urban private practice participated in this program. Information is presented on all patients who underwent a follow-up colonoscopy from 6 to 42 months after an original colonoscopy and polypectomy. Demographic information and findings from the original colonoscopy were used to stratify the risk for advanced polyps and cancer in the follow-up period. The information was collected and compared at five year intervals. Results: From 1973 to 2002, 2,090 patients had polyps or cancer identified and removed at the time of an initial colonoscopy. Follow-up colonoscopy was performed on 1,085 patients within three years of the original colonoscopy, a rate of $52 \%$. Overall compliance with follow-up was $62 \%$ in patients with advanced polyps or cancer and improved over the time of the study. Among the follow-up patients, 22 cancers were found, a rate of $1.8 \%$. Only one missed cancer was identified during the last 10 years of the study, an incidence of $0.3 \%$. Advanced polyps were identified in $10.8 \%$. The incidence of advanced polyps did not decrease dramatically over the 30 years of the study, but there was a decrease in the number of missed polyps versus those that recurred. Conclusion: Compliance with follow-up improved over the years of the study; specifically in patients with advanced polyps and cancer on initial examination. This was important because these patients are at greatest risk for missed or recurrent advanced lesions. A remarkable reduction of cancer during follow-up was identified without a similar decrease in the rate of advanced lesions. Much of the cancer prevention, we believe, was related to identifying the high risk patient and encouraging earlier follow-up colonoscopy. 


\section{W1008}

Prospective and Retrospective Study of the Effect of Oral Sodium Phosphate Prior to Colonoscopy On Renal Function

Ramy D. Abaskharoun, Stephen Vanner, Depew William

Introduction: Oral sodium phosphate (NaP) for colon cleansing prior to colonoscopy is better tolerated and is as good as or better than oral polyethylene glycol (PEG) solutions. Isolated case reports of renal failure resulting from $\mathrm{NaP}$ use have been described. We tested the hypothesis that $\mathrm{NaP}$ ingestion prior to colonoscopy is associated with renal insufficiency. Methods: In a retrospective study serum creatinine and body weight were routinely measured prior to colonoscopy from 1995-2004. We identified a cohort of consecutive patients who had serum creatinine drawn prior to colonoscopy and at least 30 days after their procedure. Development of renal insufficiency was defined by an abnormal serum creatinine at repeat measurement. Renal insufficiency was also defined as an abnormal creatinine clearance as measured by the Cockroft-Gault Equation. Medications and medical comorbid conditions were recorded. In a prospective study, 24 hour urine collections were obtained prior to and within one month post colonoscopy in a randomized trial comparing $\mathrm{NaP}$ and PEG to more accurately measure development of RI. Results: In the retrospective study 767 patients (51\%F, 49\% M $81 \% \mathrm{NaP}, 19 \% \mathrm{PEG}$ ) with normal baseline serum creatinine were identified through our database. Of these, 55 (7\%) developed renal insufficiency after colonoscopy. 42 patients (7\%) receiving NaP vs. 13 patients (9\%) receiving PEG developed renal insufficiency (Fisher's Exact test $\mathrm{p}=0$.382). Using logistic regression analysis with the choice of preparation, medications, and medical comorbid conditions as dependant variables; only age and blood pressure were predictive of the development of renal insufficiency $(\mathrm{p}=0.014$ and $\mathrm{p}=0.001$ respectively). In the prospective study, 215 patients have been randomized and 25 have completed their second urine collection. Their baseline creatinine clearance was similar. At repeat measurement their creatinine clearance was $1.99 \mathrm{ml} / \mathrm{s}$ vs $2.09 \mathrm{ml} / \mathrm{s}$ in the $\mathrm{NaP}$ and PEG group respectively ( $\mathrm{p}=0.629)$. Conclusions: The prevalence of the development of renal insufficiency between the NaP and PEG groups in this cohort of patients was similar ( $7 \%$ vs $9 \%$ respectively) in our retrospective study. Predictors of renal insufficiency were age and blood pressure independent of the preparation used. In our prospective study, preliminary data show that creatinine clearance is within the normal range and is similar between both preparations at repeat measurement. Supported by an unrestricted educational grant from C.B. Fleet Co.

\section{W1009}

Should Mixing of Sodium Phosphate with Sugared Drinks for Colonoscopy Preparation Be Avoided?

Sherman Chamberlain, James C. Balart, Kostas Sideridis, Subbaramiah Sridhar, William O. Thompson, Angelo Fernandez, Jeffrey Salek

We examined whether the addition of aspartame-containing liquids, as a dilution for oral sodium phosphate( $\mathrm{NaP})$, would provide better fluid and electrolyte balance, and provide better colonoscopy prep than the accepted clear sucrose/corn-syrup-containing liquid dilution method. NaP preps are diluted in clear sucrose-based or corn syrup-based liquid diluents for the improved patient acceptability. However, glucose-containing diluents, in combination with the NaP preps, may activate the intestinal sodium-glucose co-transporter SGLTl which would facilitate $\mathrm{Na}$ and fluid absorption leading to intravascular fluid overload and consequent poor colonoscopy prep quality. 49 in-patients undergoing colonoscopy in 2 different hospitals were prospectively randomized into 2 groups to receive different NaP colonoscopy preps. Both groups received standard pre-procedure diet instructions. Group A ingested two $45 \mathrm{~mL}$ doses of $\mathrm{NaP}$ diluted in two $720 \mathrm{~mL}$ aliquots of sucrose-containing ginger ale separated by 3 hours. Group B ingested two $45 \mathrm{~mL}$ doses of NaP diluted in two $720 \mathrm{~mL}$ aliquots of aspartame-containing ginger ale separated by 3 hours. Serum electrolytes were recorded on the morning prior to and day of colonoscopy. Primary endpoints were: quality of colonoscopy preps as rated by blinded endoscopists and serum electrolytes on the morning prior to and after preps. All patients completed the study with no adverse effects. No significant difference in colonoscopy prep quality was seen between the two groups (MantelHaenzel Chi-square $=0.7954, p=0.4844$ ) (see table). There were no significant differences in mean electrolyte shifts of $\mathrm{Na}, \mathrm{K}$, or BUN/Cr ratio between the two groups ( $\mathrm{p}=0.116,0.401$, and 0.473 , respectively). Since no significant difference was seen between the two groups with respect to quality of colonoscopy preparations or fluid and electrolyte balance, our study suggests that passive fluid transport by aquaporins was the major mediator of fluid and electrolyte shifts in the study subjects. This confirms the importance of aquaporins, and minimizes the importance of SGLT-1, in fluid and electrolyte transport in the human small intestine. Our study suggests that electrolyte-glucose fluid preparations may have little benefit in enhancing hydration status

Rating by Diluent

\begin{tabular}{|c|c|c|}
\hline Rating & Aspartame* & Glucose \\
\hline Excellent & $8(35 \%)$ & $10(38.5 \%)$ \\
\hline Good & $4(17 \%)$ & $6(23 \%)$ \\
\hline Fair & $6(26 \%)$ & $6(23 \%)$ \\
\hline Poor & $4(17 \%)$ & $4(15.5 \%)$ \\
\hline
\end{tabular}

* One patient rated as inadequate and not included in table (5\% of total aspartame group).

\section{W1010}

Comparison of Propofol and Midazolam On Psychomotor Performance Akira Horiuchi, Yoshiko Nakayama

BACKGROUND: In many countries sedative medications are routinely used for endoscopic procedures. Many patients express the desire to drive to work or home shortly after outpatient EGD. Propofol is a sedative agent that provides rapid induction of sedation and has faster recovery than midazolam. We used driving ability as measured by a driving simulator (DS 20, Mitsubishi Precision, Tokyo, Japan) as a sensitive indicator of drug effect after sedation We compared the residual effects of propofol and midazolam on driving ability and examined the relationship between driving ability and the blood concentration of propofol. METHODS: Sixty patients undergoing the diagnostic EGD were randomized to receive a bolus injection of $40 \mathrm{mg}$ of propofol $(n=30)$ or $4 \mathrm{mg}$ of midazolam $(n=30)$. Administration of sedation was performed by a registered nurse and supervised by the endoscopist. Patient satisfaction, recovery time, and complications were evaluated. Driving ability was assessed using the driving simulator. Drug levels were measured at before and at 30,60, 120, and 180 after drug administration. A control group of 20 patients who did not receive any sedative drugs were compared for post procedure driving ability. RESULTS: No major complication occurred in either group. Overall patient satisfaction on a 10-point visual scale was similar for propofol and midazolam ( 8.3 vs. $8.6 ; \mathrm{p}=0.68$ ). On average, after the procedure, the propofol patients could stand at the bedside sooner than midazolam ( 7.3 vs. $31 \mathrm{~min} ; \mathrm{p}<0.01$ ). Driving ability recovered to baseline in all patients receiving propofol within $60 \mathrm{~min}$ of administration. In contrast, $53 \%$ (16 patients) in the midazolam group had significantly impaired driving ability at $180 \mathrm{~min}$. Propofol concentrations ranged from 31 to $89 \mathrm{ng} / \mathrm{ml}$ (mean $67 \mathrm{ng} / \mathrm{ml}$ ) at 60 min whereas midazolam ranged from 0.03 to $1.2 \mathrm{mg} / \mathrm{L}$ (mean $0.43 \mathrm{mg} / \mathrm{L}$ ) at $180 \mathrm{~min}$. Driving ability recovered to the baseline when propofol levels were less than $100 \mathrm{ng} / \mathrm{ml}$. CONCLUSION: Diving ability as assessed using a driving simulator provides a sensitive measure of a drug's effect on psychomotor function. Driving or operating heavy machinery appears safe $60 \mathrm{~min}$ after receiving $40 \mathrm{mg}$ of propofol. However, driving a car should be prohibited for the day the patient receives $4 \mathrm{mg}$ of midazolam.

\section{W1011}

Trends in Colorectal Cancer, Obesity, Diet and Population Risk Factors in a Multi-Ethnic Asian Population

Mark L. Fernandes, Yiong Huak Chan, Khay Guan Yeoh

Aim: To describe the time trends in the incidence of colorectal cancer, and potential risk factors over the last 3 decades in Singapore. Methods: The incidence of cancers was obtained from the Singapore Cancer Registry, a nation-wide population-based registry. Data on the prevalence of smoking, obesity and diabetes mellitus were obtained from published national population surveys. Data on per capita meat consumption was obtained from the Agri-food and Veterinary Authority of Singapore. Results: Between 1968 and 2002, 22618 patients with colorectal cancer were registered by the Singapore Cancer Registry. The age standardized incidence (ASR) of colorectal cancer in the population rose by 107\% between 1968-1972 to $1998-2002$ from 19.3 to 39.9 per 100,000 men and by $90 \%$ from 15.3 to 19 per 100,000 women in the same period. Distal colorectal cancers increased more than proximal cancers in this period. Amongst the three main ethnic groups, the age standardized incidence of colorectal cancer was highest in Chinese (38 per 100,000 persons per year), followed by Malays (20 per 100,000 person years) and Indians ( 14 per 100,000 persons per year). In contrast, the ASR for esophageal cancer fell over the same period from 16.6 to 5.5 per 100,000 men and from 6.2 to 1.1 per 100,000 women, and the ASR for gastric cancer fell from 37.3 to 18 per 100,000 men and from 17.3 to 9.9 per 100,000 women. From 1976 to 2004 the prevalence of smoking dropped by $10 \%$. The prevalence of obesity increased by $60 \%$ between $1982-85$ to 2004 from $4.3 \%$ to $6.9 \%$. The prevalence of diabetes mellitus increased by $310 \%$ between 1975 to 2004 from $2 \%$ to $8.2 \%$ and the per capita consumption of red meat fell by $6 \%$ between 1971 to 2000 from $6.3 \mathrm{~kg}$ to $5.9 \mathrm{~kg}$. Conlusions: Colorectal cancer incidence rates have increased dramatically in Singapore in contrast to other gastrointestinal cancers such as esophagus and stomach. Distal colorectal cancers have increased more than proximal cancer. The increase in colorectal cancers are concomitantly associated with increase in the prevalence of obesity and diabetes mellitus, but were not associated with trends in smoking and red meat consumption

\section{W1012}

Proximal Shift in the Distribution of Adenomatous Colon Polyps and Associated Factors Over the Past Ten Years

Sooyoung Park, Byung Chang Kim, Sung Jae Shin, Sang Kil Lee, Tae Il Kim, Won Ho Kim

Background/Aims: In the recent reports, the incidence of right side colon cancer is increasing, and adenomatous polyp is precancerous lesion of colorectal cancer. However, there were not so many reports regarding chronologic changes in the distribution of adenomatous polyps. We aimed to study changes in the distribution of adenomatous polyps over the past ten years and to examine the factors associated with changes in the distribution of adenomatous polyps. Methods: We reviewed colonoscopic and pathologic reports of patients who underwent colonoscopy at Yonsei University. Severance Hospital between January 1995 and December 2004. We enrolled the patients who had adenomatous polyp equal or larger than $5 \mathrm{~mm}$. Of these patients, the patients who had incomplete colonoscopic examination or history of colon resection, polyp, colon cancer, polyposis syndrome, or inflammatory bowel disease were excluded. Total of 2,498 patients were enrolled and analyzed in this study. Proximal colon was defined as the colon proximal to the sigmoid-descending junction. A large adenomatous polyp $(\geq 10 \mathrm{~mm})$ or adenomatous polyp with villous component, high grade dysplasia, or cancer transformation was defined as advanced polyp. Results: Over the past 10 years, the proportion of patients who had adenomatous polyp at proximal colon significantly increased from $48.5 \%$ to $66.3 \%(\mathrm{p}<0.001)$, and that of patients with advanced polyp at proximal colon, from $42.9 \%$ to $52.6 \%(\mathrm{p}=0.003)$. The proportion of proximal colon polyps significantly increased from $48.5 \%$ to $52.6 \%(\mathrm{p}<0.001)$, and that of proximal advanced polyp significantly increased from $40.4 \%$ to $50.6 \%$ ( $\mathrm{p}=0.018$ ). Male sex, old age ( $\geq 60$ years old), non-pedunculated polyps, small size $(<10 \mathrm{~mm})$, tubular adenoma, low grade dysplasia, non-advanced polyps, and the recent years $(\geq 2000)$ were significantly associated with proximal location of polyps. Conclusions: The proportion of patients who had adenomatous polyps at proximal colon and the proportion of proximal colon polyps significantly increased over the past 10 years. 
W1013

The Utilization of Colonoscopy As a Screening Method for Colorectal Cancer in Two U. S. Metropolitan Teaching Hospitals

Mohammad A. Wehbi, Jae W. Nam, Charles G. Nesmith, Donald M. Pham, Kamil Obideen, Vincent W. Yang

BACKGROUD: Colorectal cancer (CRC) is the second leading cause of cancer death in the United States. Current guidelines advise the screening for CRC in asymptomatic individuals with average risk to begin at age 50 . Several screening modalities are available although there has been no consensus on the most appropriate method with which to screen individuals. Moreover, no study has examined the utilization of various screening strategies in detecting the ethnic or gender distribution of colorectal neoplasia. AIM: The current study is designed to examine the utilization of colonoscopy as a screening tool in asymptomatic individuals at average risk for CRC in two U.S. metropolitan teaching hospitals with different demographics. METHODS: We conducted a retrospective review of all colonoscopies performed between January 2000 and May 2005 in Emory University Hospital (EUH) and Grady Memorial Hospital (GMH). The proximal colon was defined as the segment from the cecum to splenic flexure. Advanced neoplasm was defined as tubular adenomas $>10 \mathrm{~mm}$, a polyp with tubulovillous or villous feature, a polyp with high-grade dysplasia or cancer. Statistical analysis was performed using Fisher's exact test. All confidence limits and $p$ values were two sided. RESULTS: A total of 9,737 colonoscopy records were reviewed. Among these, $2,547(26.2 \%)$ were performed to screen patients at average risk for CRC. The percent of colonoscopies conducted for screening was $33.4 \%$ and $16.5 \%$ at EUH and GMH, respectively. Among the 2,547 patients screened, $174(7 \%)$ were found to have advanced neoplasia, including 115 (66\%) tubular adenoma $>10 \mathrm{~mm}, 36(21 \%)$ tubulovillous adenoma, $7(4 \%)$ high-grade dysplasia and $16(9 \%)$ cancer. Ninety $(53 \%)$ advanced neoplasm were confined to the proximal colon. Caucasian patients had an increased risk for proximal tubular adenoma $>10 \mathrm{~mm}$ with an odds ratio of 3.50 (95\% CI 1.635 to 7.494). In contrast, African American patients had a higher risk for proximal tubulovillous adenomas with an odds ratio of $4.12(95 \% \mathrm{CI}=1.67$ to 22.76$)$. There was a trend towards a higher prevalence of proximal adenocarcinoma in African American patients ( $p$ value 0.0557 ). No differences were detected in the pathology between genders or in the regional distribution of advanced neoplasia between genders. CONCLUSION: (1) Colonoscopy as a screening tool could detect a significant percentage (53\%) of advanced colorectal neoplasia in the proximal colon, which would have been missed by flexible sigmoidoscopy. (2) Our data shows that the pathology of advanced neoplasia may differ between Caucasian and African American patients, with AA having more pathologically advanced lesions.

\section{W1014}

The Rate of Polyp Recurrence in Patients Undergoing Colonoscopy in a U.S. Metropolitan Teaching Hospital

Mohammad A. Wehbi, Jae W. Nam, Charles G. Nesmith, Donald M. Pham, Kamil Obideen, Vincent W. Yang

BACKGROUND: The majority of colorectal polyps can be divided into two groups: neoplastic (including adenomas and carcinomas) and non-neoplastic, primarily hyperplastic. It is well recognized that adenomatous polyps are precursors to colorectal cancer but the role of hyperplastic polyps is less clear. Thus, a better understanding of the natural history of the distinct histologic categories of polyps could potentially lead to better management of colorectal cancer. AIM: To examine the rate of polyp recurrence and their histological distribution among patients undergoing colonoscopy in a U.S. metropolitan teaching hospital. METHOD: We conducted a retrospective review of all colonoscopies performed between January 2000 and May 2005 in Emory University Hospital. All patients with newly documented (incident) polyps were included. The polyps were classified by location, size and histology. Recurrent polyps were defined as those detected upon a subsequent colonoscopy performed beyond three years of the initial evaluation. RESULTS: A total of 5,557 colonoscopy records were reviewed. Among these, 1,445(26\%) had newly documented polyps, including 737 female (51\%) and $708(49 \%)$ male patients with an average age of $53+6.4$ and $54+$ 6.9 , respectively. The majority $(80 \%)$ of the patients were Caucasians. Three hundred sixty one $(25 \%)$ had recurrent polyps, including 97 hyperplastic polyps (27\%), 246 tubular adenomas (68\%), and 18 tubulovillous adenomas (5\%). Patients with an incident adenomatous polyp were more likely to have recurrent polyps of the same histology $(\mathrm{p}<0.004$; $95 \% \mathrm{CI}=1.08-4.66)$. Similarly, patients with an incident hyperplastic polyp were more likely to have hyperplastic polyps at recurrence $(\mathrm{p}<0.0004: 95 \% \mathrm{CI}=3.61-18.65)$. Incident hyperplastic polyps $>8 \mathrm{~mm}$ had a statistically significant recurrence of adenomatous polyps $(p=0.007)$. Patients with an age $>56$ regardless of the histology of the incident polyp had greater recurrence of adenomatous polyps $(\mathrm{p}=0.003)$. Location of the incident polyps and patient gender did not correlate significantly with recurrence. CONCLUSION: The results of our study indicate that incident hyperplastic polyps $>8 \mathrm{~mm}$, incident adenomatous polyps and age $>56$ are associated with recurrent adenomatous polyps. Identification of these risk factors could help define surveillance strategies at follow up examinations and could contribute to prevention of colorectal cancer.

\section{W1015}

Smoking As a Risk Factor for Colorectal Neoplasia (CRN) and Hyperplastic Polyps (HP) in Japanese Patients

Fumio Omata, Fumiaki Ueno, Tetsuya Mine, Yang Chen

BACKGROUND: Smoking is a major public health issue in Japan and one of a few potentially correctable putative risk factors for colorectal polyp. Although not directly involved in the pathway to cancer, some studies suggest that HP may be a marker of a higher-risk colon. The aim of this study was to determine the association between smoking and CRN or HP among Japanese patients. METHODS: Smoking, together with other potential confounding factors and effect modifiers were investigated by questionnaire in Japanese patients presenting for colonoscopy at a single endoscopy unit in Japan. Patients with a personal history of CRN, HP, inflammatory bowel disease, or non-steroidal anti-inflammatory drug use were excluded. 870 consecutive patients (mean age [sd] 52.2 \pm 14.5 ) had a complete colonoscopy and were subsequently divided into 3 groups: $\mathrm{CRN}(\mathrm{N}=194$, mean age [sd] $60.8 \pm 10.4)$, HP $(\mathrm{N}=132$, mean age [sd] 55.3 \pm 13.2$)$ and Control $(\mathrm{N}=586$, mean age [sd] 49.2 \pm 14.6$) ; 19$ patients with colorectal cancer were included in the CRN group because the causal pathway to cancer by smoking is assumed to be the same as in adenoma. The findings at colonoscopy combined with histopathological findings served to measure the main outcome, i.e., the presence or CRN or HP. Unconditional logistic regression was performed for both univariate and multivariate analysis, using nine candidate predictors [smoking categorized in three groups (Non, Moderate and Heavy smoker), age, gender, drinking, body mass index (BMI), history of cholecystectomy, history of appendectomy. RESULTS: In univariate analysis, the increment of age by three categories of age divided by interquatile ranges $(>=43,>=53,>=$ 63 yr), gender (male), moderate and heavy smoking, alcohol use, and BMI were significantly associated with CRN, whereas, heavy smoking, gender, alcohol use and BMI were significantly associated with HP. In the final model for CRN, the adjusted odds ratio for heavy smoking was $1.720(95 \% \mathrm{CI}[1.127,2.625])$, the odds ratios for increment of age by 3 categories $(>43,>53,>63 \mathrm{yr})$ were $3.962(95 \% \mathrm{CI}[1.886,8.326]), 8.931(95 \% \mathrm{CI}[4.323,18.45]), 12.5$ $(95 \% \mathrm{CI}[6.09,25.6])$, respectively. For HP, the adjusted odds ratios for heavy smoking, BMI, drinking were 1.787 (95\%CI [1.109, 2.879]), 1.066 (95\%CI [1.002, 1.134]), 1.751 $(95 \% \mathrm{CI}[1.14,2.69])$, respectively. Increase of BMI was associated only with HP. CONCLUSIONS: Among Japanese patients in Japan, heavy smoking (over 20 pack-year) is significantly associated with both CRN and HP. Increment of age increases the risk of CRN but not of HP. Increment of BMI increases the risk of HP but not of CRN. Drinking is significantly associated with HP but not with CRN

\section{W1016}

A Study of Relation Between Sigmoid Colon Polyps and Body Fatness in Healthy Adults of Eastern Country

Young S. Park, Jeong Hoon Ji, Dong Ho Lee, Nayoung Kim, Ju Seong Kim, Hyun Chae Jung, In Sung Song

Background and aims: Obesity has been thought as a social problem in western society, but recently it is rising as a social problem in Asian people. Previous reports told us that colorectal adenoma has a positive association with obesity. However there is a few report about the relation between colorectal polyps and obesity in Asian society. Therefore we tried to find out if body fatness is related to the presence of polyps, or adenomas in general Korean populations. Method: A total of 6706 Korean patients (3564 men and 3142 women with a mean age of 51.2 years), who visited to Seoul National University Bundang hospital for screening of colorectal polyps between March 2002 and May 2005, were enrolled in this study. A retrospective analysis was performed to investigate colonic findings such as size, location, number, histopathological type by body mass index in general populations. Body mass index (BMI) was used as indices of obesity. Results: Rectosigmoid polyps were present in $29 \%(\mathrm{n}=1949)$ of patients. In patients with polyps, Age, BMI, HbAlC, and triglyceride level were significantly higher than patients without polyps. And in patients with polyps HDL-cholesterol is significantly lower than the patients without polyps. In patients with adenomatous polyps ( $16 \%, \mathrm{n}=1073)$ age, $\mathrm{BMI}, \mathrm{HbAlC}$, and triglyceride level are significantly higher than patients without adenomatous polyps. In multivariate analysis age, $\mathrm{HbAlC}$, HDL-cholesterol and LDL-cholesterol have relation with colorectal polyps. Conclusions: This study shows that BMI and HDL-cholesterol, HbAlC, and LDL-cholesterol are associated with colorectal adenomas in a Korean population. These findings suggest that obesity and its metabolic parameters are associated with an increased risk of colon adenoma in Korea.

\section{W1017}

Quality Measures for Colorectal Cancer Diagnosis: A Systematic Review Deborah A. Fisher, Christopher R. Mantyh, Michael A. Morse, Richard G. Prosnitz, Gregory P. Samsa, Meenal B. Patwardhan

Objectives: Defining and measuring quality are critical to delivering best medical care Adherence to colorectal cancer (CRC) screening has been studied extensively, while the quality of the subsequent evaluation of individuals with an abnormal screen test or who present with symptoms has not. As part of a quality assessment study of CRC, we identified and evaluated quality measures for the further evaluation of patients with positive CRC screening tests or symptoms. Methods: The primary sources were MEDLINE and the Cochrane Database. Databases were supplemented by hand searching references of included articles and relevant reviews. Only US based articles published after 1990 were included. Two investigators independently reviewed each abstract followed by full text review of included abstracts. Finally, the included articles were rated for importance and usability, scientific acceptability, and validation testing using standard methods. Results: Initially 3,429 abstracts were reviewed of which 843 articles underwent full text evaluation. Ultimately 73 articles were retained. Three measures were identified to assess the quality of CRC detection: 1. Percentage of patients with a positive fecal occult blood test (FOBT) who underwent full colon evaluation 2.Time from symptom presentation to CRC diagnosis 3. Percentage of patients with $\mathrm{CRC}$ who underwent colonoscopy as part of their evaluation. In addition, several measures pertaining to the colonoscopy itself were identified including: cecal intubation rate, complications rate, miss rate for significant neoplasia, performance by a provider with specialized training. Conclusions: Few measures were found in the US-based literature for CRC detection. The most widely used and well-developed measure was the percentage of patients with a positive FOBT who underwent appropriate evaluation. This measure was linked to mortality and validated in several populations. The other measures were intuitive but not linked to outcomes. The quality measures of colonoscopy performance were welldeveloped, but generally not validated. Notable gaps included the lack of measures for follow-up of positive flexible sigmoidoscopy or barium enema and measures of appropriate documentation. Future research should also include improvement of the existing measures by more precise numerator and denominator definitions, linkage to an outcome of interest, and additional testing for validity and reliability. 
W1018

Usefulness of Detecting K-RAS Point Mutation in Stool of Patients with Colorectal Cancer Compared to Measuring Hemoglobin and Lactoferrin in Stool

Takashi Nishikawa, Shingo Yasumoto, Yosuke Abe, Takuya Inoue, Eijiro Morita, Naoko Murano, Ken Toshina, Mitsuyuki Murano, Ryoichi Matsuse, Kazuo Uchida, Ken-Ichi Katsu

BACKGROUND and AIMS: Colorectal cancer (CRC) is one of the most common cancers in the world. The identification of high-risk individuals and the early diagnosis of CRC are crucial since a large proportion can be prevented or usually be cured either by colonoscopic resection or by surgery. In order to determine whether it could be used to screen for CRC, we examined a technique for detecting point mutations of K-ras codon 12 in stool samples using one-step polymerase chain reaction/restriction fragment length polymorphism (PCR/ RFLP) analysis compared to measurement of fecal hemoglobin and lactoferrin. METHODS: DNA was extracted from 200-mg stool specimens of 10 healthy controls and 31 CRC patients. A 107-base-pair fragment of exon 1 of K-ras was amplified by PCR using mismatched primers. PCR products were digested with Bst NI and analyzed by gel electrophoresis followed by silver staining. Specificity of one-step PCR/RFLP was examined by using synthetic oligonucleotides. The detection limit of K-ras codon 12 mutations was determined by using SW480 and HT29 cells. Further, we also measured hemoglobin and lactoferrin simultaneously from stool specimens of patients with CRC. RESULTS: The K-ras gene was successfully amplified from all healthy controls and CRC patients studied. Mutations of K-ras codon 12 were not detected in any of the healthy controls, but were identified in $41.9 \%$ of the patients with CRC. Among patients who were positive for mutations in tumor tissue, the detection rate in the stool was $84.6 \%$. Mutations were detectable in all six synthetic mutant DNAs, while none were detected among the wild type. The detection limit of this method was > or $=0.1 \%$. On the other hand, fecal hemoglobin and lactoferrin was detected $50 \%$ and $61.1 \%$ of patients with CRC respectively. CONCLUSIONS: One-step PCR/RFLP analysis to detecting K-ras point mutation could be used in mass screening for CRC, because it is highly specific, has a low detection limit, and is simpler than conventional methods for detecting genetic abnormalities. Because K-ras point mutations are associated with $40-50 \%$ of CRC, the detection sensitivity is low among cancers as a whole. However, our results suggests that the technique should provide a more sensitive tool for colorectal cancer screening, if used in combination with detection of fecal lactoferrin and hemoglobin.

\section{W1019}

Mail Return of Fecal Occult Blood Testing Is Low in a VA Population and Strongly Related to Race: A Retrospective Study

Deborah A. Fisher, Marcus R. Johnson, Nicholas J. Shaheen

Objectives: Fecal occult blood testing (FOBT) is the primary screening modality for colorectal cancer $(\mathrm{CRC})$ in the VA system. One shortcoming of FOBT is that it requires the patient to mail the completed stool cards. Our aim was to determine demographic predictors of FOBT card return in the VA system. Methods: We examined records of 500 consecutive patients at a single VA facility who had screening FOBT ordered in March 2004. All data were abstracted by trained personnel onto a standardized data abstraction form. The primary outcome variable was successful return of FOBT cards to the laboratory by 9 months after the original order (yes/no). Co-variates collected included patient age, gender, race, post office box (yes/no) and urban/rural status (by US census Metropolitan Statistical Area zip codes). Logistic regression analysis was used to evaluate potential predictors of FOBT card return. Results: The sample was 97\% men; 62\% Caucasian; 30\% African American (AA); $6 \%$ race unknown $<1 \%$ "other". We excluded the 31 patients with unknown race and the 6 patients with "other" race from analysis (final $\mathrm{N}=463$ ). The mean age was 64 years; $43 \%$ $<60 ; 26 \% 60-69 ; 23 \% 70-79 ; 7 \%>=80$. Only $46 \%$ of the patients had returned the FOBT cards at 9 months. Fifty-one percent $(51 \%)$ of Caucasians returned FOBT tests, compared to $37 \%$ of AA patients. The adjusted odds ( $95 \%$ confidence intervals) of FOBT return were $1.8(1.2-2.7)$ for Caucasian patients compared to AA patients. Logistic regression showed no significant association with FOBT return and age, gender, urban/rural status or having a post office box. Conclusions: Less than half of the primary care patients in this study sample returned FOBT cards and therefore did not benefit from CRC screening. Caucasian patients were almost twice as likely to return FOBT cards even as late as 9 months. No other patient predictors were found, including factors directly related to convenience of mail collection and delivery. Ordering an FOBT will inactivate the VA automatic CRC screening reminder for 6 months even if test is not completed, and counts the patient as successfully screened during that period. The poor return rate, particularly for AA patients, therefore inflates estimates of CRC screening utilization in the VA system.

\section{W1020}

Factors Influencing Colorectal Cancer Screening Among African-American and Caucasian Churchgoers

Christian S. Jackson, Colin W. Howden

Background: Colorectal cancer (CRC) is the second most common cause of cancer-related death in the United States. It disproportionately affects African-Americans compared to other racial and ethnic groups. One reason for this may be a lack of emphasis placed on screening by primary care physicians (PCPs). Currently, however, there are only limited data available. Methods: Several churches in the Chicago area were independently contacted. We provided questionnaires to parishioners who attended a pre-scheduled seminar on cancer screening. Parishioners did not have to complete the questionnaire to attend the seminar. All questionnaires were collected before the seminar; parishioners could not complete the questionnaire during or after the seminar. The questionnaire asked for information on age, sex, income, type of insurance, and knowledge of CRC and other cancers with high incidence and prevalence in African-Americans. The questionnaire also inquired about primary care recommendations in regards to CRC and other cancer-related screening. Results: 210 parishioners were given a questionnaire; all were collected. Of the 210 parishioners, 161 were African American and 49 were Caucasian. 117 (82 African-American and 35 Caucasian) parishioner had a PCP and were deemed eligible to receive age-appropriate CRC screening. Of the eligible African-Americans, $48 \%$ had annual income $<\$ 30,000.69 \%$ of the eligible Caucasian had annual income $>\$ 60,000$. $68 \%$ of the eligible African-Americans and $97 \%$ of the eligible Caucasians had health insurance. $59 \%$ of African-Americans and $91 \%$ of Caucasians had been offered colonoscopy as a means of CRC screening by their PCPs. $72 \%$ of AfricanAmericans and $97 \%$ of Caucasians stated that they were interested in colonoscopy as mean of CRC screening. Of the parishioners who were not eligible for age-appropriate CRC screening, $76 \%$ of African-Americans and $100 \%$ of Caucasians stated that they would interested in having colonoscopy for CRC screening. Conclusions: African-Americans were less likely to have been offered screening colonoscopy than Caucasians. PCPs, who are in position to positively influence utilization of appropriate screening practices, need to be more vigilant in offering CRC screening to African-Americans. There is high interest among African-Americans concerning appropriate CRC screening.

\section{W1021}

Is Chronic Disease a Barrier to Colorectal Cancer Screening? Maria Rosario Ferreira, Ahsan Arozullah, Marian L. Fitzgibbon, Nancy Dolan, Michael Wolf, Alfred W. Rademaker, Charles Bennett

Objectives: Colorectal cancer (CRC) screening reduces CRC-related mortality and is recommended for individuals $>=50$ years. It is not known if the presence of chronic disease affects CRC screening use by patients or providers. This study evaluated whether recommendation and adherence to CRC screening differ for veterans with chronic disease. Methods: Patients were males, >=50 years, attending a general medicine clinic, at average risk for $\mathrm{CRC}$, who had not had CRC screening. After 6-18 months from enrollment medical records were reviewed, for provider recommendations for CRC screening tests (fecal occult blood test, flexible sigmoidoscopy, or colonoscopy), completion of tests, demographics and comorbid illnesses (comorbidity index adapted from the Charlson index). Results: The mean age of 963 enrolled patients was $68,45 \%$ were white and 50\% African-American, and the average number of clinic visits per year was 3 . The Charlson index was 0 (no chronic disease) fo $31 \%$ of patients, 1 for $27 \%, 2$ for $21 \%, 3$ for $12 \%$ and 4 or higher for $9 \%$ of patients Higher Charlson scores were associated with increasing age, and greater number of yearly clinic visits, but there were no racial differences. Screening was recommended to $69.4 \%$ of patients and $32.4 \%$ completed screening. Screening recommendations and completion were lower for patients $>=75$ years. Patients with higher Charlson scores had a non-significant trend towards lower screening recommendation and completion rates. After adjustment for age, race and yearly clinic visits, patients with one or more comorbid illnesses were significantly less likely to receive a screening recommendation from their provider $(\mathrm{p}<0.01)$, compared to patients with no chronic disease. However, there was no significant difference in screening completion for patients with and without chronic illness. Implications: CRC screening recommendations declined as comorbidity scores increased, but CRC screening completion rates did not vary significantly. Based on these data it is not possible to determine whether providers and patients are making CRC screening decisions appropriately with regards to the presence of chronic illnesses.

\section{W1022}

Assessing Referral and Prioritisation Practices Using Standardised Recommendations in An Open Access Colonoscopy Service Dev S. Segarajasingam, Jacqueline M. Leber, Hooi C. Ee, Ian F. Yusoff

Introduction: Open access colonoscopy (OAC) has many potential advantages for service provision in an era of unprecedented demand for colonoscopy however inappropriate procedures and prioritisation remaining shortcomings. We aimed to i) Assess the adequacy of information provided in referrals for OAC ii) Develop standardised evidence-based recommendations for triaging patients referred for OAC iii) Validate this tool on a cohort of patients and compare this tool with current clinical practice. Methods: We reviewed the available literature and based on hospital policy we developed recommendations for i) the minimum information required to appropriately triage $\mathrm{OAC}$ referrals and ii) desirable interval to colonoscopy for common indications. Patients were classified into one of four categories with recommended interval to colonoscopy for each (Category $1:<30 \mathrm{~d}, 2: 30-90 \mathrm{~d}, 3$ $>90 \mathrm{~d}$ and 4: colonoscopy not indicated). Thereafter we used these recommendations to prospectively assess referrals and findinos at colonoscopy at our centre. Lastly we compared the performance of the standardised recommendation with current clinical practice measuring classification, interval to procedure and yield. Results: We studied 734 consecutive referrals. In total, $67.6 \%(494 / 734)$ of referrals did not contain the minimum information to allow appropriate prioritisation. The yield at colonoscopy by triage category is tabulated below The prioritisation interval given to the referral by the assessing clinician frequently varied from the recommended interval. Comparisons of Clinician versus Recommended triage categories and yield is presented below: (Insert table) Conclusions: A high proportion of referrals for $\mathrm{OAC}$ have insufficient information to allow accurate clinical prioritisation. The triage recommendation we have developed appears valid. Current clinical practice leads to heterogeneity in prioritising urgency of OAC. A standardised system for prioritisation may overcome some of these difficulties. 


\begin{tabular}{|c|c|c|c|c|c|c|}
\hline \multirow{2}{*}{$\begin{array}{c}\text { Category(Recommended } \\
\text { interval in days) }\end{array}$} & \multicolumn{4}{|c|}{$\begin{array}{c}\text { Physician triaged category } \\
\text { (Percentage of patients) }\end{array}$} & $\begin{array}{c}\text { Median } \\
\text { waiting time } \\
\text { (days) }\end{array}$ & $\begin{array}{c}\text { Significant } \\
\text { pathology(\%)* }\end{array}$ \\
\cline { 2 - 7 } & 1 & 2 & 3 & 4 & \\
\hline $1(<30)$ & $51.2 \%$ & $36.0 \%$ & $12.8 \%$ & $0.0 \%$ & 30 & 22.1 \\
\hline $2(30-90)$ & $9.0 \%$ & $41.1 \%$ & $49.9 \%$ & $0.0 \%$ & 92 & 1.4 \\
\hline $3(>90)$ & $3.5 \%$ & $20.8 \%$ & $75.7 \%$ & $0.0 \%$ & 183 & 3.9 \\
\hline 4 (No procedure) & $5.3 \%$ & $26.3 \%$ & $65.8 \%$ & $2.6 \%$ & 173 & 2.6 \\
\hline
\end{tabular}

*Significant pathology: CRC, colitis

\section{W1023}

Success Rates for Colorectal Cancer Screening Remain Disappointing Despite Open Access Colonoscopy

Shibu Oommen, Vijay Jayaraman, Catherine Messina, Joseph C. Anderson, Robert D.

\section{Shaw}

Background: Colorectal cancer (CRC) is the second most frequent cause of cancer related death in the United States. CRC screening has been proven to be effective yet less than half of eligible patients are screened. A number of factors play a role in suboptimal screening rates, including access to healthcare, poor patient acceptance, and failure of physicians to refer patients. Our VA center promotes colonoscopy as the screening test of choice and has open access for primary care referral of appropriate patients. The study was conducted in an outpatient clinic in a suburban setting. Aim: To determine CRC screening rates in response to institution of an open access program in which comprehensive screening modalities including colonoscopy, sigmoidoscopy, and FOBT testing are available to all eligible veterans. Methods: A review was performed of 1,373 patient visits to ten randomly selected primary care practitioners that occurred between 9/1/2004 and 11/31/2004. Patients were eligible for screening if they were between the ages of 50 and 80 and had no documented evidence of endoscopic screening within an appropriate interval. Referral rates for endoscopic screening and FOBT testing were recorded. Colonoscopy completion results were also recorded. Results: 196 patients were deemed to be screening-eligible at the time of a primary care visit (159 men, 37 women). All eligible veterans were given FOBT kits to return as part of CRC screening. Of the 196 patients, 104 (53\%) were not referred for endoscopic screening and $58(30 \%)$ refused. Thirty four patients were referred for endoscopic screening but only 23 actually had colonoscopy completed. Of the 173 patients who were not screened endoscopically, 49 had documentation of completion of home FOBT kits. Therefore 12\% of patients were screened endoscopically and $25 \%$ were screened by FOBT. Discussion: The surprising result of our study was that screening rates for colorectal cancer remained low despite open access to colonoscopy. It is likely that multiple factors are involved in the physician/patient interaction at the time of referral that play a role in suboptimal screening rates. Thus, dramatic improvements in colonoscopy screening rates will require a more thorough understanding of the variables patients and doctors bring to the decision making process. Exclusive emphasis on screening capacity without attention to the referral process will likely result in only modest improvements in screening rates.

\begin{tabular}{|c|c|}
\hline Patients eligible for screening & 196 \\
\hline Patients who completed colonoscopy & $23(12 \%)$ \\
\hline FOBT only completed & $49(25 \%)$ \\
\hline No screening documented & $124(63 \%)$ \\
\hline
\end{tabular}

\section{W1024}

Significance of Abnormal Findings On Abdominal CT Scan As Compared to Colonoscopy

Joseph Quagliata, Srikrishna Nagri, Krishna Kalakuntla, Anna Jagarlamudi, Laurel Bastone, Yashpal Arya, Sury Anand

AIM: To evaluate the positive predictive value of abnormal findings in the colon as detected by abdominal CT scan when compared to conventional colonoscopy. METHODS: We retrospectively reviewed 49 consecutive colonoscopies performed in adult ( $>18$ years of age) patients where the indication was the evaluation of abnormal colon findings (Colonic Wall Abnormality and Colonic Mass) during a period from April to September, 2005. RESULTS: More than 800 abdominopelvic CT scans were performed at our community hospital for various indications, 114 of which had positive findings (colonic wall abnormality or mass). Of these patients, 49 underwent colonoscopy at our endoscopy unit for inpatient/outpatient evaluation of same; $29(59.2 \%)$ female and $20(40.8 \%)$ male. The average age was 59.8 years (range, 22-88 years). The positive predictive value of the abdominopelvic CT scan findings was 55\% ( $95 \% \mathrm{Cl}=40.2-69.3$ ). This was significantly lower than the accepted rate of $80 \%(\mathrm{p}=0.0001)$. The PPV was NOT significantly different when we compared the anatomical location of the CT findings (right colon, left colon, diffuse). Biopsies of abnormalappearing colonic mucosa revealed the following: Non-specific inflammation (16), crypt abscess formation (3), pseudomembranous colitis (2), tubular adenoma (2), hyperplastic polyp (2), Invasive Adenocarcinoma (1), CMV Colitis (1). CONCLUSION: CT findings correlated with colonoscopic findings in only approximately half of the cases. While reliance on CT scan findings in planning patient management and colonoscopy may lead to unnecessary endoscopic work-ups in many patients, CT imaging is clearly a valuable tool in stratifying at-risk patients for further evaluation. Clinical suspicion and careful history, along with adjuvant and appropriate imaging, aid the clinician in determining pretest probability Positive Predictive Value (with 95\% CI) for Abdominopelvic CT as Compared to Colonoscopy

\begin{tabular}{|c|c|c|c|c|}
\hline CT DIAGNOSIS & $\begin{array}{c}\text { TOTAL } \\
\text { NUMBER }\end{array}$ & $\begin{array}{c}\text { TRUE } \\
\text { POSITIVE }\end{array}$ & $\begin{array}{c}\text { FALSE } \\
\text { POSITIVE }\end{array}$ & PPV (95\% CI) \\
\hline Colonic Mass/ Polyp & 4 & 4 & 0 & $\begin{array}{c}100 \% \\
(39.8-100)\end{array}$ \\
\hline $\begin{array}{c}\text { Colonic Wall } \\
\text { Abnormality }\end{array}$ & 45 & 23 & 22 & $\begin{array}{c}51 \% \\
(35.7-66.3)\end{array}$ \\
\hline TOTAL NUMBER & 49 & 27 & 22 & $\begin{array}{c}55 \% \\
(40.2-69.3)\end{array}$ \\
\hline
\end{tabular}

Positive Predictive Value (with $95 \% \mathrm{CI}$ ) by Location

\begin{tabular}{|c|c|c|c|c|}
\hline LOCATION & TOTAL NUMBER & TRUE POSITIVE & FALSE POSITIVE & PPV (95\% CI) \\
\hline Right Colon & 10 & 6 & 4 & $60 \%(26-88)$ \\
\hline Left Colon & 23 & 14 & 9 & $61 \%(38.5-80)$ \\
\hline Diffuse & 16 & 7 & 9 & $43.8(20-70)$ \\
\hline
\end{tabular}

\section{W1025}

Colonoscopy Demand and Practice in a Regional Hospital Over 9 Years in Hong Kong: Resource Implication for Cancer Screening Annie On On Chan, Wai Mo Hui, Chi Kuen Chan, Kam Chuen Lai, Wayne Hc Hu, Man Fung Yuen, Wai Man Wong, George Kk Lau, Chi Kin Hui, Shiu Kum Lam, Benjamin Cy Wong

Background: The incidence of colorectal cancer (CRC) in Hong Kong is rising. The trend of colonoscopy demand is uncertain. Aim: To investigate colonoscopy demand and practice in a Hong Kong regional hospital over the past nine years. Methods: Colonoscopy data from lst January 1997 to 31st August 2005 were retrieved and divided into two equal periods for comparison. Colonoscopy practice and findings between the two periods were compared. Results: There was no change in the number of endoscopists and colonoscopy sessions in the two periods. The number of colonoscopy done in the two periods was 2681 and 2871 , respectively. The indications for screening of CRC ( $2.8 \%$ vs $9.7 \%, \mathrm{P}<0.0001)$ and surveillance of colonic adenomas $(11.2 \%$ vs $25.9 \%, \mathrm{P}<0.0001)$ were increased, but decreased for diarrhea ( $18 \%$ vs $10.2 \%, \mathrm{P}<0.0001)$ and per rectal bleeding ( $19 \%$ vs $8.1 \%, \mathrm{P}<0.0001)$. The waiting time was lengthened from 2 weeks to 4 weeks $(\mathrm{P}<0.0001)$. The percentage of colonic adenomas $(19.9 \%$ vs $27.2 \%, \mathrm{P}<0.0001)$ was increased. A right-shift was observed in both CRC ( $37 \%$ vs $50 \%, \mathrm{P}=0.018$ ) and adenoma ( $21.6 \%$ vs $38.1 \%, \mathrm{P}<0.0001)$. Conclusion: The number of colonoscopies performed was governed by capacity but not demand. Ways to improve capacity for colonoscopies is needed.

\section{W1026}

Predictors of Failed Colonoscopy in Nonagenarians: A Single-Center Experience

Eliahu Shemesh, Hemda Schmilovitz-Weiss

Goals and Background: Data on the benefits of advanced medical technologies in very old patients remain limited. The aim of the study was to evaluate the success rate, safety and outcome of colonoscopy in nonagenarian patients. Study: The success and safety of colonoscopies performed during the last 5 years in our center were compared between 46 nonagenarians (group 1) and two control groups: 50 consecutive patients aged 70-79 years (group 2) and 50 consecutive patients aged 50- 59 years (group 3). The medical records were reviewed for background data, blood test results, co-morbidities, and mental and functional status at the time of colonoscopy. Chi-square test was used to detect differences in categorical variables by failure or age group. Failure was modeled using logistic regression analysis, and odds ratios with $95 \%$ confidence intervals were calculated. All tests were twosided and considered significant at $\mathrm{p}<0.05$. Results: Older age, anemia as the indication for colonoscopy, poor functional and mental status, and low serum hemoglobin and albumin levels were significantly associated with failure to complete colonoscopy. On logistic regression analysis, only serum albumin retained significance as a predictor of failed colonoscopy (OR 0.13, 95\% CI 0.02-0.6, p=0.009). Malignant findings were more frequently observed in the oldest patients. Conclusions: Serum albumin is significantly associated with failed colonoscopy in elderly frail patients. These patients should be offered other, less invasive diagnostic modalities such as virtual colography, with conventional colonoscopy reserved for therapeutic purposes. Larger, prospective, well-designed studies are needed to investigate this issue.

\section{W1027}

Natural History of CRC in France: A Population-Based Study in Private Practice

Pienkowski Patrice, Houcke Philippe, Lledo Gerard, Papazian Arsene, Richard-Mollard Bruno, Rouillon Jean-Michel, Vaillant Eric

The aim of this study was to define the natural history of Colorectal carcinoma (CRC) and its management in french population by private Gastroenterologists (PG). Material \& methods. All french PG were asked to participate the study and include all the patients seen during one week (march 14-19th, 2005) for CRC whatever the circumstances (out-patients, diagnosis, colonoscopy, follow-up) designed as "medical events". 441 PG (22,4\% of the target) included 1250 patients. The PG sample was representative according to age, sex, geographic area and medical practice; the patients sample was comparable to populationbased data from french cancer registries. Extrapolation of crude data of this 1250 patients cohort was performed to the entire population of $\mathrm{PG}$ and for one year on the basis of 44.5 worked weeks. Results. 5363 " medical events » were recorded, that is to say 238654 per year, including 29799 (12\%) for diagnosis, 15001 (6,2\%) for stagging, 31.645 (13,2\%) for 
treatment and $149044(62,4 \%)$ for follow-up. 6288 new cases were diagnosed after screening (21\%) because of familial history of CRC or polyps $(7.1 \%)$, positive Fecal Occult Blood Test $(8.3 \%)$, or mass population screening (1.5\%). The total number of colonoscopies performed each year to patients with CRC was 80058. Colonoscopy had been previously performed in 7300 incident cases (24.5\%) including 1250 cases within the year (6\%) (mean-time : 19 months). Stagging was set on ultrasonography (85.5\%), CT-scan $(64.8 \%)$, CEA $(82.4 \% \%)$ and chest X-ray $(65.7 \%) ; 62 \%$ of the medical files were discussed in pluridisciplinary sessions before treatment. Stagging was : Stage I : $34,3 \%$, stage II : $27,6 \%$, stage III : $24,8 \%$ and stage IV : $13,2 \%$. Surgery was performed in $95.4 \%$ of the patients ; adjuvant chemotherapy (CT) was performed in $68.5 \%$ of the cases (Stage III : $57,4 \%$, stage II : $30 \%$ ) and palliative CT in $55.2 \%$ (including second line CT in $17.6 \%$ ); continuous infusion of fluorouracile $(5 \mathrm{Fu})+$ leucovorine was the most common regimen $(41.3 \%)$ followed by 5Fu+Oxaliplatin 27.7\%), 5Fu+Irinotecan (19.7\%) and bolus 5Fu+leucovorin (16.4\%); oral $5 \mathrm{Fu}$ was used in $5.8 \%$ of the patients and Cetuximab in $4.8 \% ; 4.2 \%$ of the patients participated to randomized studies. Follow-up was managed by PG in $83.6 \%: 25.3 \%$ of the patients were followed every 3 months and $41.2 \%$ every 6 months. Prevalence was 203.516 patients. Conclusion. PG in France are responsible for the diagnosis of 30.000 new cases of CRC each year and for the management of 200.000 patients. These results emphasise their position in health care system and give clinical data on natural history of CRC and therapeutic strategies in a large non selected western population.

\section{W1028}

Cost-Effectiveness of mesalamine (5-aminosalicyclic acid, 5-ASA) Therapy for Maintenance of Remission in Ulcerative Colitis (UC)

Eugene F. Yen, Sunanda V. Kane, Uri Ladabaum

BACKGROUND: 5-ASA is effective in the induction and maintenance of remission in UC. The relative benefits and costs of maintenance 5-ASA therapy are uncertain. Sulfasalzine has similar efficacy to 5-ASA, but may be less well tolerated. Our aim was to evaluate the cost-effectiveness of maintenance 5-ASA therapy in UC. METHODS: We constructed a Markov model of disease activity and treatments in patients with mild to moderate UC over 2 yrs. We examined 2 arms: 1) No maintenance 5-ASA, with 5-ASA $4.8 \mathrm{~g}$ /day used after a flare to attempt to induce remission; 2) Maintenance 5-ASA $2.4 \mathrm{~g} /$ day, escalated and maintained at $4.8 \mathrm{~g} /$ day after first flare. In both arms, failure to induce remission led to other treatments as needed: prednisone, parenteral corticosteroids, cyclosporine, colectomy. After 2 courses of prednisone, 6-mercaptopurine was given. The model yielded flares/person, cost/person and cost/flare prevented. To place this estimate in perspective, we assigned health state utilities and calculated cost/quality-adjusted life-year (QALY) gained. Inputs included: risk of flare without 5 -ASA $=25 \% / 3 \mathrm{mo}$; relative risk (RR) of flare on maintenance 5 -ASA $=0.7$; flares requiring immediate hospitalization $=10 \%$; remission rate with 5 -ASA $4.8 \mathrm{~g} / \mathrm{day}=71 \%$; cost of 5 -ASA $2.4 \mathrm{~g} / \mathrm{day}=\$ 190 / \mathrm{mo}$, outpatient flare $=\$ 60$, inpatient flare $=\$ 5,500$, colectomy= $\$ 11,300$; utility of no maintenance 5 - $A S A=0.972$, maintenance 5 - $A S A=0.977$, outpatient flare $=0.793$, inpatient flare $=0.608$, status post colectomy $=0.970$. RESULTS: Without maintenance 5-ASA, mean flares/person were 1.77 and mean cost/person was $\$ 4,200$ over 2 yrs. With maintenance 5-ASA, flares/person decreased to 1.23 at a cost of $\$ 8,800 /$ flare prevented. Maintenance 5-ASA increased QALYs/person from 1.90 to 1.92 at a cost of $\$ 250,000 / \mathrm{QALY}$ gained. SENSITIVITY ANALYSES: At the lower bound for RR of flare on 5-ASA (0.51), maintenance 5-ASA cost $\$ 4,600 /$ flare prevented. If the utility of outpatient flare fell to 0.608 (equal to inpatient flare) the cost of maintenance 5-ASA decreased to $\$ 155,000 / \mathrm{QALY}$ gained. If sulfasalazine $2 \mathrm{~g}$ (or $4 \mathrm{~g}$ ) could be tolerated instead of 5 -ASA $2.4 \mathrm{~g}$ (or $4.8 \mathrm{~g}$ ) at a cost of $\$ 15$ (or $\$ 30) / \mathrm{mo}$, it cost $\$ 670 /$ flare prevented and $\$ 19,000 /$ QALY gained. CONCLUSION: Maintenance 5-ASA therapy decreases UC flares, but the cost/flare prevented may be significant, depending on society's willingness to pay. The cost/QALY gained with maintenance 5ASA therapy may be substantial unless improvements in quality of life are more dramatic than we assumed, or life expectancy is also improved. If a less costly alternative (sulfasalazine) were tolerated and yielded the same benefits, maintenance therapy is likely to be considered cost-effective

\section{W1029}

\section{Adverse Events Leading to Modification of Therapy in a Large Cohort of} Azathioprine-Treated Patients with Inflammatory Bowel Disease Ulf Hindorf, Malin Lindqvist, Hans Hildebrand, Ulrika Fagerberg, Sven Almer

Background \& aims: Adverse events (AE) leading to discontinuation or dose-reduction of thiopurine therapy occur in $9-28 \%$ of patients with inflammatory bowel disease (IBD). The aim of this retrospective study was to evaluate the influence of thiopurine methyltransferase (TPMT) status and thiopurine metabolites in a large patient population for the risk of developing AE. Methods: Three-hundred-and-sixty-four patients with IBD and present or previous thiopurine therapy were identified from a local database. Results: The AE observed in 124 patients (32\%) were more common in adults than children ( $40 \%$ vs. $15 \% ; \mathrm{p}<0.001$ ) and in low to intermediate $(\leq 9.0 \mathrm{U} / \mathrm{ml} \mathrm{RBC})$ than normal TPMT activity $(\mathrm{p}=0.02)$. Myelotoxicity developed later than the other types of $\mathrm{AE}$. Methylated thioinosine monophosphate (meTIMP) were significantly higher in myelotoxicity [2650 vs. $1500 \mathrm{pmol} / 8 \mathrm{x} 10^{\wedge} 8 \mathrm{RBC} ; \mathrm{p}=$ 0.03 ] and thioguanine nucleotide (TGN) in gastrointestinal intolerance [ $332 \mathrm{vs.} 170 \mathrm{pmol} /$ $\left.8 \times 10^{\wedge} 8 \mathrm{RBC} ; \mathrm{p}=0.02\right]$, when compared to patients without AE. An increased frequency of AE was observed in patients with TGN above 400 or meTIMP above $11450 \mathrm{pmol} / 8 \times 10^{\wedge} 8$ RBC. A shift to 6-MP was successful in $47 \%$ of AZA-intolerant patients and in all cases of AZA-induced myalgia or arthralgia. Conclusions: A pre-treatment determination of TPMT status might be validated as patients with low to intermediate TPMT activity are more prone to develop AE, while determination of metabolite levels can be useful in selected cases as myelotoxicity or gastrointestinal intolerance. 6-MP therapy should be considered in AZAintolerant patients.

\section{W1030}

The Efficacy and Remission Time of Intravenous Cyclosporin in Patients with Severe Ulcerative Colitis Are Increased When Given Immediately After An Onset Or An Exacerbation

Naoki Yoshimura, Yasuo Suzuki, Masakazu Takazoe

BACKGROUND \& OBJECTIVE: For decades patients with severe ulcerative colitis (UC) have been treated with steroids followed by colectomy in many patients who failed. However in recent years cyclosporin (CsA) has been used as an alternative medical therapy in patients with severe steroid refractory $\mathrm{UC}$ and is proved to be effective in reducing colectomy rate in patients who may need surgery, but up to $66 \%$ patients who initially respond to CsA relapsed within 9 months following start of CsA therapy. Therefore, a more sustainable CsA induced remission time is desirable. The objective of this study was to explore strategies which could increase the efficacy and the remission time of CSA in patients with severe UC. METHODS: Between 1997 and 2005, 55 patients with UC, 24 female, 31 male, mean age 34.2 years, range 13 to 75 years received intravenous (iv) CsA ( $3-4 \mathrm{mg} / \mathrm{kg} /$ day). These patient were retrospectively evaluated. Nineteen patients had received iv CsA as monotherapy (without iv steroids) or received CsA within 7 days of concomitant iv steroid, $20-40 \mathrm{mg} / \mathrm{day}$ (early CsA therapy group). Thirty-six patients who had failed to respond to at least 7 day of iv steroid (40-60 mg/day) received iv CsA with concomitant iv steroid (late CsA therapy group). The mean age and initial clinical activity scores on admission were similar in both groups. Patients whose condition did not improve after 14 days of CsA treatment underwent colectomy. Patients who responded to CsA were discharged on a regimen of oral CsA or oral CsA plus oral steroid (both were to be tapered) together with 5-aminosalicylate and were followed up clinically and endoscopically for at least 18 months. Long-term outcome was evaluated by Kaplan-Meier survival analysis. RESULTS: Overall, 36 of 55 patients (65.5\%) had a response within 14 days and avoided colectomy. Further, 17 of 19 patients (89.5\%) in the early CsA therapy group achieved remission compared with 19 of 36 patients $(52.8 \%)$ in the late CsA therapy group. Similarly, the mean remission time was 31.9 months in the early CsA therapy group versus 13.8 months in the late CsA therapy group $(\mathrm{P}<0.01)$. At 18 months, 11 of 17 patients (64.7\%) in the early CsA therapy group had maintained their remission versus 3 of $19(15.7 \%)$ in the late CsA therapy group $(P<0.01)$. CONCLUSIONS: The use of iv CSA in an early stage during severe UC has significantly better outcomes compared with waiting until conventional intensive steroid therapy has failed.

\section{W1031}

Sargramostim Improves the Health-Related Quality of Life of Crohn's Disease Patients: Sf-36, IBDQ, and VAS Eq-5d Findings Brian K. Dieckgraefe, Joshua R. Korzenik, Suzanne Laplante

INTRODUCTION: Quality of life (QOL) assessments are important for evaluating therapeutic agents in Crohn's disease (CD). Sargramostim (SRG) is an innate-immunity activator bein studied in clinical trials as a potential new CD therapy. Unlike current treatments that suppress adaptive immunity, SRG enhances the innate immune system. We investigated the effect of SRG on the QOL of CD patients (pts) using the Short-Form 36 Health Survey (SF36), the Inflammatory Bowel Disease Questionnaire (IBDQ) and the Visual Analogue Scal of the EuroQoL (VAS EQ-5D). AIM: To quantify the effect of SRG on the QOL of pts with moderately to severely active CD using the SF-36, IBDQ and VAS EQ-5D. METHODS: A placebo-controlled trial was conducted in pts with moderately to severely active CD. 124 pts were studied: 43 on placebo; 81 on SRG $6 \mu \mathrm{g} / \mathrm{kg} /$ day. The SF-36, IBDQ, and VAS EQ5D were completed at baseline; at days 15, 29, 43, and 57 of treatment; and at 30 days after treatment. Differences between responder/non-responder groups as well as remission/ non-remission groups were quantified using SF-36 and IBDQ scores (response=reduction in $\mathrm{CDAI} \geq 100$; remission=CDAI $\leq 150$ ). Differences between treatment groups were assessed using the VAS EQ-5D. RESULTS: SRG treatment yielded significant improvements to the QOL of CD pts. According to the SF-36, the physical \& mental component summary scores of responders improved by $34 \%$ ( $12 \pm 8$ points) and $27 \%$ ( $10 \pm 11$ points), respectively, vs improvements of $4 \%(1 \pm 5$ points) and $-3 \%(-1 \pm 9$ points) in non-responders. Responder improved in all individual SF-36 domains (responders:30--300\% vs non-responders:-11$9 \%)$. According to the IBDQ, responders experienced an average improvement in total scores of $42 \%(52 \pm 27$ points) vs $6 \%$ in non-responders ( $7 \pm 21$ points). The bowel, systemic, emotional \& social scores improved $36--56 \%$ in responders vs $3--10 \%$ in non responders. Similar SF-36 and IBDQ findings were observed when comparing SRG vs placebo pts (results described elsewhere) as well as remission and non-remission pts. VAS EQ-5D measurements indicated that SRG induced significant $\mathrm{QOL}$ improvements observed soon after treatment initiation. SRG pts reported significant improvements that varied 18--30\% from baseline while placebo pts reported improvements that varied $0--11 \%$. CONCLUSION: SRG is an innate immunity activator that, unlike current treatments that suppress the immune system, enhances the function of the innate immune system. SF-36, IBDQ and VAS EQ-5D measurements indicated that CD pts treated with SRG experienced significant QOL improvements The improvements were seen early and were maintained throughout the treatment and follow-up periods.

\section{W1032}

Crohn's Disease Patients Treated with Sargramostim Are More Able to Attend Work/School and Leisure/Sport Activities As Measured By Questions 4 and 12 of the IBDQ Brian K. Dieckgraefe, Suzanne Laplante, Georg Groetzbach

INTRODUCTION: Sargramostim (SRG) is an innate-immunity activator under study as a potential new therapy for Crohn's disease (CD). Unlike current treatments that suppres the immune system, SRG enhances the function of the innate immune system. In clinical trials, SRG has been found to induce clinical response and remission of symptoms in patients with moderately to severely active $\mathrm{CD}$. We report on an investigation performed to assess the effect that SRG has on two important aspects of the quality of life (QOL) of CD patients AIM: To assess the effect that SRG treatment has on the ability of $\mathrm{CD}$ patients to attend 
work/school or to perform leisure/sport activities. METHODS: The effect of SRG treatment on the QOL of patients with moderately to severely active $\mathrm{CD}$ was studied using a randomized placebo-controlled trial involving 124 patients (43 received placebo (PBO); 81 received SRG $6 \mu \mathrm{g} / \mathrm{kg} /$ day). The impact of treatment on the patients' ability to attend work $/ \mathrm{school}$ or to carry out leisure/sport activities was estimated using the Inflammatory Bowel Disease Questionnaire (IBDQ) social subscore questions 4 (work/school attendance) and 12 (leisure/ sport activities attendance). These questions were completed at baseline; at days 15, 29, 43, and 57 of treatment; and at 30 days after treatment. RESULTS. SRG induced response (CDAl reduction $\geq 100$ ) in $48 \%$ of patients and remission (CDAI $\leq 150)$ in $40 \%$ of patients by the end of the treatment period. These rates were significantly different from the rates obtained with $\mathrm{PBO}(26 \%$ response $[\mathrm{p}=0.0134]$ and $19 \%$ remission $[\mathrm{p}=0.0135])$. Changes from baseline in total IBDQ score as well as in 3 of its 4 subscores were significantly greater in the SRG group than in the PBO group ( $\mathrm{p}=0.0001-0.0397$ ). At baseline, question 4 revealed that patients were not able to attend work/school a little or some of the time while question 12 revealed that patients had some to fair amount of difficulties doing leisure/sport activities. The score to question 4 improved by up to $25 \%$ (1 point) in the SRG group compared to 0.6 points $(13 \%)$ in the PBO group. Similarly, the score to question 12 improved by up to $42 \%$ ( 1.4 points) in the SRG group compared to $23 \%$ ( 0.8 points) in the PBO group. Overall, the social score improved by 1.4 points (42\%) in the SRG group compared to 0.9 points (23\%) in the PBO group ( $\mathrm{p}=0.0288$ ). CONCLUSIONS: SRG treatment significantly improved the ability of CD patients to attend work/school and perform leisure/sport activities. SRG is an innate-immunity activator that, unlike current treatments that suppress the immune system, enhances the function of the innate immune system.

\section{W1033}

Quality of Life Indices Correlate with Clinical As Well As Endoscopic Activity in Patients with Ulcerative Colitis

Alexandra Zahn, Ulf Hinz, Max Karner, Robert Ehehalt, Wolfgang Stremmel

Background \& Aims: Inflammatory bowel disease (IBD) impairs health-related quality of life (HRQOL). Our aim was to investigate if the improvement of the Clinical Activity Index (CAI) and the Endoscopic Activity Index (EAI) is significantly correlated with the advancement of the overall HRQOL and its dimensions and to assess in what way demographic and disease-related factors influence patients experience of HRQOL. This examination was performed in the context of our recently published study of the anti-inflammatory effect of retarded release oral phosphatidylcholine (PC) in chronic active ulcerative colitis (UC). Methods: 60 patients with chronic active UC and with a CAI of $>4$ were treated with retarded release $\mathrm{PC}$ or placebo over three months. The patients had to fill in the Inflammatory Bowel Disease Questionnaire-Deutschland (IBDQ-D) concerning HRQOL before and after the study. The correlation between the CAI and EAI improvement and the quality of life scores was calculated. Demographic factors and disease-related factors were obtained. Results: A statistically significant lowering of CAI and EAI after treatment in the PC group led to a statistically significant improvement of the HRQOL $(r=-0.623, p=0.0003$ for the CAI and $r=-0.511, p=0.005$ for the EAI). Constant disease activity indices in the placebo group came along with constant HRQOL $(r=-0.747, p<0.0001$ for the CAI and $r=-0.634, p=$ 0.0002 for the EAI). Furthermore, besides a few exceptions, significant correlations between CAI and EAI and the 4 dimensions of the IBDQ-D could be shown. In our study, demographic parameters such as age, gender, disease duration and disease localization did not significantly influence the IBDQ-D scores. Conclusion: This study points out the strong relationship between clinical and endoscopic activity indices and all domains of HRQOL in patients with UC. Therefore, the IBDQ-D is a valid and reliable assessment tool that reflects changes in the health status of UC patients. It is a useful measure of therapeutic efficacy and should be used in all clinical trials in IBD.

\section{W1034}

Sick Leave, Unemployment, Disability and HRQOL in Patients with Inflammatory Bowel Disease

Tomm Bernklev, Magne Henriksen, Jorgen Jahnsen, Morten Vatn, Bjorn Moum

Background: Chronic diseases like IBD is connected with increased morbidity, like absence from work and disability pension (DP). Information regarding these issues in Norwegian IBD patients has not been available. Aim: To determine the rate of work disability, unemployment (UE) and sick leave (SL) in an unselected, Norwegian IBD-cohort and additionally to measure the effect of working status and DP on the patient's health-related quality of life. Patients and Methods: Data regarding SL last six months was collected at the 5-year follow up visit. Patients were also asked if they were unemployed or if they were receiving DP as a consequence of their IBD. Data from the Norwegian background population regarding SL, UE and DP were collected from Statistics Norway. HRQOL were determined with SF-36 and N-IBDQ. All together 495 patients were or had been in the work force during the 5 year follow up period since diagnosis and were included in the analysis, mean age 41.2 (13.7), 49\% females. Results: A total of 42 patients (8,5\%) were on DP five years after diagnosis, 18 (5,4\%) with UC and 24 (14,9\%) with CD. Females with $\mathrm{CD}$ had the highest DP rate, 17 (24,6\%). Disability rates from the background population were 8,8\%. A total of 58 patients $(11,7 \%)$ reported to be unemployed at 5 years. This was equally distributed between males and females, but more patients with UC were unemployed, $44 / 334(13,2 \%)$ compared to CD patients, $14 / 161$ (8,7\%). The unemployment rates in the Norwegian population were $4,1 \%$. SL for all causes was reported in $47 \%$ (143/306) with UC and 53\% (70/132) with CD. IBD-related SL was reported by $18 \%$ (54/306) with UC and 23\% (31/ 132) with $C D$. A majority of the patients were sick less than four weeks, while $25 \%$ contributed to a large number of the total sick leave days. UE and DP reduced HRQOL scores both in UC and CD patients. However, the most pronounced effect on HRQOL was found in patients reporting IBD-related sick leave, measured with N-IBDQ and SF-36, with reduction in scores from $9 \%$ to $40 \%$. Conclusion: Unemployment or sick leave is more common in IBD patients than in the Norwegian background population. The number of patients receiving DP is significantly increased in females with $C D$, but not in the other patient groups. Unemployment, sick leave and DP all affects the patients HRQOL in a negative way, but this effect is most pronounced in patients reporting IBD-related sick leave.

\section{W1035}

Quality of Life in Crohn's Patients with Perianal Fistulas: Influence of Fecal Diversion

Michael S. Kasparek, Joerg Glatzle, Tanja Temeltcheva, Mario H. Mueller, Alfred Koenigsrainer, Martin E. Kreis

Perianal fistulas in Crohn's disease are often refractory to medical and surgical treatment. Fecal diversion may be necessary with the potential of impaired quality of life (QOL). Our aim was to determine QOL in Crohn's patients with perianal fistulas who live with or without fecal diversion. We hypothesized that QOL is impaired in diverted patients. METHODS From 1996 through 2002, 116 Crohn's patients were treated for perianal fistulas at our institution. Four validated QOL instruments were sent to each patient, including the SF36, Gastrointestinal QOL Index (GIQLI), Cleveland Global QOL score (CGQL), Short Inflammatory Bowel Disease Questionnaire (SIBDQ). Additionally we sent our standardized questionnaire of alterations in everyday life. 77 questionnaires (66\%) were returned. Patients age (median [range]) was 41 years [21-76 y]. RESULTS: At time of evaluation, 34 of the 77 patients were diverted, while 43 were not. Diverted patients had a longer duration of Crohn's disease ( 19 years $[2-32$ y] vs. 15 years $[3-29$ y]; $\mathrm{p}<0.05$ ). Follow-up was similar in both groups (diverted 49 months [18-97 mo] vs. no diversion 44 months [14-98 mo]) No difference was observed in the SF-36 and CGQL as regards QOL in both groups. The GIQLI demonstrated a better overall QOL $($ mean \pm SEM; $68 \pm 1 \%$ vs. $60 \pm 2 \% ; \mathrm{p}<0.05)$ and better QOL on the subscale "gastrointestinal symptoms" $(81+1 \%$ vs. $67+2 \% \cdot p<0.05)$ in the diverted group. Similarly the SIBDQ also revealed a better QOL for diverted patients on the subscale "bowel" ( $81 \pm 1 \%$ vs. $67 \pm 2 \%$; $<<0.05)$. Diverted patients were less likely to complain of symptoms from their Crohn's disease (44\% vs. 79\%; p<0.05). Concerning alterations of everyday life, as evaluated by our standardized questionnaire, 10 of the 34 diverted patients reported no restrictions secondary to their diversion, and 19 experienced no difference compared to before diversion; only 5 patients reported deterioration of activities by the diversion. CONCLUSION: Overall QOL does not deteriorate when fecal diversion is required in Crohn's patients with perianal fistulas and may even improve certain subscales of QOL.

\section{W1036}

A Systematic Review of the Quality of Patient Information On Medical Treatment of Crohn's Disease and Ulcerative Colitis On the World Wide Web Morgan Langille, Chris Rodgers, Andre Bernard, Sander van Zanten

M Langille, C Rodgers, A Bernard, S Veldhuyzen van Zanten, Dalhousie University, Halifax Background: Increasing numbers of patients with inflammatory bowel disease (IBD) use the Internet to obtain health information on treatment of their disease. Study aim:to perform a systematic review on the quality of educational information on treatment of IBD for patients available on the web. Methods: medical treatments evaluated were:5-ASA, corticosteroids, azathioprine/6-MP, infliximab and other immune suppressants. A Quality Evaluation Instrument (QEI) was developed and pretested which covered indications, efficacy and common or serious side-effects. The maximum possible QEI score was 101. A global overall quality score (1-5) and reading grade level was also given. Each site was evaluated for integrity, that is information about funding and ownership. Each website was scored by two independent reviewers. Results: the top 50 sites retrieved by Google were evaluated by two independent raters. 7 sites were excluded because they were web portals. The average global score was 2.1, 2 sites received a score of 5 , and 2 sites a score of 4 . The average QEI was 36 (range $0-96), 6$ sites received a score of $>70$ and 13 sites received score $>50$. QEI scores were not related to rank order in Google. Information on infliximab was lacking in 19 of 43 websites. The average Flesch-Kincaid Grade Level was 11.4, ranging from 6.9 to 12.0. Only 24 sites gave a date when the site was last updated. Only 23 sites did disclose the funding source. Conclusion: the quality of information on treatment available for IBD patients on the Web shows marked variation and only a small number of sites achieve high quality scores. There is an urgent need for accreditation of websites that provide balanced information about treatment for IBD

\section{W1037}

Calcium and Vitamin D Supplementation for Bone Loss in Children with IBD: Does It Help?

Yoram Elitsur, Shaista Safder, Peter Chirico

Patients with inflammatory bowel disease (IBD) are at a high risk for bone fractures due to increased bone loss. Osteopenia and osteoporosis have been reported in children prior to and after steroid therapy. The current treatment protocol included calcium and Vit. D supplementation. Unfortunately, the efficacy of this treatment in children has not been established. Aim: To evaluate the efficacy of calcium and Vit. D supplementation for bone loss in children with IBD, after more than 1 year of therapy. Methods: Children with IBD were supplemented with $\mathrm{Ca}(1.5 \mathrm{~g} / \mathrm{d})$ and Vit. D $(800 \mathrm{IU} / \mathrm{d})$ for over 1 year. Bone mineral density (BMD) and bone age were performed before and after $>1$ year of therapy. Bone age was assessed radiographically, and BMD was assessed by Dual energy X-ray absorptiometry (DEXA). Osteopenia was defined as BMD Z-score between -2.5 to -1.0 , and osteoporosis as Z-score below -2.5. Serum levels of calcium, urea, and creatinine were available at baseline and at the end of the study in all patients. The BMD data was compared between baseline and at 1 year treatment for each patient separately. Results: A total of 31 children with IBD were retrospectively identified with a mean age of 15.4 years. 17 children had CD $(12 \mathrm{M} / 5 \mathrm{~F})$, and 14 had UC (7M/7F). At baseline, among the $17 \mathrm{CD}$ patients, $3(17 \%)$ had osteoporosis, 8 (47\%) osteopenia, and 6 (35\%) patients had normal BMI; and of the $14 \mathrm{UC}$ patients, 5 (36\%) had osteopenia and 9 (64\%) had normal BMI. Fourteen patients completed over 1 year of therapy $(9 \mathrm{CD} / 5 \mathrm{UC})$, of whom 3 pts $(2 \mathrm{CD}, 1 \mathrm{UC})$ had normal BMD at both measurements. Of the 11 children with abnormal BMD, 8 (73\%; 4 CD/ 4 UC) showed improved BMD, 2 (18\%; CD) worsened, and 1 (9\%; CD) was unchanged. Serum calcium, urea, and creatinine levels were normal in all patients throughout the study period. Conclusion: In $73 \%$ of our patients, calcium and Vit. D supplementations improved BMD in children with IBD. No significant difference in bone loss was observed between CD and UC patients (65\% 
vs. $36 \%$ respectively, $\mathrm{p}=0.21$ ). Larger studies will be needed to substantiate the efficacy of $\mathrm{Ca}$ and Vit. D in reversing bone loss in children with IBD.

\section{W1038}

Genotype-Phenotype Analysis in a New Inception Cohort of Inflammatory Bowel Disease in Copenhagen 2003-2004: A Study from the Danish Colitis Crohn Database

Ida Vind, Cathrine Jespersgaard, Lene Riis, Paal S. Andersen, Pia Munkholm

Background: The Danish Colitis Crohn Database (DCCD) was established in the year 2000, aiming to assure national quality of diagnosis, treatment and prognosis in patients with inflammatory bowel disease (IBD). By now, the database is implemented at all departments of gastroenterology and paediatrics in Copenhagen County and Community. Methods: All residents from this region, who were diagnosed, in accordance to strict international criteria, with Crohn's disease (CD), ulcerative colitis (UC), or indeterminate colitis (IC) during the period 1/1 2003 to 12/31 2004, were included in a prospective population based incidence cohort. The background population counted 1.21 million inhabitants. Polymorphisms were identified by single strand conformation (SSCP) analysis (CARD15 - SNP8, 12 and 13; IGR2078a_1 - rs4705950); Taqman analysis (SLC22A4 - rs1050152; DLG5 - rs2289310) or restriction fragment length polymorphism (RFLP) (SLC22A5 - rs2631367). Results: A total of 562 patients (209 CD, 326 UC, 27 IC) were diagnosed with IBD during the twoyear period. The incidences increased significantly and were 8.6/105 for CD and 13.4/105 for UC. Three hundred and forty-seven Caucasian IBD patients (156 CD and 191 UC) were available for the genetic analyses, 755 healthy persons served as controls. Fourteen percent of $\mathrm{CD}$ patients were positive for at least one CARD15 mutation vs. $11 \%$ among controls (NS). The risk associated with the TC haplotype (OCTN-TC) was only observed in homozygotes, which were significantly more frequent among CD patients vs. controls ( $24 \%$ vs. $17 \%, \mathrm{p}=0.03$; OR 1.61 ( $95 \% \mathrm{CI}: 1.06-2.43)$ ). No differences were found between UC and controls or between CD and UC. Neither did we find any differences between mutations in the DLG5 gene in CD vs. controls or vs. UC patients. Extent of $\mathrm{CD}$ at diagnosis, according to the Vienna Classification, was L1 (terminal ileum) in $31 \%$ of cases, L2 (colon) in $38 \%$, L3 (ileocolon) in 23\%, and L4 (upper GI) in $8 \%$ of cases. In CD no particular disease location was associated with mutations in CARD15 or OCTN haplotype. Conclusion: The prevalence of at least one CARD15 mutation among $C D$ patients from an unselected cohort is not different compared to healthy controls or newly diagnosed UC patients. A difference seems to exist between presence of the homozygous TC haplotype (OCTN-TC) in CD patients vs. controls. Albeit no genotype- phenotype relation was found concerning localisation.

\section{W1039}

Environmental Factors Associated with Crohn's Disease: A Population-Based Case-Control Study

Richard B. Gearry, Ann Richardson, Christopher M. Frampton, Judith A. Collett, Andrew Dodgshun, Bruce A. Chapman, Michael J. Burt, Murray L. Barclay

Introduction: The rapid increase in the incidence of Crohn's disease (CD) in developed countries is likely to be due to exposure to environmental factors in genetically susceptible individuals. Aim: To identify environmental factors associated with $C D$ in a populationbased cohort. Methods: 1421 IBD patients ( 715 CD, 668 ulcerative colitis (UC)) were recruited representing over $91 \%$ of people with inflammatory bowel disease in Canterbury, New Zealand (population 464,800). This is part of the Canterbury IBD Project, a populationbased study of genetic and environmental factors associated with IBD. Sex and age frequencymatched controls () were recruited at random from the electoral roll. Cases and controls completed a self-administered questionnaire which was posted back to the investigators. Univariate and multivariate analysis was performed. Results: Response rates were $94.4 \%$, $95.2 \%$ and $84 \%$ for CD, UC and controls, respectively. Age, sex, current and childhood socioeconomic status (SES) and smoking status were included in the multivariate model. Statistically significant adjusted odds ratios with $95 \%$ confidence intervals included: one relative with IBD 2.96 (2.15-4.08), two relatives with IBD 7.0 (3.27-14.99) smoking at diagnosis 2.01 (1.51-2.68), appendicectomy 1.67 (1.20-1.95), tonsillectomy 1.48 (1.131.95), breastfed as infant 0.55 (0.41-0.74), mother smoking in pregnancy 1.68 (1.21-2.34), OCP use at diagnosis 1.843 (1.09-3.12), antibiotic use in adolescence 2.08 (1.30-3.31), urban living in childhood 1.50 (1.09-2.07) and having a household vegetable garden in childhood 0.52 (0.37-0.72). Furthermore, a duration-response effect was observed for breastfeeding $(\mathrm{p}<0.001)$ and while high childhood SES was associated with later development of $C D(p=0.002)$, cases had lower current SES than controls $(p=0.03)$. Over sixty additional factors were not significantly-associated with $\mathrm{CD}$. Conclusions: As well as recognised factors associated with $\mathrm{CD}$, some additional factors have been reported. The reduced risk associated with breast-feeding and the increased risk associated with antibiotic use and tonsillectomy suggest an important role for faecal flora. High SES in childhood remains an important factor in this population, although associations with low SES later in life may be due to reverse causation. The association with having a vegetable garden suggests that diet early in life may play an important role in $\mathrm{CD}$ aetiology.

\section{W1040}

Juvenile Onset of Crohn's Disease: A Risk Factor for Reduced Lumbar Bone Mass

David Armstrong, Marina Mauro

Background: Bone fragility is the result of both growth related and age-related factors influencing skeletal size, its architecture and mass. Exposure to a chronic illness, such as Crohn's disease, during skeletal growth may compromise peak bone mass in early adulthood, resulting in skeletal fragility later in life. Aim: To assess the effects of the onset of Crohn's disease during skeletal growth on lumbar bone mass in adulthood. Methods: A retrospective chart review was performed to select $C D$ adult patients who had a whole body and lumbar densitometric evaluation (Hologic) between 2003 and 2005.Densitometric determinations included Bone Area, Bone Mineral Content (BMC), Bone Mineral Density (BMD), T, Z scores in lumbar area and whole body BMC, Fat Mass (FM) and Lean Mass (LM). Age, gender, weight, duration of $C D$ and age at diagnosis were assessed. Standard t-tests, simple and multiple stepwise correlation were analyzed as required. Results: Data from 39 patients (38 15 years, $M=15$ ) were analyzed. Patients with a history of onset of $C D$ before 16 years of age $(11.5+3$ years old at diagnosis, $\mathrm{n}=9)$ had low lumbar $\mathrm{T}$ (mean -1.5) and $\mathrm{Z}$ (mean -1.4) scores. They had a significantly lower weight, FM and BMC, BMD, T and Z scores in lumbar area than those who were diagnosed after the age of 16 . There was no difference in the duration of $C D$ between both groups (13.2 vs. 14.1 years) nor in total BMC and LM. There was a significant correlation between age at diagnosis of $\mathrm{CD}$, weight and LM and lumbar BMC. After multiple regression analysis age at diagnosis and LM showed to be independently associated with lumbar BMC. There was no correlation between age at diagnosis of $C D$ and total BMC. Summary: Patients who were diagnosed with CD before the age of 16 had reduced bone mass in lumbar area. The effect of the age of onset of $\mathrm{CD}$ on lumbar BMC was independent of weight and LM. Conclusions: The onset of CD during axial skeletal growth is a risk factor for reduced lumbar bone mass in adulthood.

\section{W1041}

Collagenous Colitis- a Ten Year Follow-Up Study Ahmed Madisch, Stephan Miehlke, Franziska Lindner, Birgit Bethke, Manfred Stolte Background: Collagenous colitis (CC) is a chronic disorder characterized by watery diarrhea. Data about the long-term course and prognosis of this disease are sparse. Aim: to evaluat the long-term outcome of patients with CC 10 years after the diagnosis. Patients and Methods: In 1989/1990, 65 patients were diagnosed to have collagenous colitis. Initially and after an interval of 10 years these patients were asked to complete a questionnaire on onset and duration of diarrhea, stool frequency and consistency, other gastrointestinal symptoms including weight loss, drug history, treatment success and other diseases. Diarrhea was defined as three or more loose bowel movements. Improvement of diarrhea was defined as reduction of frequency of diarrhea for at least $50 \%$. Results: After 10 years questionnaires in 47 out of 65 patients $(72.3 \%$ ) (female 40 ; mean age 68 years, range $41-95$ years) questionnaires were available for analysis. At baseline, duration of diarrhea was longer than ten years and stool frequency was reported between 5 and 10 times per day in 24 patients $(51 \%)$. Acute onset of symptoms were reported by 27 patients (57\%). After a follow-up of ten years 11 patients $(23,4 \%)$ had persistent diarrhea with no change of frequency and consistency compared to baseline. Four patients $(8,5 \%)$ showed a reduction of diarrhea frequency for at least $50 \%$. Diarrhea was resolved in 23 patients (48.9\%) during the followup period Of those, 20 patients received treatment such as corticosteroid, antibiotics, bismuth or 5-aminosalicyclic. After a complete resolution of diarrhea during the long-term follow-up, 9 patients (19.2\%) showed recurrence of symptoms. Conclusion: The long-term outcome of CC is benign with resolution of diarrhea in up to $50 \%$ of patients receiving anti-inflammatory treatments. About $30 \%$ of patients may experience persistent diarrhoe even 10 years after diagnosis. Our data confirm that $\mathrm{CC}$ is a chronic disorder with a variable course of symptoms during a long-term follow-up.

\section{W1042}

Risk Factors and Indications for First Surgery in Crohn's Disease Patients: Analysis of 289 Japanese Cases

Naofumi Morimoto, Jun Kato, Motoaki Kuriyama, Toru Nawa, Manabu Kurome, Koji Takemoto, Sakiko Hiraoka, Yasushi Shiratori

(Aim) Despite appropriate medical treatment, a large fraction of Crohn's disease (CD) patients require surgical treatments during their clinical courses. Although many reports demonstrated risk factors for recurrences after surgery in $C D$ patients, risk factors for first surgery were less frequently discussed. In this study, therefore, we aimed to identify risk factors and indications for first surgery in $C D$ patients. Moreover, which surgical indications were associated with each risk factor was also investigated. (Methods) The clinical records of 289 consecutive Japanese CD patients treated at Okayama University hospital and 22 affiliated hospitals between January 1981 and August 2003 were reviewed. Median follow-up period was 7 years. Clinical factors including gender, age at onset, year at onset, disease extent at diagnosis were examined, in order to identify the risk factors for first surgery. Indications for first surgery were also investigated, and were stratified by presence or absence of each risk factor. (Results) Of all patients, 113 (39\%) patients underwent surgery. Female gender (RR, 1.62; 95\% CI, 1.09-2.41), and disease affecting ileum (RR, 2.06; 95\% CI, 1.20-3.53) were identified as the independent risk factors for surgery by multivariate analysis using Cox proportional hazards regression model. Moreover, female patients were more likely to receive first operation due to intestinal perforation than male patients $(12 \%$ vs. $6 \%, p=$ 0.006). As for age at onset, Kaplan-Meier curves showed that patients with an onset more than 20 years were likely to receive first operation within five years after onset, while patients with onset less than 19 years were likely to receive first operation 10 years after onset. (Conclusion) Our results suggested that risk factors and indications for first surgery in CD patients varied according to patient clinical characteristics. Patients with disease affecting ileum were more likely to receive surgical treatment. Moreover, female patients were more susceptible to receiving operation, especially due to intestinal perforation. Therefore, CD patients should be consulted carefully regarding individual clinical backgrounds with the risk of operation in mind. 
W1043

Epidemiology of Inflammatory Bowel Disease in the Pediatric East Indian Population of British Columbia

Vered Pinsk, Daniel A. Lemberg, Richard Schreiber, Collin Barker, David M. Israel, Kevan Jacobson

Increasing incidence rates of ulcerative colitis (UC) and Crohn's disease (CD) have been linked to ethnicity and geography. Geographical differences, population migration and changing epidemiology suggest an environmental role in prevalence and phenotypic expression of disease. Aim: To determine incidence and phenotypic expression of IBD in the pediatric East Indian (EI) population of British Columbia (BC) diagnosed between 1985 and 2005 with comparison to the general pediatric IBD population in the same geographic area. Methods: A retrospective and prospective medical chart review was undertaken in El pediatric patients $\leq 16$ of age diagnosed with IBD at BC Children's Hospital between January 1985 and June 2005. Comparisons were made between EI pediatric patients $(n=75)$ and the rest of the pediatric IBD population $(n=504)$. Age, gender, place of birth, family history of IBD, type and extent of disease were included and analyzed. Provincial population statistics were obtained from Statistics Canada. Diagnosis and classification of disease was based on clinical, endoscopic, radiological and histological criteria. Results: EI patients represented $13.2 \%$ of IBD population, with 36 (48\%) diagnosed with CD, 25 (33.3\%) with UC and 14 (18.7\%) indeterminate Colitis (IC), in contrast to $69.2 \%, 20.2 \%$ and $10.6 \%$ respectively in the rest of the IBD population. The incidence rates for IBD between 1996 and 2001 for the EI population was $14.06 / 10^{5}\left(6.80 / 10^{5}\right.$ for $C D, 5.44 / 10^{5}$ for UC and $1.81 / 10^{5}$ for IC) compared to $5.5 / 10^{5}$ for the rest of the IBD population $\left(3.8 / 10^{5}\right.$ for $\mathrm{CD}, 0.91 / 10^{5}$ for UC and $0.79 / 10^{5}$ for IC). The EI male/female ratio was significantly different from that observed with the rest of the IBD population (2:1 vs. 1.12:1; OR 1.787 (95\% CI, 1.07 to 2.97). Of the EI patients with IBD, $92.6 \%$ were Canadian born. A family history of IBD was similar in both groups ( $35 \%$ EI children compared to $36.6 \%$ rest of IBD population). No first degree EI relatives had CD and only $22.3 \%$ of second degree relatives had CD. Irrespective of disease type, pan colitis was more common in EI patients. No significant differences were observed for age at diagnosis or duration of symptoms prior to diagnosis. Conclusions: This data suggests a significantly higher incidence of IBD in the East Indian pediatric population compared to the general pediatric population of BC, with a different pattern of phenotypic expression and male predominance. Moreover, this data indicates a difference in phenotypic expression from previously published East Indian adult IBD data. These results suggest an effect of migration, environmental and life style change on incidence and phenotypic expression of IBD.

\section{W1044}

High Rate of Crohn's Disease in Canterbury New Zealand - Results of a Population-Based Study

Richard B. Gearry, Ann Richardson, Christopher M. Frampton, Bruce A. Chapman, Michael J. Burt, Judith A. Collett, Murray L. Barclay

Introduction: Inflammatory bowel disease (IBD) has increased exponentially in industrialised nations over the last fifty years. Previous New Zealand studies have shown that IBD is less common than in other countries, however, clinical observations suggested a high incidence and prevalence of IBD in Canterbury, particularly Crohn's disease (CD). Aim: To determine the descriptive epidemiology of IBD in Canterbury, Methods: Canterbury IBD patients, recruited using multiple strategies, gave informed consent, permission for clinical record review, completed a questionnaire and were bled for DNA extraction as part of the Canterbury IBD Project. Cases were confirmed using standard criteria and completeness of recruitment was validated using capture-recapture methods. Demographic and phenotypic data were extracted from case notes. Results: 1420 patients $(715 \mathrm{CD}, 668$ Ulcerative colitis (UC)) were recruited ( $>91 \%$ of Canterbury IBD patients). In 2004, age-standardised (WHO World Standard Population) IBD, CD and UC incidence rates were 25.2,16.5, and 7.6/100,000/ year respectively. The IBD, CD and UC point prevalences on 1 June, 2005 were 308.3, 155.2 , and $145.0 / 100,000$ respectively. CD patients were more likely than UC patients to be female $(61.4 \%$ v $47.1 \%)$ and to be younger (median age: 39.9 years $\mathrm{v} 43.7$ years). $97.5 \%$ of IBD patients were Caucasian. Conclusions: IBD is at least as common in Canterbury as in other western regions. $\mathrm{CD}$ incidence and prevalence are amongst the highest ever reported and are higher than for UC. IBD population characteristics are otherwise similar to other countries. The Canterbury IBD Project will be a valuable tool for future population-based IBD epidemiology and genetics research.

\section{W1045}

Risk Factors of Ulcerative Colitis in Chinese Population: An Age- and SexMatched Case-Control Study

Bing Xia, Li Jiang, Jin Li, Mei Ye, Changsheng Deng

Background and aims The pathogenesis of inflammatory bowel disease (IBD) involves both genetic and environmental factors. Cigarette smoking, alcohol use, appendectomy, oral contraceptives and family history of IBD have all been shown to be associated with IBD, but there were no reports of risk factors for IBD in Chinese population, in which the incidence of IBD is increasing during the past decade. We conducted a case-control study to examine associations between previously reported environmental risk factors and development of ulcerative colitis (UC) in Wuhan city, central China. Methods A total of 177 patients with UC and 177 age and sex matched controls were prospectively studied in Wuhan city from January 2004 to December 2004. An age and sex matched case-control study was conducted to assess the role of smoking, alcohol use, appendectomy, childhood and other potential risk factors in the development of UC by a detailed questionnaire. The data were analyzed by $\chi 2$ test with Yates correction and multiple logistic regressions. Results Smoking was a protective factor and ex-smoking is a risk factor for UC (comparing with nonsmokers, smokers: $\mathrm{OR}=0.28,95 \% \mathrm{CI}: 0.16 \sim 0.48, \mathrm{P}=0,0001$; ex-smokers: $\mathrm{OR}=4.36,95 \% \mathrm{CI}$ : 1.46 13.04, $\mathrm{P}=0.008)$. Familial history of IBD was a risk factor $(\mathrm{OR}=4.35,95 \% \mathrm{CI}$ : $1.21 \sim 15.71, \mathrm{P}=0.025)$ whereas appendectomy was a protective factor $(\mathrm{OR}=0.24,95 \% \mathrm{CI}$ :
0.07 0.86, $\mathrm{P}=0.028$ ) for UC. There were no significant associations between $\mathrm{UC}$ and other factors examined, including alcoholic drinking, tea consumption, capsicum consumption, oral contraceptives, NSAIDs use, history of having been breast-fed in infancy, history of measles, education status, present residence and childhood residence. Conclusions The same as in western countries, smoking was a protective factor for development of UC and exsmoking and non-smoking were associated with an increase risk of UC in Chinese population. Familial history of IBD was shown a risk for UC whereas appendectomy was associated with a low risk for the disease.

\section{W1046}

Sexual Function in Men with IBD - A Survey with Controls Antje Timmer, Alexandra Bauer, Beate Bokhof, Angela Takses, Axel Dignass, Gerhard Rogler

Aims: We examined the relative frequency, type and determinants of impaired sexual functions in patients with Crohn's disease (CD) or ulcerative colitis (UC). Here, results are presented for the subgroup of men aged 18 to 65. Methods: A random sample of members of the local self-help group were surveyed using a standardized anonymous postal questionnaire $(n=500)$. Age matched friends were used as controls; in addition, controls were selected from a large health insurance cohort. Sexual function was evaluated using the International Index of Erectile Function (IIEF); a low score was defined as a score <-l on a z-transformed scale (i.e. < normative mean minus 1 SD). Other psychometric instruments used included the HADS and the SF36. Relative frequency and determinants of low function were calculated as age adjusted OR (95\% confidence intervals), using multiple logistic regression. Subgroup analyses were performed to examine the effect of the type of control (friend / health insurance) and disease activity (no or mild /moderate to high activity) on the strength of the associations Results: The response rate was $37 \%$ for cases. In $44 \%$ friend-controls were available. Overall, 153 case-control pairs were available for analysis ( $92 \mathrm{CD}, 61 \mathrm{UC})$. Most patients were in remission, there were only 26 cases with significant clinical activity. There were no differences in the proportion of persons in stable relationships (overall, $86 \%$, OR for cases 0.8 ( 0.4 to $1.6)$ or in having an active sex life ( $88 \%$, OR $1.2,0.5$ to 2.7$)$. In the sub analysis restricted to patients with active disease, OR were consistently increased: low erectile function, OR 4.3 (1.0-18.5), orgasmic function $3.0(0.7-13.2)$, sexual desire $6.3(0.7-57.9)$, sexual satisfaction $3.9(0.9$ - 16.6), overall satisfaction $5.4(1.3$ - 22.3) and total IIEF Score, 3.6 $(0.8-15.7)$. IBD in remission or mild disease was not associated with any impairment of sexual function, all OR were close to 1 . There was no difference between UC and CD disease, if controlled for disease activity. Depression and low physical quality of life score were the most important determinants of impaired sexual function across subgroups and dimensions. Conclusions: Active IBD is associated with impaired sexual function in all aspects examined. However, most patients in this cohort had no or low disease activity and did not show compromised sexual function.

\section{W1047}

Measurement of 6-Mercaptopurine Metabolites to Optimize Azathioprine Therapy in Pediatric Inflammatory Bowel Disease Is a Clinically Useful and Cost-Effective Management Strategy

Sanjoy Banerjee, Warren P. Bishop

BACKGROUND: Several studies have examined the utility of 6-mercaptopurine (6-MP) metabolite assays for management of inflammatory bowel disease (IBD). One such study, based on theoretical modeling, suggested an economic benefit (Dubinsky et al. Am J Gastroenterol 2005;100:2239). Since 2001, our group has routinely measured 6-MP metabolites in pediatric IBD patients receiving azathioprine (AZA). We felt that this resulted in better outcomes and reduced costs. We therefore chose to perform a retrospective analysis to determine whether this has resulted in actual cost reduction. METHODS: We identified two groups of patients. Group A received AZA without metabolite measurement $(n=30)$ and Group B had metabolites measured $(n=71)$. These children were on a stable dose of AZA for $\geq 4$ months and had a follow-up of $\geq 6$ months. Information gathered for each visit included disease activity scores, lab results, 6-MP metabolite levels, and drug doses. We also recorded surgeries and number and length of each hospitalization. The cost of care for both groups of patients was calculated from a payer's perspective, and was based on 2005 Iowa Medicare fees for relevant CPT codes, including laboratory tests and physician fees. Hospitalization expense was based on standard costing data provided by the accounting department. The total cost of care per 100 patient-years of follow-up was calculated for each group. The total cost included cost of disease exacerbations, outpatient (OP) visits, inpatient care, surgery, laboratory/pathology studies and 6-MP metabolite assays. We did not quantify the cost associated with morbidity related to the disease or to increased corticosteroid use RESULTS: Group B received less prednisone $(\mathrm{p}<0001)$, had a lower Pediatric Crohn's Disease Activity Index or Simple Colitis Activity Index score $(\mathrm{p}<0.05)$, and received a higher dose of AZA (median dose $2.4 \mathrm{vs} .1 .7 \mathrm{mg} / \mathrm{kg} / \mathrm{day}, \mathrm{p}<.0001$ ). The probability of remission at each clinic visit was $0.81 \pm 0.03$ in Group B vs $0.67 \pm 0.06$ in Group A ( $\mathrm{p}=$ 009). The total cost of care per 100 patient-years for Group B was $\$ 192,122$ compared to a total cost of $\$ 345,476$ in Group A. CONCLUSION: In our patient population with IBD, we found a cost benefit of using the 6-MP metabolite assay for optimizing AZA dosing. The majority of cost saving came from decreased inpatient utilization and reduced rate of disease exacerbation. We speculate that the improved outcome in Group B resulted from higher AZA dose used. EVENTS PER 100 PATIENT-YEARS 


\begin{tabular}{|c|c|c|c|c|c|}
\hline & $\begin{array}{c}\text { \# } \\
\text { Exacerbations }\end{array}$ & $\begin{array}{c}\text { \# OP } \\
\text { visits }\end{array}$ & $\begin{array}{c}\text { \# Hospitalizations/ avg } \\
\text { length of stay }\end{array}$ & $\begin{array}{c}\text { \# } \\
\text { Surgeries }\end{array}$ & $\begin{array}{c}\text { \# 6-MP metabolite } \\
\text { assays }\end{array}$ \\
\hline $\begin{array}{c}\text { Group } \\
\text { A }\end{array}$ & 63.5 & 252 & $16.2 / 6.8$ days & 3.0 & 0 \\
\hline $\begin{array}{c}\text { Group } \\
\text { B }\end{array}$ & 52.1 & 259 & $4.2 / 3.4$ days & 2.5 & 297 \\
\hline
\end{tabular}

alcohol intake in patients with IBD and to better characterize the possible relationship between alcohol consumption and GI symptoms. Methods: We recruited 121 patients - 52 patients with biopsy proven inactive Crohn's Disease (CD), 38 patients with biopsy proven inactive Ulcerative Colitis (UC) and 31 patients with normal colonoscopies who met Rome II criteria for IBS. All participants completed demographic data form, a validated questionnaire to quantify alcohol consumption both by quantity and frequency per normal week, a validated questionnaire on disease (CDAI, UCAI, or IBSS respectively), and a SF-12 quality of life measure. Patients also completed a series of questions regarding the effect of alcohol on their GI symptoms. Inactive disease in IBD was defined as a CDAI $<150$ or UCAI $<4$ Current drinkers were defined as $>12$ drinks of alcohol a year as defined by the NIAAA Pearson Chi square tests were used for analysis between the three groups. Results: Of the 121 patients surveyed, 75 were classified as current drinkers- 32 CD, 24 UC and 19 with IBS. Patients that abstained from alcohol were $38 \%$ of CD, $42 \%$ of UC, and $65 \%$ of IBS Of current drinkers, $75 \%$ of $\mathrm{CD}(\mathrm{N}=24), 75 \%$ of $\mathrm{UC}(\mathrm{N}=18)$, and $37 \%$ of $\mathrm{IBS}(\mathrm{N}=7$ reported a worsening of GI symptoms with alcohol $[X 2(2)=9.12$, p-value .01]. Mean alcohol consumption for each group was less than 1 drink per day, or light alcohol consumption as defined by the NIAAA. Mean age in the CD, UC, and IBS groups were 42,37 , and 58 respectively. Patient gender, quality of life scores, and presence of binge drinking (> 5 drink on one occasion as defined by the NIAAA) were not statistically different between the three groups. Conclusions: Current drinkers with inactive IBD are more likely to report GI symptom exacerbation with alcohol than current drinkers with IBS. The mechanism of alcohol induced symptoms in IBD is not clear, but may be one of many environmental triggers of the inflammatory cascade in the gut lumen. Further prospective studies are needed to examine the link between alcohol consumption and inflammation to establish dietary guidelines for patients with IBD. The probability, intensity and volume of utilization were estimated Results: In 2000/1, TBD patients were more likely to have an outpatient visit (RRR=1.18; 95\% CI, 1.17-1.19), and an overnight hospital stay ( $R R R=2.32 ; 95 \% \mathrm{CI}, 2.16-2.49)$. CD cases were more likely than outpatient visits. From 1990/1 to 2000/1, IBD cases experienced a significant drop in the likelihood of an outpatient surgical visit relative to non-IBD controls ( $<<0.05)$, and for those cases that were hospitalized, $\mathrm{CD}$ cases tended to be less likely than UC cases to experience IBD-specific inpatient surgery $(\mathrm{p}<0.07)$. Of the $1987 / 8$ cohort, $80 \%$ of the admissions that occurred over the follow-up period were found in the first five years following diagnosis. Conclusions: In 2000/1, health care utilization continued to be higher in IBD vs. controls and $C D$ vs. UC, however the gap in costly service utilization appeared to narrow between the latter pair

\section{W1049}

Use of Infliximab for Ulcerative Colitis in the Pre-Act I/II Era: An Analysis of 2000-2001 Commercial Insurance Claims

Patrick D. Meek, Marjorie Rosenberg, Nilay D. Shah, Bora Gumustop

BACKGROUND: Infliximab, a monoclonal antibody against tumor necrosis factor, has been used successfully for patients with Crohn's disease and rheumatoid arthritis since 1998. Small clinical-trials published in 2001 and 2002 revealed the drug's potential benefit in ulcerative colitis patients (UC) for those with inadequate relief after standard therapy. At the 2005 Digestive Disease Week meeting, data from two large controlled clinical trials (ACT I and ACT II) Gastroenterology 2005;128(4):A-104,105) were released that appear to confirm the benefit of the drug in UC. An evaluation of the patterns of infliximab use during the early years of marketing, prior to the availability of ACT I/II, may provide useful insight into the clinical decision making process during a pre-indication time period. PURPOSE: In the post ACT I/II era, with increased infliximab use for UC, the impact of the drug on subsequent health care utilization (inpatient, outpatient, concurrent prescription drug use) by patients with UC remains unknown, and requires close evaluation. This study describes the characteristics of patients and providers of infliximab use for UC during the years of 2000-2001. METHODS: The study was a retrospective analysis of inpatient and outpatient claims for persons with 12 months of continuous enrollment beyond the earliest index claim coded for inflammatory bowel disease. The source of the data was the Medstat MarketScan Commercial Claims and Encounters database. The study sample of interest consists of 5,286 patients of any age who had at least 1 claim with a diagnosis for UC (ICD9 code of 556.xx) between January 1, 2000, and December 31, 2001. Infliximab use was identified in outpatient service and prescription drug files using J1745 (J-code) and NDC 59784003001. RESULTS: (see Table) CONCLUSION: During the early pre-ACT I/II years $(2000$, 2001), insurance claims for infliximab for patients with UC were rare. This study provides information about the restricted nature of infliximab use in a privately insured population. The encounter patterns seen in the analysis highlight the need for careful risk adjustment by those evaluating resource use in UC.

Table. Characteristics of patients with ulcerative colitis by treatment group for 2000 and 2001

\begin{tabular}{|c|c|c|}
\hline & \multicolumn{2}{|c|}{ Treatment Group } \\
\hline Characteristic & Infliximab [n=57] & $\begin{array}{c}\text { Non-Infliximab } \\
{[\mathrm{n}=5229]}\end{array}$ \\
\hline Age; mean (S.D.) & $39.8(14.8)$ & $37.0(21.6)$ \\
\hline Female (\%) & 52.6 & 53.6 \\
\hline Type of UC: UC alone (\%) Indeterminate (\%) & 10.589 .5 & 82.417 .6 \\
\hline No. Annual Encounters (avg. [S.D.]) Hospitalizations & $0.75[1.21] 11.6$ & $0.28[0.77] 7.14$ \\
Office Visits & {$[15.3]$} & {$[10.49]$} \\
\hline
\end{tabular}

\section{W1050}

The Effect of Current Alcohol Consumption in Inactive Inflammatory Bowel Disease

Garth Swanson, Laurie Keefer, Mary Kwasny, Sedghi Shahriar, Ali Keshavarzian

Background: Chronic heavy alcohol use has been known to increase inflammation in a wide spectrum of disease. However, acutely alcohol has also been shown to decrease monocyte activity and immune function. Currently there is a lack of literature and no guidelines for IBD patients as to how alcohol may affect their symptoms. Our goals were to quantify

\section{W1051}

North American Inflammatory Bowel Disease (IBD) Experts and Community GI Providers Differ Significantly in Prescribing 5-ASA Products in Crohn's Disease (CD)

Eric Esrailian, Brennan M. Spiegel, Laura E. Targownik, Marla C. Dubinsky, Stephan R. Targan, Ian M. Gralnek

Background: Although the effectiveness of 5-ASA products is well established in ulcerative colitis, their effectiveness in $\mathrm{CD}$ is unclear, and the appropriateness of using 5-ASA in CD remains uncertain. We conducted a national survey of IBD experts and community GI providers to measure beliefs about the appropriateness of prescribing 5-ASA products in CD Methods: We developed a survey consisting of 4 clinical vignettes: 1) patient with newly diagnosed $C D(C D$ Dx) 2) presentation of perianal fistula 3) patient with steroid refractory $\mathrm{CD}$ and 4 ) patient status post ileocolonic (s/p IC) anastomosis for IC CD. The survey elicited provider beliefs about the appropriateness of diagnostic tests and therapies (including 5-ASA products). We measured appropriateness using the 9-point RAND Appropriateness Scale, where 1-3=inappropriate, 4-6=unsure, 7-9=appropriate and "endorsed." We calculated the RAND Disagreement Index (DI) for each set of ratings. The DI is a validated measure of provider variation, where $\mathrm{DI}>1.0=$ extreme variation, and $\mathrm{DI}<1.0=$ acceptable variation. We surveved 55 recognized North American IBD experts and a random sample of 91 AGA members. Results: Table 1 demonstrates a striking disconnect between experts and community GI providers. For all vignettes, community providers were more likely to endorse the use of 5-ASA products in CD. Moreover, both groups were internally consistent in their ratings (all DIs $<1.0$ except for experts in $\mathrm{s} / \mathrm{p}$ IC vignette). Among providers endorsing 5-ASA, there were no significant differences in the ratings of Asacol $₫$, Pentasa $₫$, or Colazal $₫$, although Dipentum $\AA$ was rated lower than the alternative formulations. In multivariable regression analysis adjusting for key practice and provider characteristics, only non-expert status independently predicted endorsement of 5-ASA products in CD Conclusions: Most community providers in this sample believe that 5-ASA products are appropriate across a variety of CD presentations. In contrast, IBD experts are significantly less likely to endorse 5-ASA products. Since intragroup beliefs are highly consistent, these data demonstrate a marked disconnect between experts and community providers, and suggest that further research and dialogue is critical to better understand the role of 5-ASA products in $C D$.

Table 1. Endorsement of 5-ASA by Experts vs Community Providers Across Vignettes. *DI $\leq 1=$ "consensus."

\begin{tabular}{|c|c|c|c|c|c|}
\hline Vignette & $\begin{array}{c}\text { \% Experts } \\
\text { Endorse }\end{array}$ & $\begin{array}{c}\text { \% Non-Experts } \\
\text { Endorse }\end{array}$ & p-value & $\begin{array}{c}\text { *Expert } \\
\text { DI }\end{array}$ & $\begin{array}{c}\text { Non-Expert } \\
\text { DI }\end{array}$ \\
\hline New CD Dx & $44 \%$ & $74 \%$ & 0.002 & 0.9 & 0.9 \\
\hline Perianal Fistula & $13 \%$ & $68 \%$ & $<0.001$ & 0.3 & 0.9 \\
\hline Steroid-refractory & $17 \%$ & $52 \%$ & $<0.001$ & 0.3 & 0.7 \\
\hline $\begin{array}{c}\text { S/P IC } \\
\text { Anastomosis }\end{array}$ & $39 \%$ & $61 \%$ & 0.01 & 1.6 & 0.8 \\
\hline
\end{tabular}

\section{W1052}

Evaluation of Densitometric Bone-Muscle Relationships in Crohn's Disease (CD)

David Armstrong, Marina Mauro

Background: Patients with CD are 1.4 to 2.5 times more likely than the normal population to sustain a fracture but the factors involved in the pathogenesis are not clearly understood. Osteopenia and osteoporosis are traditionally defined by Bone Mineral Density (BMD) parameters, but this approach disregards the influence of bone size, a main determinant of bone strength. Bone mineral content (BMC) may, therefore, provide a better measure of bone strength. Bone mass is affected both by nutrition and by muscular activity. We hypothesized that BMC would correlate better than BMD with lean mass (LM - a measure of muscular mass) and that LM would correlate better than fat mass (FM) with BMC. Aim: To assess the relationships between BMC, BMD, LM and FM, determined by whole body densitometry 
in CD patients, using LM and FM as markers of exercise and caloric intake status, respectively. Methods: A retrospective chart review was performed to select $C D$ patients who had a whole body densitometric (Hologic) evaluation between 2003 and 2005. Densitometric determinations included the BMC, Bone Area (BA), BMD, LM and FM of the whole body and lower and upper limbs. Standard t-tests, simple and multiple stepwise correlations were analyzed as required. Results: Data from 38 patients $(38.8+15.1$ years, $M=15)$ were analyzed. Significant correlations were observed between whole body and regional BMC measurements with BA ( $>0.86)$, LM $(r>0.77)$ and body weight $(r>0.55)$. In multiple regression analysis, LM was the most significant independent factor correlated with BMC in whole body and in all regions. There was no correlation between FM and BMC. LM (muscle mass) correlated significantly with BMC (bone mass) $(r>0.77$ in all regions) and BA (bone size) $(r>0.72$ in all regions). In contrast, the observed correlations between LM and BMD were relatively poor $(r<0.5$ in all regions). Summary: These findings support the hypothesis that BMC correlates better than BMD with LM and that there is a relatively poor correlation between bone mass and FM. Conclusions: These results support the use of BMC in preference to BMD as a marker of bone strength and they suggest that muscular mass and activity, rather than overall body weight, are important determinants of bone strength in Crohn's disease Thus, the management of bone loss in inflammatory bowel disease should address the effects of both nutrition and exercise on muscle mass.

\section{W1053}

Magnifying Colonoscope Findings Are Predictive of Relapse in Patients with Quiescent Ulcerative Colitis

Takafumi Ando, Yuji Nishio, Osamu Maeda, Kazuhiro Ishiguro, Osamu Watanabe, Naoki Ohmiya, Yasumasa Niwa, Hidemi Goto

Background: Relapse of ulcerative colitis (UC) is difficult to predict by routine colonoscopy. A high-resolution video-magnifying colonoscope with chromoscopy enables the observation of colorectal mucosal pit patterns. Aims: We investigated the association between pit patterns as assessed by magnifying colonoscopy with histological inflammation and mucosal chemokine activity in patients with quiescent UC, and prospectively analyzed prognostic factors that may predict exacerbations. Methods: Magnifying colonoscopy was performed in 113 UC patients in remission. Pit patterns in the rectal mucosa were classified into four magnifyingcolonoscopy (MCS) grades on the basis of size, shape, and arrangement. Mucosal interleukin8 (IL-8) activity in biopsy specimens of rectal mucosa was measured and the specimens were microscopically graded according to the system of Riley. The patients were then followed until relapse or a maximum of 12 months. Multivariate survival analysis was performed to determine independent predictors of clinical relapse. Results: A positive correlation was identified among MCS grade, histological grade and mucosal IL-8 activity. Multivariate proportional hazard model analysis showed that MCS grade was a significant predictor of relapse. Kaplan-Meier estimate of relapse during 12 months' follow-up was found to increase with increasing MCS grade. Conclusion: MCS grading is associated with the degree of histological inflammation and mucosal IL-8 activity in quiescent UC patients, and may predict the probability of subsequent disease relapse in UC patients in remission.

\section{W1054}

Careful Patient Selection May Improve Response Rates to Infliximab in Inflammatory Bowel Disease

Ian C. Lawrance, Callum B. Pearce

Introduction: Infliximab in the treatment of Crohn's Disease (CD) is accepted and it also appears to be effective in ulcerative colitis (UC). Careful patient selection, resulting in infliximab use only for patients with active disease who are truly refractory to other treatments, may improve its efficacy in inflammatory bowel disease (IBD). Methods: The aim of this study was to determine whether careful patient selection improves infliximab efficacy in IBD. Patients were recruited from a single tertiary institution with a particular interest in the management of IBD. CD or UC/IBD unclassified patients (Montreal classification) were considered for infliximab treatment only after failure of disease control with conventional therapies and confirmation of ongoing active disease. Patients with purely inflammatory disease received a single $5 \mathrm{mg} / \mathrm{kg}$ infliximab dose. Patients with fistulising disease received a course of 3 infusions of $5 \mathrm{mg} / \mathrm{kg}$ at weeks 0,2 and 6. Changes to Harvey Bradshaw (HBI) for inflammatory $\mathrm{CD}$ and Colitis Activity (CAI) for UC/IBDU were used to determine the response and remission rates 2-4 weeks after treatment. In fistulising $C D$ a remission was sustained cessation of drainage and resolution of the fistula. Findings were correlated to inflammatory marker levels. Adverse effects were analysed. Results: 70 IBD patients were treated. In CD, 85.2\% (46/54) had active luminal and 40.7\% (22/54) fistulising disease. Luminal CD responded in $91.3 \%$ (42/46) and remitted in $80.4 \%$ (37/46). Fistulising disease responded in $77.2 \%(17 / 22)$ and remitted in $50 \%(11 / 22)$. Patients with isolated colonic disease were more likely to remit $13 / 14(92.9 \%)$ compared to those with ileal involvement 23/32 (71.9\%). In UC/IBDU, 75\% (12/16) responded and 43.8\% (7/16) remitted. A total of $11.4 \%(8 / 70)$ of all patients experienced an adverse event and $5.7 \%(4 / 70)$ a serious adverse event. Conclusions: Infliximab therapy at our institution appeared to achieve a higher remission rate in CD patients (80.4\%) compared to the ACCENT 1 study (27\%). Efficacy was also found in the use of infliximab in UC/IBDU patients in line with the ACT 1 findings. These results suggest that strict patient selection may improve the observed efficacy of infliximab.

\section{W1055}

Risk Factors for Un-Investigated Dyspepsia and Non-Ulcer Dyspepsia (NUD) in a Random Adult Population-Based Endoscopic Study (Kalixanda study) Pertti Aro, Jukka Ronkainen, Tom Storskrubb, Elisabeth . Bolling-Sternevald, Nicholas J. Talley, Lars Agreus

Background: Risk factors for non-ulcer dyspepsia (NUD) in a randomly selected adult population are not well defined; smoking, H. pylori infection, use of aspirin, NSAIDs or acetaminophen, alcohol and smoking may all be relevant in inducing dyspepsia. Aim: To determine predictors for un-investigated dyspepsia (UID) and NUD in a random sample of a Swedish adult population. Methods: A representative sample $(n=2,860$, mean age 50.4 years, $52.0 \%$ male) of the adult population $(n=21,610)$ in Northern Sweden was studied. A third $(n=1001$, mean age 54.1 years, $48.8 \%$ male) were then randomly selected for esophagogastroduodenoscopy (EGD). They completed a validated Abdominal Symptom Questionnaire (ASQ) and Hospital Anxiety and Depression scale (HADS). Dyspepsia was defined according to Rome II criteria (excluding upper abdominal bloating which was not asked in the ASQ). NUD was defined as dyspepsia excluding peptic ulcer disease, esophagitis or cancer. UID without concomitant reporting of gastroesophageal reflux symptoms (GERS) or irritable bowel syndrome (IBS) was also examined in the analyses ("UID only"). The data were analyzed by multivariate logistic regression including smoking, use of alcohol, aspirin, NSAIDs and acetaminophen, obesity (body mass index BMI $\geq 30$ ), education level, H. pylori, depression and anxiety as exposure variables and adjusting for age, gender and acid reducing drugs (proton pump inhibitors (PPI), histamine-2 receptor antagonists or antacids). Results: Descriptive data of different dyspepsia groups are presented in table. Anxiety was independently associated with UID (OR=6.2, 95\% CI: 2.4-16.1) and NUD (OR=3.2, 95\% CI: 1.57.0) but depression was only associated with UID (OR=6.0,95\% CI: 1.6-22.5) as was obesity $(\mathrm{OR}=1.5,95 \% \mathrm{CI}: 1.05-2.3)$. Low education level was also a risk factor for NUD (OR=1.5, $95 \%$ CI: 1.01-2.1). Anxiety was an independent risk factor for "UID only" (OR=3.9, 95\% CI: 1.5-10.0); H. pylori was a negative predictor for "UID only" (OR=0.5, 95\% CI: 0.3-0.99). Conclusions: Anxiety and low education level are independent risk factors for NUD, but $H$. pylori infection, smoking, alcohol and NSAID use appear to be unimportant in this disorder in the community.

Descriptive data

\begin{tabular}{|c|c|c|c|c|c|c|c|}
\hline & Males & H.pylori & Education (Low) & Aspirin & Smoking & PPI use & Mean age \\
\hline UID n=376 & $38.3 \%$ & $32.8 \%$ & $56.5 \%$ & $9.0 \%$ & $20.7 \%$ & $9.8 \% \%^{\wedge}$ & 51.0 \\
\hline NUD n=298 & $33.9 \%$ & $32.0 \%$ & $56.8 \%$ & $7.1 \%$ & $19.8 \%$ & $9.7 \% *$ & 51.0 \\
\hline UID only $\mathrm{n}=71$ & $43.7 \%$ & $24.3 \%$ & $54.3 \%$ & $7.0 \%$ & $21.1 \%$ & $1.4 \% \%^{\wedge *}$ & 53.6 \\
\hline
\end{tabular}

significant differences; ${ }^{\wedge} \mathrm{p}=0.02,{ }^{*} \mathrm{p}=0.02$

W1056

Evaluation of Duodenal Acid Exposure in Healthy Subjects Using a

Radiotelemetry $\mathrm{pH}$ Monitoring System

Jason Bratten, Michael P. Jones

Introduction: Abnormal duodenal acid exposure is linked with both DU and NUD but our understanding has been limited by cumbersome recording methods. Radiotelemetry $\mathrm{pH}$ monitoring (RpHM) is a novel technology allowing minimally invasive study of $\mathrm{pH}$ throughout the GI tract. The aim of this study was to determine the feasibility of duodenal RpHM and to establish reference ranges in healthy subjs. Methods:Subjs underwent EGD to place Bravo $^{\mathrm{TM}}$ radiotelemetry capsules (RTC) in the distal duodenal bulb. RTC were secured with endoclips. RTC retention was confirmed by US after EGD and at 48hrs. RpHM was performed for $48 \mathrm{hrs}$. Standardized test meals ( $1200 \mathrm{kcal} / 50 \mathrm{gm}$ fat $/ 36 \mathrm{gm}$ protein) were eaten twice daily. Subjs were NPO other than sips of water between meals. Sleep periods were determined from diaries. Meal periods were defined as the $3 \mathrm{hr}$ period following meal initiation. Results: 25 subjs were studied. Prolonged recordings obtained in 17/25. Reasons for incomplete recordings were RTC dislodgement(5), procedural complication(1) and suspected gastric prolapse of RTC(2). RTC dislodgements occurred early in the study and were corrected by using additional endoclips to anchor the RTC. Avg EGD procedure length was $15 \mathrm{~min}$. pH (mean $\{95 \% \mathrm{CI}\}$ ) did not differ signif btwn meal (5.67\{5.42-5.93\}), sleep (5.56\{4.87-6.24\})and fasting $(5.44\{5.07-5.80\})$ periods. This was also true when analyzed by $\left[\mathrm{H}^{+}\right]$. Frequency distributions of $\mathrm{pH}$ values for the 3 periods did, however, differ signif $(\mathrm{p}<0.0001$ for all $\mathrm{pH}$ values)(Figure). $\mathrm{pH}$ mode was 6.5 during meal periods and 7.5 for sleep and fasting periods. Fasting and sleep periods also had proportionately greater periods of time with $\mathrm{pH}<2.5$. Conclusions: Prolonged duodenal RpHM can be successfully performed using an RTC secured with endoclips. Differing patterns of duodenal $\mathrm{pH}$ are seen during meals, fasting and sleep. These data provide reference ranges in healthy subjs and will inform comparisons with NUD pts currently being studied using this method. 


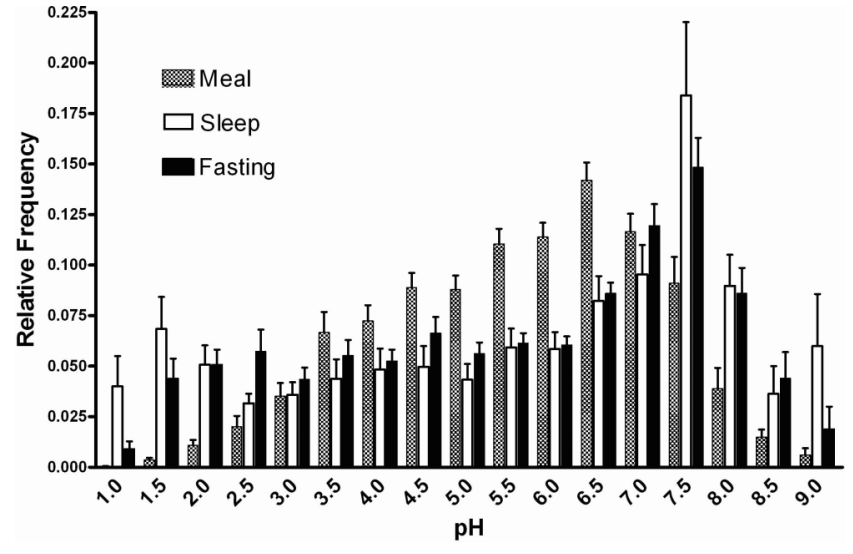

W1057

The Link Between Meal Related Symptoms and Cytokine Release in Patients with Functional Dyspepsia

Tobias Liebregts, Birgit Adam, Christoph Bredack, Alexander Roth, Susanne Heinzel, Eric Smith, Paul Drew, Gerald Holtmann

Background: Immune responses are believed to play a critical role for the manifestation of functional gastrointestinal disorders. In recent studies we have demonstrated (AJG 2004:99(4):703-10) differences in systemic cytokine levels after a meal. The current study aimed to assess the link between baseline and lipopolysacharide (LPS) stimulated cytokine levels and the symptom pattern in patients with functional dyspepsia. Methods: $22(\mathrm{H}$. pylori-negative) patients with functional dyspepsia according to ROME II (8 males, aged 2172 mean 46.3) were recruited after a standardized diagnostic work-up. Symptom pattern and severity of symptoms were assessed utilizing the BDQ (Bowel Disease Questionnaire). Peripheral blood mononuclear cells (PBMC) from $40 \mathrm{cc}$ whole blood were freshly isolated by density gradient centrifugation and cultured for 24 hours in RPMI 1640 supplemented with $10 \%$ FCS. Basal and E. coli LPS (1-100ng/ml) stimulated pro-inflammatory cytokine production (TNF-a, IL-1b, IL-6) of PBMC was measured by enzyme-linked immunosorbent assay (ELISA). Results: Overall, intensity of abdominal pain or discomfort were significantly associated with baseline TNF- $\alpha(r=0.70, \mathrm{p}<0.001), \mathrm{IL}-1 \beta(\mathrm{r}=0.54, \mathrm{p}<0.001)$ and IL-6 ( $r=$ $0.51, \mathrm{p}<0.002)$. The release of TNF- $\alpha$, IL- $1 \beta$ and IL- 6 were significantly higher in patients reporting early satiety and meal related pain (TNF- $\alpha$ p $<0.001$; IL-1 $\beta p<0.01$; IL-6 $p<0.05$ ) compared to patients without these symptoms. Cytokine release was not linked to nausea or vomiting. Summary and Conclusion: In-vitro-release of cytokines from PBMC is linked to severity of symptoms and in particular associated with meal related complaints. Based upon these data we conclude that immune responses mediated by cytokine release are linked to the symptom pattern in patients with functional dyspepsia.

\section{W1058}

Postprandial Hypersensitivity to Gastric Distension in Functional Dyspepsia Correlates with the Presence of Headache

Michele Di Stefano, Ennio Pucci, Emanuela Miceli, Paola Tana, Samanta Mazzocchi, Giorgio Sandrini, Giuseppe Nappi, Gino R. Corazza

Background and Aims: Several patients with functional dyspepsia very often present headache. Up to now, no data are available on the putative pathophysiological mechanism responsible for this association. Therefore, the aim of the present study was to assess whether headache represents a symptom associated with a peculiar pathophysiological mechanism of functional dyspepsia. Patients and Methods: Thirty-five HP-ve patients ( $22 \mathrm{~F}$, mean age $33 \pm 9$ yrs) with functional dyspepsia, diagnosed according to Rome II criteria took part in the study. Eighteen patients also suffered from migraine without aura depending on HIS criteria. As a control group, ten age- and sex-matched healthy volunteers were also enrolled. In all patients presence and severity of dyspeptic symptoms (epigastric discomfort, bloating, fullness, early satiety, nausea, vomiting, belching, epigastric burning, epigastric pain) and headache was evaluated by a $0-3$ score where $0=$ absence of symptom, $1=$ mild, $2=$ relevant, and $3=$ severe . All subjects underwent gastric barostat test according to previous studies in order to evaluate whether an alteration of gastric accommodation (1) or hypersensitivity to gastric distention (2) was present. Discomfort threshold was also determined postprandially to evaluate whether postprandial sensitisation occurs, and a relevant reduction, according to results in healthy volunteers, was defined as a threshold reduction higher than $4 \mathrm{mmHg}$. Finally, gastric emptying for solids was evaluated by 13C-octanoic acid breath test. Results: The prevalence of relevant (score $=2$ or 3 ) symptoms was not different between patients with impaired accommodation $(8 / 35 ; 22 \%)$ and patients with normal accommodation, between patients with hypersensitivity $(10 / 35 ; 28 \%)$ or normal sensitivity to gastric distention, and between patients with delayed gastric emptying $(25 / 35 ; 71 \%)$ and normal gastric emptying. On the contrary, patients with postprandial reduction of discomfort threshold $(15 / 35 ; 43 \%)$ showed a significantly higher prevalence of headache $(73 \%$ vs $35 \% ; \mathrm{p}=0.040)$. Stepwise multiple logistic analysis confirmed the significant association $(\mathrm{p}=0.01)$ between relevant headache and the risk of postprandial hypersensitivity to gastric distention. Conclusions: Among patients with functional dyspepsia, the subgroup showing an association with migraine is characterized by postprandial hypersensitivity to gastric distension.
W1059

Risk Factors Associated with Uninvestigated Dyspepsia: A Population-Based Study in Latin America

Jorge Olmos, Valeria Pogorelsky, Federico Tobal, Mariano Marcolongo, Graciela Salis, Roberto Higa, Juan Carlos Chiocca

BACKGROUND: Dyspepsia is a frequent complaint in the general population. However population-based data on risk factors for uninvestigated dyspepsia are lacking in Latin America. AIM: To assess uninvestigated dyspepsia association with potential risk factors in a population-based study in Argentina. METHODS: We conducted a cross-sectional survey of the adult population using a valid gastrointestinal symptom self-report questionnaire that measured dyspepsia symptoms and potential risk factors. The questionnaires were submitted to 1000 residents (aged 18-80 years) from 17 representative geographical areas of Argentina The samples were stratified according to age, gender, geographical areas and size of town of residence. Dyspepsia was defined as pain or discomfort (fullness/satiety) centered in the upper abdomen. Logistic regression was used to estimate the odds ratios (OR) with 95\% confidence intervals (CI) for dyspepsia symptoms associated with potential risk factors: age, gender, body mass index, educational level, psychosomatic symptoms, lifestyle habits, use of aspirin, NSAIDs, antiacids, sucralfate, prokinetics and antisecretory medication, physician visits and the existence of diseases of the esophagus or stomach in any direct family member (family history) or spouse (environment). RESULTS: 839 valid questionnaires were finally evaluated (F/M 466/373, mean age $39.9 \pm 15.4$ years). Uninvestigated dyspepsia was presen in 367 subjects $(43.2 \% ; 95 \%$ CI $39.8-46.6)$. A score $>14$ on the psychosomatic symptom scale (OR, 2.52; $95 \% \mathrm{CI}, 1.75-3.61)$, a family history (OR, $1.73 ; 95 \% \mathrm{CI}, 1.19-2.52)$ and an educational level $>12$ years $(\mathrm{OR}, 1.55 ; 95 \% \mathrm{Cl}, 1.05-2.29)$ were significantly and independently associated with uninvestigated dyspepsia. CONCLUSIONS: A score $>14$ on the psychosomatic symptom scale, a family history and educational level $>12$ years were risk factors for uninvestigated dyspepsia. The association with a higher psychosomatic symptom score suggests the role of psychological factors and somatization in this disorder.

\section{W1060}

Non-Dominant Heartburn Does Not Predict the Resolution of Symptoms in Patients with Dyspeptic Symptoms (Uninvestigated and Endoscopy-Negative) Nimish Vakil, Nicholas Talley, Johan Brun, Nigel Flook, Karsten Lauritsen, Sander Veldhuyzen van Zanten, Elisabeth Bolling-Sternevald, Tore Persson, Ewa Bjorck, Lars-Erik Svedberg

BACKGROUND: Non-dominant heartburn is common in dyspeptic patients and has led to regulatory concerns that response to acid suppression for treatment of dyspeptic symptoms is due to occult gastroesophageal reflux disease (GERD). We examine factors related to symptomatic response to 8 weeks' esomeprazole treatment in 2 clinical studies in patients with dyspeptic symptoms. METHODS: In the STARS I study (SD-NED-0021), patients (1870 years) with non-ulcer dyspepsia (dyspeptic symptoms with a sum symptom score of $\geq 5$ [scored daily: $0=$ mild to $3=$ severe] during the last 7 days of the run-in period; no predominant GERD symptoms; no GERD symptoms $>1$ day/week) and normal findings at endoscopy were randomized to either esomeprazole $40 \mathrm{mg}$ once daily (qd), twice daily (bid) or placebo for 1 week, followed by either esomeprazole $40 \mathrm{mg}$ or placebo for 7 weeks (n=1589). In the STARS II study (SD-NED-0022), Helicobacter pylori-negative primary care patients (1850 years) with dyspeptic symptoms (pain or burning centered in the upper abdomen) and without endoscopy received l-week's esomeprazole $40 \mathrm{mg}$ qd or bid, followed by 7 -weeks treatment period with either esomeprazole $40 \mathrm{mg}$ or placebo $(\mathrm{n}=1250)$. Symptoms were scored daily (none to severe) and 'response' was defined as at most one day with mild symptoms over last 7 days in the $8^{\text {th }}$ week. Patients treated with esomeprazole during both the 1-week and the 7-week period were included in this analysis. RESULTS: In STARS (non-ulcer dyspeptic patients) and STARS II (univestigated dyspeptic patients), 25.6\% o 1589 , and $38.7 \%$ of 1250 randomized patients had non-dominant heartburn at baseline. Significant prognostic factors for treatment response are shown in Table 1. CONCLUSIONS When patients with predominant or frequent heartburn are excluded, the outcome of treatment with acid inhibitors in dyspeptic patients (both in patients without significant endoscopic findings and in those not investigated with endoscopy) is not affected by the presence of background heartburn, regurgitation or burning centered in the upper abdomen Odds ratios (95\% confidence intervals) for factors predicting 8 -week treatment response 


\begin{tabular}{|c|c|c|c|c|}
\hline Factor & $\begin{array}{c}\text { Non-ulcer dyspeptic } \\
\text { patients (n=1589) }\end{array}$ & $\begin{array}{c}\mathrm{P} \\
\text { value }\end{array}$ & $\begin{array}{c}\text { Dyspeptic patients (no } \\
\text { endoscopy })(\mathrm{n}=1250)\end{array}$ & $\begin{array}{c}\mathrm{P} \\
\text { value }\end{array}$ \\
\hline Age (per year) & $1.017(1.006,1.028)$ & $\mathbf{0 . 0 0 2}$ & $1.000(0.984,1.017)$ & 0.964 \\
\hline Heartburn & $1.131(0.814,1.572)$ & 0.464 & $0.912(0.681,1.223)$ & 0.540 \\
\hline Acid regurgitation & $1.016(0.703,1.467)$ & 0.934 & $0.840(0.617,1.142)$ & 0.266 \\
\hline $\begin{array}{c}\text { Burning centred in the } \\
\text { upper abdomen }\end{array}$ & $0.795(0.580,1.088)$ & 0.152 & $1.308(0.887,1.928)$ & 0.176 \\
\hline Postprandial fullness & $1.075(0.805,1.436)$ & 0.623 & $0.613(0.458,0.821)$ & $\mathbf{0 . 0 0 1}$ \\
\hline Early satiety & $1.078(0.795,1.461)$ & 0.629 & $0.720(0.534,0.970)$ & $\mathbf{0 . 0 3 1}$ \\
\hline Nausea & $0.837(0.612,1.146)$ & 0.267 & $0.618(0.455,0.839)$ & $\mathbf{0 . 0 0 2}$ \\
\hline Bloating & $0.886(0.662,1.184)$ & 0.412 & $0.623(0.466,0.833)$ & $\mathbf{0 . 0 0 1}$ \\
\hline Belching & $1.003(0.742,1.356)$ & 0.983 & $0.726(0.542,0.972)$ & $\mathbf{0 . 0 3 1}$ \\
\hline
\end{tabular}

\section{W1061}

Meal Related Symptoms and Gastric Motor Function

Birgit Adam, Tobias Liebregts, Guru lyngkaran, F Dylan Bartholomeusz, Max Bellon, Jacob Bolwell, Gerald Holtmann

Introduction: The association of gastrointestinal symptoms during a test meal and the gastric emptying pattern has not been systematically studied. Therfore we aimed to assess and compare in healthy subjects and patients with functional dyspepsia the relation between meal related symptoms and gastric motor function. Methods: We studied 16 patients with functional dyspepsia (10 female, age 18 - 68) and 15 healthy subjects ( 8 males, aged 21 45) without a history of relevant dyspeptic symptoms. After at least an 8 hrs fast, they received every 5 minutes $200 \mathrm{ml}$ of a standardized 99mTc-labelled enteral feeding solution (EnsureR) up to a cumulative volume of $800 \mathrm{~mL}$. After each $200 \mathrm{~mL}$ drink, symptoms were evaluated with a standardized instrument that assesses 7 key symptoms (fullness, abdominal pain, retrosternal/abdominal burning, nausea, regurgitation, other) and determines peak and cumulated symptom response. The gastric nutrient distribution and emptying were assessed with a gamma camera. Data analysis: The cumulated symptom response during the 240 minute meal and post meal period was calculated separately for all symptoms. In addition, the time from the start of nutrient ingestion until the peak of the various symptoms was determined. Tlag, defined as the time at which activity was first seen in the proximal small intestine, and gastric retention at 100 min were used to characterize gastric emptying. Non parametric correlations were utilized to assess the association between symptoms and gastric emptying parameters. Results: During the test meal, patients reported significantly more severe abdominal symptoms than healthy controls (cumulated score $530 \pm 76$ vs. $325 \pm 64$, $\mathrm{p}<0.05)$. The average time, between the start of the meal until the peak symptoms occurred, were not different between patients and controls $(\mathrm{p}>0.05)$ while there were marked differences for the various symptoms (i.e. nausea $12 \mathrm{~min} \pm 9$ vs. pain $20 \mathrm{~min} \pm 23$ ). Gastric emptying (tl/2 and tlag) was not different for patient and controls. Interestingly, the cumulated fullness and pain scores were inversely correlated with the tlag $(r=0.50, p<0.02$ and $r=-0.42, p<0.05)$. In contrast, $\mathrm{t} 1 / 2$ or gastric nutrient retention at $100 \mathrm{~min}$ was not associated with any of the symptoms. Conclusions: A rapid onset of delivery of nutrients into the duodenum is associated with fullness and pain, while delayed gastric emptying was not associated with symptoms. These data suggest that duodenal mechanisms (e.g. duodenal mechano- or chemosensory afferents or nutrient clearance) play a crucial role for the manifestation of meal related symptoms.

\section{W1062}

Impact of the Rome II Criteria - Assessment of Related Comorbid Prevalence and Costs of Patients with Functional Dyspepsia

Stephen George, Nathan L. Kleinman, Richard Brook, Jim . Smeeding

Background: The etiology of functional dyspepsia (FD) is debated, but according to the ROME II Criteria, FD always presumes the absence of a structural or biochemical explanation for the symptoms. No published data exist on the associated co-morbid prevalence or costs for patients with FD. Objective: To assess the related co-morbid prevalence and costs associated with FD. Methods: A retrospective database analysis was conducted of a personcentric database derived from multiple large geographically diverse US based employers with data from 2001 through 2004. Employees with FD (ICD-9 536.8) were compared to employees without FD. Data were compared for a 12 -month period beginning 3-months prior to the first diagnosis of FD (the index date). The average index date from the FD cohort was assigned for the control group. To control and adjust for differences in the groups, we used multiple regression, Markov modeling techniques, and the Charlson Comorbidity Index. Prevalence comparisons were made using the 261 Agency of Healthcare Quality in Research (AHQR) specific diagnostic categories. Results: A total of 275,857 patients were identified, but only 1,669 patients (median age $=42$ years, $54.1 \%$ female) met the inclusion criteria for FD. Patients with FD had a higher prevalence rate of AHQR diagnostic categories 159 of $261(\mathrm{p}<0.05)$ and higher costs 141 of 261 ( $<<0.05)$. Excluding the FD diagnosis, 7 out of the top 15 most prevalent occurring AHRQ specific categories were greater in the FD group $(\mathrm{p}<0.05)$ and included abdominal pain $(46.5 \%$ vs $8.2 \%)$, other GI disorders ( $28.1 \%$ vs $4.7 \%$ ), screening suspected conditions ( $27.5 \%$ vs $20.3 \%$ ), medical exam requiring further evaluation ( $24.2 \%$ vs $15.9 \%$ ), residual codes ill defined symptoms ( $21 \%$ vs $11.6 \%$ ), nonspecific chest pain ( $20 \%$ vs $7 \%$ ), and unknown diagnosis $(19.8 \%$ vs $11.3 \%)$. The top costs differences between the two groups were for abdominal pain $(\$ 308)$, biliary tract disease (\$299), coronary atherosclerosis (\$228), stomach/duodenum disorders (\$188), esophageal disorders (\$166), gastritis and duodenitis (\$163), and nonspecific chest pain (\$139). Global health care cost comparisons for FD showed a difference of $\$ 3,421 \quad(\mathrm{p}<0.0001)$. Other cost comparisons were statistically significant for prescription drugs ( $\$ 780$ vs. $\$ 415, \mathrm{p}<0.0001$ ) and sick leave ( $\$ 462$ vs. $\$ 359, p<0.001$ ). Of interest is that only $60 \%$ of FD patients were using drug therapy. Conclusion: This study shows that FD patients present with variety of symptoms and their management is complex. There needs to be more education so that practitioners can better understand the coexistence of associated conditions and apply the ROME II criteria.

\section{W1063}

Endoscopic Findings in Patients with Dyspeptic Symptoms Who Fail Acid Suppressive Therapy

Nimish Vakil, Sander Veldhuyzen van Zanten, Nigel Flook, Karsten Lauritsen, Tore Persson, Ewa Bjorck, Johan Brun, Tore Lind, Nicholas J. Talley

BACKGROUND: The 2005 AGA guidelines on dyspepsia recommend that in low Helicobacter pylori prevalence areas $(<15 \%)$ patients should receive an empirical trial of proton pump inhibitor (PPI) therapy, with endoscopy reserved for those who fail to respond. However, there is little information on the abnormalities found in these patients. Our aim was to determine the underlying endoscopic abnormalities in patients with uninvestigated dyspepsia who fail to respond to acid suppressive therapy. METHODS: H. pylori-negative patients aged 18-50 years with intermittent or continuous epigastric pain or burning centered in the upper abdomen for $\geq 3$ months were recruited from primary care practices in 10 countries (Study SD-NED-0022). Patients were excluded if they had: undergone endoscopy or radiographic studies within the previous 6 months or on more than 2 occasions in the preceding 10 years, dominant symptoms of heartburn and acid regurgitation or alarm features (dysphagia, weight loss, bleeding), or anti-H. pylori therapy in the previous 12 months. Patients received esomeprazole for 1 week as a PPI trial, followed by esomeprazole $40 \mathrm{mg}$ or placebo for 7 weeks. Data presented here relate to patients who prematurely discontinued from the study or completed this study and had persistent symptoms. These patients underwent EGD. RESULTS: Of 1250 patients who were included in the total study population and received PPI therapy, $208(16.7 \%)$ had an EGD because of persistent gastrointestinal symptoms. Clinically significant abnormalities were found in $85(41 \%)$ of patients. The most commonly reported abnormalities are presented in the Table. No malignancies were reported in these patients. CONCLUSIONS: Although erosive esophagitis is common in H. pylori-negative uninvestigated dyspeptic patients with persistent symptoms after PPI therapy or placebo, more serious abnormalities are rare. Peptic ulcer disease is very rare in these patients. These data support the current AGA guidelines and demonstrate that serious abnormalities are not found in patients with persistent dyspeptic symptoms despite PPI therapy. Clinically significant abnormalities

\begin{tabular}{|c|c|}
\hline & Endoscopic findings \\
\hline Total patients & 208 \\
\hline Erosive esophagitis & $44(21.2 \%)$ \\
\hline Barrett's esophagus & $3(1.4 \%)$ \\
\hline Esophageal ulcers & $3(1.4 \%)$ \\
\hline Gastric erosions & $20(9.6 \%)$ \\
\hline Gastric ulcers & $1(0.5 \%)$ \\
\hline Gastritis/duodenitis & $20(9.6 \%)$ \\
\hline Duodenal ulcers & 0 \\
\hline Malignancy & 0 \\
\hline
\end{tabular}

\section{W1064}

Accelerate Reversal of Helicobacter Pylori-Induced Gastric Dysfunction By Specific Probiotics Through An Anti-Inflammatory Effect Premysl Bercik, Elena F. Verdu, Xian Xi Huang, Thomas Tompkins, Stephen M. Collins

Background/Aim: We have previously investigated the relationship between gastric dysfunction and Helicobacter pylori ( $\mathrm{Hp}$ ) eradication (E) in a murine model. Here we tested whether specific probiotics accelerate the recovery of gastric inflammation and of impaired gastric function after $\mathrm{HpE}$. Methods: Uninfected control and $\mathrm{Hp}$ infected Balb/c mice were studied before, at 2 weeks and 2 months after $\mathrm{HpE}$. Immediately after antibacterial therapy, groups of mice ( $\mathrm{n}=8$ /group) were gavaged with a probiotic product, Lacidofil, containing Lactobacillus helveticus R0052 and L. rhamnosus R0011 or, a maltodextrin placebo for 2 weeks. Gastric emptying (GE) was assessed by videofluoroscopy image analysis. Frequency of eating bouts and amount of food was recorded during 24 hours by a computer-assisted method. TNF$\alpha$ was detected by RT-PCR in gastric sections obtained 2 months after HpE. Results: Hp infected mice had delayed gastric emptying by $42 \%$ compared to uninfected mice $(p<0.05)$. Uninfected mice had $13 \pm 3.5$ eating bouts/24 hours while $H p$ infected mice had $19.5 \pm 4.8$ bouts $/ 24$ hours $(\mathrm{p}<0.05)$. Eradication therapy improved GE 2 weeks after $\mathrm{HpE}$, and normalized it at 2 months. In contrast, anti-H. pylori therapy had no effect on $\mathrm{Hp}$-induced altered eating patterns that remained abnormal 2 months after $H p$ E. Altered eating patterns after $\mathrm{HpE}$ were paralleled by a persistent $50 \%$ increase in TNF- $\alpha$ in gastric tissue when compared to uninfected mice. Prompt probiotic therapy after $\mathrm{HpE}$ accelerated normalization of GE at 2 weeks, and normalized altered feeding behavior 2 months after $\mathrm{HpE}$. Mice previously infected but treated with probiotics had decreased levels of TNF- $\alpha$ in gastric tissue by $40 \%$ vs previously infected mice treated with placebo $(\mathrm{p}=0.01)$. Conclusions: Persistent dyspeptic behavior in mice, characterized by abnormal 24-hour feeding patterns after $\mathrm{HpE}$, is normalized by prompt treatment with L. helveticus R0052 and L. rhamnosus R0052. The underlying mechanism may involve accelerated resolution of residual post-infective inflammation. Probiotics, given immediately after Hp eradication may be helpful in the management of accompanying dyspeptic symptoms. 
W1065

Dyspeptic Symptoms Improvement in Patients with Non-Erosive Reflux Disease Is Correlated with the Degree of Intragastric Acid Inhibition Giovanni Sarnelli, Luigi Russo, Ida Esposito, Francesco De Giorgi, Maria Flavia Savarese, Eleonora Efficie, Gabriele Budillon, Rosario Cuomo

Functional dyspepsia and non-erosive reflux disease (NERD) are highly prevalent functional diseases. In both the diseases symptoms overlap can be expected by chance alone, but recent data indicated that acid may be the common factor to explain symptoms generation. Indeed, esophageal acid perfusion has been recently associated with the onset of dyspeptic symptoms in healthy volunteers. Our aim was to study the effect of acid and its suppression, by PPI therapy, on NERD patients with dyspeptic symptoms. Hundred-twenty consecutive patients with reflux-associated symptoms were studied. Sixty-nine of them were selected because they were found to have non-erosive reflux disease during upper GI endoscopy and fulfilled the Rome criteria for functional dyspepsia. A standardized questionnaire, assessing the severity (effect on daily activities, from 0 to 3 ) of belching, abdominal bloating, early satiety, epigastric pain and burning, nausea, vomiting and postprandial fullness, and an esophagogastric $24 \mathrm{hr}$ pHmetry were performed before and at 4th week therapy with esomeprazole $20 \mathrm{mg}$ bid. Intragastric acid inhibition was evaluated as the $\%$ of $\mathrm{pH}>3$ and symptoms improvement was considered effective if a reduction of dyspepsia severity score (at least $50 \%)$ respect to baseline was obtained. Esophageal basal acid exposure ( $\mathrm{pH}<4>4 \%$ ) was pathological in 44 pts, whereas it was normal in 25 . No significant difference was observed in the prevalence and the severity of individual dyspeptic symptoms. At baseline the intragastric $\% \mathrm{pH}>3$ was similar in subjects with normal and abnormal acid exposure $(19 \pm 3.8$ vs $18 \pm 4.5, \mathrm{p}=\mathrm{NS}$ ). During esomeprazole therapy, reflux-associated symptoms improved in the majority of patients, but 7/69 patients had persistent pathological esophageal acid exposure. Overall PPI therapy significantly improved dyspeptic symptoms in 42/69 patients. Dyspepsia improvement was present in a higher number of patients with abnormal than in those with normal basal acid exposure (31/44 vs $11 / 25$, respectively, $\mathrm{p}<0.05)$, with the former having a longer intragastric acid suppression $(78 \pm 2.6$ vs. $65 \pm 5.4 \% \mathrm{pH}>3, \mathrm{p}<0.05)$. Our findings confirm that functional dyspepsia may coexist in patients with non-erosive reflux disease. A four-week treatment with esomeprazole is effective in reducing dyspeptic symptoms in $60 \%$ of subjects with NERD. Furthermore, a better improvement of dyspeptic symptoms seems dependent on the extent of intragastric acid suppression.

\section{W1066}

Age and Alarm Symptoms As An Indication for Endoscopy in a Resource Limited Environment

Dion A. Levin, Gillian A. Watermeyer, Sabelo J. Hlatswayo, David P. Epstein, Kd(chandu) Bardhan, David C. Metz

Introduction: AGA guidelines suggest that in the absence of alarm symptoms, endoscopy be reserved for dyspeptic patients over age 45 . However, the yield of this approach may not justify its use in a resource-limited environment such as South Africa. Aims: To compare the endoscopic yield in dyspeptic patients with and without alarm symptoms (dysphagia, anaemia, weight loss, vomiting, haematemesis or malaena) and to determine an age cut-off for endoscopy dependent on the presence or absence of alarm symptoms. Methods: A retrospective audit of 4129 upper endoscopic reports from a single tertiary-referral center between February and December 2004 yielded 1081 eligible dyspeptics [32.9\% males, mean age $49.6 \pm 15.1$ (range 13-92)]. Data validation was confirmed by review of $4 \%$ of reports. Demographic data, presenting symptoms and endoscopic findings were recorded. Statistical comparisons were made using Fischer's exact test and Student $t$ test where appropriate. Results: Patients with alarm symptoms were older than those without; $53.2 \pm 16.9$ yrs vs. $48.9 \pm 14.6$ yrs ( $p=0.0006$ ), but the two groups did not differ in terms of gender or ethnicity. Endoscopy was abnormal in 817 patients (75.6\%), [143/172 (83.1\%) of those with alarm symptoms and 674/909 (74\%) of those without alarm symptoms ( $\mathrm{p}=\mathrm{NS})$ ). Abnormal endoscopic findings included benign peptic ulcer disease in 186 patients (45 alarm, 141 no alarm, $\mathrm{p}=\mathrm{NS}$ ), carcinoma in 16 (9 alarm, 7 no alarm, $\mathrm{p}=0.0003$ ) and other findings (gastritis, oesophagitis, etc) in 615 ( 89 alarm, 526 no alarm, $\mathrm{p}=\mathrm{NS}$ ). The yield for benign peptic ulcer disease with or without alarm symptoms was unaffected by age. However, age did impact on the yield for carcinoma according to the presence or absence of alarm symptoms (see Table): Conclusion: Dyspeptic patients with alarm symptoms tend to be older with a higher carcinoma yield. In our resource limited environment, our data support reserving endoscopy for dyspeptics between the age of 45 and 60 years for those with alarm features only and performing it in all dyspeptics 60 years or older. This strategy, in addition to missing 3 cancers below 45 years of age, misses 3 additional cancers between 45 and 60 years of age $(0.28 \%$ of all dyspeptics, $0.77 \%$ of all dyspeptics between 45 and $60 \mathrm{yrs})$

\begin{tabular}{|c|c|c|c|c|c|}
\hline Comparators & $\begin{array}{l}\text { No. of } \\
\text { pts }\end{array}$ & $\begin{array}{l}\text { No. of } \\
\text { cancers }\end{array}$ & $\begin{array}{l}\text { No. OGD's } \\
\text { per cancer }\end{array}$ & $\begin{array}{l}\text { OGD's not done } \\
\text { per missed cancer }\end{array}$ & $\begin{array}{c}\text { Risk } \\
\text { Benefit } \\
\text { Ratio }\end{array}$ \\
\hline $\begin{array}{l}\text { Alarm or } 45 \mathrm{yr} \text { and older } \\
\text { No alarm and < } 45 \mathrm{yr}\end{array}$ & $\begin{array}{l}734 \\
347\end{array}$ & 133 & 57 & 116 & 2.0 \\
\hline $\begin{array}{l}\text { Alarm or } 50 \mathrm{yr} \text { and older } \\
\text { No alarm and }<50 \mathrm{yr}\end{array}$ & $\begin{array}{l}618 \\
463\end{array}$ & 124 & 51 & 116 & 2.3 \\
\hline $\begin{array}{l}\text { Alarm or } 55 \mathrm{yr} \text { and older } \\
\text { No alarm and }<55 \mathrm{yr}\end{array}$ & $\begin{array}{l}492 \\
589\end{array}$ & 115 & 45 & 118 & 2.6 \\
\hline $\begin{array}{l}\text { Alarm or } 60 \mathrm{yr} \text { and older } \\
\text { No alarm and }<60 \mathrm{yr}\end{array}$ & $\begin{array}{l}401 \\
680\end{array}$ & 106 & 40 & 113 & 2.8 \\
\hline $\begin{array}{l}\text { Alarm or } 65 \mathrm{yr} \text { and older } \\
\text { No alarm and < }<5 \mathrm{yr}\end{array}$ & $\begin{array}{l}322 \\
759\end{array}$ & 106 & 32 & 127 & 4.0 \\
\hline
\end{tabular}

\section{W1067}

A Quality Study of Self-Care Use in Patients with Dyspepsia Kerstin M. Stake-Nilsson, Maud Soderlund, Rolf Hultcrantz, Peter Unge

Background: Dyspepsia is a common condition and the lack of effective therapies may lead to the use of self-care methods. This study was conducted to elucidate self-care methods and their effects in Sandviken, a municipality in Sweden. Method: In this qualitative approach focus groups were used. Subjects with any dyspepsia according to the ROME 2 criteria and actively seeking health care institutions were included. A total of thirty-two subjects were invited. Thirteen women and twelve men gave their informed consent and were allocated into five focus groups. The interviews were conducted during April until June 2005. Data were studied systematically using thematic analyses of four self-care categories (1). The effects were classified as preventive, partial and total symptomatic relief. Results: All subjects had sometimes used self-care methods. The following self-care methods were identified to each of the four categories; Nutritional: Banana, Milk products, Bicarbonate, Soda, Rosehip soup, Whiskey, Butter, Hot water, Sweet almonds and Biscuits. All nutritional methods were associated with partial or total symptom relief if initiated at an early stage. Three of these methods were primarily used for prevention. Drug/Biologic: PPIs, H2RAs, Antacids, Silikol (mineral mix of magnesium and silicon), Aloe Vera, Molkosan (whey product), Roots from Roses, Dophilus capsules (lactobacillus) and Goat milk capsules. Drugs that achieved symptom relief were PPIs, H2RAs and antacids. A lesser effect was experienced when the drug was introduced at a late stage. PPIs and H2RAs were also regarded as preventive Spiritual Psychological: relaxation. Physical forces/devices: exercise. The effect of the latter two category methods was limited to partial symptom relief only. Conclusion: In this focus group, quality study approach, a large number of self-care methods associated with various effects were identified. All subjects had used at least one self-care method. The result from this study will be further explored in a quantitative study of patients with dyspepsia. References: 1. Murray R, Rubel A. Physicians and healers: Unwitting partners in health care. NEJM 1992;326:61-64

\section{W1068}

Distinct Dyspepsia Subgroups Exist in the General Population Rok S. Choung, Nicholas J. Talley, G R. Locke, Cathy D. Schleck, Alan R. Zinsmeister

Introduction: The heterogeneity of dyspepsia is well known. In selected tertiary referral patients, subgroups appear to exist but community data are conflicting. We postulated that dyspepsia comprises three distinct subsets, characterized by pain, early satiety or nausea. We aimed to identify and characterize these subsets of dyspepsia: 'frequent upper abdominal pain (UAP)', 'early satiety (ES)' and 'nausea/vomiting (NV)'. Methods: A population-based, cross-sectional survey study was conducted by mailing a valid questionnaire to an age- and gender- stratified random sample of residents of Olmsted County, MN, aged 30-95 years (response rate 55\%). Dyspepsia and irritable bowel syndrome (IBS) prevalence were estimated by Rome II; gastroesophageal reflux (GERD) by weekly or more frequent heartburn or acid regurgitation. Dyspepsia subgroups were categorized based on a priori defined symptoms Results: The prevalence of dyspepsia was 15\%. Of 343 dyspeptic subjects, $51 \%$ reported UAP, $20 \% \mathrm{NV}$ and $47 \%$ ES with limited overlap (figure). Among the three groups, the subjects were similar in age, education level, IBS status and overall GI symptom severity Women were more likely to have ES than UAP; those with GERD were more likely to have UAP than ES; subjects with childhood pain were more likely to have NV than UAP (table) Conclusion: Distinct subgroups of dyspepsia do exist in the general population, suggesting that separate evaluation and treatment strategies are needed. Supported in part by an unrestricted grant from Novartis.

\begin{tabular}{|c|c|c|c|c|c|}
\hline \multicolumn{6}{|c|}{ Predictors for the Symptom Groups: Odds ratios (OR [95\% CI] adjusted for age and education) } \\
\hline & $\begin{array}{c}\text { UAP } \\
\text { N=135, } \%\end{array}$ & $\begin{array}{c}\text { ES } \\
\text { N=114, } \%\end{array}$ & $\begin{array}{c}\text { NV } \\
\text { N=37, } \%\end{array}$ & $\begin{array}{c}\text { ES vs. UAP } \\
\text { OR(95\% CI) }\end{array}$ & $\begin{array}{c}\text { NV vs. UAP OR } \\
(95 \% \text { CI })\end{array}$ \\
\hline Female gender & 53 & 70 & 68 & $1.9[1.1,3.3]$ & $1.8[0.8,4.0]$ \\
\hline IBS & 18 & 27 & 30 & $1.7[0.9,3.2]$ & $2.0[0.8,4.6]$ \\
\hline GERD & 45 & 26 & 49 & $0.4[0.2,0.7]$ & $1.2[0.6,2.5]$ \\
\hline Loss of Weight & 6 & 7 & 8 & $0.99[0.3,2.9]$ & $1.6[0.4,6.7]$ \\
\hline $\begin{array}{c}\text { Childhood pain } \\
\text { (before 15) }\end{array}$ & 11 & 14 & 24 & $1.4[0.7,3.1]$ & $2.6[1.0,6.6]$ \\
\hline
\end{tabular}

$\%$ (values are column percentages) 


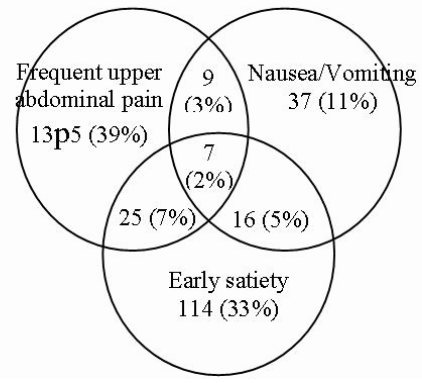

W1069

Gastrointestinal Opportunistic Infection: Is It Associated with Dyspeptic Symptoms in Immunodepressed HIV-Infected Patients Under Highly Active Antiretroviral Therapy?

Ana Luiza Werneck-Silva, Ivete B. Prado

Background: Highly active antiretroviral therapy (HAART) has dramatically reduced the incidence of opportunistic infections (OI) in HIV disease. However, gastrointestinal (GI) OI still occur, mainly in patients with marked depressed immune function. It usually affects the esophagus or intestine. Stomach is rarely involved, although many opportunistic agents have been reported to cause gastritis. Nausea and vomiting are particularly common complaints in HIV-infected patients, and may reflect a variety of different etiologies including medications. On the other hand, clinically apparent GI OI are relatively infrequent. Whether OI can cause dyspeptic symptoms is still unknown. Aim: We have done a prospective study looking for gastrointestinal OI in a large cohort of adult HIV-infected patients under HAART with and without dyspeptic symptoms, all of them with marked depressed immune function. Patients and methods: 690 HIV-infected patients (508 men, 182 women; mean age 39.71 years old) underwent upper endoscopy with gastric and duodenal biopsies. All of them were under HAART. The study conformed the principles of Declaration of Helsinki and Brazilian requirements governing. Patients were classified in two groups. Group 1: 500 patients (161 women, 339 men; mean age 38.77 years old; mean CD4 count 154.3 cells/ $\mathrm{mm} 3$ ) with dyspeptic symptoms such epigastric pain, nausea, vomiting and fullness. Group 2. 190 patients (169 men, 21 women; mean age 40.66 years old mean CD4 count 171.6 cell/mm3) without dyspepsia (in group 2 endoscopy was done to search for esophageal varices or GI Kaposi sarcoma (SK) when indicated, or as part of weight loss, anemia or halitosis investigation). Diagnosis of opportunistic infections was based on typical endoscopic and histologic findings. Results: Group 1: Ol was shown in 08 patients (1.6\%):CMV (4 cases), Cryptosporidium sp. (2 patients), Schistosoma mansoni sp. (1 patient) and Strongyloides stercoralis ( 1 patient). In 3 of them OI was detected in normal appearance gastric or duodenal mucosa. OI was diagnosed only in the stomach in 5 patients, just in the duodenum in 3 patients, and in both, stomach and duodenum, in 2 cases. Group 2. No GI OI was detected. Although GI OI were detected only in Group 1, difference between groups was not significant $(\mathrm{p}=0.07947)$. Conclusion: GI OI were shown in immunodepressed HIV-infected patients under HAART in low numbers. Despite GI OI have been detected only in dyspeptic patients, they could not be related to these symptoms, since numbers did not reach significance. To correct diagnose GI OI, multiple biopsy specimens may be necessary, even from normal appearance mucosa.

\section{W1070}

Correlation Between Electrogastrography (EGG) and Gastric Dysmotility, Duodenal Dysmotility in Patients with Functional Dyspepsia

Weihong Sha, Nonko D Pehlivanov, P Jay Pasricha, Jiande Chen

Abnormalities in gastric slow waves and motility are frequently reported in patients with functional dyspepsia. However, simultaneous assessment of the slow wave and motility abnormalities has rarely been done. The aim of this study is to assess gastric slow waves and antroduodenal motility simultaneous and determine the correlation among all measures in patients with functional dyspepsia. Methods: 31 patients $8 \mathrm{M}, 23 \mathrm{~F}$, mean age 48.7 , Range (23-79) with functional dyspepsia were assessed for severity of upper gastrointestinal symptoms, the electrogastrogram (EGG) and antroduodenal manometry. The EGG and manometry was recorded for 3-4 hours in the fasting state and for two hours after a solid meal. Computerized spectral analysis methods were utilized to compute the following EGG parameters in the fasting and fed state. Dominant frequency, Dominant power and \% of 2 4 cycles/min slow waves. Common gastrointestinal symptoms of nausea, vomiting, upper abdominal pain, bloating, early satiety and etc were assessed for symptom severity and graded. Results: 1) The EGG was abnormal in $71.0 \%$ of patients. The abnormalities included normal slow waves lower than $70 \%$ in fasting state $(51.6 \%$ of patients) and in fed state (48.4\% of patients), a decrease in dominant power in $28.9 \%$ of patients. 2) Antral motility was abnormal in $83.9 \%$ of patients and duodenal motility was abnormal in $74.2 \%$ of patients. There was a significant correlation between these two abnormalities. 3) For the EGG and antral motility, 19 of 31 patients had both abnormal EGG and abnormal antral motility; 2 of 31 patients had both normal EGG and normal antral motility. For the EGG and duodenal motility, these values were 16/31 and 2/31 respectively. 4) The patients showed high symptom scores particularly to upper abdominal pain, nausea and belch, with an average symptom score of 2.5/4 for pain; 2.5/4 for nausea and 2.3/4 for belch, respectively. The mean total score was 22/52 for all symptoms. 5) No significant correlation was noted between the symptom scores and any of the EGG or motility parameters. Conclusions: More than 2 thirds of patients with functional dyspepsia have abnormalities in the EGG and antral duodenal motility. The sensitivity of these two different methods is essentially the same Abnormalities in gastric motility and duodenal motility are correlated whereas the symptoms are not correlated with any of the EGG or motility parameters

\section{W1071}

The Effect of Helicobacter Pylori Eradication On Gastroesophageal Reflux, Gastrointestinal Symptoms and Quality of Life in Patients with Functional Dyspepsia

Irfan Soykan, Mehmet Bektas, Murat Toruner, Hulya Cetinkaya, Ali Ozden

Introduction: Although it has been postulated that reduced acid secretion related to Helicobacter pylori (H pylori) corpus gastritis might protect patients from gastroesophageal reflux disease (GERD), the pathogenesis and possible relationship between $H$ pylori and GERD remains controversial. Aims \& Methods: The aims of this study were to investigate the effect of $H$ pylori eradication on gastroesophageal reflux, gastrointestinal symptoms and quality of life in patients with functional dyspepsia (FD). $20 \mathrm{H}$ pylori positive patients (15 women, mean age:44, range: 24-66 years) diagnosed as having FD according to Rome-II criteria were enrolled into the study. H pylori status was evaluated by endoscopy before the study and at least 4 months after the completion of the eradication therapy. In each patient 3 antral and 3 corpus biopsies were obtained and biopsy specimens were subjected to $H$ pylori culture, histologic examination and rapid urease testing. Esophageal motility testing and $\mathrm{pH}$ recordings were obtained from each patient before and at the end of the study period. Each patient's gastrointestinal (GI) symptoms were recorded and graded according to Glasgow dyspepsia score. 36-Item short-form health survey (SF-36) and EDQ5 health survey were obtained form each patient in order to identify quality of life before and at least 4 months after eradication therapy. Results: Helicobacter pylori eradication was accomplished in 13 patients (65\%). Gastrointestinal symptoms improved in $H$ pylori eradicated patients (10.3 vs $7.5, \mathrm{p}<0.05)$ compared to baseline. Fasting lower esophageal sphincter pressures increased ( 21.6 vs $24.4 \mathrm{mmHg}$ ) after $H$ pylori eradication. Neither the amplitude of peristaltic contractions in the esophageal body ( 59.4 vs $57.7 \mathrm{mmHg}$ ) nor the velocity of peristaltic contractions changed before and after eradication. The percent time of esophageal $\mathrm{pH}<4(0.7$ vs 2.6 , $\mathrm{p}<0.001$ ), reflux events longer than 5 mins ( 0 vs $0.7, \mathrm{p}<0.005$ ) and total reflux number $(10.3$ vs $19.3, p<0.005)$ significantly increased after eradication therapy. $H$ pylori eradication did not influence quality of life in patients with functional dyspepsia. Conclusion: Majority of the patients experienced a significant change in esophageal acid exposure after $H$ pylori eradication. Development of GERD post $H$ pylori eradication likely reflects an increase in the acidity of the refluxate. $H$ pylori eradication significantly decreases gastrointestinal symptoms, however has no effect on quality of life in patients with functional dyspepsia.

\section{W1072}

Incident Dyspepsia in the Treatment of Peptic Ulcer Disease: A Meta-Analysis Comparing H. Pylori (HP) Eradication Versus Proton Pump Inhibitor (PPI) Therapy

Brennan M. Spiegel, Jennifer Tan, Brian Lim, Mary Farid, Inder Singh, Martijn G. van Oijen

Background: Although guidelines recommend HP "test-and-treat" (T\&T) for uninvestigated dyspepsia, it remains uncertain whether T\&T is preferable to empiric PPI therapy. Whereas PPIs are modestly effective in functional dyspepsia and highly effective in GERD, T\&T is largely ineffective in these common etiologies of dyspepsia. T\&T therefore rests on the assumption that HP eradication is superior to PPI in symptomatic peptic ulcer disease (PUD) - the remaining major etiology of dyspepsia. Yet it is unclear whether there is a difference in dyspeptic symptoms (in contrast to ulcer healing) following T\&T vs PPI in PUD, and the answer to this question has cost-effectiveness implications when selecting between strategies (Spiegel et al Gastro 2002). We performed a meta-analysis to compare the incidence of dyspeptic symptoms in patients with PUD treated with HP eradication vs PPI. Methods: We conducted a structured search of MEDLINE to identify English-language clinical trials of HP eradication or PPI in PUD that provided data about incident foregut dyspeptic symptoms, including "epigastric pain," "acid reflux," "nausea," and "dyspepsia." Independent reviewers performed title and abstract selections using a priori criteria, and subsequently abstracted data from each selected manuscript. We then calculated the sample size-weighted pooled proportion of patients with incident dyspeptic symptoms in the HP eradication and PPI arms of the included studies, and performed meta-analysis of head-tohead comparisons. We calculated stratified weighted means to adjust for PPI dose and HP eradication rates. Results: We identified 1497 titles, of which 122 (representing 260 arms) were selected for final review. The weighted incidence of dyspepsia was $18.2 \%$ (95\% CI= $13.7 \%, 23.2 \%)$ in the 148 PPI arms, and $22.8 \%(95 \% \mathrm{CI}=19.4 \%, 26.2 \%)$ in the $112 \mathrm{HP}$ eradication arms ( $\mathrm{p}<0.001$ for difference). Meta-analysis of 5 head-to-head trials revealed a higher incidence of dyspepsia with HP eradication vs PPI ( $R R=2.4 ; C I=1.6,3.8)$, with a "number needed to harm" of 10 (absolute risk increase $=10 \% ; \mathrm{CI}=5 \%, 14 \%$ ). There was less dyspepsia with high vs low-dose PPI $(\mathrm{p}<0.001)$, and with high $(>70 \%)$ vs low HP eradication rates $(\mathrm{p}<0.001)$. Conclusions: The incidence of dyspeptic symptoms is higher in PUD patients receiving HP eradication vs PPI therapy, and this difference is both statistically significant $(\mathrm{p}<0.001)$ and clinically relevant (effect size $=1.4 \mathrm{SD})$. Since previous data indicate that T\&T is inferior to PPI in functional dyspepsia and GERD, these data in PUD further the argument that empiric PPI might be preferable to T\&T in uninvestigated dyspepsia. (this independent research is unfunded) 
Acid and Weakly Acidic Reflux in 80 Patients with Unexplained Chronic Cough: An Update

Kathleen Blondeau, Lieven Dupont, Jan Tack, Jozef Janssens, Daniel Sifrim

Acid gastroesophageal reflux has been recognized as a possible cause of chronic cough. We have demonstrated that not only acid but also weakly acidic reflux can be temporally associated with episodes of cough. We aimed to further characterize the relationship between cough and both acid and weakly acidic reflux in our expanded series of 80 patients with unexplained cough. Methods: Patients with chronic unexplained cough were studied with ambulatory manometric-impedance-phmetry (Sandhill Sci). Out of these 63 [24 men, median age 55 (22-77)] were studied "off" PPI and 17 were studied "on" PPI [5 men, median age 56 (39-73)]. Esophago-gastric manometry was used for precise recognition of cough, which was defined as a rapid pressure increase occurring simultaneously in all channels. Reflux was independently assessed with impedance- $\mathrm{pH}$ monitoring and defined as acid $(\mathrm{pH}<4)$, weakly acidic ( $\mathrm{pH} 4-6.5)$ and weakly alkaline (impedance drops with $\mathrm{pH}>6.5$ ). Cough was considered "induced by" reflux, if it started in a 2 min period after a reflux event. A Symptom Association Probability (SAP) was calculated for each type of reflux in every patient and considered positive if $>95 \%$. Results: In patients studied "off" PPI, a total number of 1970 cough bursts $[25,(5-111)] /$ pat and 2782 reflux events $[42,(7-89)] /$ pat were analyzed. Reflux events were classified as acid $(\mathrm{n}=1637)$ [22(2-70)]/pat, weakly acidic $(\mathrm{n}=914)$ [13(1-44)]/ pat and weakly alkaline $(n=285)[1(0-22)] /$ pat. Twenty-three patients had an increased esophageal acid exposure. From the total number of cough, 250 (13\%) were considered "induced by" reflux (131 acid, 106 weakly acidic and 13 weakly alkaline). The per-individual analysis showed a SAP+ for acid reflux in 7 patients, weakly acidic reflux in 13 patients and for both acid and weakly acidic reflux in 3 patients. In patients studied "on" PPI, a total number of 444 cough bursts [18(5-145)]/pat and 494 reflux episodes [36(7-68)]/pat were recorded. Thirty-three reflux episodes involved acid reflux [1(1-18)]/pat, 317 weakly acidic [22(0-46)]pat and 134 weakly alkaline [3(0-26)]/pat. All patients had a normal esophageal acid exposure. In this group, 68 cough events (15.3\%) were considered to be "induced by" a reflux episode ( 3 acid, 46 weakly acidic and 11 weakly alkaline). The perindividual analysis showed 5 patients with SAP+ for weakly acidic reflux. In total 21/80 patients had a SAP+ for weakly acidic reflux. Conclusion: A significant temporal relationship between cough and weakly acidic reflux was found in $26 \%$ of the patients with chronic unexplained cough. In those patients endoscopic or surgical anti-reflux procedures might be considered.

\section{W1074}

Acid and Non-Acid Reflux After Lung Transplantation. Impedance-pH Monitoring in Patients with and Without Post-Transplant Chronic Rejection Kathleen Blondeau, Lieven Dupont, Jan Tack, Jozef Janssens, Daniel Sifrim

Pulmonary dysfunction due to Bronchiolitis Obliterans Syndrome (BOS) predisposes to a poor long term survival after lung transplantation (Ltx). It has been suggested that acid gastroesophageal reflux (GER) contributes to development of chronic rejection or BOS and preventive antireflux surgery has been proposed. The role of less acidic reflux in the pathogenesis of BOS is unknown. Aim: to determine, in a cross-sectional study, the prevalence of acid and non-acid reflux in a cohort of lung transplant recipients by means of combined esophageal 24-hr impedance-pH testing. Methods: 54 patients [27 men,age 56 (19-75)] underwent 24h impedance-pH recording (Sandill Sci) 3 years (1-9) after Ltx. Reflux episodes were identified by impedance and characterized by $\mathrm{pH}$ as acid $(\mathrm{pH}<4)$ and non-acid $(\mathrm{pH}>4)$. Three different stages of BOS were defined: BOS"0": normal pulmonary function $(\mathrm{n}=25)$; BOS "p": (FEV1 90\% of baseline)(n=14) and BOS"l" :(FEVl $\leq 80 \%$ of baseline)(n= 15). All patients were studied "on" weak acid suppression (omeprazole $20 \mathrm{mg}$ or ranitidine $300 \mathrm{mg}$ ) and most patients with BOS"p" and BOS" 1 " were "on" azithromycin to improve lung function. Azithromycin is a macrolide antibiotic with effect on gastrointestinal motility. Results: The esophageal acid exposure was normal and similar in the 3 groups. Only 2 subjects ( 1 with BOS" 0 " and 1 with BOS"1") had increased esophageal acid exposure. There was no significant difference in the mean number of total, acid or nonacid reflux events between patients with or without BOS. There was no correlation between FEVI at the time of impedance-pH testing and the esophageal acid exposure or the number of reflux events. When examined separately, patients without adequate acid suppression (gastric $\mathrm{pH}<4$ more than $50 \%)(n=21)$, those with BOS"p" and BOS"1" showed a trend to decreased acid reflux and bolus exposure. Conclusion: Our cohort of patients did not have increased acid or non-acid reflux after lung transplantation. Unlike previous reports, our patients with BOS had either similar or less reflux than those without BOS, perhaps due to an antireflux effect of the macrolide antibiotic azythromycin (under current investigation).

\begin{tabular}{|c|c|c|c|c|c|c|c|c|}
\hline & \multicolumn{4}{|c|}{ All patients } & \multicolumn{4}{c|}{ Inadequate acid suppression } \\
\hline & BOS 0 & BOS p & BOS 1 & p value & BOS 0 & BOS p & BOS 1 & p value \\
\hline Total reflux (n/24h) & 33 & 19 & 31 & 0.1 & 33 & 38 & 12 & 0.2 \\
\hline Acid reflux (n/24h) & 15 & 4 & 5 & 0.06 & 17.5 & 7 & 6 & 0.1 \\
\hline Non-acid reflux (n/24 & 18 & 16 & 16 & 0.6 & 17.5 & 17 & 7 & 0.2 \\
\hline Acid exposure (\%) & 1.1 & 1 & 0.5 & 0.1 & 2.5 & 1 & 0.5 & 0.07 \\
\hline Bolus exposure & 554 & 350 & 216 & 0.3 & 496 & 394 & 128 & 0.06 \\
\hline
\end{tabular}

\section{W1075}

Baseline Analysis of Symptom Spectrum in GERD Clinical Trial Patients: Results from the Request ${ }^{\mathrm{TM}}$ Database

Ronnie Fass, Vincenzo Stanghellini, Hubert Monnikes, Karna Dev Bardhan, Peter Berghofer, Peter Sander, David Armstrong

PURPOSE: GERD patients suffer from a broad range of symptoms, not only heartburn and acid eructation. Many of them often are not considered to be associated with the disease. The validated reflux questionnaire ReQuest ${ }^{\mathrm{TM}}$ is a dimension-orientated tool assessing the spectrum of GERD symptoms and general well-being. Here, we analyzed baseline data o these dimensions (condensing 65 symptom descriptions) as collected from GERD clinical trials that used ReQuest ${ }^{\mathrm{TM}}$. METHODS: The ReQuest ${ }^{\mathrm{TM}}$ database covers 12 erosive esophagitis (EE) as well as 2 non-erosive reflux disease (NERD) clinical trials. Baseline data of 6.810 per-protocol patients were analyzed without reference to subsequent treatment. Analyses of the symptom spectrum were done using the ReQuest ${ }^{\mathrm{TM}}$ dimensions of acid complaints, upper abdominal/stomach complaints, lower abdominal/digestive complaints, nausea, sleep disturbances, and other (extraesophageal, atypical) complaints. Subdivided into EE and NERD, mean scores of these ReQuest ${ }^{\mathrm{TM}}$ dimensions representing patients' symptom burden were calculated as weighted product of frequency $\mathrm{x}$ intensity. In addition, general wellbeing was assessed and the percentage of patients reporting complaints was calculated for each ReQuestTM dimension. RESULTS: 5.846 patients suffered from EE and 964 from NERD. The percentage of patients experiencing complaints as well as their mean symptom score was comparable in EE and NERD for each ReQuest ${ }^{\mathrm{TM}}$ symptom dimension. The mean scores for general well-being were similar in both groups (EE: 2.1 vs. NERD: 1.9) CONCLUSIONS: GERD is associated with other GI dysfunction since upper and lower abdominal symptoms are also very common. Thus, the distinction between GERD and functional disease may not be as clear as previously thought. EE and NERD have comparable symptom and well-being profiles at baseline. 'Acid complaints' is not the only symptom dimension associated with GERD. GERD therapeutic trials should evaluate the broad spectrum of GI symptoms and determine the extent to which these symptoms/dimensions respond to treatment. The present analysis further reveals that NERD patients do not have more IBSlike/functional symptoms than those with $\mathrm{EE}$, suggesting that these patients have also true GERD

\begin{tabular}{|c|c|c|}
\hline ReQuest ${ }^{\text {TM}}$ - Dimensions & $\begin{array}{c}\text { EE [n=5,846] \% of patients } \\
\text { (mean score of dimension) }\end{array}$ & $\begin{array}{c}\text { NERD [n=964] \% of patients } \\
\text { (mean score of dimension) }\end{array}$ \\
\hline Acid complaints & $91.1(2.7)$ & $96.7(2.5)$ \\
\hline $\begin{array}{c}\text { Upper abdominal / } \\
\text { stomach complaints }\end{array}$ & $79.2(2.1)$ & $82.7(2.0)$ \\
\hline $\begin{array}{c}\text { Lower abdominal / } \\
\text { digestive complaints }\end{array}$ & $60.6(1.2)$ & $60.0(1.2)$ \\
\hline Nausea & $46.2(0.9)$ & $45.3(0.9)$ \\
\hline Sleep disturbances & $66.2(1.0)$ & $57.7(0.9)$ \\
\hline Other complaints & $58.6(0.7)$ & $58.6(0.6)$ \\
\hline General well-being & $----(2.1)$ & $----(1.9)$ \\
\hline
\end{tabular}

\section{W1076}

Distinct Pathophysiological Mechanisms and Clinical Characteristics of NonErosive Reflux Disease (NERD) and GERD Related Noncardiac Chest Pain (GCP)

Justin Wu, Carrian Cheung, Vincent Wong, Joseph Sung

BACKGROUND: GERD is a common cause of noncardiac chest pain. However, it is unclea why many GCP patients do not have reflux symptoms despite abnormal esophageal pH study and symptomatic response to acid suppressant. AIM: To compare the clinical characteristics and esophageal functions of patients with GCP and non-erosive reflux disease (NERD) METHODS: We prospectively recruited consecutive patients with NERD (defined as weekly attacks of heartburn or acid regurgitation with symptom response to proton pump inhibito (PPI)) and GCP (defined as weekly attacks of noncardiac chest pain with absence of reflux symptom but complete response to PPI). All patients completed a self-administered standard questionnaire to evaluate symptom, underwent EGD to exclude erosive esophagitis, and received esophageal function tests. The tests included stationary esophageal manometr (EM), acid-perfusion and edrophonium provocation test, 2-hour postprandial EM and $\mathrm{pH}$ metry after a standard $400 \mathrm{kcal}$ test meal; and ambulatory 24-hour pH metry. RESULTS: 48 NERD and 36 GCP patients were studied. Both groups were comparable in age (NERD Vs GCP: $42.3+/-8.6$ Vs 44.1+/-10.4, p=0.17), gender (male subjects, NERD Vs GCP: 22 Vs $15, \mathrm{p}=0.7$ ) and 24-hour esophageal acid exposure (Mean DeMeester score, NERD Vs GCP 8.4+/-9.7 Vs 7.8+/-8.6, $\mathrm{p}=0.42$ ). NERD patients had significantly higher prevalence of functional dyspepsia (FD) and irritable bowel syndrome (IBS) than GCP patients (FD: 69\% Vs $22 \%, \mathrm{p}<0.001$; IBS: $44 \%$ Vs $11 \%, \mathrm{p}=0.001$ ). For EM, GCP patients had higher prevalence of ineffective esophageal motility (IEM: failed peristalsis $>/=30 \%$ ) and weak lower esophageal

. 
sphincter (LES: pressure $<10 \mathrm{mmHg}$ ), whereas NERD patients had higher prevalence of positive acid perfusion test. For 2-hour postprandial study, NERD patients had significantly more transient LES relaxation (TLESR) with and without acidic reflux, whereas zero pressure related acidic reflux predominated in GCP patients (Table). CONCLUSION: Despite similar esophageal acid exposure and response to PPI, GCP and NERD have different pathophysiological mechanisms and represent two distinct entities of GERD. The lower prevalence of positive acid perfusion test and concomitant FD and IBS in GCP patients suggest that visceral hypersensitivity plays a less important role in the pathogenesis of GCP as compared to NERD.

\begin{tabular}{|c|c|c|c|c|c|c|c|}
\hline & $\begin{array}{c}\text { IEM } \\
(\%)\end{array}$ & $\begin{array}{c}\text { LESP<10 } \\
\mathrm{mmHg} \\
(\%)\end{array}$ & $\begin{array}{c}\text { Positive acid } \\
\text { perfusiontest } \\
(\%)\end{array}$ & $\begin{array}{c}\text { Positive } \\
\text { edrophonium } \\
\text { test }(\%)\end{array}$ & $\begin{array}{c}\text { Median no. } \\
\text { of TLESR } \\
\text { without } \\
\text { reflux } \\
\text { (range) }\end{array}$ & $\begin{array}{c}\text { Median no. } \\
\text { of TLESR } \\
\text { with acidic } \\
\text { reflux } \\
\text { (range) }\end{array}$ & $\begin{array}{c}\text { Median no. } \\
\text { of acidic } \\
\text { reflux due } \\
\text { to zero } \\
\text { LESP } \\
\text { (range) }\end{array}$ \\
\hline NERD & $\begin{array}{c}6 \\
(13)\end{array}$ & $5(10)$ & $20(42)$ & $1(2)$ & $7(2-13)$ & $10(4-25)$ & $1(0-4)$ \\
\hline GCP & $\begin{array}{c}12 \\
(33)\end{array}$ & $14(39)$ & $2(6)$ & $1(3)$ & $3(1-6)$ & $2(1-9)$ & $4(0-8)$ \\
\hline P & 0.02 & 0.002 & $<0.001$ & 1 & 0.02 & 0.003 & 0.03 \\
\hline value
\end{tabular}

W1077

Increased Prevalence of Functional Dyspepsia and IBS in Proven GERD Has a Negative Effect On Health-Related Quality of Life

D.R. de Vries, Margot van Herwaarden, Andre Smout, Melvin Samsom

Background: Previous studies reported overlap between GER symptoms, functional dyspepsia (FD) and irritable bowel syndrome (IBS). To date this has not been shown among patients with proven GERD. Furthermore, the effect of this overlap on the health-related quality of life (HRQoL) has not been investigated. Aim: determine the prevalence of FD and IBS in GERD patients and the impact of both on HRQoL. Method: GERD was diagnosed using 24-hour esophageal $\mathrm{pH}$ recording (total time $\mathrm{pH}<4 \geq 6 \%$ and/or positive SAP) in 263 patients (161 male; mean age 50.6. 48 patients recruited by advertisement, 215 referred because of GERD). The patients completed a questionnaire which assessed the symptoms of FD and IBS according to Rome II. The SF-36 was used to measure HRQoL in patients and 131 ageand sex-matched healthy volunteers. Median scale scores were compared using KruskallWallis $\mathrm{H}$ and subsequently Mann-Whitney U tests. Results: FD criteria were met by 66 GERD patients (25\%, prevalence in Dutch general population 13-14\%). IBS criteria were met by 93 patients (35\%, prevalence in Dutch general population 2-6\%). Thirteen GERD patients had both FD and IBS (5\%). When the group of referred patients was compared to the group of patients recruited with an advertisement, FD and IBS prevalence were found to be higher in the referred group (FD: $26 \%$ vs $21 \%$; IBS: $39 \%$ vs $21 \%$; FD and IBS: $5 \%$ vs $4 \%$ ). When GERD patients had no concomitant FD or IBS, they scored lower HRQoL than healthy subjects on one scale only: physical functioning $(\mathrm{P}=0.001)$. GERD patients with FD scored lower than healthy volunteers on the physical and social functioning scales, the physical role limits scale, and the vitality and pain scales (all P $\leq 0.001$ ). GERD patients with IBS scored lower than healthy volunteers on all these subscales and moreover on emotional role limits and mental health (all $\mathrm{P}<0.001$ ). GERD patients with both FD and IBS scored lower on the same scales with the exception of mental health (all $\mathrm{P} \leq 0.001$ ). Patients with only GERD were compared to GERD patients who also had FD and/or IBS. GERD+FD patients scored lower on physical and social functioning, pain, vitality and general health experience (all $\mathrm{P} \leq 0.001$ ). GERD+IBS patients scored lower on all scales (all $\mathrm{P}<0.001$ ). GERD+FD+IBS patients scored lower on physical functioning, emotional and physical role limits, pain, vitality and general health experience (all $\mathrm{P} \leq 0.001$ ). Conclusion: Compared to the general population FD and IBS are more prevalent in patients with proven GERD In GERD patients who seek hospital care this prevalence is even higher. HRQoL is strikingly lowered in GERD patients who have concomitant FD or/and IBS.

\section{W1078}

Symptom Type On PPI Bid Therapy Does Not Predict Reflux Identified By Multichannel Intraluminal Impedance-pH

Inder Mainie, Radu Tutuian, Amine Hila, Amit Agrawal, Janice Freeman, Donald O. Castell

Background: Combined Multichannel Intraluminal Impedance (MII) detects gastroesophageal reflux (GER) at multiple levels of the esophagus independent of $\mathrm{pH}$ and is used in the evaluation of patients with refactory symptoms despite PPI bid therapy. Aim: To clarify if the type of symptom is a predictor of the type of reflux. Method: All patients with persistent symptoms despite proton pump inhibitor (PPI) therapy referred to the esophageal laboratory underwent combined MII-pH monitoring. Prior to the study, patients and/or the referring physician were contacted to ensure that the patient had been on at least twice daily PPI for at least a month or more prior to testing. The $2.1 \mathrm{~mm}$ catheter monitors changes in intraluminal impedance at $3,5,7,9,15$ and $17 \mathrm{~cm}$ above the LES with $\mathrm{pH}$ was at $5 \mathrm{~cm}$ above and $10 \mathrm{~cm}$ below the LES. A positive SI (+SI) was declared if $\geq 50 \%$ (i.e. at least half of the symptoms were associated with reflux). Typical GERD symptoms were heartburn, regurgitation or chest pain. Atypical symptoms were cough, hoarseness, abdominal discomfort, nausea, dysphagia throat clearing, mucous, globus, belch, catarrh, hiccup and wheeze. Results: From a total of 166 patients (Female: 119; mean age 52; range 16 to 85 years), 145 patients with symptoms during test day. 265 GERD symptoms in total were recorded; typical $(\mathrm{n}=155)$ and atypical $(n=110)$. See graph below. Conclusion: MII-pH shows presence and type of reflux related to persistent symptoms on PPI therapy. Individual symptoms, do not predict whether the results will be positive or negative. Almost two-thirds of patients with regurgitation have a positive study; however one-third of these patients did not show a temporal relationship with reflux

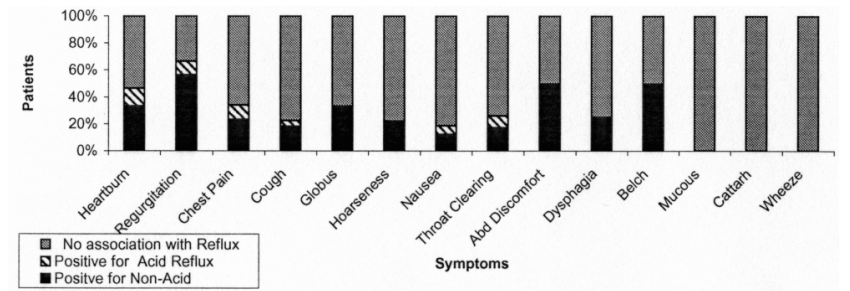

W1079

Laryngo-Pharyngeal Reflux (LPR) and GERD: A Systematic Review and MetaAnalysis of Medical and Surgical Therapies

Luigi Gatta, Nimish Vakil, Sara Zucchini, John Osborn, Giovanni Sorrenti, Dino Vaira

Aim: To evaluate the effectiveness of medical or surgical treatment for GERD in adult patients with unexplained symptoms, and/or laryngoscopic signs of LPR. Methods: Data Sources: Studies were identified by searching MEDLINE (1966-September 2005), EMBASE (1980September 2005), Cochrane Controlled Trials Register, and abstracts for the following gastroenterological and ENT meetings: DDW (1975-2005), UEGW (1992-2005), AAOHNS meeting (1995-2005); Study Selection: RCTs (parallel group and crossover designs) comparing GERD medical or surgical treatments with placebo;Data Extraction: Studies were screened for inclusion by 2 authors who independently extracted the data;Data synthesis: For the purpose of the primary analyses, we used the criteria for defining a "responder" chosen by the authors of each paper, as these differed slightly across studies. The relative risks of responding to therapy were pooled using a random-effects model, heterogeneity was assessed using chi-square test, and publication bias using the Egger regression asymmetry test. The analysis of treatment effect was performed on an intent to treat basis basis, and the number needed to treat was calculated using the reciprocal of the pooled absolute risk reduction. Results: Of the 113 paper retrieved, 6 studies (evaluating a total of 268 patients) that used proton pump inhibitors (PPIs) as intervention met the inclusion criteria and were included in the analysis. There were no controlled trials with surgical treatment as the intervention. Two studies had relief of cough as the primary object whilst the remaining four had resolution/improvement of laryngeal symptoms and/or signs as the principal endpoint. The pooled relative risk of responding to therapy was 1.20 (95\% CI: 0.82 to 1.76 ; Heterogeneity chi-squared $=4.36$ [d.f. $=5], \mathrm{p}=0.49)$, and the NNT was $28(95 \% \mathrm{CI}$ : NNTH 7.4 to infinity to NNTB 14.5). All studies but one were small and there was evidence of publication bias as the funnel plot showed statistically significant asymmetry (coefficient $1.66 ; 95 \% \mathrm{Cl}=0.38$ to $2.94 ; \mathrm{p}=0.023$ ). Sensitivity analysis was performed evaluating Jadad score, study design (parallel vs cross-over), type of symptoms assessed, type of PPI used, duration of treatment,and it did not produce any significant change in the pooled RR. Conclusions: PPIs were no more effective than placebo in resolving symptoms and/or signs of LPR. Large and well-designed studies are needed in order to better define the population of patients with LPR who respond to acid suppressive therapy

W1080

Numerous Episodes of Atypical GERD Symptoms Are Unlikely to Be Associated with Acid Or Non-Acid Reflux

Inder Mainie, Radu Tutuian, Amine Hila, Amit Agrawal, Donald O. Castell

Background: Combined Multichannel Intraluminal Impedance (MII) detects non-acid reflux (NAR) and acid reflux at multiple levels of the esophagus. Aim: To detect whether the frequency of typical or atypical GERD symptoms is associated with acid or non-acid reflux Methods:. All patients with persistent symptoms despite proton pump inhibitor (PPI) therapy referred to the esophageal laboratory underwent combined MII-pH monitoring. Prior to the study, patients and/or the referring physician were contacted to ensure that the patient had been on at least twice daily PPI for at least a month or more prior to testing. Upon completion of manometry a $2.1 \mathrm{~mm}$ MII-pH cathteter was passed transnasally with intraluminal impedance sensors at $3,5,7,9,15$ and $17 \mathrm{~cm}$ above the LES and $\mathrm{pH}$ electrodes at $5 \mathrm{~cm}$ above and $10 \mathrm{~cm}$ below the LES A positive SI $(+$ SI) was identified if $\geq 50 \%$ of symptoms were associated with reflux. Typical GERD symptoms were heartburn, regurgitation or chest pain. Atypical symptoms were cough, hoarseness, abdominal discomfort, nausea, dysphagia, mucous, globus, wheeze and throat clearing. Results: One hundred patients (Female 67; mean age 46; range 8 months to 84 years) with persistent GERD symptoms referred to our laboratory underwent 24-hour combined MII-pH monitoring while taking at least twice daily PPIs. Eighty-two patients recorded symptoms during the study. The graphs evaluate the primary symptom. Conclusion: Patients with atypical GERD symptoms are more likely to have numerous symptoms which are usually not associated with reflux. 


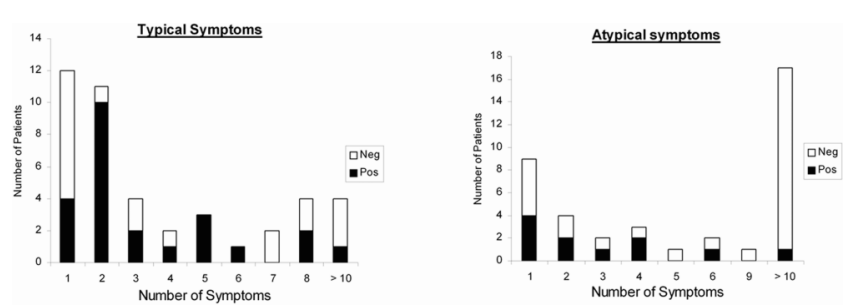

W1081

In Children with Aspiration Syndrome the Lipid-Laden Macrophage Index Is Strongly Correlated with Non-Acid Esophageal Reflux Type Osvaldo Borrelli, Fabio Midulla, Letizia Cordischi, Valeria Tromba, Lelia Lo Russo, Felicia Galos, Corrado Moretti, Salvatore Cucchiara

Background and aims. In children microaspiration of gastric contents has been suggested as a trigger of lung disease, including acute and recurrent pneumonia, bronchiectasis, interstitial disease, asthma and near-miss sudden infant death syndrome. The lipid-laden macrophage index (LLMI) in the bronchoalveolar lavage (BAL) fluid, is thought to be a sensitive marker for pulmonary microaspiration; however, its correlation with esophageal acid reflux (AR), as measured by $24-\mathrm{hr} \mathrm{pH}$-monitoring, remains controversial. Due to the availability of multichannel intraluminal impedance and $\mathrm{pH}$ monitoring (MII-pH) in detecting both acid (AR) and non-acid reflux (NAR) into the esophagus, we whished to measure prospectively in children with suspected aspiration by esophageal reflux the LLMI and to correlate it with all reflux types, as detected by MII-pH. Patients and Methods. 11 consecutive children (median age 1 year; range 6 months- 8 yr) underwent flexible fiberoptic bronchoscopy and BAL, as well as 24-hour MII-pH. LLMI was determined by evaluating the amount of lipid per single macrophage, assigning each cell a score from 0 to 4 according to the amount of lipid in the cytoplasm. The number of cells graded 0-4 was calculated for each patient, and the final LLMI was determined by evaluating 100 cells. In infants the $\mathrm{pH}$ sensor was placed $2 \mathrm{~cm}$ above the lower esophageal sphincter (LES) with impedance sensors at 2 , $3.5,5,6.5,8$ and $9.5 \mathrm{~cm}$ above the LES. In children (2-6 yrs) the pH sensor was placed $3 \mathrm{~cm}$ above the LES with impedance at $3,5,7,9,11$ and $13 \mathrm{~cm}$ above the LES. The MII$\mathrm{pH}$ parameters evaluated were: total numbers of reflux episodes (TN), numbers of $A R$, numbers of NAR, \% of time with esophageal $\mathrm{pH}<4$ (ARI), numbers of long-lasting ( $>5$ min) acid reflux episodes (LAR). Results. (means \pm SD) In all children the studies were successfully performed. LLMI was $60.73 \pm 38.53$. The MII-pH of TN, AR, NAR, ARI and LAR were $76.55 \pm 41.78,41.27 \pm 21.97,35.27 \pm 33.39,6.92 \pm 5.77$ and $2.9 \pm 2.25$ respectively. A significant correlation was found only between LLMI and TN ( $r: 0.70 ; p<0.05)$, as well as between LLMI and NAR ( $\mathrm{r}: 0.72, \mathrm{p}<0.01$ ), whereas no correlation was found between LLMI and AR, ARI and LAR. Conclusion: In children with aspiration syndrome by esophageal reflux, the LLMI is markedly correlated with gastroesophageal reflux of non acid type. The latter may have a major role in causing pulmonary disease from aspiration of gastric content refluxing into the esophagus. Our result account for the well known poor correlation between acid reflux and LLMI and recommend the MII $\mathrm{pH}$ as principal tool when investigating infants with suspected aspiration syndrome by esophageal reflux.

\section{W1082}

\section{Gastroesophageal Reflux During Obstructive Sleep Apnea}

Mari Urata, Hiroshi Fukuno, Masahiro Nomura, Tatsuro Ogata, Katuyoshi Tamaki, Susumu Ito, Kimiko Nakayasu, Yutaka Nakaya

Purpose: Patients with obstructive sleep apnea syndrome (OSAS) are frequently complicated by gastroesophageal reflux disease (GERD), because of the decreased pressure of pleural cavity during sleep apnea. However, detailed relationship between OSAS and GERD have been unclear. In the present study, gastric motility and autonomic nervous activity during sleep apnea were studied. Method: Twenty subjects with OSAS who showed 10 times/hr or more frequent sleep apnea events using a portable sleep polygraph were studied. Obese individuals with a body mass index exceeding 25.0 and those with lordosis were excluded. Percutaneous EGG was performed using an electrogastrograph (Nipro EG), and fast Fourier transformation analysis was performed. The mean amplitude of EGG was compared by EGG spectral analysis among brady-gastria $(<2.4 \mathrm{cpm})$, normo-gastria $(2.4-3.6 \mathrm{cpm})$, and tachygastria $(>3.6 \mathrm{cpm})$. Spectral analysis of heart rate variability was performed, and low frequency (LF) power $(0.04-0.15 \mathrm{~Hz})$, high frequency $(\mathrm{HF})$ power $(0.15-0.40 \mathrm{~Hz})$, and the LF/HF ratio were measured. Upper gastrofiberscopy was performed in each subject, and presence of GERD was diagnosed according to Los Angeles grade classification. Results: Normo-gastria was significantly decreased, and brady- and/or tachy-gasatria were increased during sleep apnea $(\mathrm{p}<0.01)$. Thirteen subjects $(65.0 \%)$ were diagnosed as GERD, but there was no significant relation between Los Angeles grade classification of GERD and severity of OSAS. However, LF/HF ratio during sleep apnea was significantly increased than during non-sleep apnea, which was opposite to the GERD without OSAS Decreased percutaneous arterial oxygen saturation and normo-gastria were independent risk factors in severity of GERD. Conclusions: The present study suggested that abnormal gastric motility, low oxygen, and increased sympathetic nervous activity during sleep apnea might be related to the development of GERD in addition to the decreased pressure of pleural cavity.
W1083

Small Intestinal Bacterial Overgrowth (SIBO) in Patients with Non-Erosive Reflux Esophagitis (NERD)

Alessia Cazzato, Emidio Scarpellini, Maurizio Gabrielli, Luca Santarelli, Ernesto Cristiano Lauritano, Andrea Lupascu, Lucia Sparano, Giovanni Cammarota, Giovan Battista Gasbarrini, Antonio Gasbarrini

Background and Aim: Up to $70 \%$ of patients with typical symptoms of gastro-esophageal reflux disease are affected by non-erosive esophagitis reflux disease (NERD). Possible pathophysiological causes of functional heartburn include non-acid reflux (liquid, gas, mixed), minute changes in the esophageal acidity above a $\mathrm{pH}$ of 4 , motility disorders such as sustained contractions of the longitudinal musculature, visceral hypersensitivity, and emotional and psychological abnormalities, but no definitive data are available. Moreover, overlap of reflux symptoms and irritable bowel syndrome (IBS) has been widely investigated. SIBO, a clinical condition characterized by abnormally high bacterial population level in the small intestine exceeding 106 organisms $/ \mathrm{mL}$, associated with the chronic presence of symptoms such pain bloating, diarrhoea and/or signs of malabsorption, shows a strong epidemiological association with IBS. Aim of this study is to investigate the possible pathophysiological contribution of SIBO in NERD symptoms development. Patients and Methods: Thirty consecutive patients with NERD were enrolled. NERD diagnosis was based on the concordance between symptomatologic pattern suggestive of reflux disease, evaluated using the reflux disease questionnaire (RDQ), and the absence of oesophagel mucosal breaks at upper endoscopic examination. Besides, 40 healthy volunteers were used as control group. All subjects enrolled underwent glucose breath test (GBT) to detect SIBO under standard conditions. The presence of SIBO was based on the occurrence of a peak of $\mathrm{H} 2$ values more than $12 \mathrm{ppm}$ above the basal value after $50 \mathrm{gr}$ glucose ingestion. NERD patients with positive GBT were treated with l-week course of rifaximin (1200 mg/day); GBT test was reassessed 1 month after the end of the therapy. Results: SIBO prevalence resulted higher in NERD patients (19/30; $63.33 \%$ ) when compared to control subjects $(2 / 35 ; 5.71 \%)$; the difference between group was statistically significant $(\mathrm{p}<0.05)$. One month after the end of the eradication treatment $73.68 \%$ (14/19) of NERD-SIBO positive patients, showed a negative GBT; complete heartburn relief was significantly higher in the eradicated group $(9 / 14 ; 64.24 \%$ ) compared to non eradicated patients $(1 / 6 ; 16.66 \%)(\mathrm{p}=0.05)$. Conclusion: In this study NERD patients show an higher prevalence of SIBO compared to healthy subjects. Eradication of SIBO seems reducing the occurrence of heartburn in NERD patients. The overlap of SIBO and NERD could be associated to a commom dismotility pathogenic mechanism. However, whether this is a causal or casual association has to be investigated.

\section{W1084}

Nocturnal Reflux Is Not Predominant in Children and Adolescents with Persistent Asthma and Gastroesophageal Reflux

Lucas Dalle Molle, Helena A. Goldani, Simone F. Canani, Vera G. Vieira, Sergio G. Barros, Themis R. Silveira

Introduction: The gastroesophageal reflux (GER) causal role in asthma is not well established Some studies have shown a higher frequency of nocturnal (supine) GER in patients with respiratory symptoms. This study in children and adolescents with persistent asthma aimed to evaluate prevalence of GER (supine and upright) by prolonged intraesophageal $\mathrm{pH}$ study and compare to GER symptoms and spirometry in children with and without GER. Patients and Methods: Thirty-eight patients were studied. All had diagnosis of asthma (NIH guidelines, 1991) and were chronically using medications for asthma control such as inhalated corticosteroids or leukotriene antagonists. Gastrointestinal symptoms suggestive of GER were considered as regurgitation, heartburn, and abdominal pain. Prolonged intraesophageal pH study (Digitrapper MKIII, Synetics, Sweden) and spirometry (Jaeger Flow Pro, Erich Jaeger GmbH Germany) were obtained in all patients. GER was considered positive when reflux index (RI) was higher than 5\%. Pulmonary function was evaluated by measuring FVC (forced vital capacity), FEVl (forced expiratory volume in lst second of FVC) and FEF 25-75\% (midexpiratory flow rate). Results: Median (range) age was 10 (5-15) years and $23(59 \%$ ) patients were male and 16 female. GER prevalence was $47.3 \%$. The patients were divided in 2 groups: asthma and GER (group 1, n=18); asthma without GER (group 2, n=20) Median (range) of reflux index during supine and upright periods from group 1 were $8.7 \%$ $(3.2-23.6)$ and $10.5 \%(5.2-15.0)$, without significant difference $(p=0.913)$ There was no significant difference of symptoms frequency between groups 1 and $2(\mathrm{p}=0.48)$. Nine patient $(50 \%)$ from group 1 did not have any symptom of GER. Regarding spirometry results, only FEF $25-75 \%$ was $<80 \%$ of expected value $(66.8 \%$ and $56.8 \%$ in groups 1 and 2 respectively, without significant difference, $\mathrm{p}=0.34$ ). There was no significant correlation between RI and FVC (r:-0.17, p=0.28); RI and FEVI ( $\mathrm{r}:-0.17, \mathrm{p}=0.29$ ) and RI and FEF $25-75 \%(\mathrm{r}:-0.10$ $\mathrm{p}=0.54$ ). Conclusion: A high prevalence of GER in children and adolescents with persistent asthma was found, without a predominance of nocturnal or upright GER. There was no significant correlation between results of $\mathrm{pH}$ study and spirometry.

\section{W1085}

Prevalence of Gastroesophageal Reflux Symptoms in Adults with Cystic Fibrosis

Arash A. Sabati, Robert R. Kempainen, Carlos E. Milla, Marjorie Ireland, Malcolm N Blumenthal, Sarah J. Schwarzenberg, Jordan M. Dunitz, Khalid M. Khan

OBJECTIVE: To evaluate the frequency of gastroesophageal reflux (GER) symptoms in adults with Cystic Fibrosis $(\mathrm{CF})$, identify any risk factors and impact on severity of pulmonary disease. BACKGROUND: Several studies have reported increased frequency of GER in CF The reported prevalence of symptoms in adults and children with CF has varied between $25-81 \%$. METHODS: We performed a cross-sectional survey of adult CF patients at the University of Minnesota from outpatient pulmonology clinic using two validated survey instruments, the Mayo and Gastroesophageal reflux Symptom Assessment Scale (GSAS) instruments. RESULTS: Between May 2004 and May 2005, 274 of the 331 adult CF patients

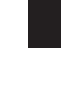


actively followed at our center were invited to participate in the study. 31 declined participation and of the 243 who received survey materials 204 (84\%) were returned. Incomplete surveys $(n=3,1.5 \%)$ were excluded from analysis leaving 201 completed surveys. The most common symptoms over the last year were heartburn $(\mathrm{n}=106,53 \%)$, acid regurgitation $(\mathrm{n}=$ $67,33 \%)$ and dysphagia $(n=36,18 \%)$ with $63 \%(n=127)$ reporting at least one of these symptoms. Gastric acid suppression was common with 122 (61\%) taking medications over the past 12 months. Sub-group analysis showed females to report worse mean GSAS symptom (4.93 vs. $4.05, \mathrm{p}=0.025$ ) and GSAS distress scores (5.63 vs. $3.75, \mathrm{p}=0.005)$ compared to males. Patients who reported weight loss over the last year $(n=51)$ had worse mean GSAS symptom scores (5.29 vs.4.24,p=0.041), worse mean GSAS distress scores (6.75 vs. 3.99, $\mathrm{p}=$ 0.012 ), lower mean FEVl (62\% vs. $71 \%, \mathrm{p}=0.045)$ and lower mean FVC ( $82 \%$ vs. $91 \%, \mathrm{p}=$ 0.019). Dividing patients into subgroups based on FEVl $(>90 \%, 70-89 \%, 40-69 \%$ and $<40 \%$ ) yielded no significant differences in GSAS scores or report of heartburn or acid regurgitation between the groups. Patients with the worst GSAS symptom scores (top quartile, GSAS symptom $\geq 7, n=49$ ) had significantly better FEVl ( $79.4 \%$ vs. $65.7, p=0.014$ ) than those reporting fewer symptoms. Similarly patients with the worst GSAS distress scores (top quartile, GSAS distress $\geq 7, \mathrm{n}=48$ ) also had a better mean FEVl (77.4 vs. 66.2, $\mathrm{p}=0.033$ ) than those with fewer symptoms. CONCLUSIONS: Over the past year $63 \%$ of patients reported at least one major GER symptom, the most common being heartburn (53\%). The relationship between severity of GER symptoms and severity of lung disease remains unclear. We cannot comment on the relationship between GER symptoms and the rate of decline in lung function. Females and patients reporting weight loss also report more symptoms. This finding requires further investigation because of the increased mortality in these patient groups.

\section{W1086}

A Realistic Mechanical Simulation of the Human Swallow in a 3D Virtual Model

Rita Yassi, Leo K. Cheng, Vijay Rajagopal, Martyn P. Nash, John A. Windsor, Andrew J. Pullan

Introduction The understanding of the role of the gastro-esophageal junction (GEJ) in normal swallowing and GERD has been limited by the inability to integrate anatomy and physiology into a unified model. The aim of this study is to reproduce a realistic propagation of a normal peristaltic wave using an anatomically-based model of the human esophagus and GEJ. Method An initial 3D computer model of the human esophagus has been constructed based on $[1,2]$. The outer and inner diameters of the model defined to be $18 \mathrm{~mm}$ and 10 $\mathrm{mm}$ respectively (wall thickness $4 \mathrm{~mm}$ ), and the length was set to $220 \mathrm{~mm}$. The model consisted of 60 nodes, 480 total degrees of freedom, and the tissue was assumed to be homogenous, isotropic and incompressible. A peristaltic wave was simulated by applying a realistic range of pressures $(60-140 \mathrm{mmHg}$; see [3]) to the inner surface of the model at different locations along the esophagus. The outer and inner wall displacements and the change in esophageal length were calculated at 5 locations in the model, during the propagation of a peristaltic wave, by solving the laws of mechanics that govern finite deformation elasticity and results were compared to experimental recordings [1,4]. Results The outer and inner wall circumferences during maximum distention were $64 \pm 3 \mathrm{~mm}$, and $43 \pm 5$ $\mathrm{mm}$ respectively (experimental; outer $84 \mathrm{~mm}$, inner $70 \mathrm{~mm}$ ). The mean circumferences of the outer and inner walls during maximum contraction were $49 \pm 2 \mathrm{~mm}$ and $19 \pm 5 \mathrm{~mm}$ respectively (experimental; outer $49 \mathrm{~mm}$, inner $15 \mathrm{~mm}$ ). The length of the esophageal model was reduced by $6.2 \%$ (experimental; $7 \%$ ). Conclusions The initial model successfully represented a qualitatively realistic swallow. Future model developments will include the addition of different muscle layers. It is envisaged that the model will help provide a better understanding of the mechanical behavior of the regions during swallowing. This work is supported by a Royal Society of New Zealand Marsden Grant and by NIH grant (ROI DK64775). A. J. Pullan is supported by James Cook Research Fellowship, which is gratefully acknowledged. 1. Gregersen, Biomechanics of the gastrointestinal tract: New perspectives in motility research and diagnostics, UK: Springer-Verlag London Ltd., 2003. 2. Meyer, Austin, Brady, Castell, "Muscle Anatomy of the Human Esophagus", J Clin Gastroenterol, 8(2) 1986. 3. Tytgat, Gastro-Oesophageal Reflux and Gastric Stasis: Pathophysiology, Diagnosis, and Therapy, UK: Adis International Ltd., 1991. 4. Edmundowciz, Clouse, "Shortening of the esophagus in response to swallowing", Am J Physiol 260 (Gastrointest Liver Physiol 23), 1991

\section{W1087}

Upper Esophageal Sphincter Dysfunction: An Alternative Explanation for Symptoms and Signs Attributed to Laryyngopharyngeal Reflux David A. Knuff, Reza Shaker, Walter J. Hogan, Benson T. Massey

BACKGROUND: Supraesophageal symptoms are increasingly being ascribed to laryngopharygeal reflux (LPR), based on laryngoscopic exam. Many patients diagnosed with LPR fail to respond to treatment, suggesting acid reflux is not the cause and that an alternative diagnosis should be sought. We hypothesized that some patients may have upper esophageal sphincter (UES) dysfunction accounting for symptoms attributed to refractory LPR. METHODS: The motility lab database was reviewed to identify patients with abnormal UES manometry (Dentsleeve), referred for further evaluation following a prior diagnosis of LPR. All diagnoses of LPR were made by otolaryngologists, based on patient symptoms and laryngoscopic findings. Charts of these patients were reviewed and the following clinical findings and test results recorded: 1) presenting symptoms 2) typical heartburn symptoms 3) esophagram 4) ambulatory pH monitoring 5) swallow study 6) EGD 7) esophageal body manometry 8) cricopharyngeal (CP) electromyography (EMG)/laryngeal nerve conduction (NCV) 9) CP pathology 10) treatment and response. RESULTS: We identified 16 patients (age $41-77 \mathrm{yr}$, $13 \mathrm{~F}$ ) with UES dysfunction (absent, incomplete, and/or short-duration deglutitive relaxation) and a prior diagnosis of LPR. They presented with the following cervical symptoms: globus 9 , hoarseness 6 , throat clearing 6 , chronic cough 5 , and/or dysphagia 4 . Eleven had no or rare heartburn and another 5 patients responded to treatment. However, cervical symptoms in all patients failed to respond to PPI therapy. While esophagram in 4 of 14 patients suggested gastroesophageal reflux, the esophagus on EGD (performed in 9) was completely normal, and ambulatory pH monitoring in 10 patients (6 with 3-site probe and 4 with 2site probe) showed normal acid exposure. Swallow study was done in 12 patients and 5 suggested abnormal pharyngeal transit while 7 were read as normal. Esophageal body manometry in 9 of 15 patients was normal. The remainder had nutcracker, nonspecific motor disorder, hypertensive lower sphincter, and/or tight wrap. CP EMG/laryngeal NCV was done in 8 patients and 5 suggested neuropathy, 2 myopathy, and 1 borderline laryngeal sensory abnormality. In one patient $\mathrm{CP}$ pathology showed neurogenic myopathy. Four of 8 patients responded at least temporarily to one of several treatments directed towards UES dysfunction (swallowing exercises, dilation, botulinum toxin, CP myotomy). CONCLUSIONS: Patients with supraesophageal symptoms and a laryngoscopic diagnosis of LPR, who do not respond to PPI, may have UES dysfunction. Such patients should have appropriate manometric evaluation before pursuing invasive anti-reflux therapy.

\section{W1088}

Prevalence and the Degree of GERD Symptoms in Japanese General Population

Hatsushi Yamagishi, Shuichi Ohara, Tomoyuki Koike, Ken Ariizumi, Yoshifumi Inomata, Katsunori Iijima, Akira Imatani, Tooru Shimosegawa, Shigemitsu Aida, Ktsuaki Kato, Daisuke Shibuya

Background and Aim: Gastroesophageal reflux disease (GERD) is a common disease in the West, which now appears to be increasing in the prevalence in Japan. However, few studies have reported the prevalence of GERD in Japanese general population. The aim of this study was to examine the prevalence of GERD symptoms in more than 100,000 unselected subjects in Japan. Subjects and Methods: A total of 145,367 Japanese (male: female, 52,694: 92,672, mean age 61.9) who underwent a group check-up on the stomach at the Miyagi cancer society between January and December 2003 were enrolled in this study. An X-ray examination was performed on all the enrolled subjects as well. Also, among these subjects, we randomly selected a total of 67,246(male : female, 26,173:41,073, mean age 62.4)for evaluating the prevalence of abdominal pain. The respective subjects were prospectively asked to complete the questionnaires concerning the symptoms of heartburn, dysphagia, and abdominal pain within one month. The subjects were requested to simply answer "yes" or " no" and if "yes", they were further questioned the frequency of symptoms (" usually" or " sometimes"). In addition, we evaluated a disease classification by X-ray examination. The values are expressed as frequency, and categorical variables were compared using the $\chi^{2}$ test. P-values $<0.05$ were considered significant. Results: The prevalences of the respective symptoms in male and female were; $16.6 \%$ (sometimes $16.1 \%$, usually $0.5 \%$ ) vs. $21.6 \%$ (sometimes $21.0 \%$, usually $0.6 \%$ ) in heartburn, $5.4 \%$ (sometimes $5.0 \%$, usually $0.4 \%$ ) vs $7.9 \%$ (sometimes $7.4 \%$, usually $0.5 \%$ )in dysphagia, and $6.6 \%$ (sometimes $6.5 \%$, usually $0.1 \%$ )vs. $9.7 \%$ (sometimes $9.6 \%$, usually $0.1 \%$ )in abdominal pain, respectively. All symptoms were significantly more common in female than in male. ( $p=0.001$ ) Among these symptoms, heartburn was particularly high comparing to other symptoms ( $\mathrm{p}=0.001$ ), and its prevalence showed a significant age and gender related difference in the patients aged 60-89 years, that is a significant increase in the prevalence with age in females, while a decrease over 60 years old in males. Heartburn was relatively frequent in male with age 30-49 years and in female with age 50-89 years. Dysphagia was also significantly more frequently seen in female, and abdominal pain was significantly more prevalent in younger age group of 30-49 years than the elderly. GERD symptoms were more common in the subjects with hiatal hernia than those without it. Conclusions: The prevalence of GERD symptoms (heartburn) was high, approximately $20 \%$, in Japanese general population, and the frequency was especially high in female with age 60-89 years old.

\section{W1089}

Premature Central Apnea and Its Relationship with Gastroesophageal Reflux Edgardo Suarez, Ivonne Cedillo-Ley, Nuria Perez y Lopez, Jesus Alejandro Insunza, Cinthia Hernandez, Moises Gerardo del Hoyo, Armando Tello, Eduardo Cardenas-Lailson

INTRODUCTION The gastroesophageal reflux and the apnea frequently occur in the premature newborns. The refluxed gastric content may reach the larynx and cause central apnea as well as airway obstruction; nevertheless, the previous studies have not been able to establish a cause-effect relationship. The aim of this study is to establish if there is a causeeffect relationship between gastroesophageal reflux and prematurity; to evaluate if the severity of reflux is associated to earlier birth. MATERIAL AND METHODS This is a retrospective, observational, comparative and longitudinal study. 32 files from premature and full term newborns from the intensive care unit at the Hospital Español de México were evaluated. They were diagnosed with central or mixed apnea. Every patient underwent polisomnography to determine the type of apnea and pHmetry for the diagnosis of GERD. Genders, gestational age, extrauterine days of life and weight were registered. RESULTS 17 (53\%) were males and $15(47 \%)$ were females. Twenty three were premature (72\%). Polisomnography showed central apnea in $59 \%$ and pHmetry showed pathological reflux in 32\%. Premature newborns have 8 times more risk of having central apnea (Odds ratio 9.92, confidence interval 95\%1.27-97.1, x2 Mantel-Haenszel=6.49, p=0-008). Patients with central apnea had lower weight (2002.89 gr) than the patients with out apnea (mean $2757.23 \mathrm{gr}$ ). T-Student -3.176 with 30 degrees of freedom, $p=0.003$. We did not find any relationship between gestational age and reflux severity $(\mathrm{p}=0.670)$. We found that newborns with reflux had twice the risk of developing central apnea by odds ratio, but the confidence interval was too wide as to affirm this. CONCLUSSION The premature and low weight newborns have higher risk of developing central apnea. Immaturity may favor gastroesophageal reflux and the physiological reflux may give rise to it. We only measured acid reflux with the 24 hour pHmetry, but the nonacid reflux could also unchain the problem. 
W1090

The Effect of Gastroesophageal Reflux (GER) On Upper Airway Obstruction in Patients with Mild Obstructive Sleep Apnea (OSA)

William C. Orr, Jennifer J. Robert, John R. Houck, Cheryl Giddens

Obesity is a risk factor for both OSA and GER, and heartburn is a common symptom noted in patients with OSA. Heartburn has been shown to be more common in patients with more severe OSA. It has been speculated that GER may alter the upper airway in a way which would facilitate the occurrence of OSA. In this investigation we have tested the hypothesis that significant acid suppression and reduction of GER would significantly reduce the rate of OSA in patients with mild OSA. METHODS: Prospective subjects were recruited from the community at large, as well as from our database of patients. All subjects underwent a sleep evaluation as well as a 24-hour esophageal $\mathrm{pH}$ evaluation. To qualify subjects had to have an apnea hypopnea index (AHI) of 15 or less, and esophageal acid contact time in the supine position of greater than $6 \%$ total or $3 \%$ supine. Qualifying subjects underwent 8 weeks of treatment with $20 \mathrm{mg}$ bid of rabeprazole. The Epworth Sleepiness Scale was completed before and after treatment. At the end of 8 weeks all subjects repeated the sleep and 24-hour esophageal $\mathrm{pH}$ evaluations. RESULTS. Subjects had significantly $(\mathrm{p}<05)$ less reflux events in both upright and supine positions and significantly less ( $\mathrm{p}<.05) \%$ acid contact time after the 8-week treatment compared to baseline. Subjects also had significantly shorter events after the 8-week treatment compared to baseline. Sleep onset latency and REM onset latency were significantly $(\mathrm{p}<05)$ shorter after the 8 -week treatment period compared to baseline. There was no difference in the apnea hypopnea index at baseline compared to the 8 -week rabeprazole treatment. Subjects reported significantly $(\mathrm{p}=.02)$ more daytime sleepiness at baseline (12) compared to the post-treatment condition (10). CONCLUSIONS: 1. Rabeprazole improved heartburn symptoms and subjective reports of daytime sleepiness in patients with mild OSA. 2. Significant acid suppression and reduction of GER did not significantly reduce the rate of OSA in patients with mild OSA.

\section{W1091}

Discriminating Non-Cardiac Chest Pain from Chest Pain with Positive Coronary Angiography: The Utility of Reflux Symptoms

Guy D. Eslick, Nicholas J. Talley

BACKGROUND: Non-cardiac chest pain (NCCP) is common and costly; discriminating this non-lethal entity from cardiac chest pain (CCP) in practice remains a major clinical issue. NCCP is most commonly caused by gastroesophageal reflux disease (GERD); whether reflux symptoms help to distinguish NCCP from cardiac pain is unknown. We aimed to determine the characteristics and predictors of a positive angiographic study among patients being investigated for chest pain. METHODS: A consecutive sample of 176 chest pain patients (cardiac and non-cardiac) aged 18 years and over who underwent coronary angiography at Nepean Hospital were enrolled in the study. A detailed history and physical examination were included in these initial procedures. Coronary artery disease was judged 'significant' at coronary angiography if there is at least a 70 percent diameter stenosis of one or more major epicardial coronary segments or at least a 50 percent diameter stenosis of the left main coronary artery. All patients completed the valid Chest Pain Questionnaire (CPQ). RESULTS: The sample consisted of 117 males and 59 females. The mean age was 63 years $(\mathrm{SD}=10$ years, range: $37-86$ years). There were $44(25 \%) \mathrm{CP}$ patients who had a positive coronary angiogram result. Univariately, the following variables were associated with a positive coronary angiogram amongst $\mathrm{CP}$ patients: advancing age (OR=1.04, 95\% CI: 1.01 1.09); male gender ( $\mathrm{OR}=0.35,95 \% \mathrm{CI}: 0.15-0.80)$; worse chest pain on cough $(\mathrm{OR}=0.06$, 95\% CI: 0.01-0.44); less frequent acid regurgitation ( $\mathrm{OR}=0.59,95 \% \mathrm{CI}: 0.35-0.98)$; less frequent dysphagia ( $\mathrm{OR}=0.66,95 \% \mathrm{CI}: 0.44-0.99)$. In multiple logistic regression models independent predictors of a positive coronary angiogram amongst the patients were: increased chest pain severity $(\mathrm{OR}=2.61,95 \% \mathrm{CI}$ : 1.16-5.85); shorter chest pain duration $(\mathrm{OR}=0.63$, 95\% CI: 0.43-0.93); worse chest pain on cough ( $\mathrm{OR}=0.06,95 \% \mathrm{CI}$ : 0.01-0.48); and less severe heartburn (OR=0.22, 95\% CI: 0.06-0.71). The model explained $10 \%$ of the variance $\left(\mathrm{R}^{2}=0.10\right)$. CONCLUSIONS: Chest pain patients with a positive coronary angiogram are more likely to have severe chest pain and have chest pain of shorter duration. These patients are less likely to have severe heartburn suggesting reflux symptoms do have some limited predictive value in identifying NCCP in this population.

\section{W1092}

Prevalence, Risk and Recurrence Risk of Esophageal Stricture in the United States

Kenneth J. Rothman, Charles E. Wentworth, Mari-Ann Wallander, Saga Johansson, Stefan Eklund

Little is known about prevalence or risk of esophageal stricture, or risk for recurrence. Using the Pharmetrics database, a large US health claims data source with over 7 million records, we identified nearly 40,000 patients who had an initial claim for repair of an esophageal stricture (procedure codes 43450 - dilate esophagus unguided, 43453 - dilate esophagus guidewire, 43455 - dilate esophagus, 43456 - dilate esophagus balloon/dilator 43458 dilate esophagus w/large balloon, 43330 - repair esophagus (Heller type), 43331 - repair of esophagus). From these numbers we estimated the prevalence of serious esophageal stricture and the risk for a first stricture serious enough to warrant repair, by age and sex. Among the approximately 10,000 patients who had a new claim for stricture repair and no history of esophageal cancer, and for whom there was prior enrollment data for at least one year, we estimated the risk of a recurrent stricture using life-table methods. The prevalence of esophageal stricture was slightly greater among males at all ages, and increased steadily by age, reaching about 3/1000 among those 60-79 years of age; the 1-yr risk of stricture among those with no stricture history was about $1 / 2000$ for both men and women age 6079 , and less for younger ages (table). The risk of recurrence was approximately $11 \%$ during the two years following the initial claim. Because the estimates of prevalence, risk and recurrence presented here are based on claims data, they are less reliable than estimates that could be derived from study populations in which the diagnoses have been validated.
On the other hand, in the absence of such validated information, estimates such as these from claims data can provide a reasonable first assessment for basic epidemiologic measures By restricting cases to those with a procedure code for stricture repair, we increased the specificity of our disease classification and thus focused on more serious strictures. Sensitivity and specificity are far from perfect when using claims databases to define disease prevalence and risk, but no classification is perfect, and the estimates obtained here are a useful start toward describing the epidemiology of esophageal stricture.

Prevalence and Risk of Stricture

\begin{tabular}{|c|c|c|c|}
\hline & Age & Prevalence (per 1000) & 1 yr Risk (per 1000) \\
\hline & $20-39$ & 0.393 & 0.642 \\
\hline Males & $40-59$ & 1.267 & 0.568 \\
\hline & $60-79$ & 3.341 & 3.741 \\
\hline & $20-39$ & 0.177 & 0.548 \\
\hline Females & $40-59$ & 0.924 & 0.507 \\
\hline & $60-79$ & 2.652 & 3.154 \\
\hline
\end{tabular}

\section{W1093}

Prevalence of GERD and Its Effect On Quality of Life in a Large Cohort of Patient with Obstructive Sleep Apnea

Bryan Green, Michael Thorn, John F. Oconnor

Purpose: Determine the prevalence of GERD and its effect on quality of life in a large cohort of patients with obstructive sleep apnea. Methods: Consecutive patients presenting for nocturnal polysomnography completed self-administered GERD questionnaires and SF-36. Results: 168 patients were analyzed. Daytime and nighttime reflux symptoms were experienced an average of 1.8 days (26\%) and 1.6 nights $(23 \%)$ respectively during the previous week. Daytime and nighttime reflux severity was moderate or severe in $24 \%$ and $31 \%$ respectively during the previous week. Obstructive sleep apnea patients had a much lower quality of life score in all subscales of the SF-36 (except mental health) than the U.S. general population. Concomitant moderate or severe nighttime reflux further worsened the quality of life. Conclusion: Reflux symptoms are common and frequently severe in patients with obstructive sleep apnea. Quality of life is severely impaired in obstructive sleep apnea and coexistent moderate/severe nighttime reflux is associated with an even worse quality of life. This study was supported by AstraZeneca.

Quality of life as measured by the SF-36 in patients with no or mild nocturnal GERD (01 on scale) compared to patients with moderate to severe nocturnal GERD (2-5 on scale)

\begin{tabular}{|c|c|c|c|}
\hline SF-36 (mean scores) & none/mild & moderate/severe & p-value \\
\hline Physical functioning & 61.5 & 48.1 & 0.0058 \\
\hline Social Functioning & 68.1 & 59.8 & NS \\
\hline Role physical & 13.2 & 11.1 & NS \\
\hline Role emotional & 15.9 & 13.0 & NS \\
\hline Mental health & 76.6 & 63.4 & 0.0033 \\
\hline Vitality & 37.4 & 21.5 & 0.0005 \\
\hline Bodily pain & 58.1 & 47.4 & 0.0126 \\
\hline General health & 53.2 & 42.6 & 0.0050 \\
\hline Physical component summary & 38.7 & 35.3 & 0.0359 \\
\hline Mental component summary & 37.7 & 33.3 & 0.0263 \\
\hline
\end{tabular}

A lower score indicates worse quality of life. U.S. population normal values for Physical and Mental Component Scores $=50$

Quality of life as measured by the SF-36 in patients with obstructive sleep apnea compared to normal values for the U.S. population.

\begin{tabular}{|c|c|c|c|}
\hline SF-36 Components (mean) & OSA & US population norms & p-value \\
\hline Physical functioning & 57.4 & 84.2 & $<0.0001$ \\
\hline Social functioning & 65.8 & 83.3 & $<0.0001$ \\
\hline Role physical & 12.7 & 80.9 & $<0.0001$ \\
\hline Role emotional & 15.1 & 81.3 & $<0.0001$ \\
\hline Mental health & 72.8 & 74.7 & 0.3693 \\
\hline Vitality & 33.0 & 60.9 & $<0.0001$ \\
\hline Bodily pain & 55.1 & 75.2 & $<0.0001$ \\
\hline General health & 50.1 & 71.9 & $<0.0001$ \\
\hline Physical component summary & 37.7 & 50.0 & $<0.0001$ \\
\hline Mental component summary & 36.6 & 50.0 & $<0.0001$ \\
\hline
\end{tabular}

A lower score indicates worse quality of life. 
W1094

Esophageal Strictures in Primary Care: Incidence, Treatment Patterns and Recurrence Rate

Ana Ruigomez, Luis A. Garcia Rodriguez, Mari-Ann Wallander, Saga Johansson, Stefan Eklund

Background and Purpose: Epidemiological data on esophageal strictures are scarce. Our study aimed to determine the incidence of esophageal strictures, and to establish its natural history in newly diagnosed primary care patients. Methods: A population-based, case-control study was conducted using the UK General Practice Research Database (GPRD). To determine the incidence rate of esophageal strictures, we identified patients aged 20-79 years who had an esophageal stricture diagnosis during the study period (1994-2000); diagnoses were confirmed by a physician-completed questionnaire. We used unconditional logistic regression analysis to compute odds ratios (OR) and $95 \%$ confidence intervals (CI). Cox proportional hazards regression was used to estimate the mortality rate and risk of recurrence of strictures between first diagnosis and December 2001. Results: From the GPRD, we identified 596 patients with a first diagnosis of an esophageal stricture. The overall incidence rate of esophageal strictures was 1.1 per 10000 person-years and increased with age to 3.5 per 10000 person-years in the 60-79 year age group. Dysphagia was present in $83 \%$ of cases and was the strongest predictor of an esophageal stricture. Most cases of stricture $(68 \%)$ were peptic strictures linked to gastroesophageal reflux disease (GERD) or esophagitis; peptic strictures had an incidence rate of 0.8 per 10000 person-years. As well as GERD (OR: 8.4 95\% CI: 6.2-11.2), peptic ulcer disease and prior alcohol use were also associated with an increased risk of an esophageal stricture. Proton pump inhibitors (PPIs) and H2-receptor antagonists were prescribed in $64 \%$ and $6 \%$ of patients, respectively, after an esophageal stricture diagnosis. More than half of esophageal stricture patients had at least one esophageal dilatation, and patients needing more than two dilatations in the year after diagnosis had an increased likelihood of a further stricture recurrence. The recurrence rate after one year among patients with peptic strictures was 6.1 per 100 person-years. The risk of esophageal stricture recurrence decreased among long-term users of PPIs (RR: 0.6; 95\% CI: 0.3-1.3). The mortality rate in patients with peptic strictures was similar to that in the general population. Conclusion. Esophageal strictures are rare, and most cases found in primary care were peptic strictures. Our data suggest that long-term PPI treatment could protect against recurrence of esophageal stricture.

\section{W1095}

Characteristics of Acid and Weakly Acidic Reflux Temporally Related to Cough

Kathleen Blondeau, Veerle Mertens, Lieven Dupont, Jan Tack, Richard H. Holloway Daniel Sifrim

Both acid and weakly acidic reflux can be temporally related to episodes of cough. In patients with heartburn, lower nadir $\mathrm{pH}$ and increased proximal extent of reflux are determinants of symptom perception. In patients with cough, such determinants remain unclear. Aim: To identify reflux characteristics underlying the reflux-cough association. Methods: 49 patients [ $24 \mathrm{M}$, age $55 \mathrm{yr}]$ with chronic unexplained cough, with at least 1 symptomatic reflux event during ambulatory impedance-pH-manometry monitoring (Sandhill Sci.) were selected for analysis. Manometry was used for precise and objective cough recognition. Reflux was detected by impedance and defined by $\mathrm{pH}$ as acid $(\mathrm{pH}<4)$ or weakly acidic (pH 4-6.5). Reflux episodes were considered symptomatic if cough occurred in a 2-min time window starting at the onset of the reflux event. Asymptomatic reflux episodes that occurred from $\mathrm{lh}$ before to $\mathrm{lh}$ after a symptomatic episode served as controls. We compared "symptomatic" vs. "asymptomatic" reflux events in 3 subsequent steps: 1) Determination of the number of reflux related cough episodes occurring during esophageal acid (pHmetry) and/or volume exposure (impedance) during the 24hs 2) Comparison of acid and volume burden, defined as total area over the curve of acid and volume exposure during $120 \mathrm{~min}$ before each reflux episode. 3) Comparison of characteristics of individual reflux events: nadir $\mathrm{pH}$, acid clearance, volume clearance, and proximal extent. Results: 250 symptomatic reflux episodes (131 acid, 119 weakly acidic) were detected. Only $6.8 \%$ of reflux-associated cough episodes occurred during esophageal acid exposure, 6.4\% during volume exposure and $9.6 \%$ during both. The majority $(76.8 \%)$ of reflux related cough events occurred when esophageal acid or volume was already cleared. The volume burden was similar for symptomatic (28000) and asymptomatic episodes (33000) whereas the acid burden was lower for symptomatic episodes ( 506 vs. $2800, p=0.07$ ). Acid clearance $(30.05 \mathrm{~s}$ vs. $31.01 \mathrm{~s}$ ), volume clearance (37. ls vs. 27.9s) and proximal extent $(9.3 \mathrm{~cm}$ vs. $9.4 \mathrm{~cm})$ did not differ significantly in symptomatic events and controls. However, the nadir $\mathrm{pH}$ was significantly higher ( $\mathrm{pH} 4.4$ vs. pH 3.4, p=0.007) in the symptomatic events. Thirty-five percent of the symptomatic events occurred during the first postprandial hour. Conclusion: Unlike in patients with heartburn, reflux episodes preceding cough were less acidic and probably had similar volume than asymptomatic reflux events. Cough within 2 min after reflux occurred outside the period of acid or volume exposure suggesting either a slow triggering mechanism or a non-acidic component.

\section{W1096}

Pepsin, a Marker of Gastric Content Is Increased in Tracheal Aspirates from Premature Infants Developing Bronchopulmonary Dysplasia

Sabeena Farhath, Zubair H. Aghai, Judy Saslow, Tarek Nakhla, Zhaoping He, Dev Mehta Background: Gastroesophageal reflux (GER) is very common in premature neonates. Chronic aspiration of gastric contents is likely to be important in the pathophysiology of bronchopulmonary dysplasia (BPD). Detection of pepsin in tracheal aspirate (TA) is a new reliable marker of gastric content and micro-aspiration. Objective: To study the association between pepsin in TA and development of BPD in premature infants. Design/Methods: Serial TA samples were collected during the first 28 days from 45 mechanically ventilated premature neonates (gestational age $<30$ weeks, birth weight $<1250$ grams). BPD was defined as oxygen requirement at 36 weeks postconception age (PCA). Moderate BPD was defined as $<30 \%$ oxygen requirement and severe BPD as $>30 \%$ oxygen requirement at 36 weeks PCA. An enzymatic assay with a fluorescent substrate was used to detect pepsin. Pepsin was also measured in 10 serum samples from 8 neonates collected at the same time of TA collection. Results: Eleven infants had no BPD (gestational age $26.4 \pm 1.4$ weeks, birth weight $927 \pm 185$

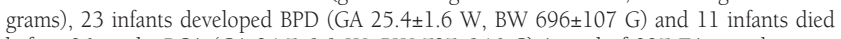
before 36 weeks PCA (GA $24.7 \pm 1.1 \mathrm{~W}$, BW $737 \pm 146 \mathrm{G}$ ).A total of $227 \mathrm{TA}$ samples were collected from 45 premature neonates (birth weight $762 \pm 166$ grams, gestational age $25.5 \pm 1.5$ weeks). Thirty seven samples were collected from infants with no BPD (average 3.3 samples per infant), 140 from infants who developed BPD (average 6 samples) and 50 samples from infants who died (average 4.5). Pepsin was detectable in 205/227(90.3\%) of TA samples and none from the serum samples. Mean pepsin level was significantly lower in infants with no BPD $(222+243 \mathrm{ng} / \mathrm{ml})$ compared to those who developed BPD $(364+320 \mathrm{ng} / \mathrm{ml}, \mathrm{P}=0.014)$ or died $(429 \pm 502 \mathrm{ng} / \mathrm{ml}, \mathrm{P}=0.03)$. Moreover, there was a trend towards higher pepsin levels in infants with severe BPD $(404 \pm 433 \mathrm{ng} / \mathrm{ml})$ compared to moderate BPD $(305 \pm 283 \mathrm{ng} / \mathrm{ml}$, $\mathrm{P}=0.07$ ) Conclusions: Pepsin, a marker of gastric content was detectable in TA from all premature ventilated neonates and none from serum samples. The level of pepsin is increased in TA from premature infants developing BPD. Chronic aspiration of gastric contents is likely to be important in the pathophysiology of BPD.

\section{W1097}

The Esophagus and Beyond: Nondigestive Symptoms in Nonerosive Reflux Disease: Nature, Prevalence and Relation to Acid Reflux Hershcovici Tiberiu, Zimmerman Joseph

Background: Some half of the patients with nonerosive gastroesophageal reflux disease (NERD) meet the criteria for irritable bowel syndrome (IBS) (1). Since nondigestive symptoms are common in IBS, it is expected that NERD patients should also experience such symptoms. The aim of the present study was to test this hypothesis. Methods: All patients referred for esophageal pH monitoring during 1977-2004 were included in this study. Nondigestive symptoms and IBS status (according to the Rome I criteria) were assessed in patients diagnosed with NERD (esophageal $\mathrm{pH}<4$ for $\geq 5 \%$ of the time on $24-\mathrm{h} \mathrm{pH}$ monitoring and normal upper endoscopy; $n=326$ ) in relation to non-patient controls $(n=174)$. Symptoms were scored using a validated, self-administered inventory including the following scales: musculoskeletal pain, urinary, sleep and neurasthenia (NA) (2). Reflux symptoms were scored on a validated scale (3). Results: The mean age was 43 and 41 in NERD and controls. The male/female ratio was similar in both groups (1:1.6), as was the proportion of smokers $(17 \%)$. NERD patients scored significantly higher than controls on all scales $(\mathrm{p}<0.001)$. After adjustment for age, gender and smoking the scores on the scales of pain, sleep and NA were significantly associated with NERD. ROC curve analysis yielded cutoff scores on these scales that were highly specific $(89,91$ and $89 \%$ respectively) in the discrimination of NERD patients from controls. Within the group of NERD patients, nondigestive symptoms were independently associated with age, reflux symptoms score and IBS status $(\mathrm{p}<0.001)$, but not with gender, smoking or acid exposure. The score on the reflux scale correlated with the scores on all the nondigestive scales $(r=0.36,0.32,0.38$ and 0.19 for NA, pain, sleep and urinary symptoms; $<<0.001$, adjusted for age, gender and smoking). $49 \%$ of the NERD patients and $18 \%$ of the controls were classified as IBS nonpatients (IBS $\left(_{+}\right)$NERD) $(\mathrm{p}<0.001) . \mathrm{IBS}(+)$ NERD patients scored significantly higher than those not meeting the IBS criteria (IBS(-)NERD) on all the nondigestive scales ( $<<0.001)$. Nevertheless, IBS(-)NERD patients did score significantly higher than controls on all these scales $(p \leq 0.005)$. Conclusions: In NERD patients, nondigestive symptoms were:l:scored significantly higher with respect to controls, and were highly specific in the differentiation between these two groups. 2: correlated with the reflux symptoms score, but not with esophageal acid exposure.3. predicted by IBS status, being more severe in the IBS(+)NERD patients. References: 1. Aliment Pharmacol Ther 2004; 20:1297-1303; 2: Dig Dis Sci, 2003; 48:743-749 3: Scand J Gastroenterol, 2004; 39:212-216.

\section{W1098}

Relationship Between Laryngopharyngeal Reflux and Symptoms and Signs Won Moon, Moo In Park, Gyung Mi Kim, Jeong Hoon Seo, Yoon Jung Kim, Ji Hun Roh, Dae Gwan Im, Kyu Jong Kim, Seun Ja Park, Hyo Sung Mun, Kang Dae Lee

Background/Aim:Laryngopharyngeal reflux is of great interest to gastroenterologists. However, its correlation with symptoms and signs is yet incompletely understood, especially in the Asians. This investigation was done to evaluate the relationship between reflux severity and symptoms and signs in Koreans. Methods:Twenty-nine, nonsmoking patients with one or more laryngeal symptoms of unknown cause, who completed a symptoms questionnaire, videostrobolaryngoscopy, esophagoscopy, 24-hour pharyngeal $\mathrm{pH}$ testing and esophageal manometry were identified retrospectively. Components of symptoms index, scored as $0-5$ at every item were the following: problem with voice, cleaning throat, excess throat mucus, difficulty swallowing, coughing after eating or lying down, breathing difficulties, troublesome cough, lump in throat, stomach acid coming up. Components of reflux finding, scored as 0-4 were the following: subglottic edema, ventricular obliteration, erythema, vocal cord edema, diffuse laryngeal edema, posterior commissure hypertrophy, granuloma, thick endolaryngeal mucus. Twelve patients (reflux group, RG) had one or more reflux episode, defined as positive reflux at 24-hour pharyngeal pH testing and remainders were 17 (non-reflux group, NRG). Results:Between RG and NRG, there was no difference in mean age (44 vs. 52) and sex (male:female, 5:7 vs. 3:14). The most predominant symptom with the highest mean score ( 2.4 vs. 2.7 ) was a problem with voice in each group. There was no difference in total scores (10 vs. 12) of symptoms questionnaire and mean duration (17 months vs. 30 months) of symptoms. Posterior commissure hypertrophy, the most predominant sign in each group was worse in RG compared to NRG ( 2.3 vs. 1.6, $\mathrm{p}=0.027)$, but no difference in total scores (3.6 vs. 3.1) of signs. There was no difference in the rate of reflux esophagitis (50\% vs. $31 \%$ ) on esophagoscopy, basal pressures of upper and lower esophageal sphincters (PUES, $68 \mathrm{mmHg}$ vs. $73 \mathrm{mmHg}$ and PLES, $31 \mathrm{mmHg}$ vs. $35 \mathrm{mmHg}$ ) and rate of normal peristalsis on 24-hour esophageal manometry. In RG, the mean number and percent time per 24 hours of pharyngeal acid reflux with pharyngeal $\mathrm{pH}<4$ were 12 and $2 \%$ respectively. None of symptoms and signs did not correlate with reflux severity. However, the time 
percent correlated significantly with duration of symptoms (coefficient $=0.509, \mathrm{p}=0.032$ ) and negatively with PLES (coefficient $=-0.547, \mathrm{p}=0.022$ ) Conclusion:Laryngeal symptoms, signs and findings of esophagoscopy and esophageal manometry did not seem to predict pharyngeal reflux. However, PLES might be related with severity of pharyngeal reflux in Korean patients. Keywords:laryngopharyngeal reflux, pH testing

\section{W1099}

Pepsin Is Detectable in Tracheal Aspirates from Premature Neonates: Relatioship with Feeding and Steroid Therapy

Sabeena Farhath, Zubair H. Aghai, Zhaoping He, Tarek Nakhla, Judy Saslow, Dev Mehta

Introduction Gastroesophageal reflux (GER) is very common in premature neonates. Aspiration of gastric contents due to GER can worsen lung disease in ventilated neonates. Aspiration of gastric contents has been shown in infants with bronchopulmonary dysplasia (BPD). Detection of pepsin in tracheal aspirate (TA) is a new reliable marker of gastric content and micro-aspiration. Objective To determine the frequency with which pepsin is detectable in TA of mechanically ventilated premature neonates and its association with feedings and steroid therapy. Material and Methods Serial TA samples (days 1,3,5,7,14,21 and 28) were collected from premature neonates on ventilator for respiratory distress syndrome. Samples were also collected before and 72 hours after starting steroids. An enzymatic assay with a fluorescent substrate was used to detect pepsin. Pepsin was also measured in 10 serum samples from 8 neonates collected at the same time of TA collection. Results A total of 227 TA samples were collected from 45 premature neonates (birth weight $762 \pm 166$ grams, gestational age $25.5 \pm 1.5$ weeks). Pepsin was detectable in 205/227(90.3\%) of TA samples and none of the serum samples. Median pepsin level of all aspirates was $248 \mathrm{ng} / \mathrm{ml}$ (range 0 -2102). Pepsin was significantly lower on day 1 (mean \pm SD, $149 \pm 174 \mathrm{ng} / \mathrm{ml}$ ) compared to all other time points (days $3,5,7,14,21$ and 28 , all $\mathrm{P}<0.01$ ). There was no significant difference in pepsin level amongst other time points. Mean concentration of pepsin was significantly lower when infants were unfed $(273 \pm 278 \mathrm{ng} / \mathrm{ml})$ compared to during feeding $(392 \pm 398 \mathrm{ng} / \mathrm{ml}, \mathrm{P}=0.007) .18$ infants received 20 courses of steroids. The mean level of pepsin significantly increased after dexamethasone therapy $(584 \pm 485 \mathrm{ng} / \mathrm{ml})$ compared to before steroids $(228 \pm 221 \mathrm{ng} / \mathrm{ml}, \mathrm{P}=0.006)$. Conclusion Pepsin, a marker of gastric content is detectable in TA from all premature ventilated neonates. The levels are increased in fed compared to unfed infants. Steroid therapy increased pepsin in TA. Chronic aspiration of gastric contents is likely to be important in the pathophysiology of BPD in premature infants.

\section{W1100}

A Sham-Controlled Study of the Influence of Radiofrequency Delivery (The Stretta Procedure) On Symptoms, Acid Exposure and Distensibility of the Gastro-Oesophageal Junction in Gord Patients

Joris Arts, Philippe Caenepeel, Rita Devos, Lieselot Holvoet, Daniel Sifrim, Toni Lerut, Paul Rutgeerts, Jozef Janssens, Jan Tack

Several studies, including one sham-controlled study, have reported symptom relief in gastrooesophageal reflux disease (GORD) patients treated with radiofrequency delivery (Stretta procedure) at the gastro-oesophageal junction (GOJ). The mechanism underlying this improvement is unclear as changes in $\mathrm{pH}$ monitoring are often inconsistent. Recently, it was proposed that decreased distensibility of the GOJ is involved in the symptomatic improvement observed after the Stretta procedure (Arts DDW 2005). The aim of the present study was to investigate the effect of Stretta on symptoms, acid exposure and GOJ distensibility in a double-blind randomised cross-over design. Methods: Consecutive GORD patients underwent two upper gastrointestinal endoscopies with 3 months interval, during which active or sham Stretta treatment was performed in a randomised double-blind fashion. The endoscopic procedure was performed by a team which was otherwise not involved in the follow-up of these patients. Before the start of the study and 3 months after the first treatment, they underwent symptom assessment, endoscopy, manometry, $24 \mathrm{~h}$ esophageal $\mathrm{pH}$ monitoring and a barostat distensibility test of the GOJ before and after administration of $25 \mathrm{mg}$ of sildenafil. Symptom scores, acid exposure, lower esophageal sphincter (LES) pressure and GOJ compliance data (mean \pm SEM) were compared using Student's $t$ test.

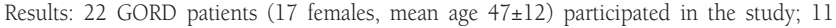
received Stretta first and 11 sham first. Three months after initial sham treatment, symptom score ( $16.8 \pm 2.6$ vs. $17.7 \pm 2.3$, NS), acid exposure $(9.0 \pm 1.3$ vs. $7.5 \pm 1.8 \%$ time, NS), LES pressure $(15 \pm 2$ vs. $13 \pm 1 \mathrm{mmHg}$, NS) and GOJ compliance $(14.1 \pm 4.5$ vs. $12.8 \pm 3.0 \mathrm{ml} /$ $\mathrm{mmHg}$, NS) were not significantly altered. Three months after initial Stretta procedure, no changes were observed in esophageal acid exposure ( $16.3 \pm 4.8$ vs. $16.5 \pm 2.7 \%$ time, NS) and LES pressure ( $11.9 \pm 1.1$ vs. $13.0 \pm 2.2 \mathrm{mmHg}, \mathrm{NS})$. In contrast, symptom score was significantly improved $(12.6 \pm 1.1$ vs. $7.5 \pm 1.8, \mathrm{p}<0.05)$ and $G O J$ compliance was significantly decreased $(15.8 \pm 2.5$ vs. $8.3+2.5 \mathrm{ml} / \mathrm{mm} \mathrm{Hg}, \mathrm{p}<0.05)$. After administration of sildenafil, an esophageal smooth muscle relaxant, GOJ compliance after Stretta was normalised to preStretta level $(13.2 \pm 1.4$ vs. $13.2 \pm 2.8 \mathrm{ml} / \mathrm{mmHg}$, NS), which rules out GOJ fibrosis as an underlying mechanism. Conclusion: In this sham-controlled study, Stretta was associated with improvement of GERD symptoms, decreased GOJ compliance and unchanged acid exposure or LES pressure. Decreased GOJ compliance, which reflects altered LES neuromuscular function, may contribute to symptomatic benefit by decreasing refluxate volume.

\section{W1101}

Predictive Factors of Long-Term Outcome After Antireflux Surgery (ARS): A Community Practice Experience

Ronan Thibault, Sylvie Sacher-Huvelin, Veronique Sebille, Stanislas Bruley des Varannes, Jean-Paul Galmiche

Background: Data about predictive factors of clinical outcome after ARS are scarce. Most studies were performed in tertiary referral centers. Quality of life (QOL) is considered as the main end-point for ARS assessment. We aimed to determine the factors predicting outcome after ARS in GERD patients managed in community practice. We choose QOL as the primary end-point for long-term assessment. Methods: We selected all consecutive patients referred to our laboratory between the 1st January 1995 and 31 December 2002 for 24-hour pH monitoring before ARS. Patients were referred by 33 surgeons, most working in district hospitals or private surgical centers. Patients were included only if there was clear evidence that acid reflux was responsible for symptoms, i.e. symptom index (SI) $\geq 50 \%$ and symptom-association probability (SAP) $\geq 95 \%$. Esophageal acid exposure (EAE) could be normal or abnormal ( $\geq 4.2 \%$ ). We included 121 patients (49 women, mean age $47 \pm 13 \mathrm{yr}$, 61 with erosive disease and 60 with non-erosive GERD). The laparoscopic approach was chosen in $96 \%$ and Nissen procedure in $85 \%$. After ARS, QOL was measured with the French validated GERD-specific questionnaire, REFLUX-QUAL. QOL was considered good or excellent when REFLUX-QUAL global score was above 80. After univariate statistical analysis, variables independently associated with a score $>80$ were determined by a stepwise logistic regression. Results: After an average $43 \pm 19$ month follow-up, $58 \%$ of patients reported a good or excellent post-operative QOL. Male gender ( $72 \%$ with score $>80$ vs $28 \%$ with score $\leq 80, \mathrm{P}<0.001$ ), abnormal EAE ( $62 \%$ vs $38 \%, \mathrm{P}=0.02$ ), preoperative regurgitation ( $54 \%$ vs $46 \%, \mathrm{P}=0.04$ ), absence of preoperative dysphagia ( $63 \%$ vs $37 \%, \mathrm{P}=0.02$ ), PPI-dependence ( $68 \%$ vs $32 \%, \mathrm{P}=0.007$ ), SI $\geq 75 \%$ ( $68 \%$ vs $32 \%, \mathrm{P}=0.02$ ) and history of GERD symptom $\geq 4 \mathrm{yr}(67 \%$ vs $33 \%, \mathrm{P}=0.04)$ were significantly associated with a postoperative QOL score $>80$. Only two factors were significantly and independently associated with an excellen post-operative QOL, namely male gender $(\mathrm{OR}=4.4 ; 95 \% \mathrm{CI}[2.00-9.80], \mathrm{P}<0.001)$ and abnormal EAE (OR=3.0; 95\% CI [1.03-8.69], $\mathrm{P}=0.04)$. Conclusion: In community practice, good or excellent surgical results are reported in less than $2 / 3$ of patients. These results are consistent with a similar study conducted in US*. Although only 2 variables were independently predictive of long-term outcome, our results strongly support the recommendation of preoperative $\mathrm{pH}$-monitoring assessment. 3) Patients with hypersensitive esophagus (normal EAE but positive SAP) are not good candidates for surgery especially those of the female gender (the most frequent situation in this case). *Vakil N et al. Am J Med 2003;114:1-5.

\section{W1102}

Quantification of Esophagogastric Junction Emptying Mechanics After Fundoplication with High-Resolution Manometry (HRM)

Eric T. Volckmann, John E. Pandolfino, Qing Zhang, Sudip K. Ghosh, Nathaniel J. Soper, Peter J. Kahrilas

AIM: Impaired esophageal emptying post-fundoplication is complex, related to both increased outflow resistance and a diminished esophageal propulsive mechanism after surgery (Ghosh SK, et al. Am J Physiol 2005; 289: G21-35). However, the manometric evaluation of postfundoplication patients has been subjective unless coupled with concurrent fluoroscopy and intense analysis. This study leveraged the enhanced spatial resolution of HRM along with recently developed automated analysis paradigms to quantify the mechanical effects of fundoplication. METHODS: 74 normal subjects (NLs) and 46 post-fundoplication patients (FPs) were studied with a solid-state 36-sensor (1 cm spacing) HRM assembly (Sierra Scientific Instruments Inc, Los Angeles, CA). Ten $5 \mathrm{ml}$ water swallows were analyzed for EGJ relaxation and peristaltic integrity. FP patients were subdivided into those with normal peristalsis (FP-NP) and those with ineffective motility (FP-IEM) using the criteria of $\geq 30 \%$ failed peristalsis. HRM data during EGJ relaxation were analyzed in custom written MATLAB programs (Pandolfino JE et al. Am J Physiol 2006; In Press) to derive: 1) the duration of time that pressure gradient from $2 \mathrm{~cm}$ above the EGJ relative to a 6 - $10 \mathrm{~cm}$ E-sleeve-type measure spanning the EGJ favored esophageal emptying [antegrade flow permissive (AFP) timel 2) the median pressure gradient during AFP time, and 3) integrated relaxation resistance (IRR) which is the median E-sleeve relaxation pressure divided by the duration of relaxation. Comparisons were made among NLs, FP-NP, and FP-IEM. RESULTS: Both groups of FP patients exhibited significantly decreased AFP time, an HRM demonstration of outflow obstruction (Table). The complaint of dysphagia was similar in both FP groups (10/29 FPNP; 5/17 FP-IEM). AFP gradient and IRR were increased only in the FP-NP group as both of these indices are influenced by peristaltic function; the genesis of the intrabolus pressure in the case of AFP gradient and of the intra-sphincteric pressure during flow in the case of IRR. CONCLUSION: HRM allows for an automated quantitative assessment of deglutitive EGJ relaxation that can identify and quantify the outflow resistance associated with FP. The symptom of dysphagia was not unique to FP-IEM patients but occurred with equal frequency in the FP-NP group suggesting a multifactorial etiology

\begin{tabular}{|c|c|c|c|}
\hline & NLs $(\mathrm{n}=74)$ & FP-NP $(\mathrm{n}=29)$ & FP-IEM $(\mathrm{n}=17)$ \\
\hline AFP time $(\mathrm{s})$ & $1.7(0.9)$ & $0.9(0.8)^{*}$ & $0.5(0.1)^{*}$ \\
\hline AFP gradient $(\mathrm{mmHg})$ & $1.3(0.3)$ & $2.9(0.3)^{*}$ & $0.9(1.0) \#$ \\
\hline IRR $(\mathrm{mmHg} / \mathrm{s})$ & $1.4(0.6)$ & $2.0(1.0)^{*}$ & $1.4(0.7) \#$ \\
\hline
\end{tabular}

All values are mean (SE); * $\mathrm{p}<0.05$ vs. NL; \# $\mathrm{p}<0.05$ vs. FP-NP

\section{W1103}

Effect of Endoscopic Gastroplication On Acid and Weakly Acidic GastroEsophageal Reflux: A Study Using Impedance Monitoring Jose M. Conchillo, Matthijs P. Schwartz, Mohamed Selimah, Albert J. Bredenoord, Melvin Samsom, Andre J. Smout

Background and aim: In the evaluation of several endoscopic antireflux procedures a discrepancy in the degree of improvement between symptoms and objective reflux parameters has been reported. We aimed to perform an in-depth analysis of the effect of endoscopic gastroplication on esophageal reflux using combined $\mathrm{pH}$-impedance recording. Methods: 10 patients with GERD ( 9 men, mean age 48 years, range $36-58$ ) and no hiatal hernia were included. They were treated with 3 endoscopic gastroplications (Endocinch ${ }^{\mathrm{TM}}$ suturing system) and underwent $24 \mathrm{hr} \mathrm{pH}$-impedance monitoring before and 3 months after treatment. Acid inhibitory drugs were discontinued 1 week before recordings. Analysis of the $\mathrm{pH}$ impedance signals included total reflux time, number of reflux episodes according to reflux content (acid, weakly acidic and weakly alkaline), gas-liquid composition and proximal

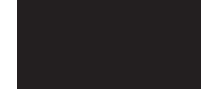


extent of the refluxate. Results: Impedance and pH-metry data before and after endoscopic gastroplication are shown in the table. Total and upright reflux times assessed by impedance were significantly decreased after treatment, but not when assessed by pH-metry. The total number of reflux episodes was significantly reduced after treatment as measured by both impedance and $\mathrm{pH}$ monitoring. Comparable reductions in acid and weakly acidic reflux episodes were observed. Mean acid and volume clearance times as well as proximal extent of the refluxate were unaffected by the procedure. Conclusions: Endoscopic gastroplication leads to a reduction in gastro-esophageal reflux of all types (acid/weakly acidic, liquid/ gaseous, upright/supine). The proximal extent of reflux is not affected by the procedure.

\section{W1104}

The Impact of Laproscopic Fundoplication On Esophageal Bolus Clearance Determined By Combined Manometry and Multi-Channel Intraluminal Impedance (man-MII)

Nam Q. Nguyen, Katrina Bland, Jenny Myers, Marcus Tippett, Glyn G. Jamieson, Richard H. Holloway

Dysphagia is a significant sequela of fundoplication and is related to impaired esophageal clearance. Assessment of clearance by manometry and/or fluoroscopy is suboptimal. MIl measures esophageal clearance directly. Aim. To assess the impact of laparoscopic fundoplication on esophageal clearance using combined man-MII. Methods. 11 patients with proven reflux disease (6M:5F; 54+4yrs) underwent combined man-MII before and 3-6 months after laparoscopic fundoplication (4-360 degree, 7 - 90 degree). Esophageal body motility and impedance were measured concurrently at 4 sites $5-\mathrm{cm}$ apart starting $2 \mathrm{~cm}$ above the lower esophageal sphincter (LES). LES pressure was recorded with a sleeve sensor. Ten, 5-ml liquid (L) boluses and $10 \times 5-\mathrm{ml}$ viscous (V) boluses were tested in each subject. Impaired clearance was defined by either prolonged bolus presence time (BPT) and/or total bolus transit time (TBTT). An individual was judged to have abnormal esophageal clearance function if there were $>2$ liquid or $>3$ viscous responses with impaired clearance. Results. Fundoplication increased nadir LES pressure, distal esophageal wave amplitude, and distal and total propagation time (Table). Fundoplication increased the proportion of responses with impaired clearance (liquid: $60 / 110$ vs $32 / 110, P=0.001$; viscous: $46 / 110$ vs. $17 / 110$, $\mathrm{P}<0.001$ ), and increased BPT in the mid and distal esophagus and TBTT. After fundoplication, more patients had abnormal bolus clearance $(9 / 11$ vs $4 / 11$ ) and the median number of impaired responses per patient increased (L: $5(2.5-8)$ vs. 2(0.5-3.5), $\mathrm{P}=0.07$; V: 4(2-6) vs. $1(0-2.5), P=0.03)$. No patient had improved bolus clearance post-operatively. There was a positive correlation between the nadir LOSP and both BPT at the most distal segment (L: $r=0.4, P<0.001 ; \mathrm{V}: r=0.2, \mathrm{P}=0.01$ ) and TBTT $(\mathrm{L}: \mathrm{r}=0.3, \mathrm{P}<0.003 ; \mathrm{V}: \mathrm{r}=0.4, \mathrm{P}<0.001$ ) Conclusions. Fundoplication impairs oesophageal clearance, predominantly at the distal $2 \mathrm{~cm}$ of the esophagus. This is related to increased resistance to flow at the gastroesophageal junction caused by the fundoplication wrap.

\begin{tabular}{|c|c|c|c|c|}
\hline \multirow{2}{*}{ * P<0.01 vs pre-fundoplication ** } & \multicolumn{2}{|c|}{ Pre-fundoplication } & \multicolumn{2}{c|}{ Post-fundoplication } \\
\cline { 2 - 5 } P $<0.001$ vs pre-fundoplication & Liquid & Viscous & Liquid & Viscous \\
\hline Nadir LOS pressure (mmHg) Distal & $1.7 \pm 0.2$ & $1.2 \pm 0.2$ & $8.3 \pm 2.0 * * *$ & $15.4 \pm 2.3 * *$ \\
Peristaltic Amplitude (mmHg) & $63.0 \pm 4.0$ & $59.0 \pm 4.0$ & $83.0 \pm 7.0 *$ & $73.0 \pm 7.0 * *$ \\
Distal propagation time (sec) Total & $1.6 \pm 0.1$ & $1.9 \pm 0.1$ & $2.1 \pm 0.2 *$ & $2.3 \pm 0.2 *$ \\
propagation time (sec) & $4.6 \pm 0.2$ & $5.3 \pm 0.1$ & $5.3 \pm 0.2 *$ & $5.7 \pm 0.2 *$ \\
\hline & $4.5 \pm 0.2$ & $3.3 \pm 0.2$ & $5.8 \pm 0.3 *$ & $5.2 \pm 0.4 * *$ \\
Mid-esophageal BPT(sec) Distal & $5.0 \pm 0.2$ & $4.7 \pm 0.2$ & $6.9 \pm 0.3 *$ & $7.1 \pm 0.5 * *$ \\
esophageal BPT (sec) TBTT(sec) & $5.7 \pm 0.3$ & $6.3 \pm 0.3$ & $9.1 \pm 0.4 * *$ & $9.8 \pm 0.4 * *$ \\
\hline
\end{tabular}

W1105

Long-Term 5-Year Follow-Up of The Stretta Procedure for Treatment of Reflux Disease

Rebecca J. Ryan, Suresh Sivanesan, William C. Tam, Mark N. Schoeman, John Dent, Richard H. Holloway

We have shown previously that radiofrequency (RFe) treatment of reflux disease (Stretta) reduces reflux symptoms, medication use and esophageal acid exposure over a 12-month follow-up (Gastroenterology 2002;122:A47). However, data on long-term efficacy are limited. Aims: To assess whether improvement in symptoms, medication usage, and esophageal acid exposure is sustained over a 5 year follow up period. Methods: Twenty patients $(10 \mathrm{M}, 10 \mathrm{~F})$ with GERD (heartburn, \%pH<4 >4\%, \pm h/o erosive esophagitis within $6 \mathrm{mths}$ ) underwent endoscopy, 24h ambulatory pH monitoring and symptom assessment (SF-36, GERD-HRQoL, dysphagia score) before and 6 months after RFe treatment. All patients were dependent on acid suppressant therapy before RFe; therapy was stopped after RFe and restarted only if symptoms relapsed. Thirteen patients $(6 \mathrm{M}, 7 \mathrm{~F})$ were available for follow-up at 5 years (56-63 mo) after treatment. These patients underwent re-evaluation with endoscopy, $24 \mathrm{~h}$ ambulatory $\mathrm{pH}$ monitoring and symptom assessment (SF-36, GERD-HRQoL, dysphagia score). Results: At 5 years, 9 of the 13 patients required ongoing acid suppression: 8 at or above the pre-treatment level; 1 had also undergone fundoplication. Only 4 patients were off therapy but all had mild symptoms. Two patients had mild (LA-A or B) erosive esophagitis but more severe than at entry; the remainder were endoscopy-negative. Esophageal acid exposure and scores for reflux symptoms and quality of life at 5 years were not statistically different from those pre-treatment or at 6 months post-treatment (Table, median (IQR)) Conclusions: The beneficial effects of the Stretta procedure that are apparent at short-term follow up are not sustained over 5 years. The role of the Stretta procedure in the management of reflux disease requires re-evaluation.

\begin{tabular}{|c|c|c|}
\hline Median (IQR) & Pre-treatment & 5 Years post-treatment \\
\hline \% time $\mathbf{p H}<\mathbf{4}($ Total) & $10.9(8.0,12.8)$ & $6.7(4.7,11.5)$ \\
\hline GERD QoL & $14.0(3.0,20.0)$ & $13.0(7.0,15.0)$ \\
\hline SF-36 (Physical) & $38.6(34.6,48.3)$ & $50.2(47.4,52.6)$ \\
\hline SF-36 (Mental) & $50.0(43.2,56.2)$ & $54.7(51.9,57.0)$ \\
\hline
\end{tabular}

W1106

Laparoscopic Nissen Fundoplication in Children with Severe Gastroesophageal Reflux Disease: A Five Year Follow-Up of Esophageal Function

Osvaldo Borrelli, Valentina Mancini, Letizia Cordischi, Felicia Galos, Marina Aloi, Manuela Cirulli, Valeria Labalestra, Ciro Esposito, Salvatore Cucchiara

Background and Aim. Despite laparoscopic surgery has become the most common operative procedure for refractory gastroesophageal reflux (GER) disease (GERD), there are few data on the esophageal functional outcome in patients with severe GERD treated with this technique. We studied esophageal function through esophageal manometry and intraluminal $\mathrm{pH}$ recording in a group of children with severe GERD who underwent laparoscopic antireflux fundoplication. Subjects and Methods. From September 1995 to October 200440 patients (median age: 8.3 years, range: $3-15$ ) were studied. Neurologic and systemic diseases had been excluded in all. Clinical assessment (symptomatic score), esophageal manometry and 24-hour esophageal $\mathrm{pH}$ test were performed in each patient before and 3, 6, 12, 24, 36, 48 and 60 months after surgery. Esophageal manometry was performed with a low compliance system to evaluate lower esophageal sphincter (LES) pressure (LESP) $(\mathrm{mmHg}), \%$ of deglutitive LES relaxation (PLESR), amplitude (Amp)( $\mathrm{mmHg}$ ) and \% of peristaltic waves (PPW). Intraesophageal $\mathrm{pH}$ was considered abnormal when the 24 -hour $\%$ of GER was $>5$. Statistical analysis was done by Anova. Results. (mean \pm DD). Surgery resulted in a marked reduction in the symptomatic score from a baseline value of $9.7 \pm 2.1$ to $4.2 \pm 0.9,4.1 \pm 1.2,3.51 \pm 1.4,4.12 \pm 1.4$, $3.8 \pm 1.9,4.2 \pm 06$ and $3.7 \pm 1.0$ at $3,6,12,24,36,48$ and 60 months, respectively ( $p<0.01$ vs baseline). A significant reduction in the intraesophageal acid exposure was detected at all the follow up study months $(3: 4.58 \pm 1.4 ; 6: 4.78 \pm 1.56 ; 12: 4.8 \pm 1.17 ; 24: 4.95 \pm 1.53$; 36: $4.86 \pm 1.86 ; 48: 4.7 \pm 1.4 ; 60: 4.6 \pm 1.7)$ as compared to baseline $(10.01 \pm 6.17, \mathrm{p}<0.01)$. Baseline values of LESP, PLESR, Amp and PPW were $8.1 \pm 2.78,89.05 \pm 7.7,38.8 \pm 15.8$, $82.0 \pm 12.24$, respectively. LESP, Amp and PPW were significantly increased at all the postoperative follow up studies $(3,6,12,24,36,48,60$ months) as compared to baseline: LESP: $16.5 \pm 3.41,16.6 \pm 3.07,16.54 \pm 3.18,17.0 \pm 2.8,16.9 \pm 2.5,17.2 \pm 3.0,16.8 \pm 2.9(\mathrm{p}<0.01)$ Amp: $51.25 \pm 14.5,51.35 \pm 14.1,50.8 \pm 14.46,55.9 \pm 15.03,57.81 \pm 14.7,56.8 \pm 16.0,58.5 \pm 16.8$ $(\mathrm{p}<0.01)$; PPW: $87.0 \pm 10.9,87.5 \pm 10.06,87.05 \pm 9.8,88.2 \pm 9.02,88.7 \pm 8.82,88.0 \pm 9.2$ $87.5 \pm 8.8(\mathrm{p}<0.05)$. The PLESR did not statistically change at the follow up evaluation. Conclusions: After 5 years of follow-up, in children with severe GERD laparoscopic Nissen fundoplication provides excellent and sustained functional results consisting in reduced esophageal acid exposure and improved esophageal motor activity.

\section{W1107}

Long-Term Results of Radio Frequency Energy Delivery for the Treatment of Gastroesophageal Reflux Disease Sustained Improvements in Symptoms, Quality of Life and Drug Use At 4-Year Follow-Up Alvaro Reymunde, Nilda Santiago

The aim of this study was to evaluate the efficacy of the Stretta procedure for GERD patients based on symptom control, quality of life and medication use. All patients underwent careful evaluation to document the diagnosis of GERD. Also the patients symptoms were assessed using standardized validated tools complemented by patient diaries and symptom logs with use of visual analog scale including GERD Symptom Score. A Quality of Life in Reflux and Dyspepsia scale was used to assess patients quality of life related to reflux disease on a scale of 0-5 with higher numbers indicating better disease control. Medication use assessment was performed, patients were specifically queried about the use of all acid reflux medications including proton pump inhibitors, $\mathrm{H} 2$ receptor antagonists, antacids and promotility agents This testing was repeated 12,36 and 48 months following the Stretta procedure. Results: 83 consecutive patients; $65 \mathrm{men}$ ( $78 \%$ ) and 17 women $(22 \%)$ have reached follow-up of 48 months. Complete, matched data sets for 80 patients (96.4\%) are reported with assessments at baseline and 12, 36 and 48 month follow-up; three patients were lost to follow-up. No serious complications were associated with the Stretta procedure.All results were statistically significant at all timepoints. GERD symptom scores improved from a mean score of 2.7 at baseline to 0.3 at 36 months and 0.6 at 48 months. $68.67 \%$ of patients showed complete resolution of symptoms with scores of $0(\mathrm{p}<0.001)$. The mean quality of life scores improved from 2.4 at baseline to 4.6 at 36 months, 4.3 at 48 months $(p<0.001)$. Use of antisecretory medications in patients following Stretta was reduced from $100 \%$ of patients using prescription anti-secretory medication at baseline to $29.4 \%$ of patients at 12 months and $12.1 \%$ of patients at 36 months and $13.75 \%$ of patients at 48 months ( $<<0.001)$. No patient who was previously on PPI BID returned to this requirement following the Stretta procedure. 90.2\% of patients on PPI or H2RA levels pre-procedure had reduced their medication to none at all. $88.75 \%$ of patients had complete elimination of the need for anti-secretory medications or a reduction in their medication usage and significant improvement in both symptom score and QOL measures. Our study demonstrates the Stretta represents a durable modality that can be offered to patients suffering from persistent GERD as a clinically viable treatment for the relief of symptoms and requirement for ongoing medical management or the potential need for an invasive surgical procedure. 


\section{W1108}

Long-Term Results of Laparoscopic Antireflux Surgery Jeroen Maljaars, Avinash D. Roopram, Jan Ringers, Ad A. Masclee

Laparoscopic (partial) fundoplication is the preferred surgical treatment for therapy resistant reflux disease. High success rates have been reported after short-term follow up of 6-12 months. In our patient population complete symptom relief occurred in $85 \%$ and normalization of $24 \mathrm{hr}$ pH metry in $80 \%$ of patients. Little is known however on long-term results and symptom relief after laparoscopic antireflux surgery. Our aim was to evaluate the longterm results of 130 patients operated at our institution between 1991 and 2001 for therapy resistant reflux disease. From 1991 to 1995 complete fundoplication was performed, thereafter partial fundoplication. We evaluated symptoms (severity score $0-3$, frequency score 0 3 , combined score 0-9), use of anti-reflux medication, patient satisfaction and quality of life (SF-36) by questionnaires. Response rate was over 70\%. Results: Mean age of patients was $54 \pm 1.4 \mathrm{yr}$ (women 53\%). The mean follow up time after surgery was $7.8 \pm 0.5 \mathrm{yrs}$. Indication for surgery was therapy resistant reflux disease or unwillingness to maintenance therapy. Reflux was documented by endoscopy (esophagitis) or $24 \mathrm{hr} \mathrm{pH}$ metry. Eight patients underwent reoperation because of recurrent reflux or dysphagia. These data were also included. Heartburn scores decreased sign. $(\mathrm{p}<0.0001)$ from $6.6 \pm 0.3$ (pre-op) to 1.6 \pm 0.3 (long term post-op), retrosternal pain from $5.0 \pm 0.4$ to $1.8 \pm 0.3(\mathrm{p}<0.0001)$ and dysphagia from $4.0 \pm 0.4$ to $2.2 \pm 0.3$ ( $\mathrm{p}<0.0001)$. Concerning antireflux medication: 60 $\%$ had no need for medication, while $31 \%$ still needed acid reducing medication on a daily basis and $9 \%$ on a weekly basis. Patient satisfaction with surgical result: $25 \%$ was not satisfied with the procedure, $15 \%$ moderately satisfied and $60 \%$ fully satisfied. When compared to age matched controls, quality of life by SF- 36 was significantly $(\mathrm{p}<0.05)$ reduced for all domains in the patients post fundoplication. In the patients with follow up of 10-15 yrs and of $5-10$ yrs, quality of life was significantly $(\mathrm{p}<0.008)$ higher versus patients with $0-5$ yrs follow up. These results were not affected by the type of surgery, nor by age or gender. In conclusion: long term follow up of patients after laparoscopic surgery shows a much less favorable outcome compared to short term follow up. One third of the patients still needs acid reducing drugs to control symptoms and 25\% is not satisfied with the procedure. Nevertheless, quality of life increases with duration of post-operative follow-up.

\section{W1109}

Effect of Diaphragm Biofeedback Training On Esophageal Acid Exposure and Proximal Gastric Volume in Patients with Gastroesophageal Reflux Disease Zhaolu Ding, Meiyun Ke, Xiaohong Sun, Zhifeng Wang, Dabo Xu

Background \& aims: Our recent study showed that diaphragm biofeedback in the fasting state could enhance EGJ pressure and reduce intake of acid inhibiting agent in patients with GERD. Since more gastroesophageal reflux occurred after a meal, which might be related with proximal gastric function impairment. The aim of our study was to investigate whether postprandial diaphragm biofeedback could reduce esophageal acid exposure and improve the proximal gastric emptying. Methods: 19 patients with GERD and 6 HS with matched demographic characteristics were enrolled. Esophageal manometry including EGJ pressure and transdiaphragmatic pressure(PDP) was performed with simultaneous esophageal $\mathrm{pH}$ recorded in a 30 min fasting period and a 120 min postprandial period. Patients were divided into three groups: 7 patients received diaphragm biofeedback training during lst hour after meal (The $500 \mathrm{kcal} / 500 \mathrm{ml}$ liquid nutrient test meal) (group BA) and 6 patients received diaphragm biofeedback training during 2 nd hour after meal (group $\mathrm{AB}$ ), whereas 6 patients received no diaphragm biofeedback training after meal (group AA). Proximal gastric emptying was measured by ultrasonography at 0 min and every $30 \mathrm{~min}$ after meal. Results: (1) Compared with group AA, the percentage time with $\mathrm{pH}<4$ in group $\mathrm{BA}$ was reduced in $120 \mathrm{~min}$ postprandial period [ $1.4 \%(0.1 \%-3.0 \%)$ vs. $8.4 \%(2.0 \%-17.5 \%), \mathrm{p}<0.05]$ and there was no significant difference of esophageal acid exposure between group $A B$ and AA. (2) At the $60 \mathrm{~min}$ and $120 \mathrm{~min}$ after the test meal, the group $B A, A B$ and $A A$ showed similar volume of proximal stomach $(\mathrm{p}>0.05)$, but significant difference was found between HS and GERD groups $(\mathrm{p}<0.05)$. (3) The crural diaphragm pressure and $E G J$ pressure were significantly increased from $11.8 \pm 2.8$ and $17.1 \pm 3.5 \mathrm{mmHg}$ at baseline to $35.3 \pm 1.7$ and $42.4 \pm 8.0 \mathrm{mmHg}$ during biofeedback training $(\mathrm{p}<0.01)$, but no alteration of LES pressure was present. (4)The increase of EGJ pressure during biofeedback training was higher than that of PDP (42.4 vs. $22.4 \mathrm{mmHg}$, p<0.05). Conclusions: The diaphragm biofeedback training during the lst hour after test meal could reduce esophageal acid exposure. The reduction in esophageal acid exposure may be due to enhanced anti-reflux barrier of the EGJ function. Therefore, diaphragm biofeedback training may provide a potential approach to conservative treatment of GERD. The effect of diaphragm biofeedback training on the proximal stomach function and transient lower esophageal sphincter relaxations (tLESRs) should be further investigated.

\section{W1110}

Mdm2 Promoter Polymorphism Associated with Susceptibility to Gastric Carcinoma and Its Prognosis

Naoki Ohmiya, Ayumu Taguchi, Nobuyuki Mabuchi, Akihiro Itoh, Yoshiki Hirooka Osamu Maeda, Takafumi Ando, Yasumasa Niwa, Hidemi Goto

Background \& Aims:Recently, a single nucleotide polymorphism in the MDM2 promoter (SNP309) has been found to lower the age of onset of tumors and increase the occurrence of multiple primary tumors in Li-Fraumeni syndrome and accelerate the development of even sporadic adult soft-tissue sarcoma. The aim of this study was to determine whether SNP 309 associates with susceptibility to gastric carcinoma and its prognosis. Methods: In a case-control study including 438 controls and 410 patients with sporadic gastric carcinoma, MDM2 SNP309 was genotyped. Serum pepsinogens (PGs) I and II were measured in 438 controls and 253 cases selected from 410 patients. Tumor tissue was immunostained with p53 and examined for mutations in exons 5-8 of p53 using polymerase chain reaction-based single strand conformational polymorphism analysis and direct sequencing. Results: The risk of overall gastric carcinoma for SNP309 (G/G) was significantly increased when compared to $\mathrm{T}$ carriers $(\mathrm{P}=0.009)$, especially carcinoma with extragastric tumors $(\mathrm{P}=0.004)$, carcinoma with severe atrophic gastritis positive for PG assay (PG I level $<70 \mathrm{ng} / \mathrm{ml}$ and PG I/II $<3.0$ ) $(\mathrm{P}=0.004)$, antral carcinoma $(\mathrm{P}=0.01)$, intestinal-type carcinoma $(\mathrm{P}=0.004)$, p53-immunopositive carcinoma $(\mathrm{P}=0.004)$, and carcinoma with $p 53$ mutations $(\mathrm{P}=0.002)$. No significan difference in age at diagnosis was observed among genotypes. SNP309 $(\mathrm{G} / \mathrm{G})$ was an independent marker of poor overall survival (hazard ratio, 2.08; $95 \%$ CI, 1.01-4.28). Conclusions This study provides evidence supporting the association of SNP 309 with gastric carcinogenesis via p53 tumor suppressor pathway, extragastric tumorigenesis, and poor prognosis.

\section{W111}

Increased Tumor Multiplicity in BALB/C Mice Congenic for Both the Min $\left(A p c^{M i n /+}\right)$ and RAG2 Knockout (Rag2 $\left.2^{\text {tmlFwa }}\right)$ Mutations

Claude Nagamine, J.N. Sohn, Elizabeth Groff, Katie Schlieper, Prashant R. Nambiar, James G. Fox, David B. Schauer

The C57BL/6-Apc Min/+ (B6-Min) mouse harbors a point mutation in its adenomatous polyposis coli gene, the same gene that is mutated in hereditary and many sporadic forms of human colorectal cancer. Therefore, the B6-Min mouse is a clinically relevant model for human colorectal cancer and can be used to dissect the molecular and genetic mechanisms underlyin colorectal carcinogenesis and progression. B6-Min mice have a reduced ( $\leq 6$ months) life span due, in part, to complications from intestinal polyposis-induced hemorrhage. This reduced life span complicates long-term studies of tumorigenesis. To circumvent this problem, we placed the $A p c^{\text {Min }}$ mutation on the BALB/c genetic background to generate the BALB-Min (C.B6-Ap $C^{\mathrm{Min} /+}$ ) congenic strain. BALB-Min mice had a lower tumor multiplicity and a concomitant increase in life span. In our colony, B6-Min mice developed an average of 100 small intestinal (SI) adenomas ( $\mathrm{n}=17$ mice, age $=3.2-6.1$ months). In contrast, BALBMin mice developed an average of 18 SI tumors $(n=15$ mice, age $=2.9-14.6$ months) Moreover, BALB-Min mice had a longer life span with some mice surviving $>1$ year. Recombination activating gene 2 (Rag2) is required for the normal development of $\mathrm{T}$ and $\mathrm{B}$ cells. To investigate the interplay of the adaptive immune system and tumorigenesis, the Rag2 knockout allele from the BALB/c congenic strain C.129S6(B6)-Rag2 ${ }^{\text {tm1Fwa }}$ was introduced into BALB-Min to generate the compound mutant strain BALB-RagMin (C. $\mathrm{Cg}-\mathrm{Apc} \mathrm{Min}^{\mathrm{A} / \mathrm{t}}$ Rag $2^{\text {tmlFwa }}$ ). Interestingly, BALB-RagMin mice showed an increase in SI tumor multiplicity relative to the parental BALB-Min strain, developing an average of 44 SI adenomas $(n=58$ mice, age $=3.1-8.4$ months). Thus, in contrast to previous studies, we found a putative dependence of SI tumor multiplicity on the adaptive immune system. We are currently characterizing the differences between B6-Min, BALB-Min, and BALB-RagMin tumors and the mechanism by which tumor multiplicity is increased in BALB-RagMin mice.

\section{W1112}

Colorectal Adenomatous Polyposis Linked to MYH Gene Mutations: Genotype and Phenotype Characteristics in a French Series of 56 Patients Guillaume Bouguen, Sylvain Manfredi, Martine Blayau, Catherine Dugast, Bruno Buecher Dominique Bonneau, Laurent Siproudhis, Veronique David, Jean-Francois Bretagne

Aim: Recent literature reports that a subgroup of patients with attenuated and classic polyposis as well as with young-onset colorectal cancer (CCR) is associated with mutations in the base excision repair gene MYH. The aim of this study was to establish the prevalence of germline MYH mutations in a French series of 56 consecutive patients with no detectable APC mutation and to describe the phenotype of those with MYH mutations. Methods: MYH mutations were screened by DNA sequencing after PCR amplification of each exon. Clinical and endoscopic characteristics (age, sex, family history, extra-colonic manifestations, numbe and types of polyps, associated cancer) and surgical data have been collected for tested patients. Results: MYH germ-line mutations were identified in 11 (19.6\%) of the 56 tested patients. The prevalence of MYH mutations was $34.4 \%$ in the subgroup of 32 patients having 10 or more adenomatous polyps. No mutation was found among patients with mixed polyposis $(n=2)$ and with CCR $(n=13)$. Among 10 patients with biallelic MYH mutations, 2 proved to be homozygotes and the other 8 were compound heterozygotes. Only one patient had a monoallelic mutation. At least one of the two mutational hot spots (Y165C and G382D) was identified in all cases, except one patient. There were 9 males and two females aged of 47 years old (ranged 36-57). Three patients presented a family history of adenomatous polyposis in siblings, but no vertical transmission was noted. The median number of colorectal adenomatous polyps was 53 (11-100) without preferential localization A CCR was associated to polyposis in 7 of the 11 patients $(63.6 \%) \cdot 2$ cancers were stage $I$, 3 stage II and 2 stage III. Gastric and duodenal adenomas with high grade dysplasia were diagnosed in one case. Ten of the 11 patients were operated on: restorative proctocolectom was performed in 4 cases, a subtotal colectomy with rectal preservation in 5 cases and a left colectomy in l case. In the latter case, polyposis was diagnosed two years after the left colectomy and total colectomy is planned at this time. Rectal adenomas were diagnosed during the follow-up in 3 patients with preserved rectum. Conclusions: 1) MYH mutations, mainly corresponding to biallelic mutations have been observed in one third of French patients who had more than 10 colorectal adenomatous polyps without APC mutation. 2) The phenotype of the disease is similar to attenuated FAP, but its transmission shows evidence on recessive or more complex inheritance. 3) The high risk of CCR suggests that prophylactic colectomy is recommended. 4) Upper GI endoscopy should be also recommended.

\section{W1113}

Role of Ultrasound for Thyroid Cancer Screening in the Familial Adenomatous Polyposis Syndrome Maite Herraiz, Giuseppe Barbesino, Gilbert Daniels, Daniel C. Chung

Background: Thyroid carcinoma (TC) is an extraintestinal manifestation of the Familial Adenomatous Polyposis (FAP) syndrome. Screening with thyroid ultrasound (TU) has been proposed, but the optimal approach to screening for TC in FAP is unknown. We sought 
to determine the role of TU in patients with FAP. Methods: We performed a retrospective chart review of patients with a proven diagnosis of FAP at a single institution. Clinical parameters including family history, Adenomatous Polyposis Coli (APC) gene testing, and TU were analyzed. Results: 50 patients were diagnosed with FAP by clinical and/or genetic criteria. Papillary TC was diagnosed in 6 female patients $(12 \%)$. The mean ages of diagnosis for FAP and TC in these 6 patients were 28 years (range 19-48 years) and 33 years (range 18-51 years), respectively. No genotype-phenotype correlations between APC mutations and risk of TC were identified. APC germ-line mutations in those with TC were located at codon 302 (exon 8), codon 564 (exon 13), codon 1935 (exon 15), and in segment 2 (between codons 686-1217, exon 15). All 6 patients had multifocal tumors and 4 had bilateral disease. On pathologic examination, some of the additional malignant foci were small (1-5 mm) None of these small foci had suspicious features of malignancy on TU. After a median follow-up of 34 months (range 13-186), all patients were alive and disease-free. Of 24 FAP patients who had at least one screening TU, 19 (79\%) had thyroid nodules; the disease was multinodular in $73 \%$. The median age of patients with nodules was 41 years (21-66); 68\% were women. The largest nodule was $\geq 9 \mathrm{~mm}$ in half of the patients. Fine-needle aspiration (FNA) was performed in 10 patients who had screening ultrasound. Two out of the 6 TC cases were discovered in this manner. Follow-up ultrasound in 10 patients after a mean of 15 months (range 12-20 months) revealed no changes in either the number or size of nodules. Conclusion: The prevalence of TC in this series of patients with FAP is $12 \%$, which is higher than previous reports of $1-2 \%$. Screening for TC with ultrasound can detect asymptomatic tumors, and the tumors are often multifocal. Nodular thyroid disease is very common among FAP patients. Because nodules $<5 \mathrm{~mm}$ may be malignant, close followup with thyroid ultrasound and FNA as needed is warranted. However, the impact of screening for TC on survival cannot be predicted at this time.

\section{W1114}

The Use of Genetic Testing in Hereditary Non-Polyposis Colorectal Cancer Families

Dewkoemar Ramsoekh, Anja Wagner, Monique van Leerdam, Dennis Dooijes, Carli Tops, Hanne Meijers-Heijboer, Ernst Kuipers

Background: Hereditary non-polyposis colorectal cancer is an autosomal dominantly inheriting cancer susceptibility syndrome. Genetic testing in subjects from known HNPCC families is of considerable medical and psychological significance. Subjects with a mutation can benefit from a medical surveillance program, i.e. surveillance endoscopy, while subjects without a mutation are relieved from anxiety and can be dismissed from further surveillance. The aim of the present study was to determine the use of genetic testing in clinical ascertained HNPCC families with a known mutation in MSH2, MLHl or MSH6. Methods: Data were collected from medical records and family pedigrees of patients originating from HNPCC amilies, who visited the department of Clinical Genetics of the Erasmus Medical Center between 1995 and August 2005. Pre-test genetic risk was defined as 100\% (diagnosed with an HNPCC related tumor), 50\% (first degree relative with an HNPCC related tumor or with a mutation) and $25 \%$ (parent with a 50\% risk). Results: Thirty-four HNPCC families with a known mutation in MSH2 $(n=10)$, MLH1 $(n=12)$ and MSH6 $(n=12)$ were included in the study. At the time of clinical ascertainment 27 of the 34 families fulfilled the Amsterdam II criteria. The 34 families consisted out of 970 living subjects (50\% male) with a 100\% $(n=108), 50 \%(n=533)$ or $25 \%(n=329)$ pre-test genetic risk of carrying the family specific mutation. Genetic testing was used by 418 (43\%) subjects, a family-specific mutation was detected in $203(49 \%)$ of them. Of the subjects with a pre-test genetic risk of $100 \%, 50 \%$ or $25 \%$ for carrying the mutation, respectively $76 \%, 53 \%$ and $16 \%$ used genetic testing (p $<0.0001$ for $100 \%$ vs. $50 \%$ risk; $\mathrm{p}<0.0001$ for $100 \%$ vs. $25 \%$ risk; $\mathrm{p}<0.0001$ for $50 \%$ vs. $25 \%$ risk). In the 970 subjects there was a significant difference in the test rate between men $(36 \%)$ and women $(50 \%), p<0.0001$. This significant difference between sexes was found in the $50 \%$ pre-test genetic risk group $(46 \%$ vs. $60 \%, p=0.001$ ), but not in the $100 \%(70 \%$ vs. $81 \%, p=0.2)$ and $25 \%$ pre-test genetic risk group ( $13 \%$ vs. $20 \%, p=$ 0.07). Conclusions: There is interest in genetic testing in subjects from HNPCC families with a known mutation. Genetic testing is used more frequently by women, and by subjects with a higher pre-test genetic risk. However, acceptance of testing is far from complete, a considerable number of subjects refrains from genetic testing. This has implications for surveillance in order to prevent cancer. Methods for improved implementation of genetic testing should be studied and optimal testing should be used as a part of the standard medical care for subjects at risk for HNPCC

\section{W1115}

Polygenic Familial Colorectal Cancers Are Associated with the E-Cadherin Polymorphism -160a/C

Frank Gruenhage, Matthias Jungck, Christine Berg, Christoph Lamberti, Ursula Becker, Constanze Pagenstecher, Michaela Mathiak, Tilman Sauerbruch, Frank Lammert

Although up to $20 \%$ of colorectal cancer (CRC) patients show some kind of familiarity, the genetic factors underlying polygenic familial CRCs have yet to be defined. However, many CRC association studies did not differentiate between sporadic and familial cancers, and controls were not evaluated for CRC precursor lesions, i.e. colorectal adenomas. Thus, our aim now was to test a group of patients with strong evidence for CRC familiarity (but exclusion of monogenic CRC syndromes) for association with polymorphisms of CRC candidate genes in comparison to patients with sporadic CRCs and healthy controls. Methods: We recruited 98 patients with familial CRC, as defined by two of the following criteria: (1) three affected relatives, one of them a first-degree relative of the others; (2) one member diagnosed with CRC before age 50; (3) two affected generations. All tumors showed regular expression of hMLH1 and hMSH2, and microsatellite instability was excluded. For comparison, we studied 95 CRC patients without inherited CRC syndromes and a negative family history as well as 220 'hyper-normal' controls who displayed no adenomatous polyps on colonoscopy. Polymorphisms in the genes encoding E-cadherin $(-160 \mathrm{~A} / \mathrm{C})$, cyclin Dl $(870 \mathrm{~A} / \mathrm{G})$, p53 (R72P), the vitamin D receptor (-8C/T) and the ileal bile acid transporter (169C/T, Al71S) were genotyped using PCR-based assays. Results: Familial cases ( $56 \pm 12$ yrs) were significantly $(\mathrm{p}<0.001)$ younger than sporadic $C R C$ cases $(65 \pm 9 \mathrm{yrs})$ and hyper-normal controls
(63 \pm 8 yrs). Hyper-normal controls were not significantly younger than sporadic CRC cases Gender distributions did not differ. The frequency of the minor allele of the E-cadherin SNP -160A/C was significantly higher in hyper-normal controls compared to familial CRC cases ( $\mathrm{p}=0.042$ ), but no such difference was detected in comparison to sporadic cases. The 160A/C allele distribution did not differ significantly between familial and sporadic CRCs Overall, carriers of the $-160 \mathrm{C}$ allele were significantly more common among hyper-normal controls, indicating a protective effect of this variant $(\chi 2=4.77, \mathrm{CI} 0.35-0.95$, OR 0.58). No differences in allele frequencies were detected for the other polymorphisms. Conclusions: In our study, the presence of the minor allele of the E-cadherin polymorphism -160A/C might be protective for the development of familial CRCs. Our findings complement the observation of a similar association in patients with sporadic CRCs, indicating that common pathways affect carcinogenesis in familial and sporadic CRCs.

\section{W1116}

Somatic Mutation As a Possible 'Second Hit' in Hereditary Diffuse Gastric Cancer Patients with E-Cadherin Germ-Line Mutations Miriam Barber, Sarah Hyland, Nicola Grehan, Cristina Bordin, Carlos Caldas, Rebecca Fitzgerald

Introduction: Germ-line mutations of the E-cadherin gene (CDH1) are causative in 30\% of Hereditary Diffuse Gastric Cancer (HDGC) cases. Although this is not a large percentage, the penetrance of the mutation is $70 \%$ and $\mathrm{CDHl}$ mutations are associated with a five-year survival rate of only $10 \%$ at the time of diagnosis. Therefore the consequences for families affected with the mutation are profound. Similar to other autosomal dominant cancer predisposing syndromes, only one of the E-cadherin alleles is mutated in the germ-line and the second allele is inactivated within the gastric tissue. Hence, E-cadherin acts as a recessive tumour suppressor and both alleles have to be inactivated to result in loss of E-cadherin function and for cancer to occur. Aim: To determine if somatic mutation could act as the possible 'second hit' in patients with known germ-line mutations of $\mathrm{CDHl}$. Methods: Five individuals ( 4 male, 1 female) from 4 mutation carrying HDGC families were analysed. The germ-line $\mathrm{CDHl}$ mutations found in these individuals are spaced throughout the gene (from exon 1 to exon 10) and there is no mutation 'hotspot' associated with HDGC. Therefore exon specific primers were used to amplify each of the 16 exons of the E-cadherin gene individually and sequencing was carried out for each exon. Any sequences alterations identified were further analysed using the TA Cloning Kit with One Shot TOP10 Chemically Competent E. coli (Invitrogen) and M13 primer sequences. Protein expression was investigated using immunohistochemistry. Two E-cadherin specific antibodies were used for this analysis; one that recognises an epitope in the extracellular domain (BD Transduction Laboratories) and one that recognises an epitope in the cytoplasmic domain (Neomarkers). Results: Two novel gene alterations were identified in addition to the germ-line mutations previously found. Both novel mutations are located in splice site regions and may therefore affect splicing. Immunohistochemical analysis showed that E-cadherin protein expression was still present in these individuals with somatic mutation, although in one case localisation of the protein had altered, with reduced, patchy membranous staining and increased cytoplasmic staining in the tumour tissue compared to strong membranous staining in the surrounding normal glands. Conclusion: Somatic mutations have been identified as the possible 'second hit' in two HDGC patients with E-cadherin germ-line mutations. Loss of heterozygosity and hypermethylation analyses are required to investigate alternative mechanisms for E-cadherin down-regulation in patients without somatic mutations.

\section{W1117}

Reproductive Decision-Making in Familial Adenomatous Polyposis: Attitudes Towards Prenatal Testing

Fay Kastrinos, Elena M. Stoffel, Judith Balmana, Sapna Syngal

BACKGROUND: Familial Adenomatous Polyposis (FAP) is an autosomal dominant syndrome associated with a $>95 \%$ risk of colorectal cancer in the absence of prophylactic colectomy. Classic FAP is most commonly associated with underlying mutations in the Adenomatous Polyposis Coli (APC) gene. In cases of FAP where there is an identifiable APC gene mutation, prenatal testing can be performed to determine whether an embryo or fetus carries the gene mutation. However, the willingness of FAP patients to undergo prenatal testing for reproductive decision-making has not been studied. We conducted a pilot study to assess attitudes towards prenatal diagnosis among individuals with FAP. METHODS: Individuals affected with FAP completed a self-administered survey containing questions on demographics, personal and family history related to FAP, and attitudes towards prenatal testing, including amniocentesis, chorionic villous sampling (CVS), and preimplantation genetic diagnosis (PGD). RESULTS: A total of 17 subjects (10 females and 7 males) completed the 40-item survey. The mean age of participants was $43.3+$ - 14.2 years (range: $28-73$ years). Of the 10 subjects who had children, 5 had children affected with FAP and 5 had children whose disease status is unknown. Four of 5 subjects with affected children had at least one child diagnosed with cancer or desmoid tumors. Sixteen of $17(94 \%)$ subjects stated that they would consider undergoing PGD and $77 \%$ would consider amniocentesis or CVS Early reassurance of having an unaffected child was cited as the most important advantage of PGD. Eleven respondents favored PGD over amniocentesis or CVS because it provides an opportunity to avoid a pregnancy termination. No participants felt that it was unethical to provide any form of prenatal testing for FAP and all four subjects reporting a "strong" religious background said they would consider PGD over other forms of prenatal testing for FAP. Lack of medical insurance reimbursement and the expensive cost of PGD was an important concern and possible deterrent for $64 \%$ of this sample. CONCLUSION: Patients with FAP are willing to consider prenatal testing approaches to prevent transmission of disease to their children. Further studies are warranted in larger study populations to evaluate optimal methods of providing information regarding prenatal testing and to evaluate ways to facilitate prenatal counseling referrals for affected patients. 


\section{W1118}

Genetic Testing Is Associated with Appropriate Colorectal Cancer Screening Among Individuals At Risk for Hereditary Nonpolyposis Colorectal Cancer (HNPCC)

Elena M. Stoffel, Rowena Mercado, Shilpa Grover, Beth Ford, Wendy Kohlmann, Kristen Shannon, Peggy Conrad, Jonathan Terdiman, Stephen Gruber, Daniel Chung, Sapna Syngal

Background: Individuals at risk for HNPCC require colonoscopy starting at age 25 at intervals of every 1-2 years for prevention of colorectal cancer (CRC). Clinical experience suggests many patients at highest risk for CRC undergo inadequate screening. Objective: To assess the prevalence of appropriate CRC screening among individuals with HNPCC and identify clinical factors associated with appropriate screening. Methods: 261 subjects who met Bethesda criteria for HNPCC recruited through 4 U.S. cancer genetics clinics completed a written questionnaire. Subjects provided information on personal and family medical history, genetic testing, and frequency of cancer screening tests. Subjects were considered to have had appropriate CRC screening if they reported undergoing colonoscopy at least every 2 years. Results: Overall, 241/261 (92\%) subjects had had at least l colonoscopy and 163 (62\%) reported undergoing colonoscopy at least every 2 years. Among the 105 subjects who had either an identifiable mismatch repair gene mutation or had inconclusive genetic test results but fulfilled Amsterdam Criteria, 90 (86\%) had CRC screening at the appropriate interval. In contrast, only 13/38 (34\%) who did not undergo genetic testing but fulfilled Amsterdam Criteria reported CRC screening that was appropriate for HNPCC $(\mathrm{p}<0.0001)$. Half $(20 /$ 40 ) of those with inadequate CRC screening practices indicated that their physician had recommended colonoscopy every 3-5 years or less frequently. In multivariable analysis controlling for subjects' age and prior history of $\mathrm{CRC}$, history of genetic testing $(\mathrm{OR}=11.6$, $95 \% \mathrm{CI}=3.4-40.0)$ and history of a first degree relative with $\mathrm{CRC}$ at age $<50(\mathrm{OR}=4.8$, $95 \% \mathrm{CI}=1.3-18.0$ ) were independent predictors of appropriate screening. Conclusions: Genetic testing is associated with appropriate CRC screening among individuals at risk for HNPCC. Patients with personal and/or family history suspicious for HNPCC should be referred for genetic evaluation so they and their physicians can be informed about the specialized screening required for cancer prevention

\section{W1119}

Patients with Diabetes More Than 15 Years Have Increased Odds of Colorectal Cancer

Donald Garrow, Brenda Hoffman, Leonard Egede

OBJECTIVE: To assess the effect of duration of diabetes mellitus on the odds of colorectal cancer within a nationally representative population. BACKGROUND: Previous studies have yielded evidence that diabetes mellitus may be a risk factor for colon cancer. Both hyperinsulinemia and hyperglycemia have been noted in vitro to be promoters of colon cancer growth. Furthermore, insulin and insulin-like growth factor-1 (IGF-1) receptors have been found on colon cancer tissue. Also, high levels of circulating IGF-l are associated with an elevated risk of colorectal adenomas and cancer. This study seeks to assess the effect of duration of diabetes on the risk of colorectal cancer. METHODS: Data collected by the 1997-2003 National Health Interview Survey (NHIS) was analyzed to assess the risk of colorectal cancer among individuals with diabetes. The NHIS is a comprehensive nationally representative survey weighted to represent the U.S. adult population. There were 226,953 subjects represented in the combined seven years of the NHIS. Duration of diabetes was divided into four groups; $0-5$ years, $5-10$ years, $10-15$ years, and $>15$ years. Multiple logistic regression was performed probing the relationship between duration of diabetes and colorectal cancer while adjusting for age, race, gender, obesity, alcohol use, tobacco use, and treatment for diabetes (insulin, pill, both, or diet only). NHIS data from 1997 - 2003 was merged with SAS v. 9.1.3. Analyses performed with STATA v. 8.0, which accounted for the complex survey design of the NHIS and generated population estimates. Multiple logistic regression was used to determine independent correlates of colorectal cancer among individuals with diabetes. RESULTS: Among the 226.953 subjects in this study, 13,399 (5.9\%) revealed a history of diabetes mellitus. Adjusted for potential confounders, individuals with diabetes $>15$ years were significantly more likely to have colorectal cancer than persons without diabetes (Odds Ratio $(\mathrm{OR})=1.76,95 \%$ Confidence Interval $(\mathrm{CI})=1.03$ to 3.00$)$. Other independent correlates with significant findings included age $>50$, history of tobacco use, and history of alcohol use. CONCLUSIONS: Individuals with diabetes more than 15 years were noted to have an increased likelihood of colorectal cancer. If this association remains positive in prospective trials, people with diabetes may require earlier and more aggressive screening for colorectal cancer than the general population. Research should be directed at understanding the pathophysiologic reasons why duration of diabetes is associated with increased odds of colorectal cancer.

\section{W1120}

Colorectal Cancer Risk Perception Is Inappropriately Low in Individuals At Risk for HNPCC with Indeterminate Genetic Test Results

Shilpa Grover, Elena M. Stoffel, Rowena C. Mercado, Beth M. Ford, Kohlmann K. Wendy, Kristen M. Shannon, Amie M. Blanco, Jonathan P. Terdiman, Stephen B. Gruber, Daniel C. Chung, Sapna Syngal

Background: Hereditary nonpolyposis colorectal cancer (HNPCC) is associated with inherited germline mutations in mismatch repair genes. When there is an identifiable mutation in a family, mutation carriers have up to an $80 \%$ lifetime risk of developing colorectal cancer (CRC), whereas individuals who do not inherit the mutation are at average population risk (true negative). A subset of individuals fulfilling clinical criteria have indeterminate genetic test results (no gene mutation found, or a mutation of unclear pathogenic significance) and should still be considered to be at high risk. There are limited data regarding patients' understanding of the complexities of genetic test results. Objectives: To examine CRC risk perception in individuals tested for HNPCC gene mutations and to identify the factors associated with an accurate cancer risk perception. Methods: 261 subjects who met the
Bethesda criteria for HNPCC were recruited through 4 U.S. cancer genetics clinics and completed a self-administered questionnaire eliciting demographic data, individual and family genetic test results, as well as personal and family cancer history. Subjects were also asked to estimate their $\mathrm{CRC}$ risk on a 5-point scale, comparing their risk to that of other individuals their age. Results: Of 165 individuals who underwent genetic testing, 98 (59\%) were found to carry an HNPCC mutation and 89/98 (91\%) correctly estimated their CRC risk as being "high" or "very high" compared to other individuals their age. Seventeen of 23 (74\%) individuals with true negative test results correctly estimated their CRC risk with respect to their personal history of CRC and polyps. However, only $27 / 44$ (61\%) individual with an indeterminate genetic test, correctly stated that their CRC risk was increased. Approximately half (8/17) of those who underestimated their cancer risk believed their risk of CRC was "below average" and 9/17 reported their cancer risk as "the same as the average population." The presence of a pathogenic germline HNPCC mutation (OR 2.7, 95\% C 1.5-4.9) and history of a family member with a known mutation (OR 2.8, 95\% CI 1.1-7.4 were strong independent predictors of accurate CRC risk perception. Conclusions: Patients at risk for HNPCC with an indeterminate genetic test may be falsely reassured. As underestimation of cancer risk may adversely affect compliance with appropriate cancer screenin recommendations, additional post-test counseling is needed to help patients interpret their genetic test results and the implications for their clinical care.

\section{W1121}

Are Colorectal Cancer (CRC) Patients with Clinical Diagnosis of Hereditary Non Polyposis Colorectal Cancer (HNPCC) Receiving Optimal Surgical Treatment?

Zohar Levi, Rachel Hazazi, Paul Rozen, Yaron Niv

Introduction: Patients with Lynch syndrome have a lifetime risk of $80 \%$ for CRC and a high incidence of synchronous or metachronous neoplasia. Therefore, once a diagnosis of CRC is made subtotal colectomy should be considered rather than segmental resection. Aim: We reviewed the surgical procedures performed on our CRC bearing patients having a clinical diagnosis of HNPCC. Methods: 20 patients having CRC and fulfilling either the Amsterdam II criteria (group A, n=9) or revised Bethesda/incomplete Amsterdam II criteria (group B, $\mathrm{n}=11$ ) were identified in our Familial Cancer Clinic. The type of surgery and subsequent follow-up were evaluated. Results: Table. Conclusion: Only 10\% of the patients with CRC and clinical (Amsterdam II) or suspected (Bethesda) criteria of HNPCC underwent primary subtotal colectomy. This requires better understanding of the syndrome by the surgeons, communication with the Familial Cancer Clinic, and more definitive surgical guidelines.

\begin{tabular}{|c|c|c|c|}
\hline & Amsterdam positive & Bethesda positive & P \\
\hline Number of patients & 9 & 11 & 0.010 \\
\hline Mutation positive & 3 & 2 & NS \\
\hline Median age at CRC diagnosis (years) & $54.8 \pm 11.3$ & $41.1 \pm 4.2$ & 0.008 \\
\hline Median years of follow-up (years) & $2(0.7-6.2)$ & $6(1-19)$ & 0.020 \\
\hline CRC diagnosed by screening & $7(77 \%)$ & $1(9 \%)$ & 0.050 \\
\hline Proximal CRC & $7(77 \%)$ & $2(18 \%)$ & NS \\
\hline Stage I CRC & $4(44 \%)$ & 0 & NS \\
\hline Synchronous CRC & $2(22 \%)$ & $1(9 \%)$ & NS \\
\hline Metchronous CRC & $1(11 \%)$ & $1(9 \%)$ & NS \\
\hline Other HNPCC-related tumors & $2(22 \%)$ & $1(9 \%)$ & NS \\
\hline Primary sub-total colectomy & $2(22 \%)$ & 0 & NS \\
\hline
\end{tabular}

\section{W1122}

Higher Gastric Mucin Secretion and Lower Gastric Acid Output in FirstDegree Relatives of Gastric Cancer Patients

Alexander Vilkin, Zohar Levi, Sara Morgenstern, Haim Shmueli, Eyal Gal, Bracha Hadad, Britta Hardi, Yaron Niv

Background: Patients infected by Helicobacter pylori who have first-degree relatives with gastric cancer have an 8-fold increased risk of developing gastric cancer themselves. Mucin are high-molecular-weight glycoproteins that play a cardinal role in the protective mechanism of the gastric epithelium. Aim: To study gastric acid and mucin secretion in dyspeptic patients with and without a family history of gastric cancer and Helicobacter pylori infection. Materials and Methods: Twenty-six dyspeptic patients underwent esophago-gastro-duodenoscopy, gastric biopsies, and acid and mucin secretory tests. The sample was divided by family history of gastric cancer and Helicobacter pylori status. Results: Patients who were infected by Helicobacter pylori had a significantly higher degree of inflammation than those who were not. Helicobacter pylori-positive patients with a positive family history had a lower basal and maximal gastric acid output than infected patients with no family history and non-infected controls, and a higher basal and maximal mucin output than infected patients with no family history. MUC5AC was the major mucin species expressed in gastric juice. Conclusions: In patients with relatives with gastric cancer, Helicobacter pylori infection is associated with a more severe inflammatory reaction consisting of decreased gastric acid secretion and increased mucin secretion. 
Characteristics of Adult Children in Families Where Both Parents Have Pancreatic Cancer

Emmy Ludwig, Jennifer Simon, Sara Olson, Robert C. Kurtz

Familial pancreatic cancer (FPC) is thought to represent about $10 \%$ of pancreatic cancers (PC). The search for predisposing genes has been centered on registries of patients and family members in loosely-defined high-risk FPC pedigrees. A goal of such registries is the identification of genetic, environmental and lifestyle factors which may play a role in the genesis of pancreatic cancer. Our FPC registry identified a small group $(n=11)$ of families where both parents died of pancreatic cancer. This report describes the characteristics of this unusual cohort. Methods: We began our FPC registry for patients and their at risk relatives in December, 2002. The Registry also is tied to an epidemiologic case-control study of environmental risk, including both familial and sporadic PC cases, a surveillance program of at-risk relatives with cross-sectional imaging and endoscopy, and banking of serum for future genetic evaluation. All enrolled participants complete an extensive questionnaire detailing factors related to known and potential PC risk factors and family history. In addition, pedigree review for other associated syndromes such as HNPCC, and BRCAl and 2, leads to formal genetic counseling. Results: We compared the 11 registry participants where both parents had PC to a control group made up of other unaffected relatives of PC patients who joined our registry based on their family history of $\mathrm{PC}(\mathrm{n}=82)$. The 11 individuals in our cohort are similar in age to controls (64\% are less than 50 years vs $54 \%$ in controls; mean age 50.3 years vs 49.3 years), show no difference in proportion exposed to secondhand tobacco smoke as a child ( $55 \%$ vs. $57 \%$ in controls); had similar BMI at age 25 (91\% with normal BMI vs $78 \%$ in controls); and are similar in race (100\% vs. $90 \%$ white nonHispanic). However, they are less likely to be Catholic (18\% vs $45 \%$ ). In addition, in all of the two-parent PC families, parents were concordant for family ancestry compared to only $52 \%$ of the controls (exact $\mathrm{p}=0.0022$ ). Conclusions: We describe a cohort of healthy individuals where both parents had PC. To our knowledge, this is the first analysis of such a group. We demonstrate that they are no more likely to have been exposed to second-hand smoke growing up than relatives of PC patients in the control group. The finding of uniform concordance for family ancestry suggests a common genetic factor yet to be identified. Planned enrollment of additional family members belonging to this cohort and gene analysis of their banked specimens may help elucidate mechanisms that lead to the development of pancreatic cancer in the parents of this unique group of individuals.

\section{W1124}

Cryoablation of Barrett's Esophagus (BE)

Mark H. Johnston, Brooks D. Cash, John D. Horwhat, Lavonne R. Johnston, Cathy A Dykes, Halisha S. Mays

Background: The aim of this study was to explore the safety and efficacy of circumferential relative to hemi-circumferential cryoablation using an endoscopic low pressure liquid N2 device (CryMed Tech. Inc, Baltimore, MD) in BE. Methods: Twenty patients with BE were enrolled. The first 10 (group I) were treated hemi-circumferentially. The next 10 patients (group II) were treated circumferentially. In both groups $4 \mathrm{~cm}$ segments were treated at a time. Each area was frozen for 20 seconds, followed by thaw and then re-frozen for 20 seconds. Results: The mean age for both groups was 58 . The mean BE length, pre-cryo, for group I was $4.5 \mathrm{~cm}$, and for group II $2.7 \mathrm{~cm}$. Post cryo the mean BE length for group I was $0.3 \mathrm{~cm}$ and for group II was $0 \mathrm{~cm}$. The mean number of treatments for group I was 4.8 versus 2.1 in group II. There was no sub-squamous specialized intestinal metaplasia (SIM) for either group at 6 months follow-up for whom there was data. There were no serious complications. Group II experienced more transient chest discomfort and dysphagia (see table). Both patient discomfort and dysphagia completely resolved. No patient developed an esophageal stricture. Conclusion: Preliminary results indicate that circumferential cryoablation compared to hemi-circumferential is similarly efficacious with respect to reversal of $\mathrm{BE}$ and absence of subsquamous SIM. It requires less than half the treatments of hemicircumferential treatment but at the cost of increased patient discomfort. Table I

\begin{tabular}{|c|c|c|c|c|c|c|c|c|}
\hline Pt\# & Age & $\begin{array}{l}\text { BE length } \\
\text { (cm) } \\
\text { pre-cryo }\end{array}$ & $\begin{array}{l}\text { BE length } \\
(\mathrm{cm}) \\
\text { post-cryo }\end{array}$ & $\begin{array}{l}\mathrm{F} / \mathrm{U} \\
(\mathrm{mo})\end{array}$ & $\begin{array}{c}\# \\
\text { Cryos }\end{array}$ & $\begin{array}{c}\text { SIM at } 6 \\
\text { mo }\end{array}$ & $\begin{array}{c}\text { Pain } \\
\text { post-cryo }\end{array}$ & Dysphagia \\
\hline 1 & 56 & 4 & 1 & 32 & 5 & + & - & - \\
\hline 2 & 51 & 1 & 0 & 31 & 6 & - & - & - \\
\hline 3 & 72 & 8 & 2 & 31 & 8 & + & - & - \\
\hline 4 & 74 & 5 & 0 & 26 & 5 & - & - & - \\
\hline 5 & 57 & 8 & 0 & 25 & 5 & - & - & - \\
\hline 6 & 60 & 4 & 0 & 20 & 1 & - & - & - \\
\hline 7 & 57 & 4 & 0 & 20 & 6 & + & - & - \\
\hline 8 & 53 & 3 & 0 & 20 & 4 & + & + & - \\
\hline 9 & 53 & 4 & 0 & 20 & 5 & - & - & - \\
\hline 10 & 50 & 4 & 0 & 19 & 3 & - & + & - \\
\hline 11 & 64 & 6 & 0 & 15 & 1 & - & + & - \\
\hline 12 & 62 & 1 & 0 & 10 & 2 & - & + & - \\
\hline 13 & 52 & 3 & 0 & 10 & 4 & - & + & + \\
\hline 14 & 59 & 7 & 0 & 10 & 4 & $\mathrm{p}$ & - & - \\
\hline 15 & 61 & 2 & 0 & 8 & 1 & - & + & + \\
\hline 16 & 41 & 1 & 0 & 9 & 1 & - & + & + \\
\hline 17 & 59 & 1 & 0 & 9 & 1 & $\mathrm{p}$ & - & - \\
\hline 18 & 67 & 1 & 0 & 8 & 2 & + & - & - \\
\hline 19 & 56 & 4 & 0 & 8 & 4 & $p$ & + & - \\
\hline 20 & 61 & 1 & 0 & 8 & 1 & + & + & - \\
\hline mean & $\begin{array}{l}\mathrm{H}=58 \\
\mathrm{C}=58\end{array}$ & $\begin{array}{l}\mathrm{H}=4.5 \\
\mathrm{C}=2.7\end{array}$ & $\mathrm{H}=0.3 \mathrm{C}=0.0$ & $\begin{array}{l}\mathrm{H}=24 \\
\mathrm{C}=10\end{array}$ & $\begin{array}{l}\mathrm{H}-4.8 \\
\mathrm{C}: 2.1\end{array}$ & $\begin{array}{l}\mathrm{H}=40 \% \\
\mathrm{C}=29 \%\end{array}$ & $\begin{array}{l}\mathrm{H}=20 \% \\
\mathrm{C}=70 \%\end{array}$ & $\begin{array}{c}\mathrm{H}=0 \% \\
\mathrm{C}=30 \%\end{array}$ \\
\hline
\end{tabular}

$\mathrm{H}=$ hemi-circumferential; $\mathrm{C}=$ circumferential; $\mathrm{p}=$ pending; $\mathrm{mo}=$ months

W1125

Safety of Fine Needle Aspiration (FNA) During Endoscopic Ultrasound (EUS): A Prospective Study

Mohammad Al-Haddad, Catherine M. Hodgens, Robin D. Toton, Seth A. Gross, Kyung

W. Noh, Surakit Pungpapong, Timothy A. Woodward, Michael B. Wallace, Massimo

Raimondo

Background: FNA is commonly performed in conjunction with EUS procedures. There is limited prospective data on complication rates. Hypothesis: In prospective assessment, FNA is a safe procedure with limited complications in a high volume EUS referral center. Methods: Approximately 1200 patients undergo EUS at Mayo Clinic Jacksonville on annual basis for different indications. Of those, about $40 \%$ will undergo FNA. Between April and October 2005, 230 patients who underwent EUS- FNA were included in this study. These patients were screened for post-procedural complications including abdominal pain, nausea, vomiting, fever, gastrointestinal bleeding and dysphagia. Complications were assessed on day 0 by direct examination and at day 30 by a telephone call. Inquiries were made about emergency room visits or hospitalizations during the same period of time. Results: Table 1 demonstrates location of FNA for all patients $(n=230)$. Complete follow up information was obtained on 207 patients (90\%). -Day 0: Three patients were admitted to the hospital for observation. Two patients had abdominal pain after pancreatic cyst FNA and one was observed after mediastinal lymph nodes FNA for chest pain. All three patients were discharged within 24 hours of admission. There was no requirement of blood transfusion and no evidence of pancreatitis or infection in any of the hospitalized patients. One patient reported to the ER after EUS-FNA and was sent home on oral analgesics after appropriate evaluation. -Day 30: Four patients expired during the first month after EUS from primary disease process ( 3 had pancreatic cancer and one had lung cancer). Five patients were hospitalized for planned elective surgeries during the same period of time. There was no unplanned morbidity or mortality attributable to EUS-FNA. Conclusion: FNA is a safe intervention in patients undergoing EUS in high volume academic center with post procedural self-limited complication rate of $1 \%$

Table 1 


\begin{tabular}{|c|c|}
\hline FNA by Location & $\mathrm{n}=230$ \\
\hline Pancreatic masses and cysts & 103 \\
\hline Mediastinal masses and lymphadenopathy & 74 \\
\hline Abdominal and pelvic lymphadenopathy & 20 \\
\hline Gastroduodenal submucosal masses & 8 \\
\hline Liver & 7 \\
\hline Rectal & 3 \\
\hline Miscellaneous & 15 \\
\hline
\end{tabular}

\section{W1126}

Confocal Microscopy: A Novel Method to Assess Aberrant Crypt Foci in APC Min+/- and APC Min+/- (PPAR $\alpha-/-)$ Mice

Anthony Shonde, Krish Ragunath, George Anagnostopoulos, David R. Bell, Andrew J. Bennett, Christopher J. Hawkey

Background: Peroxisome proliferator-activated receptor $\alpha$ (PPAR $\alpha$ ) is expressed at low levels in murine and human colon tumours. Apc min+/- mice fed with methylclofenapate (a PPAR $\alpha$ ligand) have 50\% less tumour burden in the small intestine and colon than controll. Recent studies showed that aberrant crypt foci (ACF) are the earliest morphological lesion detectable in colorectal epithelium that may lead to cancer. However it is unclear whether PPAR $\alpha$ has a role in suppressing the formation of ACF or the progression of tumour development. Dissection microscopy is the most common method used for quantifying ACF. However it is time consuming and requires a great deal of skill to obtain a good preparation. Aims: (1) To assess whether Confocal Microscopy is a viable method for identifying ACF in scientific practice. (2) Examine the role of PPAR $\alpha$ in colon carcinogenesis using APCmin+/- and APCmin+/-(PPAR $\alpha-/-)$ mice. Method: Once weaned APCmin+/- mice and APCmin+/(PPAR $\alpha-/$-) mice were fed standard chow throughout the study. Mice were weighed weekly, until mice showed signs of anaemia or lost $>20 \%$ of weight at which time they were sacrificed. ACF were assessed using Cell vizio Confocal Microscopy probe (Mauna Kea technologies, Paris) made of 30,000 optical fibers following colonic staining with acryflavine. Result: High definition images with $2.5 \mu \mathrm{m}$ lateral resolution of normal crypts and ACF were clearly identified using Confocal Microscopy. APCmin+/-(PPAR $\alpha-/-)$ mice have more ACF than APCmin+/-mice in the colon, but this failed to reach significance $(\mathrm{n}=9)$. The mean number of ACF was 5.0/ 10 fields (CI 2.10- 7.90) in APCmin+/-(PPARo-/-) mice and 2.75/ 10 fields (CI 1.22- 4.27) in APCmin+/- mice. Conclusions: Confocal Microscopy using the Cell vizio probe is a simple and quick method of accessing ACF, with the potential of refining experiments so that mice can be studied serially. PPAR $\alpha$ role in colon carcinogenesis may be to inhibit the formation of ACF rather than their progression. Greater understanding of PPAR $\alpha$ dependant genes may enable the pathogenesis of colon cancer to be further understood. 1. Potential role for peroxisome proliferator activated receptor (PPAR) in preventing colon cancer. Jackson L, et al Gut. 2003 Sep;52(9):1317-22

\section{W1127}

The Usefulness of Endoscopic Submucosal Dissection (ESD) for the Treatment of Premalignant and Malignant Gastric Neoplasm

Sun-Taek Choi, Tae-Nyeun Kim, Jung-Hoon Lee, Jong-Ryul Eun, Jun-Hwan Kim, ByungIk Jang, Heon-Ju Lee

Background/Aims; Minimally invasive treatment such as endoscopic mucosal resection (EMR) is widely used as a treatment for gastric neoplasm. But, it is difficult to obtain en bloc resection in case of large lesion with a conventional EMR techniques. Recently, ESD is reported to be a promising method to resect the lesion as an en bloc. The purose of this study was to evaluate the efficacy and safety of ESD. Methods; 78 patients referred for ESD between September 2004 and November 2005 were studied prospectively. ESD was performed using IT-knife or needle knife after submucosal injection of saline-epinephrine solution. The en bloc resection rate, complete resection rate and associated complications were recorded. Results. 83 lesions were removed by ESD in 78 patients. Indications were adenoma $56.6 \%$, dysplasia $9.6 \%$, adenocarcinoma $33.7 \%$. 3 cases of scar like mucosal lesion were resected (two cases; adenocarcinoma, one case; adenoma). The median size of the lesion and the resected specimen was $15.0 \mathrm{~mm}, 24.8 \times 18.0 \mathrm{~mm}$, respectively. The en bloc resection rate and complete resection rate were $95.2 \%, 91.6 \%$, respectively. Complication rate for bleeding after ESD was $18 \% .11$ cases $(73 \%)$ of bleeding were notified during early second look endoscopy within the first 24 hours. Hemostasis was achieved in all cases in response to endoscopic therapy. One case of perforation underwent an operation because the specimen spilt on the peritoneal cavity. The mean length of procedure time and hospitalization were 40 minutes, 5.5 days, respectively. Post-ESD histopathology was adenoma 47\%, dysplasia $12 \%$, adenocarcinoma $41 \%$. ESD resulted in upgrading of pathologic staging to adenocarcinoma or dysplasia in $22 \%$. Conclusions; ESD has high en bloc and complete resection rate in gastric neoplasm. ESD is associated with perforation and a high risk of bleeding, sufficient experience and endoscopic treatment technique are essential. Early second look endoscopy within 24 hours may be helpful to prevent bleeding from ESD. ESD changes pathologic diagnosis in a significant number of patients. Long-term follow up results of ESD are needed.

\section{W1128}

Comparision of Ultrahigh and Standard Resolution Optical Coherence Tomography (OCT) for Endoscopic Imaging of the Esophagus

Hiroshi Mashimo, Saleem Desai, Yu Chen, Aaron D. Aguirre, Pei-Lin Hsiung, Shu-Wei Huang, Joseph M. Schmitt, James G. Fujimoto

BACKGROUND/AIMS: Endoscopic optical coherence tomography (EOCT) is an emerging medical imaging technology which generates high resolution, cross-sectional images in situ, without tissue removal. Previous clinical studies using EOCT with 10-15 um axial resolution have demonstrated its capability in diagnosing Barrett's Esophagus (BE). Quantitative EOCT image analysis was promising for detecting high grade dysplasia in BE patients. We recently developed an EOCT with improved axial resolution ( $5 \mathrm{um}$ ). The goal was to compare standard and ultrahigh resolution OCT (UHR-OCT) for image quality and algorithmic detection of BE. METHODS: Images of the esophagus were obtained in vivo using UHR-OCT ( $5 \mathrm{um}$ ) and standard OCT (12 um). Image quality was compared and assessed quantitatively (speckle size). Image features of 50 endoscopic OCT images from normal and Barrett's esophagus were extracted through computational metrics including the intensity histogram (mean and standard deviation), spatial frequency analysis (2D FFT), and statistical texture analysis (center symmetric auto-correlation). These features were analyzed using principle component analysis (PCA) to reduce the vector dimension and increase the discriminative power. RESULTS: UHR-OCT images of both in vivo and ex vivo GI tissues showed improved visualization of fine architectural features over standard resolution. In addition, the quantitative image feature analysis showed reduced speckle size and enhanced discrimination of normal (o) and Barrett's ( + ) esophagus with UHR-OCT. The variance of the first 2 principle components are further separated for texture features in the UHR-OCT images (Fig. 1) CONCLUSIONS: The ability of UHR-OCT to image tissue morphology at an improved resolution could help in discerning the fine features in OCT images, which could be useful in diseases diagnosis. The enhanced classification abilities of image features using UHROCT would help in the computer aided diagnosis of GI diseases. This work demonstrates enhanced discrimination of $\mathrm{BE}$ from normal esophagus, but these results may also prove important in the discrimination of dysplasia from Barrett's tissues.

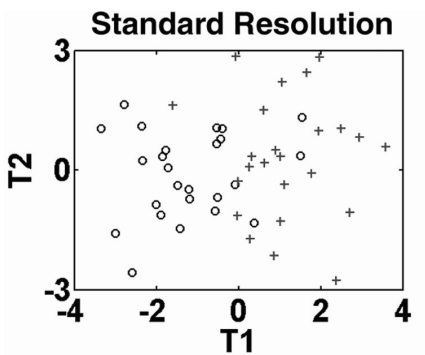

Ultrahigh Resolution

W1129

Endoscopic Optical Coherence Tomography (EOCT) with Balloon Based Catheter Probe ---for the Screening of Barrett's Esophagus (BE) Zhilin Hu, Michael V. Sivak, Andrew M. Rollins

The presentation will report a device based on endoscopic optical coherence tomography (EOCT) imaging that can accurately and safely screen patients with Barrett's esophagus (BE) for changes in the esophagus that anticipate the development of cancer of the esophagus. Barrett's esophagus (BE) is a premalignant condition that occurs in a large proportion of individuals with reflex of gastric acid into the esophagus. Once established, BE confers a $10 \%$ life-risk of esophageal cancer. Management of patients with BE is by surveillance for precancerous changes (known as dysplasia). Surveillance consists of examination of the esophagus by endoscopy at intervals of $1-2$ years with procurement of biopsies from the involved segment of the esophagus. Biopsies are taken because the precancerous changes are usually microscopic and not visible at endoscopy. Thus, biopsies are taken according to a specific sampling protocol. However, it is well established that even the best sampling protocol can overlook the presence of precancerous changes (dysplasia). Moreover, surveillance based on biopsies is time consuming and expensive. Endoscopic OCT (EOCT) was developed as an imaging modality for use at endoscopy. EOCT imaging is performed using a catheter probe inserted through the endoscope. By virtue of its high resolution and other properties, EOCT demonstrates the microscopic structure and tissues of the wall of the esophagus. In a series of clinical trials, our group has shown that EOCT has an accuracy of $70 \%$ for the detection of dysplasia in BE. If importance, the negative predictive value of EOCT for the presence of dysplasia is greater than $90 \%$. Additionally, preliminary work suggests that the accuracy can be increase by computer aided diagnosis (CAD). Unfortunately, the EOCT probe images relatively small volumes of tissue. Thus, it is unsuitable for a surveillance examination of a large surface area of tissue, which in a patient with well developed BE could be as much as 20 square centimeters or more. What is needed, therefore is a device that can provide OCT imaging data for the entire area of the esophagus involved by BE. At the presentation, we will describe the detail of our device, EOCT and a prototype of catheter probe equipped with an inflatable centering balloon that can be inserted through an endoscope and is suitable for complete imaging of long segments of the esophagus. 
infectious, autoimmune, metabolic, genetic diseases, and imaging studies to rule our cardiovascular conditions or small intestinal biopsy proven celiac sprue with no apparent neurolo-

Endoscopic Tri-Modal Imaging (ETMI) for the Detection of Dysplastic Lesions in Barrett's Esophagus

Wouter L. Curvers, Louis M. Wong Kee Song, Kenneth K. Wang, Christopher J. Gostout, Micheal B. Wallace, Herbert C. Wolfson, Krish Ragunath, Paul Fockens, Fiebo J. Ten Kate, Kausilia K. Krishnadath, Jacques J. Bergman

Background The ETMI system (Olympus, Tokyo, Japan) incorporates high-resolution endoscopy (HRE), autofluorescence imaging (AFI) and narrow band imaging (NBI) in a single device. The system uses an endoscope with 2 CCD chips; one for HRE and NBI (with an optical zoom function) and one for AFI. In the AFI mode, real-time images are constructed using a new algorithm based on total tissue autofluorescence and green reflectance. The aim of this multi-center feasibility study was to investigate the diagnostic potential of ETMI and the relative contribution of each modality for the detection of dysplasia in Barrett's esophagus (BE). Methods Pts with known BE with or without a prior diagnosis of dysplasia were recruited in the study. The esophagus was first inspected with HRE followed by AF for the detection of additional suspicious lesions. All lesions detected with HRE or AFI were subsequently inspected by NBI using the zoom mode to evaluate the presence of abnormal mucosal and microvascular patterns followed by biopsies for blinded histopathological assessment. Standard surveillance biopsies were also performed. Results Twenty-three pts have been assessed with the ETMI system to date, including 9 pts referred for inconspicuous dysplasia and 14 pts participating in a standard surveillance program. Per patient assessment: 6 pts were diagnosed as having high-grade dysplasia (HGD), and 4 of them had lesions detected with HRE and AFI. In one of these pts, AFI detected an additional lesion with HGD that was missed under HRE. In 2 pts, no abnormalities were seen with HRE and HGD was solely diagnosed with AFI. In total, 9 pts were diagnosed with low-grade dysplasia (LGD) or HGD. In 5 pts, dysplastic lesions were detected with HRE and AFI. In 4 pts, LGD or HGD was detected only with AFI. Per lesion assessment: 30 suspicious lesions were identified with AFI; 7 of these contained HGD, the remaining 23 (77\%) were found to be false-positives for HGD. With detailed characterization of these lesions using NBI, 5 of the 7 lesions containing HGD were classified as suspicious. Seven of the 23 false-positive lesions were also classified as suspicious thereby reducing the overall false positive rate to $23 \%$. Conclusion AFI appears to enhance the detection of dysplastic lesions relative to HRE However, AFI seems to be associated with a high false-positive rate, which may be reduced with the addition of NBI. The potential of ETMI for enhancing lesion detection in BE is promising but a more accurate assessment of its diagnostic performance will be obtained as more patients are enrolled in this ongoing multi-center study.

\section{W1131}

Comparison Between Multislice CT and Endoscopic Ultrasound (EUS) in the Diagnosis and Staging of Pancreatic and Periampullary Malignancy

Mansfield Stephen, John Scott, Kofi W. Oppong, David Richardson, G Sen, Bryon C. Jaques, C O'Suilleabhain, Derek Manas, Richard M. Charnley

Introduction Multislice CT, which offers improved scanning speed and resolution compared to conventional CT, was compared with EUS in patients with pancreatic and periampullary malignancy. Methods Prospective data collection on all suspected pancreatic and periampullary cancers between June 2002 and June 2004. Dynamic triple phase multislice CT and EUS were performed where clinically indicated. For quality reasons only in-house CTs were accepted for the analysis. Presence of malignancy, portal vein invasion and resectability were compared using McNemar's test of paired proportions (Table 1). Results Of 345 patients, 134 underwent in-house multislice CT, 188 EUS and 84 both Of these, 35 underwent laparotomy. The use of EUS guided fine needle aspiration was not assessed. No significant difference was demonstrated between the modalities either in the whole cohort or when small tumours $(\leq 20 \mathrm{~mm})$ were analysed separately. When the clinical impact was assessed EUS had most impact in the following groups of patients: Benign on CT - 5/21, 23.8\%; Portal vein invasion on CT - 18/37, 49\%; Uninterpretable CT findings - 9/9, 100\%. Conclusion Multislice CT is the primary imaging modality in assessing these tumours. EUS is not indicated in those patients in whom CT confidently demonstrates a resectable tumour. In those patients in whom CT suggests benign disease or borderline vascular invasion, however EUS has an important role in determining resectability.

\begin{tabular}{|c|c|c|c|c|c|}
\hline & \multicolumn{2}{|c|}{ Sensitivity } & \multicolumn{2}{c|}{ Specificty } & \multirow{2}{*}{$\mathrm{p}$} \\
\cline { 1 - 4 } & CT & EUS & CT & EUS & \\
\hline Malignancy & $97 \%$ & $95 \%$ & $87 \%$ & $52 \%$ & 0.26 \\
\hline PV invasion & $57 \%$ & $57 \%$ & $80 \%$ & $84 \%$ & 0.21 \\
\hline Resectabilty & $96 \%$ & $81 \%$ & $50 \%$ & $43 \%$ & 1.0 \\
\hline
\end{tabular}

\section{W1132}

Patients with Celiac Sprue Appear to Have An Increased Incidence of White Matter Brain Lesions On Cerebral Magnetic Resonance Imaging (MRI) Friedemann Paul, J.N. Wurfel, Bertram Wiedenmann, Axel U. Dignass, Daniel C. Baumgart

BACKGROUND: Celiac sprue causes neurological symptoms in $10 \%$ of patients. Clinical presentation can be subtle and may even occur in the absence of gastrointestinal symptoms. Common neurological manifestations of celiac disease include ataxia and polyneuropathy. White matter lesions (WML) occur in a variety of inflammatory conditions and can be detected with cerebral magnetic resonance imaging (cMRI). Their prevalence in patients with elevated gliadin serum antibody levels or celiac sprue is not known. METHODS: In this prospective, comparison study 41 adult patients with either WML of unknown etiology (group 1) despite an extensive work-up including spinal tap and serologic screening for gically symptoms (group 2) were evaluated. RESULTS: In group $1,11 \%$ of patients were positive for gliadin or transglutaminase antibodies. Two of these patients presented with atypical optical neuritis. In group 2, none of the patients had any abnormal electrophysiological findings, but there was an increased incidence (25\%) of WML on cMRI (Fig. 1) Recruitment is still ongoing. CONCLUSION: Based on the data analyzed to date, the prevalence of gliadin antibodies in patients with WML of unknown etiology appears to be similar to what has been reported for the normal population (12 to $15 \%$ ). In patients with celiac disease though, there seems to be an increased incidence of WML even in the absence of neurological symptoms. cMRI may be helpful to detect neurological abnormalities in celiac sprue patients early on

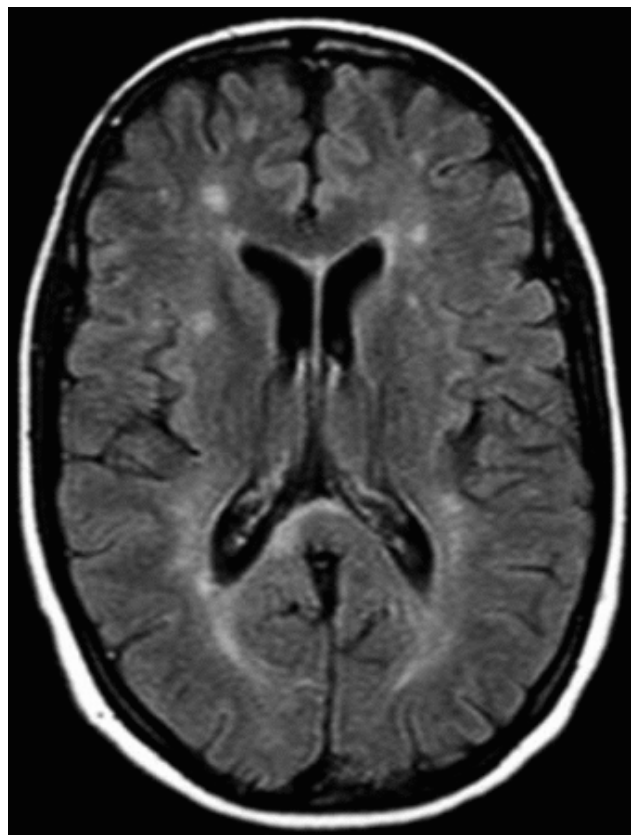

Fig. 1

\section{W1133}

Sonoct ${ }^{\circledR}$ - A New and Promising Imaging Technique That Improves On Conventional Ultrasound in the Visualisation of Pancreatic Duct Steffen Rickes, Janett Boehm, Klaus Moenkemueller, Peter Malfertheiner

Background and aims: In sonographic diagnostic investigation, evaluation of the pancreatic duct plays an important role. Evaluation frequently relates to changes to the duct, as might result from chronic pancreatitis or pancreatic carcinoma. This prospective study aimed to establish whether the new SonoCT ${ }^{\circledR}$ can improve on conventional ultrasound technique in the visualisation of the pancreatic duct. The study specifically focused on the role of the examiner in achieving good results. Patients and methods: The pancreatic duct of 14 healthy volunteers were assessed by an experienced and an inexperienced examiner, respectively, using both traditional B-mode sonography and SonoCT® (dynamic 2-5 MHz sector scanner, HDI 5000, Philips Ultrasound, Bothel, WA, USA). Assessment was based on a pre-determined set of criteria. Chi-square test was used to detect significant differences between both ultrasound methods. Results: In none of the probands, conventional B-mode sonography was able to clearly delineate the pancreatic duct. This is in marked contrast to $100 \%$ of probands using SonoCT ${ }^{\circledR}(\mathrm{p}<0.05)$. Both examiners fully concurred on this $(100 \%)$ in their respective assessments. SonoCT ${ }^{\circledR}$ also achieved significantly better results in terms of visualising borders. Whilst in conventional B-mode sonography, partial fine focusing of the Wirsungs duct could only be achieved in 4 out of 14 cases (29\%), fine focusing of the pancreatic duct was successful in all cases using SonoCT ${ }^{\circledR}(\mathrm{p}<0.05)$. Again, both examiners entirely concurred on this. Regarding clarity of representation, both examiners again agreed

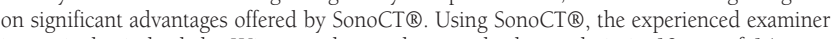
in particular judged the Wirsungs duct to be completely anechoic in 12 out of 14 cases $(86 \%)$, compared to no such rating for conventional B-mode sonography $(\mathrm{p}<0.05)$. Conclusion: SonoCT® yields markedly better results than conventional B-mode sonography in terms of delineation, visualisation of borders and artefact-free representation of the pancreatic duct. Future studies will be needed to determine whether certain diseases can be diagnosed more readily using SonoCT® rather than conventional sonographic technique.

\section{W1134}

Qualitative and Quantitative Analysis of Contrast-Enhanced Extracorporeal Ultrasonography (US) in the Diagnosis of Gallbladder Diseases Yukinari Matsumoto, Yoshiki Hirooka, Akihiro Itoh, Hiroki Kawashima, Kazuo Hara Akira Kanamori, Hiroki Uchida, Jun Goto, Koji Nonogaki, Naoki Ohmiya, Yasumasa Niwa, Hidemi Goto

Recently, contrast-enhanced US(CE-US) has been developed in digestive organs, but there are few reports of quantitative analysis. In this study, we evaluated the diagnostic usefulness of CE-US in gallbladder disorders both qualitatively and quantitatively. (Subjects and 
methods) The subjects consisted of 16 gallbladder disorders (8 carcinoma - 3 papillary adenoca., 3 tubular adenoca. and 2 adenosquamous ca.-, 1 cholesterol polyp(CP), 6 chronic cholecystitis(CC), 1 adenomyomatosis(AM)). The agent (Levobist, Nihon Schering, Japan) was adjusted to $300 \mathrm{mg} / \mathrm{ml}$ in concentration, and was injected intravenously inserted into the right median antecubital vein, at a rate of $1 \mathrm{ml} / \mathrm{sec}(8 \mathrm{ml}$ in total volume). The observation procedure was as follows: 1) The target lesions were observed by pulse inversion harmonic mode with low mechanical index (M.I.) value (0.4) for 60 seconds after injection - early phase. 2) After three minute suspension of scanning, the lesions were observed by the same mode with high M.I. value (1.3) by a sweeping scan around the lesion- late phase. In all cases, we evaluated enhancement effect between plain images and enhanced images both qualitatively and quantitatively. For the quantitative analysis, a region of interest (ROI) was established by a circle (diameter, $5 \mathrm{~mm}$ ) in the target lesion. The intensity $(\mathrm{dB})$ in the circle was measured using QLAB (Philips, USA). QLAB was provided by Philips and this software tools rapidly quantify image characteristics within ROI. In the early phase, the percentage change (max intensity / pre-enhancement intensity) was measured. The apparatus used was HDI-5000 (ATL, Philips, USA). (Result) The enhancement patterns of carcinoma were classified into three patterns: arborizing enhancement in 2 cases, dotted-rich enhancement in 2 cases and dotted-poor enhancement in 2 cases and no enhancement in 2 cases. As to papillary adenoca., two cases showed arborizing enhancement and one case was dotted-rich enhancement. As to tubular adenoca., one case showed dotted-rich enhancement and dottedpoor enhancement was seen in 2 cases. There were no enhancement effects in 2 cases of adenosquamous ca.. In 8 cases of $\mathrm{CP}, \mathrm{CC}$ and $\mathrm{AM}$, the enhancement effect was poor and could not classified into any pattern. As for quantitative analysis, the percentage change (max intensity / pre-enhancement intensity), gallbladder carcinoma (2.28) showed the higher value than that of another gallbladder diseases (1.63). (Conclusion) Although further analysis using other parameters for more lesions is needed, quantitative analysis in addition to qualitative evaluation may be helpful in the diagnosis of the gallbladder diseases.

\section{W1135}

Chromoscopic Magnifying Colonoscopy for Colorectal Cancer Screening: Is It a Better Tool?

Hulya Cetinkaya, Ali Tuzun, Mehmet Bektas, Murat Toruner, Arzu Ensari, Irfan Soykan, Ali Ozden

Background: Conventional colonoscopic examination has been reported to be one of the most commonly used techniques in colorectal cancer screening. In recent years, it has been shown that chromoscopic magnifying colonoscopy combined with chromoscopic agents permits early detection of neoplastic colorectal lesions, especially flat and depressed types which are known to be somehow difficult to detect with convensional colonoscopy. We therefore conducted a study to examine the role of chromoscopic magnifying colonoscopy in colorectal cancer screening. Subjects and Methods: 75 asymptomatic subjects over 50 years old who were admitted to Ankara University Medical School, Gastroenterology department for colorectal cancer screening were recruited into this study. Following an appropriate sedation with either propofol or midozolam, "back to back colonoscopy" was performed to each subject. Conventional pan-colonoscopy with targetted biopsy was performed initially followed by magnifying colonoscopy for chromoscopic evaluation of recto-sigmoid area. Additional biopsies were obtained as well during magnifying colonoscopy from additional suspicious lesions. Results: Thirty patients were male and forty-five subjects were female with a median age of 58 years (range: 50-60 years). Mean colonoscopy time (both first phase "conventional" and second phase "back to back" was $35.3 \pm 13.3$ minutes. Mean volume of 0.4\% Indigo Carmine was 9.9 \pm 5.3 mililiters. Overall, forty-one suspicious lesions were detected in 23 subjects (30.1\%), following indigo carmine dye spraying and 62 additional abnormalities were detected in 48 subjects $(64 \%)$. Thirteen of the additional abnormalities were serrated adenomas, while thirty lesions were flat elevated lesions, eighteen lesions were polipoid lesions and one of them was a depressed lesion. Conclusion: Chromoscopic magnifying colonoscopy might be a better technique than conventional colonoscopy in detecting colorectal malignant and/or pre-malignant lesions. However, studies sould be performed to evaluate cost effectiveness of this technique in colorectal cancer screening before using it in population screening.

\section{W1136}

Assessment of Methods to Quantify Autofluorescence Intensity in Adenomatous Polyps

Audrey L. McCallum, John T. Jenkins, Derek Gillen, Richard G. Molloy

Introduction The technology of autofluorescence (AF) endoscopy is based on tissue fluorescence of endogenous molecules and has been developed to improve detection of GI lesions. It produces a colour image with an autofluorescence intensity (AI) reading which indicates the strength of green autofluorescence and red reflectance present within the tissue. A wide variation in AI readings in colonic polyps was noticed when assessing with AF colonoscopy. Our aim was Aim To better quantify AI readings in relation to presence and degree of dysplasia of adenomatous polyps. Methods 51 patients were assessed with AF colonoscopy (Xillix LIFE-GI) with AI readings recorded of polyps, surrounding area to polyps and rectum. Biopsies of all polyps were taken. A total of 38 polyps were identified of which 20 were confirmed histologically to be adenomatous. Five different methods were used to quantify AI reading with degree of dysplasia. The first used AI polyp alone (P), the second used AI polyp minus AI surrounding tissue (P-ST), the third being AI polyp minus AI rectum (P$\mathrm{R}$ ), the fourth AI polyp/AI surrounding tissue ratio (P/ST) and finally AI polyp/AI rectum ratio $(\mathrm{P} / \mathrm{R})$. The five methods were tested statistically to determine if there was a significant difference for degree of dysplasia for any method. Results A significant statistical difference for degree of dysplasia, using Kruskal-Wallis test, was found with P/R method ( $\mathrm{p}$ value of 0.02 ). The other methods did not produce a stastically significant difference with $\mathrm{p}$ values of 0.43 for P, 0.49 for P-ST, 0.34 for P-R and 0.89 for P/ST. Conclusion AI readings can be better quantified in relation to degree of dysplasia within adenomatous polyps when a ratio is used of AI polyp/AI rectum. This new AI measurement may allow autofluorescence colonoscopy to determine the degree of dysplasia within adenomatous polyps.

\section{W1137}

Evaluation of Autofluorescence Colonoscopy for the Detection and Diagnosis of Colonic Polyps

Audrey L. McCallum, John T. Jenkins, Derek Gillen, Richard G. Molloy

Introduction: Autofluorescence (AF) has been developed to enhance conventional white light (WL) endoscopy in the diagnosis of neoplastic lesions of the GI tract. It is based on the stimulation of endogenous fluorophores and produces a pseudo-colour image of tissue. Metaplastic polyps are common and do not need to be treated, whereas adenomatous polyps carry a neoplastic potential. It would therefore be helpful to be able to distinguish between adenomatous polyps and metaplastic polyps when performing colonoscopy. Aim: To evaluate AF for the endoscopic detection and differentiation of colorectal polyps. Methods: Patients were invited to attend for colonic assessment with both AF (Xillix LIFE-GI) and WL colonoscopy. The intensity of autofluorescence (AI) is quantified automatically and readings, pictures and biopsies were recorded of any visible pathology or areas of high AF. The biopsy results were analysed and an AI reading for each biopsy site obtained by subtracting the actual Al reading from the background reading for the rectum of each patient. Results: A total of 47 patients were assessed with AF and WL colonoscopy. A total of 33 polyps were detected (19 adenomatous and 14 metaplastic polyps). It was found that adenomatous polyps had higher AI readings (median 0.53, IQR 0.15-1.05), than metaplastic polyps (median 0.09 IQR 0.06-0.10). [Mann Whitney U-test: $\mathrm{p}=0.00003$ ] Conclusion: These early data suggest that autofluorescence colonoscopy has the potential to differentiate between metaplastic and adenomatous polyps and may have a role as a new diagnostic technique for the improved detection of colonic dysplasia and early malignancy.

\section{W1138}

Randomised Prospective Study On Accuracy, Acceptability, Feasibility and Safety of Transnasal Gastroscopy Without Sedation in Comparison to Transoral Gastroscopy Under Sedation Italo Stroppa, Claudio Tosti, Cinzia Razzini, Sabrina Mazzocchi, Enrico Grasso, Omero Alessandro Paoluzi, Livia Biancone, Francesco Pallone

Background and aims - Conscious sedation is frequently required during transoral gastroscopy (TO). Transnasal gastroscopy (TN) has proven to be less invasive than TO, suggesting feasibility with no sedation. Aim of the study was to evaluate diagnostic accuracy, acceptability, feasibility and safety of TN without sedation compared to TO under sedation in a series of pts undergoing gastroscopy. Patients and methods - Thirty pts ( 16 male, mean age 48.47 \pm 13.37 years) candidate to upper GI endoscopy were randomised to undergo TN and TO on two consecutive days, by two different gastroenterologists. Pts underwent TO under conscious sedation (i.v. midazolam) and TN under local anesthesia (lidocaine). Before endoscopy, all pts were assessed for anxiety using Hamilton A scale. Diagnostic accuracy was judged comparing endoscopic findings by TN and TO. In order to assess the concordance of endoscopic patterns (inter-observer) a third endoscopist reviewed tape recordings of both examinations. Reliable histology of gastric biopsy specimens collected both during TN and TO was also considered. Acceptability was assessed on the basis of discomfort at insertion and during endoscopy and preference between TN or TO under conscious sedation. Endoscopists and nurses also gave their opinion on feasibility of TN and TO. Safety of TN and TO was evaluated comparing cardio-respiratory parameters (oxygen saturation, one-lead ECG, blood pressure) monitored during endoscopy. Results - All pts underwent both TN and TO but one who did not tolerate TO, which had to be stopped. All TN and TO, performed until the second portion of duodenum was reached, showed similar findings, the concordance between endoscopic patterns being complete. All biopsy specimens were adequate for histological assessment. Anxiety was absent in 18 pts, mild in 9, moderate in 1 and requiring treatment in 2. Mean scores of discomfort were similar in both groups, but 26/30 pts (87\%) preferred TN. Of 12 pts with anxiety, only 2 preferred TO with sedation. Feasibility was good both for TN and TO. No severe cardiac or respiratory adverse event occurred during TN and TO. Concusions - The present study demonstrates that TN without sedation is well tolerated and better accepted than TO under sedation. The high levels of diagnostic accuracy and safety suggest that TN should be taken into account as a valid alternative to TO in routine diagnostic gastroscopy.

\section{W1139}

\section{A Retrospective Analysis of Abnormal Findings On Virtual Colonoscopy Compared with Optical Colonoscopy}

Courtney Barancin, Brent Roeder, Daniel Cornett, Terrence Frick, Patrick Pfau, Andrew Taylor, David Kim, Perry Pickhardt, Mark Reichelderfer

Background: Virtual colonoscopy (VC) is a new minimally invasive method for colon polyp and cancer screening. At our institution, patients (pts) with abnormal findings on VC are often referred for optical colonoscopy (OC) which allows analysis of the accuracy of VC for detecting polyps. The aim of this study was to compare abnormal findings on VC with those on OC. Methods: We retrospectively reviewed all OCs performed from April 2004 to October 2005 in pts referred to OC following VC. VC was performed by primary 3D read with 2D resolution, as we have previously reported. Pts were uniformly referred for $\mathrm{OC}$ if they had findings on VC of a polyp or mass greater than $10 \mathrm{~mm}$. Pts with polyps between 6-9 mm were offered OC or follow up VC under an IRB-approved protocol. Patients were also refered to $\mathrm{OC}$ if a significant portion of their colon was not adequately visualized on VC. Potential lesions under $6 \mathrm{~mm}$ were not reported. The number, size, and characteristics of the polyps as well as pathology findings (if available) were recorded. Results: A total of 119 pts had OC, with 41 pts having polyps on OC that were not seen on VC. A total of 90 polyps were found with the following sizes: 46 were $<6 \mathrm{~mm} ; 13$ were from $6-9 \mathrm{~mm} ; 9$ were at least $10 \mathrm{~mm}$; 22 insufficient information. By pathology, the number of polyps identified on OC but not on VC that were adenomas were 4/46 $(<6 \mathrm{~mm}) ; 1 / 13(6-9 \mathrm{~mm}) ; 3 / 9$ (at least 10 $\mathrm{mm})$. We found a total miss rate of polyps greater than $6 \mathrm{~mm}$ to be $8.4 \%(10 / 119 \mathrm{pts})$ The total miss rate of adenomas greater than $6 \mathrm{~mm}$ was $3.4 \%$ (4/119 pts). The false positive rate was $8.4 \%$ (10/119 pts) with a total of 24 lesions that were identified as polyps on VC 
not seen or found to be a non-polypoid lesion on OC. Conclusions: 1) The majority of missed polyps found at $O C$ were less than $6 \mathrm{~mm}$ and are not reported on VC per protocol. 2) The adenoma-miss rate of polyps greater than $6 \mathrm{~mm}$ on VC was very low in our study. 3) VC with our method is a highly accurate method for colon cancer and polyp screening.

\section{W1140}

Life Events and Inflammatory Bowel Disease Relapse: A Prospective Study of Patients Enrolled in Remission

Angela Vidal, Esther Gomez Gil, Miquel Sans, Portella J. Maria, Salamero M, Pique M. Josep, Julian Panes

The impact of life events in recurrence of inflammatory bowel disease (IBD) is unclear. We sought to determine whether stressful life events are associated with IBD exacerbations, hypothesizing that the exposure of life events among patients with inactive disease will increase the risk of subsequent relapses. METHODS: In this prospective study 163 patients with inactive IBD, who had had at least one relapse in a 2-year period before entry into the study, were enrolled. The Social Readjustment Rating Scale (measuring life events), a measure of the emotional impact of these life events, and an IBD activity index were completed monthly up to the end of the study (maximum eleven months) or up to a relapse. Potential biological factors associated with an increased risk of relapse were identified in patients who relapsed. RESULTS: Fifty-one patients relapsed (32.9\%), 104 remained in remission and eight dropped out. Multivariate Cox regression analysis with time dependent variables showed that a high number of life events was not associated with an increased exacerbation rate after adjustment for significant covariates on the subsequent month (hazard ratio $=0.87$, $95 \% \mathrm{Cl}=0.67-1.11, \mathrm{p}=0.27$ ) or in the time-lagged analysis (subsequent two or three month periods). Subgroup analysis of $\mathrm{UC}$ and $\mathrm{CD}$ patients also showed a lack of association between the number of life events and risk of relapse for all lag periods studied. The emotional impact of stressful events was also not associated with the risk of relapse (hazard ratio= $0.99,95 \% \mathrm{Cl}=0.99-1.00, \mathrm{p}=0.60$ ). When patients who suffered a biological risk factor for relapsing were excluded in subsequent statistical analyses, similar results were obtained when analyzing the risk of relapse associated with the number of life events (hazard ratio= $0.91,95 \% \mathrm{CI}=0.68-1.22, \mathrm{p}=0.54$ ) or the emotional impact of these events (hazard ratio= $0.99,95 \% \mathrm{Cl}=0.99-1.00, \mathrm{p}=0.75)$.. CONCLUSIONS: Our results suggest that stressful life events do not trigger exacerbations in patients suffering from IBD.

\section{W1141}

Role of Variant Interleukin-10 Receptor 1 (IL-10R1) Signaling in Ulcerative Colitis (UC) and Cytomegalovirus (CMV) Infection

Paul Grundtner, Manuela Nemeth, Neeraja Kambham, Teri A. Longacre, Christoph Gasche

Background: Animal models link the IL-10 pathway to human inflammatory bowel disease, indicating that IL-10 regulates gut homeostasis. The IL-10 family of proteins consists of several human and viral homologs that also share receptors. One of the viral IL-10 homolog genes belongs to CMV and is thought to alter the host's response to viral infection or reactivation. Previously we have identified two variants on the IL-10RI (SNP3 and 4) that were associated with Crohn's colitis but protected from UC in Caucasian populations. Computer modeling predicted that SNP3 alters IL-10 or IL-10 homolog binding to the extracellular receptor domain (J Immunol 170:5578). SNP3 is in linkage disequilibrium with SNP4 building the SNP3+4 haplotype. In this study we asked whether these IL-10RI variants alter the likelihood of CMV reactivation in patients with colitis and thereby predispose certain populations. Methods: Genomic DNA from 78 colitis patients and 100 ethnicity matched controls (mainly normal tissue from cancer patients) was isolated from paraffin embedded tissue (colonic biopsies or colectomy samples) and small fragments (80-150bps) spanning the IL-10R1-SNP3 and IL-10R1-SNP4 were amplified by PCR, purified, and sequenced. CMV positive cases were identified from histological slides by H\&E and immunohistochemistry against CMV immediate early antigen (Am J Surg Pathol 28:365). Results: In the colitis population, the allele frequencies of IL-10R1 SNP3 and SNP4 were significantly higher than in controls (SNP3 $\mathrm{p}<0.05$, SNP4 $\mathrm{p}<0.001$, by chi square). No difference was seen between refractory and non-refractory cases, however, the CMV positive population showed the highest allele frequency of the SNP3+4 haplotype (table). Within this group, $50 \%$ of patients were heterozygous for this allele. Conclusions: The unexpected high frequency of IL-10Rl variants in this colitis population may reflect ethnic or diagnostic differences to previous studies. The enrichment of SNP3 alleles in the CMV-positive population, however, points to the presence of genetic predisposition in this cohort. These findings fit with computer modeling of SNP3 and justify further studies to elucidate the role of variant IL10 signaling in CMV reactivation.

\begin{tabular}{|c|c|c|c|c|}
\hline IL-10R1 & Alleles(n) & wt & SNP4 only & SNP3+4 \\
\hline Controls & 200 & 0.795 & 0.075 & 0.130 \\
\hline Colitis & 156 & 0.596 & 0.192 & 0.212 \\
\hline Refractory & 78 & 0.628 & 0.154 & 0.218 \\
\hline Non-refractory & 78 & 0.564 & 0.231 & 0.205 \\
\hline CMV positive & 20 & 0.650 & 0.100 & 0.250 \\
\hline CMV negative & 136 & 0.588 & 0.206 & 0.206 \\
\hline
\end{tabular}

\section{W1142}

Nonsteroidal Anti-Inflammatory Drug Use and Inflammatory Bowel Disease Relapse: Individual and Disease-Specific Susceptibility Factors Mireia Penalva, Javier P Gisbert, Olga Merino, Marta Lucia Luna, Miquel Sans, Nuria Massuet, Josep Maria Pique, Julian Panes

The effect of nonsteroidal anti-inflammatory drugs (NSAIDs) on inflammatory bowel disease (IBD) remains controversial. AIMS: To determine the relationship between NSAIDs use and IBD relapse. To explore the possible existence of disease or individual susceptibility factors. PATIENTS AND METHODS: Consecutive outpatients with established diagnosis of ulcerative colitis (UC; $n=198)$ or Crohn's disease (CD; $n=216)$ were included. Patients under medical treatment for achieving remission were excluded. Patients with active IBD as determined by the corresponding index (Harvey- Bradshaw or Truelove) were considered cases. Patients in remission were considered controls. Data about NSAIDs consumption during the last 6 months before the visit and previous history of relapse related to NSAIDs use were collected. RESULTS: 414 patients were included; 108 were cases and 306 controls. Overall, 30\% had used NSAIDs within the previous 6 months. The case-control study showed an association between NSAIDs use within the previous 30 days and IBD relapse (28\% cases, $16 \%$ controls; $\mathrm{p}=0.0054$ ), which remained significant for the period of the previous 14 days ( $22 \%$ cases, $12 \%$ controls, $\mathrm{p}=0.008)$, but not for the period of -15 to -30 days. There was no association between NSAIDs consumption within the 6 previous months and IBD activity. When examining separately $U C$ and $C D$, statistic significance was found only for UC patients ( $27 \%$ cases, $10 \%$ controls, $\mathrm{p}=0.004$ ) but not for $\mathrm{CD}(18 \%$ cases, $14 \%$ controls, $\mathrm{p}=0.40)$. Logistic regression analysis confirmed that NSAIDs use is an independent risk factor for disease relapse in UC (OR 3.93, CI 95\% 1.65-9.37, p=0.002), but not in CD (OR 1.48, CI 95\% 0.65-3.35, p= 0.34). In the subgroup of patients having consumed NSAIDs during the previous 14 days, a significant relationship was detected between disease activity at time of evaluation and a previous individual history of relapse related to NSAIDs ( $15 \%$ cases, none controls; $\mathrm{p}=$ $0.017: \mathrm{PPV}=0.9)$. CONCLUSIONS: In IBD patients, NSAIDs use within the two previous weeks is related to an increased risk of relapse. This association is observed in UC. The risk of relapse related to NSAIDs remains constant in individual patients and can be used to identify susceptible and tolerant patients.

\section{W1143}

Radiological Sacroiliitis in Crohn's Disease: A Multicenter Association Study Harald Peeters, Bert Vander Cruyssen, Herman Mielants, Kurt de Vlam, Severine Vermeire, Edouard Louis, Paul Rutgeerts, Jacques Belaiche, Martine de Vos

Background: Radiological sacroiliitis (SI), a hallmark of spondylitis, is considered to be the most frequent objective extra-intestinal manifestation (EIM) of Crohn's disease (CD). SI in $\mathrm{CD}$ can be asymmetrical and is often asymptomatic. Data on the association of SI with other clinical features of CD are scarce. A possible association with the presence of CARD15 polymorphisms has been suggested. Aim: To investigate in a cross-sectional, multicentric study the association of SI in CD patients with clinical phenotypes of the disease, other EIM and the presence of CARD15 polymorphisms. Methods: Radiographs of the sacroiliac joints were taken in a total of $236 \mathrm{CD}$ patients from 3 Belgian university hospitals (Gent, Leuven, Liège) and scored by 2 rheumatologists. Sacroiliitis was defined as the presence of at least grade 2 unilateral or bilateral changes. We recorded sex, age, age at onset of $C D$, disease duration, familial history, smoking habit, the history of ileal resections, fistulas and EIM including axial disease (inflammatory back pain or ankylosing spondylitis), peripheral arthritis, uveitis, erythema nodosum, pyoderma gangrenosum and primary sclerosing cholangitis and the CARD15 genotype (R702W, G908R and/or 1007fs). X2 and Mann-Whitney U tests were used for univariate analysis. Multivariate analysis was performed using logistic regression (SI as dependent variable), including all clinical features and CARD15 polymorphisms. Results: In 7 patients the quality of the radiographs did not allow an adequate scoring of the sacroiliac joints. Sacroiliitis was diagnosed in 63 of the remaining 229 patients (27.5\%) of whom 23 had unilateral SI grade 2 and 40 patients had bilateral changes or a higher grading. Thirty-six of the 63 patients with SI $(57.1 \%)$ had no known history of inflammatory back pain. Compared to patients with normal sacroiliac joints, patients with radiological SI had more peripheral arthritis ( $42.9 \%$ vs $24.7 \% \mathrm{P}=0.007$, OR $2.3,95 \% \mathrm{CI} 1.2-4.2$ ) and uveitis $(15.9 \%$ vs $6.6 \%, \mathrm{P}=0.03$, OR $2.7,95 \% \mathrm{CI} 1.1-6.6)$ in their history. No associations with the other recorded clinical features or with CARD15 polymorphisms were found. Only the association with peripheral arthritis was confirmed in a multivariate analysis (peripheral arthritis $\mathrm{P}=0.023$; uveitis $\mathrm{P}=0.073$ ). Conclusions: Our study confirms the high prevalence of (often asymptomatic) radiological sacroiliitis in a multicenter CD cohort. Patients with SI are more prone to develop peripheral arthritis during their disease course, suggesting a similar pathogenetic mechanism. We could not confirm the previously reported association between SI and the presence of CARD15 polymorphisms. 
W1144

Most Frequent Forms of Patient's Non-Adherence to the Treatment of Inflammatory Bowel Disease

Petr Cerveny, Martin Bortlik, Milan Lukas, Milan Nobilis, Jiri Vlcek

Objective: Non-adherence to the treatment of inflammatory bowel disease (IBD) is reported to be a quite often event and such patients are exposed to higher risk of relapse. We assessed the overall non-adherence to the treatment among patients with Crohn's disease (CD) and Ulcerative colitis (UC) and quantified it's most frequent forms. Patients and methods: 177 IBD patients were enrolled in our prospective study (84 male, 93 female, mean age 36,9, range $16-78,117 \mathrm{CD}, 60 \mathrm{UC})$. Patients were interviewed about their non-adherent behavior during the treatment. Medication, disease type and activity were completed from the documentation. Of these 177 patients, urine sample was taken from 47 patients treated by mesalamine (27 male, 20 female, mean age 38,7, range 22 - 65, $32 \mathrm{CD}, 15 \mathrm{UC}$ ) for HPLC analysis. All the data were processed with factor analysis. Results: 1.Overall voluntary nonadherence (treatment breaking, dose reducing or medication non-refilling) was reported by $36 \%$ of patients. These patients are likely to be chronically active or in relapse $(\tau=0,212$, $\mathrm{p}=0,002)$. A $18 \%$ of patients reported they minimally once in their life broke the treatment. Treatment breaking was more often among patients who ever noticed any drug adverse effect $(p=0,04)$. Reporting of adverse drug effects escalates with knowledge about disease and pharmacotherapy $(\mathrm{p}<0,01)$. Voluntary dose reducing was reported also by $18 \%$ of patients. Dose reducing was more often among patients taking themselves to be well informed about the disease $(p=0,01)$. A $14 \%$ of patients occasionally non-refill the medication in time. In voluntary non-adherence, there were no differences between male and female, disease type, undergone bowel surgery, singles or married and smokers and non-smokers. A $38 \%$ of patients reported unintentional non-adherence. Forgetting of taking daily doses paradoxically negatively correlates with number of prescribed medicines $(\tau=-0,217, p=$ $0,001)$. 2.In the group of 47 patients of which the urine was taken, in 6 cases (12\%) mesalamine was undetectable, suggesting drug holiday or non-refilling of medication in time. Of these 6 patients, 4 of them reported non-adherence in the interview. There were no statistically significant relationships among interview data on non-adherence and results of urine analysis. Conclusions: 1.Overall non-adherence was reported or analytically proved among $40 \%$ of patients. 2 . Adherence to the treatment in our cohort seems to be strongly influenced by knowledge related to disease and pharmacotherapy. Our results can stimulate discussion how to improve education of patients.

\section{W1145}

Voluntary Childlessness in IBD

Sheetal Marri, Chul Ahn, Alan L. Buchman

Introduction: Issues surrounding childbearing have received relatively little attention even though IBD predominantly affects women during their childbearing years. To date, only two studies have investigated this topic, but they provided limited/conflicting data about issues concerning childlessness. The aim of this study was to better understand patients' considerations in decision making about pregnancy. Methods: Data were obtained from specific questions in 3147 surveys mailed anonymously to all members of the statewide CCFA Illinois Carol Fisher chapter. Subjects were provided a pre-addressed, pre-stamped return envelope and instructed to return completed surveys with no identifying information. Results: The survey response rate was 20\% (637); 63\% female, 34\% male. A majority (67\%)indicated the state of their illness did not change previously held opinions on having their own children. Of the 33\% whose opinion changed, 59\%) stated they decided to have children despite their illness. Of the $27 \%$ of patients that decided against having their own children the major reasons were: concern for of genetically transmission of disease to their children $(63 \%)$, concern of being unable to care for their child (59\%),concern of added stress of raising a child (55\%), and concerns of worsening/recurrent IBD or their partner's IBD ( $46 \%$ and $41 \%$ respectively). In women who had undergone previous pregnancy, the course of those pregnancies did not change opinions of $75 \%$ of the respondents regarding future pregnancies. Of the $25 \%$ that did change their opinion, $(53 \%)$ decided to have a second pregnancy. Conclusion: This study is the largest survey to date on this issue, and contradicts previous limited data about factors contributing the pregnancy decision making process. Contrary to previous data(1), physician counseling against having children or for terminating pregnancies was not a major factor in the decision making process. Our data also indicate women with IBD do not have an increased rate of voluntary childlessness as previously thought(2. Our data suggests a positive effect of pregnancyt in patients with IBD on desire for future pregnancy. References: 1. Baird DD, Narendranathan M, Sandler RS. Increased risk of preterm birth for women with inflammatory bowel disease. Gastroenterology 1990;99:987-94 2. Mayberry JF, Weterman IT. European Survey of Fertility and Pregnancy in Women with Crohn's Diseases: A Case-Control Study by European Collaborative Group. Gut 1986 Jul;27(7):821-5.

\section{W1146}

Prothrombotic Mutations and Inflammatory Bowel Disease Charles N. Bernstein, Michael Sargent, Frits R. Rosendaal

Background and Aims: Patients with Crohn's disease (CD) and ulcerative colitis (UC) have a 3-4-fold increased risk of venous thrombosis compared with the general population. We aimed to determine if patients with CD and UC had increased likelihood of mutations in genes that increase clotting risk, in a population-based case control study. Methods: Subjects were drawn from the University of Manitoba IBD Research Registry and controls were drawn from Manitoba Health's administrative database. Cases (CD, n=327, UC, n=165) and controls $(\mathrm{n}=412)$ underwent venipuncture and DNA was purified from whole blood. Genotypes for common mutations were determined for factor II (prothrombin, G20210A), Factor V (G1691A), methylenetetrahydrofolate reductase (MTHFR, C677T), and Factor XIII (val34leu). Results: Of all participants, $1.5 \%$ were heterozygous for Factor II G20210A and $6.1 \%$ for Factor V, without differences between cases and controls. Only 1 subject was homozygous for Factor V mutation (and none were homozygous for Factor II mutation). UC patients were less likely than controls to have a mutation in MTHFR (50.3\% vs $59.4 \%$ ), with odds ratio (OR) for carrying at least one copy of the mutated C $377 \mathrm{~T}$ allele of $0.7,(95 \% \mathrm{CI}, 0.47$ 1.01). CD patients were more likely to be homozygous for the FXIII val34leu mutation than controls ( 8.7 vs $4.3 \%)(\mathrm{OR}=2.0,95 \% \mathrm{CI} 1.04-4.27)$. Age, sex, or disease phenotype did not modify these associations. Conclusions Although we found an increase in homozygosity for FXIII val34leu mutation for $C D$ vs controls, we could not explain the increased risk of venous thrombosis in $\mathrm{CD}$ and $\mathrm{UC}$ by these 4 common genotype mutations. However, this increase in mutated Factor XIII in CD should be further explored.

\begin{tabular}{|c|c|c|c|}
\hline & CD & UC & Controls \\
\hline F II GG & $98.2 \%$ & $98.8 \%$ & $98.8 \%$ \\
\hline F II AG & $1.8 \%$ & $1.2 \%$ & $1.2 \%$ \\
\hline F II AA & 0 & 0 & 0 \\
\hline F V GG & $93.6 \%$ & $95.8 \%$ & $93.9 \%$ \\
\hline F V AG & $6.1 \%$ & $4.2 \%$ & $6.1 \%$ \\
\hline F V AA & $0.3 \%$ & 0 & 0 \\
\hline MTHFR CC & $43.6 \%$ & $49.7 \%$ & $40.6 \%$ \\
\hline MTHFR CT & $43.9 \%$ & $40.4 \%$ & $47.7 \%$ \\
\hline MTHFR TT & $12.5 \%$ & $9.9 \%$ & $11.7 \%$ \\
\hline FXIII GG & $51.3 \%$ & $56.7 \%$ & $53.7 \%$ \\
\hline FXIII GT & $40.1 \%$ & $36.2 \%$ & $42.0 \%$ \\
\hline FXIII TT & $8.7 \%$ & $7.1 \%$ & $4.3 \%$ \\
\hline & & &
\end{tabular}

W1147

The Manitoba IBD Cohort Study: A Comparison of Participants' Perceptions Regarding Psychological Health, Disability, Social Supports, and Access to Health Care with a Population-Based Control

Linda Rogala, Norine Miller, Ian Clara, Patricia Rawsthorne, Lesley A. Graff, Cory McPhail, John R. Walker, Charles N. Bernstein

The Manitoba IBD Cohort Study is a longitudinal, population-based study of multiple determinants of health outcomes in IBD in those diagnosed within 7 years at enrollment In this substudy we aimed to determine participants' perceptions of their physical and psychological health, social supports, and access to health care. Methods: Interview questions were directly drawn from the Canadian Community Health Surveys 1.1 and 1.2 (CCHS) which allowed us to compare the IBD cohort to a community sample matched on age, gender, and region. All comparisons reported as significantly different were at $\mathrm{p}<0.01$. Results: IBD participants ( $\mathrm{n}=388$ )reported significantly higher levels of personal disability, with more days spent in bed and decreased activity levels at home and work. IBD subjects had higher levels of daily stress, and lower levels of general well-being. IBD participants reported significantly increased barriers to health care, including difficulties with transportation to health services, availability of health services, waiting time to see a doctor, and cost of health care. However, availability of social support (including someone who helps with household chores, someone to confide in, someone who provides love, someone who helps with worries or gives suggestions, someone who makes them feel wanted and frequency of affective support in the prior year) as well as employment levels and levels of work stress were not different between IBD and controls. Furthermore, despite significantly increased daily stress, lower well-being and less access to health care, IBD participants were no different in terms of alcohol intake and were less likely to use alternative or complementary medicines. Conclusions; The finding that IBD participants reported increased barriers to health care is surprising as this region is served by a universal health care system with no charge for physician visits or hospital services. It may be that individuals with a serious disease requiring specialized services find it difficult to obtain prompt access to the services they need relative to individuals who are more likely to be healthy and require fewer services. While IBD participants experienced more stress and disability, they are as likely to be employed as controls (although may be missing more work) and on balance have similar levels of social support. Their degree of perceived stress and general well-being may require professional intervention. Several studies have reported on complementary medicine use in IBD, but this is the first controlled study to show that this use is less than in general population.

\section{W1148}

TNF- $\alpha$ Neutralizing Antibody Improves Endothelial Dysfunction in Patients with Crohn's Disease

Alessandro Armuzzi, Francesca Schinzari, Barbara de Pascalis, Nadia Mores, Giovanni Gasbarrini, Domenico Melina, Carmine Cardillo

Background: Persistent systemic inflammation due to dysregulated cytokine activity may predispose patients with inflammatory bowel disease (IBD) to increased risk of premature atherosclerosis. Activation of the proinflammatory cytokine tumor necrosis factor- $\alpha$ (TNFa) observed in patients with IDB could be involved in the pathogenesis of endothelia dysfunction. Methods: Endothelium-dependent and -independent vasodilator responses to intraarterial infusion of increasing doses of acetylcholine (ACh) and sodium nitroprusside (SNP), respectively, were assessed by strain-gauge plethysmography in patients with Crohn's disease $(C D ; n=12)$ or ulcerative colitis $(U C ; n=12)$ and in a group of matched controls $(n=$ 12). In patients with $C D$, vasodilator function was reassessed following intraarterial infusion of infliximab, a TNF- $\alpha$ neutralizing antibody $(200 \mu \mathrm{g} / \mathrm{min}, 60 \mathrm{~min})$. Results: Both patients with $\mathrm{CD}$ and $\mathrm{UC}$ had elevated circulating levels of inflammatory mediators (C-reactive protein and interleukin- 6 ; both $\mathrm{P}<0.05$ vs. controls), without differences between the 2 disease groups (both $\mathrm{P}>0.05$ ). Circulating levels of TNF- $\alpha$ and soluble TNF receptor type 1 and 2 , in contrast, were similar among the 3 groups (all $P>0.05$ ). Endothelium-dependent vasodilation was impaired in patients with $\mathrm{CD}(\mathrm{P}=0.003$ vs. controls), but not in those 
with UC ( $\mathrm{P}=0.46$ vs. controls). By contrast, endothelium-independent vasodilation was not different among the 3 groups $(\mathrm{P}=0.69)$. In patients with $C D$, TNF- $\alpha$ neutralizing antibody markedly enhanced the responsiveness to $\mathrm{ACh}(\mathrm{P}=0.005$ vs. saline), but did not affect the response to $\mathrm{SNP}(\mathrm{P}=0.53)$. Conclusions: Despite comparable degrees of systemic inflammation in patients with $\mathrm{CD}$ and $\mathrm{UC}$, endothelial dysfunction is present only in patients with $\mathrm{CD}$. In these patients, even in the absence of increased circulating TNF- $\alpha$ activity, intravascular administration of TNF- $\alpha$ neutralizing antibody beneficially affects endothelial function. These findings suggest a predominance of disease-specific factors over inflammation per se in the pathophysiology of the atherogenic process and underscore the mechanistic role of selective cytokines activated within the vessel wall.

\section{W1149}

Post-Operative Complications Related to Surgical Approach and Medical Therapy for Inflammatory Bowel Disease

Lori A. Coburn, Brad E. Maltz, Paul E. Wise, Alan J. Herline, Roberta L. Muldoon, Tatsuki Koyama, David A. Schwartz

Introduction: Advances in medical and surgical treatment of inflammatory bowel disease (IBD) in the last decade has led to the increasing utilization of immunosuppressive agents, biologic medications, and laparoscopy (LAP). Steroids have been shown to increase the risk of post-operative complications, but the affect of other commonly used agents such as azathioprine (AZA), 6-mercaptopurine (6-MP), methotrexate (MTX), and infliximab (IFX) on post-operative complications has not been fully defined. Methods: Charts of patients (pts) with IBD who underwent surgery at an academic medical center from 2001-2005 were reviewed. Pts with follow-up $<4$ mos were excluded, therefore 124 pts were identified. Pts were categorized by IBD type [Crohn's (CD) / ulcerative colitis (UC)], meds at the time of surgery [IFX (within $8 \mathrm{wks}$ ), prednisone low ( $\leq 20 \mathrm{mg}$ daily) or high ( $>20 \mathrm{mg}$ daily) dose, AZA/ 6-MP, MTX], and type of surgery (open or LAP). Results were controlled for age, gender, smoking, timing of surgery (emergent vs. elective), disease location, and preoperative albumin level. Complications were divided into early ( $\leq 30$ days) and late ( $>30$ days). Wound infection, wound dehiscence, recurrent disease, and pneumonia were considered minor complications. Major complications included anastomotic leak, abscess/fistula, sepsis bacteremia, small bowel obstruction, thromboembolism, or death. Results: Of the 124 surgeries, 69 were LAP (56\%) and 87 had CD (70\%). Overall complication rate was $50 \%$ (2/3 were major complications). 72 (58\%) pts were on steroids (33 low dose), 74 (60\%) were on AZA/6-MP, 7 (6\%) were on MTX, and 44 (35\%) were on IFX. After controlling for confounding, there was no increase risk of complications associated with the use of any of these agents except IFX. 64\% of pts who received IFX within 8 weeks of surgery had a complication vs. $43 \%$ of pts not on IFX ( $\mathrm{p}=0.012)$, with an odds ratio of 3.7 [95\% CI (1.310.3)]. Further analysis of the IFX group revealed a trend toward a higher number of minor complications. There was no difference in complication rates based on approach (open vs. LAP) or IBD type. Conclusions: There is a significant increase in post-operative complications associated with IFX within 8 weeks of surgery, but not with steroids, AZA/6-MP, or MTX. Laparoscopy and type of IBD is not associated with an increased risk of post-operative complications. Prospective evaluation of complications associated with IFX is warranted.

\section{W1150}

Azathioprine Intolerant Crohn's Disease Patients: A Clinical Subgroup with Significantly Worse Disease Complications, Severity and Disability Ramesh Ashwathnarayan, Lydia R. Weber, Mazen Issa, Josh F. Knox, Sue Skaros, Ossama A. Hatoum, Jeanne Emmons, Mary F. Otterson, David G. Binion

Introduction: Azathioprine (AZA) is a mainstay of longterm therapy for Crohn's disease (CD) pts with moderate to severe disease. However, a subgroup of $\mathrm{CD}$ pts do not tolerate AZA, and face fewer treatment options. The natural history of AZA tolerant (TOL) and AZA intolerant (INTOL) CD pts has not been defined. We utilized a referral center database to characterize objective markers of disease complications and severity to define the natural history of pts able to receive AZA treatment vs those who were intolerant. Methods: All AZA treated CD pts followed at a referral center over a 7 -year period were reviewed, including pts referred because of drug intolerance history. Pts who discontinued or were ineligible for AZA due to side effects formed the INTOL group, while those continuing AZA comprised the TOL group. Demographic information, smoking history, $\mathrm{CD}$ duration, treatments, drug allergy/intolerance, surgeries, hospitalizations and permanent disability were recorded. Results: Among 505 CD pts who were candidates for AZA, 335 (66.3\%) were TOL and 170 $(33.7 \%)$ were INTOL. Mean age in TOL was $39 \pm 14 y(S D)$ and in INTOL was $43 \pm 14 y$ (SD; $\mathrm{p}<0.05$ ). There were $56 \%$ female in TOL and $61 \%$ in the INTOL ( $=$ NS). History of any tobacco use was significantly higher in the INTOL vs TOL group ( $38 \%$ vs $26 \%$; $\leq 0.01$ ), as was active smoking (INTOL $24 \%$ vs TOL $16 \%$; $\leq 0.03$ ). Hospitalizations were significantly higher in the AZA INTOL group (mean $2.9 \pm 2.7$ (SD)) compared with TOL (mean $2 \pm 1.8$ (SD); $\mathrm{p}<0.001$ ) as was permanent disability (INTOL $12.9 \%$ vs TOL $4.5 \% ; \mathrm{p}<0.001$ ). Mean surgeries in INTOL was $2 \pm 3.7$ (SD) compared with TOL $1.4 \pm 1.5$ ((SD); $=\mathrm{NS})$. Maintenance infliximab use was similar between the 2 groups (INTOL 27\% vs TOL 26\%) however, a higher percentage of INTOL pts had received biologic therapy ( $58 \%$ vs $49 \%$; $<<0.05$ ). The percentage of INTOL pts who experienced complications with infliximab immunogenicity was higher than the TOL group ( $27 \%$ vs $14 \%$; $<0.01)$. The percentage of AZA INTOL CD pts receiving maintenance methotrexate was $31 \%$, with a mean duration of $1.9 \pm 1.3 \mathrm{y}$ (SD) which was significantly shorter that the mean duration of AZA use in the TOL group (4.5 $\pm 3.1 y(S D)$; range $0-27 \mathrm{y} ; \mathrm{p}<0.0001$ ). Conclusions: AZA INTOL CD pts are a clinical subgroup challenged by more severe disease related complications compared with pts who may benefit from long-term purine analog treatment. Although mechanism for AZA intolerance is not clear, history of smoking appears to correlate. MTX appears to be underutilized in AZA INTOL pts. The comparison of AZA TOL vs INTOL pt subgroups suggests that long-term AZA treatment modifies the natural history of moderate to severe CD.

\section{W1151}

Vitamin D Deficiency Is Associated with Deficits in Trabecular and Cortical Bone in Incident Pediatric Crohn's Disease

Meena Thayu, Robert N. Baldassano, Babette S. Zemel, Bruce W. Hollis, Mary B. Leonard

Crohn disease (CD) is characterized by inflammation of the gastrointestinal tract leading to malabsorption, micronutrient deficiencies, and decreased bone mass. The relationships between vitamin D deficiency and musculoskeletal deficits have not been addressed in incident pediatric Crohn disease. The objectives of this prospective study were to determine the prevalence of vitamin $\mathrm{D}$ deficiency, and to identify the relationship between serum vitamin 25-hydroxy D [25(OH)D] levels and muscle and bone deficits in children with CD prior to the initiation of therapy, compared to healthy controls. Serum 25(OH)D levels were determined by $\mathrm{I}^{125}$ RIA in 72 CD subjects (57\% male, $11 \%$ black) and 160 healthy controls (50\% male, $21 \%$ black), ages 5-21. 25(OH)D insufficiency was defined as $<30 \mathrm{ng} / \mathrm{mL}$ and deficiency as $<10 \mathrm{ng} / \mathrm{mL}$. Peripheral quantitative computed tomography (pQCT, Stratec XCT 2000) scans were used to measure trabecular volumetric bone mineral density (vBMD) at the 3\% distal site, cortical bone cross-sectional area (CSA) at the $38 \%$ site, and muscle CSA at the $66 \%$ site. Height and BMI are expressed as age and sex-specific standard deviation scores (Z-scores). Bone and muscle measures are expressed as gender and race-specific Zscores derived from linear regression models in the controls. Multivariable linear regression models were used to assess relations between $C D$, vitamin D levels and pQCT Z-scores. Among non-black $\mathrm{CD}$ subjects, $80 \%$ were $25(\mathrm{OH}) \mathrm{D}$ insufficient and $5 \%$ were deficient. Among black CD subjects, $100 \%$ were $25(\mathrm{OH}) \mathrm{D}$ insufficient and $63 \%$ were deficient. 25(OH)D levels were significantly lower in CD compared with controls $(\mathrm{p}<0.05)$ adjusted for season and race. CD subjects had significant height, BMI, bone and muscle deficits, compared to controls (all p < 0.001) (Table). Within the CD subjects, trabecular vBMD Zscore was significantly associated with $25(\mathrm{OH}) \mathrm{D}$ levels $(\mathrm{p}=0.008)$, adjusted for race; each $10 \mathrm{ng} / \mathrm{mL}$ decrease in 25(OH)D was associated with a 0.43 decrease in vBMD Z-score. Cortical CSA Z-score was significantly associated with 25(OH)D levels $(\mathrm{p}<0.05)$, adjusted for race; each $10 \mathrm{ng} / \mathrm{mL}$ decrease in $25(\mathrm{OH}) \mathrm{D}$ was associated with a 0.34 decrease in cortical CSA z-score. Muscle CSA Z-scores were not significantly associated with 25(OH)D levels. 25(OH)D levels, bone, and muscle deficits were not associated with Pediatric Crohn Disease Activity Index or symptom duration. A longitudinal study of vitamin D status and changes in bone and muscle in $\mathrm{CD}$ is underway.

Crohn Disease Subject Z-scores

\begin{tabular}{|c|c|c|c|c|}
\hline Trabecular vBMD-Z* & Cortical CSA-Z*** & Muscle CSA-Z*** & Height-Z & BMI-Z \\
\hline$-1.1 \pm 1.2$ & $-0.6 \pm 1.3$ & $-0.9 \pm 1.2$ & $-0.3 \pm 1.1$ & $-0.5 \pm 1.2$ \\
\hline
\end{tabular}

*Adjusted for age **Adjusted for tibia length

W1152

Factors Predicting Postoperative Recurrence with Placebo Therapy in Patients with Crohn's Disease: A Meta-Analysis of Clinical Trials Evaluating Postsurgical Maintenance Therapy

Chinyu Su, James D. Lewis, Monina F. Pascua, Colleen Brensinger, Gary R. Lichtenstein

Background: Placebo-controlled RCTs (PC-RCTs) are the gold standard for evaluating drug efficacy for preventing postoperative recurrence (Rec) in patients with Crohn's disease (CD). Knowledge of the relapse rate on placebo (Plac) therapy and factors influencing this rate is important for designing future trials and may provide valuable information for management of postoperative $\mathrm{CD}$. Aim: To estimate the Plac relapse rate from prior $\mathrm{CD}$ PC-RCTs for postoperative maintenance therapy and to identify factors influencing this rate. Methods: PC-RCTs evaluating therapies for CD with surgically-induced remission (Rem) were identified from MEDLINE through Sept 2005. Outcomes were dichotomized into Rem v. Rec. Pooled estimates of the Plac clinical (Clin) and endoscopic (End) Rec rates were calculated using logistic regression after applying sample weights based on Plac size. Univariate logistic regression models were used to estimate the influence of design features on this rate. Results: 8 studies met the inclusion criteria. The pooled estimate of Plac End Rec rate was 0.56 $(95 \% \mathrm{Cl}=0.48-0.64$. $\mathrm{p}$ for study heterogeneity $=0.003)$. This rate was $0.56(95 \% \mathrm{Cl}=0.46$ 0.65 ) limiting analysis to 5 studies using Rutgeerts score $\geq 2$ as definition. Of the 7 studies reporting Clin outcome, the pooled estimate of Plac Clin Rem rate was $0.59(95 \% \mathrm{CI}=0.48$ $0.69, \mathrm{p}$ for heterogeneity<0.001). The table shows factors associated with the Plac outcomes. Both Clin and End outcomes were independent of study visit \#/frequency, disease duration and smoking status of Plac groups. The Clin but not End outcome was independent of disease distribution and phenotype. Conclusions: In PC-RCTs for CD postoperative prophylaxis, the Plac rates of End and Clin Rec were 56\% \& $41 \%$, respectively. Prior surgery and study duration were associated with higher cumulative relapse rates. While disease distribution and phenotype appeared to influence the Plac End Rec rates, they were not associated with Plac Clin outcome. These factors may be considered when predicting the likelihood of postoperative recurrence in patients without active intervention.

\begin{tabular}{|c|c|c|}
\hline & Clinical Remission & Endoscopic Recurrence \\
\hline Study Features & OR* (95\% CI), P-value & OR* (95\% CI), P-value \\
\hline Study duration (per 4 wk $\uparrow$ ) & $0.98(0.96-0.99) \mathrm{p}=0.003$ & $0.98(0.94-1.02) \mathrm{p}=0.37$ \\
\hline \% of Plac with prior CD surgery (per 10\% $\uparrow)$ & $0.79(0.74-0.85) \mathrm{p}<0.001$ & $1.19(1.05-1.35) \mathrm{p}=0.008$ \\
\hline$\%$ of Plac with SB disease (per 10\% $\uparrow)$ & $0.99(0.95-1.04) \mathrm{p}=0.65$ & $0.79(0.69-0.90) \mathrm{p}=0.001$ \\
\hline$\%$ of Plac with colonic disease (per 10\% $\uparrow)$ & $0.92(0.78-1.08) \mathrm{p}=0.29$ & $0.78(0.61-1.00) \mathrm{p}=0.05$ \\
\hline$\%$ of Plac with SB \& colon disease (per 10\% $\uparrow)$ & $0.91(0.75-1.11) \mathrm{p}=0.34$ & $1.20(1.09-1.33) \mathrm{p}<0.001$ \\
\hline$\%$ of Plac with fistulizing disease (per 10\% $\uparrow)$ & $1.03(0.80-1.31) \mathrm{p}=0.83$ & $1.59(1.32-1.91) \mathrm{p}<0.001$ \\
\hline$\%$ of Plac with obstructive disease (per 10\% $\uparrow)$ & $1.04(0.96-1.11) \mathrm{p}=0.35$ & $0.62(0.51-0.75) \mathrm{p}<0.001$ \\
\hline
\end{tabular}


*OR>1 indicates that the factor was associated with a higher cumulative rate of Clin Rem or End Rec in Plac-treated patients

\section{W1153}

A Large Number of Siblings Is Protective for Crohn's Disease- An Israeli Population Based Study

Eyal Klement, Joseph Lysy, Eran Goldin, Tsippy Shochat, Eran Israeli

Background: It was previously shown that subjects with a large number of siblings have lower risk for the development of asthma and atopic dermatitis. Similar case control studies for patients with Crohn's disease (CD) have failed to show conclusive results. Methods: We performed a large population based cohort study based on the computerized medical database of the Israeli Defense Forces. The association of $\mathrm{CD}$ with number of siblings was stratified by gender, place of birth and origin. For each gender 4 groups were analyzed: (1) Israel born Asian/African origin; (2) Israel born European/North American origin; (3) Israel born Israeli origin; and (4) European/North American born. Results: A population of 296,684 males and 214,942 females was included in the study. The prevalence of $C D$ was 237 per 100,000 population. Relative Risk (RR) for the first three groups of males for families of 1 4 siblings vs. families of 5 or more, were $1.09(\mathrm{CI} 195 \%=0.81-1.46), 1.67(0.71-3.92)$ and 2.07 (1.41-3.07) while for European/North American born males, a smaller number of siblings seemed to have a protective effect as RR was 0.49 (0.26-0.91). RR in African/Asian and in European/North American Israeli born females were 1.54 (0.94-2.52) and 2.63 (1.086.38) respectively, while for females included in groups 3 and 4 RR could not be calculated since there were no females with $\mathrm{CD}$, in families with 5 or more siblings. Conclusions: A high number of siblings is protective for $\mathrm{CD}$, primarily among Ashkenazi Israeli born males and females. We suggest that this association is due to the lower socioeconomic status in large Israeli families, a hypothesis that coincides with the hygiene theory.

\section{W1155}

Determinants of Progression of Indefinite Or Low-Grade Dysplasia to Advanced Dysplasia in Ulcerative Colitis

Salma Akram, Loftus V. Edward, William J. Tremaine, William A. Faubion, Darrell S. Pardi, Debra A. Jewell, William J. Sandborn

BACKGROUND: Patients (pts) with ulcerative colitis (UC) are at increased risk of colorectal cancer (CRC) and dysplasia. Recent preliminary work suggests that 5-ASA use might reduce the progression of indefinite dysplasia (ID). AIMS: To determine the risk of progression of dysplasia in UC pts evaluated at our institution over a ten-year period and to determine the influence of 5-ASA use on the clinical course of dysplasia. METHODS: We retrospectively identified 32 UC pts with either ID or low-grade (LGD) flat dysplasia diagnosed between 1994 and 2003. 5-ASA use data was obtained from the medical records. Pts who underwent colectomy within 2 months of the diagnosis (dx) of ID/LGD were excluded. RESULTS: There were $23(72 \%)$ males with median age of 53 years (yrs) (range, 29-75 yrs) at ID/LGD dx. Median duration of UC at the time of ID/LGD dx was 11 yrs (1-34 yrs). Nine pts had ID and 23 had LGD. Median follow-up from ID/LGD dx was 6.9 yrs (0.3-10.4 yrs). Progression from ID to LGD was noted in 3/9 (33\%) and from LGD to high-grade dysplasia (HGD) in $5 / 23(22 \%)$. Persistent dysplasia in 10 (31\%) and regression of dysplasia noted in 14 (44\%). Median age at $\mathrm{UC} d \mathrm{dx}$ was 33 yrs in progressive/persistent pts versus 37 yrs in regressors $(\mathrm{p}=0.04)$. Median duration of UC at ID/LGD dx was 12 yrs for progressive/persistent pts versus 8 yrs for regressors $(p=0.01)$. Sixteen pts $(50 \%)$ had colectomy with median time to surgery of $1 \mathrm{yr}(0.3-5 \mathrm{yr})$. Reasons for surgery included persistent LGD/ID in $9(56 \%)$, progression from LGD to HGD in 4 (25\%) and progression from ID to LGD in 3 (19\%). During the study period, 4 pts (13\%) died but none were from CRC. There was no difference in the average daily dose of 5-ASA prior to ID/LGD dx between progressive/persistent pts and regressors. In the post-ID/LGD period, average daily dose of 5-ASA was higher in the regressors ( $2.3 \mathrm{gm})$ compared to the progressive/persistent group $(1.5 \mathrm{gm})(\mathrm{p}=0.01)$. In the post-ID/LGD period, 5 progressive/persistent pts ( $27 \%$ ) used $>2.4 \mathrm{gm} /$ day of 5 -ASA versus 7 regressors $(50 \%)(\mathrm{p}=0.04)$. Ten progressive/persistent pts $(56 \%)$ had a history of PSC versus 4 regressors $(29 \%)(\mathrm{p}=0.01)$. Nine progressive/persistent pts $(50 \%)$ had moderately severe pancolitis vs. 7 regressors (50\%). CONCLUSION: Pts with progressive/persistent ID/ LGD were younger at UC dx, had longer UC duration and were more likely to have PSC. Extent and severity of UC were not significant predictors of progression. Post-dysplasia 5ASA dose was significantly higher in regressors compared to those with progressive/persistent dysplasia. A higher percentage of regressors were on $>2.4 \mathrm{gm} / \mathrm{d}$ of 5 -ASA. Supported by Procter \& Gamble Pharmaceuticals

\section{W1156}

Potential Contribution of Endothelin-Converting Enzyme, and ETA and ETB Receptors to Inflammatory Processes of Crohn's Disease in Humans

Takehisa Suekane, Junko Arimoto, Yoshihiro Ikura, Masahiko Ohsawa, Kenji Watanabe, Yasuhiro Fujiwara, Nobuhide Oshitani, Kazuhide Higuchi, Tetsuo Arakawa, Makiko Ueda BACKGLOUND AND AIMS: Endothelin-1 (ET-1) is a powerful vasoconstrictor and smooth muscle cell (SMC) mitogen. ET-1 can be generated locally by endothelin-converting enzyme (ECE), and the actions of ET- 1 are mediated by the specific receptors, ETA and ETB. It had been recognized that the vasoconstrictive and mitogenic properties of ET-1 are mediated mainly by binding to ETA receptors, whereas ETB receptors mediate vasodilatation. However, accumulating evidence has recently shown that ETB receptors also contribute to vasoconstrictive and mitogenic reactions. Moreover, these components of the ET system are considered to participate in inflammatory and wound healing processes. Previous reports have suggested a role for the ET-1 in the pathological mechanism of Crohn's disease (CD). However, the expression of ECE in affected intestinal tissues of $C D$ has not been studied, and precise roles of ETA and ETB receptors in the inflammatory and fibrogenic process of CD remain to be elucidated. In the present study, we immunohistochemically investigated the expression of ECE and ETA and ETB receptors in affected human intestinal tissues of CD. METHODS:
Eighteen intestinal tissue specimens were sampled from patients with CD by surgery. Reasons for the surgical resections were mostly intestinal strictures. Normal colonic tissues as control were obtained from 11 patients having surgery for colonic tumors. Frozen tissue sections cut from the samples were analyzed using immunohistochemical single and double staining techniques. For the analysis of the ET system, we used anti-ECE and anti-ETA and ETB receptors antibodies as primary antibodies. In addition, SMCs, macrophages, and endothelia cells were stained using their specific antibodies. RESULTS: In diseased intestinal tissues of $C D$, proliferation of SMCs and new vessels was frequently seen in association with inflammatory infiltration and fibrotic tissue reactions. In addition to the original SMC components such as the muscular layer and vascular SMCs, the proliferated SMCs were distinctly positive for these ET system components, ECE and ETA and ETB receptors. Vascular endothelia cells in the lesions had immunoreactivity for ECE and ETA receptor. Moreover, accumulated macrophages in the lesions also showed the expression of ECE, ETA receptors, and ETB receptors. CONCLUSION: These results strongly suggest that ECE and ETA and ETB receptors collectively play an important role in inflammatory and fibrogenic processes of $C D$. The upregulation of the ECE/ETA and ETB receptor system may contribute to SMC proliferation and enhance constrictive functions, which could lead to the development of intestinal strictures in $C D$

\section{W1157}

Cancer in Infliximab-Treated Or Untreated Crohn's Disease Patients: A 1 Year Longer Follow Up from a Multicenter Matched-Pair Study

Livia Biancone, Ambrogio Orlando, Anna Kohn, Elisabetta Colombo, Roberto Sostegni, Erika Angelucci, Fabiana Castiglione, Davide Checchin, Claudio Papi, Andrea Cocco, Gianmichele Meucci, Gabriele Riegler, Carmelina Petruzziello, Federico Mocciaro, Alessandra Geremia, Emma Calabrese, Mario Cottone, Francesco Pallone

Background and Aims. The widespread use of Infliximab (IFX) in Crohn's Disease (CD) rises concerns about cancer risk in the long term. We previously reported, in a cohort of matched-pair CD patients (pts.) followed up from April 1999 to October 2004, no differences between IFX-treated and untreated pts. in terms of new diagnoses of cancer. However, the frequency of cancer in a longer follow up needs to be assessed in this cohort of pts. Aim of the present study was therefore to assess, in a 1 year longer follow up, the frequency of new diagnoses of cancer in the same cohort of IFX-treated or untreated CD pts. Methods. In a multicenter matched-pair study, the same cohort of 404 CD pts. treated with IFX (CDIFX) matched with 404 CD never receiving IFX (CD-C) included in the previous study, was followed up for 1 more year. Cases and controls were matched for sex, age $( \pm 5$ yrs $)$ CD site, age at diagnosis ( +5 yrs), immunosuppressants (ISS) use and follow up. CD-IFX pts. were matched with $C D-C$ showing the same follow up length $( \pm 5 \mathrm{yrs})$, not necessarily requiring that CD-C were in follow up on October 2004. Therefore, CD-C and their matched CD-IFX pts. not in follow up on October 2004 were excluded from the present study. A lower proportion of CD-IFX pts. were lost to follow up on October 2005, thus reducing the number of pts. included in the present study. The following events from October $2004-$ October 2005 were recorded: new diagnoses of cancer, outcome of previously diagnosed cancer, new IFX or ISS use. Results. Matched-pair CD-IFX and CD-C still in follow up on October 2005 include 458 pts (229 CD-IFX and 229 matched-pair CD-C). Among these 458 pts, the frequency of newly diagnosed cancer from April 1999 to October 2004 was comparable in the CD-IFX ( $8 / 229$ pts. $3.49 \%)$ and in the CD-C (7/229, 3.05\%; p=1.00) group. Among the whole group of 458 pts., new diagnoses of cancer from October 2004 to October 2005 was observed in 3 pts., including: 1 breast cancer (F, age 57, no ISS use) in the CD-IFX group; 1 cutaneous T cell lymphoma (F, age 43, on AZA for 5 yrs), 1 duodenal adenocarcinoma (M, age 48, no ISS use) in the CD-C group. All these 3 pts. are currently in follow up. Conclusion. Present findings from a multicenter matched-pair study in a cohor of $\mathrm{CD}$ pts. matched for clinical variables, support previous observations from a shorter follow up period, suggesting that the frequency newly diagnosed cancer is comparable in pts. treated or untreated with IFX. However, the finding of new cases of cancer during the last year recommend the need of a longer follow up to appropriately assess the risk of cancer in this cohort of $\mathrm{CD}$ pts.

\section{W1158}

Does Infliximab Influence Surgical Morbidity Or Long-Term Outcome of Ileal Pouch-Anal Anastomosis in Patients with Ulcerative Colitis?

Stefanie J. Schluender, Eric A. Vasiliaukas, Marla Dubinsky, Konstantinos A. Papadakis, Andrew Ippoliti, Stephan Targan, Phillip Fleshner

PURPOSE: As infliximab has recently been approved for the treatment of patients with refractory ulcerative colitis (UC), physicians will be increasingly faced with operating on patients who have failed therapy with this potent immunosuppressant medication. The effects of infliximab on short-term and long-term outcomes after colectomy are unknown The aim of this study was to compare the incidence of postoperative complications and pouchitis in UC patients treated with and without infliximab prior to surgical resection METHODS: A retrospective review of patients undergoing ileal pouch-anal anastamosis (IPAA) for medically-refractory UC over the 12-year period ending October 2005 was performed. Two patient groups were identified. Group A patients $(n=17)$ had failed infliximab treatment. Group B patients $(n=134)$ were never treated with infliximab. Postoperative medical complications, surgical morbidity, and length of hospital stay (LOS) were compared between patient groups using chi-square analysis or Wilcoxon nonparametric tests. Longterm outcome was assessed prospectively and included acute pouchitis (AP) and chronic pouchitis $(\mathrm{CP})$. Pouchitis was diagnosed using clinical symptoms and confirmed in al patients with pouchoscopy. RESULTS: A two-stage IPAA was initially performed in 112 patients $(74 \%)$ and an initial subtotal colectomy (STC) with ileostomy (three-stage IPAA) was used in 39 patients (26\%). Procedures performed in Group A included 15 (88\%) IPAA and $2(12 \%)$ initial STC, and in Group B included 97 (72\%) IPAA and 37 (28\%) initia STC. There was no mortality in either patient group. Postoperative complications were observed in 43 patients (28\%), with no significant difference observed between Group A patients $(n=6,37 \%)$ and Group B patients $(n=37,27 \%)$. Overall median LOS did not differ between the two patient groups (Group A - 6 days; range, 4 to 73 days; Group B - 7 days 
range, 3 to 53 days). The incidence of either AP or $\mathrm{CP}$ was also not significantly different between Group A $(12 \%, 7 \%)$ and Group B $(19 \%, 10 \%)$ patients. CONCLUSION: These results suggest that preoperative treatment with infliximab does not significantly increase the incidence of postoperative complications or change the incidence of pouchitis in patients undergoing surgical treatment for medically-refractory UC.

\section{W1159}

High Incidence of IBD in Multiple Affected Families: Results from a Prospective Study and Association with Known Environmental and Genetic Risk Factors

Marie G. Joossens, Julien Branche, Delphine Allorge, Boualem Sendid, Herbert J. van Kruiningen, Sofie Joossens, Karolien Claes, Nele van Schuerbeek, Paul Rutgeerts, Peggy Vandewalle, Annie Standaert-Vitse, Corinne Gower, Daniel Poulain, Franck Broly, JeanFrederic Colombel, Mathias Chamaillard, Severine Vermeire

INTRODUCTION An exceptionally high frequency of familial Crohn's disease (CD) has been reported in Northern France and Belgium. We previously performed a study on environmental risk factors in 45 families with at least three members affected with CD. AIMS \& METHODS We hypothesized that environmental and genetic risk factors cluster in these multiple affected families and therefore prospectively followed up on the health status of the unaffected family members of these families. Four years after the original study, a telephone and a questionnaire survey were performed to identify if new diagnoses of IBD had arisen in the multiple affected families (including $166 \mathrm{CD}$ patients and 261 unaffected relatives) as well as in 15 matched healthy control families without IBD and 27 matched sporadic IBD families (only one case affected) who also took part in the initial study. Known genetic risk factors (CARD4, CARD8, CARD15, TLR4, DLG5) and multiple anti-microbial antibodies, anti-Saccharomyces Cerevisiae (ASCA), anti-Candida albicans (ACAA), anti-chitobioside and anti-laminaribioside carbohydrate (ACCA and ALCA) and anti-porin (OMP), were determined on blood samples of the initial visit four years ago. RESULTS All families returned our survey. Unlike sporadic and healthy control families, eight new diagnoses of IBD (6 CD and 1 UC first degree relatives and 1 CD in a fourth degree female) were made in seven multiple affected families. The age at diagnosis in these newly-diagnosed relatives varied between 12 and 44 years. Of the newly diagnosed $C D$ patients for whom full data were available, 3 patients carried a CARD15 variant (patient A Arg702Trp and patients B and C a 1007fs mutation) as well as NODl mutations (A: C27606T and C45343T; B homozygote and $C$ heterozygote for $C 32656$ TTindel). Two $C D$ patients $(C, D)$ and the new UC patient carried a CARD8 Cys10* mutation. CD patients A and B expressed positive ASCA and ALCA, A in combination with OMP antibodies, B with ACAA and ACCA. The UC patient also carried ASCA. Two of the CD patients (B, D) were ex-smokers, one $(C)$ was a current smoker. CONCLUSION Over a period of four years, we identified eight new cases of IBD in 45 multiple affected families and none in 27 simplex CD families nor in 15 healthy control families. Compared to the reported incidence of IBD of 10-15/100.000/year in our region, these findings show a 30 -fold increase in incidence. Unaffected relatives in multiple affected families are therefore at much higher risk to develop IBD. We observed a high prevalence of genetic, environmental and serological markers but the interplay between these factors in the pathogenesis of the disease remains unknown.

\section{W1160}

Familial Distribution of Serological and Genetic Markers in Crohn's Disease Patients and Their Healthy Relatives

Marie G. Joossens, Julien Branche, Boualem Sendid, Delphine Allorge, Sofie Joossens, Nele van Schuerbeek, Karolien Claes, Paul Rutgeerts, Peggy Vandewalle, Annie StandaertVitse, Corinne Gower, Franck Broly, Daniel Poulain, Jean-Frederic Colombel, Severine Vermeire, Mathias Chamaillard

BACKGROUND Crohn's disease (CD) is a chronic inflammatory disease of the gastrointestinal tract. Both genetic (e.g. CARD15) and serological (e.g. ASCA for Anti-Saccharomyces cerevisiae Antibody) markers have been associated with Crohn's disease. However the genetic determinants of the serological markers associated with $\mathrm{CD}$ remain poorly understood. AIM $\&$ METHODS In multiple affected families a high prevalence of CD patients and serological markers is observed. We hypothesized that genetic variants might influence familial expression of these markers and therefore investigated 45 multiple affected families (with at least three members with CD), 27 matched sporadic IBD families and 15 matched healthy control families. Four novel serological tests were used to determine anti-outer membrane porin (OMP; INOVA, USA), anti-mannan (gASCA), anti-chitobioside and anti-laminaribioside carbohydrate (ACCA IgA, ALCA IgG) (IBDXtm line, Glycominds Ltd., Israel) antibodies in the sera of patients and healthy individuals. The serotypes for the ASCA IgG/A/M (Sendid B et al Clin Diagn Lab Immunol, 1996) and the new tests, and genotypes for CARD4 (complex In/del), CARD8 (C10X), CARD15 (R702W, G908R and 1007fs), TLR4 (A299G) and DLG5 (R30Q) were determined. We compared data of 166 CD patients (143 and 23 CD patients from multiplex and simplex families respectively), 247 healthy relatives (181 and 66 healthy relatives from multiplex and simplex families respectively) and 83 healthy controls. RESULTS The seroprevalence of ASCA IgG/A/M (71\%;38\%;4\%), gASCA $(60 \% ; 12 \% ; 6 \%)$ and ALCA IgG $(44 \% ; 13 \% ; 13 \%)$, but not of ACCA IgA $(40 \% ; 32 \% ; 24 \%)$ and OMP IgG $(36 \% ; 27 \% ; 14 \%)$ was significantly higher in CD patients compared to their firstdegree healthy relatives (HR) and to healthy controls (HC). Whereas healthy relatives of $\mathrm{CD}$ patients showed a higher seroprevalence of ASCA IgG/A/M compared to healthy controls $(\mathrm{p}=1.10-3)$, no significant difference was found between HR and HC for gASCA and ALCA IgG. Using intraclass analysis the clustering of serum levels for the different tests was higher in multiple (0.82) compared to sporadic families (0.55). Interestingly, in multiple affected families the difference in magnitude of serological response in gASCA and ALCA IgG titres between $C D$ patients and their HR $(p=1.10-10 ; p=1.10-18$; and $p=4.10-6$ respectively) was much higher compared to single affected families $(\mathrm{p}=5.10-2 ; \mathrm{p}=5 \cdot 10-3$; and $\mathrm{p}=5.10-2$ respectively). CONCLUSION Taken together these results indicate that, among the serological markers, gASCA and ALCA IgG are the most specific markers for disease in familial CD. Correlations with genetic determinants and/or phenotypes are currently investigated.

\section{W1161}

Are Non-Inflammatory (adenomatous or metaplastic) Polyps Rare in Ulcerative Colitis?

Venkataraman Subramanian, Ashwini Banerjee, Richard Pollok

Introduction: Risk of cancer in patients with ulcerative colitis (UC) is higher than the general population. The importance of adenomatous polyps in contributing to this increased cancer risk is unclear. Recent evidence suggests that the prevalence of adenomatous polyps are less common in this patient population. We aimed to ascertain the prevalence of adenomatous and metaplastic polyps in patients with ulcerative colitis and compare it with a control population. Methods: We performed a retrospective review of endoscopic and histological records of patients with a confirmed diagnosis of UC. A control group of patients undergoing colonoscopy for altered bowel habit or abdominal pain were used as a control group. All the data was obtained from the electronic patient data records of St George's hospital. Significance was calculated using standard T-tests of proportions for independent samples. Results: 20 (5.03\%) of 397 patients with UC had adenomatous polyps. 3 of these polyps were located outside the histological extent of the disease. In contrast $77(9.3 \%)$ of 828 controls had at least one adenomatous polyp. Adenomatous polyps were significantly less common in patients with UC ( $\mathrm{p}=0.004) .9(2.27 \%)$ of patients with UC and $43(5.2 \%)$ of controls had a metaplastic polyp ( $\mathrm{p}=0.002)$. Inflammatory polyps on the other hand were significantly more common in patients with UC. Conclusions: Both adenomatous and metaplastic polyps occur less frequently in patients with UC. Adenomatous polyps therefore seem unlikely to contribute to the increased risk of colorectal cancer noted in patients with UC. The reduced incidence could be related to 5-ASA use, genetic factors or related to the disease process itself and further studies are warranted to evaluate these factors.

\section{W1162}

Nodular Regenerative Hyperplasia with Portal Hypertension in Patients with Inflammatory Bowel Disease Treated with Azathioprine

Gwenola Vernier-Massouille, Jacques Cosnes, Marc Lemann, Philippe Marteau, David Laharie, Guillaume Cadiot, Yoram Bouhnik, Martine de Vos, Arnaud Bourreille, Walter Reinisch, Jean F. Colombel

Azathioprine (AZA) and 6-mercaptopurine are commonly used to treat inflammatory bowel disease (IBD). Treatment of IBD with a closely related compound 6-thioguanine (6-TG) resulted in the frequent occurrence of a serious adverse event, nodular regenerative hyperplasia (NRH) of the liver that could lead to portal hypertension (PHTN). This observation raised the level of concern for the potential hepatotoxic effects of AZA. Aims: This retrospective study was performed to assess the characteristics, clinical course and potential risk factors of NRH in IBD patients treated with AZA in our centers. Results: Thirty cases of NRH (24 M, 6F) were identified between 1994 and 2005 in 8 centers. Twenty-seven patients had Crohn's disease (CD) and 3 had ulcerative colitis. CD location was the small bowel in 15, the colon in 8 and both the small bowel and colon in 4. IBD mean duration was 14.8 yrs (1-39). Seventeen patients had one $(n=5)$ or multiple $(n=12)$ surgical resections. The median time between the start of AZA and diagnosis of NRH was 47 mos (6-187). Prothrombotic disorders were present in 10/14 explored patients including hyperhomocysteinemia in 6 (with 2 patients homozygous for C677T variant of MTFHR gene) and protein C or S deficiency in 5. The median dose of AZA was $2 \mathrm{mg} / \mathrm{kg} / \mathrm{d}$ (1.5-3). In 6 patients in whom it was available, median 6-thioguanine nucleotide concentration was 215 pmol/8.108 erythrocytes (68-352). NRH was suspected on clinical and/or morphological evidence of PHTN in 16 , abnormal liver function tests in 9 and/or a thrombocytopenia in 11 and confirmed by a liver biopsy in all patients. PHTN was then found in all but 3 patients. AZA was stopped in all patients. After a median follow-up of 16 mos (1-138), 13 patients developed complications of PHTN with variceal haemorrhage in 9. This required insertion of a transjugular intra-hepatic porto-sytemic shunt in 2 patients and a liver transplantation in one. The three patients with no evidence of PHTN at diagnosis had no clinical complications at follow up (15, 28 and 45 mos). Conclusion: NRH can be seen in patients treated with AZA for IBD and cause severe PHTN. Male gender, small bowel resection and/or involvement, and prothrombotic conditions seem to be risk factors. Clinicians should be aware of this serious complication, closely monitor liver function tests and platelet counts, and have a low threshold to perform MRI and/or liver biopsy in their patients with IBD treated with AZA.

\section{W1163}

The Use of Immunomodulators and Biologics in Inflammatory Bowel Diseases (IBD): A Cross-Sectional French Nationwide Cohort 2004-2005

Laurent Beaugerie, Fabrice Carrat, Bouvier Anne-Marie, Nicole Brousse, Franck

Carbonnel, Jean-Frederic Colombel, Jean Faivre, Jean-Francois Flejou, Marc Maynadie, Jean-Louis Dupas, Jean-Pierre Gendre, Philippe Godeberge, Jean-Pierre Hugot, Marc Lemann, Stephane Nahon, Jean-Marc Sabate, Gilbert Tucat

Introduction: The use of immunomodulators and biologics in IBD was initially restricted to patients with refractory Crohn's disease (CD). Immunomodulators are now regarded as the reference maintenance treatment of $\mathrm{CD}$ and an alternative to surgery in patients with ulcerative colitis (UC). Few data are currently available on the use of these agents in gastroenterological practice. Patients and Methods: This cross-sectional nationwide French cohort CESAME was originally designed to prospectively assess the risk of cancers associated with the use of immunomodulators in IBD. All French gastroenterologists were invited to participate in the study. Among them, 819 (322 and 497 with hospital and private practice, respectively) included in the cohort all consecutive IBD patients seen in their practice between May 2004 and May 2005. At the end of the inclusion period, 20,919 patients were enrolled in the cohort (12,463 with CD, 7,679 with UC and 777 with indeterminate colitis). The male/female ratio was 0.8 and 1.0 for $\mathrm{CD}$ and $\mathrm{UC}$, respectively. The mean age of the patients was $40 \pm 16$ yrs $(m+S D)$. The mean time from diagnosis to the entry into the cohort was $8 \pm 8$ yrs. Results: Among patients with CD, 56\% ever received thiopurines (38\% and $18 \%$ for current and former use, respectively), $10 \%$ ever received methotrexate (MTX) (5\% and 5\%), and $15 \%$ ever received anti-TNF $\alpha$ therapy (7\% and $8 \%$ ). Among patients with UC, $26 \%$ 
ever received thiopurines (18\% and $8 \%$ ), $2 \%$ and $1 \%$ ever received MTX and anti-TNF $\alpha$ therapy, respectively. The proportion of IBD patients who ever received thiopurines according to the time between diagnosis and inclusion in the cohort increased from 11 to $50 \%$ for intervals from 1 to 5 years, then ranged between 50 and 55\% for longer intervals. Conclusions: These data show that currently in France: more than half of the $\mathrm{CD}$ patients ever received thiopurines whereas 10 and $15 \%$ of them were ever treated by MTX and antiTNF $\alpha$, respectively; one quarter of the UC patients ever received thiopurines. In both $C D$ and UC, the proportion of patients treated by thiopurines according to time from diagnosis increases progressively during the 5 first years of the disease, but does not significantly vary thereafter. The cancer incidence study on progress in the CESAME cohort should allow us to establish the relative risks of cancers associated with the current use of immunodulators and biologics.

\section{W1164}

Proton Pump Inhibitor Use and the Risk of Flare of Inflammatory Bowel Disease

Faten Aberra, James D. Lewis, Gary R. Lichtenstein, Yu-Xiao Yang

Background: Gastric acid suppression by proton pump inhibitors (PPI) and histamine receptor antagonists (h2ra) is associated with increased small bowel bacterial colonization and enhanced intestinal viability of pathogenic bacteria. Published data suggest intestinal microorganisms may play a role in the pathogenesis and course of inflammatory bowel disease (IBD). We hypothesized that hypochlorhydria may increase risk for flare of IBD by altering the intestinal bacterial flora. Our objective is to determine if gastric acid suppression by PPIs increases the risk of flare of IBD. Methods: Subjects with IBD were identified from the United Kingdom General Practice Research Database, a clinical outpatient database, from1988-2002. A case-crossover design was used. Onset of a flare was identified by a new prescription for corticosteroids (CS) or 5-aminosalicylate (5-ASA) following a remission period, validated in a prior study. A remission period started when there had been no prescription for CS or 5-ASA for $>=122$ days. We assessed exposure to a PPI within 90 days of a randomly chosen index date within each remission and the date of flare. To address potential confounding by indication we also assessed chronic PPI exposure (defined by PPI use within a 90 day window before index date, of $>=28$ days in duration, last use within 7 days before index date and $>=1$ prescription for PPI between 60-90 days before index date) and PPI dose. Conditional logistic regression was used to assess the association between PPIs and flare of disease. Potential confounders assessed were exposure to h2ra, azathioprine, NSAIDs, aspirin, antibiotics and calendar year of flare and remission. Results: There were 1134 flares/1144 remission periods among 782 Crohn's disease (CD) subjects and 1928 flares/1943 remission periods among 1235 ulcerative colitis (UC) subjects. The adjusted OR for CD flare associated with h2ra was 0.58(95\% CI:0.32-1.08). The adjusted OR for UC flare associated with h2ra was $1.55(95 \% \mathrm{CI}: 0.93-2.59)$. Any PPI exposure was associated with an adjusted OR of $1.45(95 \% \mathrm{CI}: 0.81-2.59)$ for CD flare and adjusted OR of 2.30(95\% CI:1.16-4.55) for UC flare. Chronic PPI exposure was associated with an adjusted OR 1.11(95\%CI:0.44-2.76) for CD flare and 3.23(95\%CI:1.12-9.34) for UC flare. Increased risk of flare was also appreciated with higher doses of PPI for UC (test for trend $\mathrm{p}=0.02$ ) and not for $\mathrm{CD}$ (test for trend $\mathrm{p}=0.12$ ). Conclusions: PPI exposure was associated with increased risk of flare in UC, but not in CD. Further exploration is suggested to validate these initial findings and explore the mechanism by which PPIs may increase risk of flare of disease.

\section{W1165}

Leukopenia As a Marker for Susceptibility of Infection in IBD Patients On Immunosuppressive Therapy

Scott O. Levin, Amy E. Barto, Faten N. Aberra, Chinyu Su, Gary R. Lichtenstein

Background: Leukopenia (WBC<4x103) has long been a marker for immunosuppression and a predictive value of one's susceptibility to infection. Leukopenia has not been investigated as an independent risk factor for IBD patients on immunomodulator therapy. We hypothesize that in the presence of leukopenia, patients taking immunomodulators (IMMs) alone or in combination with corticosteroids (CS), and infliximab have an increased risk of infectious complications. Methods: A computer generated list using ICD 9 codes for ulcerative colitis (UC) and Crohn's disease (CD) patients seen at our institution between 2000-2005 was compiled. Patients were divided into the following five groups: IMMs alone, IMM and CS, IMM, CS, and infliximab, IMM and infliximab, CS and infliximab, and infliximab alone. Leukopenic patients in each group were then compared to non-leukopenic patients. WBC values were recorded along with infectious complications that were acquired during the same timeline. (Severity of infection, hospitalization, duration of leukopenia, smoking and other co-morbidities were included as other possible contributing/risk factors.) Leukopenia was defined as a $W B C<4 \times 103$. Infection was defined as bacterial and viral based on clinical features documented. Results: Data was collected on 248 patients (50 UC/198 CD; 142male/ $116 \mathrm{female}$ ). Average age was 41 years (range: $19-90$ years). Average duration of disease was 14.8 years (range: $1-59$ years). $30 \%$ of UC patients and $42 \%$ of CD patients developed infections over an average follow-up time of 18 months (range: 1-70 months). Infection risk was compared for leukopenic and non-leukopenic patients within the different medication groups. The incidence rate ratio (IRR) for infections was $1.36(\mathrm{p}=0.52)$ for IMM alone, 3.70 ( $\mathrm{p}=0.24$ ) for both CS and IMMs, 2.51 ( $\mathrm{p}=0.23$ ) for CS, IMMs and infliximab, and $1.19(\mathrm{p}=0.80)$ for IMMs and infliximab. The CS and infliximab group and infliximab alone group could not be analyzed due to small sample sizes. Conclusion: Comparison of infectious risk between leukopenic and non-leukopenic patients in all medication groups showed no significant difference. A future study using severe leukopenia as a variable (WBC $<2$ x 103) may demonstrate differences between medication groups.

\section{W1166}

Ulcerative Colitis: Annual Costs of Care from a Payer Perspective Songkai Yan, Clark Paramore, Steven W. Blume, Kathy Fraeman, Mohan Bala

Objective: To assess the annual cost of care for patients with ulcerative colitis (UC) from a medical claims database. Methods: Using the PharMetrics claims database, patients with diagnosis of UC (ICD-9 code 556.x) in the year 2001 (between 1/1/2001 and 12/31/2001) were included in this analysis. Claims in 2002 (between 1/1/2002 and 12/31/2002) were used to categorize these patients into three mutually exclusive disease severity groups and conduct cost analysis. These groups were: Group 1, required hospitalization for UC; Group 2 , required chronic aggressive pharmacotherapy (i.e. corticosteroids or immnunosuppressants) for $\geqq 4$ months; Group 3, all remaining patients. Patients were matched (1:4) to controls (those without UC) based on age, gender, region, and previous time-in-plan. Direct costs based on reimbursement amount by place of service were reported. Results: 7174 patients were analyzed: $323(4.5 \%)$ in Group 1, $578(8.1 \%)$ in Group 2, and $6273(87.4 \%)$ in Group 3. Average age of patients was 47.3 years and $46.7 \%$ were men. Mean annual total cost for all patients were $\$ 7820$, compared with $\$ 3,661$ for controls. Group 1 patient incurred the highest mean cost $\$$ \$39782), while Groups 2 and 3 incurred \$12496 and $\$ 5744$, respectively (Table 1). Inpatient hospitalization costs constituted the largest component $(\$ 2860,36.6 \%)$ of the mean annual total cost for all patients. The next highest cos components were prescription medications $(\$ 1718,22.0 \%)$, outpatient visits ( $\$ 1219$, $15.6 \%$ ) and physician office visits $(\$ 1134,14.5 \%$ ). Conclusions: UC is associated with high cost of care that is more than twice of the cost for those without UC. Patients requiring hospitalization incurred the highest cost. New therapies that can reduce hospitalizations have the potential to decrease overall cost of care for UC.

Table 1. Mean 2002 total costs for patients with a UC diagnosis in 2001

\begin{tabular}{|c|c|c|c|c|}
\hline Place of service & Group 1 & Group 2 & Group 3 & All patients \\
\hline Inpatient & $\$ 29,140$ & $\$ 2,821$ & $\$ 1,510$ & $\$ 2,860$ \\
\hline Outpatient & $\$ 2,631$ & $\$ 1,771$ & $\$ 1,095$ & $\$ 1,219$ \\
\hline Physician office & $\$ 2,353$ & $\$ 2,346$ & $\$ 960$ & $\$ 1,134$ \\
\hline Prescription drug & $\$ 2,754$ & $\$ 3,985$ & $\$ 1,455$ & $\$ 1,718$ \\
\hline ER & $\$ 469$ & $\$ 118$ & $\$ 110$ & $\$ 127$ \\
\hline Other & $\$ 2,435$ & $\$ 1,455$ & $\$ 613$ & $\$ 763$ \\
\hline Total & $\$ 39,782$ & $\$ 12,496$ & $\$ 5,744$ & $\$ 7,820$ \\
\hline
\end{tabular}

W1167

The Manitoba IBD Cohort Study: Assessing the Nature of Medication Adherence

Charles N. Bernstein, Jason P. Ediger, Lesley A. Graff, Patricia Rawsthorne, Linda Rogala, Norine Miller, Kathleen Deering, Cory McPhail, John R. Walker

This study reports medication adherence data from the Manitoba IBD Cohort Study, a longitudinal, population-based study of multiple determinants of health outcomes in IBD in those diagnosed within 7 years at enrollment. Methods: Data were available from 334 subjects. Adherence was measured with a validated multi-item self-report assessment. Differences in adherence were tested across levels of disease activity (defined by Harvey Bradshaw and the Powell Tuck activity indices) and diagnosis. Further testing also explored potentia correlates such as age, gender, beliefs about medication, memory strategies, obstacles to compliance and psychological factors. Results: The most commonly reported forms of nonadherence were taking less than instructed ( $12 \%$ often/very often), altering the dose $(10 \%)$ using it only when feeling unwell (10\%), and forgetting to take it when scheduled (9\%) The most commonly reported obstacles to taking medication regularly were cost (often/very often a problem) (25\%), a sense that taking medicine is a reminder that one has a health problem (13\%), unpleasant side effects $(12 \%)$, and uncertainty about whether the medicine is helpful (11\%). Participants reporting more obstacles to medication compliance demonstrated significantly poorer adherence $(r=0.371, p<0.001)$. Participants with active disease reported more difficulty with adherence than those with inactive disease $(F(1,327)=4.30, p<$ $0.05)$. There were no significant differences in adherence between genders or IBD subtypes. Poor adherence was moderately associated with higher levels of stress $(r=0.228, p<0.001)$ lower levels of psychological well-being $(r=-0.247, p<0.001)$, and younger age $(r=$ $0.195, \mathrm{p}<0.001$ ). While $62 \%$ of respondents agreed that the medication prevented them from getting worse, $62 \%$ expressed concerns about long term effects, $30 \%$ expressed concern about becoming dependent, and 39\% reported that they worried about having to take medication. Many thought doctors used too many medicines (40\%) and placed too much trust in those medicines (27\%). The most common strategies reported to facilitate taking the medication were daily routine cues like meals or bedtime (78\%) and dispensers with daily compartments (17\%). Conclusions: Adherence to medication treatment is a problem in the treatment of IBD as in other disorders. It is helpful for the physician to ask specifically about problems with adherence in a non-judgmental way, in order to address specific concerns related to less than optimal adherence, and subsequently improve treatment outcome.

\section{W1168}

Increasing Impact of Clostridium Difficile On Inflammatory Bowel Disease: A Tertiary Referral Center Experience

Mazen Issa, Sue Skaros, Dawn B. Beaulieu, Lydia R. Weber, Aravind S. Vijayapal, Mary Beth Graham, Joshua F. Knox, Jeanne Emmons, Ossama A. Hatoum, Mary F. Otterson, David G. Binion

Introduction: Diarrhea and pseudomembranous colitis due to the anaerobic spore-forming bacillus Clostridum difficile (C. diff) is a major health problem, and recent reports suggest an increase in C. diff cases throughout the U.S. (IDSA Emerging Infections Network; www. (1) 
idsociety.org). C. diff infection can impact clinical course in pts with inflammatory bowel disease (IBD: Crohn's disease (CD); ulcerative colitis (UC)). We reviewed a tertiary referral center database regarding the detection of $\mathrm{C}$. diff and clinical outcome in patients with IBD. Methods: This was a retrospective observational study of all consecutive IBD pts followed at a single center. History of a positive stool analysis for C. diff toxin A and B (2004 - 2005) was used to identify subjects. Demographic information, diagnosis and anatomic location of disease, concomitant IBD therapy, hospitalizations and surgeries were recorded. Results: C. diff was detected in $3 \%(23 / 766)$ of the IBD pts evaluated in 2004 and $10 \%(98 / 958)$ of pts evaluated in $2005(\mathrm{p}<0.001)$. Among the 40 new C.diff diagnoses in 2005, there were $11 \mathrm{UC}$ and $29 \mathrm{CD}$, mean age $38.9 \pm 14 \mathrm{y}(\mathrm{SD})$. C. diff positive IBD patients were also more likely to be female ( $26 \mathrm{vs}$. 14). Although the number and rate of IBD hospitalizations related to $C$. diff remained unchanged between $2004(n=14)$ to $2005(n=27 ; p=N S)$ the number of colectomies in C.diff positive IBD pts decreased from 5/8 (63\%) in 2004 to $4 /$ $40(10 \%)$ in $2005(\mathrm{p}<0.001)$. Colonic involvement was found in $79 \%$ of the CD pts, and $85 \%$ of the total IBD cohort with C.diff. Maintenance immunomodulators (azathioprine, methotrexate and/or infliximab) were used by $88 \%$ of the pts who contracted C.diff coinfection. Thirteen of the pts were in remission on stable regimens of immunomodulators (10 pts) or infliximab ( 3 pts) when they experienced disease flare at the time of C.diff diagnosis. The rate of C.diff ELISA stool detection was: 1 sample (54\%), 2 samples (75\%), 3 samples (78\%), 4 samples (92\%). Conclusions: Co-infection with C. diff in IBD pts has increased 3 fold from 2004 to the present. C.diff was associated with flare activity in pts who were previously on stable maintenance regimens, including infliximab and immunomodulators. The decreased rate of colectomy noted in IBD pts infected with C.diff may reflect increased awareness and rapid detection and treatment of pts with appropriate antibiotics. C.diff appears to preferentially affect pts with prior histories of chronic inflammation in the colonic mucosa. Increased awareness of the potential for $\mathrm{C}$. diff to impact clinical course in IBD patients is warranted and aggressive diagnostic evaluation is recommended.

\section{W1169}

Increased Protein Catabolism Correlates with Increased Disease Activity in Children with Crohn's Disease

Ashish Patel, John M. Andersen, Dana Hardin

Introduction: Crohn's disease is a condition of chronic relapsing and remitting inflammation involving the gastrointestinal tract with a propensity for the ileocecal region. Growth failure is a major symptom of Crohn's disease in children. Previous studies have documented that in other chronic inflammatory diseases with impaired growth, such as cystic fibrosis, whole body protein turnover is catabolic and this increased catabolism correlates with worsened clinical status. No pediatric studies have correlated the protein turnover status of steroid naive prepubertal Crohn's disease patients with the clinical perception of disease as scored by an activity index such as the pediatric Crohn's disease activity index (PCDAI). Hypothesis: Crohn's disease negatively impacts whole body protein turnover resulting in catabolism which correlates with the clinical perception of disease activity as measured by the PCDAI Methods: Steroid naive prepubescent (Tanner I or II) patients diagnosed with Crohn's disease, without any co-morbid conditions, were eligible for this study. Measurements of whole body protein turnover were estimated using [1-13C] leucine, a previously described and validated methodology (Hardin et al, J of Clinical Endocrinology \& Metabolism 2001). The PCDAl has been validated in the pediatric Crohn's population as a reliable index of disease activity. The scoring system uses patient reported data (frequency of stools, abdominal pain, general well-being), objective data (hematocrit, erythrocyte sedimentation rate, albumin), and clinician reported data (weight, height, abdominal exam, perirectal disease, extra-intestinal manifestations). The PCDAI was calculated at the time of the study. The PCDAI score and the rate of protein turnover were correlated using Pearson's correlation. Results: All five patients studied so far displayed a marked increase in the rate of total body protein catabolism, an average rate of $321.7 \mu \mathrm{mol} / \mathrm{kg} / \mathrm{hr}$ (range 171.4-540.3 $\mu \mathrm{mol} / \mathrm{kg} / \mathrm{hr}$ ), when compared with age and Tanner matched children $(80-85 \mu \mathrm{mol} / \mathrm{kg} / \mathrm{hr})$. The correlation coefficient for PCDAl scores and protein turnover was 0.623 . Conclusion: Protein catabolism in children with Crohn's disease is markedly increased in active disease and correlates with disease activity as scored by the PCDAI. We speculate that increased protein catabolism in children with Crohn's disease contributes to growth failure.

\section{W1170}

Familial Occurrence of Inflammatory Bowel Disease: A Korean Study Suk-Kyun Yang, Jong Beom Park, Jeong-Sik Byeon, Eui-Ryun Park, Gyoo Moon, Seung Jae Myung, Won Kap Park, Seo Goo Yoon, Hyun Sik Kim, Jong Gyun Lee, Jin Ho Kim, Young Il Min, Kwang Yeon Kim

Background and Aims: Few data are available about familial aggregation of inflammatory bowel disease (IBD) in Asia. The aim of our study was to determine the risk of familial aggregation of IBD among first-degree relatives of patients with ulcerative colitis (UC) or Crohn's disease (CD) in an ethnically distinct Korean population. Methods: A total of 1440 unrelated patients with UC $(n=1043)$ or $C D(n=397)$ were enrolled in the present study. All patients were interviewed and answered to a detailed questionnaire for the evaluation of the family pedigrees and the occurrence of IBD in their first-degree relatives. Familial aggregation of IBD was evaluated in several different ways including family history, prevalence, population relative risk, and lifetime risk of IBD in first-degree relatives. Results: The male to female ratio was $0.99: 1$ among the probands with UC, whereas it was $2.13: 1$ among those with $\mathrm{CD}$. The age at diagnosis was significantly younger in the probands with $\mathrm{CD}(24.0 \pm 8.3$ years $)$ than those with $\mathrm{UC}(37.5 \pm 13.1$ years $)$. A positive first-degree family history of IBD could be confirmed in 27 probands (1.88\%): 21 (2.01\%) of 1043 with UC and $6(1.51 \%)$ of 397 with CD. Twenty-five probands had only one affected first-degree relative, and 2 had 2 , resulting in 29 first-degree relatives with IBD. The overall concordance for type of IBD among first-degree relatives was $82.8 \%$. The crude prevalence of IBD in first-degree relatives of probands with IBD was $0.31 \%: 0.10 \%$ in parents, $0.31 \%$ in offspring, and $0.45 \%$ in siblings. There was no difference in the prevalence of IBD between first-degree relatives of UC probands and those of CD probands ( $0.31 \%$ vs. $0.35 \%$ ). Also, the prevalence of UC among first-degree relatives of UC probands was similar to that of $C D$ among firstdegree relatives of $\mathrm{CD}$ probands $(0.27 \%$ vs. $0.23 \%)$. The age- and sex-adjusted population relative risk of IBD was 13.8 in first-degree relatives of probands with IBD. The age-adjusted empirical risk (i.e., lifetime risk) of developing IBD was $0.52 \%$ for all first-degree relatives of UC probands, and $0.67 \%$ for those of $\mathrm{CD}$ probands. The lifetime risk of IBD in firstdegree relatives of probands with IBD was $0.54 \%: 0.12 \%$ in parents, $0.79 \%$ in siblings, and $1.43 \%$ in offspring. Conclusions: Although a positive family history, prevalence, and lifetime risk of IBD among first-degree relatives of Korean patients are much lower than those of Western patients, the population relative risk in first-degree relatives is about the same between Koreans and Westerners. This means that a positive family history is an important risk factor for IBD in Koreans as well as in Westerners.

\section{W1171}

Preoperative Steroid Use in Patients with Inflammatory Bowel Disease and Risk of Postoperative Complications: Meta-Analysis of Observational Studies Venkataraman Subramanian, Sonia Saxena, Richard Pollok

Introduction: Patients with inflammatory bowel disease (IBD) are frequently treated with steroids. Almost a third of them will require surgery within a year of treatment with steroids Studies examining postoperative complication risk associated with use of steroids in patients with IBD have yielded conflicting results. The purpose of this meta-analysis was to estimate the risk of postoperative infectious complications following abdominal surgery in patients with IBD on steroids at the time of surgery. Methods: We searched electronic databases Pubmed, EMBASE, CINAHL, Ovid, Zetoc and Ingenta, and hand searched citations from the reference lists in all the articles identified. Studies were included if patients had been on steroids in the month preceding surgery, compared with a control group. Studies that evaluated infectious postoperative complications in the two groups with duration of postoperative follow up of a maximum of 30 days or the hospitalization period were included in the analysis. Data for calculation of odds ratios (OR) had to be provided or could be calculated from the tables or figures for the study to be included. We used the random effects model to calculate pooled odds ratio and 95\% confidence intervals. Results: All 6 studies that met the inclusion criteria were observational studies. Pooled analysis showed an increased risk of infectious postoperative complications among patients on steroids (OR $1.60,95 \%$ confidence interval 1.17-2.20). Conclusions: This is the first meta-analysis on risk of post-operative infectious complication in patients with IBD taking steroids. Our data indicates that there is an increased risk of infectious complications following the pre-operative use of steroids. This is consistent with data on pre-operative steroid use and risk of postoperative complications in other general medical patients.

\section{W1172}

Remission in Ulcerative Colitis Patients Is Associated with Improved Employment and Disability Status, Work Hours, and Productivity William J. Sandborn, Songkai Yan, Brian G. Feagan, Walter Reinisch, Paul Rutgeerts, Graham Radford-Smith, Stephen Xu, Debra Eisenberg, Allan Olson, Jean-Frederic Colombel

Purpose: To examine the impact of remission on employment and disability status, hours per week actually worked (HPWAW), and productivity, in 2 controlled clinical trials in patients (pts) with ulcerative colitis (UC). Methods: 728 pts with moderately to severely active UC were enrolled in ACT 1 and ACT 2.Pts were randomized to placebo, infliximab 5, or 10mg/ $\mathrm{kg}$. Treatment was administered at baseline, wks 2, 6, and then $\mathrm{q} 8$ wks. Pts answered a questionnaire regarding employment and disability status, and work hours in the past 8 wks, at baseline and wk 30. In ACT 1,pts completed a scale ranging from 0(not at all productive)to 100 (fully productive) to assess their productivity at work or school in the 2 wks prior to the visits.Employment and disability status was compared for pts in remission and those not in remission at wk 30 using Fisher's exact test; the mean change of HPWAW and mean change of productivity were compared using analysis of variance on van der Waerden scores. Remission was defined as a Mayo score $\leq 2$ points, with no individual subscore $>1$.Analyses were performed on ACT 1 and ACT 2 combined data except for productivity. No treatment failure rules or other imputations were applied. Results: At baseline, $35.4 \%(257 / 727)$ of pts were not employed, and 7.0\%(51/728) were receiving disability compensation. The mean baseline HPWAW was only slightly more than half-time, and the mean baseline productivity was only about $2 / 3$ of fully productive(Table 1 ). Among pts who were not employed at baseline,more patients in remission at wk 30 were employed,vs those not in remission $(20.6 \%[14 / 68]$ vs. $8.3 \%[9 / 108], \mathrm{p}<0.001)$. Among pts who were receiving disability compensation at baseline, a greater percentage of those who were in remission at wk 30 were not receiving disability compensation,vs those not in remission $(58.8 \%[10 / 17]$ vs. $20.0 \%[3 / 15], p=0.060)$. Pts in remission at wk 30 had significantly greater increases in HPWAW $(\mathrm{p}<0.001)$ and productivity $(\mathrm{p}<0.05)$ vs those not in remission (Table 1$)$. Conclusion:In UC pts,remission is associated with improved employment and disability status, hours per week actually worked,and productivity at work or school.Achieving remission has important indirect cost implications in UC.

Table 1. HPWAW and productivity by remission status at week 30: Mean baseline and change from baseline at week 30

\begin{tabular}{|c|c|c|c|c|}
\hline & \multicolumn{2}{|c|}{ HPWAW* (ACT 1 and ACT 2) } & \multicolumn{2}{c|}{ Productivity (ACT 1) } \\
\hline & In remission & Not in remission & In remission & Not in remission \\
\hline $\mathrm{n}$ & 208 & 290 & 68 & 63 \\
\hline Baseline & 22.8 & 21.1 & 68.5 & 67.6 \\
\hline Change & 4.4 & 0.2 & 23.6 & 13.3 \\
\hline $\mathrm{p}$ value & \multicolumn{3}{|c|}{$<0.001$} & \multicolumn{2}{c}{$<0.05$} \\
\hline
\end{tabular}

* Set to 0 for patients who were not employed 
W1173

Crohn's Disease, Fatigue, and Infliximab: Is There a Role for Pro-Inflammatory Cytokines in the Pathogenesis of Fatigue?

Itta M. Minderhoud, Melvin Samsom, Bas Oldenburg

Background: Fatigue is an important feature in Crohn's disease (CD). In daily practice, treatment with infliximab often seems to result in a reduction of fatigue. We studied the effect of infliximab in $C D$ patients in order to elucidate the involvement of pro-inflammatory cytokines in the pathogenesis of fatigue. Methods: Fourteen patients with proven CD were enrolled and blinded for treatment. All patients received placebo at baseline, followed by infliximab after two weeks with a follow up of 4 weeks after the last infliximab infusion. Patients completed questionnaires on fatigue, depression, quality of life, and clinical disease activity at baseline and during follow up. Blood samples were drawn every two weeks. Results: After placebo infusion, fatigue scores decreased after a few days ( $\mathrm{p} \leqq 0.01$ ), but returned to baseline values within 14 days after this infusion. In contrast, the drop of fatigue scores following infliximab infusion was sustained until the end of the study ( $\mathrm{n} \leqq 0.05$ ). The quality of life was increased at the end of the study compared to baseline values ( $p \leqq 0.005)$, whereas depression scores were significantly decreased ( $\mathrm{p} \leq 0.01$ ). We did not find a correlation between fatigue and the levels of cytokines. Conclusions: The reported rapid reduction of fatigue after treatment with infliximab in daily practice is subjective to a placebo effect. The effect of infliximab on fatigue, however, persists while the placebo effect disappears after a short period of time, suggesting involvement of inflammation-related processes. A clear role of pro-inflammatory cytokines could not be substantiated.

\section{W1174}

Combination Therapy of Infliximab \& Azathioprine Prevents Disease Progression in Crohn's Disease

Shahida Din, Claire J. Cochrane, Colin L. Noble, Jack Satsangi, Ian D. Arnott

Introduction: The Vienna classification of $\mathrm{CD}$ categorised disease behaviour into a hierarchy of inflammatory, structuring \& penetrating types. $92 \%$ of patients have been shown to progress over a 20 year period. Infliximab induces mucosal healing \& clinical remission; it is unknown whether this drug reduces the progression of disease type. We aimed to examine the impact of episodic infliximab treatment on the progression of disease behaviour in $C D$. Methods: $73 \mathrm{CD}(38 \mathrm{~F})$ patients who received episodic infliximab were assessed, together with an age and sex matched control group comprising 73 patients who had received azathioprine for at least 4 months. Both groups had 2 years follow-up during which disease progression events were identified. Demographic details, disease characteristics and treatment history were collected. Kaplan-Meier survival curve analysis, the log rank test \& multiple logistic regression were used to test for differences and identify independent variables associated with disease progression. Results: Twice as many patients experienced progression of disease type in the control (azathioprine) group (10/50) compared with the infliximab group (5/42), $\mathrm{p}=0.165, \log$ rank test. Patients on both azathioprine and infliximab $(\mathrm{n}=17)$ had less disease progression compared to those on azathioprine alone $(0 / 17 \mathrm{v} 10 / 50, \mathrm{c} 2$, $\mathrm{p}=0.045)$. Surgical rates did not differ. Disease progression was also associated with significantly more disease flares $(\mathrm{p}=0.017)$ and higher surgical rates $(\mathrm{p}=0.000)$. Multivariate analysis identified smoking at treatment induction \& concomitant usage of corticosteroids as independent predictors of disease progression. Conclusion: Combination therapy with episodic infliximab and azathioprine retards the progression of disease in $C D$ and therefore may alter the "natural" history of this disease. Smoking at treatment induction and concomitant corticosteroid therapy are independent predictors of disease progression.

\section{W1175}

Differences in Medical Treatments Between African Americans and Caucasians with Inflammatory Bowel Disease: A Retrospoective Cohort Study Mark Flasar, Sandra Quezada, Priti Bijpuria, Grishma Joshi, Sharmeel Wasan, Raymond K. Cross

Introduction: Racial disparities between African Americans (AA) and Caucasians have been reported in several diseases. There has been limited study in Inflammatory Bowel Disease (IBD), where the incidence in AA is approaching that of Caucasians. This may be due to an underestimated prevalence of Ulcerative Colitis (UC) in AA as opposed to that in Crohn's Disease (CD). Our objectives were to assess the differences between AA and Caucasians with respect to medical therapies used to treat IBD as well as response to treatment. Methods: We identified outpatients with UC and CD evaluated at the University of Maryland from 1997 to 2005. We assessed the history of medications used and corresponding disease responsiveness by race. Results: We identified 413 patients; 102 were AA (25\%). The only significant difference in demographic variables was that AA were more likely to have been evaluated at the VA compared to Caucasians; $45 \%$ vs. $32 \%(\mathrm{p}=.0062)$. There was also a trend towards a higher proportion of males in the AA cohort as compared to Caucasians; $44 \%$ vs. $33 \%(\mathrm{p}=.055)$. AA patients were less likely to receive steroids; $55 \%$ compared with $68 \%(\mathrm{p}=0.0207)$. However, there was no difference between AA and Caucasians in rates of response to treatment with steroids or rates of steroid dependant disease. No difference was found in the use of or response to oral 5-ASA preparations by race. Conversely, AA were less likely than Caucasians to be treated with $6-\mathrm{MP} ; 28 \%$ vs. $40 \%(\mathrm{p}=0.031)$. Further, AA were less likely than Caucasians to be treated with Infliximab; $10 \%$ vs. $20 \%(p=0.029)$. Despite this, there was no significant difference in rates of response to treatment with either 6-MP or Infliximab by race. After adjustment for multiple variables, only age at diagnosis $<40$ (OR 2.51, 95\% CI 1.59-3.97) and evaluation at the VA (OR 0.36, 95\% CI 0.23-0.57) were associated with use of steroids. Age at diagnosis < 40 (OR 2.99, 95\% CI 1.83-4.86) and a diagnosis of UC (OR 0.43, 95\% CI 0.28-0.67) were associated with use of 6-MP, while there was a trend towards an association with $\mathrm{AA}(\mathrm{OR} 0.61,95 \% \mathrm{CI} 0.36-1.03)$. However, AA (OR 0.45, 95\% CI 0.21-0.95), having UC (OR 0.15, 95\% CI 0.07-0.30), and age at diagnosis $<40$ (OR 3.25, 95\% CI 1.56-6.77) were all significantly associated with the use of Infliximab therapy. Discussion: After adjustment for multiple factors, being AA was significantly associated with a decreased rate of Infliximab use in an IBD cohort. Further, there was a trend to a decreased rate of 6-MP therapy in AA as compared with Caucasians. Prospective studies are needed to further examine these findings.

\section{W1176}

Characterization of Clinical Phenotypes of Crohn's Disease of the Ileal Pouch Bo Shen, Victor Fazio, Feza Remzi, Ana Bennett, Rocio Lopez, Aaron Brzezinski, JeanPaul Achkar, Ioannis Oikonomou, Kerry Sherman, Marlene Bambrick, Bret Lashner

Crohn's disease $(\mathrm{CD})$ of the pouch can occur in patients with restorative procolectomy and ileal pouch-anal anastomosis (IPAA) originally performed for a pre-operative diagnosis of ulcerative colitis or indeterminate colitis. The clinical phenotypes of $\mathrm{CD}$ of the pouch have not been described and characterized. AIM: To characterize clinical, endoscopic, histologic features, quality of life (QOL), and treatment in patients with inflammatory, fibrostenotic, and fistulizing CD of the pouch. METHODS: The study was IRB approved. 60 consecutive patients with $C D$ of the pouch seen in the Pouchitis Clinic were enrolled, including 22 inflammatory $\mathrm{CD}, 16$ fibrostenotic $\mathrm{CD}$, and 22 fistulizing $\mathrm{CD}$. The clinical phenotypes of $\mathrm{CD}$ and concurrent cuffitis or afferent limb disease were diagnosed based on a combined assessment of clinical, endoscopic, radiographic, and histologic features. Demographic, clinical, endoscopic, histologic, QOL (Cleveland Global QOL, Irritable Bowel Syndrome-QOL and Short-Form Inflammatory Bowel Disease Questionnaires), and treatment variables were prosectively studied. A multivariable logistic regression analysis with stepwise selection was performed. Three separate analyses were performed; and for each analysis, the outcome of interest was having one of the phenotypes versus not having it. RESULTS: There were no statistical differences in QOL scores between the 3 clinical phenotypes of $\mathrm{CD}$. The results of multivariable analyses were shown in Table. CONCLUSION: As time went on after IPAA, the risk for inflammatory $C D$ decreased while the risk for fibrostenotic $C D$ increased. Diarrhea was a predominant symptom in inflammatory $\mathrm{CD}$, whereas obstructive symptoms were more common in fibrostenotic CD. Females and maybe patients with a pre-op diagnosis of indeterminate colitis were prone to fistulizing disease. Concurrent cuffitis was more common in fibrostenotic and fistulizing $C D$; and the afferent limb were more often involved by inflammatory and fibrostenotic CD. IMPLICATION: Natural history, etiopathogenesis, and clinical features of the 3 phenotypes of $\mathrm{CD}$ are different.

\begin{tabular}{|c|c|c|c|}
\hline \multirow{3}{*}{$\begin{array}{c}\text { Inflammatory Crohn's } \\
\text { Disease }\end{array}$} & Factor & $\begin{array}{c}\text { Odds Ratio }(95 \% \\
\text { CI) }\end{array}$ & $\begin{array}{c}\text { P } \\
\text { value }\end{array}$ \\
\cline { 2 - 4 } & Shorter duration IPAA (yrs) & $5.84(1.62,21.06)$ & 0.007 \\
\cline { 2 - 4 } & Diarrhea & $27.66(2.74,279.37)$ & 0.005 \\
\hline \multirow{2}{*}{$\begin{array}{c}\text { Fibrostenotic Crohn's } \\
\text { Disease }\end{array}$} & Absent cuffitis & $2.20(1.23,3.92)$ & 0.008 \\
\cline { 2 - 4 } & Longer duration IPAA (yrs) & $3.33(1.26,8.83)$ & 0.02 \\
\hline \multirow{3}{*}{ Fistulizing Crohn's Disease } & Obstructive Symptoms & $10.28(1.85,57.19)$ & 0.008 \\
\cline { 2 - 4 } & Female Gender & $13.65(1.52,122.31)$ & 0.02 \\
\cline { 2 - 4 } & Absent afferent limb disease & $12.46(2.52,61.64)$ & 0.002 \\
\cline { 2 - 4 } & cong-titis & $27.23(1.04,712.39)$ & 0.047 \\
\hline & Long-term corticosteroid use & $2.79(0.75,127.66)$ & 0.08 \\
\hline
\end{tabular}

\section{W1177}

Long-Term Follow-Up of Patients Hospitalized with Ulcerative Colitis Failing Outpatient Steroid Therapy

Eric K. Ganguly, Richard Zubarik, James A. Vecchio

Aim: To describe the one, three, and five-year colectomy rates for hospitalized patients with ulcerative colitis who have failed outpatient steroid therapy. To identify clinical predictor of colectomy. Methods: Records of patients admitted with ulcerative colitis to a single, university hospital from 1990 to 2003 were reviewed. Patients 18 years or older were included if they had been taking the equivalent of at least $30 \mathrm{mg}$ of prednisone for at least 3 days prior to hospitalization. Patients were excluded if they lacked a proven diagnosis o UC, were transferred from another hospital, or were admitted directly for surgery. Clinical and laboratory data were collected from hospital and clinic charts, as well as follow-up telephone interviews. Results: 40 patients were included (24 Male, 16 Female). The average age was 35 ; the average duration of UC was 2.5 years. The mean outpatient steroid dose was $51 \mathrm{mg}$ of prednisone/day, and the mean duration of oral steroid therapy was 14 days. All patients had moderate to severe UC by Truelove and Witts criteria. The mean dose of IV steroids (in equivalent prednisone dosing) during hospitalization was $89 \mathrm{mg} /$ day (hydrocortisone or methylprednisolone). 8 patients were C. difficile toxin positive on admission, 33\% received antibiotics, and 35\% were given TPN. In total, 26 of 40 patients $(65 \%)$ had a colectomy. The 1,3 and 5 year colectomy rates after hospitalization were $60 \%, 60 \%$ and $63 \%$ respectively. One patient had a colectomy 6 years after hospitalization. Of patient undergoing colectomy, 24 of 26 (92\%) had surgery within 1 year of hospitalization. There was no significant difference between patients undergoing colectomy or not with respect to age, gender, disease duration, c. difficile toxin detection on admission, inpatient steroid dose, or inpatient use of antibiotics or TPN. Patients undergoing colectomy had a significantly higher dose of outpatient oral steroids $(52.3 \mathrm{mg} / \mathrm{d}$ vs $44.5 \mathrm{mg} / \mathrm{d}, \mathrm{p}=.027$ ), shorter outpatient steroid course ( 10.8 days vs 17.5 days, $\mathrm{p}=.038$ ), and a higher admission platelet count (450 vs $365, p=.036)$. Conclusions: Patients admitted for ulcerative colitis treatment after failing outpatient steroid have a 5 year colectomy rate of $63 \%$, with over half needing surgery within 1 year of admission. Higher platelet count, higher outpatient steroid dose, and shorter duration of outpatient steroid therapy were predictors of colectomy. 
W1178

Preterm Birth Is Associated with, But Not An Independent Risk Factor for Developing Crohn's Disease Later in Life

Barbara Sonntag, Birgit Stolze, Andreas Luegering, Jan Heidemann, Pia Lebiedz, Emilie Rijcken, A Heinecke, Wolfram Domschke, Torsten Kucharzik, Christian Maaser

Background: IBD is a heterogeneous condition the exact cause of which is still unknown. Exposure to bacterial antigens of the intestinal flora is one of the potential trigger mechanisms. Vaginal delivery is the earliest time point in life when the intestine is exposed to a microbial flora. In an initial detailed analysis of the perinatal circumstances looking at a local cohort of IBD patients we recently found a significant association of preterm delivery and the development of Crohns disease later in life. The aim of this study now was to extend our findings to a larger national cohort. Methods: A self-administered questionnaire concerning the circumstances at birth was mailed to patient members of the German Crohns and Colitis foundation (DCCV). Their partners having no IBD served as controls. Statistical analysis was performed using the Statistical Package for Social Sciences (SPSS). Comparisons of categorical and continuous variables were conducted using the Chi-Square- and t-test or analysis of variance, respectively. Odds ratios (OR) together with $95 \%$ confidence intervals (CI) were calculated to estimate the relative risk of disease status associated with various exposures. Multivariate analysis using logistic regression was performed to identify independent risk factors. Results: Information was obtained from a total of 2737 persons (763 ulcerative colitis (UC), 1096 Crohns disease (CD) 878 controls (C)). Female sex (CD: OR 3.703, CI 3.067-4.484; UC: OR 1.879, CI 1.540-2.293), smoking (CD: OR 1.229, CI 1.0091.497; UC: 0.792, CI 0.638-0.984), appendectomy (CD: OR 2.934, CI 2.334-3.689; UC: OR 0.614, CI 0.460-0.819) and maternal IBD (CD: OR 4.521, CI 1.578-12.949; UC: OR 3.705, CI 1.232-11.140) were significantly associated with disease status. In addition, preterm birth, but not caesarean section and breastfeeding was significantly associated with CD (OR 1.522, CI 1.122-2.066), but not with UC (OR 1.377, CI 0.987-1.923). Accordingly, birth weight was significantly lower in the UC and CD group ( 3341 vs. 3354 vs. $3438 \mathrm{~g}, \mathrm{p}=$ 0.017). Multivariate analysis confirmed all but preterm birth as independent risk factors. Discussion: Our results confirm a significant association of preterm birth with the development of $C D$ later in life, which may be due to alterations in the vaginal microbial flora. However, it does not prove as an independent risk factor in the multivariate analysis.

\section{W1179}

Population Based Cost Analysis of Screening for Dysplasia in Primary Sclerosing Cholangitis

Gilaad G. Kaplan, Steven J. Heitman, Robert J. Hilsden, Stefan Urbanski, Sam S. Lee, Rob P. Myers, Kelly W. Burak, Mark Swain, Remo Panaccione

Background: Patients with primary sclerosing cholangitis (PSC) and either ulcerative colitis (UC) or Crohn's disease (CD) are at risk of developing colorectal cancer (CRC). Recommendations suggest annual screening colonoscopy to detect dysplasia in patients with PSC and colitis. For a $90 \%$ confidence to detect dysplasia 33 colonic biopsies are required. Costs associated with screening PSC patients are unknown. Aims: 1) to determine the rate of dysplasia or cancer in PSC patients and 2) to assess the costs associated with screening. Methods: A population-based study was conducted between 2000 and 2004 to identify all patients with a diagnosis of PSC. PSC patients were identified using regional databases and were confirmed by chart review. Colonic histopathology was reviewed in PSC patients with colitis for: 1) the number of biopsies taken during screening colonoscopy, 2) frequency of colonoscopies conducted between 2000 and 2005, and 3) the presence of CRC, dysplasia or a dysplasia associated lesion or mass (DALM). The direct cost of a screening colonoscopy and the pathology costs incurred were determined locally. Predicted costs for the recommended annual screening colonoscopy with 33 random biopsies were compared to the actual costs accrued in our cohort using the Wilcoxon signed-rank test. All costs are reported in US dollars. Results: During the study period 45 eligible PSC patients with UC or CD were identified. Five patients ( $11.1 \%$ ) were diagnosed with low grade dysplasia $(n=2)$, DALM $(n=2)$ or $C R C(n=1)$ in the 5 year period. Two of these lesions were detected through screening and 3 because of symptomatic presentation. Of an expected 131 screening colonoscopies for dysplasia, only 56 were actually conducted. The median number of biopsies collected was 27 (IQR: 19-33). The incidence rate for detecting a lesion through screening was 3.6 events per 100 annual colonoscopies. The actual cost of screening over the 5-year study period was $\$ 42,608.71$ compared to a predicted cost of $\$ 107,108.24(p<0.001)$. The annual predicted cost of screening one PSC patient would have been $\$ 850.04$. Based on a detection rate of 3.6 lesions per 100 annual colonoscopies, $\$ 23,801.25$ would be spent to detect one case of dysplasia, DALM or cancer. Conclusion: PSC patients have a high rate of colorectal dysplasia or cancer. We have shown that screening in these patients is underperformed compared to current practice guidelines. Overall, the cost to identify one case of dysplasia, DALM or cancer in PSC patients would be $\$ 24,000$ with annual screening. This is an acceptable value given the morbidity and mortality associated with the development of colorectal neoplasia.

\section{W1180}

Thrombocytosis in IBD-Associated Anemia Is Regulated By Iron Stefanie Kulnigg, Christoph Gasche

Introduction: Thrombocytosis is a common finding in IBD, specifically when associated with iron deficiency anemia. We previously reported that iron sucrose (a semi-labile iron complex) causes an erythropoietin-independent normalization of elevated platelet counts in IBDassociated anemia. We hypothesized that this observation is caused by a direct regulatory effect of iron on megakaryopoiesis. This is specifically relevant because of the increased risk of thromboembolic complications in IBD patients. Methods: We studied the effect of a different iron compound (Vit-45, a novel, non-toxic, stable iron complex; Vifor Int., St. Gallen, Switzerland) in a cohort of 8 anemic IBD patients (mean hemoglobin 9.1g/dl, ferritin $11.3 \mu \mathrm{g} / \mathrm{l}$, transferrin saturation $3.5 \%$ ) and measured platelet counts as well as the number of circulating megakaryocytic progenitor cells (CFU-Mk). PBMC were isolated prior to iron therapy and after 4 weeks by Ficoll and cultured for 12 to 14 days using the MegaCult medium (StemCell Technologies, Vancouver, BC). CFU-Mk were stained with anti-CD4l and counted microscopically. Growth experiments were further conducted by MTT assay using two megakaryocytic cell lines (Meg-01, Dami). Iron-depleted conditions were accomplished with various iron chelators (deferoxamine [DFO]; deferiprone [L1]), FCS-dilution and addition of holo- (iron loaded) or apo- (iron free) transferrin. Results: Upon therapy with Vit-45, patients receiving a hemoglobin-adjusted mean iron dose of $1538 \mathrm{mg}$, platelet levels decreased from $421 \mathrm{G} / 1$ to $342 \mathrm{G} / \mathrm{l}$ (week $4, \mathrm{p}=0.0719$ ) and $300 \mathrm{G} / \mathrm{l}$ (week $8, \mathrm{p}=$ $0.0095)$. Small and medium sized CFU-Mk increased from 0.12 to $0.42(\mathrm{p}=0.065)$ and 0.19 to $0.43(\mathrm{p}=0.036)$, respectively. The proliferation of Meg-01 and Dami was unchanged with low concentrations of iron chelators but significantly dropped at higher doses $(>1 \mu \mathrm{M}$ for $\mathrm{DFO}$ and $>50 \mu \mathrm{M}$ for $\mathrm{L} 1)$. Both cell lines showed unchanged growth characteristics down to $1 \%$ FCS. Low concentrations of holo-transferrin $(0.4-50 \mu \mathrm{g} / \mathrm{ml})$ induced significantly better growth than higher concentrations $>50 \mu \mathrm{g} / \mathrm{ml}$. Conclusion: Vit- 45 , a novel, non-toxic intravenous iron complex, causes a normalization of elevated platelet counts in patients with IBD-associated anemia similar to what has been observed with iron sucrose. These findings support the hypothesis that the observed effect is related to ferric iron rather than the sugar complex. Circulating CFU-Mk changed inversely to platelet counts indicating that iron deficiency might inhibit megakaryocytic proliferation. Thus, thrombocytosis through iron deficiency may rather be due to an increase in megakaryocytic ploidy rather than number.

\section{W1181}

Variation in Initial 6-Mercaptopurine Dosage in Pediatric Crohn's Disease Richard B. Colletti, Robert N. Baldassano, David E. Milov, Peter A. Margolis, Pibdnet Pediatric IBD network for research and improvement

Background Variation in care can be due to overuse, underuse or misuse of diagnostic and therapeutic interventions. 6-mercaptopuprine (6MP) and its prodrug azathioprine (AZA) have efficacy in Crohn's disease (CD). Measurement of thiopurine methyltransferase (TPMT) prior to starting treatment can identify normal genotype or phenotype patients to whom the recommended dose (6MP $1.0-1.5 \mathrm{mg} / \mathrm{kg} / \mathrm{d}$ ) can be administered. Aim To determine the variation in care by pediatric gastroenterologists in administering $6 \overline{\mathrm{MP}}$ and the effect on dosage of measuring TPMT. Methods In a prospective cohort of CD patients by PIBDNet, pediatric gastroenterologists enrolled patients who were starting treatment with 6MP or AZA. They provided care as usual and completed patient encounter forms via the PIBDNet Data Collection web site. Patients Preliminary data from 37 sites are presented on 93 patients (5-17 yr, 57\% male, $90 \%$ Caucasian, 12\% Jewish). 19\% had involvement of the esophagus, $74 \%$ ileum, $60 \%$ colon, $25 \%$ perineum, and 3\% extraintestinal. $56 \%$ were underweight, $60 \%$ were taking daily prednisone, and the CRP was elevated in $61 \%$. By the Physician Global Assessment CD was inactive in $8 \%$, mild in $35 \%$, moderate in $48 \%$ and severe in $9 \%$. The Harvey Bradshaw Index was $>4$ in $39 \%$. The most common indication for 6MP/ AZA treatment was early or induction therapy. Results 24 patients $(26 \%)$ were treated with AZA and 69 patients (74\%) with 6MP. Of the 69 patients treated with 6MP, TPMT was measured in $49(71 \%)$; in 41 the TPMT was normal. Of patients in whom the TPMT was normal, the dose was between 1.0 and $1.5 \mathrm{mg} / \mathrm{kg} / \mathrm{d}$ in $51 \%$, and $23 \%$ received a dose $<1.0$ $\mathrm{mg} / \mathrm{kg} / \mathrm{d}$ (mean starting dose $1.17 \mathrm{mg} / \mathrm{kg} /$ day; range 0.68-1.89). In contrast, of patients without TPMT measurement, the dose was between 1.0 and $1.5 \mathrm{mg} / \mathrm{kg} / \mathrm{d}$ in $45 \%$, and $55 \%$ received a dose $<1.0 \mathrm{mg} / \mathrm{kg} / \mathrm{d}$ (mean starting dose $0.95 \mathrm{mg} / \mathrm{kg} / \mathrm{day}$; range $0.36-1.44$ ) (p= 0.009). Conclusion There is considerable variation in the selection of drug, measurement of TPMT and dosage prescribed. Patients in whom TPMT had not been measured were more likely to receive a dose of 6MP lower than recommended. (Supported by a grant from NASPGHAN with funds from the American Board of Pediatrics.)

\section{W1182}

Crohn's Disease Involving the Jejunum Or Proximal Ileum

Greta S. Lee, Dian Templeton, Rebecca I. Cooley, Elizabeth Fowler, Douglas Lincoln, Timothy Florin, Graham L. Radford-Smith

Background: Crohn's disease (CD) affecting the jejunum or proximal ileum (JPI) is less common than classical CD involving the terminal ileum and/or colon. Reports of its prevalence range from $3.3 \%$ to $9.5 \%$ in adults. This area of the gut, until recently, has been difficult to image and investigate. Patients with JPI disease have not been well characterized - small case series predominantly reflect patients with symptomatic clinically significant disease. Aims: To determine the prevalence and major clinical associations of patients with JPI disease in a prospectively collected cohort of adult patients with $C D$ from the population of Brisbane. Methods: Patients were recruited from the Brisbane IBD database. Information was collected prospectively by interview and questionnaire. All specific investigations for small bowel involvement were reviewed as well endoscopic, surgical, radiological and pathological reports to determine method of diagnosis of JPI disease, number/types of confirmatory investigations, extent of small bowel disease, surgical resection for small bowel disease and presence of granulomas. The cohort with JPI disease was compared with the rest of this populationbased $\mathrm{CD}$ cohort. Univariate analysis used chi-square or t-test, followed by multivariate analysis. Results are expressed as ratios, means \pm standard deviations or odds ratio (OR) with $95 \%$ confidence intervals $(\mathrm{CI})$. A significant $\mathrm{p}$-value was defined as $<0.05$. Results: Patients with JPI disease represented $9.9 \%$ of the $C D$ population. There was a highly significant male predominance in this subgroup $(\mathrm{OR}=3.1 ; 95 \% \mathrm{CI}$ : 1.8-6.4). This gender difference was not related to age at diagnosis, smoking history or any other clinical feature. The rate of surgical recurrence was significantly higher in the JPI group $(\mathrm{P}=0.018)$. Granulomas were significantly more prevalent in this group on multivariate analysis when corrected for age, gender, immunosuppression and bowel resection ( $\mathrm{OR}=1.8 ; 95 \% \mathrm{CI}: 0.9-3.6)$. Conclusion: Symptomatic CD involving the jejunum or proximal ileum, leading to investigation of this region, occurred in $10 \%$ of our patients. There was a strong male predominance in patients with JPI Crohn's disease, not explained by other potential confounders such as sex, age or smoking. Proximal small bowel CD should be considered early, especially with the number of emerging imaging modalities available. Surgical recurrence rates were almost double in patients with JPI involvement, despite high rates of immunosuppression use. This has important implications for future approaches to treatment. 
W1183

Are There Other Factors Explaining Why UC-Patients with Normal Colonoscopy Still Reports IBD-Related Symptoms?

Tomm Bernklev, Magne Henriksen, Oivind Palm, Jorgen Jahnsen, Morten Vatn, Bjorn Moum

Background: It is well known from clinical practice that IBD patients with inactive disease, judged by colonoscopy and histology, may well complain of disease-related symptoms. The lack of objective findings makes it difficult to explain this, but other, non-IBD related factors may contribute to their symptoms. Aim: To identify possible factors explaining the presence of symptoms in UC-patients with inactive disease at the time of investigation. Material and Method: All UC patients in the IBSEN cohort were offered a colonoscopy with biopsies in connection with the five-year follow-up visit. Disease symptoms were classified as none, mild, moderate/severe by the patient, judged on the last 14 days before the visit. Healthrelated quality of life was investigated with N-IBDQ and SF-36. All patients underwent a clinical examination and interview. In addition, the frequencies of fibromyalgia (FM), chronic widespread pain (CWP) and non-inflammatory joint pain (NIJP) were determined by an experienced rheumatologist. Results: All together 164 UC patients (50\%) accepted and underwent a colonoscopy, mean age 45.3yrs (15.4), 47\% female. Of these, 28 patients (17\%) complained of IBD-related symptoms but had a normale colonoscopy; mean age 47.4(14.3), 46\% female. HRQOL was significantly reduced for the dimensions stool consistency/frequency, bowel pain and worries, compared to UC patients without symptoms. Of all patients with FM/CWP in the IBSEN study, 25\% were found in this subgroup, compared to $2.7 \%$ in symptom-free UC patients. Smoking was also more frequent in this subgroup, as well as a lower educational level, compared to symptom-free UC patients,18\% vs. $8 \%$ and $36 \%$ vs. $24 \%$, respectively. Conclusion: UC patients with normal colonoscopy and symptoms are more frequently smokers, have a lower education, have an increased risk for having FM/CWP and have more bowel complains, bowel pain and worries than symptomfree UC patients.

\section{W1184}

Outcome of Surgical Versus Percutaneous Drainage of Abdominal and Pelvic Abscesses in Crohn's Disease

Alexandra Gutierrez, Hang Lee, Bruce E. Sands

Objective: Abdominal and pelvic abscesses (APA) are a common problem in the management of Crohn's disease (CD). The criteria used for selection of operative and radiographic-guided percutaneous drainage have become increasingly controversial. We studied the effect of the initial choice of therapy (surgery or percutaneous drainage (PD)) for APA on time to resolution. Methods: We recorded clinical, laboratory, and radiographic data on all adult patients with CD and APA treated at Massachusetts General Hospital between 1991 and 2001 and then followed for at least one year. Baseline characteristics were individually tested using univariate analysis to identify factors or variables associated with choice of initial modality. Statistically significant and clinically significant variables were included in a multivariate Cox's proportional hazards model to identify factors independently associated with time to resolution. Results: Of 66 episodes identified, surgery was the initial modality in 29 and PD in 37. Median time to resolution was 22 days (95\% CI 13-43), with no significant difference between those treated with surgery ( 25 days, 95\% CI 12-47) or PD (21.5 days, $95 \%$ CI 12-58). Older patients, those with longer duration of APA symptoms prior to drainage, no fistula identified radiographically, on immunomodulators and admitted to the medical service were more likely to have PD as initial modality. These factors, when incorporated in a Cox regression model, did not significantly affect the time to resolution. Days from onset of APA symptoms to radiographic diagnosis or drainage were independently associated with time to resolution of the abscess. Conclusion: Time to resolution of APA in CD is similar regardless of whether PD or surgery is performed. Earlier intervention for APA is associated with shorter time to resolution.

\section{W1185}

Mesalamine Reduces the Rate of Colorectal Dysplasia in Patients with Inflammatory Bowel Disease and Primary Sclerosing Cholangitis Who Are On Urosdeoxycholic Acid

Timothy Smith, Prabhakar Swaroop

BACKGROUND: Ursodeoxycholic acid (UDCA) has been known to reduce the risk of colonic neoplasia in patients with primary sclerosing cholangitis (PSC) and ulcerative colitis (UC) PSC-UC patients are at higher risk of developing colorectal cancer (CRC) and dysplasia compared to UC alone. This study aims to investigate the effect of mesalamine and folate supplementation on dysplasia in patients with PSC and inflammatory bowel disease (IBD) who are already on UDCA. METHODS: All patients with both PSC and IBD evaluated at St. Louis University between 1995 and 2005 were identified through a retrospective review of the medical record. Cases were required to have PSC diagnosed by cholangiography (ERCP and/or MRCP) and at least one prior colonoscopy with biopsies at our institution. Medication history was reviewed and patients who were not on UDCA were excluded. For the remaining patients, medication history and histologic data from prior colon biopsies was reviewed for dysplasia, pathology indefinite for dysplasia, or absence of dysplasia. Rates of usage of mesalamine and folic acid were compared. RESULTS: Twenty-four patients were identified ( $80 \%$ of IBD was UC). Of the 24 patients, 2 ( $8 \%$ ) had biopsies revealing dysplasia, $5(21 \%)$ had biopsies indefinite for dysplasia, and $17(71 \%)$ had no dysplasia. Nineteen patients had been on mesalamine and 5 had not. Among patients who had received mesalamine, 1/19 (5\%) had dysplasia, 3/19 (16\%) were indefinite for dysplasia, and 15/19 (79\%) had no dysplasia. Among patients who did not receive mesalamine, 1/5 (20\%) had dysplasia, $2 / 5(40 \%)$ were indefinite for dysplasia, and 2/5 (40\%) had no dysplasia. All five patients on folate supplementation had no dysplasia. Among the patients who did not receive folate, 2/19 (11\%) developed dysplasia, 5/19 (26\%) were indefinite for dysplasia, and 12/19 (63\%) had no dysplasia. These results are summarized in the table below. The relative risk of developing dysplasia in patients who were not on mesalamine was $1.9(95 \% \mathrm{CI}=0.6$ to
5.9). There was a trend toward a protective effect of folate as well. CONCLUSIONS: Mesalamine appears to provide an additional chemoprotective effect on the risk of colonic neoplasia in PSC-IBD patients on UDCA. Folate may also have an additive chemoprotective effect. In PSC-IBD patients taking UDCA, addition of both mesalamine and folate should be considered. Effect of mesalamine and folate on rate of dysplasia in patients with PSC-IBD who are on UDCA

\begin{tabular}{|c|c|c|c|c|c|}
\hline & $\begin{array}{c}\text { All } \\
\text { Patients(N=24) }\end{array}$ & Mesalamine(N=19) & $\begin{array}{c}\text { No } \\
\text { Mesalamine(N=5) }\end{array}$ & Folate(N=5) & $\begin{array}{c}\text { No Folate } \\
(\mathrm{N}=19)\end{array}$ \\
\hline Dysplasia & $2(8 \%)$ & $1(5 \%)$ & $1(20 \%)$ & $0(0 \%)$ & $2(11 \%)$ \\
\hline $\begin{array}{c}\text { Indefinite for } \\
\text { dysplasia }\end{array}$ & $5(21 \%)$ & $3(16 \%)$ & $2(40 \%)$ & $0(0 \%)$ & $5(26 \%)$ \\
\hline No dysplasia & $17(71 \%)$ & $15(79 \%)$ & $2(40 \%)$ & $5(100 \%)$ & $12(63 \%)$ \\
\hline
\end{tabular}

\section{W1186}

Comparison of Clinical Outcomes Between Intestinal Simple Ulcer and Intestinal Behcet's Disease

Yun Jin Chung, Chang-Min Cho, Young Dae Park, Won Young Tak, Seong Woo Jeon

Background/Aims: Intestinal simple ulcer, which does not meet the criteria of Behcet's disease, is not uncommon in clinics. However, its clinical courses and outcomes are not well understood. This study was performed to compare intestinal simple ulcer with intestinal Behcet's disease in clinical outcomes. Methods: We retrospectively reviewed the medical records of 52 patients who were suspected as intestinal Behcet's disease according to the patient's symptoms, colonoscopic, laboratory and pathologic characteristics. Of them, 27 patients (Group 1) met both the criteria of the International Study Group for Behcet's Disease and the Behcet's Disease Research Committee of Japan. Thirteen patients (Group 2) met only the latter and the remainder (Group 3) didn't meet any critieria. The efficacy of medica treatment was assessed by the gastrointestinal symptoms and follow-up colonoscopic or radiologic findings. Results: Mean age of the onset of gastrointestinal symptoms was $36.4 \pm 12.8$ years. Sex ratio was 1.08:1 (M:F) and mean follow-up duration was $35.2 \pm 39.5$ months Forty-six patients had gastrointestinal symptoms and abdominal pain was the most common symptom. Single, smaller than $5 \mathrm{~mm}$, round and shallow ulcer with erythematous margin, which located at ileocecal area, was the most typical colonoscopic feature. No significant differences were found in clinical manifestations and colonoscopic findings among three groups. Mean duration of medical treatment was $19.6 \pm 24.4$ months. The overall complete or partial remission was observed in 36 patients $(83.7 \%)$ and mean time to achieve remission was $12.1 \pm 25.3$ months. There were no differences among groups in the rate $(20(90.9 \%)$ vs. $9(75.0 \%)$ vs. $7(77.8 \%)$, group $1,2,3$, respectively, $\mathrm{p}=0.47)$ and mean time $(7.1 \pm 9.2$ months vs. $26.2 \pm 46.7$ months vs. $8.0 \pm 7.5$ months, group $1,2,3$, respectively, $\mathrm{p}=0.15$ ) to achieve medical remission. The overall cumulative rate of first operation and second operation was $40.5 \%$ and $71.9 \%$ in 10 years. No statistical relationship was found in the cumulative rate of operation among groups $(\mathrm{p}=0.54)$. Conclusions: Clinical courses and outcomes of the intestinal simple ulcer are not different from that of intestinal Behcet's disease. The differences of pathophysiologic mechanism between intestinal simple ulcer and intestinal Behcet's disease have not been established. However, it is desirable to regard and treat intestinal simple ulcer as intestinal Behcet's disease.

\section{W1187}

Exercise and Bone Mineral Density in Crohn's Disease

Reshma C. Rakshit, Len Almond, Farook Al-Azzawi, Keith Abrams, John F. Mayberry, Richard J. Robinson

Introduction: Bone loss is a common complication of Crohn's disease with a prevalence of $30-50 \%$. It is multifactorial in causation. The beneficial effects of a year long low-impact exercise programme were shown in a previous randomized controlled study. (1) Aim: 1.To evaluate the long-term effects of exercise on bone mineral density (bmd) in Crohn's disease 2.To compare the exercise activity indices of patients with Crohns' disease over a period of 8 years. Methods: A cohort of patients with Crohn's disease randomized to one year of low-impact exercise versus controls was followed up after 8 years. Demographic details and exercise activity levels (based on the Allied Dunbar National Fitness Survey)were collected. DEXA (Dual Energy X-Ray Absorptiometry) scans of the spine and the hip were performed at baseline and repeated after 8 years to assess bmd. Results: Data was available for 55 patients ( 30 males, 37 female patients) with a mean age of 51.9 years ( $\mathrm{sd}=11.95$ years). O these, 26 were originally randomized to exercise and 29 were controls. Independent T-test showed no statistical difference in the change in the bmd at the spine or hip over 8 year between the exercise and control groups. Chi-square testing revealed no difference between the current exercise levels of the exercise and control groups. $(\mathrm{p}=0.21)$. There is a significant difference in percentage change in bmd at the spine and hip $(\mathrm{p}=0.015 ; \mathrm{p}=0.027)$ between the different groups according to current activity level further strenghthened by post hoc analyses. A Wilcoxon test shows no significant difference in the exercise habits of patient over 8 years $(\mathrm{p}=0.81)$. No statistical difference was demonstrated in activity levels when comparing patients randomized to exercise or control groups. ( $\mathrm{p}=0.21)$. Conclusions: 1 . Short term effects of exercise on bmd do not extend over a longer time frame in patient with Crohn's disease. 2. Patients with higher levels of current activity have higher bmd at both hip and spine compared to those who are least active. 3. Short term increases in activity levels were not maintained over the 8 year period. We know that bone mineral density change in Crohn's disease is multifactorial. The effect of exercise/activity in isolation is small. Therefore, prospective studies with larger number of patients will be required to assess the overall beneficial effects of exercise on bmd in future. Reference: 1.Robinson RJ, Krzywick T, Almond L et al. Effect of a Low-Impact Exercise Program on Bone Mineral Density in Crohn's Disease: A Randomized Controlled Trial. Gastroenterology 1998;115: 36-41 
Can Clinical 'Red Flags' At the Time of Presentation with Ulcerative Colitis Predict Which Patients Will Subsequently Undergo a Change in Diagnosis to Crohn's Disease?

Gil Y. Melmed, Andrew F. Ippoliti, Gary C. Chen, Igor Nastaskin, Eric A. Vasiliauskas, Konstantinos A. Papadakis, Carol J. Landers, Robert M. Elashoff, Stephan R. Targan

Background: The diagnosis of inflammatory bowel disease often involves combining evidence rom many sources in order to correctly diagnose ulcerative colitis (UC) or Crohn's disease (CD). Some patients initially diagnosed with UC are subsequently diagnosed with $C D$. Established criteria for the differentiation of $\mathrm{UC}$ from $\mathrm{CD}$ require at least a colonoscopy with biopsies (Lennard Jones, 1989), although additional diagnostic testing may be helpful. Methods: Nested case-control study of subjects with inflammatory bowel disease entered into longitudinal database. Cases were defined as patients initially diagnosed with UC via full colonoscopy at presentation and who subsequently met Lennard-Jones criteria for CD Cases were age and sex-matched with controls ('pure' $U C$ and $C D$ ) in an approximate 1:2:2 ratio. Using modified RAND expert panel methods, clinical predictors (red flags) for CD were determined by 4 IBD experts: non-bloody diarrhea, upper GI symptoms, >10\% weight loss, active smoking, history of transfusions, family history of $\mathrm{CD}$, oral ulceration, anal skin tags, thrombocytosis, hypoalbuminemia, and elevated C-reactive protein (CRP). In addition, CD-specific serologies (ASCA IgA/lgG, OmpC, I2, CBirl) and pANCA were run in a blinded fashion from stored sera collected at the time of entry into the database. Significance threshold was adjusted for multiple testing. Results: Of 162 patients with a reported change in diagnosis, 143 had either insufficient documentation of CD criteria or only a sigmoidoscopy at presentation and were excluded. 21 subjects with change in diagnosis, $50 \mathrm{UC}$ controls, and $56 \mathrm{CD}$ controls underwent chart review to corroborate diagnosis and assess for clinical parameters at initial presentation. Those with a change in diagnosis were more likely than UC controls to have had nonbloody diarrhea $(\mathrm{p}<0.001)$ and weight loss $(\mathrm{p}<0.01)$; other parameters were not significantly different. Family history of $C D$ trended highest in those with $C D$, lowest in those with UC, and in-between among those with a change in diagnosis $(p=0.06)$. Those with a change were more likely to have at least $2(p=0.05), 3(p=0.05)$ or $4(\mathrm{p}<0.01)$ clinical 'red flags' than patients with UC. Quantitative levels of ASCA IgA, ASCA IgG, and CBirl were highest in CD controls, lowest in UC controls, and in-between among those with a change in diagnosis ( $\mathrm{p}<0.001$ for linear trend for each serology). Conclusions: Most patients with UC subsequently diagnosed with CD had insufficient workup at the time of initial diagnosis. Further diagnostics for CD may be warranted in UC patients who present with nonbloody diarrhea, weight loss, or at least 2 red flags. Prospective studies are needed.

\section{W1189}

Phenotypic Classification of Crohn's Disease (CD) and Ulcerative Colitis (UC) in Olmsted County Minnesota: Assessment of Temporal Changes Emelie F. Helou, E V. Loftus, W J. Tremaine, W S. Harmsen, A R. Zinsmeister, D A. Jewell, W J. Sandborn

Background: A simple, reliable classification system is desirable to stratify inflammatory bowel disease patients (pts) for prognosis and genetic associations. The Vienna system for $\mathrm{CD}$ categorized by age of onset, location, and behavior. Limitations include no recognition of early-onset pts, and instability in behavior over time from disease progression. A formal system for UC does not exist. The Montreal classification includes new age categories for $\mathrm{CD}$ and a system for UC. Purpose: To assess for associations among age, gender, maximal extent of disease, and time period of diagnosis in population-based cohorts of $\mathrm{CD}$ and $\mathrm{UC}$. Methods: Medical records of all 308 CD and 372 UC pts diagnosed while residing in Olmsted County, MN between 1940 and 2000 were reviewed. The Montreal system was used to reclassify age of onset and maximal disease extent. Pearson Chi-square tests were used to assess associations among age of onset, gender, disease extent, and calendar period. Results: Median age of onset was 29 years (range 4-91) for CD and 33 years (1-88) for UC. The classification is shown in Table 1 for $\mathrm{CD}$ and Table 2 for UC. For CD, age of onset changed significantly over time, with more late-onset cases recently. Ileocolonic involvement was more common in recent years. Early-onset pts were more likely to have ileal (32\%) or ileocolonic disease (64\%), while late-onset pts had more colonic involvement $(42 \%)$ $(\mathrm{p}<0.001)$. There were no significant associations between gender and extent, gender and age of onset, or gender and time period. For UC, left-sided disease and extensive involvement was more common in recent years. Early-onset pts were more likely to have extensive disease (59\%) than late-onset patients ( $39 \%, \mathrm{p}=0,01)$. Early-onset pts were more likely to be males (88\%) than late-onset pts $(59 \%, \mathrm{p}=0.01)$. No significant associations were seen between gender and extent, or gender and time period. Conclusions: In these population-based cohorts of IBD pts, involvement was more extensive in recent years. Early-onset pts are more likely to have extensive disease.

\begin{tabular}{|c|c|c|c|c|c|c|c|c|c|c|c|}
\hline $\begin{array}{c}\text { Table 1. } \\
\text { CD. }\end{array}$ & $1940-60$ & $1961-80$ & $1981-00$ & Total & $\mathrm{p}$ & $\begin{array}{c}\text { Table 2. } \\
\text { UC. }\end{array}$ & $1940-60$ & $1961-80$ & $1981-00$ & Total & $\mathrm{p}$ \\
\hline $\mathrm{N}$ & 25 & 119 & 164 & 308 & & $\mathrm{~N}$ & 37 & 147 & 188 & 372 & \\
\hline Males\% & 48 & 42 & 54 & 49 & 0.13 & Males \% & 41 & 57 & 60 & 57 & 0.08 \\
\hline $\begin{array}{c}\text { Age of } \\
\text { Onset }\end{array}$ & & & & & 0.02 & $\begin{array}{c}\text { Age of } \\
\text { Onset }\end{array}$ & & & & & 0.79 \\
\hline$<16 \%$ & 4 & 11 & 9 & 9 & & $<16 \%$ & 3 & 5 & 5 & 5 & \\
\hline $16-40 \%$ & 44 & 69 & 62 & 63 & & $\begin{array}{c}16-40 \% \\
<0\end{array}$ & 57 & 63 & 57 & 59 & \\
\hline$>40 \%$ & 52 & 20 & 30 & 28 & & $>40 \%$ & 41 & 33 & 38 & 36 & \\
\hline $\begin{array}{c}\text { Max } \\
\text { Extent }\end{array}$ & & & & & 0.02 & $\begin{array}{c}\text { Max } \\
\text { Extent }\end{array}$ & & & & & 0.001 \\
\hline $\begin{array}{c}\text { Terminal } \\
\text { Ileum \% }\end{array}$ & 36 & 25 & 31 & 29 & & $\begin{array}{c}\text { Proctitis } \\
\%\end{array}$ & 14 & 30 & 7 & 17 & \\
\hline \begin{tabular}{c} 
Colon \% \\
\hline Ileocolonic \\
$\%$
\end{tabular} & 24 & 53 & 36 & 42 & & $\begin{array}{c}\text { Extensive } \\
\%\end{array}$ & 38 & 42 & 52 & 47 & \\
\hline $\begin{array}{c}\text { Upper GI } \\
\%\end{array}$ & 0 & 0 & 0 & 0.3 & & & & & & & \\
\hline
\end{tabular}

W1190

Characteristics of Crohn's Disease Patients with History of Bowel Resection Who Had Lactulose Breath Test for Evaluation of Small Bowel Bacterial Overgrowth

Tom Mathew, Faten Aberra, Chinyu Su, Yu-Xiao Yang, Gary R. Lichtenstein

Background: Patients with Crohn's disease may have gastrointestinal symptoms that are not attributable to active disease. Small bowel bacterial overgrowth may occur in patients with Crohn's disease, particularly those with prior intestinal resections. There has been no study examining factors that predict a positive lactulose breath test (LBT) in patients with Crohn's disease who have undergone intestinal resection. Our objective was to determine the characteristics predictive of a positive LBT in this patient population. Methods: We performed a retrospective chart review of successive Crohn's disease patients with history of intestinal resection who underwent LBT over the past 2 years at a tertiary care institution. All patients had a negative colonoscopic and /or radiographic evaluation for disease recurrence. Patients underwent LBT for 3 hours with breath hydrogen monitoring in a standard fashion. Basal hydrogen levels and patterns after lactulose administration were critically analyzed. We compared patients with positive LBT to those with negative LBT based on the following characteristics: smoking, type of surgery, diseases causing hypomotility (diabetes and hypothyroidism), drugs causing hypomotility (narcotics, anticholinergics, and calcium channe blockers), specific GI symptoms or signs (abdominal pain, bloating, diarrhea, abdominal mass and general well being), Harvey-Bradshaw index and presence of a stricture. Two sided Fisher's exact test was used to compare clinical characteristics between patients with positive LBD and those with negative LBT. Results: 18 patients ( 8 men /10 women; mean age 19 years, range 25-69) with CD and prior bowel resection underwent LBT during the study period. Six of the 18 patients (33\%) had a positive LBT. Most subjects had ileocolonic resection ( $88 \%$ vs $6 \%$ ileal vs $6 \%$ colonic resection). All six patients with positive LBT had ileocolonic anastomosis, while only $6 / 16$ patients with ileocolonic anastomosis had positive LBT. Smokers were significantly more likely to have a positive LBT compared to nonsmokers ( $80 \%$ vs, $16 \%, p=0.02$ ). Conclusions: In our study, bacterial overgrowth was documented in one-third of $C D$ patients with prior intestinal resection who underwent LBT for active GI symptoms despite negative workup for disease recurrence. Smoking appeared to be a positive predictor of positive LBT in these patients.

\section{W1191}

Etiology of Anemia in Patients with Ileal Pouch-Anal Anastomosis (IPAA) Ioannis Oikonomou, Victor W. Fazio, Feza Remzi, Rocio Lopez, Sandra El-Hachem, Bret A. Lashner, Bo Shen

Background and Aim: Restorative proctocolectomy is the surgical treatment of choice in patients with medically refractory Ulcerative Colitis (UC) and Familial Adenomatous Polyposis (FAP). Even though anemia is frequently observed in patients with IPAA it has not been studied. The identification of the underlying cause is often challenging. Our aim was to define the prevalence and to identify etiologic factors for anemia in this patient population. Methods: Prospectively maintained database and medical records of all patients who had restorative proctocolectomy between 1983 and 2005 were reviewed. All patients with at least one hemoglobin value more than 6 months after IPAA between 10/1998 and 10/2005 were studied. The last reported $\mathrm{Hb}$ served as the index value. All patients with anemia ( $\mathrm{Hb}<13.5 \mathrm{~g} / \mathrm{dL}$ males, $<12 \mathrm{~g} / \mathrm{dL}$ females) were identified. A second group of randomly selected patients with normal $\mathrm{Hb}$ served as control. A thorough chart review in regards to etiologic factors included bleeding, chronic inflammation (pouchitis or other), malignancy, chronic renal insufficiency, and medications. Results: 389 patients (M:F, 214:175) had documented CBC values. 67 patients (17\%) had anemia. The prevalence of anemia was $19 \%$ and $15 \%$ in males and females, respectively. Anemia prevalence was $17 \%$ in UC patients vs. $26 \%$ in FAP patients $(\mathrm{P}=0.27)$. Mean $\mathrm{Hb}$ in the anemia group was 11.4 (median 11.7 ); 1 patient $(2 \%)$ had severe $(<7), 11(16 \%)$ moderate $(7-9.9)$, and $55(82 \%)$ mild anemia $(\geq 10)$; 1 patient $(2 \%)$ had macrocytic, $16(24 \%)$ microcytic, and $49(74 \%)$ normocytic anemia. The etiology of anemia in the disease group was unclear in 16 patients (24\%) Clinical characteristics were similar in the anemia and the control group, except of GI bleeding and bone-marrow suppressive medications which were more common in the anemia group, each affecting one fifth of the patients $(\mathrm{P}<0.05)$. Conclusion: Persistent anemia is common in patients with IPAA. Even though GI bleeding and bone-marrow suppressive 
agents were at least partially accountable, one fourth of the patients had unclear etiology. Other etiopathologic pathways should be explored.

\begin{tabular}{|c|c|c|c|}
\hline Effect & Anemia N=67 & No Anemia N=65 & P value \\
\hline Age (SD) & $46.8(15.1)$ & $45.4(12.0)$ & 0.55 \\
\hline Male (\%) & $40(59.7)$ & $28(43.1)$ & 0.056 \\
\hline UC/FAP/Others & $59 / 6 / 2$ & $59 / 1 / 5$ & 0.10 \\
\hline GI bleeding (\%) & $14(20.9)$ & $5(7.7)$ & 0.031 \\
\hline Extra-intestinal bleeding (\%) & $2(3)$ & $2(3.1)$ & 0.99 \\
\hline Malignancy (\%) & $7(10.5)$ & $1(1.5)$ & 0.062 \\
\hline Bone-marrow suppressive medication (\%) & $13(19.4)$ & $4(6.2)$ & 0.023 \\
\hline Pouchitis or Crohn's disease (\%) & $14(20.9)$ & $22(33.9)$ & 0.095 \\
\hline Primary Sclerosing Cholangitis (\%) & $4(6.0)$ & $3(4.6)$ & 0.99 \\
\hline Renal insufficiency (\%) & $3(4.5)$ & 0 & 0.24 \\
\hline
\end{tabular}

W1192

Clinical Remission Prior to Initiation of Immonusuppression with Azathioprine Positively Impacts Responsiveness in Crohn's Disease But Not in Ulcerative Colitis - Results from a Multicenter Study in 1176 Patients with IBD

Martin H. Holtmann, Frank Krummenauer, Christina Claas, Kristina Kremeyer, D.R Lorenz, Iris Vogel, Olivia Rainer, Norbert Luegering, Guido Gerken, Wolfgang Kruis, Stephan Boehm, Juergen Stein, Ulrich Boecker, Jan Schmidt, Hans Herfarth, Carsten Buening, Rainer Duchmann, Andreas Sturm, Max Reinshagen, Andreas Stallmach, Daan Hommes, Geert D'Haens, Peter Galle, Markus Neurath

Introduction: Azathioprine (AZA) is the goldstandard for maintenance therapy in IBD. In a multicenter study on 818 patients with Crohn's disease (CD) and 358 patients with ulcerative colitis (UC) from 14 european centers we were able to show contrary to preexisting data, that AZA possesses efficacy in maintenance of remission beyond 4 years in both $C D$ and $\mathrm{UC}$ and to give a quantitative assessment of the significant steroid sparing effect of AZA. In a subsequent analysis we investigated the impact of the remission status for responsiveness to AZA. Infections are a feared complication of long term immunosuppression. Additionally, we therefore analysed the rates of hospitalization due to infections under AZA treatment. Methods: Endpoints were intrainddividual changes in flare frequency and steroid need between the time periods prior to and under AZA intake. Changes of flares/year or prednisolone equivalent/month were indicated as medians with quartils in brackets. $\mathrm{P}<0.05$ in the T-test indicated statistic significance. For determining the hospitalization rates, the total number of hospitalizations were refered to the cumulative number of treatment years. Results: In $\mathrm{CD}$ patients who were put on AZA, while in remission, subsequent numbers of flares/ year and steroid consumption where significantly lower than in those patients who had disease activity at the beginning of AZA treatment. Flare incidences were 0.0/y (0-3.43) versus $0.0 / \mathrm{y}(0-6.492)(\mathrm{p}<0.001)$ and steroid consumption was $10.17 \mathrm{mg} / \mathrm{month}(0-148)$ versus $79.11 \mathrm{mg} / \mathrm{month}(0-235.59)(\mathrm{p}<0.001)$. This was not due to selection bias, because in UC there was no difference between those patients in remission and those with still active disease. Flare incidences in UC were $0 / y(0-0.818)$ versus $0 / y(0-0.572)(p=0.488)$. The corresponding number for median intake of prednisolone equivalent were $65.41 \mathrm{mg} / \mathrm{month}$ $(0-236.66)$ and $69.17 \mathrm{mg} / \mathrm{month}(0-250)(\mathrm{p}=0.455)$. In $\mathrm{CD}$ the number of hospitalizations because of infections under AZA were 23 during 2495 cumulative treatment years $(0.009$ / y); in UC there were 10 cases during 987 treatment years $(0.01 / y)$. Discussion: The divergent results regarding the role of the remission status for responsiveness to AZA in CD and UC emphasize the pathogenetic differences between these entities and might support a hit hard and early strategy in $\mathrm{CD}$. Severe infectious complications requiring hospitalization are rare and seem to be an overestimated risk of long term immunosuppression.

\section{W1193}

The Effect of Acute Treatment with Budesonide On Body Mineral Density in Greek Patients with Newly Diagnosed Mild-to-Moderate Crohn's Disease Gerassimos J. Mantzaris, Christoforos N. Kalantzis, Evangelos Kokkinakis, Anastassios Roussos, Stavroula Koilakou, Efstratios Grivas, Demetrius Tsounis, Nickolaos Raptis, Nikolaos Kalantzis, George Triantafyllou, John Kalafatas

Aim: To investigate the effect of short-term treatment with budesonide controlled ileal release (B-CIR) on Body Mineral Density (BMD) of patients with active Crohn's disease (CD). Methods: Forty-five patients [27 female, mean age 25 (17-49) y] with newly diagnosed mild-to-moderate Crohn's ileitis or ileocolitis as defined by a Crohn's Disease Activity Index (CDAI) between 200 and 400 were included in a prospective study. CD was diagnosed by ileocolonoscopy, EGD histology, and enteroclysis. Patients were treated with B-CIR $9 \mathrm{mg} /$ day for 8 weeks followed by $6 \mathrm{mg} /$ day for another 8 weeks. Routine hematology, biochemistry, and CDAI measurements were performed at baseline, and after 8 and 16 weeks of treatment. Body weight and height were recorded, and the body mass index (BMI, $\mathrm{kg} / \mathrm{m} 2$ ) was calculated. Gender, age, hormonal status (women), smoking status, time with symptoms prior to diagnosis, profession, family history of osteoporosis, and sedentary lifestyle were also evaluated at baseline. Menopaused women, patients with prior hormonal replacement therapy, or treatment with calcium, vit D, or biphosphonates were excluded. Dual energy X-ray absorptiometry scans (DXA) were performed to measure BMDs of the lumbar spine (LS, L2-L4) and the left proximal femoral neck and trochanter (FNT) at baseline and within 1 week after stopping B-CIR. LS and FNT T-scores (number of SD from a mean of normal ethnic controls) were used to evaluate the effect of treatment on BMDs. Results: All patients completed the study. The mean (SD) CDAIs at baseline were 267(58). Nine patients (20\%) worsened during treatment, 9 (20\%) did not improve and 27 (60\%) were improved (CDAI $\leq 70$ over baseline) or achieved remission $(\mathrm{CDAI}<150)$. The mean $( \pm \mathrm{SD}) \mathrm{T}$-scores at baseline were $-0.3( \pm 0.8)$. Sixteen patients $(35.5 \%)$ had mild osteopenia $(-1<\mathrm{T}$ score) but none had osteoporosis (T-scores>-2.5). Low BMIs, female gender, longstanding symptoms prior to diagnosis, and heavy smoking were associated with lower T-scores. Treatment with B-CIR for 16 weeks did not result in significant changes in LS/FNT T-scores. Nine patients whose disease worsened during treatment showed a not significant decrease in LS/FNT T-scores. T-scores were improved in 18 patients and remained unchanged in 18 patients compared with baseline. Conclusion: The majority of Greek patients in reproductive age with newly diagnosed mild-to-moderate $\mathrm{CD}$ had BMDs not significantly deviating from healthy controls which did not deteriorate after short-term treatment with B-CIR. This may be due to the effect of sunshine on vit D turnover and, consequently, to BMD. The effect of chronic BCIR treatment in these patients is under investigation.

\section{W1194}

Long Term Follow Up of Crohn's Disease Patients Treated with Infliximab Using An Episodic Strategy

Vincent Pacault, Fathia Ben Hriz, Jean-Marc Gornet, Matthieu Allez, Maria Nachury, Ravzan Iacob, Jean-Frederic Colombel, Marc Lemann

Background. Infliximab (IFX) is a treatment for luminal and fistulizing Crohn's disease (CD) Episodic ("on demand") treatment has been initially used but this strategy has been recently challenged as it could facilitate immunisation leading to more intolerance reaction and/or loss of efficacy than a scheduled strategy. Long term data in patients treated with IFX using an episodic strategy are still scarce. Patients and methods. All records of patients treated with IFX for CD in our center between January 1999 and July 2004 were retrospectively reviewed; data were updated until June 2005. In patients responder to IFX and initially treated using an episodic strategy, we estimated the probability of the following events: relapse after the induction regimen, switch to a scheduled IFX treatment and IFX discontinuation due to intolerance and/or loss of response (Kaplan-Meier method). Results. 137 patients were treated with IFX for luminal $(n=88)$ or fistulizing $(n=49) C D$; median follow up was 35 months. 125 patients (91\%) responded to the induction regimen (1-3 infusions); among them, $110(88 \%)$ were treated using an episodic strategy (on demand re-treatment). Probabilities of sustained remission after the induction regimen were $33 \%, 24 \%$ and $15 \%$ at 1,2 and 4 years, respectively. Young age (RR: 1.6; 95CI: 1.1-2.4), prior use of immunosuppressants (IS) (RR: 1.9.95CI 1.2-3.0) and smoking (RR: 1.6.95CI. 1.1-2.5) were associated with an increased risk of relapse (multivariate analysis). Probabilities of switching to a scheduled strategy due to frequent relapses were $10 \%, 23 \%$ and $41 \%$ at 1,2 and 4 years, respectively. Probabilities of discontinuation due to intolerance and/or loss of efficacy were $5 \%$ (intolerance $1 \%$ and loss of efficacy $4 \%$ ) at 1 year, $18 \%(8 \%$ and $13 \%$ ) at 2 years, and $44 \%(26 \%$ and $24 \%)$ at 4 years. Absence of continuous treatment with IS after the IFX induction regimen was the only factor associated with an increase risk of IFX discontinuation due to intolerance and/or loss of efficacy (RR: $2.2 ; 95 \mathrm{CI}: 1.1-4.8$ ). Nine severe adverse events were observed: tuberculosis $(n=1)$, severe pneumonia $(n=1)$, severe skin infection $(n=2)$, intra-abdominal abscess $(n=1)$, myocardial infarction $(n=2)$, anaphylactic shock $(n=1)$ and cancer ( $n=2$, uterus and thyroid). Two patients died of a cause unrelated to IFX. Conclusion. IFX episodic strategy is associated with a high rate of relapse and of re-treatment even when combined with IS. The rate of IFX discontinuation due to intolerance and/or loss of response was $44 \%$ at 4 years, despite a switch to a scheduled IFX treatment in $41 \%$. Continuous treatment with IS significantly reduced the rate of discontinuation.

\section{W1195}

Infliximab for Hospitalized Ulcerative Colitis Patients Failing Intravenous Corticosteroids

Miguel Regueiro, Jennifer Curtis, Scott Plevy

Aims: To evaluate the efficacy of infliximab in hospitalized ulcerative colitis (UC) pts refractory to iv corticosteroids. Background: Treatment options for corticosteroid refractory UC pts are limited and include cyclosporine and colectomy. Although two recent studies (ACT I/ II) demonstrate a benefit from infliximab in outpts with moderate to severely active UC, the utility of infliximab in hospitalized UC pts refractory to iv corticosteroids is less clear. Study: We report our open label experience with infliximab for hospitalized UC pts at the University of Pittsburgh Medical Center. Response to infliximab was defined as avoidance of colectomy and cessation of corticosteroids. A Disease Activity Index (DAI) which account for stool frequency, rectal bleeding, and the physician's rating was also used to track improvement; DAI range 0-9 with 0 denoting normal and 9 severe UC. Results: There were $12 \mathrm{UC}$ inpts refractory to iv corticosteroids and subsequently treated with infliximab. None of the pts had longstanding UC (median duration of $U C=5.5$ mos, range 1 mos-4yrs) and none had received prior immunomodulators. All of the pts had severe UC (DAI=9) and had failed to respond to outpt oral prednisone (median dose $30 \mathrm{mg}$ ). None of the pts had toxic megacolon. Nine of the 12 pts ( $75 \%$ ) failed to respond to infliximab and required a colectomy; median time to colectomy 3 mos. Three pts received only 1 dose (week 0 ) prior to colectomy, 2 pts received 2 doses (0,2, weeks), 1 pt 3 doses (0,2,6 weeks) and 3 pts 4 doses $(0,2,6,14$ weeks); none of the nonresponders had a significant drop in CAI (median CAI=8.5) after infliximab. Three of the 12 pts (25\%) did respond to infliximab (median DAI=1, range 03 ) and were able to avoid colectomy and discontinue corticosteroids. The DAI's began to drop two weeks after the first infusion of infliximab with scores of 5,7 , and 8 . Unlike the nonresponders, there was continued improvement after the second dose of infliximab and by 4 wks the median DAI was 3. Conclusions: In this open label analysis, infliximab was not effective for the majority of hospitalized UC pts refractory to iv corticosteroids. Those few pts who did improve, had a response after 2 doses of infliximab; unlike the nonresponders. Whether earlier use of infliximab will alter the natural course of UC and prevent the need for hospitalization and colectomy is uncertain. 
Corticosteroid Dose Influences Colectomy Rate in Hoospitalized IBD pts with Clostridium Difficile

Mazen Issa, Sue Skaros, Mary F. Otterson, Sarah Lundeen, Lydia R. Weber, Dawn B. Beaulieu, Josh F. Knox, David G. Binion

Background: Recent reports suggest that Clostridum difficile (C. diff) has increased in the U.S and diminished effectiveness of metronidazole has led to the increased use of vancomycin. (DM Musher Clin Infect Dis. 2005:40:1586-90). Previous reports described increasing rates of C.diff in inflammatory bowel disease (IBD) pts, noting that colectomy was needed in a high percentage of these cases. Since C.diff affect preferentially immunosuppressed individuals and treatment for severe colitis emphasizes high-dose intravenous corticosteroids, we examined the impact of decreased intravenous steroid dose on clinical outcome in IBD pts with C.diff. Methods: This was a retrospective observational study of consecutive IBD pts infected with C.diff from a tertiary referral center between 2004-05. In addition, we focused on hospitalized pts between 3/2003-11/2005 with colitis and C.diff confirmed by positive stool ELISA toxin $\mathrm{A} / \mathrm{B}$ assay. Outcome measures included colectomy and successful medical treatment. Corticosteroid dosing was examined and pts were classified into 2 groups: low dose corticosteroid (LDC; $\leq 150 \mathrm{mg} /$ day of hydrocortisone) and high dose corticosteroid (HDC; >150 $\mathrm{mg} /$ day hydrocortisone). Demographic data, smoking status, surgical outcome and use of infliximab during hospitalization were recorded. Results: Colectomies occurred in 5/8 (63\%) C diff infected IBD pts diagnosed in 2004 and decreased to $4 / 40$ pts ( $10 \%$ in 2005 ( $p<0.001)$. We then focused on specific data from 25 consecutive admissions of IBD pts ( 9 UC; 15 CD) with C.diff ( 9 male, 15 female). Colectomies occurred in 32\% of the IBD pts (5/9 UC; $3 / 15 \mathrm{CD}$ ). Active smokers with C.diff who underwent colectomy included 2 pts with UC and 1 with CD. There were 11 pts classified as HDC and 13 in the LDC group. Seven pts in the HDC required colectomy (7/11 (64\%); $<<0.01)$ and $1 \mathrm{pt}$ in the LDC group underwent colectomy $(1 / 13,8 \%)$. Oral vancomycin was used in all pts with successful medical treatment and only $4 / 8(50 \%)$ of the pts needing colectomy had received vancomycin. Infliximab was used at the time of hospitalization in 5/8 of the pts who required colectomy and in 7/16 with successful medical treatment. Conclusion: $\mathrm{C}$. diff infection is associated with poor clinical outcome in IBD colitis pts who require hospitalization. Past treatment regimens which emphasize high dose intravenous corticosteroids correlated with increased rate of colectomy. Use of lower dose corticosteroids in conjunction with oral vancomycin appears to offer the best strategy for successful medical management of C.diff in the setting of IBD colitis.

\section{W1197}

Efficacy and Safety of 4 Weeks of Adalimumab Treatment in Subjects with Active Luminal Crohn's Disease Who Lost Response Or Showed Intolerance to Infliximab

Joaquin Hinojosa, Santiago Garcia, Guillermo Bastida, Cristina Saro, Jose L. Cabriada, Miguel A. Gassull

PURPOSE To assess the efficacy and safety of adalimumab, a fully human monoclonal antibody that targets TNF- $\alpha$, in inducing clinical remission, clinical response, and fistula closure in patients with moderately to severely active Crohn's disease (CD) who have lost response or showed intolerance to infliximab. METHODS In this open-label, multi-center study, subjects with moderately to severely active CD $($ CDAI $>220)$ and intolerance or lost response to infliximab were treated with subcutaneous adalimumab: $160 \mathrm{mg}$ at Week 0,80 $\mathrm{mg}$ at Week 2, then $40 \mathrm{mg}$ every other week (eow) for 52 weeks. Subjects were assessed after 4 weeks for clinical remission $(\mathrm{CDAl}<150)$, clinical response of $\Delta 70$ or $\Delta 100$ (decrease in $\mathrm{CDAI}$ of more than 70 or 100 points compared to baseline), fistula closure, and adverse events (AE). RESULTS Of 36 subjects with mean baseline CDAI $268.3 \pm 100.6$ enrolled, $28 \%$ had fistulas $(3.8 \pm 5.5$, mean) at baseline; $42 \%(n=15)$ had previously lost responsiveness to infliximab and $58 \%(n=21)$ had developed intolerance to infliximab. Week 4 results are given in the table. Treatment-related AE were mild to moderate in severity and similar to those observed in studies of adalimumab in patients with rheumatoid arthritis. The most common AE were nausea/dizziness, vomiting, weakness, and myalgia. One patient dropped out due to self-limited fever resolved after adalimumab discontinuation. No serious AE were reported. CONCLUSIONS Adalimumab was effective and well-tolerated in inducing clinical remission and response in subjects with moderately-to-severely active luminal $C D$ who had lost response or developed intolerance to infliximab. No new safety concerns were found in patients with $\mathrm{CD}$ compared to other adalimumab-treated populations.

Remission and Clinical Response to Adalimumab at 4 Weeks

\begin{tabular}{|c|c|c|c|}
\hline & $\begin{array}{c}\text { Luminal CD, No Fistulas } \\
\mathrm{n} / \mathrm{N}(\%)\end{array}$ & $\begin{array}{c}\text { Luminal Fistulizing CD } \\
\mathrm{n} / \mathrm{N}(\%)\end{array}$ & $\begin{array}{c}\text { All Luminal CD } \\
\mathrm{n} / \mathrm{N}(\%)\end{array}$ \\
\hline $\begin{array}{c}\text { Remission } \\
\text { CDAI<150 }\end{array}$ & $8 / 26(31 \%)$ & $7 / 10(70 \%)$ & $15 / 36(42 \%)$ \\
\hline$\Delta \mathbf{7 0}$ Response & $20 / 26(77 \%)$ & $10 / 10(100 \%)$ & $30 / 36(83 \%)$ \\
\hline$\Delta \mathbf{1 0 0}$ Response & $18 / 26(69 \%)$ & $9 / 10(90 \%)$ & $27 / 36(75 \%)$ \\
\hline
\end{tabular}

Intention to treat analysis

\section{W1198}

Infliximab Is Effective As Rescue Therapy for Acute Severe Ulcerative Colitis The Initial Edinburgh Experience

Charlie Lees, Alan G. Shand, Ian D. Penman, Ian D. Arnott, Jack Satsang

Introduction. As many as $40 \%$ of patients with acute severe ulcerative colitis (UC) will fail to respond to medical therapy. Treatment of these patients has been limited to surgery or second-line medical therapy (cyclosporine). Doubts remain about the efficacy of cyclosporine and concerns over its toxicity(1). Infliximab therapy is well established for Crohn's disease, but its role in UC remains contentious(2). Most recently, Jarnerot et al demonstrated in a randomised controlled trial that infliximab as "rescue" therapy for severe UC was effective with emergency colectomy rates of $29 \%$ vs $67 \%$ for placebo $(p=0.017$, NNT<3)(3). For the first time, this trial provided evidence to support the clinical use of infliximab as rescue therapy for UC. Methods. Data were retrospectively collected from all patients treated with infliximab for acute severe UC requiring hospitalisation for intensive medical therapy between May 2005 \& September 2005. Results. 9 patients (6 male, 3 female, mean age 41) treated with infliximab for acute severe UC were identified. They were characterised by either lack of response to initial intravenous steroid therapy (6/9) or by deterioration following conversion from iv to oral steroids (3/9). Infliximab $(5 \mathrm{mg} / \mathrm{kg})$ was given an average of 7.8 days (range 4-16) after admission. 6/9 (67\%) acutely treated patients demonstrated a good clinical response to infliximab within 48 hours, were commenced on azathioprine/6-MP therapy whilst in hospital \& discharged on reducing courses of oral steroids. The median duration from infliximab to discharge was 6.5 days. All avoided colectomy to the most recent point of follow-up (median 122 days, range 73-192). One patient developed uncomplicated herpes zoster and another asymptomatic transaminitis following infliximab therapy. The other 3 patients (33\%) underwent emergency colectomy (median 5 days post-treatment). Baseline clinical data (stool frequency, CRP, albumin, calprotectin) did not predict response. Additional follow-up and steroid withdrawal data will be presented. Conclusions. These data provide further evidence that infliximab may successfully rescue acute severe UC. The treatment can function as a bridge to long-term immune-suppression and immediate colectomy can be avoided. References: 1 . Shibolet $\mathrm{O}$ et al. The Cochrane Database of Systematic Reviews 2005, Issue 1. Art. No.: CD004277.pub2. 2. D'Haens G. Gastroenterology 2005;128(7):1805-11. 3. Jarnerot et al. Gastroenterology 2005;125:1805-11.

\section{W1199}

Open Label Experience with Adalimumab in Pediatric Crohn's Disease Patients Who Lost Response Or Were Intolerant to Infliximab Colette Deslandres, Christophe Faure, Martha H. Dirks, France Gervais, Ernest G. Seidman

Adalimumab(Admab)was reported to be effective in adult Crohn's disease (CD). Aim: Test the efficacy \& safety of Admab in pediatric CD. Patients \& Methods: 9 pediatric CD patients (7 M, 2F) who had lost their responsiveness ( $n=5$, after mean of 8.6 infusions, range $4-12$, last @ 10/kg) or developed intolerance to infliximab ( $\mathrm{n}=4,4.2$ infusions, 2-9) were treated with Admab. In the acute phase for induction of remission (Harvey Bradshaw index, HBI >5) we used 80 or $160 \mathrm{mg} / 1.73 \mathrm{~m} 2$ s.c,, followed by 40 or $80 \mathrm{mg} / 1.73 \mathrm{~m} 22$ wk later ( $\mathrm{n}=$ 5 \& 3 respectively). Outcome measures: tolerance to Admab and response (@1 mo), defined as responders $(\mathrm{HBI}<4$; decrease $>50 \%$ ), partial $(\mathrm{HBI}>4$, decrease $>50 \%$ ), or non-responders As maintenance, responders received Admab $40 \mathrm{mg} / 1.73 \mathrm{~m} 2 \mathrm{q} 2 \mathrm{wk}$. The primary outcome measures were maintenance of remission, reduction in steroids, and tolerance. Secondary outcomes included changes in hgb, serum albumin \& CRP or ESR. Concomitant immunosuppressors were maintained(5 MTX, 3 on 6-MP). Results: Mean age at time of 1st treatment was 10 (range $3.1-18$ ) years. Acute phase: Among the 8 cases, 4 responded completely $(50 \%), 1$ partially $(12.5 \%)$ and 3 failed (37.5\%). Patients with loss of response to infliximab were less likely to respond to Admab ( 2 of 5 ), vs those intolerant as indication(3/3). Mean HBI scores decreased from 13.9 (range 8-24) to 5.3(0-24). 1 non-responder had serious adverse effects, including fever and a flu-like illness after each Admab. 2 complained of local pain or erythema. Maintenance phase: In 7, remission was induced with Admab, and with prednisone in 1 other. Mean follow up was 3.6 months (range 1.5-8.5). Remission was maintained (HBI < 4)in 6 patients $(75 \%)$. 2 failed to respond after $2 \& 4$ doses (HBI 16). Among 6 responders, the mean baseline prednisone was $1.03 \mathrm{mg} / \mathrm{kg} / \mathrm{d}$ (range $0.6-1.6$ ). At the time of the last visit, it was substantially reduced in all 6 , to $0.14 \mathrm{mg} / \mathrm{kg} / \mathrm{d} ; \mathrm{p}<0.05$ ). The maintenance dose of Admab had to be increased to $40 \mathrm{mg} / 1.73 \mathrm{~m} 2$ weekly in 1 case to taper steroids. That patient had an adverse event (pneumonia + pleural effusion), requiring temporary cessation of Admab. CRP and/or ESR normalized in 5/6 responders, not in 2 non-responders. Among responders, mean hob increased by $20 \mathrm{gm} / \mathrm{L}$ in 2 and was stable in the other 4. Mean serum albumin increased from 34 (range 23-42 g/L) to 36.8 (28-47), $\&$ normalized in $2 / 6$ responders. Conclusions: Our preliminary data suggest that Admab can be effective in pediatric CD with adverse events or lost response to infliximab. Although generally well tolerated, adverse events occur, and controlled trials are needed to determine efficacy and dose response.

\section{W1200}

Crohn's Disease Patients' Willingness to Accept the Risks of Serious Adverse Events in Exchange for Clinical Benefits

Bruce Sands, Corey Siegel, Hass Steve, David Miller, Jeff White, Reed Johnson, Semra Ozdemir

OBJECTIVE: To evaluate the willingness of Crohn's disease (CD) patients to accept treatment related serious adverse event (SAE) risks in exchange for improvements in their daily symptom experience. BACKGROUND: All medical interventions carry risks of adverse outcomes that must be evaluated against their clinical benefits. Information from patients regarding their benefit vs. risk trade-offs can aid clinicians and regulatory agencies in their decision-making. METHODS: An on-line panel of self-identified CD patients who were US residents $>18$ years old completed a questionnaire containing stated preference benefit-risk trade-off items. The questionnaire required patients to select between treatment alternatives with different levels of the benefit and risk attributes. The treatment attributes included the severity of daily symptoms and activity limitations, the rate of serious complications (fistulas, abscesses, bowel obstructions), the time between flare-ups, oral steroid use and varying levels of SAE risks (progressive multifocal encephalopathy [PML] death or disability, death from serious infection or lymphoma). The questionnaire also contained the short form of the Inflammatory Bowel Disease Questionnaire (SIBDQ), and items regarding patient demographics and disease and treatment history. The maximum acceptable 10-year risk (MAR) for SAE development was calculated for various levels of clinical benefit. RESULTS: A total of 357 patients completed the survey. The mean (SD) age was 45 (13) and $73 \%$ of respondents were female. There was approximately equal representation of patients in each of four categories of SIBDQ generated Total IBDQ score $(<119,120-144,145-169,>170)$. Treatment decisions were primarily driven by the desire for improvements in daily symptom severity. Higher MAR, 
indicating greater risk acceptance, was observed for trade-off tasks involving higher levels of clinical benefit, among patients with lower current SIBDQ scores and among patients reporting a low level of worry about the potential adverse events. For the PML SAE, the mean (SE) MAR for an improvement from severe daily symptoms to remission and moderate daily symptoms to remission were $7.2 \%(0.26)$ and $4.7 \%(0.18)$, respectively. The lowest observed MAR for any of the three studied SAEs is significantly above the observed rates of SAE occurrence with natalizumab or any commonly used CD medications. CONCLUSIONS: CD patients indicated they are willing to accept a defined risk of death or disability in exchange for clinical efficacy. The patient perspective on the balance between potential benefits and risks can assist in making treatment and regulatory decisions.

\section{W1201}

Relationship of Phenotype and Function of Blood T Lymphocytes to Disease Severity in Ulcerative Colitis Patients

Jacky Woo, Samuel M. Siegel, Jia Shi, Yajun Mu, Zhilan Zheng, Jianmin Li, Tanny Tsao, Lyubov Efros, James Sheridan, Jim Lowder, Uma Mahadevan, Vladimir Vexler

Background: Ulcerative colitis (UC) is an inflammatory disease of the bowel that affects the large intestine. Since dysregulated immune reactivity contributes to the disease pathogenesis, conventional treatment strategies for UC rely on reducing inflammation. However, as the disease progresses, immunosuppressive drug regimens are used. Both the disease severity and treatment can alter immune cell function. To assess immune cell function as related to disease status and therapy, the immunophenotype and in vitro function of blood T lymphocytes from healthy subjects and UC patients were evaluated. Methods: Samples from 99 UC patients, 10 in clinical remission ( 3 on 6-MP), 16 with mild-to-moderate disease (4 5-ASA or no meds, 10 steroids, 5 6-MP+steroids) and 73 inpatients with severe-fulminant disease (intravenous steroid refractory) were evaluated. Absolute blood counts of T, B, and NK cells, naive, memory and activated CD4 cells, and putative virus-controlling CD8 cell subsets were measured by flow cytometry. Functional $\mathrm{T}$ cell status was assessed by staphylococcus enterotoxin B (SEB)-induced expression of intracellular IFN-g and IL-2 by T cells. Cellmediated immunity (CMI) was assessed with ImmuKnowTM assay $($ Cylex $囚)$, which reflects the immunocompetence of peripheral CD4+ cells. Results: Reduced CD4 counts ( $<400$ cells/ $\mathrm{uL}$ ) were observed in $5 \%$ of healthy subjects, $10 \%$ of remission, $25 \%$ of mild-to-moderate, and $48 \%$ of severe-fulminant UC patients. Similar trends were observed for CD8+ T cells, NK cells and B cells. $38 \%$ of severe-fulminant UC patients demonstrated low CMI activity. In contrast, none of the mild-to-moderate UC subjects or healthy volunteers demonstrated a low CMI response. Steroids and 6-MP administration were also associated with reduced lymphocyte counts. Conclusion: Increasing impairment of immune cell function was observed with increasing disease severity (and by inference increasingly stringent treatment regimens). UC patients refractory to steroid and 6-MP therapy are significantly immunosuppressed yet still have active disease. These data suggest that treatment strategies for this patient population should avoid general immunosuppression and rather focus on therapeutics that promote mucosal-healing and restoration of the mucosal immune balance.

\section{W1202}

Open-Label Pilot Study of Sequential Treatment with Metronidazole and E. Coli Nissle 1917 for Severe, Postoperative, Endoscopic Recurrence of Crohn's Disease

Karin C. Schweiger, Mina Meshkat, Gottfried Novacek, Clemens Dejaco, Harald Vogelsang, Walter Reinisch

Background: Patients with severe endoscopic recurrence of Crohn's disease (CD) one year after ileocecal resection are at high risk of suffering from a clinical relapse within the following year. Metronidazole is effective in the postoperative prevention of $\mathrm{CD}$ relapses. For Escherichia coli strain Nissle 1917 (EcN) anti-inflammatory activities in IBD have been demonstrated. We hypothesized that sequential treatment with metronidazole and $\mathrm{EcN}$ may enhance mucosal healing in patients with postoperative endoscopic recurrence of $\mathrm{CD}$. Patients and Methods: In an open-label pilot study we included 23 patients with severe endoscopic recurrence of $\mathrm{CD}$ as defined by a Rutgeerts' score of i3 or i4 after ileocecal resection, intolerant or refractory to azathioprine. The study was designed to apply metronidazole at a daily dose of 20-30 mg per kg for 12 weeks followed by two capsules of EcN daily (each capsule containing 2,5-25 x 109 viable bacteria) for 40 weeks. Concomitant medication had to be stable throughout the study. Ileocolonoscopies were performed before and after the treatment and the endoscopic score was determined by a single investigator who was unaware of any other patient-related study data. Response was defined as a Rutgeerts' score of i2 or less, remission was defined as mucosal healing matching i0. Results: At the time of inclusion 17 of 23 patients (74\%) were rated as i4 and 6 of 23 patients (26\%) as i3. In per intentionto-treat analysis response was observed in 7 patients $(30 \%)$ and complete remission in one patient (4\%). Three patients refused follow-up ileocolonoscopy, 9 patients discontinued or changed dose of study medication mostly due to gastrointestinal adverse events. The perprotocol analysis of 11 patients revealed 3 responders (27\%) and one patient with mucosal healing ( $9 \%$ ). One patient experienced a clinical flare of CD during the study period. Conclusion: Our results suggest that sequential treatment with metronidazole and E. coli Nissle 1917 may improve the endoscopic picture of patients with severe postoperative endoscopic recurrence of $\mathrm{CD}$ in a subset of patients. However, clinical relapse in patients at high risk of disease flare-up was rarely observed.

\section{W1203}

Extractible Polyurethane-Covered Expandable Nitinol Stent (PCENS) in the Treatment of Intestinal Strictures in Crohn's Disease: Preliminary Report of Seven Cases

Yoram Bouhnik, Philippe Bulois, Kouroche Vahedi, Jean F. Colombel, Marc Lemann

Background : Intestinal strictures are a common problem in patients with Crohn's disease. Endoscopic management with hydrostatic balloon dilation is an effective alternative to surgery in patients with endoscopically accessible lesions that are shorter than $4-6 \mathrm{~cm}$. However, recurrences are noted in about $2 / 3$ of cases and need further dilations or surgery. Extractible polyurethane-covered expandable nitinol stent (PCENS) has been proposed in the treatmen of benign intestinal stenosis. Purpose: To investigate the technical feasibility and clinical effectiveness of PCENS in the treatment of Crohn's intestinal strictures. Methods: Seven patients (M-4, F-3, mean age 41 years) with $C D$ who presented with obstructive symptom were treated with PCENS. All patients were under immunosuppressive therapy (azathioprine $\mathrm{n}=6$, methotrexate $\mathrm{n}=1$ ). They had strictures from recurrent disease at the site of an ileocolonic anastomosis. All of them except one have been successfully treated with balloon dilation but relapse occurred within 4 months. The mean length of the strictures evaluated radiologically was $31 \mathrm{~mm}$ (range $10-60$ ). The procedures were performed under intravenou sedation. Extractible PCENS was introduced from the operator channel of the colonoscope (diametre $4.2 \mathrm{~mm}$ ) under fluoroscopic control in all but one patient. Informed consent was obtained in all patients before procedure. Results : Stent placement was successful in 6 patients, but failed in one patient who had a $60 \mathrm{~mm}$ length stricture. After stent placement symptoms improved in 5 of the 6 patients. Stent migration occurred in four patients 1-3 days after the procedure. One stent was systematically extracted at W3, and one patient had to be operated on at W5 for occlusion due to incarceration of the stent. In the 5 other patients, one patient was operated on for occlusion at W2 (after stent migration), one patient relapsed at M12 (in relation with multiple small intestinal stenosis) and three patients remained symptom free at $10-17$ month follow-up. Conclusion : Treatment of CD intestinal strictures with PCENS is feasible and may represent an alternative to dilatation and surgery. Further studies are needed to evaluate the long term benefit of this new treatment.

\section{W1204}

Adacolumn Selective Leukocytapheresis in Patients with Active Ulcerative Colitis: Clinical Efficacy, Effects On Plasma Interleukin-8 and the Expression of Toll Like Receptors On Granulocytes

Yasuo Suzuki, Hiroshi Aoki, Kentaro Nakamura, Yasushi Yoshimatu, Yukiko Tuda, Masaki Irie, Katuyuki Fukuda, Nobuo Hosoe, Nobuo Takada, Koji Shirai

Background: Active ulcerative colitis (UC) is often associated with elevated peripheral blood granulocytes, monocytes/macrophages (GM) which show activation behaviour, increased survival time and are found in vast numbers within the intestinal mucosa. This is widely believed to contribute to the perpetuation and exacerbation of UC. Accordingly, recently we have been able to induce remission of active $\mathrm{UC}$ by selective GM apheresis (GMA) with the Adacolumn. Our view has been that the efficacy of GMA might not be fully attributed to the depletion of GM per se. In this study, we additionally investigated the effects of Adacolumn on plasma interleukin-8 (IL-8) and the expression of toll-like receptors (TLR). TLR are transmembrane proteins expressed predominantly on myelocytic cells, with essential role in innate and adaptive immune responses, but also inappropriate TLR signaling can lead to strong inflammatory response. TLR promote overexpression of proinflammatory cytokines including IL-lbeta and IL-8. Methods: Twenty-two patients with clinical activity index (CAI) 5-17 were included. Fifteen had total colitis and 7 had left-sided colitis. Twentyone patients were on aminosalicylates, $1.5-2.25 \mathrm{~g} /$ day for over 8 weeks prior to entry and 13 were on prednisolone, $5-40 \mathrm{mg} /$ day for at least 2 weeks prior to entry, but no additional drug was given. Each patient could receive up to 10 Adacolumn sessions at 1 or 2 sessions/ week. Efficacy was assessed during the course of GMA up to 2 weeks after the last Adacolumn session. Remission was defined as $\mathrm{CAI} \leq 4$. Further, at entry and pre-Adacolumn time points, blood samples were taken for determining full blood count, the expression of TLR on leukocytes by flow cytometry and plasma IL-8. Results: An $87.5 \%$ of patients achieved remission after the 10th session $(\mathrm{P}<0.001)$; the majority after 5 sessions. C-reactive protein fell to $<1 \mathrm{mg} / \mathrm{dL}$ in all patients $(\mathrm{P}<0.001)$ and there was a very marked fall in plasma IL-8 $(\mathrm{P}<0.05)$. Also, there was a fall in circulating granulocytes $(\mathrm{P}<0.05)$, but an increase in lymphocytes $(\mathrm{P}<0.05)$. The expression of TLR2 on granulocytes was significantly downmodulated by GMA $(\mathrm{P}<0.05)$. A similar down-modulation of TLR2 was observed when human blood was exposed to the Adacolumn leukocytapheresis carriers in vitro. Conclusions: It appears that Adacolumn leukocytapheresis is an effective adjunct therapy to induce remission in the majority of patients who are then spared from excess drug therapy. The procedure, in addition to depleting excess activated peripheral blood granulocytes and monocytes/macrophages is associated with interesting immunomodulation. Control studies should strengthen these findings.

\section{W1205}

Hyperhomocysteinemia Is Associated with Osteoporosis in Patients with Crohn's Disease

Xavier Roblin, Jean Marc Phelip, Veronique Ducros, Bruno Bonaz

Background: a high prevalence of osteopenia and osteoporosis is observed in Crohn's disease (CD) $(40 \%$ and $15 \%$ respectively). Risk factors are usually age, low weight, corticosteroid treatment, inflammation, malabsorption, and malnourishment. According to recent data, homocysteine concentration, which depends on diet intake, is an important risk factor in demineralization and bone fracture. Aim: The authors had for aim to screen for an association between homocysteine concentration and low bone mineralization (LBM) in CD patients. Patients and methods: CD patients were prospectively included between September 2003 and September 2005. Bone densitometry was performed on inclusion. LBM was defined by a T-score $<-1$ and osteoporosis by a T-score $<-2.5$ at the femoral neck and/or lumbar spine level (Ll-L4). Patients were excluded from the study if presenting with kidney failure, low 
vitamin D levels, or taking drugs able to interfere with homocysteine metabolism (folates, vitamin B12, methotrexate). Postmenopausal women, men over 50, and patients previous given anti-TNF infusions were also excluded. The following parameters were analyzed: age, sex, clinical activity indexes (CDAI), duration and impact- of CD, smoking status, corticosteroid treatment, immunosuppressive drugs, plasma homocysteine, folates, and B12 concentration. Results: 92 patients (sex ratio M/F 0.87; mean age: $36.6+/-13.2$ years) were prospectively included. The prevalence of a high homocysteine concentration $(>15 \mu \mathrm{mol} / \mathrm{l})$ was $59.7 \%$. In multivariate analysis, a low level of folates was the only risk factor for a high homocysteine concentration: RR: 3.59 [1.27 - 10.17]. Osteoporosis and LBM was observed in $26(28.2 \%)$, and $60(65.2 \%)$ patients respectively. In the multivariate analysis after an adjustment to the other parameters, associated factors for osteoporosis were hyperhomocyste-

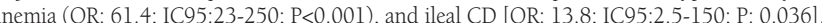
In the multivariate analysis, the associated factors with bone demineralization were: hyperhomocysteinemia [OR: 63.7; IC95:8.5-250; P<0.001] and a duration of disease of at least 5 years [OR: 11.4; IC95: 1.31-99; P: 0.039]. These results were comparable whatever the location of osteoporosis or LBM (femoral neck or lumbar vertebral). Conclusion: hyperhomocysteinemia was observed in $59.7 \%$ of our CD patients and strongly associated with LBM and osteoporosis (OR: 61.4). High hyperhomocysteinemia (> 15 $\mu \mathrm{mol} / \mathrm{l}$ ) was equally associated with bone demineralization in the hip and in the spine. The effect of folate supplementation on bone mineralization in $\mathrm{CD}$ should be of interest.

\section{W1206}

Safety and Effectiveness of Endoscopic Pneumatic Dilatation (EPD)+Infliximab Combined Therapy in Stenosing Crohn's Disease

Fausto Barberani, Sandro Boschetto, Alessandro Gigliozzi, Maurizio Giovannone, Mauro Tosoni

BACKGROUND: biological anti-TNF treatment(Infliximab-IFX)is routinely indicated in patients with fistulazing $\mathrm{CD}$ and in those refractory to other medical treatment. No definite data are currently available about IFX in stenotic patients and its modulation role in fibrogentic cytochine pathway is still controversial (Van Assch.Inflamm. Bowel. Dis. 2004; Di Sabatino Aliment. Pharmacol. Ther. 2004). EPD avoids surgery only in about $50 \%$ of these patients and frequently with relapses of stenosis and/or no complete symptoms release. AIM: to evaluate safety and effectiveness of EPD+IFX combined therapy in pts with stenotic CD refractory to other medical treatment. METHODS:between 2002 and 2005,25 pts(M:F17:8; age range 22-50)were diagnosed affected by stenosing CD.4/25 underwent to ileocolic surgery for severe stenosing disease;11/25 presented mild stenosing disease and continued 'conventional' medical therapy after successfully EPD;10/25 of these pts(moderate/severe disease-CDAI $>250$ )with unique and $<8 \mathrm{~cm}$ length ileal/ileocolic stenosis, underwent to EPD followed by IFX therapy $5 \mathrm{mg} / \mathrm{kg}$ scheduled with conventional induction timing. Endoscopic and small bowel ultrasonography (US)follow up were scheduled after 6 and 12 months and then each year, while clinical data were monitored every 8 weeks. 4 pts had associated perianal fistulas and 1 psoriasys.5/10 had anamnestic report of previous ileocolic surgical treatment for stenosis CD. RESULTS:all EPD were successfully performed with no complications; 3 pts have a follow up between 12 and 24 months, 4 between 24 and 36 months and $3>36$ months. No side effetcs or sensibilization were recordered during IFX infusion.All 10 treated pts had optimal clinical response with no significant symptoms at follow up and interrupted all other medical treatment in next 6 months.8/10 pts had no stenosis at each endoscopic and US follow up while only $2 / 10$ pts presented mild and asymptomatic stenosis at 6 months endoscopy and underwent to second EPD.8/10 pts had complete mucosal healing at each endoscopic follow up while only 2 pts presented mild flogistic activity.Only 1 pts underwent surgery for bowel sub-occlusion surgically related to fibrotic adhesion with pre-operatory endoscopy negative for stenosis or mucosal inflammation CONCLUSION:lack of obstructive complications during 2 years medium follow up of all our patients provides first evidence of safety of anti-TNF therapy in patient with stenotic CD after EPD Optimal clinical and endoscopic response to EPD+IFX in all our treated patients confirm effectiveness of each therapy and suggest this combination as new therapeutical option avoiding surgery in severe stenosing CD

\section{W1207}

Effect of Infliximab in Patients with Stricturing Crohn's Disease Frederic Marrache, Jean Marc Gornet, Vincent Pacault, Fathia Ben Hriz, Matthieu Allez, Maria Nachury, Marc Lemann

Background: Acute bowel obstruction has been reported in patients treated with Infliximab (IFX) for stricturing Crohn's disease (CD). For this reason IFX is not recommended in such patients. However, data concerning the potential risk or benefit of IFX in this setting are still scarce. The aim of this study is to report our experience with IFX in a series of patients with $C D$ strictures. Patients and methods: Among 141 patients treated between January 1999 and December 2004, 22 had 33 intestinal strictures (range 1-4) at the time of IFX infusion. Stricture-related symptoms were graded according to the following scale: 0: no symptom; 1: abdominal pain suggestive of intestinal stricture; 2: incomplete obstruction defined by nausea or vomiting, frequent abdominal pain or dyschesia; 3; complete obstruction. Location of strictures was duodenum $(n=1)$, jejunum $(n=4)$, ileum $(n=8)$, colon, $(n=$ 5), anorectum $(n=8)$ and anastomosis $(n=7)$. Evolution of stricture-related symptoms was recorded as well as the need for dilatation or surgery. Results: Median follow up was 16 months (range 0.5-53.9). Median number of IFX perfusion was 5 (range 1-18). Eight patients underwent a dilatation of stricture before IFX treatment (anus: $n=5$, anastomosis: $n=3$ ). Evolution of stricture-related symptoms is reported in the table. Complete obstruction occurred in three patients (14\%), 1, 7, and 11 months after the first IFX infusion, requiring surgical treatment $(\mathrm{n}=2)$ or dilatation of an ileorectal anastomosis $(\mathrm{n}=1)$. In addition, anorectal $(n=3)$ and anastomotic $(n=1)$ dilatations were performed due to the persistence of stricturerelated symptoms during IFX treatment in 4 patients. Conclusion: In this series of patients with stricturing Crohn's disease treated with IFX: 1) complete obstruction occurred in $14 \%$ 2) conversely, improvement of stricture-related symptoms was observed in $45 \%$ of symptomatic patients. The effect of IFX in stricturing Crohn's disease remains unclear and should be assessed in further studies
Evolution of stricture-related symptoms

\begin{tabular}{|c|c|c|c|c|}
\hline Stricture location & $\begin{array}{c}\text { Stricture-related symptoms at } \\
\text { baseline (nb of patients) }\end{array}$ & Improvement & $\begin{array}{c}\text { No } \\
\text { change }\end{array}$ & $\begin{array}{c}\text { Complete } \\
\text { obstruction }\end{array}$ \\
\hline \multirow{2}{*}{ Anorectum } & No (n=2) & - & 2 & 0 \\
\cline { 2 - 5 } & Yes (n=5) & 1 & $3^{*}$ & $1^{\circ}$ \\
\hline $\begin{array}{c}\text { Small intestine or } \\
\text { colon }\end{array}$ & No (n=9) & - & 8 & $1^{*}$ \\
\cline { 2 - 5 } & Yes (n=6) & 4 & 1 & $1^{\circ}$ \\
\hline
\end{tabular}

* dilatation; ${ }^{\circ}$ surgery

\section{W1208}

An Open Label Pilot Trial of Rifaximin in the Treatment of Patients with Refractory Pouchitis

Asher Kornbluth, Michele Hunt, James George, Peter Legnani

Introduction: Patients who undergo the ileoanal anastamosis procedure for ulcerative colitis are at risk for the development of idiopathic inflammation of the pouch, termed pouchitis. This complication occurs in at least $50 \%$ of patients followed long-term. Although there is limited randomized controlled trial data for the use of the antibiotics metronidazole and ciprofloxacin, they are the mainstay of therapy for patients with pouchitis, with approximately $80-90 \%$ success rates . However about $5-10 \%$ of patients are either refractory to these medications or require chronic suppressive antibiotic therapy. Rifaximin is a nonabsorbable antibiotic with a broad range of coverage against small bowel intestinal flora. Objective: The aim of this study was to determine the safety and efficacy of rifaximin in the treatment of patients with refractory pouchitis. Methods: 16 patients who were refractory to all therapies including metronidazole, ciprofloxacin and probiotic therapy with VSL-3, Lactobacillus, or Saccaromyces boullardi were treated with rifaximin; 6 of these patients had also failed treatment with budesonide $9 \mathrm{mg}$ /day prior to rifaximin. Patients were treated with the addition of rifaximin in doses of 600-800 mg daily. The diagnosis of pouchitis was confirmed in all patients prior to starting rifaximin treatment with pouchoscopy, and severity was defined as moderate-severe using the Pouchitis Disease Activity Index. Results: 11 patients were treated with rifaximin $400 \mathrm{mg}$ BID, and 5 patients were treated with 200 TID. 9 of 11 patients (82\%) treated with $400 \mathrm{mg}$ BID demonstrated significant clinical improvement and 4 of 5 patients $(80 \%)$ treated with $200 \mathrm{mg}$ TID demonstrated clinically significant improvement. There were no adverse effects of treatment, and all patients with clinical response improved within 3 weeks. All patients with previously medically refractory pouchitis who responded to rifaximin continued maintenance therapy with a mean dose of $400 \mathrm{mg}$ / day and sustained clinical improvement during a mean follow up of 16 weeks. Conclusions: In this open-label series of 16 patients with medically refractory pouchitis, rifaximin in doses of $600-800 \mathrm{mg} /$ day was effective in $81 \%$, without toxicity, and was effective for maintenance for a mean of 4 months, with ongoing follow-up. Prospective randomized controlled trials are warranted to define the role of rifaximin in the management of patients with pouchitis.

\section{W1209}

Short-Term Response to Infliximab in Ulcerative Colitis and Pouchitis: Results from a Single-Center Experience

Marc Ferrante, Severine Vermeire, Maja Noman, Godelieve Godefridis, Gert van Assche, Ilse Hoffman, Xavier Bossuyt, Paul Rutgeerts

BACKGROUND \& AIMS: Infliximab (IFX) is an efficacious drug for treatment of moderate to severe ulcerative colitis (UC) as shown by the recent ACTl and ACT2 randomised controlled trials. We studied the outcome of IFX treatment in UC patients in a single-center and investigated if predictors of early response or failure could be identified. METHODS: 103 patients (mean age 40 yrs (range 9-82 yr) and 50\% female) with mild to moderate UC $(n=95)$ or indeterminate colitis $(n=8)$ were treated with IFX. Eighty-seven patients $(84.4 \%)$ received $5 \mathrm{mg}$ and $15.6 \%$ received $10 \mathrm{mg}$ IFX per kg body weight. Clinical response was defined as complete if there was absence of diarrhea and blood loss, partial if there was marked improvement but still with presence of some blood. In 53 (51.5\%) patients, sigmoidoscopy (Mayo score) was performed before infusion as well as at week 4 (in case of single infusion) or week 10 (in case of three-dose induction IFX at weeks 0-2-6). Two thirds $(67.0 \%)$ of patients were on concomitant immunosuppressive therapy at the start of IFX (55 on azathioprine, 14 on methotrexate). Eleven patients (10.7\%) had a previous colectomy and were treated for refractory pouchitis. Multivariate analysis was done to identify variables influencing response. RESULTS: Overall $69(67.0 \%)$ of patients showed clinical response (45 complete and 24 partial) and $34(33.0 \%$ ) had no response. Mucosal healing (Mayo score 0 or 1 ) was observed in $3 / 14(21.4 \%)$ non-responders versus $31 / 39(79.5 \%)$ responders $(\mathrm{p}=0.0001)$. Clinical response was not different in the 40 patients receiving 0-2-6 induction therapy $(67.5 \%)$ compared to the 63 patients not given the three-dose induction $(66.7 \%)$. Although more mucosal healing was observed with the induction scheme $(69.7 \%$ versus $55.0 \%)$, this lacked significance ( $\mathrm{p}=0.28)$. Gender, age, extent of disease, smoking, baseline $\mathrm{CRP}$, concomitant immunosuppressive therapy and the presence of perinuclear antineutrophil cytoplasmic antibodies (pANCA) did not influence the short-term outcome of IFX. DISCUSSION: In this large single-center cohort of UC patients treated with infliximab, the short term response was $67.0 \%$ and mucosal healing was observed in $64.2 \%$ of patients. We could not identify clinical or serological predictors of early response or failure. Other serological and genetic markers are under investigation. Infliximab is an effective drug for the treatment of UC. 


\section{W1210}

Ciclosporin in Severe Ulcerative Colitis: Predictors of Response Garret Cullen, Denise Keegan, Hugh E. Mulcahy, Diarmuid P. O'Donoghue

Introduction: Ciclosporin (CsA) will induce a remission in severe steroid resistant ulcerative colitis (UC) in $65 \%$ of patients. Opponents of CsA argue that this merely delays surgery and does not justify the risk of adverse drug reactions. Aim: To examine the long-term outcome for patients treated with CsA in a single unit and evaluate factors associated with response. Methods: 107 consecutive UC patients were treated at this unit since 1994. Data were retrieved from a prospectively maintained database, chart review and telephone interview. All subjects had failed at least 5 days of intravenous corticosteroids. A standard regimen of ciclosporin $4 \mathrm{mg} / \mathrm{Kg}$ IV daily in 2 divided doses for lweek was used. Responders were then treated with oral CsA for a further 3 months. Treatment failure was defined as the requirement for colectomy within 6 months of commencing CsA and this was used in the primary analysis. A secondary analysis was performed using colectomy (either before or after 6 months) as the endpoint. Results: 62 patients achieved clinical remission more than 6 months in duration. The use of immunomodulatory drugs $(\mathrm{p}=0.004)$ was associated with this response as was male gender $(\mathrm{p}=0.009)$. Regression analysis showed that both of these variables were independently associated with a sustained response. 38 of these 62 patients remain in long-term remission. Immunomodulatory drug use was also associated with a favourable long-term outcome $(\mathrm{p}<0.001)$ as was previous or current cigarette smoking $(p=0.03)$. Adverse reactions to CsA were unusual and reversible. Conclusion: Only one third of patients with severe UC rescued by CsA avoid surgery. Concomitant immunomodulatory drug use has a significant beneficial impact on initial response and long-term remission in clinical practice.

\section{W1211}

The Use of Tacrolimus in Children with Ulcerative Colitis: A 5 Year Experience

David A. Ziring, William Mow, Marvin E. Ament

Aim: To review our experience in treating children with both steroid-dependent and steroidresistant ulcerative colitis with tacrolimus, and determine those factors that contributed to successful outcomes. Methods: We evaluated eighteen consecutive patients (13 with pancolitis) who were treated with oral tacrolimus at UCLA Mattel Children's Hospital, from May 1999 to October 2005, in our retrospective review. Ten patients had the diagnosis of steroidresistant UC, defined as continued symptoms of bloody diarrhea despite five days of IV steroids at a dosage of $1-2 \mathrm{mg} / \mathrm{kg}$ (up to $60 \mathrm{mg} / \mathrm{d}$ ), and eight patients were steroid-dependent, defined as inability to wean from steroids secondary to recurrence of symptoms of diarrhea, urgency, and/or bloody stools. We started patients initially on tacrolimus $0.2 \mathrm{mg} / \mathrm{kg}$ divided twice daily into equal dosage, with a goal of plasma trough level of $10-15 \mathrm{ng} / \mathrm{ml}$ for the first two weeks, and then titrated doses to achieve plasma levels between $7-12 \mathrm{ng} / \mathrm{ml}$ following induction. All patients received prophylaxis for Pneumocystis carinii pneumonia and magnesium supplementation if necessary. We monitored our patients with daily measurements of serum electrolytes, blood urea nitrogen, creatinine, magnesium, and tacrolimus trough levels on all inpatients until their lab values and plasma drug levels were stable. Results: Of the 18 patients in this study, seventeen responded positively to tacrolimus therapy, and one was a non-responder. Four patients achieved a prolonged response to tacrolimus while ten required colectomy. Three patients had only an abbreviated response (less than three months) before they required surgery. The mean time in days from initiating tacrolimus therapy until a response was noted (cessation of diarrhea) was 8.5 days. The mean duration of response was 260 days (representing those patients that had to discontinue tacrolimus and those who continue to receive tacrolimus as of the last follow-up date.) The mean time from initiation of tacrolimus therapy until colectomy was 392 days. Steroid-dependent patients have a higher likelihood of prolonged remission with tacrolimus. Conclusions: We found that using tacrolimus in pediatric patients with steroid-resistant UC was unlikely to provide a positive prolonged clinical response, and in fact, all of these patients either underwent curative colectomy or are now considering colectomy in the short term. Although we have treated only six patients with steroid-dependent UC with tacrolimus, it is possible that this therapy may be of benefit in those who have problems tolerating traditional maintenance therapy with $6 \mathrm{MP}$ or azathioprine.

\section{W1212}

Treatment of Steroid Refractory and Steroid Dependent Crohn's Disease (CD) with Leflunomide (LEF) - An Open Label, Controlled Phase I Study On Efficacy and Tolerability

Martin H. Holtmann, Anna-Lena Gerts, Arndt Weinmann, Peter R. Galle, Markus F. Neurath

Introduction: Azathioprine is the gold standard for maintenance of remission in CD. However, in a relevant proportion of patients, azathioprine is not effective or not tolerated. Equally effective treatment options are not available. Since 1999, Leflunomide is approved as disease modifying agent in rheumatoid arthritis (RA). Because of immunopathogenetic similarities between CD and RA, we studied LEF as second line therapy for maintenance of remission in CD. Methods: 24 patients with AZA refractoriness or intolerability were included. Treatment was started with $100 \mathrm{mg} / \mathrm{d}$ over $3 \mathrm{~d}$, followed by $10-20 \mathrm{mg} / \mathrm{d}$. Primary end points were reduction of CDAi and steroid requirement, given as medians with quartils as well as adverse side effects over a period of 12 months. T-testing with $\mathrm{p}<0.05$ indicated statistical significance. Results: Of 24 patients included, 8 patients completed the trial. The CDAi decreased significantly from a median of $219(160-264)$ to $87(20-127)(\mathrm{p}=0.018)$. The steroid sparing effect was significant, too, with a decrease from $25 \mathrm{mg} / \mathrm{d}(12-50)$ to $3 \mathrm{mg} / \mathrm{d}(0-20)(\mathrm{p}=0.033)$. All 9 patients with predominant arthralgias reaches partial or complete remission after 3 months. The predominant adverse side effects were skin rashes (37\%) and headaches (31\%). 20$25 \%$ of the patients reported mostly selflimiting alopecia, nausea, high blood pressure. Less than $10 \%$ developed anemia, tachycardia, tremor, insomnia, constipation, diarrhea and others. 13 out of 24 patients developed $>4$ adverse side effects. These led to discontinuation of the treatment in 15 patients. Discussion: LEF is probably effective in $C D$, in particular in the treatment of $C D$ associated arthralgias. However, the rate of adverse side effects was much higher than in the studies published for RA. The may in part be explained by the highly pre-selected group of patients characterised by sometimes multiple drug intolerabilities. Interestingly, a recent post marketing study reported a $70 \%$ discontinuation rate in RA because of lack of efficacy (38\%) and intolerability (32\%). Further studies are warranted to define the potential role of LEF in CD.

\section{W1213}

Oral Glycine Improves Symptoms in Mild to Moderate Ulcerative Colitis: An Open-Label Pilot Study

Shih-Kuang S. Hong, Christian D. Stone

PURPOSE: L-glycine is a nonessential amino acid with cytoprotective properties. In mice, dietary supplementation with glycine has been shown to effectively treat and prevent development of chemically induced colitis. This study sought to 1) determine whether oral glycine has a therapeutic benefit in subjects with mild to moderately active ulcerative colitis (UC) and 2) establish its safety in UC patients. METHODS: Seven subjects with mild to moderately active UC, defined by the Simple Clinical Colitis Activity Index (SCCAI), were treated with open-label L-glycine orally at a dose of $12 \mathrm{~g}$ three times daily for 8 weeks. An SCCAI score $\leq 4$ is defined as clinical remission; $5-8$ as mild disease; $9-12$ as moderate disease; and $\geq$ 13 as severe disease. All subjects were maintained on stable doses of concomitant medication and additional therapy for UC was not allowed during the study period. A complete clinica response is defined as a decrease of $\geq 4$ points. Patients with SCCAI score 5-12 and aged 18 to 65 were included. Patients on daily prednisone dose $>20 \mathrm{mg}$, or with history of chronic renal or hepatic disease were excluded. Subjects requiring additional therapy for UC during the treatment period were dropped from the study. The primary endpoint was change in SCCAI score at week 8. Secondary endpoints included change in IBD Questionnaire (IBDQ) and visual assessment scale (VAS) scores at week 8. Comparisons were made using a paired Student's t-test. RESULTS: Mean age was 39 (range 26-58). Five of 7 subjects completed 8 weeks of therapy. One subject was lost to follow-up and another required an increase in prednisone dose. Mean SCCAI scores at weeks 0 and 8 were 7.0 and 4.2 respectively $(\mathrm{p}=0.045)$. Three of 5 subjects achieved complete response and 2 achieved remission. Mean SCCAI scores at weeks 0 and 4 were 7.1 and 5.6, respectively $(p=0.1)$. At 4 weeks, 3 of 7 subjects achieved complete response. Mean IBDQ scores at 0 and 8 weeks were 131 and 159 , respectively $(p=0.01)$. Mean VAS scores at 0 and 8 weeks were 60 and 75 , respectively $(\mathrm{p}=0.02)$. Glycine was well tolerated by all subjects. One subject on concomitant mesalamine developed self-limited interstitial nephritis. CONCLUSIONS: Oral glycine improved symptoms and quality of life in patients with mild to moderately active UC in this open label study. Three of 5 patients achieved complete response. Ora glycine was safe and well-tolerated. These results support further study of oral glycine in $\mathrm{UC}$ in a controlled trial.

\section{W1214}

Infliximab Therapy and LFT Abnormalities in Patients with Crohn's Disease Eric Blum, Jeffry Katz

Background: Infliximab has been associated with elevations in ALT and AST in Phase III trials for Crohn's disease (CD). In post-marketing studies, infliximab has also been associated with significant hepatotoxicity, including acute liver failure, jaundice, hepatitis, and cholestasis. However, significant hepatotoxicity has not been evident in patients receiving infliximab for $\mathrm{CD}$ at our institution. Aims: The aims of this study were 1) to evaluate the change in LFTs associated with infliximab infusion and 2) to identify subgroups of infliximab-treated patients at increased risk for LFT abnormalities. Methods: 79 patients who received at leas one dose of infliximab were evaluated. Pre- and post-infusion LFTs for each patient were collected along with data on age, sex, race, use of concomitant immunomodulators, and smoking status at the time of the infusions. Analyses were done using paired sample Wilcoxon rank sum tests and multivariate linear regression models. Results: Table 1 shows the overall distribution of normal and abnormal values for each of the four LFTs. Infliximab was associated with an above normal elevation of AST in 3.8\% of patients and of ALT, alkaline phosphatase, and total bilirubin in $6.3 \%$ of patients. Table 2 shows the median for each pre and post-infusion LFT along with the associated $95 \% \mathrm{CI}$ and p-value for the comparison In multivariate linear regression models, only the pre-infusion LFT was a consistent predictor of the post-infusion LFT. No patient sub-groups could be identified with an increased risk of elevated LFTs, including patients on immunomodulators. Conclusions: Although there is a statistically significant difference in AST, ALT, and total bilirubin after infliximab infusion, the absolute differences are not clinically important. In routine clinical practice, infliximab does not appear to be associated with significant liver function abnormalities Table 1

\begin{tabular}{|c|c|c|c|}
\hline & Normal & $1-2 \times$ ULN & $>2 \times$ ULN \\
\hline AST Pre Post & 7673 & 35 & 01 \\
\hline ALT Pre Post & 7267 & 49 & 33 \\
\hline Alk Phos Pre Post & 6964 & 914 & 11 \\
\hline Total Bilirubin Pre Post & 7772 & 26 & 01 \\
\hline
\end{tabular}

ULN = upper limit of normal

Table 2 


\begin{tabular}{|c|c|c|c|}
\hline & Median & $95 \% \mathrm{CI}$ & P-value \\
\hline AST Pre Post & 20.021 .0 & -5.0 to -0.00004 & 0.04 \\
\hline ALT Pre Post & 21.029 .0 & -10.0 to -0.00003 & 0.03 \\
\hline Alk Phos Pre Post & 84.076 .0 & -4.5 to 7.5 & 0.57 \\
\hline Total Bilirubin Pre Post & 0.30 .4 & -0.2 to -0.05 & 0.001 \\
\hline
\end{tabular}

\section{W1215}

Endoscopic Polypectomy for Dysplastic Polyps in Chronic Ulcerative Colitis Rabi Kundu, Wojciech Blonski, Franz Fogt, Chinyu Su, James D. Lewis, Julius J. Deren, Gary R. Lichtenstein

Background: Malignancy in Chronic Ulcerative colitis (CUC) is believed to develop by adenoma carcinoma sequence. The management of dysplastic polyps is evolving. At least 2 small retrospective studies have suggested endoscopic polypectomy is safe for management of dysplastic polyps in CUC (Rubin, Gastro 1999;117:1295. n=30; Odze, Clin Gastro Hep 2004:2:534. n=24). This study reports outcomes of 30 pts with CUC and dysplastic polyps following colonoscopic polypectomy. Methods: Institution / pathology database searches for words dysplasia, low grade dysplasia (LGD), high grade dysplasia (HGD), ulcerative colitis, and inflammatory bowel disease from 1997-2004. All pathology reports were screened for dysplastic polyps.Patients with dysplastic polyps were evaluated in this study. Outpatients, inpatients and endoscopic data were reviewed for outcomes. Results: 87 pts had dysplastic lesions of whom 30 CUC pts had dysplastic polyps removed and are in the study. Mean age was 55 yrs (17-80 yrs). 24 pts were male. The total no of polyps resected were 54.Mean no of dysplastic polyp per patient is 1.8. Mean size of polyps $4 \mathrm{~mm}(2-13 \mathrm{~mm}) .6(11 \%)$ polyps occurred outside the area of active colitis. The index polyps were distributed 6 in rectum, 5 in sigmoid colon, 3 in descending colon, 7 in transverse colon, 10 in ascending colon and 6 in cecum. Of the 30 pts, the most advanced polyp histology was HGD in 9 and LGD in 21. Mean followup of the whole group was 38.4 mos (1-101 mos), during which 87 colonoscopies were done, mean 2.9 per pt (range: 1-11). One pt progressed from LGD to HGD and regressed to LGD (in an area outside of colitis) and one from HGD to cancer in 4 months. During surveillance 4 pts with LGD polyps formed new polyps which were removed. 7 pts (23\%) had colectomy, 6 for flat LGD and 1 for cancer. The diagnosis of cancer in one patient following removal of polyp with HGD is concerning, but the diagnosis was made in 5 months after the initial colonoscopy and was detected in early stage(well differentiated, Duke A). No complications occurred after colonoscopy or colectomy. Conclusion: In our experience polypectomy and stringent colonoscopic surveillance is an acceptable modality of treatment for dysplastic polyps in CUC, although the diagnosis of cancer in one patient following removal of a polyp with HGD is concerning. This confirms earlier observation by Odze and Rubin et al. Longer follow up is needed to clarify the safety of this strategy

\section{W1216}

Tacrolimus (FK506) Is Safe and Effective in Patients with Refractory Extraintestinal Manifestations of Inflammatory Bowel Disease

Daniel C. Baumgart, Barbara T. Tanczos, Andreas Sturm, Bertram Wiedenmann, Axel U. Dignass

BACKGROUND: Between five to twenty percent of inflammatory bowel disease patients develop extraintestinal disease manifestations (EIM), which do not always respond to conventional medical or topical therapy. Tacrolimus, a macrolide immunosuppressant inhibiting T cell activation, is currently approved for the prophylaxis of organ rejection in patients receiving allogeneic liver or kidney transplants. We have recently published the successful use of oral low dose tacrolimus in refractory inflammatory bowel disease (IBD). Here we report our experience in managing refractory EIM in patients with IBD. METHODS: In this retrospective, observational single center study the charts of 29 (16 female, 13 male) adult (mean age: $41.6 \pm 11.6 \mathrm{SD}$ years) IBD patients with steroid-dependent $(\mathrm{n}=15)$ or steroidrefractory $(n=14)$ inflammatory bowel disease (Crohn's disease, $n=7$; ulcerative colitis, $n=10$; pouchitis, $\mathrm{n}=2$ ) and extraintestinal disease manifestations (reactive arthritis $\mathrm{n}=14$, sclerosing cholangitis $=5$, pyoderma gangrenosum $n=7$, erythema nodosum $n=1$, autoimmune hemolytic anemia $\mathrm{n}=1$, autoimmune hepatitis $\mathrm{n}=1)$ were reviewed. Tacrolimus $(0.1 \mathrm{mg} / \mathrm{kg}$ body weight per day) was administered orally in all patients, aiming for serum trough levels of $4-8 \mathrm{ng} / \mathrm{mL}$. No PCP prophylaxis was administered. The mean treatment duration was $33 \pm 7.6$ SD months (range, 2.8-164.4 months). Patients were followed-up for a mean of 46 $4.3 \mathrm{SD}$ months (range, 12-164.4 months). Response was evaluated using a disease activity score (M-CAI), laboratory data, and or repeated skin inspection plus photo documentation. RESULTS: 21 patients $(72.4 \%)$ experienced a clinical and laboratory response, usually between 3 and 5 days. Tacrolimus was particularly effective in skin, blood and hepatic EIM. Mean M-CAI values dropped from 12.2 at initiation to 2.5 at 36 months follow-up. Sideeffects included a temporary rise of creatinine $(n=2,6.9 \%)$, hyperkalemia $(n=1,3.45 \%)$, and opportunistic infections $(n=13.45 \%$ ). CONCLUSION: Oral low dose tacrolimus appears to be safe and effective in refractory EIM of inflammatory bowel disease.

\section{W1217}

Dilation of Colonic Strictures By Intralesional Injection of Infliximab in Patients with Crohn's Colitis

Simon Lichtiger

Intestinal stenosis is a frequent complication of Crohn's disease, often leading to repeated bowel obstruction and surgery. The prevalence of small bowel strictures has ranged from $20-40 \%$,and $7-18 \%$ in colonic disease. Although balloon dilation is an initial approach, many patients end up with surgical resection or stricturoplasty. Infliximab, a monoclonal antibody against TNF, has been effective in the treatment of enteric as well as fistulous disease. In patients with active disease, tissue levels of TNF are elevated and may mirror activity of disease. Repeated systemic administration has been successful for active inflammatory disease, yet has been reported to be ineffective for stricturing disease. This report describes three patients with obstructive disease who had failed all medical therapy, in whom successful dilation was accomplished by injection of Infliximab intrastricturally. Methods: Two patients with colonic strictures and a patient with a rectal stricture were studied. $100 \mathrm{mg}$. of Infliximab was injected circumferentially in sclerotherapy technique into the distal end of short strictures. None of these strictures admitted a pediatric colonoscope. .Results: Within three weeks, each of the strictures were dilated leading to easy passage of the colonoscope in all three patients. Each of these patients represented with obstructive symptoms at approximately three months, and responded to reinjection. Conclusion: Intrastrictural injection of $100 \mathrm{mg}$. of Infiximab was effective in dilating short strictures which had not responded to all medical therapy. Similar to patients who respond to systemic Infliximab, these patients recurred with symptoms at approximately three month intervals, and responded to reinjection. This technique requires further investigation, and may hold some promise in the treatment of inflammatory strictures.

\section{W1218}

Allopurinol Favorably Affects AZA/6mp Metabolism By Lowering 6-Mmp Levels in Patients with Hepatoxicity from AZA/6mp Without Affecting Red Blood Cell Thiopurine Methyltransferase (TMPT) Activity

Purvi C. Panchal, Charles J. Zielman, Cynthia Schuhmacher, John F. Valentine

Background: 6-Mercaptopurine (6MP) and azathioprine (Aza) are metabolized to 6-thioguanine $(6 \mathrm{TGN})$, the putative therapeutic metabolite for the treatment of inflammatory bowel disease. However, many patients are unable to tolerate this class of medication secondary to high levels of the hepatotoxic metabolite, 6-methyl-mercaptopurine (6-MMP). 6MP is metabolized to 6MMP by TPMT and the activity of TPMT is genetically determined. We examined the effect of allopurinol on 6MMP levels and TPMT activity in patients who had hepatotoxicity on Aza or 6MP. Methods: 12 patients (9 with Crohn's disease, 3 with ulcerative colitis) who had elevated transaminases and toxic levels of 6-MMP while on 6-MP or AZA were treated with allopurinol 100mg per day and 6-MP (11) or Aza (1) at 25-30\% of their hepatotoxic doses. Weekly liver function tests and complete blood counts were monitored for potential side effects such as hepatotoxicity, leukopenia, anemia, or thrombocytopenia. At four weeks from the initiation of allopurinol, 6-TGN and 6-MMP metabolite levels, and TPMT enzyme activity levels were determined (Prometheus Labs). Results: Complete data is available of 8 subjects and one additional pt is missing TPMT data. With concomitant use of allopurinol and a lower dose of 6-MP or Aza, the mean 6-TGN levels changed from 194 to $283 \mathrm{pM} / 8 \times 10^{\wedge} 8 \mathrm{RBCs}(\mathrm{p}<0.04)$. The mean 6-MMP levels decreased from 21,143 to $598 \mathrm{pM} / 8 \times 10^{\wedge} 8 \mathrm{RBCs}(\mathrm{P}<0.0002)$. There was no statistically significant change found in red cell TPMT activity. The mean TPMT activity prior to allopurinol was $30.42 \mathrm{EU}$ and activity while on allopurinol was $32.39 \mathrm{EU}(\mathrm{P}=0.3)$. All patients liver profiles remained normal. Conclusions: In patients with demonstrated hepatoxicity from 6MP or Aza, institution of low dose 6-MP or Aza along with allopurinol $100 \mathrm{mg} / \mathrm{d}$ was able to achieve therapeutic levels of the active metabolite, 6-TGN, while significantly decreasing 6-MMP, the hepatotoxic metabolite, to low or undetectable levels. All patients maintained normal liver profiles. These data confirm that IBD patients who have been unable to tolerate 6-MP or AZA secondary to hepatoxicity, may now be able to resume 6MP or Aza at lower doses with allopurinol and effectively avoid the hepatotoxic side effects. Furthermore, the addition of allopurinol did not affect TPMT enzyme activity suggesting an alternative mechanism for allopurinol that remains to be determined.

\section{W1219}

Efficacy and Safety of Leflunomide Therapy in IBD Patients. An Uncontrolled Observational Study

Milan Lukas, Robert Donoval, Martin Bortlik

Introduction: The optimal immunosuppressive therapy in inflammatory bowel disease (IBD) patients who are unresponsive or intolerant to thiopurines is still missing. The pyrimidine analogue leflunomide has been shown to be effective in rheumatoid arthritis in patients who had been previously intolerant to thiopurine analogues. Aims of the study: 1. to evaluate efficacy of leflunomide therapy in IBD patients unresponsive to thiopurine analogues; 2 . to assess a tolerability and side effects of leflunomide therapy in IBD patients. Methods: This was an uncontrolled, observational study, performed in a tertiary referral center during years 2004-2005. Twelve IBD patients ( 7 female), who had been unresponsive or intolerant to standard immunosuppressive treatment (AZA/6-MP), were managed by leflunomide $20 \mathrm{mg}$ p.o. daily. There were 5 pts with Crohn's disease (CD), and 7 pts with ulcerative colitis (UC), mean age 40.5 years. All patients were treated by corticosteroids (mean dose $25 \mathrm{mg}$ prednisone per day) for chronic active course of $\mathrm{UC}$ (mean UC-DAI=8.8), or CD (mean $\mathrm{CDAI}=277$ ) prior to leflunomide initiation. Demographic information, concomitant therapy, disease activity, and adverse events were evaluated. Results: The mean time of leflunomide treatment was 22 weeks. Positive response, i.e. decline of disease activity index (UC-DAI>2, CDAI $>70)$, or reduction or withdrawal of corticosteroids, was observed in 5 pts $(42 \%)$ Nevertheless, no patient went to the complete clinical remission during treatment period. Adverse events due to leflunomide therapy developed in 7 pts (58\%). In 6 of them frequent liquid stool appeared within 2-8 weeks after leflunomide initiation. For three patients, the diarrhea was disqualifying to continue in the treatment with leflunomide. The skin rash in one patient and mild elevation of functional liver tests in two other cases were observed. Conclusions: 1. The positive therapeutical response of leflunomide therapy was observed in $42 \%$ of IBD patients with previously unsatisfactory course of the disease. However, no clinical remission during the mean time of 22 weeks of leflunomide treatment was achieved. 2. The adverse events were quite frequent ( $7 / 12 \mathrm{pts})$. Diarrhea, the most often disqualifying side effect, might be the major limitation for widespreading this drug in clinical practice. Futher trials with leflunomide on IBD patients unresponsive or intolerant to standard immunomodulators are needed. 
study. All patients had not responded to azathioprin therapy given in a dosage of $2 \mathrm{mg} / \mathrm{kg}$

Increased IL-10 Production By Peripheral Blood Memory T Cells Accompanies Crohn's Disease (CD) Remission and Clinical Response Induced By G-CSF Treatment

Peter Mannon, Francisco Leon, Ivan Fuss, Catherine Groden, Zhiqiong Yang, Chuli Yi, Julia Friend, Ronald Hornung, Margaret Brown, Shakuntala Gurprasad, Brian Kelsall,

Warren Strober

BACKGROUND: CD is associated with excess Thl cytokine activity. G-CSF has several properties that could modulate Thl activity including inhibition of IL-12R $\beta 2$ expression to reduce IL-12 signals and enhancing plasmacytoid dendritic cells (PDCs) to induce Th2 and T regulatory cell responses. We studied whether G-CSF effects on peripheral blood dendritic cells and memory T cell cytokine production were associated with responses to G-CSF treatment. METHODS: 9 patients with active CD (CDAI of 220-450) received G-CSF $5 \mu \mathrm{g}$ / $\mathrm{kg}$ SC QD for 29 days with follow-up (F/U) for 24 weeks after the final dose. Outcomes included changes in CDAI scores, blood DC populations (lin-DR ${ }^{+} \mathrm{CD} 11 \mathrm{c}^{+}$for myeloid DCs, lin ${ }^{-} \mathrm{DR}^{+} \mathrm{BDCA}-2^{+}$for PDCs), and CD4/CD45RO cytokine production by ELISA. RESULTS: 6 of 9 patients (baseline CDAI $313 \pm 18$, mean \pm SEM) had a clinical response (drop $\geq 100 \mathrm{CDAI}$ points) including 2 remissions $(\mathrm{CDAI}<150)$. Responses occurred by 2 weeks and were durable for 8 to 24 weeks after the last dose. CD4/CD45RO cells from responders produced significantly more IL-10 at the end of treatment vs nonresponders (NRs) $(14690 \pm 2808 \mathrm{pg} /$ $\mathrm{ml}$ vs $4574 \pm 290$, mean \pm SEM, $\mathrm{p}=0.04)$, with a trend of increased IL-4 production $(9042 \pm 3774$ $\mathrm{pg} / \mathrm{ml}$ vs $1987 \pm 983, \mathrm{p}>0.05$ ) and no differences in INF $\gamma$ or TNF $\alpha$ production. Responders showed a significantly decreased myeloid (MDC) to PDC ratio at pretreatment vs the end of treatment $(2.1 \pm 0.2 \mathrm{vs} 1.1 \pm 0.2$, mean \pm SEM MDC/PDC, $\mathrm{p}=.003)$ and kept a low ratio after 4 weeks of $F / U(1.1 \pm 0.4, p=0.05)$. NRs showed a trend of lower MDC/PDC ratio at the end of treatment $(3.5 \pm 1.7$ vs $0.9 \pm 0.3 p>2)$ rebounding after 4 weeks of $F / U(2.3 \pm 0.4 p>0.6)$. There were no significant differences in increases of PDCs between groups (responders: $19 \pm 4$ pretreatment, $29 \pm 8$ at and of treatment and $24 \pm 74$ weeks after treatment mean \pm SEM $\% \mathrm{PDC} / \mathrm{PBMC}\left(\mathrm{x10^{2 }}\right)$; NRs: $16 \pm 8$ to $28 \pm 8$ and $5.2 \pm 0$ ), NRs showing a trend of nonsustained PDCs. Corticosteroid use significantly inhibited the effect of G-CSF on the MDC/PDC ratio: steroid group $(n=4) 3.1 \pm 1.3$ to $1.4 \pm 0.1$ at the end of treatment, $p>0.2$ vs the steroid-free group $(\mathrm{n}=5) 2.2 \pm 0.3$ to $0.7 \pm 0.1 \mathrm{p}=0.001$. Steroid use also inhibited increased IL-10 production by peripheral CD4/CD45RO cells. CONCLUSIONS: Clinical responses to G-CSF therapy of $\mathrm{CD}$ are accompanied by increased production of IL-10 by blood CD4/CD45RO cells. Clinical responses were also accompanied by durable decreases in blood MPC/PDC ratios. Corticosteroid use blocked the effect on DC ratio changes and inhibited induction of IL-10-producing cells, due perhaps to reported PDC enhanced sensitivity to steroids. G-CSF may be a valuable agent in $\mathrm{CD}$ by exerting unique immunomodulatory effects.

\section{W1221}

Subcutaneous Methotrexate: A Safe and Effective Therapy in IBD Peter Gibson, Debbie Nathan, Iser John

Increased understanding of the chronically active nature of Crohn's disease in the majority of patients has led to the widespread use of long-term maintenance therapy with immunosuppressive agents. Purine analogues have been the mainstay, but more than one in three patients experience intolerance or lack of efficacy. Methotrexate (MTX) has high level evidence for efficacy but its application has been limited in part due to the impracticality of intramuscular administration and the perception of poor efficacy when used orally. Aim: To assess the response to and safety of MTX when used predominantly by the subcutaneous route in patients with IBD in a single IBD Clinic. Methods: The medical records of patients treated with MTX were evaluated with regard to the dose, duration, tolerance of and response to MTX. Response was defined as improvement in bowel symptoms and/or ability to reduce the dose of steroids. Remission was defined as improvement in symptoms with no requirement for steroids for three months, or ability to wean off steroids. Results: 65 patients, 45 Crohn's disease (CD) and 20 ulcerative colitis (UC), were identified. The median (range) age was 46 (19-79) years. $46 \%$ were men. MTX was initiated because of intolerance or resistance to azathioprine/6MP in $45(69 \%)$ and $9(17 \%)$, respectively. 8 patients were considered steroid-dependent. The initial weekly dose was $25 \mathrm{mg}$ in 29 patients, $20 \mathrm{mg}$ in $16,15 \mathrm{mg}$ in 7 or $10 \mathrm{mg}$ in $3.84 \%$ were treated subcutaneously, the remainder refused injections $(11 \%)$ or were initiated elsewhere (4\%). All received folate supplementation and blood test monitoring. Remission was achieved in 23 of $45(51 \%)$ with CD and 12 of 19 (63\%) with UC. An additional 7 (15\%) and $1(5 \%)$ showed a response. The median duration of treatment was 11 (3-36) months in responders and 6 (1.5-10) months in non-responders. Dose reduction was used in maintenance for 4 patients and was necessary due to asymptomatic elevation of ALT in 5 or other side effects in 4. The drug was tolerated well with no apparent side effects in $85 \% .7$ (11\%) ceased therapy due to intolerance. The use of subcutaneous administration was well accepted. $11 \%$ changed to oral therapy after $>6$ months in remission. Conclusions: Subcutaneously administered MTX exhibits apparent efficacy, acceptance, tolerance and safety in patients with CD or UC who are steroid-dependent and where purine analogues have been ineffective or intolerable. Such results in this more challenging group of patients suggest that MTX should be promoted from the reserve's bench to the starting line-up in immunosuppressive therapy in both CD and UC

\section{W1222}

Mobilization of Mucosa Lymphocytes By Leukocytapheresis Joerg Emmrich, Brock Peter, Gisela Sparmann, Stefan Liebe, Reinhard Klingel, Wolfgang Ramlow

Introduction: Recently published studies have suggested that leukocytapheresis (LCAP) is an useful therapy of inflammatory bowel disease after failure of conventional treatments. The mechanisms of this immunomodulatory strategy are poorly understood. Especially, the relation between the removal of blood cells through the apheresis procedure and lymphocytes localized in the gut tissue is not known. Methods: Ten patients suffering from active steroiddependent ulcerative colitis (CAI according to Rachmilewitz: 6 - 10) were included in our body weight during 6 months. The patients received leukocytapheresis therapy weekly fo five weeks using the Cellsorba FX® column system (Asahi Medical, Tokyo, Japan). Blood samples were obtained before and after each LCAP session followed by flowcytometric analysis with mouse anti-human specific monoclonal antibodies (Becton Dickinson, Oxford, England) detecting CD3, CD4, CD8, CD19, activated T cells (HLA DR), NK cells (CD16, CD56), and mucosa-derived lymphocytes (CD 103). Results: Remission of the disease (defined by CAI $\leq 4$ ) has been achieved in 6 of 10 patients by means of the LCAP strategy. LCAP-related side effects were not observed. Due to the adsorption on the Cellsorba membrane, the apheresis procedure reduced lymphocyte counts in the blood by $25 \%$ to $32 \%$. In contrast, the number of $103+$ mucosa-derived lymphocytes increased significantly in the peripheral blood after two as well as after five apheresis sessions. Moreover, there was a tendency to a positive correlation between higher numbers of CD103+ cells provoked by the apheresis sessions and the clinical improvement of the disease in response to the LCAP treatment. The other investigated species did not show significant changes comparing the composition of the lymphocyte subpopulations before and after LCAP. Conclusion: The LCAP technique can be considered as an alternative therapeutic approach inducing remission in patients suffering from steroid dependent and azathioprin refractory UC. The LCAP- caused increase of CD103+ lymphocytes suggests the mobilization of mucosa-derived lymphocytes as an immunomodulatory mechanism of the leukocytapheresis technique

\section{W1223}

An Open-Labeled Trial of Granulocyte and Monocyte Adsorption Apheresis for Pouchitis

Atsushi Sakuraba, Toshiro Sato, Yuko Iwakami, Yasuhiro Takada, Nagamu Inoue,

Masahiro Takaishi, Haruhiko Ogata, Yasushi Iwao, Toshifumi Hibi

[Background] Pouchitis is a chronic inflammatory disorder that occurs after ileal-pouch anastomosis. Antibiotics and probiotics are the most common treatments. However, some patients are resistant to conventional therapy and require pouch-excision. We have recently reported that granulocyte and monocyte adsorption apheresis (GCAP) is efficacious for active ulcerative colitis. The efficacy of GCAP for acute pouchitis has not been elucidated yet. In this pilot study the efficacy of GCAP for pouchitis was analyzed in an open-labeled uncontrolled trial. [Patients \& methods] Six patients with acute pouchitis confirmed by the Pouchitis Disease Activity Index (PDAI) were treated with GCAP for 10 sessions. The efficacy and tolerability of GCAP was assessed. [Results] Four out of 6 patients improved and had a decrease in the PDAI score. No severe side effects were observed, but 1 patient had difficulty of venous access. [Conclusion] In this pilot study GCAP was effective and safe for acute pouchitis. It may be an alternative for patients who are resistant to antibiotics and probiotics.

\section{W1224}

Appropriateness of Initial Treatment for Mild-to-Moderate Luminal Crohn's Disease: Application of EPACT Criteria to the EC-IBD European Cohort Pascal Juillerat, Valerie Pittet, Christian Mottet, Christian Felley, Vincent Wietlisbach, Jean-Jacques Gonvers, Robert W. Dubois, John-Paul Vader, Bernard Burnand, Florian Froehlich, Frank Wolters, Reinhold Stockbruegger, Pierre Michetti

Background: The appropriate use of therapy for mild-to-moderate luminal Crohn's Disease has never been formally assessed. The European Panel on the Appropriateness of Crohn's Disease Therapy (EPACT) convened in Lausanne, Switzerland to develop appropriatenes criteria. We have applied these criteria, retrospectively, to the EC-IBD prospectivelyassembled, uniformly-diagnosed European population-based inception cohort of inflammatory bowel disease (IBD) patients diagnosed between 1991 and 1993. Methods: 426 Crohn's Disease (CD) patients from 13 European participating centers (10 countries) were included at the time of diagnosis (first flare, naive patients, no maintenance treatment, not steroiddependent or -refractory). We used the EPACT definition of mild-to-moderate luminal CD (acute flare in an ambulatory patient, no documented fistula or stenosis, no surgery required) and disease location to classify patients. We analyzed the initial drug prescription of this cohort, according to the EPACT criteria ([C]: colonic location only, [IL] : ileal location +/colonic): Appropriate (A): prednisone alone or combined with sulfasalazine [C], budesonide [IL]. Uncertain (U): 5-ASA compounds, sulfasalazine [IL]. Inappropriate (I) : antibiotics, methotrexate, budesonide [C]. Results: $163(38 \%)$ of the cohort patients initially suffered from mild-to-moderate luminal CD. The disease location was ileal or ileocolonic for 92 patients $(56 \%)$ and colonic only for $71(44 \%)$. A single treatment was given in 89 cases $(55 \%)$ and 74 patients received $>1$ drug simultaneously or successively during the acute episode. 96 patients $(60 \%)$ received at least an appropriate treatment, while for 66 patients $(40 \%)$ the treatment was uncertain and in 1 case $(<1 \%)$ inappropriate. The number and percentage of patients with the various combinations of treatments are presented in detail in the table below. Conclusion: The EPACT panel criteria (www.epact.ch) were applied, retrospectively, for the first time to a European population-based (EC-IBD) IBD patien cohort. An appropriate treatment was given in the majority of cases for the initial treatment of mild-to-moderate luminal $\mathrm{CD}$, but was associated with an uncertain treatment for twothirds of the patients, thus increasing the potential risk of adverse events and decreasing cost-effectiveness. 


\begin{tabular}{|c|c|c|c|c|c|}
\hline Drug 1 & \multicolumn{5}{|c|}{ Drug 2 } \\
\hline & None & U & I & U+I & Total (approx.) \\
\hline A & $31(19 \%)$ & $59(36 \%)$ & $2(1.5 \%)$ & $4(3 \%)$ & $96(60 \%)$ \\
\hline U & $64(39 \%)$ & - & $2(1.5 \%)$ & - & $66(40 \%)$ \\
\hline I & $1(<1 \%)$ & - & - & - & $1(<1 \%)$ \\
\hline Total & $58 \%$ & $36 \%$ & $3 \%$ & $3 \%$ & 163 \\
\hline
\end{tabular}

\section{W1225}

An Open Label Study of Adalimumab in Crohn's Disease Patients with a Loss of Response to Infliximab

Malik Anwar, Maria O'Sullivan, Barbara Ryan, Niall Breslin, Humphrey O'Connor, Colm O'Morain

Introduction: Chimeric tumour necrosis factor (TNF) alpha antibody, infliximab, is an effective therapy for active Crohn's disease (CD). Patients, however, may become unresponsive to this therapy over time. The aim of this study was to determine the efficacy of adalimumab, a fully humanised anti-TNF-alpha antibody, in CD patients who become unresponsive to infliximab therapy. Methods: A total of $107 \mathrm{CD}$ patients were treated with infliximab of which $64 \%$ had a clinical response. Consecutive patients $(n=13)$ who lost response to long-term infliximab management ( $3.2 \pm 0.7$ years since first infliximab infusion) were assigned to adalimumab. Study protocol comprised a loading dose of $80 \mathrm{mg}$ of adalimumab subcutaneously and 40mg every 2 weeks. Clinical response was defined as a drop in Crohn's disease activity index (CDAI) of 70 points or more and remission as CDAI $<150$. Results: In this group, $69 \%(9 / 13)$ responded to adalimumab therapy: $62 \%(8 / 13)$ had a clinical response and 1 patient (8\%) went into complete remission. On follow up, at a median of 5.0 months, $62 \%(8 / 13)$ of patients maintained a clinical response. No significant adverse events were documented, apart from a localised reaction at the site of infusion in one patient. Conclusion: This study suggests that adalimumab is an effective option for the management of $\mathrm{CD}$ in patients who become unresponsive to infliximab therapy.

\section{W1226}

Successful Infliximab RE-Initiation in a Previously Allergic Crohn's Disease Patient Following Hematopoietic Stem Cell Transplantation (HSCT) Lydia R. Weber, Dawn B. Beaulieu, Jeanne Emmons, Josh F. Knox, Mary F. Otterson, Mazen Iss, Sue Skaros, David G. Binion

Introduction: Allergic reaction to infliximab in severely ill Crohn's Disease (CD) patients intolerant to standard immunomodulators leaves limited treatment options. HSCT has been used as a rescue strategy for $\mathrm{CD}$ patients refractory to all forms of therapy (Oyama $\mathrm{Y}$ et al. Gastroenterology. 2005;128:552-63). HSCT is believed to ablate acquired immunity which may underlie its therapeutic success in $C D$. We report a $30 \mathrm{y} / \mathrm{o}$ female $\mathrm{CD}$ pt allergic to infliximab who required long term TPN for treatment of SB/LB fistulizing CD with remission induced with HSCT. CD relapsed 18 months following HSCT and maintenance infliximab treatment was re-initiated with no associated anaphylactic reaction. Case history: $\mathrm{CD}$ was diagnosed at the age of 21 with stricturing terminal ileal and perirectal fistulizing lesions which required multiple surgical drainage procedures over the next 6 years. No response to 5-ASA agents, severe headaches with systemic corticosteroids and intolerance to purine analogues led to infliximab use. She responded to episodic infliximab infusions but was discontinued following anaphylactic reaction during dose \#7. Two unsuccessful attempts at infliximab desensitization left the drug unavailable. Failed additional therapies included $6 \mathrm{TG}$ (resulted in ascites), parenteral methotrexate, mycophenolate mofetil and leflunomide (no response). She became TPN dependent over a 16 month period, and required 17 hospitalizations for refractory $\mathrm{CD}$ as well as $2 \mathrm{SB}$ resections and a temporary diverting ileostomy. Experimental treatment with HSCT induced remission off all drugs for 18 months. CD symptoms recurred with fever, abdominal pain, diarrhea and weight loss with no response to budesonide. Given that $\mathrm{HSCT}$ ablates prior acquired immunity, and presumably drug allergies, infliximab was re-attempted. The patient tolerated the infusion and subsequent maintenance treatment over the next 15 months. She is currently stable with no active disease, receiving $5 \mathrm{mg} / \mathrm{kg}$ infusions every 7 weeks with no further hospitalizations or surgeries. Discussion: $\mathrm{HSCT}$ can induce remission in patients with refractory CD, and the mechanism is believed to involve reconstitution of acquired immunity with ablation of prior immune responses, including allergies. We report a patient with severe $\mathrm{CD}$ whose allergy to anti-TNF alpha therapy was eradicated with HSCT allowing successful treatment with infliximab following relapse of her condition.

W1227

Safety and Efficacy of Oral, Colonic-Release, Low Molecular Weight Heparin$\mathrm{Mmx}^{\mathrm{TM}}$ for the Treatment of Mild to Moderate Left-Sided Ulcerative Colitis: Preliminary Report of a Pilot Study

Simone Saibeni, Luisa Spina, Clementina Signorelli, Luca Pastorelli, Roberto De Franchis, Luigi Moro, Roberta Villa, Roberta Bozzella, Giuseppe Celasco, Maurizio Vecchi

Background: efficacy of subcutaneous heparin and low molecular weight heparins (LMWHs) has been suggested in the treatment of ulcerative colitis (UC). The multimatrix oral formulation MMX'M (Cosmo Technologies Ltd, Ireland) allows the release of active drugs to the left colon. Thus, the LMWH Parnaparin in the colon-release MMX'M formulation should be able to exert its therapeutic effect at the site of disease, avoiding systemic absorption and side effects. Aim: to assess the safety and efficacy of 3 oral dosages $(70,140,210 \mathrm{mg}$ once daily) of Parnaparin-MMXTM in mild to moderate left-sided UC. Materials and Methods: 4 UC patients (pts) were planned to be enrolled for each group. We report the results in the 8 pts enrolled so far. All pts ( 6 men, mean age 48.5 yrs) had relapsed during maintenance mesalazine therapy and had Disease Activity Index(DAI) $\geqq 4$ and $\leqq 10$ and Endoscopic Activity Index(EAI) $\geqq 2$. The study was approved by the local ethical committee.All pts, after informed consent, received the study drug while continuing mesalazine treatment at a stable dose for 8 weeks. Clinical Activity Index (CAI) was assessed at time 0 and at 1,2,4,6 and 8 weeks. EAI and DAI were assessed at time 0 and week 8 . Clinical and laboratory follow-up,including clotting parameters and anti-factor Xa activity, was performed throughout the study. Statistical analysis was performed by means of Student's paired " $\mathrm{t}$ " test. Results:1 patient of low-dose group (VM) retired from the study for clinical deterioration. No relevant side effects were observed. Slight and transient increases in CK and ALT were observed in two patients. There were no interference with hemocoagulative parameters and no increased rectal bleeding. One patient (GL) was concurrently treated for a few days with antibiotics and steroids due to an acute bacterial sinusitis. Mean ( \pm SD) final CAI and DAI are significantly lower than basal CAI $(5.0 \pm 1.6$ vs. $1.7 \pm 1.5 ; \mathrm{p}<0.007)$ and $\mathrm{DAI}(7.5 \pm 1.5$ vs. $4.7 \pm 2.6 ; \mathrm{p}<0.03)$. Basal and final EAI do not show significant difference $(8.0 \pm 1.8$ vs. $6.7 \pm 3.9)$. Conclusions: oral parnaparin-MMX ${ }^{\mathrm{TM}}$ appears to be a safe and effective treatment option in mild to moderate UC. Controlled studies are warranted to confirm its therapeutic effect.

\begin{tabular}{|c|c|c|c|c|c|c|c|}
\hline patient & dosage & Basal CAI & Final CAI & Basal EAI & Final EAI & Basal DAI & Final DAI \\
\hline $\mathrm{RF}$ & $70 \mathrm{mg}$ & 6 & 4 & 5 & 10 & 8 & 8 \\
\hline $\mathrm{VM}^{*}$ & $70 \mathrm{mg}$ & 2 & 3 (weck 4) & 3 & no & 5 & no \\
\hline ADA & $70 \mathrm{mg}$ & 5 & 3 & 9 & 9 & 8 & 7 \\
\hline $\mathrm{CB}$ & $70 \mathrm{mg}$ & 4 & 2 & 8 & 5 & 5 & 4 \\
\hline $\mathrm{AM}$ & $140 \mathrm{mg}$ & 5 & 2 & 10 & 12 & 9 & 6 \\
\hline $\mathrm{PV}$ & $140 \mathrm{mg}$ & 3 & 0 & 7 & 1 & 6 & 1 \\
\hline GC & $140 \mathrm{mg}$ & 4 & 1 & 7 & 7 & 8 & 5 \\
\hline GL* $^{*}$ & $140 \mathrm{mg}$ & 8 & 0 & 10 & 3 & 9 & 2 \\
\hline
\end{tabular}

\section{W1228}

Lymphocytic Enteritis (LESION TYPE MARSH I): Response to Gluten-Free Diet (GFD)

Merce Rosinach, Fernando Fernandez-Banares, Maria Esteve, Montse Alsina, Carme Farre, Jaume Casalots, Rebeca Santaolalla, Montse Forne, Jorge Espinos, Antonio Salas, Josep Maria Viver

Lymphocytic enteritis (LE) is the first stage of the spectrum of the gluten sensitive enteropathy (GSE). Based on the ESPGAN criteria a diagnosis of celiac disease (CD) is performed when there is villous atrophy, and only then a GFD is started. There are scarce data in the literature suggesting that LE may cause clinical symptoms similar to $C D$, which may improve with a GFD. Aim: To assess clinical, serologic and histological response to a GFD in patients with LE. Methods: 50 consecutive patients $(50 \pm 1.9$ years; $15 \mathrm{M}, 35 \mathrm{~W})$ with LE were included. Diagnostic criteria were: 1- To belong to a risk group of CD; 2- To have more than $25 \%$ intra-epithelial lymphocytes (IEL) in the mucosa from distal duodenum; and 3- To have positive HLA-DQ2/DQ8. Clinical symptoms, associated autoimmune diseases, routine blood analysis, and bone mineral density (BMD) were prospectively recorded. Serum IgA antiendomisial (AEA) and anti-transglutaminase (TGA) antibodies, and TGA in the supernatant of the duodenal mucosa culture were assessed. A clinical and histological follow-up was performed at least 7 months after starting GFD (mean: 17.3 1 months). McNemar and Student $t$ for paired data tests were used in the statistical analysis. Results: The main form of presentation was 54\% intestinal, 8\% extra-intestinal and 38\% asymptomatic or with minor symptoms. A 4\% had type I diabetes, and a 8\% thyroid disease. Clinical and biological manifestations were: $50 \%$ diarrhea $46 \%$ abdominal pain. $8 \%$ weight loss, $8 \%$ fecal incontinence, $68 \%$ gas-related symptoms, $58 \%$ abdominal distension, $32 \%$ BMD abnormalities, $30 \%$ iron deficient anemia and 12\% hypertransaminassemia. Positive AEA/TGA was observed in $12 \%$, and TGA in the supernatant in 10\% of cases. 11 patients did not accept to start GFD (9 asymptomatic and 2 with diarrhea). Response to GFD (36 patients with symptoms) was (\%before vs after GFD): diarrhea (63.9\% vs $11.1 \%$; p $<0.0005)$, abdominal pain $(61.1 \%$ vs $8.3 \%$; $<0.0005$ ), gas-related symptoms ( $80.6 \%$ vs $30.6 \% ; \mathrm{p}<0.0005$ ), abdominal distension $(77.8 \%$ vs $33.3 \%$; $p<0.0005)$ and anemia $(41.7 \%$ vs $14.3 \%$; $p=0.004)$. Overall, clinical improvement was observed in $31 / 36$ patients $(86 \%$; $95 \%$ CI, $71-94 \%$ ). At present, histological response after GFD has been evaluated in 28 patients showing improvement in $18(64.3 \%)$, with a decrease in IEL number $(42 \pm 2.5$ vs $28.9 \pm 2.9 \% ; \mathrm{p}=0.003)$. In 6 patients with clinical improvement, persisted a similar intestinal lesion. Conclusions: LE is a frequent form of GSE which causes clinical symptoms in a high percentage of patients, is often associated with a negative celiac serology, and shows in a high percentage of patients a good response to a GFD. Funded by Fundació Banc Sabadell.

\section{W1229}

Antibodies to Synthetic Gliadin-Related Peptides for Detecting Celiac Disease in a Population with High Pre-Test Probability

Sonia Niveloni, Emilia Sugai, Maria L. Moreno, Horacio Vazquez, Zulema Kogan, Ana Cabanne, Edgardo Smecuol, Roberto Mazure, Silvia Pedreira, Juan C. Gomez, Gisella S. Selvaggio, Eduardo Maurino, Julio C. Bai

Introduction: A novel antibody assays based on synthetic gliadin-related peptides (AGA II) has shown very high diagnostic yield for identification of celiac disease (CD) in selected populations. Aim: to determine the value of these antibodies for detecting $\mathrm{CD}$ in individuals with high pre-test probability undergoing intestinal biopsy due to clinical suspicion of small bowel disorder. Materials: From October 2004 to September 2005, 122 unselected consecutive patients attending the Malabsorption Clinic of our institution were prospectively enrolled in the study. We excluded patients with prior CD-related serology, intestinal histology or diagnosis of $\mathrm{CD}$ or dermatitis herpetiformis. At the time of endoscopy and biopsy, serum samples were obtained for CD-related serology which includes: AGA II IgA, AGA II IgG (QUANTA Lite Gliadin IgA and IgG II- INOVA Diagnostic Inc.; San Diego, CA), 
anti-tissue transglutaminase antibodies (a-tTG) type IgA. Diagnosis of CD and characterization of patients were based on histological criteria (Marsh's type II lesion or greater) and was supported by a-tTG and the response to a gluten-free diet in discordant patients. Results: While 57 patients (47\%) were finally diagnosed as having CD, 65 had no evidence of the disorder. Compared with controls, $\mathrm{CD}$ patients had very significantly higher mean absolute values of both AGA II antibodies ( $\mathrm{p}<0.0000$ ). Sensitivity (Sen), specificity (Spe), diagnostic accuracy (Acc), positive and negative predictive values (PPV and NPV, respectively), and positive and negative likelihood ratios (+LR and $-\mathrm{LR}$, respectively) are reported in the table 1. Although all assays exhibited outstanding results, the AGA II IgG assay had the best statistical value, even better than the well-recognized a-tTG. Conclusions: Our study based on a population of individuals with high pre-test probability has shown that the new gliadinrelated peptide antibody assays are observer-independent tests having the highest diagnostic accuracy for detecting $\mathrm{CD}$ that should be employed in the setting of high pre-test probability populations.

\begin{tabular}{|c|c|c|c|c|c|c|c|}
\hline Tests & Sen & Spe & ACC & PPV & NPV & +LR & -LR \\
\hline & $(\%)$ & $(\%)$ & $(\%)$ & $(\%)$ & $(\%)$ & & \\
\hline AGA II IgA & 98.2 & 92.3 & 95.1 & 91.8 & 98.4 & 12.7 & 0.02 \\
\hline AGA II IgG & 96.5 & 100 & 98.4 & 100 & 97.0 & 96.5 & 0.03 \\
\hline a-tTG IgA & 94.7 & 96.9 & 95.9 & 96.4 & 95.5 & 30.5 & 0.05 \\
\hline
\end{tabular}

\section{W1230}

Celiac Disease IGA Class Autoantibodies Disturb Normal Capillary Formation in Angiogenesis

Katri Lindfors, Essi Myrsky, Mari Syrjanen, Ilma Korponay-Szabo, Markku Maki, Katri Kaukinen

INTRODUCTION: A characteristic feature for celiac disease (CD) are specific IgA class autoantibodies targeted against transglutaminase 2 (TG2). These antibodies are produced locally in the small bowel and are typically deposited in the intestinal mucosa below basement membrane and around small capillaries. We have previously shown that these antibodies inhibit the mesenchymally-induced differentiation of small intestinal epithelial cells. Because of the presence of IgA in the small bowel capillaries, our aim is to study whether the angiogenic differentiation of mesenchymal cells is disturbed in CD small bowel mucosa and if CD-specific IgA class autoantibodies are responsible for abnormal angiogenesis. METHODS: The expression level of vascular mesenchymal $\alpha$-smooth muscle actin, recarded as a marker for smooth muscle cell differentiation, was evaluated in CD biopsy samples from 10 patient during active disease and on gluten free diet (GFD) by immunofluorescence stainings. The effects of CD IgA on angiogenesis "" in vitro "" were studied both in HUVEC endothelial cells cultures and in co-cultures of HUVECs and mouse mesenchymal 10T1/2 cells. The length of the tubes, the motility of the cells as well as the expression level and intracellular localisation of $\alpha$-smooth muscle actin were recorded RESULTS: Semiquantitative measurement of vascular mesenchymal cell $\alpha$-smooth muscle actin from immunostained biopsy samples showed reduced expression in the active phase of $C D$ which was normalized during GFD. In cell culture, the length of the capillary tubes in CD-IgA treated cultures was approximately half of that measured in non CD IgA treated cultures. This phenomenon caused by CD IgA was apparently due to inhibited cell motility as indicated by the conventional wound closure assay. CD IgA decreased the overall expression level of $\alpha$-smooth muscle actin, as in biopsy samples, but more importantly caused a clear deorganisation of the actin cytoskeleton essential for cell migration. CONCLUSIONS: CD-associated IgA class autoantibodies disturb mesenchymal-endothelial cell crosstalk during angiogenesis leading to the inhibition of mesenchymal cell differentiation, also seen in $\mathrm{CD}$ patient biopsy samples, as well as migration of both mesenchymal and endothelial cells due to deorganisation of the actin cytoskeleton necessary for proper cellular movement.

\section{W1231}

Splenic Hypofunction and the Spectrum of Autoimmune and Malignant Complications in Celiac Disease

Antonio Di Sabatino, Maria M. Rosado, Paolo Cazzola, Raffaele Morera, Rosanna Riboni, Federico Biagi, Rita Carsetti, Gino R. Corazza

"Background \& Aims": We investigated the prevalence of functional hyposplenism in autoimmune disorder (AID)-associated and complicated celiac disease (CD). Additionally, since the association between hyposplenism and overwhelming infections due to "Streptococcus pneumoniae" in CD is well known, we investigated whether IgM memory B cells, which are responsible for the protection against infections by encapsulated bacteria and require the spleen for their generation and/or survival, mirror the reduced splenic function in $C D$. "Methods": Peripheral blood samples were collected from 78 adult CD patients (32 with AID 36 without AID and 10 with CD-related complications, including enteropathy-associated $\mathrm{T}$ cell lymphoma, refractory sprue and ulcerative jejunoileitis, tested for T cell receptor $\mathrm{g}$ chain clonality). Thirty-four non-CD patients with AID, 35 healthy volunteers and 29 splenectomised patients were also studied. Counting of erythrocytes with membrane abnormalities (pitted red cells) was used as an indicator of splenic function, and flow cytometry was performed to analyse peripheral blood B cells. "Results": A significantly higher risk for hyposplenism was found in AID-associated CD (59\%) and complicated CD $(80 \%)$ that in uncomplicated CD without AID (19\%). In AID-associated CD, the degree of splenic function did not correlate to the duration of gluten-free diet. In AID-associated and complicated CD, the frequency of circulating IgM memory B cells was significantly lower than in $\mathrm{CD}$ patients without AID or healthy subjects. A significant inverse correlation between IgM memory B cells and pitted red cells was found in all $78 \mathrm{CD}$ patients. "Conclusions": The prevalence of splenic hypofunction is increased in CD with AID and in complicated CD, and is not related to the duration of gluten-free diet. IgM memory B cells are reduced in AID-associated and complicated CD. This defect, which is related to the impairment of splenic function, might predispose hyposplenic $\mathrm{CD}$ patients to infections by encapsulated bacteria. Polysaccharide protein conjugate vaccines, that bypass the immunological impairment due to the lack of IgM memory B cells by eliciting a T cell-dependent response, may be more appropriate than traditional 23-valent polysaccharide vaccine in reducing the risk of systemic infections in hyposplenic $\mathrm{CD}$ patients.

\section{W1232}

Evaluation of a New Simple and Fast Assay for HLA Typing of the Celiac Disease-Associated Alleles

Saverio Teresi, Sandro Drago, Eleonora Gucciardino, Mariarosaria Di Pierro, Donatella Sangiorgi, Giuseppe Stampone, Calogero Scalici, Antonio Carroccio, Giuseppe Iacono

Background: Celiac disease (CD) is an autoimmune disease, with a prevalence of 1:100 in the general population, triggered by the ingestion of gluten in genetically predisposed individuals. It has been demonstrated that all celiacs carry HLA II DQA $* 05-* 0201 / \mathrm{DQB1} * 02$ (that codify for DQ2 haplotype) and/or DQA1*03/DQB1*03 (haplotype DQ8) alleles. The subjects who have not the DQ2 or DQ8 haplotype cannot develop CD. The association between HLA DQ2/DQ8 and CD can be used to exclude the diagnosis in debate cases. However, at present, the HLA typing test commercially available are cumbersome and timeconsuming. Aims: To evaluate a new simpler and faster system for the CD-associated HLA typing (BioDQ Screen and BioDQ Typing; DiaGene S.r.l, Italy). Subjects and Methods: 23 biopsy-proven $C D$ patients and 79 their first degree relatives were enrolled. All the subject in a first step, BioDQ Screen detects in one reaction DQB1*02, DQB1*03 alleles to exclude all the subjects who are not DQ2 or DQ8. In a second step, BioDQ Typing reveals in 8 reactions the DQA1*0201, DQA1*03, DQAl*05, DQB1*02, DQB1*03, DRB1*03, DRB1*04, DRB1*07 alleles encoding for DQ2/DR3-DR7 and DQ8-DR4 haplotypes. Both kits required a rapid DNA extraction from whole blood (5 min), DNA amplification (2 hours) and electrophoresis ( $15 \mathrm{~min})$. All reactions contain primer for internal control and all reagents are provided and ready to use. In order to check the concordance of the results obtained with BioDQ system, the same samples were also typed for DQB alleles (Histo type/ DNA-SSP kit, Germany). Results: According to DQ Screen results, 23/102 subjects were not DQ2 and/or DQ8 and 79/102 DQ2 or DQ8-positives. According to the DQTyping we found 38/102 DQ2/DR3, 18/102 DQ2/DR7, 20/102 DQ8/DR4, 1/102 DQ2-DQ8/DR3-DR4, 2/10 DQ2-DQ8/DR7-DR4. All subjected resulted not DQ2 or DQ8 with BioDQ Screen were confirmed not DQ2 or DQ8 with BioDQ Typing. Moreover, the concordance of the results with those obtained with the reference test (Histo Type BAG) was 100\%. The cost of the 102 assays with the new system was $€ 2.800,00$ in comparison with a cost of $€ 5.800,00$ of the traditional system. The time needs was 3 hours for 12 samples with the new method and 18 hours for 12 samples with the traditional method. Conclusions: BioDQ system is a simple and rapid system for HLA class II typing specific for Celiac Disease and it has high sensitivity and specificity. Due to the results obtained (DQScreen false negative rate $0 \%$, we suggest to use BioDQScreen as first step in order to identify in 1 reaction DQ2 or DQ8 subjects, and successively only the positive samples (DQ2 or DQ8) with BioDQ will be assayed by BioDQ Typing

\section{W1233}

Gliadin Induces Epidermal Growth Factor Receptor (EGFR) Dependent Proliferation in Cultured Biopsies from Celiac Disease (CD) Patients Through a Delay of Endocytosis

Maria Vittoria Barone, Franco Paparo, Maria Maglio, Ribecco Maria Teresa, Giuliana Lania, Merlin Nanayakkara, Virginia Vitale, Erasmo Miele, Riccardo Troncone, Salvatore Auricchio

Background Peptic-tryptic digests of gliadin (PTG) and P31-43 gliadin peptide mimic EGFR (Epidermal Growth Factor) signaling inducing actin rearrangements and proliferation in several cell lines through a delay of the EGFR endocytosis. Here we show that gliadin effects on EGFR pathway can be reproduced in cultured biopsies from CD patients. Alexa 488 traffiking into endocytic vesicles in presence of PTG and P31-43 in biopsies from florid patients is investigated. PTG and P31-43 ability to exert EGF proliferative effects on biopsies from florid $\mathrm{CD}$ patients in colture cells was also tested. Comparison of gliadin proliferative and apoptotic effects is provided. Aim Characterization of the role of EGF signaling and traffiking in the proliferative effects of gliadin peptides on jejunal culturered biopsies from $\mathrm{CD}$ untreated patients. Methods Biopsies from untreated $\mathrm{CD}$ patients were cultured overnight in an organ culture system in the presence of peptic-triptic digest of gliadin (PTG) or of ovalbumin (PTO). In other experiments gliadin peptides P31-43 or P56-68 were used. To test the EGFR involvement in PTG and P31-43 effects on cell proliferation the bloking antibody anti EGFR 528 was employed. Bromodeoxiuridine was added to the medium to detect cells in to the S-fase of the cell cycle. Pulse and chase experiments described the endocytic alterations of the EGF-Alexa488 traffiking Results Incubation of untreated celiac biopsies in presence of PTG or P31-43 induces a delayed uptake of the EGF labelled with Alexa 488 fluorocrome: it is still visible in endocytic vesicles after 6 hours chase in treated samples, but not in prsence of media alone or P56-68 treated sample. An increase of both apoptotic and proliferating epithelial cells is detectable: with BrdU incorporation rising to $38 \%(-/+8 \%)$ versus $15 \%(-/+5 \%)$ of the control, and Tunel positive cells rising to $28 \%$ $(-/+10 \%)$ versus $8 \%(-/+4 \%)$ of the control. EGFR inhibitor anti-EGFR 528 is able to prevent the increase of BrdU incorporation obtained in presence of PTG and P31-43. In contrast PTG and P31-43 induced apoptosis was not greatly altered in presence of the EGFR inhibitors. Conclusion PTG and gliadin peptide P31-43 induce EGF accumulation in endocytic vesicles and induce EGFR activation dependent proliferation, but not apoptosis in cultured biopsies from $C D$ patients. 
W1234

Antiendomysial and Tissuetransglutaminase Autoantibodies Detection in Oral Culture Media of Untreated Coeliac Disease (CD)

S. Vetrano, M.C. Anania, C. Maffia, F. Lettieri, M. Di Tola, L. Sabbatella, S. Lanci, P. Lista, V. Libanori, O. de Pita, M.G. Sanjusto, P. Ruatti, U. Zampaletta, A. Picarelli

Aims: $C D$ is an autoimmune disorder characterised by high levels of antiendomysial (EMA) and anti-tissuetransglutaminase (anti-tTG) autoantibodies (Ab) in sera. EMA and anti-tTG $\mathrm{Ab}$ can be detected in culture supernatants of intestinal biopy from untreated $\mathrm{CD}$ patients. But it is not known whether they can be released also from other mucosal surfaces within the gut -associated lymphoid tissue (GALT). The major site of gut involvement in CD is in the jejunal mucosa, but immunological changes have been observed also in oral and rectal mucosa after local gliadin challenge. In this study we investigated whether EMA and anti-tTG Ab can also be detected in culture media from oral mucosa specimens and if the mouth could be used as an area of immunological testing for CD. Patients \& Methods: 26 adults with clinical suspicion of $C D$ were enrolled. All were submitted to serological screening (EMA and anti-tTG Ab) and to (EGDS) to diagnose CD. 15/26 patients (pts) have been diagnosed to have $C D$, whilst in the other 11 pts was excluded, and there enrolled as controls. Cheek mucosal and duodenal biopsies (organ cultures), from 15 pts with active $\mathrm{CD}$ and 11 from controls, were cultured in vitro for $48 \mathrm{~h}$ at $37^{\circ} \mathrm{C}$ in medium culture. EMA and anti-tTG Ab were detected in sera and in the supernatants of organ cultures by indirect immunofluorescence and ELISA Mann-Whitney test was used to compare anti-tTG values of CD pts vs. controls. Results: EMA and anti-tTG were positive in sera of all CD patients and in media culture from intestinal samples of $14 / 15 \mathrm{CD}$ pts, whereas they were positive in mediaculture from oral samples of 15 out of the 15 patients. No EMA and anti-tTG Ab were detected in sera and in the culture supernatants from 11 controls. The linear correlation test between oral and duodenal mucosa showed values for $\mathrm{p}>0.05$ and $\mathrm{r}=0.49$. Conclusions: Our data confirm that oral mucosa as well as intestinal mucosa is involved in $\mathrm{CD}$ and demonstrate that oral mucosa releases EMA and anti-tTG Ab in untreated CD patients. The correlation of values of tTG Ab between oral and duodenal mucosa seems to promote mouth mucosa like immunological site to detect the pathognomonic $\mathrm{Ab}$ of $\mathrm{CD}$.

\section{W1235}

Gliadin-Induced Apoptosis of Human Intestinal Epithelial Cells Is Potentiated By Enteric E. Coli/ K1

Michelle M. Pietzak, Eliza Chakraborty

Aim: An increase in DNA fragmentation is seen in the areas of villous atrophy in the biopsies of patients with untreated celiac disease. This apoptotic profile is restored to normal after the withdrawal of gluten from the diet. E. coli K1 RS218 (O18:K1:H7) has been shown in our lab to induce apoptosis in both human and rat intestinal epithelial cells. Bacterial flora, in the presence of gluten, may be a potential environmental trigger for celiac disease. We hypothesized that bacteria, in addition to gliadin, can synergistically cause apoptosis in intestinal epithelial cells. Methods: Apoptosis in T84 cells was quantified by Annexin VFITC (AV) expression and loss of propidium iodide (PI) exclusion after incubation with E. coli Kl, gliadin (Sigma, from wheat gluten), or both for 24 hours. Cells were tracked from AV- PI- (viable), to AV+ PI- (early apoptosis with membrane integrity), to AV+ PI+ (end stage apoptosis and cell death). Cells underwent FACS analysis with a Becton-Dickinson FACSCalibur flow cytometer and CellQuest Software. Results: Intestinal epithelial cells at baseline showed $27 \% \mathrm{AV}+\mathrm{PI}$ - and $30 \% \mathrm{AV}+\mathrm{PI}+$. After incubation with bacteria, AV+PI- cells increased to $51 \%$ and $\mathrm{AV}+\mathrm{PI}+$ to $54 \%$. After incubation with $5 \mathrm{ug}, 50 \mathrm{ug}, 100 \mathrm{ug}$, 500ug, and lo00ug gliadin, $\mathrm{AV}+\mathrm{PI}$ - increased to $35 \%, 32 \%, 41 \%, 55 \%$ and $70 \%$, and $\mathrm{AV}+\mathrm{PI}+$ to $43 \%$, $40 \%, 51 \%, 62 \%$, and $65 \%$ of cells, respectively. The addition of bacteria to $50 \mathrm{ug}, 500 \mathrm{ug}$ and $1000 \mathrm{ug}$ gliadin increased $\mathrm{AV}+\mathrm{PI}-$ to $39 \%, 99 \%$ and $96 \%$, and $\mathrm{AV}+\mathrm{PI}+$ to $54 \%, 94 \%$ and $76 \%$ of cells, respectively. Only 0.3 to $1.6 \%$ of cells remained viable after incubation with bacteria and 500 to 1000ug of gliadin. Conclusions: Our results suggest that enteric bacteria $\mathrm{E}$. coli $\mathrm{Kl}$ and gliadin can synergistically induce rapid, dramatic apoptosis in intestinal epithelial cells. Understanding the dietary and microbial triggers of mucosal damage and tissue remodelling in celiac disease may lead to novel therapeutics for this condition.

\section{W1236}

Positive and Negative Predictive Values of a Combination of Celiac Disease Serology Tests As Compared to Intestinal Histology Damage

Sonia Niveloni, Deborath Kryszak, Maria L. Moreno, Valeria Familiari, Anna Sapone, Emilia Sugai, Ana Cabanne, Zulema Kogan, Horacio Vazquez, Eduardo Maurino, Carlo Catassi, Alessio Fasano, Julio C. Bai

Background: Currently, the gold standard for the diagnosis of celiac disease (CD) is an upper GI endoscopy (EGD) with an intestinal biopsy. During the past few years, several non-invasive serological screening tools for $\mathrm{CD}$ have been developed. To establish the true sensitivity and specificity of these serological tests, either alone or in combination, and to develop possible non-invasive $C D$ diagnostic algorithms alternative to EGD, a study design in which an intestinal biopsy is the entry criterion is necessary. Aims: To establish the positive predicted value (PPV) and negative predicted value (NPV) of a combination of serological tests for CD using an intestinal biopsy as a gold standard. Methods: Sixty-eight consecutive patients (average age $38 \mathrm{yrs}$, range 18-80) referred by the Small Bowel disorders clinic to EGD and intestinal biopsy because of suspicion of intestinal disorders (chronic diarrhea, weight loss, abdominal distention, recurrent abdominal pain, and/or anemia) were enrolled between August 2004 and July 2005 (Group A). During the same period, a random sample of patients ( $n=98$, average age 47 yrs, range 23-73) undergoing to an EGD for symptoms or disorders not related to CD was also enrolled (Group B). All duodenal biopsies were blindly scored using the modified Marsh criteria by pathologists (AC and ZK). At the time of the procedure, a serum sample was also obtained to check for serum anti-actin antibodies (AAA), anti-tissue transglutaminase antibodies (TTG), and zonulin levels. Results: Thirty-three of the 68 patients from group A $(48 \%)$ and $1 / 98$ (1\%) from group B showed histological findings compatible with CD (Marsh IIIa-IIIc). Of the 34 CD patients identified,
27 tested positive to all three tests, 4 tested positive to TTG and zonulin, and 3 tested positive to zonulin alone. The combination of AAA, TTG, and zonulin tests showed a sensitivity of $79 \%$, a PPV of $100 \%$, and a NPV of $100 \%$. Conclusions: In subject experiencing malabsorptive symptoms, the combination of positive AAA, TTG, and zonulin can be used to rule in $C D$ without the necessity to perform an EGD. This approach would have allowed avoiding an invasive procedure in $38 \%$ of the cases of malabsorption enrolled in this study, an approach that could translate in cost saving and better patient's acceptance. The combination of negative AAA. TTG, and zonulin rules out CD without the necessity of any additional study. In these cases, an EGD could be still necessary to rule out other causes of malabsorption.

\section{W1237}

Endoscopic and Histological Findings of the Small Bowel in Patients with Celiac Disease: The Additional Value of Double-Balloon Endoscopy Muhammed Hadithi, Dimitri G. Heine, Abdulbaqi Al Toma, Adriaan van Bodegraven, Maarten A. Jacobs, Chris J. Mulder

Background and aims: The relative risk to develop enteropathy-associated T-cell lymphoma is markedly increased in patients with refractory celiac disease. Double-balloon endoscopy is an innovative endoscopy technique that allows examination of the small bowel with capabilities to take biopsy samples. We investigated the value of DBE in patients with refractory celiac disease. Methods: A total of 20 consecutive symptomatic patients with refractory celiac disease were prospectively examined by Double-balloon endoscopy to detect enteropathy-associated T-cell lymphoma that has been treated before by surgery and chemotherapy in seven of them. Another five symptomatic patients with celiac disease and without villous atrophy in duodenal histology participated in the study as controls. The small bowel was assessed for loss of folds, scalloping, nodularity of mucosa, and ulcerations. Biopsy specimens were taken from the small bowel to be evaluated according to the modified Marsh criteria and for EATL. Results: Twenty-eight double-balloon endoscopy procedures were successfully performed without complications in 25 patients. Enteropathy-associated T-cell lymphoma was found in four patients ( $25 \%$; $95 \%$ CI 6 to 44 ) as circumferential, discrete, or confluent ulcerations. Two patients had ulcerative jejunitis in absence of histological evidence of enteropathy-associated T-cell lymphoma, and only loss of folds, scalloping and nodularity were found in the other patients with refractory celiac disease. The small bowel appeared normal in control patients. Marsh III was found in two patients with enteropathyassociated T-cell lymphoma and Marsh I in another two. In patients with past history of EATL, Marsh III was found in three, Marsh I in one, and normal histology was found in one patient. Marsh III was found in all other patients with refractory celiac disease including two patients with ulcerative jejunitis. In control patients, histology was normal in three and showed changes of Marsh I in two patients. Conclusions: Small bowel examination and obtaining histology during double-balloon endoscopy in symptomatic patients with celiac disease could detect efficiently complications like ulcerative jejunitis or enteropathy-associated T-cell lymphoma. This technique should be reserved for patients with refractory celiac disease or patients with past history of enteropathy-associated T-cell lymphoma.

\section{W1238}

A Third Celiac Disease: Genotyping Reveals a Functionally Distinct Subtype Jason A. Tye-Din, Tim Beissbarth, Robert P. Anderson

Celiac disease (CD) is an enteropathy mediated by HLA-DQ2 and DQ8-restricted T cells specific for gluten peptides. Although $90 \%$ of CD is associated with the "classical" HLADQ2 heterodimer (HLA-DQA1*05 +DQB1*02, "DQ2.5") about $5 \%$ is associated with the "lower risk" HLA-DQA1*02 + DQB1*02 ("DQ2.2") combination. Does the subtle difference in HLA-DQ genotype translate to a different immunological phenotype? AIM: To compare gluten peptides recognized by T cells in DQ2.2 and DQ2.5 CD. METHODS: Amongst 250 individuals with biopsy proven $\mathrm{CD}$ on strict gluten free diet, DQ2.5 was present in 232, and DQ2.2 in only 6. Subjects ate wheat bread (200 g/d) (DQ2.2 n=2, DQ2 n=16) or barley grain $(150 \mathrm{~g} / \mathrm{d})(\mathrm{DQ} 2.2 \mathrm{n}=1, \mathrm{DQ} 2 \mathrm{n}=17)$ for three days. Interferon- $\gamma$ ELISPOT assays were performed on peripheral blood mononuclear cells (PBMC) collected on the 6th day after commencing gluten challenge to screen a comprehensive wheat gluten or hordein peptide library. Both wild-type and transglutaminase-pretreated peptides were assessed. RESULTS: PBMC from all subjects responded to gliadin with activity enhanced by deamidation. Following wheat ingestion, the most immunoreactive peptide in DQ2.2 subjects was an omegagliadin (DQ2- $\omega$-I; QPEQPFPQPEQPFPWQP) with strong responses to closely related polymorphisms. Neither subject responded (no different to medium alone) to the "immunodominant" A-gliadin peptide (p57-73 QE65; QLQPFPQPELPYPQPQS). In comparison, 13/16 DQ2.5 subjects had a dominant response to p57-73, and 10/16 responded to DQ2- $\omega-\mathrm{I}$. Following barley challenge, DQ2- $\omega$-I was immunodominant in both DQ2.2 and DQ2.5 CD subjects, and responses to p57-73 were absent or weak. CONCLUSIONS: The hierarchy of gluten T cell epitopes in DQ2.2 CD is distinct from classic DQ2.5 CD, implicating a role for HLA-DQA1*05 in determining the immuno-dominance of p57-73. Although DQ2.2 CD is rare and the numbers of subjects available is small, the immuno-phenotype of DQ2.2 CD appears distinct from DQ2.5 CD. Future studies should involve more subjects and determine whether the clinical phenotype of DQ2.2 differs from DQ2.5 CD

\section{W1239}

Flow Cytometry Patterns Specifying Celiac Disease in Lymphocytic Enteritis (MARSH I ENTEROPATHY): A Pilot Study

Anton Vrij, Alexander Martens

INTRODUCTION In celiac disease, cytology changes seem to precede more structural damage in duodenal biopsy specimens. In an early phase of celiac disease, a cytological classification could be of help in its diagnostic process. AIMS \& METHODS To study the lymphocytic characteristics of colour flowcytometry of duodenal biopsies in patients with signs of maldigestion and a disturbed sugar absorption test, suspected for celiac disease, in comparison with 
biopsies from patients with dysmotility like dyspepsia (DLD, non-inflammatory controls), and ulcerative duodenitis due to peptic ulcer disease or Crohn's disease (inflammatory controls). Parallel to histology, flow cytometry patterns were studied to characterize T cell subsets in clinically active disease in 26 patients with Marsh II-III seropositive celiac disease, 13 patients with seronegative but HLA DQ2/8 positive Marsh I enteropathy, 11 patients with seronegative as well as HLA DQ2/8 negative lymphocytic enteritis, 25 patients with DLD, as well as in 7 patients with ulcerative duodenitis. In Marsh I enteropathy. Patients with Marsh I enteropathy and Helicobacter pylori gastritis $(n=5)$ were excluded from the analysis. RESULTS In newly diagnosed and untreated patients with Marsh I, as well as Marsh II-III celiac disease, the \% of lymphocytes expressing a $C D 3+$ profile $(C D 103+, C D 3+C D 16 / 56+)$ was significantly higher, and a CD3- profile (CD3-CD16/56+, CD3-/CD7+) significantly lower, as compared to patients with idiopathic (HLA DQ2/8 negative) lymphocytic enteritis, ulcerative duodenitis or patients without duodenal inflammation (DLD). Both seronegative and seropositive celiacs had a significantly higher $\% \gamma \delta \mathrm{T}$ cells as compared to patients with idiopathic (HLA DQ2/ 8 negative) lymphocytic enteritis, ulcerative duodenitis or patients without duodenal inflammation (DLD). CONCLUSION In Marsh I celiacs, a higher \% of $\gamma \delta \mathrm{T}$ and lower \% of CD3-/ $\mathrm{CD} 7+$ was found as compared to HLA DQ2/8 negative lymphocytic enteritis, ulcerative duodenitis or DLD. The combination of a high $\%$ of $\gamma \delta \mathrm{T}$ and CD103+ cells, as well as a low \% of CD3-CD 16/56+ and CD3-/CD7+ lymphocytes seems characteristic for celiac disease.

\section{W1240}

A Practical Method for Counting Intraepithelial Lymphocytes in Duodenal Biopsies: Data from a Population-Based Endoscopic Study

Marjorie M. Walker, Robert D. Goldin, Michael Vieth, Manfred Stolte, Jukka Ronkainen, Pertti Aro, Tom Storskrubb, Joseph A. Murray, Nicholas J. Talley, Lars Agreus

Background: A raised intraepithelial lymphocyte (IEL) count in the duodenum is an important feature for the early diagnosis of celiac disease. A robust and simple method to estimate these IELs would be useful and diminish diagnostic confusion. The aims of this study were to establish the optimal site of duodenal biopsies and the number of enterocytes required to accurately count IELs to assess pathological intraepithelial lymphocytosis in the duodenum. Method: Paired duodenal (D,) biopsies from both D1 and D2 were obtained from an unselected representative sample $(\mathrm{n}=1000)$ of an adult general population in two Swedish municipalities, Kalix and Haparanda. To establish a time efficient method to count IELs 5 samples were subjected to detailed counts as follows: in each biopsy, 4 villi with epithelial nuclei aligned to the basement membrane were selected and marked. Three observers each performed IEL counts, in groups of 10 enterocytes, starting at the base of a crypt and continuing until the next base in the same 4 villi. The IEL count/100 enterocytes was extrapolated for cumulative groups of 10 enterocytes and plotted on a graph to establish at which point the counts became stable. A repeat examination of one villus per biopsy was performed to assess intraobserver variation in IEL count. CD3 immunostained duodenal biopsies from patients with known high counts of IELs were compared with H\&E counting. Results: In 200 paired D1 and D2 biopsies, 98.5\% had comparable IEL counts. In 2 of the 3 subjects where D1 counts were $>$ D2, 2 had H. pylori pangastritis and one had raised IELs with no gastric pathology. Extrapolation of IEL counts/group of 10 enterocytes showed that when 50 enterocytes have been counted there is a consistent count/100, with minor variation per villus. Importantly, both intraobserver and interobserver variation was minimal (with $70 \%$ agreement to within 5 IELs/ 100 enterocytes) and made no difference to the diagnosis. CD3 counts demonstrated more lymphocytes than H\&E generally (4/5 biopsies) but only by 3-4 IELs/100 enterocytes. Conclusions: D1 and D2 IEL counts are comparable. Counts to give a quantitative meaningful value for clinicians can be performed by counting IELs/ 50 enterocytes. There is minor intervillus variation in IEL counts so selection of two villi per biopsy is prudent. Interobserver variation is minimal in IEL counts and does not affect a diagnostic cut off value of 25 IELs/100 enterocytes. Immunostaining for CD3 counts demonstrated slightly more lymphocytes than $\mathrm{H} \& \mathrm{E}$, but is costly for everyday practice and was of no practical value.

\section{W1242}

The Prevalence of Celiac Disease Is Increasing in Process of Time Sini Lohi, Kirsi Mustalahti, Katri Kaukinen, Kaija Laurila, Pekka Collin, Harri Rissanen, Olli Lohi, Enzo Bravi, Antti Reunanen, Markku Maki

BACKGROUND Celiac disease is a gluten triggered autoimmune-mediated disease with both intestinal and extraintestinal manifestations. The prevalence of celiac disease has increased substantially during the last decades reaching $1 \%$ in the United States and many European countries. The aim of this study was to investigate whether the increase reflects a true rise of incidence in recent years or whether it is only due to a better detection rate. METHODS The prevalence of celiac disease was determined in two cross-sectional studies representing Finnish adult population in 1978-80 and 2000-01 with cohorts' sizes 8,000 and 8,028, respectively. The total prevalence of celiac disease was defined by adding together clinically diagnosed celiac disease patients and previously unrecognized celiac disease cases, which were found by tissue transglutaminase and endomysial antibody screening. RESULTS Only two $(0.04 \%)$ clinically diagnosed celiac disease cases were found in 1978-80 compared to $32(0.5 \%)$ in 2000-01. The prevalence of previously unrecognized screen-detected cases was high in the both cohorts being $1.1 \%$ and $1.4 \%$, respectively. Most importantly, the total prevalence of celiac disease increased significantly from $1.1 \%$ (CI 0.8-1.3) in 197880 to $1.9 \%$ (CI 1.6-2.2) in 2000-01. CONCLUSIONS The total prevalence of celiac disease nearly doubled in Finland during the last two decades and it cannot be explained by the better detection rate. The substantial rise in the frequency of celiac disease is in agreement with the increasing rate of other autoimmune diseases such as type 1 diabetes. The environmental factors responsible for the increasing prevalence of celiac disease in process of time are still unclear.

\section{W1243}

Baboons Affected By Hereditary Chronic Diarrhea As a Possible Non-Human Primate Model of Celiac Disease

Alessio Fasano, Debby Kryszak, Michelle Leland, Henry McGill

Background: Celiac disease (CD) represents a unique model of autoimmunity in which, in contrast to most other autoimmune diseases, a close genetic association with HLA genes (DQ2 and/or DQ8), a highly specific humoral autoimmune response (autoantibodies to tissue transglutaminase, (TTG)), and, most importantly, the triggering environmental factor (gluten), are known. Given the undisputable role of gluten in causing inflammation and immune-mediated tissue damage, $\mathrm{CD}$ could provide unique opportunities to tackle the pathogenic basis of autoimmune processes. However, the lack of an animal model of the disease represents a major limitation in reaching goals. Methods: Baboons from the pedigreed colony at the Southwest Foundation for Biomedical Research (SFBR) in San Antonio, Texas were reviewed. Those animals with chronic diarrhea ( $\geq 21$ days) and more than two clinical admissions between January 1998 and December 2003 were selected. This search yielded 163 baboons. An infective cause of the diarrhea was ruled out. CD serology and intestinal histological analysis were performed on a selected number of animals. Results: The onse of diarrhea occurred at all ages (few months- 15 years) and was analyzed for distribution among the 4,200 offspring of 57 sires of the pedigreed colony. Fifty-one of the 163 cases (31\%) of chronic diarrhea occurred among the offspring of 4 sires. Of the 15 cases studied $9(60 \%)$ tested TTG IgA and/or IgG antibodies positive, compared to $2 / 14$ (14.3\%) in healthy control baboons. Zonulin, a modulator of intestinal permeability that is up regulated in $\mathrm{CD}$ resulted elevated in $9 / 15(60 \%)$ baboons with chronic diarrhea and 0/14 controls. Ther was $100 \%$ correlation between positive TTG IgG and elevated zonulin. Duodenal samples obtained after the baboons were euthanized showed increased intraepithelial lymphocytes and, occasionally, marked lymphocytic mucosal infiltration and villi distortion and blunting Four baboons with chronic diarrhea, weight loss, anemia, and histological findings of intestinal damage compatible with CD were individually caged and fed a gluten-free diet (GFD). After 6 weeks of diet the diarrhea persisted, however the animals began to gain weight. Conclusions: These preliminary observations suggest that a gluten-dependent enteropathy is present in the pedigreed baboon colony studied, and that it is clustered among the progeny of a few sires. This sire effect is presumptive evidence that the condition is hereditary. The clinical, serological, and histological features suggest a similarity to human $C D$ and, therefore, that the progeny of this sire can be potentially used as a primate non-human model of $\mathrm{CD}$.

\section{W1244}

Evidence of Neuroautonomic Dysfunction in Celiac Disease

Roberto Zulli, Franco Nicosia, Stefania Bertolazzi, Alessandra Mora, Francesco Daminelli, Massimiliano de Vecchi, Paolo Donati, Francesco Lanzarotto, Vittorio Grassi, Alberto Lanzini

Neuroautonomic function has been reported to be altered in many inflammatory conditions. Celiac disease (CD) is a chronic inflammatory disease with highly heterogeneous clinical presentation, but little information is available on involvement of the neuroautonomic system The aim of our study was to assess the neuroautonomic function in CD by using prolonged electrocardiographic recording and analysis of the cardiac cycle variability (heart rate variability analysis, HRV) both by time and frequency domain analysis. We also evaluated the potential relationships between HRV and inflammatory parameters reflecting the severity of the disease. We enrolled 52 consecutive untreated CD patients ( 9 male, 43 female, mean age 34 years) with positive antiendomysial-ab and $\mathrm{CD}$ compatible duodenal histology, and 52 sex and age matched healthy controls with no clinical conditions potentially interfering with $\mathrm{HRV}$ analysis. The proportion of the numbers of the $\mathrm{R}$ to $\mathrm{R}$ successive intervals differing more than 50 milliseconds (pNN50) was significantly lower in CD than in controls (14.8 \pm 9.8 vs $22.1 \pm 11.7, \mathrm{p}<0.01$, respectively), and the root mean square of successive NN interval (rMSSD) was also significantly lower in $C D$ than in controls $(37.3+12.5$ vs $42.3+9.4 \mathrm{p}<0.05$, respectively). The Low Frequency power spectral band (LF, an index of sympathetic modulation) and the High Frequency power spectral band (HF, and index of parasympathetic modulation) values were significantly reduced in CD (LF $586.8 \pm 520.3 \mathrm{msec} 2 \mathrm{vs}$ 815.3 \pm 499.3 , $\mathrm{p}<0.05$, HF $453.5 \pm 687.3 \mathrm{msec} 2$ vs $681.4 \pm 555.7, \mathrm{p}<0.05)$. LF/HF ratio was higher in $\mathrm{CD}$ though the difference was not statistically significant. There was a significant relationship in CD between HRV parameters and biochemical inflammatory indexes (pNN50 rMSSD, LF, HF vs ESR, neutrophils count and fibrinogen with $r$ values ranging from -0.30 to -0.47 at Pearson's analysis). Our study suggests that neuroautonomic function is altered in $\mathrm{CD}$ as indicated by reduced HRV parameters, particularly those related to the parasympathetic modulation. This phenomenon is independent of the severity of gastrointestinal symptoms, and it is likely to be related to inflammatory changes in $\mathrm{CD}$, as reported for other inflammatory conditions and to the severity of the disease.

\section{W1245}

Anti-Yeast Antibodies Correlate with Serological Markers in Celiac Disease Sebastien Ratajczyk, Annie Standaert, Peggy Vandewalle, Boualem Sendid, Sylvain Dubucquoi, Daniel Poulain, Jean F. Colombel

Background: Antibodies against the oligomannosidic epitopes of the yeast Saccharomyces cerevisiae (ASCA) are a serological marker for Crohn's disease. Studies from our group hav shown that Candida albicans is an immunogen for ASCA (1). High prevalence of ASCA has also been reported in celiac disease. It has recently been suggested that $\mathrm{C}$.albicans could be a trigger in the onset of celiac disease through its virulence factor hyphal wall protein 1 (Hwpl) which is a transglutaminase substrate (2). Aim of the study: To look at the correlation between two yeast antibodies anti- C.albicans antibodies (ACAA) and ASCA and serological markers of celiac disease. Patients and methods: This cross-sectional study involved 81 celiac adults $(55 \mathrm{~F}, 26 \mathrm{M})$ with an average age of $34 \mathrm{yrs}$ at diagnosis and of $43 \mathrm{yrs}$ at the time of the study. IgG antibodies against gliadin (IgG-AGA), tissue transglutaminase antibodies (TTG-IgA), ASCA, ACAA and C.albicans antigenemia were determined by usual techniques At the same time, a semi-quantitative evaluation of the gluten free diet (GFD) was performed

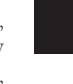


Results: The prevalence of serological markers was 49\% for IgG-AGA, 26\% for TTG-IgA $52 \%$ for ASCA and $22 \%$ for ACAA No patient had a positive C.albicans antigenemia. There was a correlation between the presence of ASCA and TTG-IgA $(p=0.037)$ and between the presence of ACAA and TTG-IgA $(p=0.008)$. Presence of ASCA and ACAA was also correlated $(\mathrm{p}=0.049)$. TTG-IgA titers were correlated with ASCA titers $(\mathrm{p}<0.01)$. Presence of TTG-IgA was correlated with adherence to GFD but no correlation was found for both anti-yeast antibodies. Conclusion: Celiac disease is frequently associated with anti-yeast antibodies. The correlations established between ASCA/ACAA and TTG-IgA presence and levels support the hypothesis that C.albicans could play a role in the pathophysiology of the disease. 1 Standaert A et al. Gastroenterology 2005; 128 (suppl 2: A508; 2 : Nieuwenhuize WF et al. Lancet 2003;361:2152-4

\section{W1246}

The Role of Indoleamine 2,3 Dioxygenase (IDO) in Celiac Disease Ram P. Janardhanam, Sherman Chamberlain, Jeffrey Lee, David Munn, Robert Schade

Purpose: IDO is the first enzyme catalyzing tryptophan degradation along the kynrenine pathway. IDO has proved to be an important immunosuppressive and tolerogenic mechanism in the immune system (Science 297: 1867, 2002). The current study addresses the hypothesis that IDO is an anti-inflammatory mechanism expressed by cells of the gut to control T-cell proliferation that results in the injury and inflammation seen in celiac disease. Methods: Archival biopsy samples obtained during upper endoscopy were retrieved. The samples selected were from biopsy-proven celiac disease and obtained at a time of histologicallyproven active disease. Overall samples were obtained from 4 patients. These included 2 patients with untreated active disease and 2 patients that included samples before and after 8 weeks of gluten free diet. Replicate sections and appropriate controls were deparaffinization and immunohistochemistry was performed using the standard avidin-biotin complex method. Affinity-purified polyclonal rabbit antibody was used for IDO staining. The samples were graded for IDO activity in lamina propria inflammatory cells and in small bowel epithelial glands in a blind fashion on a scale of $0-4+$, with $4+$ showing the highest expression of IDO. Results: IDO expression was seen in the lamina propria inflammatory cells (granulocytes, macrophages, eosinophils, and dendritic cells) and crypt epithelial cells. Three areas of the biopsy samples were scored for IDO expression: epithelial cells, inflammatory cells and dendritic cells. The epithelial cell IDO mean score was 1.75 , the inflammatory cell IDO mean score was 1.75 and dendritic cell IDO mean score was 1.5. When comparing the sets of samples that received treatment with gluten free diet, the epithelial cell IDO mean scores pre and post-treatment were 1.5, but the inflammatory cell IDO mean scores pre and post were 1.5 and 0.0 , and dendritic cells IDO mean scores pre and post were 1.5 and 0.0 . Conclusions: IDO is expressed by glandular epithelial cells and lamina propria inflammatory cells of the gastrointestinal tract in patients with celiac disease. T cell proliferation can be regulated by IDO, thus we hypothesize that IDO's presence serves as a counter-regulatory or anti-inflammtory role in these cells. The difference observed in IDO expression of inflammatory and dendritic cells by pre and post treated patients suggests that the upregulation of IDO maybe a protective response in active celiac disease

\section{W1247}

\section{Screening for Celiac Disease in Family Members: Is Follow-Up Testing} Necessary?

David S. Goldberg, Debbie Kryszak, Alessio Fasano, Peter H. Green

Background: Celiac disease (CD) occurs at an increased rate among first- and second-degree relatives of individuals with $\mathrm{CD}$. They are advised to be screened. The role of subsequent testing after an initial negative screening serologic test is unclear. The aim of our study was to determine (1) if one-time screening is sufficient and (2) the frequency of subsequent testing. Methods: The University of Maryland Celiac Research database for the previously published study of serologic screening for CD (Arch Intern Med. 2003; 163: 286-292.) was analyzed. The initial serologic testing of subjects included antigliadin antibodies (IgA,IgG) and endomysial antibodies (EMA). As the study progressed, transglutaminase antibodies (tTG) were measured. We identified those subjects who were tested on $>1$ occasion. Results: Of the 13,423 subjects in the database, 288 were tested more than once. Of these, 171 were family members who had an initial negative EMA; $3.5 \%$ (six subjects) developed a positive EMA on repeat testing ( 4 female, 2 male, ages 11-63). The average time to seroconversion was $1.7 \pm 1.2$ years ( 6 months-3 years, 2 months); statistically equivalent to the time interval between testing for those that remained EMA negative ( $1.5 \pm 1.0$ years). Of the six, one had classical CD symptoms of diarrhea, three had atypical symptoms (joint pain, heartburn, gaseousness, and abdominal pain), and two were asymptomatic. None of the patients had any change in symptoms at the time of EMA seroconversion. At initial testing, one of the six had an elevated IgA AGA, but at repeat testing, four had elevated IgA AGA levels. Of the four that had IgA tTG measured at the time of seroconversion, all had elevated levels. Four of the subjects were first-degree relatives, two second-degree. The six seroconverters and the 165 non-seroconverters were statistically equivalent in terms of age, gender, genetic relatedness to $\mathrm{CD}$ patients, and symptoms. The only statistical difference between the groups was that a higher percentage of seroconverters had abnormal levels of IgA AGA at repeat testing. None of the other groups in the database, either those at risk because of symptoms or $\mathrm{CD}$-associated diseases, seroconverted on follow-up testing. Conclusions: We found that $3.5 \%$ of family members who underwent repeat serologic testing subsequently developed a positive EMA. One-time testing of family members of those with $\mathrm{CD}$ is insufficient. A reasonable recommendation is that all first- and second-degree relatives be screened a second time, at 4 years after the initial testing, regardless of age or symptoms. Further studies are needed to determine the role of subsequent testing after that time period.

\section{W1248}

Alemtuzumab Treatment of Refractory Celiac Disease Type II Knut E. Lundin, Inger Nina Farstad, Melinda Raki, Yngve Benestad, Ole Hoie, Geir Tjonnfjord

Introduction: Celiac disease is usually a benign disorder. However, some patients fail to improve or develop villous atrophy in spite of a strict gluten free diet. This is termed celiac disease which again is divided into two categories. Type I does not, whereas Type II has a monoclonal population of premalignant intraepithelial T-lymphocytes. Type II will often develop into enteropathy-assosiated T-cell lymphoma, a condition with a poor prognosis. Patients with type II do also have a poor prognosis with a 5-year survival of well below 50 $\%$. No effective cure has so far been described. We have used the monoclonal antibody alemtuzumab for treatment of one such patient. Alemtuzumab is directed against the surface marker CD52 on T-and B-cells. Alemtuzumab is effective in treatment of various malignant disorders, including chronic lymphatic leukaemia and pro-T-cell leukemia. Patient: A now 63 year old man presented in 2003 with total villous atrophy and positive IgA-TG2. He had an initial good effect of gluten free diet but then developed weight loss, hypoalbuminaemia, diarrhoea and enlarged abdominal lymph nodes. A lymphoma was suspected and he was operated. No tumor was found. Gastroduodenoscopy, capsule endoscopy and enteroscopy showed ulcerative jejunitis. In the resected material and in small intestinal biopsies we found a monoclonal and oligoclonal population, respectively. The intraepithelial lymphocytes were phenotypically abnormal as they lacked CD8 S.c. treatment with alemtuzumab was started after careful consideration and full information of the patient. The first dose gave mild general symptoms with fever and discomfort. He had later no symptoms of the treatment that lasted for 12 weeks with $30 \mathrm{mg}$ alemtuzumab 3 times/week, total dose $1200 \mathrm{mg}$. The treatment gave full clinical remission. Profylaxis with valciklovir and Trimetoprim-Sulfa was given. Duodenal and jejunal biopsies after the treatment showed a phenotype compatible with regular celiac disease. Capsule enteroscopy and a double balloon enteroscopy showed some erosions in the ileum. Biopsies suggested persistence of the malignant monoclonal population. The patient is in these days being treated with high dose chemotherapy and total body irradiation with autologous stem cell rescue. Discussion: Refractory celiac disease type II (cryptic T-cell lymphoma) is a serious disease with a poor survival. Previously tested treatment options have not proved effective. Treatment with alemtuzumab gave clinical, histopathological and partly molecular biological remission. The treatment is promising but must be tested in larger patient series.

\section{W1249}

The Raffinose/Mannitol Sugar Absorption Test Is Related to the Severity of Celiac Histopathological Small Bowel Changes

Ivar M. Harkema, Jeroen J. Kolkman, Joop J. Vanbaarlen, Cees J. Doelman

Introduction In celiac disease, defined as partial to total villous atrophy in the duodenal biopsy, the sugar absorption test (SAT) has proven to be a reliable tool for assessment of the small bowel permeability. This test consists of ingestion of a sugar solution containing monomer (mannitol), dimer (lactose) and trimer (raffinose) saccharides. Ratio's of this saccharides are determined in the urine 5 hours after ingestion. Normal mucosa will only allow a small amount of resorption via a paracellular route. . Loss of villous architecture and "mucosal leakage", in villous atrophy increases mall bowel permeability, and therefore increased large-small sugar ratio. Several studies suggested a relation between the ratio and the severity of villous disruption, mostly comparing villous atrophy to normal mucosa. Aim To establish the relation between SAT and mucosal histopathology in patients biopsied for suspected - celiac disease, stratified according to the Marsh criteria. Method We retrospectively studied patients in whom duodenal biopsies were taken and the SAT determined. The biopsies were stained with using haematoxylin/eosin staining and immunohistochemical IEL counting (CD3), and graded according to Marsh: M-I: intraepithelial lymphocytes (IEL) >30/ 100, M-II: increased IEL plus crypt hyperplasia, M-IIIa: partial, IIIb: subtotal and IIIc: total villous atrophy . For the SAT we used $150 \mathrm{ml}$ sugar solution, with $7 \mathrm{~g}$ raffinose, $20 \mathrm{~g}$ lactose, $2 \mathrm{~g}$ mannitol and $20 \mathrm{~g}$ saccharose. Urine was collected from $0-5$ hours after ingestion. The raffinose/ mannitol ratio was calculated. The previously established normal cut-off for SAT was > 18. Results From 1997-2005 we performed 30.564 gastroscopies, and took 3454 duodenal biopsies. In 575 patients time-matched duodenal biopsies and SAT was available. M-0 M-I M-II M-IIIa M-IIIb M-IIIc No 2882054424123 Abnormal SAT 46381512 103 Mean SAT 1412203783212 Using the Spearman rank correlation we found a significant correlation between the Marsh classification and the SAT test, $r=0,20, p<0,0001$. SAT as diagnostic test for villous atrophy showed a sensitivity of $64 \%$, specificity of $82 \%$ with a likelihood ratio of $3,47(\mathrm{p}<0,001)$. Conclusion SAT is a reliable predictor of mucosal permeability and is related to the presence and severity of villous atrophy. The diagnostic accuracy seems insufficient to use it as screening tool.

\begin{tabular}{|c|c|c|c|c|c|c|}
\hline & M-0 & M-I & M-II & M-IIIa & M-IIIb & M-IIIc \\
\hline No & 288 & 205 & 44 & 24 & 12 & 3 \\
\hline Abnormal SAT & 46 & 38 & 15 & 12 & 10 & 3 \\
\hline Mean SAT & 14 & 12 & 20 & 37 & 83 & 212 \\
\hline
\end{tabular}

\section{W1250}

High Prevalence of Intraepithelial Lymphocytosis Found in Duodenal Biopsies in a Large Cohort of Patients Referred for Routine Gastroduodenoscopy Ivar M. Harkema, Joop J. van Baarlen, Jeroen J. Kolkman

Introduction The histological diagnosis of celiac disease is based on the triad of villous atrophy, crypt hyperplasia and intra-epithelial lymphocytosis according to the Marsh classification. In recent years, the required histopathological abnormalities have changed from required total villous atrophy to more subtle villous abnormalities. In this latter group several authors have shown development of overt villous atrophy with gluten challenge or resolution 
of symptoms with gluten free diet; thus suggesting that some of these patients have mild celiac disease. It is currently unclear what the prevalence of intra-epithelial lymphocytosis is among patients referred for gastroduodenoscopy. Methods From 1997 we routinely took 3-4 duodenal biopsies in patients referred for either suspected celiac disease, or patients with diarrhea, anemia, weight loss or severe dyspepsia. The duodenal biopsies were stained with haematoxylin/eosin (HE) and scored for the presence of villous atrophy, crypt hyperplasia and intra-epithelial lymphocytes (IEL's). If elevated IEL's were found on standard HE staining, immunohistochemical staining of $\mathrm{CD}-3$ positive lymphocytes was performed with morphometric measurement of IEL infiltration (expressed as lymphocyte count per 100 epithelial cells). A normal cut-off point of 30 IEL / 100 was chosen. The biopsies were classified according to Marsh. Results From January 1997 until september 2005 a total of 30.564 gastroduodenoscopies were performed. In 3454 patient (11\%) duodenal biopsies were taken. $2396(69,4 \%)$ patients showed normal mucosa, 792 patients $(22,9 \%)$ had a Marsh I lesion, 116 patients (3,4\%) Marsh II, and 150 (4,4\%) patients had villous atrophy (Marsh IIla-c). Thus, intra-epithelial lymphocytosis without villous atrophy was seen in $26,3 \%$ of patients, villous atrophy was seen in $4,4 \%$. Conclusions In patients presenting with diarrhea, anemia, weight loss and severe dyspepsia undergoing gastroduodenoscopy, intra-epithelial lymphocytosis is a common finding when immunohistochemic staining is used.

\section{W1251}

Psychiatric Alterations, Depression and Symptomatic Dimensions in Patients with Celiac Disease At Diagnosis: The Impact On Quality of Life Cristina Sfoggia, Fabio Nachman, Eduardo Maurino, Horacio Vazquez, Edgardo Smecuol, Silvia Pedreira, Sonia Niveloni, Emilia Sugai, Roberto Mazure, Maria L. Moreno, Julio C. Bai

Background/aims: Celiac disease (CD) is a chronic inflammatory enteropathy characterized by a wide clinical variability. Depression and other psychiatric symptoms have been reported as common clinical features of CD occurring in about $30 \%$ of patients. In this context, little is known about how patients perceive their health state and how depression impacts in it. Our aim was to determine the prevalence of depression and its impact on the quality of life (QoL) of patients with CD assessed at diagnosis. Methods: We prospectively enrolled a series of 134 consecutive newly diagnosed adult CD patients (117 females). At diagnosis, 100 patients evidenced classical symptoms and 34 were categorized as subclinical/silent cases. At diagnosis, all patients underwent a battery of self-administered questionnaires including the Short Form 36 Health Survey (SF-36), the Beck Depression Inventory (BDI) and the Symptom Checklist 90 Revised performed (SCL 90R). These tests allow define three levels of psychiatric morbidity. Results: At diagnosis, the $\mathrm{CD}$ population exhibited a profound alteration of the 8 items of the SF-36. The BDI showed that $50 \%$ of the overall population had evidence of depression, this finding was significantly more prevalent in patients with classical symptoms $(55 \%)$ than in those subclinical/silent cases $(21 \% ; \mathrm{p}<0.04)$. There was an inverse correlation between BDI and the eight items of SF-36 ( $\mathrm{r}:-0.50, \mathrm{p}<0.0000)$. The SCL 90 R analyses evidenced that $35(27 \%)$ of patients had no morbidity, $36(28 \%)$ had a minor psychiatric compromise and 57 (45\%) had major psychiatric morbidity. Of the nine assessed dimensions somatization $(\mathrm{n}=25)$ and hostility $(\mathrm{n}=25)$ were the most prevalents. Conclusions: According to the present findings, $\mathrm{CD}$ patients have a profound alteration of the subjective perception of QoL. Depression was present in $50 \%$ of patients being greater in those expressing classical symptom and impacts inversely on the QoL. Finally, the use of two different tests for psychiatric assessment emphasizes two still unrecognized features in adult patients such as somatization and hostility. The frequency of psychiatric alterations makes necessary a specialized support of these patients.

\section{W1252}

Is a Control Biopsy in Pediatric Celiac Disease Necessary? Anna Killander, Arnell Henrik, Hildebrand Hans, Yigael Finkel

The criteria for paediatric celiac disease (CD) rely on histopathological examination (HPath) of small bowel biopsies (SBB) and serology before and on glutenfree diet (GFD) (1). There is still controversy about the need for control SBB on GFD. Aim: To investigate the use of pre-GFD biopsies and IgA-transglutaminase antibodies (IgA-TGA) in diagnosis of pediatric CD. Method: Retrospective review of work-up for CD in children older than 18 months after one-year GFD. Children with positive IgA-TGA and pre-GFD biopsy were included, children with IgA deficiency were excluded. 71 children median age 5 (1,5-16 years) underwent gastroscopy with biopsies from bulb and distal duodenum pre-GFD (2). In 56/ 71 children, ( 34 girls), median age 5 years (1,5-16 years) data on HPath pre-GFD, IgATGA before and on GFD and Z-score for weight and height on GFD were analysed (group 1). 15/71 children (11 girls) median age 7 (1,6-11 years) underwent a second SBB on GFD (group 2). Results: HPath of SBB pre GFD showed partial, subtotal or total villous atrophy in all children. In group 1, IgA-TGA was normal after one-year GFD in 53/56, borderline in 3. Z-score for weight $(\mathrm{W})$ and height $(\mathrm{H})$ increased in 34/44 (2 missing data) young and prepubertal children aged 1,5-12 years on GFD for one-year. Median Z scores for children 1,5 -2years; $\mathrm{W}+0,74, \mathrm{H}+0,46,2-7$ years; $\mathrm{W}+0,35, \mathrm{H}+0,16,8-11$ years; $\mathrm{W}+0,32$ and $\mathrm{H}$ $+0,08$. In group 2 after one-year GFD 14/15 had normal TGA (1 missing), and HPath of SBB was normal in 13/15, while 2 children had considerably less HPath changes compared to pre-GFD SBB. A complete remission of symptoms related to CD on one-year GFD was reported in most children in both groups. Conclusion: We are confident to diagnose celiac disease in children 1,5 - 11 years on a combination of pre-GFD SBB and TGA and remission of symptoms, normalised IgA- TGA and catch-up growth on GFD. 1.Revised criteria for diagnosis of coeliac disease.Arch Dis Child. 1990;65(8):909-911. 2.Kappinen K. Arnell H, Finkel Y, Hildebrand H, Lindholm J. Villous atrophy in pediatric celiac disease may be confined to the proximal part of duodenum. J Pediatr Gastroenterol Nutr. 2005;39 Suppl $1: s 202-203$

\section{W1253}

Usefulness of the Assesment of Celiac Disease Serological Markers in Healthy Working People for the Detection of Silent Disease Among General Population Meritxell Marine, Maria Esteve, Montserrat Alsina, Carme Farre, Montserrat Cortijo, Antonio Salas, Margarita Tomas, Elias Abugattas, Merce Rosinach, Fernando Fernandez Banares, Rebeca Santaolalla, Clarisa Gonzalez, Josep Maria Viver

INTRODUCTION: Several epidemiologic studies have demonstrated that the total prevalence of $C D$ is about 1:250. Most of these cases still remain undiagnosed. Despite celiac disease (CD) has most of the WHO criteria for early disease detection (Wilson \& Jungner), mass screening of the general population is nowadays controversial. AIMS: 1- To asses the usefulness of including CD specific antibodies -antitransglutaminase antibodies (tTGA) and antiendomisium antibodies (EMA)- in healthy working population blood tests for the detec tion of the disease. 2- To reevaluate the cut off for positive values of tTGA (recommended cut-off by the supplier $>8 \mathrm{U} / \mathrm{ml}$ ). METHODS: 1781 individuals were included [1308 males, age: $36.9 \pm 0.32$ years; 473 females, age: $36.6 \pm 0.5$ years]. Blood tests were performed to al participants, including CD markers: tTGA (ELISA: Pharmacia Diagnostics) and EMA (IFI: BMD Biomedical Diagnostics). tTGA were considered positive when values $>2 \mathrm{U} / \mathrm{ml}$ were found, confirmed twice in the same sample. Duodenal biopsy and HLA DQ2/DQ8 genotyping were performed to all individuals with EMA positive values and/or tTGA. Histological damage was established using Marsh criteria. Results were expressed as $\%$ and $\mathrm{x} \pm$ SEM. RESULTS Positive antibodies were detected in 20 individuals (1.1\%): 5 with EMA+/tTGA+, 14 with tTGA+/EMA- and 1 EMA+/tTGA. Two subjects did not accept further evaluations, and 1 has not yet been evaluated (1 EMA+/tTGA+, 2 EMA-/tTGA+). We detected four Marsh II [all of them EMA and TTGA+ (values between 8.5 and $63.4 \mathrm{U} / \mathrm{ml}$ ); 3 DQ2+, 1 DQ2/DQ81, 8 Marsh I [7 EMA-/tTGA+ (values between 2 and 5 U/ml); 1 EMA+/tTGA-; 5 DQ2+, 2 with one positive allele of the DQ2 , 1 in course)], 5 Marsh 0 , two of them with $16 \%$ and $20 \%$ intraepithelial lymphocytes [EMA-/tTGA+ (values between 2 and 3.4); 5 DQ2+]. The percentage of CD (Marsh I-III) detected by histopathology was $0.67 \%$. CONCLUSIONS: 1 The percentage of $\mathrm{CD}$ found in this study (higher than other diseases where population screening is recommended) justifies the inclusion of $\mathrm{CD}$ serologic markers in healthy working population blood tests. 2 - To establish the tTGA cut off in $2 \mathrm{U} / \mathrm{ml}$ allows diagnosing more number of $C D$ type Marsh I, which can be as much symptomatic as Marsh III. 3- tTGA is more sensitive than EMA in this situation. This study has received financial support from "Banc de Sabadell" of Catalonia (Spain).

\section{W1254}

Endoscopic and Pathological Response of Ulcerative Jejunitis to Infliximab Therapy

Neil Rajoriya, Mattew Priest, Galloway Aileen, Robert Jackson, John MacKenzie, John Morris

Aim: To assess endoscopic and histological response of patients with Ulcerative Jejunitis to Infliximab infusion(monoclonal anti-TNF-alpha) therapy. Methods: Patients with Ulcerative Jejunitis complicating Coeliac disease were identified. All patients had: clinical status and Coeliac antibody status, push enteroscopy, histology and immunophenotyping studies carried out preceding and within 6 weeks post-Infliximab therapy $(5 \mathrm{mg} / \mathrm{kg})$. Results: 4 patients ( 3 male and 1 female) with Ulcerative Jejunitis were treated with Infliximab. The median age was 42.3 years (Range: 18-62 years). The time between diagnosis of Coeliac disease and Infliximab therapy was median 6 years (Range 20 months-17 years). All 4 patients had received oral corticosteroid treatment pre-Infliximab therapy. 3 were started on Azathioprine $(2 \mathrm{mg} / \mathrm{kg})$ in the peri-infusion period, and 1 patient had been on it for 7 years (disease onset at 16 months of age). Pre-treatment 3 were negative and 1 positive for Coeliac disease antibodies. All 4 had enteroscopic evidence of ulcerative jejunitis complicating Coeliac disease which was confirmed by histology. 3 patients were intra-epithelial lymphocyte $\mathrm{T}$ cell receptor $\mathrm{CD} 3+\mathrm{ve} / \mathrm{CD} 8$-ve (one was $\mathrm{CD} 8+\mathrm{ve}$ ). 2 patients were T-cell receptor CD30 +ve. PCR of intra-epithelial lymphocytes showed 3 of 4 patients were monoclonal and 1 patient oligoclonal for TCR-gamma gene rearrangement. Post-Infliximab treatment, all 4 patients had clinical improvement at 6 weeks. Repeat enteroscopies were done within 6 weeks of Infliximab therapy all showing macroscopic and histological improvement with complete healing of ulcers in 2 patients. In 2 patients there was a marked reduction in $\mathrm{CD} 30+v e$ cells, one of whom became CD30-ve. The follow up times since Infliximab have been: 3 months, 6 months, 30 months and 50 months. 2 patients had a relapse of symptoms at 6 months, one of whom died secondary to a perforated High grade T-cell Lymphoma. The 3 remaining patients are on low dose corticosteroid therapy. Conclusion: Infliximab therapy leads to short-term enteroscopic, histological

\section{W1255}

Risk Factors for Osteoporosis and Effect of Gluten Free Diet On Bone Density in Patients with Celiac Disease

Donald Duerksen, Carrie Wilhelm-Boyles, David Parry

Celiac disease (CD) is associated with osteoporosis and decreased bone density. The prevalence of decreased bone density may vary according to the population studied. The objectives of this study were to determine i) the prevalence of decreased bone density (BD) in patients with newly diagnosed $C D$ and in patients on a gluten free diet for greater than 6 month ii) the effect of clinical presentation at diagnosis on the development of osteoporosis. iii) the effect of a gluten free diet (GFD) on bone density. Methods: Adult patients with CD Group 1 (BD $<6$ mo. after diagnosis $(\mathrm{dx})$ of $\mathrm{CD}$ ) and Group 2 (BD $>6$ mo. after $\mathrm{dx}$ of $\mathrm{CD}$ ) underwent testing with $\mathrm{BD}$. Group 1 patients were divided into 3 categories based in clinical presentation at diagnosis: P1-GI symptoms and wt loss, P2-GI symptoms, P3-Silent Atypical Results: The following are the patient characteristics: Grp 1 N=51 M:F 5/46 Mean age at $\mathrm{dx}$ 42.6. Grp $2 \mathrm{~N}=61 \mathrm{M}: \mathrm{F}$ 13/48 Mean age at dx 44.3, mean duration GFD 9.42y. see Table 1 There were 42 patients who had follow-up bone density tests a mean of 2 years from the initial test. There was a $5.8 \%$ mean increase in $\mathrm{BD}$ in the spine and $6.3 \%$ mean increase in $\mathrm{BD}$ in the femur in this group. While increases in bone density were seen in

. 
73\% (spine) and 79\% (femur), significant numbers of patients had osteoporosis or osteopenia ( $48 \%$ spine and $50 \%$ femur). The effect of clinical presentation on diagnosis is summarized in Table 2 Conclusions: 1) Low bone density is common in newly diagnosed celiac patients and in those who have been on a GFD for a prolonged period of time. 2) The majority of patients have an improved bone density after 2 years of GFD with a significant increase in bone density of $\sim 6 \%$. 3) Even after a GFD of 2 years, $\sim 50 \%$ of patients are osteopenic/ osteoporosis. These patients required long-term monitoring to evaluate their risk for fractures 4) Weight loss at presentation is a significant risk factor for low bone density at diagnosis. This group should be targeted for bone density evaluation at diagnosis Table 1

\begin{tabular}{|c|c|c|c|c|}
\hline Expressed as \% & Spine T $<-2.5$ & Spine T $-1--2.5$ & Femur T $<-2.5$ & Femur T $-1--2.5$ \\
\hline Grp 1 & 25 & 25 & 10 & 45 \\
\hline Grp 2 & 18 & 23 & 25 & 8 \\
\hline
\end{tabular}

Table 2

\begin{tabular}{|c|c|c|c|c|}
\hline & Spine T & Spine Z & Femur T & Femur Z \\
\hline P1 N=13 & $-2.3^{*}$ & $-1.8^{*}$ & $-1.9^{*}$ & $-1.5^{*}$ \\
\hline P2 N=20 & -.8 & -.5 & -.7 & -.3 \\
\hline P3 N=17 & -.7 & -.3 & -.9 & -.5 \\
\hline
\end{tabular}

* $\mathrm{p}<.05$

\section{W1257}

Seroprevalence of Anti-Gliadin and Anti-Endomysium Antibodies in Mexican Adults

Nahum Mendez-Sanchez, Daniel Zamora-Valdes, Francisco Sanchez-Giron, Maria A Montano-Reyes, Lizet Lopez, Martha H. Ramos, Misael Uribe

Background: Diseases associated with a pathological immune response, such as Crohn's and celiac disease, are considered to be less prevalent in developing than in developed countries. Our concept of celiac disease have changed during the last decade, as more asymptomatic or oligosymptomatic cases are identified. There is no information concerning the prevalence of celiac disease in our country. Aim: Determine the seroprevalence of anti-gliadin and antiendomysium antibodies in a check-up unit in Mexico City. Methods: We carried out an observational study in a check-up unit of a University Hospital in Mexico City. Anthropometric, metabolic and biochemical variables were measured in subjects between 20 and 70 years. The diagnostic conclusions of the check-up were recorded. Serum was tested for total IgA (Immage, Beckman-Coulter); for gliadin IgA antibodies by micro ELISA; and for endomysium and gliadin IgA by indirect immunofluorescence (IIF) with anti-human IgA FITC-labelled and slides containing primate intestine and Gliadine Biochips. Gliadin IgA ELISA was positive if result was $>25 \mathrm{U} / \mathrm{L}$. For IIF screening dilution was $1: 10$, positivity was assessed at 100x magnification, all positives were diluted 1:100, as well as IgA antigliadin and anti-endomysium antibodies using ELISA. Results: We studied 400 subjects, $71 \%$ male and $29 \%$ female. The median age was $45 \pm 11$ years, body mass index $25.9 \pm$ 3.7, waist-to-hip ratio $0.86 \pm 0.08$, body fat $28.3 \pm 5.7 \%$. Four patients exhibited IgA $<$ $70 \mathrm{mg} / \mathrm{dL}$ and one had a below range level. We identified twelve positive cases, eleven positive for IgA anti-gliadin and two for IgA anti-endomysium. One patient had 1:100 positivity to both antibodies and four had a 1:100 positivity for anti-gliadin and 1:10 for anti-endomysium. The seropositive group were $75 \%$ male and $25 \%$ female, its median age of $47 \pm 9$ years, body mass index $25.8 \pm 4.5$, waist-to-hip ratio $0.88 \pm 0.09$ and body fat $27.5 \pm 5.4 \%$. Four patients positive to anti-gliadin antibodies were symptomatic, two presented diarrhea and two had a previous diagnosis of irritable bowel syndrome. Conclusions: We found a seroprevalence of $2.7 \%$ for anti-gliadin and $0.5 \%$ for anti-endomysium antibodies. These results are comparable to countries such as Italy and lower than countries like Sweden or the United States.

\section{W1258}

Economic Burden of a Gluten-Free Diet

Anne R. Lee, Dianna Ng, Peter H. Green

Introduction: Individuals with celiac disease must consume a gluten free diet. Gluten free foods are available at general and specialty food stores as well as via the Internet. A cost survey was conducted to evaluate the economic burden of adhering to a gluten free diet. Method: A standardized product list (regular wheat based and their gluten-free substitutes) was used to evaluate the cost differences. The differences in price between purchase venues, both type of store (general grocery store, an upscale grocery store and a health food store and four internet based grocery sites) and region was also analyzed. The stores were surveyed in the New York (New York City and Westchester County), Portland Oregon, Atlanta Georgia, Rapid City South Dakota, and Chicago Illinois. The prices comparisons are reported in price per ounce for comparative purposes. Results: In all geographical regions the gluten-free products were more expensive $(240 \%)$ than their wheat-based counterparts. The increase in cost varied for the different food types (between 140 to 350\%). The health food stores were more expensive than the regular grocery store or the upscale markets for both the gluten-free and wheat based products (by 123\%). The Internet was more expensive for the gluten free foods by 1.1 to 1.6 times the cost. Geographic location did not affect the cost comparisons as much as did choice of shopping venue. Availability varied between the regular grocery store, the health food store, the upscale market, and on line shopping. The on line sites and the health food stores carried the largest selection of gluten-free products. The variability was only 25 to $75 \%$ of the gluten-free food items on the list were carried at either the general grocery store or the upscale market compared to $100 \%$ on the Internet sites Conclusions: To date there have not been any studies in the United States evaluating the cost of gluten free foods. Our study demonstrated firstly, that the availability of glutenfree foods is limited. Not all regular grocery stores carry gluten-free products. This forces patients to purchase gluten-free products at either specialty stores or via the Internet. In addition we determined that overall gluten-free products are far more expensive than their regular wheat based gluten containing products. As more research is done on the impact of the gluten free diet on an individuals' quality of life attention should also focus on the economic burden of adhering to the diet. Additional investigation is needed to determine if the economic burden of the diet is an issue of noncompliance to the gluten-free diet regime.

\section{W1259}

High Resolution SSOP Typing of Celiac Disease-Associated HLA DQ Haplotypes

Esther Oh, Mary Barry

BACKGROUND: The involvement of HLA DQ alleles in celiac is well established. About $95 \%$ of celiacs express the DQ2 heterodimer $\mathrm{DQA1} * 05$ and $\mathrm{DQB1} * 02$, while the majority of non-DQ2 carry DQ8 (DQAl*0301/DQB1*0302). DQAl*0201/DQB1*02 has also been implicated in a small number of celiacs. The majority of the remaining patients without DQ2 or DQ8, carry at least half of the DQ2 heterodimer. The literature reports a hierarchy in the relative risk of the DQ haplotypes, with individuals homozygous for DQB1*02 at a higher risk. AIM: To evaluate and validate a high-resolution sequence-specific oligonucleotide probe (SSOP) method for determining the celiac-associated DQ haplotypes. METHOD: DNA from reference cells with known HLA DQ haplotypes was obtained from IHW and ECACC. DNA was isolated from whole blood with commercial reagents. PCR amplification was with biotinylated nucleotides in two multiplex reactions for the DQAl and DQB1 locus. Amplified product was hybridized with 35 DQA1- and 37 DQB1-specific probes immobilized on membrane strips, and detected with enzyme-conjugated streptavidin in an automated controlled water bath environment (LiPA, Innogenetics). Probe reactivity patterns were interpreted in LiRAS to determine the alleles. RESULTS: Among the 30 reference DNA tested, 13 unique DQAl and 13 DQB1 alleles were detected. Three DQAl and two DQB1 alleles differed from their known typing; sequencing results confirmed the SSOP typing, all of which were more recent alleles (Table). 25 samples previously typed as DQ2 and/or DQ8 (defined above) by SSP were also correctly identified. DQB1*0302 in the presence of certain other DQB1 alleles may result in ambiguities. All three of the potential ambiguous haplotypes include alleles that are very rare (HLA Dictionary, 2004). The DQB1*0302-030101 ambiguity with DQB1*030302-0304 was further investigated by re-amplifying the DNA bound to a probe specific for $\mathrm{DQB} 1 * 0302$, followed by hybridization to $\mathrm{DQB1}$ probes; this confirmed the presence of the DQB1*0302 allele in all 20 cases tested. Conclusion: High-resolution SSOP typing of HLA DQ alleles is accurate and specific, and provides detailed haplotype information (i.e. both DQA1 and DQB1 alleles, and the number of copies of the alleles) that may be clinically useful for assessing the risk levels, or for eliminating patients from consideration for celiac disease.

\begin{tabular}{|c|c|c|}
\hline Reference & LiPA SSOP & Sequencing \\
\hline DQA $1 * 0104,0201$ & DQA $1 * 0105,0201$ & DQA $1 * 0105,0201$ \\
\hline DQA $1 * 0301,0302$ & DQA $1 * 0301,0303$ & DQA $1 * 0301,0303$ \\
\hline DQA $1 * 0104,0501$ & DQA $1 * 0105,0505$ & DQA $1 * 0105,0505$ \\
\hline DQB $1 * 0201,0501$ & DQB $1 * 0202,0501$ & DQB $1 * 0202,0501$ \\
\hline DQB $1 * 0302,0605$ & DQB $1 * 0302,0609 / 0618$ & DQB $1 * 0302 / 0609 / 0618$ \\
\hline
\end{tabular}

\section{W1260}

Proinflammatory Cytokynes IL-1b and Tnfa Gene Polymorphisms in Celiac Disease

Alejandra Chernavsky, P A. Correa, Natalia Periolo, Sonia I. Niveloni, Eduardo Maurino, Julio C. Bai, J M. Anaya

Background/aim: Celiac Disease (CD) is a well-characterized intestinal disorder with a strong genetic predisposition in the HLA-DQ region. It is also thought that non-HLA genes are determinants of CD susceptibility. Our aim was to establish the influence of TNFA and ILIB genes polymorphisms as risk factors for developing CD. Materials and methods: We enrolled 114 patients with well-established CD and 122 healthy controls with similar gender distribution and ethnicity. Genomic DNA was extracted from anti-coagulated PBMC by standard methods. Genotyping of single nucleotide polymorphisms (SNP) of TNFA - 308(G/A) and 238(G/A), and ILlB -511(C/T) and +3953(C/T) was performed by PCR-RFLP. Hardy-Weinberg (H-W) equilibrium and linkage disequilibrium testing were performed using Arlequin software. Results: IL1B but not TNFA markers met $\mathrm{H}-\mathrm{W}$ equilibrium genotype proportions. There was linkage disequilibrium between TNFA and IL1B loci but there were no significant differences in any inferred haplotype in both controls and $C D$ patients. Compared with controls, CD patients had a significant increased prevalence both of the allele TNFA -308A (OR: 1.47, 95\% CI: 1.01-2.13) and the TNFA -308AA homozygosis (OR: 3.28, 95\% CI: 1 64-6.53). Furthermore, TNFA -308GA heterozygosis constitutes a protective factor (OR: $0.38,95 \%$ CI $0.21-0.70$ ). While the IL1B $-511 \mathrm{~T}$ allele resulted a protective factor for CD (OR: $0.66,95 \% \mathrm{CI}$ : $0.45-0.96$ ), no association was found at IL1B +3953 position. CONCLUSIONS: Our findings show that a gene polymorphism at TNFA at position -308 is a genetic marker of $\mathrm{CD}$ susceptibility in the Argentinean population. Conversely, our data on the contribution of ILIB -511 suggest a protective role for the T allele. 
W1261

Impact of Age On Outcomes of Acute Variceal Hemorrhage in Patients with Cirrhosis

William Sanchez, Jayant A. Talwalkar, Mark V. Larson, Theresa A. Nolte, Stephanie M. Johnson, Louis M. Wong Kee Song, Vijay H. Shah, W R. Kim, Patrick S. Kamath

BACKGROUND: Despite major advances in the management of gastrointestinal (GI) bleeding, older patients are often excluded from clinical trials. Few studies examine the impact of age on acute variceal hemorrhage (AVH). AIMS: 1) Determine the frequency, clinical features, and outcomes of AVH in cirrhotic patients $\geq 65$ years of age. 2) Compare short and longterm survival rates following AVH in patients $\geq 65$ years of age with younger patients. METHODS: The study cohort was identified from a prospectively collected database for all emergent endoscopies from 1/1/1989-12/31/2004. Patients with upper GI hemorrhage from varices (esophageal, gastric) were selected. Liver disease severity was defined by the MELD score. Baveno III criteria were used for defining the acute bleeding episode, control of bleed, and rebleeding. Patients were censored at the date of death, transplantation, or last followup. Vital status was ascertained using the Social Security Death Index. RESULTS: 490 patients with at least one episode of AVH comprised the study cohort. 100 patients $(20.4 \%) \geq 65$ yrs of age were identified. Mean age was 57 yrs (18-92 yrs) with $66 \%$ men. The etiology of the cirrhosis was alcohol (29\%), viral hepatitis (14\%), and cholestatic (15\%). Mean MELD score was 19 (12-25). Endoscopic and vasoactive therapies were used in 78\% and 53\% of patients, respectively. Median time to follow-up was 323 days (20-1688 days). There was no significant difference in time to recurrent AVH $(\mathrm{p}=0.66)$ or transplant-free survival $(\mathrm{p}=0.23)$ between older and younger patients. Average MELD score, use of endoscopic/ pharmacologic therapy, and ICU days were similar between subgroups ( $p>0.05$ for all). The mean \# of blood transfusions was less in older patients ( $5.9 \mathrm{vs} 8.2, \mathrm{p}=0.02$ ). Frequency of comorbid illness was significantly higher in the older cohort ( $\mathrm{p}<0.01$ ). In patients $\geq 65$ yrs of age, the presence of recurrent AVH was not associated with lower transplant-free survival $(p>0.05)$. Patients $\geq 75$ yrs of age $(n=53)$ had an increased mortality rate $(p=0.016)$ without excess recurrent $\mathrm{AVH}(\mathrm{p}=0.06)$ compared to patients less than 75 yrs old. Frequency of comorbid illness ( $p=0.004$ ) was the only variable associated with increased mortality risk in this subgroup. CONCLUSIONS: 1) In patients with AVH, an estimated $20 \%$ of individuals are $\geq 65$ years of age. 2) Transplant-free survival and frequency of recurrent bleeding is similar to younger patients regardless of MELD score or severity of bleeding. 3) Management strategies for the treatment of AVH are as effective for older patients as younger patients, and older patients should no longer be excluded from important clinical trials.

\section{W1262}

Intrahepatic Hyperphosphorylation of the Rho-Kinase-Substrate Moesin in Experimental and Human Cirrhosis

Martin Hennenberg, Jonel Trebicka, Ludger Leifeld, Michael Schepke, Erwin Biecker, Tilman Sauerbruch, Joerg Heller

Background: In cirrhosis, increased vascular intrahepatic resistance contributes to portal hypertension. The elevated intrahepatic resistance is associated with an increased susceptibility to vasoconstrictors. We have previously shown, that this is due to an upregulation of RhoA and Rho-kinase (Hepatology 2004:40(4)S1:483A). The RhoA/Rho-kinase-pathway mediates vasocontraction and is coupled to vasoconstrictor-receptors. However Rho-kinaseactivity has not been studied at protein or molecular level in cirrhotic livers so far. Therefore, we studied the phosphorylation of the Rho-kinase-substrate, moesin, in cirrhotic livers of rats with secondary biliary cirrhosis and patients with liver cirrhosis. Methods: In rats, cirrhosis was induced by bile-duct-ligation (BDL). Sham-operated rats served as controls. Human liver samples were obtained from biopsies and transplantations. Phosphorylation of moesin at the Rho-kinase-specific phosphorylation-site (Thr-558) was investigated by Western-blot-analysis with densitometric quantification and immunohistochemically using a siteand phosphospecific antibody. Results: Western-blot-analysis of whole liver homogenates revealed an increased phosphorylation of the Rho-kinase-substrate moesin (Thr-558) in livers from BDL-rats compared to sham-operated rats (BDL: $217+/-25 \%$ of sham, $\mathrm{p}<0.04$ ). Total moesin was similar between both groups (BDL: $105+/-22 \%, p=0.88$ ). As shown by immunohistochemistry, moesin-phosphorylation is elevated in the intrahepatic branches of portal veins in cirrhotic livers from BDL-rats (stained cells/vessel wall: sham 37+/-9.3\%, BDL $88+/-6.4 \%, p<0.0001$ ). The same trend to an increased moesin-phosphorylation was observed in whole liver homogenates from patients with alcohol-induced liver cirrhosis compared to non-cirrhotic patients (cirrhosis: $128+/-12 \%$ of non-cirrhotic controls). Discussion: The increased moesin-phosphorylation reflects an increased Rho-kinase activity, which presumably contributes to the increased intrahepatic resistance of cirrhotic livers, both in rat and man.

\section{W1263}

Liver Regeneration Factors After Extended Hepatic Resection with Or Without Portosystemic Shunt in a Large Animal Mode

Ruth Ladurner, Martin Schenk, Alfred Koenigsrainer

Background: The necessary liver volume after extended hepatic resection still remains to be clarified. In the literature, the most important limiting factor seems to be portal hyperperfusion in a small liver remnant. The aim of this study was the immunohistochemical analysis of liver regeneration factors after extended hepatic resection with or without portosystemic shunt. Methods: An extended left hemihepatectomy (approximately $75 \%$ of liver volume) was performed in 256 to 8 weeks old domestic pigs (body weight $25-35 \mathrm{~kg}$ ). In 15 cases liver resection was carried out after construction of a portosystemic $\mathrm{H}$-shunt between portal vein and infrahepatic vena cava. After surgery the animals were weaned from anesthesia and resumed oral feeding ad libidum after recovery. Liver biopsies were taken immediately postoperatively in the follow up until death and the factors Ki-67 and CK-7 were analysed immunhistochemically in paraffin embedded tissue sections. RESULTS: Extended left hemihepatectomy was technically feasable in all animal and hypertrophy of the remnant right lateral segment reached $250 \%$ in both groups at the end of the first postoperative week. In the animals with $\mathrm{H}$-shunt, portal blood flow decreased significantly, whereas arterial perfusion increased. The number of Ki-67 positive cells showed no substantial change in the $\mathrm{H}$-shunt group, but increased significantly in the group without shunt. With 19 versus 4 cells / 10 Glisson fields, nevertheless, Ki-67 was significantly higher in the H-shunt group within the first postoperative day. Expression of CK-7 was significantly higher in the group without shunt immediately postoperative and also in the first postoperative week. Conclusions: The expression of Ki-67 in the H-shunt group was exclusively increased within the first postoperative day, although portal hyperperfusion after extended liver resection was reduced and arterial perfusion increased. However, in the first postoperative week the regeneration of hepatocytes (level of Ki-67) and biliary regeneration (CK-7 expression) was more increased in the group without shun

\section{W1264}

Is Sedation Or Analgesia Necessary for Transjugular Liver Biopsy? A Randomized Placebo Controlled Trial

Sylvie Evrard, Micheal Adler, Axel Hittelet, Jacques Deviere, Olivier Le Moine

Background: Transjugular access to the liver is increasingly used in clinical settings either for the measurement of hepatic venous pressure gradient (HVPG) or to obtain liver tissue samples in the case of contra-indications to the percutaneous route. HVPG is an importan prognostic variable in cirrhotic patients and it might help stratifying patients to specific treatments or follow up the efficiency of medical treatments. Aim: To randomly asses patient's tolerance to transjugular liver biopsy with or without sedation/analgesia. Methods: One hundred eighty patients were included after signing informed consent. They were asked to state their level of anxiety on a scale from 1 to 10 before the procedure. Afterwards, they were randomized either to receive placebo (group $1, \mathrm{n}=60$ ), $0.02 \mathrm{mg} / \mathrm{kg}$ midazolam (group $2, \mathrm{n}=60$ ), or $0.02 \mathrm{mg} / \mathrm{kg}$ midazolam+lmg/kg pethidine (group $3, \mathrm{n}=60$ ) intravenously over 2 minutes before cervical local anesthesia. After the procedure (HVPG measurement + biopsy), patients were asked to scale from 1 to 10 their level of anxiety, pain, memory (very good or good) and willingness (yes or no) to repeat the procedure. The Mann Witney test was used to compare the groups. Results: Clinical (sex, age, etiology of liver disease, usua medication), biological (Child-Pugh scoring, creatinin) and procedure related parameter (HVPG and biopsies: length of the procedure, need to use a guidewire, number of punctures per examination, total length of samplings) were not different in the 3 groups. No significant complication occurred during or after the procedure. Overall, no significant difference was observed between the 3 groups regarding anxiety before and after the procedure, pain memory, and willingness to repeat the procedure (Table). However, patient's tolerance reflected by pain $(\mathrm{p}=0.022)$, post examination anxiety $(\mathrm{p}=0.001)$ as well as the duration of the procedure and the number of liver puncture per examination $(p=0.0001$ and 0.001 , respectively) were operator- dependent, although the evaluation of the technical difficultie assessed by the operators were not statistically different. Conclusions: Intravenous sedation or analgesia does not improve the patient's tolerance and willingness to undergo repeated transjugular liver biopsy or HVPG measurement as compared to placebo. This underscores the need for referral in highly trained centers

\begin{tabular}{|c|c|c|c|c|}
\hline & $\begin{array}{c}\text { Group 1 (plac) } \\
\mathrm{n}=60\end{array}$ & $\begin{array}{c}\text { Group 2 (MDZ) } \\
\mathrm{n}=60\end{array}$ & $\begin{array}{c}\text { Group 3 (MDZ+pethidine) } \\
\mathrm{n}=60\end{array}$ & $\mathrm{p}$ \\
\hline Anxiety before & $5(1-10)$ & $4,5(1-10)$ & $5(1-10)$ & $\mathrm{NS}$ \\
\hline Anxiety after & $1(0-7)$ & $1(1-8)$ & $1(0-8)$ & $\mathrm{NS}$ \\
\hline Pain after & $1(1-10)$ & $1(0-9)$ & $1(0-6)$ & $\mathrm{NS}$ \\
\hline $\begin{array}{c}\text { Memory after (very } \\
\text { good/good) }\end{array}$ & $50 / 10$ & $43 / 17$ & $41 / 19$ & $\mathrm{NS}$ \\
\hline Redo (yes/no) & $58 / 2$ & $59 / 1$ & $59 / 1$ & $\mathrm{NS}$ \\
\hline
\end{tabular}

\section{W1265}

Endoscopic Treatment of Esophagogastric Variceal Bleeding in Patients with Non-Cirrhotic Extrahepatic Portal Vein Thrombosis: A Long-Term Cohort Study Vmcw Spaander, S Darwish Murad, Hr van Buuren, Be Hansen, Ej Kuipers, Hla Janssen Background: Esophagogastric variceal bleeding is the most important complication of extrahepatic portal vein thrombosis (EPVT), and is usually treated endoscopically. Little is known on the prognosis of these patients. In the current cohort study, we investigated the clinical outcome and efficacy of endoscopic treatment in patients with variceal bleeding secondary to EPVT. Methods: Consecutive patients with variceal bleeding secondary to non-cirrhotic, non-malignant EPVT, who underwent endoscopic treatment at our hospital between 1982 and 2005, were enrolled in this study. Data on diagnosis, endoscopic procedures and outcome were collected by systematic chart review. Overall and re-bleeding-free survival was calculated by the Kaplan Meier method. Multivariate Cox regression, stratified for number of re-bleedings, was used to determine predictors of re-bleeding. Results: Thirtyone patients (29\% males; median age 45.6 years (range 3.5-87.5)) were followed after their first bleeding episode for a median follow-up of 8.3 years, range $0-22.4)$. Underlying diseases were protein $S$ deficiency $(n=5)$, infections ( $n=3), \operatorname{IBD}(n=1)$ and other forms of hypercoagulability $(n=10)$. A total of 164 endoscopic procedures were performed, including varicea ligation $(n=36)$, sclerotherapy $(n=125)$ or both $(n=3)$. Of these, 30 interventions were done for active bleeding, and 134 for follow-up treatment. In all patients, initial control of bleeding was obtained. Re-bleeding occurred in 13/31 (42\%) patients; in 10 (77\%) after sclerotherapy, in $1(8 \%)$ after band ligation and in $2(15 \%)$ after both modalities. Overall re-bleeding risk was $19 \%(95 \% \mathrm{Cl} 9-30)$ at 1 - and $43 \%(95 \% \mathrm{CI} 29-59)$ at 5 -years. Extension of thrombosis appeared the only independent predictor of re-bleeding, with a nearly 3-fold risk for EPVT patients with concomitant splenic and/or mesenteric vein thrombosis $(R R=2.93 ; P=0.03$ ). Two complications, both sclerotherapy-induced, were noted: esophageal stenosis needing dilatation, and ulcer bleeding leading to death. A porto-systemic shunt procedure was performed in 6 patients, in 5 for variceal (re)bleeding and in 1 for refractory ascites. Seven 
patients died, 6 due to the underlying disease causing EPVT and 1 after bleeding. Overall 5- and 10 -year survival was $100 \%$ and $65 \%$ (95\% CI 42\%-91\%), respectively. Conclusion: In patients with variceal bleeding from EPVT, endoscopic procedures, in particular band ligation, appear safe and effective with low re-bleeding risk. Bleeding related mortality is rare; survival appears primarily determined by the underlying disease causing EPVT.

\section{W1266}

Role of NOS Isoforms in the Development of Vascular Hypo-Response Following Portal Hypertension

Nicholas J. Skill, Nicholas G. Theodorakis, Janice L. Theodorakis, James V. Sitzmann

Aim: Portal hypertension (PHT) is a major complication of liver cirrhosis and accounts for the deaths of thousands of Americans annually. Predominantly there are two principle underlying causations of elevated pressure within the portal vasculature. Initially the development of cirrhosis and related increase in resistance to portal blood flow elevates portal pressure and precipitates a subsequent and secondary development of vascular dysfunction. This secondary vasculopathy manifests through an elevation of vasodilators and a reduced vascular response to vasoconstrictors, resulting in increased forward flow to the portal system and elevated portal pressure. To date the enzyme nitric oxide synthase (NOS) has been implicated in the development of vascular complications of PHT. However, the precise mechanism and the roles of individual NOS isoforms are not clear. To address this deficiency we investigated the development of vascular hypo-responsiveness to vasoconstrictors in NOS isoform gene deficient mice. Methods: Pre-hepatic PHT was generated by the partial ligation of the portal vein in wild type, endothelial (eNOS) or inducible (iNOS) gene deficient mice. Sham operated mice were used as controls. 7 day following PVL or sham surgery aortic response to vasoconstrictors was determined in-vivo and ex-vivo. In-vivo response to vasoconstrictors was determined by monitoring the abdominal aortic flow prior to and following a bolus injection (IV) of $2 \mu \mathrm{g} / \mathrm{g}$ body weight of the $\alpha \mathrm{l}$ adrenoceptor agonist methoxamine. Ex-vivo response to vasoconstrictors was determined by quantitating the contractile response of abdominal aortic rings in $10 \mathrm{nM}-1 \mu \mathrm{M}$ methoxamine. Results: Abdominal aortic flow was significantly reduced following administration of methoxamine in wild type and NOS gene deficient sham operated mice. However, in PVL operated mice reduced flow in response to methoxamine was blunted in all three mouse groups. In a similar manner, aortic ring contractility was significantly reduced 7d following PVL surgery. Discussion: The development of vascular hypo-responsiveness following PHT is not NOS isoform specific. Therefore, neither iNOS nor eNOS individually are important to the development of PHT dependant vascular hypo-responsiveness and additional studies are required to better understand PHT vasculopathy.

\section{W1267}

Therapeutic Efficacy of Balloon-Occluded Retrograde Transvenous Obliteration in Patients with Gastric Variceal Bleeding

Kyungsik Park, Byoungkuk Jang, Woojin Chung, Kwangbum Cho, Jaeseok Hwang,

Sunghoon Ahn, Younghwan Kim, Jinsu Choi, Junyoung Hwang

Backgroud/Aims: Gastric variceal bleeding is fatal complication of portal hypertension caused by end stage liver disease which is common in east Asia. But endoscopic treatment of the condition is sometimes very difficult as its hemodynamics and accessability are different with those of esophageal varix. Though transjugular intrahepatic portosystemic shunt is another important therapheutic option in gastric varix, it also has some limitations such as aggravation of hepatic encephalopathy or deterioration of hepatic function. Recently balloonoccluded retrograde transvenous obliteration(BRTO) has been used as a new procedure for gastric variceal bleeding with minimal invasiveness but reports about the results of this procedure are not enough. So we analyzed the results of our cases which we have experienced for recent 39 months. Methods: Twenty eight patients who were recevied BRTO for the purpose of primary hemostasis or secondary prevention from December 2001 to March 2005 were analyzed retrospectively. Size of gastric and esophageal varix, clinical presentations, laboratory findings were compared before and after procedure. Cumulative rebleeding and survival rate were also analyzed with Kaplan-Meier curve. Results: Twenty three men and five women were involved and mean age was $53.7 \pm 9.6$ years. Technical and clinical success rates were $89.3 \%$ and $85.7 \%$, respectively. Follows up were continued for $17.5 \pm 12.5$ months in 23 patients. Gastric varices were disappeared in $78.3 \%$ and decreased in size more than $50 \%$ in $21.7 \%$. Relapse was occurred in a(4.3\%) patient. Preexisting hepatic encephalopathy was improved in all patients. Aggravation or development of ascites, esophageal varices, portal hypertensive gastropathy were observed in $45.8 \%, 30.4 \%, 56.5 \%$, respectively. Temporary decreased albumin concentration $(\mathrm{p}=0.002)$, increased bilirubin concentration $(\mathrm{p}<0.001)$ and Child-Pugh score $(\mathrm{p}<0.001)$ was observed at 3 days after procedure but resolved at 7 days after procedure. Increased albumin concentration and decreased Child-Pugh score were maintained thereafter. Rebleeding was occurred in 3 patients and all cases were esophageal variceal bleeding. There were no factors related with rebleeding. Two-year survival rate was $54.6 \%$. Presence of hepatocellular carcinoma(HCC) $(\mathrm{p}=0.001)$ and Child-Pugh grade $\mathrm{B}(\mathrm{p}=0.036)$ and $\mathrm{C}(\mathrm{p}=0.012)$ affected survival in univariate analysis and presence of $\mathrm{HCC}$ was independent risk factor $(\mathrm{p}=0.010, \mathrm{OR}=15.837)$ in multivariate analysis. Conclusions: BRTO is effective therapheutic procedure for primary hemostasis, secondary prevention, and improvement of survival in gastric variceal bleeding patients.

\section{W1502}

Proteome-Based Identification of a Novel Interacting Protein with GLI, Transcription Factor of Hedgehog Signal

Yoshinari Asaoka, Keisuke Tateishi, Yasuo Tanaka, Motoko Seto, Bayasi Guleng, Amarsanaa Jazag, Miki Ohta, Motohisa Tada, Fumihiko Kanai, Takao Kawabe, Masao Omata

(Background and aim) The Hedgehog (Hh) family of signaling proteins play central roles driving many developmental programs from fly to humans, whereas deregulated Hh signaling results in a variety of congenital malformations and cancers. Although it is becoming increasingly clear that this pathway is also critical to gastrointestinal development and tumorigenesis, it is not unraveled which molecule in this pathway is involved. In vertebrates, three Gli proteins, Gli1, Gli2, and Gli3, function in transcriptional control of Hh target genes. Each Gli protein has unique roles. Gli3 is well investigated to behave as $\mathrm{Ci}$, the Gli transcription factor of Drosophila, which is phosphorylated and processed in the absence of Hh and is transferred to the nucleus without cleavage responding to Hh. However it is still not well defined how Hh signals regulate Glil and Gli2 transcriptional activity.Here, we identify a novel interactor with Gli transcription factor by conducting a tandem affinity purification method and mass spectrometry. (Methods) We conducted a tandem affinity purification approach coupled with MS-based proteomics technology to identify cellular proteins associated with the Glil. The purification strategy employed an N-terminal affinity tag (termed MEF) composed of two independent epitope tags, myc and FLAG, that were fused in tandem and separated by a spacer sequence containing a TEV protease cleavage site. The MEF cassette was fused to the N terminus of the Glil, and 293T cells were transfected to express the MEF-fused version of the Glil and were lysed. The expressed MEF-Glil was recovered with its binding proteins by the immunoprecipitation using agarose beads conjugated with anti-myc and anti-FLAG antibodies. The recovered proteins were analyzed by a direct nanoflow LC-MS/MS system. (Results) We identified the 14-3-3 protein as a novel Glil binding protein. We further showed that this interaction was phosphorylation- dependent as well as other 14-3-3 interactors and identified the binding site of Glil. This site is conserved in Gli2 and Gli3, so we examined their interaction with 14-3-3. Gli2 and Gli3 also bound to the 14-3-3 in a phosphorylation-dependent manner. This interaction was revealed by reporter assay to downregulate the transcriptional activity of Gli2, neither Glil nor Gli3. (Conclusion) Through a tandem affinity purification approach coupled with MSbased proteomics technology, we identified 14-3-3 as a Gli interactor. This interaction, dependent on phosphorylation by a specific kinase, downregulates the transcriptional activity of Gli. So, this reseach reveals a novel mechanism of Hh signal transduction.

\section{W1503}

EGFR Signaling Is Up-Regulated in Hyperproliferative Human Aberrant Crypt Foci: Roles of Activated ERBB2 Receptors, Activated ERK and Increased Cyclin D1

Reba Mustafi, Jeff Nathanson, Anusara Chumsangsri, Greg Cohen, Nathaniel Little, Sonia Cerda, Loren Joseph, Sharad Khare, John Hart, Maria Tretiakova, Robert Carroll, Alessandro Fichera, Marc Bissonnette

Introduction: Aberrant crypt foci $(\mathrm{ACF})$ are putative precursors of human colon cancer and the earliest detectable lesions in experimental colon cancer models. We recently examined EGFR signaling in human ACF isolated by chromoendoscopy and demonstrated that hyperproliferative ACF (PCNA mRNA $\geq 2$-fold of control) possessed upregulated transcripts for EGFR, TGF- $\alpha$, and cyclin D1 (Gastroenterology, 128: A-121, 2005). In preliminary studies we also reported that some ACF had increased PCNA protein, elevated EGFR levels, activated wild type Ras, and increased pERK or cyclin Dl by Western blotting. Since each of these proteins was not assessed within the same ACF, our conclusion that EGFR signaling is activated in hyperproliferative ACF remained inferential. Our goal in this study was to optimize our assay conditions for multiplex Western blotting to directly quantify expression and/or activation of several EGFR signal transduction components within the same ACF Methods: Using magnification chromoendoscopy in 11 patients, we collected 67 additional large ACF ( $\geq 50$ crypts/focus), as well as adjacent mucosa ( $>5 \mathrm{~cm}$ from ACF). The ACF had endoscopic features of dysplasia. We employed $4-20 \%$ gradient gels, and combinations of monoclonal and polyclonal primary antibodies, to assess expression levels of PCNA (36 kDa), EGFR (170 kDa), pErbB2 (185 kDa), pERK (42, $44 \mathrm{kDa})$, and cyclin D1 (37 kDa). Patient-matched normal biopsies and individual ACF were examined on the same blot by multiplex quantitative Western blotting, circumventing the need for stripping. Protein expression levels in ACF were expressed as fold-control and ACF classified as hyperproliferative for PCNA levels $\geq 2$-fold-control. Comparisons were made by paired Student's t-test. Results: Among large ACF, 46\% were hyperproliferative (PCNA $=4.5 \pm 0.4$-fold), $72 \%$ had increased total EGFR and $40 \%$ had activated ERK. In the hyperproliferative group, $44 \%$ possessed simultaneous up-regulations or activations in all four EGFR signaling components: EGFR (9.5 \pm 1.3 -fold, $\mathrm{p}<0.05)$, phospho-(active) ErbB2 ( $2.2 \pm 0.3$-fold, $\mathrm{p}<0.05)$, phospho(active) ERK $(3.7 \pm 0.6$-fold, $\mathrm{p}<0.05)$ and cyclin Dl $(3.4 \pm 0.4, \mathrm{p}<0.05)$. Hyperproliferative ACF with increased EGFR but lacking pERK, may be driven by other EGFR pathways. Nonhyperproliferative ACF with increased EGFR presumably lack other components, reflecting the heterogeneity of ACF. Conclusion: Thus, in the first quantitative study of EGFR signaling components within the same human ACF, we demonstrated that dysregulated EGFR signaling contributes to the increased proliferative state of dysplastic appearing ACF, the earliest visible stage of colonic malignant transformation. 
W1504

Essential Roles of AP-1 and Transcriptional Coactivator P300/CBP in the Synergistic Induction of COX-2 By Gastrin and EGF in Intestinal Epithelial Cells

Yan-Shi Guo, Ji-Zhong Cheng, Mark R. Hellmich, Courtney M. Townsend, Jr.

Cyclooxygenase (COX)-2 plays an important role during early stage of colorectal carcinogenesis. The molecular mechanisms that lead to COX-2 activation in the gut are not well defined. EGF is an inducer of COX-2 expression. We reported that gastrin stimulates expression of COX-2 mRNA, protein and its promoter activity in intestinal epithelial cells (J Biol Chem $2002 ; 277: 48755)$. The purposes of this study were to determine whether gastrin and EGF synergistically upregulate COX-2 gene expression in gut cells, and if so, what mechanisms are involved. Methods: A non-tumorigenic rat intestinal cell line (RIE/CCK $R$ R) was used that stably transfected with CCK-2 receptor and possesses EGF receptor. Cells were treated with gastrin $(100 \mathrm{nM})$ and EGF $(50 \mathrm{ng} / \mathrm{ml})$ in serum-free conditions. COX-2 mRNA, protein and promoter activity were examined by Northern blot, Western blotting, and luciferase assay, respectively. AP-1 transcription factor binding activity was determined by electrophoretic mobility shift assay. Results: Gastrin and EGF alone induced a time-dependent increase of COX-2 protein levels (3- to 5-fold) in RIE/CCK 2 R cells. The combination of both peptides synergistically stimulated the COX-2 protein expression (10- to 30- fold), COX-2 mRNA abundance and promoter activity level, as well as induced a synergistic increase of transcription factor ELK-1-dependent luciferase activity and the AP-1 binding activity. The increased COX-2 promoter activity induced by costimulation of gastrin and EGF was blocked by cfos siRNA and by cotransfection with EIA expression vector, which serves as a p300/CBP antagonist. The synergistic induction of COX-2 protein levels was abolished by p300/CBP selective inhibitor Roscovitine. Conclusions: Gastrin collaborates with EGF to synergistically induce COX-2 gene expression in intestinal epithelial cells. This effect appears to result from the synergistic induction of AP-1 transcriptional activity and the recruitment of coactivator $\mathrm{p} 300 / \mathrm{CBP}$. These findings provide new insight into the regulating mechanisms of COX2 in intestinal epithelial cells.

\section{W1505}

Interferon- $\beta$-Induced Expression of XAF1 Requires Phosphorylation of STAT1 and a Sequential Activation of Protein Kinase C and JNK in Colon Cancer Yunwei Sun, Jide Wang, Harry.H.X. Xia, Bing Zou, Marie Cm. Lin, Hsiang Fu Kung, Benjamin C. Wong

Background:XIAP associated factor 1 (XAFl) induced apoptosis and cell differentiation through antagonizing XIAP. The expression of XAFl is increased in response to IFN, but the precise mechanisms remain unclear. In this study, we investigated the regulation of XAFl expression by IFN $\beta$ through JAK-STAT pathway. Methods: Lovo and HCT1116 were treated with IFN $\beta(500 \mathrm{U} / \mathrm{ml})$. Cells were transfected with encoding constructs of ShRNASTATl, DN-JNKl; and pretreated with or without protein kinase C delta (PKCD)-specific inhibitor Rottlerin $(20 \mu \mathrm{M})$ and JNK inhibitor SP600125 (2.5 $\mu \mathrm{M})$ for 1 hour followed by IFN $\beta$ for several time points. Expressions of XAFl and STATl were detected by RT-PCR and Western blot. Dual luciferase assay was used to detect the involvement of STAT1. EMSA was used to detect the activity of STATl in ISGF complex. Results: Low and medium level of XAFl was detected in HCT116 and LOVO cells, respectively. Treatment of cells with ShRNA-STATl and Rottlerin significantly antagonized IFN $\beta$-induced XAFl expression. The influence of PKCD on IFN $\beta$-mediated induction of XAFl is dependent upon the phosphorylation of STATl at serine 727. Furthermore, PKC 8 -mediated activation of STATI requires the activation of JNK, as the inhibition of JNK activity by its specific inhibitor or transfection of its dominant negative mutant suppresses both serine phosphorylation of STATI and XAFl expression but not the activation of PKCD. It implied that JAK-STATl signaling was involved in IFN $\beta$-induced XAFl expression in colon cancer cells. Conclusion: Our results suggest that the induction of XAFl transcription by IFN $\beta$ through STATl depends upon sequential activation of PKC and JNK.

\section{W1506}

Protein Kinase C- $\zeta$ Is Down-Regulated in Human Adenomas and Pkc- $\zeta$ Expression Levels Inversely Correlate with CACO-2 Colon Cancer Cell Proliferation

Reba Mustafi, Sonia R. Cerda, Anusara Chumsangsri, Marc Bissonnette

Background: Protein kinase C-zeta (PKC- $\zeta$ ) is an atypical PKC isoform involved in the regulation of cell growth, differentiation and apoptosis. PKC- $\zeta$ is downregulated in human and experimental colon cancers. To elucidate the growth regulatory role of this isoform in human colon cancer cells, we established stable Caco-2 transfectants expressing PKC- $\zeta$ under an inducible promoter. We found that PKC- $\zeta$ up-regulation significantly inhibited proliferation, enhanced differentiation and accelerated apoptosis of Caco-2 cells grown on collagen-1. We have now extended these studies by inhibiting PKC- $\zeta$ expression or activity and assessed their consequences on Caco- 2 cell growth. We also assessed PKC- $\zeta$ expression in colonic adenomatous polyps. Methods: We used Caco-2 cells transfected with human PKC- $\zeta$ under a ponasterone-inducible eukaryotic vector. In addition, we prepared Caco-2 transfectants, stably expressing wild type or kinase dead PKC- $\zeta$ under a constitutive CMV promoter. Cells were grown on collagen-1 or in soft agar. Proliferation was measured by Coulter counter, WST-1 assay, or colony counts (soft agar). PKC- $\zeta$ expression was inhibited by PKC- $\zeta$ specific siRNA and kinase activity was blocked with a PKC- $\zeta$ pseudosubstrate. PKC- $\zeta$ expression and biochemical activity were assessed by Western blotting and immunoprecipitation kinase assay, respectively. Results: In this study, we established that PKC- $\zeta$ upregulation was accompanied by a $50 \%$ increase in insulin-stimulated kinase activity compared to EV cells $(\mathrm{p}<0.05)$. Compared to EV cells, we also demonstrated that constitutively upregulated wild type PKC- $\zeta$ inhibited Caco-2 cell growth in soft agar (82\%inhibition), whereas kinase dead PKC- $\zeta$ increased growth in soft agar(3.7-fold,p<0.05). Consistent with our previous findings that PKC- $\zeta$ upregulation inhibited proliferation, $\mathrm{PKC}-\zeta$ knock-down with siRNA ( $>70 \%$ inhibition), or kinase inhibition with the specific inhibitor, PKC- $\zeta$ myristoylated pseudosubstrate, significantly and dose-dependently increased Caco-2 cell growth on collagen-1 $(\mathrm{p}<0.05)$. In human colon, PKC- $\zeta$ was significantly decreased by more than $70 \%$ in adenomatous polyps, compared to adjacent mucosa $(n=4, p<0.05)$. Conclusions: Inhibition of PKC- $\zeta$ expression or activity increased Caco- 2 cell proliferation on either collagen-1 or soft agar. We postulate that PKC- $\zeta$ regulates cell proliferation by integrin-dependent and independent pathways. Since PKC- $\zeta$ loss occurs early in colonic tumorigenesis, and the expression of this isoform correlates inversely with cell proliferation, $\mathrm{PKC}-\zeta$ down-regulation appears to contribute to colonic malignant transformation.

\section{W1507}

Deoxycholic Acid Transactivates C-MET

Sharad Khare, Cory Holgren

Background: Bile salts have been suggested to be involved in the pathogenesis of colon cancer. Hydrophobic bile acids such as deoxycholic acid (DCA) have been shown to induce apoptosis and Cox-2 expression whereas hydrophilic ursodeoxycholic acid (UDCA) inhibit proliferation. We have shown previously that UDCA inhibits cyclooxygenase-2 (Cox-2) expression and suppresses colon cancer in rat azoxymethane (AOM) model [Cancer Research $63,3517-3523,2003]$. Further, bile acid transporters have not been identified in the colon and labeled bile acids are not taken up, in vitro, by colon cancer cells. It is presumed that these amphipathic molecules exert their effects by perturbing plasma membrane structure and function. However, the precise role of DCA in tumor promotion remains unclear. Her we demonstrate, for the first time, that DCA transactivates hepatocyte growth factor receptor (c-Met-R), a prognostic marker of colorectal cancer. Methods: HCA-7 cells were grown McCoy's 5 A media containing $10 \%$ fetal calf serum. Phosphorylation status of c-Met-R, EGFR, IGF-1R and EphA2 was assesed by immunoprecipitation and Western blotting. c-Met-R autophosphorylation and in vitro kinase activity towards an exogenous substrate was assayed with $[\gamma 32 \mathrm{P}]$ ATP. Immunoprecipitation and Western blotting assessed co-association of $\beta$ catenin with c-Met-R. Colony formation or cell growth in matrigel was also determined Cell proliferation was measured by MTT analyses. Cox- 2 expression was assesed by Western blotting. Results: Treatment of HCA-7 cells with DCA significantly increased c-Met-R phosphorylation in a time and concentration dependent manner. To determine the specificity of this activation, we also investigated other growth factor receptors EGF-R, IGF-1R and EphA2 DCA treatment had no effect on tyrosine phosphorylation status of these receptors. DCA significantly increased c-Met-R association with $\beta$-catenin. Increased association with c-Met$\mathrm{R}$ with $\beta$-catenin was accompanied by a marked increase in tyrosine phosphorylation of $\beta$ catenin. Correlating with the induction in c-Met kinase activity, HCA-7 cells had significantly increased in vitro growth rates and soft-agar colony-forming abilities in the presence of DCA. Further, k252a, a c-Met inhibitor completely abolished Cox-2 induction by DCA. Summary: Clinical studies have shown that patients with colorectal cancer have higher levels of secondary bile acids in colonic lumen. We demonstrate, for the first time, that tumor promoter DCA activates c-Met receptor and downstream signaling. The characterization of this pathway has implications for diverse disease processes including colon cancer progression and metastasis.

\section{W1508}

Intestinal Cell Kinase (ICK) Is Detected in a Subset of Crypt Cells in Intestinal Epithelium Following Radiation Injury and in the Colorectal Cancer Cell Line Colo 205

Zheng Fu, Alda Vidrich, Steven Cohn, Thomas Sturgill

Intestinal Cell Kinase (ICK) shares sequence and structure homology to both ERK2 and CDK2 at the catalytic domain. This similarity suggests that ICK may have MAPK- and CDKlike regulation and potential functions in regulation of the cell cycle, lineage specification and/or cell fate decisions. ICK was originally cloned from an intestinal crypt cDNA library by Dr. Anil Rustgi's laboratory. The ICK mRNA localizes specifically to the crypt compartmen of small intestine suggesting that ICK may play a role in lineage specification and cell fate determination in crypt epithelium. Our preliminary data indicated that ICK mRNA expression increases over 10 fold with maximal levels observed at 72 hours after irradiation corresponding to the initial appearance of regenerating crypts. When we examined small intestine and colon for ICK protein expression at this time point, we observed a high level of immunoreactive ICK in a restricted cell population more consistent with stem or progenitor cell than the more numerous transit amplifying cell population in early regenerating crypts. Additionally, our preliminary studies demonstrated that ICK is a transcriptional target for b-catenin and other regulatory proteins that control stem cell fate and differentiation in the gut. Given the surprising restricted localization of ICK protein to a small subset of undifferentiated crypt epithelial cells, our working hypothesis is that ICK regulates cell fate, replication, or function of the more restricted stem cell or progenitor cell population within the crypts. Although the common CDKs and MAPKs may regulate most of the cell cycle events in the crypts and at the crypt-villus junction during normal epithelial homeostasis under basal conditions, our preliminary data suggest that ICK may be a key component for regulating events in the crypt during epithelial regeneration following injury or during initial morphogenesis of the crypt epithelium. ICK mRNA expression is strikingly increased in human colon cancer cell line COLO 205 as compared to other cancer cell lines in the NCI60 panel (http://symatlas.gnf.org/SymAtlas/, Gene symbol ICK). Human COLO 205 colon cancer cells are highly proliferative but poorly differentiated and lack epithelial cellular polarity and columnar morphology. We used ICK antibody to analyze the protein level of ICK in COLO 205 as compared to several other cell lines. The ICK protein level is dramatically 
up-regulated in COLO 205 cells, suggesting that ICK may play a role in the regulation of cellular proliferation, differentiation and/or polarization of this colorectal cancer cell line.

\section{W1509}

Constitutive Activation of Erklb/MAP Kinase Induces Transformation of Intestinal Epithelial Crypt Cells Independently of Phosphatidylinositol 3Kinase/AKT, COX-2 and EGF Receptor Activation

Veronique Durand, Etienne Lemieux, Nathalie Rivard

Strong evidences exist for the critical involvement of Ras/Raf/MEK/ERK cascade in the regulation of intestinal epithelial cell proliferation. K-rasV12G is the most frequently mutated oncogene in colorectal cancer and targeted expression of K-rasV12G in the intestinal epithelium causes activation of the MEK/ERK pathway and tumorigenesis in mice. Moreover, blockade of the MEK/ERK pathway suppresses growth of colon tumors in vivo. However, the mechanisms by which the MEK/ERK cascade induces intestinal epithelial cell tumorigenesis remain to be clarified. Methods. Retrovirus encoding the HA-tagged MEKl wild type (wtMEK) or a constitutive active mutant of MEK1 (MEK1-S218D/S222D, caMEK) were used to infect normal intestinal epithelial crypt cells IEC-6. The effects of pharmacological inhibitors of MEK1/2 (UO126, $10 \mathrm{uM}$ ), PI-3Kinase (LY294002, $10 \mathrm{uM}$ ), COX-2 (NS-398, 10 $\mathrm{uM})$ and EGF receptor (PD153035, $10 \mathrm{uM}$ ) were examined on cell growth and morphology. Activation profiles of ERKs and Akt as well as COX-1/2 protein expression were also examined by western blotting. Results. 1) Stable expression of caMEK, but not wtMEK, in IEC-6 cells induced growth factor relaxation for DNA synthesis, enhanced migration (Boyden chambers), induced deregulation of contact inhibition cell growth, promoted morphological transformation and growth in soft agar. In addition, we found a significant up-regulation of c-myc, phosphorylated $(\mathrm{T} 58, \mathrm{~S} 62) \mathrm{c}$-myc and cyclin Dl expression levels. 2) Intriguingly, expression and activity of ERKl/2 and Akt remained comparable in wtMEK- and caMEK-expressing IEC-6 cells; however, the alternatively spliced isoform of ERKl, ERKlb, is constitutively and markedly activated in caMEK-expressing IEC-6 cells. 3) Interestingly, protein expression levels of COX1/2 were not enhanced in caMEK- versus wtMEK-expressing IEC-6 cells. 4) Pharmacological inhibition of PI-3Kinase, COX-2 or EGF receptor tyrosine kinase did not influence the transformed phenotype of caMEK-expressing IEC-6 cells. 5) By contrast, treatment of caMEK-expressing IEC-6 cells with UO126 inhibitor reduced ERK1/2 phosphorylation levels, abolished ERKlb phosphorylation levels and resulted in the reversion of the transformed phenotype of these cells. Conclusion. Our results suggest that ERKlb, an alternatively spliced isoform of ERKl, contributes to the induction of transformation of intestinal epithelial cells by MEK, independently of PI-3Kinase/Akt, COX-2 and EGF receptor activation.

\section{W1510}

Aspirin Inhibits Deoxycholate-Induced NfкB, Caspase and Protein Kinase C Signaling During Programmed Cell Death of Human Gastric Epithelial Cells Maria Redlak, Jacinda Power, Thomas Miller

We have previously shown that apoptosis is one of the major mechanisms of gastric cell death induced by deoxycholate (DC) and aspirin (ASA), and in this process the caspase cascade, mitochondria and PKC signaling play key roles. The transcription factor NFKB has been shown to modulate apoptosis by regulating the transcription of numerous pro- and anti-apoptotic genes. The aim of the present study was to examine the NFKB signaling pathway in DC- and ASA-induced apoptosis in a gastric carcinoma cell line (AGS). Methods: AGS cells were incubated with DC $(300 \mu \mathrm{M})$ or ASA $(40 \mathrm{mM})$ for varying time periods. In separate experiments cells were treated with DC with or without preincubation with aspirin (ASA, $40 \mathrm{mM}$ ) or proteasome inhibitors. NFKB (p65) activation was measured in nuclear fractions by ELISA. pIкB, caspases, PKCs, PARP and Lamin A expressions were analyzed by Western blots from cytosol fractions. Apoptotic DNA fragmentation was detected by agarose gel analysis. Results: We observed that DC activated the NFKB pathway, resulting in a time-dependent translocation of NFKB (p65) into the nuclear compartment. Cytosolic pIкB expression showed a transient increase with the maximum at $30 \mathrm{~min}$ and decreases thereafter with longer exposure to DC. Aspirin exposure, in contrast, did not affect NFKB activation; however, pre-incubation with ASA reduced DC-induced NFKB (p65) translocation into the nucleus and IKB phosphorylation in the cytosol. DC-induced NFKB (p65) translocation was dependent upon proteasome activation, since proteasome inhibitors (Proteasome Inhibitor I, Lactacystin and MG-132) significantly inhibited this process. We also observed that DC-induced IKB phosphorylation was potentiated in cells pre-treated with proteasome inhibitors. In addition, we found that aspirin (but not proteasome inhibitors) prevented DCinduced caspase- $3,-6$, and -9 activation, PARP, PKC ( $(\varepsilon), \operatorname{PKC}(\beta 1)$ and Lamin A processing, as well as DNA degradation. Conclusion: Our results indicate that DC induces the NFKB signaling pathway in gastric epithelial cells and that aspirin modulates this effect, most likely, by inhibition of IKK $\beta$. Our findings also indicate that aspirin inhibition of DC-induced apoptosis was associated with attenuation of caspase activation, PARP deactivation, PKC ( $(\varepsilon)$ and $(\beta 1)$ isoform expression, and Lamin A processing, and DNA degradation. Inhibition of the proteasome which completely inhibited DC-induced NFKB signaling, however, had no effect on other DC apoptotic targets such as caspases, PKCs, PARP, Lamin A and DNA fragmentation. (Supported by NIH Grant DK 25838).

\section{W1511}

Prostaglandin E2 Dependent Proliferation of K-RAS V12 Transduced IEC-18 Cells

Hung Pham, Benny Chong, Lee W. Slice

Background: Elevated prostaglandin E2 (PGE2) and K-Ras mutations have been observed in colorectal cancers. Transduction of Ras signaling proceeds through multiple pathways but the Ras/Raf/MEK/ERK pathway appears critical in the proliferation and transformation of cells. Aim: The aim of this study was to determine whether K-Ras signaling through MEK/
ERK increases the expression of cyclooxygenase-2 (COX-2) and microsomal prostaglandin E synthase-1 (mPGES-1) resulting in increased PGE2 and proliferation. Methods: Transduced rat ileum epithelial cell line (IEC-18) with the lentivirus containing K-RasV12-GFP (18.KRas) or GFP (18.GFP) were propagated. Real time PCR, Western blot analysis and EIA was used to measure mRNA, protein levels and PGE2, respectively. Proliferation was determined by MTT and [3H]-thymidine uptake and colony formation was determined by soft agar assay. Results: 18.K-Ras colonized in soft agar and exhibited double growth rate and higher density growth. PGE2 levels were increased and inducible by AngII and EGF in 18.K-Ras. Serum-starved 18.K-Ras showed higher levels of COX-2 and mPGES-1 mRNA whereas serum-starved 18.GFP cells produced lower levels of PGE2 as well as COX-2 and mPGES1 mRNA. Stimulation with AngII, EGF or AngII/EGF resulted in elevated PGE2 production with increased COX-2 and mPGES-1 expression. The MEK inhibitor U0126 inhibited COX2 and mPGES-1 mRNA expression in 18.K-Ras cells. The COX-2 specific inhibitor NS-398 abolished PGE2 production as well as the proliferation of 18.K-Ras cells without affecting the proliferation of 18.GFP cells. Conclusion: These findings demonstrate that active Ras/Raf/ MEK/ERK signaling leads to elevated COX-2 and mPGES-1 expression and PGE2 production, resulting in the proliferation of 18 .K-Ras.

\section{W1512}

TGF[Beta] Reverses the Phosphatidylinositol 3 Kinase /PTEN Axis in SMAD4Null Colon Cancer Cells

Jennifer Cabral, Jimmy Y.C. Chow, John M. Carethers

BACKGROUND: TGF[beta] functions as a tumor suppressor in early stages of colonic turmorigenesis, but promotes tumor growth during later tumor stages and metastasis. TGF[beta]-induced tumor suppression is thought to be mediated by the SMAD-dependent signaling pathways, but little is known about SMAD-independent regulation. Here we examined the mitogenic phosphatidylinositol 3 kinase (PI3K) and its opposing regulation by the tumor suppressor PTEN and its influence by TGF[beta]. METHODS: We used SMAD4-null SW480 colon cancer cells, which express TGF[beta] surface receptors. Cells were treated with $10 \mathrm{ng} / \mathrm{mL}$ TGF[beta] for time points up to 48 hours. Cells were then lysed for protein extraction and Western blotting. Antibodies against PTEN, p85 (a subunit of PI3K), phosphotyrosine, and SMAD2/3 were used. Total RNA was also extracted to determine PTEN mRNA expression using RT-PCR. Cell counting was performed over a 72-hour time period after TGF[beta] treatment with or without PD98059 (a MEK inhibitor) or LY298002 (a PI3K inhibitor). Luciferase assays with the p3TP plasmid were conducted to measure TGF[beta]induced SMAD-dependent promoter activity. RESULTS: TGF[beta] reduced PTEN mRNA expression maximally at 24 hours by $80 \%$, but reappeared at 48 hours, without having any effect on PTEN protein levels. TGF[beta] also induced tyrosine phosphorylation of p85, 24 and 48 hours after treatment. TGF[beta] promoted cell growth in the first 24 hrs, which was suppressed by LY294002 but not by PD98059. Despite the absence of SMAD4, TGF[beta] induced p3TP activity. CONCLUSIONS: In SMAD4-null cancer cells, TGF[beta] treatment induces activation of PI3K while simultaneously reduces PTEN mRNA, events that favor cellular proliferation. The mechanisms for reversing the PI3K/PTEN axis from tumor suppression towards favoring tumor growth likely involves SMAD-dependent (but SMAD4-indepenent) pathways as evidenced by our luciferase assay, as well as SMAD-independent pathways (through PI3K activation as revealed by $\mathrm{p} 85$ tyrosine phosphorylation). These findings could explain signaling mechanisms by which TGF[beta] uses to become a tumor promoter in cancer cells.

\section{W1513}

FOXO3A Mediates Trail Down-Regulation in HT29 Cells Salil Bose, Qingding Wang, B. Mark Evers

Phosphatidylinositol 3-kinase (PI3K) and its downstream effector protein, Akt, play a major role in intestinal cell proliferation and survival. Previously, we have shown that PI3K inhibition induces expression of TRAIL (TNF-related apoptosis inducing ligand) which plays a role in intestinal differentiation The apoptosis-inducer forkhead (FOXO) family of transcription factors, a downstream target of Akt, modulates cell apoptosis by affecting PI3K-dependent signaling. The aim of the present study was to determination whether the two FOXO isoforms, FOXOI and FOXO3a, regulate TRAIL expression in the intestine. METHODS. (i) HT29 human colon cancer cells were treated with FOXO1 and FOXO3a siRNA or nontargeting control and protein extracted for determination of TRAIL expression and expression of the Cdk inhibitor proteins $\mathrm{p} 27^{\mathrm{kip} 1}$ and $\mathrm{p} 21^{\text {wafl }}$. Additionally, RNA was extracted and real time (RT)-PCR performed to assess TRAIL mRNA levels after FOXO inhibition and treatment with sodium butyrate $(5 \mathrm{mM})$, a known differentiation inducer in transformed colon cancer cells. (ii) To further confirm a role for FOXO regulation of TRAIL, wild-type and mutant FOXO3a promoter plasmids were co-transfected with a construct containing the TRAIL promoter $(-1523 /+23)$ linked to luciferase; luciferase activity was measured by dual reporter assay with transfection efficiency controlled by Renilla luciferase. RESULTS. (i) FOXO1 inhibition by siRNA treatment decreased TRAIL and $\mathrm{p} 27^{\mathrm{kip} 1}$ protein expression. FOXO3a siRNA also decreased TRAIL mRNA levels. (ii) Additionally, co-transfection of wild-type FOXO3a plasmids with the TRAIL promoter plasmid increased relative luciferase activity compared with a FOXO3a mutant plasmid. CONCLUSIONS. FOXO3a inhibition decreased TRAIL protein and mRNA levels in HT29 cells suggesting FOXO3a regulation of TRAIL in intestinal cells. The regulation of TRAIL by FOXO3a was further confirmed by an increase in the relative TRAIL promoter activity in HT29 cells. Importantly, alterations in TRAIL protein and mRNA levels, mediated by the PI3K/Akt/FOXO3a pathway, may be a critical component in the regulation of intestinal cell differentiation and survival. 
W1514

Novel Isoform of PKC May Be Involved in the Rapid Translocation of Tight Junction Proteins to the Cell -Cell Contact By EGF in TMK-1 Gastric Cancer Cells

Kenichi Yoshida, Shigeru Kanaoka, Tetsunari Takai, Mutsuhiro Ikuma, Masayoshi Kajimura, Naoyuki Miura, Haruhiko Sugimura, Akira Hishida

Background: Tight junction (TJ) is disrupted in a number of tumor types, including stomach. Expression level of TJ proteins is involved in differentiation of gastric cancer (Am J Pathol 151: 45-54, 1997). Recently, an increasing number of growth factors have been shown to modulate TJ proteins and function. However, little is known about the effects of growth factors on TJ proteins in gastric cancer. TMK-l cells, which are diffuse type of gastric cancer, loosely contact to adjacent cells and weekly express ZO-1 and occludin proteins on the cellcell contact. We found that exposure of fresh fetal bovine serum (FBS) to TMK-l cells led to rapid translocation of ZO-1 and occludin from the cytosol to the cell-cell contact. In the present study, we examined the effects of FBS or EGF on TJ proteins of TMK-1 cells. Methods: TMK-lcells were cultured 60-80\% density. Cells were exposed to fresh medium containing 10\% FBS or 1-1000 ng/ml EGF. The effects on ZO-1 and occludin were observed by immunofluorescence (IF) and Western blot (WB) analyses. Finally, we examined intracellular signaling associating with the effects using various inhibitors. Results: IF demonstrated that ZO-1 and occludin were some weak expression at the cell-cell contact and were predominantly localized to the cytoplasm. When the medium was replaced with fresh medium containing 10\% FBS or EGF, the cells formed close contacts with the adjacent cells and ZO-1 and occludin were translocated from the cytosol to the cell-cell contact within 30 minutes. These effects were observed dose-dependently at the range of concentration of 1 to $100 \mathrm{ng} / \mathrm{ml}$ and sustained for 2 hours after the treatment of EGF without significant increasing numbers of cells. Adversely, 1,000ng/ml EGF showed weaker effects than those of $100 \mathrm{ng} / \mathrm{ml}$. WB revealed that NP-40 soluble fractions of ZO- 1 and occludin were decreased after $100 \mathrm{ng} / \mathrm{ml}$ EGF treatment. And also the ratio of NP-40 insoluble to soluble fraction of ZO-l and occludin with $100 \mathrm{ng} / \mathrm{ml} \mathrm{EGF}$ treatment were approximately 2.0-fold higher than those in the absence of EGF. Levels of ZO-1 and occludin protein in total cell extract were not changed. EGF-induced effects were inhibited PKC inhibitors, calphostin $C$ and bisindolylmaleimide I (more specific for novel type of PKC), but not another PKC inhibitor Go6976, PD98059 (MAP kinase inhibitor), LY294002 (PI3 kinase inhibitor) and KT5720 (PKA inhibitor) Conclusion: EGF induced the rapid translocation of ZO-1 and occludin from the cytosol to the cell-cell contact through the activation of PKC in diffuse type of gastric cancer cell, which is accompanied with change of cell shape similar to that of intestinal type of gastric cancer.

\section{W1515}

Modulation of Bone Morphogenetic Protein (BMP)-Induced Growth Suppression By Activated K-RAS

Stayce Beck, Barbara Jung, Eunice Del Rosario, Jessica Gomez, John M. Carethers

BACKGROUND. BMPs, part of the transforming growth factor [beta] superfamily, regulate cell differentiation, proliferation, and apoptosis through a canonical SMAD signaling cascade in mesenchymal cells. Recent evidence indicates BMPs also regulate intestinal epithelial growth in a murine model, additionally; lack of BMP signaling contributes to the formation of intestinal juvenile polyps in humans with colon cancer prone familial juvenile polyposis. As sporadic colorectal cancers appear to have intact BMP signaling, we evaluated if K-RAS, a mitogenic pathway commonly activated in colon cancer, negatively affects BMP-SMAD signaling. METHODS. We treated non-tumorigenic FET cells, which have activated K-RAS, with BMP2 (100 ng/ml) and the MEK inhibitor PD98059 and the PI3Kinase inhibitor LY294002 to inhibit potential downstream effects of K-RAS, as well as transiently transfected cells with DN-RAS to directly inhibit K-RAS. We assessed BMP-induced transcriptional activity using a SMAD-dependent BRE-luciferase construct, measured cell growth by direct cell counting and MTT metabolic assays, and measured p21 by RT-PCR, pWWP-luciferase activity, and p2l stability after cyclohexamide. RESULTS. While BMP2 treatment of FET cells increased BMP transcriptional activity 2-fold over control and modestly decreased cell growth, inhibition of K-RAS, MEK1, and PI3Kinase with BMP2 treatment further enhanced transcriptional activity by an additional 1.5 to 2 fold and improved growth suppression by $20 \%$. BMP2 did not increase the transcription of the cell cycle inhibitor p 21 , but instead increased the stability of existing p21 protein over controls. CONCLUSIONS. BMP2 is directly growth suppressive in FET colon cancer cells, which occurs simultaneously with BMP2-SMAD-specific transcriptional activity, and increases 221 protein stability K-RAS and its potential downstream effectors MEKI and PI3Kinase interfere with BMP2-induced transcriptional activity and growth suppression. These mitogenic pathways may slow SMAD cytoplasmic to nuclear translocation due to phosphorylation of the SMAD linker region as suggested in the literature. BMP2-induced transcription in colon cancer cells is modulated by activated K-RAS to reduce the growth suppression directed by this pathway.

\section{W1516}

Activin Induces Non-Transcriptional P21 Protein Stability in Colon Cancer Cells and Modulated By Mitogenic Pathways Barbara Jung, Stayce Beck, Jessica Gomez, John M. Carethers

BACKGROUND: Activin is a growth suppressive ligand of the TGF[beta] superfamily and its signaling pathway is targeted for disruption in colon cancers. The receptor encoded by ACVR2 is frameshift mutated in more than $80 \%$ of microsatellite unstable and a subset of microsatellite stable colon cancers, causing loss of ACVR2 protein expression. Activin binds to ACVR2, triggering phosphorylation of intracellular SMAD proteins that translocate to the nucleus to affect transcription of target genes to slow cellular growth. Here, we dissect the mechanism of p21 protein increase by activin as well as its modulation by mitogenic pathways. METHODS: Synchronized, activin-sensitive FET colon cancer cells were treated with activin $(25 \mathrm{ng} / \mathrm{ml})$ in conjunction with inhibitors of the RAS/MAPK and PI3kinase pathways. P21 RNA expression was assessed with RT-PCR, and protein expression by
Western blotting. Transcriptional activation was assessed using the p21-specific pWWP-Luc reporter. P21 protein degradation was monitored after inhibiting translation with cyclohexamide. RESULTS: Activin decreases cellular growth in FET colon cancer cells in association with an increase in $\mathrm{p} 21$ protein. Activin-induced growth suppression and $\mathrm{p} 21$ protein increase are further augmented with concomitant inhibition of PI3kinase or MAPK/ERK While neither p21-specific transactivation nor p21 mRNA are increased following activin treatment; activin leads to stabilization of p21 protein levels. CONCLUSIONS: Activin signaling is growth suppressive in colon cancer cells and is associated with p21 protein stabilization. This effect is augmented with inhibition of mitogenic pathways, indicating their interference with activin growth suppressive signaling. The p2l increase is due to reduced protein degradation, a novel mechanism mediating activin growth suppression in colon cancer.

W1517

Insulin-Like Growth Factor-1 Activates R-Type (CAV2.3) Channels and Chromogranin a Secreton in the Neuroendocrine Tumor Cell Line Bon Stefan Mergler, Mathias Strowski, Javier Prada, Carsten Groetzinger, Bertram Wiedenmann, Ursula Ploeckinger

BACKGROUND: Blocking voltage-operated Ca2+ channels (VOCCs) of the R-type (CaV2.3) with the tarantula toxin SNX-482 led to a decrease in intracellular calcium ([Ca2+]i) as well as chromogranin A ( $\mathrm{CgA}$ ) secretion in the human neuroendocrine tumor (NET) cell line BON (J. Membr. Biol. 2003, 194, 177-186). Insulin-like growth factor 1 (IGF-1) is a poten mitogenic and secretory factor and acts on $\mathrm{Ca} 2+$ permeable channels. It is not known, whether VOCCs play a role in the IGF-1-dependent regulation of CgA secretion in NET cells. AIM: We therefore investigated the effect of IGF-1 on individual VOCC subtypes and their role in mediating IGF-1-dependent regulation of CgA secretion in NET cells. METHODS Patch-clamp techniques as well as single cell fluorescence imaging methods were used. In addition, the IGF-1 induced effect on CgA secretion was studied in BON cells. RESULTS: Using specific modulators of VOCC subtypes, we identified the functional activity of L-, N, P/Q- and R-type channels in BON cells. IGF-1 $(10 \mathrm{ng} / \mathrm{ml})$ significantly increased the maximal VOCC inward currents (control $=2.59 \pm 0.23 \mathrm{pA} / \mathrm{pF}$ vs. IGF-1 $=3.41 \pm 0.11 \mathrm{pA}$ $\mathrm{pF}, \mathrm{n}=5$ ) resulting in a significant increase in $[\mathrm{Ca} 2+]$ i to $128 \pm 6 \%$ of its control (base level was set to $100 \%, \mathrm{n}=10$ ). This effect was reduced by the specific $\mathrm{R}$-type channel blocker SNX-482 (10 nM). IGF-1 potently induced CgA secretion, which was reduced by inhibition of R-type channel activity. CONCLUSION: R-type Ca2+ channels are expressed in NET cells and mediate both, IGF-1 receptor signalling as well as CgA secretion. Thus, pharmacological interference with R-type channels reducing Ca2+ signalling and IGF-1 dependent hypersecretion may represent a new therapeutic option in NET disease.

\section{W1518}

Protein Kinase D Stimulates Mitogenic Response and Cell Proliferation in Human Pancreatic Cancer Cells

Krisztina Kisfalvi, Cliff Hurd, Enrique Rozengurt

Ductal adenocarcinoma of the pancreas, which comprises $90 \%$ of all human pancreatic cancers, is a devastating disease with overall 5-year survival rate of only 3-5\%. The major challenge is to identify novel targets and develop strategies for its treatment. These new approaches will most likely arise from a detailed understanding of the molecular signaling pathways that stimulate the unrestrained proliferation of these cells. Consequently, the elucidation of the signaling pathways that mediate DNA synthesis and anchorage-independent growth in pancreatic cancer cells is of fundamental importance in the identification of novel targets for therapy. Protein kinase D (PKD) is implicated in the transduction of specific PKC signals, which are shown to increase growth in multiple cell types. Aim: Our hypothesi was that PKD pathway plays a role in promoting the proliferation of pancreatic cancer cells. Methods: To determine the effect of PKD on the growth of these cells we derived PANC1 cells that stably and inducibly express high levels of PKD (wild-type and kinase-dead $[\mathrm{K} 618 \mathrm{~N}]$ forms of PKD), and examined the cell proliferation in these derived cell lines. We utilized a model system in which the wild type or mutant (constitutively active or kinase dead mutants) of PKD were expressed under the control of a modified ecdysone receptor enhancer/promoter. To determine whether overexpression of PKD stimulates growth in PANC-1 cells, we measured DNA synthesis $\left(\left[{ }^{3} \mathrm{H}\right]\right.$-thymidine incorporation) and anchorage independent growth (cell number after 7-9 days in cultures growing on PolyHEMA-coated dishes) of cells treated in the absence or presence of ecdystone to induce PKD. Result: Cells overexpressing wild type PKD showed a two-fold increase in $\left[{ }^{3} \mathrm{H}\right]$-thymidine incorporation (dose-dependent of inducer). The inactive PKD mutant had no effect on DNA synthesis. On PolyHEMA-coated cultures we observed similar results, the number of cells overexpressing active PKD was significantly increased (dose-dependent of inducer) but the inactive mutant had no effect on cell proliferation. Our results demonstrate that expression of wild type but not kinase-dead mutant PKD stimulated a significant increase $(\mathrm{p}<0.005)$ in both DNA synthesis and anchorage independent growth. Conclusion: PKD overexpression leads to a mitogenic response and cell proliferation in pancreatic cancer cells. Our data suggest that PKD plays an important role in signaling proliferation of pancreatic cancer cells and thu can be a potential target for new and effective therapeutic intervention in this deadly cancer.

\section{W1519}

Gastric and Esophageal Stromal Tumors (GIST): Does Size Matter? Alan Weiss

Background: It is not clear how frequently submucosal esophagogastric stromal tumors (GIST) less than $3 \mathrm{~cm}$ in size are malignant and whether such lesions can be safely followed with endoscopy. Objective: To determine the presentation and the outcome of GIST involving the stomach and the esophagus, the relationship between the size of the tumors, the histologic 
presence of malignancy and clinical outcome was correlated. Methods: Patients with esophagogastric GIST seen at the British Columbia Cancer Agency during a ten-year period (19952004) were reviewed. Patient demographics, the presence or absence of symptoms, and the nature of the symptoms were assessed. The size and the determination of malignancy were based on pathological findings. The patient's clinical outcome was determined. Results: 54 patients were reviewed ( 25 men, 29 women). Mean age of the total population was 60.33 years (range 21-81). 45 patients were symptomatic, 6 were asymptomatic, and in 3 cases symptomatology was not recorded. 52 tumors were located in the stomach and 2 in the esophagus. Mean size of symptomatic lesions was $9.7 \mathrm{~cm}$ and mean size of incidentally found lesions was $9 \mathrm{~cm}$ (ns). Three lesions were smaller then $3 \mathrm{~cm}$ : two were symptomatic and one was found incidentally. Range of symptomatic tumors $>3 \mathrm{~cm}$ was $3.2-25 \mathrm{~cm}$ and the range of those found incidentally was $5.0-14.5 \mathrm{~cm}$. Overall, there were 40 histologically malignant tumors and 14 benign lesions. In the lesions $>3 \mathrm{cms}$, 38 were histologically malignant (76\%) and llbenign (24\%): out of this group 10 patients died and 11 had recurrences or metastases (total $47 \%$ ). In lesions $<3 \mathrm{~cm}, 2$ out of 3 had a malignant clinical course and one patient died. Conclusion: In this series, small GISTs were uncommon; however, 2/3 of the tumors were malignant and one death occurred. This experience strongly suggests that once a submucosal lesion is detected on endoscopy, it should be examined with endoscopic ultrasound and, if it is determined to be consistent with a GIST, excision rather then follow up should be considered.

\section{W1520}

The Tetraspanin Cd9 Is a Negative Regulator of EGF Receptor Signaling in Cancer Cells

Yoko Murayama, Yasuhisa Shinomura, Shinji Tamura, Norio Hayashi

Background \& Aims: CD9 is a member of the tetraspanins, and has been shown to be involved in a variety of cellular activities such as migration, proliferation and adhesion. In addition, it has been known that CD9 can associate with other proteins. Here we investigated the physical and functional association of $\mathrm{CD} 9$ with epidermal growth factor receptor (EGFR), Methods: The cell surface expression of CD9, $\beta 1$ integrin, EGFR was analyzed by flow cytometry. The association of CD9 with EGFR and/or $\beta 1$ integrin was examined by doubleimmunostaining and immunoprecipitation. Tyrosine phosphorylation of She was determined by immnoprecipitation and immunoblot. Shc and Grb2 were determined by immunoblot. Clones overexpressing CD9 was established by stable transfection with CD9/pEFBOS into a hepatocellular carcinoma cell line (HepG2) or a chinese hamster ovary cancer cell line expressing human EGFR (CHO-HER). Results: In a human gastric cancer cell line MKN28 , double-immunofluorescent staining and immunoprecipitation demonstrated that CD9 and EGFR as well as CD9 and $\beta 1$ integrin form complexes and that they co-localized on the cell surface, especially at the cell-cell contact sites. Treatment of MKN-28 cells with anti-CD9 antibody ALB6 induced a dotted or patch-like aggregation pattern of both CD9EGFR and CD9- $\beta 1$ integrin complexes, and the ALB6-treatment strengthened the association of CD9 with EGFR. It is noteworthy that the internalization of EGFR after EGF-stimulation was significantly enhanced by the treatment with ALB6. Moreover, overexpression of CD9 specifically down-regulated the surface expression of EGFR, leading to the attenuation of EGF/EGFR-signaling such as Shc tyrosine phosphorylation and Grb2 recruitment. Conclusions: These results suggest that CD9 might have an important role that attenuates EGFR signaling. Therefore, CD9 not only associates EGFR but also a new regulator, which may affect EGF-induced signaling in cancer cells.

\section{W1521}

Immunohistochemical Expression of Leptin in Colorectal Adenocarcinoma and Clinicopathological Characteristics

Dae Won Jun, Oh Young Lee, Won Sohn, Ki Seok Jang, Seung Sam Paik, Tae Wha Kim, Sun Young Yang, Sung Hee Han, Hang Lak Lee, Byung Chul Yoon, Ho Soon Choi, Joon Soo Hahm, Dong Hoo Lee, Min Ho Lee, Choon Suhk Kee

Background: Leptin, the adipocytes-derived hormone, has an important role for obesity and acts as a growth factor in several cancers including gastrointestinal malignancies. Leptin can stimulate mitogen-activated protein kinase activity in vitro and short-term infusion has been reported to have a proliferative action on the colon in vivo. First, We immunohistochemically analyzed the expression of the leptin in colon adenoma-carcinoma sequence from surgical resection at the same person, same surgical specimen (normal, adenoma and adenocarcinoma tissue respectively). Second, We investigate whether leptin expression was correlated with clinicopathological characteristics or disease outcome in colon cancer patients. Methods: This was a retrospective study of 24 colorectal adenocarcinoma patient who was taken total or hemicolectomy and adenomoatous polyp was seen in the same surgical specimen simultaneously leptin expression was assessed using immunohistochemical methods and was evaluated by grading both staining intensity $(0,+1,+2,+3)$ and staining pattern (diffuse focal, apical). The relationships between leptin expression and clinicopathological features of the patients was also evaluated. Results: Basic characteristics of 24 patients were as follow; age: 60.8 years (41-77), gender: 18 male, 6 female, Dukes' stages: $3(12.5 \%)$ at A, $8(33.3 \%)$ at B, $8(33.3 \%)$ at C, $5(20.8 \%)$ at D. Leptin expression was observed in $4.2 \%(1 / 24)$ of normal colon mucosa, $33.3 \%(8 / 24)$ of adenomatous polyps and in 50\% (12/24) of adenocarcinomas respectively $(\mathrm{p}<0.05)$. And average of immunohistochemical intensity score was also higher in adenocarcinoma $(+1.50)$ than in adenoma $(+1.25)$ and normal tissue $(+1.00)$. There was no relationships between leptin expression and tumor size $(\mathrm{tm}<$ $3 \mathrm{~cm}$ or $\mathrm{tm}>$ or $=3 \mathrm{~cm}$ ), number of metastatic lymph nodes $(<4$ or $3>$ or $=4)$, localization of the tumor (colon or rectum), distant metastasis, and vascular invasion of the tumor. However, poorly differentiated cell type was much more in leptin positive adenocarcinoma patient (poorly differentiated or moderately + well differentiated $)(p<0.05)$, but it was not associated with stage and patient survival. Conclusion: Positive rate of leptin expression and intensity have increased according to normal-adenoma-adenocarcinoma sequence in same colon cancer patient, and leptin expression correlated with histological differentiation. These results suggest leptin might play a role in colon adenoma-adenocarcinoma sequence, and tumor differentiation.

\section{W1522}

Neurotensin Receptor-1 (NTR-1) mRNA Expression in Colorectal Adenocarcinoma: An in-Situ Hybridization Study Xianyong Gui, Paul R. Dobner, Grace Guzman, Shrihari S. Kadkol

BACKGROUND \& AIM - Neurotensin (NT), a 13-AA peptide, is expressed in early fetal, but not adult colonic mucosa. However, it is re-expressed in colonic adenocarcinomas. NT was found to stimulate the in vitro growth of a few types of carcinoma cells, including colon cancer, through NT receptors (NTR). Therefore NT may be involved in the development and/or progression of colon cancer, and NTR expression patterns between normal and malignant colonic epithelium may be different. In this study we specifically characterized NTR-1 mRNA expression in normal, precancerous and carcinomatous colonic mucosa. MATERIALS \& METHODS: NTR-1 mRNA expression was determined by in-situ hybridization on formalin-fixed and paraffin-embedded tissue sections. Tissue blocks of 21 cases of colorectal adenocarcinoma (male 13, female 8, mean age 63.4 yo) were selected. A 322-bp Kspl/Pstl fragment of hNTR-l cDNA was subcloned into pBluescript II KS+ vector. Digoxigenin-labeled antisense and sense riboprobes were generated. Anti-DIG-AP conjugate and BCIP/NBT were used in chromogenic detection for hybridization signals. Negative controls included sections hybridized with sense riboprobe or with hybridization solution without probe. Representative sections treated with RNase A prior to hybridization with antisense probe were also employed to ensure specificity. Within the same tissue section, the signals were assessed in adenocarcinoma and in adjacent normal mucosa and areas of hyperplasia, dysplasia and adenoma. The signal intensity was classified as negative, weak, moderate and strong. RESULTS - In normal colonic mucosa adjacent to tumors, NTR-1 expression was absent in $74 \%(14 / 19)$ and weak in $26 \%(5 / 19)$ of the cases. In areas of hyperplasia, expression was absent in $45 \%$ (4/9) and weak in 55\% (5/9) of the cases. In areas of dysplasia, weak expression was observed in 74\% (14/19) and moderate expression in 26\% (5/19) of the cases. In 9 cases with adenomas from which adenocarcinomas arose, the adenomatous components showed weak expression in $78 \%(7 / 9)$ and moderate expression in $22 \%(2 / 9)$ of the cases. A heterogenous pattern was observed in adenocarcinomas. 24\% (5/21) showed weak, $71 \%(15 / 21)$ showed moderate and 5\% (1/21) showed strong expression. The infiltrating margins and the foci of lymphovascular invasion of tumors showed a higher intensity of expression compared with the rest of the tumor. CONCLUSION - This study suggests that enhanced expression of NTR-1 mRNA are associated with tumor progression and aggressive behavior in colonic adenocarcinoma. NTR-1 seems to play a role in the growth of human colorectal adenocarcinoma.

\section{W1523}

Activation of Survival Kinases and NF-KappaB Delays Anoikis in Primary Human Colonic Epithelial Cells

Claudia Hofmann, Monika Artinger, Johannes Grossmann, Werner Falk, Juergen Schoelmerich, Gerhard Rogler, Florian Obermeier

Background: Apoptosis induced by loss of cell anchorage - "anoikis" - plays an important role in the life cycle of colonic epithelial cells. Loss of anchorage dependency is believed to be a critical step in metastatic transformation. Recent studies with the IEC-18 cell line from normal rat intestinal epithelium showed that epithelial cells can activate a set of important kinases as well as NF- $\mathrm{kB}$, which transiently protects them from apoptosis. Therefore, we investigated the regulation of NF- $\mathrm{\kappa B}$ as well as distinct kinases known to play a role for cell survival in primary human colonic epithelial cell (CEC) anoikis. Methods: CEC were isolated as intact crypts from the mucosa of surgical specimens. Induction of anoikis was achieved by incubation of crypts in suspension for up to four hours. Reconstitution of cell-cell contacts was obtained by "pelleting" of CEC. Induction of anoikis was demonstrated by caspase- 3 activation (Western Blot, DEVD-pNA). Cell lysates were analyzed for phosphorylation of Src, EGFR, Erk1/2, p38-MAPK, JNK and c-Jun by Western Blot or ELISA. Cell culture supernatants were assessed for their content of EGF and TGF- $\alpha$ by ELISA. NF- $\mathrm{KB}$ activation was quantified by ELISA. Results: Immediately after detachment of CEC from the basal membrane a rapid increase in phosphorylation for all tested kinases was detected. Phosphorylation of EGFR, Erkl/2 and JNK reached peak levels after $60 \mathrm{~min}$, while phosphorylation of Src and p38 MAPK was still increasing thereafter. The initial activation of these kinases was observed before active caspase- 3 could be detected. CEC undergoing anoikis deliberated high amounts EGF and TGF- $\alpha$. A time-dependent activation of NF-KB also was detected after detachment of CEC. Interestingly, progress of anoikis could effectively be blocked when CEC, incubated up to $60 \mathrm{~min}$ in suspension, were "rescued" by reconstitution of their cell-cell contacts, as caspase- 3 activity was reduced by up to $50 \%(\mathrm{p}=0.04)$. Phosphorylation of Src and p38 MAPK also decreased when cell-cell contacts were reconstituted. Conclusions: Detachment of primary human CEC triggers a transient increase in the activity of antiapoptotic kinases, as well as a strong increase in growth factor secretion and NF-KB activity. By these mechanisms, CEC achieve to be transiently protected from anoikis immediately after detachment and can be "rescued" from anoikis by reconstitution of physiological cell contacts.

\section{W1524}

Chenodeoxycholic Acid Stimulates P38 MAPK Protein Expression and Activation Through Activation of PKC Alpha Bernard Bouscarel, Jianping Ming

Background and Aim: We and other have previously reported that dihydroxy bile acids and chenodeoxycholic acid (CDCA) in particular stimulate PKCalpha isoform activation not only in hepatocytes but also in non-hepatic cells, while other have reported activation of p38 MAPK by bile acids. The aim of the present study was to determine whether the increase expression and activity of p38 was PKCalpha-dependent. Methods: Human dermal fibroblasts were cultured under standard conditions. mRNA and protein expression levels were measured by RT-PCR and Western Blotting, respectively. PKCalpha translocation and activation was determine both by Western blotting and fluorescent microscopy. Results: CDCA stimulated PKCapha translocation and activation in a time-and dose-dependent manner. The maximum 
translocation to the plasma membrane was observed in around 60 min with 50 microM CDCA. Furthermore, CDCA stimulated both PKCalpha and p38 MAPK phosphorylation with a maximum observed at 60 to 150 min. Furthermore, CDCA stimulate p38 MAPK protein expression by around 4-5 fold with 100 microM. Both PKCalpha DN mutant and Gö6976 reduced the basal by $20 \%$ and the CDCA-induced p38 MAPK protein expression by over $80 \%$. PKCalpha WT over expression increased basal p38 MAPK expression by over 5 fold and that induced by CDCA by another 1.5-2 fold. Conclusion: The results of the present study underline a CDCA-induced PKCalpha-dependent increased protein expression and activation of p38 MAPK. Since the skin is one of the major non-hepatic organs that accumulate bile acids in cholestatic liver diseases and that both PKCalpha and p38 MAPK are involved in cell proliferation and apoptosis, this study highlights the possible association of bile acids and skin cancer under pathological conditions that lead to the accumulation of bile acid in the skin

\section{W1525}

High Expression of the EGFR and Phosphorylation of STAT3 On Tyrosine 705 in a Series of Colorectal Carcinoma, a Possible Link with Activated P60c-SRC Gaetan Des Guetz, Christine Lagorce, Ali Tadlaoui Hbibi, Jean-Francois Morere, Jean-Luc Breau, Philippe Wind, Antoine Martin, Bakthiar Bejou, Remi Fagard, Robert Benamouzig Introduction The cellular kinase p60c-src belongs to the family of non receptor tyrosine kinases. Src synergises with the EGF receptor (EGFR) and is required for the activation of STAT transcription factors. We previously observed in a series of colorectal cancers that the EGFR is overexpressed and phosphorylated on tyrosines, and that STAT3 is phosphorylated on tyrosine 705 , both parameters being correlated. This work was undertaken to determine the relationship between active (tyrosine 416-phosphorylated) p60c-src,the expression of EGFR, the phosphorylation of STAT3 and the clinicohistological parameters in a series of 115 colorectal cancers. Materials and Methods Inclusion blocks from 115 colorectal cancers were used. For each tumor, four cores, $0.6 \mathrm{~mm}$ in diameter, were punched in donor blocks and arrayed into the recipient paraffin block using a tissue arrayer device (Beecher Instruments). Tissue arrays were then labeled with the anti- phosphotyrosine 416-Src antibody (Cell Signaling, USA). The labeling was then graded as follows: 0, no positive cells; 1, 1-25\% labeled cells; 2, 25-50\% labeled cells; 3, > 50\% labeled cells. A composite score, ranging from 0 to 9 , was obtained by multiplying the grade by the intensity. Univariate analysis was conducted to determine the associations between the levels of P-STAT3, EGF$\mathrm{R}$ and P-EGF-R and the clinicohistological parameters. Results An high level of activation of Src (P-tyr-416) was observed in $86 \%$ of the cases of colorectal cancer. Labeling was cytoplasmic from moderate to high intensity in $61 \%$ of the cases. There was no significant relationship between the tyr-416 phosphorylation of Src, the clinicohistological parameters and the survival of patients. There was a significant correlation between the activated form of Src, the expression of EGFR ( $\mathrm{p}=0.008$ ) and the tyrosine 705 phosphorylation of STAT3 $(\mathrm{p}=0.002)$. Conclusion Our observation of a frequent detection of activated Src in colorectal cancer and its correlation with the elevated expression of EGFR and the phosphorylation of STAT3 on tyrosine 705 is in line with the synergy between Src and the EGFR and their activation of STAT3 in tumor cell lines. This correlation confirms the implication of Src in human colorectal cancer and suggests possible mechanisms involving the EGFR and STAT3. The consequences of Src activation may affect the proliferation or other aspects of cell behavior such as adhesion and migration ultimately regulating metastasis.

\section{W1526}

The Combinatorial Biological Effects of EGFR Overexpression, hTERT Activation and P53 Mutation Result in Malignant Transformation with the Induction of MMP-9 in a Novel Organotypic Culture Model

Takaomi Okawa, Claudia Andl, Carmen Michaylira, Hiroshi Nakagawa, Munenori Takaoka, Cameron Johnstone, Therese Deramaudt, Yuting Chen, Ben Rhoades, Wafik S. El-Deiry, Anil K. Rustg

Introduction: The molecular basis for malignant transformation in different types of epithelia is dependent upon the cellular and tissue microenvironment context. The stratified squamous epithelium is the most common type of epithelium in the human, and cancers that arise in this epithelium are common. Indeed, tumor cell migration and invasion is influenced by the cross talk between the epithelium and mesenchymal compartment. Methods: Primary human esophageal cells were retrovirally transduced individually and in combination with EGFR, hTERT and/or a dominant-negative p53R175H, reflecting common genetic alterations in esophageal cancer. These cells were subjected to migration (Boyden chamber) and Matrigel invasion assays. In order to mimic the tissue or tumor microenvironment, the genetically altered epithelial cells were grown on a matrix comprising type I collagen and Matrigel. Exposure to a liquid-air interface induces epithelialization (hence, organotypic culture) and phenotypes can be assessed by histopathology and immunohistochemistry. In addition, the conditioned media was analyzed by gelatin and casein zymography in order to determine matrix metalloproteinase (MMP) activity. Results: The specific combination of EGFR overexpression, telomerase activation and mutant $\mathrm{p} 53$ resulted in a statistically significant increase in cell migration and invasion, compared to controls or with 1 or 2 genetic alterations. Organotypic culture revealed a dramatic phenotype that precisely recapitulated malignant human esophageal squamous cell cancer with a severely dysplastic epithelium, invasive malignant cells into the matrix, and "pearl" formation (a marker of well-differentiated tumors). Given there was evidence of proteolytic activity of mesenchymal fibroblasts and invading cancer cells, gelatin zymography revealed induction of active MMP-9, but not MMP-2. Importantly, the invasive esophageal cancer cells were abolished with an MMP-9 inhibitor. Conclusions: In aggregate, we have established a model in which important genetic alterations (EGFR overexpression, telomerase activation and p53 mutation) conspire to recapitulate faithfully the cardinal features of invasive, malignant esophageal squamous cell cancer. Invasion through the extracellular matrix is facilitated by the induction of MMP-9, and which can be reversed with an MMP-9 inhibitor. Apart from the mechanistic insights gained, such a model permits the testing of therapeutic drugs that exert their actions on specific targets of molecular pathways critical in malignant transformation and invasion in the local tumor microenvironment.

\section{W1527}

Inhibiting Trefoil Factor Family-3 (TFF3) Expression Decreases Colon Cancer Cell Invasiveness

Christina Twyman, Yio Xianyang, Yan Zheng, Jie-Yu Zhang, Matthew Diamond, Mark Babyatsky, Lawrence Werther, Steven Itzkowitz

Background: Trefoil factor family-3 (TFF3) is a small peptide expressed by intestinal epithelial cells which contributes to mucosal healing by promoting epithelial cell migration and inhibiting apoptosis. We previously demonstrated that rat colon cancer cells that natively express TFF3 (LN cells) have a more aggressive phenotype in vivo and in vitro compared to their TFF3-negative counterpart (LP cells) (Clin Exp Metastasis 22:157, 2005). Stable transfection of the non-aggressive LP cells with TFF3 caused them to become more aggressive. Inhibiting TFF3 expression would provide more direct evidence of the role of TFF3 in cancer cell behavior. Aim: To determine the effect of inhibiting TFF3 expression on colon cancer cell invasion, a hallmark of cancer cells. Methods: Two rat TFF3-specific siRNA oligos (si365 and si78) and a scrambled siRNA oligo (negative control) were generated. LN (native TFF3 expression) and LP-CRI cells (stably expressing TFF3) were transfected with the siRNA oligos. Expression of TFF3 was analyzed by RT-PCR. Cell invasion assays were performed using modified Boyden chambers with reconstituted basement membrane (Matrigel) barriers. Results: Introducing either TFF3-specific siRNA into LN cells decreased expression of TFF3 RNA by RT-PCR. In LN cells, compared to scrambled siRNA controls, si365 resulted in a $44 \%$ decrease in invasion $(\mathrm{p}=0.01)$, and si78 reduced invasion by 53\% ( $\mathrm{p}=0.01)$ (Table) In LP-CRI cells, there was a decrease in invasion of $50 \%(\mathrm{p}=0.03)$ and $74 \%(\mathrm{p}=0.01)$ respectively. Conclusions: The decreased invasion caused by inhibiting TFF3 suggests that TFF3 contributes to the malignant behavior of colon cancer cells. Future studies will confirm this effect using an in vivo animal model and determine the signal transduction pathways involved

Number (Standard Deviation) of Invading Cells

\begin{tabular}{|c|c|c|c|c|}
\hline & Non-transfected & Scrambled siRNA Control & si365 & si78 \\
\hline LN Cells & $236(54)$ & $168(11)$ & $79(18)^{*}$ & $94(13)^{*}$ \\
\hline LP-CRI Cells & $161(11)$ & $172(23)$ & $87(21)^{* *}$ & $44(4)^{*}$ \\
\hline
\end{tabular}

* $\mathrm{p}=0.01$ vs. scrambled control $* * \mathrm{p}=0.03$ vs. scrambled control

\section{W1528}

Cross-Talk Between ELF and HFE Is Important in Suppression of Hepatocellular Carcinoma

Yi Tang, Varalakshmi Katuri, Ruihong Wang, Wilma Jogunoori, Tapas Saha, Chu-Xia Deng, David J. Brandhagen, Anton Sidawy, Bibhuti Mishra, Lopa Mishra

Hereditary hemochromatosis is associated with an increased risk for hepatocellular carcinoma (HCC). The risk had been estimated to be as high as 200 -fold increased. The Biological signals for transforming growth factor beta (TGF- $\beta$ ) play diverse and essential roles in cell proliferation and liver development. Precise modulation of TGF- $\beta$ is through Smad activation by the type I receptors, and is dependent upon adaptor proteins such as ELF, a $\beta$-spectrin. Recently, we uncovered a novel role of TGF- $\beta /$ Smad4 in regulating intestinal iron transport and iron homeostasis. Interestingly, we also found an increased incidence of hepatocellular

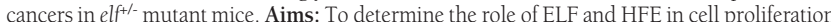
and HCC suppression. Methods and Results: 1. Prussian Blue staining results showed slightly increased level $(+)$ of iron in lf $^{+/-}$mutant muse HCC tissues when compared to the wildtype normal liver tissues. 2. Microarray results demonstrated Transferrin (TF) transcription is downregulated by two and half fold $(\mathrm{p}=0.05)$ in elf ${ }^{-}-$mouse embryonic fibroblasts (MEF) when compared to the normal MEFs. Incontrast, Ferritin transcription is increased one and half fold $(\mathrm{p}=0.04)$ in elf $^{--}$mutants. Other genes involved in iron transport and metabolism such as Hfe, Hepcidin, Ferroportin, TFR2, TFRC, Ireg, Dcytb, Dmt, Nramp and Mtp did not show a significant change in transcription in elf-/- mutants. 3. Realtime RT-PCR showed a decreased expression of elf and $\mathrm{Hfe}$ in fifty percent of hereditary hemochromatosis patient samples, which carry HFE point mutations at C282Y. 4. Co-immunoprecipitation results demonstrated endogenous association of ELF with HFE upon TGF- $\beta$ treatment. Confocal microscopy studies further confirmed colocalization of ELF and HFE. 5. Furthermore, we found $\mathrm{p} 21$ expression is dramatically decreased in the hereditary hemochromatosis patient samples that demonstrated decreased ELF and HFE expression. Conclusions: Our results show that the HFE interacts with ELF potentially regulating hepatocyte proliferation The disruption of ELF by HFE regulates p2l expression through the TGF- $\beta$ /ELF signaling and thus inhibits cell cycle progression and cell proliferation. Loss of TGF- $\beta / E L F$ function could result in the increased risk of HCC in the hereditary hemochromatosis patient carrying Hfe mutations.

\section{W1529}

Missense Mutation of the P53 Gene in Lugol-Unstained Esophageal Lesions with Non-Dysplastic Epithelium in Esophageal Cancer-Free Patients Kazuhiro Kaneko, Atsushi Katagiri, Toshinori Kurahashi, Hiroaki Ito, Taikan Yamamoto, Yosuke Kumekawa, Meiko Kuwahara, Takashi Muramoto, Yutaro Kubota, Michio Imawari Background \& Aims: Mutations of the p53 gene are detected frequently in the early stages of esophageal cancer. It is unclear whether Lugol-unstained lesions (LULs) with non-dysplastic epithelium (NDE) are precursors of esophageal squamous cell carcinoma (ESCC). Characterization of human esophageal precancerous lesions at the molecular level is of critical importance to our understanding of the etiology of this disease and to the identification of useful biomarkers for prevention studies. To investigate whether Lugol-unstained lesions (LULs) with non-dysplastic epithelium (NDE) were related to the carcinogenesis of esophageal squamous epithelium or not, the p53 mutational status in LULs-NDE was analyzed prospectively. Methods: Videoendoscopy with Lugol staining was performed prospectively in 542 consecutive esophageal cancer-free patients. After ordinary endoscopic observation, 5 to 10

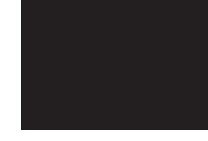


$\mathrm{ml}$ of $2.0 \%$ glycerin-free Lugol's iodine solution was sprayed from the gastroesophageal junction to the upper esophagus using a plastic spray catheter passed through the biopsy channel of the endoscope. Lugol-unstained lesions were detected in 103 patients (19\%). A total of 255 samples, including 152 Lugol-unstained lesions (NDE, 137; dysplasia, 15) and 103 paired normal-samples of normal staining epithelium, were obtained for biopsy from 103 patients. DNA was extracted from 20- $\mu$ m thick sections obtained from archival blocks of formalin fixed and paraffin embedded tissue. After polymerase chain reaction analysis, direct sequencing was used to detect p53 gene mutations in exon 5 to 8. Results: All samples of LULs-NDE had esophagitis, while 78\% of the 103 normal Lugol-staining epithelium samples had esophagitis and $22 \%$ had normal epithelium. A mutation in the p53 gene was detected in 5 of 137 samples with LUL-NDE (4\%) and 5 of 15 samples with dysplasia (33\%). A "hotspot" mutation was found in 20\% of LULs-NDE with p53 mutation and in $40 \%$ of dysplasia with p53 mutation. The hotspot mutations of LULs-NDE and samples with dysplasia were found at codon 273 and codon 175 , respectively. In contrast, no p53 mutations were found in NDE samples with normal Lugol-staining, although approximately $80 \%$ of the normal Lugol-staining epithelium samples had esophagitis. Conclusions: In biopsy samples from esophageal cancer-free individuals, a hotspot mutation in the p53 gene was found in non-dysplastic epithelium that was identified as a Lugol-unstained lesion. The findings suggest that some Lugol-unstained lesions with non-dysplastic epithelium may represent the earliest initial lesions in the carcinogenesis of esophageal squamous epithelium in our Japanese individuals.

\section{W1530}

Increased Susceptibility to Gastric Cancer Formation in Mice with Transgenic Cyclooxygenase-2 Overexpression

Wai K. Leung, Kai-Chun Wu, Christine Y. Wong, Arthur Ching, Anthony W. Chan, Minnie Y. Go, Jun Yu, Xin Wang, Yl Chui, Dm Fan, Joseph J. Sung

Background: Cyclooxygenase-2 (COX-2) overexpression is frequently detected in gastric cancer. We have previously shown that inhibition of COX-2 suppresses gastric cancer formation in animals [Hu, Gut 2004]. However, the effects of forced COX-2 overexpression on gastric carcinogenesis remain unknown. Methods \& Results: COX-2 transgenic mice were developed in C57/BL6 strain using the full length cloned human cox-2 cDNA construct. Integration of transgene was verified by PCR of tail genomic DNA. The transgenic mice line with high COX-2 expression in gastric epithelium was selected for subsequent experiments. To study the effects of COX-2 overexpression in gastric cancer formation, mice were exposed to gastric carcinogen N-methyl-N-nitrosourea (MNU). Six-week-old COX-2 transgenic or wild type (WT) mice were divided into four groups to receive MNU or vehicle alone. MNU $(240 \mu \mathrm{g} / \mathrm{L})$ were given to mice ad libitum as drinking water in five alternating cycles of 1 week MNU and 1-week normal distilled water. All mice were sacrificed at the end of 50week. Stomach was harvested and examined macroscopically and microscopically for the presence of invasive cancer. Whilst COX-2 was expressed in very low level in the stomach of WT mice, high level of COX-2 expression in gastric epithelium was confirmed in the stomach of transgenic mice by both western blot and immunohistochemistry. Gastric cancer was found in 17/29 (58.6\%) transgenic mice and 4/33 (12.1\%) WT mice at the end of 50week $(\mathrm{RR}=4.84,95 \% \mathrm{CI} 1.84$ to $12.74, \mathrm{P}=0.0001)$. None of the WT and transgenic mice treated with water alone developed gastric cancer. There was no premalignant gastric lesion noted in all animals. In normal gastric mucosa, we found no significant difference in the apoptotic and proliferation index between WT and transgenic mice. However, there was a marked increase in expression of angiogenic factor VEGF in the normal gastric mucosa of transgenic mice which is sparsely detected in WT mice, suggesting induction of angiogenic factors may play an important role on the cancer susceptibility of COX-2 transgenic mice. Conclusion: Our results suggest that COX-2 overexpression alone is insufficient for gastric tumor induction, but rather increase the susceptibility to gastric cancer formation induced by carcinogen.

\section{W1531}

In Vivo and in Vitro Evidence for Epithelial to Mesenchymal Transition (EMT) in Oesophageal Adenocarcinoma

Jonathan R. Rees, Benjamin A. Onwuegbusi, Vicki E. Save, Derek Alderson, Rebecca C. Fitzgerald

Background: Local invasion and metastasis occur early in the pathogenesis of oesophageal adenocarcinoma (AC) and result in poor long term outcome even with radical therapy. Epithelial to mesenchymal transition (EMT) has been shown to play a role in the aggressive behaviour of cancer, however data is lacking for AC. EMT is driven by a number of mechanisms and TGF $\beta 1$, a cytokine family member has been implicated in this process. Aims: 1. Determine whether there is in vivo evidence for EMT in AC. 2. Develop an in vitro model system for EMT in AC in order to elucidate the signalling pathways involved, deduce whether EMT can be reversed and identify the functional significance of these changes. Methods: 167 tumours were screened, and sections with both an invasive component and a central tumour area were identified $n=10$. Immunohistochemical characteristics of epithelial (E-cadherin, cytokeratin 18) and mesenchymal (vimentin, $\alpha$-smooth muscle actin) markers were compared at the invasion front and within the tumour in 10 tumours. Following TGF $\beta 1(0-5 \mathrm{ng} / \mathrm{ml})$ treatment of TE7 oesophageal adenocarcinoma cells, phenotypic and molecular changes were assessed using phase contrast microscopy immunofluorescence and real-time RT-PCR. The activation of cell-signalling pathways (AKT, MAPK, SMAD2, SMAD3, SIPl, snail) were assessed by real-time RT-PCR and western blotting. Functional treatmenteffects were assessed using wound healing, invasion, slow aggregation and time-lapse motility assays. We further assessed whether BMP-7 a recognised antagonist of TGFB 31 signalling and SMAD4 RNAinterference could reverse EMT. Results: At the invasive margin there was down-regulation of epithelial markers and up-regulation of mesenchymal markers, with evidence of increased TGF $\beta 1$ expression at the invasive margin. TGF $\beta 1$ induced similar molecular expression changes in vitro $(\mathrm{p}<0.05)$ and these changes were accompanied by a change from an epithelial to a mesenchymal morphology. BMP7 treatment reversed these changes, causing re-expression of epithelial markers $(\mathrm{p}<0.05)$ and return to an epithelial morphology. These changes were mediated via canonical TGF $\beta 1$ signalling with concomitant up-regulation of SIP1 $(\mathrm{P}<0.05)$. Alterations in wound healing, invasion, aggregation and cell motility parameters following TGF $\beta 1$ were consistent with a more invasive phenotype $(\mathrm{p}<0.005)$ and these changes were reversed by both BMP7 treatment and SMAD4 RNAi. Conclusions: EMT may be relevant in oesophageal carcinogenesis and targeting TGF $\beta 1$ signalling pathways may offer potential therapeutic opportunities and require further investigation.

W1532

Colorectal Cancer (CRC) Chemoprevention By Polyethylene Glycol (PEG) Involves Inhibition of Mucosal Hyperproliferation Via Modulation of Snail/ $\beta$ Catenin Signaling

Ramesh K. Wali, Dhananjay P. Kunte, Jennifer L. Koetsier, Hemant K. Roy

Experimental and epidemiological studies indicate that PEG is one of the best chemopreventive agents against CRC. [Can Epi Bio \& Prev, 2003]. However; its precise molecular mechanism is unclear. Previously we have shown that E-cadherin induction, with concomitant suppression of $\beta$-catenin activity by membrane sequestration [Can Let, 2005], are important molecular events of chemoprevention. While the mechanism of E-cadherin induction has not been well explored, we have demonstrated that the transcriptional repressor SNAIL is increased in most CRCs [Dig Dis \& Sci, 2005] resulting in diffuse mucosal hyperproliferation [Mol Can Ther, 2004]. In this study, we assess the role of SNAIL/E-cadherin/ $\beta$-catenin axis in the chemopreventive activity of PEG. Methods: 75 Fisher rats were randomized into 3 groups. Group 1 was injected saline and $\overline{2 \& 3 \mathrm{AOM}}(20 \mathrm{mg} / \mathrm{kg} /$ week x 2). Two wks later, group 3 was switched to PEG-8000 (5 mg/100g) supplemented diet. After 30 weeks colons were removed, ACF and uninvolved crypts biopsied and protein profile analyzed by IHC and Western blot. Results: PEG blocked the AOM-induced increase in the number of larger ACFs (by 64\%; $<<0.001$ ), BrDu labeling (by 78\%; p< 0.001) and Cyclin Dl expression (by $53 \% ; \mathrm{p}<0.001)$. While total $\beta$-catenin was unchanged, $\mathrm{PEG}$ reduced its nuclear localization in ACF $(50 \% ; \mathrm{p}<0.05)$. PEG blocked AOM-induced loss of E-cadherin $(\mathrm{p}<0.01)$ by inhibiting SNAIL (p< 0.05). Similarly, in HT-29 cells treated with 5\% PEG, there was a 50\% decrease in PCNA (proliferation marker), $43 \%$ decrease in $\beta$-catenin/Tcf activity (TOPflash luciferase activity; $\mathrm{p}<0.05), 70 \%$ increase in E-Cadherin expression and $52 \%$ decrease in SNAIL mRNA level. In conclusion, these studies demonstrates that PEG-mediated antiproliferative activity involves induction of E-cadherin and decrease in $\beta$-catenin activity, Snail and Cyclin Dl expressions.

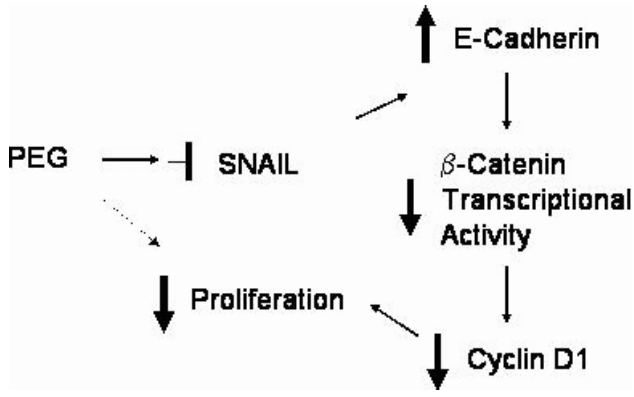

Effect of PEG on SNAIL/ $\beta$-Catenin Pathway

W1533

Proteomic Dissection of the Molecular Mechanisms Underlying Hepatic Metastasis in Colorectal Cancer

Fensong Wang, Xia Ding, Fang Wu, Zhiyou Fang, Zhihong Yang, Yong Chen, Helen Kim, Xuebiao Yao

Colorectal cancer currently accounts for $11 \%$ of all cancers in the United States with 135,000 new cases each year and is the second leading cause of cancer-related death, with the majority of deaths attributable to hepatic metastases. While surgical resection offers the best chance for long-term survival and potential for cure, few patients are deemed candidates for resection due to late-stage diagnosis. A better understanding of the molecular events of colorectal hepatic metastasis may lead to improved screening and treatment options for these patients. To delineate the molecular mechanisms underlying liver metastasis, we carried out proteomic analyses of a primary colon cancer cell line paired with liver metastatic cell lines. The protein expression patterns of these paired cell lines were analyzed in same gel using DIGE system. Proteins differentially displayed in the region of molecular masses of 21-200 kDa with isoelectric point of 3-10 were isolated, proteolyzed and finger-printed using MALDI-TOF and LC-MS/MS mass spectrometries. Ninety-seven spots corresponding to 63 different proteins served as "landmarks" for hepatic metastasis. The potential modification sites were pinpointed using LC-MS/MS, which revealed potential phospho-regulation of 13 proteins, The identified proteins included cytoskeletal proteins (i.e., ezrin; IQGAPl; Arp2/3), proteins involved in signal transduction (i.e., mTOR, S6K1, WWOX), cell proliferation and differentiation (i.e., numatrin, beta-catenin, UPLC), and metabolism (i.e., FOXO). Using a combination of siRNA-mediated protein knock-down with invasion assay, we confirmed that overexpression of 37 proteins among the list promotes the invasive activity. Currently, we are evaluating the role of these proteins in hepatic metastasis in immunodeficient mice using bioluminescence imaging. 
W1534

HMG Al Gene: A Novel Therapeutic Target for Pancreatic Adenocarcinoma Siong-Seng Liau, Stanley W. Ashley, Edward E. Whang

Background: Pancreatic adenocarcinoma has an overtly malignant phenotype. The high mobility group $\mathrm{Al}(\mathrm{HMGAl}$ ) proteins are architectural nuclear proteins which play important roles in regulating gene expression. We hypothesize that HMG Al is a determinant of malignant phenotype of pancreatic adenocarcinoma. Methods: We studied highly invasive pancreatic adenocarcinoma cell lines, MiaPaCa2 (low expression of HMGAl), BxPC3 and PANCl (overexpression of HMGAl). Two independent RNA interference (RNAi) methods were used: short interfering RNA (siHMGAl) and short-hairpin RNA (shHMGAl) expression plasmids targeting HMGAl gene. Stable shRNA expressing cell lines were developed. HMGAl-overexpressing MiaCaPa2 clones were developed following stable transfection with HMGAl cDNA vector. Effect of silencing HMGAl on cellular expression of carcinoembryonicantigen related cell adhesion molecule 6 (CEACAM6), a determinant of pancreatic adenocarcinoma invasiveness, was determined using Western analysis. Cellular invasiveness was assayed using modified Boyden chambers. Matrix metalloproteinase 9 activity assay was used. Akt kinase activity assay was performed using GSK-3 phosphorylation assay. Anchorage-independent growth in soft agar was assessed. Stable transfectants of shRNA were subcutaneously xenografted into nude mice and tumor growth was monitored weekly. Results: Using both RNAi techniques, we achieved 55-90\% silencing of HMGAl protein expression when compared to controls (scramble siRNA or shRNA) ( $\mathrm{p}<0.05$ based on densitometry). Both siHMGAl and shHMGAl transfected MiaPaCa2 and PANCl cells showed significantly lower invasive capacity compared to controls (60-84\% reduction vs. controls, $\mathrm{p}<0.05)$. MiaPaCa2 and PANCl cell lines stably expressing shHMGAl have significantly reduced MMP9 activity $(\mathrm{p}<0.05)$. In both MiaPaCa2 and PANCl cell lines, there was a significant reduction in Akt kinase activity in shHMGAl stable transfectants ( $\mathrm{p}<0.05)$. Silencing of HMGAl significantly attenuated the ability of MiaPaCa2 and PANCl cells to form soft agar colonies ( $\mathrm{p}<0.05)$. Cellular expression of CEACAM6 was reduced in MiaPaCa2 and BxPC3 with silencing of HMGAl. Forced overexpression of HMGAl in MiaPaCa2 resulted in significantly elevated levels of CEACAM6 and MMP9 expression ( $\mathrm{p}<0.05$, based on densitometry). In nude mouse xenograft models, silencing HMGAl significantly inhibited the growth of subcutaneous tumors $(\mathrm{p}<0.05)$. Conclusions: HMG Al plays a role in inducing tumor invasion and growth. This is in part modulated by CEACAM6 expression, Akt and MMP9 activity. HMGAl represents a novel therapeutic target for pancreatic cancer, warranting further investigation.

\section{W1535}

Overexpression of Angiopoietin-2 Enhances Angiogenesis and Lymphatic Spread in Pancreatic Neuroendocrine Tumors

Svenja Rieke, Annett Rexin, Petra Schulz, Sandra Rudloff, Sonja Vollmer, Peter Hauff, Michael Schirner, Bertram Wiedenmann, Stefan Rosewicz, Arne Scholz

Introduction: Angiopoietin-(Ang)-2 acting through the endothelial tyrosine kinase Tie-2 has been implicated in the regulation of tumor associated angiogenesis. To date, no data are available about the expression and biological significance of Ang-2 in well vascularized human pancreatic neuroendocrine tumors (NET). We addressed this question by analyzing human NET specimens and by experimental overexpression of Ang-2 in a novel orthotopic xenograft mouse model. Methods: 10 surgical specimens of human pancreatic NET and 8 benign pancreatic specimens were analyzed for Ang-2- and Tie-2 expression by in situ hybridization and immunohistochemistry, respectively. The human pancreatic NET cell line BON was stably transfected with Ang-2 cDNA or empty expression vector (mock control). 3 Ang-2 expressing clones and 3 mock clones with comparable growth characteristics (Alamar blue assay) were selected for orthotopic injection into the pancreas of immunodeficient mice (9-11 mice per clone). Evaluation of the biological effects in vivo was based on tumor weight, metastatic spread (macroscopically suspect lesions, human cytokeratin staining, PCR for human chromosome 17 DNA), serum human Ang-2 levels (ELISA) and microvessel density (CD34 staining). Results: Endothelial cells of malignant as well as benign human pancreatic tissues expressed both, the Tie-2 receptor and small amounts of Ang- 2 mRNA. In sharp contrast, expression of Ang-2 mRNA in epithelial cells was found exclusively in the NET tumor samples. When overexpressed in Bon cells, Ang-2 neither affected the proliferation of tumor cells in vitro nor the weight of orthotopic Bon tumors in vivo. However, animals bearing Ang- 2 clones exhibited significantly higher serum Ang-2 levels, confirming successful expression in vivo. Analysis of metastatic spread in these mice based on visual detection of macroscopically suspect lesion and subsequent confirmation via the presence of human cytokeratin revealed metastases in 25\% of the Ang-2 tumor mice versus $8 \%$ of animals bearing mock transfected clones. In good agreement, Ang- 2 enhanced the lymphatic spread of tumor cells into abdominal lymph nodes as evidenced by more frequent PCR amplification of human microsatellite DNA. The increased metastatic potential of Ang-2 overexpressing tumors was associated with a significant induction of the microvessel density. Conclusion: De novo expression of Ang-2 in human pancreatic NET promotes angiogenesis and lymphatic spread and thus defines a novel clinically relevant pathomechanism. Interference with the Ang-2/Tie-2 pathway may represent an attractive therapeutic strategy in this well vascularized tumor entity.

\section{W1536}

SRC-Dependent PI 3-Kinase Activation in Response to Extracellular Pressure Is Required for AKT and FAK Activation and FAK Translocation to the Cell Membrane: A Mechanism for Pressure-Stimulated Cell Adhesion

Vijayalakshmi Thamilselvan, David H. Craig, Marc D. Basson

BACKGROUND: Physical force induced inside-out signals may regulate tumor cell adhesion and metastasis. In particular, increased extracellular pressure stimulates integrin-mediated adhesion via FAK and Src, but how such signals modulate adhesion is not known. Since the PI 3-kinase/Akt signal pathway has been implicated in tumorigenesis, invasion and metastasis, we sought to evaluate the effect of pressure on PI 3-kinase and Akt and the interaction of these signal proteins with FAK and Src. METHODS: We subjected suspended
SW620 colon cancer cells to $15 \mathrm{~mm} \mathrm{Hg}$ increased pressure for 30 minutes. We studied adhesion to collagen I, PI 3-kinase activity, tyrosine phosphorylation of the PI 3-kinase p85 subunit, Akt phosphorylation at Ser473, and Akt membrane translocation. In some studies, we inhibited PI 3-kinase by LY294002, Akt by Akt inhibitor IV or siRNA to Aktl, Src kinases by PP2 or FAK by siRNA, with appropriate vehicle controls or non-targeting siRNA We also evaluated the interaction of the p85 subunit of PI 3-kinase with FAK and Src by coimmunoprecipitation, and FAK distribution by subcellular fractionation. RESULTS: Increased pressure $(15 \mathrm{~mm} \mathrm{Hg})$ for 30 minutes stimulated colon cancer cell adhesion and this effect was completely blocked by PI 3-kinase or Akt inhibition or $50 \%$ Aktl reduction by specific siRNA ( $\mathrm{n}>3 ; \mathrm{P}<0.05$ for all). Pressure significantly increased PI 3-kinase activity, tyrosine phosphorylation of the p85 subunit, Akt phosphorylation at Serine 473 and Akt translocation to the membrane fraction ( $\mathrm{n}>3, \mathrm{P}<0.05$ all). Pressure-induced PI 3-kinase activity and Akt phosphorylation were completely blocked by the Src inhibitor PP2 or by the PI 3-kinase inhibitor LY294002. Reducing FAK protein using specific siRNA did no affect pressure-induced PI 3-kinase activity but completely blocked Akt phosphorylation. Interaction of the p 85 subunit with Src and FAK was significantly increased by pressure, based upon coprecipitation studies. Extracellular pressure also stimulated FAK translocation $(32.0 \pm 1.0 \% ; \mathrm{n}>3 ; \mathrm{P}<0.05)$ to the membrane and this effect was blocked by Akt inhibitor IV and by PP2. CONCLUSIONS: These results suggest that pressure-induced FAK and Src are important cofactors for PI 3-kinase dependent Akt phosphorylation. Src acts as an upstream regulator of PI 3-kinase in this pathway. PI 3-kinase associates with Src and FAK, activating FAK and Akt, and eventually inducing translocation of cytosolic FAK and Akt to the membrane, where the translocated FAK may upregulate integrin binding affinity or facilitate integrin clustering. This novel pathway may play a significant role in integrinmediated colon cancer metastasis.

\section{W1537}

Biphasic Alteration of Caveolins and Its Implication in the Malignant Progression of Human Gastric Carcinoma

Hyo Jong Kim, Sung-Gil Chi, Jae Young Jang, Kwang Ro Joo, Seok Ho Dong, Byung-Ho Kim, Young Woon Chang, Il Joung Lee, Rin Chang

Background \& Aims: Reduced expression of caveolin-1 (Cav-1)has been observed in many types of human malignancies. However, elevated expression of Cav-1 was also identified to correlate with increased metastatic progression of some cancers. We investigate caveolin alteration in human gastric tumorigenesis. Methods:We characterized the expression and mutational status of Cav- 1 and Cav-2 in a series of gastric cancer cell lines and tissue specimens using quantitative RT-PCR, Western blot, and PCR-SSCP analysis. Results: Expression of Cav-1 and Cav-2 transcripts was detectable in all normal and benign tissues. In contrast, absence or extremely low levels of Cav-1 and Cav-2 transcript were detected in 40\% (6 o 15) of cancer cell lines while high levels of expression were found in $47 \%$ of cell lines. All of the 4 cell lines originated from primary cancers exhibited loss or reduction of Cav-1 and Cav-2 expression whereas 7 of 11 (64\%) cell lines derived from metastasized lesions showed elevated expression, raising the possibility that high level of caveolin expression provides tumor cells with a advantage for metastatic progression of gastric cancer. Consistent with cell lines, $43 \%$ (36 of 84) of primary carcinomas showed downregulation of Cav-1 and Cav2 expression while $14 \%$ of tumors exhibited strong expression of both genes. Mutational analysis revealed that genomic deletion of caveolin genes is infrequent but some tumor cells harbor missense mutations in Cav-1 or Cav-2. Expression of Cav-1 and Cav-2 was reactivated in nonexpressor cells by 5-aza-dC treament, and bisulfite DNA sequencing analysis revealed that hypermethylation of the promoter $\mathrm{CpG}$ islands is associated with altered expression of Cav-1. Whereas proliferation and colony forming ability of Cav-1-negative tumor cells was suppressed by restoration of wild-type Cav-1. Likewise, blockade of Cav-1 function in Cav-1-overexpressing cells by DN-Cav-1 (P132L) or siRNA-mediated knockdown of Cav1 expression led to a significant inhibition of tumor cell adhesion to endothelium. Conclusion: Our data suggest that caveolin expression might suppress early stage tumor growth but its increased expression can enhance invasive or metastatic progression of the cells.

\section{W1538}

Tumor Cells Induce MMP-9 Synthesis in Rat Pancreatic Stellate Cells -

\section{Potential Role of Emmprin}

Martin Mehrlaender, Gisela Sparmann, Robert Jaster, Heike Weber, Stefan Liebe, Joerg Emmrich

Background \& aims: It is generally accepted that pancreatic stellate cells (PSC) are decisively involved in extracellular matrix (ECM) turnover. In addition to ECM components, PSC synthesize matrix metalloproteinases (MMP). The extracellular matrix metalloproteinase inducer (EMMPRIN), a transmembrane glycoprotein preferentially expressed on tumor cells, has been reported to be a candidate in stimulating the expression of MMP in adjacent fibroblastic cells. The aim of the present study was to investigate the expression of MMP-2 and -9 in PSC in response to pancreatic cancer cells and the potential role of EMMPRIN. Methods: Primary PSC were obtained by collagenase digestion of rat pancreata and subsequent density gradient centrifugation. Cultured PSC were incubated with tumor cell membrane vesicles (TCMV) obtained by homogenization and subsequent differential centrifugation from the rat pancreatic tumor cell lines DSL-6A and AR42J. After co-incubation with TCMV, the expression of MMP-2 and -9 in PSC was analyzed on RNA- as well as protein level by competitive RT-PCR and zymography, respectively. Membrane vesicles were used to prevent contamination of PSC samples with RNA and/or proteins released from metabolically active tumor cells. EMMPRIN expression was investigated by immunocytochemistry and Western blotting. To abolish EMMPRIN glycosylation, vesicles were prepared from tunicamycintreated tumor cell lines. Studies on signal transduction pathways were performed using Western blot analysis and specific inhibitors. Results: Western blot analysis of tumor cell lysates as well as of TCMV showed heterogeneous EMMPRIN patterns indicating a differentia glycosylation of the molecule. Co-incubation with TCMV resulted in a clear induction of MMP-9 in PSC on RNA- as well as on protein level. In contrast, MMP-2 expression remained unchanged. The intracellular signal cascade in response to TCMV incubation included the phosphorylation of ERK $42 / 44$ showing the activation of the MAP kinase pathway in stellate

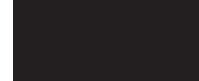


cells. Deglycosylation of EMMPRIN resulted in an inactive protein conformation with respect to MMP regulation and consequently, TCMV-mediated effects on PSC were reduced. Conclusion: Our results suggest that glycosylated transmembrane EMMPRIN of pancreatic cancer cells regulated the expression of MMP-9 in stellate cells. This mechanism may be substantially involved in the crosstalk between malignant cells and PSC facilitating tumor cell invasion and metastasis.

W1539

Prostaglandin E2-EP4 Receptor Signalling Promotes Tumorigenic Behaviour of HT-29 Human Colorectal Cancer Cells Via cAMP and ERK Signalling Gillian Hawcroft, Stanley C. Ko, Mark A. Hull

Background \& Aims: Prostaglandin (PG) E2 is the predominant pro-tumorigenic product of the cyclooxygenase (COX) pathway in the human colon. Signalling by PGE2 occurs via four G protein-coupled receptors termed EP1-4. Preliminary in vitro evidence has implicated the EP4 receptor in the pro-tumorigenic activity of PGE2 on human colorectal cancer (CRC) cells. The objective of this study was to further investigate the role of the EP4 receptor in PGE2 signalling in human CRC cells. Methods: A model of stable human EP4 receptor over-expression in HT-29 human CRC cells (HT-29-EP4) was used to investigate the role of the EP4 receptor in diverse effects of PGE2 on HT-29 cell behaviour such as resistance to apoptosis and anchorage-independent growth. The role of the EP4 receptor was confirmed using the EP4 receptor antagonist ONO-AE3-208 (EP4A). Results: PGE2 (1 $\mu \mathrm{M})$ induced resistance to spontaneous apoptosis and increased anchorage-independent growth in HT29-EP4 cells but not control transfected (HT-29-con) cells. In addition, EP4A increased apoptosis of HT-29-con cells which express low levels of endogenous EP4 receptors. PGE2 $(1 \mu \mathrm{M})$ increased intracellular cAMP levels in HT-29-EP4 cells but not in HT-29-con cells. This was associated with phosphorylation of cAMP-response element binding protein (CREB), and PKA-dependent extracellular signal-related kinase (ERK) activity, but not epidermal growth factor receptor (EGFR) trans-activation. Conclusion: PGE2-EP4 receptor signalling promotes tumorigenic behaviour of HT-29 human CRC cells. EP4 receptor activation by PGE2 in this cell line is coupled to an increase in cAMP levels, leading to phosphorylation of CREB and PKA-dependent ERK activation. Therefore, the EP4 receptor is a candidate novel target for chemoprevention of CRC. Comparision with existing literature suggests that cell type-specific differences occur in EP4 receptor coupling to downstream signalling pathways

\section{W1540}

Association of Carcinogenesis and Emergence of Spasmolytic PolypeptideExpressing Metaplasia (SPEM) in Helicobacter Pylori-Infected Mongolian Gerbils with Carcinogen

Nao Yoshizawa, Hirokazu Yamaguchi, Yoshiharu Takenaka, Sachiyo Nomura, James R Goldenring, Jeffrey R. Lee, Michio Kaminishi

Background: In previous studies, We have reported that Spasmolytic polypeptide (TFF2)expressing metaplasia (SPEM) is observed in adjacent mucosa to human gastric cancer and at the base of fundic glands showing oxyntic atrophy in rodent models such as Helicobacter felis infected mice, MT-TGF $\alpha$ transgenic mice, rats with remnant gastric cancer, and mice with DMP-777 treatment. Oxyntic atrophy is well known as a background of gastric carcinogenesis, therefore those results can set up a hypothesis that SPEM is a precursor of gastric cancer. Aim: To explore the time course changes in fundic glands and the relationships between SPEM and gastric carcinogenesis using $\mathrm{H}$. pylori $(\mathrm{Hp})$-infected and carcinogen-treated Mongolian gerbils. Materials and methods: Mongolian gerbils were assigned into four groups: Group A; with Hp infection and treatment of N-Methyl-N-Nitrosourea (MNU), Group B; with treatment of MNU, Group C; with Hp infection, Group D; control. MNU was given at $30 \mathrm{ppm}$ in drinking water at experimental week 1, 3, 5, 7, 9. At week 11, Hp colony samples were inoculated. The animals were sacrificed at $11,14,20,25,35,50$ weeks. The removed stomachs were fixed in $4 \%$ formaldehyde for 24 hours, embedded in paraffin, and cut into 4- $\mu \mathrm{m}$ sections. Replicate sections were stained with hematoxylin and eosin (H\&E). Immunohistochemistry using anti-Human TFF2 antibody and anti-human Intrinsic Factor (IF) antibody were also performed. Furthermore immunofluorescence for TFF2 and IF was performed. Results: In Group B, no SPEM and carcinoma were observed. Group C demonstrated SPEM but no neoplasm. Only Group A showed both. SPEM emerged earlier in Group C than Group A. The incidence of SPEM in Group C kept about $60 \%$ all along, however, in Group A it increased gradually up to $80 \%$. In the earlier stage, SPEM demonstrated TFF2 and IF double staining. Although number of animals with SPEM increased, SPEM showing double staining rather decreased and SPEM showing single staining by antiTFF2 was observed in the later stages. SPEM changed also morphologically. In the earlier stage SPEM organized slender and straight glands, however, in the later stage SPEM became small and distorted. Many heterotopic proliferations and adenomata were TFF2 positive. Around dysplasia SPEM was seen, however most of them are IF negative and they were small and distorted. Conclusion: SPEM was observed in the animals infected with Hp. With carcinogen and Hp, SPEM was gradually changed morphologically and immunohistochemically, and that was seen around dysplasia. These results support the hypothesis that SPEM is a candidate for a precursor of gastric cancer.

\section{W1541}

X-Ray Irradiation Promotes the Occurrence and the Multiplicity of Invasive Carcinoma in Intestinal Tract of $\mathrm{APC}^{\mathrm{Min} /+}$ Mouse

Toshiyuki Nakayama, Kazuyuki Yamazumi, Takashi Uemura, Ayumi Yoshizaki, Yuichi Yakata, Ichiro Sekine

BACKGROUNDS \& AIM: X-ray is well known to cause genetic damage and induce many types of carcinomas in human. Mouse model for human familial adenomatous polyposis (FAP), APC ${ }^{\mathrm{min} /+}$ mouse, contains a truncating mutation in APC gene and spontaneously developes intestinal adenomas. To elucidate the role of X-ray in the intestinal tumor, we examined the promotion of carcinogenesis in X-ray irradiated $\mathrm{APC}$ min/+ mouse. METHODS $\mathrm{APC}^{\mathrm{min} /+}$ mice and wild-type, C57BL/6J mice, were irradiated with a single whole body o 5 Gy X-ravs at 8 weeks of age. After 20 weeks, all animals were sacrificed. RESULTS: Total $51.9 \%$ cases (40 of 77 ) of X-ray irradiated $\mathrm{APC}$ min/+ mice were highly observed invasive carcinoma in small intestine. 54.5\% (24 of 44) in male and $48.5 \%$ (16 of 33 ) in female were showed adenocarcinoma with invasion to proprial muscle layer of small intestine. The other side, only $20.3 \%$ cases ( 13 of 64 ) of non-irradiated $\mathrm{APC}^{\mathrm{min} /+}$ mice were observed invasive carcinoma $28.1 \%$ (9 of 32) of male and $12.5 \%$ (4 of 32) of female in non-irradiated $\mathrm{APC}^{\mathrm{min} /+}$ mice were showed invasive carcinoma in small intestine. There was statistical difference of the occurrence of invasive intestinal carcinoma between X-irradiated and nonirradiated $\mathrm{Apc}^{\mathrm{min} /+}$ male or female mice $(\mathrm{p}=0.019, \mathrm{p}=0.0017$, respectively). Wild-type mice with or without X-irradiation were not occurred any invasive carcinoma. X-irradiated Apc ${ }^{\mathrm{min} /}$ + mouse showed higher number of invasive carcinoma than non-irradiated mouse in male $(\mathrm{p}=0.041) . \mathrm{APC}^{\mathrm{min} /+}$ mice presented many polyps in small and large intestine with or wthout $\mathrm{X}$-ray irradiation. The number of polyps did not show any difference between X-irradiated and non-irradiated $\mathrm{Apc}^{\mathrm{min} /+}$ mice. CONCLUSIONS: X-ray irradiated $\mathrm{Apc}^{\mathrm{min} /+}$ mice were highly observed intestinal invasive carcinoma and showed the multiplicity of invasive carcinoma. Our results suggested that X-ray irradiation promotes the invasive activity of intestinal tumor in $\mathrm{Apc}^{\mathrm{min} /+}$ mouse.

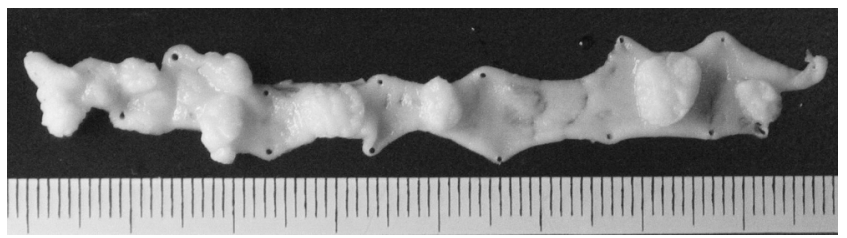

\section{W1542}

Positive Correlation Between mRNA Expression and Protein Array Results in Colorectal Cancer Sandor Spisak, Balazs Gyorffy, Tamas Zagoni, Mark Juhasz, Zsolt Tulassay

Backgrounds and aims: The relevence of the results' of mRNA expression studies on protein levels is not proven yet. Recently new whole genome mRNA expression arrays became available. Protein chips with several hundred of proteins was used to confirm mRNA results from colorectal cancer patients. Patients and methods : in 5 Dukes B and 5 Dukes stage D colorectal cancer patient malignant and normal specimen were collected and fresh frozen immediately after surgery. mRNA and proteins were isolated using Trisole reagent. mRNA expression was evaluated using a whole gebnome array (Affymetrix U133 plus 2.0) and compared to the results of a commercial protein chip analysis containing 576 proteins (Clontech, Atlay Arrays, USA) from the same specimen. Significantlly differently expressed genes were identified by $\mathrm{T}$ test using the MAS (Affymetrix, USA) programpackage and the $\mathrm{R}$ programpackage. Statistical analysis was performed concerning the coexpression of corresponding mRNA and the proteins using Spearmann's R method. Results: All the protein arrays were applicable for the expression study. The mRNa profile was in $86,5 \%$ in correlation with the protein expression array results. However in a relatively high number significant negative correlation was found (14.5\%). Between the Dukes Stage B and D cancer patients the top 5 discriminator genes were HLA-DQAl, DnaJ (Hsp40) homolog, subfamily C, member 10, cetrin 3, acetyl-Coenzyme A acetyltransferase 2, solute carrier family 39, member 8. Dukes Stage B, C and D group could classified by cluster analysis of the top 100 gene. Conlusions: Protein arrays can utilised for the verification of whole genome mRNA expression studies. Dukes stage B and D disease demonstrates a different genomics entity as it was shown in the mRNA expression profile. The difference between the protein and mRNA expresssion results could be justified by the possible mRNA splicing alternatives and protein or mRNA digestion in the sample preparation process.

\section{W1543}

Molecular Characteristics of Small Bowel Carcinomas Vary with Location Marloes Berkhout, Leonie J. Mekenkamp, Fred J. van de Molengraft, Wilbert H. Peters, Fokko M. Nagengast, Han J. van Krieken, Iris D. Nagtegaal

Background: Primary carcinomas of the small intestine are rare and their carcinogenesis remains poorly understood. The most frequent site of development is the duodenum $( \pm 50$ $\%)$, followed by the jejunum $( \pm 25 \%)$ and the ileum $( \pm 13 \%)$. It is not known whether the process of carcinogenesis differs in the different parts of the small intestine. Aim: The aim of this study is to elucidate the genetic pathways leading to the development of small intestinal carcinomas by immunohistochemical comparison of several candidate proteins in tumors of the duodenum, jejunum and ileum. Methods: The duodenal carcinomas $(n=20)$, jejunal carcinomas $(n=14)$ and ileal carcinomas $(n=6)$ were compared on Tissue Microarrays by immunohistochemical staining for the cytokeratins (CK7, CK20), DNA mismatch repair proteins (MLH1, MSH2, MSH6, PMS2), cell cycle proteins (P16, P53, cyclin D1), cell adhesion proteins (beta-catenin, E-cadherin, Ep-CAM) and SMAD4, CO-TL1, CEA. Results: In the majority of carcinomas (more than 90\%) invasion through the bowel wall was present (T3/T4). Most tumors were adenocarcinomas, the exceptions being 1 signet ring cell carcinoma in the duodenum, 1 mucinous carcinoma and 1 adenosquamous carcinoma in the jejunum and 1 mucinous carcinoma in the ileum. Approximately $50 \%$ of these tumors were poorly/undifferentiated. Both CK7 and CK20 were differentially expressed along the small bowel; with CK7 positivity in $63 \%, 28 \%$ and $0 \%$ and CK20 negativity in $35 \%, 15 \%$ and $0 \%$ of the carcinomas of the duodenum, jejunum and ileum respectively $(\mathrm{p}=0.01, \mathrm{p}=$ 0.06). P53 was overexpressed in $32 \%, 79 \%$ and $100 \%$ of the duodenal, jejunal and ileal carcinomas respectively $(\mathrm{p}=0.002)$. Moderate to high loss of extracellular E-cadherin was seen in $75 \%$ and $92 \%$ of the carcinomas of duodenum and jejunum respectively in comparison to $33 \%$ of the ileal carcinomas ( $p=0.02$ ). Conclusions: Overexpression of P53 is less frequent in the duodenal carcinomas compared with more distal small bowel carcinomas. 
Differential expression of both CK7 and CK 20 reflects preexisting differences between the upper gastrointestinal tract and the colon. We propose that in the proximal small bowel carcinogenesis reflects gastric carcinogenesis, while in distal small bowel carcinogenesis resembles colonic carcinogenesis.

\section{W1544}

Epigenetic Silencing of the Chemokine CXCL12 in Colorectal Carcinoma Enhances Metastasis

Michael K. Wendt, Priscilla A. Johanesen, Ningling Kang-Decker, Vijay H. Shah, Michael B. Dwinell

The chemokine CXCL12 and its receptor CXCR4 are co-expressed in several tissues and cell types throughout the body and play essential roles in development, where disruption of either gene leads to embryonic lethality. Not only is this signaling axis a key regulator of hematopoietic stem cell, leukocyte, and lymphocyte migration but it has also been shown to play a critical role in carcinoma cell metastasis. We have previously defined constitutive functional expression of CXCL12 and CXCR4 in the human colonic epithelium, an expression pattern absent in several colorectal carcinoma cell lines due to CXCL12 silencing by promoter hypermethylation. We hypothesized that silencing of CXCL12 in CXCR4 expressing carcinoma cells contributes to in vivo disease progression. Consistent with our hypothesis, we show aberrant methylation of the CXCL12 promoter in several primary colorectal carcinomas, a dysregulation not observed in normal colonic epithelial isolates. CXCL12 and CXCR4, in agreement with our prior reports, were co-expressed in normal colonic epithelium, while in adjacent transformed epithelium CXCL12 expression was notably absent. Importantly, CXCR4 expression in tumor tissues was maintained. Re-expression of functional, endogenous CXCL12 in colorectal carcinoma cells by stable transfection resulted in smaller tumor cell foci in soft agar. Similar results were observed in vivo, as colonic carcinoma cells re-expressing CXCL12 injected into the hepatic portal vein formed dramatically smaller liver metastases in Scid mice as compared to CXCL12 silenced control cells. These data likely reflect an increase in apoptosis in CXCL12 expressing carcinoma cells as defined using a caspase assay. Taken together, our data constitute the unique observation that silencing of CXCL12 within human colonic carcinoma cells greatly enhances their survival, contributing to increased metastasis, exacerbating disease progression.

\section{W1545}

Rho-Like Proteins in Human Colon Cancers Are Uniquely Expressed As a Function of Differentiation and Tumor Invasion Jameela Huq, Grace Guzman, Bassem Elgohary, Lubna Shakir, Sarah C. Glover

Background: Rho-like proteins i.e. Rho A, B, and C have been identified as playing distinct roles in post neoplastic transformation and metastasis in breast, bladder, hepatocellular, ovarian, lung, and prostate cancers. Specifically, Rho A promotes proliferation and neoplastic transformation while Rho $\mathrm{C}$ increases with tumor de-differentiation where it promotes invasion and metastasis. Rho B behaves in an exact opposite manner by enhancing apoptosis of tumor cells. Action of these proteins on their downstream effector, ROCK (Rho Associated Kinase), has been associated with cancer progression through its action on the actin cytoskeleton. Specific inhibition of ROCK suppresses cancer growth and spread (Nat Med 1999. 5(2): 221). While Rho has been shown to be increased in both expression and activity in colon cancer cell lysates, little is known about expression of individual Rho-like proteins or ROCK in colon cancer. Since each of these proteins may have a different impact on colon cancer cell behavior, we set out to determine the presence and location of individual Rholike proteins as well as that of their downstream effector, ROCK, in human colon cancer specimens. Methods: Ten colon cancers containing of distinct differentiation were obtained from the UIC department of pathology under an exempt IRB. All specimens were immunohistochemically probed using antibodies directed against Rho A, B, C, ROCK I, and ROCK II Antibody-specific chromogen was determined by quantitative immunohistochemistry (J. Histochem. Cytochem. 2003, 51:1041) for each cellular region. Results: Both Rho A and B were expressed in normal and dysplastic tissue adjacent to the colon cancer as well as at the leading edge of the colon cancer suggesting that they may play a role in neoplastic transformation as well as post neoplastic transformation. Rho C and ROCK II expression was dramatically increased in the cytoplasm of cells at the leading edge of the tumor and in poorly differentiated cells. The expression pattern of Rho C and ROCK II correlated with expression data from other solid tumors where overexpression of these proteins was directly linked with an increase in cancer related death due to metastatic disease (Clin Cancer Res 2003; 9(7): 2632). ROCK I expression was minimal in this same tumor specimen suggesting that it plays a lesser role. Conclusions: We show for the first time that colon cancers overexpress Rho-like family members as well as their downstream effector, ROCK II, in a unique manner and that their expression changes not only in relationship with cell differentiation but also with level of invasion.

\section{W1546}

\section{Antitumor Effect of Whole Body Hyperthermia Combined with} Geranylgeranylacetone in Mice Pulmonary Metastasis Models of Colon Cancer Tetsuya Okayama, Satoshi Kokura, Toshimitsu Okuda, Satoko Adachi, Takeshi Hattori, Nami Nakabe, Naoyuki Sakamoto, Tomohisa Takagi, Osamu Handa, Yuji Naito, Norimasa Yoshida, Toshikazu Yoshikawa

Aim: It is reported that the inhibition of NF-kB activation leads to inhibition of cancer metastasis and growth. On the other hand, heat treatment is known to initiate a wide variety of reaction through the expression of heat shock proteins (HSPs). We confirmed that heat pretreatment inhibited activation of NF-kB through the expression of heat shock proteins in a human colon cancer cell line and human endothelial cell line. Therefore, it is expected that hyperthermia inhibits NF-kB activation of a cancer cell and a vascular endothelial cell, resulting in the inhibition of cancer metastasis and cancer growth by the induction of heat shock proteins. In this study, we investigated the effect of whole body hyperthermia and a heat shock proteins-inducing drug, geranylgeranylacetone (GGA, Hsp70 inducer) in mice pulmonary metastasis models of colon cancer. Methods: Colon26 cells, a mouse colon adenocarcinoma cell line, were injected via the tail vein of male BALB/c mice and numbers of pulmonary metastases were assessed 18 days later. Colon26-bearing mice were treated with whole body hyperthermia by using far infrared rays 3 times a week. Rectal temperature was maintained for $60 \mathrm{~min}$ at 41 degrees Celsius. In some experimental groups, ora administration of GGA $(250 \mathrm{mg} / \mathrm{kg})$ was performed $2 \mathrm{hr}$ before whole body hyperthermia We investigated the therapeutic effect of whole body hyperthermia, single administration of GGA, and the combined therapy. Moreover, Hsp70 of lung tissue in each treatment group was detected by Western bloting. The activity of NF-kB in lung tissue after cancer cells in oculation was investigated by EMSA and ELISA. Results: 1) Whole body hyperthermia decreased the number of the lung metastases. 2) A single administration of GGA did no show the inhibitory effect to lung metastases. 3) The combination of whole body hyperthermia and GGA made further inhibitory effect to lung metastases. 4) GGA or whole body hyperthermia slightly induced $\mathrm{Hsp} 70$ in lung tissue $24 \mathrm{~h}$ after treatment. On the other hand, whole body hyperthermia combined with GGA administration increased the induction of Hsp70, and inhibited the activation of NF-kB in lung tissue. Conclusions: The incidence of lung metastasis was reduced by the treatment of whole body hyperthermia, and it might be suggested that heat shock proteins induced by whole body hyperthermia attenuated lung metastases because the effect of treatment is enhanced by using a Hsp70 inducer together

\section{W1547}

Association of TGF- $\beta 1$ Overexpression in Barrett's Cancer with Advanced Tumor Stage and Worse Prognosis

Burkhard H. A. Von Rahden, Mario Sarbia, Marcus Feith, Joerg Theisen, J. Ruediger Siewert, Hubert Stein

Objective. Expression of TGF- $\beta 1$, a major member of the TGF- $\beta$ superfamily and importan promoter of tumor growth, was investigated in a series of primary resected Barrett's adenocarcinomas to establish its potential clinical significance and prognostic relevance in this entity. Methods. A series of 123 primary resected adenocarcinomas of the distal esophagus (Barrett's cancer), and corresponding normal squamous epithelium ( $n=12$ ) and non-malignant Barrett's mucosa $(n=11)$, were investigated by means of quantitative RT-PCR for expression of TGF$\beta 1$, using paraffin embedded tissue samples. Gene expression levels were correlated with clinical parameters and overall survival. Results. TGF- $\beta 1$ mRNA was expressed in all tumors but relative gene expression levels varied largely among different tumors. The relative gene expression was significantly higher in tumor tissue compared to squamous epithelium ( $\mathrm{p}=$ 0.005 ) and Barrett's mucosa ( $\mathrm{p}=0.002)$, expressing only low amounts of TGF- $\beta 1$. Relative overexpression of the TGF- $\beta 1$ gene was associated with advanced UICC stage (III/ IV vs. I/ II; $\mathrm{p}=0.009$ ), depth of tumor infiltration (pT3 vs. pT1/2; $<0.001$ ), nodal involvement (pN1 vs. $\mathrm{pNO} ; \mathrm{p}=0.006$ ) and lymphatic vessel invasion ( $\mathrm{Ll} v \mathrm{vs} . \mathrm{L} 0 ; \mathrm{p}=0.011$ ). On univariate survival analysis, TGF- $\beta 1$ overexpression had a significant negative impact on survival (log rank test; $\mathrm{p}=0.0255)$. However, the prognostic impact was not independent from other strong predictors of survival (pT, pN) on multivariate survival analysis. Conclusion. Our data show that TGF- $\beta 1$ overexpression is associated with advanced stage of Barrett's cancer and implies a negative impact on survival. The TGF- $\beta$ pathway may be a potential target for molecular therapies of advanced tumors of this entity.

\section{W1548}

Atorvastatin Reduces the Attachment of Colorectal Cancer Cells to Endothelial Cells in Vitro By Down-Regulating the Endothelial Cell Surface Expression of Adhesion Molecules

Cary Aarons, Karen L. Reed, Olga Bajenova, Peter Thomas, James M. Becker, Arthur F. Stucchi

Background: Colorectal cancer (CRC) is the second most common cause of cancer mortality in the United States. Although the liver is the main organ affected by metastasis from CRC only a small percentage of patients with liver metastases are candidates for curative surgica resection. Therefore, a better understanding of the mechanisms regulating metastasis will improve the overall outcome for the metastatic cancer patient. Recent studies have shown that statins inhibit the growth of CRC cells and reduce the relative risk of colorectal cancer however, little is known about whether statins can affect metastasis. The aim of this study was to examine the role of statins in modulating two key steps in the metastasis of CRC cells: 1) endothelial cell (EC) surface expression of adhesion molecules, and 2) the attachment of CRC cells to endothelial cells. Methods: Using an in vitro model of CRC metastasis, human umbilical vein endothelial cells (HUVECs) were pretreated for 12-16 hrs with atorvastatin $(10 \mu \mathrm{M})$ and then stimulated for up to 24 hours with either TNF- $\alpha(10 \mathrm{ng} / \mathrm{ml})$ or conditioned media from differentiated THP-1 (D-THP) macrophages incubated for 24 hours with carcinoembryonic antigen (CEA) $(2 \mu \mathrm{g} / \mathrm{ml})$. As we have shown, D-THPs possess functional CEA receptors and when stimulated with CEA express a pattern of proinflammatory cytokines similar to that expressed by liver macrophages, Kupffer cells. The EC surface expression of the adhesion molecules, ICAM-1 and E-selectin was quantified by a modified ELISA. Using the same conditions as above, the attachment of HT-29 CRC cells to HUVEC was measured by a fluorescent assay. Results: Atorvastatin pretreatment reduced the TNF$\alpha$ induced upregulation of ICAM-1 and E-selectin protein expression in HUVECs by $46 \%$ $(\mathrm{p}<0.05)$ and $36 \%$, respectively, compared with controls. Atorvastatin also reduced the CEAconditioned media induced rise in E-selectin by $47 \%(p<0.05)$ compared with controls. While the incubation of HUVECs with TNF- $\alpha$ and CEA-conditioned media significantly increased the attachment of HT-29 cells to EC by $141 \%$ and $235 \%(p<0.05)$, respectively, a 60 minutes pretreatment with atorvastatin significantly inhibited this attachment by up to $132 \%(p<0.05)$. Conclusion: These data demonstrate that statins may modulate one of the early pathophysiological steps in metastasis of CRC from the colon to the liver and suggest a possible role for HMG-CoA reductase inhibitors in the prevention of CRC metastases

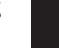


W1549

CD24 Is a Novel Oncogene in Colorectal Cancer, Detected By Microarray Profiling of Cell Transformation, and Is a Target for Immunotherapy of Cancer Eayl Sagiv, Lorenzo Memeo, Dana Yona, Adi Karin, Diana Kazanov, Jasmin Jacob, Gidi Rechavi, Hanina Hibshoosh, Nadir Arber

Aims: To identify genes that play a role in colorectal cancer (CRC) carcinogenesis by analysis of differential gene expression of normal and transformed CRC cell lines. Methods: Gene expression array analysis, (RG-U34) Genechip ${ }^{\circledR}$, was performed in normal and transformed rat intestinal epithelial cells before and after exposure to celecoxib, a CRC prevention agent (Pfizer, New York, NY, USA). In particular, we were looking for: a. altered gene expression in the transformed cells that reverts to normal following exposure to a selective COX2 inhibitor. b. Novel genes. c. Genes encoding membrane receptors or ligands. The CD24 gene fulfilled all these criteria. As a validation of the results and for human patients, immunohistochemistry was performed on 398 biological samples from the entire GI tract (normal, polyps and adenocarcinomas). Human cancer cell lines were tested for their response to anti-CD24 monoclonal antibodies. Three different antibodies were added separately to the medium at different concentrations or durations; viability was measured by Methylene blue assay. CD24 cellular levels in HT29 CRC cell line were stably down regulated using siRNA technology and changes in their proliferation capacities were examined. Results 1081 genes were differently expressed following malignant transformation; 71 genes showed alter expression that reverted to normal following treatment with celecoxib; among them was the gene to CD24. Immunohistochemistry confirmed that increased expression of CD24 is an early event in CRC carcinogenesis. It was expressed in $90.7 \%$ of adenomas and $86.3 \%$ of CRC. Very low expression was seen in normal epithelium (16.6\%). Human cancer cell lines showed growth inhibition in response to the antibodies, according to their expression levels of CD24 and in dose and time dependent manners. These results were repetitive for three different antibodies. The derivatives of HT29 that showed reduced levels of CD24 following transfections with two siRNA expression vectors that differ in their binding sites, showed a significant reduction in growth rate when compared to parental HT29 and HT29 with a control-siRNA. Conclusions: CD24 is a new gene that is overexpressed in the colonic mucosa, already at an early stage of carcinogenesis and might play a role in tumor progression. Therefore, it is likely to be a useful biomarker for early detection and might be of use as a new target in CRC therapy.

\section{W1550}

Nuclear MDM2 Accumulation: A Novel Mechanism of TP53 Inactivation in Anal Squamous Cell Carcinoma?

Heena S. Patel, John M. Northover, Andrew R. Silver

Background MDM2 is an important negative regulator of TP53 that binds and inhibits TP53 transactivation and/or results in TP53 degradation. MDM2 is overexpressed in several human tumours including cervical cancer (a human papillomavirus-associated tumour) but has not been investigated in anal squamous cell carcinoma (ASCC); cervical cancer and ASCC share similar disease aetiology. The primary mechanism of MDM2 overexpression is amplification of the gene in breast and soft tissue tumours and consequent abrogation of the pro-apoptotic functions of TP53. Interestingly, nuclear TP53 accumulation has been observed in ASCC, indicating a potential role for MDM2 in ASCC tumorigenesis. This possibility is now investigated. Methods Anal squamous carcinomas $(n=29)$ were analysed for: TP53 and MDM2 somatic mutations by direct DNA sequencing of TP53 exons $5-8$ and the MDM2 nuclear export signal (aa 90-102) and critical residues glutamine 58, cysteine 438 and 464, TP53 and MDM2 accumulation by immunohistochemistry; and MDM2 amplification by quantitative PCR. HPV status was also examined. Results One somatic TP53 mutation (frameshift resulting from $14 \mathrm{bp}$ deletion, exon 5 ,codon $176,779-792)$ was detected $(3 \%, 1 / 29) .83 \%$ (24/29) of anal SCC were positive for TP53 and 48\% (14/29) of anal SCC were positive for MDM2 by immunohistochemistry. Normal anal epithelium controls were all negative for TP53 and MDM2 immunostaining. Nuclear accumulation of TP53 and MDM2 in this group of ASCC are independent variables ( $\mathrm{p}=0.33$, Fisher's exact test). MDM2 was not amplified in our samples and no mutations were detected. A high frequency (83\%) of samples was HPV positive (24/29) of which 67\% (16/24) contained HPV 16 DNA. Nuclear MDM2 accumulation and HPV status were independent variables ( $\mathrm{p}=0.65$, Fisher's exact test) Conclusions Nuclear MDM2 accumulation was observed at high frequency in ASCC in the absence of MDM2 gene amplification or somatic mutation. This may be a feature of a novel pathway of TP53 abrogation in this tumour and adds to the known mechanisms of TP53 transcriptional inactivation and proteosomal degradation by HPV E6 and E7. Somatic TP53 mutation was rare in this study and probably has limited contribution to tumorigenesis in ASCC.

\section{W1551}

\section{Novel Interaction Between Xiap-Associated Factor 1 (XAF1) and Nuclear} Coactivator $\mathrm{Cbp}$ in Colon Cancer

Yunwei Sun, Harry.H.X. Xia, Senlin Zhu, Bing Zou, Marie Cm Lin, Hsiang Fu Kung, Benjamin C. Wong

Background: XAFl (XIAP-Associated Factor 1) is a nuclear protein that directly interacts with endogenous XIAP and results in XIAP sequestration in nuclear inclusions. cAMP response element binding protein (CREB)-binding protein (CBP) play an important role as multifunctional transcriptional coactivators. In this study, we examined the association between XAF1 and CBP in colon cancer cell. Methods: Expressions of XAF1 and CBP were detected by Western blot in colon cancer cells. The association of XAFl with CBP was confirmed in vitro by glutathione S-transferase (GST) pull-down assay and in vivo by coimmunoprecipitation. Co-localization of XAFl and CBP was demonstrated by immunofluorescence microscopy. An APl reporter assay was used to determine the functional interaction between XAFl and CBP in the presence or absence of PMA. Results: Low level of XAF1 and CBP was detected in SW1116 cells. Using GST-CBP (1-1099,1098-1758,1098-1858 and 1620-1877) bound to sepharose beads and in vitro expressed 35S-labeled XAFl, we found the XAFl binding sites on $\mathrm{CBP}$ are located in the cysteine-histidine-rich $\mathrm{C} / \mathrm{Hl}$ domains (residues 1-1098). Plasmids encoding XAFl and HA-tagged CBP were transfected into SW1116 cells alone or in combination. Immunoprecipitation of over-expressed CBP protein with anti-CBP polyclonal antibody co-precipitated XAFl was mainly located in the nucleus. CBP was located exclusively in the nucleus. XAF1-RFP and CBP-HA co-localization were seen in the nucleus after immunofluorescence staining. Reporter assays demonstrated that co-transfection with pCDNA3-XAFl and pRc/RSV-CBP led to one fold reduction of APl luciferase activity stimulated by PMA, which was further enhanced by co-transfection of CBP's homolog pCMV-P300. Conclusions: Our results identified CBP as a novel binding partner for XAFl and indicated that CBP, as a coactivator, cooperated with XAFl-mediated inhibition of APl transcription.

\section{W1552}

Significance of Eras Expression in Gastric Cancer: Resistance to Anti-Cancer Agents

E.J. Kubota, Hiromi Kataoka, Makoto Sasaki, Satoshi Tanida, Tadayuki Oshima, Naotaka Ogasawara, Tomonori Yamada, Tsuneya Wada, Yoshinori Mori, Takaya Shimura, Fumitake Fujita, Kiyofumi Asai, Takashi Joh

Background and aims: Embryonic stem (ES) cells are pluripotent, immortalized cells with a rapid growth rate that makes them attractive sources for stem cell therapies. However, when ES cells are transplanted, they produce tumors (teratomas). ERas, a novel member of the ras family, was found to be expressed in ES cells only. ERas is constitutively active without any mutations and plays a crucial role when transplanted in the transformation of ES cells to teratomas and the expression level of ERas gradually decreases when ES cells are induced to differentiate. The aim of the current study is to investigate the expression and the roles of ERas, especially resistance to chemotherapeutic agents in human gastric cancer cells. Material and Method: 1. We assessed expression of ERas in human gastric cancer and gastric cancer cell lines, including MKN28, MKN45, MKN78, KATOIII, GCIY, AGS, HGC-27, NUGC-4 and ISt-1 by western blot. 2. To analyze the functions of ERas in gastric cancer, we established stable transfectans of GCIY overexpressing ERas with pCAGMyc-human ERas-IP vector, the expression plasmid for ERas. Activation of PI3k/Akt and MAPK pathway was analyzed. 3. To assess the cytotoxicity, cells were exposed to four anticancer agents commonly used for treatment of gastric cancer: CPT-11, 5-Fluorouracil, Cisplatin and Paclitaxel and cell survival was measured by MTS assay. To evaluate apoptosis induced by anti-cancer agents, we measured caspase-3 activity using Caspase-Glo3/7 assay. 4. We investigated the effect of Rapamycin, inhibitor of mTOR which is downstream of PI3k/Akt pathway, to the cell survival. Results: 1. ERas was expressed in human gastric cancer and all gastric cancer cell lines except for AGS. 2. Overexpression of ERas did not induce the phosphorylation of MEK. However, overexpression of ERas induced phosphorylation of Akt. 3. The ERas transfectants were significantly more resistant to CPT-11 than the parent lines (survival rate: control 23.0\%, the ERas transfectants $67.4 \%, \mathrm{p}<0.001$ ), but not to 5-Fluorouracil, Cisplatin, and Paclitaxel. Overexpression of ERas significantly reduced CPT-11-induced apoptosis ( $\mathrm{p}<0.01$ ). 4. Administration of Rapamycin significantly induced cytotoxicity to the ERas transfectants (survival rate: control 93.9\%, ERas transfectant $68.5 \%$, $p<0.01$ ). Conclusion: ERas was expressed in human gastric cancer and gastric cell lines, and plays crucial roles for anti-apoptosis via phosphorylation of Akt. Gastric cancer cells which express high level of ERas might be susceptible to Rapamycin and resistant to CPT-11.

\section{W1553}

Loss of Cooperative Function of Elf, a $\beta$-Spectrin, with SMAD3 Leads to Abnormal Localization and Transcriptional Activation Upon TGF- $\beta$ Response, Which Is Essential for Gastrointestinal Tumor Suppression Sang Soo Kim, Hye Jung Baek, Varalakshmi Katuri, Tapas Saha, M. Blair Marshall, Anton Sidawy, Bibhuti Mishra, Lopa Mishra

TGF- $\beta$ /Smads regulate a wide variety of biological responses through transcriptional regulation of target genes. ELF, a $\beta$-spectrin, plays a key role in the transmission of TGF- $\beta$ mediated transcriptional response through interactions with Smad3 and Smad4. ELF was originally identified as a key protein involved in endodermal stem/progenitor cells committed to foregut lineage. Also, as a major dynamic adaptor and scaffolding protein, ELF is important for the generation of functionally distinct membranes, protein sorting and the development of polarized differentiated epithelial cells. Disruption of elf results in the loss of Smad3/ Smad4 activation and, therefore, a disruption of the TGF- $\beta$ pathway. If heterozygous, the elf mice display dramatic tumors of the digestive tract with an exacerbated phenotope on a Smad4 heterozygous background. Aim: These observations led us to pursue the interactions between ELF and Smads especially Smad3 which is directly activated by TGF- $\beta$. Utilizing deletion fragments, confocal microscopy, and luciferase assays for TGF- $\beta$ transactivation, we mapped the regions of ELF responsible for interaction, localization, and transactivation upon TGF- $\beta$ stimulation. Methods and Results: First, we found that Smad3 but not Smad2 is a major interacting partner of ELF in COS-7 cells. Our further detailed analyses showed that N-terminal MADl domain of ELF displays a strongest interaction with of Smad3. The mid-portion of ELF containing consensus repeats and nuclear localization signal (NLS) domains also shows a moderate interaction but C-terminal MAD2 region does not. Smad3 $\mathrm{MH1}$ and MH2 domains, but not the linker region, are responsible for ELF interactions. However, one mid-portion of ELF displays a dramatic nuclear translocation and transactivation upon TGF- $\beta$ stimulation. Conclusions: Taken together, these findings show ELF is finely modulated upon TGF- $\beta$ response and through its N-terminal associates with Smad3, utilizing the middle fragment for nuclear translocation, TGF- $\beta$ targets gene activation through Smad3 interaction, localization, and transactivation. Therefore, loss of ELF, frequently found in human gastric and colon cancer, prevents the proper function of TGF- $\beta$ signaling pathway which is responsible for suppression of tumorigenesis. 
W1554

T-Darpp Suppresses Apoptosis Through Up-Regulation of BCL2 in Gastroesophageal Adenocarcinomas Abbes Belkhiri, Altaf Dar, Wael El-Rifai

We have recently shown mRNA and protein overexpression of both 32-kilodalton dopamine and cyclic adenosine 3',5'-monophosphate-regulated phosphoprotein (DARPP-32) and its novel truncated form t-DARPP in majority of gastroesophageal carcinomas (GECs). In addition, we have shown that these proteins provide anti-apoptotic role, which may explain the poor response of GECs to therapy. To further study the anti-apoptotic function of t-DARPP, AGS cells stably expressing t-DARPP or control cells expressing pcDNA3 empty vector were treated with acetyl ceramide $(10 \mu \mathrm{M}, 20 \mu \mathrm{M})$ for $24 \mathrm{~h}$. Immunoblot analysis on protein extracts showed that processing of caspase 9 did not occur in t-DARPP-expressing cells following treatment with $20 \mu \mathrm{M}$ ceramide. Under the same conditions, caspase 9 was almost fully processed in control cells, indicating initiation of apoptosis. Treatment of t-DARPP cells and control cells with $10 \mu \mathrm{M}$ ceramide was not sufficient to induce apoptosis as indicated by lack of caspase 9 activation. In addition, $\mathrm{Bcl} 2$ protein expression was confirmed in ceramide-treated and -untreated t-DARPP-expressing cells, whereas $\mathrm{Bcl} 2$ protein was not detected in control cells. Quantitative real time RT-PCR analysis indicated that mRNA level of Bcl2 was 3-fold higher in t-DARPP-expressing cells than control cells. The use of siRNADARPP-32/t-DARPP significantly abolished the expression of Bcl2 at mRNA and protein levels. As Bcl2 promoter contains a CREB response element (CRE), we investigated CREB/ ATF-1 protein levels and found significantly higher in t-DARPP-expressing cells compared with control cells. Thus, we proceeded with electrophoretic mobility shift assay (EMSA) using a CREB specific probe, and detected a stronger ATF-1/CREB/CRE binding in t-DARPPexpressing cells than control cells. Binding specificity was confirmed by supershift using ATF-1 antibody. Taken together, these results show that t-DARPP suppresses apoptosis as indicated by caspase 9, through up-regulation of Bcl2 as shown by EMSA, mRNA and protein levels. These results indicate that t-DARPP may be a novel gene with potential oncogenic properties. To verify this hypothesis, transformation studies are underway.

\section{W1555}

\section{Inhibition of Biliary Duct Cancer Cell Growth By RUNX3 Induction}

Kazunori Hasegawa, Shujiro Yazumi, Toshiharu Sakurai, Masaya Kida, Junichi Yamauchi, Chouhei Sakakura, Hiroshi Ida, Yoshiaki Ito, Tsutomu Chiba

Background and Aim: RUNX3, a Runt domain transcription factor involved in TGF- $\beta$ signaling, is a candidate tumor-suppressor gene localized in $1 \mathrm{p} 36$, a region commonly inactivated by deletion and methylation in a wide variety of human tumors. The aim of this study was to elucidate the function of RUNX3 in biliary tract cancer cell line. Materials and Methods: A biliary tract cancer cell line, Mz-ChA-2, which does not express RUNX3 but has intact TGF- $\beta$ receptorII and SMAD4, was stably transfected with RUNX3 expressing plasmid (pcDNA3.0/RUNX3). Mz-ChA-2 cells stably transfected with pcDNA3.0 were used as control. RUNX3 expression was confirmed by RT-PCR and western blotting. Results: Four Mz-ChA-2/RUNX3 clones and control cells (mock) were obtained. The expression levels of RUNX3 in clones 1, 2, and 3 were 3.5, 2.4, 1.1 times as much as that in clone 4 . To better characterize the biological features of RUNX3, both cells were assessed for TGF$\beta$-induced growth inhibition assay. Although TGF- $\beta$ slightly inhibited growth of control cells, the growth inhibition by TGF- $\beta$ was significantly enhanced in clones 1 and 2 transfected with RUNX3. Moreover, we analyzed the DNA contents of the clone 1 by flow cytometry. Flow cytometry demonstrated that TGF- $\beta$-induced Gl arrest was significantly enhanced in clone 1. In contrast, TGF- $\beta$-induced apoptosis was not demonstrated in both RUNX3transfected and control cells by flow cytometry or TUNEL assay. Western blotting analysis demonstrated that although TGF- $\beta$ slightly increased p 21 expression even in control cells, it was strongly enhanced in clone 1 with simultaneous decrease of cyclins Dl and E expressions. Nude mice zenograft models disclosed that tumorigenecity was significantly decreased in the Mz-ChA-2/RUNX3 clones with inverse proportion to the expression levels of RUNX3 as compared to control cells (mock). Conclusions: Our results showed that forced RUNX3 expression enhanced TGF- $\beta$-induced Gl arrest by up-regulation of p2l in biliary tract cancer cell line. Moreover, RUNX3 might not be always necessary for TGF- $\beta$ signaling.

\section{W1556}

EGFR Reactivates Telomerase in Immortalized Squamous Epithelial Cells Through PI3K/AKT-Mediated Phosphorylation and Transcriptional Regulation Via Hif-lalpha

Steffen Heeg, Michael Quante, Erdmann Scheffer, Alexander von Werder, Gitta Goessel, Michaela Doebele, Christine Fulda, Heike Kunert, Hiroshi Nakagawa, Roderick L. Beijersbergen, Hubert E. Blum, Oliver G. Opitz

Introduction: In our cellular model of oral-esophageal carcinogenesis immortalization was induced independent of the activation of telomerase but through the recombination based alternative lengthening of telomeres (ALT). In this stepwise model additional overexpression of EGFR in immortalized cyclin D1 overexpressing/p53 inactivated cells (OKF6 D1/del.p53) led to in vitro transformation and reactivation of telomerase. Therefore, we sought to identify the cellular pathways involved in the reactivation of telomerase and the in vitro transformation induced by EGFR. Methods: We overexpressed EGFR in immortalized OKF6 D1/del.p53 cells by retroviral mediated gene transfer. Expression levels and phosphorylation status of the PI3K/AKT and MAPK/ERK pathways as well as respective downstream targets were analyzed by western blot and RT-PCR. Protein-protein-interactions were demonstrated by co-immunoprecipitation. Cells were additionally treated with respective inhibitors and analyzed for growth characteristics and telomere biology. Furthermore, analysis of the hTert promoter including site-directed-mutagenesis was performed. Results: EGFR overexpression induced a robust telomerase activity in immortalized squamous epithelial cells. The level of AKT expression was unchanged compared to OKF6 D1/del.p53 cells. Nevertheless, AKT was phosphorylated at Ser 473 and Thr 308 indicating the activation of the PI3K/AKT pathway. In contrast, ERK 1/2 levels and phosphorylation status remained unchanged. IP western blot analysis showed a direct phosphorylation of hTert as well as Hif-lalpha by AKT. Analysis of the hTert promoter indicated an additional transcriptional regulation o telomerase reactivation depending on the presence of a Hif-lalpha binding site at the hTer core promoter. Corresponding to these findings, protein levels of Hif-lalpha were increased in EGFR overexpressing cells. In addition, p21 is also a target of phosphorylation by AKT Conclusion: We were able to show that EGFR reactivates telomerase in immortalized squamous epithelial cells mediated through the PI3K/AKT pathway. This reactivation is effected by two different mechanisms, namely direct phosphorylation of hTert by AKT a well as transcriptional regulation by Hif-lalpha as key transcription factor. Surprisingly, Hiflalpha is also phosphorylated by AKT and thereby stabilized. Phosphorylated p21 is known to be translocated to the cytosol and thus it is not able to accomplish its nuclear function. Whereas immortalization is induced by ALT, in vitro transformation is dependent on telomer ase reactivation, indicating an additional role of telomerase in tumor progression besides elongating telomeres.

\section{W1557}

CDX2 Expression Is Increased in Gastric Cancers with Less Invasiveness and Intestinal Mucin Phenotype

Gwang Ha Kim, Geun Am Song, Tae Oh Kim, Jeong Heo, Dae Hwan Kang Kang, Mong Cho, Seung Keun Park, Chul Soo Song, Hee Ug Park, Sang Hyun Kim

Objective. CDX2 is an intestinal transcription factor that might be involved in the regulation of proliferation and differentiation of intestinal epithelial cells. It is well known that both gastric and intestinal phenotypic cell markers are expressed in gastric cancers. The aims of this study were to analyze the CDX2 expression and its relationship with the patients clinicopathological characteristics and the mucin phenotypes and by performing immunohistochemistry. Material and methods. A total of 259 gastric cancers (122 early and 137 advanced cancers) were evaluated histologically and phenotypically. The CDX2 expression was assessed by immunohistochemistry. Results. Increased CDX2 expression correlated with a higher proportion of intestinal-type cancers of Lauren and early gastric cancers $(\mathrm{p}<0.001$ and $p<0.001$, respectively) and a lower proportion of perineural invasion and lymph node metastasis ( $p<0.001$ and $p=0.003$, respectively). Increased intestinal mucin (MUC-2, CD10) expression and decreased gastric mucin (MUC5AC) expression was associated with an increased CDX2 expression ( $\mathrm{p}<0.001, \mathrm{p}=0.045$ and $\mathrm{p}=0.004$, respectively). MUC6 expression was not associated with the CDX2 expression. There was a significantly increased CDX2 expression in the intestinal phenotype than the other phenotypes $(p<0.001)$. Conclusions. These results suggest that CDX2 might be a useful marker to predict the clinical outcome for patients with gastric cancers.

Correlations between the expression of CDX2 and the phenotype classification in the 259 gastric cancers

\begin{tabular}{|c|c|c|c|}
\hline & & CDX2 expression & \\
\hline Phenotype & Negative $(\mathrm{n}=109)$ & Positive $(\mathrm{n}=150)$ & $\mathrm{p}$ value \\
\hline Gastric type & 40 & 34 & \\
\hline Gastrointestinal type & 35 & 43 & 0.000 \\
\hline Intestinal type & 13 & 62 & \\
\hline Null type & 21 & 11 & \\
\hline
\end{tabular}

Correlations between the expression of CDX2 and the expression of MUC2, CD10, MUC5AC and MUC6 in the 259 gastric cancers

\begin{tabular}{|c|c|c|c|}
\hline & & CDX2 expression & \\
\hline Parameter & Negative $(\mathrm{n}=109)$ & Positive $(\mathrm{n}=150)$ & $\mathrm{p}$ value \\
\hline MUC2 expression & & & \\
\hline Negative & 71 & 55 & 0.000 \\
\hline Positive & 38 & 95 & \\
\hline CD10 expression & & & 0.045 \\
\hline Negative & 95 & 116 & \\
\hline Positive & 14 & 34 & 0.004 \\
\hline MUC5AC expression & & & \\
\hline Negative & 38 & 79 & 0.799 \\
\hline Positive & 71 & 71 & \\
\hline MUC6 expression & & & \\
\hline Negative & 90 & 122 & \\
\hline Positive & 19 & & \\
\hline
\end{tabular}

W1558

Simultaneous Silencing of Key Oncogenic Pathways - A Promising Novel Approach to Colon Cancer Treatment

Johannes Fruehauf, Shuanglin Xiang, Youxin Yang, Chiang J. Li

Background Carcinogenesis of colon cancer is a multistep process involving alterations in several defined oncogenes. Gains of function and losses of function result in the acquisition of a super-phenotypical cell -the colon cancer cell- which has clear environmental advantages over normal epithelial cells, including, among others, increased proliferation, reduced apoptosis, the ability to induce new blood vessels and ability to metastasize. Hypothesis We 
hypothesized that colon cancer cells require the activity of these oncogenes not only to develop into malignant cells in the first place, but also to be able to maintain their malignant phenotype. If this is true, knocking down some of these oncogenes would result in the loss of one or more of the super-abilities and might create a means of killing cells and treating colon cancer. Methods RNA-Interference (RNAi) was used to silence oncogenic k-Ras (codon 12 GGT/GTT) and activated beta-catenin in human colon cancer cells (SW 480) in vitro and in vivo. Hairpin-RNA expressing plasmids were used to obtain gene silencing. Silencing of either oncogenic pathway resulted in marked decrease of target mRNA and protein. $\mathrm{k}$ Ras down-regulation alone resulted in $46 \%$ reduction in cell number, while beta-catenin knockdown caused $40 \%$ reduction. Simultaneous silencing of both pathways resulted in 59\% inhibition. In nude mice ( $\mathrm{n}=24)$ carrying SW 480 human colon cancer xenografts, simultaneous silencing of both $\mathrm{k}$-Ras and beta-catenin over 4 weeks lead to a significant reduction in growth speed and final tumor sizes $(\mathrm{p}<0.05)$. Tumors in treated animals were found to have lowered $\mathrm{k}$-Ras as well as beta-catenin expression on both mRNA and protein levels. The fraction of proliferating cells was significantly decreased (PCNA stain). Conclusion We conclude that cancer cells require the continued activity of diverse oncogenes to be able to maintain their super-phenotype, at least regarding the aspect of increased proliferation. Both oncogenic (mutated) k-Ras as well as activated beta-catenin are promising treatment targets for RNAi based therapy and can be successfully silenced in vitro and in vivo. Simultaneous combination treatment of key oncogenic pathways increases the efficiency of oncogenic targeting and is likely to circumvent the possible development of resitance to this type of treatment.

\section{W1559}

\section{The Expression of P53, BCL-2, MGMT, P16, BAX, GSTP, KI-67 Protein in} Neoadjuvant Chemoradiotherapy Responsiveness for Rectal Cancer Jung Hoon Lee, Jong Ryul Eun, Tae Nyeun Kim, Byeong Ik Jang, Jun Hwan Kim, Sun Taek Choi, Heon Ju Lee

<Introduction> Neoadjuvant chemoradiotherapy for rectal cancer has been shown to reduce viable tumor size, to lower local recurrence rate, to increase disease-free survival. But some case does not responed to this therapy. Therefore selection of patient is more important because neoadjuvant chemoradiotherapy for non-responder make sometimes result in progression of disease. We aimed to identified the molecular predict factors in responder patients to neoadjuvant chemoradiotherapy in rectal cancer and to apply clinical usefulness of it. <Methods> Between January 2000 and july 2003, 70 patients with rectal cancer underwent neoadjuvant chemoradiotherapy and operation were selected. Colonoscopic findings and pathologic findings pre- and post- neoadjuvant chemoradiotherapy were reviewed, and we analyzed the expression of p53, bcl-2, MGMT (O6-methylguanine-DNA methyltransferase), p16, Bax, GSTP (Glutathione S -transferase pi), Ki-67 protein by immunohistochemistry. Responsiveness of therapy was divided into non-responder and responder group, the former is less than half of tumor cell necrosis or disappearence and the latter is more than half of tumor cell necrosis or disappearence, or no viable tumor cell. <Results> Mean age of total 70 Patients (male 45, female 25) was 58.9 (range 32 to 78). Non-responsive group was $17(24.3 \%)$ and responsive group was $53(75.7 \%)$ and there was no relationship between protein expression of 553 , bcl-2, MGMT, Bax, GSTP, Ki-67 and responsiveness to neoadjuvant chemoradiotherapy. But non-responsive group had higher pl6 positive stain rate than responsive group, $9(56.3 \%)$ and $14(28.6 \%)$, respectively $(\mathrm{p}=0.018)$. <Conclusions $>$ In neoadjuvant chemoradiotherapy for rectal cancer, testing of pl6 protein expression may help to predict the responsiveness of the therapy and further more clinical study is needed.

\section{W1560}

Mutational Analysis of Braf Gene in Colorectal Mucinous Carcinoma in Associated with Histological Configuration

Naoto Yoshitake, Shigehiko Fujii, Keiichi Tominaga, Kenichiro Mukawa, Kouichi Fukui, Hideyuki Hiraishi, Akira Terano, Takahiro Fujimori

Aim: Activation of MAP kinase cascade by K-ras mutation is associated with tumor progression in colorectal carcinoma. Furthermore, because K-ras mutation has been found frequently in colorectal carcinoma with polypoid growth, a relationship between K-ras mutation and histological configuration has been clarified. Whereas, the BRAF gene, one of raf family that is a intermediate element of the MAP kinase cascade, has been recently reported to be mutated in a variety of tumors, including colorectal carcinoma. However, nothing is known bout the frequency of BRAF mutation in colorectal mucinous carcinoma (MUC). In our study, we examined the incidence of the BRAF mutation, and reviewed its relationship to K-ras mutation and histological configuration. material and method: Mutations of BRAF and K-ras were evaluated in 43 colorectal MUC by direct DNA sequencing analysis. To determine the extent of microsatellite instability (MSI), five microsatellite markers were examined. Mismatch repair deficiency was assessed by immunohistochemistry for hMLH1. Tumors were classified into two different sub types as polypoid if the tumor was elevated above the level of the adjacent normal mucosa, but nonpolypod if the tumor was at the level or below the adjacent normal mucosa. Result Seventeen of $43(39.5 \%)$ MUC studied had either BRAF or K-ras codon 12 and codon 13 mutations. BRAF mutation was detected in 4 (9.3\%) MUC and was all located exon 15. K-ras mutation was detected in 13 (30.2\%) MUC, which was located $10(76.9 \%)$ of codon 12 and $3(23.1 \%)$ of codon 13 . No BRAF and K-ras mutations were identified simultaneously in the same tumor. MSI was detected in $5(11.4 \%)$ MUC. A higher incidence $(50 \%)$ of MSI in BRAF mutated tumors was found, compared with non BRAF mutated tumors $(p=0.075)$. As well, a higher incidence $(50 \%)$ of mismatch repair deficiency in BRAF mutated tumors was found $(p=0.039)$. Consequently, a significant lower incidence of MSI and mismatch repair deficiency in K-ras mutated tumors was found (p<0.001). Of the classifiable tumors, 20 of $43(46.5 \%)$ were polypoid, and 18 of $43(41.9 \%)$ were nonpolypoid. All of 4 MUC with BRAF mutation and 9 of 13 K-ras mutation were classified as polypoid type. B-RAF or K-ras mutated tumors were higher incidence of polypoid $\mathrm{p}=0.023$ ), compared with non BRAF and K-ras mutated tumors Conclusion: Our results show that BRAF gene are associated with mismatch repair deficiency and that activation of MAP kinase cascade plays a significant role in tumor progression of colorectal MUC. Furthermore, similar K-ras mutation, BRAF mutation would associate to the polypoid growth pattern.

\section{W1561}

Suppression for Colon Cancer Cell (HT-29) Proliferation By Dominan Negative Gene Transfer of Apoptosis Signal-Regulating Kinase (ASK)-1 Hikaru Kuwamura, Kazunari Tominaga, Masayuki Siota, Reiko Ashida, Takafumi Nakao, E.J. Sasaki, Toshio Watanabe, Yasuhiro Fujiwara, Nobuhide Oshitani, Kazuhide Higuchi, Hidenori Ichijo, Hiroshi Iwao, Tetsuo Arakawa

Background: Apoptosis signal-regulating kinase (ASK)-l is identified as a molecule which mediates pro-apoptotic signaling thereby inducing apoptosis for many types of cells. On the other hand, some reports indicated that ASK-1 induced the cellular differentiation and survival. These results suggest that ASK-1 had a broad range of biological activities depending on cell types and cellular context. In the present study, we investigated the role of ASK-1 in colorectal cancer cells. Materials \& Methods: We used the well-characterized colorectal cancer cell line: HT-29 in this study. Adenovirus vector carrying dominant-negative form of ASK-1 (AD-DN-ASK-1) was produced by PCR as previously reported method. Phosphorylation of ASK-1, c-Jun N-terminal kinase (JNK), and p38 mitogen-activated protein kinase (MAPK) was analyzed by Western blot analysis. On day 5 after the transfection of AD-DNASK-1 or AD-LacZ, a control vector, the viable cell number was counted by the hemocytometer using a lightmicroscope. On day 2 after the transfection of AD-DN-ASK-1 or ADLacZ, Hoechst staining was performed to evaluate the apoptotic cell number. Results: Selective phosphorylation of ASK-1 at Thr-845, a kinase domain cite, but not Ser 83 nor 967 cites was induced in HT-29 cells by the serum stimulation in a time-dependent fashion. Its phosphorylated levels reached to the maximum levels (about 3 fold) compared to the control levels by $30 \mathrm{~min}$. Using AD-LacZ, the accuracy of the transfection techniques into HT-29 cells was confirmed by the immunostaining. Gene transfer of AD-DN-ASK-1 inhibited the serum-induced phosphorylation of JNK and p38MAPK which are downstream molecules of ASK-1. Gene transfer of AD-DN-ASK-1 at a multiplicity of infection (MOI) of 30, 50 or 100 diminished the serum-induced proliferation in a dose-dependent fashion. The number of positive cells for Hoechst staining after the AD-DN-ASK-1 transfection was significantly higher than that of AD-LacZ-transfected cells. Conclusion: These results indicated that inactivation of ASK-1 may inhibit colorectal cancer cell proliferation mediated by the apoptotic induction. DN-ASK-1 gene transfer seems to be a new strategy for the treatment of colorectal cancer

\section{W1562}

Allelic Alterations in Ileal Well-Differentiated Neuroendocrine Tumors (Carcinoid Tumors) By Genome-Wide Single Nucleotide Polymorphism Analysis

Do H. Kim, Yasuhiko Nagano, Li Zhang, James C. Yao, Jill A. White, Asif Rashid

Background: Ileal well-differentiated neuroendocrine tumors (carcinoid tumors) are uncommon indolent neoplasms. The genetic alterations of these tumors are not well characterized. Chromosomal deletions and amplifications contribute to cancer pathogenesis. We used highdensity single nucleotide polymorphism (SNP) arrays to detect copy number alterations in thirteen ileal carcinoid tumors. Methods: Frozen tumor and non-neoplastic tissue were obtained from surgical specimens of patients undergoing resections for well-differentiated neuroendocrine tumors in the frozen section laboratory of the Department of Pathology, MD Anderson Cancer Center. In this study Xbal GeneChip Mapping 50K Assay Kit (Affymetrix, Inc., Santa Clara, CA) was used. Locus-specific hybridization intensity of genomic DNA from non-neoplastic and neoplastic tissue were compared. Results: The average genotyping call rate for the normal and tumor samples were over 90\% (normal 90.4\%, tumor 91.6\%) Alleleic imbalances were identified by copy number variation from the reference set and from the copy number of the matched non-neoplastic samples. The average copy numbers for all the non-neoplastic samples was $2.0 \pm 0.05$. In contrast, tumor samples with deletions of chromosome or chromosomal loci had average copy number of 1.5 or less, and with amplifications of chromosome or chromosomal loci had average copy number of 2.5 or more. We found both known and novel genomic amplifications and deletions. This was further confirmed by comparing the SNP allelotyping status and by loss of heterozygosity status of chromosomes 9p, 11q, 16q, and 18 reported previously. Loss of chromosome 18 was the most common aberration and present in $69 \%$ (9/13) of tumors, of chromosome $9 p$ and $16 \mathrm{q}$ in $23 \%$ (3/13) each, and chromosome $2 \mathrm{q}$ in $15 \%$ (2/13). Gain of chromosomes 4 and $17 \mathrm{q}$ was the most common amplification and present in $23 \%(3 / 13)$ of tumors each, of chromosomes 5, 14 and 20 in 15\% (2/13) of tumors each. Conclusions: Our study show that genome-wide allelotyping using SNP array is a powerful new tool for the analysis of allelic imbalance in ileal well-differentiated neuroendocrine tumors and should strengthen our ability to discover cancer-causing genes.

\section{W1563}

Progression of Barrett's Metaplasia to Adenocarcinoma Is Associated with the Expression of Unphosphorylated Retinoblastoma Gene Product (pRB)

Alexander Braun, Dietmar Stueker, Thomas Kratt, Alfred Koenigsrainer

Background: Barrett's esophagus (BE) is a premalignant lesion characterized by replacement of normal squamous epithelium with columnar epithelium. Derangements in cell cycle control and apoptosis regulation might be responsible for the progression from metaplasia to intraepithelial neoplasia and adenocarcinoma. This study was undertaken to examine the expression of retinoblastoma gene product (pRB) in the carcinogenesis of Barett adenocarcinoma. Methods: A total of 30 patients with adenocarcinoma $(n=20)$ and intraepithelial neoplasia $(\mathrm{n}=10)$ in BE were included. Finally, as control group, we selected 10 patients without intraepithelial neoplasia. Samples of invasive adenocarcinoma derived from 20 oesophagectomy specimens. Endoscopic mucosal resection (EMR) was used in 20 patients (82 samples) with ( 42 samples) and without ( 40 samples) intraepithelial neoplasia. Expression of the phosphorylated / un(der)phosphorylated retinoblastoma gene product (pRB) were measured by flow cytometry. Results: 85\% (17/20) of Barrett adenocarcinoma and 60\% (6/ 10) of intraepithelial neoplasia showed an expression of un(der)phosphorylated pRB whilst $20 \%$ (4/20) of Barrett adenocarcinoma and 10\% (1/10) of intraepithelial neoplasia showed 
the phosphorylated pRB. The un(der)phosphylated pRB was found in $5 \%(2 / 40)$ in BE without intraepithelial neoplasia whilst $65 \%$ (26/40) expression of phosphorylated pRB was detected. Conclusions: Expression of un(der)phosphorylated pRB are common events in Barrett adenocarcinoma. These data adress that un(der)phosphorylated pRB may be a novel biomarker for $\mathrm{BE}$ and that its expression may help to predict the prognosis of this pathology. In the future, the use of this procedure could play an important role in the evaluation of patients with $\mathrm{BE}$.

\section{W1564}

Intestinal Epithelial Cell Migration Is Regulated By SMAD5 in Mice Impaired for BMP Signaling

Jean-Francois Schmouth, Yannick D. Benoit, Benoit A. Auclair, Lieve Umans, An Zwijsen, Deborah L. Gumucio, Nathalie Perreaul

BACKGROUND: Bone morphogenetic proteins (BMPs) are members of the TGF-Beta superfamily that play crucial roles in embryogenesis, organogenesis and homeostasis in adult tissues. The interaction of BMP proteins with their receptors triggers a signalization cascade that is driven by R-Smad transcription factors (Smad 1, 5 and 8). Smad5 null mice die at mid-gestation due to multiple embryonic defects including a vestigial gut. Despite an increasing interest in BMP signaling in the biological processes and pathologies of the gut, very little is known of the specific roles played by individual Smads in gut morphogenesis and intestinal epithelial cell homeostasis. AIM: To investigate the specific function of Smad5 within BMP signaling during intestinal organogenesis and cellular function. METHODS AND RESULTS: With the use of the Cre/loxP system we have generated two distinct mouse lines having either Bmprla or Smad5 exclusively deleted in the gut epithelium. These deletions were generated by crossing floxed Bmprla or floxed Smad5 mice with the Villin-Cre line. Histological analysis, with $\mathrm{H} \& \mathrm{E}$ staining, revealed multiplication and elongation of the crypt compartment as well as an important lengthening of the villi in the Villin-Cre;Bmprla loxP/loxP line. However, only an increase in the length of the villi was observed in the VillinCre;Smad5 loxP/loxP mice when compared to control littermates. Proliferation assays with a 90-minute BrdU pulse showed a significant increase in the number of proliferating cells in the Bmprla conditional KO mice. No significant modifications in the number or localization of proliferating cells were noticed in the Smad5 conditional KO mice when compared to control littermates. Migration assays with a 48-hour BrdU pulse showed an increase in the epithelial cell migration rate along the crypt/villus axis in both mutant mouse models. Immunostaining with an E-cadherin specific antibody revealed a relocalization of E-cadherin mainly to the basal membrane in both Bmprla and Smad5 mutant mice whereas in control littermates E-cadherin was found at the Zonula Adherens. CONCLUSION: Altogether, these results demonstrate that Smad5 plays an important role in intestinal epithelial cell migration possibly due to its implication in E-cadherin localization. Because of some differences in the phenotype of the conditional $\mathrm{KO}$ of the upstream receptor Bmprla and the downstream signal mediator Smad5, this study suggests that the various R-Smads associated with BMP signaling might have distinct functions in the gut epithelium. Further analysis will allow us to delineate the differences between the BMP related R-Smads in the gut epithelium.

\section{W1565}

Regulation of $\beta$-Catenin/TCF Complex Transcriptional Activity By Tyrosine Phosphorylation/Dephosphorylation of $\beta$-Catenin in Intestinal Epithelial Cells: Role of SRC Tyrosine Kinase and SHP-1 Tyrosine Phosphatase Melanie Simoneau, Nathalie Rivard

$\beta$-catenin plays a dual role as a key effector in the regulation of adherens junctions and as a transcriptional coactivator of the Wnt pathway in intestinal epithelial cells. We have demonstrated that the phosphatase SHP-1 negatively regulates the nuclear transcriptional function of $\beta$-catenin (Duchesne et al. J.Biol.Chem. 2003). In addition, it has been demonstrated that phosphorylation of tyrosine-654 decreases its binding to E-cadherin but stimulates the association of $\beta$-catenin to the basal transcription factor TATA-binding protein. Herein, we have further investigated the role of tyrosine phosphorylation in the regulation of $\beta$-catenin stability and transcriptional activity in intestinal epithelial cells. Methods. Transfection and Western blotting were performed in human intestinal epithelial crypt cells HIEC, Caco-2/15 colon carcinoma and HEK293 cells. We constructed specific mutants of putative tyrosine phosphorylation sites (Y86F, Y654F) and transactivation potential on the TOPFLASH reporter and on c-myc and cyclin Dl promoters was assessed. The effects of the tyrosine phosphatase inhibitor, pervanadate $(0.1 \mathrm{mM})$, and ectopic expression of a constitutive active mutant of src (Y529F,caSrc) and SHP-1 were also analyzed on phosphorylation, stability and transcriptional activity of $\beta$-catenin. Results. 1) SHP-1 expression significantly decreased by $40-50 \%$ the transactivation potential of $\beta$-catenin on the TOPFLASH reporter and on cmyc and cyclin Dl gene expression in HIEC cells; by contrast, expression of caSrc enhanced by 10 -fold the transactivation potential of $\beta$-catenin on the TOPFLASH reporter in HIEC and Caco-2/15 cells and this effect was markedly reduced by co-expression of SHP-1. 2) Overexpression of SHP-1, but not the inactive form of SHP-1 (C453S), resulted in a strong decrease in tyrosine phosphorylation of $\beta$-catenin; by contrast expression of caSRC or treatment with pervanadate strongly increased tyrosine phosphorylation levels of $\beta$-catenin. 3) Ectopic expression of caSRC significantly enhanced $\beta$-catenin stability, increasing $\beta$ catenin protein levels, an effect abolished by co-expression of SHP-1. 4) Mutations of tyrosine-86 (Y86F) and tyrosine-654 (Y654F), two putative phosphorylation sites for c-src, significantly decreased by $50 \%$ the transactivation potential of $\beta$-catenin on the TOPFLASH reporter and on target gene expression (cyclin D and c-myc) in HIEC and Caco-2/15 cells. Conclusion. Taken together, our results indicate that the transcriptional activity of $\beta$-catenin/ TCF complex is controlled by tyrosine phosphorylation of $\beta$-catenin which in turn, may be determined by Src tyrosine kinase and SHP-1 tyrosine phosphatase activities.

\section{W1566}

Stabilization of the Cytoskeleton By Transfection of Phospho-Hsp27 Restores Caveolin-Mediated Physiological Signaling Cascade Responsible for Contraction in Aged Internal Anal Sphincter Sita Somara, Jeffrey R. Martens, Khalil N. Bitar

Background: Aging-related decline in signal transduction results in decreased contraction Decline in signal transduction may be due to an age-related deficiency in the docking of signaling molecules on the membrane. Objective: Is restoration of cytoskeletal stability and caveolae formation able to reinstate smooth muscle contraction in aged smooth muscle cells (SMC)? Methods: SMC were isolated from the Internal Anal Sphincter (IAS) of adult rats (12-18 mos) and aged rats (33 mos). Aged rats SMC were transfected with either phosphoHSP27 mutant or wild type cavl cDNA. Results: 1) Whole cell lysate of IAS SMC showed the presence of all three caveolin isoforms; cavl, cav2 and cav3; 2) Significant reduction in the levels of cavl was observed in the particulate fraction of aged IAS SMC $(48.24 \pm 0.06 \%)$ as compared to adult IAS SMC (100 $\pm 0.03 \%) ; 3)$ Immunoblotting of cavl immunoprecipitates showed reduced acetylcholine-induced association of cavl with PKC $\alpha$ and with HSP27 in aged IAS SMC (PKC $\alpha \cdot 107.40 \pm 1.09 \%$ at $30 \mathrm{sec}$ and $111.75 \pm 1.03 \%$ at $4 \mathrm{~min}$ and $\mathrm{HSP} 27: 109.87 \pm 2.33 \%$ at $30 \mathrm{sec}$ and $114.47 \pm 2.46 \%$ at $4 \mathrm{~min})$ as compared to adult IAS SMC (PKC $\alpha: 177.22 \pm 0.27 \%$ at $30 \mathrm{sec}$ and $179.74 \pm 0.81 \%$ at $4 \mathrm{~min}$ and HSP27: $161.67 \pm$ $1.48 \%$ at $30 \mathrm{sec}$ and $170.30 \pm 5.91 \%$ at $4 \mathrm{~min}) ; 4$ ) Transfection of aged IAS SMC with phospho-HSP27 restored: a) cavl levels in the particulate fraction of aged IAS SMC (74.27 $\pm 0.02 \%)$; b) acetylcholine-induced association of cavl with PKC $\alpha(157.68 \pm 4.23 \%$ a $30 \mathrm{sec}$ and $159.46 \pm 4.12 \%$ at $4 \mathrm{~min}$ ) and c) acetylcholine-induced association of cavl with HSP27 $(154.39 \pm 1.12 \%$ at $30 \mathrm{sec}$ and $164.37 \pm 5.93 \%$ at $4 \mathrm{~min}) .5)$ Transfection of aged IAS SMC with cav-1 restored: a) acetylcholine-induced association of cavl with PKCo $(143.57 \pm 4.62 \%$ at $30 \mathrm{sec}$ and $148.07 \pm 4.72 \%$ at $4 \mathrm{~min})$ and b) acetylcholine-induced association of cavl with HSP27 (136.69 $\pm 0.96 \%$ at $30 \mathrm{sec}$ and $146.77 \pm 0.99 \%$ at $4 \mathrm{~min})$ Summary: Aged IAS SMC showed reduced cavl levels resulting in reduced acetylcholineinduced association of cavl with PKC $\alpha$ and with HSP27 in the particulate fraction. Transfection of aged IAS SMC with either phospho-HSP27 or with wild type cavl restored acetylcholine-induced association of cavl with PKC $\alpha$ and with HSP27 in the particulate fraction. Conclusions: Introduction of phospho-HSP27 into aged IAS SMC may restore cytoskeletal stability. A stable cytoskeleton or introduction of wild type cavl into aged IAS SMC may restore caveolae formation by maintaining cavl on the membrane, thus reinstating the physiological signaling cascade responsible for contraction in aged IAS SMC.

\section{W1567}

Differential Regulation of Pyk2 Phosphorylation At Tyr-402 and Tyr-580 in Intestinal Epithelial Cells: Roles of Calcium, SRC, Rho Kinase, and the Cytoskeleton

Steven S. Wu, Enrique Rozengurt

The calcium-dependent proline-rich tyrosine kinase Pyk 2 is activated by tyrosine phosphorylation, associable with focal adhesions, and has been linked to proliferative and migratory responses in gastrointestinal and other cell types. Pyk2 phosphorylation occurs at functionally distinct sites: Tyrosine 402 (Tyr-402) is the target for autophosphorylation, the initial event in activation, while phosphorylation of Tyr-580 within the kinase domain further enhances enzymatic activity. These activation events are thought to be tightly coupled in a sequential manner, as in the closely homologous focal adhesion kinase (FAK), though the mechanisms involved are unclear. The aim of this study was to examine the pathways mediating Pyk2 auto- and transphosphorylation in intestinal epithelial cells. Methods: Pyk2 phosphorylation at specific tyrosine sites was measured by immunoprecipitation and Western blotting with phosphospecific antibodies. IEC-18 cells were stimulated with the $G$ protein-coupled receptor (GPCR) agonists angiotensin II (Ang II) or lysophosphatidic acid (LPA), or with the $\mathrm{Ca}^{2+}$ ionophore ionomycin; selected cells were also preincubated with inhibitors of PKC, Src, Rho kinase, actin stress fibers, or $\mathrm{Ca}^{2+}$ signaling. Results: Both Tyr-402 and Tyr-580 were rapidly and transiently phosphorylated following cell stimulation by Ang II or LPA, mirroring the rapid peak ( $1 \mathrm{~min})$ of total Pyk2 tyrosine phosphorylation. However, at Tyr-402 these agonists also induced a delayed (30-60 $\mathrm{min}$ ) secondary phosphorylation peak, with no corresponding rise at Tyr-580. Other evidence showed differential phosphorylation of these two sites: Only Tyr-580 was affected by either $\mathrm{Ca}^{2+}$ inhibition (with thapsigargin+EGTA) or by $\mathrm{Ca}^{2+}$ release (via ionomycin). Conversely, Tyr-402 phosphorylation was primarily sensitive to inhibition of actin stress fibers (by cytochalasin D) or of Rho kinase, an upstream regulator of stress fiber assembly. Neither Tyr-402 nor Tyr-580 was sensitive to the Src inhibitor PP2. Conclusion: These results suggest that Pyk2 autophosphorylation is stress fiber-dependent, while transphosphorylation within the kinase domain requires $\mathrm{Ca}^{2+}$, and is Src-independent in intestinal epithelial cells. This better defines the mechanisms involved in Pyk2 activation, and contrasts with the sequentially coupled model of phosphorylation that occurs in FAK, further underlining the differences between these closely related kinases in the intestinal epithelium.

\section{W1568}

Sensitization of Human Intestinal Microvascular Endothelial Cells (HIMEC) to Ionizing Radiation By Curcumin

Aaron Rogaczewski, Pamela J. Kexel, Monica E. Theriot, Victoria M. Nelson, Mary F Otterson, David G. Binion, Parvaneh Rafiee

INTRODUCTION: Advanced colorectal adenocarcinoma is frequently treated with radiation therapy. Research into the anti-neoplastic mechanisms of external beam ionizing radiation has focused primarily on direct effects against tumor cells. Recent investigation suggests that the therapeutic effect of radiation may also involve the destruction of microvessels supplying blood and $\mathrm{O} 2$ to tumors. This possibility has prompted new investigation into the effects of ionizing radiation on endothelial cells. The aim of this study is to investigate the radiosensitizing effects of curcumin, a promising anti-inflammatory agent, on primary cultures of HIMECs compared to HIMECs that were irradiated alone. Curcumin is a spice which

. 
exhibits growth inhibitory effects on a broad range of tumors. METHODS: Serum-deprived HIMEC cultures (passage $8-12$ ) with and without curcumin were exposed to 2-10 Gy ionizing radiation and incubated for $1-48 \mathrm{~h}$. We tested the effect of a combination of curcumin and radiation on cell survival, PI3K/Akt proteins, MAPK, NFKB activation, induction of apoptosis and COX-2 expression. Analyses were performed using RT-PCR, Western blotting, in vitro Kinase activity, and Tunnel assay. RESULTS: Curcumin in combination with radiation significantly abolished PI3k/Akt activities and enhanced HIMEC apoptosis. Ionizing radiation alone induced HIMEC growth which was associated with PI3K/Akt activation and JNK activity. Ionizing radiation also induced NFKB transcription factor activation in HIMEC, resulting in increased expression of bcl-2 protein. Curcumin treatment of HIMEC combined with radiation abolished JNK activity and inhibited NFKB activity, resulting in down-regulation of bcl-2 protein and enhanced radio-sensitization. COX-2 mRNA and protein were upregulated in irradiated HIMEC in a dose and time-dependent manner. Curcumin treatment of HIMEC abolished COX-2 mRNA and protein expressions in irradiated HIMEC. CONCLUSIONS: The combination of radiation and curcumin abolished PI3k/Akt activities and enhanced apoptosis in HIMEC. This data suggests that curcumin sensitizes HIMEC by causing the radiation-induced pro-survival gene expression. Curcumin inhibited COX-2 via suppression of JNK and NFKB in irradiated HIMEC. We concluded that curcumin sensitizes HIMEC by modulating the mitogenic, anti-proliferative and apoptotic pathways. Thus curcumin may have potential as a new radiosensitizing strategies in radiation therapy.

W1569

Increased Arginase II and Decreased NO Synthesis in Human Microvascular Endothelial Cells (HIMEC) of IBD Patients

Scott Horowitz, Aaron Rogaczewski, Victoria Nelson, Zelmira Lazarova, Christopher Andrekopouis, Balaraman Kalyanaraman, Mary F. Otterson, David G. Binion, Parvaneh Rafiee

Introduction: Inflammatory bowel disease (IBD) is characterized by microvascular dysfunction in areas of chronically inflamed bowel. IBD microvascular dysfunction is linked to impaired endothelial generation of nitric oxide (NO), normally a homeostatic mechanism in vascular physiology. Mechanisms underlying diminished microvascular endothelial generation of NO in IBD are not fully defined. The arginase enzymes (ARI, ARII) deplete L-arginine levels in the intracellular environment, preventing NOS isoforms from accessing the essential substrate for NO generation. We hypothesized that arginase expression is increased in chronically inflamed IBD microvessels and their endothelial lining, which contributes to decreased NO production in the chronically inflamed gut microvasculature. Methods: Freshly resected human gut tissues and HIMEC cultures (passage 8 - 12) from control and IBD pts were used for all studies. L-arginine (L-Arg) acted as substrate for both NO synthase (NOS isoforms) and arginase (ARI, ARII), producing either NO or urea respectively. AR and NOS was assessed in both HIMEC and gut tissues by immunostaining, RT-PCR, western blotting and enzymatic assays. Results: TNF- $\alpha$ /LPS increased ARII mRNA and protein in HIMEC. The arginase inhibitor L-Val $(30 \mathrm{mM})$ decreased urea and increased NO production in both control and TNF- $\alpha /$ LPS activated HIMEC, while addition of excess substrate $(10 \mathrm{mM} \mathrm{L}$ Arg) increased both urea and NO production. Combination of both excess substrate (L$\mathrm{Arg}$ ) and AR inhibitor (L-Val) decreased urea production compared with L-Arg alone and increased NO production compared with L-Val alone. These data suggest that competition for intracellular L-Arg by AR may be involved in the regulation of NOS activity in control HIMEC and in response to TNF- $\alpha /$ LPS treatment. Finally, immunostaining of freshly resected control and IBD tissues revealed marked increase in perivascular arginase protein expression in IBD tissues. Discussion: Loss of NO production in IBD microvessels is linked to enhanced levels of AR in endothelial cells exposed to chronic inflammation in vivo. The enhanced expression of AR in passaged HIMEC, which are removed from the chronic inflammatory environment suggests acquired pattern of arginase overexpression in response to TNF- $\alpha /$ LPS. These data suggest that increased arginase expression is an epigenetic alteration in endothelial gene expression in human endothelial cells exposed to chronic inflammation in IBD.

\section{W1570}

Comparison of Kinase Profiles in Barrett's Esophagus, Normal Squamous Esophagus and Normal Gastric Cardia Proves That Barrett's Esophagus Has a High Glycolytic Activity

Jantine van Baal, Sander H. Diks, Agnieszka M. Rygiel, Francesca Milano, Jacques J. Bergman, Ronald J. Wanders, Maikel P. Peppelenbosch, Kausilia K. Krishnadath

BACKGROUND: Barrett's esophagus (BE) is the metaplastic process in which the normal squamous epithelium (SQ) is replaced by columnar lined epithelium. To gain more insight into the cellular mechanisms in the development of $\mathrm{BE}$, the aim of this study was to create a comprehensive description of cellular kinase activity occurring in BE, SQ and gastric cardia (GC). MATERIAL AND METHODS: Peptide arrays exhibiting 1176 specific consensus sequences for protein kinases were used to produce a global analysis and comparison of cellular kinase activity of BE, SQ and GC. Differences in kinase activity were validated by conventional technology in tissue samples of $27 \mathrm{BE}$ patients. RESULTS: Three unique kinome profiles were compared. Validation by immunoblotting showed that in BE the MAPK signaling is decreased probably due to increased inhibition of c-Raf through Rab. One of the most prominent differences between $\mathrm{BE}$ and $\mathrm{SQ}$ was the significant decreased phosphorylation of Src consensus substrates. Furthermore, we found that EGF receptor activity is decreased in $\mathrm{BE}$ compared to SQ, but its activity is increased compared to GC. In addition kinome analysis demonstrated that glycolysis is an important process in $\mathrm{BE}$ as validated by pyruvate kinase activity confirming that this activity is significantly up-regulated in BE compared to SQ and GC (see Figure below). CONCLUSIONS: Using a peptide array we demonstrated that BE has several specific kinase activities when compared to the kinome profiles of SQ and GC. This study contributes to a better understanding of kinase activities, and several signal transduction pathways and cellular functions that are associated with BE. Future manipulations of these pathways will help us to treat BE and prevent the associated esophageal adenocarcinoma

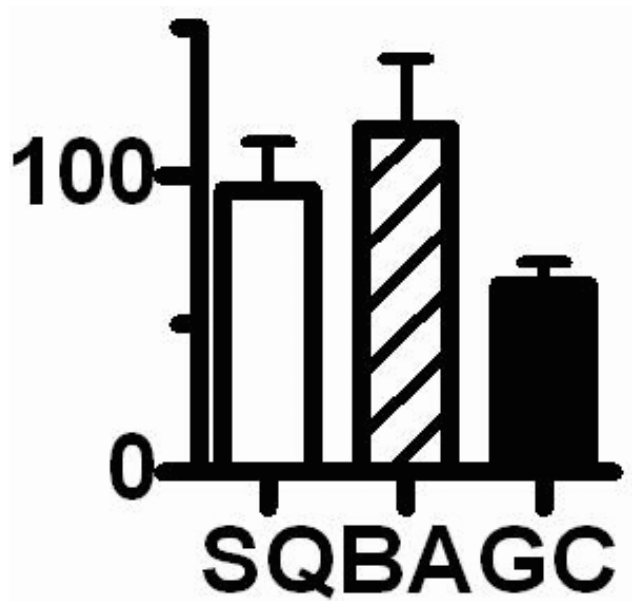

W1571

Myosin Vi Regulates Surface Expression of CFTR and Its Endocytosis from the Apical Plasma Membrane in the Intestine in Vivo Nadia Ameen, Franca G. Bisello, Hannes Kohnke, Gerard Apodaca

The cystic fibrosis transmembrane conductance regulator (CFTR) is present in organelles that participate in clathrin-mediated endocytosis in the intestine, but the physiologic mechanisms regulating its endocytosis are not understood. Myosin VI is a unique inwardly directed actin motor that regulates apical endocytosis in polarized cells and its distribution overlaps that of CFTR in the small intestine. We hypothesized that Myosin VI would regulate surface expression of CFTR, its endocytosis from the apical plasma membrane and function in the small intestine Methods: Intestinal tissues from Myosin VI -/- mice were examined by electron microscopy. CFTR distribution in the intestine was examined by immunofluorescence confocal microscopy and surface biotinylation. CFTR endocytosis was determined following surface biotinylation in vivo Results: Scanning and transmission electron microscopy revealed disordered and shorter microvilli in Myosin VI -/- mice compared to littermate controls ( $.82 \mu \mathrm{m}$ vs. $1.18 \mu \mathrm{m}, \mathrm{n}=14$ cells, $\mathrm{p}<0.005$ ). Quantification of CFTR fluorescence in immunolabeled sections revealed a two-fold increase in apical CFTR. Surface biotinylation confirmed a 3-fold increase of CFTR cell surface expression in Myosin VI-/- intestine and endocytosis of CFTR was reduced by $50 \%$ in Myosin VI -/- mice. Conclusions: Myosin VI regulates surface expression of CFTR and its endocytosis from the plasma membrane in the native small intestine. Functional studies of CFTR anion transport in Myosin VI-/- intestine will determine the physiologic relevance of these novel observations.

\section{W1572}

Involvement of the Apelin-Apj System in Intestinal Epithelial Proliferation During Developmen

Guiyun Wang, Song Han, Xiang Qi, Ella W. Englander, George H. Greeley

Apelin is the endogenous ligand for the APJ receptor. Apelin and APJ are expressed in the GI tract and mammary gland. In the rat, breast apelin expression increases $\sim 7$ - to 20-fold during pregnancy and lactation. In breast milk and stomach lumen of nursing rats, apelin peptide levels are exceptionally high $(300-600 \mathrm{ng} / \mathrm{ml})$ suggesting that luminal apelin plays a role in regulation of GI development. The aim of these studies was to define expression levels of apelin and APJ receptor in the developing rodent gut. Additionally, the effect of exogenous apelin on GI epithelial proliferation was tested. Methods. Apelin and APJ mRNA levels in the developing mouse and rat GI tracts were measured by real time RT-PCR. Enteric apelin peptide levels were examined by immunohistochemistry (IHC). The effect of apelin ( $50 \mu \mathrm{g}, 3 \mathrm{x} /$ day for 3 days, SC) on stomach, duodenal and colonic epithelial proliferation was assessed in mice by giving BrdU $(290 \mathrm{mg} / \mathrm{kg}) 90 \mathrm{~min}$ before sacrifice. BrdU incorporation was examined by IHC. Results. Apelin mRNA levels in the rat and mouse stomach, duodenum and colon were highest at birth and declined after weaning into adulthood (see Table, mouse colon values shown only). IHC showed that enteric apelin peptide levels were low perinatally and increased only after weaning APJ mRNA levels in the gut were highest at birth and decreased dramatically before weaning into adulthood. Apelin administration decreased the colonic proliferation rate by $50 \%$ (control BrdU cells: $149 \pm 23$ vs apelin treated BrdU cells $67 \pm 16 /$ field; $\mathrm{N}=5$ mice/group, $\mathrm{P}<0.05$ ); the stomach and duodenum were unaffected. Discussion and Conclusions. Ingestion of apelin in breast milk suggests a role for lumenal apelin in regulation of the GI tract during development. Despite high enteric apelin expression during development, marginal amounts of apelin peptide are produced in the perinatal gut indicating a dependence upon milk-borne apelin. The importance of luminal apelin and the GI apelin-APJ system is reinforced by the high APJ expression levels in the gut at birth and the perinatal period. The finding that apelin treatment lowers colonic, but not small intestine and stomach epithelial proliferation indicates apelin exerts a selective modulatory role on colonic epithelial proliferation during developmen

\begin{tabular}{|c|c|c|c|c|}
\hline Colon & Fetal & $6 \mathrm{~d}$ pup & $16 \mathrm{~d}$ pup & Adult \\
\hline Apelin mRNA 18S mRNA & $1.1 \pm 0.1^{1}$ & $1.4 \pm 0.06$ & $1.1 \pm 0.1$ & $0.25 \pm 0.02^{*}$ \\
\hline APJ mRNA 18S mRNA & $0.91 \pm 0.09$ & $0.71 \pm 0.03^{*}$ & $0.4 \pm 0.2^{*}$, & $0.19 \pm 0.01^{*,}$ \\
\hline
\end{tabular}

${ }^{*}=\mathrm{P}<0.05$ vs fetal, ${ }^{\dagger}=\mathrm{P}<0.05$ vs fetal, $6 \mathrm{~d}$ pup $\mathrm{l}=$ mean $\pm \mathrm{SEM}, \mathrm{N}=5$ mice/group 
W1573

Novel Mechanisms of Beta-Catenin Regulation By Tumor Suppressor APC Jun Yang, Wen Zhang, Paul M. Evans, Chunming Liu

Background: The majority of colorectal cancers have mutations of the APC (adenomatous polyposis coli) gene or beta-catenin gene. These mutations result in abnormal beta-catenin accumulation and activation of beta-catenin target genes which leads ultimately to cancer. In normal cells, beta-catenin degradation is initiated by serine/threonine phosphorylation at its amino terminal region. Our previous studies have shown that, without Wnt signaling, glycogen synthase kinase-3 (GSK-3), casein kinase I alpha (CKIalpha) in complex with the scaffold protein Axin, carries out beta-catenin phosphorylation. Moreover, we have shown that ubiquitin ligase beta-Trcp recruits the phosphorylated beta-catenin for ubiquitin/proteasome degradation. beta-catenin with serine/threonine mutations escapes CKIalpha or GSK-3 phosphorylation and beta-Trcp recognition, resulting in constitutive beta-catenin activation that leads to cancer. APC is required for beta-catenin degradation, however, the molecular mechanisms regulating APC mediated beta-catenin degradation are not known. Method: Colon cancer cell lines, HT29, SW480, DLD-1 and HCT116 were used for this study beta-catenin phosphorylation was analyzed with phospho-specific antibodies that recognize GSK3 or CKlalpha phosphorylation sites of beta-catenin. beta-catenin ubiquitin/ degradation was analyzed by treating the cell with proteasome inhibitor, MG132. APC fragments were delivered to cells by adenovirus. Results: APC has two types of beta-catenin binding domains, $15 \mathrm{aa}$ repeats and 20aa repeats. APC also has Axin binding domains. It was suggested that APC is involved in the Axin complex and regulates beta-catenin phosphorylation. Surprisingly, we found that the APC mutations do not prevent beta-catenin phosphorylation in colon cancer cells. We also found that, in the colon cancer cell line SW480, APC truncations block beta-catenin ubiquitination regardless of beta-catenin phosphorylation. After introducing functional APC by adenovirus, we found that the beta-catenin ubiquitin/degradation was rescued in SW480 cells, suggesting that APC regulates betacatenin ubiquitin/degradation in addition beta-catenin phosphorylation. The functions of different APC domains were analyzed in this study. We found that at least three 20aa repeats of APC were required for beta-catenin ubiquitin/degradation. The effects of APC on betacatenin subcellular localization were also analyzed. We hypothesize that APC regulates betacatenin phosphorylation and ubiquitination by distinct domains and by separate molecular mechanisms.

\section{W1574}

Induction of Trail Expression By the PKC $\theta / \mathrm{PKD} / \mathrm{NFAT}$ Pathway in Human Intestinal Cells

Qingding Wang, Yuning Zhou, Suimin Qiu, B. Mark Evers

TNF-related apoptosis-inducing ligand (TRAIL; Apo2) has been shown to promote intestinal cell differentiation. Recently, we found that PKCO (a novel PKC isoform), PKD (serine/ threonine kinase structurally distinct from the PKC proteins), and the nuclear factor of activated T cells (NFAT) are involved in the regulation of intestinal cell differentiation. The purpose of this study was to determine the upstream regulation of TRAIL associated with intestinal differentiation. METHODS. (i) The human colon cancer cell lines HT29, SW480 and Caco- 2 were used. Cells were treated with interferon- $\gamma$ (IFN- $\gamma$ ), which induces TRAIL expression, in the presence or absence of cyclosporine A (CSA), which inhibits NFAT signaling through inhibition of calcineurin; TRAIL expression was assayed by Western blotting. To further determine the role of NFAT, cells were transfected with the NFAT reporter construct, NFAT siRNA or constructs overexpressing NFAT. (ii) To determine the possible role of $\mathrm{PKC} \theta$ or PKD, cells were co-transfected with the control vector or the plasmid overexpressing the active form of PKC $\theta$ or PKD, in combination with a $1.3 \mathrm{~kb}$ TRAIL promoter construct. TRAIL promoter activity was determined by luciferase assays using a dual reporter assay system; transfection efficiency was controlled by Renilla luciferase. (iii) Expression of PKD (total and phosphorylated) and TRAIL was analyzed in human colon mucosa by immunohistochemistry. RESULTS. (i) Inhibition of NFAT by CsA or transfection with NFAT siRNA attenuated IFN $\gamma$-induced TRAIL expression in HT29 and SW480 cells, whereas overexpression of NFAT increased TRAIL promoter activity in Caco-2 cells. Consistent with these results, five putative NFAT binding sites were identified in the proximal TRAIL promoter. (ii) Overexpression of active PKC $\theta$ increased TRAIL promoter activity as well as NFAT reporter activity. Activation of PKD by PKCO was noted by increased PKD phosphorylation in cells overexpressing PKCO. Moreover, overexpression of active PKD increased NFAT reporter activity as well as TRAIL promoter activity and protein expression. (iii) TRAIL, total PKD and phospho-PKD demonstrated similar expression patterns with localization to the upper crypts (ie, most differentiated region) of the colonic mucosa. CONCLUSIONS. Our results are the first to identify TRAIL as a downstream target of the PKCO/PKD/NFAT pathway. Importantly, the PKC $/$ /PKD/NFAT signaling pathway may regulate intestinal cell differentiation through the regulation of TRAIL expression.

\section{W1575}

Transforming Growth Factor-Beta Signaling May Play a Protective in Preventing Liver from Insulin Induced Steatosis By Regulating Insulin Driven AKT Inactivation of Forkhead Transcriptional Factor Proteins

Krit Kitisin, Tang Yi, Varalakshmi Katuri, Nady Golestneh, Wilma Jogunoori, Kirti Shetty, Lopa Mishra, Lynt B. Johnson

Non-alcoholic fatty liver disease (NAFLD) represents a progression of liver fibrosis, liver injury, and possibly development of hepatocellular carcinoma. Disruption of the transforming growth factor-beta (TGF-beta) signaling cascade, which plays a critical role in hepatic homeostasis and normal liver development, might also contribute to initiation of hepatic steatosis. Precise modulation of TGF-beta through Smad activation by the type I receptors is dependent upon adaptor proteins such as ELF, a beta-spectrin. Normal biliary epithelial development is absent in the elf-/-and in the Smad2+/-/3+/- mutant embryos. Both elf-/and $S$ mad $2+/ / 3+/-$ mice die at mid-gestation from defective liver development. Mice with elf + - mutant are viable but as many as 35\% (7/20) develop hepatocellular carcinoma (HCC) with significant centrilobular steatosis. Aside from the TGF-beta signaling pathway, forkhead transcriptional proteins, particularly FOXA2 and FOXO1, are essential in gluconeogenesi and fatty acid oxidation during starvation. Mice with either FOXA2 or FOXO1 knockou develop insulin-resistance and diabetes. Aims: 1 . To investigate a possible cross-talk between TGF-beta signaling and forkhead protein regulation under PI3 kinase signaling. Methods and Results: Embryonic liver tissues isolated from normal (wt), elf-/- and Smad2+/-/3+/mutants were isolated and cultured in a large range of growth factor combinations and conditions to determine their growth properties. 1. Explants from Smad2+///3+/- mutan embryo treated with insulin demonstrated a dramatic increase in steatosis. 2. Western blot analysis of cell lines derived from elf $+/$ - and elf $+/ / / S \operatorname{mad} 4+/$ - mutants revealed significan increases in p-AKT level compared to the wild type cell line. 3. p-AKT levels in these mutan cell lines decrease with restoration of EIF protein. 4 . Immunohistochemical labeling of hepatocellular cancer taken from elf $+/$ - mice revealed increased cytoplasmic labeling of FOXO protein. Conclusions: In Smad2 $+/-/ 3+/-$ and elf $+/-$ mutant embryo explants, the addition of insulin resulted in severe steatosis. The mechanisms of these findings could be secondary to the alteration in forkhead protein transcrintional factors level whereby forkhead proteins are sequestered by Insulin driven Akt, and are unable to partner with Smads and ELF to modulate expression of forkhead target genes. More importantly, in adult tissues these derangements may promote fibrosis, steatosis and hepatocarcinogenesis.

\section{W1576}

Histamine Stimulates EGF Receptor Ligand Production from Gastric Parietal Cells

E.J. Nakamura, Ryo Hatazawa, Takuya Endo, Kikuko Amagase, Hideki Taniguchi, Susumu Okabe, Koji Takeuchi

Background \& Aims: We previously reported that both $\mathrm{H}_{2}$ receptor-knockout $\left(\mathrm{H}_{2} \mathrm{R}-\mathrm{KO}\right)$ mice and histidine decarboxylase-knockout (HDC-KO) mice exhibit marked gastric mucosal hyperplasia due to hypergastrinemia. However, the degree of hyperplastic changes in HDC$\mathrm{KO}$ mice was much less than that of $\mathrm{H}_{2} \mathrm{R}-\mathrm{KO}$ mice, and there was an apparent difference in the levels of gastric mucosal histamine contents between these two groups. In the process to clarify the mechanisms underlying such different phenotypes between $\mathrm{H}_{2} \mathrm{R}-\mathrm{KO}$ and $\mathrm{HDC}$ $\mathrm{KO}$ mice, we found that the marked gastric hyperplastic changes were induced by not only histamine overexpressed by hypergastrinemia but also chronically administered histamine mediated via EGF receptor pathway in vivo. Thus, in the present study, we further performed the more detailed analyses to characterize the source cells of EGF receptor ligand(s) stimulated by histamine in the gastric mucosa. Methods: Immunohistochemical analyses to detect TGF$\alpha$ amphiregulin and HB-EGF as well as betacellulin were performed in the gastric mucosa of C57/BL6J mice treated with histamine (10 mg/kg, subcutaneously) or vehicle (saline). In addition, to exclude the involvement of the systemic factor, the isolated gastric mucosae incubated with or without histamine was also examined immunohistochemically. Cell characterization was performed for parietal cells (anti- $\mathrm{H}^{+}, \mathrm{K}^{+}$-ATPase), enterochromaffin-like cells (ECL; anti-HDC), chief cells (anti-pepsinogen), G cells (anti-gastrin) and D cells (antisomatostatin). Western blotting was used for the quantitative analysis for TGF- $\alpha$ and amphiregulin expression. Results: The expression of amphiregulin and TGF- $\alpha$ proteins was observed relatively potently in the parietal cells and weak in the surface cells in vehicle-treated mice. Exogenous histamine increased the expression of amphiregulin in a time-dependent manner Famotidine, an histamine $\mathrm{H}_{2}$-receptor antagonist, had no effect on the stimulatory effect o histamine on the expression of amphiregulin in the parietal cells. Conclusion: These results indicate that histamine stimulates the expression of EGF receptor ligand in the parietal cells and this action may be independent of the activation of histamine $\mathrm{H}_{2}$-receptors

\section{W1577}

The BRK Tyrosine Kinase Confers Resistance to Dextran Sodium Sulfate Induced Colitis

Katherine Jelinek, Wenjun Bie, Andrea Haegebarth, Angela L. Tyner

Brk (also called Sik or Ptk6) is an intracellular epithelial specific tyrosine kinase expressed in normal epithelial linings of the gastrointestinal tract. Previously it was reported that Brk expression is increased in colon tumors. Since chronic inflammation and cancer are often linked in the colon, we investigated functions of Brk in a murine colitis model. We subjected wild-type and Brk-/- mice to 3\% dextran sodium sulfate (DSS) in drinking water ad libitum for 5 days, followed by a recovery period with water for 3 days. Mice were closely monitored for weight loss and rectal bleeding. At 5 or 8 days post initiation of DSS treatment, mice were sacrificed and colons were isolated. Colon sections were stained with hematoxylin and eosin and evaluated by light microscopy. Brk-deficient colons had more severe and extensive denudation of the surface epithelium and goblet cell depletion than wild-type control mice. Furthermore, Brk-deficient mice had a greater amount of inflammatory infiltrate in the submucosa and lamina propria and more crypt distortion. Although Brk-/- mice had more localized inflammation in their distal colons following DSS administration, this did not have an impact on their immediate survival. Total RNA was isolated from untreated and DSS treated colons of wild-type and Brk-/- mice and pro-inflammatory cytokine gene expression was analyzed by multiprobe RNase protection assays. After DSS treatment, expression of RNAs encoding several pro-inflammatory cytokines was detected in both wild-type and Brk knockout mice. However, a significantly greater increase in induction of IL-1 alpha/beta, and IL-6 mRNA expression was observed in Brk-deficient mice when compared with wildtype control mice. In contrast, the severe denudation of the epithelium in the Brk-/- colon led to decreased epithelial IL-18 mRNA expression. Our results demonstrate that Brkdeficient mice develop more severe acute colitis in response to DSS, and suggest that Brk tyrosine kinase signaling plays a protective role in the colonic epithelium. 


\section{W1578}

KLF5 Transcriptionally Upregulates Integrin $\beta 1$ and Ilk and Activates the Mek/ ERK Pathway in Non-Transformed Primary Esophageal Cells

Yizeng Yang, Bree G. Goldstein, Yuliya Yermolina, Jonathan P. Katz

Background and aims: Klf5 (IKLF; BTEB2), a member of the Krüppel-like factor (KLF) family, is expressed in epithelia of the gastrointestinal tract as well as several other organs. Klf5 is found in regions of active cell proliferation and promotes proliferation, mediates the transforming activity of oncogenic H-ras, and negatively regulates Klf4 (GKLF). Klf5 is expressed in the basal layer of the esophagus, where interactions with the extracellular matrix (ECM) are critical. Integrins, heterodimeric transmembrane receptors, and integrinlinked kinase (ILK) are also expressed mainly in basal cells. Integrins and ILK transduce signals from the ECM to downstream pathways and are crucial for numerous cellular processes, including proliferation, migration, and survival. We hypothesized that Klf5 promotes proliferation and migration in esophageal epithelial cells by upregulating integrin signaling. Methods: We stably infected primary mouse esophageal keratinocytes with retrovirus overexpressing Klf5 or siRNA directed against Klf5. Control cells and those with overexpression or suppression of Klf5 were infected with adenovirus to express wild-type or dominant negative ILK. Cells were examined for growth characteristics, and migration was assessed by transwell assays. Immunohistochemistry, including staining for markers of proliferation, differentiation, and apoptosis, was performed, and DNA, RNA, and protein were isolated for real-time PCR. Western blots, protein kinase assays, and chromatin immunoprecitation (ChIP). Results: Overexpression of Klf5 in primary esophageal cells increased proliferation and migration, while suppression of Klf5 inhibited proliferation and migration. Klf5 upregulated mRNA and protein levels of integrin $\beta 1$ and ILK and increased ILK kinase activity. ChIP assay demonstrated Klf5 binding directly to the integrin $\beta 1$ and ILK promoters. Overexpression of Klf5 increased phosphorylation of MEK and ERK. In contrast, total MEK and ERK, as well as total and phosphorylated Akt and GSK3 $\beta$ were not altered. As Akt and GSK3 $\beta$ are ILK targets in other tissues, these data suggest that ILK may act specifically on MEK/ERK signaling in esophageal epithelia. Conclusions: Klf5 is crucial for normal proliferation and migration in esophageal epithelial cells. Klf5 transcriptionally upregulates integrin $\beta 1$ and ILK and increases ILK activity, leading to specific activation of the MEK/ ERK pathway. These data provide evidence of a critical link between Klf5 and integrin signaling in non-transformed primary esophageal cells, providing a new paradigm for the regulation of proliferation and migration by Klf5 in esophageal basal cells.

\section{W1579}

Glucagon-Like Peptide-2 Intracellularly Stimulates eNOS Phosphorylation and Specifically Induces Submucosal Arteriole Vasodilation Via a Sheer StressIndependent, Local Neural Mechanism

Xinfu Guan, Keying Zhang, Heidi Karpen, John Bukowski, Xiaochun Bian, James Galligan, Douglas Burrin

Glucagon-like peptide-2 (GLP-2) is a nutrient-responsive neuropeptide that exerts diverse actions in the gastrointestinal tract, including enhancing mucosal cell survival and proliferation, mucosal blood flow, luminal nutrient uptake, and suppressing gastric motility and secretion. We have shown that GLP-2-stimulated mucosal blood flow is associated in vivo with increased activation of endothelial nitric oxide synthase (eNOS), but not neuronal nitric oxide synthase (nNOS), in the neonatal pig intestine. We have also shown that the GLP-2 receptor (GLP-2R) is co-localized with eNOS in enteric neurons. However, it is well established that blood flow-induced sheer stress is a key stimulator of endothelial eNOS activity. Thus, the GLP-2-induced increase in blood flow may indirectly activate endothelial eNOS. We hypothesized that GLP-2R activation intracellularly stimulates eNOS phosphorylation in enteric neurons and is independent of blood flow-mediated sheer stress. Our aim was to establish whether the GLP-2-mediated activation of eNOS occurs intracellularly via a neural-dependent mechanism, independent of blood flow-induced sheer stress. We first studied an ex vivo submucosal preparation from the young guinea-pig jejunum to quantify individual submucosal arteriole diameter with a computer-aided, real-time videomicroscopy system. Under pre-constriction with $\mathrm{KCl}(60 \mathrm{mM})$, super-perfusion of human GLP-2 (at $100 \mathrm{nM}$ ) immediately increased the submucosal arteriole dilation by approximately $30 \%$, but did not alter mesenteric artery dilation. However, GLP-2-induced vasodilation was completely blocked with co-superfusion of either a NOS inhibitor (NG-nitro-L-arginine at $300 \mu \mathrm{M}$ ) or a neuronal $\mathrm{Na}$ channel blocker (tetrodotoxin at $300 \mathrm{nM}$ ), implying that the GLP-2-mediated vascular action was localized specifically on the submucosal arteriole (the final and major resistance vessel in the gut) via a nitric oxide-dependent local neural mechanism. We next defined whether GLP-2R activation activates eNOS intracellularly in HEK 293T cells transiently transfected with full-length cDNA clones of human GLP-2R and eNOS. GLP-2 treatment dose-dependently induced eNOS phosphorylation on Serl177 in the double transfected HEK 293T cells and this was maximal within 15 min at $20 \mathrm{nM} \mathrm{GLP}$ 2. Thus, we concluded that GLP-2-induced submucosal arteriole dilation is locally mediated via a neural-dependent mechanism, independent of blood flow-mediated sheer stress. Moreover, GLP-2R activation intracellularly leads to stimulation of eNOS phosphorylation, independently from sheer stress. (Supported by NIH HD33920 and USDA/ARS 2533703361).

\section{W1580}

Transcriptional Regulation of Acidic pH Induced Interleukin-6 Expression and Secretion in Human Esophageal Epithelial Cells (HET-1) Via Mapks Parvaneh Rafiee, Aaron Rogaczewski, Victoria M. Nelson, Irshad Ali, David G. Binion, Reza Shaker

INTRODUCTION: Inflammation is a complex biologic process involving a highly orchestrated interaction among several cells of different origin and function. Human esophageal epithelial cells contribute to esophageal inflammation by expressing and secreting a variety of cytokines and chemokines in response to various stressors. IL- 6 is a pleiotropic cytokine, which regulates immune defense and inhibits apoptosis. Involvement of IL-6 in various diseases including carcinogenesis has been established. We have recently demonstrated that acidic exposure stimulates IL-6 production in HET-1 cells via COX-2. However, mechanisms underlying IL-6 expression and secretion in response to acidic pH exposure are complex, involving multiple protein kinases and transcriptional factors. The aim of present study is to investigate MAPK signaling pathways in transcriptional regulation of IL-6 expression and secretion in HET-1 cells following acidic pH stimulation. METHODS: HET-1 cells monolyer were exposed to acidic $\mathrm{pH} 4.5$ for $1 \mathrm{~h}$, then incubated in regular culture media for $1,3,6$, 12 and 24. To determine the role of MAPKs family member the cells were pretreated with PD98059 (ERK), SP600125 (JNK) and SB203580 (p38 MAPK) inhibitors 30 min prior to acidic exposure. IL-6 mRNA expression was done by RT-PCR. ELISA and Western blotting were utilized to analyze IL-6 protein secretion and expression respectively. NFKB activity was measured by ELISA based DNA binding nuclear protein assay and Western blotting. RESULTS: Exposure of HET-1 to acidic pH 4.5 enhanced IL- 6 secretion, mRNA and protein expression as measured by ELISA, RT-PCR and Western blotting respectively. Acidic pH also resulted in phosphorylation and activation of ERK, p38 MAPK and JNK in HET-1 cells. The IL-6 expression was blocked by the ERK inhibitor PD98059, and by the JNK inhibitor SP600125, but not by the $\mathrm{p} 38$ MAPK inhibitor, SB203580. Acidic $\mathrm{pH}$ enhanced the transcriptional activity of the IL- 6 gene by activation of the transcriptional factors NF- $\mathrm{KB}$, which was attenuated by PD98059. JNK phosphorylation was inhibited by SP600125 without blocking either $\mathrm{p} 38$ MAPK or ERK. CONCLUSIONS: These results demonstrates an independent role for ERK and JNK in acidic pH induced IL-6 expression and secretion via NF-KB in HET-1 cells. Our data suggests that acidic pH induction of the IL-6 in esophageal epithelial may contribute to pathophysiologic mechanisms in GERD

\section{W1581}

Increased Human Intestinal Microvascular Endothelial Arginase Activity Mediated By RHOA Pathway: Mechanisms Underlying IBD Endothelial Dysfunction

Parvaneh Rafiee, Scott Horowitz, Aaron Rogaczewski, Victoria Nelson, Balaraman Kalyanaraman, Mary F. Otterson, David G. Binion

BACKGROUND: Arginase competes with endothelial nitric oxide synthase (eNOS) for the substrate L-arginine and decreases nitric oxide (NO) production. NO plays a critical role in vascular physiology, and production is decreased in blood vessels from chronically inflamed bowel in human inflammatory bowel disease (IBD). Mechanisms underlying the decreased production of NO in IBD microvascular endothelial dysfunction are not fully defined. We sought to define the signaling pathways involved in the microvascular endothelial expression of arginanse. Previously we have demonstrated that MAPK activation occurs during TNF$\alpha$ and LPS-induced endothelial activation in human intestinal microvascular endothelial cells (HIMEC). Because GTP-binding proteins have been implicated in MAPK activation we hypothesized that Rho a GTP-binding protein is a mediator of TNF- $\alpha /$ LPS induced arginase activity in HIMEC and IBD tissues. METHODS: HIMEC monolayers passage 8-14 stimulated with TNF- $\alpha$ and LPS, were used to examine arginase expression by western blotting and fluoresence microscopy. MAPK activity was assessed using a phospho-specific in vitro kinase assay. p38 MAPK inhibitor (SB203580), p44/42 MAPK inhibitor (PD098059), RhoA inhibitor (Clostridium botulinum C3 exoenzyme) and Rho-associated coiled-coil kinase (ROCK) inhibitor Y-27632 were used to demonstrate the involvement of these signaling pathways in TNF$\alpha /$ LPS induced arginase expression and activity. Control and IBD tissues were also analyzed using Western blot analysis. RESULTS: Arginase enzymatic activity was increased in a concentration and time dependent fashion in response to $100 \mathrm{U} / \mathrm{ml}$ of TNF- $\alpha$ and $1 \mu \mathrm{g} / \mathrm{ml}$ of LPS for 24 hours, peaking at a 2 fold increase. TNF- $\alpha /$ LPS activation of HIMEC resulted in a rapid, sustained increase in endothelial p38 MAPK and p44/42 MAPK activity. Inhibition of p38 MAPK and p44/42 MAPK with SB203580 and PD098059, respectively, decreased TNF- $\alpha /$ LPS induced arginase in HIMEC. C3 exoenzyme RhoA inhibitor and Y-27632 ROCK inhibitor both attenuated TNF- $\alpha /$ LPS induced MAPK activation and blocked arginase expression in HIMEC. Moreover, a significantly higher arginase II activity and increased RhoA protein level were observed in IBD tissues surrounding microvessels as compared with normal control tissue. CONCLUSIONS: TNF- $\alpha$ /LPS enhances arginase activity via RhoA/ ROCK in HIMEC. Rho acts upstream of MAPKs in mediating TNF- $\alpha /$ LPS induced arginase activity. Higher arginase expression and enzymatic activity appears to play a key role in IBD endothelial dysfunction. Targeting vascular arginase may represent a novel therapeutic strategy for inflammatory bowel disease.

\section{W1582}

Role of Mapk in Activation of Signal Transducers and Activators of Transcription (STATs) Proteins in VEGF Stimulated Human Intestinal Microvascular Endothelial Cells (HIMEC)

Parvaneh Rafiee, Monica Theriot, Mary F. Otterson, David G. Binion

INTRODUCTION: VEGF effects on endothelial cells, preventing apoptosis as well as inducing migration, growth, proliferation and differentiation. VEGF activates various signaling cascades (i.e. MAPKs, PI3K/Akt) in HIMEC. STAT proteins modulate cell growth responses, and play a role in endothelial proliferation, but have not been characterized in human intestinal microvascular angiogenesis. We investigated the contribution of STATs signaling pathway in tissues from control and IBD pts as well as control and disease specific HIMEC following VEGF activation. METHODS: Mucosa from control and IBD (UC and Crohn's disease(CD)) pts were homogenized in hypotonic buffer and nuclear proteins were prepared. Control and IBD HIMEC monolayers were treated with VEGF, and nuclear translocation of STAT proteins was detected by immunofluorescence staining. An ELISA-based oligonucleotide binding assay containing an immobilized STAT consensus binding site was used to demonstrate the DNA binding in nuclear protein extracts. Phospho-STAT proteins were detected by Western bloting using specific phospho-antibodies. Inhibitors of p38 MAPK and p44/ 42 MAPK were used to identify the signaling pathways. The specific VEGFR2 antibody was used to determine whether STATs activation in HIMEC were mediated through VEGFR2/ KDR receptor. RESULTS: Western bloting revealed phosphorylated STATl and STAT3 in both UC and CD intestinal tissues but not the control and uninvolved mucosal specimens. Similarly DNA-nuclear proteins assay demonstrated increased level of STATI and STAT3 but not STAT5A and 5B. VEGF induced tyrosine phosphorylation and nuclear translocation 
of STATl $\alpha$, STAT3, STAT5A and STAT 5B in HIMEC. VEGF activated IBD HIMEC ( $\mathrm{n}=4)$ demonstrated enhanced STATl and STAT3 compared with control HIMEC $(n=3)$. Pretreatment of HIMEC with VEGFR2 antibody suppressed STATs phosphorylation and nuclear translocation. The $\mathrm{p} 38$ MAPK inhibitor SB203580 decreased STATl $\alpha$ activation, whereas PD098059 (MEK/p44/42 MAPK inhibitor) suppressed both STATl $\alpha$ and STAT3 in HIMEC. CONCLUSIONS: Differential STAT activation characterizes intestinal inflammation in IBD tissues and HIMEC.VEGF activates STATs in HIMEC through the VEGFR2/KDR receptor. MAPK family members play a differential role in STATs activation. These findings suggest a role for the STATs in the regulation of gene expression associated with chronic inflammation and the angiogenic effects of VEGF in gut specific microvascular endothelial cells

\section{W1583}

Identification of Ser58 in C-Jun As a Novel Phosphorylation Site Targeted By Protein Kinase D

Richard T. Waldron, Julian P. Whitelegge, Enrique Rozengurt

Background: The protooncogenic transcription factor c-Jun, a major component of AP-1 complexes, regulates gene expression critical for development, inflammation, growth and proliferation. Furthermore, c-Jun has been recently found to participate in colorectal carcinoma via interaction with the Wnt signaling pathway. Protein kinase D (PKD), a target of GI peptides, attenuates c-Jun N-terminal kinase (JNK)-c-Jun signaling and forms complexes with JNK. We previously demonstrated that PKD directly phosphorylates the c-Jun Nterminus in vitro at Ser/Thr sites distinct from JNK, but did not identify the site(s). Methods: Here, we have used isolated PKD in kinase assays with glutathione S-transferase (GST)-cJun fusion proteins as substrates to identify novel PKD-mediated phosphorylation sites within c-Jun. Results: PKD was incubated with GST-c-Jun (1-89) and tryptic fragments derived from the phosphorylated protein were analyzed using LC-MS/MS. Using this approach, we obtained a phosphopeptide containing phospho-Ser58. PKD-mediated phosphorylation of a protein comprising GST fused to the first 135 amino acids of c-Jun was diminished by approximately $40 \%$ in a S58A point mutant. In contrast, PKD phosphorylation of a S63A mutant was equal to that of wild type GST-c-Jun(1-135). Interestingly, PKD also robustly phosphorylated a GST-c-Jun(1-72) fusion protein, to levels comparable to GST-c-Jun(1135), and this was again reduced by approximately $40 \%$ in a S58A mutant but not a S63A mutant. These results substantiate the specificity of Ser 58 phosphorylation and suggest the presence of at least one additional PKD phosphorylation site within the c-Jun amino terminal (aal-72). Notably, incubation with activated PKD in an in vitro kinase assay did not prevent subsequent binding of GST-c-Jun(1-72) to JNK2. Accordingly, phosphorylation by PKD was not appreciably altered by S37A, a mutation within the JNK-docking motif of GST-c-Jun(172). In COS-7 cells, myc-tagged, full-length, wild-type c-Jun was transfected either alone or together with PKD to examine its effect on c-Jun phosphorylation in vivo. Using metabolic labeling with 32P, we found that cotransfection with PKD significantly increases both basal and serum-stimulated total c-Jun phosphorylation, suggesting that PKD can phosphorylate c-Jun in vivo. Luciferase reporter gene assays indicate that c-Jun-myc promotes the expression of AP-1-containing genes in HEK-293 cells. Conclusions: PKD-mediated phosphorylation of c-Jun at distinct and novel sites in vivo including Ser58 may affect gene expression either directly, or by regulating the binding of c-Jun to its upstream regulators/transcriptional coactivators.

\section{W1584}

N-Acetyl-L-Cysteine Is a Potent Secretagogue for Intestinal Cholecystokinin Release

George H. Greeley, Feng Qin, Jason Reed, Ella W. Englander

Administration of N-acetyl-L-cysteine (NAC), an antioxidant and substrate for endogenous glutathione (GSH) synthesis, to diabetic animals improves hyperglycemia, glucose intolerance and defective insulin secretion. The notion that NAC increases secretion of gut hormones that stimulate insulin secretion and glucose disposal (ie, incretins) has not been tested. Our aim was to define influence of NAC on secretion of intestinal cholecystokinin (CCK), an incretin. Methods. For in vivo experiments, adult male SD rats were given NAC acutely either into the stomach $(5 \mathrm{ml}, 100 \mathrm{mg} / \mathrm{ml})$ or duodenal $(1 \mathrm{ml}, 100 \mathrm{mg})$ lumen by gastric lavage or direct duodenal administration. Plasma was collected 15 min later for measurement of CCK levels by immunoassay. For in vitro experiments, intestinal STC-1 cells were exposed to NAC $(10 \mathrm{mM})$ for different periods $(4,7,24 \mathrm{~h})$, media were then replaced with fresh media alone or media containing bombesin $\left(10^{-9} \mathrm{M}\right)$, a CCK secretagogue. Media and cells were harvested $30 \mathrm{~min}$ later for CCK measurements. Cell density was monitored. Results. Administration of NAC into the gastric or duodenal lumen caused a significant elevation in plasma CCK levels (CCK level [pg/ml]: basal: $1 \pm 1$ vs stomach: $35 \pm 6$ or duodenal: $36 \pm$ 14, $\mathrm{P}<0.05, \mathrm{~N}=6$ rats/group). In vitro exposure of STC-1 cells to NAC, for 4-24 h, enhanced basal and bombesin-stimulated CCK secretion significantly (1.3- to 4-fold, $\mathrm{P}<0.05$ ) (see Table, $24 \mathrm{~h}$ NAC exposure). NAC exposure for $24 \mathrm{~h}$ also increased cellular CCK content 4-fold (control: $2823 \pm 151$ vs NAC treated: $9143 \pm 275$ pg/ml, $\mathrm{P}<0.05$ ). Conclusions and Discussion. NAC is a potent secretagogue for CCK secretion. In vitro findings show that NAC can act directly on enteroendocrine cells to cause CCK release and synthesis. The finding that NAC exposure for $24 \mathrm{~h}$ enhanced basal CCK release to a greater magnitude ( 4 -fold) when compared to acute ( $3,7 \mathrm{~h}$ ) exposure (1.3- to 1.5-fold) suggests involvement of increased GSH levels in stimulation of CCK secretion and synthesis. These findings suggest that beneficial effects of NAC on insulin secretion and glucose levels are mediated partly by increased CCK secretion.

\begin{tabular}{|c|c|c|}
\hline & \multicolumn{2}{|c|}{ CCK secretion (fg/1000 cells) } \\
\hline Treatment & Basal & 30 min post-bombesin \\
\hline Vehicle & $63 \pm 15^{1}$ & $160 \pm 10$ \\
\hline NAC, $24 \mathrm{~h}$ & $224 \pm 36^{*}$ & $336 \pm 35^{*}$ \\
\hline
\end{tabular}

$1=$ mean SEM, $\mathrm{N}=6$ dishes/group. $*=\mathrm{P}<0.05$ vs vehicle

\section{W1585}

Interruption of Transforming Growth Factor-Beta Signaling By Curcumin Negatively Regulates Gene Expression of Peroxisome Proliferator-Activated Receptor-Gamma in Rat Hepatic Stellate Cells Anping Chen, Shizhong Zheng

BACKGROUND: Activation of hepatic stellate cells (HSC), the major effectors of hepatic fibrogenesis, is coupled with sequential alterations in gene expression, including an increase in receptors for transforming growth factor- $\beta$ (TGF- $\beta$ ) and a dramatic reduction in the peroxisome proliferator-activated receptor-gamma (PPAR $\gamma$ ). The casual relationship remain obscure. We previously demonstrated that curcumin induced gene expression of PPAR $\gamma$ in activated HSC, leading to reducing cell proliferation, inducing apoptosis and suppressing the expression of ECM genes. The underlying molecular mechanisms are largely unknown. We recently observed that stimulation of PPAR $\gamma$ activation by curcumin suppressed gene expression of TGF- $\beta$ receptors in activated HSC, leading to the interruption of TGF- $\beta$ signaling. [Zheng and Chen Am J Physiol Gastrointest Liver Physiol (November 23, 2005). doi:10.1152/ajpgi.00450.2005]. This observation partially supported our assumption of the existence of an antagonistic relationship between PPAR $\gamma$ activation and TGF- $\beta$ signaling in HSC. AIM AND HYPOTHESIS: The current study is to further elucidate the mechanisms of curcumin in induction of PPAR $\gamma$ gene expression and to evaluate the effect of TGF- $\beta$ signaling on regulating PPAR $\gamma$ gene expression in activated HSC. We hypothesize that TGF$\beta$ signaling might negatively regulate gene expression and activation of PPAR $\gamma$ in activated HSC. RESULTS: The present report demonstrates that exogenous TGF- $\beta 1$ inhibits gene expression of PPAR $\gamma$ in activated HSC. Pretreatment of cells with curcumin eliminates the inhibitory effect likely by interrupting TGF- $\beta$ signaling. Transfectection assays further indicates that blocking TGF- $\beta$ signaling by dominant negative type II TGF- $\beta$ receptor increases the promoter activity of PPAR $\gamma$ gene. Promoter deletion assays, site-directed mutageneses and gel shift assays reveal two Smad binding sites (SBS) within -1522 to -1492bp of the PPAR g gene promoter. The two SBS act as the curcumin response elements and negatively regulate the promoter activity of PPAR $\gamma$ in passaged HSC. CONCLUSION: Results in this study demonstrate that interruption of TGF- $\beta$ signaling by curcumin induces gene expression of PPARy in activated HSC in vitro. Our studies provide novel insights into the molecular mechanisms of curcumin in induction of PPARy gene expression and in inhibition of HSC activation. [The work was supported by the grant RO1 DK047995 from NIH/NIDDK and the Bridging Award from LSUHSC-S to A. Chen].

\section{W1586}

Magi-3 Negatively Regulates Signaling By the Lpa2 Receptor Huanchun Zhang, Randy A. Hall, Chris Yun

Lysophosphatidic acid (LPA) mediates multiple cellular responses by activating G proteincoupled receptors, LPAl-4. We and others have shown that LPA2 is overexpressed in several types of cancers, including ovary, colon, and testis. LPA2 receptor has been shown to interact with PDZ domain-containing proteins, NHERF2 and rhoGEF. To further identify other PDZ proteins that interact with LPA2, we screened a PDZ domain proteomic array that contains 96 distinct PDZ domains expressed and purified as His-tagged proteins. This blot was overlaid with C-terminal (45 amino acids) GST fusion proteins of LPA2 receptor. In contrast to GST alone, the C-terminus of LPA2 interacted with several of the PDZ domains on the array, including NHERF2, NHERF1, MAGI-2, MAGI-3, and neurabin. In the current work, we report the findings on the role of MAGI-3 in LPA2-mediated signaling. The interaction between LPA2 and MAGI-3 was confirmed by far-Western blot where individual PDZ domains of MAGI-3 were overlaid with GST-LPA2 or GST as a control. The strongest binding of GST-LPA2 was observed with the 5th PDZ domain. This binding was confirmed by copurification of LPA2 and MAGI-3 from transiently transfected SW480 cells. To determine a role of MAGI-3 in LPA-mediated signaling, we stably over-expressed V5-tagged human MAGI-3 in SW480 cells. SW480 transfected with V5-MAGI-3 or pcDNA vector were treated with $10 \mathrm{uM} \mathrm{LPA}$ for up to $24 \mathrm{~h}$. The activation of Erkl/2, as determined by Western blot using anti-phospho-Erk1/2 antibodies, was not significantly affected by over-expression of MAGI-3. Because MAGI-3 has previously shown to affect the JNK pathway of Frizzled, we next determined whether MAGI-3 affected LPA-mediated JNK pathway. In control SW480/ pcDNA cells, LPA maximally activated JNKs at $2 \mathrm{~h}$ without any appreciable change in the total amount of JNKs. In contrast, LPA-mediated JNK activation was drastically attenuated in SW480/MAGI-3 cells. This down-regulation of the JNK pathway by MAGI-3 was confirmed by similar attenuation in phosphorylation of c-Jun, a known target of JNKs, in SW480/ MAGI-3 compared with SW480/pcDNA. In addition to c-Jun phosphorylation, LPA greatly increased the amount of c-Jun protein in SW480/pcDNA, whereas this stimulation in c-Jun expression was significantly inhibited in SW480/MAGI-3 cells. Our results demonstrate that MAGI-3 specifically interacts with LPA2 receptor and down-regulates activation of the JNK pathway by the LPA2 receptor.

\section{W1587}

Intracellular Activation of Epidermal Growth Factor Receptor and Phosphatidylinositol 3-Kinase Regulate Human Gastric Epithelial Cell Migration

Marie-Pier Tetreault, Nathalie Rivard, Daniel Menard

The integrity of the gastric epithelium is maintained through its highly-dynamic renewal capacity. Following stem cell division in the isthmus, cell migrating upward will differentiate into surface mucous cells (mucine-5) while those migrating downward will differentiate into mucous neck cells (mucine-6), chief cells (human gastric lipase, pepsinogen) and parieta cells $(\mathrm{HCl})$. Our previous studies established that growth factors (EGF/TGFa, HGF) differently regulate the migration of human gastric epithelial cells (Exp Cell Res.306(1):285-297, 2005). However, the intracellular signaling pathways that transmit extracellular cues and that regulate basic and stimulated gastric epithelial cell migration are still unclear. Aim: To investigate the involvement of EGFR(EGF receptor)/phosphatidylinositol-3-kinase (PI3K)

. 
signalling pathways in the regulation of human gastric epithelial migration. Methods: Confluent HGE-17 cell monolayers were wounded with a razor blade and the migration was quantified by counting the number of cells across the wound margin. The effects of pharmacologic inhibitors LY294002 (PI3K), PD153035 (EGFR), PP2 (Src) and of a neutralizing antibody against EGFR were examined. Wounded cultures were lyzed at different time intervals and the activation profiles of Akt, Src and EGFR were analyzed. Results: The kinase Akt was stimulated in a PI3K-dependent manner by wounding and TGFa treatment. Moreover the addition of LY294002 strongly inhibited (40-60\%) basal and TGFa-induced responses and this inhibition was already significant after only 60 min exposure. Accordingly, treatments with phosphatidylinositol $(3,4,5)$-triphosphate greatly accelerated cell migration by $230 \%$. Pharmacological inhibition of EGFR tyrosine kinase activity decreased basal migration by $73 \%$ while the prevention of EGF binding with an EGFR neutralizing antibody had no effect on basal migration. Furthermore, treatment of wounded monolayers with the EGFR neutralizing antibody has no effect on the Akt activation whereas treatment with the EGFR inhibitor completely inhibited Akt activation. Wounding also stimulated the Src activity and the Src inhibitor PP2 significantly diminished (72\%) the number of cells across wound margin. Pre-treatment of cells with PP2 completely blocked the phosphorylation levels of Akt. Conclusion: Activation of PI3K signalling pathway is a central event in the modulation of gastric epithelial migration. Moreover, a ligand-independent intracellular mechanism mediated by Src/EGFR inhibits the basal migration, this transactivation being responsible for the PI3K/Akt signaling pathway activation in human gastric epithelial cell migration.

\section{W1588}

Down-Regulation of Soluble Guanylyl Cyclase (sGC) Expression and Activity By Interleukin-1 $\beta$ in Colonic Smooth Muscle Cells Is Mediated By PKGDependent Activation of JNK

Jiean Huang, Wenhui Hu, Sunila Mahavadi, Karnam S. Murthy

Pro-inflammatory cytokines such as interleukin- $1 \beta$ (IL-1 $\beta$ ) and tumor necrosis factor- $\alpha$ induce expression of iNOS in immune cells, endothelial cells, and smooth muscle cells. NO generated from iNOS activates soluble guanylyl cyclase $(s G C)$ to generate cGMP. Despite the sustained increase in NO resulting from iNOS activity, the initial increase in cGMP reverts to basal levels; in addition, the cGMP response to nitrovasodilators is attenuated. We postulated that the decrease in cGMP response reflected down-regulation of soluble GC Aim. The aim of the study was to identify the mechanism by which IL-1 $\beta$ inhibits SGC expression and activity in smooth muscle. Methods. Colonic circular muscle strips were incubated in DMEM in the absence or presence of IL-1 $\beta(10 \mathrm{ng} / \mathrm{ml})$ for $48 \mathrm{~h}$. Muscle cells isolated from these strips were used for measurement of sGC activity and expression. Expression of iNOS and sGC was determined by immunoblot analysis, and sGC activity was measured by cGMP formation in response to Na nitroprusside (SNP) in the presence of a PDE5 inhibitor. JNK phosphorylation (activation) was determined using phosphospecific antibody in the presence or absence of a PKG inhibitor. Results. In muscle cells isolated from cultured smooth muscle strips in the absence of IL-1 $\beta$, sGC expression and $\mathrm{SGC}$ activity were similar to those of freshly dispersed muscle cells. In cells isolated from strips pretreated with IL-1 $\beta$, iNOS expression was induced, whereas SGC expression and SNP-induced sGC activity were decreased by $65 \%$ and $72 \%$, respectively, and JNK activity was increased. Treatment of the tissue with the PKG activator, pCPT-cGMP in the absence of IL-1 $\beta$, also decreased SNP-stimulated SGC activity and increased JNK activity. Addition of the NOS inhibitor, L-NNA, the PKG inhibitor, 8-pCPT-cGMP[S], or the JNK inhibitor SP600125 restored SNP-stimulated SGC activity (cGMP levels). Conversely, JNK activity induced by treatment with IL-1 $\beta$ was inhibited by L-NNA and the PKG inhibitor, 8-pCPTcGMP[S]. The results implied that inhibition of sGC expression and activity were mediated by PKG-activated JNK. Conclusion. IL-1 $\beta$ augmented iNOS expression, but the sustained formation of $\mathrm{NO}$ and activation of $\mathrm{sGC}$ and PKG were abrogated by PKG-mediated activation of JNK, which triggered a feedback mechanism that resulted in inhibition of SGC expression and activity.

\section{W1589}

Effect of High Cholesterol (Ch) Levels On the Genomic Effects Induced By Progesterone (PG) in Muscle Cells from Human Gallbladder (GB)

Cong Ping, Piero Biancani, Jose Behar

The incidence of gallbladder complications is increased during pregnancy due to higher $\mathrm{Ch}$ concentrations in bile and GB stasis. It has been suggested that they are caused by lithogenic bile with $\mathrm{Ch}$ worsened by rising levels of estrogens in the first two trimesters and to impaired muscle contraction induced by PG in the last two trimesters. PG impairs muscle contraction induced by agonists that are receptor (CCK-8) or G protein (GTP $\gamma S$ ) dependent due to down regulation of $G$ proteins that mediate contraction ( $\mathrm{Gi} 3$ ) and up regulation of $G$ proteins that mediate relaxation (Gs). In contrast high levels of caveolar Ch impairs the contraction induced by receptor dependent agonists (CCK-8) and by $\mathrm{KCl}$ without affecting $\mathrm{G}$ protein patterns. These studies therefore were aimed at examining the effect of high caveolar $\mathrm{Ch}$ on those PG actions that is the actual setting in human pregnancy. The PG genomic effects were examined in enzymatically dissociated muscle cells from GB with high caveolar $\mathrm{Ch}$ (specimens with $\mathrm{Ch}$ stones) and normal caveolar $\mathrm{Ch}$ (specimens with pigment stones). The cells were pre-treated with buffer, Ch-rich liposomes or Ch-free liposomes for $90 \mathrm{~min}$ and then treated with PG $\left(10^{-5} \mathrm{M}\right)$ for $6 \mathrm{~h}$. The Ch-rich liposomes increased $\mathrm{Ch}$ levels and the $\mathrm{Ch}$-free liposomes removed the excess $\mathrm{Ch}$ from caveolae. High levels of caveolar $\mathrm{Ch}$ in muscle cells from GB with $\mathrm{ChS}$ or incubated with Ch-rich liposomes blocked the actions of PG. The contraction patterns in muscle cells incubated with Ch-rich liposomes followed by treatment with PG were similar to that observed in muscle cells incubated with Ch-rich liposomes alone. Antibodies against caveolin-3 protein (CAV3) also blocked PG genomic effects. Similar results were obtained in muscle cells transfected with CAV3 siRNA in tissue culture for 24 h. CAV3 siRNA decreased CAV3 expression by $60 \%$. High Ch levels, caveolin 3 protein antibodies or CAV3 siRNA significantly reduced the incorporation of 3H-PG into he caveolae at 10,30 and $60 \mathrm{~min}$. It is concluded that $\mathrm{PG}$ is internalized through caveolar domains with CAV3 facilitating its transport; and, that high levels of caveolar Ch inhibits PG induced genomic effects by blocking its incorporation into the caveolae These results suggest that PG is unlikely to contribute to the high incidence of GB complications during pregnancy, which may instead be due to abnormal $\mathrm{Ch}$ incorporation into gallbladder muscle cells

\section{W1590}

Gastrointestinal Hormones Cause C-Met Receptor Downregulation Via a Clathrin-Mediated Endocytosis and Lysosome Dependent Mechanism Karl M. Hoffmann, Jose A. Tapia, Marc Berna, Michelle Thill, Till Braunschweig, Samuel Mantey, Terry Moody, Robert T. Jensen

The receptor tyrosine kinase c-Met when activated by hepatocyte growth factor (HGF) has potent growth effects on many normal tissues and tumors. C-Met expression is regulated by HGF exposure, but it is unknown if it can be regulated by gastrointestinal (GI) hormones This question is important because c-Met is found in many GI tissues/tumors that possess GI hormone receptors. To investigate this question we studied rat pancreatic acinar cells that possess c-Met as well as numerous GI hormone receptors using Western blotting and immunofluorescence-histochemistry (IF-IHC). Incubation of rat pancreatic acini with CCK8 , carbachol and bombesin caused a time-dependent decrease in c-Met which at 45 min decreased by $85 \pm 5,32 \pm 9$ and $26 \pm 10 \%$ of control, respectively. Secretin or VIP had no effect. Since CCK-8 caused the largest decrease its effect was studied in detail. Maximal c-Met downregulation occurred with $100 \mathrm{nM} \mathrm{CCK-8} \mathrm{(IC50} 5 \mathrm{nM}$ ) and at $45 \mathrm{~min}$ (t0.5 $17.5 \mathrm{~min}$ ). CCK-JMV $(1 \mu \mathrm{M})$ had no effect. CCK-8 $(100 \mathrm{nM})$ treatment for up to $90 \mathrm{~min}$ did not decrease cell viability or the ability of EGF to cause tyrosine (Y) phosphorylation of Y1068 of the EGF receptor in pancreatic acini. GF109203X (PKC inhibitor), thapsigargin (Ca++ inhibitor) or a combination of both partially reversed the effect of CCK-8 $(31 \pm 3,40 \pm 9$, $60 \pm 5 \%$ of control, respectively). CCK-8 abolished HGF induced Y1234/1235c-Met phosphorylation and that of $\mathrm{Y} 627 \mathrm{Gabl}$, which is crucial for c-Met signaling. In situ IF-IHC studies showed a decrease in c-Met receptor staining with CCK-8 treatment. Prolonged HGF exposure ( $>3 \mathrm{~h}$ ) caused a $46 \%$ decrease in c-Met, a process that was sensitive to the proteasome inhibitor lactacystin and the lysosome inhibitor concanamycin A. CCK-8's effect was prevented by concanamycin A and by inhibitors of clathrin-mediated endocytosis (sucrose, phenylarsine oxide, concanavalin A), but not by lactacystin. CCK-8 induced pancreatic cMet downregulation also occurred in-vivo in rats injected intraperitoneally with CCK-8. In conclusion we describe for the first time that GI hormones down-regulate c-Met and that this regulation can occur in vitro and in vivo. CCK- 8 differed from HGF's downregulation of c-Met in that it had a greater effect ( $85 \%$ vs $46 \%$ decrease), was more rapid, was insensitive to proteasome inhibition and was dependent on clathrin-mediated endocytosis and degradation through the lysosome. These results indicate a new mechanism of influencing c-Met expression and signaling which could be important for its regulation in normal as well as in neoplastic tissue in the GI tract.

\section{W1591}

Cholecystokinin-2 Receptor Splice Variant Stimulates Hif-1 $\alpha$ and VEGF Expression and Promotes Tumor Growth in Vivo Celia Chao, Elizabeth Goluszko, Courtney M. Townsend, Jr., Mark R. Hellmich

Introduction: Colorectal, pancreatic, and esophageal cancers express a variant of the $\mathrm{CCK}_{2}$ receptor $\left(\mathrm{CCK}_{2} \mathrm{R}\right)$, called the $\mathrm{CCK}_{2 \mathrm{i} 4 \mathrm{sv}} \mathrm{R}$. Unlike $\mathrm{CCK}_{2} \mathrm{R}, \mathrm{CCK}_{2 \mathrm{i} 4 \mathrm{sv}} \mathrm{R}$ can constitutively activate the proto-oncogene $\mathrm{Src}$, which regulates receptor subcellular distribution and increases its rate of resensitization. We have shown that expression of $\mathrm{CCK}_{2 i 4 s v} \mathrm{R}$, unlike $\mathrm{CCK}_{2} \mathrm{R}$, enhances HEK tumor growth in vivo, in part, through a Src-dependent mechanism in the absence of exogenous gastrin. The aim of this study was to determine the role of constitutive receptor activity and the molecular mechanism of $\mathrm{CCK}_{2 i 4 s \mathrm{~s}} \mathrm{R}$-stimulated tumor growth in vivo Methods: To assess tumor growth in vivo, nu/nu mice were injected subcutaneously with $4 \times 106$ cells transfected with $\mathrm{CCK}_{2} \mathrm{R}, \mathrm{CCK}_{2 \mathrm{i} 4 \mathrm{~s}} \mathrm{R}$, or vector, randomized into 4 groups ( $\mathrm{n}=$ 6) for each receptor, and treated with $\mathrm{NaCl}, \mathrm{CR} 2945$ (CR) antagonist, pentagastrin (PG), or both PG and CR, at $30 \mathrm{mg} / \mathrm{kg} /$ day. On day 21 , tumors were weighed. To determine in vitro cell proliferation, growth assays were performed in triplicate over 8 days. Levels of hypoxia-inducible factor-1 (HIF-l $\alpha$ ) expression were assessed by Western blot. Vascular endothelial growth factor (VEGF) upregulation was demonstrated by Northern blot. Results: There was a 16.5 -fold increase in the mass of $\mathrm{CCK}_{2 \mathrm{i} 4 \mathrm{sv}} \mathrm{R}(297 \pm 118 \mathrm{mg})$ compared with the $\mathrm{CCK}_{2} \mathrm{R}$ tumors $(18 \pm 10)$ injected with $\mathrm{NaCl}$. PG induced greater $(2-3 \mathrm{x})$ tumor mass for both receptor variants $(660 \pm 245$ and $62 \pm 30 \mathrm{mg}$, respectively). CR blocked the stimulatory effects of $P G$ on both receptor-expressing tumors, but had no effect on basal growth when used alone. Thus, $\mathrm{CCK}_{2 \mathrm{i} 4 \mathrm{sv}} \mathrm{R}$ expression promotes tumor growth in both a constitutive and agonistdependent manner, whereas, $\mathrm{CCK}_{2} \mathrm{R}$-mediated tumor growth requires agonist. Since in vitro growth studies revealed no statistical differences between the doubling times for $\mathrm{CCK}_{2 \mathrm{i} 4 \mathrm{sv}} \mathrm{R}$ and $\mathrm{CCK}_{2} \mathrm{R}$-expressing cells ( $47 \pm 5 \mathrm{~h}$ vs. $38 \pm 4 \mathrm{~h}$, respectively), we reasoned that the increased

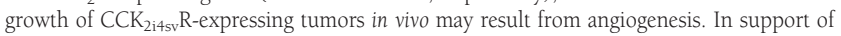
this hypothesis, basal HIF-l $\alpha$ protein levels were elevated in both $\mathrm{CCK}_{24}$ svv $_{\mathrm{R}}$-expressing cells in vitro and in tumor tissue when compared with $\mathrm{CCK}_{2} \mathrm{R}$. Concomitant with the increased HIF-l $\alpha$ was an increase in the mRNA levels of VEGF. Conclusions: Previously, we have shown that expression of $\mathrm{CCK}_{2 \mathrm{i} 4 \mathrm{sv}} \mathrm{R}$ promotes tumor growth in vivo, in part, via the constitutive activation of Src. Overexpression or activation of Src is known to promote tumor growth/survival by induction of HIF-l $\alpha$ target genes such as VEGF. Since $\mathrm{CCK}_{2 \mathrm{i} 4 \mathrm{sv}} \mathrm{R}$ does not increase cell growth in vitro, we suggest that the mechanism in vivo may be the upregulation of proangiogenic factors. 
W1592

Pacap Activation of Secretory and Proliferative Signaling Pathways in Bon Cells Is Mediated Through PKA, Calmodulin and RAS

Sandy N. Lieu, David S. Oh, Nils Lambrecht, Iskandar Yakubov, George Sachs, Joseph R. Pisegna, Patrizia M. Germano

Introduction: BON is an established human neuroendocrine tumor cell line, in which we have shown the expression of the receptor for PACAP, PACl, and that PACAP induces intracellular $\mathrm{Ca} 2+$, and cAMP increase, serotonin release, and growth. However, the signaling cascades mediating these events in BON are unclear. Aims: To characterize the intracellular pathways mediating PACAP's effects in BON by microarray analysis. Methods: Total RNA was isolated from BON cells, unstimulated, or activated by PACAP for 24h. The RNA was assessed for purity and stability, fluorescently labeled with Cy3- or Cy5-CTP and then amplified. Labeled cRNA from unstimulated BON cells and BON cells stimulated with PACAP were combined and hybridized to a $44 \mathrm{~K}$ human oligonucleotide expression array. The microarray was then scanned and the intensities normalized. Results: Microarray gene expression specific to $G$ protein-coupled signaling pathways were examined in response to PACAP. The gene expression of Gaq pathway regulators, revealed down regulation of Gaq subunits and PLC $(0.55+0.35,0.49+0.33$, respectively). Furthermore, DAG $(1.07+0.04)$, PKC isoforms $(1.34+0.28)$, and MAPK $(1.04+0.38)$ displayed generally neutral responses to PACAP activation, further suggesting that PACAP does not strongly activate the PKC pathway in BON cells. However we found highly significant increased expression of calmodulin (2.56), which is consistent with our previous finding of PACAP eliciting a Ca2+ response in BON cells. Subsequently, rRAS gene expression was also significantly enhanced $(2.14+0.05)$. We next examined regulators pertinent to the GaS pathway and found a three-fold activation of adenylyl cyclase (AC) 7 in BON cells, and a significant activation of PKA. These results and our previous biochemical studies indicate that PACAP, acting at the $\mathrm{PACl}$ receptor, predominantly activates the GaS pathway, but may also activate Gaq and $\mathrm{Ca} 2+$ signaling. These data further establishes that PACAP and PACl induced signaling pathways are coupled to proliferation in neuroendocrine tumoral BON cells. Conclusion: PACAP and PACl triggering leads to the activation of calmodulin, rRAS, AC, and PKA genes in BON cells, as determined by our microarray analysis studies. This methodology provides a useful tool for further defining signaling pathways that can be integrated with secretion and proliferation in $\mathrm{BON}$ cells to permit a greater understanding of the role of PACAP in the regulation of neuroendocrine tumors.

\section{W1594}

PKC Mediates Constitutive $\mathrm{CCK}_{2 \mathrm{i} 4 \mathrm{sv}} \mathrm{R}$ Internalization and the Steady-State Phosphorylation of the 3il Domain

Jeseong Park, Kirk L. Ives, Courtney M. Townsend, Jr., Mark R. Hellmich

Introduction: Cancers of the colon, pancreas, and esophagus express a splice variant of the $\mathrm{CCK}_{2}$ receptor $\left(\mathrm{CCK}_{2} \mathrm{R}\right)$, called $\mathrm{CCK}_{2 \mathrm{i} 4 \mathrm{y}} \mathrm{R}$. Compared to $\mathrm{CCK}_{2} \mathrm{R}, \mathrm{CCK}_{2 \mathrm{i} 4 \mathrm{sy}} \mathrm{R}$ has 69 additional amino acid residues in its 3rd intracellular loop (3il) domain, and shows distinct signaling properties, including stimulation of tumor growth in nude mice, agonist-independent activation of Src kinase, constitutive internalization, and an accelerated rate of resensitization. Since phosphorylation of the 3il domain of $G$ protein-coupled receptors plays a critical role their regulation, the aims of this study were to determine the phosphorylation state of the 3il domain of $\mathrm{CCK}_{2 \mathrm{i} 4 \mathrm{sv}} \mathrm{R}$ and to identify both the protein kinase(s) and the specific phosphorylation site(s) involved. Methods: Phosphorylation of the 3il domain was assessed by labeling cells with $\left[{ }^{32} \mathrm{P}\right]$ ortho-phosphate followed by immunoprecipitation of EGFPtagged 3il protein with an anti-EGFP antibody. To identify the protein kinases involved in 3il phosphorylation, cells were treated with either the PKC inhibitor, GF109203X, the Src kinase inhibitors, SU6656 or PP2, the EGF receptor inhibitor, AG1478, or the mitogenactivated kinase kinase inhibitor, PD98059. Specific phosphorylation sites within the 3 il domains of $\mathrm{CCK}_{244 \mathrm{sv}} \mathrm{R}$ and $\mathrm{CCK}_{2} \mathrm{R}$ were identified by Matrix-Assisted Laser Desorption Ionization Time-of-Flight/Time-of-Flight Mass Spectrometry (MALDI-TOF/TOF MS) analyses. To assess the effect of receptor phosphorylation on subcellular distribution, EGFPtagged receptor-expressing cells were analyzed using Laser Scanning Confocal Microscopy (LSCM). Results: There was an increased level of basal (steady-state) phosphorylation of the 3il domain of $\mathrm{CCK}_{2 \mathrm{i} 4 \mathrm{sv}} \mathrm{R}$ compared to $\mathrm{CCK}_{2} \mathrm{R}$. Inhibiting PKC with either GF109203X or overnight treatment with PMA $(1 \mu \mathrm{M})$ blocked the steady-state phosphorylation of $\mathrm{CCK}_{2 \mathrm{i} 4 \mathrm{sv}} \mathrm{R}$, whereas, treatment with $100 \mathrm{nM}$ PMA for $30 \mathrm{~min}$ (a condition that activates PKC) further increased (2-fold) the level of 3il phosphorylation. Treatment with SU6656, PP2 AG1478, or PD98059 did not affect the steady-state levels of 3il domain phosphorylation of either receptor variant. MALDI-TOF/TOF MS analyses identified two peptide fragments with the same sequence (KLEMELS312WER), but which differed in their mass by approximately 30 Daltons, suggesting phosphorylation of \$312. S312 is located within the 69 amino acid insertion unique to $C_{C K}$ R. Treatment of cells expressing $C_{C K}$. $R$ for $24 \mathrm{~h}$ with PMA caused a 2.5-fold increase in the level of plasma membrane-associated receptor. Conclusions: Constitutive $\mathrm{CCK}_{2 i 4 s v} \mathrm{R}$ phosphorylation and internalization is regulated, in part, by a PMAsensitive PKC isozyme.

\section{W1595}

CGRP and SP Differentially Modulate Epithelial Cell Restitutioin Via Expression of TGF $\beta$ in Fibroblasts and Mastcells in Vitro Kerem Bulut, Peter Felderbauer, Karoline Hoeck, Susanne Deters, Wolfgang E. Schmidt, Peter Hoffmann

Background: Substance P (SP) and calcitonin gene related peptide (CGRP) are neurotransmitters of the afferent sensory nervous system. In experimental models of colitis in rats and rabbits, a protective role of SP and CGRP on intestinal mucosa was presumed. The mucosal protection in part depends on a SP and CGRP mediated modulation of mucosal blood flow after injury. We demonstrate a mastcell and fibroblast mediated effect of SP and CGRP on epithelial cell restitution in vitro. Methods: Mastcell and rat kidney fibroblast (NRK-49F) cell- lines were exposed to CGRP or SP in various concentrations. After incubation, the cell culture supernatants were taken from the mastcell of fibroblast cultures and were directly applied to IEC-18 or Caco-2 monolayers, which were wounded with a razor blade as described previously $24 \mathrm{~h}$ prior to the experiments. Epithelial cell migration was assessed by counting cells across the wound edge and epithelial cell proliferation was assessed using the MTT- test. Results: CGRP significantly induced epithelial cell migration and proliferation in vitro via stimulation of mastcells but had no effect on epithelial cells via stimulation of fibroblasts or when applied directly to epithelial cells. SP significantly induced epithelia cell migration and inhibited epithelial cell proliferation via stimulation of fibroblasts but had no effects via stimulation of mastcells or when applied directly to epithelial cells in vitro. The effects on epithelial cell migration were abolished after neutralizing anti-TGFP was added to the cell cultures. Conclusion: CGRP and SP modulate epithelial cell restitution in vitro mediated either by mastcells or by fibroblasts. The epithelial cell migration depends on a TGF $\beta$ release from CGRP stimulated mastcells or SP stimulated fibroblasts. Thi observation underlines an important role for the afferent sensory nervous system in mucosal defence and repair and in keeping the mucosal homeostasis.

\section{W1596}

Myd88-Dependent Phosphatidylinositol 3-Kinase (PI-3K) Activation in Flagellin/Toll-Like Receptor 5-Mediated Signaling Sang Hoon Rhee, Kristopher S. Strach, Charalabos Pothoulakis

Background \& Objectives: PI-3K activation is essential for intestinal epithelial cell proliferation in vitro and in vivo. Bacterial flagellin, released from commensal and enteroinvasive microbes in the human gut, stimulates Toll-like receptor 5 (TLR5) leading to proinflammatory responses and activation of PI-3K in colonic epithelial cells. However, the molecular mechanisms by which TLR5 activates PI-3K have not been well investigated. Here we determined the signaling pathways involved in flagellin/TLR5-induced PI-3K activation in human colonocytes. Methods: $\mathrm{C} 3 \mathrm{H} / \mathrm{HeJ}$ mice received water or dextran sulfate sodium (DSS)(2.5\%) followed by intracolonic administration of flagellin-containing enema (0.8mg/day). After 7 days total colonic protein extracts were analyzed by immunoblot analysis to evaluate flagellininduced PI-3K activation. To determine PI-3K activation in vitro, expression of TLR5 or its adaptor molecule MyD88 was silenced by stable transfection of TLR5 or MyD88 siRNA constructs, respectively in non-transformed NCM460 human colonocytes. Several TLR5 mutants harboring point-mutated tyrosine residues, including SH-2-domain binding motif 'YXXM' in the cytoplasmic TIR domain of TLR5, were generated by site-directed mutagenesis. Co-immunoprecipitation assays were performed to determine the association between TLR and MyD88 or PI-3K. Results: Flagellin administration in DSS-disrupted colon resulted in PI-3K activation, while intact colon did not respond to flagellin. Flagellin exposure to NCM460 cells transiently activated PI-3K within 10-30 min, while blocking PI-3K reduced IL-8 production in colonocytes. TLR5 silencing blocked PI-3K activation by flagellin, indicating that TLR5 specifically mediates PI-3K activation in colonocytes. Immunoprecipitation studies showed that TLR5 recruits the p85 regulatory subunit of PI-3K to its cytoplasmic TIR domain following flagellin stimulation. Moreover, following flagellin stimulation, MyD88 interacted with TLR5 in a time-dependent manner. Immunoprecipitation studies with TLR mutant constructs demonstrated that the SH-2 binding 'YXXM' motif in the TIR domain of TLR5 is not involved in $\mathrm{p} 85$ recruitment, implying that p85 is indirectly recruited to TLR5. Moreover, silencing MyD88 expression in colonocytes disrupted PI-3K activation and blocked the association between TLR5 and the p85 regulatory subunit. Lastly, co-immunoprecipitation experiments showed that MyD88 binds directly to 885 in response to flagellin. Conclusions: PI-3K activation in TLR5-associated signaling in human colonocytes is MyD88-dependent. Supported by a Senior Research Award (CP) and a Research Fellowship Award (SHR) from the CCFA

\section{W1597}

Histamine H4 Receptor Antagonism Attenuates Toll-Like Receptor Signaling and Inhibits Experimental Colitis in the Rat

Paul J. Dunford, Csaba Varga, Robin L. Thurmond, Brendan J. Whittle

The histamine H4 Receptor (H4R) is a newly described G-protein coupled receptor with little homology to the pro-inflammatory histamine $\mathrm{H} 1$ receptor or the $\mathrm{H} 2$ receptor. Expression appears limited to immune cells of hematopoietic lineage such as mast cells, dendritic cells, T cells and eosinophils. We have previously demonstrated the involvement of H4R in tolllike receptor (TLR)-mediated acute inflammation (JPET. 2004:309:404-13) and here describe the activity of a novel H4R antagonist both in vitro, against bacterial derived TLR ligand mediated events and in vivo, in a hapten-mediated colitis model in the rat. Hapten-mediated IBD models share a similar proposed enteric bacteria (and possibly TLR) mediated etiolog and pathology with human IBD. Daily oral dosing of rats with the highly selective H4R antagonist, JNJ 10191584 (10-100ma/kg bid) commencing l day prior to intra-colonic trinitrobenzene sulfonic acid (TNBS) challenge caused a dose dependent inhibition of macroscopic damage, inhibition of TNBS-elevated myeloperoxidase (MPO) and a reduction in histologically assessed neutrophil influx by $40 \pm 8,79 \pm 6$ and $70 \pm 9 \%$, respectively, $n=$ $10 ; \mathrm{P}<0.001$ at the highest dose) as well as attenuation of the mucosal/sub-mucosal thickening determined after 3 days. Analysis of cytokine levels in TNBS diseased tissues indicated elevations in TNF- $\alpha$ and IL- 6 that were significantly inhibited by administration of JNJ 10191584 (by $67 \pm 6$ and $75 \pm 5 \%$ at the highest dose respectively, $n=10, P<0.01$ ). As dendritic cells are intimately associated with the recognition and signaling of TLR ligands we investigated the role of H4R in TLR signaling on in vitro cultures of CDll+ dendritic cells. Pre-treatment of these cultures with $10 \mu \mathrm{M}$ H4R antagonist markedly attenuated the production of pro-inflammatory cytokines in response to stimulation with Pam3Cys ( $\mathrm{ng} /$ $\mathrm{ml}$ ) and lipopolysaccharide (LPS, $1 \mathrm{ng} / \mathrm{ml}$ ), ligands of TLR2 and TLR4, respectively. Most notably, TLR2-mediated TNF- $\alpha$ and IL- 6 release was reduced by $59 \%$ and $50 \%$, respectively. Irregular expression and/or signaling of TLRs has been genetically and clinically implicated in the pathogenesis of human IBD. From the present findings in vitro and in vivo, we conclude that H4R antagonists may have a potential therapeutic role in the treatment of IBD through inhibition of aberrant TLR signaling and pro-inflammatory cytokine production. 
W1598

Efficacy of An Estrogen Receptor- $\beta$ (ER- $\beta$ )Agonist, But Not An ER- $\alpha$ Agonist Or ER Antagonist, in the Mdrla-/- Murine Colitis Model

Silvia C. Resta-Lenert, Kim E. Barrett

Background: We have shown that estrogens have important roles in the physiology and pathophysiology of the gut and that an ER- $\beta$ agonist modulated ion transport and barrier function in colitic mdrla-/- mice. We hypothesized that ER agonists may also have a role in preventing mucosal injury in this model of intestinal barrier dysfunction. Methods: 4 and 12 wk old mdrla-/- (KO) mice (8-10 mice/group, $\mathrm{M}=\mathrm{F}$ ), were treated with an ER- $\beta$ agonist, diarylpropionitrile (DPN), an ER- $\alpha$ agonist, propylpyrazole triol (PPT), 17 $\beta$-estradiol (E), the ER antagonist, tamoxifen (TMX), or placebo for 4 weeks. Basal and stimulated ion transport and transepithelial resistance (TER), histopathology and inflammation markers (iNOS, COX-2, MPO, TNF $\alpha$ ) were examined in harvested colonic tissues by Ussing chamber methodology, microscopy, Western blotting, or immunoassays, respectively. Results: 4 wk old $\mathrm{KO}$ mice were clinically normal whereas $12 \mathrm{wk}$ old $\mathrm{KO}$ mice showed evidence of severe colitis, as expected. 4 wk old $\mathrm{KO}$ mice treated with placebo developed colitis over the 4 wk treatment period. Colitis in all animals was associated with increase in basal ion transport, diminished ion transport responses to forskolin, and impaired barrier function. E- and DPNtreated 4 wk old KO mice showed improved basal $(50.0 \pm 16.5$ and $30.3 \pm 3.5$ vs $84.0 \pm 3.3$; $\mathrm{p}<0.01$, respectively), and forskolin-stimulated $(155.5 \pm 6.0$ and $165.7 \pm 5.0$ vs $77.7 \pm 4.0$, $\mathrm{p}<0.01$, respectively) colonic ion transport, measured as change in short circuit current in $\mu \mathrm{A}$ $\mathrm{cm} 2$, and increased TER $(68.5 \pm 5.0$ and $69.8 \pm 3.0$ vs $55.3 \pm 3.0 \Omega . \mathrm{cm} 2 ; \mathrm{p}<0.05$, respectively) compared to placebo-treated KO mice. Colonic tissues from 4 wk old E- and DPN-treated KO mice maintained their census of goblet cells, and showed decreased neutrophilic infiltration compared with controls. DPN and E also significantly reduced iNOS, COX-2, MPO and TNF $\alpha$ in 4 wk old mice, effectively abrogating the onset of colitis. In 12 wk old KO mice, colitis is already established. While E and DPN did not reverse this colitis completely, electrophysiological, histological and inflammatory parameters were all significantly improved. In contrast, PPT and TMX were without effects on colitis in either age group, despite evidence of bioavailability. Conclusions: Activation of ER- $\beta$ prevented the onset of colitis in 4 wk old KO mice and improved colitis in older mice with established disease. Our data support the hypothesis that estrogen may play important roles in the physiology and pathophysiology of the gut, and that selective ER agonists devoid of effects on reproductive physiology may represent therapeutic alternatives in inflammatory bowel diseases.

\section{W1600}

Up-Regulation of Rgs4 Expression Induced By Interleukin-1 $\beta$ in Colonic Smooth Muscle Cells Is Mediated By Nf- $\mathrm{KB}$

Wenhui Hu, Fang Li, Jiean Huang, Karnam S. Murthy

Initial $\mathrm{Ca}^{2+}$-dependent contraction of intestinal smooth muscle mediated by $\mathrm{G}_{\mathrm{q}}$-coupled receptors is attenuated by the intrinsic GTPase activity of $G \alpha_{\mathrm{q}}$. GTPase activity is accelerated by a protein regulator of G-protein signaling (RGS4), resulting in rapid inactivation of $\mathrm{G} \alpha_{\mathrm{q}}$. Our recent studies have shown that treatment of colonic muscle cells with the proinflammatory cytokine, interleukin-1 $\beta$ (IL-1 $\beta$ ) inhibits initial acetylcholine-stimulated contraction by increasing the expression of RGS4. The signaling pathways involved in transcriptional regulation of RGS4 are not known. Aim. To determine whether up-regulation of RGS4 expression by IL-1 $\beta$ is mediated by transcription factor NF-KB. Methods. Rabbit colonic smooth muscle cells were cultured in DMEM and experiments were performed in first passage. RGS4 mRNA levels were determined by quantitative RT-PCR. IL-1 $\beta$-induced NF-KB activity was measured by electrophoretic mobility shift assay (EMSA), luciferase reporter gene assay, and by immunoblot using phospho-specific antibody to IKK (Ser178/ 180) or p65 (Ser536). The involvement of NF-KB pathway in RGS4 expression was analyzed using kinase-inactive IKK2 mutant (K44A), phosphorylation-deficient IKB $\alpha$ mutant (S32A) S36A) and by the use of selective inhibitors for IKK2 (IKKIV) and NF-KB (NF-KB activation inhibitor). Results. Exposure of smooth muscle cells to IL-1 $\beta$ induced activation of NF-KB as demonstrated by EMSA, reporter gene assay, I $\mathrm{\kappa} \mathrm{\alpha}$ degradation, and by phosphorylation of IKK2 at Ser178/180 and p65 at Ser536. IL-1 $\beta$ induced both rapid (1 h) and sustained 15-fold increase in RGS4 mRNA expression after 3 days. RGS4 expression was blocked by selective inhibitors of IKK2 or NF-KB and in cells expressing dominant negative mutants of IKK2(K44A) or IKB $\alpha(\mathrm{S} 32 \mathrm{~A} / \mathrm{S} 36 \mathrm{~A})$. The results imply that the increase in RGS4 expression caused by IL-1 $\beta$ is mediated by the canonical NF-KB pathway, which involves phosphorylation of IKB $\alpha$ by IKK2 and its degradation via a proteasomal pathway resulting in activation of NF- $\mathrm{KB}$. Conclusion. Up-regulation of RGS4 expression in response to IL-1 $\beta$ is mediated by transcription factor NF-KB. The increase in RGS4 expression accelerates deactivation of $\mathrm{G \alpha}_{\mathrm{q}}$ and results in inhibition of $\mathrm{Ca}^{2+}$-dependent muscle contraction.

\section{W1601}

\section{Sepsis Upregulates Enteric Apelin Expression Through An ERK Pathway} Guiyun Wang, Ella W. Englander, George H. Greeley

Apelin, the endogenous ligand for the APJ receptor, shows a widespread distribution in the body. Apelin is expressed in the vasculature, brain, kidney, adipose tissue, heart, lung and GI tract. Apelin signaling can influence the cardiovascular system, water and food intake. These effects of apelin may play a role in the pathophysiology of sepsis. The aim of these studies was to investigate the influence of sepsis on apelin expression in GI tract, the role of increased apelin transcriptional activity in the elevated apelin expression during sepsis, and to identify the signaling pathway behind LPS-increased apelin transcriptional activity. Methods. Sepsis was induced by lipopolysaccharide (LPS, $100 \mu \mathrm{g} / \mathrm{kg}$, IP) administration to rats. Gut tissues were harvested for measurement of apelin mRNA levels by Northern blotting. The effect of LPS on apelin transcriptional activity was tested in transient transfection assays using a mouse macrophage cells (RAW264.7) transfected with either rat or human apelin 5'-upstream fragment-luciferase reporter gene constructs. Involvement of p38, JNK, ERK or NFKB signaling in LPS-induced activation of apelin promoter activity was tested by means of pharmacologic inhibitors: (SB203580 [20 $\mu \mathrm{M}$ ], JNK inhibitor II [20 $\mu \mathrm{M}$ ], PD98059 [40 $\mu \mathrm{M}]$, and SN-50 NFKB $[10 \mu \mathrm{M}])$. EMSAs were done to define the extent to which LPS alters binding of a consensus AP1 oligonucleotide for a nuclear protein extract of RAW264.7 cells A putative APl binding site occurs in the rat and human apelin core promoters. Results. Apelin expression increased 2 to 3-fold in the rat stomach, duodenum and ileum 8 and 24 $\mathrm{h}$ after LPS treatment (stomach-control: $0.5 \pm .05$ vs LPS: $1.2 \pm 0.2$; duodenum-control $0.3 \pm .03$ vs LPS: $1.1 \pm .01$; ileum-control: $0.3 \pm .03$ vs LPS: $1.3 \pm 0.3$; $\mathrm{P}<0.05)$. Transient transfection experiments showed LPS $(100 \mathrm{ng} / \mathrm{ml})$ stimulated rat and human apelin promoter activity $\sim 3$-fold maximally $24 \mathrm{~h}$ after LPS. Apelin promoter activity was increased in a doseand time-dependent manner. Blockade of ERK activity but not p38, JNK and NFKB signaling lowered LPS activation of apelin promoter activity. EMSAs showed that LPS exposure increased APl binding activity. Conclusions and Discussion. LPS treatment increased apelin expression in the GI tract significantly. Enhanced apelin expression may be due to increased apelin transcriptional activity. LPS may stimulate apelin transcription via an ERK pathway since inhibition of ERK activity blocked LPS induced apelin promoter activity and LPS increased APl binding activity. These changes in GI apelin expression may have a role in the pathophysiology of the wasting syndrome during sepsis.

\section{W1602}

H. Pylori Caga Protein Down-Regulates TGF- $\beta$ Signal Pathway and Attenuates TGF- $\beta$-Induced Rgml Cell Growth Inhibition

Hai Ying Fu, Kayoko Asahi, Yujiro Hayashi, Tsutomu Nishida, Hiroaki Murata, Hiroshi Eguchi, Takeshi Azuma, Sunao Kawano

Background and Aim: CagA, a toxic protein of H.pylori, is injected into host cells via a type IV secretion system of bacteria and undergoes tyrosine phosphorylation forming complex with SHP-2 in the cells, thereby modifying cellular signal transduction. We have reported that CagA protein increased cell growth via MAPK pathway. On the other hand, TGF- $\beta$ is reported to inhibit the cell growth of normal gastric epithelial cells and negatively to regulate Thl cell development. Here, we investigated the effect of CagA expression on TGF- $\beta$ and Smad signal pathway. Materials and Methods: We transfected a cagA gene into rat gastric mucosal (RGM1) cells and established cell lines, in which CagA expression can be controlled under the Tet-off system. Smad7 expression induced by CagA protein was analyzed by RTPCR. The expression of CagA, Smad7, Smad2/3 and the phosphorylation of Smad2/3 were investigated by Western blot analysis. EMSA was used to examine the nuclear import of Smad 2/3/4 complex. The effect of CagA expression on TGF- $\beta$-induced cell growth inhibition was investigated with WST- 8 reagent. Results: In cagA gene-transfected RGM1 cells, the increased Smad7 expression and decreased Smad2/3 phosphorylation were found after CagA expression. Moreover, the nuclear import of Smad2/3/4 complex was inhibited in CagA expressing cells, compared with those in CagA unexpressing cells and mock cells. Furthermore, the addition of TGF- $\beta$ into cell culture medium showed inhibition of RGMl cell growth. Conclusion: CagA from H.pylori, expressed in RGMI cells, increased Smad7 expression, decreased Smad2/3 phosphorylation and inhibited the nuclear import of Smad2/3/4 complex. Moreover, TGF- $\beta$-induced RGMl cell growth inhibition was blocked by CagA expression. The results indicate that CagA protein down-regulates TGF- $\beta$ and Smad signal pathway, attenuating TGF- $\beta$-induced cell growth inhibition.

\section{W1603}

Secretory Responses to Substance P (SP) Are Mediated Via Several Neurokinin (NK) Receptor Subtypes in the Mucosa of the Chronically Inflamed Rat Colon Caroline B. Appleyard, Marangelie Morales, Cariluz Santiago

We have previously shown that SP-induced mucosal responses of the normal healthy rat colon are region-specific, with NKI receptors contributing proportionately more in all regions. Changes in SP and its receptors have been documented in colitis representing a possible therapeutic target. AIM: To systematically characterize the contributions of NK receptor subtypes to the responses induced by SP in chronically inflamed mucosa along the length of the rat colon. METHODS: Chronic colitis was induced in Sprague-Dawley rats by intracolonic administration of TNBS, reactivated 6 weeks later. Following euthanasia segments of proximal, mid and distal colon $(4-5 \mathrm{~cm})$ were stripped of muscularis propria and mounted in Ussing chambers for the measurement of short circuit current. Data were expressed as a percentage of each tissue's maximum response to acetylcholine (Ach). The effects of selective agonists and antagonists were assessed. RESULTS: Visible colonic macroscopic damage was found in all animals, with $\sim 35 \%$ having diarrhea at the time of sacrifice. Each region of the rat colon mucosa exhibited a concentration-dependent secretory response to Ach $\left(10^{-9}-10^{-3} \mathrm{M}\right)$ and SP $\left(3.3 \times 10^{-10}-3.3 \times 10^{-6} \mathrm{M}\right)$. The largest SP responses occurred in the proximal region $(50.2 \pm 10.5 \%, \mathrm{n}=8)$, and this could be attenuated by pre-treatment with TTX $(10 \mu \mathrm{M}, \mathrm{p}<0.01)$. Indomethacin pretreatment had no effects. In all regions the selective $\mathrm{NKl}$ agonist $\mathrm{Pro}^{9} \mathrm{SP}\left(7.2 \times 10^{-10}-7.2 \times 10^{-6} \mathrm{M}\right)$ elicited concentration-response curves that were more potent than for SP. The NK2 agonist $\left[\mathrm{Nle}^{10}\right]$-Neurokinin A $\left(1.3 \times 10^{-10}-1.3 \times 10^{-6} \mathrm{M}\right)$ produced less potent responses reaching a significantly smaller maximum in both the proximal and distal colon $(15.9 \pm 5.8 \%$ and $13.3 \pm 7.3 \%$ respectively, $n=5 \mathrm{p}<0.05)$. The NK3 agonist [MePhe ${ }^{7}$-Neurokinin $\mathrm{B}\left(7.8 \times 10^{-10}-7.8 \times 10^{-6} \mathrm{M}\right)$ produced little response in any region until the highest concentration. The selective NK1, NK2 and NK3 antagonists (SR140333, SR48968 and SR142801 respectively) each significantly attenuated SP-induced responses of the distal colonic mucosa $(10 \mu \mathrm{M} ; \mathrm{p}<0.01)$. SR142801 also significantly reduced the proximal mucosal responses to SP $(10 \mu \mathrm{M} ; \mathrm{p}<0.05)$. CONCLUSIONS: In the chronically inflamed rat colon NK1 receptors play a greater role in the mucosal response than either NK2 or NK3. These neurokinin-mediated secretory responses appear to be region specific, and to be mediated via both direct and indirect mechanisms. The use of selective agonists revealed the presence of all three NK receptor subtypes, and selective antagonism confirmed that each appears to mediate responses to endogenous SP in the chronically inflamed distal rat colon. Supported in part by SO6GM08239 and RR03050. 
W1604

Nitric Oxide Derived from Neuronal Nitric Oxide Synthase Regulates Relation of Ghrelin and Insulin

Tatsuhiro Masaoka, Hidekazu Suzuki, Yohei Nomoto, Hiroshi Hosoda, Toshihro

Nishizawa, Eisuke Iwasaki, Makoto Suematsu, Kenji Kangawa, Toshifumi Hibi

Background Ghrelin is a gastrointestinal peptide with physiological roles, including stimulation of growth hormone release, food intake and gastric motility. We previously reported increased plasma ghrelin levels in rats with streptozotocin-induced diabetes (FEBS Lett. 541:64,2003),H.pylori-infected Mongolian gerbils (Gut 53:187, 2004) and rats with cysrteamine-induced duodenal ulcers (Am J Physiol. 289:G138. 2005). Although in normal obese individuals, decreased plasma ghrelin levels have been reported, in patients with PraderWilli syndrome (PWS) characterized by hyperphagia and obesity, increased plasma and gastric ghrelin levels have been reported (J Clin Endocrinol Metab. 90:5441, 2005). This could be attributable to the lower level of insulin resistance in these patients as compared with that in patients with simple obesity. Since nitric oxide (NO) induces adaptive relaxation of the lower esophageal sphincter or gastric fundus, NO is considered to be an conditioning factor for meal ingestion. Thus, a relationship between NO and ghrelin is suggested. Using neuronal nitric-oxide-synthase-deficient $\left(\right.$ nNOS $\left.^{-/}\right)$mice, we evaluated whether NO derived from nNOS contributed to the mechanism of ghrelin production and secretion. Method Twelve-week old male nNOS ${ }^{-1}$ mice $(n=10)$ and wild-type (WT) mice $(n=10)$ were examined after eighteen hours fasting. The plasma and gastric ghrelin levels were measured by radioimmunoassay, and the preproghrelin mRNA expression level was measured by quantitative RT-PCR. Ghrelin-immunoreactive cells in the stomach were evaluated by immunohistochemistry. Serum leptin and insulin levels were measured by ELISA. Results In the $\mathrm{nNOS}^{-1-}$ mice, the body weight, food intake, plasma levels of active and total ghrelin, gastric levels of active and total ghrelin, gastric preproghrelin mRNA expression levels and numbers of ghrelinimmunoreactive cells in the gastric fundus were significantly increased as compared with the corresponding values in the WT mice. Furthermore, while the serum levels of leptin, which reflect the amount of adipose tissue, tended to be higher in the nNOS ${ }^{-1}$ mice as compared with those in the WT mice (WT mice: $647.3 \pm 157.9 \mathrm{ng} / \mathrm{ml}^{\mathrm{vs}} \mathrm{nNOS}{ }^{-/}$mice: $1480.7 \pm 370.6 \mathrm{ng} / \mathrm{ml}, \mathrm{p}=0.06$ ), there were no significant differences between the two groups of mice in the serum levels of insulin. Conclusion The production and secretion of ghrelin from the stomach were significantly increased in $\mathrm{nNOS}^{-/}$mice, which manifest significant body weight increase and lower levels of insulin resistance, as reported for patients with PWS. The results suggest that NO derived from nNOS may regulate the relation between ghrelin and insulin.

\section{W1605}

Teduglutide (ALX-0600), a Glucagon-Like Peptide-2 (GLP-2) Analogue Inhibits Contractility in the Colon Ex Vivo

Hong Wang, Lidia L. Demchyshyn

BACKGROUND: Administration of teduglutide, a dipeptidyl peptidase IV resistant peptide analogue of human GLP-2, increases intestinal mass, promotes nutrient transport activity and enhances barrier function in the gastrointestinal epithelium. Numerous studies have demonstrated that teduglutide reduces epithelial damage and mortality in animal models of intestinal injury. The physiological effects of GLP-2 and teduglutide are mediated by the GLP-2 receptor (GLP-2R). OBJECTIVE: The following study examined the effects of teduglutide and GLP-2 on the contractility of rodent colon ex vivo. METHODS \& RESULTS: Segments of colon from Sprague Dawley rats were suspended in organ bath chambers containing Krebs' solution and maintained at $37^{\circ} \mathrm{C}$ with $95 \% \mathrm{O} 2$ and $5 \% \mathrm{CO} 2$. Basal tone, contractility rate and peak height were used to measure the properties of spontaneous and ligand-mediated colonic contractions. Segments were initially loaded to a tension of $2 \mathrm{~g}$ and allowed to equilibrate for 60 minutes. During this period, segments were repeatedly washed every 10 minutes. GLP-2 analogues were subsequently incubated with each tissue segment for 5 to 10 minutes. The tissues were allowed to re-equilibrate with repeated washes following each experiment. Teduglutide and GLP-2 inhibited muscle contractility in a concentrationdependent manner with an estimated IC50 of $4.9 \pm 1.9 \mathrm{nM}(\mathrm{n}=6)$ and $13.5 \pm 5.4 \mathrm{nM}(\mathrm{n}=$ 9), respectively. Contractions did not recover to the original amplitude following wash-out and teduglutide re-challenge suggesting GLP-2R desensitization. While having no effect alone, GLP 2(3-33), the primary breakdown product of GLP-2, blocked the inhibitory effects of teduglutide and GLP-2. In the presence of $10 \mu \mathrm{M}$ GLP-2(3-33), teduglutide and GLP-2 inhibited colon contractility with an IC50 of $59.9 \pm 11.9 \mathrm{nM}(\mathrm{n}=6)$ and $142.7 \pm 55.9 \mathrm{nM}$ $(n=5)$ respectively. The rank orders of potency of GLP-2 peptide analogues were similar to that previously reported for peptide-induced increases in cAMP levels in cell lines recombinantly expressing the rGLP-2R. CONCLUSIONS: Teduglutide reproducibly and significantly reduced the contractility of rat colon in a concentration-dependent manner. GLP-2(3-33) exhibited no inhibition on contractility alone, however blocked the effects of teduglutide and GLP-2, acting as a GLP-2R antagonist. The rank order of potency of GLP-2 analogues on the inhibition of colon contractility was comparable to the rank order of increased cAMP accumulation in heterologous cells expressing GLP-2R. This study demonstrates the ability of teduglutide and GLP-2 to directly affect native GLP-2R-mediated contractility in colon tissue.

\section{W1606}

Dual Mechanism for Inhibition of cAMP By Interleukin-1 $\beta$ in Smooth Muscle: Inhibitory S-Nitrosylation of Adenylyl Cyclase V/Vi Via iNOS-Derived NO and Stimulation of Pde4 Expression and Activity

Jiean Huang, Sunila Mahavadi, Karnam S. Murthy

Exposure of smooth muscle to the pro-inflammatory cytokine, IL-1 $\beta$ induces iNOS expression leading to sustained release of nitric oxide (NO) that triggers feedback inhibition of soluble guanylyl cyclase (sGC) expression and activity and a decrease in cGMP formation. IL-1 $\beta$ also causes a decrease in cAMP levels, but the mechanism(s) for this decrease is/are unknown. The decrease in cAMP levels could reflect down-regulation of adenylyl cyclase V/VI (AC V/ VI) and/or up-regulation of cAMP-specific phosphodiesterase, PDE4D5, both of which are specifically expressed in smooth muscle. Aim. To characterize the expression and activities of AC V/VI and PDE4D5 in smooth muscle treated with IL-1 $\beta$. Methods. Colonic circular muscle strips were incubated for $48 \mathrm{~h}$ in DMEM with or without IL-1 $\beta(10 \mathrm{ng} / \mathrm{ml})$. Muscle cells were then isolated from these strips and used for determination of AC V/VI and PDE4D5 expression and activity. S-nitrosylation of AC V/VI was determined by the biotin-switch method. Results. IL-1 $\beta$ induced iNOS expression, and increased AC V/VI expression deter mined by immunoblot and RT-PCR. However, isoproterenol- and forskolin-stimulated cAMP formation (measured in the presence of IBMX to eliminate the effect of PDEs) was decreased, suggesting that IL-1 $\beta$ inhibited AC V/VI activity. Addition of NOS inhibitor (L-NNA), bu not the sGC inhibitor (ODQ) or the PKG inhibitor (R-pCPT-cGMP[S]), reversed the effect of IL-1 $\beta$ on cAMP formation, implying that the effect was mediated directly via iNOS derived NO. Treatment of muscle tissue with the NO donor S-nitroglutathione (GSNO), but not with a cGMP analog (8-Br-cGMP) or a PKG activator (CPT-cGMP), also inhibited isoproterenol- or forskolin-stimulated cAMP formation, providing further support for a direc inhibitory effect of NO. IL-1 $\beta$ or GSNO induced inhibitory S-nitrosylation of AC V/VI: a thiol reducing agent, DTT, blocked S-nitrosylation and reversed the inhibition of cAMP formation. In addition to its effect on cAMP formation, IL-1 $\beta$ increased cAMP degradation by stimulating PDE4D5 expression (determined by immunoblot) and activity (determined by degradation of ${ }^{3} \mathrm{H}$-cAMP). These effects were also evident by the decrease of cAMP levels in response to isoproterenol or forskolin (measured in the presence of L-NNA to eliminate the inhibitory effect of NO on AC V/VI and in the absence of IBMX). Conclusion. IL-1 $\beta$ inhibits cAMP formation via S-nitrosylation of adenylyl cyclase V/VI by iNOS-derived NO, and augments CAMP degradation by stimulating the expression and activity of cAMPspecific PDE4D5

\section{W1607}

Gastric Secretory Response to a Meal and Modulation of Somatostatin and Gastrin Immunoreactive Cells During Fasting and Feeding in Somatostatin Receptor Type 2 (SSTR2) Knockout Mice

Lixin Wang, Laura Piqueras, Yvette Tache, Vicente Martinez

The prandial state differentially regulates somatostatin and gastrin gene expression in the rat stomach. We previously established the role of SSTR2 in mediating somatostatin antisecretory effects using SSTR2 knockout (KO) mice (N-S Arch Pharmacol 370:510, 2004). AIM: To examine the distribution and regulation of gastric somatostatin- and gastrin-immunoreactive (IR) cells by the prandial status and the acid response to a meal in conscious SSTR2 KO mice. METHODS: Gastrin- and somatostatin-IR cells were quantified, after staining with anti-gastrin (CURE 05109.1) and anti-somatostatin (CURE S.6) antibodies, in the fundic and antral mucosa in adult SSTR2 KO and wild-type (WT) mice fasted for 18-20 h or fed ad libitum. Microscopic gastric morphology was also assessed in H-E stained sections. Gastric secretory responses (intragastric $\mathrm{pH}$ and acid content) to a standard meal were also determined. RESULTS: Fasting increased the number of fundic, but not antral, somatostatinIR cells by 2 - and 7-fold in STTR2 KO and WT respectively (Table). In SSTR2 KO mice the number of antral somatostatin-IR cells was decreased by $53 \%$ and $58 \%$ in fed and fasted state, respectively, compared with WT (Table). Gastrin-IR cells were present in the antral but not fundic mucosal glands. Their density was similar in KO and WT mice and not altered by fasting (Table). Stomach weight (WT: $186 \pm 8 \mathrm{mg}$; KO: $201 \pm 5 \mathrm{mg}$, each $\mathrm{n}=20$ ) and morphometric analysis of the antral and fundic mucosa, submucosa or muscular revealed no differences between $\mathrm{KO}$ and WT mice. Basal intragastric $\mathrm{pH}$ and acid content were similar in fasted WT $(3.5 \pm 0.2$ and $6.9 \pm 0.4 \mu \mathrm{mol}, \mathrm{n}=5)$ and $\mathrm{KO}$ mice $(3.1 \pm 0.1 ; 8.4 \pm 1.2 \mu \mathrm{mol}, \mathrm{n}=$ 5). The acid response to re-feeding for $1 \mathrm{~h}$ was similar in both groups (WT: $4.6 \pm 0.1 ; 8.6 \pm 1.1$ $\mu \mathrm{mol}$; KO: $4.8 \pm 0.3 ; 9.8 \pm 1.0 \mu \mathrm{mol}$; each $\mathrm{n}=5$ ). CONCLUSIONS: SSTR2 KO mice had a 2 fold reduction in the number of antral somatostatin-IR cells vs WT, while gastrin-IR was not altered. The unchanged acid response to feeding in $\mathrm{KO}$ mice indicates that functional somatostin-SSTR2 pathways do not modulate normal postprandial secretory responses. Compensatory changes to the absence of SSTR2-dependent inhibitory pathways at functional and/or ultrastructural levels might explain the apparent normal gastric morphology and functionality of SSTR2 KO mice.

\begin{tabular}{|c|c|c|c|c|}
\hline & \multicolumn{2}{|c|}{ Wild-type } & \multicolumn{2}{c|}{ SSTR2 KO } \\
\hline & Fasted & Fed & Fasted & Fed \\
\hline Fundic somatostatin-IR cells & $14 \pm 4$ & $2 \pm 1 *$ & $10 \pm 1$ & $5 \pm 2$ *\# \\
\hline Antral somatostatin-IR cells & $15 \pm 2$ & $12 \pm 2$ & $7 \pm 1 \#$ & $5 \pm 1 \#$ \\
\hline Antral gastrin-IR cells & $11 \pm 1$ & $10 \pm 2$ & $10 \pm 1$ & $9 \pm 1$ \\
\hline
\end{tabular}

Data are mean \pm SEM and represent IR cells/field. *:P $<0.05$ vs cell density in fasted mice; \# $\mathrm{P}<0.05$ vs corresponding value in WT mice

\section{W1608}

Effect of Female Sex Hormone Supplementation and Withdrawal On Gastrointestinal and Colonic Transit in Postmenopausal Women Jonathan Gonenne, Tuba Esfandyari, Michael Camilleri, Lorraine Fitzpatrick, Duane D Burton, Debra Stephens, Kari Baxter, Alan R. Zinsmeister

Females are disproportionately affected by constipation, which is often aggravated during pregnancy and the luteal phase of the menstrual cycle. Aims: To evaluate effects of acute administration of $400 \mathrm{mg} /$ day micronized progesterone, $0.2 \mathrm{mg} /$ day estradiol, combination of the same doses of progesterone and estradiol, or placebo on gastric emptying (GE), small bowel transit, and colonic transit (CT) in healthy postmenopausal subjects; and to determine whether withdrawal of the hormones was associated with a change in transit. Methods: 48 postmenopausal females were randomized (12 per group) and underwent scintigraphy using a ${ }^{99 \mathrm{~m}} \mathrm{Tc}$-egg meal and ${ }^{111} \mathrm{In}$-charcoal. All kept daily stool diaries. After withdrawal of medications, transit measurement was repeated. Treatment assignment was double-blinded; treatment groups were balanced on age. Primary endpoints were ascending colon emptying 
$\mathrm{tl} / 2$ (hr) and colonic geometric center (GC) at 24 hours. Secondary variables were colon GC at 4 and 48 hours, GE at 4 hours, colonic filling at 6 hours, and stool frequency, consistency (Bristol stool form scale) and ease of passage. Results: A statistically significant effect of progesterone on colonic transit was evident by shorter ascending colon emptying tl/2 (Fig.) and significantly accelerated overall transit shown by colonic geometric center at 48 hours. Other transit endpoints were not significantly altered by progesterone. No transit endpoints were altered by estradiol or combined hormonal treatment relative to placebo. Withdrawal of the hormone supplement was not associated with any significant alteration in transit. Progesterone $(\mathrm{p}=0.024)$ and estradiol $(\mathrm{p}=0.04)$ were associated with looser stool consistency. Conclusion: Micronized progesterone accelerates colonic transit in postmenopausal females. This has implications for the pathophysiology and management of slow transit constipation in postmenopausal females.

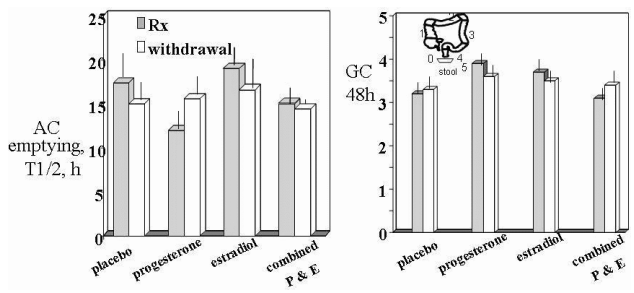

W1610

Intracerebroventricular, But Not Intravenous, Stresscopin Acts By CRF Type 2 Receptor to Inhibit Pengastrin-Induced Stimulation of Gastric Acid Secretion in Rats

Chih-Yen Chen, Full-Young Chang, Shou-Dong Lee

Background \& Aims: Stresscopin, a novel 40-amino acid peptide, was recently identified and characterized. Stresscopin represents endogenous CRF2 receptor ligand for maintaining homeostasis after stress. Although stresscopin has been shown to suppress food intake, delay gastric emptying and decrease heat-induced edema in mice, no previous studies have examined the effects of this novel stress-related peptide on pentagastrin-induced stimulation gastric acid output (GAO) in rats. Methods: We first examined the effects of intracerebroventricular (ICV) or intravenous (IV) administration of stresscopin on pentagastrin-stimulated $\mathrm{GAO}$ in urethane-anesthetized rats. The novel selective CRF2 receptor antagonist, astressin2$\mathrm{B}$, was either ICV or IV injected before stresscopin given. The responsible receptor sites in the brain as well as periphery were investigated. IV injection of omeprazole served as the wellknown inhibitory control group of GAO under pentagastrin-induced stimulation. Results: IV injected stresscopin, even at the very high dose $(4.58 \mathrm{nmol})$, failed to suppress pentagastrinstimulated (16 ug/kg/hr, IV) GAO, whereas IV injected omeprazole (345 ug) significantly inhibited pentagastrin-induced stimulation. In contrary, ICV injected stresscopin $(0.3,1$ and $3 \mathrm{nmol}$ ) dose-dependently inhibited pentagastrin-stimulated GAO. Pre-treatment with ICV injection of the selective CRF2 receptor antagonist, astressin2-B, completely blocked ICV injected stresscopin-induced inhibition of pentagastrin-stimulated GAO. However, pre-treatment with IV injection of astressin2-B did not have effects on ICV injected stresscopininduced inhibition on pentagastrin-stimulated GAO. Conclusions: The results suggest that ICV but not IV injection of stresscopin inhibits pentagastrin-induced stimulation of GAO in rats. In the brain, CRF receptor subtype 2, is involved in this acid inhibitory mechanism.

\section{W1611}

Gastric Emptying in CRF-Overexpressing and CRF Receptor 2 Knockout Mice Lixin Wang, Million Mulugeta, Vincent S. Wu, Pu-Qing Yuan, Honghui Liang, Mary P. Stenzel-Poore, Pauline M. Jamison, Yvette Tache

Background: Previously we showed in rodents that CRF and related peptides inhibit gastric emptying (GE) through $\mathrm{CRF}$ receptor type $2\left(\mathrm{CRF}_{2}\right)$ while post-operative gastric ileus does not occur in $\mathrm{CRF}_{1}$ knockout mice or is prevented by $\mathrm{CRF}_{1}$ unlikely by IP $\mathrm{CRF}_{2}$ antagonist (Peptides 2004, 25:1733; Gastroenterology 2003, 125:654). The $\mathrm{CRF}_{1}$-medaited action may involve the central sympathetic pathways. Aim: To characterize GE in transgenic mice with altered CRF signaling system: $\mathrm{CRF}$-overexpressing $(\mathrm{OE})$ and $\mathrm{CRF}_{2}$ deficient (-/-). Methods: Female adult CRF-OE and $\mathrm{CRF}_{2}-$ - mice or their wide type littermates (WT or $\overline{\mathrm{CRF}_{2}+/+}$ ) fasted for $16-18 \mathrm{~h}$ were anesthetized for $10 \mathrm{~min}$ and underwent abdominal surgery (celiotomy and $30 \mathrm{~s}$ cecum palpation), or no surgery (sham). The $20 \mathrm{~min}$ GE was determined by gavaging the mice with a non-nutrient liquid at $100 \mathrm{~min}$ after the surgery. Brains from CRFOE mice were processed $2 \mathrm{~h}$ after surgery for Fos immunoreactivity (IR) and the stomach for expression of CRF receptors and urocortin 2 using RT-PCR. Results: CRF-OE mice had higher basal GE than WT mice and developed delayed GE after abdominal surgery that had similar magnitude of decrease from respective basal values ( $\Delta$ changes: $33.4 \%$ in CRF-OE v $30.7 \%$ in WT) (Table). Abdominal surgery induced expression of Fos-IR in selective brain areas such as the paraventricular nucleus of the hypothalamus (PVN), central nucleus of the amygdale, lateral parabrachial nucleus, ventrolateral medulla and nucleus of the solitary tract. However, there was no significant difference between CRF-OE and WT mice. Expression of transcripts of $\mathrm{CRF}_{2} \beta$ (the functional subtype) and urocortin $2\left(\mathrm{CRF}_{2}\right.$ ligand) in the gastric corpus did not show differences among groups. No CRF or CRFl transcript was detected in the stomach of both CRF-OE and WT mice in this study. Abdominal surgery inhibited significantly GE in both $\mathrm{CRF}_{2}-/$ and $\mathrm{CRF}_{2}+/+$ compared to sham (Table), while interleukin$1 \beta\left(10 \mu \mathrm{g} / \mathrm{kg}\right.$, IP) did not inhibit $\mathrm{GE}$ in $\mathrm{CRF}_{2}+/+$ as it did in $\mathrm{CRF}_{2}-/$. Conclusions: Peripheral $\mathrm{CRF}_{2}$ is not involved in post-operative gastric ileus but may contribute to impaired GE induced by cytokines. The negative feedback of glucocorticoid on CRF containing neurons in the PVN in CRF-OE mice (Peptides 2001, 22:733) and metabolic changes may override the $\mathrm{CRF}$ signaling system, resulting higher GE.

Table. Gastric emptying rate (\%) of a non-nutrient liquid meal in $\mathrm{CRF}-\mathrm{OE}$ and $\mathrm{CRF}_{2-\digamma_{-}}$mice after abdominal surgery.

\begin{tabular}{|c|c|c|c|c|}
\hline & WT & CRF-OE & $\mathrm{CRF}_{2+/+}$ & $\mathrm{CRF}_{2---}$ \\
\hline Sham & $53.4 \pm 3.7$ & $74.7 \pm 7.9 \#$ & $82.7 \pm 7.7^{*}$ & $61.0 \pm 8.8$ \\
\hline Abdominal Surgery & $22.7 \pm 6.5^{*}$ & $41.3 \pm 12.2^{*}$ & $28.2 \pm 9.4 *$ & $16.1 \pm 4.6^{*}$ \\
\hline
\end{tabular}

*: $\mathrm{P}<0.05$ vs the same genotype sham; \#: $\mathrm{P}<0.05$ vs WT sham

W1612

PI3K/Akt Signaling Regulates Stem Cell Activation in Colitis-Induced Cancer Goo Lee, Ramanarao Dirisina, Navdha Mittal, Jennet Manjali, Gery Grimm, Yueming Tang, Xi C. He, Linheng Li, Terrence A. Barrett

Data in human colitis-induced cancer $(\mathrm{ClC})$ indicate that neoplastic transformation occurs via distinct mechanism from sporadic colorectal cancer. To examine the mechanisms involved CIC, we utilized piroxicam model of colitis in IL10-/- mice which induces severe transmural colitis followed by indolent period of chronic inflammation associated with elevation in proinflammatory cytokine and chomokine mRNA. Current study is focused on epithelial stem cell response to chronic inflammation. Using BrdU incorporation as an indicator for epithelial proliferation, we found that crypt proliferation increased by 2 fold during early period of colitis but areas of high grade dysplasia seen on D35-42, BrdU index increased by 6 fold with levels as high as 10 folds above normal in invasive cancer observed on D70. Staining for nuclear (activated) beta catenin (Bcat-N) was used to enumerate activated stem cells in tissue(He et al Nat genet 2004). Real time PCR analyses were performed to detect induction of Bcat target genes (cmyc, cox2, mmp7, fra-1, enc-1). This study indicated that number of stem cells increased by 4 fold in colitis and 12 fold in area of dysplasia with areas of invasive cancer with 15 fold. Activate stem cells in areas of dysplasia and carcinoma were remarkably identified outside basally located stem cell zone. Western blot analysis revealed that PI3K signaling was induced with 20-50\% higher levels of P-Akt and P-GSk3 beta seen in colitis and $100 \%$ elevated levels of phosphrylated intermediates in dysplasia and tumor nodules. To examine the effect of PI3K inhibition on stem cell activation in colitis and cancer, mice were treated 3d with systemic LY294002 (30mg/Kg/day). Data revealed that numbers of activated stem cells were reduced significantly with the greatest effect $(90 \%$ reduction) detected in cancer nodules without significant change of pro-inflammatory cytokine mRNA level. Western blot analysis of Bcat-N levels in isolated epithelial cells revealed that PI3k inhibition reduced Bcat-N levels by $30-50 \%$ in colitis and dysplasia whereas levels were reduced by $90 \%$ in areas of carcinoma. These data correlated well with reduction of proliferation in these tissues as well as dramatic amelioration of cMyc expression in LY294002treated mice. Taken together these findings suggest that stem cell activation is a critical step for induction of CIC. Furthermore, aberrant activation of PI3K/Akt pathway was required for persistent stem cell activation in colitis and during neoplastic transformation. Most notably, inhibition of this pathway showed most pronounced effect in areas of carcinoma suggesting that this may be a target for future therapeutic intervention.

\section{W1613}

IL-7 Overexpression: Role for Intestinal EC-Derived IL-7 in Formation of Intraepithelial and Lamina Propria Lymphocyte Populations

Hua Yang, Deborah L. Gumucio, Blair Madison, Daniel H. Teitelbaum

Background: We have previously shown that loss of IL-7 results in significant derangements in intestinal lymphocyte phenotypes including a decline in intestinal lymphocyte numbers. Because IL-7 plays a crucial role in controlling T-cell development and homeostasis, we investigated the effect of intestinal specific over-expression of IL-7 on intestinal mucosal lymphocytes. Meathod: We generated an intestinal epithelial cell specific over-expressing IL-7 transgenic mouse model (IL-7vill), by cloning mouse IL-7 cDNA behind an intestinespecific villin promoter (P12.4 KVill). IL-7 vill mice were sacrificed at 8 weeks of age. Wildtype C57BL/6J (WT) mice were used as control. Intestinal derived IL-7 expression was detected using RT-PCR and Western blot. Serum IL-7 levels were detected by ELISA. To further confirm the transgenic IL-7 specific expression was only from intestinal mucosa, thymocytes and splenocytes, as well as hepatic and kidney tissue were also harvested. Phenotype changes of intraepithelial lymphocytes (IEL) and lamina propria lymphocytes (LPL) were studied with flow cytometry. Results: RT-PCR showed IL-7 expression to be expanded exclusively in the small and large intestine. Intestinal mucosal IL-7 protein expression in IL-7vill mice increased 3-fold compared to WT mice. No serum IL-7 was detectable in either WT and IL-7vill mouse. Numbers of IEL and LPL in IL-7vill mice were significantly $(\mathrm{P}<0.05)$ higher than WT mice (Table). $\mathrm{CD} 8 \alpha \beta+$, presumably thymic-dependent, increased 5.6-fold in IEL, CD4+CD8- IEL increased 6.5-fold, whereas CD4+CD8- LPL increased 2fold. TCR- $\alpha \beta+$ IEL increased 2.5-fold, mature CD4+ IEL (denoted by CD44+) increased 1.5-fold, mature CD4+ LPL increased 2-fold; and activated CD4+ IEL (CD4+,CD69+) increased 7.6-fold compared to WT mice, activated CD4+ LPL increased 3.7-fold. Systemic lymphoid populations did not change in IL-7vill mice. Conclusions: IL-7 over-expression by intestinal epithelial cells significantly affected both IEL and LPL populations, resulting in over-expansion of the CD4+ and CD8 $\alpha \beta+$ IEL subtypes, as well as an increase in IEL and LPL maturation and activation. These results offer further insight into the role of epithelial cell-derived IL-7 in IEL and LPL development.

\begin{tabular}{|c|c|c|c|c|c|c|c|}
\hline & LPL & & & IEL & & \\
\hline & $\begin{array}{c}\text { Cell } \\
\times 106\end{array}$ & $\begin{array}{c}\text { CD4+CD8- } \\
(\%)\end{array}$ & $\begin{array}{c}\text { CD4+CD44+ } \\
(\%)\end{array}$ & $\begin{array}{c}\text { Cell } \\
\text { Number } \\
\times 106\end{array}$ & $\begin{array}{c}\text { CD4+CD8- } \\
(\%)\end{array}$ & $\begin{array}{c}\text { CD4+CD44+ } \\
(\%)\end{array}$ & $\begin{array}{c}\text { CD8 } \alpha \beta+ \\
(\%)\end{array}$ \\
\hline WT & $4.8 \pm 1.2$ & $26.5 \pm 4.5$ & $34.3 \pm 3.5$ & $8.1 \pm 1.7$ & $3.7 \pm 1.5$ & $6.5 \pm 1.7$ & $6.5 \pm 1.7$ \\
\hline IL-7vill & $14.6 \pm 4.5^{*}$ & $59.8 \pm 6.0^{*}$ & $42.8 \pm 6.0^{*}$ & $21.0 \pm 2.4^{*}$ & $24.6 \pm 4.6^{*}$ & $64.5 \pm 16.7 *$ & $45.2 \pm 8.8^{*}$ \\
\hline
\end{tabular}

Results are mean $\pm \mathrm{SD} .{ }^{*} \mathrm{P}<0.05$ using t test 
W1614

Quercetin Inhibits TNF-Induced IRF-1 But Not NF-KB Recruitment to the IP10 Gene Promoter in Intestinal Epithelial Cells Through the Modulation of Histone Acetyl Transferase Activity

Pedro A. Ruiz, Annett Braune, D.R. Haller

Background \& Aim. The molecular understanding of nutritional factors in the process of host factor-mediated activation of the intestinal epithelium may play an important role for assessing adjunct nutritional therapy of chronic intestinal inflammation. In this study, we characterized the molecular mechanisms of the polyphenolic compound quercetin and its enteric bacterial metabolites taxifolin, alphitonin and 3, 4-dihydroxy-phenylacetic acid to inhibit TNF-induced pro-inflammatory gene expression in the murine small intestinal epithelial cell (IEC) line Mode-K. Methods. Mode-K cells were stimulated with TNF (5 pg/ml) for various times in the presence and absence of $100 \mu \mathrm{M}$ of the polypehenolic compounds followed by the analysis of IP- 10 protein secretion, NF- $\mathrm{\kappa B}$ reporter gene activity, Akt and IKB/NF- $\mathrm{KB}$ phosphorylation/degradation, caspase 3 activation, IP-10 gene promoter binding activity. Oral application of quercetin ( $10 \mathrm{mg} / \mathrm{day} / \mathrm{kg}$ body weight) were applied to heterozygous TNFAARE mice. Results. Quercetin inhibits TNF-induced IP-10 gene expression with an effective inhibitory concentration of $39 \mu \mathrm{M}$. Interestingly, taxifolin, alphitonin and 3, 4dihydroxy-phenylacetic acid completely failed to inhibit TNF responses in IEC, suggesting that the colonic microbial transformation of quercetin completely abolished its anti-inflammatory effect. Interestingly and in contrast to 3'-hydroxy-flavone, quercetin completely failed to inhibit TNF-induced NF- $\kappa \mathrm{B} / \mathrm{I}-\kappa \mathrm{B}$ phosphorylation and I $\kappa \mathrm{B}$ degradation as well as TNF-

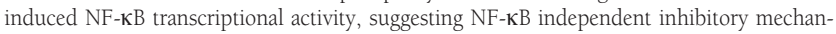
isms. Chromatin immunoprecipitation (ChIP) revealed inhibitory effects of quercetin on the recruitment of the transcriptional co-activator interferon regulated factor (IRF)-1 but not NF- $\mathrm{kB}$ to the IP-10 gene promoter. Most important for the molecular understanding of this inhibitory mechanism, quercetin inhibited histone acetyl transferase (HAT) activity and the phosphorylation/acetylation of histone 3 at the IP-10 gene promoter. In addition, quercetin inhibited TNF-induced survival pathways by blocking Akt phosphorylation associated with the late induction of pro-apoptotic mechanisms in IEC. The physiological role of quercetin in modulating experimental ileitis is currently evaluated in heterozygous TNF $\triangle$ ARE mice. Conclusion. These studies support an anti-inflammatory effect of quercetin on the small intestinal epithelium through mechanisms that inhibit co-factor recruitment at the chromatin level by modulating the histone acetyl transferase activity.

\section{W1615}

PI3-Kinase Regulates T Cell Activation-Induced SDF-1/CXCR4 mRNA Expression in Intestinal Epithelial Cells Yueming Tang, Gery Grimm, Goo Lee, Terrence Barret

Background and Aims: The intestinal stem cell differentiates into 4 main lineages of epithelial cells, namely, enterocytes, goblet cells, enteroendocine cells, and Paneth cells. Our recent studies show that $\mathrm{Wnt} / \beta$-catenin signaling pathway plays an important role in intestinal stem cell activation in T cell activation and colitis-induced cancer model. Intestinal stem cells were activated by T cell or colitis to proliferate into terminally differentiated cells or cancer cells through PI3-Kinase/ $\beta$-catenin signaling. Stromal cell-derived factor-1 (SDF-1) and its receptor (CXCR4) are important regulators of stem cell homeostasis in different physiology or pathology conditions such as inflammation and cancer. Recent research has indicated that SDF-1 is up-regulated in malignant tissues and CXCR4 is involved in increasing the metastatic potential of colon cancer. Therefore, inhibition of SDF-1/CXCR4 axis could be a strategy for the prevention of colon caner. In current study, we use T cell activation model to study the effects of T cell activation on SDF-1/CXCR4 expression and the role of PI3kinase in the mechanisms. Methods and Results: To activate T cells, 6-8 week-old C57BL/ 6 mice were injected intraperitoneally with 200 $\mathrm{g}$ anti-CD3. Mice were sacrificed for tissue harvest at 3 hours after anti-CD3 injection. Intestinal epithelial cell were isolated using a modification of a previously published method. SDF-1/CXCR4 mRNA expression were assessed by quantitative real time PCR. The data show that T cell activation induced SDF1 mRNA increased $1.9 \pm 0.20$ folds, CXCR4 mRNA increased $2.75 \pm 0.32$ folds. Pretreatment with the PI3kinase inhibitor LY-294002, the T cell activation-induced SDF-1/CXCR4 mRNA expression were inhibited $64 \%$ and $76 \%$. Conclusions: Our data show that SDF-1/CXCR4 is up-regulated by $\mathrm{T}$ cell induced PI3kinase signaling. Since T cell-induced PI3kinase/ $\beta$ catenin signaling plays an important role in the mechanisms of IBD and colon cancer, our results suggest that SDF-1/CXCR4 might be a general target for inhibition of intestinal epithelial cell proliferation. SDF-1/CXCR4 inhibitors have potential as anticancer agents to suppress outgrowth of micrometastases.

\section{W1616}

\section{Carcinoembryonic Antigen Expressing Cells Preferentially Activate CD8+ T} Cells

Xianyang Yio, Caroline Korsten, Constantin Friedman, Mee Sook Park, Jiang Yio, Franziska Roth-Walter, Lilani Perera, Oke Kamalu, Lloyd Mayer

Background: Previous studies in our lab have demonstrated that the intestinal epithelial cell (IEC) is able to activate CD8+ regulatory T cells, termed TrE. Evidence suggests that the induction of TrE cells results from interactions of the gp 180/CDld complex and CD8/T cell receptor. Protein sequencing data concluded that gpl 80 belongs to the carcimoembryonic antigen (CEA) family. This finding raises the spectre of immune suppression by tumor cells expressing CEA. Aim: To study whether CEA/gpl80 affects CD8+ and CD4+ T cell proliferation in antigen non-specific and alloantigen stimulated T cell proliferation systems. Methods: 1) PHA was used to stimulate peripheral blood $T$ cells, $C D 8+T$ cells, and CD4+ T cells, in the presence or absence of gpl 80 or CEA. 3H-thymidine incorporation measured cell proliferation. 2) T cells were co-cultured with a human melanoma cell line transfected with either human CDld cDNA (FO1.D5), the CDld and CEA (FO1.D5-CEA), or FO1.D5 pulsed with CEA. T cell proliferation was assessed by $3 \mathrm{H}$-thymidine incorporation. 3) PARP western blot was performed to assess the induction of apoptosis. Results: gp180/CEA suppressed total T cell proliferation in PHA stimulated T cell in a dose dependent fashion. Apoptosis was absent by PARP western blot. This suppression was specifically inhibited by addition of monoclonal antibody to gp180 (mAb B9), suggesting gpl80/CEA exerts an inhibitory effect on T cell proliferation. Addition of gp 180 enhanced the proliferation of CD8+ T cells but suppressed CD4+ T cell proliferation. Interestingly, FO1.D5 cells were able to induce CD4+ T cell proliferation while CEA expressing FO1.D5-CEA failed to induce such proliferation, suggesting an inhibitory effect of CEA on CD4+ T cells. In contrast, the CEA expressing FO1.D5-CEA cell resulted in better CD8+ T cell proliferation as compared to FOl.D5 cell alone. These results suggest that CEA/gp180, either in soluble or membrane bound form, stimulates CD8+ T cell proliferation. These results are consistent with our previous finding that gp 180 is involved in the induction of CD8+ suppressor T cells. Conclusions: It appear that cells expressing CEA/gpl80 stimulate CD8+ T cell proliferation while inhibiting CD4+ T cells. Further studies are required to characterize and compare the proliferating CD8+ cells stimulated by the soluble or membrane form of CEA/gp180 in the presence or absence of CDld. (Supported by the AGA Centocor Excellence in IBD Clinical Research Fellowship and CCFA grant).

\section{W1617}

Dose-Dependent Inhibition of Stem Cell Activation 5 ASA: A Possible Explanation for the Chemo Preventative Effect of 5 ASA Therapy in ColitisInduced Cancer

Gery Grimm, Goo Lee, Ramanarao Dirisina, Navdha Mittal, Elizabeth Managlia, Jennet Manjali, Yueming Tang, Jeffrey Brown, Terrence Barrett

Intro/Aims:Data suggest that 5ASA medications reduce the risk of colitis-induced cancer(CIC). The AOM/DSS mouse model of $\mathrm{CIC}$ was utilized to examine whether 5ASA reduces the risk for CIC in colitic mice by impairing epithelial stem cell activation. Methods: C57BL 6J mice were given azoxymethane (AOM-8 $\mathrm{mg} / \mathrm{kg}$ ip), fed $2 \%$ dextran sulfate sodium(DSS) $\mathrm{x} 7 \mathrm{~d}$ followed by $\mathrm{H} 2 \mathrm{O}$ xl4d for 2 cycles. 5ASA dosages $100 \mathrm{mg} / 250 \mathrm{~g}$ chow (low dose-LD) or $300 \mathrm{mg} / 250 \mathrm{~g}$ chow (high dose-HD) were chosen based on their partial $(\sim 25 \%, \mathrm{LD})$ and moderate $(\sim 75 \%, \mathrm{HD})$ inhibition of T cell-induced mucosal iNOS, CXCL-10 and COX-2 mRNA. 2 treatment groups were used to assess the ability of 5ASA to prevent CIC. Initially, we confirmed reports that long term 5ASA reduces dysplasia by giving mice 5ASA from day 1 of the protocol for 50d. Mice were given 5ASA after the initial bout of colitis to study the treatment effects of 5ASA(d21-42). Colitis (grade 0-4), levels of low grade dysplasia (LGD) and high grade dysplasia (HGD), and cancer (Ca) were measured (Cooper et al, Lab Invest, 1993) at sacrifice. Epithelial stem cell activation was assessed by IHC of nuclear beta-catenin (Xi et al, Nature Genetics, 2004). RESULTS: Long term LD and HD 5ASA reduced levels of dysplasia by $50-60 \%$ (LD 5ASA) and by $>90 \%$ (HD 5ASA). To determine effects of 5ASA used as a treatment for active colitis, mice were examined after 5ASA was given to mice with severe colitis (Fig.1). Summary: 1) A dose-dependent drop in rates of HGD was seen in mice given 5ASA x 3wk after the onset of severe colitis. 2) LD and HD 5ASA blocked the progression of CIC. 3) Severe colitis dramatically increased rates of stem cell activation and crypt cell proliferation. 4) 5ASA treatment reduced stem cell activation and proliferation in a dose-dependent manner. Conclusions: These data suggest that 5ASA inhibition of aberrant stem cell activation explains its chemopreventative effect in CIC.

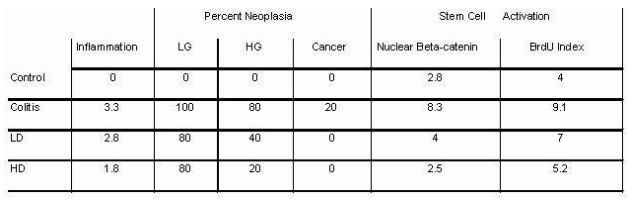

\section{W1618}

The Differentiation of Intestinal Epithelial Cells (IECs) Is Regulated By the Crosstalk Between IECs and Lamina Propria Lymphocytes Stephanie Dahan, Giulia Roda, Franziska Roth-Walter, Lloyd Mayer

The interactions between luminal contents, epithelial cells and mucosal T cells likely play a significant role in maintaining intestinal homeostasis. Data from many systems suppor crosstalk between IECs and T cells. In this study, we asked whether Normal or Crohn's Disease (CD) lamina propria lymphocytes (LPL) could provide signals to IECs promoting their growth and differentiation. METHODS: T84, Caco-2 and HT29 subclone 16E cells were grown polarized or non-polarized and co-cultured with freshly isolated LPL for varying periods. After removal of LPL, IECs were lysed and subjected to i) Western blot analysis for MAPK and Akt activation; ii) quantification of alkaline phosphatase (AP) activity; iii) RT-PCR for MUC2 and TFF3 gene expression. In vitro findings were corroborated in vivo using immunohistochemistry (MAPK and Akt activation) on human colonic tissue sections. RESULTS: Phospho-MAPK (ERK1/2, p38, and JNK) and phospho-Akt were seen as early as 30 min after co-culture persisting for at least 3 hours. Interestingly, the level of the MAPK activation was greater in T84 and Caco-2 cells co-cultured with CD LPL than with Normal LPL. There was an increase in epithelial P-ERK1/2 and P-JNK staining of the surface colonic mucosa in active $C D$ vs inactive $C D$ or normal mucosa. In contrast, P-Akt had a differen localization pattern, ie in the basolateral aspect of the upper $2 / 3$ of the surface IEC in normal mucosa, which extended into the crypt in inactive $C D$, but not in active $C D$ mucosa. $A P$ activity was slightly increased in T84 cells (and slightly decreased in HT29 16E cells ) cocultured for 8 days with Normal LPL $(82 \pm 54$ in T 84 alone vs $219 \pm 194 \mathrm{nmol} \mathrm{pNP} / \mathrm{mg}$ prot) In contrast, AP activity was significantly increased in both T84 and HT29 16E cells cocultured with CD LPL $(86 \pm 84$ in T84 alone vs $686 \pm 18 \mathrm{nmol} \mathrm{pNP} / \mathrm{mg}$ prot; and $249 \pm 24$ in HT29 16E alone vs $337 \pm 8 \mathrm{nmol} \mathrm{pNP} / \mathrm{mg}$ prot). Despite this, the co-culture of either Normal or CD LPL with HT29 16E for 4 days shut down the MUC2 mRNA expression. CONCLUSION: The MAPK and PI-3K signaling pathways are involved in cell survival, growth, and differentiation. LPL provide signals to IECs, which tend to favor IEC differentiation. Dysregulated IEC differentiation in CD may be explained by the disturbance of this dialogue in vivo. 
W1619

Curcumin Modulates Neutrophil Migration At Multiple Levels Claire Billerey-Larmonier, Monica T. Midura-Kiela, Anna J. Midura, Fayez K. Ghishan, Pawel R. Kiela

Polymorphonuclear neutrophils (PMN) are the first cells recruited at the inflammation site. These cells play a key role in the innate immune response by recognizing, ingesting and destroying many pathogens without the support of the adaptive immune response. However ransepithelial migration of neutrophils in intestinal inflammation results also in reduction of epithelial barrier function, perpetuation of inflammatory processes, and tissue destruction via oxidative damage and the release of proteases. Curcumin (diferulolylmethane) has been shown to display a protective role in mouse models of IBD, a phenomenon accompanied by a dramatic reduction of neutrophilic infiltration in the colon. We therefore hypothesized that this molecule may modulate PMN recruitment and function. The effects of dietary curcumin-mediated attenuation of colonic inflammation in BALB/c mouse model of TNBSinduced colitis were measured via microarray and real-time PCR analysis, with a particular focus on expression of genes involved in neutrophil chemotaxis. These analyses identified a list of genes intimately involved in this process which were normalized by dietary curcumin. This list included CXCL-2 (MIP-2; mouse IL-8 homologue), CXCL-13, CCL-9, IL1 $\beta$, formyl peptide receptor-like 1 (Fprl1), colony stimulating factor 2 receptor (Csf2r $\beta 1), C 3$ complement, calgranulins A and B, and SSP1 (osteopontin). Curcumin-mediated attenuation of LPS-stimulated transcription and secretion of MIP- 2 and IL1 $\beta$ was further confirmed in YAMC colonic epithelial cells and macrophages (RAW264.7 and resting peritoneal macrophages). In vitro chemotaxis assays with calcein-stained bone marrow-derived mouse PMN demonstrated profound effects of curcumin when conditioned medium from control, LPS or LPS/curcumin treated peritoneal macrophages, RAW264.7 or YAMC cells was used as chemoattractants. This inhibition corresponded with the dramatically decreased concentration of MIP-2 in the medium. Although curcumin had only a moderate effect on PMN chemotaxis toward recombinant MIP-2, it markedly inhibited random migration of PMN in the absence of chemoattractants, suggesting an effect on neutrophil motility and chemokinesis. This finding was further confirmed with PMN pre-treated with increasing concentrations of curcumin, which had dramatically reduced random and MIP-2 directed translocation in vitro, with a maximal effect at $10 \mu \mathrm{M}$ of curcumin. In summary our results indicate that curcumin could interfere with the early phase of colonic inflammation partly through inhibition of neutrophil chemotaxis, both directly as well as indirectly via inhibition of expression of chemotactic molecules and their receptors.

\section{W1620}

Differential Regulation of the IL-18/IL-18bp System in Human Intestinal Epithelial Cells

Stephan Haas, Melanie Gruber, Darko Gosenca, Peter Feick, Manfred V. Singer, Ulrich Boecker

Background: An imbalance of cytokines on the mucosal level is believed to contribute to the immunopathogenesis of chronic inflammatory bowel diseases. Biological effects of interleukin-18 may promote intestinal inflammation if insufficiently counterbalanced by their natural antagonists, the IL-18 binding proteins. Aim: To determine whether expression and release of IL-18 and IL-18bp are differentially regulated in intestinal epithelial cells. Methods: Cultivated intestinal epithelial cell lines HT-29 and Caco-2 were exposed to different stimuli in vitro in the presence or absence of specific inhibitors of signal transduction. Expression of IL-18, IL-18bp and Caspase-1 mRNA and protein was determined by PCR, Western blot analysis and ELISA. Caspase-1 activity was assessed by a functional assay. Presence of IL-18, IL-18bp and caspase-1 in primary human intestinal epithelial cells was analyzed by immunohistochemistry on mucosal biopsies from Crohn's disease patients and controls. Results: Both IL-18 and Il-18bp mRNA were constitutively expressed in intestinal epithelial cells. Only the $24 \mathrm{kDa}$ proform of the IL-18 protein was detectable in unstimulated cells. Butyrate enhanced the expression of IL-18, while the basal level of IL-18bp remained unchanged. By contrast, up-regulation of IL-18bp was achieved by exposure to IFN- $\gamma$ and augmented by co-incubation with TNF- $\alpha$, but abrogated in the presence of butyrate. Dosedependent induction of IL-18bp by IFN- $\gamma$ was completely suppressed when protein kinase $\mathrm{C}$ activity was inhibited by RO 31-8220. As opposed to the differential effects of IFN- $\gamma$, exposure to gram-positive Staphylococcus aureus (SAC) increased both the protein content of IL-18 and IL-18bp in the intracellular compartment; only IL-18bp was synergistically enhanced by SAC and IFN- $\gamma$. Expression and functional activity of caspase-1 was markedly augmented by IFN- $\gamma$ and SAC, respectively. In the presence of chronic inflammation expression of IL-18, IL-18bp and caspase-1 was up-regulated in the epithelium of mucosal biopsies. Conclusions: Expression of IL-18 and IL-18bp is differentially regulated by IFN- $\gamma$, TNF- $\alpha$ and SAC in human intestinal epithelial cells. A better understanding of the signal transduction processes involved may enable a selective shift of the IL-18/IL-18bp ratio in chronic inflammatory bowel disease.

\section{W1621}

Crosstalk Between Intraepithelial Lymphocytes (IELs) and Epithelial Cells (ECS) Increases IL-8 Production Through the CD2-CD58 Interaction with Regulation By Interferon (IFN) $\gamma$ Asit Panja, Ellen Ebert

Introduction: IL-8 is produced in large quantities by intestinal ECs. Since IELs have poten chemotactic activity with IL-8, they are drawn into the epithelium. We hypothesize that IL8 production by ECS and IELs is modulated by interactions between the two cell types. Methods: IELs were isolated from jejunal mucosa of patients undergoing gastric bypass operations for morbid obesity. HT-29 cells were used as a model of ECs. IELs and ECs were co-cultured without a stimulus at a 5:1 ratio for 18 hrs. IL-8 was measured by ELISA. Results: Both IELs and HT-29 cells produced IL-8. There was a 1.6-fold synergistic increase in IL-8 when these two cell types were cocultured. When IELs were cultured in an insert overlying a monolayer of HT-29 cells, IL-8 still synergistically increased but not to the same extent as when the cells were cultured together. This suggests the involvement of cell contact as well as a soluble factor. Antibody blocking experiments revealed that the synergistic increase in IL-8 with coculture of IELs and HT-29 cells was dependent on the CD2-CD58 rather than the TCR-MHC class I interaction. When neutralizing various cytokines with specific antibody, the most marked change in IL-8 production was a 1.7 -fold increase with anti-IFN $\gamma$. When cells were cultured separately, IFN $\gamma$ surprisingly reduced while anti-IFN $\gamma$ increased IL- 8 production by IELs but not by HT- 29 cells. In addition, IFN $\gamma$ increased CD58 expression on HT-29 cells, potentially up-regulating IFN $\gamma$ release by IELs through the CD2 pathway. Conclusion: This study shows a synergistic increase in IL-8 production when IELs and HT-29 cells were cultured together. This increase depended on the CD2-CD58 interaction, a potent stimulus for IFN $\gamma$. IFN $\gamma$, in turn, up-regulated CD58 expression on HT-29 cells, potentially stimulating further IFN $\gamma$ release through the CD2 pathway in IELs. Finally, IFN $\gamma$ suppressed IL-8 production, not by HT-29 cells, but by IELs. IFN $\gamma$ as an inhibitor of IEL function is a novel, and surprising, finding that serves to control IL-8 production.

W1622

Regulation of Matrix Metalloproteinases Synthesis By Interleukin-21 (IL-21) in Human Intestinal Fibroblasts

Ilaria Peluso, Daniele Fina, Roberta Caruso, Carmine Stolfi, Valentina Gioia, Massimo C. Fantini, Thomas T. MacDonald, Francesco Pallone, Giovanni Monteleone

Background and Aim: Activated lamina propria T cells play a central role in the pathogenesis of tissue damage in Inflammatory Bowel Disease (IBD). Although, the exact mechanism by which $\mathrm{T}$ cells mediate mucosal injury during chronic intestinal inflammation remains unknown, there is evidence that $\mathrm{T}$ cell derived-cytokines stimulate stromal cells to synthesize matrix metalloproteinases (MMPs), thereby promoting mucosal degradation. We have previously shown that, in IBD, mucosal inflammation associates with high production of interleukin (IL)-21, a T cell-derived cytokine. In this study we have extended our analysis of IL21 biological activity in the gut, and first examined whether intestinal fibroblasts express IL-2l receptor (IL-21R), and second investigated the effect of IL-2l on MMP production. Methods: IL-21R was evaluated in normal and IBD fibroblasts cultured in the presence or absence of inflammatory cytokines by RT-PCR and Western blotting. To examine the effect of IL-21 on MMPs, IBD fibroblasts were either left untreated or treated with graded doses of IL-2I in the presence or absence of TNF- $\alpha$, and then MMPs synthesis evaluated by RTPCR, Western blotting and zymography. Finally, we evaluated the effect of a neutralizing IL-21R fusion protein (IL-2lR/Fc) on the induction of MMPs in fibroblasts stimulated with Crohn's disease (CD) lamina propria mononuclear cell (LPMC) supernatants. Results. A constitutive expression of both IL-2l receptor (IL-21R) and common gamma-cytokine chain, which are necessary for IL-21-driven signaling, was seen in intestinal fibroblasts isolated from normal and IBD mucosa. Importantly, TNF- $\alpha$ but not IFN- $\gamma$ enhanced IL-21R expression. Stimulation of both normal and IBD fibroblasts with IL-2l resulted in enhanced synthesis of interstitial collagenase, stromelisyn-1, gelatinases A and B, whereas expression of tissue inhibitors of MMP- 1 and -2 remained unchanged. Importantly, the effect of IL-21 on MMPs secretion was dose-dependent and enhanced by TNF- $\alpha$. Finally, we showed that CD LPMC supernatants stimulate MMP secretion by intestinal fibroblasts, and that this effect is partly inhibited by IL-21R/Fc, clearly indicating that CD LPMC-derived IL-2l is functionally capable of regulating MMP production. Conclusions: These results suggest that fibroblasts are a potential target of IL-2l in the gut and that, in IBD, IL-2l can contribute to the tissue damaging immune response by driving MMPs production.

\section{W1623}

IL-7 Is Essential for the Development and Persistence of Chronic Colitis Teruji Totsuka, Takanori Kanai, Yasuhiro Nemoto, Motomi Yamazaki, Shin Makita, Mamoru Watanabe

Background: IL-7 has recently emerged as a key cytokine involved in controlling the survival of peripheral resting memory $\mathrm{CD} 4^{+} \mathrm{T}$ cells and their homeostatic turnover. We have previously demonstrated that mucosal CD4 $4^{+} \mathrm{T}$ cells expressing high levels of IL-7 receptor (IL$7 \mathrm{R}^{\text {high }}$ ) are pathogenic cells responsible for chronic colitis. Methods \& Results: To investigate the role of IL-7 in the development and the persistence of chronic colitis, we transferred normal CD $4^{+} \mathrm{CD} 45 \mathrm{RB}^{\text {high }} \mathrm{T}$ cells or colitogenic lamina propria (LP) $\mathrm{CD} 4^{+}$memory $\mathrm{T}$ cells into $\mathrm{IL}-7^{+/+} \times \mathrm{RAG}_{-1}-^{-1-}$ and IL- $7^{-1-} \times \mathrm{RAG}-1^{-1-}$ mice. After the adoptive transfer of $\mathrm{CD} 4{ }^{+} \mathrm{CD} 45 \mathrm{RB}^{\text {high }}$ splenocytes into syngeneic ${\mathrm{IL}-7^{+/+}}^{+} \mathrm{RAG}-\mathrm{I}^{-/}$mice, the recipients develop massive inflammation of the large intestinal mucosa concurrent with massive expansion of Thl cells. In contrast, clinical and histological signs of disease are completely absent in IL- $7^{-/} \times$RAG$1^{-/-}$mice transferred with normal $\mathrm{CD} 4^{+} \mathrm{CD} 45 \mathrm{RB}^{\text {high }}$ splenocytes. Furthermore, IL- $7^{+/+} \times \mathrm{RAG}-$ $1^{-/}$, but not $\mathrm{IL}-7^{-/-} \times \mathrm{RAG}-1^{-/}$, mice re-transferred with the colitogenic LP $\mathrm{CD}^{+} \mathrm{CD} 44^{\text {high }} \mathrm{CD} 62 \mathrm{~L}^{-}$effector-memory $\mathrm{T}\left(\mathrm{T}_{\mathrm{EM}}\right.$ ) cells obtained from colitic $\mathrm{CD} 4{ }^{+} \mathrm{CD} 45 \mathrm{RB}^{\text {high }}$ transferred RAG- ${ }^{-1}$ mice, developed colitis. Surprisingly, rapid proliferation of donor colitic $\mathrm{LP} \mathrm{CD} 4^{+}$memory $\mathrm{T}_{\mathrm{EM}}$ cells was observed in the in IL-7-1- $\times \mathrm{RAG}^{-1^{-/}} \mathrm{LP}$ to a similar extent of those in ${\mathrm{IL}-7^{+/+}} \times \mathrm{RAG}_{-1} \mathrm{1}^{-1} \mathrm{LP} \mathrm{CD} 4^{+}$memory $\mathrm{T}_{\mathrm{EM}}$ cells 7 days after the transfer into RAG$1^{-1-}$ mice using CFSE dilution method. In contrast, a massive LP CD4+ $\mathrm{T}$-cell apoptosis was observed in IL- $7^{-/} \times$RAG- $1^{-/}$, but not in IL- $7^{+/+} \times \mathrm{RAG}_{-1} 1^{-/}$, mice. Conclusions: Taken together, IL-7 is essential for the development and persistence of chronic colitis as a critical survival factor rather than growth factor for colitogenic $\mathrm{LP} \mathrm{CD} 4^{+}$memory $\mathrm{CD} 4^{+} \mathrm{T}$ cells, suggesting that therapeutic approaches targeting IL-7/IL-7R signal pathway may be feasible in the treatment of inflammatory bowel disease. 
W1624

Mechanism of Interferon-Gamma (IFN- $\gamma$ ) Induced Increase in Intestinal Epithelial Tight Junction Permeability: Cross-Talk Between PI-3 Kinase and NF-KB Pathways

Praveen K. Roy, Michel Boivin, Angela Bradley, John C. Kennedy, Thomas Y. Ma

Background: Interferon gamma (IFN- $\gamma$ ) induced increase in intestinal epithelial tight junction (TJ) permeability has been proposed as an important pathogenic mechanism contributing to intestinal inflammation. However, the intracellular pathways that mediate the IFN- $\gamma$ induced increase in intestinal TJ permeability remain poorly understood. Previous studies have shown that IFN- $\gamma$ may activate PI-3 kinase or NF-kB in various cell types. Thus, the purpose of this study was to investigate the possible role of the PI-3 kinase pathway and the intracellular mechanism involved in mediating the IFN- $\gamma$ induced increase in intestinal tight junction permeability using filter-grown T84 intestinal epithelial cells. Methods: T84 TJ permeability was assessed by measuring the trans-epithelial electrical resistance (TER) and paracellular permeability. PI-3 kinase activation was assessed by translocation of PI-3 kinase (p85) subunit from the cytoplasm (soluble) to the membrane (insoluble) fraction by western blot. NF- $\mathrm{KB}$ activation was assessed by DNA binding ELISA assay and NF-KB responsive SEAP promoter assay. Results: IFN- $\gamma$ produced a concentration and time-dependent decrease in T84 TER ( $80 \%$ decrease at 72 hours). The drop in TER correlated with a three-fold increase in paracellular permeability to inulin. IFN- $\gamma$ induced drop in T84 TER was also associated with a decrease in occludin protein expression and disruption in junctional localization. IFN- $\gamma$ treatment resulted in rapid activation of PI-3 kinase (within 5 minutes); and inhibition of PI-3 kinase activation by selected inhibitors, LY 294002 and Wortmanin, prevented the IFN- $\gamma$ induced drop in T84 TER and decrease in occludin expression, suggesting that PI-3 kinase activation was required for the IFN- $\gamma$ induced increase in T84 TJ permeability. IFN- $\gamma$ caused a delayed activation of NF- $\mathrm{\kappa B}$ pathway ( $8 \mathrm{~h}$ after treatment); and inhibition of NF- $\mathrm{KB}$ activation with NF- $\mathrm{\kappa B}$ inhibitor PDTC prevented the IFN- $\gamma$ drop in TER and down-regulation of occludin expression. Moreover, PI-3 kinase inhibition also prevented NF- $\mathrm{KB}$ activation. Conclusion: Our results suggest for the first time that the IFN- $\gamma$ induced increase in intestinal TJ permeability and disturbance in occludin protein were mediated by the activation of PI-3 kinase pathway. Our findings indicate that IFN- $\gamma$ leads to upstream activation of PI-3 kinase, which in turn leads to a step-wise activation of NF- $\mathrm{\kappa B}$ pathway, down-regulation of occludin protein expression and a functional opening of the T84 TJ barrier

\section{W1625}

Histidine Inhibits LPS-Induced Pro-Inflammatory Cytokine Secretion from Macrophages Via Suppression of NF- $\kappa$ B Activation

Ayatoshi Andou, Tadakazu Hisamatsu, Susumu Okamoto, Hiroshi Chinen, Nobuhiko Kamada, Masaki Hashimoto, Hideaki Kihara, Toshifumi Hibi

BACKGROUND: Elemental diet (ED) therapy for Crohn's disease is efficacious in the induction and maintenance of remission. Although some of its therapeutic effects are postulated due to its low antigenic load and low fat content, the mechanisms remain unclear. ED contains a high amount of free amino acids, some of which were recently reported to contribute to the modulation of gut inflammation in a colitis model. In an IL-10 knock-out (KO) transfer model, dietary histidine significantly reduced local inflammation. AIM: To investigate the mechanism of the ameliorating effect of histidine on experimental colitis. METHODS: In the IL-10 KO transfer model (Ikenoue et al. Int Immunopharmacol. 2005), the severity of colitis was evaluated pathologically, the wet colon was weighed. Colonic mRNA expression of TNF- $\alpha$ was detected by quantitative RT-PCR. The localization of macrophages in the colon was confirmed by frozen section immunohistochemistry. In vitro, thioglycolate-induced mouse peritoneal exudate cells (TG-PEC) or human peripheral blood derived $\mathrm{CD}_{1} 4^{+}$monocytes were incubated with LPS and amino acids. The concentration of cytokines in the supernatant was determined by enzyme-linked immuno-sorbent assay. Cell viability was determined using a colorimetric assay (WST-8). Degradation of IKB- $\alpha$ was comfirmed by immunoblotting analysis. RESULTS: In the IL-10 KO transfer model, dietary $5 \%$ histidine, but not $5 \%$ alanine, reduced histological damage, colonic weight (38\% inhibition, $\mathrm{p}<0.05$ ), and colonic mRNA expression of TNF- $\alpha$. Since immunohistochemical analysis revealed a significant increase of $\mathrm{F} 4 / 80^{+}$macrophages in the inflamed lamina propria in this model, we hypothesized that histidine directly inhibits TNF- $\alpha$ secretion from the intestinal macrophage. In vitro, histidine inhibited LPS-induced TNF- $\alpha$ and IL-6 secretion from mouse macrophages (TG-PEC) in a dose-dependent manner and the inhibition rate reached $80 \%$ at a concentration of $5 \mathrm{mM}$ (basal medium; $0.2 \mathrm{mM}$ histidine), whereas alanine had no such effect. Neither amino acid was toxic to the cells at concentrations up to $25 \mathrm{mM}$.

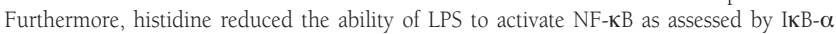
proteolysis. Finally, histidine also suppressed TNF- $\alpha$ production in human monocytes. CONCLUSIONS. These results suggested that histidine reduced NF-kB activation, and this effect was attributable to its down-regulatory action on pro-inflammatory cytokine production in macrophages. The anti-inflammatory effects of amino acids might reveal a new aspect of the action mechanism of ED therapy for Crohn's disease

\section{W1626}

A New Transcription Factor That Regulates TNF- $\alpha$ Gene Expression, LITAF (Lipopolysaccharide Induced TNF- $\alpha$ Factor) Is Increased in Ileal and Colonic Tissues from Patients with Crohn's Disease and Ulcerative Colitis Karen L. Reed, Arthur F. Stucchi, Michael J. O'Brien, Sandra R. Cerda, Charles Andrews, Adam C. Gower, Kristen N. Bushell, Susan E. Leeman, Salomon Amar James M. Becker

Background: The proinflammatory cytokine TNF- $\alpha$ plays a key role in the pathogenesis of Crohn's disease (CD) and ulcerative colitis (UC). Recently, a new transcription factor, LITAF, was shown to mediate TNF- $\alpha$ gene transcription in human macrophages by binding directly to the TNF- $\alpha$ promoter. Since the initial studies implicating LITAF in TNF- $\alpha$ gene expression were performed in a macrophage cell line, the aim of this study was to determine LITAF expression in vivo in ileal and colonic tissues resected from patients with $\mathrm{CD}$ and $\mathrm{UC}$. We also sought to determine the relative levels of LITAF message and protein in inflamed compared to non-inflamed areas of these tissues. Methods: Human ileal and colonic tissue from patients with $C D$ and UC were collected at the time of surgery. LITAF protein was localized by immunohistochemistry and quantified by Western blot analysis, while LITAF mRNA was measured by real time PCR. Results: COLON: LITAF immunostaining was localized to lamina propria macrophages and was markedly increased in UC and CD tissues compared to non-IBD controls. Non-inflamed CD tissues expressed 5-times more LITAF mRNA compared with non-IBD controls $(\mathrm{p}<0.05)$. A further $60 \%$ increase in LITAF mRNA was observed in inflamed $C D$ tissues vs. to non-inflamed areas $(p<0.08)$. In UC, LITAF mRNA levels from non-inflamed areas of the colon were elevated 15 -fold $(\mathrm{p}<0.05)$ above non-IBD controls. However a further increase was not observed in tissues resected from inflamed areas. Similar to PCR results, Western blot analysis showed a marked increase in LITAF protein in inflamed areas compared with non-inflamed areas of the colon $(\mathrm{p}<0.05)$ from patients with $C D$. In tissues obtained from patients with UC, LITAF protein level were similar in non-inflamed and inflamed areas. The relative levels of TNF- $\alpha$ mRNA and protein in these tissues paralleled those of LITAF. ILEUM: Similarly, in inflamed ileal tissues from CD patients, LITAF was localized to lamina propria macrophages, and LITAF mRNA and protein levels were significantly increased in inflamed ileal tissues compared with noninflamed areas. Conclusions: This is the first demonstration of LITAF expression in colonic and ileal tissues from patients with either CD or UC. LITAF is readily detectable in these tissues and is significantly elevated above controls. It is localized to macrophages, a majo source of TNF- $\alpha$. These data provide strong evidence for a role for LITAF in the pathophysiologic regulation of the TNF- $\alpha$ gene and underscore the potential value of anti-LITAF strategies in the clinical management of these diseases.

\section{W1627}

A STAT3 Dependent Transcriptome Is Up-Regulated in Pediatric Crohn's Colitis At Diagnosis

Rebecca Carey, Xiaonan Han, Lee Denson

The IL-6 dependent transcription factor STAT3 has been implicated in the pathogenesis of IBD. We have reported that growth hormone $(\mathrm{GH})$ down regulates STAT3 activation in Crohn's disease (CD)and reduces STAT3 activation and mucosal inflammation in IL-10 deficient murine colitis. However, a global assessment of STAT3 target genes which may promote chronic colitis has not been reported. We hypothesized that STAT3 target genes regulating leukocyte recruitment, cell proliferation and survival, and tissue remodeling would be up-regulated in $\mathrm{CD}$ at diagnosis, and that $\mathrm{GH}$ would exert an anti-inflammatory effect in murine colitis via down regulation of these genes. Methods: Colon biopsies and serum were obtained from eight children with $\mathrm{CD}$ at diagnosis and eight healthy controls. RNA was prepared from colon biopsies and gene expression was determined using the Affymetrix Human Genome U133 array and analyzed with GeneSpring TM software. Serum cytokine and chemokine levels were measured using the Bioplex ${ }^{\mathrm{TM}}$ assay $\mathrm{CD}$ colon biopsies were exposed to GH in short term culture and localization of activated STAT3 was determined. IL-10 deficient mice with colitis were treated with GH and colon chemokine expression were determined. Results: Bioplex analysis of serum cytokines and chemokines showed consistent up regulation of IL-6. Moreover, multiple IL-6/STAT3 target genes were up regulated in affected $\mathrm{CD}$ colon. These included chemokines regulating neutrophil (CXCL2, 3,5, and 6) and T cell (CXCL11) recruitment, several collagen (COLlAl and COL4Al) and matrix metalloproteinase (MMP1, 3, and 10) subtypes, and transcription factors regulating cell proliferation and survival including C-FOS, JUNB, and C/EBPD. The degree of up regulation of these genes was proportional to the degree of histological injury, as measured by the Crohn's Disease Histological Index of Severity (CDHIS). Short term exposure to GH in tissue culture reduced STAT3 activation in CD colon biopsies in both CEC and LP mononuclear cells. mRNA expression of the murine homologues of the T cell chemokines CXCL9 and CXCL11 was increased in IL-10 deficient mice and localized primarily to CEC by immunohistochemistry. Chronic GH administration partially reduced expression of these chemokines, in association with reduced STAT3 activation and mucosal inflammation. Conclusions: A pro-inflammatory IL-6/STAT3 dependent transcriptome which regulates leukocyte recruitment, cell proliferation and survival and tissue remodeling is activated at diagnosis in pediatric Crohn's colitis. Therapeutic approaches which reduce STAT3 activation are likely to promote mucosal healing in Crohn's colitis.

\section{W1628}

Pro-Inflammatory Cytokines IL-1 $\beta$ and TNF- $\alpha$ Repress Transcriptional Activation of the Enterocyte Differentiation Marker Intestinal Alkaline Phosphatase Gene

Madhu S. Malo, Shaluk Biswas, Mario A. Abedrapo, Lisa Yeh, Alexander Chen, Richard A. Hodin

INTRODUCTION: Gut inflammatory diseases such as IBD are associated with an alteration in the phenotype of the intestinal epithelial cells, most notably a loss in expression of the brush border marker, intestinal alkaline phosphatase (IAP). In the present study we investigated the molecular mechanisms responsible for this IAP gene silencing. METHODS: HT-29 cells were treated with $5 \mathrm{mM}$ sodium butyrate $(\mathrm{NaBu})$ to induce villus-like differentiation. The cells were exposed to the cytokines IL-1 $\beta$ and TNF- $\alpha$ prior to the differentiation stimulus in order to simulate the inflammatory conditions seen in vivo. Northern analysis, IAP-luciferase reporter assays, western blotting, and chromatin immunoprecipitation (ChIP) were performed to examine the mechanism of the effects of cytokines on NaBu-induced IAP gene activation RESULTS: Northern blot analyses confirmed the marked induction in IAP expression after $24 \mathrm{~h}$ of $\mathrm{NaBu}$ treatment, whereas pre-treatment $(6 \mathrm{~h}$ or $18 \mathrm{~h}$ ) with cytokines showed a dose-dependant decrease in IAP mRNA levels (approximately $35 \%$ for IL-1 $\beta, 25 \%$ for TNF$\alpha$ at $10 \mathrm{ng} / \mathrm{ml}$ of cytokines). The transfection studies revealed that IAP-Luc activity was markedly induced by $\mathrm{NaBu}$ (approximately 5 -fold), but the cells pre-treated with cytokines showed significant inhibition of IAP activation (90\% and $60 \%$ with IL-1 $\beta$ and TNF- $\alpha$, respectively). Western analyses on purified histones showed that $\mathrm{NaBu}$ induced a dramatic $\mathrm{H} 4$ and $\mathrm{H} 3$ acetylation, consistent with its known activity as a histone deacetylase inhibitor. This global histone hyperacetylation by $\mathrm{NaBu}$ was unaffected by pre-treatment with IL-1 $\beta$

\section{-}


or TNF- $\alpha$. ChIP assays showed that $\mathrm{NaBu}$ induced both $\mathrm{H} 4$ and $\mathrm{H} 3$ acetylation in the IAP proximal promoter region $(\mathrm{H} 4>\mathrm{H} 3)$. This local $\mathrm{H} 4$ and $\mathrm{H} 3$ acetylation was selectively reduced by pre-treatment with IL-1 $\beta$, but not by TNF- $\alpha$. CONCLUSION: We conclude that the cytokine inhibition of IAP gene expression is not due to a blockade of global histone hyperacetylation, but in the case of IL- $1 \beta$, it may be due to a specific inhibition of acetylation within the proximal promoter region. It is likely that the altered enterocyte phenotype associated with the inflammatory cytokines results from distinct inhibitory pathways.

\section{W1629}

Blockade of Interleukin (IL)-21 Enhances Lamina Propria T Lymphocytes Apoptosis in Patients with Inflammatory Bowel Disease (IBD)

Daniele Fina, Ilaria Peluso, Roberta Caruso, Valentina Gioia, Carmine Stolfi, Massimo C. Fantini, Fabio Andrei, Gian C. Naccari, Salvatore Bellinvia, Francesco Pallone, Giovanni Monteleone

Background \& Aim. In IBD, lamina propria T lymphocytes (T-LPL) are resistant to apoptosis, but the mechanism underlying such a defect is not fully understood. We have recently shown that IL-21 is produced in excess in IBD. As IL-21 signals through the $\gamma$-chain receptor, and $\gamma$-chain-mediated signals inhibit T cell apoptosis, we evaluated whether blockade of IL21 enhances IBD T-LPL death. Materials \& Methods. IBD CD3+ and CD4+ LPL were cultured with a neutralizing IL-2l antiserum or control serum (1:500-4000 final dilution), and the number of Annexin V (AV) and/or propidium iodide (PI) cells was evaluated by flow cytometry. Loss of mitochondrial potential was assessed by DIOC6 staining. Total extracts were prepared from either untreated or treated LPL and analyzed for caspase-3 by Western blotting. Results. After 20 hours culture, the anti-IL-21 dramatically reduced the survival of CD3+ LPL. Flow cytometry analysis revealed that the anti-IL-21 significantly enhanced the percentages of $\mathrm{AV}+(28,5 \pm 8), \mathrm{PI}+(7 \pm 1,4)$ and $\mathrm{AV}+\mathrm{PI}+(41 \pm 7,6)$ in comparison to unstimulated (AV: $11 \pm 1$; PI: $1,5 \pm 0,16$; AV-PI:20,8 $\pm 5,7$ ) or control serum-treated (AV 11,6 $\pm 0,69$; PI: $2 \pm 0,7$; AV-PI: $21 \pm 5,77)$ cells $(P<0.01)$. The anti-IL-2l also enhanced the percentage of AV and PI in CD4+LPL, thus excluding the possibility that its effect occurred via an antibody-mediated cytotoxicity. These effects were associated with a time- and dosedependent loss of mitochondrial potential and caspase- 3 activation. Conclusions: Data indicate that neutralization of IL-21 activity dramatically reduces the survival of IBD LPL thus suggesting a role for this cytokine in maintaining the ongoing $\mathrm{T}$ cell-mediated inflammation in IBD.

\section{W1630}

Differential Interaction of Cmvil-10 with Variants of the IL-10 Receptor Chain 1 (IL-10R1)

Sabine G. Gruber, Paul Grundtner, Gloria Luciani, Christoph Gasche

Background: CMV reactivation in $\mathrm{UC}$ is commonly associated with therapy refractory disease, which might be caused by virus-host interactions such as cmvIL-10 (a viral IL-10 homolog) Affinity measurements had shown a similar affinity of human IL-10 and cmvIL-10 to the IL-10RI (PNAS 99:9404). Genetic variations of the IL-10RI (SNP3 and 4) are associated with Crohn's colitis but protect from ulcerative colitis (UC) in Caucasian populations. Computer modeling predicted that IL-10RI SNP3 changes IL-10 or IL-10 homolog binding to the extracellular receptor domain (J Immunol 170:5578). Together with the high frequency of the IL-10R1-SNP3+4 haplotype in a CMV-positive colitis population, these findings indicate that SNP3 may differentially alter human and viral IL-10 signaling and thereby influence virus-host interactions. We therefore studied cmvIL-10 signaling through the variant IL-10RI receptors. Methods: HeLa cells were transfected with pIRESpuro constructs of various IL-10R1 alleles (wt, SNP3, SNP4, or SNP3+4) or the empty vector. Stably transfected single cell clones expressing the various IL-10Rl were chosen based upon receptor density (as measured by flow cytometry and Western blot) and IL-10-dependent STAT3 phosphorylation. Cells were incubated with various concentrations of cmvIL-10 or human IL-10 (0.01 to $10 \mathrm{ng} / \mathrm{ml}$; both R\&D). After 30 min, cells were harvested and protein extracts were analyzed for STAT3 and pSTAT3 (Y705) phosphorylation by Western blotting. Blots were quantified by densitometry (ImageJ; http://rsb.info.nih.gov/ij), ratios between pSTAT3 and STAT expression levels were calculated, and the results of cmvIL-10 stimulation were normalized to human IL-10 for each variant clone. Results: From a large number of clones, one clone of each IL-10R1 variant was selected based on moderate IL-10RI expression and similar (human) IL-10-induced STAT3 phosphorylation. In the IL-10R1-wt clone, cmvIL10 was about 100-fold more active than human IL-10. In the IL-10RI-SNP3 and IL-10RISNP4 clones, cmvlL-10-induced STAT3 phosphorylation was slightly reduced (IL-10R1SNP3: $75-83 \%$, IL-10R1-SNP4: 81-87\% of IL-10R1-wt), while the IL-10RI-SNP3+4 clone showed significant reductions (42-49\% of IL-10Rl-wt). Minimal STAT3 phosphorylation $(6-10 \%)$ was observed in the mock-transfected HeLa Conclusion. The IL-10RI-SNP3+4 displays a loss of cmvIL-10 signaling in relation to human IL-10. This functional change might explain why patients that carry this allele are at higher risk to reactivate CMV in UC and why they may become refractory to anti-inflammatory therapy.

\section{W1631}

The Novel IL-10 Related Cytokine IL-26 Is Increased in Active Inflammatory Bowel Disease and Intestinal Epithelial Cells Express the Functional IL-26 Receptor Complex

Julia Dambacher, Florian Beigel, Rosa Golluscio, Torsten Olszak, Kathrin Zitzmann, Soeren T. Eichhorst, Jan-Michel Otte, Julia Seiderer, Helmut Diepolder, Christoph J. Auernhammer, Thomas Ochsenkuehn, Burkhard Goeke, Stephan Brand

Background: Human interleukin-26 (IL-26) signals through a receptor complex consisting of IL-20R1 and IL-10R2. Preliminary studies suggest T lymphocytes as primary source of this cytokine. Aim: The purpose of this study was to analyze IL-26 receptor expression, signal transduction and specific biological functions of this cytokine system in intestinal epithelial cells (IEC). Methods: Expression studies were performed by RT-PCR and quantitative PCR. Signal transduction was analyzed by Western blot experiments and ELISA. Cell proliferation was measured by MTS assay and Fas induced apoptosis by flow cytometry. Results: The IEC lines Caco-2, SW480, HCT116 and HT-29 express both IL-26 receptor subunits IL-20R1 and IL-10R2. IL-26 binding to its receptor complex activates ERK-1/2 and SAPK/JNK MAP kinases, and Akt. IL-26 also induced phosphorylation of STATl and STAT3. IL-26 slightly decreased cell proliferation while it had no effect on Fas-ligand induced apoptosis. Moreover, IL-26 stimulation increased IL-8 protein expression levels in ELISA assays up to 3.7-fold. IL-26 mRNA expression was increased in inflamed colonic lesions compared to non-inflamed tissue in 8 out of 12 patients with Crohn's disease (CD; average 5.0-fold increase) and in 7 out of 10 patients with ulcerative colitis (UC; average 3.4-fold increase) and correlated highly with the IL-8 expression in these lesions ( $r=0.659$ for CD patients; $r=0.788$ for UC patients). Conclusion: IEC express the functional IL-26 receptor complex. Binding of IL-26 to its receptor results in phosphorylation of MAP kinases and STAT proteins and an increased expression of proinflammatory cytokines. Moreover, our data indicate a role for this cytokine system in intestinal inflammation as seen in patients with inflammatory bowel disease.

\section{W1632}

Anti-TNF Antibody Induced Stimulated T Lymphocyte Apoptosis Depends On the Concentration of the Antibody and Etanercept Induces Apoptosis At Rates Equivalent to Infliximab and Adalimumab At 10 Micrograms Per Ml Concentration

Rakesh Chaudhary, Matt Butler, Raymond J. Playford, Subrata Ghosh

Background: Infliximab, but not etanercept, has been reported to cause apoptosis in stimulated peripheral blood and lamina propria T lymphocytes in healthy controls and Crohn's disease patients. We aim to investigate whether higher concentrations of etanercept might induce lymphocyte apoptosis similar to infliximab and adalimumab. Methods: Peripheral CD4 T lymphocytes were isolated from healthy subjects $(n=6)$. Medium with or without anti-TNF antibody (Ab) was added at the time of antibody stimulation. Infliximab, adalimumab and etanercept were added at concentrations of $0.1,1$ and $10 \mathrm{ug}$ per ml. Human Ig G 1 antibody was used as a negative control. Apoptosis was detected using annexin following 7 days of stimulation by flow cytometry. Topro3 was used as dead cell discriminator. Baseline apoptosis was taken as the rate of apoptosis of stimulated lymphocytes with medium alone. Results: All 3 anti-TNF Abs were found to cause an increase in CD4 lymphocyte apoptosis. The mean increase in lymphocyte apoptosis rates compared to baseline with $0.1 \mathrm{ug} / \mathrm{ml}$, lug/ $\mathrm{ml}$ and $10 \mathrm{ug} / \mathrm{ml}$ for infliximab was $50 \%, 99 \%$ and $132 \%$. For adalimumab the rates were $42 \%, 102 \%$ and $109 \%$ for $0.1 \mathrm{ug} / \mathrm{ml}, 1 \mathrm{ug} / \mathrm{ml}$ and $10 \mathrm{ug} / \mathrm{ml}$ doses. Corresponding apoptosis rates with etanercept were $30 \%, 40 \%$ and $77 \%$. Infliximab and adalimumab were significantly better than etanercept at $\operatorname{lug} / \mathrm{ml}(\mathrm{p} \leq 0.05)$ at inducing lymphocyte apoptosis but not so at $10 \mathrm{ug} / \mathrm{ml}(\mathrm{p}=\mathrm{NS})$. Conclusion: In our in-vitro model not only infliximab and adalimumab but also etanercept causes apoptosis of stimulated lymphocytes. Anti-TNF antibody induced apoptosis was found to be concentration dependant, and at higher concentrations the difference between etanercept and infliximab / adalimumab tends to diminish. These invitro observations bring into question whether lack of apoptosis by etanercept is an integral property of the molecule. If apoptosis is an important mechanism of action then the possibility that a higher dose of etanercept might be clinically effective cannot be excluded.

\section{W1633}

A New Transcription Factor That Regulates TNF- $\alpha$ Gene Expression, Litaf (Lipopolysaccharide Induced TNF- $\alpha$ Factor) Is Increased in Lamina Propria Macrophages After Trinitrobenzene Sulfonic Acid (TNBS)-Induced Colitis in Mice

Kristen N. Bushell, Susan E. Leeman, Salomon Amar, Karen L. Reed, Adam C. Gower, Arthur F. Stucchi, James M. Becker

Background: Crohn's Disease (CD) is a chronic inflammatory bowel disease known to involve the dysregulation of proinflammatory cytokines, primarily TNF- $\alpha$. The chronic exposure of the GI tract to bacterial endotoxins, such as lipopolysaccharide (LPS), leads to the upregulation of cytokines in inflammatory cells which exacerbate the inflammation and destroy tissue structure. LITAF is a newly discovered transcription factor that binds to the TNF- $\alpha$ promoter and has been shown to regulate TNF- $\alpha$ gene expression in a cell line of differentiated human THP-1 macrophages. Since the initial studies implicating LITAF in the regulation of TNF- $\alpha$ gene expression were performed in a macrophage cell line, the aim of this study was to determine the relative levels of LITAF message and protein in lamina propria macrophage cells (LPMC) isolated from colonic tissues in a murine model of CD. Methods: C57BL/6 mice were rectally administered TNBS (1.5 mg in 50\% EtOH vehicle) and were monitored for up to 7 days. Body weights were measured daily. Myeloperoxidase (MPO) levels were measured in isolated colon tissue extracts. LITAF protein was measured by Western blot analysis and LITAF mRNA quantified by real-time-PCR (RT-PCR) in whole cell extracts of LPMC. Results: Body weights fell significantly on days one and two in TNBS treated mice compared with controls. By day 4, MPO levels were increased 3-fold in colon tissue extracts from TNBS treated mice compared to controls $(\mathrm{p}<0.05)$. LITAF protein levels in LPMC extracts were increased by $75 \%$ following TNBS administration compared to controls. RT-PCR analyses of colon tissue at day 7 showed a two-fold increase $(\mathrm{P}<0.05)$ in LITAF message in TNBS treated mice compared to controls. Conclusions: These data provide evidence that this transcription factor, LITAF, which is associated with an increase in the expression of several other proinflammatory cytokines in addition to TNF- $\alpha$, responds to the induction of $\mathrm{CD}$ in this murine model and underscores the potential value of anti-LITAF strategies in the therapeutic management of this disease. 


\section{W1634}

Neutralization of Soluble and Membrane Tumor Necrosis Factor- $\alpha$ (TNF- $\alpha$ ) By Infliximab, Adalimumab, Or Certolizumab Pegol Using P55 Or P75 TNF- $\alpha$ Receptor-Specific Bioassays

Amie Gramlick, Gianluca Fossati, Andrew M. Nesbit

Background: The pro-inflammatory cytokine tumor necrosis factor- $\alpha$ (TNF- $\alpha$ ) plays a key role in Crohn's disease and rheumatoid arthritis. Biologic agents that neutralize TNF- $\alpha$ have been developed for these inflammatory conditions. The two TNF- $\alpha$ receptors $-\mathrm{p} 55$ and p75 - are both widely expressed on many cell types, and most bioassays do not differentiate between signaling via each of the receptors. To address this limitation, we aimed to engineer two cell lines that could be activated following specific binding of TNF- $\alpha$ to either the p55 or the $\mathrm{p} 75$ receptor, respectively. These cell lines could be used to assess the ability of the anti-TNF- $\alpha$ agents infliximab, adalimumab and certolizumab pegol (CDP870) to neutralize signaling instigated by the binding of soluble or membrane TNF- $\alpha$ to either the p55 or p75 receptor. Methods: The A549 human lung carcinoma cell line was transfected with a luciferase reporter system that allowed the measurement of TNF- $\alpha$ signaling via the p55 receptor only. Similarly, a chimeric p75 receptor construct was transfected into the Jurkat human $\mathrm{T}$ cell line to allow the measurement of $\mathrm{p} 75$-specific signaling, again using a luciferase reporter gene. The ability of infliximab, adalimumab, or certolizumab pegol to neutralize human recombinant soluble TNF- $\alpha$ and membrane TNF- $\alpha$ signaling via $\mathrm{p} 55$ or $\mathrm{p} 75$ receptorspecific pathways was assessed in vitro using these cell lines. In addition, an NSO cell line transfected with non-cleavable human membrane TNF- $\alpha$ was used to measure the neutralization of membrane TNF- $\alpha$ by the anti-TNF agents. Results: All three anti-TNF agents were equally potent at neutralizing membrane TNF- $\alpha$ signaling via either the p55 or the p75 receptor. However, certolizumab pegol was at least 2 -fold more potent than adalimumab or infliximab at neutralizing soluble TNF- $\alpha$ signaling via either receptor. Conclusion: Certolizumab pegol, infliximab, and adalimumab were equally potent at neutralizing membrane TNF- $\alpha$ signaling via either the $\mathrm{p} 55$ or the $\mathrm{p} 75$ receptor. However, certolizumab pegol was more potent than infliximab or adalimumab at neutralizing the signaling induced by soluble TNF- $\alpha$ through each receptor.

\section{W1635}

Induction of in-Vitro Apoptosis of Stimulated Cd4 T Lymphocytes Can Be Demonstrated with Not Only Infliximab and Adalimumab But Also Etanercept If Anti-TNF Antibodies Are Added Simultaneously with Anti-CD3 and AntiCD28 Antibodies

Rakesh Chaudhary, Matt Butler, Raymond J. Playford, Subrata Ghosh

Background: Apoptosis is considered an important mechanism of action of anti-TNF therapy in Crohn's disease. Infliximab, but not etanercept, has been reported to cause apoptosis in stimulated peripheral blood and lamina propria T lymphocytes in healthy controls and Crohn's disease patients. We report on the induction of stimulated peripheral T lymphocyte apoptosis by infliximab, adalimumab and etanercept in relation to the timing of addition of anti-TNF antibody (Ab) in-vitro. Methods: Peripheral CD4 T lymphocytes were isolated from healthy subjects and stimulated with anti-CD3 \& anti-CD28 antibodies. Medium with or without anti-TNF Ab was added either simultaneously $(n=7)$ or after stimulation for 72 hours ( $\mathrm{n}=5)$. Control antibody was used as a negative control and cisplatin as a positive control. Apoptosis was detected after 7 days of stimulation using annexin $\mathrm{V}$ and topro-3 by flow cytometry. Three anti-TNF Abs- infliximab, adalimumab and etanercept- were used at a concentration of $10 \mathrm{ug} / \mathrm{ml}$. Apoptosis was expressed as a percentage $( \pm$ SEM) of the background apoptosis of stimulated lymphocytes i.e. rate of apoptosis of stimulated lymphocytes following the addition of medium alone. Results: Following anti-TNF Ab addition to lymphocytes stimulated for 72 hours the apoptosis rates compared to background apoptosis were $92 \%( \pm 5 \%)$ for infliximab, $93 \%( \pm 8 \%)$ for adalimumab and $91 \%( \pm 8 \%)$ for etanercept $(\mathrm{p}=\mathrm{NS})$. With simultaneous lymphocyte stimulation and anti-TNF Ab addition lymphocyte apoptosis compared to background was $232 \%( \pm 18 \%), 203 \%( \pm 16 \%)$ and $172 \%( \pm 11 \%)$ with infliximab, adalimumab and etanercept respectively. This increase in apoptosis following synchronous anti-TNF Ab addition and stimulation was significant for all 3 anti-TNF Abs compared with control $\mathrm{Ab}(\mathrm{p}<0.0001)$ but the difference between the 3 anti-TNF Abs was not significant $(\mathrm{p}=0.08)$. Conclusion: The manner of lymphocyte stimulation determines the apoptosis read out. When anti-TNF Abs is added simultaneously at the time of stimulation all anti-TNF Abs including etanercept cause a significant increase in lymphocyte apoptosis. The difference between the 3 anti-TNF Abs did not reach statistical significance. In contrast, if drug is added following 72 hours of stimulation then there is no detectable increase in apoptosis. We conclude that under appropriate conditions all 3 anti-TNF Abs may induce apoptosis of stimulated T lymphocytes though there is a trend towards slightly lower rates with etanercept (not reaching statistical significance).

\section{W1636}

Antigenic Soluble Factors (ASF) in Patients with Crohn's Disease According to Their Pathological Behaviour

Id Pousa, J Mate, X Salcedo, R Moreno-Otero, Javier P. Gisber

INTRODUCTION: CD can be classified according to their behaviour in the Vienna's classification into three subgroups: inflammatory, fistulizing and fibrostenotic. The angiogenic process is closely related to the inflammation and to the fibrosis, and therefore it could be assumed that the expression of some angiogenic soluble factors (ASF) could be altered in the inflammatory or fibrostenotic subgroups of these patients. AIMS: To evaluate the differences in ASF concentrations according to the disease's behaviour of patients with $\mathrm{CD}$ in remission. METHODS: Thirty patients with $C D$ in remission classified according to their behaviour: inflammatory (mean age $48 \pm 14 \mathrm{yr}$ ), fistulizing $(49 \pm 17$ ), fibrostenotic ( $41 \pm 11$ ); and 10 healthy controls $(41 \pm 12)$ were studied. Serum levels of vascular endothelial growth factor (VEGF) and its receptors (Flt-1 y KDR), placental growth factor (PlGF), basic fibroblast growth factor (bFGF), angiopoietin 2 (Ang-2) and its receptor (Tie-2) were determined by ELISA. Statistical analysis: ANOVA one-way. RESULTS: Overall, significantly increased VEGF,
Flt-1, PlGF, Ang-2 and Tie-2 serum levels were observed in patients with CD in remission, when compared with healthy controls (table 1). A tendency to increased VEGF, FGFb and Ang-2 levels was observed in patients with fibrostenotic CD compared to those with inflammatory or fistulising behaviour. In patients with inflammatory $C D$, an increase of PIGF and Tie-2 levels was demonstrated in comparison with those with another disease's behaviour. However, these tendencies did not reach statistical significance, probably due to the limited sample size. CONCLUSIONS: 1) Patients according to their disease's pathologica behaviour showed differences in the levels of several ASFs. 2) In the patients with fibrostenotic $\mathrm{CD}$, an increase of VEGF, FGFb and Ang-2 levels was observed, whereas those with inflammatory $\mathrm{CD}$ showed a higher expression of PlGF and Tie-2. 3) Further and more extensive studies are necessary to determine if these ASFs could be useful to predict the evolution of patients with $C D$.

Means \pm SD of ASF concentracions $[\mathrm{pg} / \mathrm{mL}] .{ }^{*} \mathrm{p}<0.05 .{ }^{* *} \mathrm{p}<0.01 . \AA<0.001$. Controls vs. CD's subgroup.

\begin{tabular}{|c|c|c|c|c|}
\hline VEGF & $\begin{array}{c}\text { Healthy controls } \\
(\mathrm{N}=10)\end{array}$ & $\begin{array}{c}\text { Inflammatory CD } \\
(\mathrm{N}=10)\end{array}$ & $\begin{array}{c}\text { Fibrostenotic CD } \\
(\mathrm{N}=10)\end{array}$ & $\begin{array}{c}\text { Fistulizing CD } \\
(\mathrm{N}=10)\end{array}$ \\
\hline PIGF & $261 \pm 82$ & $439 \pm 213$ & $610 \pm 237^{* * *}$ & $432 \pm 268$ \\
\hline FGFb & $27 \pm 1$ & $38 \pm 18^{* *}$ & $35 \pm 5^{*}$ & $35 \pm 6^{*}$ \\
\hline Flt-1 & $126 \pm 15$ & $26 \pm 3$ & $31 \pm 10$ & $27 \pm 5$ \\
\hline KDR & $2047 \pm 397$ & $2071 \pm 326$ & $2005 \pm 413$ & $2029 \pm 499$ \\
\hline Ang-2 & $519 \pm 103$ & $1034 \pm 233^{*}$ & $1449 \pm 528 *$ & $1143 \pm 489^{* *}$ \\
\hline Tie-2 & $2.7 \pm 0.7$ & $3.8 \pm 0.4 *$ & $3.6 \pm 0.5^{* *}$ & $3.5 \pm 0.4^{*}$ \\
\hline
\end{tabular}

W1637

Generation of Ceramide in Tight Junctions Increases Transepithelial Permeability

Juergen A. Bock, Gerhard Liebisch, Joachim Schweimer, Gerd Schmitz, Juergen Schoelmerich, Gerhard Rogler

Background: The sphingolipid ceramide, generated by the action of acid sphingomyelinase (ASM), has emerged as a second messenger of stimuli as diverse as ionizing radiation, chemotherapy, ligation of various receptors or infection with some bacteria and viruses. Many signaling molecules involved in intestinal inflammation (e.g. TNF, INF- $\gamma$, PAF), also activate the ASM. Recently we had shown that hydrolysis of sphingomyelin to ceramide by exogenous sphingomyelinase (Smase) contributes to an increased permeability of intestinal epithelial cells. Therefore, we now aimed to characterize the occurring lipid modifications and localize the underlying mechanism. Methods: Sphingolipid-rich membrane microdomains were isolated by discontinuous sucrose gradients and characterized for their association with TJ-proteins by western-blot. The contents of ceramide, sphingomyelin and cholesterol were analysed by tandem mass spectrometry. Localization of ceramide was determined by immunofluorescent staining. Permeability of Caco-2 monolayers was determined by measurement of transepithelial flux and transepithelial resistance (TEER). Results: Incubation of Caco- 2 cell monolayers with Smase induced a rapid accumulation of ceramide in sphingolipid-enriched microdomains with concomitant decrease of sphingomyelin and cholesterol. Using $0.25 \mathrm{U} / \mathrm{ml}$ Smase, ceramide increased from $1 \%$ to $6 \%$ of analysed lipids within 10 min. In comparison the increase of ceramide was only $0.75 \%$ in the total cell lysate Accumulation of ceramide occurred in the same membrane fractions containing occludin and claudin-4, which represent TJ-proteins. Immunofluorescent staining of ceramide confirmed clustering of ceramide at the sites of cell-cell contact. Investigation of transepithelial flux after incubation with $0,25 \mathrm{U} / \mathrm{ml}$ Smase led to an increase of permeability by more than 50 $\%$ after $6 \mathrm{~h}$ with moderate decrease of TEER, indicating an increase of TJ-permeability. Neutralization of surface ceramide completely prevented the permeability-increase induced by platelet-activating factor. Conclusion: These findings indicate that the geometry of tightjunctional ceramide, sphingomyelin and cholesterol regulate transepithelial permeability which might be responsible for loss of barrier integrity upon various cellular stimuli.

\section{W1638}

Downregulation of the Ubiquitin-Proteasome Machinery in Normal Colonic Lamina Propria Macrophages and RE-Induction in Inflammatory Bowel Disease Mucosa

Andrea M. Brandl, Monika Seidl, Katrin Menzel, Juergen Schoelmerich, Hans Herfarth, Gerhard Rogler, Martin Hausmann

Background: The ubiquitin-proteasome machinery is a large multiprotein complex that degrades cellular proteins. Ubiquitin is required for ATP-dependent, nonlysosomal intracellular degradation of proteins with a rapid turnover. Intestinal lamina propria macrophages (IMACs) in normal mucosa are anergic and have a tolerance inducing and tolerance preservin function. Inhibition of the ubiquitin-proteasome system can suppress the activation of transcription factor nuclear factor-kappa B (NF-KB) by stabilizing the inhibitory protein IкB Regulating the ubiquitin system has important functions in the immune and inflammatory response. Methods: IMACs were isolated and purified from intestinal mucosa of surgica specimens with the help of immunomagnetic beads armed with a CD33 antibody. mRNA from in vitro differentiated macrophages and IMACs from normal mucosa and IBD mucosa was isolated and Affymetrix@ oligonucleotide array analysis was applied. Quantitative Taq man-PCR for five proteasomal and five ubiquitinylation genes was performed. Ubiquitin B in the intestinal mucosa was analyzed by immunohistochemistry. Results: Affymetrix analysis showed a downregulation of all represented 25 proteasomal and 22 ubiquination mRNAs in IMACs compared to in vitro differentiated macrophages and monocytes. By Taqman-PCR up to ten fold higher mRNA expression of 5 ubiquitination-associated genes (ubiquitinconjugating enzyme E2A, E2D2, E2L6, ubiquitin specific peptidase 14 and ubiquitin B) in 
in vitro differentiated macrophages compared to IMACs was demonstrated. Immunohistochemistry confirmed lack of ubiquitin B in intestinal mucosa of control patients and patients suffering from diverticulitis. In contrast, a significant increase in ubiquitin B protein was revealed in the intestinal mucosa of patients with Crohn's disease and ulcerative colitis. Using double labelling technique CD68 positive cells in those specimens showed co-staining with ubiquitin B. Upregulation (re-induction) of ubiquitin B mRNA in patients with inflammatory bowel disease was confirmed by Taqman-PCR. Conclusion: Reduced expression of subunits of the ubiquitin-proteasome machinery in IMACs from control patients supports the concept of a non-reactive, anergic macrophage phenotype present in the gut under normal conditions. Re-induction in IMCAs from IBD mucosa reflects activated IMACs that present antigenic peptides and support inflammation.

\section{W1639}

Beneficial Effect of Mesalamine on Microvascular Dysfunction in Ulcerative Colitis

Ossama A. Hatoum, Hiroto Miura, Rebecca M. Selle, Brandon T. Larsen, Doron

Kopelman, Mary F. Otterson, David D. Gutterman, David G. Binion

Introduction: Treatment with mesalamine derivatives has formed the mainstay of therapy for uncomplicated mild to moderate inflammatory bowel diseases (IBD). Although precise mechanism of action is not known, the beneficial effects of mesalamine in IBD have been attributed to its anti-inflammatory and antioxidant properties. We have previously demonstrated microvascular dysfunction in vessels isolated from chronically inflamed IBD intestine, which is characterized by sustained increase in oxyradical species. The aim of this study was to investigate the effect of mesalamine on microvascular dysfunction found in ulcerative colitis (UC) and Crohn's disease (CD) patients. Methods: Freshly isolated arterioles (140 \pm $32 \mu \mathrm{m})$ were dissected from IBD (UC + CD) human bowel specimens collected at the time of intestinal surgery. The vessels were cannulated with glass micropipettes and pressurized at $60 \mathrm{mmHg}$ in a vessel chamber and perfused with physiological salt solution. Changes in the internal diameter were recorded using videomicroscopy. Reactive oxygen species (ROS) production was assessed using dihydroethidine and DCF-DA staining. Results: After constriction (30-50\%) with endothelin-1, graded doses of Ach (10-9-10-4M) were administered to $\mathrm{UC}$ and $\mathrm{CD}$ vessels with and without mesalamine (dose equivalent to $4.8 \mathrm{~g} / \mathrm{day}$ ). Improvement in Ach dilation was observed in UC vessels with mesalamine ( $\max$ dilation $(\mathrm{MD})=70.4 \pm 5.7 \%$, compared to UC vessels without mesalamine $23.5 \pm 6.7 \%, n=6, p=<0.01$ ). However, mesalamine did not improve dilation of $C D$ vessels to Ach, as vasorelaxation remained at the low levels typically seen in $\mathrm{CD}$ (MD $25.4 \pm 5.8 \%, \mathrm{n}=4, \mathrm{~N} . \mathrm{S}$ ). Furthermore, clinically relevant concentrations of mesalamine (equivalent to $0.6,1.2,2.4,3.6,4.8$, and 6 g/day) caused a significant dose-dependent enhancement of dilation in response to Ach in vitro $(17.72 \pm 3.2$, $21.25 \pm 2.77,33.25 \pm 2.8,50.26 \pm 6.1,70.4 \pm 5.7$ and $78.51 \pm 4.5$, respectively, $\mathrm{n}=4, \mathrm{p}<0.05)$. Using dihydroethidine and DCF-DA staining to assess ROS production, we observed a significant reduction in superoxide production $(322.56+19.4343$ vs. $206.6 \pm 14.7, n=5$, $\mathrm{p}<0.05)$, but not in hydrogen peroxide production in mesalamine-treated microvessels from UC patients compared to non-treated vessels. Conclusions: IBD is associated with acquired microvascular endothelial dysfunction in the human gut. Mesalamine promotes the rapid re-establishment of dilation to Ach in vitro in $U C$ but not in $C D$ patients, by reducing superoxide generation. These results suggest that, in addition to its anti-inflammatory properties, mesalamine may also improve microvascular function in UC patients through its antioxidant effects.

\section{W1640}

Lipopolysaccharide Activates Innate Immune Responses in Murine Intestinal Myofibroblasts Through Multiple Signaling Pathways Kristen L. Walton, Leisha Collins, R Balfour Sartor

Background: Myofibroblasts (MF) play an important role in wound healing in the intestine. A compromised epithelial barrier exposes intestinal subepithelial MF to luminal bacterial products. However, the responses of murine intestinal MF to bacterial adjuvants are not well defined. We investigated the responsiveness of primary murine $\mathrm{MF}$ to lipopolysaccharide (LPS) and the signaling pathways activated by this bacterial adjuvant. Methods: Multiple primary murine MF lines were established from the ileum or colon of $129 \mathrm{SvEv}$ mice following collagenase digestion and mechanical disruption. Expression of toll-like receptors (TLR) $-2,-4,-5$, and -9 were detected by RT-PCR. Intracellular responses to $20 \mathrm{ng} / \mathrm{ml} \mathrm{LPS}$ were assessed by western blotting for the phosphorylated p65 subunit of NFKB, I $\mathrm{KB} \alpha$ phosphorylated Akt, ERK1/2, p38 MAP kinase, and cyclooxygenase-2 (COX-2). Secretion of prostaglandin E2 (PGE2), interleukin (IL)-6, IL-10, and the neutrophil chemoattractant KC/CXCLl after stimulation with E. coli LPS or E. coli lysate was measured by ELISA. Results: All intestinal MF lines tested expressed TLR-2, TLR-4, TLR-5, and TLR-9 mRNA. LPS caused rapid phosphorylation of NFKB p65 and degradation of IKB $\alpha$. Phosphorylation of Akt, p38 MAPK, and ERK 1/2 were also detected after LPS stimulation. Maximal phosphorylation of Akt was detectable by 15 min of LPS stimulation, while phosphorylation of p38 MAPK and ERK $1 / 2$ were maximal at 30-60 minutes. COX-2 protein was increased in MF after 8 or $24 \mathrm{hr}$ of LPS treatment. Inhibition of the PI3-kinase pathway by wortmannin reduced LPS-induced COX-2 protein expression in MF. A similar attenuation of COX-2 expression occurred when cells were co-incubated with LPS and SB203580, an inhibitor of the p38 MAPK pathway. Both LPS and E. coli lysate induced secretion of PGE2 $(1.8 \pm 0.2$ fold above unstimulated cells, $\mathrm{n}=5, \mathrm{p}<0.05)$, IL-6 $(3.5 \pm 0.8$ fold induction, $\mathrm{p}<0.05)$, and $\mathrm{KC}$ $(3.25 \pm 0.5$ fold induction, $\mathrm{p}<0.01)$, but not IL-10. Conclusions: Primary murine intestinal MF respond to a bacterial adjuvant, evidenced by LPS- stimulated activation of NFKB, PI3kinase and MAPK signaling pathways and secretion of the proinflammatory molecules $\mathrm{KC}$, IL-6, and PGE2. Inhibition of the PI3-kinase and p38 MAPK pathways attenuated LPSdependent COX-2 expression, indicating that multiple signaling pathways are involved in activation of MF by LPS. These data support the hypothesis that MF are a component of the innate immune system that may regulate adjacent epithelial and immune cell responses in a paracrine fashion by responding to luminal bacterial adjuvants.

\section{W1641}

The Thrombomodulin-Activated Protein C-Endothelial Protein C Receptor Pathway: A Novel Anti-Inflammatory System in Inflammatory Bowel Disease Silvio Danese, Franco Scaldaferri, Cristina Graziani, Alessandro Sgambato, Bruce Gerlitz, Alfredo Papa, Riccardo Ricci, Miquel Sans, Brian Grinnell, Giuseppe Fedeli, Achille Cittadini, Antonio Gasbarrin

Coagulation and inflammation are intimately interdependent processes. Endothelial protein $C$ receptor (EPCR) and thrombomodulin (TM) are expressed at high levels selectively in the microvasculature, and convert protein $\mathrm{C}$ (PC) to its activated form (APC), which is a potent anti-coagulant and anti-inflammatory molecule. In several chronic inflammatory conditions, the protein $C$ pathway is down regulated, leading to an impaired generation of APC. The aim of this study was to explore the protein $C$ pathway in inflammatory bowel disease (IBD), and to assess the therapeutic implication of manipulating this system in experimental colitis. The levels of TM and EPCR expression was evaluated by immunohistochemistry in colonic sections from controls, Crohn's disease (CD) and ulcerative (UC) subjects, and by flow cytometry in human intestinal microvasculature endothelial cells (HIMEC), at baseline and after treatment with TNF- $\alpha$, TGF- $\beta$ and IL- 10 . The capacity to generate APC by HIMEC was measured by colorimetric assay at baseline and after treatment with TNF- $\alpha$. The anti-inflammatory effect of recombinant APC was studied on TNF- $\alpha$ stimulated HIMEC, and expression of VCAM-1 and ICAM-1 assessed by flow cytometry, and ENA-78 and IL-8 measured by ELISA. Adhesion assays were used to evaluate the capacity of APC to inhibit T-cell adhesion to HIMEC. In vivo, the anti-inflammatory effect of APC was tested in the $2.5 \%$ dextran sodium sulphate (DSS) model of colitis in mice. Both CD and UC patients expressed lower levels of TM and EPCR in intestinal microvasculature than controls. In cell culture, TNF- $\alpha$ significantly $(\mathrm{p}<0.001)$ down regulated HIMEC expression of EPCR and TM, leading to impaired capacity ( $\mathrm{p}<0.05)$ to generate APC. Recombinant APC had a potent anti-inflammatory effect, being able to significantly $(\mathrm{p}<0.01)$ downregulate TNF- $\alpha$-dependent VCAM- 1 and ICAM-1 expression, as well as ENA-78 $(\mathrm{p}<0.01)$ and IL-8 $(\mathrm{p}<0.001)$ production. APC was able to significantly $(\mathrm{p}<0.01)$ inhibit T-cell adhesion to HIMEC. Finally, APC was very effective in preventing experimental colitis in the DSS model, as assessed by weight loss $(\mathrm{p}<0.05)$, disease activity index $(\mathrm{p}<0.01)$ and histological colitis $(p<0.05)$. The protein $C$ pathway is strongly down regulated in the inflamed intestine in IBD and represents a new system involved in microvascular inflammation in the gut. Restoring APC conversion may represent a new therapeutic approach to down play intestinal inflammation by inhibiting endothelial cell adhesion molecule expression and chemokine production in intestinal microvasculature, thus avoiding leukocyte recruitment into the inflamed gut.

\section{W1642}

Induction of Resistin-Like Molecule Beta (Relm- $\beta /$ Fizz2) Expression During Initiation of Ileitis in Samp1/FC Mice Does Not Depend On Th2 Cytokines Or Luminal Flora

Sean L. Barnes, Alda Vidrich, Giorgos Bamias, Fabio Cominelli, Steven M. Cohn

RELM- $\beta$ is a goblet-cell-secreted, cysteine-rich-peptide with immunomodulatory function that is normally expressed in the colon. SAMP1/Fc mice develop ileitis similar to human Crohn's disease beginning at about age 6 wks. We previously found that RELM- $\beta$ is highly expressed in the ileum of adult SAMP1/Fc mice with ileitis. Previous studies (W. He et al 2003; Gastroenterology 125:1388) have shown that RELM- $\beta$ expression in the colon is dependent on bacterial colonization and is induced by Th2 cytokines. AIM: To determine whether luminal bacteria and/or Th2 cytokines expressed in the inflammatory milieu mediate the induction of RELM- $\beta$ in the ileum of SAMP1/Fc mice. METHODS: RNA was isolated from the ileum of specific pathogen free (SPF), germ free (GF) SAMP1/Fc mice and SPF AKR controls. Some SPF SAMP1/Fc mice received weekly injections of anti-mouse IL 4 MAb starting at age 3 wks. Levels of RELM- $\beta$, IL4, IL5, and IL13 mRNA were determined by quantitative real-time RT-PCR. Histologic assessment of ileitis was performed. RESULTS: RELM- $\beta$ is highly induced in the ileum of the SAMPI/Fc mice beginning at age 5-6 wks coincident with the initial histologic appearance of ileitis. In contrast, IL5 and IL13 mRNA levels began to increase between age 8 and $10 \mathrm{wks}$, well after the observed induction of RELM- $\beta$ and reached peak levels (179 fold and 1420 fold, respectively) during the chronic phase of inflammation. A modest increase ( 3.9 fold) in ileal IL4 mRNA was seen at age 4 wks compared to AKR controls, suggesting this cytokine might drive the induction of RELM$\beta$. However, RELM- $\beta$ mRNA levels and inflammatory scores were not altered by treatment with anti-mouse IL $4 \mathrm{MAb}$. GF SAMP1/Fc mice had a modest degree of ileitis relative to SPF mice $(6 \pm 0.1$ vs $18 \pm 0, p<0.0001)$. RELM- $\beta$ mRNA levels were increased in the ileum of $80 \%$ of GF SAMP1/Fc mice examined relative to SPF AKR mice of similar age. Although levels of ileal RELM- $\beta$ mRNA in GF mice were modestly decreased compared to those of SPF SAMP1/FC mice, these levels were still 13 to 154 -fold higher compared to SPF AKR controls. CONCLUSIONS: Neither induction of RELM- $\beta$ expression nor ileal inflammation requires the presence of viable intestinal flora. However, intestinal flora may modulate the level of RELM- $\beta$ once inflammation is established. Th2 cytokines do not appear to mediate the induction of RELM- $\beta$ in the ileum of SAMP1/Fc mice. The regulation of RELM- $\beta$ expression in the intestine is complex, likely involving both Th2-dependent and independent mechanisms. We speculate that early induction of RELM- $\beta$ in the ileum may be involved in the initiation of inflammation in SAMP1/Fc mice.

\section{W1643}

Poly a Element Is Involved in TNF $\alpha$ Mediated Decreases in Phex Gene Transcription

Jennifer K. Uno, Helayne R. Feferman, Olga I. Kolek, Hua Xu, Pawel R. Kiela, Fayez K. Ghishan

Reduced bone mass is a common complication of IBD; however the mechanisms contributing to osteopenia are not fully understood. TNF $\alpha$ is a major target in the successful treatment of IBD and has been shown to inhibit bone mineralization. Disruption of Phex gene, expressed 
primarily in osteoblasts, results in defective bone mineralization. Phex is thought to be down-regulated by a variety of factors including vitamin $\mathrm{D}_{3}, \mathrm{PTH}$, and TNF $\alpha$. Recent work on Phex transcriptional regulation implicates the involvement of a polyadenine element in the proximal Phex promoter. Studies suggest that this poly A region (nt -117/-100) plays a significant role in the basal regulation of Phex gene transcription. The aim of this study was to determine if the mechanism involved in TNF $\alpha$-mediated down-regulation of Phex gene transcription. Methods: Chimeric promoter constructs were made in the pTAL-Luc vector which is designed for assaying enhancer sequences cloned upstream of a TATA-like promoter region form the Herpes simplex virus thymidine kinase promoter. These constructs were transfected into UMR106 osteoblast-like cells treated with PBS or TNF $\alpha$. Electromobility shift assays (EMSA) were performed with nuclear protein from UMR106 cells treated with PBS or $10 \mathrm{ng} / \mathrm{ul}$ TNF $\alpha$ for 72 hrs using probes from predicted putative binding sites. Mutated oligos were also used to specify the site of protein-DNA interactions. Results: Reporter gene assay with a series of 5 ' deletion constructs $(-133 /-2,-98 /-2,-74 /-2)$ showed the $-133 /-2$ construct was functional in UMR106 cells and was down-regulated by TNF $\alpha$. Conversely, the -98/-2 and -74/-2 constructs, which lack the poly A region of the Phex promoter, inhibited luciferase activity below basal levels suggesting the presence of a repressive element. Luciferase activity with these two constructs was not altered by TNF $\alpha$. EMSAs performed with a probe spanning -134/-84 confirmed a protein-DNA complex which was diminished in TNF $\alpha$ treated cells. Further, unlabeled poly A probe, but not its mutated form, successfully competed for binding with the radio-labeled wild-type probe. Competition with unlabeled 134/-84 probe with mutated poly A region did not affect the decreased binding in response to TNF $\alpha$. Conclusions: The poly A region of the Phex promoter plays an important role in TNF $\alpha$-mediated down regulation of the murine Phex gene transcription. Identification of the putative trans-factor binding to the poly A region and its complex interactions within the Phex promoter will provide better insight into the mechanisms of Phex gene transcription and how its down-regulation contributes to the metabolic bone disease associated with IBD.

\section{W1644}

Chitinase 3-Like-1 (CHI3L1) Elicits the Production of Proinflammatory Cytokines and Chemokines in Colonic Epithelial Cells and Exacerbates TNBSInduced Colitis

Mayumi Kawada, Yuriko Hachiya, Emiko Mizoguchi

Background \& Aims: Many complicated factors are actively involved in the development of IBD. Our group recently demonstrated that a Chitinase 3-like-1 (CHI3Ll/YKL40/HC-gp39) molecule, which is expressed by colonic epithelial cells (CEC) and lamina proprial macrophages, is involved in the development of acute and chronic colitis. However, the pathogenic mechanisms of CHI3L1 have not been fully defined. Methods: SW480 and HT29 colonic epithelial cell lines were stimulated with purified human CHI3Ll protein at different concentration. The effect was evaluated by NF-кB lucifease reporter assay, ELISA, and RT-PCR. To examine the functional role of $\mathrm{CHI} 3 \mathrm{Ll}$, anti-CHI3Ll Ab was administrated into TNBSinduced colitis model. Results: CHI3Ll, a mammalian chitinase, shows high sequential homology with human Chitinase 3-like -3 (also known as eosinophil chemotactic factor-L: ECF-L) and acidic mammalian chitinase (AMCase), both of which possess strong stimulatory effect on chemokine production. To examine the pro-inflammatory effect of CHI3L1, SW480 and HT29 (transfected with 10 ng PIV-NF-KB-luciferase and $1 \mathrm{ng}$ renilla plasmids) were stimulated with or without purified human CHI3L1 (Quidel, San Diego, CA) at the concentration of $5-80 \mathrm{ng} / \mathrm{ml}$ for 5 hours. NF-KB luciferase assay showed that CHI3Ll stimulation significantly activates the NF-KB transcription in SW480 (3.2 fold) and HT29 (9.2 fold) cells compared to these cells without CHI3Ll stimulation at the dose of $80 \mathrm{ng} / \mathrm{ml}$. CHI3Ll also significantly stimulated IL-8 (CXCL8) production in the cell lines. In addition, CHI3L1 significantly enhanced the production of TNF $\alpha$, RANTES, and exotaxin in SW480 and HT29 cells with a dose-dependent manner. IKB $\alpha$-phosphorylation has been detected by western blot as well as immunofuorescence analyses after stimulated with $80 \mathrm{ng} / \mathrm{ml}$ of CHI3Ll in SW480 cells. In vivo administration of anti-CHI3L1 Ab (on days -1, 0, and 1) significantly enhanced the recovery from TNBS induced colitis as indicated by the improvement of body weight loss, clinical and histological score, and colonic epithelial damage (evaluated by BrdU-incorporation). One time injection of the $\mathrm{Ab}$ on day - 1 was not significantly effective compared to three times injection. Of note, anti-CHI3Ll Ab treated mice showed significantly less inflammatory infiltration ( $T$ cells, macrophages, and neutrophils) and chemokine production in colonic mucosa. Conclusions: CHI3Ll plays a crucial role in the pathogenesis of colitis by enhancing the production of proinflammatory cytokines and chemokine of CEC. Inhibition of CHI3Ll activity would provide a rationale to develop new therapeutic strategy for human IBD

\section{W1645}

Concordant Expression of Secretory Leukocyte Protease Inhibitor (SLPI) in Colonic Tissue of IBD and Animal Models of Colitis

Stephen M. Prouty, John R. Mabus, Cynthia Smith, Edward S. Kimball, Craig R.

Schneider, Nathaniel H. Wallace, Paul R. Wade, Meghan Towers, Sergey Ilyin, Michael D'Andrea, Anton Bittner, Andrew Carmen, Dhammika Amaratunga, Oscar Go, Pamela J. Hornby

Background: Successful drug development for IBD relies on animal models that are predictive of the pathophysiology in IBD patients. Comparison of changes in mRNA expression in animal models and humans can identify genes on conserved signaling pathways that could be used for drug targets and/or biomarkers. Therefore, patterns of gene expression were compared between murine colitis induced by 1) dextran sulfate sodium (DSS), 2) oil of mustard (OM), and in human ulcerative colitis (UC) and Crohn's colitis (CC). One example of conserved gene expression revealed by these studies is that of Secretory Leukocyte Protease Inhibitor (SLPI), a serine protease inhibitor with antibacterial activity, and is of interest given the role of proteinase-activated receptors in infectious colitis. Methods: For DSSinduced colitis, mice were treated with 5\% DSS in drinking water for 7 days, whereas controls received normal drinking water. For OM-induced colitis, $0.5 \% \mathrm{OM}$ was applied intracolonically, and colitis allowed to develop for 2, 6, 24, or 72 hours. Human colonic biopsies (diseased and adjacent "normal") were obtained by informed consent from patients diagnosed with UC ( $n=6)$ or CC $(n=2)$. Total RNA, isolated from murine full-thickness dista colon and from human biopsies, was analyzed by microarray and quantitative (q) RT-PCR Cell-type expression was determined with immunohistochemistry (IHC). Results: Microarray analysis of DSS-treated mice and human UC/CC revealed increased SLPI mRNA with colitis (9.6 fold, $\mathrm{P}<2.9 \times 10-8$ and 3.4 fold, $\mathrm{P}<4.0 \times 10-6$, respectively). QRT-PCR validated these elevations (DSS: 19.4 fold increase, $\mathrm{P}<0.0001$; UC/CC: 3.16 fold increase, $\mathrm{P}<0.0001$ ). Ther was a correlation between SLPI mRNA levels and macroscopic damage scores in OM colitis. SLPI mRNA began to rise at 6 hours ( 2.1 fold) and peaked at 24 hours ( 4.38 fold increase, $\mathrm{P}<0.001$ ), correlating with time-dependent increases in macroscopic damage scores. In normal mouse and human colon, by IHC, SLPI protein expression occurs in the upper hal of glandular epithelia, as well as in cells in the lamina propria. In human colon, SLPI was expressed in a subset of enteroendocrine (EE) cells. In colitis tissues, epithelial expression increased in the lower half of the glands. Conclusions: Increased SLPI expression is component of a fundamental signaling pathway in experimental colitis and in human IBD which likely functions to limit serine proteolytic activity and/or bacterial invasion, both o which are events associated with IBD. SLPI expression in EE cells suggests a protective role in the human colon and its potential for use as a biomarker in human and experimental colitis.

\section{W1646}

AP-1 and PPAR- $\gamma$ Are Involved in Peptidoglycan-Mediated Immune Activation of Enteric Glial Cells (EGC)

Eike Hollenbach, Shyam Kavuri, Manfred Neumann, Peter Malfertheiner, Anne Ruhl

NF-KB and activator protein-1 (AP-1) are major transcription factors inducing mainly proinflammatory responses. Our previous work has suggested a role for EGC in intestinal immune responses via NOD and NF-אB. Here, we have investigated if bacterial peptidoglycan (PGN) challenge induces activation of AP- 1 and if PPAR- $\gamma$ receptor is involved in NF-KB activation in EGC. Rat EGC were incubated with $\mathrm{H}$. pylori to model intracellular translocation of PGN. A radioactive in-vitro kinase assay for c-Jun N-terminal kinase (JNK) activity using immunoprecipitated JNK and GST-coupled c-jun as substrates demonstrated a marked upregulation with a peak of JNK activity at 30-60 min after exposure of EGC to an $H$. pylori strain with an intact type IV secretion system. In contrast, only weak JNK activation could be detected after EGC infection with a mutant $H$. pylori strain that is unable to convey PGN translocation. JNK activation was completely blocked by pre-treatment with SP600125 $(5 \mu \mathrm{M}$ for $30 \mathrm{~min}$ ). Results of the in-vitro kinase assay were confirmed by AP-1 electrophoretic mobility shift assay (EMSA). Supershift analysis revealed that the activated AP-1 complex consists mainly of c-jun and only to a minor extent of c-fos. We further investigated the role of PPAR- $\gamma$ in glial NF- $\mathrm{KB}$ activation. Expression of PPAR- $\gamma$ in EGC was determined by FACS analysis using intracellular staining of the intracellular receptor. The expression of PPAR- $\gamma$ was significantly upregulated 8 hours after infection with the PGN secretion-competent $H$. pylori strain, whereas the mutant $H$. pylori strain did only induce a weak PPAR- $\gamma$ upregulation. 30-60 min after exposure of EGC to the wild-type $H$. pylori strain, immunoblot analysis revealed a strong peak of I $\mathrm{KB} \alpha$-degradation and nuclear translocation of $\mathrm{p} 65$ as a measure of NF-KB activation, which was significantly attenuated by a $30 \mathrm{~min}$ pre-incubation period of EGC with the PPAR- $\gamma$ agonist Ciglitazone $(15 \mu \mathrm{M})$ or the endogenous ligand $15 \mathrm{~d}$ PGJ2 (20uM). These findings were confirmed by EMSA, revealing a reduction of NF-KB activation by the PPAR- $\gamma$ agonists. In contrast, RICK (a downstream kinase of the NOD receptor) activity - which is dramatically upregulated $60 \mathrm{~min}$ after infection - was unaffected by PPAR- $\gamma$ agonist treatment. These results provide further evidence for an immunoregulatory role of EGC in the intestine. Glial stimulation by bacterial products like PGN results in a

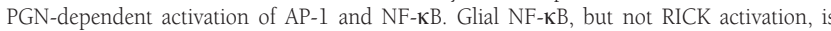
attenuated by PPAR- $\gamma$ agonists, indicating a contribution of IKB $\alpha$ stabilization, probably via IKK inhibition. Supported by Broad Medical Research Program IBD-0105R

\section{W1647}

Expression of the IGF-I Splice Variants, IGF-IEC and IGF-IEB, Encoding Mechano Growth Factor By Smooth Muscle Cells Is Upregulated in Crohn's Disease and TNBS Colitis

John F. Kuemmerle, Jennifer G. Bowers

Endogenous Insulin-like Growth Factor-I (IGF-I) regulates the growth of intestinal smooth muscle cells. IGF-I expression in muscle is upregulated in Crohn's Disease and TNBS colitis, and regulates muscle hyperplasia. The IGF-I gene is alternatively spliced with the main isoform, IGF-I, encoded by the IGF-IEa splice variant. An IGF-I splice variant, IGF-IEc in human (IGF-IEb in rat), has been identified that encodes a distinct protein, Mechano Growth Factor (MGF) which mediates skeletal and cardiac muscle hypertrophy post injury. Aim To identify the effect of inflammation on expression of the IGF-IEc in humans, and IGFIEb splice variants, in rats. Methods: Intestinal smooth muscle cells were isolated from regions of active Crohn's Disease or normal resection margins and from TNBS or vehicle treated rat colons 7 days post TNBS instillation. Isolated muscle cells were used to prepare total RNA or placed into primary culture. Expression of IGF-I splice variants was quantified with real time PCR using primers specific for IGF-IEa, IGF-IEc and IGF-IEb. Changes in expression were determined using $\beta$-actin as control. IGF-I receptor activation by IGF-I and MGF was measured by phosphotyrosine immunoblotting and growth by $\left[{ }^{3} \mathrm{H}\right]$ thymidine incorporation. Results: The IGF-IEa and IGF-IEc splice variants were expressed in human intestinal muscle cells. IGF-IEa expression increased $2.4 \pm 0.2$ fold and IGF-IEc expression increased $4.2 \pm 0.3$ fold in the inflamed muscle of Crohn's Disease. IGF-IEa and IGF-IEb splice variants were expressed in rat smooth muscle cells. IGF-IEa expression increased 1.9 \pm 0.2 fold and IGF-IEb expression increased $5.8 \pm 0.4$ fold in the TNBS treated rats compared to control. IGF-I $(100 \mathrm{nM})$ increased IGF-I receptor phosphorylation by $210 \pm 12 \%$ and $360 \pm 30 \%$ in normal human and rat intestinal smooth muscle cells, respectively, and increased $\left[{ }^{3} \mathrm{H}\right]$ thymidine incorporation by $280 \pm 15 \%$ and $209 \pm 14 \%$, respectively. The effects of IGF-I on receptor phosphorylation and $\left[{ }^{3} \mathrm{H}\right]$ thymidine incorporation were inhibited by $70-95 \%$ by the of IGF-I receptor blocking antibody, $1 \mathrm{H} 7(10 \mu \mathrm{g} / \mathrm{ml})$. MGF $(100 \mathrm{nM})$ had no effect on IGF-I receptor phosphorylation or $\left[{ }^{3} \mathrm{H}\right]$ thymidine incorporation in either species. Conclusions: Expression of IGF-IEc (human) and IGF-IEb (rat) splice variants that encode Mechano Growth Factor is upregulated in Crohn's Disease and in TNBS colitis 
concomitantly with expression of IGF-IEa that encodes IGF-I. Increased MGF expression may mediate smooth muscle cell hypertrophy, and jointly with increased IGF-I expression, which mediates smooth muscle hyperplasia, contribute to stricture formation in the chronically inflamed intestine.

\section{W1648}

Phosphatidylcholine Can Therapeutically Be Used in IBD. It Modulates Membrane Dependent Reactions and Inhibits TNF $\alpha$ Induced NFKB Activation Robert Ehehalt, Irina Treede, Mark Kuehnel, Thomas Giese, Annika Braun, Alexandra Zahn, Joachim Fuellekrug, Gareth Griffiths, Wolfgang Stremmel

Introduction: Intestinal mucosal cells are protected against injurious contents of the gastrointestinal lumen by a surface barrier, which consists in part of a continuous, hydrophobic and adherent mucus layer. A major lipid of this mucus layer is phosphatidylcholine (PC). We could recently show that mucus from patients with ulcerative colitis (UC) is characterized by an abnormally low level of the lipid PC while clinical studies reveal that therapeutic addition of PC to the colonic mucus using slow release preparations is beneficial to UC patients (Scand J Gastroenterol (2004) 39: 737-742; Gut (2005) 54: 966-971). The positive role of PC in this disease is completely elusive. However, we now could show that PC is involved in signaling networks that inhibit pro-inflammatory signaling in membranes. Methods: Different phospholipids with various fatty acid side chains (including PC with saturated, monounsaturated, polyunsaturated, short chain, long chain and combinations thereof) were tested on isolated phagosomal membranes in vitro and at the cellular level using intestinal epithelial cells. The main experimental read outs were actin assembly from phagosomal membranes as well as NFKB activation and pro-inflammatory gene transcription. Results: All PC species inhibited actin assembly in vitro. Most effective were PCs with very long fatty acid side chains with exception of those with arachidonic acid side chains. The in vitro results were consistent with the findings for a cellular system. Experiments on the intestinal cell line $\mathrm{CaCo}-2$ revealed that application of a low concentration of $\mathrm{PC}(<50 \mu \mathrm{M})$ could inhibit TNF $\alpha$ induced translocation of NFKB from the cytoplasm into the nucleus (dose dependent effect) and thus prevents pro-inflammatory gene transcription. Conclusion: PC can inhibit cell signaling responses induced by inflammation at the level of cell membranes. These effects are upstream of NFKB induced gene transcription. This is a potential mechanism how PC can diminish inflammation in ulcerative colitis. Further studies are needed to better characterize the membrane networks involved in this process

\section{W1649}

Intestinal Epithelial Inflammatory Challenge Modulates Chemokines Expression in Neurons: Induction of Neuronal Chemotactic Properties Emmanuelle Tixier, Jean-Paul Galmiche, Michel Neunlist

INTRODUCTION: Although recent evidences suggest that enteric neurons can secrete chemokines during intestinal inflammation, the cellular mechanisms and functional consequences of this phenomenon remain unknown. Therefore, the aims of the study were (1) to investigate the role of neuro-epithelial interactions in the modulation of chemokines production by neurons and (2) to determine the ability of neurons to induce the chemotaxis of immune cells. METHODS. (1) Neuro-epithelial interactions were studied using a coculture model composed of human Caco-2 intestinal epithelial cells, and human NT2-N neuronal cells. Before being placed in coculture with NT2-N, Caco-2 were inflamed or not with TNFalpha and IFNgamma. Pharmacological studies were performed using IL-lreceptor antagonist (IL-lra) and direct effect of IL-lbeta upon neuronal chemokines expression was studied in NT2-N cells and human specimens of submucosa. IL-8 or MIP-1beta expression was measured by real time RT-PCR, ELISA or immunohistochemistry. (2) Neuronallyinduced chemotaxis was studied using a coculture model of NT2-N neurons and human peripheral blood mononuclear cells (PBMC). Before being placed in coculture with PBMC, neurons were inflamed or not with IL-1beta. RESULTS: (1) The coculture of inflamed Caco2 with NT2-N induced a 4.6-fold increase in IL-8 mRNA expression $(n=12, p<0.001)$ and a 6.9 -fold increase in MIP-lbeta mRNA expression $(\mathrm{n}=10, \mathrm{p}<0.005)$ in NT2-N compared to control. Neuronal induction of IL-8 and MIP-lbeta mRNA expression was blocked by IL-1ra $(\mathrm{n}=4, \mathrm{p}<0.05)$. In addition, IL-lbeta induced IL-8 and MIP-1beta expression both in NT2-N and human submucosal neurons. (2) Following IL-lbeta treatment, NT2-N induced a 2 -fold increase in the chemotactic response of PBMC compared to control $(n=10, p<0.05)$. Neuronally-induced chemotaxis was reduced by a specific neutralizing anti-IL-8 antibody $(\mathrm{n}=7, \mathrm{p}<0.05)$ but not by a specific neutralizing anti-MIP-lbeta antibody. CONCLUSION: Direct neuro-epithelial interactions can modulate chemokines production in neurons via an IL-lbeta-dependent pathway. Furthermore neurons can induce the chemotaxis of immune cells via IL-8 neuronal secretion. This study could set new basis for the development of novel pharmacological approaches in enteric inflammatory neuropathies.

\section{W1650}

Inflammatory Bowel Disease Induces Heparanase (HP) Expression By Colonic Epithelial Cells

Matti Waterman, Neta Ilan, Gabriel Groisman, Rami Eliakim, Israel Vlodavsky, Ofer BenIzhak

Background: $\mathrm{Hp}$ is an endoglycosidase that specifically cleaves heparan sulfate (HS) side chains of HS proteoglycans (HSPG), yielding HS fragments of still appreciable size ( $5-7$ $\mathrm{kDa}$ ) and biological potency. HSPG consist of a protein core to which HS side chains are covalently attached. These complex macromolecules are highly abundant in the extracellular matrix (ECM) and are thought to play an important structural role, contributing to ECM integrity and insolubility. Hp activity has been traditionally correlated with cellular invasion associated with cancer metastasis, as is now also supported by compelling clinical studies. Similarly, Hp activity was implicated in dissemination of immune cells during the course of inflammation. Aim: To study Hp expression in patients with inflammatory bowel disease Methods: We applied immunohistochemistry on archived paraffin-embedded colon sections obtained from patients with Crohn's disease ( $C D, n=17$ ), ulcerative colitis (UC, $n=17$ ), infectious colitis $(n=8)$ and normal-looking mucosa distant from the tumor margins, collected from 17 patients who underwent colon resection for colorectal carcinoma. Immunostaining was performed with anti-heparanase polyclonal antibodies that preferentially recognize the $50 \mathrm{kDa}(\# 733)$, and $8 \mathrm{kDa}(\# 810)$ subunits of the active Hp heterodimer vs. the latent 65 kDa pro-enzyme, thus closely reflecting Hp activity. Immunostaining was assessed by an expert pathologist in a blind manner, and staining intensity was classified as (0): no staining; (2): weak; (4): moderate; and (6): strong staining. Results: Hp expression was barely detected in normal-looking colon specimens (mean score $=0.65$ ). In contrast, expression was markedly induced in tissue specimens obtained from patients with UC and CD (mean score $=3.47$, 2.82 , respectively, $\mathrm{p}<0.001)$. Surprisingly, the colonic epithelial cells, rather than inflammatory cells, were strongly stained with both anti-heparanase antibodies, and staining intensities correlated with inflammation severity. Additionally, significantly low enhancement of $\mathrm{Hp}$ expression was observed in specimens of infectious colitis patients (mean score $=0.88$, $\mathrm{p}<0.008$ ). Conclusions: Our results suggest, for the first time, that chronic, rather than acute inflammatory conditions, induce Hp expression by epithelial cells and may contribute to inflammation severity. This possibility is supported by studies demonstrating that unfractionated heparin, as well as low molecular weight heparin, which inhibit Hp activity, are therapeutically effective in the treatment of IBD patients.

\section{W1651}

Inhibition of the PI3kinase Pathway Abrogates Poly I:C-Stimulated Hyaluronan-Mediated Human Mucosal Smooth Muscle Cell Binding of U937 Monocytic Cells

Sudip K. Bandyopadhyay, Carol A. de la Motte, Scott A. Strong

Background: The origin of inflammatory bowel disease (IBD) is unknown and likely multifac torial. Our laboratory has established that cellular stress induced by virus or viral mimic double stranded RNA (poly I:C) in human mucosal smooth muscle cells (M-SMCs) increases cell surface hyaluronan (HA) deposition and formation of long cable-like structures of HA that are important for leukocyte attachment. Since hyperplasia of M-SMCs and accumulation of mononuclear leukocytes are characteristic pathological changes observed in the intestinal mucosa of IBD patients, and the PI3Kinase/Akt signaling pathway has an established role in cell survival, we investigated whether this pathway is involved in this unique HA-mediated leukocyte attachment. Methods: Colonic M-SMC cultures were derived from human surgical specimens and all experiments were performed within the first three passages. M-SMCs were either untreated or treated with poly $\mathrm{I}: \mathrm{C}$ for $22 \mathrm{~h}$ in presence or absence of PI3Kinase inhibitor (Ly294002) and leukocyte binding of M-SMCs was assayed using 51Cr-labeled U937 cells. Cellular extracts were prepared by standard method for Western blot analysis using specific antibody and immunohistochemical analyses were performed for HA staining. Results: Poly I:C-stimulated M-SMCs monolayers bound substantially more U937 cells than unstimulated cells and this response was inhibited in a dose-dependent manner by treatment with the PI3Kinase inhibitor, LY294002. Treatment of M-SMCs with LY294002 alone had no effect on U937 cells binding. Since Akt is a critical downstream regulator of PI3Kinase, we investigated the response of Akt in M-SMCs after treatment with poly I:C for $1 \mathrm{~h}$ followed by Western blot analysis using an antibody specific to the phosphorylated 473 -serine residue of Akt. A phosphorylated Akt band was observed under these conditions and, as expected, the phosphorylated-Akt band was undetectable in LY 294002- plus poly I:C-treated extracts. Confocal microscopy of M-SMCs stained for HA revealed HA cable formation after poly I:C treatment that was abrogated by LY 294002. Conclusions: Poly I:C-stimulated M-SMC cultures express phosphorylated Akt, demonstrate HA cable formation, and promote leukocyte adhesion through a PI3Kinase-dependent manner.

\section{W1652}

Serotonin Synthesis Is Increased in Crohn's Disease Patients in Remission with Irritable Bowel Syndrome-Like Symptoms

Itta M. Minderhoud, Bas Oldenburg, Marguerite E. Schipper, Jose J. Ter Linde, Melvin Samsom

Background: Symptoms suggestive of irritable bowel syndrome (IBS) are frequently reported in Crohn's disease (CD) patients in remission. Studies of the mucosal content of serotonin, which is a pivotal neurotransmitter in the gut, suggest that serotonin availability is altered in IBS patients. We aimed to study the role of serotonin in IBS-like symptom generation in $\mathrm{CD}$ patients in remission. Material and methods: Ileal- and colonic biopsies were obtained from $20 \mathrm{CD}$ patients in remission, 10 with and 10 without IBS-like symptoms, and 11 healthy controls. Enterochromaffin (EC) cells were counted, and mRNA expression levels of tryptophan hydroxylase (TpH)-1 and the serotonin reuptake transporter (SERT) were determined. Results: The levels of mucosal SERT expression were significantly higher in the ileum than in the colon, in all groups studied $(\mathrm{p}<0.02)$. Analyzing ileum and colon separately, TpH-1 expression in the colon of CD patients with IBS-like symptoms was found to be significantly higher compared to the two other studied groups (controls: $\mathrm{p}<0.005$; $C D$ patients without IBS-like symptoms: $p<0.01$ ). The number of $E C$ cells per gland was comparable for the patient groups in ileum and colon. Discussion: CD patients in remission with IBS-like symptoms have elevated mucosal TpH-1 levels in the colon, suggesting that increased serotonin biosynthesis in the colon plays a role in the generation of IBS-like symptoms. As it is known that inflammation affects the serotonergic pathway, our data implicate that these changes persist in the subset of $C D$ patients in remission with IBSlike symptoms. 
W1653

Effect of Immunosuppressing Drugs On the Migration of Human Primary Colonic Lamina Propria Fibroblasts (CLPFs)

Julia Brenmoehl, Carmen Epp, Sandra N. Leeb, Werner Falk, Juergen Schoelmerich, Gerhard Rogler

BACKGROUND: A central event during wound repair is the migration of activated myofibroblasts (colonic lamina propria fibroblasts, CLPFs) to the wound area. Recently, we have shown that Crohn's disease(CD)-CLPFs have a reduced migratory potential compared to control CLPFs. A permanent reduction of the migratory potential of CLPF from control mucosa could be induced by 3 day incubation with IFN- $\gamma$ and TNF. Most immuno-suppressants used in the therapy of CD cause a reduction of the secretion of TNF, IFN- $\gamma$, IFN- $\alpha$ and other interleukins. We therefore investigated whether drugs typically used in IBD therapy modulate the migration and wound contracting ability of CLPFs. METHODS: Primary CLPF were isolated from patients with $C D$ and from healthy controls. The influence of 5aminosalicylic acid (0.01-1 mg/mL), azathioprine $(0.001-1 \mu \mathrm{g} / \mathrm{mL})$, budesonide $(0.001-10$ $\mu \mathrm{g} / \mathrm{mL})$, cyclosporine A $(0.1-50 \mu \mathrm{g} / \mathrm{mL})$, infliximab $(0.001-3 \mu \mathrm{g} / \mathrm{mL})$, methotrexate $(0.001$ $10 \mu \mathrm{g} / \mathrm{mL})$, prednisolone $(0.001-100 \mu \mathrm{g} / \mathrm{mL})$ and tacrolimus $(0.1-50 \mathrm{ng} / \mathrm{mL})$ on the migration of CLPFs was determined in migration assays in the modified 48-well Boyden chamber. CLPF-mediated contraction was tested in collagen matrices with control-CLPFs. The CLPFs were incubated for $14 \mathrm{~h}$ with tacrolimus, cyclosporine, and azathioprine. Matrix diameters were measured every $24 \mathrm{~h}$ for 7 days. RESULTS. 5-aminosalicylacid, azathioprine, budesonide, prednisolone, and methotrexate had no effect on the migration of control-and CD-CLPFs. Cyclosporine A reduced the migratory potential of CD- and control-CLPFs significantly. 50 $\mu \mathrm{g} / \mathrm{mL}$ cyclosporine reduced the migration of $\mathrm{CD}$ - and control-CLPFs to $5 \%$ of untreated CLPFs. Infliximab reduced only the migration of control-CLPFs significantly at a concentration of $100 \mathrm{ng} / \mathrm{mL}$ and $1 \mu \mathrm{g} / \mathrm{mL}$ to $72 \%$ and $69 \%$, respectively. The migration of $\mathrm{CD}$ - and control-CLPFs was significantly decreased by $50 \mathrm{ng} / \mathrm{mL}$ and $100 \mathrm{ng} / \mathrm{mL}$ tacrolimus to 36 and $38 \%$, respectively. After 7 days the matrix diameters of azathioprine incubated CLPFs were $34 \%$ lower than of untreated control-CLPFs indicating higher contracting activity. Cyclosporin A treated CLPFs contracted 33\% more and tacrolimus incubated CLPFs contracted 58\% more compared to control-CLPFs. CONCLUSION: Cyclosporine, tacrolimus, and infliximab reduced the migration of control- and CD-CLPF at pharmacological concentrations. Azathioprine as well as cyclosporine A and tacrolimus increased matrix contraction by CLPFs. These results may provide a first explanation for the mechanism of fistulae closure by these drugs.

\section{W1654}

S100- $\beta$ Secretion and Enteric Glial Cells Alterations During Inflammatory Bowel Disease (IBD)

Arnaud Bourreille, Emmanuel Coron, Claudine Azoulay, Tanguy Chaumette, Julien Chevalier, Claude Masliah, Jean-Paul Galmiche, Michel Neunlist

Introduction : Enteric Glial Cells (EGCs) represent an important component of the enteric neurons system which exerts major physiological functions. They express several proteins among which $S 100-\beta$ is secreted into the extra-cellular compartment and therefore in the serum. In animal models, glial alteration results in severe intestinal damage. The aims of our study were to assess modifications in $S 100-\beta$ secretion according to the severity of the disease and to characterize EGCs phenotype in colonic mucosa of IBD patients. Methods: 43 IBD patients (23 Crohn's disease (CD), 20 ulcerative colitis (UC)) were studied at different phases of their disease. Serum concentrations of S100- $\beta$ and C-Reactive Protein (CRP) were measured by electro-chemoluminescence and nephelemetry respectively. Biopsy samples were obtained in inflammatory and non-inflammatory areas in 6 patients with active disease and the EGCs network was characterized by immunohistochemistry using an anti-S100- $\beta$ monoclonal antibody. In seven active CD patients, S100- $\beta$ concentrations were measured after infliximab therapy after achieving clinical remission. Results: 1) S100- $\beta$ serum concentrations were significantly lower during active phase of IBD irrespectively of the type of IBD (i.e. CD or UC). 2) After anti-TNF- $\alpha$ therapy induced remission, S100- $\beta$ significantly increased $(0.058 \pm 0.016$ vs $0.071 \pm 0.017 ; p=0.005 ; t$ test $)$. 3$)$ S100 $-\beta$ protein concentrations were inversely correlated to CRP concentrations $(r=-0.256 ; \mathrm{p}=0.03$; Spearman's test $)$. 4) At immunohistochemistry, EGCs showed important alterations with a reduced expression of S100- $\beta$ staining in inflammatory segments compared to non-inflammatory segments. Conclusions: S100- $\beta$ protein changes are detected in the colonic mucosa and in peripheral blood of IBD patients during an active phase of their disease. These changes are reversible after treatment and non-specific for CD or UC. Although these data are not in favor of a primary deficiency of S100- $\beta$, they support an important role of EGCs in human IBD.

\section{W1655}

Modulation of the Migration of Colonic Lamina Propria Fibroblasts By Elevation of Intracellular Cyclic AMP

Florian Rieder, Julia Brenmoehl, Monika Artinger, Anita Zuegner, Katrin Menzel, Martina Georgieva, Juergen Schoelmerich, Werner Falk, Gerhard Rogler

Introduction: Regulation of the migration of colonic lamina propria fibroblasts (CLPF) and their synthesis of extracellular matrix (ECM) is an important mechanism during wound healing and fistula formation in Crohn's disease (CD). Prostaglandin E2 (PGE2) leads to an elevation of intracellular cyclic AMP (cAMP) by ligation of its EP2-receptor and is elevated in $C D$. However its role on CLPF migration has not been investigated so far. Methods: Primary cultures of CLPF were isolated from the mucosa of surgical specimens of patients with active $\mathrm{CD}$ (CD-CLPF), CD fistulas (FI-CLPF) and control persons (CO-CLPF). Migration assays were perfomed in the 48-well Boyden chamber (number of migrated CLPF counted per four high power fields at a magnification of 400x) and PGE2, EP2-receptor and cAMP were determined by ELISA, immunohistochemistry and Western Blot. Results: PGE2 and the EP2-receptor agonist butaprost significantly and dose-dependently reduced the migration of CO-CLPF, CD-CLPF and FI-CLPF starting at a dose of 10nM PGE2 and 5 $\mu \mathrm{M}$ butaprost $\left({ }^{*} \mathrm{p}<0,05\right)$. The effect of PGE2 on migration could be partly reversed by treatment with the
EP2-receptor antagonist AH6809. The EP2-receptor was present on all CLPF tested with an increase after stimulation with IL-1 $\beta$. Initial experiments indicate a higher expression in CD-CLPF compared to controls with maximum levels in FI-CLPF. In addition to this the EP2-receptor was stronger expressed in the submosa of CD-patients and patients with fistulas compared to controls as detected by immunohistochemistry. Forskolin, a direct activator of adenylatecyclase, dose dependently reduced migration in CO-CLPF $\left({ }^{*} \mathrm{p}<0,05\right)$. PGE2, butaprost and forskolin led to an increase in intracellular cAMP in CLPF. In addition to this CLPF spontaneously secreted PGE2 $(430 \mathrm{pg} / \mathrm{ml})$ with a dramatic increase after stimulation with IL-1 $\beta$ (20100 pg/ml). The EP1/EP3-receptor agonist sulprostone did not alter CLPF migration. Conclusion: Elevation of intracellular cAMP reduced the migration of CO-CLPF CD-CLPF and FI-CLPF. This effect is partly mediated by the EP2-receptor. As CLPF secrete significant amounts of PGE2 in colonic inflammation this might be one possible mechanism for the impairment of intestinal wound closure and fistula formation in $\mathrm{CD}$.

\section{W1656}

Galectin-3 Differentially Induces Migration of Intestinal Fibroblasts Elisabeth Lippert, Julia Brenmoehl, Werner Falk, Juergen Schoelmerich, Gerhard Rogler

Background: Recently we identified galectin-3 (gal-3) as the most potent activator of intestina (myo)fibroblasts secreted or deliberated by colonic epithelial cells (CEC). In addition, we showed that gal-3 is strongly expressed in normal and inflamed mucosa, however gal-3 protein was demonstrated to be reduced in Crohn's disease (CD) fistula and strictures. As fibroblast migration plays a central role in wound closure and is believed to be relevant for fistulae and stricture formation, we investigated the influence of gal-3 on the migration on mucosa-fibroblasts Material and Methods: Colonic lamina propria fibroblasts (CLPFs) were isolated from surgical specimens. CEC were isolated from specimens without inflammation CEC-conditioned medium containing gal-3 was used after incubation for $24 \mathrm{~h}$. Recombinan gal-3 was applied in concentrations between 1, 5 and $10 \mu \mathrm{g} / \mathrm{ml}$. Migration assays of CLPFs were performed in a modified 48-well Boyden chamber (with or without addition of fibronectin) with CEC-conditioned media, fibronectin as positive control and unconditioned media as negative control for $6 \mathrm{~h}$. Migration was determined as cells/microscopic field. Each experiment was repeated 8 to 12 times per patient. Results: In cultures of control and CD fibroblasts from inflamed mucosa gal-3 induced significant less migration compared to fibronectin (control CLPF: gal-3: $8.4+$ +/ 4.5 vs fibronectin: $29.8+$ +/ 11.5 cells/hpf). In fibroblasts derived from ulcerative colitis mucosa gal-3 incubation was followed by a higher migration, which was not significantly different to fibronectin $(29.8+/-5.9$ vs $38.0+/-5$. cells/hpf). In CPLF derived from CD fistula gal-3 induced a strong migration comparable to that induced by fibronectin $(51.1+/-9.9$ vs $64.1+/-13.3$ cells/hpf). Discussion: Besides induction of cytokine secretion gal-3 mediated an induction of fibroblast migration comparable to fibronectin, which so far was regarded as strongest activator of migration. However the extent of migration-induction was strongly dependent on the source of CLPF (fistula or UC versus control or inflammation)

\section{W1657}

The Angiogenic Chemokine Granulocyte Chemoattractant Protein-2 (GCP-2) Is Selectively Expressed By Endothelial Cells in Ulcer Beds in Chronic Inflammatory Bowel Diseases (IBD)

Klara Gijsbers, Gert De Hertogh, Gert van Assche, Paul Rutgeerts, Jo van Damme, Karel Geboes

Background Chemokines are small proteins released by many cell types upon stimulation with inflammatory mediators. By attracting and activating immune cells, chemokines play a crucial role in the onset and perpetuation of inflammatory reactions. Overexpression o chemokines causes an uncontrolled immune response that leads to chronic inflammation IBD is characterized by a constant, proinflammatory cytokine and chemokine-mediated influx of leukocytes in diseased tissues. Aim GCP-2 is an angiogenic chemokine that selectively attracts neutrophils. Because these are abundantly present in zones of active inflammation, the expression of GCP-2 was investigated in intestinal tissue sections of IBD subjects. Methods Transmural bowel biopsies from 29 Crohn's disease (CD) patients and 31 ulcerative colitis (UC) patients were stained immunohistochemically for GCP-2. Fourteen biopsies from macroscopically normal bowel in colorectal cancer patients and 6 normal conjunctiva samples were used as non-inflammatory controls. Two inflamed appendices and 13 biopsies from vernal keratoconjunctivitis served as intestinal and extra-intestinal inflammatory controls, respectively. Results Microscopic analysis of non-involved tissues from IBD patients showed only a small number of GCP-2 positive mucosal leukocytes. The number of GCP-2 positive leukocytes in the lamina propria was increased in pathological areas in IBD and correlated with the disease activity observed in the biopsy samples. In addition, pronounced GCP-2 positive staining was observed in endothelial cells (Factor VIII+, CD34+) of blood vessels located in ulcer beds in IBD patients. No GCP-2 positive endothelial cells were observed in inflamed areas without epithelial or mucosal defects. There was also no endothelia GCP-2 staining in inflammatory and non-inflammatory controls. Conclusion The selective expression of GCP-2 by endothelial cells at sites of ulceration in IBD is probably important for the attraction of neutrophils into inflamed ulcer beds. This may be an essential defense mechanism in these lesions, which are constantly in close contact with numerous microbial and dietary antigens. 
Subclinical Inflammation Causes Alterations in Serum Antioxidant and Fatty Acid Levels in Quiescent Inflammatory Bowel Disease (IBD)

Thomas Koernicke, Lennart Schaper, Luzia Valentini, Susanne Hengstermann, RalphJoachim Schulz, Sabine Buehner, Brigitte M. Winklhofer-Roob, Carsten Buening, Herbert Lochs

Aim: The aim of this project was to assess the nutritional and inflammatory status in IBD patients in clinical remission and compare the results to those of healthy controls. Methods: Inflammatory status (C-reactive protein (CRP), Orosomucoid and faecal calprotectin, a noninvasive marker of intestinal inflammation) was assessed in 52 IBD patients $(37 \pm 10 \mathrm{yrs}$; $22,3 \pm 2,1 \mathrm{~kg} / \mathrm{m}^{2}$ ) and 52 healthy controls pair-matched for sex-, age- and BMI. All IBD patients were in clinical remission (32 Crohn disease (CD), CDAI $65 \pm 41 ; 20$ ulcerative colitis (UC), CAI 3,3 $\pm 1,8$ ). Furthermore, plasma levels of protein, fatty acid patterns (FA), antioxidants (lutein/zeaxanthin, retinol, $\beta$-cryptoxanthin, $\alpha$-and $\gamma$-tocopherol, lycopene, $\alpha$ and $\beta$-carotene) were compared. None of the patients was steroid-dependent. Results are expressed as median and interquartile range. Results: In total, $88 \%$ of IBD patients were well nourished according to subjective global assessment, body mass index and serum albumin $>40 \mathrm{~g} / \mathrm{l}$. However, we observed differences in the inflammatory status with respect to CRP, orosomucoid and faecal calprotectin of CD patients (see table). In addition, $\omega-3$, $\omega-6$ and total FA levels were significantly higher in CD patients, especially $\alpha$-linolenic, arachidonic, docosatetranoic and docosapentanoic acid (see table). We also found increased protein values $(77[73 ; 81]$ vs $74[72 ; 77] \mathrm{g} / \mathrm{l}, \mathrm{p}=0.013)$. Plasma antioxidant concentrations were comparable to healthy controls except for decreases in carotene levels (see table). UC patients only showed higher calprotectin levels $(17.4 \mathrm{mg} / \mathrm{l}$ vs. $0.0 \mathrm{mg} / \mathrm{l}, \mathrm{p}=0.05)$ but no significant changes with respect to all other values compared to controls. Conclusion: CD patients even when they are in disease remission show clear signs of systemic and intestinal inflammation which is associated by increases in serum polyunsaturated FA levels and higher oxidative stress. Further studies are warranted to investigate the potential impact of our findings on disease relapse.

\begin{tabular}{|c|c|c|c|}
\hline & $\mathrm{CD}(\mathrm{n}=32)$ & $\operatorname{CON}(\mathrm{n}=32)$ & $\mathrm{p}$ \\
\hline \multicolumn{5}{|c|}{ Inflammatory status $(\mathrm{mg} / \mathrm{l})$} \\
\hline CPR & $1.80[0.55 ; 6.35]$ & $1.00[0.60 ; 1.45]$ & 0.007 \\
\hline Orosomucoid & $795[6020 ; 1065]$ & $620[515 ; 755]$ & 0.015 \\
\hline Calprotectin & $18.8[6.4 ; 36]$ & $0.0[0.0 ; 0.0]$ & 0.008 \\
\hline \multicolumn{5}{|c|}{ Plasma fatty acids $(\mu \mathrm{mol} / \mathrm{l})$} \\
\hline$\alpha$-linolenic acid & $65[44 ; 83]$ & $46[42.4 ; 51]$ & 0.02 \\
\hline arachidonic acid & $527[454 ; 611]$ & $430[392 ; 467]$ & 0.007 \\
\hline docosatetratronic acid & $13[11 ; 15]$ & $10[8 ; 11]$ & 0.001 \\
\hline docosapentanoic acid & $28[24 ; 32]$ & $25[20 ; 27]$ & 0.003 \\
\hline \multicolumn{5}{|c|}{ Plasma antioxidants $(\mu \mathrm{mol} / \mathrm{l})$} \\
\hline$\alpha$-carotene & $0.110[0.069 ; 0.189]$ & $0.305[0.179 ; 0.528]$ & 0.022 \\
\hline$\beta$-carotene & $0.611[0.371 ; 1.123]$ & $1.261[0.918 ; 1.486]$ & 0.032 \\
\hline
\end{tabular}

W1659

Novel Effects of Pkc-Lambda (Pkc- $\lambda$ ) Isoform Activation On Intestinal Monolayers: Carbonylation and Nitration of Cytoskeletal Proteins and Cytoskeletal and Barrier Disruption Following NO Generation in Intestinal Epithelium

Ali Banan, Lijuan Zhang, Jeremy Z. Fields, Ashkan Farhadi, Maliha Shaikh, Christopher B. Forsyth, Ali Keshavarzian

Despite a decade of attempts at unraveling the role of PKC isoform signaling in the GI tract, the structures \& $\%$ or targets of PKC isoforms are unclear. Using monolayers of intestinal cells, we reported that the $\lambda$ isoform of PKC (a member of the atypical PKC subfamily) is required for barrier instability, but the mechanisms underlying this unique phenomenon are unknown. Since inducible NO Synthase (iNOS) is key to cell injury, we hypothesized that PKC- $\lambda$ activation induces iNOS upregulation and NO overproduction, leading to cytoskeletal oxidation / disarray \& barrier disruption. Methods: Intestinal cells were transfected to create new clones over- or under-expressing PKC- $\lambda$. These innovative cells were incubated \pm oxidants or PKC activators/inhibitors. Wild type (WT) cells were treated similarly. We assessed cytoskeletal integrity \{confocal microscopy\}, cytoskeletal oxidation / nitration \{carbonylation/ nitrotyrosination of actin, PAGE fractionation\}, cytoskeletal assembly / disassembly \{F-actin / G-actin ratio, SDS-PAGE\}, barrier permeability \{fluorometry\}, PKC- $\lambda$ subcellular activity / distribution \{immunoprecipitation, in vitro kinase\}, iNOS / NO levels \{scintillation counting, PAGE, chemiluminescence\}, $n=6 /$ grp. Results: Exposure of WT cells to oxidant stress or transfection of cells to overexpress PKC- $\lambda$ disrupted the cytoskeleton $\&$ increased oxidative injury as indicated by native PKC- $\lambda$ activation, iNOS pathway upregulation [iNOS activity/ protein levels, NO levels, oxidative stress/quenchable fluorescence, actin oxidation/nitration], actin pool instability (reduced F-actin, increased G-actin), structural disruption of the actin cytoskeleton, \& monolayer barrier dysfunction. Disruption induced by PKC- $\lambda$ synergized with disruption by oxidants. In oxidant-treated transfected or WT cells, PKC- $\lambda$ was active $\&$ complexed with actin. Dominant mutant inactivation of endogenous PKC- $\boldsymbol{\lambda}$ prevented all measures of iNOS upregulation $\&$ its injurious consequences to the cytoskeleton. Conclusions: We have discovered a new pathophysiological process in gut epithelium. 1) PKC- $\lambda$ is required for oxidant-induced iNOS upregulation $\&$ the consequent nitration $\&$ oxidation of cytoskeletal proteins. 2) PKC- $\lambda$ activation causes cytoskeletal injury following upregulation of NO driven processes. 3) Molecular events underlying the destabilizing effects of PKC- $\lambda$ are increases in carbonylation \& nitration of the subunit components of the cytoskeleton. The ability to promote NO overproduction \& cytoskeletal nitration / oxidation is a novel
W1660

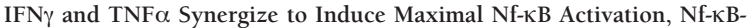
Mediated Trail Expression and Apoptosis of Colonic Epithelial Cells Kenneth Nally, Darren O'Brien, John MacSharry, Emer Bairead, Gerald C. O'Sullivan, Joe O'Connell, Fergus Shanahan

Background and Aims: IFN $\gamma$ and TNF $\alpha$ are potent cytokines with pleiotropic activities such as induction of inflammation, cell cycle arrest and apoptosis. While both are proinflammatory, they have opposing roles in tumorigenesis, with IFNy being primarily a tumor suppressor and TNF $\alpha$ a tumor promoter. TNF $\alpha$ mediates its effects through activation of the transcription factor NF- $\mathrm{\kappa B}$. As inappropriate activation of NF- $\mathrm{\kappa B}$ promotes both chronic inflammation and tumor progression, modulation of its function is seen as a potential strategy for both diseases. Because inhibition of NF-KB function is known to sensitize cells to TNF $\alpha$ triggered cell death, we investigated the involvement of NF- $\kappa B$ in IFN $\gamma$-mediated sensitization of colonic epithelial cells to TNF $\alpha$-triggered apoptosis. Methods and Results: IFN $\gamma+\mathrm{TNF} \alpha$ synergized to induce apoptosis of HT29, WIDR, SW480 and Colo-205 cell lines. In the HT29 cell line, IFN $\gamma$ treatment did not block TNF $\alpha$-induced phosphorylation or degradation of the NF- $\kappa B$ inhibitory protein, I $\kappa B \alpha$. In contrast to other published studies, IFN $\gamma$ treatment did not inhibit the nuclear translocation, DNA binding or transactivation function of NF$\kappa \mathrm{B} /$ RelA. In fact, compared to TNF $\alpha$ treatment alone, IFN $\gamma+\mathrm{TNF} \alpha$ significantly increased DNA binding of RelA between 4 and 16 hours. Quantitation of the NF- $\mathrm{\kappa B}$ target genes RelA, p50, RIP2K, SOD2, cIAP2 and IL-8, demonstrated that IFN $\gamma+$ TNF $\alpha$ synergized to induce maximal expression of all these genes. In addition, compared to IFN $\gamma$ or TNF $\alpha$ treatment alone, IFN $\gamma+\mathrm{TNF} \alpha$ synergized to induce massive expression of TRAIL, as quantitated by PCR and ELISA. However, siRNA-mediated knockdown of TRAIL receptor - DR5 but not DR4 - expression only provided modest protection from IFN $\gamma+\mathrm{TNF} \alpha$-triggered apoptosis. Interestingly, siRNA-mediated knockdown of RelA in HT29 cells did not sensitize them to TNF $\alpha$-triggered apoptosis. It did, however, increase the degree of apoptosis induced by IFN $\gamma+\mathrm{TNF} \alpha$. Finally, quantitative PCR analysis of cDNA from HT29 cells - transfected with siRNA to knockdown RelA expression - demonstrated, that RelA is absolutely required for the synergistic increase in expression of cIAP2, SOD2, IL-8 and TRAIL by IFN $\gamma+$ TNF $\alpha$. Conclusions: These data show that IFN $\gamma$ and TNF $\alpha$ synergize to induce maximal NF-

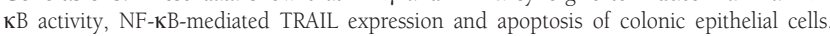
Surprisingly, they also demonstrate that inhibition of NF- $\mathrm{kB} /$ RelA expression does not sensitize these cells to TNF $\alpha$-triggered apoptosis. Finally, they show that NF- $\mathrm{KB}$ is required for the synergistic induction of both anti-apoptotic and pro-apoptotic genes by IFN $\gamma+$ TNF $\alpha$ in colonic epithelial cells.

\section{W1661}

Characterization of a Novel TNF-Alpha Signalling Cascade Involving Autocrine EGF Receptor Trans-Activation in Human Intestinal Epithelial Cells Hb Jijon, A Buret, K Fines, S Mokashi, H Parsons, Paul Beck

Background: Tumor Necrosis Factor-alpha (TNF- $\alpha$ ) is a key inflammatory mediator in the pathogenesis of Inflammatory Bowel Diseases. Intestinal epithelial cells are in a key position to interact with the intestinal lumen and communicate with the intestinal immune system, thus it is important to understand how TNF- $\alpha$ signaling in enterocytes elicits changes in enterocyte function. We have previously demonstrated that TNF- $\alpha$ activates multiple mitogen activated protein kinase (MAPK) cascades in intestinal epithelial cells. As the EGFR is a potent activator of MAPKs, we hypothesized that the EGFR may participate in TNF-dependent MAPK activation. Aims: In this study we examined the possible interaction between the TNF and EGFR's in HT-29 cells. Methods and Results: Immunoprecipitation of the EGFR and subsequent immunoblotting revealed that the EGF receptor is phosphorylated on tyrosine following a 15 minute treatment of intestinal epithelial cells (HT-29) with $10 \mathrm{ng} / \mathrm{ml} \mathrm{TNF}$ $\alpha$. This event is dependent on the intrinsic kinase activity of the EGFR as tyrosine phosphorylation of the EGFR was blocked by the EGFR kinase inhibitor AGl478 (200nM-luM) but not by the platelet-derived growth factor (PDGF) receptor kinase inhibitor AG1298 (10 $\mu \mathrm{M})$. Autophosphorylation of the EGFR was also inhibited by both the Src-kinase inhibitor PP2 $(10 \mu \mathrm{M})$ and the tyrosine kinase inhibitor genistein $(100 \mu \mathrm{M})$, suggesting a role for a Src-family kinase in EGFR phosphorylation. In addition, EGFR autophosphorylation was inhibited by the matrix metalloproteinase (MMP) inhibitor BB94 (10 $\mu \mathrm{M})$. Conclusions: These results suggest that activation of the TNF receptor leads to the activation of a Srcrelated kinase, leading to the activation of an MMP, potentially the release of a membrane bound EGF receptor ligand ultimately resulting in the activation of the EGFR tyrosine kinase in a manner similar to what has been described for $G$ protein-coupled receptor-mediated transactivation of the EGFR. These results reveal a previously unrecognized interaction between the TNF receptor and the EGFR and suggest that the latter may act to relay signals emanating from TNF receptors.

\section{W1662}

SRC Kinase and Racl Gtpase Signals Mediate the Mitogenic Downstream Activation of Focal Adhesion Kinase and Mitogen-Activated Protein Kinases ERK1/2 By Repetitive Deformation in Human CACO-2 Intestinal Epithelial Cells

Lakshmi S. Chaturvedi, H. Michael Marsh, Marc D. Basson

Intestinal epithelial cells are subject to repetitive deformation during normal gut peristalsis and villous motility, while the mucosa atrophies during sepsis or ileus when such stimuli are absent. We have previously shown that rhythmic mechanical strain stimulates intestinal epithelial cell proliferation and promotes differentiation. Activation of focal adhesion kinase (pp125FAK) and mitogen-activated protein kinases ERK1/2 appears required for the strain effect in human Caco-2 intestinal epithelial cells. However, the upstream mediators of these 
effects are unknown. We investigated whether c-Src and the small GTPase Racl mediate stretch-induced FAK phosphorylation and proliferation in Caco-2 cells. Cells were cultured on collagen I-precoated membranes and subjected to an average $10 \%$ repetitive deformation at 10 cycles/minute, consistent with the deformation which the mucosa experiences in vivo. Cells were harvested for Western blot analysis, in vitro kinase assay, subcellular fractionation, crystal violet staining, or cell counting. Mechanical strain activated c-Src within 2 minutes and caused translocation of Racl GTPase from cytosol to membrane within 5 minutes after the initiation of strain in a time-dependent manner. $(n=4, p<0.05)$. Furthermore, strain induced both rapid and sustained (up to $\mathrm{l} \mathrm{h}$ ) increases in phosphorylation of c-Src tyrosine kinase at Tyr416, Racl-cdc42 at Ser71, FAK at Tyr576 and Tyr397, and ERK1/2 at Thr202/ Tyr204 $(n=5, p<0.05)$. Twenty four hours of cyclic strain increased cell numbers compared with static conditions $(n=5, p<0.005)$. The Src kinase inhibitor PP2 $(10 \mu \mathrm{mol} / \mathrm{l})$ or the Racl specific inhibitor NSC23766 (50 $\mu \mathrm{mol} / \mathrm{l})$ blocked strain-induced mitogenicity. The c-Src kinase or Racl inhibition also prevented strain-induced FAK576 and ERK1/2 phosphorylation, but not FAK397 phosphorylation $(n=4, p<0.05)$. Reducing total FAK by $70 \%$ using specific siRNA attenuated stretch-induced ERKl/2 phosphorylation but not c-Src tyrosine kinase or Racl-cdc42 phosphorylation $(\mathrm{n}=6, \mathrm{p}<0.05)$. The Src kinase inhibitor PP2 blocked strain-induced pRacl-cdc42 phosphorylation $(n=5, p<0.05)$. In contrast, the Racl specific inhibitor NSC23766 did not inhibit $c-S r c$ phosphorylation $(n=4, p<0.05)$. In conclusion, these data suggest that repetitive deformation induced by peristalsis or villus motility may support the gut mucosa by a pathway involving c-Src kinase, the small GTPases Racl and cdc42, FAK, and ERK1/2. Aberrations in this signal pathway during ileus or fasting may contribute to mucosal atrophy despite adequate parenteral nutrition and may represent an important target for therapeutic intervention designed to support the mucosa.

\section{W1663}

PIAS-1 Promotes GATA4 Sumoylation and Potentiates Gata4 Mediated Transcriptional Activation of Intestinal Promoters

Narasimhaswamy S. Belaguli, Mao Zhang, Muhammad Aftab, David H. Berger

Introduction: Expression of several gut epithelial differentiation marker genes is regulated by the gut epithelium-enriched GATA factor, GATA4. Tissue-specificity and promoterspecificity of GATA4 appears to be due to its ability to interact with various ubiquitously expressed and gut epithelium-restricted cofactors. We have used the yeast two hybrid system to identify gut tissue-expressed GATA4 cofactors. Methods: Zinc finger and the C-terminal domains of GATA4 fused in frame with the GAL4 DNA binding domain was used as bait. The bait and the GAL4 activation domain-fused human small intestinal cDNA library were cotransformed into yeast Y187 strain and selected for growth on ade/his/leu/trp quadruple dropout media. For coimmunoprecipitation experiments, lysates prepared from HCT116 cells transiently transfected with HA epitope tagged GATA4 and FLAG epitope tagged PIAS1, were used. For GST pulldown experiments, bacterially expressed, GST fused GATA4 and PIAS-1 proteins and 35 [S] methionine labeled in vitro translated mutants of GATA4 and PIAS-1 were used. Site directed mutagenesis of GATA4 and PIAS-1 were performed using QuickChange II XL kit (Stratagene). Transient transfection experiments were performed by cotransfecting subconfluent HCT116 cells with $0.2 \mathrm{ug}$ of IAP and IFABP promoter-luciferase vectors with $0.2 \mathrm{ug}$ to $0.4 \mathrm{ug}$ of GATA4 and PIAS-1 expression vectors. Results: Protein inhibitor of activated STAT-1 (PIAS-1) was identified as a GATA4 cofactor by yeast two hybrid analysis. Coimmunoprecipitation experiments were used to demonstrate that GATA4 and PIAS-1 interact in vivo in mammalian cells. Using GST protein pulldown experiments, the second zinc finger domain and the immediately C-terminal basic region of GATA4 were identified as regions mediating the physical interaction between GATA4 with PIAS-1. PIAS1 stimulated sumoylation of GATA4 and mutation of SUMO acceptor site (K365) of GATA4 or mutation within the RING finger region of PIAS-1 (C350) affected GATA4 sumoylation. In transient cotransfection assays GATA4 synergistically activated IAP and IFABP promoters in the presence of wild type PIAS-1 but not the SUMO ligase activity deficient mutant of PIAS-1 (C350A). Conclusions: PIAS-1 is a GATA4 cofactor that promotes GATA4 sumoylation and synergizes with GATA4 in transcriptional activation of IAP and IFABP promoters.

\section{W1664}

Induced PDKI Kinase Activity Suppresses Apoptosis in Intestinal Epithelial Cells By Activating AKT Signaling Following Polyamine Depletion Kaspar M. Keledjian, Jaladanki N. Rao, Tongtong Zou, Lan Liu, Bernard S. Marasa, Aihong Zhang, Eric D. Strauch, Jian-Ying Wang

Apoptosis plays a critical role in maintenance of gut mucosal homeostasis and is highly regulated by numerous factors including polyamines. Our previous studies have demonstrated that decreasing cellular polyamines promotes the resistance of intestinal epithelial cells (IECs) to apoptosis by increasing Akt kinase activity, but the exact mechanisms by which polyamine depletion activates Akt remain unknown. The 3-phosphoinositide-dependent protein kinase-1 (PDK1), a kinase functioning downstream of PI3K and upstream of Akt, serves as a major regulatory point in Akt signaling and regulates various cellular functions. The current study tests the hypothesis that polyamine depletion induces PDKl kinase activity resulting in Akt activation in IECs. Methods: Studies were conducted in IEC6 cells, derived from rat small intestinal crypts. Apoptosis was induced by exposure to tumor necrosis factor- $\alpha$ (TNF $\alpha$ ) plus cycloheximide (CHX). PDKl activation was examined by measuring levels of phosphorylated PDKl (pPDKl) and its kinase activity. Functions of PDKl were investigated by using small interfering RNA targeting PDKl (siPDK1). Results: Depletion of cellular polyamines by $\alpha$-difluoromethyl-ornithine (DFMO) (specific inhibitor of polyamine synthesis) induced phosphorylation of PDKl and increased PDKl kinase activity, which were completely prevented by exogenous polyamine spermidine. Levels of pPDKl and its kinase activity were increased by $\sim 3$ times the control values 6 days after treatment with DFMO. Induced PDKl activation following polyamine depletion was associated with a significant increase in phosphorylated Akt (pAkt) and Akt kinase activity. Levels of pAkt in polyamine-deficient cells were $\sim 6$ times the control value, while Akt kinase activity was increased by $\sim 10$ times. This activated PDKl/Akt was also associated with decreased caspase- 3 activation and increased resistance to TNF $\alpha / C H X$-induced apoptosis. In contrast, polyamine depletion did not alter levels of total PDK1, total Akt, PTEN, p85, and p110 (subtypes of PI3K) proteins. Inactivation of PDKl in polyamine-deficient cells by either treatment with PI3K inhibitor LY294002 or transfection with siPDK1 not only prevented the induced Akt activation but also blocked the increased resistance to TNF $\alpha / \mathrm{CHX}$ induced apoptosis. Conclusions: These results indicate that induced PDKl activation mediates Akt phosphorylation and is crucial for the increased resistance to apoptosis following polyamine depletion.

\section{W1665}

Keratins Modulate Mitochondrial HMG-CoA Synthase in the Colon Diana M. Toivola, Aida Habtezion, Jian Liao, Qin Zhou, Eugene C. Butcher, M B. Omary

Background: Keratin 8 (K8) was the first intermediate filament (IF) protein targeted for deletion in transgenic mice. In addition to a liver injury susceptibility phenotype, K8-null mice develop, within 2-3 weeks of birth, colonic hyperproliferation and colitis with diarrhea that respond to antibiotic therapy, and enterocyte mistargeting of ion transporters. Aim: We hypothesized that a proteomic analysis will provide molecular insight regarding the function of keratins in the colon, and the colitis that is induced by keratin absence. Methods: Wildtype (WT) and K8-null colons were removed followed by isolation of colonic crypts using calcium-free conditions. Protein homogenates were prepared and equal fractions were labeled with Cy3 (WT) and Cy5 (K8-null). Labeled fractions were analyzed using 2-Dimensional Differential In Gel Electrophoresis (2-D DIGE) coupled with mass spectrometry to identify differentially regulated proteins. Results: In K8-null enterocytes, eleven major non-keratin proteins were down-regulated which include selenium binding protein, 3-hydroxy-3-methyl glutaryl Coenzyme A (HMG-CoA) synthase and carbonic anhydrase I. Fourteen major proteins were up-regulated including dimethylglycine dehydrogenase, and argininosuccinate synthase 1. We focused on HMG-CoA synthase, an important mitochondrial regulator of ketogenesis, whose four isoforms were downregulated 3-6 fold in K8-null colonic crypts The decreased expression of HMG-CoA synthase was confirmed at the protein (by immunoblotting and immunofluorescence staining) and RNA levels. Changes in colonic HMG-CoA synthase were specific to the colon in that they were not found in the liver. Peroxisome proliferator-activated receptor gamma (PPAR gamma), an HMG-CoA synthase transcriptiona activator was also down-regulated in K8-null colon. Conclusion: Our results suggest that colonic keratins may be involved in lipid and ketone body metabolism, which is likely relevant to the colitis phenotype since bacteria are the major source of colonic butyrate.

\section{W1666}

Norepinephrine Enhances Radiosensitivity in Rat Ileal Epithelial Cells Mutsumi Matsuu-Matsuyama, Kazuko Shichijo, Kumio Okaichi, Toshiyuki Nakayama, Masahiro Nakashima, Ichiro Sekine

BACKGROUND: We previously reported that after X-irradiation the apoptosis index in jejunal crypt cells in spontaneously hypertensive rats, a well-known model for essential hypertension in humans, was significantly greater than in control Wistar-Kyoto rats. Also, the administration of reserpine to induce sympathetic dysfunction of spontaneously hypertensive rats or Wistar-Kyoto rats resulted in a significant suppression of apoptosis. (J. Radiat. Res, 41, 55-65, 2000). We hypothesized that hyperfunction of the sympathetic nervous system is involved in the high susceptibility of the jejunal crypt cells to radiation-induced apoptosis. To test this hypothesis, the effect of norepinephrine (NE) on cell survival was examined using the clonogenic assay and on apoptosis after X-ray irradiation in rat ileal epithelial cells (IEC-18) in vitro. METHODS: Rat ileal epithelial cells (IEC-18) in serumfree medium were treated for $2 \mathrm{~h}$ with $\mathrm{l} \mathrm{MM} \mathrm{NE}$ or without NE, then exposed to $8 \mathrm{~Gy} \mathrm{X}$ ray irradiation, and followed by the colony formation assay. Apoptotic fragments were confirmed by TUNEL staining at $6 \mathrm{~h}$ after $8 \mathrm{~Gy}$ irradiation. In another series of experiments, cells were incubated with prazosin $(5 \mu \mathrm{M})$, yohimbine $(5 \mu \mathrm{M})$ and propranolol $(5 \mu \mathrm{M})$, which are alphal, alpha2, and beta-adrenergic receptor antagonists respectively, for 30 min before $\mathrm{NE}$ treatment, and colony formation assays were examined after $8 \mathrm{~Gy}$ irradiation. Cleaved caspase- 3 expression was examined by western blot analysis at $3 \mathrm{~h}$ after irradiation. RESULTS $\mathrm{NE}$ treatment in rat ileal epithelial cells resulted in the decrease of cell survival in a dose dependent manner. A combination of $\mathrm{NE}$ and radiation significantly decreased the surviving fraction at 8 Gy from $24.1 \%$ to $6.4 \%$ ( $<<0.05)$. NE concentration of $1 \mu \mathrm{M}$ resulted in significantly increasing the apoptotic rate from $3.5 \%$ to $6.0 \% 6 \mathrm{~h}$ after 8 Gy irradiation $(\mathrm{p}<0.05)$. The survival fraction of prazosin $(130 \%)$ and yohimbine $(141 \%)$ treated IEC-18 cells increased compared to non-treated cells after $8 \mathrm{~Gy}$ irradiation. Enhanced cell sensitivity to radiation by NE was attenuated by alphal-adrenergic antagonist (prazosin) but was not affected by beta-adrenergic antagonist (propranolol). Cleaved caspase-3 (active form) expression in NE treated cells was increased compared to non-treated cells $3 \mathrm{~h}$ after $8 \mathrm{~Gy}$ irradiation, but was reduced by prazosin treatment. CONCLUSIONS: The results of this study suggest that norepinephrine enhanced the radiosensitivity including radiation-induced apoptosis of rat ileal epithelial cells and that the alphal-adrenergic receptor may be involved in the process.

\section{W1667}

Differential Promoter Usage and Activity May Regulate Function of RNA Binding Protein CUGBP2 in Intestinal Epithelial Cells Satish Ramalingam, Courtney W. Houchen, Brian K. Dieckgraefe, Shrikant Anant

RNA binding protein CUGBP2 is a ubiquitously expressed protein that is located in the nucleus under physiological conditions. When subjected to physiological stress, such as exposure to radiation, CUGBP2 expression is significantly induced. Furthermore, the protein translocates to the cytoplasm, where it is found bound to AU-rich sequences in the 3'untranslated region of cyclooxygenase-2 (COX-2) mRNA. Following binding to 3'UTR, the protein stabilizes COX-2 mRNA but inhibits its translation. CUGBP2 contains two N-terminal RNA recognition motif (RRM) domains, one C-terminal RRM domain, and alanine and glutaminerich region between the second and third RRM domains. SAGE analyses for ten norma human tissues, calculated as the number of appearances of the corresponding tag divided 
by the total number of tags in libraries derived from that tissue and subsequently then normalized by multiplying by $1.2 \mathrm{M}$ demonstrated highest levels of expression in the brain and spinal cord, but high levels were also seen in liver, kidney and lungs. We have also observed expression of CUGBP2 in the intestine, but the expression is significantly suppressed in cancer tissues. Alternative promoter usage, and alternative splicing results in three transcript variants encoding slightly different CUGBP2 isoforms. Isoform 1 encodes the smallest protein of 490 amino acids, while the isoforms 2 and 3 include additional 24 and 19 amino acids in the N-terminus of the protein, respectively. Based on RT-PCR analyses, we have determined that isoform 1 is the dominant form of CUGBP2 mRNA that is expressed in human colon cancer cell lines, HT-29, HCT-116 and SW480. Furthermore, the protein encoded by isoform 1 is localized in the nucleus. We have previously shown that CUGBP2 expression is significantly induced in HT-29 cells following radiation, while its expression is lost following treatment with prostaglandin $\mathrm{E}_{2}\left(\mathrm{PGE}_{2}\right)$. We have now determined that isoform 1 is significantly induced in the cells following radiation exposure. Furthermore, expression of isoform 1 is suppressed following treatment of the cells with either $\mathrm{PGE}_{2}$ or epidermal growth factor (EGF). In contrast, expression of isoforms 2 and 3 were lost following treatment of cells with either radiation, $\mathrm{PGE}_{2}$ or EGF. Ectopic overexpression on isoform 1 induces cells to undergo apoptosis. Together these data suggest that radiation treatment results in expression of an isoform that is involved in apoptosis.

\section{W1668}

Juvenile Polyps Express Muc5ac Which May Be Related to Reduced SMAD4 and $\mathrm{Cdx} 2$ Expression

Raquel Almeida, Rita Barros, Jose Almeida, Nuno Mendes, Isabelle van Seuningen, Celso Reis, Filipe Santos-Silva, Carme De Bolos, Leonor David

Mucin core proteins are used as cell differentiation markers. MUC5AC is a marker of gastric foveolar differentiation, MUC6 is a marker of gastric glandular differentiation and MUC2 is a marker of intestinal goblet cells. Aberrant expression of mucins occurs in cancer and in lesions that are benign or pre-neoplastic: MUC2 is expressed in gastric intestinal metaplasia (IM), whereas MUC5AC is expressed in the colon in hyperplastic and serrated polyps. Cdx2 is the master gene responsible for intestinal differentiation and is involved in the transcription of several intestinal genes including MUC2. Transgenic mice with Cdx2 ectopic expression in the gastric mucosa develop IM and conversely $\mathrm{Cdx} 2+/-$ mice develop hamartomatous polyps in the colon with gastric differentiation. Interestingly, Smad4+/- mice also develop hamartomatous lesions in the colon. Based on these observations we hypothesized that loss of CDX2 and/or Smad4 might contribute to generate hamartomatous lesions of the colon, with a switch from colonic to gastric phenotype, including juvenile polyps that in familial and sporadic cases can occur in the presence of Smad4 mutations. Based on the same line of thought we hypothesized that Smad4 might regulate Cdx2 expression. We characterized 18 juvenile polyps by immunohistochemistry for gastric mucins expression, MUC5AC (MoAb $\mathrm{CLH} 2$ ) and MUC6 (MoAb CLH5), intestinal mucin expression, MUC2 (MoAb PMH1) and expression of Cdx2 (Biogenex) and Smad4 (B8, SantaCruz Biotechnology). MUC2 expression was also assessed by In Situ Hibridization. Transactivation of Cdx2 by Smad4 was assessed using Luciferase reporter assays. We observed that $83 \%$ of the polyps express de novo MUC5AC, none of the cases express MUC6 and all the cases express MUC2 with a "patchy", heterogeneous pattern, with areas of absence of immunostaining and negativity by ISH. Both $\mathrm{Cdx} 2$ and Smad4 expression were observed in all cases, with a "patchy" pattern including negative areas. Luciferase reporter assays showed that Smad4 transactivates Cdx2 promoter. In conclusion, acquisition of a foveolar gastric differentiation, indicated by the presence of MUC5AC, is a common feature of juvenile polyps, as previously demonstrated for hyperplastic and serrated colon polyps. This aberrant gastric differentiation is associated with loss of colonic differentiation, namely MUC2, and with reduced Cdx2 and Smad4 expression. The observed transactivation of $\mathrm{Cdx} 2$ by Smad4 may contribute to explain, in the absence of a functional Smad4 pathway in juvenile polyposis, the onset of a gastric phenotype.

\section{W1669}

Functional Characterization and Transcriptome Analysis of Mastomys Enterochromaffin Cells

Mark Kidd, Irvin M. Modlin, Geeta N. Eick, Manish C. Champaneria

Although the enterochromaffin (EC) cell is one of the primary neuroendocrine (NE) regulatory cells of the small intestine, the lack of a purified cell system has precluded its characterization. We have developed methodology to produce a pure population of Mastomys ileal EC cells utilizing density gradient centrifugation and FACS sorting, and have investigated its functional regulation and transcriptome. Mastomys ilea $(n=10)$ were everted, end-ligated, digested with pronase/collagenase, Nycodenz gradient centrifuged, and EC cells collected by FACS sorting of acridine orange-labeled cells. Enrichment was confirmed by immunostaining of specific EC cell markers, serotonin content, EC cell marker gene expression and electron microscopy. PACAP, somatostatin, and gastrin receptor expression was determined by real-time RT-PCR. FACS sorted EC cells were cultured and the effects of forskolin, isoproterenol, acetylcholine, GABAA, PACAP-38 and gastrin on serotonin secretion (ELISA) assessed. The EC cell transcriptome was derived from GeneChip Affymetrix profiling of FACS-sorted cells. FACS sorting produced a $>70$-fold enrichment of EC cells with a serotonin content of $240+22 \mathrm{ng} /$ mg protein. Preparations were $99+0.7 \%$ pure by immunostaining for tryptophan hydroxylase (TPH). The contaminating cell type was NE (CgA/neurotensin-positive). Somatostatin cells were absent in FACS preparations. VPACI and SSTR2 receptors were present but PACl and CCK2 receptors were undetectable. Forskolin, isoproterenol and PACAP-38 stimulated serotonin secretion at EC50 values of $5 \times 10-10 \mathrm{M}, 4.5 \times 10-10 \mathrm{M}$ and $1.2 \times 10-9 \mathrm{M}$, respectively. Octreotide, acetylcholine and GABAA inhibited serotonin secretion with IC50 values of 3x10-11M, 3x10-10M, 2.9x10-10M, respectively. Gastrin had no effect on serotonin secretion. Analysis of the EC cell transcriptome revealed highly expressed ( $>22.6$-fold) EC cell marker genes (TPH, substance P and guanylin), the absence of marker genes for other small intestinal cell types (villin, mucins, defensins, CD4, inflammatory gene complexes), and a receptor profile that included cholinergic (M4), adrenergic (b1), dopaminergic (D2), serotoninergic (5-HT2C), GABAergic (A a $2 \& \mathrm{~b})$, and prostaglandin receptors (E1,4 \& F). The isolation of a homogeneous preparation $(>99 \%)$ of live EC cells for regulatory characterization and transcriptome assessment indicates that functional characterization of the human ileal EC cell is feasible. This will enable physiologic assessment of the role of the EC cell in conditions such as inflammatory/irritable bowel disease and investigation of its role in the development of carcinoid tumors.

\section{W1670}

The Glycine Transporter GLYT1 Is Required for Glycine-Mediated Protection of Intestinal Cells and Is Up-Regulated in Response to Stress Alison Howard, Imran Tahir, Sarah M. Waring, Barry H. Hirs

In the intestine glycine attenuates cell damage caused by ischemia/reperfusion (IR), prevents or reverses colitis induced by 2,4,6-trinitro-benzene sulphonic acid (TNBS) and dextran sulphate, and inhibits apoptosis during IR. The aim of this study was to investigate the mechanism of glycine cytoprotection in the intestinal cell lines, HCT-8 and Caco-2. In both cell lines, glycine uptake at the basal membrane was approximately five fold greater than at the apical membrane and the specific glycine transporter GLYTl accounted for 30-40\% of this. Monolayers of cells were subjected to oxidative stress by incubation for $24 \mathrm{~h}$ in medium containing $150 \mu \mathrm{M}$ tert-butylhydroperoxide (t-BOOH). This induced approximately $50 \%$ cell death (cell viability controls, $100 \pm 1.98 \%$; $\mathrm{t}-\mathrm{BOOH}$ treated, $53.5 \pm 4.9 \%, \mathrm{n}=8$; $\mathrm{p}<0.05$ ). Pre-incubation of the cells in medium containing 1 or $5 \mathrm{mM}$ glycine protected the cells from injury and reduced cell death to $20-30 \%$ (cell viability, $72.5+4.9$ and $81.2+8.1 \%$ of control respectively, $\mathrm{n}=8 ; \mathrm{p}<0.05$ compared to $\mathrm{t}-\mathrm{BOOH}$ treated cells both concentrations). Glycine added into the medium during $\mathrm{t}-\mathrm{BOOH}$ treatment had no effect on the decrease in cell viability. Including the GLYTl inhibitor N-[3-(4'-fluorophenyl)-3-(4'-phenylphenoxy)propyl] sarcosine (ALX-5407) in the pre-incubation medium completely blocked the protective effects of glycine (cell viability, $48.5 \pm 2.0 \%, \mathrm{n}=8$ ). Glycine pre-treatment preserved cellular glutathione concentration during exposure to $\mathrm{t}-\mathrm{BOOH}$ (glutathione concentration in control cells, $1.94 \pm 0.45 \mu \mathrm{g} / \mathrm{mg}$ protein, $\mathrm{n}=9 ; \mathrm{t}-\mathrm{BOOH}$ treated, $0.32 \pm 0.20, \mathrm{n}=9 ; 5 \mathrm{mM}$ glycine $+\mathrm{t}-\mathrm{BOOH}$ treated; $1.36 \pm 0.22, \mathrm{n}=9$ ). To study the effect of stress on GLYTl mRNA levels, cells were incubated in medium lacking amino acids or supplemented with diethyl maleate, to deplete glutathione, or tunicamycin, to invoke ER stress and the unfolded protein response. Abundance of mRNA relative to $18 \mathrm{~S}$ rRNA was measured by real-time RT-PCR. GLYTl mRNA levels were increased significantly by all treatments (relative mRNA level: controls, $1.00 \pm 0.85$ arbitrary units; amino acid starved, $3.18 \pm 1.09$; tunicamycin, $2.39 \pm$ 0.62 ; DEM $2.46 \pm 0.12 . \mathrm{n}=9, \mathrm{p}<0.05$ for all). Similar increases were seen in mRNA encoding the cystine-glutamate exchanger xCT. Expression of the peptide transporter, PepTl, was unchanged. These results indicate that GLYTl activity is required for glycine protection of intestinal cells and that abundance of its mRNA is increased during intestinal cell response to stress. Corresponding increases in GLYTl and xCT mRNA levels suggest that this response may lead to increased supply of amino acids leading into glutathione synthesis.

\section{W1671}

The Transcriptome and Function of Normal and Neoplastic Human EC Cells Irvin M. Modlin, Mark Kidd, Geeta N. Eick, Manish C. Champaneria, Roswitha Pfragner

The neuroendocrine (NE) regulation of small intestinal (SI) function is ill-understood since pure NE cells are unavailable. Similarly, the biological basis of SI carcinoid tumors-of enterochromaffin (EC) cell origin - is unknown since neoplastic human EC cells are unavailable. We purified EC cells from ilea and characterized a malignant EC cell carcinoid cell line (KRJ-1) to define the functional regulation and transcriptome of naive and neoplastic EC cells. Human ilea $(n=12)$ from $R$. hemicolectomies were pronase/collagenase digested, Nycodenz gradient centrifuged, and EC cells FACS sorted after acridine orange-labeling. Enrichment was defined by immunostaining, gene expression, serotonin (5-HT) content and real-time RT-PCR. Naïve FACS-sorted EC and KRJ-1 cells were cultured and 5-HT secretion was measured after stimulation with forskolin, isoproterenol (Iso), acetylcholine (ACh), GABAA, PACAP-38 and gastrin. The normal and neoplastic EC cell transcriptome was acquired by Affymetrix profiling (U133A). FACS produced $100 \pm 0.3 \%$ (CgA staining) and $99+0.7 \%$ pure (TPH) EC cells with $>67$-fold enrichment and a 5 -HT content of $180+18 \mathrm{ng} /$ mg protein (mucosa $=3.5+0.9$ ). The $<1 \%$ contamination was neurotensin-positive cells. NE/ EC-specific (CgA, VMATl, TPH 14-21-fold >than mucosa), as well as subst P, guanylin and SERT transcripts were detected. VPACI and SSTR2 receptors were present on both cell types but $\mathrm{PACl}$ and $\mathrm{CCK} 2$ were undetectable. Forskolin and Iso-stimulated 5-HT secretion was $10-100 \mathrm{x}>$ potent for naive $(\mathrm{EC} 50=1.8 \times 10-9 \mathrm{M} ; 5.1 \times 10-9 \mathrm{M})$ vs neoplastic cells $(\mathrm{EC} 50=$ 2. $1 \times 10-7 \mathrm{M} ; 8.1 \times 10-8 \mathrm{M})$ but PACAP-38 was similar $(\mathrm{EC} 50=1 \times 10-7 \mathrm{M})$. Iso stimulated cAMP levels $1.6 \pm 0.1$-fold vs basal $(E C 50=2.7 \times 10-9 M)$. ACh inhibited normal EC cell 5 -HT secretion more than neoplastic (IC50=3.2x10-9M vs $1.6 \times 10-7 \mathrm{M})$, while GABAA was more potent in neoplastic cells (IC50=3.9x10-10M vs $4.4 \times 10-9 \mathrm{M}$ ). Octreotide inhibited normal but not neoplastic basal 5-HT secretion; its inhibitory effect on Iso-stimulated secretion (IC50= 1.3x10-8M and 5.3x10-9M) was not significantly different. Gastrin had no effect on 5-HT secretion in normal or KRJ-1 cells confirming the absence of a CCK2 receptor. Comparison of transcriptomes revealed shared NE (CgA, VMATl) and EC cell specific (TPH, subst P, guanylin) marker genes. Neoplastic cells expressed somatostatin SSTR2/3, serotonin 5-HT2A, adrenergic alC/bl, glutaminergic Ag3 receptors vs SSTR2/5, 5-HTlA and B/2C, bl, and Aa6/b2 receptors in naive cells. The putative carcinoid malignancy genes (MTAl, MAGED2) are unique to the EC cell neoplastic transcriptome. Further delineation will allow identification of novel targets to control secretion and proliferation of carcinoids. 
W1672

Specific Upregulation of Pancreatic Enzymes in Rat Duodenal Mucosa By Duodenal Ulcerogens: Gene Expression Indicates New Elements in Pathogenesis

Longchuan Chen, Xiaoming Deng, Klara Osapay, Tetyana Khomenko, Sandor Szabo

Chemically induced duodenal ulcers in animal models have been widely used to study the pathogenesis and healing of duodenal ulcers. Our previous structure-activity studies have identified the functional groups of potent ulcerogenic chemicals (PNAS, 1991, 88:62986302 ), e.g., cysteamine (HS-CH2-CH2-NH2) is a potent ulcerogen while its close analog ethanolamine (HO-CH2-CH2-NH2) fails to induce duodenal ulcers in rats. Propionitrile and mepirizole are also potent duodenal ulcerogens. Using these chemicals, we have performed DNA microarray analysis of gene expression in rat duodenal mucosa to identify gene expression changes specifically associated with duodenal ulceration. Goals: to identify common and distinct molecular pathways leading to duodenal ulcer formation induced by various ulcerogens. Methods and Results: Groups of unfasted Sprague-Dawley female rats (180-200g) were given cysteamine $(25 \mathrm{mg} / 100 \mathrm{~g}$, once or $\mathrm{x} 3$, every $4 \mathrm{hr})$ or equimolar amount of ethanolamine $(21.47 \mathrm{mg} / 100 \mathrm{~g}$, once or $\mathrm{x} 3$, every $4 \mathrm{hr}$ ) by gavage, or propionitrile $(5 \mathrm{mg} /$ $100 \mathrm{~g}, \mathrm{x} 3$ ) injected subcutaneously. Rats were euthanized by CO2 inhalation at $2 \mathrm{hr}$ or 12 hr after first dose of chemicals. Proximal duodenal mucosa was scraped after pancreatic tissue was carefully removed. Total RNA was extracted from mucosal samples and further processed for DNA microarray analysis using Affymetrix Rat 2302.0 chip (30,000 transcripts). Results from two independent experiments were analyzed. Real-time PCR was performed to confirm candidate genes that were upregulated. A cluster of pancreatic enzymes such as chymotrypsinogen B, carboxypeptidase A2, chymotrypsin-like protein, pancreatic colipase, pancreatic trypsin 1 , phospholipase $\mathrm{A} 2$, and a secretory granule protein syncollin were among the genes that were upregulated (4-100 fold) by cysteamine (at both $2 \mathrm{hr}$ and 12 $\mathrm{hr}$ ) and propionitrile but not affected by ethanolamine (at either $2 \mathrm{hr}$ or $12 \mathrm{hr}$ ). Conclusions: 1) We have identified a group of genes upregulated by duodenal ulcerogens but not by nonulcerogenic analog in the duodenal mucosa. 2) The unconventional upregulation of these genes especially proteases and lipases in the duodenal mucosa may point to a new mechanism of ulcer formation and organ-specific tissue injury. 3) Thus, gene and protein expression studies may help to identify new pathways in the pathogenesis of duodenal ulceration.

\section{W1673}

The Molecular Mechanism of Tumor Necrosis Factor- $\alpha$ (TNF- $\alpha$ ) Modulation of Intestinal Tight Junction (TJ) Permeability: Regulation of Myosin Light Chain Kinase (MLCK) Promoter Activity

Dongmei Ye, Thomas Y. Ma

TNF- $\alpha$ induced increase in intestinal epithelial TJ permeability has been proposed as an important mechanism contributing to intestinal inflammation. Our previous studies have indicated that the TNF- $\alpha$ increase in Caco-2 TJ permeability was mediated by an increase in MLCK promoter activity, leading to a step-wise increase in MLCK expression and activity, and an increase in Caco-2 TJ permeability. TNF- $\alpha$ increase in Caco-2 TJ permeability also required NF-KB activation.(AJP 2004; 286:G367-76) The aim of this study was to delineate the regulatory mechanisms that mediated the TNF- $\alpha$ modulation of MLCK promoter activity. Methods: A 2091 bp MLCK promoter was cloned (Gene ID DQ 090939). Various molecular and biochemical methods were used. Results: A series of 5' deletion constructs of MLCK promoter were generated and the minimal MLCK promoter region identified (-448 to -18). Two functionally active NF- $\mathrm{KB}$ binding sites were identified on the promoter region (-84/75 and $-456 /-447$ ). The $\mathrm{KB}$ motif at $-84 /-75$ was located within the minimal promoter region (cis-element) and the $\kappa \mathrm{B}$ motif at $-456 /-447$ was located outside the minimal promoter region (trans-element). MLCK promoter deletion constructs encoding only cis- or trans- $\mathrm{KB}$ site were generated. TNF- $\alpha$ caused an increase in the promoter activity of the DNA construct encoding the cis- $\mathrm{\kappa B}$ site (but lacking the trans- $\mathrm{\kappa B}$ site). In contrast, TNF- $\alpha$ caused a small decrease in the promoter activity of the DNA construct encoding only the trans- $\mathrm{KB}$ site. These results indicated that the two $\mathrm{\kappa B}$ sites on the MLCK promoter have opposite regulatory actions. Electrophoretic mobility shift assay indicated that NF-KB p50/p65 heterodimer binds to the cis- $\mathrm{KB}$ site, while $\mathrm{p} 50 / \mathrm{p} 50$ homodimer binds to the trans- $\mathrm{KB}$ site, suggesting that the binding of $\mathrm{p} 50 / \mathrm{p} 65$ to cis-KB site induces an up-regulation while binding of p50/p50 to trans-кB site induces a down-regulation of the promoter activity. Lastly, the depletion of NF- $\mathrm{KB}$ p65 expression in Caco-2 cells by siRNA prevented the TNF- $\alpha$ induced up-regulation of promoter activity caused by the cis- $\mathrm{KB}$ site (binds to $\mathrm{p} 50 / \mathrm{p} 65$ ), but did not affect the down-regulation by the trans-KB site (binds to p50/p50). Conclusions: Our results indicate that the TNF- $\alpha$ induced activation of MLCK promoter is selectively regulated by binding of specific NF- $\mathrm{KB}$ dimer type to the cis and trans-KB motifs on the MLCK promoter. These studies provide important new insight into the molecular mechanism of TNF- $\alpha$ regulation of MLCK promoter activity and Caco-2 TJ barrier function.

\section{W1674}

Glycogen Synthase Kinase 3 Regulates Cytokine and LipopolysaccharideInduced Gene Expression in Intestinal Epithelia

Eleana M. Harmel, Kris A. Steinbrecher

Background: While glycogen synthase kinase $3 \alpha$ (GSK-3 $\alpha$ ) and GSK- $3 \beta$ control numerous signaling pathways, little is known about the role of these potential therapeutic targets in intestinal inflammatory diseases. Initial studies suggest that GSK-3 is able to regulate the activity of specific nuclear factor $\mathrm{\kappa B}$ (NF- $\mathrm{\kappa B}$ ) and activator protein 1 (AP-1) subunits that are integral to both cytokine and Toll-like receptor (TLR)-mediated intestinal epithelial cell (IEC) gene expression. We hypothesize that blockade of GSK-3 activity in IECs results in deregulated gene expression following tumor necrosis factor- $\alpha$ (TNF $\alpha$ ) or lipopolysaccharide (LPS) stimulation. Methods: We used the pharmacological inhibitor SB216763 or stable integration of short hairpin-expressing RNAi constructs to decrease GSK-3 activity in a nontransformed intestinal epithelial cell line (IEC-18). Kinase assays and/or western blotting were used to evaluate the status of relevant signal transduction molecules including NF-KB activation pathway intermediates and mitogen-activated protein kinases following TNF $\alpha$ or LPS stimulation. Gene expression studies were performed using realtime RT-PCR. Results: Inhibition of GSK-3 in IEC-18 cells greatly decreased GSK-3-mediated phosphorylation of $\beta$-catenin without increasing $\beta$-catenin levels. The ability of IEC-18 cells to use alternative $\beta$-catenin regulation pathways provides a system to investigate the function of GSK-3 withou the potentially confounding high levels of $\beta$-catenin found in many transformed IEC lines. Similar to that seen in TNF $\alpha$-signaling decreased GSK-3 activity in IEC-18 cells did no effect the initial steps in LPS-induced activation of NF- $\mathrm{KB}$. Western blotting and kinase assays suggested that TNF $\alpha$ - or LPS-mediated activation of JNK and phosphorylation of $\mathrm{c}-$ Jun were sustained in cells lacking full GSK-3 activity. This effect on JNK regulation was specific as we saw no change in ERKI/2 activity in GSK-3-blocked IECs. Regulation of numerous NF-KB and AP-1 target genes was altered in cells lacking GSK-3 activity. While I $\mathrm{B} \alpha \mathrm{mRNA}$ levels were unaffected, interleukin 6 expression was greatly diminished. Conversely, TNF $\alpha$ or LPS stimulation resulted in increased CXCLl and MCP-1 expression after loss of GSK-3 activity. Conclusions: Based on these data, we suggest that GSK-3 proteins regulate cytokine and TLR-mediated gene expression in IECs. Furthermore, transcriptional control of these genes appears to be highly promoter specific. These data and our previous work support the concept of an important function of GSK-3 in modulating NF-KB and AP-1 function that may be especially relevant to intestinal inflammatory diseases.

\section{W1675}

Transcriptional Regulation of Adenosine 2b Receptor By IL-6 Vasantha L. Kolachala, Tracy S. Obertone, Brittani Kridler, Adel Driss, Lixin Wang, Didier Merlin, Shanthi V. Sitaraman

Background and Significance: We have previously shown that adenosine, generated during intestinal inflammation through neutrophil-epithelial cross-talk, induces transcriptionallymediated synthesis of IL-6 by intestinal epithelial cells. We also demonstrated that IL-6 receptors are present basolaterally in intestinal epithelial cells and IL-6 induces the activation of NF-kB and STAT signaling in epithelial cells. In this study we addressed the effects of IL- 6 on the regulation of epithelial adenosine $2 \mathrm{~b}$ receptor (A2bR). Methods: Model colonic epithelial cells, T84, were stimulated with IL-6 $(100 \mathrm{ng} / \mathrm{ml}$, basolateral). Homo sapiens chromosome 175 was obtained from a PAC library (P1 derived artificial chromosome) clone and PCR was performed to obtain a 1.4-kb fragment upstream of A2bR coding region which was subsequently cloned into a pGL3 luciferase vector. The luciferase vector containing insert was transfected into COS7 cells. 72 hours after transfection the luciferase activity was measured. The transcription start site was determined using rapid amplification of cDNA ends (RACE). The potential regulatory elements within the promoter region were identified by sequence analysis and by transcriptional element search system. Results: Pretreatment of intestinal epithelial cells with IL-6 increased A2b receptor mRNA (two-fold) and protein expression (66\% increase). The increase in IL-6-induced A2bR mRNA was seen starting at 2 hours and protein levels were increased after 12 hours of pretreatment with IL-6. In order to further understand the transcriptional regulation of A2bR by IL- 6 , we cloned and characterized the $1.4 \mathrm{~kb} 5$ ' flanking region of the human A2bR gene. The transcriptional start site was seen at $119 \mathrm{bp}$ upstream of the translational start site. There were multiple IL-6 response element binding sites (IL-6 RE-BP) as well as STAT binding site in the A2bR promoter. Transfection of COS-7 with the A2bR promoter resulted in 6-fold increase in activity. Deletion and mutation studies are ongoing in the laboratory. Conclusions: We demonstrate that IL-6 induces a transcriptionally regulated synthesis of A2bR, likely through activation of IL-6RE-BP/STAT sites in the A2bR promoter. Along with our previous observation that IL- 6 is induced by adenosine in epithelial cells, these data suggest autocrine regulation of $\mathrm{A} 2 \mathrm{~b}$ receptor by the epithelial-derived IL- 6 .

\section{W1676}

SP1 and SP3 Mediate the Effects of Histone Deacetylase Inhibitor, TSA, On the Induction of the Human NHE3 Promoter Activity in C2BBEl Cells Jaleh Malakooti, Leda Ghannad, Ricardo Sandoval, Pradeep K. Dudeja, Krishnamurthy Ramaswamy

$\mathrm{NHE} 3$ is the major $\mathrm{Na}+\mathrm{H}+$ exchanger isoform in the ileum where it plays a critical role in $\mathrm{Na}+$ and water absorption. Previous reports indicate that sodium butyrate and trichostatin A (TSA), two histone deacetylase inhibitors, enhance the NHE3 mRNA expression and transport activity both in vivo and in vitro. However, the mediators of these effects are poorly understood. To examine the molecular events involved in these processes, we investigated the effect of TSA, a specific histone deacetylase, on the transcriptional regulation of the human NHE3 promoter. Transient transfection of a series of 5'-deletion NHE3 promoter-luciferase constructs in $\mathrm{C} 2 \mathrm{BBe}$ l cells revealed that the TSA-responsive element is located in a GCrich region $\sim 85$ bp upstream from transcription initiation site. The promoter construct containing the TSA-response element showed a 4-fold increase in reporter gene activity, subsequent to exposure to TSA ( $100 \mathrm{ng} / \mathrm{ml}$ and $24 \mathrm{~h}$ ). In gel mobility shift assays, transcription factors Spl, Sp3 and AP-2 $\alpha$ bind to the GC-rich sequence and co-transfection with the Spl and $\mathrm{Sp} 3$ expression vectors elevate the NHE3 promoter activity in SL2 cells. However neither the protein expression level nor DNA binding affinity of Spl and Sp3 were affected by TSA treatment as judged by Western blot analysis and GMSA. Mutational analyses confirmed that the TSA response element coincides with the GC-box in the core promoter region, as deletions or point mutations of the Spl/Sp3 and AP-2 binding sites reduced the basal and TSA-stimulated NHE3 promoter activity. P300, an acetyltransferase, has been shown to function with both Spl and Sp3 in GC-box-dependent transcription. Therefore, we examined the transactivation property of a P300 expression vector on the NHE3 core promoter containing the GC-box in $\mathrm{C} 2 \mathrm{BBel}$ cells. These results showed that transfection of P300 expression vector enhanced the NHE3 promoter activity in a dose-dependent manner, with maximum activity of $\sim 4$-fold in the highest concentration of the P300 expression construct. Thus the TSA-responsive GC-box is also involved in mediating the functional effect of P300. In conclusion, induction of NHE3 expression by TSA appears to be mediated via interactions with a GC-box, involving the co-activator P300 and Spl/ Sp3 transcription factors 
W1677

Sodium Butyrate Increases Human NHE3 Gene Expression Via Sp1/Sp3 Transcription Factors and Blocks the Repressive Effects of IFN- $\gamma$ and TNF- $\alpha$ in C2BBEl Cells

Md Ruhul Amin, Jaleh Malakooti, Pradeep K. Dudeja, Krishnamurthy Ramaswamy

Diarrhea associated with inflammatory bowel disease has been attributed to stimulated secretion of pro-inflammatory cytokines like IFN- $\gamma$ and TNF- $\alpha$, which have been shown to down-regulate the expression of the sodium hydrogen exchanger3 (NHE3) gene. NHE3 isoform is localized to the apical pole of the intestinal epithelial cells where it plays a major role in regulating water and sodium ion absorption. Previously, we have shown that IFN$\gamma$ and TNF- $\alpha$ down-regulate the expression of the human NHE3 gene by phosphorylating $\mathrm{Spl}$ and Sp3 transcription factors in $\mathrm{C} 2 \mathrm{BBel}$ cells. It is known that sodium butyrate (NaB) stimulates NHE3 gene expression in intestinal cells, and this stimulation is mediated by PKA. However, further details of the mechanisms how NaB up-regulates NHE3 gene expression are not known. In this study, we have investigated the mechanisms involved in up-regulation of NHE3 gene expression by $\mathrm{NaB}$ and its effect on the down-regulation of NHE3 gene by combined IFN- $\gamma(30 \mathrm{ng} / \mathrm{ml})$ and TNF- $\alpha(20 \mathrm{ng} / \mathrm{ml})$ treatment. In transient transfection studies, $\mathrm{NaB}(5 \mathrm{mM})$ led to $~ 10$-fold stimulation of NHE3 promoter activity after incubation for 24 hours. Using $5^{\prime}$-deletion constructs, the $\mathrm{NaB}$ responsive region was mapped to the NHE3 core promoter region (-95 to +5$)$, which contains a number of cis-elements including $\mathrm{Spl}$ binding sites and mediates the repressive effects of IFN- $\gamma$ and TNF- $\alpha$ on the NHE3 promoter. The stimulatory effect of $\mathrm{NaB}$ on the NHE3 promoter was reduced by $60 \%$ in the presence of these cytokines. Gel mobility shift assays with nuclear proteins from $\mathrm{NaB}$ or cytokine-treated cells showed enhanced and diminished binding of Spl and Sp3 to the NHE3 promoter, respectively, compared to control. The stimulatory effect of $\mathrm{NaB}$ on the NHE3 promoter was partially blocked by phosphatase inhibitors, Fostriecin (100 nM) and Okadaic acid $(200 \mathrm{nM})$ in transient transfection assays. $\mathrm{NaB}$ or cytokine treatment showed no effect on Spl and Sp3 mRNA and protein expression as assessed by RT-PCR and Western blot analyses. In conclusion, our data suggest that $\mathrm{NaB}$ treatment may result in dephosphorylation of $\mathrm{Spl}$ and $\mathrm{Sp} 3$ proteins leading to their increased affinity for binding to the NHE3 promoter in C2BBel cells and resulting in increased transcription of NHE3.

\section{W1678}

Human Gene Transcriptional Activation By Vitamin D Studied in Small Intestinal Endoscopic Biopsies in short Term Culture

Sonia Sangha, Sara Balesaria, Julian R. Walters

Background: Knowledge of the regulation of gene expression in the human small intestine has been impaired by the lack of a suitable differentiated cell-line. Differences may exist when compared with animal model systems. Consequently we aimed to develop a human small intestinal explant system which could be used to determine transcriptional regulation of gene expression. Changes in expression of calcium transport genes were studied in response to the active hormonal form of vitamin $\mathrm{D}, 1,25(\mathrm{OH})_{2} \mathrm{D}_{3}$. Methods: Volunteers undergoing diagnostic upper endoscopy were recruited after giving informed consent for studies, approved by the local research ethics committee. Subjects with various specific disorders were not included. After diagnostic biopsies had been obtained, 8-10 additional duodenal mucosal biopsies were taken and placed in pre-oxygenated tissue culture medium. Biopsies in groups of 2-4 were placed on microporous membrane inserts and incubated in tissue culture medium with either hormone or vehicle control. After culture, biopsies were placed in RNAlater and then stored frozen. RNA and cDNA were prepared using standard methodology. Real-time quantitative RT-PCR (Taqman) was used to assay gene expression, which was standardized to expression of the housekeeping gene, GAPDH. Results: Experiments were performed after $6 \mathrm{~h}$ exposure to $1,25(\mathrm{OH})_{2} \mathrm{D}_{3}\left(10^{-9} \mathrm{M}\right)$, or control. The expression of transcripts for the brush-border membrane calcium channel, TRPV6, increased significantly ( $<<0.001$, Wilcoxon signed rank test; TRPV6/GAPDH ratios $1.50 \pm 0.21$ (mean \pm SEM) in controls, and $5.38 \pm 1.00$ in the treated group). 29 out of 30 subjects showed an increase; the median change in expression ratios was 3.10. Similar changes occurred in men and women and were not related to age. Transcripts for the basolateral calcium-pumping ATPase, PMCAl, increased significantly ( $<<0.02$, median change 1.34) but the cytoplasmic calcium binding protein, calbindin-D9k, did not change significantly. Sucrase transcripts were also unaffected. Conclusion: A robust human small intestinal explant system has been developed. This was able to show a marked increase by $6 \mathrm{~h}$ in expression of transcripts for the TRPV6 calcium transporter and PMCAl with physiological doses of $1,25(\mathrm{OH})_{2} \mathrm{D}_{3}$. It has the potential to study other agents that may regulate gene expression including other hormones and drugs.

\section{W1679}

MDP-Mediated Activation of NALP3/NOD2 Cooperates with Tlr2 in Secretion of IL-8 in Intestinal Epithelial Cell

Tatjana Eigenbrod, Johann Federhofer, Christian Huy, Manuela Gunckel, Julia Brenmoehl, Werner Falk, Juergen Schoelmerich, Gerhard Rogler

Background: The NODs and the NALPs are members of the NLR family of cytoplasmic pattern recognition receptors (PRRs) which are involved in activation of the innate immune system. Mutations of NALPs and NODs are associated with inflammatory diseases. To date, 14 different NALPs have been identified. Activation of NALP1 and NALP2/3 finally results in assembly of the 'inflammasome', a multiprotein platform which mediates the autocatalytic cleavage of the inflammatory caspases- 1 and -5 . Subsequently, the active caspases induce proteolytic cleavage and maturation of IL-1b and IL-18. While most NALP ligands are still unknown, Muramyldipeptid (MDP) has only recently been identified as a ligand for NALP3. MDP is also known to activate NOD2, resulting in NF-kB mediated production of IL-8 and pro-IL-lb. Therefore, NOD2 and NALP3 might be coworkers for the induction of innate immune responses. Furthermore, a synergistic as well as an antagonistic cross-talk of MDPmediated signalling and TLR2-signalling has been reported in monocytic cells but has not yet been investigated in intestinal epithelial cells (IEC). Methods: Expression patterns of NALP 2-14 were assessed in different epithelial cell lines (Caco-2, HT29, SW480, T84) and in primary IEC from inflamed and noninflamed mucosa by quantitative Taqman PCR Primary IEC isolated from noninflamed mucosa were stimulated with varying concentrations of MDP and/or Pam3Cys, a synthetic TLR2 ligand, and IL-8 was measured in the supernatant. Results: NALP7 was not detected in any cell type and NALP5 was not expressed in Caco2 and HT29. NALP12 was only expressed in SW480 and in T84. The other investigated NALPs were detected in all tested cell types. The highest expression of NALP2 was observed in Caco-2 (4- to 64-fold higher than in the other tested cells), whereas all other NALPs were in average 4- to 32-fold higher expressed in primary IEC than in the investigated cell lines. Primary IEC isolated from inflamed mucosa did not show an upregulation of NALP mRNA. Stimulation of primary IEC with MDP or Pam3Cys resulted in a dose-dependent secretion of IL-8. In order to investigate a potential cross-talk of NALP3/NOD2- and TLR2signalling, cells were simultaneously stimulated with MDP and Pam3Cys in varying concentrations. In these co-stimulation experiments, a synergistic effect of MDP- and Pam3CysSignalling on IL-8 secretion could be detected. Conclusions: NALPs show different expression patterns in epithelial cell lines and in primary IEC. Activation of the NALP3/NOD2 signalling pathway by MDP cooperates with TLR2-mediated induction of proinflammatory cytokine IL-8 in primary IEC

\section{W1680}

\section{Monocyte Chemotactic Protein-1 Polymorphism Is Associated with Acute} Recurrent Pancreatitis

Giulia M. Cavestro, Simone Bertolini, Stefano Okolicsanyi, Giuliana Sereni, Luca Frulloni, Lucas Cavallaro, Elena Masselli, Alessandra Protti, Paolo Del Rio, Paola Zanelli, Mario Sianesi, Giorgio Cavallini, Angelo Franze, Francesco Di Mario

Pancreatitis is associated with an intense inflammatory response. Monocyte chemotactic protein-1 (MCP-1) is a chemokine that plays a central role establishing and maintainig the inflammatory process. Polymorphism $-2518 \mathrm{G}$ of the regulatory region of monocyte MCP affects the level of expression of this chemokine increasing the inflammatory response. Aim To determine wether: 1)MCP1 $2518 \mathrm{G}$ allele is associated with the severity of acute pancreatitis (AP) 2)MCP1 $2518 \mathrm{G}$ polymorphism is associated to acute recurrent pancreatitis (ARP) Patients amd Methods: Determination of MCP1 $2518 \mathrm{G}$ polymorphism was achieved by PCR/RFLP in 93 patients affected from a single episode of biliary AP $(61.3 \% \mathrm{M}) ; 63$ patients affected from ARP $(58.7 \% \mathrm{M})$ and 73 healthy controls of the same ethnical group $(56.3 \%$ M). The severity of AP and ARP was based upon the Atlanta criteria: 119 patients had mild pancreatitis (76.3\%), 37 had severe pancreatitis $(25.7 \%)$. MCP1 $2518 \mathrm{G}$ polymorphism is associated with ARP (Fisher's exact test, $\mathrm{p}=0.005, \mathrm{OR}=2.97, \mathrm{CI}=[1.38-5.21]$ ). Table 1 shows the heterozygous polymorphism of $\mathrm{MCPl}(\mathrm{A} / \mathrm{G})$ was found in $32 \mathrm{ARPs}(50.8 \%)$; the homozygous polyomorphism $(\mathrm{G} / \mathrm{G})$ was found in 6 ARPs $(9.5 \%)$ and 25 ARPs did not present the $G$ allele (A/A; 39.7\%). $45 \mathrm{APs}(48.4 \%$ ) were found $\mathrm{A} / \mathrm{G}$ positive; $1 \mathrm{AP}(1.1 \%)$ was $\mathrm{G} / \mathrm{G}$ positive and 43 APs did not present the G allele (50.5\%). In control group, 21 subjects (28.8\%) were positive for $\mathrm{A} / \mathrm{G}$ genotype; $\mathrm{G} / \mathrm{G}$ genotype was found in 5 controls $(6.8 \%)$ and 47 subjects did not present the G allele (64.4\%). No association was observed between MCPI $\mathrm{G}$ polymorphism and $\mathrm{AP}(\mathrm{p}=0.27)$. No association between MCPl $\mathrm{G}$ polymorphism and severity of AP was detected ( $\mathrm{p}=0.9$ ): table 2 shows $\mathrm{A} / \mathrm{G}$ genotype was found in 60 mild APs (50.4\%); G/G was found in 5 mild APs (4.2\%) and 54 mild APs did not present the G allele (45.4\%). A/G genotype was found in 17 severe APs (45.9\%); G/G was found in 2 severe APs (5.4\%) and 18 severe APs did not present the G allele (48.6\%). MCP-1 polymorphism is associated with ARP. This polymorphism could increase the inflammatory response leading to a recurrence of the disease and possibly to chronic pancreatitis.

Table 1: MCP genotype in Acute Pancreatitis (AP) and Acute Recurrent Pancreatitis (ARP)

\begin{tabular}{|c|c|c|c|}
\hline & MCP genotype A/A (\%) & MCP genotype A/G (\%) & MCP genotype G/G (\%) \\
\hline AP $(\mathrm{n}=93)$ & $47(50.5 \%)$ & $45(48.4 \%)$ & $1(1.1 \%)$ \\
\hline ARP $(\mathrm{n}=63)$ & $25(39.7 \%)$ & $32(50.8 \%)$ & $6(9.5 \%)$ \\
\hline CONTROLS $(\mathrm{n}=73)$ & $47(64.4 \%)$ & $21(28.8 \%)$ & $5(6.8 \%)$ \\
\hline
\end{tabular}

MCP genotype in Mild Acute Pancreatitis (AP) and Severe Acute Pancreatitis (ARP)

\begin{tabular}{|l|c|c|c|}
\hline & MCP genotype A/A (\%) & MCP genotype A/G (\%) & MCP genotype G/G (\%) \\
\hline MILD AP $(\mathrm{n}=119)$ & $54(45.4 \%)$ & $60(50.4 \%)$ & $5(4.2 \%)$ \\
\hline SEVERE AP $(\mathrm{n}=37)$ & $18(48.6 \%)$ & $17(45.9 \%)$ & $2(5.4 \%)$ \\
\hline
\end{tabular}

\section{W1681}

Activated Protein C Up-Regulates TM and EPCR Expression to Attenuates the Severity of Severe Acute Pancreatitis in Rats

Ping Chen, Yaozong Yuan, Yongping Zhang, Mingming Qiao

Aim $\&$ backgroud: The protein $C$ pathways were recognized for their role in regulating thrombin activity and generation, but they were increasingly understood to have diverse regulatory functions in inflammation. However, the exact of mechanisms of which remained unclear in severe acute pancreatitis (SAP). The aim of our studies was the investigation of the effect of pre-treatment activated protein $C$ (APC) on endothelial protein $C$ receptor (EPCR) and thrombomodulin (TM) expressions to expose the mechanisms of attenuating the severity of SAP in rats. Methods: Sixty SD rats were randomly allocated four groups as follows: In SAP group, rats were retrograde infused with $5 \%$ sodium taurocholate $(1.5 \mathrm{ml} /$ $\mathrm{kg}$ ) into the pancreatic duct to stimulate severe acute pancreatitis $(\mathrm{n}=20)$; In treatment groups, rats were received different dose of APC just before the induction of SAP (50 ug/ $\mathrm{kg}, 10 \mathrm{ug} / \mathrm{kg}, \mathrm{n}=15$, i. v., respectively); In control group, rats only given sham operation $(\mathrm{n}=10)$. Blood sample and pancreatic tissue were harvested at 16 hours after the onset of induction. The severity of pancreatitis was evaluated by histological scoring, serum amylase level and pancreatic dry/wet weight ratio. RT-PCR was performed to quantify the intrapancreatic EPCR, TM and matrix metalloproteinase 9 (MMP-9) mRNA levels. The protein expression 
of EPCR, TM and MMP-9 were investigated by the method of western blotting. Meanwhile, immunohistochemistry located pancreatic EPCR and TM expression and was performed semi-quantitive analysis. Serum TNF- $\alpha$ and IL-8 levels were determined by ELISA Results: The expression of pancreatic tissue MMP-9 at mRNA and protein levels were significantly reduced in high-dose and low-dose groups compared to SAP group $(\mathrm{p}<0.01$ or $<0.05$, respectively). Meanwhile, serum TNF- $\alpha$ and IL- 8 levels showed significant decrease in the treatment groups $(\mathrm{p}<0.01)$. On the other hand, the expression of intrapancreatitic EPCR or TM at mRNA and protein levels were significantly up-regulated in treatment groups compared with those in SAP group and control group ( $\mathrm{p}<0.01$ or $<0.05$, respectively). Histological scoring, serum amylase level and pancreatic dry/wet weight ratio in treatment groups were also decreased in comparison with SAP group $(\mathrm{p}<0.01)$. Conclusion: The finding suggested that APC up-regulated EPCR and TM expression by suppressed the expression of MMP-9 and decreased the release of TNF- $\alpha$ and IL-8. We speculate that the administration of exogenous APC in induction of SAP could augment the anti-coagulation and anti-inflammatory activity of the protein $C$ pathways, and could attenuate the following pancreatitic damage.

\section{W1682}

Involvement of Endogenous Growth Hormone and Insulin-Like Growth Factor1 in the Protective Effect of Ghrelin in Necrotizing Acute Pancreatitis Zygmunt Warzecha, Artur Dembinski, Piotr Ceranowicz, Wieslaw W. Pawlik, Ryszard Sendur, Romana Tomaszewska, Beata Kusnierz-Cabala, Jerzy Naskalski, Peter C. Konturek, Atsukazu Kuwahara, Ikuo Kato

Background: Ghrelin, an endogenous ligand for the growth hormone secretagogue receptor, was primary isolated from the human and rat stomach. It stimulates growth hormone secretion, food intake and exhibits gastroprotective properties. In the previous study, we have shown the protective effect of ghrelin against edematous caerulein-induced pancreatitis. This study has been undertaken to check whether ghrelin administration protects the pancreas against ischemia/reperfusion-induced necrotizing pancreatitis and, if so, what is the role of growth hormone $(\mathrm{GH})$ and insulin-like growth factor-1 (IGF-1) in this effect. Methods: Two weeks after sham-operation or hypophysectomy, acute pancreatitis was induced in Wistar rats by clamping of inferior splenic artery for $30 \mathrm{~min}$ followed by reperfusion. Ghrelin was administered twice ( $30 \mathrm{~min}$ prior to start of ischemia and after next $3 \mathrm{~h}$ ) at the doses: 4,8 or $16 \mathrm{nmol} / \mathrm{kg} /$ dose. After 6 -h reperfusion animals were sacrificed. Results: In rats with intact pituitary, treatment with ghrelin increased serum level of ghrelin and attenuated the development of ischemia/reperfusion-induced pancreatitis, what was demonstrated in histological examination as the reduction in pancreatic edema, inflammatory infiltration, necrosis, hemorrhages and the decrease in vacuolization of pancreatic acinar cells. Also, ghrelin administration decreased the activity of lipase and poly-C ribonuclease, and concentration of pro-inflammatory IL-1 in the serum, whereas the pancreatitis-evoked fall in pancreatic DNA synthesis was partly reversed. Treatment with ghrelin was without effect on serum level of anti-inflammatory IL-10 and the pancreatitis-related fall of pancreatic blood flow. Ischemia/reperfusion-induced pancreatitis strongly reduced serum concentration of GH and IGF-1, and ghrelin administration reversed this effect. Ghrelin at the dose 8 $\mathrm{nmol} / \mathrm{kg}$ exhibited maximal beneficial effect. Hypophysectomy lowered serum concentration of growth hormone under the detection limit and reduced serum level of IGF-1 by $90 \%$, but increased serum level of ghrelin. These effects were associated with augmentation of pancreatic damage after induction of acute pancreatitis. Administration of ghrelin was without significant effect on the pancreatic damage evoked by ischemia/reperfusion-induced pancreatitis in hypophysectomized rats. Conclusions: (1) Administration of ghrelin attenuates pancreatic damage evoked by ischemia/reperfusion-induced pancreatitis; (2) Protective effect of ghrelin administration on the pancreas is related to its action on the pituitary and the release of GH and IGF-1.

\section{W1683}

Protective Effects of Vascular Endothelial Growth Factor On Intestinal Epithelial Cell Apoptosis and Endotoxin/Bacterial Translocation in Experimental Severe Acute Pancreatitis

Takahiro Nakajima, Takashi Ueda, Yoshifumi Takeyama, Takeo Yasuda, Hidehiro Sawa, Yoshikazu Kuroda

Background and Aim: Endotoxin/bacterial translocation (E/BT) plays an important role for systemic complications in severe acute pancreatitis (SAP). Breakdown of intestinal integrity is considered to be implicated in E/BT. We recently demonstrated that accelerated apoptosis of intestinal mucosa may be participated in E/BT. On the other hand, vascular endothelial growth factor (VEGF) is a glycoprotein with potent angiogenic, mitogenic and vascular permeability-enhancing activities specific for endothelial cells. We recently reported that serum VEGF levels are significantly elevated in patients with SAP, and that VEGF functions as a protective factor via an antiapoptotic effect against the renal and liver injuries in rat experimental SAP. This study aimed to investigate effects of VEGF on intestinal epithelial cell apoptosis and E/BT in experimental SAP. Methods: Male Wistar rats weighing 250-300 $\mathrm{g}$ were used. Only laparotomy was performed in sham-operated rats (Sham group). Severe hemorrhagic necrotizing pancreatitis was induced by retrograde injection of $3 \%$ sodium deoxycholate into the biliopancreatic duct (SAP group). Recombinant rat VEGF $(2 \mu \mathrm{g} / \mathrm{ml}$, $1 \mathrm{ml}$ ) was injected from jugular vein, and immediately SAP was induced as described above (VEGF+SAP group). Eight hours after induction of SAP, serum amylase and lipase levels were measured, and apoptosis of ileal mucosa was evaluated biochemically by DNA fragmentation ELISA and histochemically by TUNEL staining. After 18 hours, histological examination of the terminal ileum was conducted by HE staining, and villous height and crypt depth were measured. Furthermore, blood endotoxin level was determined, and bacterial culture of the mesenteric lymph nodes was examined. Results: Eight hours after induction of SAP, serum amylase and lipase levels were significantly elevated in SAP group than those in Sham group. VEGF did not affect them. Apoptosis of ileal mucosa was accelerated in SAP group compared with Sham group. VEGF significantly improved the increasing apoptosis. Eighteen hours after induction of SAP, villous height and crypt depth were significantly lower in SAP group than that in Sham group. VEGF significantly improved them. Blood endotoxin levels were significantly elevated and bacterial translocation occurred in SAP group. VEGF significantly improved the elevation of blood endotoxin and prevented the bacterial translocation. Conclusion: These results suggest that breakdown of intestina mucosa via accelerated apoptosis causes E/BT in SAP, and that VEGF inhibits intestinal epithelial cell apoptosis and following E/BT

\section{W1684}

Galanin Antagonism Reduces Hyperenzymemia Associated with Acute Pancreatitis (AP) in a Possum Model

Mayank Bhandari, Anthony C. Thomas, Colin J. Carati, Masahiko Kawamoto, Mark Brook-Smith, James Toouli, Gino Saccone

Background/Aim: Severe AP is associated with significant morbidity and mortality, and is characterized by microcirculatory and secretory disturbances, leading to pancreatic necrosi and plasma hyperenzymemia. To date, there is no specific treatment for AP. We have shown that the neurotransmitter galanin (GAL) is abundant in the pancreas of the Australian possum, and affects pancreatic vascular perfusion. In this study, we investigated the effect of GAL, and its antagonist galantide (GT) on plasma enzymes, in an AP possum model. Methods: AP was induced in anaesthetized possums by pancreatic duct ligation and simultaneous hyperstimulation with i.v. cholecystokinin and secretin for $6 \mathrm{~h}$. In separate experimenta groups $(\mathrm{n}=5-10)$, GAL $(1-10 \mathrm{nmol} / \mathrm{h})$ or GT $(3-30 \mathrm{nmol} / \mathrm{h})$ was administered as bolus injection $15 \mathrm{~min}$ prior to duct ligation followed by a continuous i.v. infusion for the duration of experiment (prophylactic dose). In order to simulate a clinical situation, GT was also administered $2 \mathrm{~h}$ post AP onset (therapeutic dose) in separate animals. Blood was collected hourly for $8 \mathrm{~h}$ and analyzed for plasma lipase and amylase activity. The pancreas was harvested at the end of the experiment (8h) for histological assessment. Statistical analysis used ANOVA. Results: AP was associated with significantly increased plasma lipase which reached a plateau at $3 \mathrm{~h}$ (Figure). This change was exacerbated by prophylactic GAL and significantly ameliorated by prophylactic GT. At $8 \mathrm{~h}$ therapeutic GT also significantly reduced the plasma lipase activity (mean \pm SE, $589 \pm 54$ IU) compared with the AP group (mean \pm SE, $942 \pm 71$ IU). Statistically significant similar effects were noted for plasma amylase levels $(\mathrm{P}<0.05)$. Conclusion: Prophylactic and therapeutic administration of GT significantly decreased the plasma lipase and amylase levels, perhaps by ameliorating the severity of AP. Ongoing histological studies are assessing the effect of GT on pancreatic necrosis in AP.

Lipase Activity - AP, Prophylactic GAL and GT

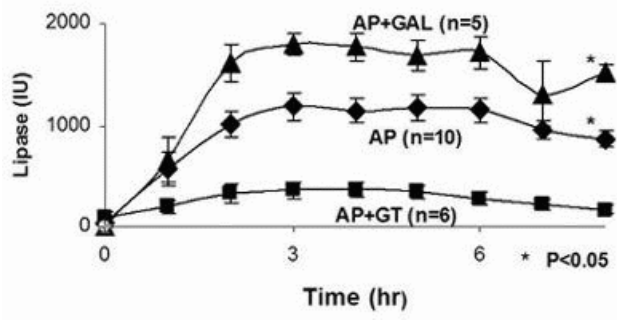

W1685

Pseudorabies Virus Infection Stimulates Pancreatic Ductal HCO3- Secretion Viktoria Venglovecz, Zoltan Rakonczay, Balazs Ordogh, Tamas Takacs, Janos Lonovics, Annamaria Szabolcs, Andras Varro, Andras Toth, Gyula J. Papp, Mike A. Gray, Barry E. Argent, Zsolt Boldogkoi, Peter Hegyi

Background. We have previously shown that hypersecretion can be observed during the early phase of experimental oedematic and necrotizing pancreatitis. However the source and the role of hypersecretion are unknown. Several viruses have been reported to cause acute pancreatitis, but we have no information concerning the effect of viral infection on pancreatic secretion. Our aim in this study was to examine the effect of acute infection caused by a herpesvirus (pseudorabies virus; PRV) on pancreatic ductal secretion. Methods. Ba-DupGreen (BDG), a genetically modified replicating strain of PRV was used in this study. The green fluorescent protein (GFP) gene was inserted into the PRV antisense promoter region located in the inverted repeat segment of the virus. Small intra/interlobular duct were isolated from the pancreas of guinea pigs. Ducts were incubated in McCoy's based culture medium containing BDG virus (107 plaque forming units $/ \mathrm{ml}$ ) for 6 hours, while the control ducts were incubated only with the culture media. The ducts were then cultured for further 18 hours. The rate of $\mathrm{HCO}$ - secretion was determined as described earlier (Hegyi et al. Am J Physiol Cell Physiol 285:C268-C276, 2003). All the experiments were performed in $\mathrm{HCO}$--buffered Ringer solution at $370 \mathrm{C}$. Viral structural proteins were visualized by immunohistochemistry. Native GFP expression and immunofluorescence signals were recorded by a confocal laser scanning microscope. Results and Conclusions. The virus infected all accessible epithelial cells of the duct as judged by the appearance of GFP and viral antigens in the ductal cells. PRV infection stimulated ductal HCO3- secretion about 4-5-fold after 24h. We suggest that this response of pancreatic ducts to PRV infection could represent a defence mechanism against invasive pathogens to avoid pancreatic injury. This work was supported by The Wellcome Trust and OTKA. 
W1686

Arginine Induced Oxidative Stress Is Required for the Initiation of Endoplasmic Reticulum Stress Signaling and Acute Pancreatitis in Rats Constanze H. Kubisch, Thiruvengadam Arumugam, Craig D. Logsdon

Background: We previously found that all major ER stress signalling pathways are activated early in arginine induced acute pancreatitis. In the current study, we investigated the mechanisms involved in this response and the relationship between the extent of ER stress and disease severity. Methods: Pancreatitis was induced in rats by ip injection of $4.0 \mathrm{~g} / \mathrm{kg}$ bw arginine. Pancreatitis severity was assessed by analysis of serum amylase, pancreatic trypsin activity, water content and histology. ER stress related molecules PERK, XBP-1 were analyzed. We examined the influence of the NADPH oxidase inhibitor diphenylene iodonium (DPI) on ER stress and acute pancreatitis. Results: Arginine treatment induced pancreatitis, as indicated by increased serum amylase, pancreatic edema, and acinar cell damage within 4h. Arginine treatment also activated ER stress, as shown by phosphorylation of PERK ( $268 \%$ increase after $4 \mathrm{~h}$ ) and XBP-1 splicing (152\% increase after 24h). Inhibition of NADPH oxidase by DPI profoundly inhibited the activation of ER stress mechanisms. PERK phosphorylation was inhibited by $60 \%$ at $4 \mathrm{~h}$ and $55 \%$ at the $24 \mathrm{~h}$. XBP- 1 splicing which occurred later was reduced by $49 \%$ at $24 \mathrm{~h}$. The development of acute pancreatitis was also significantly reduced by NADPH inhibitor treatment. Serum amylase levels were reduced by $54 \%$ and $66 \%$ and tissue water content was reduced by $9 \%$ and $14 \%$ after 4 and $24 \mathrm{~h}$, respectively Moreover, treatment with NADPH inhibitor dramatically reduced pancreatic histological damage. Conclusions: These results indicate that inhibition of the oxidative stress mechanisms involving NADPH oxidase prevent arginine induced ER stress and acute pancreatitis. These data are consistent with a model in which arginine causes oxidative stress leading to ER stress and subsequent acute pancreatitis.

\section{W1687}

Effect of SiRNA Mediated Gene Knockdown of Pancreatitis-Associated Protein (PAP) in Experimental Pancreatitis

Yin Yao Lin, Martin Bluth, Albert Stanek, Hazal Drew, Tamar A. Smith-Norowitz,

Domenico Viterbo, Cathy M. Mueller, Michael E Zenilman

Background: The pancreatitis-associated protein (PAP) family of genes is induced in acute pancreatitis. We have previously demonstrated that antisense mediated gene knockdown of PAP in vivo decreased PAP gene expression and worsened pancreatitis. Here we investigated the effect of a more specific inhibition of PAP using siRNA gene knockdown using in vitro and in vivo models of experimental pancreatitis Methods: In vitro, pancreatic acinar cell line, AR42J, was cultured with IL6 and dexamethasone (IL6/dex) to induce expression of PAP. Following transfection of siRNA into stimulated AR42J cells, culture supernatants were analyzed for amylase and lipase levels at 12,24, and $48 \mathrm{hr}$. In vivo, acute pancreatitis was induced in Sprague Dawley rats by retrograde infusion of $4 \%$ sodium taurocholate (NaT) into the pancreatic duct with subsequent subcapsular administration of PAP specific siRNA. Controls included administration of scrambled siRNA (scRNA) or vehicle alone. After 24hr. pancreata were harvested and assessed for worsening pancreatitis by histopathology; serum was analyzed for PAP (ELISA), amylase and lipase protein levels. In both models endogenous PAP (PAPI, PAPII, PAPIII) gene expression was assessed at 24 hrs using real time RT-PCR. Data are presented as mean + SEM, $\mathrm{P}<0.05$, Student's t-test. Results: In vitro, PAP isoform (PAPI, PAPII, PAPIII) mRNA expression was reduced (PAPI: 76\%, PAPII: 8\%, PAPIII: $24 \%$ ) in cells treated with PAP siRNA when compared with controls. Amylase and lipase levels were also reduced at all time points $(23 \%+7,36 \%+8$, respectively) in cells treated with PAP siRNA when compared with controls. In vivo, induction of pancreatitis was confirmed by histopathology, serum amylase and lipase levels. PAP isoforms expression was reduced (PAPI: $36 \%$, PAPII: $49 \%$, PAPIII: $66 \%$ ) in siRNA treated rats, compared with controls. However serum PAP protein levels were similar between siRNA and control groups. Serum amylase levels decreased after administration of PAP siRNA compared with vehicle control $(3013+317$ $\mathrm{U} / \mathrm{L}$ vs. $1583+312 . \mathrm{U} / \mathrm{L})(\mathrm{p}<0.05)$. In addition, serum lipase levels decreased after administration of PAP siRNA compared with vehicle control $(478+125 \mathrm{U} / \mathrm{L}$ vs. $162+42 \mathrm{U} / \mathrm{L} ; \mathrm{p}=0.08)$. There was a trend of less tissue damage (histopathology) with siRNA administration compared with controls $(\mathrm{p}=0.06)$. Conclusions: siRNA mediated gene knockdown of PAP appeared to improve pancreatitis severity and demonstrated an opposite effect of antisense gene knockdown. This observed difference may be due to increased specificity, transfection stability, and/or different mechanisms of action for siRNA gene knockdown compared with antisense technology.

\section{W1688}

Role of MCP-1 On the Blood Brain Barrier Permeability Increase in Rats with Pancreatitis

Zhen Ding, Rong Lin, Wei Qian, Xiaohua Hou

Background \& Aims: Monocyte chemotactic protein-1(MCP-1) is known as a kind of Chemokine which can attract leukocytes especially monocyte and T cell. Our aim was to value the relationship between the expression of MCP-1 and the increase of blood brain barrier (BBB) permeability in the rats which had been induced experimental pancreatitis. Methods: Male Wistar rats were divided 8 groups in terms of sampling time and the grade of acute pancreatitis, including control group, mild acute pancreatitis (MAP, 2- and 6-h group), severe acute pancreatitis (SAP, 2-, 6-, 12-, 24- and 48-hour group). Each group had 6 rats Acute pancreatitis was induced by intrapancreatic ductal injection of choleate sodium, which concentration was $0.5 \%$ and $5 \%$ in inducing MAP and SAP respectively. Immunohistochemistry straining was used to detect the expression of MCP-1 in rats' pancreas and brain. Evans' blue was a matter to detect the permeability of BBB. Result: (1) There were no significant increases in BBB permeability in rats with MAP than in control rats (MAP $2 \mathrm{~h} 1.67 \pm 0.13$ $\mathrm{mg} / \mathrm{dl}$ vs. $1.54 \pm 0.25 \mathrm{mg} / \mathrm{dl} \mathrm{p}=0.389$; MAP $6 \mathrm{~h} 1.71 \pm 0.13 \mathrm{mg} / \mathrm{dl}$ vs. $1.54 \pm 0.25 \mathrm{mg} / \mathrm{dl} \mathrm{p}=$ 0.205 ). (2) BBB permeability was increased in all of SAP rats (SAP2h $1.97 \pm 0.17$; SAP6h $2.75 \pm 0.15$; SAP12h $2.95 \pm 0.45$; SAP24 $3.24 \pm 0.23$; SAP48h $1.86 \pm 0.09$ ). However, in the comparison of group SAP2h and SAP48h, it was much higher in groups SAP6h, SAP12h, and SAP24. (3) MCP-1 expressed in all SAP groups in brains, but there was no MCP-1 expression in controls and MAP rats. Conclusion: BBB permeability is significantly increased in SAP rats and this change is time dependent, but no change in MAP rats. At the same time, MCP-1 expressed in the brain only in SAP group. Therefore, MCP-1 expression may be an early event in the pathogenesis of pancreatic encephalopathy and play an important role in the increasing of BBB permeability because of its ability of attracting leukocytes.

W1689

Oral Administration of a Pancreatic- and Neutrophil- Elastase Inhibitor (ZD0892) Ameliorates Caerulein Induced Pancreatitis Julia Mayerle, Manuel Ruthenbuerger, Frank-Ulrich Weiss, Walther Halangk, Markus M. Lerch

Introduction: Circulating serum levels of neutrophil-elastase (PMN-elastase) are known to correlate with the severity of clinical pancreatitis and are closely associated with the extent of leukocyte transmigration into inflamed tissue. We tested whether prophylactic administration of a novel, orally available peptidyl trifluoromethyl ketone inhibitor of PMN-elastase (ZD0892), with cross-specificity for pancreatic elastase, can ameliorate experimental pancreatitis. Methods: Male Wistar rats (250-300gr) received gavage tube feeding of ZD-0892 (at a dose of $240 \mathrm{mg} / \mathrm{kg}$ per day) at $3 \mathrm{~h}$ and $\mathrm{lh}$ before the induction of acute caerulein pancreatitis for 4 and 12 hrs. Pancreatic and lung tissue as well as serum were collected for further analysis of clinical and morphological severity markers. Isolated pancreatic acini were incubated with ZD-0892 and protease activity after supramaximal caerulein stimulation was monitored employing specific fluorogenic substrates for trypsin, elastase, cathepsin B and cathepsin L. Results: Oral administration of the elastase inhibitor ZD-0892 significantly reduced the serum level of amylase, the extent of pancreatic edema, as well as leukocyte infiltration in the pancreas and lungs during caerulein-induced pancreatitis. Incubation of isolated pancreatic acini with ZD-0892 and subsequent supramaximal CCK stimulation reduced pancreatic elastase activity to below 20\%, whereas neither trypsin, nor cathepsin B nor cathepsin L activity were affected. Conclusion: Prophylactic administration of a novel, orally available PMN-elastase inhibitor with cross-specificity for pancreatic elastase improves the severity of acute experimental pancreatitis. ZD-0892 therefore represents the first orally active drug with treatment potential for patients at risk of developing pancreatitis such as those before ERCP, those with exogenous risk factors (e.g. ethanol abuse) and those with inherited predispositions.

\section{W1690}

Description and Characterization of An Experimental Model of Severe Acute Pancreatitis in Mice Induced By Infusing Na-Taurocholate Into the Pancreatic Duct

Gijs J. Van Acker, Eric R. Weiss, Michael L. Steer, George Perides

Background/Aim: The availability of genetically modified mouse strains makes that species an ideal candidate for mechanistic pancreatitis studies but, unfortunately, clinically relevant models of pancreatitis in mice have not been described. Rather, the current mouse models depend upon supramaximal secretagogue stimulation with caerulein, exposure to toxic doses of arginine, or administration of a choline-deficient ethionine-supplemented diet. Since clinical pancreatitis is not triggered by these events, the clinical relevance of each of these models has been questioned. We now describe techniques which have enabled us to adapt the well-described rat model of bile salt-induced biliary pancreatitis for studies involving mice. Methods: Following hilar occlusion of the bile duct, the pancreatico-biliary duct was cannulated with a 30G needle passed trans-duodenally. A 0-5\% Na-taurocholate/saline solution (10-50 $\mathrm{l}$ ) was infused into the duct at a rate of $1-5 \mu \mathrm{l} / \mathrm{min}$. The needle was removed and, in some animals, the duct was ligated. The animals were returned to their cages, given water ad libitum, and sacrificed at intervals of up to 24 hours. Pancreatitis severity was evaluated histologically. Results: Infusion of saline alone did not cause histologic changes of pancreatitis but it did cause mild, transient pancreatic edema. In contrast, infusion of saline followed by duct ligation for $24 \mathrm{~h}$ resulted in mild pancreatic injury and inflammation. Infusion of Na-taurocholate in the absence of duct ligation caused extensive pancreatic necrosis and inflammation and the severity of that pancreatitis was directly related to the volume of the infusion as well as the concentration of Na-taurocholate being infused. Ligation of the pancreatic duct for $24 \mathrm{~h}$ after Na-taurocholate infusion markedly worsened the severity of Na-taurocholate induced pancreatitis. None of the animals died within 24 hours of duct infusion, with or without duct ligation. Conclusion: We have developed a highly reproducable mouse model of biliary acute pancreatitis which is induced by retrogradely infusing the pancreatic duct with Na-taurocholate. The severity of this pancreatitis can be manipulated by altering the amount of the infusion and the concentration of Na-taurocholate or by ligating the pancreatic duct. This model will be of great value for studies using genetically altered mouse strains to probe mechanistic questions regarding pancreatitis. Supported by NIH grant R01-31396. THIS PRESENTATION, IF GIVEN IN ORAL FORM, WILL BE ACCOMPANIED BY A VIDEO THAT DEMONSTRATES THE ANATOMICAL LANDMARKS AND SURGICAL PROCEDURES THAT ARE INVOLVED IN CREATING THIS MODEL.

\section{W1691}

Application of Caerulein-Induced Pancreatitis in the Recessive Yellow (e/e) Mouse Strain Reveals a New Role of Melanocortin 1-Receptor in the Development of An Acute Inflammation

Tobias Meister, D.R. Wennmann, Thomas Brzoska, Wolfram Domschke, Juergen Schnekenburger

Introduction: Melanocortin-1-receptors (MClR) are mainly expressed on melanocytes but also on the surface of dendritic cells, granulocytes, monocytes and endothelial cells. The main ligand of MClR is alpha-melanocyte-stimulating-peptide (a-MSH). It has been shown, that a-MSH exerts anti-inflammatory properties and that a dysfunction of MCIR increases the lethal outcome in mice with an experimentally induced colitis. In the present study we 
analyzed the course of a caerulein-induced pancreatitis in a mouse strain defective for a functional MClR. Materials and methods: Experimental pancreatitis was induced with 7 intraperitoneal injections of caerulein $(50 \mu \mathrm{g} / \mathrm{kgBW})$ in wildtype mice and e/e-mice, who express a truncated $\mathrm{MClR}$ without any biological activity. After this procedure mice were sacrified. Pancreas, lung and blood serum were taken and standard parameters of pancreatitis were measured. The severity of pancreatitis was determined by measuring amylase and trypsin activity in serum and pancreas homogenates by means of fluorometry. Paraffinembedded sections of pancreatic tissue were stained with hematoxylin/eosin. The localization of MClR was detected by immunofluorescence microscopy using MClR specific antibodies. Additionally RNA was purified from pancreas homogenates and isolated acinar cells for mRNA-expression studies. All animal experiments were in accordance with the guiding principles of the American Physiological Society. Results: Supramaximal stimulation with cerulein caused a significant slower increase in serum-amylase activity in e/e-mice compared to wildtype mice. Trypsin activity in pancreas homogenates was not significantly altered in cerulein-treated e/e-mice compared to wildtype mice. The analysis of hematoxylin/eosin stained sections of caerulein-treated wildtype mice showed clear signs of pancreatitis with typical zymogen-granula, infiltration of macrophages and edematous tissue while caeruleintreated e/e-animals had only mild signs of pancreatitis. MClR expression was only detected in infiltrating leukocytes after supramaximal caerulein stimulation. Conclusions: In contrast to comparable animal models of acute gastric inflammations the dysfunction of MClR in e/e-mice reduced the severity of a caerulein-induced pancreatitis. The fact that a-MSH does not induce a signalling cascade once bound on MClR in e/e mice suggests that either a-MSH has no anti inflammatory function during an acute pancreatitis or that the anti inflammatory properties must be mediated through other receptors, possibly the MC3R.

\section{W1692}

The Systemic Cytokine Response During Experimental Acute Pancreatitis Impact of Enteral Probiotics

Harro M. Timmerman, L. Paul van Minnen, Wilco de Jager, Sergey R. Konstantinov,

Femke Lutgendorff, Andre Verheem, Wil Harmsen, Maarten R. Visser, Hauke Smidt, Hein G. Gooszen, Louis M. Akkermans, Ger T. Rijkers

Background: In the course of severe acute pancreatitis, mortality usually occurs early (< 1 week) or late ( $>2$ weeks). Early mortality is often a consequence of the severe systemic inflammatory response syndrome (SIRS), whereas late mortality can be attributed to secondary infection of pancreatic necrosis. Translocation of intestinal microorganisms is primarily held responsible for infection of pancreatic necrosis during the late phase, but may also contribute to SIRS in the early phase. Selected probiotic bacteria are able to modulate intestinal flora and prevent bacterial translocation. Aims of this study were: 1) to correlate plasma levels of various cytokines and chemokines during early and late phase of pancreatitis with clinical outcome, and 2) assess the effect of probiotics on cytokine profiles. Materials and methods: Acute pancreatitis was induced in male Sprague-Dawley rats by intraductal bile salt infusion (glycodeoxycholate, $15 \mathrm{mM}$ ) followed by pancreatic hyperstimulation (intravenous cerulein, $5 \mu \mathrm{g} / \mathrm{kg} / \mathrm{hr}$, for 6 hours). Probiotics or placebo were administered daily via a permanent gastric cannula, from five days prior to until seven days after induction of pancreatitis. Plasma cytokine levels were determined before, and $6 \mathrm{hrs}, 24 \mathrm{hrs}$ and 7 days after induction of pancreatitis. Bacteraemia was assessed by blood cultures, performed on day 7 after induction of pancreatitis. Cox regression analysis was used to correlate plasma cytokine levels and probiotic treatment with mortality and bacteraemia. Results: In the course of severe acute pancreatitis specific 'cytokine signatures' could be detected which were associated with differential clinical outcome: 1) Early mortality $(<24 \mathrm{~h})$ was preceded by significantly higher plasma levels of IL-6, IL-10 and CXCL1, 2) Elevated levels of TNF- $\alpha$ and IL-1 $\beta$ were predictive for the onset of bacteraemia potentially causing late mortality, 3) an early and self-resolving IL-10 response was found in animals protected from early mortality, whereas sustained elevated IL-10 levels (24h) were associated with bacteraemia. Clinical outcome, being mortality or bacteraemia at day 7 , was improved by probiotic treatment (Hazard ratio $0.37, \mathrm{P}=0.039$ ). Probiotic treatment resulted in a modest reduction of plasma levels of pro-inflammatory cytokines and significantly reduced CXCLl levels (24h), preventing unfavourable neutrophil activation and associated remote organ damage. Conclusions: Specific pro-inflammatory and regulatory cytokine combinations are predictive for mortality and bacteraemia during severe acute pancreatitis. Prophylactic treatment with probiotics reduced pro-inflammatory cytokine overexpression.

\section{W1693}

Pancreatic Secretory Trypsin Inhibitor Is a Target Antigen in Experimental Autoimmune Pancreatitis in Mice

Masanori Asada, Akiyoshi Nishio, Keiichi Kiriya, Kazuyuki Saga, Jyunya Tanaka, Katsuhiro Kasahara, Masahiro Kido, Satoru Ueno, Norimitsu Uza, Satoko Inoue, Hirosh Kitamura, Shinya Ohashi, Toshiro Fukui, Minoru Matsuura, Norihiko Watanabe, Hiroshi Nakase, Kazuichi Okazaki, Tsutomu Chiba

Background \& Aim: Autoimmune pancreatitis (AIP) is a unique type of chronic pancreatitis. Hypergammaglobulinemia, increased serum levels of total $\operatorname{Ig} G$ or $\lg G 4$, and various autoantibodies are often observed in patients with AIP. However, the autoimmune mechanisms leading to AIP still remains unclear. We previously reported that autoantibodies against pancreatic secretory trypsin inhibitor (PSTI) are specifically detected in patients with AIP. (DDW 2004, New Orleans) In this study, we aimed to investigate the role of PSTI as an autoantigen in the pathogenesis of murine experimental model of AIP. Materials \& Methods: Six-week old female MRL/+ mice were injected with polyinosinic polycytidylic acid (poly $\mathrm{I}: \mathrm{C})$ intraperitoneally at a dose of $5 \mathrm{mg} / \mathrm{kg}$ of body weight twice a week up to 12 weeks. Control mice were injected with phosphate-buffered saline (PBS). Mice were serially sacrificed and the severity of the pancreas was graded based on the histological scoring system ( 0 to 4$)$. Immunohistochemical examinations were also performed. The serum levels of autoantibodies including anti-PSTI antibody, were measured by ELISA method. Results: All mice developed pancreatitis 12 weeks with poly I:C treatment, but controls did not. Histological examinations demonstrated destruction of the pancreatic acini and fatty degeneration accompanied by intense infiltration of inflammatory cells. Histological score of the pancreatitis increased according to the treatment period. ( $6 \mathrm{wk} ; 1.8 \pm 0.4,12 \mathrm{wk} ; 3.3 \pm 0.6$ ) Immunohistochemistry revealed that $\mathrm{B} 220+\mathrm{B}$ cells were diffusely infiltrated into the pancreatic parenchyma bu CD4+ T cells and CD8+ T cells were mainly observed around the pancreatic ducts. Poly $\mathrm{I}: \mathrm{C}$ administered mice highly produced anti-PSTI antibody (91.7\%) in contrast with the relative low frequency of anti-lactoferrin antibody $(45.8 \%)$ or anti-carbonic anhydrase-Il antibody (33.3\%). Conclusions: Anti-human PSTI antibody is highly detected in poly I:C administered MRL/+ mice. PSTI may be involved in the pathogenesis of experimental autoimmune pancreatitis model in mice.

\section{W1694}

Overexpression of SMAD6 Enhances Pancreatic Fibrosis in Transgenic Mice Tamao Miyamoto, Hayato Nakamura, Hiroshi Asaumi, Yoko Nomiyama, Shiro Watanabe, Yoshikuni Nagashio, Mituo Tashiro, Makoto Ostuki

Introduction: We have developed transgenic ( $\mathrm{Tg}$ ) mice overexpressing Smad6 in the pancreas and demonstrated that acute pancreatitis induced in these mice by repeated intraperitoneal injections of cerulean $(\mathrm{Cn})$ or by feeding choline-deficient, ethionine-supplemented diet was severer than that in control C57BL/6N (Wt) (Kaku M, et al. Gastroenterology 2005;128:A 379). In the present study, we examined whether overexpression of Smad6 has any influences on pancreatic fibrosis after chronic pancreatic injury. Method: Chronic pancreatic injury was induced in $\mathrm{Tg}$ and $\mathrm{Wt}$ mice by six hourly intraperitoneal injections of $50 \mathrm{ug} / \mathrm{kg}$ body weight $\mathrm{Cn}$, three times a wk for 4 wks. Pancreatic wet weight and body weight were measured at wk $0,1,2,3$, and 4 . Serum amylase activity and amylase and trypsinogen contents in the pancreas were determined. Pancreatic fibrosis was evaluated by histological examination after $\mathrm{H} \& \mathrm{E}$ and Azan staining and image analysis, and by measuring hydroxyproline contents in the pancreas. Results: There were no significant differences in body weight, pancreatic wet weight, and amylase activity in serum and the pancreas between the two groups throughout the observation periods. Pancreatic trypsinogen contents in $\mathrm{Tg}$ mice at baseline were similar to those in Wt mice, but significantly increased in $\mathrm{Tg}$ mice after induction of pancreatic injury compared with those in Wt mice (Fig 1). Histological grade of fibrosis and fibrotic area by image analysis in $\mathrm{Tg}$ mice were significantly higher than those in Wt mice. Hydroxyproline contents in the pancreas in $\mathrm{Tg}$ mice were significantly higher than those in Wt mice at wk 1 and 2 (Fig 2). Conclusion: Our present results demonstrate that overexpression of Smad6 in the pancreas enhances the development of pancreatic fibrosis induced by chronic pancreatic injury, probably due to decreased exocytosis of trypsinogen.

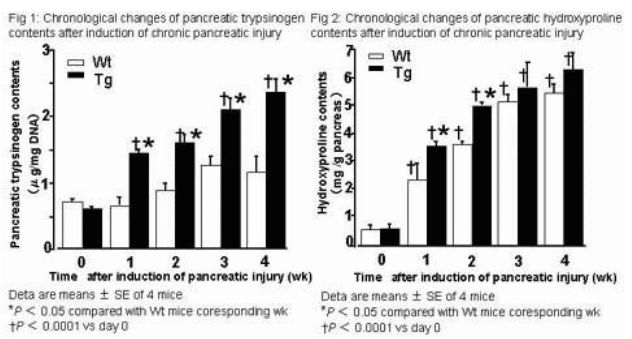

W1695

Autophagic Cell Death of Pancreatic Acinar Cells in Serine Protease Inhibitor Kazal Type 3 Deficient Mice

Masaki Ohmuraya, Masahiko Hirota, Hideo Baba, Ken-Ichi Yamamura

Background \& aims: Acute pancreatitis is a severe inflammatory disorder of the pancreas, which is believed to be caused by an autodigestion of the pancreas by its own digestive enzymes. Digestive enzymes, however, are secreted from the pancreas as inactive precurso zymogens and are normally activated only in the duodenum. Thus, premature protease activation should occur in the pancreas to trigger pancreatitis. Because the main trigger is conversion of trypsinogen to trypsin, it is important to know how trypsin activation is inhibited not to develop pancreatitis. Serine protease inhibitor Kazal Type l (SPINK1), which is structurally similar to epidermal growth factor, can bind to trypsin to inhibit its activity. Point mutations in the SPINKl gene seem to predispose to human pancreatitis; however, the clinical significance of SPINK1 mutations remains unclear. This study aimed to elucidate the role of SPINK1. Methods: We generated Spink3-deficient (Spink3-/-) mice by gene targeting in mouse embryonic stem cells. Embryonic and neonatal pancreases were analyzed morphologically and molecularly. Specific probes were used to examine the typical autophagy occurring during acinar cell death. Results: In Spink3-/- mice, the pancreas developed normally up to embryonic day (E) 15.5. However, autophagic degeneration of acinar cells started from E16.5; and rapid onset of cell death, but not of necrosis and apoptosis, in the pancreas and duodenum within a few days after birth, resulting in death by 14.5 days. Although the trypsin activation in the pancreas of Spink3-/- mice was observed when examined by very sensitive method, it seems to be too low to induce autophagy There was no sing of acinar cell regeneration despite the fact that residual duct-like cells strongly expressed pancreatic duodenal homeodomain containing protein at 7.5 days after birth. Conclusions: Inactive trypsinogen can be activated to trypsin in the absence of Spink3, suggesting that the mutation of SPINKl gene may result in pancreatitis due to trypsin activation or due to potentiation of the harmful effect of other genetic and environmental insults. In addition, Spink3 may have direct roles in autophagosome formation and acinar cell regeneration, and hence the lack of Spink3 may result in the progressive disappearance of acinar cells. Taken together, Spink3 has essential roles for maintenance of integrity and regeneration of acinar cells.

. 
W1696

Hydrogen Peroxide and Nicotine Induces P-ERK 1/2 and P-Akt Activation, Increases Lipid Peroxidation and Decreases Stimulated Cell Function in AR42J Cells

Parimal Chowdhury, Azida Walker, Kodetthoor Udupa

One of the hallmarks of pathophysiological basis of pancreatic injury is the oxidative stress. The current study was conducted in AR42J cells to evaluate the direct effect of an oxidative marker on pancreatic acinar cell. In earlier studies we have established nicotine dose and time of $100 \mu \mathrm{M}$ and $3 \mathrm{~min}$ incubation at which p-ERK $1 / 2$ and p-Akt were activated. In this study we have established dose and time response curve for hydrogen peroxide of 20 $\mu \mathrm{M}$ and $15 \mathrm{~min}$ of incubation at which significant induction of these signals occurred. Methods: AR42J cells were grown in F-12 nutrient medium at 370C and 5\% CO2. At log phase, they were separated, sub cultured for 3 days at specified dose and time with hydrogen peroxide, nicotine and the combination of both. The cells were lysed and cellular extracts were subjected to Western blotting employing antibodies to ERK 1/2, and Akt. Lipid peroxidation assay was conducted employing malondialdehyde (MDA) as a standard biomarker of lipid peroxidation. For functional assay, AR42J cells were incubated with either $20 \mu \mathrm{M}$ of hydrogen peroxide for $15 \mathrm{~min}$, or with $100 \mu \mathrm{M}$ of nicotine for $3 \mathrm{~min}$ or the combination of both for the same time and concentration in serum free medium. At the end of the incubation period, the cells were washed, re-suspended and then incubated with CCK-8 $(10-10 \mathrm{M})$ for $30 \mathrm{~min}$ at $370 \mathrm{C}$. Cell function was assessed by the amount of amylase released in the media with procion yellow starch as substrate Results: Incubation of AR42J cells for 15 min with hydrogen peroxide $(20 \mu \mathrm{M})$ or with nicotine $(100 \mu \mathrm{M})$ induced 3-4 fold activation of phospho-ERK $1 / 2$ and p-Akt when compared to untreated cells $(\mathrm{p}<0.05)$. This response was associated with significant increase in MDA levels in the cell lysate $(p<0.05)$ Incubation of cells with hydrogen peroxide $(20 \mu \mathrm{M})$, CCK-stimulated release of amylase was not different from that of control cells, however, the cells treated with $100 \mu \mathrm{M}$ of nicotine showed a significant increase in stimulated cell function as compared to control and hydrogen peroxide treated cells. Incubation of cells with combination of both hydrogen peroxide and nicotine decreased stimulated cell function by ( $25 \%)$ when compared to the stimulated amylase release by nicotine-treated cells. Conclusion: These data suggest that in AR42J cells, mitogen activated protein kinase (MAPK) signaling is induced by hydrogen peroxide in concert with nicotine treatment as reported earlier with an increase in lipid peroxidation and subsequent decrease in cell function. The data implies a plausible mechanism by which oxidative stress may implicate cell injury leading to its compromised function.

\section{W1697}

Existence of Autocrine Loop Between Interleukin-6 and TGF- $\beta_{1}$ in Activated Rat Pancreatic Stellate Cells

Hiroyoshi Aoki, Hirohide Ohnishi, Kouji Hama, Satoshi Shinozaki, Kentaro Sugano

Background and Aims: Pancreatic stellate cells (PSCs) are activated in chronic pancreatitis and promote pancreatic fibrosis by producing and secreting extracellular matrix. Interleukin6 (IL-6) is a pro-inflammatory cytokine assumed to participate in the progression of pancreatic fibrosis by stimulating pancreatic stellate cells. Although activated PSCs secrete multiple cytokines, it is still unclear whether activated PSCs secrete IL-6. In this study, we examined IL-6 secretion from culture-activated rat PSCS and its regulatory mechanism. Methods: PSCS isolated from rat pancreata using Nycodenz gradient centrifugation were culture-activated during 2-3 passages. The mRNA expression and peptide secretion of IL- 6 and TGF- $\beta_{1}$ was determined with RT-PCR and ELISA, respectively. TGF- $\beta_{1} / S$ mad signaling was investigated with adenovirus(AdDNSmad2/3)-mediated expression of dominant-negative Smad2/3. Extracellular signal-regulated kinase (ERK) dependent signaling pathway was examined using MEKI inhibitor PD98059. Results: Activated PSCs express IL-6 mRNA and secrete IL-6 peptide. Since autocrine TGF- $\beta_{1}$ plays central roles in the functional regulation of PSCs, we examined the effect of autocrine TGF- $\beta_{1}$ on the IL- 6 expression and secretion by activated PSCs. TGF- $\beta$ intracellular signaling is mainly mediated by Smad2/3-dependent pathways. The infection with AdDNSmad2/3 expressing dominant-negative Smad2/3 did not alter TGF$\beta_{1}$ mRNA expression or peptide secretion of activated PSC. However, when autocrine TGF$\beta_{1}$ signaling was blocked by the infection with AdDNSmad2/3, IL- 6 expression and secretion of activated PSCS was inhibited. Furthermore, exogenous TGF- $\beta_{1}$ added in the culture medium enhanced IL- 6 expression and secretion of PSCs. In contrast, exogenous TGF- $\beta_{1}$ could not increase IL-6 expression or secretion of PSCs infected with AdDNSmad2/3. These data suggest that autocrine TGF- $\beta_{1}$ enhances IL- 6 expression and secretion of PSCs through Smad2/3-dependent pathway. We also examined the reciprocal effect of PSC-secreted IL-6 on the TGF- $\beta_{1}$ secretion from PSCs. Anti-IL- 6 neutralizing antibody added in the culture medium inhibited TGF- $\beta_{1}$ secretion from PSCs in a dose dependent manner. In addition, exogenous IL- 6 enhanced TGF- $\beta_{1}$ expression and secretion of PSCs. Preincubation of cells with 10 nM PD98059, an ERK-dependent pathway inhibitor, attenuated basal and IL-6stimulated TGF- $\beta_{1}$ expression and secretion of PSCs. Conclusion: These data indicates the existence of autocrine loop between IL- 6 and TGF- $\beta_{1}$ through ERK- and Smad2/3-dependent pathways in activated PSCs.

\section{W1698}

Pancreatic Stellate Cells Express Toll-Like Receptors Atsushi Masamune, Kazuhiro Kikuta, Noriaki Suzuki, Tooru Shimosegawa

Background \& Aims: Activated pancreatic stellate cells (PSCS) play a pivotal role in the pathogenesis of pancreatic fibrosis. Upon activation, PSCs acquire proinflammatory as well as profibrogenic properties. Toll-like receptors (TLRs) are proteins involved in the recognition of foreign pathogen-associated molecular patterns and activation of processes leading to innate immune recognition. This study aimed to clarify whether PSCs expressed TLRs and responded to foreign pathogen-associated molecular patterns. Methods: PSC were isolated from rat pancreas tissue and used in their culture-activated, myofibroblast-like phenotype. Expression of TLRs was examined by reverse transcription-PCR and Western blotting. PSCs were treated with lipoteichoic acid (LTA from Staphylococcus aureus, a ligand for TLR2), polyinosinic-polycytidylic acid (poly I:C; a ligand for TLR3), lipopolysaccharides (LPS from E. coli; a ligand for TLR 4), or flagellin (from Salmonella munchen, a ligand for TLR5) Activation of mitogen-activated protein (MAP) kinases was assessed by Western blotting using antiphosphospecific antibodies. Activation of transcription factors was examined by electrophoretic mobility shift assay. Chemokine production was examined by enzyme-linked immunosorbent assay. Expression of inducible NO synthase (iNOS) was examined by Western blotting. Fluid-phase and mannose receptor-mediated endocytosis was assessed by uptake of fluorescein isothiocynate-labeled low-molecular weight $(10 \mathrm{kDa})$ and high-molecular weight $(70 \mathrm{kDa})$ dextran. Results: PSCs expressed TLR2, 3, 4, and 5 as well as CD14 and $\mathrm{MD} 2$. Ligands of TLRs activated activator protein-1, nuclear factor- $\mathrm{\kappa} B$, and MAP kinases (extracellular signal-regulated kinase, c-Jun N-terminal kinase, and p38 MAP kinase). TLR ligands induced the expression of monocyte chemoattractant protein-1 and iNOS. TLR ligands-induced monocyte chemoattractant protein-1 production was abolished in the pres-

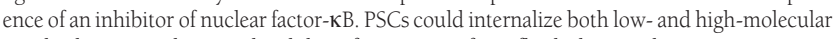
weight dextran, indicating the ability of PSCs to perform fluid-phase and mannose receptormediated endocytosis. Conclusion: Activated PSCs expressed a variety of TLRs, and responded to foreign pathogen-associated molecular patterns followed by the induction of chemokines and iNOS. In addition, activated PSCs had a powerful endocytic activity, suggesting that PSCs might participate in the immune functions of the pancreas.

\section{W1699}

Smad7-Dependent TGF- $\beta_{1}$ Negative-Feedback Is Constitutively Attenuated Through Nf- $\kappa B$ in Activated Pancreatic Stellate Cells Kouji Hama, Hirohide Ohnishi, Hiroyoshi Aoki, Satoshi Shinozaki, Kentaro Sugano

Background and Aim: During chronic pancreatitis, pancreatic stellate cells (PSCs) are activated and transformed to myofibroblast-like cells, and play central roles in pancreatic fibrogenesis by producing and secreting extracellular matrix such as collagen and fibronectin. Autocirne TGF- $\beta_{1}$ accelerates the activated PSC functions promoting pancreatic fibrosis. Smad7 is an inducible antagonist of TGF- $\beta_{1}$ signaling that physiologically acts as TGF- $\beta_{1}$ negative-feedback regulator. However, activated PSCs are continuously stimulated by autocrine TGF- $\beta_{1}$ and promote fibrogenesis in chronic pancreatitis tissue. Therefore, we conducted this study to examine whether Smad7-operated TGF- $\beta_{1}$ negative-feedback machinery exists in activated PSCs. Methods: PSCs were isolated from rat pancreas using Nycodenz gradient centrifugation and activated during passages 2-3. Smad proteins function was examined with their adenovirus-mediated overexpression. Smad7 transcription activity and mRNA expression was determined by Smad7-Luciferase assay and RT-PCR, respectively. NF- $\mathrm{kB}$-dependent pathway was examined by blocking NF- $\mathrm{KB}$ activity using N-tosyl-L-phenylalanine chloromethyl ketone

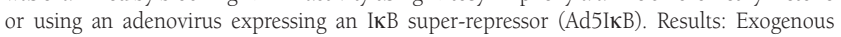
TGF- $\beta_{1}$ added in the culture medium increased Smad7 transcription and mRNA expression in activated PSCs in a dose-dependent manner concomitantly with increasing $\alpha$-smooth muscle actin ( $\alpha$-SMA) expression, a parameter of PSC transformation. Although adenovirusmediated overexpression of Smad proteins did not alter the amount of autocrine TGF- $\beta_{1}$, overexpression of the combination of Smad3 and Smad4 enhanced Smad7 transcription and mRNA expression in activated PSCs. These data indicate that excess TGF- $\beta_{1}$ stimulation can induce Smad7 expression through Smad3- and Smad4-dependent pathway in activated PSCs. Moreover, overexpression of Smad7 inhibited basal and TGF- $\beta_{1}$-stimulated $\alpha$-SMA expression of PSCs, indicating that excess Smad7 expression can attenuate TGF- $\beta_{1}$ stimulation in activated PSCs. On the other hand, when NF-KB activity in activated PSCs was blocked by the preincubation with N-tosyl-L-phenylalanine chloromethyl ketone or by the infection with Ad5IкB enhanced basal and TGF- $\beta_{1}$-stimulated Smad7 transcription and mRNA expression. These results indicate that Smad7 transcription is constitutively attenuated via NF-KB dependent pathway. Conclusion: Smad7-dependent TGF- $\beta_{1}$ negative-feedback machinery exists in activated PSCs but is constitutively inhibited through NF-אB dependent pathway.

\section{W1700}

Isolation of Pancreatic Sensory Neurons: 1, 1'-Dioctadecyl-3, 3,3' 3'Tetramethylindocarbocyanine Perchlorate (DiI) Retrograde Tracing and Immunohistochemisty in Rat Dorsal Root Ganglia (DRG)

Sarah Grahn, Helge Eilers, Elizabeth Wick, Mark Schumacher, Kimberly S. Kirkwood

Experimental pancreatitis induces inflammatory hyperalgesia as evidenced by an increase in fos protein expression at the T9-T10 levels in the spinal cord. Recent studies suggest that the vanilloid receptor TRPVI may be activated by inflammatory mediators resulting in pancreatic hyperalgesia. Our understanding of pancreatic nociception is limited by our inability to study sensory neurons from the pancreas. The purpose of the present study was to use a vital dye to isolate pancreatic afferent neurons, quantify the distribution of these neurons within DRGs at different levels and identify these pancreatic neurons in cultured DRGs. The vital dye DiI was injected into pancreatic parenchyma of anesthetized rats as a retrograde nerve tracer. After 10 days the rats were killed and the DRGs removed for either cell counting, immunohistochemical staining or culture. Injection of DiI into the pancreas resulted in labeling of small to medium diameter neurons in the T7-L2 DRGs. The greatest number of labeled neurons were in the T9-Tll DRGs (Figure 1). Among pancreatic (DiI+) neurons $75 \%$ were TRPVI positive by immunohistochemical staining (Figure 2). Pancreatic afferents labeled with DiI were easily identified among DRG neurons in short term culture. Thus we have developed a technique by which we identified and quantified specific pancreatic afferent neurons in rat DRGs using a vital dye and these neurons have been isolated in short term culture. These small-medium fiber neurons are TRPV1 positive and therefore represent potential pancreatic nociceptors which may be activated during acute pancreatitis leading to pancreatic hyperalgesia. 

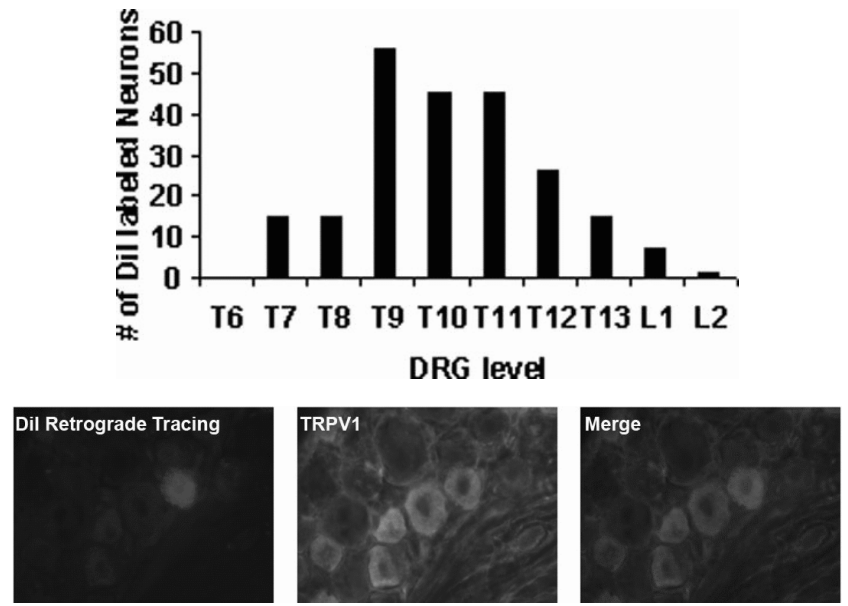

Co-localization of Dil and TRPVl in a Tll DRG neuron showing cell body with central nucleus and cytoplasmic staining (40X)

\section{W1701}

Pioglitazone Restores CCK Stimulated Pancreatic Secretion \& Normalizes Insulin Resistance in eNOS (-/) Mice

Yibai Hao, Raj Reddy, Sae-Hong Lee, Owyang Chung, Matthew J. Dimagno

BACKGROUND: We recently showed that eNOS isoform modulates pancreatic secretion; NOS inhibition or eNOS gene deletion reduced in vivo murine pancreatic secretion by 40 $45 \%$ in response to carbachol and CCK-8. eNOS also modulates organ perfusion, and eNOS $(-/-)$ mice exhibit insulin resistance. Thus, our aims were to detemine if exocrine pancreatic secretion is linked to pancreatic blood flow (PBF) in WT mice and to determine if pioglitazone (an insulin sensitizer) reduces insulin resistence and improves exocrine pancreatic secretion. METHODS: We used microspheres to measure PBF in WT mice in response to graded CCK8 doses. WT \& eNOS (-/-) mice were gavaged with pioglitazone $(20$ or $50 \mathrm{mg} / \mathrm{kg} / \mathrm{d})$ or vehicle $\mathrm{x} 5$ days. Then mice were anesthetized and either the bile-pancreatic duct cannulated to study in vivo pancreatic secretion in response to maximal stimulatory CCK-8 (160 pmol/ $\mathrm{kg} / \mathrm{h}$ ) or we measured pancreatic digestive enzyme mRNA content, hepatic insulin receptor protein expression \& serum insulin concentration (by RIA). RESULTS: In WT mice, baseline PBF was unaffected by physiologic CCK-8 (40-160 pmol/kg/h). Supramaximal CCK-8 (400 \& $1600 \mathrm{pmol} / \mathrm{kg} / \mathrm{h})$ increased PBF 1.6 and 2.4 fold respectively $(\mathrm{p}<0.05)$. Pioglitazone augmented CCK-8 $(160 \mathrm{pmol} / \mathrm{kg} / \mathrm{h})$ stimulated protein output in eNOS $(-/-)(\mathrm{p}<0.05)$ but not in WT mice; in response to vehicle, $20 \& 50 \mathrm{mg} / \mathrm{kg}$ pioglitazaone increased CCK-8 stimulated output in eNOS (-/-) mice by $195+/-29 \%, 266+/-39 \%$ and $357+/-47 \%$, repectively and in WT mice by $328+/-35 \%, 336+/-46 \%$ and $284+/-48 \%$, respectively. Also, during physiologic CCK stimulation, pioglitazone $(50 \mathrm{mg} / \mathrm{kg})$ normalized the 2 -fold greater serum insulin values observed in eNOS $(-/-)$ mice receiving vehicle $(\mathrm{p}<0.05)$ vs WT groups and secondly, augmented cytosolic and membrane hepatic insulin receptor expression by $35+/$ $10 \%$ and $26+/-5 \%$ respectively. Although insulin increases protein synthesis, pancreatic mRNA expression of multiple digestive enzymes was unaffected by eNOS genotype. CONCLUSION: Physiologic CCK-8 evoked pancreatic secretion in WT mice is not coupled to increased PBF. Pioglitazone restores pancreatic secretion in CCK-8 stimulated eNOS (-/) mice without affecting these parameters in WT mice. Secondly Pioglitazone reverses hyperinsulinemia in CCK-8 stimulated eNOS (-/-) mice, and increases hepatic insulin receptor expression, which might reduce hyperinsulinemia by increasing receptor dependent hepatic insulin uptake and clearance. Insulin resistance may reduce pancreatic secretion in eNOS $(-/-)$ as well as in humans with diabetes mellitus, and be reversible by pioglitazone.

\section{W1702}

Regulatory Interaction Between Apical Na+-H+ Exchange and Cystic Fibrosis Transmembrane Conductance Regulator in Mouse Pancreatic Duct Cells Akiko Yamamoto, Hiroshi Ishiguro, Takaharu Kondo, Satoru Naruse

Background and Aim: Na+-H+ exchangers (NHE) are localized in both basolateral and apical membranes of various epithelia and 8 isoforms have been identified. NHE3 is localized in the apical membrane and mediates $\mathrm{HCO} 3-$ absorption in kidney proximal tubule and $\mathrm{H+}$ coupled dipeptide absorption in the small intestine. In the present study, to investigate the role of apical NHE in $\mathrm{HCO}$ - secretion from pancreatic duct cells, we examined the activity of apical NHE in interlobular pancreatic duct segments isolated from normal and $\Delta \mathrm{F}$ mice, a cystic fibrosis mouse model in which the $\Delta \mathrm{F} 508$ mutation was introduced in the mouse CFTR (cystic fibrosis transmembrane conductance regulator) and pancreatic $\mathrm{HCO} 3$ - secretion is impaired. Methods: Interlobular duct segments (diameter: 100 $\mu \mathrm{m}$ ) were isolated by collagenase digestion and microdissection. The ducts were superfused with HCO3--free Hepes-buffered solutions at $37 \mathrm{oC}$ and the lumen was microperfused separately. Intracellular $\mathrm{pH}(\mathrm{pHi})$ was measured by microfluorometry in ducts loaded with $\mathrm{pH}$-sensitive fluoroprobe BCECF. The duct cells were acid-loaded with a 2-min pulse of $20 \mathrm{mM} \mathrm{NH} 4+$, which was followed by a Na+-free solution in both the bath and lumen. The rate of pHi recovery after re-addition of $\mathrm{Na}+$ to the luminal solution was calculated as a measure of the activity of apical $\mathrm{Na}+-\mathrm{H}+$ exchange. Results: The rate of $\mathrm{pHi}$ recovery $(\mathrm{dpH} / \mathrm{dt}$ ) (dependent on luminal $\mathrm{Na}$, independent of HCO3-) was $0.12 \pm 0.01 \mathrm{pH}$ unit $/ \mathrm{min}$ (mean $\pm \mathrm{SD}, \mathrm{n}=8$ ) in wild type (WT/WT) ducts, which was completely inhibited by $100 \mu \mathrm{M} \mathrm{HOE642,} \mathrm{an} \mathrm{inhibitor}$ of NHE. Forskolin $(1 \mu \mathrm{M})$, an activator of adenylate cyclase, reduced the apical NHE activity to $0.05 \pm 0.01 \mathrm{pH}$ unit/min $(\mathrm{n}=9, \mathrm{p}<0.01)$. The apical NHE activity in cystic fibrosis
$(\Delta \mathrm{F} / \Delta \mathrm{F})$ ducts was $0.20 \pm 0.01 \mathrm{pH}$ unit/min $(\mathrm{n}=6)$, which was significantly $(\mathrm{p}<0.01)$ higher than that in wild type ducts and was accelerated to $0.66 \pm 0.11 \mathrm{pH}$ unit/min (n $6, \mathrm{p}<0.01$ ) by application of forskolin. Conclusions: In mice pancreatic duct cells, the activity of apical NHE was suppressed by functional CFTR and it was stimulated by cAM in the absence of functional CFTR. These data suggest that the inhibitory regulation of apica NHE by CFTR does not work in cystic fibrosis pancreatic duct, which may lead to acidification of pancreatic juice.

\section{W1703}

Lack of in Vivo Evidence for a Constitutive Secretory Pathway for Pancreatic Trypsinogen in Fasted Healthy Human Subjects

Stephen J. O'Keefe, Justin M. Kiswardy, Wen Zhou

Experimental evidence, based on in vitro animal studies, suggested that there are 2 primary mechanisms for the acinar cell secretion of newly synthesized enzymes, the 'constitutive' pathway where enzymes are discharged from the acinar cell after the trans Golgi network and the 'regulated' pathway which becomes operational only during food-stimulation where newly synthesized enzymes are only secreted following equilibration with the stored zymogen pool. The finding of the early release of low quantities of highly labeled enzymes durin fasting lead investigators to conclude that secretion during fasting was chiefly supported by the constitutive pathway. To test this hypothesis in human physiology, we studied healthy volunteers during fasting $(\mathrm{n}=5)$ and feeding $(\mathrm{n}=7)$ by in vivo labeling of pancreatic trypsinogen with prime, continuous 6-h IV infusions of ${ }^{13} \mathrm{C}$-leucine and isolation of trypsin form duodena secretions (AJPhysiol 1994). To determine whether trafficking was disrupted during acute pancreatitis, 9 patients with severe necrotizing disease were studied in the same manner. Results: Figure shows that the isotope enrichment of secreted trypsin followed a similar pattern during fasting and feeding, but that the time of first appearance of labeled enzyme was significantly faster during feeding (75(7) vs. 94(11) minutes, $\mathrm{p}=0.044$, Mann-Whitney NP test). Measurements in pancreatitis patients showed the early release of labeled enzymes with disturbance of the isotope enrichment curve. In conclusion, our results fail to support the presence of a constitutive pathway in healthy fasted humans as the isotope enrichment curves fitted the 'regulated' model described by Jameson \& Palade (1967), only the process is accelerated during feeding. In patients with acute pancreatitis, the capacity to synthesize enzymes is conserved, but post-Golgi trafficking is disrupted.

\section{Isotope Enrichment of Secreted Trypsin}

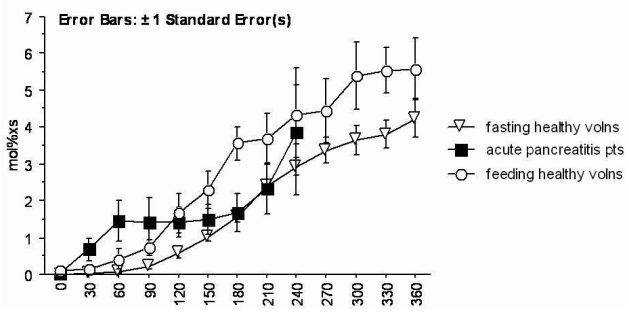

W1704

High Glucose Inhibits Fluid Secretion and Na+-Hco3- Cotransport in Rat Pancreatic Duct Cells

Sachiko Futakuchi, Hiroshi Ishiguro, Satoru Naruse, Shigeru Ko, Miyuki Nakakuki, Toshiyuki Yoshikawa, Akiko Yamamoto, Takaharu Kondo, Hidemi Goto

Pancreatic exocrine dysfunction is commonly found in diabetic patients, in whom not only enzyme secretion (acinar function) but also fluid and $\mathrm{HCO} 3$ - secretion (ductal function) is impaired. To clarify the mechanisms by which ductal secretion decreases in diabetes, we examined (1) effects of glucose concentration on fluid secretion and Na+-HCO3-cotransport in pancreatic ducts isolated from normal and streptozotocin-induced diabetic rats, and (2) mRNA expression of glucose transporters in pancreatic ducts. Interlobular ducts (diameter $\sim 150 \mu \mathrm{m}$ ) were isolated from the pancreas obtained from normal and diabetic rats. The ducts were cultured overnight in either a normal-glucose ( $10 \mathrm{mM})$ or high-glucose $(44 \mathrm{mM})$ solution, during which time both ends of the ducts sealed spontaneously. Fluid secretion into the closed luminal space was measured by videomicroscopy and expressed as secretory rate per unit area of epithelium. Intracellular $\mathrm{pH}$ (pHi) was measured by microfluorometry using BCECF to estimate Na+-HCO3- cotransport activities. The mRNA expression of Na+dependent and -independent glucose transporters (SGLT1, 2 and GLUT1, 2, 3, 4, 8) in isolated ducts was examined by RT-PCR. Isolated ducts were superfused with HCO3buffered solution (glucose $10 \mathrm{mM}$ ) at $37 \mathrm{oC}$ and stimulated with secretin $(1 \mathrm{nM})$. The fluid secretory rate in ducts from normal rats was $1.74 \pm 0.14 \mathrm{nl}$ min- $1 \mathrm{~mm}-2, \mathrm{n}=8$, mean \pm $\mathrm{SE})$. When glucose in the perfusate was raised to $44 \mathrm{mM}$, the secretory rate decreased by $41.4 \%(p<0.05)$. The addition of mannitol $(34 \mathrm{mM})$ did not affect fluid secretion. Isolated ducts from diabetic rats kept in the high-glucose condition secreted fluid at a significantly $(\mathrm{p}<0.05)$ lower rate $(0.70 \pm 0.07, \mathrm{n}=8)$ than those in the normal condition. When the diabetic ducts were kept in the normal-glucose condition, the secretory rate recovered by $37.4 \%(\mathrm{p}<0.05)$. The rate of pHi recovery after acid-loading with NH4+-pulse was significantly $(\mathrm{p}<0.05)$ reduced in diabetic ducts and in normal ducts under the highglucose condition. SGLT1, GLUT1, GLUT2, GLUT3 and GLUT8 mRNAs were abundantly expressed in pancreatic ducts. High glucose reversively inhibits fluid secretion from rat pancreatic duct cells probably by inhibiting $\mathrm{HCO} 3-$ uptake via $\mathrm{Na}+-\mathrm{HCO} 3-$ cotransport Under a high-glucose condition, the entry of $\mathrm{Na}+$ via SGLT may reduce the transmembrane $\mathrm{Na}+$ gradient and thus may inhibit the activity of $\mathrm{Na}+-\mathrm{HCO} 3-$ cotransport. 
W1705

Phosphatidylinositol 3-Kinase Facilitates Bile Acid-Induced Ca2+ Responses in Pancreatic Acinar Cells

Lars Fischer, Anna S. Gukovskaya, Josef M. Penninger, Helmut Friess, Ilya Gukovsky, Stephen J. Pandol

Background \& Aims: Bile acids induce Ca2+ responses in the pancreatic acinar cell; however, the underlying mechanisms are poorly understood. These responses may play a role in bile acid-induced experimental acute pancreatitis. We have recently shown that phosphatidylinositol 3-kinase (PI3K), and in particular its gamma isoform, regulates changes in free cytosolic $\mathrm{Ca} 2+([\mathrm{Ca} 2+] \mathrm{i})$ elicited in pancreatic acinar cells by neurohormones such as cholecystokinin (CCK). We showed that PI3K negatively regulates CCK-induced Ca2+ reloading into endoplasmic reticulum (ER) stores by inhibiting sarco/endoplasmic reticulum Ca2+-ATPase (SERCA). The present study sought to determine whether PI3K regulates bile acid-induced $[\mathrm{Ca} 2+]$ i responses in the pancreatic acinar cell. Results: In isolated pancreatic acini, pharmacologic inhibition of PI3K with LY294002 or wortmannin markedly inhibited [Ca2+]i responses (measured with Fura-2) induced by taurolithocholic acid 3-sulfate (TLC-S) and taurochenodeoxycholate (TCDC). Furthermore, genetic deletion of PI3K gamma isoform also decreased $[\mathrm{Ca} 2+]$ i responses to bile acids. Depletion of CCK-sensitive intracellular $\mathrm{Ca} 2+$ pools or application of caffeine, a blocker of inositol 1,4,5-trisphosphate (IP3) receptor, inhibited bile acid-induced $[\mathrm{Ca} 2+]$ i signals, indicating that bile acids release $\mathrm{Ca} 2+$ from agonistsensitive ER stores via IP3-dependent mechanism. The PI3K inhibitors increased the amount of $\mathrm{Ca} 2+$ releasable from the intracellular stores during exposure of acinar cells to bile acids. This suggests that PI3K negatively regulates the SERCA-dependent Ca2+ reloading into ER during bile acid stimulation. Further, in permeabilized acinar cells bile acids inhibited $\mathrm{Ca} 2+$ reloading into the ER, and this effect was augmented by phosphatidylinositol 3,4,5trisphosphate (PIP3), a major product of PI3K. These results indicate that both bile acids and PI3K act synergistically to inhibit SERCA. Conclusions: Our results indicate that PI3K and its product, PIP3, facilitate bile acid-induced $[\mathrm{Ca} 2+]$ i responses in pancreatic acinar cells through inhibition of SERCA-dependent $\mathrm{Ca} 2+$ reloading into the ER. The findings have important implications for the mechanism of acute pancreatitis since $[\mathrm{Ca} 2+] \mathrm{i}$ increases mediate key pathologic processes in this disorder.

\section{W1706}

Development of An Efficient Inducible Acinar Cell Specific Transgenic Model (BAC-Elastase-Cre-Er)

Baoan Ji, Jian Song, Craig D. Logsdon

Background: Pancreatic acinar cells play critical roles in gastrointestinal physiology as the primary source of digestive enzymes. These cells are also central to the initiation of pancreatitis and may also be involved in pancreatic cancer.Acinar cell specific gene promoters have been widely utilized to control acinar cell specific transgene expression with the rat pancreatic elastase promoter being the most widely utilized.However, the partial sequence $(531 \mathrm{bp})$ typically utilized for this purpose is not highly efficient nor is its expression restricted to fully differentiated acinar cells.Aim: In order to develop an improved system for pancreatic acinar cell specific transgene expression we utilized a full-length pancreatic elastase promoter using bacterial artificial chromosome(BAC) technology. Furthermore, for maximum flexibility and utility in studies of pancreatic biology, we developed a mouse line in which this BAC elastase promoter is used to express a tamoxifen regulated Cre (BAC-El-Cre-Er).This allows acinar cell specific and tamoxifen regulated Cre recombination that when used in conjunction with a gene controlled by a loxp-flanked stop sequence allows highly efficient and specific regulated expression in acinar cells. Method: We used an E. coli-based chromosome engineering system developed by Dr. Copeland (NCI). A BAC carrying the intact elastase I gene was modified to express Cre-Er. An IRES-Cre-Er-frt-Kan-frt PCR fragment was produced by replacing the EGFP-Cre with Cre-Er in plasmid pICGN21. To either side of this expression cassette was added $~ 50$ bp DNA fragments homologous to the sequence of elastase I gene between the last exon and the polyA signal. Using homologous recombination, the IRES-Cre-Er-frt-Kanfrt cassette was inserted into the elastase I gene. The Kan selection marker was then removed by flp-mediated recombination and the insert site and orientation were verified by sequencing. Transgenic mice were produced by pronuclear microinjection. Results:Founders were identified by PCR using Cre primers and crossed with Rosa26 reporter mice. Double transgenic mice were injected with tamoxifen $3 \mathrm{mg} / 40 \mathrm{~g} / \mathrm{d}$ for three days before the pancreas was removed for X-gal staining. We found that nearly $100 \%$ of acini from double transgenic mouse expressed $\beta$-gal after, but not before, tamoxifen treatment, indicating highly efficient regulated recombination. Initial analysis indicates that the expression was completely specific for acinar cells.Conclusion: A BAC-El-Cre-Er transgenic mouse has been developed. This model of pancreatic acinar specific conditional gene expression will be a powerful tool for future transgenic, knockout and cell linage studies.

\section{W1707}

RHOA Directly Interacts with TRPCl and Regulates Capacitative $\mathrm{Ca}^{2+}$ Entry During Intestinal Epithelial Restitution After Wounding Rao N. Jaladanki, Tongtong Zou, Lan Liu, Bernard S. Marasa, Kaspar M. Keledjian, AiHong Zhang, Lan Xiao, Jie Chen, Jian-Ying Wang

Early mucosal restitution occurs as a consequence of epithelial cell migration to resealing of superficial wounds after injury. An increase in cytosolic $\mathrm{Ca}^{2+}$ concentration $\left(\left[\mathrm{Ca}^{2+}\right]_{\mathrm{cyt}}\right)$ is crucial for initiating cellular response and consists of an initial phase that reflects $\mathrm{Ca}^{2+}$ release from intracellular stores and a second phase that results from $\mathrm{Ca}^{2+}$ influx through $\mathrm{Ca}^{2+}$ permeable ion channels. We have recently reported that TRPCl (canonical transient receptor potential-1) functions as a store-operated $\mathrm{Ca}^{2+}$ channel mediating capacitative $\mathrm{Ca}^{2+}$ entry (CCE) in intestinal epithelial cells (IECs) and that increasing $\mathrm{Ca}^{2+}$ influx through CCE plays an important role in early epithelial restitution. However, the exact mechanisms by which TRPCl channels are activated after $\mathrm{Ca}^{2+}$ store depletion in IECs remain unclear. This study determined if RhoA, a small GTP-binding protein, interacts with and regulates TRPCl during epithelial restitution. Methods: Studies were conducted in the IEC-6 cells, derived from rat intestinal crypts. Association of RhoA with TRPCl was measured by immunoprecipitation (IP), while $\left[\mathrm{Ca}^{2+}\right]_{\mathrm{cyt}}$ was measured by fluorescence digital imaging analysis. Functions of RhoA were investigated by using its specific inhibitor exoenzyme $\mathrm{C} 3$ transferase (C3) and transfection with dominant negative mutant RhoA (DNMRhoA) or wild-type RhoA (WTRhoA). Cell migration was measured in a model that mimics cell division-independent epithelial restitution. Results: Stable TRPCl-transfected IEC-6 cells (IEC-TRPC1) exhibited increased TRPCl expression, and levels of TRPCl protein were $\sim 5$-folds the value of parental IEC-6 cells. Consistently, sustained $\mathrm{Ca}^{2+}$ influx through CCE induced by store depletion was increased by $\sim 2$-folds in IEC-TRPCl cells. RhoA interacted with TRPCl and formed the RhoA/TRPCl complexes. RhoA/TRPCl complexes in IEC-TRPCl cells were increased by $~ 30 \%$ compared with those observed in parental IEC- 6 cells. Inactivation of endogenous RhoA by either exposure to $\mathrm{C} 3$ or ectopic expression of DNMRhoA not only reduced RhoAV TRPCl complexes but also inhibited $\mathrm{Ca}^{2+}$ influx through CCE. RhoA/TRPCl complexes were decreased by $\sim 40 \%$ in IEC-TRPC 1 cells treated with C 3 or transfected with DNMRhoA, while $\mathrm{Ca}^{2+}$ influx through CCE was decreased by $~ 50 \%$. Cell migration was also inhibited by $40 \%$ after C3 treatment or DNMRhoA transfection. In contrast, ectopic expression of WT-RhoA induced RhoA/TRPCl complexes, increased $\mathrm{Ca}^{2+}$ influx through CCE, and promoted cell migration after wounding. Conclusions: These findings indicate that RhoA directly interacts with and regulates TRPCl, thus stimulating cell migration as a result of increase in $\mathrm{Ca}^{2+}$ influx.

\section{W1708}

Expression and Glucocorticoid Regulation of Multiple Corticotropin-Releasing Factor (CRF) Receptor Type 2 Variants in the Rat Esophagus Shuping V. Wu, Pu-Qing Yuan, Lixin Wang, Yenlin Peng, Yvette Tach

Background: Corticotropin-releasing factor (CRF) and three related urocortin (Ucn) peptides act on two distinct $G$ protein-coupled receptors: CRF1 and CRF2 to exert stress-inducing and coping effects. Both CRF receptor subtypes are expressed in multiple isoforms in the central and peripheral tissues. In the gastrointestinal tract, the involvement of CRF receptors in gastric emptying and colonic motility has been well documented. However, the presence of CRF ligands and receptors, and their functional roles in the esophagus are not yet determined. Aims: This study was designed to determine: 1) whether functional CRF receptors and their ligands are present in rat esophagus, 2) which CRF receptor isoforms are expressed in specific esophageal cell types that can be regulated by corticosterone treatment, and 3) the effects of CRF and Ucn on cultured esophageal cell signaling. Methods: The upper, middle and lower esophagus including lower esophageal sphincter (LES) from naïve, vehicleand corticosterone-treated rats were collected. Mucosal and muscle sections were obtained by laser capture microdissection and cells were isolated by enzyme/EDTA dispersion and cultured in serum-containing medium. RT-PCR and DNA sequencing were performed to detect and confirm the presence of transcripts of CRF receptors and ligands in the whole tissues, laser-captured sections and cultured cells. Activation of ERK1/2 was assessed by Western blot in cultured esophageal cells. Results: In rat esophagus, CRFI was detectable but present at very low levels, while multiple CRF2 variants were abundantly expressed. CRF2 $\beta$ was predominantly found in the epithelial and circular muscle layer while CRF2 $\alpha$ was present throughout the esophagus. In addition to wild type CRF $2 \alpha$, two novel CRF $2 \alpha$ variants were identified: the first variant CRF2 $\alpha$-desQ106, encodes a functional membranebound receptor, the second variant CRF $2 \alpha-13$ containing unspliced intron 3, is a truncated soluble receptor. All three urocortins, but not CRF, were detected in both mucosa and muscle sections. Acute injections of corticosterone decreased the esophageal expression of CRF $2 \alpha-13$ variant but caused no significant changes in wild type CRF $2 \alpha$ and CRF $2 \beta$ levels after 6 h. Exogenous Ucn stimulated dose-dependent activation of ERK1/2 in cultured esophageal cells. Conclusion: These data demonstrate that multiple and functional CRF2 receptor isoforms and their cognate ligand urocortins are expressed in the esophagus and LES, suggesting that CRF2 system may play important roles in regulating mucosal blood flow, muscle contraction and relaxation, and maintenance of epithelium and muscle integrity in the esophagus.

\section{W1709}

The Roles of Prostaglandin EP2 Receptors, cAMP, P-CREB, VEGF and Angiogenesis in Esophageal Ulcer Healing: Signaling Pathways and Effect of PGEl Analog

Andrzej S. Tarnawski, Dolgor Baatar, Zsuzsanna Sandor, Rama Pai

Previous studies demonstrated that esophageal ulceration induces Cox2 expression in the ulcer margin and clinical studies indicate that prostaglandins E (PGEs) may facilitate healing of tissue injury and ulcers. The roles of PGEs, their EP receptors, signaling pathways and their relation to VEGF and angiogenesis during esophageal ulcer healing remain unknown. We examined in rats: whether esophageal ulceration alters EP1-4 receptors expression and cAMP response element-binding protein (CREB) activity; and whether PGEl analog, misoprostol (MIS), agonist of EP receptors, affects cAMP concentration, CREB activity, VEGF expression, angiogenesis in granulation tissue and ulcer healing. METHODS: Esophageal ulcers were induced in rats by focal application of $100 \%$ acetic acid. Rats were treated with either placebo or MIS i.g. $50 \mu \mathrm{g} / \mathrm{kg}$ b.i.d. STUDIES at 3, 6 and 9 days after ulcer induction: 1) ulcer size; 2) quantitative histology; 3) cell proliferation; 4) microvessel density; 5) VEGF mRNA and 6) EP1-4, CREB, P-CREB and VEGF protein expression by Western blotting. In cultured human esophageal epithelial HET-1A cells (derived from normal human esophageal epithelium), we examined expression of EP1-4 receptors and the effects of MIS, Rp-cAMP (PKA inhibitor) and Sp-cAMP (PKA activator) on cAMP levels, P-CREB and VEGF expression. RESULTS: Esophageal ulceration triggered EP2 (but not EP1, 3 or 4) receptor overexpression at day 3 ( $>200 \% \mathrm{p}<0.01)$, increases in cAMP, CREB phosphorylation (4.5-fold increase; $p$ $<0.01$ ) and VEGF mRNA and protein by $\sim 2$ - and 6 -fold, respectively, at day 3 (both $\mathrm{p}<$ 0.01 ). MIS treatment further enhanced in ulcerated esophageal mucosa CREB activation and VEGF expression (in squamous epithelium) and angiogenesis in granulation tissue (by $50 \%$ and $60 \%$, respectively, at day 6 and 9) and accelerated ulcer healing. In cultured human HET-1A cells, MIS increased intracellular cAMP levels (by 163-fold; $\mathrm{p}<0.001$ ), significantly increased P-CREB and VEGF expression. PKA inhibitor inhibited all these MIS actions, while 
PKA activator had similar to MIS action. CONCLUSIONS: 1) Esophageal ulceration triggers overexpression of EP2 (but not EP1, 3 or 4) receptors, CREB phosphorylation and VEGF upregulation in squamous epithelium bordering ulcer; 2) EP2/cAMP/protein kinase A pathway mediates stimulatory effects of MIS on CREB activity and VEGF expression in ulcerated esophageal mucosa, which in turn activates angiogenesis and ulcer healing; 3) Increased VEGF production by epithelial cells activates VEGF receptors in endothelial cells and thus angiogenesis, reflecting local epithelial-mesenchymal interactions and paracrine regulation.

\section{W1710}

Downregulation of Tff1 (pS2) Expression By SOX2 in Gastric Epithelial Cells Tadahito Shimada, Takero Koike, Kyoko Tabei, Takashi Namatame, Akihiro Tajima, Masashi Yoneda, Hideyuki Hiraishi

Background and Aim: SOX2, a SRY-related HMG box protein, is a transcription factor expressed in a wide variety of tissues. SOX2 is considered to be critically involved in organ development and cell differentiation. In the gastrointestinal tract, previous studies showed the expression of SOX2 in gastric epithelial cells. However, little is known about the function and the target genes of SOX2 in gastric epithelial cells. Trefoil factor family is a group of small peptides that play important roles in the mucosal defense of the gastrointestinal tract. Among TFF subtypes (TFF1, TFF2, and TFF3), TFFl and TFF2 are expressed at high levels in the gastric mucosa. In this study, we examined the expression of SOX2 in gastric cell lines and investigated whether SOX2 affects the expression of TFF. Methods: MKN45, Kato3, and AGS, cell lines derived from human gastric cancers, were used. COS-7 cells were also used in some experiments. TFF1, TFF2, TFF3, and SOX2 mRNA expression levels were examined by real-time RT-PCR. Beta-actin mRNA measurement was also performed for standardization. SOX2 protein expression was examined by Western blot analysis. TFF reporter genes were made by inserting PCR-amplified human TFFl gene promoter sequence $(-956$ to +36$)$, TFF2 gene promoter sequence $(-912$ to +24$)$, and TFF3 gene promoter sequence $(-956$ to +12$)$ into the pGL3-basic vector. SOX2 expression vector was made by inserting the cording sequence of human SOX2 gene into pcDNA3.1 vector. Results: (1) SOX2 mRNA and proteins were expressed in MKN45, Kato3, and AGS cells at a significant level. (2) In COS-7 cells, the overexpression of SOX2 slightly upregulated the transcription of TFF1, TFF2, and TFF3 reporter genes. (3) In contrast, in gastric cell lines, the overexpression of SOX2 significantly downregulated the transcription of TFFl reporter gene. (4) The transcription of TFF2 reporter gene was slightly suppressed by SOX2 overexpression and that of TFF3 reporter gene was not significantly affected by SOX2 overexpression in gastric cell lines. (5) Consistent with this, endogenous TFFl mRNA expression was found to be downregulated by the overexpression of SOX2 in gastric cell lines. (6) In a series of reporter gene experiments, SOX2 responsive element was mapped between -456 and -326 from the transcription start site of the human TFFl gene. Conclusions: These results suggest that SOX2 negatively regulates TFFl expression in gastric epithelial cells. Since SOX2 overexpression caused opposite effect on the transcription of TFFl reporter gene in COS-7 cells, other gastric cell specific factors might also be involved in the action of SOX2 in gastric epithelial cells.

\section{W1711}

Involvement of the Pineal Gland in Gastroprotection and Ulcer Healing By Endogenous Melatonin and Its Precursor, L-Tryptophan

Iwona Brzozowska, Danuta Drozdowicz, Peter C. Konturek, Krystyna Zwirska-Korczala, Tomasz Brzozowski, Michal Pawlik, Stanislaw J. Konturek, Wieslaw W. Pawlik, Eckhart G. Hahn

Melatonin, a major hormone of pineal gland, exhibits gastroprotective and ulcer healing actions, but the mechanism of anti-ulcerogenic beneficial activity of this indole has been little studied. In this study, using rats without or with removed pineal gland (pinealectomy), we compared the effects of i.g. or i.c.v. treatment of melatonin and its precursor, L-tryptophan, with or without concurrent treatment with luzindole, a selective antagonist of melatonin $\mathrm{MT}_{2}$ receptors, indomethacin or rofecoxib to suppress cyclooxygenase (COX-1) and COX2 activity, respectively on gastric lesions induced by water immersion restraint stress (WRS) and healing of preexisting gastric ulcers (acetic acid method; ulcer area $=28 \mathrm{~mm}^{2}$ ). At $3.5 \mathrm{~h}$ after the end of WRS or 9 days after ulcer induction, gastric lesions and ulcers were measured by planimetry, gastric blood flow (GBF) was determined by $\mathrm{H}_{2}$-gas clearance technique, plasma melatonin and gastrin levels and mucosal $\mathrm{PGE}_{2}$ generation were traced by specific RIA and expression of mRNA for COX-1 and COX-2 was assessed by RT-PCR. Melatonin (1.25-10 mg/kg i.g.) or (1.25-10 $\mu \mathrm{g} /$ rat i.c.v.) dose-dependently attenuated the WRS-induced gastric damage and accelerated ulcer healing while raising GBF, plasma melatonin and gastrin levels and mucosal generation of $\mathrm{PGE}_{2}$. Pinealectomy, which suppressed plasma melatonin levels, aggravated the gastric lesions induced by WRS and significantly delayed ulcer healing and these effects were counteracted by treatment of pinealectomized rats with exogenous melatonin or L-tryptophan. Luzindole abolished the gastroprotective ulcer healing effects of melatonin and L-tryptophan and attenuated significantly the rise in GBF evoked by the indole and its precursor. Indomethacin and rofecoxib which diminished $\mathrm{PGE}_{2}$ biosynthesis by $\sim 90 \%$ and $75 \%$, respectively, significantly attenuated melatonin- and L-tryptophaninduced protection, ulcer healing and the rise in the GBF. COX-1 mRNA was detected by RT-PCR in the intact and melatonin-treated gastric mucosa, while COX-2 mRNA, which was undetectable in the intact gastric mucosa, appeared as a strong signal in melatonintreated animals. We conclude that 1 ) exogenous melatonin and its precursor, L-tryptophan, attenuate WRS-induced gastric lesions via interaction with melatonin $\mathrm{MT}_{2}$ receptors and an enhancement of gastric microcirculation, probably mediated by $\mathrm{PGE}_{2}$ derived from COX-2 overexpression and activity, and 2) the pineal gland plays an important role in the limitation of WRS-induced gastric lesions and acceleration of ulcer healing via releasing melatonin, which exerts gastroprotective and ulcer healing actions.

\section{W1712}

Title Role of Reg I for Regeneration After Gastric Mucosal Injury: A Lesson from Transgenic Mice

Hiroyuki Fukuhara, Yasunori Kadowaki, Takayuki Ose, Syunji Ishihara, Yoshikazu Kinoshita

Background and Aim Reg I (regenerating gene product I) was originally identified as a growth factor involved in pancreatic regeneration, and was suggested also to be a controller of gastric mucosal regeneration, based on its temporal and spatial expression pattern around the gastric ulcers. Recently, we created RegI-transgenic ( $\mathrm{Tg}$ ) mice to demonstrate that Reg has an activity to stimulate the growth of the gastric stem cells, as well as an activity to direct the proper differentiation(Oncogene 2004;23:3572). In the current study, we utilized the Regl-transgenic mice as a model system to provide the direct in vivo evidence that Reg I is involved in the regeneration process after gastric mucosal injury. Method Gastric mucosa injury was experimentally induced in large array of littermate pairs (86 pairs of Wild type $(\mathrm{Wt})$ and $\mathrm{Tg}$ ) by administration of $\mathrm{HCl} /$ ethanol. Each animal was sacrificed $6 \mathrm{hr}$ (injury phase), $12 \mathrm{hr}, 18 \mathrm{hr}$ or $24 \mathrm{hr}$ (recovery phase) after the administration, and the ulcer index was measured and statistically processed. The gastric specimen from each time point was subjected to northern blot analysis or immunohistochemistry to examine the expression levels of several proliferation markers. Results There was no difference in the ulcer index between Wt and Tg in the injury phase (6hr: Wt $32 \mathrm{~mm}+/-5 \mathrm{~mm}$, Tg $39 \mathrm{~mm}+/-8 \mathrm{~mm}$ N.S). However, in the recovery phase, level of gastric injury of Tg-mice was significantly decreased compared with that of wild-mice. (12hr: Wt $36 \mathrm{~mm}+/-5 \mathrm{~mm}$, Tg $23 \mathrm{~mm}+/-2 \mathrm{~mm}$ $\mathrm{p}=0.0044)(18 \mathrm{hr}:$ Wt $19 \mathrm{~mm}+/-3 \mathrm{~mm}, \mathrm{Tg} 9 \mathrm{~mm}+/-3 \mathrm{~mm} ; \mathrm{p}=0.0207)(24 \mathrm{hr}:$ Wt $12 \mathrm{~mm}+/$ $3 \mathrm{~mm}, \mathrm{Tg} 5 \mathrm{~mm}+/-1 \mathrm{~mm} ; \mathrm{p}=0.0178)$. Expression levels of $\mathrm{c}$-myc and $\mathrm{c}$-fos, which were employed as proliferation markers, in Tg became significantly higher than those in wildtype mice in the recovery phase. Conclusion Reg I-Tg mice were less susceptible to $\mathrm{HCl} /$ ethanol-induced gastric mucosal injury than Wt mice. Based on the data from the time course study, we concluded that the overexpressed Reg I protein contributed to the phenotype, not by enhancing mucosal defense, but rather by enhancing the cell growth required in regeneration process of the mucosal architecture.

\section{W1713}

Simultaneous Effects of Nitric Oxide and Growth Factors On Wound Healing in Cultured Gastric Mucosal Epithelium

Outi Nylander-Koski, Harri Mustonen, Tuula Kiviluoto, Pauli Puolakkainen, Eero Kivilaakso

High levels of nitric oxide can be produced by inducible nitric oxide synthase during infection and inflammation. For example, during chronic Helicobacter pylori infection a constant nitric oxide production is likely to occur, which may lead to cell and tissue, and even DNA damage, thus increasing the risk of cancer development. Helicobacter infection also promotes apoptosis, possibly via induction of iNOS. Since Helicobacter infection occurs commonly in the stomach we have here investigated the simultaneous effects of growth factors and nitric oxide on cell migration and apoptosis, with an attempt to simulate the conditions of epithelial repair in inflamed gastric mucosa. METHODS: Isolated primary cultured rabbi gastric epithelial cells were cultured to confluency in appropriate media $\left(5 \% \mathrm{CO}_{2}, 37^{\circ} \mathrm{C}\right)$. The cells were treated by hepatocyte growth factor (HGF), transforming growth factor alph (TGF $\alpha$ ) and keratinocyte growth factor (KGF), with or without sodium nitroprusside (SNP NO donor). Caspase-3 (promoter of apoptosis) enzyme activity was measured with time resolved fluorescence based assay and cell viability was measured with tetrazolium salt based assay. Migration rate after wounding was calculated from the decreasing wound area. RESULTS: HGF was the most potent growth factor to stimulate migration, increasing migration speed from $15.6 \pm 0.2$ to $27.6 \pm 1.1 \mu \mathrm{m} / \mathrm{h}(\mathrm{p}=<0.001, \mathrm{~N}=21,10 \mathrm{ng} / \mathrm{ml})$, which was significantly faster than the TGF $\alpha(10 \mathrm{ng} / \mathrm{ml})$ stimulated migration speed $(20.2 \pm 0.4 \mu \mathrm{m} / \mathrm{h}$, $\mathrm{p}<0.001)$ or $\mathrm{KGF}(10 \mathrm{ng} / \mathrm{ml})$ stimulated migration speed $(16.1 \pm 0.7 \mu \mathrm{m} / \mathrm{h}, \mathrm{p}<0.001)$. SNP dose dependently decreased the migration speed from $17.6 \pm 0.3$ to $11.8 \pm 0.3 \mu \mathrm{m} / \mathrm{h}(\mathrm{p}<0.001$ $\mathrm{N}=144,1 \mathrm{mM}$ SNP). The SNP retarded migration $(12.6 \pm 0.5 \mu \mathrm{m} / \mathrm{h})$ was returned to normal values by HGF $(22.8 \pm 1.3 \mu \mathrm{m} / \mathrm{h}, \mathrm{p}<0.001, \mathrm{~N}=15)$ and TGF $(16.1 \pm 0.8 \mu \mathrm{m} / \mathrm{h}, \mathrm{p}<0.001, \mathrm{~N}=$ 12 ), whereas KGF had no effect. SNP also induced caspase- 3 activity $(1.50 \pm 0.08$ fold control, $\mathrm{p}<0.001, \mathrm{~N}=12)$, which was inhibited by HGF $(0.98 \pm 0.05$ fold control, $\mathrm{p}<0.001, \mathrm{~N}=12$ ) and TGF $\alpha(1.10 \pm 0.03$-fold control, $\mathrm{p}<0.001, \mathrm{~N}=12)$. CONCLUSION: Our findings indicate that growth factors inhibit apoptosis signaling and that they can also overcome the nitric oxide induced retardation of migration. This inhibition of apoptosis might also contribute to the observed stimulatory effect of growth factors on SNP retarded wound healing, since as the number of surviving cells is increased, they can cover the wound faster.

\section{W1714}

Cyclooxygenase-2/Prostaglandins Accelerate Healing of Gastric Ulcers Through Up-Regulation of VEGF Via EP4 Receptors

Ryo Hatazawa, Akiko Tanaka, Yuka Takahira, Mayu Tanigami, Koji Takeuchi

Background/Aim: It is known that selective cyclooxygenase (COX)-2 inhibitors impair the healing of pre-existing gastric ulcers and that endogenous prostaglandin $\mathrm{E}_{2}\left(\mathrm{PGE}_{2}\right)$ derived from COX-2 play a pivotal role in the mechanism of ulcer healing. However, it remains still unexplored which type of EP receptor subtypes is responsible for the healing promoting action of $\mathrm{PGE}_{2}$, and how $\mathrm{PGE}_{2}$ contributes to ulcer healing. In the present study, we investigated the effects of subtype selective EP agonists and antagonists on the healing of gastric ulcers and investigated the possible mechanism involved in this action. Methods: Male SD rats were used. Gastric ulcers were produced by thermocauterization $\overline{\left(70^{\circ} \mathrm{C}, 20\right.}$ $\mathrm{sec}$ ) under ether anesthesia, and the animals were killed 3,7 and 14 days later. Indomethacin, rofecoxib or SC-560 was given $\mathrm{PO}$ once daily for 10 days starting 3 days after ulceration. Various EP agonists were given SC together with or without indomethacin. Gastric fibroblasts were isolated from rat stomachs. The stomachs were minced and incubated in collagenase/ EDTA in PBS. The obtained fibroblasts were incubated in D-MEM/Ham's F-12 supplemented

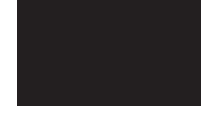


with $10 \%$ fetal calf serum. Results: Ulcer healing was significantly delayed by daily administration of indomethacin and rofecoxib but not by SC-560. Mucosal PGE 2 content was markedly increased after ulceration, and this response was significantly suppressed by administration of rofecoxib but not SC-560. The delayed healing caused by indomethacin was significantly

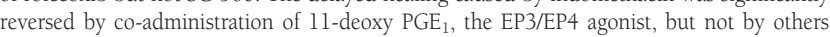
including EP1, EP2 and EP3 agonists. Daily administration of the EP4 antagonist also significantly impaired the healing of gastric ulcers, as effectively as rofecoxib did. The expression of VEGF protein was markedly up-regulated in the ulcerated mucosa. The intensity of the VEGF expression was further enhanced by the EP4 agonist while the intensity was apparently suppressed by indomethacin and rofocoxib as well as the EP4 antagonist, but not SC-560. On the other hand, the expression of VEGF protein in gastric fibroblasts in vitro was also increased by the pre-incubation with $\mathrm{PGE}_{2}$ as well as the EP4 agonist, and these changes were inhibited by the co-incubation with the EP4 antagonist. Conclusion: These results demonstrated that $\mathrm{COX}-2 / \mathrm{PGE}_{2}$ plays an important role in the healing process of gastric ulcers, and further suggested that this action of $\mathrm{PGE}_{2}$ is associated with the stimulation of VEGF expression in the gastric fibroblasts mediated by the activation of EP4 receptors.

\section{W1715}

Methylnicotinamide Counteracts the Augmentation of Aspirin- and StressInduced Gastric Lesions Under Diabetic Conditions in Rats

Tomasz Brzozowski, Peter C. Konturek, Slawomir Kwiecien, Urszula Szczyrk, Stefan Chlopicki, Jakub Cieszkowski, Stanislaw J. Konturek, Wieslaw W. Pawlik

Gastric mucosa of diabetic rats is highly vulnerable to the damage induced by variety of damaging agents but the influence of methylnicotinamide (MNA), an amide derivative of witamin Bl, that has been shown to exhibit anti-inflammatory properties in gastrointestinal tract, on aspirin (ASA)- and stress-induced gastric lesions under diabetic conditions has not been elucidated. In this study, streptozocin ( $70 \mathrm{mg} / \mathrm{kg}$ injected intraperitoneally) was used to induce diabetes mellitus and non-diabetic and diabetic rats were pretreated $30 \mathrm{~min}$ prior to exposure to ASA ( $150 \mathrm{mg} / \mathrm{kg}$ in $0.1 \mathrm{~N} \mathrm{HCl}$ i.g.) or $3.5 \mathrm{~h}$ of water immersion and restraint stress (WRS) with 1) vehicle (saline) or 2) MNA (2.5 - $50 \mathrm{mg} / \mathrm{kg}$ i.g.). The area and number of gastric lesions was determined by planimetry, gastric blood flow (GBF) was examined by $\mathrm{H}_{2}$-gas clearance technique and mieloperoxidase (MPO) activity and activity of superoxide dismutase (SOD) and the malonyldialdehyde (MDA) concentration as an index of lipid peroxidation were determined in gastric mucosa using ELISA. The gene expression IL-1 $\beta$ and TNF- $\alpha$ in the gastric mucosa and their plasma levels were evaluated by RT-PCR and ELISA, respectively. ASA and WRS caused typical gastric lesions and reduced significantly GBF (by 35\% and 28\% from basal) and increased MPO activity (by 2-3 folds) and gastric mucosal MDA content and these effects were significantly augmented in diabetic rats (plasma glucose $\leq 400 \mathrm{mg} / \mathrm{dL}$ ). The ulcerogenic effects of ASA or WRS were accompanied by the fall in the GBF and the overexpression of mRNAs for TNF- $\alpha$ and IL-1 $\beta$ and the marked increase in their plasma levels. MNA dose-dependently attenuated ASA- and WRS-induced gastric erosions in diabetic and non-diabetic animals while raising GBF in intact and injured gastric mucosa by about $22 \%$ and $33 \%$, respectively, and caused downnregulation of IL$1 \beta$ and TNF- $\alpha$. The mucosal SOD activity was significantly decreased while the MDA activity was significantly increased in ASA- and WRS-exposed animals without or with diabetes and these effects were markedly attenuated by MNA. We conclude that 1) diabetes enhances the susceptibility of gastric mucosa to the damage induced by ASA and WRS via mechanism involving fall in GBF, suppression of SOD activity, an increase in lipid peroxidation and overexpression of proinflammatory cytokines IL- $1 \beta$ and TNF- $\alpha$, and 2) MNA affords protection against ASA- and WRS-induced damage under diabetic conditions, possibly due to enhancement in the GBF mediated by attenuation of both, the oxidative stress and proinflammatory cytokine expression and release.

\section{W1716}

Intracellular $\mathrm{pH}$ and $\mathrm{Ca}++$ Measurements in the Mouse Esophagus Solange M. Abdulnour-Nakhoul, Stephanie M. Busque, John P. Geibel

Introduction: Mouse esophagus is lined by keratinized squamous epithelium which has a stratum germinativum layer and two to three layers of cells in the stratum spinosum and corneum. The availability of transgenic mice makes this tissue a very good model for the elucidation of ion transport mechanisms in different genotypes and disease models caused by specific mutations aimed at ion transport proteins. We examined the effect of $\mathrm{Ca}$-sensing receptor (CaSR) activation on intracellular $\mathrm{pH}(\mathrm{pHi})$ regulation and intracellular $\mathrm{Ca}++(\mathrm{Cai}++)$ in the mouse esophagus. Immunofluorescence methods using an antibody to CaSR were used to localize the protein in cryosections of esophageal tissue. Methods: Wild type (CaSR+/ $+/ \mathrm{GCM} 2+/+)$ mice were anesthetized and the esophagus was dissected out and stripped from its muscularis propria in cold HEPES-buffered Ringer. Thin sections $(\sim 200 \mu \mathrm{m})$ were transversely cut, mounted on cover slips and loaded with BCECF $(10 \mu \mathrm{M})$ for pHi measurements or Fluo-4 $(5 \mu \mathrm{M})$ for Cai++ measurements. Changes in Cai++ were estimated from changes in fluorescence signal measured in arbitrary units (AFU). Results: Immunofluorescence showed the presence of CaSR on the basolateral membrane of the basal cells. Reduction of extracellular Ca++ to $0.5 \mathrm{mM}$ reduced $\mathrm{AFU}$ by $3 \pm 0.8 \%$ while increasing $\mathrm{Ca}++$ to $5 \mathrm{mM}$ caused an increase in AFU of $5 \pm 0.9 \%$. When extracellular Ca++ was removed (+EDTA) there was a $20 \pm 5.2 \%$ decrease in arbitrary fluorescence units (AFU). Mean pHi in 17 different tissues was $7.34 \pm 0.05$. When the cells were acid-loaded by pre-treatment with $\mathrm{NH} 4+$ in nominally $\mathrm{HCO} 3$ - free Ringer pHi measurements showed a pHi recovery (rate $0.22 \pm 0.03$ $\mathrm{pH} / \mathrm{min}$ ) that was totally dependent on the presence of extracellular Na. Activation of the CaSR with neomycin and/or increases in extracellular $\mathrm{Ca}++$ did not significantly affect the rate of $\mathrm{pHi}$ recovery from an acid load. Conclusion: The mouse esophagus expresses a basolateral Na-H (NHE) exchanger whose activity appears to be independent of both extracellular and intracellular $\mathrm{Ca}++$. Furthermore this protein is not modulated by activation of the CaSR. We postulate that the mouse esophageal NHE plays a role in cell pH homeostasis. CaSR expressed in this tissue may be less involved in regulation of $\mathrm{pHi}$ or ion transport than in other cell functions such as growth and differentiation. Supported in part by a VA Merit grant.

\section{W1717}

Role of Monocarboxylate Transporter 1 in the Recovery of Wounds Induced in the Gastric Epithelial Cells

Shusaku Hayashi, E.J. Nakamura, Yoshikazu Kubo, Susan J. Hagen, Koji Takeuchi

Background/Aim: During the early healing process of gastric mucosal lesions, the intact epithelial cells migrate to promptly reseal the wounded area. It has been suggested that the regulation of cell volume and intracellular $\mathrm{pH}$ through ion transporters plays a role in cell migration. However, the role of ion transporters in cell migration after injury in the gastric mucosa remains still unclear. We have previously reported that DIDS, a stilbene compound, reportedly known to block $\mathrm{Cl}^{-} / \mathrm{HCO}_{3}^{-}$exchangers (AE), $\mathrm{Na}^{+} / \mathrm{HCO}_{3}{ }^{-}$co-transporters (NBC) and monocarboxylate transporter (MCT), completely abolished the recovery of injury both in the rat gastric epithelial cell line RGMl monolayers and the isolated guinea pig gastric mucosa. In the present study, we determine which ion transporters are involved in the repair process of epithelial cells after injury. Methods: After RGMl cell monolayer being confluent, a round artificial wound with constant size was induced in the center of the cell monolayer using a pencil-type mixer with a rotating silicon tip. The restoration process was monitored 0,4 and $8 \mathrm{~h}$ after the induction of wound. In the guinea-pig gastric mucosa mounted in Ussing chamber, injury was produced by mucosal application of $0.5 \%$ Triton$\mathrm{X}$ for $5 \mathrm{~min}$, and the restitution was evaluated by recovery of the electrical resistance (R) and morphology. We also examined the messenger RNA and protein expression of various ion transporters in RGMl cells. Results: In the vehicle-treated group, the size of cell-free areas was decreased with time after induction of the wound. Phloretin, an inhibitor of MCT, significantly suppressed the repair of wound in a concentration-dependent manner. Replacement of the physiological buffered solution with $\mathrm{Na}^{+}, \mathrm{Cl}^{-}$or $\mathrm{HCO}_{3}^{-}$-free buffer significantly suppressed the recovery of wound. Addition of phloretin to ion-free buffer potentiated the suppression of wound recovery observed in each ion-free buffered solution. Concerning the MCT subtypes, RGMl cells potently expressed MCTl mRNA and protein. In addition, RGMl cells also expressed both AE2a and $\mathrm{NBCl}$. Conclusion: These results suggest that $\mathrm{MCTl}$, in addition to $\mathrm{AE} 2 \mathrm{a}$ and $\mathrm{NBCl}$, plays an important role in the recovery of the gastric mucosa by regulating the migration of epithelial cells after injury.

\section{W1718}

Lipopolysaccharide Injury to the Stomach May Promote Remote Organ Pathogenesis

Michelle Lopez-Storey, Lenard Lichtenberger, Elizabeth Dial

Background: Previously we reported that systemically administered bacterial lipopolysaccharide (LPS, E. coli 0111:B4) induced a reflux of intestinal bile acid into the stomach, a reduction of gastric surface hydrophobicity, and the appearance of bleeding into the stomach (Shock $17: 77,2002$ ). While the presence of blood in the gastric lumen suggested there was local injury to the mucosa, the status of the gastric mucosal barrier was not fully known. Therefore, we decided to directly investigate the effect of LPS on the permeability of the stomach to sucrose, as an indice of the integrity of the barrier. We also assessed a role for another potential luminal damaging agent, that of intestinal protease activity. Methods: Fasted Sprague-Dawley rats were administered LPS $(0,5$, and $20 \mathrm{mg} / \mathrm{kg}$, ip) four hours before gastric contents were collected for analysis of bile acid, protease activity, and hemoglobin levels. In a separate study, rats were similarly dosed with LPS and then were administered sucrose orally ( $\mathrm{lg} /$ $1.5 \mathrm{ml} / \mathrm{rat}$ ) 3.5 hours later. The absorption of intact sucrose has been shown to reflect damage to the gastric mucosal barrier (Gastroenterology 104:1619, 1993). After an overnight collection of urine from these animals, sucrose was analyzed by a precise colorimetric method (Analytical Biochemistry 244:103, 1997). Results: As shown in the table below LPS treatment induced a dose-dependent increase in bile acid, protease activity and hemoglobin in the stomach. LPS also caused a significant increase in gastric permeability to sucrose. Conclusion: LPS-induced injury to the gastric mucosa may involve the reflux of multiple intestinal factors, including bile acid and proteolytic enzymes. The accompanying attenuation in the barrier property of the stomach, to both small $(\mathrm{HCl})$ and potentially large (toxins) molecules, may contribute to further pathogenesis of the mucosa or to that of remote organs. (Supported by NIGMS P50 GM38529).

\begin{tabular}{|c|c|c|c|c|}
\hline $\begin{array}{c}\text { LPS } \\
(\mathrm{mg} / \mathrm{kg})\end{array}$ & $\begin{array}{c}\text { Bile acid } \\
\text { (nmol/stomach) }\end{array}$ & $\begin{array}{c}\text { Protease activity (trypsin } \\
\text { units/stomach) }\end{array}$ & $\begin{array}{c}\text { Hemoglobin } \\
\text { (ug/stomach) }\end{array}$ & $\begin{array}{c}\text { Sucrose }(\% \\
\text { excreted) }\end{array}$ \\
\hline 0 & 67 & 0.9 & 140 & 0.10 \\
\hline 5 & 197 & 19.5 & 373 & 0.25 \\
\hline 20 & 1285 & 52.1 & 891 & 0.56 \\
\hline
\end{tabular}

\section{W1719}

DSKB-2, a Newly Synthesized NF-KappaB Inhibitor, Ameliorates Gastric Mucosal Damage Induced By Ischemia and Reperfusion in Rats

Hiroshi Ichikawa, Norimasa Yoshida, Tomohisa Takagi, Osamu Handa, Satoshi Kokura, Yuji Naito, Takeshi Okanoue, Youichi Nunokawa, Toshikazu Yoshikawa

Objective: Nuclear factor kappa B (NF-kappaB) is activated by several factors, which increase the inflammatory response, and this activation leads to the expression of several genes such as cytokines, and may play an important role in ischemia-reperfusion injury. DSKB-2, a new NF-kappaB inhibitor, is an indan derivative with inhibition of NF-kappaB transcriptional activation, and it has been reported that this compound suppressed the production of TNFalpha and of nitric oxide in RAW 264.7 cells stimulated with LPS. Furthermore DSKB2 also ameliorated carrageenan-induced rat paw edema formation in vivo. Aim: The aim of the present study is to evaluate the anti-inflammatory effect of DSKB-2 on reperfusion-induced gastric mucosal injury in rats. Methods: Gastric ischemia was induced for $30 \mathrm{~min}$ by applying a small clamp to the celiac artery in male Sprague-Dawley (SD) rats weighing $190-210$ grams. Reoxygenation was produced by removal of the clamp. Just before removal of the vascular clamp, DSKB-2 at a dose of $0.1-1 \mathrm{mg} / \mathrm{kg}$ was injected intraperitonealy. Sixty minutes 
after reperfusion, the rats were killed by exsanguinations, and stomachs were removed. Results: The area of gastric mucosal erosion or ulcerative lesion (erosion index) significantly increased from mean basal levels after $60 \mathrm{~min}$ of reperfusion. This erosion index was significantly inhibited by pretreatment with DSKB-2 dose-dependently. The concentration of thiobarbituric acid-reactive substances (TBA-RS) in the gastric mucosa, an index of lipid peroxidation, was also significantly higher than basal level after $60 \mathrm{~min}$ of reperfusion. This increase in TBA-RS in the gastric mucosa after ischemia-reperfusion was inhibited by the pretreatment with DSKB-2. Furthermore, myeloperoxidase (MPO) activity in the gastric mucosa, an index of neutrophil infiltration, significantly increased by ischemia reperfusion, and pretreatment of DSKB-2 significantly reduced this increase of MPO activity in gastric mucosa. The content of both mucosal TNF-alpha and CINC-1 in the control group was significantly increased compared with the levels of those in the normal group. These increases of the levels of TNF-alpha and CINC-1 were significantly inhibited by the treatment with DSKB-2. Conclusion: These findings suggest that the activation of NF-kappaB plays an important role in the pathogenesis of ischemia-reperfusion injury, and that the NF-kappaB inhibitor, DSKB-2, may become a new therapeutic agent for reperfusion injury.

\section{W1720}

Taurocholate Potentiates Ethanol Induced NfאB Activation and Inhibits Caspase-3 Activity in Cultured Rat Gastric Epithelium Harri Mustonen, Tuula Kiviluoto, Pauli Puolakkainen, Eero Kivilaakso

We have previously shown that ethanol induces NFKB activation and apoptosis in rat gastric epithelium. However, it is not known how concomitant exposure to other ulcerogenic agents modulates these actions. This study investigates NFKB activation and apoptosis in cultured gastric epithelium following concomitant exposure to ethanol and taurocholate. METHODS: Rat gastric mucosal cells (RGM-1) were grown in cell culture flasks to confluent monolayers at $37^{\circ} \mathrm{C}$ in humidified atmosphere containing $5 \% \mathrm{CO}_{2}$ in air. The cells were exposed apically for different time periods to ethanol and taurocholate. For assessment of NFKB activation nuclear protein extracts were analyzed with ELISA based transcription factor assay. Caspase3 (promoter of apoptosis) enzyme activity was measured with time resolved fluorescence based assay. Cell viability was measured with tetrazolium salt based assay and cell membrane integrity with lactate dehydrogenase based assay. RESULTS. $2 \%$ and $5 \%$ ethanol induced NFKB activation dose dependently $1.6 \pm 0.2(\mathrm{p}<0.01, \mathrm{~N}=8)$ fold and $3.7 \pm 0.4(\mathrm{p}<0.01, \mathrm{~N}=8)$ fold as compared to control. Exposure to $5 \%$ ethanol with simultaneous exposure to $5 \mathrm{mM}$ or $10 \mathrm{mM}$ taurocholate increased $\mathrm{NFKB}$ activation from $3.4 \pm 0.4$ to $7.9 \pm 0.9$ fold control $(\mathrm{p}<0.01, \mathrm{~N}=8)$ and from $3.4 \pm 0.4$ to $8.5 \pm 1.7$ fold control $(\mathrm{p}=0.01, \mathrm{~N}=8)$, respectively. Taurocholate exposure alone had no effect on $\mathrm{NFKB}$ activation. Exposure to taurocholate (10 $\mathrm{mM}$ ) decreased caspase- 3 activity from $1.0 \pm 0.1$ to $0.43 \pm 0.1$ fold control $(\mathrm{p}<0.01, \mathrm{~N}=12$ ). Taurocholate $(10 \mathrm{mM})$ decreased the ethanol (5\%) induced caspase-3 activity from $2.0 \pm 0.3$ to $0.88 \pm 0.2$ fold control $(\mathrm{p}=0.02, \mathrm{~N}=16)$. Also the ethanol $(5 \%)$ induced decrease in cell viability was opposed by simultaneous exposure to taurocholate $(10 \mathrm{mM})$ from $93+2 \%$ to $105 \pm 2 \%(\mathrm{p}<0.01, \mathrm{~N}=20$, control cells $=100 \%$, lysed cells $=0 \%)$. The ethanol induced cell membrane damage was also opposed by low concentrations $(10 \mathrm{mM})$ of taurocholate from $7.7 \pm 0.3 \%$ to $0.6 \pm 0.3 \%(\mathrm{p}<0.01, \mathrm{~N}=24$, intact membrane $=0 \%$, totally damaged membrane $=$ $100 \%$ ). Larger concentrations than $10 \mathrm{mM}$ of taurocholate with ethanol resulted gradually in severe cell membrane damage and decrease in cell viability. CONCLUSIONS. Although taurocholate itself does not induce $\mathrm{NF} \kappa \mathrm{B}$ activation at low concentrations, it potentiates ethanol induced NFKB activation, inhibits ethanol induced apoptosis and increases cell viability lowered by ethanol. Larger concentrations of taurocholate result in cell membrane damage with ensuing loss of cell viability.

\section{W1721}

Mechanism Underlying Impaired Healing By Alendronate of Gastric Ulcers in Rats

Kikuko Amagase, Shusaku Hayashi, Eitaro Aihara, Kaoru Nishikawa, Koji Takeuchi

Background/Aim: Bisphosphonates (BPPs) such as alendronate have been developed as antiresorptive agents capable of treating diseases related to bone remodeling. These drugs have untoward effects on the upper gastrointestinal tract in humans such as gastroesophageal inflammation and ulceration. However, the influence of these drugs on the healing of the pre-existing gastric ulcers has not been much studied. In the present study, we examined the effect of alendronate on the healing of acetic acid-induced gastric ulcers in rats and investigated the mechanism involved in this action. We also examined the effect of alendronate on the epithelial restitution after injury, using the rat gastric epithelial cell line (RGM1). Methods: 1) Male SD rats and RGMl cells were used. Gastric ulcers were induced in rats by serosal application of acetic acid $(99 \%, 100 \mu \mathrm{l})$ for $60 \mathrm{sec}$, using a small cup. Alendronate ( $60 \mathrm{mg} / \mathrm{kg}, \mathrm{PO})$ or indomethacin $(2 \mathrm{mg} / \mathrm{kg}, \mathrm{SC})$ was given once daily for 7 days, starting 3 days after acid application. Prostaglandin (PG) $\mathrm{E}_{2}$ content was measured by EIA. Expression of growth factor protein was determined by western blotting. 2) After RGMl cell monolayer being confluent, a round artificial wound was induced in the center of the cell monolayer using a pencil-type mixer with a rotating silicon tip, and the restitution process was monitored up to $8 \mathrm{~h}$ thereafter. Results: Gastric ulcers induced by acetic acid healed spontaneously with up-regulation of $\overline{\mathrm{COX}-2}$ expression and $\mathrm{PGE}_{2}$ production as well as the expression of VEGF and bFGF in the ulcerated mucosa. The healing of the ulcers was significantly impaired by the repeated administration of indomethacin or alendronate. Indomethacin significantly inhibited the production of $\mathrm{PGE}_{2}$ in the mucosa while aledronate did not. However, alendronate decreased the protein expression of both VEGF and bFGF in the ulcerated mucosa, similar to indomethacin. On the other hand, the size of cell-free areas in RGMl cells in vitro decreased with time after the wound induction, the process being due to a migration of intact cells, and this healing was significantly promoted by EGF (10 ng/ml). Co-incubation with alendronate $(1 \mathrm{mM})$ did not affect the spontaneous healing but significantly suppressed the accelerated wound healing caused by EGF. Conclusion: These results suggest that 1) alendronate delays the healing of acetic acid gastric ulcers in rats, and 2) the healing impairment effect may be related to the down-regulation of growth factors such as VEGF and bFGF, important for the vascularization/granulation, and the suppression of the stimulatory action of EGF on the epithelial proliferation/migration.

\section{W1722}

Dietary Nitrate and Luminal Nitrite Decrease Taurocholate and Diclofenac Induced Gastric Mucosal Damage

Joel Petersson, Mia Phillipson, Emmelie A. Jansson, Jon O. Lundberg, Lena Holm

Background: Most of the dietary nitrate originates from green leafy vegetables. Bacteria in the oral cavity reduce nitrate to nitrite. In the acidic stomach nitrite is further reduced to nitric oxide (NO). We have earlier shown that acidified nitrite increases gastric mucosa blood flow (GMBF) in both rats and mice, and that this is a direct effect of luminal NO We wanted to investigate the gastro-protective role of acidified nitrite administrated luminally as well as dietary intake of nitrate, by measuring the mucosal permeability and GMBF before, during and after a provoked damage caused by diclofenac and taurocholate. Methods: Rats were anesthetized with inactin (120 $\left.\mathrm{mgxg}^{-1} \mathrm{ip}\right)$ and the gastric mucosa was exteriorized for intravital microscopy. Mucosal permeability was measured as blood to lumen clearance of ${ }^{51} \mathrm{Cr}$-EDTA. During the experiments (130 min), the gastric mucosa was covered with 10 $\mathrm{mM} \mathrm{HCl}$. Diclofenac $\left(5 \mathrm{mgxkg}^{-1} \mathrm{iv}\right)$ was given $20 \mathrm{~min}$ after start and 30 min later $20 \mathrm{mM}$ taurocholate was applied luminally for $40 \mathrm{~min}$. The luminal solution was replaced every 10 min for analyses of ${ }^{51} \mathrm{Cr}$-EDTA. One group of animals received $2.0 \mathrm{mM} \mathrm{NaNO}$, luminally $10 \mathrm{~min}$ after start and then throughout the entire experiment. A second group was pretreated with $\mathrm{NaNO}_{3}(0.1 \mathrm{mgxkg}-1$ daily) in the drinking water for 7 days before the experiment. A third group served as controls. GMBF (Laser-Doppler flowmetry) and blood pressure were measured during the experiments and vascular resistance was calculated. Luminal NO was measured with chemiluminescence. Results: Animals given nitrite and nitrate had significantly higher GMBF and lower vascular resistance than the control group. Permeability did not significantly differ between the groups before taurocholate and diclofenac administration, but was significantly less increased during the provocation in the nitrite and nitrate treated animals (Table 1 ). Animals pretreated with nitrate had greatly increased luminal NO levels $(6280 \pm 1995 \mathrm{ppb})$, compared to control animals $(127 \pm 40 \mathrm{ppb})$. Conclusions: Nitrite and nitrate pretreatment decreases taurocholate and diclofenac induced mucosal damage The gastro-protective effect might depend on a higher basal mucosal blood flow due to NO production. NO produc
Table 1

\begin{tabular}{|c|c|c|c|c|c|c|}
\hline & $\begin{array}{l}\text { Cr-EDTA } \\
\text { Clearence }\end{array}$ & & $\begin{array}{l}\text { GMBF } \\
\text { (PFU) }\end{array}$ & & $\begin{array}{l}\text { Vascular } \\
\text { Resistance }\end{array}$ & \\
\hline & $\begin{array}{l}\text { Before } \\
\text { Damage }\end{array}$ & $\begin{array}{c}\text { After } \\
\text { Damage }\end{array}$ & $\begin{array}{l}\text { Before } \\
\text { Damage }\end{array}$ & $\begin{array}{l}\text { After } \\
\text { Damage }\end{array}$ & $\begin{array}{l}\text { Before } \\
\text { Damage }\end{array}$ & $\begin{array}{c}\text { After } \\
\text { Damage }\end{array}$ \\
\hline Control & $0.11 \pm 0.07$ & $0.92 \pm 0.12$ & $106 \pm 11$ & $129 \pm 12$ & $1.02 \pm 0.14$ & $0.73 \pm 0.10$ \\
\hline $\begin{array}{c}\text { Nitrite } \\
\text { Luminally }\end{array}$ & $0.07 \pm 0.02$ & $0.42 \pm 0.07 *$ & $132 \pm 12 *$ & $149 \pm 10$ & $0.79 \pm 0.08 *$ & $0.65 \pm 0.07$ \\
\hline $\begin{array}{l}\text { Nitrate Pre } \\
\text { treatment }\end{array}$ & $0.07 \pm 0.01$ & $0.56 \pm 0.14 *$ & $179 \pm 17 *$ & $131 \pm 11$ & $0.73 \pm 0.08^{*}$ & $0.67 \pm 0.05$ \\
\hline
\end{tabular}

*p $<0,05$ vs Control, Clearence ( $\mathrm{ml} / \mathrm{min} 100 \mathrm{~g}$ tissue) Resistance (mmHg/PFU) Values are mean \pm SEM

\section{W1723}

Da-9601, a Standardized Extract of Artemisia Asiatica, Blocks TNF- $\alpha$-Induced

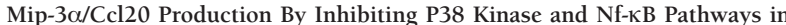
Human Gastric Epithelial Cells

Tae Young Oh, Chang-Duk Jun, Chang Yell Shin, Suck-Chei Choi, Eun-Ju Choi, HyunMee Oh, Eun-Young Choi, Myeung-Su Lee, Yong-Il Shin, Suck-Jun Choi, Won-Jung Lee, Jae-Sik Park

CCL20 appears to link innate and acquired immunity by attracting immature dendritic cells, effector memory T cells and B cells via its unique receptor CCR6. Artemisia asiatica has been used in traditional Asian medicine for the treatment of inflammatory and other disorders The formulated ethanol extract (DA-9601) of A. asiatica have pronounced anti-oxidative and anti-inflammatory activities and exhibit cytoprotective effect againsts gastrointestinal damages. Here we have investigated whether or how DA-9601 inhibits TNF- $\alpha$-induced CCL20 production in gastric epithelial AGS cells. Treatment of AGS cells with DA-9601 reduced TNF- $\alpha$-induced CCL20 promoter activity as well as the gene expression and protein release. Although all three MAP kinase family members were phosphorylated in response to TNF- $\alpha$, only SB203580, a selective inhibitor of P38 kinase, could inhibit both NF-KBdependent transcriptional activity and CCL20 production, suggesting that p38 kinase is functionally linked to NF-אB-dependent pathways in regulating CCL20 induction. Interestingly, DA-9601 also selectively inhibited p38 kinase phosphorylation induced by TNF- $\alpha$ Moreover, DA-9601 blocked NF-KB-dependent transcriptional activity, NF-KB p65 nuclear translocation, and I-אB degradation in TNF- $\alpha$-treated AGS cells or HEK293T cells. Collectively, we conclude that DA-9601 potently inhibits TNF- $\alpha$-mediated CCL20 production by blocking p38 kinase pathway and/or a signal leading to NF-KB-dependent pathways.

\section{W1724}

Epigenetic Inactivation of Wnt Inhibitory Factor-1 Is Frequently Observed in Human Gastrointestinal Cancers

Hiroyuki Yamamoto, Hiroaki Taniguchi, Katsuhiko Nosho, Yasushi Adachi, Takao Endo, Kohzoh Imai, Yasuhisa Shinomura

Aberrant activation and upregulation of the Wnt pathway is a key feature of many cancers Wnt antagonists have recently attracted wide attention. Wnt inhibitory factor-1 (WIF-1) is a secreted antagonist that can bind to Wnt proteins directly and inhibit Wnt signaling pathway. It has been reported that WIF-1 expression is down regulated in several solid tumors and that WIF-1 is silenced by promoter hypermethylation in lung and colorectal cancer. By using RT-PCR, bisulfite sequence analysis, and methylation-specific PCR, we

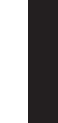


analyzed expression and methylation of WIF-1 in cancer cell lines and freshly resected cancer tissues of the esophagus, stomach, colorectum, and pancreas. Downregulation of WIF-1 mRNA expression was observed in 61 (91.0\%) of 67 cancer cell lines, $16(80.0 \%)$ of 20 esophageal, $23(74.2 \%)$ of 31 gastric, $41(82.0 \%)$ of 50 colorectal, and six $(75.0 \%)$ of eight pancreatic cancer tissues. Downregulation of WIF-1 expression was also observed at protein level. No significant association between WIF-1 downregulation and clinicopathological characteristics was found, suggesting that downregulation of WIF-1 expression is an early event in carcinogenesis of these cancers. Indeed, downregulation of WIF-1 expression was observed in $32(72.7 \%)$ of 44 colorectal adenoma tissues and 18 (78.2\%) of 23 early mucosal or submucosal colorectal carcinoma tissues. CpG island hypermethylation in the WIF-1 promoter region correlated with downregulation of WIF-1 expression in cancer cel lines and tissues. Treatment with demethylating agent, 5-aza-2'-deoxycytidine (5-aza-dC), restored WIF-1 expression in cancer cell lines. A combined treatment of 5-aza-dC and a histone deacetylase inhibitor, trichostatinA, restored WIF-1 expression synergistically, indicating the role of cytosine methylation and histone deacetylation in the silencing of the WIF-1 gene. Transfection of the WIF-1 gene construct into TE-1 esophageal cancer cell lines or SW48 colon cancer cell lines lacking WIF-1 expression resulted in a significant inhibition on colony formation, cell proliferation, anchorage-independent growth in soft agar. TOPflash assay showed WIF-1 inhibits Wnt canonical signaling in these cell lines. These results suggest tumor suppressive function of WIF-1, due to its ability to inhibit Wnt signaling. Our results suggest that WIF-1 silencing due to promoter hypermethylation is an important mechanism underlying aberrant activation of the Wnt signaling pathway in carcinogenesis of the digestive organs. Modulation of the Wnt pathway, through reversal of WIF-1 silencing by demethylating agents, is a potential target for treatment and/or prevention of gastrointestinal cancers.

\section{W1725}

Epigenetic Silencing of Axin2 in Colorectal Carcinoma with Microsatellite Instability

Koji Koinuma, Hideo Nagai, Hiroyuki Mano

Mutation or epigenetic silencing of mismatch repair genes, such as MLH1 and MSH2, results in microsatellite instability (MSI) in the genome of a subset of colorectal carcinomas (CRCs). However, little is yet known of genes that directly contribute to tumor formation in such cancers. To characterize MSI-dependent changes in gene expression, we have now compared transcriptomes between fresh CRC specimens positive or negative for MSI ( $\mathrm{n}=10$ for each) with the use of high-density oligonucleotide microarrays harboring $>44000$ probe sets. Correspondence analysis of the expression patterns of isolated MSI-associated genes revealed that the transcriptome of MSI(+) CRCs is clearly distinct from that of MSI(-) CRCs. Such MSI-associated genes included that for AXIN2, an important component of the WNT signaling pathway. AXIN2 was silenced, apparently as a result of extensive methylation of its promoter region, specifically in $\mathrm{MSI}++$ CRC specimens. Forced expression of AXIN2, either by treatment with 5 '-azacytidine or by transfection with AXIN2 cDNA, resulted in rapid cell death in an MSI(+) CRC cell line. These data indicate that epigenetic silencing of AXIN2 is specifically associated with carcinogenesis in MSI(+) CRCs.

\section{W1726}

A Cpg Island Within the P-Cadherin Promoter Is Hypomethylated Early During Colorectal Tumourigenesis: Biological Consequences

Stuart McDonald, Paul A. Atherfold, Jolanta Obszynska, Robert G. Hardy, Scott Sanders, Rebecca Harrison, Andrew Rowan, Ian P. Tomlinson, Nicholas A. Wright, Janusz A. Jankowski

Cadherins mediate cell-cell adhesion in tissues of higher organisms. Our group and others have shown that in colorectal tumourigenesis, altered patterns of cadherin expression are associated with neoplastic progression. Specifically, P-cadherin, normally expressed only in stratified epithelia (and not normal colon), is intimately associated with early Aberrant Crypt Foci (ACFs) and subsequent colorectal carcinoma development. Neither mutation within nor chromosomal amplification of the P-cadherin gene accounts for such ectopic expression, and this study therefore assessed whether changes in P-cadherin promoter methylation correlate with changes in expression and what the functional implications might be. Using methylation-specific PCR (MSP), the methylation status of the P-cadherin promoter was analysed in 19 samples of normal colorectal mucosa, 20 samples of normal colorectal mucosa adjacent to carcinoma, $15 \mathrm{ACF}$ and 15 carcinomas. P-cadherin $\mathrm{mRNA}$ and protein expression was confirmed by RT-PCR and immunohistochemistry. In DNA from 4 of 39 'histologically normal' colon samples, the P-cadherin promoter was fully unmethylated, but only these cases, which were derived from tissue adjacent to colorectal cancer, had detectable P-cadherin mRNA expression. In the remaining samples, both methylated and unmethylated DNA was detected. Microisolation of enterocytes from non-expressing normal colonic mucosa showed that these cells were fully methylated. All ACFs and carcinomas analysed possessed a fully unmethylated promoter, and expressed P-cadherin mRNA, indicating demethylation during or prior to tumourigenesis. Forced P-cadherin mRNA expression in non-constitutively expressing cells increased S-phase cell cycle fractions. siRNA experiments, in colorectal cancers cell lines without promoter methylation demonstrated that down regulation of Pcadherin expression increased G0/Gl cell cycle fractions and decreased total cell counts. These results demonstrate that demethylation of the P-cadherin promoter can occur at very early stages of tumourigenesis. Our data suggest that this mechanism can lead to the Pcadherin gene being ectopically expressed, increasing entry into the cell cycle and contributing to hyperplasia, in ACFs and neoplasia.

\section{W1727}

Laterally Spreading Type of Colorectal Adenoma Exhibits a Unique Methylation Phenotype and K-RAS Mutations

Sakiko Hiraoka, Jun Kato, Joichiro Horii, Keita Harada, Hideyuki Fujita, Tamiya Morikawa, Hidenori Shiraha, Yasushi Shiratori

Background and aims: Laterally spreading tumors (LST) of the colon, which are characterized by lateral extension along the luminal wall with a low vertical axis, were initially reported by Kudo et al. (World J Surg 1997). A previous report indicated that LST were found in approximately $6 \%$ of early stage colorectal cancers. LST were further classified into granular type LST (G-LST) and flat type LST (F-LST) according to the morphology of the surface (Hurlstone DP. et al. Gut 2004). G-LST were composed of superficially spreading aggregates of nodules forming a flat based lesion with a granulonodular and uneven surface, while FLST have a flat smooth surface, with an absence of granulonodular formation. Although these types of tumors were initially recognized only in Japan, they have also been detected and accepted in Western countries in recent years. However, genetic and epigenetic characteristics of these tumors were scarcely reported. The aim of this study is to determine methylation phenotypes and K-ras mutations of LST in comparison with those of conventional protruded adenomas. Methods: A total of 108 sporadic colorectal adenomas larger than $1 \mathrm{~cm}$ were collected. These tumors were classified into conventional protruded-type (73 lesions, 68\%), G-LST (23 lesions, 21\%), and F-LST (12 lesions, 11\%) according to the macroscopic appearance of adenomas, based on colonoscopy observations. CpG island methylator phenotype (CIMP) was determined by examination of methylation status at p16, MINT 1, 2, 12, and 31 loci. CIMP-high was defined as adenomas with concordant methylation of two or more sites out of five regions examined. K-ras codon 12 and 13 point mutations were also examined. The relationship between macroscopic appearance and CIMP status or K-ras mutations was analyzed. Results: $\mathrm{CpG}$ island methylation involving two or more loci (CIMPhigh) was more likely to be observed in G-LST $(14 / 23 ; 61 \%)$ than in conventional protrudedtype adenomas $(18 / 73 ; 25 \%)(\mathrm{p}=0.002)$. The prevalence of K-ras mutations in G-LST (18/ $23 ; 78 \%)$ was significantly higher than that in protruded-type adenomas $(18 / 73 ; 25 \%)$ (p $<0.0001$ ). Moreover, the prevalence of CIMP-high and K-ras mutations in G-LST located in the proximal colon was much higher (11/13; $85 \%$, and $12 / 13 ; 92 \%$, respectively). In contrast, F-LST exhibited low prevalence of CIMP-high (1/12; $8 \%$ ) and K-ras mutations (2/ 12; 16\%). Conclusions: High prevalence of CIMP-high and K-ras mutations in G-LST, especially in the proximal colon, could strongly suggest that G-LST appearance is associated with a unique genetic and epigenetic carcinogenic pathway.

\section{W1728}

Genome-Wide Analysis of Allelic Imbalance By Using High-Density Single Nucleotide Polymorphism (SNP) Array in Colorectal Cancer

Motohisa Tada, Fumihiko Kanai, Yasuo Tanaka, Lian-Jie Lin, Keisuke Tateishi, Osamu Yokosuka, Masashi Sanada, Yasuhito Nannya, Seishi Ogawa, Masao Omata

(Background and Aim) Most human cancers are characterized by genetic instabilities. These genetic instabilities in colorectal cancer can be divided into two distinct types, with over $85 \%$ of tumors showing chromosomal aberrations, and $10 \%$ of tumors demonstrating microsatellite instability. Though comparative genomic hybridization (CGH) was initially used to detect chromosomal copy number changes, it was difficult to identify key molecule for carcinogenesis because of low resolution (about $20 \mathrm{Mbp}$ ). In this study, we sought to identify the key molecule in colorectal cancer pathogenesis by using high-density SNP array. (Materials and Methods) We analyzed 35 colorectal cancer cell lines and 20 primary colorectal cancer for areas of DNA copy number changes and loss of heterozygosity (LOH) through genome-wide analysis of 116, 204 SNPs distributed across all human chromosomes with Affymetrix GeneChip Human Mapping $100 \mathrm{~K}$ Set (mean intermarker distance 23.6 Kbp). Copy Number Analyzer for GeneChip (CNAG), our original analysis tool that automates the detection of copy number and regions of $\mathrm{LOH}$, was used for data analysis. The results from the array analysis were verified by PCR, quantitative real-time PCR and Fluorescent In Situ Hybridization. (Results)Over all, in both cell lines and primary tumors, gain of $8 \mathrm{q}$, $13 q, 20 p$ and $20 q$, loss of $8 p$ and $18 q$ and $L O H$ of $5 q, 14 q, 17 p$ and $18 q$ were detected with high frequency. Moreover, allelic homozygosity was detected at 5q, 14q, 17p and 18q frequently in both cell lines and primary tumors. In detail, narrow (less than $1 \mathrm{Mbp}$ ) 22 regions of high grade amplification $(n \geqq 5$ ) were found in at least two cell lines. The size of minimal common amplified region was $42 \mathrm{Kbp}$. Thirty-two regions of high grade amplification ( $n \geqq 4$ ) were detected in at least one primary tumor. Twenty-four regions of homozygous deletion were detected in at least one cell line and the size of minimal deletion was $11 \mathrm{Kbp}$. Homozygous and hemizygous deletions were detected in 6 and 29 regions in at least one primary tumor, respectively. In addition to known genetic abnormality, homozygous deletion of Chr.4q22.1 region was newly identified. (Conclusion) Affymetrix GeneChip Human Mapping $100 \mathrm{~K}$ Set and CNAG revealed that allelic homozygosity may be one of the mechanisms of colorectal carciniogenesis and by using these methods, we could detect minimal region of copy number alteration, which had never been able to detect by other methods because of its limitation of resolution. The novel amplified and deleted regions discovered in this study may be significant and useful for early diagnosis and novel therapy based on molecular targeting in colorectal cancer.

\section{W1729}

Mgmt Promoter Hypermethylation in Barrett's Adenocarcinomas and Its Premalignant Lesions

Regine Schneider-Stock, Doerthe Kuester, Dar Altaf, Michael Vieth, Ulrich Peitz, Brigitte Peters, Christopher C. Moskaluk, Frank Meyer, Manfred Stolte, Peter Malfertheiner, Hans Lippert, Albert Roessner, Wael El-Rifai

Transcriptional silencing of tumor suppressor genes by promoter $\mathrm{CpG}$ island hypermethylation plays a role in the multistep-carcinogenesis of many tumors of the gastrointestinal tract. Reports on methylation status of the premalignant lesions of Barrett's adenocarcinoma (BA) are limited. The DNA-repair gene O6-methylguanine-DNA methyltransferase (MGMT) 
transfers alkyl groups at the O6-position of guanine to a cysteine residue avoiding specific mutagenic effects of DNA damage. Thus, MGMT inactivation is associated with $G: C$ to A:T mutations in the $\mathrm{p} 53$ gene. It was reported that $\mathrm{p} 53$ mutations and p53-LOH are highly frequent events in BA even in the absence of dysplasia and adenocarcinomas. Therefore, we studied the MGMT promoter methylation status as well as MGMT mRNA and protein expression in a group of $61 \mathrm{BA}$ and their corresponding preneoplastic lesions and normal esophageal mucosae (NT). Separation of the different histological stages of Barrett's carcinogenesis was done on hematoxylin-eosin slices of formalin-fixed, paraffin-embedded tissue and partly performed by laser microdissection. Furthermore, we studied the immunohistochemical MGMT protein expression using tissue microarrays of additional $66 \mathrm{BA}$ and correlated these findings with clinicopathological data. Using methylation-specific PCR we revealed MGMT hypermethylation (low/high) in 21.4\%/0\% of NT, 22.2\%/66.7\% of Barrett's mucosa (BM), $6.7 \% / 93.3 \%$ of intraepithelial neoplasia, and $17.9 \% / 59 \%$ of BA. MGMT promoter methylation correlated significantly with a decrease in MGMT mRNA expression $(\mathrm{p}=0.048)$ and with a decrease in MGMT immunohistochemical protein expression $(\mathrm{p}<0.01)$. There was a tendency for BA with progressed GERD status to have MGMT promoter methylation more frequently. We noted a frequent loss of MGMT protein expression with advanced depth of tumor invasion and tumor stages $(\mathrm{p}=0.04)$. There was no correlation between MGMT inactivation and sex, age, tumor grading and gastric Helicobacter pylori status. We report an increased frequency of MGMT hypermethylation with tumor progression of BA and consider MGMT promoter hypermethylation an early event in Barrett's carcinogenesis.

\section{W1730}

Helicobacter Pylori Infection, Interleukin 1 Beta Polymorphism and Predisposition to Gastric Cancer Through the Cpg Island Methylation Pathway

Annie On On Chan, Kent Man Chu, Suet Yi Leung, Camy Huang, Yun Wei Sun, Samuel Ko, Man Fung Yuen, Harry Xia, Chi Hin Cho, Wai Mo Hui, Shiu Kum Lam, Asif Rashid, Benjamin Cy Wong

Background: The association of HP infection and IL- $1 \beta$ polymorphism increases the risk of developing gastric cancer. Gastric mucosa of patients with Helicobacter pylori (HP) infection have $\mathrm{CpG}$ island methylation at tumor suppressor genes. Interleukin lbeta (IL-1 $\beta$ ) increases gene methylation at $\mathrm{CpG}$ islands. We postulate that HP infection and IL-1 $\beta$ polymorphism predispose to gastric cancer through $\mathrm{CpG}$ island methylation pathway. Aim: To investigate if an association exists between IL-1 $\beta$ polymorphism, HP infection and methylation and in patients with gastric cancer. Methods: We determined IL-1B-511/-31 and IL-1RN genotypes in 98 patients with gastric cancer. Methylation of MGMT, E-cadherin, DAPK and pl6 were assessed in these patients using methylation-specific PCR and confirmed by sequencing. HP status was confirmed by histology and serology. Results: HP infection was present in $65.3 \%$ (64) patients. The $\mathrm{T}$ and $\mathrm{C}$ alleles at the -511 locus of the IL-1B gene were in near total linkage disequilibrium with the $\mathrm{C}$ and $\mathrm{T}$ alleles at the -31 locus. Hence only -511 locus was assessed. The allele frequencies at IL-1B-511 were 16\% (16), 59\% (58), and 25\% (24) for $\mathrm{C} / \mathrm{C}, \mathrm{C} / \mathrm{T}$, and T/T genotypes, respectively. Allele 2 of the IL-1RN locus was not detected in any patients. Methylation of MGMT, E-cadherin, DAPK and pl6 were present in 30\% (29), 59\% (58), 43\% (42) and 45\% (44) of patients, respectively. Methylation of two or more genes was present in 84\% (20) of patients with the IL-1B-511 T/T genotype, 44\% (7) with $\mathrm{C} / \mathrm{C}$ genotype, and $74 \%$ (43) with $\mathrm{T} / \mathrm{C}$ genotype $(\mathrm{p}=0.02)$. This association was present in patients with HP infection: $88 \%$ (15) with T/T genotype, $38 \%$ (3) with C/C genotype, and $77 \%(30)$ with $\mathrm{T} / \mathrm{C}$ genotype $(\mathrm{p}=0.02)$, but not present in patients without HP infection $(\mathrm{p}=0.6)$. Conclusion: We postulate that the patients with HP infection and IL-1B-511 T/T genotype may be predisposed to gastric cancer through the CPG island methylation pathway

\section{W1731}

A Negative Correlation Between IL-6 and DNA Methylation in Patients with Gastric Cancer

Li Ping Tang, Chi Hin Cho, Wai Mo Hui, Camy Huang, Ching Lung Lai, Harry Hx Xia, Shiu Kum Lam, Asif Rashid, Benjamin Cy Wong, Annie On On Chan

Background: Serum IL-6 levels have been shown to have prognostic significance in gastric cancer. CpG island methylation is an important mechanism for gene silencing and has been demonstrated in gastric cancer. It has been shown that DNA methylation is a non-redundant repressor of the Th2 effector system. Aim: To investigate the association between serum IL6 levels and CPG island methylation at pl6, DAPK, MGMT and E-cadherin in patients with gastric cancer. Methods: Methylation status was studied using methylation specific polymerase chain reaction (MSP) in surgical specimens of 75 patients with gastric adenocarcinoma. $\mathrm{CpG}$ island methylator phenotype (CIMP) was defined as positive if methylation was present in more than one marker. IL- 6 serum levels were measured by chemiluminescent enzyme immunoassay (CLEIA) in sera of these patients. Concentration of IL-6 was expressed in median (range). The comparison of IL-6 in relation to methylation and clinical data was assessed by Mann Whitney test. Results: Methylation of p16, DAPK, MGMT, and E-cadherin were present in $53 \%, 48 \%, 32 \%$, and $59 \%$ of patients. Patients with tumors methylated at p16 and DAPK had lower serum levels of IL- 6 compared to patients with unmethylated tumors $(1.8 \mathrm{pg} / \mathrm{ml}$ (range: $0.6-15.6 \mathrm{pg} / \mathrm{ml}$ ) vs $4.8 \mathrm{pg} / \mathrm{ml}$ (range: $0.3-206.3 \mathrm{pg} / \mathrm{ml}$ ), p=0.01 for $\mathrm{pl}$, and $1.5 \mathrm{pg} / \mathrm{ml}$ (range: $0.6-100.8 \mathrm{pg} / \mathrm{ml}$ ) vs $6.2 \mathrm{pg} / \mathrm{ml}$ (range: $0.3-206.3 \mathrm{pg} / \mathrm{ml}$ ), p= 0.0001 for DAPK), but there was no difference with MGMT and E-cadherin methylation status. Lower concentration of IL-6 was also observed in patients with CIMP+ tumors: 2.2 $\mathrm{pg} / \mathrm{ml}$ (range: $0.3-206.3 \mathrm{pg} / \mathrm{ml}$ ) vs $7.0 \mathrm{pg} / \mathrm{ml}$ (range: $1.2-34.7 \mathrm{pg} / \mathrm{ml})(\mathrm{p}=0.016)$. Serum IL6 levels were also associated with TNM stage ( $\mathrm{p}=0.001)$, depth of tumor invasion $(\mathrm{p}=0.002)$, lymphatic invasion $(\mathrm{p}=0.01)$, vascular invasion $(\mathrm{p}=0.008)$, metastasis $(\mathrm{p}=0.002)$ and signet cell histology $(\mathrm{p}=0.001)$. Conclusion: Low serum IL-6 levels were associated with tumor methylation in patients with gastric cancer, which may be due to simultaneous methylation at the IL-6 gene, or that inflammatory cytokines can modulate tumor methylation.

\section{W1732}

Is Risk for Sporadic Colorectal Adenoma with Higher Calcium and Vitamin D Intakes Modulated By XRCC1 Base Excision Repair Genotype? Aasma Shaukat, Woody J. Coker, Roberd M. Bostick

BACKGROUND: Despite a strong rationale and support from basic science and animal models, findings in humans for an association between calcium and vitamin D intake and risk for colorectal neoplasms have been inconsistent, and the underlying reasons for this inconsistency are unclear. The XRCCl gene is involved in DNA base excision repair. Calcium and vitamin D intake may protect colonocytes from DNA damage by reducing free bile acids in the gut. However, an interaction of calcium and vitamin D with XRCCl genotype on risk for colorectal neoplasms has not been investigated. AIMS: To investigate whether the capacity for calcium and vitamin D intake to reduce risk for incident sporadic colorectal adenomas depends upon XRCCl base excision DNA repair genotypes. METHODS: We analyzed data from a community/colonoscopy-based case-control study of incident sporadic colorectal adenomatous polyps. Average risk patients $30-74$ years of age were scheduled for elective, outpatient usual care colonoscopies and completed questionnaires, including a food frequency questionnaire, prior to colonoscopy. To determine XRCCl genotypes, 167 pathology-confirmed cases and 214 adenoma-free controls underwent PCR-RFLP analyses for C194T (exon 6), G280A (exon 9), and G399A (exon 10) polymorphisms of the XRCC1 gene. Calcium and vitamin D intake was categorized above and below the median intake in controls. RESULTS: Overall, risk for adenomas was lower with higher intakes of calcium (OR 0.58, 95\% CI 0.36-0.93), but not vitamin D (OR 0.71, 95\% CI 0.71-1.12). In analyses stratified by XRCCl genotypes risk for adenomas with higher calcium intake was more pronounced among those with one or more variant alleles at exons 9 (OR $0.23,95 \%$ C $0.04-1.43)$ and 10 (OR $0.30,95 \% \mathrm{CI} 0.15-0.60)$, but it was statistically significant only with exon $10(\mathrm{p}=0.001)$. In fact, any combination of exon 9 and 10 genotypes that contained even one allele with a GA or AA genotype was associated with lower risk for adenoma with higher calcium intake (OR $0.34,95 \% \mathrm{CI} 0.18-0.64)$. The results for vitamin D were similar but less pronounced and not statistically significant. CONCLUSION: These results suggest that possible preventive effects of calcium and perhaps vitamin D may depend on XRCCl genotype, and provide a possible explanation for inconsistent associations between calcium and vitamin D intake and risk for colorectal adenomas.

\section{W1733}

Inactivation of Differential Tumor Suppressor Genes in Neuroendocrine Tumors of the Gastrointestinal Tract

Christian Arnold, Andrea Sosnowski, Rudolf Arnold, Hubert E. Blum

Introduction: Little is known about the molecular mechanisms leading to neuroendocrine tumors (NET) of the gastrointestinal system. We have recently demonstrated the role of promoter methylation of tumor suppressor genes (TSG) in NET. In this study we investigated the differential inactivation of TSG and the functional significance of genes silenced by promoter methylation which are believed to be frequently inactivated in NET. METHODS In this study 118 well differentiated fore- and midgut NET from 71 patients were investigated The methylation status of hMLH1, pl6, APC, O6-MGMT, PTEN, HICl, e-cadherin, RASSFla TIMP3 and MEN1 was examined by methylation specific PCR. The expression profiles of pl6-, APC- and MENIN were investigated by immunohistochemistry. TSG expression and methylation status was correlated with the ki-67 proliferation index. RESULTS: Promoter hypermethylation was a prominent feature. Apart from a difference in promoter hypermethyl ation of the runx3-gene in functional vs. non-functional NET $(p=0,023)$, no differences in the promoter methylation profile of the tumors were evident. Tumors with high ki-67 index $(>10 \%)$ were significantly more often methylated than NET with low ki-67 index (< $2 \%)(p=0,034)$. We observed a significant loss of $\mathrm{p} 16$-expression $(\mathrm{p} \leq 0,05)$ in insulinomas vs. pancreatic non-functional and non-functional NET. pl6-expression in functional vs non-functional NET was also significantly decreased $(p \leq 0,01)$. APC was expressed significantly less in gastrinomas $(p \leq 0,01)$ and functional NET $(p \leq 0,05)$ vs. non-functional tumors. APC-expression was strongly decreased in insulinomas vs. pancreatic non-functional tumors $(p=0,017)$. NET with the carcinoid syndrome $(p=0,029)$ and non-functional NET $(p=0,019)$ expressed MENIN significantly less than insulinomas. In general, NET with the carcinoid syndrome had a decreased MENIN-expression than pancreatic NET $(p=0,033)$. Discussion: This is the first study demonstrating differential expression of $\mathrm{p} 16, \mathrm{APC}$ and MENIN in NET. In general, functional NET of the pancreas showed a decreased expression of pl6 and APC, while MENIN was expressed less in NET with the carcinoid syndrome Promoter methylation was a common feature in all tumor types from various origins with only few differences. Tumor with a high proliferation index demonstrated a high promoter methylation status compared with tumors with a low ki-67 index indicating a prognostic significance for promoter methylation in NET.

\section{W1734}

Microsatellite Instability in Human Colorectal Cancer (CRC): Effect of EGFR Overexpression

Nijez Aloulou, Marie-Therese Chaumette, Amel Sekour, Karen Leroy, Mohammad Abolhassani, Iradj Sobhani

EGFR is expressed in colorectal tumours. In the experimental models, EGF like peptides exert either trophic effect and control anti tumoral immune response in CRC. The most immunogenic tumours have high frequency of MSI-H. The aim of this work was to analyze EGFR expression in colon tumours with respect to the mismatch repair (MMR) status. Patients-methods. Data of 130 consecutive patients [57 W, $73 \mathrm{M}$; median age 70 (29 to 100) yrs] were collected. MSI-H was determined using Fluorescent PCR Pentaplex and Immunohistochemistry (IHC) on the basis of at least 3 instable sequences out of 5 monomorphic microsatellite markers, and lack of hMLH1 or hMSH2, respectively, when tumoral tissue was compared to the normal homologous mucosa. EGFR expression was similarly determined via IHC. Membrane expression of EGFR was evaluated semi quantitatively as $\mathrm{E}$ (extension) x I (intensity), each item varying from 0 (negative or absent) to 4 (extended or 
very strong). The "E x I" score (0 to 16) was used to define two classes with weak $(\mathrm{Wk}<9)$ or high (ST>or=9) EGFR expression with MaxE x I being $=9$ in normal tissues. The statistical analyses were carried out by X2 or Anova and multiple regression logistic tests and survival KM curve. Results. Tumours were of stage I (4), II (54), III (49) and IV (23) in pTNM classification with 55 tumours displaying high EGFR expression (ST). There was no difference on ST status concerning the type of surgery, pTNM, and tumour size at baseline, the number and the type of adjuvant or palliative chemotherapy afterward. Among the 24 tumours with MSI-H, 2 did not express hMSH2 and 21 hMLHl on IHC with 16 of them $(66 \%)$ being ST (vs $36 \%$ in MSS; $\mathrm{p}=0.01$ ) and $6 \mathrm{MSI}-\mathrm{H}$ tumours displaying a score of 16 . The univariate analysis showed a significant relationship between ST of EGFR, and age $<60(48+/-38$ vs $62+/-26 ; \mathrm{P}=0.01$ ), right-sided ( $58 \%$ vs $32 \%$ left and $34 \%$ rectum; $\mathrm{P}=0.017$ ), characteristics of tumours ( $66 \%$ vs $45 \%$ ulcerated or $26 \%$ perforated; $P=0.009$ ), the presence of mucoid component (54\% vs 34\%; $\mathrm{P}=0.03)$, and a more important lymphoid infiltration ( $49 \%$ vs $30 \% ; \mathrm{P}=0.04$ ) whereas the number of lymph node, vascular or nervous infiltrations were not significantly related. There was no difference in term of survival according to the EGFR expression although the time to relapse was longer ( 21 month) in patients with tumours (of stage II) with strong expression than for the tumours with weak EGFR expression (19 month; $\mathrm{P}=0.05)$. In multivariate analysis only MSI-H status appeared as independent factor (Odds Ratio $=2.7,95 \%$ IC 1.1-7.2; P=0.04). Conclusion: The high EGFR expression in colorectal tumours may be linked to the mismatch repair alteration.

\section{W1735}

Aberrant Promoter Methylation Pattern in Ulcerative Colitis-Associated Carcinomas

Doerthe Kuester, Wael El-Rifai, Thomas Guenther, Arndt Hartmann, Andrea Tannapfel, Frank Meyer, Ulrich Bohr, Matthias Pross, Michaela Guenther, Frauke Bataille, Petra Ruemmele, Andrew Silver, Peter Malfertheiner, Hans Lippert, Albert Roessner, Regine Schneider-Stock

Ulcerative colitis (UC) is a disease that is characterized by chronic inflammation, rapid cell turnover, and a substantial risk of colorectal cancer. Little is known about the significance of promoter hypermethylation in inflammatory bowel disease. It is not known if the promoter methylation pattern can serve as a criterion for differentiating UC-associated colorectal cancer from sporadic colorectal cancer. Thus, we compared a group of $37 \mathrm{UC}$-associated with 80 sporadic colorectal cancers, investigating a panel of genes from different signal cascades. These markers included CHFRl (check point with FHA and ring finger), GpX3 (glutathione peroxidase 3), GSTP1 (glutathione S-transferase 1), DAPK (death-associated protein kinase), and pl6. CHFRl is a stress-related mitotic checkpoint regulation gene. GpX3 and GSTP1 are major scavengers of ROS and play an important role in protecting the gastrointestinal epithelium from cytotoxic and carcinogenic agents. DAPK is a serin-threonine kinase involved in apoptotic cell death. P16 is a cyclin-dependent kinase inhibitor regulating the Gl/S checkpoint of the cell cycle. Methylation-specific PCR from formalin-fixed, paraffin-embedded carcinoma tissue revealed the following methylation pattern of the UC-associated/ sporadic colorectal carcinoma: CHFR1 (71.4\%/41.2\%), GpX3 (40\%/50\%), GSTP1 (13.5\%/ $6 \%$, DAPK $(17.6 \% / 56 \%)$, and pl6 (15.8\%/29.4\%). Immunohistochemical protein expression of all markes were investigated by tissue microarray analysis. Here, promotor methylation status correlated significantly with a decrease or loss of protein expression. In UC-associated colorectal cancer, $40 \%$ of cases did not show any hypermethylation, whereas more than $30 \%$ of cases showed simultaneous methylation in 2 or more genes investigated. In sporadic colorectal cancer, only $10 \%$ of the cases did not show methylation in any of the genes investigated. In more than $60 \%$ of the cases 2 or more genes were methylated simultaneously. Whereas CHFRl and GpX3 seem to be important markers, GSTP1 is only weakly involved in the carcinogenesis of both carcinoma types. Furthermore, there is a different methylation pattern for both carcinoma types, with high methylation (more than $40 \%$ ) for CHFRl/GpX3 in UC-associated colorectal cancer and CHFR1/GpX3/DAPK in sporadic colorectal cancer. Transcriptional silencing by promoter hypermethylation seems to be critical in the inflammation-associated development of carcinomas in UC. Furthermore, we suggest that differences in the frequency and the pattern of hypermethylation reflect multiple pathways for the development of UC-associated and sporadic colorectal cancer

\section{W1736}

What Is a Crucial Factor Related to CPG Islands Hypermethylation of TumorRelated Genes in H.pylori-Infected Gastric Mucosa?

Mitsuru Kaise, Jin Yonezawa, Takuji Yamasaki, Jun Miwa, J.N. Tashiro, Takashige Tomita, Yasuo Matsubara, Hisao Tajiri

Background and aim: $\mathrm{CpG}$ islands hypermethylation (CIHM) of tumor-suppressor genes involves gastric carcinogenesis. We have demonstrated that $H$.pylori eradication significantly reduces CIHM, and the organism may act as a carcinogen via inducing CIHM (Gastroenterology. 128 suppl2;A134, 2005). However, the levels of CIHM may be different among H.pyloriinfected individuals because H.pylori can elicit diverse clinical disorders. The aim of our study is to elucidate crucial factors related to CIHM in H.pylori-infected gastric mucosa. Methods: Two samples from non-cancerous corpus mucosa were obtained in H.pyloriinfected patients with duodenal ulcer ( $D U, n=22)$, gastric ulcer $(G U, n=20)$, and gastric cancer (GCA, $\mathrm{n}=25$ ). One sample was pathologically examined, and morphological parameters such as polymorphoneutrophil infiltration (PMN), mononuclear cell infiltration (Mono), atrophy, intestinal metaplasia (IM) and H.pylori density were scored according to the updated Sydney system (score 0 to 3 equating to normal, mild, moderate and marked). Genomic DNA retrieved from the other sample was subjected to bisulfite modiciation. We examined CIHM status of p16, E-cadherin (Ecad) and death-associated protein kinase (DAPK) gene promoters by and methylation-specific PCR. Levels of CIHM were compared between diseases, and between morphological parameters. Results: (1) Methylation rates of DAPK, Ecad and p16 promoters were significantly $(\mathrm{p}<0.001)$ higher in GCA $(96 \%, 96 \%, 83 \%)$ than in GU $(55 \%$, $50 \%, 15 \%)$ and in DU $(74 \%, 30 \%, 9 \%)$. (2) Methylation rates of DAPK, Ecad and pl6 promoters were significantly $(\mathrm{p}<0.01)$ higher in PMN-positive samples $(90 \%, 76 \%, 47 \%)$ than in PMN-negative ones $(39 \%, 17 \%, 11 \%)$. Those were neither different between IMpositive and IM-negative samples, and nor between atrophy-positive and atrophy-negative samples. Methylation rates of DAPK were significantly higher in H.pylori-abundant samples (score $>1$ ) than in H.pylori-scanty ones $($ score $=1$ or $<1$ ), but those of Ecad and pl6 were not different. Those of DAPK were significantly higher in Mono-abundant samples (score>1) than in Mono-scanty ones (score $=1$ or $<1$ ), but those of Ecad and pl6 were not different. Conclusions: H.pylori significantly induces CIHM of tumor-related genes in non-cancerous gastric mucosa bearing GCA as compared to mucosa bearing DU or GU. PMN in H.pyloripositive mucosa was a crucial factor linked with CIHM, suggesting that PMN-induced events or factors such as superoxide may elicit CIHM.

\section{W1737}

Aberrant DNA Methylation Associates with the Initiation and Progression of Colon Adenomas and Is Maintained in Colon Adenocarcinomas

Young-Ho Kim, Slavomir Dzieciatkowski, Joseph Willis, M. Kay Washington, Steven C. Stain, Will Chapman, William M. Grady

Background: The aberrant methylation of the 5' promoter regions of genes is a recently appreciated epigenetic mechanism for silencing tumor suppressor genes in colon cancer. Aberrant DNA methylation has been observed as early as the aberrant crypt focus step of the adenoma-carcinoma sequence, but its role in affecting genes that mediate initiation and progression events in the colon cancer formation process is not well defined, particularly regarding the metastatic spread of colon cancer Aim: To clarify the role of epigenetic silencing by promoter hypermethylation in the full range of the adenoma-carcinoma progression sequence through the assessment eight genes implicated in cancer formation and progression, MGMT, HLTF, hMLH1, p14ARF, p16/CDKN2A, TIMP3, THBS1, and CDH1 Methods: DNA, extracted from formalin-fixed, paraffin-embedded tissues obtained from individuals with colon adenomas $(n=41)$, adenocarcinomas $(n=80)$ and metastases $(n=28)$, was subjected to treatment using sodium bisulfite and then methylation specific PCR using primers that assess the methylation status of the genes listed above. Among 28 cases of hepatic metastasis, 14 cases could be matched with primary colorectal cancer. Results: The average number of methylated genes per case in the primary cancers was significantly higher than in the adenomas $(2.04 \pm 1.35$ vs $1.59 \pm 1.70, p=0.04)$, but there was no significant difference between primary cancers and metastatic cancers $(2.04 \pm 1.35 \mathrm{vs} 1.75 \pm 1.20, \mathrm{p}>0.05)$. In the adenomas, the average number of methylated genes in the advanced adenomas was higher than in the non-advanced adenomas $(2.25 \pm 1.73$ vs $0.70 \pm 1.22, \mathrm{p}=0.03)$. There were differences in the frequency of hypermethylation between individual genes between the adenomas, primary cancers, and metastatic cancers. Statistical analysis of these differences is ongoing to determine if these trends are statistically significant. Finally, the methylation pattern of metastatic cancer was similar, but not identical to that of matched primary cancer. Conclusions: Epigenetic silencing by promoter hypermethylation of the genes assessed in this study is associated with the progression of adenomas to adenocarcinomas but not of early adenocarcinomas to late adenocarcinomas. The frequency of gene methylation in the different stages of the adenomas and adenocarcinomas varied between genes suggesting that there are unique gene-specific selective pressures that influence the frequency of the methylation of the genes in these histological steps of colon cancer formation.

\section{W1738}

Evidence for BRAF Mutation and Variable Levels of MSI in a Sporadic Colorectal Cancer (CRC) in African Americans (AA) Hassan Ashktorab, Hassan Brim, Serwat Siddiqi, Francis Giardiello, Ed Lee, Duane T. Smoot

Background: An alternative pathway of tumorigenesis has been identified in the CRC associated with serrated precursor lesions, variable levels of microsatellite instability (MSI), and driven in part by activating mutations in the BRAF proto-oncogene (V600E). We have assumed that BRAF mutations might be associated with hMLHl methylation status rather than MSI status. Therefore, we studied the sporadic CRC on the status of the methylation of mismatch repair genes hMLH1, hMSH2, MSI, BRAF (V600E mutation) and explored the molecular profiles of CRC in AA that are known to be at higher risk of CRC. Methods: We used five different sets of primers (BAT25, BAT26, NR21, NR22 and NR24) for MSI in CRC samples from 94 patients ( 55 females/39 males). HMLHl and hMSH2 gene expression was checked using an immunohistochemical (IHC) and MSP technique. BRAF V600E mutation was determined using Mutector technique. The mean age for carcinoma diagnosis was 65.7 years (69.4 years for female patients and 60.1 years for male patients). Results: Of the 94 patients, $28(30 \%)$ were MSI-H $8(8.5 \%)$ were MSI-L and $58(61.5 \%)$ were MSS. Of the $28 \mathrm{MSI}-\mathrm{H}, 19(67.8 \%)$ were in the right colon while $7(25 \%)$ were in the left colon (2 were unknown). V600E was observed in 7 of 94 (7.4\%) cancers. Of the $26 \mathrm{MSI}-\mathrm{H}, 6$ were BRAF V600E (23\%) and 1 of 8 MSI-L (12.5\%). Of five with BRAF V600E mutation, four (80\%) were expressing (by IHC) hMLHl while one didn't (20\%). All of BRAF V600E mutation except one was located in the right side of colon. In addition, of five of BRAF V600E mutation, four $(80 \%)$ were methylated for hMLHI while one was not $(20 \%)$. The MSI-H tumors were of three different tumor stages with 10 tumors at stage II, 13 tumors at stage III and one tumor at stage IV. Most tumors were moderately differentiated (19 of 28 MSI$\mathrm{H}$ and 6 of 8 MSI-L). Eight MSI-H and one MSI-L were poorly differentiated while only one MSI-H was well differentiated. Mucin production was noted in $13 \mathrm{MSI}-\mathrm{H}$ and $2 \mathrm{MSI}-$ L tumors. HMLHl promoter was methylated in 30 of 34 (88\%) tumors of which $13(87 \%)$ CRCs demonstrated MSI-H. In total, $56 \%$ and $42 \%$ of tumors assayed by IHC showed loss of staining for hMLH1 and hMSH2, respectively. In addition, loss of hMLH1 and hMSH2 staining was observed in $66 \%$ and $38 \%$ of MSI-H tumors, respectively. These findings suggest that BRAF activation may participate in the carcinogenesis of sporadic CRCs with hMLHl hypermethylation in the proximal colon, and it may be dependent on the MSI status. Conclusion: Overall, the prevalence of MSI-H colorectal tumors was 2 fold higher and the defect in the MMR genes (hMLH1 and hMSH2) and BRAF in AA patients may play important role in CRC 
W1739

Promoter Methylation of Helicase-Like Transcription Factor Is Associated with the Early Stages of Gastric Cancer with Family History

Jae J. Kim, Seung Won Chung, Jung Hwan Kim, Sung Kim, Sang Yong Song, Joobae Park, Duk-Hwan Kim

Background: The aberrant methylation of the $\mathrm{CpG}$ island at the promoter region of the helicase-like transcription factor (HLTF) gene has been detected in approximately 20-50\% of primary gastric cancers, suggesting that HLTF methylation is a frequently-encountered feature of tumorigenesis in the stomach. The aim of this study is to investigate the clinicopathological significance of promoter methylation of HLTF in primary gastric cancer. Methods: Two-hundred fifty six patients participated in this study. Methylation status of HLTF gene was evaluated in fresh-frozen tissues by methylation-specific polymerase chain reaction. All statistical analysis were two-sided, with a 5\% type I error rate. Results: Aberrant methylation of HLTF was found in 98 (38\%) of 256 gastric cancer patients. HLTF methylation was significantly associated with family history in the early stages of gastric cancer, regardless of histologic types. In the intestinal-type cases, HLTF methylation occurred in $15(56 \%)$ of 27 patients with family history, and in $26(31 \%)$ of 85 patients without family history ( $\mathrm{P}=$ 0.02). In the diffuse-type cases, patients with family history were also found to exhibit a higher prevalence of HLTF methylation than those without family history $61 \%$ vs. 34\%; $\mathrm{P}=0.009$ ). HLTF methylation in both of the histologic types occurred in about $70-90 \%$ of the early stage cases in which the patient had a family history and in 15-30\% of cases in which the patient did not have a family history. In the multivariate logistic regression analysis, the stage I, II cases with family history were determined to carry a higher risk of HLTF methylation than did the stage III, IV cases without family histories in both the intestinal-type $(\mathrm{OR}=6.01,95 \% \mathrm{CI}=1.20-30.01, \mathrm{P}=0.02)$ and the diffuse-type cancers $(\mathrm{OR}=8.25,95 \% \mathrm{CI}=1.67-40.86, \mathrm{P}=0.009)$. Conclusions: These results suggest that HLTF methylation may play a crucial role in the early stages of gastric carcinogenesis in patients with family history and may prove to be a valuable susceptible marker for the risk of gastric cancer in individuals with family history.

\section{W1740}

Genomwide Methylation of CPG Sites in Patients with Gastric Cancer and in Patients with Increased Risk to Develop Gastric Cancer

Andreas Leodolter, Sergio Alonso, Matthias Ebert, C Roecken, Thomas Wex, Ulrich Peitz, Peter Malfertheiner, Manuel Perucho

Hypomethylation of genome wide $\mathrm{CpG}$ sites is observed in tumor tissues from cancer patients. The Correa model of development of gastric cancer (GC) assumed a cascade of changes in gastric mucosa mainly caused by $H$. pylori infection. The aim of this study was to evaluate the methylation level of $\mathrm{CpG}$ sites in patients with $\mathrm{GC}$ and compare these levels with those of patients with advanced stages of gastritis before and after $H$. pylori eradication therapy, first degree relatives and controls. Methods: Gastric DNA was analyzed from patients with GC ( $\mathrm{n}=95$ adenocarcinomas, diffuse/intestinal type, tumor and non-tumor tissues), high risk gastritis (corpus-predominant/pangastritis w or w/o intestinal metaplasia $(n=14)$ with $H$. pylori infection, previous high risk gastritis after eradication therapy $(n=24)$, first degree relatives of gastric cancer patients $(n=16)$ and controls $(n=17)$ without H. pylori infection. In all patients a M. Sss enzyme assay for methylation of $\mathrm{CpG}$ sites with s-adenosylL-[methyl-3H]methionine as methyl donor was performed using 100ng of genomic DNA at a time. For standardization of DNA amount and to calculate the percentage of methylated CpG sites a dam enzyme assay (methylates A in GATC sites) was performed using equal test conditions. All experiments were repeated three times. Results: The mean value of methylation of $\mathrm{CpG}$ sites differed significantly in patients with GC compared to all other investigated groups. The non-tumor tissue revealed a significant lower amount of methylated CpG sites compared to tissue of patients without tumor. By establishing a cut-off for severe hypomethylation based on the results of the healthy controls, 39 cases (41\%) with gastric adenocarcinoma had a severe hypomethylation and even in 22 cases (23\%) this was detectable in the non-tumor tissue. In the groups with high risk gastritis 36\% had severe hypomethylation and $33 \%$ in the groups with previous $H$. pylori eradication therapy and high risk gastritis (ns). The portion of patients with severe hypomethylation in the group of lst degree relatives was lower (13\%). Conclusions: The decreased methylation level of non-tumor tissue in GC patients compared to healthy controls suggests an early occurrence of the tumor associated epigenetic alterations. Severe genomewide hypomethylation is already detectable in patients with high risk gastritis and might be not reversible by $H$. pylori eradication therapy. For parts of the epigenetic alterations the point of no return might be already reached for those patients with severe $H$. pylori associated gastritis

\section{W1741}

Epigenetic Regulation of the 11p15 Mucin Genes (MUC2, MUC5AC, MUC5B, MUC6) in Epithelial Cancer Cell Lines and Tissue Patients

Audrey Vincent, Jean-Luc Desseyn, Christophe Mariette, Marie-Christine Copin, Pasca Pigny, Isabelle van Seuningen

Introduction: The human mucin genes MUC2, MUC5AC, MUC5B and MUC6 are clustered on the p15.5 arm of chromosome 11 in a GC-rich area known to be a hot spot of methylation in cancer. These four genes encode large secreted gel-forming mucins that participate in protection of underlying epithelia. Alteration in their expression in cancers will have consequences for epithelium homeostasis and maintenance of an efficient barrier to various aggressions. Aim: The organization as a cluster in a hot spot of methylation and the structure of their promoters led us to evaluate the influence of DNA methylation and histone acetylation on their expression in epithelial cancer cell lines and tissue patients. Methods: Influence of methylation and histone acetylation was evaluated by treating pre- and post-confluent cells with the demethylating agent 5-aza-2'-deoxycytidine $(5 \mu \mathrm{M}, 72 \mathrm{~h})$ or with the histone deacetylase inhibitor, trichostatin A $(0.3 \mu \mathrm{M}, 24 \mathrm{~h})$, respectively. Level of mucin mRNA and protein expression were measured by RT-PCR and immunochemistry, respectively. Mapping of the methylated cytosines in the promoters was performed by MS-PCR and bisulfite-treated
DNA sequencing both in cell lines and tissue patients. Results: MUC2 and MUC5B are highly submitted to epigenetic regulation in epithelial cancers cells and their level of methylation is directly correlated to the state of differentiation of the cells. The promoter of MUC2 is characterized by site-specific methylation of a few CpG sites whereas MUC5B is silenced by extensive hypermethylation throughout its promoter. Compared to MUC2 and MUC5B the promoter of MUC5AC is not very rich in $\mathrm{CpG}$ sites and was shown to be rarely regulated by methylation. We showed that the MUC6 promoter possesses a highly methylated dista region that does not interfere with its expression. Moreover, the proximal $\mathrm{CpG}$ island located upstream of the transcription start site was not submitted to methylation. Correlation between the methylation of mucin gene promoters and repression of their expression in bronchial tumors from patients with lung cancers confirmed our in vitro data. Conclusion: This firs wide study about the influence of methylation and acetylation on the expression of the four mucin genes belonging to the $11 \mathrm{p} 15$ cluster points out an important role for epigenetics in regulating the $11 \mathrm{p} 15$ mucin genes in epithelial cancers. Evaluation of methylation status of mucin genes may thus become a promising tool as a diagnostic and/or prognostic marker in epithelial cancers.

\section{W1742}

Microsatellite Instability Is Associated with Reduced Proliferative Indices in Human Colorectal Cancers

Frank A. Sinicrope, Rafaela L. Rego, Kevin C. Halling, Nathan Foster, Daniel J. Sargent, Amy French, Megan Garrity, Richard M. Goldberg, John A. Laurie, Lawrence J. Burgart, Stephen N. Thibodeau, Thomas E. Witzig

BACKGROUND. Colorectal cancers with microsatellite instability (MSI) are reported to have a favorable clinical outcome, yet prognostic factors underlying this observation are poorly understood. We studied differences in biomarkers between MSI-H and MSS/MSI-L tumors including DNA ploidy and proliferative indices (PI), chromosome 17p allelic imbalance (AI) p53 expression, and patient survival. MATERIALS/METHODS. We examined Dukes' stage $B 2$ and $C$ primary colon $(n=305)$ and rectal $(n=25)$ carcinomas from patients enrolled in five 5 -fluorouracil-based adjuvant trials conducted by the North Central Cancer Treatment Group. MSI and AI were determined using 11 microsatellite markers. hMLH1, hMSH2 and p53 proteins were evaluated by immunostaining. DNA ploidy and PI $\left(S\right.$ phase $\left.+G_{2} M\right)$ were analyzed by flow cytometry. Correlations between markers and associations with overall survival (OS) were determined. RESULTS. MSI-H ( $\mathrm{n}=61)$ vs. MSS/MSI-L tumors were more frequently Dukes' B than C ( $\mathrm{p}=0.0008)$ and had fewer metastatic lymph nodes $(\mathrm{p}=0.005)$. MSI-H tumors were more frequent in female patients (vs. MSS/MSI-L; $p=0.0003$ ), and were highly correlated with proximal site, high grade histology, diploidy and reduced p53 expression (vs. MSS/MSI-L; all p<0.0001). Median overall PI (15.8; mean 16.2 \pm 7.6) was lower in MSI-H compared to MSS/MSI-L tumors (11.7 versus $16.8, \mathrm{p}=0.001)$. Lower PI was associated with diploid DNA content, reduced p53 expression, and intact 17p (Table 1). Of 44 MSI-H cases tested, 41 showed loss of hMLHl $(n=39)$ or hMSH2 $(n=2)$ proteins Overall survival was related to tumor stage $(\mathrm{p}=0.002)$, DNA ploidy $(\mathrm{p}=0.03)$, histologic grade $(\mathrm{p}=0.0003)$, loss of mismatch repair proteins $(\mathrm{p}=0.02)$, and number of lymph nodes $(p<0.0001)$. MSI status ( $p=0.07)$, and PI $(p=0.07)$, were of borderline significance whereas $17 \mathrm{p} \mathrm{AI}$ and $\mathrm{p} 53$ were not related to patient survival. CONCLUSIONS. Reduced proliferative indices are a component of the MSI-H phenotype in human colorectal cancers. Lower proliferative indices, indicative of reduced tumor growth rates, may contribute to the earlier stage at presentation of MSI-H tumors.

\begin{tabular}{|c|c|c|c|}
\hline & $\mathrm{PI} \leq 15^{1}$ & $\mathrm{PI}>15^{1}$ & P-value ${ }^{2}$ \\
\hline $\begin{array}{l}\text { MSI } \\
\text { MSI-H } \\
\text { MSSIMSI-L }\end{array}$ & $\begin{array}{l}31 \\
72\end{array}$ & $\begin{array}{c}13 \\
104\end{array}$ & 0.0004 \\
\hline $\begin{array}{l}\text { p53 } \\
\text { Negative } \\
\text { Positive }\end{array}$ & $\begin{array}{l}52 \\
50\end{array}$ & $\begin{array}{l}43 \\
74\end{array}$ & 0.03 \\
\hline $\begin{array}{l}17 \mathrm{p} \\
\text { No Allelic Imbalance } \\
\text { Allelic Imbalance }\end{array}$ & $\begin{array}{c}9 \\
12\end{array}$ & $\begin{array}{c}6 \\
25\end{array}$ & 0.07 \\
\hline $\begin{array}{l}\text { DNA Ploidy } \\
\text { Ane uploid/Te traploid } \\
\text { Diploid }\end{array}$ & $\begin{array}{l}20 \\
83\end{array}$ & $\begin{array}{c}109 \\
8\end{array}$ & $<0.0001$ \\
\hline $\begin{array}{l}\text { Histologic Grade } \\
1,2 \\
3,4\end{array}$ & $\begin{array}{l}65 \\
38\end{array}$ & $\begin{array}{l}82 \\
35\end{array}$ & 0.27 \\
\hline $\begin{array}{l}\text { Number of Nodes } \\
0 \\
1-3 \\
>3\end{array}$ & $\begin{array}{l}18 \\
54 \\
27\end{array}$ & $\begin{array}{l}23 \\
58 \\
30\end{array}$ & 0.89 \\
\hline
\end{tabular}

W1743

Molecular Subtyping of Colorectal Cancers with Microsatellite Instability Luigi Laghi, Paolo Bianchi, Massimo Roncalli, Alberto Malesci

Background. Colorectal cancers (CRC) with mismatch repair (MMR) defects and microsatellite instability (MSI) are heterogeneous as to hereditary (HNPCC) or sporadic features and promoter methylation. Aim. To find correlations between molecular and phenotypic features of MSI CRC. Materials and methods. In a cohort of 88 (M/F, 50/38) MSI CRC staged by AJCC criteria (stage $1-2 / 3-4,59 / 29)$, comprising HNPCC $(n=25)$ and sporadic $(n=63)$ cases, we determined MMR defects, hMLH1 and P16 methylation, BRAF mutations and frameshift mutations in target genes (FMT). Results. Out of 88 MSI CRC, 59 (67\%) had methylation of hMLH1 (M/F, 28/31; p=0.01), and $45(51 \%)$ of P16 (M/F, 29/16). hMLH1 methylation only occurred in $21(24 \%)$ CRC $(\mathrm{M} / \mathrm{F}, 6 / 15 ; \mathrm{p}=0.003)$ and of $\mathrm{P} 16$ only in $7(8 \% ; \mathrm{M} / \mathrm{F}, 7 /$ $0 ; \mathrm{p}=0.01$ ). Patients with $h M L H 1$-methylated CRC were older than those with un-methylated 
ones (70.6 \pm 12.1 vs $60.1 \pm 15.7 \mathrm{yr}$, respectively; $\mathrm{p}=0.001)$, a difference not observed for P16. The age of patients with hMLH1-methylated CRC did not differ as to gender, but as to BRAF status (mutated, $\mathrm{n}=33,76.1 \pm 7.1$ yr vs wild-type $63.5 \pm 13.5 \mathrm{yr} ; \mathrm{p}<0.001$ ). In MSI CRC, the median FMT was 4 , the only 5 MSI CRC with no frameshifts lacked hMSH6 or PMS2. hMLH1-methylated MSI CRC had higher FMT (median=4) than un-methylated (median=2, $\mathrm{p}=0.03)$ ones. Stage 3-4 CRC were more frequent in males than females $(\mathrm{M} / \mathrm{F} 21 / 8$; $\mathrm{p}=$ $0.03)$, in proximal than distal tumors $(27 / 69$, vs $2 / 19 ; \mathrm{p}=0.02)$, in $P 16$-methylated than unmethylated CRC $(21 / 45$, vs $8 / 43 ; \mathrm{p}=0.005)$ and in MSI CRC with $\mathrm{FMT} \leq 3$ than in $\mathrm{CRC}$ with FMT $\geq 4(\mathrm{p}=0.037)$. P16 status was associated with advanced stages even in sporadic CRC (4/26 in un-methylated vs 19/37 in methylated CRC; $p=0.003$ ). Upon multivariate analysis, proximal site, FMT $\leq 3$ and P16 methylation were independently associated with advanced tumor stage in all cases, but only P16 in sporadic disease $(\mathrm{p}=0.016$; OR 3.8 , 95\%CL 1.4-10). In females we observed 7 stage 3-4 cancers out of 16 with P16 and hMLH1 methylation, but only $1(7 \%)$ in 15 with $h M L H 1$ methylation only $(\mathrm{p}=0.03)$. Conclusions. hMLH1-methylated MSI CRC are frequent in females and in old patients, P16 is more frequently methylated in stage 3-4 than in stage 1-2 MSI CRC. The combination of female gender and $h M L H 1$-only methylation can identify a sub-set of patients with a profuse mutator phenotype and less advanced MSI CRC. In contrast, P16 methylation is associated with advanced disease, and tumors with MMR defects other than hMLH1 and hMSH2 do not require alterations of the mutator pathway.

\section{W1744}

Microsatellite Instability Accounts for Tumor Site-Related Differences in Prognostic Markers in Human Colon Cancers

Frank A. Sinicrope, Rafaela L. Rego, Nathan Foster, Daniel J. Sargent, Amy French, Megan Garrity, Richard M. Goldberg, John A. Laurie, Lawrence J. Burgart, Thomas E. Witzig, Stephen N. Thibodeau

BACKGROUND. Evidence indicates that proximal and distal sporadic colon cancers have a different pathogenesis and prognosis. We compared cellular and molecular marker expression in relation to tumor site, microsatellite instability (MSI), DNA mismatch repair proteins, DNA ploidy and p53 expression. MATERIALS \& METHODS. Dukes' stage B2 $(n=158)$ and $C(n=370)$ primary colon adenocarcinomas were analyzed from patients enrolled in six 5 fluorouracil-based adjuvant therapy trials conducted by the North Central Cancer Treatment Group. Tumors were analyzed for MSI using 11 microsatellite markers and MSI was classified as instability at $>$ or equal $30 \%$ (MSI-H) or $<30 \%$ of loci (MSS/MSI-L). Immunostaining for hMLH1 / hMSH2 ( $\mathrm{n}=329)$, and $\mathrm{p} 53$ proteins was also performed. DNA ploidy was analyzed by flow cytometry and categorized as diploid versus aneuploid/tetraploid. RESULTS. Patients with proximal cancers were older ( $\geq 65$ years) and proximal tumors were more likely to be MSI-H, diploid, and to have high histologic grade compared to distal tumors (Table 1). Nuclear p53 staining was more frequent in distal tumors $(p=0.001)$ that were generally aneuploid/tetraploid $(\mathrm{p}=0.008)$. Patients with MSI-H tumors $(n=95)$ were more likely to be female (vs. MSS/MSI-L; $\mathrm{p}=0.0002$ ), and were also more frequently proximal, diploid, and more likely to have a high grade histology (vs MSS/MSI-L; all $\mathrm{p}<0.0001$ ). When MSI$\mathrm{H}$ tumors were excluded, no differences were found for any of the variables between proximal versus distal MSS/MSI-L tumors, except for p53 expression which remained significant $(\mathrm{p}=0.039)$. CONCLUSIONS. MSI-H tumors account for differences in prognostic markers between proximal and distal colon cancers, and to a lesser extent for p53. Therefore, previously described differences in the pathogenesis and prognosis of colon cancers based upon tumor site appear to be predominantly explained by the inclusion of MSI-H cases.

\begin{tabular}{|c|c|c|c|}
\hline Variable & Proximal & Distal & P-value* \\
\hline Age & & & \\
\hline$<65$ & 117 & 121 & 0.0375 \\
\hline$>65$ & 152 & 108 & \\
\hline DNA Ploidy & & & \\
\hline $\begin{array}{l}\text { Aneuploid/Tetr aploid } \\
\text { Diploid }\end{array}$ & $\begin{array}{l}141 \\
128\end{array}$ & $\begin{array}{c}147 \\
82\end{array}$ & 0.0080 \\
\hline $\mathrm{p} 53$ & & & \\
\hline Positive & 86 & 82 & 0.0012 \\
\hline $\begin{array}{l}\text { Negative } \\
\text { MSI }\end{array}$ & & & \\
\hline $\begin{array}{l}\text { High } \\
\text { Stable/Low }\end{array}$ & $\begin{array}{c}81 \\
188\end{array}$ & $\begin{array}{c}7 \\
222\end{array}$ & $<0.0001$ \\
\hline $\begin{array}{l}\text { Histologic Grade } \\
1,2\end{array}$ & 178 & 181 & 0.0014 \\
\hline 3,4 & 91 & 48 & \\
\hline
\end{tabular}

\section{W1745}

Posttraumatic Sclerosing Cholangitis (PTSC): A Novel Biliary Disease Dorothee H. Dorlars, Jenny Gebhardt, Juergen Pausch

Background: To date, we have increasingly observed a new entity of severe progressive cholestasis syndromes mimicking post-traumatic sclerosing cholangitis (PTSC). These patients usually have healthy livers prior to trauma or ICU treatment. PTSC is different from non-alcoholic fatty liver disease following total parenteral nutrition and can, however, rapidly progress to the point of requiring transplantation. Patients and methods: From 5/2001 to 11/2005, we observed 13 patients presenting with post-traumatic cholestasis syndrome. All were men, aged 21 to 66 yrs (mean 48). All had required long-term mechanical ventilation (10- 74 days, $\mathrm{n}=8$ for more than 4 weeks) and a longer stay at the ICU (13-164 days, mean 48). 2 patients had a 2 nd degree craniocerebral injury. 11 patients suffered polytrauma: one had multiple fractures followed by ARDS, 10 cases suffered fractures and a primary thoracic trauma (lung contusion, hematopneumothorax), 4 of them in combination with a craniocerebral injury. We analyzed common clinical findings, risk factors and the natural history of PTSC. Results: Upon admission to hospital, the liver function tests (LFTs) were normal in all patients. Overall, the cholestasis parameters in $12 / 13$ patients increased between day 4 and 10 of their ICU stay. The AP values peaked at 695 - $5073 \mathrm{U} / \mathrm{l}$ (mean 3193), the GGT values at $360-2509 \mathrm{U} / \mathrm{h}$ (mean 1181), bilirubin values at $0.8-28 \mathrm{mg} \%$ (mean 10). AST and ALT were only slightly elevated. The ERC showed the typical signs of sclerosing cholangitis or vanishing bile duct syndrome. 6 of 13 patients were treated endoscopically (removal of sludge etc.). The histological evaluations showed sclerosing cholangitis and varying degrees of liver fibrosis and inflammation. Follow-up: All patients are being treated with UDCA $20-25 \mathrm{mg} / \mathrm{kg}$. To date, 1 patient has received a liver transplant, and 2 patients are currently listed for transplant. In 6 patients the disease is progressively worsening 3 of these are ineligible for transplant. Finally, 4 patients are clinically and histologically stable. Conclusions: PTSC is a not entirely rare, but usually unrecognized disease with a poor prognosis. Risk factors are male gender, polytrauma with thoracic trauma, craniocerebral injury, extended ventilation and (acc. to literature) burns. Laboratory findings include early elevated AP more than GGT. The ERC findings also suggest an etiological role for ITBL (ischemic-type biliary lesions). Recommended modes of treatment include regular endoscopic interventions, high-dose UDCA therapy and early assessment for liver transplant. Future research must aim to identify high risk patients and possible prophylactic treatments.

\section{W1746}

Low Dietary Cholic Acid Administration to ABCB4-/- Mice Is Sufficient to Unravel Critical Phenotypic Features Mimicking the Severe Consequences of $A B C B 4$ Mutations in Humans

Majorie Martel, Veronique St-Hilaire, Beatriz Tuchweber, Marie-Eve Bourbonnais, Guylaine Bouchard

Mutations in the canalicular phospholipid translocator $\mathrm{ABCB} 4$ contribute to a large array of liver diseases. In humans, the most severe complications of ABCB4 genetic variants result in progressive familial intrahepatic cholestasis type 3 (PFIC-3), with affected children often exhibiting growth failure and progression of liver disease necessitating transplantation. Albeit Abcb4-/- mice develop spontaneous cholangiopathy and hepatic liver disease, they do not suffer from growth failure or highly premature death rate. In search of a more valuable experimental model that could mimic these severe complications, we here tested the response of $A b c b 4-/$ - mice to long-term low cholic acid (CA) feeding. Methodology: Post-weaning Abcb4-/- mice and wild-type controls (FVB/J) were fed a semi-synthetic diet supplemented or not with $0.025 \% \mathrm{w} / \mathrm{w}$ CA. Body weight was followed weekly for the first month and then monthly. A small saphene vein puncture enabled to follow serum liver enzymes monthly. Mice were sacrificed after 4 months of treatment and liver histology (HPS, Masson Trichrome) was performed and scored in a blind fashion. Results: Body weight gain in Abcb4-/- and +/ + mice not fed CA was undistinguishable despite a specific and progressive elevation in serum liver enzymes in -/- mice. At sacrifice, -/- mice exhibited significant ductular proliferation, moderate portal inflammation and fibrosis, with a clear progression of all these features compared to $-/$ - mice sacrificed at the post-weaning stage. In contrast to +/+ mice where no significant influence was observed on all parameters assessed, addition of low dietary CA to -/- mice markedly changed the severity of the phenotype as exposed by lack of body weight gain as well as more rapid and sustained elevations of serum ALT, AST, ALP, GGT, bilirubin and total bile salts. After 3-4 months of CA treatment, Abcb4-/- mice started to exhibit severe signs of physical distress, culminating in a $40 \%$ death rate. In the surviving $-/$ mice, gross appearance of the liver was drastically changed and severe bridging portal fibrosis and ductular proliferation were now observed, with portal inflammation level however remaining similar to -/- mice not receiving CA. Conclusion/Speculation: Although Abcb4-/mice spontaneously develop cholangiopathy, they do not fully express the severe phenotypic consequences of $\mathrm{ABCB} 4$ defects observed in human. However, a pathologic profile mimicking severe cases of human $\mathrm{ABCB} 4$ deficiency can be recreated by very low supplementation with CA. Further studies using this strategy shall now enable a better comprehension of the severe mechanistic consequences of $\mathrm{ABCB} 4$ malfunction.

\section{W1747}

Hepatic Aquaporin-8 (AQP8) Water Channels and Oxidative Stress Are Early Affected During Extrahepatic Cholestasis in Bile Duct Ligated (BDL) Rats Piero Portincasa, Ignazio Grattagliano, Mario Testini, Nicola Marinelli, Michele Persichella, Antonio Moschetta, Giuseppe Palasciano, Maria Svelto, Giuseppe Calamita

Background: Interruption of bile flow during extrahepatic cholestasis may result in liver failure. Here we investigate for the first time if both AQP8-mediated osmotic water movement and oxidative stress might be early affected in an animal model of cholestasis. Materials and methods: Wistar rats underwent proximal BDL or sham operation; at days 1,3 , and 7 we measured liver histology, AQP8 (expression, subcellular localization), oxidative stress as total, oxidized glutathione (GSH, GSSG), and protein sulphydril (PSH, cytosolic/mitochondrial fractions). Results: BDL livers showed progressive intrahepatic bile duct dilatation, bile aggregates, inflammatory changes, cell degeneration, mitochondrial swelling. At day 3 , intracellular but not plasma membrane AQP8 was decreased by $75 \%(\mathrm{P}<0.001)$. At day 7 , AQP8 was decreased both intracellularly $(-67 \%)$ and in plasma membranes $(-56 \%)(\mathrm{P}<$ $0.05)$ suggesting defective translocation or membrane insertion of AQP8 Cytosolic GSH and PSH gradually decreased while GSSG increased ( $\mathrm{P}<0.01$, ANOVA) after BDL. Specifically, GSH and P-SH levels declined and GSSG increased earlier in mitochondria than cytosol. Conclusions: Water transport is greatly affected by BDL; here we observed an early downregulation of hepatocyte AQP8 protein expression which could contribute to bile secretory dysfunction of cholestatic hepatocytes. Also, early cholestatic liver shows associated remarkable changes of GSH content and redox status, especially in hepatocyte mitochondria.

\section{W1748}

Enhanced Expression of Caveolin-2 of the Proliferating Bile Ductules At Protein and Gene Levels in Primary Biliary Cirrhosis

Hiroaki Yokomori, Masaya Oda, Toshifumi Hibi

BACKGROUND AND AIMS: An increase in bile ductular structures is observed in diverse human liver diseases, especially in primary biliary cirrhosis (PBC). These structures harbor the progenitor cell compartment of the liver. Caveolins (CAV) are cholesterol-binding proteins involved in regulating of several intracellular process, including cholesterol transport. In this study, immunohistochemistry, Western blotting and in situ hybridization were performed 
on human liver specimens from patients with primary biliary cirrhosis (PBC) and normal liver specimens. MATERIALS AND METHODS: Ten wedged liver biopsies of PBC (10 cases each of stage 2 and stage 3) were studied. As controls, wedge biopsy specimens from normal portions of the liver were obtained from 10 patients ( 8 males and 2 female; aged from 44 to 73 years with a mean of 57.3 years) who underwent surgical resection for metastatic liver carcinoma (4 colonic carcinoma and 1 gastric carcinoma). Immunostaining was performed on serial sections. Four-micrometer sections were cut from paraffin blocks of formalin-fixed tissue, deparaffinized with xylene, and rehydrated by graded ethanol The expression of CAV-1 and -2 was determined using specific antibodies. Western blotting was conducted using fresh control and PBC liver tissues. In situ hybridization was done to examine the messenger RNA expression of CAV-1 and -2 on formalin-fixed, paraffin-embedded sections using peptide nucleic acid probes and the catalyzed signal amplification technique. RESULTS: In normal liver samples, there was scarce staining for CAV-1 and 2 on bile ducts. In PBC liver samples, CAV-2 expression was strongly increased on proliferative bile ductules, especially in stage 2-3 specimens. Especially, regenerating bile ductules at the interface of portal tracts and necrotic areas stained intensely for CAV 2. While CAV-2 was stained intensely in the cytoplasm, CAV-1 was stained mainly on the plasma membrane. CAV-1 and 2 protein expression was confirmed by Western blot. In situ hybridization demonstrated CAV-1 and 2 mRNA expression in bile ductules. CAV-2 mRNA was especially strongly expressed on infiltrating bile ductules. CONCLUSION: The present results demonstrate increased expression of caveolin-2, and to a less extent caveolin-1, in proliferating bile ductules in primary biliary cirrhosis, which may be related to the homeostasis of cholesterol transport in the regenerating bile ductules in $\mathrm{PBC}$ liver.

\section{W1749}

Is Helicobacter Pylori Associated with Primary Sclerosing Cholangitis? Alyssa M. Krasinskas, Yuan Yao, Parmjeet Randhawa, Antonia R. Sepulveda

Helicobacter pylori (Hp) DNA has been identified in human livers and has been implicated in chronic liver disease and liver cancer. Hp DNA can be isolated from the blood of patients infected with the organism; hence it is not clear whether Hp DNA detection in the liver represents a "contaminant" from the circulation in hepatic blood vessels or in Kupffer cells, or whether bacteria or bacterial products reach the biliary epithelium through bile reflux from the duodenum and cause a chronic inflammatory response similar to that seen in gastritis. Data from different populations and different countries are conflicting. To better understand the potential role of $\mathrm{Hp}$ in primary sclerosing cholangitis (PSC), an idiopathic inflammatory disease with unknown etiology, Hp infection status was studied in a group of patients with PSC from the US. Methods: Explanted livers from 25 patients with endstage PSC and 33 age-matched controls with nonbiliary tract disease were studied. Hilar hepatic ducts and limited adjacent liver parenchyma was microdissected from formalinfixed, paraffin-embedded tissue sections. Genomic DNA was extracted and a 398 bp fragment was amplified with primers for the Hp $16 \mathrm{~S}$ rDNA. The PCR products were resolved by gel electrophoresis. Serum was available on 14 patients and was tested for Hp anti-IgG by ELISA. Immunohistochemical (IHC) staining for $\mathrm{Hp}$ was performed on all specimens in step sections of the slides used for microdissection. Results: Hp was detected in 9 of the 58 (16\%) patients in this study; Hp was detected by PCR in 8 cases and by ELISA in 2 cases ( 1 case was also PCR positive); 6 of the 25 (24\%) patients with PSC and 3 of the 33 (9\%) controls (nonalcoholic steatohepatitis, $\mathrm{n}=2$ and acetaminophen toxicity, $\mathrm{n}=1$ ) were positive for Hp ( $\mathrm{p}=0.15)$. No Hp organisms were identified in the livers by IHC. Conclusion: Hp was detected in $16 \%$ of explanted livers from patients with biliary and nonbiliary liver disease. A higher percentage of patients with PSC had evidence of Hp infection (24\%) compared to the controls (9\%). In all but one Hp-positive case, Hp was detected in microdissected hilar biliary epithelium, supporting the hypothesis that the organisms or bacterial products might reach the biliary tract through bile reflux from the duodenum, in addition to circulating through the blood. Our findings support a possible contributory role of $\mathrm{Hp}$ in some PSC patients.

\section{W1750}

\section{Response to Steroid Therapy in Patients with Sclerosing Cholangitis} Associated with Elevated IgG4 Level

Ryo Terada, Hirofumi Kawamoto, Yasuhiro Miyake, Kohsaku Sakaguchi, Yoshiaki Iwasaki, Akinobu Takaki, Haruhiko Kobashi, Yoshiaki Kobayashi, Shin-Ichiro Nakamura, Kazuhide Yamamoto, Yasushi Shirator

Background and Aims: Multiple fibrosing inflammatory strictures of the biliary tree can be caused by both primary and secondary screlosing cholangitis. Recently, it is reported that bile duct lesions were associated with autoimmune pancreatitis or immunoglobulin(Ig) G4related cholangitis. We investigated the clinical course of patients who demonstrated bile duct lesions resembling primary sclerosing cholangitis(PSC) by cholangiography and evaluated the effect of steroid administration. Patients and Method: The subjects were 33 patients (21 male, 12 female) who demonstrated PSC-like bile duct stricture by endoscopic retrograde cholangiography or magnetic resonance cholangiopancreatography at our university or its affiliated hospitals between December 1988 and June 2005. The median age at the diagnosis was 47 years (range 14-70) and the median observation period was 37 months (range 4163). Results: Out or 33 cases, 28 patients had PSC, one patient had cholangiocellular carcinoma, 3 patients had autoimmune pancreatitis with biliary duct lesions and one patient had sclerosing cholangitis associated with marked eosinophoilia (WBC 20450/mm3, Eo $70 \%$ ). Since this patient with eosinophilia had no apparent pancreatic lesions and elevated serum IgG4 level (218 mg/dl, normal range 4.8-105), he was considered as IgG4-related cholangitis. Five of 28 PSC patients were treated with steroid therapy. However, no patients showed the improvement of the bile duct lesions. On the other hand, three patients with autoimmune pancreatitis and one patient with IgG4-related cholangitis were responded to steroid therapy and showed prompt amelioration of liver-function tests and improvement of bile duct lesions. The serum IgG4 level of these four patients were relatively high (Mean \pm S.D. $300 \pm 224 \mathrm{mg} / \mathrm{dl}$ ), as compared with $38.3 \pm 25.1 \mathrm{mg} / \mathrm{dl}$ in patients with PSC and cholangiocellular carcinoma. Conclusion: Patients with autoimmune pancreatitis and IgG4related cholantitis need to be distinguished from PSC, as they can be treated with steroid therapy. The serum IgG4 concentrations and eosinophilia may be a useful marker for diagnosis of these "steroid-responsive" sclerosing cholangitis.

\section{W1751}

The Incidence of Cholangiocarcinoma in Primary Sclerosing Cholangitis During Longtime Treatment with Ursodeoxycholic Acid Adolf Stiehl, Petra Kloeters-Plachky, Gerda Rudolph

Introduction. Cholangiocarcinoma (CCA) represents a serious complication of primary sclerosing cholangitis (PSC). In two recent studies with 604 and 161 patients followed over a median time of 5.7 and 11.5 years the reported incidence rate of CCA was 13.3 and 6.8 \% (A Bergquist et al., J Hepatol 2002;36:321-327; K Burak et al., Am J Hepatol 2004:99:523526). Ursodeoxycholic acid (UDCA) possibly may influnce the incidence of CCA in man (B Brandsaeter et al. J Hepatol 2004:40:815-822). Aim of study. The aim of the present study was to evaluate the incidence rate of CCA in PSC patients treated with ursodeoxycholic acid. Patients and methods. The present study was started in May 1987 and data up to May 2005 were considered in the evaluation. A total of 150 patients (105 men and 45 women) with PSC but without evidence of CCA at entry were included in the study. Of the patients included 101 had inflammatory bowel disease (93 ulcerative colitis and 6 Crohn's disease) Altogether 120 patients were treated for 1 year, 107 for 2 years, 97 for 3 years, 83 for 4 years, 72 for 5 years, 62 for 6 years and 57 for $7-18$ years. Up to 1995 the patients were treated with UDCA in a dose of $750 \mathrm{mg} / \mathrm{d}(8.8-15.3 \mathrm{mg} / \mathrm{kg})$, starting from 1997 with 13.8 $17.4 \mathrm{mg} / \mathrm{kg}$ and starting from 2001 with $18-20 \mathrm{mg} / \mathrm{kg}$. A minority of 63 patients who were included in a study of UDCA absorption intermittently received higher doses of up to 30 $\mathrm{mg} / \mathrm{kg}$ for up to 3 months. All 72 patients with dominant stenoses were treated by repeated endoscopic dilatation. Diagnosis of CCA was established in each case by histologic examination of tissue obtained at laparotomy or in the explanted liver at the time of liver transplantation. Results. The median treatment time of the 150 patients was 6.4 years. Altogether 5 patients developed a CCA during UDCA treatment yielding an incidence rate of $3.3 \%$. The calculation of CCA per patient years revealed 0.58 CCA per 100 patient years in years 0 2.5, 0.49 CCA in years 2.5-8.5, and no CCA thereafter up to 18 years after entry into the study. The Kaplan Meier estimate of CCA incidence during UDCA treatment reached a plateau after 8.3 years. All 5 patients with CCA belonged to the subgroup of patients with colitis and in addition to the subgroup of patients with dominant stenosis. Summary and conclusion. The annual incidence rate of CCA in PSC treated with UDCA is low and decreases with time of treatment. Compared with the CCA incidence rates reported in studies on the natural course of the disease, the CCA incidence after UDCA treatment was lowe than expected.

\section{W1752}

Does Maternal Immunization Using Rotavirus Virus-Like Particles Prevent Rotavirus-Associated Biliary Atresia in Neonatal Mouse Pups? Paula M. Hertel, Sue E. Crawford, Finegold J. Milton, Estes K. Mary

BACKGROUND: Biliary atresia (BA) is a progressive, irreversible obliterating disease of bile ducts that results in severe childhood liver disease and is the indication for more than $50 \%$ of pediatric liver transplants. Rotavirus infection in the very early neonatal period is suspected to be the cause of some cases of BA. We hypothesized that: a) inoculation of neonatal mice with live rotavirus will cause biliary atresia, but inoculation with inactivated virus will not; and b) maternal immunization using recombinant VP 2/6/7 rotavirus virus-like particles (VLPs), which contain immunogenic rotavirus capsid proteins but are noninfectious, will prevent rotavirus-associated biliary atresia in pups. METHODS: To assess if live virus is required to cause biliary atresia, Balb/c mouse pups were inoculated intraperitoneally (ip) with 4x107 pfu live, purified rhesus rotavirus (RRV), with comparable doses of inactivated RRV, or with saline on day of life 2. Stool color was assessed daily using a graded color scale. Cholestasis (blockage of bile flow into the small intestine) was defined as pale stools Mice were euthanized at 1,2 , or 3 weeks post-inoculation (pi). To assess maternal immunization as a preventive measure against BA, serum rotavirus antibody titers were determined in Balb/c dams with varying rotavirus exposure histories. Dams were then given intramuscular injections with either 20ug rotavirus VLPs plus adjuvant or adjuvant alone before mating When litters were born, maternal serum antibody titers were repeated. Pups were inoculated with 107 pfu live RRV ip on the 2nd day of life, and assessed 5-11 dpi for cholestasis. Al were euthanized at 11 dpi. RESULTS: $0 \%$ of pups inoculated with saline or with inactivated virus developed cholestasis, whereas $100 \%$ of live-RRV-infected pups born to unexposed, unimmunized mothers developed cholestasis. Pups born to previously unexposed dams immunized with rotavirus VLPs + adjuvant did not develop cholestasis following rotaviru infection, whereas pups born to dams immunized only with adjuvant developed cholestasis. Pups born to dams with high rotavirus antibody titers prior to immunization were protected whether dams were immunized with VLPs + adjuvant or with adjuvant alone. CONCLUSIONS: Infection of neonates with live virus is required to cause biliary atresia; inactivated virus is insufficient. High maternal rotavirus antibody titers can protect offspring against biliary atresia following rotavirus infection in the neonatal period. Maternal immunization of previously nonimmune mothers confers protection to offspring against biliary atresia following neonatal rotavirus infection.

\section{W1753}

Paclitaxel Inhibits LPS-Induced Collagen Formation By Rat Bile Duct Fibroblasts By Preventing PDGF-BB Synthesis in Rat Cholangiocytes Jong Ho Moon, Christopher E. Savard, Ho Soon Choi, Tae Hyun Kim, Igino Rigato, Rahul Kuver, Sum Lee

Background/Aim: The pathogenesis of proliferative cholangitis in chronic biliary disease is unknown. Platelet-derived growth factor (PDGF)-BB is an important signal in the process of hepatic fibrosis. Paclitaxel (Taxol) is a demonstrated anti-fibrotic agent. The present study investigated the role of PDGF-BB on biliary fibrosis and to examine the effect of paclitaxe 
on this process. Methods: Rat cholangiocytes and bile duct fibroblasts were isolated and cultured. Cells in serum-free medium were treated with E. coli lipopolysaccharide (LPS), with or without addition of paclitaxel (1nM) one hour earlier. Collagen content in cells and medium was assessed by colorimetric assay and gel electrophoresis. mRNA of PDGF-BB, and TGF- $\beta 1$ was measured by relative quantitative real-time PCR. Results: Fibroblasts treated with LPS increased collagen in cells by $76.6 \%$ and in medium by $41.1 \%$ compared to controls; paclitaxel reversed this process. mRNA of PDGF-BB was expressed by cholangiocytes and fibroblasts, but its receptor (PDGFR- $\beta$ ) mRNA was only expressed by fibroblasts. LPS increased mRNA of PDGF-BB levels 20X in cholangiocytes and fibroblasts. In cholangiocytes, but not in fibroblasts, this response was almost completely suppressed by paclitaxel. mRNA of TGF- $\beta 1$ was not increased significantly with LPS. Conclusions: Our data suggest that PDGF-BB is involved in biliary fibrosis. Binding of PDGF-BB to PDGFR- $\beta$ on subepithelial fibroblasts may cause collagen deposition leading to fibrosis. Inhibition by paclitaxel of PDGF-BB formation by cholangiocytes might provide a new approach to limiting collagen synthesis and fibrosis in the biliary tract.

\section{W1754}

Usefulness of Contrast-Enhanced Ultrasonography in Differential Diagnosis of Gallbladder Diseases

Kazuo Inui, Junji Yoshino, Hironao Miyoshi, Masashi Hattori, Kazumu Okushima, Yuta Nakamura, Toshihiko Chikaishi

We investigated usefulness of contrast-enhanced ultrasonography for differential diagnosis of gallbladder diseases in 71 patients, comparing contrast-enhancement patterns with pathologic findings. Surgical pathologic diagnosis $(n=30)$ or clinical diagnosis $(n=41)$ patients included gallbladder carcinoma in 27 , adenoma in 2 , benign polyp in 33 , and adenomyomatosis in 9. We used two ultrasonographic instruments developed by TOSHIBA (Tokyo, Japan, SSA$370 \mathrm{~A}$ and SSA-770A). A galactose-based contrast agent (Levovist, Nippon Schering, Japan) was resolbilized to represent a dose of $300 \mathrm{mg} / \mathrm{ml}$. Seven milliliters of solution was injected intravenously as a bolus at a rate of $1 \mathrm{ml} / \mathrm{sec}$. We monitored vascular flow with Advanced Dynamic Flow for $60 \mathrm{sec}$, constructing a time/intensity curve (TIC) with flash-echo imaging (FEI) after administration of an additional $7 \mathrm{ml}$ of Levovist. We investigated the diagnostic capability for gallbladder diseases of contrast-enhanced patterns and TIC. We also compared number of vessels, degree of inflammation, and vessel diameter determined by contrastenhancement patterns and by pathologic examination using CD31 immunohistochemistry and hematoxylin and eosin staining in five fields at $\times 400$. Contrast-enhancement patterns were classified as linear (type L), scattered (type S), diffuse (type D), or branched (type B). Among carcinomas type B was seen in 4 and type $D$ in 15 ; among adenomas type $S$ in 1 and type D in 1 ; among case of adenomyomatosis, type $\mathrm{S}$ in 8 and type $\mathrm{D}$ in 1 ; and among benign polyps, type $\mathrm{L}$ in 12 , type $\mathrm{S}$ in 14 , and type $\mathrm{D}$ in 7 . When type $\mathrm{D}$ and type $\mathrm{B}$ were considered indication of cancer, accuracy was $84.5 \%$; sensitivity $100 \%$; and specificity $76.9 \%$. When only type B was so considered, accuracy was $100 \%$. In gallbladder carcinoma, the TIC rose from no contrast to early-phase contrast sooner than in adenoma or adenomyomatosis. The TIC in benign gallbladder polyps rose gradually from the early to the late phase. In adenocarcinoma, high-intensity values persisted at $120 \mathrm{sec}$ after injection, which was unusual in other diseases. With an intensity of 90 or greater at $120 \mathrm{sec}$ taken as an indication of cancer, accuracy was $89.7 \%$; sensitivity $89.5 \%$; and specificity $89.7 \%$. Vessels were significantly more numerous in type D cases than in those with other pattern. Type D and type B included the greatest number of cases with advanced inflammation. Vessel diameter was greatest in type D and type B patterns, both differing significantly from type L. In conclusions, ultrasonographic contrast-enhancement patterns show characteristic associations with pathologic findings and serve as valuable adjunct in diagnosis of gallbladder diseases

\section{W1755}

Modified Criteria of Fatty Meal Hepatobiliary (HIDA) Scan for Diagnosis of Biliary Dyskinesia

Abdullah Rashdan, Kusai Al-Muqbel, Mohammad Bani-Hani

Background: The utilization of fatty meal HIDA scan for evaluating acalculus right upper quadrant (RUQ) abdominal pain of suspected biliary origin is not well recognized. We conducted a study with modefication of diagnostic criteria of fatty meal HIDA scan for diagnosis of biliary dyskinesia. Methods: Patients with chronic acalculus RUQ pain were investigated with $40 \mathrm{gm}$ fatty meal HIDA scan. Biliary dyskinesia was considered if the gallbladder ejection fraction (GBEF) was $\leq 50 \%$ [compared to the standard criteria of GBEF $\leq 35 \%$ ] or delayed biliary-bowel transit time beyond 60 minutes of the filling phase. Subjects with abnormal study were offered laparoscopic cholecystectomy versus medical management of functional pain. Symptomatic improvement were recorded prospectively and correlated with the degree of dyskinesia and histologic findings. Results: 117 patients enrolled, 68 (59\%) had abnormal HIDA scan. 52 subjects underwent cholecystectomy 48 patients (92\%) had complete or partial relief of symptoms on long-term follow up (mean of 3 years). Chronic cholecystitis was found in 50 (96\%) of the cases. No correlation was between degree of dyskinesia and symptomatic relief. The current criteria has $90 \%$ sensitivity with specificity of $86 \%$ and overall accuracy of $88 \%$. Conclusion: Modified criteria using fatty meal HIDA scan appears to be an accurate diagnostic tool in patients with suspected biliary dyskinesia

\section{W1756}

Clinical Features of Patients with Choledocholithiasis Showing High Levels of Aminotransferases

Won Joong Jeon, Joung Ho Han, Seon Mee Park, Hee Bok Chae, Sei Jin Youn

Aims: We aimed to determine the clinical features of patients with common bile duct (CBD) stones who showed very high serum levels of AST or ALT. Methods: A retrospective review of 93 patients with CBD stones was completed. Clinical characteristics, CBD diameters, and pre-endoscopic retrograde cholangiopancreatography (ERCP) diagnoses were assessed between the two groups with $\geq 400 \mathrm{IU} / \mathrm{L}$ (group 1) or $\leq 100 \mathrm{IU} / \mathrm{L}$ (group 2) of serum AST or ALT levels. Results: The numbers of patients were 19 and 17 in group 1 and group 2, respectively. The most common symptom was abdominal pain in both groups. Group 1 was about 14 years younger than group $2(\mathrm{p}=0.003)$. The duration of symptoms in group 1 and group 2 were 4.1 and 36.8 days, respectively $(\mathrm{p}=0.005)$. The $C B D$ diameter was smaller in group $1(11.4 \mathrm{~mm})$ than that of group $2(16.3 \mathrm{~mm})(\mathrm{p}=0.001)$. Most patients were diagnosed to have $\mathrm{CBD}$ stones by abdominal ultrasound or computed tomography before ERCP, except two patients in group 1 who diagnosed as hepatitis initially. All recovered by stone removal during ERCP and antibiotics treatment. There were inverse correlations between CBD diameters and AST or ALT levels ( $r=-0.517, p=0.002 / r=-0.504, p=0.002)$. Conclusion: The common bile duct stones with high AST or ALT levels are frequently observed in the younger patients with shorter duration of symptoms and smaller CBD diameter. In these patients, it is possible to misdianose as acute hepatitis. Therefore, special caution is needed in diagnosis of the patients with CBD stones who show very high AST or ALT level.

Clinical Features between the Groups

\begin{tabular}{|c|c|c|c|}
\hline Parameters & Group 1 $(\mathrm{n}=19)$ & Group 2 $(\mathrm{n}=17)$ & $\mathrm{p}$-value \\
\hline Sex (M:F) & $8: 11$ & 12.5 & 0.505 \\
\hline Age (years) & $53.5 \pm 13.5$ & $67.2 \pm 10.8$ & 0.003 \\
\hline Symptom duration (days) & $4.1 \pm 4.4$ & $36.8 \pm 39.9$ & 0.005 \\
\hline Fever & $52.6 \%(10 / 19)$ & $11.8 \%(2 / 17)$ & 0.046 \\
\hline WBC $(/ \mu \mathrm{l})$ & $8499 \pm 3412$ & $9061 \pm 3962$ & 0.739 \\
\hline Total bilirubin $(\mathrm{mg} / \mathrm{dL})$ & $4.4 \pm 3.3$ & $3.1 \pm 2.8$ & 0.156 \\
\hline Alkaline phosphatase $(\mathrm{IU} / \mathrm{L})$ & $685.8 \pm 622.4$ & $376.3 \pm 216.8$ & 0.063 \\
\hline Amylase $(\mathrm{IU} / \mathrm{L})$ & $312.7 \pm 1043.3$ & $153.6 \pm 309.7$ & 0.515 \\
\hline CBD diameter $(\mathrm{mm})$ & $11.4 \pm 3.8$ & $16.3 \pm 3.7$ & 0.001 \\
\hline
\end{tabular}

WBC, white blood cell; $C B D$, common bile duct. All data represent mean \pm standard deviation

\section{W1757}

Role of Endoscopic Ultrasound in Prevention of Unnecessary ERCP: A Prospective Follow-Up Study of 150 Patients Rasoul Sotoudehmanesh, Shadi Kolahdoozan, Morteza Khatibian, Ali Ali Asgari, Sanaz Ainechi, Reza Malekzadeh

Background \& Aims: The aim of this study was to determine the impact of substituting endoscopic ultrasonography for endoscopic retrograde cholangiography in cases of low to intermediate risk for choledocholithiasis. Methods: During 16 months patients who referred for suspected choledocholithiasis, biliary colic or acute biliary pancreatitis on the basis of slight alteration in liver enzymes with or without gallbladder stone seen in transabdominal ultrasound were included. Patients with common bile duct stone on endoscopic ultrasonography were subjected to endoscopic retrograde cholangiography and those with cholecystolithiasis sent for cholecystectomy. Other cases underwent EUS and were followed up to one year post-procedure by phone. Results: During 16 months, a total of 150 patients ( 55 male, 95 female with a mean age of $49.6 \pm 17.5)$ were included. Thirty three cases $(22 \%)$ were diagnosed as choledocholithiasis by endoscopic ultrasonography. Of these, 31 underwent endoscopic retrograde cholangiography and the diagnosis was confirmed in 24 patients $(77.4 \%)$. Fifty one cases had normal common bile duct and follow up for one year showed no abnormality. No complications were encountered by endoscopic ultrasound. Endoscopic retrograde cholangiography was avoided by this approach in 111 cases (74\%). Conclusion: When the pretest probability for choledocholithiasis is low, EUS before ERCP can preclude the need for endoscopic retrograde cholangiography in most cases.

\section{W1758}

Carbon Dioxide Mdct Cholangio-Pancreatography Providing Synchronous Virtual Angiography

Maki Sugimoto, Hideki Yasuda

PURPOSE: The authors investigated the feasibility of a new software system employing 3dimensional (3D) virtual anatomic reconstruction using MDCT with carbon dioxide (CO2) gas as a negative contrast agent, and intra and extra luminal virtual exploration for detection of preoperative visualization of the cholangio-pancreatic $(\mathrm{CP})$ anatomy. MATERIALS AND METHODS: Total of 70 patients who were thought to have $\mathrm{CP}$ disease underwent therapeutic endoscopic drainage were included in this study. 16-slice MDCT was performed with the infusion of 5-50 cc CO2 via endoscopic naso-pancreaticobiliary drainage (ENBD, EPS). Threedimensional MDCT CP images (CO2 MDCT-CP) were produced using volume rendering for virtual $\mathrm{CP}$ endoscopy . Capability of $\mathrm{CO} 2$ to pass the obstruction and delineate distal parts of the CP duct was evaluated. 1. CO2 MDCT-CP All procedures had 100\% technical success; complication had a $0 \%$ morbidity rate. The correct diagnosis was made on CO2 MDCT-CP for cholelithiasis, adenomyomatosis of the gallbladder, xantogranuromatous cholecystitis, bile duct malignancies, over $2 \mathrm{~mm}$ cystic lesions in IPMN, and invasive pancreatic cancer. $\mathrm{CO} 2$ could go through the narrowing cystic duct and obstructed bilio-pancreatic duct, additional distal segments of the occluded bile duct induced the impact stone and bile duct cancer occlusion. 2. CO2 Virtual endocopy Virtual CP endoscopy using CO2 MDCT revealed excellent and moderate endoluminal visualization to detect stenosis, obstruction, and detailed protruding lesions over $2 \mathrm{~mm}$. 3. Syncro CO2 MDCTA-CP Generally MDCT angiography (MDCTA) with CP imaging has to be needed multiphase fusion scanning. Our CO2 MDCT$\mathrm{CP}$ can provide the entire synchronous $\mathrm{CP}$ imaging by $\mathrm{CO} 2$ with virtual angiography initiated after the IV infusion of contrast agent (CO2 MDCTA-CP) because of the high contrast interface between soft tissue and gas. This provides valuable information for planning surgery, 
including the location of the obstruction and its relationship to the surrounding vessels. CONCLUSION: Our preliminary results emphasize that synchronous CO2 MDCTA-CP is a newer safe tecnique providing visualization of $\mathrm{CP}$ disorder and detailed preoperative reconstruction of $\mathrm{CP}$ anatomy. Because of its low viscosity, $\mathrm{CO} 2$ is a useful contrast medium to visualise the extrahepatic biliary system before performing percutaneous drainage in biliary obstruction. Unnecessary intraductal manipulations may be reduced. Newer software developments may further enhance its accuracy, so that CO2 MDCTA-CP might challenge or replace more invasive diagnostic measures in the near future.

\section{W1759}

The Clinical Outcome of Palliative Drainage Methods (Percutaneous T-Stent Internal Drainage versus Endoscopic Retrograde Biliary Drainage) in Klatskin Tumors

Dae Han Kang, Gwang Ha Kim, Tae Oh Kim, Joo Ho Lee, Jeong Heo, Geun Am Song, Mong Cho, Chang Won Kim, Jeong Ho Heo, Jae Seung Lee

Background/Aims : Curative surgery is not possible in majority of Klatskin tumors. Palliation with endoscopic or percutaneous internal drainage is a well-established procedure in such a irresectable biliary tumor. We evaluated the clinical efficacy of bilateral internal drainage by means of a newly designed $\mathrm{T}$-configured dual stent placement. We compared the clinical outcome of percutaneous T-stent with that of endoscopic retrograde unilateral biliary drainage. Methods: From 2001 to 2004, 36 patients with Klatskin tumors were treated with percutaneous transhepatic placement of two self-expandable metallic endoprostheses in a $\mathrm{T}$ configuration through a single transhepatic access. We undertook a retrospective chart review of all 29 Klatskin tumors which drained by unilateral ERBD during the period same as the above. Results: The patients' characteristics are outlined in Table 1. Klatskin tumor grades according to the Bismuth classification differ among both study groups ( $\mathrm{T}$ stent group: 8 type II, 23 type III, and 5 type IV versus ERBD group: 11 type I, 14 type II, and 4 type III). To compensate the differences, we corrected the obtained clinical outcome data (Table 2) according to the covariance analysis. The frequency of cholangitis in $\mathrm{T}$ stent group was lower than that in ERBD group, but the difference was not statistically significant. Also there were no significant differences in survival and stent patency among both T-stent and ERBD groups. Conclusions: T stent placement can be used effectively and safely to achieve bilateral internal drainage in biliary bifurcation tumors and is technically simple. In the more advanced hilar tumors, it can be considered as the first palliative drainage method. But, in the not advnced hilar tumors unilateral drainage by ERBD can achieve sufficient palliation as bilateral drainage.

Table 1. Clinical characteristics of the patients who have Klatskin tumors which drained by T-stent or ERBD.

\begin{tabular}{|c|c|c|}
\hline & T stent $(\mathrm{n}=36)$ & ERBD $(\mathrm{n}=29)$ \\
\hline Age (years) & 67.8 (range 41 to 85$)$ & 68.5 (range 37 to 87$)$ \\
\hline Gender (M/F) & $22 / 14$ & $17 / 12$ \\
\hline Pre-treatment TB & $11.25($ SD 7.732$)$ & $11.90($ SD 5.595$)$ \\
\hline Post-treatment TB & 1.93 (SD 1.430) & 1.61 (SD 1.349) \\
\hline Stent obstruction during survival & $61.1 \%(22$ of 36$)$ & $58.6 \%(17$ of 29$)$ \\
\hline Survival more than 180 days & $69.4 \%(25$ of 36$)$ & $62.1 \%(18$ of 29$)$ \\
\hline Mortality within 30 days & $5.6 \%(2$ of 36$)$ & $3.4 \%(1$ of 29$)$ \\
\hline
\end{tabular}

Table 2. Clinical outcome data of the Klatskin tumors in both study groups.

\begin{tabular}{|c|c|c|c|}
\hline & T stent $(\mathrm{n}=36)$ & ERBD $(\mathrm{n}=29)$ & $\mathrm{p}$ value \\
\hline Survival time (dsys) & 284.64 & 313.24 & 0.592 \\
\hline Stent patency time (days) & 209.69 & 198.48 & 0.757 \\
\hline Reduction of TB after procedure & -9.316 & -10.298 & 0.528 \\
\hline Incidence of cholangitis (\%) & $8.3(3 / 36)$ & $17.2(5 / 29)$ & 0.450 \\
\hline
\end{tabular}

W1760

Percutaneous Biliary Drainage: Influence of Microbiology On Antibiotic Strategy and Therapeutic Management

Olivier Corcos, Frederique Maire, Oana Zamfir, Annie Sibert, Magaly Zappa, Philippe Levy, Valerie Vilgrain, Marie-Helene Nicolas-Chanoine, Dermot O'Toole, Philippe Ruszniewski

Percutaneous biliary drainage has proven to be efficacious in symptomatic hilar or intrahepatic biliary stenosis. However, drainage is often complicated by biliary super-infection (the latter which is complicated by bilio-digestive drains and/or stents and their lavage; indeed digestive flora contamination appears frequent) and antibiotic treatment is therefore difficult. Here we describe the type of germs and antibiotic strategies employed in a large cohort of patients treated in a single centre. Patients/method: 64 consecutive patients (mean age 64 years) underwent percutaneous biliary drainage between July 2004 and March 2005. The cause of biliary stenosis was cholangiocarcinoma $(n=27)$, pancreatic adenocarcinoma $(n=7)$, benign disease of the CBD $(n=5)$, biliodigestive anastomosis $(n=7)$, post liver transplantation $(n=8)$ compression from metastases $(n=5)$. Indications of drainage were: ascending cholangitis $(n=$ 21 ), pruritus $(n=13)$, jaundice $(n=17)$, obstruction of pre-existing biliary stent $(n=5)$ or prior to surgery $(\mathrm{n}=10)$. Microbiologic analysis of bile was performed during drainage and subsequent follow-up. Results: In 35 patients $(55 \%)$ the lst bile aspirate was sterile (20 patients were on antibiotics, 11 for ascending cholangitis). In 29 patients with a positive culture of lst bile aspiration antibiotic treatment prior to drainage had administered in

17 (58\%), 10 for ascending cholangitis: the aspiration showed a single bacteria species
(Enterococcus, E. coli, Proteus mirabilis and other species of Enterobacteria, naturally resistant to amoxicillin and clavulanic acid namely Enterobacter, Citrobacter and Morganella species). Bile which was subsequently analysed in the internal/external drains $(\mathrm{n}=56)$ was positive in $99 \%$ of cases with a multitude of digestive micro-organisms including bacteria and fungi. Such colonisation was clinically symptomatic in 18 cases (33\%). Conclusion: 1 ) The initial bile aspirate during percutaneous drainage is sterile in the over half of patients whether pre-treated or not with antibiotics; 2) The bile sample is contaminated in a majority of patients with ascending cholangitis treated by antibiotics $(59 \%) ; 3$ ) subsequent bile samples are frequently contaminated by digestive micro-organisms but a minority have a clinical infection; 4) Antibiotic prophylaxis using amoxicillin and clavulanic acid, frequently employed in these patients, does not treat most of the Enterobacter species encountered.

\section{W1761}

Clonorchiasis Is Associated with Cholangiocarcinoma, Not with Biliary Stone or Hepatocellular Carcinoma: Nation-Wide Multicenter Survey in Korea Ho Gak Kim, Myung-Hwan Kim, Seung-Ok Lee, Sung-Jae Park, Chan-Guk Park, YoungEun Joo, Gwang Ha Kim, Chang-Hun Yang, Jin Bong Kim, Kyu Taek Lee, Dongki Lee, Taehyo Kim, Hyun Soo Kim, Jae Seon Kim, Young-Soo Moon, Chang-Min Cho, Woo Jin Lee, Kyo-Sang Yoo, Gabjin Cheon, Jong Ho Moon, Tae Nyeun Kim, Yong Woo Choi, Byeong Moo Yoo, Ji Kon Ryu, Yong Bum Yoon

Background/Aim: Clonorchiasis has been recognized as one of the most important endemic diseases in Korea. Infection rate of Clonorchis sinensis(CS) was not reduced despite of Praziquantel because of the custom of eating raw freshwater fish. We investigated nationwide infection rate of CS in patients with gastrointestinal(GI) symptoms, and the relation of CS with hapato-biliary diseases prospectively in 26 secondary and tertiary hospitals in Korea. Patients and Methods: Consecutive patients who were admitted in Gastrointestina Department with GI symptoms were enrolled from March to April 2005 in 26 nation-wide hospitals. Present and past CS infection was diagnosed by one of stool direct smear, serologic test for IgG clonorchis circulating antibody (CAb), intradermal test for CS, bile cytology, and typical radiological findings. Epidemiology, correlation between CS infection and hepatobiliary diseases were surveyed. Results: Among the 3081 patients with GI diseases $396(12.9 \%)$ were infected with CS at present or in the past. 1131 patients had history of eating raw freshwater fish and 235(20.8\%) patients infected with CS. $161(8.4 \%)$ of 1925 patients who had no history of eating raw freshwater fish also infected with CS. Cholangiocarcinoma $(\mathrm{CCa})$ was more prevalent in CS infected patients than CS not-infected patients \{ $27 /$ $396(6.8 \%), 102 / 2685(3.8 \%)$ respectively, $p=0.038\}$. CS infected patients were more common in the patients who ate raw fish in the basins of 5 rivers in southern part of Korea than those who ate raw fish in the basins of 5 rivers in middle part of Korea $\{182 / 578(31.5 \%)$, $38 / 464(8.2 \%)$ respectively, $p=0.000$. CCa was more prevalent in the patients who ate raw fish in the basins of rivers in southern Korea than the patients who ate raw fish in the basins of rivers in middle Korea \{28/353(7.9\%), 8/232(3.4\%) respectively, $\mathrm{p}=0.027\}$. CCa and CS infection have significant positive cross-relation each other $(\mathrm{p}=0.008)$. Alcoholic pancreatitis was more common in CS infected patients than CS not-infected patients $\{19(4.8 \%), 71(2.6 \%)$ respectively, $\mathrm{p}=0.018$. It is explained by that Korean people have a custom of eating raw fish at drinking parties. Choledocholithiasis, cholecystolithiasis, cholangitis, and hepatocellular carcinoma were not related with CS infection. Intradermal test for CS has the highes sensitivity $(75.1 \%)$ compared with serologic CAb test $(42.9 \%)$ and stool direct smear( $17.1 \%)$ Conclusions: Infection rate of CS is high in patients with GI diseases in Korea, and is not reduced for recent two decades. CCa is related with CS infection and is more common in the areas of CS prevalent rivers, so CS can be the possible etiology of CCa.

\section{W1762}

Management Strategies of the Gallbladder Polyps Joo Kyung Park, Yong Bum Yoon, Yong Tae Kim, Ji Kon Ryu, Won Jae Yoon, Jun Kyu Lee, Sang Hyub Lee, Min Jung Park

Introduction Polypoid lesions of the gallbladder(GB) encompass a wide variety of pathologies. Although most the lesions are benign, problems remain in selecting patients for surgery or regular follow up. Aims and Methods The aim of this study is to describe the natural course of $\mathrm{GB}$ polyps and to propose reasonable factors for making a decision in favor of surgery or regular follow-up. We studied the epidemiology of GB polyps in Health Care Center o Seoul National University Hospital from Oct. 2003 to July 2005. In 24,617 healthy individuals who underwent abdominal ultrasonography (USG), the prevalence of GB polyps was $7.1 \%$ and $4.8 \%$ for men and women, respectively. The 740 patients with GB polyps documented by abdominal USG from January 1988 to December 2004 at Seoul National University Hospital were randomly enrolled, but 69 patients were excluded due to such comorbidities congestive heart failure, chronic renal failure, coronary heart disease, liver cirrhosis or malignancy. Results There were 399 men and 272 women in the total of 671 patients. The median age was 47 years old, and the median follow up duration was 51 months. The mean diameter of initially diagnosed polyp was $6 \mathrm{~mm}$ (range from 2 to $30 \mathrm{~mm}$ ). Two hundred thirty six patients $(35 \%$ ) had polyps below $5 \mathrm{~mm}, 312$ patients ( $47 \%$ ) from 5 to $9 \mathrm{~mm}$, and 123 patients ( $18 \%$ ) above $10 \mathrm{~mm}$. Four hundred patients (60\%) had single polyp and 271 (40\%) patients had two or more polyps (range from 2 to 10). There were 57 patients with accompanying gallstones. The majority of patients experienced no change in the GB polyp $(496 ; 74 \%)$ or decreased GB polyp size by more than $3 \mathrm{~mm}(69 ; 10 \%)$. Pathologic reports were obtained on the 163 patients who underwent surgery. There were 7 patients (1\%) who had adenocarcinomas of GB, and all of them had polyps with size of more than $10 \mathrm{~mm}$ (range from $10.0 \mathrm{~mm}$ to $24 \mathrm{~mm}$ ). There were 44 patients $(7 \%)$ who had adenomas of $\mathrm{GB}$, and the size of polyps was variable (range from 3 to $20 \mathrm{~mm}$ ). We performed $\chi 2$ test to find risk factors of precancerous lesions (adenoma) and cancer: sex, age, symptoms, associated stones, size, shape and multiplicity of polyps. Among them, size of the polyp $(\geqq 10 \mathrm{~mm}$ ) was the only statistically significant risk factors of cancer and adenoma ( $\mathrm{p}=$ $0.007,0.04$ ). Conclusion The GB polyp is fairly common finding in clinical practice, and it will be found more often as the medical screening examinations increase. Among the 
clinical factors, the size of the polyp ( $\geqq 10 \mathrm{~mm}$ ) was the most important risk factor of precancerous lesion (adenoma) and cancer, and such a polyp should be carefully observed. Also, more investigations should be performed to look for indications of surgery.

\section{W1763}

Role of Pro-Hepcidin in PSC Patients with Bacterial Infections of Bile Ducts Hasan Kulaksiz, Adolf Stiehl

Background \& Aims: Primary sclerosing cholangitis (PSC) is characterized by progressive fibrotic inflammation and strictures of the biliary system. Recently, we have shown that bacterial infections are a frequent finding in PSC patients with dominant stenoses of the bile ducts (Pohl et al. Eur J GastroenterolHepatol 2005). Since the newly discovered hepatic peptide hormone hepcidin has antibacterial and antifungal effects, we have analyzed prohepcidin in bile of PSC patients with infections of the bile ducts. Methods: Bile probes were obtained during endoscopic retrograde cholangiographie (ERC) in 42 PSC patients (26 patients with dominant stenosis and bacterial infections of the bile ducts; 16 controls with dominant stenosis but no infections). Immunoblot analysis with specific antibodies were performed to detect pro-hepcidin in bile fluids. A sensitive ELISA assay was developed for determination of pro-hepcidin in bile probes. Results: The antibacterial and antifungal peptide pro-hepcidin is present in bile. In PSC patients with dominant stenoses but no infections of the bile ducts the mean pro-hepcidin level was $22.2 \mathrm{ng} / \mathrm{ml}$. Enhanced levels of prohepcidin (mean $67.9 \mathrm{ng} / \mathrm{ml}$ ) were found in PSC patients with dominant stenoses and infections of the bile ducts. High levels of pro-hepcidin were associated with elevated serum bilirubin. A significant correlation was also found between pro-hepcidin and serum Creactive protein and leucocytes. Conclusion: Detection of pro-hepcidin in the bile and its association with bacterial infections of bile ducts implicates a possible local antibacterial activity of this peptide in PSC patients with dominant stenosis. This finding underlines the major impact of dominant stenoses in inflammatory processes of the bile ducts.

\section{W1764}

Sacral Extracorporeal Magnetic Stmulation Is the Same Effective Treatment for Pelvic Floor Dyssynergia As Biofeedback Therapy

Joon Seong Lee, Hee Hyuk Im, Hyun Ku Jung, Young Dae Kim, In Seop Jeong, Su Jin Hong, Jin Oh Kim, Joo Young Cho, Moon Sung Lee, Chan Sup Shim, Boo Sung Kim

Background: Recent development of extracorporeal magnetic stimulation (ECMS) which uses current-changing magnetic fields allows the induction of electrical stimulation in the desired deep tissue. Preliminary study shows that ECMS of sacral nerve can modify pelvic floor function and expel rectal balloon in patients with pelvic floor dyssynergia (PFD). Aim: To evaluate the effect of ECMS compared with biofeedback therapy(BF) in patients with PFD. Methods: Thirty-eight patients who fulfilled Rome II criteria for PFD by colon transit time and anorectal function tests, were randomly treated with 8 sessions of ECMS (2/weeks; $\mathrm{n}=19)$ or $\mathrm{BF}(2 /$ weeks; $\mathrm{n}=19)$. Stimulation parameters were set at $50-80 \%$ of maximum intensity, 10 and $50 \mathrm{~Hz}$ frequency, $3 \mathrm{~s}$ burst length with 3 and $6 \mathrm{~s}$ off using arm-typed stimulator (BioCom-1000, Mcube Co., Korea). Symptom scores for constipation, anorectal function test were repeatedly measured after each treatment. Response was defined as $50 \%$ or more decreased symptom score after treatment (partial response: $30-50 \%$, poor: $<30 \%$ ). Results: Fifteen patients (age 49.1 $\pm 13.4 \mathrm{yr}$, mean \pm SD; 4 men) completed 8 session of BF and 14 patients $(54.5 \pm 17.6 \mathrm{yr}, 3 \mathrm{men})$ completed 8 session of ECMS. Total symptom scores

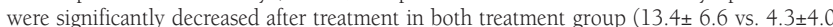
for $\mathrm{BF}, \mathrm{p}=0.009 ; 14.9 \pm 5.6$ vs. $3.4 \pm 4.0$ for ECMS, $\mathrm{p}<0.001$ ). Thirteen out of 15 patients showed response in BF group and 12 out of 14 showed good response in ECMS group. Conclusion: ECMS is as effective as BF for the treatment of PFD. Long-term effect of ECMS need to be evaluated. Key words: magnetic stimulation, pelvic floor dyssynergia, biofeedback

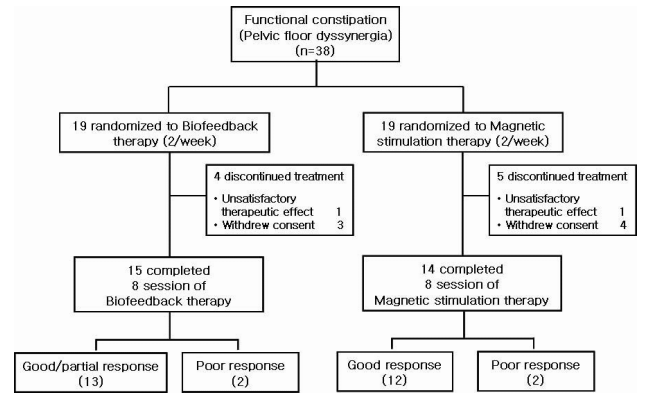

\section{W1765}

Role of Rho Kinase in the Basal Tone in the Internal Anal Sphincter (IAS) Smooth Muscle

Chirag A. Patel, Marcio A. De Godoy, Satish Rattan

Background \& Aims: Increase in Rho kinase (ROK) activity has been associated with the agonist-induced sustained contraction of the smooth muscle. However the role of ROK in the truly tonic smooth muscle in the basal state is not known. Methods: The present studies examined the effects of ROK inhibitor Y27632 in the tonic smooth muscle of the IAS vs. in the flanking phasic smooth muscle of the rectum (RSM) using computerized recording of force measurements. The studies were performed in the tissues from rat, an appropriate animal model for human anorectal studies. In addition, studies were performed to determine the relationship between the changes in the basal IAS tone following Y 27632 vs. ROK and protein kinase $\mathrm{C}$ (PKC) activities. We also determined changes in free intracellular $\left.\mathrm{Ca}^{2+}\right]<$ $\left(\left[\mathrm{Ca}^{2+}\right]_{\mathrm{i}}\right)$. The levels of RhoA/ROK in these diverse smooth muscles were determined using
Western Blots and reverse transcriptase-polymerase chain reaction (RT-PCR). In order to investigate the cellular distribution of RhoA/ROK in the IAS vs. the RSM, we performed confocal microscopy studies in the isolated smooth muscle cells (SMC). Results: The ROK inhibitor caused several-fold and significantly more potent relaxation of the IAS as compared with the RSM. In this regard, $27632(1 \mu \mathrm{M})$ causing $50 \%$ inhibition $\left(\mathrm{IC}_{50}\right)$ in the basal IAS tone had minimal effect on the RSM phasic activity $(\mathrm{p}<0.05 ; \mathrm{n}=7)$. The relaxant effect of Y 27632 was directly at the SMC because different neurohumoral antagonists (including NOS inhibitor L-NNA $(0.3 \mathrm{mM})$ and tetrodotoxin $(1 \mu \mathrm{M})$ ) did not modify this effect. In addition, a similar relaxation was observed in the IAS SMC. The IAS relaxation by Y 27632 correlated directly with the decrease in ROK activity without significant changes in the levels of $\left[\mathrm{Ca}^{2+}\right]_{\mathrm{i}}$ and PKC activity. ROK activity assays, Western Blot and RT-PCR studies revealed that in the basal state, the IAS had distinctly higher levels of RhoA/ROK as compared to the RSM. Confocal microscopy studies verified relatively higher distribution of RhoA/ROK towards the periphery of the SMC of the IAS in contrast to the RSM. In in vivo studies, the lower doses of Y 27632 caused potent and selective fall in the IAS pressures (IASP) without any adverse systemic effects. 5 nmoles/kg of Y 27632 caused maximal fall in the IASP while $25 \mu$ moles $/ \mathrm{kg}$ (5,000 times) of the ROK inhibitor was required to produce a similar fall in the mean systolic blood pressure. Conclusions: 1). Rho kinase plays an important role in the maintenance of the basal tone in the IAS; and 2). ROK inhibitors have a therapeutic potential in the IAS dysfunction especially characterized by the hypertensive IAS.

\section{W1766}

Fecal Incontinence: Doctors Don't Ask and Patients Don't Tell Sara K. Hawes, Afkhamossadat Merikhi, Ayaz Matin, Elango Vinjirayer, James C. Reynolds, Asyia Ahmad

Fecal incontinence (FI) is a severely debilitating and embarrassing disorder rarely reported by patients. Previous studies have only elicited a history of FI by mailed surveys or questionnaires given to patients upon arrival at their doctor's office. The value of a direct patientdoctor interview to identify the prevalence of patients with FI has never been evaluated. Our aim was to determine the prevalence of FI in patients presenting to a gastroenterology (GI) office by including FI questions in a thorough gastroenterology review of systems. METHODS: Two hundred random outpatient charts were retrospectively reviewed for any documentation pertaining to fecal incontinence during the initial doctor-patient interview. We then prospectively asked 150 patients in our GI office a thorough review of systems including if s/he experienced the escape of flatus or soiling with liquid or formed stool. Patients that answered yes were entered into our study and administered a questionnaire regarding their demographic and medical history as well as the validated Rockwood FIQOL (quality of life) and FISI (fecal incontinence severity index). Patients were also asked if the topic of FI had ever been addressed by their health care provider. RESULTS: Retrospective chart review revealed that only 2 out of 200 (1\%) outpatient GI patients had documentation of any discussion regarding FI. In the prospective arm of our study $29(19 \%)$ of patients interviewed had a history of FI and $80 \%$ of these patients had never been questioned about FI by any doctor. Only one of these patients had ever been asked by a primary care doctor about FI, yet $86 \%$ of our FI patients had a medical history significant for well known risk factors for FI. Of the 29 patients with FI, only 2 of them specifically came to our office for that reason, yet $55 \%$ had $>1$ episode of FI per day and $67 \%$ had symptoms for $>1$ year. Furthermore 38\% percent of patients had poor QOL and $66 \%$ of patients had high FISI. Regression analysis revealed a direct correlation between FISI and QOL $(\mathrm{R}=0.77)$ in women. Surprisingly there was no correlation between FISI and QOL in men. Our study differs from previous studies in that $65 \%$ of our patients were women. Interestingly, $50 \%$ of our patients had an education level beyond high school. CONCLUSIONS: Nineteen percent of patients seen in gastroenterology offices with FI will be missed unless specifically questioned. Unfortunately, despite greatly diminished QOL, high SI scores and advanced education level, patients rarely bring up FI with their doctor. To elicit a history of FI it is imperative for gastroenterologists to specifically and routinely question their patients about FI.

\section{W1767}

Mode of Delivery Affects Anal Sphincter Innervation Heiko Franz, Heidemarie Hinninghofen, Axel Kowalski, Roberto Merletti, Paul Enck

Introduction: Surface-EMG (S-EMG) of external anal sphincter (EAS) by multi-electrode arrays (MEA) was used for a large series of gynecologic patients for the first time. We wished to determine the degree of asymmetry of sphincter innervation, its determinants, and the relationship to fecal incontinence. Material and Methods: In 129 continent women following childbirth and in 40 patients with fecal incontinence recruited in a gynecological hospital, S-EMG of the EAS was performed by 16-channel MEA during maximal voluntary contraction, and generation and propagation of individual motor unit actions potentials (MUAP) were recorded in the anal circumference at 3 levels within the anal canal. Innervation zones (IZ) of MUAP, their circular distribution in the quadrants as measure of symmetry, as well as the statistical characteristics of amplitude (average rectified values,ARV; root mean square, RMS) and mean frequency (MNF) of MUAP firing were compared between and across subgroups (age, continence status, number and mode of deliveries) by ANOVAs. Results: All S-EMG characteristics were best identified in the proximal and distal anal canal, but IZ and ARV were overall lower in incontinence, and differences between the three levels were less pronounced in incontinent patients. Age reduced independently the number of IZ ( $\mathrm{p}=$ .017 ) and the amplitude (ARV, $\mathrm{p}=0.046$ ). In incontinent subjects, lower amplitudes (RMS) $(\mathrm{p}=.084)$ and higher firing frequency $(\mathrm{MNF})(\mathrm{p}<0.001)$ of EMG were found. Significant asymmetry of innervation was found for dorsal versus ventral, but not for left versus right comparison of amplitudes (RMS, $\mathrm{p}=.004$ ) and frequency (MNF, $\mathrm{p}=.001$ ), and was more pronounced in the distal anal canal for IZ distribution $(\mathrm{p}=.007)$ and in the proximal canal for EMG amplitude (ARV, $p=.007$ ). The asymmetry of firing frequency (MNF) was increased with more deliveries, especially in incontinent women $(\mathrm{p}=.026)$. Episiotomy was asociated with significant left/right asymmetry of IZ distribution ( $\mathrm{p}=.075)$, as was vacuum extraction $(\mathrm{p}=.04)$ and forceps delivery $(\mathrm{p}=.04)$, but not cesarian section (n.s.). Conclusions: Asymmetry of innervation of the EAS seems to play a role in incontinence, with independent contribution 
of age, number of childbirth, and the mode of delivery. (Supported by grants from the EU and the Fresenius Foundation, Germany)

\section{W1768}

Length Tension Relationship of the Puborectalis Muscle in Women Bikram S. Padda, Sung-Ae Jung, Delores Pretorius, Jianmin Liu, Debbie Denboer, Ravinder K. Mittal

Aims and Hypothesis: Role of puborectalis muscle (PRM) in fecal continence remains unclear because of the lack of an adequate recording technique to monitor PRM function. Recent study suggests that the vaginal high-pressure zone (VHPZ) may be related to PRM contraction. If that were true, increasing PRM length should increase pressure in the vaginal HPZ (length tension function) Methods: 15 nullipara females were studied. Effect of vaginal distension, using a $30 \mathrm{~mm}$ bag, on PRM length was determined using 3D-transperineal ultrasound imaging. VHPZ was recorded using probes of diameters $5,10,15,10,25, \& 30 \mathrm{~mm}$. Probes of diameter $>10 \mathrm{~mm}$ had a linear groove to accommodate a $5 \mathrm{~mm}$ diameter sleeve sensor for pressure measurement. Pressures were recorded with the sleeve sensor facing the anterior and posterior position, at rest and maximal voluntary squeeze. Results: 3D US images show the entire loop of the PRM from which PRM length were determined using a computer and software. Increase in bag diameter increased the PRM length. VHPZ shows a resting pressure, which increases with voluntary squeeze. A linear increase in the resting, squeeze and delta (difference between rest and squeeze pressures) pressure in the VHPZ was recorded with the increase in the probe size. There is a wide variability in the maximal pressures recorded among different subjects (max squeeze pressure of $250-800 \mathrm{mmHg}$ ). Anterior VHPZ pressures are 2-4 times greater than posterior pressures. Conclusions: Pressure increase in the VHPZ with the increase in PRM length further proves that the VHPZ is related to PRM contraction. Future studies should determine the role of PRM length-tension function in fecal continence. Effect of PRM Distension on Vaginal Pressure Recording

\begin{tabular}{|c|c|c|c|c|}
\hline & \multicolumn{2}{|c|}{ Anterior Pressure $(\mathrm{mm} \mathrm{Hg})$} & \multicolumn{2}{c|}{ Posterior Pressure $(\mathrm{mm} \mathrm{Hg})$} \\
\hline Probe Diameter $(\mathrm{mm})$ & Rest & Contraction & Rest & Contraction \\
\hline 5 & $18 \pm 4$ & $46 \pm 7$ & $15 \pm 3$ & $36 \pm 4$ \\
\hline 10 & $30 \pm 9$ & $96 \pm 19$ & $31 \pm 6$ & $79 \pm 11$ \\
\hline 15 & $48 \pm 8$ & $150 \pm 31$ & $29 \pm 5$ & $75 \pm 9$ \\
\hline 20 & $86 \pm 13$ & $281 \pm 54$ & $37 \pm 7$ & $108 \pm 10$ \\
\hline 25 & $201 \pm 54$ & $431 \pm 76$ & $41 \pm 5$ & $138 \pm 25$ \\
\hline 30 & $286 \pm 85$ & $550 \pm 75$ & $53 \pm 8$ & $149 \pm 17$ \\
\hline
\end{tabular}

\section{W1769}

Rectocele and Intrarectal/Anal Intussusception Are Not Related with Impaired Rectal Transit and Emptying, in Functional Constipation

Federico Alghisi, Danilo Badiali, Roberto Cappuccio, Fiorella Anzini, Enrico Corazziari, Fortunee Irene Habib

Rectocele and intra-rectal/anal intussusception are frequent findings in chronic constipation, and their surgical repair has been proposed for the treatment of obstructed defecation syndrome. However, it is not established whether rectocele and intra-rectal/anal intussusception, per se, affect rectal transit and/or emptying. Aim of this study was to assess the relationship between rectocele and intra-rectal/anal intussusception with rectal transit and/ or emptying. Materials and methods: 146 consecutive patients (F 130, mean age $45.8 \pm 13.6$ yrs), affected by functional chronic constipation according to ROME II criteria, were submitted to defecography and measurement of rectal transit time by means of radio-opaque markers assessed at 72-hrs intervals on abdominal antero-posterior x-ray. To identify the rectum, a latero-lateral radiogram, including sacrum and coccyx, was added when markers reached the pelvis. At defecography the presence of significant rectocele $(>3.5 \mathrm{~cm})$, intrarectal/anal intussusception, post-evacuative residue were assessed. Results: Rectal transit was delayed in 45 pts $(30.8 \%)$. The following defecographic findings were found: rectocele $32.2 \%$, intra-rectal/anal intussusception $41.8 \%$. The frequency of prolonged rectal transit did not differ in patients with, and without, rectocele $(\chi 2=0.12$, n.s. $)$ and intra-rectal/anal intussusception $(\chi 2=0.01, n$.s. $)$. A moderate/severe post-evacuative residue was observed in $66.4 \%$ of the patients, with no statistical difference between patients with and without defecographic changes or delayed rectal transit. Conclusions. The results of this study suggest that both rectocele and intra-rectal/anal intussusception, per se, scarcely affect rectal emptying and do not delay transit through the rectum.

\section{W1770}

Comparison of Water-Perfused (WPM) and Manoscan ${ }^{\mathrm{TM}}$ High Resolution (HRM) Anorectal Manometry (ARM)

Jennifer Post, Sarah Wessinger, Michael P. Jones

Introduction: ARM is used to evaluate fecal incontinence and dysynergic defecation. It is poorly standardized and agreement btwn ARM and other tests of anorectal function is often poor. HRM is a new stationary recording technique using closely spaced solid state transducers. In esophageal studies, HRM provides superior resolution of motility compared with WPM. We compared WPM and HRM in patients undergoing ARM. Methods: Consecutive pts referred for ARM underwent simultaneous WPM and ARM. WPM was performed using a $6 \mathrm{~cm}$ sleeve assembly with sideholes spaced at $3 \mathrm{~cm}$ intervals. HRM was performed using the ManoScan ${ }^{\mathrm{TM}}$ system consisting of a $4.2 \mathrm{~mm}$, 36-channel catheter with sensors spaced at $1 \mathrm{~cm}$ intervals. Space-time pressure data were displayed in topographic form using ManoView ${ }^{\mathrm{TM}}$ analysis software that is part of the ManoScan ${ }^{\mathrm{TM}}$ system. Balloon distension was performed using a random distension protocol with volumes between $20-100 \mathrm{ml}$. Results: 18 pts were studied. Indications were constipation in 13 and incontinence/urgency in 5 .
Good correlation existed between the two methods for anal sphincter (AS) pressure at rest, with voluntary contraction and during $40 \mathrm{ml}$ rectal balloon distension (table). Correlation of AS/intrarectal(IR) pressure ratios during pseudodefecation were also good. HRM provided greater resolution of AS. The figure demonstrates paradoxical puborectalis contraction durin pseudodefecation seen with HRM but not WPM. Conclusions: HRM and WPM were highly correlated. HRM provides greater anatomic detail allowing superior discrimination of anorectal function. HRM may be of particular value in the evaluation of suspected dysynergic defecation.

\begin{tabular}{|c|c|c|c|c|}
\hline mean \pm SEM & AS:resting & AS:squeeze & AS:relax & AS/IR \\
\hline $\mathrm{WPM}(\mathrm{mmHg})$ & $66 \pm 5$ & $138 \pm 14$ & $36 \pm 5$ & $2.22 \pm 0.36$ \\
\hline $\mathrm{HRM}(\mathrm{mmHg})$ & $55 \pm 6$ & $103 \pm 10$ & $39 \pm 5$ & $1.92 \pm 0.31$ \\
\hline $\mathrm{r}(\mathrm{p})$ & $0.57(0.011)$ & $0.66(0.002)$ & $0.59(0.013)$ & $0.64(0.004)$ \\
\hline
\end{tabular}

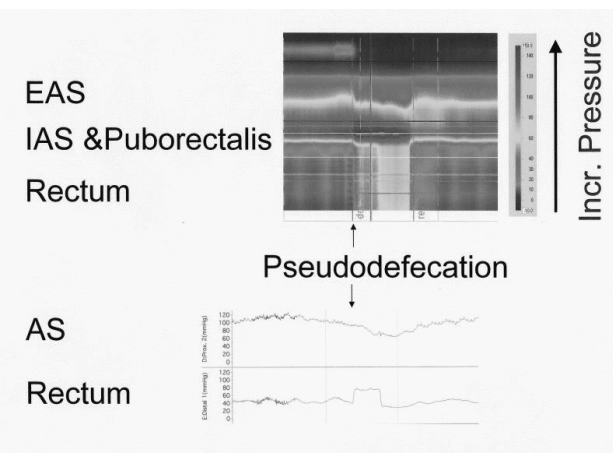

AS=anal sphincter EAS=external anal sphincter IAS-internal anal sphincter

W1771

Rectosigmoid Compliance and Visceral Perception in Spinal Cord Injury Patients

Beatrice Salvioli, Roberto De Giorgio, Vincenzo Stanghellini, Giovanni Barbara, Mauro Menarini, Roberto Corinaldesi, Gabriele Bazzocchi

Background and Aim: In studies assessing anorectal motor function in traumatic complete or incomplete spinal cord injury (SCI), various continence mechanisms have been shown to be disturbed, resulting in either constipation or incontinence. Rectal or colonic compliance may be normal or reduced in SCI patients compared to controls. Data indicate that the colonic response to food is preserved in patients with higher T6 SCI. Theoretically, patients with cervical lesions, classified as having a complete injury according to clinical criteria (American Spinal Injury Association, ASIA), do not experience pelvic sensation. Nevertheless, some evidence reports a preservation of sensory functions in these patients. The origin o this sensation has not been determined yet and data on physiological features of rectosigmoid compliance and perception in SCI patients are lacking. The scope of this study was to investigate recto-sigmoid compliance and perception in chronic SCI patients. Methods: We evaluated recto-sigmoid responses to fixed tension distensions applied by means of tensostat in 6 patients $(6 \mathrm{M}, 42 \pm 4$ yrs) with complete transsection of the spinal cord (high$\mathrm{SCI} ; 5$ tetraplegic $\mathrm{C} 5-\mathrm{C} 7$ and 1 paraplegic T4-T6) classified as ASIA-A and impaired evacuation (i.e., constipation). Results were compared to those obtained in 10 healthy subjects $(6 \mathrm{M}, 25 \pm 1 \mathrm{yrs})$. Results: SCI patients had higher rectosigmoid compliance at the highes distension level than controls $(10.3 \pm 2.4 \mathrm{~mL} / \mathrm{mmHg}$ vs. $5.1 \pm 0.8 \mathrm{~mL} / \mathrm{mmHg} ; \mathrm{P}<0.05)$ and reduced maximal tension tested $(54 \pm 14 \mathrm{~g}$ vs $85 \pm 4 \mathrm{~g}$; $\mathrm{P}<0.05)$. Perception scores at firs sensation were higher in patients $(2.3 \pm 0.7$ vs. $1.1 \pm 0.1 ; P<0.05)$, but were not different at the highest distension levels ( $3.7 \pm 0.8$ vs. $3 \pm 1$; NS). In 2 patients, rectosigmoid distension induced autonomic dysreflexia. Compared to controls, the most commonly reported sensation by patients was distension/bloating ( $70 \%$ vs. 54\%, respectively; NS). Patients reported sensations less commonly to the hypogastrium (the sub-umbelical region) compared to controls (29\% and $88 \%$; NS). Conclusion: Patients with ASIA-A SCI have: 1) increased recto-sigmoid compliance; 2) maintenance of visceral perception, which appears differently referred from that defined by controls. These data indicate that increased compliance is an underlying mechanism of abnormal evacuation in these patients. The preservation of visceral sensations, although abnormally referred, implies the occurrence of sensory input remodelling at spinal level.

\section{W1772}

Effects and Mechanisms of Vaginal Electrical Stimulation On Rectal Tone and Anal Sphincter Pressure: Therapeutic Potentials for Fecal Incontinence Gengqing Song, Hongbing Zhu, Jiande Chen

Aims: The aims of this study were: 1) to evaluate the effect of vaginal electrical stimulation (VES) on rectal tone and compliance and anal sphincter pressure (APS); 2) to explore possible mechanisms involved in the effects of VES on the rectal tone in conscious dogs. Methods: 8 female hound dogs inserted with a VES polyacetal probe with two rings electrodes were studied. The study included two experiments. The first experiment was composed of 2 separate sessions: rectal tone and compliance; and ASP. Each session included three sub sessions: VES with long pulses (500ms, $6 \mathrm{~mA}, 12 \mathrm{cpm})$, VES with trains of short pulses $(0.3$ $\mathrm{ms}, 6 \mathrm{~mA}$, and $40 \mathrm{~Hz}$ with $2 \mathrm{~s}$ on, $3 \mathrm{~s}$ Off), and VES with trains of long pulses ( $4 \mathrm{~ms}, 6 \mathrm{~mA}$ and $40 \mathrm{~Hz}$ with $2 \mathrm{~s}$ on, 3s Off). The second experiment was performed in two sessions (long-pulse or long-pulse train VES with guanethidine). In each session, rectal tone was sequentially recorded for 30-40 min at baseline, $20 \mathrm{~min}$ after intravenous infusion of 
guanethidine ( $3 \mathrm{mg} / \mathrm{kg}$ for 5-min), $20 \mathrm{~min}$ during VES and $20 \mathrm{~min}$ after the termination of VES. Results: 1) VES with long pulses or trains of long pulses but not trains of short pulses was able to significantly decrease rectal tone $(\mathrm{P}<0.05$, ANOVA). VES significantly increased the intra-balloon volume from $27.6 \pm 5.3 \mathrm{ml}$ at baseline to $38.2 \pm 6.6 \mathrm{ml}$ with long pulses $(\mathrm{P}=$ 0.0077 ) or $35.6 \pm 5.9 \mathrm{ml}$ with trains of long pulses ( $\mathrm{P}=0.023)$, respectively. 2) VES with long pulses or trains of long pulses but not trains of short pulses was capable of significantly increasing ASP $(\mathrm{P}<0.05$, ANOVA). In comparison with ASP at baseline $(57.7 \pm 8.2)$, VES significantly increased ASP with long pulses $(79.3 \pm 10.1, \mathrm{P}=0.014)$ or with trains of long pulses $(71.5 \pm 9.6, \mathrm{P}=0.027)$. 3) Neither method of VES altered rectal compliance $(\mathrm{P}>0.05)$. 4) The inhibitory effect of VES with trains of long pulses but not long pulses on rectal tone was abolished by guanethidine. After the infusion of guanethidine, the intra-balloon volume with long-pulse VES was significantly increased in contrast to that without VES $(36.5+5.4$ $\mathrm{ml}$ vs. $30.4 \pm 6.2 \mathrm{ml}, \mathrm{P}=0.041$ ). Conclusion: VES with long pulses or trains of long pulses but not trains of short pulses reduces rectal tone and increases ASP. The inhibitory effect of VES with trains of long pulses is mediated by the sympathetic pathway. These findings suggest that VES may be a potential treatment for fecal incontinence.

\section{W1773}

Anorectal Dysfunction Persists and Adversely Impacts Daily Activities 5 Years After Radiation Treatment for Prostate Carcinoma Addolorata C. Di Matteo, Rochelle J. Botten, Richard H. Holloway, Robert J. Fraser, Eric Yeoh

Data on the late sequelae of radiation proctitis in patients following external beam radiation therapy (RT) for prostate carcinoma $(\mathrm{CaP})$ are limited, despite its major impact on $\mathrm{QOL}^{1}$. We have shown that symptoms such as urgency of defecation and fecal incontinence persist at least 2 years following RT despite the introduction of newer techniques of treatment such as 3-D conformal $\mathrm{RT}^{2}$. However, the long term effects of RT on anorectal function are unknown. Aim: To determine the symptomatology and pathophysiology of anorectal dysfunction and the effect on daily activities 5 years after completion of RT. Methods: Anorectal symptoms and function were prospectively evaluated in 20 patients (59-76 years) with localized $\mathrm{CaP}$ (T1 and T2, N0, M0 on TNM classification). Studies were performed pretreatment and annually up to 5 years after its completion. The following parameters were evaluated (i) symptoms including impact on daily activities (validated questionnaire), (ii) anorectal motility (perfused sleeve sidehole manometry), (iii) sensory function (response to graded balloon distension) and (iv) anal sphincter morphology (endoanal ultrasound). Data are mean \pm SEM, analysed using two way repeated measures ANOVA. Results: The increase in frequency and urgency of defecation after RT was still present 5 years after treatment, although increases in fecal incontinence and rectal bleeding seen 1 and 2 years after RT were not sustained. The decrease in anal sphincter pressures and increase in rectal sensation seen at 1 and 2 years after RT were still present at 5 years. There was a progressive reduction in rectal compliance at 5 years (see Table: ${ }^{*} \mathrm{p}<0.05{ }^{* *} \mathrm{p}<0.01,{ }^{*} \mathrm{p}<0.001{ }^{\# \#} \mathrm{p}<0.0001$ ) External but not internal anal sphincter thickness $(\mathrm{mm})$ was also reduced after RT. Altered anorectal function had an adverse impact on the daily activities of $45 \%$ of patients. Conclusion: Anorectal dysfunction persists for up to 5 years after RT for $\mathrm{CaP}$ even in the absence of rectal bleeding. The resultant symptoms have significant impact on the daily activities of patients. This information needs to be provided to patients with $\mathrm{CaP}$ when considering their treatment options. ${ }^{1}$ Yeoh et al, Am J Gastro, 2004, 99920:361-369, ${ }^{2}$ AC Di Matteo et al, Gastroenterology 2005, 128(4) A:265

\begin{tabular}{|c|c|c|}
\hline & Pre-treatment & 5 years after RT \\
\hline Bowel actions/week & $11 \pm 1$ & $13 \pm 1^{*}$ \\
\hline Urgency of defecation & $0.6 \pm 0.3$ & $2 \pm 0.4^{*}$ \\
\hline Anal squeeze pressure $(\mathrm{mmHg})$ & $131 \pm 15$ & $106 \pm 8^{*}$ \\
\hline Rectal volume for perception of sensation $(\mathrm{ml})$ & $27 \pm 2$ & $16 \pm 1^{\# \#}$ \\
\hline Rectal volume for desire to defecate $(\mathrm{ml})$ & $73 \pm 10$ & $58 \pm 7^{\#}$ \\
\hline Rectal compliance $(\mathrm{ml} / \mathrm{mmHg})$ & $8 \pm 0.7$ & $5 \pm 0.6^{\#}$ \\
\hline External anal sphincter thickness $(\mathrm{mm})$ & $10 \pm 0.5$ & $9.9 \pm 0.6^{\#}$ \\
\hline
\end{tabular}

\section{W1774}

The Cytokinesis Blocked Micronucleus Assay Correlates with Acute Anorectal Dysfunction After Radiation Therapy (RT) for Carcinoma of the Prostate and May Predict Individual Radiosensitivity

Addolorata C. Di Matteo, Rochelle J. Botten, Julie F. Butters, Michael Fenech, Ian Olver, Eric Yeoh

RT for carcinoma of the prostate ( $\mathrm{CaP}$ ) is associated with anorectal dysfunction which persists and impairs the daily activities of patients 2 years $l a t e r^{1}$. Patients receive the same radiation dose despite wide variations in individual susceptibility to normal tissue damage ${ }^{2}$. The Cytokinesis Blocked Micronucleus (CBMN) assay, which quantifies radiosensitivity based on ex-vivo irradiation of peripheral blood lymphocytes (PBL), has been correlated with symptoms of anorectal damage after $\mathrm{RT}$ for $\mathrm{Ca}^{2}$. However, there has been no prospective study to examine the relationship between the CBMN index and anorectal dysfunction after RT. Aim: To determine whether the CBMN assay predicts individual susceptibility to anorectal dysfunction after standard dose ( $70 \mathrm{~Gy} / 35$ fractions/ 7 weeks) 3D conformal RT for localized CaP. Methods: Each patient had fasting venous blood taken before RT and the CBMN index of PBL determined at baseline and after 3Gy ex-vivo irradiation ${ }^{3}$. GI symptom scores (LENTSOMA based questionnaire ${ }^{4}$ ), anorectal motor and sensory function (perfused sleeve anorectal manometry and graded rectal balloon distension ${ }^{1}$ ) and anal sphincteric morphology (endoanal ultrasound) were evaluated before RT and after its completion. GI symptom scores and motor and sensory function variables of 12 patients (55-78 years) who have completed evaluation at 1 month were analysed using paired t-test. Linear regression was used to examine the relationships among the baseline and irradiated CBMN index data and (i) LENTSOMA symptom scores, (ii) motor and sensory function and (iii) the total volume of rectum irradiated. All statistical analyses were 2 tailed and a p value of $\leq 0.05$ considered significant. Results: LENT-SOMA GI symptom scores but not anorectal motor and sensory function variables increased 1 month after RT. Baseline CBMN index was directly related to (i) urgency of defecation $(r=0.66, p<0.05)$ and (ii) diarrhea $(r=0.62, p<0.05)$ scores at 1 month. Exvivo irradiated CBMN index was inversely related to rectal compliance $(\mathrm{ml} / \mathrm{mmHg})$ in the patients 1 month after RT $(r=-0.63, p<0.05)$. Conclusions: Mis-repair of spontaneous and radiation-induced genomic damage are likely to be important underlying pathogenetic mechanisms of acute anorectal dysfunction after RT for carcinoma of the prostate. Baseline and ex-vivo irradiated CBMN index may form the basis for individualizing patient RT. ${ }^{1} Y e o h$ et al Amer J Gastrointerol 2004 2: 361-369 ${ }^{2}$ Lee et al Int J Radiat Oncol Biol Phys 2003, 57 : 222-229 ${ }^{3}$ Fenech Mutation Research 2000, 455: 81-95 ${ }^{4}$ Yeoh et al Int J Radiat Oncol Biol Phys 2003, 55: 943-955

\section{W1775}

Antegrade Continent Enema for Chronic Constipation in Adults: Long Term Results

Sushil K. Maslekar, Jill Marshall, Graeme S. Duthie

Introduction: Slow transit chronic constipation in adults can be very difficult to treat and defaecation can be often painful. Total colectomy with ileorectal anastomosis is offered conventionally but is associated with persisting constipation in $10-30 \%$ patients. Ileostomy brings relief but is not universally acceptable. Antegrade continent enema (ACE) procedure has previously been shown to be effective in approximately half of these patients in the short term, but longer-term results are unknown. The aim of this study was to evaluate the long-term results of the ACE procedure in chronic constipation and faecal leakage, including its impact on quality of life. Methods: All patients undergoing ACE procedure from 1996 to 2000 were included in the study. Telephonic interview was conducted following which questionnaires were sent to all the patients. These questionnaires included SF-36 type questions, faecal incontinence quality of life, Wexner score type and general questions about the procedure. Retrospective review of the case notes was done to obtain clinical, demographic and anorectal physiology data. Results: 24 patients ( 22 females) with a mean age of 37.9 years underwent the ACE procedure, out of which 17 were done laparoscopically. 18 patients had chronic refractory constipation, 3 patients had faecal leakage and 3 patients had mixed symptoms preoperatively. At a median follow up of 72 months (minimum 5 years follow up), 14 patients $(60 \%)$ were still successfully irrigating. 8 of the 24 patients needed revision of their conduits. After ACE reversal in the remaining 10 patients, 5 patients $(50 \%)$ underwent ileostomy. No serious complications occurred in any patient. $75 \%$ patients had demonstrated initial improvement in symptoms and $52 \%$ showed satisfactory improvement in bowel function at 72 months follow up. Quality of life showed significant improvement in the mean mental score on theSF-36 form from 34.6 preoperatively to 51 ( $\mathrm{p}=0.04$; Mann-Whitney $\mathrm{U}$ test) and an improvement in mean physical component score from 38.3 to 44.3 ( $\mathrm{p}=0.08)$. The type of symptoms or type of conduit did not influence the outcomes. Conclusion This is the longest follow up of adults undergoing ACE procedures. The procedure is technically simple and effective, and affords an improvement in the quality of life in patients with chronic constipation and faecal leakage.

\section{W1776}

Is There Any Correlation Between Viscero-Motor Response and Sensation in Man?

Alfred Bayati, Martin Karpefors, Per Jerndal, Kristina Jordo, Louis M. Akkermans

Viscero motor response (VMR) to colorectal distension has been used in animals for some time as a marker for noxious stimuli. This response is believed to represent discomfort and pain which otherwise can not be measured in animals. In order to see if this hypothesis can be tested in man we sought to test firstly if this response can be measured in man, secondly if this response is indeed related to discomfort/pain and thirdly if there is any difference between healthy volunteers and IBS patients in this respect. A total of 69 healthy volunteers (43 females) and 97 IBS patients (66 females) were included in these studies with ramp tonic distension. In a smaller group of 10 healthy volunteers and 9 IBS patients an ascending method of limit paradigm (AML) was performed using a barostat. Abdominal muscle EMG was measured using surface electrodes and VMR was calculated as the area under the curve of the rectified EMG signal. Discomfort and pain was measured continuously using a newly developed electronic analogue scale (EAS). It is shown that during AML, VMR could be measured in all subjects. The VMR increased by increasing pressure showing a good linear correlation to the distension pressure. Discomfort felt by the subjects measured by EAS was completely related to the pressure and VMR changes. During ramp tonic distension, VMR could be measured in 54\% and 44\% of healthy volunteers and IBS patients respectively. The threshold was calculated to $34 \pm 2$ and $40 \pm 2 \mathrm{mmHg}(\mathrm{p}=0.1)$ and the maximum AUC to $0.97 \pm 0.09$ and $0.6 \pm 0.07 \mathrm{~V}^{*} \mathrm{~s}(\mathrm{p}=0.009)$ in healthy volunteers and IBS patients respectively. In the rest of the subjects VMR response was, probably, blocked by cortical control soon after its start. It is concluded that VMR can be measured in man in the same way as it is measured in animals and it is very well correlated to the sensation felt by the subjects and differed between healthy volunteers and IBS patients.

\section{W1777}

Influence of Naloxone On Rectal Sensitivity and Accommodation of the Rectum After Consecutive Distensions in Health Brecht Geeraerts, Lukas van Oudenhove, Rita Vos, Georgios Karamanolis, Jan Tack Background: Failure of descending anti-nociceptive pathways (with opioids as the main candidate neurotransmitters) has been suggested to contribute to the pathogenesis of irritable bowel syndrome (IBS). Rectal balloon distention studies in IBS patients have revealed hypersensitivity to rectal balloon distention (Whitehead 1990) and impaired rectal accommodation 
to repeat balloon distention (Kwan 2004). The aim of this study is to investigate the effect of suppression of endogenous opioids by naloxone on rectal sensorimotor function in health. Methods: Thirteen healthy subjects (age $26 \pm 1$ years) participated in a randomized, placebocontrolled, double blind rectal barostat study. Sensorimotor function was evaluated during 2 consecutive stepwise distensions ( $4 \mathrm{mmHg}$ steps at 1.5 minute intervals, until discomfort or pain) separated by 30 minutes of basal volume recording. Continuous infusion of saline or naloxone ( $20 \mu \mathrm{g} / \mathrm{kg} / \mathrm{h}$ after $0.4 \mathrm{mg}$ bolus) was started 15 minutes before the $2 \mathrm{nd}$ distension. VAS scales were used to register relevant symptoms. Upper abdominal sensation was scored on a $0-6$ graded scale $(0=$ no sensation, $6=$ maximal $)$ at the end of every distension step. Results: Naloxone did not alter the pressure-perception curve compared to saline or to baseline. Consequently, the pressure needed to induce first perception ( $12.9 \pm 1.3 \mathrm{vs} .12 .9 \pm 1.8$ $\mathrm{mmHg}$, NS) or discomfort $(34.0 \pm 1.4$ vs. $34.6 \pm 1.6 \mathrm{mmHg}$, NS) and corresponding intraballoon volumes (respectively $108 \pm 21$ vs. $93 \pm 19 \mathrm{ml}$, and $248 \pm 16$ vs. $258 \pm 17 \mathrm{ml}$, both NS) did not differ between saline and naloxone. Comparison of VAS scores between naloxone and saline did not reveal altered intensities of pain (AUC $17.3 \pm 8.8$ vs. $19.4 \pm 9.1 \mathrm{~mm} * \mathrm{mmHg}$, NS) or discomfort (AUC $35.9 \pm 12.2$ vs. $33.1 \pm 16.2 \mathrm{~mm} * \mathrm{mmHg}$, NS). During the second distention, the pressure-volume curve was shifted to the left in the placebo condition but not in the naloxone condition: compared to the baseline distension, a significant adaptive increase in compliance occurred during the second distension after saline $(7.3 \pm 0.8$ vs. $10.0 \pm 0.6 \mathrm{ml} / \mathrm{mmHg}, \mathrm{p}=0.008$ ), as previously reported (Kwan 2004). This adaptive increase in rectal compliance did not occur after naloxone $(8.7 \pm 0.8$ vs $9.2 \pm 0.9 \mathrm{ml} / \mathrm{mmHg}$, NS), and the increase in compliance between both distentions was significantly suppressed after naloxone ( $\Delta$ compliance $2.6 \pm 0.8$ vs. $0.5 \pm 0.7 \mathrm{ml} / \mathrm{mmHg}, \mathrm{p}=0.003$ ), thereby mimicking previous findings in IBS (Kwan 2004). Conclusion: Naloxone does not alter rectal sensitivity but it abolishes rectal accommodation in response to repeat balloon distention. These observations suggest that the endogenous opioid system is involved in control of rectal tone rather than rectal sensitivity.

\section{W1778}

Anorectal Inhibitory Reflex (RAIR) Can Be Assessed When Children Are Sedated with Etomidate

Reinaldo Garcia, Michael Reed, Lia Lowrie, Gisela Chelimsky

Background: The manometric evaluation of the anorectal area is a noninvasive, inexpensive and simple test that is often used as a diagnostic tool in Hirschsprung's disease. In nonsedated patients the rectoanal inhibitory reflex may be masked by patients' lack of cooperation. To avoid this technical obstacle, effective sedation of the younger or uncooperative child undergoing anorectal manometry is essential. We have been using etomidate at our institution to sedate children undergoing anal manometry. Etomidate is an imidazole sedative-hypnotic agent with a very rapid onset of action (5-20 seconds) and rapid recovery time following single dose administration. Methods: Age, sex, etomidate dose, number of doses, total amount administered, length of the procedure, drug-associated complications, other medications used, and the presence of RAIR were obtained from the procedural records of children who underwent sedated anal manometry at Rainbow Babies and Children's Hospital from 1/00 to $12 / 02$. Results: Twenty-four children, ages 3 months to 8.8 years (mean 2.99 years, SD + - 2.26) underwent an etomidate-sedated anorectal manometry. All but 2 were effectively sedated with etomidate. Individual etomidate doses ranged from 0.06 to $0.6 \mathrm{mg} / \mathrm{kg} / \mathrm{dose}$ with the total amount of etomidate per procedure ranging from 0.4 to $1.4 \mathrm{mg} / \mathrm{kg}$. All but one child tolerated etomidate without difficulty (one subject with Down's syndrome experienced airway obstruction, resolved with repositioning). No other significant complications were observed. One subject had inconsistent RAIR, but had ganglion cells present on rectal biopsy. In 4 patients we were unable to immediately determine their rectoanal inhibitory reflex since they were too deeply sedated. Despite their level of sedation the total amount of medication given in $\mathrm{mg} / \mathrm{kg}$ did not differ from the other subjects. Successful determination of the rectoanal reflex was later obtained in this subset of subjects as the sedation was wearing off. Average sedation time: 29 minutes (range from 13 to 68 minutes (> $50 \mathrm{~min}$ when other medications were added). Two patients had oral midazolam $(0.33 \mathrm{mg} / \mathrm{kg}$ ) prior to the procedure; two subjects had inadequate sedation with etomidate and required additional medications to be given (propofol, pentothal). All of these subjects demonstrated RAIR. Conclusion: Anal manometry is an integral component of a patient's evaluation for Hirschsprung's disease. Our data supports that etomidate can be safely and effectively used in the setting of anal manometry without inhibiting the ability to assess for the rectoanal inhibitory reflex.

\section{W1779}

Validation of a Novel Surface EMG Probe for Assessing Anal Sphincter Innervation

Juila Traue, Jasper Daube, William Litchy, Nima Mowzoon, Paul Enck, Adil Bharucha

Background-Needle electromyography (nEMG) is the only technique that can accurately evaluate for neural injury affecting the anal sphincter. Because nEMG examination requires considerable technical expertise, a novel surface EMG (sEMG) probe with 16 circumferential electrodes to characterize anal sphincter innervation was developed (Digestion 2004;69:112). Aims-The aims of this study were to validate the sEMG probe against nEMG exam for assessing anal sphincter innervation. Methods-Anal sphincter innervation was assessed simultaneously in a 2 channel recording of $n E M G$ and sEMG in 18 healthy, nulliparous women [mean age 40 years (range 19-75 years)] without a functional GI disorder and 6 women with fecal incontinence (FI). Sphincter activity was recorded by nEMG in each of 4 quadrants at rest (60 seconds) and during squeeze (2 repetitions, 15 seconds each). SEMG tracings were reviewed to characterize the site of onset and propagation of surface motor unit potentials (SMUP). Because the sEMG signal represents a composite of several SMUP, individual SMUP were identified by spike triggered averaging (STA) off-line. MUP were isolated from the needle recording by both manual isolation with trigger windows (Bromberg) on a Nicolet Viking EMG machine and by decomposition quantitative EMG (DQEMG) (Doherty \& Stashuk) on a Neuroscan Comperio EMG machine. Needle MUP triggered an average of the surface signal to identify the SMUP. Propagation of individual STA SMUP around the sphincter was confirmed. Data Analysis-For validation, we assessed the proportion of total and propagated SMUP also identified by the needle electrode. Data were averaged across both squeeze maneuvers. A 2-sided Wilcoxon signed rank test was used to assess i these proportions differed from that anticipated due to chance alone (i.e., 0.5). Results-At least 1 sMUP was recorded in 15 women at rest and in all women during squeeze. $50 \%$ o sMUPs at rest and $78 \%$ of sMUPs during squeeze were propagated. The proportion of sMUP that were also recorded by the needle was greater than expected by chance alone (Table) Conclusions-Propagated and non-propagated MUAPs in the anal sphincter generally correspond to identical motor units recorded by needle EMG in controls and FI. Less well-defined propagation can be due to superimposition of more than one needle MUP in generating the SMUP. These findings validate the use of SEMG for investigating anal sphincter innervation in health and disease.

\begin{tabular}{|c|c|c|c|c|c|}
\hline Maneuver & $\begin{array}{c}\text { Needle } \\
\text { MUP }\end{array}$ & $\begin{array}{c}\text { Surface } \\
\text { MUP }\end{array}$ & $\begin{array}{c}\text { Combined (i.e., } \\
\text { needle and surface } \\
\text { MUP) }\end{array}$ & $\begin{array}{c}\text { Percentage of } \\
\text { SMUP also } \\
\text { identified by needle }\end{array}$ & $\begin{array}{c}\text { Percentage of } \\
\text { propagated sMUP also } \\
\text { identified by needle(\%) }\end{array}$ \\
\hline Rest & $6.7(0.8)$ & $2.4(0.3)$ & $2.0(0.2)$ & $88 \%(5)^{*}$ & $90 \%(6)^{*}$ \\
\hline Squeeze & $5.1(0.6)$ & $2.5(0.3)$ & $1.6(0.2)$ & $67 \%(6)^{*}$ & $78 \%(7)^{*}$ \\
\hline
\end{tabular}

Values are mean number (SE) or mean $\%(\mathrm{SE}) ; * \mathrm{p}<0.01$

\section{W1780}

Effect of Hysterectomy On Anorectal Functions - A Prospective Study Barbara M. Seide, Christopher J. Klingele, John B. Gebhart, Alan R. Zinsmeister, Adil Bharucha

Background - Hysterectomy ranks as the second commonest surgical procedure among women in the USA with over $>600,000$ procedures performed annually One in three women will have a hysterectomy by the age of 60 years. Dissection of the rectovaginal septum can also damage the extensive network of nerve fibers surrounding the rectum, cervix, and lateral vaginal surfaces during a hysterectomy. While a radical hysterectomy is known to impair anorectal functions, the effects of a "simple" (i.e., vaginal or abdominal) hysterectomy on anorectal functions are poorly understood. Aims - To prospectively assess the effects of a simple hysterectomy on anorectal sensori-motor functions. Methods - In this ongoing study, anorectal functions are assessed before and 2 months after a hysterectomy [vaginal (14) or abdominal (2)] in 16 women [(age $47 \pm 3$ years (Mean \pm SEM)] without significant GI symptoms. Assessments include: (i) anal resting and squeeze pressures and the traction required to expel a latex balloon filled with $60 \mathrm{ml}$ of water; (ii) rectal compliance (balloon volume response to $4 \mathrm{mmHg}$ stepwise increments from 0-44 $\mathrm{mmHg}$ ); (iii) rectal sensation, measured by thresholds for first sensation, desire to defecate (DD), and urgency during the staircase distention and a 100-mm VAS scale for gas, urgency and pain during phasic colonic balloon distentions of 8,16 , and $24 \mathrm{mmHg}$ above operating pressure delivered in random order; and (iv) rectal evacuation assessed by the external traction (gm) required to expel a balloon filled with $60 \mathrm{ml}$ water. Analyses - Staircase distentions were summarized as $\operatorname{Pr}_{1 / 2}$ (the pressure at 1/2 max volume) and rectal capacity (i.e., volume at $32 \mathrm{mmHg}$ ). Anorectal functions were compared before and after a hysterectomy by 2 -tailed paired parameteric or nonparametric tests. Results - Table shows mean (SEM) for parameters before vs after hysterectomy. Also, rectal volumes, but not VAS scores tended to be lower during phasic distentions (data not shown). Conclusions - These preliminary observations suggest that rectal evacuation and capacity decline 2 months after a hysterectomy. Long term assessments, currently in progress, are necessary to identify the risk factors for anorecta dysfunctions after a hysterectomy.

Effect of Hysterectomy on Anorectal Functions

\begin{tabular}{|c|c|c|c|c|c|c|c|}
\hline Epoch & $\begin{array}{c}\text { Anal } \\
\text { Resting Pr } \\
(\mathrm{mmHg})\end{array}$ & $\begin{array}{c}\text { Anal } \\
\text { Squeeze Pr } \\
(\mathrm{mmHg})\end{array}$ & $\begin{array}{c}\text { Rectal } \\
\text { Compliance } \\
\mathrm{Pr}_{\text {half }}(\mathrm{mmHg})\end{array}$ & $\begin{array}{c}\text { Rectal } \\
\text { Capacity } \\
(\mathrm{ml})\end{array}$ & $\begin{array}{c}\text { Balloon } \\
\text { Expulsion } \\
(\mathrm{wt})\end{array}$ & $\begin{array}{c}\text { DD Vol } \\
(\mathrm{ml})\end{array}$ & $\begin{array}{c}\mathrm{DD} \mathrm{Pr} \\
(\mathrm{mmHg})\end{array}$ \\
\hline Before & $62 \pm 5$ & $129 \pm 15$ & $14.9 \pm 0.8$ & $293 \pm 10$ & $175 \pm 54$ & $169 \pm 20$ & $16.8 \pm 1.9$ \\
\hline After & $62 \pm 5$ & $125 \pm 12$ & $15.4 \pm 0.6$ & $281 \pm 8^{*}$ & $361 \pm 66^{*}$ & $138 \pm 16 \#$ & $17.3 \pm 1.7$ \\
\hline
\end{tabular}

$* \mathrm{p}=0.02, \# \mathrm{p}=0.1$ by paired analysis

\section{W1781}

Sacral Nerve Stimulation in Fecal Incontinence: Are There Factors Associated with Failures?

Syrine Gallas, Guillaume Gourcerol, Francis Michot, Philippe Denis, Anne-Marie Leroi

Background: Sacral nerve stimulation has been used successful in treating faecal incontinence. The effectiveness of the procedure has been tested by temporary nerve stimulation. Nevertheless, some patients show deterioration in primary outcome measures. Our aim was to determine parameters associated with these failures. Methods: A total of 29 patients (26 women) (median age 56.5 years old, range 33-77) with refractory faecal incontinence, underwent permanent sacral nerve stimulation for a median of 12 months (range 6-72). The results of sacral nerve stimulation were evaluated based on daily bowel-habits diaries over a 3-weeks period. More than $50 \%$ improvement of faecal incontinence and/or urgency episodes was considered successful sacral nerve stimulation. The factors compared between the two groups (success and failures) were patients' age and sex, diagnosis and characteristics of faecal incontinence, previous surgery, quality of life scores, anorectal manometry, endoanal ultrasound, electrophysiological test performed before implantation and rate of success of temporary nerve stimulation. Results: After permanent implantation, 31\% of patients did not achieve the effect of the screening phase in the number of episodes of faecal incontinence or urgency (failures). It appeared that the left bulbo(clitorido)-cavernosus reflex was more frequently delayed in the success group than in the others $(60.5+21.4$ msec versus $44.3+12$. msec, $p=0.03$ ). Chronic diarrhoea refractory to medical treatment was related with poor 
results observed on faecal urgency $(r=0.48 ; \mathrm{p}=0.02)$. Conclusion: Diarrhoea and electrophysiological results evaluated before implantation could be factors of prognosis for the treatment by sacral nerve stimulation.

\section{W1782}

Sacral Nerve Neuromodulation As Primary Treatment for Faecal Incontinence with Disrupted Anal Sphincters: Medium and Long-Term Results Sushil K. Maslekar, Angela B. Gardiner, Graeme S. Duthie

Introduction The standard treatment for faecal incontinence secondary to obstetric sphincter damage is anal sphincter repair. However, the results of this procedure deteriorate with time. Sacral nerve stimulation (SNS) has become an established therapy for faecal incontinence in patients with intact sphincter muscles, with good medium term results. We assess the medium and long-term results of SNS as first line treatment in patients with obstetric-related faecal incontinence. Methods: All patients with obstetric-related faecal incontinence and demonstrated external and internal sphincter disruption on anal endosonography were included in the study. These patients would have normally undergone anterior anal sphincter repair. Patients underwent temporary stimulation followed by assessment at 2 weeks. Permanent stimulator was then implanted in patients with significant improvement. Post implantation patients were followed up $1,3,6,12,36$, and 48 months. Demographic, clinical, operative and anorectal physiology data was collected prospectively. Results: 20 consecutive women with a median age of 42 (35-62) years completed temporary screening and 19 went on to permanent implantation. Faecal incontinence improved in all patients from Wexner scores of median $16(10-20)$ preoperatively to median $5(1-11)$ at 2 weeks. There was further improvement in faecal incontinence with median Wexner scores of $3,3.5,4,4,3.5 \& 3$ at $1,3,6,12,36 \& 48$ months respectively $(\mathrm{p}<0.0001)$. The remaining patient was followed up at 2 weeks post temporary SNS and demonstrated significant improvement in faecal incontinence (Wexner score 19 pre-procedure to 3 post-procedure), but was lost to follow up thereafter. There was significant improvement in general and mental health, emotional role, social function and vitality in all patients. There were no major complications and no implants were removed. Conclusion: This is the first study showing long-term results of SNS for primary treatment of faecal incontinence with damaged anal sphincter. We have shown that sacral nerve neuromodulation is a safe and effective minimally invasive first line treatment for such patients. The improvement in faecal incontinence achieved in short term is sustained over a follow up of 4 years

\section{W1783}

Effect of Acute Fasting Hyperglycemia and Duodenal Glucose On Rectal Perception and Compliance in Healthy

Hermann Harder, Ana C. Hernando-Harder, Heinz-Juergen Krammer, Manfred V. Singer

Background: Acute changes in blood glucose concentration have major reversible effects on gastrointestinal motor and sensory function. Aims: To test influences of intravenous glucose (hyperglycemic hyperinsulinemia) duodenal glucose (euglycemic hyperinsulinemia) and duodenal saline (as control) on rectal perception and compliance. Methods: In 8 healthy subjects, responses to fixed tension rectal distension applied by means of an electronic tensostat were compared on 3 separate days in randomized order during a marked hyperglycemic clamp ( 13 mmol/l), duodenal glucose at $2 \mathrm{Kcal} / \mathrm{min}$, or duodenal saline as control. Results: At perception of normal urge to defecate (score 3) on the 0 - 5 rectal scale, perception of rectal distension was significantly lower during hyperglycaemia $(83 \pm 4 \mathrm{~g}$ tolerance) whereas perception of rectal distension was significantly higher during duodenal glucose ( $45 \pm \mathrm{g}$ tolerance), both reaching significance as compared to control $(64 \pm 6 \mathrm{~g}$ tolerance; $\mathrm{p}<0.05)$. At the former perception level, no relevant variations of rectal compliance were seen, which were $9.5 \pm 1 \mathrm{ml} / \mathrm{mmHg}$ for the group with hyperglycemia, $10.3 \pm 1 \mathrm{ml} /$ $\mathrm{mmHg}$ during duodenal glucose ( $>0.05$ vs. control for both) and $9.7 \pm 2 \mathrm{ml} / \mathrm{mmHg}$ in the control studies. Overall rectal compliance ranged between $8.3 \pm 2 \mathrm{ml} / \mathrm{mmHg}$ and 11 $\pm 1 \mathrm{ml} / \mathrm{mmHg}$ ( $>0.05$ for all). Conclusion: Acute hyperglycemia contributes to rectal hyposensitivity whereas duodenal glucose provokes rectal hypersensitivity. Despite different rectal tensosensitivity, compliance remains unchanged.

\section{W1784}

Morphologic and Mechano-Sensitive Characteristics of the Rectum After Prostate Carcinoma Treatment with High-Precision Radiotherapy Agustin Balboa, Meritxell Molla, Antonio Salas, Antonia Perello, Monica Perona, Raimond Miralbell, Fermin Mearin

Radiotherapy is very effective in the treatment of prostate carcinoma but it may cause late complications in the rectum, both inflammatory and mechano-sensitive (chronic radiation proctitis). OBJECTIVE: To investigate whether new high-precision radiotherapy with modulated intensity and extracranial stereotactic positioning system lead on not to late rectal alterations, both morphologic (endoscopic or histologic) and mechano-sentive (rectal compliance and sensitivity). METHODS: In 29 patients that had received radiotherapy for prostate carcinoma at least 15 months prior to procedure, we evaluated endoscopic lesions (mucosal edema, telangiectasis, ulcers, stenosis and necrosis), histologic abnormalities (epithelial lesions, crypts distortion, vascular edema, lamina propria fibrosis, and inflammatory activity) and mechano-sensitive characteristics with a rectal barostat (rectal compliance and sensitivity thresholds) RESULTS: Both, endoscopic and histologic scores were low $(1.3+0.2$ over 5 and $2.5 \pm 0.3$ over 15 , respectively), with a good correlation between both scores $(r=0.63 ; 95 \%$ IC: $0.34-0.81 ; \mathrm{p}<0.05)$. We didn't find any correlation between morphological scores and total radiation doses. Rectal compliance was normal $(5.3 \pm 0.4 \mathrm{ml} / \mathrm{mmHg})$ whereas rectal sensitivity was low compared to healthy control values: first perception threshold: $12.0 \pm 1.7$ vs $6.2 \pm 0.8 \mathrm{mmHg}, \mathrm{p}<0.05$; tenesmus threshold: $23.5 \pm 1.6$ vs $20.4 \pm 3.0 \mathrm{mmHg}$; $=\mathrm{NS}$; discomfort threshold: $36.0 \pm 2.9$ vs $20.7 \pm 2.1 \mathrm{mmHg} ; \mathrm{p}<0.05$. No correlation was found among sensitivity thresholds and histologic or endoscopic scores. CONCLUSION: Minimal morphological alterations of the rectum are observed after prostate carcinoma treatment with high-precision radiotherapy; rectal compliance does not change whereas rectal sensitivity is decreased.

\section{W1785}

Comparison of Rectal Contractile Responses During Inflation, Sustained Distention, and Deflation in Health and Fecal Incontinence Christopher N. Andrews, Barbara M. Seide, Alan R. Zinsmeister, Adil Bharucha

Background-Rectal distention paradigms focus on perception thresholds during rectal inflation. An exaggerated contractile response to distention may partly explain increased rectal perception in IBS (Corsetti, CGH 2004). Current concepts suggest that the rectum generally accommodates to sustained distention in health. However, patients often complain of persistent rectal urgency, perhaps due to a persistent contractile response to sustained distention. It is also unknown if rectal deflation can induce a contractile response, akin to the off response described in esophageal smooth muscle (Goyal, AJP 1984). Aim-To compare the contractile response and perception during inflation, sustained distention, and deflation in health and fecal incontinence (FI). Methods-Rectal balloon pressure and volume were recorded during barostat-driven rectal distention in 51 FI patients $(60 \pm 2$ years; mean \pm SEM) and 20 healthy controls (age $61 \pm 2$ years). Three ramp distentions $(25,50,100 \mathrm{~mL}$ min from $0-200 \mathrm{~mL}$ ) were performed in random order. Each distention was comprised of inflation, sustained distention (at $200 \mathrm{~mL}$ for $1 \mathrm{~min}$ ) and deflation. Contractions were identified by a $\geq 2 \mathrm{mmHg}$ change in baseline pressure for at least 5 seconds. The number of contractions during inflation (I), sustained distention (SD) and deflation (D) in controls and FI were analyzed by a repeated measures analysis of variance assessing for effects of group (i.e., control or FI) and rate. Results-Modulation of rectal volume evoked rectal contractions not only during I, but also during SD and during D (Table). The contractile response during $\mathrm{SD}$ and $\mathrm{D}$ was more prominent $(\mathrm{p} \leq 0.01)$ during faster distention in $\mathrm{FI}$ and controls. During I, a rate effect was observed in controls, but not FI ( $\mathrm{p}$ for group*rate interaction < 0.05). Group effects were not significant. Conclusions-Rectal distention induced a contractile response not only during I, but also during SD and to a lesser degree during D in controls and FI. This contractile response may increase rectal pressures and contribute to rectal urgency.

Effect of Rectal Distention on Rectal Contractions

\begin{tabular}{|c|c|c|c|c|c|c|}
\hline \multirow{2}{*}{} & \multicolumn{3}{|c|}{ Controls } & \multicolumn{3}{c|}{ FI } \\
\cline { 2 - 7 } & I & SD & D & I & SD & D \\
\hline $\mathbf{2 5} \mathbf{~ m L / m i n ~}$ & $0.46(0.13)$ & $0.51(0.18)$ & $0.07(0.04)$ & $0.67(0.13)$ & $0.86(0.18)$ & $0.18(0.05)$ \\
\hline $\mathbf{5 0} \mathbf{~} \mathbf{m L} / \mathbf{m i n}$ & $0.82(0.20)$ & $1.45(0.25)$ & $0.10(0.05)$ & $0.64(0.12)$ & $1.37(0.25)$ & $0.22(0.05)$ \\
\hline $\mathbf{1 0 0} \mathbf{~ m L} / \mathbf{m i n}$ & $0.98(0.23)$ & $1.42(0.38)$ & $0.29(0.09)$ & $0.65(0.14)$ & $1.78(0.26)$ & $0.37(0.11)$ \\
\hline
\end{tabular}

All values are mean (SEM) for contractions/minute

\section{W1786}

Baroreceptor Sensitivity in Irritable Bowel Syndrome Patients and Healthy Volunteers During Colorectal Distension

Robert Spaziani, Keith Redmond, Harjeet S. Bajaj, Alfred Bayati, Karen Miranda, Hakan Larsson, Subhas Ganguli, Steven Collins, Markad Kamath

Background: Arterial baroreflex provides an immediate adjustment of blood pressure following a perturbation. Baroreceptor sensitivity (BRS) analysis $(\Delta \mathrm{R}-\mathrm{R} / \Delta \mathrm{BP})$ using systolic blood pressure (SBP) provides an index of baroreceptor control of heart rate (HR) by autonomic mechanisms. Aim. To examine the BRS response to colorectal distension (CRD) in Irritable Bowel Syndrome patients (IBS) compared with healthy controls (HC). Materials and Methods: After a 20-min. baseline, a barostat-controlled rectal balloon (max. volume $863 \mathrm{~mL}$ ) was distended $(0.3 \mathrm{mmHg} / \mathrm{s})$, to a max. pressure of $70 \mathrm{mmHg}$, or $50 \%$ tolerable discomfort (whichever came first), and maintained in this tonic state for 3 mins. A 20-min. recovery period was recorded. Forty-six HC (19 females; $30 \pm 10$ yrs.) and 32 patients ( 28 females; $40 \pm 11$ yrs.) with IBS were studied. BRS was computed during baseline, ramp, tonic, and post-distension periods using 3 or more increasing sequences within continuous HR and systolic BP time series. Group means were compared using Student's t-test. Results: IBS patients and $\mathrm{HC}$ reached similar CRD pressures $(51 \pm 15 \mathrm{mmHg}$ vs. $52 \pm 14 \mathrm{mmHg}$ ); patients experienced more discomfort $(69 \pm 20 \%$ vs. $58 \pm 11 \%, \mathrm{p}=0.01)$. With tonic distension, both HR (HC: $65 \pm 8$ to $70 \pm 10 \mathrm{BPM}, \mathrm{p}=0.01$ vs. IBS: $64 \pm 9$ to $71 \pm 11 \mathrm{BPM}, \mathrm{p}=0.007$ ), and SBP (HC: $124 \pm 15$ to $144 \pm 19 \mathrm{mmHg}, \mathrm{p}<0.001$ vs. IBS: $129 \pm 20$ to $157 \pm 23 \mathrm{mmHg}, \mathrm{p}<0.001$ ) increased significantly. IBS patients had a larger SBP response to CRD compared to $\mathrm{HC}(\mathrm{p}=$ 0.006). HR (HC: $65 \pm 8$ BPM; IBS: $63 \pm 9$ BPM) and SBP (HC: $131 \pm 16 \mathrm{mmHg}$; IBS: $138 \pm 20$ $\mathrm{mmHg}$ ) decreased in the post-distension phase. Table 1 compares BRS in IBS vs HC. Conclusions: BRS was consistently diminished in IBS patients compared with HC at rest and throughout the CRD paradigm CRD induced a sympathetic response in both $\mathrm{HC}$ and IBS patients with increased HR and SBP. IBS patients demonstrated the greatest SBP perturbation, suggesting insufficient baroreceptor control. Attenuated baroreceptor responsiveness suggests an underlying autonomic abnormality in patients with IBS. Funded by: AstraZeneca, NSERC and DeGroote Foundation.

Table 1: BRS (msec./mmHg) in IBS vs. Healthy Controls with CRD

\begin{tabular}{|c|c|c|c|c|}
\hline & Baseline & Ramp & Tonic & Post \\
\hline Healthy Controls & $12.75 \pm 2.37$ & $8.48 \pm 2.67 * * \dagger$ & $8.15 \pm 2.39 * * \dagger$ & $10.81 \pm 2.58^{* *}$ \\
\hline IBS Patients & $7.20 \pm 2.53^{*}$ & $6.00 \pm 2.50^{* \dagger} \dagger$ & $7.08 \pm 2.44 \dagger$ & $8.80 \pm 2.45^{*}, * *$ \\
\hline
\end{tabular}

* $\mathrm{p}<0.05$ when compared to controls ** $\mathrm{p}<0.05$ when compared to baseline $\uparrow \mathrm{p}<0.05$ when compared to post 
W1787

Development of a Validated Questionnaire to Identify Idiopathic Fecal Incontinence

Alexandra Ilnyckyj, Jennifer Laforce, Sandra Allford

Background: The epidemiology of fecal incontinence(FI) remains incompletely understood; prevalence studies have not found consensus. This may stem from a lack of validated measures and poor discrimination of idiopathic FI from FI ocurring within a known GI condition such as irritable bowel syndrome (IBS) or inflammatory bowel disease (IBD). Aim: Develop and validate a brief self-report questionnaire to assess the prevalence of idiopathic FI. Methods: A 10 item FI measure, including validated and not validated questions used in previous prevalence studies were administered to 95 participants, who were recruited from GI and Internal Medicine ambulatory clinic. All participants were assessed by one gastroenterolgist and/or GI nurse clinician to define status of bowel function and GI diagnosis. Participants were classifed into one of 6 groups 1)idiopathic FI (no IBS, IBD or other known organic disease to explain FI) 2)IBS with FI 3)IBD with FI 4)Normal bowel function 5)IBS no FI 6) IBD no FI. Results: Six items were found to accurately identify participants with FI and 2 items were found which discriminated those with idiopathic FI from those with FI due to a known cause. Thus, from the 10 items, 2 scales emerged; 1) FI scale: a 6 item scale measuring FI and 2)abdominal distress scale: a 2 item scale discrinating FI secondary to IBS/IBD. The use of these two scales was supported by a Maxiumum Likelihood factor analysis with Oblique Rotation in which the two factor solution accounted for $55 \%$ of the scale variance. Both scales are internally consistant, Cronbach's alpha $=.83$ and .84 for scale 1 and 2 respectively. Participants with FI scored higher on the FI scale (M=6.52,SD 1.53) than did those with $\mathrm{GI}$ conditions but no $\mathrm{FI}(\mathrm{M}=3.76, \mathrm{SD}=1.79)$. Both groups scored higher than healthy participants $(\mathrm{M}=2.13, \mathrm{SD}=0.79), \mathrm{F}(2,93)=66.26, \mathrm{p}<.001$. Within the sample of participants with FI, the abdominal distress scale was able to discriminate those with FI due to a known cause vs idiopathic FI. The group with idiopathic FI $(\mathrm{M}=4.34, \mathrm{SD}=2.37)$ scored lower on this scale than the groups with FI with a known cause $(M=7.23, S D=2.54$, $\mathrm{t}(36)=-3.55, \mathrm{p}=.001$. Conclusion: The FI scale and abdominal distress scale are robust instruments to measure FI and differentiate those with FI secondary to known conditions. The items are brief making them suitable for application in a prevalence study.

\section{W1788}

Influence of Sildenafil On Rectal Sensorimotor Function in Irritable Bowel Syndrome (IBS) Patients

Maria Teresa G. Medeiros, Armenio A. Santos, Ricardo B. Oliveira, Monica Colares O. Lima, Geraldo M. Macedo, Ana Paula M. Maia, Miguel Angelo N. Souza

Rectal hypersensitivity is frequently found in IBS. Pharmacologically-induced reduction of rectal tone may change both the mechanical and the sensitivity patterns of the rectum. The aim of the present study was to evaluate the effects of sildenafil, a phosphodiesterase type5 inhibitor, on the sensorimotor function of the rectum in IBS patients. METHODS: Twelve informed IBS patients, (Rome II criteria), aged 27-60 yr, 4 males, took part in the study. Four presented constipation, 3 diarrhea, and 5 had both diarrhea and constipation. Anxiety and depression were assessed by means of Hamilton's scores. Fourty minutes before experiments, each patient received either sildenafil $(50 \mathrm{mg}$ tablet diluted in standardized orange juice) or 15 days apart, placebo (standardized orange juice) in a single dose, double-blind design, crossover, randomized order. Rectal ramp distension (Synectics), were performed in each experiment using a $600 \mathrm{~mL}$ PVC bag (12-cm cross-sectional radius) attached to a single lumen $16 \mathrm{~F}$ catheter. The rectal pressure $(\mathrm{mmHg})$, volume $(\mathrm{mL})$ and wall tension $\left(\mathrm{g} x \mathrm{~cm}^{-1}\right)$ thresholds for the first sensation, first desire to defecate, pain, and maximum tolerated distension were determined. A perfect spherical bag shape was assumed for wall tension calculation. Data (mean \pm SEM) were analyzed by Student's t test and Pearson correlation. RESULTS: In the sildenafil study day, rectal wall tensions at $8,12,16,20 \mathrm{mmHg}$ distending pressures were higher than in the placebo study day $(19.7 \pm 2.3$ vs $22.3 \pm 2.4, \mathrm{p}=$ $0.01 ; 40.5 \pm 3.6$ vs $44.5 \pm 3, \mathrm{p}<0.001 ; 65.5 \pm 2.8$ vs $69.5 \pm 1.6$, $\mathrm{p}<0.001 ; 89.9 \pm 2.4$ vs $92.3 \pm 1.6$, $\mathrm{p}<0.001$; respectively), denoting a higher rectal cross-sectional radius and relaxation. The thresholds for the first desire to defecate were higher after sildenafil $(15.8 \pm 1.3 v \mathrm{~s} 26.1 \pm 3.8$ $\mathrm{mmHg}, \mathrm{p}=0.01 ; 127.6 \pm 15.9$ vs $185.4 \pm 14.9 \mathrm{~mL}, \mathrm{p}=0.004 ; 66.3 \pm 7.4$ vs $126.9 \pm 20.4 \mathrm{gxcm}^{-1}$ $\mathrm{p}=0.008)$; higher at nonsignificant levels for the first sensation $(13.8 \pm 1.5$ vs $17.6 \pm 1.9 \mathrm{mmHg}$, $\mathrm{p}=0.11 ; 111.8 \pm 19.9$ vs $144.8 \pm 12.7 \mathrm{~mL}, \mathrm{p}=0.12 ; 55.3 \pm 8.9$ vs $76.9 \pm 8.9 \mathrm{gxcm}^{-1}, \mathrm{p}=0.12$ ); and similar to placebo for pain and maximum tolerated volume. Sildenafil and placebo minimal distending pressures were similar $(3.7 \pm 0.7$ vs $3.6 \pm 0.7 \mathrm{mmHg})$. Pressure, volume, and wall tension thresholds for pain correlated inversely to anxiety score after sildenafil $(r=-0.69, \mathrm{p}=$ $0.03 ; \mathrm{r}=-0.71, \mathrm{p}=0.02 ; \mathrm{r}=-0.68, \mathrm{p}=0.03$; respectively) but not after placebo $(\mathrm{r}=-0.01, \mathrm{p}=$ $0.98 ; \mathrm{r}=-0.42, \mathrm{p}=0.23 ; \mathrm{r}=-0.14, \mathrm{p}=0.7 ;$ respectively). CONCLUSION: In IBS, rectal relaxation by sildenafil diminished the perception to defecate and possibly the first rectal sensation. Remarkably, it also unfolded anxiety trait associated pain threshold lowering.

\section{W1789}

Effect of Atropine On the Esophago-Gastric Junction Opening Function Assessed Using Intraluminal Ultrasound Imaging

Valmik Bhargava, Ibrahim Dogan, Torahiko Takeda, Jianmin Liu, Ravinder K. Mittal

Relaxation and opening of the esophago-gastric junction (EGJ) are two distinct and crucial functions for esophageal transport across EGJ. It is well known that atropine causes a significant reduction (relaxation) in the lower esophageal sphincter pressure but there is limited information on its effect on the EGJ opening. Methods: Eight healthy subjects were studied before and after atropine ( $15 \mu \mathrm{gm} / \mathrm{kg} \mathrm{I.V.)}$ to assess its effect on the EGJ opening function using a novel ultrasound (US) methodology. A high frequency intraluminal US probe, a solid-state pressure transducer and an infusion tube were placed inside a $20 \mathrm{~mm}$ diameter cylindrical bag, which was placed across the EGJ. Bag was incrementally inflated with pressures between 10 and $60 \mathrm{mmHg}$ and at each bag pressure US transducer was pulled across the bag, inside its sheath, using a motorized puller at a constant speed. Bmode US images were digitized and converted into M-mode images to display the entire region of the esophagus, EGJ and stomach. From these M-mode images, the narrowes region of the EGJ was identified and its corresponding cross-sectional area (CSA) determine from B-mode image. Results: Our study shows that at low bag-pressures $(<20 \mathrm{mmHg})$ EG but not esophagus and stomach, is collapsed around the bag. With an increase in the bag pressure, there is a gradual opening of EGJ, starting from its distal end. The region of the EGJ surrounded by crural diaphragm is least distensible. Mean bag pressure for the EG opening was $18 \mathrm{mmHg}$. We observed a linear relationship between bag-pressure and EG CSA. Bag pressures to open $50 \%$ and $80 \%$ of the maximal bag diameter were $37 \pm 4$ and $56 \pm 6 \mathrm{mmHg}$ respectively. Atropine reduced opening bag pressure and shifted the EGJ pressure-CSA curve upward slightly without affecting the slope of the line. Conclusion: EG opening curve shows two distinct components, the first component (initial EGJ opening), which is likely to be related to the tonic EGJ contraction and a second component, related to its viscoelastic properties. Atropine has minimal effects on the EGJ opening function

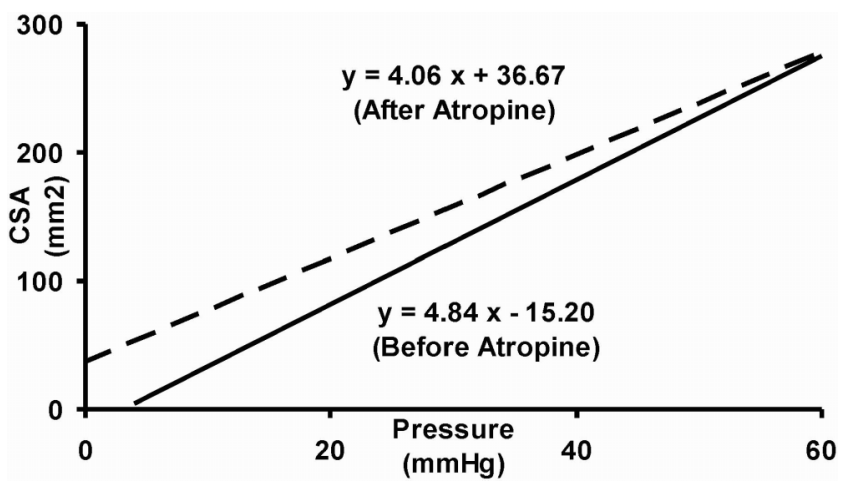

W1790

Effect of Phonation On the Intraluminal Pressures of the Esophagus and Its Sphincters

Lilani Perera, Krisna Chai, Candy Hofmann, Tanya Rittmann, Mark Kern, Einat Liebenthal, Reza Shaker

Recent studies have documented the existence of a reciprocal influence between the upper GI and the aerodigestive tracts. Phonation is known to be accompanied by a significant increase in subglottal tracheal pressure that is believed to be generated by contraction of the diaphragm, simultaneously causing an increase in intra-abdominal pressure. This phenomenon, especially during high intensity vocalization such as seen with professional singers, can predispose to gastroesophageal reflux, a recognized clinical problem in this group. The mechanisms of protection against esophageal or pharyngeal reflux of gastric content during this function have not been systematically studied. AIM: To determine and characterize the effect of phonation on the luminal pressures of the esophagus and its sphincters. METHODS: We studied 7 healthy volunteers (3M, 4F) by concurrent high resolution manometry (Manoscan, Sierra Scientific) and voice analysis (Praat, University of Amsterdam). We tested high and low pitch vowels for durations of 10 seconds. Findings were verified in 2 subjects by UES manometry using a sleeve device. RESULTS: High and low pitch vowels had significantly different frequencies $(\mathrm{Hz})(\Delta \mathrm{P}<0.05)$ (Table). UES pressures during phonation were significantly higher compared to the resting baseline for all of the studied pitches ( $69 \pm 6$ vs $140 \pm 26,92 \pm 9,185 \pm 31,104 \pm 11 \mathrm{mmHg}$, respectively) Pressure changes during phonation compared to those immediately prior to phonation showed similar patterns and are shown in the table. Findings were similar for studies using a sleeve device $(214 \pm 22,297 \pm 15 \mathrm{mmHg}$, high pitches A and E, respectively) CONCLUSIONS: Phonation affects UES intraluminal pressure differently from the esophageal body and LES, in that, UES pressure change due to phonation is pitch-dependent. During high pitch phonation UES pressure increase is significantly higher than that of esophageal and gastric pressures, providing a barrier against potential pharyngeal reflux of gastric content. Supported in part by NIH grants T32 DK61923 and RO1 DK25731.

\begin{tabular}{|c|c|c|c|c|c|}
\hline \multicolumn{7}{|c|}{ Change over Pre-Phonation Pressure $(\mathrm{mmHg})$ Mean \pm SE } \\
\hline & Pitch (Hz) & Gastric & LES & Distal Esophagus & UES \\
\hline High pitch E & $309 \pm 24 \Delta$ & $5 \pm 3$ & $11 \pm 14$ & $2 \pm 2$ & $62 \pm 50^{*}$ \\
\hline Low pitch E & $152 \pm 24$ & $4 \pm 2$ & $10 \pm 6$ & $4 \pm 3$ & $6 \pm 17$ \\
\hline High pitch A & $301 \pm 40 \Delta$ & $5 \pm 3$ & $1 \pm 8$ & $5 \pm 3$ & $101 \pm 52^{*}$ \\
\hline Low pitch A & $158 \pm 27$ & $5 \pm 3$ & $-2 \pm 5$ & $4 \pm 2$ & $23 \pm 20$ \\
\hline
\end{tabular}

* $\mathrm{p}<0.05$ UES vs gastric, esophagus

\section{W1791}

Mast Cells Recruit Eosinophils in the Guinea Pig Esophagus After Antigen Inhalation Challenge Via a Histamine Pathway

Shaoyong Yu, Edward Stahl, Qing Li, Ann Ouyang

Introduction: Mast cells (MC) play an important role in the inflammatory responses in the esophagus. We have previously shown that antigen challenge induces mast cell degranulation and histamine release in the esophagus. Eosinophils (Eos) have been observed to increase in the esophagus after allergen inhalation in a mouse model (J Clin Invest 2001; 107:8390). Aims: To test the hypothesis that antigen inhalation induces esophageal mast cell activation resulting in the recruitment of eosinophils in the esophagus, via a histaminemediated pathway. Methods: Guinea pigs were actively sensitized (S) by three i.p. injections 
of ovalbumin $(10 \mathrm{mg} / \mathrm{kg})$. Three weeks after the third injection, groups of animals received either inhalation of $0.1 \%$ ovalbumin for 2-10 minutes or repeated inhalation challenge daily for three days. After 1 and 24 hours with one inhalation challenge (C-1 and C-24) or 1 hour after the three repeated inhalations $(\mathrm{C}-72(\mathrm{x} 3 \mathrm{C})$ ), esophageal tissues were removed, processed and sectioned for immunofluorescent-staining of mast cell tryptase (Tryp-LI) and eosinophil major basic protein (MBP-LI). An additional group of animals received thioperamide (histamine $\mathrm{H} 3 / \mathrm{H} 4$ receptor antagonist, $5 \mathrm{mg} / \mathrm{kg}$, i.p.), 30 mins prior to, and were sacrificed 24 hours after challenge (C-24 (T)). Total Tryp-LI and MBP-LI cells were counted as well as the percent of positive cells in the epithelial layer of the mucosa. Results: Esophageal MC and Eos are mainly located in the sub-epithelial layer of the mucosa. Total number of Tryp-LI cells did not change in challenged groups, but the \% of mast cells in the epithelium increased significantly in C-1 and C-72(x3C) groups. MBP-LI cells increased in $\mathrm{C}-1$ and $\mathrm{C}-72(\mathrm{x} 3 \mathrm{C})(\mathrm{p}=0.07)$ groups, and the percentage of Eos migrating to the epithelium increased in the C-24 and C-72 (x3C). Thioperamide inhibited Eos migration into the epithelium. Conclusion: Inhalation with antigen induces mast cell and Eos to migrate towards and into the esophageal epithelium via a pathway involving either (or both) the $\mathrm{H} 3 \mathrm{or} \mathrm{H} 4$ histamine receptor.

Antigen inhalation induced mast cells and eosinophils responses in the guinea pig esophagus

\begin{tabular}{|c|c|c|c|c|}
\hline & Tryp(+)total & Tryp(\% Ep) & MBP(+)total & MBP(\% EP) \\
\hline sensitized & $40.7 \pm 5.9$ & $11.9 \pm .16$ & $14.4 \pm 2.6$ & $11.9 \pm 1.7$ \\
\hline C-1 & $46.3 \pm 7.7$ & $21 \pm 2.2 *$ & $38.5 \pm 5.3 *$ & $6.4 \pm 2.1$ \\
\hline C-24 & $43.2 \pm 8.4$ & $10.4 \pm 2.4$ & $16.2 \pm 3$ & $24.8 \pm 1.6 *$ \\
\hline C-24(T) & $48.8 \pm 5.78$ & $10.6 \pm 1.6 \#$ & $11.9 \pm 0.4 \#$ & $8.7 \pm 2.9 \# \#$ \\
\hline C-72(x3C) & $60 \pm 10.13$ & $49.7 \pm 1.8 * *$ & $40.7 \pm 11.2$ & $52.8 \pm 7.3 * *$ \\
\hline$* P<0.03$ & vs sensitized alone & & & \\
\hline$* * \mathrm{P}<0.0003$ & vs sensitized alone & & & \\
\hline$\# \mathrm{P}<0.03$ & $\mathrm{C}-24(\mathrm{~T})$ vs C-1 & & & \\
\hline$\# \# \mathrm{P}<0.01$ & $\mathrm{C}-24(\mathrm{~T})$ vs C-24 & & & \\
\hline
\end{tabular}

W1792

Vanilloid Receptor TRPV1-Induced PAF Production Mediates Acid-Induced Esophageal Inflammation

Ling Cheng, Karen M. Harnett, Weibiao Cao, Jose Behar, Piero Biancani

The transient receptor potential channel, vanilloid subfamily member-1 (TRPV1), was originally described in primary sensory neurons as a receptor for capsaicin and related natural irritants (also called vanilloids). TRPV1 is believed to function as a molecular integrator of noxious stimuli, including heat, acids, and endogenous pro-inflammatory substances. To test whether this acid-sensitive receptor may be a candidate as the initial mediator of acidinduced inflammation in the esophagus, a tubular segment of normal mucosa was removed from the esophagus, tied at both ends, forming a mucosal sac. The sac was filled with $0.01 \mathrm{~N}$ $\mathrm{HCl}$ (or normal Krebs' for control) and kept in oxygenated Krebs' solution at $37 \mathrm{C}$. The supernatant outside the $\mathrm{HCl}$-filled mucosal sac was collected after $3 \mathrm{hr}$ incubation and applied to circular muscle strips. The supernatant of the $\mathrm{HCl}$-filled sac almost abolished contraction of normal muscle strips in response to electric field (i.e. neural) stimulation (EFS). This supernatant-induced inhibition was reversed by application of the TRPVl antagonist IRTX inside the mucosal sac, confirming presence of TRPVI receptors in the mucosal wall. Similarly, EFS-induced contraction of esophageal circular muscle was almost abolished by supernatant of capsaicin $(1 \mu \mathrm{M})$ - filled mucosal sac. The reduction induced by supernatant of capsaicin- filled sac was reversed by application of the selective TRPVI antagonist IRTX inside the mucosal sac, confirming presence of capsaicin-sensitive TRPVI receptors in the mucosal wall. The reduction in contraction caused by supernatant of capsaicin- filled sac was also abolished by pre-exposure of the muscle to the PAF receptor antagonist CV9388. This is consistent with release of PAF by mucosa in response to activation of TRPVI by capsaicin. Measurements of PAF in mucosa tissue and in mucosa supernatant indicate that $\mathrm{PAF}$ levels increase 4-5 times in the $\mathrm{HCl}$-filled mucosal sac and in its supernatant when compared to control (Kreb's filled mucosal sac at pH7.4). The HCl-induced increase in PAF was abolished by the TRPVI receptor antagonist IRTX The data indicate that acid-induced activation of TRPVI receptors in the mucosa, induces production and release of the proinflammatory mediator PAF that affects contraction of circular muscle in response to electrical field stimulation.

\section{W1793}

Manometry in the 21st Centry: A Novel Fibre Optic Based Technology for Multi-Channel Intraluminal Manometry

Taher Omari, John Arkwright, Simon Doe, Michal Szczesniak, Nathalie Rommel, Philip Dinning, Ian Cook

INTRODUCTION: Mutichannel intraluminal pressure measurement is routinely used for the measurement of gut motility and diagnosing motility disorders. Perfused manometric assemblies are inexpensive, but technically demanding, require luminal infusion of water, are subject to movement artefact, baseline drift and hydraulic dampening of the pressure signal. Solid state manometric assemblies are expensive, fragile and require low temperature chemical sterilisation. Neither of these technologies are amenable to single use. We have developed a novel fibre optic technology that offers the potential for low cost, high fidelity, multi-channel intraluminal manometric recordings from a large number of pressure sensing elements based on a single optical fibre. The number of sensing elements is limited only by the available optical hardware and can theoretically exceed 50 discrete elements. We report our initial results from a pilot study of single and multiple sensor assemblies (OD 3.5mm). METHODS: Multiple pressure sensing elements were fabricated on a single optical fibre using multiple fibre Bragg gratings (FBG) designed to reflect a unique optical wavelength.
Each FBG was encased in a pressure sensitive diaphragm such that distortion of the membrane resulted in a proportionate change in the pitch of the FBG and therefore a change in reflected optical wavelength. Wavelength division multiplexing (WDM) allows simultaneous tracking of changes at each wavelength and therefore any variation in pressure along an array of sensors can be monitored in real time. In healthy adult volunteers, we recorded esophageal pressure waves initiated during bolus swallows using FBG assemblies and compared these with simultaneous side-by-side recordings made using a solid state transducer assembly (GaelTech Ltd) RESULTS. A total of $20 \times 10 \mathrm{ml}$ and $20 \times 20 \mathrm{ml}$ bolus swallows were analysed. The peak amplitudes of esophageal pressure waves recorded by the FBG assemblies and solid state assembly were similar $(85.2 \pm 24.9 \mathrm{mmHg}$ vs $84.5 \pm 24.2 \mathrm{mmHg})$ and there was a significant agreement between the two devices $(\mathrm{r} 2=0.95, \mathrm{p}>0.0001$, using Pearson correlation) CONCLUSIONS. Fibre optic manometric assemblies incorporating FBG sensor elements are capable of high fidelity manometric recordings of intraluminal pressure. This technology offers the potential of single use manometric assesmblies with large pressure sensor arrays using a single optical fibre and therefore without the need to increase the external dimension of the catheter or the complexity of the recording system as the sensor count increases.

\section{W1794}

Patients with Successfully Treated GERD Are Persistently Hypersensitive to Esophageal Balloon Distension

Joel H. Rubenstein, Borko Nojkov, Sheryl Korsnes, Beth Weinman, John M. Inadomi, William D. Chey

Background \& Aims: Patients with erosive esophagitis are hypersensitive to esophageal acid perfusion. Some evidence suggests that patients who have undergone a healing course of proton pump inhibitors (PPIs) have resolution of their chemosensitivity to acid perfusion. Less evidence is available regarding mechanosensation to balloon distension. We aimed to compare esophageal sensation to acid perfusion and balloon distension in patients with typical gastroesophageal reflux disease (GERD) that has responded to PPI therapy to healthy volunteers without GERD. Methods: 19 healthy volunteers (heartburn less than 3 times a month), and 16 subjects with a history of heartburn at least 3 times a week prior to PPI and no heartburn after at least 8 weeks of PPI, underwent esophageal perfusion with 0.1 normal $\mathrm{HCl}$ at $10 \mathrm{~mL} / \mathrm{min}$ for 10 minutes and stepwise intraesophageal balloon distension in random order. Subjects rated their sensation and unpleasantness on validated $20 \mathrm{~cm}$ scales. Acid sensation scores, and acid unpleasantness scores were calculated ( $\mathrm{cm} x$ time of sensation / 100). Subjects also underwent ambulatory esophageal $\mathrm{pH}$ monitoring. Results: GERD patients after PPI treatment had lower threshold pressure for weak sensation with balloon distension than controls ( $12.8 \mathrm{vs} .17 .3 \mathrm{mmHg}, \mathrm{p}=0.01$ ), and tended to have lower threshold pressure for discomfort (18.3 vs. $23.8 \mathrm{mmHg}, \mathrm{p}=0.08$ ). In mixed models of sensation over various pressures of balloon distension, male gender and increasing age tended toward lower sensation. Similar associations were found for unpleasantness. Controlling for gender and age, GERD patients had higher sensation $(+3.8 \mathrm{~cm}, \mathrm{p}=0.01)$ and higher unpleasantness $(+$ $3.2 \mathrm{~cm}, \mathrm{p}=0.02$ ) at any given pressure than controls. GERD patients tended to have higher sensitivity to acid ( 34.6 vs. $27.5 \mathrm{~cm}-\mathrm{s}, \mathrm{p}=0.89$ ), and unpleasantness with acid perfusion ( 29.2 vs. $23.3 \mathrm{~cm}-\mathrm{s}, \mathrm{p}=0.96$ ), but neither was statistically significant. Neither the results of ambulatory $\mathrm{pH}$ monitoring, nor the order of testing affected the results. Conclusions: Despite resolution of symptoms with PPI therapy, patients with GERD have persistent hypersensitivity to balloon distension. This could be due to allodynia induced by prior GERD. This study was supported by an unrestricted grant from Janssen Pharmaceutica, a unit of Ortho-McNeil and Esai Inc., and a greant from the Univ. of Michigan General Clinical Research Center. JHR was funded by NIH Training Grants T32 DK07367 and DK062708, and GlaxoSmithKline Digestive Health Clinical Research Award.

\section{W1795}

The Role of Esophageal Mast Cells in Patients with Symptoms of Heartburn Shaoyong Yu, Deborah M. Bethards, Allen C. Myers, Ann Ouyang

Introduction: The mast cell is known to be an important inflammatory cell in the GI tract. Our previous pre-clinical animal studies show that mast cell activation results in the release of inflammatory mediators in the esophagus with resulting stimulation of nociceptors. Our hypothesis is that heartburn may be a symptom similarly generated by the effect of mast cell activation on esophageal nociceptors in humans. Aims: To identify mast cells in the esophagus and review their correlation with symptoms of heartburn. Method: Sixteen patients were recruited from all patients who were referred for upper endoscopy. Exclusion criteria included asthma, atopic skin disease, chronic inflammatory conditions (RA, SLE), food allergies, known celiac disease, recent steroid treatment, ischemic heart disease or esophageal varices. During upper endoscopy biopsies were obtained from the lower esophagus and the mucosal appearance of the esophagus was noted and graded by the endoscopist. Mast cell tryptase staining was performed using a standard immunofluorescence method by an investigator blinded to the clinical information and the number of mast cells per mm2 was calculated. The sections were also stained with antibodies to TRPVI (capsaicin receptor) and PGP9.5. The patients' symptoms of heartburn were categorized using a scale from 1-5 for severity, duration and frequency (giving a maximal score of 15); this information was obtained by questionnaire completed prior to the procedure. Five patients had a symptom score of 0-4, one had a score between 5-9 and ten had a score between 10-15. The esophageal mast cell count was compared between patients with symptoms scores of 0-4 and those with scores of 10-15. Results: In the majority of patients no significant macroscopic esophageal mucosal change was noted on endoscopy. The number of mast cells was found to be significantly elevated in those patients with a heartburn score of 10-15 compared to those with a score of $0-4(119.4 \pm 37.8$ vs $42.8 \pm 19.1$ cells $/ \mathrm{mm} 2$, mean \pm SEM, $\mathrm{p}<0.03)$. The TRPVl positive nerve fibers were identified in the esophageal mucosa in proximity to mast cells. Conclusion: In a patient population in which allergic and inflammatory conditions have been excluded there is a significant correlation between heartburn symptom score and number of mast cells in lower esophageal biopsies. We propose that mast cells release inflammatory mediators that are responsible for the development of heartburn symptoms in these patients without macroscopic esophagitis. 
W1796

PAF Induced Activation of NADPH Oxidase Is Mediated By Arachidonic Acid and the Myeloid-Related Proteins S100a8/A9

Ling Cheng, Karen M. Harnett, Weibiao Cao, Jose Behar, Piero Biancani

In a cat model on in vivo-induced esophagitis by acid perfusion repeated over three days and in a human esophageal specimen with histologically confirmed erosive esophagitis, we have shown that PAF is produced and released by esophageal mucosa and present in esophageal circular muscle, where $\mathrm{PAF}$ induces production of $\mathrm{H}_{2} \mathrm{O}_{2}$ and reduces contraction in response to electrical field stimulation (EFS). We therefore examined mechanisms of PAFinduced production of $\mathrm{H}_{2} \mathrm{O}_{2}$. In esophageal circular muscle $\mathrm{PAF}\left(10^{-5} \mathrm{M}, 2\right.$ hour) increased $\mathrm{H}_{2} \mathrm{O}_{2}$ level 2.8 times over that of control. PAF-induced production of $\mathrm{H}_{2} \mathrm{O}_{2}$ was confirmed by examining esophageal muscle contraction in response to electrical field stimulation (EFS). EFS-induced contraction was reduced by PAF, and the reduction was reversed by the $\mathrm{H}_{2} \mathrm{O}_{2}$ scavenger catalase, indicating that the reduction was mediated through production of $\mathrm{H}_{2} \mathrm{O}_{2}$. The PAF-induced reduction was reversed by the NADPH oxidase inhibitor apocynin, by the 138 MAK kinase inhibitor SB203589, and by the CPLA2 inhibitor AACOCF3, indicating that formation of $\mathrm{H}_{2} \mathrm{O}_{2}$ depends on activation of the $\mathrm{p} 38 \mathrm{MAK}$ kinase and of the cytosolic PLA2, with formation of arachidonic acid, and activation of NADPH oxidase. This conclusion was confirmed by direct measurements of $\mathrm{H}_{2} \mathrm{O}_{2}$ in response to PAF. The PAF-induced increase in $\mathrm{H}_{2} \mathrm{O}_{2}$ was inhibited by apocynin, by the p38 MAK kinase inhibitor SB203589, and by the CPLA2 inhibitor AACOCF3, indicating that activation of the $\mathrm{p} 38$ MAK kinase and of the cytosolic PLA2, with formation of arachidonic acid, may be required steps for activation of NADPH oxidase and formation of $\mathrm{H}_{2} \mathrm{O}_{2}$. It has been shown that arachidonic acid promotes activation of NADPH oxidase by binding to the myeloid-related proteins S100A8/A9 which then interact with the p47-p67 NADPH components promoting interactions between the oxidase subunits and enabling oxidase activation. We therefore examined a possible role of S100A8 in PAF induced production of $\mathrm{H}_{2} \mathrm{O}_{2}$ in cat esophageal circular muscle. Enzymatically isolated smooth muscle cells in primary culture were electroporated in the presence of S100A8 small interfering RNA (siRNA) or scrambled siRNA. With scrambled siRNA, PAF increased $\mathrm{H}_{2} \mathrm{O}_{2} 2.5$ times, and arachidonic acid increased $\mathrm{H}_{2} \mathrm{O}_{2} 3.2$ times, when compared to control. When the electroporated cells were incubated with S100A8 siRNA, S100A8 protein expression decreased by more than half, as measured by Western blot analysis and the increase in $\mathrm{H}_{2} \mathrm{O}_{2}$ in response to PAF and arachidonic acid was reduced by more than half. The data indicate that PAF-induced production of $\mathrm{H}_{2} \mathrm{O}_{2}$ is mediated through arachidonic acid interaction with S100A8 to activate NADPH oxidase.

\section{W1797}

Novel, Automated High-Resolution Manometry (HRM) Paradigms for Quantifying EGJ Relaxation: Integrated Relaxation Resistance (IRR) and Median Flow Permissive Pressure (mFPP) John E. Pandolfino, John Rice, Sudip K. Ghosh, Qing Zhang, John P. Norvell, Peter J. Kahrilas

Aim: Post-deglutitive esophageal emptying depends on the balance between EGJ resistance and intrabolus pressure proximal to the EGJ, making these ideal measures of EGJ function. The enhanced spatial resolution of HRM make it possible to quantify both in an automated fashion. This study aimed to establish normal values for IRR, mFPP, and E-sleeve pressure during post-deglutitive EGJ relaxation and compare their performance in assessing 400 consecutive patients. Methods: 75 controls were studied with a 36-channel solid state HRM assembly ( $1 \mathrm{~cm}$ spacing) to establish normal values for: 1 ) the lowest $3 \mathrm{~s}$ mean residual pressure after swallow (E-sleeve), 2) mFPP, and 3) IRR. mFPP was the median pressure it would take to overcome post-deglutitive EGJ resistance (10 s or until the arrival of the contractile complex). IRR was the mFPP divided by the interval between the swallow and the arrival of the contractile complex (if present). Data from 400 consecutive patients who underwent HRM were then analyzed to compare the performance of the E-sleeve and IRR in assessing EGJ relaxation with the mFPP being the final adjudicator of abnormal function. All measurements were made by computer analysis, either in ManoScan or custom written Matlab programs. Results: The 95th percentile of normal for EGJ relaxation parameters amongst the 75 controls were: $15 \mathrm{mmHg}$ for the E-sleeve; $18 \mathrm{mmHg}$ for mFPP; and 3.0 $\mathrm{mmHg} / \mathrm{s}$ for the IRR. 90 patients were abnormal by E-sleeve. Of these, 69 were also abnormal by IRR confirming EGJ dysfunction. Of the remaining 21 , the mFPP was abnormal in 12 patients most of whom were aperistaltic ( $\mathrm{mFPP} 18.0-27.2 \mathrm{mmHg}$ ) also verifying EGJ dysfunction. However, in an additional 8 patients, the E-sleeve value was deceptively abnormal because of interference with either the crural diaphragm or the heart, neither of which led to spurious mFPP or IRR calculations. Only one patient (with aperistalsis) exhibited normal values of IRR $(1.9 \mathrm{mmHg} / \mathrm{s})$ and $\mathrm{mFPP}(10.6 \mathrm{mmHg})$ with an abnormal E-sleeve (19.7 mmHg). 310 patients were normal by E-sleeve criteria. Of these 13 had abnormal IRR or mFPP. Further scrutiny of these patients showed that all 13 patients had EGJ pathology (variant achalasia or post-fundoplication dysphagia) suggesting that the E-sleeve misclassified them. Conclusion: IRR and mFPP are novel HRM parameters for assessing deglutitive EGJ relaxation demonstrating superior clinical performance to E-sleeve relaxation pressure.

\section{W1798}

The Effect of a Novel 5-HT 3 Receptor Partial Agonist (DDP733) On Feline Esophageal Peristalsis and Lower Esophageal Sphincter Pressure Kalina Venkova, Anthony C. Johnson, Matthew O. Fraser, Karl B. Thor, Beverley Greenwood-van Meerveld

The present study investigated whether DDP733, a new 5-HT 3 receptor partial agonist, affects lower esophageal sphincter pressure (LESP), esophageal peristalsis and distal esophageal $\mathrm{pH}$ in a feline model. We hypothesized that activation of $5-\mathrm{HT}_{3}$ receptors, known to cause excitation of mucosal vagal afferents and local enteric neurons, may increase LESP and present a novel approach to the treatment of gastroesophageal reflux disease (GERD). Methods: In anesthetized cats, esophageal contractility and LESP were recorded manometrically via a water-perfused catheter assembly system. Changes in $\mathrm{pH}$ in the distal esophagus were monitored continuously throughout the experiment using a computerized Orion II system. Mean LESP, the number of spontaneous dry swallows (SDS) and the SDS-induced LES relaxation were monitored. Each cat received the vehicle or DDP733 $(0.1-10 \mathrm{mg} / \mathrm{kg})$ administered intravenously (i.v.) or intragastrically (i.g.) in separate recording sessions performed 7 days apart. Results: Preliminary studies revealed that following i.v. dosing DDP733 induced an immediate and dose-dependent increase in LESP. To simulate oral dosing in our anesthetized cat model, DDP733 (at single doses of $0.1,1$ or $10 \mathrm{mg} / \mathrm{kg}$ ) or the vehicle were administered i $g$. DDP733 induced a dose-dependent increase in LESP (Table 1). The increase in LESP reached a maximum within 20-45 min of treatment and remained elevated 60-min after drug administration. There was no significant difference between the numbe of SDS recorded during the 1-h following treatment with DDP733. Despite the increase in LESP induced by DDP733, each SDS was associated with a normal esophageal peristalsis through the striated and smooth muscle portion of the esophagus. DDP733 did not alter the ability of the LES to relax in response to SDS. Esophageal pH data recorded simultaneousl with the esophageal pressure suggested a trend of DDP733 to decrease the duration of lower esophageal exposure to harmful $\mathrm{pH}$ levels $(\mathrm{pH}<4)$ during LES relaxation. Conclusion: Our results demonstrate that activation of $5-\mathrm{HT}_{3}$ receptors promotes an increase in LESP withou altering the ability of the LES to relax in response to a swallow. Overall, the findings suggest that oral administration of DDP733 may be useful in the treatment of GERD.

Table 1

\begin{tabular}{|c|c|c|c|c|c|}
\hline Treatment & \multicolumn{5}{|c|}{ Changes in LESP (\% from basal) } \\
\hline (i.g.) & $0-5 \mathrm{~min}$ & $10-15 \mathrm{~min}$ & $25-30 \mathrm{~min}$ & $40-45 \mathrm{~min}$ & $55-60 \mathrm{~min}$ \\
\hline vehicle & -8.6 & -14.6 & -11.3 & -27.1 & -33.1 \\
\hline DDP773 $0.1 \mathrm{mg} / \mathrm{kg}$ & -3.9 & -5.0 & -0.4 & -15.4 & -7.3 \\
\hline DDP773 $1 \mathrm{mg} / \mathrm{kg}$ & $11.5^{*}$ & $6.8^{*}$ & $20.5^{*}$ & $38.6^{*}$ & $22.2^{*}$ \\
\hline DDP773 $10 \mathrm{mg} / \mathrm{kg}$ & $24.6^{*}$ & $31.7^{*}$ & $69.4^{*}$ & $47.4^{*}$ & $49.9^{*}$ \\
\hline
\end{tabular}

Mean values from 5-6 experiment in different animals. $* \mathrm{p}<0.05$ compared to vehicle.

\section{W1799}

Apnea-Associated Inhibition of Esophageal Body Motility and Lower Esophageal Sphincter Tone in Premature Infants

Taher Omari

INTRODUCTION: The association of gastroesophageal reflux (GER) and apnea is controversial. A large number of studies have investigated this association and have demonstrated both a relationship and no relationship. Esophageal motility studies in premature infants indicate that the lower esophageal sphincter (LES) is a competent anti-reflux barrier and that GER episodes require LES relaxation, usually transient LES relaxation. LES pressure during apnoea has not been described in infants. The aim of this study was to characterise pharyngo-esophageal motility during episodes of significant (life threatening) apnea in premature infants. METHODS: A retrospective review was undertaken of all combined manometric and physiological monitoring studies performed in premature infants at the WCH between 1994-2002. Significant apnoea was defined as a respiratory pause of $>20 \mathrm{sec}$, which was associated with bradycardia to a HR of $<100$ beat $/ \mathrm{min}$ and desaturation to a $\mathrm{SaO} 2<90 \%$. For each apneic event identified, pharyngeal swallowing, oesophageal motility and LES pressure was assessed before, during and after apnea. RESULTS: Twelve episodes of significan apnea (mean duration $44 \mathrm{sec}, \mathrm{HR} 64$ beats/min, $\mathrm{SaO} 272 \%$ ) were identified in 7 of 156 infants who had undergone esophageal motility studies. All were predominantly central apneic events incorporating short periods of obstructed breaths at the onset and/or offse of apnea and therefore were defined as 'mixed' apnea episodes. A sequence of swallows was observed during the period of apnea with the first swallow occurring within $2 \mathrm{sec}$ before to $6 \mathrm{sec}$ after apnea onset (mean $1.3 \pm 0.7 \mathrm{sec}$ after). The median [IQR] number of swallows occurring before, during and after apnea was $0[0,0], 4[2,7](\mathrm{p}<0.05)$ and $1[0,1]$ swallows respectively. The esophageal body was refractory during apnea. Only 19\% of swallows triggered during apnea initiated normal antegrade and complete oesophageal peristalsis, compared while $82 \%$ of swallows after apnoea. LES pressure dropped during apnea from a basal pressure of $15.6 \pm 2.7 \mathrm{mmHg}$ at apnea onset to a nadir of $4.1 \pm 0.8 \mathrm{mmHg}(\mathrm{p}<0.0005)$ an average of $6.0 \pm 1.1 \mathrm{sec}$ after apnea onset. Inhibition of LES tone was maintained for the duration of apnea. CONCLUSION: Rare episodes of significant apnea are associated with an increase in pharyngeal swallowing coupled with a refractory esophageal body and a decrease in LES tone. These observations suggest that a reduction in vagal drive at the onset of apnea also impacts upon the motility of the upper GI tract. LES inhibition coupled with esophageal inhibition during apnea may make triggering of GER more likely during apnea episodes.

\section{W1800}

Effects of Electroacupuncture On Esophageal Motility and Serum Hormones in Cats with Esophagitis Xiaowei Shuai, Pengyan Xie, Jianxiang Liu, Yanshu Xiang, Jiang Li

AIM: To investigate effects of electroacupuncture (EA) applied to the Zusanli point (36S) on esophageal motility, and compare the effect of EA on serum gastrin (GAS), motilin (MTL) and vasoactive intestinal peptide (VIP). METHODS: Thirty-two cats were randomly divided into 4 equal groups. All animals underwent a Heller myotomy. 6-8 weeks after the operation, endoscopy confirmed if reflux esophagitis had been developed. Two frequencies (2-15 Hz/ 2-100 Hz) of EA (2-4 mA) were delivered in two different groups through acupuncture needles inserted bilaterally into Zusanli acupoints on hind limb (low-frequency EA group, high frequency EA group). Animals submitted to EA $(2-15 \mathrm{~Hz} / 2-100 \mathrm{~Hz})$ on a non-point region (EANP) were used as controls respectively in the other two groups (low-frequency EANP group, high-frequency EANP group). Thirty minutes of electric stimulation was applied in each group. Esophageal motility was continuously monitored using water-perfusion manometry system before, during, and after the EA/EANP. Serum levels of GAS, MTL and VIP 
were also measured. RESULTS: The LESP decreased significantly after Heller myotomy compared with that before the operation $(19.4 \pm 4.66$ v.s. $35.8 \pm 5.12 \mathrm{mmHg}, \mathrm{P}<0.01)$, and there were no significant differences among groups. Mild to moderate esophagitis developed following Heller myotomy in all the cats. The LESP decreased in both low-frequency EA cats and low-frequency EANP cats, and in EA cats the pressure decreased more $(12.1 \pm 5.06$ v.s. $15.1 \pm 5.99 \mathrm{mmHg}$ ). On the contrary, the LESP increased in high-frequency EA group $(30.0 \pm 10.36 \mathrm{mmHg})$, which was significantly higher than that in EANP group $(24.6 \pm 15.86 \mathrm{mmHg}, \mathrm{P}<0.05)$. EA had no effect on contraction duration. Although highfrequency EA significantly increased the peak amplitude in esophageal peristalsis, lowfrequency EA did not decreased it. There was a decrease in serum GAS and MTL in lowfrequency EA cats compared with low-frequency EANP cats (GAS $14.5 \pm 3.51$ v.s. $26.6 \pm 4.55$ $\mathrm{pg} / \mathrm{ml}$, MTL $53.5+17.38$ v.s. $95.2+13.26 \mathrm{pg} / \mathrm{ml}$, both $\mathrm{P}<0.01)$. GAS and MTL were significantly higher in high-frequency EA group than in high-frequency EANP group (GAS 51.0 \pm 5.18 v.s. $30.7 \pm 8.79 \mathrm{pg} / \mathrm{ml}$, MTL $162.7 \pm 14.87$ v.s. $113.4 \pm 23.86 \mathrm{pg} / \mathrm{ml}$, both $\mathrm{P}<0.01$ ). Serum VIP decreased in high-frequency EA group $(14.0 \pm 4.83$ v.s. $50.0 \pm 8.49 \mathrm{pg} / \mathrm{ml}, \mathrm{P}<0.05)$, while increased in low frequency EA group $(82.6 \pm 8.11$ v.s. $43.6 \pm 8.74 \mathrm{pg} / \mathrm{ml}, \mathrm{P}<0.05)$, compared with EANP groups respectively. CONCLUSION: EA with a high frequency at Zusanli might enhance the esophageal motility, while EA with a low frequency might weaken it. The effect of acupuncture is acu-point-specific, and this effect appears to be mediated through GAS, MTL, and VIP

\section{W1801}

Effect of Atropine and L-NAME On LESP in Rats By Electro-Acupuncture At Zusanli Acupoint

Xiangdong Mu, Pengyan Xie, Jianxiang Liu, Huhong Wang

AIM: To observe the effect of atropine and N-nitro-L-arginine methyl ester (L-NAME) on the pressure of lower esophageal sphincter (LESP) by electro-acupuncturing (EA) at Zusanli acupoint (He-Sea, st 36) of stomach meridian and the neural mechanism of EA. METHODS: Forty-eight rats were divided into six groups: control group, EA group, atropine group atropine+EA group, L-NAME group and L-NAME+EA group. LESP was observed and recorded by using three-channel perfusion manometric measurement system. RESULTS: (1) The basic LESP of EA group was $10.7+/-2.1 \mathrm{mmHg}$ : LESP increased significantly under EA or $0-20,21-40 \mathrm{~min}$ after $\mathrm{EA}(\mathrm{P}<0.01)$, the variation degrees were respectively $62.77+1$ $27.02 \%, 131.01+/-36.42 \%$ and $122.6+/-25.9 \%$. (2) LESP of atropine+EA group significantly decreased $16.0+/-7.7 \%(\mathrm{P}<0.01)$ after atropine intraperitoneal injection; LESP under or after EA contrast to those before EA (after atropine i.p.) increased respectively $28.5+/-19.7 \%$ $(\mathrm{P}<0.01)$ and $31.2+/-28.5 \%(\mathrm{P}<0.05)$, which were significantly lower than those of EA group no significances were observed between the LESP under EA or after EA $(\mathrm{Pl}=0.155, \mathrm{P} 2=$ 0.252 ) contrast to basic LESP. (3) LESP of L-NAME+EA group significantly increased 30.5+/$17.4 \%(\mathrm{P}<0.01)$ after L-NAME intraperitoneal injection; LESP under or after EA contrast to those before EA (after L-NAME i p) increased respectively 39.4+/-28.6\% and 48.6+/-4l.4\%, which were significantly lower than those of EA group $(\mathrm{P}<0.01)$; LESP under or after EA contrast to basic LESP increased respectively $82.0+/-45.0 \%$ and $93.0+/-58.0 \%(\mathrm{P}<0.01)$, no significances were observed between L-NAME+EA group and EA group. CONCLUSIONS: (1) LESP increases significantly under or after EA at Zusanli acupoint. (2) Cholinergic M receptor blocker partly abolishes the influence of EA on LESP, but EA can restore the decreased pressure of cholinergic M receptor blocked rats. (3) Nitric oxide synthase (NOS) inhibitor increases LESP, and EA can make it higher. (4) The main efferent pathway of regulating effect of EA at Zusanli acupoint on LESP is possibly via the cholinergic vagus nerve.

\section{W1802}

Prevalence of Increased Esophageal Muscle Thickness in Patients with Esophageal Motor Disorders

Ibrahim Dogan, James L. Puckett, Bikram S. Padda, Ravinder K. Mitta

Background and aims: Patients with achalasia, diffuse esophageal spasm and nutcracker esophagus have a thicker muscularis propria than normal subjects. The goal of our study was to determine the prevalence of increased muscle thickness (MT) in a group of unselected patients referred to the esophageal functions laboratory for esophageal symptoms. Methods: We studied 40 normal subjects (mean age $41.8 \pm 14.4$ yr; 25 males, range 21-75 yr) and 94 consecutive patients with esophageal symptoms (mean age $53.6 \pm 15.3 \mathrm{yr} ; 46$ males, range 20-81 yr). Manometry and ultrasound images of the esophagus were recorded concurrently, using a special custom-built catheter. Esophageal MT and muscle cross sectional area (MCSA) were measured at 2 and $10 \mathrm{~cm}$ above the lower esophageal sphincter (LES). Patients were assigned manometric diagnosis and determination was made if they had increased MT and MCSA. Results: Esophageal baseline MT $(1.45 \pm 0.31 \mathrm{~mm})$ and MCSA $(65.08+9.51 \mathrm{~mm} 2)$ in normal subjects, at $2 \mathrm{~cm}$ was greater than MT $(1.24 \pm 0.23 \mathrm{~mm})$ and MCSA $(54.49 \pm 8.61$ $\mathrm{mm} 2)$ at $10 \mathrm{~cm}$ above the LES ( $<<0.05)$. Based on mean+2SD value in normal subjects, baseline MT of greater than $2.08 \mathrm{~mm}$ and $1.71 \mathrm{~mm}$, at 2 and $10 \mathrm{~cm}$ sites respectively were considered to be abnormal. Corresponding normal values for MCSA at 2 and $10 \mathrm{~cm}$ sites were $84.11 \mathrm{~mm} 2$ and $71.72 \mathrm{~mm} 2$ respectively. All patients with achalasia, $7 / 8$ subjects with diffuse esophageal spasm and 2/2 patients with nutcracker esophagus showed an increase in the MCSA at $2 \mathrm{~cm}$ site. Nearly all patients with well-defined spastic motor disorders, i.e. achalasia, diffuse esophageal spasm and nutcracker esophagus revealed an increase in MT or MCSA, 2; increase in esophageal MT/MCSA was seen, albeit at a lower prevalence rate, in patients with less well-characterized manometric abnormalities, i.e., hypertensive LES (1/ 1), impaired LES relaxation (1/1), ineffective esophageal motility (7/11) and hypotensive LES (5/9), 3; 24\% of patients with esophageal symptoms but normal manometry were also found to have an increase in MCSA. Dysphagia was more likely, and heartburn less likely in patients with increased MT/MCSA, but there were no differences in chest pain and regurgitation symptoms between the groups. Conclusion: An increase in esophageal MT/ MCSA is sensitive but not specific marker of manometrically defined esophageal motor disorders. Further studies are needed to determine the cause of increase in esophageal MT/ MCSA in patients with esophageal symptoms.

\section{W1803}

The Effect of Lower Esophageal Sphincter (LES) Electrical Stimulation On LES Pressure

Claudia P. Sanmiguel, Masanobu Hagiike, Martin P. Mintchev, Ryan B. Dela Cruz, Edward H. Phillips, Scott A. Cunneen, Jeffrey L. Conklin, Edy E. Soffer

Background: Electrical stimulation (ES) of the stomach has been shown to modulate LESP Electrical stimulation, using neural high frequency stimulation (NGES) can induce contractions of the smooth muscle of the gut. The purpose of this study was to determine if electrical stimulation of the LES can affect LESP. Methods: Four female hound dogs, weight: 20-25 $\mathrm{kg}$, underwent an esophagostomy that allowed the introduction of a sleeve manometry catheter into the esophagus. They were also implanted with a pair of electrodes along the longitudinal axis of the LES. After 3 weeks of recovery, they underwent esophageal manometry recording during control and ES, performed randomly on separate days, using 4 different stimulations: 1-Low frequency: freq: 6 cycles/min, pulse: 350 milisec, amp: 5 mAmp; 2 Highfrequency: freq: $50 \mathrm{~Hz}$, pulse: 1 milisec, amp: $5 \mathrm{mAmp}$; 3 - NGES: freq: $50 \mathrm{~Hz}$, pulse:20milisec amp:10 volts; 4- High-frequency, circular: freq: $20 \mathrm{~Hz}$, pulse:lmilisec, amp:5 mAmp. Al recordings were performed 1 hour after consumption of 3 ounces of canned dog food, to prevent fluctuations in LESP and under mild sedation (acepromazine $0.5 \mathrm{~m} / \mathrm{kg}$ ). Tests consisted, during ES days, of 3 periods of 20 minutes each: control, stimulation and post stimulation. The effect of NGES was also tested under anesthesia and following administration of L-NAME $50 \mathrm{mg} / \mathrm{kg}$ IV. and also atropine $0.05 \mathrm{mg} / \mathrm{kg}$ IV. Analysis: area under the curve (AUC) and pressure were compared among the 3 periods. Data shown as mean \pm SD, ANOVA and t-test, $\mathrm{p}<0.05$. Results: Sustained increase in LESP was observed during low frequency stimulation, $32.1 \pm 12.8$ vs. $42.4 \pm 18.0$ vs. $50.1 \pm 23.6$, control vs. stimulation vs. post stimulation respectively, $\mathrm{p}=0.013$. AUC also significantly increased during and after stimulation, $39,320 \pm 15,722$ vs. $51,294 \pm 21,826$ vs. $59,823 \pm 28,198 \mathrm{mmHgxsec}$, control vs. stimulation vs. post stimulation respectively, $\mathrm{p}=0.01$. There was no significant change with other types of ES. NGES induced an initial rise in LESP followed within few seconds by relaxation with slow resumption of pressure over a 1 minute period. L-NAME increased LESP and augmented the initial rise in LESP following NGES but markedly diminished or abolished the relaxation phase. Atropine lowered LESP and abolished the initial rise in LESP induced by NGES. Conclusions: Low frequency ES of the LES increases LESP in conscious dogs. NGES has dual effect on LESP: an initial stimulation, cholinergically mediated, followed by relaxation mediated by nitric oxide.

\section{W1804}

Protection Against Pharyngeal Reflux of Gastric Content During Coughing: Another Role for the Upper Esophageal Sphincter (UES)

Lilani Perera, Krisna Chai, Candy Hofmann, Tanya Rittmann, Mark Kern, Reza Shaker

Respiratory functions such as coughing induces significant increase in intra-abdominal pressure causing a circumstance conducive to development of gastroesophageal reflux. Indeed this phenomenon is documented frequently during $\mathrm{pH}$ monitoring studies. However, these reflux events do not occur during every cough event and not in every individual suggesting the existence of anti-reflux mechanism(s). The nature of this mechanism preventing gastroesophageal/pharyngeal reflux events, however, has not been systematically studied. Our aim was to determine and characterize the gastroesophageal, UES and LES pressure phenomena during high and low amplitude cough in healthy individuals. METHODS: We studied 7 healthy volunteers (3M, 4F) by a concurrent manometric (Manoscan, Sierra Scientific) and voice analysis technique (Praat, University of Amsterdam) and quantified and correlated upper GI pressures during low and high intensity coughing. Each event was studied x 3 . RESULTS: Amplitudes of tested coughs were $42 \pm 3 \mathrm{~dB}$ for weak and $59 \pm 2 \mathrm{~dB}$ for strong cough. Intragastric, Intraesophageal, LES and UES pressures during coughing were significantly higher than pre-cough pressures $(\mathrm{p}<0.001)$. UES pressure increases for both cough intensities were significantly higher than those of the esophagus, LES, and stomach $\left({ }^{*} \mathrm{p}<0.001\right)$. There was a direct relationship between intensity of the cough and intragastric LES, intraesophageal and UES pressure increases $(\# p<0.05)$. CONCLUSION: Different cough intensities induce different changes in pressures of the esophagus and its sphincters. Coughinduced LES and intraesophageal pressures are similar to that of intragastric pressure and as such may not be effective in preventing GE reflux. Cough-induced UES pressure, on the other hand, is significantly higher than intragastric, LES and intraesophageal pressure, providing a strong barrier against pharyngeal reflux of gastric content. Supported in part by NIH grants T32 DK61923 and RO1 DK25731.

\begin{tabular}{|c|c|c|c|c|}
\hline \multicolumn{5}{|c|}{ Cough-Induced Pressure Changes over Pre-cough Values $(\mathrm{mmHg})(\mathrm{Mean} \pm \mathrm{SE})$} \\
\hline & Gastric Pressure $\Delta$ & LESP $\Delta$ & Esoph Pressure $\Delta$ & UESP $\Delta$ \\
\hline Weak cough & $37 \pm 4$ & $31 \pm 5$ & $39 \pm 5$ & $196 \pm 29 *$ \\
\hline Strong cough & $114 \pm 15 \#$ & $121 \pm 16 \#$ & $111 \pm 15 \#$ & $326 \pm 15 \# *$ \\
\hline
\end{tabular}

LESP: lower esophageal sphincter pressure; UESP: upper esophageal sphincter pressure

W1805

The Upper Esophageal Sphincter Contractile Response to Reflux Events Is Not Acid Sensitive

David A. Knuff, Tanya Rittman, Reza Shaker

BACKGROUND Prior studies have shown that the upper esophageal sphincter (UES) contracts in response to reflux events. However, the effect of reflux acidity on the UES contractile response (UESCR) is unknown. Our aim was to compare the UESCR to acid (ARE) and non acid (NARE) reflux events. METOHDS: Five subjects with gastroesophageal reflux (GER) were studied. In order to avoid the stimulatory effect of perfused manometry a Manoscan (Sierra Scientific Instr. Los Angeles) catheter was placed transnasally such that the pressure sensors covered the entire length of the UES and esophagus. In addition a two site $\mathrm{pH}$ probe 
was placed transnsally. After confirming intragastric $\mathrm{pH}$ the $\mathrm{pH}$ sensors were positioned $5 \mathrm{~cm}$ and $15 \mathrm{~cm}$ above the manometrically determined lower esophageal sphincter. Subjects then ingested a standard $1000 \mathrm{kcal}$ meal and were placed in the supine position. Simultaneous data were then continuously recorded with the Manoscan system and $\mathrm{pH}$ probe for 90 minutes. Each subject was studied off of acid suppressive therapy for at least 24 hours and again on acid suppressive therapy for at least 72 hours. We determined the occurrence of common cavities and their characteristics, UES pressure prior to and during common cavities, presence of secondary peristalsis response, as well as corresponding esophageal $\mathrm{pH}$. RESULTS: 36 ARE $(\mathrm{pH}<4.0)$ and 45 NARE $(\mathrm{pH}>4.0)$ were identified. All events, irrespective of $\mathrm{pH}$, were associated with a UESCR. The common cavity characteristics and UES parameters were not significantly different between ARE and NARE. Table (mean \pm SE). Frequency occurrence of UESCR was significantly more than that of secondary peristalsis response $*(\mathrm{P}<0.05)$. CONCLUSION: The UESCR to gastroesophageal events is mediated by esophageal distension and is insensitive to refluxate $\mathrm{pH}$. The UESCR, but not secondary peristalsis response, to GER events is all or none.

\begin{tabular}{|c|c|c|c|c|c|c|}
\hline & $\begin{array}{c}\text { UES Baseline } \\
\text { Pressure }\end{array}$ & $\begin{array}{c}\text { UESCR Peak } \\
\text { Pressure }\end{array}$ & $\begin{array}{c}\text { UESCR } \% \\
\text { Increase }\end{array}$ & $\begin{array}{c}\text { UESCR } \\
\text { Duration }\end{array}$ & $\begin{array}{c}\text { Induced } \\
\text { Secondary } \\
\text { Peristalsis }\end{array}$ & $\begin{array}{c}\text { Induced } \\
\text { UESCR }\end{array}$ \\
\hline ARE & $35 \pm 9 \mathrm{mmHg}$ & $\begin{array}{c}172 \pm \\
32 \mathrm{mmHg}\end{array}$ & $503 \pm 70 \%$ & $8.5 \pm 4 \mathrm{~s}$ & $63 \pm 28 \%$ & $100 \% *$ \\
\hline NARE & $55 \pm 11 \mathrm{mmHg}$ & $\begin{array}{c}162 \pm \\
21 \mathrm{mmHg}\end{array}$ & $277 \pm 55 \%$ & $9.5 \pm 4 \mathrm{~s}$ & $59 \pm 26 \%$ & $100 \% *$ \\
\hline P-value & NS & NS & NS & NS & NS & NS \\
\hline
\end{tabular}

W1806

Oesophageal Solid Bolus Transit: Studies Using Concurrent Videofluoroscopy and High-Resolution Manometry

Mark Fox, Michael Fried, Werner Schwizer

INTRODUCTION: Esophageal solid bolus transit is less efficient than that of liquids in health and disease. Esophageal function is complex and current investigations do not collect data that fully addresses the relationship between motor function and bolus transport. A recent study has shown that high-resolution manometry (HRM) resolves the segmental anatomy of the esophagus and predicts the success of liquid bolus transport more accurately than conventional techniques. AIM: Concurrent HRM and videofluoroscopy (VF) were performed to study esophageal solid bolus transport. METHOD: 18 healthy subjects were studied on swallowing $10 \mathrm{ml}$ liquid barium and $10 \mathrm{~mm}$ barium marshmallow bolus in a supine posture. HRM and VF were analyzed separately and in a blinded fashion. The characteristics of esophageal peristalsis on HRM were assessed and related to the success of solid bolus transport by covariant analysis. RESULTS: Data was available for 75 swallows (median $4 /$ subject). Esophageal clearance was complete for $88 \%$ liquid and $65 \%$ solid bolus swallows $(\mathrm{p}<0.01)$. For successful swallows, peristalsis and bolus transport from pharynx to stomach took more time for solid than liquid bolus ( $8.7 \mathrm{~s}$ vs. $8.2 \mathrm{~s} ; \mathrm{p}<0.05)$ mainly due to slower peristaltic velocity in the proximal esophagus ( $2.9 \mathrm{vs} .3 .4 \mathrm{~cm} / \mathrm{s} ; \mathrm{p}<0.05)$; velocity in the mid- ( 4.7 vs. $4.8 \mathrm{~cm} / \mathrm{s} ; \mathrm{p}=\mathrm{ns}$ ) and lower- $(2.7 \mathrm{vs} .2 .8 \mathrm{~cm} / \mathrm{s} ; \mathrm{p}=\mathrm{ns})$ esophagus was not affected. Contraction pressures were higher during solid than liquid bolus transport in the proximal ( 75 vs. $67 \mathrm{mmHg}$; $<0.05$ ), mid- (90 vs. $70 \mathrm{mmHg}$; $<0.01$ ) and lower- (119 vs. $102 \mathrm{mmHg} ; \mathrm{p}<0.01)$ esophagus. The pressure gradient across the gastro-esophageal junction was greater during solid bolus transit $(\mathrm{p}<0.01)$. Failures of solid bolus transport usually $(86 \%)$ occurred at the pressure trough between the proximal- and mid- segments of the esophagus. This 'transition zone' was wider when bolus escape occurred $(2.9 \mathrm{vs} .1 .7 \mathrm{~cm}$, $\mathrm{p}<0.01$ ). Contraction pressure in the proximal esophagus ( 68 vs. $70 \mathrm{mmHg}, \mathrm{p}=0.62$ ) and nadir pressure in the 'transition zone' ( 21 vs. $25 \mathrm{mmHg}, \mathrm{p}=0.18$ ) were not affected. Below the level of bolus escape in the distal esophagus contraction pressure was reduced $(90 \mathrm{mmHg}$ vs. $107 \mathrm{mmHg}, \mathrm{p}<0.04$ ). Only 'transition zone' width was independently associated with failure of solid bolus transport (i.e. other effects were the result rather than the cause of bolus escape). CONCLUSION: The physical properties of the bolus alter esophageal function. When bolus escape occurs in healthy subjects it is usually in the 'proximal transition zone' Coordination between segmental contractions is more important than contraction pressure to the success of solid bolus transport.

\section{W1807}

High Resolution Manometry (HRM) of the Upper Esophageal Sphincter (UES): An Accurate Automated Method for Quantifying Deglutitive Function Sudip K. Ghosh, Qing Zhang, John E. Pandolfino, Jennifer M. Post, Peter J. Kahrilas

Aim: Pathophysiological studies of the UES suggest that sphincter pathology is clinically manifest by increased intrabolus pressure (IBP) during trans-sphincteric flow (Williams et al., AJP, 281:1290, 2001). Those studies utilized concomitant manometry and fluoroscopy to track sphincter movement and localize intra-sphincteric pressure. Though possible, such studies are cumbersome and we sought to determine whether the enhanced spatial resolution and interpolation techniques of HRM allow for an accurate assessment of these clinically relevant attributes of UES function in an automated fashion and without fluoroscopy. Methods: 75 asymptomatic controls (40 male, ages 19-48) were studied. A solid-state manometric assembly with 36 circumferential sensors spaced $1 \mathrm{~cm}$ apart was positioned to record from the hypopharynx to the stomach (Sierra Scientific Instruments Inc., Los Angeles, CA). All subjects performed $105 \mathrm{ml}$ water swallows and 1 each of 1,10 , and $20 \mathrm{ml}$. Pressure profiles across the UES were displayed in isobaric contour format and analyzed using an automated computational algorithm written in Matlab (The Mathworks Inc, Natick, MA) that measured: 1) the relaxation interval (RI) defined as the onset of maximal relaxation to the instant that the sphincter recovered to $50 \mathrm{mmHg}$, 2) the median intrabolus pressure (mIBP) during the RI, and 3) the integrated relaxation resistance (IRR) defined as mIBP/RI. Mean values were compared using ANOVA. Results: Mean \pm SD RI for the 1, 5, 10 and $20 \mathrm{ml}$ swallows were $0.30 \pm 0.09 \mathrm{~s}, 0.40 \pm 0.09 \mathrm{~s}, 0.46 \pm 0.09 \mathrm{~s}$ and $0.52 \pm 0.12 \mathrm{~s}$ respectively $(\mathrm{p}<0.01)$. Mean \pm SD mIBP for the $1,5,10$ and $20 \mathrm{ml}$ swallows were $5.99 \pm 3.42 \mathrm{mmHg}$, $6.42 \pm 2.18 \mathrm{mmHg}, 8.99 \pm 3.40 \mathrm{mmHg}$ and $11.13 \pm 3.01 \mathrm{mmHg}$ respectively $(\mathrm{p}<0.01)$ The IRR across volumes were $22.4 \mathrm{mmHg} / \mathrm{sec}, 21.3 \mathrm{mmHg} / \mathrm{sec}, 21.0 \mathrm{mmHg} / \mathrm{sec}$ and 22.7 $\mathrm{mmHg} / \mathrm{sec}$ for increasing swallow volumes; none of these differences were significant. Peak pharyngeal contraction during the return to the resting state post-swallow was 265.42 $\mathrm{mmHg}, 284.75 \mathrm{mmHg}, 291.97 \mathrm{mmHg}$ and $297.43 \mathrm{mmHg}$ for 1, 5, 10 and $20 \mathrm{ml}$ respectively; none of these differences were significant. Conclusions: 1) The enhanced spatial resolution of HRM allows for the accurate, automated assessment of UES relaxation and intrabolus pressure characteristics, in both cases confirming the volume-dependent effects and absolute values of these parameters demonstrated by detailed analysis of concurrent manometry/ fluoroscopy data in previous studies. 2) A new HRM parameter, the IRR, was developed that normalized the volume-dependent variability of the mIBP and RI, potentially providing an index to quantify functional obstruction at the UES

\section{W1808}

Effects of Positive Modulation of Gabab Receptors On Gastro-Esophageal Reflux (GER), Basal Lower Esophageal Sphincter Pressure (BLESP) and Esophageal Motility in Conscious Dogs

Marie-Laure Rouzade-Dominguez, Ludovic Perrot, Elisabeth Braun, Hans-Juergen Pfannkuche

Introduction: $\mathrm{GABAB}$ receptor agonists, such as baclofen reduce the incidence of Transien Lower Esophageal Sphincter Relaxations (TLESRs) in men and dogs and decrease swallows. Positive modulators of $\mathrm{GABAB}$ receptors act synergistically with an agonist by enhancing the activation of the receptor, but have no or only little intrinsic agonistic activity. Aim: To investigate the role of GABA tone on GER, BLESP, swallows and esophageal motility using a positive modulator of GABAB receptors (CGP7930) alone or in combination with a threshold dose of baclofen. Method: In Beagle dogs $(n=6)$ with chronic esophagostomies, TLESRs were triggered by gastric infusion of a liquid caloric meal $(\mathrm{pH} 3,100 \mathrm{ml} / \mathrm{min}, 30 \mathrm{ml} / \mathrm{kg}$ ) followed by air infusion ( $50 \mathrm{ml} / \mathrm{min}, 20 \mathrm{~min})$. CGP7930 (3 mg/kg, i.v.) or vehicle were tested with and without a threshold dose of baclofen $(1.2 \mu \mathrm{mol} / \mathrm{kg}$, s.c.). TLESRs and acid reflux were counted during food and air infusion in the stomach. BLESP was averaged every 2 min (excluding swallows and TLESRs), the number of swallows were counted and esophageal motility (contraction duration and amplitude, propagation speed) was evaluated in baseline conditions, following compounds administration and during gastric distension. Data were represented as mean \pm SEM. Results: The control number of TLESRs $(6.2 \pm 0.5)$ was significantly $(\mathrm{p}<0.05)$ reduced by baclofen $(4.3 \pm 0.6)$, but not by CGP7930 $(6.2 \pm 0.7)$. With the combination of CGP7930 and baclofen, TLESRs were significantly $(\mathrm{p}<0.05)$ reduced vs control but not vs baclofen $(4.2 \pm 0.4)$. Control acid reflux $(2.2 \pm 0.4)$ was not changed by baclofen $(2.5 \pm 0.6)$ or CGP7930 alone $(2.2 \pm 0.8)$ but was significantly $(\mathrm{p}<0.05)$ reduced by the combination $(1.0 \pm 0.3)$. BLESP was not modified by any compound administered. The control number of swallows/10 min during distension in the vehicle experiment (8.6 $\pm 0.6)$ was not changed by baclofen $(7.0 \pm 1.2)$ or CGP7930 ( $8.2 \pm 0.7)$ but was significantly $(\mathrm{p}<0.05)$ reduced with the combination $(4.7 \pm 1.7)$. No major effect on esophageal motilit were found for any treatment. Doses of CGP7930 higher than $3 \mathrm{mg} / \mathrm{kg}$ were not tolerated Conclusion: In our dog model, at the dose tested, CGP7930 did not reduce TLESRs and did not potentiate the effects of a threshold dose of baclofen. However, the reduction of acid reflux and swallows with the combination of baclofen and CGP7930 are suggestive of a positive modulation of GABAB receptors. Swallowing pathways seems to be under GABAergic control. The apparent discrepancy between effects on TLESRs and on acid reflux might suggest a dual regulation of gastro-esophageal reflux mechanisms.

\section{W1809}

Effect of Tegaserod On the Topography of Esophageal Motility in Subjects Receiving OMEPRAZOLE: A Study Using High-Resolution Manometry (HRM) Ray E. Clouse, Laura Haroian, Mikhail Rojavin, David L. Earnest

Tegaserod has gastric and intestinal prokinetic effects, but its impact on esophageal motility is less understood. Preliminary findings suggest tegaserod can favorably influence transit by an unknown mechanism. The mid esophagus (2nd topographic segment in the peristaltic chain as defined by HRM) is under dominant cholinergic control with potential susceptibility to a 5HT4 agonist. This segment also may be important in the peristaltic pump. Subjects: 8 asymptomatic volunteers (age $38.4 \pm 10.9 \mathrm{yr}$, age range $35-47 \mathrm{yr}, 5$ female) who had taken omeprazole $20 \mathrm{mg}$ BID for l week underwent HRM to see if an effect of tegaserod could be detected, particularly in this region of interest. Methods: Following overnight fast, subjects were studied with a 21-lumen water-perfused system (1-cm recording port spacing) designed for use in HRM and for display of topographic 3-dimensional (isocontour) pressure data (MMS, Enschede, Holland). The catheter was anchored distally so that the majority of the esophageal body including both smooth-muscle peristaltic segments (segments $2 \& 3$ ) and LES after contraction (segment 4) was present. 10 baseline 5-ml water-bolus swallows were taken followed by a $12 \mathrm{mg}$ PO dosage of tegaserod. Swallows were repeated at $30 \mathrm{~min}, 60$ min, and 90 minutes following the dose, and all data were analyzed without knowledge of the sequence or subject. The primary outcome measure was segment 2 pressure volume (mmHg.cm.s) at $60 \mathrm{~min}$, measured using a previously described on-screen assessment tool. Additional measures (segment length, peak segment amplitude) also were extracted from the topographic isocontour maps. Results: Tegaserod increased segment 2 volume at each time point and to a significant degree by $60 \mathrm{~min}(1171 \pm 997 \mathrm{vs} 1364 \pm 965 \mathrm{mmHg} . \mathrm{cm} . \mathrm{s}, \mathrm{p}=$ 0.032 ), whereas volumes of segments 3 and 4 were unaffected. Tegaserod lengthened segmen 2 at each time point $(6.3 \pm 1.1$ vs $7.3 \pm 1.4 \mathrm{~cm}$ at $60 \mathrm{~min}, \mathrm{p}=0.036)$ and increased peak amplitude within the segment $(89 \pm 53$ vs $99 \pm 51 \mathrm{~mm} \mathrm{Hg}$ at $60 \mathrm{~min}, \mathrm{p}=0.007)$. Segment 3 decreased in length $(8.3 \pm 2.8$ vs $7.6 \pm 2.9 \mathrm{~cm}$ at $60 \mathrm{~min} ; \mathrm{p}=0.06)$ in response to lengthening of segment 2 , but there were no other changes with tegaserod on the topographic analyses. Conclusions: As demonstrated by HRM, a single $12 \mathrm{mg}$ PO dose of tegaserod selectively increases contractile strength in the mid esophagus in subjects who had been taking omeprazole $20 \mathrm{mg}$ BID. Tegaserod may enhance esophageal clearance through this mechanism. 
W1810

The Manometric Common Cavity Phenomenon Is An Unreliable Indicator of Gastroesophageal Reflux

Marissa C. Aanen, Albert J. Bredenoord, Melvin Samsom, Andre J. Smout

Introduction The manometric common cavity phenomenon is characterized by a simultaneous intra-esophageal pressure rise to gastric pressure level and is considered indicative of gastroesophageal reflux. There are differences in opinions on the sensitivity and the specificity of the common cavity phenomenon as reflux detector, as well as on the nature of reflux events that are associated with a common cavity. Therefore we tested the value of reflux detection by means of common cavity analysis using combined impedance and $\mathrm{pH}$ recording as the gold standard. Methods Ten healthy male subjects (mean age 28 year range 20-46) underwent combined stationary pressure, $\mathrm{pH}$ and impedance recording for 4.5 hours. After 75 minutes of recording, a reflux-eliciting meal was consumed (McDonald's Quarter Pounder hamburger, onions 20 grams, chips 44 grams, and orange juice $475 \mathrm{ml}$; total of $967 \mathrm{kCal}$ ). A common cavity was defined as a pressure increase in at least two esophageal tracings to gastric level within ls and remaining at that level for $>0.5 \mathrm{~s}$. Reflux episodes were classified as liquid, mixed or gaseous reflux and the acidity of liquid and mixed reflux episodes was determined. The chi-square test was used for statistical analysis. Results A common cavity was found in 96 (43\%) of the 223 reflux events detected by impedance, while 7 common cavities were unrelated to a reflux episode. In $54 \%$ of the reflux events detection of a common cavity was obscured by either contractile activity or artifacts caused by posture changes. The type of reflux associated with a common cavity (liquid $60 \%$, mixed $31 \%$, gas $9 \%$ ) and without a common cavity (liquid 59\%, mixed 29\%, gas $12 \%$ ) did not differ, nor did the acidity of the reflux episodes (with common cavity: $\mathrm{pH}<467 \%, \mathrm{pH}>433 \%$; without common cavity $\mathrm{pH}<458 \%, \mathrm{pH}>4$ 42\%). Discussion The manometric common cavity phenomenon is specific but not sensitive as marker of gastroesophageal reflux. Furthermore, a common cavity is not specific for a particular type of reflux.

\section{W1811}

A Practical in-Vitro Model for Physiological and Pharmacological Studies of Esophageal Muscle Function Samuel K. Arthur, Yousif Al-Serkal, David F. Evans

We describe a model for studying separately, the contraction of esophageal muscles without anatomically separating them. Preloaded, whole esophageal segments from rats $(\mathrm{N}=36)$ were mounted in organ baths with Krebs-Ringer solution (KRS) at 37 Celsius gassed with 95\% oxygen and 5\% CO2. Maximal carbachol-induced longitudinal contraction of the segments was achieved with a dose of $10 \mathrm{uM}$ carbachol. This was followed by tetanic electric field stimulation (EFS) with stimulus parameters $40 \mathrm{~V}, 0.2 \mathrm{~ms}, 25 \mathrm{~Hz}$ for $2 \mathrm{~s}$. The resulting lonitudinal isometric force of contraction was superimposed on the carbachol stimulated contraction. In the presence of calcium-free KRS, ouabain, dantrolene or barium chloride, esophageal segments were also stimulated by EFS. Carbachol stimulated contraction was abolished completely by $l \mathrm{uM}$ atropine and by $100 \mathrm{uM}$ hexamethonium. EFS resulted in a biphasic isometric contraction. The second phase of contraction was abolished completely by $l \mu \mathrm{M}$ atropine and by $100 \mu \mathrm{M}$ hexamethonium but the first phase was not. Ouabain did not affect either phase of EFS induced contraction. However, $\mathrm{BaCl} 2$ abolished the second phase with topographical differences in the dose of $\mathrm{BaCl} 2$ required. The first phase was also abolished by $\mathrm{BaCl} 2$ but at a higher dose than required for the second phase. $\mathrm{BaCl} 2$ inhibition of the second phase was greater in low-potassium (1mM) KRS than in normal potassium $(4.16 \mathrm{mM})$ KRS. The second phase EFS induced contraction disappeared in calcium- free KRS and was restored by adding $2.5 \mathrm{mM}$ calcium. The first phase contraction was not affected by calciumfree KRS. However, dantrolene significantly reduced the first phase contraction without affecting the second phase. We have shown that the physiology of esophageal smooth and skeletal muscles can be studied separately without disrupting the anatomical integrity of the esophagus. It is evident that postganglionic nicotinic receptor activation, activation of acetylcholine gated ion channels and calcium entry into the smooth muscle cell are involved in the initiation of rat esophageal smooth muscle contraction. This model has potential for use in the study of the dynamics of calcium signalling in esophageal muscle contraction. It can also be used to study topographical differences in anatomy and function. Because anatomical integrity is preserved it is a good model for studying enteric neural transmission. Tissue viability can be maintained for up to th with this model. Finally, this model is promising for the development of new prokinetic drugs.

\section{W1812}

Upper Esophageal Sphincter (UES) Responsiveness to Esophageal Stimulation in Infants with Chronic Lung Disease of Infancy

Sudarshan R. Jadcherla, Alankar Gupta, Soledad Fernandez, Reza Shaker

BACKGROUND: Chronic lung disease of infancy (CLDI) is defined as oxygen requirement beyond 36 wks corrected gestational age(GA). UES is an important barrier that confers airway protection. UES function with esophageal provocation in CLDI is not understood. OBJECTIVE: To identify the characteristics of Esophago UES Contractile Reflex (EUCR) responses with esophageal stimulation with air and water infusions in CLDI and compare with control infants. HYPOTHESIS: EUCR is impaired in infants with CLDI. METHODS: We recorded pharyngo-esophageal motility using a micromanometric pneumohydraulic water perfusion system, a specially designed pharyngo-esophageal catheter assembly with 5-sideports, UES sleeve, concurrent with submental EMG to document swallow, with MMS motility system, in 6 healthy neonates $(36 \pm 2 \mathrm{wk}, \mathrm{GA})$ and 6 infants with CLDI $(28 \pm 5 \mathrm{wk}$ GA) at a corrected GA of $39 \pm 2$ wks and $52 \pm 11$ wks respectively. Graded volumes (0.1$2.0 \mathrm{ml}$ )of air and water infusions were given thrice via the mid-esophageal infusion port. Sensory and motor characteristics of UES responses were evaluated upon stimulation with 268 esophageal infusions. All values are stated as mean $\pm \mathrm{SD}$ or $\%$. ANOVA and t-test were performed. RESULTS: In contrast to controls, subjects with CLDI required longer $(* \mathrm{P}<$ $0.01)$ respiratory support $(4 \pm 5 \mathrm{vs} 200 \pm 170$ days $*)$, gavage feeds $(27 \pm 37$ vs $188 \pm 174$ days*) and length of stay $(29 \pm 26$ vs $197 \pm 171$ days*). Threshold volumes of air $(1.6 \pm$
1.8 vs $1.3 \pm 0.6 \mathrm{ml})$ and water $(0.7 \pm 0.3$ vs $0.8 \pm 0.3 \mathrm{ml})$ were similar. Frequency of EUCR was $68 \%$ vs $26 \%$ with air and $49 \%$ vs $18 \%$ with water (both, $P<0.001$ ). Response time to EUCR with air was $1.6 \pm 0.6$ vs $3.2 \pm 2.2 \mathrm{sec}$ and with water $1.4 \pm 0.9$ vs $4.7 \pm 2.1 \mathrm{sec}$ (both, $\mathrm{P}<0.05$ ). EUCR response was volume dependent (table) with air in controls and CLDI (both, $\mathrm{P}<0.05)$; and with water in controls only $(\mathrm{P}<0.05)$ but not in CLDI. Comparison between the groups was significant with both air and water $(\mathrm{P}<0.05)$. CONCLUSIONS: Threshold volumes to evoke EUCR with air and water were similar. Response time was longer in CLDI with both media. Volume dependent recruitment of EUCR was significantly different between the groups. The proximal aerodigestive tract sensory-motor functions are impaired in response to esophageal stimuli in CLDI and may contribute to increased resource utilization.

\% EUCR, stimulus response relationship: Controls vs CLDI

\begin{tabular}{|c|c|c|c|c|}
\hline & $0.1 \mathrm{ml}$ & $0.5 \mathrm{ml}$ & $1.0 \mathrm{ml}$ & $2.0 \mathrm{ml}$ \\
\hline Control, air* & 0 & 64 & 72 & 75 \\
\hline CLDI, Air* & 0 & 0 & 42 & 61 \\
\hline Control, Water** & 0 & 43 & 47 & 100 \\
\hline CLDI, Water & 0 & 17 & 25 & Not given \\
\hline
\end{tabular}

$* \mathrm{P}<0.05, \uparrow \mathrm{P}<0.05$ between groups

\section{W1813}

Influence of Sleep Stages On Glottal Function: Implications for Nighttime Airway Safety

Jasmohan Bajaj, Tanya Rittmann, David Knuff, Kulwinder Dua, Reza Shaker

BACKGROUND: Airway inlet is controlled by the striated adductor/abductor glottal muscles Deeper sleep stages, particularly REM sleep, are associated with varying degrees of muscular hypotonia of striated muscles; however, the effect of various sleep stages (ST) on glottal function has not been systematically evaluated. AIM: To quantify and compare respirationinduced movements of the vocal cords during various sleep stages. METHODS: Six healthy volunteers $(31 \pm 5$ yrs, $4 \mathrm{M})$ were studied during awake state and for at least 4 hours during various stages of sleep in a sleep laboratory. Glottal function was assessed by direct video recording of the vocal cords through a transnasal (3mm OD) video-endoscope(Pentax FNL $\mathrm{OAP}$ ) and sleep parameters were measured by concurrent polysomnography (REMbrandt, Amsterdam), electromyography (EMG) and pulse oximetry (SpO2). Glottal function during inspiration (in) and expiration (ex) was studied by measuring its intra-luminal lateral (LA), antero-posterior (AP) diameters and glottal inlet area (Area). Findings were compared to those measurements in the awake state. RESULTS: Glottal function was studied in all subjects during ST $1 \& 2$, three subjets during delta sleep and 2 during REM sleep. There was no significant difference in the respiratory rate or $\mathrm{O} 2$ saturation between conditions $(15 \pm 2$, $96 \pm 1 \%$, respectively). Vocal cord movements were maintained during REM sleep despite generalized muscle hypotonia. However, LA, AP \& Area were affected by deeper sleep stages in that area of glottal inlet was reduced in delta sleep and increased in ST 1, $2 \&$ REM compared to awake state (Table). Area(in) Delta sleep was significantly reduced compared to ST $\mathrm{l}(\mathrm{p}=0.003)$ and trended towards significance compared to Stage $2(\mathrm{p}=0.06)$ and during expiration. CONCLUSION: Generalized hypotonia during REM sleep does not prevent vocal cord inspiratory/expiratory movements. Sleep stages affect glottal function differently; while Delta sleep seems to reduce the size of the glottal inlet, other stages are accompanied by an increase in laryngeal inlet compared to the awake state.

\begin{tabular}{|c|c|c|c|c|}
\hline & ST1 $(\% \Delta)$ & ST2 $(\% \Delta)$ & Delta $(\% \Delta)$ & REM $(\% \Delta)$ \\
\hline LA (ex) & $2 \pm 20$ & $-3 \pm 14$ & $-24 \pm 38$ & $-42 \pm 3$ \\
\hline LA (in) & $6 \pm 11$ & $8 \pm 10$ & $-24 \pm 16$ & $4 \pm 7$ \\
\hline AP (ex) & $18 \pm 13$ & $28 \pm 17$ & $15 \pm 11$ & $21 \pm 12$ \\
\hline AP (in) & $11 \pm 19$ & $31 \pm 13$ & $17 \pm 11$ & $25 \pm 1$ \\
\hline Area (ex) & $25 \pm 15$ & $21 \pm 26$ & $-23 \pm 10$ & $1 \pm 17$ \\
\hline Area (in) & $27 \pm 22$ & $38 \pm 37$ & $-27 \pm 8$ & $31 \pm 19$ \\
\hline
\end{tabular}

$\% \Delta$ : percent change compared to awake state

\section{W1814}

Manometric and Endoscopic Findings in the Symptomatic Post-Fundoplication and Post-Redo-Fundoplication Patients: Do They Differ Significantly? Moises Garcia, Benson Massey, Reza Shaker, Walter Hogan

Fundoplication is associated with postoperative morbidity in a significant minority of patients.Redo fundoplication does not assure a successful outcome either. Aim of study: Define the differences in symptoms,manometric and endoscopic findings in post-fundoplication(Post-F)and post-redo fundoplication(Post-redo)patients from a single referral center Methods:94 patients(64F;30M;age $47.7 \pm 12.8$ yrs)were referred to the MCW Dysphagia Institute from January 2000 to July 2005 for evaluation of post-operative symptoms. There were 57 Post-F patients and 37 post-redo patients.All Post-F patients had manometry(abnormal criteria: wrap pressure elevated $(>25 \mathrm{mmHg}$ ) or low $(<7 \mathrm{mmHg}$ );ramp pressure $>25 \mathrm{mmHg}$ or peristalsis frequency $<60 \%$ of swallows) 48 of 57 Post-F patients ( $84 \%$ ) had endoscopy(abnormal criteria:inflammation,slipped,improperly placed,disrupted or too tight wrap). All 37 Post-Redo patients had manometry study; 32(86\%)had endoscopy.Symptoms were recorded by an experienced investigator prior to manometry study. Results:Dysphagia was the primary symptom in both groups, with a frequency in the Post-redo(24/37:65\%) versus Post-F patients $(26 / 57 ; 46 \%)(\mathrm{p}=0.106)$. Heartburn was the major symptom in 17 Post- $\mathrm{F}(30 \%)$ versus 7 Post-redo patients $(19 \%)(\mathrm{p}=0.346)$. Manometry abnormalities were detected in 53\% Post$\mathrm{F}$ (decreased peristalsis 35\%) compared to 59\% Post-redo patients.A too-tight wrap(elevated 
wrap or ramp pressures)was found on $10 / 57$ post-F;13/37 post-redo patients. Endoscopy detected abnormalities in $60 \%$ of Post-F(wrap abnormalities $45 \%$ )and $69 \%$ of Post-redo patients $(31 \%$ deformed wrap) $(\mathrm{p}=0.284)$. Intubation of the wrap was considered snug in 10 / 32 post-redo and 3/48 Post-F patients $(\mathrm{p}<0.008)$,but this did not correlate manometricaly. Esophagitis was more frequent on endoscopy in Post-F(35\%)versus Post-redo patients $(19 \%)(\mathrm{p}=0.737)$ Conclusions:manometry and endoscopy findings in symptomatic post-F and post-redo patients were not significantly different. Dysphagia was the major postop symptom in both groups.Heartburn was more common in Post-F patients reflecting the higher incidence of inflammation seen on endoscopy.Manometry and endoscopy are complimentary in assessing Post-Fundoplication and Post-redo-fundoplication symptoms. When one or both diagnostic modalities are abnormal,a plausible explanation is present in greater that $80 \%$ of these patients.

Diagnostic results post- $\mathrm{F}(\mathrm{n}=57)$ vs. post-redo( $\mathrm{n}=37)$

\begin{tabular}{|c|c|c|}
\hline Results & Post-F \% & Post-redo $\%$ \\
\hline Normal & 14 & 10 \\
\hline Abn. Manometry & 53 & 59 \\
\hline Abn. Endoscopy & 60 & 69 \\
\hline Abn. any modality & 83 & 88 \\
\hline
\end{tabular}

Not significant difference.

\section{W1815}

Different $\mathrm{Ca}^{2+}$ Stores Mediate Acetylcholine (ACh)-Induced Contraction of Cat LES and Esophageal Circular Smooth Muscle

Weibiao Cao, Karen M. Harnett, Ling Cheng, Jose Behar, Piero Biancan

We have previously shown that in smooth muscle cells from the circular layer of cat esophagus (ESO) ACh-induced contraction is $\mathrm{IP}_{3}$ and calmodulin-independent and mediated through a protein kinase $\mathrm{C}$ (PKC)-dependent pathway, involving a calcium insensitive PKCE. We have also shown that in ESO calcium is needed to activate phospholipases and produce second messengers. In contrast, in the lower esophageal sphincter (LES) contraction in response to a maximally effective dose of acetylcholine (Emax ACh) is mediated through $\mathrm{IP}_{3}$-induced $\mathrm{Ca}^{2+}$ release from intracellular stores, and activation of a calmodulin-dependent pathway. In LES fura 2-loaded cells, Emax ACh caused a cytosolic $\mathrm{Ca}^{2+}$ increase from $93 \pm 8 \mathrm{nM}$ to $537 \pm 54 \mathrm{nM}$ in normal $\mathrm{Ca}^{2+}$ buffer and from $59 \pm 7 \mathrm{nM}$ to $483 \pm 57 \mathrm{nM}$ in $\mathrm{Ca}^{2+}$-free

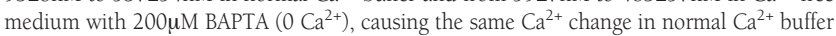
$(444 \pm 47 \mathrm{nM})$ and in $0 \mathrm{Ca}^{2+}(424 \pm 50 \mathrm{nM})$. These data confirm that the $\mathrm{ACh}$ induced $\mathrm{Ca}^{2+}$ signal in LES is mainly caused by $\mathrm{Ca}^{2+}$ release from intracellular stores. This $\mathrm{Ca}^{2+}$ signal was significantly reduced by the $\mathrm{IP}_{3}$ receptor antagonist xestospongia $\mathrm{C}(1 \mu \mathrm{M})$ and by the $\mathrm{PIP}_{2}$-specific phospholipase $\mathrm{C}$ inhibitor U73122 $(1 \mu \mathrm{M})$, but not by ruthenium red $(5 \mu \mathrm{M})$, an inhibitor of ryanodine sensitive $\mathrm{Ca}^{2+}$ channels, or by the phosphatidylcholine-specific phospholipase inhbitor D609 $(10 \mu \mathrm{M})$, confirming that in LES ACh causes $\mathrm{Ca}^{2+}$ release from $\mathrm{IP}_{3}$-sensitive stores. In ESO fura 2-loaded cells Emax ACh increased intracellular $\mathrm{Ca}^{2+}$ from $100 \pm 10 \mathrm{nM}$ to $547 \pm 84 \mathrm{nM}$ in normal $\mathrm{Ca}^{2+}$ buffer and from $56 \pm 7 \mathrm{nM}$ to $190 \pm 69 \mathrm{nM}$ in $0 \mathrm{Ca}^{2+}$, thus the ACh-induced $\mathrm{Ca}^{2+}$ change was significantly higher $(\mathrm{p}<0.05$, ANOVA) in normal $\mathrm{Ca}^{2+}$ buffer $(447 \pm 87 \mathrm{nM})$ than in $0 \mathrm{Ca}^{2+}(134 \pm 65 \mathrm{nM})$, suggesting that both $\mathrm{Ca}^{2+}$ influx and $\mathrm{Ca}^{2+}$ release contribute to the ACh induced $\mathrm{Ca}^{2+}$ signal in ESO. In $0 \mathrm{Ca}^{2+}$ the $\mathrm{KCl}$ induced $\mathrm{Ca}^{2+}$ signal was abolished, confirming that incubation in this medium $\left(0 \mathrm{Ca}^{2+}\right)$ abolishes influx of extracellular $\mathrm{Ca}^{2+}$. ACh induced $\mathrm{Ca}^{2+}$ signal in normal $\mathrm{Ca}^{2+}$ buffer was significantly reduced by ruthenium red, and by D609, but not by xestospongia $\mathrm{C}$, or by $10 \mathrm{uM}$ AACOCF3 (cPLA2 inhibitor), or $10 \mu \mathrm{M}$ NPPB (a chloride channel blocker), suggesting that in ESO ACh causes $\mathrm{Ca}^{2+}$ release from ryanodine sensitive stores. This $\mathrm{Ca}^{2+}$ release is not mediated by arachidonic acid or by chloride channel activation, and is different from intestinal muscle cells as reported by others. The data suggest that the ACh-induced calcium signal in ESO depends in part on ryanodine-sensitive $\mathrm{Ca}^{2+}$ stores, and in $\mathrm{LES}$ on $\mathrm{IP}_{3}$-sensitive $\mathrm{Ca}^{2+}$ stores.

\section{W1816}

NADPH Oxidase-Derived $\mathrm{H}_{2} \mathrm{O}_{2}$ Contributes to Maintenance of Human Lower Esophageal Sphincter (LES) Tone

Weibiao Cao, Karen M. Harnett, Ling Cheng, Jose Behar, Piero Biancani

We have previously shown that a low molecular weight group I PLA $A_{2}$ mediates cat and human in vitro LES tone by producing arachidonic acid, which is metabolized to $\mathrm{PGF}_{2} \alpha$ and thromboxane $A_{2}$. These arachidonic acid metabolites act on receptors linked to $G_{i 3}$ and $\mathrm{G}_{\mathrm{q}}$ proteins to activate phospholipases, producing second messengers, and maintaining LES tone. In cat the non-selective cyclooxygenase inhibitor indomethacin abolishes tone, confirming a role of arachidonic acid metabolites in tone maintenance. Indomethacin, however, was less effective in reducing tone of vitro human LES circular muscle, causing approximately a $50 \%$ reduction, and suggesting that other mechanisms may also contribute to maintenance of tone. We have previously shown that high levels of $\mathrm{H}_{2} \mathrm{O}_{2}$ are present in esophagitis and reduce LES tone through several pathways, including release of calcium from intracellular stores, resulting in depletion of releasable calcium. In the present study we examined a possible role of low levels $\mathrm{H}_{2} \mathrm{O}_{2}$ in maintenance of human LES tone. $\mathrm{H}_{2} \mathrm{O}_{2}$ in low doses $\left(10^{-8}-10^{-6} \mathrm{M}\right)$ increased spontaneous tone of in vitro human LES circular muscle strips, and the membrane-permeable hydrogen peroxide scavenger tiron dose-dependently decreased LES tone, suggesting that low levels of $\mathrm{H}_{2} \mathrm{O}_{2}$ may contribute to maintenance of human LES tone. The NADPH oxidase inhibitor apocynin dose-dependently decreased LES tone, indicating that NADPH oxidase-derived reactive oxygen species may play a role in maintenance of tone. The apocynin-induced reduction in LES tone was not affected by the nitric oxide synthase inhibitor L-NNA, indicating that inhibition of NADPH oxidase and reduction in basal levels of $\mathrm{H}_{2} \mathrm{O}_{2}$ relaxes $\mathrm{LES}$ muscle through an $\mathrm{NO}$-independent mechanism, which is different from the role of NADPH oxidase in maintenance of vascular tone. The sustained contraction induced by $\mathrm{H}_{2} \mathrm{O}_{2}$ was abolished by the Rho kinase inhibitor $\mathrm{Y} 27632$ and was significantly decreased by the MEKl inhibitor PD98059 and by the P38 MAP kinase inhibitor SB203580 in LES muscle strips and in isolated muscle cells. These data are consisten with a role of low levels $\mathrm{H}_{2} \mathrm{O}_{2}$ in mediating sustained contraction of human LES circular muscle, in contrast to high levels of $\mathrm{H}_{2} \mathrm{O}_{2}$, that reduce LES tone through multiple pathways.

\section{W1817}

Assessment of Dietary Nutrients That Influence Cortical Perception of IntraEsophageal Acid Reflux Events

Michael Shapiro, Colleen N. Green, Jimmy M. Bautista, Roy Dekel, Sara Risner-Adler, Robin Whitacre, Ellen Graver, Ronnie Fass

Background: Gastroesophageal reflux disease (GERD) symptoms are most commonly reported postprandially, suggesting that some diet components are likely to induce symptoms more than others. Aims: To determine which of the various dietary nutrients is a strong predictive factor for symptom generation in association with a sensed acid reflux even (reflux event that is associated with GERD-related symptom). Methods: Subjects with typica heartburn symptoms were evaluated by the GERD Symptom Checklist and a demographics questionnaire. Thereafter, all patients underwent an upper endoscopy to assess for the presence of esophageal inflammation. Subsequently, ambulatory 24-hour esophageal pH monitoring was carried out using a four-sensor $\mathrm{pH}$ probe. During the $\mathrm{pH}$ study, patients completed a detailed 24-hour dietary intake record using the University of Minnesota Nutrition Data System Research (NDS-R) version. This is a micro-computer based system for collection and analysis of dietary data that prompts the user to describe detailed food intake. Results: Fifty GERD patients (22 with erosive esophagitis, M/F 37/13, mean age 51.5, mean BMI 27.9) were enrolled into the study. A total of 112 (78\%) symptoms were considered as sensed reflux event. BMI did not correlate with having sensed reflux event through all regressions $\left(R^{2}=0.005, \beta=0.05, t\right.$-value $\left.=0.55\right)$. Patients who consumed more cholesterol, saturated fatty acids and had more percentage calories from fat were significantly more likely to experience a sensed reflux event $(\mathrm{r}=0.22,0.16,0.18$, respectively, $\mathrm{p}<0.01$ ). Regression analysis and beta coefficient were specifically significant for cholesterol $\left(\mathrm{R}^{2}=0.03\right.$, F-change $=3.3, \beta=0.0004, \mathrm{t}$-value $\left.=2.5, \mathrm{p}<0.01\right)$. Alcohol consumption demonstrated negative correlation with having a sensed reflux event $(r=-0.2, p<0.01)$. Al other dietary nutrients, including monounsaturated fatty acids, polyunsaturated fatty acids, caffeine, vegetable protein, animal protein, total protein, total carbohydrates and total fat did not correlate with a sensed reflux event. Also, \% calories from fat, carbohydrates or protein were not correlated with a sensed reflux event. Conclusions: Of all dietary nutrients and components, cholesterol enhances the most the perception of intra-esophageal acid reflux event in patients with GERD.

\section{W1818}

Does Esophageal Acid Infusion in Healthy Subjects Cause Esophageal Thermal Sensitivity?

Philip J. Matthews, Lowri Gwyn, Qasim Aziz

Background A proportion of gastroesophageal reflux disease sufferers have heartburn symptoms which may be due to esophageal hypersensitivity (EH) to acid. An experimental model of $\mathrm{EH}$ has been developed in which distal esophagus acid infusion induces hypersensitivity to electrical stimuli in the proximal (non-acid exposed) esophagus [1]due to central sensitisation. However electrical stimulation activates all sensory afferent esophageal nerves. We chose to test whether esophageal thermoreceptors are specifically sensitised by esophageal acid infusion in the model. Aims To determine whether the distal esophageal acid infusion (DEAI) could induce hypersensitivity to thermal stimuli (a specific receptor mediated sensation) in the proximal esophagus and chest wall of healthy volunteers. Method 7 healthy subjects were studied. After esophageal intubation, thermal stimuli were delivered in the proximal esophagus using a specially designed solid state thermal stimulating catheter. Subjects recorded their response on a modified Visual Analogue Scale. Chest wall thermal stimuli were delivered using a somatic thermode. After baseline readings the subject were randomised to an esophageal infusion of either $0.15 \mathrm{M} \mathrm{HCL}$ or $\mathrm{N}$ saline(control) which was delivered into the distal esophagus. Readings were taken periodically for 2 hours thereafter. Results DEAI caused significant proximal oesophageal thermosensitivity compared to control. Mean AUC for saline was 21.6 (95\% C.I. - 19 to 62) compared with 364.7 for acid (95\% C.I. 172 to $556(\mathrm{p}<0.007))$. Chest wall thermosensitivity remained unchanged after acid infusion in comparison to saline infusion. Conclusion This study shows that esophageal thermosensitivity is increased by esophageal acid exposure and this may be relevant to the development of esophageal hypersensitivity in susceptible individuals. We have previously demonstrated the presence of the acid / thermosensitive receptor TRPVI in esophageal mucosal biopsie of normal subjects and patients with esophagitis (in whom it is increased)[2]. With the advent of specific antagonists to TRPVl receptors; esophageal hypersensitivity to acid might be usefully explored using this model in future drug trials. 1. Sarkar, S., et al., Contribution of central sensitisation to the development of non-cardiac chest pain. Lancet, 2000 356(9236): p. 1154-9. 2. Matthews, P.J., et al., Increased capsaicin receptor TRPV1 nerve fibres in the inflamed human oesophagus. Eur J Gastroenterol Hepatol, 2004. 16(9): p. 897-902

\section{W1819}

Outstanding Change in Esophageal Muscle Thickness and Cross Sectional Area Using High-Frequency Intraluminal Ultrasound 6 Months Later After Pneumatic Dilation in Patients with Achalasia

Jeong Hwan Kim, Poong-Lyul Rhee, Yong Sung Choi, Hee Jung Son, Jae J. Kim, Jong Chul Rhee

Background: All forms of treatment for achalasia focus on reducing the pressure gradient across lower esophageal sphincter (LES). LES pressure can be reduced by forceful pneumatic dilation of the gastroesophageal junction, which is considered the main procedures for treating achalasia. However, it is known that any treatment can little restore the muscular 
activity of the denervated esophagus. Recently, it has been reported that an increase in esophageal muscle thickness using a high-frequency intraluminal ultrasound (HFIUS) is an important feature of patients with primary esophageal motility disorder such as achalasia. We aimed to compare the clinical features, the radiological and manometric findings, and the esophageal mean muscle thickness and cross sectional area (CSA) between pre and post pneumatic dilation states in patients with achalasia. Methods: Fifteen patients ( 8 men and 7 women, mean age $39 \pm 15$ years) with achalasia treated by pneumatic dilation were enrolled in this study. The assessment of the clinical features, the maximal diameter of esophageal body on barium esophagogram, the LES resting pressure on manometry, and the esophageal mean muscle thickness and CSA at $3 \mathrm{~cm}$ above LES on HFIUS was performed before pneumatic dilation for baseline assessment. And these were repeated 6 months later after pneumatic dilation. Results: In post pneumatic dilation state, enrolled patients exhibited distinctly different features from pre pneumatic dilation state; an improvement of dysphagia symptom, a recovery of weight, a reduction of maximal diameter of the esophageal body, and a decrease of LES resting pressure. These differences between the two states were statistically significant. Esophageal muscle thickness and CSA were observed to be significantly lesser in post pneumatic dilation state than in pre pneumatic dilation state at $3 \mathrm{~cm}$ above LES (mean muscle thickness $(\mathrm{mm})$; pre vs. post $=2.04 \pm 1.02$ vs. $1.67 \pm 0.54$, CSA $(\mathrm{mm} 2)$; pre vs. post $=138 \pm 44$ vs. $90 \pm 45, \mathrm{P}<0.05$, respectively). Conclusions: Esophageal muscle thickness and CSA was recoveried after pneumatic dilation as was the clinical, radiological, and manometric findings in patients with achalasia in this study. This result may be aided to understand the functional motor disturbance of achalasia

\section{W1820}

Acid Induced Symptom Generation (Heartburn) May Not Be Mediated By Esophageal Muscle Contraction

Toshihiko Tomita, Chizuko Hayashi, Yongmin Kim, Kazuhiko Nakajima, Noriyasu Yamamoto, Takashi Sakagami, Takayuki Matsumoto, Hiroto Miwa

(Background and Aim)Heartburn and regurgitation is considered as typical symptom of acid reflux, yet the symptom resolution is not always achieved by acid inhibition treatment, suggesting acid itself does not cause the symptoms directly. However the mechanisms of the symptom generation remain unknown. Accordingly we observed esophageal muscle contraction in esophageal acid perfusion test, by which we can monitor the physiological changes of the esophagus during heartburn symptom generation. (Subjects and Methods) Eight healthy subjects ( 6 males, mean age $26+3.5 y r s$, ranged 23 to 34 ) underwent acid perfusion test. Briefly, a double rumen gastric tube was inserted via nasal route and placed at the middle portion of the esophagus $(15 \mathrm{~cm}$ above LES) where the acid $(0.1 \mathrm{M})$ was applied in the rate of $8 \mathrm{ml}$ per min for 12 minutes (after two minutes application of saline). The ultrasonographic transducer was put and placed from the other rumen at $5 \mathrm{~cm}$ above LES, by which the thickness of the esophageal muscle was monitored every 5 seconds and video recorded. The esophageal muscle thickness was measured on the image analyzing system. The intensity of the heartburn was evaluated real-timely using visual analogue scale. The mean baseline thickness (that of first two minutes during saline perfusion) and that of 10 to 12 minutes (last two minutes) was measured both in subjects with none or mild and moderate or severe heartburn. (Results) Of eight subjects, four had moderate to severe heartburn while the rest of four subjects had no or mild symptoms. The relative esophageal muscle thickness at last two minutes compared to the initial two minutes was $1.14+0.25$ and $0.98+0.0($ mean $+S D)$ in the subjects with and without heartburn, respectively $(\mathrm{p}=0.231)$. Contraction amplitude as well as contraction duration was not significantly altered during acid perfusion both in the subjects with and without symptoms. (Conclusion) Our results indicate that acid induced symptom generation (heartburn) may not be mediated by esophageal muscle contraction.

\section{W1821}

The Virtual Esophagus: An Interactive Model of Esophageal Physiology, Pathophysiology, and Simulation of Esophageal Motility Disorders George Fantry, Marjorie McShane, Stephen Beale, Joshua Forman, John Raczek, Sergei Nirenburg, Bruce Jarrell

Background: A computer model simulating esophageal physiology and demonstrating the symptoms and test results of motility disorders would enhance trainee understanding of esophageal diseases and allow rehearsal of medical decision-making prior to patient care. Aim: To develop an interactive virtual esophagus that demonstrates automatic behavior reflective of underlying normal and diseased physiological functions and models motility and clinical management strategies. Methods: Knowledge engineers and subject matter experts collaborated to elicit knowledge rich in normal esophageal physiology, pathophysiology and clinical management. This included modeling normal esophageal concepts: anatomy, physiology and neuromuscular function, and abnormal concepts: acid reflux, inflammation, LES dysfunction, esophageal dysmotility and associated symptoms. The knowledge was encoded into machine tractable language using existing techniques developed by the collaborators. Computational methods were developed to reason with the encoded knowledge to achieve a virtual esophagus that functions in a realistic manner. Results: The knowledge and reasoning capacity successfully created an esophagus with automatic behavior capable of generating multiple qualities: (1) Intrinsic normal organ function such as basal lower esophageal sphincter pressure and lower esophageal sphincter relaxation and peristalsis in response to swallowing, (2) Symptoms of esophageal diseases such as heartburn and dysphagia, (3) Associated diagnostic test results on barium swallow, endoscopy, manometry, and $24 \mathrm{hr}$ pH monitoring such as bird's beak, stricture, aperistalsis, and DeMeester score, (4) Natural disease progression such as progressive dysphagia as the esophageal lumen narrows, (5) Disease response to various treatments such as resolution of heartburn with a proton pump inhibitor or improvement of dysphagia with dilation of a stricture. The accumulation of these qualities provides a functioning virtual esophagus capable of demonstrating the normal esophagus, achalasia, gastroesophageal reflux disease (GERD), GERD with erosive esophagitis, Barrett's metaplasia, or adenocarcinoma. Conclusions: We have designed the backbone of an interactive virtual esophagus that models esophageal physiology, pathophysiology, and clinical management for common esophageal diseases. Use of the virtual esophagus may provide a novel and optimal teaching opportunity to allow a trainee to query the esophagus, diagnose esophageal diseases, perform treatments, and observe the results of treatments. This model will be further refined with addition of other esophageal pathology and expanded to include other organs.

\section{W1822}

Vigorous Achalasia and Classic Achalasia: Two Similar But Separate Entities? Amit Agrawal, Amine Hila, Radu Tutuian, Marcelo Vela, Donald O. Castell

BACKGROUND: Achalasia is primarily defined manometrically by an aperistaltic esophagus. Traditionally, hypertensive LES, and incomplete lower esophageal sphincter (LES) relaxation are considered part of the manometric definition. Vigorous achalasia is identified in a subset of patients having distal esophageal amplitude (DEA) greater than $37 \mathrm{mmHg}$, while still having total absence of peristalsis. Combined multichannel intraluminal impedance and manometry (MII-EM) allows both a functional and a manometric evaluation of esophageal motility and also identifies chronic fluid retention. AIM: To compare manometric and MII characteristics in patients with achalasia and vigorous achalasia. METHODS. Review of 73 MII-EM tracings (38 females; mean age 53.5; range 14 - 95 years) from patients with achalasia and 73 patients with normal motility (49 females; mean age 54; 22-82 years) Manometric and MII characteristics were compared during 10 liquid and 10 viscous swallows. Patients were divided into 3 groups: classic achalasia, vigorous achalasia, and normal RESULTS: There were $59(81 \%)$ patients with classic achalasia and $14(19 \%)$ patients with vigorous achalasia. EM and MII details are shown in Tables 1 and 2 respectively. In 73 patients with normal motility, the mean baseline MII was $1955+229$ ohms, $1985+232$ ohms after 10 liquid swallows and $2102+246$ ohms after 10 viscous swallows. There was also a significant difference $(\mathrm{p}<0.05)$ between the baseline MII in patients with both classic and vigorous achalasia and normals. CONCLUSION: Patients with vigorous achalasia are likely to have a hypertensive LES, higher number of swallows with complete bolus transit for liquid and viscous swallows, and higher impedance suggesting less fluid retention than those with classic achalasia. Classic and vigorous achalasia are similar but have distinct features. Combined MII-EM helps confirm the diagnosis.

Tablel: Manometric data

\begin{tabular}{|c|c|c|c|}
\hline & Distal esophageal amplitude & LES pressure & LES residual pressure \\
\hline Classic & 22 & $36 \pm 4.3$ & $10.4 \pm 1.4$ \\
\hline Vigorous & 79 & $47 \pm 5.6$ & $11.4 \pm 3.1$ \\
\hline $\mathrm{P}$ & $<0.05$ & $<0.05$ & NS \\
\hline
\end{tabular}

Table 2: Impedance data

\begin{tabular}{|c|c|c|c|c|c|}
\hline & $\begin{array}{c}\text { CBT (median) } \\
\text { (liquid) }\end{array}$ & $\begin{array}{c}\text { CBT (median) } \\
\text { (viscous) }\end{array}$ & $\begin{array}{c}\text { Baseline MII } \\
\text { (ohms) }\end{array}$ & $\begin{array}{c}\text { Liquid MII } \\
\text { (ohms) }\end{array}$ & $\begin{array}{c}\text { Viscous MII } \\
\text { (ohms) }\end{array}$ \\
\hline Classic & $0(0-4)$ & $0(0-3)$ & 689 & 389 & 343 \\
\hline Vigorous & $1.5(0-6)$ & $0(0-6)$ & 1265 & 812 & 650 \\
\hline $\mathrm{p}$ & $<0.05$ & $<0.05$ & $<0.05$ & $<0.05$ & $<0.05$ \\
\hline
\end{tabular}

$\mathrm{CBT}=$ complete bolus transit; MII measured at baseline, post 10 liquid and viscous swallows.

W1823

Novel MII-pH Methods in the Evaluation of Physico-Chemical and Spatiotemporal Characteristics and Peristaltic Clearance Mechanisms with Reflux Events (RE) in Infants with Chronic Lung Disease of Infancy Sudarshan R. Jadcherla, Alankar Gupta, Soledad Fernandez, Carlo Dilorenzo BACKGROUND The role of physico-chemical properties of GER in prematurely born infants with CLDI (oxygen need at $>36$ wk corrected gestation age, GA ) is not well understood. OBJECTIVES To characterize the association between the physical (gas, mixed, liquid) chemical (acid, minor-acid, non-acid) and spatiotemporal (height of refluxate and duration) characteristics of RE with aerodigestive symptoms and clearance mechanisms (primary peristalsis, PP and secondary peristalsis, SP). METHODS Six neonates ( $27 \pm 1 \mathrm{wk}$ GA) with CLDI associated with dysphagia were studied. First, esophageal manometry was performed to determine the segmental lengths of the aerodigestive tract. Next, a 24-hr concurrent MII$\mathrm{pH}$ recording was performed following manometry. Clinical symptoms were documented in real time. The physical and chemical nature and the extent of the refluxate were determined for each RE. Symptoms were considered associated if they occurred during, before and after 1-min of RE. Clearance mechanism (PP, SP, and none) with each RE was recorded based on the proximal extent of the refluxate and aboral propagation. Proportions were compared between the RE characteristics, and symptom sensitivity index (SSI = \# of RE with symptoms/ total RE *100). RESULTS A total of $318 \mathrm{RE}$ (gas $36 \%$, mixed 19\%, liquid $45 \%$ ) were analyzed. Of these, $23 \%$ were acid $\mathrm{pH}<4.0,10 \%$ minor acid as $\mathrm{pH}$-decrease by 1 unit, and $67 \%$ non-acid events. Proximal extent of all RE was up to pharynx (PX, 76\%), proximal esophagus (PE, 17\%), mid esophagus (ME, 7\%), and distal esophagus (DE, 1\%). The bolus clearance time for mixed events was $17 \pm 18 \mathrm{sec}$, liquids $19 \pm 29 \mathrm{sec}(\mathrm{P}=\mathrm{NS})$. Response time for peristaltic reflex for gas RE was $3.6 \pm 5.6 \mathrm{sec}$, mixed RE $32 \pm 18 \mathrm{sec}$, and liquid $16 \pm$ $13 \mathrm{sec}$ ( ANOVA P<0.001). 134 symptoms were noted with $318 \mathrm{RE}$; of which $20 \%$ with gas, $28 \%$ with mixed, $52 \%$ with liquid. CONCLUSIONS The symptoms associated with RE and the clearance mechanisms are dependent on the physical properties and proximal extent of the refluxate. The gas and mixed RE constitute the majority of the RE with symptoms. Response times for clearance reflex responses were dependent on the physical property of refluxate. Occurrence of PP (esophago-deglutition response) with refluxate stimulating DE, $\mathrm{ME}$, and $\mathrm{PE}$ are noted.

Peristaltic clearance reflexes and SSI based on physical and spatiotemporal parameters 


\begin{tabular}{|c|c|c|c|c|c|c|}
\hline Extent & $\begin{array}{c}\text { Gas: } \\
\text { PP-SP-None* }\end{array}$ & Gas-SSI & $\begin{array}{c}\text { Mixed: } \\
\text { PP-SP-NR* }\end{array}$ & Mixed-SSI & $\begin{array}{c}\text { Liquid: } \\
\text { PP-SP-NR* }\end{array}$ & $\begin{array}{c}\text { Liquid- } \\
\text { SSI }\end{array}$ \\
\hline PX & $58-00-42$ & 23 & $66-00-34$ & 48 & $60-06-34$ & 38 \\
\hline PE & $33-00-67$ & 0 & $13-12-75$ & 7 & $38-29-33$ & 10 \\
\hline ME & $00-00-100$ & 0 & $33-00-67$ & 5 & $29-47-24$ & 2 \\
\hline DE & NONE & 0 & $50-00-50$ & 0 & $00-00-100$ & 0 \\
\hline
\end{tabular}

Chi Square and ANOVA, ${ }^{*} \mathrm{P}<0.05$, All values in $\%$

\section{W1824}

Syntaxin 1A Paradoxically Increases Esophageal Smooth Muscle Kv1.2 Membrane Trafficking to the Plasma Membrane

Leila Neshatian, Yuk M. Leung, Xiaodong Gao, Robert G. Tsushima, Herbert Y. Gaisano, Nicholas E. Diaman

Background: Kvl.2 is an important delayed rectifier potassium channel in esophageal smooth muscle (ESM) and is involved in setting the resting membrane potential. In secretory cells, SyntaxinlA (Syn1A), a t-SNARE protein, regulates Kv2.1 current density, in part by reducing the trafficking of these channels to the plasma membrane. During exocytosis, Syn $1 \mathrm{~A}$ undergoes a conformational change from the 'inactive' closed form to an 'activated' open configuration capable of binding and activating SynlA-binding proteins to effect their actions. Remarkably, we have found that in ESM, wild-type SynlA paradoxically decreased Kvl.2 current density more effectively than the open-form mutant. Hypothesis: SynlA-induced inhibition of Kvl.2 current density is in part by inhibition of trafficking of Kvl.2 to the ESM plasma membrane surface. Methods: Using streptavidin resin, cell-surface biotinylation was performed on Kyl 2- expressing HEK293 cells cotransfected with SynlA wild-type or open-form. Results: Wild type SynlA coexpression caused plasma membrane Kvl.2 protein levels to increase by $72 \%$, whereas open-form SynlA co-expression had no effect. Total Kvl.2 protein content in the total lysates did not change, indicating that neither SynlA proteins had any effect on Kvl 2 protein synthesis. Conclusion: Wild-type SynlA paradoxically promotes Kv1.2 ESM plasma-membrane surface expression, while open-form SynlA has no effect. That is, wild-type SynlA, presumably in its closed conformation, facilitates trafficking of Kvl.2 to the ESM cell membrane despite its strong and direct inhibition of the channel. These results indicate that reduced Kvl.2 current amplitude by wild-type SynlA must be due entirely to a primary and specific effect of the wild-type SynlA protein on the channel. These effects of SynlA on the non-secretory ESM are opposite to those transduced by SynlA in secretory cells, and may indicate that SynlA modulation of Kvl.2 current differs depending on the functional role involved, e.g. setting resting membrane potential vs. exocytosis

\section{W1825}

Evidence for Extrinsic Autonomic Dysregulation in the Spastic Esophageal Motility Disorders (SMDs)

Gregory S. Sayuk, Clinton T. Snedegar, Laura R. Haroian, Phyllis K. Stein, Robert M. Carney, Patrick J. Lustman, Ray E. Clouse

SMDs, including diffuse esophageal spasm (DES) and nonspecific SMDs, are esophageal motor disorders commonly detected in symptomatic subjects, but their pathogenesis is poorly understood. Imbalance between cholinergic and non-adrenergic, non-cholinergic intramural mechanisms is likely; whether this results from an intrinsic esophageal process or extrinsic autonomic dysregulation is unknown. Heart rate variability (HRV) was measured concurrently with esophageal manometric testing to determine if specific spectral patterns could predict SMDs and support a component of extrinsic autonomic dysregulation in their pathogenesis. Methods: Consecutive patients referred for clinical esophageal manometry without confounding conditions or contraindications underwent HRV testing during manometry. Manometric patterns were analyzed independently of HRV and categorized using an established classification scheme. HRV, including high-frequency (HF, parasympathetic) and low-frequency (LF, sympathetic) spectra, in addition to the LF:HF ratio, was examined in relation to both motility pattern and individual manometric features representing the SMDs. Results: 57 non-achalasic subjects ( $48 \pm 14$ yr; 39 F) with either normal manometry (NORM, $\mathrm{n}=14$ ), hypomotility (HYPO, $\mathrm{n}=23$ ) or SMDs (DES, $\mathrm{n}=1$; nonspecific SMDs, $\mathrm{n}=19$ ) were evaluated. HF was reduced in SMDs compared with HYPO ( $184 \pm 28$ vs. $422 \pm 74, \mathrm{p}<0.01)$ and in SMDs compared with NORM ( $184 \pm 28$ vs. $430 \pm 104, p=0.01)$, whereas HYPO and NORM did not differ $(\mathrm{p}=0.99)$. LF did not differ across groups. LF:HF ratios were increased in SMDs compared with both NORM $(8.5 \pm 1.3$ vs. $3.6 \pm 0.7, \mathrm{p}<0.01)$ and HYPO $(8.5 \pm 1.3$ vs. $4.4 \pm 0.6, p<0.01)$, whereas HYPO and NORM again did not differ $(\mathrm{p}=0.18)$. Logistic regression analyses controlling for intergroup differences in age and sex confirmed that decreased HF predicted the presence of an SMD (OR 2.7, 95\% CI: 1.3-5.9; $\mathrm{p}=0.02$ ) over other manometric patterns and that an elevated LF:HF ratio portended a nearly five-fold increased odds of SMDs over other motility patterns (OR 4.6, 95\%CI: 1.4-15.6; $\mathrm{p}<0.01$ ). Correlations of HF and LF:HF ratio with individual parameters that collectively represent the SMDs were nonsignificant. Conclusions: Reduced parasympathetic tone and increased sympathetic-to-parasympathetic ratio using HRV testing predicted the presence of SMDs over other manometric patterns. These findings support the presence of extrinsic autonomic dysregulation in the production of the SMDs. SMDs may share a pathophysiological basis with other conditions, e.g., irritable bowel syndrome, that demonstrate a similar HRV pattern.

\section{W1826}

Presence of Acid Reduces the Threshold for Elicitation of Esophago-UES Contractile Reflex and Secondary Peristalsis During Sleep But Not in Awake State

Jasmohan Bajaj, David Knuff, Tanya Rittmann, Kulwinder Dua, Reza Shaker

Background: Earlier studies have shown the activation of esophago-upper esophageal sphincter contractile reflex (EUCR) and secondary peristalsis (2P) by minute infusion of water in the proximal esophagus. It is not however, known whether presence of acid results in sensitization of these reflexes. Aim: To compare the threshold volume (Tvol) for elicitation of EUCR and 2P by proximal esophageal infusion of $0.1 \mathrm{~N} \mathrm{Hcl}$ and water during awake and stage II sleep states. Methods: Four healthy volunteers ( $32 \pm 4$ yrs; $3 \mathrm{M}$ ) were studied in supine position during awake and sleep stage II using polysomnography (REMbrandt) and electromyography (EMG) in the sleep laboratory. Esophageal manometry was assessed concurrently by a pneumohydraulic manometry apparatus(Dentsleeve,Arndorfer Specialit ies)passed transnasally with a posteriorly-directed injection port below the upper esophagea sphincter (UES). Infusion of water and $0.1 \mathrm{~N} \mathrm{Hcl}$ were carried out at a rate of $2.7 \mathrm{ml} / \mathrm{min}$ until swallow, cough or elicitation of EUCR and 2P occurred.A 10 minute water washout water was performed after each $\mathrm{Hcl}$ infusion. Subjects were blinded to the nature of the infusions and a visual analog scale was used 1 minute after each injection to evaluate ches discomfort in awake state. Tvol for EUCR and 2P elicitation were recorded. Results: Al subjects were monitored for at least 4 hours during sleep and none reported chest discomfor during the study. EUCR and 2P were successfully elicited in all during awake state and Stage II sleep. Acid perfusion resulted in a significant reduction in Tvol for both EUCR and 2P during stage II sleep but not the awake state (Figure/Table). Conclusion: Presence of acid influences the elicitation of EUCR and 2P differently in awake and sleep states. While in stage II sleep, presence of acid reduces the Tvol for triggering of EUCR and $2 p$, in awake state its influence is not significant.

\begin{tabular}{|c|c|c|c|c|}
\hline $\mathrm{ml} \pm \mathrm{SD}$ & Awake Water & Awake Acid & St II water & St II acid \\
\hline EUCR & $2.1 \pm 0.8$ & $0.9 \pm 0.6$ & $5.6 \pm 3.9$ & $0.9 \pm 0.2^{*}$ \\
\hline $2 \mathrm{P}$ & $2.4 \pm 0.4$ & $1.1 \pm 0.5$ & $5.9 \pm 3.9$ & $1.5 \pm 0.8^{*}$ \\
\hline
\end{tabular}

*significant compared to StII water; St:Stage
T vol 2P

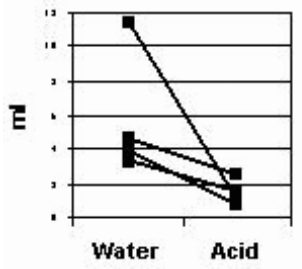

T VolEUCR

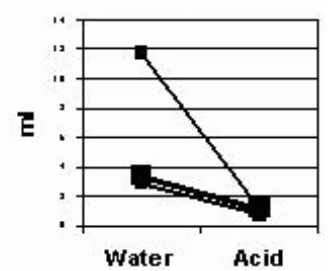

W1827

High Viscous Liquid Manometry Unmasks Esophageal Dysmotility in Patients with Early Chagas' Disease

Lucilene R. Westmore, Roberto Dantas, George Triadafilopoulos, Clovis Kuwahara, Pedro Leite, Miriam Zebian

Introduction: Patients with early esophageal Chagas' disease may present with dysphagia without megaesophagus. After demonstration that changes in bolus viscosity may alter esophageal contractility, highly viscous substances have been used during esophageal manometry (EM) to unmask defects not otherwise revealed by dry and water swallows. Aims: To evaluate the esophageal motility in symptomatic patients with early Chagas' disease by dry swallows and by liquids of different viscosities (water and sugar cane syrup) swallows. Methods: Fifteen healthy volunteers ( 9 male, 6 female; median age: 42 years, range: 26 60 ) and 10 patients with Chagas' disease, with dysphagia but without megaesophagus ( 5 male, 5 female; median age: 43 years, range: $36-63$ ), were submitted to perfusion EM. Basa lower esophageal sphincter (LES) pressure was measured by the rapid pull-through. With the catheter positioned with 1 lumen opening in the LES, and the others at 5, 10 and 15 $\mathrm{cm}$ above the LES, 10 dry swallows, 10 swallows of $5 \mathrm{~mL}$ of water, and 10 swallows of 5 $\mathrm{mL}$ of sugar cane syrup were performed. Amplitude, duration and velocity of contractions and the LES relaxation duration were measured. Morphology, peristalsis and failed contractile waves were also studied. Results: There was no difference $(p>0.05)$ between patients and controls in LES pressure and duration of contractions for dry, water as well as sugar cane syrup swallows. Patients had shorter LES relaxation duration than controls only after dry ( $\mathrm{p}=$ $0.0005)$ and sugar cane syrup $(\mathrm{p}=0.04)$ swallows. They also had more synchronus contractions than controls only for dry $(\mathrm{p}=0.01)$ and sugar cane syrup $(\mathrm{p}=0.04)$ swallows. Patients had lower amplitude of contractions only after sugar cane syrup swallows at $10 \mathrm{~cm}(\mathrm{p}=0.05)$ and $5 \mathrm{~cm}$ above LES $(\mathrm{p}=0.04)$. They also had more multipeaked contractions than controls only for sugar cane syrup swallows $(\mathrm{p}=0.0002)$. Patients had tendency to present higher velocity of contractions only after sugar cane syrup swallows from 15 to $10 \mathrm{~cm}$ above LES ( $\mathrm{p}=0.09$ ) as well as 10 to $5 \mathrm{~cm}$ above LES $(\mathrm{p}=0.08)$. Failed contractions were more frequent in patients than controls for dry $(\mathrm{p}=0.003)$, water $(\mathrm{p}=0.02)$, and sugar cane syrup $(\mathrm{p}=0.05)$ swallows. Conclusion: Patients with symptomatic early esophageal Chagas' disease may exhibit esophageal dysmotility, more likely following high viscous substance swallows than after water and dry swallows. High viscous liquid manometry may be the preferred approach in patients with early esophageal Chagas' disease. 
Evaluation of Transit in the Transition Zone On Multichannel Intraluminal Impedance (MII) Allows Detection of Transit Abnormalities in More Patients with Abnormal Motility

Amine Hila, Amit Agrawal, Wojciech Blonski, Inder Mainie, Janice Freeman, Donald O. Castell

BACKGROUND: Combined MII and esophageal manometry (MII-EM) allows both functional and manometric evaluation of esophagus. MII-EM catheter has 4 impedance segments $(5,10,15,20 \mathrm{~cm}$ above LES) and 5 pressure sites (at LES and $5,10,15,20 \mathrm{~cm}$ above LES) Manometric diagnoses are based on data provided by sites in distal $10 \mathrm{~cm}$ of esophagus; i.e. smooth muscle. Site at $15 \mathrm{~cm}$ above the LES is not included as it may contain smooth and striated muscle (transition area). Evaluation of swallows with MII is assessed for complete or incomplete transit, defined by bolus entry at $20 \mathrm{~cm}$ and bolus exit in 15,10 and $5 \mathrm{~cm}$ segments. Does inclusion of the $15 \mathrm{~cm}$ segment, which is in the transition zone, overly detects abnormalities in patients with normal manometry? AIM: To assess the effect of using impedance data from the transition zone on accuracy of MII-EM interpretation. METHODS: 200 consecutive MII-EM studies ( $65 \%$ females; mean age $=54 \mathrm{yrs}$ ). All patients given 10 saline and 10 viscous swallows while recumbent. All tracings initially read defining complete transit for each swallow based on bolus entry at $20 \mathrm{~cm}$ and bolus exit at 15,10 and $5 \mathrm{~cm}$. Then, all tracings read a 2nd time with complete transit defined by bolus entry at $20 \mathrm{~cm}$ and bolus exit at 10 and $5 \mathrm{~cm}$, thus excluding transition zone. Manometric and transit diagnoses from these 2 different reading methods were compared. RESULTS: Excluding transition zone from impedance analysis changed transit diagnosis from incomplete to complete transit in 33 patients (17\%): 16 changed in liquid transit, 16 in viscous transit and 1 in both. 1 st table summarizes prevalence of manometric diagnoses in all patients (group A), and in the 33 where transit diagnosis changed (group B). In patients where transit diagnosis changes from normal to abnormal with addition of transition zone, a higher percentage of patients have abnormal manometric finding. In all studies, there were 3962 swallows (group C), of which 226 swallows (group D) changed diagnosis. 2nd table summarizes prevalence of manometric diagnoses by swallows. In swallows where transit diagnosis changes from normal to abnormal with addition of transition zone, a higher percentage have abnormal manometry. CONCLUSION: Using findings from the transition zone in the transit diagnosis detects transit abnormalities in more patients with abnormal manometry than in patients with normal manometry.

\begin{tabular}{|c|c|c|c|c|c|}
\hline & Normal & IEM & Spasm & Achalasia & Nutcracker \\
\hline Group A & $58 \%$ & $18 \%$ & $15 \%$ & $7 \%$ & $2 \%$ \\
\hline Group B & $40 \%$ & $36 \%$ & $24 \%$ & 0 & 0 \\
\hline
\end{tabular}

\begin{tabular}{|c|c|c|c|}
\hline Swallows & Normal & Ineffective & Simultaneous \\
\hline Group C & $64 \%$ & $28 \%$ & $8 \%$ \\
\hline Group D & $43 \%$ & $41 \%$ & $16 \%$ \\
\hline
\end{tabular}

W1829

Manometric and Impedance Characteristics of Achalasia: Facts and Myths Amit Agrawal, Amine Hila, Wojciech Blonski, Radu Tutuian, Inder Mainie, Marcelo Vela, Donald O. Castel

BACKGROUND: Achalasia is defined manometrically by an aperistaltic esophagus. Variations in the manometric findings occur in achalasia suggesting that all manometric features should not be required to diagnose achalasia. Combined multichannel intraluminal impedance and EM (MII-EM) allows both a functional and a manometric evaluation of esophageal motility and identifies chronic fluid retention. AIM. To compare manometric and MII characteristics in patients with achalasia. METHODS: Review of 73 MII-EM tracings ( 38 females; mean age 53.5; range 14 - 95 years) from patients with achalasia were compared with 73 patients with normal manometry (49 females; mean age 54; $22-82$ years). Manometric and MIl characteristics were compared during 10 liquid and 10 viscous swallows. RESULTS: In 2/ $73(2.7 \%)$ achalasia patients, the catheter would not pass the lower esophageal sphincter (LES). 23/71 (32\%) of the achalasia patients had hypertensive LES. Mean LES pressure for the 71 patients with achalasia was $37.9+4.5 \mathrm{~mm} \mathrm{Hg}$ compared to $27.3+3.2 \mathrm{~mm} \mathrm{Hg}$ $(\mathrm{p}<0.05)$ in patients with normal motility. Elevated intraesophageal pressure (IEP) was noted in $45 / 73(61.6 \%)$. Mean LES pressure with elevated IEP was $41.1+6.1 \mathrm{~mm} \mathrm{Hg}$ compared to $32.5+6.1 \mathrm{~mm} \mathrm{Hg}(\mathrm{p}<0.05)$ with normal IEP. Mean LES residual pressure in this group was $11.9+1.5(\mathrm{p}<0.05)$ compared to $8.5+.6 \mathrm{~mm} \mathrm{Hg}$ with normal IEP. Mean baseline MIl was $801+94$ ohms compared $(\mathrm{p}<0.05)$ to $523+61$ ohms after 10 liquid swallows and $417+48$ ohms after 10 viscous swallows. In 73 patients with normal motility, mean baseline MII was $1955+229$ ohms, $1984+232.4$ ohms after 10 liquid swallows and $2102+246$ ohms after 10 viscous swallows. There was a significant difference $(\mathrm{p}<0.05)$ between the MII $(p<0.05)$ in patients with achalasia and normals. CONCLUSION: Most patients with achalasia have elevated IEP, elevated LES residual pressure, normal LES pressure, and low baseline impedance. All manometric features should not be required to diagnose achalasia. Patients with an elevated IEP are likely to have an elevated LES pressure and LES residual pressure. Low MII values identify chronic fluid retention and helps confirm the diagnosis. Table l summarizes the manometric findings

\begin{tabular}{|c|c|}
\hline 1. Absent Peristalsis & $73(100 \%)$-required \\
\hline 2. Incomplete LESRP & $45(63 \%)$ \\
\hline 3. Elevated resting LESP & $23(32 \%)$ \\
\hline 4. 1, 2, and 3 & $16(22.5 \%)$ \\
\hline 5. Increased intraesophageal pressure & $45(62 \%)$ \\
\hline 6. All of the above & $3(4.2 \%)$ \\
\hline
\end{tabular}

\section{W1830}

Esopahgeal Function Test (EFT) in the Evaluation of the Effectiveness of Peristalsis: Viscous Vs. Liquid Swallows

Pernilla Lundgren, Gisela Ringstrom, Hasse Abrahamsson, Magnus Simren

The term ineffective peristalsis (IP) is based on videomanometry recordings, where peristaltic waves with amplitude $<30 \mathrm{mmHg}$ in the distal esophagus often failed to move a barium swallow normally. In previous studies the number of swallows studied was limited by the use of radiation. With combined manometry and intraluminal impedance (EFT) this limitation is overcome. Moreover, recent investigations have added viscous swallows to the test of esophageal function. The additional value of this in the assessment of the effectiveness of peristalsis needs to be further evaluated. Methods: EFT was performed in 35 asymptomatic volunteers (16 M; mean age 37 (21-63) years) and 31 patients with dysphagia without a specific esophageal motility disorder (12 M; mean age 47 (18-71) years). Pressure sensors were located in the lower esophageal sphincter (LES) and 20,15,10 and $5 \mathrm{~cm}$ above the LES, and impedance segments centered 20,15,10 and $5 \mathrm{~cm}$ above LES Subjects were investigated in supine position: 20 swallows (5ml), 10 liquid (saline) and 10 viscous material. IP was defined as contraction amplitude $<30 \mathrm{mmHg}$ in at least one of the two most distal recordings in the esophageal body. Results: With liquid swallows IP was found in 102/676 swallows, compared with 101/638 swallows with viscous material (NS). Incomplete bolus transit was more prevalent with viscous than with liquid swallows (19\% vs. $10 \% ; \mathrm{p}<0.0001$ ). Swallows with incomplete bolus transit had lower contraction amplitudes at all recording sites in the esophageal body, both with viscous and liquid swallows $(p<0.0001$ for all comparisons). Also the propagation velocity (onset) of the contractions waves were faster in swallows with incomplete bolus transit, both with liquid ( $5.7 \pm 5.4$ vs. $4.8 \pm 2.9 ; \mathrm{p}=0.04)$ and viscous swallows $(5.7 \pm 5.3$ vs. $4.5 \pm 3.5 ; \mathrm{p}=0.007)$, whereas the LES pressure was similar. The mean pressure in swallows with incomplete bolus transit tended to be higher at all levels in the esophageal body with viscous vs. liquid swallows. Conclusion: The effectiveness of peristalsis should be determined with impedance rather than with manometry alone. The use of viscous as compared with liquid swallows increases the proportion of incomplete bolus transit without affecting the proportion of ineffective peristalsis based on characteristics of the pressure waves. By combining manometry and impedance additional valuable information regarding esophageal function is obtained, and the addition of viscous material seems to be a greater challenge for the esophagus.

\section{W1831}

An Automated Measurement and Diagnostic System for Esophageal Motility Disorders

Hongbing Zhu, Zhijun Weng, Jiande Chen

Esophageal motility disorders are common among patients ranging from gastroesophageal reflux diseases (GERD) to dysphagia. Measurement and diagnosis of various esophageal motility disorders, however, require extensive knowledge and expertise. The aim of the study was to develop a fully automated measurement and diagnostic system for esophageal manometry. Methods: The novel automated measurement and diagnostic system was composed of 1) man-machine interface modular for interactive performance of the esophageal motility procedure. This modular automatic determines the location of each channel and provides step-by-step guidelines for the measurement procedure. 2) automated identification of all esophageal motility parameters, including lower esophageal sphincter (LES) pressure at resting and relaxation during wet swallows, amplitude and propagation patterns of esophageal contractions during wet swallows as well as profile of upper esophageal sphincter. 3) automated diagnosis of various esophageal motility disorders. A rule-based algorithm has been developed. Based on the esophageal motility profile and patient's symptoms, the final diagnosis is provided by the system including one of the followings: normal, achalasia, hypotensive or hypertensive LES, diffuse esophageal spasma, ineffective esophageal motility disorder and non-specific esophageal motility disorders. 4) database. All patient information and their motility profiles are stored in a database. The database can be searched based on patient symptoms or diseases, or procedures or any specific esophageal motility disorders. Results: 1) The interactive navigating measurement system was tested in 10 patients and accurate guidelines were provided regarding the detection of the LES location. Each time when the catheter was passed through the LES, the tracings were accurately highlighted enabling users to identify LES. It was found extremely useful in patients with hypotensive LES pressure. 2) Automated identification of motility profiles was confirmed by simultaneous visual examination of tracings in 20 patients. The identification accuracy was higher than $90 \%$. 3) Comparing with experts' diagnosis, the automated system provided $94 \%$ matched diagnosis in 50 patients with various esophageal motility disorders. Conclusions: The newly developed automated system provides interactive guidelines during the procedure, which is extremely useful for new users or users with little experience. The automated analysis of motility parameters is accurate and does rarely need users' interventions, suggesting the feasibility of complete automated quantitative analysis and diagnosis. 


\section{W1832}

Altered Counts of Small Bowel Bacteria in Patients with Irritable Bowel Syndrome (IBS)

Iris Posserud, Per-Ove Stotzer, Einar S. Bjornsson, Hasse Abrahamsson, Magnus Simren

Background: Small intestinal bacterial overgrowth (SIBO), diagnosed using the lactulose hydrogen breath test (LHBT), has been proposed to be common in IBS. This has not been confirmed using culture of jejunal aspirate with a standard definition of SIBO $(\geq 100,000$ colony forming units $(\mathrm{cfu}) / \mathrm{ml})$. However, the presence of minor alterations in small bowel bacterial flora in IBS remains unclear. Aim: To further investigate alterations in the jejunal bacterial flora in IBS and correlation with symptom profile, results on LHBT, and altered small bowel motility. Method: We included 165 IBS patients (mean age 38 years, range 19$68 ; 122$ females) and 26 healthy controls (mean age 40 years, range 20-79; 15 females) The bacterial flora in the small bowel was evaluated by cultures from jejunal aspirate obtained from all subjects during an antroduodenojejunal manometry. Presence of small intestinal dysmotility was assessed by an experienced investigator based on internationally accepted criteria and normal values obtained in healthy volunteers at our lab. Forty-three patients also underwent a LHBT. Patients were divided into subgroups based on ROME II criteria resulting in 51 patients with diarrhea predominant IBS, 37 constipation predominant, and 77 with alternating diarrhea and constipation. Results: There were no significant differences in the prevalence of $\geq 100,000 \mathrm{cfu} / \mathrm{ml}$ between IBS patients and healthy controls $(6 \% \mathrm{vs}$. 4\%). However, mildly elevated counts of small bowel bacteria (respiratory flora excluded) were more common in patients compared with controls. Cultures with $\geq 10,000 \mathrm{cfu} / \mathrm{ml}$ were found in $24 \%$ of patients compared with $4 \%$ in controls ( $\mathrm{p}=0.02$ ), and cultures with $\geq 5,000 \mathrm{cfu} / \mathrm{ml}$ were observed in $43 \%$ vs. $12 \%$ ( $\mathrm{p}=0.002$ ). Signs of small intestinal dysmotility were found in $41 \%$ of all patients and were more frequently observed in patients with $\geq 50,000 \mathrm{cfu} / \mathrm{ml}$ of colonic type bacteria $(\mathrm{p}=0.02)$. Bacterial counts did not differ between the different subgroups based on predominant bowel habit. LHBT was positive in $16 \%$ of the patients, but the results from the LHBT did not reflect the results from the jejunal cultures, since there was no difference in bacterial counts between patients with positive and negative LHBT. Conclusion: A substantial number of IBS patients have mildly elevated counts of small intestinal bacteria compared with controls. Signs of small intestinal dysmotility are found in some patients but there is no association with a specific bowel habit and the clinical relevance remains unclear. The LHBT does not seem to reflect altered counts of bacteria in the upper part of the small bowel in IBS

\section{W1833}

A New Non-Scintigraphic Method for Measuring Gastrointestinal Transit in Gastroparetic Patients

Irene Sarosiek, Kenneth Koch, Kathy Selover, Braden Kuo, Henry Parkman, William Halser, Wo M. John, Sitrin Michael, William D. Chey, Jeffrey Lackner, David D'Andrea, Richard W. McCallum

Background: Clinical diagnosis and therapy of motility disorders could be enhanced by better methodology for assessing Gastric Residence Time (GRT) as well as GI Total Transit Time (TTT) providing opportunity to improve correlation of symptoms with transit tests. The aim of the study: The aims of this study were to compare TTT and its major components GRT and small/large bowel transit time (SLBTT), measured with a new diagnostic capsule (SmartPill) in asymptomatic volunteers (Controls, C) and compare with corresponding values recorded in patients with gastroparesis (GP). Methods: 60 patients with GP: 23 diabetics (DM), 37 idiopathic (ID); $50 \mathrm{~F} \& 10 \mathrm{M}$, mean age of 42 (range 19-66) mean BMI 26.3 (range 16.8-41.8) and 87 asymptomatic controls (C) 32F \& 55M, mean age of 31 (range 19-57 years), with mean BMI 25.8 (range 17.9-41.1) participated in this multicenter study. After an overnight fast all subjects swallowed a wireless GI monitoring capsule (SmartPill) equipped with sensors that measured pressure, $\mathrm{pH}$, and temperature. After initial data gathering at the study site, subjects returned to their normal ambulatory environment with parameters being captured by a portable recording device. Gastric Residence Time (GRT) was defined as the time from ingestion to an abrupt sustained rise in $\mathrm{pH}$ to $>4$ and at least a rise of $3 \mathrm{pH}$ units from the baseline. GRT $<30$ was excluded from calculations. The TTT was measured from the time of ingestion of the wireless capsule until a drop in temperature or abrupt loss of signal associated with a bowel movement confirmed by patients' diary. By subtracting GRT from TTT, SLBTT was calculated. Mann-Whitney Rank Sum Test was used to address statistical data. Results: In GP the median TTT was significantly longer than in controls ( $48.3 \mathrm{~h}$ vs $26.9 \mathrm{~h}, \mathrm{P}<0.001$ ), as was GRT (10.2 vs $3.7 \mathrm{~h}, \mathrm{P}<0.001)$ as well as SLBTT ( 29.5 vs $22.2 \mathrm{~h}, \mathrm{P}<0.001$ ). Additionally, the TTT in patients with GP of diabetic etiology was significantly prolonged compared to patients with ID GP (60.1 vs $32.1 \mathrm{~h}, \mathrm{P}=0.036)$. As was the SLBTT in DM GP compared to ID GP (53.1 vs $28.2 \mathrm{~h}, \mathrm{P}=0.014)$. TTT, GRT, and SLBTT were similar for both genders among gastroparetics. Conclusions: 1. Significant differences in GRT, TTT, and SLBTT were observed between GP and controls by utilizing non-scintigraphic capsule technology. 2. Diabetic GP patients exhibit a prolonged SLBTT consistent with contributions from neuropathy, hyperglycemia, and other factors. 3. SmartPill has research and diagnostic potential for assessment of GI tract function in health and disease including physiologic and pharmacologic studies.

\section{W1834}

Modulation of $\mu$-Opioid Agonist-Induced Inhibition of Gastrointestinal Transit By $\delta$-Opioid Receptor Antagonism

Beverley A. Moore, Rachel King, Sandra L. McKenney, Pamela J. Hornby, Paul R. Wade

Opioid analgesics are used to manage severe acute or chronic pain and produce analgesia by acting as full agonists at $\boldsymbol{\mu}$ opioid receptors (ORs) and as partial agonists at $\delta O R s$ in the CNS. Activation of ORs in the enteric nervous system (ENS) inhibits propulsive gastrointestinal (GI) motility, resulting in constipation - a common side effect of opioid analgesics. Selective inhibition of $\delta \mathrm{ORs}$ has been shown to accelerate colonic transit in vitro (Foxx-Orenstein AJP $275: 38, G 979,1998)$. We tested the hypothesis that combination of an $\mu \mathrm{OR}$ agonist

with an $\delta O R$ antagonist will ameliorate the inhibitory effects of $\mu \mathrm{OR}$ agonists on ENS mediated GI smooth muscle contraction in vitro and on GI transit in vivo. Methods: Segment of guinea pig ileum were mounted in a myobath apparatus to record longitudinal contractions evoked by electrical field stimulation (EFS), normalized as \% of contraction to $1 \mu \mathrm{M}$ acetylcholine $(\mathrm{ACh})$. Selective $\mu \mathrm{OR}$ agonists or $\delta \mathrm{OR}$ antagonists were applied to the bath and their effects on the EFS-evoked twitch recorded. GI transit was determined in vivo in CDmice by calculating the geometric center (GC) of a FITC-labeled dextran $(70 \mathrm{kD}$; in $0.5 \%$ methylcellulose) marker $45 \mathrm{~min}$ after oral ( $\mathrm{po}$ ) administration. Results: The effects of the $\mu \mathrm{OR}$ agonist [D-Ala2, N-Me-Phe4, Gly5-ol] enkephalin (DAMGO) and the $\delta \mathrm{OR}$ antagonis naltrindole (Naltr) on the ileal twitch were concentration-dependent: DAMGO inhibited (61 $\pm 4.2 \% ; \mathrm{EC} 50=1.8 \mathrm{nM})$ and Naltr enhanced $(145 \pm 5.5 \% ; \mathrm{EC} 50=18.4 \mathrm{nM})$ ileal twitch Combination of the ligands at their EC50s restored the twitch to $94 \pm 5 \%$ of ACh control $(\mathrm{P}<0.001$ vs DAMGO alone; $\mathrm{N}=4)$. GI transit was dose-dependently $(1-10 \mathrm{mg} / \mathrm{kg}$, p.o. $)$ inhibited by morphine or loperamide (Lop; a peripherally restricted, selective $\mu \mathrm{OR}$ agonist) $\mathrm{GC}$ at $10 \mathrm{mg} / \mathrm{kg}$ : morphine $=4.5 \pm 0.27$; $\mathrm{Lop}=3.7 \pm 0.3$; vehicle treated controls $=8.3 \pm$ $0.22 ; \mathrm{n}=5, \mathrm{P}<0.0001$ vs controls). Naltr alone ( $3-100 \mathrm{mg} / \mathrm{kg}$, s.c.) did not alter GI transit however, when given $1 \mathrm{hr}$ prior to Lop, Naltr dose-dependently attenuated the inhibitory effect of Lop. This effect of Naltr was also observed when given p.o. ( $30 \mathrm{mg} / \mathrm{kg}$ ) in combination with either Lop or morphine ( $3 \mathrm{mg} / \mathrm{kg}$ ) $1 \mathrm{hr}$ prior to measuring GI transit (GC: Lop, $4.3 \pm$ 0.23 vs Lop+Naltr $5.7 \pm 0.30, \mathrm{P}<0.01$; morphine, $5.1 \pm 0.13$ vs morphine+Naltr $7.2 \pm 0.04$ $\mathrm{P}<0.01 ; \mathrm{N}=6$,). Conclusions: These data show that selective antagonism of $\delta \mathrm{OR}$ reduces the inhibitory effects of $\mu \mathrm{OR}$ agonists on GI contractility; moreover these same actions have now been demonstrated on propulsive motility in vivo, and suggest that $\delta O R$ antagonism may attenuate constipation associated with opioid analgesics.

\section{W1835}

Proteinase-Activated Receptor 2 Agonist Prevents Mesenteric Ischemia/ Reperfusion Injury in Rats: Partial Involvement of Substance P (SP) and Calcitonin-Gene Related Peptide (CGRP)

Fiore Cattaruzza, Elisabetta Barocelli, Mariannina Impicciatore, Vigilio Ballabeni, Paola Ghizzardi, Nathalie Vergnolle, Catia Sternini

Background and Aims: In an experimental model of rat intestinal ischemia/reperfusion (I/ $\mathrm{R})$ we previously observed that the exogenous stimulation of Proteinase-Activated Receptor 2 by the PAR2-activating peptide (AP), SLIGRL-NH2, prevented motility and inflammatory disorders in part through the involvement of mast cells and extrinsic visceral afferents. A role for PAR-2 as signalling system in the neuro-immune communication in intestinal ischemic injuries was proposed, but the mechanism remained to be clarified. Hence the aim of the study was to investigate the effects of blockade of receptors for CGRP and SP, neuropeptides colocalized in visceral afferents, on PAR2-AP induced gastrointestinal transi (GIT) acceleration and anti-inflammatory response in I/R rats. Methods: Mesenteric ischemia (lh) induced by occlusion of the superior mesenteric artery, was followed by $6 \mathrm{hr}$ reperfusion (I/R) in Wistar rats. Sham operated (SO) animals served as controls. CGRP receptor antagonist, CGRP (8-37) (150 $\mu \mathrm{g} / \mathrm{kg}$, i.v. repeated 4 times every 2 hours) or, RP $67580(1 \mathrm{mg} / \mathrm{kg}$ i.v followed by $0.5 \mathrm{mg} / \mathrm{kg}$ after 3 hours) antagonist of NKl, the preferred receptor for SP, was administered in different groups of rats starting from $15 \mathrm{~min}$ before I/R or SO. The PAR2 AP, SLIGRL-NH2 $(3.5 \mathrm{mg} / \mathrm{kg})$ or saline was intraduodenally administered at the beginning of reperfusion. GIT was measured by charcoal gavage and expressed as \% of the distance travelled by the charcoal in the small bowel. Leukocytes infiltration, as index of tissue inflammation, was evaluated by measuring myeloperoxidase activity (MPO) of jejunal tissue and expressed as $\mathrm{mU} / \mathrm{mg}$ proteins. Results: PAR2-AP stimulatory effect on GIT in I/R $(76.99 \pm 3.09 \%$ vs. saline $\mathrm{I} / \mathrm{R} 42.69 \pm 3.63 \% ; \mathrm{P}<0.001)$ was reversed by CGRP receptor antagonist $(38.37 \pm 8.9 \%$ vs. $76.99 \pm 3.09 \% ; \mathrm{P}<0.001)$ and by NKl antagonist $(43.06 \pm 5.96 \%$ vs $76.99 \pm 3.09 \% ; \mathrm{P}<0.001$ ). The increase of MPO activity induced by $\mathrm{I} / \mathrm{R}$ was prevented by the administration of SUIGRL-NH2 (1.82 \pm 1.00 vs. $8.46 \pm 1.78 \mathrm{mU} / \mathrm{mg})$. This PAR2-AP protective effect was not altered by CGRP receptor antagonist- $(1.73 \pm 0.42$ vs. $1.82 \pm 1.00 \mathrm{mU} / \mathrm{mg})$, nor by NKl antagonist-pretreatment $(3.26 \pm 0.74$ vs. $1.82 \pm 1.00 \mathrm{mU} / \mathrm{mg} \mathrm{P}=\mathrm{NS})$. Conclusions This study supports the hypothesis that CGRP and SP mediate GIT acceleration induced by PAR2-AP in I/R rats while the apparent anti-inflammatory effect of PAR-2 pharmacological activation might involve different mechanisms. Supported by PRIN (MIUR2004) grants, NIH grants DK51455, $57037 \& 41301$, \& Crohn's \& Colitis Foundation of Canada

\section{W1836}

Sodium Channel Mutations and Polymorphisms in People with IBS-Potential Role for An Ion Channelopathy

G. Richard Locke, David Tester, Yuri Saito, Smita Halder, Nicholas Talley, Michael Ackerman, Gianrico Farrugia

Background: The SCN5A-encoded $\mathrm{Na}_{\mathrm{V}} 1.5$ sodium channel is expressed in human intestinal circular muscle layer interstitial cells of Cajal and smooth muscle cells. Mutations in SCN5A result in distinct cardiac channelopathies. Recently, we reported that patients with mutations in SCN5A predisposing to either SCN5A-precipitated long QT syndrome or Brugada syndrome were more likely to report GI symptoms, especially abdominal pain. This led to the hypothesis that a possible pathogenic mechanism for abdominal pain associated with irritable bowel syndrome (IBS) may be defective sodium channels. Aim: To evaluate SCN5A as a candidate gene involved in the pathogenesis of IBS. Methods: Comprehensive mutational analysis of the 27 translated exons of SCN5A was performed on genomic DNA obtained from 47 subjects who were seen in an academic practice for IBS and who reported chronic (more than two years), and at least moderately severe abdominal pain. Mutation/polymorphism data derived from over 800 healthy controls served as the reference group. Results: In the 47 cases, the distribution of genotypes for the H558R-SCN5A common polymorphism was $\mathrm{HH}=55 \%$, $\mathrm{HR}=34 \%$ and $\mathrm{RR}=11 \%$ as compared to $60 \%, 35 \%$ and $5 \%$ in controls, respectively. Although the frequency of the $\mathrm{R}$ allele was numerically greater than control this was no statistically significant ( $\mathrm{p}$ value $=0.18$ ). However, one patient hosted a loss-of-function, missense mutation, G298S. This mutation has been previously published in one patient with cardiac conduction disease and has not been observed in over 3000 reference alleles worldwide. The IBS patient with the G298S missense mutation is a 43 year old female. Her

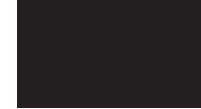


EKG showed normal sinus rhythm, with a QT of 376 ms and a QTc of 439 ms. Conclusions: This pilot data provides molecular and functional evidence that perturbations in SCN5A may confer susceptibility to the development of IBS. While further studies are necessary, the concept of an IBS-predisposing channelopathy in the GI tract deserves ongoing exploration. Supported by a grant from the Miles and Shirley Fiterman Foundation and NIH DK52766

\section{W1837}

Effects of Intestinal Electrical Stimulation On Postprandial Small Bowel Motility and Transit in Fed Dogs

Huibin Qi, Jiande Chen

Background and aims: Intestinal electrical stimulation (IES) with long pulses has been reported to inhibit small intestinal motility yet accelerate intestinal transit of continuous perfusion. However, it is unknown whether there is a correlation between the IES-induced alterations in motility and transit and unknown whether the effect of IES on the transit of a bolus is different from that of continuous perfusion. The aims of this study were therefore to investigate the effects of IES on small bowel motility and transit and their correlations in dogs. Methods: The study was performed in 2 postprandial sessions (control and stimulation) in 5 hound dogs with 1 pair of electrodes chronically implanted on the serosal surface of the proximal jejunum and with 2 intestinal cannulas, one $20 \mathrm{~cm}$ from the pylorus and the other $150 \mathrm{~cm}$ distance from the first cannula. IES was performed using repetitive long pulses (pulse width: $200 \mathrm{~ms}$, amplitude: $6 \mathrm{~mA}$, frequency: 20 pulses/min). Intestinal motility was recorded manometrically via a water-perfused catheter inserted via the proximal intestinal cannula. A bolus of phenol red was injected into the intestine via the proximal cannula and collected through the distal cannula. Small bowel transit time was expressed as the time from the phenol red injection to the complete clearance of phenol red assessed from the distal cannula. Results: 1. In comparison with the control session, IES significantly decreased the motility index $(14.3 \pm 1.0$ vs. $6.7 \pm 0.9, P<0.01)$. 2 . IES significantly increased small bowel transit time compared with the control $(170.0 \pm 27.3 \mathrm{~min}$ vs. $38.0 \pm 12.1 \mathrm{~min}, \mathrm{P}<0.001) .3$. A significant negative correlation (Pearson correlation $\mathrm{r}=-0.89, \mathrm{p}<0.001$ ) was found between motility index and small bowel transit time. Conclusion: IES with long pulses inhibits small bowel motor activity and delays small bowel transit of a bolus. A significant negative correlation exists between motility index and small bowel transit time during IES. It seems IES has different effects on the transit of a bolus and that of continuous perfusion.

\section{W1838}

Excitatory Effects of Synchronized Intestinal Electrical Stimulation On Small Intestinal Motility in Dogs

Jieyun Yin, Jiande Chen

Our previous studies showed that synchronized gastric electrical stimulation enhanced antral contractions, accelerated gastric emptying in dogs. It has never been reported whether synchronized electrical stimulation could improve small intestinal motility. The aim of this study was to investigate the effects of synchronized intestinal electrical stimulation (SIES) on small bowel motility in both fasting and fed states in dogs. Methods: Five healthy female dogs $(18-24 \mathrm{Kg})$ were equipped with a duodenal cannula for the measurement of small bowe motility using manometry. Two pairs of bipolar electrodes were implanted on the small intestinal serosa with an interval of $25 \mathrm{~cm}$; the first one was $10 \mathrm{~cm}$ beyond the pylorus and used for stimulation. The experiment was consisted of four sessions in each dog with a randomized order. In the fasting state, $20 \mathrm{~min}$ after occurrence of phase III, SIES was initiated and maintained for $45 \mathrm{~min}$, small bowel motility was recorded during the entire experiment, and no stimulation was performed in the control session. In other two sessions, dogs were fed with solid meal at the beginning of the experiment; glucagon $(0.1 \mathrm{mg} / \mathrm{kg})$ was injected $20 \mathrm{~min}$ after feeding, SIES was initiated at the same time for $20 \mathrm{~min}$ followed by $20 \mathrm{~min}$ recovery period. The stimulus was composed of train of pulses with on-time of $0.5 \mathrm{~s}$, frequency of $20 \mathrm{~Hz}$, pulse width of $2 \mathrm{~ms}$ and amplitude of $4 \mathrm{~mA}$. Results: 1). In the fasting state, SIES induced small intestinal contractions during phase I. The motility index was $5.2 \pm 0.6$ in the control session and significantly increased to $10.3 \pm 0.7$ with SIES $(\mathrm{P}=0.003)$. 2). In the fed state, glucagon substantially and significantly inhibited small intestinal motility. The motility index was $11.3 \pm 0.7$ after feeding and reduced to $3.4 \pm 0.5$ with glucagon injection $(\mathrm{P}<0.001)$. SIES significantly enhanced glucagon-induced small intestinal postprandial hypomotility. The motility index was $3.4 \pm 0.5$ in the control session and increased to 6.0 \pm 0.3 with the presence of SIES $(P=0.03)$. Conclusions: Intestinal electrical stimulation synchronized with intestinal slow waves induces intestinal contractions during phase I and enhanced small intestinal postprandial hypomotility induced by glucagon. SIES may have the therapeutic potential for treating small intestinal motility disorders. (Supported by a grant from American Diabetes Association

\section{W1839}

Large Propagating Contractions in Pig Ileum Are Myogenic and Inhibited By Intrinsic Nerves: Evidence from Spatio-Temporal Mapping

Bridget R. Southwell, Julie E. Bines, Kathleen B. Neal, Joel C. Bornstein

Studies in mice have shown that Interstitial cells of Cajal (ICC) act as pacemaker cells producing electrical slow waves and act as intermediates in transmission from nerves to muscle. Previous studies also suggest that nerves inhibit and modulate slow wave activity. This study used computer analysis of video recordings to determine the relative roles of ICC and intrinsic nerves in producing and controlling propagating waves of contraction in pig ileum. Methods: Computer analysis of video images of contracting intestine was used to investigate motor patterns in intact segments of juvenile pig intestine. Ten $\mathrm{cm}$ segments of ileum from 5 landrace pigs (8-wk old) were cannulated, placed in horizontal water bath, and perfused and superfused with Krebs physiological saline. Mesenteric arteries were cannulated and perfused to help preserve mucosal integrity. A video camera was mounted $30 \mathrm{~cm}$ above the segment, images recorded ( 10 frames/sec) onto a computer and software used to analyse intestinal motor activity. The intestinal diameter was converted to greyscale then plotted against distance along the segment and elapsed time to create spatio-temporal maps of intestinal motility. These reveal motor patterns that are difficult to detect with other techniques. Decanoic acid and alanine were perfused lumenally, carbachol via the mesenteric artery and tetrodotoxin (TTX) was added to the bath (serosal surface). Results: Ileum infused with Krebs solution, decanoic acid or alanine was largely quiescent and small localised constrictions (diameter $20 \mathrm{~mm}$, amplitude $2 \mathrm{~mm}$ ). Addition of 10-5M carbachol (cholinergic agonist) via the mesenteric artery caused constriction (diameter reduced from 20 to $10 \mathrm{~mm}$ ) followed by oscillating contractions/relaxations at the narrower diameter. Addition of 10-6 M TTX to block all neural activity produced large rhythmic oscillations (amplitude $10 \mathrm{~mm}$, frequency of $9.6 / \mathrm{min}$ ) that propagated orally or anally for the length of the preparation. Carbachol added in the presence of TTX reduced the diameter, reduced the amplitude of oscillations and eliminated orally propagating constrictions leaving only anally propagating constrictions. Conclusions: Blocking neural activity in intact segments of pig ileum revealed large rhythmic propagating oscillations (constrictions and relaxations) that occur at the frequency reported for slow waves in the pig. The oscillations are suppressed by activity within the enteric neural circuitry and hence are myogenic, presumably arising from activity of interstitial cells of Cajal. Neural activity altered the diameter of the intestine, and the amplitude, frequency and direction of propagating constrictions.

\section{W1840}

Serotonin Transporter Expression Is Reduced in Celiac Disease Stephen Foley, Nicholas Coleman, Geoffrey Holmes, Eugene Campbell, Andrew Bennett, Robin Spiller

Background We have previously shown that T-cell inflammation in celiac disease is associated with increased mucosal 5-HT content, and enhanced 5-HT release. The sodium dependant serotonin transporter (scl6a4) is primarily expressed in gut epithelial cells and is downregulated in inflammatory states including experimental colitis, and ulcerative colitis. We examined expression of serotonin transporter in duodenal mucosa in patients with celiac disease (Marsh Grade III-IV) and healthy controls. Methods Duodenal (D2) biopsies were taken from 14 patients with celiac disease and 11 controls. Biopsies were snap frozen in liquid nitrogen and stored at -80C. The biopsies were homogenised and RNA purified by Guanidine thiocyanate/ phenol extraction. RNA was reversed transcribed using Superscript II reverse transcriptase (Invitrogen). Quantitative real time PCR was performed using Platinum Mastermix kits (Invitrogen) and an ABI Prism 7700 PCR machine. Primers and probes for Cytokeratin 20 (CK20) (as an epithelial cell-specific control) and Serotonin transporter(SERT) were designed using Primer3 software. The primer and probe sequences were as follows CK20 Forward TGCTCAACTGCAAAATGCTC Reverse, CCTTGGAGATCAGCTTCCAC, Probe CTGGCTGCTGAGGACTTCAGACTGAA SERT Forward TAGATGCAGCCGCTCAGAT Reverse GATGACAAATCCCGAAACG Probe GGGTCCTGCTGGCTTTTGCTAGCT. 18s RNA was measured using the eukaryotic 18s endogenous control kit (Applied Biosystems). Relative quantification was calculated using the standard curve method. Conclusions Cytokeratin 20 expression is reduced in celiac disease, presumably reflecting the loss of epithelial cells. Serotonin transporter expression was reduced in celiacs relative to controls when normalised with either 18s or CK20. Thus SERT expression is lower in celiac patients than in control even when reduction in epithelial cell number is taken into account. These findings add further evidence that SERT is downregulated in inflammatory states. This reduction in SERT expression may contribute to the 5-HT excess in celiac disease that plays a role in the diarrhoea and dyspeptic symptoms. This work was supported by an educational grant from Novartis Pharma

\begin{tabular}{|c|c|c|}
\hline Gene expression ratio (data as mean \pm SEM) & Celiacs & Controls \\
\hline CK20 expression relative to 18S RNA & $24.28 \pm 14.82 *$ & $91.7 \pm 34.63$ \\
\hline SERT expression relative to 18S RNA & $0.042 \pm 0.021 *$ & $1.21 \pm 0.44$ \\
\hline SERT expression relative to CK20* & $0.0066 \pm 0.003 *$ & $0.019 \pm 0.004$ \\
\hline
\end{tabular}

$* \mathrm{p}<0.05$

\section{W1841}

Impaired Intestinal Gas Clearance During Marked Hyperglycemia in Patients with Functional Abdominal Bloating

Hermann Harder, Ana C. Hernando-Harder, Frank Riddinger, Heinz-Juergen Krammer, Manfred V. Singer

BACKGROUND: We have previously demonstrated an acceleration of intestinal gas clearance in healthy by acute fasting hyperglycaemia (Digestion 2005 Jun 2;71(3):179-186) and this physiological mechanism may fail in patients with functional abdominal bloating and distension. METHODS: In 14 healthy subjects and 14 patients with functional abdominal bloating we compared, by paired studies, effects of acute fasting hyperglycemia during intravenous glucose infusion (hyperglycemic clamp; 12mmol/l) and euglycemia during intravenous saline infusion (control studies; $2 \mathrm{ml} / \mathrm{min}$ ) on intestinal gas dynamics and tolerance. Gas was infused into the jejunum ( $12 \mathrm{ml} / \mathrm{min}$ ) for 120 minutes while rectal gas evacuation was continuously measured; perception and abdominal girth changes were separately evaluated. RESULTS: In healthy, rectal gas expulsion after 1 hour jejunal gas infusion was significantly accelerated during acute fasting hyperglycemia. No relevant change in abdominal perception or of abdominal perimeter was observed. In contrast, acute hyperglycemia failed to increase 1 hour gas expulsion and significant gas retention developed during hyperglycemia and euglycemia, being maintained until the end of the 120-minute study period. Gas retention was accompanied by an increase in abdominal perception and perimeter (Table 1). CONCLUSION: In patients with abdominal bloating, retention of intestinal gas and missing acceleration of intestinal gas clearance by acute hyperglycemia contribute to abdominal complaints. 


\begin{tabular}{|c|c|c|c|c|}
\hline & Healthy & & $\begin{array}{c}\text { Functional abdominal } \\
\text { bloating }\end{array}$ & \\
\hline & $\begin{array}{c}\text { Saline } \\
\text { (control) }\end{array}$ & hyperglycaemia & Saline (control) & hyperglycaemia \\
\hline gas retention $(\mathrm{ml})$ & $137 \pm 65$ & $-98 \pm 53^{*}$ & $424 \pm 70^{* *}$ & $412 \pm 116^{* * *}$ \\
\hline $\begin{array}{c}\text { abdominal } \\
\text { perception }(0-6 \text { score })\end{array}$ & $1.5 \pm 0.5$ & $1.5 \pm 0.5$ & $3.3 \pm 0.5^{* *}$ & $3.6 \pm 0.6^{* *}$ \\
\hline girth increment $(\mathrm{mm})$ & $0.0 \pm 0.1$ & $0.0 \pm 0.0$ & $0.5 \pm 0.1^{* *}$ & $0.5 \pm 0.2^{* *}$ \\
\hline
\end{tabular}

Table 1: 1-hour intestinal gas retention, abdominal perception and girth increment during jejunal gas infusion $(12 \mathrm{ml} / \mathrm{min})$ and hyperglycemia $(\sim 12 \mathrm{mmol} / \mathrm{l})$ or normoglycemia (control studies) in 14 healthy subjects and 14 patients with functional abdominal bloating (* p< 0.05 vs. control; $* * \mathrm{p}<0.05$ vs. healthy).

\section{W1842}

\section{Alterations of Neuropeptid Expression in Human Gut Myenteric Neurons} During Sepsis

Petra Jacob, Jutta Jahn, Udo Nagele, Joerg Hennenlotter, Arnulf Stenzl, Alfred Konigsrainer, Jorg Glatzle

Background: It is well recognized that gastrointestinal motility is significantly reduced during sepsis. However the pathomechanism is poorly understood. We therefore investigated the expression of pro- and anti-kinetic neuropeptides in the neurons of the myenteric plexus during sepsis in human gut tissue. Aims: To investigate the expression of SP and VIP in human small intestinal myenteric plexus neurons during sepsis. Methods: Tissue samples of human ileum were gathered from either healthy patients undergoing ileum conduit operation or patients with severe peritonitis. In fixed, whole mount sections of ileum immunohistochemistry for myeloperoxidase (MPO), and the neuropeptides substance-P (SP) and vasoactive intestinal peptide (VIP) was performed using commercial antibodies (Dianova, Biotrend and Fitzgerald). To determine the inflammation of the gut, MPO positive cells were counted in intestinal muscularis whole-mounts. SP and VIP immunoreactivity was analyzed in myenteric plexus of whole mount sections, using the Leica Quantimed system at a magnification of 20x. A threshold for the immunostaining of either neuropeptide was set in contrast to the background staining. The area of the immunostaining within a myenteric plexus was analyzed in relation to the total area of the plexus. In the following immunoreactivity is expressed as a percentage independently of the size or shape of a myenteric plexus. Results: During sepsis inflammatory MPO positive cells were significantly increased it the intestinal musculature compared to healthy control tissue (MPO positive cells per optical section, control vs. sepsis: $18 \pm 6.5$ vs. $66 \pm 23 ; \mathrm{p}<0.01$ ). Further the immunoreactivity for SP was significantly reduced by approximately $80 \%$ in myenteric plexus neurons during sepsis (SP immunoreactivity, control vs. sepsis: $32.3 \pm 5.8 \%$ vs. $6.5 \pm 1.4 \%$; $<<0.05$ ). In contrast the immunoreactivity for VIP was significantly increased by more than 2 -fold in myenteric plexus neurons during sepsis (VIP immunoreactivity, control vs. sepsis: $5.1 \pm 1 \%$ vs. $11.1 \pm 0.8 \%$; $p<0.05$ ). Conclusions: We have shown in human small intestinal tissue that under control conditions about $30 \%$ of myenteric plexus nerve fibers are immunoreactive for SP, known as a pro-kinetic neuropeptide and only 5\% of the fibers express VIP, known to inhibit small intestinal motility. During sepsis the neuropeptide expression changes dramatically; only about $7 \%$ of the fibers express SP and $11 \%$ of the fibers express the antikinetic neuropeptide VIP; this may contribute to the hypomotility during sepsis. Supported by DFG GL 311/3-1.

\section{W1843}

Use of High Resolution Manometry to Characterize the Human PYLORIC Sphincter

Joshua Desipio, Annapurna Korimilli, Henry P. Parkman, Joel E. Richter, Robert S. Fisher, Benjamin Krevsky, Frank K. Friedenberg

Background: Measurement of pyloric pressure has been elusive due to difficulty in securing manometric assemblies across the channel for prolonged periods. There are inherent limitations to established techniques using either station pull through or sleeve devices. Our purpose was to demonstrate the feasibility of prolonged pyloric measurement using a solid state catheter system. Methods: 5 healthy subjects ( 3 male, age 24-40) were recruited. A catheter (4.2 mm OD) with 36 circumferential manometric leads spaced $1 \mathrm{~cm}$ apart (ManosScan ${ }^{360}$,Sierra Scientific)was placed transnasally and advanced to the duodenum. Endoscopic and fluoroscopic confirmation of position was performed. After 30 min recovery, subjects underwent $180 \mathrm{~min}$ of recording followed by $250 \mathrm{cc}$ of Ensure ${ }^{\circledR}$ and another 60 min of recording. The catheter was thermally calibrated upon removal. Recordings were analyzed using Sierra's ManoView Analysis software after zeroing to atmospheric pressure. The pylorus was identified using all of the following criteria: 1.Fluoroscopic position 2.Pressure wave form spatially located between antral and small bowel pressure tracings during Phase III MMC 3.Manometric morphology representing a hybrid of antral and duodenal morphology 4.Persistence as a high pressure zone throughout study as identified using the color contour feature of ManoView. Results: The catheter's distal sensor was placed a mean of $9.4 \pm 2.1 \mathrm{~cm}$ beyond the pylorus (range $6.0-13.0$ ). Mean pyloric axial length was $2.5 \pm 0.4$ $\mathrm{cm}(2.2$ to 3.0$)$. Table 1 shows data for resting pressures in the fasting and fed states during Phase I. Mean pyloric pressure increased in the fed state $(12.5 \pm 8.4$ to $19.8 \pm 7.7 ; \mathrm{P}=$ 0.043 ,Wilcoxon ranks). In 3 subjects complete pyloric relaxation was observed during antral contractile events of Phase III MMC's. Conclusion: With high-resolution manometry we were able to identify and record pyloric pressures. We found remarkable consistency in pyloric channel axial length. Resting pyloric pressure was consistently increased relative to gastric and duodenal pressures, and pressures within all three regions increased after a liquid nutrient meal. Pyloric relaxation during antral contractile events of Phase III MMC's was easily identified.

Table 1. Phase I MMC Pressure Data

\begin{tabular}{|c|c|c|c|c|c|c|}
\hline \multicolumn{7}{|c|}{ Fasting $(\mathrm{mmHg})$....................................Post-Ensure (mmHg) } \\
\hline Subject & Antrum & Pylorus & Duodenum & Antrum & Pylorus & Duodenum \\
\hline 1 & 2.1 & 4.7 & 4.2 & 11.2 & 17.2 & 13.7 \\
\hline 2 & 7.0 & 9.2 & 1.4 & 12.2 & 13.8 & 16.0 \\
\hline 3 & 24.0 & 26.6 & 16.6 & 31.7 & 32.9 & 29.8 \\
\hline 4 & 6.0 & 9.2 & 2.8 & 18.5 & 20.3 & 20.0 \\
\hline 5 & 8.2 & 12.7 & 7.5 & 15.0 & 14.9 & 14.0 \\
\hline Mean \pm SD & $9.5 \pm 8.4$ & $12.5 \pm 8.4$ & $6.5 \pm 6.1$ & $17.7 \pm 8.3$ & $19.8 \pm 7.7$ & $18.7 \pm 6.7$ \\
\hline
\end{tabular}

W1844

Smartpill As a Novel Non-Scintigraphic, Ambulatory Method for Assessing GI Transit: Results in Normal Subjects

Irene Sarosiek, Richard W. McCallum, Michael Sitrin, John Wo, Henry Parkman, Braden Kuo, William Hasler, William D. Chey, Judy Hooker, Beverly Landrigan, Kenneth Koch

Background: Although the clinical tests for gastric residence time (GRT) have been evolving, estimation of the small/large bowel transit time (SLBTT) and gastrointestinal total transi time (TTT) poses more of a challenge for imaging technology. The aim of the study: To evaluate a new methodology for assessing the GRT, SLBTT, and TTT based on a diagnostic capsule (SmartPill) in asymptomatic volunteers. Methods: Eighty seven asymptomatic subjects (32F \& 55M), mean age of 31 (range 19-57 years); mean BMI of 25.8 (range 17.941.1); after an overnight fast swallowed a wireless GI monitoring capsule (SmartPill ACTI) equipped with sensors that measured pressure, $\mathrm{pH}$, and temperature. After initial data gathering at the study site asymptomatic subjects returned to their usual daily environment with parameters being captured by a portable recording device. Gastric residence time (GRT) was defined as the time from ingestion to a sudden rise in $\mathrm{pH}$ to $>4$ of at least $3 \mathrm{pH}$ units from the baseline GRT $<30$ min was excluded from calculations. The TTT was measured from the time of ingestion of the wireless capsule until there was a drop in temperature or abrupt loss of signal associated with a bowel movement confirmed by patients' diary. By subtracting GRT from TTT we calculated SLBTT. T-test compared TTT female vs males; Mann-Whitney Rank Sum Test calculated GRT and SLBTT in regard to gender and Pearson PMC test addressed correlations between BMI and TTT. Data are presented as mean \pm 1 SD or median. Results: All normal subjects had a mean GRT of $4.67 \pm 4.47$ hours, mean SLBTT of $25.1 \pm 14.1$ hours and mean TTT of $29.4 \pm 14.3$ hours. Mean TTT among females was longer $34.8 \pm 17.7$ hours, compared to males $26.3 \pm 11.0$ hours $(\mathrm{P}=0.020)$. Median GRT for females and males was similar, 3.8 vs 3.67 hours. SLBTT of 25.3 hours in females was prolonged compared to 21.4 hours in males $(\mathrm{P}=0.05)$. There were correlations between BMI and TTT $(\mathrm{r}=-0.323 ; \mathrm{p}=0.0086)$, BMI and SLBTT $(\mathrm{r}=-0.26 ; \mathrm{p}=0.035)$, but not for BMI and GRT $(\mathrm{r}=-0.092 ; \mathrm{p}=0.42)$. Conclusions: 1 . The SmartPill capsule represents a novel, nonscintigraphic advance for assessing not only gastric residence, but also small bowel and colon transit times. 2. In normal subjects small/large bowel transit time was longer in females than males. 3. Longer small/large bowel transit time correlates with higher BMI in both genders. 4. This technology has potential for clinical and research applications in neuromuscular gut disorders.

\section{W1845}

Diagnosis of Intestinal Motor Abnormalities By Endoluminal Image and Displacement Analysis: A Novel Approach

Carolina Malagelada, Fosca de Lorio, Fernando Azpiroz, Panagiota Spyridonos, Petia Radeva, Juan R. Malagelada

Gold standard diagnosis of intestinal motor disorders is based on intestinal manometry and full thickness intestinal histopathology. Our aim was to apply a non-invasive diagnostic method to the evaluation of these patients, based on an original technological approach. METHODS. In 11 patients with small bowel dysmotility diagnosed by manometric criteria $(\mathrm{n}=10)$ and / or abnormal full thickness biopsy $(\mathrm{n}=5)$ and 14 healthy subjects, the Pillcam capsule (Given Imaging) was administered. When the capsule reached the jejunum, a test meal ( Ensure HN, $300 \mathrm{ml}, 1 \mathrm{kcal} / \mathrm{ml}$ ) was ingested. Endoluminal images of the gut were analysed using a semisupervised computer program. Contractions were defined as partial or total luminal occlusion. Intercontractile phenomena included tunnel pattern defined as static, large blob area by La Placian filtering, and secretory component defined by color histogram analysis based on self organizing maps using a support vector machine. The program also analyses and correlates data on displacement (velocity) of the capsule within the abdomen. RESULTS. Endoluminal image and displacement analysis identified several abnormalities in patients as compared to healthy subjects. Patients exhibited: 1) Reduced contractility $(1.7 \pm 4$ contractions/ min vs $4.0 \pm 0.2$ in health; $p<0.01) ; 2$ ) Slower velocity $(16 \%$ lower displacement over time than healthy subjects; $\mathrm{p}<0.05)$, whereas total displacement covered by the capsule was similar; 3) Frequent endoluminal images of static tunnel reflecting quiescence $(17 \pm 5 \%$ of the recording time vs $5 \pm 2 \%$ in health; $p<0.05)$; 4) More frequent secretory component $(44 \pm 10 \%$ of the time vs $18 \pm 3 \%$ in health; $p<0.05)$; 5) Impaired postprandial response with blunted postprandial increment in contractility and propulsion, as opposed to healthy subjects. Based on the above criteria, only $2 / 14$ healthy subjects exhibited one parameter out of the normal range, while all patients exhibited one or more abnormalities (mean 2.2). CONCLUSION. Endoluminal image and displacement analysis identifies abnormal parameters of motor function, establishing an objective, noninvasive, positive diagnosis in patients with small bowel motility disorders. (Supported in part by a Research Grant from Given Imaging Ltd). 
W1846

The Prevalence of Small Bacterial Overgrowth in Type 1 Diabetic Patients Veronica Ojetti, Dario Pitocco, Emidio Scarpellini, Franco Scaldaferri, Alessio Migneco, Francesco Zaccardi, Giovanni Gasbarrini, Giovanni Ghirlanda, Antonio Gasbarrini Objective: Gastrointestinal motility disorders are often present in diabetic patients (pts). Impaired intestinal motility is often followed by small-bowel bacterial overgrowth (SBBO), but only few studies evaluated the relationship between autonomic neuropathy, impaired intestinal motility and SBBO in diabetic pts. Intestinal motility dysfunction in diabetes has been attributed to autonomic neuropathy even though the pathophysiological mechanisms are not completely defined. Autonomic neuropathy in diabetic pts is normally evaluated through cardiovascular autonomic tests (score $<2$ ). Aim of our study was to compare the prevalence of small-intestine bacterial overgrowth and oro-cecal transit time between type 1 diabetic pts showing autonomic cardiovascular dysfunction and type 1 diabetic pts without autonomic dysfunction. None of these pts had previously known diabetes-related gastrointestinal disorders. Methods: $25 \mathrm{pts}$ (13males, $12 \mathrm{females}$; mean age $44.2+/-7$ duration of diabetes $22.3 \pm 1.3$ years, HbAlc $7.06 \pm 0.3 \%$ ) affected by IDDM with normal cardiovascular autonomic test (group A) and 25 IDDM pts matched for sex and age, with abnormal cardiovascular autonomic test (group B) were submitted to H2-lactulose breath test. Exclusion criteria were gastric or pancreatic surgery, celiac disease, inflammatory bowel disease, lactose intolerance, scleroderma, hypothyroidism. Pts took only insulin, but no other drugs. They did not use laxatives or antibiotics eight weeks before the test. Results: 2 out of 25 (8\%) showed bacterial overgrowth among group A pts, while 11 out of 25 (44\%) showed bacteria overgrowth among group B pts ( $\mathrm{p}<0.01) .16$ of $25(64 \%)$ pts of group A and 19/25 (76\%) pts of group B showed delayed transit time. Conclusions: All diabetic pts showed a high prevalence of delayed transit time, but only pts with altered cardiovascular autonomic tests showed high prevalence of $\mathrm{SBBO}$. This is probably due to the fact that delayed transit time may be considered a risk factor for the development of secondary SBBO. Our study shows a significant association between SBBO and autonomic neuropathy diagnosed by pathological cardiovascular reflex tests, probably because these pts suffer from a more severe degree of intestinal motility disturbance.

\section{W1847}

Voltage-Dependent Changes in Cholinergic Component of Nerve-Stimulated Contractions in Diabetic Guinea Pigs

Kathy J. Lepard, Joseph Cellini, Ryan Pommie

Diabetes mellitus can result in neuropathy of autonomic neurons, including enteric neurons, resulting in abnormal gut motility. These studies investigated contractions due to pharmacologic activation of muscarinic and tachykinin receptors and nerve-stimulated neurotransmitter release. Contractions were recorded using whole ileal strips from control guinea pigs and animals made diabetic by streptozotocin. Drugs were added to the tissue bath or nerves were stimulated to release endogenous substances. (*p<0.05). Activation of muscarinic receptors by an agonist (bethanechol, 1-1000 $\mathrm{\mu M}$ ) or by inhibiting acetylcholine (Ach) breakdown (neostigmine, $0.05-1.0 \mu \mathrm{M}$ ) produced stronger contractions in diabetic tissues [Bethanechol (g contraction, $\mathrm{n}=40$ ): $1 \mu \mathrm{M}$ : Control, $0.46 \pm 0.07$; Diabetic, $0.86 \pm 0.1^{*} ; 10 \mu \mathrm{M}$

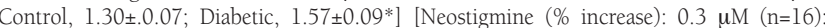
Control, $69+7 \%$ : Diabetic, $119+16 \% * 1$. Substance P (1-1000 nM)-induced maximal contractions were increased in ileum from diabetic animals at the lowest dose only $[\mathrm{g}$ contraction $(\mathrm{n}=16-24)$ : $1 \mathrm{nM}$ : Control, $0.76 \pm 0.04$; Diabetic, $0.96 \pm 0.09 * 1$. In contrast, electrical fieldstimulated contractions (6V) were decreased in diabetic tissues [ $\mathrm{g}$ contraction $(\mathrm{n}=60)$ : Control, $2.11 \pm 0.04$; Diabetic, $1.84 \pm 0.07^{*} 1$. At $6 \mathrm{~V}$, contractions were more noncholinergic as peak amplitudes were reduced only $29 \pm 5 \%$ (diabetic, $n=21$ ), as compared to $42 \pm 4 \%$ (control, $\mathrm{n}=21, \mathrm{p}=0.06)$, by the $\mathrm{M} 3$ receptor antagonist 4-DAMP $(1 \mu \mathrm{M})$. For $6 \mathrm{~V}$, the concentration of Ach in the tissue bath was similar (nM Ach/100 $\mu \mathrm{l} / \mathrm{mg}$ tissue wt: Control, $0.33 \pm 0.06$; Diabetic, $0.42 \pm 0.08)$. At $4 \mathrm{~V}$, contractions were similar in amplitude [g contraction $(\mathrm{n}=40)$ : Control, $1.69 \pm 0.05$; Diabetic, $1.64 \pm 0.08$ ], but were more cholinergic in diabetic tissues. Peak amplitude was reduced $63 \pm 8 \%$ * (diabetic, $n=21$ ) and $39 \pm 7 \%$ (control, $n=21$ ) by 4 DAMP $(1 \mu \mathrm{M})$. Again Ach was not different (nM Ach/100 $\mu \mathrm{l} / \mathrm{mg}$ tissue wt: Control 0.44 \pm 0.07 ; Diabetic, $0.60 \pm 0.13)$. Even though Ach remained the same, in diabetic tissues contractions were more cholinergic at $4 \mathrm{~V}(63 \pm 8 \%)$ as compared to $6 \mathrm{~V}(29 \pm 5 \%)$. The role of substance $\mathrm{P}$ in mediating nerve-stimulated, noncholinergic contractions was investigated using $\mathrm{CP}$ $96,345$, a neurokinin receptor antagonist. CP-96,345 (30 $\mu \mathrm{M})$ equally reduced the peak amplitude of nerve-stimulated contractions [\% decrease ( $\mathrm{n}=24)$ : 4V: Control, 26 $\pm 7 \%$; Dia-

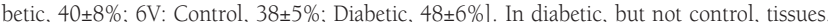
the proportion of the total contraction due to Ach varied depending on the voltage. Substance $\mathrm{P}$ may mediate the 4-DAMP-resistant portion of the total contraction. Supported by College of Health Sciences and NS047106.

\section{W1848}

Electrogastrography Differentiates Enteric Neuropathy from Myopathy in Adults with Severe Motility Disorders of the Gut

Shahab Abid, Greger Lindberg

Chronic intestinal pseudo-obstruction, enteric dysmotility and slow transit constipation are severe motility disorders of the gut that usually are associated with an underlying enteric neuropathy or myopahty. Electrogastrography is a non-invasive technique that records gastric myoelectric activity. Aim of the study. To determine whether EGG can differentiate enteric neuropathy from myopathy as the primary pathology in patients with severe motility disorders of the gut. Material and methods. This is a retrospective analysis of patients with various motility disorders of the gut that underwent full thickness small bowel biopsy. A single bipolar channel measured the EGG. The ability of EGG to differentiate between neuropathy and myopahty was tested by comparing EGG parameters between the groups. EGG parameters analysed were; (i) Percentage of time with a dominant frequency (DF) in normogastric $(2-4 \mathrm{cpm})$, tachygastric $(4-10 \mathrm{cpm})$ and bradygastric $(0.5-2 \mathrm{cpm})$ frequency bands, (ii)
EGG power attributed to the three frequency bands (normogastria, tachygastria and bradygastria), (iii) the ratio of postprandial to fasting power of the DF, (iv) the overall DF in fasting and postprandial periods, (v) variability of the dominant frequency (DFIC) for the fasting and postprandial periods, and (vi) variability of the dominant power (DPIC) in the fasting period and postprandial periods. Results. A total of 38 patients, 35 (92\%) females, mean age $42 \pm 13$ years, were analysed. Twenty patients had enteric dysmotility ( 19 with neuropathy and 1 with myopathy), 12 had slow transit constipation (10 with neuropathy and 2 with myopathy), and 6 had chronic intestinal pseudo-obstruction ( 3 with neuropathy and 3 with myopathy). Patients with myopathy showed higher percentages of fasting time with DF in bradygastric and tachygastric frequency bands and a higher postprandial DF (Table) than did patients with neuropathic motility disorders. Conclusions. Patients with visceral myopathy exhibited more arrythmia during fasting and a higher DF following the test meal and this indicates that myopathies are associated with more electrical disturbances than neuropathies. EGG may be considered for differentiating between these two disease entities. Comparison of EGG parameters in visceral neuropathy and myopathy

\begin{tabular}{|c|c|c|c|}
\hline EGG parameter & Neuropathy $\mathbf{n}=\mathbf{3 2}$ & Myopathy $\mathbf{n}=\mathbf{6}$ & p-value \\
\hline${\text { Fasting bradygastric DF\% }{ }^{\dagger}}^{\dagger}$ & $0(0-15)$ & $9(3-14)$ & $<0.001$ \\
\hline Fasting tachygastric DF\% ${ }^{\dagger}$ & $8(0-69)$ & $24(3-42)$ & $<0.05$ \\
\hline Fasting normogastric DF\% ${ }^{\dagger}$ & $88(31-100)$ & $56(19-94)$ & $<0.001$ \\
\hline Postprandial DF & $3.1 \pm 0.1$ & $3.9 \pm 2.5$ & $<0.05$ \\
\hline
\end{tabular}

$\mathrm{DF}=$ dominant frequency; $\dagger=$ median and range

\section{W1849}

Effects of Itopride Hydrochloride On Ileal and Colonic Motility of Guinea Pig in Vitro

Hyojin Park, Lim Jung Hyun, Young Gyun Kim

Backgrounds: Itopride has antiacetylcholinesterase activity as well as dopamine D2 receptor antagonist activity and is used for the treatment of functional dyspepsia. However, there are limited data showing the stimulatory action on small bowel and colonic motility. Aim: Therefore, we investigated the effects of itopride on ileal peristalsis and colonic trnasit in the guinea pig. Methods: A specially designed peristaltic bath was used to investigate the effect of itopride on peristaltic waves induced by intraluminal perfusion. The mechanical activity of the circular muscle was measured with a clip attached, via the three sites of serosal surface, to the underlying circular muscle of ileum in the peristaltic chamber. For the measurement of colonic transit time study, artificial feces were inserted into the oral side of lumen, and moved toward the anal side by intraluminal perfusion via a peristaltic pump. Total $10 \mathrm{CM}$ was observed and the time taken for moving about each $2 \mathrm{CM}$ was measured. Results: Itopride (10-9 10-7M) increased the amplitude of contraction and area under curve at anal side, and it increased the peristaltic velocity in a concentration dependent manner. Itopride also shortened the transit time of colon. Neostigmine significantly decreased the colonic transit time than control. Dopamine decreased the peristaltic velocity of ileum, but dopamine plus itopride significantly increased the velocity. Dopamine increased the colonic transit time than control, and dopamine plus itopride significantly decreased the colonic transit time. Conclusions: We concluded that itopride exerted prokinetic effect on the ileum and colon of guinea pig by both dopamine receptor antagonistic activity and antiacetyl cholinesterase activity. We suggest the potential use of itopride for functional bowel disorders, such as constipation or postoperative ileus, in human.

\section{W1850}

What Is the Relevance of Small Intestinal Motor Abnormalities in Patients with Irritable Bowel Syndrome (IBS)?

Magnus Simren, Amanda Ersryd, Pia Agerforz, Gisela Ringstrom, Iris Posserud, Hans Strid, Hasse Abrahamsson

Background: Altered gastrointestinal motility is considered to be important in IBS, but its relevance for specific symptoms remains unclear. Some studies have proposed a link between small bowel motor abnormalities and symptoms (Kellow et al Gastroenterology 1987), whereas others have failed to confirm these findings (Gorard et al Gut 1994). However, the majority of previous studies have been performed in small groups of patients, potentially limiting their usefulness. Methods: We included 95 patients with IBS according to the Rome II criteria (68 females; mean age 36 (19-68) years). They underwent a stationary antroduodenojejunal manometry with fasting recording for 3 hours and recording for 1 additional hour after intake of a standard 500kcal meal. The presence of dysmotility was assessed by an experienced investigator (blinded to patient characteristics) based on internationally accepted criteria and normal values obtained in healthy control subjects at our lab. All patients completed the Gastrointestinal Symptom Rating Scale (GSRS) to assess severity of GI symptoms and the Hospital Anxiety and Depression scale (HAD) to evaluate psychological symptom severity. Results: Small intestinal dysmotility was observed in $53 \%$ of the subjects, with moderate to severe alterations in $16 \%$, the rest having mild abnormalities. The most common abnormalities found were isolated bursts (24\%), postprandial discrete clustered contractions (DCCs) lasting more than $30 \mathrm{~min}$ (13\%) and frequent long clusters outside the normal range in healthy controls (19\%). Less commonly seen abnormalities were abnormal appearance or propagation pattern of phase III $(7 \%$ ) generalized hypocontractility (amplitude $<20 \mathrm{mmHg}$ ) (3\%) and late postprandial phase III-like activity (2\%). The GI and psychological symptom severity assessed with GSRS and HAD did not differ between IBS patients with or without small intestinal dysmotility. The presence of small intestinal dysmotility was similar in IBS subgroups based on the predominant bowel pattern. DCCs in the fasting state was common $(32 \%)$, but not regarded as abnormal if it was the only alteration demonstrated. Patients with DCCs had more severe abdominal pain (4.0 1.1 vs. $3.3 \pm 1.0$; $\mathrm{p}=0.01)$ and satiety $(3.0 \pm 1.5 \mathrm{vs} .2 .2 \pm 1.4 ; \mathrm{p}=0.05)$ as compared with patients without DCCs. Conclusion: Small intestinal dysmotility of mild to moderate severity is commonly seen in 
patients with irritable bowel syndrome. However, its direct relevance for the symptoms in these patients remains questionable. Discrete clustered contractions may have some relevance for pain in IBS.

\section{W1851}

Effect of Tegaserod On Antroduodenal and Jejunal Motility Issam Nasr, Ashok Attaluri, Robert Summers, Satish Rao, Syed Hashmi

BACKGROUND \& AIM: Tegaserod, a 5-HT4 agonist, has been shown to accelerate gastric and small bowel transit in healthy males. However, there is little information on the effects of Tegaserod on antral and small bowel activity in humans. In an open- labeled study, we investigated the effects of Tegaserod on antral, duodenal and jejunal motor activity in subjects with suspected upper GI dysmotility. METHODS: 24 patients $(\mathrm{M} / \mathrm{F}=4 / 20$; mean age $=$ 37) with symptoms of upper gut dysmotility underwent prolonged Antro-Duodeno-Jejunal manometry by placement of a 6 sensor solid state probe, with 2 sensors located in each segment. $18 \mathrm{hrs}$ after probe placement, in the fasted state, baseline motor activity was recorded for $30 \mathrm{~min}$. Subsequently, $12 \mathrm{mg}$ of Tegaserod was administered orally and motility recordings were continued for 3 hours. The Area Under the Curve (AUC) of pressure waves was calculated as an index of overall motility. RESULTS: When compared to the baseline period, Tegaserod significantly $(\mathrm{p}<0.05)$ increased pressure wave activity in the antrum, duodenum and jejunum (table). Following Tegaserod administration, a 'fed- response' like pattern was seen, with intermittent, phase II- like waves at 2-3 cycles/min in the antrum and 10-14 cycles/min in the small bowel. The highest activity $(\mathrm{p}<0.05)$ occurred in the jejunum and in the 3rd hour ( $<<0.05$, table). Post drug, phase III MMCs were seen in 10 patients ( $41 \%$ ). During the study, 6 patients reported nausea (27\%). Prior to the procedure, patients reported the following symptoms on a questionnaire: fullness (91\%), bloating (91\%), nausea $(79 \%)$, abdominal pain (79\%), vomiting (70\%), diarrhea (63\%), indigestion (58\%) and weight loss $\geq 10 \mathrm{lbs}(62 \%)$. CONCLUSIONS: Tegaserod induces a gradual but sustained increase in upper gut motility, starting within 60 minutes. This effect is more pronounced in the jejunum than the antrum. These findings suggest that Tegaserod has a prokinetic effect in the upper gut, is well tolerated and may be useful in the treatment of patients with upper gut dysmotility. Supported in part by NIH R01 DK5700-03

\begin{tabular}{|c|c|c|c|c|}
\hline \multirow[t]{2}{*}{ Segment } & \multicolumn{4}{|c|}{ AUC $\left(\left(\mathrm{mm} H \mathrm{H}, \mathrm{sec} \times 10^{3}\right)\right.$ (\%) increase over baseline) } \\
\hline & Bascline & 60 min & $120 \mathrm{~min}$ & $180 \mathrm{~min}$ \\
\hline Antrum & 21 & $27\left(128 \varepsilon_{0}\right)$ & $28\left(13^{\circ}\right)$ & $30\left(22 \varepsilon_{6}\right)$ \\
\hline Duodenum & 22 & $29(6 \%)$ & $31(14 \%)$ & $33(2356)$ \\
\hline Jejunum & 18 & $22(20 \%)$ & $25\left(366^{\circ}\right)$ & $28\left(566^{\circ}\right)$ \\
\hline
\end{tabular}

\section{W1852}

In Search for New Parameters of Intestinal Motor Activity in Humans Fosca de Lorio, Carolina Malagelada, Fernando Azpiroz, Fernando Vilarino, Jordi Vitria, Anna Accarino, Juan R. Malagelada

Current evaluation of intestinal motility relays on manometric detection of occlusive contractions, but these phasic events account only for a minor fraction of recording time, and quiescence is assumed by exclusion. Our aim was to explore the potential use of other parameters, related to intercontractile phenomena, and hence, manometrically silent events. METHODS. Twenty six healthy subjects were studied modelling two experimental conditions: prokinetic stimulation was induced by ingestion of a meal (Ensure HN, $300 \mathrm{ml}, 1 \mathrm{kcal} /$ $\mathrm{ml} ; \mathrm{n}=13$ ) and motor inhibition was induced by glucagon administration $(4.8 \mu \mathrm{g} / \mathrm{kg}$ bolus plus $9.6 \mu \mathrm{g} / \mathrm{kg} \mathrm{h}$ infusion for $\mathrm{l} \mathrm{h} ; \mathrm{n}=5$ ). Pos-treatment events were compared to basal conditions (fasting; $\mathrm{n}=8$ ). Intestinal motor activity was evaluated through analysis of images emitted by the Pillcam capsule (Given Imaging) and recorded by external detectors fixed to the abdominal wall. Endoluminal images of the gut were analysed using a semisupervised computer program. Contractions were defined as partial or total luminal occlusion. Intercontractile phenomena included tunnel pattern, defined as static, large blob area by La Placian filtering, and secretory component defined by color histogram analysis based on self organizing maps using a support vector machine. The program also correlates data on displacement (propulsion) of the capsule within the abdomen. RESULTS. The meal stimulus increased the number of contractions $(5.1 \pm 0.4$ contraction $/ \mathrm{min})$, while motor inhibition suppressed contractile activity ( 0 contractions/ min) as compared to basal conditions $(2.5 \pm 0.5$ contractions/min; $<<0.05$ for both). Key parameters reflecting motor activity were also significantly affected by the experimental manipulation: 1) Propulsion of the capsule increased by $118 \%$ during prokinetic stimulation, and decreased by $73 \%$ during inhibition. 2) During motor inhibition the percentage of time when the capsule was completely still was much longer than during basal conditions $(65 \pm 6 \%$ of the time vs $6 \pm 2 \%$, respectively; p <0.05). 3) Conversely, prokinetic stimulation reduced the time during which the gut presented a static tunnel appearance ( $3 \pm 1 \%$ of the time vs $14 \pm 8 \%$ during basal conditions), and the this was not due to reduced visibility by intraluminal contents (secretion), which were only detected during $7 \pm 2 \%$ of the time (vs $23 \pm 7 \%$ during basal conditions; p $<0.05$ ). CONCLUSION. Intestinal motility can be non-invasively evaluated by endoluminal image computer analysis, that quantifies parameters of the dynamic and static components that determine gut motor function. (Supported in part by a Research Grant from Given Imaging Ltd).

\section{W1853}

Bran-Induced Small Bowel Secretion: Assessment By Novel Non-Invasive MRI Technique

Luca Marciani, John J. Totman, Caroline L. Hoad, Steve Evans, Amisha Mistry, Penny A. Gowland, Robin C. Spiller

Background: Bloating, abdominal distension and pain are frequent symptoms in Irritable Bowel Syndrome (IBS) which can often be aggravated by bran, but the underlying mechanisms are currently unknown. We have previously shown using scintigraphy that both bran and plastic pellets accelerated small bowel transit. However it was not feasible to use the then available perfusion techniques to demonstrate the hypothesised increase in small bowe secretions. Recent developments in magnetic resonance imaging (MRI) allow high resolution, non-invasive, monitoring of small bowel water content. Aims: To test the hypothesis that bran acts by stimulating gastrointestinal secretion using MRI. Methods: 9 healthy volunteers attended on 2 separate days, having fasted overnight. They ate a standard 331 kcal rice pudding with or without added $15 \mathrm{~g}$ of coarse wheat bran. Serial MRI imaging was performed at $45 \mathrm{~min}$ intervals for 8 hours. A pasta and dessert meal $(1007 \mathrm{kcal})$ with $250 \mathrm{ml}$ water was consumed at $\mathrm{t}=7$ hours. The volume of fluid in the small bowel at each time point after the meal was calculated by integrating all image pixels containing water signal above a given threshold. Results: (mean \pm SEM) Bran increased the small bowel water content which was significantly higher at time $\mathrm{t}=225 \mathrm{~min}$ after the bran meal $(202+48 \mathrm{ml})$ than after the contro meal $(135 \pm 54 \mathrm{ml}), \mathrm{n}=9$, paired Student's $\mathrm{p}<0.05$. The figure below shows 5 contiguous coronal slices acquired across the abdomen of a volunteer at time $\mathrm{t}=225 \mathrm{~min}$ for both meals. The images were thresholded to blank out all pixels with intensity below water. Conclusion We have shown for the first time that bran markedly increases small intestinal water content probably by stimulating intestinal secretion.

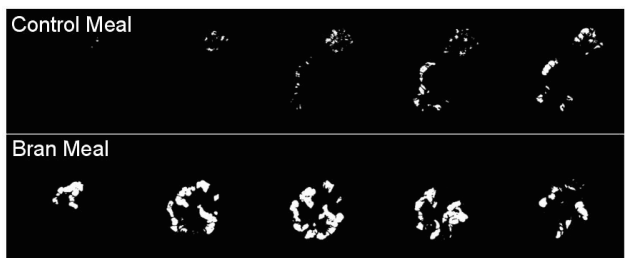

\section{W1854}

Small Bowel Motility and Quality of Life in Patients with Severe Irritable Bowel Syndrome Compared with Chronic Intestinal Pseudo-Obstruction Jutta Keller, Kai Kobarg, Christiane Fibbe, Peter Layer

Background: Intrinsic neuropathies disturbing small bowel motility have not only been demonstrated in chronic intestinal pseudo-obstruction (CIPO) but also in severe irritable bowel syndrom (IBS). Moreover, both diseases are known to markedly impair quality of life (QoL). However, neither small bowel motility nor QoL of patients with severe IBS or CIPO have been compared directly, so far. Methods: Small bowel motility, gastrointestinal symptoms and QoL were prospectively evaluated in 12 healthy subjects and 54 patients with severe abdominal symptoms using manometry and standardised questionnaires (SF36 for QoL). Eight-channel antro-duodeno-jejunal manometry was performed using a waterperfused system for 3 to $4 \mathrm{~h}$ during fasting and for $2 \mathrm{~h}$ following a $450 \mathrm{kcal}$ meal. Manometry was classified as pathological if at least two parameters (motility index (MI), phase IIIcharacteristics, preterm postprandial phase III, number of clusters, bursts and giant contractions) were found to be abnormal. Nine patients were classified as having severe IBS (constipation-predominant in 7 subjects) and 6 as having CIPO without taking manometric findings into account. Data from patients with other diagnoses (e.g. slow transit constipation, functional dyspepsia, mechanical subocclusion and known neurological diseases including known extrinsic neuropathies) were not analysed in this study. Results: Overall, IBS patients tended to have a lower duodenal MI than healthy subjects $(\mathrm{p}=0.04)$, but, compared with IBS, duodenal MI was even more decreased in CIPO ( $\mathrm{p}=0.02)$. Absence of phase III during up to $4 \mathrm{~h}$ of fasting or pathological propagation was less frequent in IBS than CIPO. According to manometric criteria, $5 \mathrm{CIPO}$ patients had neuropathic disease. QoL was markedly and similarly decreased in IBS and CIPO compared with healthy subjects (tab.). Conclusions Patients with severe IBS show similar but less marked alterations of small bowel motility compared with CIPO. In particular, signs of intrinsic neuropathy are less frequent. Thus, our findings may support the hypothesis that certain cases of severe IBS might be a mitigated form of CIPO. However, other mechanisms appear to contribute to the decrease in $\mathrm{QoL}$ in severe IBS which is as marked as in CIPO patients.

\begin{tabular}{|c|c|c|c|c|}
\hline & Path. MI (1) & Path. Phase III (2) & Path. Manometry & QoL (SF-36) \\
\hline Healthy subjects & $0 \%$ & $8 \%$ & $0 \%$ & $139+/-1$ \\
\hline IBS & $56 \%$ & $33 \% *$ & $67 \% * *$ & $75+/-7 * *$ \\
\hline CIPO & $83 \% * *$ & $83 \% * *$ & $100 \% * *$ & $85+/-7 * *$ \\
\hline
\end{tabular}

(1) at $>1$ recording site (2) absence or pathological propagation * $\mathrm{p}=0.078$ vs. CIPO ** $\mathrm{p}<0.01$ vs. healthy subjects

\section{W1855}

A Physiological Rate of Butyrate (B) Entry Into the Cecum of Piglets Causes Increased Cell Proliferation (CP) in Cecum, Distal Colon, and Jejunum Without Affecting Cecal pH Or Inducing Injury, But the Prebiotic, Inulin (IN), Inhibits This Effect

C. Lawrence Kien, Ruth Blauwiekel, Wendy Frankel, Rhonda Maple, Karen Everingham Increased colonic $\mathrm{CP}$ may be detrimental to the risk of cancer, but increased gut $\mathrm{CP}$ may be beneficial in patients post gut resection. Previously, we showed that lactulose feeding caused decreased cecal CP and increased cecal B synthesis. Thus, we hypothesized that cecal infusion of $\mathrm{B}$ at a rate equal to the abnormal increment in $\mathrm{B}$ production (during lactulose feeding) also would cause decreased CP in cecum, colon (distal colon), and perhaps jejunum. Since pre- and/or co-feeding IN seemed to attenuate the decreased cecal CP during lactulose feeding, we also hypothesized that the same effect would be seen when B was exogenously "produced". Piglets ( $\mathrm{N}=24,11-13 \mathrm{~d}$ age) were all fed sow milk replacement formula and randomized into 4 equal groups: (I) cecal phosphate buffered saline infusion (PBS); (II) cecal B infusion (2.3 $\mu \mathrm{mol} / \mathrm{kg} / \mathrm{min})$; (III) IN (3 g/L formula) + PBS; (IV) IN + B. After $6 \mathrm{~d}$ 
of oral feeding ( \pm IN), a cecal catheter was inserted, and B or PBS was infused for 4d. On $\mathrm{d} 4$ post-op, cecal, colon, and jejunal CP (fractional proliferative index, entire crypt or villus) was assessed using BrdU, and injury/inflammation was scored. Data were analyzed by two way analysis of variance. Cecal wet/dry weight ratio was affected by the IN treatment ( $\mathrm{P}=$ .018 , no interaction): I $(8.7 \pm 0.7)$; II $(7.6 \pm 0.8)$; III $(5.9 \pm 0.4)$; IV $(6.7 \pm 1.0)$, but there was only a borderline IN effect on the frequency of loose stools $(\mathrm{P}=0.08)$. Cecal pH also was affected by IN $(\mathrm{P}=0.007)$ but not $\mathrm{B}$ (no interaction): I $(7.04 \pm 0.11)$; II $(7.01 \pm 0.17)$; III $(6.61 \pm 0.19)$; IV $(6.51 \pm 0.13)$. There were no group effects on weight gain, breath concentration of $\mathrm{H} 2$ or $\mathrm{CH} 4$, cecal or colon crypt depth, jejunal villus height, or injury score for cecum, colon, or jejunum. IN per se did not affect CP in cecum, colon, or jejunum. $\mathrm{B}$ caused increased $\mathrm{CP}$ in the cecum, colon, and jejunum but only in group I: Cecum: $\mathrm{P}=$ 0.003 , interaction, $\mathrm{P}=0.003$, I $(0.19 \pm 0.02)$, II $(0.42 \pm 0.02)$, III $(0.26 \pm 0.05)$, IV $(0.27$ \pm 0.03 ; Colon: $\mathrm{P}=0.024$, interaction, $\mathrm{P}=0.018$, I $(0.09 \pm 0.01)$, II $(0.18 \pm 0.02)$, III $(0.14$ $\pm 0.02)$, IV $(0.14 \pm 0.02)$; Jejunum: $P=0.013$, interaction, $P=0.002$, I $(0.10 \pm 0.01)$, II $(0.22 \pm 0.01)$, III $(0.17 \pm 0.02)$, IV $(0.16 \pm 0.03)$. Thus, in contrast to the association, during lactulose feeding, of increased synthesis of $B$ with decreased $C P$, increased exogenous $\mathrm{B}$ in the cecum causes increased $\mathrm{CP}$, both locally and distally/systemically(distal colon and jejunum), but IN blocks this effect in an analogous but opposite effect to how it attenuates decreased CP during lactulose feeding. (Support: DK61775).

\section{W1856}

Butyrate Increased the Growth Activity and Showed An Anti-Inflammatory Effect in An Intestinal Epithelial Cell Line

Osamu Kanauchi, Keiichi Mitsuyama, Akira Andoh, Yoshiaki Matsumoto

Background: There is increasing evidence that intestinal microflora play an important role in the pathogenesis of inflammatory bowel disease. In our previous study, a prebiotic, germinated barley foodstuff, significantly increased the number of Eubacterium limosum and production of its major metabolite butyrate in experimental colitis and patients with ulcerative colitis. This study was carried out to examine the effects of certain organic acids that are metabolites of Eucabterium limosum on a colonic epithelial cell line in vitro. Methods: HT-29 (ATCC No. HTB-38) was cultured in modified Eagle medium with butyrate, propionate, acetate, lactate, and succinate $(0,0.1,0.5,1.0$, and $2.0 \mathrm{mM})$. Cell growth activity was determined by the activity of succinate dehydrogenase (SUDH) using a commercial kit. For evaluation of the anti-inflammatory effects of organic acids, IL-8 production with stimulation of TNF-alpha $(50 \mathrm{ng} / \mathrm{ml})$ was determined by ELISA. The expression of toll-like receptor 4 (TLR4) mRNA was determined in T-84 (ATCC No. CCL-248) as well as in HT29. These two expressions were treated with TNF-alpha $(25 \mathrm{ng} / \mathrm{ml})$ with sodium butyrate $(0,0.5,1.0$, and $2.0 \mathrm{mM})$, then the total RNA was extracted and RT-PCR was performed. Results: Butyrate (at 0.1 and $0.5 \mathrm{mM}$ ), propionate (at 2.0 and $5.0 \mathrm{mM}$ ), acetate (at $2.0 \mathrm{mM}$ ), and lactate (at $0.1 \mathrm{mM}$ ) significantly increased the SUDH activity. In IL-8 production, butyrate (at $0.5,1.0$, and $2.0 \mathrm{mM}$ ), propionate (at $0.5,1.0$, and $2.0 \mathrm{mM}$ ) and lactate (at $2.0 \mathrm{mM}$ ) showed significant decreases. Succinate had no significant change in both SUDH activity and IL-8 production. Interestingly, the expressions of TLR4 mRNA in both HT-29 and T-84 were increased by TNF alpha stimulation, and this increase was significantly attenuated by butyrate (at 1.0 and $2.0 \mathrm{mM}$ ) in both cell lines. Conclusion: The major metabolite of Eubacterium limosum, butyrate, is considered to have multifunctional roles that accelerate growth activity, decrease inflammatory cytokine (IL-8) production, and modulate the mucosal barrier function via TLR4. The new mechanism of the expression of TLR4 may contribute to new treatments for inflammatory bowel disease in view of the interaction between epithelium and bacteria in the intestinal tract.

\section{W1857}

Experimental Examination About the Usefulness of the Water-Soluble Dietary Fiber On Metabolic Syndrome

Satomi Akagiri, Hiroshi Ichikawa, Yuji Naito, Osamu Handa, Tomohisa Takagi, Satoshi Kokura, Norimasa Yoshida, Toshikazu Yoshikawa

Aim: The change of the lifestyle in Japanese eating habits caused the rapid increase in the metabolic syndrome which makes obesity an underlying disease. Lifestyle habit is also exert a deep influence on digestive diseases including fatty liver, nonalcoholic steatohepatitis, and irritable bowel syndrome. Furthermore these diseases are closely related to obesity or metabolic syndrome. The aim of this study is to evaluate the influence of the water-soluble dietary fiber using metabolic syndrome animal models. Methods: We used the male Otsuka Long-Evans Tokushima Fatty (OLETF) rat which is a model of human non-insulin-dependent diabetes mellitus (NIDDM). OLETF rats are characterized by late onset of hyperglycemia at 18 weeks of age, followed by insulin-dependent diabetes mellitus at 65 weeks of age. In this study, at 5 weeks of age, OLETF rats were fed a control diet (CRF-1) or a high-fiber diet (5\% of the Guar gum origin water-soluble dietary fiber) for 30 weeks. This dietary fiber was prepared by selectively cleaving the mannan backbone-chain of guar gum by using endo- $\beta$-D-mannanase and is composed of galactomannan with a molecular weight of about 20,000 Da. Body weight, food intake, oral carbohydrate tolerance test, plasma insulin levels, urine glucose and urine protein were measured every week. As an indicator of insulin resistance, the homeostasis model assessment for insulin resistance (HOMA-R) was applied. Histological search of liver was carried out after the end of the study. Results: There was no significant difference between 2 groups in the increase in body weight and in daily food intake. All OLETF rats in control diet group have developed glucose tolerance or diabetes within 30 weeks of age. The insulin resistance evaluated by the HOMA-R index was significantly improved by the water-soluble dietary fiber ingestion. The levels of urine glucose and of urine protein were significantly lower in high-fiber diet group than in control diet group. Histological findings of the liver showed that the severe fatty infiltration at the periphery of central vein was found in control diet group but not in high-fiber diet group. Conclusion: This indicates conclusively that ingestion of water-soluble dietary fiber prevents development of symptoms of metabolic syndrome and insulin resistance. Furthermore, it has been demonstrated that dietary fiber intake prevent the onset of fatty liver.

\section{W1858}

Dose-Response Effect of Arabinoxylanoligosacharides On the Colonic $\mathrm{Nh}_{3}$ Metabolism Using Lactose- $\left({ }^{15} \mathrm{~N},{ }^{15} \mathrm{~N}\right)$-Ureide in Healthy Volunteers Lieselotte Cloetens, Paul Rutgeerts, Katrien Swennen, Christophe M. Courtin, Jan A. Delcour, Kristin Verbeke

Introduction: Arabinoxylanoligosacharides (AXOS), with a variable degree of substitution (DS) and polymerisation (DP) can be obtained by enzymatic treatment of arabinoxylans from cereals. AXOS are non-digestible in the upper gastrointestinal tract and may exert prebiotic effects in the colon. Lactose- $\left({ }^{15} \mathrm{~N},{ }^{15} \mathrm{~N}^{\prime}\right)$-ureide $\left({ }^{15} \mathrm{~N}\right.$-LUR $)$ has previously been evaluated as a biomarker to quantify the effectiveness of prebiotics by measuring their influence on the colonic $\mathrm{NH}_{3}$-metabolism. ${ }^{1}$ Upon stimulation of bacterial growth and/or metabolic activity, a shift from urinary ${ }^{15} \mathrm{~N}$-excretion towards faecal ${ }^{15} \mathrm{~N}$-excretion is expected. The influence of different doses AXOS (DP of 15, DS of 0.27) has been investigated. Materials

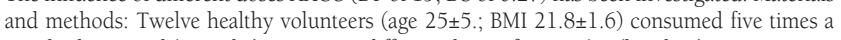
standard test meal (pancake) containing different doses of AXOS (0g (baseline), 0.25g, 0.75g, $2.25 \mathrm{~g}$ and $5 \mathrm{~g}$ ) in random order with an interval of at least one week. Each test meal contained $75 \mathrm{mg}{ }^{15} \mathrm{~N}$-LUR to measure the influence of $\mathrm{AXOS}$ on the $\mathrm{NH}_{3}$-metabolism in the colon and $185 \mathrm{kBq}{ }^{3} \mathrm{H}-\mathrm{PEG}$ as an inert marker to measure total transit. After each test meal, a $48-\mathrm{h}$ urine collection and a 72-h faeces collection were performed. The ${ }^{15} \mathrm{~N}$ enrichment in the samples was measured by combustion-IRMS. All results were expressed as $\%$ of administered dose. Faecal data were corrected for total transit. Results: The ${ }^{15} \mathrm{~N}$-excretion in the 48 -h urine collection was $42.53 \pm 8.84$ at baseline. Upon addition of $0.25 \mathrm{~g}$ or $0.75 \mathrm{~g}$ AXOS, no differences were observed in the urinary ${ }^{15} \mathrm{~N}$-excretion (respectively $40.15 \pm 9.89$ and $42.92 \pm 10.09)$. On the contrary, administration of $2.25 \mathrm{~g}(34.08 \pm 6.16)$ or $5 \mathrm{~g}(35.50 \pm 6.76)$ AXOS resulted in a significantly decreased urinary ${ }^{15} \mathrm{~N}$-excretion (respectively $\mathrm{p}=0.008$ and $\mathrm{p}=0.035$ ). Faecal ${ }^{15} \mathrm{~N}$-excretion was significantly increased after the intake of $2.25 \mathrm{~g}$ $(23.89 \pm 6.42 ; \mathrm{p}=0.034)$ and $5 \mathrm{~g}(23.90 \pm 7.13 ; \mathrm{p}=0.019)$ AXOS as compared to baseline values $(15.38 \pm 6.43)$ whereas lower doses of AXOS, $0.25 \mathrm{~g}(20.54 \pm 17.34)$ and $0.75 \mathrm{~g}(17.61 \pm 3.98)$, did not influence the faecal ${ }^{15} \mathrm{~N}$-excretion significantly. The shift from urinary to faecal ${ }^{15} \mathrm{~N}$ excretion after addition of $2.25 \mathrm{~g}$ and $5 \mathrm{~g}$ AXOS suggests a lower generation of ammonia in the colon and/or a higher uptake of ${ }^{15} \mathrm{~N}$ due to an increased bacterial metabolism and growth and provides an indication for prebiotic properties of AXOS. Conclusion: AXOS administered at a dose of minimally $2.25 \mathrm{~g}$ significantly influences the colonic ammonia metabolism in human volunteers, providing (preliminary) evidence for AXOS prebiotic properties. ${ }^{1}$ De Preter et al., Br J Nutr 2004; 92,439-446

\section{W1859}

Differential Effect of Long-Term Dietary Intake of Lactulose and OligofructoseEnriched Inulin On the $\mathrm{Nh}_{3}$-Metabolism and Predominant Microbiota in the Colon of Healthy Volunteers

Vicky de Preter, Tom Vanhoutte, Geert Huys, Jean Swings, Paul Rutgeerts, Kristin Verbeke Introduction: Dietary intervention with prebiotics can cause changes in the ecological balance of intestinal microbiota and hence influence the microbial metabolic activities in the colon. In the present study, the influence of lactulose and oligofructose-enriched inulin (OF-IN) on the relative composition of the predominant faecal microbiota was studied. Changes in metabolic activity especially the metabolic fate of $\mathrm{NH}_{3}$, were investigated by means of lactose- $\left[{ }^{15} \mathrm{~N},{ }^{15} \mathrm{~N}\right]$-ureide (LU). Methods: The effect of lactulose and OF-IN was evaluated in a randomized study. At the start of the study and at the end of the 4-week treatment period, healthy volunteers consumed a test meal containing $75 \mathrm{mg} \mathrm{LU}$. During the study period, they received $10 \mathrm{~g}$ lactulose (b.i.d.) group $1(\mathrm{n}=29)$ or $10 \mathrm{~g}$ OF-IN (b.i.d.) group 2 $(\mathrm{n}=19)$. Urine and faeces were collected during 48-h and 72 -h, respectively. Samples were analysed for ${ }^{15} \mathrm{~N}$-content by combustion-IRMS and results were expressed as $\%$ of administered dose. A culture-independent approach using PCR-DGGE population fingerprinting was applied to determine the composition of the predominant faecal microbiota, using a universal primer targeting the $\mathrm{V} 3$ region of the $16 \mathrm{~S}$ rRNA gene. Results: In group 1, a significant decrease in the urinary ${ }^{15} \mathrm{~N}$ content was found after lactulose intake, as compared to the baseline values (from $44.12 \pm 13.13$ to $36.52 \pm 12.81 ; \mathrm{p}=0.011$ ). This decrease was compensated by an increase of the faecal ${ }^{15} \mathrm{~N}$ excretion (from $19.94 \pm 6.48$ to $26.29 \pm 8.01$; $\mathrm{p}=0.032$ ). Concurrently, comparison of DGGE banding patterns indicated the appearance or intensification of a specific DGGE band after lactulose intake in $75 \%$ of the subjects, which was assigned to bifidobacterial organisms. In group 2, OF-IN significantly reduced the urinary ${ }^{15} \mathrm{~N}$-excretion from $41.72 \pm 8.76$ to $24.59 \pm 12.27$ ( $\left.\mathrm{p}<0.001\right)$, whereas the faecal excretion of the label remained unchanged $(19.03+6.43$ vs $22.18 \pm 5.21$; $\mathrm{p}=\mathrm{NS})$. As a consequence, long-term administration of OF-IN resulted in a decreased total ${ }^{15} \mathrm{~N}$-excretion (from $60.30 \pm 9.50$ to $46.34 \pm 13.92, p=0.001$ ), suggesting that a higher fraction of ${ }^{15} \mathrm{~N}$ is retained within the human body. In DGGE profiling, no universal changes were observed after OF-IN intake. Possibly, the observed retention of the marker in the colon may be linked to the fact that OF-IN primarily stimulates mucosa-associated microbiota rather than the microbiota in the lumen of the colon. Conclusions: Administration of lactulose has a favourable effect on the colonic $\mathrm{NH}_{3}$-metabolism and was accompanied by an overall stimulation of the bifidobacterial population. In contrast, administration of OF-IN only resulted in a reduction of the urinary ${ }^{15} \mathrm{~N}$-excretion.

\section{W1860}

Enteropathogenic Infection in a Human Enterocyte Zinc Deficiency Model Is Modulated By Probiotic Factor(S)

Ilse J. Broekaert, N Nanda Nanthakumar, W Allan Walker

Background: In developing countries infectious diarrhea is frequently associated with malnutrition and an impaired immune status. Previous observations have shown that infants with infectious diarrhea become zinc ( $\mathrm{Zn}$ )-deficient causing altered intestinal epithelial functions. Both probiotic bacteria and $\mathrm{Zn}$-supplementation have been shown to improve diarrhea. However, there are no studies addressing the effect of probiotic bacteria on enteropathogenic infection in Zn-deficiency. Aim: The aim is to investigate the probiotic effect of different 
Lactobacilli species and Bifidobacterium bifidum on enteropathogenic invasion (Salmonella typhimurium and Listeria monocytogenes) and the epithelial response in a Zn-deficiency model. Methods: Human intestinal cell lines (Caco-2 and T84) were grown either in Znsufficient or deficient growth media for 1 week. Then they were infected with Gram-negative S. typhimurium or Gram-positive L. monocytogenes in the presence or absence of the supernatant of several lactobacilli or B. bifidum or purified probiotic bacteria. Pathogenic invasion in enterocytes grown in $\mathrm{Zn}$-deficient versus sufficient media was quantified by standard gentamycin assay and IL-8 response was measured by ELISA. To characterize the putative probiotic factor(s) the supernatant was treated with acids, proteases, DNAses, and RNAses and exposed to heat. Results: Probiotic supernatants but not purified probiotic bacteria reduce both pathogenic invasion and IL-8 production of intestinal epithelial cells grown in Zn-sufficient and deficient media. Zn-deficiency allows greater pathogenic invasion and enhanced IL-8 secretion of intestinal epithelial cells. The properties of the probiotic factor(s) are resistant to acid, proteases, DNAses, RNAses and to heat. Conclusions: Pathogenic invasion and IL-8 production is significantly enhanced in enterocytes grown in Zn-deficient media. Probiotic supernatants of several lactobacilli and of B. bifidum significantly reduce the increased invasion with S. typhimurium and L. monocytogenes and the enhanced IL-8 response in enterocytes grown in $\mathrm{Zn}$-deficient media, suggesting that the effect of $\mathrm{Zn}$ and probiotics may be synergistic. The inhibiting factor(s) is neither a protein nor a nucleic acid and is stable with acid, $\mathrm{pH}$ and heat changes. Ongoing studies will definitively characterize the nature of the probiotic factor(s). Our observations suggest that probiotics may reduce the duration and severity of infectious diarrhea found in Zn-deficiency by enhancing the innate immune response of the intestinal epithelium. Therefore, probiotics and $\mathrm{Zn}$ might be inexpensive tools in the prevention and treatment of infectious diarrhea.

\section{W1861}

The Effects of the Water-Soluble Dietary Fiber On Gene Expression in Intestinal Cells in Otsuka Long Evans Tokushima Fatty Rats Using a HighDensity Oligonucleotide Array

Hiroshi Ichikawa, Yuji Naito, Satomi Akagiri, Ktsura Mizushima, Osamu Handa, Tomohisa Takagi, Satoshi Kokura, Norimasa Yoshida, Toshikazu Yoshikawa

Aim: Although the gene expression patterns during the development of diabetes have been studied in both rodent models and humans, only a small portion of the mRNAs expressed in the intestinal cells has been characterized. The purpose of the present study is to evaluate the biological effects of the water-soluble dietary fiber on gene expression in intestinal cells by using cDNA microarray analysis in experimental rat models of type 2 diabetes in vivo. Methods: We used 5-week-old male Otsuka Long-Evans Tokushima Fatty (OLETF) rat which is a model of human non-insulin-dependent diabetes mellitus. At 5 weeks of age, OLETF rats were fed a control diet (CRF-1) or a high-fiber diet ( $5 \%$ of the Guar gum origin water-soluble dietary fiber) for 30 weeks. As an indicator of insulin resistance, the homeostasis model assessment for insulin resistance (HOMA-R) and oral carbohydrate tolerance test were applied every week. Intestinal cells were obtained from the terminal ileum of OLETF rats by laser capture microdissection. Preparation of cRNA and target hybridization were performed according to the Affymetrix Gene Chip Eukaryotic Small Sample Target Labeling Assay Protocol (version II). The gene expression profile was determined by the Rat Genome 230-2.0 GeneChip (Affymetrix). Results: All OLETF rats in control diet group have developed glucose tolerance or diabetes within 30 weeks of age. The insulin resistance evaluated by the HOMA-R index was significantly improved by the water-soluble dietary fiber ingestion. Microarray analysis revealed that by comparison between control diet group and high-fiber diet group, 1747 probes that increased in expression with ingestion of dietary fiber and 1182 probes that decreased in intestinal cells were identified. It was found that the decreased gene expression for enzymes involved in lipid or bile metabolism, such as FABP6, solute carrier family 10 and bile acid-Coenzyme A in intestinal cells by ingestion of water-soluble dietary fiber. Furthermore, the increased expression of genes for enzymes, such as PPARgamma (coactivator lalpha) and PPARalpha were recognized in intestinal cells. As for gastrointestinal hormone related genes, we could see the decreased expression of PYY precursor and secretin, and also see the enhanced expression of ghrelin precursor, ACEl and VIP. Additionally, the upregulated expression of genes involved in anti-inflammation, such as caspase 6, MIF and estrogen-related receptor alpha, were found. Conclusion: We suggest that the preventive effect of water-soluble dietary fiber on diabetes was involved in the gene expression for lipid metabolism, gastrointestinal hormone and anti-inflammation.

\section{W1862}

Comparison of the Effect of Long-Term Administration of Of-in Ingested in Combination with Lactobacillus Casei Shirota with of-in Alone On the Colonic Fate of Protein Fermentation Metabolites $\mathrm{NH}_{3}$ and Phenols Vicky de Preter, Tamara Coopmans, Paul Rutgeerts, Kristin Verbeke

Introduction: Dietary intervention with probiotics or prebiotics may cause changes in the relative populations of colonic bacteria and hence in microbial metabolic activities. Their synbiotic combination could stimulate not only the growth of the endogenous bacterial strains, but also the growth of the exogenous ones and perhaps favour their implantation. In the present study the influence of long-term administration of the synbiotic combination of Lactobacillus casei Shirota cells with oligofructose-enriched inulin (OF-IN) on the colonic fate of $\mathrm{NH}_{3}$ and phenolic compounds (phenol and p-cresol) was compared with the administration of OF-IN alone. Methods: A randomized, cross-over study was performed in 9 healthy volunteers. The lactose- $\left[{ }^{15} \mathrm{~N},{ }^{15} \mathrm{~N}\right]$-ureide biomarker was used to study the fate of $\mathrm{NH}_{3}(1)$. At the start of the study and at the end of each 4-week treatment period, urine was collected during 48 -h. Each treatment period was separated by a 14-day wash-out period. In the prebiotic test period, the volunteers received $10 \mathrm{~g}$ OF-IN (b.i.d.) and in the synbiotic test period they received $6.5 \times 10^{9} \mathrm{~L}$. casei Shirota cells $+10 \mathrm{~g}$ OF-IN (b.i.d.). The urinary p-cresol and phenol content was determined by means of GC-MS (mg phenols/24-h). The ${ }^{15} \mathrm{~N}$ content was analysed by combustion-IRMS (\% of administered dose). Results: Long-term administration of OF-IN resulted in a statistically significant reduction of the urinary ${ }^{15} \mathrm{~N}$ content compared to baseline (from 39.47 (IQR 33.83-49.39) to 24.60 (IQR 10.81-31.74) $(\mathrm{p}<0.001)$. Also after synbiotic intake the ${ }^{15} \mathrm{~N}$-excretion significantly decreased from 39.47
(IQR 33.83-49.39) to 15.43 (IQR 5.95-27.67) ( $<<0.001$ ). The urinary p-cresol conten decreased from 24.43 (IQR 15.28-39.72) at baseline to 14.70 (IQR 7.07-31.34) after OFIN intake ( $\mathrm{p}=0.015$ ), respectively to 9.79 (IQR 4.56-27.03) after synbiotic administration $(\mathrm{p}=0.021)$. No significant effect on the urinary phenol content was observed after long-term administration of the substrates. Conclusions: The results of this study demonstrate that OF-IN and the synbiotic combination of $L$. casei Shirota cells with OF-IN are able to modulate the colonic environment and the metabolic activity of the intestinal flora, with a favourable effect on the colonic protein metabolites $\mathrm{NH}_{3}$ and phenols. A tendency to more pronounced effects was observed after synbiotic intake compared to OF-IN alone. (1)De Preter V et al. Br J Nutr (2003)

\section{W1863}

Amino Acid Fermentation in the Colon Is Impaired Upon Simultaneous Administration of Fermentable Carbohydrates: Results of a Direct Instillation Study Using ${ }^{13} \mathrm{C}$-Leucine

Karen Geboes, Anja Luypaerts, Vicky de Preter, Paul Rutgeerts, Kristin Verbeke

Introduction : Stimulation of carbohydrate fermentation is believed to prevent accumulation of toxic products from amino acid metabolism. In this study, the influence of inulin on the colonic fermentation of ${ }^{13} \mathrm{C}$-leucine has been evaluated using direct instillation. Methods Eleven healthy volunteers swallowed a narrow polyvinyl tube which progressed down the intestine overnight. On the following morning, radiographic screening ensured the position of the tube in the right colon. Baseline breath and serum samples were retrieved before bolus administration of a $40-\mathrm{ml} \mathrm{NaCl} 0.9 \%$ solution containing $200 \mathrm{mg}{ }^{13} \mathrm{C}$-leucine in the first experiment and $200 \mathrm{mg}{ }^{13} \mathrm{C}$-leucine with $2 \mathrm{~g}$ of inulin (Raftilin $\mathrm{HP} 囚$ ) in the second After instillation, breath samples for analysis of ${ }^{13} \mathrm{C}$-enrichment were obtained every 15 min and serum samples were obtained every 30 min during 6 hours. ${ }^{13} \mathrm{C}$-leucine serum concentrations $(\mu \mathrm{g} / \mathrm{ml})$ were calculated from the ${ }^{13} \mathrm{C}$-leucine enrichment and the leucine concentratrion which were both measured using gas-chromatography combustion isotope ratio mass spectrometry (GC-C-IRMS, Delta Plus, Thermo Electron). ${ }^{13} \mathrm{C}$-breath content was analyzed using a stable isotope ratio mass spectrometer (IRMS, PDZ). Four hours after the instillation, all volunteers received a sandwich with ham or cheese. Results: The ${ }^{13} \mathrm{CO}$ excreted in breath is a measure for the total oxidation of ${ }^{13} \mathrm{C}$-leucine and originates from colonic bacterial metabolism of ${ }^{13} \mathrm{C}$-leucine with subsequent diffusion of ${ }^{13} \mathrm{CO}_{2}$ through the colonic wall or from oxidation of absorbed ${ }^{13} \mathrm{C}$-leucine in the liver. In the presence of inulin, a clear, although statistically not significant decrease in ${ }^{13} \mathrm{CO}_{2}$-excretion in breath was seen (cum $\%{ }^{13} \mathrm{CO}_{2} / 6 \mathrm{~h}: 36+/-12 \%$ versus $29+/-9 \%$, mean $+/-\mathrm{SD}, \mathrm{n}=11, \mathrm{p}=0.14$ ). The absence of an effect on serum ${ }^{13} \mathrm{C}$-leucine concentration favours the hypothesis that the lower ${ }^{13} \mathrm{CO}_{2}$ excretion in breath is due to a lower ${ }^{13} \mathrm{CO}_{2}$-generation in the colon and hence, a lower degree of fermentation of ${ }^{13} \mathrm{C}$-leucine in the presence of inulin. Therefore, the results of this study are in agreement with the generally accepted idea that stimulation of carbohydrate fermentation through administration of inulin impairs amino acid fermentation. The absence of a clear change in amino acid absorption, suggests another fate for the remaining ${ }^{13} \mathrm{C}$ leucine. Presumably, more of the amino acid is incorporated by bacteria and used for microbial growth. Conclusion: We demonstrated in a direct instillation study that stimulation of carbohydrate fermentation through administration of inulin decreases fermentation of ${ }^{13} \mathrm{C}$-leucine in the colon.

\section{W1864}

The Modest Addition of Prebiotics to An Elemental Liquid Diet Alleviates the Intestinal Compromise Associated with Weaning Syndrome in Piglets Kristin L. Reifsteck, Brian M. Chung, Kristy L. Powell, Kelly Tappenden

A prebiotic is a non-digestible food ingredient that beneficially affects the host by selectively stimulating the growth and/or activity of one or a limited number of bacteria in the colon, thus improving host health. Using a preclinical model for intestinal compromise in human infants, this study tested the hypothesis that intestinal injury caused by weaning syndrome is reduced by the consumption of enteral formula containing prebiotics. Forty-eight $17-$ day-old piglets were randomized into one of three experimental groups: 1) sow-fed negative control (SOW); 2) early weaned, onto a low-residue complete whey-based, peptide liquid diet (WEAN-), or; 3) early weaned, onto an identical peptide diet containing $3.6 \mathrm{~g}$ prebiotics/ $\mathrm{L}(\mathrm{WEAN}+$ ). Piglets were further randomized to be euthanized at experimental day (D) 2 or D4. While initial body weight (BW) did not differ between groups, SOW BW was increased $(\mathrm{p}<0.0001)$ compared to WEAN- and WEAN + on Dl-D4 consistent with weaning syndrome. Daily formula intake (mL/kg BW) did not differ between WEAN- and WEAN+ over the course of the study. Structural and functional attributes of the duodenum, jejunum, ileum and colon were measured on D2 and D4. Jejunoileal length $(\mathrm{cm} / \mathrm{kg} \mathrm{BW})$ was lower $(\mathrm{p}=$ 0.0007 ) in WEAN- than either SOW or WEAN+ on D2, but both WEAN groups were higher $(\mathrm{p}=0.0007)$ than SOW on D4. Duodenal $(\mathrm{p}=0.0131)$ and colonic $(\mathrm{p}=0.0167)$ length did not differ between groups at D2, but were higher than SOW in both WEAN groups on $\mathrm{D} 4$. At D2 and D4, intestinal weight ( $\mathrm{g} / \mathrm{kg} \mathrm{BW}$ ) was greatest in SOW compared to eithe WEAN group. Consistent with weaning syndrome, transmural resistance in the duodenum $(p<0.0001)$, jejunum $(p<0.0001)$ and ileum $(p<0.0001)$ were lower in both WEAN groups than SOW. However, transmural resistance in the colon did not differ between SOW and WEAN+, but was lower ( $\mathrm{p}=0.0062)$ in WEAN-. Sodium-dependent glucose transport did not differ between either WEAN group in any intestinal segment studied, but was higher than SOW in the jejunum $(\mathrm{p}=0.0005)$ and ileum $(\mathrm{p}=0.013)$. Duodenal $(\mathrm{p}=0.005)$, jejunal $(\mathrm{p}=0.020)$, and colonic $(\mathrm{p}=0.024)$ glutamine transport on $\mathrm{D} 4$ was higher in WEAN-, whereas WEAN+ did not differ from SOW. In summary, the data obtained in this study indicate that the modest addition of prebiotics to a whey-based, peptide liquid diet can alleviate some complications associated with weaning syndrome in piglets. These beneficial effects impact both structural and functional attributes of the intestine and occur throughout the entire gastrointestinal (GI) tract. Further research examining the potential benefits of prebiotic consumption in children with GI compromise is warranted.

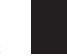


W1865

Effect of short- and Long-Term Administration of Lactobacillus Casei Shirota and Bifidobacterium Breve On the Colonic Fate of Protein Fermentation Metabolites Phenol and P-Cresol

Vicky de Preter, Tamara Coopmans, Paul Rutgeerts, Kristin Verbeke

Introduction: The presence of saccharolytic species such as lactobacilli and bifidobacteria in the colon has been reported to be benefial to health. Dietary intervention with those species may cause changes in the relative populations of colonic bacteria and hence in microbial metabolic activities. The aim of present study was to investigate in vivo whether short- and long-term intervention with Lactobacillus casei Shirota (LacS) and Bifidobacterium breve would reduce the concentration of $\mathrm{p}$-cresol and phenol in the colon. $\mathrm{p}$-Cresol and phenol are unique bacterial metabolites of aromatic amino acid-containing proteins, which are either excreted in the faeces or absorbed through the colonic wall and, after sulphateor glucuronide-conjugation in the mucosa or liver, urinary excreted. Phenols have been implicated in the pathogenesis of bladder and bowel cancers. Methods: To evaluate the immediate influence of administration of $L$. casei Shirota or B. breve on the excretion of phenol and p-cresol, the volunteers received a pancake test meal to which in the first test no substrate and in the second test respectively $6.5 \times 10^{9} \mathrm{LacS}(\mathrm{n}=19)$ or $10^{9}$ B. breve cells $(n=10)$ were added. To study the influence of long-term administration of the substrates, the same volunteers received either $2 \times / \mathrm{d} 6.5 \times 10^{9}$ LacS or $2 \times / \mathrm{d} 10^{9} \mathrm{~B}$. breve cells during 1 month and consumed a pancake test meal at the end of the intake period. For each test, the volunteers performed a 48-h urine collection. The urinary p-cresol and phenol content was determined by means of GC-MS. The results were expressed as mean p-cresol/phenol content excreted in urine $(\mathrm{mg} / 24 \mathrm{~h})$. Results: Simultaneous administration of LacS or B. breve cells with the test meal resulted in a decreased excretion of $\mathrm{p}$-cresol, respectively $\mathrm{p}=0.013$ for LacS and $\mathrm{p}=0.028$ for B. breve. Also long-term dietary intervention with LacS or B. breve cells resulted in a significant decrease in p-cresol excretion compared to baseline values ( $\mathrm{p}=$ 0.005 (LacS) and $\mathrm{p}=0.001$ (B. breve)). No changes in the excretion of phenol were observed after short- or long-term probiotic intake. Conclusions: The results of this study demonstrate that Lactobacillus casei Shirota and Bifidobacterium breve are able to modulate the metabolic activity of the intestinal flora, with a favourable effect on the colonic p-cresol and phenol metabolism.

\begin{tabular}{|c|c|c|c|c|}
\hline \multicolumn{2}{|c|}{ Short- + long-term effects } & Baseline & Short-term & Long-term \\
\hline \multirow{3}{*}{ L. casei Shirota } & p-cresol & $29.11+/-10.86$ & $21.69+/-12.15^{*}$ & $21.27+/-13.01^{*}$ \\
\cline { 2 - 5 } & phenol & $7.77+/-3.99$ & $7.16+/-3.01$ & $7.60+/-5.87$ \\
\hline \multirow{3}{*}{ B. breve } & p-cresol & $27.46+/-17.49$ & $19.74+/-11.66^{*}$ & $17.42+/-8.78^{*}$ \\
\cline { 2 - 5 } & phenol & $7.40+/-4.68$ & $8.42+/-6.11$ & $7.45+/-4.72$ \\
\hline
\end{tabular}

* Significantly different from baseline

\section{W1866}

Quantitative Analysis of hTERT mRNA in Pancreatic Juice Is More Useful for Differentiating Pancreatic Cancer from IPMN Than from Chronic Pancreatitis Kenoki Ohuchida, Kazuhiro Mizumoto, Eishi Nagai, Hiroyuki Konomi, Koji Yamaguchi, Masao Tanaka

Backgrounds. Although telomerase activity is a promising diagnostic marker for pancreatic cancer, clinical introduction of this marker for cancer diagnosis is still problematic due to the lack of means of evaluating sample quality. hTERT, one of the subunits of telomerase, is also a promising diagnostic marker. Aim To determine the feasibility of quantitative analysis of hTERT mRNA for diagnosis of pancreatic cancer. Methods. hTERT expression was determined in 88 pancreatic juice samples and carcinoma cells, intraductal papillary mucinous neoplasm (IPMN) cells, and chronic pancreatitis-affected cells obtained by microdissection. Results. Quantitative analyses of hTERT mRNAs in pancreatic juice were more useful in discriminating carcinoma from IPMN than from chronic pancreatitis. When the specificity was set at $100 \%$, the sensitivity for differentiation between carcinoma and IPMN was $43.5 \%$, whereas the sensitivity of cytologic examination was $22.0 \%$. There were significant differences in hTERT expression between carcinoma cells, IPMN cells, and normal ductal cells isolated from pancreatic tissues by microdissection. Lymphocytes and hyperplastic epithelial cells isolated from tissues with the histologic appearance of pancreatitis showed various expression levels of hTERT. Conclusion. Our results suggest that quantitative analysis of hTERT mRNA in pancreatic juice is advantageous over cytologic analysis for differentiation between carcinoma and IPMN but probably not for differentiation between carcinoma and chronic pancreatitis.

\section{W1867}

Endoscopic Biliary Stenting for Pancreatic Cancer and Biliary Obstruction: Predictive Factors of Stent Patency and Patient Survival

Cristina Gomez, Carlos Guarner, Candid Villanueva, Xavi Cusso, Antonio Farre, Joaquin Balanzo

Endoscopic biliary stenting is a well established palliation treatment for patients with biliary stricture caused by nonresecable pancreatic cancer. Stent clogging is the predominant late complication, ocurring more frequently with plastic stents. The use of self-expanding metallic stent (SEMS) however is not current practice due to their high cost. It would be important to select patients in our area with a better predicted survival, who could benefit from a SEMS, in order to be more cost-effective. OBJECTIVES: To evaluate the efficacy and complications of palliative endoscopic treatment for biliary obstruction caused by pancreatic cancer, and to investigate predictive factors of stent patency and patient survival. PATIENTS AND METHODS: Prospective, non-randomized trial in patients with nonresectable pancreatic cancer and biliary obstruction treated with endoscopic plastic or SEMS between January 2000 and May 2005. RESULTS: One hundred and twenty-six patients with nonresectable pancreatic cancer and biliary obstruction were treated with 151 endoscopic stents (plastic= 111 , SEMS $=40$ ). Technical success was $90 \%$. Early complications were observed in $17 \%$ of cases (cholangitis 6\%, early stent obstruction $6.5 \%$, haemorraghe $2.5 \%$ and migration $2.7 \%$ ). The most common late complication was stent clogging (25.7\%). Median stent patency (period between stent placement insertion and obstruction or death) was 4 months. The type of stent (plastic vs. SEMS) was identified in a multivariate analysis as the only independent predictive factor of patency. Comparing plastic versus SEMS, the actuarial probability of patency at 6 months was the $12 \%$ and $50.1 \%(\mathrm{p}<0.05)$ respectively. In a multivariate analysis, the absence of metastases (median survival $=6.2$ months versus 4 months with metastasic disease) and coadjuvant chemotherapy (median survival= 13 months versus 4 months without chemotherapy) were independent predictive factors of better prognosis for survival ( $>4$ months). CONCLUSION: Patients with nonresecable pancreatic cancer and biliary obstruction without metastases are the ideal candidates for self-expanding metallic biliary stent insertion.

\section{W1868}

Nationwide Survey of the Contemporary Management of Pancreatic Cancer Kolitha Goonetilleke, Ajith K. Siriwardena

Introduction: Outcome after pancreatic cancer surgery is better in specialist, high-volume units. In the United Kingdom this has led to concentration of pancreatic surgery in specialist units. However, there remains no consensus on many aspects of management including optimal staging and pre-operative biliary drainage. This questionnaire-based survey addresses aspects of practice volume, resection volume and outcome, technical aspects of pancreaticoduodenectomy and terminology for post-operative complications. Methods: A postal questionnaire survey was undertaken in 2005 of the 517 members of the Association of Upper Gastrointestinal Surgeons of Great Britain (AUGIS). Of 162 respondents, 57 surgeons stated that they undertook pancreatic resection. This figure was cross-checked with the UK Pancreatic Society (a pancreatic specialists forum) which estimated 64 pancreatic surgeons. A further cross-check was carried out by comparing numbers of PD stated as being carried out by respondents with Government Data. A total of 734 PD were reported by respondents compared to 822 PD for financial year 2003/2004 for UK NHS hospitals. Results: The modal case volume per annum was $6-10$. Thirteen $(23 \%)$ undertook more than 15 procedures each and 1 more than 25 . There were 24 in-hospital deaths in 732 resections (3\%) mortality. For individual respondents the modal percentage mortality was $5 \%$ ( 0 to $16 \%$ ). All clinicians with mortality in excess of $10 \%$ did less than 10 resections per annum. In terms of staging: contrast CT is used by all. Endoscopic ultrasound by $27(47 \%)$ and laparoscopy by $27(40 \%)$. Laparoscopic ultrasonography was used by 11 (19\%). Positron emission tomography (PET) was used by 2 (4\%). No respondents utilised mesenteric angiography. Pre-operative biliary drainage was undertaken by $29(51 \%)$. In terms of operative detail, $60 \%$ undertook intraoperative frozen section, $96 \%$ reconstructed by pancreaticojejunostomy and 36\% used a post-operative feeding jejunostomy. A range of descriptors was made available for definition of post-pancreaticoduodenectomy pancreatic fistula and $96 \%$ favoured the following term: amylase rich discharge ( $>3 \mathrm{x}$ serum amylase) beyond 7 th post-operative day Conclusion: Bearing in mind that survey results can be affected by biased or idealized responses, this major survey captures the majority of pancreatic resectional activity in the UK for 2004. It is clear that there remain volume-related effects on mortality and important variations in staging and biliary drainage practice. For the first time, there is evidence of a national consensus on the choice of terminology for definition of post-resection pancreatic fistula.

\section{W1869}

Malignancies Associated with Intraductal Papillary Mucinous Neoplasm of the Pancreas

Terumi Kamisawa, Naoto Egawa, Hitoshi Nakajima, Kouji Tsuruta, Atsutake Okamoto

Background and Aim: Intraductal papillary mucinous neoplasm (IPMN) of the pancreas is a distinct entity characterized by intraductal papillary growth and thick mucus secretion. IPMN is a slow-growing tumor and many cases of branch duct type IPMN have been followed. As IPMN has a favorable prognosis, associated malignancies have potential significance in these patients. We examined the incidence and characteristics of preexisting, coexisting and subsequent malignancies in patients with IPMN. Patients and Methods: Seventy-nine cases ( 54 males and 25 females, average age 68.5) of IPMN were diagnosed by detection of mucus in the pancreatic duct during endoscopic retrograde pancreatography. Twenty-three patients underwent surgical resection. Histological diagnosis was confirmed in 30 cases (adenoma $(n=19)$ and adenocarcinoma $(n=11))$. Other primary malignancies associated with IPMN, occurring in the prediagnostic or postdiagnostic period, were investigated. Average postdiagnostic follow-up period was 3.3 years (range, 0.2-20). Results: Other 40 malignancies occurred in $28(35 \%)$ of 79 patients with IPMN. They were found before $(n=15)$, at $(n=$ 19) and after $(n=6)$ the diagnosis of IPMN. Major associated malignancies were gastric cancer $(n=12)$, colonic cancer $(n=7)$, esophageal cancer $(n=4)$, pulmonary cancer $(n=4)$, and independent pancreatic cancer $(n=3)$. Three other organs were involved in 3 patients, and two organs were in 4 . All of 15 other malignancies occurring $1-10$ years before the diagnosis of IPMN were resected surgically or endoscopically. Fifteen of 19 other malignancies diagnosed simultaneously with IPMN were resected surgically or endoscopically. Only 1 other malignancy diagnosed after IPMN was surgically resected. Fourteen patients died of associated malignancies. Three IPMNs associated with independent pancreatic cancer were of branch type located in the pancreatic head and ranged from 1.5 to $3 \mathrm{~cm}$, and the lesion of IPMN was proximal to pancreatic cancer in 2 patients and distal in the other. Two patients had the two lesions synchronously, and pancreatic cancer occurred 3 years after diagnosis of IPMN in one patient Development of other malignancies was related to age (71.9 vs. 66.8 , $\mathrm{p}<0.05)$, but not to gender, follow-up period, site of IPMN, or resection of IPMN. Conclusion: IPMN is associated with a high incidence of other malignancies, particularly gastric and colonic cancers. Common genetic mechanisms between IPMN and other associated malignancies might be present. Clinicians should pay attention to the possibility of associated malignancies in preoperative screening and follow-up of patients with IPMN. 


\section{W1870}

Predicting Success of Endoscopic Aspiration for Suspected Pancreatic Cystic Neoplasms

Richard M. Walsh, Gregory Zuccaro, John Vargo, John Dumot, Nancy Brown

The analysis of cyst fluid contents can play a valuable role in the management of patients with suspected pancreatic cystic neoplasms. It has an increasing role in evaluating small incidental cysts when observation is being considered. We reviewed our experience with cyst aspiration of suspected pancreatic cystic neoplasms to determine the size and location of cysts which resulted in a successful cyst fluid analysis. Over a five-year period ending December 2004, 235 patients enrolled in a prospective protocol for evaluation of suspected pancreatic cystic neoplasms. The route for all cyst aspirations was by endoscopic ultrasound (EUS). A successful aspiration analysis was defined by the ability to determine up to three variables: cytology including qualitative assessment of extracellular mucin, CEA and amylase. The distribution of cystic lesions was: 87 (37\%) in the head, 77 (33\%) tail, 42 (18\%) body, 22 (9\%) uncinate, and 7 patients with multiple cysts. EUS was performed in 210 patients (89\%) for cysts with a mean size of $3.4 \mathrm{~cm}$. An aspirate was obtained in 194 patients (92\%) with a mean volume of $10 \mathrm{cc}$. There was a significant correlation between cyst size and volume aspirated (Spearman correlation of $0.60, \mathrm{p}<0.0001$ ). The ability to achieve a complete aspirate analysis (all three variables) significantly correlated with cyst size (Spearman correlation of $0.31, \mathrm{p}<0.0001$ ). All three variables were successfully obtained in 67 patients (35\%) with a mean cyst size of $4.3 \mathrm{~cm}$, two or more variables in 81 patients (42\%) with a mean size of $4.3 \mathrm{~cm}$, and one or more variables in 149 patients (77\%) with a mean size of $3.8 \mathrm{~cm}$. Logistic regression curves predict the likelihood of success based on cyst size, e.g. aspiration of a $2 \mathrm{~cm}$ cyst will be successful in $73 \%$ to determine one variable, $33 \%$ for two variables, and $26 \%$ for three variables. There was no significant difference between location of a cyst and successful aspiration $(\mathrm{p}=0.14)$, although there was a trend towards an unsuccessful aspiration and fewer cysts with a complete aspirate analysis for uncinate cysts when compared to all other locations ( $44 \%$ versus $20 \%$ and $18 \%$ versus $37 \%$, respectively). Twenty-five patients did not undergo EUS and their cysts averaged $1.9 \mathrm{~cm}$, and $16(8 \%)$ had an unsuccessful attempt at EUS aspiration with cysts that averaged $2.4 \mathrm{~cm}$. There was one EUS complication of a duodenal perforation in a patient with a serous cystadenoma who survived emergent surgical repair. In summary, there are a predictable number of variables that can be obtained from an EUS aspiration from a pancreatic cyst based on cyst size which is independent of location.

\section{W1871}

Incidence of Other Malignancies and Overall Prognosis in the Patients with Intraductal Papillary Mucinous Neoplasms (IPMN) of the Pancreas Manabu Osanai, Hiroyuki Maguchi, Akio Katanuma, Kuniyuki Takahashi, Satoshi Tanno, Yutaka Kohgo

Background/Aims: The intraductal papillary-mucinous neoplasms (IPMN) of the pancreas is a relatively new and increasingly reported entity. An IPMN is characterized a low potential for malignancy, and a slow-growing tumor with a better prognosis than other pancreatic malignancies. In addition, IPMN often found in elderly people, so their prognosis would be sometimes affected by other disease. However, little is known about pancreatic cancer and extrapancreatic malignancies in patients with IPMN. We evaluated the incidence of other malignancies and overall prognosis in IPMN patients. Methods: 307 patients with IPMN were diagnosed in our two hospitals. There were 181 males and 126 females, with a mean age of 68.3 years (35-87). Fifteen patients were main duct type and 292 patients were branch duct type. Eighty-three patients who had underwent surgery (hyperplasia 7 adenoma 41, non-invasive adenocarcinoma 26, invasive adenocarcinoma 8) and 224 patients were follow up cases. Result: Seventy-one patients (23.1\%) had extrapancreatic malignancies and 10 patients $(3.3 \%)$ had both IPMN and independent pancreatic cancer before $(n=31)$, at $(\mathrm{n}=28)$, and after $(\mathrm{n}=22)$ the diagnosis of IPMT. These 71 cases included colon cancer $(n=19)$, gastric cancer $(n=18)$, lung cancer $(n=10)$, Esophagial cancer $(n=5)$, and others. Fifty-three IPMN patients died. Among them, 6 patients (11.3\%) died of invasive malignant IPMN, 11 patients $(20.8 \%)$ died of extrapancreatic malignancies, 10 patients $(18.9 \%)$ died of pancreatic cancer, 26 patients $(49.0 \%)$ died of others. Conclusions: IPMN is highly associated with other malignancies, especially during follow-up period. Cause of death from other malignancies accounted for $39.7 \%$ of all deaths, which was higher than the death rate of $11.3 \%$ from the IPMN. In IPMN patients, even in follow up cases, systemic surveillance is necessary for detection of the other malignancies.

\section{W1872}

Predictors of Malignancy in Branch Duct Intraductal Papillary Mucinous Neoplasms (IPMN-Br): A Study of 62 Resected IPMN-Br

Mario Pelaez-Luna, Thomas C. Smyrk, Jonathan E. Clain, Michael J. Levy, Randall K. Pearson, Bret T. Petersen, Mark D. Topazian, Santhi Swaroop Vege, Michael B. Farnell Suresh T. Chari

Background/Aims: The prevalence of carcinoma at diagnosis in main duct IPMN (IPMN-M) is high ( $57 \%$ to $92 \%$ ). This has led to recommendation that all mucin-producing neoplasms need resection, a view tempered by rapidly increasing frequency of diagnosis of IPMN-Br and its uncertain natural history. The aim of our study was to describe clinical, imaging and histological characteristics of a large cohort of resected IPMN-Br and determine preoperative characteristics that distinguish benign from malignant IPMN-Br. Methods: Demographic, clinical, imaging and histological data were collected from medical records of 62 patients identified from the surgical pathology database who underwent surgical resection for IPMN-Br between 2000 and 2004 at our institution. On imaging, all patients had $\geq 1$ cystic lesion of the pancreas without main duct dilation $(<6 \mathrm{~mm})$. Cysts with ovarian-type stroma were not included in the study. Results: The mean age at surgery for IPMN-Br was $64 \pm 11 \mathrm{yr}$ and $55 \%$ were women. Only $56 \%$ of patients had symptoms definitely or possibly related to the cysts (abdominal pain, acute pancreatitis, weight loss) and the remaining were asymptomatic or had non-specific symptoms. Over half (55\%) the cysts were in the uncinate process and head of pancreas. Twenty eight (45\%) patients had multiple IPMN-Br and they tended to be older than those with single lesions $(66 \pm 11$ vs $62 \pm 11$ years, $p=0.06)$. O pathology, $54(87 \%)$ were benign (adenoma [n=39] or borderline $[\mathrm{n}=15])$ and $8(13 \%)$ were malignant (carcinoma in situ $[n=3]$ or invasive carcinoma $[n=5]$ ). Patients with benign and malignant IPMN-Br did not differ in age, gender, size of lesions $(28 \pm 16 \mathrm{~mm}$ vs $28 \pm$ $25 \mathrm{~mm}$ ), presence of multiple IPMN-Br, cyst fluid CEA levels or presence of symptoms. Presence of one or more of the following identified all patients with malignancy: cyst related symptoms, solid mass lesion on CT, suspicious cytology in cyst fluid or intra-mural nodules on endoscopic ultrasound. However $71 \%$ of benign IPMN-Br also met one or more of these criteria. Conclusions: Compared to reported prevalence of malignancy in main duct IPMN prevalence of malignancy in resected IPMN-Br is much lower $(<15 \%)$. Clinical symptoms, imaging (CT and endosonography) characteristics and analyses of cyst aspirate can help identify malignancy in IPMN-Br; however, these criteria have low specificity.

\section{W1873}

Pancreatic Cyst: Depiction On Magnetic Resonance Cholangiopancreatography and Relationship to Pancreatic Cancer

Saburou Matsubara, Minoru Tada, Hiroyuki Isayama, Masaaki Akahane, Kuni Otomo, Takashi Sasaki, Toshihiko Arizumi, Osamu Togawa, Natsuyo Yamamoto, Yousuke Nakai, Naoki Sasahira, Kenji Hirano, Takeshi Tsujino, Nobuo Toda, Haruhiko Yoshida, Takao Kawabe, Masao Omata

Background \& AIM: Widespread use of magnetic resonance cholangiopancreatography (MRCP) has led to the identification of increasing numbers of patients with asymptomatic pancreatic cysts. The purpose of our study was to examine whether pancreatic cyst could be a risk factor for pancreatic cancer by checking incidence of pancreatic cyst detected by MRCP. Patients \& Methods: Frequency of pancreatic cyst and divisum by MRCP was compared between patients with and without pancreatic cancer. MRCP was obtained in 85 patients with pancreatic cancer and 532 controls between 1998 and 2005. Controls were consisted of patients with biliary diseases or suspicion of biliary symptoms. All the images were reviewed for the presence of pancreatic cysts and pancreatic divisum. Pancreatic cysts smaller than $5 \mathrm{~mm}$ in diameter were excluded in this study. Result: Pancreatic cysts were detected in $41(48.2 \%)$ patients in cancer group, and in $41(7.7 \%)$ in controls, respectively $(\mathrm{OR}=11.1, \mathrm{p}<0.01)$. In cancer group, median size of cysts was $18 \mathrm{~mm}$. Proportion of single number of cyst and simple cyst was $27 / 41(65.9 \%)$ and $16 / 41(39.0 \%)$, respectively. In 17 of $41(41 \%)$ patients, these cysts were occupying the pancreas proximal to the carcinoma which suggested that these cysts had existed prior to the development of cancer. In the control group, median size of cysts was $8 \mathrm{~mm}$. Proportion of single number of cyst and simple cyst was $27 / 41(65.9 \%)$ and $14 / 41(34.1 \%)$, respectively. Pancreatic divisum was observed in one (1.4\%) patient with cancer, and $11(2.1 \%)$ in controls, respectively ( $\mathrm{p}=$ n.s.). Conclusion: High incidence of pancreatic cyst in patients with pancreas cancer suggested that pancreatic cyst could be a risk factor for the carcinoma. Picking up and follow-up of patients with asymptomatic pancreatic cysts may lead to early diagnosis of pancreatic cancer. In contrast, panceatic divisum was not associated with pancreatic cancer.

\section{W1874}

Twist, a Novel Oncogene, Is Up-Regulated in Pancreatic Cancer; Clinical Implication of Twist Expression in Pancreatic Juice Kenoki Ohuchida, Kazuhiro Mizumoto, Eishi Nagai, Hiroyuki Konomi, Koji Yamaguchi, Masao Tanaka

Backgrounds. Despite accumulation of evidences that Twist, a highly conserved basic helix loop-helix transcription factor, is a novel oncogene, there is no report on Twist expression in pancreatic cancer. Intraductal papillary mucinous neoplasm (IPMN) is the representative cystic neoplasm of the pancreas, which is being detected with increasing frequency due to application of diagnostic imaging technologies, such as computed tomography and magnetic resonance. Aim. To investigate Twist mRNA expression levels in pancreatic cancer, IPMN and normal or pancreatitis-affected pancreas. Method. We performed quantitative analysis of Twist mRNA in bulk tissues, microdissected cells, and pancreatic juice samples. Results. We found significant differences in Twist expression between bulk pancreatic cancer an IPMN tissues, but did not between bulk pancreatic cancer and normal pancreatic tissues There were significant differences in Twist expression between cancer cells, IPMN cells, and pancreatitis-affected cells isolated by microdissection. In pancreatic juice analysis, we found a significant difference in Twist expression between cancer- and IPMN- juice samples, but not between cancer- and chronic pancreatitis-juice samples. ROC curve analysis showed that quantitative analyses of Twist mRNA are more useful in discriminating cancer from PMN than from chronic pancreatitis. Conclusion. Our results suggest that Twist is a novel oncogene of pancreatic cancer and that quantitative analysis of Twist mRNA in pancreatic juice may be useful for differentiation of pancreatic cancer from IPMN 